\title{
REFERENCE
}

NIST

PUBLICATIONS

\section{Publications of the}

National Institute of

Standards and Technology

1994-1996 Catalog

QC

100

.057

N0.305

Supl.26

1997
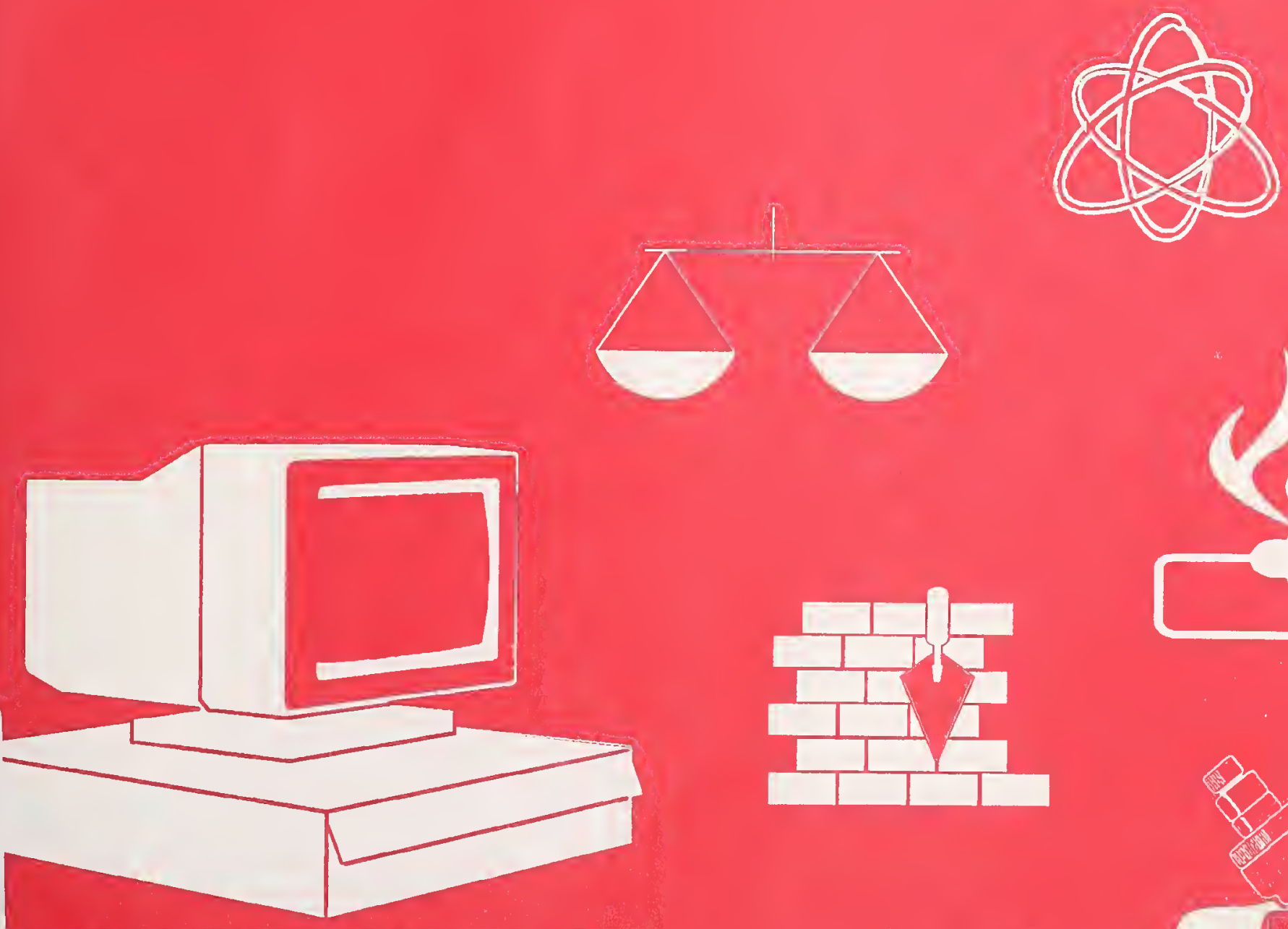

U.S. Department of Commerce Technology Administration

National Institute of Standards and Technology

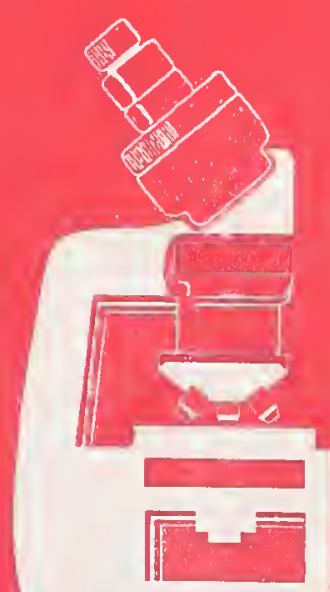


he National Institute of Standards and Technology was established in 1988 by Congress to "assist industry in the development of technology ... needed to improve product quality, to modernize manufacturing processes, to ensure product reliability ... and to facilitate rapid commercialization ... of products based on new scientific discoveries."

NIST, originally founded as the National Bureau of Standards in 1901, works to strengthen U.S. industry's competitiveness; advance science and engineering; and improve public health, safety, and the environment. One of the agency's basic functions is to develop, maintain, and retain custody of the national standards of measurement, and provide the means and methods for comparing standards used in science, engineering, manufacturing, commerce, industry, and education with the standards adopted or recognized by the Federal Government.

As an agency of the U.S. Commerce Department's Technology Administration, NIST conducts basic and applied research in the physical sciences and engineering, and develops measurement techniques, test methods, standards, and related services. The Institute does generic and precompetitive work on new and advanced technologies. NIST's research facilities are located at Gaithersburg, MD 20899, and at Boulder, CO 80303. Major technical operating units and their principal activities are listed below. For more information contact the Publications and Program Inquiries Desk, 301-975-3058.

Office of the Director

- National Quality Program

- International and Academic Affairs

\section{Technology Services}

- Standards Services

- Technology Partnerships

- Measurement Services

- Technology Innovation

- Information Services

\section{Advanced Technology Program}

- Economic Assessment

- Information Technology and Applications

- Chemical and Biomedical Technology

- Materials and Manufacturing Technology

- Electronics and Photonics Technology

\section{Manufacturing Extension Partnership}

Program

- Regional Programs

- National Programs

- Program Development

\section{Electronics and Electrical Engineering}

Laboratory

- Microelectronics

- Law Enforcement Standards

- Electricity

- Semiconductor Electronics

- Electromagnetic Fields ${ }^{\prime}$

- Electromagnetic Technology ${ }^{1}$

- Optoelectronics'

\section{Chemical Science and Technology}

\section{Laboratory}

- Biotechnology

- Physical and Chemical Properties ${ }^{2}$

- Analytical Chemistry

- Process Measurements

- Surface and Microanalysis Science
Physics Laboratory

- Electron and Optical Physics

- Atomic Physics

- Optical Technology

- Ionizing Radiation

- Time and Frequency

- Quantum Physics'

\author{
Materials Science and Engineering \\ Laboratory \\ - Intelligent Processing of Materials \\ - Ceramics \\ - Materials Reliability \\ - Polymers \\ - Metallurgy \\ - NIST Center for Neutron Research
}

\section{Manufacturing Engineering Laboratory \\ - Precision Engineering \\ - Automated Production Technology \\ - Intelligent Systems \\ - Fabrication Technology \\ - Manufacturing Systems Integration}

\section{Building and Fire Research}

Laboratory

- Structures

- Building Materials

- Building Environment

- Fire Safety Engineering

- Fire Science

\section{Information Technology Laboratory}

- Mathematical and Computational Sciences ${ }^{2}$

- Advanced Network Technologies

- Computer Security

- Information Access and User Interfaces

- High Performance Systems and Services

- Distributed Computing and Information Services

- Software Diagnostics and Conformance Testing 


\section{Publications of the National Institute of Standards and Technology 1994-1996 Catalog}

Debby King, Editor

Office of Information Services

National Institute of Standards and Technology

Gaithersburg, MD 20899-0001

Issued July 1997

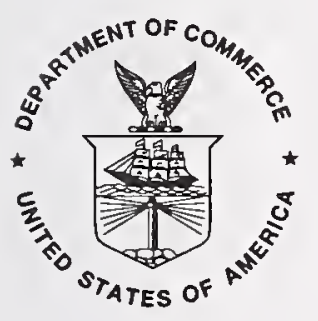

U.S. Department of Commerce

William M. Daley, Secretary 
National Institute of Standards and Technology Special Publication 305 Supplement 26

to accompany National Bureau of Standards / NIST Special Publication 305 and its Supplements 1 through 25 Natl. Inst. Stand. Technol. Spec. Publ. 305 Suppl. 26, 1,147 pages (July 1997)

CODEN: NSPUE2

\section{U.S. GOVERNMENT PRINTING OFFICE} WASHINGTON: 1997 


\section{CONTENTS}

About the National Institute of Standards and Technology inside front cover

Catalog structure and use. iv

Availability and ordering information iv

NIST publications announcements

Indexes

Personal author

Keyword

Title.

NTIS order/report number

Appendixes

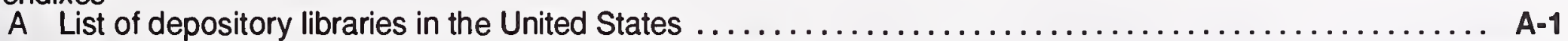

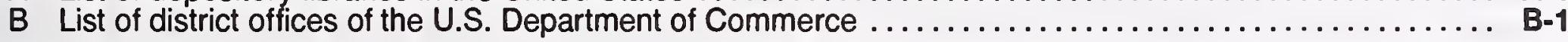

Order forms.

NIST technical publications program

inside back cover NTIS subject categories 


\section{CATALOG STRUCTURE AND USE}

Full bibliographic citations including keywords and abstracts for National Institute of Standards and Technology (NIST) papers published and entered into the National Technical Information Service (NTIS) collection are cited in the "NIST Publications Announcements" section of this catalog. (Also included are papers published prior to 1994 but not reported in previous supplements of this annual catalog.) Entries are arranged by NTIS subject classifications which consist of 37 broad subject categories (see back cover) and over 350 subcategories. Within a subcategory, entries are listed alphanumerically by NTIS order number.
Four indexes are included to allow the user to identify papers by personal author, keywords, title, and NTIS order/ report number. Each entry lists the appropriate title, the NTIS order number, and the abstract number.

Papers may also be identified by searching the NTIS database either online via commercially available systems such as DIALOG, or in the issues of NTIS'S Governmerit Reports Announcements and Index and its Government Reports Annual Index.

\section{AVAILABILITY AND ORDERING INFORMATION}

The highest quality and least expensive copies of NIST publications published as Government documents are available from the Superintendent of Documents, U.S. Government Printing Office, Washington, DC 20402. Publications cited with stock numbers (SN) should be ordered by these numbers. GPO will accept payment by check, money order, VISA, MasterCard, or deposit account. For availability and price, write to the GPO or telephone (202) 783-3238. Should a NIST publication be out of print at the GPO, its continued availability is assured at NTIS which sells publications in microfiche or paper copy reproduced from microfiche.

If an entry has a price code, such as PC A04/MF A01, the publication may be ordered from NTIS in paper copy (PC) or microfiche (MF) or both if both codes are given. Order from the National Technical Information Service, 5285 Port Royal Road, Springfield, VA 22161. A copy of the latest price code schedule is available from NTIS. NTIS will accept payment by check, money order, VISA, American Express, MasterCard, or deposit account. NTIS is the sole source of Federal Information Processing Standards (FIPS), Interagency Reports (IRs), and Grant/Contract Reports (GCRs). For more information call (703) 4874650.

Papers noted "Not Available NTIS" may be obtained directly from the author or from the external publisher cited. Such papers are not for sale by either the GPO or NTIS.

Two other sources for NIST publications are depository libraries (libraries designated to receive Government publications) and Department of Commerce District Offices. The depository libraries listed in Appendix A receive selected NIST publications (see inside back cover for a description of the various NIST publication series). While not every Government publication is sent to all depository libraries, certain depositories designated as Regional Depositories receive and retain one copy of all Government publications made available. Contact the depository library in your area to obtain information on what is available and where.

Department of Commerce District Offices listed in Appendix $B$ provide ready access at the local level to publications, statistical data and summaries, and surveys. Each District Office serves as an official sales agency of the Superintendent of Documents, U.S. Government Printing Office. A wide range of Government publications can be purchased from these offices. In addition, the reference library of each District Office contains review copies of many Government publications. 


\section{SAMPLE ENTRY}

BUILDING INDUSTRY TECHNOLOGY

Building Equipment, Furnishings, \& Maintenance

00,123

PB94-194339

PC A03/MF A01

National Inst. of Standards and Technology (BFRL),

Gaithersburg, MD. Fire Science Div.

Performance Parameters of Fire Detection Systems

R. L. Smith. Jun 94, 20p

NISTIR-5439

Keywords: "Fire detectors, "Buildings, Fire prevention, Parameters, Fire extinguishers, Warning systems, Probability theory, Smoke detectors, Mathematical models, Fires.

This report is a formal, functional analysis of fire detection systems' requirements. The performance parameters of fire detection systems are given as conditional probabilities. These parameters are identified by the objective analysis of the functions of a fire detection system. It is demonstrated that using the false alarm rate to specify the malfunctioning of a threshold detection system is inadequate. The principal function of fire detection systems is identified as the notification of anti-fire agents of the probability of an unwanted fire. The evaluation of the information provided by a detector system is central to its worth.
NTIS Subject Category

\author{
NTIS Subcategory \\ Abstract Number \\ NTIS order number Availability Price Codes \\ Corporate or performing organization
}

\section{Report Title}

Personal authors Report date Page count

Report Number

Contract or grant number

Keywords: * indicates keyword index entry

Abstract

\section{ADMINISTRATION \& MANAGEMENT}

\section{Management Information Systems}

\section{0,001}

PB94-163383 PC A06/MF A02

National Inst. of Standards and Technology, Gaithersburg, MD.

Putting the Information Infrastructure to Work: Report of the Information Infrastructure Task Force Committee on Applications and Technology. Special pub.

May 94, 114p, NIST/SP-857, ISBN-0-16-043188-3. Also available from Supt. of Docs. as SNOOH-00303267-1.

Keywords: "Information systems, "National interests, Commerce, Manufacturing, Industries, Health care, Education, Environmental monitoring, Libraries, Gov- ernment agencies, Public participation, Citizen participation, *Nil(National Information Infrastructure), Information superhighways.

The goal of this document is to express how improvements in the technical foundation upon which modern communications rests can benefit all Americans. The authors call this platform the National Information Infrastructure (NII), meaning the facilities and services that enable efficient creation and diffusion of useful information. They wish to focus the public debate on the uses of the NII and the benefits to be derived by applications of advanced computing and communications technologies.

\section{0,002}

PB96-146360 PC A04/MF A01

National Inst. of Standards and Technology (CSL), Gaithersburg, MD. Systems and Software Technology Div.

Open System Environment (OSE): Architectural Framework for Information Infrastructure.

Special pub.

F. Schulz. 1995, 54p, NIST/SP-500/232.

Also available from Supt. of Docs. as SNOO3-00303380-4.

Keywords: "Computer networks, "Information systems, * Systems management, Distributed processing. Computer applications, Application program(Computers),
Computer architecture, Software engineering, Operating systems(Computers), Communication networks, Information services, Computer program portability, Computer program transferability, Systems engineering, Information management, Electronic security, Human-computer interface, ${ }^{*}$ Open System Environment, National Information Infrastructure.

This document identifies a set of interfaces, services, and formats which are to be provided to users of an information infrastructure, and the methods for accessing these services. This document provides a context and guidance for standards selection, but does not identify standards for use within an information infrastructure. The document may, however, refer to standards in order to illustrate aspects of the services which are important in making selections among the candidate specifications. This discussion will provide a basic set of terminology and concepts to support discussion and resolution of policy issues. Finally, technical guidance is provided to agencies which intend to use information infrastructure for exchange of government information and services within government, and to deliver government information and services to citizens and organizations outside of government. Gaithersburg, MD 


\author{
Manager's Guide for Monitoring Data Integrity in \\ Financial Systems. \\ Special pub. \\ R. Sies. Feb 96, 72p, NIST/SP-500-233. \\ Also available from Supt. of Docs. as SN003-003- \\ 03387-1. See also PB95-216602.
}

Keywords: "Financial management, "Monitoring, "Accounting, "Automation, Data integrity, Computer security, Data processing security, Electronic data exchange, Management information systems, Software tools, Federal information systems.

This guide will enable the manager to identify and justify inclusin of software tools and techniques into new or existing financial systems. The result would be a Monitoring Module consisting of a set of software routines and an easy-to-use report generator. The users of financial and accounting systems and the users of systems that feed data into financial and accounting systems could use the Monitoring Module in a proactive way to ensure the accuracy, completeness and timeliness of input to the financial systems. The Monitoring Module if included in new or existing systems would help ensure consistency of data across the organization, and provide management with more ability to test and report the level of success with which their management goals are being met. In an environment where most financial transactions are automated, there may be no 'papes trail.' Furthermore, implementation of highly automated financial systems may expose organizations to increased risks of waste, fraud or abuse if system controls fail to be effective. It is imperative, therefore, that automated monitoring capabilities as sophisticated as the automated financial operations themselves be available to managers. This guide is a step in that direction.

\section{Management Practice}

\section{0,004}

PB95-126025 Not available NTIS

National Inst. of Standards and Technology (NEL) Gaithersburg, MD. Applied and Computational Mathe matics Div.

Development and Validation of Multicriteria Ratings: A Case Study.

Final rept.

S. I. Gass, and S. R. Torrence. 1991, 10p.

Pub. in Socio-Economic Planning Sciences 25, n2 p133-1421991.

Keywords: "Ratings, Conferences, Complexity, Costs, Services, Decision making, Criteria, Calibrating. Models, Reprints, "Multicriteria, AHP(Analytic Hierarchy Process), US NIST.

Using the hierarchical structure and methodology of the Analytic Hierarchy Process (AHP), a procedure was developed for the rating of conferences in terms of their complexity that enabled the Public Information Division of the National Institute of Standards and Technology to assess the cost of its staff's services. A meeting's complexity is measured by a multicriteria process that involves both the time and expertise of the Division's staff. The validity of the model, that is the calibration of the weights of the decision criteria, was established by showing that the weighting procedure of the AHP replicated the previous year's decisions.

\section{0,005}

PB96-161237 Not available NTIS

National Inst. of Standards and Technology (CSL), Gaithersburg, MD.

Guidance of the Legality of Keystroke Monitoring. Final rept.

S. M. Radack. 1993, 4p.

Pub. in Computer Systems Laboratory Bulletin, p1-4 Mar 93.

Keywords: "Keyboards, "Data processing terminals, "Monitoring, Man machine systems, Human-compute interface, Users, Legal aspects, Liability, Computer security, Electronic security, Reprints, "Legality.

This bulletin advises federal system administrators that keystroke monitoring during computer sessions may be found illegal in certain circumstances and that notice of such monitoring should be given to users

\section{Productivity}

00,006

PB97-124135 PC A99/MF E08

National Inst. of Standards and Technology,

Standards Activities of Organizations in the United

States.

Special pub. (Final).

R. B. Toth. Sep 96,788 p.

Contract NIST-43NANB514538

Also available from Supt. of Docs. as SN003-003. 03427-4. See also PB91-177774

Keywords: "Standardization, "Organizations, "United States, "Directories, Sources, Standards, Trade associations, National government, Specifications, Military organizations, Technical societies, Recommendations, Test methods, Coding.

The directory is a guide to mandatory and voluntary standards activities in the United States at the Federal level and by nongovernment (trade associations, technical and other professional societies). It excludes proprietary (company) standards and those of local levels of government. Superseding the 1991 edition (NBS SP 806 ), 'Standards Activities of Organizations in the United States', it includes standards distributors, libraries, and information centers. It also lists organizations that no longer develop standards or have become defunct since the previous directory was issued. Over 700 current descriptive commentaries are formatted, with subject headings to facilitate access to specific information. The main sections cover developers of formal and informal nongovernment studies; Federal agencies which develop standards; sources of standards documents and information; a subject index and related listings covering acronyms and initials, defunct bodies, and those organizations with name changes. Organizations have been included if they develop standards or contribute to the standardization process, whether voluntary of mandatory, or are sources of standards documents or information. An introductory section provides general information on Federal (including military) standards activities, a list of 20 major nongovernment standards developers, and an overview of U.S. (National) standardization activities.

\section{Public Administration \& Government}

\section{0,007}

PB95-216602 PC A03/MF A01

National Inst. of Standards and Technology (CSL) Gaithersburg, MD. Systems and Software Technology Div

\section{Self Monitoring Accounting Systems.}

Special pub.

R. F. Sies. Mar 95, 18p, NIST/SP-500/226.

Also available from Supt. of Docs. as SN003-00303324-3.

Keywords: "Financial management, "Auditing, *National government, Computer systems programs, Chief Financial Officers(CFO) Act of 1990.

The Federal Government is in need of financial systems that provide more complete, consistent, reliable, and timely information. This report recommends a service for use by management in performing self au dits of financial systems. Tools used in the past by Electronic Data Processing Auditors to audit accounting systems are examined, and the similarities between these tools and the service. The use of this service is then described, as it would be used, within the accounting system. How the service can provide more internal control and financial information usualiy obtained from analysis performed by financial consultants is explained.

\section{Research Program Administration \& Technology Transfer}

00,008

DE94-017162 PC A05/MF A02

Department of Energy, Washington, DC
Economic, Energy, and Environmental Impacts of the Energy-Related Inventions Program.

M. A. Brown, C. R. Wilson, C. A. Franchuk, S. M. Cohn, and D. Jones. 31 Jul 94, 100p, ORNLICON381.

Contract AC05-84OR21400

Keywords: "Economic impact, "Inventions, Inventions/ Environmental impacts, Commercialization, US DOE Grants, Patents, Program evaluation, ERIP (Energy Related Inventions Program), US NIST.

The report provides information on the economic, en ergy, and environmental impacts of inventions supported by the Energy-Related Inventions Program (ERIP)--a program jointly operated by the US Department of Energy and the National Institute of Standards and Technology (NIST). It describes the results of the latest in a series of ERIP evaluation projects that have been completed since 1980 . The period of interest is 1980 through 1992. The evaluation is based on data collected in 1993 through mail and telephone surveys of 253 program participants, and historical data collected during previous evaluations for an additional 189 participants

00,009

\section{PB94-157831 PC A12/MF A03}

Bio En-Gene-Er Associates, Inc., Wilmington, DE. Opportunities for Innovation: Biotechnology. R. M. Busche. Sep 93, 275p, NIST/GCR-93/633. Grant NANB2D1219

Sponsored by National Inst. of Standards and Technology (CSTL), Gaithersburg, MD. Biotechnology Div.

Keywords: "Biotechnology, "Technology innovations "Commercial development, "US NIST, Health care, Farm crops, Chemical industry. Electronics industry Mining, Pollution control, Plastics industry, Drug industry, Small businesses, Research and development, Government/industry relations, Food processing, Animal husbandry, Genetic engineering, Renewable resources, Ethanol fuels, Biodegradability, Biomass, Synthetic fuels, Bioconversion, Agribusiness.

The basic purpose of this project is to help small businesses get on the fast track in biotechnology research and development leading to the spin off of viable commercial businesses, probably with the help of larger companies having the resources for commercialization that are lacking in a smali enterprise. Such resources could include financing, and positions in marketing. manufacturing, regulatory affairs, and raw material supply, to name a few. In general, biotechnology can be expected to have a major impact on fundamental human needs engendered in the market segments of: health care, agriculture, forestry, food ingredients, industrial chemicals, plastics, energy, mining, pollution control, and bioelectronics.

00,010

PB94-166212 PC A08/MF A02

National Research Council, Washington, DC. Commission on Engineering and Technical Systems.

Learning to Change: Opportunities to Improve the Performance of Smaller Manufacturers.

G. Markovits, W. J. Brill, J. P. Cooper, S. P.

Garretson, H. G. Hall, B. E. Hamilton, A. L. Heald, D.

F. Kocaoglu, J. H. Mize, R. D. Nelson, R. A. Pritzker,

P. D. Rimington, W. B. Rouse, I. Feller, and B. M.

Fossum. 1993, 154p, ISBN-0-309-04982-2.

Contract NIST-50SBNB2C7187

Library of Congress catalog card no. 93-86600. Sponsored by National Inst. of Standards and Technology, Gaithersburg, MD.

Keywords: "Manufacturing, "Small businesses, "Public policies, Technology transfer, Performance evaluation, Technology assessment. Advisory services, Regulations, Government/industry relations, US NIST, National government, State government, Operating costs, Quality, Improvement, Competition, Industrial sector, Technical assistance, "Modernization, MTC(Manufacturing Technology Centers), Manufacturing extension centers.

State and local governments have created industrial assistance services, the federal government has multiple programs aimed at helping small businesses, and there is strong interest in the Clinton administration in creating a national network of industrial assistance centers (Clinton and Gore, 1993). Expanding the $\mathrm{Na}$ tional Institute of Standards and Technology (NIST) Manufacturing Technology Centers (MTCs) program is one mechanism for creating such a national assistance network. Given this context--rapid changes in manu- 
facturing, growth in the numbers of smaller manufacturers, and their apparent lag in modernization effort-NIST asked the Manufacturing Studies Board to form a committee to examine the barriers to manufacturing improvement in smaller firms and to identify the appropriate role of the MTCs in addressing those barriers. In addition to discussing barriers facing smaller manufacturers, both company representatives and assistance providers discussed opportunities to help firms overcome these barriers and to improve significantly their production costs, quality, and market responsiveness. The recommendations of the committee reflect the changed circumstances that have resulted from the emergence in early 1993 of substantial additional federal funding for industrial assistance activities, as well as President Clinton's proposals for the creation of a national network of manufacturing extension centers.

\section{0,011}

PB $94-185360 \quad$ Not available NTIS

National Inst. of Standards and Technology (EEEL), Gaithersburg, MD. Electronics and Electrical Engineering Lab. Office.

Transfer of Technology from Defense to Civilian Sectors.

Final rept.

R. Hebner. 1994, 3p.

Pub. in Institute of Electrical and Electronics Engineers Power Engineering Review, p9-11 May 94

Keywords: "Technology transfer, "Defense industry, "Governmentindustry relations, Reprints, Civilian population, Economic development, Technology utilization, Science, Electricity.

Changes occurring today within government with respect to its science and technology policies reflect the close linkage that has existed between technology and defense. In the past, the best minds of the times were set to solve technology problems at the government's behest to provide for national defense. Today, the Cold War has ended. The situation provides the U.S. with a window of opportunity to redefine its technology investment strategy. New forms of government-industry interaction are being explored to help technology make a difference in the civilian economy like it has in national defense.

\section{0,012}

\section{PB94-207750 PC A04/MF A01}

National Inst. of Standards and Technology (CSL), Gaithersburg, MD. Computer Security Div.

Preliminary Functional Specifications of a Prototype Electronic Research Notebook for NIST. S. J. Chang, E. Fong, J. Foti, and B. Rosen. Apr 94, 65p, NISTIR-5395

Keywords: "Research and development, "Information management, "Data storage, "Multimedia, Information retrieval, Computer security, Digital techniques, Requirements, Technology assessment, ERNs(Electronic Research Notebooks), US NIST.

This report is a preliminary study on the feasibility and possible use of electronic research notebooks (ERNs) at the National Institute of Standards and Technology (NIST). The goal of this project is to determine the requirements for ERN and to assess current technologies for the design of a prototype ERN for NIST scientists. The project involves the determination and specifications of functional requirements of the ERN. NIST scientists were interviewed to survey current notekeeping practices and identify specific needs for the ERN. The survey identified a set of basic and enhanced ERN features. Current technologies and products were also assessed in order to see how these requirements may be met. A potential system configuration is proposed where functional specifications for a basic ERN are defined.

\section{0,013}

PB94-211836 Not available NTIS

National Inst. of Standards and Technology, Gaithersburg, MD. Office of the Director.

Differences in Competitive Strategies between the United States and Japan.

Final rept.

H. Hellwig. 1992, 2p.

Pub. in Institute of Electrical and Electronics Engineers Transactions on Engineering Management $39, n 1$ p77. 781992

Keywords: "Technology innovation, *Competition, "United States, "Japan, Technology transfer, Research and development, Commercialization, Businesses,
Product development, Human resources, Production, Reprints.

The subject is introduced with a taxonomy (called product genesis) of the process of bringing an idea from the laboratory to the marketplace. A discussion of the competitive threshold follows: in a competitive fashion, the traditional strategic approach in both countries is discussed in the areas of human resources, production processes, and business objectives. These strategies are mapped against the various stages of product genesis. The hypothesis is developed that the competitive threshold for Japanese firms is much further downstream than the onset of competitive worries in U.S companies; i.e., U.S. companies already protect thei applied research activities whereas their Japanese counterparts still cooperate at this stage and become protective only before design or prototyping of products and processes takes place.

00,014

PB95-200747 PC A17/MF A04

National Inst. of Standards and Technology (TS), Gaithersburg, MD. Office of Information Services.

Publications of the National Institute of Standards and Technology 1992 Catalog.

Special pub.

D. I. King. Mar 95, 388p, NIST/SP-305/24

Also available from Supt. of Docs. as SN003-003 03323-5. See also report for 1991, PB92-217579.

Keywords: “Catalogs(Publications), "Bibliographies, *National Institute for Standards and Technology.

The 24th Supplement to Special Publication 305 contains full bibliographic citations including key words and abstracts for National Institute of Standards and Technology (NIST) 1992 papers published and en tered into the National Technical Information Service (NTIS) collection. (Also included are NIST papers published prior to 1992 but not reported in previous supplements of this annual catalog.) Four indexes are included to allow the user to identify NIST papers by personal author, key words, titles, and NTIS order/report number.

\section{0,015}

PB95-209854 PC A05/MF A0

National Inst. of Standards and Technology (MEL) Gaithersburg, MD. Office of Manufacturing Programs. Automated Manufacturing Research Facility 1994 Annual Report.

D. C. Stieren, and C. F. Albus. Nov 94, 78p, NISTIR5613.

Sponsored by Office of Naval Research, Arlington, VA. Manufacturing Science and Technology Div.

Keywords: ${ }^{*}$ Computer aided manufacturing *Research facilities, US NIST, Navy, Reporting requirements, Robotics, Annual reports.

The Automated Manufacturing Research Facility (AMRF) was created at the National Institute of Stand ards and Technology (NIST) in 1982 in order to provide a national testbed for research and development in computer integrated manufacturing. Since its founding, the AMRF has achieved several significant accomplishments. Among these include the development of over 25 commercial products, roughly 20 patents, and more than 25 national and international standards. Most of the AMRF efforts have been jointly funded by NIST, the Navy ManTech Program, other agencies, and industry. AMRF technical projects are conducted prima rily within the Manufacturing Engineering Laboratory (MEL) of NIST.

00,016

PB96-182266 PC A14/MF A03

National Research Council, Washington, DC. Computer Science and Telecommunications Board.

Unpredictable Certainty. Information Infrastructure through 2000

c1996, 298p, ISBN-0-309-05432-X

Grant NSF-IRI-9421465

Library of Congress catalog card no. 96-67383. Sponsored by National Science Foundation, Arlington, VA Advanced Research Projects Agency Arlington, VA and National Inst. of Standards and Technology, Gaithersburg, MD

Keywords: *Computer networks, "Telecommunication, "Government policies, "Meetings, Technology assessment, Technology transfer, Trends, Information technology, Economic models, Financing, Television, Investment, Electronics industry, Communication net- works, United States, Telephones, Wireless communications, Public sector, Private sect

The authors have available an impressive array of in formation technology. They can transmit literature, movies, music, and talk Government, businesses, and individuals are eager to go on-line to buy, sell, teach, learn, and more. The Unpredictable Certainty explores the national information infrastructure (NII) as the collection of all public and private information services. How will more and better services reach the home, small businesses, and remote locations. The Unpredictable Certainty examine swho will finance the NII, exploring how technology companies decide to invest in deployment and the vain search of 'killer apps' (applications that drive markets). It discusses who will pay for ongoing services and how they will pay, looking at past cost/price models relevant to the future. The Unpredictable Certainty discusses the underlying technologies, appliances, and services needed before the NII becomes a reality; reviews key features of important technologies, and analyzes current levels of deployment in telephone, cable and broadcast television, and wireless systems, and the difficulties in interconnection. The volume explores the challenge of open interfaces that stimulate new applications but also facilitate competition, the trend toward the separation of infrastructure from specific services, the tension between mature services and new contenders, the growth of the Internet, and more.

00,017

PB96-183215 PC A11/MF A03

Nationa' Inst. of Standards and Technology (TS), Gaithersburg, MD. Office of Information Services.

Publications of the National Institute of Standards and Technology 1993 Catalog.

D. I. King. Apr 96, 216p, NIST/SP-305/25.

Also available from Supt. of Docs. as SN003-003-

03409-6. See also report for 1992, PB95-200747.

Keywords: "Catalogs(Publications), "Bibliographies, Sciences, Technologies, Research management, Materials, Chemistry, Electronics, Physics, Manufacturing, Buildings, Fire research, Computer systems, Engineering, "National Institute of Standards and Technology.

The 25th Supplement to Special Publication 305 contains full bibliographic citations including key words and abstracts for National Institute of Standards and Technology (NIST) 1993 papers published and entered into the National Technical Information Service (NTIS) collection. (Also included are NIST papers published prior to 1993 but not reported in previous supplements of this annual catalog.) Four indexes are included to allow the user to identify NIST papers by personal author, key words, titles, and NTIS order/report number.

\section{0,018}

PB96-210059 PC A07/MF A02 National Inst. of Standards and Technology,
Gaithersburg, MD.

Working Conference on Global Growth of Technology: Is America Prepared. Held in Gaithersburg, Maryland on December 7, 1995.

Special pub.

G. Mulholland, J. Fassett, and C. Ehrlich. Jun 96

$108 p$, NIST/SP-897.

Errata sheet inserted. Also available from Supt. of Docs, as SN003-003-03413-4. Prepared in cooperation with President's Council on Competitiveness, Washington, DC.

Keywords: "Technology innovation, *United States Economic growth, Government policies, Government industry relations, Meetings, International trade, Investments, Productivity, Living standards, "Global economy.

The purpose of this conference 'Global Growth of Technology: Is America Prepared' - was to articulate the issues behind the question and to address these issues from the broadest range of perspectives. The impetus for the conference was provided by the $\mathrm{Na}$ tional Institute of Standards and support of the Council on Competitiveness (CoC) for organizing this meeting. NIST and the CoC are appropriate partners to host this conference: NIST's programs are defined by its core mission of promoting U.S. economic growth by working with industry to develop technology, measurements, and standards; the CoC focuses on the key issues related to competitiveness such as trade, productivity, investment, and the U.S. standard of living. 


\section{AERONAUTICS \& AERODYNAMICS}

\section{Aerodynamics}

00,019

PB94-213170 Not available NTIS

National Inst. of Standards and Technology (CAML) Gaithersburg, MD. Statistical Engineering Div.

Recent Approaches to Extreme Value Estimation with Application to Wind Speeds. Part 1. The Pickands Method.

Final rept.

J. A. Lechner, S. D. Leigh, and E. Simiu. 1992, $11 \mathrm{p}$. Engineering ar

dynamics 41-44, p509-519 1992.

Keywords: "Wind velocity, "Wind pressure, "Estimating "Extreme-value problems, Statistical analysis, Wind effects, Functions(Mathematics), Dynamic loads, Wind measurement, Reprints, "Extreme value estimation.

The last 15 years have seen the development of a new body of theory and of a new generation of statistical estimation procedures applicable to extreme data. Essentially these approaches emphasize the primacy of the information concerning the largest of the extremes (the tails), as opposed to information inherent in the bulk of the extreme data (the body of the distribution) We consider the application of a terminal CME (conditional mean exceedance) slope estimator, inspired by Bryson (1974), to yearly extreme wind data samples obtained from more than 100 stations in the conterminous United States. Next we apply our own implementation of the Pickands method, serially fitting exponential and Generalized Pareto (or Generalized Extreme Value) Distribution models to the conditiona exceedance CDF's of the wind data, starting with each ordered sample value in turn, and plotting the fitted tail index versus the order number.

\section{Aeronautics}

\section{0,020}

PB95-180279 Not available NTIS

National Inst. of Standards and Technology (BFRL) Gaithersburg, MD. Fire Science Div.

Suppression Effectiveness of Extinguishing Agents under Highly Dynamic Conditions.

Final rept.

G. Gmurczyk, W. Grosshandler, and D. Lowe. 1994, $12 p$.

Sponsored by Department of the Air Force, Wright-Patterson AFB, OH

Pub. in Proceedings of International Symposium on Fire Safety Science (4th), Ottawa, Ontario, Canada June 13-17, 1994, p925-936.

Keywords: "Fire extinguishing agents, "Fire suppression, "Aircraft fires, Effectiveness, Fire protection, Fire fighting, Ignition, Combustion, Aviation safety, Fire safety, Reprints, Halon 1301.

Alternatives to halon 1301 are sought which are effective fire suppressing agents and which do not create unacceptable safety, environmental, or systems compatibility problems. Investigations of eleven chemica compounds using a deflagration/detonation tube have revealed a great potential for the technique to study the fire suppression process. The facility is used to evaluate new suppressants, establishing their dynamic characteristics as well as elucidating complex suppression mechanisms occurring in fires under highly dy namic conditions typical of fast turbulent flames, explosions and detonations. The deflagration/detonation tube arrangement has been successfully employed to clearly discriminate among the dynamic characteristics of the eleven alternative agents, revealing new unexpected effects. The results have been used to help select among the alternatives for full-scale testing in simulated aircraft dry bay fires.
00,021

PB95-181095 Not available NTIS

National Inst. of Standards and Technology (BFRL), Gaithersburg, MD. Fire Science Div.

Suppression of Elevated Temperature Hydraulic Fluid and JP.8 Spray Flames.

Final rept.

I. Vazquez, W. Grosshandler, W. Rinkinen, M.

Glover, and C. Presser. 1994, 11p.

Sponsored by Department of the Air Force, Wright-Patterson AFB, $\mathrm{OH}$.

Pub. in Proceedings of the International Symposium on Fire Safety Science (4th), Ottawa, Ontario, Canada, June 13-17, 1994, p1255-1265.

Keywords: "Hydraulic fluids, "JP-8 jet fuel, "Fire extinguishing agents, "Aircraft fires, Fire suppression, Fire fighting. Aviation safety, Aviation fuels, Fire safety, Concentration(Composition), Fire protection, Fuel sprays, Flames, Combustion, Reprints, Halon 1301.

A coaxial turbulent spray burner was used to determine the suppression characteristics of twelve different fire fighting agents in elevated temperature hydraulic fluid and jet fuel (JP-8) spray flames. The effectiveness of the gaseous agents, being considered as alternatives to halon 1301, was compared based upon the mass required for suppression and the equivalent storage volume, normalized by the amount of halon $1301 \mathrm{re}$ quired to suppress the flame. The elevated temperature results were compared to measurements previously obtained with the incoming air and JP-8 at ambient temperature. No statistically significant difference in relative agent performance was found between the heated hydraulic flame and the previous JP-8 experiments.

\section{0,022}

PB95-202420 Not available NTIS

National Inst. of Standards and Technology (BFRL), Gaithersburg, MD. Fire Science Div.

Flow of Alternative Agents in Piping.

Final rept.

T. G. Cleary, W. L. Grosshandler, and J. C. Yang.

1994, 11p.

Sponsored by Department of the Air Force, Wright-Patterson AFB OH.

Pub. in Proceedings of Halon Options Technical Working Conference, Albuquerque, NM., May 3-5, 1994 , p105-115.

Keywords: "Fire extinguishing agents, "Fire suppression, "Fluid flow, "Nacelles, "Aircraft fires, Engine inlets, Flow measurement, Aircraft safety, Fire fighting, Piping systems, Two phase flow, Fire protection, Pressure gradients, Reprints, HFC-227ea, Halon 1301.

As part of the USAF, Army, Navy and FAA sponsored halon replacement project, the pipe flow characteristics of selected alternative agents for engine nacelle fire protection are being studied. Due to the remote location of the agent storage bottle, piping is required to transport the agent to various locations in an engine nacelle. The pipe flow from an agent bottle is characterized as a transient, two-phase flow. Since the selected alternative agents have thermophysical properties different from halon 1301, the flow characteristics will be different, which may require system design changes. An experimental apparatus to study the flow characteristics of the alternative agents was designed and is described. Preliminary results on the flow characteristics of HFC-227ea and halon 130! are presented.

\section{Aircraft}

\section{0,023}

\section{PB94-203403 PC A99/MF E08}

National Inst. of Standards and Technology (BFRL), Gaithersburg, MD. Fire Science Div.

Evaluation of Alternative In-Flight Fire Suppres. sants for Full-Scale Testing in Simulated Aircraft Engine Nacelles and Dry Bays.

Special pub.

W. L. Grosshandler, R. G. Gann, and W. M. Pitts Apr $94,857 \mathrm{p}$, NIST/SP-861.

Also available from Supt of Docs, as SN003-00303268-9. Sponsored by Department of the Air Force, Wright-Patterson AFB, OH., Naval Air Systems Command, Washington, DC., Aviation and Troop Command (Army), St. Louis, MO., and Federal Aviation Administration Technical Center, Atlantic City, NJ.
Keywords: "Aircraft fires, "Halon alternatives, "Fire suppression, Fluorocarbons, Thermodynamic properties, Fluid dynamics, Combustion chemistry, Reaction kinetics, Fire research, "Halon 1301, HCFCs(Hydrochlorofluorocarbons)

FCs(Fluorocarbons), HFCs(Hydrofluorocarbons), Discharge rate, National Institute of Standards and Technology.

Civilian and military aircraft suppliers and operators are searching for alternatives to the discontinued chemical Halon 1301 (CF3Br) for protecting aircraft against inflight fires. This study identifies the best two or three candidates from among a list of twelve fluorocarbons (FCs), hydrofluorocarbons (HFCs), and hydrochlorofluorocarbons(HCFCs) for full-scale testing in the engine nacelle and dry bay simulators at WrightPatterson Air Force Base. An assessment of the potential for a powder, sodium bicarbonate ( $\mathrm{NaHCO}$ ), and other realistic gaseous candidates was also requested. The primary recommendations for the dry bay application were FC-218 and HFC-125; partial testing of CF3 was also recommended. Details of all aspects of the research program and the rationale for making these recommendations are provided in this special publication.

\section{0,024}

PB95-242368 PC A03/MF A01

National Inst. of Standards and Technology (MSEL), Gaithersburg, MD. Metallurgy Div.

Fracture Testing of Large-Scale Thin-Sheet Aluminum Alloy.

R. deWit, R. J. Fields, S. R. Low, D. E. Harne, and T. Foecke. May 95, 32p, NISTIR-5661

Sponsored by Federal Aviation Administration, Washington, DC

Keywords: *Aluminum alloys, "Fracture tests, "Aircraft, Toughness, Fracture properties, Crack propagation, Plastic properties, Models, Fuselages, Monocoque construction, Aircraft panels, R-curve, MSD(Multiple Site Damage), US FAA.

A series of fracture tests on large-scale, pre-cracked aluminum alloy panel was carried out to examine and to characterize the process by which cracks propagate and link up in this material. Extended grips and test fixtures were specially designed to enable the pane specimens to be loaded in tension in a $1780-\mathrm{kN}$-capacity universal testing machine. Using existing information, a test matrix was set up to explore regions of failure controlled by fracture mechanics, with additional tests near the boundary between plastic collapse and racture. In addition, a variety of multiple site damage (MSD) configurations were included to distinguish beween various proposed linkage mechanisms. All tests but one used anti-buckling guides. The data were analyzed by two different procedures, (1) the plastic zone model based on the residual strength diagram, and (2) the R-curve. The first three tests were used to determine the basic material properties, and these results were then used in the analysis of the subsequent tests with MSD cracks.

\section{0,025}

PB96-160601 Not available NTIS

National Inst. of Standards and Technology (BFRL) Gaithersburg, MD. Fire Science Div.

Computer-Aided Molecular Design of Fire Resistant Aircraft Materials.

Final rept.

M. R. Nyden, and J. E. Brown. 1993, 93p.

Also available as N94-10779 (Order as N94-10766) Pub. in International Conference for the Promotion of Advanced Fire Resistant Aircraft Interior Materials, Atlantic City, NJ., February 9-11, 1993, p147-158.

Keywords: "Computer-aided design, "Aircraft construction materials, "Flammability testing, "Fire resistan materials, Aircraft fires, Honeycomb structures, Ignition, Burning rate, Commercial aircraft, Nonflammable materials, Molecular structure, Heat treatment, Heat transmission, Flame calorimeters, Reprints.

Molecular dynamic simulations and Cone Calorimeter measurements were used to assess the effects of electron beam irradiation and heat treatments on the flammability of the honeycomb composites used in the sidewalls, ceilings and stowage bins of commercial aircraft. The irradiation of this material did not result in any measureable changes. A dramatic reduction in the peak rate of heat release, however, was observed in samples that had been heated overnight at $250 \mathrm{deg}$ 


\section{Avionics}

00,026

AD.A278 782/8 PC A03/MF A01

National Bureau of Standards, Gaithersburg, MD Handbook Preferred Circuits Navy Aeronautica Electronic Equipment. Supplement Number 3. 1 Apr 60, 18p.

See also Supplement 2, AD-A278 783.

Keywords: "Circuits, "Electronic equipment, *Handbooks, Navy, Aeronautics, Standardization, Vacuum apparatus, Transistors, Direct current, Power supplies, Cathode followers, Amplifiers, Multivibrators, Trigger circuits, Pulses, Automatic frequency control, Noise reduction, Crystal oscillators, Silicon, Tubes, Regulators, Squelch, Servoamplifiers, "Preferred circuits, N-44599.

No abstract available.

\section{0,027}

AD-A278 783/6 PC A03/MF A01

National Bureau of Standards, Gaithersburg, MD Handbook Preferred Circuits Navy Aeronautical Electronic Equipment. Supplement Number 2. 1 Apr 59 46P BUAER-16-1-519-SUPP-2.

See also Supplement No. 3 AD-A278 782 and Supplement No. 1, AD-A278 784.

Keywords: "Circuits, "Electronic equipment, "Transistors, "Handbooks, Navy, Aeronautics, Powe supplies, Receivers, Airborne, Fire control computers, Crystal oscillators, Trigger circuits, Delay circuits, Cathode followers, Automatic frequency control, Cathode ray tubes, "Preferred circuits, N44599, Blocking oscillators, Frequency dividers, Distance mark generators, Video mixers.

No abstract available.

\section{0,028}

\section{AD-A278 784/4 PC A05/MF A0}

National Bureau of Standards, Gaithersburg, MD.

Handbook Preferred Circuits Navy Aeronautical Electronic Equipment. Supplement Number 1.

1 Aug 58, 94p, BUAER-16-1-519-SUPP-1.

See also Supplement No. 2, AD-A278 783 and Supplement No. 3, AD-A278 782.

Keywords: *Circuits, *Electronic equipment, "Handbooks, Navy, Aeronautics, Direct current, Video amplifiers, Oscillators, Noise reduction, Airborne, Fire control computers, Regulators, Multivibrators, "Preferred circuits, N-44599.

No abstract available.

00,029

PB94-145711 PC A05/MF A01

National Inst. of Standards and Technology (EEEL) Boulder, CO. Electromagnetic Fields Div.

Aperture Excitation of Electrically Large, Lossy Cavities.

Technical note

D. A. Hill, J. W. Adams, M. T. Ma, M. L. Crawford, R T. Johnk, A. R. Ondrejka, and B. F. Riddle. Sep 93, $81 p$, NIST/TN-1361.

Also available from Supt. of Docs. as SN003-00303238-7.

Keywords: "Electromagnetic interference, "Electromagnetic shielding, *Apertures, Cavities, O factors, Reverberation, Transmission, Mathematical models, Scaling, Absorption, Polarization(Waves) Computer software, US NIST, Avionics, Lossy materials, "Aircraft electronics, "Cavity Q.

The document presents a theory based on power balance for aperture excitation of electrically large, lossy cavities. The theory yields expressions for shielding ef fectiveness, cavity $Q$, and cavity time constant. In shielding effectiveness calculations, the incident field can be either a single plane wave or a uniformly random field to model reverberation chamber or random field illumination. The $Q$ theory includes wall loss, $a b-$ sorption by lossy objects within the cavity, aperture leakage, and power received by antennas within the cavity. Extensive measurements of shielding effectiveness, cavity $Q$, and cavity time constant were made on a rectangular cavity, and good agreement with theory was obtained for frequencies from 1 to $18 \mathrm{GHz}$.
National Inst. of Standards and Technology (EEEL), Boulder, CO. Electromagnetic Fields Div.

Measurements of Shielding Effectiveness and Cavity Characteristics of Airplanes.

D. A. Hill, M. L. Crawford, R. T. Johnk, A. R. Ondrejka, and D. G. Camell. Jul 94, 63p, NISTIR5023

Keywords: "Avionics, "Electromagnetic shielding, "Electromagnetic interference, ${ }^{*}$ Electromagnetic measurement, Electromagnetic fields, Time domain, Electromagnetic wave propagation, Leakage, Frequencies, Electronic warfare.

We present measured data for shielding effectiveness, cavity $Q$, and cavity time constant of three small (twinengine) airplanes for frequencies from $400 \mathrm{MHz}$ to 18 $\mathrm{GHz}$. Both $\mathrm{cw}$ and time-domain measurement methods were used, and the time-domain method yields higher values of cavity $Q$. Both methods yield $Q$ values below a theoretical upper bound determined by window leak. age losses. The measured shielding effectiveness is quite variable, but averages about $15 \mathrm{~dB}$. The measured time constants are also variable and average about $15 \mathrm{~ns}$. This short time constant is a result of the low $Q$ of the aircraft cavities.

\section{0,031}

PB95-175675 Not available NTIS

National Inst. of Standards and Technology (EEEL), Boulder, CO. Electromagnetic Fields Div.

Aperture Excitation of Electrically Large, Lossy Cavities.

Final rept. $T$. D. A. Hill, M. T. Ma, A. R. Ondrejka, R.
Riddle, and M. L. Crawford. 1994, 10p.

Pub. in Institute of Electrical and Electronics Engineers Transactions on Electromagnetic Compatibility $36, n 3$ p169-178 Aug 94.

Keywords: "Cavities, *Apertures, "Electromagnetic shielding. "Lossy media Avionics, Polarization(Waves). Electromagnetic interference, Electromagnetic wave transmission, Electromagnetic absorption, Q factors, Reverberation, Reprints.

We present a theory based on power balance for aperture excitation of electrically large, lossy cavities. The theory yields expressions for shielding effectiveness, cavity $Q$, and cavity time constant. In shielding effectiveness calculations, the incident field can be either a single plane wave or a uniformly random field to model reverberation chamber or random field illumination. The $\mathrm{Q}$ theory includes wall loss, absorption by lossy objects within the cavity, aperture leakage, and power received by antennas within the cavity. Extensive measurements of shielding effectiveness, cavity $\mathrm{Q}$, and cavity time constant were made on a rectangular cavity, and good agreement with theory was obtained for frequencies from 1 to $18 \mathrm{GHz}$.

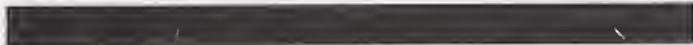 \\ AGRICULTURE \& FOOD}

\section{Agricultural Equipment, Facilities, \& Operations}

\section{0,032}

\section{PB94-154390 PC A03/MF A01}

National Inst. of Standards and Technology (MSEL) Gaithersburg, MD. Metallurgy Div.

Corrosion Resistance of Materials for Renovation of the United States Botanic Garden Conservatory. E. Escalante, and R. E. Ricker. Jan 94, 29p, NISTIR5360.

Sponsored by Architect of the Capitol, Washington, DC.

Keywords: *Greenhouses, *Renovating, "Corrosion resistance, Washington DC, Botany, District of Columbia, Trusses, Steel structures, Moisture, *United States Botanic Garden.

The purpose of the report is to evaluate the corrosivity of the greenhouse environments and their effect on materials considered for restoration of the Botanic Garden Conservatory. On the basis of this evaluation, rec- ommendations are made on materials which will provide the best performance in these environments. To accomplish this goal of materials selection, the range of conditions anticipated in the greenhouses, in combination with the various herbicides, pesticides, and fertilizers normally used are considered. Special corrosion control considerations, where necessary, are pointed out. The information presented here is intended to serve as a general guideline to the architects and engineers responsible for designing the details of the botanic garden conservatory. Within the time limits of the review, the authors can only address the major problems that the designers will encounter and some of the more important details that will lead to a successful design.

\section{Animal Husbandry \& Veterinary Medicine}

\section{0,033}

PB94-200524 Not available NTIS

National Inst. of Standards and Technology (CSTL) Gaithersburg MD Organic Analytical Research Div.

Individual Carotenoid Content of SRM 1548 Total Diet and Influence of Storage Temperature, Lyophilization, and Irradiation on Dietary Carotenoids.

Final rept.

N. E. Craft, S. A. Wise, and J. H. Soares, 1993, 6p Pub. in Jnl. of Agricultural and Food Chemistry 41, n2 p208-2131993.

Keywords: "Animal feed, "Food analysis *Carotenoids, "Food storage, Liquid chromatography, Temperature, Lyophilization, Reprints, "Standard ref erence materials.

A modified version of the AOAC procedure for the extraction of carotenoids from mixed feeds was coupled with an isocratic reversed-phase liquid chroma tography (LC) method to measure individua carotenoids in SRM 1548 total diet and in a high-carotenoid mixed diet (HCMD). The major carotenoids identified in SRM 1548 were lycopene, beta-carotene lutein, alpha-carotene, and zeaxanthin in descending order of concentration. The concentration of al carotenoids in SRM 1548 decreased as storage temperature increased. Significant differences in carotenoid concentrations occurred between -80 and $4 \mathrm{C}$ storage temperatures. Lyophilization of the HCMD significantly decreased beta-carotene and lycopene concentrations and produced an apparent increase in xanthophyll concentrations. Exposure to gamma-irradiation significantly decreased alpha-carotene and betacarotene concentrations and led to an apparent in crease in beta-cryptoxanthin. SRM-1548 was found to be unsuitable for use as a reference material for carotenoid measurements, while HCMD has greater potenial as a reference material.

\section{Fisheries \& Aquaculture}

\section{0,034}

PB94-198157 PC A03/MF A01

Environmental Research Lab., Gulf Breeze, FL Mistopathology, Blood Chemistry, and Physiological Status of Normal and Moribund Striped Bass ('Morone saxatilis') Involved in Summer Mortality ('Die-Off') in the Sacramento-San Joaquin Delta of California.

Journal article

G. Young, C. L. Brown, R. S. Nishioka, J. R

Cashman, H. A. Bern, L. C. Folmar, and M.

Andrews. C1994, 22p, EPA $600 / J-94 / 339$

Pub. in Jnl. of Fish Biology 44, p491-512 1994. Prepared in cooperation with California Univ. Berkeley., Crockett Sports Fishing Center, CA., and IGEN Research Inst., Seattle, WA.

Keywords: "Striped bass, *Animal physiology, "Blood chemical analysis, "Mortality, Reprints, Marine fishes, Marine biology, Toxicology, Histology, Pathology, Pacific Ocean, Assays, Statistical analysis, "Sacramento(California), "Carquinez Strait, "San Francisco Bay, Summary die-off, Morone saxatilis.

Summer mortalities of juvenile and adult striped bass (Morone saxatilis) occur annually in the San Francisco 
Bay-Delta system, most commonly in the lower delta area. Preliminary work identified pathological changes in the liver of moribund animals. This study represents a comprehensive survey of tissue pathology, blood chemistry and physiological status of moribund animals in comparison to wild and hatchery-reared bass

\section{Food Technology}

\section{0,035}

PB94-198322 Not available NTIS

National Inst. of Standards and Technology (TS) Gaithersburg, MD. Standard Reference Materials Program.

Recently Developed NIST Food Related Standard Reference Materials.

Final rept.

R. Alvarez. 1990, 3p

Pub. in Fresenius Jnl. of Analytical Chemistry 338, n4 p466-468 1990

Keywords: "Food analysis, "Chemical composition, *Toxic substances, *Food contamination *Nutrients, Reprints, Chemical analysis, Certification, Cholesterol, Vitamins, Quality control, Trace elements, Organic compounds, Inorganic compounds, *Standard re erence materials, NIST(National Institute of Standards and Technology), Recommended Dietary Allowances.

NIST issues food related, chemical composition Stand ard Reference Materials (SRMs) for validating food analyses. SRMs certified for inorganic constituents are: Non-Fat Milk Powder (SRM 1549), Oyster Tissue (SRM 1566a), Bovine Liver (SRM 1577a), Wheat Flou (SRM 1567a), Rice Flour (SRM 1568a), and Total Die SRM 1548). The certificate of analysis for the total die SRM also provides a certified concentration for cholesterol. Oyster tissue, a renewal SRM, is certified for 25 elements including $6(\mathrm{Al}, \mathrm{Cl}, \mathrm{I}, \mathrm{P}, \mathrm{S}$, and $\mathrm{V})$ that had not been certified in the previously issued SRM 1566 The elemental certified concentrations are based on concordant results of two or more independent analyt cal methods. The chemical compositions of the six food matrix SRMs are tabulated. Three food matrix SRMs certified for organic constituents are: Cholesterol and Fat-Soluble Vitamins in Coconut Oil (SRM 1563), Cholesterol in Whole Egg Powder (SRM 1845) and Organics in Cod Liver Oil (SRM 1588). Serum and urine matrix SRMs are also available that may be use ful for metabolic and bioavailability studies.

\section{0,036}

PB94-199650 Not available NTIS

National Inst. of Standards and Technology (NML) Gaithersburg, MD. Ionizing Radiation Div.

Estimation of the Absorbed Dose in RadiationProcessed Food. 2. Test of the EPR Response Function by an Exponential Fitting Analysis.

Final rept.

M. F. Desrosiers. 1991, 3p

Pub. in Applied Radiation and Isotopes-International Jnl. of Radiation Applications and Instrumentation 42 n7 p617-619 1991. See also Part 3, PB94-199684

Keywords: "Food processing, "Dosimetry, "Ionizing radiation, Meat, Electron paramagnetic resonance, Dose-response relationships, Reprints.

The use of electron paramagnetic resonance (EPR) spectrometry to accurately estimate the absorbed dos to radiation-processed bones (and thus meats) is ex amined. Additive re-irradiation of the bone produced a dose response which was used to determine the initial dose by back-extrapolation. It was found that an exponential fit (versus linear or polynomial) to the data pro vided improved accuracy of the estimated dose. These data as well as the protocol for the additive dose method are presented.

\section{0,037
PB94-199684 Not available NTIS}

National Inst. of Standards and Technology (PL), Gaithersburg, MD. Ionizing Radiation Div

Estimation of the Absorbed Dose in RadiationProcessed Food. 3. The Effect of Time of Evaluation on the Accuracy of the Estimate.

Final rept.

M. F. Desrosiers, and F. G. Le. 1993, 4p.

Pub. in Applied Radiation and Isotopes 44, n1/2 p439442 1993. See also Part 2, PB94-199650.

Keywords: "Food processing, "Ionizing radiation, *Dosimetry, Durapatite, Electron paramagnetic reso- nance, Meat, Chickens, Bones, Dose-response relationships, Reprints.

Electron paramagnetic resonance (EPR) spectrometry is evaluated as a method to retrospectively assess the abserbed dose to radiation-processed chicken (containing bone). Decay of the hydroxyapatite paramagnetic center EPR signal intensity was monitored at three different dose levels $(0.5,3.0,7.0 \mathrm{kGy})$ up to 20 days, and the dose was assessed for each level at 1, 8, and 20 days after irradiation. It was determined that the time of evaluation (up to 20 days postirradiation) did not adversely affect the estimate for 0.5 and 3.0 kGy bone, and only moderately affected the 7.0 kGy estimates.

00,038

PB94-199692 Not available NTIS

National Inst. of Standards and Technology (PL), Gaithersburg, MD. Ionizing Radiation Div.

Estimation of the Absorbed Dose in RadiationProcessed Food. 4. EPR Measurements on Eggshell.

Final rept.

M. F. Desrosiers, F. G. Le, P. M. Harewood, E. S.

Josephson, and M. Montesalvo. 1993, 5p.

Pub, in Agricultural Food Chemistry 41, p1471-1475 1993. See also Part 3, PB94-199684.

Keywords: "Food processing, "Ionizing radiation, *Dosimetry, Egg shell, Electron paramagnetic resonance, Salmonella, Dose-response relationships, Eggs, Reprints.

Fresh whole eggs were treated with ionizing radiation for Salmonellae control testing. The eggshell was then removed and examined by electron paramagnetic resonance (EPR) spectroscopy to determine if EPR could be used to (1) distinguish irradiated from unirradiated eggs and (2) assess the absorbed dose. No EPR signals were detected in unirradiated eggs, while strong signals were measurable for more than 200 days after irradiation. Although a number of EPR signals were measured, the most intense resonance $(g=2.0019)$ was used for dosimetry throughout the study. This signal was observed to increase linearly with dose (up to approximately $6 \mathrm{kGy}$ ), which decayed approximately $20 \%$ within the first 5 days after irradiation and remained relatively constant thereafter. The standard added-dose method was used to assess, retrospectively, the dose to eggs processed at $0.2,0.7$, and 1.4 kGy. Relatively good results were obtained when measurement was made on the day the shell was reirradiated; with this procedure estimates were better for shell processed at the lower doses.

\section{0,039}

PB94-199718 Not available NTIS

National Inst. of Standards and Technology (NML) Gaithersburg, MD. Ionizing Radiation Div.

Estimation of the Absorbed Dose in RadiationProcessed Food. 1. Test of the EPR Response Function by a Linear Regression Analysis. Final rept.

M. F. Desrosiers, G. L. Wilson, C. R. Hunter, and D. R. Hutton. 1991, 4p.

Pub, in Applied Radiation and Isotopes-Internationa $\mathrm{Jnl}$ of Radiation Applications and Instrumentation 42 n7 p 613-616 1991. Sponsored by Monash Univ., Clayton (Australia). Dept. of Physics.

Keywords: "Food processing, "Bones, "Dosimetry, "Cesium 137 Electron paramagnetic resonance, Chickens, Meat, Regression analysis, Reprints.

Free radicals produced in chicken bone tissue by (137) Cs gamma rays were measured using electron paramagnetic resonance (EPR) spectroscopy. The yield of radicals was proportional to the absorbed dose. Additive re-irradiation of previously irradiated bones is the basis of a method to estimate the absorbed dose in radiation-processed foods. The ability of the method to accurately provide estimates for a range of doses $(0.5-7.4 \mathrm{kGy})$ is tested here. It was determined that a linear fit to the data yielded good dose estimates for bone irradiated $<2 \mathrm{kGy}$, but failed at the higher doses using a linear approximation to the dose response. These data and their implications are discussed.

\section{0,040}

PB95-175204 Not available NTIS

National Inst. of Standards and Technology (CSTL), Boulder, CO. Thermophysics Div.
Supercritical Fluid Extraction of Biological Products.

Final rept

J. Bruno, C. A. Nieto de Castro, J. F. Hamel, and A. M. F. Palavra. 1993, 52p

Pub. in Recovery Processes for Biological Materials, Chapter 11, p303-354 1993

Keywords: "Separation processes, "Biotechnology, "Pharmacology, Materials recovery, Supercritical fluids, Solubility, Bioprocessing, Extraction, Food processing, Drugs, Reprints, *Supercritical fluid extraction, *Bioproducts.

In this review, the application of supercritical fluid extraction to biological products is discussed. The review begins with a general discussion of the supercritical fluid state, covering pure fluid and mixture properties, solubility, transport phenomena, and phase diagram modeling. The major experimental measurement are discussed, especially the determination of solute solubility in supercritical fluids. Some recent applications in the areas of pharmaceuticals and foods are described in detail. Finally, an overview of current work and future prospects is presented.

00,041

PB95-180675 Not available NTIS

National Inst. of Standards and Technology (PL), Gaithersburg, MD. Ionizing Radiation Div.

Food Irradiation Dosimetry.

Final rept.

W. L. McLaughlin. 1994, 4p.

See also PB88-175179.

Pub. in Transactions of the American Nuclear Society 71, p8-11 1994.

Keywords: "Radiation dosage, "Food irradiation, "Dosimetry, Gamma rays, X rays, Absorption, Doseresponse relationships, Reprints.

Measurements of absorbed dose, dose rate, and dose distribution in food products are vital to the success of treatment of food by ionizing radiation ( $x$ - and gamma radiation and electron beams). Dosimetry is used not only for regulatory purposes and quality control, but as documentation of research leading to each food treatment process and for setting proper doses and acceptable dose limits. For regulatory purposes as well as for economic viability of food irradiation, such documentation is thoroughly dependent on dosimetry. It must include process characterization, dosimeter calibration data, dose mapping results, routine dosimetry data and associated process parameters and control for each run, readout equipment maintenance and standard operating procedures, statistical data, product loading and dosimeter locations, dose uniformity, and evaluations of probability that the process specifications have (or have not) been met.

\section{0,042}

PB96-161690 Not available NTIS

National Inst. of Standards and Technology (PL), Gaithersburg, MD. Ionizing Radiation Div. Inter-Laboratory Trials of the EPR Method for the Detection of Irradiated Meats Containing Bone.

Final rept.

M. F. Desrosiers, F. G. Le, and W. L. McLaughlin. 1994, 7p.

Pub. in International Jnl. of Food Science and Technology, v29 p153-159 1994

Keywords: "Food, *Labelling compliance, *Meats, Reprints, Test evaluation, Irradiaton, Bone, "Foreign technology, "Electron paramagnetic resonance.

Three international trials on the use of electron paramagnetic resonance (EPR) to detect irradiated meats containing bone were sponsored as part of a coordinated research programmed by the International Atomic Energy Agency. In these trials, 11 laboratories used EPR to examine a total of 154 bone samples from three different meats. In one of the trials food irradiation processing conditions were simulated. The results were $100 \%$ successful for identifying both irradiated and unirradiated bones. A protocol for the EPR method is provided.

00,043

PB96-164124 Not available NTIS

National Inst. of Standards and Technology (PL), Gaithersburg, MD. Ionizing Radiation Div. 
Commentary on 'Optimization of Experimental Parameters for the EPR Detection of the Cellulosic Radical in Irradiated Foodstuffs'.

Final rept.

M. Desrosiers, D. Bensen, and D. Yaczko. 1995, 6p. Pub. in International Jnl. of Food Science and Technology, v30 p675-680 1995

Keywords: "Food processing, Reprints, Standards, Protocols, "Foreign technology, "EPR, Spectrometer parameters.

After many interlaboratory trials, several EPR methods for the detection of irradiated foods are being prepared as standard protocols. The works of Goodman et al (1994) and Raffi et al. (1992) present conflicting general recommendations for optimum parameter sets in the EPR protocols. Here we demonstrate that shortcomings of recommending general parameter sets for the varying types of food samples and the differen spectrometer configurations employed to examine them.

\section{ASTRONOMY \&} ASTROPHYSICS

\section{General}

00,044

PB96-123278 Not available NTIS

National Inst. of Standards and Technology (PL), Boulder, CO. Quantum Physics Div.

Goddard High Resolution Spectrograph: Instrument, Goals, and Science Results.

Final rept.

J. L. Linsky. 1994, 19p.

Pub. in Publications of the Astronomical Society of the Pacific, v106 p890-908 Aug 94.

Keywords: "Spaceborne astronomy, "Ultraviolet astronomy, Spectrographs, Spectral resolution, Stellar physics, Stellar activity, Stellar spectra, Sky surveys(Astronomy), Hubble Space Telescope, Reprints, "Goddard High Resolution Spectrograph.

The Goddard High Resolution Spectrograph (GHRS), currently in Earth orbit on the Hubble Space Telescope (HST), operates in the wavelength range 1150-3200 A with spectral resolutions of approximately $2 \times 10$ to the 3rd power, $2 \times 10$ to the 4th power, and $1 \times 10$ to the 5th power. The instrument and its developmen from inception, its current status, the approach to operations, representative results in the major areas of the scientific goals, and prospects for the future are described.

\section{0,045}

PB96-123328 Not available NTIS

National Inst. of Standards and Technology (PL), Boulder, CO. Quantum Physics Div.

Scientific Rationale and Present Implementation Strategy for the Far Ultraviolei Spectrograph Explorer (FUSE)

Final rept.

J. L. Linsky. 1993, 11p.

Pub. in International Workshop on 'Space Project to Probe the Internal Structure and Magnetic Activity of the Sun and Sun-like Stars', Catania, Italy, November 5-8, 1991, p323-332

Keywords: *Spectrographs, "Sky surveys(Astronomy) Ultraviolet astronomy, Cool stars, Far ultraviolet radiation, Ultraviolet spectra, High resolution, Project administration, Reprints, "FUSE(Far Uitraviolet Spectrograph Explorer), "Far Ultraviolet Spectrograph Explorer

FUSE will observe a broad range of astrophysical sources, including cool stars, with high sensitivity and high spectral resolution in the far ultraviolet $(912$ 1205A). It will also observe the $100-1600 \mathrm{~A}$ region with high throughput and modest resolution. The author summarizes the development and present status of FUSE.

\section{Astrophysics}

00.046

AD-A278 521/0 PC A05/MF A02

National Bureau of Standards, Boulder, $\mathrm{CO}$

Aerodynamic Phenomena in Stellar Atmospheres - A Bibliography.

Technical note.

R. N. Thomas. Sep 59, 98p.

Keywords: "Aerodynamics, "Stellar atmospheres, "Bibliographies, Spectroscopy, Bibliographies, Gas dynamics, Interstellar matter, Sun.

This is an attempt to provide a working bibliography for particular use in preparation for the Fourth Symposium on Cosmical Gas Dynamics: Aerodynamic Phenomena in Stellar Atmospheres.

\section{0,047}

AD-A280 291/6 PC A03/MF A0t

National Bureau of Standards, Gaithersburg, MD. Papers on the Symposium on Collision Phenomena in Astrophysics, Geophysics, and Masers. M. J. Seaton, A. Dalgarno, and C. Pecker. Dec 61, $35 \mathrm{p}$, NBS-TN-124.

Keywords: "Astrophysics, Symposia, Solar atmosphere, Particle collisions, Nebulae, Earth atmosphere Upper atmosphere.

This publication comprises three papers on astrophysi$\mathrm{cal}$ and geophysical problems that were presented at a special symposium at the National Bureau of Stand ards Boulder (Colo.) Laboratories in June 1961. The speakers spoke on the question: What are the most important atomic and molecular data needed by theoreticians for progress in astrophysics, geophysics, and gas lasers. The papers are entitled 'Astrophysical problems' by Michael Seaton; 'Collision processes in the high atmosphere' by A. Dalgarno; and 'Some problems connected with the analysis of the structure of the solar atmosphere' by Charlotte Pecker.

\section{0,048}

PB94-185212 Not available NTIS

National Inst. of Standards and Technology (PL), Boulder, CO. Quantum Physics Div.

Four Years of Monitoring alpha Orionis with the VLA: Where Have All the Flares Gone.

Final rept

S. A. Drake, J. A. Bookbinder, D. R. Florkowski, R E. Stencel, J. L. Linsky, and T. Simon. 1992, 3p.

Pub. in Proceedings of Cambridge Workshop on Cool Stars, Stellar Systems, and the Sun (7th), Tucson, AZ. October 1991, p455-457 1992

Keywords: "Supergiant stars, "Red giant stars, Stellar chromospheres, Stellar winds, Radio astronomy, Reprints, "Alpha Orionis star, Very large array(VLA).

We observed this prototypical $M$ supergiant using the VLA at centimeter wavelengths about once a month over a 4-year period from July 1986 to September 1990, resulting in a cumulative exposure time of about 120 hours. There is no evidence for radio flaring at any of the observing wavelengths. Low-level variability (about $=$ or $<25 \%$ ) does appear to be present at the shorter wavelengths $(2$ and $3.6 \mathrm{~cm}$ ) and may be also occurring at $6 \mathrm{~cm}$, and is usually in the form of temporary decreases of the radio flux densities from their usual levels. The timescale for these radio dips is not well-determined by the present data set, but significant variability on a timescale of $=$ or $<1$ month is observed on occasion. There is no correlation between the variations observed at the different wavelengths.

\section{0,049}

PB94-185220 Not available NTIS

National Inst. of Standards and Technology (PL), Boulder, CO. Quantum Physics Div.

Distant Future of Solar Activity: A Case Study of Beta Hydri. 3. Transition Region, Corona, and Stellar Wind.

Final rept.

D. Dravins, P. Linde, T. R. Ayres, T. Simon, F.

Wallinder, J. L. Linsky, and B. Monsingnori-Fossi. 20 Jan $93,14 p$

Contract NASA-NAG5-82

Pub in Astrophysical Jnl. 403, p412-425, 20 Jan 93. Sponsored by National Aeronautics and Space Administration, Washington, DC

Keywords: "Solar activity, "Stellar evolution, Stellar coronae, Stellar winds, Ultraviolet spectra, X-ray spec- tra, Atmospheric models, Late stars, IUE, Reprints, *Beta Hydri star.

The secular decay of solar-type activity with age is studied through a detailed comparison of the present Sun (G2 V) with the very old (9-to Gyr) solar-type star beta Hydri (G2 IV), taken as a proxy of the future Sun. Analyses of successive atmospheric layers are made, and this Paper III in the series treats the outermost parts. The far-UV emission lines from the transition zone are among the faintest so far seen in any solartype star. The significance of the deduced fluxes for the weak and marginally detected lines is tested through two independent reductions of IUE data. The coronal soft $X$-ray spectrum was measured through different filters on EXOSAT and compared to simulated $X$-ray observations of the Sun seen as a star. The flux from beta Hydri is weaker than that from the solar corona and has a different spectrum.

00,050

PB94-185626 Not available NTIS

National Inst. of Standards and Technology (PL), Boulder, CO. Quantum Physics Div.

Rotational Modulation and Flares on RS Canum Venaticorum and BY Draconis Stars. XVI. IUE Spectroscopy and VLA Observations of C1182(=V 1005 Orionis) in October 1983.

Final rept

M. Mathioudakis, J. G. Doyle, M. Rodono, S

Avgoloupis, J. L. Linsky, D. Gary, L. N. Mavridis, P.

Varvoglis, D. M. Gibson, and P. B. Byrne. 1991, 1 tp Pub. in Astron. Astrophys. 244, p155-165 1991.

Keywords: *Stellar flares, Ultraviolet spectra, Microwave spectra, Radiative transfer, IUE, Reprints, Very large array (VLA)

A large flare was detected simultaneously with IUE and VLA on Gl 182 on 5 October 1983, this event showing the largest C IV flare enhancement yet observed by IUE. A smaller flare was also detected on 4 October. although only with the IUE satellite. We use line ratio and emission measure techniques to derive various physical parameters of the flares, i.e., the electron pressures and the dimensions of the emitting plasma. The radio data suggest that the flare was an opticallythick gyro-resonance or gyro-synchrotron event. There is no evidence that the event was circularly polarized.

00.051

PB94-185915 Not available NTIS

National Inst. of Standards and Technology (PL), Boulder, CO. Quantum Physics Div.

High Sensitivity Survey of Radio Continuum Emission in Herbig Ae/Be Stars.

Final rept.

S. L. Skinner, A. Brown, J. Linsky, and R. T. Stewart. 1992, 3p.

Contract NASA-W-17772

Pub. in Proceedings of Cambridge Workshop on Cool Stars, Stellar Systems, and the Sun (7th), Tucson, AZ. October 1991, p331-333 1992. Sponsored by NASA Center for AeroSpace Information, Baltimore-Washington International Airport, MD.

Keywords: "Pre-main sequence stars, Radio sources(Astronomy), Radio emission, High sensitivity, Surveys, Reprints, Very large array(VLA).

We present results of a high sensitivity VLAVAustralia Telescope survey of radio continuum emission from the 57 Herbig Ae/Be stars (and candidates) cataloged by Finkenzeller and Mundt (1984). Twelve stars were detected at the primary observing wavelength of 3.6 $\mathrm{cm}$, with an additional five stars possibly detected. Upper limits for undetected stars are typically $0.1 \mathrm{mJy}$ ( 3 sigma). Follow-up observations in the wavelength range $2-20 \mathrm{~cm}$ give spectral index estimates for 9 stars. Preliminary analysis suggests that the radio emission is of thermal origin in most cases, although nonthermal emission is a possibility for Tr CrA and MWC 137.

\section{0,052}

PB94-198652 Not available NTIS

National Inst. of Standards and Technology (PL), Boulder, CO. Quantum Physics Div.

Discovery of an X-Ray Selected, Radio-Loud Quasar at $z=3.9$.

Final rept

R. H. Becker, D. J. Helfand, and R. L. White. 1992, $4 \mathrm{p}$.

Pub. in Astronomical Jnl. 104, n2 p531-534 Aug 92 Sponsored by Department of Energy, Washington, DC Office of Energy Research. 


\section{Astrophysics}

Keywords: "X-ray sources, *Quasars, Radio sources(Astronomy), Emission spectra, Red shift, Reprints.

A flux-limited catalog of radio sources in the northern sky has been used to select about 600 weak x-ray sources from a database of about 10 (sup 5) 2-4 sigma fluctuations derived from Einstein Observatory Imaging proportional counter $x$-ray images. Optical spectroscopy of only six of these sources resulted in the discovery of a quasar with an emission-line redshift of 3.87 , the highest redshift $x$-ray source yet discovered. Subsequent VLA observations reveal the source to have a flat-spectrum radio core plus an unresolved radio lobe 2.5 seconds away with a spectral index of alpha=1.45. The lobe is connected to the core by a weak jet. The integrated flux density of the source at $20 \mathrm{~cm}$ is $0.66 \mathrm{Jy}$. The $\mathrm{V}$ magnitude of the quasar in 19.5, and the tentative $x$-ray source has a very soft spectrum. The authors discuss the properties of the object in the context of known high-redshift radio sources and existing x-ray selected quasar samples, and comment on the potential of our discovery technique for exploring the properties of very distant active galaxies.

\section{0,053 \\ PB94-199437 Not available NTIS}

National Inst. of Standards and Technology $(P L)$, Boulder, CO. Quantum Physics Div.

IUE Observations of Solar-Type Stars in the Pleiades and the Hyades.

Final rept.

J. P. Caillault, O. Vilhu, and J. L. Linsky. 20 Dec 91, $8 p$

Contracts NASA-NAG5-82, NASA-INT-89-00202

Pub. in Astrophysical Jnl. 383, p594-601, 20 Dec 91 Sponsored by NASA Center for AeroSpace Information, Baltimore-Washington International Airport, MD. and National Science Foundation, Arlington, VA.

Keywords: *Pleiades cluster, ${ }^{*} \mathrm{~F}$ stars, ${ }^{*} \mathrm{G}$ stars, Stellar chromospheres, Stellar spectra, Satellite observation, Ultraviolet spectra, Late stars, Magnesium ions, Carbon ions, IUE, Reprints, *Hyades cluster.

The authors have made the first extensive set of IUE observations of solar-type stars (spectral types F5-G5) in the Pleiades. Spectra were obtained in 1988 January and August for both the transition region (C IV 1550 A; low-resolution) and chromospheric (Mg II $2800 \mathrm{~A}$; high-resolution) emission wavelength regions, respectively. The Pleiades solar-type stars lie at the extreme limit of what IUE can observe in very long exposures. The authors were able to detect Mg II fluxes for two out of three Pleiades stars and interesting C IV upper limits for two of these stars. Long-wavelength, highresolution spectra were also obtained for previously unobserved solar-type stars in Hyades. With the inclusion of spectra of additional Hyades stars obtained from the IUE archives, they have calculated surface fluxes and fractional luminosities for both clusters' solar-type stars. These values provide a better estimate for the $\mathrm{Mg}$ II saturation line for single (as opposed to contact-binary) stars.

\section{0,054}

\section{PB94-199528 Not available NTIS}

National Inst. of Standards and Technology (PL), Boulder, CO. Quantum Physics Div.

First Results from the Goddard High-Resolution Spectrograph: The Chromosphere of Tauri.

Final rept.

K. G. Carpenter, R. D. Robinson, G. M. Wahlgren, J. L. Linsky, A. Brown, F. M. Walter, T. B. Ake, and D. C. Ebbets. 1991, 4p.

Contract NASA-S-56460-D

Pub. in Astrophysical Jnl. 377, pL45-L48, 10 Aug 91. Sponsored by NASA Center for AeroSpace Information, Baltimore-Washington International Airport, MD.

Keywords: *Stellar chromospheres, Stellar winds, Stellar spectra, Ultraviolet spectra, Emission spectra, Late stars, $\mathrm{K}$ stars, Copper ions, Silicon ions, Iron ions, Nickel ions, Cobalt ions, Reprints, Alpha Tavri star.

The K5 III star alpha Tau was observed with the Goddard High Resolution Spectrograph on 1990 November 27 as part of the Science Assessment Program for the Hubble Space Telescope. The observations include spectra in both the Large and Small Science Apertures in modes G270M at $2345 \mathrm{~A}$ and echelle $\mathrm{B}$ at $2325 \mathrm{~A}$. The spectra show intersystem and permitted chromospheric emissions lines of C II (right bracket), Si II (right bracket), Fe II, Fe I, Ni II, and Co II. Resolved profiles of the C II (right bracket) lines indicate a complex chromospheric turbulent velocity distribution with mean value of approx. $24 \mathrm{~km} / \mathrm{s}$ (right bracket), while their observed wavelengths indicate a $4 \mathrm{~km} / \mathrm{s}$ downflow of the $\mathrm{Cll}$ (right bracket) plasma. Twenty-five new emission lines have been found in the 2320-2370 A region, 17 of which have been identified with the aid of Skylab data obtained above the solar limb including four lines from Co II (UV 8) and an Fe I (UV 12) line.

\section{0,055}

PB94-199601 Not available NTIS

National Inst. of Standards and Technology (PL), Boulder, CO. Quantum Physics Div.

ROSAT All-Sky Survey of Active Binary Coronae. 2. Coronal Temperatures of the RS Canum Venaticorum Systems.

Final rept

R. C. Dempsey, J. L. Linsky, J. H. M. M. Schmitt,

and T. A. Fleming. 1993, 6p.

Contract NASA-NAG5-1797

Pub. in Astrophysical Jnl. 413, p333-338, 10 Aug 93. Sponsored by NASA Center for AeroSpace Information, Baltimore-Washington International Airport, MD.

Keywords: *Binary stars, *Stellar coronal, *Stellar temperature, X-ray spectra, ROSAT mission, Reprints, Sky surveys(Astronomy).

The authors present the results from an analysis of $X$ ray spectra of $44 \mathrm{RS} C V \mathrm{n}$ systems obtained during the ROSAT All-Sky Survey with the Position Sensitive Proportional Counter (PSPC). Thermal plasma models with two temperature components are found to reproduce the observations better than single or continuous temperature models. The authors typically find that a bimodal distribution of temperatures centered near 2 $\times 10$ (sup 6) and $1.6 \times 10$ (sup 7) $\mathrm{K}$ fit the data best They show that the PSPC temperatures agree well with those from similar low-resolution measurements, although differences exist, primarily due to differing detector bandpasses. After comparing coronal characteristics--temperature or emission measure--with stellar parameters including rotation period and dynamo number, the authors find no compelling relationship. Including a gravity term, as suggested by previous investigators, leads to a deceptive relationship, because gravity is a strong function of rotation period for these systems. Similarly, the height integrates emission measures of the components in the two-temperature models, including a gravity term, are found to be well correlated with temperature.

\section{0,056}

PB94-211067 Not available NTIS

National Inst. of Standards and Technology (PL), Boulder, CO. Quantum Physics Div.

Dynamic Phenomena on the RS Canum Venaticorum Binary II Pegasi in August 1989. 1. Observational Data.

Final rept.

J. G. Doyle, B. J. Kellett, C. J. Butler, A. Brown, D. Fox, J. E. Linsky, P. B. Byrne, and J. E. Neff. 1992, 23p.

Pub. in Astronomy and Astrophysics Supplement Series 96, p351-373 1992

Keywords: *Binary stars, *Stellar flares, X-ray spectra Visible spectra, Balmer lines, GINGA satellite, IUE, Reprints, RS CVn stars, Raymond-Smith models.

Results are presented for two flares detected by the $X$-ray satellite GINGA the International U/traviolet EXplorer satellite, ground-based Johnson U-band photometry, and optical spectroscopy from the Isaac Newton telescope for the RS CVn star II Peg.

\section{0,057}

PB94-211083 Not available NTIS

National Inst. of Standards and Technology (PL), Boulder, CO. Quantum Physics Div.

Radio Continuum and X-Ray Properties of the Coronae of RS Canum Venaticorum and Related Active Binary Systems.

Final rept.

S. A. Drake, T. Simon, and J. L. Linsky. 1992, 11 p. Contract NASA-W-17777

Sponsored by NASA Center for AeroSpace Information, Baltimore-Washington International Airport, MD. Pub. in Astrophysical Jnl. Supplement Series 82, n1 p311-321 Sep 92.

Keywords: "Binary stars, *Stellar coronae, Radio emission, Continuous radiation, Circular polarization, $X$ rays, Reprints, RS CVn stars.

We present some new radio continuum and X-ray data on the coronae of RS CVn and related active binary systems which complement those included in the previous Drake, Simon, and Linsky study of the radio emission properties of these stars. The bulk of these new radio data consists of $6 \mathrm{~cm}$ circular polarization measurements of 28 binaries for which we can set significant constraints (approx $=$ or $<25 \%$ ) on this quantity. Seven sources showed definite circular polarization at levels from $2 \%$ to $13 \%$, and two others showed possible circular polarization. We also present $2 \mathrm{~cm}$ flux density and circular polarization measurements for 29 Dra, lambda And, and sigma Gem on dates for which we also have $6 \mathrm{~cm}$ observations.

\section{0,058}

PB94-211802 Not available NTIS

National Inst. of Standards and Technology (PL), Boulder, CO. Quantum Physics Div.

Interstellar Disk-Halo Connection in Galaxies: Review of Observational Aspects.

Final rept.

C. Heiles. 1991, 16p

Pub. in Proceedings IAU Symposium 144, v51 p4334481991.

Keywords: *Galactic halos, Interstellar space, Magnetic fields, Cosmic rays, Neutral gases, Ionized gases, Reviews, Reprints.

The review is organized as follows. First, the author discusses the various gas components in rough orde of increasing scale height. Section 1 discusses neutral gas, section 2 the 'warm' and 'not-so-warm' ionized gas, section 3 the $\mathrm{T}$ approx $=10$ (sup 5) $\mathrm{K}$ component at higher $z$ that is detected in UV absorption and emission, section 4 the high-velocity neutral gas, section 5 the cosmic-ray halo as revealed by synchrotron emission, and section 6 the magnetic field. Next, section 7 covers the interaction between the low- $z$ gas and the halo, which is the main topic of this symposium; and finally, section 8 discusses some aspects of the interstellar medium that are relevant to this interaction, with emphasis on the uncertainties.

\subsection{9}

PB94-212214 Not available NTIS

National Inst. of Standards and Technology (PL), Boulder, CO. Quantum Physics Div.

Opacity Project and the Practical Utilization of Atomic Data.

Final rept.

D. G. Hummer. 199199.

Contract NASA-NAGW-766, Grant NSF-AST88-

02937

Sponsored by NASA Center for AeroSpace Information, Baltimore-Washington International Airport, MD. and National Science Foundation, Washington, DC.

Pub. in Stellar Atmospheres: Beyond Classical Models, p431-439 1991.

Keywords: "Stellar envelopes, "Stellar atmospheres, "Radiative transfer, Ionization cross sections, Transition probabilities, Computer applications, Photoionization, Reprints, Opacity project, Atomic data.

The Opacity Project was organized to compute new opacities for stellar envelopes, but has produced as a secondary product a vast amount of atomic radiative data for almost all elements of astrophysical interest. The photoionization cross sections in many cases show extensive resonance structure, which introduces complications when they are used in stellar modeling Possible ways of dealing with this problem are discussed. Finally, a method of presenting atomic data in standardized subroutines in order to eliminate the need for large, special purpose data sets is proposed.

\section{0,060}

PB94-213022 Not available NTIS

National Inst. of Standards and Technology (PL), Boulder, CO. Quantum Physics Div.

Radiation-Driven Winds of Hot Luminous Stars $X$ The Determination of Stellar Masses Radii and Distances from Terminal Velorities and Mass-Loss Rates.

Final rept.

R. P. Kudritzki, D. G. Hummer, A. W. A. Pauldrach,

J. Imhoff, J. Puls, and F. Najarro. 1992, 8p.

Pub. in Astronomy and Astrophysics 257, p655-662 1992.

Keywords: "Hot stars, "Stellar mass, "Stellar winds, Stellar mass ejection, Massive stars, Terminal velocity, Reprints, *Stellar distances, *Stellar radii.

A new, purely spectroscopic method to determine masses, radii and distances of massive, luminous hot 
stars is presented. This method is based on the theory of radiation-driven winds and uses terminal velocity, mass-loss rate and effective temperature as observational quantities determined from the spectrum. The potential of this method is discussed.

00,061
PB94-213287 Not available NTIS

National Inst. of Standards and Technology (PL), Boulder, CO. Quantum Physics Div.

Astrophysical Aspects of Neutral Atom Line Broadening.

E. L. Lewis. $1990,12 p$

Pub. in Proceedings of International Conference on Spectral Line Shapes (10th), Austin, TX., June 25-29, 1990, p541-552.

Keywords: "Atom-atom collisions, "Line broadening, Resonance lines, Spectral lines, Neutral atoms, Hydrogen, Reprints, Redistribution functions.

Recent work on aspects of line shapes which are relevant to astrophysical spectra are discussed, namely (i) the impact broadening of strong resonance lines by atomic hydrogen, (ii) the effects on line profiles of collisional redistribution of radiation in the impact region and (iii) the influence of velocity changing collisions on impact region redistribution.

\section{0,062}

PB94-213402 Not available NTIS

National Inst. of Standards and Technology (PL), Boulder, CO. Quantum Physics Div.

Atomic Data Needed for Far Ultraviolet Astronomy with HUT and FUSE.

Final rept.

J. L. Linsky. 1993, 10p.

Pub. in Atomic and Molecular Data for Space Astronomy: Needs and Availability, Chapter 3, p33-42 1993.

Keywords: *Ultraviolet astronomy, Extreme ultraviolet spectra, Far ultraviolet radiation, Spaceborne astronomy, Astronomical satellites, Electron collisions, Oscillator strengths, Cross sections, Photoionization, Wavelengths, Reprints, Atomic data, FUSE satellite.

The author will summarize the spectroscopic capabilities of existing and planned space experiments, including HUT and FUSE, that will obtain spectra of astronomical sources at wavelengths shorter than Lymanalpha. The important atomic and molecular data needed to analyze far and extreme ultraviolet spectra that will be obtained with these instruments include accurate wavelengths, oscillator strengths, photoionization cross sections for six important molecules, and, especially, electron collisional excitation cross sections for both low and high stages of ionization.

\section{0,063}

\section{PB94-213410 Not available NTIS}

National Inst. of Standards and Technology (PL), Boulder, CO. Quantum Physics Div.

FUSE: The Far Ultraviolet Spectrograph Explorer.

J. L. Linsky. 1992, 7p.

Pub. in Proceedings of Cambridge Workshop on Cool Stars, Stellar Systems, and the Sun (7th), Tucson, AZ., October 1991, p622-628 1992

Keywords: "Astronomical satellites, Extreme ultraviolet spectra, Far ultraviolet radiation, Ultraviolet astronomy, Cool stars, Satellite observation, Spaceborne astronomy, Reprints, "FUSE satellite, FUSE project.

The author will summarize the past developments and present status of the FUSE Project. FUSE will observe a broad range of astrophysical sources, including cool stars, with high sensitivity and high spectral resolution in the far ultravioiet (912-1250A). It will also study broad band spectra (100-1600A) with high throughput and modest resolution.

\section{0,064 \\ PB94-213428 Not available NTIS}

National Inst. of Standards and Technology (PL), Boulder, CO. Quantum Physics Div.

Ultraviolet Observations of Stellar Coronae: Early Results from HST.

Final rept

J. L. Linsky. 1992, 13p.

Contract NASA-S-56500-D

Sponsored by NASA Center for AeroSpace Information, Baltimore-Washington International Airport, MD Pub. in Proceedings of IAU Joint Commission Meeting on Solar and Stellar Coronae, Buenos Aires, Argentina, July 31, 1991, p577-5891992.
Keywords: "Stellar coronae, "Ultraviolet spectra, Hubble Space Telescope, Steilar spectra, Giant stars, Late stars, Binary stars, Emission spectra, Electron density, Stellar chromospheres, Reprints, "Capella star, "Garnma Draconis star.

I report on the first GHRS (Goddard High Resolution Spectrograph) spectra of two very different late-type giant stars - Capella and gamma Dra. Capella is a 104 day period binary system consisting of two stars (G9 III and GO III) each of which shows bright emission lines formed in solar-like transition region and coronae. By contrast, gamma Dra is a hybrid-chromosphere star with very weak emission lines from high-temperature plasma Low-dispersion spectra of these stars covering the 1160 to $1717 \mathrm{~A}$ spectral range show unresolved emission lines from neutral species through $\mathrm{N} V$. The very different surtace fluxes detected in the spectra of these stars suggest different types of heating mechanisms.

00,065

PB94-213436 Not available NTIS

National Inst. of Standards and Technology (PL), Boulder, CO. Quantum Physics Div.

$X$-rays from Stellar Flares.

Final rept.

J. L. Linsky. 1991, 14p.

Contract NASA-NAGW-1480

Sponsored by NASA Center for AeroSpace Information, Baltimore-Washington International Airport, MD. Pub. in Mem. Soc. Astron. Ital. 62, n2 p307-320 1991. Keywords: "Stellar flares, X-ray sources, Binary stars, Algol, Reprints, "Stellar x-rays, RS CVn stars.

I will summarize the $x$-ray observations of flares on $\mathrm{dMe}$, active spectroscopic binaries, and young stars. Questions of particular interest include the energy associated with the $x$-ray emission and its relation to other components of the flare energy budget, the time behavior of the flaring plasma as revealed by the $x$ ray emission, and comparisons of stellar flare parameters with solar compact and two ribbon flares.

\section{0,066}

PB94-213444 Not available NTIS

National Inst. of Standards and Technology (PL), Boulder, CO. Quantum Physics Div.

Goddard High-Resolution Spectrograph Observations of the Local Interstellar Medium and the Deuterium/Hydrogen Ratio along the Line of Sight Toward Capella.

Final rept.

J. L. Linsky, A. Brown, K. Gayley, T. R. Ayres, W. Landsman, S. N. Shore, S. R. Heap, A. Diplas, and B. D. Savage. $1993,16 \mathrm{p}$.

Sponsored by NASA Center for AeroSpace Information, Baltimore-Washington International Airport, MD Pub. in The Astrophysical Jnl. 402, p694-709, 10 Jan 93.

Keywords: *Interstellar matter, Far ultraviolet radiation, Hubble Space Telescope, Ultraviolet spectra, Binary stars, Line of sight, Giant stars, Late stars, Hydrogen, Deuterium, Magnesium ions, Iron ions, Ratios, Reprints, Capella star.

We analyze HST Goddard High-Resolution Spectrograph observations of the 1216,2600 , and $2800 \mathrm{~A}$ spectral regions for the spectroscopic binary system Capella, obtained at orbital phase 0.26 with $3.27-3.57$ $\mathrm{km} / \mathrm{s}$ resolution and high signal-to-noise ratio. We infer the column densities of $\mathrm{HI}, \mathrm{DI}, \mathrm{Mg} \mathrm{II}$, and $\mathrm{Fe}$ II for the local interstellar medium along the $12.5 \mathrm{pc}$ line of sight, together with estimates of the temperature and turbulent velocity If we assume that the intrinsic Ly(alpha) lines of the component stars in the Capella system can be approximated as scaled solar lines (with self-reversals), which is consistent with the observed Ly(alpha) profile of the high radial velocity star delta Lep, then the average interstellar neutral hydrogen number density $=0.047 / \mathrm{cc}$.

00,067

PB94-213451 Not available NTIS

National Inst. of Standards and Technology (PL), Boulder, CO. Quantum Physics Div.

Class of Radio-Emitting Magnetic B Stars and a Wind-Fed Magnetosphere Model.

Final rept.

J. L. Linsky, S. A. Drake, and T. S. Bastian. 1992

$3 p$

Contract NASA-W-17772, Grant NGL-06-003-057 Sponsored by NASA Center for AeroSpace Information, Baltimore-Washington International Airport, MD.
Pub. in Proceedings of Cambridge Workshop on Coo Stars, Stellar Systems, and the Sun (7th), Tucson, AZ. October 1991, v26 p325-327 1992.

Keywords: "Magnetic stars, "Radio stars, Radio sources(Astronomy), Stellar magnetospheres, Stellar winds, Peculiar stars, B stars, A stars, Reprints.

We have detected a total of 16 magnetic Bp-Ap stars at $6 \mathrm{~cm}$, out of 61 observed, including both $\mathrm{He}$-strong and $\mathrm{He}$-weak/Si-strong stars with $\log \mathrm{L}(6)=14.7$ to 17.9 , but none of the classical (SrCrEu-type) Ap stars at a detection limit of $\log L(6)<14.9$. We believe that the radio-emitting $\mathrm{CP}$ (chemically peculiar) stars form a distinct class of radio stars that differs from both the hot star wind sources and the active late-type stars. For the detected CP stars, we find that $L(6)$ is proportional to $M($ sup 0.5 ) B(rms), produced by optically thick nonthermal gyrosynchrotron emission. In our model the electrons are accelerated in current sheets located $10-20$ radii from the star.

00,068

PB94-213469 Not available NTIS

National Inst. of Standards and Technology (PL), Boulder, CO. Quantum Physics Div.

Radio Emission from Chemically Peculiar Stars.

Final rept

$16 p$

Linsky, S. A. Drake, and T. S. Bastian. 1992

Sponsored by NASA Center for AeroSpace Informa tion, Baltimore-Washington International Airport, MD Pub. in The Astrophysical Jnl. 393, n1 p341-356, $1 \mathrm{Ju}$ 92

Keywords: "Magnetic stars, "Radio stars, Radio sources(Astronomy), Stellar magnetospheres, Radio emission, Electron trajectories, Stellar winds, Peculiar stars, B stars, A stars, Very large array(VLA), Reprints

We have extended the initial survey of radio emission from magnetic $\mathrm{Bp}-\mathrm{Ap}$ stars by Drake et al, in five subsequent very large array (VLA) observing runs. A total of 16 sources have been detected at $6 \mathrm{~cm}$ out of 61 observed, giving a detection rate of $26 \%$. Of these stars, three are also detected at $2 \mathrm{~cm}$, four at $3.6 \mathrm{~cm}$, and five at $20 \mathrm{~cm}$. In addition to the three He-strong and two He-weak/Si-strong stars previously reported as radio sources, we have detected 11 new stars as radio sources with spectral types B5-AO that are Heweak and Si-strong. We have not yet detected any of the classical (SrCrEu-type) Ap stars despite many attempts. We find a wide range of high $6 \mathrm{~cm}$ radio luminosities for the detected stars, with $\mathrm{log} L(6)=14.7$ 17.9. We show that all of the observed properties of radio emission from these stars may be understood in terms of optically thick gyrosynchrotron emission from a nonthermal distribution of electrons. We propose a model in which the electrons are produced in curren sheets forming 10-20 radii from the star in this mode the electrons travel along magnetic fields to smalle radii and higher magnetic latitudes where they mirro and radiate microwave radiation.

00,069

PB94-213477 Not available NTIS

National Inst. of Standards and Technology (PL), Boulder, CO. Quantum Physics Div.

First Results from a Coordinated ROSAT, IUE, and VLA Study of RS CVn Systems.

Final rept

J. L. Linsky, D Fox, A. Brown, J. H. M. M. Schmitt,

T. Fleming, M. Rodono, I. Pagano, J. E. Neff, G.

Bromage, R. Dempsey, and C. Schmitt. 1992, 3p.

Contracts NASA-W17-772, NAG5-82

Sponsored by NASA Center for AeroSpace information, Baltimore-Washington International Airport, MD. Pub in Proceedings of Cambridge Workshop on Cool Stars, Stellar Systems, and the Sun (7th), Tucson, AZ. October 1991, p106-108 1992

Keywords: ROSAT mission, Ultraviolet spectra, $X$ ray spectra, Radio emission, Binary stars, IUE, Very large array(VLA), Reprints, "RS CVn stars.

This is a preliminary report on our extensive set of coordinated observations of RS CVn systems obtained during the ROSAT all-sky survey. Simultaneous with the PSPC and WFC observations, we observed five RS CVn systems with IUE and 15 systems with the VLA, typically at three wavelengths. We list the times of the coordinated observations and show our derived fluxes for one system - TY Pyx. 


\section{Astrophysics}

National Inst. of Standards and Technology (PL), Boulder, CO. Quantum Physics Div.

Rapid Decline in the Optical Emission from SN $1957 \mathrm{D}$ in M83.

Final rept.

K. S. Long, P. F. Winkler, and W. P. Blair. 1992, 5p. Pub. in Astrophysical Jnl. 395, p632-636, 20 Aug 92.

Keywords: "Supernova remnants, Emission spectra, Oxygen ions, Sulfur ions, $H$ alpha line, Nucleosynthesis, Reprints, "Supernova 1957D.

As part of a search for the supernova remnants in M83, we have obtained new interference filter images of the site of SN 1957D and new spectra of the young supernova remnant (SNR) itself. Images were obtained in the light of $\mathrm{H}$ (alpha) (O III), (S II), and several continuum bands using the prime focus CCD camera on the CTIO $4 \mathrm{~m}$ telescope. An inspection of the images shows that the flux from the SNR, as observed through the (O III) filter, has decreased by at least a factor of 5 compared with observations made at Las Campanas 4 years earlier. There is no indication of a change in the emission from the SNR in any other filter. Spectroscopic observations conducted at the position of the SN yield an (O III) flux in 1991 April, down by a factor of 3 from measurements with the same instrument 2 years earlier.

00,071

PB95-125738 Not available NTIS

National Inst. of Standards and Technology (NML), Gaithersburg, MD. Molecular Physics Div.

Infrared Spectra of van der Waals Complexes of Importance in Planetary Atmospheres.

Final rept.

G. T. Fraser, A. S. Pine, and W. J. Lafferty. 1990,

$5 p$.

Pub. in Proceedings of International Conference on Laboratory Research for Planetary Atmospheres (1st), p64-68 May 90.

Keywords: "Planetary atmospheres, "Infrared spectra "Complexes, Van der Waals forces, Near infrared radiation, Vibrational spectra, Venus atmosphere, Mars atmosphere, Predissociation, Argon complexes, Trimers, Dimers, Reprints, "Carbon dioxide complexes.

It has been suggested that (CO2)2 and $\mathrm{Ar}-\mathrm{CO} 2$ are important constituents of the planetary atmospheres of Venus and Mars. Here, we present recent results on the laboratory spectroscopy of $\mathrm{CO} 2$-containing van der Waals complexes which may be of use in the modeling of the spectra of planetary atmospheres. Sub-Doppler infrared spectra have been obtained for (CO2)2 (CO2)3, and rare-gas-CO2 complexes in the vicinity of the CO2 Fermi diad (nu(1) + nu(3), 2nu(2) + nu(3)) at 2.7 micrometers using a color-center-laser optothermal spectrometer. From the spectroscopic constants, the geometries of the complexes have been determined and van der Waals vibrational frequencies have been estimated. The equilibrium configurations are $C(2 h)$, $\mathrm{C}(3 \mathrm{~h})$, and $\mathrm{C}(2 \mathrm{v})$, for $(\mathrm{CO} 2) 2$, (CO2)3, and the raregas- $\mathrm{CO} 2$ complexes, respectively. The linewidths of the rovibrational transitions are $=$ or $<22 \mathrm{MHz}$, indicating that predissociation is as much as four orders of magnitude faster than radiative processes for vibrational relaxation in these complexes.

00,072

PB95-152070 Not available NTIS

National Inst. of Standards and Technology (NML), Boulder, CO. Quantum Physics Div.

IRAS Spectroscopic Observations of Young Planetary Nebulae.

Final rept.

C. Y. Zhang, and S. Kwok. 1990, 16p.

Pub. in Astronomy and Astrophysics 237, p479-494 1990.

Keywords: "Planetary nebulae, "Interstellar matter "Cosmic dust, Intermediate infrared radiation, Infrared astronomy, Infrared Astronomy Satellite, Stellar evolution, Silicates, Oxygen, Carbon, Reprints.

The 18 micrometer silicate dust feature is detected in 11 young planetary nebulae selected from radio properties. The presence of the 18 micrometer silicate feature suggests that oxygen-rich planetary nebulae are more common than previously believed. The silicate dust probably originates from the remnants of the circumstellar envelopes of the nebulae's asymptoticgiant-branch progenitors and remains visible in young planetary nebulae. Most interestingly, there are six young planetary nebulae that show both oxygen- and carbon-rich dust features. It is possible that a planetary nebula that descends from an oxygen-rich progenitor star will be classified as carbon rich as the silicate feature diminishes and the ultraviolet-excited polycycllc aromatic hydrocarbon bands become more prominent as the nebula ages. We suggest that a true chemical origin of planetary nebulae can only be reliably determined from young planetary nebulae.

\section{0,073}

PB95-152195 Not available NTIS

National Inst. of Standards and Technology (NML), Gaithersburg, MD. Time and Frequency Div.

Laboratory Measurements for the Astrophysical Identification of $\mathrm{MgH}$.

Final rept.

L. R. Zink, D. A. Jennings, K. M. Evenson, and K. R. Leopold. 1990, 2p.

Pub. in Astrophysical Jnl. 359, n2 pL65-L66 1990.

Keywords: "Magnesium hydrides, "Rotational spectra, Electron transitions, Interstellar matter, Reprints.

The frequencies of the $\mathrm{N}=1<\cdots 0$ transition of $\mathrm{MgH}$ are reported. They will serve as a basis for astrophysical searches for the molecule.

\section{0,074}

PB95-153441 Nol available NTIS

National Inst. of Standards and Technology (PL), Boulder, CO. Quantum Physics Div.

Distant Future of Solar Activity: A Case Sturjy of lar Wind.

Final rept.

D. Dravins, P. Linde, T. R. Ayres, T. Simon, F

Wallinder, J. L. Linsky, and B. Monsingnori-Fossi.

1993, 14p.

Contract NAG5-82

Sponsored by NASA Scientific and Technical Information Facility, Baltimore, MD.

Pub. in Astrophysical Jnl. 403, p412-425, 20 Jan 93.

Keywords: "Solar activity, "Stellar evolution, "Stellar coronae, Stellar winds, Uitraviolet spectra, X-ray spectra, Atmospheric models, Late stars, EXOSAT satellite, IUE, Reprints.

The secular decay of solar-type activity with age is studied through a detailed comparison of the present Sun (G2 V) with the very old (9-10 Gyr) solar-type star beta Hyi (G2 IV), taken as a proxy of the future Sun. Analyses of successive atmosphenic layers are made and this Paper III in the series treats the outermost parts. The far-UV emission lines from the transition zone are among the faintest so far seen in any solartype star. The significance of the deduced fluxes for the weak and marginally detected lines is tested through two independent reductions of IUE data. The coronal soft $X$-ray spectrum was measured through different filters on EXOSAT and compared to simulated $X$-ray observations of the Sun seen as a star. The flux from beta Hyi is weaker than that from the solar corona and has a different spectrum. Implications are discussed.

\section{0,075}

PB95-202263 Not available NTIS

National Inst. of Standards and Technology (PL), Boulder, CO. Quantum Physics Div.

Hydrogen Lyman-alpha EmIsslon of Capella.

Final rept.

T. R. Ayres, A. Brown, K. G. Gayley, and J. L.

Linsky. 1993, $11 p$

Contract NASA-S- 56500

Sponsored by NASA Scientific and Technical Information Facility, Baltimore, MD.

Pub. in Astrophysical Jnl. 402, p710-720, 10 Jan 93.

Keywords: "Lyman alpha radiation, Lyman spectra, Emission spectra, Ultraviolet spectra, Binary stars. IUE, Reprints, "Capella star.

We describe the hydrogen Ly(alpha) emission of the spectroscopic binary Capella (G8 III + GO III) recorded at $0.1 \mathrm{~A}$ resolution by the International Ultraviolet $\mathrm{Ex}$. plorer (IUE). The overt changes in the composite line shape with orbital phase are controlled by the active GO III star and permit a dissection of the stellar components despite the obliteration of the central portion of the profile by atomic hydrogen and deuterium absorption along the $12.5 \mathrm{pc}$ sightline. The Ly(alpha) line shape of the active GO III star is surprisingly asymmetnic and possibly is variable. Both characteristics suggest a stellar wind of moderate excitation, a key component of the coronal evolution scenario of Simon and Drake for the Hertzsprung-gap giants.
00,076

PB95-202321 Not available NTIS

National Inst. of Standards and Technology (PL), Boulder, CO. Quantum Physics Div.

Observatlons of 3C 273 with the Goddard High Resolution Spectrograph on the Hubble Space Telescope.

Final rept.

1993, 16p.

Pub. in Astronomical JnI. 105, n3 p831-846 Mar 93.

Keywords: "Quasars, Hubble space telescope, Spaceborne astronomy, Interstellar matter, Absorption spectra, Ultraviolet spectra, Nickel ions, Galaxies, Reprints, " 3 C 273 radlo source.

The observations of the quasar $3 \mathrm{C} 273$ taken with the Goddard High Resolution Spectrograph in February 1991 are presented here. We have included both the reduced raw data, and smoothed and deconvolved spectra. Also, a list of observed absorption lines is presented. The data comprise 11 spectra, including 1 low resolution observation and 10 medium resolution observations. The wavelength region covered ranged from about 1,150 to $2,820 \mathrm{~A}$, but was not all inclusive. The procedures used to obtain and reduce the data, including corrections for fixed pattem noise, compensation for the effects of spherical aberration in the HST primary mirror, and objective detection of weak absorption lines, are described. We have also included a short discussion on the detection of galactic Ni II and Virgo cluster metal lines.

\section{0,077}

PB95-202479 Not available NTIS

National Inst. of Standards and Technology (PL), Boulder, CO. Quantum Physics Div.

ROSAT All-Sky Survey of Active Binary Coronae. 1. Qulescent Fluxes for the RS Canum Venaticorum Systems.

Final rept.

R. C. Dempsey, J. L. Linsky, T. A. Fleming, and J. H. M. M. Schmitt. 1993, 11 p.

See also Part 2, PB94-199601. Sponsored by NASA Scientific and Technical Information Facility, Baltimore, MD.

Pub. in Astrophysical Jnl. 86, n2 p599-609 Jun 93.

Keywords: "Binary stars, "Stellar coronae, $\mathrm{X}$ ray astronomy, Spaceborne astronomy, ROSAT mission, Sky surveys(Astronomy), Reprints.

We present observations of 136 RS CVn active binary systems obtained with the ROSAT Position Sensitive Proportional Counter (PSPC) during the all-Sky Survey phase of the mission. Of this sample, 112 targets were detected in exposures of about $600 \mathrm{~s}$ or less. This represents the largest sample of RS CVn systems observed to date at any wavelength, including $X$-rays. Furthermore, since the entire sky was surveyed, these data do not suffer from any biases other than those present in the methods used to discover the RS CVn binaries. Comparison of the $X$-ray properties of the RS CVn systems with $6 \mathrm{~cm}$ radio and C IV ultraviolet emission is also presented.

\section{0,078}

PB95-202669 Not available NTIS

National Inst. of Standards and Technology (PL), Boulder, CO. Quantum Physics Div.

Sobolev Approximation for Line Formation with Partlal Frequency Redistribution.

Final rept.

D. G. Hummer, and G. B. Rybicki. 1992, 10p. Contract NASA-NAGW-766, Grant NSF-AST8802937

See also PB85-226058. Sponsored by National Science Foundation, Washington, DC. and National Aeronautics and Space Administration, Washington, DC.

Pub. in Astrophysical JnI. 387, p248-257, 1 Mar 92.

Keywords: "Spectral lines, Radiative transfer, Reprints, Sobolev approximation, Expanding atmospheres.

The formation of a spectral line in a uniformly expanding infinite medium is investigated in the Sobolev approximation with particular attention to the various mechanisms for frequency redistribution. Numerical and analytic solutions of the transfer equation are presented for a number of redistribution functions and their approximations, including type I and type II partial redistribution, coherent scattering and complete redistribution, and the Fokker-Planck and uncorrelated ap- 
proximation to the $R$ (II) function. The solutions for the mean intensity are shown to depend very much on the type of redistribution mechanism, while for the fre quency-weighted mean intensity $\mathrm{J}$ (bar), which enters the rate equations, this dependence is weak. This implies that the use of Sobolev escape probabilities based on complete redistribution can be an adequate approximation for many calculations for which only the radiative excitation rates are needed. However, it is shown that the criteria for applicability of Sobolev the ory may be difficult to meet when transfer occurs primarily in the Voigt wings, especially for complete redis tribution.

\section{0,079}

PB95-202677 Not available NTIS

National Inst. of Standards and Technology (PL), Boulder, CO. Ouantum Physics Div.

Recombination Line Intensities for Hydrogenic Ions-III. Effects of Finite Optical Depth and Dust. Final rept.

D. G. Hummer, and P. J. Storey. 1992, 14p.

Contract NASA-NAGW-766

See also Part 2, PB89-107148. Sponsored by NASA Scientific and Technical Information Facility, Baltimore, MD

Pub. in Monthly Notices of the Royal Astronomical Society 254, p277-2901992.

Keywords: "Hydrogen, Lyman lines, Cosmic dust, Element abundance, Universe, Radiative recombination, Radiative transfer, Radio astronomy, Helium, Reprints We explore systematically the effect on the recombination spectrum of hydrogen arising from: (i) finite optical thickness in the Lyman Lines; (ii) the overlapping of Lyman lines near the series limit; (iii) the absorption of Lyman lines by dust or photoionization, and (iv) the long-wave radiation emitted by dust. Full account is taken of electron and heavy particle collisions in redis tributing energy and angular momentum. We find tha each of these deviations from the classical Case $B$ leads to observable effects, and that dust influences the recombination spectrum in characteristic ways that may make possible new observational constraints on dust properties in nebulosities. On the basis of these calculations, we believe the uncertainty in the determination of the helium-to-hydrogen abundance ratio in the Universe may be larger than currently claimed.

\section{0,080}

PB95-202834 Not available NTIS

National Inst. of Standards and Technology (PL), Boulder, CO. Quantum Physics Div.

Stellar Coronal Structures.

Final rept

J. L. Linsky. 1994, 10p.

Sponsored by National Aeronautics and Space Administration, Washington, DC.

Pub. in IAU Colloq. 144 'Solar Coronal Structures' Tatronska Lomnica, Slovak Rep., September 20-24, 1993, p641-6501994.

Keywords: "Stellar coronae, Stellar winds, Binary stars, Dwarf stars, Peculiar stars, Magnetic fields, Re prints.

Large magnetic structures in the coronae of stars conaining gas at a wide variety of temperatures are now being studied in $X$-rays, radio wavelengths, and $H$ (alpha). Here the author summarizes what we are learning about coronal structures in three types of stellar systems: the magnetic chemically peculiar stars the RS CVn binary systems containing G-and K-type subgiants, and active solar-type dwarfs like V471 Tauri and $A B$ Doradus.

\section{0,081}

PB95-202842 Not available NTIS

National Inst. of Standards and Technology (PL), Boulder, CO. Ouantum Physics Div.

Deuterium in the Local Interstellar Medium: Its Cosmological Significance.

Final rept.

J. L. Linsky, A. Diplas, B. Savage, C. Andrulis, and

A. Brown. 1994, 4 p.

Contract NASA-S-56500-D

Sponsored by National Aeronautics and Space Administration, Washington, DC.

Pub. in Frontiers of Space and Ground-Based Astronomy, p301-304 1994.

Keywords: "Interstellar matter, "Deuterium, "Hydrogen, "Isotope ratio Hubble space telescope Lyman alpha radiation, Line of sight, Galactic evolution, Cosmology, Reprints, Procyon star.
We report on our ongoing program to measure the deuterium/hydrogen $(\mathrm{D} / \mathrm{H})$ ratio and interstellar gas properties along many lines of sight through the local interstellar medium using the Hubble Space Telescope (HST) Goddard High-Resolution Spectrograph. For the line of sight towards Capella $(12.5 \mathrm{pc})$ we had previously found $\mathrm{D} / \mathrm{H}=1.65(+0.07,-0.18) \times 10(\exp -5)$, $T=7,000 \mathrm{~K}$, and turbulent velocity $1.66 \mathrm{~km} / \mathrm{s}$. These quantities were determined by modeling the interstellar hydrogen and deuterium Lyman alpha lines and the resonance lines of $\mathrm{Fe} \mathrm{II}$ and $\mathrm{Mg} \mathrm{II}$ against the background stellar emission-line profiles. We now report on our preliminary analysis of these spectra! lines for the line of sight toward Procyon $(3.5 \mathrm{pc})$. We find that $\mathrm{D} /$ $\mathrm{H}=1.40+$ or $-0.05 \times 10(\exp -5)$. Further analysis of this and other lines of sight are planned to determine whether the $\mathrm{D} / \mathrm{H}$ ratio varies within the local interstellar medium. We infer the primordial value of $\mathrm{D} / \mathrm{H}$ from $\mathrm{Ga}$ lactic evolution models and comment on the derived baryon density of the Universe.

\section{0,082}

PB95-202859 Not available NTIS

National Inst. of Standards and Technology (PL), Boulder, CO. Quantum Physics Div.

Peeking Through the Picket Fence: What Astrophysical Surprises May Be Present in the 100-1200 Angstrom Region.

Final rept.

J. L. Linsky, and D. G. Luttermoser. 1991, 10p. Contracts NASA-NAG5-82, NASA-H-80531

Sponsored by NASA Scientific and Technical Information Facility, Baltimore, MD.

Pub. in Advances in Space Research 11, n11 p(11)5(11) 141991.

Keywords: "Ultraviolet astronomy, Extreme ultraviolet radiation, Far ultraviolet radiation, Ultraviolet spectrometers, Ultraviolet spectra, Spaceborne astronomy, Stellar coronae, Flare stars, G stars, Repnnts.

In anticipation of more sensitive EUV and FUV spectroscopic instruments, we simulate spectra, including interstellar absorption, of solar-like, RS CVn, and flare stars as folded through the instrument parameters of the Extreme Ultraviolet Explorer (EUVE), Lyman/Far Ultraviolet Spectroscopic Explorer (FUSE) Phase $A$, and a desirable next-generation spectrometer. We find that even the relatively insensitive EUVE spectrometer will be able to detect sufficient spectral lines from many active binary and $\mathrm{dMe}$ stars to determine their coronal emission measure distributions. The Lyman/FUSE or next-generation spectrometers are needed to study solar-type stars or flaring stars with high time resolution. The high throughput and effective area of a next-generation spectrometer is needed for Doppler imaging studies, stellar wind and downflow measurements, and high time and spectral resolution of stellar flares.

\section{0,083}

PB95-203501 Not available NTIS

National Inst of Standards and Technology (PL), Boulder, CO. Quantum Physics Div.

New High-Redshift Damped Lyman-alpha Absorption Systems and the Redshift Evolution of Damped Absorbers.

Final rept.

R. L. White, A. L. Kinney, and R. H. Becker. 1993,

$14 p$

Pub. in Astrophysical Jnl. 407, n2 p456-469, 20 Apr 93.

Keywords: "Lyman alpha radiation, Galactic evolution, Absorption spectra, Red shift, Cosmology, Galaxies, Reprints.

We have discovered two high-redshift $(Z(a b s)=3.388$ and 2.908) damped Ly(alpha) absorption systems and a third possible damped system $(Z$ (abs) $=3.080$ ) while obtaining spectra of optical countemarts from a sample of flat spectrum radio sources. These three systems all have higher redshifts than any previously known damped Ly(alpha) absorption systems in the spectra of radio-loud quasars, and they are among the highest redshift damped Ly(alpha) systems known. This is the first good statistical evidence for evolution in the number (or size) of damped Ly(alpha) absorbers at high redshift. We argue that the most likely explanation for the rapid disappearance of damped Ly(alpha) absorbers between $z=3.5$ and $z=3$ is that we are observing the epoch of galaxy formation.
National Inst. of Standards and Technology (PL), Boulder, CO. Quantum Physics Div.

CCD Mosaic Images of the Supernova Remnant 3C 400.2.

Final rept.

P. F. Winkler, T. M. Olinger, and S. A. Westerbeke. 1993, $11 p$

Sponsored by National Science Foundation, Washing ton, DC. and National Aeronautics and Space Adminisration, Washington, DC

Pub. in Astrophysical Jnl. 405, p608-613, 10 Mar 93. Keywords: "Supernova remnants, Charge coupled devices, $\mathrm{H}$ alpha radiation, Nitrogen ions, Oxygen ions, Sulfur ions, Planetary nebulae, Reprints.

We have constructed CCD mosaic images of the old Galactic supernova remnant $3 \mathrm{C} 400.2$ in lines of $\mathrm{H}($ alpha $)+(\mathrm{N} \mathrm{II}),(\mathrm{S} \mathrm{II})$, and (O III), plus a continuum band. These are the first CCD images covering the full extent of this remnant, and they reveal significantly more nebulosity than the deepest photographic plates. Comparison with radio and $X$-ray images indicates dramatically different morphology in the three regimes. The optical images both in $\mathrm{H}$ (alpha) + (N II) and in (S I) show an almost complete, irregular shell of emission, with a diameter of about $16^{\prime}$, little over half that of the radio shell, while the X-ray structure is a centrally peaked ellipsoid. We also report a previously uncataloged planetary nebula southwest of 3C 400.2 .

\section{0,085}

PB95-203535 Not available NTIS

National Inst. of Standards and Technology (PL), Boulder, CO. Ouantum Physics Div.

G203.2-12.3: A New Optical Supernova Remnant in Orion.

Final rept.

P. F. Winkler, and B. Reipurth. 1992,6p.

Pub. in Astrophysical Jnl. 389, pL25-L28, 10 Apr 92 Keywords: "Supernova remnants, $\mathrm{H}$ alpha radiation, Sulfur ions, Nebulae, Reprints.

We report the discovery of what appears to be a supernova remnant at unusually high latitude near the Galactic anticenter. CCD images in the light of both (S II) and $\mathrm{H}$ (alpha) show patchy filaments scattered over a $3^{\prime}$ region, but with relative strengths in the two lines which vary by a factor of 20 from one filament to anther. Spectra confirm the line identifications and variable intensities. The most extreme filaments have line flux ratios (S II)/H(alpha) approx $=5$, among the highest observed in remnants. The position of $\mathrm{G} 203.2-12.3$ coincides with that of a 'guest star' recorded by Chinese astronomers in A.D. 483

\section{0,086}

PB96-102033 Not available NTIS

National Inst. of Standards and Technology (PL), Boulder CO Ouantum Physics Div. Far-Ultraviolet Flare on a Pleiades G Dwarf

Final rept.

T. R. Ayres, J. R. Stauffer, T. Simon, G. S. Basri, J A. Bookbinder, A. Brown, G. A. Doschek, J. L. Linsky, L. W. Ramsey, F. M. Walter, R. A. Stern, and S. K. Antiochos. 1994, 4p.

Sponsored by National Aeronautics and Space Administration. Washington, DC.

Pub. in Astrophysical Jnl. 420, pL33-L36, 1 Jan 94.

Keywords: "Stellar flares, "G stars, "Dwarf stars, "Brightness, Stellar mass ejecta Ultraviolet spectra Far ultraviolet radiation, Stellar luminosity, Luminous intensity, Stellar magnetosphere, Pleiades cluster, Reprints.

The HST/FOS recorded a remarkable transient brightening in the C IV (gamma-gamma) 1548,50 emissions of the rapidly rotating Pleiades $\mathrm{G}$ dwarf $\mathrm{H} \| \mathrm{I}^{314}$. on the one hand, the 'flare' might be a rare event luckily observed; on the other hand it might be a bellwether of the coronal heating in very young solar-mass stars. If the latter, flaring provides a natural spin-down mechanism through associated sporadic magnetospheric mass loss

\section{0,087}

PB96-102249 Not available NTIS

National Inst. of Standards and Technology (PL), Boulder, CO. Quantum Physics Div.

Search for Radio Emission from the 'Non-Magnetic' Chemically Peculiar Stars.

Final rept.

S. A. Drake, J. L. Linsky, and J. A. Bookbinder.

1994, 4p.

Sponsored by National Aeronautics and Space Admin-

istration, Washington, DC. 
Pub. in Astronomical Jnl. 108, n6 p2203-2206 Dec 94. Keywords: "Peculiar stars, "Radio emission, "Stellar magnetic fields, Radio sources(Astronomy), Stellar magnetosphere, Steller luminosity, Stellar temperature, Very large array(VLA), Reprints.

The authors observed 23 members of the Am and $\mathrm{HgMn}$ subclasses of chemically peculiar (CP) stars with the VLA to search for nonthermal radio emission at levels comparable to those found for the Si and $\mathrm{He}$ peculiar subclasses of the CP stars be Linsky et al. (ApJ, 393, 341 (1992)). The authors detected none of the Am and HgMn stars as radio emitters with upper limits typically $<0.20 \mathrm{mj}$ y. Applying a correlation between radio luminosity, surface magnetic field, and effective temperature derived from previous radio studies of the Si and He peculiar CP stars, the authors find that the predicted radio luminosities of alpha-And (an $\mathrm{HgMn}$ star) and Sirius (a hot Am star) are more than an order of magnitude larger than the observed upper limits, indicating that these stars lack magnetospheres, and, by inference, surface magnetic fields.

\section{0,088}

PB96-102256 Not available NTIS

National Inst. of Standards and Technology (PL), Boulder, CO. Quantum Physics Div.

$\mathrm{X}$-ray Emission from Chemically Peculiar Stars.

Final rept.

S. A. Drake, J. L. Linsky, J. H. M. M. Schmitt, and C.

Rosso. 1994, 5p.

Contract NASA-W-17772

Sponsored by NASA Scientific and Technical Information Facility, Baltimore, MD.

Pub. in Astrophysical Jnl. 420, p387-391, 1 Jan 94.

Keywords: "X ray emission, "Peculiar stars, "Stellar magnetic fields, Binary stars, Companion stars, Steller magnetospheres, $x$ ray sources, Radio sources(Astronomy), Steller luminosity, $X$ ray astronomy, Rosat mission, Reprints.

The authors have searched the ROSAT All-Sky Survey (RASS) database at the positions of approximately 100 magnetic Bp-Ap stars of the helium-strong heliumweak, silicon, and strontium-chromium subclasses. The authors detect $X$-ray sources at the positions of 10 of these stars; in four cases the X-ray emission presumably arises from an early-type companion with a radiatively driven wind, while the authors believe that the magnetic chemically peculiar (CP) star is the most likely $\mathrm{X}$-ray source (as opposed to a binary companion) in at least three and at most five of the six remaining cases. The authors discuss the $\mathrm{X}$-ray and radio emission properties of their sample of CP stars, and argue that both types of emission may be magnetospheric in origin; however, there is clearly not a simple one-toone correspondence between them, since many of the magnetic stars that are detected radio sources were not detected as X-ray sources in the present survey.

00,089

PB96-102322 Not available NTIS

National Inst. of Standards and Technology (PL), Boulder, CO. Ouantum Physics Div.

Rotational Modulation and Flares on RS Canum Venaticorum and BY Draconis Stars. XVIII. Coordinated VLA, ROSAT, and IUE Observations of RS CVn Binaries.

Final rept.

D. C. Fox, J. L. Linsky, A. Veale, J. E. Neff, I.

Pagano, M. Rodono, G. E. Bromage, M. Kurster, J.
H. M. M. Schmitt, R. C. Dempsey, and A. Brown.

1994, 14p.

See also PB94-185626. Sponsored by National Aeronautics and Space Administration, Greenbelt, MD. Goddard Space Flight Center. Pub. in Astronomy and Astrophysics 284, p91-104
1994.

Keywords: "Binary stars, "Steller flares, "Radio sources(Astronomy), "X ray sources, Radio emission, Stellar coronae, Stellar chromospheres, Stellar radiation, Radio spectra, Ultraviolet spectra, Variable stars, Rosat mission, Very large array(VLA), IVE, Radio astronomy, Reprints.

As part of a coordinated program of multi-wavelength observations of RS CVn close binary systems, the authors observed 15 systems with the VLA and 10 systems with IUE, simultaneously or nearly simultaneously with the ROSAT All Sky Survey observations of these stars. Of the 22 systems observed with ROSAT, three were observed both by IUE and the VLA. The prinicipal aim of this program was to check the validity of the existing empirical correlations between the radio and soft $X$-ray emissions of their coronae, and between the chromospheric/transition region and coronal emissions.

\section{0,090}

PB96-102504 Not available NTIS

National Inst. of Standards and Technology (EEEL), Boulder, CO. Electromagnetic Technology Div.

Extended $\mathrm{CO}(7$ yields 6 ) Emission from Warm Gas in Orion.

Final rept.

J. E. Howe, D. T. Jaffe, E. N. Grossman, G. J.

Stacey, W. F. Wall, and J. G. Mangum. 1993, 9p.

Pub. in Astrophysics Jnl., v410 p179-187, 10 Jun 93.

Keywords: "Orion nebula, "Interstellar gas, "Molecular clouds, Carbon oxides, Interstellar matter, Cosmic dust, Cosmic gases, Stellar luminosity, Brightness temperture, Line spectra, Emission spectra, Reprints. We mapped the quiescent emission from the $807 \mathrm{GHz}$ $J=7$ yields 6 transition of $\mathrm{CO}$ in Orion along a strip in R.A. extending from $0.7 \mathrm{pC}$ west to $1.2 \mathrm{pC}$ east of Theta(sup 1) C Orionis. Orion KL outflow shows that the luminosity of shock-excited $\mathrm{CO}(7$ yields 6 ) emission in Orion is only a few percent of the luminosity of the widespread quiescent $\mathrm{CO}(7$ yields 6$)$ emission.

00,091

PB96-102694 Not available NTIS

National Inst. of Standards and Technology (PL), Boulder, CO. Ouantum Physics Div.

High-Velocity Plasma in the Transition Region of AU Microscopii: Evidence for Magnetic Reconnection and Saturated Heating during Quiescent and Flaring Conditions.

Final rept.

J. L. Linsky and B. E. Wood. 1994, 9p.

Contract NASA-S-56500-D

Sponsored by National Aeronautics and Space Administration, Washington, DC.

Pub. in Astrophysical Jnl. 430, p342-350, $20 \mathrm{Jul} 94$

Keywords: "Flare stars, "Ultraviolet spectra, "Chromospheres, Reprints, Emission spectra.

We analyze high-resolution HST spectra of the dMOe flare star AU Mic, including the profiles of the C IV 1548.2 A, 1550.8 A and Si IV 1393.8 A, 1402.8 A lines obtained with the G160M grating of the Goddard HighResolution Spectrograph. The quiescent profiles of the C IV and Si IV lines are broad, and not simple Gaussian in shape. Flux in the C IV and Si IV lines, for example, can be measured reliably out to about + or - $200 \mathrm{~km} \mathrm{~s}-1$ from line center. Each of the C IV and Si IV profiles can be fitted accurately by two Gaussians (one narrow and the other broad) centered on nearly the same wavelength, with the narrower component accounting for roughly $60 \%$ of the total integrated flux. The narrow components have similar line widths to those observed in solar active and quiet region. The broad Gaussian components of the AU Mic line profiles are reminiscent of the broad C IV profiles observed in solar transition region explosive events, which are thought to be associated with emerging magnetic flux regions where field reconnnection occurs.

\section{0,092}

PB96-102777 Not available NTIS

National Inst. of Standards and Technology (PL), Boulder, CO. Ouantum Physics Div.

Observing Stellar Coronae with the Goddard High Resolution Spectrograph. I. The dMe Star AU Microscopii.

Final rept.

S. P. Maran, R. D. Robinson, S. N. Shore, B. E. Woodgate, J. L. Linsky, A. Brown, P. B. Byrne, M. R Kundu, S. White, J. C. Brandt, J. W. Brosius, and K G. Carpenter. 1994, 9p.

Sponsored by National Aeronautics and Space Administration, Washington, DC

Pub. in Astrophysical Jnl. 421, p800-808, 1 Feb 94

Keywords: "Stellar coronae, "Stellar spectra, "Spectral resolution, Noise reduction, Emission spectra, Stellar flares, Stellar chromospheres, Ultraviolet spectra, Late stars, Reprints, HST(Hubble Space Telescope), High Resolution Spectrography.

The authors report on an observation of AU Mic taken with the Goddard High Resolution Spectrograph, aboard the Hubble Space Telescope. The data consist of a rapid sequence of spectra covering the wavelength range 1345-1375 Angstroms with a spectral resolution of 10,000 . The observations were originally in tended to search for spectral variations during flares. No flares were detected during the $3.5 \mathrm{hr}$ of monitoring. A method of reducing the noise while combining the individual spectra in the time series is described which resulted in the elimination of half of the noise while rejecting only a small fraction of the stellar signal. The resultant spectrum was of sufficient quality to allow the detection of emission lines with an integrated flux of $10(\exp -15)$ ergs $/ \mathrm{sq} \mathrm{cm} / \mathrm{s}$ or greater. Lines of $\mathrm{C} \mathrm{I,} \mathrm{O}$ I, O V, CI I, and Fe XXI were detected, This is the first indisputable detection of the 1354 Angstroms Fe XXI line, formed at $\mathrm{T}$ about $10(\exp 7) \mathrm{K}$, on a star other than the Sun. The line was well resolved and displayed no significant bulk motions or profile asymmetry.

00,093

PB96-103189 Not available NTIS

National Inst. of Standards and Technology (PL), Boulder, CO. Ouantum Physics Div.

Volume-Limited ROSAT Survey of Extreme UItra. violet Emission from all Nondegenerate Stars within 10 Parsecs.

Final rept.

B. E. Wood, A. Brown, J. L. Linsky, S. T. Hodgkin, J. P. Pye, B. J. Kellett, and G. E. Bromage. 1994, 21 p. Contracts NAGW-2904, NAG501792

Sponsored by National Aeronautics and Space Administration, Washington, DC

Pub. in Astrophysical Jnl. Supplement Series 93, p287307 Jul 94

Keywords: "Extreme ultraviolet radiation, "Sky surveys(Astronomy), "Late stars, Ultraviolet emission, Ultraviolet spectra, Emission spectra, Stellar luminos ity. Stellar coronae, M stars, X ray stars, Binary stars, Rosat mission, Ultraviolet astronomy, Reprints, "Nondegenerate stars.

We report the results of a volume-limited ROSAT Wide Field Camera (WFC) survey of all nondegenerate stars within 10 pc. Of the 220 known star systems within 10 $\mathrm{PC}$, we find that 41 are positive detections in at least one of the two WFC filter bandpasses (S1 and S2), while we consider another 14 to be marginal detections. We compute $X$-ray luminosities for the WFC detections using Einstein Imaging Proportional Counter (IPC) data, and these IPC luminosities are discussed along with the WFC luminosities throughout the pape for purposes of comparison. Extreme ultraviolet (EUV) luminosity functions are computed for single stars of different spectral types using both $S_{1} 1$ and $S_{2}$ luminosities, and these luminosity functions are compared with $\mathrm{X}$-ray luminosity functions derived by previous authors using IPC data.

00,094

PB96-112016 Not available NTIS

National Inst. of Standards and Technology (PL), Boulder, CO. Ouantum Physics Div.

GHRS Observations of Cool, Low-Gravity Stars. 1 The Far-Ultraviolet Spectrum of alpha Orions (M2 lab).

Final rept.

K. G. Carpenter, R. D. Robinson, G. M. Wahlgren, J. L. Linsky, and A. Brown. 1994, $16 \mathrm{p}$.

Pub. in Astrophysical Jnl., v428 p329-344, 10 Jun 94.

Keywords: "Far ultraviolet radiation, ${ }^{*} \mathrm{Cool}$ stars *Ultraviolet spectra, "Stellar spectra, M stars, Supergiant stars, Red giant stars, Late stars, Stellar chromospheres, Stellar atmospheres, Stellar temperature, Line spectra, Ultraviolet astronomy, Reprints, "Alpha Orionis star.

We present far-UV (1200-1930 A) observations of the prototypical red supergiant star alpha Ori, obtained with the Goddard High Resolution Spectrograph (GHRS) on the Hubble Space Telescope. The observations, obtained in both low- (G14OL) and medium(G160/200M) resolution modes, umamibiguously confirm that the UV 'continuum' tentatively seen with IUE is in fact a true continuum and is not due to a blend of numerous faint emission features or scattering inside the IUE spectrograph. This continuum appears to originate in the chromosphere of the star, at temperatures ranging from $3000-5000 \mathrm{~K}$, and we argue that it is not related to previously reported putative companions or to bright spots on the stellar disks.

00,095

PB96-119474 Not available NTIS

National Inst. of Standards and Technology (PL), Boulder, CO. Ouantum Physics Div. 
Stars, Atmospheres, Radiative Transfer. Final rept.

D. G. Hummer. 1992, 2p.

Pub. in Astronomy and Astrophysics Encyclopedia, p703-704 Mar 92.

Keywords: *Stellar atmospheres, "Radiative transfer, "Transition probabilities, Stellar spectra, Stellar winds, Stellar temperature, Stellar mass, Gases, Radiation, Stars, Flow rate, Stellar models, Reprints.

Radiative transfer in stellar atmospheres refers to the quantitative treatment of the flow of radiant energy through the outer layers of stars and the interaction of radiation with the gas in these regions. In addition to radiant energy, in certain types of stars a small amount of energy is transported through the atmosphere by the large-scale motion of the gas itself; both forms, of course, arise ultimately from thermonuclear processes deep inside the stars. The division between these two modes is not fixed, as radiation can transfer momentum to the gas, causing outflows known as stella winds, and flow energy can be transformed into radiation by shocks. Radiation undergoes repeated interactions with the gas, which collectively determine the rate at which it can escape from the atmosphere. Because the rate at which energy is produced deepin the star is essentially fixed. These interactions establish the equilibrium distribution of temperature and density in the atmosphere. In addition to this constructive role, radiative transfer also plays a diagnostic role, for the spectrum of the escaping radiation carries with it all that we can possibly learn about the internal physical conditions of the atmosphere and the underlying lay ers.

00,096

PB96-119532 Not available NTIS

National Inst. of Standards and Technology (CAML) Gaithersburg, MD. Statistical Engineering Div.

Discussion: Statistical Signal Processing of Quasiperiodicities.

Final rept.

W. Liggett. 1992, 3p.

Pub. in Statistical Challenges in Modern Astronomy, Chapter 16, p378-380 1992.

Keywords: "Binary stars, "Stellar mass accretion, "X ray spectra, Stellar spectra, Signal processing, $X$ ray astronomy, Statistical analysis, Stellar physics, Reprints.

The authors summarize what has been inferred about the physics of binary stars through a variety of statistical signal processing techniques. They affirm certain aspects of the most widely accepted model for accreting binary stars, conclude that clumps of matter must accrete at more than 400 per second, and poin to aspects of the observed $x$-ray intensities that seem unexplained.

\section{0,097}

PB96-119540 Not available NTIS

National Inst. of Standards and Technology $(P L)$, Boulder, CO. Quantum Physics Div

Relationship between Radiative and Magnetic Fluxes for Three Active Solar-Type Dwarfs.

Final rept

J. L. Linsky, C. Andrulis, S. H. Saar, T. R. Ayres, and M. S. Giampapa. 1994, 3p.

Pub. in Cool Stars, Stellar Systems, and the Sun, Cambridge Workshop (8th), ASP Conference Series, Athens, GA., October 1993, p438-440 Sep 94.

Keywords: "Dwarf stars, "Stellar magnetic fields, "Radiative transfer, "Magnetic flux, Stellar activity, Stellar magnetospheres, Stellar radiation, Magnetic field configurations, Functional analysis, Reprints, Spatial analysis.

We present some preliminary results from our coordinated campaign of IUE and McMath Telescope magnetic field measurements of three active solar-type dwarf stars: 59 Vir, xi Boo A and HD 131511. We observed the three stars mearly every day from May 9 to May 25, 1993, covering between 1 and 3 rotations. We explore the functional and spatial relationship between magnetic and radiative fluxes.

\section{0,098}

PB96-122817 Not available NTIS

National Inst. of Standards and Technology (PL), Boulder, CO. Quantum Physics Div.
Sleuthing the Dynamo: HST/FOS Observations of UV Emissions of Solar-Type Stars in Young Clusters.

Final rept.

T. Ayres, G. Basri, T. Simon, S. Antiochos, J.

Bookbinder, A. Brown, G. Doschek, J. Linsky, L.

Ramsey, F. Walter, J. Stauffer, and R. Stern. 1994

3p.

Pub. in Proceedings: Cool Stars, Stellar Systems, and the Sun Workshop (8th) ASP Conterence Series, Athens, Georaia, October 1993, v64 p53-55 Sep 94.

Keywords: *Ultraviolet spectra, *Stellar spectra, "Late stars, Stellar coronae, Stellar chromospheres, Emission spectra, Open clusters, Hubble Space Telescope Reprints, Stellar evolution.

HST/FOS spectra of young solar-type stars are provid ing new clues to the early evolution of subcoronal activ. ity.

\section{0,099}

PB96-122882 Not available NTIS

National Inst. of Standards and Technology (PL), Boulder, CO. Quantum Physics Div.

Efficient Way of Identifying New Active Stars: A VLA Survey of X-ray Selected Active Stellar Can. didates.

Final rept.

S. A. Drake, T. Simon, J. L. Linsky, and N. E. White. 1994, 3p.

Contract NASA-NAG5-2075

Sponsored by National Aeronautics and Space Administration Washington DC.

Pub. in Cool Stars, Stellar Systems, and the Sun, Cam bridge Workshop (8th), ASP Conference Series, Athens, GA., October 1993, v64 p690-692.

Keywords: "X ray sources, "Stellar radiation, "Sky surveys(Astronomy), Binary stars, Stellar coronae, Radio sources(Astronomy), Very large array(VLA), $X$ ray astronomy, Radio astronomy, Reprints.

Source confusion makes it difficult to identify active stars, such as the RS CVn binaries, with X-ray sources detected in low angular resolution surveys. Short (15 minute) VLA radio continuum observations of candidates having appropriate spectral types and $X$-ray to visual flux ratios obtained from an $X$-ray selected sam. ple, combined with other information, has enabled us either to confirm or rule out proposed late-star counterparts for 27 out of $32 \mathrm{X}$-ray sources selected from the Einstein Slew Survey.

\section{0,100}

Not available NTIS

National Inst. of Standards and Technology (PL), Boulder, CO. Quantum Physics Div.

Magnetic Fields in Star-Forming Regions: Observations.

Final rep

C. Heiles, A. A. Goodman, C. F. McKee, and E. G. Zweibel. 1993, 25p.

Pub. in Protostars and Planets III, p279-326 1993.

Keywords: "Magnetic fields, "Orion nebula, "Spectral lines, Interstellar gas, Cosmic gases, Molecular clouds, Interstellar clouds, Cosmic dust, Zeeman effect, Polarization, Emission spectra, Reprints.

The authors review the observational aspects of magnetic fields in dense, star-forming regions. First the authors discuss ways to observe the field. Next, they discuss selected observational results, focusing on detailed discussions of a small number of points rather than a generalized discussion that covers the watertront Next the authors discuss the derivation of the complete magnetic vector, including both the systematic and fluctuating component, from a large sample of Zeeman and linear polarization measurements for the L204 dark cloud. Third, they discuss the virial theorem as it applies to dark clouds in general and one dark cloud. Finally the authors critically discuss the numerous claims for alignment of cloud structural features with the plane-of-the-sky component of the magnetic field and find that many of these have not been definitively established.

00,101

PB96-123286 Not available NTIS

National Inst. of Standards and Technology (PL), Boulder, CO. Quantum Physics Div.

A-type and Chemically Peculiar Stars.

Final rept

J. L. Linsky. 1993, 10p.

Contract NAG5-1797

Sponsored by NASA Scientific and Technical Information Facility, Baltimore, MD
Pub. in Physics of Solar and Stellar Coronae, p257266 Sep 93.

Keywords: "Peculiar stars, "A stars, "Stellar temperature, Stellar spectra, Emission spectra, Radio emission, $X$ ray emission, Stellar luminosity, Stellar coronae, Steller magnetospheres, Reprints.

The authors will show that the magnetic chemically peculiar (CP) stars hotter than about spectral type A2 display many of the phenomena seen in the most active late-type stars. In particular, many CP stars are luminous nonthermal radio sources and ROSAT confirms that many are also luminous coronal $x$-ray sources like the RS CVn systems. A wind-fed magnetosphere model has been proposed to explain both the nonthermal radio and the x-ray emission. In this model, the stellar wind plays the role of a mechanical energy source analogous to the role played by convection in the active late-type stars.

00,102

PB96-123294 Not available NTIS

National Inst. of Standards and Technology (PL), Boulder, CO. Quantum Physics Div.

High Velocity Plasm in the Transition Region of Au Mic: A Stellar Analog of Solar Explosive Events.

Final rept.

J. L. Linsky, and B. E. Wood. 1994, 3p

Contract NASA-S-56500-D

Sponsored by National Aeronautics and Space Administration, Washington, DC

Pub. in Cool Stars, Stellar Systems, and the Sun, Cambridge Workshop (8th), ASP Conference Series, Athens, GA., October 1993, v64 p441-443.

Keywords: "Late stars, "Stellar mass ejecta, "Ultraviolet spectra, Stellar flares, Line spectra, Emissions spectra, Stellar intensity flux, Carbon isotopes, Silicon isotopes, High resolution, Reprints.

High-resolution GHRS spectra of the dMOe flare star AU Mic shows CIV and Si IV line profiles that are unexpectedly broad and can be fit accurately by two Gaussians (one narrow and the other broad), The broad Gaussian components of the AU Mic line profiles resemble closely the C IV profiles observed in solar explosive events in emerging flux regions (EFRs). The authors therefore propose that the EFR is the solar analog of the most active component of the transition region of this and presumably other dMe flare stars.

00,103

PB96-123302 Not available NTIS

National Inst. of Standards and Technology (PL), Boulder, CO. Quantum Physics Div.

Radio and X-ray Emissions from Chemically Peculiar B- and A-Type Stars: Observations and a Model.

Final rept.

J. L. Linsky. 1993, 10p.

Contracts NASA-W17772, NASA-H-04630

Sponsored by NASA Scientific and Technical Informa tion Facility, Baltimore, MD.

Pub. in Peculiar versus Normal Phenomena in A-Type and Related State P, IAU Colloq. 138, Trieste, Italy, July 1993, v44 p507-516.

Keywords: "Peculiar stars, *A stars, "Stellar temperature, Magnetic stars, Radio stars, Stellar spectra Radio emission, $X$ ray emission, Stellar luminosity, Stellar coronae, Stellar magnetosphere, Reprints.

The author argues that the magnetic chemically peculiar (CP) stars hotter than about spectral type A2 display many of the activity phenomena seen in the most active late-type stars. In particular, many CP stars are luminous nonthermal radio and coronal $x$-ray sources like the RS CVn systems. A wind-fed magnetosphere model has been proposed to explain both the nonthermal radio and the $x$-ray emission. In this model the stellar wind plays the role of a mechanical energy source analogous to the role played by convection in the active late-type stars.

\section{0,104}

PB96-123310 Not available NTIS

National Inst. of Standards and Technology (PL), Boulder, CO. Quantum Physics Div.

Redshifts in Stellar Transition Regions.

Final rept.

J. L. Linsky, B. E. Wood, and C. Andrulis. 1994, 3p. Contract NASA-S-56560-D

Sponsored by National Aeronautics and Space Administration, Washington, DC

Pub. in Cool Stars, Stellar Systems, and the Sun, Cam. bridge Workshop (8th), ASP Conference Series, Athens, GA., October 1993, v64 p59-61. 


\section{Astrophysics}

Keywords: "Red shift, "Stellar spectra, "Spectral resoIution, Stellar chromospheres, Stellar coronae, Late stars, Ultraviolet spectra, Emission spectra, Transition probabilities, Reprints.

The authors present moderate resolution GHRS spectra of the stars Procyon (F5 IV-V), AU Mic (MO Ve) Beta Gem (Ko III), Capella (GO III + G8 III), alpha TrA (K2 $\|-\| \|)$, and Beta Dra (G2 $\|-\| b)$ to study the phenomenon of redshifted transition region lines first observed by IUE. The authors' GHRS data show redshifts for all of these stars except alpha TrA, but they find no correlation between redshift and stellar activity.

\section{0,105}

PB96-123336 Not available NTIS

National Inst. of Standards and Technology (PL), Boul-

der, CO. Quantum Physics Div.

Transition Regions of Capella.

Final rept.

J. L. Linsky, B. E. Wood, A. Brown, T. R. Ayres, C.

Andrulis, and P. Judge. 1994, 3p.

Contract NASA-S-56460-D

Sponsored by National Aeronautics and Space Administration, Washington, DC.

Pub. in Cool Stars, Stellar Systems, and the Sun, Cambridge Workshop (8th), ASP Conference Series, Athens, GA., v64 p62-64 Oct 93.

Keywords: "Binary stars, "Transition probabilities, "Stellar chromospheres, *Ultraviolet spectra, Emission spectra, Low resolution, Moderate resolution, High resolution, Red shift, Electron density, Hubble Space Telescope, Reprints, "Capella star.

The authors report on an extensive set of observations of Capella obtained with GHRS on HST at orbital phase 0.26 . The data set consisting of low, moderate, and high resolution spectra, allows the authors to separate the contributions of each star to many of the emission lines and to measure line centroid redshifts. The authors estimate the electron density in the transition region of the hotter star and construct an emission measure distribution.

\section{0,106}

PB96-135264 Not available NTIS

National Inst. of Standards and Technology (PL), Boulder, CO. Quantum Physics Div.

Recalibration for the Final Archive of the international Ultraviolet Explorer (IUE) Satellite.

Final rept.

J. L. Linsky, and J. Nochols-Bohlin. 1993, 8p

Pub. in Proceedings of the Workshop on the Vacuum Ultraviolet Calibration of Space Experiments (9th), Boulder, CO., March 1993, p197-204.

Keywords: "IUE, "Ultraviolet spectra, "Calibration, "Data storage systems, Flux density, Signal to noise ratio, Ultraviolet radiation, Ultraviolet astronomy, Astronomical satellites, Reprints.

For the past several years, the IUE Project has been developing the software needed to reprocess the entire data set into a form suitable for its Final Archive. The object of the Final Archive Project is to produce a uniform archive of all spectra, including enhanced signalto-noise and corrections for fixed pattern noise and scattered light, and new algorithms for sensitivity changes with time and spectrography temperature. The Final Archive will use the new Signal Weighted Extraction Technique (Kinney, Bohlin, and Neill 1991) to extract spectra with the highest feasible signal/noise and will use the bet available absolute flux calibration. The present brief report will summarize the absolute flux calibration approach used in preparing the Final Archive.

00,107

PB96-176706 Not available NTIS

National Inst. of Standards and Technology (PL), Boulder, CO. Quantum Physics Div.

Developments in Stellar Coronae.

Final rept.

J. L. Linsky. 1995, 4p.

See also PB87-223798.

Pub. in Transacions of IAU, Theory of Stellar Atmospheres, p414-417 Sep 95.

Keywords: "Stellar coronas, "Observation, "Radio sources(Astronomy), "X ray sources, Binary stars, Extraterrestrial radiowaves, $X$ ray $s p e c t r a, X$ rays, Astronomical observatories, Spaceborne astronomy, ROSAT mission, Hubble space Telescope, Extreme Ultraviolet Explorer Satellite, Astro missions(STS), Japanese spacecraft, HEAO 2, Reprints.
The report summarizes the major developments lobservational and theoretical) in the study of stella coronae driven in large part by the analysis of the new data sets from ROSAT, EUVE, ASCA, Yohkoh, and HST, together with the continuing analysis of Einstein data and radio observations.

\section{0,108}

PB96-176714 Not available NTIS

National Inst. of Standards and Technology (PL), Boulder, CO. Ouantum Physics Div.

Transition Regions of Capella (1995)

Final rept.

J. L. Linsky, B. E. Wood, P. Judge, T. R. Ayres, A.

Brown, and C. Andrulis. 1995, 20p.

Contract NASA-S-56500-D

See also PB96-123336. Sponsored by National Aeronautics and Space Administration, Washington, DC.

Pub. in Astrophysical Jnl., v442 p381-400, 20 Mar 95

Keywords: "Binary stars, "Transition probabilities, Stellar chromospheres, "Ulltraviolet spectra, Emission spectra, High resolution, Red shift, Electron density, Hubble Space Telescope, Stellar coronas, Norma density functions, Reprints, "Capella star.

The authors have used the Goddard High Resolution Spectrometer (GHRS) to observe the spectroscopic binary system Capella (G8 III + G1 III)

\section{0,109}

PB96-200217 Not available NTIS

National Inst. of Standards and Technology (PL), Boulder, CO. Quantum Physics Div.

Riass Coronathon: Joint X-ray and Ultraviolet $\mathrm{Ob}$ servations of Normal F-K Stars.

Final rept.

T. R. Ayres, T. A. Fleming, A. Brown, R. C.

Dempsey, and J. L. Linsky. 1995, 32p.

Pub. in Astrophysical Jnl. Supplement Series, v96 p223-259 Jan 95.

Keywords: "X ray astronomy, "Ultraviolet astronomy, "Sky surveys(Astronomy), "Stellar atmospheres, $\mathrm{X}$ ray stars, Late stars, F stars, G stars, K stars, Stellar coronas, Chromosphere, Extreme ultraviolet radiation, UItraviolet emission, Emission spectra, ROSAT mission IUE, Reprints.

Between 1990 August and 1991 January the ROSAT/ IUE All Sky Survey (RIASS) coordinated pointings by the International Ultraviolet Explorer with the continuous $X$-ray/EUV mapping by the Roentgensatellit. The campaign provided an unprecedented multiwavelength view of a wide variety of cosmic sources. We repor findings for F-K stars, a large proprtion of the RIASS targets. Forty-eight of our 91 'Coronathon' candidates were observed by the IUE during the campaign. For stars missed by the IUE, we supplemented the ROSAT survey fluxes with archival UV spectra and/or follow on observations. In addition to the coordinated work we examined the UV emission histories of the Coronathon stars.

\section{0,110}

PB96-200621 Not available NTIS

National Inst. of Standards and Technology (PL), Boulder, CO. Quantum Physics Div.

Accurate Measurements of the Local Deuterium Abundance from HST Spectra.

Final rept.

L. Linsky. 1996, 4 p.

Contract NASA-S-56460-D

Sponsored by National Aeronautics and Space Admin istration, Washington, DC

Pub. in Examining the Big Bang and Diffuse Background Radiations, p529-532 1996

Keywords: "Deuterium, *Abundance, "Ultraviolet stars, Hydrogen, Isotope ratio, Interstellar matter, Intergalactic media, Line spectra, Absorption spectra, Baryons, $H$ lines, D lines, Lyman alpha radiation, Line of sight, Cosmology, Galactic evolution, Hubble space telescope, Reprints.

An accurate measurement of the primordial value of $\mathrm{D} / \mathrm{H}$ would provide a critical test of nucleosynthesis models for the early universe and the baryon density. I briefly summarize the ongoing HST observations of the interstellar $\mathrm{H}$ and D Lyman-alpha absorption for lines of sight to nearby stars and comment on recent reports of extragalactic D/H measurements.
National Inst. of Standards and Technology (PL), Boulder, CO. Ouantum Physics Div.

Deuterium and the Local Interstellar Medium: Properties for the Procyon and Capella Lines of Sight. Final rept.

J. L. Linsky, A. Diplas, B. E. Wood, B. D. Savage, A Brown, and T. R. Ayres. 1995, 17p

Contract NASA-S-56500-D

See also PB95-202842. Sponsored by National Aeronautics and Space Administration, Washington, DC

Pub. in Astrophysical Jnl., v451 p335-351 Sep 95.

Keywords: "Deuterium, "Ultraviolet star, "Line of sight, *Interstellar matter, Stellar spectra, Line spectra, D lines, H lines, Hydrogen, Stellar composition, Abundance, Isotope ratio, Lyman alpha radiation, Emission spectra, Absorption spectra, Reprints, Pryocyon star, Capella star.

We present Goddard High-Resolution Spectrograph observations of the interstellar H I and D | Ly(alpha) lines and the $\mathrm{Mg}$ II and Fe II resonance lines formed along the lines of sight toward the nearby stars Procyon ( $3.5 \mathrm{pc}, \mathrm{l}=214$ degrees, $b=13$ degrees) and Capella ( 12.5 pc, $\mid=163$ degrees, $b=5$ degrees). New observations of Capella were obtained at orbital phase 0.80 , when the radial velocities of the intrinsic Ly(alpha) emission lines of each star were nearly reversed from those of the previous observations at phase 0.26 (analyzed by Linsky et al.). For the analysis of the Procyon line of sight we assumed that $(\mathrm{D} / \mathrm{H})$ (sub LISM) $=1.6 \times 10$ to the -5 th power, the same value as for the Capella line of sight, and we modified the broadened solar profile to achieve agreement between the simulated and observed line profiles.

\section{0,112}

PB97-122295 Not available NTIS

National Inst. of Standards and Technology (CSTL), Gaithersburg, MD.

Experimental Determination of the Rate Constant for the Reaction of $\mathrm{C} 2 \mathrm{H} 3$ with $\mathrm{H} 2$ and Implications for the Partitioning of Hydrocarbons in Atmospheres of the Outer Planets.

Final rept.

A. Fahr, P. S. Monks, L. J. Stief, and A. H. Laufer. 1995,80

Pub, in ICARUS, v116 p415-422 1995

Keywords: "Gas giant planets, "Planetary atmospheres, *Hydrocarbons, Atmospheric chemistry, Chemical reaction kinetics, Partition, Absorption spectroscopy, Gas chromatography, Hydrogen ions, Vinyl radicals, Jupiter atmosphere, Photolysis, Laser pumping, Reprints, Rate constants.

The reaction between $\mathrm{C}_{2} \mathrm{H}_{3}$ and $\mathrm{H}_{2}$ has been suggested to be potentially important in accounting for observational data on the abundance of low-molecular weight hydrocarbons in the atmospheres of the giant planets. In the work, the room temperature rate constant for the reaction of the vinyl radical with molecular hydrogen has been determined by employing laser hotolysis coupled to a kinetic-absorption spectroscopic technique and separately via a gas chromatographic product analysis technique.

\section{General}

\section{0,113}

PB96-175666 PC A07/MF A02

National Inst. of Standards and Technology, Gaithersburg, MD

Journal of Research of the National Institute of Standards and Technology, January/February 1996. Volume 101, Number 1.

1996, 115p

See also PB96-175674 through PB96-175690 and PB96-159215. Also available from Supt of Docs, as SN703-027-00068-7.

Keywords: "Marine atmospheres, "Experimental mechanics, "Electron beams, "Calibration, Reprint. 
Contents:

An International Marine-Atmospheric 222Rn Measurement Intercomparison in Bermuda Part I:

NIST Calibration and Methodology for Standardized Sample Additions;

An International Marine-Atmospheric 222Rn Measurement Intercomparison in Bermuda Part II:

Results for the Participating Laboratories;

Theory of Electron Beam Moire.

00,114

PB96-175674 (Order as PB96-175666, PC A07) MF A02)

National Inst. of Standards and Technology, Gaithersburg, MD.

International Marine-Atmospheric (222)Rn Measurement Intercomparison in Bermuda. Part 1. NIST Calibration and Methodology for Standardized Sample Additions.

R. Colle, M. P. Unterweger, P. A. Hodge, and J. M. R. Hutchinson. 1996, 19p.

Included in Jnl. of Research of the National Institute of Standards and Technology, v101 n1 p1-19 Jan/Feb 96.

Keywords: "Marine atmospheres, "Calibration, Environment, Reprints, Intercomparison, Measurement, Standards, Radium-226, Radon-222.

As part of an international 222Rn measurement intercomparison conducted at Bermuda in October 1991, NIST provided standardized sample additions of known, but undisclosed ('blind') 222Rn concentrations that could be related to U.S. national standards. The standardized sample additions were obtained with a calibrated 226Ra source and a specially-designed manifold used to obtain well-known dilution factors from simultaneous flow-rate measurements. The additions were introduced over sampling periods of several hours (typically $4 \mathrm{~h}$ ) into a common streamline on a sampling tower used by the participating laboratories for their measurements. The standarized 222Rn activity concentrations for the intercomparision ranged from approximately 2.5 Bq.m-3 to 35 Bq. m-3 (of which the lower end of this range approached concentration levels for ambient Bermudian air) and had overall uncertainties, approximating a 3 standard deviation uncertainty interval, of about $6 \%$ to $13 \%$. This paper describes the calibration and methodology for the standardized sample addition.

00,115

PB96-175682 (Order as PB96-175666, PC A07)

MF A02)

National Inst. of Standards and Technology, Gaithersburg, MD.

International Marine-Atmospheric (222)Rn Measurement Intercomparison in Bermuda. Part 2. Results for the Participating Laboratories.

R. Colle, M. P. Unterweger, J. M. R. Hutchinson, B. Ardouin, J. G. Kay, J. P. Friend, B. W. Blomquist, W. Nadler, T. T. Dang, R. J. Larsen, A. R. Hutter, S. Whittlestone, and G. Polian. 1996, 25p.

Prepared in cooperation with Australian Nuclear Science and Technology Organisation, Sutherland. Centre des Faibles Radioactives, Gif-sur-Yvette (France)., Drexel Univ., Philadelphia, PA. Dept. of Chemistry. and Department of Energy, New York. Environmental Measurements Lab.

Included in Jnl. of Research of the National Institute of Standards and Technology, v101 n1 p21-46 Jan/ Feb 96.

Keywords: "Marine atmospheres, Environment, Air, Reprints, Intercomparison, Measurement, Radon-222.

As part of an international $222 \mathrm{Rn}$ measurement intercomparison of instruments used to measure atmospheric 222Rn, four participating laboratories made nearly simultaneous measurements of 22Rn activity concentration in commonly sampled, ambient air over approximately a 2 week period, and three of these four laborato ries participated in the measurement comparison of 14 introduced samples with known, but undisclosed ('blind') 222Rn activity concentration. The exercise was conducted in Bermuda in October 1991.

\section{Aeronomy}

00,116

AD-A292 039/5 PC A01/MF A01

National Bureau of Standards, Washington, DC. Inst for Basic Standards.

Comparison of Meteor Activity with Occurrence of Sporadic E Reflections.

V. C. Pineo. 14 Jul 50, 2p

Pub. in Science, v112 n2898 p50-51, 14 Jul 50.

Keywords: "Meteors, "Ionization trails, "Meteor burst communications, lonosphere, Reprints, Electromagnetic wave reflections, E region, Sporadic e layer.

In a previous paper, the writer presented evidence to show that the character of the echo obtained from a trail of ionization produced by a meteor is different in character from the echoes frequently obtained on onosphere recorders and described as sporadic $E$ reflections. Since the presentation of that paper a statistical study has been carried out on the frequency of reflections obtained with the meteor equipment in operation by the Central Radio Propagation Laboratory of the National Bureau of Standards, and the frequency of occurrence of sporadic $E$ reflections observed over the same hiterval with the automatic multifrequency ionosphere recorder operated by the laboratory. By sporadic $E$, also sometimes called abnormal $E$, is meant what Lovell referred to as long duration abnormalities in the $E$ region as distinguished from what $A p$ pleton and Naismith referred to as ionization bursts. In the scaling of sporadic $E$ data from the vertical incidence ionosphere records, extreme eare was taken to see that only reflections of the sporadic $E$ type were scaled. (MM).

00,117

PB96-148101 Not available NTIS

National Inst. of Standards and Technology (CSTL), Gaithersburg, MD. Chemical Kinetics and Thermodynamics Div.

Free Radical Chemistry of the Atmospheric Aqueous Phase.

Final rept.

R. E. Huie. $1995,46 p$

See also PB90-218207

Pub. in Free Radical Chemistry of the Atmospheric Aqueous Phase, Chapter 10, p374-419 1995

Keywords: "Atomspheric chemistry, "Free radicals, Electron transfer, lonizing radiation, Aerosols, Fog, Clouds(Meteorology), Chemical reactions, Reprints, *Aqueous phase, Gas phase.

The chemical environment of the atmospheric aqueous phase: clouds, fogs, aerosols, and raindrops, has a rich chemistry strongly dependent upon the surrounding gas phase. For many atmospheric species, this environment is quite different than the gas phase; in water, they may hydrate, protonate, deprotonate, or dissociate. Most important, the aqueous phase opens up the possibility of an entirely new class of chemica reaction: electron transfer. In this review, the author will discuss the aqueous-phase chemistry of a number of free radicals of possible importance to the aqueous phase of the atmosphere.

00,118

PB97-112577 Not available NTIS

National Inst. of Standards and Technology (CSTL), Gaithersburg. MD Chemical Kinetics and Thermodynamics Div.

Atmospheric Lifetimes of HFC-143a and HFC 245fa: Flash Photolysis Resonance Fluorescence Measurements of the $\mathrm{OH}$ Reaction Rate Constants. Final rept.

V. L. Orkin, R. E. Huie, and M. J. Kurylo. 1996, 6p. Pub. in Jnl. of Physical Chemistry, v100 p8907-8912 1996.

Keywords: "Atmospheric chemistry, "Chemical reaction kinetics, "Hydroxyl radicals, "Hydrofluorocarbons, Photolysis, Fluorescence, Temperature, Arrhenius equation, Error analysis, Constants, Reprints.

Rate constants for the reactions of hydroxyl radicals with Ch3CF3 (HFC-143a) and $\mathrm{CHF}_{2} \mathrm{CH} 2 \mathrm{CF} 3$ (HFC 245fa) have been measured using the flash photolysis resonance fluorescence technique over the temperature range $273-370 \mathrm{~K}$. A data analysis procedure is presented which should minimize rate constant errors introduced by the possible effects of radical diffusion.

\section{Dynamic Meteorology}

00,119

PB94-143427 PC A03/MF A01

Massachusetts Inst. of Tech., Cambridge. Dept. of Mechanical Engineering.

Computational Model for the Rise and Dispersion of Wind-Blown, Buoyancy-Driven Plumes. Part 2. Linearly Stratified Atmosphere.

Final rept.

X. Zhang, and A. F. Ghoniem. Dec 93, 49p, NISTGCR-93-637

Contract 60NANBOD1036

Sponsored by National Inst. of Standards and Technology (BFRL), Gaithersburg, MD.

Keywords: "Wind direction, "Dispersions, "Plumes, "Buoyancy, Mathematical models, Atmospheric composition, Stratification, Dynamics, Atmospheric density, Vorticity, Height, Lagrangian functions, Baroclinic vorticity.

A multi-dimensional computational model of wind blown, buoyancy-driven flows is applied to study the effect of atmospheric stratification on the rise and dispersion of plumes. The model utilizes Lagrangian transport elements, distributed in the plane of the plume cross section normal to the wind direction, to capture the evolution of the vorticity and density field, and another set of elements to model the dynamics in the atmosphere surrounding the plume. Solutions are obtained for a case in which atmospheric density changes linearly with height. Computational results show that, similar to the case of a neutrally stratified atmosphere, the plume acquires a kidney-shaped cross section which persists for a long distance downstream the source and may bifurcate into separate and distinct lumps.

00,120

PB95-220471 PC A03/MF A01

National Inst. of Standards and Technology (BFRL) Gaithersburg, MD. Structures Div.

Extreme Wind Estimates by the Conditional Mean Exceedance Procedure.

J. L. Gross, N. A. Heckert, J. A. Lechner, and E.

Simiu. Apr $95,20 p$, NISTIR-5531.

Keywords: "Wind velocity, "Estimating, Functions(Mathematics), Mathematical models.

We describe work aimed at improving procedures for the estimation of non-tornadic extreme wind speeds regardless of their direction, in regions not subjected to hurricanes. Using the Generalized Pareto Distribution (GPD) approach and the Conditional Mean Exceedance (CME) estimation method, we analyze 115 17-year to 52-year sets of largest annual speeds and sets drawn from 48 15-year to 26-year records of maximum daily wind speeds. Based on this analysis we attempt an assessment of the widely held belief that the Gumbel distribution with site-dependent location and scale parameters is a universal model of extreme wind speeds. Some of our results suggest that the reverse Weibull distribution is a more appropriate model This would result in more reasonable estimates of wind-induced failure probabilities and wind load factors than the corresponding estimates based on the Gumbel distribution. However, our assessment is so far only tentative owing to uncertainties inherent in our results. Future work based on lower thresholds (larger data samples) and alternative estimation methods is planned.

\section{Meteorological Data Collection, Analysis, \& Weather Forecasting}

00,121

AD-A295 319/8 PC A02/MF A01

National Bureau of Standards, Gaithersburg, MD.

Low-Temperature Performance of Radiosonde Electric Hygrometer Elements.

Research paper.

A. Wexler. Jul 49,8p, RP2003

Pub. in Jnl. of Research of the National Bureau of Standards, v43 p49-56, Jul 49.

Keywords: "Radiosondes, "Hygrometers, Reprints, Low temperature, Electric power, Calibration, Range(Extremes), Humidity, Atmospheric sounding.

The performance of radiosonde electric hygrometer elements was investigated in the temperature range 
from 0 to $400 \mathrm{C}$. It was found that an element indicated relative humidity with an average deviation of +2.4 percent relative humidity from the average calibration for all of the elements tested. The maximum deviation in indication of any element did not exceed 10.5-percent relative humidity. The lag in response was found to increase markedly with decrease in temperature, to depend upon the magnitude and direction of relative humidity change, and the relative humidity from which the change was made.

\section{0,122}

PB95-108791 Not available NTIS

National Inst. of Standards and Technology (NEL) Gaithersburg, MD. Fire Measurement and Research Div.

Global Climatic Effects of Aerosols: The AAAR Symposium.

Final rept.

J. E. Penner, and G. W. Mulholland. 1991, 2p

Pub. in Atmospheric Environment A 25, n11 p243324341991

Keywords: "Meetings, "Aerosols, "Climatic changes, "Global aspects, "Air pollution, Nuclear war, Globa warming, Ozone depletion, Reprints, "American Association of Aerosol Research.

The American Association of Aerosol Symposium on global climatic effects of aerosols included papers on the aerosol-related phenomena for the following three topics: Climatic Effect of Nuclear War; Global Warming; and The Ozone Hole. This introduction to the symposium volume highlights key findings presented at the meeting.

\section{Meteorological Instruments \& Instrument Platforms}

\section{0,123}

AD-A278 851/1 PC A03/MF A01

National Bureau of Standards, Gaithersburg, MD

Methods of Measuring Humidity and Testing Hygrometers.

A. Wexler, and W. G. Brombacher. 28 Sep 51, 22p,

NBS-512.

Keywords: "Humidity, "Hygrometers, "Measuring instruments, Water, Test and evaluation, Gases, Atmospheres, Calibration, Dew point, Mechanical properties, Electrical properties, Gravimetry, Thermal conductivity, Pressure, Volume, Dielectrics, Psychrometers, Meteorology, Earth sciences, Climate, Index of refraction.

No abstract available.

\subsection{4}

PB95-150132 Not available NTIS

National Inst. of Standards and Technology (BFRL), Gaithersburg, MD. Fire Science Div

Radiometric Model of the Transmission Cell-Reciprocal Nephelometer.

Final rept.

G. W. Mulholland, and N. P. Bryner. 1994, 15p.

Pub. in Atmospheric Environment 28, n5 p873-887 1994

Keywords: "Nephelometers, "Aerosols, *Light scattering, *Radiometric correction, *Atmospheric scattering, Aerosol generators, Microspheres, Polystyrene, Absorptivity, Optical measuring instruments, Atmospheric optics, Reprints.

A radiometric model has been developed to assess the effects of angular truncation, finite size of the detector, and angle response characteristics of the cosine sensor on the measurement of the total scattering coefficient by a transmission cell-reciprocal nephelometer. These effects are computed for monodisperse polystyrene spheres over the size range $0.02-8$ micrometers based on Mie theory and for smoke agglomerates ranging from 10 to $10(\exp 7)$ primary units based on the Fisher-Burford approximation. The accuracy of the model calculations is determined by comparison with exact solutions for the case of a detector with an infinitesimal area and for a finite area detector with a diffuse scattering function. The predicted results are compared with measured results for six different sizes of monodisperse polystyrene sphere aerosols with particle diameters in the range $0.1-2.35$ micrometers. The measurements were carried out as a function of the distance between the laser beam and detector for 1.3 and $2.7 \mathrm{~cm}$ diameter cosine sensors. A table of design parameters for making accurate total scattering measurements is obtained for both spheres and agglomerates. An accuracy of + or $-5 \%$ was obtained for spherical particles with diameters less than or equal to 1.1 micrometers with our TCRN, and we estimate that similar performance would be obtained for smoke agglomerates with up to $3 \times 10(\exp 3)$ primary spheres per agglomerate.

\subsection{5}

\section{PB96-214648 PC A04/MF A01}

National Inst. of Standards and Technology (PL) Gaithersburg, MD. Optical Technology Div. Report on USDA Ultraviolet Spectroradiometers. E. A. Early, and A. Thompson. Jul 96, 36p, NISTIR5871 .

Keywords: "Spectroradiometers, "Uitraviolet spectroradiometers, Radiometers, Linearity Responsivity, Slit-scatter, Stray-light, Wavelength.

Two ultraviolet spectroradiometers manufactured by Research Support Instruments, Inc., and the Atmos pheric Science Research Center for the U.S. Department of Agriculture monitoring network were characterized. From measurements of the linearity of the instru ments, the dead time of the photon counting system was approximately 12 ns. From spectral scans of the emission lines of a $\mathrm{Hg}$ lamp, the wavelength precision of the instrument of $0.02 \mathrm{~nm}$, while the accuracy is 0.04 $\mathrm{nm}$. Spectral scans of the $325 \mathrm{~nm}$ line of a HeCd lase showed that the bandwidth of the instruments is 0.3 $\mathrm{nm}$ and that the monochromators have a stray light rejection of 10 to the minus 8 power. The spectral irradiance responsivity, determined with FEL-type irradiance standards lamps, changes both with movement of the instruments and with time.

\section{Physical Meteorology}

00,126

PB94-198215 Not available NTIS

National Inst. of Standards and Technology (NML) Gaithersburg, MD. Chemical Thermodynamics Div.

Thermodynamic Properties of Gas Phase Species of Importance to Ozone Depletion.

Final rept.

S. Abramowitz, and M. W. Chase. 1991, 6p

Pub. in Pure and Applied Chemistry 63, n10 p1449. 14541991.

Keywords: "Ozone depletion, "Thermodynamic properties, "Vapor phases, "Atmospheric chemistry, "Spectrum analysis, Chlorine oxides, Reaction kinetics, Stratosphere, Air pollution, Reprints.

Thermodynamic and spectroscopic data have been evaluated for several chlorine-oxygen gas phase spe cies of interest in the study of ozone depletion models. The evaluated data have been used to compute JANAF Thermochemical Tables for these species. The data will be discussed and applied to several proposed models for ozone depletion. The recent catalytic cycle involving ozone loss by $\mathrm{ClO}$ and $\mathrm{Cl} 2 \mathrm{O} 2$ in the Antarctic stratosphere is discussed.

\section{0,127}

PB95-219416 PC A05/MF A01

National Inst. of Standards and Technology (BFRL), Gaithersburg, MD. Structures Div.

Extreme Wind Distribution Tails: A 'Peaks Over Threshold' Approach.

Building science series.

E. Simiu, and N. A. Heckert. Mar 95, 80p.

Also available from Supt. of Docs. Prepared in co operation with Johns Hopkins Univ., Baltimore, MD. Dept. of Civil Engineering. Sponsored by National Science Foundation, Arlington, VA.

Keywords: "Wind velocity, "Extreme value problems, Wind effects, Wind pressure, Wind loads, Dynamic Loads, Winds, Structural engineering, Statistical analysis, Weibull distribution, Graphs(Charts), "Peaks ove threshold methods, Load factors, Extreme winds, Probabilistic models.

The authors seek to ascertain whether the reverse Weibull distribution is an appropriate extreme wind speed model by performing statistical analyses based on the 'peaks over threshold' approach. The data are taken principally from records of the largest daily wind speeds obtained over periods of 15 to 26 years at 44 U.S. weather stations in areas not subjected to małure hurricane winds. From these records the authors create samples with reduced mutual correlation among the data. In our opinion, the analyses provide persuasive evidence that extreme wind speeds' are described predominantly by reverse Weibull distributions, which unlike the Gumbel distribution have finite upper tail and lead to reasonable estimates of wind load factors. Instructions are provided for accessing the data and attendant programs.

\section{BEHAVIOR \& SOCIETY}

\section{General}

\section{0,128}

FIPS PUB 10-4 PC E05

National Inst. of Standards and Technology (CSL), Gaithersburg, MD.

Countries, Dependencies, Areas of Special Sovereignty, and Their Principal Administrative Divisions. Category: Data Standards and Guidelines; Subcategory: Representation and Codes.

6 May 93, 56p.

Supersedes FIPS PUB 10-3.

Keywords: "Geocoding, "Geography, "Classification, "Foreign countries, Territories, Data processing, Data elements, Data codes, Administrative divisions, Dependencies, Provinces, Data standards and guidelines, Representation and codes, Federal Information Processing Standards.

This Standard provides a list of the basic geopolitical entities in the world, together with the principal divisions that comprise each entity. Each basic geopolitical entity that was listed in FIPS PUB 10-3, Countries, Dependencies, and Areas of Special Sovereignty, as updated, is included; it is represented by the same two-character alphabetic country code. Each principal administrative division is identified by a four-character code consisting of the two-character country code followed by a two-character administrative division code.

00,129

PB95-502563 CP T05

National Inst. of Standards and Technology (CSL), Gaithersburg, MD.

Codes for Named Populated Places, Primary County Divisions, and Other Locational Entities of the United States (FIPS PUB 55-3) (on Magnetic Tape). Data file.

Nov 94, mag tapes.

This product contains text only. Customers must provide their own search and retrieval software. Also available in paper copy, order as FIPS PUB 55-3-DC. SUpersedes PB87-142436.

Available in 9-track, ASCII character set tape, 1600 bpi, $6250 \mathrm{bpi}$, or 3480 cartridge. Documentation included; may be ordered separately as FIPS PUB 55-

Keywords: "Data file, "Communities, "Counties, "Federal information processing standards, United States, Coding, Urban areas, Villages, Census tracts, Reservations, Airports, Military facilities, National parks, Postal service, Geographic areas, Magnetic tapes.

This guideline implements ANS X3 47-1993, Information Systems-Codes, Structure and Data Requirements for the Identification of Named Populated Places, Primany County Divisions, and Other Locational Entities of the United States and its Outlying and Associated Areas for Information Interchange, as amplified herein, as a Federal Information Processing Standard (FIPS). This publication, which supersedes FIPS PUB 55-2 and 55DC-4, provides a 2-character FIPS State code and a 5-character FIPS numeric code to uniquely identify each entity contained in a list of names of incorporated places, other communities and 
settlements, primary county divisions (such as townships, New England towns, and census county divisions), American Indian and Alaska Native areas, air. ports, military bases, national parks, post offices, and other locational entities (except natural or physical features). A 2-character class code distinguishes the different types of geographic entities. The purpose of the codes is to promote the interchange of formatted, machine-sensible data.

00,130
PB95-503504 Diskette $\$ 30.00$

National Inst. of Standards and Technology, Gaithersburg, MD

Countries, Dependencies, Areas of Special Sovereignty, and Their Principal Administrative Divisions (for Microcomputers).

Data file.

Sep 95,1 diskette

This product contains text only. Customers must provide their own search and retrieval software. WordPerfect 5.1 can be used but is not contained on diskette. Supersedes PB87-222859.

The datafile is on one $31 / 2$ inch DOS diskette, $1.44 \mathrm{M}$ high density. File format: ASCII text. Also available as paper copy, order number FIPSPUB 10-4.

Keywords: "Data file, "Geocoding, "Geography, "Classification, "Foreign countries, Territories, Data processing, Diskettes, Data elements, Data codes, Administrative divisions, Dependencies, Provinces, Data standards and guidelines, Representation and codes, Federal Information Processing Standards.

This standard provides a list of the basic geopolitical entities in the world, together with the principal divisions that comprise each entity. Each basic geopolitical entity that was listed in FIPS PUB 10-3 'Countries, Dependencies, and Areas of Special Sovereignty', as updated, is included; it is represented by the same two-character alphabetic, "country code." Each principal administrative division is identified by a four-character code consisting of the Two-character 'country code' followed by a two-character 'administrative division code.' This Standard supersedes FIPS 103 in its entirety.

\section{Education, Law, \& Humanities}

\section{0,131}

ED-376 $823 \quad$ Not available NTIS

National Inst. of Standards and Technology, Gaithersburg, MD.

Information Infrastructure: Reaching Society's Goals. A Report of the Information Infrastructure Task Force Committee on Applications and Technology.

NIST-S-PUB-868

Available from ERIC Document Reproduction Service (Computer Microfilm International Corporation), 3900 Wheeler Ave., Alexandria, VA 22304-5110

Keywords: "Computer mediated communication "Policy formation, "Public policy, "Technological advancement, Access to information, Computer networks, Copyrights, Criminology, Disabilities, Emergency programs, Government role, Humanities, Physical environment, Social change, Standards, Traffic safety, Transportation, "National Information Infra structure, Electronic Libraries, Internet, Telecommuting.

Intended for public comment and discussion, this document is the second volume of papers in which the Information Infrastructure Task Force has attempted to articulate in clear terms, with sufficient detail, how improvements in the National Information Infrastructure (NII) can help meet other social goals. These are not plans to be enacted, but the material with which the citizens and their government may have a structured conversation, a purposeful interaction, and deliberation on the issues raised in the evolution of more capable means of information processing and human communications. Eight papers are presented in this volume: (1) NII: An Investment in People with Disabilities; (2) Supply and Demand of Electric Power and the NII; (3) Improving Transportation: The NII and Intelligent Transportation Systems; (4) Promoting Telecommuting: An Application of the NII; (5) The Elfect of NII on Local, State, and Federal Emergency Management; (6) Public Empowerment with Environ- mental Information; (7) Arts, Humanities, and Culture on the NII: and (8) Public Safety and the NII: Supporting Law Enforcement and Criminal Justice. Six of the papers include references. (BBM).

\section{2}

P894-173028 Not available NTIS

National Inst. of Standards and Technology (MEL), Gaithersburg, MD. Precision Engineering Div.

Investing in Education to Meet a National Need for a Technical-Professional Workforce in a Post-Industrial Economy.

Final rept.

D. A. Swyt. 1990, $10 p$

Pub. in Keynote Paper NATO Advanced Research Conference, Eindhoven, Netherlands, October 9-12, 1990.

Keywords: "Education, "Fixed investment, "Labor force, Technology, Productivity, Economy, United States, Reprints.

Workforce data show that the U.S. is shifting from blue collar production to technical-professional service as the basis of its post-industrial economy. The lesson of increased effectiveness with increased capital per person has not been lost on U.S. service industries which have for the last decade lead the U.S. economy in cap ital spending. In contrast, what may be the most important service industry -- basic public education -- has undergone a steep fifteen-year-long decline in its growth in capital investment per student. This suggests that means to focus on capital spending in basic education, such as instituting capital-equipment budgets are in order.

\section{0,133}

\section{PB94-188463 PC A03/MF A01}

National Inst. of Standards and Technology (CSL) Gaithersburg, MD. Systems and Software Technology

User Profile for Researchers Studying Objects: Implications for Computer Systems.

See also PB-235 952

Keywords: "Humanities, "Information processing, User needs, Artifacts, Computers, Information systems, Data acquisition, Requirements, Research projects, Imaging, Data base management, Computer communications, Documents, Information retrieval, Guidelines, Numismatics.

A group of twenty-five researchers who study objects was observed over a four year period. A variety of methods of data collection was used: observation interview, questionnaires, and analysis of published materials. This report identifies the features needed in computer systems to facilitate the research of those who study objects. The researchers need to link obects with text, work with a variety of data types, and collaborate with colleagues over a computer network.

\section{0,134}

P896-159769 Not available NTIS

National Inst. of Standards and Technology (TS), Gaithersburg, MD. Director's Office Technology Services.

Montgomery Education Connection and Resource Education Awareness Partnership Making Connections between Local Schools and NIST Volunteers. Final rept.

D. R. Johnson. $1991,8 p$

Pub. in Editorial in $R$ and D Magazine.

Keywords: "Information resources, Cooperation, Information dissemination Resource assessment Schools, Education, Reprints, *US NIST, "Public-private partnerships, Montgomery Education Connection, Montgomery County(Maryland)

Establised in the Spring of 1984, the Montgomery Education Connection (MEC) is a non-profit public-private partnership formed specifically to link Montegomery County Public Schools and the county's employers. The mission is simply to share private sector resources to improve the education of our children and future employees. The objectives of the organization are to identify the school system needs, to identify community resources to meet those needs, and to identify business needs to which the school system can respond. The Resource Educational Awareness partnership (REAP) is the NIST in-house program that works with the MEC. The goal is to share NIST resources through presenters who visit the schools, through science kits and materials to loan, and through lab visits and special activities.
00,135

PB96-160874 Not available NTIS

National Inst of Standards and Technology (CSL) Gaithersburg, MD. Systems and Software Technology Div.

Using a Multi-Layered Approach to Representing Tort Law Cases for Case-Based Reasoning.

Final rept.

B. B. Cuthill, and R. McCartney. 1993, 7p.

Pub in Case-Based Reasoning Papers from the 1993 Workshop, Washington, DC., July 11-12, 1993, p4147

Keywords: "Litigation, "Information retrieval, Lawsuits, Comparisons, Knowledge representation, Information systems, Integrated systems, Reprints, "Case-based reasoning, Case indexing, Case representation, Case retrieval, Multi-layer systems

This paper presents a multi-layered case representa tion for addressing the problem of comparing and indexing cases in a case-based reasoning system using both the facts and the underlying themes associated with those cases. In many domains, experts discuss problems in terms of multiple layers of abstract principles, classifying problems by conflicts, strategies, or themes important to the domain. Representing the case as a single flat frame does not support reasoning about the underlying themes in the case because it does not represent interconnections among the facts and themes of the case. Instead, a case-based rea soner should use a multi-layered representation includ ing both these facts and interconnections. This representation has implications for much of the casebased reasoning process including case comparison, selection and retrieval mechanisms. The paper will describe the CHASER case-based reasoning system which uses a multi-layered case representation approach to reason about tort law cases.

00,136

P896-179502 Not available NTIS

National Inst. of Standards and Technology (TS) Gaithersburg, MD. Office of Standards Services. Consensus Process in Standards Development. Final rept.

B. L. Collins. 1995, 17p.

Sponsored by National Center for Education Statistics, Washington, DC

Pub. in Proceedings of Joint Conference on Standard Setting for Large-Scale Assessments, Washington, DC., October 5-7, 1994, v2 p203-219 Oct 95.

Keywords: "Education, "Standards, "Agreements, Procedures, Requirements, Process control, Product de velopment, Systems engineering, Ouality assurance, SO, Reprints, US NIST, American National Standards Institute.

The American National Standards Institute (ANSI), al major standards developers in the United States, the National Institute of Standards and Technology (NIST) and the International Organization for Standardization (ISO) have each defined procedures for developing consensus. Although these procedures are generally applied to the development of product and process standards to ensure quality, safety, health, and/or environmental integrity, they may provide a useful model for evaluating the effectiveness of procedures and standards developed in the field of education. The general consensus process is described with particula focus on relevance for developing standards for education.

\section{Job Training \& Career Development}

\section{0,137}

P896-123161 Not available NTIS

National Inst. of Standards and Technology (CSL), Gaithersburg, MD. Systems and Software Technology Div.

Proposed International Interactive Courseware Standard.

Final rept.

W. F. Thode, L. A. Welsch, J. Keck, and S. Lewis.

1991, 10p

Pub. in International Training Equipment Conference (ITEC) (2nd), Maastricht, Netherlands, April 15-17, 1991, 10p.

Keywords: "Video recording, "Training aids "Standards, "Computer assisted instruction, Teaching 
methods, Computer programs, Computer graphics, Military training, Interoperability, Interactive systems, Man computer interface, Reprints.

Millions of dollars are invested in the development of interactive courseware training to be delivered by microcomputer-based training systems, often with interactive video included. Significant development and delivery costs can be avoided if new interactive courseware materials can be made to operate on multiple delivery platforms. A standard that uses a virtua device interface is a flexible approach to interactive courseware portability. The US Department of Defense is adopting this standard as a requirement for future purchases of interactive courseware by the uniformed services. The interface is also under study by the US National Institute for Standards and Technology as a potential component of a general multimedia courseware architecture.

\section{BIOMEDICAL TECHNOLOGY \& HUMAN FACTORS ENGINEERING}

\section{Biomedical Instrumentation \& Bioengineering}

00,138

PB94-172012 Not available NTIS

National Inst. of Standards and Technology (MSEL) Gaithersburg, MD. Polymers Div.

Influence of Tempering Method on Residual Stress in Dental Porcelain.

Final rept.

K. Asaoka, N. Kuwayama, and J. Tesk. 1992, 5p.

Keywords: "Dental materials, "Residual stress, "Porcelain, Thermophysical properties, Cooling curves, Computer simulation, Metal strips, Reprints.

The porcelain component of a porcelain-fused-to-metal restoration is strengthened by residual (tempering) stresses which are induced by cooling procedures followed in dental laboratories. The thermophysical properties of materials and cooling rate are the main factors which determine the residual stress. In this paper, the temperatures in the midplane of body-porcelain disks were measured from a heat-soak temperature (1000 C) to room temperature during two different cooling procedures: slow cooling in air and forced-air cooling. Experimental results approximated exponential cooling wherein the cooling rates could be represented by a linear equation of temperature. Residual stresses, as affected by the tempering method and thickness of a porcelain disk, were calculated by computer simulation for regions away from the edges. The cooling rate dependencies of the glass transition temperature and the temperature distribution during cooling were also included. The cooling rates used in this simulation were derived from the tempering data.

\section{0,139}

PB94-172608 Not available NTIS

National Inst. of Standards and Technology (MSEL). Gaithersburg, MD. Polymers Div.

Adsorption of Low-Molecular-Weight Sodium Polyacrylate on Hydroxyapatite.

Final rept.

D. N. Misra. Oct $93,5 p$

Pub. in Jnl. of Dental Research 71, n10 p1418-1422 Oct 93. Sponsored by American Dental Association Health Foundation, Chicago, IL.

Keywords: *Molecular weight, "Polyacrylates, "Hydroxyapatites, "Adsorption, "Dental cements, Minerals, Electrostatics, Osmolar concentration, Phosphates, Teeth, Isotherms, Reprints.

Adsorption of low-molecular-weight sodium polyacrylate from aqueous solution onto synthetic hydroxyapatite was studied at room temperature so that the mechanism of adhesion of polyacrylate cements to tooth mineral could be elucidated. The ad. sorption isotherm of sodium polyacrylate was Langmuirian in shape and was thus qualitatively different from that of polyacrylic acid (Misra, 1991), which exhibited an adsorption maximum. The self-association of the molecules that probably causes the maximum to occur with polyacrylic acid was effectively absent for the relatively well-ionized, electrostatically repelling polyacrylate ions of the salt. With the adsorption of acrylate jons, the concentration of phosphate ions increased monotonically, while the concentration of calcium ions showed a minimum. The adsorption of sodium polycrylate was irreversible, as it was for polyacrylic acid.

\section{0,140}

PB94-172616 Not available NTIS

National Inst. of Standards and Technology (MSEL), Gaithersburg, MD. Polymers Div.

Interaction of Some Coupling Agents and Organic Compounds with Hydroxyapatite: Hydrogen Bonding, Adsorption and Adhesion.

Final rept.

D. N. Misra. 1994, 13p.

Pub. in Jnl. of Adhesion Science and Technology, v8 n2 p87-99 1994. Sponsored by American Dental Association Health Foundation, Chicago, IL.

Keywords: "Hydroxyapatites, "Adsorption, "Hydrogen bonding, "Organic compounds, "Adhesion, "Dental materials, Solutions, Cross-linking reagents, Bone cements, Dental cements, Osmolar concentration, Reprints.

Adhesion to hydroxyapatite (which is the structural prototype of bone or tooth mineral) is a first step towards the efficacious application of restorative composites and bone cements to teeth and bones. The key medium to effect chemical adhesion between the mineralized substrate and a composite resin is a coupling agent. On the basis of many adsorption studies, primarily by the author, which are reviewed in this paper, it is proposed that a potential coupling agent suitable for dental adhesion can develop strong and durable bonds with a substrate through its hydrogen-bonding functional groups. The coupling agents must also be polyfunctional or possess some hydrophobic moieties to be hydrolytically stable. The criterion defining the capability for adhesion of a compound to hydroxyapatite is determined through a study of its adsorption characteristics from a particular solvent. The stability of this bond in aqueous environments may be determined by desorbing the adsorbed compound with water. The surface orientation of a coupling agent and whether it is reversibly or irreversibly adsorbed from a solvent primarily depend on the balance between the type, location, and number of hydrophilic and hydrophobic moieties in the agent molecule. In the case of watersoluble ionic compounds as coupling agents, the uptake of the compounds will also be influenced by the concentrations of calcium, phosphate, and hydrogen ions in the solution.

00,141

PB94-172723 Not available NTIS

National Inst. of Standards and Technology (MSEL), Gaithersburg, MD. Polymers Div.

Effect of Two Initiator/Stabilizer Concentrations in a Metal Primer on Bond Strengths of a Composite to a Base Metal Alloy.

Final rept.

N. D. Richards, F. Eichmiller, S. V. Dickens, and F V. Simoni. Mar 93, 4p.

Pub. in Dental Materials 9, p91-94 Mar 93. Sponsored by American Dental Association Health Foundation, Chicago, IL

Keywords: "Dental materials, "Chemical bonds, *Composite materials, "Alloys, Additives, Amines, Peroxides, Stabilizers(Agents), Polymers, Polymerization, Adhesives, Reprints, Peroxide/benzoyl, Toluidine/ $\mathrm{N}$-N-dimethyl, Butylated hydroxytoluene, Pyrometallic glycerol dimethacrylate.

The study examined the effect of three additives, amine, peroxide and stabilizer, in two concentrations in a metal primer on the adhesion between a cast metal alloy and a resin composite using a 23 factorial statistical design. The additives, benzoylperoxide (BPO) used at $1 \%$ or $2 \% \mathrm{w} / \mathrm{W}$ and $\mathrm{N}, \mathrm{N}$-dimethyl-p-toluidine (DMPT) at $0.5 \%$ or $1.8 \% \mathrm{w} / \mathrm{w}$, are polymerization initiators. The third additive, butylated hydroxytoluene (BHT) at $0.01 \%$ or $0.03 \% \mathrm{w} / \mathrm{w}$, is used as a stabilizer. $\mathrm{BPO}$ and $\mathrm{BHT}$ were dissolved in an acetone solution containing $20 \%$ of the adhesive resin pyromellitic glyceroldimethacrylate (PMGDM). DMPT was in a separate acetone solution. Bonding resin and composite were applied over the primer and stored overnight in water. Bond strengths were determined by shearing the composite from the metal at a cross head speed of $0.5 \mathrm{~mm} / \mathrm{min}$

00,142

PB94-172871 Not available NTIS

National Inst. of Standards and Technology (MSEL) Gaithersburg, MD. Polymers Div.

Dental Materials.

Final rept.

J. A. Tesk, J. M. Antonucci, F. C. Eichmiller, R. W. Waterstrat, A. C. Fraker, L. C. Chow, L. A. George, J. W. Stansbury, E. E. Parry, J. R. Kelly, and N. W. Rupp. 1993, 77p.

Pub. in Kirk-Othmer Encyclopedia of Chemical Technology, (Fourth Edition), v7 p946-1022 1993.

Keywords: "Dental materials, "Equipment and supplies, Dentistry, Composites, Porcelain, Metals, Glasses, Ceramics, Alloys, Polymers, Dental cements, Temporary dental restoration, Permanent dental restoration, Cost analysis, Reprints.

Dental therapy includes the replacement of hard and soft oral tissues lost through disease using inert materials that may be metallic, ceramic, or organic, or composites employing combinations of these three classes. The operative restorations and prostheses are made of amalgam, precious and nonprecious alloys, special cements, synthetic polymers, porcelain, and glass-ceramics. All must withstand the rigors of the oral environment. The accessory materials needed in the fabrication procedures include synthetic polymers, synthetic and natural gums and waxes, hydrocolloids, gypsums, and refractories. The total value of dental supplies and equipment manufactured in the United States in 1992 is estimated at about $\$ 1.2$ billion. These materials are used by about 170,000 practicing dentists (1992) and $>8,500$ commercial dental laboratories employing about 40-50,000 technicians. The dental materials market is limited because the public does not buy directly except for toothpastes, mouthwashes, tooth brushes, denture aids, etc.

00,143

PB94-198397 Not available NTIS

National Inst. of Standards and Technology (MSEL), Gaithersburg, MD. Polymers Div.

Effect of Transformation of Alloy on Transient and Residual Stresses in a Porcelain-Metal Strip.

Final rept.

K. Asaoka, and J. A. Tesk. 1992, 6p.

Pub. in Residual Stresses - III, Science and Technology, v1 p626-631 1992.

Keywords: "Dental materials, "Phase transformations, "Alloys, "Stress analysis, "Computer simulation, "Composite materials, Thermal expansion, Residual stress, Porcelain, Metal strips, Ceramics, Transient loads, High strength, Reprints.

High strength dental alloys a re usually strengthened by phase transformations which affect their coefficients of thermal expansion. These transformations can affect the stresses developed in porcelain-fused-to-metal (PFM) restorations. In order to aid the understanding of these effects, we conducted computer simulations of stresses developed in a PFM strip. Pd alloys were chosen as Pd-based alloys form the basis for some major alternatives to high cost gold alloys, yet can maintain many of the desirable characteristics. The PdCu alloy system which has a super lattice at 40.3 atomic $\% \mathrm{Cu}$ displays a typical first-order transformation feature. The transient stresses in the porcelains and alloy thermal contraction and curvature of a PFM beam were computed for cooling from a heat soak temperature to room temperature. The residual stress distribution in the composite was also computed. Effects of the transformation of the alloy on transient and residual stresses were revealed from the results of the simulations.

\section{0,144}

PB94-199049 Not available NTIS

National Inst. of Standards and Technology (MSEL), Gaithersburg, MD. Polymers Div.

Adhesion of Composites to Dentin and Enamel.

Final rept.

R. L. Bowen, and W. A. Marjenhoff. 1993, 4p.

Pub. in California Dental Association Jnl. 21, n6 p1922 Jun 93. Sponsored by American Dental Association Health Foundation, Chicago, IL 
Keywords: "Adhesives, "Dental materials, "Dentin, Reprints.

Extrapolations based on the history of the development of composites and an adhesion system for bonding dental resins and composites to hard tooth tissues can rightfully be the basis for strong optimism regarding future improvements in esthetic and conservative treatment modalities. Improved understanding of mechanisms of action and the clinical application steps common to current adhesion systems will certainly lead to improved oral health care.

\section{0,145}

PB94-199056 Not available NTIS

National Inst. of Standards and Technology (MSEL), Gaithersburg, MD. Polymers Div.

Development of an Adhesive Bonding System.

Final rept

R. L. Bowen, and W. A. Marjenhoff. 1992, 6p

Pub. in Operative Dentistry, Supplement 5, p75-80

1992. See aiso PB91-236539. Sponsored by American

Dental Association Health Foundation, Gaithersburg MD. Paffenbarger Research Center

Keywords: "Acid bonded reaction cements. "Adhesives, "Dental materials, Enamels, Dentin, Teeth, Inorganic silicates, Composite materials, Aqueous solutions, Reprints.

Building on findings concerning adhesion to enamel $R$. L. Bowen and his colleagues at the Paffenbarger Research Center, National Institute of Standards and Technology, began addressing and solving problems associated with (1) silicate cements and unfilled resins (2) bonding in an aqueous environment, and (3) the development of an adhesion system for both dentin and enamel that could withstand various stresses. This article reviews the development of an adhesion system for bonding dental composites to dentin and enamel.

00,146
PB94-211240 Not available NTIS

National Inst. of Standards and Technology (MSEL), Gaithersburg, MD. Polymers Div

Clinical Perspective on Dentin Adhesives.

Final rept.

F. C. Eichmiller. 1993, 3p.

Pub. in JnI. of Indiana Dental Association 72, n5 p2224 1993. Sponsored by American Dental Association Health Foundation, Chicago, IL.

Keywords: "Dental materials, "Adhesive bonding, "Dentistry, "Polymers, Dentin, Monomers, Acids, Surfaces, Reprints, Restorative materials.

The newer generation of dentin adhesives has changed the practice of restorative dentistry. Two features common to most of the bonding restorative materials are the ability to alter, through the use of acidic etching agents, the surface of the dentin and the ability to completely wet and re-infiltrate the altered dentin surface with a hydrophilic polymerizable monomer. The acidic etchants remove or break up the smear layer, partialiy demineralize and/or alter the mineral in the first few microns of the dentin surface, and expose the collagen fibril network. The hydrophilic adhesive monomers that contain both acidic and methacrylate groups on the same molecule are bifunctional in nature. These monomers are able to infiltrate the demineralized dentin due to their hydrophilic nature and polymerize to encapsulate the exposed collagen fibers. Available products can be divided into two broad categories: systems that contain a low concentration of adhesive monomer diluted in a volatile solvent and systems that use adhesive resins of moderate to high viscosity. Knowledge of the features and properties of dentin adhesives provides the needed rationale for the protocol of application and the proper choice of materials in restorative dentistry.

\section{0,147}

PB94-211257 Not available NTIS

National Inst. of Standards and Technology (MSEL), Gaithersburg, MD. Polymers Div.

Selective Inhibition of Crystal Growth on Octacalcium Phosphate and Nonstoichiometric Hydroxyapatite by Pyrophosphate at Physiological Concentration.

Final rept.

N. Eidelman, W. E. Brown, and J. L. Meyer. 1991

$10 \mathrm{p}$.

Sponsored by American Dental Association Health Foundation, Chicago, IL
Pub. in Jnl. of Crystal Growth 113, p643-652 1991.

Keywords: "Calcium phosphates, "Pyrophosphates, "Crystal growth, "Inhibitors, Apatites, Hydroxy compounds, Dental materials, Reprints, Hydroxyapatite, Biominerals.

Octacalcium phosphate, $\mathrm{Ca} 8 \mathrm{H} 2(\mathrm{PO} 4) 6$ (dot) $5 \mathrm{H} 2 \mathrm{O}$ $(\mathrm{OCP})$, appears to be a precursor in biomineral formation. The formation of OCP as the precursor is sup ported by the observation that stoichiometric hydroxyapatite, Ca5(PO4)3OH (OHAp), cannot form directly because of the presence of its growth inhibitors in serum. Therefore, the effects of the physiological concentration of pyrophosphate (P2O7(4-)), one of the most important calcium phosphate growth inhibitors in blood, on calcium phosphate growth rates on OCP and nonstoichiometric OHAp (apatite) seeds were measured. The amounts of seed crystals used to initiate the growth were adjusted by trial and error so that the control growth rates (in the absence of $\mathrm{P} 2 \mathrm{O} 7(4-)$ ) were the same on both OCP and apatite seeds at a given supersaturation. The crystal growth on both kinds of seed crystals from supersaturated solutions in the presence of I microM P2O7 (4-) added once ('one-time' addition) at constant $\mathrm{pH}(7.4)$ and $25 \mathrm{C}$ was determined by $\mathrm{KOH}$ titration and decreases in $\mathrm{Ca}$ and $\mathrm{PO} 4$ concentrations in the solutions. Crystal growth on OCP seed crystals in the presence of a constant concentration of I microM P2O7(4-) was measured. The results of this study show that: (1) P2O7(4-) ions inhibited the growth on the apatite seeds more than on the OCP seeds; (2) apparently OCP precipitated on both types of seeds, followed by its hydrolysis to a more apatitelike phase; (3) slower crystal growth was observed on OCP seeds in the presence of a constant physiological concentration of $\mathrm{P} 2 \mathrm{O} 7(4-)$ (1 microM) than in the 'onetime' addition of $\mathrm{P} 2 \mathrm{O} 7(4$-)

00,148

PB94-211513 Not available NTIS

National Inst. of Standards and Technology (NEL), Gaithersburg, MD. Automated Production Technology Div.

NIST Power Reference Source.

Final rept.

S. E. Fick. 1993, 15p.

in Ultrasonic Exposimetry, Chapter 6, p169-183 1993.

Keywords: "Laboratory equipment, "Ultrasonic tests, Clinical medicine Ultrasonic wave transducers, $\mathrm{Re}$ prints, "Power reference sources, Standard reference materials

This chapter addressed ways in which laboratory in struments used to evaluate clinical ultrasonic equip ment can themselves be characterized using a source of well known levels of ultrasonic power. A detailed presentation of the design and working characteristics of NIST SRM 1855, an ultrasonic absolute powe transfer standard, is preceded by an exposition o methods by which clinical and research ultrasonic equipment should be tested before being put into service.

\section{0,149}

\section{PB94-212008 Not available NTIS}

National Inst. of Standards and Technology (MSEL), Gaithersburg, MD. Polymers Div

Periapical Tissue Reactions to a Calcium Phos phate Cement in the Teeth of Monkeys.

Final rept.

Y. C. Hong, J. T. Wang, C. Y. Hong, W. E. Brown, and L. C. Chow. 1991,14p.

Sponsored by American Dental Association Health Foundation, Chicago, IL.

Pub. in Jnl. of Biomedical Materials Research 25, p485-498 1991.

Keywords: "Dental materials, "Calcium phosphates, "Biocompatible materials, "Biological effects, "Root canal filling materials, Laboratory animals, Monkeys, Tissues(Biology), Dental pulp cavity, Comparison, Per formance evaluation, Reprints, Grossman sealer, Sargenti N2.

A calcium phosphate cement, Grossman sealer, and Sargenti N2 were compared under conditions where root canals of monkey incisors were deliberately overfilled and the apical tissue responses were evaluated histologically. The periapical tissues exposed to Sargenti N2 revealed severe irritation at all times through the 6-month experimental period. The reactions to Grossman sealer were milder but persisted throughout the observation perioo. The calcium-phos- phate-cement treated animals showed mild tissue irritation after 1 month, but thereafter the adverse tissue reactions were minimal. The compatibility of calcium phosphate cement with the periapical tissue suggests that the cement may have other applications in dentistry and medicine.

\section{0,150}

PB94-216090 Not available NTIS

National Inst. of Standards and Technology (MSEL), Gaithersburg, MD. Polymers Div.

Effect of Three Sterilization Techniques on Finger Pluggers.

Final rep

W. D. Luper, F. C. Eichmiller, W. Doblecki, D.

Campbell, and S. H. Li. 1991, 4p.

Sponsored by American Dental Association Health Foundation, Chicago, IL

Pub. in Jnl. of Endodontics 17, n8 p361-364 Aug 91 Keywords: *Sterilization, Dental materials Fatigue(Materials), Fracture(Materials), Autoclaving, Heat, Steam, Reprints, "Finger pluggers

The effects of different sterilization methods on fatigue life of finger pluggers was investigated. Four sizes ( $A$, $B, C, D)$ of finger pluggers were subjected to 1,8 , or 15 cycles of steam autoclave, dry heat, or bead sterilization. Controls were not sterilized. Finger pluggers were subjected to cyclic bending until fracture. Only the autoclave 8-cycles subgroup of the A finger pluggers had a significantly lower number of cycles to failure compared to controls. The following subgroups had significantly greater cycles before failure when compared to their controls: autoclave 15-, bead 1-, and bead 15-cycles subgroups of B finger pluggers; autoclave 1-, 8-, and 15-, dry heat 1-, and bead 15-cycles subgroups of $C$ finger pluggers; and the bead 15-cycles subgroup of D finger pluggers. The others were not different. Since all but one sterilized group had cyclic lifetimes that were not significantly shorter than nonsterilized controls, clinicians can generally use any of the 3 sterilization methods without a detrimental effect on fatigue life of finger pluggers.

00,151

PB94-216355 Not available NTIS

National Inst. of Standards and Technology (MSEL), Gaithersburg, MD. Polymers Div.

Paffenbarger Research Center: The Cutting Edge of Dental Science.

Final rept.

W. A. Marjenhoff, and L. A. George. 1992, 4p. Sponsored by American Dental Association Health Foundation, Chicago, IL

Pub. in Jnl. of the American College of Dentists 59, n4 p6-9 1992

Keywords: "Dental materials, *Dentistry, "Technology innovation, Adhesion, Dental cements, Dentin, Dental enamel, Licenses, Protective coatings, Calcium phosphates, Composite resins, Reprints, "Paffenbarger Research Center, National Institute of Standards and Technology.

Science Research (CEMSR) and Paffenbarger Research Center (PRC) at the National Institute of Standards and Technology (NIST) has been in the forefront of transferring new and improved dental technologies to the profession since 1928. The list of PRC/NIST innovations is long and impressive. During the past five years, dentistry has seen the commercialization of a PRC adhesion system for bonding composites to dentin and enamel simultaneously and glass-ceramic inserts that improve the properties of composite restorations. Licenses are currently being negotiated with dental manufacturers for a variety of other products based on American Dental Association Health Foundation (ADAHF) patents. Current research includes the further development of calcium phosphate cements for various dental and medical applications; resins that exhibit minimal dimensional change on hardening; protective coatings for dentin, as well as enamel; and synthetic dentin.

\section{0,152}

PB94-216561 Not available NTIS

National Inst. of Standards and Technology (MSEL), Gaithersburg, MD. Polymers Div

Crystal Structure of Dicalcium Potassium Trihydrogen Bis(pyrophosphate) Trihydrate. Final rept.

M. Mathew, L. W. Schroeder, and W. E. Brown.

$1993,5 p$

Sponsored by American Dental Association Health

Foundation, Chicago, IL. 


\section{Biomedical Instrumentation \& Bioengineering}

Pub. in Jnl. of Crystallographic and Spectroscopic Research 23, n8 p657-661 1993.

Keywords: "Crystal structure, "Calcium phosphates, "Pyrophosphates, "Potassium phosphates, Dental materials, Layers, X-ray diffraction, Reprints.

The crystal structure of $\mathrm{Ca} 2 \mathrm{KH} 3(\mathrm{P} 2 \mathrm{O} 7) 23 \mathrm{H} 20$ has been determined by single crystal $X$-ray diffraction. Crystals are monoclinic, space group $\mathrm{P} 2($ sub 1$) / n$ with $a=10.518(3), b=19.253(9), c=7.340(3) \mathrm{A}$, Beta $=$ $90.07(2) \mathrm{deg}$, and $Z=4$. The structure was refined to $R=0.048$ and $R($ sub $w)=0.044$ for 1,839 reflections with $\mathrm{I}>$ than or $=3$ sigma(l). The structure consists of a compact assembly of $\mathrm{Ca}, \mathrm{K}, \mathrm{HP} 2 \mathrm{O} 7$, and $\mathrm{H} 2 \mathrm{P} 2 \mathrm{O} 7$ ions and three water molecules arranged in layers perpendicular to the b-axis. The two independent $\mathrm{Ca}$ ions and the $\mathrm{HP} 2 \mathrm{O} 7$ ion comprise one layer; $\mathrm{K}$ and $\mathrm{H} 2 \mathrm{P} 2 \mathrm{O} 7$ ions and the three water molecules form an interstitial layer. Coordinations of the two independent $\mathrm{Ca}$ ions are quite similar, but the environments of HP2O7 and $\mathrm{H} 2 \mathrm{P} 2 \mathrm{O} 7$ ions are quite different, probably due to their locations in different layers. The general structural features are quite similar to those of $\mathrm{Ca}(\mathrm{NH} 4) \mathrm{HP} 2 \mathrm{O} 7$.

00,153

PB94-216579 Not available NTIS

National Inst. of Standards and Technology (MSEL), Gaithersburg, MD. Polymers Div.

Crystal Structure of Calcium Adipate Monohydrate. Final rept.

M. Mathew, S. Takagi, and H. L. Ammon. 1993, 5p. Sponsored by American Dental Association Health Foundation, Chicago, IL

Pub. in Jnl. of Crystallographic and Spectroscopic Research 23, n8 p617-6211993.

Keywords: *Crystal structure, Dental materials, X-ray diffraction, Polymers, Chemical bonds, Layers, Monocrystals, Hydrates, Least squares method, Reprints, "Calcium adipate monohydrate, "Calcium dicarboxylate.

The crystal structure of calcium adipate monohydrate, $\mathrm{Ca}(\mathrm{C} 6 \mathrm{H} 8 \mathrm{O} 4)(\mathrm{H} 2 \mathrm{O})$, has been determined by single crystal X-ray diffraction study. The structure was refined by full-matrix least-squares techniques to $R=$ $0.040, R($ sub $w)=0.058$ for 1,283 reflections with $\mid>$ or $=3$ sigma $(1)$. Ca is coordinated to seven oxygen atoms and the coordination polyhedron is best described as pentagonal bipyramid. The coordinations of the two carboxylate groups in adipate ion are quite different. One carboxylate group binds three different $\mathrm{Ca}$ ions forming a four-membered chelate ring with one $\mathrm{Ca}$ ion and unidentate bridge bonds to two other $\mathrm{Ca}$ ions. The other carboxylate group links to two $\mathrm{Ca}$ ions through unidentate bonds. The structure is highly polymeric, but with a layer-type structure parallel to (001) with the hydrocarbon chains sandwiched between the polar regions consisting of $\mathrm{Ca}$, carboxylate and water molecules.

\section{0,154}

PB95-125613 Not available NTIS

National Inst. of Standards and Technology (MSEL), Gaithersburg, MD. Polymers Div.

Evaluation of Fracture Toughness and Residual Stress in Dental Porcelain by IndentationMicrofracture Method.

Final rept

K. Asaoka, J. A. Tesk, and J. R. Kelly. 1994, 6p.

Pub. in International Conference on Residual Stresses (4th), Baltimore, MD., June 8-10, 1994, p854-859.

Keywords: "Dental materials, "Porcelain, "Residual stress, "Fracture strength, Surfaces, Toughness, Study estimates, Crack propagation, Reprints, *Indentation-microfracture method.

The indentation-microfracture (IM) method was applied to estimate both the fracture toughness, $\mathrm{K}$ (sub Ic), and residual stress in the surface of $2.0 \times 10.0 \mathrm{~mm}$ disks of two dental porcelains ( $A$ and $B$ ) that were either slowly cooled or tempered by two different treatments. The shape parameter, $m$, for Weibull plots of the K/sub Ic)'s that were measured by IM method, was calculated to be within the range of 7.1 to 9.1 for the slowly cooled specimens. Tempering had no significant effect on the Weibull shape parameters. The surface residual stresses for porcelain A were estimated to be $26-28$ and 64-88 $\mathrm{MPa}$ in compression for specimens cooled in ambient air and with forced air, respectively, as calculated from the median/radial crack length after indentation. The estimated residual stresses were in good agreement with the results of computer simulations by Asaoka et al. It was concluded that the IM method can be applied for measurement of the fracture toughness and residual stress in the near surface region (100-400 micrometers deep) for dental metal-ceramic porcelains that have a micro structure that is unaffected by cooling rate.

00,155

PB95-126173 Not available NTIS

National Inst. of Standards and Technology (PL), Gaithersburg, MD. Ionizing Radiation Div.

Image Information Transfer Properties of X-Ray Intensifying Screens in the Energy Range from 17 to $320 \mathrm{keV}$.

Final rept.

A. Ginzburg, and C. E. Dick. 1993, 9p

Pub. in Medical Physics 20, n4 p1013-1021 Jul/Aug 93

Keywords: "Biomedical radiography, "Fluorescent screens, " $X$-ray fluorescence, $\mathrm{KeV}$ range 10-100, KeV range 100-1000, $X$-ray absorption, Image intensifiers, Ouantum efficiency, Calcium tungstates, Rare earths, Phosphors, Reprints.

The image information transfer properties of a number of $x$-ray fluorescent screens have been measured for $x$-ray energies from 17 to $320 \mathrm{keV}$. The detective quantum efficiency of the screens at each $x$-ray energy has been determined by separate measurements of the $x$ ray absorption efficiency and the statistical factor associated with the emission of optical photons upon absorption of an incident $x$ ray. Data have been recorded for both rare-earth phosphor screens and calcium tungstate screens. The value of the statistical factor for optical photon emission tends toward a constant value as the incident energy increases. Comparisons of the image information transfer properties are presented for several screens, which have been measured over a ten year interval. The utility of the screens for high-energy radiography is discussed.

\section{0,156}

PB95-140448 Not available NTIS

National Inst. of Standards and Technology (MSEL) Gaithersburg, MD. Polymers Div.

Effects of Surface-Active Resins on Dentin/Composite Bonds.

Final rept.

G. E. Schumacher, F. C. Eichmiller, and J. M.

Antonucci. 1992, 5p

Sponsored by National Inst. of Dental Research, Bethesda, MD.

Pub. in Dental Materials 8, p278-282 Jul 92.

Keywords: "Dentin, *Dental materials, "Surfactants, "Chemical bonds, Polymerization, Monomers, Feasibility studies, Reprints, "Pyromellitic dianhydride, "Methacrylate/hydroxyethyl, Chemical reaction mechanisms.

Effective dentin bonding systems based on paraPMDM diadduct of pyromellitic dianhydride and 2 hydroxyethyl methacrylate (HEMA) have been developed. Para-PMDM, a solid of limited solubility, is usually applied from an acetone solution to dentin that has been preconditioned with acid and $\mathrm{N}$-phenylglycine The feasibility of using a liquid, surface-active bonding resin to substitute for or to supplement para-PMDM was explored. The results suggest that solutions based on Mono(2-methacryloyloxy)ethyl phthalate (MMEP) and/or para-PMDM in acetone or in other monomers, especially those containing HEMA, can effectively promote bonding to dentin. A new mechanism for the observed self-polymerization of MMEP or para-PMDM with $\mathrm{N}$-phenylglycine is proposed.

\section{0,157}

PB95-151080 Not available NTIS

National Inst. of Standards and

Gaithersburg, MD. Polymers Div. Effects of Calcium Phosphate Solutions on Dentin Permeability.

Final rept.

Pashley. 1993, 5p.

Sponsored by American Dental Association Health Foundation, Chicago, IL

Pub. in Jnl. of Endodontics 19, n8 p383-387 Aug 93.

Keywords: *Calcium phosphates, "Dentin permeability, *Dental materials, Precipitation(Chemistry), pH, X-ray diffraction. Scanning electron microscopy, Solutions, Kinetics, Reprints, “Dental tubules.

Calcium phosphate solutions at various concentrations and $\mathrm{pH}$ levels were used to obstruct the dental tubules.
The effects were evaluated by measurements of permeability through dentin discs and by scanning electron microscopy. Precipitation kinetics were followed by $\mathrm{pH}$ changes in the solutions and products were determined by $X$-ray powder diffraction. The solutions were applied in two ways: (a) calcium and phosphate solutions were mixed before application and (b) one solution (calcium or phosphate) was applied first followed by the other solution.

\section{0,158}

PB95-151163 Not available NTIS

National Inst. of Standards and Technology (MSEL), Gaithersburg, MD. Polymers Div.

Modified Surface-Active Monomers for Adhesive Bonding to Dentin.

Final rept.

S. Venz, and B. Dickens. 1993, 5p.

Sponsored by American Dental Association Health Foundation, Chicago, IL.

Pub. in Jnl. of Dental Research 72, n3 p582-586 Mar 93

Keywords: "Adhesive bonding, "Dentin, "Monomers, *Adhesives, Performance evaluation, Reliability, Curing, Synthesis(Chemistry), Surface properties, Reprints, "PMGDM, PMDM, Pyromellitic dianhydride, Glycerol dimethacrylate.

PMGDM, a PMDM-type adhesive monomer, was synthesized from pyromellitic dianhydride and glycerol dimethacrylate. Only the para isomer of PMDM, a solid, is used in dental adhesives. The adhesive mono mer PMGDM is a liquid and consists of a mixture of para- and meta isomers. This study shows that PMGDM has several advantages over PMDM. The adhesive bonds were tested in both shear and tensile modes. PMDM was used as the control. The reliability of the bonds, as judged from the Weibull modulus and Weibull characteristic strength, was improved by (1) use of more concentrated solutions of PMGDM adhesive, (2) use of an adhesive thickness of about $25 \mathrm{mi}$ crometers and (3) modification of PMGDM with a diluent monomer which is expected to enhance the degree of cure and/or the dentin-wetting properties of the adhesive resin.

00,159

PB95-152831 Not available NTIS

National Inst. of Standards and Technology (MSEL), Gaithersburg, MD. Polymers Div.

Evaluation of Fracture Toughness and Residual Stress in Dental Porcelain by IndentationMicrofracture Method.

Final rept.

K. Asaoka, J. A. Tesk, and J. R. Kelly. 1994, 6p.

Pub. in Proceedings of International Conference on Residual Stresses (4th), Baltimore, MD., June 8-10, 1994, p854-859.

Keywords: "Dental materials, "Residual stress, "Porcelain, "Fracture strength, *Surfaces, Study estimates, Microstructure, Computerized simulation, Crack propagation, Reprints, "Indentationmicrofracture method.

The indentation-microfracture (IM) method was applied to estimate both the fracture toughness, $\mathrm{K}$ (sub Ic) and residual stress in the surface of $2.0 \times 10.0 \mathrm{~mm}$ disks of two dental porcelains ( $A$ and $B$ ) that were either slowly cooled or tempered by two different treatments. The shape parameter, $m$, for Weibull plots of the K(sub Ic)'s that were measured by IM method, was calculated to be within the range of 7.1 to 9.1 for the slowly cooled specimens. Tempering had no significant effect on the Weibull shape parameters. The surface residual stresses for porcelain A were estimated to be $26-28$ and 64-88 MPa in compression for specimens cooled in ambient air and with forced air, respectively, as calculated from the median/radial crack length after indentation. The estimated residual stress were in good agreement with the results of computer simulations by Asaoka et al. It was concluded that the IM method can be applied for measurement of the fracture toughness and residual stress in the near surface region (100-400 micrometers deep) for dental metal-ceramic porcelains that have a micro structure that is unaffected by cooling rate.

00,160

PB95-153417 Not available NTIS

National Inst. of Standards and Technology (PL), Gaithersburg, MD. Quantum Metrology Div. 
Noninvasive High-Voltage Measurement in Mammography by Crystal Diffraction Spectroscopy. Final rept.

R. D. Deslattes, J. C. Levin, M. D. Walker, and A

Henins. 1994, 4p

Pub. in Medical Physics 21, n1 p123-126 Jan 94.

Keywords: "Mammography, "X-ray spectrometers, "Radiology, High voltage, Medical equipment, $R e$ prints.

Wavelength dispersive crystal diffraction spectrometry has been applied to the measurement of the accelerating voltage on an $x$-ray source in a prototype experiment in the mammographic source. The results indicate that this noninvasive approach can yield determinations of such voltages within $0.1 \mathrm{kV}$, a level of imprecision that appears adequate for high-level standardization of such potentials.

00,161

PB95-161972 Not available NTIS

National Inst. of Standards and Technology (MSEL), Gaithersburg, MD. Polymers Div.

Wear of Human Enamel against a Commercial Castable Ceramic Restorative Material.

Final rept.

D. S. Palmer, M. T. Barco, G. B. Pelleu, and J. E.

McKinney. 1991, 4p.

Pub, in Jnl. of Prosthetic Dentistry 65, n2 p192-195 Feb 91.

Keywords: "Dental enamel, "Wear tests, "Ceramics, *Dental restoration, "Dental materials, Comparison, Casting, Porcelain, Reprints.

This study determined the effect of castable ceramic with and without shading porcelain applied, on ename wear. The wear produced by conventional dental porcelain was used as a control. Cusp tips from extracted human third molars were precision-machined into cones of enamel approximately $1 \mathrm{~mm}$ long. Three groups of nine cones each were abraded against rotating disks of (1) castable ceramic with shading porcelain, (2) castable ceramic without shading porcelain, and (3) conventional dental porcelain. Enamel wear was calculated from microscopic measurements of the enamel cones before and after abrading. Significant differences were found between castable ceramic with and without shading porcelain and between conventional dental porcelain and castable ceramic with shading porcelain ( $p<0.001$ ANOVA and 0.05 Scheffe's test). These findings suggest that castable ceramic with shading porcelain should not be used in regions that will function against opposing natural teeth.

00,162

PB95-162251 Not available NTIS

National Inst. of Standards and Technology (MSEL), Gaithersburg, MD. Polymers Div.

Ring-Opening Dental Resin Systems Based on Cyclic Acetals.

Final rept.

B. Reed, J. Stansbury, and J. Antonucci. 1994, 7p.

Pub. in Polymers of Biological and Biomedical Significance, Chapter 15, p184-1901994.

Keywords: "Dental materials, "Polymerization, "Monomers, "Cyclic compounds, "Acetals, Free radicals, Mechanical properties, Resin matrix composites, Molecular structure, Reprints.

For monomers of comparable size, ring-opening polymerization results in less shrinkage than that which accompanies 1,2-vinyl addition polymerization. Two monomer types were synthesized, nonvinyl (NVCA) and vinyl (VCA) cyclic acetals. The goals of this study were to assess the potential for reduced shrinkage through free radical ring-opening polymerization of NVCA and VCA type monomers, and to test the mechanical strength of dental resin composites formulated with these novel monomers. Homo- and copolymerizations were conducted with several NVCAs and VCAs to evaluate their potential as comonomers in dental polymeric composites. Composite specimens were formulated with PBMD, a VCA derived from terephthaldehyde, and EBPADM, an ethoxylated bisphenol A dimethacrylate, and tested for their mechanical strength.

\section{0,163}

PB95-163507 Not available NTIS

National Inst. of Standards and Technology (MSEL), Gaithersburg, MD. Polymers Div. Effect of Ethanol on the Solubility of Dicalcium
Phosphate Dihydrate in the System $\mathrm{Ca}(\mathrm{OH}) 2-$ H3PO4-H2O at 37C

Final rept.

M. S. Tung, and T. J. O'Farrell. 1993, 7p

Sponsored by American Dental Association Health Foundation, Chicago, IL.

Pub. in Jnl. of Molecular Liquids 56, p237-243 1993.

Keywords: "Dental materials, *Calcium phosphates, "Solubility, "Ethanol, Mixtures, Dielectric properties, Born approximation, Reprints, "Dicalcium phosphate dihydrate.

Solubility values of dicalcium phosphate dilhydrate were determined in $0,7.86,16.04,24.53$, and $33.38 \%$ by weight ethanol-water mixtures at $37 \mathrm{C}$. The decreased solubility of DCPD, expressed as solubility product ( $\mathrm{K}(\mathrm{sub} \mathrm{sp})$ ), from $\mathrm{pK}(\mathrm{sub} \mathrm{sp})=6.69$ at $0 \%$ ethanol to 8.91 at $33.38 \%$ ethanol, was mainly due to the decrease in the dielectric constant. The data were fitted to the simple Born equation as follows: pK(sub $\mathrm{sp})-6.69=575.2(1 /$ epsilon -0.0135$)$

\section{0,164}

PB95-164661 Not available NTIS

National Inst. of Standards and Technology (MSEL) Gaithersburg, MD. Polymers Div.

Evaluation of Methylene Lactone Monomers in Dental Resins.

Final rept.

J. W. Stansbury, and J. M. Antonucci. 1992, 4p.

Sponsored by National Inst. of Dental Research, Bethesda, MD.

Pub. in Dental Materials 8, p270-273 Jul 92

Keywords: *Dental materials, "Monomers, "Polymerization, Mechanical properties, Free radicals, Synthesis(Chemistry), Cyclic compounds, Curing, Chemical bonds, Methacrylates, Reprints, *Methylene lactones, Methylene butyrolactone.

alpha-Methylene-gamma-butyrolactone (MBL), which can be described as the cyclic analog of methyl methacrylate, exhibits greater reactivity in free radical polymerizations than conventional methacrylate monomers. Unfilled resin formulations composed of Bis-GMA/MBL or Bis-GMATTEGDMA/MBL were lightcured. The effect of the more reactive methylene lactone monomer on mechanical properties and the degree of conversion of the polymers was examined The infrared absorption bands for the carbon-carbon double bonds of MBL and the methacrylate monomers are well resolved and allow the conversion of each component to be calculated individually. The synthesis and polymerization of several substituted methylene lactones was also studied.

00,165

PB95-168712 Not available NTIS

National Inst. of Standards and Technology (MSEL), Gaithersburg, MD. Polymers Div

Behavior of a Calcium Phosphate Cement in Simulated Blood Plasma In vitro.

Final rept.

K. Ishikawa, S. Takagi, L. C. Chow, K. Asaoka, Y Ishikawa, and E. D. Eanes. 1994, 7p.

Sponsored by American Dental Association Health Foundation, Chicago, IL

Pub. in Dental Materials 10, p26-32 1994

Keywords: "Calcium phosphate, "Dental cements, *Biocompatibility In vitro analysis Dental materials, Hardness, Physical properties, Blood plasma, Synthetic materials, Biochemistry, Performance evaluation, Reprints, Hydroxyapatite.

The purpose of the study was to gain a better under standing of the integration of calcium phosphate cement (CPC) implants in biological tissue An invitro continuous flow system was employed to examine the protracted behavior of disc-shaped specimens of this bioactive material under sustained physiological-like solution conditions. The results suggest that under in vivo conditions, CPC implants would not dissolve in phyisological fluids. Carbonate hydroxyapatite (OHAp) coatings may form on the implants, which may en hance bonding of implants to bone by mechanically strengthening the interface between them.

00,166

PB95-168894 Not available NTIS

National Inst. of Standards and Technology (MSEL), Gaithersburg, MD. Polymers Div.
Octacalcium Phosphate Carboxylates. 5. Incorporation of Excess Succinate and Ammonium lons in the Octacalcium Phosphate Succinate Structure. Final rept.

M. Markovic, B. O. Fowler, and W. E. Brown. 1993,

Sponsored by American Dental Association Health Foundation, Chicago, IL

Pub. in Proceedings of Materials Research Society Symposium, Hydroxyapatite and Related Compounds, San Francisco, CA., April 13-15, 1993, p139-144 1994. Keywords: "Dental materials, "Ions, "Crystal structure, $X$ ray diffraction, Hydration, Chemical analysis, Infrared spectroscopy, Reprints, "Octacalcium phosphate succinate, Octacalcium phosphate, Octacalcium phosphate carboxylates.

Octacalcium phosphate succinate Ca8(HPO4) 1.07(succ)0.93(PO4)4-6H2O (OCP. SUCC), has an approx $50 \%$ larger hydrated layer volume than that of its parent compound, octacalcium phosphate, Ca8(HPO4)2(PO4)4-5H2O (OCP). The incorporation of structurally excess ions in OCP-SUCC was established by chemical analysis, X-ray powder diffraction, and infrared spectroscopy. Both excess succinate and counter ammonium ions were found to incorporate in the hydrated layer of the OCP-SUCC structure; this incorporation caused a slight expansion in the alpha-axis of up to $3 \%$. Removal of these excess ions resulted in contraction of the alpha-axis to near that of OCP-SUCC. OCP-SUCC and particularly other OCP-carboxylates (OCPCs) that have larger hydrated layer volumes have a potential to incorporate various molecules and ions; these OCPCs could serve as vehicles to facilitate slow release of these additives into aqueous solutions.

\section{0,167}

PB95-168928 Not available NTIS

National Inst. of Standards and Technology (MSEL), Gaithersburg, MD. Polymers Div

Crystal Structure of Calcium Succinate Monohydrate.

Final rept.

M. Mathew, S. Takagi, B. O. Fowler, and M.

Markovic. 1994, 4p.

Sponsored by American Dental Association Health Foundation, Chicago, IL

Pub. in Jnl. of Chemical Crystallography 24, n7 p437 4401994

Keywords: "Dental materials, "Crystal structure, Calcium compounds, $X$ ray diffraction, Hydrates, Chemical bonds, Monocrystals, Polymers, Reprints, *Calcium succinate monohydrate, Calcium adipate monohydrate.

The crystal structure of calcium succinate monohydrate, $\mathrm{Ca}(\mathrm{C} 4 \mathrm{H} 4 \mathrm{O} 4)-\mathrm{H} 2 \mathrm{O}$, has been determined by single crystal $X$-ray diffraction. The crystals are monoclinic. The structure was refined by full-matrix least-squares techniques to $R=0.027, R($ sub $w)=$ 0.040 for 829 reflections with $\mathrm{I}>$ or $=3$ sigma(I). Ca is coordinated to seven oxygen atoms, and the coordination polyhedron is best described as a pentagonal bipyramid. One carboxylate group in the succinate ion is bonded to three different $\mathrm{Ca}$ ions, forming a fourmembered chelate ring with one $\mathrm{Ca}$ ion and unidentate bridge bonds to two other $\mathrm{Ca}$ ions. The other carboxylate group is bonded to two $\mathrm{Ca}$ ions through unidentate bonds. The structure is highly polymeric. The general structural features are nearly identical to those of calcium adipate monohydrate.

\section{0,168}

PB95-169074 Not available NTIS

National Inst. of Standards and Technology (MSEL), Gaithersburg, MD. Metallurgy Div.

Ambient Temperature Synthesis of Bulk Intermetallics.

Final rept.

M. Ratzker, D. S. Lashmore, and M. P. Dariel. 1994, $6 p$.

Sponsored by American Dental Association Health Foundation, Chicago, IL.

Pub. in Materials Research Society Symposia Pro ceedings, v350 p41-46 1994

Keywords: *Intermetallic compounds, "Dental materials, "Ambient temperature, "Synthesis(Chemistry) Interfaces, Thin films, Binary systems(Materials), Melting points, Diffusion, Indium alloys, Silver alloys, Tin alloys, Reprints.

Room-temperature intermetallic compound formation occurs when one of the component metals has a very 


\section{Biomedical Instrumentation \& Bioengineering}

low melting point or when two metals in close contact interdiffuse very rapidly. Compound formation at room temperature at the interface of superposed thin films has been observed in several instances, often in systems relating to electronic materials. The overall amount of compound produced in such configurations, however, is limited, due to the intrinsic limitations involved in the thin layer geometry. Bulk quantities of intermetallic can be produced at ambient temperature in solids by increasing the interface area between the components that interdiffuse rapidly. This condition can be achieved by having small size powder particles of one component coated with a layer of the second component. The very large interface area leads to rapid formation of bulk quantities of compounds even at ambient temperature. By appropriate control of the initial constituents and the coating parameters, it is possible to custom-prepare various intermetallic compounds present in binary systems such as silver-tin, gold-tin and silver-indium in which fast interdiffusion takes place.

\section{0,169}

PB95-169231 Not available NTIS

National Inst. of Standards and Technology (MSEL), Gaithersburg, MD. Polymers Div.

Effect of Ethanol on the Solubility of Hydroxyapatite in the System $\mathrm{Ca}(\mathrm{OH}) 2 \cdot \mathrm{H} 3 \mathrm{PO}$ 4$\mathrm{H} 2 \mathrm{O}$ at $25 \mathrm{C}$ and $33 \mathrm{C}$

Final rep

M. S. Tung, C. Lin, T. H. Chow, and P. Sung. 1993

$7 \mathrm{p}$.

Sponsored by American Dental Association Health Foundation, Chicago, IL

Pub. in Proceedings of Materials Research Society Meeting: Hydroxyapatite and Related Materials, San Francisco, CA., April 13-15, 1993, p145-151 1994.

Keywords: "Ethanol, "Dental materials, "Solubility, Physicochemical properties, Aqueous solutions, Calcium phosphates, Reprints, "Apatite/hydroxy.

Hydroxyapatite ( $\mathrm{HA}), \mathrm{Ca} 5(\mathrm{PO} 4) 3 \mathrm{OH}$, is the prototype of the inorganic constituent found in tooth and bone. Its physico-chemical properties have wide biological and industrial importance. Hence, its solubility in aqueous solutions has been extensively studied. Although ethanol has been known to increase the association constants of ionic compounds and decrease their solubilities, the effect of ethanol on the solubility of $\mathrm{HA}$ has not been studied. Recently, ethanol has been reported to affect the precipitation of HA and other calcium phosphates. This effect of ethanol may be due to the increase in precipitation rate or the effect on the solubility of HA or both. Therefore, the purpose of this study is to investigate the effect of ethanol on the solubility of $H A$

\section{0,170}

PB95-175261 Not available NTIS

National Inst. of Standards and Technology (MSEL) Gaithersburg, MD. Polymers Div

Formation of Hydroxyapatite in Cement Systems. Final rept.

L. C. Chow, S. Takagi, and K. Ishikawa. 1994, 11p.

Contract NIH-DE05030

Sponsored by American Dental Association Health Foundation, Chicago, IL

Pub. in Proceedings of Conference on Hydroxyapatite and Related Materials, San Francisco, CA., April 13 15, 1993, p127-1371994.

Keywords: "Dental cements, "Sealing compounds, "Physicochemical properties, Calcium phosphates, Acid bonded reaction cements, Biocompatible materials, Sealants, Surface chemistry, Hardness, Physical properties, Dental materials, Reprints, "Hydroxyapatite, "Cement systems, Self setting.

Among the self-setting calcium phosphate cement systems, those cements that consist of tetracalcium phos phate (TTCP) and dicalcium phosphate anhydrous (DCPA) or dicalcium phosphate dihydrate (DCPD) form OHAp as the only reaction product. The paper reports the effects of the TTCP/DCA ratio and the phosphate concentration in the cement liquid on the formation of OHAp in the TTCP+DCPA system. Also reported is the formation of OHAp in new cement compositions comprising TTCP and alpha-tricalcium phosphate (alpha-TCP), octacalcium phosphate (OCP), amorphous calcium phosphate (ACP), or monocalcium phosphate monohydrate (MCPM).
National Inst. of Standards and Technology (MSEL), Gaithersburg, MD. Polymers Div.

Physical and Chemical Properties of Resin-Reinforced Calcium Phosphate Cements.

Final rept.

S. Dickens-Venz, S. Takagi, L. C. Chow, B. Dickens,

R. L. Bowen, and A. D. Johnston. 1994, 7p.

Sponsored by American Dental Association Health Foundation, Chicago, IL.

Pub. in Dental Materials 10, p100-106 Mar 94

Keywords: "Dental materials, "Calcium phosphates "Dental cements, "Physical properties, *Chemica properties, Dental pulp capping, Dental pulp cavity, Dental cavity lining, Hydroxyapatites, Chemical reac tions, Monomers Hydrophylic polymers, Reprints, "Self-setting, Apatite/hydroxy, Cement polymer composites.

The purpose of this study was to improve the handling and physical properties of a self-setting, water-base calcium phosphate cement by combining it with polymerizable resins and to study the setting reactions involved. Dual-cured composite cements were prepared from a calcium phosphate cement powder and dental monomers that contain carboxylated hydrophilic resins or resin/water mixtures. The hydrophilic acidic resins allows mixing with water and/or allow rapid diffusion of water into the resinous cement so that the oissolution and reprecipitation processes required for the conversion of the calcium phosphate components to hydroxyapatite can occur. The characteristics of the resulting composite cements suggest that the materials may be useful in pulp capping and/or cavity lining.

\section{0,172}

PB95-180626 Not available NTIS

National Inst. of Standards and Technology (MSEL) Gaithersburg, MD. Polymers Div.

Diagnosis and Treatment of an Oral Base-Metal Contact Lesion Following Negative Dermatologic Patch Tests.

Final rept.

W. A. Lyzak, C. M. Flaitz, R. S. McGuckin, F.

Eichmiller, and R. S. Brown. 1994, 5p.

Sponsored by American Dental Association Health Foundation, Chicago, IL

Pub. in Annals of Allergy 73, p161-165 Aug 94.

Keywords: "Dental materials, "Immunologic diseases, Allergic diseases, "Nickel alloys, Base metal, Reprints.

We report a confirmed case of intraoral contac mucositis secondary to nickel dental alloy hypersensitivity. The lesion resolved after remova! of the offending prosthesis. The patient responded nagatively to dermatologic patch tests, but a positive intraoral rechallenge confirmed the mucositis diag nosis. A nonreactive, gold alloy prosthesis was in serted for a successful result.

00,173

PB95-180642 Not available NTIS

National Inst. of Standards and Technology (MSEL), Gaithersburg, MD Polymers Div.

Formation of Hydroxyapatite in a Polymeric Calcium Phosphate Cement.

Final rept.

Matsuya, S. Matsuya, J. M. Antonucci, S. Takagi, and L. C. Chow. 1994, 2p.

See also PB95-175261. Sponsored by American Dental Association Health Foundation, Chicago, IL

Pub. in Proceedings of International Conference on Composites Engineering, New Orleans, LA., August 28-31, 1994, p861-862

Keywords: "Dental cements, "Calcium phosphates, "Polymers, "Hydroxyapatites, $X$ ray diffraction, Chemical reactions, Reprints, Apatite/hydroxy, Poly(maleic acid-ether/methyl vinyl), Tetracalcium phosphate, Dicalcium phosphate anhydrous.

Recently it was shown that the reaction of aqueous solutions of poly(alkenoic acids) with mixtures of tetracalcium phosphate (TTCP) and dicalcium phos hate anhydrous (DCPA) formed strong polymeric calcium phosphate cements. Surprisingly, $x$-ray diffraction XRD) analysis of these cements, even after prolonged storage in water, failed to reveal the presence of hydroxyapatite (OHAp). In this study poly(methyl vinyl ether-maleic acid), (PMVE-Ma) was reacted with only TTCP to form polymeric calcium phosphate cements. TTCP samples ground for different lengths of time were used as the base powder. Grinding enhanced the reactivity of TTCP with PMVE-Ma and yielded cements with higher diametral tensile strengths than similar cements formulated with unground TTCP. XRD analysis of the ground TTCP/PMVE-Ma cements also showed the presence of significant amounts of OHAp.

00,174

PB'96-102405 Not available NTIS

National Inst. of Standards and Technology (MSEL), Gaithersburg, MD. Polymers Div.

Reduction of Marginal Gaps in Composite Restorations by Use of Glass-Ceramic Inserts.

Final rept.

L. A. George, N. D. Richards, and F. C. Eichmiller $1995,4 p$

Sponsored by American Dental Association Health Foundation, Chicago, IL.

Pub. in Operative Dentistry 20, p151-1541995.

Keywords: "Dental materials, "Inserts, "Composite materials, "Shrinkage, Glass, Ceramics, Polymerization, Monomers, Gaps, Teeth, Reprints.

The objective of this study was to evaluate the effects of glass-ceramic inserts on reducing the marginal gaps caused by polymerization shrinkage in composite restorations. A light microscope was used to measure the largest gap at margins around restorations made in glass cylinders and tooth cavities with and without adhesion promoters. Where the cylinder was not silanated, the average gap was less in samples containing an insert than in those without. Two preparations were made in the dentin of 20 human molars. In each molar one cavity was restored with a dentin bonding agent and composite and the other with a dentin bonding agent and an inserted seated in the composite. The average maximum gap width of restorations containing inserts was statistically less than for those with only composite (paired t-test, $P<0.0001$ ). When considering the volume of composite displaced by the insert, these results indicate that the use of a glassceramic insert decreased the marginal gaps resulting from polymerization shrinkage.

00,175

PB96-122536 Not available NTIS

National Inst. of Standards and Technology (MSEL), Gaithersburg, MD. Polymers Div.

Failure of All-Ceramic Fixed Partial Dentures 'In vitro' and 'In vivo': Analysis and Modeling.

Final rep

J. R. Kelly, J. A. Tesk, and J. A. Sorensen. 1995, 6p. Pub. in Jnl. of Dental Research, v74 n6 p1253-1258 1995

Keywords: "Dental materials, "Failure analysis, In vitro analysis, Reprints, Prosthodontics

Hertzian cone cracks visible at the loading site of 20 all-ceramic fixed partial dentures (FPDs), tested in vitro, led to the hypotheses that failure was due to the propagation of localized contact damage crack systems (Hertizian stress state) and that such damage was an unlikely clinical failure mode. Fractographic analysis of the 20 laboratory-failed and nine clinicallyfailed all-ceramic FPDs allowed for definitive testing of these hypotheses and a comparison between in vitro and in vivo failure behavior. In all cases, failure occurred in the FPD connectors (none from contact damage), with approximately 70 to $78 \%$ orginating from the interface between the core and veener ceramics. The coincidence between failure origins provides strong evidence that the in vitro test modeled aspects of structural behavior having clinical importance. The ractographic observations, coupled with the in vitro failure load data, furnished very specfic boundary conditions which were applied to constrain mathematical models of FPD connector failure. Finite element analysis (FEA) of the laboratory PPDs found that maximum principal tensile stresses would occur at locations consistent with the fractographic observations only if (1) there were appropriate elastic moduli differences be tween the ceramics; and (2) a small amount of abutment rotation was allowed. Weibul! failure probability (Pf) calculations, incorporating FEA stress profiles, very closely replicate the veneer interface was much lower than that for the free veneer surface (i.e, the interface is of lower quality with regard to defects).

00,176

PB96-122635 Not available NTIS

National Inst. of Standards and Technology (EEEL), Gaithersburg, MD. Electricity Div.

Overview of Bioelectrical Impedance Analyzers.

Final rept.

N. M. Oldham. 1994, 3p.

Pub. in Proceedings of the Technology Assessment Conference on Bioelectrical Impedance Analysis in 


\section{Prosthetics \& Mechanical Organs}

Body Composition Measurement, NIH, Bethesda, MD., December 12, 1994, p1-3.

Keywords: "Bioinstrumentation, "Electrical impedance, "Body composition, Humans, Algorithms, Lipids, Bodywater, Height, Body weight, Age, Physical fitness, Electrical measuring instruments, Reprints, "Bioelectrical impedance analyzers, BIAs, Body fat.

The class of instruments referred to as bioelectrical impedance analyzers (BIAs) are designed to measure human body impedance-a parameter that has been used to estimate body composition based on algonithms that also include height, weight, gender, age, and level of physical activity. The algorithms have been described in the literature, as have evaluations of commercial BIAs on human subjects. This paper describes a project to investigate the properties of these instruments as electrical impedance meters.

00,177

PB96-122940 Not available NTIS National Inst. of Standards and Technology (MSEL),
Gaithersburg, MD. Polymers Div.

Dental Applications of Ceramics.

Final rept.

L. A. George, and F. C. Eichmiller. 1995, 16p.

Pub. in Proceedings of American Ceramic Society, Bioceramics: Materials and Applications, Indianapolis, IN., April 24-28, 1994, v48 p157-172.

Keywords: "Dental materials, "Biocompatible materials, "Reconstruction, Ceramics, Porcelain, Glasses, Dental cements, Temporary dental restoration, Permanent dental restoration, Teeth, Reprints.

Properties of ceramics that make them appropriate for use in dental applications include compressive strength, durability, radiopacity, and inertness towards the oral environment. The principle dental use of ceramics is in the aesthetic restoration of missing teeth or tooth structure. Aesthetic dental ceramics are primarily manufactured from two classes of materials, beneficiated feldspathic minerals, and glass-ceramics. Processing restorations with feldspathic materials generally involves stacking powder-water slurries upon a metal framework and subsequent sintering. Glass-ceramics used in restorative parts involves the casting or forming of complex shapes that are then cerammed.

00,178

PB96-123229 Not available NTIS

National Inst. of Standards and Technology (MSEL), Gaithersburg, MD. Polymers Div.

Properties and Mechanisms of Fast-Setting Calcium Phosphate Cements.

Final rept.

K. Ishikawa, S. Takagi, L. C. Chow, and Y. Ishikawa. 1995, 6p.

Pub. in Jnl. of Materials Science: Materials in Medicine, v6 p528-533 1995.

Keywords: "Dental cements, "Calcium phosphates, Phosphate additive, Tetracalcium phosphate, Reprints, Dicalcium phosphate, Fast setting cement, Hydroxyapatite.

The setting time of a calcium phosphate cement consisting of tetracalcium phosphate (TTCP) and dicalcium phosphate anhydrous (DCPA) was reduced from 30 to 5 min by use of a cement liquid that contained a phosphate concentration of $0.25 \mathrm{~mol} / \mathrm{l}$ or higher. The diametral tensile strength and conversion of the cement ingredients to hydroxyapatite (OHAp) during the first $3 \mathrm{~h}$ were also significantly increased by the phosphate. However, the phosphate produced no significant effects on the properties of the 24-h cement samples. Results from additional experiments in a slurry system verified that the high phosphate concentration in the solution accelerated the formation of OHAp in the TTCP-DCPA system, and this reaction could explain the fast-setting properties of the cements.

00,179

PB 96-158001 Not available NTIS

National Inst. of Standards and Technology (MSEL), Gaithersburg, MD. Polymers Div.

Reinforcement of Cancellous Bone Screws with Calcium Phosphate Cement.

Final rept.

L. E. Mermelstein, L. C. Chow, C. Friedman, and J. J. Crisco. 1996, 6p.

Pub. in Jnl. of Orthopaedic Trauma, v10 n1 p15-20 1996.

Keywords: "Cancellous bone, "Biomaterials, Reprints, "Calcium phosphate cement, "Screw fixation.
The ability of calcium phosphate cement (CPC) to reinforce cancellous screws placed in previously stripped holes was studied in vitro. The distal end of canine femurs were harvested. A total of fifteen screws were placed in six femurs. The pullout strength (failure force), failure displacement, stiffness and energy absorbed were determined for the screws in the intact cancellous bone. Next, these stripped screw holes were packed with CPC. The pullout test was repeated and the results were compared using a paired, Student's $t$ test. We found that the CPC was able to reinforce the previously stripped holes and significantly increase the pullout strength (1159 plus or minus 278 $\mathrm{N}$ vs. 678 plus or minus $297 \mathrm{~N})$ and the stiffness $(1,900$ plus or minus $569 \mathrm{~N} / \mathrm{mm}$ vs. 1,519 plus or minus 609 $\mathrm{N} / \mathrm{mm}$ ) of the constructs, as well as the energy absorbed by the constructs until failure ( 467 plus or minus $180 \mathrm{~N} / \mathrm{mm}$ vs. 278 plus or minus $140 \mathrm{~N} \mathrm{~mm}$ ). There was no difference in the failure displacement $(0.94$ plus or minus 0.23 vs. 0.85 plus or minus $0.51 \mathrm{~mm}$ ). This study documents the ability of CPC to acutely reinforce cancellous bone screws in a region with no or poor quality cancellous bone

\section{0,180}

PB96-164264 Not available NTIS

National Inst. of Standards and Technology (MSEL) Gaithersburg, MD. Polymers Div.

Polymeric Calcium Phosphate Cements Derived from Poly(methyl vinyl ether-maleic acid).

Final rept.

Y. Matsuya, J. M. Antonucci, S. Matsuya, S. Takagi, and L. C. Chow. 1996, 6p.

Pub. in Dental Materials, v12 p2.7 Jan 96

Keywords: "Dental materials, "Acid bonded reaction cements, "Polybasic organic acids, "Calcium phosphates, Cements, Reprints.

The purpose of this study was to assess the feasibility of forming polymeric calcium phosphate cements from a mixed powder of dicalcium phosphate/tetracalcium phosphate or only tetracalcium phosphate and poly(methyl vinyl ether-maleic acid) (PMVE-Ma), and to study their setting reaction. The setting reaction process of the polymeric cements was evaluated by mechanical strength tests, infrared spectroscopy and $x$-ray diffraction analysis and compared with that of a water-setting calcium phosphate cement. The machanical strength data were analyzed using ANOVA and Scheffe's multiple comparisons test.

00,181

PB97-118780 Not available NTIS

National Inst. of Standards and Technology (EEEL) Gaithersburg, MD. Electricity Div.

Overview of Bioelectrical Impedance Analyzers.

Final rept.

N. M. Oldham. $1996,8 p$.

See also PB96-122635.

Pub. in American Jnl. of Clinical Nutrition, v64 n3 p405S-412S Sep 96.

Keywords: *Bioinstrumentation, "Electrical impedance, "Body composition, Humans, Algorithms, Liquids, Bodywater, Physical fitness, Electrical measuring instruments, Reprints, "Bioelectrical impedance analyzers, Body fat.

Six commercial bioelectrical impedance analyzers were evaluated to determine their accuracy as impedance meters, their sensitivity to contact impedance, and other operating parameters such as maximum current amplitude and test waveform. Over a range of impedances that simulate human body impedance, analyzer errors varied from less than $1 \%$ to nearly $20 \%$ Larger errors were observed when the contact impedance was at the limits of the operating range of the analyzer. Body models, sources of error, and severa simple tests that the user can perform are also discussed.

\section{Bionics \& Artificial Intelligence}

00,182

PB94-172277 Not available NTIS

National Inst. of Standards and Technology (CSL), Gaithersburg, MD. Advanced Systems Div.
Analysis of a Biologically Motivated Neural Network for Character Recognition.

Final rept.

M. D. Garris, R. A. Wilkinson, and C. L. Wilson

1991, 16p.

Pub, in Conference of Analysis of Neural Network Ap plications, ANNA-91, Fairfax, VA., May 29-31, 1991, p160-175.

Keywords: "Character recognition, "Neural nets, Image processing, Feature extraction, Pattern recognition, Fonts, Accuracy, Reprints, Back propagation, Gabor functions.

A neural network architecture for size and local shape invariant digit recognition has been developed. The network is based on known biological data on the structure of vertebrate vision but is implemented using more conventional numerical methods for image feature extraction and pattern classification. The input receptor field structure of the network uses Gabor function feature selection. The classification section of the network uses back propagation. Using these features as neurode input, an implementation of back propagation on a serial machine achieved $100 \%$ accuracy when trained and tested on a single font size and style while classifying at a rate of $2 \mathrm{~ms}$ per character.

00,183

\section{PB95-220505 PC A03/MF A01}

National Inst. of Standards and Technology (MEL), Gaithersburg, MD. Intelligent Systems Div.

Texture-Independent Vision-Based Closed-Loop Fuzzy Controllers for Navigation Tasks.

S. R. Kundur, and D. Raviv. Apr 95, 40p, NISTIR-

5637

Grant NSF-IRI-9115939

Prepared in cooperation with Florida Atlantic Univ. Boca Raton. Sponsored by National Science Foundation, Arlington, VA. Div. of Information, Robotics and Intelligent Systems.

Keywords: "Fuzzy logic, "Computer vision "Controllers, "Autonomous navigation, Computerized simulation, Cameras, Image processing, Visual perception, Real time operations.

The paper deals with vision-based closed-loop contro schemes for collision avoidance as well as maintenance of clearance in a-priori unknown textured environments. These control schemes are base on fuzzy logic and employ a visual motion cue, they call the Visual Threat Cue (VTC) that provides some measure for relative change in range as well as clearance between 3D surface and a fixated observer in motion. II is a collective measure obtained directly from the raw data of gray level images, is independent of the 3D surface texture and needsno optical flow information, 3D reconstruction, segmentation, feature tracking or preprocessing. This motion cue is scale-independent, rotation independent and is measure in (time-1) units. Design of a closed-loop conventional controller for vision based navigation tasks pose a problem as the system is complex and ill-defined. On the other hand fuzzy control which is closer in spirit to human thinking and can implement linguistically expressed heuristic contrl policies directly without any knowledge about the dynamics of the complex process. The fuzzy controllers were tested in real time using a 486-based Personal Computer and a camera capable of undergoing 6 -DOF motion. Results are highly encouraging.

\section{Prosthetics \& Mechanical Organs}

00,184

PB94-172863 Not available NTIS

National Inst. of Standards and Technology (MSEL) Gaithersburg, MD. Polymers Div.

Physicochemical Properties of Calcific Deposits Isolated from Porcine Bioprosthetic Heart Valves Removed from Patients Following 2-13 Years Function.

Final rept

B. B. Tomazic, W. E. Brown, and F. J. Schoen. 1994 $13 p$.

Pub. in Jnl. of Biomedical Materials Research 28, p3547 1994. Sponsored by American Dental Association Health Foundation, Chicago, IL

Keywords: "Calcium phosphates, "Heart valve prosthesis, "Prosthesis failure, "Chemical properties, "Physical properties, Humans, Mineralization, Fourie 


\section{Prosthetics \& Mechanical Organs}

transform infrared spectroscopy, Scanning electron microscopy, X-ray diffraction, Chemical analysis, Swine, Reprints.

The purpose of the study was to characterize the physicochemical properties of calcific deposits that cause the failure of tissue-derived heart valve bioprosthese. This was done in an effort to understand the mecha nism of pathologic biomineralization in the cardiovascular system and potentially prevent deterioration of bioprosthese. Calcific deposits taken from 10 failed bioprosthetic valves that had been implanted in patients for 2-13 years were characterized by chemica analysis, x-ray diffraction, FTIR spectroscopy, scanning electron microscopy, polarized light microscopy and solubility measurements. The combined results identified the biomineral as an apatitic calcium phosphate salt with substantial incorporation of sodium, magnesium and carbonate. The average Ca/PO4 ratio for this young pathologic biomineral was approximately 1.3 , considerably lower than approximately 1.7 found in mature atherosclerotic plaque biomineral and mature skeletal biomineral, both of which approximate hydroxyapatite in composition. Deproteinated calcific deposits from bioprosthese had thermodynamic solubilities comparable to those of both atherosclerotic plaque, typical pathologic biomineral and hydrolyzed octacalcium phosphate, a proposed precursor phase to biomineral apatite. This suggests an approach to ward prevention of bioprosthetic tissue calcification through control of the formation of the kinetically favored OCP precursor and/or its transformation into bioapatite

\section{5}

PB94-185238 Not available NTIS

National Inst. of Standards and Technology (MSEL) Gaithersburg, MD. Polymers Div.

Tapered Cross-Pin Attachments for Fixed Bridges Final rept.

F. C. Eichmiller, and E. E. Parry. 1994, 4p.

Pub. in Operative Dentistry 19, p7-10 1994. Sponsored by American Dental Association Health Foundation Chicago, IL.

Keywords: "Dental prosthesis design, Dental cements, Partial denture, Alignment, Pins, Reprints.

The design and fabrication of multi-unit fixed pros theses where abutment teeth are misaligned have been difficult te thnical challenges for dentists and dental technicians. There have been a number of methods developed to attain a common path of insertion, includ ing modified preparation designs, telescopic copings placed on abutments to correct alignment, adhesively retained bridge frameworks, and mechanical precision attachments between sections of a segmented prosthesis. This paper describes a method of fabricating a precision attachment on a segmented prosthesis that can be rigidly fixed after cementation. The technique involves the use of a cast tapered pin to permanently attach the prosthesis segments together. All parts can be fabricated by conventional lost-wax techniques with a minimum of instrumentation. The technique has the added advantage of being reversible should the pros thesis ever need to be separated for repair or recementation.

\section{0,186}

PB95-150959 Not available NTIS

National Inst. of Standards and Technology (MSEL) Gaithersburg, MD. Polymers Div.

Critical Evaluation of the Purification of Biominerals by Hypochlorite Treatment

Final rept.

B. B. Tomazic, W. E. Brown, and E. D. Eanes. 1993, $9 p$.

Sponsored by American Dental Association Health Foundation, Chicago, IL.

Pub. in Jnl. of Biomedical Materials Research 27, p217-225 1993

Keywords: *Heart valve prosthesis, "Purification, "Mineralization, Extraction, X-ray diffraction, Crystallization, Calcification, Performance evaluation, Com parison, Reprints, "Hypochlorite treatment, "Deproteination, *Hydrazine treatment.

The quantitative deproteination of calcific deposits from surgically explanted heart valve bioprostheses was carried out by both hypochlorite and hydrazine extraction to establish which is better procedure for preparing purified mineral suitable for detailed chemical and structural characterization. Hypochlorite treatment resulted in a material with a higher $\mathrm{Ca} / \mathrm{PO} 4$ ratio than that of the untreated deposits. The hydrazine treatment did not produce such an effect. A systematic comparison of $x$-ray diffraction patterns of calcific deposits showed an increase in crystallinity of hypochloritetreated versus native material, while the crystallinity of hydrazine-treated materials did not change. The deproteination is preferable to hypochlorite extraction in isolating pathologic mineral deposits from bioprosthetic materials for further study.

\section{0,187}

PB96-156039 Not available NTIS

National Inst. of Standards and Technology (MSEL), Gaithersburg, MD. Polymers Div.

Physicochemical Characterization of Natural and Bioprosthetic Heart Valve Calcific Deposits: Implications for Prevention.

Final rept.

B. B. Tomazic, W. E. Edwards, and F. J. Schoen.

1995, 6p.

See also PB94-172863. Sponsored by American Dental Association Health Foundation, Chicago, It.

Pub. in Proceedings of the International Symposium Cardiac Bioprostheses (6th), Vancouver, Canada, July $29-31,1994$, The Annals of Thoracic Surgery, v60 pS322-S327 1995 .

Keywords: "Heart valve prothesis, "Prothesis, Cardio vascular system, Precursors Solubility, Reprints, "Foreign technology, Bioapatities, Calcific deposits, Octacalcium phosphate hydrolyzate.

This investigation was performed to provide a com prehensive physicochemical characterization of clacific deposits (CDs) that form on human heart valves under various pathological conditions. The authors examined and characterized $\mathrm{C}[$ ss associated with aortic stenosis on congenitally bicusipid vállves $(n=10)$, degenerative aortic stenosis on valves with previously normal anatomy $(n=10)$, an Rheumatic aortic $(n=10)$ and mitra $(n=10)$ stenosis. Native and deproteinated CDs underwent chemical analysis and structural characterization, whereas deproteinated CDs were measured for thermodynamic solubility. The CDs in valvular heart disease were microcrystalline apatitic products containing substantial amounts of sodium, magnesium, carbonate, fluoride, and organic fraction.

\section{0,188}

PB97-113120 Not available NTIS

National Inst. of Standards and Technology (MSEL), Gaithersburg, MD

International Standards and Reference Materials.

Final rept.

J. A. Tesk. 1996, 4p.

See also PB90-217894.

Pub. in Proceedings, Conference on Implant Retrieval and Analysis, Buffalo, NY., June 2-3, 1996, p1-4

Keywords: "Standards, Biocompatible materials, Certification, Interlaboratory comparisons, Chemical analysis, Reprints, "Standard reference materials, "Medical implants, Implant retrieval.

International organization for Standardization Definitions of reference materials and NIST definitions are given and compared. The question of needs for special reference materials for retrieved implant analysis is raised for discussion.

\section{Protective Equipment}

00,189

PB95-151171 Not available NTIS

National Inst. of Standards and Technology (MSEL), Gaithersburg, MD. Polymers Div.

NIR-Spectroscopic Investigation of Water Sorption Characteristics of Dental Resins and Composites. Final rept.

S. Venz, and B. Dickens. 1991, $18 p$.

Sponsored by American Dental Association Health Foundation, Chicago, IL

Pub. in Jnl. of Biomedical Materials Research 25 p1231-1248 1991

Keywords: *Dental materials, "Composite materials, *Water, "Absorption, Moisture content, Sorption, $\mathrm{Hy}$ drogen bonds, Reprints, "Near infrared spectroscopy.

A near infrared (NIR) method using the $5200 / \mathrm{cm}$ absorption of water has been employed to examine water absorbed in photopolymerized dental resins and com- posites in the form of $0.01 \mathrm{~cm}$ - to $0.15 \mathrm{~cm}$-thick specimens. Water sorption was determined gravimetrically and correlated to the absorbance in the NIR spectrum. The NIR absorptivity, epsilon, of water absorbed in a polymeric medium was found to be inversely related to the degree of hydrophilicity and hydrogen bonding capability of the polymer. The presence of water clusters in a polyethylene oxide methacrylate polymer was inferred from convex-up curvature in the plot of epsilon vs. water content.

00,190

PB96-190301 Not available NTIS

National Inst. of Standards and Technology (EEEL), Boulder, CO. Optoelectronics Div.

Optical Density Measurements of Laser Eye Protection Materials.

Final rept.

T. R. Scott, R. J. Rockwell, and P. Batra. 1993, top.

Pub. in Proceedings of the International Laser Safety Conference, Cincinnati, $\mathrm{OH}$., December 1-4, 1992, p87-8-16 1993.

Keywords: "Laser hazards, "Eye safety, "Optical density Laser beams, Laser safety, Protective equipment, Optical filters, Optical properties, Vision, Configurations, Wavelengths, Reprints, "Protective eyewear, Eye protectors.

Various types of protective eyewear are available for use in shielding the user's eyes from the harmful effects of intense laser radiation. Proper protective eyewear is chosen by selecting materials whose inherent transmission characteristics are appropriate for the expected laser operating conditions (e.g. wavelength, power level, etc). For convenience, the transmittance values of protective eyewear are typically expressed in terms of optical density (i.e. the logarithm to the base 20 of the reciprocal of the transmittance) for specific laser wavelengths. These density values are usually stamped or printed on the eyewear assemblies. The paper describes the measurement methods and results of the optical density measurements performed on designated protective eyewear samples at several specific laser wavelengths. The measurement configurations and associated measurement error sources will also be reviewed.

\section{BUILDING INDUSTRY TECHNOLOGY}

\section{General}

00,191

PB94-145976 PC A04/MF A01

National Inst. of Standards and Technology (BFRL), Gaithersburg, MD.

Use of Computer Models to Predict Temperature and Smoke Movement in High Bay Spaces. K. A. Notarianni, and W. D. Davis. Dec 93, 64p NISTIR-5304.

Sponsored by National Aeronautics and Space Administration, Greenbelt, MD. Goddard Space Flight Center. and Public Buildings Service, Washington, DC. Keywords: "Bays(Structural units), "Fire tests, *Computerized simulation, Warehouses, Smoke, Industrial buildings, Plumes, Hangars.

Large spaces, such as those found in warehouses, historical buildings, atriums, and aircraft hangars, represent some of the most difficult fire protection challenges since they are frequently of historical significance, contain large quantities of fuel, have unique geometries, and/or present special life safety problems. Accurate detector activation predictions are important in these large spaces, as timely detection of a fire is more difficult due to the distance heat and products of combustion must travel to reach sprinklers and detectors. The Building and Fire Research Laboratory (BFRL) was given the opportunity to make measurements during fire calibration tests of the heat detection system in an aircraft hangar with a nominal $30.4 \mathrm{~m}$ (100 ft) ceiling height near Dallas, TX. Three closeddoor tests were conducted. 
00,192

PB94-164191 PC A03/MF A01

National Inst. of Standards and Technology (BFRL), Gaithersburg, MD.

BFRL Fire Publications, 1993.

N. H. Jason. Apr $94,38 \mathrm{p}$, NISTIR-5397.

See also PB94-121050.

Keywords: "Fire tests, "Bibliographies, Combustion, Smoke, Soot, Fire hazards, Fire protection, Technology transfer, Carbon monoxide, Models, "Building fires.

The document contains references to the publications prepared by the members of the Building and Fire Research Laboratory (BFRL) fire research staff, by other National Institute of Standards and Technology (NIST) personnel for BFRL, or by external laboratories under contract or grant from the BFRL during the calendar year 1993.

\section{0,193}

PB94-199270 Not available NTIS

National Inst. of Standards and Technology (BFRL) Gaithersburg, MD. Fire Safety Engineering Div.

Analysis of the Happyland Social Club Fire with HAZARO I.

Final rept.

R. W. Bukowski, and R. C. Spetzler. 1992, 14p.

Pub. in Jnl. Fire Prot. Engr. 4, n4 p117-130 1992

Keywords: "Fire hazards, "Buildings, Fires, Fire safety, Egress, Fire protection, Safety engineering, Sprinklers, Finishes, Reprints, "Happyland Social Club Fire, HAZ ARD I model, Fire analysis.

The paper presents the reconstruction of the Happyland Social Club fire using the HAZARD I fire hazard assessment method along with an examination of four potential mitigation strategies: automatic sprinklers, a door at the base of the stairway to the second floor, a second means of egress from the second floor or a noncombustible interior finish. The paper concludes that the traditional second means of egress might have not eliminated the observed fatalities, and that the noncombustible interior finish or sprinkler options would, with the former being the more cost effective approach

\section{0,194}

\section{PB94-205952 PC A03/MF A01}

National Inst. of Standards and Technology (BFRL)

Gaithersburg, MD.

Fire Growth Analysis of the Fire of March 20, 1990 Pulaski Building, 20 Massachusetts Avenue, N.W. Washington, DC

H. E. Nelson. Jun 94, 42p, NISTIR-4489.

See also PB92-132984. Sponsored by Corps of Engineers, Washington, DC.

Keywords: "Office buildings, "Fires, Smoke, District of Columbia, Flames, Flashover, "Building fires, Pulask building.

An analysis of an office building fire was made using fire modeling techniques. The data to conduct the analysis was obtained through on-site inspection and inte views. The analysis describes a rapid fire developing in easily ignited boxing materials that flashed over in about six minutes from flame initiation, causing failure of the ceiling system, venting of fire products in the ple num system above the ceiling, and rapid filling of the entire flow area with smoke. The report suggests a likely source of ignition and provides analysis of the impac that several fire protection systems would have had were they pressent at the time of the fire.

\section{0,195}

\section{PB94-206356 PC A04/MF A01}

National Inst. of Standards and Technology (BFRL), Gaithersburg, MD. Fire Safety Engineering Div.

Evaluating Small Board and Care Homes: Sprinklered vs. Nons prinklered Fire Protection. S. Deal. Nov $93,63 p$, NISTIR-5302.

Sponsored by Fire Administration, Emmitsburg, MD.

Keywords: "Fire safety, "Nursing homes, Evacuating(Transportation), Computerized simulation, Sprinklers, Egress, Fire hazards.

This report studied the effectiveness of sprinklered and nonsprinklered fire protection options in small Board and Care homes. The tools used to compare the effec tiveness of these fire protection options were mathe matical fire models, experimental data and docu mented fire incidents. The mathematical models estimated fire protection effectiveness through a margin of safety analysis. The margin of safety is defined in this report as the excess time an evacuee has to reach a point of safety before that evacuee's exit path becomes untenable. The margin of safety calculations considered fire growth, detection/alarm activation, evacuee egress movement and smoke tenability analysis. Two egress movement plans were simulated; one plan reflected necessary movement in a one-exit home, the second plan reflected movement in a twoexit home. Two fast-growing, large flashover fires (with high and low CO production rates) and a small smoldering fire were modeled. Two sets of full-scale spinklered and post-flashover fire experiments, as well as 61 documented fire incidents were included in the study of fire protection system effectiveness.

\section{0,196}

PB94-501988 Diskette $\$ 250.00$

National Inst. of Standards and Technology, Gaithersburg, MD

HAZARD I Fire Hazard Assessment Method (Version 1.2) (for Microcomputers).

Software.

Jun 94, 3 diskettes, NIST/SW/DK-94/001

System: IBM PC or compatible; MS DOS 3.0+ operating system. Language: FORTRAN and Assembly. Hardware requirements: 386 processor or later with 3 megabytes of RAM. Supersedes PB92-500420.

The software is available on three $31 / 2$ inch diskettes, $1.44 \mathrm{M}$ high density.

Keywords: "Software, "Fire hazards, "Buildings, Fire damage, Fire losses, Injuries, Casualties, Fire safety, Fires, Evacuation, Building materials, Furniture, Diskettes, "Hazard assessment, HAZARD I computer program, Fire models.

A method for quantifying the hazards to occupants of buildings from fires, and the relative contribution of specific products (e.g., furniture, wire insulation) to those hazards is presented. The method, called HAZARD I, combines expert judgement and calculations to estimate the consequences of a specified fire. These procedures involve four steps: (1) defining the content, (2) defining the scenario, (3) calculating the hazard, and (4) evaluating the consequences. Steps 1,2, and 4 are largely judgemental and depend on the expertise of the user Step 3, which involves use of the extensive computer software, requires considerable expertise in fire safety practice. The heart of HAZARD $I$ is a sequence of computer software procedures which calculate the development of hazardous conditions over time, calculate the time needed by building occupants to escape under those conditions, and estimate the resulting loss of life based on assumed occupant behav. ior and tenability criteria.

00,197

PB94-501996 CP D99

National Inst. of Standards and Technology, Gaithersburg, MD

HAZARD I Fire Hazard Assessment Method, Version 1.2 (Upgrade Package) (for Microcomputers). Software.

Jun 94, 3 diskettes, NIST/SW/DK-94/002

System: MS DOS $3.0+$ operating system, 640K. Language: FORTRAN, Assembly. Hardware requirements: 386 processor or later with 3 megabytes of RAM. Available to previous buyers of PB92-500438 only. One copy per registered buyer. Supersedes PB92-500438. See also PB94-501988.

The software is on three $31 / 2$ inch DOS diskettes, 1.44M high density.

Keywords: "Software, "Fire hazards, "Buildings, Fire damage, Fire losses, Injuries, Casualties, Fire safety, Fires, Evacuation, Building materials, Furniture, Diskettes, "Hazard assessment, HAZARD I computer program, Fire models.

A method for quantifying the hazards to occupants of buildings from fires, and the relative contribution of specific products (e.g., furniture, wire insulation) to those hazards is presented. The method, called HAZARD I, combines expert judgement and calculations to estimate the consequences of a specified fire. These procedures involve four steps: (1) defining the content, (2) defining the scenario, (3) calculating the hazard, and (4) evaluating the consequences. Steps 1,2, and 4 are largely judgmental and depend on the expertise of the user. Step 3, which involves use of the extensive computer software, requires considerable expertise in fire safety practice. The heart of HAZARD $I$ is a sequence of computer software procedures which cal- culate the development of hazardous conditions over ime, calculate the time needed by building occupants to escape under those conditions, and estimate the resulting loss of life based on assumed occupant behav or and tenability criteria.

00,198

PB95-161188 Not available NTIS

National Inst. of Standards and Technology (BFRL), Gaithersburg, MD. Fire Safety Engineering Div. Locating Fire Engineering Information.

Final rept.

N. H. Jason. 1993, 4p.

Pub. in Society of Fire Protection Engineers Bulletin, p5-8 Sep/Oct 93

Keywords: "Fire research, "Information systems, "Databases, Fire protection, Fire hazards, Fire safety, Fire prevention, Fire damage, Fire fighting, Safety engineering, Fires, Online systems, Information retrieval Information dissemination, Reprints.

Various information resources are discussed FIREDOC, the online bibliographic database of the Building and Fire Research Laboratory's literature colection, is discussed in depth and its role for the fire protection engineer.

\section{0,199}

PB95-164588 Not available NTIS

National Inst. of Standards and Technology (NEL), Gaithersburg, MD. Center for Fire Research Quantitative Evaluation of Building Fire Safety New Tools for Assessing Fire and Building Code Provisions.

Final rept.

J. E. Snell. 1989, 12p.

Pub. in Proceedings of Pacific Rim Conference on Building Officials, Honolulu, HI., April 9-13, 1989, p1-

Keywords: "Fire hazards, "Building codes, "Risk assessment, Fire safety, Safety engineering, Fire prevention, Fire damage, Fire losses, Buildings, Computerized simulation, Reprints. HAZARD I computer program, Hazard assessment.

The National Institute of Standards and Technology has developed a quantitative method for evaluation of fire hazard, HAZARD I, built around a computer software package designed for use on a personal computer. This system permits the user to simulate the consequences, in terms of loss of life, injury, or ultimately property damage of a specified fire in a prescribed building. The paper outlines HAZARD I and recent improvements to it, and reports the results of trial uses of this new tool. The steps envisioned for the effective transfer of this new technology within the fire safety community are examined and the implications of it for fire and building code officials are drawn.

\section{0}

PB95-175220 Not available NTIS National Inst. of Standards and Technology (BFRL), Gaithersburg, MD. Fire Safety Engineering Div Developing Rational Performance-Based Fire Safety Requirements in Model Building Codes.

Final rept.

R. W. Bukowski, and V. Babrauskas. 1994, 19p Pub. in Fire and Materials 18, p173-191 1994

Keywords: *Building codes, "Fire safety, "Performance standards, Fire protection, Risk analysis, Design standards, Buildings, Performance tests, Fire tests Japan, United States, Reprints.

The technical and philosophical basis for performancebased assessment of building fire performance is reviewed. A strategy for the evolutin of a performance code is described Current efforts toward the development of performance codes in the USA and Japan are reviewed. Recommendations for critical steps necessary to advance the development and acceptance of performance codes are presented. The table of conents of the Japanese risk methodology for assessing 'Article 38 equivalencies' is included in an appendix.

\section{0,201}

PB95-175238 Not available NTIS

National Inst. of Standards and Technology (BFRL), Gaithersburg, MD. Fire Safety Engineering Div. Earthquake and Fire in Japan: When the Threat Became a Reality.

Final rept.

R. W. Bukowski, and C. Scawthorn. 1994, $6 p$.

Pub. in NFPA Jnl. 88, n3 p89-92, 94, 96, May/Jun 94. 


\section{General}

Keywords: "Earthquakes, "Fires, "Emergency preparedness, "Risk analysis, "Japan, "United States, Fire hazards, Fire safety, Fire departments, Emergency
plans, Fire protection, Warning systems, Disasters, Seismic effects, Earthquake engineering, Buildings, Reprints.

San Francisco Fire Department released a report that identified problem areas encountered following the quake. Despite all of the problems, the fire department of 1989 was better prepared for a disaster than the department of 1906. The department and the city learned from the destruction and made many needed changes.

\section{0,202}

PB95-175808 Not available NTIS

National Inst. of Standards and Technology (BFRL), Gaithersburg, MD. Fire Safety Engineering Div. Fire and Smoke Control: An Historical Perspective. Final rept.

J. H. Klote. 1994, 5p

Pub. in American Society of Heating, Refrigeration and Air-Conditioning Engineers Jnl. 36, n7 p46-50 Jul 94.

Keywords: "Fire prevention, "Air flow, "Ventilation, "Historical aspects, Smoke abatement, Fire safety, Fire protection, Safety engineering, Buildings, Reprints, "Smoke control.

In commemoration of the ASHRAE Centennial, the paper is a brief history of ASHRAE's activities in fire and smoke control. The first ASHRAE fire related symposium marked the start of a period of ASHRAE fire related activity that is ongoing. This was followed by formation of a Technical Committee 5.6 on Fire and Smoke Control. In those early years, the committee also found time to develop a chapter for the ASHRAE handbook and to play a vital role in member education. In later years, the committee became well focused and highly efficient at most every aspect of technical committee effort.

\section{0,203}

PB95-217162 PC AO4/MF A01

Virginia Polytechnic Inst. and State Univ., Blacksburg. Dept. of Mechanical Engineering.

Compartment Fire Combustion Dynamics. Annual Report, September 1, 1993-September 1, 1994.

U. Vandsburger, B. Y. Lattimer, and R. J. Roby. Dec 94, 57p. NIST/GCR-95/666.

Grant NIST-60NANB1D1176

See also PB92-156744. Prepared in cooperation with Hughes Associates, Inc., Columbia, MD. Sponsored by National Inst. of Standards and Technology, Gaithersburg, MD.

Keywords: "Fires, "Combustion products, "Buildings, Carbon monoxide, Soot, Toxicity, Gases, Tests.

The overall scope of this research is to investigate the phenomena that control the generation and oxidation of compartment fire exhaust gases, specifically carbon monoxide (CO), total unburned hydrocarbons (THC) and soot, which are transported down an adjacent corridor. The first year of the investigation concentrated on the formation of exhaust gases, mainly $\mathrm{CO}$, within the compartment. In the second year, a hallway was attached to the compartment in such a manner that the gases from the compartment entered at the ceiling of the corridor. The oxidation of the exhaust gases down the hallway was studied for a corridor having no soffits at either end. During the past year three additional hallway soffit combinations were added to see the results of varying the fluid mechanics of the exhaust gases within the hallway.

\section{0,204}

\section{PB95-269817 PC A03/MF A01}

Civil Engineering Research Foundation, Washington, DC

National Construction Sector Goals: Industry Strategies for Implementation.

Jul 95, 44p, NISTIR/GCR-95/680.

Contract NIST-50SBNB56C8506

Sponsored by National Inst. of Standards and Technology (BFRL), Gaithersburg, MD.

Keywords: "Residential buildings, "Public works, ${ }^{*}$ Construction industry, Goals, Objectives, Cost engineering, Strategic analysis, Research and development, Technology transfer.

The strategy outlined in this report is an important first step towards the success of this bold national construction goals initiative for public works infrastructure. The process has begun with this initial strategy; it will be continued through the monthy (or more often) meetings of the public works oversight group, commencing in July with the first meeting of the four organizations who have stepped forward to provide the initial leadership for this vital national initiative.

\section{0,205}

PB96-102108 Not available NTIS

National Inst. of Standards and Technology (BFRL)

Gaithersburg, MD. Fire Safety Engineering Div.

Fire Codes for Global Practice.

Final rept.

R. W. Bukowski. 1995, 3p.

Pub. in Progressive Architecture, p117-119 Jun 95

Keywords: "Fire safety, "Building codes, "Design standards, Fire codes, Safety design, Safety engineering. Fire research, Fire hazards, Buildings, Design analysis, Design criteria, Risk management, Compliance, Global, Reprints.

Architecture in a world economy, with multinational clients and a global range of building materials and systems, demands fire codes based on performance. The International Council for Building Research is now working on methods to verify compliance under performance-based fire codes. Performance codes will have several advantages: code objectives clearly stated and understood by all parties, and analytical methods, data, and assumptions formallized in a single code of practice.

\section{0,206}

PB96-102116 Not available NTIS

National Inst. of Standards and Technology (BFRL), Gaithersburg, MD. Fire Safety Engineering Div.

How to Evaluate Alternative Designs Based on Fire Modeling.

Final rept.

R. W. Bukowski. 1995, 6p

Pub. in NFPA Jnl., v89 n2 p68-70, 72-74 Mar/Apr 95

Keywords: "Buildings, "Design analysis, "Fire safety, Fire research, Fire hazards, Safety design, Design standards, Safety engineering, Building codes, Fire codes, Alternatives, Regulations, Reprints, Fire models.

Fire models and other predictive methods are becoming common means of supporting the design and arrangement of building fire safety features to code officials where strict compliance with prescriptive requirements is not possible. This paper provides code officials faced with such analyses guidance on the key elements which should be addressed to ensure credibility. Guidelines are presented covering the selection of appropriate models and methods, design fires, evacuation calculations, sensitivity and uncertainty, and documentation. Some comments on establishing an independent review process for alternative design analyses and on certification of appropriate methods are included

\section{0,207}

\section{PB96-102934 Not available NTIS}

National Inst. of Standards and Technology (BFRL), Gaithersburg, MD. Fire Safety Engineering Div.

Santa Ana Fire Department Experiment at 1315 South Bristol, July 14, 1994. (Reprint).

Final rept.

A. D. Putorti, W. D. Walton, W. H. Twilley, S. Deal,

and J. C. Albers. 1995, 3p.

See also PB95-188868.

Pub. in Fire Technology, v31 n1 p62-67 1995.

Keywords: "Residential buildings, "Fire tests, Fire protection, Fire detectors, Sprinklers, Flammability, Construction materials, Temperature measurement, Fire safety, Smoke, Reprints, "Santa Ana(California)

This report of test addresses a fire experiment conducted on July 14, 1994 in a vacant single family dwelling at 1315 South Bristol Street in Santa Ana, California. Fire phenomena measured included: temperatures within various rooms, the velocity and temperature of outflowing cases, smoke detector activation times, and time to full room involvement.

\section{0,208}

PB96-102967 Not available NTIS

National Inst. of Standards and Technology (CAML), Gaithersburg, MD

Pressure Equations in Zone-Fire Modeling.

Final rept.

R. G. Rehm, and G. P. Forney, 1994, 13p.

See also PB92-238617.
Pub. in Fire Science and Technology, v14 n1-2 p61 731994

Keywords: "Fire tests, "Pressure dependence, *Ordinary differential equations, Compartment analysis, Heat transfer, Exothermic reactions, Combustion Pressure gradients, Mathematical models, Numerica analysis, Phase plane analysis, Thermodynamics, Reprints, Fire models, Zone fires, Singular perturbation analysis.

The nonadiabatic nature of low-speed combustion and fire, in which strongly exothermic reactions produce large temperture variations but only mild pressure variations, can cause difficulty when integrating zone mod els of enclosure fires. Examples of simple zone fire models are examined to illustrate the analytical nature of the problems encountered. These difficulties arise in the solution of the equations for the pressure in general enclosures because the pressure equilibrates much more rapidly than other dynamical variables. Singular perturbation methods and phase plane analyses, together with numerical integration of the nondimensionalized equations, are employed to study the stiff nature of the equations. We conclude tha many of the difficulties associated with numerical integration of zone fire models may be circumvented by appropriate analysis of the zone fire model equations.

00,209

PB96-117916 PC A04/MF A01

National Inst. of Standards and Technology (BFRL) Gaithersburg, MD.

Post-Earthquake Fire and Lifelines Workshop. Held in Long Beach, California on January 30-31, 1995. Proceedings.

Special pub.

R. M. Chung. N. H. Jason, B. Mohraz, F. W. Mowrer, and W. D. Walton. Aug 95, 56p, NIST/SP-889.

Keywords: "Earthquake damage, "Fire hazards, *Emergency planning *Meetings, Earthquakes, Fires, Damage assessment, Pipelines, Highways, Wate supply, Public utilities, Natural gas, Electric power Telecommunication, Transportation sector, Liquid fuels, Sprinkler systems, Fire damage, Fire alarm systems, Fire protection, Fire fighting, Fire suppression Fire research, Seismic design.

A postearthquake fire and lifeline workshop sponsored by the Building and Fire Research Laboratory, National Institute of Standards and Technology, was held January $30-31,1995$, in Long Beach, California. The objec tive of the workshop was to identify technology development and research needs that will be used in developing recommendations to reduce the number and se verity of postearthquake fires. The workshop participants included leaders in the fire service, fire protection engineering, codes and standards, insurance, transportation systems, and water, gas, power distribution, and telecommunication utilities systems with experience in dealing with consequences of earthquakes. The workshop participants developed a list of priority project areas where further research, technology de velopment, or information collection and dissemination would serve as a vital step in the future reduction of the losses from postearthquake fires.

00,210

PB96-146790 Not available NTIS National Inst. of Standards and Technology (BFRL),
Gaithersburg, MD. Fire Science Div.

Global Equivalence Ratio Concept and the Formation Mechanisms of Carbon Monoxide in Enclosure Fires.

Final rept

W. M. Pitts. $1995,41 p$

See also PB94-207511

Pub. in Progress in Energy and Combustion Science, v21 p197-2371995.

Keywords: "Crabon monoxide, "Fire hazards, ${ }^{\star}$ Combustion products, Compartment fires, Enclosures, Flame propagation, Combustion kinetics, Chemical reaction kinetics, Polymers, High temperature, Mathematical models, Reprints, `Global Equivalence Ratio.

This report summarizes a large number of investigations designed to characterize the formation of carbon monoxide $(\mathrm{CO})$ in enclosure fires--the most importan factor in fire deaths. It includes a review analysis of the studies which form the basis for the global equivalence ratio (GER) concept. Based on the findings, two completely new mechanisms for the formation of $\mathrm{CO}$ in addition to the quenching of a fire plume by a rich 
upper layer, which is described by the GER concept, are identified.

00,211

PB96-148119 Not available NTIS

National Inst. of Standards and Technology (BFRL), Gaithersburg, MD. Fire Safety Engineering Div. Information Resources for the Fire Community.

Final rept.

N. H. Jason. 1995, $6 p$.

Pub. in Proceedings of the International Conference of Fire Research and Engineering, Orlando, FL., September 10-15, 1995, p469-474.

Keywords: *Fire research, "Information retrieval, "Data bases, Information systems, Fire protection. Fire safe ty, Safety engineering, Information dissemination, Fire prevention, Reprints.

As information technology expands, information has become more readily available but the sources of this information have become more diverse. Within the field of fire research and engineering, it has become more challenging to find critical information because traditional sources of information have been augmented by a variety of electronic sources. Looking for specific information used to be performed by brute force, i.e. checking the library card catalog. With the explosion in computer communication, information retrieval has become an art form. The fire scientists or engineer must develop a familiarity with a wide range of old and new methods for acquiring information as well as staying abreast of latest developments. Success in the new world order of information science will go to the well informed user. An overview of print and electronic resources that are described in this paper can provide a starting point for responding to any information request.

\section{0,212}

PB96-148192 Not available NTIS

National Inst. of Standards and Technology (BFRL), Gaithersburg, MD. Fire Safety Engineering Div.

Heights of Wall-Fire Flames.

Final rept.

H. E. Mitler. 1995, 5p.

Pub. in Proceedings of the International Conference on Fire Research and Engineering, Orlando, FL., September 10-15, 1995.

Keywords: "Fire research, "Walls, "Flames, Height, Fires, Pyrolysis, Reprints, Line fire, Wall fires.

A correlation between the visible height of a flame and the power output of the flame is useful for a number of reasons. Thus, observation of a fire can permit one to estimate the rate of heat release and therefore the fuel flow rate. Again, an expression for the flame height is needed in order to calculate the upward flame spread rate on walls. It is therefore important that such an expression be reliable. The heights of flames from line burners adjacent to walls have been correlated by a number of workers.

00,213

PB96-151394 Not available NTIS

National Inst. of Standards and Technology (BFRL), Gaithersburg, MD. Building and Fire Research Lab.

Fire Safety Engineering Research in the United States.

Final rept.

J. E. Snell. 1992, 9p.

Pub. in International Fire Safely Engineering Conference: The Concepts and the Tools, Kings, Cross, Sydney, NSW, Australia, October 18-20, 1992, Session 3, p1-9.

Keywords: "Fire research, "Fire safety, "Salety engineering, Fire prevention, Fire codes, Fire losses, Research programs, Technology innovation, United States, Reprints, US NIST.

The paper describes fire research in the United States and particularly the fire research program at the $\mathrm{Na}$ tional Institute of Standards and Technology. First, it comments on the background to this program, and then describes the context in which it functions, i.e., the building regulatory and product approval systems and other fire research activities in the USA. Next, it outlines the rationale for NIST fire research and notes some of the research products and their impacts.

\subsection{4}

PB96-151402 Not available NTIS

National Inst. of Standards and Technology (BFRL), Gaithersburg, MD. Building and Fire Research Lab.
Elements of a Framework for Fire Safety Engineering.

Final rept.

J. E. Snell. 1993, 10p

Pub. in International Fire Conference (6th), Interflam '93, Oxford, England, March 30-April 1, 1993, p447456.

Keywords: "Fire safety, "Safety engineering, "Design criteria, Fire prevention, Fire codes, Fire research, Risk analysis, Decision making, Standards, Reprints

The paper lays out a framework for fire safety engineering based on scientific tools for fire safety design and decision-making. These tools include computerbased models for fire safety hazard and risk prediction, measurement methods to provide data for such methods, databases and expert systems to provide access to them, and the ultimate integration of these tools with other elements of computer-aided design, construction and conformity assessment. The paper suggests needs, roles and actions required to bring fire safety engineering to the level of sophistication enjoyed in most other areas of engineering practice. The need for international cooperation and public-private collabora tion is stressed.

\section{0,215}

PB96-154968 PC A03/MF A01

Clemson Univ., SC

Sensitivity Analysis for Mathematical Modeling of Fires in Residential Buildings.

Final rept. Oct 94-Sep 95

M. M. Kostreva. Feb $96,15 p$, NIST/GCR-95/683.

Grant NIST-NANB4D1649

Sponsored by National Inst. of Standards and Technology (BFRL), Gaithersburg, MD

Keywords: "Fires, "Residential buildings, "Mathematical models, "Sensitivity analysis, Rooms, Gases, Smoke, Parameters, Heat flux, Flame propagation, Differential equations, Fires safety.

The Building and Fire Research Laboratory engages in research and development of mathematical models of fires in residential buildings together with human egress of the building occupants. The research here analyzes the existing approach of HAZARD I, together with the likely modifications incorporated into HAZARD II (CONRAD2) and establishes a prototype sensitivity analysis equipped fire model computer program, thereby evaluating and demonstrating recently obtained results on the mathematical foundations of fire models.

\section{0,216}

PB96-155767 Not available NTIS

National Inst. of Standards and Technology (BFRL), Gaithersburg, MD. Structures Div.

Spectrum of the Stochastically Forced DuffingHolmes Oscillator.

Final rept.

E. Simiu, and M. Frey. 1993, 4p.

Pub. in Physics Letters A, v177 p199-202 1993.

Keywords: "Noise, "Chaos, Building technology, Dy namics, Stochastic processes, Structural dynamics, Reprints, "Foreign technology

The Brudsen-Holmes method of power spectrum estimation for the Duffing-Holmes oscillator is applied to the case of weak quasiperiodic excitation and then extended to weak colored noise excitation with any specified spectral density. A novel model of near-Gaussian noise is introduced to achieve this extension. The results obtained by this approach coincide with and ex tend those obtained by Stone and Holmes' application of the Fokker-Planck equation. In particular, our results confirm Stone's conjecture that the expression for the mean time between successive maxima in the case of colored noise similar to that for white noise.

\section{0,217}

PB96-156120 Not available NTIS

National Inst. of Standards and Technology (BFRL), Gaithersburg, MD. Siructures Div.

Noise-Induced Transitions to Chaos.

Final rept.

M. Frey, and E. Simiu. 1995, 16p.

Contract ONR-N-00014-93-F-0028

Sponsored by Office of Naval Research, Arlington, VA Pub. in Proceedings of the NATO Advanced Research Workshop on Spatio-Temporal Patterns in Non-Equilibrium Complex Systems, p529-544 1995.

Keywords: "Noise, *Chaos, Building technology, Dynamical systems, Reprints, Melnidov transforms, Multi- stable systems, Phase space flux. Stochastic equations

Additive noise is shown to induce chaotic motion with sensitive dependence on initial conditions in multistable dynamical systems. This establishes a fundamental connection between two fields hitherto viewed as distinct: deterministic chaos and stochastic differential equations modeling the dynamics of multistable systems. Our results for additive noise are then generalized to multiplicative noise. Using a newly introduced model of shot noise, these results for multiplicative noise are applied to the Duffing oscillator with shot noise-like dissipation.

\section{0,218}

PB96-156138 Not available NTIS

National Inst. of Standards and Technology (BFRL) Gaithersburg, MD. Structures Div

Deterministic and Stochastic Chaos.

Final rept.

M. Frey, and E. Simiu. 1993, 22p

Pub. in Computational Stochastic Mechanics, Chapter 9, p195-216 1993.

Keywords: "Chaos, "Noise, Dynamical systems, Reprints, "Foreign technology, Nonlinear systems, Shot noise, Stochastic systems.

Slochastic differential equations and classical technique related to the Fokker-Planck equation are standard bases for the analysis of nonlinear systems perturbed by noise. An alternative, complementary ap proach applicable to systems featuring heteroclinic or homoclinic orbits uses phases space flux as a measure of noise-induced chaotic dynamics. We continue our development of this method extending our previous treatment additive noise to the more general case of multiplicative noise. This extension is used with a new model of shot noise to treat the Duffing oscillator with shot noise-like dissipation.

00,219

PB96-156146 Not available NTIS

National Inst. of Standards and Technology (BFRL), Gaithersburg, MD. Structures Div.

Experimental and Numerical Chaos in Continuous Systems: Two Case Studies.

Final rept.

G. R. Cook, and E. Simiu. 1991, 4p.

Pub. in Proceedings of the ASCE Engineering Me chanics Specialty Conference, Columbus, OH., May 20-22, 1991, p786-789.

Keywords: "Chaos, "Buckled columns, Structural engineering, Reprints, Fluid elasticity, Galloping oscillators

Motivated by recent numerical investigations according to which certain types of deep-water compliant offshore structures may experience undesirable chaotic motions, two types of experimental structural systems capable of exhibiting chaotic or apparently chaotic behavior were studied. The first type of system is a harmonically forced buckled column with a concentrated mass at midspan and with pretensioned continuous springs. For this system a model with pretensioned continuous springs. For this system a model with ac ceptable predictive capabilities can be constructed if the continuous springs are represented by a sufficien number of lumped masses with discrete stiffnesses. The second type of system consists either of a galloping square bar or of a pair of parallel, elastically coupled galloping square bars. Our results suggest that the behavior of this type of system, including apparently chaotic behavior, can be described at least to a first approximation by conventional fluidelastic models. However, the predictive capabilities of such models are poor, i.e., in the present state of the art the existence of certain types of hydroelastic behavior may be alto gether missed at the design stage. The incorporation of stochastic excitations in the models to account for small flow irregularities may result in improved prediction capabilities.

00,220

PB96-156153 Not available NTIS

National Inst. of Standards and Technology (BFRL) Gaithersburg MD. Building and Fire Research Lab. Internationalization of Fire Safety Engineering Re search and Strategy.

Final rept.

J. E. Snell. 1992, 12p.

Pub. in Proceedings of the International Flre Saloty En.

gineering Conference: The Concept of Tools, Klngs

Cross, Sydney, NSW, Australia, October 18-20, 1992

Session 1, p1-12 


\section{General}

Keywords: "Fire safety, "Safety engineering, "Research programs, Fire research, Fire hazards, Fire protection, Fire suppression, Fire provention, International cooperation, Reprints.

This paper address this new era of fire safety engineering the trends and other factors driving it, what it means to how we provide fire safety in our societies, and why an international strategy for its further development is i our mutual interest. The role of the Forum for International Cooperation on Fire Research is discussed and actions are suggested.

\section{0,221}

PB96-156179 Not available NTIS

National Inst. of Standards and Technology (BFRL) Gaithersburg, MD. Fire Safety Engineering Div.

Fire Protection Engineering Tools. Simple Tools: The Equations.

Final rept.

R. W. Bukowski. 1992, $6 p$

See also PB92-132984.

Pub. in Proceedings of International Fire Safety Engineering Conference, 'The Concept and the Tools', Sydney, NSW, Australia, October 18-20, 1992, Session 4, p1-6 Oct 92.

Keywords: "Fire safety, "Software tools, "Computer programs, Fire protection, Fire hazards, Fire tests, Pisk analysis, Mathematical models, Reprints, Fire models.

The paper reviews the development and current state of calculational tools for fire safety engineering, particularly algebraic equations and simple models, in relation to the more complex zone and field models also being used in practice. Examples of some of the more common relations are presented along with a discus sion of their important limitations. Some comments on accuracy and precision are included.

\section{0,222}

PB96-156195 Not available NTIS

National Inst. of Standards and Technology (BFRL) Gaithersburg, MD. Fire Safety Engineering Div.

Review of Internatioanl Fire Risk Predictions Meth ods.

Final rept

R. W. Bukowski. 1992, 9p.

Pub. in Proceedings of International Fire Safety Engineering Conference 'The Concept and the Tools', Sydney, NSW, Australia, October 18-20, 1992, Session 7 p1-9.

Keywords: "Flame propagation, "Risk assessment, "Fire hazards, Fire damage, Fire safety, Fire research, Performance evaluation, Fire codes, Computer programs, Fire tests, Reprints, Fire models, Fire growth.

In the 1980's, computer models and other predictive methods were increasingly applied to a broad range of practical problems in fire safety. Incertainties in the models' predictions were no greater than those associated with the traditional, but much more expensive fullscale experimental studies. Seperate, multi-year research projects in Japan and the United States resulted in the publication of prototype fire hazard analy sis systems which demonstrated the ability to account for the complex interaction of the fire, building, active protection systems, occupant actions, and detailed outcomes including damage estimates and fatality counts.

\section{0,223}

PB96-159660 Not available NTIS

National Inst. of Standards and Technology (BFRL) Gaithersburg, MD. Fire Safety Engineering Div.

International Organization for Standardization: Current Activities in Fire Safety Engineering.

Final rept.

A. J. Fowell. 1993, 3p

Pub. in NFPA/SFPE Annual Meeting, Orlando, FL. May 20-28, 1993, 3p.

Keywords: "Fire safety, "Safety engineering. "Standards, Buildings, Fire tests, Risk assessment, Fire hazards, Fire protection, Fire codes, International cooperation, ISO, Reprints

The International Organization for Standardization's Technical Committee on Fire Tests on Building Components and Structures (ISO TC92) has performed a new Subcommittee, SC4 - Fire Safety and Engineering to extend the scope of the Technical Committee to include calculation methods in addition to standardized testing. This paper outlines the scope, organization, di- rection, and current progress on each of these efforts. The UK Code of Practice on the Application of Fire Engineering Principles to Fire Safety of Buildings, currently under development, is being offered as a strawman for a logical way to consider the many parameters and interactions involved in fire risk assessment.

\section{0,224}

PB96-175708 Not available NTIS

National Inst. of Standards and Technology (BFRL), Gaithersburg, MD. Fire Science Div.

Scaling Compartment Fires: Reduced- and FullScale Enclosure Burns.

Final rept.

N. P. Bryner, E. L. Johnsson, and W. M. Pitts. 1995

$6 \mathrm{p}$.

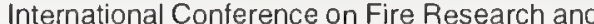
Engineering, Orlando, FL., September 10-15, 1995. p9-14.

Keywords: "Fire tests, "Combustion chemistry, ${ }^{*}$ Combustion products "Scale models, Carbon monoxide, Toxic agents, Flashover. Air flow Ventilation, Concentration(Composition), Temperature, Fuel-air ratio, Mathematical models, Reprints, "Compartment fires, Room fires.

This work extends the earlier reduced-scale enclosure (SRE) study to a full-scale enclosure (FSE) and focuses on comparing the gas concentrations and temperatures of the upper layers and the ventilation behaviors of the two compartments. The study is part of a larger research effort which is designed to develop a better understanding and a predictive capability for the generation of carbon monoxide, the major toxican in fires. The findings will be incorporated into realistic fire models and used in the development of strategies for reducing the number of deaths attributed to carbon monoxide.

\section{0,225}

\section{PB96-177373 PC A13/MF A03}

National Inst. of Standards and Technology (BFRL), Gaithersburg, MD. Fire Science Div.

Innovation in the Japanese Construction Industry: A 1995 Appraisal.

Special pub.

R. G. Gann. Mar 96, 262p, NIST/SP-898

Also available from Supt. of Docs. as SN003-003 03401-1.

Keywords: "Japan, "Construction industry, "Research and development, Technology innovation, Construc tion materials, Safety engineering, Construction management.

The objective of the project is to provide an evaluation of the state-of-the-art of the various aspects of the Japanese building and construction industry, featuring comparison with the U.S. counterparts. To accomplish this task, a team of experts was assembled in the technical areas to be included in the assessment. The ex perts were selected from the industry, academia, and research laboratories.

\subsection{6}

\section{PB96-183074 PC A08/MF A02}

National Inst. of Standards and Technology (BFRL), Gaithersburg, MD. Fire Safety Engineering Div

Publications 1995: NIST Building and Fire Research Laboratory.

Special pub.

N. H. Jason. Apr 96, 140p, NIST/SP-838/9

Also available from Supt. of Docs. as SN003-003-003 03408-8. See also PB95-270047.

Keywords: "Research laboratories, "US NIST, "Bibliographies, Research programs, Buildings, Fire safety, Standards, Documents, Hazards.

Building and Fire Research Laboratory Publications, 1995 contains references to the publications prepared by the members of the Building and Fire Research Laboratory (BFRL) staff, by other National Institute of Standards and Technology (NIST) personnel for BFRL, or by external laboratories under contract or grant from the BFRL during the calendar year 1995 .

\section{0,227}

PB96-190137 Not available NTIS

National Inst. of Standards and Technology (BFRL) Gaithersburg, MD. Fire Safety Engineering Div.

Locating Fire Information.

Final rept.

N. H. Jason. 1996, $8 p$

See also PB95-161188.
Pub. in INTERFLAM '96, International Interflam Conference (7th), Cambridge, England, March 26-28, 1996, p691-698.

Keywords: "Fire research, "Computer systems programs, "Databases, Information retrieval, Information systems, Bibliographies, Fire protection, Fire safety, Fires, Fire hazards, Online systems, Information dissemination, Reprints, Electronic resources.

Locating the best reference or answer to a specific question in fire science may be difficult There are standard reference books and journals that may be used. New sources continue to become available, in particular, electronic tools that are available internationally. This discussion will center on developing an information tool kit identifying resources from the print media, multitopic bibliographic databases, fire databases, and the World Wide Web, to enable one to find the answer from either the traditional print media or an electronic resource.

\section{0,228}

PB96-193743 PC A05/MF A01

Washington State Univ., Pullman. Dept. of Mechanical and Materials Engineering.

Development of an Economical Video Based Fire Detection and Location System.

O. A. Plumb, and R. F. Richards. Jul 96, 73p, NIST/ GCR-96/695.

Grant NIST-60NANB2D1290

Sponsored by National Inst. of Standards and Technology, Gaithersburg, MD

Keywords: "Fire detection systems, "Video recording Smoke detectors, Position(Location), Computerized simulation.

In the report we present the results of work directed toward the development of a working prototype of a system that can automatically detect, locate, and size an accidential fire. The fire detection system uses a video camera to gather temperature data from an array of temperature-sensitive, color-changing sensors distributed around a compartment and a personal computer based inverse problem solution algorithm to determine the fire's location and heat release rate using the gathered temperature data. Such a system offers great promise for use in industrial facilities such as warehouses and factor floors which combine significant fire risks with minimal human monitoring for extended periods.

00,229

PB96-195508 PC A06/MF A01

Virginia Polytechnic Inst. and State Univ., Blacksburg.

Dept of Mechanical Engineering.

Dynamics, Transport and Chemical Kinetics of Compartment Fire Exhaust Gases.

Annual rept. Sep 94-Sep 95

U. Vandsburger, and R. J. Roby. Jun 96, 77p, NIST/ GCR-96/688

Grant NANB1D1176

See also PB95-231700. Prepared in cooperation with Hughes Associates, Inc., Baltimore, MD. Sponsored by National Inst. of Standards and Technology, Gaithersburg, MD.

Keywords: "Combustion kinetics, "Thermodynamics, "Flame propagation, Gases, Smoke, Fires, Combus tion products, Burning rate, Thermochemistry, Air flow, Fire chemistry, Fire research, "Compartment fires, Hallways.

The investigation focuses on the transport of carbon monoxide (CO) away from a burning compartment and the conditions necessary for the existence of fatally high concentrations of $\mathrm{CO}$ at remote locations. During the past year, the research has concentrated on the transport of CO away from a reduced-scale burning compartment located on the side of the end of a hallway. High levels of $\mathrm{CO}$ were transported to remote locations by limiting the air entrainment into the plume of compartment fire gases entering the hallway. The non-uniform transport of combustion gases down the hallway explains the locations of fatalities in previously reported fires.

\section{0,230}

PB96-202221 PC A07/MF AO2

National Inst. of Standards and Technology (BFRL) Gaithersburg, MD. Office of Applied Economics 
Benefits and Costs of Research: Two Case Studies

in Building Technology. NISTIR-5840.

Keywords: "Buildings, "Research management, Cost benefit analysis, Case studies, Economic analysis, Construction management, Energy conservation, Life cycle costs.

This report is the outgrowth of a series of microstudies prepared by NIST's Building and Fire Research Laboratory (BFRL). This report has four major purposes. First, it examines five standardized methods for evaluFirst, it examines five standardized methods lor evalu-
ating existing and past research projects. Second, it establishes a framework for identifying, classifying, quantifying, and analyzing the benefits and costs of a research project, of a research program, or of a new technology. Third, it presents a generic format and a set of quidelines for summarizing the economic impacts of alternative research investments. Fourth, it itlustrates--by way of two case studies--how the framework and standardized methods would be applied in practice.

\section{0,231}

\section{PB96-202247 PC A04/MF A01}

National Fire Protection Association, Quincy, MA. Fire Analysis and Research Div.

Enhancement of EXIT89 and Analysis of World Trade Center Data.

Final rept.

R. F. Fahy. Jun 96, 48p, NIST/GCR-95/684.

Grant NIST-NANB4D1584

Sponsored by National Inst. of Standards and Technology (BFRL), Gaithersburg, MD.

Keywords: "Evacuation, "Fire safety, "Human behavior Fire hazards, Smoke Escape(Abandonment), Exits, Egress, Decision making, Office buildings, Surveys, Computerized simulation, "EXIT89 computer model, Emergency escape, World Trade Center, New York City(New York).

The features of an enhanced model for egress from fires in non-residential occupancies is presented along with a users manual describing the use of the model. The enhancements to the model include analysis of locations of safety, smoke blockages, disabled occupants, and delays in egress. Comparisons with some pants, and delays in egress. Comparisons with some
available field measurements is presented. Further analysis of human behavior during a fire in the World Trade Center is presented. While previous human behavior studies have shown that people will move through smoke, this incident demonstrated that people will not only move through smoke, but also through worsening conditions. Implications for evacuation and training are discussed.

\section{0,232}

\section{PB96-202288 PC A06/MF A01}

National Inst. of Standards and Technology (BFRL), Gaithersburg MD Office of Applied Economics.

Benefits and Costs of Research: A Case Study of the Fire Safety Evaluation System.

Final rept.

R. E. Chapman, and S. F. Weber. Jul 96, 93p, NISTIR-5863.

See also PB96-202221.

Keywords: "Fire safety, "Research management, Benefit cost analysis, Case studies, Investments, Buildings, Life cycle costs, Hospitals, Nursing homes.

This report focuses on a critical analysis of the economic impacts from past BFRL research efforts leading to the development and introduction of the perform. ance-based Fire Safety Evaluation System (FSES) for hospital and nursing home occupancies. The FSES was developed as an alternative to prescriptive compliance to the Life Safety Code for hospitals and nursing homes participating in the Medicare and Medicaid programs. The case study estimates the cost saving from using FSES-based modifications to promote fire safety in existing hospitals and nursing homes over the period from 1975 through 1995

00,233

PB97-114235 PC A04/MF A01

Maryland Univ., College Park. Dept. of Fire Protection Engineering

Survey of Fuel Loads in Contemporary Office Buildings.

T. C. Caro, and J. A. Milke. Sep 96, 35p, NIST/GCR96/697

Grant NIST-60NANB4D1625

Sponsored by National Inst. of Standards and Technology (BFRL), Gaithersburg, MD.
Keywords: "Office buildings, "Fire safety, Federa buildings, Combustion, Flammability, Surveys, Furniture, Books, Papers.

The method, used in the latest study performed in 1975 , for surveying offices to determine fuel load estimates is presented. The frequency distribution for the estimates of the fuel load found in the study are presented. Two methods for determining movable fuel load are utilized in this study. Movable fuel load is con sidered to be the furniture, equipment, and other items brought in for the service of the occupants after construction of the building. Direct weighing techniques are utilized in both methods. In one method, the office contents are taken from their operational location and weighed. The second method, weighs the office contents when packaged for either relocation or remodeling purposes. Surveys were conducted in buildings at the University of Maryland College Park and at the General Services Administration (GSA) Headquarters Building in Washington, D.C.

\section{0,234}

PB97-114250 PC A04/MF AO

National Inst. of Standards and Technology (BFRL), Gaithersburg, MD.

Summary of Federal Construction and Building $\mathbf{R}$ and $D$ in 1994.

S. McGaraghan. Sep 96, 38p, NISTIR-5849.

Prepared in cooperation with Department of Energy Washington, DC.

Keywords: *Federal building, "Construction, *Research and development, Tables(Data), Contracts, Databases, US DOA, US DOC, US DOD, US DOE US EPA, US DOT, US HHS, NSF(National Science Foundation)

In 1994, the federal government supported approximately $\$ 70$ billion of research and development (R\&D) in many different areas through a wide variety of government agencies and contractors. Because the work is spread among so many different entities, it can be difficult to develop a clear picture of the work being done in any one area. In response to this, a new database has been developed which tracks funding from the appropriation down to the project award level, allowing for comprehensive searches. The study, found that approximately $\$ 566$ million was spent on R\&D for construction and building in 1994. Two agencies, the Department of Defense and the Department of Energy, together account for nearly $75 \%$ of the total. In all, 12 agencies were found to have supported R\&D in this area.

\section{0,235}

PB97-114482 PC A07/MF A02

National Inst. of Standards and Technology (BFRL) Gaithersburg, MD. Fire Safety Engineering Div.

Fire Safety Engineering in the Pursuit of Performance-Based Codes: Collected Papers.

R. W. Bukowski. Oct 96, 109p, NISTIR-5878.

Keywords: "Fire safety, "Building codes, "Research and development, Safety engineering, Smoke, Fire hazards, Risk, Performance standards.

This is a collection of papers on the application of modern fire safely engineering concepts to the evaluation of building performance in the context of performancebased codes.

\section{0,236}

PB97-116081 PC A04/MF A01

Maryland Univ., College Park. Dept. of Fire Protection Engineering

Full-Scale Room Fire Experiments Conducted at the University of Maryland.

Test rept.

J. A. Milke, and S. M. Hill. 25 Jun 96, 34p, NIST/ GCR-96/703.

Sponsored by National Inst. of Standards and Technology (BFRL), Gaithersburg, MD

Keywords: "Fire tests, "Residential buildings, Furniture, Chairs, Desks, Tables, Bedding equipment, Mattresses, Flashover.

Two full size furnished bedrooms were burned, June 5 and June 6 , at the University of Maryland Fire and Rescue Institute Facilities. These burns were performed for two cooperating agencies; The Alcohol, Tobacco and Firearms Agency of the Treasury Department, who used them as part of their Certified Fire Investigator training, and the Building and Fire Research Laboratory of NIST, who used them for forensic re- search. It was intended that these two burns be identical, to see if close analysis of the results would find differences. There were differences, possibly due to small differences in the inflow of ventilation air. In both cases, ignition was caused by burning newspaper on an upholstered chair. This report describes the test arrangement and instrumented results.

00,237

PB97-116131 PC A10/MF A02

Maryland Univ., College Park. Dept. of Mechanical En-

Fire Protection Foam Behavior in a Radiative Environment.

Final rept. Sep 95-Sep 96

C. F. Boyd, and M. di Marzo. Oct 96, 184p, NIST/

GCR-96/702

Grant NIST-60NANB5D0136

Sponsored by National Inst. of Standards and Technology (BFRL), Gaithersburg, MD.

Keywords: *Fire protection, "Foam, FORTAN, Numerical analysis, Mathematical models, Thermal radiation.

The overall objective of the current research program is to identify the parameters associated with the performance of fire-fighting foams used to protect structures from heat and fire damage. Specifically, the current research focuses on the destruction of a fire-fighting foam subjected to heat radiation. A numerical model which predicts the foam's properties during the destruction process is sought. The model is developed using experimental measurements and observations as a guide. Experimental results and the numerical model are used to identify the role of the various pa. rameters which govern the foam's behavior.

00,238

PB97-116222 PC A03/MF A01

Maryland Univ., College Park. Dept. of Fire Protection Engineering.

Evaluation of Survey Procedures for Determining Occupant Load Factors in Contemporary Office Buildings.

J. A. Milke, and T. Caro. Jun 96, 25p, NIST/GCR-96/ 698

Grant NIST-60NANB4D1625

Sponsored by National Inst. of Standards and Technology (BFRL), Gaithersburg, MD.

Keywords: "Office buildings, "Fires, Furniture, Fuels, Surveys, Flammability, Federal buildings

The development of survey methods for determining the occupant load in office buildings (business occupancies) is described. Considerations involved in formulating the survey methods are presented. The type of data to be collected and data collection techniques are discussed. The two survey methods utilized to collect the population counts within contemporary office building are a building walk-through and a telephone survey. Occupant load data obtained from the survey methods applied in 23 office buildings located in the Washington, DC area are presented. Data are presented on the magnitude and distribution of the loads. The building data is sorted according to the following groups: open plan office designs versus well-compartmented office designs, and government (federal and county) versus privater sector tenants. Statistical summaries of the data are presented.

\section{Architectural Design \& Environmental Engineering}

00,239

PB94-145653 PC A07/MF A02

National Inst. of Standards and Technology (BFRL) Gaithersburg, MD. Building and Fire Research Lab. Office

Manual for Ventilation Assessment in Mechanically Ventilated Commercial Buildings.

A. K. Persily, Jan 94, 128p. NISTIR-5329.

See also PB92-145374 and PB93-113595 Sponsored by Environmental Protection Agency, Washington, DC. Office of Air and Radiation, and Bonneville Power Administration, Portland, OR.

Keywords: "Office buildings, "Air quality, "Ventilation, Space HVAC systems, Indoor air pollution, Air flow, Ducts, Comfort.

The manual describes procedures for assessing ventilation system performance and other aspects of build- 


\section{Architectural Design \& Environmental Engineering}

ing ventilation in mechanically ventilated commercial buildings. These procedures are intended to provide basic information on building ventilation for comparing ventilation performance to standards, guidelines and building design values and for investigating indoor air quality problems. The procedures in the manual are based on established measurement techniques and available instrumentation and provide practical means for obtaining reliable information on ventilation performance.

00,240

PB94-165982 PC A04/MF AO

National Inst. of Standards and Technology (CAML), Gaithersburg. MD Office of Applied Economics.

Least-Cost Energy Decisions for Buildings: Part 2. Uncertainty and Risk Video Training Workbook. H. E. Marshall. Apr 93, 53p. NISTIR-5178.

See also PB89-129522 and PB90-232810. Sponsored by Federal Energy Management Program, Washington, DC

Keywords: "Buildings, "Investments, "Economic analysis, "Energy conservation, "Risk, Training films, Decision making, Cost analysis, Financing.

This workbook accompanies the video training film Uncertainty and Risk: Least-Cost Energy Decisions fo Buildings'. The workbook and its companion video are the second in a series of training video-workbook packages designed to assist you in using economic analysis to improve the long-run economy of your buildings. The workbook supports the video in providing some of the fundamentals for measuring, describing, and inter preting uncertainty and risk in economic evaluations of energy conservation and other projects.

00,241

PB94-172160 Not available NTIS

National Inst. of Standards and Technology (BFRL) Gaithersburg, MD. Building Environment Div.

Psychological Aspects of Lighting: A Review of the Work of CIE TC 3.16.

Final rept.

B. L. Collins. $1990,10 p$

Pub. in Proceedings of Psychological Aspects of Architectural Lighting Symposium, University Park, PA., Oc tober 26-28, 1990, p1-10

Keywords: "Lighting systems, "Psychological effects, *Buildings, "Human factors engineering, Illuminance, Emotions, Glare, Architecture, Color, Brightness, Disorientation, Reprints.

The work of Commission Internationale de l'Eclairage (CIE) TC 3.16 on 'Psychological Response to Lighting' is described. The committee is charged with reviewing the literature on the psychological response to lighting and preparing a report for CIE. The committee has identified about 260 research documents, developed a framework for review. prepared an outline, abstracted about $60 \%$ of the documents and developed an initial draft. Tentative output from the draft is described in the area of preferred task illuminance, glare, adaptation level, luminance distributions, light source position and extent, light source characteristics such as colo emperature, color rendering and daylight, and dynamic temporal effects such as flicker, variation, and photobiology. A list of references is included.

\section{0,242}

PB94-173077 Not available NTIS

National Inst. of Standards and Technology (NEL) Gaithersburg, MD. Center for Fire Research.

Fire Hazard and Risk: Evaluating Alternative Technologies.

Final rept.

J. E. Snell. 1990, 20p.

Pub. in Proceedings of Symposium Sprinklers in Residential and Commercial Buildings, Charleston, SC August 19-20, 1990, 20p

Keywords: "Fire hazards, "Risk assessment, *Buildings, Fire protection, Sprinklers, Fire safety, Fire detectors, Fire resistance, Design, Reprints.

Fire safety decisions typically involve difficult choices between safety, cost and functionality. Obviously, buildings could be made completely fire safe by making sure they, and their contents simply do not burn or by providing technologies that would clearly overpower any possible fire threat. Unfortunately, few would like, could afford or would be comfortable in such surroundings. Typically, the cost and functionality aspects of designs and products are well understood and effectively communicated. However, until recently means have not been available to quantify the fire hazard or risk people face, or to measure the effectiveness of fire protection technologies in specific real world situations. The paper reviews the status and potential of new tools for evaluating fire hazard and risk, cites examples of recent applications, and offers observations relevant to the current debate on the best means to provide fire safety in residential and commercial buildings.

\section{0,243}

PB94-185766 Not available NTIS

National Inst. of Standards and Technology (BFRL), Gaithersburg, MD. Building Environment Div.

Air Change Effectiveness Measurements in Two Modern Office Buildings.

Final rept.

A. K. Persily, W. S. Dols, and S. J. Nabinger. 1994,

$16 p$.

Pub. in Indoor Air 4, p40-55 1994. Sponsored by Department of Energy, Washington, DC. Office of Buildings Energy $R$ and $D$.

Keywords: *Office buildings, *Indoor air quality, ${ }^{*}$ Tracer techniques, "Ventilation, Flow measurement, Air circulation, Air flow, Indoor air pollution, Pollution monitoring, HVAC systems, Test methods, Reprints, "Air change effectiveness.

Local age of air and air change effectiveness were determined in two office buildings using tracer gas techniques to study the applicability of the associated measurement procedures in the mechanically ventilated office buildings. Measurement issues examined include the establishment of a uniform tracer gas concentration at the start of the test and the relationship of ventilation system configuration and system operation to the test procedure. Air change effectiveness was determined at locations in the occupied space based on the local age of air at that location and the age of air in the corresponding ventilation system return duct. These tests provide data on air change effectiveness to supplement the limited database on mechanically ventilated office buildings in the U.S. In addition, the experience obtained with the measurement procedures will assist in the development of a standardized approach to measuring air change effectiveness in the field.

\section{0,244}

PB94-200383 Not available NTIS

National Inst. of Standards and Technology (NEL), Gaithersburg, MD. Building Environment Div.

Papers Presentations Shine.

Final rept.

B. L. Collins. $1990,3 p$

Pub. in Lighting Design and Application, p21-23 Oct 90.

Keywords: "Lighting systems, "Luminaires, "Energy conservation, "Energy efficiency, "Daylighting, Illuminating, Light sources, Computer graphics, Visibility, Lighting equipment, Highway lighting, Reprints.

The technical highlights from the recent IESNA Conference in Baltimore, MD are summarized in some detail. At the session 48 papers on lighting research, technology, design and application were presented. Topics discussed included light sources and conservation issues for displays; efficiency standards for lamps; daylight measurement and modeling; lamp performance including fluorescent, incandescent, metal halide high pressure sodium, and special sources; tunnel and roadway lighting; measurement and controls; modeling and lighting geometry; calculations; visibility and visua performance; computer graphics; and VDT's. Issues relating to energy conservation, such as efficiency standards, daylighting, and new lamp technology generated considerable attention, as did papers on lighting and conservation as well as computer graphics as a design tool

\section{0,245}

PB94-212735 Not available NTIS

National Inst. of Standards and Technology (NEL) Gaithersburg, MD. Building Environment Div.

Using Emulator/Testers for Commissioning EMCS Software, Operator Training, Algorithm Development, and Tuning Local Control Loops.

Final rept.

G. E. Kelly, C. Park, and J. P. Barnett. 1991, 10p.

Sponsored by Department of Energy, Washington, DC. Pub. in American Society of Heating, Refrigerating and Air-Conditioning Engineers Transactions, pt1 p6696781991
Keywords: *Buildings, *Energy management, "Control systems, Simulation, Simulators, Real time, Computer graphics, Man computer interface, Heating, Air conditioning, Algorithms, Reprints, EMCS(Energy Management and Control System), US NIST, HVACSIM+ computer program.

A Building Emulator/EMCS Tester emulates a building and its mechanical system in real time. The Emulator/ Tester is connected to the Energy Management and Control System (EMCS) in place of the regular sensors and actuators. The EMCS then controls the simulated building as it would a real building, while the Emulator/ Tester evaluates the EMCS performance. An Emulator/Tester has been developed by the National Institute of Standards and Technology (NIST). Two studies were performed. The first involved a model of a building operated in the heating mode, while the second consisted of the building air handling system sup. plying conditioned air to a single building zone during summer operation. Both studies showed good agreement between the simulation and emulation results. An exercise using the Emulator/Tester to facilitate the tuning of local control loops was performed. Two different tuning methods were explored. Information is also presented on how the Emulator/Tester can be used for training, commissioning EMCS software, and developing new control algorithms.

00,246

PB94-219367 (Order as PB94-219326, PC A05) MF A02)

National Inst. of Standards and Technology, Gaithersburg, MD. Lamps.

J. H. Walker, and A. Thompson. 1994, 7p.

Included in Jnl. of Research of the National institute of Standards and Technology, v99 n3 p255-261 May/ Jun 94.

Keywords: "Lamps, "Standards, "Radiance, Irradiance, Radiometry.

As radiometric lamp standards improve, the need to set lamp current to specific values becomes more important. Commercially available power supplies typically provide 12 bit internal digital-to-analog logic which permits current control with a relative expanded uncertainty of about 1 part in 4096 , corresponding to an ex. panded uncertainty of the current of about $2 \mathrm{~mA}$ at 8 $A$ (in this paper, expanded uncertainties are given as 2 standard deviations). For the FEL-type standard spectral irradiance lamp, this corresponds to a spectral irradiance difference of $0.12 \%$ at $655 \mathrm{~nm}$. The authors have developed a technique using 16 bit digital-to-analog conversion which permits current control with a relative expanded uncertainty of about 1 part in 65536 , corresponding to an expanded uncertainty of the current of about $0.1 \mathrm{~mA}$ at $8 \mathrm{~A}$.

00,247

PB95-108833 Not available NTIS

National Inst. of Standards and Technology (NEL) Gaithersburg, MD. Building Environment Div.

Field Measurements of Ventilation and Ventilation Effectiveness in an Office/Library Building.

Final rept.

A. K. Persily, and W. S. Dols. 1990, 21p.

Sponsored by Department of Energy, Washington, DC Pub. in Proceedings of the AIVC Conference on Ventilation System Performance (11th), Belgirate, Italy, September 18-21, 1990, p294-313.

Keywords: "Ventilation, "Office buildings, "Indoor air poliution, "Air flow, "Libraries, "Thermal comfort, Field tests, District of Columbia, Tracer techniques, Spatial distribution, Performance evaluation, Environmental engineering, Reprints.

Mechanical ventilation system performance involves the provision of adequate amounts of outdoor air, uniform distribution of ventilation air within the occupied space, and the maintenance of thermal comfort. Standardized measurement techniques exist to evaluate thermal comfort and air exchange rates in mechanically ventilated buildings; field techniques to evaluate air distribution or ventilation effectiveness are still being developed. The paper presents field measurements of air exchange rates and ventilation effectiveness in an office/library building in Washington D.C. The tracer gas decay technique was used to measure whole building air exchange rates. Ventilation effectiveness was investigated at several locations within the building through the measurement of local tracer gas decay rate and mean local age of air. The ventila- 
tion effectiveness measurements serve as an investigation of the applicability of the measurement procedures employed, providing insight into the measurement issue of establishing initial conditions, the spatia variation in tests results within a building, and the repeatability between tests.

00,248

PB95-125951 Not available NTIS

National Inst. of Standards and Technology (TS), Gaithersburg, MD. Lab. Accreditation Program. Water Efficient Plumbing Fixtures through Standards and Test Methods.

Final rept.

L. S. Galowin, and J. A. Swaffield. 1990, 5p.

Pub. in Proceedings of CONSERV 90 - National Conference and Exposition Offering Water Supply Solutions for the 1990s, Phoenix, AZ., August 12-16, 1990, p179-183.

Keywords: "Standards, "Test methods, "Waste consumption, "Sewage, "Building codes, "Piping systems, Drainage, Waste disposal, Buildings, Water flow, Conservation, Reprints, "Low flow plumbing fixtures.

Concern exists for low volume fixture flows from water conserving plumbing fixtures and devices discharged into the drainage system to perform the functions for waste removal, transport and clearing the drains. Re duced flow rates and volumetric limits on water consumption for plumbing fixtures and appliances for the water saving plumbing products are proposed in national legislation introduced in the U.S. Congress. Re search, experiments and test measurements in both laboratories and actual field installments are reviewed. Conditions included measured solid waste transpor from water closet discharges into drainlines and loading conditions for a variety of laboratory type simulants applicable for test methods in standards. Data shows that sweeping of wastes through the drainage piping with low flows can be accomplished. The application of selected research results in the preparation of test method procedures for plumbing standards is discussed, e.g., drainline waste transport test of low flush water closets. Computer modeling calculations and data show the 'carry out' of the waste solids from the building to the sewer, or septic system, may occur in a series of successive carries and not necessarily from a single discharge of a fixture.

00,249

PB95-135596 PC A06/MF A02

National Inst. of Standards and Technology (BFRL) Gaithersburg, MD. Building and Fire Research Lab. Of fice.

Indoor Air Quality Impacts of Residential HVAC Systems, Phase 1 Report: Computer Simulation Plan.

S. J. Emmerich, and A. K. Persily. Feb 94, 123p, NISTIR-5346.

Sponsored by Consumer Product Safety Commission Washington, DC. Directorate of Engineering Science.

Keywords: "Indoor air poltution, "Residential buitdings, Computerized simulation, Design criteria, HVAC systems, Air flow, Combustion products, Air quality, Ai pollution control, Ventilation, Contaminants.

NIST has completed the first phase of a project to study the impact of HVAC systems on residential in door air quality and to assess the potential for using residential forced-air systems to control indoor pollutant levels. This project will use computer simulations to assess the ability of modifications to central forcedair heating and cooling systems to control the con centrations of selected pollutants in single-family residential buildings. The first phase consisted of three major efforts: conducting a literature review, developing a plan for computer analysis, and holding an exper workshop to discuss the plan. The second phase of the project will involve performing the computer simula tions and analyzing the results. This report details the results of the Phase I efforts. The objective of the literature review was to obtain information for planning computer simulations that will be performed in Phase II of the project.

00,250

PB95-150991 Not available NTIS

National Inst. of Standards and Technology (BFRL) Gaithersburg, MD. Building Environment Div.

Lighting and HVAC.

Final rept.

S. J. Treado. 1991, 6p.

Pub. in Lighting Design and Application, p18-23 Jul 91.
Keywords: "Lighting systems, "Space HVAC systems, "Energy consumption, "Commercial buildings, "Cost analysis, Interior lighting, Fluorescent lamps, Lighting loads, Heat transfer, Thermal analysis, Energy use, Performance evaluation, Reprints.

Lighting in commercial buildings is the single largest user of electric energy, typically ranging from 25 to 50 percent of total building electrical energy requirements. The performance of the dominant commercial light source, the fluorescent lamp, is strongly dependent on thermal conditions: Both lamp light output and power consumption vary with minimum lamp wall temperature, as much as 20 percent under typical conditions. Proper control of room thermal conditions can ensure that the lamps are operating at thier most efficient level.

00,251

PB95-151007 Not available NTIS

National Inst. of Standards and Technology (BFRL), Gaithersburg, MD. Building Environment Div.

NIST Lighting and HVAC Interaction Test Facility. Final rept.

S. J. Treado. 1991, 3p.

Pub. in American Society of Heating, Refrigeration and Air-Conditioning Engineers Jnl., p47-48, 50, Apr 91.

Keywords: "Test facilities, "Lighting systems, "Space HVAC systems, "Research programs, Commercial buildings, Interior lighting, Fluorescent lamps, Lighting loads, Energy consumption, Energy efficiency, Thermal analysis, Heat transfer, Cost analysis, Reprints.

Lighting in commercial buildings is the single largest user of electric energy, typically ranging from 25 to 50 percent of total building electrical energy requirements. The performance of the dominant commercial light source, the fluorescent lamp, is strongly dependent on thermal conditions. Both lamp light output and power consumption vary with minimum lamp wall temperature by as much as 20 percent under typically encountered conditions. Proper control of room thermal conditions can ensure that the lamps are operating at their most efficient level.

00,252

PB95-151783 Not available NTIS

National Inst. of Standards and Technology (NEL), Gaithersburg, MD. Building Environment Div.

Lighting Quality and LIght Source Size.

Final rept.

J. A. Worthey. $1990,7 p$

Pub. in Jnl. of the Illuminating Engineering Society 19, n2 p142-148 1990.

Keywords: "Daylight, "Light sources, "Optical properties, "Size(Dimensions), Lighting equipment, Color, Luminance, Sunlight, Reflection, Reprints.

The sun covers only about $10(\exp -5)$ of the sky dome, while a luminous ceiling in effect presents an entire bright sky. Through its effects on veiling reflections and highlights, source size affects the range of colors and luminances in a scene. Some new calculations of these phenomena are presented.

\section{0,253}

PB95-151791 Not available NTIS

National Inst. of Standards and Technology (EEEL), Gaithersburg, MD. Electronics and Electrical Engineering Lab. Office.

Lighting Research and Theory Can Create Business Prospects.

Final rept.

J. A. Worthey. 1991, 4p

Pub. in Lighting Design and Application 21, n9 p1417 Sep 91 .

Keywords: "Lighting systems, "Theories, "Research projects, "Technology transfer, Illumination, Luminance, Light sources, Design, Economic analysis, $R e-$ prints.

The topics of the article are: What can theory really do for the business of lighting; and What benefits can come from a more theoretical approach to lighting research and design. The answer proposed will be this: Better theory can give better lighting system designs and, in particular, new and better luminaires.

00,254

PB95-153276 Not available NTIS

National Inst. of Standards and Technology (NEL), Gaithersburg, MD. Building Environment Div.
Psychological Aspects of Lighting: A Review of the Work of CIE TC 3.16

Final rept.

B. L. Collins. $1990,10 p$.

Pub. in Proceedings of Psychological Aspects of Architectural Lighting Symposium, University Park, PA., Oc tober 26-28, 1990, p1-10.

Keywords: "Lighting equipment, "Psychological ef ects, "Workplace layout, Glare, Illuminance, Color, Lu minance, Visibility, Workloads(Psychophysiology), Human reactions, Stress(Psychology), Emotions, Comfort, Reprints.

The work of Commission Internationale de l'Eclairage (CIE) TC 3.16 on 'Psychological Response to Lighting' is described. The committee is charged with reviewing the literature on the psychological response to lighting and preparing a report for the CIE. The committee has identified about 260 research documents, developed a framework for review, prepared an outline, abstracted about $60 \%$ of the documents and developed an initial draft. Tentative output from the draft is described in the area of preferred task illuminance, glare, adaptation level, luminance distributions, light source position and extent, light source characteristics such as color temperature, color rendering and daylight, and $d y$ namic temporal effects such as flicker, variation, and photobiology. A list of references is included.

\section{0,255}

PB95-162079 Not available NTIS

National Inst. of Standards and Technology (BFRL) Gaithersburg, MD. Building Environment Div. Assessing Ventilation Effectiveness in Mechanically Ventilated Office Buildings.

Final rept.

A. K. Persily. 1993, $12 p$

Sponsored by Department of Energy, Washington, DC Pub. in Proceedings of International Symposium on Room Air Convection and Ventilation Effectiveness, Tokyo, Japan, July 22-24, 1992, p201-212 1993.

Keywords: "Office buildings, "Ventilation systems, "Ai circulation, "Indoor air quality, Air infiltration, Air flow, Air intakes, Intake systems, Vents, Space HVAC systems, Test and evaluation, Reprints.

Mechanica| ventilation systems are designed and op erated to bring outdoor air into buildings, distribute ventilation air within the occupied space, remove internally generated contaminants, and maintain thermal comfort. The paper presents a general discussion of ven. tilation effectiveness in mechanically ventilated office buildings as the ability of the ventilation system to provide ventilation air in a manner consistent with the design goals of the system. The design and performance of air distribution systems are discussed on a range of scales, from the air handler to the individual work space, as are the means for assessing ventilation ef fectiveness on each of these scales. Various ap proaches to the assessment of mixing within ventilated spaces, the most common conception of ventilation ef fectiveness, are presented and discussed in relation to their use in mechanically ventilated office buildings.

00,256

PB95-162087 Not available NTIS

National Inst of Standards and Technology (BFRL) Gaithersburg, MD. Building Environment Div.

Modeling Radon Transport in Multistory Residential Buildings.

Final rept.

A. K. Persily. 1993, 17p.

Pub. in Modeling of Indoor Air Quality and Exposure ASTM STP 1205, p226-242 1993.

Keywords: "Residential buildings, "Radon, "Radionuclide migration, *Flow models, Air flow, Air circulation, Spatial distribution, Air infiltration, Leakage Radioecological concentration, Indoor air pollution, Ai pollution monitoring, Computerized simulation, $\mathrm{Re}$ prints.

Radon concentrations have been studied extensively in single-family residential buildings, but relatively little work has been done in large buildings, including multistory residential buildings. The paper presents the re. sults of a computer simulation of airflow and radon transport in a twelve-story residential building. Interzone airflow rates and radon concentrations were predicted using the multizone airflow and contaminan dispersal program CONTAM88. Limited simulations were conducted to study the influence of two different radon source terms, indoor-outdoor temperature difference and exterior wall leakage values on radon transport and radon concentration distributions. 


\section{0,257
PB95-175006 PC A11/MF A03}

National Inst. of Standards and Technology (CAML), Gaithersburg, MD. Office of Applied Economics.

Life-Cycle Costing Workshop for Energy Conservation in Buildings: Student Manual.

Final rept.

S. K. Fuller, and S. R. Petersen. Oct 94, 236p,

NISTIR-5165

Supersedes PB93-198984. See also PB93-208460. Sponsored by Federal Energy Management Program, Washington, DC

Keywords: "Federal buildings, "Energy conservation, "Life cycle costs, "Training, Space HVAC systems, Facilities management, Operation and maintenance, Operating costs, Cost analysis, Energy efficiency, Computer applications, Education, "BLCC computer pro. gram, BLCC(Building Life-Cycle Cost).

The Student Manual for the Life-cycle Costing Workshop for Energy Conservation in Buildings is a workbook for a two-day course on life-cycle cost (LCC) analysis. The purpose of the workshop is to provide professionals concerned with energy conservation in federal buildings with the knowledge and skills they need to perform economic analyses quickly and correctly. The Student Manual presents the criteria and methods that govern LCC analysis for energy conservation in federal buildings; treats basic economic concepts; gives step-by-step instructions for performing LCC analyses; and introduces computer software and hands-on exercises for performing LCC analyses both manually and on PCs.

\section{0,258}

PB95-175329 Not available NTIS

National Inst. of Standards and Technology (TS), Gaithersburg, MD. Office of Standards Services.

Performance of Compact Fluorescent Lamps at Different Ambient Temperatures.

Final rept.

B. L. Collins, S. J. Treado, and M. J. Ouellette. 1994, $14 \mathrm{p}$.

Pub. in Jnl. of the Illuminating Engineering Society 23, n2 p72-85 1994 .

Keywords: "Fluorescent lamps, "Temperature effects, "Performance evaluation, *Luminous intensity, Light sources, Power factor, Energy efficiency, Energy conservation, Reprints.

Compact fluorescent lamps are used to replace incandescent lamps to aid in energy conservation in commercial and domestic lighting applications. In conjunction with the Institute for Research in Construction (IRC) at the National Research Council, Canada (NRCC), the Lighting Group at the National Institute of Standards and Technology (NIST) conducted an experimental evaluation of twelve sets of different types of compact fluorescent lamps at six different ambient temperature conditions. An additional set of incandescent lamps was also evaluated for comparison. A total of three lamps were tested for each of the thirteen lamp types, both compact fluorescent and incandescent

\section{0,259}

PB95-175774 Not available NTIS

National Inst. of Standards and Technology (BFRL), Gaithersburg, MD. Building Environment Div.

Using Emulators to Evaluate the Performance of Building Energy Management Systems.

Final rept.

G. E. Kelly, W. B. May, J. Y. Kao, and C. Park. 1994, $12 p$

Sponsored by Department of Energy, Washington, DC. Building Systems and Materials Div.

Pub. in American Society of Heating, Refrigerating and Air-Conditioning Engineers Transactions 100, pt1 p1482-14931994.

Keywords: "Space HVAC systems, "Computerized simulation, "Performance tests, Control systems, Heating systems, Ventilation, Air conditioning, Energy consumption, Comfort, Performance evaluation, Reprints, "Building Energy Management Systems, Emulators

The performance of a building energy management system (BEMS) is directly related to the amount of energy consumed in a building and the comfort of the building's occupants. The paper describes using emulators to evaluate a BEMS. Major topics include setting up a BEMS and an emulator, evaluating system/command and DDC software, and methodologies for lesting BEMS application algorithms. Consider- ations are presented for evaluating the programming capabilities of a BEMS, DDC control loop performance, and rating different aspects of BEMS performance. A brief discussion of BEMS software is also included.

\section{0,260}

PB95-175980 Not available NTIS

National Inst. of Standards and Technology (BFRL) Gaithersburg, MD. Building Environment Div. Reproducibility of Tests on Energy Management and Control Systems Using Building Emulators.

Final rept.

H. C. Peitsman, S. Wang, S. Karki, C. Park, and P. Haves. 1994, 10p.

Sponsored by Department of Energy, Washington, DC. Office of Conservation and Renewable Energy.

Pub. in American Society of Heating, Refrigerating and Air-Conditioning Engineers Transactions 100, pt1 p1455-1464 1994.

Keywords: *Energy management systems, "Control systems, "Performance tests, "Computerized simulation, *Space HVAC systems, Ventilation, Air conditioning, Heating systems, Comfort, Real time, Performance evaluation, Energy consumption, Predictions Test methods, Reprints

An emulator consists of a real-time simulation of a building and its HVAC system and a hardware interface that allows the simulation to be connected to a real control system. As part of a research project, an experiment involving four different emulators with two different (EMCS) was performed to study the reproducibility of the emulation method. The paper presents the results of this experiment in the form of a comparison of the predictions of energy consumption, thermal comfort, and control activity obtained from tests of the EMCS using emulators based on different design and different simulation programs.

\section{0,261}

PB95-182259 PC A04/MF A01

National Inst. of Standards and Technology (BFRL), Gaithersburg, MD

Algorithm to Describe the Spread of a Wall Fire under a Ceiling

H. E. Mitler. Nov 94, 62p, NISTIR-5547.

Keywords: "Algorithms, "Ceilings(Architecture), *Buildings, *Flame propagation, Thermal radiation, Convection(Heat transfer), Plumes, Ignition, Mathematical models, Combustion, Flames, Burning rate, "Wall fires, SPREAD computer program.

After some discussion of wall fires, the effects that a ceiling has on a wall fire are analyzed and discussed qualitatively. There are two kinds of effects: first, when the upper sill of the ventilating opening lies below the ceiling (as is usual), a layer of hot gas is trapped, which heats the walls by convection and radiation; the heated ceiling also radiates to the walls. A calculation which uses the wall-fire computer model SPREAD is carried out to demonstrate how these effects can be calculated now. Second, when the flame impinges on the ceiling it bifurcates and spreads horizontally, rather than vertically. A simple algorithm is presented which calculates the spread rate of these horizontal flame extensions as well as the modified pyrolysis rate, due to the modified radiation feedbacks.

\section{0,262}

PB95-182309 PC A04/MF AO1

National Inst. of Standards and Technology (BFRL), Gaithersburg, MD

Indoor Air Quality Commissioning of a New Office Building.

W. S. Dols, A. K. Persily, and S. J. Nabinger. Jan 95, 60p, NISTIR-5586.

See also PB90-164484. Sponsored by Nuclear Regulatory Commission, Washington, DC.

Keywords: *Office buildings, "Indoor air quality, "Ventilation systems, "Test and evaluation, Space HVAC systems, Indoor air pollution, Air quality stand ards, Specifications, Cooling and ventilating equipment, Building materials, Air flow, Air infiltration, Comfort.

Commissioning does not always include the verifica. tion of an 'acceptable' indoor environment for the building occupants, including factors related to indoor air quality $(\mathrm{AQ})$. In light of growing concern about indoor air quality. IAQ commissioning could become an important part of building commissioning programs. The program described in this report consisted of three tasks: (1) evaluate the mechanical ventilation system design from an IAQ perspective; (2) develop a set of environmental performance parameters and associated reference values that will be used to evaluate $I A Q$ in the building; and, (3) measure these environmental parameters in the building and compare them with the reference values developed in Task 2 .

\section{0,263}

PB95-190682 PC A06/MF A02

National Inst. of Standards and Technology (BFRL), Gaithersburg, MD

BLCC: The NIST 'Building Life-Cycle Cost' Program, Version 4.21. User's Guide and Reference Manual.

S. R. Petersen. Jan 95, 103p, NISTIR-5185-2.

Sponsored by Federal Energy Management Program, Washington, DC.

Keywords: "Buildings, "Energy efficiency, "Life cycle costs, User manuals, Investments Computer applications, Energy conservation, Operating costs, Economic analysis, Benefit cost analysis.

The NIST Building Life-Cycle Cost (BLCC) computer program provides economic analysis of proposed capital investments that are expected to reduce long-term operating costs of buildings or building systems/components. It is especially useful for evaluating the costs and benefits of energy conservation projects in buildings. Two or more alternative designs can be evaluated to determine which has the lowest life-cycle cost and therefore is most economical in the long run. Economic measures, including net savings, savings-to-investment ratio, adjusted internal rate of return, and years to payback can be calculated for any design alternative relative to the designated base case. BLCC can be used for evaluating federal (including Department of Defense), state, and local government projects as well as projects in the private sector. It complies with ASTM standards related to building economics as well as FEMP and OMB Circular A-94 guidelines for economic analysis of federal building projects. BLCC has new capabilities for using demand charges and block rate calculations for computing annual electricity costs. While BLCC is primarily intended for the economic evaluation of building systems, it can be applied to a wide range of project investments which are intended primarily to reduce future operating-related costs.

00,264

PB95-210944 PC A04/MF A01

National Inst. of Standards and Technology (BFRL) Gaithersburg, MD. Building Environment Div.

Meas urements of Outdoor Air Distribution in an Office Building.

W. S. Dols, and A. K. Persily. Jun 94, 58p, NISTIR5320 .

Sponsored by Bonneville Power Administration, Portland, OR.

Keywords: "Office buildings, "Air circulation, *Ventilation, "Indoor air quality, Air infiltration, Air flow rates, Flow measurement, Air sampling, Tracer techniques, Environmental surveys, Outdoor air.

The National Institute of Standards and Technology (NIST) has performed a study of methods to measure the local outdoor air delivery rates in an office building. This is a follow-up study of one which was performed in the same building to measure outdoor aifflow rates to the building as a whole. This study focuses on the delivery of outdoor air to smaller sections of the building. Airflow rates were measured to various zones of the building which range in size from that served by an entire air handler to individual workstations served by a single supply air diffuser. Comparisons were made between the use of different procedures used to measure airflow rates to the same zones in terms of relative accuracies and levels of effort involved in performing the measurements. The use of tracer gas techniques to measure local ages of air was also demonstrated. The local ages of air were then used to determine air change effectiveness of the ventilation system at the measurement locations. Air change effectiveness provides a measure of the uniformity of outdoor air delivery and mixing in a space.

\section{0,265}

PB95-253597 PC A04/MF AO1

National Inst. of Standards and Technology (BFRL). Gaithersburg, MD. 
Least-Cost Energy Decisions for Buildings. Part 3. Choosing Economic Evaluation Methods. Video Training Workbook

H. E. Marshall. May $95,56 p$, NISTIR-5604.

See also PB94-165982. Sponsored by Federal Energy Management Program, Washington, DC

Keywords: "Buildings, "Energy efficiency, "Cost benefit analysis, Environmental engineering, Value engineering, Facilities management, Operation and maintenance, Energy conservation, Life cycle costs, Operating costs, Return on investment, Cost analysis, Economic analysis, Alternatives, Workbooks, Savings-toinvestment ratio.

The workbook and its companion video are the third in a series of training video-workbook modules designed to assist you in using economic analysis to improve the long-run economy of your buildings. This module describes the types of investment decisions that you will have to deal with when you evaluate energy conservation projects-.-decisions to accept or reject a project, what design to choose, and what priority to assign candidate projects. Then it tells you how to match the different types of investment decisions with the appropriate economic methods. The workbook supports the video with expanded descriptions of technical material shown in the video; figures and tables presented in the video; formulas for computing economic measures used in the video; exercises to give you practice; and a glossary of technical terms used by the video instructors.

\section{0,266}

PB95-501953 CP D02

National Inst. of Standards and Technology, Gaithersburg, MD.

Building Life Cycle Cost Computer Program (BLCC), Version 4.2-95 (for Microcomputers).

Software.

1995, diskette, NIST/SW/DK-94/003

System: MS DOS operating system. Open READ.ME file for installation instructions. Supersedes PB94500055. See also PB94-500097 (ERATES).

The software is on one $31 / 2$ inch DOS diskette, $1.44 \mathrm{M}$ high density. File format: ASCII text. Documentation included; may be ordered separately as PB93-208460 and PB91-167288.

Keywords: "Software, "Buildings, "Life cycle costs, "Energy conservation, Economic analysis, Long range(Time), Operating costs, Return on investment, Savings, Prices, Benefit cost analysis, Diskettes.

Product provides economic analysis of proposed capital investments that are expected to reduce long-term operating costs of buildings or building systems/components. It is especially useful for evaluating the costs and benefits of energy conservation projects in buildings. Two or more alternative designs can be evaluated to determine which has the lowest life-cycle cost and therefore is most economical in the long run. Economic measures, including net savings, savings-to-investment ratio, adjusted internal rate of return, and years to payback can be calculated for any design alternative relative to the designated base case. BLCC can be used for evaluating federal (including Department of Defense), state, and local government projects as well as projects in the private sector. It complies with ASTM standards related to building economics as well as FEMP and OMB Circular A-94 guidelines for economic analysis of federal building projects. It has new capabilities for using demand charges and block rate calculations for computing annual electricity costs. While it is primarily intended for the economic evaluation of building systems, it can be applied to a wide range of project investments which are intended primarily to reduce future operating-related costs.

00,267
PB95-502779 CP D02

National Bureau of Standards, Gaithersburg, MD. Building Life Cycle Cost Computer Program (BLCC) Version 4.21-95 (for Microcomputers). Software.

1995, diskette, NIST/SW/DK-95/002

MS DOS operating system. Open READ.ME file for installation instructions. Supersedes PB95-501953. See also PB94-500097 (ERATES).

The software is on one $1.44 \mathrm{M}, 31 / 2$ inch disc. File format: ASCII text. Documentaion included; may be ordered separately as PB95-169652.

Keywords: "Software, "Buildings, "Life cycle costs, "Energy conservation, Economic analysis, Benefit cost analysis, Operating costs, Long range(Time), Return on investment, Savings, Prices, Project management, National government, State government, Local govern ment, Standards, Diskettes.

The product provides economic analysis of proposed capital investments that are expected to reduce longterm operating costs of buildings or building systems/ components. It is especially useful for evaluating the costs and benefits of energy conservation projects in buildings. Two or more alternative designs can be evaluated to determine which has the lowest life-cycle cost and therefore is most economical in the long run. Economic measures, including net savings, savings-to-in vestment ratio, adjusted internal rate of return, and years to payback can be calculated for any design alternative relative to the designated base case. Building Life Cycle Cost (BLCC) can be used for evaluating federal (including Department of Defense), state and local government projects, as well as projects in the private sector. It complies with ASTM standards related to building economics as well as FEMP and OMB Circular A-94 guidelines for economic analysis of federal building projects. It has new capabilities for using demand charges and block rate calculations for computing annual electricity costs. While it is primarily in tended for the economic evaluation of building sysems, it can be applied to a wide range of project in vestments which are intended primarily to reduce future operating-related costs.

\section{0,268}

PB95-503397 CP D02
National Inst. of Standards and Technology, Gaithersburg, M

Building Life Cycle Cost Computer Program (BLCC) Version 4.22-95 (for Microcomputers).

(B)

1995, 1 diskette, NIST/SW/DK-95/002

MS DOS operating system. Open READ.ME file for in stallation instructions. Supersedes PB95-502779. See also PB94-500097 (ERATES)

The software is on one $31 / 2$ inch DOS diskette $1.44 \mathrm{M}$ high density. File format: ASCII text. Documentation included; may be ordered separately as PB95-169652.

Keywords: "Software, "Buildings, "Life cycle costs, "Energy conservation, "Benefit-cost analysis, OperatSavings, Prices, Project management, National gov ernment, State governments, Local governments, Standards, Diskettes, Documentation.

Product provides economic analysis of proposed capital investments that are expected to reduce long-term operating costs of buildings or building systems/components. It is especially useful for evaluating the costs and benefits of energy conservation projects in buildings. Two or more alternative designs can be evaluated to determine which has the lowest life-cycle cost and therefore is most economical in the long run. Economic measures, including net savings, savings-to-investment ratio, adjusted internal rate of return, and years to payback can be calculated for any design alcan be used for evaluating federal (including Department of Defense), state, and local government projects as well as projects in the private sector. It complies with ASTM standards related to building economics as well as FEMP and OMB Circular A-94 guidelines for economic analysis of federal building projects. It has new capabilities for using demand charges and block rate calculations for computing annual electricity costs. While it is primarily intended for the economic evaluation of building systems, it can be applied to a wide range of project investments which are intended primarily to reduce future operating-related costs.

\section{0,269}

PB96-111901 Not available NTIS

National Inst. of Standards and Technology (BFRL) Gaithersburg, MD. Building Environment Div. Use of Building Emulators to Evaluate the Performance of Building Energy Management Systems. Final rept.

H. Vaezi-Nejad, E. Hutter, P. Haves, P. Nusgens, S. Wang, A. L. Dexter, and G. Kelly. 1994, 6p.

See also PB95-175774. Sponsored by Department of Energy, Washington, DC.

Pub. in Proceedings of Building Simulation Conference, Nice, France, August 20-22, 1991, p1-6.

Keywords: "Space HVAC systems, "Computerized simulation, "Performance tests, Control systems, Heating systems, Ventilation, Air conditioning, Energy consumption, Comfort, Performance evaluation, Reprints, *Building Energy Management Systems, Emulators.
Three complementary approaches may be used in the performance of building control systems-simulation, emulation and field testing. In emulation a real-time simulation of the building and HVAC plant is connected to a real building energy management system (BEMS) via a hardware interface. Emulation has the advantage of allowing controlled, repeatable experiments whils testing real devices that may contain proprietary algorithms. Building emulators have been developed by the authors in the context of IEA Annex 17, which is concerned with the use of simulation to evaluate the per formances of BEMS. The paper discusses different ap proaches to the design of building emulators and describes the different architectures, hardware and software used by the authors. The problem of evaluating the overall performance of BEMS is discussed and results are presented that illustrate the use of emulators to investigate the influence of the tuning of local loop controls on building performance.

\section{0,270}

PB96-122932 Not available NTIS

National Inst. of Standards and Technology (TS) Gaithersburg, MD. Lab. Accreditation Program.

Laboratory Accreditation for Testing Energy Efficient Lighting.

Final rept.

L. Galowin, W. Rossiter, R. Runkles, and R. Brown 1994, 12p.

Pub. in Proceedings: Technology Research, Develop ment and Evaluation, Asilomar Conference Center Monterey, CA., August 28-September 3, 1994, v3 p3.77-3.88 1994

Keywords: "Lighting equipment, "Energy efficiency, "Accreditation, "Laboratories, Lamps, Energy conservation, Procedures, Requirements, Comparative evaluations, Standards, Tests, Reprints.

NEMA requested establishment of the accreditation program for laboratory testing of lamps and luminaires from concensus standards prior to enactment of EPACT. NVLAP conducts regulatory and voluntary ac creditation programs. Impacts cited by NEMA for NVLAP were; third-party objective accreditation programs for establishment of credible testing data, avoid ance of duplicated efforts by different Federal govern ment agencies, avoidance of confusion in the marke place, improving product quality, and assistance to utilities/conservation planners. Following public comment by interested organizations, NVLAP developed the En ergy Efficient Lighting Program for laboratories con ducting standard test methods for electrical, photometric, and color measurements. Industry recommendations at FTC hearings supported NVLAP aCcredited laboratory data for labeling

\section{0,271}

PB96-138508 Not available NTIS

National Inst. of Standards and Technology (BFRL) Gaithersburg, MD. Building Environment Div. Improving the Evaluation of Building Ventilation. Final rept.

A. K. Persily. $1995,15 p$

Pub. in Annual Meeting of the Air and Waste Manage ment Association (88th), San Antonio, TX., June 18 23, 1995, p1-15

Keywords: "Commercial buildings, "Ventilation systems, "Air circulation, Performance evaluation, Test and evaluation, Standardization, Air flow, Air infiltration, Research projects, Reprints, Mechanical ventilation.

A project is being conducted at the National Institute of Standards and Technology (NIST) to identify ap proaches to improve these evaluations, to develop seected ventilation assessment protocols, and to identify research needed to make further advances in the field. This project has included a characterization of the applications of ventilation assessment in buildings, an identification of the objectives addressed by differen approaches to ventilation assessment, and a review of existing protocols. This paper discusses the characterzation of ventilation evaluation that was performed as part of this project and is organized into the following sections: Uses of Ventilation Evaluation; Specific Eval uation Objectives; Existing Evaluation Protocols; and Current Inadequacies: Problems, Reasons and Solutions.

00,272

PB96-141353 Not available NTIS

National Inst. of Standards and Technology (BFRL) Gaithersburg, MD. Building Environment Div. 
Optimal Control of Building and HVAC Systems. Final rept.

J. M. House, and T. F. Smith. 1995, 5p

Pub. in Proceedings of the American Control Conference, Seattle, WA., June 21-23, 1995, p4326-4330.

Keywords: "Space HVAC systems, "Optimal control, "Buildings, Comfort, Energy efficiency, Energy consumption, Control systems, Reprints.

An optimal control strategy is compared with a conventional strategy to demonstrate the capability of optimal control to maintain comfort while reducing the utility cost for a two-zone building and HVAC system. The optimal control strategy yields a reduction in the utility cost of 11 percent for the conditions considered. A new-optimal control strategy that requires less implementation effort is also discussed.

00,273
PB96-154463 PC A04/MF A01

National Inst. of Standards and Technology (BFRL), Gaithersburg, MD.

Workplan to Analyze the Energy Impacts of Envelope Airtightness in Office Buildings.

S. J. Emmerich, A. K. Persily, and D. A

VanBronkhorst. Dec 95, 34p, NISTIR-5758. See also PB93-183770. Sponsored by Department of
Energy, Washington, DC.

Keywords: "Office buildings, "Space HVAC systems, *Ventilation systems, Air flow, Air infiltration, Thermal insulation, Energy efficiency, Energy consumption, Commercial buildings, Temperature control, Heating systems, Air conditioning, Standards, Computerized simulation, Mathematical models, “Air tightness.

This report describes the impact of building airflows on energy consumption in multi-zone buildings and the analysis approaches that can be used to account for the energy associated with these airflows. Plans to link a multi-zone network airflow analysis program with a building energy analysis program are disussed. An initial estimate of the energy associated with infiltration in U.S. office buildings, based on a simplified analysis approach, is presented.

\section{0,274}

PB96-155593 Not available NTIS

National Inst. of Standards and Technology (BFRL), Gaithersburg, MD. Building Environment Div.

Study of Ventilation Measurement in an Office Building.

W. Stuart Dols, and A. K. Persily, 1995, 24p.

See also PB93-113595.

Pub. in Airflow Performance of Building Envelopes, Components, and Systems, ASTM STP 1255, p23-46 1995.

Keywords: "Office buildings, "Air flow, "Ventilation, Commercial buildings, Air infiltration, Air circulation, Flow measurement, Monitoring, Indoor air pollution, Tracer techniques, Reprints, Air exchange rates.

The National Institute of Standards and Technology has conducted a study of ventilation and ventilation measurement techniques in the Bonneville Power $\mathrm{Ad}$ ministration (BPA) Building in Portland, Oregon. The project involved in the use of the following outdoor air ventilation measurement techniques: tracer gas decay measurements of whole-building air change rates, the determination of air change rates based on peak carbon dioxide (CO2) concentrations, the determination of percent outdoor air intake using tracer gas (Sulfur hexafluoride and occupant-generated $\mathrm{CO} 2$ ), and direct airtlow rate measurements with in the air handling sys tem. In addition, air change rate measurements made approximately three years apart with an automated tracer gas decay system were compared.

\section{0,275}

PB96-155601 Not available NTIS

National Inst. of Standards and Technology (BFRL), Gaithersburg, MD. Building Environment Div.

Development and Application of an Indoor Air Quality Commissioning Program in a New Office Building.

W. Stuart Dols, A. K. Persily, and S. J. Nabinger.

1994, 11p.

Pub. in Indoor Air Quality Engineering Indoor Environments, p33-43 1994.

Keywords: "Office buildings, "Indoor air quality, "Environmental surveys, Ventilation systems, Building materials, Air flow, Air circulation, Air infiltration, Air intakes, Baseline measurements, Monitoring, Air sampling, Flow measurement, Design analysis, Reprints.

An indoor air quality commissioning program has been developed and is being implemented in a new office building in Rockville, Maryland. New buildings can have an increased potential for indoor air quality (IAQ) problems due to new building materials and deficiencies in mechanical ventilation system operation during construction and initial occupancy. This IAQ commissioning effort is being implemented to reduce the potential for such problems in this building. This commissioning program consists of three tasks: (1) evaluate the mechanical ventilation system design, (2) develop a set of environmental parameters and associated reference values that will be used to evaluate $\mid A Q$ in the building, and (3) measure various environmental parameters for comparison with the reference values deveioped in task 2 .

\section{0,276}

PB96-164181 Not available NTIS

National Inst. of Standards and Technology (BFRL), Gaithersburg, MD. Building Environment Div.

Distributed Architecture for Standards Processing. Final rept.

H. Kiliccote, J. H. Garrett, B. Choi, and K. A. Reed. $1995,8 p$

Sponsored by Carnegie-Mellon Univ., Pittsburgh, PA. Pub. in Proceedings of the International Conference on Computing in Civil and Building Engineering (6th), Berlin, Germany, July 12-15, 1995, p343-350.

Keywords: "Computer-aided design, "Design criteria, Architecture, Design analysis, Building codes, $R e$ quirements, Standards compliance, Reprints, "Building design, Standards processing.

An approach to providing computer-aided support for using design standards in design system is presented. The standards processing system we developed is composed of five major components that interact with each other using the Internet: standards processing servers which evaluate a given design to check whether it satisfies the requirements of a specific design standard; the standards processor broker which is used by the designer to identify applicable design standards; the data server which acts as a front-end between the database of the design system and the standards processing activities, multiple standards can be dealt with by the design system and the design system is insulated from changes in standards.

\section{0,277}

PB96-502794 CP D02

National Inst. of Standards and Technology, Gaithersburg, MD

Building Life Cycle Cost Computer Program (BLCC) Version 4.22-95 (for Micrcomputers).

Model-Simulation.

1995, 2 diskettes.

MS DOS operating system. Supersedes PB95-502779 and PB95-503397.

The software is on two $31 / 2$ inch DOS diskettes, $1.44 \mathrm{M}$ high density. Documentaion included; may be ordered separately as PB96-199229.

Keywords: "Software, "Buildings, "Life cycle costs, "Energy conservation, Economic analysis, Cost benefit analysis, Operating costs, Return on investment Savings, Prices, Project management, Long range(Time), Federal government, State government, Standards, Diskettes.

The software provides economic analysis of proposed capital investments that are expected to reduce longterm operating costs of buildings or building systems/ components. It is especially useful for evaluating the costs and benefits of energy conservation projects in buildings. Two or more alternative designs can be evaluated to determine which has the lowest life-cycle cost and therefore is most economical in the long run. Economic measures, including net savings, savings-to-investment ratio, adjusted internal rate of return, and years to payback can be calculated for any design alternative relative to the designated base case. BLCC can be used for evaluating federal (including Department of Defense), state, and local government projects as well as projects in the private sector. It complies with ASTM standards related to building economics as well as FEMP and OMB Circular A-94 guidelines for economic analysis of federal building projects. It has new capabilities for using demand charges and block rate calculations for computing annual electricity costs. While it is primarily intended for the economic evalua. tion of building systems, it can be applied to a wide range of project investments which are intended primarly to reduce future operating-related costs.

00,278

PB97-106843 PC A05/MF A01

National Inst. of Standards and Technology (BFRL), Gaithersburg, MD

Mathematical Analysis of Practices to Control Moisture in the Roof Cavities of Manufactured Houses.

D. M. Burch, G. A. Tsongas, and G. N. Walton. Sep 96, 63p, NISTIR-5880.

Prepared in cooperation with Portlanj State Univ., OR Dept. of Mechanical Engineering.

Keywords: "Moisture, "Roofs, "Prefabricated buildings, Mathematical models, Vents, Cavities, Venting, Mobile homes.

This report presents a mathematical model to simulate the performance of a double-wide manufactured house constructed in compliance with HUD Standards. An interior vapor retarder was installed in the ceiling construction and ventilation openings were installed in the roof cavity consistent with HUD's 1/300 Rule. The effect of passive and mechanical ventilation, as well as a wide range of other factors on the roof sheathing moisture content was investigated as a function of time. The weekly average moisture content of the lower surface of the plywood sheathing was analyzed in several cold climates, while the relative humidity at the lower surface of the ceiling insulation was analyzed in a hot and humid climate.

00,279

PB97-110142 Not available NTIS

National Inst. of Standards and Technology (BFRL), Gaithersburg, MD. Building Environment Div.

Indoor Air Quality Commissioning of a New Office Building.

W. S. Dols, A. K. Persily, and S. J. Nabinger. 1995 $13 p$.

See also PB95-182309 and PB96-155601.

Pub. in Indoor Air Quality (IAQ) '95: Practical Engineering of IAQ, Denver, CO., October 22-24, 1995, p2941 Dec 95

Keywords: "Office buildings, "Indoor air quality, *Environmental surveys, Ventilation systems, Building materials, Air circulation, Monitoring, Air sampling, Design analysis, Reprints.

New buildings can have an increase potential for indoor air quality problems due to new building materials and deficiencies in mechanical ventilation system performance during construction and initial occupany. In order to decrease the potential for such problems, an indoor air quality commissioning program was developed and implemented in a new office building for the U.S. Nuclear Regulatory Commission (NRC). The in door air quality commissioning effort consisted of three tasks: (1) evaluation of the mechanical ventilation system design, (2) development of a set of environmental parameters and associated reference values to be used in evaluating the building indoor air quality, and (3) measurement of these environmental parameters in this building and comparison of them with the reference values developed in task 2 . The evaluation of the mechanical ventilation system design was based on the recommendations of the 1987 BOCA mechanical code and ASHRAE Standard 62-1989. The design evaluation showed that the system ventilation rates were consistent with the recommendations of both documents. The environmental parameters identified in task 2 address ventilation system performance, indoor pollutant levels, and thermal comfort. The reference values for these parameters were based on available standards and guidelines as well as on the results of previous indoor air quality research. In task 3 , these environmental parameters were measured in three phases of building construction: after completion of interior buildout, after the installation of the systems furniture, and roughly one month after occupancy. The measured values were within the project reference values with only a few exceptions, and these exceptions were usually attributed to a correctable circumstance.

\section{0,280}

PB97-111298 Not available NTIS

National Inst. of Standards and Technology (BFRL), Gaithersburg, MD. Building Environment Div. 
Post-Occupancy Evaluation of the Forrestal Building.

Final rept

P. A. Sanders, and B. L. Collins. 1996, 15p.

Pub. in Jnl. of the Illuminating Engineering Society, p89-103, Summer 1996

Keywords: "Office buildings, "Work environments, "Surveys, Workplace layout, Comfort, Human reactions, Reprints.

Post-occupancy evaluation techniques provide a means for evaluating occupant responses to changes in an environment and linking this response to physical measures of that environment. Post-occupancy evaluations use a battery of tests to assess environmental conditions in the facility, including questionnaire surveys of the occupants, physical measures, personal observations, and individual interviews. The post-occupancy evaluation techrique is designed to provide in formation about the occupants' reaction to their work spaces and document the physical conditions to which they are responding, usually on a pre- and post-retrofit basis.

00,281

PB97-112221 Not available NTIS

National Inst. of Standards and Technology (MEL), Gaithersburg, MD. Manufacturing Systems Integration Div.

HVAC CAD Layout Tools: A Case Study of University/Industry Collaboration.

Final rept.

J. Cagan, R. Clark, P. Dastidar, S. Szykman, and P.

Weisser. 1996, 10 p.

Pub. in Proceedings of the American Society of Mechanical Engineers Design Engineering Technical Conference and Computers in Engineering Conference, Irvine, CA., August 18-22, 1996, p1-10.

Keywords: "Space HVAC systems, "Computer-aided design, "Heat pumps, Tubes, Energy efficiency, Online control systems, Heat transfer, Computer tools, Computerized simulation, Reprints.

An effective partnership betvveen industry and the university resulted in the system of design tools for th layout of HVAC systems presented in the paper and illustrated with the design of a heat pump. The system provides tools to assist in the placement of components and routing of tubes between the components. The paper provides insight on both the collaborative research interaction and the resulting set of tools.

00,282

PB97-112544 Not available NTIS

National Inst. of Standards and Technology (PL), Gaithersburg, MD. Radiometric Physics Div.

Characterization of Modified FEL Quartz-Halogen Lamps for Photometric Standards.

Final rept.

Y. Ohno, and J. K. Jackson. 1996, 4p.

Pub. in Metrologia, v32 p693-696 1995/96.

Keywords: "Photometry, "Standards, Luminous intensity, Illuminance, Lumens, Light sources, Luminous flux, Flux density, Color, Spectrum analysis, Optical measurement, Reprints, "Halogen lamps, "Incandescent lamps

The stability of luminous intensity and color temperature of modified FEL-type, $1000 \mathrm{~W}$ quartz-halogen lamps has been tested at various color temperatures to investigate the suitability of these lamps for use as photometric transfer standards. Characteristics of the amps have also been investigated for operation a $2000 \mathrm{~K}$ to $3100 \mathrm{~K}$, for reversed polarity, and for longterm storage.

00,283

PB97-121321 Not available NTIS

National Inst. of Standards and Technology (BFRL) Gaithersburg, MD. Building Environment Div.

Fault Diagnosis of an Air-Handling Unit Using Artificial Neural Networks.

Final rept.

W. Y. Lee, and C. Park. 1996, 10p

Pub. in American Society of Heating, Refrigerating and Air-Conditioning Engineers Transactions: Symposia, v102 pt1 p540-549 Jun 96.

Keywords: "Neural nets, "Fault detection, "Space HVAC systems, Air conditioning, Heating systems, Ventilation systems, Control systems, Error analysis, Error detection codes, Reprints, Air handling systems. The objective of the study is to describe the application of artificial neural networks to the problem of fault diag- nosis in an air-handling unit. Initially, residuals of system variables that can be used to quantify the dominant symptoms of fault modes of operation are selected. Idealized steady-state patterns of the residuals are then defined for each fault mode of operation. The study-state relationship between the dominant symptoms and the faults is learned by an artificial neural network using the backpropagation algorithm. The trained neural network is applied to experimental data for various faults and successfully identifies each fault.

\section{0,284}

PB97-500342 CP D02

National Inst. of Standards and Technology, Gaithersburg, MD

Building Life Cycle Cost Computer Program (BLCC) Version 4.4-97 (for Microcomputers).

Model-Simulation.

Sep 96, 2 diskettes.

MS DOS operating system. Documentation is in Word Perfect 5.1. Supersedes PB95-502779, PB95-503397 and PB96-5002794

The software is on two $31 / 2$ inch DOS diskettes, 1.44M high density. File format: ASCII text.

Keywords: "Software, "Buildings, "Life cycle costs, "Energy conservation, "Benefit-cost analysis, Operating costs, Economic analysis, Return on investment, Savings, Prices, Project management, National government, State government, Local government, Standards, Diskettes, Documentation.

The software provides economic analysis of proposed capital investments that are expected to reduce longterm operating costs of buildings or building systems/ components. It is especially useful for evaluating the costs and benefits of energy conservation projects in buildings. Two or more alternative designs can be evaluated to determine which has the lowest life-cycle cost and therefore is most economical in the long run. Economic measures, including net savings, savings-to-investment ratio, adjusted internal rate of return, and years to payback can be calculated for any design alternative relative to the designated base case. BLCC can be used for evaluating federal (including Department of Defense), state, and local government projects as well as projects in the private sector. It complies with ASTM standards related to building economics as well as FEMP and OMB Circular A-94 guidelines for economic analysis of federal building projects. It has new capabilities for using demand charges and block rate calculations for computing annual electricity costs. While it is primarily intended for the economic evaluation of building systems, it can be applied to a wide range of project investments which are intended primarily to reduce future operating-related costs.

\section{Building Equipment, Furnishings, \& Maintenance}

\section{0,285}

PB94-139193 PC A03/MF A01

National Inst. of Standards and Technology (BFRL) Gaithersburg, MD

Some Factors Affecting Design of a Furniture Calorimeter Hood and Exhaust.

L. Y. Cooper. Dec 93, 26p, NISTIR-5298.

Keywords: "Calorimeters, "Fire tests, "Furniture, Exhaust systems, Plumes, Hoods, Flames, Flow rate, Mathematical models, Design criteria.

This work considers factors affecting the design of an effective and versatile furniture calorimeter hood and exhaust system. The purpose of the furniture calorimeter, design functions, and inherent limitations of a particular design are discussed. The interactions between the hood structure and the fire and its plume are analyzed in the context of avoiding: flame impingement on the hood; enhanced combustion of a test article, over and above that of a free-burn; loss of combustion product plume gases due to 'spill-over' below the hood; unacceptable dilution of plume gases in the measurement section of the exhaust duct. The concept of the ideally designed hood is introduced, where, throughout the course of the burn of a test article the hood is always immediately above the flame tip and the exhaust rate always exactly matches the hood-ceiling-elevation plume-flow rate. Methods to partially or completely achieve the ideal design are presented. These include the combined features of adjustable hood evaluation and adjustable hood exhaust rate. The ideas and results of analyses developed are applied in examples relevant to the existing furniture calorimeter hood and exhaust system of the NIST Fire Research Laboratory Building 205. Recommendations for improvements to this facility are presented.

\section{0,286}

PB94-160876 PC A10/MF A03

National Inst. of Standards and Technology (NEL) Gaithersburg, MD. Center for Fire Research.

Summaries of Center for Fire Research In-House Projects and Grants: 1990

S. M. Cherry. Oct 90, 224p, NISTIR-4440.

See also report for 1989, PB90-127101.

Keywords: "Combustion, "Furniture, Flammability, Carbon monoxide, Grants, Research projects, Toxicity, Ignition, Soot, Fire hazards, Fire safety.

This report describes the research projects performed in the Center for Fire Research (CFR) and under its grants program during FY 1990 . Topics considered include the following: Turbulent Combustion; Soot For mation; CO Prediction; Polymer Gasification; Flame Spread; Toxic Potency; Furniture Flammability; Building Fire Modeling and Smoke Transfer; Fire Hazard Assessment; Engineering Analysis System and Fire Reconstruction; Suppression; Cone Calorimeter Development; Fire/Modeling Interactions; and Fire Protection Technology.

\section{0,287}

PB94-163441 PC A07/MF A02

National Inst. of Standards and Technology (BFRL), Gaithersburg, MD

Feasibility and Design Considerations of Emergency Evacuation by Elevators.

J. H. Klote, D. M. Alvord, B. M. Levin, and N. E

Groner. Sep 92, 127p, NISTIR-4870

See also PB92-164771 and PB92-238641. Prepared in cooperation with George Mason Univ., Fairfax, VA Sponsored by Public Buildings Service, Washington, DC. Office of Real Property Management and Safety.

Keywords:

*Elevators(Lifts)

"Evacuating(Transportation), Design criteria, Handicapped workers, Feasibility, Human factors engineer ing, Fire safety, Emergencies, Computerized simula tion, Smoke, Egress.

Throughout most of the world, warning signs next to elevators indicate they should not be used in fire situations, and today's elevators have not been designed for fire evacuation and should not be used for fire evac uation. However, the idea of using elevators to speed up fire evacuation and to evacuate persons with dis abilities has gained considerable attention. The potential of elevator evacuation is so significant that the U.S General Services Administration (GSA) has sponsored a research project at NIST to develop techniques fo occupant use of elevators during building evacuations. This paper is the final report of that project, and it ad dresses fundamental system considerations, engineering design considerations, design analysis, and human behavior. This paper shows that use of elevators in addition to stairs during a fire emergency allows occu pants and firefighters an additional system of vertical transportation

\section{0,288}

PB94-194339 PC A03/MF AO

National Inst. of Standards and Technology (BFRL) Gaithersburg, MD. Fire Science Div. Performance Parameters of Fire Detection Systems.

R. L. Smith. Jun 94, 20p, NISTIR-5439.

Keywords: "Fire detectors, "Buildings, Fire prevention, Parameters, Fire extinguishers, Warning systems Probability theory, Smoke detectors, Mathematical models, Fires.

This report is a formal, functional analysis of fire detection systems' requirements. The performance parameters of fire detection systems are given as conditiona probabilities. These parameters are identified by the objective analysis of the functions of a fire detection system. It is demonstrated that using the false alarm rate to specify the malfunctioning of a threshold detec tion system is inadequate. The principal function of fire detection systems is identified as the notification of anti-fire agents of the probability of an unwanted fire. The evaluation of the information provided by a detector system is central to its worth. 


\section{Building Equipment, Furnishings, \& Maintenance}

\section{0,289}

PB94-198454 Not available NTIS

National Inst. of Standards and Technology (BFRL), Gaithersburg, MD. Fire Safety Engineering Div. Toxicity, Fire Hazard and Upholstered Furniture. Final rept.

V. Babrauskas. 1992, 9p

Pub. in Proceedings of European Conference on Furniture Flammability (3rd), Brussels, London, November $24-25,1992, \mathrm{p} 125-133$

Keywords: "Fire hazards, "Toxicity, "Furniture, Fires Combustion products, Burning rate, Fire safety, Safety engineering, Reprints, "Upholstery.

Fire fatalities associated with upholstered furniture fires commonly involve the toxic effects of fire gases Extensive results, however, both from experiments and from modeling, are presented in this paper that demonstrate that occupant life safety can only be ensured by assuring that furniture fires do not reach a high heat release rate. Differences among commercial products associated with toxicity effects are not significant. A very remote possibility exists that someone could produce furniture having combustion products' toxicity significantly greater than exists in the present marketplace. A combustion toxicity test does exist which would allow the accurate screening out of such prod ucts. Any usable combustion toxicity test method requires the use of test animals; no non-animal-based test is possible which can successfully identify products of unusual or extreme toxicity.

\section{0,290}

PB94-199262 Not available NTIS

National Inst. of Standards and Technology (BFRL) Gaithersburg, MD. Fire Safety Engineering Div

Studies Assess Performance of Residential Detectors.

Final rept.

R. W. Bukowski. 1993, 7p

Pub. in National Fire Protection Association Jnl. 87, n1 048-54 Jan/Feb 93

Keywords: "Residential buildings, "Fire detectors, "Fire safety, Performance, Fire protection, Smoke detectors, Warning systems, Literature surveys, Reprints, "Building fires, "Heat detectors.

Current thinking on the performance of residential heat detectors compared to smoke detectors has recently been challenged. The paper was prepared to assemble a comprehensive picture from the literature of what is known about the relative performance of these two detector types in residential fires. The paper reviews 10 independent studies, conducted over two decades, in four countries, involving 206 tests in houses and apartments, typically burning actual items (furniture, mattresses, boxes of trash, clothing, etc.) and using real heat and smoke detectors representative of devices being sold at the time. In each case, the study is summarized and the conclusions relative to heat and smoke detector performance are quoted. All of the studies reach essentially identical conclusions, that: (1) either the ionization or photoelectric smoke detectors when located outside bedrooms and on each level of a house provides adequate warning to allow occupants to evacuate through their normal egress routes for most residential fire scenarios; and (2) heat detectors, even when located in the room of fire origin (effectively requiring locating a heat detector in every room) do not provide adequate warning in most fire scenarios.

\section{0,291}

PB94-212883 Not available NTIS

National Inst. of Standards and Technology (NEL), Gaithersburg, MD. Fire Science and Engineering Div. Smoke Control Systems for Elevator Fire Evacu. ation.

Final rept.

J. H. Klote, and G. T. Tamura. 1991, 12p

Pub. in Proceedings of American Society of Mechanical Engineers Symposium on Elevators and Fire, Baltimore, MD., February 19-20, 1991, p83-94.

Keywords: "Elevators(Lifts), "Evacuation, "Smoke, *Fire safety, Buildings, Handicapped persons, Emergencies, Pressurizing, Ventilation, Fire tests, Stairways, Reprints, “Smoke control, "Elevator fires, Piston effect.

Some people cannot use stairwells because of phys cal disabilities, and for these people fire evacuation is a serious problem. A potential solution of this problem is the use of elevators for fire evacuation. A joint project of the U.S. National Institute of Standards and Technology (NIST) and the National Research Council of Canada (NRCC) was formed to evaluate the feasibility of using elevators for the evacuation of the handicapped during a fire. This project consisted of conceptual studies, full scale fire experiments, and theoretical analysis. This paper summarizes the findings of the joint project that are relevant to the design of smoke control systems for elevators. A method of dealing with elevator piston effect is discussed. All other things being equal, piston effect is considerably greater for single car hoistways than for multiple car hoistways. Different approaches to deal with the pressure fluctuations due to opening and closing of building doors are presented. A method of design analysis is presented with an example analysis. Results indicate that there are many types of elevator smoke control systems which can be designed to provide acceptable levels of pressurization even under severe conditions of doors opening and closing.

\section{0,292}

PB94-213717 PC A07/MF AO2

National Inst. of Standards and Technology (BFRL), Gaithersburg, MD

Measurement of Room Conditions and Response of Sprinklers and Smoke Detectors during a Simulated Two-Bed Hospital Patient Room Fire.

K. A. Notarianni. Jul 93, 142p, NISTIR-5240.

See also PB88-164223. Sponsored by National Institutes of Health, Bethesda, MD. Div. of Engineering Services.

Keywords: "Sprinklers, "Smoke detectors, *Fire protection "Beds, Fire safety, Heat flux, Temperature, Safety equipment, Furniture, Time dependence, "Hospital fires.

A series of experiments are reported in which a wood crib was burned within a simulated two bed hospital patient room in order to measure the activation times of various types of quick and standard response sprin klers and ionization and photoelectric smoke detectors at several locations in the room simulating multiple options for protection of the space. Gas and surface temperatures, heat flux, carbon dioxide, carbon monoxide and oxygen concentrations were continuously meas ured in order to access the tenability of the room. Of the parameters measured, temperature was the best indicator of the tenability of the space. Temperature at time of activation of the quickl response sprinklers was at or below $77 \mathrm{deg} C$ (171 deg F) at the five foot leve and at or below $48 \mathrm{deg} C(118 \mathrm{deg} F)$ at the three foot level between the patient beds, in all tests with the exception of the shielded fire scenario where temperaiures at the five and three foot levels reached 111 deg $C(232 \operatorname{deg} F)$, and $78 \operatorname{deg} C$ ( 172 deg $F$ ) respectively. An initial detector activation was received between 232-377 seconds prior to activation of the first sprinkler, and $552-722$ seconds prior to activation of the QR-EC sidewall sprinkler.

\section{3}

PB94-216181 Not available NTIS

National Inst. of Standards and Technology (BFRL), Gaithersburg, MD. Fire Science Div.

Sprinkler Fire Suppression Algorithm.

Final rept.

D. Madrzykowski, and R. L. Vettori. 1992, 14 p.

Pub. in Jnl. of Fire Prot. Engr. 4, n4 p151-164 1992.

Keywords: "Sprinklers, "Fire suppression, *Algorithms, "Furniture "Buildings, Fire protection, Fire hazards, Models, Activation, Burning rate, Time dependence, Reprints, "Building fires.

A study was conducted to develop a sprinkler fire suppression algorithm for use with sprinkler activation time models. Large-scale experiments were performed to determine the heat release rate (HRR) of selected office fuel packages with and without sprinklers operating. Eight different fuel packages were evaluated. The results from these experiments were used to develop a time dependent HRR reduction factor. The sprinkler fire suppression algorithm consists of multiplying the HRR reduction factor by the HRR at the time of sprinkler activation, yielding an expected upper bound to the HRR at a given time after sprinkler activation for office furnishing fires that are not heavily shielded. This sprinkler fire suppression algorithm can be thought of as a 'zeroth order' fire suppression model for 'light hazard' occupancies with a sprinkler spray density of $0.07 \mathrm{~mm} /$ $\mathrm{s}(0.1 \mathrm{gpm} / \mathrm{sq} \mathrm{ft})$ or greater

00,294

PB95-126330 Not available NTIS
National Inst. of Standards and Technology (BFRL), Gaithersburg, MD. Fire Science Div.

Assessment of Technologies for Advanced Fire Detection.

Final rept.

W. Grosshandler. 1992, $10 p$

Pub. in Proceedings of a Conference on Heat and Mass Transfer in Fire and Combustion Systems, Anaheim, CA., November 8-13, 1992, p1-10.

Keywords: "Fire alarm systems, "Fire detectors, "Technology assessment, "Buildings, Fire safety, Microelectronics, Fire protection, False alarms, Flame propagation, Infrared detectors, Ignition, Reprints.

The majority of fires are sensed either by the heat or the smoke they produce at a set location in space, with an alarm signal being issued when a threshold temperature or particulate level is exceeded. Many of these sensors are inexpensive and perfectly suitable for certain applications, but issues such as false alarms and increased performance can necessitate alternative sensing techniques. Advances in sensor technology, in microelectronics, and in our understanding of ignition and flame spread provide an opportunity to greatly enhance the performance of fire detection systems in traditional applications. Techinological advances have also led to new situations with unique protection requirements at a time when environmental considerations have eliminated our most effective suppressants (halons), making the early detection of a fire even more critical. This article describes some of these developments, suggests possible applications, and indicates limitations to the technologies which need to be overcome before exploitation is feasible.

\section{0,295}

PB95-155560 PC A04/MF A01

National Inst. of Standards and Technology (TS), Gaithersburg, MD. National Voluntary Lab. Accreditation Program.

National Voluntary Laboratory Accreditation Program: Carpet and Carpet Cushion.

S. Galowin, W. J. Ros siter, W. A. Hall, and L. I. Knab. Oct $94,74 \mathrm{p}$, NIST/HB-150/6.

Also available from Supt. of Docs as SN003-00303300-6. See also PB95-155552.

Keywords: "Carpets, *Laboratories, "Certification, Materials testing, Procedures, Requirements, Calibration standards, Standardization, Licensure, Methodology, Accuracy, Performance evaluation, Carpet cushion, Carpet pads, National Voluntary Laboratory Accreditation Program, US NIST.

NIST Handbook 150-6 presents the technical requirements of the National Voluntary Laboratory Accreditation Program (NVLAP) for Carpet and Carpet Cushion (formerly called the Carpet Testing program). It is intended for information and use by staff of accredited laboratories, those laboratories seeking accreditation, other laboratory accreditation systems, users of laboratory services, and others needing information on the requirements for accreditation under the Carpet and Carpet Cushion program. This publication supplements NIST Handbook 150 (PB94-178225) 'NVLAP Procedures and General Requirements' which contains Part 285 of Title 15 of the U.S. Code of Federal Regulations (CFR) plus all general NVLAP procedures, criteria, and policies. Handbook 150-6 contains information that is specific to the Carpet and Carpet Cushion program and does not duplicate information contained in the Procedures and General Requirements. It is organized to cross-reference with Handbook 150

\section{0,296}

PB95-164810 Not available NTIS

National Inst. of Standards and Technology (BFRL) Gaithersburg MD Fire Science Div.

Acoustic Emission of Structural Materials Exposed to Open Flames.

Final rept.

W. Grosshandler, and M. Jackson. 1994, 20p.

See also PB93-138980.

Pub. in Fire Safety Jnl. 22, p209-228 1994.

Keywords: "Fire tests, "Acoustic emission, "Construction materials, "Fire detectors, Heat flux, Signal processing, Fires, Plywood, Wallboard, Flames, Fire safety, Transducers, Reprints.

The use of acoustic emission (AE) as an early indicator of structural materials exposed to a flame has been investigated and found to be possible. Piezoelectric 
transducers have been mounted directly on $0.5 \mathrm{~m}$ long simply supported beams of aluminum, gypsum board, wood and plastic, and have been used to record ultrasonic events resulting from a small flame placed under the beam. The number of $A E$ events in a minute and the cumulative energy released during the heating cycle provide a good measure of the overheated state of some of these materials even before a temperature increase is indicated. The measured signals varied in energy and number with the type of material, the thickness of the specimen and heat flux. Wood was particularly susceptible to acoustic emission, producing more than 1000 events/min in a solid fir board and $30 / \mathrm{min}$ in $13 \mathrm{~mm}$ thick plywood when the flame exceeded 1 $\mathrm{kW}$ Some critical issues which remain to be investigated before this technique can be adapted to practical fire detection are mentioned.

00,297

PB95-180162 Not available NTIS

National Inst. of Standards and Technology (BFRL), Gaithersburg, MD. Fire Science Div.

Influence of Ignition Source on the Flaming Fire Hazard of Upholstered Furniture. (NIST Reprint). Final rept.

T. G. Cleary, T. J. Ohlemiller, and K. Villa. 1994,

$24 p$.

See also PB92-205384

Pub. in Fire Safety Jnl. 23, p79-102 1994

Keywords: "Fire hazards, "Ignition, *Upholstery, Fires Combustion, Furniture, Calorimeters, Residential buildings, Fabrics, Chairs, Reprints, "Home fires.

A set of upholstered chairs constructed from five different fabric/foam combinations was subjected to a variety of ignition sources suggested by fire statistics. The sources included a cigarette, a small match-like flame, an incandescent lamp, a space heater, and a large flame source (TB 133 ignition source). The tests were performed in a furniture calorimeter where heat release rate and species production rates were obtained. For any chair type, the time to the peak heat release rate depended on the ignition sequence, but the magnitude of the peak did not, within the scatter of the data for any given chair. HAZARD 1, the fire hazard assessment method developed at the National Institute of Standards and Technology (NIST), was used to quantify the hazard posed by the different ignition scenarios.

00,298

PB95-180311 Not available NTIS

National Inst. of Standards and Technology (BFRL),

Gaithersburg, MD. Fire Science Div.

Early Detection of Room Fires Through Acoustic Emission. (NIST Reprint).

Final rept.

W. Grosshandler, and E. Braun. 1994, 12p.

See also PB94-112257.

Pub. in Proceedings of International Symposium on Fire Safety Science (4th), Ottawa Ontario, Canada, June 13-17, 1994, p773-784.

Keywords: "Fire detectors, "Acoustic emissions, Warning systems, Signal processing. Fire alarm systems, Acoustic detectors, Fire safety, Transducers, Acoustic signals, Reprints.

Acoustic emission (AE) has been shown to be a viable concept for the early indication of an open flame impinging on various structural materials. To assess its effectiveness in a more realistic environment, experiments have been performed in a $2.4 \mathrm{~m}$ cubical room constructed of gypsum board and wood. AE transducers were mounted on top of a ceiling joist and on a wall stud. The threats examined were a natural gas fire producing a thermal load up to $125 \mathrm{~kW}$, and a charring condition achieved by attaching a $550 \mathrm{~W}$ electrical heater to a wall stud. A signal discernable above the background was recorded from at least one $A E$ sensor in six of nine situations. The conclusion is that $\mathrm{AE}$ emission appears to be sufficiently sensitive to detect two particular threats, and that an overheated condition in a wall or ceiling can be detected if it is not more than $3 \mathrm{~m}$ from the transducer.

00,299

PB95-182267 PC A03/MF A01

National Inst. of Standards and Technology (BFRL), Gaithersburg, MD.
Comparison of Fire Sprinkler Piping Materials: Steel, Copper, Chlorinated Polyvinyl Chloride and Polybutylene, in Residential and Light Hazard Installations.

K. A. Notarianni, and M. A. Jackson. Jun 94, 48p NISTIR-5339.

Sponsored by Fire Administration, Emmitsburg, MD.

Keywords: "Sprinklers, "Vinyl plastics, "Piping systems, "Steels, "Buildings, "Mechanical properties, *Copper, Design criteria, Corrosion, Pipes, Polyvinyl chloride, Bending, Fire protection, Specifications, Degradations, Thermal expansion, "Chlorinated polyviny chloride, "Polybutylene.

A literature based study was conducted at the Building and Fire Research Laboratory of the National Institute of Standards and Technology to compare characteris tics and usage of steel, copper, chlorinated polyviny chloride and polybutylene fire sprinkler pipe primarily related to residential and light hazard installations. This report addresses key variables, such as material properties, usage criteria and limitations, design and installation requirements, economics and maintenance. This study was sponsored by the United States Fire Admin istration, and presents information useful for the selection of a sprinkler pipe material.

00,300

PB95-189452 PC A03/MF A01

National Inst. of Standards and Technology (BFRL), Gaithersburg, MD. Fire Science Div.

Review of Measurements and Candidate Signatures for Early Fire Detection.

W. L. Grosshandler. Jan 95, 44p, NISTIR-5555.

Keywords: *Fire detectors, "Infrared signatures, "Fire protection, Combustion, Smoke, Fires, Acoustic meas urement, Transport properties, Flames, Carbon monoxide, Test methods.

The physical and chemical transformations associated with a burgeoning fire are discussed and the results of past experimental measurements of these transformations are summarized. Standard test methods for the current generation of fire detectors and recent developments in detection technologies for which existing standards may not be suitable are described. The literature has been reviewed to determine the extent to which fires have been characterized in their early phase. One finds dramatic variations in the measured magnitude and rate of growth of $\mathrm{CO}$ concentration in a variety of standard fires. The variation is also large between repeat runs of the same tests. When scaled by estimated mass consumed of fuel, the different standard fires group a bit more systematically. Additional measurements of species, temperature and velocity just above the flame are suggested to get a more complete footprint of each fire type. Similar measurements of non-fire nuisance sources are required in order to discriminate between a fire and non-threatening situation with a high degree of certainty. The concept of a universal fire emulator/detector evaluator ( $\mathrm{FE}$ DE) is introduced. The objective is to have a facility that will eliminate the unavoidable run-to-run variations associated with full-scale tests, and to allow more well controlled environments. Computational fluid dynamics could then be used to insert the fire source into the space being protected to guide detector placement and to predict system performance, as well as to compare alternative systems and new concepts on a level, realistic playing field.

00,301

PB95-231585 PC A05/MF A01

National Inst. of Standards and Technology (BFRL), Gaithersburg, MD. Fire Science Div.

Behavior of Mock-Ups in the California Technica Bulletin 133 Test Protocol: Fabric and Barrier Etfects.

T. J. Ohlemiller, and J. R. Shields. May 95, 80p NISTIR-5653.

Keywords: "Furniture, "Flammability testing, "Hea transmission, Upholstery, Fabrics, Buring rate, Hea flux Ignition, Heat transmission, Flame propagation, Heat transfer, Calorimeters, Fire tests, "California Technical Bulletin 133.

Twenty-seven material combinations (seven fabrics, four barriers and two polyurethane foams) were tested in four cushion mock-up form in accord with California Technical Bulletin 133 using a furniture calorimeter. Both mock-up and Cone sample behavior were recorded on video to facilitate behavioral comparisons of the samples; distinct differences were noted for ther- moplastics fabrics. The mock-up behavior always comprised at least a heat release peak during the $80 \mathrm{sec}-$ ond gas burner exposure; it often included a later and larger peak as well. A statistical fit of the available data to these more complex types of correlations appears to work best for charring fabrics; it helps improve the correlation for all types of fabrics but at least two matenal combinations were outliers.

\section{0,302}

PB96-102181 Not available NTIS

National Inst. of Standards and Technology (BFRL) Gaithersburg, MD. Fire Safety Engineering Div.

Some Factors Affecting the Design of a Calorimeter Hood and Exhaust.

Final rept.

L. Y. Cooper. 1994, 14p

See also PB94-139193.

Pub. in Jnl. of Fire Protection Engineering, v6 n3 p99 1121994

Keywords: "Plumes, "Calorimeters, "Fire tests "Furniture, Exhaust systems, Hoods, Flames, Walls, Flow rate, Design criteria, Mathematical models, Combustion, Reprints.

This paper considers factors affecting the design of an effective and versatile calorimeter hood and exhaust system. The purpose of the calorimeter, design functions, and inherent limitations of a particular design are discussed. The interactions between the hood structure and the fire and its plume are analyzed in the context of avoiding: flame impingement on the hood; en hanced combustion of a test article, over and above that of a free-burn; loss of combustion product plume gases due to 'spill-over' below the hood; and unaccept able dilution of plume gases in the measurement sec tion of the exhaust duct. Methods to partially or completely achieve the ideal design are presented. These include the combined features of adjustable hood ele. vation and adjustable hood exhaust rate.

00,303

PB96-128095 PC A06/MF A02

National Inst. of Standards and Technology (BFRL) Gaithersburg, MD. Fire Science Div.

Study of Technology for Detecting Pre-Ignition Conditions of Cooking-Related Fires Associated with Electric and Gas Ranges and Cooktops, Phase 1 Report.

E. L. Johnsson. Oct 95, 117p, NISTIR-5729.

Contract CPSC-IAG-95-1145

Sponsored by Consumer Product Safety Commission, Bethesda, MD.

Keywords: "Fires, "Cooking devices, "Ignition, Residential buildings, Kitchens, Reviews, Technlogy innovation.

A significant portion of residential fires stem from kitchen cooking fires. Existing fire data indicate that cooking fires primarily are unattended and most often involve oil or grease. The purpose of this investigation was to ascertain the existence of one or more common features or characteristics of the pre-ignition environment that could be used as input to a sensor in a pre-fire detection device. The ultimate goal of this continuing study is to evaluate the feasibility of incorporating such a device into the range that would react to a pre-fire condition and reduce the occurrence of unwanted kitchen fires.

00,304

PB96-141056 Not available NTIS

National Inst. of Standards and Technology (BFRL)

Gaithersburg, MD. Fire Safety Engineering Div.

Evaluation of Sprinkler Activation Prediction Meth-

ods.

Final rept

D. Madrzykowski. $1995,8 p$

Pub. in International Conference on Fire Science and Engineering (1st), ASIAFLAM '95, Kowloon, Hong Kong, March 15-16, 1995, p211-218.

Keywords: “Sprinklers, "Activation, "Time lapse, Fire suppression, Flame propagation, Fire detectors, Gas burners, Fire tests, Computer models, Reprints, Com partment fires, Heat release rate, Thermal detectors.

The objective of this study was to evaluate the ability of sprinkler activation models to predict activation time Large scale compartment fire tests were used to obtain activation times for four different types of sprinklers. The tests were conducted in an $18.9 \mathrm{~m}$ by $2.35 \mathrm{~m}$ high compartment using floor based, gas burner fires with constant heat release rates of $115,155,215,290$, and 


\section{BUILDING INDUSTRY TECHNOLOGY}

\section{Building Equipment, Furnishings, \& Maintenance}

$520 \mathrm{~kW}$. Non-dimensional sprinkler radial positions, $r$ $H$ of 0.67 and 1.3 were evaluated. In addition to sprinkler activation times, ceiling jet temperature, velocity, and radiation measurements were made. The study in cluded: (1) a review of public domain, personal-computer based, single-compartment thermal-detector activation models; (2) an analysis of predicted vs. experimental sprinkler activation times; and (3) a method to determine the applicability of current sprinkler activation models.

\section{0,305}

PB96-154810 PC A11/MF A03

National Inst. of Standards and Technology (BFRL), Gaithersburg, MD

Santa Ana Fire Department Experiments at South Bristol Street.

W. D. Walton, A. D. Putorti, W. H. Twilley, and J. C

Albers. Feb 96, 220p, NISTIR-5776

See also PB95-188868 and PB96-102934. Prepared in cooperation with Santa Ana City Fire Dept., CA. Fire Safety Div.

Keywords: "Residential buildings, "Fire tests, Furniture, Flammability, Rooms, Smoke detectors, Sprinklers, Temperature measurement, Velocity measure ment, Flame propagation, Heat flux, Santa Ana(California), Fuel loads, Mass loss.

A series of fire experiments were conducted in vacan single family dwelling on South Bristol Street in Santa Ana, California. Fire experiments were conducted in bedrooms and living rooms. Fuel consisted of either home furnishings or a propane burner. Fire phenomena measured included: temperatures within various rooms, wall jet velocity, fuel mass dring burning, heat flux smoke detector activation time, sprinkler activation time, oxygen concentration, and time to full room involvement

00,306

PB96-155411 Not available NTIS

National Inst. of Standards and Technology (BFRL), Gaithersburg, MD. Fire Science Div.

Quantifying the Ignition Propensity of Cigarettes. Final rept.

T. J. Ohlemiller, K. M. Villa, E. Braun, J. R. Lawson, R. G. Gann, K. R. Eberhardt, and R. H. Harris. 1995 ,

Pub. in Fire and Materials, v19 p155-169 1995

Keywords: "Ignition, "Flammability testing, "Fire hazards, Upholstery, Fabrics, Furniture, Combustion, Tobacco products, Fire tests, Test products, Statistical analysis, Reprints, "Cigarettes.

Research funded under the Fire Safe Cigarette Act of 1990 (United States Public Law 101-352) has led to the development of two test methods for measuring the ignition propensity of cigarettes. The Mock-Up Ignition Test Method uses substrates physically similar to upholstered fumiture and mattresses: a layer of fabric over padding. The measure of cigarette performance is ignition or non-ignition of the substrate. The Ciga rettes Extinction Test Method replaces the fabric/pad ding assembly with multiple layers of common filter paper. The measure of performance is full-length burning or self-extinguishment of the cigarette. Routine measurement of the relative ignition propensity of cigarettes is feasible using either of the two methods. Using the two methods, some current commercial cigarettes are shown to have reduced ignition propensities relative to the current best-selling cigarettes.

\section{0,307}

PB96-156187 Not available NTIS

National Inst. of Standards and Technology (BFRL),

Gaithersburg, MD. Fire Safety Engineering Div.

Protecting Your Family from Fire.

Final rept.

R. W. Bukowski. 1993, 19p

Sponsored by Fire Administration, Emmitsburg, MD.

Pub. in U.S. Fire Administration Public Fire Safety Educational Booklet, p1-19 Feb 93.

Keywords: "Fire safety, "Residential buildings, "Fire protection, Smoke detectors, Fire detectors, Fire detection systems, Warning systems, Fire alarm systems, Sprinklers, Reprints, Heat detectors.

This fire safety educational booklet provides information on residential fires, especially involving children and older persons. The proper use of smoke detectors heat detectors, fire alarm systems, and residentia sprinklers is presented. Guidelines on the preparation of a hcme escape plan is included.

\section{0,308}

PB96-193800 PC A06/MF A01

National Inst. of Standards and Technology (BFRL), Gaithersburg, MD. Structures Div.

State of the Art Report on Seismic Design Requirements for Nonstructural Building Components. L. T. Phan, and A. W. Taylor. Jun 96, 78p, NISTIR5857 .

Keywords: "Earthquake engineering, "Building codes, "Lighting equipment, "Ceilings, "Sprinkler systems, Seismic design, United States, New Zealand, Japan, Seismic effect, Earthquake damage, Damage assessment, Dynamic response, Loads(Forces), Displacement, Requirements, Nonstructural components.

Seismic design requirements for nonstructural building components of five major building codes, including the 1994 Uniform Building Code, the 1994 Standard Building Code, the 1994 NEHRP Recommended Provisions for Seismic Regulations for New Buildings, the New Zealand Building Code, and the Japanese Building Code, were reviewed in this study. Comparisons of codes reveal wide variation in seismic force and displacement requirements, both in terms of levels of stringency and levels of details. The study also found a lack of focused investigations, dedicated to mitigating seismic damage to nonstructural building components, even though widespread damage to nonstructural building components continues to be observed in recent earthquakes. Based on the findings of this review, areas of needed research were identified.

\section{0,309}

PB97-111546 Not available NTIS

National Inst. of Standards and Technology (BFRL). Gaithersburg, MD. Building Environment Div.

Experimental Verification of a Moisture and Heat Transfer Model in the Hygroscopic Regime. Final rept.

D. M. Burch, R. R. Zarr, and A. H. Fanney. 1995,

$10 \mathrm{p}$.

Pub. in 'Thermal VI' Thermal Performance of the Exterior Envelopes of Buildings VI, Clearwater Beach, FL., December 4-8, 1996, p273-282 1995.

Keywords: "Moisture, "Heat transfer, Building envelopes, Calibrated hot box, Manufactured housing, Analysis, Transfer, Reprints, MOIST.

The National Institute of Standards and Technology (NIST) has developed a personal computer model called NOIST, for predicting the transient moisture and heat transfer within building envelopes. The paper summarizes selected results from a comprehensive laboratory experiment conducted to verify the accuracy of the computer model in the hygroscopic regime. The paper discusses three different multilayer wall specimens installed in a calibrated hot box. The exterior surfaces of the wall specimens were first exposed to both steady and time-dependent winter conditions, while their interior surfaces were maintained at 21 degrees $\mathrm{C}$ ( 70 degrees $\mathrm{F}$ ) and $50 \%$ relative humidity.

\section{0,310}

PB97-111553 Not available NTIS

National Inst. of Standards and Technology (BFRL), Gaithersburg, MD. Building Environment Div.

Testing Conformance and Interoperability of BACnet (Trade Name) Building Automation Products.

Final rept

S. T. Bushby. 1996, 7p

See also PB92-181221. Sponsored by General Services Administration, Washington, DC and Department of Energy, Washington, DC

Pub. in CIBSE/ASHRAE Joint National Conference, Harrogate, United Kingdom, September 29-October 1, $1996, \mathrm{p} 1-7$

Keywords: "Buildings, "Automation, "Control systems design, Automatic control, Computer communications, Communication networks, Protocol(Computers), Energy management systems, Space HVAC systems, Digital systems, Conformance, Interoperability, Reprints.

The BACnet standard defines classes of conformance and other collections of protocol functionality. The paper describes BACnet conformance classification issues and the work of the NIST consortium in developing testing tools and applying them to test products made by member companies.
National Inst. of Standards and Technology (BFRL), Gaithersburg, MD. Building Environment Div.

Factors Affecting the Energy Consumption of Two Refrigerator-Freezers.

Final rept.

J. Y. Kao, and G. E. Kelly. 1996, 11p.

Sponsored by Department of Energy, Washington, DC. Office of Codes and Standards.

Pub. in American Society of Heating. Refrigerating and Air-Conditioning Engineers Transaction Annual Meeting, San Antonio, TX., June 23-26, 1996, v102 pt2 p111.

Keywords: "Refrigerators, "Freezers, "Energy consumption, "Performance evaluation, Temperature measurement, Room temperature, Humidity, Doors, Performance tests, Reprints.

Two refrigerator-freezers, one with a top-freezer and one with side-by-side doors, were tested in the laboratory to determine the sensitivity of their energy consumption to various operational factors. Room temperature, room humidity, door openings, and the setting of the anti-sweat heater switch were the factors examined. The results indicated that the room temperature and door openings had a significantly greater effect on energy consumption than the other two factors. More detailed tests were then performed under different room temperature and door opening combinations. The relationship of door openings and the equivalent test room temperature were established. Finally, the effect on energy of different temperature settings were studied. Test results are presented and discussed.

\section{Building Standards \& Codes}

00,312

PB94-185196 Not available NTIS

National Inst. of Standards and Technology (BFRL), Gaithersburg, MD.

Standards Development in North America for Performance of Whole Buildings and Facilities.

Final rept

G. Davis, and J. G. Gross. May 93,6p

Pub. in Some Examples of the Application of the Performance Concept in Building, CIB Report 157, p6166 May 93

Keywords: *Standards, "Building codes, Residential buildings, Office buildings, School buildings, Energy consumption, Quality, Maintenance, Performance(Engineering), Reprints, "Building operation and maintenance, Building performance, ASTM E06

This paper reviews the activities of ASTM Committee E06 on Performance of Buildings and the Subcommittee E06.25 on Whole Buildings and Facilities. Planned standards activities are outlined for 14 task groups. Under development are standards related to objective rating of quality and functionality of office facilities, area measurements, scales for comparing the operation and maintenance of facilities, rating scales for comparing the functionality and quality of educational facilities, behavioral measures, measurement of serviceability, rating buildings for their overall energy performance and monitoring and comparing end-use electrical consumption of commercial buildings and monitoring the energy use of residential buildings. An overview of the full ASTM Committee E06 on Performance of Buildings is provided.

\section{0,313}

PB94-207511 PC A08/MF A02

National Inst. of Standards and Technology (BFRL) Gaithersburg, MD

Global Equivalence Ratio Concept and the Prediction of Carbon Monoxide Formation in Enclosure Fires.

W. M. Pitts. Jun 94, 172p, NIST/MONO-179.

Also available from Supt. of Docs. as SN003-003 03271-9. See also PB89-200091 and PB90-209602.

Keywords: "Carbon monoxide, "Fire hazards, "Buildings, Mortality, Combustion products, Global, Standards, Natural gas, Rooms, Exhaust gases, Enclosures, Safety engineering, Reaction kinetics, Pyrolysis, Ventilation, Polymers, Plywood, Mathematica models. Flow distribution, Reduced scale enclosure, Oxygenated polymers.

This report summarizes investigations of the formation of carbon monoxide $(\mathrm{CO})$ in enclosure fires--the most 
important factor in fire deaths. It contains the first complete review and analysis of the literature available on the global equivalence ratio (GER) concept. The results of a number of recent investigations (including work during the past fiscal year) which were either supported by the BFRL Grants Program or carried out inhouse are then discussed. Based on the findings, two completely new mechanisms for the formation of $\mathrm{CO}$, in addition to the quenching of a fire plume by a rich upper layer covered by the GER concept, are identified. The first involves reaction of rich flame gases with air which is entrained directly into the high-temperature upper layer of an enclosure fire. The second results from the pyrolysis of oxygenated polymers (such as wood) which are located in a rich, high-temperature upper layer. The findings of these studies form the basis of an analysis which yields the conditions for which use of the GER concept is appropriate for predicting CO formation in enclosure fires.

\section{0,314}

PB95-126306 Not available NTIS

National Inst. of Standards and Technology (BFRL), Gaithersburg, MD. Building and Fire Research Lab. Office.

Growing Significance of ClB.

Final rept.

J. G. Gross. 1992, 1p.

Pub. in CIB Newsletter 'Information', p1 Nov/Dec 92. Keywords: "Building codes, "Research, Buildings, Standards, Standardization, Construction materials, Fire safety, Reprints, "Building technology, International Council for Building Research Studies and Documentation.

This editorial addresses the need for worldwide cooperation in building research. Activities and opportunities are cited where the International Council for Building Research, Studies and Documentation (CIB) can provide a forum for facilitating cooperative activities.

00,315

PB95-231858 PC A05/MF A02

Green (Melvyn) and Associates, Inc., Torrance, CA

Comparison of the Seismic Provisions of Model Building Codes and Standards to the 1991 NEHRP Recommended Provisions.

May 95, 98p, NIST/GCR-95/674.

Sponsored by National Inst. of Standards and Technology (BFRL), Gaithersburg, MD. and Federal Emergency Management Agency, Washington, DC. Mitigation Directorate.

Keywords: "Building codes, "Earthquake resistant structures, Federal building, Structural design, Seismic waves, Design standards, Earthquake engineering, Standards, Design criteria, Regulations, Tables(Data).

The intent of this study is to review the seismic provisions of the current editions the BOCA National, SBCCI Standard and the ICBO Uniform Codes to determine whether the codes provide an equivalent level of safety to that contained in the 1991 Edition of the NEHRP Provisions. In addition the provision of the CABO One and Two Family Dwelling Code and ASCE 7-93 are reviewed.

\section{0,316}

PB96-141239 Not available NTIS

National Inst. of Standards and Technology (BFRL), Gaithersburg, MD. Fire Safety Engineering Div. Predicting the Fire Performance of Buildings: Establishing Appropriate Calculation Methods for Regulatory Applications.

Final rept.

R. W. Bukowski. 1995, 10p

Pub. in Proceedings of the International Conference on Fire Science and Engineering (1st), ASIAFLAM '95, Kowloon, Hong Kong, March 15-16, 1995, p9-18.

Keywords: "Fire safety, "Design standards, "Safety factors, Fire codes, Building codes, Regulations, Risk analysis, Fire protection, Performance standards, Fire hazards, Models, Reprints.

This paper explores questions from the perspective of the fire scientist, the practicing engineer, and the regulatory official. The fir scientist needs to be explicit about the impact of assumptions on the applicability of the results. The engineer needs to utilize methods and assumptions which are justified by the application and to assess the sensitivity and uncertainty implications. The regulatory officials are insisting on appropriate and properly documented methods. There is a need for models and calculations incorporated into codes of practice, handbooks, or the codes themselves to be reviewed, verified, documented, and approved for use in specific manners and by qualified persons. There are international efforts to define levels of risk acceptable to society in specific occupancies.

\section{Construction Management \& Techniques}

00,317

PB94-185998 Not available NTIS

National Inst. of Standards and Technology (BFRL) Gaithersburg, MD. Building and Fire Research Lab. Of-

Infratechnologies: Tools for Innovation.

Final rept

R. N. Wright. $1993,2 p$

Pub. in Civil Engineering 63, n11 p68-69 Nov 93.

Keywords: "Construction, "Technology innovation Quality assurance, Building codes, Standards, Computer aided design, Technology assessment, Technology utilization, Reprints.

The quest for innovation and quality assurance in consiruction makes it important to recognize the need for technologies far beyond those in use today. Although we now check for conformance to codes and standards, gather and document product information, use computer-aided design (CAD) and engineering, and apply the principles that help ensure quality, there will be better ways to do these tasks in the future. We call them infratechnologies.

\section{0,318}

PB94-186004 Not available NTIS

National Inst. of Standards and Technology (BFRL) Gaithersburg, MD. Building and Fire Research Lab. Of-

Status of Construction and Construction Technologies.

R. N. Wright. 1993, 9p

Pub. in Proceedings Symposium on Advanced Build ing Technologies, Troy, NY., April 10, 1992, p10-18 1993.

Keywords: "Construction industry, "Competition "North America, Automation, Productivity, Buildings, Design, Construction materials, Maintainability, Market research, Reprints, "Building technology, Intelligent buildings, Constructability

Trends in building technology for North America will be dominated by advances in building process technologies: advanced computation and automation These will facilitate effective responses to demands for increasing the international competitiveness of North American commerce and industry, supporting new industries, commerce and life styles, improving safety and health, and conserving energy and the environment. Advanced information technologies will affect organization of the building process to allow better attention to design to issues such as constructability, main tainability and productivity of constructed facilities. Automation will advance in design, construction and operation (intelligent buildings) of constructed facilities.

\section{0,319}

PB94-193646 PC A03/MF A01

National Inst. of Standards and Technology, Gaithersburg, MD

Program of the Subcommittee on Construction and Building.

R. N. Wright, A. H. Rosenfeld, and A. J. Fowell. Jun 94, 50p, NISTIR-5443.

Prepared in cooperation with Department of Energy, Washington, DC. Assistant Secretary for Energy Efficiency and Renewable Energy.

Keywords: "Construction, "Competitiveness, "Technology innovation, Health, Automation, Construction materials, Buildings, Energy efficiency, Productivity, Research, Safety, "Building technology.

The National Science and Technology Council (NSTC), a cabinet-level group charged with setting Federal technology policy, has established the Committee on Civilian Industrial Technology (CCIT), to enhance the international competitiveness of U.S. indus try. The Subcommittee on Construction and Building
C\&B) of CCIT deals with Federal technology policies and programs related to the industries that conduct $R \& D$ and produce, operate and maintain constructed facilities including buildings and infrastructure. The report provides some background on the construction industry, describes the role, the goals, and milestones of the program, and lists the Federal agencies participating in the Subcommittee. The goals of better buildings and improved health and safety of construction workers have been strongly endorsed in a white paper developed and published by the Civil Engineering Research Foundation. The white paper is included as an appendix

\section{0,320}

PB94-216405 Not available NTIS

National Inst. of Standards and Technology (CAML), Gaithersburg, MD.

Standards in Building Economics: Why We Need Them and How to Write Them.

Final rept.

H. E. Marshall. 1992, 7p

Pub. in Proceedings of 1992 American Association of Cost Engineers Transactions, Orlando, FL., June 28 July 1,1992 , pL.5.1-L.5.7.

Keywords: "Buildings, "Economic analysis, "Standards, Construction management, Budgeting Life cycle costs, Profits, Benefit cost analysis, Reprints.

Standard methods, guides, and classifications are available for use in the economic evaluation of buildings and building components. Standards help owners, consultants, designers, and managers to reduce project costs and enhance profits by providing a con sistent basis for comparing the economic worth of building alternatives. This applies to government and private projects undertaken domestically and abroad. The paper explains how sometimes conflicting vested interests can work as a team in the standards process to create consensus standards. It surveys and explain the application of specific ASTM standard methods, such as life-cycle costing and the savings-to-investment ratio methods. It explains the role that business, government agencies, and professional societies such as the American Association of Cost Engineers (AACE) play in the promulgation of standards. The paper concludes that organizations need to participate in or at least be abreast of the latest developments in standard methods to reduce expenditures, keep competitive, and maximize profits.

00,321

PB95-122537 PC A03/MF A01

National Inst. of Standards and Technology Gaithersburg, MD

Program of the Subcor
Building (July 1994)

Building (July 1994).

R. N. Wright, A. H. Rosent

Supersedes PB94-193646. Prepared in cooperation with Department of Energy, Washington, DC. Assistant Secretary for Energy Etficiency and Renewable Energy

Keywords: "Construction, "Competitiveness, "Technology innovation, Health, Buildings, Construction materials, Safety, Automation, Research and development, Productivity, Energy efficiency.

The President has established the National Science and Technology Council (NSTC), a cabinet-level group charged with setting Federal science and technology policy, to coordinate and prioritize R\&D and deployment strategies across a broad cross-section of public and private interests. It has established nine research and development committees, including the Committee on Civilian Industrial Technology (CCIT) to collaborate with the private sector in developing a comprehensive national technology policy. The purpose of CCIT is to enhance the international competitiveness of U.S. industry through Federal technology policies and programs. The Subcommittee on Construction and Building coordinates and defines priorities for Federal re search, development and deployment related to the industries that produce, operate and maintain constructed facilities, including buildings and infrastruc ture. The Subcommittee on Construction and Building has studied research priorities including those expressed by the construction industry and defined two priority thrusts; better constructed facilities and health and safety of the construction workforce.

00,322

PB95-154704 PC A07/MF A02 
National Inst. of Standards and Technology (BFRL), Gaithersburg, MD.

Rationale and Preliminary Plan for Federal Research for Construction and Building.

R. N. Wright, A. H. Rosenfeld, and A. J. Fowell. Nov 94, 140p, NISTIR-5536.

Also available from Supt. of Docs. Sponsored by Department of Energy, Washington, DC. Assistant Secretary for Energy Efficiency and Renewable Energy.

Keywords: "Research, "Buildings, "Construction, "Technology assessment, Construction materials, Government/industry relations, Technology transfer. Competitiveness, Project management, Occupational safety, Civil engineering.

The National Science and Technology Council (NSTC), a cabinet-level group charged with setting federal technology policy, coordinates R\&D strategies across a broad cross-section of public and private in terests. It has established nine research and development committees, including the Committee on Civilian Industrial Technology (CCIT), to collaborate with the private sector in developing a comprehensive national technology policy. The purpose of CCIT is to enhance the international competitiveness of U.S. industry through federal technology policies and programs. The Subcommittee on Construction and Building (C\&B) of dustries that produce, deployment related to the in dustries that produce, operate and maintain con-
structed facilities, including buildings and infrastructure.

00.323

PB96-122593 Not available NTIS

National Inst. of Standards and Technology (CAML), Gaithersburg, MD

Economic Methods and Risk Analysis Techniques for Evaluating Building Investments: A Survey. Final rept.

H. E. Marshall. 1991, 55p.

See also PB90-241589.

Pub. in CIB Report. Published by the International Council for Building Research, Studies and Documentation, n136 55p 1991.

Keywords: "Buildings, "Construction management, "Life cycle costs, "Cost benefit analysis, Cost engineering, Investments, Budgeting, Interest rate of return, Return on investment, Cost estimation, Economic analysis, Project administration, Risk management, Decision making, Reprints.

Traditional economic methods--life cycle costing, benefit-to-cost ratio, net benefits analysis, adjusted internal rate of return, and discounted payback--are described for evaluating building decisions about accepting or rejecting a given building, and the economically efficient combination of projects competing for a limited budget. Appropriate applications for each economic method are described. Technically correct formulas for the methods are presented. Techniques are described that to some extent account for uncertainty in input variables and in some cases risk exposure and risk attitude. The techniques are conservative benefit and cost estimating, sensitivity analysis, the risk-adjusted discount rate, mean-variance criterion, coefficient of variation, decision analysis, simulation, mathematical analytical technique, and portfolio analysis. Advantages and disadvantages of each are described. Guid ance is provided for selecting the appropriate technique for any given investment problem.

\section{0,324}

PB96-137104 PC A06/MF A02

National Inst. of Standards and Technology (BFRL), Gaithersburg, MD.

National Planning for Construction and Building $R$ and $\mathrm{D}$.

R. N. Wright, A. H. Rosenfeld, and A. J. Fowell. Dec 95, 105p, NISTIR-5759.

Prepared in cooperation with National Science and Technology Council, Washington, DC. Subcommittee on Construction and Building. Sponsored by Department of Energy, Washington, DC. Assistant Secretary for Energy Efficiency and Renewable Energy,

Keywords: "Construction industry, "Building materials, "Research and development, "Government/industry relations, Construction materials, Research projects, Cooperation, Coordination, Research management, Competitiveness, Technology innovation, Goals, Human factors engineering. Automation, Information systems, Regulations, Private sector.

This planning report is a resource document designed to provide the private sector with a straw man on a di- rection and strategy for construction and building research, development, and demonstration to achieve the National Construction Goals, and the products likely to be produced by that effort. The report also will provide federal agencies with information on each other's R\&D programs to facilitate coordination of effort. The Federal and private sector plans will be coordinated to form an industry-led National Plan to meet the National Construction Goals. This report provides background on each of the goals, the measures by which progress can be gauged, and research needed. The industry perspective on relative importance of the different goals by the various sectors of the construction industry is reported, and the intitial strategy proposed by some of those sectors to provide a platform for the National Plan is outlined.

\section{0,325}

PB96-158670 PC A06/MF A01

National Inst. of Standards and Technology (BFRL), Gaithersburg, MD. Office of Applied Economics.

Multiattribute Decision Analysis Method for Evaluating Buildings and Building Systems.

Final rept.

G. A. Norris, and H. E. Marshall. Sep 95, 90p,

NISTIR-5663.

See also PB93-146017.

Keywords: "Buildings, "Life cycle costs, "Cost benefit analysis, Building materials, Construction, Fabrication, Architecture, Space HVAC systems, Energy management systems, Ranking, Project administration, Operating costs, Cost engineering, Investments, Return on investment, Economic analysis, Alternatives, Decision making, MADA(Multiattribute Decision Analysis).

Multiattribute decision analysis (MADA) methods consider non-financial attributes (qualitative and quantitative) in addition to common financial worth measures when evaluating project alternatives. The report reviews 14 classes of methods for performing MADA. It summarizes their usefulness for screening, ranking, and choosing among projects; their data input requirements; and how each method scores project alternatives. Two methods--the analytical hierarchy process (AHP) and non-traditional capital investment criteria (NCIC)--are described in detail. Assumptions, procedures, strengths, and limitations are described for each.

\section{Construction Materials, Components, \& Equipment}

\section{0,326}

\section{AD-A310 426/2 PC A03/MF A01}

National Bureau of Standards, Gaithersburg, MD. Polyvinyl Chloride (PVC) Plastic Drain, Waste, and Vent Pipe and Fittings.

Apr 65, 28p.

Availability: Document partially illegible.

Keywords: "Pipes, "Drainage, "Plastics, "Vents, *Polyvinyl chloride, Requirements, Quality assurance, Wastes, Fittings.

The purpose of this Commercial Standard is to establish, on a national basis, standard dimensions and significant quality requirements for polyvinyl chloride (PVC) plastic drain, waste, and vent (DWV) pipe and fittings. It is also intended to inform producers, distributors, engineers, code officials, and users of the significant qualities of this product to assist buyers and vendors in obtaining and vending quality merchandize for the benefit of the user, and to promote understanding among all these groups concerning commercially available PVC plastic DWV pipe and fittings.

\section{0,327}

\section{AD-A310 724/0 PC A03/MF A01}

National Bureau of Standards, Gaithersburg, MD. Acrylonitrile-Butadiene-Styrene (ABS) Plastic Drain, Waste, and Vent Pipe and Fittings.

Commercial standard

Apr 65, 27p, CS270-65.

Availability: Document partially illegible.

Keywords: "Styrenes, "Acrylonitrile polymers, "Pipes, *Drainage, "Wastes, "Butadienes, "Vents, "Fittings, Organic compounds, Standards, Quality assurance, Plastics, Abs(Acrylonitrile- butadiene-styrene), Dvw(Drain-vent-waste).
The purpose of this Commercial Standard is to establish, on a national basis, standard dimensions and significant quality requirements for acrylonitrile-butadiene-styrene (ABS) plastic drain, waste, and vent (DWV) pipe and fittings. It is also intended to inform producers, distributors, engineers, code officials, and users of the significant qualities of this product, to assist buyers and vendor in obtaining and vending quality merchandise for the benefit of the user, and to promote understanding among all these groups concerning commercially available ABS plastic DWV pipe and fittings.

\section{0,328}

PB94-139722 PC A03/MF A01

California Univ., Berkeley. Dept. of Mechanical Engineering.

Fire Induced Thermal Fields in Window Glass I: Theory.

Rept. for 31 Aug 91-31 Aug 92.

A. A. Joshi, and P. J. Pagni. Nov 93, 35p, NISTGCR-93-634.

Grant 60NANB1D1168

Sponsored by National Inst. of Standards and Technology (BFRL), Gaithersburg, MD

Keywords: "Glass, "Windows, "Fires, "Thermal analysis, Heat transfer, Buildings, Heat flux, Mathematical models, Construction materials, Temperature distribution.

Window glass breaking plays an important role in compartment fire dynamics as the window acts as a wall before breaking and as a vent after breaking. Previous work suggested a model for the time to breakage of a window glass exposed to a particular fire. In this paper, the glass thermal fields obtained using that model are examined in detail. The temperature field dependence on heat transfer coefficients, radiative decay length and flame radiation is explored. The results show that the glass surface temperature increases with a decrease in the decay length and in creases with an increase in flame radiation heat flux. Early in the fire, the glass temperature may be higher than the hot layer temperature due to direct impingement of flame radiation. Later the glass temperature lags the hot layer temperature. The variation of the time to breakage as a function of the shading width and decay length is also presented and the results indicate that the breaking time decreases with an increase in the shading width and decreases with a decrease in decay length. Heat flux maps for typical conditions indicate that most of the heat influx is stored in the glass, increasing its temperature.

\section{0,329}

\section{PB94-160751 PC A03/MF A01}

National Inst. of Standards and Technology (BFRL), Gaithersburg, MD

Performance Approach to the Development of Criteria for Low-Sloped Roof Membranes.

W. J. Rossiter G. J C. Frohnsdorff, L. W. Masters, and J. W. Martin. Jul 91, 35p, NISTIR-4638.

Keywords: "Roofs, "Membranes, "Waterproofing, "Industrial buildings, "Commercial buildings, Composite materials, Polymers, Bitumens, Thermoplastic resins, Insulation boards, Barner coatings, Vapor deposition, Requirements, Diagrams, Test methods Performance evaluation, United States, Low-sloped roof system, Structural deck

Waterproofing of roofs of commercial and industria buildings in the United States is prima rily accomplished using low-sloped membrane roofing systems. Estimates indicate that approximately 300 million square meters ( 3 billion square feet) of membrane roofing is installed annually. The three main types of membranes are built-up bituminous, single-ply elastomeric and thermoplastic, and polymer-modified bitumens. In addition to the membrane, the other main components are insulation boards and the structural deck, with elements such as vapor retarders and membrane surfacings used in some systems.

\section{0,330}

PB94-160777 PC A03/MF A01

National Inst. of Standards and Technology (BFRL), Gaithersburg, MD 
Microstructural Features of Some Low Water/Solids, Silica Fume Mortars Cured at Different Temperatures.

P. E. Stutzman, and J. R. Clifton. Apr 92, 20p, NISTIR-4790.

Keywords: "Mortars(Materials), "Microstructure, Silicon dioxide, Portland cements, Plasticizers, Concretes, *HPC(High Performance Concrete)

The microstructure of mortars with water/solids ratios (w/s) of 0.36 and 0.29 , cured under water at 7,23 , and $40 \mathrm{C}$, were studied by scanning electron microscopy and X-ray powder diffractometry. The mortars contained silica fume and a superplasticizer. The degree of hydration and extent of pozzolanic reaction was estimated after quantifying the residual unhydrated cement by image analysis and the mass percent calcium hydroxide by X-ray powder diffraction. Their hydroxide by X-ray powder diffraction. Their
microstructures were fairly homogeneous in both the microstructures were fairly homogeneous in both the
bulk paste and at the past-aggregate transition zone. In all mortars, the outer 250 micrometers was hydrated to about 85 percent and highly microcracked. The degree of hydration decreased rapidly beyond the outer zone to about 69 percent and less microcracking was observed. A temperature effect on the reactivity of silica fume was found.

00,331

PB94-164407 PC A03/MF A01

National Inst. of Standards and Technology (MSEL),

Gaithersburg, MD. Metallurgy Div.

Analysis of Failed Dry Pipe Fire Suppression Sys. tem Couplings from the Filene Center at Wolf Trap Farm Park for the Performing Arts.

M. R. Stoudt, J. L. Fink, and R. E. Ricker. Mar 94, $16 \mathrm{p}$, NISTIR-5389.

Sponsored by Wolf Trap Farm Park for the Performing Arts, Vienna, VA.

Keywords: *Pipe joints, *Sprinkler systems, "Brittle fracturing, Pipe bends, Cast iron, Metallurgical analysis, Freezing, Wolf Trap Farm Park(Virginia).

A detailed metallurgical analysis was performed on a number of gray cast iron couplings taken from a dry pipe fire suppression system at the Filene Center to verify the source of failure. The results of the analysis indicated that all of the specimens failed in a brittle manner due to the stresses induced by the expansion of freezing water. The available background information indicated that the ambient air temperature on the night of the failures was low enough to induce freezing in a relatively short time period.

00,332

PB94-172418 Not available NTIS

National Inst. of Standards and Technology (BFRL), Gaithersburg, MD. Fire Safety Engineering Div

Improvement in Predicting Smoke Movement in Compartmented Structures.

Final rept.

W. W. Jones, and G. P. Forney. 1993, 29p.

Pub. in Fire Safety Jnl. 21, p269-297 1993.

Keywords: *Fires, "Flow models, "Buildings, Fire safety, Radiative transfer, Ventilation, Smoke, Transport properties, Buoyancy, Reprints, "Smoke propagation, "Compartment fires, CFAST Model.

This paper describes improvements which have been made in the CFAST model of fire growth and smoke transport for compartmented structures. In particular we are interested in the ability to model the movement of toxic gases from the room of origin of a fire to a distant compartment. The newest phenomena in the model are vertical flow and mechanical ventilation. $\mathrm{Fi}$ nally, we have improved the radiation transport scheme which affects energy distribution, and therefore the buoyancy forces. These are very important in actual situations relevant to fire growth and smoke propagation, as is demonstrated.

00,333

PB94-173051 Not available NTIS

National Inst. of Standards and Technology (BFRL)

Gaithersburg, MD. Building Materials Div.

Quantitative Phase Abundance Analysis of Three Cement Clinker Reference Materials by Scanning Electron Microscopy

Final rept.

P. E. Stutzman. $1990,12 p$

Pub. in Proceedings of Conference on Advances in Cementitious Materials, Gaithersburg, MD., July 2226, 1990, p199-210 1991.

Keywords: "Cements, "Scanning electron microscopy, "Image analysis, Test methods, $X$ rays,
Backscattering, Microstructure, Grain size, Brightness, Construction materials, Reprints, "Portland cement clinker, "Reference materials.

Three portland cement clinker reference materials are available from the Standard Reference Materials Program at the National Institute of Standards and Technology. They are the first clinker reference materials for phase abundance analyses and were selected as representative of North American clinker production with respect to the range of phase abundance, grain size, and distribution. They are intended for use in the development and testing of methods for quantitative phase abundance analyses of portland cement clinker. Portland cement clinker microstructures were characterized by backscattering electron imaging in the scan. ning electron microscope. Clinker phases were identified on the basis of their brightness, grain morphology, occurrence within the clinker, and composition Quantitative phase abundance analyses by point counting, and by image analysis of backscattered electron images and, for periclase, of X-ray maps, generally agreed with the reference material values. With its capability to clearly image polished clinker sections at a wide range of magnifications, the scanning electron mi-
croscope with $X$-ray analysis and with image analysis capabilities is a powerful tool for quantitative cement clinker microscopy.

\section{0,334}

PB94-185774 Not available NTIS

National Inst. of Standards and Technology, Gaithersburg, MD

Proficiency Testing as a Component of Quality Assurance in Construction Materials Laboratories.

Final rept.

J. H. Pielert. 1990,11p.

Pub. in Proceedings of International Symposium Test Quality and Quality Assurance in Testing Laboratories for Construction Materials and Structures, Saint-Remyles-Chevreuse, France, October 15-17, 1990, p60-70. rials, Philadelphia, PA.

Keywords: "Laboratories, "Construction materials, "Quality assurance, *Interlaboratory comparisons, Standards, Test facilities, Quality control, Standardization, Sampling, Concretes, Accuracy, Precision, Reprints, *Proficiency testing, Construction Materials Reference Laboratories.

Proficiency testing is a procedure for using results generated in interlaboratory test comparisons for the purpose of assessing the technical competence of participating testing laboratories. This gives users of laboratory services confidence that a testing laboratory is capable of obtaining reliable results. Interlaboratory testing involves the organization, performance and evaluation of tests on the same or similar materials by two or more different laboratories in accordance with predetermined conditions. Interlaboratory testing may also be used for checking the individual performance of laboratory staff, evaluating the effectiveness of a test method, and determining characteristics of a material or product. Programs in the United States which use proficiency testing to evaluate laboratory performance are discussed along with international programs which have been identified by RILEM Technical Committee 91-Cement Reference Laboratories. The U.S. Construction Materials Reference Laboratories which distributes over 6,000 samples of 13 different construction materials is highlighted, along with ASTM standardization activities related to proficiency and interlaboratory testing.

\section{0,335}

PB94-187283 PC A04/MF AO1

National Inst. of Standards and Technology (BFRL), Gaithersburg, MD.

Calculating Flame Spread on Horizontal and Vertical Surfaces.

G. N. Ahmed, M. A. Dietenberger, and W. W. Jones. Apr $94,58 p$, NISTIR-5392.

Prepared in cooperation with Portland Cement Association, Skokie, IL. and Forest Products Lab., Madison, WI.

Keywords: "Algorithms, "Flame propagation, Fires, Buildings, Heat flux, Pyrolysis, Geometry, Ignition, Mathematical models, Soot, "Compartment fires, "Fire models.

The flame spread model described in this paper is a new algorithm which provides the capability to calculate a self-consistent fire based s:ibstantially on bench scale fire data. The flame spread model simu- lates object fire growth and burnout of a slab in a room and produces acceptable predictions of the spread of fire, smoke and production of both toxic and nontoxic gases. The purpose of the flame spread model is to allow a fire to grow realistically, possibly making a hole in the material surface. This is one mechanism for barrier penetration. The algorithm is based on empirical data, gathered from standard test apparatus, including flame spread test method). The objective of including the flame spread model is to predict the accelerative growth of a fire from ignition to a peak value and then the gradual termination normally seen in a fire. The intent of the project was to develop an algorithm which could be utilized in a complete model of a fire in a building. The three-dimensional aspects of the flame spread model include: first, panels made of combustible materials with different thicknesses and at various orientations; second, flames of two basic types, pool fire and purely wall fire; third, a radiation heat exchange between objects, flames, and gases. The pool fire has flame spreading polygon on a horizontal panel and the wall fire is used either for inclined or vertical panels.

00,336

PB94-193620 PC A03/MF AO1

Factory Mutual Research Corp., Norwood, MA

Prediction of Fire Dynamics.

Quarterly rept. 28 Jun-28 Aug 92 (Final).

R. L. Alpert, and J. de Ris. Sep 92, 39p, NIST/GCR-

$94 / 642$

Grant NIST-60NANB1D1177

Sponsored by National Inst. of Standards and Technology (BFRL), Gaithersburg, MD.

Keywords: "Combustion physics, "Buildings, Fires, Heat flux, Radiative heat transfer, Accuracy, Flame propagation, Building codes, Mathematical models, Al-

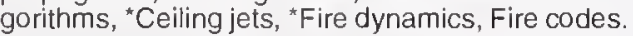

The report summarizes accomplishments of a Factory Mutual Research Corporation (FMRC) project on the Prediction of Fire Dynamics for the NIST grant period August 1991 through August 1992. Work performed under a subcontract by Professor H.W. Emmons on Ceiling-Jet Dynamics and on Development of Strategies for Performance Fire Codes is first described under Task 1 . The accomplishment of three tasks performed at FMRC are then presented in summaries of Tasks 2-4. All of this work is aimed at the development of subroutines or algorithms that can be used in NIST/ BFRL comprehensive computer models. During the past year, there has been further progress in the develepment of predictive models for flame radiant heat flux and in the development of a practical laboratory test method for measuring the smoke point of solid noncharring or charring materials. This progress should allow continuing improvements in the accuracy and applicability of fire propagation theories.

\section{0,337}

PB94-193778 PC A05/MF AO2

Clemson Univ., SC. Dept. of Mathematical Sciences. Mathematical Modeling of Human Egress from Fires in Residential Buildings.

Final technical rept.

M. M. Kostreva. Jun 94, 100 ,

Grant NIST-60NANBOD 1023

See also PB-273 166. Sponsored by National Inst. of Standards and Technology (BFRL), Gaithersburg, MD. Keywords: "Residential buildings, "Mathematical models, "Egress, "Human behavior, "Fires, "Fire safety, Algorithms, Reprints, Human reactions, Decision mak ing, Injury prevention, Escape(Abandonment), HAZARD I Computer program.

The Building and Fire Research Laboratory has been developing mathematical models for predicting the en vironmental conditions which occur as a fire develops and spreads. These models form a significant part of the computer program entitled HAZARD I. Human egress modeling has been implemented in HAZARD by means of simulation of actual human movement, invoking psychological theories and heuristic methodologies to create paths which the humans in the residential building under consideration would likely have taken. The research supported by this grant has been rather successful at finding mathematical models, ap propriate solution techniques, coding the techniques, and making sample applications. In seven different research papers the results of the research have been documented. Six of these papers have appeared in print, while the seventh is submitted for publication. The full copies of the papers are included as appendices of this report, while summaries are presented 
here for the reader's convenience. The research performed has advanced the state of the art in fire egress analysis, which forms an important part of fire hazard analysis and modeling as embodied in HAZARD I. In doing so, some new mathematical ideas, algorithms and theories have also been advanced. It is particularly satisfying to have new mathematics derived in support of such an important national priority as fire safety, and, as the mathematical knowledge is general, it may be of service to others in the future.

\section{0,338}

PB94-199320 Not available NTIS

National Inst. of Standards and Technology (BFRL), Gaithersburg, MD. Building Environment Div. Analysis of Moisture Accumulation in a Wood. Frame Wall Subjected to Winter Climate.

Final rept.

D. M. Burch, and W. C. Thomas. 1992, 13p

Pub. in Proceedings of Conference on Thermal Performance of the Exterior Envelopes of Buildings $V$, Clearwater Beach, FL., December 7-10, 1992, p467479. See also PB92-116334. Sponsored by Department of Energy, Washington, DC

Keywords: "Wood products, "Moisture content, "Walls, "Mathematical models, "Environmental effects, Winter, Performance evaluation, Construction materials, Mois ture resistance, Capillary flow, Diffusion, Heat transfer Water vapor, Finite difference method, Framed structures, Permeability, Humidity, Reprints.

A transient, one-dimensional, finite-difference model is presented that predicts that coupled transfer of heat and moisture in a multilayer wall under nonisothermal conditions. The model can predict moisture transfer in the diffusion through the capillary flow regimes. It has a provision to account for convective moisture transfer by including embedded cavities that may be coupled to indoor and outdoor air. The model is subsequently used to predict the time-varying average moisture content in the sheathing and siding of a wood-frame wall as a function of time of year. The effect of several construction parameters on the winter moisture accumulation is investigated. The parameters include the interior vapor retarder permeance, sheathing permeance, exterior paint permeance, indoor air leakage, and the amount of insulation.

\section{0,339}

\section{PB94-206091 PC A03/MF A01}

Maryland Univ., College Park. Dept. of Fire Protection Engineering.

Development of the Fire Data Management System. Final rept.

F. W. Mowrer. Aug 93, 37p, NIST/GCR-94/639.

Grant NIST-60NANB1D1164

See also PB94-198462. Sponsored by National Inst. of Standards and Technology (BFRL). Gaithersburg. MD.

Keywords: "Data base management systems, "Information systems, "Fire tests, "Buildings, Construction materials, Furniture, Data bases, Fire safety. Flammability, Ignition, Burning rate, "Fire Data Man agement System.

The Fire Data Management System (FDMS) is being developed under international collaboration to provide uniform means for storing fire test data. The purpose of the present work has been to aid the development of the FDMS for practical use by fire safety design professionals. To be of practical use by fire safety design professionals, the FDMS must contain relevant data and these data must be readily accessible. Currently, the data fields in the FDMS are too general and too restricted to be of practical design use. Additional data fields are needed in the FDMS to assist the design professional find flammability test data relevant to the design of different combustible objects in buildings. To help identify the types of additional data fields that are needed, a series of morphological charts have been developed to describe the types, elements and attributes of combustible objects in buildings. These morphological chants represent a first effort to describe a common syntax for identifying appropriate objects and their attributes for flammability analyses. Further refinement of the charts is needed.

00,340

PB94-206299 PC A03/MF A01

Kentucky Univ., Lexington. Dept. of Mechanical Engineering.
Upward Flame Spread along the Vertical Corner Walls (October 1993).

Final rept.

C. Qian, H. Ishida, and K. Saito. Oct 93, 46p, NIST/ GCR-94/648.

Grant NIST-NANB2D1295

See also PB89-214787. Sponsored by National Inst. of Standards and Technology (BFRL), Gaithersburg, MD

Keywords: "Walls, "Pyrolysis, "Flame propagation, Burning rate, Fire safety, Fires, Heat flux, Mathematical models, Temperature measuring instruments.

Flame spread behavior and the pyrolysis region spread characteristics along vertical corner walls were studied in detail with an automated infrared imaging temperature measurement technique (IR technique). The technique was recently developed for the measurement of transient pyrolysis temperature on both charring and non-charring materials. Temporal isotherms on PMMA samples were successfully obtained, from which the progress rate of the pyrolysis front was automatically deduced. It was found that the pyrolysis front shape was always $M$-shaped, i.e., no spread along the corner, and the maximum spread is in a few centimeters away from the corner. Understanding of the mechanism of the $M$-shape formation is important in developing a prediction model of the spread rate. Four possible mechanisms were identified and flame displacement effects are found to be the principal mechanism. Transient total heat flux distributions above the M-shape pyrolysis peak for a spreading fire were measured. Using these values, it was shown that the upward spread rate is predictable from a simple, one-dimensional, thermal model.

\section{0,341}

\section{PB94-207388 PC A05/MF A01}

Pennsylvania State Univ., University Park. Dept. of Mechanical Engineering.

Turbulent Upward Flame Spread on a Vertical Wall under External Radiation.

Annual rept. 30 Sep 91-15 Jan 93

A. K. Kulkami, E. Brehob, S. Manohar, and R. Nair

Feb 94, 93p, NIST/GCR-94/638.

Grant NIST-NANB8D0849

See also PB92-112531. Sponsored by National Inst. of Standards and Technology (BFRL), Gaithersburg, MD.

Keywords: "Walls, "Turbulent flow, "Flame propagation, Heat flux, Buming rate, Data bases, Radiant flux density, Mathematical models.

Progress made on NIST grant number 6ONANB8D0849 for the period September 30, 1991 to January 15,1993 is reported. The overall objective is to understand the upward flame spread phenomenon under simulated surrounding fire conditions by establishing a data base for upward flame spread under external radiation, developing a mathematical model, measuring the relevant basic material properties need ed, and checking the validity of the model by comparing its results with data. Emphasis is placed on studying and predicting the behavior of practical wall materals used in building and vehicle interiors, and textiles. In the past year, the authors measured flame spread on several different materials under a range of externa radiant fluxes of up to $15 \mathrm{~kW} / \mathrm{sq} \mathrm{m}$. A model for describing the upward flame spread process was developed and numerical results were compared with data. The model needed input of certain properties, such as the burning rate characteristics and sufface radiation properties. A series of supporting studies were undertaken which provided the needed input properties to the model and other useful material property data.

\section{0,342}

PB94-207404 PC A03/MF AO1

National Inst. of Standards and Technology (BFRL), Gaithersburg, MD

Comparison of Wall-Fire Behavior With and Without a Ceiling.

H. E. Mitler, and K. D. Steckler. Nov 93, 34p,

NISTIR-5380.

See also PB85-133973.

Keywords: "Burning rate, "Walls, "Ceilings, "Flame propagation, Heat flux, Fire tests, Mathematical models, Fires, FIRST room fire model, SPREAD wall fire model.

This paper demonstrates that the effects of the ceiling on the progress of a wall fire are quite significant, and that a project to quantify and model the effects of a ceiling on the progress of a wall fire is indeed justified Experimental results from the open literature are used for this purpose, as well as previously unpublished ex perimental results obtained at NIST. The wall-fire SPREAD and the room-fire model FIRST are used senatim to show that part of this effect can be calculated now.

00,343

PB94-207495 PC A08/MF A02

National Inst. of Standards and Technology (BFRL), Gaithersburg, MD.

Project Summaries 1994: NIST Building and Fire Research Laboratory

Special pub.

N. J. Raufaste. Jun $94,161 p$, NIST/SP-838/5

Also available from Supt. of Docs. as $\mathrm{SNOO3-003-}$ 03273-5. See also PB94-113420.

Keywords: "Buildings, "Fires, "Research management, "Testing laboratories, Building codes, Fire safety, Flammability testing, Standards, Construction materials.

The report summarizes the Building and Fire Research Laboratory's research for 1994 . The report is arranged by its research programs, structural engineering, matenals engineering, mechanical and environmental systems, fire science and engineening, and fire measurement and research. Each summary lists the project title, point of contact, sponsor, research, and results. BFRL's mission is to increase the usefulness, safety and economy of constructural facilities, and reduce the human and economic costs of unwanted fires in buildings.

\section{0,344}

PB94-210077 PC A03/MF AO1

National Inst. of Standards and Technology (BFRL), Gaithersburg, MD.

Combined Buoyancy- and Pressure-Driven Flow Through a Shallow, Horizontal, Circular Vent.

L. Y. Cooper. Apr 94, 50p, NISTIR-5384.

L. Y. Cooper. Apr 94, 50 p
See also PB94-103694.

Keywords: "Vents, "Air flow, "Buoyancy, "Flame propagation, Fire protection, Buildings, Boundary value problems, Boundary conditions, Mathematical models, Flow rate, "Compartment fires, "Fire models.

Combined buoyancy- and pressure-driven (i.e., forced) flow through a horizontal vent is considered where the vent-connected spaces near the elevation of the vent are filled with fluids of different density in an unstable configuration, with the density of the top space larger than that of the bottom space. With zero-to-moderate cross-vent pressure difference, delta $p$, the instability leads to a bi-directional exchange flow between the two spaces. For relatively large delta $p$, the flow through the vent is unidirectional, from the high- to the low-pressure space. Previously published experimental data and results of an analysis of the relevant boundary value problems are used to develop a flow model which takes all of these effects into account. The result is a uniformly valid algorithm to calculate flow through shallow (small depth-to-span ratio), horizontal, circular vents under high-Grashof number conditions. This is suitable for general use in zone-type compartment fire models (e.g., an ambient temperature environment above the vent and a hot smoky environment below). The algorithm is used in example applications where steady rate-of-burning in a ceiling-vented room is estimated as a function of room temperature, vent area, and oxygen concentration. Results of the analysis are seen to be consistent with previously published data involving ceiling-vented fire scenarios.

00,345

PB94-210127 PC AO4/MF AO1

National Inst. of Standards and Technology (BFRL) Gaithersburg, MD. Fire Safety Engineening Div.

VENTCF2: An Algorithm and Associated FORTRAN 77 Subroutine for Calculating Flow through a Horizontal Ceiling/Floor Vent in a ZoneType Compartment Fire Model.

L. Y. Cooper. Aug 94, 71p, NISTIR-5470.

Keywords: "Fire protection, "Vents, "Algorithms, "Subroutines, "Flame propagation, Air flow, Fire safety, Enthalpy, Mass flow, Mathematical models, Buildings, "Compartme

An algorithm and associated FORTRAN 77 subroutine, called VENTCF2 is presented for calculating the of fects on two-layer compartment fire environments of 
BUILDING INDUSTRY TECHNOLOGY

Construction Materials, Components, \& Equipment

the quasi-steady flow through a circular, shallow (i.e., small ratio of depth to diameter), horizontal vent connecting two spaces. The two spaces can be either two inside rooms of a multi-room facility or one inside room and the outside ambient environment local to the vent. The description of the flow through the vent is determined by combining considerations of the uni-directional-type of flow driven by a cross-vent pressure difference and, when appropriate, the combined pressure- and buoyancy-driven flows which occur when the density configuration across the vent is unstable, i.e., a relatively cool, dense gas in the upper space overlays a less dense gas in the lower space. In the algorithm, calculation of the rates of flow exchange between the two spaces is based on previously reported model equations. Characteristics of the geometry and the instantaneous environments of the two spaces are assumed to be known and specified as inputs. The outputs calculated by the algorithm/subrou. tine are the rates and the properties of the vent flow at the elevation of the vent as it enters the top space from the bottom space and/or as it enters the bottom space from the top space. Rates of mass, enthalpy, and products of combustion extracted by the vent flows from upper and lower layers of inside room environments and from outside ambient spaces are determined explicitly.

\section{0,346}

PB94-211372 Not available NTIS

National Inst. of Standards and Technology (BFRL), Gaithersburg, MD. Fire Science Div.

Suppression Research: Strategies.

Final rept.

D. D. Evans. 1992, 15p

Pub. in Proceedings of International Conference on Fire Suppression Research (1st), Stockholm, Sweden, May 5-8, 1992, p21-35

Keywords: "Fire extinguishers, "Fire tests, Burning rate, Fire fighting, Fires, Diffusion, Sprinklers, Mathematical models, Furniture, Reprints, *Extinction time

Millions of unwanted fires occur each year. More effec tive fire suppression is a means to minimizing property damage and life loss due to fire. The Damkohler number, which is the ratio of the characteristic time for flow or diffusion of reactants to the time required for reaction, is the basis for a fire extinction criterion that may provide insight for many fire suppression situations. A correlation of wood crib fire extinction data is used to show the predictive capabilities that can be developed from experimental results alone, even without complete and general understanding of the fire suppression phenomena. The use of computer field modeling techniques is discussed as a means to generate insight into the complex physical interactions that occur during fire suppressiorı in an enclosure.

\section{0,347}

PB94-212859 Not available NTIS

National Inst. of Standards and Technology (NEL), Gaithersburg, MD. Fire Science and Engineering Div. Full Scale Smoke Control Tests at the Plaza Hotel Building.

H. Klote. 1989, 3p.

Pub. in Jnl. of American Society of Heating, Refrigeration and Air-Conditioning Engineers 3i, n4 p28-30 Apr 89.

Keywords: "Fire tests, "Buildings, Ventilation, Fire safety, Smoke, Pressurization, Air flow, Pressure gradients, Stairways, Reprints, "Smoke control, "Hotel fires.

No zoned smoke control system has been tested under real fire conditions. This paper presents a general overview of a project of full scale fire experiments to be conducted at the Plaza Hotel Building in Washington, DC. The objectives of these experiments are to evaluate the current approach concerning minimum pressure difference and to achieve smoke control for zoned smoke control systems with and without stairwell pressurization. Also, the interaction between smoke control and the fire will be studied. Air will be exhausted at six changes per hour from the fire floor to evaluate the effect of this common exhaust rate on the fire energy release rate.

00,348

PB94-212867 Not available NTIS

National Inst. of Standards and Technology (BFRL)

Gaithersburg, MD. Fire Science and Engineering Div.
Overview of Smoke Control Technology.

Final rept.

J. H. Klote. $1988,11 \mathrm{p}$

Pub. in American Society of Heating, Refrigerating and Air-Conditioning Engineers Transactions 94, pt 1 p1211-1221 1988

Keywords: "Buildings, "Fire safety, "Smoke, Evacuation, Stainways, Elevators(Lifts), Leakage, Fire protection, Ventilation, Reprints, *Smoke control, "Building fires.

Considerable advances in smoke control technology have occurred in the last few decades. However smoke control is just beginning to take its proper place as a fire protection tool. This paper provides an overview of this technology, including discussions of the fundamental principles, stainwell pressurization, zoned smoke control, elevator smoke control, system activation and acceptance testing. In addition the problems of smoke purging are addressed.

\section{0,349}

PB94-212875 Not available NTIS

National Inst. of Standards and Technology (BFRL), Gaithersburg, MD. Fire Safety Engineering Div.

Preview of ASHRAE's Revised Smoke Control Manual.

Final rept

J. H. Klote. 1992, 4p.

Pub. in American Society of Heating, Refrigeration and

Air-Conditioning Engineers Jnl., p34-37 Nov 92

Keywords: "Buildings, "Fire safety, "Smoke, Manuals, Elevators(Lifts), Stainways, Pressurization, Air flow, Ventilation Toxicity, Reliability Reprints, "Smoke control, "Building fires, ASHRAE(American Society of Heating Refrigerating and Air Conditioning Engineers).

The American Society of Heating, Refrigerating, and Air-Conditioning Engineers (ASHRAE) smoke control manual has been extensively revised and expanded. This manual consolidates and systematically presents data and calculational procedures for use by smoke management system designers. Fundamental issues of smoke management include reliability, activation, smoke obscuration, toxicity, and the driving forces of smoke movement. The mechanisms of compartmentation, dilution, air flow, pressurization, tion to manage smoke conditions in fire situations. The new manual presents systems for stainwell pressurization, elevator smoke control, zoned smoke control, and atrium smoke management.

\section{0,350}

\section{PB94-213253 Not available NTIS}

National Inst. of Standards and Technology (BFRL), Gaithersburg, MD. Building and Fire Research Lab. Office.

Development of a New Small-Scale Smoke Toxicity Test Method and Its Comparison with Real-Scale Fire Tests.

Final rept.

B. C. Levin. 1992, 8p.

Pub. in Toxicology Letters 64/65, p257-264 1992.

Keywords: "Smoke, "Toxicity, "Test methods, Computerized simulation, Fire hazards, Fire safety, Heat flux, Combustion products, Buildings, Reprints, "Building fires.

A comprehensive methodology has been developed for obtaining and using smoke toxicity data for fire hazard analysis. This bench-scale method can simulate diverse fire conditions and identify extremely toxic smoke under both pre- and post-flashover conditions. However, incidence data show that most of the fire deaths in the U.S. occur outside the room of fire origin from smoke and toxic gases that are generated from a fire under post-flashover conditions. Therefore, the most relevant real-scale combustion conditions to simulate in the bench-scale apparatus would be the postflashover conditions which are achieved by using radiant heat, a high heat flux, and correcting the benchscale carbon monoxide $(\mathrm{CO})$ results to agree with $\mathrm{CO}$ yields observed in real-scale post-flashover fires. The number of test animals (Fischer 344 male rats) is minimized by using the $\mathrm{N}$-Gas Model to estimate the LC50 value from the chemical analysis of the smoke. The prediction is checked with a smail number of animal tests and an approximate LC50 value is determined. The bench-scale results have been validated with fullscale room wall burns of a limited number of materials of widely differing characteristics chosen to challenge the system. The toxic potency values are assessed to determine if the smoke from a material or product is unusually or extremely toxic and can then be used in computations of fire hazard.

\section{0,351}

PB94-213261 Not available NTIS

National Inst. of Standards and Technology (NEL) Gaithersburg, MD. Fire Science and Engineering Div. and Actions in Residential Fires.

Final rept.

B. M. Levin. 1989, 10p

Pub. in Proceedings of International Symposium on Fire Safety Science (2nd), Tokyo, Japan, June 13-17, 1988, p561-570 1989

Keywords: *Residential buildings, *Computerized simulation, "Evacuation, "Decision making, Fire hazards, Smoke, Fire safety, Emergencies, Human behavior, Reprints, *Residential building fires, EXITT computer program, Escape time.

EXITT is a discrete event simulation of occupant decisions and actions in a postulated fire. To run the model the physical descriptions of a residence, a specific fire in that residence, and the assumed occupants of the residence are entered into the computer. Based on a large set of decision rules, the occupants make decisions which are a function of the smoke conditions in the building, the characteristics and status of the occupants (including their capabilities), and the available travel routes. The occupants investigate the fire, aler and assist others, and evacuate the building. The simulation ends when all the occupants are either out of the building or are trapped by the smoke.

\section{2}

PB94-217486 PC A14/MF A03

National Inst. of Standards and Technology (BFRL) Gaithersburg, MD. Structures Div.

Hollow Clay Tile Prism Tests for Martin Marietta Energy Systems: Task 2 Testing.

C. W. C Yancey 1993, 324p NISTIR-5328.

Color illustrations reproduced in black and white.

Keywords: "Compressive strength, "Modulus of elasticity, "Clays, "Tiles, "Poisson ratio, Masonry, Construction materials, Mechanical properties, Loads(Forces), Displacement, *Hollow clay tile prisms.

Forty-one Hollow Clay Tile prisms were tested in monotonic, uniaxial compression to failure as the second task in a two-task prism test program for the Department of Energy (DOE). Twenty prisms were nominally $330 \mathrm{~mm}(13 \mathrm{in})$ thick and twenty-one were nominally $200 \mathrm{~mm}(8 \mathrm{in})$ thick. Twenty-one prisms were tested with the compressive load applied normal to the axis of the hollow cores and the other twenty were subjected to compressive load acting parallel to the axis of the cores. The objectives of the Task 2 test series were to: (1) obtain the compressive strength, (2) determine the modulus of elasticity in compression, (3) determine Poisson's ratio for the prisms, (4) study and understand the behavior of this type of masonry prism, (5) determine whether workmanship during construction of the prisms significantly affects the strength and (6) compare the results of using load control versus displacement control while loading the prisms. Test results are presented in tabular form to report the gross and net area compressive strengths, and Secant Mod ulus of Elasticity on the gross and net areas. Load-displacement plots are presented to graphically report on the output from vertical and horizontal Linear Variable Differential Transformers attached to the prism faces.

00,353

PB94-218583 PC A03/MF AO

National Inst. of Standards and Technology (BFRL) Gaithersburg, MD. Building Materials Div.

Survey of Recent Cementitious Materials Research in Western Europe.

D. P. Bentz. Aug 94, 35p, NISTIR-5480.

Keywords: "Cements, "Concretes, "Hydration, *Research management, Microstructure, Shrinkage, Cracking(Fracturing), Concrete durability.

The report summarizes recent research on cementitious materials in western Europe, based on a perspective gained during a six month stay at the Centre Scientifique et Technique du Batiment in Grenoble, France. During this time period, the author visited six teen laboratories in seven different European countries. Numerous publications, all included in the reference list of this report, were obtained during these visits. Research is grouped into topical areas with a 
brief description of separate activities at each institution. Emphasis has been placed on those research topics of interest to the Building Materials Division of NIST. A list of researchers working on cementitious materials in western Europe is also provided for those readers wishing to obtain further information on specific topics.

\section{0,354}

\section{PB94-219052 PC A06/MF A02}

National Inst. of Standards and Technology (BFRL), Gaithersburg, MD. Structures Div.

NIST Research Program on the Seismic Resistance of Partially-Grouted Masonry Shear Walls.

A. E. Shultz. Jun 94, 112 p, NISTIR-5481.

See also PB92-116342.

Keywords: "Masonry. "Walls, Shear stress, Cyclic loads, Tests, Seismic design, Earthquake engineering, Buildings.

A review of the current status of research on masonry structures at the Building and Fire Research Laboratory of the National Institute of Standards and Technology (NIST) is presented, and an ongoing project on partially-grouted masonry shear walls is summarized. The report draws from previous work conducted at NIST including a comprehensive literature review, simulated seismic load tests of unreinforced and reinforced masonry walls, and numerical analyses employing empirical formulations and finite element models. The previous NIST research culminates with a preliminary draft outlining a research program on partiallygrouted masonry walls and numerical analyses.

00,355

PB95-105136 PC A03/MF A01

National Inst. of Standards and Technology (BFRL), Gaithersburg, MD.

Controlling Moisture in the Walls of Manufactured Housing.

Technical note.

D. M. Burch, and A. TenWolde. Dec 92, 42p,

NISTIR-4981, HUD-0006080

See also AD-A139 637. Sponsored by Department of Housing and Urban Development, Washington, DC. Office of Policy Development and Research and Forest Products Lab., Madison, WI.

Keywords: "Walls, "Moisture, "Permeability "Construction materials, "Housing(Dwellings), Humid ity, Boundary conditions, Diffusion, Condensation Wallboard, Ventilation, "Manufactured housing.

This report contains the results of a detailed computer analysis to investigate the effectiveness of three alternative practices for controling moisture accumulation in the walls of manufactured housing during the winter. The three practices included: (1) providing a vapor retarder, (2) using permeable sheathing and siding, and (3) providing an outdoor ventilated cavity. The current Manufactured Home Construction and Safety Standards do not require a vapor retarder for practices 2 and 3 . The analysis was carried out for a cold winter climate (Madison, WI), an intermediate winter climate (Boston, MA), a mild winter climate (Atlanta, GA), and a Pacific northwest climate (Portiand, OR). The practice of providing a vapor retarder was found to be effective in all four climates. The moisture content of the siding was always considerably below fiber saturation. On the other hand, the practice of using permeable sheathing and siding and the practice of providing an outdoor ventilated cavity were not always effective in colder climates.

\section{0,356}

PB95-107397 Not available NTIS

National Inst. of Standards and Technology (BFRL), Gaithersburg, MD. Building Environment Div.

Measurements of Moisture Diffusivity for Porous Building Materials.

Final rept.

R. F. Richards. 1992, $11 \mathrm{p}$.

Sponsored by Department of Energy, Washington, DC. $\mathrm{Pub}$. in Proceedings of Conference on Thermal Performance of the Exterior Envelopes of Buildings $V$, Clearwater Beach, FL., December 7-10, 1992, p501511.

Keywords: "Construction materials, "Porous materials, *Diffusivity, "Moisture, Mass transfer, Flux(Rate), Pressure, Steady state, Moisture content, Reprints.

Moisture diffusivities of several common porous building materials were determined experimentally. Both wood-based and inorganic materials were included in the study. A steady-flux method was used that involved determining the one-dimensional moisture content profile produced in a sample through which a known constant flux of water is flowing. The transient mass fluxes through samples upon exposure to water, steady-state mass fluxes, one-dimensional moisture content profiles, and capillary pressure curves for low suction pressures, as well as the moisture diffusivities for a range of moisture contents, are presented for each material. Results of the study are compared to previously published measurements for similar materials.

\section{0,357}

PB95-108718 Not available NTIS

National Inst. of Standards and Technology (BFRL), Gaithersburg, MD. Fire Science Div.

Verification of a Model of Fire and Smoke Transport.

Final rept.

R. D. Peacock, W. W. Jones, and R. W. Bukowski.

$1993,41 p$

Pub. in Fire Safety Jnl. 21, p89-129 1993.

Keywords: "Fires, "Smoke, Flame propagation, Fire tests, Computer programs, Combustion products, Mathematical models, Algorithms, Reprints, "Fire models, "Smoke transport, "Room fires.

A set of comparisons between a comprehensive room fire model and a range of real-scale fire experiments is presented. For these comparisons, a zone-based model, CFAST ("consolidated fire and smoke transport' model) is used. The model predicts the evolution of a fire in a room and the subsequent transport of the smoke and toxic gases which result from this fire. These comparisons serve two purposes: to determine, within limits, the accuracy of the predictions for those quantities of interest to the users of the models (usually those extensive variables related to hazard), and to highlight the strengths and weaknesses of the underlying algorithms in the models to guide future improvements in this and other models. The predicted variables selected for comparison deal with both of these purposes. Although differences between the mode and the experiments were clear, they can be explained by limitations of the model and of the experiments.

\section{0,358}

PB95-125795 Not available NTIS

National Inst. of Standards and Technology (NEL) Gaithersburg, MD. Building Materials Div.

Suggestions for a Logically-Consistent Structure for Service Life Prediction Standards.

Final rept.

G. Frohnsdorff, and L. Masters. 1990, 14p.

Pub. in Proceedings of International Conference on Durability of Building Materials and Components (5th) Brighton, UK., November 7-9, 1990, p113-126.

Keywords: "Service life, "Construction materials, "Aging(Materials), "Standards, Degradation, Life(Durability). Buildings, Prediction analysis techniques, Reprints, "Environmental characterization.

Ability to predict the service life of building materials, components, and systems is needed to improve the selection process. Evaluation of durability using existing standards does not give adequate service life information. Because service life prediction is more complex than current durability evaluations, its standardzation will require a new body of standards to be put in place. The standards must define a general methodology, and essential components of the methodology. These are environmental characterization, characterization of the item whose service life is to be predicted, identification of the mechanisms and kinetics of the degradation processes, development of mathematical models of degradation, application of the models in service life prediction, and reporting of the results. It is proposed that the needed standards must comprise a hierarchy with the highest level being the general methodology, the second level defining the essential components of the methodology, and the third and lower levels describing the application of the generic standards to specific materials, components, or systems.

\section{0,359}

PB95-125845 Not available NTIS

National Inst. of Standards and Technology (NEL), Gaithersburg, MD. Building Materials Div.
Application of Thermal Analysis Techniques to the Characterization of EPDM Roofing Membrane Materials.

Final rept.

G. D. Gaddy, W. J. Rossiter, and R. K. Eby. 1990 ,

$16 p$.

Sponsored by National Roofing Contractors Association, Rosemont, IL. and Johns Hopkins Univ., Baltimore, MD.

Pub. in Proceedings of a Symposium on Roofing Research and Standards Development, ASTM STP 1088, San Francisco, CA., June 17, 1990, v2 p37-52.

Keywords: "Thermal analysis, "Membranes, "Roofs, "Test methods, Construction materials, Elongation, Calorimetry. Thermogravimetric analysis, Heat meas4637, Dynamic mechanical analysis.

This study was conducted to provide data on the feasibility of using thermal analysis (TA) methods for the characterization of roofing membrane materials. TA methods have never been widely applied to such materials. The TA methods used were thermogravimetry (TG), differential scanning calorimetry (DSC), and dynamic mechanical analysis (DMA). Three black (carbon black filled), two white (titanium dioxide pigmented), and one white on black laminate ethylene propylene diene terpolymer (EPDM) membrane materials were analyzed before and after exposure to the heat, ozone and UV conditions given in ASTM D 4637, and also to outdoor exposure. Load-elongation tests were conducted to compare the results with those of the TA methods. The results indicated that: (1) TA techniques can be used to analyze EPDM membrane materials; (2) both the black and white membrane materials showed only slight property changes under exposure, as determined using the TA methods; and (3) in contrast to the TA results, the load elongation values displayed relatively large changes.

00,360

PB95-125852 Not available NTIS

National Inst. of Standards and Technology (NEL), Gaithersburg, MD. Building Materials Div.

Use of Thermal Mechanical Analysis to Characterize Ethylene-Propylene-Diene Terpolymer (EPDM) Roofing Membrane Materials.

Final rept

G. D. Gaddy, W. J. Rossiter, and R. K. Eby. 1991, $8 p$.

Sponsored by National Roofing Contractors Association, Rosemont. IL. and Johns Hopkins Univ., Baltimore, MD.

Pub. in Proceedings of a Conference on Materials Characterization by Thermomechanical Analysis,
ASTM STP 1136, Philadelphia, PA., March 19-20, 1990, p168-175 1991

Keywords: *Roofs, "Membranes, "Test methods, Thermal analysis, Construction materials, Elongation, Heat measurement, Reprints, "EPDM rubber roofing, "Thermal mechanical analysis, ASTM D 4637.

This study was conducted to provide baseline data on the applicability of thermal mechanical analysis (TMA) for characterizing EPDM roofing membrane materials. TMA has not been used extensively for the analysis of such materials. Three black and three white membrane materials were analyzed before and after exposure to the heat, ozone and UV conditions described in ASTM D 4637, as well as to outdoor exposure. Loadelongation was conducted as a comparison to the TMA results. The results indicated that: (1) TMA can be applied to the characterization of EPDM membrane materials showing the differences in glass transition temperature between various brand name products; (2) changes in glass transition temperatures induced by the exposure conditions were relatively low and covered the same range for both the black and white membrane materials; and (3) in contrast to the TMA testing, the percent elongation values displayed relatively large changes.

00,361

PB $95-125977$ Not available NTIS National Bureau of Standards (NEL), Gaithersburg,
MD. Fire Measurement and Research Div. Relating Bench-Scale and Full-Scale Toxicity Data. Final rept.

R. G. Gann, E. Braun, and B. C. Levin. 1988, 4p Pub. in Proceedings of Joint Panel Meeting UJNR Panel on Fire Research and Safety (10th), Tsukuba, Japan, June 9-10, 1988, p50-53.

Keywords: "Fires, "Toxicity, "Test methods, "Buildings, Exposure(Physiology), Fire tests, Fire hazards, 
Smoke, Combustion products, Construction materials, Reprints.

This work proposes criteria for comparing the toxic potency of fire smokes as measured by bench-scale toxicity tests with that measured in real-scale fire tests. A first set of experiments finds that this protocol is practicable, and that the NBS toxicity test method shows only partial correlation.

\section{0,362
PB95-125985 Not available NTIS}

National Inst. of Standards and Technology (NEL), Gaithersburg, MD. Building Materials Div

Computer Simulation of the Diffusivity of CementBased Materials.

Final rept.

E. J. Garboczi, and D. P. Bentz. 1992, 10 p.

Sponsored by Northwestern Univ., Evanston, IL. Center for Advanced Cement-Based Materials.

Pub. in Jnl. of Materials Science 27, n8 p2083-2092 1992.

Keywords: "Portland cement, "Microstructure, *Computerized simulation, *Diffusivity, "Chlorides, Hydration, Durability, Construction materials, Transport properties, Image analysis, Porosity, Reprints.

A digital-image-based model of the microstructure of cement paste coupled with exact transport algorithms, is used to study the diffusivity of portland cement paste. The principal preparation and material variables are water:cement ratio, degree of cement hydration, and capillary porosity. Computational methods are described in some detail, and diffusivity results are presented and found to agree within experimental error with the available experimental measurements. Model cement pastes prepared with different water:cement ratios, and having different degrees of cement hydration, are found to have diffusivities that lie on a single master curve when plotted as a function of capillary porosity. Using percolation ideas, the diffusivity of cement paste is mapped out as a function of capillary porosity for any water:cement ratio cement paste.

\section{0,363}

PB95-125993 Not available NTIS

National Inst. of Standards and Technology (NEL), Gaithersburg, MD. Building Materials Div.

Digital Simulation of the Aggregate-Cement Paste Interfacial Zone in Concrete.

Final rept.

E. J. Garboczi, and D. P. Bentz. 1991, 6p.

Sponsored by Northwestern Univ., Evanston, IL. Dept. of Civil Engineering.

Pub. in Jnl. of Materials Research 6, n1 p196-201 1991

Keywords: "Concretes, "Interfaces, "Aggregates, *Computerized simulation, Hydration, Curing, Portland cement, Image analysis, Porosity, Diffusion, Construction materials, Scanning electron microscopy, Reprints, "Interfacial zone.

Back-scattered scanning electron microscopy, along with quantitative image analysis techniques, have clearly demonstrated the existence of a highly porous interfacial region between the aggregate and the hardened cement paste mairix in ordinary Portland cement concrete. This paper presents the results of a digitalimage-based simulation model of this interfacial zone. A dissolution-diffusion-reaction cycle of hydrating cement particles is directly simulated using cement particles packed around a simple non-reactive aggregate particle. The model is two-dimensional, as we are comparing to experimental results obtained on images of polished sections. The qualitative features seen in the experiment, such as large amounts of porosity and calcium hydroxide in the interfacial zone, are faithfully reproduced. The new mechanism of one-sided growth is proposed, along with the usual particle-packing ideas, as an explanation of the origin of the characteristic features of the interfacial zone.

\section{0,364}

\section{PB95-126009 Not available NTIS}

National Inst. of Standards and Technology (NEL), Gaithersburg, MD. Building Materials Div.

Fundamental Computer Simulation Models for $\mathrm{Ce}$ ment-Based Materiais.

Final rept.

E. J. Garboczi, and D. P. Bentz. 1991, 29p.

Pub. in Mater. Sci. Concr., v2 p249-277 1991.

Keywords: "Cements, "Microstructure, "Construction materials, "Mechanical properties, ${ }^{*}$ Computerized sim- ulation, Hydration, Admixtures, Curing, Durability, Litrature surveys, Mathematical models, Reorints.

The report is a critical review of fundamental computersimulation models that have been applied to cementbased materials, to relate microstructure to properties. The report also contains a significant literature survey.

\section{0,365}

PB95-126264 Not available NTIS

National Inst. of Standards and Technology (BFRL) Gaithersburg, MD. Building Environment Div.

Comparison of Heat-Flow-Meter Tests from Four Laboratories.

Final rept.

R. S. Graves, D. L. McElroy, R. G. Miller, D. W

Yarbrough, and R. R. Zarr. 1992, 4p.

Pub. in Jnt. of Thermal Insulation 15, p354-357 Apr 92

Keywords: "Thermal conductivity, "Heat measurement, "Test methods, "Laboratories, "Therma! insulation, Heat transmission. Transport properties, Calibration, Heat transfer, Reprints, "Heat flowmeters, ASTM C 518.

A recent comparison of apparent thermal conductiv ities measured at the Oak Ridge National Laboratory (ORNL), Jim Walter Research Corp (JWRC), Tennessee Technological University (TTU), and the National Institute of Standards and Technology (NIST) has been discussed in an ORNL Report. The four laboratories used equipment built and operated in accord ance with ASTM C 518, and used different calibration materials and procedures. These tests are discussed in this paper.

\section{0,366}

PB95-130845 PC A10/MF A03

National Inst of Standards and Technology (BFRL) Gaithersburg, MD. Fire Safety Engineering Div.

Summaries of BFRL Fire Research in-House Projects and Grants, 1994

N. H. Jason. Oct 94, 213p, NISTIR-5504

See also report for 1993, PB94-121050.

Keywords: "Fire safety, "Fire resistant materials Standards, "Fires, "Fire suppression, "Furniture, "Buildings, "Industrial plants, Flames, Flame propagation, Combustion, Models, Turbulent flow, Combustion products, Fire extinguishing agents, Smoke, Fire alarm systems, Fire protection, Construction materials, Fire hazards.

This report describes the fire research projects performed in the Building and Fire Research Laboratory (BFRL) and under its extramural grants program during Fiscal Year 1993. Included are research pertormed both with funds appropriated to the BFRL Fire Research Program and under contract to outside organizations. The BFRL Fire Research Program has directed its efforts under four program thrusts. The in house priority projects, extramural grants, and externally-funded efforts thus form an integrated, focussed ensemble. This publication is organized along those lines: (1) Performance-based Fire Standards; (2) Fire Safe Materials and Products; (3) Advanced Fire Sensing and Suppression; (4) Large/Industrial Fires. For the convenience of the reader, an alphabetical listing of all grants is contained in the Part 2.0.

\section{0,367}

PB95-140885 Not available NTIS

National Inst. of Standards and Technology (NEL), Gaithersburg, MD. Fire Science and Engineering Div. FPETOOL: Fire Protection Tools for Hazard Estimation. An Overview of Features.

Final rept.

H. E. Nelson. 1990, 8p

See also PB92-132984.

Pub. in Proceedings of the International Fire Conference Interflam ' 90 (5th), Canterbury, England, September 3-6, 1990, p85-92.

Keywords: "Fire hazards, "Buildings, *Fire protection, "Fire safety Computer programs, Fires, Computerized simulation, Fire detectors, Heat transfer, Risk assessment, Flame propagation, Sprinklers, Reprints, *FPETOOL computer prcgram.

FPETOOL is a computerized package of relatively simple engineering equations and models. It contains engineering tools useful in estimating potential fire hazard and the response of the space and fire protection systems to the developing hazard. The computations use established engineering relationships, which are discussed in this paper for the benefit of engineers and others interested in using the tools in the package.
00,368

PC A03/MF A01

National Inst. of Standards and Technology (BFRL) Gaithersburg, MD. Fire Safety Engineering Div. Simulating Smoke Movement through Long Vertical Shafts in Zone-Type Compartment Fire Models. L. Y. Cooper. Nov 94, 40p, NISTIR-5526.

Keywords: "Smoke, "Air flow, "Buoyancy, "Two phase flow, Turbulence, Vents, Plumes, Diffusion, Elevators(Lifts), Density, Mathematical models, "Zonetype compartment fire models, "Building fires.

A limitation of traditional zone-type compartment fire modeling concepts identify; namely, the inadequacy of two-layer quasi-steady-buoyant-plume analyses to simulate the fire-generated environment in room configurations with large height-to-span ratios, e.g., elevator shafts and long, vertical, ventilation shafts and ducts. A possible means of removing this limitation is developed. This involves a method of analysis and as sociated model equations that can be implemented and used to advance zone-type models. The model equations simulate time-dependent flows in a long ventilated, vertical shaft/duct with an arbitrary vertica density distribution, including one or more intervals along the shaft/duct length where the vertical distribution of the average cross-section density may be unstably stratified, i.e. density increasing with increas ing elevation. The model equations are partially verified by favorable comparisons between solutions and previously published data from unsteady experiments in long vertical tubes involving initially unstable configurations: salt-water over fresh-water and heavy-gas over light-gas. Additional verification of the proposed equation set with cold-air over hot-air systems and with fire-driven smoke flows, both of which involve gas-tosurface heat transfer, is required before this model can be used with confidence in professional practice.

00,369

PB95-143202 PC A06/MF AO2

National Inst. of Standards and Technology (BFRL) Gaithersburg, MD. Fire Safety Engineering Div. Building and Fire Research Laboratory Publications, 1993.

Special pub

N. H. Jason. Sep 94, 113p, NIST/SP-838/6

Also available from Supt. of Docs. as SN003-003 03295-6. See also report for 1992, PB93-188845.

Keywords: "Buildings, "Fires, "Bibliographies, Research, Combustion, Fire protection, Abstracts, Flammability, Construction materials, Heat transfer, Building codes, Fire resistance, Ignition.

Building and Fire Research Laboratory Publications, 1993 contains references to the publications prepared by the members of the Building and Fire Research Laboratory (BFRL) staff, by other National Institute of Standards and Technology (NIST) personnel fo BFRL, or by external laboratories under contract or grant from the BFRL during the calendar year 1993

00,370

PB95-150439 Not available NTIS

National Inst. of Standards and Technology (BFRL) Gaithersburg, MD. Building Materlals Div. Percolation and Pore Structure in Mortars and Concrete.

Final rept.

D. Winslow, M. Cohen, D. Bentz, K. Snyder, and E. Garboczi. 1994, 13p.

Sponsored by Center for Advanced Cement-Based Materials, Evanston, IL

Pub. in Cement and Concrete Research 24, n1 p25371994

Keywords: "Building materials, "Concretes, "Motars, "Pore structure, "Percolation, Computerized simulation, Microstructure, Interfaces, Durability, Transport properties, Reprints, Mercury intrusion porosimetry Silica fume.

The cement paste in concrete and mortar has been shown to have a pore size distribution different than that of plain paste hydrated without aggregate. For mortar and concrete, additional porosity occurs in pore sizes larger than the plain paste's threshold diamete as measured by mercury intrusion. Based on the as sumption that these larger pores are essentially present only in the interfacial zones surrounding each aggregate, an experimental program was designed in which the volume fraction of sand in a mortar was varied in a systematic fashion and the resultant pore sys 


\section{BUILDING INDUSTRY TECHNOLOGY}

\section{Construction Materials, Components, \& Equipment}

tem probed using mercury intrusion porosimetry. The intrusion characteristics were observed to change drastically at a critical sand content. Similar results are observed for a series of mortar specimens in which the cement paste contains $10 \%$ silica fume. To better inter pret the experimental results, a hard core/soft shell computer model has been developed to examine the percolation characteristics of these interfacial zone pores. Using the model, interfacial zone percolation in concretes is also examined. Finally, the implications of interfacial zone percolation for transport properties and durability of mortar and concrete are discussed.

\section{0,371}

PB95-150462 Not available NTIS

National Inst. of Standards and Technology (BFRL), Gaithersburg, MD. Building Environment Div.

Effects of Humidity and Elevated Temperature on the Density and Thermal Conductivity of a Rigid Polyisocyanurate Foam Co-Blown with CCl3F and co2.

Final rept

R. R. Zarr, and T. Nguyen. 1994, 21p

Pub. in Jnl. of Thermal Insul. and Bldg. Envs. 17, p330 350 Apr 94.

Keywords: "High temperature tests, "Humidity, "Foams, "Thermaluctivity, "Density(Mass/volume), measurement, Thermodynamic properties, Laminates, Thermophysical properties, Temperature dependence Test facilities, Reprints, "Rigid polyisocyanurate foam

Measurements of density and apparent thermal conductivity are presented for specimens cut from rigid polyisocyanurate foam co-blown with trichlorofluoromethane and carbon dioxide. Eight specimens, nominally 580 by 580 by $27 \mathrm{~mm}$, were pre pared from two boards ( 1.2 by $2.4 \mathrm{~m}$ by $50 \mathrm{~mm}$ ) of foam were placed in an ambient condition of $22 \mathrm{C}$ and $40 \%$ relative humidity $(\mathrm{RH})$. The other four specimens were each placed in one of the following environments: (1) $60 \mathrm{C}$ and less than $10 \% \mathrm{RH}$; (2) $60 \mathrm{C}$ and $40 \% \mathrm{RH}$ (3) $60 \mathrm{C}$ and $60 \% \mathrm{RH}$; and (4) $60 \mathrm{C}$ and $75 \% \mathrm{RH}$ Measurements of apparent thermal conductivity at 24 C were conducted over a period of 372 days at approximately 50-day intervals. Curves of specimen mass, volume, density, and thermal conductivity ver sus time are presented, and the implications of changes in these properties are discussed.

00,372

PB95-151338 Not available NTIS

National Inst. of Standards and Technology (NEL) Gaithersburg, MD. Building Materials Div.

Effects of Adhesive Thickness, Open Time, and Surface Cleanness on the Peel Strength of Adhesive-Bonded Seams of EPDM Rubber Roofing Membrane.

Final rept.

H. Watanabe, and W. J. Rossiter. 1990, 16p.

Pub. in Roofing Research and Standards Develop ment: ASTM STP 1088, v2 p21-36 1990.

Keywords: "Roofing, "Adhesion, "Peel strength, "Seams(Joints), Adhesive bonding, Surface properties, Construction materials, Roofs, Contamination, Failure modes, Rubber, Membranes, Curing Strength(Mechanics), Cleanliness, Reprints, “EPDM Rubber Roofing Membrane, Open time.

A laboratory study was undertaken to examine the effects of adhesive thickness, open time, and surface cleanness on T-peel strength of EPDM rubber seam specimens. Seam specimens bonded with butyl-based contact adhesive were tested after a 2 -week cure time. The peel strength generally showed a positive depend ency on the adhesive thickness except that it tended towards a plateau value for thick adhesive layers. The leveling of peel strength at large thickness might result from the presence of small voids in the adhesive layer. The peel strength was not dependent on the open times used in this study. Increased levels of surface contamination lowered the peel strength and changed the failure mode from cohesive to interiacial.

\section{0,373}

Pot available NTIS

National Inst. of Standards and Technology (BFRL) Gaithersburg, MD. Building Environment Div.
Effects of Humidity and Elevated Temperature on the Density and Thermal Conductivity of a Rigid Polyisocyanurate Foam.

Final rept.

R. R. Zarr, and T. Nguyen. 1992, 9p.

Pub. in Proceedings of Society of the Plastics Industry Annual Technical/Marketing Conference (34th) $1992, \mathrm{p} 422-430$.

Keywords: "Humidity, "High temperature tests, "Density(Mass/volume), "Thermal conductivity, * Thermal insulation, Test methods, Heat measurement, Temperature dependence, Thermal resistance, Thermodynamic properties, Thermophysical properties, Test facilities, Reprints, *Rigid polyisocyanurate foam

Measurements of apparent thermal conductivity are presented for specimens of rigid polyisocyanurate (PIR) foam cut from a commercial insulation product and aged in air $60 \mathrm{C}$ and different humidities. Eight specimens, nominally 600 by $600 \mathrm{~mm}$, were prepared from two boards ( 1.2 by 2.4 by $0.05 \mathrm{~m}$ ) of rigid PIR foam blown with trichlorofluoromethane and having permeable organic-inorganic facers. Facers and excess foam were removed by sanding the specimens to a thickness of $27.9+$ or $-0.1 \mathrm{~mm}$. Four specimens were placed in ambient conditions of $22 \mathrm{C}$ and $40 \%$ relative humidity $(\mathrm{RH})$. Measurements of apparent thermal conductivity were conducted at $24 \mathrm{C}$ and a temperature difference of $22 \mathrm{C}$ using a heat-flow-meter apparatus conforming to ASTM Test Method C 518. Measurements were conducted for a period of 357 days at approximately 50-day intervals. Aging curves of specimen mass, volume, density, and thermal conductivity for rigid PIR foam are presented and implications of changes in these properties are discussed in the paper.

\section{0,374}

PB95-152039 Not available NTIS

National Inst. of Standards and Technology (NEL) Gaithersburg, MD. Building Environment Div.

Room Temperature Thermal Conductivity of Fumed-Silica Insulation for a Standard Reference Material.

R. R Zarr, T A. Somers, and D F. Ebberts. 1991 $23 \mathrm{p}$. 23p. in Improved Thermal Insulation Problems and Perspectives, Chapter 5, p97-1191991.

Keywords: "Thermal conductivity, "Thermal insulation, "Silicon dioxide, Conductive heat transfer, Thermal resistance, Heat measurement. Thermodynamic properties, Test facilities, Reprints, "Standard reference materials.

Thermal conductivity of fumed-silica insulation board was measured using the National Institute of Standards and Technology 1-meter Guarded Hot Plate. Measurements were conducted for the following range of parameters: bulk density, 304.5 to $325.4 \mathrm{~kg} / \mathrm{cu} \mathrm{m}$, mean temperature, 283.1 to $311.0 \mathrm{~K}$; and barometric pressure, 97.51 to $103.43 \mathrm{KPa}$. The effect of moisture content on room-temperature measurements was minimized by prior conditioning of the specimen at 100 $C$ for 24 hours. Seventy-five samples 600 by 600 by $25.4 \mathrm{~mm}$ ) were transferred to the Office of Standard Reference Materials in Gaithersburg, Maryland, USA. The material is offered as a Standard Reference Material having a low thermal conductivity at room temperature.

00,375

PB95-152864 Not available NTIS

National Inst. of Standards and Technology (BFRL), Gaithersburg, MD. Fire Science Div.

Effects of Specimen Edge Conditions on Heat Release Rate.

Final rept.

V. Babrauskas, W. H. Twilley, and W. J. Parker.

1993, 13p.

Pub. in Fire and Materials 17, n2 p51-63 1993.

Keywords: *Calorimeters, "Fire tests, "Edges, "Construction materials, "Heat flux, Burning rate, Heat transmission, Flammability, Plastics, Wood, Heat measurement, Reprints, "Reaction-to-fire tests.

When bench-scale specimens are tested for heat release rate, it is generally of interest that the behavior of the specimen simulate, as much as is possible, that of a real-scale product performing in a real fire. A number of issues have been raised recently by workers try- ing to understand the optimal conditions of specimen preparation and mounting. In the present study a large number of materials were explored in the Cone Calorimeter to determine the effect of edge conditions and edge frames. It was found that by the use of an insu lated edge frame, heat release rate values can be obtained which are slightly closer to expected true values. The testing procedure, however, is significantly more complicated. This makes the insulated edge frame useful for collecting specialized data for fire modeling, but not for conducting routine reactions-to-fire tests. For routine testing use, it is recommended: (1) that no edge frame needs to be used unless the test specimen presents special difficulties, such as due to intumescence; (2) that in those cases where the use of the steel edge frame is found necessary, the results should be reported on the basis of an effective exposure area of $0.0081 \mathrm{sq} \mathrm{m}$. When reported on such a basis, the heat release rate results do not show a systematic bias, compared to results with no edge frame.

\section{0,376}

PB95-154746 PC A05/MF A0

National Inst. of Standards and Technology (BFRL), Gaithersburg, MD. Fire Safety Engineering Div. Method of Predicting Smoke Movement in Atria with Application to Smoke Management.

J. H. Klote. Oct $94,96 \mathrm{p}$, NISTIR-5516.

Also available from Supt. of Docs.

Keywords: "Smoke, "Air flow, "Fires, "Buildings, "Atria, Unsteady flow, Fire tests, Temperature distribution, Mathematical models, Architecture, Building codes, Mass flow, Steady flow, "Smoke management, "Fire models, ASMET computer program, ASMET (Atria Smoke Management Engineering Tools).

The paper presents information that can be used for predicting smoke movement for design of atrium smoke management systems. NFPA 92B (1991) and the smoke control design book by Klote and Milke (1992) define smoke as the airborne solid and liquid particulates and gases evolved when a material undergoes pyrolysis or combustion, together with the quantity of air that is entrained or otherwise mixed into the mass. In recent years, approaches to smoke management in atria have been introduced into codes and engineering guides (BOCA; UBC 1993; NFPA 92B 1991; Klote and Milke 1992; Hansel and Morgan 1994). While these basic approaches differ in many respects, they all have the zone fire model concept as a common foundation and consist of a collection of algebraic equations intended for design calculation. For simplicity, discussion of the basic approaches is limited to those of NFPA 92B. However, much of this applies to the other approaches as well. The paper explains the physical concepts behind NFPA 92B including system limitations. The conservative nature of this approach is investigated, and the unique design challenge of atrium smoke detection is discussed. The application of zone computer fire models to atrium design is addressed. This paper also presents a computer program entitled Atria Smoke Management Engineering Tools (ASMET) that consists of a set of equations that may be of help for conventional applications. Appendices tions.

\section{0,377}

PB95-162491 Not available NTIS

National Inst. of Standards and Technology (NEL) Gaithersburg, MD. Building Materials Div.

Characteristics of Adhesive-Bonded Seams Sampled from EPDM Roof Membranes.

Final rept.

W. Rossiter, J. Seiler, W. Spencer, J. Lechner, and P. Stutzman. 1991, $13 p$.

Pub. in Proceedings of International Symposium on Roofing Technology (3rd), Montreal, Canada, April 1719, 1991, p167-179.

Keywords: "Failure analysis, "Seams(Joints), "Adhesive bonding, *Roofing, "Characterization, Rubber, Membranes, Construction materials, Adhesives, Scanning electron microscopy, Sampling, Nondestructive tests, Mechanical properties, Defects, Reprints, "EPDM roof membranes.

This study reports on the characterization of seam samples cut from EPDM roof systems. The availability of data on seam characteristics and failure analysis is beneficial to furthering the understanding of seam performance and to developing performance criteria for seams. Forty-eight samples were cut from EPDM roofs whose seams were rated as having provided satisfactory or unsatisfactory performance. The samples were 
subjected to laboratory tests which included identification of the adhesive, measurement of adhesive thickness, and determination of the strength and mode of failure in peel. Comparisons were made between the test results and performance ratings. It was found that the majority of the unsatisfactorily performing seams contained neoprene-based adhesives and were exposed on roofs for 45 months or more when sampled. Of the samples subjected to SEM analysis, the majority showed evidence of release agent on the rubber and adhesive surfaces analyzed. This finding provided evidence that a field method to judge rubber surface cleanness before application of the adhesive is needed.

\section{0,378}

\section{PB95-163150 Not available NTIS}

National Inst. of Standards and Technology (NEL), Gaithersburg, MD. Building Materials Div.

Rheology of Fresh Cement Paste.

Final rept.

L. J. Struble. 1991, 23p.

Sponsored by National Science Foundation, Washington, DC. and Northwestern Univ., Evanston, IL. Center for Advanced Cement-Based Materials.

Pub. in Ceram. Trans. 16, p7-29 $199 t$

Keywords: "Cements, "Rheology, "Microstructure, Construction materials, Binders(Materials), Curing,
Particulates, Dispersions, Flow theory, Reprints.

Rheology provides considerable information about the microstructure of dense particulate suspensions, and an important reason for studying the flow behavior of fresh cement paste is to infer the extent to which parfresh cement paste is to infer the extent to which par-
ticles are flocculated, how densely they are picked, and how their microstructure is modified by hydration reactions prior to set. These aspects of the fresh paste microstructure influence both the initial flow behavior and the ultimate performance of concrete. The rheology of fresh cement paste and other dense particulate suspensions is reviewed. There has been much progress in formulating relationships between flow behavior and microstructure of disperse suspensions, and recent advances in dense, flocculated suspensions have been assisted in part by new experimental techniques. This recent understanding offers encouragement as well as promising research directions in developing direct relationships between flow behavior and microstructure for flocculated cement particles in fresh paste.

\section{0,379}

PB95-163168 Not available NTIS

National Inst. of Standards and Technology (NEL), Gaithersburg, MD. Building Materials Div.

Cement and Concrete Characterization by Scanning Electron Microscopy.

Final rept.

P. E. Stutzman. 1991, 5p

Pub. in Proceedings of American Society of Civil Engineers Engineering Mechanics Specialty Conference, Columbus, OH., May 20-22, 1991, p1087-1091.

Keywords: "Concrete, "Portland cement, "Characterization, "Scanning electron microscopy Microstructure, Image analysis, X-ray analysis, Petrography, Nondestructive tests, Microanalysis, Inspection, Reprints.

The scanning electron microscope (SEM) is a powerful tool for the microstructural and chemical characterization of cement and concrete. Imaging techniques such as secondary electron, backscattered electron and $X$ ray imaging provide information about surface texture, microstructure, aggregates, and the air void system. Xray microanalysis is useful for qualitative and quantitative chemical analysis and $X$-ray mapping of element distribution. Image processing and analysis is used for quantification of microstructural features. The linking of these operations will provide automated, quantitative, and consistent analysis of portland cement and concrete.

\section{0,380}

PB95-163481 Not available NTIS

National Inst. of Standards and Technology (NEL) Gaithersburg, MD. Fire Science and Engineering Div. Wall Flame Heights with External Radiation.

Final rept.

K. M. Tu, and J. G. Quintiere. 1991, $9 p$.

Pub. in Fire Technology 27, n3 p195-203 Aug 91 Keywords: "Walls, "Burning rate, "Fire tests,
"Fiberboard, Heat flux, Wallboard, Heat transfer, Con:- struction materials, Fire safety, Wood particle boards, PMMA, Reprints, "Upward flame spread.

This work was an experimental study of upward flame spread of vertically oriented wall material with external radiation. Its inherent potentials of high fire growth rate and spreading to surrounding materials makes this study a very challenged task. An existing flame hea transfer fire testing apparatus was used to study the upward flame spread potential of two kinds of wall ma terials: (1) the PMMA (Polymethylmethacrylate) and (2) the Douglas Fir Particle Board. PMMA is non-charring whereas Douglas Fir Particle Board is a charring material. Various levels of external radiant heat flux ranging from $1.8 \mathrm{~W} / \mathrm{sq} \mathrm{cm}$ to $3.4 \mathrm{~W} / \mathrm{sq} \mathrm{cm}$ were $\mathrm{im}$ posed on to the wall samples for carrying out the serie of fire tests. Relevant parameters measured were fue mass burning rate, wall fire flame height, external radiant heat flux level and flame heat transfer, etc. Observations and data measurements of these experimental results were employed to evaluate flammability characteristics of wall materials as well as validating the application of ignition and flame spread theory on predicting material performance.

\section{0,381}

PB95-163804 Not available NTIS

National Inst. of Standards and Technology (NEL) Gaithersburg, MD. Building Materials Div.

Pulse-Echo Ultrasonic Evaluation of the Integrity of Seams of Single-Ply Roof Membranes.

Final rept.

H. Watanabe, and W. J. Rossiter. 1991, 12p.

Pub. in Proceedings of 1991 International Symposium on Roofing Technology, p72-83

Keywords: "Roofing, "Ultrasonic tests, "Seams(Joints) "Adhesive bonding, Membranes, Defects, Voids, Non destructive tests, Construction materials, Reprints "Single-ply roof membranes, "Pulse-echo ultrasonic evaluation

This paper summarizes results of a study to develop an ultrasonic pulse-echo method for evaluating the in tegrity of seams of single-ply roofing membranes. A prototype pulse-echo apparatus (the field scanner), which was designed to scan across seams of roofs while maintaining acoustic coupling to the seam surface was developed. Results of initial laboratory inves tigations showed that voids in seams could be distinguished from well-bonded sections using the intensity of the echo from the adhesive layer. This echo was relatively weak for well-bonded seams, and relatively strong for voids. Seams of existing EPDM single-ply membranes were examined by roof-top scanning to evaluate the performance of the field scanner in prac tice. The findings indicated that the field scanner was sensitive to detecting micro-cavities that could be created in the adhesive layer at the time of seam fabrication, thus resulting in false positive readings. It was concluded that, at least in its present form, the field scanner had limited applicability to resolving microcavities from voids and delaminations in solvent-adhesive seams.

\section{0,382}

PB95-164711 Not available NTIS

National Inst. of Standards and Technology (BFRL) Gaithersburg, MD. Fire Safety Engineering Div

Fire-Plume-Generated Ceiling Jet Characteristics and Convective Heat Transfer to Ceiling and Wall Surfaces in a Two-Layer Fire Environment: Uniform Temperature Ceiling and Walls.

Final rept.

L. Y. Cooper. 1993, 17p.

See also PB92-123074. Sponsored by Nuclear Regu latory Commission, Washington, DC.

Pub. in Fire Science and Technology 13, n1-2 p1-17 1993.

Keywords: "Fires, "Plumes, "Convective heat transfer, "Ceilings(Architecture), "Walls, Jet flow, Heat flux, Temperature distribution, Algorithms, Velocity distribu tion, Subroutines, Mathematical models, Reprints CEILHT subroutine.

This work presents a model to predict the instanta neous rate of convective heat transfer form fire plume gases to the overhead ceiling surface in a room of fire origin. The room is assumed to be a rectangular parallelopiped and, at times of interest, ceiling temperatures are simulated as being uniform. Also pre sented is an estimate of the convective heat transfer due to ceiling-jet-driven wall flows, to both the uppe and lower portions of the walls. The effect on the heat transfer of the location of the fire within the room is taken into account. Finally presented is a model of the velocity and temperature distributions in the ceiling jet. The model equations were used to develop an algorithm and associated modular computer subroutine to carry out the indicated heat transfer calculations. The subroutine is written in FORTRAN 77 and called CEILHT. The algorithm and subroutine are suitable for use in two-layer zone-type compartment fire mode computer codes. The subroutine was tested for a variety of fire environments involving a $10(\exp 7) \mathrm{W}$ fire in a $8 \mathrm{~m} \times 8 \mathrm{~m} \times 4 \mathrm{~m}$ high enclosure. While the calculated results were plausible, it is important to point out that CEILHT simulations have not been experimentally validated.

\section{0,383}

PB95-178885 PC A03/MF A01

National Inst. of Standards and Technology (BFRL), Gaithersburg, MD

Manufactured Housing Walls That Provide Satisfactory Moisture Performance in All Climates.

D. M. Burch, C. A. Saunders, and A. TenWolde. Jan 95, 36p, NISTIR-5558

See also PB95-105136. Prepared in cooperation with Forest Products Lab., Madison, WI. Sponsored by Department of Housing and Urban Development, Washington, DC. Office of Policy Development and Research.

Keywords: "Construction materials, "Walls, "Moistureproofing, "Prefabricated buildings, Moisture, Diffusion, Plywood, Wallboard, Cold regions, Mathematical models, Humidity, Thermal insulation, Per meability, Hot regions, *Moisture transfer.

We used the MOIST Computer Model to conduct a de tailed analysis of the moisture performance of one wall typical of current construction practice in manufactured housing, and two new alternative wall designs with potential for better moisture performance in a wider var ety of climates. The analysis showed that the currentpractice wall with an interior vapor retarder performed acceptably in a cold climate (Madison, WI), but poorly in a hot and humid climate (Miami, FL). The alternative wall designs both exhibited satisfactory moisture performance in the cold climate and the hot and humid climate, even with moderately severe indoor conditions. The alternative wall designs also performed satisfactorily in a mixed climate (Little Rock, AR). These alternative wall designs should be of interest to the manufactured housing industry, which distributes homes to all climatic regions of the United States.

00,384

PB95-180063 Not available NTIS

National Inst. of Standards and Technology (BFRL), Gaithersburg, MD. Fire Science and Engineering Div. Mathematical Modeling and Computer Simulation of Fire Phenomena.

Final rept.

H. R. Baum, K. B. McGrattan, and R. G. Rehm

1994, 9p

Pub. in Proceedings of International Symposium on Fire Safety Science (4th), Ottawa, Ontario, Canada, June 13-17, 1994, p185-193.

Keywords: *Transport properties, "Convective flow, "Computational fluid dynamics, Computerized simulation. Mathematical models, Finite difference theory, Combustion, High Reynolds number, Fires, Buoyancy, Navier-Stokes equations, Reprints, "Enclosure fires.

A method of studying the large scale transport of smoke and hot gases induced by fires in enclosures is described. The approach is based on solving the governing equations directly (if approximately) by decomposing the fire-driven flow field into large scale convective and small scale combustion components. In this work, results involving large scale convective transport generated by flow fields associated with typical fire scenarios are presented. The large scale flow is studied using finite difference techniques to solve large eddy simulations of the Navier-Stokes equations at high Reynolds numbers.

00,385

PB95-180139 Not available NTIS

National Inst. of Standards and Technology (BFRL),

Gaithersburg, MD. Structures Div.

Nondestructive Testing of Concrete: History and Challenges.

Final rept.

N. J. Carino. $1994,56 p$

Pub. in Proceedings of $V$. Mohan Malhotra Symposium on Concrete Technology: Past, Present, and Future, San Francisco, CA., March 21-23, 1994, p623-678. 
Keywords: "Concretes, "Nondestructive tests, Ultrasonic tests, Statistical analysis, Test methods, Durability, Mechanical properties, Infrared spectroscopy, Thermography, Reprints.

A brief history of nondestructive testing of hardened concrete over the past 50 years is presented. The contributions of V.M. Malhotra towards the development and promotion of nondestructive testing are emphasized. The underlying principles and inherent limitations of the methods are reviewed, and historical highlights of their development are presented. Test methods are grouped into those which assess in-place strength and those which evaluate non-strength characteristics, such as flaws and deterioration. The paper concludes with a discussion of the challenges for the 21 st century in the area of nondestructive testing.

00,386

PB95-181194 Not available NTIS

National Inst. of Standards and Technology (BFRL), Gaithersburg, MD. Building Environment Div.

Control Stability of a Heat-Flow-Meter Appartus.

Fina! rept.

R. R. Zarr. 1994, 12p.

Pub. in Jnl. Thermal Insul. and Bldg. Envs. 18, p116127 Oct 94

Keywords: "Thermal insulation, "Heat meters, *Calibration, Heat transmission, Errors, Steady state, Precision, Measuring instruments, Reprints.

Calibration measurements of a commercial heat-flowmeter apparatus are presented. The apparatus has been calibrated using the same speciment of high-density fibrous-glass board over a period of four years, from 1989 to 1993. Seventy-three tests have been conducted, generally at ambient conditions of $24 \mathrm{C}$ with a moderate temperature difference of either 15,22 , or $27 \mathrm{C}$ across the specimen. Variations within a set of data for each test have been examined to verify underlying assumptions of randomness, normal frequency distribution of errors, repeatability, and stability of the data. Variations between test data indicate a smal drift, on the order of one percent over four years, in the calibration factor of the apparatus. A model has been developed to describe the small drift with time. The analysis of variations between test data has also identified intermittent shifts in the precision of the calibration factor of the apparatus.

00,387

PB95-181202 Not available NTIS

National Inst. of Standards and Technology (BFRL), Gaithersburg, MD. Building Environment Div.

Intra-Laboratory Comparison of a Line-HeatSource Guarded Hot Plate and Heat-Flow-Meter Apparatus.

Final rept.

R. R. Zarr. 1991, 18p.

Sponsored by Department of Energy, Washington, DC and Oak Ridge National Lab., TN.

Pub in Insulation Materials: Testing and Applications, ASTM STP 1116, p502-5191991.

Keywords: "Heat meters, "Thermal insulation, "Thermal conductivity, Heat transmission, Heat flux, Measuring instruments, Calibration, Standards, Thickness, Comparison, Transducers, Test methods, Tes facilities, Reprints, "Line heat sources, "Guarded hot plate, Standard reference materials, Fibrous-glass board.

The apparent thermal conductivity and thermal resistance of several building insulations were determined at the National Institute of Standards and Technology (NIST) using three apparatus. Reference values were determined with NIST's 1-meter Line-Heat-Source Guarded Hot Plate (GHP). The other two apparatus were heat-flow-meter (HFM) apparatus calibrated with a Standard Reference Material of fibrous-glass board. Deviations from reference values of the GHP were calculated for the HFM apparatus. The apparatus, test procedure, and analysis of the deviations are described in the paper.

\section{0,388}

B 95-181210 Not available NTIS

National Inst. of Standards and Technology (BFRL)

Gaithersburg, MD. Building Environment Div.
Effect of Environmentally Exposures on the Properties of Polyisocyanurate Foam Insulation: Thermal Conductivity Measurements.

Final rept.

R. R. Zarr, and T. Nguyen. 1991, $15 p$.

Pub. in Proceedings of International Workshop on the Long-Term Thermal Performance of Cellular Plastics (2nd), Ontario, Canada, June 5-7, 1991, 15p.

Keywords: "Thermal conductivity, *Thermal insulation, "Aging(Materials), "Test methods, Heat meters, Thermophysical properties, Heat transmission, Humidity, Foams, Measuring instruments, Expanded plastics, Reprints, *Polyisocyanurate, ASTM C 518.

Measurements of apparent thermal conductivity are presented for specimens of rigid polyisocyanurate (PIR) foam conditioned in one of four environments. Measurements were conducted at $24 \mathrm{C}$ and a temperature difference of $22 \mathrm{C}$ at monthly intervals for six months using a heat-flow-meter apparatus (ASTM Tes Method C 518). Four specimens (nominally 610 by 610 $\mathrm{mm}$ ) were prepared from one commercial board (nominally 1.2 by $2.4 \mathrm{~m}$ ) of rigid PIR foam blown, with R11 and having permeable organic/inorganic facers. For the six-month period, the increase in apparent therma conductivity for the four specimens ranged from 11.5 to $14.2 \%$. Aging curves of thermal conductivity for each specimen are described in the paper.

\section{0,389}

PB95-188868 PC A03/MF A01

National Inst. of Standards and Technology (BFRL), Gaithersburg, MD. Fire Safety Engineering Div.

Santa Ana Fire Department Experiment at 1315 South Bristol, July 14, 1994.

A. D. Putorti, W. D. Walton, W. H. Twilley, S. Deal,

and J. C. Albers. 31 Aug 94, 23p, FR-3995.

Prepared in cooperation with Santa Ana City Fire Dept., CA. Fire Safety Div.

Keywords: "Residential buildings, "Fire tests, "Fire protection, "Fire detectors, Sprinklers, Flammability, Construction materials, Temperature measurement, Fire safety, Smoke, *Santa Ana(California).

The Santa Ana Fire Department of Santa Ana, California conducted a series of fire experiments in residences on South Bristol Street in the City of Santa Ana in July, 1994. The National Institute of Standards and Technology (NIST) provided technical support, consisting of measurements of fire phenomena to the fire department during these experiments. The experiment addressed in this report occurred on July 14, 1994, at 1315 South Bristol Street. The measurements included: temperatures within various rooms, the velocity and temperature of outflowing gases, smoke detector activation time, sprinkler activation times, and time to full room involvement. Data were recorded every 5 seconds with a computerized acquisition system.

00,390

PB95-188918 PC A04/MF A01

Nationai Inst. of Standards and Technology (BFRL), Gaithersburg, MD. Fire Safety Engineering Div.

Fire Performance of an Interstitial Space Construction System.

J. R. Lawson, E. Braun, L. DeLauter, and G

Roadarmel. Feb 95, 67p, NISTIR-5560.

See also PB86-106002. Sponsored by Office of the Chief of Engineers (Army), Washington, DC. Medical Facilities Office.

Keywords: "Floors, "Fire tests, "Steel structures, Fire resistance, Test methods, Surface temperature, Construction materials, Structural members, Fire protection, Fire safety, "Interstitial space construction systems.

An interstitial space building construction assembly, consisting of a walk-on deck suspended from above by structural steel which also supported a functional floor, reproduce a design planned for use in a new hospital complex at Elmendorf Air Force Base, Alaska. This interstitial space assembly was built in the multistory steel test structure at the National Institute of Standards and Technology. The construction assembly was tested by the same protocol used to evaluate the Veteran's Administration interstitial space construction assembly, tested in 1984. This protocol followed the National Fire Protection Association's NFPA 251 Fire Tests of Building Construction and Materials Standards, 1990 edition. Fire testing of the interstitial space system was carried out during the summer of 1994. This construction assembly met the requirements for a 2 hour fire endurance rating. The maximum surface temperature on the unexposed functional floor above the interstitial space reached $33 \mathrm{C}(91 \mathrm{~F})$ at the end of the two hour period. The maximum structural steel temperature inside of the interstitial space was $123 \mathrm{C}(253 \mathrm{~F})$. The structural assembly was evaluated for a total of 2 hours and 30 minutes before the test was terminated.

00,391

\section{PB95-200655 PC A05/MF A01}

National Inst. of Standards and Technology (BFRL), Gaithersburg, MD. Building Environment Div.

Heat and Moisture Transfer in Wood-Based Wall Construction: Measured versus Predicted.

Building science series.

R. R. Zarr, D. M. Burch, and A. H. Fanney. Feb 95 92p, NIST/BSS-173.

Also available from Supt. of Docs. as SN003-00303325-1.

Keywords: "Walls, "Heat transfer, "Moisture content, Wood, Construction materials, Buildings, Wall temperature, Humidity, Heat flux, Thermal insulation, Wooden structures, Steady state, Thermodynamic properties, MOIST computer program.

This report describes a comprehensive laboratory study to verify the accuracy of MOIST for 12 different wall specimens. The rate of heat transfer through each of the 12 wall specimens was measured. The moisture content of the exterior construction materials was measured for eight of the twelve wall specimens. For the remaining four walls, the relative humidity level was measured at the interior side of the exterior sheathing. The measured heat transfer rates, moisture content levels and relative humidities were compared to the predictions of MOIST. In general, the agreement between MOIST and the experimental measurements was good. The moisture content predicted by MOIST was within one percent of the measured values for seven of the eight walls that contained moisture ,ontent sensors. The measured relative humidities for two of the remaining four walls agreed well with the MOIST predictions. The relative humidity measurements from the other two walls could not be compared to MOIST since the walls were constructed with vapor retarder defects that introduced two-dimensional effects. The heat flux predicted by MOIST was within ten percent of the values measured under steady-state conditions.

00,392

PB95-208757 PC A09/MF A02

National Inst. of Standards and Technology (BFRL), Gaithersburg, MD.

Optical Performance of Commercial Windows.

S. J. Treado, and J. W. Bean. Jan 92,184 p, NISTIR4711.

Keywords: "Windows, "Absorptance, "Privacy, Contrast, Window glazing, Visibility, Performance.

The role of window system characteristics on privacyrelated issues was examined. The optical characteris tics of various window materials were measured and compared to determine the best candidates for enhancing building occupant privacy. Strategies for inducing privacy are discussed, along with related performance characteristics of window systems.

\section{0,393}

PB95-216990 PC A07/MF A02 Maryland Univ., College Park Burning Rate and Flame Heat Flux For PMMA in the Cone Calorimeter.

Master's thesis.

B. T. Rhodes. Dec 94, 130p, NIST/GCR-95/664.

Grant NIST-60NA2D1266

Sponsored by National Inst. of Standards and Technology (BFRL), Gaithersburg, MD. Fire Science Div.

Keywords: "Calorimeters, "Buming rate, "Flames, "Heat flux, Ignition temperature, Ignition time, Models, Thermoplastic resins, Polymethyl methacrylate, Fire tests, Theses, Black Polycast PMMA, Cone calonimeter.

Ignition and burning rate data are developed for thick $(25 \mathrm{~mm}$.) black Polycast PMMA in a Cone Calorimeter heating assembly. The objective is to establish a testing protocol that will lead to the prediction of ignition and burning rate from Cone data. This is done for a thermoplastic like PMMA. For black PMMA, ignition temperatures of 250 to $350 \mathrm{deg} C$ and vaporization temperatures of approximately 325 to $380 \mathrm{deg} C$ were measured over irradiance levels of 15 to $65 \mathrm{~kW} / \mathrm{sq}$ m. The incident flame heat flux for irradiation levels of 0 
to $75 \mathrm{~kW} / \mathrm{sq} \mathrm{m}$, is found to be approximately $37 \mathrm{~kW} /$ sq $\mathrm{m}$ for black PMMA. Its constancy is shown due to the geometry of the Cone flame.

\subsection{4}

PB95-217147 PC A08/MF A02

Maryland Univ., College Park. Dept. of Mechanical Engineering.

Water Droplet Evaporation from Radiantly Heated Solids.

Final rept. Sep 92-May 94

S. Tinker, and M. di Marzo. Dec 94, 157p, NIST/

GCR-95/665

Contract NIST-70NANB1H1173

Sponsored by National Inst. of Standards and Technology (BFRL), Gaithersburg, MD. Fire Safety Engineering Div.

Keywords: "Drops(Liquids), "Evaporative cooling, "Radiant heating "Solids, Evaporation, Heat transfer Models, Sprinkler systems, Water sprays, Dissolved gases, Computer programs, Surface temperature Thermal conductivity, Vaporizing, Contact angle.

A model describing the configuration of a water droplet evaporating on the surface of a radiantly heated sem infinite solid is developed. A shape factor and the solidliquid-vapor contact angle described the transient droplet shape, though the initial value of the latter parameter is found to have a negligible effect on the dropet's evaporation. The droplet shape model and a modified radiation heat term are incorporated into a pre viously-developed computer model to predict the evaporation of a single droplet on a semi-infinite solid subjected to radiant heat input. The code predicts transient temperature profiles that agree well with experiment.

\section{0,395}

\section{PB95-220455 PC A03/MF AO}

National Inst. of Standards and Technology (BFRL)

Gaithersburg, MD. Building Materials Div.

Testing of Selected Self-Leveling Compounds for Floors.

C. F. Ferraris. Jan $95,36 p$, NISTIR-5633.

Contract GSA-862-2500

Sponsored by General Services Administration, Washington, DC.

Keywords: "Lightweight concretes, "Construction materials, "Floors, Bonding, Office buildings, Technology utilization, Tests, Concretes, Shrinkage, Adhesives, Properties, Substitutes, Self-leveling compounds.

During the past year, a severe odor has developed in some floors of the Silver Spring Metro Center I build ing. The odor has been attributed to interactions among a self-leveling compound, carpet adhesive, and the carpet. The owner of the building, General Service Administration (GSA), wants to ascertain if by removing the existing self-leveling compound and replacing it with a compound of a different composition will elminate the odor. The National Institute of Standards and Technology (NIST) was asked to evaluate the properties of selected self-leveling compounds for use at the Silver Spring Metro Center I building. Lightweight concrete was also to be tested for possible use as a substrate for the self-leveling compounds. The report gives the test results obtained on the self-leveling com pounds alone or in combination with regular concrete or lightweight concrete. It also gives the results obtained on lightweight concrete alone.

\section{0,396}

PB95-220489 PC A04/MF A01

National Inst. of Standards and Technology (BFRL), Gaithersburg, MD. Building Materials Div.

Survey of Concrete Transport Properties and Thei Measurement.

N. S. Martys. Feb 95, 52p, NISTIR-5592.

Keywords: *Concretes, “Transport properties, "Service life, "Standards. Permeability, Microstructure, Test methods, Chlorides, Porosity, Tomography, $X$ ray anal-
mate, ysis.

In this report we present a survey of the current knowledge of the transport properties of concrete. The basi theory and measurement methods are discussed. Em phasis is placed on transport properties, such as diffusion, permeability, and capillary flow, that may play a large role in degradation processes in high performlarge role in degradation processes in high perform-
ance concrete. It is concluded that standard test meth-
ods used to predict the service life of concrete via ods used to predict the service life of concrete via measurement of transport properties, especially in high need further development.
00,397

PB95-220497 PC A03/MF A01

National Inst. of Standards and Technology (BFRL), Gaithersburg, MD.

Compositional Analysis of Beneficiated Fly Ashes. P. E. Stutzman, and L. Centeno. May 95, 28p, NISTIR-5598.

Keywords: "Fly ash, "Concretes, "Physical properties, Admixtures, Particle size.

The study is part of a study of the material properties of fly ashes and other mineral admixtures to identify factors that control the performance of mineral admixtures in concrete. The study includes the determination of the kinetics of reactions, development of microstructure, and simulation modelling. The development of microstructure, and simulation modelling. The overall goal is understanding of how the material properties of fly ash affect the properties and durability of concrete. The report examines the composition of a set of fly ashes segregated by particle size, and comments on the effects of compositional and physical attributes on the performance of concrete.

\section{0,398}

PB95-226684 PC A07/MF A02

National Inst. of Standards and Technology (BFRL), Gaithersburg, MD. Fire Safety Engineering Div.

Building and Fire Research Laboratory Publications, 1994.

Special pub.

N. H. Jason. May $95,132 p$, NIST/SP-838/7

Also available from Supt. of Docs. See also report for 1993, PB95-143202.

Keywords: "Buildings, "Fires, "Bibliographies, "Research, Combustion, Fire protection, Abstracts, Flammability, Construction materials, Heat transfer.

Building and Fire Research Laboratory Publications, 1994 contains references to the publications prepared by the members of the Building and Fire Research (BFRL) staff, by other National Institute of Standards and Technology (NIST) personnel for BFRL, or by external laboratories under contract or grant from the BFRL during the calendar year 1994

\section{0,399}

\section{PB95-251617 PC A03/MF A01}

National Inst. of Standards and Technology (BFRL), Gaithersburg, MD. Building Environment Div.

Water-Vapor Measurements of Low-Slope Roofing Materials.

D. M. Burch, and A. O. Desjarlais. 1995, 34p, NISTIR-5681.

Sponsored by Oak Ridge National Lab., TN.

Keywords: "Moisture, "Isotherms, "Roofing, "Permeability, Water vapor, Moisture content, Humidity, Construction materials, Test methods.

New measurement methods recently developed at the National Institute of Standards and Technology were used to measure the sorption isotherm and permeability of several low-slope roofing materials at a mean temperture of $24 \mathrm{deg} C(75 \mathrm{~F})$. The materials included: fireboard, perlite board exierior-grade plywood, polyisocyanurate board insulation with glass-matt facers, and glass-fiber insulation with a facer. For the sorption isotherm measurements, the materials were placed in various ambient relative humidities ranging from a dry to a saturated state. The equilibrium mois. ture content plotted versus ambient relative humidity at 24 deg $C(75 \mathrm{~F})$ gave the sorption isotherm. Separate sorption isotherms were obtained for specimens initially dry (adsorption isotherm) and specimens initially saturated (desorption isotherm). For the permeability measurements, a series of cup measurements was performed, and the permeability was plotted as a function of the mean relative humidity across the specimen.

\section{0,400}

\section{PB95-270047 PC A10/MF A03}

National Inst. of Standards and Technology (BFRL), Gaithersburg, MD

Project Summaries 1995: NIST Building and Fire Research Laboratory.

Special pub.

N. J. Raufaste. Aug 95, 218p, NIST/SP-838/8. Also available from Supt. of Docs. as SN003-003. 03350-2. See also report for 1994, PB94-207495.

Keywords: "Building, "Fires, "Research management, "Testing laboratories, Building codes, Fire safety,
Flammability testing, Standards, Construction materials.

NIST's Building and Fire Research Laboratory (BFRL), one of NIST's eight Laboratories, enhances the competitiveness of U.S. industry and public safety through performances prediction and measurement technologies and technical advances that improve the life cycle quality of constructed facilities. BFRL's efforts are closely coordinated with complementary activities of industry, professional and trade organizations, academe, and other agencies of government. This report summarizes BFRL's research for 1995 . The report is arranged by its research programs; structural engineering, materials engineering, mechanical and environmental systems, fire safety and engineering, fire science, and applied economics. Each summary lists the project title, the BFRL point of contact, sponsor, research, and recent results.

00,401

PB96-109541 PC A04/MF A01

National Inst. of Standards and Technology (BFRL), Gaithersburg, MD. Fire Safety Engineering Div.

CFAST Output Comparison Method and lts Use in Comparing Different CFAST Versions.

See also PB93-174902.

Keywords: "Computer models, "Fires, "Flame propagation "Building, Smoke, Plums, Fire test, Combustion products, Transport properties, Algorithms, Variables, Computerized simulation, Comparison, CFAST computer model.

A multiple step method was developed to compare the output of CFAST simulations, produced either by the same version of CFAST, or by different versions of the model. Scenarios to be compared are run with CFAST before the method is used, producing files containing a history of the model results. The first step of the comparision method produces a text file of important output variables from each of these history files, corresponding to significant fire phenomena occuring during the course of each fire simulation. The next step of the method is used to compare two such text files, and store their differences. Finally, the last step summarizes the difference information found in one or more files from the previous step. The comparison method can be used to find differences between CFAST runs, and track changes in the CFAST model and detect if they perform as anticipated.

00,402

PB96-122130 (Order as PB96-117767, PC A08/

MF A02)

National Inst of Standards and Technology, Gaithersburg, MD.

Study on the Reuse of Plastic Concrete Using Extended Set-Retarding Admixtures.

C. Lobo, W. F. Guthrie, and R. Kacker. 1995, 15p.

Prepared in cooperation with National Ready Mixed

Concrete Association, Silver Spring, MD.

Included in Jnl. of Research of the National Institute of Standards and Technology, v100 n5 p575-589 Sep/ Oct 95

Keywords: "Plastic concrete, "Admixtures, Recycling, Shrinkage, Process variables, Stabilizers(Agents), Environmental issues, Strength, Reprints, NIST(National Institute of Standards and Technology).

The disposal of ready mixed concrete truck wash water and returned plastic concrete is a growing concern of the ready mixed concrete indusiry. Recently, extended set-retarding admixtures, or stabilizers, which slow or stop the hydration of portland cement have been introduced to the market. In a statistically designed experiment, the properties of blended concrete containing stabilized plastic concrete were evaluated. The variables in the study included (1) concrete age when stabilized, (2) stabilizer dosage, (3) holding period of the treated (stabilized) concrete prior to blending with fresh ingredients, and (4) amount of treated concrete in the blended batch. The setting time, strength, and drying shrinkage of the blended concretes were evaluated.

00,403

PB96-123419 Not available NTIS

National Inst. of Standards and Technology (BFRL) Gaithersburg, MD. Building Materials Div.

Hydraulic Radius and Transport in Reconstructed Model Three-Dimensional Porous Media.

Final rept.

D. P. Bentz, and N. S. Martys. 1994, 18p.

in Transportation in Porous Media, v17 p221-238 1995 


\section{Construction Materials, Components, \& Equipment}

Keywords: "Building technology, "Porous media, "Hydraulic radius, Conductivity, Critical diameter, Permeability, Reconstruction, Reprints, "Foreign technology.

Methods for reconstructing three-dimensional porous media from two-dimensional cross sections are evaluated in terms of the transpont properties of the reconstructed systems. Two-dimensional slices are selected at random from model three-dimensional microstructures, based on penetrable spheres, and processed to create a reconstructed representation of the original system. Permeability, conductivity, and a critial pore diameter are computed for the original and reconstructed microstructures to assess the validity of the reconstruction technique. A surface curvature algorithm is utilized to further modify the reconstructed systems by matching the hydraulic radius of the reconstructed three-dimensional system to that of the twodimensional slice. While having only minor effects on conductivity, this modification significantly improves the agreement between permeabilities and critical diameters of the original and reconstructed systems for porosities in the range of $25-40 \%$. For lower porosities, critical pore diameter is unaffected by the curvature modification so that little improvement between original and reconstructed permeabilities is obtained by matching hydraulic radii.

\section{0,404 \\ Not available NTIS}

National Inst. of Standards and Technology (BFRL), Gaithersburg, MD. Fire Safety Engineering Div.

Computing the Effect of Sprinkler Sprays on Fire Induced Gas Flow.

Final rept.

G. P. Forney, and K. B. McGrattan. 1995, 6p.

Pub. in Proceedings of the International Conference of Fire Research and Engineering, Orlando, FL., September 10-14, 1995, p59-64.

Keywords: "Sprinklers, "Time lapse, "Fire suppression, Fire protection, Gas flow, Fluid flow, Fire tests, Simulation, Walls, Flame propagation, Smoke detectors, Fire fighting, Reprints.

Over the past twenty years there has been much debate concering the interaction of sprinklers and draft curtains in large storage facilities. At issue is whether or not the two fire protection systems are mutually beneficial. Draft curtains inhibit the spread of hot gases near the ceiling. In some cases this may accelerate the activation of sprinklers and in others it could delay the activation of sprinklers needed to suppress the fire. The intent is not necessarily to simulate in detail the two phase interaction of droplets and air from a single sprinkler, nor to predict the suppression of the fire itself, but rather to study the effect of dozens of sprinklers on a fire-driven flow field in enclosures up to 60 meters on a side and 10 meters high. The sprinkler spray serves to cool the upper layer hot gases by mechanical mixing with cooler gases below and absorption of heat by the droplets.

\section{0,405}

PB96-154836 PC A06/MF A01

National Inst. of Standards and Technology (BFRL), Gaithersburg, MD. Structures Div

Simplified Design Procedure for Hybrid Precast Concrete Connections.

G. S. Cheok, W. C. Stone, and S. D. Nakaki. Feb 96 91p. NISTIR-5765.

Sponsored by Concrete Research Council, Detroit, MI

Keywords: "Earthquake resistant structures, "Precast concrete, "Construction joints, "Beams(Supports), Design criteria, Buildings, Dynamic response.

A design procedure is presented to compute the maximum (plastic) moment, and the story drift capacities of a hybrid precast moment-resisting beam-to-column connection. The hybrid connections consist of mild steel which is used to dissipate energy by yieldirig and high strength prestressing steel which is used to provide the shear resistance through friction developed at the beam-column interface by the post-tensioning force. The design procedure is based on three $1 / 3$ scale hybrid precast beam-to-column connections tested at the National Institute of Standards and Technology (NIST). The simplified procedure relies on the stess-strain behavior of mild steel up to its ultimate strength and is based on equilibrium equations at the beam-column joint. The appendices include a proposed evaluation criteria for this hybrid connection, sample calculations using the design procedure, and other calculations used to develop the design criteria.

\section{0,406}

PB96-156161 Not available NTIS National Inst. of Standards and Technology (BFRL),
Gaithersburg, MD. Building Materials Div.

Application of Digital-Image-Based Models to Microstructure, Transport Properties, and Deg. radation of Cement-Based Materials.

Final rept.

D. P. Bentz, E. J. Garboczi, and N. S. Martys. 1996, $19 p$.

Pub. in Modelling Microstructure and Its Potential for Studying Transport Properties and Durability, p1671851996.

Keywords: "Cements, "Construction materials, Percolation, Transport properties, Microstructure, Durability, Reprints, "Foreign technology

As multi-phase composites, cement-based materials have physical properties that are strongly influenced by the volume fractions and topologies of the individua phases. Because of their inherent random nature, these materials often defy a simple geometrical de scription. The use of digital-image-based models al lows one to realistically represent this class of materials, as resultant microstructures can be quickly quantified with respect to the volume fraction and interconnectivity or percolation of each phase or any combination of phases. In addition, physical properties such as diffusivity and permeability can be conveniently computed using finite-difference or finite-elemen techniques. These computer modelling techniques will be demonstrated for microstructural models of these materials at two scales: hydrated cement past at the micrometer level and calcium silicate hydrate gel at the nanometer level. The properties computed for the ge at the nanometer level can be used as input for the micrometer-level model.

00,407

PB96-159652 Not available NTIS

National Inst. of Standards and Technology (BFRL) Gaithersburg, MD. Fire Safety Engineering Div.

Fire Hazard Model Developments and Research Efforts at NIST.

Final rept.

A. J. Fowell. 1993, 7p

Pub. in Fire Retardant Chemicals Association Meeting Proceedings on Fire Safety, New Orleans, LA., March $21-24,1993,7 p$

Keywords: "Fire hazards, "Computerized simulation, Fire tests, Fire research, Fire safety, Combustion, Ventilation, Flammability testing, Burning rate, Materials tests, Standards, Reprints, "US NIST, "Fire Models.

Exposure conditions such as location, orientation, ventilation, and proximity to other materials can influence the fire performance of the material, so these parameters must be addressed by the models and/or the flammability characteristics measurements. Before the combination of fire models and bench scale measure ment methods become universally accepted as tools for assessing the fire safety suitability of materials for specific aplication, the models must be shown to be valid, some of the shortcomings in the measurement methods need to be addressed and a usable data base needs to be assembled.

\section{0,408}

PB96-164066 Not available NTIS

National Inst. of Standards and Technology (BFRL), Gaithersburg, MD. Fire Science Div.

Heat Transfer in an Intumescent Material Using a Three-Dimensional Lagrangian Model.

Final rept.

K. M. Butler, H. R. Baum, and T. Kashiwagi. 1996,

Pub. in International Conference on Fire Research and Pub. in International Conference on Fire Research and
Engineering, Orlando, FL., September 10-15, 1995, p261-266.

Keywords: "Bubbles, "Intumescence, Mathematical models, Numerical analysis, Thermoplastics, Reprints.

Intumescence is a property of an important class of fire-resistant materials. In the presence of fire a succession of chemical reactions results in melting, the generation of multiple tiny bubbles causing the material to swell, and solidification into a thick multicellular char layer. An intumescent coating protects the underlying substrate from the fire through two mechanisms: heat is absorbed by the endothermic chemical reactions that produce the bubbles, and the low thermal conductivity of the bubbles provides an insulating layer.
The chemical mechanism causes a plateau in the plot of substrate temperature vs. time, and the decreased effective thermal conductivity slows the temperature increase with time for the final char layer.

\section{0,409}

PB96-164108 Not available NTIS

National Inst. of Standards and Technology (BFRL), Gaithersburg, MD. Fire Safety Engineering Div.

Calculating Combined Buoyancy- and PressureDriven Flow Through a Shallow, Horizontal, Circular Vent; Application to Problem of Steady Burning in a Ceiling-Vented Enclosure.

Final rept.

L. Y. Cooper. 1995, $6 p$.

See also PB96-164116.

Pub. in International Conference on Fire Research and Engineering Proceedings, Orlando, FL., September 10-15, 1995, p321-326

Keywords: "Vents, "Air flow, "Buoyancy, "Flame propagation, Fire protection, Buildings, Mathematical models, Flow rate, Reprints

A model was developed previously for calculating combined buoyancy- and pressure-driven (i.e., forced) flow through a shallow, circular, horizontal vent where the vent-connected spaces are filled with fluids of different density in an unstable configuration (density of the top fluid is larger than that of the bottom). In this paper the model equations are summarized and then applied to the problem of steady burning in a ceiling-vented enclosure where normal atmospheric conditions characterize the upper space environment. Such fire scenarios are seen to involve a zero-to-relatively-moderate cross-vent pressure difference and bi-directional exchange flow between the enclosure and the upper space. A general solution to the problem is obtained. This relates the rate of energy release of the fire to the area of the vent and to the temperature and oxygen concentration of the upper portion of the enclosure environment. The solution is seen to be consistent with previously-published data involving ceiling-vented fire scenarios

\section{0,410}

PB96-164116 Not available NTIS

National Inst. of Standards and Technology (BFRL), Gaithersburg, MD. Fire Safety Engineering Div. Combined Buoyancy and Pressure-Driven Flow Through a Shallow, Horizontal, Circular Vent. Final rept.

L. Y. Cooper. 1995, 9p.

See also PB96-164108 and PB94-210077.

Pub. in American Society of Mechanical Engineers International Mechanical Engineering Congress and Exhibition, Chicago, IL., November 6-11, 1994, Jnl. of Heat Transfer, v117 p659-667 Aug 95.

Keywords: "Vents, "Air flow, "Buoyancy, "Flame propagation, Boundary conditions, Reprints, Fire protection, Buildings, Mathematical models, Flow rate, Boundary value problems.

Combined buoyancy and pressure-driven (i.e., forced) flow through a horizontal vent is considered where the vent-connected spaces are filled with fluids of different density in an unstable configuration (density of the top is larger than that of the bottom). With zero-to-moderate cross-vent pressure difference, delta $p$, the instability leads to bidirectional exchange flow between the two spaces, e.g., as in the emptying from the bottom of a liquid-filled can with a single vent opening. For relatively large delta $p$, the flow through the vent is unidirectional, from the high to the low-pressure space e.g., as is the case when the can has a large enough second vent at the top. Problems of a commonly used unidirectional orifice vent flow model, with Bernoulli's equation and a constant flow coefficient, $\mathrm{Cd}$, are discussed.

00,411

PB96-193651 PC A03/MF A01

National Inst. of Standards and Technology (BFRL) Gaithersburg, MD. Building Materials Div.

Warping of Terrace Pavers at the U.S. Capitol Building.

C. F. Ferraris, P. Stutzman, and J. Clifton. May 96 , 26p, NISTIR-5847.

Keywords: Construction materials, Aggregates, $\mathrm{Ce}$ ments, Concretes, Alkali aggregate reactions, Concrete durability, Chemical reactivity, Composition, Silicon dioxide, Environmental effects, Expansion, Shrinkage, Overlays, Substrates, Washington DC, "Pavers, 
*United States Capitol, "Warping, Alkali-silica reactions

The terraces of the U.S. Capitol are covered with cement-based pavers designed to emulate the granite pavers used elsewhere on the Capitol grounds. These pavers are composed of two layers; an upper, decorative white-cement-based mortar with crushed micaceous quartz aggregate supported by a base of conventional concrete. Field inspection and laboratory testing indicates that warping is probably due to the high cement content, environmental exposure conditions, and possibly differences in hydraulic length changes of two layers comprising the pavers. Alkalisilica reactivity (ASR) tests indicate that the base layer aggregate is marginally reactive and the upper layer aggregate is non-reactive.

00,412

PB96-193693 PC A04/MF A01

National Inst. of Standards and Technology (BFRL),

Gaithersburg, MD. Building Environment Div.

Room-Temperature Thermal Conductivity of Expanded Polystyrene Board for a Standard Reference Material.

R. R. Zarr, M. W. Davis, and E. H. Anderson. May 96, 45p, NISTIR-5838.

Keywords: "Thermal insulation, "Standards, *Polystyrene, Building materials, Tests, SRM(Standard Reference Material).

Thermal conductivity measurements at room temperature are presented as the basis for certified values of SRM 1453, expanded polystyrene board. The measurements have been conducted in accordance with a randomized full factorial experimental design with two variables, bulk density and temperature, using NIST's one-meter line-heat-source guarded hot plate apparatus. Uncertainties of the measurements, consistent with current ISO guidelines, have been prepared. The thermal conductivity measurements were conducted over a range of bulk density of 37.4 to $45.8 \mathrm{~km} / \mathrm{cu} \mathrm{m}$ and mean temperature of 281 to $313 \mathrm{~K}$. Statistical analyses of the physical properties of the SRM are presented and include variations between boards, as well as within boards. Measurements of the foam's compressive properties and microstructure are presented.

00,413

PB96-202239 PC A09/MF A02

National Inst. of Standards and Technology (BFRL), Gaithersburg, MD

NIST Construction Automation Program Report No. 2. Proceedings of the NIST Construction Automation Workshop. Held in Gaithersburg, Maryland on March 30-31, 1995

W. C. Stone. May 96, 159p, NISTIR-5856.

Keywords: "Meetings, "Buildings, "Automation, "Robotics, "Construction, Excavation, Data exchange, Laser metrology, Global positioning system, Telemetry, Virtual reality, Display systems, Wireless communications, Optical detectors, Standards, "Helmet mounted displays.

A two-day workshop on Construction Automation was hosted at NIST from March 30-31, 1995 . Research programs actively underway at NIST in this area include the development of sensing systems, hardware, and software algorithms for advanced real-time construction site metrology; wide band telemetry and data acquisition (the ability to track many sensors at once through wireless communications); virtual site simulation and object representation standards (development of robust virtual reality models for construction site objects and machines); person-in-loop systems including head-up displays, virtual simulators, tele-operations workstations, and portable database interrogators which provide information-on-demand to construction site personnel; and semi-autonomous machine operations. These topics, and the need for database and machine intertacing standards, were discussed by workshop participants representing industry, government, and academe. Specific invited presentations included laser distancing, non-line-of-sight and kinematic GPS metrology, automated data exchange standards, real-time kinematic modeling, military helmet-mounted displays, virtual reality displays, construction robotics, automated excavation, virtual site representation, and automated building construction.

00,414

PB96-202338 PC A04/MF A01

National Inst. of Standards and Technology (BFRL), Gaithersburg, MD. Building Materials Div.
Measurement of Rheological Properties of High Performance Concrete: State of the Art Report. C. F. Ferraris. Jul 96, 41p, NISTIR-5869.

Keywords: "Concretes, "Rheological properties, Test methods, Viscosity, Cements, Slumping, Workability.

The rheology or flow properties of concrete in general and of high performance concrete (HPC) in particular is important, because many factors such as ease of placement, consolidation, durability, and strength depend on the flow properties. This report gives an overview of the flow properties of a fluid or a suspension followed by a critical review of the most often used tests for concrete rheology. Particular attention is given to tests that could be used for HPC. Tentative definitions of terms such as workability, consistency, and rheological parameters are provided. An overview of the most promising tests and models for cement paste is given.

00,415

PB97-111892 Not available NTIS

National Inst. of Standards and Technology (BFRL), Gaithersburg, MD. Building Environment Div.

Test Procedures for Advanced Insulation Panels. Final rept.

A. H. Fanney, C. A. Saunders, and S. D. Hill. 1995, $13 p$.

Pub. in Superinsulations and the Building Envelope, Washington, DC., November 14, 1995, p149-161

Keywords: "Panels, "Thermal insulation, "Heat transfer, "Test methods, Wallboard, Calorimeters, Flowmeters, Moisture proofing. Humidity, Procedures, Reprints.

The National Institute of Standards and Technology (NIST) has undertaken a research program to develop thermal measurement techniques appropriate for ad vanced insulation panels. The paper describes the design of a calorimetric apparatus, compares the calorimetric results to measurements made using a heat flow meter apparatus for homogenous materials, and describes the procedure used to determine the thermal resistance of an advanced insulation panel. Finite-element modeling results are presented which show the effect of various physical parameters on the overall thermal resistance of a metal-clad powder-filled vacuum insulation system.

00,416

PB97-111991 Not available NTIS

National Inst. of Standards and Technology (BFRL) Gaithersburg, MD. Building Environment Div.

Empirical Validation of a Transient Computer Model for Combined Heat and Moisture Transfer. Final rept

C. Rode, and D. M. Burch. 1995, 13p.

Pub. in 'Thermal Vl' Thermal Performance of the Exte ror Envelopes of Buildings VI, Clearwater Beach, FL. December 4-8, 1996, p283-295 1995

Keywords: "Wallboards, "Heat transfer, "Moisture content, "Computer models, Panels, Building materials, Water vapor, Condensation, Diffusion, Transient response, Transfer functions, Reprints.

A computer program for transient modeling of combined heat and moisture transfer in building constructions is introduced. The model's predictions are compared against moisture content and heat flux data oblained for six typical North American lightweight wall constructions that have been exposed to climatic conditions in a calibrated hot box. The experiment, and thus the validation, was restricted to diffusive transport mechanisms taking place in the hygroscopic region.

\section{0,417}

PB97-118996 Not available NTIS

National Inst. of Standards and Technology (BFRL), Gaithersburg, MD. Building Environment Div.

Line-Heat-Source Guarded-Hot-Plate Apparatus. Final rept.

R. R. Zarr, and M. H. Hahn. 1995, 32p.

See also PB95-181202.

Pub. in Line-Heat-Source Guarded-Hot-Plate Apparatus, ASTM C 1043, 32p Dec 95.

Keywords: "Design, "Construction, Heat meters, Thermal insulation, Thermal conductivity, Heat transmission, Thermodynamic properties, Thermal measurement, Reprints, "Line heat sources, "Guarded hot plate.

The adjunct describes the line-heat-source guardedhot-plate apparatus fabricated by the National Institute of Standards and Technology. It is intended as a guide in the design and construction of a guarded hot plat having circular line heat sources. The essential re quirements for steady-state testing of heat insulators are described in ASTM Test Method C 177 and the essential requirements for the design of guarded hot plates having circular line-heat sources are covered in ASTM Practice C 1043

00,418

PB97-121339 Not available NTIS

National Inst. of Standards and Technology (BFRL)

Gaithersburg, MD. Building Materials Div.

Computer Simulations of Binder Removal from 2 $D$ and 3-D Model Particulate Bodies.

Final rept.

J. A. Lewis, M. A. Galler, and D. P. Bentz. 1996,

$12 p$.

Pub. in Jnl. of the American Ceramic Society, v79 n5 p1377-13881996.

Keywords: "Binders, "Thermoplastic resins, "Particulates, "Removal, Plasticizers, Microstructure Liquid phases, Porosity, Transport properties, Iso thermal processes, Two dimensional models, Three di mensional models, Computerized simulation, Reprints.

A series of computer simulations were developed to investigate the remoral of multicomponent, thermoplastic binders from two- and three-dimensio lal model particulate bodies. Monosized particles with varying diameters were randomly placed in such systems, and all unoccupied pixels were assigned to the binder phase at ratios of $1: 9,1: 2$ or $1: 1$ plasticizer (volatile) to polymeric (nonvolatile) species. Simulations were carried out under isothermal conditions to study the influence of liquid-phase transport processes, i.e., plasticizer diffusion in the binder-filled pore network and capillary-driven redistribution of the binder phase, on plasticizer removal rates. Plasticizer diffusion was modeled by a random-walk algorithm, and nonplanar pore development arising from capillary-driven binder redistribution was modeled by an invasion percolation algorithm. For comparison, simulations were also carried out on systems in which binder redistribution was not permitted.

00,419

PB97-122535 Not available NTIS

National Inst. of Standards and Technology (BFRL)

Gaithersburg, MD. Building Environment Div.

Parametric Study of Wall Moisture Contents Using a Revised Variable Indoor Relative Humidity Version of the 'Moist' Transient Heat and Moisture Transfer Model.

Final rept.

G. Tsongas, D. Burch, C. Roos, and M

Cunningham. 1995, 13p.

Pub. in 'Thermal VI' Thermal Performance of the Exterior Envelopes of Buildings VI, Clearwater Beach, FL December 4-8, 1996, p307-319 1995

Keywords: "Relative humidity, "Moisture content "Walls, Water vapor, Wallboards, Panels, Condensation, Diffusion, Heat transfer, Building materials, Win ter, Computer models, Computerized simulation, Reprints, "Indoor environments, Moisture transfer, MOIST computer model.

The authors modified the model to calculate the hourly indoor relative humidity during the heating season as a function of outdoor weather conditions, indoor air temperature, building size and airtightness, and indoo moisture generation rate. This enhanced version of MOIST was subsequently used to investigate moisture accumulation in a $5-\mathrm{cm}$ by $15-\mathrm{cm}(2-\mathrm{in}$. by 6 -in.) wood framed wall exposed to a number of different winter climates. Predictions with a constant indoor relative hu midity were compared to those with a 'floating' or variable indoor relative humidity. In addition, the variable indoor relative humidity program was used to analyze the effect of building airtightness, the indoor moisture generation rate, and the existence of exfiltration. The need for an interior vapor retarder in walls exposed to cold climates also was examined. Moreover, the effects of exterior insulating sheathing and an exterior vapor retarder were modeled. Results and findings are presented along with pertinent conclusions regarding appropriate building construction techniques in winter heating climates. 


\section{Structural Analyses}

00,420

PB94-159779 PC A04/MF AO1

National Inst. of Standards and Technology (BFRL), Gaithersburg, MD. Center for Building Technology Seismic Instrumentation of Existing Buildings.

Final rept.

L. T. Phan. Oct 90,62p, NISTIR-4419.

Contract GSA/PBS-87-03

Sponsored by Public Buildings Service, Washington, DC. Office of Real Property Development.

Keywords: "Federal buildings, "Earthquake engineering, US GSA, Seismic design, Dynamic structural analysis, Instruments, Computerized simulation, Two existing GSA buildings, one in Long Beach, California, and one in Portland, Oregon, were subjected to low-level vibration tests to determine their dynamic properties and response frequencies. The measured dynamic properties of the buildings were incorporated into the computer models of the buildings and time-history analyses using these models were performed. The purpose of the analyses is to reveal building response under these realistic earthquake excitations, so that logical seismic instrumentation schemes can be developed for these buildings. The results of the analyses suggest that the response of the Portland building is influenced more by torsional and rocking motions, while the response of the Long Beach building is influenced mainly by translational modes. From the observed behavior of the buildings, a seismic instrumentation scheme is developed for each bullding, and a general guideline for seismic instrumentation in existing building is recommended.

\section{0,421}

PB94-161114 PC A09/MF A02

National Inst. of Standards and Technology (BFRL), Gaithersburg, MD. Structures Div.

Northridge Earthquake, 1994. Performance of Structures, Lifelines and Fire Protection Systems. D. Todd, N. Carino, R. M. Chung, W. D. Walton, H. S. Lew, and A. W. Taylor. Mar 94, 176p, NISTIR-

Keywords: "Earthquakes, "Damage assessment, "California, Buildings, Bridges(Structures), Office buildings, Freeways, Apartment buildings, Los Angeles(California), Northridge(California).

A magnitude 6.8 (MS) earthquake centered under the community of Nonthridge in the San Fernando Valley shook the entire Los Angeles metropolitan area at 4:31 a.m. local time Monday, January 17, 1994. Moderate damage to the built environment was widespread; severe damage included collapsed buildings and highway overpasses. A total of 58 deaths were attributed to the earthquake by the Los Angeles Coroner. About 1,500 people were admitted to hospitals with major in juries; another 16,000 or so were treated and released. Estimates indicated that this will be the United States' most costly natural disaster ever. A multi-agency team, organized under the auspices of the Interagency Committee on Seismic Safety in Construction and headed by the National Institute of Standards and Technology, arrived at the earthquake site within days of the event to document the effects of the earthquake. The team focused on the effects to the built environment, with the goal of capturing perishable data and quickly identifying situations deserving in-depth study. The report includes a summary of the team's observations. While most structural damage occurred in buildings and bridges of construction type and vintage known to be vulnerable to earthquake shaking, there were some unexpected failures. Notable among these were the collapses of relatively modern parking structures and a bridge that appeared to be adequate by today's standards. Recommendations are made for further studies of the Northridge earthquake that can lead to improved mitigation of earthquake effects.

00,422

PB94-161734 PC A03/MF A01

National Inst. of Standards and Technology (BFRL),

Gaithersburg, MD. Structures Div

Draft Guidelines for Quality Control Testing of

Elastomeric Seismic Isolation Systems.

H. W. Shenton. Feb 94, 33p, NISTIR-5345.

See also PB92-139732.

Keywords: "Vibration isolators, "Buildings, "Earthquake engineering, Seismic design, Quality control, Design criteria, Tests, Lateral forces.
Seismic isolation systems designed according to the 1991 Uniform Building Code, or the 1991 AASHTO Guide Specification for Seismic Isolation Design are required to undergo a series of prototype and quality control tests before being installed in the structure. At the present time standards do not exist for conducting these tests and results are subject to unknown variability. The document represents the initiation of the process to develop standards for quality control testing of seismic isolation systems built in the U.S. The guidelines are devoted specifically to quality control testing of elastomeric systems. The guidelines address material and component tests to be conducted during production, and tests on completed isolation units.

00,423

PB94-172947 Not available NTIS

National Inst. of Standards and Technology (BFRL), Gaithersburg, MD. Structures Div.

Draft Guideline for Testing and Evaluation of Seismic Isolation Systems.

Final rept.

H. W. Shenton. 1993, 6p

Pub. in Proceedings of a Seminar on Seismic Isolation, Passive Energy Dissipation, and Active Control, San Francisco. CA., March 11-12, 1993, p349-354.

Keywords: "Vibration isolators, "Earthquake resistant structures, "Building codes, "Test methods, Standards, Structural vibration, Earthquake engineering, Damping, Energy dissipation, Reprints, "Seismic isolation systems.

The Building and Fire Research Laboratory of the $\mathrm{Na}$. tional Institute of Standards and Technology is developing a draft guideline for testing and evaluation of seismic base isolation systems. This is a multi-year effort with the goal being the adoption of the guidelines by model code organizations and the Interagency Committee on Seismic Safety in Construction (ICSSC). A five member Oversight Committee has been formed to guide NIST in the development of the guidelines. The committee includes individuals from the research and private sectors with expertise in the design, fabrication and testing of seismic isolation components and hardware, and in the design of structures which incorporate seismic isolation. The draft guideline is intended to be general and comprehensive; it will be applicable to an isolation system regardless of the superstructure; it will be applicable to all viable systems, whether they be elastomeric, sliding or hybrid; and will be applicable to systems which consist of multiple isolator units, or incorporate different components that are distributed throughout the isolation interface. It will cover all pertinent structural evaluation tests of the isolation device. The objective and scope of the effort are described, along with a preliminary multi-year schedule. Progress to date is summarized.

00,424

PB94-176278 PC AO8/MF AO2

National Inst. of Standards and Technology (BFRL), Gaithersburg, MD.

Evaluation and Strengthening Guidelines for Federal Buildings: Identification of Current Federal Agency Programs

Final rept.

C. D. Poland, W. T. Holmes, J. O. Malley, D.

Provencher, and J. Soulages. Mar 94, 153p, NIST/

GCR-94/649.

Prepared in cooperation with Interagency Committee on Seismic Safety in Construction. and Degenkolb (H.J.) Associates, San Francisco, CA. Sponsored by Federal Emergency Management Agency, Washington, DC.

Keywords: "Federal buildings, "Earthquake engineering, Seismic design, Standards, Building codes.

The National Institute of Standards and Technology (NIST), by order of the President, is developing seismic evaluation and strengthening guidelines (Guidelines for Federal Buildings) for federally owned and leased buildings. The project is overseen by the Interagency Committee on Seismic Safety in Construction (ICSSC) and funded by the Federal Emergency Management Agency (FEMA). The report develops Task 1, the identification of seismic mitigation programs. The report in cludes a detailed work plan and schedule for the entire project, a list of ICSSC member contacts, the results of telephone conversations with all ICSSC committee members to identify existing seismic strengthening programs, the results of detailed meetings with seven federal agencies and four private sector organizations selected for in-depth study, and summaries of the performance objectives for all agencies and organizations.
00,425

PB94-181856 PC A08/MF A02

National Inst. of Standards and Technology (BFRL) Gaithersburg, MD.

Evaluation and Strengthening Guidelines for Federal Buildings: Assessment of Current Federal Agency Evaluation Programs and Rehabilitation Criteria and Development of Typical Costs for Seismic Rehabilitation.

Final rept.

C. D. Poland, W. T. Holmes, J. R. Soulages, and D. L. Provencher. Mar 94, 162p, NIST/GCR-94/650.

Prepared in cooperation with Degenkolb (H.J.) Associates, San Francisco, CA. Sponsored by Federal Emergency Management Agency, Washington, DC.

Keywords: "Federal buildings, "Earthquake engineering, "Design standards, Seismic design, Public Law 101-614.

The National Institute of Standards and Technology (NIST), in accordance with Public Law 101-614, is developing seismic evaluation and strengthening guidelines (Guidelines for Federal Buildings) for federallyowned and leased buildings. The project is overseen by the Interagency Committee on Seismic Safety in Construction (ICSSC) and funded by the Federal Emergency Management Agency (FEMA). The report develops Task 2, (see Appendix A for complete scope of work) assessment of current federal agency evaluation programs and rehabilitation criteria and Task 3 development of typical costs for seismic rehabilitation. Pan I of the Task 2 report includes a qualitative and quantitative comparison of six federal agency programs to the most recent versions of the NEHRP Evaluation Handbook and the NEHRP Techniques Handbook

\section{0,426}

PB94-187648 PC A03/MF A01

National Inst. of Standards and Technology (BFRL), Gaithersburg, MD

Strengthening Methodology for Lightly Reinforced

Concrete Frames-II. Recommended Calculation Techniques for the Design of Infill Walls.

L. T. Phan, D. R. Todd, and H. S. Lew. May 94, 46p, NISTIR-5421.

See also PB94-161354.

Keywords: "Reinforced concrete "Reinforcement(Structures), "Dynamic structural analysis, "Frames, Concrete structures, Loads(Forces), Shear strength, Earthquake enginee

Empirical equations were developed for estimations of ultimate lateral shear strength, story drift ratio at ultimate load, and ductility factor of existing lightly reinforced concrete frames (bare frames), existing monolithic shear walls, and reinforced concrete frames strengthened either by cast-in-place infilled walls or by single or multiple precast concrete panels. These equations were derived based on experimental results of many independently conducted test programs. Estimations of the ultimate shear stress, ultimate story drift ratio (story drift at ultimate load divided by story height), and ductility factor using the empirical equations compared favorably with the experimental results. The estimations confirm many observations made independently in individual test programs. The empirical expressions also provide benchmark values or ranges for these important parameters and thus provide a useful means for quick estimations of seismic capacity of bare, monolithic, and strengthened lightly reinforced concrete frames.

00,427

PB94-199981 Not available NTIS

National Inst. of Standards and Technology (BFRL),

Gaithersburg, MD. Structures Div.

Dynamic Characteristics of Five Tall Buildings during Strong and Low-Amplitude Motions.

Final rept.

M. Celebi, L. T. Phan, and R. D. Marshall. 1993,

$15 p$.

Pub.

Keywords: "Structural vibration, "Buildings, "Dynamic response "Seismic effects, *Soil-structure interactions, Earthquake damage, Earthquake engineering, Dynamic structural analysis, Data acquisition, Accelerometers, Earth movements, Reprints.

The objectives of the paper are to present (1) a comparison of dynamic characteristics of five buildings de- 
termined from recorded strong-motion response data and from low-amplitude (ambient vibration) tests, and (2) a description of the low-amplitude ambient testing and PC-based data-acquisition approach that is integrated with the permanent strong-motion instrumentation in the five buildings. All five buildings are within the San Francisco Bay area and the strong-motion dynamic characteristics are extracted from the October 17 1989 Loma Prieta earthquake response records. Ambient vibration tests on the same five buildings were conducted in September 1990. Analyses of strong-motion response and low-amplitude test data have been performed by many investigators. The present study differs from numerous previous investigations because (1) in the study, accelerometers in the five permanently-instrumented buildings were used during the low-amplitude testing, and (2) rapid screening of the strong-motion response data was achieved with a concerted use of system identification software. The results show for all cases that the fundamental periods and corresponding percentages of critical damping determined from low-amplitude tests are appreciably lower than those determined from strong-motion response records.

\section{0,428}

\section{PB94-206125 PC A06/MF A02}

National Inst. of Standards and Technology (BFRL), Gaithersburg, MD

Wind Load Provisions of the Manufactured Home Construction and Safety Standards: A Review and Recommendations for Improvement.

R. D. Marshall. May 93, 107p, NISTIR-5189.

See also PB-297 463. Sponsored by Department of Housing and Urban Development, Washington, DC.

Keywords: "Wind loads, "Prefabricated buildings, Hurricanes, Florida, Damage assessment, Building codes, Computerized simulation, *Mobile homes, Hurricane Andrew, Dade County(Florida).

Limited wind speed measurements obtained during landfall of Hurricane Andrew in south Florida and wind speed estimates obtained from a computer-based model and from analyses are summarized and compared with code-specified design speeds for the affected area. Published reports of wind damage to manufactured homes and to conventional wood-framed dwellings are reviewed to identify modes of failure and intensity of damage. In general, manufactured homes which were built subsequent to issuance of the Manufactured Home Construction and Safety Standards (MHCSS) suffered less damage than did units built prior to issuance of the MHCSS

\section{0,429}

\section{PB94-216421 Not available NTIS}

National Inst. of Standards and Technology (NEL)

Gaithersburg, MD. Structures Div.

Lessons Learned by a Wing Engineer.

Final rept.

R. D. Marshall. 1991, $10 p$

Pub. in Proceedings of Symposium and Public Forum on Hurricane Hugo One Year Later, Charleston, SC. September 13-15, 1990, p160-1691991.

Keywords: "Wind pressure, "Building codes, "Damage assessment, Hurricanes, Wind effects, Structural analysis, Gust loads, Dynamic loads, Wind measurement, Wind velocity, Reprints, Hurricane Hugo.

Surface wind speeds during the passage of Hurricane Hugo through the U.S. Virgin Islands and Puerto Rico are described. Although damage to the affected areas was extensive, an assessment of relevant data indicates that the actual wind speeds were far lower than those reported by the news media. The consequences of overstating the wind speeds are examined and actions to improve the accuracy of measuring and reporting wind speeds are outlined.

\section{0,430}

PB95-108841 Not available NTIS

National Inst. of Standards and Technology (BFRL), Gaithersburg, MD. Structures Div.

Seismic Strengthening of Reinforced Concrete Frame Buildings.

Final rept.

L. T. Phan, D. R. Todd, and H. S. Lew. 1993, 10p

Pub. in Proceedings of the National Earthquake Con ference, Memphis, TN., May 2-5, 1993, v2 p235-244.

Keywords: "Reinforced concrete, "Reinforcement(Structures), "Earthquake resistan structures, "Structural response, Earthquake engineer- ing, Structural analysis, Concrete structures, Seismic effects, Dynamic response, Frames, Reprints.

Most reinforced concrete (RC) frame structures in the mid-western and eastern regions of the United States are built mainly for gravity loads without proper seismic design, and thus are vulnerable to severe damage in the event of a major earthquake in these regions. While the need to retrofit these RC frame structures is recognized and different strengthening techniques have been laboratory-tested and applied in practice worldwide, ability to assess the relative merits of different strengthening schemes or to predict the improved per formance of a strengthened structure is still lacking. The paper describes the development of experimental based hysteresis models of unstrengthened and strengthened concrete frames by using the system identification method. The analytical models are validated by comparing the analytical results with results of experiments on strengthened RC frames.

\section{0,431}

PB95-130209 PC A06/MF A02

National Inst. of Standards and Technology (BFRL) Gaithersburg, MD. Structures Div.

Standards of Seismic Safety for Existing Federally Owned or Leased Buildings and Commentary

D. R. Todd. Feb 94, 110p, NISTIR-5382.

See also PB92-205343. Prepared in cooperation with Interagency Committee on Seismic Safety in Construc tion. Sponsored by Federal Emergency Management Agency, Washington, DC. Mitigation Directorate.

Keywords: "Federal buildings, "Seismic design, Earthquake engineering, Building codes, Design standards, Safety engineering, Retrofitting.

These seismic evaluation and mitigation standards, Standards of Seismic Safety for Existing Federally Owned or Leased Buildings and Commentary, were developed for use by the Federal government by the Interagency Committee on Seismic Safety in Construction (ICSSC) in conjunction with the National Institute of Standards and Technology (NIST). The project was funded by the Federal Emergency Management Agency (FEMA). The intent of this document is to provide Federal agencies with minimum standards for the evaluation and mitigation of seismic hazards in their build ing inventories.

\section{0,432}

PB95-143129 PC A04/MF A01

National Inst. of Standards and Technology (BFRL) Gaithersburg, MD. Structures Div.

Manufactured Homes: Probability of Failure and the Need for Better Windstorm Protection through improved Anchoring Systems.

Final rept.

R. D. Marshall. Nov 94, 64p, NISTIR-5370.

Contract DU100193000037

See also PB94-206125. Sponsored by Department o Housing and Urban Development, Washington, DC

Keywords: "Wind loads, "Prefabricated buildings, * Mobile homes, Hurricanes, Florida, Damage assess ment, Building codes, Computerized simulation, Dade County(Florida)

The report describes a continuation of earlier studies carried out by the National Institute of Standards and Technology (NIST) at the request of the Departmen of Housing and Urban Development (HUD) following the devastation caused by Hurricane Andrew in south Florida on August 24, 1992. In the earlier work, damage to manufactured homes was examined in light o the probable maximum wind speeds in the affected area and the wind load provisions of selected codes and standards used for structural design in hurricaneprone regions were compared. On the basis of that work, it was recommended that ASCE 7-88 (Minimum Design Loads for Buildings and Other Structures should be the primary resource document for updating and improving the wind load provisions of the Manufac tured Home Construction and Safety Standards (MHCSS)

\section{0,433}

PB95-147385 PC A99/MF A06 National Inst. of Standards and Technology (BFRL)
Gaithersburg, MD.
Wind and Seismic Effects. Proceedings of the U.S. Japan Cooperative Program in Natural Resource Panel on Wind and Seismic Effects (26th). Held in Gaithersburg, Maryland on May 17-20, 1994.

Special pub. (Final)

N. J. Raufaste. Sep 94, 732p, NIST/SP-871.

Also available from Supt. of Docs. as SN003-003 03297-2. See also PB93-120152.

Keywords: "Seismic effects, "Wind effects, *Structura engineering, *Dynamic structural analysis, "Meetings, Earthquake engineering, Design standards, Design cri teria, Research programs, Dynamic loads, Structura vibration, Wind pressure, Wind loads, Earthquakes, Tsunamis, Storm surges, Foundations(Structures) Highway engineering, Bridges, Dams, Retrofitting, Earthquake resistant structures, Wind engineering.

The publication is the Proceedings of the 26th Joint Meeting of the U.S.-Japan Panel on Wind and Seismic Effects. The Proceedings include the program, list of members, panel resolutions, task committee reports, and 45 technical papers. The papers were presented under five themes: (1) Wind Engineering, (2) Earthquake Engineering, (3) Storm Surge and Tsunamis, (4) Joint Cooperative Research Program, and (5) Northridge Southern California and Hokkaido NanseiOki Earthquakes.

00,434

PB95-150918 Not available NTIS

National Inst. of Standards and Technology (BFRL) Gaithersburg, MD. Structures Div.

Evaluation and Retrofit Standards for Existing Fed erally Owned and Leased Buildings.

Final rept

D. Todd. $1993,5 p$

Pub. in Proceedings of National Earthquake Conference, Memphis, TN., May 2-5, 1993, v2 p25-29.

Keywords: *Federal buildings, "Reinforcement(Structural), "Earthquake engineering, *Building codes, "Design standards, Earthquake resist ant structures, Retrofitting, Seismic design, Reinforcing materials, Safety engineering, Design criteria, Dynamic structural analysis, Reprints, "Seismic safety.

Public Law 101-614 requires the Interagency Commit tee on Seismic Safety in Construction to develop "standards for assessing and enhancing the seismic safety of existing buildings constructed for or leased by the Federal Government.' The paper describes the process that is being used to develop the standards presents the results of an Issues Workshop held fo potentially affected Federal agencies, at which philo sophical and administrative concerns were addressed and describes the anticipated content of the standard.

\section{0,435}

PB95-150926 Not available NTIS

National Inst. of Standards and Technology (BFRL) Gaithersburg, MD. Structures Div.

Some Basics on Who's Who and What's What in Seismic Safety.

Final rept.

D. Todd. 1992, 11p.

Pub. in Federal Construction Council Technical Report 120. Seismic Safety Technology and Regulations: A Look At the Near Future, p1-11 1992

Keywords: "Earthquake engineering, *Safety engineer ing, "Acronyms, Seismic design, Earthquake resist ance, Design criteria, Building codes, Design stand ards, Seismic effects, Dynamic structural analysis, $R e$ prints, *Seismic safety, NEHRP(National Earthquake Hazards Reduction Program).

The tendency of both governments and engineers to form acronyms seems to reach an apex in the U.S. earthquake engineering community. The paper defines organization acronyms and presents short idiomatic and official titles of earthquake-related documents. The text uses a description of the Federal earthquake program, the world of building codes, and the history of the NEHRP Recommended Provisions to illustrate how many of these organizations and documents are related.

\section{0,436}

PB95-151809 Not available NTIS

National Inst. of Standards and Technology (BFRL) Gaithersburg, MD. Building and Fire Research Lab. Of fice. 


\section{Structural Analyses}

Implementation of Executive Order 12699: Seismic Safety of Federal and Federally Assisted or Regulated New Building Construction.

Final rept.

R. N. Wright. 1992, 3p

Pub. in Phenomenal News 3, n2 p12-14 Jan 92.

Keywords: "Federal buildings, "Public buildings, "Earthquake engineering, "Seismic design, "Safety engineering, Earthquake resistant structures, Federal aid, Building codes, Design standards, Design criteria, Structural response, Reprints, Seismic safety.

Losses of property approaches $\$ 10$ billion, losses of 62 lives, and severe disruption of human activities from the October 17, 1989, Loma Prieta, California, earthquake reminded the United States of the severe threats posed by great earthquakes. Unless enhanced efforts are made to reduce earthquake hazards, a large earthquake close to a major metropolitan area can kill tens of thousands of people, cause tens of billions of dollars of direct property losses, and, through consequent losses, severely disrupt economic activity for the whole nation. 46 of the 50 states are vulnerable to strong earthquakes. In the eastern half of the country, the area that would be affected by an earthquake could be 10 times as large as for a similar earthquake in California.

00,437

PB95-151817 Not available NTIS

National Inst. of Standards and Technology (BFRL), Gaithersburg, MD. Building and Fire Research Lab. Office.

Structural Analysis in Context.

Final rept

R. N. Wright. 1993, $6 p$

Pub. in Proceedings of Structures Congress 93. Structural Engineering in Natural Hazards Mitigation, Irvine, CA., April 19-21, 1993, v2 p1657-1662.

Keywords: "Structural analysis, "Education, Structural engineering, Structural design, Design analysis, Students, Engineers, Reprints.

The issues of what students should learn about structural analysis and how it should be taught are explored considering the purposes of structural analysis in engineering practice and the motivation of structural engineering students. Structural analysis is considered to be the process by which the structure and its environment are modeled to predict structural response. Structural analysis is addressed as a process linked integrally to professional responsibilities such as design, review for safety and serviceability, construction, condition assessment of existing structures, performance investigations, modification and renovation.

00,438

PB95-153094 Not available NTIS

National Inst. of Standards and Technology (BFRL), Gaithersburg, MD. Structures Div.

Model Precast Concrete Beam-to-Column Connections Subject to Cyclic Loading.

Final rept.

G. S. Cheok, and H. S. Lew. 1993, 13p

Pub. in PCl Jnl. 38, n4 p80-92 Jul/Aug 93.

Keywords: "Cyclic loads, "Precast concrete, *Columns(Supports), "Beams(Structural), "Structural analysis, "Joints(Junctions), "Model tests, Earthquake resistant structures, Dynamic loads, Concrete structures, Structural components, Energy dissipation, Structural design, Strands, Ductility, Displacement, Structural failure, Dynamic response, Reprints, "Beamcolumn joints.

Experimental results of eight $1 / 3$-scale model precast concrete beam-to-column connections are presented. The test specimens consisted of interior connections designed in accordance with the 1985 Uniform Building Code provisions for Seismic Zones 2 and 4 . These tests constitute the second and third phases of a multiyear test program being conducted at the National Institute of Standards and Technology. The objective of the test program is to develop guidelines for an economical precast beam-to-column connection for regions of high seismicity. Variables considered in the research program include location of the post-tensioning steel, the use of post-tensioning bars vs. prestressing strands and fully bonded vs. partially bonded strands. Specimens were subjected to reversed cyclic loading according to a prescribed displacement history. Comparison of results with the monolithic test specimens indicates that the post-tensioned olithic test specimens indicates that the post-tensioned
precast concrete specimens had comparable connec- tion strengths, higher ultimate displacement ductilities and total energy dissipation to failure, but lower energy dissipation per cycle.

00,439

PB95-153102 Not available NTIS

National Inst. of Standards and Technology (BFRL) Gaithersburg, MD. Structures Div.

Partially Prestressed and Debonded Precast Concrete Beam-Column Joints.

Final rept.

G. S. Cheok, W. C. Stone, and H. S. Lew. 1992, 9p. Sponsored by Concrete Research Council, Detroit, MI. Pub. in Proceedings of Meeting of the U.S.-Japan Join Technical Coordinating Committee (3rd) on Precas Seismic Structural Systems, La Jolla, CA., Novembe 18-20, 1993, p1-9.

Keywords: "Precast concrete, "Prestressing Beams(Structural), "Columns(Supports), "Model tests, *Structural analysis, "Cyclic loads, Joints(Junctions), Concrete structures, Reinforcing materials, Structural components, Structural failure, Structural design, Strands, Earthquake resistant struc ures, Dynamic response, Energy dissipation, Ductility, Dynamic loads, Reprints, "Beam-column joints.

The experimental test results from three 1/3-scale model precast concrete beam-to-column connections is summarized. These tests are part of a multi-phased test program being conducted at the National Institute of Standards and Technology. The objective of the tes program is to develop guidelines for an economical precast beam-to-column connection for regions of high seismicity. The test specimens were interior connections designed using the Uniform Building Code (ICBO 1985 and 1988) criteria for seismic Zones 2 and 4 as design guidelines. Partially debonded strands were employed in two of the specimens and while the third combined low strength steel with post-tensioning steel Specimens were subjected to reversed cyclic loading according to a prescribed displacement history. Comparisons are made between the behavior of these pre cast specimens with previous precast and monolithic specimens which were tested earlier. The comparisons were based on connection strength, connection ductility, and energy absorption characteristics.

\section{0,440}

PB95-153110 Not available NTIS

National Inst. of Standards and Technology (BFRL) Gaithersburg, MD. Structures Div.

Seismic Performance Behavior of Precast Concrete Beam-Column Joints.

Final rept.

G. S. Cheok, W. C. Stone, and H. S. Lew. 1993, 6p. Pub. in Proceedings of American Society of Civil Engineers Structures Congress '93, Irvine, CA., April 19 21, 1993, v1 p83-88.

Keywords: "Precast concrete, "Prestressing "Beams(Structural), "Columns(Supports), "Model tests, "Structural analysis, "Cyclic loads, "Joints(Junctions), Concrete structures, Reinforcing materials, Structural components, Structural failure Structural design, Strands, Earthquake resistant struc tures, Dynamic response, Energy dissipation, Ductility, Dynamic loads, Reprints, "Beam-column joints.

The experimental test program being conducted at the National Institute of Standards and Technology on $1 /$ 3-scale model precast concrete beam-to-column connections is summarized. The objective of the test program is to develop guidelines for an economical pre cast beam-to-column connection for regions of high seismicity. The test specimens were interior connections designed using the Uniform Building Code (ICBO, 1985 and 1988) criteria for seismic Zones 2 and 4 as guidelines. To date, fifteen specimens have been test ed. Variables in the study include location of the posttensioning steel, the use of post-tensioning bars versus prestressing strands, fully bonded versus partially bonded strands, and the combination of low strength steel and post-tensioning. Specimens were subjected to reversed cyclic loading according to a prescribed displacement history Comparisons are made between the behavior of these precast specimens with previous precast and monolithic specimens. The comparisons were based on connection strength, connection ductility, and energy absorption characteristics.

\section{0,441}

PB95-161360 Not available NTIS

National Inst. of Standards and Technology (CAML) Gaithersburg, MD. Statistical Engineering Div.
Assessment of 'Peaks Over Threshold' Methods for Estimating Extreme Value Distribution Tails. Final rept.

J. A. Lechner, E. Simiu, and N. A. Heckert. 1993,

Pub. in Structural Safety 12, p305-314 1993.

Keywords: "Structural analysis, "Wind effects, "Extreme-value problems, Statistical analysis, Methodology, Comparison, Monte Carlo method, Simulation, Reprints, Peaks over threshold methods, CME(Conditional Mean Exceedance) methods.

In the past twenty years a vast new body of extreme value theory was developed, referred to as 'peaks over threshold modeling.' This theory allows the use in the analysis of all data exceeding a sufficiently high threshold, a feature that may result in improved extreme value estimates. The application of the theory depends upon the performance of methods for estimating the distribution parameters corresponding to any given set of extreme data. We present a comparative assessment of the performance of three such methods. The assessment is based on Monte Carlo simulations from populations with four distributions: Gumbel, Weibull, generalized Pareto, and normal. The simulation results showed that the de Haan and the Conditional Mean Exceedance (CME) methods performed consistently better than the Pickands method (NIST implementation). For the distributions, parameter values, and mean recurrence intervals assumed in this work, the CME method outperiormed the de Haan method only when the percent estimation errors were about one percent or smaller, a case unlikely to be encountered in wind engineering practice.

\section{0,442}

PB95-164091 Not available NTIS

National Inst. of Standards and Technology (BFRL), Gaithersburg, MD. Building and Fire Research Lab. Office.

Lessons from the Loma Prieta Earthquake.

Final rept.

R. N. Wright. $1993,18 p$

Pub. in Proceedings of Joint Meeting UJNR Wind and Seismic Effects (25th), Japan, May 17-20, 1993, p769786

Keywords: "Earthquake engineering, "Structural engineering, "Design standards, Seismic design, Structural design, Safety engineering, Dynamic structural analysis, Earthquake resistance, Building codes, Public policy, Reprints, "Seismic safety, Loma Prieta Earthquake.

The Loma Prieta, California earthquake (LPE) of October 17,1989 , has had profound influence on both policies and practices for earthquake hazards reduction in the United States. The paper describes lessons in public policy as well as those in earth, engineering and soil sciences and practices resulting from the earthquake.

00,443

PB95-174488 PC A04/MF AO1

National Inst. of Standards and Technology (BFRL), Gaithersburg, MD. Structures Div.

Performance of HUD-Affiliated Properties during the January 17, 1994 Northridge Earthquake.

D. Todd, E. Anderson, N. Carino, J. Gross, L. Phan, A. E. Schultz, H. W. Shenton, A. Taylor, C. W. C Yancey, G. Cheok, and R. Chung. Aug 94, 70p, NISTIR-5488

Contract DU1001940000027

Portions of this document are not fully legible. Sponsored by Department of Housing and Urban Development, Washington, DC

Keywords: "Residential buildings, "US HUD, "Earthquake damage, "Damage assessment, Seismic effects, Dynamic structural analysis, Soil structure interactions, Dynamic response, Structural vibration, Multifamily housing, Performance evaluation, California, Northridge Earthquake, Southern Region(California), Structural damage, Nonstructural damage.

The magnitude 6.8 January 17, 1994 Northridge Earthquake was centered under the densely populated San Fernando Valley northeast of Los Angeles, California. At the request of the Department of Housing and Urban Development (HUD), the Building and Fire Research Laboratory (BFRL) of the National Institute of Standards and Technology (NIST) conducted field observations of multi-family residences three stories or more in height in the affected area for the purposes of identifying common damage states in residential construc- 
tion. Sixty-nine HUD-affiliated sites, totalling 425 buildings and over 10,000 living units, were visually examined from the exterior and interior. By collecting information primarily on damaged buildings, it was possible to identify typical types and degrees of damage to residential buildings.

\section{0,444}

PB95-179024 PC A04/MF A01

National Inst. of Standards and Technology (BFRL) Gaithersburg, MD.

Performance of 1/3-Scale Model Precast Concrete Beam-Column Connections Subjected to Cyclic Inelastic Loads. Report No. 4.

G. S. Cheok, and W. C. Stone. Jun 94, 72p, NISTIR5436.

Errata sheet inserted. See also PB93-227502 and PB94-101813.

Keywords: "Earthquake resistant structures, "Construction joints, "Precast concrete, "Cyclic loads, *Beams(Supports), Model tests, Concrete structures, Earthquake engineering, Structural analysis, Structural failure, Stress analysis, Failure modes, Compressive strength.

Test results of four hybrid post-tensioned concrete beam-to-column connections are presented. The objective of the test program is to develop guidelines for the design of moment resistant precast connections in regions of high seismicity. The hybrid connections consist of mild steel used as energy dissipators and posttensioning steel used to provide the required shear resistance. Variables examined were different amounts and type of mild steel. The amount of post-tensioning steel was kept constant. The specimens were subjected to reversed cyclic loading in accordance with a prescribed displacement history. The performances of the connections were evaluated based on comparisons of energy dissipation capacity, connection strength, and drift capacity with previous NIST tests. The results show that a hybrid precast connection can be designed so that it matches the performance of a monolithic connection in terms of energy dissipation, strength, and drift capacity.

00,445

PB95.179040 PC A07/MF A02

National Inst. of Standards and Technology, Gaithersburg, MD.

Effects of Testing Variables on the Measured ComEffects of Testing Variables on the Measured Com-
pressive Strength of High-Strength (90 MPa) Concrete.

N. J. Carino, and W. F. Guthrie. Oct 94, 147p,

NISTIR-5405.

Sponsored by Federal Highway Administration, McLean, VA. Office of Advanced Research.

Keywords: "Compressive strength, "Test methods, Loads(Forces), Stress strain relations, Test facilities, Aggregates, Construction materials, Experimental design, Statistical analysis, Standards, Mechanical properties, "High strength concrete, ASTM C 39, AASHTO T 22.

A review is presented on the factors affecting the measured compressive strength of concrete specimens, with particular emphasis on the testing of highstrength concrete. A full factorial experiment was designed to examine the effects of cylinder size, end preparation, stress rate and type of testing machine on the measured compressive strength. Two concrete mixtures (45 $\mathrm{MPa}$ and $90 \mathrm{MPa}$ ) were used to determine whether there were interactions between strength level and the other factors. In addition, a 65-MPa mixture was required to allow testing four combinations of specimen size and testing machine. The cylinder sizes were $100 \times 200 \mathrm{~mm}$ and $150 \times 300 \mathrm{~mm}$. The ends of the cylinders were either capped with sulfur mortar or ground flat The stress rate was either $0.14 \mathrm{MPa} / \mathrm{s}$ or $0.34 \mathrm{MPa} / \mathrm{s}$, which are limits currently specified in ASTM C 39 (AASHTO T 22). Besides the main test series, supplementary tests were done to investigate the effects of a defective spherically-seated bearing block. The defective bearing block had a concave depression within the central $100 \mathrm{~mm}$. The maximum value of the depression was more than $0.2 \mathrm{~mm}$, compared with the value of $0.025 \mathrm{~mm}$ currently allowed by ASTM C 39 (AASHTO T 22). Comparative tests with $68-\mathrm{MPa}$ concrete showed no difference in mean strength due to the defective bearing block. Analysis of dispersion showed that the $100-\mathrm{mm}$ cylinders had higher within-test variability, but the differences were not statistically significant. Recommendations for modifications to testing standards and future research are provided.
00,446

PB95-180469 Not available NTIS

National Inst. of Standards and Technology (BFRL)

Gaithersburg, MD. Structures Div.

Gust Factors Applied to Hurricane Winds.

Final rept.

W. R. Krayer, and R. D. Marshall. 1992, 7p.

Pub. in Bulletin of the American Meteorological Society 73, n5 p613-617 May 92.

Keywords: "Wind loads, "Hurricanes, *Structural analysis, "Buildings, "Gust loads, Structural engineering, Stress analysis, Dynamic response, Wind pressure, Statistical analysis, Wind velocity, Reprints, "Gust factors.

An important consideration in the design of structures is their response to extreme winds. This is especially true in regions affected by hurricanes. In this research gust factors derived from hurricane wind-speed records are compared with those derived by Durst and others from open-scale records obtained in well-developed, extratropical storms. Based on records obtained from four hurricanes and 11 different recording stations, it is concluded that an upward adjustment of the Durst gust factors for the estimation of hurricane gust speeds may be in order. Anomalously high gust factors observed for hurricane winds in inland areas suggest the need for additional study. Also, it is concluded that a reexamination of the statistics of gust factors obtained from extratropical storm data would be useful in clearly identifying the appropriate probability distribution function.

\section{0,447}

PB95-182291 PC A03/MF A01

National Inst. of Standards and Technology (BFRL), Gaithersburg, MD. Structures Div.

Seismic Safely of Federal Buildings. Initial Program: How Much Will It Cost.

D. R. Todd. Apr $94,40 p$, NISTIR-5419.

See also PB94-176278, PB94-181856, and PB95130209. Sponsored by Federal Emergency Management Agency, Washington, DC. Mitigation Directorate.

Keywords: *Federal buildings, "Earthquake engineer ing, "Reinforcement(Structures), "Cost estimates, Seismic design, Design standards, Design criteria, Structural design, Earthquake resistant structures, Retrofitting, Building codes, Cost analysis, "Seismic safety.

A proposed Executive order titled 'Seismic Safety of Existing Federally Owned or Leased Buildings' sets forth an initial program aimed at laying the foundation of achieving the long-term goal of seismic safety in all Federal buildings. This paper identifies direct costs associated with adoption of the proposed Executive Order and develops an estimate of those costs. It does not consider the value of the benefits associated with adopting the proposed order. The estimated costs represent the aggregate impact on the Federal budget Note that the estimate of that is presented in this paper does not include the costs of continuing already-existing seismic rehabilitation programs, but considers only budget.

\section{0,448}

\section{PB95-189528 PC A05/MF A01}

National Inst. of Standards and Technology (BFRL), Gaithersburg, MD. Structures Div.

Proceedings: Workshop on Research Needs in Wind Engineering. Held in Gaithersburg, Maryland on September 12-13, 1994.

R. D. Marshall. Feb 95, 78p, NISTIR-5597.

Keywords: "Meetings, "Wind effects, "Wind pressure, * Structural failure Loads(Forces), Wind(Meteorology), Wind tunnel tests, Storms(Meteorology), Buildings, Damage.

This report presents findings and recommendations developed at a workshop on research needs in wind engineering. Representatives from universities, the private sector, and Federal agencies presented program overviews and participated in working group sessions addressing various aspects of wind engineering research and wind disaster mitigation. Research needs and topics for technology transfer were identified and prioritized. It was concluded that current funding of wind engineering research in the United States falls far short of what is needed to effectively address the problem of spiraling losses due to wind damage. There is, however, considerable wind engineering knowledge now a vailable for implementation by the model building codes and by the building industry in general.

00,449

PB95-203428 Not available NTIS

National Inst. of Standards and Technology (BFRL), Gaithersburg, MD. Structures Div.

World of Building Codes.

Final rept.

D. Todd. 1992, 3p.

Pub. in Phonomenal News 3, n3 p1-3 Apr 92

Keywords: "Building codes, "Design standards, *Earthquake resistant structures, Earthquake engineering, Structural engineering, Design criteria, Struc tural design, Reprints, "Seismic design.

Improvements to the seismic provisions of the legally enforceable building codes implemented by states, counties, and localities derive from improvements to the three major model building codes. The model codes draw from national standards and resource documents for improvements. The NEHRP Recommended Provisions and the SEAOC Blue Book are the two major resource documents for seismic design and construction in this country. Both documents are periodically updated to include new research and investigation results.

\section{0,450}

PB95-210928 PC A07/MF A02

California Univ., Davis. Dept. of Civil and Environmental Engineering.

Assessment of Site Response Analysis Procedures

I. M. Idriss. Jul 93, 148p, NIST/GCR-95/667.

Sponsored by National Inst. of Standards and Technology (BFRL), Gaithersburg, MD.

Keywords: *Site characterization, "Soil-structure interactions, "Ground motion, "Seismic waves, Seismic velocity, Seismic effects, Soil dynamics, Soil mechanics, Shear waves, Shear strain, Shear stress, Seismographs, Accelerometers, Seismological station, Spectrum analysis, Subsurface investigations, Yerba Buena Island, California, "San Francisco Airport, *Treasure Island Naval Station, "Loma Prieta Earthquake, San Francisco(California), Seismic response.

The Loma Prieta earthquake occured on October 17 1989 at 5:04 pm Pacific daylight time along a $45-\mathrm{km}$ long segment of the San Andreas fault in the Santa Cruz Mountains. The earthquake triggered by far the largest number of instruments ever triggered by an earthquake and recordings were obtained at well over 200 locations, including free-field stations, small build ings, high rise structures and dams. The records obtained from the free-field stations are summarized in Appendix $A$ of this report. These include 31 stations at rock sites, nine stations at soft soil sites and 48 stations at other soils sites. The results of subsurface investigations at Treasure Island site and at the San Francisco Airport site were used in the study to calculate the horizontal components of site reponse at these two sites and to assess the procedures used for conducting such response calculations.

00,451

PB95-211918 PC A09/MF A02

National Inst. of Standards and Technology (BFRL) Gaithersburg, MD. Structures Div

Survey of Steel Moment-Resisting Frame Buildings Affected by the 1994 Northridge Earthquake.

N. F. G. Youssef, D. Bonowitz, and J. L. Gross. Apr 95, $176 \mathrm{p}$, NISTIR-5625

Prepared in cooperation with Nabih Youssef and Associates, Los Angeles, CA.

Keywords: "Buildings, "Steel structures, "Frame structures, "Earthquake damage, Damage assessment, Seismic effects, Structural members, Dynamic response, Dynamic structural analysis, Construction joints, Cracking(Fracturing), Moments, Surveys, *Northridge Earthquake, Los Angeles(California).

The January 1994 Northridge earthquake caused unexpected widespread damage to steel moment-resisting frame (MRF) buildings throughout greater Los Angles. The report presents results of a survey of MRF's inspected for connection damage since the earthquake. The survey is intended to provide an overall view of the greater Los Angeles steel frame population, as well as a single-source building-specific record of observed conditions. A computerized database was developed to track submittals, compile basic survey data, and generate summary tables. Principal conclu- 
sions from the survey data support the observation that MRF connection damage is not well correlated to any single structural characteristic. On the contrary, the survey data show that connection performance may be best understood in probabilistic, not deterministic, terms with emphasis on construction and inspection quality.

\section{0,452}

PB95-231601 PC A05/MF A01

University of Central Florida, Orlando.

Enhancements to Program IDARC: Modeling Inelastic Behavior of Welded Connections in Steel Moment-Resisting Frames.

S. K. Kunnath. May 95, 80p, NIST/GCR-95/673.

See also PB93-227502. Sponsored by National Inst. of Standards and Technology (BFRL), Gaithersburg. MD.

Keywords: "Earthquake engineering, "Reinforced concrete, "Farmed structures, "Computerized simulation Steels, Damage analysis, Walls, Retrofitting, Computer programs, Hysteresis, IDARC computer program.

An existing computer code, IDARC, is enhanced to permit the modeling of steel moment-resisting frames (SMRFs) with the potential for weld failures at beamto-column connections. The steel member model is derived from flexibility formulations in order to allow complex degrading hysteresis behavior to be incorporated. A panel zone element is developed to account for inelastic shear deformations in the beam-to-column connection region. Finally, a new conceptual hysteresis model is developed to represent the force-deformation characteristics at a welded connection, before and after weld fallure. The results of the study indicate that the enhanced program, referred to as IDASS, is capable of adequately reproducing observed behavior of SMRFs and can be used as an effective tool to inves tigate the effects of weld failure in steel structures under earthquake loading.

\section{0,453}

PB95-231775 PC A03/MF A01

National Inst. of Standards and Technology (BFRL) Gaithersburg, MD. Structures Div.

Performance of Federal Buildings in the January 17,1994 Northridge Earthquake.

D. Todd, and A. Bieniawski. Jan 95, 28p, NISTIR 5574 .

Keywords: "Federal buildings, "Earthquake damage "Seismic effects, Damage assessment, Dynamic structural analysis, Structural response, Structural vibration US DOC, US DOD, NASA, US DOA, US DOE, US DOI, US GSA, US DOJ, US Postal Service, "Northridge Earthquake, Southern Region(California), Structural damage, Federal Bureau of Prisons, Department of Veterans Affairs.

On January 17, 1994, a magnitude 6.8 earthquake struck Northridge, California, in the northeast suburbs of Los Angeles. This report summarizes information collected by the Interagency Committee on Seismic Safety in Construction (ICSSC) on the performance of federally-owned buildings in the Northridge earthquake. Ten agencies reported that they owned buildings in the affected area. Collectively, over 4000 federally-owned buildings were shaken; approximately 100 were damaged by the quake. Only two sites suffered major damage; most damage was minor.

00,454

\section{PB95-260725 PC A04/MF A01}

National Inst. of Standards and Technology (BFRL), Gaithersburg, MD. Structures Div.

Strengthening Methodology for Lightly Reinforced Concrete Frames: Recommended Design Guide lines for Strengthening with Infill Walls.

L. T. Phan, G. S. Cheok, and D. R. Todd. May 95 ,

$66 p$, NISTIR-5682

See also PB94-187648.

Keywords: "Reinforced concrete, "Reinforcement(Structures), "Structural design, "Frames, Concrete structures, Design analysis, Structural analysis, Earthquake engineering, Displacement, Dynamic response, Design criteria, Structural failure Shear strength, Loads(Forces), "Infilled walls.

A study of the sensitivity of the behavior of lightly reinforced concrete frames strengthened using the infil wall method to certain variables was conducted. These variables include the infill wall type (cast-in-place and precast), wall thickness, and the amount of ancho area and anchor type. The hysteretic behavior of the frames were predicted using three parameters and equations proposed in previous NIST work. Both quasi-static and transient dynamic analyses were performed using the program IDARC. General design guidelines are proposed based on these analyses and on observations gathered from existing experimental tests

00,455

\section{PB96-106901 PC A09/MF A02}

Cladding Research Inst., Emeryville, CA

Literature Review on Seismic Performance of Building Cladding Systems.

\section{Feb $95,181 p$, NIST/GCR-95/681}

Sponsored by National Inst. of Standards and Technology (BFRL), Gaithersburg, MD.

Keywords: "Cladding, "Construction, "Buildings, "Earthquake resistance, "Literature surveys, Precast concrete, Reinforced concrete, Steels, Seismic design, Structural members, Structural analysis, Structural response, Seismic effects, Ground motion, Building codes, Earthquake engineering, Seismic isolation, Soil-structure interactions.

Chapter 1 is an introduction that includes definitions, cladding panel configurations, details of architectural precast concrete cladding systems in the U.S.A., New Zealand, Japan, and Canada. Chapter 2 describes the current practice for seismically isolated precast concrete cladding panels and connections, including U.S. codes and their interpretation and foreign codes. Chapter 3 offers information on the structural utilization of precast concrete cladding panels and connections. including a historical overview, levels of contribution in seismic response, architectural implications for structural cladding, conditions for effective structural cladding, and issues of responsibility. Chapter 4 contains abstracts and informational highlights from research on the structural utilization of precast concrete cladding panels and connections, including eleven sets of research projects from the U.S.A., one project from Canada, and one project from Japan. Chapter 5 outlines other cladding materials for heavy panels, including prefabricated panel systems, GFRC panels, new types of reinforcement, a new type of RC sandwich panels, and steel and steel alloy panels.

\section{0,456}

PB96-112198 Not available NTIS

National Inst. of Standards and Technology (BFRL), Gaithersburg, MD. Structures Div.

Effects of Testing Variables on the Strength of High-Strength ( $90 \mathrm{Mpa}$ ) Concrete Cylinders.

Final rept.

N. J. Carino, W. F. Guthrie, E. S. Lagergren, and G M. Mullings. 1994, 44p.

Pub. in Proceedings of the American Concrete Institute International Conference on High-Performance Concrete, Sinapore, November 15-18, 1994, p589-632.

Keywords: "Compressive strength, "Test methods, Reprints, Meetings, Loads(Forces), Stress strain relations, Test facilities, Aggregates, Construction materials, Statistical analysis, Test equipment, "High strength concrete.

A full factorial experimental design was used to investigate the effects of the following variables on cylinder strength: end preparation (sulfur capping versus grinding), cylinder size (100 versus $150 \mathrm{~mm}$ diameter), type of testing machine (1.33-MN capacity versus a 4.45 . MN capacity), and nominal stress rate ( 0.14 versus $0.34 \mathrm{Mpa} / \mathrm{s}$ ). Two !evels of strength were used ( 45 and $90 \mathrm{Mpa})$, and three replicates were tested for each run. Specific gravities were measured to check on the consistency of cylinder fabrication. Statistical analyses indicated that all the factors had significant effects on the measured compressive strength. There were significant interactions among the factors, so that the effects were greater than the average values for particular factor settings. For example, the effect of end preparation depended on the strength level. Recommendations for modifications to testing standards are provided.

\section{0,457}

PB96-119607 Not available NTIS

National Inst. of Standards and Technology (BFRL), Gaithersburg, MD. Structures Div

Response of Buildings to Ambient Vibration and the Loma Prieta Earthquake: A Comparison.

Final rept.

L. T. Phan, R. D. Marshall, and M. Celebi. 1992, 4p Pub. in Structures Congress '92, San Antonio, TX. April 13-15, 1992, p583-586.
Keywords: "Structural vibration, "Seismic waves, Dynamic response, Structural dynamics, Seismic effects, Structural response, Buildings, Ground motion, Earthquakes, San Francisco(CA), Vibration damping, Seismic waves, Spectrum analysis, Soil-structure interactions, Reprints, Loma Prieta Earthquakes, Seismic spectra.

Structural response characteristics of five existing buildings in the San Francisco bay area, obtained from ambient vibration testing conducted after the Loma Prieta earthquake (LPE), are compared with the response characteristics observed during the LPE. The purpose is to provide an assessment of the applicability of ambient vibration testing as a means to obtain dynamic properties for use in earthquake design. The comparisons show that, for all five buildings, the response frequencies measured from ambient vibration testing are higher than those obtained from the LPE, and damping estimates computed from ambient vibration response records are smaller than those from the LPE response records.

00,458

PB96-122601 Not available NTIS

National Inst. of Standards and Technology (BFRL), Gaithersburg, MD. Structures Div.

Some Notable Hurricanes Revisited.

Final rept.

R. Marshall. 1992, 4p.

Pub. in Structures Congress '92, San Antonio, TX., April 13-15, 1992, p250-253.

Keywords: "Wind velocity, "Hurricanes, "Damage assessment, Tropical storms, Storm damage, Gust loads, Wind loads, Buildings, Dynamic response, Structural analysis, Structural engineering, Reprints.

Recorded hurricane wind speeds, uncorrected for instrument height, averaging time or local wind exposure, can be very misleading. This is particularly true when such records are used to assess the performance of structures exposed to extreme winds. Only when proper corrections are applied can the true distribution of surface wind speeds in a hurricane be ascertained. Recent improvements to adjustment procedures and resulting corrections to wind speed records from some notable hurricanes are described.

00,459

PB96-128103 PC A03/MF A01

National Inst. of Standards and Technology (BFRL) Gaithersburg, MD. Structures Div.

ICSSC Guidance on Implementing Executive Order 12941 on Seismic Safety of Existing Federally Owned or Leased Buildings.

D. Todd, and A. Bieniawski. Oct 95, 27p, NISTIR-

5734

Prepared in cooperation with Interagency Committee on Seismic Safety in Construction. Sponsored by Fed eral Emergency Management Agency, Washington, DC. Mitigation Directorate.

Keywords: "Federal buildings, "Seismic design "Design standards, "Earthquake resistant structures, Retrofitting. Reinforcement(Structural), Structural stability, Seismic waves, Earthquake engineering, Vuinerability, Building codes, Cost estimates, Government policies, Federal government.

In this guidance document, the Interagency Committee on Seismic Safety in Construction (ICSSC) recommends appropriate approaches for Federal departments and agencies to use in implementing the inventorying and cost estimating requirements of Executive Order 12941. The ICSSC recommends that all Federally-owned buildings be included in an electronic inventory database of specified format. Buildings are to be identified as either exempt or non-exempt from the seismic standards adopted by the order. All exceptionally high risk buildings are to be seismically evaluated, and estimates of the cost of their rehabilitation developed. Additionally, agencies are to perform seismic evaluations on a representative sample of their non-high-risk, non-exempt buildings, and use this information to estimated the vulnerability of that population and the cost of achieving adequate seismic safety.

00,460

PB96-128285 PC A05/MF A01

National Inst. of Standards and Technology (BFRL), Gaithersburg, MD. Structures Div. 
Recommended Performance-Based Criteria for the Design of Manufactured Home Foundation Systems to Resist Wind and Seismic Loads.

R. D. Marshall, and F. Y. Yokel. Aug 95, 77p, NISTIR-5664

Contract DU100193000037

Sponsored by Department of Housing and Urban Development, Washington, DC. Office of Research Evaluation and Monitoring.

Keywords: "Wind effects, "Seismic effects, *Prefabricated buildings, "Building codes, "Design criteria, Earthquake engineering. Wind loads, Hurricanes Structural vibrations, Dynamic structural analysis, Soi structure interactions, Foundations(Structures), Anchors(Structural), Soils, Performance evaluation, Wind engineering.

This report addresses the issue of tornado wind speeds as a basis for the design of manufactured homes and compares base shears due to earthquake excitation with base shears due to wind loa ding for various seismic and wind zones. In view of the accepted probabilities of attaining or exceeding design limit states for ordinary buildings, it is concluded that tornadoes should not be a part of the wind load design criteria for manufactured homes. Also, it is concluded that base shears due to wind loading will always govern the design of anchor and tiedown systems in the direction normal to the axis for a manufactured home, regardless of seismic zone. There are several alternative systems on the market or under development that show considerable promise for providing the required resistance to wind and earthquake loads. Finally, a set of performance-based criteria for anchoring manufactured homes against wind and earthquake loads is proposed.

\section{0,461}

PB96-131552 PCA10/MF A03

National Inst. of Standards and Technology (BFRL), Gaithersburg, MD

How-To Suggestions for Implementing Executive Order 12941 on Seismic Safety of Existing Federal Buildings, A Handbook.

A. Bieniawski, and D. Todd. Nov 95, 210p, NISTIR5770 .

Also pub. as Interagency Committee on Seismic Safety in Construction rept. no. ICSSC/TR-17. See also PB95-130209. Prepared in cooperation with Inter agency Committee on Seismic Safety in Construction. Sponsored by Federal Emergency Management Agency, Washington, DC. Office of Mitigation and Research.

Keywords: "Federal buildings, "Retrofitting, "Earthquake engineering, Reinforcement(Structures), Earthquake resistance, Seismic design, Structural design, Building codes, Safety engineering, Cost estimates, Cost analysis, Design standards, Inventories, Regulatory guides.

This document supplements 'ICSSC guidance on Im. plementing Executive Order 12941 on Seismic Safety of Existing Fede'ally Owned or Leased Buildings' (RP5). This Handoook offers two additional levels of guidance beyound that presented in RP5: (1) detailed methodologies for fulfilling the RP5 recommendations for inventorying $\dot{c}$ nd cost estimating, which can be followed as default procedures by agencies which do not have agency-sperific programs in place and which do not wish to develo s agency-specific programs, and (2) detailed specifications for preparing and submitting the cost estimate and supporting documentation called for in the Executive Order. While the first type of guidance presented in Sections 1-4 of this Handbook, is optional, the second type, presented in Section 5 , is to be considered mandatory in order to ensure uniform reporting.

\section{0,462}

PB96-141148 Not available NTIS

National Inst. of Standards and Technology (BFRL), Gaithersburg, MD. Structures Div.

De Facto Microzonation through the Use of Soils Factors in Design Triggers.

Final rept.

D. Todd, and J. R. Harris. 1995, 8p.

Pub. in Proceedings of the International Conference on Seismic Zonation (5th), Nice, France, October 17-19, 1995, p510-517.

Keywords: "Soil-structure interactions, "Seismic design, Soil mechanics, Soil profiles, Seismic events, Earthquake resistant structures, Design criteria, Design standards, Dynamic structural analysis, Earth- quake engineering, Reprints, "Microzonation, Soil factors.

The 1994 edition of the National Earthquake Hazard Reduction Program (NEHRP) Recommended Provisions for the Development of Seismic Regulations for New Buildings takes a step toward becoming a microzonation-based design guideline by including soils factors in its design control factors (triggers). This paper discusses the effect of the changes adopted in the 1994 edition of the NEHRP Recommended Provisions, and examines the impact of full conversion to soils-factor-based control factors.

00,463

PB96-154901 PC A04/MF A01

National Inst. of Standards and Technology (BFRL), Gaithersburg, MD

Summary and Results of the NIST Workshop on Proposed Guidelines for Testing and Evaluation of Seismic Isolation Systems. Held in San Francisco, California on July 25, 1994.

H. W. Shenton. Jan $96,44 p$, NISTIR-5785.

See also PB94-161734, PB94-161940 and PB94 161957.

Keywords: "Vibration isolators, "Earthquake resistan structures *Building codes, "Test methods, *Meetings, Seismic design, Quality control, Earthquake engineering, Design criteria, Standards, Structural vibration, Prototypes, Dynamic response, Damping, Model tests Guidelines, Compression tests, Aging, 'Seismic isolation systems.

The Building and Fire Research Laboratory (BFRL) of the National Institute of Standards and Technology (NIST) has published comprehensive draft guidelines for testing and evaluating seismic isolation systems. The procedures outlined in the guidelines encompass all the required tests of the isolation systems, from the early stages of development to final production tests. The principal mechanism for soliciting feedback on the draft guidelines was a workshop held on July 25, 1994 in San Francisco. The purpose of the workshop was to provide a forum for review and discussion of the draft guidelines. This report is a summary of the workshop discussions. Topics which received the most attention in the discussions were scale model testing performance criteria, quality control testing, factors of safety, aging of isolation systems, and the sustained compression test for elastomeric systems. Rec ommendations were made regarding third party inspection of the test procedure, a test to evaluate the re-centering capability of the isolation system, a direct shear test for elastomeric systems, and fire rating.

00,464

PB96-155783 Not available NTIS

National Inst. of Standards and Technology (BFRL) Gaithersburg, MD. Structures Div.

Development of Computer-Based Models of Standards and Attendant Knowledge-Base and Procedural Systems.

Final rept.

E. Simiu, J. H. Garrett, and K A. Reed. 1993, 6p. Pub. in Proceedings of the Structures Congress, Irvine CA., April 19-21, 1993, p841-846.

Keywords: "Knowledge-based systems, "Standards, "Extreme-value problems, Building codes, Wind loads, Wind velocity, Wind effects, Data bases, Objectorieted programming, Expert systems, Reprints.

We propose the development of computer-based mod els of standards, and of knowledge-based and procedural systems to be incorporated in or used in conjunction with such models. Pilot projects should demonstrate the significant potential for inprovement in de sign and construction productivity inherent in such a new generation of standards. Wind loading examples are used for illustration.

\section{0,465}

PB96-156021 Not available NTIS

National Inst. of Standards and Technology (BFRL), Gaithersburg, MD. Structures Div.

Executive Order 12941. Seismic Safety of Existing Federally Owned or Leased Buildings: It's History, Content and Objectives.

Final rept.

D. Todd. $1995,4 p$.

See also PB96-128103 and PB96-131552.

Pub. in EERI Technical Seminar, 'The Kobe Earth-

quake: Impact on the Executive Order for Existing Buildings', Alexandria, VA., December 5, 1995, 4p.
Keywords: "Federal buildings, "Seismic design, "Earthquake engineering, Earthquake resistance, Retrofitting, Reinforcement(Structural), Structural stability, Vulnerability, Safety engineering, Inventories, Cost estimates, Reprints.

Work by the Interagency Committee on Seismic Safety in Construction (ICSSC) is expected to lead to the eventual development of a systematic program of seis mic upgrading for Federally owned buildings. Steps that have been taken to date include (1) the develop ment of seismic evaluation and rehabilitation standards, (2) the drafting of an Executive Order which adopts the technical standards and calls for a seismic inventory and cost estimate, and (3) the issuance of guidance on how to efficiently and consistently invencosts of mitigating unacceptable seismic risks.

\section{0,466}

PB96-158050 Not available NTIS

National Inst. of Standards and Technology (BFRL), Gaithersburg, MD. Structures Div

Strengthening Methodology for Lightly Reinforced Concrete Frames.

Final rept.

L. T. Phan, D. R. Todd, and H. S. Lew. 1993, 8p

See also PB95-260725.

Pub. in Wind and Seismic Effect: Proceedings of the Joint Meeting (25th), UJNR, Japan, May 1993, p265 272

Keywords: "Reinforcement(Structures), "Dynamic structural analysis, "Buildings, "Frames, Concrete structures, Reinforced concrete, Load bearing capac ity, Shear strength, Earthquakes, Design criteria Hysteresis, Computerized simulation, Mathematical models, Reprints, Infilled walls.

An analytical method for evaluating the inelastic dy namic structural response of lightly reinforced concrete (RC) frames strengthened by infilled shear walls was developed. This method involved the development of hysteresis failure models for existing and strengthened $R C$ frames and the incorporation of the models into the computer program IDARC for analysis. The hysteresis models were developed in terms of the stiffness degradation parameter the strength degradation parameter and the pinching parameter. The results of the analyses showed that (1) hysteresis models developed using one-story, one-bay frames can be incorporated into IDARC for the analysis of frames with more than one-story height, and (2) reasonable predictions of structural behavior, both in terms of ultimate load capacity and in absorbed energy on a per cycle basis can be determined using the hysteresis models.

00,467

PB96-159645 Not available NTIS

National Inst. of Standards and Technology (BFRL), Gaithersburg, MD. Structures Div.

Comparison of Responses of a Select Number of Buildings to the 10/17/1989 Loma Prieta (California) Earthquake and Low-Level Amplitude Test Results.

Final rept

M. Celebi, L. T. Phan, and R. D. Marshall. 1991 ,

25p.

Pub. in Proceedings, UJNR Joint Meeting (23rd), USJapan Panel on Wind and Seismic Effects, Tokyo, Japan, May 12-25, 1991, p475-499.

Keywords: "Earthquake damage, "Damage assessment, "Buildings, "Dynamic response, Dynamic structural analysis Ground motion, Structural vibration, Seismic discrimination, Amplitudes, Seismic effects, Damping, Reprints.

This paper summarizes dynamic characteristics of five buildings within the San Francisco Bay area. The dynamic characteristics are extracted from the October 17, 1989 Loma Prieta earthquake response records and from ambient tests conducted in November 1990. Dynamic characteristics determined for two of the five buildings prior to the Loma Prieta earthquake are also included for comparison. The preliminary results show, in some cases, that low-level test are useful and, in other cases, they show considerable differences with those determined from strong-motion response records

00,468

PB96-159686 Not available NTIS

National Inst. of Standards and Technology (BFRL) Gaithersburg, MD. Structures Div. 


\section{Structural Analyses}

Extreme Winds Estimation by 'Peaks Over Threshold' and Epochal Methods.

Final rept.

J. L. Gross, N. A. Heckert, J. A. Lechner, and E.

Simiu. 1994, 6p.

See also PB96-159694

Pub. in Structures Congress XII, Atlanta, GA., April 2428, 1994, v2 p1472-1477.

Keywords: "Wind loads, "Extreme-value problems, "Estimation, Wind effects, Wind velocity, Wind pressure, Wind profiles, Data analysis, Statistical analysis, Mathematical models, Monte Carlo method, Reprints, Peaks over threshold method, Extreme winds, Wind engineering.

With a view to applying the 'peaks over threshold' method to the estimation of extreme wind speed data, we perform Monte Carlo simulations for which the parameters of the population distributions were estimated from sets of actual extreme wind speed data. We summarize results concerning (1) the relative efficiency of several estimation procedures used in such methods,

(2) the optimal threshold for any given set of data, and (3) estimates based on the 'peaks over threshold' method as compared to estimates based on the epochal approach.

\section{0,469}

PB96-159694 Not available NTIS

National Inst. of Standards and Technology (BFRL), Gaithersburg, MD. Structures Div.

Modeling of Extreme Loading by 'Peaks Over Threshold' Methods.

Final rept.

J. L. Gross, N. A. Heckert, J. A. Lechner, and E.

Simiu. $1993,8 p$.

See also PB96-159686.

Pub. in Dynamic Response and Progressive Failure of Special Structures, Charlottesville, VA., June 6-9, 1993, p135-147.

Keywords: "Wind loads, "Extreme-value problems, *Statistical analysis, Wind effects, Wind velocity Wind profiles, Wind pressure, Gumbel distribution, Weibull distribution, Estimation, Monte Carlo method, Mathematical models, Reprints, Peaks over threshold methods, Extreme winds, Load factors.

In this work, we seek to apply the "peaks over threshold' theory to the estimation of extreme wind speeds. The studies presented are based on Monte Carlo simulations for which the parameters of the population distributions were estimated from sets of actual extreme wind speed data. Results are presented concerning (1) the relative efficiency of several estimation procedures used in such methods, (2) the optimal threshold for any given set of data and (3) estimates based on the 'peaks over threshold' method as compared to estimates based on the epochal approach using largest yearly wind speeds.

\section{0,470}

PB96-159702 Not available NTIS

National Inst. of Standards and Technology (BFRL) Gaithersburg, MD. Structures Div.

Workgroup Summary Report: Plastic Hinge-Based Techniques for Advanced Analysis.

Final rept.

J. L. Gross. 1993 3p.

Pub. in Plastic Hinge-Based Methods for Advanced Analysis and Design of Steel Frames, Pittsburgh, PA. April 5, 1992, p175-177 Mar 93

Keywords: "Construction joints, "Steel structures, "Structural analysis, Framed structures, Structural members, Buckling, Loads(Forces), Moments, Plasticity, Mechanical properties, Dynamic response, Reprints.

The purpose of this workgroup was to demonstrate, clarify, and discuss the current capabilities and limitations of contemporary plastic hinge-based methods for advanced analysis and design of steel frames. The approach taken was to discuss and comment on whether capabilities of current methods are essential to advanced analysis, beneficial (and under what circumstances), or whether there is insufficient information for immediate application of the capability. Some capabilities were assumed to be beyond the present scope and were not addressed.

\section{0,471}

PB96-162540 PC A04/MF A01

National Inst. of Standards and Technology,

Gaithersburg, MD.
Estimates of Hurricane Wind Speeds by the 'Peaks Over Threshold' Method.

Technical note.

E. Simiu, N. A. Heckert, and T. Whalen. Feb 96, 49p, NIST/TN-1416.

Grant NSF-CMS-9411642

Also available from Supt. of Docs. as SN003-003 03396. See also PB95-219416. Prepared in coopera tion with Johns Hopkins Univ., Baltimore, MD. Dept. of Civil Engineering. Sponsored by National Science Foundation, Arlington, VA. and North Atlantic Treaty Organization, Brussels (Belgium)

Keywords: "Hurricanes, "Wind velocity, "Estimation Wind effects, Wind loads, Wind pressure, Dynamic loads, Structural reliability, Structural engineering, Design standards, Building codes, Extreme valve problems, Weibull distribution, Graphs(Charts), "Peaks over threshold method, Load factors.

We report results that lend support to the hypothesis that extreme hurricane wind speeds are described predominantly by reverse Weibull distributions, which have limited upper tails. The results are based on the analysis of hurricane wind speed data obtained in an earlier project and used for the development of the ASCE 7-83 and ASCE 7-93 Standard wind speed map. According to our results, wind load factors should be larger in hurricane-prone regions than the load facto specified in current standard provisions. However, the requisite increases are smaller than would be the case in the distributions were assumed to have infinite uppe tails, as has been done so far in all principal studies of hurricane winds in the United States.

00,472

PB96-165949 PC A04/MF AO

National Inst. of Standards and Technology (BFRL), Gaithersburg, MD. Structures Div.

Modified Optimal Algorithm for Active Structura Control.

F. Sadek, and B. Mohraz. Jan 96, 36p, NISTIR-5782. Sponsored by Southern Methodist Univ., Dallas, TX. School of Engineering and Applied Science.

Keywords: "Optimal control, "Active control, *Algorithms, Structural dynamics, Seismic isolation, Vibration isolators, Control systems, Buildings, Degrees of freedom, Structureal response, Seismic waves, Dynamic response, Excitation, *Base isolation, Gain mat rices, Seismic loads.

This study presents a modification to two linear optima control algorithms, namely classical and instantaneous, to achieve a greater reduction in structural displacements and control forces. The modification consists of building a library of gain matrices and selecting the gain matrix that would result in the maximum control force without exceeding the control system capacity. The modification was used to compute the response of several single-degree-of-freedom (MDOF) system, and a base isolated structure. The study shows that the external excitation influences the selection of the control system parameters such as controler capacity and gain matrices. These parameters, therefore, should be determined according to the seismic excitation intensity expected at the site.

00,473

PB96-167820 PC A04/MF A01

Southern Methodist Univ Dallas, TX

Method of Estimating the Parameters of Tuned Mass Dampers for Seismic Applications.

F Sadek, B. Mohraz, A. W Taylor, and R. M.

Chung. Apr 96, 36p, NISTIR-5806

Sponsored by National Inst. of Standards and Technology (BFRL), Gaithersburg, MD. Structures Div.

Keywords: *Dampers, *Vibration damping, *Structura vibration, Passive systems, Energy dissipation, Buildings, Structural response, Dynamic response, Seismic waves, Seismic effects, Loads(Forces). Tuning, Degrees of treedom, Earthquake engineering, "Passive control, Seismic loads.

The optimum parameters of tuned mass dampers TMD) that result in considerable reduction in the response of structures to seismic loading are presented. The criterion used to obtain the optimum parameters is to select, for given mass ratio, the frequency (tuning) and damping ratios that would result in equal and larger modal damping in the first two modes of vibration The parameters are used to compute the response of several single and multi-degree-of-freedom structures with TMDs to different earthquake excitations. The results indicate that the use of the proposed parameters reduces the displacement and acceleration responses significantly. It is shown that as a result of selecting the parameters as proposed in this paper, significant reduction in the response of tall buildings can be achieved

00.474

PB96-183223 PC A04/MF A01

National Inst. of Standards and Technology (BFRL), Gaithersburg, MD. Structures Div.

Probabilistic Estimates of Design Load Factors for Wind-Sensitive Structures Using the 'Peaks Over Threshold' Approach.

Technical note.

T. M. Whalen. Apr $96,34 \mathrm{p}, \mathrm{NIST} / \mathrm{TN}-1418$

Also available from Supt. of Docs. as SN003-00303407-0. See also PB96-162540.

Keywords: "Hurricanes, "Wind velocity, "Probabilistic estimation, Wind effects, Wind loads, Wind pressure, Dynamic loads, Structural reliability, Structural engineering, Design standards, Building codes, Extreme value problems, Weibull distribution, "Peaks over threshold method, Load factors.

The 'peaks over threshold' method is used to estimate ratios of wind-induced loads with various long mean recurrence intervals to loads with a 50-year mean recurrence interval. The results support the conclusion that the load factor value of 1.3 specified in the ASCE Standards 7-93 and 7-95 is adequate for extratropical storm regions. However, for hurricane-prone regions, the results imply that the standard value of the load factor (even after being augmented by an importance factor specified in the standards) leads to nominal ultimate wind loads with considerably shorter mean recurrence intervals than is the case for extratropical regions. This suggests that the 1.3 load factor value specified in the ASCE Standards 7-93 and 7-95 is inadequate for wind-sensitive structures in hurricane-prone regions.

00,475

PB97-104160 PC A99/MF A06

National Inst. of Standards and Technology (BFRL),

Gaithersburg, MD.

January 17, 1995 Hyogoken-Nanbu (Kobe) Earthquake. Performance of Structures, Lifelines and Paper.

Special pub.

R. Chung. Jul 96, 578p, NIST/SP-901.

Also available from Supt. of Docs. as SNO03-05303412-6. Also pub. as Interagency Committee on Seismic Safety in Construction rept. no. ICSSC/TR-18.

Keywords: "Earthquake damage, "Damage assessment, "Seismic effects, "Soil-structure interactions, "Dynamic structural analysis, Hokkaido Island, Earthquake engineering, Airports, Bridges, Highways, Buildings, Public utilities, Gas utilities, Pipelines, Subways, Transportation sector, Harbors, Electric power, Sewer systems, Water treatment plants, Sewage treatment plants, Steel structures, Reinforced concrete, Fires, Liquefaction, "Hyogoken-Nanbu Earthquake, Kobe(Japan), Communication links, Lifelines, Wood structures.

The National Institute of Standards and Technology's Building and Fire Research Laboratory (BFRL) dispatched an advance team of three BFRL members, followed by a larger team of 18 members made up of individuals from a number of federal agencies and others affiliated with national earthquake engineering research centers. The teams were to observe, document, and summarize important lessons from this earthquake.

00,476

PB97-104376 PC A99/MF A06

National Inst. of Standards and Technology (BFRL). Gaithersburg, MD.

Wind and Seismic Effects: Proceedings of the Joint Meeting of the U.S.Japan Cooperative Program in Natural Resources Panel on Wind and Seismic Effects (28th). Held in Gaithersburg, Maryland on May 14-17, 1996.

Special pub.

N. J. Raufaste. Aug 96, 648p, NIST/SP-904.

Also available from Supt. of Docs. as SN003-00303424-0. See also PB95-147385.

Keywords: *Seismic effects, "Wind effects, *Structural engineering, "Dynamic structural analysis, "Meetings, Earthquake engineering, Geotechnical engineering, 
Design standards, Design criteria, Research programs, Dynamic loads, Structural vibration, Wind pressure, Wind loads, Earthquakes, Tsunamis, Storm surges, Risk assessment, Foundations(Structures), Highway engineering, Bridges, Dams, Repairing, Retrofitting, Earthquake resistant structures, Wind engineering, Lifelines.

This publication is the Proceedings of the 28th Joint Meeting of the U.S.-Japan Panel on Wind and Seismic Effects. The Proceedings include the program, list of members, panel resolutions, task committee reports, and the 46 technical papers written for this joint meeting. The papers were presented within five themes: (1) Storm Surge and Tsunamis, (2) Earthquake Engineering, (3) Joint Cooperative Research Program, (4) Wind Engineering, and (5) Summaries of Task Committee Workshop Reports (oral presentations only).

00,477

PB97-113245 Not available NTIS

National Inst. of Standards and Technology (BFRL), Gaithersburg, MD. Structures Div.

Dynamics of Multi-DOF Stochastic Nonlinear Systems.

Final rept.

T. M. Whalen. 1996, 4p.

Pub. in Proceedings of the Specialty Conference on Probabilistic Mechanics and Structural Reliability, Worchester, MA., August 7-9, 1996, p82-85.

Keywords: *Column buckling, "Multidimensional systems, Building technology, Chaos, Feedback control, Gaussian noise, Reprints, ‘Melnikov's method.

Melnikov's method for finding necessary conditions for homoclinic chaos is extended to stochastically forced multi degree of freedom nonlinear systems. The stochastic forcing induces a stochastic Melnikov process, from which information can be obtained on probabilities of escape and mean exit rates from regions of phase space as well as parameter bounds for nonchaotic motion. Applications are presented to the dyhnamics of a feedback controlied buckled column.

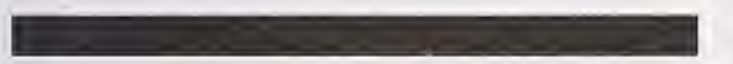

\section{BUSINESS \& ECONOMICS}

\section{General}

00,478

PB94-173002 Not available NTIS

National Inst. of Standards and Technology,

Gaithersburg, MD. Program Office.

Functions of Technology Infrastructure in a Competitive Economy.

Final rept.

G. Tassey. 1991, 17p

Pub. in Research Policy 20, p345-361 1991.

Keywords: "Economic models, "Technology innovation, Economic growth, Economy, Competition, Research and development, Laboratories, Government policies, Standards, US NIST, Reprints, "Infrastructure.

Governments of industrialized nations play varied but important roles in providing the diverse technology infrastructure that supports a modern, competitive economy. The required technology infrastructure policy model is derived from a microeconomic model of the typical technology-based industry. Existing national strategies have demonstrated the importance of efficiency at the production and market development stages as well as at the R\&D stage, but the trend is towards promoting simultaneous efficiency at all these stages. That is, the model and the derived infrastructure functions must cover all stages and interfaces in the technology and related product life cycles.

00,479

PB94-210150 PC A04/MF A01

National Inst. of Standards and Technology (TS),

Gaithersburg, MD. Office of Standards Services.
Survey on the Implementation of ISO/IEC Guide 25 by National Laboratory Accreditation Programs. Final rept.

M. Breitenberg. Jul 94, 51p, NISTIR-5473

Keywords: International organizations, Laboratories, Test methods, Calibration, Standards, Meetings, Surveys, " ISO/IEC guide 25, "Laboratory accreditation "Accreditation, NVLAP Program.

The International Organization for Standardization International Electrotechnical Commission (ISO/IEC) Guide 25, 'General requirements for the competence of calibration and testing laboratories,' has been used by many laboratory accreditation programs worldwide to establish accreditation requirements designed to promote confidence in the calibrations and testing results of laboratories which conform to the guide's requirements. National delegations to the Internationa Laboratory Accreditation Conference (ILAC), an international conference of national organizations interested in laboratory accreditation, were surveyed to collect information on the implementation and supplementation of the requirements of ISO/IEC Guide 25 within the context of their countries' laboratory accreditation programs. This report summarizes the results of that survey. The report also includes a bibliographic list of publications concerned with ISO/IEC Guide 25 implementation compiled from the information submitted by the national delegations.

00,480

PB94-219102 PC AO3/MF A01

National Inst. of Standards and Technology (NCSL) Gaithersburg, MD. Systems and Network Architecture

Analyzing Electronic Commerce.

Special pub.

L. Gebase, and S. Trus. Jun 94, 43p, NIST/SP-500/ 218

Also available from Supt. of Docs. as SNOO3-00303270-1.

Keywords: "Commerce, "Telecommunication, "Data management, Electronic mail, Data processing security, Models, Computer networks, Man machine systems, Data base management systems, Distributed computer systems, "Electronic commerce, EDI(Electronic Data Interchange)

This report begins, after citing some important technological advances, by defining electronic commerce and identifying the scope of issues to be addressed within this paper. It then proceeds to examine some ways in which electronic commerce is presently being conducted, most notably with Electronic Data Interchange (EDI). An architectural model for supporting services necessary to conduct electronic commerce is then de fined. The chapters that follow provide further detail on the model's components. The final chapter draws some conclusions about the benefits of deploying electronic commerce.

00,481

PB96-189444 PC A07/MF A02

National Research Council, Washington, DC. Commission on Engineering and Technical Systems.

Financing Tomorrow's Infrastructure: Challenges and Issues. Proceedings of a Colloquium. Held in Washington, DC. on October 20, 1995.

c1996, 115p, ISBN-0-309-05543-1.

Grant NSF-CMS-9505733

Library of Congress catalog card no. 96-69107. Sponsored by Economic Development Administration, Washington, DC., National Science Foundation, Arlington, VA. and National Inst. of Standards and Technology, Gaithersburg, MD.

Keywords: "Meetings, "Finance, Financing, Investments, Economic development, United States, *Infrastructure.

The colloquium was an attempt to explore these issues in a social, political, and fincancial context, to examine models for successfully financing infrastructure projects, and to discuss new and innovative ways of dealing with contemporary realities.

\section{Consumer Affairs}

00,482

PB94-178969 PC A08/MF A02
National Inst. of Standards and Technology (TS) Gaithersburg, MD. National Voluntary Lab. Accreditation Program.

National Voluntary Laboratory Accreditation Program 1994 Directory.

Special pub.
V. R. White. Mar 94, 161p, NIST/SP-810-ED-1994

Supersedes PB93-156644. Also available from Supt. of Docs. as SNO03-003-03259-0.

Keywords: "Laboratories, "Directories, Acoustic measurement, Computer applications, Construction materials, Electromagnetic compatibility, Thermal insulation, Lighting equipment, Seals(Stoppers), Asbestos, Carpets, Dosimetry, Paints, Paper, Plastics, Plumbing, Sealers Telecommunication, States(United States), Tests, "National Voluntary Laboratory Accreditation Program, NVLAP program, Product testing.

The Directory is published annually and provides a listing of laboratories accredited by the National Institute of Standards and Technology, National Voluntary Laboratory Accreditation Program (NVLAP). Approximately 700 laboratories in 20 fields of accreditation are included in the 1994 edition. The Directory lists the name, address, contact person, phone number, accreditation renewal data and scope of accreditation for each accredited laboratory. A brief description of the NVLAP program, a summary of laboratory participation, and user instructions are provided. The Directory consists of four indexes which are cross-referenced by NVLAP Lab Code: Index A, Listing by Laboratory Name; Index B, Listing by Field of Accreditation; Index C, Listing by State/Country; and Index D, Listing by NVLAP Lab Code. A listing of the test methods (Scope of Accreditation) is provided for each laboratory in Index D.

\section{0,483}

\section{PB95-174454 PC A08/MF A02}

National inst. of Standards and Technology (TS), Gaithersburg, MD. National Voluntary Lab. Accreditation Program

National Voluntary Laboratory Accreditation Program 1995 Directory.

Special pub.

V. R. White, and C. L. Monti. Jan 95, 165p, NIST/SP. 810-ED-1995

Supersedes PB94-178969. Also available from Supt. of Docs. as SN003-003-03315-4

Keywords: "Laboratories, "Directories, Asbestos, Calibrating, Computer applications, Dosimetry, Electromagnetic compatibility, Standards, Tests, States(United States), "National Voluntary Accreditation Program, "National Institute of Standards and Technology, NVLAP Program, Product testing.

The Directory is published annually and provides a listing of laboratories accredited by the National Institute of Standards and Technology, National Voluntary Laboratory Accreditation Program (NVLAP). Over 700 laboratories in 17 fields of accreditation are included in the 1995 edition. The Directory lists the name, address, contact person, phone number, accreditation renewal date and scope of accreditation for each accredited laboratory. A brief description of the NVLAP program, a summary of laboratory participation, and user instructions are provided. The Directory consists of four indexes which are cross-referenced by NVLAP Lab Code: Index A, Listing by Laboratory Name; Index $\mathrm{B}$, Listing by Field of Accreditation; Index C, Listing by State/Country; and Index C, Listing by State/County; and Index D, Listing by NVLAP Lab Code. A listing of the test methods (Scope of Accreditation) is provided for each laboratory in Index $D$.

\section{0,484}

PB95-182226 PC A04/MF AO1

National Inst. of Standards and Technology (TS), Gaithersburg, MD. Weights and Measures Program.

Checking the Net Contents of Packaged Goods as Adopted by the 79th National Conference on Weights and Measures, 1994, Third Edition, Supplement 4.

C. S Brickenkamp, K. Butcher, and T. Coleman. Oct 94, 64p. NIST/HB-133-ED-3-SUP-4, ISBN-0-16045384-4

Also available from Supt. of Docs. as $\mathrm{SNOO}^{-003}$ 03299-9. See also PB93-124956.

Keywords: *Packaging, "Commodities, "Labels, Handbooks, Revisions, Requirements, Sampling, Tests, Procedures, Changes, US NIST, Handbook 133.

Only minor additions and revisions to the Natlonal Instltute of Standards and Technology (NIST) Handbook 


\section{Consumer Affairs}

133, Third Edition, 'Checking the Net Contents of Packaged Goods,' were adopted by the 79th National Conference on Weights and Measures in 1994. A few editorial changes have also been made. This document consists of change pages to be added to Hand book 133, Third Edition, as amended by the 1993 Supplement.

\section{0,485}

\section{PB96-162714 PC A10/MF A03}

National Inst. of Standards and Technology (TS), Gaithersburg, MD. National Voluntary Lab. Accreditation Program.

National Voluntary Laboratory Accreditation Program 1996 Directory.

Special pub.

V. R. White. Jan 96, 193p, NIST/SP-810-ED-1996 Supersedes PB95-174454. Also available from Supt of DoCS. as SN003-003-03388-0.

Keywords: "Laboratories, "Directories, Asbestos, Calibrating, Computer applications, Dosimetry. Electromagnetic compatibility, Standards, Tests, United States, Acoustic measurement, "National Voluntary Laboratory Accredition Program(NVLAP) "NVLAP(National Voluntary Laboratory Accredition Program), "National Institute of Standards and Technology Product Testing.

The Directory is published annually and provides a listing of laboratories accredited by the National Institute of Standards and Technology, National Voluntary Laboratory Accreditation Program (NVLAP). Approximately 700 laboratories in 19 fields of accreditation are included in the 1996 edition. The Directory lists the name, address, contact person, phone and fax num bers, accreditation renewal date, and scope of accreditation of each accredited laboratory. The Directory contains a description of the NVLAP program, a summary of laboratory participation, and user instructions, followed by five laboratory indexes which are cross-referenced by NVLAP Lab Code: Index A. Listing by Laboratory Name; Index B, Listing by Field of Accreditation; Index C, Listing by State/Country; Index D. Listing of Testing Laboratories by NVLAP Lab Code; Index E, Listing of Calibration Laboratories by NVLAP Lab Code. The Scopes of Accreditation are provided for testing and calibration laboratories in Indexes $\mathrm{D}$ and $\mathrm{E}$, respectively. Current accreditation statuses of participating laboratories may be verified by calling or writing NVLAP.

00,486

PB97-110183 Not available NTIS

National Inst. of Standards and Technology (TS), Gaithersburg, MD

Evaluation and Accreditation of State Calibration Laboratories.

Final rept.

G. L. Harris. 1993, 16p.

Pub. in National Conference of Standards Laboratories Managing Quality to Improve Profitability Workshop and Symposium, Albuquerque, NM., July 25-29 1993, p395-410.

Keywords: "Calibration, "Laboratory accreditation, Measurement control, Metrology training, Interlaboratory testing, Accreditation, Assessments, Reprints.

The primary function of any accreditation process should be the evaluation of laboratory competency. Can the laboratory do what it claims. However, the as sessment process should also evaluate the degree of proficiency (skill) that the laboratory demonstrates. As. sessment of laboratory facilities is usually straighforward when using documented criteia and checklists. Since accuracy of measurements is an important attribute of any laboratory, how do we evaluate measurement accuracy. We often use the phrase, 'traceable to NIST standards' to convey accuracy relative to national standards, but how do we verify measurement traceability.

\section{Domestic Commerce, Marketing, \& Economics}

\section{0,487}

PB94-168390 PC A19/MF A04

National Inst. of Standards and Technology (CSL), Gaithersburg, MD. Advanced Systems Div.
Design and Development of an Information Retrieval System for the EAMATE Data. Volume 2 of 2. Appendices.

N. Willman, and L. L. Downey. Apr 94, 448p,

NISTIR-5394.

Sponsored by Social Security Administration, Baltimore, MD.

Keywords: *Information retrieval, "Wages, "Optical disks, Records management, Automation, Systems engineering, Man machine systems, Prototypes, Computer programs, "EAMATE system.

The Social Security Administration maintains records of wages earned by every person who has a social security number. Currently, all reports are converted to and maintained on microfilm. When a person's wage record must be verified, the process is cumbersome as the film is difficult to read and employees are listed in no particular order. The EAMATE system automates this manual process through the use of cost effective optical disk technology. The volume contains the appendices to the report on the information retrieval system for EAMATE. It includes user instructions, installation and configuration, system error messages, listing of the code, scout comments from EAMATE testing, and preliminary employer report statistics.

00,488

PB95-111514 PC A05/MF A01

National Inst. of Standards and Technology, Gaithersburg, MD.

NIST Industrial Impacts: A Sampling of Successful Partnerships.

Special pub.

Sep $94,77 p$, NIST/SP-872

Also available from Supt. of Docs. as SN003-00303293-0.

Keywords: "Industries, "Technical assistance, "Government/industry relations, Organizational structure, Technology innovation, Manufacturing, Labora. tories, Corporations, Businesses, *US NIST, Malcolm Baldrige National Quality Awards.

The National Institute of Standards and Technology (NIST) has a mission to work in partnership with industry toward the shared goal of strengthening the nation's technological and economic bases. This booklet briefly chronicles recent cases in which U.S. companies and NIST have crossed paths to their mutual benefit. It includes: Organizational Contacts, Advanced Technology Program Impacts, Manufacturing Extension Partnership Impacts, Laboratory Impacts, Malcolm Baldrige National Quality Award Impacts, Index of Companies, and Index of Applications.

\section{0,489}

\section{PB95-209193 PC A05/MF A01} National Inst. of Standards and Technology,

NIST Industrial Impacts: A Sampling of Successful Partnerships (Revision, March 1995).

Special pub.

Mar 95, 89p, NIST/SP-872-REV-1-95.

See also PB95-111514

Keywords: "Industries, "Technical assistance *Government/industry relations, Organizational structure, Technology innovation, Manufacturing, Laboratories, Corporations, Businesses, "US NIST, Malcolm Baldridge National Quality Awards.

The National Institute of Standards and Technology (NIST) has a mission to work in partnership with industry toward the shared goal of strengthening the nation's technological and economic bases. It includes: Organizational Contacts; Advanced Technology Program Impacts; Manufacturing Extension Partnership Impacts; Laboratory Impacts; Baldrige National Quality Program Impacts; Index of Companies.

\section{0,490}

\section{PB95-219325 PC A08/MF A02}

New Jersey Inst. of Tech., Newark. Center for Manufacturing Systems.

Network Brokers Handbook: An Entrepreneurial Guide to Cooperative Strategies for Manufacturing Competitiveness.

C. R. Hatch. Jan 95, 164p, NIST/GCR-94/663.

C. R. Hatch. Jan 95, $164 p$,
Grant NIST-70NANBIH1146

Sponsored by National Inst. of Standards and Technology, Gaithersburg, MD. Manufacturing Extension Partnership.

Keywords: "Manufacturers, "Networks, "Small businesses, "Manufacturing, Technical assistance, Eco- nomic development, Industries, Productivity, Entrepreneurship.

Contents:

Five Ways to Launch Manufacturing Networks;

Introduction;

Section One:

Broker Skills;

Section Two:

Targeting Firms;

Section Three:

Network Focus Groups:

Section Four:

Resource Access;

Section Five:

Pilot Network Projects:

Section Six:

Building a Strong Network Culture;

and Appendices.

00,491

PB96-190046 Not available NTIS

National Inst. of Standards and Technology (TS),

Gaithersburg, MD. Office of Standards Services.

Helping to Reduce Technical Barriers to Trade.

Final rept.

B. L. Collins. 1995, $6 p$.

Pub. in Bringing Standards Together: An International Framework Conference, Washington, DC., July 18 1995, p48-53.

Keywords: "Trade, "Barriers, "Standards, Consumer products, Marketing, International trade, Regulations, Conformity, Reprints, "Technical barriers, World trade organization, TBTs(Technical barriers to trade).

Dramatic changes are occurring in the international markets that form the background for U.S standards and conformity assessment activities. Last year the United States exported about $\$ 700$ billion worth of goods and services. These goods and services were sold into an increasingly competitive global market containing many barriers to trade. Having a large domestic market, good quality and reasonable prices are not longer guarantees of market access for a product. Technical barriers to trade (TBTs) almost always must be overcome or dealt with constructively to gain access to a market before any product can be traded. Trade experts have indicated that additional exports worth $\$ 20$ to $\$ 40$ billion could be produced right now if we could overcome all technical barriers to trade.

\section{Foreign Industry Economic Development}

\section{0,492}

PB94-212461 Not available NTIS

National Inst. of Standards and Technology (NML), Gaithersburg, MD

Aid for Smaller Businesses.

Final rept.

D. R. Johnson. 1990, 2p.

Pub. in Issues in Science and Technology VI, n4 p781990 .

Keywords: "Economic development, "Regional development, "Europe, Industries, Competition, Manufacturing, Productivity, United States, Small businesses, Reprints, US NIST.

This letter comments on the article, 'Regional Development European Style.' It expresses agreement that the U.S. can learn from European programs and experiences in technically oriented economic development and describes several new activities at the National Institute of Standards and Technology

00,493

PB97-110126 Not available NTIS

National Inst. of Standards and Technology (TS) Gaithersburg, MD. Office of Standards Services.

Stacking the Cards in Europe: One Company's Story.

Final rept

H. D. Delaney. 1996, 4p.

Pub in American Society for Testing and Materials Standardization News, v24 n8 p34-37 Aug 96

Keywords: "Barriers, Europe, Directives, Performance, Standards, Trade, Reprints, "Conformity assessments. A U.S. manufacturer of connector hoses for gas appliances has encountered a series of difficulties in re. 
maining in European markets as a consequence of shifting interpretations of an EU Directive and the applicability of the standards and conformity assessment requirements of individual European nations. Critical issues include the European use of design, rather than performance, standards and lack of access to the European technical committees and working groups. The staff of the U.S. Mission to the European Union, including the NIST standards expert, are assisting American manufacturers and exporters to resolve standards-related trade barriers.

\section{International Commerce, Marketing, \& Economics}

\section{0,494}

\section{PB94-185832 Not available NTIS}

National Inst. of Standards and Technology (CSL), Gaithersburg, MD.

Planning the Infrastructure for Global Electronic Commerce.

Final rept.

R. G. Saltman. 1993, 5p.

Pub. in EDI Forum: Jnl. of Electronic Data Interchange 6, n3 p52, 59-62 1993.

Keywords: "International trade, "Telecommunication, Commerce, Data transmission, Computer networks, Government'industry relations, Standards, Reprints, "Electronic commerce, "Infrastructure.

The components of global electronic commerce and the matrix of concepts underlying its feasibility are elucidated. Some of the issues and choices facing decision-makers on the direction of its development are discussed. Electronic commerce has implications for the way business is conducted, and may in the long run affect the ability of nations to compete effectively in international trade. It is proposed that governmentprivate sector coordination may help provide a sound basis for U.S. representation in forums deciding the future of global electronic commerce. Many of these fo. rums are multi-governmental; they concern lechnica standards and the administration of international trade. An aim of such representation would be to maximize the probability of outcomes at such forums favorable for U.S. competitiveness.

\section{0,495}

\section{PB95-103461 PC A03/MF A01}

National Inst. of Standards and Technology Gaithersburg, MD. Office of Standards Code and Information.

Questions and Answers on Quality, the ISO 9000 Standard Series, Quality System Registration, and Related Issues. More Questions and Answers on the ISO 9000 Standard Series and Related Issues M. Breitenberg. Apr 93, 40p, NISTIR-4721, NISTIR-

\section{5 .}

Supersedes PB93-152080 and PB93-140689.

Keywords: "International trade, "Quality assurance, "Standards, Quality control, Test facilities, Assess. ments, Reliability, Inspection, Specifications *ISO(International Organization for Standardization) "International Organization for Standardization, ISO 9000 , US NIST, Registration.

The report provides information on the development, content and application of the ISO 9000 standards to readers who are untamiliar with these aspects of th standards. It attempts to answer some of the mos commonly asked questions on quality; quality systems the content, application and revision of the ISO 9000 standards: quality system approval/registration; European Community requirements for quality system ap proval/registration; and sources for additional help.

\section{0,496}

\section{PB95-255881}

PC A11/MF A03

National Inst. of Standards and Technology (TS), Gaithersburg, MD. Office of Standards Services. Proceedings of the Meeting of the Intergovern mental U.S.-Russian Business Development Committee's Standard Working Group (4th). Held in New York City, New York on March 27-29, 1995 and in Northbrook, Illinois on March 30-31, 1995. Internal rept.

E. E. Zulfugarzade. Jul 95, 247p, NISTIR-5688. See also PB93-179968.
Keywords: "Russian Federation, "Standards, Proceedings, Metrology, Standardization, International trade Automobile industry, Commerce, Intergovernmental relations, Commercial development, Certification, ASME(American Society of Mechanical Engineers), Gosstandart, Harmonization.

The fourth meeting of the U.S.-Russia Business Development Committee's Standards Working Group took place on March 27-29, 1995 in New York City, New York hosted by the American Society of Mechanical Engineers International (ASME), and on March 30-31, 1995 in Northbrook, Illinois hosted by Underwriters Laboratories, Inc. (UL). The Russian delegation consisted of representatives of the Committee of the Russian Federation for Standardization, Metrology and Certification (GOSSTANDART). The U.S. delegation included representatives from government agencies and the private sector, including testing and certification bodies, individual companies, and trade associations. The meeting resulted in an exchange of information regarding standards and conformity assessment programs and practices in areas of mutual interest, such as Boilers and Pressure Vessels, occupational and food and drug safety, automotive and telecommunications standards, and electrical and fire safety of consumer and industrial products. Of particular significance was the signing of a Joint Statement on Conformity Assessment between GOSSTANDART and ASME International, a Joint Statement of Information Exchange between GOSSTANDART and FDA, and a working statement between GOSSTANDAR and UL.

\section{0,497}

PB96-106935 PC A03/MF A01

National Inst. of Standards and Technology (TS), Gaithersburg, MD. Office of Standards Services.

GATT Standards Code Activities of the National Institute of Standards and Technology 1994.

J. R. Overman. Aug 95, 39p. NISTIR-5697.

See also report for 1991, PB92-187095.

Keywords: *International trade, "Multilateral agreements *Standards Governments, Regulations, Foreign government, U.S. Government, Certification, Experts, Technical assistance, GATT(General Agreement on Tariffs and Trade).

This report describes the General Agreement on Tariffs and Trade (GATT) Standards Code activities conducted by the National Institute of Standards and Technology (NIST), for calendar year 1994. NIST received and processed 508 notifications of proposed technical regulations; reported 67 proposed U.S. technical regulations to the GATT Secretariat; respond to 409 inquiries for GATT notification information; participated in various bilateral and multilateral standards-related trade discussions; and responded to inquiries on the existence, source and availability of standards-related trade discussions; and responded to inquiries on the existence, source and availability of standards and standards-related information.

\section{0,498}

PB96-160361 Not available NTIS

National Inst. of Standards and Technology (TS), Gaithersburg, MD Office of Standards Services

International Challenges in Defining the Public and Private Interest in Standards.

Final rept.

S. I. Warshaw, and M. H. Saunders. 1995, 8p.

Pub. in SPRU-OECD International Workshop on

Standards, Innovation, Competitiveness and Policy,

Brighton, England, November 10-12, 1993, p67-74 1995.

Keywords: "Standards, "Regulations, "International agreements, Conformity, Competition, Global, Market analysis, ISO, European Communities, Reprints, Competitiveness, IEC(International Electrotechnical Commission), GATT(General Agreement on Tariffs and Trade)

Increasing global trade and technological integration raise important issues concerning: (1) existing infrastructures and their efficacy for developing internationally accepted standards; (2) the interaction of conformity assessment activities and systems among marketled economies; and (3) the changing prerogatives of governments. As a basis for world trade, manufactures and processors are demanding single globally acceptable technical standards and conformance tests. However, national governments must accommodate domestic health, safety and environinental goals that often differ dramatically between cc.nities and l'egions.
00,499

PB97-104178 PC A04/MF A01

National Inst. of Standards and Technology (TS) Gaithersburg, MD. Office of Standards Services. TBT Agreement Activities of the National Institute of Standards and Technology, 1995. Annual rept.

J. R. Overman. Sep 96, 35p, NISTIR-5898 See also PB96-190046.

Keywords: "International trade, "Barriers, "Standards, "Multilateral agreements, Conformity, Consumer products, Regulations, United States, Marketing, Notifications, Foreign government, Federal government, Certifications, "TBT(Technical barriers to trade) "Technical barriers to trade(TBT), World trade organization.

This report describes the World Trade Organization (WTO) Agreement on Technical Barriers to Trade (TBT Agreement) activities conducted by the National Institute of Standards and Technology (NIST), for calendar year 1995. NIST received and processed 378 notifications of proposed technical regulations; reported 20 proposed U.S. technical regulations to the WTO Secretariat; responded to 329 inquiries for notification inormation: participated in various bilateral and multilateral standards-related trade discussions; and responded to inquiries on the existence, source and availability of standards and standards-related information. In 1995, NIST also served as the U.S. inquir point under the Agreement on the Application of Sanitary and Phytosanitary Measures (SPS Agreement).

\section{General}

\section{0,500}

AD-A278 140/9 PC A03/MF A01

National Bureau of Standards, Boulder. CO.

Standard Materials. A Descriptive List with Prices. 12 Mar 62, 39p, NBS-MR-241.

Keywords: "Standardization, "Chemical composition "Metals, "Ceramic materials, "Chemicals, "Hydrocarbons, Procurement, Composite materials, Measurement, Accuracy, Tables(Data), "National Bu reau of Standards, Descriptive listing, Fees, Certification

No abstract available.

\section{0,501}

PB94-185535 Not available NTIS

National Inst. of Standards and Technology (CAML), Gaithersburg, MD. Statistical Engineering Div

Experimental Optimization of Peak Shape with Application to Aerosol Generation.

Final rept.

W. S. Liggett, and K. Ehara. 1993, 9p.

Pub. in Proceedings of the Society of Physical and Engineering Science, Rochester, NY., October 1993, p $744-182$.

Keywords: "Aerosol generators, "Particle size distribution, Hermite polynomials, Laser applications, Optimization, Measurement, Polystyrene, Spheres, Reprints. Chemometrics, Nebulizers.

Consider measuring instruments that respond to a single-component experimental material with a function having a single peak. In the experimental optimization of such instruments, one task is the minimization of peak width. This paper presents an approach to this task based on Hermite function approximation of the peaks observed under various instrument configurations. The approach requires at least two experimental materials with different peak locations and at least two different strengths (concentrations) of each material so that both the horizontal and vertical scale of the instrument response can be adjusted in the comparison of instrument configurations. The approach is applied to the optimization of an aerosol generator that nebulizes : $0^{\prime \prime}$ styrene latex spheres with; nearly identical sizes. 


\section{General}

In the experiment, the size distribution of the particles in the resulting aerosol is measured with a laser particle counter. The results show that the size distribution in the aerosol changes with time and that various nebulizer adjustments have an important effect on the peak width observed after the nebulizer has run for some period.

O0,502
PB94-199213 Not available NTIS

National Inst. of Standards and Technology (NML), Boulder, CO. Thermophysics Div.

Summary of the Patent Literature of Supercritical Fluid Technology.

Final rept.

T. J. Bruno. 1991, 50p.

Pub. in Supercritical Fluid Technology: Reviews in Modern Theory and Applications, p525-574 1991.

Keywords: "Supercritical fluids, Technology utilization, Literature surveys, Inventions, Abstracts, Uses, Reprints, Patenting.

The major patents issued in the field of supercritical fluid technology between 1982 and 1989 are summarized. In each case, the title, the names of the inventors, the critical dates and the assignee are provided. The abstract of each patent is also supplied.

\section{0,503}

PB94-200664 Not available NTIS

National Inst. of Standards and Technology (NEL), Gaithersburg, MD. Precision Engineering Div.

Report of Density Intercomparisons Undertaken by the Working Group on Density of the CCM.

Final rept.

R. S. Davis. $1990,6 p$

Pub. in Metrologia 27, n3 p139-144 1990.

Keywords: *Density measurement, Interlaboratory comparisons, International cooperation, Stainless steels, Metrology, Silicon, Reprints, Intercomparison.

The results of international comparisons of density measurements are reported. Participants include national laboratories of Australia, the Federal Republic of Germany, Italy, Japan, the United Kingdom, and the United States, as well as the Bureau International des Poids et Mesures. The comparisons involved hydrostatic density determinations of samples made of single-crystal silicon and samples made of stainless steel. Agreement among laboratories is generally better for samples of silicon than for samples of stainless steel. Overall discrepancies do not, however, appear to be serious with respect to scientific and metrological needs.

\section{0,504}

PB94-200672 Not available NTIS

National Inst. of Standards and Technology (MEL) Gaithersburg, MD. Automated Production Technology Div.

Mass and Density Determinations.

Final rept.

R. S. Davis, and W. F. Koch. 1992, 99p.

Pub. in Physical Methods of Chemistry, Chapter 1, v6 p1-99 1992.

Keywords: *Density measurement, Weight measurement, Densitometers, Dilatometers, Pycnometers, Balances, Buoyancy, Accuracy, Surveys, Reprints, *Mass measurement

Basic concepts of mass and density measurements are presented as well as a survey of practical techniques. Ample references are provided for the interested reader to find the basic work in a given area and a sampling of recent uses of the techniques discussed Emphasis is placed on the suitability of each method for specific problems, the major experimental difficulties, and the accuracy which can be obtained. The presentation is aimed at advanced undergraduates and graduate students in chemistry and related sciences.

\subsection{5}

PB95-125654 Not available NTIS

National Inst. of Standards and Technology (NCSL), Gaithersburg, MD. Information Systems Engineering Div.

Application of Expert System to Select Data Sources from Chemical Information Databases.

Final rept.

E. N. Fong, and C. E. Dabrowski. 1988, 11p

Pub. in Proceedings of American Society of Mechani-

cal Engineers International Computers in Engineering
Conference and Exhibition, San Francisco, CA., July 31-August 4, 1988, p107-117.

Keywords: "Chemical engineering, *Information systems, "Data base management systems, "Expert systems, Data acquisition, Knowledge bases(Artificial intelligence), Knowledge representation, Data bases, Vapor pressure, Reprints.

This paper describes a research project of building a prototype expert system called 'Automated Advisor.' This expert system conducts dialog with the engineers and recommends a list of data sources from chemical information databases.

\section{0,506}

\section{PB95-170387 PC A07/MF A02}

National Inst. of Standards and Technology (MEL), Gaithersburg, MD. Precision Engineering Div. Workshop Summary Report: Industrial Applications of Scanned Probe Microscopy. A Workshop Co-sponsored by NIST, SEMATECH, ASTM, E42.14, and the American Vacuum Society. Held in Gaithersburg, Maryland on March 24-25, 1994.

Conference proceedings.

J. A. Dagata, A. C. Diebold, C. K. Shih, and R. J.

Colton. Dec 94, 131p, NISTIR-5550.

See also AD-A245 300. Prepared in cooperation with SEMATECH, Austin, TX. and Texas Univ. at Austin.

Keywords: "Microscopy, "Industries, *Technology utilization, "Meetings, Research and development, Manufacturing, Metrology, Standards, Calibrating, Test methods, Scanning, Probes, Measuring instruments, US NBS, Critical dimension metrology, Electrical characterization, Microroughness, National Institute of Standards and Technology.

This report is a summary outcome statement for the Industrial Applications of Scanned Probe Microscopy (SPM) workshop which was held at National Institute of Standards and Technology (NIST) Gaithersburg on March 24-25, 1994. The meeting, co-sponsored by NIST, SEMATECH, ASTM E42.14, and the American Vacuum Society, was attended by over one hundred SPM users, suppliers, researchers and program managers from industry, government, and academia. The focus of the workshop was on fostering a common understanding of the roles of each of these groups in evolution of applied SPM, to achieve a consensus view on standard practices of SPM-based measurements, and to establish the basic features required of future generations of commercially available SPMs required for quantitative measurement. This report assesses the effectiveness of the workshop format and concludes with specific recommendations for a second, follow-up workshop.

\section{0,507}

\section{PB95-210225 PC A08/MF A02}

National Inst. of Standards and Technology (CSTL), Gaithersburg, MD. Process Measurements Div. NIST Workshop on Gas Sensors: Strategies for Future Technologies. Proceedings of a Workshop. Held in Gaithersburg, Maryland on September 8-9, 1993.

Special pub.

S Semancik. Dec 94, 172p, NIST/SP-865.

Also available from Supt. of Docs. as SN003-003 03327-8.

Keywords: "Gas detectors, "Air pollution monitoring, "Safety, "Meetings, Technology innovation, Commercial development, Indoor air pollution, Process control, Manufacturing, Occupational safety and health, Research and development, Performance, Transductance.

This Proceedings issue describes a Workshop on gas sensing held in Gaithersburg, MD, September, 8-9, 1993. Future gas sensors with improved response characteristics, higher reliability, and lower cost can be expected to have increased application in diverse areas, including environmental monitoring, process control, and personal safety. This Workshop brought together gas sensor manufacturers, present and potential users, and researchers to explore ways to incorporate research discoveries and emerging technologies more efficiently into new, high-performance sensors. The Proceedings includes publications on invited and contributed presentation, reports on discussions of Emerging Technologies and Commercialization and Standards issues, and reports summarizing breakout sessions.

\section{0,508}

PB95-232518 PC A03/MF A01
National Inst. of Standards and Technology (TS), Gaithersburg, MD. Standard Reference Materials Program.

NIST Standard Reference Materials (Trade Name) Catalog 1995-1996.

Special pub.

N. M. Trahey. Jan $95,40 p$, NIST/SP.260

Also available from Supt of Docs, as SN003-003 03326-0. See also PB92-181163.

Keywords: "Catalogs(Publications), "Standards, Chemical analysis, Chemical composition, Ouality as surance, Ouality control, Callibrating, Concentration(Composition), Standardization, Measurement, "Standard reference materials, "Reference materials, Certified reference materials.

The document contains the description of NIST SRM Program and comprehensive listing of all $1300+$ Stand ard Reference Materials (SRMs) and Reference Materials (RMs) developed, certified and distributed by NIST.

\section{0,509}

PB96-131602 PC A07/MF A02

National Inst. of Standards and Technology (MEL), Gaithersburg, MD

Summary Report: Workshop on Industrial Applications of Scanned Probe Microscopy (2nd). A Workshop Co-Sponsored by NIST, SEMATECH, ASTM E42.14, and the American Vacuum Society. Held in Gaithersburg, Maryland on May 2-3, 1995

J. A. Dagata, A. C. Diebold, C. K. Shih, and R. J.

Colton. Nov 95,140 , NISTIR-5752

See also PB95-170387. Prepared in cooperation with SEMATECH, Austin, TX., Texas Univ. at Austin. Dept. of Physics. and Naval Research Lab., Washington DC.

Keywords: "Microscopy, "Industries, "Technology utilization, "Meetings, Magnetic recording, Polymers, Coatings, Semiconductor devices, Tools, Standards, Calibrating, Tests, Surface properties, Metrology *Scanned probe microscopy, National Institute of Standards and Technology.

The Second Workship on Industrial Applications of Scanned Probe Microscopy (IASPM) was held at the National Institute of Standards and Technology (NIST) Gaithersburg, MD on May 2-3, 1995. The meeting, cosponsored by NIST, SEMATECH, the American Society for Testing and Materials (ASTM) E.42.14 Subcommittee, and the Manulacturing Science and Technology Group of the American Vacuum Society, was attended by approximately one hundred scanned probe microscopy (SPM) users, suppliers, and researchers from industry, government, and academia This Summary Report presents an overview of industrial applications of SPM in the areas of magnetic recording technology, polymers and coatings, and semiconductors, and reviews recent progress on SPM standardization and tool development.

00,510

PB96-161773 Not available NTIS

National Inst. of Standards and Technology (CSTL), Gaithersburg, MD. Biotechnology Div.

Conformational Alterations of Bovine Insulin Adsorbed on a Silver Electrode.

Final rept.

V. Reipa, A. Gaigalas, and S. Abramowitz. 1993

$16 \mathrm{p}$.

Pub. in Jnl. of Electroanalytical Chemistry, v348 p4134281993.

Keywords: "Insulin, “Electrodes, "Silver electrodes, Bovines, Reprints, Disulfide reduction, Protein adsorption, SERS.

Surface enhanced Raman spectra of bovine insulin, adsorbed on the silver electrode from aqueous solutions of micromolar concentrations, are presented for the potential range -0.2 to $-1.2 \mathrm{~V} / \mathrm{AgCl}$. The data suggest that insulin is bound to silver through ionized tyrosine residues and carboxy terminal groupings. Disulfide linkages are reduced sequentially upon increasing negative potential: $\mathrm{A} 7-\mathrm{B} 7$ at -0.3 to $-0.5 \mathrm{~V}$, and $\mathrm{A} 20$. $\mathrm{B} 19$ at -0.5 to $-0.6 \mathrm{~V}$. Rupture of disulfide bonds increased the portion of beta/disordered conformation at the expense of the alpha helix.

00,511

PB96-163654 Not available NTIS

National Inst. of Standards and Technology (CSTL), Gaithersburg, MD. Biotechnology Div. 
Feasibility of Fluorescence Detection of Tetracycline in Media Mixtures Employing a Fiber Optic Probe.

Final rept.

S. A. Glazier, and J. J. Horvath. 1995, 18p.

Pub. in Analytical Letters, v28 n15 p2607-2624 1995.

Keywords: "Fiber optics, "Fluorescence, "Tetracycline, "Fermentation, Media, Reprints.

The work assesses the feasbility of fluorescence detection of tetracycline in very optically dense mixtures of highly fluorescent media ingredients used in tetracycline production by fermentation. The fluorescence measurements are accomplished with a fiber optic measurements are accomplished with a fiber optic this study. Each one contained a nonfluorescent based on nutrients and salts along with one of the following media ingredients at $5 \mathrm{~g} / 100 \mathrm{~mL}$ : cottonseed flour, corn gluten meal, soybean flour, distiller's grains and solubles, corn steep liquor, brewer's yeast, and molasses. The concentration of tetracycline was varied in each mixture and flourescence measurements were made at every concentration step. Excitation light of $390 \mathrm{~nm}$ was used to probe the samples, and emission spectra were obtained over the wavelength range from 400 to $600 \mathrm{~nm}$. In most of the samples studied, the fluorescence intensity in the wavelength range corresponding to background media fluorescence (420$480 \mathrm{~nm}$ ) decreased as the tetracycline concentration increased. The decreases in the short wavelength range might be explained by the absorption by tetracycline of $390 \mathrm{~nm}$ excitation light (in competition with absorption by the media) and/or by absorption of background media fluorescence by tetracycline. Frequently, the maximum emission of the mixtures shifted to longer wavelengths. The maximum approached that of tetracycline (approximately $520 \mathrm{~nm}$ ). Plots of integrated fluorescence intensity, in the emission wavelength regions of $420-480 \mathrm{~nm}$ and $500-560 \mathrm{~nm}$, versus tetracycline hydrochloride concentration reflect these shifts. The authors have found that the changes in fluorescence intensity in these two wavelength regions during tetracycline addition depend on the indentity of the media component in the mixture.

00,512

PB96-163662 Not available NTIS

National Inst. of Standards and Technology (CSTL). Gaithersburg, MD. Biotechnology Div.

Novel Amperometric Immunosensor for Procainamide Employing Light Activated Labels. Final rept.

S. A. Glazier, and H. H. Weetall. 1994, 1p.

Pub. in Third World Congress on Biosensors, New Orleans, LA., June 1-3, 1994, 1p.

Keywords: *Immunoassays, "Amperometric, Reprints, "Electrochemical immunoassays, *Background currents, Anthraquinone, Procainamide.

Electrochemical immunoassays are often complicated by the presence of electroactive interferences which cause high current backgrounds. The authors are studying a method to correct for background currents in homogeneous immunoassays. The assays are conducted with an immunosensor employing 9,10anthraquinone derivatives as electroactive labels for the model analyte, procainamide. Anthraquinones have the ability to be photochemically reduced by light to their hydroquinone analogues. In the assay, background correction may possibly be achieved by sub. tracting the oxidation current of a sample containing the anthraquinone-procainamide conjugate from the current obtained after photoreduction. This scheme relies of preferential reduction of the anthraquinone by proper wavelength selection, and the greater electroactivity of the hydroquinone form at oxidative potentials relative to the parent anthrquinone.

\section{0,513}

PB96-163712 Not available NTIS

National Inst. of Standards and Technology (BFRL), Gaithersburg, MD. Fire Science Div.

Inhibition of Premixed Methane-Air Flames by Iron Pentacarbonyl.

Final rept.

G. T. Linteris, and G. Gmurczyk. 1995, 5p.

Sponsored by Department of the Air Force, Wright-Patterson AFB, OH

Pub. in International Colloquium on the Dynamics of Explosions and Reactive Systems, Boulder, CO., July 30-August 4, 1995, 5p.

Keywords: *Chemical inhibition, *Flame chemistry, Reprints, Flame models, Retardants, Flame speed.
Brominated fire suppressants are effective and widely used. Due to their destruction of stratospheric ozone however, the production of these chemicals was halted in January 1994. Although testing and development of possible substitutes is occurring, a replacement with all of the desirable properties of CF3Br (the most common fire suppressant) has yet to be identified. Consequently, the Fire Science Division at the National Institute of Standards and Technology (NIST) is conducting research to identify new chemical inhibitors, understand the mechanisms of inhibition of known or widely used agents, and evaluate the performance of proposed agents.

\section{0,514}

PB96-163720 Not available NTIS

National Inst. of Standards and Technology (BFRL) Gaithersburg, MD. Fire Science Div.

Parametric Study of Hydrogen Fluoride Formation in Suppressed Fires.

Final rept.

G. Linteris, and G. Gmurczyk. 1995, $12 p$.

Pub. in Halon Options Technical Working Conference, Albuquerque, NM., May 9-11, 1995, 12p.

Keywords: "Chemical inhibition, "Fire research, "Flame chemistry, Reprints, Flame models, Retardants, Halon alternatives.

Some of the proposed replacements for $\mathrm{CF} 3 \mathrm{Br}$, the fluorinated hydrocarbons, are required in higher concentrations to extinguish fires and contain more halogen atoms per molecule. Since they decompose in the flame, they produce correspondingly more hydrogen fluoride than $\mathrm{CF} 3 \mathrm{Br}$ when suppressing a fire. Recen laboratory experiments with burners using heptane propane, and methane have indicated that the amoun of HF formed in steady state can be estimated within about a factor of two for diffusion flames and within $10 \%$ for premixed flames based on equilibrium thermodynamics. In this model for HF formation, the inhibito molecule is transported to the reaction zone by convection and diffusion and is consumed in the flame sheet to form the most stable products (usually HF, $\mathrm{CO} 2$, and COF2). In the present work, the equilibrium model is used to estimate the upper limit of HF formation in suppressed fires. The effects of fuel and agent type, fuel consumption rate, and agent injection rate are included in the model, as are room volume, humidity, and concentration of inhibitor necessary to extinguish the fire.

\section{0,515}

PB96-163738 Not available NTIS

National Inst. of Standards and Technology (CSTL), Boulder, CO. Thermophysics Div.

Molar Heat Capacity at Constant Volume for Air from 67 to $300 \mathrm{~K}$ at Pressures to $35 \mathrm{MPa}$.

Final rept.

J. W. Magee. 1994, 13p

Pub. in International Jnl. of Thermophysics, v15 n5 p849-861 Sep 94.

Keywords: "Air, "Calorimeters, "Heat capacity, Reprints, High pressure, Isochoric.

Measurements of the molar heat capacity at constant volume $\mathrm{Cv}$ for air were conducted with an adiabatic calorimeter. Temperatures ranged from 67 to $300 \mathrm{~K}$ and pressures ranged up to $35 \mathrm{MPa}$. Measurements were conducted at 17 densities which ranged from gas to highly compressed liquid states In total, $227 \mathrm{Cv}$ values were obtained. The air sample was prepared gravimetrically from research purity gases resulting in a mole fraction composition of $0.78112 \mathrm{~N} 2+0.20966 \mathrm{O} 2$ $+0.00922 \mathrm{Ar}$. The primary sources of uncertainty are the estimated temperature rise and the estimated quantity of substance in the calorimeter Overall, the uncertainty of the $\mathrm{Cv}$ values is estimated to be less than plus or minus $2 \%$ for the gas and plus or minus $5 \%$ for the liquid.

\subsection{6}

\section{PB97-109(}

MF A03)

Cambridge Crystallographic Data Centre (England). Cambridge Structural Database (CSD): Current ACtivities and Future Plans.

D. G. Watson. 1996, 3p.

Included in Jnl. of Research of the National Institute of Standards and Technology, v101 n3 p227-229 May/ Jun 96.

Keywords: "Crystallography, "Organic compounds, "Organometallic compounds, "Information systems,
Crystal structures, Polymers, Searching, Information retrieval, Data analysis, Chemical structure, Computer graphics, Chemical composition, Numeric data.

This paper reviews the search and analysis software packages OUEST3D and VISTA, also the databasebuilding program Pre Quest. The relationship between the CSD and the Protein Data Bank is discussed and development plans are outlined.

00,517

PB97-109060 (Order as PB97-109011, PC A11/ $\mathrm{MF}$ A03)

Brookhaven National Lab, Upton, NY.

Protein Data Bank: Current Status and Future Challenges.

E. E. Abola, N. O. Manning, J. Prilusky, D. R.

Stampt, and J. L. Sussman. 1996, 11p.

Prepared in cooperation with Weizmann Inst. of Science, Rehovoth (Israel)

Included in Jnl. of Research of the National Institute of Standards and Technology, v101 n3 p231-141 May/ Jun 96.

Keywords: *Crystallography, "Proteins, "Information systems, Crystal structure, Three dimensional, Software tools, Data base management.

The Protein Data Bank (PDB) is an archive of experimentally determined three-dimensional structures of proteins, nucleic acids, and other biological macromolecules with a 25 year history of service to a global community. PDB is being replaced by 3DB, the Three-Dimensional Database of Biomolecular Structures that will continue to operate from Brookhaven $\mathrm{Na}$ tional Laboratory. 3DB will be a highly sophisticated knowledege-based system for archiving and accessing structural information that combines the advantages of object oriented and relational database systems.

\section{0,518}

PB $97-109078$

MF A03)

Rutgers - The State Univ., Piscataway, NJ. Dept. of Chemistry

Nucleic Acid Database: Present and Future.

H. M. Berman, A. Gelbin, L. Clowney, J. Westbrook S. H. Hsieh, and C. Zardecki. 1996, 15p.

Included in Jnl. of Research of the National Institute of Standards and Technology, v101 n3 p243-257 May/ Jun 96

Keywords: "Crystallography, "Nucleic acids, *Information systems, Chemical structure, Crystal structure, Three dimensional, Information retrieval, DNA, RNA, Data base management.

The Nucleic Acid Database is a relational database containing information about three-dimensional nucleic acid structures. The methods used for data processing, structure validation, database management and information retrieval, as well as the various services available via the World Wide Web, are described. Plans for the future include greater reliance on the Macromolecular Crystallographic Information File for both data processing and data management.

00,519

PB97-10914

MF A03)

Kentucky Univ., Lexington. Dept. of Chemistry.

Investigations of the Systematics of Crystal Packing Using the Cambridge Structural Database.

C. P. Brock. 1996, 5p

Included in Jnl. of Research of the National Institute of Standards and Technology, v101 n3 p321-325 May/ Jun 96

Keywords: "Crystallography, "Molecular structure, *Information systems, Crystal packing

Several studies that used the Cambridge Structural Database to elucidate principles of packing in molecular crystals are described. Some possible sources of bias in the statistical distributions are discussed

00,520

PB97-110225 Not available NTIS

National Inst. of Standards and Technology (CSTL),

Gaithersburg, MD. Process Measurements Div.

Thin-Film Ruthenium Oxide - Iridium Oxide Thermocouples.

Final rept.

K. G. Kreider. 1991, $8 p$

Pub. in Proceedings of the Materials Research Society

Symposium, Anaheim, CA., April 29-May 3, 1991. v234 p205-212. 


\section{General}

Keywords: "Iridium oxide, "Ruthenium oxide, "Thin films, Sputtering, Temperature measurement, Thermocouples, Reprints.

Ruthenium oxide and iridium oxide have outstanding resistance to corrosion. These oxides are also excellent electrical conductors and have been used as blochemical charge injection electrodes. Their unique electrical and electrochemical properties have also led to their consideration as high temperature $\mathrm{pH}$ electrodes. Thin films are the most useful form for these applications as they permit the miniaturization of fast response sensors and electrodes. The study was used to characterize the thermoelectric and electrical conductance parameters of ruthenium and iridium oxide sputtered thin films. The electric and thermoelectric properties of the thin films were found to be sensitive to the annealing temperature of the sputtered oxides. The properties of the film are related to the microstructure, stoichiometry and crystal structure as determined by $x$-ray diffraction. Heat treatments were used to stabilize the thermoelectric response and the thermal coefficient of resistivity.

00,521

PB97-112460 Not available NTIS

National Inst. of Standards and Technology (CSTL) Gaithersburg, MD. Chemical Kinetics and Thermodynamics Div.

Ferric Ion Assisted Photooxidation of Halocetates. Final rept.

P. Maruthamuthu, and R. E. Huie. 1995, 9p.

Pub. in Chemosphere, v30 n11 p2199-2207 1995.

Keywords: "Haloacetates, "Photooxidation, Photodecarboxylation, Hydroxyl radical, Hydrofluorocarbons, Hydrochlorofluorocarbons, Reprints, *Foreign technology.

Aqueous solutions containing ferric ions and chloro- or fluoroacetates were photolyzed by light with wavelengths greater than $300 \mathrm{~nm}$. Significant variation in the extent of reaction was observed, with the totally halogenated acetates degraded very little in the course of the experiments. The relative rates of degradation were found to correlate very well with the relative rates of reaction of the haloacetate with the hydroxyl radical

\section{Analytical Chemistry}

\section{0,522}

DE94018563 PC A02/MF AO

National Inst. of Standards and Technology, Gaithersburg, MD.

Improvement of Ultrasensitive Techniques Isotopic Biasing in the RIS Process Ionization Efficiencies and Selectivities.

Progress rept.

1989, 10p, DOE/ER/60447-T3

Contract Á105-86ER60447

Sponsored by Department of Energy, Washington, DC

Keywords: *Resonance Ionization Mass Spectroscopy, Isotope Ratio, Progress Report, Sensitivity, Trace Amounts, EDB/400102.

Work in improvement of ultrasensitive (RIMS) techniques, RIS schemes and atomic data, and development of isotopic ratio standards and standards for ultratrace analysis is reported.

\section{0,523}

DE94018565 PC A02/MF A01

National Inst. of Standards and Technology, Gaithersburg, MD.

I: Improvement of Resonance Ionization Spectroscopy (RIS) Techniques; II: Atomic Data for RIS; III Standards for Ultratrace Analysis. Progress Re port.

1991, 6p, DOE/ER/60447-T5.

Contract Al05-86ER60447

Sponsored by Department of Energy, Washington, DC

Keywords: *Resonance Ionization Mass Spectroscopy, Isotope Ratio, Progress Report, Trace Amounts, EDB 400102.

I: Work focused on converting the VG SIMS system into a dual pumose instrument for analysis in SIMS or SIRIS mode, and on a glow discharge source for the NIST magnetic sector mass spectrometer. Ionization efficiencies and selectivities are being studied. II: The first two groups of 10 elements each have been published; draft data sheets have been completed for a third group of 5 elements; the data base of published and unpublished RIS work has been expanded. III: Dilution checks are being performed on the (sup 36$) \mathrm{Cl}$ standard.

\section{0,524}

PB94-140563

MF A02)

National Inst. of Standards and Technology, Gaithersburg, MD.

$36 \mathrm{Cl} / \mathrm{Cl}$ Accelerator-Mass-Spectrometry Standards: Verification of Their Serial-Dilution-Solution Preparations by Radioactivity Measurements.

R. Colle, and J.W. L. Thomas. 1993, 25p

Included in Jnl. of Research of the National Institute of Standards and Technology, v98 n6 p653-677 Nov/ Dec 93.

Keywords: "Mass spectrometry, "Chlorine 36 * Standards, Proportional counters, Liquid scintillators, Beta decay, Gravimetric analysis, Isotope dilution, Isotope ratio, Radioactivity, Radioisotopes, Metrology, Solutions, "Accelerator mass spectroscopy, Dilution factors.

A consortium of accelerator-mass-spectrometry (AMS) laboratories recently prepared a series of $(36) \mathrm{Cl} / \mathrm{Cl}$ isotopic ratio AMS standards by an eight-step serial gravimetric dilution scheme. Of the resulting nine solutions, only the latter six could be assayed by AMS to confirm the gravimetric dilution factors. The paper provides the results of relative radioactivity measurements on the first four solutions to verify the first three dilution factors. The fourth solution was the only dilution capable of being directly measured by both AMS and radionuclidic metrology of $(36) \mathrm{Cl}$, and therefore its assay by radioactivity counting was deemed of considerable importance.

\subsection{5}

PB94-163003 PC A02/MF AO1

National Inst. of Standards and Technology (CSTL), Gaithersburg, MD. Surface and Microanalysis Science Div.

Airborne Asbestos Method: Standard Test Method for High Precision Counting of Asbestos Collected on Filters. Version 1.0

S. Turner, and E. B. Steel. Mar 94, 10p, NISTIR

5350 .

Keywords: "Asbestos, "Counting techniques ${ }^{*}$ Particles, ${ }^{*}$ Chemical analysis, Transmission electron microscopy, Standards, Regulations, Air pollution detection, Water pollution detection, Filters, Procedures.

The analysis of asbestos by transmission electron microscopy (TEM) is important for the determination of the cleanliness of air or water and for research purposes. Counting rules are used to determine the amount of asbestos observed by TEM. This method describes a high precision set of counting rules. For this method, all qualifying particles are counted as one structure; the number of asbestos fibrils within a particle does not affect the number of structures assigned to the particle.

\section{0,526}

PB94-163045 PC A03/MF A01

National Inst. of Standards and Technology (CSTL) Gaithersburg, MD. Surface and Microanalysis Science Div.

Airborne Asbestos Method: Standard Test Method for Verified Analysis of Asbestos by Transmission Electron Microscopy. Version 2.0.

S. Turner, and E. B. Steel. Mar 94, 26p, NISTIR-

5351.

See also PB94-113578.

Keywords: "Asbestos, *Chemical analysis, Air pollution detection, Transmission electron microscopy, Quality assurance, Water pollution detection, Standard, Procedures, "Verified analysis.

The analysis of asbestos by transmission electron microscopy is important for determination of the cleanliness of air or water and for research purposes. Verified analysis provides a method for determining the quality of the analyses. Verified analysis is a procedure in which a grid opening is independently analyzed for as bestos by two or more transmission electron microscope (TEM) operators and in which a comparison and evaluation of the correctness of the analyses are made by a verifying analyst. Detailed information - including absolute or relative location, a sketch, orientation, sized (length, width), morphology, analytical informa tion and structure identification - is recorded for each observed asbestos structure. Comparisons of the analyses are made on a structure-by-structure basis and the percentage of true positives, false positives and false negatives are determined for each TEM operator. Verified analyses can be used as part of a quality assurance program for asbestos analyses and as a training procedure. This report describes a method for conducting a verified analysis. The method is reported in ASTM format. This version contains a revised procedure, examples and flow charts.

00,527

PB94-172095 Not available NTIS

National Inst. of Standards and Technology (CSTL),

Gaithersburg, MD. Inorganic Analytical Research Div. Local Area Networks in NAA: Advantages and Pitfalls.

Final rept.

M. Blaauw, and R. M. Lindstrom. 1993, 10p.

Pub. in Jnl. of Radioanalytical and Nuclear Chemistry 169, n2 p443-452 1993

Keywords: "Gamma ray spectroscopy, "Activation analysis, "Local area networks, "Computer networks, Data acquisition, Workstations, Reprints.

Both at IRI and at NIST, Local Area Networks (LANs) are being used to acquire and process data from multiple gamma-ray spectrometers. In this paper, differences and similarities between three systems are discussed, resulting in recommendations for new systems to be set up.

\subsection{8}

PB94-185063 Not available NTIS

National Inst. of Standards and Technology (CSTL) Gaithersburg, MD. Inorganic Analytical Research Div. Classical Analysis: A Look at the Past, Present, and Future.

Final rept.

C. M. Beck. 1994, 14p.

Pub. in Analytical Chemistry 66, n4 p224A-239A, 15 Feb 94.

Keywords: "Analytical techniques, ${ }^{*}$ Chemical analysis Measuring instruments, Comparison, Performance evaluation, Laboratories, Testing procedures, Reprints, "Classical analysis.

Despite the fact that instrumental analysis has rightfully assumed an overwhelmingly major role in the analytical laboratory, there remains a limited, although important, need for classical analysis. Instrumental analysis is most useful for elemental determinations at minor and trace levels (about $1 \%$ all the way down to 1 atom), and in this range classical analysis performs either poorly or not at all. However, instrumental analysis generally does not give high precision and accuracy at major levels (about $1 \%$ up to $100 \%$ ), and in this range classical analysis does perform well. Moreover, instrumental and classical analysis complement each other and can be used in tandem to the analyst's advantage.

\section{0,529}

PB94-185071 Not available NTIS

National Inst. of Standards and Technology (CSTL), Gaithersburg. MD. Inorganic Analytical Research Div. Preparation and Certification of a Rhodium Stand. ard Reference Material Solution.

Final rept.

C. M. Beck, M. L. Salit, R. L. Watters, T. A. Butler, and L. J. Wood. 1993, 4p.

Pub. in Analytical Chemistry 65, n20 p2899-2902, 15 Oct 93.

Keywords: "Rhodium, *Standards, "Chemical analysis, Solutions, Comparison, Certification, Sample preparation, Gravimetric analysis, Spectrum analysis, Solubility, Inorganic salts, Quality assurance, Reprints, "Standard Reference Materials, SRM 3144

A dual-path approach has been developed for the production of an accurate rhodium solution standard. First a water-soluble rhodium salt was assayed for rhodium by gravimetry. Second, a different water-soluble rhodium salt was synthesized from a known mass of highpurity rhodium metal. The agreement between both rhodium solution standards, each based on gravimetry, and subject to different random and systematic errors, confirmed the accuracy of their rhodium concentrations. Based on this work an accurate rhodium solution standard (NIST Standard Reference Material (SRM) 3144) was prepared by dissolving in water a weighed 
portion of an accurately assayed, homogeneous, water-soluble modium salt, which had been dried to constant weight.

\section{0,530 \\ PB94-185253 Not available NTIS}

National Inst. of Standards and Technology (CSTL), Gaithersburg, MD. Organic Analytical Research Div. Liquid Chromatography: Laser-Enhanced Ionization Spectrometry for the Speciation of Organolead Compounds.

Final rept.

K. S. Epler, T. C. O'Haver, and G. C. Turk. 1994, 4p. Pub. in Jnl. of Analytical Atomic Spectrometry 9, p7982 Feb 94.

Keywords: "Liquid chromatography, *Chromatographic analysis, "Organometallic compounds, "Lead compounds, Chemical analysis, Ion spectroscopy, Trace amounts, Reprints, Laser enhanced ionization, Standard reference materials.

Liquid chromatography (LC) and laser-enhanced ionization spectrometry (LEI) have been combined to provide a very sensitive method for the measurement of organolead species. Measurement is possible in environmental and biological matrices, which often contain high levels of easily ionized elements that normally interfere with LEI measurements. However, LC is able to resolve the interferents from the analyles, and LEI is sensitive enough to counterbalance the increase in detection limits that results from the use of LC. The method has been applied to the determination of organolead compounds in National Institute of Standards and Technology Standard Reference Material $1566 a$, Oyster Tissue. Trace levels of trimethyllead were observed in the Oyster Tissue, but concentrations were variable among the samples tested.

\section{0,531}

\section{PB94-185261 Not available NTIS}

National Inst. of Standards and Technology (CSTL), Gaithersburg, MD. Inorganic Analytical Research Div. Comparative Strategies for Correction of Interferences in Isotope Dilution Mass Spectrometric Determination of Vanadium.

Final rept.

J. D. Fassett, E. S. Beary, X. Xiong, and L. J. Moore. 1994, 5p.

Pub. in Analytical Chemistry 66, n7 p1027-1031, 1 Apr 94.

Keywords: "Resonance ionization mass spectroscopy, "Mass spectroscopy, "Vanadium, Quantitative chemical analysis, Interference, Chromium, Titanium, Reprints, "Thermal ionization mass spectroscopy, "Isotope dilution mass spectroscopy, Standard reference materials.

Vanadium has been determined in SRM 1573a, Tomato Leaves, by isotope dilution mass spectrometry using thermal ionization (TIMS) and resonance ionization (RIMS). The capabilities of the two techniques to compensate for interferences from chromium and titanium are compared. Results are discussed.

\section{0,532}

PB94-185337 Not available NTIS

National Inst. of Standards and Technology (CSTL), Gaithersburg, MD. Inorganic Analytical Research Div. Frozen Human Serum Reference Material for Standardization of Sodium and Potassium Measurements in Serum or Plasma by Ion-Selective Electrode Analyzers.

Final rept.

P. Gunaratna, W. Koch, R. Paule, N. Greenberg, K. O'Connell, A. Malenfant, A. Okorodudu, R. Miller, D. Kus, G. Bowers, A. Cormier, and P. D'Orazio. 1992, $7 p$. Pub. in Clinical Chemistry 38, n8 p 1459-1465 1992. Keywords: "Sodium, "Potassium, "Blood analysis, Blood serum, Standards, Medical laboratories, Interlaboratory comparisons, lon selective electrode analysis, Atomic spectroscopy, Emission spectroscopy, Reprints, "Standard reference materials.

Three interlaboratory round-robin studies (RR1, RR2, and RR3) were conducted to identify a serum-based reference material that would aid in the standardization of direct ion-selective electrode (ISE) measurements of sodium and potassium. Ultrafiltered frozen serum reference materials requining no reconstitution reduced between-laboratory vaniability (the largest source of imprecision) more than did other reference materials. ISE values for RR3 were normalized by the use of two points at the extremes of the clinical range for sodium (i.e., 120 and $160 \mathrm{mmol} / \mathrm{L}$ ), with values assigned by the flame atomic emission spectrometry (FAES) Re erence Method.

\section{0,533}

PB94-185980 Not available NTIS

National Inst. of Standards and Technology (CSTL), Gaithersburg, MD. Inorganic Analytical Research Div. Fourier Transform Atomic Emission Studies Using a Glow Discharge as the Emission Source.

Final rept.

M. R. Winchester, J. C. Travis, and IVI. L. vam. ,993, $13 p$.

Pub. in Spectrochimica Acta 485, „11 p1325-1337 1993.

Keywords: "Glow discharges, "Emission specuuscupy, Measurement, Precision, Reprints, Fourier transform atomic emission spectroscopy. Photon noise.

The glow discharge (GD) is investigated as a possible atomic emission source for Fourier transform atomic emission spectroscopy (FT-AES). Noise power spectra are presented to demonstrate that GD emission is primarily characterized by photon noise, although a drif noise component exists at extremely low frequencies. The photon noise character is important, as photon noise limited sources are expected to outperform source flicker noise limited sources in terms of measurement precision. The implementation of bandpass restriction and dual channel subtractive noise cancellation as possible means of improving measurement precision are also presented. In both cases, the improvements were found to be minimal, a fact attributed to the probable suitability of the GD for FT-AES.

\section{0,534}

PB94-187564 PC A03/MF AO1

National Inst. of Standards and Technology, Gaithersburg, MD.

Application of the Electronic Balance in High Precision Pycnometry.

R. M. Schoonover, M. S. Hwang, and W. E. Crupe. May $94,18 p$, NISTIR-5422.

Keywords: "Pycnometers, "Electronic equipment, "Weight indicators, Laboratory equipment, Calibrating, Standardization, Density(Mass/Volume), Measuring instruments, Glassware, Liquids, Volume, Precision, Mass, Electronic balance, Liquid density.

Pycnometers are used to measure the density of fluids. Usually pycnometer volume is determined by measuring the mass and temperature of contained water and thereafter is used to determine the density of contained fluid by further weighing. The calibration and use of the pycnometer can be achieved on a modern electronic balance without the use of the usual set of mass standards. This paper explores the electronic balance application to pycnometry, presents supporting data with analysis and discusses a pycnometer design.

\section{0,535}

\section{PB94-188836 PC A07/MF A02}

National Inst. of Standards and Technology (CSTL), Gaithersburg, MD. Surface and Microanalysis Science Div.

Proficiency Tests for the NIST Airborne Asbestos Program, 1990.

S. Turner, S. S. Doorn, E. B. Steel, K. K. Starner, J. M. Phelps, and E. S. Windsor. May 94, 146p,

\section{NISTIR-5431.}

Prepared in cooperation with Research Triangle Inst. Research Triangle Park, NC. Center for Environmental Systems.

Keywords: "Asbestos, "Air pollution detection, "Chemical analysis, "Standards, Transmission electron microscopy, Performance evaluation, Laboratories, Sampling, Personnel development, Quality assurance, Quality control, "National Voluntary Accreditation Program, "Proficiency tests.

The National Voluntary Accreditation Program (NVLAP) at the National Institute of Standards and Technology (NIST) has since 1990 had a program to accredit those laboratories involved in the analysis of airborne asbestos by transmission electron microscopy. As a part of that program, laboratories are sent proficiency tests twice yearly to evaluate their ability to correctly analyze samples and to test the genera knowledge of laboratory personnel. The results of the tests are sent to the participating laboratories in the form of a summary report. This NIST Internal Report (NISTIR) contains the instructions and summary re- ports issued for the proficiency tests in 1990 (PT901, PT90-2).

\section{0,536}

PB94-188844 PC A10/MF A03

National Inst. of Standards and Technology (TS) Gaithersburg, MD. Office of Measurement Services.

Standard Reference Materials: Glass Filters as a Standard Reference Material for Spectrophotometry S Selection, Preparation, Special pub. (Final)

R. Mavrodineanu, R. W. Burke, J. R. Baldwin, J. C.

Travis, J. C. Colbert, M. V. Smith, and J. D. Messman. Mar 94, 207p, NIST/SP-260/116. Also available from Supt. of Docs. as SNO03-00303256-5. See also PB-246 437

Keywords:

"Spectrophotometers, *References(Standards), "Optical filters, Performance evaluation, Procedures, Certification, Accuracy, Precision, Calibrating, Electromagnetic absorption, Transmittance, Reproducibility, Wavelengths, "Standard ref erence materials, SRM 930, SRM 1930.

This publication describes the various factors that can affect the proper functioning of a spectrophotomete and suggests procedures to assess and control these factors. Particular consideration is given to the longand short-term stability of a spectrophotometer, wave length accuracy, spectral bandpass, stray radiation, and the accuracy of the transmittance or absorbance scale. A description is given of the Standard Reference Materials (SRMs) that can be used to control these factors. The methods for the preparation, certification, and use of two such materials (SRM 930 and SRM 1930) are also presented. The results obtained in the actua use of the SRMs are examined in some detail. An Appendix contains the reproduction of several publications and Certificates relevant to the subject discussed in this publication.

00,537

PB94-193828 PC A04/MF AO

National Inst. of Standards and Technology (CSTL) Gaithersburg, MD. Surface and Microanalysis Science Div.

Proficiency Tests for the NIST Airborne Asbestos Program - 1991.

S. Turner, E. B. Steel, S. S. Doom, and S. B. Burris. May 94,52 , NISTIR-5432.

See also PB94-188836. Prepared in cooperation with Research Triangle Inst., Research Triangle Park, NC. Center for Environmental Measurements and Quality Assurance.

Keywords: "Asbestos, "Air pollution detection "Chemical analysis, "Transmission electron microscopy, Performance evaluation, Sampling, Quality control, Quality assurance, Comparison, "Proficiency tests, National Voluntary Accreditation Program.

The National Voluntary Accreditation Program (NVLAP) at the National Institute of Standards and Technology (NIST) has since 1990 had a program to accredit those laboratories involved in the analysis of airborne asbestos by transmission electron microscopy. As a part of that program, laboratories are sent proficiency tests twice yearly to evaluate their ability to correctly analyze samples and to test the general knowledge of laboratory personnel. The results of the tests are sent to the participating laboratories in the form of a summary report. This NIST Internal Report (NISTIR) contains the instructions and summary reports issued for the proficiency tests in 1991 (PT91. 1, PT91-2).

\section{0,538}

PB94-193885 PC A03/MF A01

National Inst. of Standards and Technology (MSEL) Gaithersburg, MD. Polymers Div.

Preparation and Monitoring of Lead Acetate Containing Drinking Water Solutions for Toxicity Studies.

Final rept

W. R. Blair K L. Jewett, F. W. Wang, and S. B. Schiller. May 94, 280, NISTIR-5388.

See also PB86-189875 and PB92-126499. Sponsored by Maryland Univ. at Baltimore.

Keywords: "Drinking water, "Lead inorganic compounds, "Animal husbandry, Water pollution monitoring, Concentration(Composition), Exposure, Toxicity, Absorption spectra, Atomic spectroscopy, Chemica analysis, US NBS, Dosage, "Lead acetate, 
FAAS(Flame Atomic Absorption Spectrophotometry), Lead nephropathy, NIST(National Institute of Standards and Technology).

The protocols developed and implemented to provide lead acetate containing drinking water solutions for animal toxicity studies are described in detail. The procedure involved preparation of 20 liter batches of high concentration lead acetate solution $(20,000 \mathrm{ppm})$ at National Institute of Standards and Technology (NIST). Conventional flame atomic absorption spectrophotometry (FAAS) was employed to confirm that the lead concentration of the solution was within five percent of the target value. This solution was then dispensed into containers of appropriate volume such that upon dilution at the animal research facility, drinking water solutions containing lead concentrations of 50,250 and $1000 \mathrm{ppm}$ were produced. Samples of these diluted solutions and samples taken from animal cage water bottles were returned to NIST for followup FAAS determination of lead concentration to insure that animal exposure to lead was meeting experimental goals.

\subsection{9}

PB94-194362 PC A03/MF A01

National Inst. of Standards and Technology (CSTL), Gaithersburg, MD. Surface and Microanalysis Science Div

Proficiency Tests for the NIST Airborne Asbestos Program - 1992.

S. Turner, E. B. Steel, S. S. Doorn, and S. B. Burris. May 94, 48p, NISTIR-5433.

Prepared in cooperation with Research Triangle Inst. Research Triangle Park, NC. Center for Environmental Measurements and Quality Assurance.

Keywords: *Asbestos, *Air pollution detection, "Chemical analysis, "Transmission electron microscopy, Performance evaluation, Sampling, Quality control, Quality assurance, Comparison, "Proficiency tests, National Voluntary Accreditation Program.

The National Voluntary Accreditation Program (NVLAP) at the National Institute of Standards and Technology (NIST) has since 1990 had a program to accredit those laboratories involved in the analysis of airborne asbestos by transmission electron microscopy. As a part of that program, laboratories are sent proficiency tests twice yearly to evaluate their ability to correctly analyze samples and to test the general knowledge of laboratory personnel. The results of the tests are sent to the participating laboratories in the form of a summary report. This NIST Internal Report (NISTIR) contains the instructions and summary reports issued for the proficiency tests in 1992 (РT921, PT92-2).

\section{0,540}

PB94-198710 Not available NTIS

National Inst. of Standards and Technology (NML), Gaithersburg, MD. Inorganic Analytical Research Div. Laser Ablation of Thin Films as a Free Atom Source for Pulsed RIMS.

Final rept

L. Bengtsson, J. Travis, T. B. Lucatorto, K. Kreider, and L. Brown. 1989, 4p.

Contract DEAIO586ER60446

Pub. in Proceedings of the International Symposium on Resonance Ionization Spectroscopy and Its Applications (4th), Gaithersburg, MD., April 10-15, 1988, p167-170 1989. Sponsored by Department of Energy, Washington, DC.

Keywords: "Resonance ionization mass spectroscopy, Chemical analysis, Laser ablation, Pulsed lasers, Trace elements, Thin films, Reproducibility, Sampling, Reprints, Atom sources.

The efficient application of pulsed-laser resonance ionization mass spectrometry (RIMS) to ultrasensitive analysis requires conservation of samples during the analysis dead time. Laser ablation of solids has been shown to provide vapor plumes of appropriate spatial and temporal properties, but free atom fractions suffer from particle formation due to thermal stress fracturing. The authors report here preliminary studies with semitransparent thin (about $50 \mathrm{~A}$ ) films, intended to improve the reproducibility and atom yield of laser ablation sampling.

\section{0,541}

PB94-198736 Not available NTIS

National Inst. of Standards and Technology (NML), Gaithersburg, MD. Gas and Particulate Science Div.
Relative Sensitivity Factors and Useful Yields for a Microfocused Gallium Ion Beam and Time-ofFlight Secondary Ion Mass Spectrometer.

Final rept.

J. Bennett, and D. Simons. 1991, $6 \mathrm{p}$

Pub. in Jnl. of Vacuum Science and Technology A 9, n3 p1379-1384 1991.

Keywords: "Ion sources, "Gallium ions, Secondary ion mass spectroscopy, Time-of-flight method, Mass spectrometers, Liquid metals, Thin films, Sensitivity, Yield, Magnesium, Calcium, Glass, Reprints.

Relative sensitivity factors for several elements in micrometer size glass particles, bulk glasses and glass thin films have been determined for a $\mathrm{Ga}(1+)$ liquid metal ion gun combined with a time-of-flight secondary in mass spectrometer. The reproducibility of the values within and among glasses of various compositions is better than $20 \%$. Reproducibility is improved by sput ter-cleaning the sample prior to data collection. Relative sensitivity factors have also been obtained from bulk glasses using pulsed, low-energy electrons to neutralize charge build-up on the sample. Useful yields for $\mathrm{Mg}$ and $\mathrm{Ca}$ in a thin glass film were calculated to be a $3.4 \times 10$ (sup -4) and $8.0 \times 10$ (sup -4), respectively.

\section{0,542}

PB94-199114 Not available NTIS

National Inst. of Standards and Technology (NML) Gaithersburg, MD, Gas and Particulate Science Div. Concentration Histogram Imaging: A Scatter Diagram Technique for Viewing Two or Three Related Images.

Final rept

D. S. Bright, and D. E. Newbury. 1991, $7 \mathrm{p}$.

Pub. in Analytical Chemistry 63, n4 p243A-244A p246A-250A, 15 Feb 91

Keywords: "Image processing, "Histograms, *Concentration(Composition), "Microanalysis, Reprints, Scatter diagrams, Quantitative analysis, Chemical analysis, $X$-ray analysis, "Concentration histogram imaging, X-ray maps.

Quantitative compositional mapping involves the production of images, which directly depict the concentrations of the elemental constituents present in a specimen. A problem is encountered when numerical concentration information is to be presented in the form of an image--the authors wish to simultaneously present both spatial position information and numerical concentration data for two or more constituents. The authors have developed the Concentration Histogram Image (CHI) which is a modified scatter diagram where compositional maps are transformed from rea space to concentration space. Unlike the scatter diagram, the CHI is displayed by encoding the frequency information with a thermal color scale. The authors have implemented the $\mathrm{CHI}$ on various computers to view the $\mathrm{CHI}$ for selected areas of the sample and show the correspondence between features (clumps or bands of points) in the $\mathrm{CHI}$ with specific areas in the original maps. The $\mathrm{CHI}$ is a useful diagnostical tool for detecting inhomogeneities in materials, checking quantitative corrections of $x$-ray maps, evaluating sampling and measurements statistics, and selecting features of interest for further analysis.

\section{0,543}

\section{PB94-199155 Not available NTIS}

National Inst. of Standards and Technology, Gaithersburg, MD. Occupational Health and Safety Div.

Germanium Detector Optimization of MDA for Efficiency vs. Low Intrinsic Background.

Final rept

D. R. Brown, and L. A. Slaback. 1988, 7p.

Pub. in Proceedings of Health Physics Society Midyea Topical Meeting on Instrumentation (22nd), San Anto nio, TX., December 4-8, 1988, p46-52.

Keywords: "Detectors, "Germanium, Samples, Spectrum analysis, Standards, Performance evaluation, Reprints, "Minimum detectable activity.

The paper will discuss research performed in selecting a high purity germanium detector of between 20 and 45 percent relative efficiency. The primary goal will be to op umize the minimum detectable activity (MDA) of the detector. Several factors will be evaluated that contribute to MDA; such as, relative background nuclides in the detector structural material, relative detector efficiency, and shield design. Results to be presented include efficiencies for various sample geometries, MDA for several detector/sample configurations, and gamma spectrum analysis of detector background counts.

\section{0,544}

PB94-199171 Not available NTIS

National Inst. of Standards and Technology (CSTL) Boulder, CO. Thermophysics Div

Applications of the Vortex Tube in Chemical Analysis.

Final rept

T. J. Bruno. 1992, 13p.

Pub. in Process Control and Quality 3, p 195-207 1992.

Sponsored by Gas Research Inst., Chicago, IL.

Keywords: "Hilsch tubes, Heat transfer, Laboratory equipment, Chemical analysis, Reprints, "Vortex tubes.

The vortex tube is a unique heating and cooling device that has no moving parts, but operates only with a stream of compressed air. Although first described nearly six decades ago, it has only recently been 'discovered' by scientists and engineers outside of the heat transfer fraternity. The vortex tube can provide air streams at temperatures as low as $-40 \mathrm{C}$, and as high as $190 \mathrm{C}$. It is especially useful for heating and cooling the small, irregularly shaped instrument components and devices that are so common in chemical analysis. In this paper, the operational essentials of the vortex tube are presented, and several applications in chemical analysis are discussed.

\section{0,545}

PB94-199197 Not available NTIS

National Inst. of Standards and Technology (CSTL), Boulder, CO. Thermophysics Div.

Simple and Efficient Low-Temperature Sample Cell for Infrared Spectrophotometry.

Final rept.

T. J. Bruno. 1992, 2p.

Pub. in Review of Scientific Instruments $63, n 10$ p4459-4460 Oct 92. Sponsored by Gas Research Inst., Chicago, IL.

Keywords: "Optical equipment, "Infrared spectrophotometers, Hilsch tubes, Solvents, Refrigerants, Design, Reprints, "Low temperature cell, Vortex tubes.

It is often helpful in infrared spectrophotometry to contain a liquid sample in a cell that is maintained at a subambient or nearly cryogenic temperature. Volatile samples and solvents being studied can vaporize and form bubbles in conventional liquid sample cells, especially under the influence of the infrared radiation from the source in a continuous-wave instrument. This will result in the formation of spikes on the spectra. In addition, some analytes are thermally labile and must be protected from warming while the spectrum is recorded. An easily constructed cell cooled with a vortex tube can solve many of these problems, and is in many ways an improvement over commercial devices. In this note, such a cell is described and its application to volatile alternative refrigerants is discussed.

\section{0,546}

PB94-199205 Not available NTIS

National Inst. of Standards and Technology (CSTL), Boulder, CO. Thermophysics Div.

Simple, Inexpensive Apparatus for Sample Concentration.

Final rept.

T. J. Bruno. 1992, 2p.

Pub. in Jnl. of Chemical Education 69, n10 p837-838 Oct 92. Sponsored by Gas Research Inst., Chicago,

Keywords: "Concentrating, "Laboratory equipment, "Design, Reprints, "Sample concentrators, Vortex heating and cooling

In many synthetic and analytical procedures, it is often necessary to concentrate samples by controlled evaporation of the solvent. Several glass concentrators are available (such as the micro or Kuderna Danish type), consisting of a solution vessel with a collection cold finger directly below, and a reflux tube above. In conventional practice, the solution vessel section is electrically heated in an aluminum block, while the cold finger is kept in ambient air at a lower temperature. An alternative to this technique that provides several important advantages is to use a Ranque-Hilsch vortex tube to both heat the solution vessel and cool the cold finger. In this note, the construction and application of such an apparatus is described. 
00,547

PB94-199460 Not available NTIS

National Inst. of Standards and Technology (NML), Gaithersburg, MD. Surface Science Div.

Performance of a Reflectron Energy Compensating Mirror.

Final rept.

P. P. Camus, and A. J. Melmed. 1991, 5p.

Pub. in Surface Science 246, n1-3 p415-419 1991.

Keywords: Surface analysis, Performance, Resolution, Mirrors, Reprints, "Atom probes, Einzel lenses, Reflectrons.

This paper describes the performance characteristics of a first order reflectron energy compensating lens system on a voltage-pulsed atom probe. The performance of the instrument using the electrostatic mirror is compared to that obtained using the same electronics and a linear flight path, and the effect of an einzel lens on the performance is presented.

\section{0,548}

PB94-199726 Not available NTIS

National Inst. of Standards and Technology (NML), Gaithersburg, MD. Inorganic Analytical Research Div. Establishing Quality Measurements for Inorganic Analysis of Biomaterials.

Final rept.

J. R. DeVoe. 1991, $6 p$.

Pub. in American Chemical Society Symposium Series - Biol. Trace Elem. Res., v445 p101-106 1991.

Keywords: "Biocompatible materials, "Chemical analysis, "Inorganic compounds, "Trace elements, Diseases, Quality assurance, Interlaboratory comparisons, Reprints.

While there have been a number of excellent studies showing the effects of trace element concentrations in biomaterials in a number of diseases and other healthrelated processes, the inconsistencies of quantitative analytical data still limit progress. It is suggested tha a well integrated system of laboratories that is dedicated to analytical chemistry quality assurance can provide an interlaboratory structure that will improve the quality of the measurements. Quantitative measurement of the elemental-bound compound appears to be greatly needed, and significant effort needs to be expended in this most difficult area. Progress can be greatly enhanced by including the analytical chemist in the biomedical research team.

00,549

PB94-200516 Not available NTIS

National Inst. of Standards and Technology (CSTL), Gaithersburg, MD. Organic Analytical Research Div.

Carotenoid Reversed-Phase High-Performance Liquid Chromatography Methods: Reference Compendium.

Final rept.

N. E. Craft. 1992, 21p.

Pub. in Methods in Enzymology Chapter 17, v213 p185-205 1992. Sponsored by National Cancer Inst. Bethesda, MD.

Keywords: *Carotenoids, "Chemical analysis, High pressure liquid chromatography, Solvents, Temperature, Test methods, Reprints.

A survey of reversed-phase liquid chromatography (LC) methods for the separation of carotenoids is tabulated. The tables include carotenoid sources, individual carotenoids separated, LC stationary phase, LC mobile phase, flow rate, and column temperature. The influence of various stationary (pore diameter, carbon load, mode of synthesis, end-capping) and mobile phase (modifiers, buffers) parameters on the separation and recovery of carotenoid compounds are discussed and illustrated. Both isocratic and gradient reversed-phase LC methods developed at NIST for the separation of carotenoids are presented.

\section{0,550}

PB94-200599 Not available NTIS

National Inst. of Standards and Technology (NML) Gaithersburg, MD. Gas and Particulate Science Div. Importance of Chemometrics in Biomedical Measurements

Final rept.

L. A. Currie. $1991,27 \mathrm{p}$

Pub. in American Chemical Society Symposium Series 445, p74-100 1991.

Keywords: "Biomedical measurements, Trace elements, Multivariate analysis, Quality control, Mathe- matics, Statistics, Environmental pollutants, Diet, Reprints, "Chemometrics.

Chemometrics as a discipline blends modern mathematical and statistical techniques with chemical knowledge for the design, control, and evaluation of chemical measurements. For complex systems, such as those involving biomedical trace element research the multi-disciplinary efforts toward problem formulation and measurement process design and evaluation can be substantially aided by exploratory chemometric approaches. Following a brief overview of potential chemometrics contributions, primary attention is to be given to exploratory multivariable data analysis techniques, which can capture the essence of a complex data set in a few, visualizable dimensions. Such techniques are appropriate because nuclear-related measurements, quality control samples and data, global dietary intakes, and biological compositions all comprise multiple chemical species, frequently exhibiting correlated behavior. Applications of some of the more powerful techniques, such as principal component factor analysis, are illustrated by multivariable interlaboratory quality control, assessment of pollutant origins, and exploration of daily dietary intake data.

\section{0,551}

PB94-200607 Not available NTIS

National Inst. of Standards and Technology (NML), Gaithersburg, MD. Gas and Particulate Science Div. Metrological Measurement Accuracy: Discussion of 'Measurement Error Models' by Leon Jay Gleser. Final rept.

L. A. Currie. 1991, 9p.

Pub. in Chemom. Intell. Lab. Syst. 10, n1-2 p59-67 1991.

Keywords: "Metrology, Multivariate analysis, Factor analysis, Error analysis, Accuracy, Matrices, Reprints, ${ }^{*}$ Chemometrics.

The paper represents a contribution to the chemist statistician colloquy on multivariate measurement and model error. Specifically, it is a discussion of 'measurement error models' by Leon Jay Gleser (Dept. of Mathematics and Statistics, Univ, of Pittsburgh). Basic topics addressed include the 'errors in $x(-)$ and $y(-)$ ', extended to the multivariable domain, as realized in factor analysis and errors in variables regression. The distinction between structural and functional models for statistical relationships is considered, as well as their asymptotic character. Most importantiy, approaches are suggested for complementing the important advances in mathematical statistics (as reviewed by Gleser) with chemical knowledge and insight. These take three forms: chemical limitations imposed on the several statistical assumptions of the measurement error models (especially those involving the diagonal and off-diagonal elements of the variance covariance matrix); chemically feasible approaches to measurement refinement, such as tracer and unique variable techniques for reducing multi-collinearity; and linear and non-linear model refinement, based on scientific knowledge of the system under investigation.

\section{0,552}

PB94-210168 PC A03/MF A03

National Inst. of Standards and Technology (CSTL), Gaithersburg, MD. Surface and Microanalysis Science Div.

Airborne Asbestos Method: Standard Practice for Recording Transmission Electron Microscopy Data for the Analysis of Asbestos Collected onto Filters. Version 1.0.

E. S. Windsor, S. Turner, and E. B. Steel. Feb 94 , 14p, NISTIR-5358.

Keywords: *Asbestos, "Test methods, *Chemical analysis, Air pollution detection, Quality assurance Water pollution detection, Electron microscopy, Standard, Procedures, US NBS, "TEM(Transmission electron microscopy), Transmission electron microscopy, Verified analysis, National Institute of Standards and Technology.

This is a standard practice that describes the procedure for recording information obtained during a Transmission Electron Microscope (TEM) analyses of asbestos. Included in this practice is a TEM analysis form that serves as a record and work sheet for the analyst. Forms completed according to this practice allow for the verification of asbestos structures determined by two or more analysts. The practice is designed to give a uniform recording format so that analytical data may be compared among laboratories.
00,553
PB94-211620 Not available NTIS

National Inst. of Standards and Technology (CSTL), Gaithersburg, MD. Biotechnology Div.

L-threo-beta-Hydroxyhistidine, an Unprecedented Iron(III) Ion-Binding Amino Acid in a Pyoverdinetype Siderophore from Pseudomonas fluorescens 244.

Final rept.

D. K. Hancock, B. Coxon, S. Y. Wang, J. M.

Bellama V. E. White, and D. J. Reeder. 1993, 3p.

Pub. in Jnl. of Chem. Soc., Chemical Communications 5, p468-470 1993.

Keywords: *Nuclear magnetic resonance, *Mass spectrometry, "Amino acids, "Pseudomonas fluorescens, lons, Binding sites, Reprints, "L-threo-betahydroxyhistidine, Pyoverdine.

Nuclear magnetic resonance spectroscopy and mass spectrometric analysis of a unique pyoverdine-type siderophore isolated from the culture filtrate of Pseudomonas fluorescens 244 reveals a new natura amino acid, L-threo-beta-hydroxyhistidine, that functions as an iron(III) bidentate ligand.

\section{0,554}

PB94-212289 Not available NTIS

National Inst. of Standards and Technology (NML) Gaithersburg, MD. Inorganic Analytical Research Div. Determination of Boron and Lithium in Diverse Biological Matrices Using Neutron Activation - Mass Spectrometry (NA-MS).

Final rept.

G. V. lyengar, W. B. Clarke, and R. G. Downing

$1990,5 p$.

Pub. in Fresenius Jnl. of Analytical Chemistry 338, n4 p562-566 1990.

Keywords: "Neutron activation analysis, "Mass spectrometry, "Boron, "Lithium, "Chemical analysis, Trace elements, Food, Tissues(Biology), Reprints

Essential features of the Neutron Activation-Mass Spectrometry (Na-MS) technique are described. Applicability of this technique for the simultaneous determination of boron and lithium is demonstrated for a diverse group of biomaterials. This is a non-destructive type of analytical technique, and dynamic in nature since its coverage extends to a broad range of concentration levels. At the post-irradiation stage, extraneous contamination by natural lithium or boron is nonexistent, since only the radioactive products are the analytes assayed. Coupling the nuclear activation phenomenon which generates (4) $\mathrm{He}$ and (3) $\mathrm{He}$ (from (10)B and (6) $\mathrm{Li}$, respectively), with the high accuracy potential of mass spectrometry forms the principle of this technique. Under ideal conditions the detection limit is extendable to $\mathrm{pg} / \mathrm{g}$ concentration ranges and therefore, it is extremely well suited to investigate the natural concentration levels of boron and lithium in biomaterials. The potential of this method for the determination of lithium in biomedical trace element research investigations is of special significance since determination of sub-ppb levels of lithium faces serious analytic difficulties by other analytical techniques due to mainly contamination control and in some cases also to detection limit problems.

\section{0,555 Not available NTIS}

National Inst. of Standards and Technology (NML), Gaithersburg, MD. Inorganic Analytical Research Div. High-Sensitivity Determination of lodine Isotopic Ratios by Thermal and Fast Neutron Activation.

Final rept.

R. M. Lindstrom, G. J. Lutz, and B. R. Norman. 1991, $12 p$.

Pub. in Jnl. of Trace Microprobe Technology 9, n1 p21321991.

Keywords: "Neutron activation analysis, "Activation analysis, *lodine 129, *lodine 127, Radioactive contaminants, High sensitivity, Neutron irradiation, Fission products, Isolope ratio, Thermal neutrons, Fast neutrons, Reprints.

In order to study the transformations of radioiodine in the environment, the analytical method used for the determination of iodine-129 concentrations and of (129)I/ (127)! ratios must be capable of measuring concentrations down to the present-day background level, of order 100 million atoms/gram, with acceptable precision and low blank. A neutron activation procedure has been developed to perform reliable concentration 


\section{Analytical Chemistry}

measurements at this level in a few grams of sample. $(129) \mathbf{I} /(127)$ I ratios as low as $6 \times 10$ (sup -10) have been measured. The procedure described uses oxygen combustion both before and after irradiation to separate iodine from other elements. Irradiation is performed with optimized fluxes of both thermal and fast neutron. Bromine-82 and other interferences are removed by a gas-solid reaction with Hydrated Manganese Dioxide. High-resolution gamma-ray assay permits small amounts of induced (130)I to be measured simultaneously with (126)I produced from stable iodine by the $(n, 2 n)$ reaction.

\section{0,556}

\section{PB94-213394 Not available NTIS}

National Inst. of Standards and Technology (CSTL), Gaithersburg, MD. Inorganic Analytical Research Div. Neutron Capture Prompt Gamma-Ray Activation Analysis at the NIST Cold Neutron Research Facility.

Final rept

R. M. Lindstrom, R. Zeisler, D. H. Vincent, E. A.

Mackey, D. L. Anderson, D. D. Clark, R. R.

Greenberg, and C. A. Stone. 1993, 6p.

Pub. in Jni. of Radioanalytical and Nuclear Chemistry 167, n1 p121-126 1993.

Keywords: "Activation analysis, Radiation measuring instruments, Neutron capture gamma rays, Prompt gamma radiation, Chemical analysis, Cold neutrons, NBSR reactor, Radiation shielding, Reprints, CNRF facility, US NIST.

An instrument for neutron capture prompt gamma-ray activation analysis (PGAA) has been constructed as MW National Institute of Standards and Technology Research Reactor. The neutron fluence rate (thermal equivalent) is $1.5 \times 10$ (sup 8$) \mathrm{n} / \mathrm{sq} \mathrm{cm} / \mathrm{s}$ with negligible fast neutrons and gamma-rays. With compact geometry and hydrogen-free construction, the sensitivity is sevenfold better than an existing thermal instrument. Hydrogen background is thirtyfold lower.

\section{0,557 \\ 00,557
PB94-216140 Not available NTIS}

National Inst. of Standards and Technology (CSTL), Gaithersburg, MD. Inorganic Analytical Research Div. Scattering and Absorption Effects in Neutron Beam Activation Analysis Experiments.

Final rept.

E. A. Mackey, and J. R. D. Copley. 1993, 6p

Pub. in Jnl. of Radioanalytical and Nuclear Chemistry 167, n1 p127-1321993.

Keywords: *Neutron activation analysis, "Activation analysis, Monte Carlo method, Prompt gamma radiation, Neutron absorption, Neutron scattering, Sensitivity, Simulation, Corrections, Reprints.

We have investigated the effects of scattering and absorption in neutron beam activation analysis experiments, both by direct measurement and by Monte Carlo simulation. Significant sensitivity enhancements occur for thin disks placed at $45 \mathrm{deg}$ to the beam but very much smaller effects occur for spheres. The agreement between measurement and calculation is generally good.

\section{0,558}

PB94-216157 Not available NTIS

National Inst. of Standards and Technology (NML), Gaithersburg, MD. Inorganic Analytical Research Div. Effects of Target Shape and Neutron Scattering on Element Sensitivities for Neutron-Capture Prompt Gamma-ray Activation Analysis.

Final rept.

E. A. Mackey, G. E. Gordon, R. M. Lindstrom, and D. L. Anderson, 1991, 5p.

Pub. in Analytical Chemistry 63, n3 p288-292 1991.

Keywords: "Activation analysis, Neutron capture gamma rays, Neutron scattering, Sensitivity, Hydrogen, Reprints, Target shape.

A study of the effects of neutron scattering by hydrogen on elemental sensitivities of in-beam neutron capture prompt gamma-ray activation analysis (PGAA) is presented. Elemental sensitivities (counts/s/mg) for $\mathrm{H}, \mathrm{B}$, $\mathrm{Na}, \mathrm{Cl}, \mathrm{K}, \mathrm{Mn}, \mathrm{Br}, \mathrm{Ag}, \mathrm{Cd}, \mathrm{I}, \mathrm{Sm}$, and $\mathrm{Gd}$, increase linearly with $H$ density $(\mathrm{g} / \mathrm{mL})$. Nine of the twelve elements studied undergo $1.69+$ or $-0.18 \%$ enhancement per percent increase in $\mathrm{H}$ density. The enhancement of the sensitivity for $H$ itself varies with matrix composition. In a scattering matrix, elemental sensitivity is also a function of target shape. For several series of disk-shaped hydrogenous targets, elemental sensitivities increase with decreasing target thickness until, at some limiting thickness the probability for multiple interactions begins to decrease and this trend is reversed. Consistent with theory, sensitivities meas ured for spherical hydrogenous targets show no enhancement.

\section{0,559}

\section{PB94-216348 Not available NTIS}

National Inst. of Standards and Technology (NML) Gaithersburg, MD. Gas and Particulate Science Div. Study of Diffusion Zones with Electron Microprobe Compositional Mapping.

Final rept.

R. B. Marinenko, D. S. Bright, C. A. Handwerker, and J. J. Mecholsky. 1990, 3p

Pub. in Microbeam Analysis 25, p190-192 1990.

Keywords: "Electron microprobe analysis, X-ray analysis, Reprints, “Diffusion zones, *Compositional mapping, Metal-metal interfaces, Glass-metal interfaces, $\mathrm{X}$-ray mapping.

Electron microprobe $x$-ray compositional mapping has been used to study diffusion zones between metalmetal and glass-metal interfaces. Image analysis techniques including gray-level enhancement, product and quotient images, and concentration-histogram images have proven to be extremely useful in visualizing the extent of diffusion. The qualitative and quantitative in formation provided by these digital acquisition and display techniques far exceeds what conventional line scan and $x$-ray dot maps could provide.

\section{0,560}

PB94-219250 (Order as PB94-219219, PC A06/

MF A02

National Inst. of Standards and Technology Gaithersburg, MD.

Measurement and Uncertainty of a Calibration Standard for the Scanning Electron Microscope.

J. Fu, M. C. Croarkin, and T. V. Vorburger. 1994, 9p. Included in Jnl. of Research of the National Institute of Standards and Technology, v99 n2 p191-199 Mar/ Apr 94

Keywords: "Scanning electron microscopy, *Catibration standards, Precision, Data covariances, Interferometers, Measuring instruments, Random error, Standard error, Uncertainty, SRM(Standard ref erence material), SRM-484, National Institute of Standards and Technology.

Standard Reference Material 484 is an artifact for calibrating the magnification scale of a Scanning Electron Microscope (SEM) within the range of $1000 \times$ to 20000 $x$ Seven issues, SRM-484, and SRM-484a to SRM484 i have been certified between 1977 and 1992. This publication documents the instrumentation, measurement procedures and determination of uncertainty fo SRM-484 and illustrates with data from issues 484 e and 484

\section{0,561}

PB94-219383 (Order as PB94-219326, PC. A05/ MF A02)

Mississippi State Univ., Mississippi State.

Theoretical Analysis of the Coherence-Induced Spectral Shift Experiments of Kandpal, Vaishya and Joshi.

J. T. Foley, and M. Wang. 1994, 14p.

Included in Jnl. of Research of the National Institute of Standards and Technology, v99 n3 p267-280 May/ Jun 94

Keywords: "Spectroscopy, "Radiometry, "Spectral shift, Optus, Coherence, Wolf shifts

The optical system used by Kandpal, Vaishya, and Joshi in their experiments on coherence-induced spectral shifts is analyzed theoretically. An approximate form for the cross-spectral density in the secondary source plane is obtained, and it is shown that, contran to the assertions of Kandpal, Vaishya, and Joshi, the corresponding complex degree of spectral coherence in this plane is wavelength dependent. After making some assumptions about the behavior of the inter erence filter used in the system, an approximate form or the spectrum of the light on-axis in the observation plane is obtained. It is shown that the peak wave lengths of this spectrum do not agree with those reported by Kandpal, Vaishya, and Joshi. Possible reasons for this disagreement are discussed

\section{0,562
PB95-107165 Not available NTIS}

National Inst. of Standards and Technology (NML), Gaithersburg, MD. Gas and Particulate Science Div. Electron Probe X-Ray Microanalysis.

Final rept.

D. E. Newbury. 1992, 17 p.

Pub. in Encyclopedia of Materials Characierization: Surfaces, Interfaces, Thin Films, Chapter 3.5, p1751911992.

Keywords: "Electron probes, "Microanalysis, "X-ray anlaysis, Electron scanning, Electron microscopy, Spatial resolution, Digital systems, Quantitative analysis, Qualitative analysis, Reprints, Elemental analysis, Scanning electron microscopy, Compositional maps.

Electron probe $x$-ray microanalysis (EPMA) is an elemental analysis technique based upon bombarding a specimen with a focussed beam of energetic electrons (beam energy from $5-30 \mathrm{keV}$ ) to induce emission of characteristic $x$-rays (energy range from $0.1-15 \mathrm{keV}$ ). The energy (wavelength) of the $x$-rays identifies the elements present in the specimen (qualitative analysis), and the $x$-ray intensity can be related to the quantity present (quantitative analysis). Elemental coverage extends from beryllium to the actinides. Detection limits are approximately 100 parts per million for elements with atomic numbers greater than 10 . Spatial distributions of elemental constituents can be visualized qualitatively by $x$-ray area scans (dot maps) and quantitatively by digital compositional maps.

\section{0,563}

PB95-107173 Not available NTIS

National Inst. of Standards and Technology (NML), Gaithersburg, MD. Gas and Particulate Science Div. Microanalysis to Nanoanalysis: Measuring Composition at High Spatial Resolution.

Final rept.

D. E. Newbury. $1990,28 p$

Pub. in Nanotechnology $1, \mathrm{n} 2$ p103-130 Oct 90.

Keywords: *Spectrochemistry analysis, *Microanalysis, "Measuring instruments, "Spatial resolution, Mass spectrometers, Electron probes, X-ray analysis, Auger electron spectroscopy, Electron scanning. Electron microscopy, High resolution, Reprints, * Nanoanalysis, Laser microbe mass spectrometry, Secondary ion mass spectrometry, Field ion microscopy, Composition.

Spatially-resolved analysis of elemental, isotopic, and molecular constituents is possible with microanalysis and nanoanalysis techniques. At the micrometer scale of spatial resolution, electron probe $x$-ray microanalysis provides elemental coverage from $\mathrm{Be}$ to the actinides with detection limits in the range from 100-1000 parts per million (ppm). Secondary ion mass spectrometry and laser microprobe mass spectrometry detect all elements and isotopes with sensitivity from parts per billion to parts per million, and molecular signals can also be detected. Molecular microanalysis is also performed with photon detection techniques, including infrared, fluorescence, and Raman spectroscopies. Analytical electron microscopy and time-of-flight secondary ion mass spectrometry extend spatial resolution to the $100-n m$ spatial level.

\section{0,564}

PB95-107181 Not available NTIS

National Inst. of Standards and Technology (CSTL), Gaithersburg, MD. Surface and Microanalysis Science Div

Design of a Protocol for an Electron Probe Microanalyzer k-Value Round Robin.

Final rept.

D. E. Newbury, and R. B. Marinenko. 1989, 3p.

Pub. in Microbeam Analysis 24, p257-259 1989.

Keywords: "X-ray analysis, "X-ray spectroscopy, Microanalysis, Matrices, Correction, Quantitative analysis, Reprints, "Electron probe microanalysis, "k-value.

The basic measurement in quantitative electron probe microanalysis is the determination of the 'k-value', which is the ratio of the characteristic $x$-ray intensity measured for an unknown to that measured for a standard. Models for quantitative matrix corrections are tested on suites of k-value measurememts which are not standardized. A $\mathrm{k}$-value round robin is planned which will attempt to establish a new base of measurements with sufficient accuracy and precision to permit adequate testing of the models. A proposal for the measurement protocol for the k-value round robin is described. This protocol is critical if adequate measurements are to be obtained. 
00,565

PB95-107199 Not available NTIS

National Inst. of Standards and Technology (NML) Gaithersburg, MD. Gas and Particulate Science Div. Compositional Mapping of the Microstructure of Materials

Final rept.

D. E. Newbury, R. B. Marinenko, R. L. Myklebust, and D. S. Bright. 1991, 19p.

Pub. in Images of Materials, Chapter 10, p290-308 1991.

Keywords: "Microstructure, "Mass spectrometers, *Image processing, Reprints, Microanalysis, Electron probes, Mapping, Chemical analysis, Digital systems, Compositional mapping, Secondary ion mass spectrometry, Microbeam analysis.

Recent developments in electron probe microanalysis and secondary ion mass spectrometry techniques enable the analyst to prepare compositional maps of the microstructure of materials. The technique of compositional mapping involves performing a complete quantitative analysis at each beam location in the imaged field of view on the specimen. The images directly convey the sense of the spatial distribution and compositional interrelationships of the elemental constituents in the microstructure. The images are supported at every picture element by numerical values of the concentrations, as well as data on the statistical precision of the measurement. The combination of images and conventional numerical concentration data provides a powerful tool for microstructural analysis.

00,566

PB95-107249 Not available NTIS

National Inst. of Standards and Technology (NML),

Gaithersburg, MD. Organic Analytical Research Div.

Separation and Identification of Organic Gunshot and Explosive Constituents by Micellar Electrokinetic Capillary Electrophoresis.

Final rept.

D. M. Northrop, D. E. Martire, and W. A. MacCrehan $1991,5 p$.

Pub. in Analytical Chemistry 63, n10 p1038-1042 1991.

Keywords: "Electrophoresis, "Forensic science, "Chemical explosives, Capillaries, Micellar systems, Sodium sulfates, Parameters, Chromatography, Reprints, "MECC(Micellar electrokinetic capillary chromatography), "Micellar electrokinetic capillary chromatography, Gunshot residue, Sodium dodecylsulfate, Capillary zone electrophoresis.

Micellar electrokinetic capillary chromatography (MECC) was used to effect a rapid and efficient separation of some common constituents of gunshot and explosive residues. A separation of 26 constituents of interest was accomplished in under 10 minutes with efficiencies typically between 200,000 and 400,000 . The effects of various experimental parameters were studied. Sodium dodecylsulfate (SDS) concentration, $\mathrm{pH}$, addition of a tetraalkylammonium salt, capillary diameter, and injection times were all examined in order to optimize the system. It was found that capillary diameter and injection time played an important role in improving detection limits and efficiencies. This method was applied to extracts from swabbings of a .38 caliber and a .45 caliber shell casing demonstrating the presence of gunshot residues.

00,567

PB95-107272 Not available NTIS

National Inst. of Standards and Technology (TS), Gaithersburg, MD. Office of Measurement Services.

Certification, Development and Use of Standard Reference Materials.

Final rept.

S. D. Rasberry, and T. E. Gills. 1991, $6 p$.

Pub. in Spectrochimica Acta B 46, n12 p1577-1582 1991.

Keywords: "Chemical analysis, *Quality assurance, "Standards, Accuracy, Certification, Concentration(Composition). Procedures, Environmental tests, Industries, Clinical medicine, Nutrition, Construction materials, Reprints, "Standard reference materials.

Analytical measurements are increasingly important to industrial quality assurance (QA) and to critical decision making in such fields as clinical, nutritional, and environmental chemistry. Several factors are important to attaining analyses that are reliably accurate. Some of these include well-designed methods, trained staff, adequate instrumentation, reliable reference materials, laboratory QA procedures, and periodic proficiency testing. The paper will focus on the role of reference materials in accurate chemical analysis. Fundamental attributes of reference materials will be described together with details on how limits of uncertainty are es tablished for certified values. Examples will be drawn from materials recently certified at NIST. Besides in dustrial examples, such as new alloys and construction materials such as cement and glass, recent clinical. nutritional, and environmental certifications will be cited. Matrices for these types of materials are very wide-ranging and includes human serum (for vitamins in serum, in whole egg powder, and in diet material), lead in reference fuel, organics in cod liver oil, and several others.

\section{0,568}

PB95-108536 Not available NTIS

National Inst. of Standards and Technology (CSTL), Gaithersburg, MD. Surface and Microanalysis Science Div.

Formation of Technical Committee 201 on Surface Chemical Analysis by the International Organization for Standardization.

Final rept.

C. J. Powell, and R. Shimizu. 1993, 4p.

Pub. in Surface and Interface Analysis 20, p322-325 1993.

Keywords: "Chemical analysis, "Surface analysis, Standards, Reprints, International Organization for Standardization, Technical Committee 201

The International Organization of Standardization (ISO) recently created a new Technical Committe (ISO/TC 201) on Surface Chemical Analysis in response to a proposal from Japan. As of February 1993 en national standards bodies had indicated willing ness to become participating members of ISO/TC 201 (Austria, China, Germany, Italy, Japan, Russia, Sweden, Turkey, the United Kingdom and the United States of America) and fifteen national standards bodes had indicated willingness to become observe members (Australia, Belgium, Egypt, Finland, France, India, Ireland, Korea, Norway, Philippines, Poland, Romania, Singapore, South Africa and Switzerland!. The following subcommittees are planned: Terminology General Procedures; Data Management and Treat ment; Depth Profiling; Auger Electron Spectroscopy Secondary Ion Mass Spectrometry and X-ray Photoelectron Spectroscopy. ISO/TC 201 will develop international standards and will consider standards and documents prepared by other groups as potential international standards. Information is given in this article on the purpose, structure and planned work of ISO TC 201.

\section{0,569}

PB95-108742 Not available NTIS

National Inst. of Standards and Technology (NML) Gaithersburg, MD. Gas and Particulate Science Div. Addition of $M$ and L-Series Lines to NIST Algorithm for Calculation of X-Ray Tube Output Spectral Distributions.

Final rept.

P. A. Pella, L. Feng, and J. A. Small. 1991, 2p.

Pub. in X-ray Spectrometry 20, n3 p109-110 1991.

Keywords: "X-ray fluorescence analysis, " $X$-ray tubes, Spectral energy distribution, Computation, Tungsten, Gold, Reprints, M lines, L lines, NIST algorithm.

Characteristic $M$ x-ray tube target lines and some minor $L$-series lines have been added to a previously developed National Institute of Standards and Technology (NIST) algorithm for calculation of the output spectral distributions from x-ray tubes. This algorithm is used in first principle calculations for correction of interelement effects in quantitative $x$-ray fluorescence analysis. The $M$ lines from $x$-ray tube targets such as $W$ and $A u$ can be important for excitation of low atomic number elements in some applications. Also, minor Lseries lines have been added to give a more complete description of the L-series in the calculated output spectral distribution.

\section{0,570}

PB95-126108 Not available NTIS

National Inst. of Standards and Technology (NML) Gaithersburg, MD. Gas and Particulate Science Div.
Use of Kinetic Energy Distributions to Determine the Relative Contributions of Gas Phase and Surface Fragmentation in KeV Ion Sputtering of a Quaternary Ammonium Salt.

Final rept.

G. Gillen. 1991, 10p.

Pub. in International Jnl. of Mass Spectrometry and Ion Processes 105, n3 p215-224 1991.

Keywords: "Ammonium compounds, "Mass spectroscopy, "Secondary ion mass spectroscopy, "Quaternary ammonium salts, Organic nitrogen compounds, Kinetic energy, KeV range, Fragmentation, Sputtering, Reprints.

Kinetic energy distributions have been used to evaluate the extent to which gas phase decompositions contribute to the production of fragment ions in organic secondary ion mass spectra. Molecular kinetic energy distributions demonstrate that many fragment ions have a prominent gas phase component which may account for as much as $50 \%$ of the observed fragment ion signal obtained in a double focussing mass spectrometer.

00.571

PB95-126124 Not available NTIS

National inst. of Standards and Technology (NML), Gaithersburg, MD. Gas and Particulate Science Div. Molecular lon Imaging and Dynamic Secondary Ion Mass Spectrometry of Organic Compounds.

Final rept.

G. Gillen, D. S. Simons, and P. Williams. 1990, 9p Pub. in Analytical Chemistry 62, n19 p2122-2130 1990.

Keywords: "Secondary ion mass spectroscopy, "Mass spectroscopy, "Imaging techniques, Quaternary ammonium salts, Aromatic hydrocarbons, Amino acids, Molecular ions, Radiation prints, "Ion imaging.

An ion microscope equipped with a resistive anode encoder imaging system has been used to acquire molecular secondary ion images, with lateral resolutions on the order of 1 micrometer, from several quaternary ammonium salts, an amino acid, and a polynuclear aromatic hydrocarbon, which were deposited onto copper transmission electron microscope grids. All images were generated using the secondary ion signal of the parent molecular species. The primary ion dose dependence of parent and fragment molecular ion signal decays indicate that, for many bulk organic compounds, bombardment-induced fragmentation of parent molecules reaches a saturation at primary ion doses of 1-8 $\times 10$ (sup 14) ions/sq $\mathrm{cm}$. Subsequent ion impacts do not significantly contribute to further accumulation of damage in the sample, resulting in desorption of intact parent molecular ions even after prolonged ion bombardment. This saturation process allows molecular images to be obtained at high primary ion doses and allows depth profiles to be obtained from simple molecular/metal test structures.

00.572

PB95-140141 Not available NTIS

National Inst. of Standards and Technology (NEL), Gaithersburg, MD. Statistical Engineering Div.

Combining Data from Independent Chemical Analysis Methods.

Final rept.

S. Schiller, and K. R. Eberhardt. 1991, 7p Pub. in Spectrochimica Acta B 46, n12 p1607-1613 1991

Keywords: "Chemical analysis, Statistical analysis Data analysis, Inhomogeneity, Certification, Uncertainty, Reprints, Standard reference materials, Systematic errors, Weighted mean

Often, data from several different chemical analysis methods must be combined to form an overall mean value and uncertainty. However, the data from these different methods frequently do not agree when a statistical comparison is done, so taking a simple averag of all of the individual observations is inappropriate. Computation of a weighted mean and corresponding uncertainty that take into consideration the random measurement error within each method as well as the disagreement between methods will be described. Ex amples of this approach, applied to the certification of NIST's Standard Reference Materials, will be given.

00.573

P1395-140604 Not available NTIS

National Inst. of Standards and Technology (NML). Gaithersburg, MD. Organic Analytical Research Div. 
Device for Subambient Temperature Control in Liquid Chromatography.

Final rept.

L. C. Sander, and N. E. Craft. 1990, 3p.

Pub. in Analytical Chemistry 62, n 14 p1545-1547 1990.

Keywords: "Liquid column chromatography, "Temperature control, "Cooling systems, Heat exchangers, Selectivity, Design, Performance evaluation, Reprints, "Subambient temperature control.

The construction of a device for regulating column temperature in liquid chromatography (LC) is described. The device is based on the thermoelectric cooling principle, and utilizes four thermoelectric heat exchangers placed in contact with an aluminum block. This block is machined to accept commercial LC columns. In the cooling mode, temperatures in the range .25 to $17 \mathrm{C}$ are possible using 'tap water' (17 C) at a flow rate of $1.25 \mathrm{~L} / \mathrm{min}$. Although the main purpose of this device is cool LC columns, elevated temperatures are possible (maximum temperature, $99 \mathrm{C}$ ) by reversing the polarity of the power supply output. The column cooling device provides an economical, compact and portable means of controlling column temperature. The design allows for maximum flexibility in use and permits the device to be moved between instruments with little effort, as the need arises.

00,574

PB95-140976 Not available NTIS

National Inst. of Standards and Technology (NML),

Gaithersburg, MD. Organic Analytical Research Div.

Comparison of the Liquid Chromatographic Behavior of Selected Steroid Isomers Using Different Reversed-Phase Materials and Mobile Phase Compositions.

Final rept.

M. Olsson L Sander, and S. Wise 1991, 11p.

Pub. in Jnt. of Chromatography 537, n1-2 p73-83 1991.

Keywords: "Liquid column chromatography,
"Chromatographic analysis, "Isomers, Comparison, "Chromatographic analysis, "Isomers, Comparison, crystals, Phase change materials, Polymers, Aromatic polycyclic hydrocarbons, Separation, Reprints, "Mobile phase compositions, "Selection rules, "Retention.

Reversed-phase liquid chromatographic selectivity for steroid isomers was found to change as a function of both temperature and mobile-phase composition. The influence of the stationary phase was studied by investigating retention and selectivity on a number of different monomeric and polymeric $\mathrm{C} 18$ stationary phase materials, as well as beta-cyclodextrin based phases. Three steroid isomers, i.e., equilin, 17 alpha- and 17 beta-estradiol, were chosen as probe solutes to illustrate the dramatic effect of changes in mobile-phase composition. Selectivity of androstane standards on a polymeric $\mathrm{C} 18$ phase was compared to selectivity on liquid crystalline phase in supercritical fluid chromatography (SFC). Although liquid crystalline and polymeric $\mathrm{C} 18$ phases have similar shape-selectivity for certain classes of solutes, such as polycyclic aromatic hydrocarbons (PAHs), different trends were observed for androstane isomers.

\section{0,575}

PB95-150199 Not available NTIS

National Inst. of Standards and Technology (CSTL), Gaithersburg, MD. Inorganic Analytical Research Div. Automated, High-Precision Coulometric Titrimetry. Part 1. Engineering and Implementation.

Final rept

K. W. Pratt. 1994, 10p

See also Part 2, PB95-150207.

Pub. in Analytica Chimica Acta 289, p125-134 1994.

Keywords: "Coulometers, "Chemical analysis, "Primary standards, "Automatic control, Standard deviation, Statistical analysis, Error analysis, Electrochemistry, Benzoic acids, Chromates, Reprints.

Automated constant-current coulometry, based on Faraday's Laws, achieves uncertainties (relative standard deviation) of less than 1 part in 20,000 without chemical standardization. It is applicable to acidbase, redox, and precipitation titrations of high-purity compounds and solutions. Automation of the technique permits unsupervised operation and reduces operatordependent errors. Initial setup and sample introduction are the sole manual steps. Each assay consists of a main titration at high, constant current, bracketed by the initial and final endpoint routines, each at a lower current. The coulometric assay is analogous to a conventional titration in which two different concentrations of the titrant are used to attain optimum accuracy. The initial endpoint determination corresponds to the blank determination in a classical titration. Each titration in cludes a statistical analysis of the random and systematic uncertainties associated with the analysis. Individual steps in the procedure are performed by a hierarchical series of subroutines to reduce program com plexity. Results are presented for $\mathrm{K} 2 \mathrm{Cr} 2 \mathrm{O} 7$, benzoic acid, and solutions of strong acids.

\section{0,576}

PB95-150207 Not available NTIS

National Inst. of Standards and Technology (CSTL) Gaithersburg, MD. Inorganic Analytical Research Div. Automated, High Precision Coulometric Titrimetry. Part 2. Strong and Weak Acids and Bases.

Final rept

K. W. Pratt. 1994, 8p

See also Part 1, PB95-150199.

Pub. in Analytical Chimica Acta 289, p135-142 1994

Keywords: "Coulometers, "Chemical analysis, "Benzoic acids, "Sodium carbonates, "Hydrochloric acid, "Automatic control, Standard deviation, Precision, Titration, Reprints.

Automated constant-current coulometric acidimetry based on Faraday's Laws, is uncertain to less than part in 20,000 (relative standard deviation) and requires no chemical standardization. It is applicable to strong and weak acids and bases, with bases back titrated after addition of excess strong acid. Initial setup and sample introduction are the sole manual steps. Assays of $\mathrm{HCl}$, benzoic acid, $\mathrm{Na} 2 \mathrm{CO} 3$, and tris(hydroxymethyl)aminomethane are presented. In the endpoint determination procedure, a generalized titration equation yields the theoretical charge remaining to the endpoint before each charge addition. The ratio of the experimental to the theoretical charge for the preceding aliquot corrects for experimental deviations.

\section{0,577}

PB95-150231 Not available NTIS

National Inst. of Standards and Technology (CSTL) Gaithersburg, MD. Inorganic Analytical Research Div. Integrating Automated Systems with Modular Architecture.

Final rept.

M. L. Salit, F. R. Guenther, G. W. Kramer, and J. M Griesmeyer. 1994, 7p

Pub. in Analytical Chemistry 66, n6 p361 A-367 A, 15 Mar 94.

Keywords: "Chernical analysis, "Modules, "Automatic control, Chemical laboratories, Interfaces, Design, Re prints, "Chemistry workcell, "Laboratory automation, System integration.

An architecture has been developed to facilitate the creation of automated chemical analysis systems. This architecture is based upon the functionality of the traditional analytical chernistry organization, with automated entities specified to anthropomorphically fill the roles of the client for the chemical information, the expert analyst, the laboratory manager and the bench chernist. Standard interfaces between these entities are in the process of being designed. These interfaces when coupled with standard entity behaviors, will allow or a modular 'plug-and-play' systems integration environment. This paper describes the architecture as it is currently understood and outlines the plan for its further evolution.

\section{0,578}

PB95-150249 Not available NTIS

National Inst. of Standards and Technology (CSTL), Gaithersburg, MD. Organic Analytical Research Div

Development of Engineered Stationary Phases for the Separation of Carotenoid Isomers.

Final rept

L. Sander, K. Sharpless, N. Craft, and S. Wise.

1994, 8p.

Pub. in Analytical Chemistry 66, n10 p1667-1674, 15 May 94.

Keywords: "Carotene, "Isomerization, "Separation processes, "Distillation equipment, Ligands, Substrates, Carotenoids, Standards, Phase separation(Materials), Retaining, Aromatic polycyclic hydrocarbons, Nutrients, Reprints.

A variety of bonded phase parameters (endcapping phase chemistry, ligand length, and substrate param- eters) were studied for their effect on column retention and selectivity toward carotenoids. Decisions were made on how each of these variables should be optimized based on the separation of carotenoid and polycyclic aromatic hydrocarbon test probes. A column was designed with the following properties: high absolute retention, enhanced shape recognition of structured solutes, and moderate silanol activity. The effectiveness of this 'carotenoid phase' was demonstrated for the separation of a mixture of structuraliy similar carotenoid standards, an extract of a food matrix Stand. ard Reference Material, and a beta-carotene dietary supplement under consideration as an agent for cancer intervention/prevention.

\section{0,579}

PB95-150256 Not available NTIS

National Inst. of Standards and Technology (CSTL), Gaithersburg, MD. Organic Analytical Research Div.

Shape Selectivity Assessment of Stationary Phases in Gas Chromatography.

Final rept.

L. C. Sander, M. Schneider, S. A. Wise, and C. Woolley. 1994, 11p.

Pub. in Jnl. of Microcol. Sep. 6, p115-125 1994.

Keywords: "Gas chromatography, "Separation processes, "Distillation equipment, Solutes, Aromatic polycyclic hydrocarbons, Liquid crystals, Phase stability(Materials), Reprints, "Shape selectivity, Methyi polysiloxane.

Column selectivity is examined for a series of smectic liquid crystalline columns and is compared with methyl and $\mathrm{C} 18$ polysiloxane columns for the separation of polycyclic aromatic hydrocarbon (PAH) isomers. A set of extended and condensed solute probes is described that provides a sensitive indication of variations in column shape selectivity. Examples of shape selectivity differences are presented for smectic liquid crystalline columns and $5 \%$ phenyl polysiloxane columns using various PAH isomer sets. Variations in selectivity have been observed among different smectic liquid crystalline columns, and this problem appears more significant than for methyl polysiloxane columns. The selectivity ratio for tetraphenylmethane and p-terphenyl provides a sensitive indication of column shape selectivity, with a change in elution order occurring between ordered (smectic liquid crystalline) columns and non-ordered (methyl polysiloxane) columns. Shape selectivity differences indicated by this test mixture are apparent for more complex PAH isomer mixtures. Despite stationary phase selectivity variability. smectic liquid crystalline columns offer considerable potential for solving difficult separation problems involving structured solutes.

00,580

PB95-150603 Not available NTIS

National Inst of Standards and Technology (EEEL), Gaithersburg, MD. Semiconductor Electronics Div.

Tin Oxide Gas Sensor Fabricated Using CMOS Micro-Hotplates and In-situ Processing

Final rept.

J. S. Suehle, R. E. Cavicchi, M. Gaitan, and S. Semancik 1993, 3p.

Pub in Institute of Electrical and Electronics Engineers Electron Device Letters 14, n3 p118-120 Mar 93.

Keywords: "Gas detectors, "Tin oxides, Monolithic structures(Electronics). Micromachining, Fabrication, Hydrogen, Oxygen, Substrates, Silicon, Arrays, Films, CMOS, Reprints, Microhotplates.

We report the first monolithic tin oxide ( $\mathrm{SnO} 2$ ) gas sensor realized by commercial CMOS foundry fabrication (MO-SIS) and post-fabrication processing techniques. The device is composed of a sensing film that is sputter-deposited on a silicon micromachined hotplate. The fabrication technique requires no masking and utilizes in-situ process control and monitoring of film resistivity during film growth. Gas sensor responses of pure $\mathrm{SnO} 2$ films to $\mathrm{H} 2$ and $\mathrm{O} 2$ with an operating temperature of $350 \mathrm{C}$ are reported. The fabrication methodology allows integration of an array of gas sensors of various films with separate temperature control for element in the array, and circuits for a low-cost CMOS-based gas sensor system.

00,581

PB95-150835 Not available NTIS

National Inst. of Standards and Technology (NML), Gaithersburg, MD. Radiometric Physics Div 
Standards for Corrected Fluorescence Spectra. Final rept.

A. Thompson, and K. Eckerle. 1989, 6p

Pub. in Proceedings of Society of Photo-Optical Instrumentation Engineers: Fluoresc. Detect. 3, v1054 p20251992

Keywords: *Spectral emission, "Standards, "Fluorescence spectroscopy, Interlaboratory comparisons, Near infrared radiation, Visible radiation, Phosphors, Mixtures, Fluorimeters, Fluorometers, Reprints, *Fluorescence standards, *Standard reference materials, Polytetrafluorethylene, Spectrofluorimeters, Spectrofluorometers.

A set of four fluorescent standards has been produced and calibrated at the National Institute of Standards and Technology (NIST) for corrected relative spectral emission over the wavelength range from 400 to 740 $\mathrm{nm}$. This new Standard Reference Material (SRM) 1931 has been produced in the form of sintered mix tures of inorganic phosphors and polytetrafluorethylene (PTEE) powder for spectral response calibration of spectrofluorimeters used in biology, medicine and studies of molecular structure. They provide a means for correcting spectrofluorimeter errors due to wavelength dependencies of optics, monochromators, and detectors, thus permitting meaningful intercomparison of results obtained in different laboratories.

\section{0,582}

PB95-150876 Not available NTIS

National Inst. of Standards and Technology (CSTL), Gaithersburg, MD. Thermophysics Div.

Characteristics of Partial Pressure Analyzers.

Final rept.

C. R. Tilford. 1992, 11p.

Pub. in Proceedings of Society of Photo-Optical Instrumentation Engineers: Damage to Space Optics and Properties and Characteristics of Optical Glass, San Diego, CA., July 20, 22-23, 1992, v1761 p119-129.

Keywords: "Gas analysis, "Partial pressure, "Mass spectrometers, Calibration, Sensitivity, Vacuum apparatus, Measuring instruments, Pressure measurement, Reprints, "Partial pressure analyzers, Residual gas analyzers.

Partial pressure or residual gas analyzers are often in valuable for materials and contamination studies. However, some instrument design features and combination of operating parameters can cause performance features quite different from what many users expect These unexpected performance characteristics can include pressure-dependent sensitivities, the sensitivity for one gas depending on the pressures of other gases, and time-dependent sensitivities after operation with gases such as oxygen or water. These characteristics can greatly complicate instrument calibration and can cause errors as large as two orders of magnitude. This paper discusses measured performance characteristics for different partial pressure analyzers that itlustrate a wide range of performances from the different instruments, as well as several features that seem to be common to most instruments.

PQ,583

National Inst. of Standards and Technology (CSTL) Gaithersburg, MD. Organic Analytical Research Div. High-Performance Liquid Chromatography of Phytoplankton Pigments Using a Polymeric Reversed-Phase $\mathrm{C} 18$ Column.

Final rept.

L. Van Heukelem, A. J. Lewitus, T. M. Kana, and N. E. Craft. 1992, 6p

Pub. in Jnl. of Phycology 28, p867-872 1992.

Keywords: "Phytoplankton, "Pigments, "Purification, High pressure liquid chromatography, Molecular structure, Chlorophyll, Carotenoids, Solvents, Reprints, Zeaxanthin Neoxanthin, 19'hexanoyloxyfucoxanthin, HPLC columns.

A high-performance liquid chromatographic (HPLC) method is described that allows improved resolution of several chemotaxonomically significant phytoplankton pigments. The protocol, which employs two pumps and a modified Mantoura and Llewellyn (1983) solvent system, can be easily adapted for many HPLC systems currently in use. The most unique aspect of the method is the use of a polymeric $\mathrm{C} 18$ reversed-phase HPLC column (VYDAC 201TP). In comparison to the monomeric $\mathrm{C} 18$ columns typically used in the characterization of phytoplankton pigments, polymeric $\mathrm{C} 18 \mathrm{col}$ - umns offer superior selectivity for structurally similar compounds. The protocol was evaluated for the ability to resolve most of the phytoplankton pigments of diagnostic importance using algal cultures from nine classes. Pigment pairs that were resolved by the method include (1) lutein and zeaxanthin, (2) neoxanthin and 19'-hexanoyloxyfucoxanthin, and (3) alpha-carotene and beta-carotene, and partial resolution of chlorophyll $\mathrm{C} 1$ and chlorophyll $\mathrm{C} 2$.

\section{0,584}

PB95-151197 Not available NTIS

National Inst. of Standards and Technology (CSTL), Gaithersburg, MD. Surface and Microanalysis Science

Factorial Design Techniques Applied to Optimization of AMS Graphite Target Preparation.

Final rept.

R. M. Verkouteren, and G. A. Klouda. 1992, 9p Pub. in Radiocarbon 34, n3 p335-343 1992.

Keywords: *Mass spectroscopy, *Carbon 14, "Particle accelerator targets, "Factor analysis, Graphite, Error analysis, Methane, Experimental design, Reduction(Chemistry), Monte Carlo method, Carbon dioxide, Reprints, “Accelerator mass spectrometry.

Many factors influence the preparation and quality of graphite targets for (14)C accelerator mass spectrometry (AMS). We identified four factors (sample size, H2 pressure, catalyst temperature and pretreatment time) as potentially critical, and investigated their effects on two particular characteristics: the integrated rates of $\mathrm{CO} 2$ reduction (to graphite) and methane production. We used a 2-level fractional factorial experimental design and determined chemical reduction yield rates through manometry and partial pressure monitoring of residual gases by mass spectrometry. Chemical reduction yield rates ranged from $0.2 \%$ to $6.2 \%$ per hour. With respect to their influence on percent yield rate, the factors we studied were ordered as: sample size $>$ level of hydrogen > pretreatment of the catalyst. The temperature of the catalyst, and the sample size $x$ hydrogen (2-factor) interaction, were only marginally influential. We estimated uncertainty in the order of influence and magnitudes of the effects by the Monte Carlo method of error propagation. We observed significant methane production in only one experiment, which suggests that methane originates from indigenous carbon in untreated iron catalyst only in the presence of hydrogen and only at thermodynamically favorable temperatures. This exploratory investigation indicates that factorial design techniques are a useful means to investigate multivariate effects on the preparation and quality of AMS graphite targets.

\section{0,585}

PB95-151650 Not available NTIS

National Inst. of Standards and Technology (CSTL), Gaithersburg, MD. Organic Analytical Research Div.

Determination of Polycyclic Aromatic Mydrocarbons by Liquid Chromatography.

Final rept.

S. A. Wise, L. C. Sander, and W. E. May. 1993, 21p. Pub. in Jnl. of Chromatography 642, p329-349 1993.

Keywords: "Liquid column chromatography, Polycyclic aromatic hydrocarbons, "Quantitative spectroscopy Chromatographic analysis, Pollution sampling, Measurement, Reference standards, Reprints, "Environmental samples, Standard reference materials.

Reversed-phase liquid chromatography (LC) using fluorescence detection is a powerful analytical technique for the measurement of polycyclic aromatic hydrocarbons (PAHs) in environmental samples. The NIST experience in the use of $L C$ for the determination of PAHs in environmental samples is summarized in the paper including: selection of the appropriate column, approaches to analyzing complex PAH mixtures, and the accurate quantitation of PAHs in environmental samples.

\section{0,586}

PB95-151668 Not available NTIS

National Inst. of Standards and Technology (CSTL). Gaithersburg, MD. Organic Analytical Research Div.
Standard Reference Materials for the Determination of Polycyclic Aromatic Hydrocarbons in Environmental Samples - Current Activities.

Final rept.

S. A. Wise, M. M. Schantz, B. A. Benner, L. C.

Sander, B. J. Koster, S. N. Chesler, W. E. May, R

M. Parris, and R. E. Rebbert. 1993, 5p

Pub. in Fresenius Jnl. of Analytical Chemistry 345, p325-329 1993

Keywords: "Polycyclic aromatic hydrocarbons ${ }^{*}$ Pollution sampling, ${ }^{*}$ Chemical analysis, Water pollution, Marine environments, Sediments, Animal tissues, Mussels, Bioassay, Air pollution, Calibration standards, Reference standards, Reprints, "Environmental samples, "Standard reference materials, Mutagenicity.

Recent activities at the National Institute of Standards and Technology (NIST) related to the development of standard reference materials (SRMs) for the determination of polycyclic aromatic hydrocarbons (PAHs) are described. These activities include: (1) the development of four new calibration solution SRMs, a ma rine sediment SRM, and a frozen mussel tissue SRM (2) noncertified measurements of PAHs for two additional sediment SRMs: and (3) the establishment of reference Ames bioassay mutagenicity values on three existing SRMs. Activities in progress include the recertification of the existing air particulate and diesel particulate SRMs and the preparation of a new diese particulate extract SRM

\section{0,587}

PB95-151676 Not available NTIS

National Inst. of Standards and Technology (CSTL) Gaithersburg, MD. Organic Analytical Research Div. Development of Frozen Whale Blubber and Liver ganic and Inorganic Contaminants.

Final rept.

S. Wise, M. Schantz, B. Koster, R. Greenberg, M. Burow, P. Ostapczuk, T. Lillestolen, R. Demiralp, and E. Mackey. 1993, 8p.

Sponsored by National Oceanic and Atmospheric Administration, Washington, DC

Pub. in Fresenius Jnl. of Analytical Chemistry 345 p270-277 1993.

Keywords: "Water pollution effects(Animals), "Whales, "Biological Accumulation, "Animal tissues, Bioassay, Trace elements, Polycyclic aromatic hydrocarbons, Pesticides, Liver, Neutron activation analysis, Voltammetry, Atomic spectroscopy, Bioaccumulation, Environmental monitoring, Reference standards, Reprints, *Standard reference materials, Blubber.

Fresh frozen homogenates of pilot whale blubber and liver tissue were prepared for use as control materials for the determination of organic and inorganic contaminants in marine mammal tissue analyses. The blubber material was analyzed to determine 30 polychlorinated biphenyl congeners and 16 chlorinated pesticides using gas chromatography with electron capture detection and gas chromatography-mass spectrometry. A total of 39 trace elements and methyl-mercury were determined in the liver homogenate using instrumental neutron activation analysis, voltammetry and cold vapor atomic absorption spectroscopy. The preparation and analysis of these two tissue materials are part of the development of marine mammal tissue reference materials.

00,588

PB95-151924 Not available NTIS

National Inst. of Standards and Technology (NML) Gaithersburg, MD. Ionizing Radiation Div.

Pattern-Recognition Analysis of Low-Resolution $X$ Ray Fluorescence Spectra.

Final rept.

L. I. Yin, and S. M. Seltzer. 1990, 7p.

Pub. in Nuclear Instruments and Methods in Physics Research A299, p571-577 1990

Keywords: "X-ray fluorescence analysis, "Pattern recognition, Proportional counters, Correlation coefficients, X-ray spectra, Spectrum analysis, Chemical composition, Reprints, Geological samples.

Using a high-resolution Si(Li) spectrometer to perform $\mathrm{X}$-ray fluorescence (XRF) analysis of various geological, alloy, and paint samples, we have demonstrated that in situations where quantitative information is no the primary concern, a pattern-recognition approach may be used to obtain qualitative results very quickly and efficiently. Specifically, the pattern-recognition technique uses a single parameter, the normalized cor- 
relation coefficient, to identify and select samples with similar chemical compositions from their raw XRF spectra. The algorithm can be easily implemented on a personal computer; typically it takes only a few seconds to perform the pairwise comparison of 9 or 10 spectra. We report here the results of our attempt to extend the pattern-recognition technique to the analysis of low-resolution XRF spectra from a proportional counter where the spectral information is considerably poorer than that of the $\mathrm{Si}(\mathrm{Li})$ detector. Analyzing the XRF spectra obtained from a set of geological samples both in air and in vacuum, we find that even with a proportional counter the pattern-recognition technique can nevertheless satisfactorily identify samples with similar chemical compositions.

\subsection{9}

PB95-152047 Not available NTIS

National Inst. of Standards and Technology (CSTL) Gaithersburg, MD. Inorganic Analytical Research Div. Determination of Inorganic Constituents in Marine Mammal Tissues.

Final rept.

R. Zeisler, R. Demiralp, B. J. Koster, P. Ostapczuk, S. A. Wise, P. R. Becker, and M. Burow. 1993, 22p Pub. in Science of the Total Environment 139/140, p365-386 1993.

Keywords: "Marine mammals, "Animal tissues, "Inorganic compounds, "Water pollution effects(Animals), "Bioaccumulation, Tissue banks, Liver, Kidney, Muscles, Seals(Mammals), Whales, Methyl mercury, Trace elements, Biological accumula tion, Bioassay, Neutron activation analysis, Environmental monitoring, Reference standards, Reprints.

Analyses of selected tissues from the Alaska Marine Mammal Tissue Archival Project (AMMTAP) have provided comprehensive information related to levels of 36 trace elements and methyl-mercury in marine mammal tissues. Liver, kidney and muscle tissues from two northern fur seals, four ringed seals and six belukha whales were analyzed. The bulk of the investigated tissues and additional tissues from a total of 65 marine mammals are banked in the AMMTAP. The results are compared to literature values for trace element concentrations in marine mammal tissues and their relevance to environmental studies is discussed.

\section{0,590}

PB95-152799 Not available NTIS

National Inst. of Standards and Technology (CSTL) Gaithersburg, MD. Biotechnology Div.

Resolution of DNA in the Presence of Mobility Modifying Polar and Nonpolar Compounds by Discontinuous Electrophoresis on Rehydratable Polyacrylamide Gels

Final rept.

R. Allen, B. Budowle, and D. Reeder. 1993, 9p.

Pub. in Applied and Theoretical Electrophoresis 3 p173-1811993.

Keywords: „Deoxyribonucleic acid, "Polyacrylamide gel electrophoresis, *Hydration, Monosaccharides, Alanine, Buffers, Serine, Disaccharides, Alcohols, Glycerol, Reprints.

Ultrathin-layer rehydratable gels were surface loaded and run in the horizontal position to study effects of mobility modification of DNA. Mobility modification of DNA fragments was achieved by the addition of nonpolar monosaccharides and their corresponding sugar alcohols as well as glycerol and ethylene glyco in the leading ion buffer. These compounds show little effect when included in the trailing ion buffer. Disaccharides show no mobility modification. Trailing ions such as serine and members of the Good buffer series reduced also the $R$ (sub $F$ ) of double- or singlestranded DNA. While beta-alanine had no effect, serine and members of the Good buffer series, particularly MOPSO showed a marked ability to decrease the $\mathrm{R}$ (sub $\mathrm{F}$ ), presumably due to changing the unstacking limits. Rapid separation of sequencing gels with high resolution was achieved with discontinuous buffer sys tems. The potential methodology for high-resolution scanning of gels as DNA zones unstack from moving boundary is suggested.

\subsection{1}

PB95-152815 Not available NTIS

National Inst. of Standards and Technology (PL), Boulder, CO. Quantum Physics Div.
Single-Photon Ionization and Detection of $\mathrm{Ga}, \mathrm{In}$, and As(sub n) Species in GaAs Growth.

Final rept.

A. L. Alstrin, P. G. Strupp, L. Cook, and S. R. Leone. 1993,110

Contract AFOSR90-0166

Pub. in Proceedings of Society of Photo-Optical Instrumentation Engineers: Laser Techniques for State-Se lected and State-to-State Chemistry Los Angeles, CA. January 21-23, 1993, v1858 p367-377. Sponsored by Air Force Office of Scientific Research, Bolling AFB, DC.

Keywords: "Molecular beam epitaxy, "Gallium arsenides, "Indium, "Gallium, "Arsenic, "Mass spectroscopy, Time-of-flight method, Far ultraviolet radiation, Real time operations, Monitoring, Ionization, Laser radiation, Desorption, Silicon, Reprints.

Single photon ionization time-of-flight mass spectros copy (SPI-TOFMS) is used to monitor chemical fluxes of $\mathrm{In}, \mathrm{Ga}$, and $\mathrm{As}(\mathrm{n})$, relevant in molecular beam epitaxy of GaAs. With single photon ionization at 118 $\mathrm{nm}(10.5 \mathrm{eV})$, the photon energy is large enough to ionize the species, but not sufficient to ionize and fragment. The lack of molecular dissociation of species such as As2 and As4 greatly simplifies the interpretation of mass spectra. SPI-TOFMS provides the ability to measure densities, and hence fluxes, of multiple chemical species above a substrate noninvasively and in real time during conventional molecular beam epitaxy. The relative ionization efficiencies of $\mathrm{Ga}$ and the As(n) species at $118 \mathrm{~nm}$ are determined. Additionally, this laser probing technique is used to study the isothermal and temperature programmed desorption of arsenic from Si(100). The catalytic cracking of As4 on $\mathrm{Si}$ is also examined and discussed. This technique promises to be a valuable in-situ optical diagnostic for III-V and II-VI molecular beam epitaxy.

\section{0,592}

PB95-153383 Not available NTIS

National Inst. of Standards and Technology (TS), Gaithersburg, MD. Standard Reference Materials Program

Reference Materials by Isotope Dilution Mass Spectrometry.

Final rept.

P. DeBievre, J. R. DeLaeter, H. S. Peiser, and W. P. Reed. 1993, 30p.

Pub. in Mass Spectrometry Review 12, p143-172 1993

Keywords: "Mass spectroscopy, Geochronology, Geology, Metrology, Precision, Accuracy, Reprints, *Reference materials, Isotope dilution mass spectroscopy, Measurement evaluation program.

This is a review of the role of inorganic mass spectrometers in Chemical Metrology. It discusses measurements by isotope dilution mass spectrometry, and how it contributes to precision and accuracy in Chemical Metrology and the Certification of Reference Material It also reviews reference materials available for geology and geochronology

00,593

PB95-153599 Not available NTIS

National Inst. of Standards and Technology (CSTL), Gaithersburg, MD Organic Analytical Research Div.

Liquid Chromatographic Method for the Determination of Carotenoids, Retinoids, and Tocopherols in Human Serum and in Food. Final rept.

K. S. Epler, R. G. Ziegler, and N. E. Craft. 1993, 12p. Pub. in Jnl. of Chromatography 619, p37-48 1993.

Keywords: "Liquid chromatography, "Carotenoids, *Retinoids, "Tocopherol, "Chemical analysis, "Food analysis, Ultraviolet spectroscopy, Solvents, Methodology, Reprints.

A liquid chromatographic (LC) has been developed for the quantitative measurement of the six major carotenoids in human serum (lutein, zeaxanthin, betacryptoxanthin, lycopene, alpha-carotene, and beta-carotene) as well as retinol retinyl palmitate, alpha-tocopherol, gamma-tocopherol, and delta-tocopherol. Several polar carotenoids, 2',3'-anhydrolutein, alphacryptoxanthin, and geometric isomers of lycopene and beta-carotene are also separated. Retinoids and carotenoids are monitored using a programmable ultraviolet-visible detector, while tocopherols are monitored using a fluorescence detector. The method uses a gradient containing acetonitrile, methanol, and ethyl acetate. Ammonium acetate is introduced with the methanol to minimize carotenoid losses on the LC column aggravated by the use of acetonitrile and ethyl acetate. The method is also applicable to the analysis of foods.

\section{0,594}

PB95-161162 Not available NTIS

National Inst. of Standards and Technology (NML), Boulder, CO. Chemical Engineering Science Div.

Enzyme and Protein Mass Transfer Coefficient in Aqueous Two Phase Systems. 1. Spray Extraction Columns.

Final rept.

K. R. Jafarabad, S. B. Sawant, J. B. Joshi, and S. K. Sikdar. 1992, 12p.

Pub. in Chemical Engineering Science 47, n1 p57-68 1992

Keywords: *Proteins, "Enzymes, "Mass transfer, Purification, Bovine serum albumin, Salts, Polyethylene glycol, Reprints, "Spray extraction columns, Amyloglucosidase

Fractional dispersed phase hold-up and dispersed side mass transfer coefficients for bovine serum albumin (BSA) and amyloglucosidase were measured in 22, 34 $50,56,70$ and $95 \mathrm{~mm}$ i.d. spray columns using saltpolyethylene glycol (potassium phosphate-PEG and sodium sulphate-PEG) systems. The effect of distributor design and column height was investigated. The effect of phase compositions of the aqueous phase system was also studied. Empirical and semiempirical correlations have been developed for fractional dispersed phase hold-up and dispersed side mass transfer coefficients.

\section{0,595}

\section{PB95-162228 Not available NTIS}

National Inst. of Standards and Technology (NML), Boulder, CO. Chemical Engineering Science Div.

Protein Extraction in a Spray Column Using a Polyethylene Glycol Maltodextrin Two-Phase Polymer System.

Final rept.

K. S. M. S. Raghav Rao, D. S. Szlag, S. K. Sikdar, J. B. Joshi, and S. B. Sawant. 1991, 7p.

Pub. in Chemical Engineering Jnl. 46, n3 pB75-B81 1991.

Keywords: "Proteins, "Polyethylene glycol, *Purification, Bovine serum albumin. Mass transfer, Water, Reprints, *Maltodextrin.

Recovery of a model protein, bovine serum albumin (BSA), was demonstrated in a spray column contractor using immiscible aqueous phases, composed of polyethylene glycol (PEG) and maltodextrin (MDX). Dispersed phase (PEG) hold-up and protein transfer coefficients were measured in a $22 \mathrm{~mm}$ and a $32 \mathrm{~mm}$ i.d. spray columns using several designs of spargers. Protein transfer coefficients were greater in the PEG/ maltodextrin system than those encountered in PEG/ dextran systems. Empirical correlations have been proposed for mass transfer coefficients and PEG-holdups.

00,596

PB95-162608 Not available NTIS

National Inst. of Standards and Technology (CSTL),

Gaithersburg, MD Organic Analytical Research Div.

Shape Selectivity in Reversed-Phase Liquid Chromatography for the Separation of Planar and NonPlanar Solutes.

Final rept.

L. C. Sander, and S. A. Wise. 1993,17p.

Pub. in Jnl. of Chromatography A 656, p335-351 1993.

Keywords: "Liquid column chromatography, "Liquid chromatography, "Aromatic polycyclic hydrocarbons, * Solutes, Separation, Mixtures, Isomers, Reviews, Reprints, Shape selectivity, Solute retention.

Solute retention in reversed-phase liquid chromatography is the result of a variety of complex interactions between solute, mobile phase and stationary phase species. An understanding of the parameters that influence retention is useful in the development of separation methods with existing columns. Such knowledge is even more important for the design of new bonded stationary phases with engineered chromatographic properties. This review will examine some of the factors that affect retention and selectivity with alkyl-modified sorbents, particularly for the separation of solutes with well defined, rigid structure (e.g., polycyclic aromatic hydrocarbons). The 
basis of molecular structure, namely 'shape selectivity" will be studied in terms of contributions from bonded phase morphology, and in terms of operational conditions. An emphasis is placed on practical choices that are available to control selectivity and optimize separations for isomers and related mixtures.

\section{0,597}

PB95-163101 Not available NTIS

National Inst. of Standards and Technology (NML) Gaithersburg, MD. Inorganic Analytical Research Div. Trace Elements Associated with Proteins. Neutron Activation Analysis Combined with Biological Isolation Techniques.

Final rept.

S. F. Stone, R. Zeisler, G. E. Gordon, R. P. Viscidi, and E. H. Cerny 1991, 13p.

Pub. in American Chemical Society Symposium Series Biol. Trace Elem. Res. 445, p265-277 1991.

Keywords: "Neutron activation analysis, "Phosphoproteins, "Trace elements, Polyacrylamide gel electrophoresis, Antigen-antibody reactions, Casein, Milk, Eggs, Immunoassay, Gold colloid, Reprints, Phosvitin.

Combinations of biological techniques with neutron activation analysis (NAA) can be employed for protein quantification in biological samples. A protein of interest can be isolated physically with a high resolution separation technique such as polyacrylamide gel electrophoresis (PAGE) or by immunochemical properties, such as with a specific antibody-antigen reaction. NAA is then used for quantification of the isolated protein by determining either an element that is structurally intrinsic, or one that has been introduced as a label. An example of the former method is the determination of phosphoproteins by PAGE/NAA. This method is discussed and results for two phosphoproteins, alpha-casein and phosvitin, are presented for several materials, including an alpha-casein concentration of $26 \mathrm{mg} / \mathrm{mL}$ in low-fat milk, and a phosvitin concentration of $4.7 \mathrm{mg} / \mathrm{mL}$ in whole egg powder (lyophilized). The second method presented is an immunoassay combining a colloidal gold tag with detection by NAA.

\section{0,598}

PB95-163424 Not available NTIS

National Inst. of Standards and Technology (NML), Gaithersburg, MD. Inorganic Analytical Research Div. Trace Detection in Conducting Solids Using LaserInduced Fluorescence in a Cathodic Sputtering Cell.

Final rept.

J. C. Travis, G. C. Turk, R. L. Watters, L. J. Yu, and J. L. Blue. 1991, 11p.

Pub. in Jnl. of Analytical Atomic Spectrometry 6, n4 p261-271 1991.

Keywords: "Laser induced fluorescence, "Atomic spectroscopy, "Chemical analysis, Glow discharges, Trace amounts, Background noise, Background radiation, Sensitivity, Samplers, Brasses, Iron, Reprints, Cathodic sputtering.

A cathodic sputtering atom reservoir designed for atomic absorption spectroscopy of conducting solid samples is examined as a potential sampling device for trace and ultra-trace detection using laser-induced fiuorescence (LIF) spectrometry. The analytical results are promising, with sub-(micro) $\mathrm{g} / \mathrm{g}$ sensitivity for the model analyte $(\mathrm{Fe})$ in brass samples, and with reasonable precision and accuracy (+ or - 15\%) for the pulsed laser system used, but with far less sensitivity than might be predicted. Noise studies clearly indicate that laser-induced background fluorescence is the principal limiting noise source. Fundamental material transport studies indicate that diffusional loss of atomic number density to the walls is of much less importance than the background fluorescence in determining the sensitivity of the system. Extrapolations based on cell/experiment design to maximize number density and minimize background emission and fluorescence promise $\mathrm{ng} / \mathrm{g}$ sensitivities for future implementations.

\section{0,599}

PB95-164653 Not available NTIS

National Inst. of Standards and Technology (CSTL), Gaithersburg, MD. Biotechnology Div.
Enhanced Detection of PCR Products Through Use of TOTO and YOYO Intercalating Dyes with Laser Induced Fluorescence - Capillary Electrophoresis. Final rept.

K. Srinivasan, S. C. Morris, J. E. Girard, M. C. Kline, and D. J. Reeder. 1993, 5p.

Pub. in Applied and Theoretical Electrophoresis 3, p235-239 1993.

Keywords: "Polymerase chain reaction, "Fluorescence spectroscopy, *Dyes, "Intercalating agents, *Chemical analysis, Reprints, "Capillary electrophoresis, "Thiazole orange, *Oxazole orange.

Recent developments in the chemical synthesis of DNA-binding dyes have enhanced detection of polymerase chain reaction (PCR) products by capillary electrophoresis. These dyes are dimers of thiazole or ange (TOTO) or oxazole orange (YOYO) and have a very high binding affinity for DNA. These dyes show enhanced fluorescence signals when they bind to double-stranded DNA and their fluorescence in the unbound state is almost zero, making them extremely useful in detecting minute ( $\mathrm{fg}$ ) quantities of DNA. We report here the utility of these dyes in DNA typing applications using a laser-induced fluorescence detector in conjunction with a capillary electrophoresis system.

\section{0,600}

PB95-175485 Not available NTIS

National Inst. of Standards and Technology (PL), Boulder, CO. Time and Frequency Div.

Diode Laser as a Spectroscopic Tool.

Final rept.

R. W. Fox, C. S. Weimer, L. Hollberg, and G. C.

Turk. 1993, 9p.

Sponsored by National Aeronautics and Space Administration, Washington, DC. and Air Force Office of Scientific Research, Bolling AFB, DC

Pub. in Spectrochimica Acta Rev. 15, n5 p291-299 1993.

Keywords: "Laser spectroscopy, "Semiconductor lasers, "Spectroscopic analysis, Near infrared radiation, Aluminum gallium arsenides, Laser cavities. Tunable lasers, Nonlinear optics, Visible radiation, Red(Color) Line width, Reprints.

The properties of diode lasers that make them attractive as spectroscopic sources are discussed, along with the use of extended cavities to enhance their tun ing range and reduce their linewidths. Semiconductors can now provide efficient, tunable, narrow linewidth laser light over much of the red and near infrared re gion of the spectrum. The progress in using nonlinear optics to extend the useful wavelength range of diodes lasers is also considered. Our recent analytical work with diode laser spectroscopy provides examples of their application

\section{0,601}

PB95-175709 Not available NTIS

National Inst. of Standards and Technology (CSTL), Gaithersburg, MD. Biotechnology Div.

Fluorescence Measurements of Tetracycline in High Cell Mass for Fermentation Monitoring.

Final rept.

J. J. Horvath, and S. A. Glazier. 1993, 2p.

Pub. in American Biotechnology Laboratory 11, n7 2p Jun 93.

Keywords: "Tetracycline, ${ }^{*}$ Chemical analysis, *Cells(Biology), "Fluorescence spectroscopy, Fer mentation, Streptomyces aureofaciens, Fiber optics, Soybeans, Reprints

This work describes a new spectroscopic optical fiber rod sensor for in situ real time measurement of product concentrations in bioreactors. The variable excitation emission wavelength capability of this sensor allows for species selective measurement during fermentations. Product concentrations (tetracycline) have been measured in high cell mass fermentation media of Streptomyces aureofaciens with $5 \%(w / v)$ soybean meal. By using multiple excitation wavelengths (390 and $413 \mathrm{~nm}$ ) the linear measurement range was extended to $8160 \mathrm{microgram} / \mathrm{ml}$ at cell densities of greater than $50 \mathrm{~g} / \mathrm{l}$ dry weight. The sensor is robust, able to undergo many cycles of in situ steam sterilization without degradation, and its fiuorescence signal is linear with concentration for all species studied in this work.

\section{0,602}

PB95-175964 Not available NTIS

National Inst. of Standards and Technology (CSTL), Gaithersburg, MD. Inorganic Analytical Research Div.
Cold Neutron Prompt Gamma Activation Analysis at NIST: A Progress Report.

Final rept.

R. L. Paul, R. M. Lindstrom, and D. H. Vincent. 1994 $7 p$.

Pub. in Jnl. of Radioanalytical and Nuclear Chemistry 180, n2 p263-269 1994.

Keywords: "Activation analysis, Neutron capture gamma rays, Allende meteorite, Cold neutrons, Progress report, Sensitivity, Reprints, CNRF facility, US NIST.

An instrument for prompt gamma-ray activation analy sis is now in operation at the NIST Cold Neutron Research Facility (CNRF). The cold neutron beam is relatively free of contamination by fast neutrons and reactor gamma rays, and the neutron fluence rate is 1.5 (center dot) $10(\exp 8) / \mathrm{sq} \mathrm{cm}$ (center dot) s (thermal equivalent). As a result of a compact target-detector geometry, the sensitivity is better by a factor of as much as seven than that obtained with an existing ther mal instrument, and hydrogen background is a facto of 50 lower. We have applied this instrument to multielement analysis of the Allende meteorite and other materials.

00,603

PB95-175972 Not available NTIS

National Inst. of Standards and Technology (CSTL), Gaithersburg, MD. Inorganic Analytical Research Div. Neutron Scattering by Hydrogen in Cold Neutron Prompt Gamma-Activation Analysis.

Final rept.

R. L. Paul, and E. A. Mackey. 1994, 13p.

Pub. in Jnl. of Radioanalyticai and Nuclear Chemistry 181, n2 p321-3331994

Keywords: "Activation analysis, "Neutron scattering, "Hydrogen, Neutron capture gamma rays, Cold neutrons, Sensitivity, Reprints.

The effects of neutron scattering by hydrogen within targets for cold neutron prompt gamma-ray activation analysis (CNPGAA) have been characterized. For most targets studied, the probability for neutron absorption, and hence CNPGAA sensitivities (counts (center dot) $1 / \mathrm{s}$ (center dot) $1 / \mathrm{mg}$, decrease with increasing $\mathrm{H}$ content and with target thickness. Comparisons with results from thermal neutron PGAA indicate that the effects of cold neutron scattering differ from those of thermal neutron scattering. CNPGAA sensitivities for ' $1 / \mathrm{nu}^{\prime}$ ' nuclides show similar sensitivity decreases, while Sm sensitivities show smaller decreases.

\section{0,604}

PB95-180113 Not available NTIS

National Inst. of Standards and Technology (CSTL), Boulder, CO. Thermophysics Div.

Chromatographic Cryofocusing and Cryotrapping with the Vortex Tube.

Final rept.

T. J. Bruno. 1994, 4p.

Contract GRI-5088-260-1700

Sponsored by Gas Research Inst., Chicago, IL

Pub. in Jnl. of Chromatographic Science 32, p112-115 1994

Keywords: "Chromatographic analysis, "Cryotrapping, "Cryogenic cooling, "Hilsch tubes, Vortices, Solutes, iniections, Desorption, Gas chromatography, Reprints, "Cryofocusing.

Cryofocusing and cryotrapping techniques are the anaiytical-scale preconcentration or collection of solutes using low temperature, with subsequent solute removal and analysis. Although these methods have been used with many analytical procedures including spectroscopic techniques, the major applications have been in chromatographic methods that involved a wide variety of sample mixtures. We describe a simple, entirely pneumatic approach to chromatographic sample cryofocusing--cryotrapping. The two airstreams from a vortex tube (cold and hot, respectively) are used to both chill the cryofocus region of the injector and then to thermally desorb the collected solutes for injection.

\section{0,605}

PB95-180246 Not available NTIS

National Inst. of Standards and Technology (CSTL) Gaithersburg, MD. Inorganic Analytical Research Div. Final rept

J.D. Fassett, and R. L. Watters. 1994, 7p.

Pub. in Reviews on Analytical Chemistry: Euroanalysis VIII, p13-19 1994 
Keywords: "Chemical analysis, Interlaboratory comparisons, Primary standards, Metrology, Reprints, Isotope dilution mass spectroscopy, Traceability.

The CIPM has established a Working Group on Metrology in Chemistry to address the specific problem of traceability of chemical measurements. One of the terms of reference of the Working Group is to draw up an exploratory program of cooperative work among leading national chemical metrology laboratories. This program is designed to test the hypothesis that coordinated analysis of a few key reference materials, using one or two reference methods of wide application, will provide such laboratories with a base from which to extend international comparability to a wider range of methods and reference materials. NIST is coordinating the details of the first interlaboratory exercise. Isotope dilution mass spectrometry (IDMS) has been chosen as the reference method for the initial comparison. The design and status of the interlaboratory exercise is summarized.

00,606

PB95-180758 Not available NTIS

National Inst. of Standards and Technology (CSTL), Gaithersburg, MD. Organic Analytical Research Div. Instrument for Evaluating Phase Behavior of Mix. tures for Supercritical Fluid Experiments.

Final rept.

S. H. Page, J. F. Morrison, R. G. Christensen, and S. J. Choquette. 1994, 5p

Pub. in Analytical Chemistry 66, n21 p3553-3557, 1 Nov 94.

Keywords: "Supercritical fluids, "Phase studies, "Instrumentation, Supercritical fluid chromatography, Light scattering, Binary mixtures, Carbon dioxide, Methanol, Water, Reprints.

Fluid phase behavior has a profound impact on supercritical fluid chromatography performance. The effect of fluid phase behavior on supercritical fluid ex traction performance has not been studied. Generally methods available to generate phase diagrams of fluid mixtures are time-consuming. This paper describes relatively easily constructed instrumentation for the rapid screening of fluid mixtures to determine whether or not they are single phase. Either scattered or transmitted light can be used to probe the phase behavior of the fluids. Examples using CO2/methanol and $\mathrm{CO} 2 /$ water are presented. The phase behavior of multicomponent mixtures of $\mathrm{CO} 2$ or Freon (R22, chlorodifluoromethane) with methanol/triethylaminel water was determined.

00,607

PB95-180824 Not available NTIS

National Inst. of Standards and Technology (CSTL), Gaithersburg, MD. Surface and Microanalysis Science Div

Activities of ISO Technical Committee 201 on Surface Chemical Analysis.

Final rept.

C. J. Powell, and R. Shimizu. 1994, 400p.

Pub. in Surface and Interface Analysis 21, p615-620 1994.

Keywords: "Chemical analysis, "Surface chemistry, Standards, ISO, Reprints.

A status report is given on activities of ISO Technical Committee 201 (ISO/TC 201) on Surface Chemical Analysis. The committee has seven subcommittees which met for the first time in 1993. Information is given on the working groups that have been established for specific purposes. We show national participation in ISO/TC 201 and its subcommittees, and we identify the chairmen and secretariats of the subcommittees and the conveners of the working groups.

\section{0,608}

\section{PB95-242319 PC A16/MF A03}

National Inst. of Standards and Technology (CSTL), Gaithersburg, MD.

CSTL Technical Activities, 1994.

H. G. Semerjian. 1994, 374p, NISTIR-5584

Presented to the Board on Assessment of NIST Programs, National Research Council, March 22-23, 1995. See also report for 1993, PB95-160602, report for 1992, PB93-173482 and report for 1991, PB94160769

Keywords: "Chemistry, "Research and development, Chemical engineering, Biotechnology, Measurements, Metrology, Models, Measurement traceability, Process technology, Reference data.
This report summarizes the scientific and engineering activities of the Chemical Science and Technology Laboratory (CSTL) for fiscal year 1994. Activity reports are grouped into Divisions of CSTL Biotechnology, Chemical Kinetics and Thermodynamics, Inorganic Analytical Research, Organic Analytical Research, Process Measurements, Surface and Microanalysis Science, and Thermophysics. In addition to the technical reports, outputs and interactions are listed for reach Division, which include publications, talks, CRADAs, patents, SRMs, SRDs, calibrations, committee assignments, editorships, seminars, conferences, and workshops. The overall program is summarized and yearly highlights are given.

\section{0,609}

PB96-102488 Not available NTIS

National Inst. of Standards and Technology (MSEL) Gaithersburg, MD. Ceramics Div.

Standard Reference Material for the Measurement of Particle Mobility by Electrophoretic Light Scattering.

Final rept.

V. A. Hackley, R. S. Premachandran, S. G. Malghan, and S. B. Schiller. 1995, 16p.

Pub. in Colloids and Surfaces A 98, p209-224 1995

Keywords: "Electrophoresis, "Light scattering, -Calibration standards, Particle motion, Goethite Phosphates, Statistical quality control, Interlaboratory comparisons, Reprints.

A standard reference material (SRM) was developed for the calibration and evaluation of light scattering equipment used to measure electrophoretic mobility. SRM 1980 is a positive mobility standard containing $500 \mathrm{mg} / \mathrm{cubic} \mathrm{dm}$ microcrystalline goethite (alpha$\mathrm{FeOOH}$ ) and $100 \mathrm{micromol} / \mathrm{g}$ phosphate in $0.05 \mathrm{~mol} /$ cubic dm sodium perchlorate electrolyte solution at $\mathrm{pH}$ 2.5. The suspension is diluted prior to use. A certified mobility value $(2.53+$ or -0.12 micrometer $\mathrm{cm} / \mathrm{v} / \mathrm{s})$ was determined from a statistical analysis of round robin data from five laboratories according to NIST quidelines. This paper documents the development, characterization and evaluation of this material. Also discussed are the various contributions to measurement uncertainty and inconsistency, and appropriate protocols for dealing with these problems.

\section{0,610}

\section{PB96-106463 PC A03/MF A01}

National Inst. of Standards and Technology (CSTL) Gaithersburg, MD. Surface and Microanalysis Science Div.

Proficiency Tests for the NIST Airborne Asbestos Program, 1993.

S. Turner, E. B Steel, O. S. Crankshaw, S. Silberstein, and H. M. Richmond. Jun $95,45 p$ NISTIR-5680.

See also report for 1992, PB94-194362. Prepared in cooperation with Research Triangle Inst., Research Triangle Park, NC. Center for Environmental Measurements and Quality Assurance.

Keywords: "Asbestos, "Materials testing, "Laboratories, Requirements, Certification, Air pollution detection, Calibration standards. Standardization, Test methods, Procedures, Transmission electron microscopy, Chrysotile, Amosite, Crocidolite, Amphiboles, Performance evaluation, Forms(Paper), *Proficiency tests, National Voluntary Laboratory ACcreditation Program, Anthophyllite, Actinolite, Tremolite.

NIST has published proficiency test instructions and summary reports for the airborne asbestos program since 1990. The reports provide a historical record of proficiency testing materials sent to the laboratories so that they can be referenced in other publications and background material can be given to those laboratories entering the accreditation program. This report contains the instructions and summary reports issued for 1993 proficiency tests.

\section{0,611}

PB96-111653 Not available NTIS

National Inst. of Standards and Technology (CSTL) Gaithersburg, MD. Biotechnology Div.

Overview of Reference Materials Prepared for Standardization of DNA Typing Procedures.

Final repi

D. J. Reeder, M. C. Kline, and K. L. Richie. 1995, 4p. Pub. in Fresenius Jnl. of Analytical Chemistry, v352 p246-249 1995.

Keywords: "Calibrating standards, "Quality assurance, chemistry, Laboratories, Reprints, DNA typing,
SRM(Standard Reference Material). NIST(National Institute of Standards and Technology).

Although DNA typing is an accurate, precise, and robust procedure, quality assurance is enhanced by availability of a suitable reference material. The $\mathrm{Na}$ tional Institute of Standards and Technology (NIST) recently released a Standard Reference Material (SRM) that meets the calibration and quality assurance needs of laboratories that perform DNA typing. Each step of the analytical process of DNA typing maybe verified by one or more of twenty different components of the SRM. As newer, more sensitive methods for DNA typing have been introduced into the human identification laboratory repertoire, new SRMs will be required for quality assurance.

00,612

PB96-111877 Not available NTIS

National Inst. of Standards and Technology (CSTL), Gaithersburg, MD. Inorganic Analytical Research Div. Measuring Hydrogen by Cold-Neutron PromptGamma Activation Analysis.

Final rept.

R. M. Lindstrom, R. L. Paul, D. H. Vincent, and R. R. Greenberg. 1994, 5p.

Pub. in Jni. of Radioanalytical and Nuclear Chemistry, v180 n2 p271-275 1994.

Keywords: "Activation analysis, "Hydrogen, Long wavelength, Prompt gamma radiation, Chemical analysis, Reprints, Cold neutrons, *Foreign technology.

By irradiating with cold neutrons and avoiding hydrogenous materials of construction, we have developed a PGAA instrument at the Cold Neutron Research Facility at NIST with hydrogen detection limits in the microgram range in many materials. Quantities of 510 micrograms $\mathrm{H} / \mathrm{g}$ are presently measureable in gram-sized samples of silicon or quartz, and of order 0.01 wt \% can be quantitatively measured in complex silicate rocks.

\subsection{3}

PB96-111968 Not available NTIS

National Inst. of Standards and Technology (CSTL), Gaithersburg, MD. Organic Analytical Research Div.

Stability of Compressed Gas Mixtures Containing Low Level Volatile Organic Compounds in Aluminum Cylinders.

Final rept.

W. R. Miller, and G. C. Rhoderick. 1995, 9p.

Pub. in Fresenius Jnl. of Analytical Chemistry, v351 p221-229 1995 .

Keywords: "Gas cylinders, "Compressed gas, Stability, Reprints, Gases, Mixtures, "Foreign technology, "Volatile organic compounds.

Compressed gas mixtures containing up to twenty-six volatile organic compounds (VOCs) in a balance of nitrogen have been prepared and analyzed at the $\mathrm{Na}$ tional Institute of Standards and Technology (NIST). The mixtures are contained in aluminum cylinders and the hydrocarbons included are aromatic or aliphatic, both saturated and unsaturated and some containing a halogen, oxygen or nitrogen atom. The individual compounds are present at concentrations ranging from $0.1-3000 \mathrm{nmol} / \mathrm{mol}$ and the relative standard uncertainty in the concentration of each is between plus or equal to $2-5 \%$. The stability of the mixtures over various time intervals is discussed.

00,614

PB96-112099 Not available NTIS

National Inst. of Standards and Technology (CSTL), Gaithersburg, MD. Analytical Chemistry Div.

Spectral Interference in the Determination of Arsenic in High-Purity Lead and Lead-Base Alloys Using Electrothermal Atomic Absorption Spectrometry and Zeeman-Effect Background Correction.

Final rept.

M. S. Epstein, G. C. Turk, and L. J. Yu. 1994, 8p. Pub. in Spectrochimica Acta, v49B, n12-14 p168116881994.

Keywords: "Spectrometry, "Lead(Metal), "Absorption spectrometry, Metals, Reprints, Arsenic, Lead alloys, "Spectral interference, Zeeman effect background correction.

Arsenic, antimony, and tellurium are determined at part-per-million concentration levels in bullet lead, lead-base alloy, and high purity lead using 
electrothermal atomization atomic absorption spectrometry (ETAAS) with Zeeman-effect background correction. A spectral interface by lead absorption lines resulting from excited state transitions is observed on both primary arsenic lines, at $193.696 \mathrm{~nm}$ and 197.197 $\mathrm{nm}$. Analytical bias caused by the interference at $197.197 \mathrm{~nm}$ is eliminated by using temperature programming and temporal resolution.

00,615

PB96-112107 Not available NTIS

National Inst. of Standards and Technology (CSTL), Boulder, CO. Thermophysics Div.

Applications of the Vortex Tube in Chemical Analysis. Part 2. Applications.

Final rept.

T. J. Bruno. 1993, 9p.

See also PB94-199171

Pub. in American Laboratory, v25 n14 p16-24 1993

Keywords: "Hilsch tubes, Reprints, Heat transfer, Laboratory equipment, Chemical analysis, "Vortex tubes. Many applications of the vortex tube in chromatographic analysis have been devised in recent years mainly because of the usefulness of low-temperature chromatographic techniques. One of the most important is GC column temperature control, especially in subambient ranges. The operation of a GC column tow temperatures began in the mid-1950's, but has received renewed attention for the trace analysis of pririty pollutants in air and in the analysis of alternative refrigerant fluids. Subambient temperature GC generally refers to procedures in which the column temperature is between -100 and 0 degrees C, but many separations are greatly enhanced at temperatures no lower than -40 degrees $\mathrm{C}$.

00,616

PB96-112131 Not available NTIS

National Inst. of Standards and Technology (CSTL), Gaithersburg, MD. Inorganic Analytical Research Div. Effects of Target Temperature on Analytical Sen sitivities of Cold-Neutron Capture Prompt gammaray Activation Analysis.

Final rept.

A. Mackey. 1994, 6p.

Pub. in Biological Trace Element Research, p103-108 1994.

Keywords: "Activation analysis, "Neutron scattering, Reprints, Neutron capture gamma rays, Cold neutrons, Sensitivity, " Foreign technology.

Cold-neutron prompt gamma-ray activation analysis sensitivities are often decreased because of an in crease in the average neutron energy on scattering within room temperature targets. Experiments were within room temperature targets. Experiments were performed to determine whether target cooling would creased hydrogen sensitivity by as much as $25 \%$. Target cooling decreases those effects of neutron scattering on CNPGAA sensitivities that are the result of an increased average neutron energy. However, coldneutron scattering may aiso change the average path ength traveled, and this effect on sensitivity is not alleviated by controlling temperature.

00,617

PB96-112149 Not available NTIS

National Inst. of Standards and Technology (CSTL), Gaithersburg, MD. Inorganic Analytical Research Div. SUM and MEAN: Standard Programs for Activation Analysis.

Final rept.

R. M. Lindstrom. $1994,7 p$

Pub. in Biological Trace Element Research, p597-603 1994

Keywords: *Activation analysis, "Computer programs, Reprints, Emission spectra, Weighted means, Peak integration, Gamma ray spectrometry.

Two computer programs in use for over a decade in the Nuclear Methods Group at NIST illustrate the utility of standard software: programs widely available and widely used, in which (ideatly) well-tested public algorithms produce results that are well understood, and thereby capable of comparison, within the community of users. SUM interactively computes the position, net area, and uncertainty of the area of spectral peaks, and can give better results than automatic peak search programs when peaks are very small, very large, or unusually shaped. MEAN combines unequal measurements of a single quantity, tests for consistency, and obtains the weighted mean and six measures of its uncertainty.
00,618

PB96-119458 Not available NTIS

National Inst. of Standards and Technology (CSTL) Gaithersburg, MD. Analytical Chemistry Div.

Comparison of Selectivities for PCBs in Gas Chro matography for a Series of Cyanobiphenyl Stationary Phases.

Final rept.

B. R. Hillery, J. E. Girard, M. M. Schantz, M. L. Lee,

S. A. Wise, and A. Malik. 1995, 10p.

Pub. in Jnl. of Microcolumn Separations, v7 n3 p221 2301995.

Keywords: *Chlorinated aromatic hydrocarboris, "Gas chromatography, Columns(Process engineering) Phase, Planar structures, Selectivity, Shapes, Re prints, "Cyanobiphenyl phases, Stationary phases.

Selectivity for polychlorinated biphenyl (PCB) congeners was examined for a series of cyanobipheny stationary phases, and the results were compared to a nonpolar $5 \%$ phenyl methylpolysiloxane phase and a shape-selective smectic liquid crystalline phase. For all of the columns studied, the degree of ortho substitution of the congener was found to be a significan factor controlling chromatographic retention of PCBs. The retention pattern on six different cyanobipheny phases followed the same general pattern as on the liquid crystalline column, though the trends were more exaggerated on the liquid crystalline phase. While the general retention pattern was similar for all of the cyanobiphenyl phases studied, subtle differences were observed, resulting in significant selectivity differences.

\section{0,619}

PB96-123435 Not available NTIS

National Inst. of Standards and Technology (CSTL), Gaithersburg, MD. Chemical Kinetics and Thermodynamics Div.

All-Metal Collection System for Preparative-Scale Gas Chromatography: Purification of Low-BoilingPoint Compounds.

Final rept.

T. J. Buckley, and K. A. Gillis. 1995, 8p.

Pub. in Jnl. of Chromatography A, v702 p243-250 1995

Keywords: "Gas chromatography, "Collection systems, "Purification, Low boiling point, Reprints, Preparative scale gas chromatography.

We describe a purification system based on a commercial preparative-scale chromatograph with a customdesign condenser, collector, and fraction handling sys tem. In our fraction collector design, all the wetted surfaces were either 316 stainless-steel or nickel. The collectors and the integrated gas-handling manifold were designed to be used down to liquid nitrogen temperature and up to $7 \mathrm{MPa}$ of pressure to accommodate low boiling-point compounds, such as refrigerants. The design, operation, and performance of this apparatus are presented.

\section{0,620}

\section{PB96-123443 Not available NTIS}

National Inst. of Standards and Technology (CSTL), Gaithersburg, MD. Surface and Microanalysis Science Div

Ultrafast Time-Resolved Infrared Probing of Energy Transfer at Surfaces.

Final rept.

R. R. Cavanagh, T. A. Germer, and J. C.

Stephenson. 1995, 7p

Pub. in Vibrational Spectroscopy, v9 p77-83 1995.

Keywords: "Infrared spectrometry, "Energy transfer, "Absorbates, Surfaces, Carbonyl groups, Reprints, "Foreign technology.

Picosecond and femtosecond infrared techniques are used to characterize vibrational energy transfer rates and provide novel insights into the relaxation of non equilibrium energy distributions. For absorbates on metal surfaces, it is essential to determine the absolute coupling strengths between different degress of freedom, i vibrational modes and low-lying electronic states. In the present work, the spectral shift of the CO stretch has been used to follow the temporal evolution of the adlayer response as a consequence of ultrashor infrared, visible and ultraviolet excitation of the underly ing metal. Results from CO/Pt(111) and $\mathrm{CO} / \mathrm{Cu}(100)$ will be presented, Their differences will be discussed and comparison will be made to current theoretical models of the damping rates.

00,621
PB96-123682 Not available NTIS
National Inst. of Standards and Technology (CSTL). Gaithersburg MD Organic Analytical Research Div. Influence of Stationary Phase Chemistry on Shape Recognition in Liquid Chromatography.

Final rept.

L. C. Sander, and S. A. Wise. 1995, 9p.

Pub. in Analytical Chemistry, v67 n18 p3284-3292 7995.

Keywords: "Bonded phase, "Liquid chromatography, Selectivity, Reprints, Polymeric stationary phase, Retention mechanism, Self-assembled monolayer.

Molecular shape recognition is examined for a series of C18 columns prepared using a variety of synthetic approaches. Mono-, di-, and trifunctional silanes are used to prepare stationary phases through monomeric and poly meric surface modification procedures, including an approach employing self-assembled monolayer technology. Shape discrimination properties of the columns were investigated with various nonplanar, planar, and linear polycyclic aromatic hydrocarbon solute probes. Chromatographic retention behavior is examined in the context of recently proposed statistical mechanical 'interphase' retention models.

00,622

PB96-138581 Not available NTIS

National Inst. of Standards and Technology (CSTL).

Gaithersburg, MD. Analytical Chemistry Div.

Selectivity Trends in Packed Column Supercritical Fluid Chromatography with C18 Stationary Phases.

Final rept.

K. L. Williams, L. C. Sander, S. H. Page, and S. A

Wise. $1995,6 p$

Pub. in Jnl. of High Resolution Chromatography, v18 p477-482 1995 .

Keywords: "Chromatography, Polycyclic aromatic hydrocarbons, Isomers, Reprints, "Shape selectivity, Coupled columns, Coal ta

The retention behavior of polycyclic aromatic hydrocarbons (PAHs) in packed-column supercritical fluid chromatography (SFC) is studied for monomeric and polymeric C18 columns. Molecular shape discrimination (shape selectivity) is assessed through the use of Standard Reference Materials (SRMs), and changes in selectivity are studied as a function of temperature pressure, and mobile phase composition. Examples of separations of complex PAH isomer mixtures are presented, and guidelines are provided for modification and optimization of shape selectivity in SFC.

00,623

PB96-146766 Not available NTIS

National Inst. of Standards and Technology (CSTL) Gaithersburg. MD. Analytical Chemistry Div.

Amperometric Measurement of Moisture in Trans. former Oil Using Karl Fischer Reagents.

Final rept.

S. A. Margolis. $1995,8 p$

Keywords: "Moisture content, "Electrical measurement, Calibration standards, Coulometers, Mineral oil, Insulating oil Volumetric analysis, Reprints, Karl Fisher reagents, RMs(Reference Materials).

Moisture was measured in two oil Reference Materials (RMs), transformer oil (RM 8506) and mineral oil (RM 8507 ), by the volumetric and coulometric Karl Fisher methods. A variety of analysis conditions were used, including the solvent composition of the titration vessel. The maximum amount of moisture was obtained when the chloroform content of the titration vessel was above 65 percent after the solvent had been titrated to dry. ness. The highest moisture titers were measured by the volumetric method using either the Hydranal or Pyridine-based Karl Fisher reagents or by the coulometric method using Hydranal AG-H reagent. Other coulometric reagents measured lower amounts of moisture even after the addition of organic solvents.

\section{0,624}

PB96-155536 Not available NTIS

National Inst. of Standards and Technology (CSTL), Gaithersburg, MD. Organic Analytical Research Div.

Population Distributions and Intralaboratory Reproducibility for Fat-Soluble Vitamin-Related Compounds in Human Serum.

Final rept.

K. S. Sharpless, and D. L. Duewer. 1995, 7p

Pub. in Analytical Chemistry, v67 n23 p4416-4422 Dec 95. 


\section{Analytical Chemistry}

Keywords: *Carotenoids, "Vitamins, Histograms, Liquid chromatography, Statistics, Reprints, "Bivariate distributions.

Concentrations of alpha and beta-carotene, lycopene, beta-cryptoxanthim, zeaxanthin, lutein, alphagamma-, and delta-tocopherol, retinol, and retinyl palmitate have been determined in over 1400 human sera from two epidemiologial studies. Complete adult population distributions for these analytes, and for a chromatographically defined 'total carotenids' component, are detailed as data-defined histograms. The dis tributions for retinol and alpha-tocopherol concentrations are much narrower than those for the other analytes. Information provided by analysis of control samples facilitated intercomparison of the two studies and provided univariate and bivariate and bivariate estimates of the intralaboratory measurement reproduc ibility.

\section{0,625}

PB96-158068 Not available NTIS

National Inst. of Standards and Technology (PL) Gaithersburg, MD. Electron and Optical Physics Div. Resonance Ionization Spectroscopy/Resonance Ionization Mass Spectrometry Data Service. V-Data Sheets for $\mathrm{Ga}, \mathrm{Mn}, \mathrm{Sc}$, and TI.

Final rept.

E. B. Saloman. 1994, 31p.

Pub. in Spectrochimica Acta, v49B n3 p25\}-281 1994.

Keywords: *Analytical chemistry, "Atomic data, "Data service, Elemental analysis, Spectroscopy, Reprints, "Foreign technology, Resonance ionization, Mass spectrometry.

A data service has been established at the National Institute of Standards and Technology to provide the necessary information to apply to the techniques of resonance ionization spectrocopy (RIS) and resonance ionization mass spectrometry (RIMS) to routine use in analytical chemistry. This service collects and calculates the relevant atomic data, chooses appropriate resonance ionization schemes, and indicates pertinent operating details of successful RIMS studies The first group of data sheets was published previously covering the elements As, B, Cd, C, Ge, Au, Fe, Pb $\mathrm{Si}$ and $\mathrm{Zn}$. The second group published covered the elements $\mathrm{Al}, \mathrm{Ca}, \mathrm{Cs}, \mathrm{Cr}, \mathrm{Co}, \mathrm{Cu}, \mathrm{Kr}, \mathrm{Mg}, \mathrm{Hg}$ and $\mathrm{Ni}$. The third group published covered the elements $\mathrm{Sb}$, $\mathrm{Bi}, \mathrm{P}, \mathrm{Na}$ and $\mathrm{Sn}$. The fourth group published covered the elements $\mathrm{Be}, \mathrm{In}, \mathrm{Li}, \mathrm{K}, \mathrm{Rb}, \mathrm{Ag}, \mathrm{Ti}$ and $\mathrm{V}$. The fifth group of data sheets is presented here. It covered the elements Ga, Mn, Sc and TI.

\section{0,626}

\section{PB96-160833 Not available NTIS}

National Inst. of Standards and Technology (CSTL) Gaithersburg, MD. Process Measurements Div

Development of the NIST Transient Pressure and Temperature Calibration Facility.

Final rept.

V. E. Bean, G. J. Rosasco, W. S. Hurst, and W. J Bowers. 1991, 5p.

Pub. in Proceedings of the Conference of the National Conference of Standards Laboratories, Albuquerque, NM., August 18-22, 1991, p197-201.

Keywords: "Calibration, "Raman spectroscopy, Shock tube, Transducers, Standards, Pressure, Temperature, Reprints, *Foreign technology.

NIST is developing the capability transient-pressure transducers and transient-temperature transducers. The source for both of these calibrations is a chock tube capable of generating reflected shock pressures up to $20 \mathrm{MPa}$ and temperatures in the range of 1200 $K$ with submicrosecond rise times. The shock tube will be calibrated to avoid the assumptions and uncertain ties involved in calculating the pressure and temperature from shock tube theory. The primary standard for the calibration of the shock tube is a sample of diatomic gas. Diatomic gas molecules have a fundamental vibrational motion whose frequency is affected by pressure in a simple way. These molecules also have well defined rotational energy leve/s whose populations provide a reliable measure of temperature. The vibrational frequencies and the populations of the rotational energy levels can be determined by laser spectroscopy. As the time required for the spectroscopic meas urements can be reduced to nanoseconds, spectra can be obtained under shock conditions and these spectra can be evaluated in terms of spectra obtained under known static pressure and temperature conditions.

National Inst. of Standards and Technology (CSTL), Gaithersburg, MD. Process Measurements Div. ITS-90 Calibration Facility.

Final rept.

R. S. Kaeser, and G. F. Strouse. 1992, 10p.

Pub. in National Conference of Standards Laboratories, Washington, DC., June 18, 1992, $10 p$.

Keywords: "Bridge, "Furnace, "Thermometers, Computers, Laboratory, Resistance, Standards, Calibration, Reprints, International Temperature Scale of 1990, SPRT.

A platinum resistance thermometer calibration laboratory is described that provides ten of the defining fixed points of the New International Temperature Scale of 1990 (ITS-90)1, from the argon triple point up to the gold freezing point. Data are taken by computer using the IEEE-488 bus to access resistance bridges, digital multimeters, and scanners for thermometer selection and measurement. Details include laboratory layout, furnace controls and performance, measuring instruments, and a description of the software.

\section{0,628}

PB96-161286 Not available NTIS

National Inst. of Standards and Technology (CSTL), Gaithersburg, MD. Process Measurements Div.

Direct Comparison of Three PTB Silver Fixed-Point Cells with the NIST Silver Fixed-Point Cell.

Final rept.

G. F. Strouse, B. W. Mangum, H. G. Nubbemeyer

and H. J. Jung. 1993, $9 p$

Pub. in Comite Consultatif de Thermometrie (CCT), 18 , Paris, France, September 1993, 9p.

Keywords: "Silver, "Freezing point, "Thermometers, Reprints, "Foreign technology, International Temperature Scale of 1990, ITS-90, HISPRT.

An accurate realization of the International Temperature Scale of 1990 (ITS-90) requires a measurement assurance program for the thermometric fixed-point cells of the defining fixed points. A previous comparison in 1991 of the scales realized at PTB and NIST, through the use of a high-temperature standard platinum resistance thermometer (HTSPRT) calibrated in fixed-point cells in the range between 0 degrees $C$ and 961.78 degrees $\widehat{O}$ at NIST and at PTB, indicated that the differences between the freezing-point temperatures, as realized on the plateaus of the freezing curves, of Sn, Zn and Al fixed-point cells of NIST and PTB were all approximately $1 \mathrm{~m}$ degrees $\mathrm{C}$. For silver, however, the maximum temperature difference between the freezing plateaus of the PTB and NIST silver cells appeared to be approximately $18 \mathrm{~m}$ degrees $\mathrm{C}$. This extremely large difference was estimated from independent calibrations of the same HTSPRT at PTB and at NIST. In order to deiermine more accurate values of the temperature differences at the silver point, cell to cell comparisons were carried out recently at NIST.

\section{0,629}

PB96-161294 Not available NTIS

National Inst. of Standards and Technology (CSTL), Gaithersburg, MD. Process Measurements Div.

Investigation of High-Temperature Platinum Resistance Thermometers at Temperatures Up to 962C, and, in Some Cases, $1064 \mathrm{C}$.

Final rept

G. F. Strouse, B. W. Mangum, A. I. Pokhodun, and N. P. Moiseeva. 1992, $6 \mathrm{p}$

Pub. in Temperature: Its Measurement and Control in Science and Industry, v6 pt1 p389-394 1992

Keywords: "Thermometers, Stability, Annealing, Reprints, "High temperature thermometry, International Temperature Scale of 1990.

The stability of high temperature standard platinum resistance thermometers, having nominal resistances of 0.25 to $2.5 \mathrm{ohms}$, and their use in the realization of the International Temperature Scale of 1990 over the range of 0 degrees $C$ to 961 degrees $C$ were investigated. Special procedures were employed for their use above 500 degrees $\mathrm{C}$. These techniques involved annealing and protection against contamination. A sodium heat-pipe furnace was used to realize the freezing points of aluminum, silver, and gold. The results to be presented yielded information on non-uniqueness, subrange inconsistencies and the effects of heating above 600 degrees $C$.

\section{0,630}

PB96-161302 Not available NTIS
National Inst. of Standards and Technology (CSTL), Gaithersburg, MD. Process Measurements Div. Investigation of the ITS-90 Subrange Inconsistencies for 25.5 Omega SPRTs.

Final rept.

G. F. Strouse. 1992, 4p.

Pub. in Temperature: Its Measurement and Control in Science and Industry, v6 pt1 p165-168 1992.

Keywords: "Thermometry, Reprints, "Foreign technology, "Water triple point cells, International Tem. perature Scale of 1990, Platinum resistance.

Large variations in observations over extended time were recently reported on water triple point (WTP) cells prepared by using liquid nitrogen (LN) cooled rods to treeze the ice mantle. Experiments were conducted to test the results by using the same technique for preparing the WTP cell ice mantle and by using, for comparison, two other techniques -- solid-CO2 and immersioncooler methods. Because of the strains that are introduced in preparing the ice mantle by the three methods, results that have been obtained at the National Institute of Standards and Technology (NIST) thus far indicate some variations in the observations during the first few days. The LN-cooled-rod technique caused the ice mantle to crack and the observations on WTP cells prepared by this technique indicate more variations than were observed in cells with mantles prepared by other techniques.

00,631

PB96-161310 Not available NTIS

National Inst. of Standards and Technology (CSTL), Gaithersburg, MD. Process Measurements Div.

NIST Assessment of ITS-90 Non-Uniqueness for 25.5 Ohm SPRTs at Gallium, Indium and Cadmium Fixed Points.

Final rept.

G. F. Strouse. 1992, 4p.

Pub. in Temperature: Its Measurement and Control in Science and Industry, v6 pt1 p175-178 1992.

Keywords: "Thermometry, "Fixed points, Temperature, Reprints, International Temperature Scale of 1990, Platinum resistance.

The use of temperature subranges in the definition of the International Temperature Scale of 1990 (ITS-90) permits standard platinum resistance thermometers (SPRTs) to be measured at defining fixed points of a particular subrange as well as at fixed points not utilized in that subrange. These redundant fixed points allow analysis of the non-uniqueness of the scale. Specifically, with $25.5 \mathrm{ohm}$ SPRTs at gallium, indum, and cadmium fixed points have been made and examined to determine the non-uniqueness at these points. The results of this investigation indicate that the levels of non-uniqueness of the ITS-90 at the three redundant fixed points does not contribute significantly to the total error associated with the calibration of an SPRT.

\section{0,632}

PB96-161328 Not available NTIS

National Inst. of Standards and Technology (CSTL) Gaithersburg, MD. Process Measurements Div. NIST Implementation and Realization of the ITS-90 Over the Range $83 \mathrm{~K}$ to $1235 \mathrm{~K}$ : Reproducibility, Stability, and Uncertainties.

Final rept.

G. F. Strouse. 1992, $6 p$

Pub. in Temperature: Its Measurement and Control in Science and Industry, v6 pt1 p169-174 1992.

Keywords: "Thermometry, "Fixed points, Temperature, Reprints, International Temperature Scale of 1990 Platinum resistance.

The National Institute of Standards and Technology is using thermometric fixed points to realize the International Temperature Scale of 1990 (ITS-90) and disseminate the temperature scale through the calibrations of standard platinum resistance thermometers over the range of $83.8058 \mathrm{~K}$ to $1234.93 \mathrm{~K}$. This work involved the optimization of experimental techniques and measurement procedures to reduce uncertainty in the data. The realization of the scale allows for an indepth investigation of reproducibility and stability of the fixed points user? to define the :arious temperature subranges of the ITS 90 Additionally, propagation of errors associated with tile ITS-90 is discussed.

PB96-161336 Not available NTIS

Naticin: inst. of Standards and Fechnology (CSTL), Gaithers'surg, MD. Process Measurements Div. 
NIST Measurement Assurance of SPRT Calibrations on the ITS-90: A Quantitative Approach. Final rept

G. F. Strouse, and B. W. Mangum. 1993, 17p. Pub. in Measurement Science Conference, Anaheim CA., January 21-22, 1993, 17p

Keywords: "Thermometry, "Fixed points, High temperature, Reprints, HTSPRT, International Tempera ture Scale of 1990 , Platinum resistance.

The National Institute of Standards and Technology NIST) is responsible for realizing, maintaining and dis seminating the International Temperature Scale of 1990 (ITS-90) for the United States of America. One of the methods used for the dissemination of the scale is the calibration of standard platinum resistance thermometers (SPRTs) and high-temperature SPRTs HTSPRTS), using thermometric fixed points. At NIST, the calibration of these thermometers as defining, interpolating devices for the ITS-90 includes various internal quality control checks in order to minimize the uncertainty associated with a calibration. The internal measurement assurance program incorporated a NIST includes the use of check (HT)SPRTs, contro charts, redundant fixed points, and statistical process control. This program, as well as some results showing fixed-point reproducibility and calibration uncertainties, will be discussed.

\section{0,634}

PB96-161344 Not available NTIS

National Inst. of Standards and Technology (CSTL)

Gaithersburg, MD. Process Measurements Div.

Preliminary Results of a Comparison of Water Triple-Point Cells Prepared by Different Methods.

Final rept.

G. F. Strouse, G. T. Furukawa, and B. W. Mangum.

1993, 23p.

Pub. in Comite Consultatif de Thermometrie (CCT), 18 Paris, France, September 1993, 23p.

Keywords: "Thermometry, "Fixed points, Temperatures, Reprints, International Temperature Scale of tures, Reprints, Internation
1990, Platinum resistance.

The International Temperature Scale of 1990 provides greater flexibility than the International Practice Tem perature Scale of 1968. Amended Edition 1975 through the use of more fixed points and more temperature subranges. The present investigation examined the ranges of $83.8058 \mathrm{~K}$ to $273.16 \mathrm{~K}$ and 273.15 $\mathrm{K}$ to $933.473 \mathrm{~K}$ for 25.5 omega standard platinum re sistance thermometers. These two ranges have multiple subranges that partly overlap, creating inconsist encies. The magnitude of the inconsistencies in the interpolation between the appropriate defining fixed points in the temperature overlap regions were invespoints in the temperature overlap regions were inves binations of subranges, there were no significant diferences between manufacturer/models and that the inconsistencies in most cases are within estimated measurement uncertainties.

\subsection{5}

PB96-164041 Not available NTIS

National Inst. of Standards and Technology (CSTL) Boulder, CO. Thermophysics Div.

Simple and Efficient Methane-Marker Devices for Chromatographic Samples.

Final rept.

T. J. Bruno. 1996, $8 p$

Pub. in Jnl. of Chromatography A, v721 p157-164 1996.

Keywords: "Chromatography, Reprints, "Hold-up times, "Methane-marker devices, Retention times.

Calculation of the most useful gas chromatographic retention parameters such as net and specific retention volumes, relative retentions, and retention indices, requires that raw retention data be corrected for gas hold-up time or volume (sometimes called dead time or volume) of the chromatographic system. When a flame-ionization detector is used, a common technique (where it is physically appropriate) is to introduce methane into the sample as a marker to approximate unretained species to correct for hold-up. This is often done by bubbling methane (or natural gas) through the sample just before injection. In this paper, we describe two easily constructed devices that provide continuous release of methane into liquid samples, sustainable for several weeks or even montlis. This long-ierm feature makes the techniques especialy suitable to extensive reter:tion studies done at multiple temperatures as several stationary phases. Moreover, one of the devices described is applicable to commercially available auto matic sampler vials. After a detailed description of the consiruction of the devices is provided, some applications are discussed.

00,636

PB96-164272 Not available NTIS

National inst of Standards and Technology (CSTL) Gaithersburg, MD. Inorganic Analytical Research Div Certifying the Chemical Composition of a Biological Material: A Case Study.

Final rept.

R. Zeisler, D. A. Becker, and T. E. Gills. 1995, $5 p$.

Pub. in Fresenius Jnl. of Analytical Chemistry, n352 p111-1151995.

Keywords: "Chemical compositions, *Biological materials, "Trace elements, Comparative evaluations, Reprints, Food, *Foreign technology, Analytical Quality Control Services.

A worldwide laboratory intercomparison was organized by the Intel, ational Atomic Energy Agency's Analytical Quality Control Services (AOCS) involving the determination of trace elements in plant materials used for human consumption. The National Institute of Standards and Technology (NIST) Standard Reference Materials Program donated $5 \mathrm{~kg}$ of spinach designated for the production of the future Standard Reference Material SRM 1570a to this intercomparison: the AQCS provided a similar amount of cabbage. For the study, 150 units of each material were distributed and 114 laboratories reported results on both materials to AQCS. The results for the spinach, encompassing more than forty elements, have been compiled and evaluated; estimates of the elemental concentrations were made based on statistical evaluations, principles of analytical procedures and the laboratory performance indicated by the results on the cabbage material. Satisfactory estimates were obtained for 27 elements. Comparison with IAEA laboratory and NIST reference data did not reveal any significant bias that might have been introduced by the intercomparison approach or its evalua tion.

\section{0,637}

PB96-167168 Not available NTIS

National Inst. of Standards and Technology (CSTL). Gaithersburg. MD. Analytical Chemistry Div.

Supercritical Fluid Extraction-Immunoassay for the Rapid Screening of Cocaine in Hair.

Final rept.

J. F. Morrison, S. N. Chesler, and J. L. Reins. 1996 , $9 p$.

Plu $\mathrm{Jnl}$ of Microcolumn Separations, v8 n1 p37451996.

Keywords: "Cocaine, "Hair, "Radioimmunoassay, Reprints, Supercritical fluid extraction.

Supercritical fluid extraction-radioimmunoassay (SFERIA) was evaluated as a rapid screening tool for the detecion of cocaine residues in human hair. SFE was performed using carbon dioxide modified with triethylamine (TEA) and water, with off-line collection of extracted cocaine in methanol. Extracts were analyzed for the presence of cocaine using a commercially available solid-phase RIA kit. In order to develop a suitable RIA calibration method for reliable measurement of cocaine in SF extracts, calibration data was compared for methanolic standards both with and without the presence of the SF modifier. Methanol had only a minor impact on immunoassay performance, producing an $11 \%$ decrease in maximum binding counts. In contrast, the presence of TEA/H2O profoundly degraded assay performance, producing a $60 \%$ suppression in assay counts. To preserve RIA sensitivity, SF extracts were evaporated under nitrogen to remove the modifier and reconstituted in menthanol for RIA analysis. Incorporation of the evaporation step permitted the use of modifier-free cocaine calibrators in memthanol. Calibration data was fit to a four-parameter logistic model. SFE-RIA analysis of a series of drug-free hair samples established an RA cut-off value for distinguishing between a negative and presumptive positive cocaine sample at an SF extract concentration of 1.2 $\mathrm{ng} / \mathrm{mL}$, or a hair concentration of $0.07 \mathrm{ng} / \mathrm{mg}$. The robustness of the SFE-RIA method was demonstrated by the analysis of a variety of hair samples from both users and non-users. The quantitative SFE-RIA findings correlated well with the values obtained by an acid incubation/GC-MS method.

\section{0,638}

PB96-167283 Not available NTIS
National Inst of Standards and Technology (CSTL), Gaithersburg, MD. Inorganic Analytical Research Div. Resolution of Discrepant Analytical Data in the Certification of Platinum in Two Automobile Catalyst SRMs

Final rept.

D. A. Becker. 1995, 3p.

Pub. in Fresenius Jnl. of Analytical Chemistry, v352 p224-226 1995 .

Keywords: "Automobile catalyst, Analytical interference, Accuracy, Reprints, Quality assurance, technology, Standard reference materials, Plantinum determination.

The National Institute of Standards and Technology (NIST) recently developed two recycled automobile catalyst materials as standard reference materials SRMS), in response to a request from the Internationa Precious Metals Institute. Results of analyses for platinum by two different analytical techniques did not agree well, with the mean differing up to $6 \%$. The palladium results for these same two techniques in the same materials showed much better agreement. Finally, it was determined that on technique (instrumental neutron activation analysis - INAA) had a small, random interference due to a tantalum impurity in/on the acid washed polyethylene bag used to hold the finely powered sample. This $\mathrm{Ta}$ was not present in any of the empty bags used to evaluate the bag blank. Once this problem was identified, the unique characteristics of the INAA technique allowed the magnitude of the interference to be accurately determined for each sample, data recalculated, and a final result obtained which agreed well with the second technique.

\section{0,639}

PB96-167291 Not available NTIS

National Inst. of Standards and

Permeation Tube Approach to Long-Term Use of Automatic Sampler Retention Index Standards.

Final rept.

T. J. Bruno. 1995, 6p.

Pub.

Keywords: "Alternative refrigerants, "Chromatographic analysis, Reprints, Hydrocarbons, Permeation tubes, Chromatographic samples.

A permeation tube that is sealed internally in a commercially-available automatic sampler vial provides a simple and convenient method of preparing, using, and storing standard retention index samples for long periods of time. The approach is especially suited to the handling of volatile organic compounds that are ver important in fuels research, alternative refrigerant research and in many environmental analyses. It also provides the very desirable feature of dispensing sample that is at very low concentration or even at infinite dilution since no commercial automatic sampler is currently capable of doing this for the analyst. The device is very simple, and can be constructed and prepared with a liquid sample in a few minutes. It requires the use of minimal quantities of sample, and substantially descreases the hazards associated with handling volatile organics in the laboratory.

00,640

PB96-190061 Not available NTIS

National Inst. of Standards and Technology (CSTL) Gaithersburg, MD. Analytical Chemistry Div.

solation and Structural Elucidation of the Pre dominant Geometrical Isomers of alpha-Carotene. Final rept.

C. Emenhiser, G. Englert, L. C. Sander, B. Ludwig, and S. J. Schwartz. 1996, 11 p.

Pub. in Jnl. of Chromatography A, v719 p333-343 May 96.

Keywords: "Carotene, "Isomers, "Stereochemistry Isomerization, Chromatographic analysis, Carotenoids, Polymers, Carbon 30, Spectroscopic analysis, Reprints, Liquid chromatography, Geometrical isomers, Shape selectivity.

The recent development and application of a polymeric C30 stationary phase have given unique separations t cis-trans carotenoid isomers in reversed-phase (RP) liquid chromatography (LC) owing to the expectiona shape selectivity of this stationary phase. In the present research, several geometrical isomers of alpha-carotene were at least partially resolved from a photo-isomerized mixture when chromatographed on 


\section{Analytical Chemistry}

a 3-micro m polymeric $\mathrm{C} 30$ column. Confirmation of the structures of geometrical alpha-carotene isomers will aid further studies on the possible physiological roles of these compounds in biological tissues.

\section{0,641}

\section{PB96-190277 Not available NTIS}

National Inst. of Standards and Technology (CSTL), Gaithersburg, MD. Analytical Chemistry Div.

Embossable Grating Couplers for Planar Waveguide Optical Sensors.

Final rept.

B. L. Ramos, S. J. Choquette, and N. F. Fell. 1996 ,

Pub. in Analytical Chemistry, v68 n7 p1245-1249 1996.

Keywords: "Grating coupling, "Optical sensors, *Embossable grating, Reprints, Planar waveguide.

Planar optical waveguides are an attractive tool for use in analytical chemistry and spectroscopy. Although similar to fiber optics, planar waveguides have been slow to be commercially accepted due to the difficulty of coupling light into the guide. Generally, prism coupling is the method of choice in the laboratory, as efficiencies approaching $80 \%$ can be reached. However prisms are impractical for routine use for several reasons: expensive positioning equipment is required coupled power is sensitive to environmental fluctuations, and prism coupling prohibits the fabrication of a truly planar device. The use of thin gratings on the surface of the waveguide allows for a two-dimensional structure to be maintained, while providing enough efficiency to be useful as a sensor. The research efforts focus on developing a technique to make inexpensive, reproducible gratings that are easy to fabricate. By chemically modifying the surface of a commercial grating with a suitable release agent, it is possible to emboss replica gratings onto a variety of waveguide types. The fabrication of embossed gratings will be de scribed, and their performance on glass, ion-diffused, polymer, and semiconductor waveguides will be presented.

\section{0,642}

PB96-200951 Not available NTIS

National Inst. of Standards and Technology (CSTL), Gaithersburg, MD. Analytical Chemistry Div.

Flow Immunoassay Using Solid-Phase Entrapment.

Final rept.

L. Locascio-Brown, L. Martynova, R. G. Christensen, and G. Horvai. 1996, 6p.

Pub. in Analytical Chemistry, v68 n9 p1665-1670 May 96.

Keywords: "Flow injection, "Immunoassay, Sorbents, Separation, Assays, Reprints, "Solid-phase entrapment

A flow injection immunoassay was performed using a column packed with reversed-phase sorbents to effect separation of the immunoreacted species by entrapping free analyte and allowing antibody-conjugated analyte to pass unretained. Fluorescein-labeled analyte was measured in a competitive assay for the anticonvulsant drug phenytoin. The simplicity of the assay was the greatest advantage of the technique, which allowed for measurement of phenytoin in a 2 min assay time. The reliable detection limit for the assay was $5 \mathrm{nmol} \mathrm{L}$ to the minus 1 power of phenytoin in serum. The columns were regenerated with periodic injections of ethanol solutions to remove the entrapped analyte and prepare the column for subsequent analyses.

\section{0,643}

\section{PB96-201041 Not available NTIS}

National Inst. of Standards and Technology (CSTL) Gaithersburg, MD. Chemical Kinetics and Thermodynamics Div.

UV Absorption Cross Sections of and Liquid Phase Measurements.

Final rept.

A. K. Nayak, M. J. Kurylo, and A. Fahr. 1995, 5p.

Pub. in Jnl. of Geophysical Research, v100 nD6 p11,185-11,189 Jun 95.

Keywords: "Tungsten silicide, "Formation enthalpy, *Calorimetry, Analytical chemistry, Reprints, "Foreign technology, Methylchloroform.

The absorption cross sections for methylchloroform $(\mathrm{CH} 3 \mathrm{CCl} 3$ ) have been measured in the gas phase (from 160 to approximately $240 \mathrm{~nm}$ ) and in the liquid phase (from approximately 235 to $260 \mathrm{~nm}$ ) over the temperature range $220-330 \mathrm{~K}$. The liquid phase results were converted into effective gas phase cross sections using a wavelength shift procedure described and verified in earlier work. The results are compared with other available data in the wavelength region 185-240 $\mathrm{nm}$. The multiplicity of measurements in different laboratories lends increased confidence in the quantification of uncertainties in the atmospheric (photolysis and kinetic) lifetime calculations for this important chemical species.

\section{0,644}

PB96-210786 PC A04/MF A01

National Inst. of Standards and Technology (CSTL), Gaithersburg. MD. Analytical Chemistry Div.

NIST Traceable Reference Material Program for Gas Standards: Standard Reference Materials.

Special pub

F. R. Guenther, W. D. Dorko, W. R. Miller, and G. C. Rhoderick. Jul 96, 44p, NIST-SP-260-126.

Also available from Supt. of Docs. as SN003-00303419-3.

Keywords: "Gas analysis, "Calibration standards, *Tracer techniques, Gas mixtures, Samples, Concentration(Composition), Certification, Validation Maintenance, Interlaboratory comparisons, Data quality, Quality assurance, "Standard Reference Materials. A program is described by which the concentration of commerically produced gas mixtures may be related to gaseous primary standards maintained by the $\mathrm{Na}$ tional Institute of Standards and Technology (NIST) The responsibilities of the producer and NIST are detailed along with recommended procedures the producer should follow during production and analysis of the mixtures. Procedures also are included for the maintenance of NTRM batches. Appendices are included for the preparation of NTRMs related to various Standard Reference Materials.

\section{0,645}

PB96-210877 PC A05/MF A01

National Inst of Standards and Technology (CAML). Gaithersburg, MD. Statistical Engineering Div.

Statistical Aspects of the Certification of Chemical Batch SRMs. Standard Reference Materials. Special pub.

S. B. Schiller. Jul 96, 52p, NIST-SP-260-125

Also available from Supt. of Docs. as SN003-00303416-9. See also PB95-143087.

Keywords: "Chemical analysis, "Calibration standards, "Certification "Statistical analysis, Analytical methods, Sampling, Chemical composition, Homogeneity, Uncertainty, Confidence level, Accuracy, Precision, Quality control, Quality assurance, "Standard reference materials

The accurate determination of chemical analytes in batches of material is the principal requirement in chemical Standard Reference Material (SRM) certification. Many measurement made for chemical constituent batch SRMs are destructive, and batches are usually large, so selecting a random but representative sample from the batch is vital to inference about the material. Homogeneity assessment must be done to verify the materials suitability for sale, and to determine what type of statistical interval will make an appropriate summary for the certificate. Finally, results from more than one independent chemical method often must be combined in a statistically meaningful way to arrive at a realistic estimate of the uncertainty of the results achieved. This paper provide guidelines for addressing these statistical issues. The motivations behind those guidelines are also explained to facilitate understanding of them.

\section{0,646}

PB96-214614 PC A04/MF A01

National Inst. of Standards and Technology (CSTL) Gaithersburg, MD. Surface and Microanalysis Science Div

Airborne Asbestos Method: Bootstrap Method for Determining the Uncertainty of Asbestos Concentration. Version 1.0.

S. Turner, R. Myklebust, B. B. Thorne, S. D

Leigh, and E. B. Steel. Aug 96, 36p, NISTIR-5723.

Sponsored by Environmental Protection Agency, Washington, DC

Keywords: "Asbestos, "Test methods, "Chemical analysis, "Statistical analysis, "Standards, Electron microscopy, Air pollution detection, Quality assurance, Air filters, Air sampling, "Transmission electron microscopy.
The determination of the uncenainty of the analytical value for the concentration of asbestos in air is of practical use for: (1) comparison of analyses obtained by different operators or laboratories on the same sampling area and (2) providing an indication of how an analytical value compares with values set by government regulations. This test method describes a procedure for determining the component of uncertainty that is due to variation in sampling of a population of values (corresponding to the Type A evaluation of uncertainty described in NIST Technical Note 1297). The test method describes use of a bootstrapping procedure for determination of a $95 \%$ confidence interval for the concentration of asbestos deposited onto filters.

\section{0,647}

PB96-214630 PC A10/MF A03

National Inst. of Standards and Technology (CSTL), Gaithersburg, MD.

CSTL Technical Activities, 1995.

H G Semeriian, and W. F. Koch, 1995, 200p,

NISTIR-5828.

See also report for 1994, PB95-242319.

Keywords: "Chemistry, "Research and development, Chemical engineering, Biotechnology, Measurements, Metrology, Models, Measurement traceability, Process technology, Reference data.

This report summarizes the research and services provided by the Chemical Science and Technology Laboratory of the National Institute of Standards and Technology for Fiscal Year 1995. The report includes: a general overview of the laboratory's activities, a summary of the technical accomplishments in the six CSTL divisions - Biotechnology Chemical Kinetics and Thermodynamics. Process Measurements, Surface and Microanalysis, Thermophysics and Analytical Chemistry, and a description of 130 selected technical projects.

00,648

PB97-109037

MF A03)

Gmelin Inst. for Inorganic Chemistry of the Max Plank Society, Frantfurt (Germany).

Inorganic Crystal Structure Database (ICSD) and Standardized Data and Crystal Chemical Characterization of Inorganic Structure Types (TYPIX): Two Tools for Inorganic Chemists and Crystallographers.

E. Fluck. 1996, 4p

Included in Jnl. of Research of the National Institute of Standards and Technology, v101 n3 p217-220 May/ Jun 96

Keywords: *Crystallography, "Inorganic compounds, *Information systems, "Chemical composition, Data base management.

The two databases ICSD and TYPIX are described, ICSD is a comprehensive compilation of crystal structure data of inorganic compounds (about 39,000 entries). TYPIX contains 3600 critically evaluated data sets representative of structure types formed by inorganic compounds.

\section{0,649}

PB97-109045 (Order as PB97-109011, PC A11/ MF A03)

Bonn Univ. (Germany, F.R.). Inst. for Inorganic Chemistry.

Evaluation of Crystallographic Data with the Program DIAMOND.

G. Bergerhoff, M. Berndt, and K. Brandenburg. 1996, $5 p$.

Included in Jnl. of Research of the National Institue of Standards and Technology, v101 n3 p221-225 May' Jun 96

Keywords: "Crystallography, "Chemical composition, "Information systems, "Inorganic compounds, Information retrieval, Crystal structure, Computer graphics, Database management.

The new crystal structure information system DIAMOND is presented. It handles all kinds of crystallographic databases on PCs including SHELX files and Chemical Information Files (CIF's). DIAMOND, because of its graphics capability, is a powerful tool for establishing structural relationships and for evaluating crystallographic data. 
National Inst. of Standards and Technology (CSTL), Gaithersburg, MD. Analytical Chemistry Div.

Role of Certified Reference Materials in Trace Analysis Quality Assurance.

Final rept.

D. A. Becker. 1995, 4p.

Pub. in Proceedings of Polish Conference on Analytical Chemistry (5th), Gdansk, Poland, September 3-8, 1995, p39-42.

Keywords: "Standards, Chemical analysis, Reprints, "Foreign technology, "Certified reference materials, * Standards reference materials.

The quest for accuracy in trace element chemical analysis requires a constant effort by the analyst in the 10cation, elucidation, determination and elimination of error in any of its many forms. At NIST, the authors obviously have a high degree of interest in obtaining the highest accuracy and precision practicable, for certification of elemental content in NIST Standard Reierence materials (SRM). The paper will discuss the author's concept of the role of Certified Reference Materials (CRM) in trace analysis quality assurance, with specific illustrations from the author's work in neutron activation analysis (NAA).

\section{0,651}

PB97-111975 Not available NTIS

National Inst. of Standards and Technology (CSTL), Gaithersburg, MD. Chemical Kinetics and Thermodynamics Div.

Oxidation of Caffeic Acid and Related Hydroxycinnamic Acids.

Final rept.

P. Hapiot, A. Neudeck, J. Pinson, C. Rolando, H.

Fulcrand, and P. Neta. 1996, $8 \mathrm{p}$

Pub. in Jnl. of Electroanalytical Chemistry, v405 p1691761996.

Keywords: "Oxidation, "Caffeic acid, Electrooxidation, Ferulic acid, Pulse radiolysis, Reprints, 4-coumaric acid.

Oxidation of caffeic acid (3,4-dihydroxycinnamic acid) $1 \mathrm{H} 3$ has been studied by electrochemical methods and by pulse radiolysis in aqueous and organic solvents. The results have been compared with the behavior of 4-coumaric acid $2 \mathrm{H} 2$ and ferulic acid $3 \mathrm{H} 2$. The first oxidative intermediates have been characterized by their UV spectra and oxidation potentials. In case of $2 \mathrm{H} 2$ and $3 \mathrm{H} 2$, the initial radicals decay by a second order process indicating a radical-radical coupling mechanism. On the contrary, for caffeic acid the oxidation leads to the formatuion of the corresponding o-quinone through disproportionation of the initial semiquinone radical.

\section{0,652}

PB97-112437 Not available NTIS

National Inst. of Standards and Technology (CSTL), Gaithersburg, MD. Analytical Chemistry Div.

Use of Neutron Beams for Chemical Analysis at NIST.

Final rept.

E. A. Mackey, D. L. Anderson, H. Chen-Mayer, G. P. Lamaze, R. M. Lindstrom, D. F. R. Mildner, R. L. Paul, R. G. Downing, and R. R. Greenberg. 1996, $15 p$.

Pub. in Jnl. of Radioanalytical and Nuclear Chemistry, Articles, v203 n2 p413-427 1996.

Keywords: "Quantitative chemical analysis, "Neutron capture gamma rays, Cold neutrons, Neutron activation analysis, Neutron scattering, Reprints, Neutron focusing, Neutron depth profiling, Target irradiation.

At the National Institute of Standards and Technology, there are two techniques for chemical analysis that use neutron beams from the reactor for target irradiation: neutron depth profiling (NDP) and prompt gamma-ray activation analysis (PGAA). The paper includes a brief description of the facilities, the measurement capabilities of each, some recent applications of NDP and PGAA, and neutron focusing as applied to these techniques.

\section{0,653}

PB97-113260 Not available NTIS

National Inst. of Standards and Technology (CSTL).

Gaithersburg, MD. Analytical Chemistry Div.
Use of a Naphthylethylcarbamoylated- betaCyclodextrin Chiral Stationary Phase for the Separation of Drug Enantiomers and Related Compounds by Sub- and Supercritical Fluid Chromatography. Final rept.

K. L. Williams, L. C. Sander, and S. A. Wise. 1996,

Pub. in Chirality, v8 p325-331 Sep 96

Keywords: "Enantiomeric separation, "Chrial recognition, Derivatized cyclodextrin, Chromatography, Drug analysis, Reprints.

Enantiomeric separation of a variety of drugs and relat ed compounds was achieved on an (S) NEC-CD) chiral stationary phase (CSP) using sub-and subcritical fluid chromatography (SFC). Compounds previously resolved on native or derivatized cyclodextrin CSPS in liquid chromatography (LC) using reversed phase or polar organic mobile phase modes could be resolved in SFC using a simple carbon diox ide/methanol eluent. Resolution of cromakalim, which is not possible on the S-NEC-CD column in LC, was readily accomplished in SFC. The importance of modifier, temperature, and pressure was assessed in relation to retention, selectivity, and resolution. The nature of the modifier and the modifier concentration were found to be crucial parameters.

00.654

PB97-119002 Not available NTIS

National Inst. of Standards and Technology (MSEL) Gaithersburg, MD.

Segmental Concentration Profiles of End-Tethered Polymers with Excluded-Volume and Surface Interactions.

Final rept.

M. Adamuti-Trache, W. E McMullen, and J.F.

Douglas. 1996, 14p.

Pub. in Jnl. of Chemical Physics, v105 n11 p47984811 Sep 96.

Keywords: *Grafted polymer layer, "Excluded volume, "Surface interaction, Concentration profiles, Reprints, End-tethered polymers.

The segmental concentration profile $p$ of end-tethered flexible polymer chains is calculated for comparison with recent measurements on polymer layers having a low surface grafting density omega. Moments of $p$ are also calculated as these quantities, rather than the ull concentration profiles, are usually measured experimentally. Exact calculations of $p$ are summarized for ideal flexible chains with an arbitrary short-range polymer-surface interaction. Though technical difficulties restrict the renormalization group (RG) calcula tions for swollen chains to limiting values of the polymer-surface interaction, the analysis indicates that the relatively simple, closed-form, Gaussian-chain expression for $p$ provides a good approximation to the $R G$ results if the average size of the Gaussian chain is replaced by its swollen-chain analog. This approximate concentration profile for chains with excluded volume and variable polymer-surface interaction should prove useful when interpretating experimental results for lowgrafting-density polymer layers formed in good solvents.

00,655

PB97-119242 Not available NTIS

National Inst. of Standards and Technology (CSTL) Gaithersburg, MD Process Measurements Div.

Comparisons of Some NIST Fixed-Point Cells with Similar Cells of Other Standards Laboratories. Final rept.

B. W. Mangum, E. R. Pfeiffer, G. F. Strouse, T. I Yeh, $P$. Marcarino, R. Dematteis, $Y$. Liu, $Q$. Zhao, A. T. Ince, F. Cakiroglu, H. G. Nubbemeyer, H. J. Jung. J. Valencia-Rodriguez, and J. H. Lin. 1996, 11 p. Pub. in Metrologia, v33 n3 p215-255 1996

Keywords: "Fixed points, "Temperature measurement, Interlaboratory comparisons, Gallium, Water, Tin, Indium, Zinc, Aluminum, Silver, Thermometry, Calibra tion standards, Uncertainty, Reprints, International Temperature Scale of 1990

In the paper, the authors present results of international comparisons of fixed-point cells of some of the defining fixed-point materials of the International Temperature Scale of 1990 . These comparisons involved cells from seven national laboratories, although in some cases only one type of fixed-point material from the various laboratories was to within $1 \mathrm{mK}$.

\section{Basic \& Synthetic Chemistry}

\section{0,656}

\section{AD-A286 620/0 PC A03/MF A01}

National Bureau of Standards, Gaithersburg, MD.

Fuzed-Quartz Fibers. A Survey of Properties, Applications and Production Methods.

25 Jan 56, 30p, NBS-569.

Keywords: "Fused silica, "Fibers, "Quartz, Fabrication, Hardness, Silica glass, Thermal expansion, Viscosity, Elastic properties, Surveys.

Fused-silica fibers have an important function in many measuring instruments used in scientific research. Much of the information on the production and fabrication methods and on the properties of the fibers is widely scattered throughout the technical literature. This Circular is a survey of this literature and a summary findings. A bibliography of pertinent references on the subject is included to provide the sources of more complete and detailed information necessary for specific applications. (Author)

\section{0,657}

\section{AD-A297 265/1 PC A03/MF A01}

National Bureau of Standards, Gaithersburg MD. Physical Properties of Some Purified Aliphatic Hydrocarbons.

Research paper.

D. B. Brooks, F. L. Howard, and H. C. Crafton. Jan $40,13 p$

Pub in Jnl of Research of the National Bureau of Standards, v24.

Keywords: "Physical properties, "Refractive index, "Aliphatic hydrocarbons, Density, Temperature, High rate, Methyl radicals, Efficiency, Alkanes, Purification, Freezing, Pressure, Aviation fuels, Butenes, Ethyl radicals, Butanes, Distillation, Pentanes, Olefin polymers, Fractionation, Heptanes, Reprints, *Paraffin hydrocarbons, *Oiling point, Dimethylbutene, Dimethylbutane, Ethylpentane, Heptane.

In an investigation of the suitability of various paraffin hydrocarbon constituents of aviation fuel, which is being conducted for the National Advisory committee for Aeronautics, the Navy Bureau of Aeronautics, and the Army Air Corps, four olefin and seven paraffin hydrocarbons have been obtained in a state of high purity. Eight of these materials were synthesized, on was isolated from a commercial synthetic crude, and two were obtained from commercial sources. All were purified by distillation in automatically controlled fractionating columns of high efficiency. The measured physical properties of these materials included freezing point, oiling point and its variation with pressure, refrac tive index and density and their variations with temperature. jg p. 1

\section{0,658}

\section{PBg4-211208 Not available NTIS}

National Inst. of Standards and Technology (MSEL) Gaithersburg, MD. Polymers Div.

Proteoglycan Inhibition of Calcium Phosphate Pre cipitation in Liposomal Suspensions.

Final rept.

E. D. Eanes, A. W Hailer R J Midura, and V C.

Hascall. 1992, 8p.

Sponsored by National Inst. of Dental Research, Bethesda, MD

Pub. in Glycobiology 2, n6 p571-578 1992

Keywords: "Proteoglycans, "Liposomes, "Calcium phosphates, Precipitation, Cartilage, Calcification, Chondroitin sulfate, Calcium, Reprints, Aggrecan.

The major proteoglycan in cartilage (aggrecan) is a complex macromolecule with numerous chondroitin sulphate, keratan sulphate, and oligosaccharide substituents. It has been proposed that this macromolecule has an important role in regulating mineralization in this tissue a process which is initiated by the deposition of apatite in matrix vesicles. We have used a liposome-centered endogenous precipitation method as a model for matrix vesicle mineralization to study the effect of the rat chondrosarcoma aggrecan and its chondroitin sulphate and core protein components on apatite formation from solution. Aggrecan $(0.5 \%)$ in the suspending medium had no effect on intraliposomal precipitation, but severely reduced (ap proximately $70 \%$ reduction at $24 \mathrm{~h}$ ) its subsequen spread into the medium. The chondroitin sulphate and core protein were similarly inhibitory. The degree to which aggrecan and its constituent parts inhibited pre- 
cipitation correlated with their capacity to bind $\mathrm{Ca}(2+)$ ions. These findings suggest that functional groups in aggrecan blocked apatite growth by linking via $\mathrm{Ca}(2+)$ bridges to growth sites on the crystal surfaces. Similar Ca-mediated interactions may well have a critical regulatory role in cartilage mineralization.

\section{0,659}

\section{PB95-151981 Not available NTIS}

National Inst. of Standards and Technology (NML), Gaithersburg. MD. Chemical Process Metrology Div. Flame Synthesis of High Tc Superconductors.

Final rept.
M. R. Zachariah, and S. Huzarewicz. 1991, 4p.

Pub. in Combustion and Flame 87, n1 p100-103 1991.

Keywords: "High temperature superconductors, "HighTC superconductors, "YBCO superconductors, Diffusion flames, Particle size distribution, Reprints, "Flame synthesis, "Yttrium barium cuprates.

High temperature superconducting particles of the 1:2:3 Yttrium-Barium-Copper oxide system have been synthesized in an inverted configuration coannular diffusion flame. The particles were produced from a spray pyrolysis technique, employing aerosolized nitrate salts of $\mathrm{Y}, \mathrm{Ba}$ and $\mathrm{Cu}$. Particles produced showed a transition temperature of $92 \mathrm{~K}$ as determined by magnetic susceptibility measurements. The particles were shown to have a wide particle size distribution, ranging from 10 to $1000 \mathrm{~nm}$. It was also observed that due to the effects of water vapor reactions at high temperatures, only a diffusion flame successfully produced the correct phase.

\section{0,660}

PB95-161535 Not available NTIS

National Inst. of Standards and Technology (MSEL), Gaithersburg, MD. Polymers Div.

Octacalcium Phosphate Carboxylates. 1. Preparation and Identification.

Final rept.

M. Markovic, B. O. Fowler, and W. E. Brown. 1993,

$5 \mathrm{p}$

See also Part 2, PB95-161543. Sponsored by American Dental Association Health Foundation, Chicago, Pub. in Chem. Mater. 5, n10 p1401-1405 1993.

Keywords: "Chemical preparation, *Chemical analysis, Dentistry, Calcium phosphates, X-ray diffraction, Infrared spectroscopy, Raman spectroscopy, Ions, Molecular structure, Precipitation(Chemistry), Minerals, Calcification, Reprints, "Octacalcium phosphate carboxylates.

The formation of octacalcium phosphate carboxylates, $\mathrm{Ca} 8(\mathrm{HPO} 4) \mathrm{m}$ (carboxylate)n(PO4)4-yH2O, by conversion of alpha-Ca3(PO4)2 in solutions of 19 ammonium carboxylates (monocarboxylates; saturated, unsaturated, hydroxy, keto, and amino dicarboxylates; and tricarboxylates) was investigated. The various solid phases formed, depending on initial pH's and conversion times, were determined by $X$-ray diffraction and infrared and Raman spectroscopy. Octacalcium phosphate carboxylates containing structurally incorporated malonate, succinate, adipate, suberate, sebacate, fumarate, malate, and citrate ions were formed and identified. Octacalcium phosphate carboxylates were also formed from pyruvate and alpha-ketoglutarate solutions but with uncertain carboxylate ion structures. All of these identified compounds are structurally similar to octacalcium phosphates, Ca8(HPO4)2(PO4)4$5 \mathrm{H}_{2} \mathrm{O}$, but have expanded a-axis unit-cell dimensions that generally increased with increasing number of carbon atoms in the carboxylate ion. Among these compounds, of special importance are those containing carboxylates that are present as intermediates in the Krebs cycle. The possible precipitation of these octacalcium phosphate carboxylates in mitochondria and their possible role as precursors in calcified tissue formation are discussed.

\section{0,661}

PB95-163317 Not available NTIS

National Inst. of Standards and Technology (CSTL), Gaithersburg, MD. Biotechnology Div.

Equilibrium and Calorimetric Investigation of the Hydrolysis of L-Tryptophan to (Indole + Pyruvate + Ammonia)

Final rept.

Y. B. Tewari, and R. N. Goldberg. 1994, 18p

Pub. in Jnl. of Solution Chemistry 23, n2 p167-184 1994

Keywords: "Calorimetry, "Hydrolysis, "Tryptophan, *Indoles, *Ammonia, "Pyruvates, "Chemical reactions,
*Equilibrium, High pressure liquid chromatography, $\mathrm{pH}$, Temperature, Enthalpy, Catalysis, Reprints

Apparent equilibrium constants and calorimetric enthalpies of reaction have been measured for the reaction L-tryptophan (aq) $+\mathrm{H} 2 \mathrm{O}(\mathrm{l})=$ indole $(\mathrm{aq})+$ pyruvate(aq) + ammonia(aq) which is catalyzed by Ltryptophanase. High-pressure liquid-chromatography and microcalorimetery were used to perform these measurements. The equilibrium measurements were performed as a function of $\mathrm{pH}$, temperature, and ionic strength. The results have been interpreted with a chemical equilibrium model to obtain thermodynamic quantities for the reference reaction: L-tryptophan(aq) $+\mathrm{H} 2 \mathrm{O}(\mathrm{l})=$ indole $(\mathrm{aq})+$ pyruvate $(-)(\mathrm{aq})+\mathrm{NH} 4(+)(\mathrm{aq})$ At $\mathrm{T}=25 \mathrm{C}$ and $\mathrm{I}(\mathrm{sub} \mathrm{m})=0$ the results for this reaction are: $K($ sup o $)=(1.05+$ or -0.13$)$ times ten to the minus fourth power, Delta(sub r) G(sup o) $=(22.71+$ or -0.33$)$ $\mathrm{kJ} / \mathrm{mol}$, Delta(sup r) $\mathrm{H}$ (sup o $)=(62.0+$ or -2.3$) \mathrm{kJ} / \mathrm{mol}$ and Delta(sub r)S(sup o) $=(132+$ or -8$) \mathrm{J} / \mathrm{K} / \mathrm{mol}$ These results have been used together with thermo dynamic results from the literature to calculate standard Gibbs energies of formation, standard enthalpies of formation, standard molar entropies, standard molar heat capacities, and standard transformed formation properties for the substances participating in this reaction.

\section{0,662}

PB96-167333 Not available NTIS

National Inst. of Standards and Technology (CSTL) Boulder, CO. Thermophysics Div.

Structure and Rheology of Hard-Sphere Systems. Final rept

S. M. Clarke, J. Melrose, A. R. Rennie, P. J. Mitchell, H. J. M. Hanley, G. C. Straty, R. H. Ottewill, and D. Heyes. 1994, 5p.

Pub. in Jnl. Phys. Condens. Matter, v6 pA333-A337 1994.

Keywords: "Colloidal dispersion "Shear thinning "Rheology, Computerized simulation, Reprints, "Foreign technology, "Small angle neutron scattering

There is need to understand the structure of hardsphere dispersions under flow in order to develop models for colloid rheology since changes in structure are related to non-Newtonian behavior. Small-angle neutron scattering (SANS) data for polymer lattices, behaving as nearly hard spheres, at shear rates through the region of the shear thinning are presented. Intensity distributions from these new and detailed low angle scattering experiments are compared with the in tensities calculated directly from computer simulations employing a Rouse model.

\section{0,663}

PB96-167374 Not available NTIS

National Inst. of Standards and Technology (CSTL), Gaithersburg, MD. Thermophysics Div.

Small Angle Neutron Scattering Study of a Clay Suspension Under Shear.

Final rept.

H. J. M. Hanley, G. C. Straty, and F. Tsvetkov. 1994, $3 p$.

Pub. in Langmuir, v10 p3362-3364 1994.

Keywords: *Clay, Shear, Suspension, Disks, Reprints, *Small angle neutron scattering.

The most useful expression for the orientational probability of cylinders on a suspension subjected to a shear field is that of Hayter and Penfold, reported in 1984. Here an alternative equation is proposed to ac count for the shear induced orientation of disks. Our equation is then used to interpret SANS (small angle heutron scattering) intensity data from dilute aqueous suspensions of Na-montmorillonite. Parenthetically we note that SANS studies on dilute clay suspensions in equilibrium are well-known but that investigations of clay suspensions under shear are not in the literature.

\section{0,664}

PB96-200225 Not available NTIS

National Inst. of Standards and Technology (MSEL), Boulder, CO. Materials Reliability Div.

Accurate Modeling of Size and Strain Broadening in the Rietveld Refinement: The 'Double-Voigt' Approach.

Final rept.

D. Balzar, and H. Ledbetter. 1995, 8p.

Pub. in Advances in X-ray Analysis, v38 p397-404 1995.

Keywords: "Lattice strain, " $X$ ray diffraction, Peak profile, Peak broadening, Reprints, "Rietveld refinement voight function.
In the 'double-Voight' approach, an exact Voigt function describes both size- and strain-broadened profiles. The lattice strain is defined in terms of physically credible mean-square strain averaged over a distance in the diffracting domains. Analysis of Fourier coefficients in a harmonic approximation for strain coefficients leads to the Warren-Averbach method for the separation of size and strain contributions to diffraction line broadening. The model is introduced in the Rietveld refinement program in the following way: Line widths are modeled with only four parameters in the isotropic case. Varied parameters are both surface- and volume-weighted domain sizes and root-mean-square strains averaged over two distances. Refined parameters determine the physically broadened Voigt line profile. Instrumental Voigt line profile parameters are added to obtain the observed (Voigt) line profile. To speed computation, the corresponding pseudo-Voigt function is calculated and used as a fitting function in refinement. The approach allows for both fast computer code and accurate modeling in terms of physically identifiable parameters.

\section{0,665}

PB96-200613 Not available NTIS

National Inst. of Standards and Technology (PL), Boulder, CO. Quantum Physics Div.

Kinetic-Energy-Enhanced Neutral Etching. Final rept.

S. R. Leone. 1995, 10p.

Contract DAAL03-91G-0191, Grant NSF-PHY90-

12244

Sponsored by Army Research Office, Research Triangle Park, NC. and National Science Foundation, Arlington, $V A$.

Pub. in Japan Jnl. of Applied Physics, v34 pt1 n4B p2073-2082 Apr 95

Keywords: *Silicon, *Etching, "Dry processes, Kinetics, Chlorine, Energy, Reprints, "Foreign technology.

A review is presented of the emerging field of neutralspecies kinetic-energy-enhanced etching of silicon. As the gate oxide thickness of metal oxide semiconductor field-effect transistors (MOSFET) is decreased to dimensions of 50 Angstroms or less, the potentially damaging effects of high-kinetic-energy ions in plasma processing become important. New methods are required to remove material in a more refined, selective manner. In this review, the motivations of studies of neutral-species enhanced-kinetic-energy etching are described. Currently available sources of neutral reactive species with enhanced kinetic energies of $1-10 \mathrm{eV}$ are discussed, and published experimental and theoretical investigations of enhanced-kinetic-energy neutral etching are reviewed. Problems associated with neutral species etching are also considered, and some possible future developments in the field are summarized.

\section{0,666}

PB97-122568 Not available NTIS

National Inst. of Standards and Technology (MSEL), Gaithersburg, MD. Reactor Radiation Div.

Determination of Anomalous Superexchange in $\mathrm{MnCl} 2$ and Its Graphite Intercalation Compound.

Final rept.

D. G. Wiesler, M. Suzuki, I. S. Suzuki, and N. Rosov. 1995, 4p.

Pub. in Physical Review Letters, v75 n5 p842-945 Jul 95.

Keywords: "Intercalation compound "Neutron diffractions, Molecular structure, Synthesis, Chemical properties, Reprints, *Graphite, Manganese chloride.

The low-temperature magnetic structure of $\mathrm{MnCl} 2$ graphite intercalation compound has been studied by neutron diffraction. Magnetic peaks occur at wave vectors incommensurate with the $\mathrm{MnCl} 2$ and graphene sublattices. The in-plane spin configuration is explained by an exchange Hamiltonian that includes three shells of nearest neighbors in the plane. The nearest-neighbor exchange is ferromagnetic but anamalously weak, and the magnetic behavior is dominated instead by the antiferromagnetic third-neighbor interaction. The exchange parameters are used to explain the spin configuration of bulk $\mathrm{MnCl} 2$ after adding an interplanar coupling. 


\section{Industrial Chemistry \& Chemical Process Engineering}

\section{0,667}

DE95013079 PC A03/MF A01

National Inst. of Standards and Technology (CSTL), Boulder, $\mathrm{CO}$

Distributed measurements of tracer response on packed bed flows using a fiberoptic probe array. Final report.

PROGRESS REPT.

M. C. Jones, R. Nassimbene, J. Wolfe, and N

Frederick. 28 Oct 94, 28p, DOE/ER/13770-T3.

Contract A105-87ER 13770

Sponsored by Department of Energy, Washington, DC. Keywords: "Extraction, *Packed Beds, Chromatography, Columns(Process engineering), Column packings, Dyes, Experimental Data, Fiber Optics, Flow Models, Flow Visualization, Fluid Flow, Fluorescence, Mass Transfer, Progress Report, Tracer Techniques, Tables(Data), EDB/400105.

Scale-up of packed bed processes, particularly those involving chromatographic separations, is made difficult by a seemingly inevitable increase in dispersion due to packing nonuniformity. To provide a suitable characterization, the authors measured the spatial discharacterization, the authors measured the spatial dis-
tribution of dispersion and mixing in packed beds of uniform impervious spherical glass particles by a tracer impulse technique. The key feature in this work is the use of a fiberoptic array at the exit plane to obtain a time-resolved spatially distributed response All experiments were in the creeping flow regime. The authors used a fluorescent dye with laser excitation through the fiber terminations in the bed. The fluoresced radiation was collected through the same fibers. They analyzed the data by the use of indices of the extent of micromixing based on Danckwerts's original degree of segregation and an additional index of structural uniformity. The computations involve a moment analysis of the individual and average probe responses. A simple model gives expressions for the indices in terms of the Peclet number and is shown to provide a useful limiting case. The computed indices are also shown to be very sensitive to adsorption of dye on the surface of the glass. However, for some of the experiments with the largest spheres using Pyrex glass, the effects of adsorption are indiscernible. This technique successfully separates the contribution of micromixed fluid to overall bed dispersion from the contribution due to the transverse variation of the flow residence time.

00,668

PB94-199221 Not available NTIS

National Inst. of Standards and Technology (NML), Boulder, CO. Thermophysics Div.

Thermophysical Property Data for Supercritical Fluid Extraction Design.

Final rept.

T. J. Bruno. 1991, 32p.

Pub. in Supercritical Fluid Technology: Reviews in Modern Theory and Applications, Chapter 7, p293-324 1991.

Keywords: *Thermophysical properties, *Supercritical state, "Extraction, Reprints, Vapor pressure, Solubility, Stability, Equations of state, Diffusivity, "Supercritical fluid extraction, Chemical stability.

The design of any industrial-scale separation process requires some degree of knowledge about the thermophysical and chemical properties of the materials to be separated. This knowledge can take the form of experimentally measured data, particular for that system, or predictions obtained from a suitable mathematical model such as an equation of state or empirical data correlation. It is generally considered better to use predicted properties, since calculations are far more economical to perform than are experimental studies. Unfortunately, there are relatively few reliable predictive methods currently available that have sufficient accuracy to be used in the design of supercritical fluid extraction (SFE) processes. This is because such processes usually involve high pressures and large, often polar, solutes. In addition, SFE processes often require operation near solvent critical points, where even good models of well-studied systems become marginal. This lack of data is slowly improving as more experimental and applied theoretical studies are completed. In the chapter, the more important thermophysical properties needed for supercritical fluid extraction design are discussed from the experimental point of view. Both the solvent and solute are discussed in terms of equilibrium and transport properties.
00,669

PB94-211745 Not available NTIS

National Inst. of Standards and Technology (MSEL) Gaithersburg, MD. Metallurgy Div.

Vapor Transport in Materials and Process Chemistry.

inal rept.

J. W. Hastie, and J. P. Hager. 1990, 24p.

Pub. in Proceedings of Elliott Symposium on Chemical Process Metallurgy, Cambridge, MA., June 10-13 1990, p301-324

Keywords: "Extractive metallurgy, "Metal vapors, "Materials recovery, Thermochemistry, Halides, Ox transport, "Materials chemistry.

As was effectively illustrated by $\mathrm{H}$. $\mathrm{H}$. Kellogg in his 1966 Extractive Metallurgy Lecture to AIME vaporization chemistry can play a very significant role in extractive metallurgy as well as in other materials processes. The field of high temperature chemistry, with its emphasis on vapor phase material transport, has continued its rapid growth over the almost twenty five yea period since Kellogg's milestone lecture. This presentation reviews the results and implications of this growth, and makes recommendations concerning fufure directions in the research and application of vapor transport to process metallurgy. Particular emphasis is given to advances in measurement and diagnostic techniques and to the development of process models Representative examples, taken primarily from the authors' respective laboratories, are given for the metallurgically important halide, oxide and sulfide systems.

\section{0,670}

PB95-151031 Not available NTIS

National Inst. of Standards and Technology (NML) Gaithersburg, MD. Chemical Kinetics Div.

Resonance Enhanced Multiphoton Ionization Spectroscopy of 2-Butene-1-yl (C4H7) between $455-485 \mathrm{~nm}$

Final rept.

B. P. Tsai, R. D. Johnson, and J. W. Hudgens. 1989 $4 p$

Pub. in Proceedings of International Symposium on Resonance Ionization Spectroscopy and Its Applications (4th), Gaithersburg, MD., April 10-15, 1988 p129-132 1989 .

Keywords: "Free radicals, *Butenes, "Electronic spectra, "Spectrum analysis, Barriers, Potential energy, Energy levels, Molecular spectroscopy, Chemical reactions, Reprints, Resonance enhanced multiphoton ionization spectroscopy.

Resonance enhanced multiphoton ionization (REMPI) studies of 2-butene-1-yl (1-methylallyl) radicals from two geometric isomers of $\mathrm{C} 4 \mathrm{H} 8$ precursors yield two distinct REMPI spectra. Analysis of these spectra reveal: (1) that geometric structure is preserved in this chemical reaction due to the existence of a rotational barrier between the cis- and trans-1-methylallyl radcals; (2) a Rydberg-like 3p doublet B(sub 1) intermediate state in each of the isomeric radicals through which REMPI occurs; and (3) an upper limit to the adiabatic ionization potential for the cis-1-methylallyl radical.

00,671

PB95-151965 Not available NTIS

National Inst. of Standards and Technology (NML) Gaithersburg, MD. Chemical Process Metrology Div.

Modeling Ceramic Sub-Micron Particle Formation from the Vapor Using Detailed Chemical Kinetics: Comparison with In-situ Laser Diagnostics.

Final rept.

M. R. Zachariah. $1990,8 p$

Pub. in Chemical Engineering Science 45, n8 p255125581990.

Keywords: "Silicon dioxide, "Particle production,

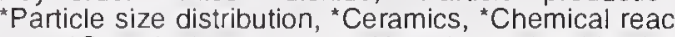
tors, "Chemical vapor deposition, "Reaction kinetics, Mathematical models, Ionization, Spectrum analysis, Silicon, Hydroxyl radicals, Nucleation, Reprints, "Laser diagnostics, Multiphoton ionization spectroscopy.

In this paper we review the in-situ measurements and modeling of an aerosol flame reactor producing submicron silica aerosols as a generic ceramic. Silica powders are produced in a hydrogen-oxygen counter flow diffusion flame reactor and interrogated with light scattering methods for determination of the time-temperature history of particle growth. In addition laser-induced fluorescence and multi-photon ionization have shown to be applicable as diagnostics in a heavily particle laden flow. These diagnostics have been used 10 measure the $\mathrm{OH}$ radical and silicon atoms. These experimental results have been used in conjunction with detailed chemical kinetics and aerosol dynamic models for particle growth. The results show the effects of rity of the resulting solids.

00,672

PB95-151973 Not available NTIS

National Inst. of Standards and Technology (NML) Gaithersburg, MD. Chemical Process Metrology Div. Controlled Nucleation in Aerosol Reactors for Supression of Agglomerate Formation.

Final rept

M. R. Zachariah, and P. Dimitriou. 1990, 13p. Pub. in Aerosol Science and Technology 13, n4 p4134251990

Keywords: "Aerosols, "Nucleation, *Retarding, *Agglomeration, "Sintering, Particle size, Monomers, Reprints, "Controlled nucleation.

The formation of agglomerated particles can be a detriment to the production of highly sinterable materials. It has been seen experimentally that agglomerates are almost exclusively constructed from $10-30 \mathrm{~nm}$ primary particles. This paper develops criteria for the avoidance of these small primary particles through control of the nucleation rate. The relationship between particle size and number density can be used to obtain regions of stable and unstable particle growth. It is seen that the critical source rate for the prevention of runaway nucleation can be scaled without knowledge of the monomer number density.

00,673

PB95-152005

National Inst. of Standards and Technology (NML), Gaithersburg, MD. Chemical Process Metrology Div. Experimental and Numerical Studies of Refractory Particle Formation in Flames: Application to Silica Growth.

Final rep

M. R. Zachariah, and H. G. Semerijian. 1989, 13p.

Pub. in High Temperature Science 28, p113-125 1989

Keywords: "Particle production, "Silicon dioxide, "Diffusion flames, Mathematical models, Vapor phases, Nucleation, Density(Number/volume), Temperature gradients, Light scattering, Optical measurement, Silanes, Particle size distribution, Ceramics, Computerized simulation, Reprints.

Recent interest in high temperature materials have focused attention on the processing requirements as well as the fundamentals of submicron particle formation and growth. In this study, silica particle growth in flames was followed by in-situ light scattering dissymmetry methods for the measurement of particle size and number density. In addition, temperature profiles for the flames were obtained by thermocouple thermometry. The majority of the experiments conducted have used silane as the source of silicon, although a limited number of experiments using organo-silicon compounds have been tried. The results have shown that the silicon source concentration and the temperature are the two dominant variables controlling particle growth and morphology. Two particle growth models have been applied, which account for the consumption of the gas precursor, nucleation, and subsequent growth by surface condensation and coalescence. Comparison with experiment have shown good agreement. Finally, numerical simulations of the gas phase chemistry of silicon have shown the presence of multicomponent nucleation under some conditions, evidence for which has been observed by laser extinction measurements.

00,674

PB95-152013 Not available NTIS

National Inst. of Standards and Technology (NML),

Gaithersburg, MD. Chemical Process Metrology Div.

Simulation of Ceramic Particle Formation: Com. parison with In-situ Measurements.

Final rept.

M. R. Zachariah, and H. G. Semerijian. 1989, 10p. Pub. in American Institute of Chemical Engineers Jnl. 35, n12 p2003-2012 Dec 89

Keywords: "Particle production, "Silicon dioxide, "Ceramics, "Vapor phases, "Computerized simulation, Particle size, Particle size distribution, Density(Number/volume), Diffusion flames, Light scat- 
tering, Chemical reactors, Chemical vapor deposition, Mathernatical models, Reprints.

Ceramic particle formation processes have been studied using $\mathrm{SiO}_{2}$ as a model compound. Silica particles have been synthesized in a counterpropagating diffusion flame reactor, in which in-situ measurements of particle size and number density have been made. In addition, the time-temperature history of the particle field has been calculated from a flame simulation. Numerical simulations using moment and sectional methods for particle formation have been applied and compared to the experimental measurements. The simulations for the particle formation assume a kineticallyconstrained approach, allowing a simple representation of nucleation, surface growth and coagulation. The results suggest that, if the source rates are known well enough, particle formation of low vapor pressure species can be predicted. Both models do well in predicting the gross features of particle formation (number density and mean particle size), although the moment solution does a poor job of predicting the polydispersity effects during periods of high monomer generation rates.

\section{0,675}

PB95-161212 Not available NTIS

National Inst. of Standards and Technology (NML), Boulder, CO. Chemical Engineering Science Div.

Continuous Counter-Current Two Phase Aqueous Extraction.

Final rept

J. B. Joshi, S. B. Sawant, K. S. M. S. Raghava Rao, S. K. Sikdar, T. A. Patil, and K. M. Rostami. 1990 ,

$14 \mathrm{p}$

Pub. in Bioseparation 1, n3-4 p311-324 1990.

Keywords: "Proteins, "Extraction columns, *Columns(Process engineering), Purification, Separation, Polymers, Reprints, "Two phase aqueous extraction systems, Packed columns, Spray columns, York Scheibel columns.

Continuous counter-current column operation has been shown to provide operating convenience for contacting two-phase aqueous partitioning systems for protein extraction. The authors discuss in detail the important parameters for designing spray, packed, plate and York-Scheibel columns for protein recovery using both polymer-polymer and polymer-salt two-phase aqueous systems. The authors compare the various contactors for their operating and extraction efficiency. The work also provides a step-by-step design proce dure, and specific recommendations for future data needs.

\section{0,676}

PB95-161998 Not available NTIS

National Inst. of Standards and Technology (NEL), Boulder, CO. Chemical Engineering Science Div.

Two Phase Aqueous Extraction: Rheological Properties of Dextran, Polyethylene Glycol, Bovine Serum Albumin and Their Mixtures.

Final rept.

A. Pandit, S. B. Sawant, J. B. Joshi, R. A. Perkins,

and S. K. Sikdar. 1989, 6p.

Pub. in Biotechnology Techniques 3, n2 p125-130 1989.

Keywords: "Polyoxyethylene, "Serum albumin, "Dextran, "Viscosity, "Aqueous solutions, Mixtures, Viscometers, Temperature dependence, Rheological properties, Shear properties, Reprints, "Polyethylene glycol.

Rheological properties have been measured for aqueous solutions of dextran, polyethylene glycol and bovine serum albumin. Mixtures of these materials have also been studied. A rotating concentric cylinder viscometer was used to study the rheological properties of these materials over the temperature range 10 to $40 \mathrm{C}$. Over the range of concentrations, molecular weights, temperature and shear rates covered in this work, all aqueous solutions exhibited Newtonian behavior. Correlations have been reported for viscosities of dextran, polyethylene glycol, and bovine serum albumin. The viscosity of mixtures of these materials is not linear with respect to concentration.

\section{0,677}

PB96-201199 Not available NTIS

National Inst. of Standards and Technology (CSTL), Gaithersburg, MD. Thermophysics Div.
Vapor Pressure of Pentafluorodimethyl Ether.

Final rept.

L. A. Weber, and D. R. Defibaugh. 1996, 4p.

Pub. in Jnl. of Chemical and Engineering Data, v41 n3 p382-3851996.

Keywords: "Pentafluorodimethyl ether, "Vapor pressure, Thermophysical properties, Vaporization, Reprints, Ebulliometry.

Vapor pressures of pentafluorodimethyl ether (E125) have been measured from (228 to 331 ) $\mathrm{K}$ using two ebulliometers. Experimental pressures ranged from (65 to 2024) $\mathrm{kPa}$. Thermodynamic calculations were used to estimate pressures down to $140 \mathrm{~K}(16.5 \mathrm{~Pa})$ and a vapor pressure curve is given which is valid from $140 \mathrm{~K}$ to the critical point $(354.49 \mathrm{~K})$. The normal boiling temperature was determined to be $238.06 \mathrm{~K}$. The enthalpy of vaporization has been calculated from (140 to 300$) \mathrm{K}$.

\section{0,678}

PB96-204029 Not available NTIS

National Inst. of Standards and Technology (CSTL), Gaithersburg, MD. Chemical Kinetics and Thermodynamics Div.

Enthalpy Increment Measurements from 4.5 to 350 $\mathrm{K}$ and the Thermodynamic Properties of Titanium Disilicide(cr) to $1700 \mathrm{~K}$.

Final rept.

D. G. Archer, M. S. Sabella, S. E. Stillman, and E. J. Cotts. 1995,5 p

Pub. in Jnl. of Chemical and Engineering Data, v40 p1237-12411995.

Keywords: “Thermodynamic properties, Reprints, Enthalpy, Temperature scales, Measurements, *Titanium disilicide.

Enthalpy increments for titanium disilicide were measured from 4.5 to $350 \mathrm{~K}$ with an adiabatic calorimeter. The enthalpy increments were combined with previous differential scanning calorimetry values to $500 \mathrm{~K}$ Kopp's rule estimates for $500-800 \mathrm{~K}$, and previous enthalpy increment measurements for temperatures to $1700 \mathrm{~K}$ in order to give the thermodynamic properties of titanium disilicide to $1700 \mathrm{~K}$. Values of the Dibye temperature, theta(D), and the coefficient for the hea capacity of the conduction electrons, lambda(ei), were determined from the model. Thermodynamic properties for formation from the elements at $298.15 \mathrm{~K}$ were also given.

\section{0,679}

PB96-204037 Not available NTIS

National Inst. of Standards and Technology (CSTL) Gaithersburg, MD. Chemical Kinetics and Thermodynamics Div.

Enthalpy Increment Measurements from $4.5 \mathrm{~K}$ to $350 \mathrm{~K}$ and the Thermodynamic Properties of the Titanium Silicide Ti5Si3(cr).

Final rept.

D. G. Archer, D. Filor, E. Oakley, and E. J. Cotts

1996, 5p.

Pub. in Jnl. of Chemical and Engineering Data, v41 n3 p571-575 1996.

Keywords: "Thermodynamic properties, Reprints, Enthalpy, Temperature scales, Measurements, *Titanium silicides.

Enthalpy increments for $\mathrm{Ti}(5) \mathrm{Si}(3)(\mathrm{cr})$ were measured from $4.5 \mathrm{~K}$ to $350 \mathrm{~K}$ with an adiabatic calorimeter. From a representation of these measurements the enthalpy relative to $\mathrm{O} k$ the entropy, and the heat capacity of $\mathrm{Ti}(5) \mathrm{Si}(3)(\mathrm{cr})$ to $350 \mathrm{~K}$ were calculated. Values of the Debye temperature, $O$ sub $D$, and the coefficient for the heat capacity of the conduction electrons gamma(ei), were determined from the model. Thermodynamic properties for formation from the elements at $298.15 \mathrm{~K}$ were also given. Our previous value of $\mathrm{O}$ sub $\mathrm{D}$ for TiSi(2) was revised due to improvement in the method of representation.

\section{0,680}

PB97-111447 Not available NTIS

National Inst. of Standards and Technology (CSTL), Gaithersburg, MD. Chemical Kinetics and Thermodynamics Div

Thermodynamic Properties of Synthetic Otavite CdCO3(cr): Enthalpy Increment Measurements from $4.5 \mathrm{~K}$ to $350 \mathrm{~K}$

Final rept.

D. G. Archer. 1996, 7p

Pub. in Jnl. of Chemical and Engineering Data, v41 p852-858 1996 .
Keywords: "Otavite, "Thermodynamics, "Enthalpy, Entropy, Heat capacity, Reprints.

Enthalpy increments for cadmium carbonate were measured for temperatures from $4.5 \mathrm{~K}$ to $350 \mathrm{~K}$ with an adiabatic calorimeter. These measurements were used to compute the entropy, enthalpy relative to $0 \mathrm{~K}$, and the heat capacity of cadmium carbonate. A small anomaly was observed for the sample in the temperature region from approximately $175 \mathrm{~K}$ to $265 \mathrm{~K}$. Comparison of the calculated entropy for $298.15 \mathrm{~K}, 103.88$ J.K-1.mol- 1 , is made with other previously published reference values to arrive at a more precise and consistent set of thermodynamic properties for otavite.

00,681

PB97-113047 Not available NTIS

National Inst. of Standards and Technology (CSTL), Gaithersburg, MD. Process Measurements Div.

In-situ Studies of a Novel Sodium Flame Process for Synthesis of Fine Particles.

Final rept.

K. L. Steffens, M. R. Zachariah, D. P. Dufaux, and R. L. Axelbaum. 1996, 6p.

Pub. in Proceedings of the Materials Research Society Symposium, Boston, MA., November 27-30, 1995, v400 p71-76 Jul 96

Keywords: "Flames, "Particles, *Sodium, Synthesis, Boron, Nanoparticles, Titanium, Fluorescence, Reprints.

The study focuses on the optical characterization of a novel method of forming nanoscale titanium and boron particles, which can be used to form ceramic precursors such as $\mathrm{TiB} 2$. $\mathrm{TiCl} 4$ or $\mathrm{BCl} 3$ reacts with heated $\mathrm{Na}$ vapor in a counterflow diffusion flame reactor. After $\mathrm{Na}$ strips the $\mathrm{Ti}$ or $\mathrm{B}$ of its $\mathrm{Cl}$ atoms, nanosize $\mathrm{Ti}$ or $\mathrm{B}$ particles form and become encased in $\mathrm{NaCl}$, which helps to prevent agglomeration and oxidation. The twodimensional spatial distribution of the $\mathrm{Na}$ dimer has been optically interrogated using planar laser-induced fluorescence (PLIF) to clarify the influence of the concentration distributions and transport on particle formation rates.

\section{0,682}

PB97-113054 Not available NTIS

National Inst. of Standards and Technology (CSTL),

Gaithersburg, MD. Process Measurements Div.

Optical and Modeling Studies of Sodium/Halide Reactions for the Formation of Titanium and Boron Nanoparticles.

Final rept.

K. L. Steffens, M. R. Zachariah, D. P. DuFaux, and

R. L. Axelbaum. 1996, 10p.

Pub. in Chemistry of Materials, v8 n8 p1871-1880 1996.

Keywords: *Titanium, "Boron, *Sodium, Nanostructural materials, Fluorescence, Reprints.

The study focuses on the optical characterization of a method for the formation of nanoscale titanium and boron particles. The versatile method can also be used to form a variety of metals as well as ceramic powders such as TiB2. The gas-phase chemical process, given by $(m n) \mathrm{Na}+(n) \mathrm{MClm} \rightarrow(\mathrm{M}) \mathrm{n}+(\mathrm{mn}) \mathrm{NaCl}$, should be generic to many metal chlorides or mixtures of metal chlorides. In the study, either $\mathrm{TiCl} 4$ or $\mathrm{BCl} 3$ is reacted with $\mathrm{Na}$ vapor in a counterflow diffusion flame reactor. After the $\mathrm{Cl}$ is stripped from the metal chloride by the $\mathrm{Na}$ vapor, nanosize $\mathrm{Ti}$ or $\mathrm{B}$ particles form and, under certain thermodynamic circumstances, become encased in $\mathrm{NaCl}$, which helps to prevent agglomeration and postflame oxidation. The two-dimensional spatial distribution of $\mathrm{Na} 2$ has been optically interrogated using planar laser-induced fluorescence under various conditions to clarify the influence of concentration and transport on particle formation. Reactant concentration and time available for reaction were found to dramatically influence the reactive flow.

00,683

PB97-113153 Not available NTIS

National Inst. of Standards and Technology (CSTL),

Gaithersburg, MD. Thermophysics Div.

Critical Evaluation of Thermal Mass Flow Meters. Final rept.

S. A. Tison. 1996, 10p

Pub. in Jnl. of Vacuum Science and Technology A, v14 n4 p2582-2591 Jul/Aug 96.

Keywords: "Flow meters, "Thermal mass flow meters, Gas admission, Correction factors, Reprints.

Many semiconductor processes require that stable and known flows of gas be delivered to the processing 
chamber. The thermal mass flow meter (TMFM) is used almost exclusively in the semiconductor industry for the admission of process gases. While TMFM's have been used in the semiconductor industry for over twenty years, much still remains to be understood about their behavior. The abundance of TMFM manufacturers that make instruments which are supposedly interchangeable complicates the use of TMFM's because the instruments generally have different designs and performance. While some attempt has been made via written standards to address the specifications of the instruments, these standards do not address all performance issues and cannot eliminate the systematic errors in the original manufacturers calibration of the TMFM's. Further, the TMFM's used to measure the process gases are generally calibrated with nitrogen and 'corrected' for other gases, but the correction factors are not well understood and are of questionable reliability. It is also important to understand how the TMFM's perform under conditions that differ from the laboratory conditions where they were calibrated and the measurement errors that are introduced as a result of these different operating conditions. This article presents data on the performance of five low-flow TMFM's, from different manufacturers, with full scale ranges of $1.5 \times 10(-6)-3.7 \times 10(-6) \mathrm{mol} / \mathrm{s}(2-5 \mathrm{sccm})$. The manufacturers' calibration of the TMFM's with nitrogen as compared to the National Institute of Standards and Technology (NIST) measured values differed by up to $17 \%$. Three of the five testeo' TMFM's were within the manufacturers' stated tolerance of + or $-1 \%$ of full scale. While some of the instruments' initial calibration was poor, all of the TMFM's were stable to within + or - $1 \%$ of full scale over the test interval of nine months.

\section{0,684}

PB97-113237 Not available NTIS

National Inst. of Standards and Technology (CSTL), Gaithersburg, MD. Thermophysics Div.

Vapor Pressure of 1,1,1,2,2-Pentafluoropropane. Final rept.

L. A. Weber, and D. E. Defibaugh. 1996, 3p.

Pub. in Jnl. of Chemical and Engineering Data, v4 1 n4 p762-764 1996 .

Keywords: "Vapor pressure, "Pentafluoropropane, Ebulliometry, Refrigerants, Reprints.

The authors have used a comparative ebulliometer to measure the vapor pressure of $1,1,1,2,2$ pentafluoropropane (HFC245ch) in the temperature range (248 to 326 ) K. Pressure?s ranged from $(74$ to 995) $\mathrm{kPa}$. The data were adjusted for impurities in the sample. The temperature of the normal boiling point was found to be (255.11 plus or minus 0.10$) \mathrm{K}$, and the Pitzer acentric factor was calculated to be 0.297 . An estimate for the critical pressure is given, $\mathrm{PC}=(3148$ plus or minus 15) $\mathrm{kPa}$

\section{Photochemistry and Radiation Chemistry}

00,685

PB94-185170 Not available NTIS

National Inst. of Standards and Technology (PL), Gaithersburg, MD Ionizing Radiation Div.

Long-Term Stability of Carrier-Free Polonium Solution Standards.

Final rept.

R. Colle. 1993, 11p.

Pub. in Radioactivity and Radiochemistry 4, n2 p20351993.

Keywords: "Polonium 208, "Solutions, "Stability, "Trace elements, Radiochemistry, Hydrochloric acid, Concentration(Composition), Scintillation counters, Reprints.

The long-term solution stability of trace quantities of polonium in dilute hydrochloric acid has been investigated. Polonium solutions at trace concentrations, under various alkaline, neutral, or weakly acidic conditions are known to be unstable: being readily hydrolyzed, chemically deposited, or volatilized; exhibiting 'radiocollodial' behavior; and undergoing 'plateout' or adsorption onto glass surfaces. Although stored polonium solutions are generally considered to be stable in the acid range of 0.1 to 1.0 normality $(N)$, scant data exist on any possible long-term effects, particularly for very dilute, aged solutions. In this study, pre- viously standardized carrier-free solutions of (208)Po activity concentration, ranging in age from 1.2 to nearly 9 years, and with acid concentrations from about 0.1 to $2.0 \mathrm{~N} \mathrm{HCl}$ were re-assayed to determine the remaining soluble fraction of polonium. The results indicate that at acid concentrations of a few tenths of $1 \mathrm{~N}$, the solutions are clearly unstable. In the range of 0.3 to $0.5 \mathrm{~N}$, the results are somewhat equivocal. Only in the range at or above $1 \mathrm{~N}$ do the solutions appear to be stable over many years, approaching a decade. All measurements were performed by 4 pi-alpha liquid scintillation counting of gravimetrically-determined aliquots of the standardized, aged solution samples.

\section{0,686}

PB94-185733 Not available NTIS

National Inst. of Standards and Technology (CSTL) Gaithersburg, MD. Biotechnology Div.

Application of Photochemical Reaction in Electrochemical Detection of DNA Intercalation.

Final rept.

P. C. Pandey, and H. H. Weetall. 1994, $6 \mathrm{p}$

Pub. in Analytical Chemistry 66, n8 p1236-1241, 15 Apr 94

Keywords: "Electrochemistry, "DNA damage, *Intercalating agents, "Photochemistry, Anthraquinones, Flow injection analysis, Oxidation, Deoxyribonucleic acids, Reduction(Chemistry), Reprints.

A flow injection analysis (FIA) system for the detection of the compounds that intercalate within DNA is re ported. A derivative of 9,10-anthraquinone has been used as the reference compound for photoelectrochemical detection. The sodium salts of 9,10-anthraquinone-2,6-disulfonic acid and 9,10anthraquinone-2 sulfonic acid are photochemically activated and then reduced in the presence of an electron donor (glucose). The electrochemical signal is based on the measurement of the anodic current resulting from the oxidation of the reduced form of 9,10 anthraquinone. The reduced form of the 9,10anthraquinone is oxidized through a mediated mecha nism at the surface of a tetracyanoquinodimethane(TCNQ)-modified graphite paste electrode covered by a Nucleopore membrane. TCNQ acts as an efficient mediator for the oxidation of reduced 9,10anthraquinone. Cyclic voltammetry, photocyclic voltammetry, and the photoelectrochemical FIA response of 9,10-anthraquinone are reported. Experimental results show that these anthraquinones can be intercalated within the helix of double-stranded calf thymus DNA.

00,687

PB94-199288 Not available NTIS

National Inst. of Standards and Technology (CSTL) Gaithersburg, MD. Surface and Microanalysis Science Div.

Photodecomposition Dynamics of $\mathrm{Mo}(\mathrm{CO}) 6 / \mathrm{Si}(111)$ 7x7: CO Internal State and Translational Energy Distributions.

Final rept.

S. A. Buntin, R. R. Cavanagh, and L. J. Richter

1993, 4p

Pub. in Jnl. of Chemical Physics 98, n9 p7651-7654

1 May 93. Sponsored by Department of Energy, Washington, DC

Keywords: "Carbon monoxide, Reprints, Rotational states, Metal carbonyls, Photodecomposition, Absorption, Photolysis, Silicon, Hexacarbonylmolybdenum, Photodesorption, Translations.

The rotational state and translational energy distributions of $\mathrm{CO}$ photodesorption products resulting from the $266 \mathrm{~nm}$ photolysis of $\mathrm{Mo}(\mathrm{CO}) 6$ absorbed on Si(111) $7 \times 7$ with coverages in the multilayer regime are reported State-resolved measurements show two desorption components with highly disparate energy dispositions. Results for different surface temperatures indicate that the energy content in one component reaches quasi-equilibration with the surface temperature, which is attributed to collisional relaxation of nascent photodecomposition products within the adlayer. The other component exhibits disparate rotational and translational 'temperatures' that are significantly great er than, and independent of, the surface temperature. These nascent photodecomposition products are influenced by both energy quenching effects and dynamical constraints imposed by the existence of the adlayer.

00,688
PB94-199965 Not available NTIS
National Inst. of Standards and Technology (CSTL) Gaithersburg, MD. Surface and Microanalysis Science Div.

Dynamics of Nonthermal Reactions: Femtosecond Surface Chemistry.

Final rept.

R. R. Cavanagh, D. S. King, J. C. Stephenson, and

T. F. Heinz. 1993, 13p

Pub. in the Jnl. of Physical Chemistry 97, n4 p786-798 1993. Sponsored by Department of Energy, Washington, DC

Keywords: *Surface reactions, Laser radiation, Light pulses, Energy transfer, Reviews, Reprints,

Optically driven surface reactions are attracting an increasing level of attention in the physical chemistry community. Not only have there been recent advances in establishing the viability of laser driven surface reac tions, but there has also been an increased awareness of the need to understand the underlying reaction mechanisms. The necessity of accounting for energytransfer processes that occur on the femtosecond time scale is now apparent. In this review the experimental and theoretical basis of our current understanding is surveyed, and prospective areas of advancement are considered.

\section{0,689}

PB94-212255 Not available NTIS

National Inst. of Standards and Technology (PL) Gaithersburg, MD. Ionizing Radiation Div.

Role of the Office of Radiation Measurement in Quality Assurance.

Final rept.

K. G. W. Inn, B. M. Coursey, E. Eisenhower, H. T.

Heaton, K. C. Duvall, M. D. Walker, and J. C.

Humphreys. 1993, 11p.

Pub. in Science of the Total Environment 130/131 p497-507 1993

Keywords: "Quality assurance, "Ionizing radiation "Calibrating, Radiation dosage, Laboratories, Dosimetry, Radiation protection, Radiation measuring instruments, Environmental monitoring. Standards, Radon, Health physics, Bioassay, Radiochemistry, Reprints.

Over the past ten years the National Institute of Stand ards and Technology has, through its Office of Radi ation Measurement, developed several national Secondary Laboratories systems in the field of ionizing radiation. These Secondary Laboratories systems provide the necessary calibrations and quality assurance testing to support and affirm the caliber of the meas urements in special areas of ionizing radiation. The areas that are supported by the program include State Radiation Protection, Personnel Dosimetry, Survey Instrument Calibration, High-level Dosimetry, Radiation Therapy, Bioassay, Survey Instrument Testing, Ioniz ing Radiation, Environmental Radioactivity, Radioactivity Standards, and Radon.

00,690

PB95-108726 Not available NTIS

National Inst. of Standards and Technology (PL), Boulder, CO. Quantum Physics Div.

Laboratory Studies of Low-Temperature Reactions of $\mathrm{C}_{2} \mathrm{H}$ with $\mathrm{C}_{2} \mathrm{H}_{2}$ and Implications for Atmospheric Models of Titan.

Final rept.

J. O. P. Pedersen, B. J. Opansky, and S. R. Leone.

$1993,8 \mathrm{p}$

Contract NAGW-2438

Sponsored by National Aeronautics and Space Administration, Washington, DC

Pub. in Jnl. of Physical Chemistry 97, n26 p6822-6829 1993

Keywords: "Satellite atmospheres, *Acetylene, *Titan, Temperature range 0065-0273-K, Temperature range 0273-0400 K, Temperature dependence, Photochemical reactions, Chemical radicals, Laboratory tests, Reprints, Acetylene radicals.

Rate coefficients for the reaction $\mathrm{C} 2 \mathrm{H}+\mathrm{C} 2 \mathrm{H}_{2}->\mathrm{C} 4 \mathrm{H}_{2}$ $+\mathrm{H}$ are measured over the temperature range 170-350 $K$. The reactions are carried out in a temperature variable flow cell. $\mathrm{C} 2 \mathrm{H}$ radicals are produced by pulsed laser photolysis of $\mathrm{C} 2 \mathrm{H} 2$, and a tunable infrared color center laser is used to probe the transient removal of $\mathrm{C} 2 \mathrm{H}$ in absorption to derive the rate coefficients. The results show that the rate coefficient is independent of temperature over the range $170-350 \mathrm{~K}$ and equal to $(1.1+$ or -0.2$) \times 10(\sup -10) \exp ((28+$ or -20$) / T) \mathrm{cc} /$ molecule/s. The reaction studied is of central impor- 
tance for models of the photochemistry of the atmospheres of the outer planets, in particular for the satellite Titan, and the implications of the present results for these models are discussed.

00,691

PB95-125720 Not available NTIS

National Inst. of Standards and Technology (NML) Gaithersburg. MD. Molecular Physics Div.

Vibrational Predissociation Dynamics of OvertoneExcited HN3.

Final rept.

B. R. Foy, M. P. Casassa, J. C. Stephenson, and D.

S. King. $1989,3 p$

Pub. in American Institute of Physics Conference Proceedings on Advances in Laser Science-IV, Atlanta, GA., October 2-7, 1988, n191 p612-614 1989.

Keywords: "Photodissociation, Photochemical reactions, Molecular spectroscopy, Rotational spectra, Vibrational states, Visible spectra, Predissociation, Reprints, "Hydroazoic acid, Intramolecular dynamics.

Vibrational overtone photodissociation is used to examine rotationally resolved spectra of $\mathrm{HN} 3$ at $\mathrm{E}(\mathrm{vib})=$ $15120 / \mathrm{cm}$ and $17670 / \mathrm{cm}$. The spectra exhibit extensive coupling of the NH stretching overtones to background vibrational states. Lifetimes for vibrational predissociation, HN3(X tilde) $\rightarrow \mathrm{HN}(X)+\mathrm{N} 2(X)$, are found to be $210 \mathrm{nsec}$ and $0.95 \mathrm{nsec}$ for the levels $\mathrm{v}(\mathrm{NH})=5$ and 6 , respectively.

\section{0,692}

PB95-151643 Not available NTIS

National Inst. of Standards and Technology (NML), Gaithersburg, MD. lonizing Radiation Div.

Unusual Spin-Trap Chemistry for the Reaction of Hydroxyl Radical with the Carcinogen NNitrosodimethylamine.

Final rept.

D. A. Wink, and M. F. Desrosiers. 1991, 6p.

Pub. in Radiation Physics and Chemistry 38, n5 p4674721991

Keywords: "Hydroxyl radicals, "Radiolysis, Radiochemistry, Carcinogens, Reprints, *Chemical reaction mechanisms, "Amine/ $\mathrm{N}$-nitrosodimethyl, "Spin trap, Benzene sulfonate/dibromo-nitroso, EPR(Electron Paramagnetic Resonance).

The reaction of the potent carcinogen $\mathrm{N}$ nitrosodimethylamine (NDMA) with hydroxyl radical generated via radiolysis was studied using EPR techniques. Attempts to spin trap NDMA radical intermediates with 3,5-dibromo-4-nitrosobenzene sulfonate (DBNBS) produced only unusual DBNBS radicals. One of these radicals was shown to be gen erated by both reaction of DBNBS with nitric oxide, and direct oxidation of DBNBS with an organic oxidant $(\mathrm{Br} 2(-))$. Another DBNBS radical was identified as a sulfite spin adduct resulting from the degradation of DBNBS by a NDMA reactive intermediate. In the absence of DBNBS, hydroxyl radical reaction with NDMA gave the dimethylnitroxide radical. Unexpectedly, addition of DBNBS to a solution containing dimethylnitroxide produced an EPR spectrum nearly identical to that of NDMA solutions with DBNBS added before radiolysis. A proposed mechanism accounting for these observations is presented.

\section{0,693}

PB95-175790 Not available NTIS

National Inst. of Standards and Technology (CSTL) Gaithersburg, MD. Surface and Microanalysis Science Div.

Comparative Study of Fe-C Bead and Graphite Target Performance with the National Ocean Sciences AMS (NOSAMS) Facility Recombinator Ion Source. Final rept.

D. B. Klinedinst, A. P. McNichol, L. A. Currie, K. F vonReden, R. M. Verkouteren, G. A. Jones, R. J. Schneider, and G. A. Klouda. 1994, 6p.

Pub. in Nuclear Instruments and Methods in Physics Research B 92, p166-171 1994.

Keywords: "Carbon 14 target Concentration(Composition), Sampling, Environmenta materials, Performance evaluation, Poisson equation, Reprints, "Accelerator mass spectrometry.

An accelerator mass spectrometry (AMS) experiment was designed to investigate (14)C target performance for two target types over a range of isotopic concentrations and sample sizes, with a special focus on the ability to measure (14) C in environmental samples having only microgram amounts of carbon. The findings were positive, showing that precision, accuracy, and stability were adequate to determine $(14) \mathrm{C}$ to $1 \%$ or better in samples containing as little as 25 micrograms carbons. Satisfactory Poisson uncertainty and target stability were demonstrated down to a level of 7 micrograms carbon, but experimental data showed that accurate measurements at that level require detailed knowledge of blank variability and mass dependence of the modern carbon calibration factor.

00,694

PB96-146899 Not available NTIS

National Inst. of Standards and Technology (CSTL), Gaithersburg, MD. Chemical Kinetics and Thermodynamics Div.

Experimental Determination of the Ionization Energy of $1 O(X($ sup 2) $I($ sub 3/2)) and Estimations of Delta(sub f) $\mathrm{H}$ (sup deg)(sub 0$)(\mathrm{IO}($ sup -$))$ and PA(IO).

Z. Zhang, P. S. Monks, L. J. Stief, S. C. Kuo, R. B.

Z. Znang, P. S. Monks, L. J. Stief, S. C. Kuo, R. B.
Klemm, J. F. Liebman, and R. E. Huie. 1996, 6p.

Pub. in Jnl. of Physical Chemistry, v100 n1 p63-68 1996.

Keywords: "Photoionization, "lodine oxide, Mass spectrometers, Reprints, "Ionization energy.

Photoionization efficiency (PIE) spectra of 10 (X2/li) were measured over the wavelength range lambda = $115.0-130.0 \mathrm{~nm}$ and in the jonization threshold region lambda $=126.0-130.0 \mathrm{~nm}$, using a discharge flowphotoionization mass spectrometer apparatus coupled to a synchrotron radiation source. Iodine oxide was generated by the reactions of $O(3 \mathrm{P})$ atoms with 12 and ior. 3I. The PIE spectra displayed step-function behav-

\section{0,695}

PB96-160346 Not available NTIS

National Inst. of Standards and Technology (PL), Gaithersburg, MD. Ionizing Radiation Div.

New and Revised Half-Life Measurement Results. Final rept.

M. P. Unterweger, D. D. Hoppes, and F. J. Schima. 1992, 4p.

Pub. in Nuclear Instruments and Methods in Physics Research, Section A, nA312 p349-352 1992.

Keywords: "Radioisotopes, "Half-life, "Measurement, Counting techniques, Comparisons, Cobalt 60 . Krypton 85, Cesium 137, Sodium 22, Barium 133, Bismuth 207, Europium 152, Europium 154, Europium 155, Antimony 125 , Reprints

The results of these measurements for many long-lived radionuclides such as Co-60, Cs-137, Kr-85, Na-22, $\mathrm{Ba}-133, \mathrm{Bi}-207, \mathrm{Eu}-152, \mathrm{Eu}-154, \mathrm{Eu}-155$, and $\mathrm{Sb}-125$ have been recently revised. The results for the halflives of the many radionuclides measured over the last three decades are tabulated. Comparisons with recommended values from the International Atomic Energy Agency Coordinated Research Program (IAEA CRP) are given for the long-lived radionuclides.

\section{0,696}

PB96-164157 Not available NTIS

National Inst. of Standards and Technology (CSTL), Gaithersburg, MD. Analytical Chemistry Div.

Isotopic and Nuclear Analytical Techniques in Biological Systems: A Critical Study. 10. Elemental Isotopic Dilution Analysis with Radioactive and Stable Isotopes (Technical Report).

Final rept.

J. D. Fassett. 1995, $7 p$

Pub. in Pure and Applied Chemistry, v67 n11 p194319491995.

Keywords: "Isotope dilution, "Chemical analysis, "Biological systems, Reprints, Accuracy, "Foreign technology, Nuclear analytical techniques.

Isotope dilution is a method of chemical analysis based on the mixing (or dilution) of a radioisotope or a separated stable isotope with its natural isotope(s) in the sample. The activity or isotopic ratio of the mixture defines the concentration of the analyte, which is a tremendous advantange for measurement since quantitative separation of the analyte is not required. The technique has a multitude of variations and has been combined with many classical and instrumental procedures used in aralytical chemistry. The technique is noted for its accuracy. This review focuses on the application of the technique in the determination of elemental concentrations in biological systems.
00,697

PB96-167101 Not availabie NTIS

National Inst. of Standards and Technology (CSTL), Gaithersburg, MD. Inorganic Analytical Research Div. Dead Time, Pileup, and Accurate Gamma-Ray Spectrometry.

Final rept.

R. M. Lindstrom, and R. F. Fleming. 1995, 7p.

Pub. in Radioactivity and Radiochemistry, v6 n2 p20271995

Keywords: "Gamma ray spectrometry, "Pulse pileup Dead times, Reprints, Radioactive decay, "Foreign technology, Random summing.

The accuracy of gamma-ray spectrometric measurements is ultimately limited by the precision of Poisson counting statistics. With careful attention to detail, all other sources of error in the ratio of activities of two sources of a radionuclide in small samples can be made insignificant, even when the statistical limit is well below one percent. An important source of error comes from the dinite time required by the counting electronics to detect and process pulses. Dead-time losses (mostly in the analog-digital converter) are usually compensated very well by the pulse-height analyzer, but pileup losses (mostly in the amplifier) may not be. Errors of $10 \%$ or more may result. Several methods are available for detecting and correcting rate-related losses. These methods are sufficiently reliable and well understood that a decaying source can be measured with acceptably small errors even at count rates as high as tens of thousands per second.

\section{0,698}

PB96-167242 Not available NTIS

National Inst. of Standards and Technology (CSTL) Gaithersburg, MD. Inorganic Analytical Research Div. Determination of 21 Elements by INAA for Certification of SRM $1570 a$, Spinach.

Final rept.

D. A. Becker. $1995,8 p$.

Pub. in Jnl. of Radioanalytical and Nuclear Chemistry, Articles, v193 n1 p25-32' 1995.

Keywords: "Neutron activation analysis, "Spinach, Elemental analysis, Reprints, Accuracy, "Foreign technology, "Botanical reference material, Standard reference materials.

Analysis for certification have been made by instrumental neutron activation analysis (INAA) for the determination of 21 elements in the Natiaonl Institute of Standards and Technology (NIST) Spinach renewal reference material, SRM 1570a. Elements determined included ones with short halflife products (AIV, Ca, $\mathrm{Mg})$, intermediate halflife products ( $\mathrm{Mn}, \mathrm{Na}, \mathrm{K}, \mathrm{La})$ and long halflife products ( $\mathrm{Ba}, \mathrm{Co}, \mathrm{Cr}, \mathrm{Cs}, \mathrm{Eu}, \mathrm{Fe}, \mathrm{Rb}, \mathrm{Sb}$, $\mathrm{Sc}, \mathrm{Se}, \mathrm{Sr}, \mathrm{Th}$, and $\mathrm{Zn}$ ). For the first time a new robotic samplechanger was used in the counting of long halflife indicator isotopes for certification of an SRM. Uncertainties obtained averaged plus or minus $1.80 \%$ for the four major and minor constituents ( $\mathrm{Ca}, \mathrm{K}, \mathrm{Mg}$, $\mathrm{Na}$; ; plus or minus $3.14 \%$ for elements with concentrations from 1 to $400 \mathrm{mg} / \mathrm{kg}$ ( $\mathrm{Al}, \mathrm{Ba}, \mathrm{Cr}, \mathrm{Fe}, \mathrm{Mn}, \mathrm{Rb}, \mathrm{Sr}$, and $\mathrm{Zn}$ ); and plus or minus $8.31 \%$ for the ultra trace elements (less than $\mathrm{mg} / \mathrm{kg}$ ) (Co, Cs, Eu, La, Sb, Sc, $\mathrm{Se}, \mathrm{Th}$, and $\mathrm{V}$ )

\section{0,699}

PB96-200811 Not available NTIS

National Inst. of Standards and Technology (CSTL) Gaithersburg MD. Process Measurements Div.

Unique Quality Assurance Aspects of INAA for Reference Material Homogeneity and Certification.

Final rept.

D. A. Becker. 1993, 4p

Pub. in Fresenius Jnl. of Analytical Chemistry, v345 p298-3011993.

Keywords: "Neutron activation analysis, "Radiation detection, "Quality assurance, Neutron detection, Radiochemistry, Incident radiation, Excitation, Measurement, Certification. Trace elements, Analytical methods, Reprints, "Standard reference materials, Emitted radiation.

Instrumental neutron activation analysis (INAA) has become one of the primary analytical techniques for certification of elemental content in biological Standard Feference Materials (SRMS) at the National Institute of Standards and Technology (NIST). One importan reason why INAA has become so widely used and valuable in the certification of NIST SRMs is that INAA has unique inherent quality assurance (QA) character- 
istics which provide the capability for accurate analysis and which often allow the analytical values obtained to be internally evaluated and cross checked. While the spectroscopic techniques, the specific characteristics include uniform activation, long and well-documented excited states, highly penetrating emitted radiation, and an excited state decay process which is statistically random in nature.

00,700

PB97-111603 Not available NTIS

National Inst. of Standards and Technology (PL),

Gaithersburg, MD. Ionizing Radiation Div.

63Ni Half-Life: $A$ New Experimental Determination and Critical Review.

Final rept.

R. Colle, and B. E. Zimmerman. 1996, 15p

Pub. in Applied Radiation and Isotopes, v47 n7 p6776911996.

Keywords: "Nickel 63, "Standardization, *Calibration standards, *Half life, Liquid scintillators, Calorimetry, Gravimetric analysis, Radiation measurement, Tritium, Reprints.

The Ni-63 half-life has been determined to be 101.06 + or - 1.97 years based on three independent measurements, conducted over the past 27 years, of the massic activity of gravimetrically-related $\mathrm{Ni}-63$ sources. The present result is the first and only determination of the Ni-63 half-life which is based on actually following the radioactive decay of Ni-63. Based on a critical evaluation of the extant data set, a Ni-63 halflife value of $T=101.1+$ or -1.4 years is recommended.

\section{0,701}

PB97-1118.19 Not available NTIS

National Inst. of Standards and Technology (PL), Gaithersburg, MD. Ionizing Radiation Div.

Nickel-63 Standardization: 1968-1995.

Final rept.

R. Colle, and B. E. Zimmerman. 1996, 16p.

See also PB92-236553.

Pub. in Radioactivity and Radiochemistry, v7 n2 p1227 Aug 96.

Keywords: "Nickel 63, ${ }^{*}$ Calibration standards, *Standardization, Radiation measurement, Liquid scintillators, Spectroscopy, Calorimetry, Tritium, Beta decay, Beta particles, Emanation, Reprints, Efficiency tracing.

The radionuclide Ni-63 is widely employed as a relatively long-lived, low-energy beta-particle-emitting calibration standard. Between 1968 and 1995, the National Insititute of Standards and Technology (NIST), formerly the National Bureau of Standards (NBS), actively engaged in the preparation and calibration of $\mathrm{Ni}$ 63 solution standards. The chronicle summarizes these NIST/NBS standardization activities and highlights the remarkably consistent measurement results obtained on the standards over the past 27 years.

\section{0,702}

PB97-119119 Not available NTIS

National Inst. of Standards and Technology (CSTL), Gaither'sburg, MD. Chemical Kinetics and Thermodynamics Div.

Resonance Enhanced Multiphoton Ionization Spectroscopy of the PF Radical.

Final rept.

J. Howe, M. N. R. Ashfold, C. M. Western, and J. W. Hudgens. 1996, 3p.

Pub. in Jnl. of Chemical Physics, v104 n8 p2789-2800 1996.

Keywords: "Spectroscopy, "Ionization spectroscopy, "Multiphoton, Resonance, Reprints, “PF radicals.

PF radicals in both their ground and metastable electronic: states have been produced by the gas phase reaction of $F$ atoms with phosphine in a discharge flow reactor and detected by mass selective resonance enhanced multiphoton ionization (REMPI) spectroscopy in the wavelength range $410-225 \mathrm{~nm}$. Analysis of the longer wavelength end of this spectrum has enabled identification and spectroscopic characterization of five hitherto unknown Rydberg states of this radical.

00,703

PE397-119291 Not available NTIS

National Inst. of Standards and Technology (PL),

Gaithersburg, MD. lonizing Radiation Div.
Calibration and Performance of GafChromic DM100 Radiochromic Dosemeters.

Final rept.

B. J. Mincher, M. K. Zaidi, R. E. Arbon, W. L.

McLaughlin, and G. L. Schwendiman. 1996, 4p.

Pub. in Solid State Dosimetry, Budapest, Hungary July 10-14, 1995, Radiation Protection Dosimetry, v66 n1-4 p233-236 1996

Keywords: *Dosemeters, *Radiochromic films, Gamma radiation, Organic chemistry, Radiation chemistry, Calibration, Reprints, *Foreign technology.

GafChromic DM-100 dosemeters were used to measGre absorbed doses in liquid samples exposed to a $137 \mathrm{Cs}$ gamma ray source. The initial calibration of the commercially available film was performed using $60 \mathrm{Co}$ gamma rays. A comparison of simultaneously irradiated bare dosemeters mocked to simulate the liquid samples shows the importance of iraddiation of dosemeters under conditions as nearly identical to that of the actual sampels as possible. In addition, the dosemeter response to absorbed dose for two differen batches of GafChromic film, to different temperatures at the time of irradiation and to delay before readout. was examined. the reproducibility of the GarChromic dosemeter is also discussed.

\section{Physical \& Theoretical Chemistry}

\section{4}

AD-A234 043/8 PC A03/MF A01

National Inst. of Standards and Technology (NML), Gaithersburg, MD. Molecular Spectroscopy Div.

Production and Spectroscopy of Small Polyatomic Molecular Ions Isolated in Solid Neon. (Reannouncement with New Availability Information).

M. E. Jacox, and W. E. Thompson. 1990, 11p, ARO25664.2- $\mathrm{CH}$

Contract ARO-MIPR-120-90

Pub. in High Temperature Science, v28 p225-234

Keywords: *Molecular ions, Absorption, Spectroscopy Neon, Infra red spectra, Reprints, "Matrix isolation, Molecular clusters, Cluster ions, Solidified gases.

No abstract available.

\section{0,705}

AD-A238 415/4 PC A02/MF A01

National Inst. of Standards and Technology, Gaithersburg MD.

Vibrational Spectra of Molecular lons Isolated in Solid Neon. 6. $\mathrm{CO} 4(-)$. (Reannouncement with New Availability Information)

M. E. Jacox, and W. E. Thompson. 4 Apr 91, 8p,

ARO-25664.9- $\mathrm{CH}$

Contract ARO-MIPR-120-90

Pub. in Jnl. of Physical Chemistry, v95 n7 p2781-2787, 4 Apr 91.

Keywords: "Molecular vibration, Molecular spectroscopy, Carbonates, Carbon dioxide, Oxygen, Nitrogen Reprints, "Neon matrices, "Vibrational spectra, *Molecularions.

No abstract available.

\section{0,706}

AD-A239 729/7 PC A03/MF A01

National Inst. of Standards and Technology (NML), Gaithersburg, MD. Molecular Physics Div.

Vibrational Spectra of Molecular lons Isolated in Solid Neon. 7. $\mathrm{CO}(+), \mathrm{C} 2 \mathrm{O} 2(+)$, and $\mathrm{C} 2 \mathrm{O} 2(-)$ (Reannouncement with New Availability Information).

W. E. Thompson, and M. E. Jacox. 15 Jul 91, 11p ARO-25664.10-CH.

Pub. in Jnl. of Chemical Physics, v95 n2 p735-745, 15 Jul 91.

Keywords: "Neon, "Carbon monoxide, "Ion molecule interactions, "Infrared spectra, "Molecular ions, *Vibrational spectra, Photoionization, lonized gases, Vapor deposition, Absorption, Atomic beams, Electron capture, Reprints, Solids, Penning ionization, Low temperature research.

No abstract available.

00,707

AD-A253 551/6 PC A03/MF A01
National Inst. of Standards and Technology, Gaithersburg, MD.

Vibrational Spectra of Molecular lons Isolated in Solid Neon: HCCH+ and HCC-. (Reannouncement with New Availability Information).

D. Forney, M. E. Jacox, and W. E. Thompson. 1992 13p, ARO-25664.12-CH

Contract ARO-MIPR-120-90

Pub. in Jnl. of Molecular Spectroscopy, v153 p680-691 1992.

Keywords: "Neon, "Solids, "Molecular ions, "Vibrational spectra, "Acetylenes, Reprints, Cations, Excitation, Carbon, Ground state, Argon, Chemical bonds, Microwave discharges, Polarizability, Stretching

When a $\mathrm{Ne}: \mathrm{C} 2 \mathrm{H} 2$ sample is codeposited at approximately $5 \mathrm{~K}$ with a beam of neon atoms that has been excited in a microwave discharge, a sharp, prominen absorption assigned to Upsilon(3) of $\mathrm{HCCH}(+)$ appears at $3137.6 \mathrm{~cm}(-1)$, very close to the previously reported gas-phase band center. Experiments on carbon-13 and deuterium substituted samples support this assignment and permit the identification of all of the infrared-active $\mathrm{CH}$ - and $\mathrm{CD}$-stretching fundamentals of the isotopically substituted acetylene cations, as well as the determination of the stretching and stretching-interaction force constants. The absorptions of the carbon13 substituted acetylene cations have also been identified in the analogous argon-matrix experiments, bu exhibit a matrix shift of approximately $30 \mathrm{~cm}(-1)$, pos sibly because of the larger polarizability of argon.

00,708

AD-A275 828/2 Not available NTIS

National Inst. of Standards and Technology (NML) Gaithersburg, MD. Molecular Physics Div.

Vibrational Spectra of Molecular lons Isolated in Solid Neon. 11. NO2(+), NO2(-), and NO3(-).

D. Forney, W. E. Thompson, and M. E. Jacox. 15

Nov 93, 12p, ARO-30094.3-CH.

Availability: Pub. in Jnl. of Chemical Physics, v99 n10 p7393-7403, 15 Nov 93

Keywords: "Infrared spectra, "Cations, "Vibration, "Solids, "Molecular ions, "Neon, "Anions, Electron capture, Ionization, High energy, Nitrites, Deposition, Reprints, "Vibrational spectra, Photodetachment.

When a NE:NO2 or a Ne:NO:O2 sample is codeposited at approximately $5 \mathrm{~K}$ with a beam of neon atoms that have been excited in a microwave discharge, infrared absorptions of $\mathrm{NO}_{2}+$ NO2-, and $\mathrm{NO}_{3}$ appear. Detailed isotopic substitution studies suppor the assignment of prominent absorptions to $v 3$ of $\mathrm{NO} 2+$ and NO2- and of weak to moderately intens absorptions to the $v 1+v 3$ combination band of each of these species. When the contribution of anharmonicity is considered, the positions of the $\mathrm{NO} 2$ absorptions are in satisfactory agreement with the valves for the stretching fundamentals obtained in a recent gas-phase study of that species. When the sample is exposed to $240-420 \mathrm{~nm}$ mercury-arc radiation the initially present absorptions of NO3- trapped in sites with a small residual cation interaction diminish in intensity, and the unsplit v3 (e') absorption of isolated NO3- grows. The mechanism responsible for this growth in the absorption of isolated NO3- is considered. Electron capture, Infrared spectrum, Ionization, Neon matrix, $\mathrm{NO} 2+, \mathrm{NO} 2-, \mathrm{NO}_{3}-$, Photodetachment.

\section{9}

AD-A278 131/8 PC A03/MF A01

National Bureau of Standards, Boulder, $\mathrm{CO}$

Ultraviolet Multiplet Table. Finding List for Spectra of the Elements Molybdenum to Lanthanum $(Z=42$ to 57): Hafnium to Radium ( $\mathrm{Z}=72$ to 88 ).

C. E. Moore. 6 Apr 62, 37p.

Keywords: *Ultraviolet spectra, *Transition metals, Tables(Data), Chemical elements, "Multiplet table, "Finding list, "Molybdenum to lanthanum, "Hafnium to radium, $\mathrm{N}-2309$

No abstract available.

\section{0,710}

AD-A278 446/0 PC A08/MF A02

National Bureau of Standards, Gaithersburg, MD.

Ultraviolet Multiplet Table.

C. E. Moore. 28 Apr 50, 169p.

Keywords: "Ultraviolet spectra, Chemical elements, Hydrogen, Helium, Lithium, Beryllium, Boron, Carbon, Nitrogen, Oxygen, Fluorine, Neon, Sodium, Magne- 


\section{CHEMISTRY}

\section{Physical \& Theoretical Chemistry}

sium, Aluminum, Silicon, Phosphorus, Sulfur, Chlorine, Argon, Potassium, Calcium, Scandium, Titanium, Vanadium, "Finding list, "Multiplet table, Tables(Data). No abstract available.

\section{0,711}

AD-A278 517/8 PC A03/MF A01

National Bureau of Standards, Gaithersburg, MD

Density of Solids and Liquids.

P. Hidnert, and E. L. Peffer. 15 Mar 50, 34p, NBS CIRC-487.

Keywords: "Liquids, "Solids, "Density, Thermal expan-

No abstract available.

\section{0,712}

\section{AD-A278 956/8 PC A04/MF A0t}

National Bureau of Standards, Gaithersburg, MD Table of Dielectric Constants of Pure Liquids. A. A. Maryott, and E. R. Smith. 10 Aug 51, 51p, NBS-514.

Keywords: "Liquids, 'Dielectric properties, Tables(Data), Low frequencies, Temperature control Constants, Methanols, Nitrobenzenes, Water, Liquid hydrogen, Inorganic compounds, Organic compounds. No abstract available.

\section{0,713}

\section{AD-A279 951/8 PC A05/MF A01}

National Bureau of Standards, Boulder, $C O$

Tabulation of the Thermodynamic Properties of Normal Hydrogen from Low Temperatures to 300K and from 1 to 100 Atmospheres.

Technical note no. 12

ง. W. Dean. Nov 61, 76p.

Keywords: "Enthalpy, "Entropy, "Hydrogen, *Thermodynamic properties, Temperature, Barometric pressure, Specific volume, Hydrogen gas.

Pressure, volume, temperature, internal energy, enthalpy, and entropy of normal hydrogen gas have been tabulated along isobars in $1 \mathrm{deg} \mathrm{K}$ temperature steps. The range covered is from the saturation temperature to $300 \mathrm{deg} K$ and from a pressure of 1 to 100 atmospheres. The source of data is the Research Paper 1932 of the National Bureau of Standards Journal of Research. The method is described by which the data presented in Research Paper 1932 is reduced to properties directly useful for engineering calculations. A method is also described for estimating the effect of ortho-para compositions upon the tabulated properties. Tabular values are presented in the dimensional units of the metric system. The tabulations are also available in the dimensional units of the British system as Technical Note No. 120, Supplement A

\section{0,714}

AD-A280 279/1 PC A13/MF AO3

National Bureau of Standards, Gaithersburg, MD

Atomic Energy Levels. As Derived From the Analyses of Optical Spectra. Volume 3.

C. E. Moore. 1 May 58, 284p

Keywords: "Atomic energy levels, "Optics, "Spectra, "Atomic spectra, Standards, Molybdenum, Lanthanum *Molybdenum-lanthanum.

No abstract available.

\section{0,715}

\section{AD-A280 293/2 PC A20/MF AO4}

National Bureau of Standards, Gaithersburg, MD Tables of Chemical Kinetics Homogeneous Reactions.

15 Sep 61, 464p, NBS-34

Keywords: "Chemical reactions, "Tables(Data), *Reaction kinetics.

No abstract available.

\section{0,716}

AD-A286 603/6 PC A05/MF A01

National Bureau of Standards, Boulder, $C O$.

Compilation of the Physical Equilibria and Related Properties of the Hydrogen-Carbon Monoxide System.

Technical note.

D. E. Drayer, and T. M. Flynn. May 61, 96p.

Keywords: "Hydrogen, "Carbon monoxide, "Chemical equilibrium, Physical properties, Solid phases, Vapor phases, Liquid phases, K Factor.
Literature data have been used to calculate K-factors for the hydrogen-carbon monoxide system over the range of 68.2 to $122.2 \mathrm{deg} K$ and 10 to 225 atmospheres. K-factors are presented graphically for eight isotherms over this range. Published data on the solid-vapor region are presented separately as composition versus pressure at constant temperature. A bibliography of approximately 450 references is also presented on related properties for this system and for the pure components.

\section{0,717}

AD-A286 648/1 PC A03/MF A0 1

National Bureau of Standards, Gaithersburg, MD

High Temperature Reactions of Uranium Dioxide with Various Metal Oxides.

S. M. Lang, F. P. Knudsen, C. L. Fillmore, and R. S. Roth. 20 Feb 56, 30p, NBS-CIRC-568

Keywords: "Metals, "Oxides, Uranium, Dioxides, Oxides, Chemical reactions. Phase diagrams, Chemical equilibrium, Cations, Valence, Oxygen, "Uranium oxides

No abstract available.

\section{0,718}

\section{AD-A295 411/3 PC A03/MF A01}

National Bureau of Standards, Gaithersburg, MD

Arc Spectra of Gallium, Indium, and Thallium.

W. F Meggers, and R. J. Murphy. 4 Apr 52, 11 .

Pub. in Jnl. of Research of the National Bureau of Standards, v48 n4 p334-344 Apr 52.

Keywords: "Gallium, "Indium, "Infrared spectra, *Thallium, Reprints, Optical properties, Spectra, Visi ble spectra, Atomic energy levels, "Arc spectra.

This research was inspired by the compilation of Atom ic energy levels as derived from the analyses of optical spectra While compiling the data on gallium spectra, C. E. Moore noted that the first spectrum of gallium (Ga I) was very incompletely investigated. In particular, no Ga I lines had been observed with wavelengths greater than $6414 \mathrm{~A}$ in the red, although two infrared lines (11904 and 12096 A) were predicted in 1914 from spectral-series formulas. These predicted infrared lines have now been recorded photographically, and, in addition, 37 new Ga I lines have been discovered. These observations have led to revision and extension of the known atomic-energy levels is derived from the analysis of the Ga I spectrum. jg p.1.

\section{0,719}

AD-A296 377/5 PC A03/MF A01

National Inst. of Standards and Technology, Gaithersburg, MD.

Microwave Spectrum and Structure of $\mathrm{CH}_{3} \mathrm{NO} 2$ H20.

F J. Lovas, N Zobov, G. T. Fraser, and R. D.

Suenram. 1995, 12p, ARO-29596.1-CH.

Contract ARO-MIPR-126-94

Pub. in Jnl. of Molecular Spectroscopy, v171 p189-199 1995

Keywords: "Structural properties, "Microwaves, "Spectra, "Nitromethane, Fourier transformation, Mirrors, Reprints, Water Mass, Ouadrupole moment Fabry perot interferometers, Separation, Molecula structure, Rotation, Fourier spectrometers, Moment of inertia, Dipole moments, Nuclear electric moments Pulsed beams.

The microwave spectrum of the nitromethane-water complex ( $\left.\mathrm{CH} 3 \mathrm{NO} 2-\mathrm{H}_{2} \mathrm{O}\right)$ has been studied with a pulsed-beam Fourier-transform Fabry-Perot-cavity spectrometer. Both a-type and b-type transitions were observed for the A state of the complex with the b-type transition being more intense by a factor of 2 . Critical to the rotational assignments were well resolved (14)N nuclear electric quadrupole transitions, and the incorporation of the pulsed nozzle in one of the mirrors which provided a beam coaxial with the cavity axis to attain linewidths of about $2 \mathrm{kHz}$ (full-width at half maximum-FWHM). To provide additional structural information, the spectra of the HDO, D2O, and Cd3NO2 substituents were assigned. The molecular structure derived from the moments of inertia has a center of mass separation of $3.506(7) \mathrm{A}$. The moments of inertia can not distinguish between two possible forms of the complex, one with the dipole moment vectors aligned and the other with them antialigned. jg p.2.

\section{0,720}

AD-A296 498/9 PC A03/MF AO 1
National Bureau of Standards, Gaithersburg, MD Molecular Microwave Spectra Tables P. Kisliuk, and C. H. Townes. Jun 50, 31p, NBS-RP. 2107

Pub. in Jnl. of Research of the National Bureau of Standards, v44 p611-641, Jun 50. Available only to DTIJ users. No copies furnished by NTIS

Keywords: "Microwaves, "Spectra, "Molecular properties, Coupling(Interaction), Frequency, Reprints, Vibration, Molecules, Quantum theory, Intensity, Eigenvalues, Cavities, Ouadrupole moment, Measuring instruments, Tables(Data), Absorption, Rotation, Moment of inertia, Dipole moments, Wavemeters.

This paper presents a group of tables that give the frequencies, assignment of quantum numbers, and intensities of over 700 microwave absorption lines. The best available values of other pertinent molecular data, such as moments of inertia, dipole moments, quadrupole coupling constants, and rotation-vibration constants are also included. The frequencies are listed once for each molecule and again in consecutive as cending order of frequency. References are given for all data included. Frequencies listed to the nearest megacycle were generally measured with a cavity wave meter and may be in error by as much as 10 megacycles, whereas those given to a fraction of megacycle are generally known to an accuracy of about 0.1 megacycle. jg p. 1 .

\section{0,721}

AD-A297 943/3 PC A03/MF A01 National Inst. of Standards and Technology,
Gaithersburg, MD.

Crystal Diffraction Spectrometry for Accurate, Non-Invasive kV/Spectral Measurement for Improvement of Mammographic Image Quality Annual rept. 18 Apr 94-31 Mar 95.

R. D. Deslattes, and L. Hudson. $30 \mathrm{Apr} \subseteq 5,23 p$. Contract MIPR-94MM4539

Document partially illegible.

Keywords: "Spectrometry, "Image registration, Breast cancer, Setting(Adjusting), Detection, Clinical medicine, Prototypes, Crystals, $X$ rays, Radiography, Diffraction, Quantum efficiency, Spectrometers, Solid state electronics, Pharmacology, Mammography, Retrofitting, Abrasives, Image tubes, Dental equipment.

Phase one of this contract has been devoted to the development and demonstration of the MST prototype crystal spectrometer which was designed to provide accurate measurement of $x$-ray source voltage and full spectral characterization of the radiation ernitted from mammographic $x$-ray sources. A major advance has been the movement from film to solid state image registration. Our current systems take advantage of recently introduced large area digital radiography sensors which are now being used in place of clental film. Initial clinical trials indicated the need for improved detection quantum efficiency. This has been overcome primarily by increasing the crystal bandpass with an air abrasive treatment of the surface and retrofitting the sensor with a thicker scintillating material. The significance of these advances is that we now have an instrument which: measures kVp to an accuracy in excess of clinical requirements, is easily adaptable to the clinical setting, records the entire spectral profile, including the effects of both inherent and added filtration, and is sensitive enough to obtain these data within the time of a typical mammographic exposure. Not only will this contribute to the quality control of mammogra!phy but potentially to refinements in technique.

\section{0,722}

DE94017816 PC A02/MF A01

National Inst. of Standards and Technology, E3oulder,

Measurement of the Thermal Properties of Electrically Conducting Fluids Using Coated Transient Hot Wires

R. A. Perkins. 1994, 9p, CONF-9404137-6.

Contract Al05-89ER13992

Symposium on Energy Engineering Sciences (12th) Argonne, IL (United States), 27-29 Apr 1994. Sponsored by Department of Energy, Washington, DC.

Keywords: "Argon, "Fluorinated Aliphatic Hydrocarbons, "Probes, "Thermal conductivity, Design, Electrical Insulation, Experimental Data, Performance, Protective Coatings, Refrigerants, Supercritical Sitate, Tantalum Oxides, Tables(data), EDB/360606, EDB/ 440500.

Measurements of fluid thermal properties using the transient hot-wire technique are described. When bare 
hot wires are used in electrically conducting fluids there are additional measurement uncertainties due to the formation of electric double layers on the surfaces of the wires and the cell wall. If the electrical conductivity of the fluid is large enough there is also significant power generation in the fluid. These measurement uncertainties can be eliminated by electrically insulating the hot wires with a thin film. The use of tantalum hot wires with an anodized layer of tantalum pentoxide is demonstrated with measurements on nonpolar argon and polar 1,1,1,2 tetrafluorethane (R134a). Although coated tantalum hot wires have been used previously in a transient mode to measure the thermal conductivity of liquids, this work is the first demonstration of the use of coated wires to measure thermal conductivity in the liquid, vapor, and supercritical gas phases.

\section{0,723}

\section{DE94018562 PC A02/MF A01}

National Bureau of Standards, Gaithersburg, MD. Atom-counting standards and Doppler-free resonance ionization mass spectroscopy. (Progress report).

J. M. R. Hutchinson, and K. G. W. Inn. 1988, 9p DOE/ER/60447-T2

\section{Contract Al05-86ER60447}

Sponsored by Department of Energy, Washington, DC.

Keywords: "Resonance Ionization Mass Spectroscopy,

Beryllium Isotopes, Molybdenum Isotopes, Progress

Report, Trace Amounts, Uranium, EDB/400102.

This program has two components: quantification and improvement of ultrasensitive techniques, and development of isotopic ratio standards in the ultrasensitive range of 10(sup (minus)10) and lower. Data sheets were developed.

\section{0,724}

N95-15839/0 (Order as N95-15827/5, PC A07/

$\mathrm{MF}$ A02)

National Inst. of Standards and Technology, Boulder,

$\mathrm{CO}$.

Investigating the 3.3 Micron Infrared Fluorescence from Naphthalene Following UItraviolet Excitation. R. M. Williams, and S. R. Leone. May 94, 6p.

In NASA Ames Research Center, the Diffuse Interstellar Bands: Contributed Papers p 59-64.

Keywords: "Infrared spectra, "Naphthalene, Absorption spectra, Fluorescence, Molecular excitation, Vapor phases, Excimer lasers, Fourier transformation, Polycyclic aromatic hydrocarbons, Spectrometers, UItraviolet lasers, Intermediate infrared radiation.

Polycyclic aromatic hydrocarbon (PAH) type molecules are proposed as the carriers of the unidentified infrared (UIR) bands. Detailed studies of the 3.3 micrometer infrared emission features from naphthalene, the simplest $\mathrm{PAH}$, following ultraviolet laser excitation are used in the interpretation of the 3.29 micrometer (3040 cm(sup -1)) UIR band. A time-resolved Fourier transform spectrometer is used to record the infrared emission spectrum of gas-phase naphthalene subsequent to ultraviolet excitation facilitated by an excimer laser operated at either $193 \mathrm{~nm}$ or $248 \mathrm{~nm}$. The emission spectra differ significantly from the absorption spectrum in the same spectral region. Following $193 \mathrm{~nm}$ excitation the maximum in the emission profile is redshifted $45 \mathrm{~cm}($ sup -1$)$ relative to the absorption maximum; a $25 \mathrm{~cm}($ sup -1) red-shift is observed after 248 $\mathrm{nm}$ excitation. The red-shifting of the emission spectrum is reduced as collisional and radiative relaxation removes energy from the highly vibrationally excited molecules. Coupling between the various vibrational modes is thought to account for the differences between absorption and emission spectra. Strong visible emission is also observed following ultraviolet excitation. Visible emission may play an important role in the rate of radiative relaxation, which according to the interstellar PAH hypothesis occurs only by the slow emission of infrared photons. Studying the visible emission properties of PAH type molecules may be useful in the interpretation of the DIB's observed in absorption.

\section{0,725}

PB94-112695 PC A15/MF A03

National Inst. of Standards and Technology (MSEL), Gaithersburg, MD. Ceramics Div.
Electroacoustics for Characterization of Particulates and Suspensions. Proceedings of a Workshop. Held in Gaithersburg, Maryland on February 3-4, 1993.

Special pub.

S. G. Malghan. Sep 93,343p, NIST/SP-856

Also available from Supt. of Docs. as SNO03-00303237-9.

Keywords: "Meetings, "Electroacoustics, "Laboratory equipment, "Particulates, "Colloids, Ceramics, Electrokinetics, Suspensions, Measuring instruments, tions, Surface chemistry, Coatings, Polarity.

The electroacoustic technique based on physical affects described by Debye, is relatively new and the laboratory instrumentation has become available only in the last decade. This new instrumentation by Matec Applied Sciences and Pen Kem Inc. is based on the application of either electrical fields or ultrasound as external fields to the particles and measurement of the resulting electrical or ultrasonic response. These in struments, while addressing the primary drawbacks of electrophoresis instruments, allow the determination of signals that are related to the electrokinetic zeta potential for colloidal suspensions in polar and nonpola media. In recent years, the electroacoustic technique has been extensively utilized for the characterization of a variety of particulates in dense suspensions of polar and nonpolar liquids with great success. As a result of this surge in activity, a number of technical is sues have surfaced. In order to discuss these issues and develop a common understanding of the fundamentals of electroacoustics, a workshop was organized at the suggestion of the user community. The volume contains all but two papers presented at the workshop.

\section{0,726}

PB94-140571 (Order as PB94-140555, PC A06/

MF A02)

Los Alamos National Lab., NM

Pressure-Volume-Temperature Relations in Liquid and Solid Tritium.

E. R. Grilly. 1993, 12p.

Included in Jnl. of Research of the National Institute of Standards and Technology, v98 n6, p679-690 Nov/ Dec 93.

Keywords: "Tritium, "Thermodynamic properties, Temperature range 0013-0065 K, Temperature dependence, Pressure dependence, Solidified gases, Liquefied gases, Deuterium, Hydrogen.

PVT relations in liquid and solid T2 near the melting curve were measured over $20.5 \mathrm{~K}-22.1 \mathrm{~K}$ and $0 \mathrm{MPa}$ $7 \mathrm{MPa}$ ( 0 bar-70 bar) with a cell that used diaphragms for pressure and volume variation and measurement. Because of ortho-para self conversion, the melting pressure $P(m)$ and the liquid molar volume $V(I m)$ in creased with time. Measurements of the volume change on melting and the thermal expansion and compressibility for liquid T2 were consistent with those for H2 and D2. Impurities such as H2, HT, DT, and (3) He were removed by a technique using an adsorption column of cold activated alumina. Corrections for (3) He growth during an experiment were adequate except near the triple point.

\section{0,727}

PB94-151842 PC A06/MF A02 National Inst, of Standards and Technology,
Gaithersburg, MD.

NIST Standard Reference Data Products Catalog, 1994.

Special pub.

M. W. Chase, and J. C. Sauerwein. Jan 94, 117p,

NIST/SP-782-1994ED.

Supersedes PB93-173409. Also available from Supt. of Docs. as SNOO3-003-03247-6.

Keywords: "Catalogs(Publications), "Data bases, Chemical analysis Atomic physics, Biotechnology Reaction kinetics, Molecular structure, Molcular spectroscopy, Thermophysical properties, Thermochemistry, Thermodynamics. Numerical data, Software tools, Fluids.

The National Institute of Standards and Technology's (NIST) Standard Reference Data Program provides reliable well-documented data to scientists and engineers for use in technical problem-solving, research, and development. This catalog lists published data compilations and current databases in the Standard Reference Database Series. The activities concentrate in the following disciplines: Analytical Chemistry; Atomic and Molecular Physics; Biotechnology; Chemical Kinetics; Materials Properties; Process Design; Thermodynamics and Thermochemistry; and Thermophysical Properties of Fluids.

00,728

PB94-160769 PC A16/MF A03

National Inst. of Standards and Technology (CSTL), Gaithersburg, MD

CSTL Technical Activities 1991

H. S. Hertz, and B. I. Diamondstone. Feb 92, 353p. See also report for 1992. PB93-173482. Presented to the Board on Assessment of NIST Programs, National Research Council, February 11-12, 1992.

Keywords: "Research and development, "Chemistry, Biochemistry, Biotechnology, Chemical engineering, Analytical chemistry, Reaction kinetics, Metrology, Thermodynamics, Inorganic compounds, Particulates, Organic compounds, Technology transfer, Surface chemistry, Measurement, Thermophysics, Processing Microanalysis, Ouality assurance, US NIST, "Chemical Science and Technology Library.

The report summarizes the technical activities of the Chemical Science and Technology Laboratory at the National Institute of Standards and Technology. It emphasizes activities over the Fiscal Year 1991 in the Biotechnology Division, the Chemical Engineering Division, the Chemical Kinetics and Thermodynamics Division, the Inorganic Analytical Research Division, the Organic Analytical Research Division, the Process Measurements Division, the Surface and Microanalysis Division and the Thermophysics Division.

\section{0,729}

PB94-160975 Not available NTIS

American Chemical Society, Washington, DC

Journal of Physical and Chemical Reference Data, Volume 22, No. 1, January/February 1993.

Bimonthly rept.

J. W. Gallagher. c1993, 289p.

See also PB94-160983 through PB94-161007 and PB93-149136. Prepared in cooperation with American Inst. of Physics, New York. Sponsored by National Inst. of Standards and Technology, Gaithersburg, MD. Available from American Chemical Society, 1155 Sixteenth St., NW, Washington, DC. 20036-9976.

Keywords: "Physical properties, "Chemical properties, "Physical chemistry, Alkaline earth metals, Thermodynamics, Oxygen, Reaction kinetics, Liquid phases, Tables(Data), Spectroscopy, Periodicals, ${ }^{*}$ Reference materials.

Table of Contents:

Thermodynamic Properties of the Group IIA Elements:

Spectroscopy and Structure of the Lithium Hydride Diatomic Molecules and Ions:

Ouantum Yields for the Photosensitized Formation of the Lowest Electronically Excited Singlet State of Molecular Oxygen in Solution; Cumulative Listing of Reprints:

Cumulative Listing of Supplements, Monographs, and Special Issues.

00,730

PB94-160983 Not available NTIS

Notre Dame Univ., IN. Center for Sensor Materials. Thermodynamic Properties of the Group IIA Elements.

C. B. Alcock, M. W. Chase, and V. Itkin. c1993, 85p. Prepared in cooperation with National Inst. of Stand ards and Technology (NML), Gaithersburg, MD. Standard Reference Data. and Toronto Univ. (Ontario). Dept. of Metallurgy and Materials Science.

Included in Jnl. of Physical and Chemical Reference Data, v22 n1 p1-85 Jan/Feb 93. Available from American Chemical Society, 1155 Sixteenth St., NW, Washington, DC. 20036-9976.

Keywords: "Research, "Physical chemistry, Alkaline earth metals. Thermodynamics, Gibbs free energy, Specific heat, Enthalpy, Phase transformations, Chemical equilibrium, Heat of fusion, Heat of transformation

The thermodynamic properties of the alkaline earth metals in the condensed state have been critically reassessed, and recommended values for all of the relevant thermodynamic properties are given. These values are compared with those published in recent reviews by the staff at the Institute for High Temperatures (Moscow) and the National Institute of Standards and 
Technology (Washington, DC), and the reasons for any differences are discussed in detail. A direct result of this review is a set of recommendations for experimental studies which should enhance the reliability of the thermodynamic results

00,731

PB94-160991 Not available NTIS

lowa Univ., lowa City.

Spectroscopy and Structure of the Lithium Hydride Diatomic Molecules and Ions.

W. C. Stwalley, and W. T. Zemke. c1993, 26p.

Prepared in cooperation with Wartburg Coll., Waverley, IA. Dept. of Chemistry.

Included in Jnl. of Physical and Chemical Reference Data, v22 n1 p7-112 Jan/Feb 93. Available from American Chemical Society, 1155 Sixteenth St., NW, Washington, DC. 20036-9976

Keywords: "Research, "Physical chemistry, Lithium hydride, Hydrides, Reaction kinetics, Dissociation energy, Molecular spectroscopy, Molecular structure, Isotope effect, Potential energy, Born-Oppenheimer approximation.

All significant experimental measurements and many theoretical calculations of the spectroscopy and structure of the isotopic lithium hydrides $((6) \mathrm{LiH},(7) \mathrm{LiH}$, (6) LiD, (7) LiD) are identified and reviewed. Published molecular constant determinations from conventional and laser spectroscopy are evaluated; recommended spectroscopic constants for the $X(1)$ sigma $(+1)$, $A(1)$ sigma(+1) and $B(1) \|$ states are tabulated. Potential energy curves (RKR, IPA and hybrid) for the $X(1)$ sigma( +1$), A(1)$ sigma $(+1)$ and $B(1) \|$ states are evaluated and recommended curves are tabulated. Adiabatic corrections to the Born-Oppenheimer approximation are also reviewed. Calculations on $\mathrm{LiH}(+1)$ and $\mathrm{LiH}(-1)$ are also listed and described briefly.

\section{0,732}

PB94-161007 Not available NTIS

Loughborough Univ. of Technology (England). Dept. of Chemistry.

Quantum Yields for the Photosensitized Formation of the Lowest Electronically Excited Singlet State of Molecular Oxygen in Solution.

F. Wilkinson, W. P. Helman, and A. B. Ross. c1993, 150p.

Prepared in cooperation with Notre Dame Univ., IN Radiation Lab.

Included in Jnl. of Physical and Chemical Reference Data, v22 n1 p113-262, Jan/Feb 93. Available from American Chemical Society, 1155 Sixteenth St., NW, Washington, DC. 20036-9976

Keywords: "Research, "Physical chemistry, Data processing. Energy transfer, Oxygen, Quantum efficiency, Quantum chemistry, Photosensitivity, Atomic energy levels, Solutions, Decay.

Quenching of excited singlet and triplet states of many substances by ground state molecular oxygen produces singlet oxygen, the lowest electronically excited singlet state of molecular oxygen, O2(1 delta sub g). The fractions of singlet and triplet states quenched which produce singlet oxygen and the quantum yields of formation of singlet oxygen in fluid solutions have been critically compiled. Methods for determining yield parameters have been reviewed. Data have been com. piled from the literature through 1991. Photosensitizers are included in Table 1. Porphyrins and phthalocyanines are included in Table 2. Other materials which have been investigated for singlet oxygen production, such as dyes and drugs, are collected in Table 3 along with heterogeneous systems such as polymer-bound photosensitizers.

\section{0,733}

\section{PB94-162211 Not available NTIS}

American Chemical Society, Washington, DC

Journal of Physical and Chemical Reference Data, Volume 22, No. 2, March/April 1993.

Bimonthly rept.

J. W. Gallagher. c1993, 322p.

See also PB94-162229 through PB94-162252 and PB94-160975. Prepared in cooperation with American Inst. of Physics, New York. Sponsored by National Inst. of Standards and Technology, Gaithersburg, MD Available from American Chemical Society, 1155 Sixteenth St., NW, Washington, DC. 20036-9976.

Keywords: "Physical properties, "Chemical properties, "Physical chemistry, Chemical reactions, Wavelengths, Thermodynamics, Reaction kinetics, Vapor phases, Table(Data), Periodicals, "Reference materials.

Table of Contents:

Wavelengths and Energy Level Classifications for the Spectra of Sulfur (S I through S XVI);

Thermodynamic Properties of Alkenes (MonoOlefins Larger Than C(4))

The Thermodynamic Behavior of the $\mathrm{CO}_{2}-\mathrm{H}_{2} \mathrm{O}$ System from 400 to $1000 \mathrm{~K}$ up to $100 \mathrm{MPa}$ and $30 \%$ Mole Fraction of CO2

Thermodynamics of Enzyme-Catalyzed Reactions:

Part 1. Oxidoreductases;

Cumulative Listing of Reprints;

Cumulative Listing of Supplements, Monographs, and Special Issues.

\section{0,734}

PB94-162229 Not available NTIS National Inst. of Standards and Technology,

Wavelengths and Energy Level Classifications for the Spectra of Sulfur (SI through S XVI).

V. Kaufman, and W. C. Martin c1993, 96p.

Included in Jnl. of Physical and Chemical Reference Data, v22 n2 p279-375, Mar/Apr 93. Available from American Chemical Society, 1155 Sixteenth St., NW Washington, DC. 20036-9976.

Keywords: "Research, "Physical chemistry, Atomic energy levels, Ions, Spectra, Wavelengths, Frequencies, Sulfur, Infrared radiation, Ultraviolet radiation, Tables(Data)

Wavelengths and their classifications have been compiled for the spectra of the atom and all positive ions of sulfur $(Z=16)$. The selections of data are based on the compilations of energy levels by Martin Zalubas, and Musgrove in 1990, with some updating from the more recent literature. Wavelengths (or wavenumbers) calculated from the differences of the energy levels are given along with the observed values for all classified lines; these calculated wavelength should in general be more accurate than the observed values wherever the two values differ significantly. No limitation has been imposed on the wavelength range of the classified lines, except for the omission of $x$-ray transitions in the neutral atom. Two finding lists are also included, one for S I through S III and the othe for S IV through S XVI.

\section{0,735}

PB94-162237 Not available NTIS

National Inst. for Petroleum and Energy Research, Bartlesville, OK

Thermodynamic Properties of Alkenes (MonoOlefins Larger Than C4)

W. V. Steele, and R. D. Chirico. c1993, 53p.

Included in Jnl. of Physical and Chemical Reference Data, v22 n2 p377-430, Mar/Apr 93. Available from American Chemical Society, 1155 Sixteenth St, NW Washington, DC. 20036-9976.

Keywords: "Research, "Physical chemistry, Alkene compounds, Thermodynamic properties, Entropy, Gibbs free energy, Specific heat, Vapor pressure, Heat of vaporization, Heat of formation, Ideal gas, Density(Mass/Volume)

The thermodynamic properties of the mono-olefins with carbon numbers greater than $\mathrm{C} 4$ were reviewed. Properties included critical properties, vapor pressures, densities, second virial coefficients, enthalpies of vaporization, heat capacities, and enthalpies of com bustion. Enthalpies of formation for the liquid, gas, and ideal-gas state at $298.15 \mathrm{~K}$ were calculated for $47 \mathrm{com}$ pounds based on the experimental values. Gaps in the available data were identified and recommendations for additional experiments are made. Evidence for errors in several of the original experimental results is presented, and revised values are suggested.

\section{0,736}

PB94-162245 Not available NTIS

National Inst. of Standards and Technology (CSTL), Gaithersburg, MD. Thermophysics Div.

Thermodynamic Behavior of the $\mathrm{CO} 2-\mathrm{H} 2 \mathrm{O}$ System from 400 to $1000 \mathrm{~K}$, up to $100 \mathrm{MPa}$ and $30 \%$ Mole Fraction of $\mathrm{CO} 2$.

J. S. Gallagher, R. Crovetto, and J. M. H. L.

Sengers. c1993, 82p.

Included in Jnl. of Physical and Chemical Reference

Data, v22 n2 p431-513, Mar/Apr 93. Available from American Chemical Society, 1155 Sixteenth St., NW, Washington, DC. 20036-9976.
Keywords: "Research, "Physical chemistry, Thermodynamic properties, Carbon dioxide Water, Enthalpy, Equations of state, Volume, Fugacity, Henrys law, Mixtures, Steam, Critical point, FORTRAN, Phases, Boundaries, Computer programs.

A model is presented for the thermodynamic properties of the aqueous mixture of carbon dioxide, up to 30 mol\% composition, in a large range of temperatures (400-1000 K) and pressures (0-100 MPa) around the critical point of water. The model for the Helmholtz tree energy of the mixture is based on the principle of generalized corresponding states, with the NBS/NRC Steam Tables as the reference state for pure water. Input to the model are data for the critical line of the mixture, apparent molar volume and pVTx data in supercritical water, phase boundaries, excess enthalpies and mixture second virial coefficient data. Comparisons are presented with those data, with Henry's constants and with other formulations available for this system. The Fortran codes for generating these properties are listed in Appendix B.

\section{0,737}

PB94-162252 Not available NTIS

National Inst. of Standards and Technology (CSTL), Gaithersburg, MD. Biotechnology Div.

Thermodynamics of Enzyme-Catalyzed Reactions: Part 1. Oxidoreductases.

R. N. Goldberg, Y. B. Tewari, D. Bell, K. Fazio, and E. Anderson. c1993, 68p.

Prepared in cooperation with Food and Drug Administration, Rockville, MD.

Included in $\mathrm{Jnl}$. of Physical and Chemical Reference Data, v22 n2 p515-582, Mar/Apr 93. Available from American Chemical Society, 1155 Sixteenth St., NW, Washington, DC. 20036-9976

Keywords: "Research, "Physical chemistry, Thermodynamics, Enthalpy, Enzymes, Chemical reactions, Catalysts, Oxidoreductases, Heat of reaction, Chemical equilibrium.

Equilibrium constants and enthalpy changes for reactions catalyzed by oxidoreductases have been compiled. For each reaction the following information is given: the reference for the data; the reaction studied; the name of the enzyme used and its Enzyme Commission number; the method of measurement; the conditions of measurement (temperature, $\mathrm{pH}$, ionic strength, and the buffer(s) and cofactor(s) used); the data and an evaluation of it; and, sometimes, commentary on the data and on any corrections which have been applied to it. The data from 205 references have been examined and evaluated. Chemical Abstract Service Registry Numbers have been assigned to the substances involved in these various reactions. There is a cross reference between the substances and the Enzyme Commission numbers of the enzymes used to catalyze the reactions in which the substances participated.

\section{0,738}

PB94-162260 Not available NTIS

American Chemical Society, Washington, DC

Journal of Physical and Chemical Reference Data, Volume 22, No. 3, May/June 1993.

Bimonthly rept.

J. W. Gallagher. c1993, 216p.

See also PB94-162278 through PB94-162302 and PB94-162211. Prepared in cooperation with American Inst. of Physics, New York. Sponsored by National Inst. of Standards and Technology, Gaithersburg, MD. Available from American Chemical Society, 1155 Sixteenth St., NW, Washington, DC. 20036-9976.

Keywords: "Physical properties, "Chemical properties, "Physical chemistry, Thermodynamics, Nitrogen organic compounds, Temperature, Reaction kinetics, Vapor phases, Tables(Data), "Reference materials.

Table of Contents:

Estimation of the Heat Capacities of Organic Liquids as a Function of Temperature Using Group Additivity. I. Hydrocarbon Compounds;

Estimation of the Heat Capacities of Organic Liquids as a Function of Temperature Using Group Additivity. II. Compounds of Carbon, Hydrogen, Halogens, Nitrogen, Oxygen, and Sulfur;

Thermodynamic and Thermophysical Properties of Organic Nitrogen Compounds. Part II. 1and 2-Butanamine, 2-Methyl-1-Propanamine, 2-Methyl-2-Propanamine, Pyrrole, 1-, 2-, and 3-Methylpyrrole, Pyridine, 2-, 3-, and 4Methylpyridine, Pyrrolidine, Piperidine, Indole, 
Quinoline, Isoquinoline, Acridine, Carbazole, Phenanthridine, 1- and 2- Naphthalenamine, and 9-Methylcarbazole;

Intemational Equations for the Saturation Properties of Ordinary Water Substance. Revised According to the Intemational Temperature Scale of 1990. Addendum to Joumal of Physical and Chemical Reference Data 16, 893 (1987)

Cumulative Listing of Reprints;

Cumulative Listing of Supplements, Monographs, and Special Issues.

\section{0,739}

PB94-162278 Not available NTIS

National Inst. of Standards and Technology (CSTL), Gaithersburg, MD. Chemical Kinetics and Thermodynamics Div.

Estimation of the Heat Capacities of Organic Liquids as a Function of Temperature Using Group Additivity. I. Hydrocarbon Compounds.

V. Ruzicka, and E. S. Domalski. c1993, 22p

Included in Jnl. of Physical and Chemical Reference Data, v22 n3 p597-618, May/Jun 93. Available from American Chemical Society, 1155 Sixteenth St., NW, Washington, DC. 20036-9976.

Keywords: "Research, "Physical chemistry, Specific heat, Estimating, Liquid phases, Hydrocarbons, Temperature dependence, Least squares method, Organic compounds, Group additivity, Organic liquids.

A second-order group additivity method has been developed for the estimation of the heat capacity of liquid hydrocarbons as a function of temperature in the range from the melting temperature to the normal boiling temperature. The temperature dependence of group contributions and siructural corrections has been represented by a polynomial expression. Recommended heat capacities from a large compilation of critically evaluated data that contains over 1300 organic liquids served as a database both for the development and testing of the method.

\section{0,740}

PB94-162286 Not available NTIS

National Inst. of Standards and Technology (CSTL) Gaithersburg, MD. Chemical Kinetics and Thermodynamics Div.

Estimation of the Heat Capacities of Organic Liquids as a Function of Temperature Using Group Additivity. II. Compounds of Carbon, Hydrogen, Halogens, Nitrogen, Oxygen, and Sulfur.

V. Ruzicka, and E. S. Domalski. c1993,39p.

Included in Jnl. of Physical and Chemical Reference Data, v22 n3 p619-657, May/Jun 93. Available from American Chemical Society, 1155 Sixteenth St., NW, Washington, DC. 20036-9976.

Keywords: "Research, "Physical chemistry, Specific heat, Estimating, Liquid phases, Hydrocarbons, Temperature dependence, Least squares method, Organic compounds, Nitrogen organic compounds, Oxygen organic compounds, Sulfur organic compounds, Halogen organic compounds, Group additivity, Organic liquids.

A second-order group additivity method has been developed for the estimation of the heat capacity of liquid organic compounds containing carbon, hydrogen, halogens, nitrogen, oxygen, and sulfur. The method permits the estimation of the heat capacity as a function of lemperature in the range from the melting temperature to the normal boiling temperature. Group contributions and structural corrections have been made temperature dependent by the use of a polynomial expression. The work has drawn information for both the deveiopment and testing of the method from a large compilation of critically evaluated heat capacity data for over 1300 organic liquids.

00,741

PB94-162294 Not available NTIS

Texas $A$ and $M$ Univ., College Station. Thermodynamics Research Center.

Thermodynamic and Thermophysical Properties of Organic Nitrogen Compounds. Part II. 1- and 2Butanamine, 2-Methyl-1-Propanamine, 2-Methyl-2Propanamine, Pyrrole, 1-, 2-, and 3-Methylpyrrole, Pyridine, 2-, 3-, and 4-Methylpyridine, Pyrrolidine, Piperidine, Indole, Quinoline, Isoquinoline, Acridine, Carbazole, Phenanthridine, 1- and 2Naphthalenamine, and 9-Methylcarbazole.

A. Das, M. Frenkel, N. A. M. Gadalla, A. S. Rodgers, R. C. Wilhoit, S. Kudchadker, and K. N. Marsh.

$c 1993,124 p$

See also PB91-192542.
Included in Jnl. of Physical and Chemical Reference Data, v22 n3 p659-782, May/Jun 93. Available from American Chemical Society, 1155 Sixteenth St., NW, Washington, DC. 20036-9976.

Keywords: "Research, *Physical chemistry, Thermodynamic properties, Nitrogen organic compounds, Enthalpy, Heat of vaporization, Heat of combustion, Specific heat, Ideal gas, Thermochemistry, Vapor pressure, Condensed phase.

The thermodynamic and thermophysical properties of 1- and 2-butanamine, 2-methyl-1-propanamine, 2methyl-2-propanamine, pyrrole, 1-, 2- and 3methylpyrrole, pyridine, 2-, 3-, and 4-methylpyridine, pyrrolidine, pipenidine, indole, quinoline, isoquinoline, acridine, carbazole, phenanthridine, 1- and 2naphthalenamine, and 9-methylcarbazole have been evaluated. Recommended values are given for the following properties: normal boiling, freezing and triple point temperatures, critical constants, thermodynamic properties in the solid and liquid phases, vapor pressure, enthalpy of vaporization, density, second virial coefficients, and enthalpy of combustion. Ideal gas thermodynamic properties have been calculated by statistical mechanical methods.

\section{0,742}

\section{PB94-162302 Not available NTIS}

Ruhr Univ., Bochum (Germany, F.R.). Inst. fuer Thermo- und Fluiddynamik.

International Equations for the Saturation Properties of Ordinary Water Substance. Revised According to the International Temperature Scale of 1990. Addendum to Journal of Physical and Chemical Reference Data 16, 893 (1987).

W. Wagner, and A. Pruss. c1993, $5 p$.

Included in Jnl. of Physical and Chemical Reference Data, v22 n3 p783-787, May/Jun 93. Available from American Chemical Society, 1155 Sixteenth St., NW, Washington, DC. 20036-9976.

Keywords: "Research, "Physical chemistry, Enthalpy, Entropy, Water saturation, Density(MassNolume) Temperature, Vapor pressure, Correlation IAPWS(Intemational Association for the Properties of Water and Steam), Saturated liquid density, Saturated vapor density.

In 1987, consistent with the latest experimental data and the intemationally recommended values for the critical parameters, the authors published compact and accurate correlation equations for properties on the saturation line of ordinary (light) water substance. (A Saul and W. Wagner, J. Phys. Chem. Ref. Data 16 893 (1987)). As an addendum to this 1987 paper the present paper brings all temperature values and adjusted coefficients in all correlation equations given in the 1987 paper into agreement with the International Temperature Scale of 1990 (ITS-90). The new equations form the basis of the 'Revised Supplementary Release on Saturation Properties of Ordinary Water Substance' issued by the International Association for the Properties of Water and Steam (IAPWS). This revised release which contains all equations and coefficients adjusted with regard to the ITS-90 is the main part of this paper.

\section{0,743}

PB94-162310 Not available NTIS

American Chemical Society, Washington, DC

Journal of Physical and Chemical Reference Data, Volume 22, No. 4, July/August 1993.

Bimonthly rept.

J. W. Gallagher. c1993, 380p

See also PB94-162328 and PB94-162260. Prepared in cooperation with American Inst. of Physics, New York. Sponsored by National Inst. of Standards and Technology, Gaithersburg, MD

Available from American Chemical Society, 1155 Sixteenth St., NW, Washington, DC. 20036-9976.

Keywords: "Physical properties, *Chemical properties "Physical chemistry, Thermodynamic properties, Solid phases, Liquid phases, Vapor phases, Tables(Data), Reference materials.

Table of Contents:

Estimation of the Thermodynamic Properties of C$\mathrm{H}-\mathrm{N}-\mathrm{O}-\mathrm{S}-\mathrm{Hal}$ ogen Compounds at $298.15 \mathrm{~K}$; Cumulative Listing of Reprints;

Cumulative Listing of Supplements, Monographs, and Special Issues.

00,744

PB94-162328 Not available NTIS
National Inst. of Standards and Technology (CSTL), Gaithersburg, MD. Chemical Kinetics and Thermodynamics Div.

Estimation of the Thermodynamic Properties of C. $\mathrm{H}-\mathrm{N}-\mathrm{O}-\mathrm{S}-\mathrm{Hal}$ ogen Compounds at $298.15 \mathrm{~K}$

E. S. Domalski, and E. D. Hearing. c1993, 354p.

Included in Jnl. of Physical and Chemical Reference Data, v22 n4 p805-1159, Jul/Aug 93. Available from American Chemical Society, 1155 Sixteenth St., NW, Washington, DC. 20036-9976

Keywords: *Research, "Physical chemistry, Specific heat, Entropy, Enthalpy, Thermodynamic properties, Organic compounds, Hydrocarbons, Halogen organic compounds, Nitrogen organic compounds, Oxygen organic compounds, Sulfur organic compounds, Solid phases, Liquid phases, Vapor phases, Estimating.

An estimation method, which was developed by S. W. Benson and coworkers for calculating the thermodynamic properties of organic compounds in the gas phase, has been extended to the liquid and solid phases for organic compounds at $298.15 \mathrm{~K}$ and $101,325 \mathrm{~Pa}$. As with a previous paper dealing with hydrocarbon compounds, comparisons of estimated enthalpies of formation, heat capacities, and entropies with literature values show that extension of the Benson's group additivity approach to the condensed phase is easy to apply and gives satisfactory agreement. This work covers 1512 compounds containing the elements: cabon, hydrogen, oxygen, nitrogen, sulfur, and halogens in the gas, liquid, and solid phases. About 1000 references are provided for the literature values which are cited.

00,745

PB94-162336 Not available NTIS

American Chemical Society, Washington, DC

Journal of Physical and Chemical Reference Data,

Volume 22, No. 5, September/October 1993.

Bimonthly rept.

J. W. Gallagher. c1993, 266p

See also PB94-162344 through PB94-162369 and PB94-162310. Prepared in cooperation with American Inst. of Physics, New York. Sponsored by National Inst. of Standards and Technology, Gaithersburg, MD. Available from American Chemical Society, 1155 Sixteenth St., NW, Washington, DC. 20036-9976.

Keywords: "Physical properties, "Chemical properties, "Physical chemistry, Atomic properties, Oxygen, Germanium, Chromium, Spectrum analysis, Tables(Data), Atomic energy levels, Periodicals, "Reference materials.

Table of Contents:

A Compilation of Energy Levels and Wavelengths for the Spectrum of Singly-lonized Oxygen (O II);

Energy Levels of Germanium, Ge I through Ge XXXII;

Spectral Data and Grotrian Diagrams for Highly lonized Chromium, $\mathrm{Cr} V$ through $\mathrm{Cr}$ XXIV;

Cumulative Listing of Reprints

Cumulative Listing of Supplements, Monographs, and Special Issues.

00,746

PB94-162344 Not available NTIS

National Inst. of Standards and Technology (PL) Gaithersburg, MD. Physics Lab. Office.

Compilation of Energy Levels and Wavelengths for the Spectrum of Singly-lonized Oxygen (O II).

W. C. Martin, V. Kaufman, and A. Musgrove. c1993, 33p.

Included in Jnl. of Physical and Chemical Reference Data, v22 n5 p1179-1212, Sep/Oct 93. Available from American Chemical Society, 1155 Sixteenth St., NW, Washington, DC. 20036-9976.

Keywords: "Research, "Physical chemistry, Atomic energy levels, Oxygen, Wavelengths, Frequencies, Ionization potentials, Atomic structure, Atomic spectra.

The authors have assembled a complete list of the most accurately measured wavelengths for all classified lines of singly-ionized oxygen (O II). The data are based mainly on recent extensions of the observations and analysis of this spectrum carried out at the University of Lund, Sweden. They denived new optimal values for the energy levels using a computer code and the observed wavelengths for all classified lines. Relevant astrophysical wavelength measurements, appropriately weighted, were included in the level-optimization calculation. 
National Inst. of Standards and Technology (PL), Gaithersburg, MD. Atomic Physics Div.

Energy Levels of Germanium, Ge I through Ge XXXII.

J. Sugar, and A. Musgrove. c1993, 65p.

Included in Jnl. of Physical and Chemical Reference Data, v22 n5 p1213-1278, Sep/Oct 93. Available from American Chemical Society, 1155 Sixteenth St., NW, Washington, DC. 20036-9976.

Keywords: "Research, "Physical chemistry, "Germanium, "Atomic energy levels, "Ions, "Spectra, *Tables(Data).

Atomic energy levels of germanium have been compiled for all stages of ionization for which experimental data are available. No data have yet been published for Ge VIII through Ge XIII and Ge XXXII. Very accurate calculated values are compiled for $G e X X X \mid$ and XXXII. Experimental g-factors and leading percentages from calculated eigenvectors of levels are given. A value for the ionzation energy, either experimental when available or theoretical, is included for the neutral atom and each ion.

\section{0,748}

PB94-162369 Not available NTIS Japan Atomic Energy Research Inst. Tokai.

Spectral Data and Grotrian Diagrams for Highly lonized Chromium, $\mathrm{Cr} V$ through $\mathrm{Cr}$ XXIV.

T. Shirai, Y. Nakai, T. Nakagaki, J. Sugar, and W. L. Wiese. c1993, 144p.

Prepared in cooperation with National Inst. of Standards and Technology, Gaithersburg, MD.

Included in Jnl. of Physical and Chemical Reference Data, v22 n5 p1279-1423, Sep/Oct 93. Also available from American Chemical Society, 1155 Sixteenth St. NW, Washington, DC. 20036-9976.

Keywords: "Research, "Physical chemistry, Chromium, Spectrum analysis, Wavelengths, Spectral lines, Atomic energy levels, lons, Diagrams.

Wavelengths, energy levels, ionization energies, line classifications, oscillator strengths, and atomic transition probabilities for $\mathrm{Cr} V$ to $\mathrm{Cr}$ XXIV are tabulated. A short review of the line identifications and wavelength measurements is given for each stage of ionization. Grotrian diagrams are given to provide graphical overviews. The literature has been surveyed through December 1991.

\section{0,749}

P894-168556 Not available NTIS

American Chemical Society, Washington, DC

Journal of Physical and Chemical Reference Data, Volume 22, No. 6, November/December 1993.

Bimonthly rept

J. W. Gallagher. c1993, 178p.

See also PB94-168564 through PB94-168606 and PB94-162336. Prepared in cooperation with American Inst. of Physics, New York. Sponsored by National Inst. of Standards and Technology, Gaithersburg, MD. Available from American Chemical Society, 1155 Sixteenth St., NW, Washington, DC. 20036-9976.

Keywords: "Physical properties, "Chemical properties, "Physical chemistry, Atomic properties, Chemical reactions, Thermodynamics, Reaction kinetics, Vapor phases, Tables(Data), "Reference materials.

Contents:

Thermodynamic Properties of Synthetic Sapphire (alpha-Al2O3), Standard Reference Material 720 and the Effect of Temperature-Scale Differences on Thermodynamic Properties

Thermodynamic Properties of Gaseous Silicon Monotelluride and the Bond Dissociation Enthalpy DM(SiTe) at T $\rightarrow 0$ :

The Disilicides of Tungsten, Molybdenum, Tantalum, Titanium, Cobalt, and Nickel, and Platinum Monosilicide:

A Survey of Their Thermodynamic Properties;

Evaluated Bimolecular Ion-Molecule Gas Phase Kinetics of Positive lons for Use in Modeling Planetary Atmospheres, Cometary Comae, and Interstellar Clouds;

Atomic Weights of the Elements 1991

Index of Properties and Classes of Materials Volumes 21-22 (1992-1993)

Author Index - Volumes 21-22 (1992-1993) Cumulative Listing of Reprints:

Cumulative Listing of Supplements, Monographs, and Special Issues.
National Inst. of Standards and Technology (CSTL), Gaithersburg, MD. Chemical Kinetics and Thermodynamics Div.

Thermodynamic Properties of Synthetic Sapphire (alpha-Al2O3), Standard Reference Material 720 and the Effect of Temperature-Scale Differences on Thermodynamic Properties.

D. G. Archer. c1993, 12p.

Included in Jnl. of Physical and Chemical Reference Data, v22 n6 p 1441-1453, Nov/Dec 93. Available from American Chemical Society, 1155 Sixteenth St., NW, Washington, DC. 20036-9976.

Keywords: "Research, "Physical chemistry, Thermodynamic properties, Aluminum oxide, Specific heat, Enthalpy, Temperature scales, Calibration standards, SRM720(Standard Reference Material 720), Synthetic sapphire.

Comparison of the National Institute of Standards and Technology's Standard Reference Material 720 certificate values for heat capacity with those obtained from recent experimental determinations indicated the possibilty of a systematic error in the certificate values. Selected experimental determinations of enthalpy increments and heat capacities were fitted in order to obtain a representation of the thermodynamic properties of alph-Al2O3, a sample of which is the standard reterence material (SRM720) for calibration of some types of calorimeters. The fitted equation and calculated values of the heat capacity, the relative enthalpy, and the entropy are given. The new values are more accurate and result from a better representation of the experimental values than did the 1982 SRM720 certificate values.

00,75

PB94-168572 Not available NTIS

National Inst. of Standards and Technology (CSTL). Gaithersburg, MD. Chemical Kinetics and Thermodynamics Div.

Thermodynamic Properties of Gaseous Silicon Monotelluride and the Bond Dissociation Enthalpy $\mathrm{D}($ sub $\mathrm{m})(\mathrm{SiTe})$ at T approaches 0 .

P. A. G. O'Hare. c1993, 4p.

Included in Jnl. of Physical and Chemical Reference Data v22 n6 p1455-1458. Nov/Dec 93. Available from American Chemical Society, 1155 Sixteenth St., NW Washington, DC. 20036-9976.

Keywords: "Research, "Physical chemistry, Statistical analysis, Thermodynamic properties, Silicon compounds, Enthalpy, Entropy, Gibbs free energy, Silicon monotelluride, Dissociation enthalpy, Enthalpy of formation.

Statistical-thermodynamic calculations have been combined with the results of high-temperature Knudsen-effusion studies of the vaporization of $\mathrm{Si} 2 \mathrm{Te} 3$ to calculate the thermodynamic properties of $\mathrm{SiTe}(\mathrm{g})$ from $T \rightarrow 0$ to $T=2000 \mathrm{~K}$. The dissociation enthalpy $\mathrm{D}($ sub $\mathrm{m})(\mathrm{SiTe}, \mathrm{T}->0)$ is $(448+$ or -8$) \mathrm{kJ} / \mathrm{mol}$; its value is discussed vis-a-vis the other silicon monochalcogenides.

\section{0,752}

PB94-168580 Not available NTIS

Houston Advanced Research Center, The Woodlands, TX. Materials Science Research Center.

Disilicides of Tungsten, Molybdenum, Tantalum, Titanium, Cobalt, and Nickel, and Platinum Monosilicide: A Survey of Their Thermodynamic Properties.

M. S. Chandrasekharaiah, J. L. Margrave, and P. A. G. O'Hare. c1993, 10p.

Prepared in cooperation with National Inst. of Stand ards and Technology (CSTL), Gaithersburg, MD Chemical Kinetics and Thermodynamics Div.

included in Jnl. of Physical and Chemical Reference Data v22 n6 p1459-1468, Nov/Dec 93 Available from American Chemical Society, 1155 Sixteenth St. NW, Washington, DC. 20036-9976.

Keywords: "Research, "Physical chemistry, Thermodynamics, Enthalpy, Gibbs free energy, Molybdenum silicides, Tungsten silicides, Titanium silicides, Tantalum silicides, Platinum silicides, Cobalt silicides, Nicke silicides, Evaluated properties.

A critical evaluation is presented of the thermodynamic properties of six disilicides and one monosilicide that are important in the manufacture of very large scale integrated circuits. Values of the standard molar enthalpies of formation HM at $\mathrm{T}=298.15 \mathrm{~K}$ and $\mathrm{p}=$ $0.1 \mathrm{MPa}$ are recommended as follows: WSi2, - $(79+$ or - 5) $\mathrm{kJ} / \mathrm{mol}, \mathrm{MoSi} 2,-(137+$ or - 4) $\mathrm{kJ} / \mathrm{mol}$, TiSi2
$-(171+$ or - 11) kJ/mol CoSi2 $-(103+$ or - 15) kJ/mol $\mathrm{NiSi} 2,-(88+$ or -12$) \mathrm{kJ} / \mathrm{mol}$, TaSi2, $-(120+$ or -20$)$ $\mathrm{kJ} / \mathrm{mol}$, and PtSi, $-(119+$ or -7$) \mathrm{kJ} / \mathrm{mol}$. Equations are given for the molar enthalpy increments of some of the silicides along with a few evaluated standard Gibbs free energies of formation. Brief descriptions of methods of synthesis and limited structural information have also been included.

00,753

PB94-168598 Not available NTIS

Jet Propulsion Lab., Pasadena, CA.

Evaluated Bimolecular Ion-Molecule Gas Phase Kinetics of Positive lons for Use in Modeling Planetary Atmospheres, Cometary Comae, and Interstellar Clouds.

V. G. Anicich. C1993, 100p.

Included in Jnl. of Physical and Chemical Reference Data, v22 n6 p1469-1569, Nov/Dec 93. Available from American Chemical Society, 1155 Sixteenth St., NW, Washington, DC. 20036-9976.

Keywords: *Research, "Physical chemistry, Planetary atmospheres, Comets, Cosmic gas dynamics, Interstellar matter, Reaction kinetics, Cations, Chemical composition, Tables(Data), Evaluated results, Product distributions, lon-molecule reactions.

Recommendations of reaction rate coefficients and product distributions for bimolecular positive ion-molecule reactions of importance in planetary atmospheres, cometary comae, and interstellar clouds are presented. Two publications Anicich and Huntress, Astrophys. J. Suppl. Ser. 62, 553 (1986) and Anicich, Astrophys. J. S!.jppl. Ser. 84, 215 (1993) served as the basis for this evaluation, which covers the literature from 1965 through 1991 with some additional citations missed in the original surveys.

00,754

PB94-168606 Not available NTIS

Commission on Atomic Weights and Isotopic Abundances, Reston, VA

Atomic Weights of the Elements 1991.

c1992, 14p.

Included in Jnl. of Physical and Chemical Reference Data, v22 n6 p1571-1584, Nov/Dec 93. Available from American Chemical Society, 1155 Sixteenth St., NW, Washington, DC. 20036-9976.

Keywords: "Research, "Physical chemistry, Isotope ratio Atomic mass, Atomic properties, Radioactive isotopes. Elements, Periodic system, Abundance, IUPAC (International Union of Pure and Applied Chemistry).

The biennial review of atomic weight, $\operatorname{Ar}(E)$, determinations and other cognate data has resulted in changes for the standard atomic weight of indium from 114.82 + or -0.01 to $114.818+$ or -0.003 , for tungsten from $183.85+$ or -0.03 to $183.84+$ or -0.01 and for osmium from $190.2+$ or -0.1 to $190.23+$ or -0.03 due to new high precision measurements. Recent investigations on silicon and antimony confirmed the presently accepted Ar values. The footnote ' $g$ ' was added for carbon and potassium because it has come to the notice of the Commission that isotope abundance variations have been found in geological specimens in which these elements have an isotopic composition outside the limits for normal material. Because many elements have a different isotopic composition in non-terrestrial materials, recent data on non-terrestrial material are included in this report for the information of the interested scientific community

00,755

PB94-172178 Not available NTIS

National Inst. of Standards and Technology (MSEL), Gaithersburg, MD. Reactor Radiation Div.

Rotational Dynamics of Solid C70: A Neutron-Scattering Study.

Final rept.

C. Christides, T. J. S. Dennis, K. Prassides, J. R. D. Copley, R. L. Cappelletti, and D. A. Neumann. 1994, $7 \mathrm{p}$. Pub. in Physical Review B 49, n4 p2897-2903, 15 Jan Keywords: "Fullerenes, Neutron diffraction, Neutron scattering, Inelastic scattering, Temperature dependence, Reprints, Rotational dynamics.

The authors report the results of neutron-diffraction and low-energy neutron-inelastic-scattering experiments on high-purity solid C7 be found to accompany 
structural changes both on cooling and on heating. The observed diffuse scattering intensity does not change with temperature. At $10 \mathrm{~K}$ broad librational peaks are observed at 1.82(16)meV (full width at half maximum $=1.8(5) \mathrm{meV}$ ). The peaks soften and broaden further with increasing temperature. At and above room temperature, they collapse into a single quasielastic line. At $300 \mathrm{~K}$, the diffusive reorientational motion appears to be somewhat anisotropic, becoming less so with increasing temperature. An isotropic rotational diffusion model, in which the motions of adjacent molecules are uncorrelated, describes well the results at $525 \mathrm{~K}$. The temperature dependence of the rotational diffusion constants is consistent with a thermally activated process having an activation energy of $32(7) \mathrm{meV}$.

\section{0,756}

PB94-172244 Not available NTIS

National Inst. of Standards and Technology (MSEL), Gaithersburg, MD. Polymers Div.

Octacalcium Phosphate. 3. Infrared and Raman Vibrational Spectra.

Final rept

B. O. Fowler, M. Markovic, and W. E. Brown. 1993, $7 p$.

Pub. in Chem. Mater. 5, n10 p1417-1423 1993. See also PB89-131288. Sponsored by American Dental Association Health Foundation, Chicago, IL.

Keywords: "Raman spectra, "Vibrational spectra, Water, Binding sites, Dentistry, Reprints, "Octacalcium phosphate.

Infrared and Raman band assignments are given for powdered samples of the biologically importan compound, octacalcium phosphate, in the 4,000-300$\mathrm{cm}$ (sup -1) range. Specific assignments were made for bands of the two crystallographically independent acidic phosphate groups. The numbers of observed bands were markedly less than those predicted by facto group analysis; additional unresolved bands are probably present. Infrared spectra indicate that two polymorphs of octacalcium phosphate may exist whose structures differ mainly in acidic phosphate and water bonding and in possibly different contents of water. Polymorphic forms of octacalcium phosphate are structurally plausible because of possible differences in bonding of its acidic phosphate groups and water molecules and in number of water molecules. The wavenumber positions of Raman bands, especially acidic phosphate bands, were sensitive to laser excitation power.

00,757

Not available NTIS

National Inst. of Standards and Technology (NML) Gaithersburg MD Gas and Particulate Science Div. Complementary Molecular Information on Phthalocyanine Compounds Derived from Laser Microprobe Mass Spectrometry and Micro-Raman Spectroscopy.

Final rept.

R. A. Fletcher, E. S. Etz, and S. Hoeft. 1990, 4p.

Pub, in Proceedings on Annual Conference of the Microbeam Analysis Society (25th), Seattle, WA., August 12-18, 1990, p89-92.

Keywords: "Phthalocyanines, "Molecular structure, "Spectrum analysis, Raman spectroscopy, Mass spectroscopy, Vibrational spectra, Reprints, Laser microprobe mass spectrometry.

The purpose of this work is to show the complementaiy information about molecular structural characteristics of metal phthalocyanine compounds provided by laser microprobe mass spectrometry (LAMMS) and microRaman spectroscopy. The vibrational frequencies ound in micro-Raman spectroscopy indicate molecular structure, and mass spectrometry indicates the mass $(\mathrm{m} / \mathrm{z}$ value) of the molecule, as well as certain structural features. The characteristic fragment peaks that result during the ionization process contain additional information about the molecular species being analyzed.

00,758

PB94-172285 Not available NTIS

National Inst. of Standards and Technology (CSTL) Gaithersburg, MD. Surface and Microanalysis Science Div.

Hot Carrier Excitation of Adlayers: Time-Resolved Measurement of Adsorbate-Lattice Coupling.

Final rept.

T. A. Germer, J. C. Stephenson, E. J. Heilweil, and R. R. Cavanagh. 1993, 4p.

Pub. in Physical Review Letters 71, n20 p3327-3330, 15 Nov 93
Keywords: *Carbon monoxide, Infrared absorption, PIcosecond pulses, Laser pumping, Adsorbates, ExCitation, Phonons, Copper, Reprints, Hot carriers.

Picosecond time-resolved infrared absorption measurements of $\mathrm{CO} / \mathrm{Cu}(100)$ following visible and ultraviolet laser pumping allow a determination of the time scales for photoexcited carriers and lattice phonons to couple to the $\mathrm{CO}$ frustrated translation vibrational mode.

\section{0,759}

PB94-172343 Not available NTIS

National Inst. of Standards and Technology (MSEL), Gaithersburg, MD. Metallurgy Div.

Recent Experimental and Modeling Developments in High Temperature Thermochemistry.

Final rept.

J. W. Hastie, and D. W. Bonnell. 1989, 15p.

Pub. in Proceedings of Symposium on Thermochemistry and Chemical Processing Kalpakkam, India, p179-193 1989.

Keywords: "High temperature tests "Thermochemistry, Vapor phases, Mass spectroscopy Thermodynamics, Experimental design, Mathematical models, Slags, Glass, Dolomite, Reprints.

The application of thermochemical principles to high Themperature processes requires advanced measurement and modeling techniques. Recent work in this area, primarily from the authors' laboratory, is reviewed. Emphasis is on the vapor phase, present in equilibrium with complex liquid and solid processmedia such as salts, slags, glasses, minerals, etc Examples of the thermochemical characterization of several practical systems are given, including silicate glasses, a nuclear waste borosilicate glass, a coal-derived slag and the mineral dolomite. The experimental echniques described include, a modified Knudsen effusion mass spectrometric method and the relatively novel methods of transpiration mass spectrometry and laser vaporization mass spectrometry. The modeling approach considered involves the multicomponen equilibrium calculation of phase compositions and activities using an ideal mixing of complex components, or liquid associate model.

\section{0,760}

PB94-172517 Not available NTIS

National Inst. of Standards and Technology (MSEL) Gaithersburg, MD. Metallurgy Div.

Electrodeposition.

Final rept.

D. S. Lashmore. $1990,3 p$

Pub. in Electrochemistry and Solid State Science in the Electrochemical Society, p12-14 1990

Keywords: "Electrodeposition, "Metal ions, Metals, Electrochemistry, Amorphous state, Electroforming, Metal matrix composites, Substrates, Alloys, Coatings, Catalysis, Reprints.

Electrodeposition involves reducing metal ions electrochemically from aqueous, inorganic and fused salt electrolytes to form coherent metals and alloys on various substrates. These coatings may be removed from the substrate to constitute free standing parts using process called electroforming. The reduction of the ions to metal is usually a result of an external electroIytic process; however, autocatalytic processes exist in which the reducing agent is incorporated into the electrolyte. One of these processes, the autocatalytic nickel phosphorus process, is now in wide commercial use.

\section{0,761}

\section{PB94-172525 Not available NTIS}

National Inst. of Standards and Technology (NEL) Boulder, CO. Electromagnetic Technology Div. Use of Ion Scattering Spectroscopy to Monitor the $\mathrm{Nb}$ Target Nitridation during Reactive Sputtering. Final rept.

D. J. Lichtenwalner, A. C. Anderson, and D. A.

Rudman. 1991, 6p.

Pub. in Proceedings of Surface Chemistry and Beam Solid Interactions Symposium, Boston, MA., Novem ber 26-29, 1990, p613-618 1991.

Keywords: "Niobium nitrides, Surface reactions, Surface chemistry, Thin films, Monitoring, Nitrogen, Targets, Reprints, Ion beam reactive sputtering, lon scattering spectroscopy.

Ion scattering spectroscopy (ISS) has been used to directly monitor the nitrogen coverage of a niobium tar- get during ion-beam reactive sputtering. The measurement shows that the fully reacted target is nearly completely covered with nitrogen, revealing a major difference in the target surface state when reactive sputtering versus the sputtering of a compound target. The functional dependence of the nitrogen coverage on the nitrogen pressure has also been obtained. Taking the fractional target coverage to be a steady-state equilibrium between nitrogen sputter removal and thermal $\mathrm{N} 2$ arrival, the measured target coverage has been accurately modeled. The results indicate that the nitridation reaction is controlled by the thermal $\mathrm{N} 2 \mathrm{~mol}-$ ecules, which stick to the target with a high probability. This firmly establishes the fact that the molecular N2 flux can control the target reaction. The target coverage model has also been applied to fit the measured deposition rate as a function of the nitrogen pressure, and shows that the deposition rate does not accurately reflect the target coverage.

00,762

PB94-172574 Not available NTIS

National Inst. of Standards and Technology (NML), Gaithersburg, MD. Surtace Science Div.

Role of Adsorbed Alkalis in Desorption Induced by Electronic Transitions.

Final rept.

T. E. Madey, S. A. Joyce, and C. Benndorf. 1989,

$10 p$.

Pub. in Physics and Chemistry of Alkali Metal Adsorption, p185-194 1989. Sponsored by Department of Energy, Washington, DC

Keywords: "Surface chemistry, "Alkali metals, Adsorption, Desorption, Ions, Reprints, Electron stimulated desorption ion angular distribution

DIET processes (desorption induced by electronic transitions) involving atoms and molecules on metal surfaces are strongly influenced by coadsorption with alkali metal atoms. Angular distributions and yields of positive ions, negative ions and electronically excited neutrals from adsorbed species are affected by alkali coadsorption, due to a combination of molecular reorientation and electronic effects.

00,763

PB94-172673 Not available NTIS

National Inst. of Standards and Technology (MSEL),

Gaithersburg, MD. Reactor Radiation Div.

Inelastic Neutron Scattering Studies of Rotational Excitations and the Orientational Potential in C60 and $\mathrm{A} 3 \mathrm{C} 60$ Compounds.

Final rept.

D. A. Neumann, J. R. D. Copley, D. Reznik, R. L.

Paul, R. M. Lindstrom, W. A. Kamitakahara, and J. J. Rush. 1993, 14p.

Pub. in Jnl. of Physics and Chemistry of Solids 54, n12 p1699-1712 1993

Keywords: "Fullerenes, Alkali metal compounds, Rotational states, Neutron scattering, Inelastic scattering, tional potentials, Interatomic potentials.

The authors describe neutron scattering studies of rotational excitations of the $C(60)$ molecule in pure $C(60)$ and in $A 3 C(60)(A=$ alkali metal) compounds. Welldefined peaks due to librations are observed below the orientational ordering transition temperature in $C(60)$ itself, and at all temperatures at which measurements were made (up to $675 \mathrm{~K}$ in $\mathrm{K} 3 \mathrm{C}(60)$ ) in the compounds. The energies of these excitations have been used to extract information about orientational potentials and reorientation mechanisms. For the systems studied so far, the authors find that the size of the ion occupying the tetrahedral site correlates with the librational energy demonstrating that the repulsive part of the A. $C$ interaction makes a significant contribution to the interatomic potential.

\subsection{4}

PB94-172699 Not available NTIS

National Inst. of Standards and Technology (CSTL), Gaithersburg, MD. Surface and Microanalysis Science

Inelastic Interactions of Electrons with Surfaces: Application to Auger-Electron Spectroscopy and $X$-ray Photoelectron Spectroscopy.

Final rept.

C. J. Powell. 1994, 15p

Pub. in Surface Science 299/300, p34.48 1994.

Keywords: * $X$ ray photoelectron spectroscopy, "Auger electron spectroscopy, "Electron scattering, "Surface 


\section{CHEMISTRY}

\section{Physical \& Theoretical Chemistry}

analysis, Mean free path, Inelastic scattering, Reprints, Surface composition.

Electron-based probes of surface properties are used frequently in surface science since strong inelastic scattering for electron energies between about 50 and 2000 eV ensures high surface sensitivity. An overview is given of developments in the understanding of inelastic electron scattering in solids with emphasis on importan ( surface properties. Auger-electron spectroscopy (AES) and $X$-ray photoelectron spectroscopy (XPS) are the two techniques most commonly used for measurements of surface composition. The authors give a brief description of the development of AES and XPS and then proceed to describe the role of inelastic electron scattering in AES and XPS. Attention is given to the measurement of electron attenuation lengths, to the calculation of electron inelastic mean free paths, and to intensity measurements for quantitative AES and XPS.

\section{0,765}

PB94-172798 Not available NTIS

National Inst. of Standards and Technology (NML), Gaithersburg, MD. Inorganic Analytical Research Div. Absolute Determination of Electrolytic Conductivity for Primary Standard $\mathrm{KC} 1$ Solutions from 0 to $50 \mathrm{C}$.

Final rept.

Y. C. Wu, and W. F. Koch. 1991,11p.

Pub. in Jnl. of Solution Chemistry 20, n4 p391-401 1991

Keywords: "Potassium chloride, "Conductivity, "Aqueous electrolytes, "Primary standards, Electrical resistivity, Temperature dependence, Reprints.

An absolute determination of aqueous electrolytic conductivity has been made for primary standards 0.01 demal (D) and 0.1 D potassium chloride solutions over the temperature range of 0 to $50 \mathrm{C}$ in 5 degree intervals. A cell with a removable center section of accurately known length and area was used for measurements. Values were adjusted to be in conformity with the ITS-90 temperature scale. The overall uncertainty over the entire temperature range is estimated to be $0.03 \%$. Values at $25 \mathrm{C}$ for $0.01 \mathrm{D}$ and $0.1 \mathrm{D}$ are $0.00140841 \mathrm{~S} / \mathrm{cm}$ and $0.0128506 \mathrm{~S} / \mathrm{cm}$, respectively.

\section{0,766}

PB94-172822 Not available NTIS

National Inst. of Standards and Technology (CSTL), Gaithersburg, MD. Surface and Microanalysis Science Div.

Environmental Scanning Electron Microscope Imaging Examples Related to Particle Analysis.

Final rept.

S. A. Wight, and C. J. Zeissler. 1993, 5p.

Pub. in Microscopy Research and Technique 25, p3933971993

Keywords: "Scanning electron microscopy, "Particles, "Environmental monitoring, Air pollution sampling, Particulates, Image processing, Filters, Surface energy, Reprints.

The work provides examples of some of the imaging capabilities of environmental scanning electron microscopy applied to easily charged samples relevant to particle analysis. Environmental SEM (also referred to as high pressure or low vacuum SEM) can address uncoated samples that are known to be difficult to image. Most of these specimens are difficult to image by conventional SEM even when coated with a conductive layer. Another area where environmental SEM is particularly applicable is for specimens not compatible with high vaccum, such as volatile specimens. Samples from which images were obtained that otherwise may not have been possible by conventional methods included fly ash particles on an oiled plastic membrane impactor substrate, a one micrometer diameter fiber mounted on the end of a wire, uranium oxide particles embedded in oil-bearing cellulose nitrate, teflon and polycarbonate filter materials with collected air particulate matter, polystyrene latex spheres on cellulosic filter paper, polystyrene latex spheres 'loosely' sitting on a glass slide, and subsurface tracks in an etched nuclear track-etch detector. Surface charging problems experienced in high vacuum SEMS are virtually eliminated in the low vacuum SEM, ex tending imaging capabilities to samples previously difficult to use or imcompatible with conventional methods.

\section{0,767}

PB94-173036

Not available NTIS
National Inst. of Standards and Technology (CSTL), Gaithersburg, MD. Chemical Kinetics and Thermodynamics Div.

Thermal Decomposition of Hydroxy- and MethoxySubstituted Anisoles.

Final rept.

M. M. Suryan, S. A. Kafafi, and S. E. Stein. 1989, 7p Pub. in Jnl, of American Chemical Society 111, n4 p1423-1429 1989

Keywords: "Anisole, "Aryl ethers, "Reaction kinetics, "Pyrolysis, "Binding energy, Chemical bonds, Organic oxygen compounds, Isomers, Reprints. *Hydroxyanisoles, "Methoxyanisoles, Bond homolysis

Rates of decomposition of anisole and the three isomeric hydroxyanisoles and methoxyanisoles were determined in a very low pressure pyrolysis apparatus. The predominant reaction was O-methyl bond homolysis. Therefore, relative rates provide a quantitative measure of the effects of hydroxy and methoxy substituents on bond strengths in anisoles. The 0 hydroxy effect is unusually large $(7 \mathrm{j} \mathrm{kcal} / \mathrm{mol}$ bond strength weakening). Rate ratios of ethyoxybenzene (phenetole) and o-hydroxyphenetole were similar to the anisole/o-hydroxyanisole pair, confirming the large o-hydroxy effect. The p-hydroxy group, however, only weakened the anisole 0 -methyl bond by $2.5 \mathrm{kcal} / \mathrm{mol}$ and meta substitution had little effect. Both 0 - and $p$ methoxy groups weakened the O-methyl bond by 4 $\mathrm{kcal} / \mathrm{mol}$ and $\mathrm{m}$-methoxy weakened this bond by 1 $\mathrm{kcal} / \mathrm{mol}$. Rates of these reactions, extrapolated to lower temperatures, were similar to rates of condensed phase thermolysis reactions, indicating that homolysis is a key step in the liquid phase reactions.

00,768

PB94-185014 Not available NTIS

National Inst of Standards and Technology (PL), Boulder CO. Quantum Physics Div.

Can Quantum Mechanical Description of ElectronSodium Collisions $\mathrm{Be}$ Considered Complete. Present Status and Future Prospects for $3 s<->3 p$ Transitions.

Final rept.

N. Andersen, and K. Bartschat. 1993, 32p.

Pub. in Comments on Atomic and Molecular Physics 29, n3 p157-188 1993. Sponsored by National Science Foundation, Washington, DC

Keywords: "Electron-atom collisions, "Sodium, Electron scattering Electron transitions, Quantum mechanics, Polarization(Spin colarization), Reprints, Electron spin polarization.

In the spirit of Bederson's 'Perfect Scattering Experiment,' we generalize the existing description of collision induced atomic $S$ <-> P transitions by spin-polar zed electrons on spin-polarized target atoms. Analysis of existing experimental and theoretical data on the 3 s --> 3p transitions in $\mathrm{Na}$ determines to what extent the 'perfect scattering experiment' has been achieved to date. Using theoretical data, we demonstrate to what extent additional missing parameters can be extracted from present experiments. Finally, new experimental arrangements are suggested to remove remaining ambiguities

00,769

PB94-185030 Not available NTIS

National Inst. of Standards and Technology (PL), Boulder, CO. Quantum Physics Div.

Low-Energy Electron Scattering from Caesium Atoms: Comparison of a Semirelativistic BreitPauli and a Full Relativistic Direc Treatment.

Final rept.

K. Bartschat. 1993, 15p.

Grant NSF-PHY-9014103

Pub. in Jnl. of Phys. B: At. Mol. Opt. Phys. 26, p35953609 1993. Sponsored by National Science Founda tion, Washington, DC

Keywords: "Electron-atom collisions, "Cesium, Electron scattering, Differential cross sections, Total cros sections, Relativistic effects, Hamiltonians, $R$ matrix Comparison, Reprints, Electron spin polarization.

The discrepancies between a previous Breit-Pauli Rmatrix calculation by Scott et al and a recent calculation using the Dirac Hamiltonian by Thumm and Norcross are further analyzed and partially resolved. When comparable methods are applied to obtain the best possible target description in both approaches, reduced Breit-Pauli formulation, with only the one-body spin-orbit operator included to account for relativistic effects explicitly, seems sufficient for an accurate de- scription of most of the low-energy collision processes in this system. There remain, however, some interesting differences between the Breit-Pauli and the Dirac results for spin polarization and asymmetry functions, as well as for very sensitive resonance parameters. To shed more light on the possible origin of these differences, the effect of including additional close-coupling channels on the results is investigated.

\section{0,770}

PB94-185089 Not available NTIS

National Inst. of Standards and Technology (PL), Boulder, CO. Quantum Physics Div.

Electron-Impact Ionization of $\operatorname{In}(+)$ and $\mathrm{Xe}(+)$. Final rept

E. W. Bell, N. Djuric, and G. H. Dunn. Dec 93, 6p. Contract DE-A105-86ER53237

Pub. in Physical Review A 48, n6 p4286-4291 Dec 93. Sponsored by Department of Energy, Washington, DC.

Keywords: "Electron-ion collisions, "Indium ions, "Xenon ions, Ionization cross sections, EV range 1001, 000 , Colliding beams, Reprints.

Absolute ionization cross sections for $\ln (1+)$ and $\mathrm{Xe}(1+)$ by electron impact have been measured from below threshold to $200 \mathrm{eV}$ using the crossed-beams technique. The cross sections for $\ln (1+)$ were possibly enhanced by indirect ionization processes. The excitation of the ion from the $4 \mathrm{~d}(10) 5 \mathrm{~s}(2)$ ground state to the $4 d(9) 5 s(2) 5 P$ state followed by autoionization has been postulated. Experimental measurements are compared to configuration-averaged distorted-wave calculations (M. S. Pindzola et al, J. Phys. B 16, L355 (1983)) the semiempirical formula of Lotz ( $Z$. Phys. $216,241(1968))$, and, in the case of $\mathrm{Xe}(1+)$, previous experimental results ( $C$. Achenbach et al, j. Phys. B 17, 1405 (1984)). Also presented are ionization-rate coefficients and fitting parameters for both ions for temperatures in the range $10,000 \mathrm{~K}=$ or $<\mathrm{T}=$ or $<10$ million $\mathrm{K}$.

00,771

PB94-185097 Not available NTIS

National Inst. of Standards and Technology (PL), Boulder, CO. Time and Frequency Div.

Measurement of the $\mathrm{J}=2$ less than 1 Fine-Structure Interval for (28)Si and (29)Si in the Ground (3)P State.

Final rept.

J. M. Brown, L. R. Zink, and K. M. Evenson. 1994

$8 p$.

Contract NASA-W15-047

Pub in Astrophysical Jnl. 423, pL151-L.154, 10 Mar 94. Sponsored by National Aeronautics and Space Administration, Washington, DC.

Keywords: "Infrared spectra, "Silicon 28, *Silicon 29 Far infrared radiation, Interstellar matter, Ground state, Fine structure, Reprints, Laser magnetic resonance.

We report the first observation of the far-infrared laser magnetic resonance spectrum associated with the $\mathrm{J}=$ $2<-1$ fine-structure interval of (28) Si and (29) Si in the triplet $\mathrm{P}$ ground state. In (28) $\mathrm{Si}$ this separation is $4378.3280(2) \mathrm{GHz}$, and for (29)Si it is $4378.3306(6)$ $\mathrm{GHz}$. The magnetic hyperfine parameter $\mathrm{A} 2$ has also been determined for (29) Si, apparently for the first time. Zero-field transition frequencies for the hyperfine components of the $\mathrm{J}=2<-1$ transition in (29) Si are determined and will aid in their identification in interstellar sources.

\section{0,772}

PB94-185204 Not available NTIS National Inst. of Standards and Technology (CSTL), Gaithersburg, MD. Thermophysics Div.

Thermodynamic Properties of Difluoromethane.

Final rept

D. R. Defibaugh, G. Morrison, and L. A. Weber. Apr 94, 8p.

Contract DE-FG02-91CE23810

Pub. in Jnl. of Chemical and Engineering Data 39, n2 p333-340 Apr 94. Sponsored by Department of Energy, Washington, DC

Keywords: "Fluorohydrocarbons, "Thermodynamic properties, Pressure, Volume, Refrigerants, Density(Mass/volume), Temperature, Equations of state, Liquid phases, Vapor phases, Saturation, Reprints, "Methane/difluoro, R32, Burnett apparatus.

The pressure-volume-temperature (PVT) behavior of difluoromethane (R32) has been measured using a vibrating tube densimeter apparatus and a Burnett/ isochoric apparatus. Liquid PVT data from the vibrating 
tube densimeter ranged in temperature from 242 to $348 \mathrm{~K}$ with a pressure range of $2,000-6,500 \mathrm{kPa}$. Data from the Bumett apparatus consisted of 11 isochores for the gas and supercritical fluid, along with vapo pressure measurements. The temperature ranged from 268 to $373 \mathrm{~K}$. The gas-phase data are correlated with a virial equation of state. The compressed liquid data are fit with an abbreviated form of the modified Benedict-Webb-Rubin (mBWR) equation. A table of thermodynamic properties is presented for the saturated liquid and vapor states.

00,773

PB94-185303 Not available NTIS

National Inst. of Standards and Technology (PL), Boulder, CO. Quantum Physics Div.

Quantum Dynamics of Renner-Teller Vibronic Coupling: The Predissociation HCO.

Final rept.

E. M. Goldfield, S. K. Gray, and L. B. Harding. 1993 ,

$16 p$

Pub. in Jnl. of Chemical Physics 99, n8 p5812-5827, 15 Oct 93.

Keywords: Reprints, Wave packets, Quantum theory, Predissociation, Hamiltonians, "Formyl radicals, Renner-Teller coupling, Potential energy surfaces, $A b$ initio calculations.

A Hamiltonian model and parity-adapted wave packet representation are developed to describe a rotating triatomic system with two Renner-Teller coupled poential surfaces, and $\mathrm{HCO}$ predissociation is studied. New configuration interaction calculations on $\mathrm{HCO}$ are performed to determine its excited (A bar) ((sup 2)A(double prime)) potential surface, and Bowman Bittman, and Harding ground potential surface is employed. The properties of many resonances, correlating with stretch/bend excitations on the $A$ (double prime) surface, are determined.

\section{0,774}

PB94-185444 Not available NTIS

National Inst. of Standards and Technology (PL), Boulder, CO. Quantum Physics Div.

Selected Ion Flow Tube-Laser Induced Fluores cence Instrument for Vibrationally State-Specific Ion-Molecule Reactions.

Final rept.

S. Kato, M. J. Frost, V. M. Bierbaum, and S. R.

Leone. 1993, 13p.

Contract FA9620-92-J-0072

Pub. in Review of Scientific Instruments $64, n 10$ p2808-2820 Oct 93. Sponsored by Air Force Office of Scientific Research, Washington, DC

Keywords: "Ion molecule interactions, "Nitrogen ions, Laser induced fluorescence, Vibrational states, Rotational states, Molecular ions, Charge transfer, Oxygen, Argon, Reprints, lon flow tubes.

A selected ion flow tube apparatus is coupled with laser induced fluorescence (LIF) detection for the selective monitoring of ion vibrational states and their reactions. Mass selected ions are injected into a flow tube with a venturi inlet using $\mathrm{He}$ carrier gas. A 200 $\mathrm{Hz}$ dye laser system provides sensitive LIF detection $\mathrm{Hz}$ dye laser system provides sensitive LIF detection of the injected ions at densities as low as $100,000 / \mathrm{cc}$ for $\mathrm{N} 2(1+)$. The rotational temperatures of the $\mathrm{N} 2(1+$ detection, while the vibrational temperatures can be high and may be varied by the injection potentials. Vibrationally state-selected ion-molecule reactions of $\mathrm{N} 2(1+)(\mathrm{nu}=0,1$, and 2$)$ are studied with $\mathrm{N} 2, \mathrm{Ar}$, and $\mathrm{O} 2$ at themal kinetic energies $(\mathrm{E}(\mathrm{lab})<0.1 \mathrm{eV})$, where translation-to-vibration energy transfer is negligible. Isotopically specific charge-transfer reactions of (15) N2 $(1+)(n u)$ with (14)N2 are also studied. The ability to mass select ions and characterize their vibrational states and those of their reaction products allows novel studies of state-to-state ion chemistry.

\section{0,775}

PB94-185584 Not available NTIS

National Inst. of Standards and Technology (PL), Boulder, CO. Quantum Physics Div.

Charge Transfer and Collision-Induced Dissociation Reactions of $\mathrm{CF}(2+)$ and $\mathrm{CF} 2(2+)$ with the Rare Gases at a Laboratory Collision Energy of $49 \mathrm{eV}$.

Final rept.

M. Manning, S. D. Price, and S. R. Leone. 1993

$10 \mathrm{p}$

Contract AFOSR-F49620-92-J-007

Pub. in Jnl. of Chemical Physics 99, n11 p8695-8704

1 Dec 93. Sponsored by Air Force Office of Scientific Research, Bolling AFB, DC.
Keywords: "lon-atom collisions, "Carbon fluorides, *Rare gases, *Charge transfer, Molecular ions, EV range 10-100, Chemical radicals, Cross sections, Dissociation, Reprints, Dications.

Multiple product channels are observed for the reactions of $\mathrm{CF}(2+)$ and $\mathrm{CF}_{2}(2+)$ with the rare gases at a laboratory collision energy of $49+$ or $-1 \mathrm{eV}$. A dication beam is produced in an electron impact ion source and mass selected using a quadrupole mass spectrometer. The ion beam is focused into a collision region and a time-of-flight mass spectrometer is used to monitor the reaction products. Reactions of $\mathrm{CF}(2+)$ produce $C F(1+), C(1+)$, and $F(1+)$ ions and reactions of $\mathrm{CF} 2(2+)$ result in $\mathrm{CF}(2+), \mathrm{CF}(1+), \mathrm{C}(1+)$, and $\mathrm{F}(1+)$ ion formation accompanied by the corresponding rare gas ions when charge transfer occurs. The relative yields of these products are measured directly. For reactions of both dications, there is a substantial increase in the total reaction cross section as the rare gas collision partner changes from $\mathrm{He}$ to $\mathrm{Xe}$. The trends in charge transfer reactivity are successfully modeled using Landau-Zener theory.

\section{0,776}

PB94-185600 Not available NTIS

National Inst. of Standards and Technology (MSEL), Gaithersburg, MD. Polymers Div.

Octacalcium Phosphate Carboxylates IV. Kinetics of Formation and Solubility of Octacalcium Phosphate Succinate.

Final rept.

M. Markovic, B. O. Fowler, and W. E. Brown. 1994,

Pub, in Jnl. of Crystal Growth 135, p533-538 1994 Sponsored by American Dental Association Health Foundation, Chicago, IL.

Keywords: "Reaction kinetics, "Thermodynamics, "Solubility, pH, Chemical analysis, X-ray diffraction, Infrared spectroscopy, Phase transformations, Reprints, "Octacalcium phosphate succinate, Octacalcium phosphate carboxylates.

Kinetic and thermodynamic studies of octacalcium phosphate succinate (OCP-SUCC). $\mathrm{Ca8}(\mathrm{HPO} 4)(2$ $\mathrm{x}$ )(succ) $\mathrm{x}$ (PO4)4' $\mathrm{yH} 2 \mathrm{O}$, where $0.8<\mathrm{x}<1.0$ and 5.5 $<y<6.5$, broaden the present knowledge in the chemistry of octacalcium phosphate carboxylates. The kinetics of formation of OCP-SUCC by conversion of alphatricalcium phosphate (alpha-TCP), alpha-Ca3(PO4)2, in ammonium succinate solutions was followed at varied initial $\mathrm{pH}, 37 \mathrm{C}$. The changes in the liquid phase were monitored by $\mathrm{pH}$ measurements. Solid phases were characterized by means of chemical analyses, $X$ ray diffraction and infrared spectroscopy. The rate of conversion decreased with increasing initial $\mathrm{pH}$. In the solubility experiments the concentrations of calcium, phosphate, succinate, ammonium and $\mathrm{pH}$ were determined in solutions equilibrated with OCP-SUCC (6.1 $<\mathrm{pH}<7.4$ )

\section{0,777}

PB94-185691 Not available NTIS

National Inst. of Standards and Technology (PL), Boulder, CO. Quantum Physics Div.

High Resolution IR Studies of Polymolecular Clusters: Micromatrices and Unimolecular Ring Opening.

Final rept.

D. J. Nesbitt. 1994 15p.

Contracts NSF-CHE90-00641, NSF-PHY90-12244

Pub. in Reaction Dynamics in Clusters and Condensed Phases, p137-151 1994. Sponsored by National Science Foundation, Arlington, VA

Keywords: Van der Waals forces, Intermolecular forces, Infrared spectroscopy, High resolution, Matrix isolation. Hydrogen bonds, Argon complexes, Reprints, *Molecular clusters, Hydrogen fluoride complexes.

A long standing goal in the field of cluster research has been to elucidate the transition between the properties of isolated monomer species in the gas phase and the corresponding properties in the condensed phase. One of the most exacting probes of small clusters in this transition region has recently been offered by high resolution spectroscopies, particularly in the microwave and IR region of the spectrum. Such high resolution studies at rotational resolution can provide detailed information on the distribution of the masses in the cluster, as well as clearly distinguish between various low lying isomeric structures of the same molecular composition. High resolution spectroscopy in the IR also provides the opportunity to study dynamical phe- nomena such as predissociation, isomerization, and ivR channels that can be accessed by quantum state selected vibrational excitation of the clusters.

00,778

PB94-185725 Not available NTIS

National Inst. of Standards and Technology (PL), Boulder, CO. Quantum Physics Div.

Angular Distributions for Near-Threshold $(e, 2 e)$ Processes for $\mathrm{Li}$ and $\mathrm{Mg}$.

Final rept.

C. Pan, and A. F. Starace. Mar 93, 4p.

Contract NSF-PHY-9108002

Pub. in Physical Review A 47, n3 p2389-2392 Mar 93. Sponsored by National Science Foundation, Arlington, VA.

Keywords: "Electron-atom collisions, "Lithium, *Magnesium, Differential cross sections, Angular distribution, Electron impact, lonization, Reprints.

Distorted-wave calculations of the triply differentia cross sections for electron-impact ionization of $\mathrm{Li}$ and $\mathrm{Mg}$ are presented for the coplanar, theta $(12)=\mathrm{pi} \mathrm{ge}$ ometry in which the final-state electrons share $2 \mathrm{eV}$ of excess energy equally. Our theoretical approach, described in detail elsewhere (C. Pan and A. F. Starace, Phys Rev A 45, 4588 (1992)), employs a partial-wave expansion of initial- and final-state wave functions treats direct and exchange interactions of initial- and final-state electrons with the target core, and treats the final-state interaction between the two continuum electrons by a screening potential. $\mathrm{Li}$ and $\mathrm{Mg}$ targets are found to have more complex (e,2e) angular distributions than either $\mathrm{H}$ or $\mathrm{He}$ targets, stemming in large part from significant $p$-wave and higher one-electron phase shifts in the former elements.

\subsection{9}

Not available NTIS

National Inst. of Standards and Technology (CSTL), Gaithersburg, MD. Surface and Microanalysis Science Div.

Compositional Analyses of Surfaces and Thin Films by Electron and lon Spectroscopies.

Final rept.

C. J. Powell. 1993, 19p.

Pub. in Critical Reviews in Surface Chemistry 2, n1 2 p17-35 1993

Keywords: "Surface analysis, "Electron spectroscopy, *Ion spectroscopy, Secondary ion mass spectroscopy, Auger electron spectroscopy, lon scattering analysis Rutherford scattering, $X$-ray photoelectron spectroscopy, Chemical composition, Thin films, Limitations, Reviews, Reprints, Sputtered neutral mass spectroscopy.

An overview is given of the current capabilities, advantages, and limitations of the six techniques in common use for compositional analyses of surfaces and thin films: Auger electron spectroscopy (AES), X-ray photoelectron spectroscopy (XPS), secondary ion mass spectrometry (SIMS), sputtered-neutral mass spectrometry (SNMS), ion-scattering spectroscopy (ISS), and Rutherford backscattering spectroscopy RBS). Information is also given on efforts to improve hese techniques and on the potential of other methods for determining surface compositions during processing.

00,780

PB94-185808 Not available NTIS

National Inst. of Standards and Technology (PL), Boulder, CO. Quantum Physics Div.

Collision-Induced Neutral Loss Reactions of Molecular Dications.

Final rept.

S. D. Price, M. Manning, and S. R. Leone. 1993, 6p. Contract AFOSR-F49620-92-J-0249

Pub. in Chemical Physics Letters 214 n6 p553-558, 19 Nov 93. Sponsored by Air Force Office of Scientific Research, Bolling AFB, DC.

Keywords: "Ion-atom collisions, ${ }^{*}$ Carbon fluorides *Sulfur fluorides, *Rare gases, Molecular ions, EV range 10-100, Chemical radicals, Reprints, Neutral loss reactions, Dications.

Collision-induced neutral loss reactions are observed to be a major product channel for reactions of $\mathrm{CF} 3(2+)$ SF4(2+), SF3(2+) and SF2(2t) with the rare gases a $49 \mathrm{eV}$ laboratory collision energy. This reactivity, which involves the formation of doubly charged molecula daughter ions, differs markedly from that observed for other molecular dications. The doubly charged produc 
ion yield is largest for systems in which charge transfer does not compete effectively with the collision-induced process.

\section{0,781}

PB94-185816 Not available NTIS

National inst. of Standards and Technology (CSTL), Gaithersburg, MD. Biotechnology Div

Thermodynamic and NMR Study of the Interactions of Cyclodextrins with Cyclohexane Derivatives.

Final rept.

M. V. Rekharsky, F. P. Schwarz, Y. B. Tewari, Y. Yamashoji, R. N. Goldberg, and M. Tanaka. 1994 $6 p$.

Pub. in Jnl. of Physical Chemistry 98, n15 p4098-4103 1994

Keywords: "Cyclodextrins, "Cyclohexanols, "Heat of reaction, "Equilibrium, Specific heat, Temperature dependence, Calorimetry, Thermodynamic properties, Nuclear magnetic resonance, Spectrum analysis, Entropy, Reprints.

Equilibrium constants and standard molar enthalpies of reaction have been determined for a series of cyclohexanols and other cyclohexane derivatives with alpha-cyclodextrin (a-cyclodextrin) and betacyclodextrin (b-cyclodextrin) by titration calorimetry. For reactions involving cyclohexanol, standard molar heat capacity changes as a function of temperature were also determined. The equilibrium constants for the reactions of the compounds were always larger with b-cyclodextrin than with a-cyclodextrin. The stand ard molar enthalpies of reaction were similar for both cyclodextrins; however, the standard molar entropies of reaction with b-cyclodextrin were substantially more positive than for the reactions with a-cyclodextrin. The hydrophilic nature of the groups on the cyclohexane ring as well as steric effects were found to influence the thermodynamics of these reactions. NMR spectral analysis indicated that while the proximity of these cyclohexane derivatives to the walls of the two cyclodextrins were comparable, the cyclohexane derivatives penetrate deeper into the larger bcavity.

\section{0,782}

PB94-185972 Not available NTIS

National Inst. of Standards and Technology (MSEL), Gaithersburg, MD. Reactor Radiation Div

X-Ray Diffraction from Anodic TiO2 Films: In situ and Ex situ Comparison of the Ti(0001) Face.

Final rept.

D. G. Wiesler, M. F. Toney, O. R. Melroy, C. S

McMillan, and W. H. Smyrl. 1994, 9p.

Pub. in Surface Science 302, p341-349 1994

Keywords: *Titanium dioxide, X-ray diffraction, Syn chrotron radiation, Single crystals, Thin films, Crysta structure, Epitaxy, Anodizing, Anatase, Reprints.

$X$-ray diffraction with synchrotron radiation is used to study the structure and epitaxy of a thin (about $250 \mathrm{~A}$ ) anodic film grown slowly on the basal plane of singlecrystal Ti. Anatase crystallites, roughly $130 \mathrm{~A}$ in diameter, are observed, with no indication of other forms of $\mathrm{TiO} 2$ or films with lower oxidation state. The oxide is more orientationally disordered than films grown on the $(11-20)$ and (10-10) faces of $\mathrm{Ti}$, but it exhibits weak six- and twelve-fold texturing by the metal sublayer. In situ and ex situ measurements are qualitatively similar, suggesting that the oxide does not change appreciably upon emersion.

\section{0,783}

\section{PB94-198280 PC A02}

National Inst. of Standards and Technology (NML) Gaithersburg, MD. Chemical Kinetics Div.

Temperature Dependence of the Rate Constants for Reaction of Inorganic Radicals with Organic Reductants.

Z. B. Alfassi, R. E. Huie, P. Neta, and L. C. T.

Shoute. 1990, 6p

Pub. in Jnl. of Physical Chemistry 94, n25 p8800-8805 1990.

Keywords: "Chemical radicals, "Phenols, "Ascorbic acid, "Reaction kinetics, "Temperature dependence Reprints, Activation energy, Chemical reactions, Molecularions, Halogens.

Rate constants for the reactions of several inorganic radicals with several organic reductants in aqueous solutions have been measured by pulse radiolysis as a function of temperature, generally between 5 and 75 deg $C$. The reactions studies were those of the radicals $\mathrm{N} 3, \mathrm{NO} 2, \mathrm{Br} 2(-), \mathrm{I} 2(-),(\mathrm{SCN}) 2(-)$ reacting with several phenols and ascorbate. Rate constants were also measured for the reactions of $\mathrm{Cl} 2(-)$ with phenol and of $\mathrm{ClO} 2$ with $\mathrm{p}$-methoxyphenolate. The rate constants measured were in the range of 100,000 to nearly 10 to the 10th power and the calculated Arrhenius activation energies ranged from 7 to $41 \mathrm{~kJ} / \mathrm{mol}$. The pre-exponential factors also varied considerably, with $\log A$ ranging from 9.2 to 13.9 . The temperature dependence of these reactions do not seem to relate to their exothermicities. Variations in rate constants appear to be more strongly dependent on changes in preexponential factors rather than on changes in activation energy.

\section{0,784}

PB94-198314 Not available NTIS

National Inst. of Standards and Technology (PL), Boulder, CO. Quantum Physics Div.

Vibrational Distributions of As2 in the Cracking of As 4 on $\mathrm{Si}(100)$ and $\mathrm{Si}(111)$.

Final rept.

A. L. Alstrin, R. V. Smilgys, P. G. Strupp, and S. R

Leone. 1992, 7p.

Contract AFÓSR90-0166

Pub. in Jnl. of Chemical Physics 97, n9 p6864-6870,

1 Nov 92. Sponsored by Air Force Office of Scientific Research, Bolling AFB, DC.

Keywords: "Silicon, *Arsenic, *Desorption, Reprints, Laser induced fluorescence, Vibrational states, Surface temperature, Temperature dependence, Chemisorption, Dimers

The desorption dynamics of arsenic from $\mathrm{Si}(100)$ and $\mathrm{Si}(111)$ are studied by measuring the vibrational population distributions of desorbed As2 using laser-induced fluorescence. In these measurements, a steady state flux of desorbing As2 is produced by continuously dosing a heated Si surface with a beam of As4 from a conventional molecular beam epitaxy oven. Measurements of the fluxes of As2 as a function of surface temperature suggest that the As2 may be kinetically formed in two distinct steps: The As4 first chemisorbs to form atoms on the surface which then recombine to desorb as dimers. However, there may also be direct dissociation of As4 at the hot surface. The vibrational populations of As2 desorbed from $\mathrm{Si}(100)$ are Boltzmann and indicate a vibrational temperature that is nearly $350 \mathrm{~K}$ lower than the surface temperature. The vibrational populations of As2 desorbed from Si(111) are not Boltzmann, but also have an effective vibrational temperature $400 \mathrm{~K}$ colder than the surface temperature. The observed lack of accommodation in the desorption from both surfaces is discussed in terms of the possible mechanisms.

\section{0,785}

PB94-198371 Not available NTIS

National Inst. of Standards and Technology (NML), Gaithersburg, MD. Chemical Kinetics Div

Oxidation of 10-Methylacridan, a Synthetic Analogue of NADH and Deprotonation of Its Cation Radical. Convergent Application of Laser Flash Photolysis and Direct and Redox Catalyzed Electrochemistry to the Kinetics of Deprotonation of the Cation Radical.

Final rept.

A. Anne, P. Hapiot, J. Moiroux, P. Neta, and J. M.

Saveant. 1991, $8 p$

Pub. in Jnl. of Physical Chemistry 95, n6 p2370-2377 1991

Keywords: "Nitrogen heterocyclic compounds, *Oxidation, *Decomposition reactions, "Reaction kinetics, "Chemical radicals, Radiolysis, Chemical reactions, Electrochemistry, Photolysis, Reprints, *10 Methylacridan, Cation radicals, Deprotonation.

Photolysis of 10-methylacridan (AH) in acetonitrile solutions containing $\mathrm{CCl} 4$ led to quantitative oxidation of $\mathrm{AH}$ to $\mathrm{A}(+)$. Laser flash photolysis of the solution with 248 or $308 \mathrm{~nm}$ light yielded the cation radical $\mathrm{AH}$ (sup dot, + ) which decays by deprotonation to give the neutral radical $A$ (sup dot). There is a good agreement between the values determined for $k(s u b H)$ here and, previously, by direct electrochemistry using ultramicroelectrodes. The vast majority of the data points in a plot of logk(sub H) vs the pKa of the bases, covering $18 \mathrm{pKa}$ units and rate constants up to the diffusion limit, fall on the same Bronsted line in spite of the fact that they involve bases of quite different struc. ture: pyridines, aliphatic nitrogen bases and carboxylates. Pulse radiolysis experiments in acidic aqueous solutions showed that $\mathrm{AH}$ is oxidized by $\mathrm{Cl}$ (sup dot,-) to give $\mathrm{AH}$ (sub dot, + ), which decays with a first order rate constant of $900 / \mathrm{s}$. Reduction of $\mathrm{Al}+$ gave $A$ (sup dot) which did not undergo protonation even at $2 \mathrm{M} \mathrm{HClO}_{4}$, but reaction of $\mathrm{A}(+)$ with $\mathrm{H}$ atoms resulted in partial addition of $\mathrm{H}$ to the 9 position to give $\mathrm{AH}$ (sup dot, + )

\section{0,786}

PB94-198504 Not available NTIS

National Inst. of Standards and Technology (CSTL) Gaithersburg, MD. Surface and Microanalysis Science Div.

Energy Dependence of Collision Characteristics in Molecule-Surface Collisions.

Final rept.

V. Balasubramanian, A. Bahel, I. P. Dubey, N.

Sathyamurthy, and J. W. Gadzuk. 1992, 4p.

Pub. in Jnl. of Physical Chemistry 96, n20 p7870-7873 1992.

Keywords: "Molecule collisions, Surface reactions, Diatomic molecules, Chemisorption, Fractals, Chaos, Reprints, Surface dynamics, Translations.

For a model diatomic molecule-surface collision, we show that the extent of chaoticity, as evidenced in ac tion-angle and lifetime plots, decreases dramatically with an increase in the translational energy of the molecule In addition to the existence of a threshold for the dissociative chemisorption, there is also an antithreshold at higher energies. The implications for the existence (or lack thereof) of chaos/fractals in molecule-surface collisions are also discussed, particularly as related to translational-vibrational energy redistribution.

00,787

PB94-198546 Not available NTIS

National Inst. of Standards and Technology (NML) Gaithersburg, MD. Molecular Physics Div.

Density Matrix Calculation of Population Transfer between Vibrational Levels of Na2 by Stimulated Raman Scattering with Temporally Shifted Laser Beams.

Final rept

Y. B. Band, and P. S. Julienne. 1991, 8p.

1991.

Keywords: *Sodium, Reprints, Vibrational states, Electron transitions, Density matrix, Raman spectra, Bloch equations, Laser radiation.

We compare the results of full density matrix calcula tions with recently reported experimental results describing the population transfer in Na2 using Raman scattering with the fundamental laser beam temporally shifted relative to the Stokes laser beam. Our calculations confirm the conclusion that aimost total transfer of the population into the terminal level of the Raman transition can be achieved when the laser frequencies are tuned to resonance and the fundamental lase beam is temporally delayed relative to the Stokes shifted laser beam but still partially overlapping it. We analyze our results when the frequencles of the lasers are tuned off resonance as a function of the Rabi frequencies and the detuning, and compare with experimental and theoretical results. The alignment of the terminal level as a function of time displacement of the Stokes and pump beams is predicted.

00,788

PB94-198595 Not available NTIS

National Inst. of Standards and Technology (NML), Gaithersburg, MD. Quantum Metrology Div.

Strong Hydrogen Bond in the Formic Acid-Formate Anion System.

Final rept.

H. Basch, and W. J. Stevens. 1991, 7p

Pub. in Jnl. of the American Chemical Society 113, n1 p95-101 1991. Sponsored by Bar-llan Univ., Ramat Gan (Isràel).

Keywords: *Hydrogen bonds, "Formic acid, *Molecular orbitals, Mathematical models, Dimerization, Formates, Bonding strength, Quantum chemistry, Reprints, Formate anion.

Hydrogen bond energies and geometric structures of several symmetric and asymmetric formic acid-formate anion dimer complexes have been determined. Ab initio gradient optimization at the self-consistent field (SCF) level in a double zeta + polarization + diffuse Gaussian function basis set was followed by a series of second order Moller Plesset (MP2) single point cal- 
culations for the lowest energy structures. The equilibrium asymmetric dimer with the (anti)formic(syn) forma co SCF and MP2 levels at 28.8 and 33 $\mathrm{kcal} / \mathrm{mole}$, respectively. Experimentally, a gas phase heat of dimerization value of $36.8 \mathrm{kcal} / \mathrm{mole}$ has been reported for the biformate anion. The calculated stability of this anti dimer includes a $\mathrm{C}-\mathrm{H}-\mathrm{O}$ interaction which is estimated to contribute approximately 2.4 (SCF) or approximately 1.8 (MP2) $\mathrm{kcal} / \mathrm{mole}$ to the dimer binding energy. The corresponding proton-transferred (syn)formic-(anti)formate complex is calculated less stable by 5.6 (SCF) or 3.7 (MP2) kcal/mole, even though in the isolated monomer syn formic acid is the lower energy conformer by 5.8 (SCF) or 5.4 (MP2) $\mathrm{kcal} / \mathrm{mole}$ in the same basis set. The greater stability of the anti dimer can be attributed both to less interfragment exchange repulsion and to more coulomb attraction compared with the syn dimer conformation.

00,789

PB94-198603 Not available NTIS

National Inst. of Standards and Technology (NML), Gaithersburg, MD. Chemical Thermodynamics Div. Structure of Glycine-Water H-Bonded Complexes. Final rept.

H. Basch, and W. J. Stevens. 1990, 6p.

Pub. in Chemical Physics Letters 169, n4 p275-280 1990.

Keywords: *Glycine, "Hydrogen bonds, "Water, "Molecular structure, Reprints, Molecular orbitals Quantum chemistry, Amino acids, *Glycine-water complex, Moller-Plesset perturbation theory, Self consistent field optimization.

$A b$ initio calculations have been carried out on several cyclic hydrogen-bonded structures of giycine with water. Single configurations self-consistent-field energy-gradient optimization methods were used to obtain the structures using double-zeta plus polarization basis sets. Second-order Moller-Plesset perturbation theory was used to determine the effect of correlation on the hydrogen-bonded distances. The most stable structure is a double hydrogen-bonded structure with the water accepting a proton from the $\mathrm{O}-\mathrm{H}$ of the carboxyl groups while donating a proton to the carbonyl group. This structure is bound by $8.6 \mathrm{kcal} / \mathrm{mol}$ and should be observable experimentally.

\section{0,790 \\ PB94-198611 Not available NTIS}

National Inst. of Standards and Technology (PL), Boulder, CO. Quantum Physics Div.

Femtosecond Time-Resolved Wave Packet Motion in Molecular Multiphoton Ionization and Fragmentation.

Final rept.

T. Baumert, B. Buhler, M. Grosser, E. Wiedenmann, G. Gerber, R. Thalweiser, and V. Weiss. 1991, 8p. Pub. in Jnl. of Physical Chemistry 95, n21 p8103-8110 1991.

Keywords: "Molecular dynamics, "Sodium, Reprints, Multi-photon processes, Molecular beams, Molecula ions, Time dependence, Laser radiation, Diatomic molecules, Photoelectron spectroscopy, Mass spectroscopy, Dye lasers, Photoionization, Fragmentation, Sodium ions, Wave packets, "Multiphoton ionization, Femtosecond pulses.

The dynamics of molecular multiphoton ionization and fragmentation of a diatomic molecule (Na2) have been studied in molecular beam experiments. Femtosecond laser pulses from an amplified colliding-pulse modelocked (CPM) ring dye laser are employed to induce and probe the molecular transitions. The final continuum states are analyzed by photoelectron spectros copy, by ion mass spectrometry, and by measuring the kinetic energy of the formed ionic fragments. Pumpprobe spectra employing 70-fs laser pulses have been measured to study the time dependence of molecular multiphoton ionization and fragmentation. The oscillatory structure of the transient spectra showing the dynamics on the femtosecond time scale can best be understood in terms of the motion packets in bound molecular potentials. The transient $\mathrm{Na2}(1+)$ ionization and the transient $\mathrm{Na}(1+)$ fragmentation spectra show that contributions from direct photoionization of singly ex cited electronic state and from excitation and autoionization of a bound doubly excited molecula state determine the time evolution of molecular multiphoton ionization.
National Inst. of Standards and Technology (MSEL), Gaithersburg, MD. Reactor Radiation Div. Neutron Powder Diffraction Study of a $\mathrm{Na}$, Cs-Rho Zeolite.

Final rept.

W. H. Baur, A. Bieniok, R. D. Shannon, and E. Prince. 1989, 14p.

Pub. in Zeitschrift fur Kristallographie 187, p253-266 1989 .

Keywords: "Zeolites, *Crystal structure, Reprints, Neutron diffraction, Deuterium compounds. Hydrates, Powder(Particles). Temperature range 0273-0400 K, Sodium cesium aluminum silicates, Rho zeolites, Rietveld method.

The crystal structure of a Na, Cs-Rho zeolite was studied at $373 \mathrm{~K}$ ( $\mathrm{Na}(8.4) \mathrm{Cs}(3.2) \mathrm{Si}(36.4) \mathrm{Al}(11.6) 096$. $5 \mathrm{D} 20$ ) by neutron powder diffraction and Rietveld profile analysis. It crystallizes in the noncentrosymmetric space group I (bar 4)3m and has a unit cell constant and $\mathrm{R}($ wp $)=8.01 \%$. The distortion of the (Al, Si) O2framework of this zeolite fits into the pattern established for other noncentrosymmetric Rho zeolites. Particularly, the ellipticity of the eight-ring and the pore size are strictly a function of the cell constant of the zeolite. The mean distance (Al,Si)-O is observed to be $1.636 \mathrm{~A}$, in excellent agreement with the mean (Al, Si)$\mathrm{O}$ distance of $1.638 \mathrm{~A}$ calculated for the Si and Al content of our sample.

\section{0,792}

PB94-198637 Not available NTIS

National Inst. of Standards and Technology (NML), Boulder, $\mathrm{CO}$. Time and Frequency Div.

Rotational Spectrum of Copper Hydride Using Tunable Far Infrared Radiation.

Final rept.

S. P. Beaton, and K. M. Evenson. 1990, 4p.

Pub. in Jnl. of Molecular Spectroscopy 142, n2 p3363391990.

Keywords: "Copper hydrides, "Rotational spectra, Far infrared radiation, Molecular spectra, Electron transitions, Interstellar matter, Copper 63, Reprints.

We have detected and measured five low-J rotational transitions of (63) CuH between 40 and $125 / \mathrm{cm}$ using coherent tunable far infrared radiation. A least squares fit to the data yields two orders of magnitude improvement in the values for the rotational molecular constants. The $1<-0$ transition of astronomical interest is predicted to be 468652.2 (2) $\mathrm{MHz}$.

\section{3}

\section{PB94-198678 Not available NTIS}

National Inst. of Standards and Technology (NML), Gaithersburg, MD. Molecular Physics Div.

Tunneling-Rotation Spectrum of the Hydrogen Fluoride Dimer.

Final rept

S. P. Belov, E. N. Karyakin, I. N. Kozin, M. Y. Tretyakov, N. F. Zobov, R. D. Suenram, W. J. Lafferty, A. F. Krupnov, and O. L. Polyansky. 1990, $19 p$.

Pub. in Jnl. of Molecular Spectroscopy 141, n2 p2042221990.

Keywords: "Hydrogen fluoride, "Microwave spectra, Far infrared radiation, Submillimeter waves, Millimeter waves, Rotational spectra, Electron transitions, Ground state, Dimers, Reprints.

The tunneling-rotation spectrum of the ground state of the hydrogen fluoride dimer, HF.-.-HF, has been extended to the submillimeter wavelength region with the RAD-3 submillimeter spectrometer at Gorky in the frequency range $180-380 \mathrm{GHz}$ at $210 \mathrm{~K}$ and pressure from 0.5 to 1.5 Torr. The spectrum has been reinvestigated at lower frequencies with a conventional Stark spectrometer at pressures between 0.1 to 0.2 Torr at NIST. Lines of the a-type $K=3$ subband have been observed for the first time. The tunneling frequency for the $\mathrm{K}=3$ state is 114306.35 (53) $\mathrm{MHz}$. An atlas of all a- and b-type far infrared and microwave ground state tunneling-rotation transitions for $\mathrm{K}$ up to 3 giving frequency, uncertainty, and relative intensity has been prepared.

\section{0,794}

PB94-198892 Not available NTIS

National Inst. of Standards and Technology (PL), Gaithersburg, MD. Molecular Physics Div.
Infrared and Microwave Spectroscopy of the Argon - Propyne Dimer.

Final rept.

T. A. Blake, D. F. Eggers, S. H. Tseng, R. Beck, R.

. Watts, F. J. Lovas, M. Lewerenz, and R. P. Swift.

1993, 13p.

Grants NSF-CHE-8703766, NSF-CHE-9015765

Pub. in Jnl. of Chemical Physics 98, n8 p6031-6043, 15 Apr 93 . Sponsored by National Science Foundation, Arlington, VA. and Petroleum Research Fund, Washington, $D C$.

Keywords: *Argon complexes, Reprints, Van de Waals forces, Infrared spectroscopy, Microwave spectroscopy, Molecular structure, Dipole moments, Hamiltonians, Dimers, ${ }^{*}$ Propyne complexes.

Microwave and infrared measurements are reported for the van der Waals complex $\mathrm{ArCH} 3 \mathrm{CCH}$ and its isotopomers. The structure is $T$ shaped with equilibrium center-of-mass separation of $3.73 \mathrm{~A}$ and an angle of $82 \mathrm{deg}$. between the molecule symmetry axis and the van der Waals bond. The infrared and microwave spectra are complex due to the effects of a slightly hindered internal rotor. Analysis of the spectral data shows that the dipole moment is almost parallel to the dimer $b$ axis. A modified vibration/rotation Hamiltonian that includes an internal rotor potential is used to show that the barrier to internal rotation is near $10.8 / \mathrm{cm}$.

\section{0,795}

PB94-199189 Not available NTIS

National Inst. of Standards and Technology (CSTL), Boulder, CO. Thermophysics Div.

Measurement of Diffusion in Supercritical Fluid Systems: A Review.

Final rept.

T. J. Bruno. 1993, 7p

Pub. in Proceedings of Aerospace Sciences Meeting and Exhibit (31st), Reno, NV., January 11-14, 1993 p1-7.

Keywords: *Supercritical state, *Diffusion coefficient, "Aviation fuels, "Chromatographic analysis, Reprints, Diffusion, Thermophysical properties, Chemical analysis, "Supercritical fluid solutions.

In the paper, the experimental procedures that are applicable to the measurement of diffusion in supercritica fluid solutions will be reviewed. This topic is of great importance to the proper design of advanced aircraft and the turbine fuels these aircraft will use. The main reason for this is that the fuels on these high performance aircraft may sometimes operate unde supercritical fluid conditions when they are used as a heat sink, for the cooling of friction-heated surfaces These conditions may produce unfavorable chemica reactions in the fuel. The decomposition products so produced will therefore be a major cause of heat ex changer fouling, and this must be addressed in the early stages of fuel and component design. Since transport properties are needed for these designs, the authors will consider measurements of one of the most critical: the binary interaction diffusion coefficient. Afte a brief introduction to the concept of diffusion, the authors will discuss in detail the use of chromatographic methods, and then briefly treat light scattering, nuclea magnetic resonance spectra, and physical methods.

00,796

PB94-199452 Not available NTIS

National Inst. of Standards and Technology (PL) Gaithersburg, MD. Molecular Physics Div.

Experimental Studies of Line Shapes from a BalleFlygare Spectrometer.

Final rept.

E. J. Campbell, and F. J. Lovas. 1993, $6 p$

Pub. in Review of Scientific Instruments 64, n8 p2173 2178 Aug 93.

Keywords: "Microwave spectrometers, "Line shape, Fourier transformation, Carbon oxysulfide, Molecula beams. Reprints, “Balle-Flygare spectrometers, Carbonyl sulfides.

The line shape problem for the Balle-Flygare-type microwave spectrometer is reexamined experimentally using the method of sine and cosine Fourier transforms with a phase correction.

00,797

PB94-199544 Not available NTIS

National Inst. of Standards and Technology (NML) Gaithersburg, MD. Chemical Kinetics Div. 


\section{CHEMISTRY}

\section{Physical \& Theoretical Chemistry}

\author{
New Rydberg States of Aluminum Monofluoride \\ Observed by Resonance-Enhanced Multiphoton \\ Ionization Spectroscopy \\ Final rept \\ D. V. Dearden, R. D. Johnson, and J. W. Hudgens. \\ 1991, 6p. \\ Pub. in Jnl. of Physical Chemistry 95, n11 p4291-4296 \\ 1991.
}

Keywords: "Aluminum fluorides, "Rydberg states, "Electronic spectra, "Vibrational spectra, "Chemical reactions, Metastable state, Aluminum, Spectrum analysis, Ionization potentials, Reprints, "Aluminum monofluoride, Resonance enhanced multiphoton ionization spectroscopy.

AIF has been generated in the gas phase by high temperature reduction of $\mathrm{AlF}_{3}$ by $\mathrm{Al}$, and detected and characterized using resonance-enhanced multiphoton ionization. Five Rydberg series comprised of thirteen new Rydberg states have been identified for the first time. Least-squares fitting of the Rydberg series to the Rydberg equation yields the adiabatic ionization potential of AIF, IP (sub a) $=9.729+$ or -0.001 Ev. Vibrational intervals for all of the new Rydberg states are about $20 \%$ greater than those of AIF (X(sup 1)Sigma (sup +$)$ ) with most lying between $930-980 / \mathrm{cm}$. The vibrational frequencies are in agreement with an ionic bonding model for AIF.

\section{0,798}

PB94-199569 Not available NTIS

National Inst. of Standards and Technology (CSTL), Gaithersburg, MD. Thermophysics Div.

Thermodynamic Properties of CHF2-OCHF2,Bis(difluoromethyl) Ether.

Final rept.

D. R. Defibaugh, K. A. Gillis, M. R. Moldover, G.

Morrison, and J. W. Schmidt. 1992, 21p.

Contract N0002490MP33493

Pub. in Fluid Phase Equilibria 81, p285-305 1992. Sponsored by Department of the Navy, Washington, DC.

Keywords: "Fluorine organic compounds, "Ethers, "Refrigerants, "Thermodynamic properties, Reprints, Substitutes, Equations of state, Interfacial tension, Specific heat, Critical temperature, Boiling points, "bis(Difluoromethyl)ether, "E134, Speed of sound

The authors have measured the thermodynamic properties of bis(difluoromethyl) ether, a candidate alternative refrigerant that is also known as E134. From the data the authors obtained the coefficients of a Carnahan-Starling-DeSantis equation of state and a polynomial representation of the ideal-gas heat capacity. This representation of the thermodynamic properties of E134 is consistent with the computer package REFPROP distributed by the National Institute of Standards and Technology to represent the properties of many candidate refrigerants. The representation is based on measurements of the refractive index of the saturated liquid and vapor, and the speed of sound of the dilute vapor. These measurements provide the boiling point, critical parameters, and the ideal-gas heat capacity of E134. Measurements on less pure samples were used to estimate the density of saturated liquid E134 and compressed liquid E134, and the interfacial tension. The pure samples appeared to be stable during the measurements; under similar conditions impure samples were not. Azeotropy in mixtures of $\mathrm{E} 134$ with $\mathrm{CHF} 2 \mathrm{CH} 2 \mathrm{~F}$ (also known as R143) was discovered.

\section{0,799}

PB94-199577 Not available NTIS

National Inst. of Standards and Technology (PL), Gaithersburg, MD. Radiometric Physics Div.

Vibrational Autoionization in $\mathrm{H} 2$ : Vibrational Branching Ratios and Photoelectron Angular Distributions Near the $v(+)=3$ Threshold

Final rept.

J. L. Dehmer, P. M. Dehmer, J. B. West, A. C. Parr, M. A. Hayes, and M. R. F. Siggel. 1992, 7p.

Pub. in Jnl. of Chemical Physics 97, n11 p7911-7917. 1 Dec 92 . Sponsored by Department of Energy, Washington, DC

Keywords: "Hydrogen, "Photoionization, Reprints, Vibrational states, Branching ratio, Angular distribution, Autoionization, Photoelectrons, Asymmetry.

Vibrational branching ratios and photoelectron angular distributions are reported for photoionization of normal (the equilibrium ortho/para mixture) hydrogen in the region just above the nu( +$)=3$ ionization limit. This re- gion contains a number of vibrationally autoionizing Rydberg states, and the goal of the work was to ob. serve experimentally the characteristic behavior associated with the decay of these states that was predicted by Raoult and Jungen (J. Chem. Phys. 74,3388 (1981)) on the basis of an MQDT (multichannel quantum defect theory) calculation on para- $\mathrm{H}_{2}$ ( $\mathrm{J}$ double prime $=0$ ). We have indeed observed that vibrational autoionization strongly favors the ionization channel representing the minimum change in the vibrational state of the ion core, as predicted. We also observed a sharp reduction in the photoelectron asymmetry of the $n u(t)=3$ (and, to a lesser extent, the nu(t) = 2) ionization channel for resonant photoionization. Hence, qualitative agreement with theory is observed however, a quantitative comparison now requires an extended calculation that includes the additional rotational levels that were populated under the present experimental conditions.

\section{0,800}

PB94-199619 Not available NTIS

National Inst. of Standards and Technology (MSEL)

Gaithersburg, MD. Reactor Radiation Div.

Maximum Entropy as a Tool for the Determination of the C-Axis Profile of Layered Compounds.

Final rept.

P. Depondt, D. A. Neumann, and S. F. Trevino.

1993, 6p.

Pub. in Acta Crystallographica B49, p153-158 1993.

Keywords: "Organometallic compounds, Reprints, Molybdenum compounds, Maximum entropy method Room temperature, Neutron scattering, Structure fac tors, Deuterium compounds, Intercalation, Graphite Layered compounds.

A simple procedure for the determination of the struc ture normal to the basal plane of layered compounds based on the now ubiquitous maximum-entropy meth od is presented. It is illustrated by the analysis of roomtemperature $(0 \mathrm{OI})$ elastic neutron-scattering experi ments performed on two graphite intercalation compounds, stage $3 \mathrm{C}(44.6) \mathrm{MoCl}(5)$ and stage KC24(ND3) 4.3. The former example is quite simple requiring only a crude heuristic model to determine the structure-factor phases. The latter shows good sensitivity to the orientation of the ND3 threefold axis with respect to the basal plane, thus providing its first direct determination.

\section{0,801}

P894-199817 Not available NTIS

National Inst. of Standards and Technology (NML), Gaithersburg, MD. Chemical Thermodynamics Div. Calibration Standards for Differential Scanning Calorimetry. 1. Zinc Absolute Calorimetric Meas urement of Enthalpy of Fusion and Temperature of Fusion HM.

Final rept.

D. A. Ditmars. 1993, 13p.

Pub. in Jnl. of Chemical Thermodynamics 22, n7 p6396511990.

Keywords: "Zinc, "Heat of fusion, "Fusion(Melting) "Enthalpy, Reprints, Specific heat, Transition temperature, Calorimetry, Measurement, Standard reference materials, Method of mixtures

The temperature and enthalpy of fusion of a pure zinc specimen chosen from NIST SRM 2221a have been measured for the first time simultaneously in a highprecision, method-of-mixtures, isothermal, phasechange calorimeter. The measured temperature of fusion $(692.745+$ or -0.010$) K$ was obtained with a new partial-fusion technique described herein. This result agrees well with the IPTS-68 temperature for the freezing point of pure zinc. The measured enthalpy of fusion $(7026+$ or -40$) \mathrm{J} / \mathrm{mol}$ differs substantially from previous values in the evaluated literature. Detailed examination of the original zinc literature data and of the sev eral published attempts to derive from them the enthalpy of fusion has reinforced the author's conclusion that the present result for the enthalpy of fusion of zinc is of higher accuracy than any value currently given in evaluated data compilations.

\section{0,802}

PB94-199908 Not available NTIS

National Inst. of Standards and Technology (NML) Gaithersburg, MD. Molecular Physics Div.
Fragment Energy and Vector Correlations in the Overtone-Pumped Dissociation of HN3X(1)A'. Final rept.

M. P. Casassa, B. R. Foy, J. C. Stephenson, and D. S. King. 1993, 12p.

Pub. in Jnl. of Chemical Physics 94, n1 p250-261 1991.

Keywords: "Hydrazoic acid, "Dissociation *Photochemical reactions, Vibration, Energy levels, Molecular structure, Reprints, Vibrational predissociation.

$\mathrm{NH}$ stretching overtone and combination states in the HN3 singlet XA' state were excited by IR-visible double resonance pumping and by direct overtone pumping in the range $17670 / \mathrm{cm}$ to $20070 / \mathrm{cm}$. NH fragments in the singlet a-delta and triplet $X$ sigma(sup-1) states were detected by laser induced fluorescence with subDoppler resolution to determine branching ratios, correlated fragment rotational state and kinetic energy dis tributions, and fragment vector correlations. The spinforbidden triplet channel was accessible to all states excited, while the threshold for the singlet channel was determined to lie in the range 18190 to $18755 / \mathrm{cm}$. Vector correlations and lambda-doublet propensities show that nonplanar dissociation processes influence the $\mathrm{NH}$ rotations, but become less important for higher $\mathrm{NH}$ rotational states.

00,803

PB94-199957 Not available NTIS

National Inst. of Standards and Technology (CSTL), Gaithersburg, MD. Surface and Microanalysis Science

Time-Resolved Probes of Surface Dynamics.

Final rept.

R. R. Cavanagh, E. J. Heilweil, and J. C.

Stephenson. 1993, 7p.

Pub. in Surface Science 283, p226-232 1993.

Keywords: "Carbon monoxide, Reprints, Vibrational spectra, Density matrix, Transient response, Excited states, Line shape, Infrared radiation, Absorption, Surfaces, Platinum, Femtosecond pulses

The excited vibrational state dynamics of $\mathrm{CO}$ absorbed on $\mathrm{Pt}(111)$ are explored using subpicosecond intrared pump-probe techniques. The response for a $\mathrm{CO}(\mathrm{nu}=$ 1) adlayer for coverages of $0.1,0.3$, and 0.5 monolayers is measured as a function of time delay and probe wavelength. The observed transient $\mathrm{CO}$ vibrational spectra reveal a shift to lower frequency of the CO internal stretch mode as the degree of excitation of the adlayer is increased. These spectra are discussed in terms of density matrix models which address the conerence time of the adlayer $(n u=1)$ excitation and the excited state spectral characteristics of strongly-coupled anharmonic oscillators. The two-tothree picosecond recovery time of the transient response is consistent with relaxation through electronhole pair creation. The relationship of the measured $T(1)$ decay to the observed lineshape is addressed.

00,804

PB94-199973 Not available NTIS

National Inst. of Standards and Technology (CSTL),

Gaithersburg, MD. Process Measurements Div.

Reactivity of Pd and Sn Adsorbates on Plasma and Thermally Oxidized SnO2(110).

Final rept

R. Cavicchi, and S. Semancik. 1991, 9p. Pub. in Surface Science 257, p70-78 1991

Keywords: "Tin oxides, *Surface analysis, Reprints, Surface reactions, Metal films, Semiconductors, Chemisorption, Absorbates, Interfaces, Palladium.

The surface accumulation layer that forms as a result of oxygen vacancies near the surface of an oxide semiconductor can be used as an extremely sensitive probe of phenomena occurring at the surface. By incorporating a UHV-compatible, four-point conductance measurement in our surface analysis apparatus, we have investigated the formation of metal-semiconductor interfaces on $\mathrm{SnO} 2(110)$ with varying oxygen stoichiometry. For only a 0.1 monolayer equivalent (ML) coverage of $\mathrm{Sn}$ deposited onto a high-oxygen-content surtace prepared by oxygen plasma treatment, we observe a 500-fold increase in conductance. Our results suggest that at this coverage, Sn reacts with the excess chemisorbed oxygen and also creates point defects by abstracting oxygen from the lattice. In contrast, $P d$ reacts only with chemisorbed species, as indicated by a smaller conductance change and by differences in the size-effect shifted core levels observed at the 
same coverage for thermal and plasma oxidized surfaces. Oxygen plasma treatment of an $8 \mathrm{ML}$ Pd film is shown to be an effective room temperature oxidation procedure and is used as a basis of comparison for studies of the reaction of submonolayer coverages of Pd on plasma and thermally oxidized surfaces.

\section{0,805}

PB94-200037 Not available NTIS

National Inst. of Standards and Technology (CSTL), Gaithersburg, MD. Surface and Microanalysis Science Div.

Pure Element Sputtering Yield Data: Appendix 4. Final rept.

G.P. Chambers, and J. Fine. 1992, 16p.

Pub. in Practical Surface Analysis, v2 p705-720 1992.

Keywords: "Surface analysis, "Sputtering, Ion bombardment, Argon ions, Xenon ions, EV range 100$1000, \mathrm{KeV}$ range $01-10, \mathrm{KeV}$ range $10-100$, Yield, Reprints, Depth profiles.

An evaluated compilation of absolute sputtering yield data has been prepared for those parameters specific to surface analysis and depth profiling. This compilation was done for elemental targets bombarded with ion species of $\mathrm{Ar}$ and $\mathrm{Xe}$ at energies from 0.5 to 20 $\mathrm{keV}$. Data evaluation was based on the target surface cleanliness during measurement and on the total ion dose. Calculated yields were fitted to the evaluated data to obtain evaluated sputtering yields. We have shown this approach to be consistent and reliable.

00,806

PB94-200219 Not available NTIS

National Inst. of Standards and Technology (MSEL), Gaithersburg, MD. Reactor Radiation Div.

Neutron-Scattering Study of C6O(n-) $(n=3,6)$ Librations in Alkali-Metal Fullerides.

Final rept

C. Christides, D. A. Neumann, K. Prassides, M. J.

Rosseinsky, D. W. Murphy, R. C. Haddon, J. R. D.

Copley, and J. J. Rush. 1992, 4p.

Pub. in Physical Review B 46, n18 p12 088-12 091, 1 Nov 92.

Keywords: "Superconductors, Rubidium compounds, Potassium compounds, Room temperature Neutron scattering, Inelastic scattering, Doped materials, Librations, Fullerenes, Reprints, "Fullerides.

We have measured the low-energy inelastic neutron scattering spectra of superconducting $\mathrm{K} 3 \mathrm{C} 60$ and insulating Rb6C60. Well-defined peaks are observed at room temperature at $3.59(4) \mathrm{meV}$ (full width at half maximum (FWHM) $=1.11(15) \mathrm{meV}$ ) and $5.49(38) \mathrm{meV}$ $(F W H M=3.41(44) \mathrm{meV})$, respectively. They harden with decreasing temperature and the dependence of their intensities on the scattering vector shows that they are due to small-amplitude librational motion. No anomalous behavior of the librational peak was observed on cooling through the superconducting transition temperature, indicating that electron-librational coupling is weak. The energy barrier for reorientation is estimated to be at least twice as large in $\mathrm{K} 3 \mathrm{C} 60$ as in $\mathrm{C} 60$. Substantial disorder persists for $\mathrm{K} 3 \mathrm{C} 60$ in the superconducting state.

\section{0,807}

PB94-200318 Not available NTIS

National Inst. of Standards and Technology (MSEL), Gaithersburg, MD. Ceramics Div.

Introduction of a NIST Instrument Sensitivity Standard Reference Material for X-Ray Powder Diffraction.

Final rept.

J. P. Cline, S. B. Schiller, and R. Jenkins. 1992, 12p. Pub. in Advances in X-Ray Analysis, v35 p341-352 1992.

Keywords: "Sample preparation, "X-ray diffraction, "Diffractometers, "Powder(Particles), Interlaboratory comparisons, Aluminum oxide, Chemical analysis, Performance evaluation, Design criteria, Error analysis, Certification, Sensitivity, Reprints, "Standard reference materials, NIST(National Institute of Standards and Technology), SRM 1976.

Improvements in sample preparation methods have resulted in increased accuracy in $\mathrm{x}$-ray powder diffraction intensity measurements. This improvement has focused scrutiny on the instrument as a potential source of error. NIST Standard Reference Material, SRM 1976 consists of a sintered alpha alumina, corundum, plate certified with respect to 12 relative intensity values from 25 to 145 degrees 2 theta. Its function is to allow for standardization of powder diffraction intensity as a function of 2 theta angle (instrument sensitivity). An increase in the accuracy of interlaboratory comparisons of diffraction intensity and related determinations will result. Utilization of the SRM requires the user to collect intensity data from the test instrument in a manner which conforms to that used in the certification. Graphical interpretation of the ratio of these data to those on the certificate will allow for appropriate judgment as to the condition of the test instrument.

\section{0,808}

\section{PB94-200466 Not available NTIS}

National Inst of Standards and Technology (NML) Gaithersburg. MD. Molecular Spectroscopy Div.

2-Tunneling Path Internal-Axis-Method-Like Treatment of the Microwave Spectrum of Divinyl Ether. Final rept.

L. H. Coudert. 1988, 22p.

in Jnl. of Molecular Spectroscopy 132, n1 p13341988

Keywords: "Ethers, "Spectrum analysis, "Microwave spectra, Molecular structure, Rotational spectra, Isomers, Stereochemistry, Reprints, "Divinyl ether

The microwave spectrum of the cis-trans conformer of divinyl ether, previously measured by Hirose and coworkers, has been fit using a multidimensional Internal Axis Method-tike (IAM-like) treatment which accounts for the $27 \mathrm{MHz}$ tunneling splitting displayed by this molecule. This multidimensional IAM-like treatment begins by first determining the various feasible tunneling path(s) connecting the two frameworks of the molecules. For the tunneling process corresponding to an antigeared rotation of each vinyl unit about axes coinciding with the respective $\mathrm{CO}$ bonds, two limiting cases are considered: if the molecule goes through a plana configuration during the tunneling, only one tunneling path arises; if the intermediate configuration is not planar, two equivalent tunneling paths occur. The consequences for the $J$ and $K$ dependence of the splitting area are examined, and using this formalism the microwave data are fitted with an RMS deviation of 0.17 $\mathrm{MHz}$. The parameters to the $J$ and $K$ dependence of the splitting are also determined and their values are interpreted in favor of a two tunneling path system. A comparison with the values obtained theoretically for those parameters is also carried out.

\section{0,809}

\section{PB 94-200508 Not available NTIS}

National Inst. of Standards and Technology (NML). Gaithersburg, MD. Organic Analytical Research Div.

Two-Dimensional POMMIE Carbon-Proton Chemical Shift Correlated (13)C NMR Spectrum Editing Final rept.

B. Coxon. $1990,6 \mathrm{p}$

1990.

Keywords: "Triacetyloleandomycin, "Nuclear magnetic resonance, "Carbon 13, "Spectrum analysis, Reprints, Molecular structure, Computer programs, Protons, Chemical shift.

Two pulse sequences are described for acquisition of two-dimensional carbon-proton chemical shift correlated, (13)C NMR spectra by the 'phase oscillations to maximize editing technique'. One of these sequences provides two-dimensional, carbon-proton chemical shift correlated spectra in which the (1) $\mathrm{H}-(1) \mathrm{H}$ coupling constants are present in the (1) $\mathrm{H}$ chemical shift dimension, whereas the other sequence includes a bilinear rotation decoupling unit that removes the vicinal (1) $\mathrm{H}-(1) \mathrm{H}$ couplings in this dimension. Extensions of these techniques to generation of two-dimensional carbon-proton chemical shift correlated $\mathrm{CH}, \mathrm{CH} 2$, and $\mathrm{CH}$, (13)C NMR subspectra from linear combinations of three, two-dimensional data sets are described. Decreased residual signals in the edited 2D subspectra have been achieved by Pascal programs that include six floating point coefficients, and a method for their calibration is discussed. Results are reported for troleandomycin (1).

\section{0,810}

PB94-200581 Not available NTIS

National Inst. of Standards and Technology (NML) Gaithersburg, MD. Chemical Kinetics Div.

Rate Constants for Hydrogem Atom Attack on Some Chlorinated Benzenes at High Temperature. Final rept.

J. P. Cui, Y. Z. He, and W. Tsang. 1989, 4p.

Pub. in Jnl. of Physical Chemistry 93, n2 p724-727 1989
Keywords: *Reaction kinetics, "Chlorobenzenes, "High emperature, "Hydrogen, "Displacement reactions, Re prints, Temperature dependence, Pressure dependence, Dissociation, Shock tubes, "Hydrogen atoms.

Hydrogen atoms from the decomposition of hexamethylethane were reacted with chlorobenzene o-dichlorobenzene, $\mathrm{p}$-dichlorobenzene and 1,2,3 trichlorobenzene in the presence of toluene or $1,3,5$ trimethylbenzene in single pulse shock tube experiments. Rate laws for the reactions were written using the rate expression for the displacement of the methy group from the methylated aromatics as standards for mperatures of 1050-1150 K and pressures between 2.5 to $3.2 \mathrm{~atm}$. In this temperature range the relative rate constants on a per chlorine basis for the reactions are $1: 1.15: 1.05: 1.8: 2.4$

\section{0,811}

PB94-200680 Not available NTIS

National Inst. of Standards and Technology (PL), Boulder, CO. Time and Frequency Div.

Extension of Heterodyne Frequency Measurements on OCS to $87 \mathrm{THz}(2900 \mathrm{~cm}(-1))$.

Final rept

A. Dax, M. Murtz, J. S. Wells, W. Urban, M

Schneider, and E. Bachem. 1992, 6p.

Pub. in Jnl. of Molecular Spectroscopy 156, p98-103 1992.

Keywords: *Carbon oxysulfide, "Frequency measurement, Reprints, Transition radiation, Carbon monoxide lasers, Infrared radiation, Calibration, Frequencies, Tables(Data), "Carbonyl sulfide.

Heterodyne frequency measurements have been extended to $87 \mathrm{THz}$ by using the $\mathrm{CO}$ overtone laser as a transfer oscillator. Measurements were made on the 11(sup 1) 1-01(sup 1) 0 band of OCS at $2900 / \mathrm{cm}$. Fre quency differences were measured between a tunable diode laser (TDL) which was locked to OCS absorption lines and a stabilized overtone laser which was referenced to stabilized $\mathrm{CO} 2$ lasers. These measurements have been combined with earlier Fourier transform (FT) measurements and a comprehensive set of data from frequency and FT measurements on lowe lying transitions in OCS. This permits obtaining accurate values of transition frequencies for calibration of spectra in the 3.4-micrometer region.

\section{0,812}

PB94-211125 Not available NTIS

National Inst. of Standards and Technology (NML) Gaithersburg, MD. Molecular Physics Div. Multichannel Quantum Defect Half Collision Analy sis of K2 Photodissociation Through the B1Pi(sub u) State.

Fina! rept

R. L. Dubs, and P. S. Julienne. 1991, 11p.

Pub. in Jnl. of Chemical Physics 95, n6 p4177-4187 1991.

Keywords: "Photodissociation, "Potassium, Polarization(Waves), Fluorescence, Alignment, Reprints, Multichannel quantum defect theory, Half collision analysis.

Recent experiments by Zafiropulos et al (Phys. Rev. Lett. 61, 1485 (1988)) indicate that K2 photodissociation through the B singlet $\mathrm{Pi}(u)$ state results in fluorescence polarization which is strongly de pendent on excitation wavelength. To understand these results, we have studied the $\mathrm{K} 2$ system quantum mechanically using a half collision analysis derived from the generalized form of multichannel quantum defect theory. An approximation called the Adiabatic (a$>c) /$ Recoil $(c->$ e) approximation is developed for the half collision matrix which reproduces quantitatively the exact half collision results projected for the close-coupied wavefunction. The quantum mechanical expression for the polarization as a function of $J(0)$ and tota energy $E$ is found to be extremely simple in this approximation, depending only on $\mathrm{J}(0)$ and the singlechannel $P, Q$ and $R$ phase shifts for the adiabatic reference states corresponding to the $\mathrm{B}$ singlet $\mathrm{Pi}(\mathrm{u})$ state at short distance.

00,813

PB94-211133 Not available NTIS

National Inst. of Standards and Technology (NML) Gaithersburg, MD. Molecular Physics Div. 
Intersystem Crossing in Collisions of Aligned $\mathrm{Ca}(4 \mathrm{~s} 5 \mathrm{p}(1) \mathrm{P})+\mathrm{He}: \mathrm{A}$ Half Collision Analysis Using Multichannel Quantum Defect Theory.

Final rept.

R. L. Dubs, P. S. Julienne, and F. H. Mies. 1990, 9p. Pub. in Jnl. of Chemical Physics 93, n12 p8784-8792 1990

Keywords: "Atom-atom collisions, "Atomic collisions, Factorization, Scattering, Alignment, Calcium, Helium, Reprints, Intersystem crossings, Multichannel quantum defect theory, Half collision analysis.

A half collision analysis of alignment effects on intersystem crossing in the collisions of $\mathrm{Ca}(4 \mathrm{~s} 5 \mathrm{p}$ singlet $\mathrm{P}$ ) with He has been performed using generalized Multichannel Quantum Defect Theory (MCQDT) The theory provides a rigorous analytical representation of the numerically exact close-coupled scattering wavefunctions. The half collision analysis results in a factorization of the fuli quantum collision problem into a number of simpler quantum mechanical problems which reflect different regions of development during the collision. A WKB-assisted, frame transformation approximation to the incoming half collision matrix is tested numerically and is found to be useful in project ing out information on the 'locking results' concept.

\section{0,814}

PB94-211331 Not available NTIS

National Inst. of Standards and Technology (MSEL) Gaithersburg, MD. Polymers Div.

Total Surface Areas of Group IVA Organometallic Compounds: Predictors of Toxicity to Algae and Bacteria.

G. Eng, E. J. Tierney, G. J. Olson, F. E. Brinckman, and J.M. Bellama. 1991, 5p.

Pub. in Appl. Organomet. Chem. 5, n1 p33-37 1991

Keywords: "Toxicity, "Organometallic compounds, "Surface area, "Algae, "Bacteria, Structure-activity relationships, Group 4A compounds, Metals, Solubility, Organotin compounds, Organosilicon compounds, Reprints, Organogermanium compounds, Organolead compounds.

There exists a high correlation between molecular surface area (TSA) of triorganotin and triorganolead compounds and their toxicity towards an algae (E. coli) and a bacterium (S. capricornutum). Parallel attempts to correlate other Group IVA organometals incorporating silicon or germanium were unsuccessful. It was further demonstrated, however, that a high correlation was obtainable between certain series of compounds with the same organic substituent but different metal centers in volving all Group IVA elements. In both instances, the inability to obtain a quantitative structure-activity relationship (QSAR) for all systems studied appears to be a function of the solubility of the compounds. While organotin TSA values have been found to correlate well with their toxicities toward various organisms, this study clearly suggests that this type of QSAR can be readily extended to include other organometal sys tems, provided that there is no solubility problem and the toxicity is a function of the hydrophobicity of the organometal compounds.

\section{0,815}

PB94-211398 Not available NTIS

National Inst. of Standards and Technology (NML), Gaithersburg, MD. Chemical Kinetics Div.

Reaction Rate Determinations of Vinyl Radical Reactions with Vinyl, Methyl, and Hydrogen Atoms. Final rept.

A. Fahr, A. Laufer, R. Klein, and W. Braun. 1991, 7p. Pub. in Jnl. of Physical Chemistry 95, n8 p3218-3224 1991

Keywords: "Reaction kinetics, "Free radicals, Photolysis, Atoms, Hydrogen, Reprints, "Vinyl radicals, Kinetic spectroscopy.

Laser photolysis-kinetic spectroscopy, end-product analysis, and detailed modeling were used to inves tigate three reaction systems in detail. Included were vinyl-vinyl, vinyl-methyl, and vinyl-methyl-hydrogen atoms. Product ratios, as a function of the rate constants, could be approximated using simple expressions. This greatly simplified the precise numerical modeling of these complex systems and also permitted a realistic error analysis. Reactions and rate constants at $25 \mathrm{C}$ are summarized.
National Inst. of Standards and Technology (PL), Boulder, CO. Quantum Physics Div.

High-Resolution Infrared Overtone Spectroscopy of ArHF via Nd:YAG/Dye Laser Difference Frequency Generation.

Final rept.

J. T. Farrell, O. Sneh, A. Mcllroy, A. E. W. Knight, and D. J. Nesbitt. 1992, 12p.

Contracts NSF-PHY90-12244, NSF-CHE90-00641

Sponsored by National Science Foundation, Washing ton, DC.

Pub. in Jnl. of Chemical Physics 97, n11 p7967-7978, 1 Dec 92.

Keywords: *Argon complexes, "Infrared spectroscopy, Van der Waale forces, Near infrared radiation, Infrared absorption, High resolution, Difference frequency, $\mathrm{Ne}$ odymium lasers, Reprints, "Hydrogen fluoride complexes, Potential energy surfaces, Overtone spectroscopy, Supersonic expansion.

The first high-resolution spectra of ArHF excited to the $\mathrm{nu}(\mathrm{HF})=2<-0$ manifold near $7,800 / \mathrm{cm}$ are recorded via direct infrared absorption in a slit supersonic expansion. The tunable difference frequency light is generated via nonlinear subtraction of a cW Nd:YAG laser from a tunable $\mathrm{cW}$ ring dye laser in temperature phase matched LiNbO3, and permits continuous single-mode access to the 1-2 micrometer near-IR region. In conjunction with previous results from the nu(HF) $=1$ and nu $(\mathrm{HF})=0$ vibrational levels, these studies provide the necessary data for fitting an atom + diatom potential energy surface as a function of all intermolecular and intramolecular internal degrees of freedom.

\section{0,817}

PB94-211463 Not available NTIS

National Inst. of Standards and Technology (NML), Gaithersburg, MD. Molecular Physics Div.

P-Type Doubling in the Infrared Spectrum of NOHF.

Final rept

W. M. Fawzy, G. T. Fraser, J. T. Hougen, and A. S. Pine. 1990, 13p

Pub. in Jnl. of Chemical Physics 93, n5 p2992-3004 1990

Keywords: "Hydrogen bonds, "Nitrogen oxides, *Hydrogen fluoride, "Infrared spectra, Spectrum analysis, Rotational states, Vibrational spectra, Reprints, "Van der Waals complex.

The HF stretching band of the NO-HF open-shell complex has been recorded using a molecular-beam optothermal spectrometer. The spectrum exhibits P. type doubling indicative of an unpaired electron spin coupled to the rotational angular momentum of a bent complex with substantially quenched electron orbital angular momentum. From $B^{\prime}=0.111316(28) / \mathrm{cm}$, the zero-point center-of-mass separation is estimated to be 3.439 (3) A. The HF trequency shift of $84 / \mathrm{cm}$ indicates that the complex is hydrogen bonded, and the spectral intensities imply that the HF axis is aligned closely to the center-of-mass axis and the NO is off axis by $30+$ or $-15 \mathrm{deg}$. The Renner-Teller-like orbital quenching parameter is somewhat larger than the spin-orbit constant in the free NO molecule and increases substantially upon vibrational excitation. The transitions in this band exhibit vibrational predissociation broadening of $200+$ or $-40 \mathrm{MHz}$ (FWHM), similar to that observed for a number of closed-shell hydrogen-bonded HF complexes.

\section{0,818}

PB94-211661 Not available NTIS

National Inst. of Standards and Technology (NML), Gaithersburg, MD. Chemical Kinetics Div.

Radiation Chemistry of Cyanine Dyes: Oxidation and Reduction of Merocyanine $\mathbf{5 4 0}$.

Final rept.

A. Harriman, and P. Neta. 1991, 6p.

Pub. in Jnl. of Physical Chemistry 95, n6 p2415-2420 1991

Keywords: "Reaction kinetics, "Oxidation reduction reactions, "Antiviral agents, "Radiolysis, "Cyanine dyes, Decomposition reactions, Free radicals, Leukemias, Reprints, "Merocyanine 540.

Merocyanine 540 (MC) shows promise as a treatment for certain types of leukemia. It is shown that MC readily undergoes one-electron reduction under pulse radiolytic conditions. The pi-radical anion, produced by reduction with hydrated electrons and 2-hydroxypropyl radicals, disproportionates rapidly under anaerobic conditions but reduces $\mathrm{O} 2$ to superoxide ions in aer- ated solution. The dye reacts with trichloromethylperoxyl radicals to form several prod ucts, one of which is believed to be an adduct formed by addition of $\mathrm{CCl} 3 \mathrm{OO}$ (dot) to the bridgehead carbonatom of the benzoxazole subunit. This species decays via first-order kinetics under pulse radiolytic conditions to form cleavage products. A second primary product is believed to arise from addition of $\mathrm{CC}$ 13O2(dot) to the polymethine chain to form an alpha-amino carbon-centered radical capable of reducing $\mathrm{O} 2$ to superoxide ions. Preliminary studies indicate that the breakdown products are cytotoxic and could be important intermediates for the known antiviral activity of MC.

\section{9}

PB94-211703 Not available NTIS

National Inst. of Standards and Technology (NML) Gaithersburg, MD. Thermophysics Div.

Supercritical Solubility of Solids from Near-Critical Dilute-Mixture Theory.

Final rept.

A. H. Harvey. $1990,4 p$

Pub. in Jnl. of Physical Chemistry 94, n22 p8403-8406 1990.

Keywords: "Critical point, *Solubility, "Supercritical fluids, "Phase diagrams, "Solids, Henrys law, Phase transformations, Thermodynamics, Reprints, "Nearcritical Dilute Mixture Theory.

A recently derived asymptotic theory for Henry's Law constants near the critical point of a solvent is adapted to the description of the solubility of solids in supercritical fluids. When solubility data for several systems are examined in the manner suggested by the theory, all the experimental iostherms for a given system (with the exception of a few near-critical points where we attribute the deviations to finite-concentration effects) collapse onto a single curve which is linear over a substantial range of solvent densities. This work provides a theoretical justification for some often-noted empirical relationships between solubilities and en hancement factors and the density of the supercritical solvent.

\section{0,820}

PB94-211810 Not available NTIS

National Inst. of Standards and Technology (NML), Gaithersburg, MD. Molecular Spectroscopy Div.

Vibrational Relaxation Measurements of Carbon Monoxide on Metal Clusters.

Final rept.

E. J. Heilweil, R. R. Cavanagh, and J. C.

Stephenson. 1988, 3p.

Pub. in Ultrafast Phenomena VI, Springer Series in Chemical Physics, v48 p447-449 1988.

Keywords: "Carbon monoxide, "Solid clusters, *Adsorbates, Picosecond pulses, Light pulses, Infrared radiation, Tunable lasers, Transition metals, Metal carbonyls, Energy transfer, Measurement, Reprints, Vibrational relaxation.

Measurements using tunable picosecond infrared pulses of $\mathrm{CO}(\mathrm{v}=1)$ vibrational population relaxation for carbon monoxide bound to transition metal cluster compounds and supported metal particles give the first time-resolved evidence for vibrational-to-electronic energy damping of adsorbates on metals.

\section{0,821}

Not available NTIS

National Inst. of Standards and Technology (MSEL) Gaithersburg, MD. Reactor Radiation Div.

Discontinuous Volume Change at the Orientational-Ordering Transition in Solid $\mathrm{C} 60$.

Final rept.

P. A. Heiney, G. B. M. Vaughan, J. E. Fischer, J. R

D. Copley, D A Neumann, W A Kamitakahara, K.

M. Creegan, D. M. Cox, J. P. McCauley, A. B. Smith, N. Coustel, and D. E. Cox. 1992, 4p

Pub. in Physical Review B 45, n8 p4544-4547, 15 Feb 92

Keywords: "Buckminsterfullerene, "Fullerenes, Crystal-phase transformations, $X$-ray diffraction, Neutron diffraction, Lattice parameters, Volume, Reprints.

$X$-ray and neutron-diffraction measurements have been used to study the evaluation of the lattice parameter of solid $C(60)$ through the orientational-ordering transition. The lattice parameter jumps by $+0.044+$ or - $0.004 \mathrm{~A}$ on heating, indicating a strongly first-order transition. The average isobaric volume thermal-expansion coefficient both above and below the transition is $6.2+$ or $-0.2 \times 10$ (sup -5 ) $/ \mathrm{K}$. We observe phase 
coexistence over a 5-K range, but little if any hysteresis.

\section{0,822}

PB94-211901 Not available NTIS

National Inst. of Standards and Technology (NML), Gaithersburg, MD. Chemical Kinetics Div.

International Conference on Chemical Kinetics (2nd). Held in Gaithersburg, Maryland on July 2427, 1989.

Final rept.

J. T. Herron, S. E. Stein, W. Tsang, and D. M.

Golden. 1990, 2p.

Pub. in Jnl. of Physical Chemistry 94, n8 p3229-3230 1990.

Keywords: "Reaction kinetics, "Meetings, Physical chemistry, Reprints

This is a brief report on 'The Second International Conference on Chemical Kinetics', held July 24-27, 1989 at NIST, Gaithersburg, MD.

\section{0,823}

PB94-212032 Not available NTIS

National inst. of Standards and Technology (NML) Gaithersburg, MD. Molecular Physics Div.

Use of Extended Permutation-Inversion Groups in Constructing Hyperfine Hamiltonians for Symmetrical-Top Internal Rotor Molecules Like H3C-SiH3. Final rept.

J. T. Hougen, W. L. Meerts, and I. Ozier. 1991, 41p. Pub. in Jnl. of Molecular Spectroscopy $146, \mathrm{n} 1 \mathrm{p} 8-48$ 1991.

Keywords: "Molecular beams, Selection rules, Hamiltonians, Silicon compounds, Reprints, Permutation groups.

The $\mathrm{m}$-fold extended group $\mathrm{G}$ (sub 18, sup $\mathrm{m}$ ), corresponding to the permutation-inversion group $\mathrm{G}$ (sub 18) for molecules like $\mathrm{H} 3 \mathrm{C}$ - $\mathrm{SiH} 3$, has been obtained In this group, $m$ is the smallest integer for which $m$ (rho) is also an "integer," where rho is the usual ratio of the moment of inertia of the top about $A$ axis to the moment of inertia of the molecule about the $A$ axis. The extended group has $18 m$ elements, divided into $(9 m+3)$ 2 or $(9 m+6) / 2$ classes, for odd and even values of $m$ respectively. It is possible to rationalize the pattern of observed and unobserved avoided-crossing signais in recent molecular beam studies of symmetric top internal rotor molecules. With the understanding, it also proved possible to detect for the first time one of the 'missing' avoided-crossing signals in $\mathrm{CH} 3 \mathrm{SiH} 3$.

00,824

PB94-212115 Not available NTIS

National Inst. of Standards and Technology (NML) Gaithersburg, MD. Chemical Kinetics Div.

Kinetics of the Reaction of $\mathrm{CCl} 3-\mathrm{Br}-2$ and the Thermochemistry of $\mathrm{CCl} 3$ Radical and Cation.

Final rept.

J. W. Hudgens, R. D. Johnson, R. S. Timonen, J. A Seetula, and D. Gutman. 1991, 6p.

Pub. in Jnl. of Physical Chemistry 95, n11 p4400-4405 1991

Keywords: "Reaction kinetics, "Free radicals, "Temperature dependence, "Thermodynamic properties, Entropy, Specific heat, Heat of formation Chemical equilibrium, Thermochemistry, Reprints, *Trichloromethyl radicals.

The rate constant of the $\mathrm{CCl} 3+\mathrm{Br} 2 \rightarrow \mathrm{CCl} 3 \mathrm{Br}+\mathrm{Br}$ reaction was determined as a function of temperature between 300 and $532 \mathrm{~K}$ and fit to an Arrhenius expression. The reaction was studied in a tubular flow reactor using laser photolysis to produce the $\mathrm{CCl} 3$ reactant and photoionization mass spectrometry to monitor $\mathrm{CCl} 3$ in time resolved experiments. Previously published kinetic data were evaluated to calculate $k$ (sub1), the rate constant for the reverse reaction, and recent spectroscopic data were used to calculate accurate entropies and heat capacities. The rate constants were used in a Third Law thermodynamic calculation to obtain values for the standard enthalpies of formation of $\mathrm{CCl} 3$ and $\mathrm{CCl} 3(1+), 17.7$ and $205.9 \mathrm{kcal} / \mathrm{mol}$, respectively.

\section{0,825}

PB94-212123 Not available NTIS

National Inst. of Standards and Technology (CSTL) Gaithersburg, MD. Chemical Kinetics and Thermodynamics Div.
Resonance Enhanced Multiphoton Ionization Detection of GeF and GeCl Radicals.

Final rept.

J. W. Hudgens, R. D. Johnson, and B. Tsai. 1989,

$4 p$.

Pub. in Inst. Phys. Conf. Ser. Reson. Ioniz. Spectrosc 94, p125-128 1989 .

Keywords: "Electron transitions, "Rydberg states, *Germanium, *Electronic spectra, Energy levels, Excited states, Chemical radicals, Laser spectroscopy, Spectrum analysis, Reprints, "Germanium fluoride radicals, "Germanium chloride radicals, Multiphoton ionization.

Resonance enhanced multiphoton ionization (REMPI) spectra of $\mathrm{GeF}$ and $\mathrm{GeCl}$ radicals were observed be iween 400-500 nm. Numerous vibrational bands of several electronic states were observed through $2+1$ $1+2$, and $1+1+1$ REMPI excitation mechanisms. The use of REMPI spectroscopy enabled observation of several one photon forbidden transitions.

00,826

PB94-212131 Not available NTIS

National Inst. of Standards and Technology (NML) Gaithersburg, MD. Chemical Kinetics Div.

Experimental and Abinitio Studies of Electronic Structures of the CCI3 Radical and Cation.

Final rept.

J. W. Hudgens, R. D. Johnson, B. P. Tsai, and S. A.

Kafafi. 1990, 10p.

Pub. in Jnl. of the American Chemical Society 112, n15 p5763-5772 1990 .

Keywords: "Electron transitions, "Rydberg states, "Electronic spectra, "Free radicals, Energy levels, Spectrum analysis, Molecular orbitals, Optical spectra Molecular structure, Reprints, "Trichloromethyl radicals, Multiphoton ionization

The structures and optical spectroscopy of the $\mathrm{CCl} 3$ radical and cation were studied by ab initio molecular orbital calculations and by experiment. The structures of the $X$ singlet $A^{\prime}$ (sub 1) state of the $\mathrm{CCl}^{3}$ cation and the $X$ doublet $A($ sub 1 ) and $X$ doublet $A($ sub 2)(sup double prime) structures of the $\mathrm{CCl} 3$ radical were optimized by ab initio calculations using the 6-31G(star) basis set. The ground state of the $\mathrm{CCl} 3$ radical is of $X$ doublet $A($ sub 1$)$ symmetry with $r($ sub e) $(C-C)=$ $1.7142 \mathrm{~A}$ and $\mathrm{Cl}-\mathrm{C}-\mathrm{Cl}$ bond angle $=117.10 \mathrm{deg}$. Vibra tional frequencies for each $\mathrm{CCl} 3$ species were computed In experiments the electronic spectrum of (35)C $\mathrm{Cl} 3$ radicals was observed between $336-440 \mathrm{~nm}$ using mass resolved resonance enhanced multiphoton ionzation (REMPI) spectroscopy. The spectrum arose from two-photon resonances with planar Rydberg states.

\section{0,827}

PB94-212164 Not available NTIS

National Inst. of Standards and Technology (CSTL) Gaithersburg, MD. Chemical Kinetics and Thermodynamics Div.

Kinetics of the Self-Reaction of Hydroxymethylperoxyl Radicals.

Final rept

R. E. Huie, and C. L. Clifton. 1993, 5p.

Pub. in Chemical Physics Letters 205, n2,3 p163-167, 9 Apr 93.

Keywords: "Reaction kinetics, "Peroxy radicals, Temperature dependence, Chemical reactions, Methanol, Reprints, "Hydroxymethyl peroxy radicals, "Methanol peroxy radical, Sulfate radicals.

The rate constant for the self-reaction of the peroxyl radical derived from methanol, $\mathrm{HOCH} 2 \mathrm{OO}($ dot), has been measured over the temperature range 5-53 C. The radical has a maximum absorptivity of $970 \mathrm{l} / \mathrm{mol} /$ $\mathrm{cm}$ at $240 \mathrm{~nm}$ and an absorptivity of about $510 \mathrm{l} / \mathrm{mol} /$ $\mathrm{cm}$ at $280 \mathrm{~nm}$. From a modified Arrhenius fit to the data, the rate constant at $25 \mathrm{C}$ was calculated to be $\mathrm{K} 298 \mathrm{~K}=((7.4+$ or -0.1$) \times 10$ to the 8th power $) \mathrm{l} / \mathrm{mol} /$ $\mathrm{s}$ and the temperature dependence of the reaction to be $E / R=1395+$ or $-66 \mathrm{~K}$ As part of this study the rate constant for the recombination of the sulfate radical, SO4(1-), was found to be $((4.4+$ or -0.4$) \times 10$ to the 8 th power) $\mathrm{l} / \mathrm{mol} / \mathrm{s}$, independent of temperature over the range 12 to $53 \mathrm{C}$.

00,828

PB94-212172 Not available NTIS

National Inst. of Standards and Technology (NML) Gaithersburg, MD. Chemical Kinetics Div.
Temperature Dependence of the Rate Constants for Reactions of the Sulfate Radical, SO4-, with Anions.

Final rept.

R. E. Huie, and C. L. Clifton. 19430, 7p

Pub. in Jnl. of Physical Chemistry' 94, n23 p8561-8567 1990

Keywords: "Reaction kinetics, "Sulfates, "Chemical radicals, "Anions, Activation energy, Temperature dependence, Electron transfer, Chemical reactions, $\mathrm{Re}-$ prints, "Sulfate radicals.

Rate constants have been measured as a function of temperature over the range 10 to $60 \mathrm{C}$ for the reactions of the sulfate radical, SO4(1-) with cizide, chloride, cyanate, cyanide, acetate, and carbonate anions. Room temperature rate constants ranged from abou $5,000,000$ to 5 times 10 to the 11 th $\mathrm{power} / \mathrm{M} / \mathrm{s}$. The variation of the rate constant with substrate depended mainly on the Arrhenius pre-exponential factor and less on the activation energy. For the reaction of SO4(1-) with $\mathrm{Cl}(1-)$, the dependence of the rate constant on ionic strength was determined to be in agreement with the theoretical prediction.

\section{0,829}

PB94-212180 Not available NTIS

National Inst. of Standards and Tec:hnology (NML), Gaithersburg, MD. Chemical Kinetics [)iv.

Electron Transfer Reaction Rates and Equilibria of the Carbonate and Sulfate Radical A nions.

Final rept.

R. E. Huie, C. L. Clifton, and P. Neta. 1991, 5p. Pub. in Radiation Physics and Chemistry 38, n5 p477 4811991

Keywords: "Electron transfer, "Oxidatior reduction re actions, "Free radicals, "Reaction kinetiss, "Chemical equilibrium, Radiolysis, Anions, Electric potential, Elec trodes, Reprints, "Carbonate radicals, "Sulfate radicals, Reduction potentials.

Electron transfer reactions of the carbonate radical anion with azide, bromide, and hypochlorite ions have been studied by pulse radiolysis. From the equilibrium $\mathrm{Br} 2(1-)(\mathrm{dot})+\mathrm{CO} 3(2-)=2 \mathrm{Br}(1-)+\mathrm{CO} 3(-)(\mathrm{dot})$ and the known redox potential for the $\mathrm{Br} 2(1-)(\mathrm{dot}), 2 \mathrm{Br}(1-)$ hal reaction, the redox potential for the $\mathrm{ClO}(1 \mathrm{dot}) / \mathrm{ClO}(1-)$ half reaction was derived. The equilibrium reaction $\mathrm{SO} 4(1-)($ dot $)+\mathrm{Cl}(1-)=\mathrm{SO} 4(2-)+\mathrm{Cl}($ dot $)$ vvas studied by laser photolysis using $284 \mathrm{~nm}$ excitation of $\mathrm{S} 208(2$ ) to produce $\mathrm{SO} 4(1-)$ (dot) by examining the rate of re action of this radical with $\mathrm{Cl}\left(1_{-}\right)$in the presence of vary ing concentrations of SO4(2-). By modeling the reactions occurring in this system, the rate coristant and equilibrium constant for the reaction of $\mathrm{Cl}$ (dot) with SO4(2-) were derived.

00,830

PB94-212198 Not available NTIS

National Inst. of Standards and Technology (CSTL) Gaithersburg, MD. Chemical Kinetics and Thermodynamics Div.

Reaction of NO with Superoxide.

Final rept.

R. E. Huie, and S. Padmaja. 1993, 5p

Sponsored by Department of Energy, Washington, DC Pub. in Free Rad. Res. Comms. 18, n4 p195-19() 1993.

Keywords: "Reaction kinetics, "Nitric oxide *Superoxide radicals, Free radicals, Catalysts. Formates, Reprints, "Peroxynitrite radicals.

The rate constant for the reaction of NO witl the superoxide anion was determined to be $((6.7+$ or - 0.9$)$ $X 10$ to the 9 th power $) \mathrm{l} / \mathrm{mol} / \mathrm{s}$, considerably higher than previously reported. Rate measurements were made from pH 5.6 to 12.5 both by monitoring the loss of the superoxide anion and the formation of the procuct peroxynitrite radical. The decay rate of the peroxynitrite radical, in the presence of $0.1 \mathrm{~mol} / /$ formate, ranges from $1.2 / \mathrm{s}$ at $\mathrm{pH} 5$ to about $0.2 / \mathrm{s}$ in strong base, the latter value probably reflecting catalysis by formate.

\section{0,831}

PB94-212206 Not available NTIS

National Inst. of Standards and Technology (NML) Gaithersburg, MD. Chemical Kinetics Div.

Temperature Dependence of the Rate Constants for Reactions of the Carbonate Radical with Oi. ganic and Inorganic Reductants.

Final rept.

R. E. Huie, L. C. T. Shoute, and P. Neta. 1991, 12p.

Pub. in International Jnl. of Chemical Kinetics $23, \mathrm{n} 6$ p541-552 1991 . 
Keywords: "Reaction kinetics, "Chemical radicals, *Carbonates, "Anions, "Temperature dependence, *Organic ions, Activation energy, Oxidation reduction reactions, Radiolysis, Reprints, “Carbonate radicals.

Rate constants have been measured by pulse radiolysis for the reactions of the carbonate radical with a number of organic and inorganic reactants as a function of temperature, generally over the range 5 to 80 C. The reactants include the substitution-inert cyano complexes of $\mathrm{Fe}(\mathrm{II}), \mathrm{Mo}(\mathrm{IV})$, and $\mathrm{W}(\mathrm{IV})$, the simple inorganic anions $(\mathrm{SC} 3)(2-),(\mathrm{ClO} 2)(1-),(\mathrm{NO} 2)(1-), 1(1-)$ SCN (1-), several phenolates, ascorbate, tryptophane, cysteine, cystine, methionine, triethylamine, and allyl alcohol. The measured rate constants ranged from less than 100,000 to $(3 \times 10$ to the 9 th $p o w e r) / M / s$ and the activation enelgies ranged from -11.4 to $18.8 \mathrm{~kJ} /$ mol. The activation energies for the metal complexes and inorganic anicons generally decrease with increasing driving force for the reaction, as expected for an outer sphere electron transfer. For highly exothermic reactions, however, the activation energy appears to increase, probably reflecting the temperature dependence of diffusion. For many of the organic reactants, the activation energies were low and independent of driving force, suggesting that the oxidation is via an inner sphere meshanism.

\section{0,832}

PB94-212297 Not available NTIS

National Inst. of Standards and Technology (CSTL), Gaithersburg, MD. Surface and Microanalysis Science Div.

Formalism arıd Parameters for Quantitative Surface Analysis by Auger Electron Spectroscopy and $X$-Ray Photoelectron Spectroscopy.

Final rept.

A. Jablonski, and C. J. Powell. 1993, 16p.

Pub. in Surfaice and Interface Analysis 20, p771-786 1993.

Keywords: "Surface analysis, X-ray photoelectron spectroscopy, Auger electron spectroscopy, Elastic scattering, Electron scattering, Electron transport, Quantitative: analysis, Reprints.

It has been realized during the last 5 years that quantitative surfiace analyses by Auger electron spectroscopy (AE:3) and $x$-ray photoelectron spectroscopy elastic elesctron greatly complicated by the effects of viously besen largely ignored. We review here the effects of elastic scattering in the formalism of quantitative su rface analysis by AES and XPS and discuss the vario'us definitions of terms (inelastic mean free path, attennuation length and escape depth) that have been useed to describe inelastic scattering and the surface serisitivities of various electron spectroscopies. We show how a realistic theoretical model of electron transport that includes elastic electron scattering can be related, for some analytical situations, to the common sirnple formalism of AES and XPS in which elastic scattering is neglected. We consider specifically measurements of overlayer thickness, determination of surface cromposition for a homogeneous surface region and esitimation of the average depth of analysis and we inclicate which parameter describing inelastic scattering can be used in the simple formalism for these appliciations.

\section{$00,8: 33$}

PB94-212305 Not available NTIS

National Inst. of Standards and Technology (MSEL), Gaithersburg, MD. Polymers Div.

Glass Transition of Organic Liquids Confined to Small Pores.

Final rept.

C. L. Jackson, and G. B. McKenna. 1991, 4p

Pub. in Jnl. of Non-Crystalline Solids 131, p221-224 1991.

Kezywords: *Organic compounds, *Porous materials, "Cilass, "Glass transition temperature, Phase transfermations, Benzyl alcohols, Calorimetry, Reprints, "Organic liquids, o-Terphenyl.

The glass transition temperatures, $\mathrm{Tg}$, of organic liquids confined to small pores were studied by differential scanning calorimetry (DSC). The Tg was measured as a function of pore size in controlled pore glasses having pore diameters in the range of $40-730 \mathrm{~A}$. The surface of the glass was treated with hexamethyldisilazane to promote wetting by the organic liquids studied (o-terphenyl and benzyl alcohol). Glasses formed in the pores had a lower Tg than in the bulk and the reduction in Tg increased as the pore size decreased. For example, the depression of the glass transition temperature, delta $\mathrm{Tg}$, of benzyl alcohol in $40 \mathrm{~A}$ and $85 \mathrm{~A}$ pores was $7.2 \mathrm{~K}$ and $3.1 \mathrm{~K}$ respectively. The magnitude of delta $\mathrm{Tg}$ also depends on the material, e.g. for o-terphenyl in the $85 \mathrm{~A}$ pores delta Tg was $8.8 \mathrm{~K}$ versus $3.1 \mathrm{~K}$ for benzyl alcohol. In general, it was noted that delta $\mathrm{Tg}$ was considerably less than for the depression of the crystalline melting point delta Tm, studied in related work. For example, for benzyl alcohol in the $85 \mathrm{~A}$ pores, delta Tm was approximately $25 \mathrm{~K}$ and delta $\mathrm{Tg}$ was approximately $3 \mathrm{~K}$.

00,834

PB94-212313 Not available NTIS

National Inst. of Standards and Technology (MSEL), Gaithersburg, MD. Polymers Div.

Melting Behavior of Organic Materials Confined in Porous Solids.

Final rept.

C. L. Jackson, and G. B. McKenna. 1990, 10p.

Pub. in Jnl. of Chemical Physics 93, n12 p9002-9011 1990.

Keywords: "Melting points, "Organic compounds, "Porous materials, "Glass, Phase transformations, Heat of fusion, Cyclohexane, Benzene, Naphthalene, Heptanes, Chlorobenzenes, Reprints, *Melting point depression, "Organic liquids, Crystal size.

The solid-liquid phase transition temperatures and heats of fusion (HOF), of non-polar organic solids confined in the pores of controlled pore glasses were measured by differential scanning calorimetry. The pore diameters, $d$, were in the range of $40-730 \mathrm{~A}$ and the organics studied were cis-decalin, trans-decalin, cyclohexane, benzene, chlorobenzene, naphthalene and heptane. In accordance with previous reports on studies of inorganic materials, the melting point of the pore solid, $T(d)$, decreased with decreasing pore diameter. In addition, a large reduction in the bulk HOF of the pore solid was measured, which has not been studied in detail by other workers. A linear correlation was found between the melting point depression and the reciprocal diameter, in accordance with the GibbsThomson equation. The calculated values of the solid liquid interfacial energy, using a modification of the Gibbs-Thomson equation were in good agreement with values reported in the literature based on other methods of measurement.

00,835

PB94-212487 Not available NTIS

National Inst. of Standards and Technology (NML), Gaithersburg, MD. Chemical Kinetics Div.

Multiphoton Ionization of $\mathrm{SiH} 3$ and SiD3 Radicals. 2. Three Photon Resonance-Enhanced Spectra Observed between 460 and $610 \mathrm{~nm}$.

Final rept

R. D. Johnson, and J. W. Hudgens, 1991, 10p

Pub. in Jnl. of Chemical Physics 94, n8 p5331-5340 1991.

Keywords: "Electron transitions, "Rydberg states, *Electronic spectra *Free radicals, Energy levels, Vibrational spectra, Isotope effect, Spectrum analysis, Reprints, *Silyl radicals, Multiphoton ionization.

The electronic spectra of silyl radicals, $\mathrm{SiH} 3$ and $\mathrm{SiD} 3$ were observed between 460-610 nm $(49,200-65,200$ $\mathrm{cm}$ ) by resonance enhanced multiphoton ionization (REMPI) mass spectroscopy. The spectra were pro duced through a $3+1$ REMPI mechanism. Spectra of four new planar Rydberg states were observed and as signed. In $\mathrm{SiH} 3$ the observed states and spectroscopic constants are $D$ doublet $A^{\prime}($ sub 1) (3d): To = $49787(30)$, omega(sub 2) $=808(31) / \mathrm{cm}$; I' $(4 \mathrm{~d})$ : To $=$ $56253(30)$, omega(sub 2) $=814(25) / \mathrm{cm} ; \mathrm{J}$ (4d): To $57726(30)$ omega(sub 2) $=835(26) / \mathrm{cm}$; and $\mathrm{K}^{\prime}(5 \mathrm{~d})$ : To $=59615(30) / \mathrm{cm}$, omega $($ sub 2) $=835(26) / \mathrm{cm}$. In SiD3, the observed states and spectroscopic constants are $D$ doublet $A^{\prime}($ sub 1) (3d): To $=49787(30)$, omega $($ sub 2) $=604(28) / \mathrm{cm}$; l' (4d): To = 56253(30) omega(sub 2) $=604(17) / \mathrm{cm}$; and J' $(4 \mathrm{~d})$ : To $=$ $57726(30)$, omega $($ sub 2) $=599(20) / \mathrm{cm}$.

\section{0,836}

PB94-212495 Not available NTIS

National Inst. of Standards and Technology (NML), Gaithersburg, MD. Chemical Kinetics Div.

New Electronic States of NH and ND Observed from 258 to $288 \mathrm{~nm}$ by Resonance Enhanced Multiphoton Ionization Spectroscopy.

Final rept.

R. D. Johnson, and J. W. Hudgens. 1990, 6p.

Pub. in Jnl. of Chemical Physics 92, n11 p6420-6425 1990.
Keywords: "Electron transitions, ${ }^{\star} R y d b e r g$ states, "Electronic spectra, "Free radicals, Energy levels, Excited states, Rotational spectra, Vibration, Photolysis Hydrazoic acid, Spectrum analysis, Reprints, *Imidogen radicals, Multiphoton ionization.

Four new electronic states of NH and ND have been observed and analyzed by REMPI (Resonance Enhanced Multiphoton Ionization) Spectroscopy in the region of 258 through $288 \mathrm{~nm}$. The NH (ND) was produced by the photolysis of HN3 (DN3) (hydrazoic acid) in the same wavelength region. The observed two-photon transitions are from the a singlet delta state to $3 \mathrm{~s}$ and $3 p$ Rydberg states. The new state assignments are : e singlet $\mathrm{Pi}$ (3s sigma) at $82857 / \mathrm{cm}$, f singlet $\mathrm{Pi}$ (3p sigma) at $86378 / \mathrm{cm}$, g singlet delta (3p pi) at $88141 / \mathrm{cm}$, and $\mathrm{h}$ singlet Sigma (3p pi) at $89151 / \mathrm{cm}$. Rotational constants (B and D) and, where possible, vibrational spacings for the twelve observed bands are also determined.

00,837

PB94-212503 Not available NTIS

National Inst. of Standards and Technology (NML), Gaithersburg, MD. Chemical Kinetics Div.

Multiphoton lonization of $\mathrm{SiH} 3$ and SiD3 Radicals: Electronic Spectra, Vibrational Analyses of the Ground and Rydberg States, and lonization Potential.

Final rept.

R. D. Johnson, B. P. Tsai, and J. W. Hudgens. 1989, 20p.

Pub. in Jnl. of Chemical Physics 91, n6 p3340-3359, 15 Sep 89.

Keywords: "Electron transitions, *Rydberg states, "Electronic spectra, "Free radicals, Energy levels, Spectrum analysis, Reprints, ”Silyl radicals.

The electronic spectra of silyl radicals, $\mathrm{SiH} 3$ and SiD3, were observed between 310 and $430 \mathrm{~nm}(46,000$ $64,000 / \mathrm{cm})$ by resonance enhanced multiphoton ionization (REMPI) mass spectroscopy The spectra were generated through a $2+1$ REMPI mechanism. Two Rydberg series originating from planar, $\mathrm{D}$ (sub $3 \mathrm{~h}$ ) point group states were observed. One series, of quantum defect delta $=1.45(2)$, is comprised of the $E$ doublet $A$ (sub 2)(sup double prime) (4p), J doublet A(sub 2) (sup double prime) (5p), and $M$ doublet $A$ (sub 2)(sup double prime) (6p) Rydberg states which have origins at nu(sub $0-0)=48,438,56,929$, and $60,341 / \mathrm{cm}$ in $\mathrm{SiH} 3$ and at nu(sub o-0) $=48,391,56,874$, and $60,267 /$ $\mathrm{cm}$ in SiD3. In SiD3, the P doublet A(sub 2) (sup double prime) (7p) Rydberg origin was observed at nu(sub oo) $=62,002 / \mathrm{cm}$. The $\mathrm{H}, \mathrm{K}$ and $\mathrm{N}$ states observed in the SiD3 spectrum comprise the second Rydberg series, delta $=2.09$, and were tentatively assigned as ns doublet $A^{\prime}($ sub 1) Rydberg states $(n=5,6,7)$.

\section{0,838}

PB94-212792 Not available NTIS

National Inst. of Standards and Technology (PL), Gaithersburg, MD. Molecular Physics Div.

Product Kinetic Energies, Correlations, and Scattering Anisotropy in the Bimolecular Reactor $\mathrm{O}((1) \mathrm{D})+\mathrm{H} 2 \mathrm{O}$ yields $2 \mathrm{OH}$.

Final rept.

D. S. King, D. G. Sauder, and M. P. Casassa. 1992, $4 p$.

Sponsored by Air Force Office of Scientific Research, Bolling AFB, DC

Pub. in Jnl. of Chemical Physics 97, n8 p5919-5922, 15 Oct 92

Keywords: *Hydroxyl radicals, Chemical reactions, Internal energy, Kinetic energy, Oxygen 16, Oxygen 18, Anisotropy, Correlations, Ozone, Water, Reprints, Doppler spectroscopy.

Doppler spectroscopy of the (16) $\mathrm{OH}$ and (18)OH products of the (16) O(singlet $\mathrm{D})+\mathrm{H} 2(18) \mathrm{O}$ reaction reveals scattering anisotropy: (16) $\mathrm{OH}$ scatters in the hemisphere containing the (16) O-atom velocity vector. Internal energies of geminate $\mathrm{OH}$ fragments are correlated: fragments of high internal energy form with cofragments of low internal energy.

\section{0,839}

PB94-212826 Not available NTIS

National Inst. of Standards and Technology (NML), Gaithersburg, MD. Center for Atomic, Molecular and Optical Physics. 
Molecular-Beam Optothermal Spectrum of the $\mathrm{OH}$ Stretching Band of Methanol.

Final rept.

I. Kleiner, G. T. Fraser, J. T. Hougen, and A. S. Pine. 1991, 18p

Pub. in Jnl. of Molecular Spectroscopy 147, n1 p1551721991.

Keywords: *Methanol, Molecular spectroscopy, Molecular beams, Perturbation, Line width, Reprints, Vibrational relaxation.

The $\mathrm{OH}$ stretching fundamental band, nu(1), of $\mathrm{CH} 3 \mathrm{OH}$ has been recorded with sub-Doppler resolution using a molecular-beam optothermal spectrometer. The low effective temperature (about $6 \mathrm{~K}$ ) of the molecular beam greatly reduces the spectral congestion from hot torsional vibrations and high rotational levels seen at room temperature, permitting a reasonably complete assignment, a fit to a global hindered internal rotor Hamiltonian, and identification of several perturbations and their likely perturbers. The observed inewidths are about $15 \mathrm{MHz}$, yielding an upper limit to any intramolecular vibrational relaxation rate.

\section{0,840}

PB94-212834 Not available NTIS

National Inst. of Standards and Technology (NML), Gaithersburg, MD. Molecular Physics Div.

Fundamental Torsion Band in Acetaldehyde.

Final rept

Kleiner, M. Godefroid, M. Herman, and A. R. W. McKellar. 1990, 16p.

Pub. in Jnl. of Molecular Spectroscopy 142, n2 p2382531990.

Keywords: "Acetaldehyde, Far infrared radiation, Infraed spectra, Band spectra, Rotational states, High resolution, Microwave spectra, Reprints, Fourier transform spectroscopy.

High resolution and low temperature experimental conditions allowed us to carry out the rotational analysis of the nu(15) fundamental band of acetaldehyde, observed around $150 / \mathrm{cm}$. Some 1,000 lines of $A$ and $E$ ypes have been assigned to the main band and some 90 A-type lines have been identified in the first overtone of the torsion mode. A simultaneous fit, using the non rigid internal axis method, of a very severely se. lected set of unblended FIR data, together with microwave data published in the literature, allowed us to obtain accurate molecular parameters describing internal and overall rotation in acetaldehyde. In particular, the Fourier coefficients $V(3)$ and $V(6)$ of the barrier for internal rotation as well as the rotationless origins of the nu(15) fundamental band and its first overtone were determined with improved accuracy, compared to the data previously available in the literature. Some difficulties in treating $v(t)=1$ microwave data lead us to suspect problems in the theoretical model adopted or in the present $v(t)=1$ microwave data set.

00,841

PB94-212891 Not available NTIS

National Inst. of Standards and Technology (CSTL), Gaithersburg, MD. Thermophysics Div.

Determination of Osmotic Pressure and Fouling Resistances and Their Effects on Performance of Ultrafiltration Membranes.

Final rept.

M. K. Ko, and J. J. Pellegrino. 1992, 17

Pub. in Jnl. of Membrane Science 74, p141-157 1992.

Keywords: *Osmotic pressure, *Ultrafiltration, *Artificial membranes, "Proteins, Adsorption, Bovine serum albumin, Lactoglobins, pH, Sodium chloride, Polarization, Reprints, "Fouling resistance.

A study was made of the effects of material and geometric properties of ultrafiltration (UF) membranes on flux decline due to concentration-polarization (osmotic pressure) and adsorption (fouling) resistances. UF experiments with solutions of beta-lactoglobulin and bovine serum albumin on commercial membranes of poly(vinylpyrrolidone)-coated polycarbonate (PC) and regenerated cellulose (RC) were carried out. $A$ threestage experimental strategy based on an osmotic pressure-adsorption model was used to separately determine the effective hydraulic membrane resistance osmotic pressure, and fouling resistance. By a normalization procedure, the performance of membranes with varying material and geometric characteristics could be compared and evaluated in terms of the extent of flux reduction individually due to concentration-polarization and fouling. Under all the conditions studied, the total flux reduction for the $P C$ membrane was main- ly controlled by the fouling resistance and for the RC membrane by the osmotic pressure build-up due to concentration-polarization. The effect of bulk protein concentration, transmembrane pressure, crossflow velocity, $\mathrm{pH}$, and $\mathrm{NaCl}$ concentration on the individual, relative contributions to flux reduction by osmotic pressure and fouling were found to depend on membrane material properties.

\section{0,842}

\section{PB94-212909 Not available NTIS}

National Inst. of Standards and Technology (CSTL), Gaithersburg, MD. Thermophysics Div.

Characterization of the Adsorption-Fouling Layer Using Globular Proteins on Ultrafiltration Membranes.

Final rept.

M. K. Ko, J. J. Pellegrino, R. D. Nassimbene, and P. Marko. 1993, 20p.

Pub. in Jnl. of Membrane Science 76, p101-120 1993

Keywords: "Adsorption, "Ultrafiltration, "Proteins, *Artificial membranes, Lactoglobins, Bovine serum albumin, Osmotic pressure, Reprints, "Fouling resistance.

The mechanisms of membrane fouling and the effects of properties and structure of the adsorbed-protein layer on membrane fouling were studied using bovine serum albumin (BSA) and beta-lactoglobulin (beta-LG) as test proteins, and poly(vinylpyrrolidone)-coated polycarbonate (PC) and regen membranes. Ultrafiltration (UF) experiments were modeled with a 3-parameter resistance model to separately determine resistances due to osmotic pressure and adsorption. An analytical method was also developed to determine protein loadings under UF and static conditions. The dynamic behavior of osmotic pressure or interfacial concentration is discussed on the basis of the material balance around the interface. Derjaguin-Landau and Verwey-Overbeek (DLVO) theory and the concept of a rate-limiting step are applied to phenomena that occur at the interface during protein adsorption. A steric hindrance repulsion due to PVP tails on the PC membrane has a pronounced effect on protein loading. The hydrophilic matrix of RC may act as a water reservoir that maintains continuous hydration of the adsorbed layer, which can be dehydrated during protein adsorption. We believe that hydration results in a lower fouling resistance. Additionally, the size of the pores relative to the protein molecule has a pronounced effect on the properties and structure of the adsorbed layer and its fouling resistance.

\section{0,843}

\section{PB94-212974 Not available NTIS}

National Inst. of Standards and Technology (NML), Gaithersburg, MD. Molecular Physics Div.

Active Site lonicity and the Mechanism of Carbonic Anhydrase.

Final rept

M. Krauss, and D. Garmer. 1991, 10p.

Pub. in Jnl. of the American Chemical Society $113, \mathrm{n} 17$ p6426-6435 1991.

Keywords: *Carbonic anhydrase "Surface properties, Ligands, Protons, Hydrolysis, Bicarbonates, Mathematical models, Bonding strength, Reprints, "Active site ionicity, "Cluster models.

We report ab initio calculations on a cluster model for the active site of the enzyme carbonic anhydrase which also considers the effect of the ionicity of the site on the energetics of ligand binding and reaction behavior. Hydrolysis occurs by a direct attachment of the $\mathrm{Zn}$ bound hydroxyl oxygen on the carbon atom of $\mathrm{CO} 2$ with a small activation energy. The final product state in the cluster model has bicarbonate bound by one short and one long metal to oxygen bond. Detailed mechanisms for the initial attachment of $\mathrm{OH}(1-)$ on $\mathrm{CO} 2$ and proton movement out to the free oxygen atom were studied by locating intermediates and transition states. Two mechanisms for rearrangement of bound bicarbonate to its most stable form are found to have low barriers. The first involves rocking of the bicarbonate from the initial intermediate. The second accomplishes this shift by a cycle exchange of protons with a hydrogen-bound water or Thr-119. Both pathways with a small barrier may be required to explain (18)O exchange experiments.

\section{0,844}

\section{PB94-212982 Not available NTIS}

National Inst. of Standards and Technology (NML), Gaithersburg, MD. Molecular Physics Div.
Dipole Moments in Rare Gas Interactions.

Final rept.

M. Krauss, and B. Guillot. 1989, 3p.

Pub. in Chemical Physics Letters 158, n1-2 p142-144

1989

Keywords: *Rare gase's, "Dipole moments, Self-consistent field, Atomic interactions, Perturbation theory, Dimers, Trimers, Neon, Argon, Krypton, Xenon, Reprints.

A hybrid model is usedl to calculate the dipole moments of rare gas dimers and trimers. The exchange and polarization interactions are obtained with a single-configuration self-consistent-field (SCF) calculation and the dispersion contribution to the dipole obtained by perturbation theory. The SCF dipoles for the trimers are found to compare well with values modeled by an exchange quadrupole induced dipole mechanism. The dispersion dipole for the Arkr dimer is found to be significant compared to the exchange contribution even to the region of the: equilibrium separation. Values of the $\mathrm{B}$ hyperpolarizability are evaluated for $\mathrm{Ne}, \mathrm{Ar}, \mathrm{Kr}$. and $\mathrm{Xe}$ which allow the perturbation calculation of the dispersion dipole for any pairs of rare gas atoms.

\section{0,845}

\section{PB94-212990 Not available NTIS}

National Inst. of Standards and Technology (NML), Gaithersburg, MD. Molecular Physics Div.

Analysis of Prot.ein Metal Binding Selectivity in a Cluster Model.

Final rept.

M. Krauss, and W. J. Stevens. 1990, 7p.

Pub. in Jnl. of the American Chemical Society 112, n4 p1460-1466 1990

Keywords: "Calcium ions, "Molecular orbitals, * Subtilisins, Clusiters, Water Formamides, Formates, Proteins, Ligands, Hydrations, Cations, Reprints, "Metal-ligand binding, Binding sites.

$A b$ initio molecsular orbital calculations of the binding energy of metall cations to octahedral clusters of water formamide, and formate ligands are used to analyze $\mathrm{Ca}$ binding sittes in proteins. The intrinsic energetics of the first coordination shall provide a basis for evaluating the conformation behavior and the selectivity of cation binding. The enthalpies of binding are modeled by estimatingy the environmental polarization energy relative to the model cluster of the first shell. Cluster reaction enthalpies are calculated for transferring $\mathrm{Mg}$, $\mathrm{Ca}$, and $\mathrm{Na}$ cations from a water cluster to the protein model cluster as a function of cluster size which is found to strongly affect cation binding selectivity between catioris of the same and different charges. The selectivity is a function of both steric and electrostatic interactions. As is well known, selectivity between cations of thie same charge is dependent on steric factors but there is also an electrostatic component independent of steric influences which is selective between cations of clifferent charge. The data is applied to an analysis of the binding sites in the protein, Subtilisin BPN.

\section{0,846}

PB94-213006 Not available NTIS

National Inst. of Standards and Technology (NML), Gaithersburg, MD. Molecular Physics Div.

Final rept.

M. Krausis, and W. J. Stevens. 1990, 10p.

Pub. in .InI. of Chemical Physics 93, n12 p8915-8924 1990

Keywords: "Solid clusters, "Gallium arsenides, "Cesium, Van der Waals forces, Polarization characteristics, Charge transfer, Surface reactions, Reprints. The biriding of $\mathrm{Cs}$ atoms to cluster models of the (110) surface of GaAs is examined for the polarization charge transfer, and dispersion interactions. Singleconfiguration self-consistent-field binding energies to GaAs per Cs atom for two or three Cs atom clusters are calculated to be less than $0.1 \mathrm{eV}$. Orbital and tota charge density plots exhibit a polarized alkali valence charge weakly bound between the alkali atoms and to the Ga atom. A London analysis of the correlation energy between the Cs and GaAs clusters or van de Waals interaction energy finds it is large compared to the difference in binding energies between gas-phase or polyhedral clusters and the quasi-linear clusters that are experimentally observed on the GaAs surface. The large VDW energy is due to the large polarizabilities for quasi-linear chains of Cs atoms those Iongitudinal component increases approximately with the square of the chain length. 
00,847

PB94-213071 Not available NTIS

National Inst. of Standards and Technology (NML), Gaithersburg, MD. Molecular F'hysics Div.

Spectroscopic Constants for the 2.5 and $3.0 \mathrm{mi}$ crometer Bands of Acetylene.

Final rept.

W. J. Lafferty, and A. S. Pine. $1990,8 p$.

Pub. in Jnl. of Molecular Spectroscopy 141, n2 p2232301990.

Keywords: "Acetylene, Near infrared radiation, Laser spectroscopy, Infrared spectrosicopy, Band spectra, Rotational states, Vibrational states, Reprints, Fourier transform spectroscopy.

Fourier-transform interferometer and difference-frequency laser spectra of acetylene have been recorded in the 2.5 and 3.0 micrometer regions, yielding improved rotation-vibration constants for the nu2+nu4+nu5, for their associated hot bands originating in the nu 4 and nu5 bends, and for the $\mathrm{C}-\mathrm{H}$ stretchbend combination bands. An analysis of the Fermi resonance gives more reliable estimates for the deperturbed constants and the antrarmonic coupling.

\section{0,848}

PB94-213139 Not available NTIS

National Inst. of Standards and Technology (PL), Boulder, CO. Quantum Physics Div.

Laser-Induced Fluorescence Measurements of Rotationally Resolved Velocity Listributions for $\mathrm{CO}(+)$ Drifted in $\mathrm{He}$.

Final rept.

C. P. Lauenstein, M. J. Bastian, V. M. Bierbaum, S.

M. Penn, and S. R. Leone. 1991, 9p.

Contract AFOSR89-0073

Sponsored by Air Force Office of Scientific Research,

Bolling AFB, DC. and National Science Foundation,

Washington, DC.

Pub. in Jnl. of Chemical Physics 94, n12 p7810-7818, 15 Jun 91

Keywords: "Carbon monoxide, Laser induced fluorescence, Rotational states, Molecular ions, Ionic mobility, prints.

Measurements of ion-velocity distributions of $\mathrm{CO}(1+)$ in a $\mathrm{He}$ buffer gas are presented as a function of an applied electric field. The distributions are: obtained by single frequency, laser-induced fluorescence from various initial rotational states with the laser loeam propagating parallel and perpendicular to the drift velocity vector. All distributions are well represented by a Maxwellian for the observed $\mathrm{E} / \mathrm{N}$ range of $0-13 \mathrm{Td}$. From the width of the Doppler profiles, translational 'temperatures' are calculated, which are compared to simple attractive and repulsive Maxwell models as a function of the field. The measured values disagree with the predictions, which are well established for atomic ion systems. The differences are discussed in terms of rotationally inelastic energy transfer in the collisions, which is predicted by kinetic theory models.

\section{0,849}

PB94-213295 Not available NTIS

National Inst. of Standards and Technology (NML), Gaithersburg, MD. Electron and Optical Phy.sics Div. RIS Studies of Autoionization in Calcium.

Final rept.

Q. Li, T. J. Mcllrath, E. B. Saloman, and T. B.

Lucatorto. 1991, 4p.

Pub. in Institute of Physics, Conference Series on Resonance Ionization Spectroscopy, v114 p55-58 1:991.

Keywords: "Autoionization, "Calcium, Resonance ionization mass spectroscopy, Time-of-flight spectrometers, Laser isotope separation, Atomic energy levels, Photoionization, Reprints, Two photon processes.

Autoionization levels belonging to configurations of $3 p(6) 3 \mathrm{dnl}$ are being studied as part of an effort to find efficient ionization pathways applicable to ultrasensitive isotopic analysis and laser isotope $\mathrm{s}$ : ration. The studies are performed in a RIMS (RESOnance Ionization Mass Spectroscopy) apparatus vvith a time-of-flight mass spectrometer and simple therrnal filament atomization source. Estimates of two-photon transition rates and excited state photoionization cross sections show that the $4 S(2)$ singlet $S(0)-\cdots>-\cdot>3045 x$ singlet $D(2)-\rightarrow 3 d 7 p$ singlet $P(1)$ (autoionizing) ionization pathway might be efficient enough to allow effective isoptically selective ionization with $\mathrm{cw}$ Ti:sapphire lasers.
00,850

PB94-216058 Not available NTIS

National Inst. of Standards and Technology (PL), Boulder, CO. Quantum Physics Div

Spectroscopic Puzzle in ArHF Solved: The Test of a New Potential.

Final rept.

C. M. Lovejoy, J. M. Hutson, and D. J. Nesbitt. 1992 $10 \mathrm{p}$.

Pub. in Jnl. of Chemical Physics 97, n11 p8009-8018, 1 Dec 92.

Keywords: "Argon complexes, Van der Waals forces, Infra red spectroscopy, Intermolecular forces, High resolution, Predissociation, Reprints, "Hydrogen fluoride complexes, Supersonic expansion, Potential energy surfaces.

The perturbed $(n u, b, k, n)=(1210),(1113)<-(0000)$ band of ArHF is observed in a tunable laser/slit supersonic expansion spectrometer. The (1210) level correlates with $\mathrm{j}=2$ rotation of the $\mathrm{HF}$ within the complex and therefore provides a test of high-order terms in a Legendre expansion of the intermolecular potential. Transitions to (1113) are observed due to intensity sharing with (1210), induced by a strong homogeneous ( $J$-independent) perturbation that is analyzed quantitatively. The (1113) level has three quanta of Van der Waals stretch and thus probes the radial dependence of the potential close to the dissociation limit. The vibrational and rotational assignment is made possible by predictions based on the new $\mathrm{H} 6(4,3,2)$ intermolecular potential of Hutson (J. Chem. Phys. 96 6752 (1992)), which agree nearly quantitatively with experiment.

00,851

PB94-216108 Not available NTIS

National Inst. of Standards and Technology (CSTL), Boulder, CO. Thermophysics Div.

Modified Leung-Griffiths Model of Vapor-Liquid Equilibrium: Extended Scaling and Binary Mixtures of Dissimilar Fluids.

Final rept.

J. J. Lynch, and J. C. Rainwater. 1992, 15p.

Pub. in Fluid Phase Equilibria 75, p23-37 1992. Sponsored by Department of Energy, Washington, DC

Keywords: "Liquid-vapor equilibrium, "Carbon dioxide "Butanes, Binary mixtures, Simplex method, Critical point, Reprints, Leung-Griffiths model, Wegner correction.

Extended scaling or the Wegner correction has been added to a model of vapor-liquid equilibrium (VLE) designed for the extended critical region. The model, that of Leung and Griffiths as modified by Moldover, Rainwater and co-workers, has heretofore incorporated only simple scaling. The revised formalism has been applied to the abundant data on carbon dioxide $+n-$ butane where versions of the model with and without extended scaling have been optimized by the simplex method. With extended scaling, the average thermodynamic 'distance' between a measured and cal culated dew or bubble point decreases by approximately 30 percent.

\section{0,852}

PB94-216165 Not available NTIS

National Inst. of Standards and Technology (CSTL), Gaithersburg, MD. Surface and Microanalysis Science Div.

Structure of Molecules on Surfaces as Determined Using Electron-Stimulated Desorption.

Final rept.

T. E. Madey, S. A. Joyce, and A. L. Johnson. 1990,

$18 \mathrm{p}$.

Pub. in Interaction of Atoms and Molecules with Solid Surfaces, Chapter 14, p459-476 1990.

Keywords: "Surface chemistry, "Molecular structure, Reprints, Electron stimulated desorption.

The structure of molecules on surfaces is an area of great importance in surface science, and a variety of surface-sensitive methods have been applied to structural problems. Many of these techniques (including Angle Resolved Ultraviolet Photoemission Spectroscopy (ARUPS) $X$-ray absorption near-edge structure (XANES), surface extended $X$-ray absorption fine structure (EXAFS), high-resolution electron energy loss spectroscopy (HREELS), and photoelectron diffraction (PD)) are discussed in other chapters in this volume.

\section{0,853}

PB94-216173 Not available NTIS
National Inst. of Standards and Technology (NML), Gaithersburg, MD. Surface Science Div.

Desorption Induced by Electronic Transitions. Final rept.

T. E. Madey, S. A. Joyce, and J. A. Yarmoff. 1990,

Pub. in Springer Ser. Surf. Sci. Chem. Phys. Solid Surf. 8, v22 p55-68 1990

Keywords: "Surfaces, "Desorption, Electron transitions, Electron beams, Radiation damage, Reprints, Electron stimulated desorption, Photon stimulated desorption.

We summarize the principles and mechanisms of DIET (desorption induced by electronic transitions) with emphasis on ESD (electron stimulated desorption) and PSD (photon stimulated desorption). We describe applications of ESDIAD (electron stimulated desorption ion angular distributions) to surface structure, and provide examples of electron beam damage at surfaces.

00,854

PB94-216199 Not available NTIS

National Inst. of Standards and Technology (NML), Boulder, CO. Thermophysics Div.

Thermophysical Properties of $\mathrm{CO} 2$ and $\mathrm{CO} 2$-Rich Mixtures.

Final rept.

J. W. Magee. 1991, 9p.

Pub. in Supercritical Fluid Technology: Reviews in Modern Theory and Applications, Chapter 8, p326-334 1991.

Keywords: "Carbon dioxide, "Thermophysical properties, Thermal conductivity, Supercritical fluids, Density(Mass/volume), Transport properties, Specific heat, Viscosity, Mixtures, Bibliographies, Reviews, Reprints

Thermophysical properties data, including thermodynamic and transport properties, are fundamental to the clear understanding of supercritical fluid processes and technology. Benchmark experimental measurements provide the best check of theoretically-based predictive models for the thermophysical properties of supercritical fluids, including $\mathrm{CO} 2$ and $\mathrm{CO} 2$-rich mixtures. For this reason, a review of published data will be given herein. Only highly accurate thermophysical property data, capable of testing predictive models, has been considered in this context.

00,855

PB94-216223 Not available NTIS

National Inst. of Standards and Technology (NML), Gaithersburg, MD. Molecular Physics Div.

High-Resolution Measurements of the nu2 and 2nu2-nu2 Bands of (34)S(16)O2.

Final rept.

A. G. Maki, and Y. A. Kuritsyn. 1990, 2p.

Pub. in Jnl. of Molecular Spectroscopy 144, n1 p2422431990.

Keywords: "Sulfur dioxide, Infrared spectroscopy, Molecular spectroscopy, Band spectra, Air pollution, Acid rain, Sulfur 34 , Oxygen 16 , Reprints

Infrared measurements have been made on (34) SO2 between 450 and $600 / \mathrm{cm}$ with a resolution of $0.004 /$ $\mathrm{cm}$. The B-type bands due to the bending transitions 010-000 and 020-010 have been assigned and analyzed. Revibrational constants are given that fit the current measurements and the pure rotational transitions reported in the literature.

00,856

PB94-216231 Not available NTIS

National Inst. of Standards and Technology (NML), Gaithersburg. MD. Molecular Physics Div.

FTS Infrared Measurements of the Rotational and Vibrational Spectrum of $\mathrm{LiH}$ and LiD.

Final rept.

A. G. Maki, W. B. Olson, and G. Thompson. 1990 $12 \mathrm{p}$.

2681990.

Keywords: "Lithium deuterides, "Lithium hydrides, Vibrational spectra, Rotational spectra, Vapor phases, High resolution, Measurement, Reprints, Fourier transform spectroscopy, Dunham constants.

Fourier transform spectra have been measured for the rotational (Delta $v=0$ ) and vibrational (Delta $v=1$ ) spectrum of lithium hydride and lithium deuteride in the gas phase. Dunham potential constants and 
rovibrational constants are given for $\mathrm{LiH}$ and LiD. Difficulties were encountered in attempts to fit all four isotopic species to a single modified Dunham potential function. Even data for a single isotopic species could not be fit to a Dunham potential function without significant systematic deviations. These problems are attributed to break down in the Born-Oppenheimer approximation.

00,857

PB94-216371 Not available NTIS

National Inst. of Standards and Technology (PL) Gaithersburg, MD. Molecular Physics Div.

Self Broadening in the nu1 Band of $\mathrm{NH} 3$.

Final rept.

V. N. Markov, A. S. Pine, G. Buffa, and O. Tarrini.

1993, 12p

Sponsored by National Aeronautics and Space Administration, Washington, DC. Upper Atmospheric Research Program.

Pub. in Jnl. of Quantitative Spectroscopy and Radiative Transfer 50, n2 p167-178 1993

Keywords: *Ammonia, Band spectra, Laser spectrometers, Pressure dependence, Reprints, Self broadening, Pressure shifts, Dicke narrowing.

Self-broadening coefficients, pressure shifts and integrated intensities have been measured for $Q$ - and $R$ branch transitions in the nu(1) fundamental band of ammonia using a difference-frequency laser spectrometer. A strong, systematic $J$ and $K$ dependence of the broadening coefficients, reminiscent of the groundstate inversion transitions, is observed and compared with semiclassical line broadening calculations. Dicke narrowing is evident at intermediate pressures for the sharpest lines, primarily the $\mathrm{R}(\mathrm{J}, \mathrm{O})$ transitions. Incipient line mixing is apparent in the $Q$ branch at pressures above about 0.1 bar.

\section{0,858}

PB94-216454 Not available NTIS

National Inst. of Standards and Technology (NML) Gaithersburg, MD. Chemical Kinetics Div.

Precision and Accuracy in Tandem Mass Spectrometry Measurements: A Kinetics-Based Protocol for Instrument-Independent Measurements of Collision-Activated Dissociation in RF-Only Quadrupoles.

Final rept

R. I. Martinez. $1990,2 p$

Pub. in Jnl. of the American Society for Mass Spectroscopy 1, n3 p272-273 1990.

Keywords: "Mass spectroscopy, Reaction kinetics, Measurement, Precision, Accuracy, Protocols Quadrupoles, Particle collisions, Reprints, Collisionally induced dissociation.

This is a news item to announce NIST protocol for MS/ MS measurements.

\section{0,859}

PB94-216462 Not available NTIS

National Inst. of Standards and Technology (NML) Gaithersburg, MD. Chemical Kinetics Div.

Kinetics and Mechanism of the Collision-Activated Dissociation of the Acetone Cation.

Final rept.

R. I. Martinez, and B. Ganguli. 1992, 18p.

Pub. in Jnl. of the American Society for Mass Spectrometry 3, n4 p427-444 1992.

Keywords: Branching ratio, Reprints, "Acetone cations Acetyl cations, Methyl cations, Collisionally induced dissociation, Tandem mass spectrometers.

For center-of-mass collision energies $E(\mathrm{~cm})=1-60 \mathrm{eV}$ the major fragment ions for the collision-activated dissociation (CAD) of the acetone cation are the acetyl cation ( $\mathrm{m} / \mathrm{z} 43$; absolute branching ratios of $0.96-0.60$ ) and the methyl cation ( $\mathrm{m} / \mathrm{z} 15$; absolute branching ratios of $0.02-0.26$ ); the absolute total cross-sections were 24-35 A(sup 2). The breakdown curves (viz, plots of the absolute branching ratios versus $E(\mathrm{~cm})$ ) show complex, complementary energy dependences for production of $\mathrm{MeCO}(1+)$ and $\mathrm{Me}(1+)$, indicating apparen closure of the $\mathrm{Me}(1+)$ channel for $\mathrm{E}(\mathrm{cm})>30 \mathrm{eV}$. Our observations are consistent with a competition be tween three fast, primary (direct) reactions, each of which opens sequentially at its respective threshold energy.
National Inst. of Standards and Technology (NML), Gaithersburg, MD. Chemical Kinetics Div.

Carbon Acidities of Aromatic Compounds. 1. Effects of In-Ring Aza and External Electron-Withdrawing Groups.

Final rept.

M. Mautner, and S. A. Kafafi. 1988, $7 p$

Pub. in Jnl. of the American Chemical Society 110, n19 p6297-6303 1988 .

Keywords: "Acidity, "Carbon, "Diazines, "Benzene, Structural chemical analysis, Aromatic compounds, Heterocyclic compounds, Chemical reactivity, Anions, Hydrogen ions, Reprints, "Electron withdrawing groups, "Carbon acidity, Topological charge distribution, Gas phase acidity.

We report experimental intrinsic carbon acidities and theoretical AM1 results on benzene derivatives and nitrogen heterocyclics. When benzene and pyridine are compared, in-ring aza substitution increases the acidity, i.e decreases the acid dissociation energy by 9.7 $\mathrm{kcal} / \mathrm{mol}$. Further aza substitution in 1,2-and 1,3-diazine increases the acidity by an additional 8.6 and 5.8 $\mathrm{kcal} / \mathrm{mol}$, respectively. However, in 1,4-diazine, where the deprotonated carbon must be adjacent to nitrogen, lone-pair repulsion decreases the acidity below pyridine. The in-ring aza-substitution effects are qualitatively similar in those observed in azoles by Taft et al., but their parameters for one-pair repulsion and electronegative substituents do not reproduce the effects quantitatively. The electron-withdrawing substituents, $F$, acidity of benzene by $13.5,13.6,17.5$, and $46.5 \mathrm{kcal} /$ $\mathrm{mol}$, and $\mathrm{CHCH} 2$ increases it by $9.7 \mathrm{kcal} / \mathrm{mol}$. The effects of $F$ and $\mathrm{CHCH} 2$ demonstrate that the ring acidity is affected by the sigma rather than pi donating or withdrawing ability of substituents. Ab initio calculations at the MP2=FC/6-31G(star)/ $/ \mathrm{RHF} / 6-31 \mathrm{G}$ level were performed on pyridine and diazines. The computed ab initio DPEs were consistently higher than the experimental values by about $22 \mathrm{kcal} / \mathrm{mol}$. Semiempirical computations using Dewar's AM1 method for 1,4-diazine suggest that a correction of $11 \mathrm{kcal} / \mathrm{mol}$ is needed for adjacent lone-pair repulsion. With this correction, AM1 predicts qualitatively sensible deprotonation sites for diazines and $\mathrm{F}_{-}, \mathrm{CN}_{-}, \mathrm{CF}_{3}$, and $\mathrm{CHCH}_{2}$-substituted benzenes and reproduces the deprotonation energies within $6 \mathrm{kcal} / \mathrm{mol}$.

00,861

PB94-216603 Not available NTIS

National Inst. of Standards and Technology (NML), Gaithersburg, MD. Chemical Kinetics Div.

Proton Affinity Ladders from Variable-Temperature Equilibrium Measurements. 1. A Re-Evaluation of the Upper Proton Affinity Range.

Final rep

M. Meot-Ner, and L. W. Sieck. 1991, 13p.

Pub. in Jnl. of the American Chemical Society $113, \mathrm{n} 12$ p4448-4460 1991.

Keywords: "Proton transport, "Thermodynamic equilibrium, Thermochemistry, Temperature dependence, Reaction kinetics, Reprints, "Proton affinity, Ionic equilibria.

An interlocking ladder of relative proton affinities (PA) of 43 compounds, over a range of $55 \mathrm{kcal} / \mathrm{mol}$, was obtained from the temperature dependence of proton transfer equilibria. From C3H6 $(\mathrm{PA}=179.5 \mathrm{kcal} / \mathrm{mol})$ to i-C4H8 (PA-195.9 kcal/mol) the results agree well with tabulated values, but for the upper PA range the present values are increased significantly. For example, the present vs. literature PA values are: $\mathrm{NH}_{3}$, $208.3+$ or -1 vs. $204.0 \cdot \mathrm{CH} 3 \mathrm{NH}_{2} \cdot 219.6$ vs 214.1 . $\mathrm{t}-\mathrm{C} 4 \mathrm{H} 9 \mathrm{NH} 2,229.2 \mathrm{vs} .220 .8 \mathrm{kcal} / \mathrm{mol}$ (all relative values + or $-1 \mathrm{kcal} / \mathrm{mol}$, related to PA(i-C4H8) $=195.9$ $\mathrm{kcal} / \mathrm{mol})$. The new value for the latter is also confirmed by the association thermochemistry of t-C $4 \mathrm{H} 9(1+)$ with $\mathrm{NH} 3$.

\section{0,862}

PB94-216751 Not available NTIS

National Inst. of Standards and Technology (PL), Boulder, CO. Quantum Physics Div.

High-Resolution IR Laser-Driven Vibrational Dynamics in Supersonic Jets: Weakly Bound Complexes and Intramolecular Energy Flow.

Final rept.

A. Mcllroy, and D. J. Nesbitt. 1991, 29p.

Grants NSF-PHY86-04504, NSF-CHE86-05970

Sponsored by National Science Foundation, Washington, DC.

Pub. in Advances in Molecular Vibrations and Collision Dynamics, v1A p109-1371991.
Keywords: " Molecular vibration, ${ }^{*}$ Complexes, Van der Waals forces, Infrared spectroscopy, Infrared absorption, Molecular dynamics, Line broadening, Excited states, High resolution, Predissociation, Reprints, Intermolecular potentials, Supersonic expansion.

The combination of low temperatures from supersonic jet expansions and high-resolution laser IR absorption methods provides a nearly ideal probe of quantum state resolved unimolecular dynamics in vibrationally excited molecules under collision-free conditions. In this paper we present three classes of examples of such studies from our laboratory. (1) First, we discuss how precise $(<10$ (sup -4$) / \mathrm{cm})$ rotation-vibration spectroscopy can be used to obtain intermolecular potentials for weakly bound complexes. (2) Next we analyze data on van der Waals systems where the internal vibrational energy in the complex is sufficient to photofragment the weak bond and thereby lead to predissociative line broadening in the spectra. (3) Finally, we consider vibrational dynamics in the limit of chemically bound, stable molecules such as terminal acetylenes, where excess fine structure in a high-resolution spectrum can be used to infer rates of intramolecular energy flow.

\section{0,863}

PB94-219268

MF A02)

Commission of the European Communities, Geel (Belgium). Inst. for Reference Materials and Measurements.

New Values for Silicon Reference Materials, Certified for Isotope Abundance Ratios. Letter to the Editor.

P. De Bievre, S. Valkiers, and H. S. Peiser. 1994, 2p. Prepared in cooperation with National Inst. of Stand ards and Technology, Gaithersburg, MD.

Included in Jnl. of Research of the National Institute of Standards and Technology, v99 n2 p201-202 Mar/ Apr 94.

Keywords: "Silicon, "Isotope ratios, "Atomic mass, Abundance Cyclotron frequency, Silicon tetrafluoride, Penning effect, Penning trap, Reference materials, National Institute of Standards and Technology.

New isotope abundance and relative atomic mass (atomic weight) values-with low, hitherto unattained uncertainty-are reported for two previously described silicon reference materials using a well-known method with an improved isotope-ratio mass spectrometer These new values are directly traceable to the SI, more specifically to the unit for amount of substance, the mole, and independent of the SI unit of mass and of the Avogadro constant. Besides, the residual massspectrometric uncertainties, these new values depend in effect only on a recently published direct comparison of the cyclotron frequency in a Penning trap of (28) $\mathrm{Si}(+1)$ with that of $(12) \mathrm{C}(1+)$

00,864

PB95-107017 Not available NTIS

National Inst. of Standards and Technology (NML) Gaithersburg, MD. Chemical Kinetics Div.

Changes in the Redox State of Iridium Oxide Clusters and Their Relation to Catalytic Water Oxidation: Radiolytic and Electrochemical Studies.

Final rept.

G. S. Nahor, P. Hapiot, P. Neta, and P. Harriman 1991, 6p

Pub. in Jnl. of Physical Chemistry 95, n2 p616-621 1991

Keywords: "Iridium oxides, "Catalysts, "Water "Photochemical reactions, "Oxidation, Reaction kinetics, Oxidation reduction reactions, Electrochemistry Radiolysis, Optical properties, Reprints.

Radiolytically prepared iridium oxide (IrOx.nH2O) clus ters have been shown to catalyze the photochemical oxidation of water. These catalysts have been oxidized by radiolytic or electrochemical methods and the changes in their optical absorption and redox state have been studied. The clusters contain 4-5 Ir atoms in a mixture of Ir(III) and Ir(IV) states, formally described as $\operatorname{Ir}(3.2+)$. Time-resolved pulse radiolytic studies revealed three processes in the sub-second time-scale. The same species were observed in gamma-radiolysis and in spectroelectrochemical experiments. Further oxidation of the cluster leads to oxidation of water to $\mathrm{O} 2$. The kinetics of all the above processes are $\mathrm{pH}$ dependent and involve acid-base equilibria in the various oxidation states.

\section{0,865}

PB95-107058 Not available NTIS 
National Inst. of Standards and Technology (NML), Gaithersburg, MD. Chemical Kinetics Div.

One-Electron Oxidation of Nickel Porphyrins. Effect of Structure and Medium on Formation of Nickel(III) Porphyrin or Nickel(II) Porphyrin pi-Radical Cation.

Final rept.

G. S. Nahor, P. Neta, P. Hambright, and L. R.

Robinson. 1991, 4 p.

Pub. in Jnl. of Physical Chemistry 95, n11 p4415-4418 1991.

Keywords: "Nickel compounds, "Porphyrins, "Oxidation, Photochemical reactions, Spectrum analysis, Cations, Radicals, Transition element compounds, Reprints, One-electron oxidation, Pi-radical cations.

The oxidation of several nickel(II) porphyrins by various radicals has been studied by pulse radiolysis in different media. Photochemical oxidation was also examined in some cases. The absorption spectrum of the oxidation product was monitored within several microseconds after the pulse. Two types of differential spectra were observed, a broad absorption at 640-700 $\mathrm{nm}$ ascribed to the pi-radical cation, or a sharp absorption at $560-580 \mathrm{~nm}$ ascribed to nickel(III) porphyrin. $\mathrm{Ni}(\mathrm{II}) \mathrm{TPP}$ (tetraphenylporphyrin) in several organic solvents, protic and aprotic, was oxidized to Ni(III)TPP.

\section{0,866}

\section{PB95-107066 Not available NTIS}

National Inst. of Standards and Technology (NML),

Gaithersburg, MD. Chemical Kinetics Div.

Site of One-Electron Reduction of Ni(II) Porphyrins. Formation of Ni(I) Porphyrin of Ni(II) Porphyrin piRadical Anion.

Final rept.

G. S. Nahor, P. Neta, P. Hambright, L. R. Robinson, and A. Harriman. 1990, 5p. Pub. in Jnl. of Physical Chemistry 94, n17 p6659-6663
1990 .

Keywords: "Radiolysis, "Reduction(Chemistry), "Nickel compounds, "Porphyrins, Spectroscopic analysis, Reaction kinetics, Radicals, Anions, Electrochemistry, Reprints, One-electron reduction, Pi-radical anions.

The products (Ni(II)P) have been examined by radiolytic reduction in protic media to determine whether (Ni(I)P) or the pi-radical anion Ni(II)P(1-) is formed. Kinetic spectrophotometric detection was utilized to record absorption spectra of products immediately after one-electron reduction and gamma-radiolysis was used for recording the spectra of the stable reduction products. The initial reduction product was dependent on the nature of substituents on the porphyrin but not on the redox potential; a meso-4-pyridyl group seems to direct the reduction toward the porphyrin ring whereas, in most other cases, reduction occurred on the metal. On longer time-scales (minutes) all porphyrins produced the two-electron reduced products, chlorin or phlorin anion.

\section{0,867}

PB95-107074 Not available NTIS

National Inst. of Standards and Technology (NML),

Gaithersburg, MD. Chemical Kinetics Div.

Reduction of Dinitrogen to Ammonia in Aqueous Solution Mediated by Colloidal Metals.

Final rept.

G. S. Nahor, L. C. T. Shoute, P. Neta, and A.

Harriman. 1990, 6p.

Pub. in Jnl, of the Chemical Society $86, n 23$ p3927. 39331990.

Keywords: "Reduction(Chemistry), "Nitrogen, "Colloids, Reprints, Metals, Radicals, Radiolysis, Photolysis, Ammonia, Rubidium, Rhenium, Platinum, PVA(Polyvinyl alcohol), PB(Polybrene), PSS(Polystyrene sulfonate).

Reduction of $\mathrm{N} 2$ to $\mathrm{NH} 3$ was investigated by radiolysis and photolysis in N2-saturated aqueous solutions containing colloidal dispersions of Ru, Pt, or Rh stabilized either by neutral (poly(vinyl alcohol), PVA) or charged (polybrene, PB, or polystyrene sulfonate, PSS) polymers. The yield depends on the irradiation time, colloid concentration, type of reducing radical, and $\mathrm{pH}$. At longer irradiations the concentration of $\mathrm{NH} 3$ increased at a much slower rate until the reaction stopped. The results suggest fast and efficient reduction of any $\mathrm{N} 2$ that is pre-absorbed at highly reactive, possibly metal oxide, sites on the surface of the colloid.
National Inst. of Standards and Technology (NML), Gaithersburg, MD. Chemical Kinetics Div.

Metalloporphyrin Sensitized Photooxidation of Water to Oxygen on the Surface of Colloidal Iridium Oxides. Photochemical and Pulse Radiolytic Studies.

Final rept

G. S. Nahor, A. N. Thompson, P. Neta, P.

Hambright, and A. Harriman. 1989, 7p

Pub. in Jnl. of Physical Chemistry 93, n16 p6181-6187 1989.

Keywords: "Porphyrins, "Photolysis, "Water, - Oxidation, Colloids, Radicals, Cations, Photochemical reactions, Reaction kinetics, Radiolysis, Transition metals, Iridium, Reprints, Iridium oxide.

Derviatives of TSPP (tetrakis(4sulfonatophenyl)porphyrin) were prepared and tested as photosensitizers for oxidation of water to oxygen on the surface of colloidal iridium oxide. Triplet quantum yields, energies, and lifetimes were measured by laser flash photolysis. Rate constants for quenching the porphyrin triplet state with 02 and with persulfate ions were also determined. The rate of interaction between the porphyrin radical cations and colliodal Ir02 particles were measured by pulse radiolysis for several of the compounds. Illumination of a porphyrin in the presence of sodium perfulfate and an IrO2 colloid resulted in generation of 02 in a process that was strongly dependen upon $\mathrm{pH}$ and upon the nature of the photosensitizer in the same manner as the kinetics determined by pulse radiolysis. The rate of 02 production under any conditions could be explained on the basis of thermodynamic criteria relating to either of the individual quenching or water oxidation steps.

00,869

PB95-107116 Not available NTIS

National Inst. of Standards and Technology (NML), Gaithersburg, MD. Time and Frequency Div.

Determination of the Molecular Parameters of $\mathrm{NiH}$ in Its (2)Delta Ground State by Laser Magnetic Resonance.

Final rept

T. Nelis, S. P. Beaton, K. M. Evenson, and J. M.

Brown. 1991, 17p.

Pub. in Jnl. of Molecular Spectroscopy 148, n2 p4624781991.

Keywords: "Molecular structure, "Nickel hydrides, Laser spectroscopy, Magnetic resonance, Rotational states, Far infrared radiation, Radicals, Transition metals, Ground state, Infrared spectra, Zeeman effect, Reprints, "LMR(Laser magnetic resonance), "Laser magnetic resonance.

The rotational spectrum of the $\mathrm{NiH}$ radical in the $\mathrm{nu}=0$ level of its X(2)Delta state has been studied by lase magnetic resonance (LMR) at far-infrared wavelengths. Transitions have been detected for all 5 isotopes of nickel and within both the lower ((sup2)Delta 5/2) and upper ((sup 2)Delta 3/2) spin components. Nuclear hyperfine splittings for both the proton and $(61) \mathrm{Ni}(1=3 / 2)$ are observed. The effective Zeeman Hamiltonian for a molecule in a $(2 s+1)$ Delta state is developed and used to fit all the data together with the similar magnetic resonance observations of the $(1,0)$ vibration-rotation band. There are several indications that this exercise is only just possible in the case of $\mathrm{NiH}$ in its X(2)Delta state.

\section{0,870}

PB95-107124 Not available NTIS

National Inst. of Standards and Technology (MSEL), Gaithersburg, MD. Reactor Radiation Div.

Crystal Structure of a New Sodium Zinc Arsenate Phase Solved by 'Simulated Annealing'.

Final rept.

T. M. Nenoff, W. T. A. Harrison, G. D. Stucky, J. M. Nicol, and J.M. Newsam. 1993, 5p.

Sponsored by National Science Foundation, Arlington, VA.

Pub. in Zeolites 13, p506-510 Sep/Oct 93.

Keywords: "Crystal structure, "Powder metals, "Phase studies, Simulation, Annealing, Crystallography, Neu tron diffraction, $X$-ray diffraction, Sodium compounds, Zinc compounds, Reprints, *Sodium zinc arsenate, Simulated annealing, Sodalite analogs, Rietvelt profile refinement.

The framework structure of a new sodium zinc arsenate, produced by dehydration of the $\mathrm{Na} 6(\mathrm{ZnAsO} 4) 6$ $8 \mathrm{H} 2 \mathrm{O}$ sodalite analog, has been solved by a simulated annealing method. This method utilizes typical tetra- hedral-atom bonding schemes with possible space group and unit cell dimensions to randomly generate possible continuous frameworks and their calculated energies. The Rietveld refinement of the $\mathrm{Na6}(\mathrm{ZnAsO4}) 6$ solution phase was initially performed on room temperature $X$-ray data and then continued on low-temperature constant wavelength neutron data. The sodium zinc phosphate is a hexagonal polycrystalline material. The tridymite structural analog undergoes a room-temperature transformation to the open-framework sodalite structure.

\section{0,871}

PB95-107132 Not available NTIS

National Inst. of Standards and Technology (PL), Boulder, CO. Quantum Physics Div.

Mode Specific Vibrational Predissociation Dynamics in Fragile Molecules.

Final rept.

D. J. Nesbitt. 1991, $14 p$.

Grant NSF-CHE86-05970

Sponsored by National Science Foundation, Washington, DC

Pub. in Mode Selective Chemistry, p113-126 1991.

Keywords: "Infrared spectroscopy, "High resolution, "Hydrogen bonds, Energy transfer, Van der Waals forces, Quantum chemistry, Frequencies, Slits, Supersonic jet flow, Reprints, Mode specific, Predissociation, Sub-Doppler.

State resolved vibrational dynamics in weakly bound complexes is investigated via high resolution IR absorption spectroscopy in a slit supersonic jet expansion. The tunable frequency near IR source can readily excite the high frequency intramolecular vibrations of the subunits, as well as the low frequency, intermolecular vibrations via combination band excitations built on the high frequency modes. These upper state energies lie far above the van der Waals binding energy limit, and yet predissociate typically on the nsec time scale or longer (i.e. many millions of vibrational periods). This exceptional metastability in such small systems is highly non-statistical, and proves to be a rich arena of vibrational mode mixing and mode-specific predissociation behavior. The authors select recent examples from their group effort to illustrate these concepts.

\section{0,872}

PB95-107140 Not available NTIS

National Inst. of Standards and Technology (PL), Boulder, CO. Quantum Physics Div.

Vibration, Rotation, and Parity Specific Predissociation Dynamics in Asymmetric OH Stretch Excited ArH2O: A Half Collision Study of Resonant V-V Energy Transfer in a Weakly Bound Complex.

Final rept

D. J. Nesbitt, and R. Lascola. 1992, 15p.

Grant NSF-PHY90-12244

Sponsored by National Science Foundation, Washington, DC

1 Dec 92

Keywords: "Infrared spectroscopy, "Water, "Argon, "High resolution, Quantum chemistry, Energy transfer, Van der Waals forces, Spectrum analysis, Frequencies, Supersonic jet flow, Kinetics, Collisions, Reprints, Local mode, Vibrational predissociation.

Para ArH2O complexes are detected in the near IR via slit jet direct absorption spectroscopy using $\mathrm{cw}$ difference frequency generation of high resolution tunable infrared radiation (IR) in the $3780 \mathrm{~cm}(-1) \mathrm{V} 3=10$ asymmetric $\mathrm{OH}$ stretch region. Frequency shifts in the $f$ parity levels ( $Q$ branch), and both frequency shifts and predissociation broadened linewidths in the e parity levels (P/R branch) are evidenced in the spectrum, unambiguously characteristic of an avoided crossing in the vicinity of $\mathrm{J}^{\prime}=6$ with a second, near resonant vibration-internal rotation state in ArH2O. The data establish a clear upper limit on the dissociation bond strength for para $\mathrm{ArH} 2 \mathrm{O}$, and place spectroscopic constraints on the promoting internal rotor state in ArH2O (v1=1). A simple local mode theory of half-collision induced mixing between symmetric and asymmetric stretch excitation in $\mathrm{H} 2 \mathrm{O}$ is presented.

00,873

PB95-107157 Not available NTIS

National Inst. of Standards and Technology (NML), Gaithersburg, MD. Chemical Kinetics Div. 
Solvent Effects in the Reactions of Peroxyl Radicals with Organic Reductants. Evidence for Proton Transfer Mediated Electron Transfer.

Final rept.

P. Neta, P. Maruthamuthu, S. Steenken, and R. E.

Huie. $1989,6 p$

Pub. in Jnl. of Physical Chemistry 93, n22 p7654-7659 1989

Keywords: "Electron transfer, "Radiolysis, "Radicals, "Solvents, Protons, Reduction(Chemistry), Reaction kinetics, Solvent properties, Reprints, "Peroxyl radicals, Proton transfer.

Absolute rate constants for the reactions of substituted methylperoxyl radicals with ascorbate, urate, trolox (6hydroxy-2,5,7,8-tetramethylchromane-2-carboxylic acid), and TMPD (N,N,N',N'-tetramethyl-pphenylenediamine) have been determined by pulse radiolysis in different solvents. In water-alcohol or waterdioxane solutions the rate constants for trihalomethylperoxyl radicals generally increase with increasing water content. The rate constants were found to correlate well with a two-parameter equation which includes the dielectric constant of the solvent and the coordinate covalency parameter, a measure of the proton-transfer basicity of the solvent.

00,874

PB95-107223 Not available NTIS

National Inst. of Standards and Technology (IMSE), Gaithersburg, MD. Reactor Radiation Div.

Inelastic Neutron Scattering Studies of Nonlinear Optical Materials: p-Nitroaniline Adsorbed in ALPO-5.

Final rept

J. Nicol, T. J. Udovic, J. J. Rush, S. D. Cox, and G D. Stucky. 1990, 6p

Pub. in Materials Research Society Symposia Proceedings Neutron Scattering Materials Science, v166 p367-372 1990 .

Keywords: "Vibrational spectra, "Analines, Reprints, Optical materials, Nonlinear optics, Nitro compounds, Molecular sieves, Hydrogen bonds, Neutron scattering, Inelastic scattering, Adsorption, "Nitroanilines. Inelastic neutron scattering has been used to characterize the vibrational spectroscopy below $220 \mathrm{meV}$ of para-nitroaniline adsorbed in the molecular sieve ALPO-5. Samples at loadings of both 3 and 13 weight $\%$, which represent the onset of and the maximum in the nonlinear optical properties respectively, were studied. The torsional vibration of the amino $\left(\mathrm{NH}_{2}\right)$ group has been identified at ca. $50 \mathrm{meV}$. The splitting and structure of this mode is sensitive to the loading level. This can be related to differences in the nature of the hydrogen bonding in these materials.

\subsection{5}

PB95-107264 Not available NTIS

National Inst. of Standards and Technology (NML), Gaithersburg, MD. Thermophysics Div.

Fluctuation Dominated Recombination Kinetics with Traps.

Final rept.

J. C. Rasaiah, J. Zhu, J. B. Hubbard, and R. J.

Rubin. $1990,7 \mathrm{p}$

Pub. in Jnl. of Chemical Physics 93, n8 p5768-5774, 15 Oct 90

Keywords: "Recombination reactions, "Fluctuations, Annihilation reactions, Computerized simulation, Kinetics, Diffusion, Trapping, Exponential functions, Reprints, Donsker-Varadhan Model, Lattices.

Theoretical and computer simulation studies of annihilation reactions with traps on two and in three dimensional lattice systems are discussed. Several remarkable features of these reactions, some predicted by theory and others observed in computer simulations are pointed out. The stretched exponential behavior of the Donsker-Varadhan formula $A(t)$ approximately equal to e raised to the $-t(\operatorname{supd} /(d+2))$ power, wher $d$ is the dimensionality, already found for the reactan decay in A-A annihilation reactions with traps on a square lattice (Rasaiah et al. - J. Phys. Chem., in press), was tested for universality by studying triangular and hexagonal lattices in two dimensions (2d) and a cubic lattice in three dimensions (3d). The same behavior is also observed when the free particle annihilation is turned off.

\section{0,876}

PB95-108445 Not available NTIS

National Inst. of Standards and Technology (PL), Gaithersburg, MD. Molecular Physics Div.
Self-, N2- and Ar-Broadening and Line Mixing in $\mathrm{HCN}$ and $\mathrm{C} 2 \mathrm{H} 2$.

Final rept.

A. S. Pine. 1993, 18p.

Sponsored by National Aeronautics and Space Administration, Washington, DC

Pub. in Jnl. of Quantitative Spectroscopy and Radiative Transfer 50, n2 p149-166 1993

Keywords: "Hydrogen cyanide, "Acetylene, Intermediate infrared radiation, Pressure broadening, Band spectra, Tunable lasers, Nitrogen, Argon, Reprints Collisional energy transfer, $Q$ branches, Self broadening

Self-, N2- and Ar-broadening coefficients have been measured for the stretch-bend infrared combination bands nu(sub 1) + nu(sub 2, sup 1) $(4004 / \mathrm{cm})$ of $\mathrm{HCN}$ and nu(sub 1) + nu(sub 5 , sup 1) $(4091 / \mathrm{cm})$ of $\mathrm{C}_{2} \mathrm{H} 2$ using a tunable difference-frequency laser. At atmospheric pressures, the $\mathrm{Q}$ branches of these bands ex hibit significant rotational narrowing or line mixing. The broadening coefficients have been fit with empirical rotationally inelastic collision rate laws, which are then used to model the line mixing in the overlapped $\mathrm{Q}$ branch profiles. Simple energy gap fitting laws appear to be suitable for the shorter-range intermolecular quadrupole-quadrupole and induction forces, whereas an energy-corrected-sudden scaling law works better for the longer-range dipole-dipole and dipolequadrupole collision partners. In all cases, the line-coupling coefficients are substantially reduced from the rotationally inelastic rates fit to the broadening coefficients, indicating that $35-70 \%$ of the broadening may be due to other collisional mechanisms such as crossrelaxation to the degenerate $(\mathrm{Pi})$ state vibrational level.

\section{0,877}

PB95-108452 Not available NTIS

National Inst. of Standards and Technology (PL), Gaithersburg, MD. Molecular Physics Div.

Decoupling in the Line Mixing of Acetylene Infrared Q Branches.

Final rept.

A. S. Pine, and J. P. Looney. 1990, 12p

Pub. in Jnl. of Chemical Physics 93, n10 p6942-6953 1990.

Keywords: "Acetylene, Near infrared radiation, Infrared spectroscopy, Pressure broadening, Line shape, Reprints, $Q$ branches.

The Q-branch profiles of the nu(1) + nu(5), nu(3) + nu(4) and nu(2) + 2nu(4) + nu(5) II(u) - Sigma(g) combination bands in the 25 micrometer $\mathrm{C}$ - $\mathrm{H}$ stretch-bend region of acetylene have been recorded with a difference-frequency laser spectrometer at pressures from 1 to 500 Torr $(0.13$ to $66.7 \mathrm{kPa})$. The broadening coefficients, obtained from the nu(1) + nu(5) band at pressures low enough to avoid significant spectral overlap can be well fit with empirical rotationally-inelastic energy-gas scaling laws or satisfactorily modeled with semiclassical line broadening theory using known intermolecular potential parameters.

\section{0,878}

PB95-108460 Not available NTIS

National Inst. of Standards and Technology (CSTL) Gaithersburg, MD. Biotechnology Div.

Self-Assembled Phospholipid/Alkanethiol Biomimetic Bilayers on Gold.

Final rept.

A. L. Plant. 1993, 4p.

Pub. in Lagmuir 9, n11 p2764-2767 Nov 93.

Keywords: "Phospholipids, "Lipid bilayers, "Gold, *Electrochemistry, Melittin, Substrate specificity, Solvents, Artificial membranes, Reprints, "Alkanethiols.

Alkanethiols can act as hydrophobic monolayer substrates for the formation of phospholipid-containing bilayers. Phospholipid vesicles were allowed to fuse to alkanethiol monolayers, resulting in stable, solventfree lipid bilayers on gold electrodes. The capacitance of these belayers has been determined by impedance measurements, and the effect of the pore-forming peptide melittin on the bilayer has been determined by the measurement of faradaic current. These supported self-assembling phospholipid/alkanethiol bilayers demonstrate properties consistent with those of fluid membranes, and provide a useful way to study the electrical characteristics of membranes in the absence of solvent.
National Inst. of Standards and Technology (CSTL), Gaithersburg, MD. Biotechnology Div. Fokker-Planck Description of Multivalent Interactions.

Final rept

A. L. Plant, M. Gray, and J. B. Hubbard. 1993, 15p.

Pub. in Biophysical Chemistry 48, p75-89 1993.

Keywords: *Binding sites, "Kinetics, "Chemical models, Liposomes, Reprints, "Fokker-Planck descriptions.

A dynamic model is presented which uses a mean first passage time to characterize multivalent binding as the continuum limit of a one-step stochastic process. The use of a stochastic model instead of a model based on rate equations permits consideration of fluctuations in the number of bonds formed. The importance of fluctuations to the problem of multivalent interactions, especially low affinity interactions, is discussed. Assumptions for the theory are based on data in which liposomes were used as a model system for multivalency. The data suggest a linear sequential process of bond rupture, and thus a high degree of cooperativity in the microscopic events associated with multivalent liposomal binding.

00,880

PB95-108494 Not available NTIS

National Inst. of Standards and Technology (PL), Boulder, CO. Quantum Physics Div.

Photoelectron Spectroscopy of Negatively Charged Bismuth Clusters: $\mathrm{Bi}(-) 2, \mathrm{Bi}(-) 3$, and $\mathrm{Bi}(-$ 14.

Final rept

M. L. Polak, J. Ho, G. Gerber, and W. C. Lineberger 1991, 11p

Grant NSF-CHE88-19444

Sponsored by National Science Foundation, Washington, DC

Pub. in Jnl. of Chemical Physics 95, n5, p3053-3063, 1 Sep 91 .

Keywords: "Photoelectron spectroscopy, "Atomic clusters, Negative ions, Electron affinity, Excited states, Ground state, Reprints, *Bismuth clusters.

The authors have recorded the $351 \mathrm{~nm}$ photoelectron spectra of $\mathrm{Bi}(-) 2, \mathrm{Bi}(-) 3$, and $\mathrm{Bi}(-) 4$. The spectrum of Bi(-)2 shows transitions to at least seven electronic states of $\mathrm{Bi} 2$ neutral, four of which are observed with vibrational resolution. Term energies, bond lengths, and vibrational frequencies are obtained for the anion ground state and for the first three excited states of Bi2. These results are compared to previous spectroscopic measurements and to the ab initio calculations presented in the accompanying paper. The photoelectron spectrum of $\mathrm{Bi}(-) 3$ reveals some of the electronic structure of $\mathrm{Bi} 3$ and the results are discussed in comparison to recent theoretical work. Adiabatic electron affinities are obtained for Bi2 (1.271(8) $\mathrm{eV})$ and for $\mathrm{Bi} 3(1.60(3) \mathrm{eV})$. The electron affinity of $\mathrm{Bi} 4$ is estimated from the onset of photodetachment to be $1.05(10) \mathrm{eV}$

00,881

PB95-108528 Not available NTIS

National Inst. of Standards and Technology (CSTL), Gaithersburg, MD. Surface and Microanalysis Science Div.

Activities of the ASTM Committee E-42 on Surface Analysis.

Final rept

C. J. Powell. 1992, 4p

Pub. in Surface and Interface Analysis 19, p237-240 1992.

Keywords: "Surface analysis, "Standards, Surface chemistry, Reprints, ASTM committees, Reference materials.

The ASTM Committee E-42 on Surface Analysis was formed in 1976 to advance the field of surface analysis and the quality of surface analyses through the development of appropriate standards (reference procedures, reference materials and reference data) round robins, symposia, workshops and publications. A major function of the Committee is the development of documentary standards or reference procedures. This article contains a brief summary of Committee activities, with emphasis on the reference procedures that have been published and those that are under development.

00,882

PB95-108593 Not available NTIS

National Inst. of Standards and Technology (MSEL), Gaithersburg, MD. Reactor Radiation Div. 


\section{CHEMISTRY}

Physical \& Theoretical Chemistry

Construction of Maximum-Entropy Density Maps, and Their Use in Phase Determination and Extension.

Final rept.

E. Prince. 1993, 5p

Pub. in Acta Cryst. D49, p61-65 1993.

Keywords: "Electron density(Concentration), "Molecular structure, Fourier analysis, Macromolecules, Reprints, Phase determination, Maximum entropy.

Methods for constructing everywhere-positive electron-density maps with Fourier amplitudes matching those for arbitrarily large sets of observed data, using dual-function methods for maximization of entropy, are described. Possible strategies for using these maps for the determination and extension of phases in macromolecular structure determination are suggested, and problems are discussed.

\section{0,883}

PB95-108619 Not available NTIS

National Inst. of Standards and Technology (PL), Boulder, CO. Quantum Physics Div.

Vibrational Energy Transfer in $\mathbf{s 1} p$ Difluorobenzene. A Comparison of Low and Room Temperature Collisions.

Final rept.

C. J. Pursell, and C. S. Parmenter. 1993, 7p.

Pub. in Jnl. of Physical Chemistry 97, n8 p1615-1621 1993.

Keywords: "Atom-molecule collisions, Temperature range 0013-0065 K, Room temperature Vibrational states, Energy transfer, Difluoro compounds, Helium, Argon, Reprints, “Difluorobenzene.

Vibrational energy transfer in S(1) p-difluorobenzene has been studied at low temperature within a supersonic free jet. Quantitative relative cross sections for the state-to-state vibrational energy transfer channels (i.e. flow patterns) from the 8(sup 2) vibrational level (epsilon(vib) $=346 / \mathrm{cm}$ ) were obtained for low energy collisions ( $\mathrm{T}$ approx $=20-35 \mathrm{~K}$ ) with $\mathrm{He}$ and Ar. They are qualitatively similar to analogous flow patterns obtained earlier from the 0 (sup 0) level from room temperature $(300 \mathrm{~K})$ collisions with $\mathrm{He}$ and $\mathrm{Ar}$. Both the $300 \mathrm{~K}$ and the low temperature flow patterns show that the vibrational energy transfer is selective among the possible channels and that the competition among vibrational channels is distinctive for each collisional partner. The low and room temperature flow patterns differ however in quantitative detail. A treatment of the standard SSH-T vibrational energy transfer model describes semiquantitatively the flow patterns at both low and room temperature and for collisions with $\mathrm{He}$ and Ar. The success of a single model suggests that vibrational energy transfer in S(1) p-difluorobenzene is governed by the same mechanism over the entire span of collision energies associated with temperatures from about 20 to $300 \mathrm{~K}$.

00,884

PB95-108627 Not available NTIS

National Inst. of Standards and Technology (NML), Gaithersburg, MD. Chemical Process Metrology Div.

Measurement of the Self Broadening of the $\mathrm{H} 2 \mathrm{Q}(\mathrm{O}$ 5) Raman Transitions from 295 to $1000 \mathrm{~K}$ Final rept.

L. A. Rahn, R. L. Farrow, and G. J. Rosasco. 1991,

Pub. in Physical Review A 43, n11 p6075-6088 1991.

Keywords: "Raman spectroscopy, "Hydrogen, Temperature range 0273-0400 $\mathrm{K}$, Temperature range 0400-1000 K Temperature dependence, Pressure dependence, Vibrational states, Line broadening, High resolution, Reprints, Self broadening, Q branches.

High-resolution inverse Raman spectroscopy (IRS) has been used to measure the self-broadening coefficients of the Raman Q-branch transitions in pure (natu ral) hydrogen. Measurements of six lines $(Q(0)-Q(5)$ were made at pressures from 2 to 50 atmospheres and from 295 to $1000 \mathrm{~K}$. The dependence of the derived broadening coefficients on temperature and rotational quantum number is analyzed using the energy-corrected-sudden scaling law (A. E. DePristo, J. Chem. Phys. 73, 2145 (1980)). The authors discuss three models for the J-dependence of the pure-vibrational dephasing and find that models with little or no J-dependence in the pure-vibrational dephasing are preferred.

00,885

PB95-108635 Not available NTIS
National Inst. of Standards and Technology (CSTL), Boulder, CO. Thermophysics Div. Calculation of Enthalpy and Entropy Differences of Near-Critical Binary Mixtures with the Modified Leung-Griffiths Model.

Final rept.

J. C. Rainwater, and D. G. Friend. 1993, 10 p.

Pub. in Jnl. of Chemical Physics 98, n3 p2298-2307. 1 Feb 93.

Keywords: "Liquid vapor equilibrium, "Binary mixtures, "Benzene, "Alkanes, "Entropy, "Enthalpy, Thermodynamic properties, Phase transformations, Fugacity, Free energy, Mathematical models, Reprints, "Clapeyron equation, "Leung-Griffiths Model.

In previous applications of the Leung-Griffiths model as modified by Moldover and Rainwater, many nearcritical vapor-liquid equilibrium surfaces have been described successfully in the space of pressure, temperature, density, and composition, but calorimetric properties such as entropy and enthalpy have not been examined. Such calculations are difficult in general because of the need to determine a parameter that is usually unknown in the definition of an independent field variable (the fugacity fraction). However, a generalization for mixtures of the Clapeyron equation is available which allows for the calculation of differences in entropy, enthalpy, and free energies between a pair of (noncoexisting) dew and bubble points at the same temperature and composition. A calculation of entropy differences from a correlation of carbon dioxide + propylene agrees well with results inferred indirectly from experimental data. As a more direct check, calculation of enthalpy differences from a correlation of n-pentane + benzene agrees with an interpolation of enthalpy data provided by calorimetric measurements of Lenoir and Hipkin. The generalized Clapeyron equation appears to be singular at the maxcondentherm point, but it is shown that the singular terms cancel one another and that the leading-order finite term is consistent with a thermodynamic Maxwell relation.

\section{0,886}

PB95-108817 Not available NTIS

National Inst. of Standards and Technology (CSTL), Boulder, CO. Thermophysics Div.

Polarized Transient Hot Wire Thermal Conductivity Measurements.

Final rept.

R. A. Perkins, A. Laesecke, and C. A. Nieto de

Castro. 1992, 12p.

Sponsored by Department of Energy, Washington, DC $\mathrm{Pub}$. in Fluid Phase Equilibria 80, p275-286 1992.

Keywords: "Thermal conductivity, "Fluorohydrocarbons, Liquids, Refrigerants, Substitutes, Reprints, "1, "1-chloro-1, "R134a, "R142b, 1, 1, 2-Tetrafluoroethane, 1-difluoroethane, Transient hot wire instruments.

Additional experimental uncertainty is introduced in thermal conductivity data obtained with the transient hot-wire technique when bare hot wires are used in polar liquids. The use of a dc polarization voltage applied between the hot wires and the cell wall greatly reduces this uncertainty for polar liquids. Differences between polarized and nonpolarized experiments are described for the alternative refrigerants $1,1,1,2$ tetrafluoroethane (R134a) and 1-chloro-1,1difluoroethane $(R 142 b)$. Comparisons are made between data from the polarized transient hot-wire technique and other experimental techniques for $\mathrm{R} 142 \mathrm{~b}$. The polarization technique enables existing transient hot-wire instruments with bare wires to study the thermal conductivity of moderately polar liquids with confidence.

\section{0,887}

PB95-125621 Not available NTIS

National Inst. of Standards and Technology (PL), Gaithersburg, MD. Molecular Physics Div.

Reanalysis of the (010), (020), (100), and (001) Rotational Levels of (32)S(16)O2.

Final rep

J. M. Flaud, A. Perrin, L. M. Salah, W. J. Lafferty, and G. Guelachvili 1993, 7p.

Sponsored by National Aeronautics and Space Administration, Washington, DC

Pub. in Jnl. of Molecular Spectroscopy 160, p272-278 1993.

Keywords: "Sulfur dioxide, "Rotational states, Infrared spectra, Band spectra, High resolution, Oxygen 16, Sulfur 32, Reprints, Molecular constants.

Using high-resolution infrared spectra, a reanalysis of the nu(sub 2), 2nu(sub 2)-nu(sub 2), nu(sub 1), and nu(sub 3) bands of (32)S(16)O2 has been performed. These infrared data, combined with the available heterodyne and microwave data, were fitted within their respective experimental accuracies. For the (010) levels it was sufficient to use a Watson-type Hamiltonian, whereas it proved necessary to consider explicitly interaction terms for the $(020)(100)(001)$ states. More precisely, a (Delta) $\mathrm{K}=2$ quadratic distortion-correction to the Fermi interaction term was used to treat the interactions between (020) and (100) and a (Delta)K $=3$ Coriolis-type term to treat the interactions between (100) and (001).

\section{0,888}

PB95-125662 Not available NTIS

National Inst. of Standards and Technology (PL), Gaithersburg, MD. Molecular Physics Div.

Mid- and Near-Infrared Spectra of Water and Water Dimer Isolated in Solid Neon.

Final rept.

D. Forney, M. E. Jacox, and W. E. Thompson. 1993

Sponsored by Army Research Office, Research Triangle Park, NC.

Pub. in Jnl. of Molecular Spectroscopy 157, p479-493 1993.

Keywords: "Water, "Infrared spectra, Temperature range 0000-0013 K Intermediate infrared radiation, Near infrared radiation, Deuterium compounds, Matrix isolation, Oxygen 16, Oxygen 18, Dimers, Reprints, Solid neon.

Spectra have been obtained between 700 and $8000 /$ $\mathrm{cm}$ for $\mathrm{H} 2(16) \mathrm{O}$, and between 700 and $5000 / \mathrm{cm}$ for deuterium and/or oxygen-18-enriched water, trapped in solid neon at approximately $5 \mathrm{~K}$. Samples with Ne:water mole ratios between 400 and 6400 were studied. As in the heavier rare-gas solids, isolated water molecules can undergo relatively free rotation in solid neon, and nuclear spin equilibration is slow. The observed spectra can be explained by postulating excitation from the two lowest rotational levels of the water molecule. Absorptions of nonrotating water are also present in the spectrum. The matrix shifts for water isolated in a neon matrix are much smaller than those reported for water in matrices of the heavier rare gases. Absorptions contributed by the $\mathrm{H}$ - or $\mathrm{D}$-donor moiety of $(\mathrm{H} 2 \mathrm{O}) 2$, ( $\mathrm{HDO}) 2$, and (D2O)2 have also been identified and assigned.

\section{0,889}

PB95-125670 Not available NTIS

National Inst. of Standards and Technology (PL) Gaithersburg, MD. Molecular Physics Div.

Vibrational Spectra of Molecular Ions Isolated in Solid Neon. X. H2O(+), $\mathrm{HDO}(+)$, and D2O(+).

Final rept.

D. Forney, M. E. Jacox, and W. E. Thompson. 1993,

Sponsored by Army Research Office, Research Triangle Park, NC.

Pub. in Jnl. of Chemical Physics 98, n2 p841-849, 15 Jan 93.

Keywords: "Molecular ions "Vibrational spectra, * Water. Temperature range 0000-0013 K Infrared absorption, Infrared spectra, Deuterium compounds, Matrix isolation, Photodetachment, Reprints, Solid neon.

When a $\mathrm{Ne}: \mathrm{H} 2 \mathrm{O}=$ or $>200$ sample is codeposited a approximately $5 \mathrm{~K}$ with a beam of neon atoms that have been excited in a microwave discharge, new infrared absorptions appear close to the gas-phase band centers of the three vibrational fundamentals of $\mathrm{H} 2 \mathrm{O}(1+)$. Detailed isotopic substitution studies confirm this assignment and provide assignments for all of the vibrational fundamentals of $\mathrm{HDO}(1+)$ and $\mathrm{D} 2 \mathrm{O}(1+)$ When ions are present in the neon matrix, rotation of a significant fraction of the water molecules is inhibited. Electrons produced by the photodetachment of anions, which must be present to maintain overall charge neutrality of the deposit, accelerate nuclear spin equilibration of water in the matrix. As the concentration of $\mathrm{H} 2 \mathrm{O}(1+)$ is decreased by capture of the photodetached electrons, the absorptions assigned to nonrotating water are also reduced in intensity. The nature of the other ionic species which may be present in the sample is considered.

\section{0,890}

PB95-125688 Not available NTIS

National Inst. of Standards and Technology (PL), Gaithersburg, MD. Molecular Physics Div. 
Vibrational Spectra of Molecular lons Isolated in Solid Neon. XI. NO2(+), NO2(-), and NO3(-).

Final rept.

D. Forney, W. E. Thompson, and M. E. Jacox. 1993, $11 p$.

Sponsored by Army Research Office, Research Triangle Park, NC

Pub. in Jnl. of Chemical Physics 99, n10 p7393-7403, 15 Nov 93

Keywords: "Molecular ions, "Nitrogen dioxide, "Vibrational spectra, Temperature range 0000-0013 K Infrared absorption, Infrared spectra, Electron capture, Matrix isolation, Reprints, "Solid neon, "Nitrite ions, Nitrate ions.

When a Ne:NO2 or a Ne:NO:O2 sample is codeposited at approximately $5 \mathrm{~K}$ with a beam of neon atoms that have been excited in a microwave discharge, intrared absorptions of NO2(1+), NO2(1-), and NO3(1-) appear. Detailed isotopic substitution studies support the assignment of prominent absorptions to nu(sub 3 ) of $\mathrm{NO} 2(1+)$ and NO2(1-) and of weak to moderately inense absorptions to the nu(sub 1) + nu(sub 3) combination band of each of these species. When the contribution of anharmonicity is considered, the positions of the $\mathrm{NO} 2(1+)$ absorptions are in satisfactory agreement with the values for the stretching fundamentals obtained in a recent gas-phase study of that species. When the sample is exposed to $240-420 \mathrm{~nm}$ mercuryarc radiation, the initially present absorptions of NO3(1-) trapped in sites with a small residual cation interaction diminish in intensity, and the unsplit nu(sub 3) (e') absorption of isolated $\mathrm{NO}^{\prime}(1-)$ grows. The mechanism responsible for this growth in the absorption of isolated $\mathrm{NO} 3\left(1^{-}\right)$is considered.

\section{0,891}

PB95-125779 Not available NTIS

National Inst. of Standards and Technology (CSTL), Boulder, CO. Thermophysics Div.

Thermodynamic Properties of the Methane-Ethane System.

D. G. Friend, and J. F Ely, 1992, 12p

Sponsored by Department of Energy, Washington, DC and Gas Research Inst., Chicago IL.

Pub. in Fluid Phase Equilibria 79, p77-88 1992.

Keywords: "Thermophysical properties, "Methane "Ethane, "Binary mixtures, Thermodynamic properties, Liquid phases, Equations of state, Critical point, Specific heat, Phase stability(Materials), Pressure, Volume, Temperature, Acoustic velocity, Reprints, "Extended corresponding states.

Our recently completed wide-ranging correlations for he thermophysical properties of methane and ethane fluids have enabled us to improve the extended corresponding states calculations for the properties of the binary mixtures. The pure fluid equations are based on the analytic Schmidt-Wagner equation of state and give improved results in the general region of the critical point. We use these pure fluid equations (and a reference fluid for which an equivalent equation is known) to calculate properties and compare with experimental PVT data, isochoric heat capacities, and sound speeds of binary mixtures of methane and ethane. We also ex amine the properties at the vapor-liquid boundary and compare the representation from the classical ex ended corresponding states calculations with quantities derived from a scaling theory model. The shape of the binary mixture phase boundary is improved with this model, however our new representation of the single phase properties provides only a slight improvement over models based on modified Benedict-WebbRubin pure fluid equations of state.

\section{0,892}

PB95-125803 Not available NTIS

National Inst. of Standards and Technology (NML) Gaithersburg, MD. Chemical Process Metrology Div. Conductance Response of $\mathrm{Pd} / \mathrm{SnO} 2(110)$ Model Gas Sensors to $\mathrm{H} 2$ and $\mathrm{O} 2$.

Final rept.

T. B. Fryberger, and S. Semancik. 1990, 5p

Pub. in Sensors and Actuators B 2, n4 p305-309 1990

Keywords: "Gas detectors, "Tin oxides, "Palladium, "Hydrogen, "Oxygen, Metal oxide semiconductors, Electrical conductivity, Gas analysis, Surface analysis Adsorption, Reprints.

Gases can influence the conductance of tin oxide sensors by a variety of mechanisms. In general, it is difficult to distinguish between different sensing mecha- nisms because so little is known about the sensor surface during operation. We have used surface analytical techniques together with in situ gas conductance response measurements to study gas sensing mechanisms on well-characterized Pd/SnO2(110) model sensor surfaces. In this letter, we report results for $\mathrm{H} 2$ and $\mathrm{O} 2$ adsorption and coadsorption: hydrogen was chosen because of its importance both as a gas to be monitored and as an adsorption product of hydrocarbons and alcohols, and oxygen because it is nearly always abundant in the sensor ambient (often, air)

\section{0,893}

PB95-125860 Not available NTIS

National Inst. of Standards and Technology (CSTL), Gaithersburg, MD. Surface and Microanalysis Science Div.

Single-Atom Point Source for Electrons: FieldEmission Resonance Tunneling in Scanning Tunneling Microscopy.

Final rept.

J. W. Gadzuk. 1993, 8p.

Pub. in Physical Review B 47, n19 p12 832-12 839, 15 May 93.

Keywords: "Scanning tunneling microscopy, * Resonant tunneling. Field emission, Atomic clusters, Point sources, Electron sources, Atoms, Reprints, Tunneling spectroscopy.

Many years ago spectroscopic evidence based on a single-electron process, namely, field-emission resonance tunneling, was reported showing the electronic structure of single atoms adsorbed on metal surfaces. Huge enhancements in the highly collimated tunneling current through the virtual states of the adparticle made the single-atom spectroscopy feasible when the field-emission spectrometer was operated in the 'probe-hole mode.' A related effect is currently popular in today's scanning tunneling microscopy and also in nanometer-cluster spectroscopy. The phenomenology and theory of the effect is presented, and the current activity is considered in light of what has been uncovered in the field-emission work.

\section{0,894}

PB95-125878 Not available NTIS

National Inst. of Standards and Technology (CSTL) Gaithersburg, MD. Biotechnology Div.

Aggregation Kinetics of Colloidal Particles Destabilized by Enzymes.

Final rept.

A. K. Gaigalas, and H. H. Weetall. 1993, $7 \mathrm{p}$

Pub. in Analyitical Biochemistry 213, p329-335 1993.

Keywords: "Colloids, "Kinetics, "Enzymes, Radiation scattering, Light, Diffusion, Particle density(Concentration), Chemical models, Agglomeration, Proteins, Carbohydrates, Photons, Reprints.

Dynamic light scattering was used to measure enzymatic activity by monitoring the rate of destabilization of colloidal particles coated with proteins or carbohydrates. The photon autocorrelation spectrum was analyzed using a cumulant expansion or direct inversion. In all cases, the measured average diffusion coefficient and the standard deviation of the diffusion coefficient decreased with time. A kinetic model with a bimodal particle distribution function was used to represent the time dependence of the cumulants. The cumulants are very sensitive to the details of the particle distribution.

\section{0,895}

PB95-126041 Not available NTIS

National Inst. of Standards and Technology (CSTL), Gaithersburg, MD. Surface and Microanalysis Science Div.

Picosecond Measurement of Substrate-to-Adsorbate Energy Transfer: The Frustrated Translation of CO/Pt(111).

Final rept.

T. A. Germer, J. C. Stephenson, E. J. Heilweil, and R. R. Cavanagh. $1993,9 p$

Pub. in Jnl. of Chemical Physics 98, n12 p9986-9994, 15 Jun 93

Keywords: "Carbon monoxide, "Adsorbates, Vibrational states, Temperature dependence, Picosecond pulses, Infrared radiation, Energy transfer, Excitation, Substrates, Platinum, Phonons, Reprints, Translations.

The transient infrared response of $\mathrm{CO} / \mathrm{Pt}(111)$ following picosecond visible excitation is reported. A spectrally broad decrease in reflectivity correlates with heat- ing of the Pt lattice, and an observed shift in the $\mathrm{CO}(\mathrm{nu}$ $=0 \cdots>1$ ) transition is interpreted as heating of the $60 /$ $\mathrm{cm}$ in-plane frustrated translational mode. A phenomenological three temperature model that assumes the adsorbate vibrational temperature exclusively couples oither the electronic temperature (with a time constant tau(e)) or to the lattice temperature (with a time constant tau(lat)) describes the temporal response of the adsorbate vibrations. The lattice phonon temperature and measured temperature dependence of the optical constants predict the observed spectrally broad reflectivity change. Density matrix methods model the infrared response of the transiently heated molecule. Limits of tau(e) $=2$ plus or minus 1 ps or tau(lat) 1 ps are established by comparison of predicted spectra and the data.

\section{0,896}

PB95-126140 Not available NTIS

National Inst. of Standards and Technology (PL) Gaithersburg, MD. Molecular Physics Div.

Rotational Spectrum and Structure of a Weakly Bound Complex of Ketene and Acetylene.

Final rept

C. W. Gillies, J. Z. Gillies, F. J. Lovas, and R. D.

Suenram. 1993, 10p.

Pub. in Jnl. of the American Chemical Society 115, n20 p9253-9262 1993

Keywords: Van der Waals forces, Microwave spectros copy, Rotational spectra, Deuterium compounds, Electric moments, Dipole moments, Hamiltonians Reprints, "Acetylene complexes, "Ketene complexes, Fourier transform spectroscopy.

Rotational spectra of $\mathrm{CH} 2 \mathrm{CO}-\mathrm{C} 2 \mathrm{H} 2, \mathrm{CD} 2 \mathrm{CO}-\mathrm{C}_{2} \mathrm{H} 2$ $\mathrm{CH} 2 \mathrm{CO}-\mathrm{C} 2 \mathrm{HD}$, and $\mathrm{CH} 2 \mathrm{CO}-\mathrm{C} 2 \mathrm{D} 2$ were observed with a pulsed-beam Fabry-Perot cavity Fourier-transform microwave spectrometer. The b-type transitions were split into four states for $\mathrm{CH} 2 \mathrm{CO}-\mathrm{C} 2 \mathrm{H} 2$ and $\mathrm{CD} 2 \mathrm{CO}-\mathrm{C} 2 \mathrm{H} 2$, while two states were assigned for $\mathrm{CH} 2 \mathrm{CO}-\mathrm{C} 2 \mathrm{HD}$ and $\mathrm{CH} 2 \mathrm{CO}-\mathrm{C} 2 \mathrm{D} 2$. All states were fit individually to a quartic Watson Hamiltonian. The electric dipole moment of $\mathrm{CH} 2 \mathrm{CO}-\mathrm{C} 2 \mathrm{H} 2$ was measured.

00,897

PB95-126157 Not available NTIS

National Inst. of Standards and Technology (NML) Gaithersburg, MD. Chemical Thermodynamics Div.

Biological Macromolecular Crystallization Database: A Tool for Developing Crystallization Strategies.

Final rept.

G. L. Gilliland, and D. Bickham. 1990, $6 p$.

Pub. in Methods 1, n1 p6-11 1990

Keywords: "Crystallization, "Data bases, X-ray diffraction, Reprints, "Biological macromolecules.

The Biological Macromolecule Crystallization Database (BMCD) contains the crystallization conditions and crystal data for crystal forms of all classes of biological macromolecules which have had the unit cell dimensions and space group determined by singlecrystal $x$-ray diffraction analysis. The BMCD provides a menu driven interface which allows searching the data for a value or a range of values for any of nineteen different parameters, individually or in any combination. The results of a search may be displayed or printed in a variety of different formats. Besides providing the information necessary to crystallize a particular macromolecule, the BMCD may be utilized to design strategies for the crystallization of molecules which have not previously been crystallized. Strategies for the crystallization of modified (or mutant) biological macromolecules, biological macromolecules which are homologous to previously crystallized macromolecules, and unique biologica tallized are discussed.

00,898

PB95-126181 Not available NTIS

National Inst. of Standards and Technology (MSEL) Gaithersburg, MD. Reactor Radiation Div.

Characterization of Chemically Modified Pore Surfaces by Small Angle Neutron Scattering.

Final rept.

C. J. Glinka, L. C. Sander, S. A. Wise, and N. F

Berk. 1990, 6p

Pub. in Materials Research Society Symposia Proceedings, v166 p415-4201990.

Keywords: "Surface chemistry, "Silicon dioxide "Porous media, "Small angle scattering, "Molecular 
structure, "Particles, Neutron scattering, Adsorbates, Hydrocarbons, Grafted polymerization, Chemical bonds, Liquid column chromatography, Reprints.

Small angle neutron scattering has been used to characterize the structure of linear hydrocarbon chains chemically grafted to the internal pore surfaces of microporous silica particles. The aim of this work has been to relate the structure of the bonded adsorbate layers in these particles to their performance in, for example, reverse-phase liquid chromatography. By filling the pore space in the modified silica with a solution that matches the scattering density of the silica framework, the scattering from the adsorbate layers is enhanced and provides a sensitive probe of the effective thickness, uniformity and degree of solvent penetration in the layers. Results are presented for both monomeric and polymeric phases of alkyl chains ranging from $\mathrm{CB}$ to $\mathrm{C} 30$ bonded to silica particles with a mean pore size of $100 \mathrm{~nm}$.

\section{0,899}

PB95-126231 Not available NTIS

National Inst. of Standards and Technology (CSTL), Gaithersburg, MD. Thermophysics Div.

vapor Pressure of 1,1-dichloro-2,2,2trifluoroethane (R123).

Final rept.

A. R. H. Goodwin, D. R. Defibaugh, G. Morrison, and L. A. Weber. 1992, 11 p

Pub. in International Jnl. of Thermophysics 13, n6 p999-1009 Nov 92

Keywords: "Vapor pressure, "Refrigerants, Fluorohydrocarbons, Freons, Thermodynamic prop erties, Ebulliometry, Static tests, Reprints, "Ethane/ dichloro-trifluoro, Freon 123.

The vapor pressure of 1,1-dichloro-2,2,2trifluoroethane (R123) has been measured at temperatures between 256.4 and $453.8 \mathrm{~K}$ by ebulliometric and static techniques. These results have been combined to obtain a correlation for the vapor pressure from $256.4 \mathrm{~K}$ to the critical temperature.

00,900

PB95-140133 Not available NTIS

National Inst. of Standards and Technology (NML),

Gaithersburg, MD. Thermophysics Div.

Formulation of the Refractive Index of Water and Steam.

Final rept.

? Schiebener, J. Straub, J. M. H. Levelt Sengers,

and J. S. Gallagher. 1990, 8p

Pub. in Properties of Water and Steam, p103-110 1990.

Keywords: "Water vapor, "Water, "Steam, "Refractive index. Equations of state, Temperature dependence Infrared radiation, Visible radiation, Ultraviolet radiation, Supercooling, Reprints, Density dependence.

A new formulation is presented of the refractive index of water and steam over large ranges of temperature and density, and in the visible, infared and ultraviolet up to the first resonances. The formulation makes use of the fact that the molar refractivity of water and steam, at constant wavelength, varies no more than a few percent, so that temperature and density dependence require very few adjustable parameters. The NBS/NRC equation is used to convert experimental pressures to temperatures. It is demonstrated that the formulation, in conjunction with the best refractive index data, can be used to discriminate between various highly accurate equations of state for water.

\section{0,901}

PB95-140166 Not available NTIS

National Inst. of Standards and Technology (NML), Gaithersburg, MD. Thermophysics Div.

Near Critical Fluid Interfaces: A Comparison of Theory and Experiment.

Final rept.

J. W. Schmidt. 1991, 13p.

Pub. in Physica A 172, n1-2 p40-52 1991.

Keywords: "Interfaces, "Liquids, "Reflectivity, "Elliptical configuration, Capillary waves, Temperature dependence, Density(Mass/Volume), Phase transformations, Reprints, Consolute points.

Measurements of the ellipticity and reflectivity of liquidliquid interfaces in mixtures near their consolute points are compared with theories of the interface. Both sets of measurements yield the same interfacial thickness within experimental error when scaled appropriately by theories that combine both the density-profile and cap illary-wave aspects of the interface. The theories correctly predict the temperature dependence of the thick ness, but systematically overestimate the thickness itself. When the few comparable data for pure fluids are scaled to the same theories, they show an unexpected temperature dependence of the thickness which is not consistent with the mixture results.

\section{0,902}

PB95-140174 Not available NTIS

National Inst. of Standards and Technology (CSTL), Gaithersburg, MD. Thermophysics Div.

Structure of the Vapor-Liquid Interface Near the Critical Point.

Final rept.

J. W. Schmidt, and M. R. Moldover. 1993, 8p.

Pub. in Jnl. of Chemical Physics 99, n1 p582-589, 1 Jul 93

Keywords: "Liquid-vapor interfaces, "Ellipsometry, "Critical point, Carbon dioxide, Sulfur hexafluoride Fluroalkanes, Phase diagrams, Refractivity, Reprints Trifluoromethane.

We measured the thicknesses of the vapor-liquid interfaces near the critical points of carbon dioxide (CO2) sulfur hexafluoride (SF6), and trifluoromethane (CHF3), using ellipsometry. The data (when scaled by the refractive index difference and the correlation length) are in agreement with other ellipticity data for binary and pseudobinary mixtures at low pressures. Fully constrained theories of the interface correctly predict the temperature dependence and scaling of the thickness but systematically overestimate the thickness itself by $15 \%-20 \%$. The theory can be brough into agreement with experiment when an intrinsic interfacial stiffness is added to the theory. A novel feature of the present measurements is that the effects from pressure-induced window strain were measured and mitigated by using a cylindrically-symmetric pressure cell with floating seals.

\section{0,903}

PB95-140182 Not available NTIS

National Inst of Standards and Technology (CSTL), Gaithersburg, MD. Thermophysics Div.

Operational Mode and Gas Species Effects on Rotational Drag in Pneumatic Dead Weight Pressure Gages.

Final rept.

J. Schmidt, B. E. Welch, and C. D. Ehrlich. 1993 9p.

Pub. in Meas. Sci. Technol. 4, p26-34 1993.

Keywords: "Relaxation(Mechanics), "Rotation, "Pressure gages, " Gas dynamics, "Helium, "Hydrogen *Nitrogen, „Sulfur hexafluoride, Pistons, Mathematica models, Low pressure, Spin, Annuli, Reprints, -Rotational drag, Spin time.

Rotational dissipation in a low-pressure pneumatic dead weight piston gauge has been measured for four gases: He H2 N2 and SF6. Significant differences in the rotational dissipation were observed between the four gas species. Even larger differences were observed between two operational modes (gauge and absolute). The measured results are interpreted by a model for the rotational dissipation due to the gas in the annular region between the piston and cylinder. Good agreement was found between the measured and modeled results for all four gas species with essentially no adjustable parameters.

\section{0,904}

PB95-140414 Not available NTIS

National Inst. of Standards and Technology (NEL), Gaithersburg, MD. Precision Engineering Div.

Scanning Tunneling Microscopy and Fabrication of Nanometer Scale Structures at the Liquid-Gold Interface.

Final rept.

J. Schneir, H. H. Harary, J. A. Dagata,

Hansma, and R. Sonnenfeld. 1989, 6p.
Pub. in Scanning Microscopy 3, n3 p719-724 1989.

Keywords: "Liquid-solid interfaces, "Scanning tunneling microscopy, "Metal surfaces, "Gold, Atomic force microscopy, Imaging techniques, Electrochemistry, Electroplating, Fabrication, Lithography, Reprints, Nanostructures.

The Scanning Tunneling Microscope (STM) can image gold surfaces covered with a variety of liquids. This paper reviews the results obtained using the STM to image gold surfaces covered with liquid. These results include the creation of $10 \mathrm{~nm}$ structures, images of the electrochemical process of electroplating, and the production of atomically flat Au (111) surfaces. We conclude that in the future STM will find further application in the area of nanostructure fabrication and electrochemistry. The trend in the field is toward greater control of the electrochemical environment.

00,905

PB95-140463 Not available NTIS

National Inst. of Standards and Technology (CSTL), Gaithersburg, MD. Thermophysics Div.

Flow of Microemulsions through Microscopic Pores.

Final rept.

D. Ripple, and R. F. Berg. 1992, 5p.

Pub. in Jnl. of Chemical Physics 97, n10 p7761-7765, 15 Nov 92.

Keywords: "Viscosity, "Microemulsions, Drops(Liquids), Volume, Water, Decanes, Porosity, Reprints, Dioctylsulfosuccinate, AOT, Ternary systems.

The flow of the microemulsion dioctylsulfosuccinate (AOT)/decane/water through 0.1 and 1.0 micrometer diameter pores was studied for water droplet volume fractions ranging from 0 to 0.5 . The viscosity of these microemulsions, as measured in a capillary viscometer with a bore of approximately $1 \mathrm{~mm}$ diameter, exceeds by as much as a factor of 4 theoretical predictions of the viscosity of suspensions of hard or liquid spheres. If droplet clustering causes this viscosity enhancement, then flow of the microemulsions through pores with diameter small compared to the characteristic cluster size should display a large finite-size effect. The apparent viscosity of the microemulsions in pores differed from the viscosity measured in the capillary viscometer by less than $40 \%$ for 0.1 micrometer diameter pores and by less than $8 \%$ for 1.0 micrometer diameter pores. These differences are of the same scale as estimates of two effects: adsorption of droplets on the pore wall and the enhanced flow of suspensions near a wall. The absence of larger finite-size effects implies that clustering of droplets on length scales of 0.1 micrometer or larger does not contribute substantially to the microemulsion viscosity.

\section{0,906}

PB95-140489 Not available NTIS

National Inst. of Standards and Technology (NML), Gaithersburg, MD. Chemical Process Metrology Div. Michaelis-Menten Equation for an Enzyme in an Oscillating Electric Field.

Final rept.

B. Robertson, and R. D. Astumian. 1990, 6p.

Pub. in Biophysical Jnl. 58, p969-974 Oct 90.

Keywords: "Enzymes, "Electric fields, Oscillations, Protein conformation, Catalysis, Thermodynamics, $R e$ prints, *Michaelis-Menten equation.

The electric charges on an enzyme may move concomitantly with a conformational change. Such an enzyme will absorb energy from an oscillating electric field. If, in addition, the enzyme has a larger association constant for substrate than for product, as is often true, it can use this energy to drive the catalyzed reaction away from equilibrium. Approximate analytical expressions are given for the field-driven flux, electrical power absorbed, free-energy produced per unit time, thermodynamic efficiency, and zero-flux concentrations. The field-driven flux is written as a generalized Michaelis-Menten equation.

\section{0,907}

PB95-140992 Not available NTIS

National Inst. of Standards and Technology (EEEL), Gaithersburg, MD. Electricity Div.

Associative Electron Attachment to S2F10, $\mathrm{S} 2 \mathrm{OF} 10$, and $\mathrm{S} 2 \mathrm{O} 2 \mathrm{~F} 10$

Final rept.

J. K. Olthoff, K. L. Stricklett, R. J. Van Brunt, I.

Sauers, J. H. Moore, and J. A. Tossell. 1993, 6p

Sponsored by Department of Energy, Washington, DC Office of Electrical Systems.

Pub. in Jnl. of Chemical Physics 98, n12 p9466-9471. 15 Jun 93.

Keywords: "Electron-molecule collisions, "Sulfur fluonides, *Electron attachment *Gas dissociation, Mill eV Range, EV range 01-10, Fluorine ions, Negative ions, Cross sections, Resonance, Reprints, "Sulfur oxofluorides.

The absolute cross sections for dissociative electron attachment to the molecules S2F10S2OF10, and 
$\mathrm{S} 2 \mathrm{O} 2 \mathrm{~F} 10$ were measured in an electron transmission experiment. The corresponding negative-ion fragments were identified in a separate mass spectrometric measurement. For $\mathrm{S} 2 \mathrm{~F} 10$, the attachment of thermal electrons (energy less than $0.1 \mathrm{eV}$ ) appears to result primarily in the formation of $F(-)$ and $S F 5(-)$ with possibly a small fraction of SF4(-) and SF6(-). The ions $\mathrm{F}(-)$ and SF5(-) are also produced from two attachment resonances at electron energies of about 4.5 and 9.5 $\mathrm{eV}$. Both S2OF10 and $\mathrm{S} 2 \mathrm{O} 2 \mathrm{~F} 10$ have unusually large dissociative attachment cross sections at energies near $0.1 \mathrm{eV}$. Electron attachment to $\mathrm{S} 2 \mathrm{OF} 10$ yields primarily SOF5(-), while S2O2F10 yields both SF5(-) and SOF5(-) with possible minor fractions of $F(-)$ and SOF3(-).

\section{0,908}

PB95-141032 Not available NTIS

National Inst. of Standards and Technology (PL), Gaithersburg, MD. Molecular Physics Div

Infrared Spectrum of OCIO in the $2000 / \mathrm{cm}(-1) \mathrm{Re}$ gion: The 2(nu sub 1 ) and (nu sub $1+$ nu sub 3 ) Bands.

Final rept.

J. Ortigoso, R. Escribano, J. B. Burkholder, and W.

J. Lafferty. 1993, 10p.

Sponsored by National Aeronautics and Space Administration, Washington, DC. Upper Atmospheric Research Program.

Pub. in Jnl. of Molecular Spectroscopy 158, p347-356 1993

Keywords: "Chlorine oxides, "Infrared spectra, Atmospheric composition, High resolution, Infrared spectroscopy, Band spectra, Dioxides, Chlorine 35, Chlorine 37, Reprints, Molecular constants

Founer Transform Infrared (FTIR) spectra of the 2nu(sub 1) and nu(sub 1) + nu(sub 3) of $\mathrm{ClO} 2(\mathrm{OClO})$ have been recorded in the $2000 / \mathrm{cm}$ region, with an instrumental resolution of about $0.004 / \mathrm{cm}$. The spectra have been analyzed using a Hamiltonian which includes spin-rotation terms to provide the first high-resolution analysis of these bands. Around 1900 transitions of (35) ClO2 (plus about 600 of (37) ClO2) have been assigned in 2nu(sub 1), and 1100 transitions of (35) $\mathrm{ClO} 2$ (plus about 450 of (37) $\mathrm{ClO} 2$ ) have been identified in nu(sub 1) + nu(sub 3). By making use of all available data, accurate band centers, rotational constants, spin rotational constants, and a number of the chi(sub rs) anharmonicity constants as well as alpha and gamma parameters have been obtained.

\section{0,909}

\section{PB95-141040 Not available NTIS}

National Inst. of Standards and Technology (PL), Gaithersburg, MD. Molecular Physics Div.

Intensities and Dipole Moment Derivatives of the Fundamental Bands of (35) $\mathrm{ClO} 2$ and an Intensity Analysis of the nu1 Band.

Final rept.

J. Ortigoso, R. Escribano, J. B. Burkholder, and W.

J. Lafferty. 1992, 9p.

Pub. in Jnl. of Molecular Spectroscopy 156, p89-97 1992.

Keywords: "Chlorine oxides, "Infrared spectra, Dipole moments, Band spectra, Luminous intensity, Brightness, Chlorine 35, Dioxides, Reprints, Herman-Wallis factors.

Integrated band intensities of all the fundamental bands of OCIO as well as the 2nu(sub 1) overtone and the nu(sub 1) + nu(sub 3) combination band have been measured. Dipole moment derivatives with respect to the normal coordinates have been derived for these bands. For the nu(sub 1) band (35) $\mathrm{ClO} 2$ individual relative lines intensities have been obtained for many high K(sub alpha) (sup r) R branch lines, and a systematic deviation from intensities calculated from a zero order model, in which rotation-vibration interactions are not included, has been found. The deviation can be explained with the use of a transformed dipole moment operator. We have compared these results with those obtained using an expression derived by Watson for symmetric tops.

\section{0,910}

PB95-141107 Not available NTIS

National Inst. of Standards and Technology (CSTL), Gaithersburg. MD. Chemical Kinetics and Thermodynamics Div.
Reaction of Nitric Oxide with Organic Peroxyl Radicals.

Finai rept.

S. Padmaja, and R. E. Huie. 1993, 6p.

Pub. in Biochemical and Biophysical Research Communications 195, n2 p539-544 1993.

Keywords: "Peroxyl radicals, "Nitric oxide, "Reaction kinetics, Free radicals, Aqueous solutions, Biochemistry, Reprints, Alcoholic solutions.

Nitric oxide and organic peroxyl radicals are key reactive radicals implicated in a wide range of biological processes. We have measured rate constants for the reactions of several organic peroxyl radicals with nitric oxide in aqueous solution and found that they are all fast, with $\mathrm{k}=1-3 \times 10(\exp 9) \mathrm{L} / \mathrm{mol} / \mathrm{s}$. The reactions lead to the formation of an intermediate, identified as the organic peroxynitrite, which decayed with a rate constant of $\mathrm{k}=0.1-0.3 / \mathrm{s}$. Possible products of this decay include the more reactive alkoxyl and nitrogen dioxide free radicals, or the less reactive organic nitrate. In alcoholic solutions, the rate constants of the initial reactions were similar, but the product decay rates were about 200 times faster. Because of the ubiquity of both nitric oxide and organic peroxyl radicals, their interaction could make a significant contribu tion to many physiological processes.

\section{0,911}

PB95-141131 Not available NTIS

National Inst. of Standards and Technology (MSEL), Gaithersburg, MD. Ceramics Div.

Ca4Bi6013: A Compound Containing an Unusually Low Bismuth Coordination Number and Short Bi Bi Contacts.

Final rept.

J. B. Parise, C. C. Torardi, M. H. Whangbo, B. P

Burton, C J Rawn and R. S. Roth. 1990, 5 p.

Pub. in Chemistry of Materials 2, n4 p454-458 1990.

Keywords: ${ }^{*}$ Crystal structure, Second harmonic generation, Orthorhombic lattices, Molecular orbitals. Bismuth oxides, Calcium oxides, Reprints, ${ }^{\star}$ Calcium bismuthates, ${ }^{*}$ Calcium bismuth oxides.

Single crystals and powder samples of Ca4Bi6O13 have been synthesized and studied by $x$-ray diffraction. This compound crystallizes in the orthorhombic space group C2mm, with $a=5.9368(7), b=17.356(4), c=$ 7.206(4)A. The absence of a center of symmetry was confirmed by the presence of a second harmonic signal some 60 times that observed for quartz. A weak superstructure exists along $c$ due to alternation of oxy gen and vacancies along the c-axial direction. The structure consists of ribbons of edge-linked $\mathrm{BiO} 5$ square pyramids running paralle! with the c-axis These chains are linked via a 3 -coordinate $\mathrm{Bi}$ atom to form semicylinders stacked along the a-axial direction.

00,912

PB95-141180 Not available NTIS

National Inst. of Standards and Technology (CSTL) Gaithersburg, MD. Thermophysics Div.

Interatomic Potential of Argon.

Final rept.

S. J. Boyes. 1994, 6p.

Pub. in Chemical Physics Letters 221, p467-472 1994 Keywords: "Argon, Virial coefficients, Predictions, Acoustics, Reprints, "Intera tomic potentials.

The recent literature abounds with proposed interatomic potential functions for argon. Of these the Hartree-Fock dispersion (HFD) type functions have been very successful and are considered to be among the best characterizations of the argon interaction to date. It is the purpose of this Letter, however, to show that of the proposed HFD-type potentials, none predic the recent acoustic virial coefficients measured by Moldover et al. In view of this, an alternative interatomic potential has been constructed for argon. The potential is of the Hartree-Fock dispersion individually damped (HFD-ID) type and represents a subtle but significant improvement for the interaction.

\section{0,913}

PB95-141198 Not available NTIS

National Inst. of Standards and Technology (CSTL), Gaithersburg, MD. Surface and Microanalysis Science Div.

Time-Resolved Measurements of Energy Transfer at Surfaces.

Final rept.

R. R. Cavanagh, E. J. Heilweil, and J. C

Stephenson. 1994, 13p.

Pub. in Surface Science 299/300, p643-655 1994.
Keywords: "Surfaces, "Adsorbates, Solid surfaces, Liquid surfaces, Metal surfaces, Energy transfer, ExDielectrics, Électronic states, Measurement, Reprints, Femtosecond pulses.

Developments in time-resolved measurements of en ergy transfer at surfaces are reviewed. Picosecond and femtosecond measurements of vibrational and electronic relaxation at surfaces are highlighted. Experimental results for vibrational relaxation of simple adsorbates on metals, semiconductors, and insulators are reviewed, and relaxation mechanisms such as electron-hole pair formation, multiphonon relaxation and image dipole damping are considered. Energy transfer involving excited electronic states of molecules on liquid and solid dielectric surfaces and the relaxation of surface electronic states on semiconductors and of image states on metal surfaces are discussed.

\section{0,914 \\ PB95-150090 Not available NTIS}

National Inst. of Standards and Technology (MSEL) Gaithersburg, MD. Reactor Radiation Div.

Phase Transitions in Solid C70: Supercooling, Met astable Phases, and Impurity Effect.

Final rep

A. R. McGhie, J. E. Fischer, P. A. Heiney, D. A. Neumann, W. H. Mueller, H. Mohn, H. U. ter Meer P. W. Stephens, and R. L. Cappelletti. 1994, 5p. Contract DEFG-0291-ER45231, Grant NSF-DMR91. 20668

Sponsored by National Science Foundation, Arlington, VA. and Department of Energy, Washington, DC

Pub. in Physical Review B 49, n18 p12 614-12 618, 1 May 94

Keywords: "Fullerenes, *Phase transformations, Orientation(Direction) Metastable state Supercooling Impurities, Reprints, Differential scanning calorimetry Modulated differential scanning calorimetry of sublimed $C(70)$ shows clear evidence for four firstorder transitions, two strong and two weak, which we associate with the two different molecular rotational degrees of freedom in majority (equilibrium) and minority (metastable) phases. The latter correspond to the two different stacking sequences of close-packed layers, and their relative contributions of the thermal data correlate well with high-temperature $\mathrm{x}$-ray powder-diffraction results. The upper of the thermal data correlate well with high-temperature $x$-ray powder-diffraction results. The upper (long axis tumbling) transition in the majority phase exhibits $50 \mathrm{~K}$ supercooling when scanned at $2 \mathrm{~K} / \mathrm{min}$. C(60) impurities at the few \% level depress the transition temperatures substantially.

00,915

PB95-150124 Not available NTIS

National Inst. of Standards and Technology (CSTL), Gaithersburg, MD. Thermophysics Div.

Simulations of Glass Forming Liquids: What Has Been Learned.

Final rep

R. D. Mountain. 1994, 3p.

Pub. in International Jnl. of Modern Physics C 5, n2 p247-249 1994.

Keywords: Computerized simulation, Molecular dynamics, Amorphous state. Thermodynamic properties, Supercooling, Glass, Reprints, "Supercooled liquids.

Molecular dynamics simulations of supercooled fluid mixtures of soft-spheres and of Lennard-Jones particles have revealed the existence of a kinetic transition that occurs above the glass transition temperature. This transition appears to be thermodynamic in origin. It is associated with a change in the local mobility of the particles. The basis of these conclusions is dis cussed.

\section{0,916}

PB95-150355 Not available NTIS

National Inst. of Standards and Technology (CSTL), Gaithersburg, MD. Surface and Microanalysis Science Div.

Calculations of Electron Inelastic Mean Free Paths. 5. Data for 14 Organic Compounds over the 50 . 2000 eV Range.

Final rept.

S. Tanuma, C. J. Powell, and D. R. Penn. 1993, 12p. Pub. in Surface and Interface Analysis 21, p165-176 1993

Keywords: "Mean free path, "Electron scattering, 


\section{CHEMISTRY}

\section{Physical \& Theoretical Chemistry}

$1000, \mathrm{KeV}$ range 1-10, Inelastic scattering, Paraffins Adenines, Carotene, Albumins, Diphenyl compounds, Guanines, Polyacetylene, Polyethylene, Polymethyl methacrylate, Polystyrene, DNA, Surface analysis, Electron spectroscopy, Reprints, Kapton(Trademark)

We report calculations of electron inelastic mean free paths (IMFPs) of 50-2000 eV electrons for a group of 14 organic compounds: $26-n-p a r a f f i n$, adenine, betacarotene, bovine plasma albumin, deoxyribonucleic acid, diphenyl-hexatriene, guanine, kapton, polyacetylene, poly(butene-1-sulfone), polyethylene, polymethylmethacrylate, polystyrene and poly(2 vinylpyridine). The computed IMFPs for these compounds showed greater similarities in magnitude and in the dependences on electron energy than was found in our previous calculations for groups of elements and inorganic compounds (Papers II and III in this series) Comparison of the IMFPs for the organic compounds with values obtained from our predictive IMFP formula TPP-2 showed systematic differences of approx $40 \%$ These differences are due to the extrapolation of TPP 2 from the regime of mainly high-density elements (from which it had been developed and tested) to the low-density materials such as the organic compounds We analyzed the IMFP data for the groups of elements and organic compounds together and derived a modified empirical expression for one of the parameters in our predictive IMFP equation. The modified equation, denoted TPP-2M, is believed to be satisfactory for estimating IMFPs in elements, inorganic compounds and organic compounds.

\section{0,917}

PB95-150421 Not available NTIS

National Inst. of Standards and Technology (MSEL) Gaithersburg, MD. Reactor Radiation Div

Neutron Reflectometry Studies of Surface Oxidation.

Final rept.

D. G. Wiesler, and C. F. Majkrzak. 1994, 6p.

Pub. in Physica B 198, p181-1861994.

Keywords: "Titanium oxides, "Oxide films, "Oxidation, Anodic coatings, Film thickness, Aqueous electrolytes, Surface chemistry, Corrosion resistance, Reprints, Neutron reflectometry, Film density, Hydrogen loading

We have employed neutron reflectometry to study the thickness and density of thin oxide films of Ti in contac with electrolyte. Such films play an important role in corrosion resistance and surface chemistry in aqueous environments. We compare the structures of such ox ides formed by either ramping the potential or by step polarization. The effect of subsequent cathodic bias on the films is explored, and the time evolution of the hy drogen content in the oxide and metal is investigated

\section{0,918}

PB95-150561 Not available NTIS

National Inst. of Standards and Technology (CSTL) Gaithersburg, MD. Thermophysics Div.

Contrast Matched Studies of a Sheared Binary Colloidal Suspension.

Final rept

G. C. Straty, J. Pieper, and H. J. M. Hanley. 1991 , $6 p$.

Sponsored by Department of Energy, Washington, DC Pub. in Molecular Physics 72, n1 p241-246 1991

Keywords: "Suspensions, "Dispersions, Small angle scattering. Neutron scattering, Shear stress, Structure factors, Couette flow, Polystyrene, Colloids, Mixtures, Silica, Latex, Heavy water, Reprints, "Colloidal suspensions.

A method is proposed to measure by neutron scattering the partial scattered intensities from a suspension mixture in an $\mathrm{H} 2 \mathrm{O} / \mathrm{D} 2 \mathrm{O}$ solvent that will match out on of the components. Total and partial neutron-scattered intensities from an aqueous colloidal suspension mixture (volume fraction phi $=0.15$ ) of $91 \mathrm{~nm}$ polystyrene atex and $54 \mathrm{~nm}$ silica particles in water are reported. Results are given for the mixture at rest and unde shear. We comment on the standard assumption that structure factors of components in a mixture should scale with the particle radii.

00,919

PB95-150611 Not available NTIS

National Inst. of Standards and Technology (NML) Gaithersburg, MD. Molecular Physics Div.
Microwave Spectra of van der Waals Complexes of Importance in Planetary Atmospheres.

Final rept.

R. D. Suenram, and F. J. Lovas. 1989, 7p.

Pub. in Proceedings of International Conference on Laboratory Research for Planetary Atmospheres (1st), Bowie, MD., October 25-27, 1989, p78-84.

Keywords: "Microwave spectra, "Planetary atmospheres, "Complexes, Van der Waals forces, Hydrogen bonds, Nitrogen complexes, Oxygen complexes, Rotational spectra, Trimers, Reprints, "Wate dimers, Carbon dioxide complexes, Carbon monoxide complexes, Hydrogen sulfide complexes, Sulfur dioxide complexes, Water complexes, Ozone complexes.

The Fourier-transform Fabry-Perot pulsed-molecularbeam microwave spectrometer at NIST has been used to study the microwave spectra of a number of molecular dimers and trimers that may be present in planetary atmospheres. The weak van der Waals bonds associated with these species usually give rise to rotationaltunneling splittings in the microwave spectra. We have used the microwave spectrum of the water dimer species to illustrate the complications that can arise in the study of the rotational spectra of these loosely bound species. In addition to the water dimer species, the microwave spectra of the following hydrogen-bonded and van der Waals complexes have been studied: (CO2)2 $\mathrm{H} 2 \mathrm{O}, \mathrm{CO} 2(\mathrm{H} 2 \mathrm{O}) 2, \mathrm{CO} 2 \mathrm{H} 2 \mathrm{~S}, \mathrm{~N} 2 \mathrm{H} 2 \mathrm{O}, \mathrm{CO}$ $\mathrm{H} 2 \mathrm{O}, \mathrm{SO}_{2} \mathrm{H} 2 \mathrm{O}$, and $\mathrm{O} 3 \mathrm{H} 2 \mathrm{O}$.

00,920

PB95-150678 Not available NTIS

National Inst. of Standards and Technology (PL), Boulder, CO. Quantum Physics Div.

High-Resolution Infrared Spectroscopy of DF Trimer: A Cyclic Ground State Structure and DF Stretch Induced Intramolecular Vibrational Coupling.

Final rept.

M. A. Suhm, J. T. Farrell, S. Ashworth, and D. J. Nesbitt. 1993, 5p.

Pub. in Jnl. of Chemical Physics 98, n7 p5985-5989, 1 Apr 93.

Keywords: "Deuterium fluorides, *Hydrogen fluoride, "Molecular clusters, "Infrared spectroscopy, Molecula vibration, Absorption spectra, Hydrogen bonds, High resolution, Deuterium compounds, Fine structure, Ground state, Trimers, Reprints, Supersonic expansion.

We present high resolution, infrared laser absorption spectra of (DF) 3 in a slit supersonic jet expansion. In contrast to previous structureless near IR spectra of (HF) 3, the (DF) 3 data reveal clear rotational structure characteristic of a cyclic, 6-membered 'ring,' and therefore provide the first accurate experimental evidence for the equilibrium geometry of any hydrogen fluoride oligomer beyond the well-studied dimer. Furthermore, the spectra display homogeneous rotational fine structure 2-3 orders of magnitude in excess of what could be anticipated from a single vibrational band. Analysis of this fine structure elucidates a novel IVR mechanism which involves single hydrogen bond cleavage, and consequent opening of the (DF) 3 ring on the 40 ps time scale.

00,921

PBg5-150694 Novailable NTIS

National Inst. of Standards and Technology (PL), Boulder, CO. Quantum Physics Div.

Laser Double Resonance Measurements of the Quenching Rates of $\mathrm{Br}((2) \mathrm{P} 1 / 2)$ with $\mathrm{H} 20, \mathrm{D} 20$, $\mathrm{HDO}$, and $\mathrm{O} 2$.

Final rept.

C. A. Taatjes, C. M. Lovejoy, B. J. Opansky, and S. R. Leone. 1991, 6p.

Grants NSF-WLK-1-043, NSF-CHE87-13646

Sponsored by National Science Foundation, Washington, DC.

Pub. in Chemical Physics Letters 182, n1 p39-44, 19 Jul 9

Keywords: Laser applications, Room temperature, Excited states, Heavy water, Quenching, Oxygen, Reprints, "Bromine atoms, Rate constants.

Quenching rate constants for spin-orbit excited $\mathrm{Br}^{*}$ atoms (doublet $\mathrm{P}(1 / 2)$ ) with $\mathrm{H} 2 \mathrm{O}, \mathrm{D} 2 \mathrm{O}, \mathrm{HDO}$, and $\mathrm{O} 2$ are measured at $300 \mathrm{~K}$ using a two-laser pump-probe technique. The rate constants and error bars for the water species are given. The relative rates for the water species are in qualitative agreement with models for electronic-to-vibrational energy transfer based upon dipole-quadrupole coupling. Quenching of $\mathrm{Br}^{*}$ by oxygen is found to be extremely inefficient, which is in disagreement with previously reported results by four orders of magnitude.

\section{0,922}

PB95-150728 Not available NTIS

National Inst. of Standards and Technology (CSTL), Gaithersburg, MD. Surface and Microanalysis Science Div.

Calculations of Electron Inelastic Mean Free Paths (IMFPs). 4. Evaluation of Calculated IMFPs and of the Predictive IMFP Formula TPP-2 for Electron Energies between 50 and $2000 \mathrm{eV}$.

Final rept.

S. Tanuma, C. J. Powell, and D. R. Penn. 1993, 13p. Pub. in Surface and Interface Analysis 20, p77-89 1993.

Keywords: "Mean free path, "Electron scattering, *Electron collisions, EV range 10-100, EV range 100$1000, \mathrm{KeV}$ range 1-10, Inelastic scattering, Aluminum oxides, Gallium arsenides, Uncertainty, Reprints.

We have made additional evaluations of the electron inelastic mean free paths (IMFPS) and of the predictive IMFP formula TPP-2 presented in papers II and III of this series. Comparisons have been made with other formulae for the IMFPS and electron attenuation lengths (ALs). We find substantial differences between our IMFP results for 27 elements and 15 inorganic compounds and the AL formulae of Seah and Dench; these differences include different dependences on electron energy and on material parameters. We present IMFP calculations for $\mathrm{Al} 2 \mathrm{O} 3$ and $\mathrm{GaAs}$ from TPP-2 in which each parameter of the formula is varied in some physically reasonable range about the true value for each compound; these results show the sensitivity of the computed IMFPs to the choices of parameter values. Finally, we give a summary of sources of uncertainty in the IMFP algorithm, in the experimental optical data from which IMFPs are calculated and of the TPP-2 formula. We conclude that TPP-2 is robust and useful for predicting IMFPs for electron energies and material parameter values in ranges for which the formula was developed and tested.

\section{0,923}

Not available NTIS

National Inst. of Standards and Technology (CSTL), Gaithersburg, MD. Process Measurements Div.

Silver Metalization of Octadecanethiol Monolayers Self-Assembled on Gold.

Final rept.

M. J. Tarlov, $1992,10 p$

Pub. in Langmuir 8, n1 p80-89 1992.

Keywords: "Thiols, "Monomolecular films, "Silver, "Gold "Surface chemistry, "Vapor deposition, Atomic energy levels, Temperature dependence, $X$-ray analysis, Ultraviolet spectroscopy, Reprints, "Octadecanethiol, "Self assembled monolayers, "Silver deposition.

The interaction between evaporated silver and self-assembled monolayers of octadecanethiol (ODT) on gold was studied at temperatures of 300 and $90 \mathrm{~K}$ using X-ray photoelectron spectroscopy (XPS), ultraviolet photoelectron spectroscopy (UPS), and ion scattering spectroscopy (ISS). Equivalent Ag coverages ranging from submonolayers to multilayers were examined. Ag deposited at $300 \mathrm{~K}$ penetrates the ODT monolayer and resides at the ODT/gold interface. The attenuation behavior of the XPS C 1 s and Au $4 f$ signals reveals that $\mathrm{Ag}$ nucleates as clusters underneath the ODT monolayer. UPS and ISS data are in accord with XPS results and, furthermore, indicate that the deposited $\mathrm{Ag}$ does not significantly alter the ODT monolayer film structure. In contrast to the behavior observed at 300 $\mathrm{K}$, results from $\mathrm{Ag}$ deposition at $90 \mathrm{~K}$ indicate that $\mathrm{Ag}$ forms clusters on the ODT monolayer surface. In this case the $\mathrm{Ag}$ 3d core level exhibits a shift to higher binding energy relative to that of metallic silver that is attributable to a final state Coulombic effect characteristic of metal clusters on relatively poorly conducting substrates. There is apparently little chemical interaction between $\mathrm{Ag}$ and the hydrocarbon chains at both 90 and $300 \mathrm{~K}$, although at $300 \mathrm{~K} \mathrm{Ag} \mathrm{3d}$ XPS data suggest that $\mathrm{Ag}$ bonds to sulfur head groups. The low-temperature Ag/ODT/Au structure is apparently a metastable phase because warming of the sample to room temperature results in the migration of the Ag to the ODT/ Au interface and possibly further clustering.

00,924

PB95-150751 Not available NTIS 
National Inst. of Standards and Technology (CSTL), Gaithersburg, MD. Process Measurements Div. UV-Photopatterning of Alkylthiolate Monolayers Self-Assembled on Gold and Silver.

Final rept.

M. J. Tarlov, D. R. F. Burgess, and J. G. Gillen.

$1993,2 p$.

Pub. in Jnl. of the American Chemcial Society $115, \mathrm{n} 12$ p5305-5306, 16 Jun 93.

Keywords: "Thiols, "Monomolecular films, "Gold, *Silver, *Uitraviolet radiation, "Coating processes, Surface properties, $X$-ray analysis, Mass spectroscopy, Surface chemistry, Reprints, "Alkylthiolates, *Self assembled monolayers, "Photopatterning.

A method of photopatterning alkylthiolate self-assembled monolayers (SAMS) on gold and silver is described. A pattern of alkylsulfonates is first formed on the alkylthiolate SAMs by ultraviolet (UV) irradiation through a mask. The sample is then immersed in a dilute solution of a different alkylthiol and the alkylsulfonates in the exposed areas are displaced resulting in the incorporation of the second alkylthiol. $X$ ray photoelectron spectroscopy (XPS) and secondary ion mass spectrometry (SIMS) imaging were used to characterize the UV-irradiated and exchanged monolayers. XPS spectra of the $S 2 p$ region indicate the formation of sulfonate species upon UV irradiation the formation of sulfonate species upon Un irradiation
and their displacement by thiolates following immersion. SIMS images of photopatterned and thiol-exchanged SAMS confirm the existence of two molecularly distinct assemblies with faithful reproduction of the mask pattern and resolution of features as small as 15 micrometers.

00,925

PB95-150819 Not available NTIS

National Inst. of Standards and Technology (CSTL), Gaithersburg, MD. Thermophysics Div.

Activated Dynamics, Loss of Ergodicity, and Transport in Supercooled Liquids.

Final rept.

D. Thirumalai, and R. D. Mountain. 1993, 11p. Sponsored by National Science Foundation, Washington, DC

Pub. in Physical Review E 47, n1 p479-489 Jan 93.

Keywords: Ergodic processes, Molecular dynamics, Self-diffusion, Computerized simulation, Temperature dependence, Shear properties, Supercooling, Viscosity, Mixtures, Glass, Reprints, *Supercooled liquids.

The dynamics of the transition from supercooled liquid to glass is examined in terms of several probes: ergodic measures, self-diffusion coefficients, the Van Hove self-correlation functions, and the shear viscosity. Constant-pressure molecular-dynamics calculations at several temperatures are performed for a LennardJones mixture and binary mixtures of soft spheres. The temperature dependence of the ergodicity diffusion parameters for both systems follow the Vogel-Fulcher law. On the other hand the self-diffusion coefficients exhibit Arrhenius behavior for the soft-sphere system, but Vogel-Fulcher behavior for the Lennard-Jones system. These observations suggest that loss of effective ergodicity may be the universal feature of glass-forming substances. Various probes of the dynamics of the mixtures studied here suggest that the mechanism for mass transport dramatically changes from a simple diffusive process to one that involves activated transitions.

\section{0,926}

PB95-151015 Not available NTIS

National Inst. of Standards and Technology (MSEL) Gaithersburg, MD. Reactor Radiation Div.

Methyl Torsional Levels of Solid Acetonitrile (CH3CN): A Neutron Scattering Study.

Final rept.

S. F. Trevino, C. S. Choi, and D. A. Neumann. 1993 $5 p$.

Pub. in Jnl. of Chemical Physics 98, n1 p78-82, 1 Jan 93.

Keywords: "Acetonitrile, Neutron scattering, Neutron diffraction, Crystal structure, Deuterium compounds, Rotational states, Activation energy, Methyl radicals, Reprints.

Neutron powder diffraction has been used to obtain the thermal parameters for the deuterium atoms and to confirm the crystal structure of acetonitrile-d(3) at $4 \mathrm{~K}$. confirm the crystal structure of acetonitrile-d(3) at $4 \mathrm{~K}$,
Inelastic neutron scattering from both isotopic species is used to determine the energies of the first and second rotational levels of the methyl group. These four levels are reasonably reproduced by a threefold potential with $V(3)=125 \mathrm{meV}$. The activation energy derived from this potential is in agreement with that previously obtained from the temperature dependence of $T(1)$ in proton magnetic resonance measurements.

00,927

PB95-151023 Not available NTIS

National Inst of Standards and Technology (NML) Gaithersburg, MD. Chemical Kinetics Div.

Electronic Spectra of $\mathrm{CF} 2 \mathrm{Cl}$ and $\mathrm{CFCl} 2$ Radicals Observed by Resonance Enhanced Multiphoton Ionization.

Final rept

B. P. Tsai, J. W. Hudgens, and R. D. Johnson. 1989

Pub. in Jnl. of Physical Chemistry 93, n14 p5334-5336 1989

Keywords: "Rydberg States, "Fluoroalkanes, "Free radicals, *Vibrational states, "Electronic spectra Spectrum analysis, Energy levels, Reprints, "Multiphoton ionization.

$\mathrm{CFCl} 2$ and $\mathrm{CF} 2 \mathrm{Cl}$ radicals were observed between $365-410 \mathrm{~nm}$ using resonance enhanced multiphoton ionization spectroscopy. Both spectra were generated by two-photon resonances with planar 3p Rydberg states. A third laser photon ionized the radicals. The CF2Cl spectrum displayed a vibrational progression assigned to the out-of-plane bending nu'(sub 4) b(sub 1) (OPLA) mode (omega' (sub 4$)=745 / \mathrm{cm}$ ) and a origin at $406.2 \mathrm{~nm}$ (nu(sub 0-0) $=49,230 / \mathrm{cm}$ ). Assignments for the $\mathrm{CFCl} 2$ radical included the nu'(sub 4) b(sub 1 ) OPLA (omega'(sub 4) $=590 / \mathrm{cm}$ ) and the nu'(sub 2) a(sub 1) CCl symmetric stretch (omega'(sub 2) $870(30) / \mathrm{cm})$ modes. An observed origin was assigned to lie at $49,260 / \mathrm{cm}$.

00,928

PB95-151049 Not available NTIS

National Inst. of Standards and Technology (NML), Gaithersburg, MD. Chemical Kinetics Div.

Homogeneous Gas Phase Decyclization of Tetralin and Benzocyclobutene.

Final rept.

W. Tsang, and J. P. Cui. 1990, 7p.

Pub. in Jnl. of the American Chemical Society 112, n5 p1665-1671 1990.

Keywords: "Tetralin, "Cyclobutene compounds *Reaction kinetics, "Vapor phases, Temperature dependence, Shock tubes, Chemical reactions, Aromatic hydrocarbons, Styrene, Lasers, Pyrolysis, Reprints 'Benzocyclobutene, "Decyclization, Unimolecular reactions.

Teiralin has been decomposed in single pulse shock tube experiments in the temperature range of 1,000 $1,400 \mathrm{~K}$. The initial unimolecular decomposition chan nels are the formation of benzocyclobutene and oallytoluene. Benzocyclobutene decomposes to form styrene. The main products from the hydrogen atom induced decomposition of tetralin are dihydronaphthalenes, styrene, indene and napthalene. Rate constant expressions for these reactions were determined. Comparisons are made with published re sults from the laser powered pyrolysis and shock tube studies, and it is concluded that in both cases the initial unimolecular channels are accessed. The temperatures in the laser experiments are at least as high as in the shock tube studies. The rate expressions for tetralin decomposition are compatible with a biradical mechanism and follow the general patterns for related cyclic compounds.

00,929

PB95-151064 Not available NTIS

National Inst. of Standards and Technology (CSTL) Gaithersburg, MD. Chemical Kinetics and Thermodynamics Div.

Mechanism and Rate Constants for the Reactions of Hydrogen Atoms with Isobutene at High Tem-

peratures.

W. Tsang, and J. A. Walker. 1989, 8p.

Pub. in Proceedings of Conference International Symposium on Combustion (22nd). National Institute of Standards and Technology, p1015-1022 1989

Keywords: "Butenes, "Hydrogen, "Reaction kinetics, *Decomposition reactions, *2-Methylpropene Shock tubes, Temperature dependence, High temperature Pyrolysis, Reprints, "Hydrogen atoms, "Isobutene, Hydrogen abstraction.
Hydrogen atoms from the thermal decomposition of 3,3 trimethylbutanol-2 have been used to induce the decomposition of isobutene in single pulse shock tube experiments. We have been able to observe products arising from all of the high temperature reaction channels for hydrogen attack on isobutene. The following rate expressions have been determined: $\mathrm{k}(\mathrm{H}$ isobutene $\rightarrow$ propylene $\left.+\mathrm{CH}_{3}\right)=1.72 \times 10$ (exp 10) $\operatorname{Exp}(-1808 / \mathrm{T}) \mathrm{l} / \mathrm{mol}-\mathrm{s}$ and $\mathrm{k}(\mathrm{H}+$ isobutene $->2$-methyl2 -allyl $+\mathrm{H} 2)=1.72 \times 10(\exp 11) \operatorname{Exp}(-4024 / \mathrm{T}) \mathrm{I} / \mathrm{mol}$ $\mathrm{s}$ in the temperature range of $1,000-1,180 \mathrm{~K}$ and at 2.5 3 atmospheres of pressure. The rate constants for the abstraction of vinylic hydrogens are not more than $8 \%$ of that for abstraction of the allylic hydrogens. Comparisons are made with rate constants of similar processes involving branched aromatics and lower tem perature results on isobutene. Interesting correlations are observed and lead to the possibility of estimating rate expressions for reactions involving other olefins.

\section{0}

PB95-151148 Not available NTIS

National Inst. of Standards and Technology (CSTL) Boulder, CO. Thermophysics Div.

Experimental Method for Obtaining Critical Densities of Binary Mixtures: Application to Ethane + n-Butane.

Final rept.

L. J. Van Poolen, V. G. Niesen, and J. C. Rainwater $1991,25 p$.

Pub. in Fluid Phase Equilibria 66, p161-185 1991.

Keywords: "Binary mixtures, "Ethane, "Butanes, "Density(Mass/volume). Phase transformations, Vapor phases, Liquid phases, Thermophysical properties, Reprints, "Critical density, *Rectilinear diameter, Van Poolen-Rainwater procedure, Leung-Griffiths theory.

According to the experimental and analytical proce dure proposed by Van Poolen and Rainwater, rectilinear diameters based on measured vapor-liquid coexistence densities along a path of constant overall density and composition have been determined and used to obtain critical densities of the binary mixture ethane + n-butane. Seven experimental runs for the mixture were made at compositions ranging from 21.65 to $80.75 \mathrm{~mol} \%$ ethane. The procedure has been explored as a new way to determine critical densities of mixtures. Results are compared with densities generated from a modified Leung-Griffiths correlation of the extensive vapor-liquid equilibrium data of Kay. The critical densities obtained are in agreement with the correlation within the overall uncertainty of the method, thereby verifying the proposed technique.

00,931

PB95-151361 Not available NTIS

National Inst. of Standards and Technology (CSTL), Gaithersburg, MD. Thermophysics Div.

Ebulliometric Measurement of the Vapor Pressure of Difluoromethane.

Final rept.

L. A. Weber, and A. R. H. Goodwin. 1993, 3p.

Sponsored by Department of Energy, Washington, DC. DC.

Pub. in Jnl. of Chemical and Engineering Data 38, n2 p254-256 Apr 93

Keywords: "Vapor pressure, "Fluorohydrocarbons, *Refrigerants, Ebulliometry, Temperature depend ence, Mathematical models, Boiling points, Reprints, ${ }^{*}$ R32, "Difluoromethane, Antoine equation.

We have used a comparative ebulliometer to make accurate measurements of the vapor pressure of difluoromethane (R32) in the range 49-214 kPa, which corresponds to temperatures on ITS-90 between 208 and $237 \mathrm{~K}$. The results are represented with an Antoine equation and are compared with literature values. We have combined our results with literature values, and we present an interpolating equation for the vapor pressure at temperatures between $190 \mathrm{~K}$ and the critical temperature $(351.36 \mathrm{~K})$. We also tabulate thermodynamic properties for R32 on the saturation boundary between 200 and $250 \mathrm{~K}$

00,932

PB95-151387 Not available NTIS

National Inst. of Standards and Technology (PL), Boulder, CO. Quantum Physics Div. 


\section{CHEMISTRY}

\section{Physical \& Theoretical Chemistry}

Van der Waals Bond Lengths and Electronic Spectral Shifts of the Benzene-Kr and Benzene-Xe Complexes.

Final rept

T. Weber, E. Riedle, H. J. Neusser, and E. W

Schlag. 1991, 7p.

Pub. in Chemical Physics Letters 183, n1-2 p77-83, 23

Aug 91.

Keywords: "Krypton complexes, "Xenon complexes "Chemical bonds, "Spectral shift, Van der Waals forces, Electronic spectra, Ultraviolet spectra, Ground states, Excited states, Reprints, *Benzene complexes.

Rotationally resolved UV-spectra are presented for the 6 (sub 0 , sup 1) bands of benzene- $K r$ and benzene- $X$ complexes yielding precise rotational constants and van der Waals bond lengths for the ground and excited vibronic state, and electronic band shifts. These values complement the previously published data for the othe rare gases and the various quantities have now been determined for all the benzene-rare gas complexes. Measured values of the bond length were used to calculate the band shifts from recent theoretical predictions. They are compared with the experimental values of this work.

\section{0,933}

PB95-151395 Not available NTIS

National Inst. of Standards and Technology (PL), Boulder CO. Quantum Physics Div.

N2(a'(sup 1)Sigma(sub g)(sup +)) Metastable Collisional Destruction and Rotational Excitation Transfer by $\mathrm{N} 2$.

Final rept.

A. B. Wedding, J. Borysow, and A. V. Phelps. 1993 , $8 p$

Contract USAF-FY1455-84-N0641

Sponsored by Phillips Lab., Kirtland AFB, NM

Pub. in Jnl. of Chemical Physics 98, n8 p6227-6234 15 Apr 93

Keywords: "Molecular collisions, "Nitrogen, Molecula energy levels, Molecular excitation, Metastable state, Rotational states, Electric discharges, Optical pumping, Time dependence, Laser applications, Reprints, Rate coefficients.

Quenching and rotational coupling rate coefficients have been measured for the $\mathrm{J}=4-10$, nu $=0$ levels of the a(double prime) singlet Sigma $(g)(1+)$ metastable state of N2 in collisions with ground state N2. Lase absorption is used to monitor the population of rotational levels of the a(double prime) single Sigma $(g)(1+)$ state following depletion of the popu ation of one or more levels by optical pumping to othe states. The observed time dependence of the recovery of population of the perturbed level and the collision induced growth and decay of the populations of adjacent levels are interpreted in terms of quenching to other electronic levels and excitation exchange among adjacent rotational levels.

\section{0,934}

PB95-151437 Not available NTIS

National Inst. of Standards and Technology (NML)

Boulder, CO. Quantum Physics Div.

Nonadiabatic Effects in the Photoassociation of H2S.

Final rept.

K. Weide, V. Staemmler, and R. Schinke. 1990, 2p.

Pub. in Jnl. of Chemical Physics $93, n 1$ p861-862,

Jul 90.

Keywords: "Hydrogen sulfide, "Photodissociation, Nonadiabatic conditions, Vibrational states, Excited states, Reprints, *Photoassociation.

The photodissociation of $\mathrm{H} 2 \mathrm{~S}$ in the $195 \mathrm{~nm}$ band has been extensively investigated in the last decade. Although the major part of the experimental data indicates an overall dissociation dynamics similar to $\mathrm{H} 2 \mathrm{O}$ pronounced differences have been noted. The centra question is whether $\mathrm{H} 2 \mathrm{~S}$ dissociates via a single electronic state, like $\mathrm{H} 2 \mathrm{O}$, or if two states are involved. This question concerns also the origin of the very diffuse vibrational structure. Using the CEPA method we calculated the potential energies of the two lowest excited singlet states for a fixed bond angle of $92 \mathrm{deg}$. In $C(2 n u)$ symmetry these states are singlet $B(1)$ and singlet $A(2)$

\section{0,935}

PB95-151601 Not available NTIS

National Inst. of Standards and Technology (EEEL)

Boulder, CO. Electromagnetic Technology Div.
Studies of the Higher Order Smectic Phase of the Large Electroclinic Effect Material W317.

Final rept.

P. A. Williams, L. Komitov, A. G. Rappaport, D. M Walba, G. W. Day, B. N. Thomas, and N. A. Clark

1993, $11 p$

Pub. in Liquid Crystals 14, n4 p1095-1105 1993

Keywords: "Liquid crystals, Temperature dependence, Electric fields, Reprints, "Electroclinic effect, "W317 liquid crystal, Smectic phases.

We present studies of the large electroclinic effect material W317. We found via X-ray scattering, calorimetry and optical observation that quenching from the smectic A phase results in several higher order phases including an orthogonal (hexatic) smectic with short range in-layer translational order and no interlayer order. We characterize the electroclinic response in the quenched phase, and determine its magnitude and response time as a function of electric field amplitude and temperature.

\section{0,936}

PB95-151759 Not available NTIS

National Inst. of Standards and Technology (MSEL), Gaithersburg, MD. Ceramics Div.

Crystal Chemistry and Phase Equilibria Studies of the $\mathrm{BaO}(\mathrm{BaCO} 3)-\mathrm{R} 2 \mathrm{O} 3-\mathrm{CuO}$ Systems. 4. Crystal Chemistry and Subsolidus Phase Relationship Studies of the CuO-Rich Region of the Ternary Diagrams, $R=$ Lanthanides.

Final rept.

W. Wong-Ng, B. Paretzkin, and E. R. Fuller. 1990

$16 \mathrm{p}$.

See also PB95-151742, Part 2, PB95-151734 and Part

5. PB95-151718

Pub. in Jnl. of Solid State Chemistry 85, n1 p117-132 1990.

Keywords: *Barium oxides, ${ }^{*}$ Yttrium oxides, ${ }^{\star}$ Copper oxides, "Rare earth compounds, "Phase diagrams, *Crystal chemistry High temperature superconductors, YBCO superconductors, Solid solutions, Ternary systems, Crystal phase transformations, Reprints, Yttrium barium cuprates, Phase equilibrium.

A general trend of phase formation, solid solution formation and phase relationship is found to be correlated with the size of $\mathrm{R}$ in the $\mathrm{BaO}-1 / 2 \mathrm{R} 2 \mathrm{O} 3-\mathrm{CuO}$ systems, where $\mathrm{R}=$ lanthanides and yttrium. There is a progressive change in the phase relationship and appearance of these ternary diagrams in the CuO rich region, from the La through the $\mathrm{Nd}, \mathrm{Sm}, \mathrm{Eu}, \mathrm{Gd}, \mathrm{Y}$ to the Er system, with samples prepared in air at $950 \mathrm{C}$. Firstly, the La system has the greatest number of ternary compounds Secondly the superconductor materials, Ba2RCu3O $(6+x)$, of the first half of the f-block elements, e.g. R=La, Nd, Sm, Eu and Gd, which are retatively larger in size, exhibit solid solution of $\mathrm{Ba}(2-$ z) $R(1+z) C u 3 O(6+x)$ with range of formation according to the size of $\mathrm{R}$. These solutions terminate at $\mathrm{Gd}$, after which the superconductor phase presumably assumes a point stoichiometry. Thirdly, a trend regarding the tieline connection between the phases $\mathrm{Ba}(2$ x) $\mathrm{R}(1+\mathrm{x}) \mathrm{Cu} 3 \mathrm{O}(6+\mathrm{x})$, CuO, BaR2CuO5 and binary phase R2CuO4/R2Cu2O5 is observed.

\section{0,937}

PB95-151775 Not available NTIS

National Inst. of Standards and Technology (PL), Boulder, CO. Quantum Physics Div.

Photodissociation of Ammonia at $193.3 \mathrm{~nm}$ : Rovibrational State Distribution of the $\mathrm{NH2}(\mathrm{A}(2) \mathrm{A} 1)$ Fragment.

Final rept.

E. L. Woodbridge, M. N. R. Ashfold, and S. R.

Leone. 1991, 10p

Contract DE-FG02-883ER-13860, Grant NSF-

PHY86-04504

Sponsored by Department of Energy, Washington, DC Office of Energy Research. and National Science Foundation, Washington, DC.

Pub. in Jnl. of Chemical Physics 94, n6 p4195-4204, 15 Mar 91.

Keywords: *Ammonia, "Photodissociation, Far ultraviolet radiation, Rotational states, Vibrational states, Infrared spectroscopy, Emission spectroscopy, Room temperature, Reprints, "Ammonia radicals, Fourier transform spectroscopy.

The rovibrational state distribution of the nascent $\mathrm{NH} 2$ (A(tilde) doublet $\mathrm{A}(1)$ ) fragments generated by $193.3 \mathrm{~nm}$ photodissociation of a room temperature sample of $\mathrm{NH} 3$ is determined through an analysis of a major portion $(6000-13000 / \mathrm{cm})$ of the $\mathrm{NH} 2$ (A(tilde) doublet $A(1) \rightarrow$ X(tilde) doublet $B(1))$ near infrared emission spectrum obtained by time-resolved Fourier transform infrared emission spectroscopy. The essential features are entirely consistent with a direct carry over, into the fragment, of the out-of-plane bending vibrational motion introduced in the parent molecule by the photoexcitation process.

00938

PB95-152112 Not available NTIS

National Inst. of Standards and Technology (CSTL), Gaithersburg, MD. Thermophysics Div.

Susceptibility Critical Exponent for a Nonaqueous Ionic Binary Mixture Near a Consolute Point.

Final rept.

K. C. Zhang, M. E. Briggs, R. W. Gammon, and J. M. H. Levelt Sengers. 1992,6p.

Contract NASA-NAS3-25370

Sponsored by National Aeronautics and Space Administration, Washington, DC.

Pub. in Jnl. of Chemical Physics 97, n11 p8692-8697 Dec 92.

Keywords: "Nonaqueous electrolytes, Temperature dependence, Phenyl ether, Binary mixtures, Ammonium compounds, Reprints, Phase separation(Materials), Critical exponents, Consolute points.

We report turbidity measurements of a nonaqueous ionic solution of triethyl n-hexylammonium triethyl nhexylboride in diphenyl ether. A classical susceptibility critical exponent gamma $=1.01+$ or -0.01 is obtained over the reduced temperature range $10(\exp -4)=$ or $<t=$ or $<10(\exp -1)$. The best fits of the sample transmission had a standard deviation of $0.39 \%$ over this range. Ising and spherical model critical exponents are firmly excluded. The correlation length amplitude zeta(0) from fitting is $1.0+$ or $-0.2 \mathrm{~nm}$ which is $\mathrm{m}: \mathrm{ch}$ larger than values found in neutral fluids and sc.ne aqueous binary mixtures.

00,939

Pot available NTIS

National Inst. of Standards and Technology (NML) Gaithersburg, MD. Chemical Kinetics Div.

Gas Phase Reactivity Study of $\mathrm{OH}$ Radicals with 1,1-Dichloroethene and cis-1,1-Dichloroethene and Trans-1,2-Dichloroethene over the Temperature Range 240-400 K.

Final rept.

Z. Zhang, R. Liu, R. E. Huie, and M. J. Kurylo. 1991, $3 p$.

Pub. in Jnl. of Physical Chemistry 95, n1 p194-196 1991.

Keywords: "Vapor phases, "Reaction kinetics, *Hydroxyl radicals, ${ }^{*}$ Chlorohydrocarbons, Temperature, Fluorescence, Reprints, ”Ethylene/dichloro.

Rate constants have been measured for the reactions of hydroxyl radicals with the three dichloroethenes over the temperature range 240 to $400 \mathrm{~K}$ using the flash photolysis resonance fluorescence technique. The Arrhenius equations derived from the data are given. There was no apparent effect of pressure on the rate constant for the reaction of $\mathrm{OH}$ with trans-1,2dichloroethene over the range 5 to 50 torr (with $\mathrm{Ar}$ ).

\section{0}

PB95-152153 Not available NTIS

National Inst. of Standards and Technology (NML), Gaithersburg, MD. Chemical Kinetics Div.

Rate Constants for the Gas Phase Reactions of the $\mathrm{OH}$ Radical with CF3CF2CHCl2 (HCFC-225ca) and CF2CICF2CHCIF (HCFC-225cb).

Final rept

Z. Zhang, R. Liu, R. E. Huie, and M. J. Kurylo. 1991, $3 p$

Pub. in Geophysical Research Letters 18, n1 p5-7 1991.

Keywords; "Reaction kinetics, "Hydroxyl radicals, * Vydrochlorofluorocarbons.

Rate constants have been measured for the gas phase reactions of the hydroxyl radical $(\mathrm{OH})$ with the hydrochlorofluorocarbons CF3CF2CHCl2 (HCFC$225 \mathrm{ca}$ ) over the temperature range 270 to $400 \mathrm{~K}$ and CF2CICF2CHCIF (HCFC-225cb) over the temperature range 298 to $400 \mathrm{~K}$

00,941

PB95-152187 Not available NTIS 
National Inst. of Standards and Technology (NML), Gaithersburg, MD. Time and Frequency Div.

Rotational Far Infrared Spectrum of (13)CO.

Final rept.

L. R. Zink, P. De Natale, F. S. Pavone, M. Inguscio, M. Prevedelli, and K. M. Evenson. 1990, 7p.

Pub. in Jnl. of Molecular Spectroscopy 143, n2 p3043101990.

Keywords: "Carbon monoxide, "Rotational spectra, *Infrared spectra, Far infrared radiation, Carbon 13 Reprints, Fourier transform spectroscopy.

The pure rotational spectrum of (13) CO between 0.66 and $3.3 \mathrm{THz}$ has been measured with a tunable far infrared spectrometer. Revised values for $\mathrm{B}(\mathrm{O}), \mathrm{D}(\mathrm{0})$, and $H(0)$ have been obtained with a $50 \mathrm{kHz} 1$ sigma standard deviation of the fit. Additional measurements were performed with a Fourier transform spectrometer, and a $1 \mathrm{MHz}(3.3 \times 10(\exp -5) / \mathrm{cm})$ measurement accuracy is demonstrated with this device. The rotational spectrum from $\mathrm{J}$ double prime $=0$ to $\mathrm{J}$ double prime $=30$ is calculated and gives the frequencies with an accuracy (one sigma) of better than $120 \mathrm{kHz}$.

\section{0,942}

PB95-152211 Not available NTIS

National Inst. of Standards and Technology (NML), Gaithersburg, MD. Molecular Physics Div.

Microwave and Submillimeter Spectroscopy of Ar$\mathrm{NH} 3$ States Correlating with $\mathrm{Ar}+\mathrm{NH} 3(\mathrm{j}=1, \mathrm{k}=1)$.

Final rept

E. Zwart, H. Linnartz, W. L. Meerts, W. Klemperer

G. T. Fraser, and D. D. Nelson. 1991, 11p.

Pub. in Jnl. of Chemical Physics 95, n2 p793-803 1991.

Keywords: *Argon complexes, "Microwave spectroscopy, Van der Waals forces, Submillimeter waves, Microwave spectra, Hamiltonians, Anisotropy, Reprints, "Ammonia complexes, Intermolecular potentials.

Microwave and submillimeter transitions for $\mathrm{Ar}-\mathrm{NH} 3$ have been observed and assigned for the two Sigma and two Pi states correlating asymptotically with $\mathrm{Ar}+$ $\mathrm{NH} 3(j=1, k=1)$. The Sigma states are found to lie below the $\mathrm{Pi}$ states and are separated by approximately the inversion splitting of free $\mathrm{NH} 3$. For the $\mathrm{Pi}$ states the $\mathrm{NH} 3$ inversion tunneling is nearly quenched, being only weakly allowed through Coriolis interactions with the nearby Sigma states. The observed microwave and submillimeter spectra also allow the determination of $(14) \mathrm{N}$ quadrupole coupling constants and relative submillimeter absorption intensities. All the above results are interpreted using a model internalrotation inversion Hamiltonian, leading to detailed information about the anisotropy of the intermolecular potential.

00,943

PB95-152823 Not available NTIS

National Inst. of Standards and Technology (NML), Gaithersburg, MD. Center for Chemical Technology. Crystal Packing Interactions of Two Different Crystal Forms of Bovine Ribonuclease A.

Final rept.

L. A. Svensson, J. Dill, L. Sjolin, D. Bacon, J. Moult, B. Veerapandian, G. L. Gilliand, A. Wlodawer and M. Toner. 1991, 12p

Pub. in Jnl. of Crystal Growth 110, n1-2 p119-130 1991

Keywords: "Pancreatic ribonuclease, "Crystals, $\mathrm{pH}$ Hydrogen bonding. Electrostatics, Van der Waals forces, Binding sites, Solvents, Cattle, Reprints.

The crystal packing interactions of two crystal forms of bovine ribonuclease $\mathrm{A}$ are compared. An analysis of the crystal packing interactions, including solvent bridging, indicates that the high-alcohol crystal form of ribonuclease $A$ has a balance of hydrogen bonding, electrostatic and van der Waals interactions. In contrast, the high-salt crystal packing forces are dominated by van der Waals interactions, but at the dimer interface of the molecules in this crystal form the interactions have a large electrostatic component. The electrostatic interactions of the dimer may be due to a rearrangement in the charge-charge interactions at the active site, a direct result of the presence of the inhibitor, deoxythymidine, covalently bound to His 12 .

00,944

PB95-152930 Not available NTIS

National Inst. of Standards and Technology (MSEL) Gaithersburg, MD. Office of Intelligent Processing of Materials.
Memory Function Approach to the Shape of Pressure Broadened Molecular Bands.

Final rept

G. Birnbaum. 1994, 14p.

Pub. in Molecular Physics 81, n3 p519-532 1994.

Keywords: "Molecular absorption, "Absorption spectra, "Pressure broadening, Molecular collisions, Quantum mechanics, Spectral bands, Line spectra, Spectros copy, Reprints, "Memory function, Far wings, Line interference.

A unified treatment of the shape of pressure-broadened molecular absorption bands from near resonance to the far wings is achieved by an approach using memory functions. Empirical models for these functions are employed that interpolate between known short and long time behavior. This treatment includes the following band shaping mechanisms: line intererence, finite duration of collision, molecular torques, and detailed balance. Line interference can produce a very sharp decrease in absorption in the periphery of a band, e.g., as in the nu(sub 3) band of carbon dioxide. Absorption in the far wings, which in this band exhibits a much less rapid decrease with increasing frequency, is attributed to the effect of molecular torques acting during collisions. By using a model memory function, a simple analytical expression is obtained for the far wing absorption. The parameters in this mode are related to known molecular quantities. The computed and measured far wing absorption of the nu(sub 3) band of carbon dioxide broadened by argon at 296 $\mathrm{K}$ and $613 \mathrm{~K}$ are compared.

00,945

PB95-152989 Not available NTIS

National Inst. of Standards and Technology (PL) Gaithersburg, MD. Radiometric Physics Div.

Interatomic Potential of Argon.

Final rept.

S. J. Boyes. 1994, 6p

Pub. in Chemical Physics Letters 221, p467-472 1994

Keywords: "Argon, Virial coefficients, Interatomic forces, Reprints, "Interatomic potentials.

The recent literature abounds with proposed interatomic potential functions for argon of these at Hartree-Fock dispersion (HFD) type functions have been very successful and are considered to be among the best characterizations of the argon interaction to date. It is the purpose of this Letter, however, to show that of the proposed HFD-type potentials, none predict the recent acoustic virial coefficients measured by Moldover et al. In view of this, an alternative interatomic potential has been constructed for argon. The potential is of the Hartree-Fock dispersion individually damped (HFD-ID) type and represents a subtle but significant improvement for the interaction.

00,946

PB95-153011 Not available NTIS

National Inst. of Standards and Technology (PL), Gaithersburg, MD. Molecular Physics Div.

Product State Correlations in the Reaction of $\mathrm{O}((1) \mathrm{D})$ and $\mathrm{H} 2 \mathrm{O}$ in Bimolecular Collisions and in 3. 20 Clusters.

Final rept.

M. P. Casassa, D. G. Sauder, and D. S. King. 1993 $7 p$.

Sponsored by Air Force Office of Scientific Research, Bolling AFB, DC

Pub. in Proceedings of Society of Photo-Optical Instrumentation Engineers: Laser Techniques for State-Selected and State-to-State Chemistry, Los Angeles, CA., January 21-23, 1993, v1858 p256-262.

Keywords: "Molecular collisions, "Molecular clusters, "Oxygen, "Ozone, "Water, Hydroxyl radicals, Chemical reactions, Oxygen 16, Oxygen 18, Reprints, Translations.

Measurements of the translational energy and internal state distributions of $\mathrm{OH}$ products produced in the bimolecular reaction (16) O(singlet $\mathrm{D})+\mathrm{H} 2(18) \mathrm{O}-->$ (16) $\mathrm{OH}+(18) \mathrm{OH}$ are reviewed. These detailed measurements reveal relationships between the states of geminate fragments produced in individual reaction events. Preliminary measurements on the same reaction initiated in $\mathrm{O} 3$ (dot) $\mathrm{H} 2 \mathrm{O}$ clusters formed in free jet expansions are reported. The dynamics of the cluster reaction are dramatically different than those of the bimolecular reaction. The results indicate that the third body present in the cluster (O2), carries a significant amount of the energy released in the reaction.
00,947

PB95-153037 Not available NTIS

National Inst. of Standards and Technology (CSTL), Gaithersburg, MD. Surface and Microanalysis Science Div.

Time-Resolved Measurements of Energy Transfer at Surfaces.

Final rept

R. R. Cavanagh, E. J. Heilweil, and J. C.

Stephenson. 1994, 13p

Pub in Surface Science 299/300, p643-655 1994

Keywords: *Surface chemistry, "Adsorbates, Molecular relaxation, Picosecond pulses, Time dependence, Excited states. Energy transfer, Semiconductors, Dielectrics, Metals, Reprints, Femtosecond pulses, Vibrational relaxation.

Developments in time-resolved measurements of en ergy transfer at surfaces are reviewed. Picosecond and femtosecond measurements of vibrational and electronic relaxation at surfaces are highlighted. Ex perimental results for vibrational relaxation of simple adsorbates on metals, semiconductors, and insulators are reviewed, and relaxation mechanisms such as electron-hole pair formation, multiphonon relaxation and image dipole damping are considered. Energy transfer involving excited electronic states of molecules on liquid and solid dielectric surfaces and the relaxation of surface electronic states on semiconductors and of image states on metal surfaces are discussed.

00.948

PB95-153201 Not available NTIS

National Inst. of Standards and Technology (MSEL),

Gaithersburg, MD. Reactor Radiation Div.

Rotational Dynamics of $\mathrm{C} 60$ in Na2RbC 60

Final rept.

C. Christides, K. Prassides, D. A. Neumann, K.

Tanigak, I. Hirosawa, T. W. Ebbesen, J. R. D.

Copley, and J. Mizuki. 1993, 6p.

Pub. in Europhysics Letters 24, n9 p755-760 1993

Keywords: "Buckminsterfullerene, "Fullerenes, Temperature range 0013-0065 K, Temperature range order transformations, Rubidium compounds, Sodium compounds, Neutron scattering, Inelastic scattering Superconductors, Reprints, "Sodium rubidium fullerides, Rotational dynamics.

We have measured the low-energy neutron inelasticscattering (NIS) spectra of superconducting $\mathrm{Na} 2 \mathrm{RbC}(60)$ in the temperature range $50-350 \mathrm{~K}$. Welldefined librational peaks are observed at $50 \mathrm{~K}$ at 2.83(17) meV (FWHM = 1.7(5) meV). They soften and broaden with increasing temperature. Their behavio mimics that found in solid $C(60)$ and differs markedly from $K 3 C(60)$. The rotational barrier for $C(60)$ reorientations in $\mathrm{Na} 2 \mathrm{RbC}(60)$ is somewhat higher than in pristine $\mathrm{C}(60)$ and approximately half as large as in $\mathrm{K} 3 \mathrm{C}(60)$. An order-disorder transition is anticipated at a temperature higher than that found in $\mathrm{C}(60)$

00,949

PB95-153219 Not available NTIS

National Inst. of Standards and Technology (MSEL)

Gaithersburg, MD. Reactor Radiation Div.

Rotational Dynamics of Solid C70: A Neutron-Scattering Study.

Final rept.

C. Christides, T. J. S. Dennis, K. Prassides, J. R. D Copley, R. L. Cappelletti, and D. A. Neumann. 1994 $7 p$.

Pub. in Physical Review B 49, n4 p2897-2903, 15 Jan 94.

Keywords: *Fullerenes, Temperature range 0000-0013 $\mathrm{K}$, Temperature range 0013-0065 K, Temperature range 0065-0273 K, Temperature range 0273-0400 K Temperature range 0400-1000 K Temperature dependence Neutron diffraction, Neutron scattering, Inelastic scattering, Low energy, Reprints, Rotational dynamics.

We report the results of neutron-diffraction and low-energy neutron-inelastic-scattering experiments on highpurity solid $C(70)$ between 10 and $640 \mathrm{~K}$. Therma hysteresis effects are found to accompany structural changes both on cooling and on heating. The observed diffuse scattering intensity does not change with temperature. At $10 \mathrm{~K}$ broad librational peaks are observed at $1.82(16) \mathrm{meV}$ (full width at half maximum $=1.8(5)$ $\mathrm{meV})$. The peaks soften and broaden further with in- 
creasing temperature. At and above room temperature, they collapse into a single quasielastic line. At 300 $K$, the diffusive reorientational motion appears to be somewhat anisotropic, becoming less so with increasing temperature. An isotropic rotational diffusion model, in which the motions of adjacent molecules are uncorrelated, describes well the results at $525 \mathrm{~K}$. The temperature dependence of the rotational diffusion constants is consistent with a thermally activated process having an activation energy of $32(7) \mathrm{meV}$.

\section{0,950}

PB95-153292 Not available NTIS

National Inst. of Standards and Technology (MSEL), Gaithersburg, MD. Reactor Radiation Div.

Structure and Dynamics of Buckyballs

Final rept.

J. R. D. Copley, W. I. F. David, and D. A. Neumann.

1993, 9p.

Pub. in Neutron News 4, n4 p20-28 1993.

Keywords: "Buckminsterfullerene, "Fullerenes, Molecular structure, X-ray diffraction, Neutron scattering, Inelastic scattering, Molecular dynamics, Reviews, Reprints, US NIST.

$\mathrm{C}(60)$ (buckminsterfullerene) is the most well-known, the most stable, the most readily available, and in many respects the most interesting member of a recently discovered class of pure carbon molecules known as the fullerenes. A method of making macroscopic quantities of fullerene mixtures was described in late 1990, and since that time many scientists have been investigating the properties of $C(60)$, the higher fullerenes, and their various compounds and derivatives. To date most of the neutron scattering research on these materials has been carried out either at the $20 \mathrm{MW}$ research reactor of the National Institute of Standards and Technology (NIST), or at the ISIS spallation source, Rutherford-Appleton Laboratory. In this article we shall describe the research that has interested and occupied us the most, namely diffraction and low energy inelastic scattering studies of solid $\mathrm{C}(60)$. Much of the work that we omit is discussed in two recently published reviews.

00,951

PB95-153300 Not available NTIS

National Inst. of Standards and Technology (MSEL),

Gaithersburg, MD. Reactor Radiation Div.

Neutron and $X$-Ray Scattering Cross Sections of Orientationally Disordered Soild $\mathrm{C} 60$.

Final rept.

J. R. D. Copley, and K. H. Michel. 1993, 18p

Pub. in Jnl. of Phys: Condens. Mater. 5, p4353-4370 1993.

Keywords: "Buckminsterfullerene, "Fullerenes, Differential cross sections, Scattering cross sections, $X$ ray scattering, Neutron scattering, Orientation(Direction), Single crystals,
Powder(Particles). Distribution functions, Crystal fields, Reprints.

Differential cross sections for neutron and $\mathrm{x}$-ray scattering have been derived for the orientationally disordered phase of solid $\mathrm{C}(60)$. Interaction centers are placed at nuclei and at the centers of interatomic bonds. Bragg and diffuse scattering cross sections, for single crystals and for powders, are formulated using symmetry-adapted rotator functions. Thermal averages are calculated taking account of crystal field effects. Thermally averaged orientational distribution functions have also been calculated.

00,952

PB95-153730 Not available NTIS

West Coast Information System, Walnut Creek, CA.

Dielectric Studies of Fluids with Reentrant Reso-

nators.

Final rept.

A. R. H. Goodwin, and M. R. Moldover, 1993, 10p

Contract DE-Al05-88ER13823

Sponsored by Department of Energy, Washington, DC. Pub. in Proceedings of Symposium on Energy Engineering Sciences (11th), Argonne, IL., May 3-5, 1993, p139-148.

Keywords: "Fluorohydrocarbons, "Refrigerants, "Dielectric properties, "Cavity resonators, Radio waves, Dipole moments, Binary mixtures, Ethane, Carbon dioxide, Argon, Calibration, Helium, Mathematica models, Reprints, "1,1,1,2,3,3-hexafluoropropane, *R236ea, Phase boundaries, Clausius-Mossotti equation.
We have used a reentrant radio-frequency ( $r$ ) cavity as a resonator operating near $375 \mathrm{MHz}$ to measure changes in the dielectric constant of fluids within it. The utility of these measurements was demonstrated by determining the dipole moment of $1,1,1,2,3,3$ hexafluoropropane, a candidate replacement refrigerant (denoted R236ea) and by detecting the phase boundaries in the mixture $((1-x) \mathrm{C} 2 \mathrm{H} 6+x \mathrm{CO} 2)$, for the mole fraction $x=0.492$. The densities of the coexisting phases of the mixture were determined using the Clausius-Mossotti relation which has errors on the order of $0.5 \%$ in this application. To test the accuracy of the present techniques, the if resonator was calibrated with helium and then used to redetermine the molar polarizability A(sub e) of argon. The results were in excellent agreement with published values. Our design of the reentrant resonator makes it suitable for use with corrosive fluids at temperatures up to $400 \mathrm{C}$.

00,953

PB95-160602 PC A17/MF A04

National Inst. of Standards and Technology (CSTL), Gaithersburg, MD.

CSTL Technical Activities, 1993.

Feb 94, 385p, NISTIR-5303.

Presented to the Board on Assessment of NIST Pro grams, National Research Council, February 1-2, 1994. See also report for 1992, PB93-173482 and report for 1991, PB94-160769.

Keywords: "Research and development, "Chemistry, Biochemistry, Biotechnology, Chemical engineering, Reaction kinetics, Thermodynamics, Inorganic compounds, Technology transfer, Standards, Thermophysics, Organic compounds, Surface chemistry, Microanalysis, Measurement, Quality assurance, Processing, "Chemical Science and Technology Library, Standard reference materials, US NIST, National Institute of Standards and Technology.

The expanded responsibilities the authors were given nearly six years ago in becoming the National Institute of Standards and Technology have provided us with a significant challenge. In response to this challenge, the authors have expanded their interactions with U.S industry to meet their needs and help improve their global competitiveness, while continuing to provide the national system of chemical and physical measurements, the fundamental research base for tomorrow's chemical science and technology, and a national reference laboratory to address critical problems related to public health and safety. With these goals in mind, the report contains research from the following divisions: Biotechnology, Chemical Engineering, Chemical Kinetics and Thermodynamics, Inorganic Analytical Research, Organic Analytical Research, Process Measurements, Surface and Microanalysis Science, and Thermophysics.

\section{0,954}

PB95-161097 Not available NTIS

National Inst. of Standards and Technology (MSEL) Gaithersburg, MD. Reactor Radiation Div.

Neutron Powder Diffraction Study of the Nuclear and Magnetic Structures of the Oxygen-Deficient Perovskite YBaCuCoO5

Final rept.

O. Huang, P. Karen, V. L. Karen, A. D. Mighell, I. N. Sora, N. Rosov, A. Santoro, A. Kjekshus, and J. W. Lynn. 1994, 7p.

Pub. in Jnl. of Solid State Chemistry 108, p80-86 1994

Keywords: "Crystal structure, Barium oxides, Cobalt oxides, Copper oxides, Yttrium oxides, Perovskites, Room temperature, Tetragonal lattices, Lattice parameters, Neutron diffraction, Magnetic moments, Re prints, "Yttrium barium cuprate cobaltates, "Magnetic ordering.

The nuclear and magnetic structures of the oxygen-deficient perovskite $\mathrm{YBaCuCoO} 5$ have been determined by neutron powder diffraction at room temperature. The nuclear structure has the symmetry of space group $P 4 / \mathrm{mmm}$ and lattice parameters $\mathrm{a}=3.8679(1)$ $c=7.5674(2) \mathrm{A}$. Copper and cobalt atoms are completely disordered in this compound and the oxygen vacancies are located on the layer of the yttrium atoms. As a consequence of this configuration, the $\mathrm{Co} / \mathrm{Cu}$ atoms have fivefold, pyramidal coordination. The magnetic structure is based on a unit cell related to that of the nuclear structure by an axis transformation of matrix $(1,-1,0 / 1,1,0 / 0,0,2)$. The magnetic origin of the extra reflections observed experimentally was es tablished by polarized neutron diffraction measure ments. If we assume that the magnetic structure has tetragonal symmetry, then the magnetic moments have to assume an orientation parallel to the $\mathrm{c}$ axis of the unit cell.

\section{0,955}

PB95-161543 Not available NTIS

National Inst. of Standards and Technology (MSEL), Gaithersburg, MD. Polymers Div.

Octacalcium Phosphate Carboxylates. 2. Characterization and Structural Consideration.

Final rept.

M. Markovic, B. O. Fowler, and W. E. Brown. 1993 ,

110.

See also Part 1, PB95-161535. Sponsored by American Dental Association Health Foundation, Chicago, IL.

Pub. in Chem. Mater. 5, n10 p1406-1416 1993.

Keywords: "Molecular structure, "Physicochemical properties, Calcium phosphates, Ions, Dentistry, X-ray diffraction, Infrared spectroscopy, Raman spectroscopy, Crystallography, Crystal lattices, Reprints *Octacalcium phosphate carboxylates

Detailed physicochemical characterization of octacalcium phosphate carboxylates (OCPCs) with structurally incorporated succinate, adipate, suberate, sebacate, fumarate, and citrate ions is reported. Compositional formulas of the OCPCs were derived from $\mathrm{Ca}, \mathrm{P}, \mathrm{C}, \mathrm{H}$, and $\mathrm{H} 2 \mathrm{O}$ analyses. $\mathrm{X}$-ray diffraction patterns of OCPCS show structural similarity with the parent compound octacalcium phosphate (OCP); the $b$ and $c$ axes of the unit cells were nearly the same as those of OCP but the a axes were progressively expanded concomitant with carbon chain length. Infrared and Raman assignments were made for nearly all bands of these six OCPCs; an OCP-succinate containing the deuterated succinate ion was prepared to facilitate band assignments. Spectra of the OCPCs, as compared to that of OCP, showed the presence of carboxylate groups, changes in water bonding, only slight changes in $\mathrm{PO} 4$ environments, and preferential reduction in HPO4(5) content. OCP has two crystallographically nonequivalent HPO4 groups (designated 5 and 6 ); the preferential replacement of HPO4(5) by the dicarboxylate ion is plausible considering lattice geometry. By utilizing combined data from the different methods, the possible positions of carboxylate ions in the OCPC structures are discussed.

\section{0,956}

PB95-161873 Not available NTIS

National Inst. of Standards and Technology (PL), Boulder, CO. Ouantum Physics Div.

Slit Jet Infrared Spectroscopy of Hydrogen Bonded N2HF Isotopomers: Rotational Rydberg-KleinRees Analysis and H/D Dependent Vibrational Predissociation Rates.

Final rept.

D. J. Nesbitt, T. G. Lindeman, J. T. Farrell, and C. M. Lovejoy. 1994, 11p.

Grant NSF-PHY90-12244

Sponsored by National Science Foundation, Arlington, VA.

Pub. in Jnl. of Chemical Physics 100, n2 p775-785, 15 Jan 94.

Keywords: "Nitrogen complexes, "Infrared spectroscopy Vibrational states, Rotational states, Absorption spectra, High resolution, Hydrogen bonds, Infrared lasers, Nitrogen 14, Nitrogen 15, Isomers, Reprints, "Hydrogen fluoride complexes, "Deuterium fluoride complexes, "Isotopomers, Vibrational predissociation, Supersonic expansion, Potential energy surfaces.

High resolution IR laser direct absorption spectra in a slit jet are presented and analyzed for nitrogen ((15)N (14)N-HF, (14)N (15)N-HF, (15)N(15)N-HF, and deuterium ((14)N(14)N-DF) substituted N2HF isotopomers. Both (14) N(15) N-HF and (15) N(14) N-HF isomers are observed, indicating a sufficiently deep minimum in the hydrogen bonding potential energy surface to quench internal rotation of the N2. The vibrationally averaged stretching potentials for each substituted species are recovered from rotational Rydberg-Klein-Rees (RKR) analysis. Features of the one-dimensional (1D) potential surface such as hydrogen bond length, harmonic force constant, and well depth are then tested for isotopic invariance by direct comparison of the different isotopomers. Agreement among the various $\mathrm{N}$ substituted species for HF based complexes for either nu(HF) $=0$ or 1 is excellent, and provides effective $1 \mathrm{D}$ potentials for the stretching $\mathrm{CO}$ ordinate between 3.39 and $3.75 \mathrm{~A}$. 
00,957

PB95-162152 Not available NTIS

National Inst. of Standards and Technology (EEEL), Boulder, CO. Electromagnetic Fields Div.

Diffusion of Copper into Gold Plating.

Final rept.

S. P. Pucic. 1993, 4p.

Pub. in Proceedings of Institute of Electrical and Electronics Engineers Instrumentation and Measuremen Technology Conference, Irvine, CA., May 18-20, 1993 p114-117.

Keywords: "Copper alloys, "Gold alloys, "Diffusion coefficient, "Electroplating, "Electrical resistivity, "Microwaves, "Interfaces, Electromagnetic radiation, Copper oxides, Surface properties, Electrical engineering, Nickel, Skin effects, Ambient temperature, Reprints.

The value of the room temperature copper - gold interdiffusion coefficient derived by extrapolating from high temperature measurements is an underestimate by several orders of magnitude. Once the full thickness of the gold film is penetrated, copper accumulates on the surface, and a layer of high concentration of copper exists immediately below the gold/air interface. Electrical resistivity of an alloy is much higher than the resistivity of either component. This high resistivity layer may be localized within the skin depth of propagating electromagnetic waves; in cases where copper has reached the surface, it is permanently within the skin depth at any frequency. A nickel 'diffusion barrier.' commonly applied between copper and gold, is unsuitable in many microwave and millimeter-wave applications because of ferromagnetism of nickel at room temperature. The compound that forms on the surface of untreated copper at room temperature in a reasonably clean atmosphere is cuprous oxide. Its properties make it a better alternative to gold in microwave and millimeter-wave engineering.

00,958

PB95-162269 Not available NTIS

National Inst. of Standards and Technology (MSEL), Gaithersburg, MD. Reactor Radiation Div.

Neutron-Scattering Study of Librations and Intramolecular Phonons in Rb2.6K0.4C60.

Final rept.

D. Reznik, W. A. Kamitakahara, D. A. Neumann, R

M. Strongin, M. A. Cichv, A. B. Smith, J. R. D.

Copley, and J. E. Fischer. 1994, 6p.

Pub. in Physical Review B 49, n2 p1005-1010, 1 Jan 94.

Keywords: "Fullerenes, Neutron scattering, Inelastic scattering. Temperature dependence, MeV range 1 . 10, Superconductors, Librations, Potentials, Phonons Reprints, "Rubidium potassium fullerides, Density of states.

We report the results of inelastic neutron-scattering measurements on $\mathrm{Rb}(2.6) \mathrm{K}(0.4) \mathrm{C}(60)$. Librationa modes were observed as broad peaks with maxima between 4.1 and $4.7 \mathrm{meV}$, as the temperature is lowered from 300 to $12 \mathrm{~K}$. As in $\mathrm{K} 3 \mathrm{C}(60)$, no change in the width or position of the librational peak was observed when the sample was cooled through the superconducting transition. Thus any coupling of the librations to electronic states is small. The magnitude of the orientational potential barrier was estimated from the librational peak frequency. A flat background observed in the low-energy inelastic-scattering spectra is ascribed to two-phonon scattering. The density of states of intramolecular modes is similar to that of previously studied $\mathrm{M} 3 \mathrm{C}(60)$ compounds; modes at 53 and $66 \mathrm{meV}$ in pure $\mathrm{C}(60)$ are not observed in $\mathrm{Rb}(2.6) \mathrm{K}(0.4) \mathrm{C}(60)$

\section{0,959}

PB95-162319 Not available NTIS

National Inst. of Standards and Technology (CSTL), Gaithersburg, MD. Surface and Microanalysis Science Div.

Laser-Induced Desorption of NO from Si(111): Effects of Coverage on NO Vibrational Populations. Final rept.

L. J. Richter, S. A. Buntin, D. S. King, and R. R.

Cavanagh. $1990,10 p$

Contract DE-Al05-84ER13150

Sponsored by Department of Energy, Washington, DC Pub. in Jnl. of Electron Spectroscopy and Related Phenomena 54/55, p181-190 1990

Keywords: "Nitric oxide, "Desorption, Near infrared radiation, Visible radiation, Vibrational states, Surface chemistry, Substrates, Silicon, Reprints, "Laser induced desorption.

Laser-induced desorption of NO from Si(111) has been investigated using state-specific detection techniques. The observed energy partitioning in the desorbed NO varies significantly with the NO coverage. Characterization of energy partitioning with desorption-laser wavelengths in the range 355-1907 nm indicates that different substrate electronic excitations are responsible for the desorption at low NO coverage vs saturation coverage. The relationship between the substrate electronic excitation and observed vibrational-statepopulation distributions is explored.

\section{0,960}

PB95-162368 Not available NTIS

National Inst. of Standards and Technology (CSTL), Gaithersburg MD Thermophysics Div.

Viscosity of the Saturated Liquid Phase of Six Halogenated Compounds and Three Mixtures.

Final rept

D. Ripple, and O. Matar. 1993, $5 p$

Pub. in Jnl. of Chemical and Engineering Data 38, n4 p560-564 1993.

Keywords: "Kinematic viscosity, "Fluorohydrocarbons, "Binary mixtures, Viscometers, Temperature dependence, Refrigerants, Reprints, *1,1,1,2Tetrafluoroethane, "Bis(difluoromethyl) ether," "2Difluoromethoxy-1,1,1-trifluoroethane

"Pentafluoroethane,

" 1 -Chloro-1,2,2,2

tetrafluoroethane, "Difluoromethane, "R134a, "RE134, "RE245, "R125, "R124, "R32.

Data are reported for the viscosity of six saturated liquids and three mixtures of these liquids over a temperature range from 250 to $330 \mathrm{~K}$. The liquids studied are the halogenated compounds, $1,1,1,2-$ tetrafluoroethane (R134a), bis(difluoromethyl) ether (RE134), 2-(difluoromethoxy)-1,1,1-trifluoroethane (RE245), pentafluoroethane (R125), 1-chloro-1,2,2,2tetrafluoroethane (R124), and difluoromethane (R32). The mixtures studied are R125 + R134a, R32 + R134a, and R32 + R124, all at approximately $50 \%$ mole fraction. A capillary viscometer constructed of stainless steel and sapphire was used to obtain the data. The measurements are accurate to $3-5 \%$ of the kinematic viscosity. A free volume model of viscosity was used to correlate the data.

00,961

PB95-162459 Not available NTIS

National Inst. of Standards and Technology (NML), Gaithersburg, MD. Chemical Process Metrology Div.

Simultaneous Forward-Backward Raman Scatter ing Studies of D2 Broadened by D2, $\mathrm{He}$, and Ar. Final rept.

G. J. Rosasco, W. J. Bowers, W. S. Hurst, A. D.

May, J. P. Looney, and K. C. Smyth. 1991, 9p.

Pub. in Jnl. of Chemical Physics 94, n12 p7625-7633, 15 Jun 91

Keywords: "Raman spectra, "Deuterium, "Hydrogen, Atom-molecule collisions, Atom-atom collisions, Line broadening, Line shape, Colliding beams, Helium, Argon, Reprints.

Beam crossings within a spherical-mirror, multipasscell provide the opportunity to measure, simultaneously, the Raman spectrum for both the forward and backward scattering geometries. The analyses necessary to quantitatively account for such forward-backward spectra are summarized. These simultaneous forward-backward spectra enable unique experimental tests of lineshape functions used for the description of the Raman Q-branch spectrum under conditions where Doppler contributions and Dicke narrowing are significant. Results for the D2:D2 and D2:He systems support the well-known Galatry, or soft collision, lineshape function. For the case of $\mathrm{D} 2: \mathrm{Ar}$, evidence is advanced for the need to employ the more general complex soft collision function. In addition, these studies have provided data on linear-with-density line broadening (previously published) and line shifting coefficients (reported here) for these molecular systems.

\section{0,962}

PB95-162509 Not available NTIS

National Inst. of Standards and Technology (NML), Gaithersburg, MD. Radiometric Physics Div.
Vibronic Coupling and Other Many-Body Effects in the 4 sigmag $(-1)$ Photoionization Channel of CO2. Final rept.

P. Roy, R. J. Bartlett, W. J. Trela, S. H. Southworth, J. E. Hardis, V. Schmidt, J. L. Dehmer, T. A. Ferrett, and A. C. Parr. 1991, $8 \mathrm{p}$

Pub. in Jnl. of Chemical Physics 94, n2 p949-956 1991.

Keywords: "Carbon dioxide, "Photoionization, EV range 10-100, Photoelectron spectroscopy, Branching ratio, Vibrational states. Angular distribution, Forbidden transitions, Autoionization, Reprints.

Vibrational branching ratios and photoelectron angular distributions were measured for $4($ sigma)g Of particular interest are three vibrational components of the resulting $\mathrm{CO} 2(1+) \mathrm{C}($ tilde) doublet Sigma(sub $\mathrm{g}$, sup +) state--the allowed (000) and (100) bands and the forbidden (101) band. The wavelength dependence of the beta parameter for the forbidden band deviated significantly from that of the two allowed bands, showing instead a strong resemblance to that of the $B$ (tilde) doublet Sigma(sub u, sup +) state. This behavior suggests that vibronic coupling to the $B$ (tilde) doublet Sigma(sub $u$, sup + ) state is responsible for the appearance of the forbidden (101) band in the $\mathrm{C}$ (tilde) doublet Sigma(sub g, sup +) state photoelectron spectrum. We also observe evidence for other many-body effects--shape-resonance-induced continuum-continuum coupling and doubly-excited autoionizing resonances--in the present data.

\section{0,963}

PB95-162731 Not available NTIS

National Inst. of Standards and Technology (NML) Gaithersburg, MD. Chemical Process Metrology Div. Fundamental Studies of Gas Sensor Response Mechanisms: Palladium on SnO2(110).

Final rept.

S. Semancik, and T. Fryberger. 1990, 4p.

Pub. in Proceedings of Internationa! Meeting on Chemical Sensors (3rd), Cleveland, OH., September 24-26, 1990, p23-26.

Keywords: "Surface chemistry, "Gas detectors "Palladium, Adsorption, Oxygen, Hydrogen, Planar devices, Electrical properties, Tin oxides, Surface properties, Reprints, Coadsorption.

Surface analytical techniques and in situ conductance measurements have been used to study the sensing action of low coverage Pd dispersed on a SnO2(110) crystal. The Pd coverage dependence and the tem. perature dependence for $\mathrm{H} 2$ detection are discussed for this system. The authors also examine the role of coadsorbate reactions between hydrogen and oxygen at the $\mathrm{Pd} / \mathrm{SnO} 2$ surfaces in controlling interfacial electrical properties. Results from this model study are being used to guide the fabrication of prototype conductance sensors consisting of thin (epitaxial) oxide films with metal layers

00,964

PB95-162756 Not available NTIS

National Inst. of Standards and Technology (NML), Gaithersburg, MD. Chemical Kinetics Div.

Temperature Dependence of the Rate Constants for Reaction of Dihalide and Azide Radicals with Inorganic Reductants.

Final rept.

L. C. T. Shoute, Z. B. Alfassi, P. Neta, and R. E.

Huie. 1991, 5 p.

Pub. in Jnl. of Physical Chemistry 95, n8 p3238-3242 1991.

Keywords: "Radiolysis, "Radicals, "Temperature, ${ }^{*}$ Reaction kinetics, Inorganic azides, Chemical reactions, Inorganic compounds, Reduction(Chemistry) Radiation chemistry, Activation energy, Reprints, Rate constants.

Rate constants for several reactions of inorganic radicals with inorganic reductants in aqueous solutions have been measured by pulse radiolysis as a function of temperature, generally between 5 and $75 \mathrm{deg} C$. The temperature dependence of the reaction rate constant is correlated to the reaction exothermicity for the metal complexes, which apparently react by outersphere electron transfer. The simple anions, however, have lower activation energies, which do not correlate well with the exothermicities, suggesting that these anions probably react by an inner-sphere mechanism. 


\section{0,965}

PB95-162764 Not available NTIS

National Inst. of Standards and Technology (NML), Gaithersburg, MD. Chemical Kinetics Div.

lodine Atoms and lodomethane Radical Cations: Their Formation in the Pulse Radiolysis of lodomethane in Organic Solvents, Their Complexes, and Their Reactivity with Organic Reductants.

Final rept.

L. C. T. Shoute, and P. Neta. 1991, 4p.

Pub. in Jnl. of Physical Chemistry 95, n11 p4411-4414 1991

Keywords: "Radiolysis, "Iodine, "Iodine organic compounds, "Reaction kinetics, Atoms, Cations, Chemical reactions, Organic solvents, Radicals, Radiation chemistry, Chemical reactivity, Reprints, Pulse radiolysis

Pulse radiolysis of iodomethane in various organic solvents leads to formation of iodine atoms or iodomethane radical cations, which in turn form complexes with iodomethane or with the solvent. Radiolysis in cyclohexane gives $\mathrm{CH} 3 \mathrm{II}$, which exhibits an absorption peak at $390 \mathrm{~nm}$, whereas radiolysis in benzene forms the solvent complex, C6H6.I, which exhibits an intense broad absorption centered at $490 \mathrm{~nm}$. Replacing the methyl group of iodomethane radical cation with ethyl or isopropyl decreases the reactivity, whereas trifluoromethyl increases the reactivity. These oxidation reactions proceed via an intermediate complex between the iodine species and the organic reductant.

\section{0,966}

PB95-162814 Not available NTIS

National Inst. of Standards and Technology (NML), Gaithersburg, MD. Chemical Kinetics Div.

Ionization Energy of Sulfur Pentafluoride and the Sulfur Pentafluoride-Fluorine Atom Bond Dissociation Energy.

Final rept.

L. W. Sieck, and P. J. Ausloos. 1990, 5p.

Pub. in Jnl. of Chemical Physics 93, n11 p8374-8378 1990.

Keywords: "Thermochemistry, "Mass spectroscopy, *Ionization potentials, Thermodynamic properties Atomic properties, Chemical reactions, Chemical equilibrium, Sulfur hexafluoride, Fluorides, Reprints, Ionmolecule reactions.

The techniques of Fourier Transform Spectroscopy and High Pressure Mass Spectrometry have been used to investigate the ionization potential (I.P.) of SF5 via electron exchange reactions. An I.P. of $9.60+$ or $-0.05 \mathrm{eV}$ has been derived from this data, which is in disagreement with estimates from flowing afterflow measurements (I.P. > or $-10.15 \mathrm{eV}$ ). In addition, no evidence has been found for a fluoride transfer equilibrium involving $\mathrm{CF} 3(1+)$ and SF6, which was though to interrelate the heats of formation of $\mathrm{CF} 3(1+)$ and SF5(1+).

\section{0,967}

PB95-163051 Not available NTIS

National Inst. of Standards and Technology (NML)

Gaithersburg, MD. Chemical Kinetics Div.

Diamond and Graphite Precursors: Comments.

Final rept.

S. E. Stein. 1990, 1 p.

Pub. in Nature 346, n6284 p517 1990

Keywords: "Diamonds, "Graphite, "Precursors, Thermodynamics, Nucleation, Stability, Reprints Nanostructures.

The letter 'Nanometre-Sized Diamonds are More Stable Than Graphite' by Badziag et al. (BVEG) attempts to provide a basis for the increasingly common observation that diamond is a product in carbon-forming reaction systems. In a plot of bonding energy versus $\mathrm{H}$ / $\mathrm{C}$ ratio they find diamond precursors to be of lower energy than graphite precursors. They suggest that this implies that diamond precursors are more 'stable' than graphite precursors, hence, from a thermodynamic standpoint, homogeneous diamond nucleation is no more difficult than graphite nucleation.

\section{0,968}

PB95-163465 Not available NTIS

National Inst. of Standards and Technology (NML), Gaithersburg, MD. Chemical Kinetics Div.
Shock Tube Techniques in Chemical Kinetics.

Final rept.

W. Tsang, and A. Lifshitz. 1990, 41p.

Pub. in Annual Review of Physical Chemistry 41, p5595991990

Keywords: "Shock tubes, "Reaction kinetics, *Reviews, High temperature, Chemical reactions, Ignition, Reprints.

The review is concerned with the use of shock tubes to study the kinetics of high temperature gas phase chemical processes. It will emphasize reports on investigations published during the past ten years. The last Annual Review article on this subject was published in 1969 by Belford and Strehlow. A book 'Shock Waves in Chemistry', published in 1981, covers much of the literature during the intervening years. There are many reasons for interest in high temperature chemical kinetics. Increasing the temperature opens new reaction channels and thus expands the range of phenomena that can be studied. In conjunction with lower temperature results, the nature of the chemical reaction can be more properly defined. Many important industrial processes occur at high temperatures. With the increasing role of computer simulations for the understanding of such complex processes, there is a great need for fundamental quantitative kinefic information which are the necessary inputs for such programs. The shock tube provides a unique means of generating controlled and chemically well defined high temperature environments.

\section{0,969}

PB95-163473 Not available NTIS

National Inst. of Standards and Technology (NML),

Gaithersburg, MD. Chemical Kinetics Div.

Hydrogen Atom Attack on Perchloroethylene.

Final rept.

W. Tsang and J. A. Walker. 1991, 7p.

Pub. in Proceedings of International Symposium on Combustion, v23 p139-145 1991.

Keywords: "Tetrachloroethylene, "Hydrogen, *Reaction kinetics, "Combustion kinetics, Shock tubes, High temperature, Temperature dependence, Chemi$\mathrm{cal}$ reactions, Reprints, "Hydrogen atoms, Perchloroethylene.

Hydrogen atoms have been reacted with perchloroethylene under the high temperature conditions of a single pulse shock tube. Rate expressions over the temperature range $950-1100 \mathrm{~K}$ and at pressures near 2.5 atm were determined. Comparisons are made with results from other olefinic, aromatic and related systems and the effects on hydrogen reactivity with olefins upon chlorine substifution are deduced.

\section{0,970}

PB95-163523 Not available NTIS

National Inst. of Standards and Technology (MSEL), Gaithersburg, MD. Reactor Radiation Div.

Neutron Spectroscopic Comparison of Rare-Earth/ Hydrogen alpha-Phase Systems.

Final rept

T. J. Udovic, J. J. Rush, N. F. Berk, P. Vajda, O

Blaschko, I. S. Anderson, and J. N. Daou. 1993, 9p Pub. in Zeitschrift fuer Physikalische Chemie 179 , p349-357 1993.

Keywords: "Lutetium hydrides, "Scandium hydrides, "Yttrium hydrides, "Vibrational spectra, Neutron spectroscopy, Neutron scattering, Solid solutions, Deuterides, Reprints.

Incoherent inelastic neutron scattering was used to study the vibrational spectroscopy of hydrogen (and deuterium) in alpha-phase solid solution with Sc, Lu, and $Y$. Low-resolution spectra of the tetrahedrallybound $\mathrm{H}$ (and $\mathrm{D}$ ) in the three rare-earth metals exhibit similar behavior: a soft c-axis-polarized vibration about 25-30\% lower in energy than the doubly-degenerate vibrations polarized along the basal plane. Previous diffuse elastic neutron scattering studies of these systems indicate the existence of short-range ordering of metal-atom-bridged hydrogen pairs into $\mathrm{c}$-axis directed chains. High-resolution spectra of the broad $\mathrm{C}$-axis vibrations reveal a temperature- and $\mathrm{H}$-concentrationdependent splitting believed to represent local 'optic' and 'acoustic' bands associated with the dynamical coupling of these hydrogen pairs.

PB95-163531 Not available NTIS

National Inst. of Standards and Technology (PL), Gaithersburg, MD. Radiometric Physics Div.
Photoelectron Study of Electronic Autoionization in Rotationally Cooled N2: The $n=6$ Member of the Hopfield Series.

Final rept.

K. Ueda, J. B. West, M. A. Hayes, J. L. Dehmer, M.

R. F. Siggel, and A. C. Parr. 1993, 6p.

Contract W-31-109-ENG-38

Sponsored by Department of Energy, Washington, DC. and Science and Engineering Research Council, Swindon (England).

Pub. in Jnl. of Physics B: Atomic and Molecular Optical Physics 26, pL601-L606 1993.

Keywords: *Nitrogen, "Autoionization, Molecular energy levels, Rotational states, Angular distribution, Synchrotron radiation, Electron spectroscopy, Cross sections, Photoionization, Photoelectrons, Asymmetry, Reprints, Ab initio calculations.

We report preliminary results of a study of the Hopfield series in rotationally cooled molecular nitrogen. By using angle resolved electron spectroscopy in combination with synchrotron radiation, partial cross sections and asymmetry parameters for formation of the vibrationally resolved $\mathrm{X}$ and $\mathrm{A}$ states of $\mathrm{N} 2(1+)$ were measured in the region of the $n=6$ member of the Hopfield bands. The results are compared with earlier ab initio MQDT calculations and reflect qualitative agreement for the $X$ doublet Sigma(sub $g$, sup + ) blet Pi(sub mu) decay channel.

\section{0,972}

PB95-163812 Not available NTIS

National Inst. of Standards and Technology (CSTL), Gaithersburg, MD. Thermophysics Div.

Criteria for Establishing Accurate Vapor Pressure Curves.

L. A Weber. 1994,6p.

Sponsored by Department of Energy, Washington, DC. Pub. in Rev. Int. Froid 17, n2 p117-122 1994.

Keywords: "Vapor pressure, "Fluorohydrocarbons, "Refrigerants, Specific heat, Thermodynamic properties, Reprints, ${ }^{*} \mathrm{R} 123,{ }^{*} \mathrm{R} 134 \mathrm{a}$.

The authors used standard thermodynamic relationships to demonstrate the accuracy of new vapor pressure measurements by using them in the calculation of liquid-phase heat capacities and comparing the results with recently published experimental values. The results for new alternative refrigerants R134a and R123 are used as examples.

00,973

PB95-164059 Not available NTIS

National Inst. of Standards and Technology (MSEL), Gaithersburg, MD. Ceramics Div.

Molecular Orbital Calculations of Bond Rupture in Brittle Solids.

Final rept.

W. Wong-Ng, G. S. White, and S. W. Freiman. 1990, 3p.

Pub. in Proceedings of Conference on Frontiers of Chemistry (2nd), Columbus, $\mathrm{OH}$., November 11-14, 1990, v2 p21-23.

Keywords: "Molecular orbitals, "Chemical bonds, "Solids, "Brittleness, "Environmental effects, Silicon dioxide, Numerical analysis, Crack propagation, Covalent bonds, Self consistent field, Strains, Reprints.

Based on crack growth rate data, it is known that most brittle solids, ranging from completely ionic (MgF2) to mixed ionic/covalent ( $\mathrm{SiO} 2, \mathrm{Al} 2 \mathrm{O} 3$ ), undergo environmentally enhanced bond rupture. In order to further our understanding of the bond rupture mechanism, we have carried out molecular orbital calculations (MO) using the ab initio self-consistent field technique. The work to date has involved investigations of the effects of strain on the atomic charges, bond overlap population and ionic character of the Si-O bond at the crack tip of silica. Silica was chosen as the model material because of the extensive experimental data available on it.

\section{0,974}

Not available NTIS

National Inst. of Standards and Technology (PL), Boulder, CO. Quantum Physics Div.

Laser Flash Photolysis/Time-Resolved FTIR Emission Study of a New Channel in the Reaction of $\mathrm{CH} 3+\mathrm{O}$ : Production of $\mathrm{CO}(\mathrm{v})$.

Final rept.

P. W. Seakins, and S. R. Leone. 1992, 8p.

Sponsored by Department of Energy, Washington, DC. Office of Energy Research. 
Pub. in Jnl. of Physical Chemistry 96, n11 p4478-4485 1992

Keywords: "Methyl radicals, "Oxygen atoms, "Carbon monoxide, Vibrational spectra, Chemical reactions, Photolysis, Reprints, Fourier transform infrared spectroscopy.

The reaction of $\mathrm{CH} 3$ radicals and $\mathrm{O}$ atoms is studied using laser flash photolysis/time-resolved Fourier transform infrared emission spectroscopy. In addition to observing formaldehyde vibrational $\mathrm{CH}$ emission, molecular CO vibrational emission is also detected. Experiments are performed to confirm that $\mathrm{CO}$ is a primary product from the title reaction. The observed CO vibrational distribution $(n u=1-8)$ can be fit with a vibrational temperature of $12700+$ or $-1400 \mathrm{~K}$, and the fraction of the reaction exothermicity released into $\mathrm{CO}$ vibration is 0.22 . The branching fraction to $\mathrm{CO}$ is estimated to be $0.40+$ or -0.10 .

\section{0,975}

PB95-164307 Not available NTIS

National Inst. of Standards and Technology (CSTL), Gaithersburg, MD.

Brownian Diffusion of Hard Spheres at Finite Concentrations.

Final rept.

M. S. Selim, M. A. Al-Naafa, and M. C. Jones. 1993 ,

$14 \mathrm{p}$

Pub. in American Institute of Chemical Engineers Jnl. 39, n1 p3-16 Jan 93.

Keywords: "Viscosity, "Spheres, "Brownian movement, "Diffusion coefficient, Dispersions, Silica, Concentration(Composition), Colloids, Reprints, Concentration dependence.

The authors investigated the effect of concentration on the Brownian diffusion of uncharged rigid spheres. Monosize silica spheres were prepared according to the method of Stober (1968). The particles were sterically stabilized by chemisorption of stearic alcohol at their surface by the method developed by van
Helden (1981). Particle radius was $14.5 \mathrm{~nm}$ from elecHelden (1981). Particle radius was $14.5 \mathrm{~nm}$ from elec-
tron micrographs of the coated particles. Osmotic pressure measurements of the sterically stabilized particles dispersed in cyclohexane showed that the particles behaved as hard spheres. The measurements agreed well with predictions from the Carnahan-Starling equation over the concentration range $0.0458<\mathrm{phi}<0.37$ where phi is the volume fraction of the particles in the suspension. Viscosity measurements of silica dispersions were made over the concentration range $0<$ phi $<0.25$. The Brownian diffusion coefficient of the particles dispersed in cyclohexane was measured over the concentration range $0.0055<$ phi $<0.248$ using Taylor's hydrodynamic stability method.

\section{0,976}

\section{PB95-164315 Not available NTIS}

National Inst. of Standards and Technology (PL), Boulder, CO. Quantum Physics Div.

Bonding in Doubly Charged Diatomics.

Final rept.

J. Senekowitsch, S. ONeil, and W. Meyer. 1992, 9p. Contracts AFOSR-NSFGO, AFOSR890074

Sponsored by Air Force Office of Scientific Research, Bolling AFB, DC.

Pub. in Theoretica Chimica Acta 84, p85-93 1992.

Keywords: "Chemical bonds, *Atomic ions, Potential energy, Wave functions, Reprints, Dication potentials.

The potential energy of interacting atomic ions $A(+)+$ $B(+)$ often shows a shallow local minimum separated by a broad potential barrier from the dissociation products at much lower energy. Early interpretations of dication potential shapes were based on the similarity of the electronic structure between isoelectronic neutral and ionic species and led to a picture of a chemical bond superimposed on a repulsive Coulomb potential. More recently, barriers in dication potentials have commonly been interpreted as avoided curve crossings involving covalent and ionic structures. In this paper, we demonstrate that the former model is the appropriate one except in cases with very small asymptotic ionic/ covalent energy splittings. By denving dication wavefunctions from their neutral isoelectronic counterparts, we obtain upper bound dication potential curves which show all the characteristic features. By further modeling induction effects, we arrive at an almost quantitative fit of accurate $a b$ initio dication potentials.
National Inst. of Standards and Technology (CSTL), Gaithersburg, MD. Thermophysics Div.

Application of the Taylor Dispersion Method in Supercritical Fluids.

Final rept.

J. M. H. L. Sengers, U. K. Deiters, U. Klask, P.

Swidersky, and G. M. Schneider. 1993, 30p.

Pub. in International Jnl. of Thermophysics 14, n4 893-922 Jul 93.

Keywords: "Supercritical fluids, Diffusion coefficient Light scattering, Critical point, Carbon dioxide, Benzene, Toluene, Diffusivity, Reprints, Taylor dispersion.

This paper describes some of the experimental and theoretical problems encountered when the Taylor dispersion method is applied to the measurement of diffusion coefficients near gas-liquid critical points. We have used our own measurements of diffusion of benzene and toluene in supercritical carbon dioxide, along with measurements from several other sources, to illustrate some of the experimental challenges. Special attention is given to the peak shape. The intercomparisons are greatly simplified by comparing the experimental data as functions of density, rather than pressure. We find large and unexplained discrepancies between the various experimental sources. We discuss the theoretical predictions for the relationships between the diffusion coefficients and diffusivities obtained from Taylor dispersion and dynamic light scattering in fluids near critical points. We conclude that there is no strong reason to press for Taylor dispersion measurements near the gas-liquid critical point of the carnier gas.

\section{0,978}

PB95-164331 Not available NTIS

National Inst. of Standards and Technology (CSTL). Gaithersburg, MD. Thermophysics Div.

Critical Behavior of lonic Fluids.

Final rept.

J. M. H. L. Sengers, and J. A. Given. 1993, 15p.

Pub. in Molecular Physics 80, n4 p899-913 1993.

Keywords: "Electrolytes, Thermodynamic properties, Critical point, Reviews, Reprints, lonic fluids, Phase separation.

The past 25 years have seen detailed experimental confirmation, in many fluids, of concepts from the the ory of critical phenomena. It has been demonstrated that fluids belong to the universality class of the $3 \mathrm{D}$ Ising model, with critical exponents as given by renormalization group calculations. Nevertheless, there is an important class of fluid systems, those with ionic components, for which it is not clear that the notions of critical behavior developed for uncharged systems apply. We will review the available experimenta evidence, and assess the state of theoretical knowledge.

\section{0,979}

PB95-164349 Not available NTIS

National Inst. of Standards and Technology (CSTL), Gaithersburg, MD. Thermophysics Div.

Standard States, Reference States and Finite-Concentration Effects in Near-Critical Mixtures with Applications to Aqueous Solutions.

Final rept.

J. M. H. L. Sengers, A. H. Harvey, R. Crovetto, and

J. S. Gallagher. 1992, 23p.

Pub. in Fluid Phase Equilibria 81, p85-107 1992.

Keywords: "Aqueous solutions, "Critical point, Critical experiments, Liquid-gas mixtures, Debye-Huckel theory, Supercritical fluids, Helmholtz free energy, Concentration(Composition), Activity coefficients, Carbon dioxide, Dilution, Reprints.

The excess Gibbs free energy reference states of both solvent and solute, as commonly used for aqueous solutions, behave anomalously near the critical point of water. This leads to a concentration-dependence of the partial and apparent molar properties that is stronger than usually assumed for nonelectrolyte as well as for electrolyte solutions, and affects a large range of pressure-temperature space where water is highly compressible. As a consequence extrapolation techniques developed for extracting infinite-dilution properties from data at finite concentration will fail. Examples from various experimental sources, including recent volume measurements in supercritical dilute aqueous $\mathrm{CO} 2$, will be used to make this point. The anomalous initial concentration dependence of apparent molar properties interferes with the determination of the Debye-Huckel limiting law description, conventionally incorporated in the excess Gibbs free energy, fails when the com pressibility of the solvent changes rapidly. A more appropriate frame of reference will be proposed.

00,980

PB95-164414 Not available NTIS

National Inst. of Standards and Technology (NML) Gaithersburg, MD. Electron and Optical Physics Div. Surface Geometry of BaO on W(100): A Surface-Extended X-Ray-Absorption Fine-Structure Study.

Final rept.

A. Shih, C. Hor, W. Elam, J. Kirkland, and D.

Mueller. 1991, 9p.

Pub. in Physical Review B 44, n11 p5818-5826, 15 Sep 91 .

Keywords: "Barium oxides, "Monomolecular films, Surface analysis, Substrates, Single crystals, Adsorption, Tungsten, Reprints, X-ray absorption fine structure.

A surface-extended $x$-ray-absorption fine-structure study of ordered monolayers of coadso bed barium and oxygen on a single-crystal $W(100)$ surface is described. A (square root of 2) $X$ (square root of 2 ) R45(degrees) structure with a stoichiometric bariumto-oxygen ratio, and a (2(square root of 2)) $X$ (square root of 2) R45(degrees) structure with a nearly 1:2 barium-to-oxygen atomic ratio both form on W(100). The surface-extended $x$-ray absorption fine-structure results indicate that all of the barium and oxygen atoms are nearly coplanar in the (square root of 2 ) $X$ (square root of 2) R45(degrees) overlayer. The Ba-to-O distance in this overlayer is $3.20+$ or $-0.05 \mathrm{~A}$ and beta $=82 \mathrm{deg}+$ or $-5 \mathrm{deg}$, where beta is the angle between the Ba-O internuclear axis and the surface normal. For the (2(square root of 2)) $X$ (square root of 2 ) R45(degrees) overlayer, there are two types of oxygen sites. Oxygen atoms, nearly coplanar with the barium atoms are also present in these films.

00,981

PB95-164422 Not available NTIS

National Inst. of Standards and Technology (CSTL), Gaithersburg, MD. Surface and Microanalysis Science Div.

Observation of a Stable Methoxy Intermediate on $\operatorname{Cr}(110)$.

Final rept.

N. D. Shinn. $1992,9 p$.

Sponsored by Department of Energy, Washington, DC. Pub. in Surface Science 278, p157-165 1992

Keywords: "Surface chemistry, Photoelectron spec troscopy, Ultraviolet spectroscopy, Chemical dissociation, Temperature range 0065-0273 K, Temperature range 0273-0400 K, Stability, Work functions, Photoemission, Methanol, Chromium, Reprints, "Methoxy radicals.

Ultraviolet photoelectron spectroscopy (UPS) and work function changes are used to identify a stable methoxy intermediate, $\mathrm{CH} 3 \mathrm{O}$ (ads), on $\mathrm{Cr}(110)$ at $90 \mathrm{~K}$ following low exposures of methanol, $\mathrm{CH} 3 \mathrm{OH}(\mathrm{g})$. Higher methanol exposures at $90 \mathrm{~K}$ add subsequent layers of physisorbed methanol which desorb above $120 \mathrm{~K}$. Methoxy dissociation into atomic fragments occurs between 250 and $400 \mathrm{~K}$. The absence of a stable $\mathrm{CO}$ (ads) dissociation intermediate indicates that methyl group dehydrogenation is rate limiting on $\operatorname{Cr}(110)$. As was previously found on $\mathrm{Fe}(100)$ and $\mathrm{Mo}(100)$, the stability of $\mathrm{CH} 3 \mathrm{O}$ (ads) over $\mathrm{CO}$ (ads) on $\mathrm{Cr}(110)$ is opposite of that proposed by Sexton based upon relative heats of adsorption. Consideration of the $\mathrm{CH} 3 \mathrm{O}$ :metal and CO:metal bonding mechanisms leads to the proposition that substrate electronic properties, rather than steric or substrate morphological factors, are responsible for the anomalous methoxy stability on these surfaces.

\section{0,982}

PB95-164430 Not available NTIS

National Inst. of Standards and Technology (PL) Gaithersburg, MD. Molecular Physics Div.

Fragment State Correlations in the Dissociation of NO. $H F(v=1)$.

Final rept.

J. H. Shorter, M. P. Casassa, and D. S. King. 1992

Pub. in Jnl. of Chemical Physics 97, n3 p1824-1831, 1 Aug 92.

Keywords: "Chemical dissociation, Chemical bonds Excited states, Vibrational states, Rotational states, Fragments, Dimers, Reprints, ${ }^{*}$ Nitric oxide complexes, "Hydrogen fluoride complexes, "Vibrational predissociation. 
The $N O(n u, J)$-fragment population distributions and recoil energies were measured for the vibrational predissociation of NO (dot) HF following excitation of the H-F stretch. Most of the available energy appears in NO vibration and/or HF rotation. There is little recoil momentum. The data support two possibilities for the $\mathrm{NO}$ (dot) HF dimer bond energy: (1) $\mathrm{D}(0)=448+$ or - $5 / \mathrm{cm}$ with coincident pairs of fragments $\mathrm{NO}(\mathrm{nu}=0)$ $+\mathrm{HF}(\mathrm{J}=12)$ and $\mathrm{NO}(\mathrm{nu}=1)+\mathrm{HF}(\mathrm{J}=8)$; (2) $\mathrm{D}(0)$ $=1769+$ or $-10 / \mathrm{cm}$ with $\mathrm{J}(\mathrm{HF})=9$ and 2 , respectively.

\section{0,983}

PB95-164471 Not available NTIS

National Inst. of Standards and Technology (PL)

Gaithersburg, MD. Radiometric Physics Div.

Shape-Resonance-Enhanced Continuum-Continuum Coupling in Photoionization of $\mathrm{CO} 2$.

Final rept.

M. R. F. Siggel, J. B. West, M. A. Hayes, I. Iga, A. C. Parr, and J. L. Dehmer. 1993, 8p.

Pub. in Jnl. of Chemical Physics 99, n3 p1556-1563, 1 Aug 93

Keywords: "Carbon monoxide, "Photoionization, EV range 10-100, Angular distribution, Branching ratio, Electronic states, Photoelectrons, Asymmetry, Reprints.

We have measured photoionization branching ratios and photoelectron asymmetry parameters for photoionization of $\mathrm{CO} 2$ leading to the first four electronic states of $\mathrm{CO} 2(1+)$ over the photon energy range from 20 to $50 \mathrm{eV}$. The motivation for this work was the prediction by Lucchese (J. Chem. Phys. 92, 42.03 (1990)) that the sigma(sub mu) shape resonance in the (4 sigma(sub g))(sup -1) C(tilde) doublet Sigma(sub g, sup +) ionization channel would influence the photoionization dynamics in the other valence-shell continua through continuum-continuum channel interaction, with the main effect occurring in the (3 sigma(sub mu))(sup -1) B(tilde) doublet Sigma(sub mu, sup +) channel. Indeed, clear evidence for this phenomenon is observed in this channel, the most prominent indication being a broad, shallow minimum in the asymmetry parameter at approx $40 \mathrm{eV}$. Comparisons of the present results with theory and other measurements reflect good overall agreement and provide some guidance regarding the effectiveness of alternative approximations used in the theoretical calculations.

\section{0,984}

PB95-164489 Not available NTIS

National Inst. of Standards and Technology (CSTL),

Gaithersburg, MD. Thermophysics Div.

Ebulliometric Measurement of the Vapor Pressure of 1-Chloro-1,1-Difluoroethane and 1,1Difluoroethane

Final rept.

A. M. Silva, and L. A. Weber. 1993, 3p.

Pub. in Jnl. of Chemical and Engineering Data 38, n4 p644-646 1993.

Keywords: "Vapor pressure, "Fluorohydrocarbons, "Refrigerants, Ebulliometry, Temperature dependence, Boiling points, Reprints, 1-Chloro-1,

The vapor pressures of 1-chloro-1,1-difluoroethane (R142b) and 1,1-difluoroethane (R152a) have been measured at temperatures between 224.8 and 284.7 $\mathrm{K}$ for R142b and between 219.9 and $273.1 \mathrm{~K}$ for R152a by a comparative ebulliometric technique. Our results have been combined with selected published results to provide a smoothing equation for the vapor pressure. For R142b, our equation is valid from 200 to 300 $K$ while for R152a the temperature range goes from $215 \mathrm{~K}$ to the critical temperature, near $386 \mathrm{~K}$.

\section{0,985}

PB95-164513 Not available NTIS

National Inst. of Standards and Technology (PL),

Gaithersburg, MD. Molecular Physics Div.

2nu9 Band of Propyne-d 3 .

Final rept.

K. Singh, G. Rajappan, V. A. Job, W. B. Olson, V. B. Kartha, and A. Weber. 1993, 12p.

Pub. in Jnl. of Molecular Spectroscopy 157, p467-478 1993.

Keywords: "Propyne, "Infrared spectra, Molecular spectra, Vibrational spectra, Rotational spectra, Band spectra, Temperature range 0065-0273 K, Room temperature, Deuterium compounds, High resolution, Reprints, Fourier transform spectroscopy.
High-resolution Fourier transform spectra of $\mathrm{CD} 3 \mathrm{CCH}$ at room temperature and at $195 \mathrm{~K}$ have been recorded in the region of the $2 \mathrm{nu}(\mathrm{sub} 9)$ band $(1225-1285 / \mathrm{cm})$ at an apodized resolution of $0.004 / \mathrm{cm}$. Approximately 1000 vibration-rotation transitions in the $P$ and $R$ branches of 2nu(sub 9) (A(sub 1)) have been fitted with a standard deviation of $0.00035 / \mathrm{cm}$. Two vibration-rotation interactions have been identified and analyzed. The parameters of the 2nu(sub 9) (A(sub 1)) state and some of the those of the interacting states have been delermined.

00,986

PB95-164596 Not available NTIS

National Inst. of Standards and Technology (CSTL), Boulder, CO. Chemical Engineering Div

Phase Composition, Viscosities, and Densities for Aqueous Two-Phase Systems Composed of Polyethylene Glycol and Various Salts at $25 \mathrm{C}$.

Final rept.

S. M. Snyder, K. D. Cole, and D. C. Szlag. 1992, 7p. Pub. in Jnl. of Chemical and Engineering Data 37, n2 p268-274 Apr 92

Keywords: "Polyoxyethylene, "Inorganic salts, "Phase diagrams, Density(Mass/volume), Viscosity, Sodium carbonates, Sodium sulfates, Magnesium sulfates, Potassium phosphates, Ammonium sulfate, Phase transformations, Reprints, "Polyethylene glycol.

Phase diagrams of aqueous two-phase systems composed of polyethylene glycol and various salt solutions were measured. The densities and viscosities of these phase systems were also measured. Polyethylene glycol was used with three average molecular masses of 1000,3350 , and 8000 . The salts used were magnesium sulfate sodium sulfate, sodium carbonate, ammonium sulfate, and potassium phosphate. Phase diagram data, as well as the densities and viscosities of the phases, were measured at $25 \mathrm{C}$.

\section{0,987}

\section{PB95-164638 Not available NTIS}

National Inst. of Standards and Technology (CSTL), Gaithersburg, MD. Process Measurements Div

Characterization of Cytochrome c/Alkanethiolate Structures Prepared by Self-Assembly on Gold.

Final rept

S. Song, R. A. Clark, E. F. Bowden, and M. J.

Tarlov. 1993, 9p.

Pub. in Jnl. of Physical Chemistry 97, n24 p6564-6572. 1993.

Keywords: "Cytochrome c, "Monomolecular films, "Gold, "Adsorption, Electron transfer, Electrodes, Spectrum analysis, Kinetics, Surface properties, Biochemistry, Reprints, *Alkane thiolates.

Composite monolayer structures comprised of cytochrome c strongly adsorbed to alkanethiolate selfassembled (SA) monolayers on sputter-deposited gold film electrodes, i.e., cyt c/HOOC $\left(\mathrm{CH}_{2}\right) \mathrm{nS} / \mathrm{Au}(\mathrm{n}=5,10$, 15), were examined using cyclic voltammetry (CV), electrochemical impedance spectroscopy (EIS), and $X$-ray photoelectron spectroscopy (XPS). Monolayer coverage of cytochrome $c$ in a stable, functional, electroactive state was obtained in neutral phosphate electroactive state was obtained in neutral phosphat
buffer of low ionic strength for the thickest film $(n=15)$ Somewhat lower electroactive coverages were generally observed with the thinner SA monolayers $(n=$ $5,10)$ The surface formal potential of cytochrome c, $+215 \mathrm{mV}$ vs NHE, is nearly identical to values pre viously reported for cytochrome $c$ bound to physiological membranes. The potential usefulness of these composite monolayers for investigating biological electron transfer is highlighted.

\section{0,988}

PB95-168704 Not available NTIS

National Inst. of Standards and Technology (CSTL), Boulder, CO. Thermophysics Div.

Thermodynamic Properties of $R 134 \mathrm{a}(1,1,1,2-$ Tetrafluoroethane).

Final rept.

M. L. Huber, and M. O. McLinden. 1992, 10p.

Pub. in Proceedings of International Refrigeration Conference, Purdue University, July 14-17, 1992, 10p

Keywords: "Fluorohydrocarbons, "Thermodynamic properties, "Refrigerants, Temperature dependence, Pressure dependence, Specific heat, Density(Mass/ volume), Vapor pressure Acoustic velocity, Vapor phases, Liquid phases, Phase transformation, Equations of state, Volume, Reprints, "R134a, ${ }^{*} 1,1,1,2$. Tetrafluoroethane, Benedict-Webb-Rubin equation.
The thermodynamic surface of R134a is expressed in terms of a 32-term modified Benedict-Webb-Rubin (MBWR) equation of state. Coefficients for this equation and for the ancillary equations representing the saturated liquid and vapor densities and the vapor pressure are presented. Temperatures are given on the new International Temperature Scale of 1990 (ITS 90). The MBWR coefficients were obtained using a multi-property fit that used experimental data for PVT properties, isochoric heat capacity, second-virial coefficients, speed of sound, and coexistence properties, including new measurements not used in previous formulations. The formulation is applicable to both the liquid and vapor phases at pressures up to $70 \mathrm{MPa}$, and for a temperature range from the triple point to $450 \mathrm{~K}$ The accuracy of the equation of state is estimated to be + and $-0.2 \%$ in density, + and $-1.0 \%$ in constantvolume heat capacities, and + and $-0.6 \%$ in the sound velocity, except in the critical region, based on comparisons with experimental data. In addition, a comprehensive bibliography of experimental data for $\mathrm{R} 134 \mathrm{a}$ is presented.

00,989

PB95-168860 Not available NTIS

National Inst. of Standards and Technology (CSTL) Boulder, CO. Thermophysics Div.

High-Temperature Adiabatic Calorimeter for Constant-Volume Heat Capacity Measurements of Compressed Gases and Liquids.

Final rept

J. W. Magee. 1991, 5p

Sponsored by Department of Energy, Washington, DC Pub. in Proceedings of Symposium on Energy Engineering Sciences (9th), Argonne, IL., May 13-15, 1991 $5 p$.

Keywords: *Specific heat, "Catorimeters, Calorimetry Compressed gases, High pressure, High temperature Reprints, "Constant volume heat capacity, "Adiabatic calorimeters.

A high-temperature adiabatic calorimeter has been developed to measure constant-volume molar heat capacity. The chief design feature is its nearly identical iwin cells which allow accurate measurement of energy differences without large corrections for energy losses due to thermal radiation fluxes. Operating conditions for the calorimeter are temperatures from 300 to 700 $\mathrm{K}$ at pressures from 0 to $20 \mathrm{MPa}$. The calorimeter is designed to measure heat capacities of both gases and liquids, especially fluids of interest to emerging energy technologies.

00,990

PB95-169249 Not available NTIS

National Inst. of Standards and Technology (CSTL), Gaithersburg, MD. Thermophysics Div.

Critical Scaling Laws and a Classical Equation of State.

Final rept.

A. van Pelt, G. X. Jin, and J. V. Sengers. 1994, 11p. Contract DE-FG05-883413902

Sponsored by Department of Energy, Washington, DC Pub. in International Jnl. of Thermophysics 15, n4 p687-697 Jul 94.

Keywords: "Equations of state, "Critical point, "Scaling laws, Mathematical models, Thermodynamic properties, Thermophysical properties, Ideal gas law, Reprints, Carnahan-Starling-DeSantis equation of state. In this paper we present a method which modifies a classical equation of state by incorporating the nonclassical critical behavior. As an example we have applied our procedure to the Carnahan-StarlingDeSantis (CSD) equation of state. The resulting equation reproduces the universal scaling behavior near the critical point and reduces to the universal ideal-gas behavior at low densities. We show that the renormalized CSD equation yields an improved and consistent representation of both mechanical and caloric thermodynamic properties. In addition, the suppression of the critical temperature due to the critical fluctuations is clearly demonstrated.

00,991

PB95-171948 PC A03/MF AO

National Inst. of Standards and Technology (CSTL), Gaithersburg, MD. Thermophysics Div.

Neighbor Tables for Molecular Dynamics Simulations.

R. D. Mountain. Dec 94, 15p, NISTIR-5545.

Keywords: "Condensed matter physics, "Molecular dynamics, "Computerized simulation, Monte Carlo meth- 
od, Thermodynamic properties, Transport properties, Computer programs, Particle interactions, Sorting, Liquids, Solids, "Neighbor tables, Fortran 77 program. ming language, Cell index method, Method of shadows, Particle simulations.

Two methods for constructing a neighbor table for use in Molecular Dynamics simulations are discussed. A linked-list based method is shown to require time for generation of the table that is roughly proportional to the number of particles, $N$, in the system when the range of forces is small compared to the volume of the system. An alternative method that requires O (N(sup 2)) operations is also discussed and problems where it may be preferable to the linked-list method are mentioned. Source code listing both methods is included in appendices.

00,992

PB95-175048 Not available NTIS

National Inst. of Standards and Technology (PL), Boulder, CO. Time and Frequency Div.

Rotational Spectroscopy of the $\mathrm{CoH}$ Radical in Its Ground (3)Phi State by Far-Infrared Laser Magnetic Resonance: Determination of Molecular Parameters.

Final rept.

S. P. Beaton, K. M. Evenson, and J. M. Brown. 1994 , $21 \mathrm{p}$.

Contract NASA-W15-047

Sponsored by National Aeronautics and Space Administration, Washington, DC

Pub. in Jnl. of Molecular Spectroscopy 164, p395-415 1994.

Keywords: "Rotational spectra, Far infrared radiation, Hyperfine structure, Chemical radicals, Chemical bonds, Ground states, M1-transitions, E2-transitions, Reprints, "Cobalt hydrides, Laser magnetic resonance.

Five rotational transitions of $\mathrm{CoH}$ in its ground triplet Phi state have been detected by far-infrared laser magnetic resonance, three in the lowest Omega $=4 \mathrm{com}$ ponent and two in the Omega $=3$ component. All of the Zeeman transitions show an octet hyperfine pattern due to the $(59)$ Co nucleus $(I=7 / 2)$. The much smaller proton doubling was also resolved for most transitions. The data have been fitted to experimental accuracy by an effective Hamiltonian for a molecule in an isolated triplet Phi state. The electron orbital and spin $\mathrm{g}$ factors Jetermined by the data confirm conclusively that the ground state of $\mathrm{CoH}$ is a triplet Phi state. The accurate measurement of the rotational constant allows the equilibrium bond length to be determined. The cobalt hyperfine splittings have been fitted to determine magnetic dipole and electric quadrupole parameters.

00,993

PB95-175162 Not available NTIS

National Inst. of Standards and Technology (PL), Boulder, CO. Time and Frequency Div.

Fine-Structure Intervals of (14)N(+) By Far-Infrared Laser Magnetic Resonance.

Final rept.

J. M. Brown, and A. L. Cooksy. 1994, 4p.

Pub. in Astrophysical Jnl. 428, pL37-L40, 10 Jun 94.

Keywords: *Nitrogen ions, Far infrared radiation, Laser spectroscopy, Hyperfine structure, Fine structure, Ground states, Nitrogen 14, Reprints, Laser magnetic resonance.

The far-infrared laser magnetic resonance spectra associated with both fine-structure transitions in $(14) N(1+)$ in its ground triplet $P$ state have been recorded. This is the first laboratory observation of the $\mathrm{J}=1<--0$ transition and its frequency has been determined two orders of magnitude more accurately than previously. The measurement of the $\mathrm{J}=2<--1$ spectrum revealed a small error in the previous laboratory measurements. The fine-structure splittings (free of hyperfine interactions) determined in this work are (Delta) $\mathrm{E}(10)=1461.13190$ (61) GHz, (Delta) $\mathrm{E}(21)=$ $2459.38006(37) \mathrm{GHz}$. Zero-field transition frequencies which include the effects of hyperfine structure have also been calculated. Refined values for the hyperfine constants and the $g(\mathrm{~J})$ factors have been obtained.

00,994

PB95-175170 Not available NTIS

National Inst. of Standards and Technology (PL), Boulder, CO. Time and Frequency Div.
Laser Magnetic-Resonance Measurement of the (3)P1 - (3)P2 Fine-Structure Splittings in (17)O and (18)O.

Final rept

J. M. Brown, K. M. Evenson, and L. R. Zink. 1993,

Pub. in Physical Review A 48, n5 p3761-3763 Nov 93

Keywords: "Oxygen atoms, "Oxygen 17, "Oxygen 18 "Isotope effects, Far infrared radiation, Laser spectroscopy, Fine structure, Reprints, Laser magnetic resonance.

The triplet $P(1)$ - triplet $P(2)$ fine-structure splittings of (17) $\mathrm{O}$ and (18)O have been measured by far-infrared laser magnetic-resonance spectroscopy. The signals for (17)O were detected with a sample in its natural abundance $(0.038 \%)$. The isotopic shifts from the corresponding interval in (16) O are $+13.0 \mathrm{MHz}$ for (17) O and $+23.5 \mathrm{MHz}$ for $(18) \mathrm{O}$, in accord with theoretical expectations.

\section{0,995}

PB95-175287 Not available NTIS

National Inst. of Standards and Technology (CSTL) Boulder, CO. Thermophysics Div.

Vapor-Liquid Equilibria of Mixtures of Propane and Isomeric Hexanes.

Final rept.

S. W. Chun, W. B. Kay, and J. C. Rainwater. 1993 ,

$8 p$.

Sponsored by Department of Energy, Washington, DC. Pub. in Jnl. of Chemical and Engineering Data 38, n4 p494-501 1993.

Keywords: "Liquid-vapor equilibrium, "Hexanes, "Propanes, "Phase diagrams, Phase transformations, Temperature, Critical pressure, Pressure dependence, Concentration(Composition), Density(Mass/volume) Thermodynamic properties, Reprints, "Dew bubble curves, "Leung-Griffiths theory, "Ternary mixtures.

Vapor-liquid equilibrium data extending to critical pressures are reported for mixtures of propane with each of the hexane isomers, n-hexane, 2-methylpentane, 3methylpentane, 2,2-dimethylbutane, and 2,3dimethylbutane. Dew-bubble curves for five different compositions are tabulated for each of the five mixtures. The data in pressure, temperature, and density are successfully correlated by the Leung-Griffiths model as modified by Moldover, Rainwater, and coworkers. The coexistence surfaces are all quite simila with only subtle differences due to the different shapes of the isomeric hexane molecules.

00,996

PB95-175618 Not available NTIS

National Inst. of Standards and Technology (CSTL) Boulder, CO. Thermophysics Div.

Partial Scattered Intensities from a Binary Suspension of Polysiyrene and Silica.

Final rept.

H. J. M. Hanley, G. C. Straty, and P. Lindner. 1994 $8 p$.

Sponsored by Department of Energy, Washington, DC Pub. in LANGMUIR 10, n1 p72-79 1994

Keywords: "Neutron scattering, "Suspensions, "Binary systems(Materials), "Silicon dioxide, "Polystyrene, Small angle scattering, Latex, Particles, Mixtures, Water, Structure factors, Reprints, "Colloidal suspensions.

The paper reports SANS (small angle neutron scattering) experiments on aqueous binary suspension mixtures of charged polystyrene latex and silica particles characterized by the size ratio, gamma = sigma(sub si) sigma(sub pa) approximately 0.72 , with a nominal polystyrene diameter, sigma(sub pa) $=91 \mathrm{~nm}$. The scattered intensities are reported over the wave vector $(\mathrm{O})$ range $0.025<Q / n m($ sup -1$)<0.4$. Partial scattered in tensities were obtained by contrast matching the constituents with an appropriate $\mathrm{D} 2 \mathrm{O} / \mathrm{H} 2 \mathrm{O}$ solvent. Results are presented. The partial scattered intensity of polystyrene differs significantly from that of silica at a given value of $n$, and the partial scattered intensity of a component in a mixture suspension can differ markedly from the equivalent scattered intensity of the component in a pure suspension. The variation of the intensity with $Q$ for silica in a $1 / 1$ mixture suggests long range silica order at low $\mathrm{O}$. There is some evidence that the larger component, polystyrene, may form a polycrystalline fcc phase, in agreement with the density functional theory. The effect of multiple scattering on the data is discussed.
00,997
PB95-175626 Not available NTIS

National inst. of Standards and Technology (CSTL) Boulder, CO. Thermophysics Div.

Solubility Measurement by Direct Injection of Supercritical-Fluid Solutions into a HPLC System. Final rept.

B. N. Hansen, and T. J. Bruno. 1993, 4p.

Pub. in Jnl. of Supercritical Fluids 6, p229-232 1993. Keywords: "Solubility, "Supercritical fluids, "Chromatography, "Solutes, Naphthalene Thermophysical properties, Carbon dioxide, Test facilities, Measuring instruments, Reprints.

A new apparatus has been built for measuring solubility of solutes in supercritical fluids by direct injection of saturated supercritical-fluid solutions into a high-performance liquid chromatograph. To test the system, we measured the solubility of solid naphthalene in supercritical carbon dioxide at $55 \mathrm{C}$ for pressures between 6.58 and $10.23 \mathrm{MPa}$. The system was designed to operate at pressures and temperatures up to 34 $\mathrm{MPa}$ and $140 \mathrm{C}$.

\section{0,998}

PB95-175634 Not available NTIS

National Inst. of Standards and Technology (CSTL), Boulder, CO. Thermophysics Div

High-Pressure Equilibrium Cell for Solubility Measurements in Supercritical Fluids.

Final rept.

B. N. Hansen, A. F. Lagalante, R. E. Sievers, and T. J. Bruno. 1994, 3p.

Pub. in Review of Scientific Instruments 65, n6 p2112 2114 Jun 94.

Keywords: "Supercritical fluids, "Solubility, "Ferrocenes, "Measuring instruments, Carbon dioxide, Thermophysical properties, Solutes, Test facilities, Concentration(Composition), Reprints, "High pressure cells.

This paper describes the design, construction, and operation of an ultraviolet-visible light-transmission cell for high-pressure fluids. The cell can be operated at pressures as high as $70 \mathrm{MPa}$ and at temperatures up to $100 \mathrm{C}$. This instrument was specifically designed to measure the solubility of solutes in supercritical fluids. The cell has an internal saturator and fluid circulator that are easy to remove and clean. The solubility of ferrocene in supercritical carbon dioxide was measured in the high-pressure cell described here. At $40 \mathrm{C}$, the mole fraction of ferrocene in carbon dioxide in creased from 0.00005 to 0.0015 as the solvent density was increased from 5 to $17 \mathrm{~mol} / \mathrm{L}$.

00,999

PB95-175683 Not available NTIS

National Inst. of Standards and Technology (CSTL), Boulder, CO. Thermophysics Div.

Critical Properties and Vapor-Liquid Equilibria of the Binary System Propane + Neopentane.

Final rept.

D. W. Hissong, W. B. Kay, and J. C. Rainwater.

1993, 8p

Pub. in Jnl. of Chemical and Engineering Data 38, n4 p486-493 1993

Keywords: "Propane, "Pentanes, "Liquid-vapor equilibrium, "Binary mixtures, "Phase diagrams, Phase transformations, Temperature, Critical pressure, Pres sure dependence, Density(Mass/volume), Thermo dynamic properties, Reprints, "Leung-Griffiths theory, Dew bubble curves.

Vapor-liquid equilibrium data extending to critical pressures are reported for five mixtures of propane with neopentane. The data in pressure, temperature, and density are correlated to high accuracy by the LeungGriffiths model as modified by Moldover, Rainwater and co-workers. In addition, a complete bibliography of similar experiments on binary mixtures from the laboratory of W.B.K. is presented.

\section{1,000}

PB95-175758 Not available NTIS

National inst. of Standards and Technology (CSTL), Gaithersburg, MD. Surface and Microanalysis Science Div.

Elastic-Electron-Scattering Effects on Angular Dis. tributions in X-ray Photoelectron Spectroscopy.

Final rept.

A. Jablonski, and C. J. Powell. 1994, 10p.

Pub. in Physical Review B 50, n7 p4739-4748, 15 Aug 94. 
Keywords: "X ray photoelectron spectroscopy, "Electron scattering, Monte Carlo method, Angular distribution, Surface analysis, Elastic scattering, Photoionization, Photoemission, Simulation, Gold, Reprints.

Electron trajectories in $\mathrm{x}$-ray photoemission from solids are partially randomized by elastic collisions, and thus the angular distribution of photoelectrons leaving the surface is different from that for isolated atoms. This problem is approached in the present work oy extensive Monte Carlo simulations of electron trajectories re sulting from photoionization of the gold $4 s, 4 p(3 / 2)$, $4 d(5 / 2)$, and $4 f(7 / 2)$ subshells by $M g$ characteristic $x$ rays. Calculations were made for the full range of angles of $x$-ray incidence and for all possible positions of the electron energy analyzer. In comparisons with intensities predicted from the common formalism in which elastic scattering is neglected, it was found that the elastic-scattering effects can be accounted for with two correction factors. These factors are, to a large extent, independent of experimental geometry for certain ranges of angles.

\section{1,001}

PB95-175766 Not available NTIS

National Inst. of Standards and Technology (CSTL) Gaithersburg, MD. Surface and Microanalysis Science Div.

Grazing Incidence X-ray Photoemission and Its Implementation on Synchrotron Light Source X-ray Beamlines.

Final rept.

T. Jach, M. J. Chester, and S. M. Thurgate. 1994,

3p.

Pub. in Nuclear Instruments and Methods in Physics Research A 347, p507-509 1994.

Keywords: "X ray photoelectron spectroscopy, Synchrotron radiation sources, $X$ ray sources, Grazing incidence, KeV range 1-10, Chemical composition, Oxide films, Surface layers, Photoemission, Germanium, Reprints, Surface composition.

Grazing incidence X-ray photoemission spectroscopy provides a method of obtaining information about surface chemical composition as well as the variation of composition with depth. Photoemission spectra are taken as $\mathrm{X}$-rays are directed onto a surface over a range of incidence angles near the critical angle for total external reflection. The technique is particularly suited to the study of surface layers in the thickness range 10-40 A, using $X$-rays in the energy range of 1 $2 \mathrm{keV}$. We have implemented the technique in a geometry that minimizes distortion of the spectral lineshape by keeping a fixed relationship between the sample and the electron spectrometer. We present data taken in the laboratory that illustrate its application to the study of oxide films on $\mathrm{Ge}$. The counting time for a spectrum would be shortened considerably by implementing the method on a soft $X$-ray beamline at a synchrotron light source. We present a method for doing so that retains the advantages of a fixed geometry between the sample and the electron spectrometer.

\section{1,002}

PB95-175816 Not available NTIS

National Inst. of Standards and Technology (CSTL), Boulder, CO. Thermophysics Div.

Correlation of the Ideal Gas Properties of Five Aromatic Hydrocarbons.

Final rept

A. Laesecke. 1993, 3p

Pub. in Ind. Eng. Chem. Res. 32, p759-761 1993

Keywords: "Benzene, "Toluene, "Xylenes, "Ideal gas "Specific heat, "Enthalpy, "Entropy, "Empirical equations, Thermodynamic properties, Temperature dependence, Mathematical models, Statistical mechanics, Reprints, "Temperature functions.

The ideal gas thermodynamic properties specific heat capacity, specific entropy, and specific enthalpy have been correlated for benzene, toluene, o-xylene, $m$ - $x y$ lene, and p-xylene by a uniform, semiempirical function of temperature. The correlation is based on literature values calculated from statistical mechanics for temperatures up to $1500 \mathrm{~K}$ for benzene and up to 3000 $\mathrm{K}$ for the other molecules. The temperature function chosen is more accurate than that used for previous correlations, and can be used in a wider temperature range.
National Inst. of Standards and Technology (CSTL), Boulder, CO. Thermophysics Div.

Composition Dependence of a Field Variable Along the Binary Fluid Mixture Critical Locus.

Final rept.

J. C. Rainwater, and D. G. Friend. 1994, 7p.

Sponsored by Department of Energy, Washington, DC. Pub. in Physics Letters A 191, p431-437 1994.

Keywords: Binary mixtures, Liquid-vapor equilibrium, Critical point Concentration(Composition), Temperature, Phase transformations. Phase diagrams, Thermodynamic properties, Fluids, Reprints, "Field variables, Critical line.

There are several classes of field variables used to extend the principle of critical-point universality to binary fluid mixtures. In particular, one can construct a field variable with sufficient flexibility to allow linearity in composition along the critical locus for the large class of binary systems with a critical line which is monotonic in temperature. This choice of hidden field variable has been used extensively in studies of vapor-liquid equilibrium, but a recent study of an alternative class of field variables suggests that linearity in composition along the critical locus cannot, in principle, be imposed exactly. It is demonstrated that several arguments presented by proponents of this proposition do not, in fact, apply to the field variable constructed in our studies.

\section{1,004}

\section{PB95-176046 Not available NTIS}

National Inst. of Standards and Technology (CSTL), Boulder, CO. Thermophysics Div.

Equilibrium Pair Distribution Function of a Gas: Aspects Associated with the Presence of Bound States.

Final rept.

J. C. Rainwater, and R. F. Snider. 1993, 11p

Sponsored by Department of Energy, Washington, DC. Pub. in Jnl. of Chemical Physics 99, n11 p9111-9121, 1 Dec 93.

Keywords: *Distribution functions, "Potential energy, *Dimers, "Gases, Momentum, Chemical equilibrium, Thermodynamic equilibrium, Moments, Mathematical models, Atoms, Reprints, "Potential functions.

At thermal equilibrium the momentum distribution of atoms in a gas is usually assumed to be Maxwellian, whether classically or quantally. However, if an atom is bound in a diatomic molecule, the atom's momentum distribution is non-Maxwellian. This paper explores the consequent singlet and pair particle distribution functions in a gas having both unbound atoms and bound pairs of atoms. Comment is made on the range of behavior associated with whether the chemical equilibrium constant for diatom formation is small or large. Calculations of distribution functions and their moments for atoms which are members of dimers are presented for some specific model potentials.

\section{1,005}

PB95-176194 Not available NTIS

National Inst. of Standards and Technology (PL), Boulder, CO. Time and Frequency Div.

Pure Rotational Spectra of CuH and CuD in Their Ground States Measured by Tunable Far-Infrared Spectroscopy.

Final rept.

T. D. Varberg, and K. M. Evenson. 1994, 5p.

Pub. in Jnl. of Molecular Spectroscopy 164, p531-535 1994

Keywords: "Copper hydrides, "Rotational spectra, * Infrared spectra, Molecular spectroscopy, Far infrared radiation, Ground states, Copper 63, Copper 65, Reprints, "Copper deuterides.

Pure rotational transitions of $\mathrm{CuH}$ and CuD within the Chi singlet Sigma( + ) (upsilon $=0$ ) state were measured over the ranges $\mathrm{J}$ (double prime $)=1$ to 10 for $\mathrm{CuH}$ and $J$ (double prime) $=2$ to 18 for CuD including both the (63) $\mathrm{Cu}$ and $(65) \mathrm{Cu}$ isotopes. The rotational parameters $B(0), D(0), H(0)$, and $L(0)$ were separately determined for each of the four isotopomers by least-squares fitting to the observed transitions. For (65) CuH, (63) CuD and (65) CuD these parameters are about two orders of magnitude more accurate than those determined by previous workers. Accurate calculated frequencies of all four isotopomers which will be useful in astronomical studies of $\mathrm{CuH}$ and $\mathrm{CuD}$ are given.

\section{1,006}

PB95-176202 Not available NTIS

National Inst. of Standards and Technology (PL), Boulder, CO. Time and Frequency Div.
Rotational Spectrum of $\mathrm{OH}$ in the $\mathrm{v}=0-3$ Levels of Its Ground State.

Final rept.

T. D. Varberg, and K. M. Evenson. 1993, 13p.

Sponsored by National Aeronautics and Space Admin istration, Washington, DC

Pub. in Jnl. of Molecular Spectroscopy 157, p55-67 1993.

Keywords: "Hydroxyl radicals, "Hydroxyl emission, "Rotational spectra, *Infrared spectra Far infrared ra diation, Frequency shift, Spectral shift, Fine structure, Ground states, Pressure dependence, Helium, Re prints.

Rotational and fine structure transitions have been observed between low rotational levels in the upsilon = $0-3$ levels of the $\mathrm{OH}$ Chi doublet Pi state by tunable far-infrared spectroscopy. The rotational and fine structure constants of the ground state have been refined by least-squares fitting to the observed data in combination with far-infrared and microwave measurements by other authors. Pressure-induced frequency shifts with helium as a buffer gas are also reported for some of the upsilon $=0$ transitions.

\section{1,007}

PB95-180105 Not available NTIS

National Inst. of Standards and Technology (PL), Boulder, CO. Time and Frequency Div.

Atomic Sulfur: Frequency Measurement of the $J=0$ inversely maps 1 Fine-Structure Transition at 56.3 Microns by Laser Magnetic Resonance.

Final rept.

J. M. Brown, K. M. Evenson, and L. R. Zink. 1994,

Pub. in Astrophysical Jnl. 431, pL147-L149, 20 Aug 94 Keywords: "Infrared spectra, Far infrared radiation Electron transitions, Fine structure, Ground state, Re prints, "Sulfur atoms, Laser magnetic resonance.

The $J=0<-1$ fine-structure transition in atomic sulfur $(S I)$ in its ground triplet $P$ state has been detected in the laboratory by far-infrared laser magnetic resonance. The fine-structure interval has been measured accurately as $5,322,492.9+$ or $-2.8 \mathrm{MHz}$ which corresponds to a wavelength of $56.325572+$ or 0.000030 micrometers.

\section{1,008}

PB95-180121 Not available NTIS

National Inst. of Standards and Technology (MSEL), Gaithersburg, MD. Polymers Div.

Cyclic Polyamine Ionophore for Use in a Dibasic Phosphate-Selective Electrode.

Final rept

C. M. Carey, and W. B. Riggan. 1994, 5p

Sponsored by American Dental Association Health Foundation, Chicago, IL.

Pub. in Analytical Chemistry 66, n21 p3587-3591, Nov 94.

Keywords: "Polyamines, "Phosphates, Electrodes, Anions, Cyclic compounds, Reprints, "Selective electrodes, Cyclodecane-dione/decyl-triaza, Ionophore.

A cyclic polyamine, 3-decyl-1,5,8-triazacyclodecane2,4-dione (N3-cyclic amine), was used as the ionophore for a dibasic phosphate-selective electrode. This electrode exhibited a linear response between 1.0 micromol/L and $0.1 \mathrm{~mol} / \mathrm{L}$ dibasic phosphate activity with a near-Nernstian slope of approximately $-29 \mathrm{mV}$ per activity decade. The electrode selectivity for dibasic phosphate over other commonly occurring anions was evaluated. A mechanism for the selectivity of the electrode toward $\mathrm{HPO} 4(-2)$ ions is postulated to be a function of the size and charge of the N3- cyclic amine ionophore relative to the size and charge of HPO4(2) ions. The electrode's superior selectivity and sensitivity make possible the direct measurement of phosphate activity in a wide variety of applications.

\section{1,009}

PB95-180154 Not available NTIS

National Inst. of Standards and Technology (CSTL), Gaithersburg, MD. Surface and Microanalysis Science

Div. State-Resolved Evidence for Excited State Relaxation and Quenching.

Final rept

P. M. Chu, S. A. Buntin, L. J. Richter, and R. R Cavanagh. 1994, 11p.

Contract DE-AI02-93ER14330

Sponsored by National Science Foundation, Arlington, $V A$. and Department of Energy, Washington, DC 
Pub. in Jnl. of Chemical Physics 101, n4 p2929-2939, 15 Aug 94

Keywords: "Metal carbonyls, "Photodecomposition, Temperature range 0065-0273 K, Photochemical reactions, Surface chemistry, Ultraviolet radiation, Excited states, Silicon, Reprints, "Hexacarbonylmolybdenum, Laser induced desorption.

State-resolved detection techniques have been used to characterize the ultraviolet photodecomposition dy namics of Mo(CO) 6 on Si(111) $7 \times 7$ at $100 \mathrm{~K}$. Details of the excitation/fragmentation mechanism including adsorbate energy transfer were examined by measuring the cross sections and the internal and translational energies of the photoejected $\mathrm{CO}$ from submonolayer through multilayer coverage regimes. The CO energy distributions are found to be independent of $\mathrm{Mo}(\mathrm{CO}) 6$ coverage, and can be characterized by two components with markedly different mean energies. In contrast to the coverage independence of the measured energy disposal, the cross section was found to decrease by a factor of 3 from multilayer coverages to submonolayer coverages.

\section{1,010}

PB95-180667 Not available NTIS

National Inst. of Standards and Technology (CAML) Gaithersburg, MD. Applied and Computational Mathematics Div.

Rayleigh instability for a Cylindrical Crystal-Melt nterface.

Final rept.

G. B. McFadden, S. R. Coriell, and B. T. Murray

1993, 11p.

Sponsored by National Aeronautics and Space Administration, Washington, DC. and Defense Advanced Research Projects Agency, Arlington, VA

Pub. in Variational and Free Boundary Problems, p159-169 1993

Keywords: Binary systems(Materials), Temperature gradients, Temperature effects, Rayleigh Taylor instability, Cylindrical configuration, Capiliarity, Metals, Reprints, "Crystal-melt interface, Two phase systems.

The temperature of a crystal-melt interface at equilibrium is given by the Gibbs-Thomson equation, which relates the interface temperature to the mean Curvature of the interface. As isothermal cylindrical cryslal-melt interface is subject to capillary instabilities o the type originally studied by Rayleigh. The dependence of this instability on the materials properties of the system is described for a two-phase, single-component system with isotropic surface energy. The ef fect of radial temperature gradients on the instability lect of radial temperature gradients on the instability be important at micrometer-sized length scales, but become insignificant at macroscopic (millimeter-sized) length scales. Heating the liquid causes stabilization, and the instability is completely suppressed for large enough temperature gradients in the liquid.

\section{1,011}

PB95-180691 Not available NTIS

National Inst. of Standards and Technology (CSTL), Gaithersburg, MD. Thermophysics Div.

Quantitative Measure of Efficiency of Monte Carlo Simulations.

Final rept.

R. D. Mountain, and D. Thirumalai. 1994, $8 p$.

Contract AFOSR-F496209410106

Sponsored by Air Force Office of Scientific Research,

Bolling AFB, DC

Pub. in Physica A 210, p453-460 1994.

Keywords: "Monte Carlo method, Lennard-Jones potential, Computerized simulation, Triple point, Efficiency, Sampling, Liquids, Algorithms, Reprints.

An easily applied, physically motivated algorithm for determining the efficiency of Monte Carlo simulations is introduced. The theoretical basis for the algorithm is developed. As an illustration, I apply the method to he Lennard-Jones liquid near the triple point. We show hat an acceptance ratio of 0.2 is twice as efficient for the purpose of generating a satisfactory sample as is an acceptance ratio of 0.5 . There is a strong correlation between the efficiency measure and the diffusion rate of liquid particles during the simulation. We argue that he optimal value of the acceptance ratio is calculable rom short Monte Carlo simulations. The method is very general and is applicable to Monte Carlo simulations involving arbitrary potentials.
National Inst. of Standards and Technology (MSEL), Gaithersburg, MD. Reactor Radiation Div.

Structural and Chemical Investigations of $\mathrm{Na} 3(\mathrm{ABO} 4) 3.4 \mathrm{H} 2 \mathrm{O}$-Type Sodalite Phases.

Final rept.

T. M. Nenoff, W. T. A. Harrison, T. E. Gier, V. I

Srdanov, J. M. Nicol, G. D. Stucky, N. L. Keder, and

C. M. Zaremba. 1994, 9p.

Grant NSF-DMR91-20007

Sponsored by Office of Naval Research, Arlington, VA and National Science Foundation, Arlington, VA Pub. in Inorganic Chemistry 33, p2472-2480 1994.

Keywords: "Crystal structure, Synthesis(Chemistry), Neutron diffraction, $\mathrm{X}$ ray diffraction, Cubic lattices, Precursor, Hydrates, Sodalite, Reprints, Sodium aluminum silicates, Sodium gallium silicates, Sodium aluminum germanates, Sodium gallium germanates, Sodium zinc arsenates.

The sodalite cage containing only an electron as a nonframework 'anion' is well adapted for the study of solvated 'electride' Wigner lattices and quantitative mapping of the intra- and intercage electronic potential surfaces. In this paper we report the direct synthesis of anion free cage structures of the form $\mathrm{Na} 3$ )(ABO4)3(center dot)nH2O (A: B = Al.Ga:Si.Ge) as possible precursors for sodalite electride synthesis. A novel, low-temperature sodalite dehydroxylation methOd is presented for the preparation of $\mathrm{Na3}$ (
(AISiO4) 3 (center $4 \mathrm{H} 2 \mathrm{O}$ $\mathrm{Na} 4(\mathrm{OH})$ (AlSiO4) 3 (center dot) $1.72 \mathrm{H} 2 \mathrm{O}$. Results from a neutron diffraction study of $\mathrm{Na} 3$ (ZnAsO4)3(center dot) $4 \mathrm{H} 2 \mathrm{O}$ give evidence of hydrogen bonding between the framework oxygen atoms and the Beta-cage substructure and aid in explanation of analog unit cell trends. All of the investigated sodalites have an ordered array of their framework tetrahedral cations and crystallize in the cubic space group $P($ bar 4$) 3 n, Z=2$.

\section{1,013}

PB95-180741 Not available NTIS

National Inst of Standards and Technology (MSEL). Gaithersburg, MD. Reactor Radiation Div.

Characterization of the Interaction of Hydrogen with Iridium Clusters in Zeolites by Inelastic Neutron Scattering Spectroscopy.

Final rept.

J. M. Nicol, T. J. Udovic, R. R. Cavanagh, T. Mure,

B. C. Gates, Z. Xu, and S. Kawi. 1994, 6p.

Pub. in Matierals Research Society Symposia Pro ceedings, v351 p189-194 1994

Keywords: *Metal clusters, "Iridium, "Hydrogen, Atom ic clusters, Neutron scattering, Inelastic scattering, Catalysis, Zeolites, Chemical bonds, Interactions, Hydrides, Reprints.

Incoherent inelastic neutron scattering (IINS) measurements of hydrogen bound to Ir6 clusters in the nanoscale pores of zeolite $\mathrm{NaY}$ and similar clusters in $\mathrm{KL}$ zeolite are reported. On the basis of hydrogen up take measurements and the observation of peaks in the IINS spectra, we infer that hydrogen is bound to the metal nanoclusters in the zeolite pores. The IINS data for both zeolites are similar and consistent with the presence of $\mathrm{I} \mathrm{H} 2 \mathrm{species}$

\section{1,014}

PB95-180766 Not available NTIS

National Inst. of Standards and Technology (MSEL), Gaithersburg, MD. Reactor Radiation Div.

Inelastic-Neutron-Scattering Studies of Poly(pphenylene vinylene)

Final rept.

P. Papanek, J. E. Fischer, J. L. Sauvajol, M. J. Winokur, F. A. Karasz, A. J. Dianoux, and G. Mao. 1994, top.

Grant NSF-DMR91-20668

Sponsored by National Science Foundation, Arlington, $\checkmark A$

Pub. in Physical Review B 50, n21 p15 668-15 677 , 1 Dec 94.

Keywords: Time-of-flight spectrometers, Polarization characteristics, Vibrational states, Deuterium compounds, Neutron scattering, Inelastic scattering, Films, Reprints, "Poly(p-phenylene vinylene), Density o states, Lattice modes.

Mode polarization and energy spectra for the lattice modes of poly ( $p$-phenylene vinylene) are obtained by inelastic-incoherent-neutron-scattering methods. Experiments were performed on stretch-oriented, highly crystalline films with time-of-flight and filter-analyzer spectrometers. By measuring all-hydrogen and vinyl- ene-deuterated samples, we show that almost all of the modes can be attributed to phenylene motions. The low-energy features in the vibrational density of states consist of a longitudinal $2.5-\mathrm{meV}$ mode assigned to a damped translational motion along the chain axis, a 7-meV phenyl ring in-phase librational mode (transverse with respect to the chain axis), and strong bands at 15 and 25 meV showing mixed polarization the latter also displaying negative frequency shift with increasing temperature. Higher-frequency modes are in excellent agreement with published infrared and Raman studies, as well as with the results of our vibrational analysis based on the semiempirical AM1 method

\section{1,015}

PB95-180865 Not available NTIS

National Inst. of Standards and Technology (MSEL), Gaithersburg, MD. Reactor Radiation Div.

Powder Neutron Diffraction Investigation of Structure and Cation Ordering in $\mathrm{Ba} 2+x \mathrm{Bi} 2-x \mathrm{O} 6-\mathrm{y}$.

Final rept.

K. P. Reis, A. J. Jacobson, and J. M. Nicol. 1993,

$16 \mathrm{p}$.

Pub. in Jnl. of Solid State Chemistry 107, p428-443

Keywords: "Crystal structure, Time-of-flight method, Ambient temperature, Neutron diffraction, Monoclinic lattices, Cubic lattices, Cations, Reprints, "Barium bismuthates, ${ }^{*}$ Barium bismuth oxides.

The structures and cation ordering of several barium bismuth oxide phases, $\mathrm{Ba}(2+x) \mathrm{Bi}(2-x) \mathrm{O}(6-\mathrm{y})(x=0.22$ $0.28,0.40,0.67$ ) , have been determined at ambient temperature from constant wavelength and time-offlight neutron powder diffraction data. The end member of the series, Ba2Bi(III)Bi(V)O6, has a monoclinic distortion of the simple cubic perovskite structure with an ordered arrangement of the $\mathrm{Bi}(3+)$ and $\mathrm{Bi}(5+)$ cations on the $B$ sites. When additional barium atoms are introduced into the structure, substitution of $\mathrm{Ba}$ for $\mathrm{Bi}(\mathrm{III})$ occurs to give phases with the composition $\mathrm{Ba} 2((\mathrm{Bi})(1$ x) $\mathrm{Ba}(\mathrm{x})>\mathrm{Bi}) \mathrm{O} 6$ when $\mathrm{x}<0.6$. The monoclinic structure at $x=0$ changes first to a rhombohedral structure and then to cubic as $x$ is increased. At $x=0.67$, the structure remains cubic but the cation distribution changes to give an unusual arrangement, with $\mathrm{Bi}(3+)$ cations on the perovskite $\mathrm{A}$ sites and complete $\mathrm{Ba}(2+) / \mathrm{Bi}(5+)$ or dering on the $B$ sites.

01.016

PB95-180873 Not available NTIS

National Inst. of Standards and Technology (CSTL), Boulder, CO. Biotechnology Div.

Thermodynamic Study of the Reactions of Cyclodextrins with Primary and Secondary Aliphatic Alcohols, with D- and L-Phenylalanine, and with L-Phenylalanineamide.

Final rept

M. V. Rekharsky, F. P. Schwarz, Y. B. Tewari, and R. N. Goldberg. 1994, 7p

Pub. in Jnl. of Physical Chemistry 98, n40 p1028210288,6 Oct 94

Keywords: "Thermodynamic properties "Cyclodextrins, "Alcohols, "Chemical reactions, Heat of reaction, Equilibrium, Specific heat, Entropy, Calorimetry, Volumetric analysis, Gibbs free energy, Aliphatic compounds, Reprints

Equilibrium constants and standard molar enthalpies of reaction have been determined by titration calorimetry for the reactions of 1-propanol, 2-propanol, 1-butanol, (R)-(+)-2-butanol, (S)-(-)-2-butanol, (+or -)butanol, (R)-(+)-2-pentanol, (S)-(-)-2-pentanol, (R)-(+)2-hexanol, (S)-(-)-2-hexanol, (R)-(+)-2-heptanol, (S)-( -2-heptanol D-phenylalanine, L-phenylalanine, and Lphenylalanineamide with alpha-cyclodextrin and Betacyclodextrin. The standard molar Gibbs energies, standard molar enthalpies, and standard molar entropies for these reactions correlate well with respect to the number of carbon atoms in the chemical formula of the alcohol and form a series of distinct curves for the different types of alcohols. The results are also discussed in relation to hydrophobic, steric, and charge effects and the exchange reaction for a ligand from alpha-cyclodextrin to Beta-cyclodextrin. With the exception of the results for the standard molar enthalpies of reaction of the 2-butanols with alpha-cyclodextrin, the results obtained in this study show that, within the indicated uncertainties, there are no differences in any of the thermodynamic quantities for the reactions of these ligands with either alpha- or Beta-cyclodextrin due to the change in the location of a hydrogen atom on an optically active carbon atom. 


\section{1,017}

PB95-181012 Not available NTIS

National Inst. of Standards and Technology (MSEL), Gaithersburg, MD. Reactor Radiation Div.

Local-Mode Dynamics in YH2 and YD2 by IsotopeDilution Neutron Spectroscopy.

Final rept.

T. J. Udovic, J. J. Rush, and I. S. Anderson. 1994

$5 p$.

Pub. in Physical Review B 50, n21 p15 739-15 743, 1 Dec 94.

Keywords: "Yttrium hydrides, Neutron scattering, Inelastic scattering, Vibrational spectra, Phonons, Dynamics, Reprints, "Yttrium deuterides, Isotope dilution neutron spectroscopy, Density of states

Isotope-dilution neutron spectroscopy was used to investigate the dynamics of the $\mathrm{H}$ and $\mathrm{D}$ optic vibrations densities of states of the tetrahedrally coordinated $H$ and $\mathrm{D}$ atoms in the isotopically pure materials collapse to single, sharp, triply degenerate features when the $H$ and $D$ atoms are sufficiently diluted with their companion isotopes. This demonstrates that the vibrational dispersion for the pure materials is due to the presence of significant $\mathrm{H}-\mathrm{H}$ and $\mathrm{D}-\mathrm{D}$ dynamic-coupling interactions. Moreover, a spectral doublet (with 5-meV splitting) superimposed on the sharp D-defect-mode feature for $Y(H(0.9) D(0.1)) 2$ is suggestive of localized acoustic and optic branches of dynamically coupled, near-neighbor $\mathrm{D}-\mathrm{D}$ pairs isolated in the predominantly hydrided material. The magnitude of the splitting implies a D-D/Y-D force-constant ratio of about $6 \%$. The dynamics of both light $(H)$ and heavy $(D)$ mass defects are in general agreement with simple mass-defect theory.

\section{1,018 Not available NTIS}

National Inst. of Standards and Technology (MSEL), Gaithersburg, MD. Reactor Radiation Div.

Neutron Spectroscopic Evidence of Concentration-Dependent Hydrogen Ordering in the Octahedral Sublattice of beta-TbH $2+x$.

Final rept.

T. J. Udovic, J. J. Rush, and I. S. Anderson. 1994 3p. 94.

Keywords: "Terbium hydrides,

Keywords: "Terbium hydrides, Concentration(Composition), Quantity ratio, Neutron scattering, Inelastic scattering, Reprints, ${ }^{*}$ Hydrogen ordering, Density of states.

The octahedrally coordinated hydrogen $(H(O))$ of the superstoichiometric rare-earth dihydrides beta- $\mathrm{TbH}(2$ $+x)(0.03<x<0.25)$ has been probed by incoherent inelastic neutron scattering. The $H(O)$ sublattice arrangements and associated optical vibrations are sensitive to the value of $x$. For $x=0.03$ at low temperature, the majority of the low-concentration o-site hydrogens are isolated in a local cubic environment and exhibit a relatively sharp vibrational density of states at 80.8 $\mathrm{meV}$, in accord with the presence of triply degenerate normal modes. In contrast, for $x=0.25$, a broad bimodal band peaking at about 76.7 and $83.1 \mathrm{meV}$ is evident, consistent with the type of long-range order that is known to occur in the $\mathrm{H}(\mathrm{o})$ sublattice at this higher concentration. Increasing the temperature above the long-range ordering transition leads to a more disordered $H(0)$ sublattice and a density of states similar to that at low values of $x$ but broadened, most likely by the persistence of some short-range order.

\section{1,019}

PB95-181038 Not available NTIS

National Inst. of Standards and Technology (MSEL),

Gaithersburg, MD. Reactor Radiation Div.

Vibrations of Hydrogen and Deuterium in Solid Solution with Lutetium.

Final rept.

T. J. Udovic, J. J. Rush, I. S. Anderson, J. N. Vajda, and O. Blaschko. 1994,6p.

Pub. in Physical Review B 50, n6 p3696-3701, 1 Aug 94.

Keywords: "Vibrational spectra, "Hydrogen, "Deuterium, "Lutetium, Solid solutions, Neutron scattering, Inelastic scattering, Temperature dependence, Reprints.

The vibrational spectroscopy of hydrogen isotopes in alpha-phase solid solution with Lu was probed by inco- herent-inelastic-neutron-scattering methods Low-resolution spectra of $\mathrm{H}$ and $\mathrm{D}$ located at tetrahedral interstices are qualitatively similar to those of other rareearth/hydrogen alpha phases, indicating a vibrational mode along the $c$ direction that is about $30 \%$ softer and significantly broader than the doubly degenerate vibrational modes in the basal plane. High-resolution spectra of these broad c-axis vibrations reveal temperature- and concentration-dependent line shape believed to consist of local acoustic and optic bands associated with the dynamical coupling of hydrogen atoms paired on either side of a metal atom along the c direction. Previous diffuse-elastic-neutron-scattering studies indicate the existence of short-range ordering of these hydrogen pairs into c-axis-oriented chains possessing interchain correlations. The shape and width of these bands at low temperature near the alpha/Beta-phase boundary compared to those of the analogous $S c$ and $Y$ alpha-phase systems suggest that the extent of ordering in alpha- $\mathrm{LuH}(\mathrm{x})$ is intermediate with respect to that in the (less ordered) alpha- $\mathrm{ScH}(\mathrm{x})$ and (more ordered) alpha- $Y H(x)$ solid solutions.

\section{1,020}

PB95-181053 Not available NTIS

National Inst. of Standards and Technology (EEEL) Gaithersburg, MD. Electricity Div.

Plasma Chemical Model for Decomposition of SF6 in a Negative Glow Corona Discharge.

Final rept.

R. J. Van Brunt, and J. T. Herron. 1994, 21p.

Sponsored by Electric Power Research Inst., Palo Alto CA. and Department of Energy, Washington, DC.

Pub. in Physica Scripta T53, p9-29 1994.

Keywords: "Sulfur hexafluoride, "Corona discharges, "Glow discharges, "Plasma chemistry, Decomposition reactions, Gas mixtures, Electron impact, Oxygen fluorides, Oxidation, Reprints.

A zonal plasma chemical model is proposed to account for the observed oxidation and decomposition of sulfur hexafluoride (SF6) by a negative, point-to-plane glowtype corona discharge in pressurized SF6/O2/H2O gas mixtures. The model yields dependencies of stable neutral oxidation by-products such as SOF2, SO2F2, SOF4, S2F (10), and SO2 on time, discharge current and $\mathrm{O} 2$ and $\mathrm{H} 2 \mathrm{O}$ concentrations which are consisten with measured results. Electron-impact-induced dissociation of SF6 in the glow region of the discharge is the decomposition rate-controlling process. The relative roles played by different reactions involving neutral free radicals and ions in different zones of the discharge are examined, and in some cases, reaction rate coefficients have been adjusted within reasonable limits to give best fits to observed production rates of various by-products.

\subsection{1}

PB95-181087 Not available NTIS

National Inst. of Standards and Technology (PL), Boulder, CO. Time and Frequency Div.

Detection of $\mathrm{OH}+$ in Its a(1)Delta State by Far Infrared Laser Magnetic Resonance.

Final rept.

T. D. Varberg, K. M. Evenson, and J. M. Brown 1994, 5p.

Pub. in Jnl. of Chemical Physics 100, n4 p2487-2491, 15 Feb 94

Keywords: "Hydroxyl radicals, Far infrared radiation, Laser spectroscopy, Electronic structure, Metastable state, Rotational spectra, Infrared spectra, Positive ions, Reprints, Laser magnetic resonance.

The spectrum associated with the $J=3<-2$ transition of $\mathrm{OH}(1+)$ in the alpha singlet Delta $(\mathrm{nu}=0)$ state has been observed by far infrared laser magnetic resonance spectroscopy. A new microwave discharge source enabled the detection of this spectrum, which is the first observation of the rotational spectrum of an ion in a metastable state. Assignment and leastsquares fitting of the observed transitions have determined the following molecular constants: $\mathrm{B}(0)=$ 494.420388 (22) GHz, the proton hyperfine parameter alpha $=74.84$ (32) $\mathrm{MHz} g^{\prime}(\mathrm{L})=1.000915$ (15) and $g(r)=-0.001815(18)$, with the 1 (sigma) uncertainties of the last digits in parentheses. The relationship of these parameters to the geometric and electronic structure of $\mathrm{OH}(1+)$ is discussed.

\section{1,022}

PB95-181178 Not available NTIS

National Inst. of Standards and Technology (MSEL) Gaithersburg, MD. Polymers Div.
Water Adsorption at Polymer/Silicon Wafer Interfaces.

Final rept.

W. L. Wu. 1994,5 p

Contract N00014-92-F-0036

Sponsored by Office of Naval Research, Arlington, VA. Pub. in Materials Research Society Symposia Proceedings, v338 p565-569 1994.

Keywords: "Interfaces, "Water, "Adsorption, "Neutron scattering, "Wafers, Monocrystals, Reflectivity, Polymers, Silicon, Polyimides, Epoxy resins, Reprints.

Neutron reflectivity (NR) was applied to measure the concentration of water at the buried interfaces between polymer and silicon single crystal wafers. In the polyimide samples excess water was discovered within 30 A of the silicon/polymer interface, where the water concentration reached $17 \%$ (by volume) for the samples without a coupling agent and $12 \%$ for the ones with a coupling agent. Beyond the interface, the water concentration was measured at $2-3 \%$, which is typical of bulk polyimide. Excess water was also found at the silicon/epoxy interface; more than $30 \%$ water was found within $150 \mathrm{~A}$ of the interface in the samples with their interface precoated with a coupling agent. The equilibrium moisture content in bulk epoxy used in this study is about $6 \%$. The above results demonstrate conclusively the unique power of NR in determining water concentration near a buried interface, and provide the first quantitative evidence for a water concentration profile which peaks in the interface region.

\section{1,023}

PB95-202222 Not available NTIS

National Inst. of Standards and Technology (PL), Boulder, CO. Ouantum Physics Div.

In-situ Monitoring of Molecular Beam Epitaxial Growth Using Single Photon lonization.

Final rept.

A. L. Alstrin, A. K. Kunz, and S. R. Leone. 1994, 9p. Contract ACS-PRF-15190-AC6, Grant NSF-PHY9012244

Sponsored by National Science Foundation, Arlington, VA. and Petroleum Research Fund, Washington, DC. Pub. in Proceedings of Society of Photo-Optical Instrumentation Engineers: Laser Techniques for Surface Science, Los Angeles, CA., January 27-29, 1994, v2125 p42-50

Keywords: "Molecular beam epitaxy, "Gallium arsenides, "Crystal growth, "Gallium, "Arsenic, Real time operations, Semiconducting films, Laser radiation, Desorption, Reprints, Time-of-flight mass spectroscopy, Reflection high energy electron diffraction, In situ monitoring, Optical probes

Single photon laser ionization time-of-flight mass spectroscopy (SPI-TOFMS) is used to monitor the gaseous fluxes of Ga, As? and As4 which are relevant in molecular beam epitaxy (MBE) of GaAs. This noninvasive and real-time technique measures densities, and hence fluxes, of multiple chemical species impinging on or scattered from a substrate during conventional MBE. With single photon ionization at $118 \mathrm{~nm}(10.5$ $\mathrm{eV}$ ), the energy is sufficient to ionize the species, but insufficient to ionize and fragment. The lack of molecular fragmentation greatly simplifies the interpretation of mass spectra. Additionally, the probe geometry permits simultaneous film growth monitoring using RHEED Results will be presented on the probing of scattering and desorption of III-V MBE species during GaAs growth. This technique promises to be a valuable in-situ diagnostic for III-V and II-VI MBE.

01,024

PB95-202230 Not available NTIS

National Inst. of Standards and Tec

der, CO. Quantum Physics Div.

Direct Detection of Atomic from Si(100).

Final rept.

A. L. Alstrin, P. G. Strupp, and S. R. Leone. 1993

3p.

Contract AFOSR90-0166

Sponsored by Air Force Office of Scientific Research, Bolling AFB, DC

Pub. in Applied Physics Letters 63, n6 p815-817, 9 Aug

Keywords: "Arsenic, "Silicon, "Desorption, Molecular beam epitaxy, Far ultraviolet radiation, Gallium arsenides, Photoionization, Lase Time-of-flight mass spectroscopy.

Application of the $118 \mathrm{~nm}$ single photon laser ionization technique to a molecular beam epitaxy machine is 
used for the first time to demonstrate direct desorption of As atoms from Si(100). Both As2 and As are the desorbing species from $1 \mathrm{ML}$ of arsenic on silicon above $1,000 \mathrm{~K}$. This is in contrast to previously reported models that considered only dimer desorption. With a continuous flux of As4, the scattered and desorbing arsenic species from $\mathrm{Si}(100)$ are examined as a function of surface temperature $(650-1200 \mathrm{~K})$. Atomic desorption is large, $75 \%+$ or - $19 \%$, above $1,000 \mathrm{~K}$, and complete conversion of As4 to As2 and As occurs at $1,200 \mathrm{~K}$. The species selectivity of laser ionization time-of-flight mass spectroscopy has broader implications for GaAs growth.

01,025

PB95-202313 Not available NTIS

National inst. of Standards and Technology (PL), Boulder, CO. Quantum Physics Div.

Location of a (1)A(sub g) State in Bithiophene.

Final rept.

D. Birnbaum, and B. E. Kohler. 1992, 4p

Pub. in Jnl. of Chemical Physics 96, n4 p2492-2495, 15 Feb 92

Keywords: "Thiophenes, Temperature range 0065$0273 \mathrm{~K}$, Molecular excitation, Fluorescence spectroscopy, Oligomers, Hexanes, Reprints, "Bithiophenes, Polythiophenes, Two photon processes, Dilute solutions.

The 0-0 excitation energy of a singlet $A(\mathrm{~g})$ state of 2,2'bithiophene has been determined by measuring the two-photon fluorescence excitation spectrum of a dilute solution of this molecule in crystalline $n$-hexane at $77 \mathrm{~K}$. Because the 0-0 energy is what would have been predicted by extrapolating previously measured alpha, omega-dithienylpolyene 2 singlet $A(g)$ 0-0 energies to zero polyene chain length, it is assigned to the 2 singlet $A(g)$ state. The $0-0$ band is centered at $36,173 / \mathrm{cm}$, approximately $6,570 / \mathrm{cm}$ above the $0-0$ of the 1 singlet $A(g)$ to 1 singlet $B(u)$ transition. This order of bithiophene excited singlet states is opposite to that of the linear polyene with the same number of double bonds but may reverse for chains longer than six repeat units.

01,026

PB95-202339 Not available NTIS

National Inst. of Standards and Technology (PL), Boulder, CO. Quantum Physics Div.

Lowest Excited Singlet State of Isolated 1-phenyl1,3-butadiene and 1-phenyl-1,3,5-hexatriene.

Final rept.

W. J. Buma, B. E. Kohler, J. M. Nuss, T. A. Shaler, and K. Song. 1992, 9p.

Sponsored by National Science Foundation, Washington, DC. and National Institutes of Health, Bethesda, MD.

Pub. in Jnl. of Chemical Physics 96, n7 p4860-4868, 1 Apr 92

Keywords: "Polyenes, Molecular spectra, Excited states, Multi-photon processes, Supersonic jet flow, Reprints, "Phenylbutadiene, "Phenylhexatriene, Supersonic expansion, Multiphoton ionization, Singlets.

We report vibrationally resolved $S(0)$--> $S(1)$ excitation spectra and vibronic level decay times for the phenylsubstituted polyenes 1-phenylbutadiene and 1phenylhexatriene seeded in supersonic $\mathrm{He}$ expansions. This information was obtained using one- and two-color resonance-enhanced multiphoton ionization techniques. The shift in the excitation energy of the lowest excited singlet state upon deuteration of the phenyl ring demonstrates that in 1-phenylbutadiene $S(1)$ is mainly an excitation of the benzene ring while in 1-phenylhexatriene $S(1)$ is mainly the 2 singlet $A(g)$ state of hexatriene. Analysis of the excitation spectrum of 1-phenylhexatriene shows that the spectrum contains contributions from two species, the trans, trans and the trans,cis isomers, whose 2 singlet $A(g)$ state excitation energies differ by about $155 / \mathrm{cm}$.

01,027

PB95-202537 Not available NTIS

National Inst. of Standards and Technology (PL), Boulder, CO. Quantum Physics Div.

Plasma Chemistry in Silane/Germane and Disilane/ Germane Mixtures.

Final rept.

J. R. Doyle, D. A. Doughty, and A. Gallagher. 1992, $12 p$.

Contract SERI-XG-1-1216

See also PB90-187659. Sponsored by Solar Energy Research inst., Golden, CO.
Pub. in Jnl. of Applied Physics 71, n10 p4727-4738, 15 May 92

Keywords: "Glow discharges, "Plasma chemistry, "Germanes, "Silanes, Silicon solar cells, Photovoltaic cells, Amorphous silicon, Gas mixtures, Reprints Disilanes.

A detailed kinetic study of silane-germane glow discharges is presented. Stable gas decomposition and production rates have been measured using mass spectrometry and a kinetic model for the plasma chemistry is developed. It is found that germane depletes about four times faster than silane, nearly independ ently of their relative fractions. Germane is found to be much more reactive than silane with silylene, germalyn, and atomic hydrogen, and the silylene-germane reaction leads in large part to film, rather than stable gases. The spatial characteristics of the discharge are studied, using optical emission and fibe deposition profiles. From these it is deduced that the present, low-power discharge operates in a "hybrid" alpha-gamma regime, and that ion effects are important near the electrodes.

\section{1,028}

PB95-202586 Not available NTIS

National Inst. of Standards and Technology (PL), Boulder, CO. Quantum Physics Div.

Vibrations of $\mathrm{S1}((1) \mathrm{B} 2 \mathrm{U})$ p-Difluorobenzene - d4 S1-So Fluorescence Spectroscopy and ab Initio Calculations.

Final rept.

H. J. Elston, E. R. Davidson, F. G. Todd, and C. S.

Parmenter. 1993, 13p.

Sponsored by National Science Foundation, Washington, DC.

Pub. in Jnl. of Physical Chemistry 97, n21 p5506-5518 1993.

Keywords: "Vibrational spectra, Fluorescence spec roscopy, Molecular spectroscopy, Molecular vibration Fermi resonance, Supersonic jet flow, Reprints, "Difluorobenzene, Ab initio calculations.

The $S(1)$ (singlet $B(2 u)$ )-S(0)(singlet $A(g)$ ) spectros copy of p-difluorobenzene-d(4) (C6D4F2 pDFB-d(4)) cooled in a supersonic free jet expansion has been characterized by fluorescence excitation (FE) and dis persed fluorescence. The 0 (sub 0 , sup 0 ) band lies a $36,987+$ or $-1.0 / \mathrm{cm}$ (vacuum). The FE band assign ments have provided the values of $11 \mathrm{~S}(1)$ fundamentals. The pattern of Franck-Condon allowed vibrationa activity and of vibronically induced transitions is simila to that of pDFB-h(4). An exception concerns the a(g) mode nu(4) that is a prominent source of progression in $d(4)$ on account of harmonic mode scrambling but inactive in $\mathrm{h}(4)$. Bands involving an out-of-phase mode (nu(8)) that undergoes large frequency change in many aromatics upon formation of rare gas van de Waals complexes have anomalously large intensity when S(1) overtones are excited but normal FranckCondon intensity for $S(0)$ overtones. This singular behavior occurs in both $\mathrm{h}(4)$ and $\mathrm{d}(4)$. Four Fermi resonances have been detected.

\section{1,029}

PB95-202685 Not available NTIS

National Inst. of Standards and Technology (PL), Boulder, CO. Quantum Physics Div.

Vibrational Dependence of the Anisotropic Intermolecular Potential of $\mathrm{Ar}-\mathrm{HCl}$.

Final rept.

J. M. Hutson. 1992, $11 p$

See also PB95-202693.

Pub. in JnI. of Physical Chemistry 96, n11 p4237-4247 1992.

Keywords: "Argon complexes, Van der Waals forces Intermolecular forces, infrared spectroscopy, High resolution, Microwave spectroscopy, Anisotropy, Reprints, "Hydrogen chloride complexes, "Deuterium chloride complexes, "Intermolecular potentials, Potential energy surfaces, Vibrational dependence.

A new intermolecular potential for $\mathrm{Ar}-\mathrm{HCl}$ is obtained by fitting to results from high-resolution microwave, farinfrared, and infrared spectroscopy. The new potentia is substantially more accurate than any previous Ar$\mathrm{HCl}$ potential and is the first to include the dependence of the potential on the $\mathrm{HCl}$ monomer vibration: it is a function of the diatom mass-reduced vibrational quantum number, as well as the intermolecular distance and angle. The functional form used has 19 adjustable parameters, which are determined from the spectroscopic data. The resulting potential, designated
H6 $4,3,0)$, reproduces all of the available spectroscopic data for levels of $\mathrm{Ar}-\mathrm{HCl}$ and $\mathrm{Ar}-\mathrm{DCl}$ correlating with $\mathrm{HCl}$ nu $=0$ and 1 and $D C l$ nu $=0$ and 1. The trends in intermolecular potentials for the series $\mathrm{Ar}-\mathrm{HF}, \mathrm{Ar}-\mathrm{HCl}$, and $\mathrm{Ar}-\mathrm{HBr}$ are discussed.

\section{1,030}

PB95-202693 Not available NTIS

National inst. of Standards and Technology $(\mathrm{PL})$, Boulder, CO. Quantum Physics Div.

Vibrational Dependence of the Anisotropic Intermolecular Potential of Ar-HF.

Final rept.

J. M. Hutson. 1992, 16p.

See also PB95-202685.

Pub. in Jnl. of Chemical Physics 96, n9 p6752-6767, 1 May 92.

Keywords: "Argon complexes, Van der Waals forces intermolecular forces, Microwave spectroscopy, Infra red spectroscopy, High resolution, Anisotropy, $R$ prints, "Hydrogen fluoride complexes, "Deuterium fluoride complexes, "Intermolecular potentials, Potentia energy surfaces, Vibrational dependence.

A new intermolecular potential for Ar-HF is obtained by fitting to results from high-resolution microwave, farinfrared, and infrared spectroscopy. The new potential designated $\mathrm{H} 6(4,3,2)$ is a function of the diatom massreduced vibrational quantum number as well as the intermolecular distance $\mathrm{R}$ and angle theta, and has 22 adjustable parameters. It reproduces all of the available spectroscopic data for levels of Ar-HF correlating with $\mathrm{HF}$ nu $=0,1$, and 2 , and DF, nu $=0$ and 1 . The $H 6(4,3,2)$ potential is qualitatively similar to previous potentials, with a linear Ar-H-F equilibrium geometry and a secondary minimum at the linear Ar-F-H geometry. Predictions of additional spectroscopic properties that would test the new potential are given, including far-infrared and overtone spectra of Ar-DF and dipole moments of excited states of Ar-HF and Ar-DF.

\section{1,031}

PB95-202743 Not available NTIS

National Inst. of Standards and Technology (MSEL), Gaithersburg, MD. Polymers Div.

Generalized Stokes-Einstein Equation for Spherical Particle Suspensions.

Final rept.

A. Kholodenko, and J. Douglas. 1995, 10p

Pub. in Physical Review E 51, n2 p1081-1090 Feb 95

Keywords: "Suspensions, Diffusion coefficient, Cou pled modes, Sum rules, Polymers, Droplets, Spheres, Reprints, Stokes-Einstein equations.

The cooperative diffusion coefficient $D(c)$ for spherical particle suspensions is calculated using a 'mode-coupling' method which extends previous calculations of $D(c)$ for critical fluids and semidilute polymer solutions. The renormalization of the viscosity in the velocity-velocity correlation function from the solvent to the suspension viscosity leads to a generalized Stokes-Einstein (SE) equation in which the suspension viscosity eta replaces the solvent viscosity eta(0) and the correlation length xi (related to the osmotic compress ibility) replaces the sphere radius $R$ at nonvanishing suspension concentrations. Insertion of the leading order hard sphere virial expansions for eta and the osmotic compressibility into our generalized SE equation gives a virial expansion for $\mathrm{D}(\mathrm{c})$ which is consistent with theoretical estimates obtained by alternative methods. These leading order virial expansions are also consistent with experiments on model ("hard sphere') suspensions, Results are given for the virial expansion of spherical liquid droplets interacting via a contact attractive interaction. Further experiments are required to test the generalized SE equation at higher suspension concentrations.

\section{1,032}

Not available NTIS

National Inst. of Standards and Technology (PL), Boulder, CO. Quantum Physics Div

Single Photon Ionization, Laser Optical Probe Technique for Semlconductor Growth.

Final rept

A. K. Kunz, A. L. Alstrin, S. M. Casey, and S. R.

Leone. 1994, 8p.

Sponsored by National Science Foundation, Arllngton, VA and Petroleum Research Fund, Washington, DC Pub. in Proceedings of Society of Photo-Optleal Instrumentation Engineers: Optical Characterizatlon Techniques for High-Performance Microolectronle Devlce Manufacturing, Austin, TX., October 20, 1994, v2337 p20-27 
Keywords: "Molecular beam epitaxy, "Surface reactions, "Gallium arsenides, "Crystal growth, Real time operations, Semiconducting films, Laser radiation, Monitoring, Desorption, Arsenic, Silicon, Substrates, Reprints, Time-of-flight mass spectroscopy, Reflection high energy electron diffraction, Optical probes.

Single Photon Ionization Time-of-Flight Mass Spectroscopy (SPI-TOFMS) is used as an in situ optical characterization technique to monitor chemical reactions occurring at semiconductor surfaces during molecular beam epitaxial (MBE) growth by delecting gaseous species. In this approach, $118 \mathrm{~nm}$ (10.5 eV) laser photons are generated and passed in front of a semiconductor substrate in the ultra-high vacuum (UHV) chamber. Here, the photons ionize the gaseous scattered and desorbed growth species which are detected by time-of-flight mass spectroscopy. SPI-TOFMS has been used to study $\mathrm{As}(\mathrm{n}) / \mathrm{Si}(100)$ desorption kinetics and, more recently, MBE growth of GaAs. Results are presented on the real-time monitoring of $G A(n)$ and As $(n)$ growth species. Simultaneous monitoring of growth with Reflection High-Energy Electron Diffraction (RHEED) is also discussed. Future work includes SPI-TOFMS studies of Si delta-doping in GaAs and surfactant-enhanced epitaxy of Ge on Si. SPI-TOFMS is an in situ UHV optical probe used to study the growth chemistry of semiconductor surfaces. This non-intrusive, species-specific real-time monitor of growth can be applied to increase the quality of device manufacturing.

\section{1,033}

PB95-202792 Not available NTIS

National Inst. of Standards and Technology (PL), Boulder, CO. Quantum Physics Div.

Slit-Jet Near-Infrared Spectroscopy and Internal Rotor Dynamics of the ArH2O van der Waals Complex: An Angular Potential-Energy Surface for Internal H2O Rotation.

Final rept.

R. Lascola, and D. J. Nesbitt. 1991, 16p.

Grants NSF-CHE90-00641, NSF-DJNB

Sponsored by National Science Foundation, Washington, DC.

Pub. in Jnl. of Chemical Physics 95, n11 p7917-7932, 1 Dec 91

Keywords: "Argon complexes, Van der Waals forces, Supersonic jet flow, Near infrared radiation, Infrared spectroscopy, High resolution, Rotational states, Reprints, "Water complexes, Potential energy surfaces. Near-infrared vibration-rotation spectra of jet-cooled Ar-H2O complexes are detected for the first time via direct absorption of tunable difference frequency infrared radiation in a slit supersonic expansion source. Transitions from both the lowest para and ortho complexes are observed which correlate to 0 (sub 00) and 1 (sub 01 ) rotational levels of free $\mathrm{H} 2 \mathrm{O}$, respectively, and permit spectroscopic characterization of the complex in both the ground (nu(3) = 0) and asymmetric stretch excited $(n u(3)=1$ ) levels. Information obtained in the study permits determination of a two-dimensional (2D) angular potential-energy surface of the complex as a function of the $\mathrm{H} 2 \mathrm{O}$ orientation.

\section{1,034}

PB95-202800 Not available NTIS

National Inst. of Standards and Technology (PL), Boulder, CO. Quantum Physics Div.

Orbital Stereochemistry: Discovering the Symmetries of Collision Processes.

Final rept.

S. R. Leone. 1992, 6p

Grants NSF-PHY90-12244, NSF-CHE84-08403

Sponsored by National Science Foundation, Washington, DC.

Pub. in Accounts of Chemical Research 25, n2 p7176 Feb 92.

Keywords: "Stereochemistry, Molecular orbitals, Directional control, Energy transfer, Laser radiation, Polarization, Alignment, Symmetry, Collisions, Reprints.

One of the most exciting recent aspects of laser chemistry is the ability to prepare highly aligned, or directional, reagents using the polarization of lasers. It is now possible to investigate chemical processes with directional control in a variety of new measurements. The results provide a means of visualizing the important symmetries of the chemical process with unprecedented detail. Many mechanistic interpretations of chemical transformations are built upon compelling pictures of orbital directionality, electron overlap and transfer, and symmetry arguments. Chemists can ex- ploit the methods of directional control to verify these pictures and to broaden our understanding of chemical stereospecificity.

\section{1,035}

PB95-202875 Not available NTIS

National Inst. of Standards and Technology (PL), Boulder, CO. Quantum Physics Div.

Preferential In-Plane Rotational Excitation of $\mathrm{H} 2 \mathrm{O}$ (001) by Translational-to-Vibrational Transfer from $2.2 \mathrm{eVH}$ Atoms.

Final rept.

C. M. Lovejoy, L. Goldfarb, and S. R. Leone. 1992, 3p.

Contract DE-FG02-883413860, Grant NSF-CHE8713646

Sponsored by National Science Foundation, Washing ton, DC. and Department of Energy, Washington, DC Office of Energy Research.

Pub. in Jnl. of Chemical Physics 96, n9 p7180-7182. 1 May 92.

Keywords: "Atom-molecule collisions, Water, EV range 1-10, Rotational states, Energy transfer, Excitation, Reprints, Fourier transform infrared spectroscopy, $\mathrm{Hy}$ drogen atoms.

The translational-to-vibrational and rotational (T-V,R) excitation of $\mathrm{H} 2 \mathrm{O}$ with $2.2 \mathrm{eV}$ hydrogen atoms is studied by time-resolved Fourier-transform infrared spectroscopy. Up to $2900 / \mathrm{cm}$ of rotational excitation is observed in (001), with a strong preference for in-plane rather than out-of-plane rotation for $\mathrm{J}=$ or $>7$, which implies a unique collision process leading to rotational alignment.

\section{1,036}

PB95-202933 Not available NTIS

National Inst. of Standards and Technology (PL), Boulder, CO. Quantum Physics Div.

Large Amplitude Skeletal Isomerization as a Promoter of Intramolecular Vibrational Relaxation in CH Stretch Excited Hydroca rbons.

Final rept.

A. Mcllroy, and D. J. Nesbitt. 1994, 15p.

Grants NSF-PHY90-12244, NSF-CHE90-00641

Sponsored by National Science Foundation, Arlington VA.

Pub. in Jnl. of Chemical Physics 100, n5 p3421-3435, 1 Sep 94

Keywords: "Butenes, Supersonic jet flow, Molecular vibration, Vibrational states, Infrared spectra, High resolution, Isomerization, Reprints.

The high resolution, slit jet cooled infrared nu $=1<-$ 0 methyl asymmetric stretch spectra of trans-2 butene and 1-butene are reported. Both of these molecules are singly unsaturated butene chains, have 30 vibrational degrees of freedom, and yield nearly equiv alent vibrational state densities at $\mathrm{CH}$ stretch levels of excitation. The key difference between these two molecules is the presence of a large amplitude C-C-C skeletal torsional coordinate in 1-butene corresponding to a low barrier, internal isomerization pathway which is completely absent in trans-2-butene. The trans-2butene asymmetric $\mathrm{CH}$ stretch (nu 16) spectrum is fully discrete at $0.002 / \mathrm{cm}$ resolution, and the coarse structure readily assigned to zero order rovibrational transitions in an asymmetric top. Fragmentation of these zero order transitions into spectral 'clumps' of fine structure provides direct evidence for coupling of the $\mathrm{CH}$ stretch to vibrational bath states

\section{1,037}

PB95-202941 Not available NTIS

National Inst. of Standards and Technology (PL), Boulder, CO. Quantum Physics Div.

Sub-Doppler, Infrared Laser Spectroscopy of the Propyne 2nul Band: Evidence of z-Axis Coriolis Dominated Intramolecular State Mixing in the Acetylenic CH Stretch Overtone.

Final rept.

A. Mcllroy, D. J. Nesbitt, E. Kerstel, G. Scoles, B. H. Pate, and K. K. Lehmann. 1994, 16p.

Grant NSF-PHY90-12244

Sponsored by National Science Foundation, Arlington, VA.

Pub. in Jnl. of Chemical Physics 100, n4 p2596-2611, 15 Feb 94.

Keywords: "Propyne, Infrared spectroscopy, Laser spectroscopy, Molecular relaxation, Vibrational states, Absorption spectra, Reprints.

The eigenstate-resolved 2nu(1) (acetylenic $\mathrm{CH}$ stretch) absorption spectrum of propyne has been observed for
$J^{\prime}=0-11$ and $K=0-3$ in a skimmed supersonic molecular beam using optothermal detection. Radiation near 1.5 micrometers was generated by a color center laser allowing spectra to be obtained with a full-width at halfmaximum resolution of $6 \times 10(\exp 4) / \mathrm{cm}(18 \mathrm{MHz})$. Three distinct characteristics are observed for the perturbations suffered by the optically active (bright) acetylenic $\mathrm{CH}$ stretch vibrational state due to vibrational coupling to the nonoptically active (dark) vibrational bath states.

\section{1,038}

PB95-202966 Not available NTIS

National Inst. of Standards and Technology (MSEL), Gaithersburg, MD. Reactor Radiation Div.

Determination of Complex Structures from Powder Diffraction Data: The Crystal Structure of La3Ti5Al15037.

Final rept.

R. E. Morris, J. J. Owen, J. K. Stalick, and A. K.

Cheetham. 1994, 6p.

Grant NSF-DMR-9123048

Sponsored by National Science Foundation, Arlington, VA.

Pub. in Jnl. of Solid State Chemistry 111, p52-57 1994.

Keywords: "Crystal structure, Synchrotron radiation, Neutron diffraction, X ray diffraction, Complex systems, Reprints, "Lanthanum titanium aluminates, Rietveld method, Powder diffraction.

The applicability of powder diffraction techniques to structure determination has improved substantially in recent times, but it has only been successfully utilized in the solution of relatively simple structures of up to 29 atoms in the asymmetric unit. The structure La3Ti5Al15037, which has 60 atoms in the asymmetric unit, has been solved using a combination of synchrotron $X$-ray and neutron powder diffraction. This represents a considerable advance in the size of structure that has been solved using powder diffraction techniques. The structure of La3Ti5Al15O37 consists of small regions of simpler structure types in the $\mathrm{La}$ $\mathrm{Ti} / \mathrm{Al} / \mathrm{O}$ system, interleaved to form a complex $3 \mathrm{D}$ network

\subsection{9}

PB95-202982 Not available NTIS

National Inst. of Standards and Technology (PL), Boulder, CO. Quantum Physics Div.

Acceleration of Intramolecular Vibrational Redis. tribution by methyl Internal Rotation. A Chemical Timing Study of p-fluorotoluene and pfluorotoluene-d3.

Final rept

D. B. Moss, and C. S. Parmenter. 1993, 9p

Pub. in Jnl. of Chemical Physics 98, n9 p6897-6905, 1 May 93.

Keywords: "Molecular vibration, Vibrational spectra, Vibrational states, Rotational states, Fluorescence spectroscopy, Chemical bonds, Deuteration, Reprints, "Fluorotoluenes, Methyl groups.

Time-integrated, frequency-resolved fluorescence spectroscopy has been used to determine rates of intramolecular vibrational energy redistribution (IVR) from the vibrational levels 3(sup I) (epsilon (vib) approx $=1,200 / \mathrm{cm}$ ) and 3(sup I) 5(sup I) (epsilon (vib) approx $=2,000 / \mathrm{cm}$ ) in both p-fluorotoluene and $p$ fluorotoluene-d(3) for comparison with each other and with comparable levels in p-difluorobenzene. Methyl substitution increases the rate of IVR by roughly two orders of magnitude, while deuteration of the methyl rotor produces at most a small (two- to fourfold) further increase in the rate of IVR. It is argued that the IVR response to methyl substitution is a consequence of the methyl internal rotation without significant influence from the methyl vibrations.

\section{1,040}

PB95-203006 Not available NTIS

National Inst. of Standards and Technology (PL), Boulder, CO. Quantum Physics Div.

High-Resolution, Direct Infrared Laser Absorption Spectroscopy in Slit Supersonic Jets: Intermolecular Forces and Unimolecular Vibrational Dynamics in Clusters.

Final rept.

D. J. Nesbitt. 1994, 33p.

Grant NSF-PHY90-12244

Sponsored by National Science Foundation, Arlington,

VA.

Pub. in Annual Review of Physical Chemistry 45, p3673991994. 
Keywords: "Infrared spectroscopy, Absorption spectroscopy, Laser spectroscopy, Near infrared radiation, Supersonic jet flow, Van der Waals forces, Intermolecular forces, Hydrogen bonds, Argon complexes, High resolution, Predissociation, Reviews, Reprints, Potential energy surfaces, Hydrogen fluoride complexes, Deuterium fluoride complexes.

The organization of this review will be as follows: In Sections 2 and 3 , some basic experimental considerations are presented to highlight the specific advantages of direct absorption near IR laser methods, as well as the role of pulsed 'slit' jet expansions in obtaining the requisite path lengths and sensitivities. In Section 4 , an overview of van der Waals spectroscopy of rare gas hydrogen halides is presented, illustrating the transition between a nearly 'rigid' to a nearly 'free' internal rotor dynamics. In Section 5 , we discuss the role of large amplitude motion and intermolecular orientation on energy flow and bond breaking dynamics, focusing on internal rotor predissociation and $V-V$ transfer between the monomer subunits. In Sections 6 and 7 , we look to two ongoing areas of investigation of larger clusters. Section 6 presents experimental and theoretical results on the series of $\operatorname{Ar}(n)-H F$ and $\operatorname{Ar}(n)-D F$ clusters ( $n=1,2,3$ and 4$)$, addressing the $n$-dependent vibrational red shifts, multibody effects, and intermolecular vibrational modes supported by these 'micromatrices.' Section 7 describes recent explorations into the spectroscopy and dynamics of (DF)3, which provides direct evidence for a cyclic ring 'structure,' and elucidates the hydrogen bond breaking dynamics that lead to ring opening on the 40 psec time scale.

\section{1,041}

PB95-203014 Not available NTIS

National Inst. of Standards and Technology (PL), Boulder, CO. Ouantum Physics Div.

Rotational-RKA Inversion of Intermolecular Stretching Potentials: Extension to Linear Hydrogen Bonded Complexes.

Final rept.

D. J. Nesbitt, and M. S. Child. 1993, 9p.

Grant NSF-PHY90-12244

Sponsored by National Science Foundation, Washington, DC.

Pub. in Jnl. of Chemical Physics 98, n1 p478-486, 1 Jan 93.

Keywords: "Nitrogen complexes, Near infrared radiation, Infrared spectroscopy, Rotational spectra, Van der Waals forces, High resolution, Hydrogen bonds, Reprints, "Hydrogen fluoride complexes, Interatomic potentials, RKR method.

A Rydberg-Klein-Rees (RKR)-based method is described which determines effective 1D intermolecular stretching potentials for polyatomic linear complexes from high precision rotational data alone. This extends the 'rotational RKR' inversion method from pseudodiatomic van der Waals clusters with only two nonhydrogenic atoms to much larger complexes with several heavy atoms. Sample inversion of rotational eigenvalues generated from a model 1D potential reproduces the model potential to approx $=$ or $<0.13 /$ $\mathrm{cm}$ accuracy and correctly predicts harmonic frequencies, force constants, and dissociation energies to approx $=$ or $<0.1 \%$. In contrast, the commonly used 'pseudodiatomic' approximation lead to quite significant $(10 \%-20 \%)$ errors, even for exact model potentials for which these approximations were developed. The method is further tested on high resolution near IR spectroscopic data of (14)N(14)N-HF, which determines the vibrationally averaged hydrogen bond stretching potential from 3.39 approx $=$ or $<R(\mathrm{~cm})$ approx $=$ or $<3.85 \mathrm{~A}$. The RKR data yield a hydrogen bond length of $R(\mathrm{~N}-\mathrm{H})=2.106 \mathrm{~A}(2.079 \mathrm{~A})$ and predict a van der Waals stretching frequency of $86.9 / \mathrm{cm}(90.7)$ $\mathrm{cm})$ for $n u(H F)=0(n u(H F)=1)$.

01,042

PB95-203022 Not available NTIS

National Inst. of Standards and Technology (PL), Boulder, CO. Quantum Physics Div.

Rigid Bender Analysis of van der Waals Complexes: The Intermolecular Bending Potential of a Hydrogen Bond.

Final rept.

D. J. Nesbitt, and C. M. Lovejoy. 1992, 14p.

Grants NSF-DJNB1, NSF-DJNC

Sponsored by National Science Foundation, Washing. ton, DC.

Pub. in Jnl. of Chemical Physics 96, n8 p5712-5725,

15 Apr 92.
Keywords: "Hydrogen bonds, Van der Waals forces Supersonic jet flow, Intermolecular forces, Rotational spectra, Vibrational spectra, High resolution, Predissociation, Reprints, "Hydrogen fluoride complexes, Intermolecular potentials, Potential energy surfaces.

High resolution IR data on weakly bound OCOHF complexes formed in a slit supersonic expansion reveal a progression of extremely low frequency vibrational levels associated with the bending of the OCO-HF hydrogen bond. In a previous paper (J Chem Phys 93, 7716 (1990)), we presented a spectroscopic analysis of the fundamental, combination and hot bands observed corresponding to transitions between nu(bend)(sup I) $=0(\sup 0), 1(\sup 1), 2(\sup 0), 2(\sup 2)$, and $3(\sup 1)$ where nu(bend)(sup I) denotes quanta of OCOHF skeletal bend excitation with I units of vibrational angula momentum. In this paper, we analyze the rotationally resolved data in terms of the rigid bender formalism of Hougen, Bunker and Johns to determine an explicit angular potential, $\mathrm{V}$ (theta), for the OCOHF complex in both the HF ground (nu(HF) $=0$ ) and vibrationally excited $(\mathrm{nu}(\mathrm{HF})=1)$ state.

\section{1,043}

PB95-203055 Not available NTIS

National Inst. of Standards and Technology (PL), Boulder, CO. Quantum Physics Div.

Kinetics of the Reaction $\mathrm{C} 2 \mathrm{H}+\mathrm{O} 2$ from 193 to 350 $\mathrm{K}$ Using Laser Flash Kinetic Infrared Absorption Spectroscopy.

Final rept.

B. J. Opansky, P. W. Seakins, J. O. P. Pedersen

and S. R. Leone. 1993, 7p.

Contract NASA-NAGW-2438

Sponsored by NASA Scientific and Technical Information Facility, Baltimore, MD

Pub. in Jnl. of Physical Chemistry 97, n33 p8583-8589 1993.

Keywords: "Reaction kinetics, "Oxidation, Temperature range 0065-0273 $\mathrm{K}$, Temperature range 0273$0400 \mathrm{~K}$, Temperature dependence, Absorption spectroscopy, Infrared spectroscopy, Photolysis, Oxygen Reprints, "Acetylene radicals, Color center lasers, Rate coefficients, Ethynyl radicals.

Rate coefficients for the reaction $\mathrm{C} 2 \mathrm{H}+\mathrm{O} 2$ are measured from 193 to $350 \mathrm{~K}$ by using transient abso:ption spectroscopy with an infrared color center laser. Ethynyl radicals are produced by pulsed laser photolysis of $\mathrm{C} 2 \mathrm{H} 2$ in a temperature variable flow cell and tunable color center laser probes the transient removal of $\mathrm{C} 2 \mathrm{H}$ in absorption. The rate coefficient has a slight negative temperature dependence over the range 193 $350 \mathrm{~K}$, and an expression is obtained. The lack of pressure dependence under our experimental conditions strongly suggests that the $\mathrm{HCCOO}^{*}$ complex is short lived with respect to encountering a collision and rapidly dissociates to products without competition from collisional stabilization to $\mathrm{HCCOO}$ or collisionally induced dissociation to reactants.

\section{1,044}

PB95-203121 Not available NTIS

National Inst. of Standards and Technology (PL), Boulder, CO. Ouantum Physics Div.

Photodissociation Dynamics in Quantum State-Selected Clusters: A Test of the One-Atom Cage Effect in $\mathrm{Ar}-\mathrm{H} 2 \mathrm{O}$

Final rept.

D. Plusquellic, O. Votava, and D. J. Nesbitt. 1994 ,

$3 p$.

Sponsored by National Science Foundation, Arlington

Pub. in Jnl. of Chemical Physics 101, n7 p6356-6358, 1 Oct 94.

Keywords: "Argon complexes, Van der Waals forces Supersonic jet flow, High resolution, Excimer lasers, Parametric oscillators, Photodissociation, Photolysis, Reprints, "Water complexes.

High resolution IR overtone pumping with an injection seeded optical parametric oscillator (OPO) is used in conjunction with excimer laser photolysis to investigate the state-resolved dynamics of quantum state-selected van der Waals clusters in a slit supersonic expansion The narrow band IR light source $(160 \mathrm{MHz}, 5 \mathrm{~mJ}$ ) preselects a specific upper state via the internal roto band of $\mathrm{Ar}-\mathrm{H} 2 \mathrm{O}$ which correlates to a transition in $\mathrm{H} 2 \mathrm{O}$ monomer. At fixed UV photolysis and probe wavelength, scanning the high resolution OPO yields the overtone action spectrum of Ar-H2O complexes.
01,045

PB95-203139 Not available NTIS

National Inst. of Standards and Technology (PL), Boulder, CO. Quantum Physics Div.

Photoelectron Spectroscopy of Small Antimony Cluster Anions: $\mathrm{Sb}(-), \mathrm{Sb} 2(-), \mathrm{Sb} 3(-)$, and Sb4(-). Final rept.

M. L. Polak, G. Gerber, J. Ho, and W. C. Lineberger. 1992, 11p.

Grants NSF-PHY90-12244, NSF-CHE88-19444 Sponsored by National Science Foundation, Washing ton, DC

Pub. in Jnl. of Chemical Physics 97, n12 p8990-9000, 15 Dec 92.

Keywords: "Photoelectron spectroscopy, "Atomic clusters, Near ultraviolet radiation, Ultraviolet spectra, Electronic structure, Electron affinity, Excited states Negative ions, Reprints, *Antimony clusters.

We report the $351 \mathrm{~nm}$ photoelectron spectra of $\mathrm{Sb}(1$ ), Sb2(1-), Sb3(1-), and $\mathrm{Sb} 4(1-)$. The electron affinity of atomic $\mathrm{Sb}$ is measured to be $1.046(5) \mathrm{eV}$. The Sb2(1-) photoelectron spectrum displays rich vibrational and electronic structure. For the photoelectron spectra of Sb3(1-) and Sb4(1-), the observed electronic structure is explained in terms of recently reported ab initio calculations. We report photoelectron angular distributions for all of the observed spectra, and find that the autodetaching resonance causes unusual angular distributions for Sb2(1-) photodetachment. Finally, electron affinity trends for group $V$ atoms, dimers, and small clusters are discussed in light of the present study.

\section{1,046}

PB95-203196 Not available NTIS

National Inst. of Standards and Technology (PL), Boulder, CO. Ouantum Physics Div.

High Resolution, Jet-Cooled Infrared Spectroscopy of $(\mathrm{HCl}) 2$ : Analysis of nu1 and nu2 $\mathrm{HCl}$ Stretching Fundamentals, Interconversion Tunneling, and Mode-Specific Predissociation Lifetimes. Final rept.

M. D. Schuder, C. M. Lovejoy, R. Lascola, and D. J. Nesbitt. 1993, 17p.

Grants NSF-PHY90-12244, NSF-CHE90-0064 Sponsored by National Science Foundation, Washington, DC.

Pub. in Jnl. of Chemical Physics 99, n6 p4346-4362, $15 \operatorname{Sep} 93$

Keywords: Supersonic jet flow, Near infrared radiation Temperature range 0000-0013 K Absortion spectra, Infrared spectroscopy, High resolution, Reprints *Hydrogen chloride dimers, Vibrational predissociation.

An extensive series of near-infrared absorption spectra are recorded for jet-cooled (6-14 K) hydrogen chloride dimer $(\mathrm{HCl}) 2$. Both Delta $\mathrm{K}($ alpha $)=0$ and Delta $\mathrm{K}$ (alpha) $=+$ or -1 bands are observed for both the free (nu(1)) and bonded (nu(2)) $\mathrm{HCl}$ stretches; all three chlorine isotopomers $(\mathrm{H}(35) \mathrm{Cl}-\mathrm{H}(35) \mathrm{Cl}, \mathrm{H}(35) \mathrm{Cl}$ $\mathrm{H}(37) \mathrm{Cl}$, and $\mathrm{H}(37) \mathrm{Cl} \cdot \mathrm{H}(37) \mathrm{Cl})$ are observed and analyzed for $\mathrm{K}$ (alpha) (double prime) $=$ or $<2$. The slit jet spectrum extends significantly the previous cooled cell infrared study of this complex and provides a measure of tunneling splittings for K(alpha) $=0$ and 1 for each of the $\mathrm{HCl}$ ground $(\mathrm{nu}=0)$ and excited $(\mathrm{nu}=1)$ states. Mode specific vibrational predissociation is observed via analysis of the absorption line shapes, with Lorentzian contributions to the line profiles of Delta nu(1) approx $=$ or $<1.6 \mathrm{MHz}$ and Delta nu(2) $=5.1$ + or - 12 (2 sigma) $\mathrm{MHz}$ full width at half-maximum for nu(1) and nu(2) excitation, respectively.

\section{1,047}

PB95-203204 Not available NTIS

National Inst. of Standards and Technology (PL), Boulder. CO. Ouantum Physics Div.

Slit-Jet Near-Infrared Diode Laser Spectroscopy of (DCI)2: nu1, nu2 DCl Stretching Fundamentals, Tunneling Dynamics, and the Influence of Large Amplitude 'Geared' Intermolecular Rotation.

Final rept.

M. D. Schuder, D. D. Nelson, and D. J. Nesbitt. 1993, 16p.

Grants NSF-PHY90-12244, NSF-CHE90-00641

Sponsored by National Science Foundation, Washing ton, DC

Pub. in Jnl. of Chemical Physics 99, n7 p5045-5060, 1 Oct 93.

Keywords: "Hydrogen chloride, Supersonic jet flow, Near infrared radiation, Infrared spectroscopy, Laser 
spectroscopy, High resolution, Hydrogen bonds, Vibrational spectra, Chlorine 35, Chlorine 37, Reprints "Deuterium chloride dimers, Vibrational predissociation.

The first high resolution spectra of (DCI)2 are reported using direct IR laser absorption spectroscopy in a slit supersonic expansion. The spectral data are analyzed to obtain vibrational frequencies, rotational constants, and tunneling (interconversion) level splittings for isotopically symmetric $(D(35) \mathrm{Cl}) 2$ and $(\mathrm{D}(37) \mathrm{Cl}) 2$, and mixed $D(35) C I-D(37) C l$ dimers. Six dimer absorption bands are observed and analyzed for both (D(35)Cl)2 and $\mathrm{D}(35) \mathrm{Cl}-\mathrm{D}(37) \mathrm{Cl}$.

\section{1,048}

PB95-203212 Not available NTIS

National Inst. of Standards and Technology (PL), Boulder, CO. Quantum Physics Div.

High Resolution Near Infrared Spectroscopy of $\mathrm{HCl}-\mathrm{DCl}$ and $\mathrm{DCl}-\mathrm{HCl}$ : Relative Binding Energies, Isomer Interconversion Rates, and Mode Specific Vibrational Predissociation.

Final rept

M. D. Schuder, and D. J. Nesbitt. 1994, 18p. Grants NSF-PHY90-12244, NSF-CHE80-00641 Sponsored by National Science Foundation, Arlington, VA.

Pub. in Jnl. of Chemical Physics 100, n10 p7250-7267 15 May 94

Keywords: Supersonic jet flow, Near infrared radiation Infrared spectroscopy, Laser spectroscopy, Binding energy, Hydrogen bonds, Energy transfer, High resolution, Isomers, Dimers, Reprints, "Hydrogen chloride complexes, "Deuterium chloride complexes, Vibrational predissociation.

Both $\mathrm{D}$ - and $\mathrm{H}$-bonded isomers of the mixed dimers formed between $\mathrm{HCl}$ and $\mathrm{DCl}$ are investigated via high resolution infrared difference frequency and diode laser spectroscopy in the 2,885 and $2,064 / \mathrm{cm}$ regions. From an analysis of the relative integrated absorption intensities, the D-bonded complex (i.e., $\mathrm{HCl}-\mathrm{DCl}$ ) is determined to be more stable by $16+$ or $-4 / \mathrm{cm}$ than the $\mathrm{H}$-bonded (i.e., $\mathrm{DCl}-\mathrm{HCl}$ ) species. All four chlorine isotopic combinations of the lower energy $(\mathrm{HCl}-\mathrm{DCl}) \mathrm{com}$ plex are probed via excitation of both $\mathrm{HCl}$ and $\mathrm{DCl}$ stretches. Additionally, two chlorine isotopomers of the higher energy $(\mathrm{DCl}-\mathrm{HCl})$ complex are investigated through $\mathrm{HCl}$ excitation. Compared to the facile tunneling observed in both $(\mathrm{HCl}) 2$ or $(\mathrm{DCl}) 2$ complexes these mixed dimers exhibit more rigid behavior characteristic of two distinct isomeric species. However, the relatively small energy difference $(16+$ or $-4 / \mathrm{cm})$ be tween the two isomers still allows the wave functions for both species to sample both the $\mathrm{HCl}-\mathrm{DCl}$ and $\mathrm{DCl}$ $\mathrm{HCl}$ local minima on the potential surface.

\section{1,049}

PB95-203238 Not available NTIS

National Inst. of Standards and Technology (PL), Boulder, CO. Quantum Physics Div.

Laser Flash Photolysis, Time-Resolved Fourier Transform Infrared Emission Study of the Reaction $\mathrm{Cl}+\mathrm{C} 2 \mathrm{H} 5$ yields $\mathrm{HCl}(\mathrm{v})+\mathrm{C} 2 \mathrm{H} 4$.

Final rept.

P. W. Seakins, E. L. Woodbridge, and S. R. Leone

1993, 10p.

Contract DE-FG02-883413860

See also PB95-164281. Sponsored by Department of Energy, Washington, DC.

Pub. in Jnl. of Physical Chemistry 97, n21 p5633-5642 1993.

Keywords: "Chlorine, Chemical reactions, Hydrogen chloride, Ethylene, Emission spectroscopy, Infrared spectroscopy, Vibrational states, Chemiluminescence Photolysis, Reprints, "Atom radical reactions, "Ethy radicals.

The atom-radical reaction $\mathrm{Cl}+\mathrm{C} 2 \mathrm{H} 5$-.> $\mathrm{HCl}(\mathrm{nu})+$ $\mathrm{C} 2 \mathrm{H} 4$ is studied using laser flash photolysis, time-resolved Fourier transform infrared emission spectros copy and broad band infrared chemiluminescence The $\mathrm{Cl}$ atoms and ethyl radicals are produced from number of different precursors using one or two lasers. The initial $\mathrm{HCl}$ vibrational distribution is determined. The vibrational distribution is characteristic of an addition-elimination mechanism and can be reproduced using modified statistical theories of energy partitioning within the $(\mathrm{C} 2 \mathrm{H} 5 \mathrm{Cl})$ intermediate. The time evolution of the $\mathrm{HCl}(\mathrm{nu}=4)$ emission is used to estimate a rate coefficient for this reaction of $(3.0+$ or -1.0$) \times 10$ (exp -10) cc/molecule/s.
01,050

PB95-203311 Not available NTIS

National Inst. of Standards and Technology (PL), Boulder, CO. Quantum Physics Div.

Laser-Induced Desorption of In and Ga from Si(100) and Adsorbate Enhanced Surface Damage. Final rept.

P. G. Strupp, A. L. Alstrin, B. J. Korte, and S. R

Leone. 1992, 6p

Contract AFÓSR90-0166

Sponsored by Air Force Office of Scientific Research, Bolling AFB, DC

Pub. in Materials Research Society Symposia Proceedings, v236 p27-32 1992.

Keywords: "Indium, "Gallium, Laser induced fluorescence, Neodymium lasers, YAG lasers, Pulsed lasers, Laser radiation, Radiation damage, Surface reactions, Ultrahigh vacuum, Adsorbates, Silicon, Reprints, "Laser induced desorption, Surface damage.

Laser-induced desorption (LID) of In and Ga from $\mathrm{Si}(100)$ under ultra-high vacuum conditions is investigated. The frequency doubled $532 \mathrm{~nm}, 2-6 \mathrm{~ns}$ output of a Nd:YAG laser is focussed to $0.14+$ or $-0.03 \mathrm{~J} /$ $\mathrm{sq} \mathrm{cm}$ on the $\mathrm{Si}$ surface to induce desorption Desorbed In or $\mathrm{Ga}$ atoms are detected by laser-induced fluorescence initiated by a pulsed dye laser propagating in front of the surface. Adsorbate-enhanced laser-induced surface damage is also observed; only 0.2 monolayer of In reduces the number of laser pulses required to observe damage by greater than a factor of 30

\section{1,051}

PB95-203329 Not available NTIS

National Inst. of Standards and Technology (PL), Boulder CO. Quantum Physics Div.

Pulsed Laser Irradiation at $532 \mathrm{~nm}$ of In and Ga Adsorbed on Si(100): Desorption, Incorporation, and Damage.

Final rept.

P. G. Strupp, A. L. Alstrin, B. J. Korte, and S. R

Leone. 1992, 7p.

Contract AFOSR90-0166

Sponsored by Air Force Office of Scientific Research, Bolling AFB, DC

Pub. in Jnl. of Vacuum Science and Technology A 10. n3 p508-514 May/Jun 92.

Keywords: "Indium, "Gallium, Laser induced fluorescence, Radiation damage, Surface reactions, Ultrahigh vacuum, Neodymium lasers, YAG lasers, Pulsed lasers, Laser radiation, Silicon, Adsorbates, Reprints, "Laser induced desorption, Surface damage.

Laser-induced desorption (LID) of In and $\mathrm{Ga}$ from Si(100) under ultrahigh vacuum conditions is investigated. The frequency-doubled $532 \mathrm{~nm}, 2-6 \mathrm{~ns}$ output of $\mathrm{Nd}: Y A G$ laser is focused to $0.14+$ or $-0.03 \mathrm{~J} / \mathrm{sq}$ $\mathrm{cm}$ on the Si surface to induce desorption. Desorbed In or $\mathrm{Ga}$ atoms are detected by laser-induced fluorescence initiated by a second pulsed laser propagating in front of the surface. LID occurs by thermal desorption with approximate desorption energies and preexponential factors in agreement with the literature values obtained previously by isothermal desorption measurements. Experiments at higher coverages suggest that desorption occurs predominantly from the two-dimensional (2D) adlayer with little desorption occurring from the adsorbate islands directly into the vacuum. The 2D layer is resupplied by either diffusion out of adsorbate islands or by diffusion of incorporated adsorbate out of the bulk. Adsorbate-enhanced laser-induced surface damage is also observed; only 0.2 monolayer of In reduces the number of laser pulses required to observe damage by greater than a factor of 30 .

\section{7,052}

PB'95-203337 Not available NTIS

National Inst. of Standards and Technology (PL), Boulder, CO. Quantum Physics Div.

Single-Photon Laser lonization Time-of-Flight Mass Spectroscopy Detection in Molecular-Beam Epitaxy: Application to As4, As2, and Ga.

Final rept.

P. G. Strupp, A. L. Alstrin, R. V. Smilgys, and S. R. Leone. 1993, 5p.

Contract AFOSR90-0166

Sponsored by Air Force Office of Scientific Research, Bolling AFB, DC

Pub. in Applied Optics 32, n6 p842-846, 20 Feb 93. Keywords: "Molecular beam epitaxy, "Gallium arsenides, "Gallium, "Arsenic, Real time operations,
Semiconducting films, Gas ionization, Far ultraviolet radiation, Laser radiation, Neodymium lasers, YAG lasers, Reprints, Time-of-flight mass spectroscopy.

Single-photon laser ionization time-of-flight mass spectroscopy (TOF-MS) is used to monitor fluxes of As4 As2, and $\mathrm{Ga}$, species that are important in molecularbeam epitaxy of GaAs. With this technique, fluxes of multiple chemical species above a substrate can be measured noninvasively and in real time during conventional molecular-beam epitaxy. Additionally, the geometry of the single-photon ionization TOF-MS permits simultaneous film-growth monitoring by using techniques such as reflection high-energy electron diffraction (RHEED). Here gas-phase arsenic and gallium beams are ionized by a single 118-nm $(10.5-\mathrm{eV})$ photon and detected with a TOF-MS. The 118-nm photons are produced by frequency tripling 355-nm light from a pulsed Nd:YAG laser in Xe. With single-photon ionization less than $0.4 \%$ of the As $4(1+)$ signal fragments to As2(1+). Neither As4(1+) nor As2(1+) fragments to As $(1+)$ at $118 \mathrm{~nm}$. The relative ionization probability of As4/As2 at $118 \mathrm{~nm}$ is approximately $4: 1$. This technique promises to be a powerful tool for analyzing most III-V and II-VI molecular-beam epitaxy growth species.

\section{1,053}

PB95-203386 Not available NTIS

National Inst. of Standards and Technology (CSTL) Gaithersburg, MD. Biotechnology Div

Thermodynamics of the Hydrolysis of N-Acetyl-L phenylalanine Ethyl Ester in Water and in Organic Solvents.

Final rept

Y. B. Tewari, M. M. Schantz, P. C. Pandey, M. V. Rekharsky, and R. N. Goldberg. 1995, 8p.

Pub. in Jnl. of Physical Chemistry 99, n5 p1594-1601, 2 Feb 95.

Keywords: "Thermodynamics, "Hydrolysis, Water, Organic solvents, Equilibrium, Gibbs free energy, Entropy, Enthalpy, Reprints, *Phenylalanine/N-acetyl(ethyl-ester).

Equilibrium measurements have been performed on the alpha-chymotrypsin-catalyzed hydrolysis reaction of $\mathrm{N}$-acetyl-L-phenylalanine ethyl ester to ( $\mathrm{N}$-acetyl-Lphenylalanine + ethanol) with carbon tetrachloride dichloromethane, toluene, and aqueous phosphate buffer as solvents for the reactants and products. Apparent equilibrium constants were measured as a function of temperature for this reaction in all four solvents. Calorimetric measurements were also performed for this reaction in aqueous phosphate buffer. The rathe limited range of values that was found for the equilibrium constants is significant. The very limited amount of information available from the literature is also suggestive of the rule that equilibrium constants for hydrolysis reactions in different solvents are comparable if the reaction refers to neutral species and the concentration of water is included in the formulation of the equilibrium constant. Also, the standard molar en thalpy of reaction was found to be a linear function (slope $=313 \mathrm{~K}$ ) of the standard molar entropy of reaction. This is indicative of an enthalpy-entropy compensation effect.

\subsection{4}

PB95-203485 Not available NTIS

National Inst. of Standards and Technology (PL), Boul der, CO. Quantum Physics Div.

Signatures of Large Amplitude Motion in a Weakly Bound Complex: High-Resolution IR Spectroscopy and Quantum Calculations for $\mathrm{HeCO} 2$.

Final rept.

M. J. Weida, J. M. Sperhac, D. J. Nesbitt, and J. M. Hutson. 1994, 13p.

Sponsored by National Science Foundation, Arlington, VA.

Pub. in Jnl. of Chemical Physics 101, n10 p8351-8363, 15 Nov 94.

Keywords: "Helium complexes, Molecular dynamics, Infrared spectroscopy, High resolution, Van der Waals forces, Supersonic jet flow, Reprints, "Carbon dioxide complexes, Potential energy surfaces

The infrared spectrum of the $\mathrm{HeCO} 2$ van der Waals molecule is recorded in the region of the $\mathrm{CO} 2 \mathrm{nu}(3)$ asymmetric stretch via direct absomtion of a tunable $\mathrm{Pb}$-salt diode laser. $\mathrm{HeCO} 2$ is formed in a slit jet supersonic expansion; the slit valve and the stagnation gas must be precooled to $-35 \mathrm{C}$ before substantial formation of the complex is observed. Sixty-six rovibrational transitions are recorded by exciting the nu(3) asymmetric stretch of the $\mathrm{CO} 2$ monomer within the complex. 
Forty-three of these transitions can be assigned using intemally consistent combination differences as a btype band of a T-shaped asymmetric rotor. There are several indications that large amplitude motion is significant to $\mathrm{HeCO} 2$, including the poor quality of the fit to an asymmetric rotor model and the large positive inertial defects of Delta $=8.54$ and $10.98 \mathrm{u}$ (Angstrom squared) in the ground and excited states, respectively. However, a hindered rotor analysis based on these inertial defects demonstrates that the $\mathrm{CO} 2 \mathrm{mo}$ tion within the complex is far from the free rotor limit. No evidence of predissociation broadening is observed, indicating a lifetime for the complex of tau > $6 \mathrm{~ns}$.

\section{1,055}

PB95-251732 PC A03/MF A01

National Inst. of Standards and Technology (CSTL), Gaithersburg, MD. Process Measurements Div.

Standard Reference Material 1744: Aluminum Freezing-Point Standard.

Special pub.

G. F. Strouse. May 95, 41p, NIST/SP-260-124

Also available from Supt. of Docs. as SN003-00303342-1.

Keywords: "Aluminum, "Melting points, "Calibration standards, Temperature, Phase transformations, Scale(Ratio), Standardization, Interlaboratory comparisons, "Standard Reference Material 1744, SRM 1744 International Temperature Scale of 1990, ITS-90, Fixed-point cell.

The freezing point of aluminum (660.323 deg. C) is a defining fixed point of the International Temperature Scale of 1990 (ITS-90). Realization of this freezing point is performed using a fixed-point cell containing high punty (greater than or equal to $99.9999 \%$ pure) aluminum. The aluminum constituting Standard Reference Material 1744 (SRM 1744) has been evaluated, and certified as suitable for use in the realization of the freezing-point temperature of aluminum for the ITS-90. Based on results obtained with three fixed-point cells containing random samples of SRM 1744, the plateau temperature of a freezing curve of the SRm 1744 aluminum is expected to differ by not more than $1 \mathrm{~m}$ deg $C$ from the ITS-90 assigned temperature. In the document, the methods used for the construction of the aluminum freezing-point cells and for the evaluation of SRM 1744 are described.

01,056

\section{PB95-260618 PC A09/MF A02}

National Inst. of Standards and Technology (CSTL), Boulder, CO. Process Measurements Div.

Thermochemical and Chemical Kinetic Data for Fluorinated Hydrocarbons.

Technical note.

D. R. F. Burgess, M. R. Zachariah, W. Tsang, and P.

R. Westmoreland. Jul $95,184 p$, NIST/TN-1412

Also available from Supt. of Docs. as SNO03-003-

03343-0. Prepared in cooperation with Massachusetts Univ., Amherst. Dept. of Chemical Engineering.

Keywords: "Halohydrocarbons, "Fluorination, "Thermochemistry, "Reaction kinetics, Molecular orbitals, Molecular theory, Transport properties, Models, Environmental chemical substitutes, Chemical extinguishers.

A comprehensive, detailed chemical kinetic mechanism was developed for fluorinated hydrocarbon destruction and flame suppression. Existing fluorinated hydrocarbon thermochemistry and kinetics were compiled and evaluated. For species where no or incomplete thermochemistry was available, these data were calculated through application of ab initio molecular orbital theory. Group additivity values were determined consistent with experimental and ab initio data. For reactions where no or limited kinetics was available, these data were estimated by analogy to hydrocarbon reactions, by using empirical relationships from other fluorinated hydrocarbon reactions, by ab initio transition state calculations, and by application of RRKM and QRRK methods. This report provides the thermochemical and chemical kinetic data used in this work.

01,057

PB95-260808 PC A03/MF A01

National Inst. of Standards and Technology (TS), Gaithersburg, MD. Standard Reference Data Program.
NIST Standard Reference Data Products Catalog, 1995-96. Achieve with Standard Reference Data. Special pub.

J. C. Sauerwein. Jul 95, 45p, NIST/SP-782

Supersedes PB94-151842. Also available from Supt. of Docs. as SN003-003-03346-4.

Keywords: "Catalogs(Publications), "Data bases Chemistry, Chemical analysis, Atomic physics, Bio technology, Reaction kinetics, Numeric data, Physics, Fluids, Thermochemistry, Molecular structure, Molecular spectroscopy, Software tools, Compilations, Character recognition, Evaluated data, Materials.

The National Institute of Standards and Technology's (NIST) Standard Reference Data Program provides re liable, well-documented data to scientists and engineers for use in technical problem-solving, research and development. This catalog lists published current databases and published data in the National Standard Reference Database Series. This edition of the catalog contains many new databases and updates curren ones. These data compilations have been subdivided into ten categories. Prices and ordering information are located at the back of the document.

01,058

PB95-261962 (Order as PB95-261897, PC A07)

MF A02

National Inst. of Standards and Technology, Gaithersburg, MD

New IUPAC Guidelines for the Reporting of Stable Hydrogen, Carbon, and Oxygen Isotope-Ratio Data. Letter to the Editor.

T. B. Coplen. 1995, $1 \mathrm{p}$

Included in Jnl. of Research of the National Institute of Standards and Technology, v100 n3 p285 May/Jun 95

Keywords: "Isotope ratio, "Abundance, "Hydrogen isotopes, "Carbon isotopes, "Oxygen isotopes, Chemical elements, Atomic mass, Sea water, Standards, IUPAC guideliness.

To eliminate possible confusion in the reporting of isotopic abundances on noncorresponding scales, the Commission on Atomic Weights and Isotopic Abundances of the Internationl Union of Pure and Allied Chemistry (IOPAC) recommended at the IUPAC 37 th General Assembly in August 1993 at Libson, Portugal that (1) $2 \mathrm{H} / 1 \mathrm{H}$ relative ratios of all substances be expressed relative to VSMOW (Vienna Standard Mean Ocean Water) on a scale such that $2 \mathrm{H} / 1 \mathrm{H}$ of SLAP (Standard Light Antarctic Precipitation) is 0.572 times that of VOSMOW; (2) $13 \mathrm{C} / 12 \mathrm{C}$ relative ratios of al substances be expressed relative to VPDB (Vienna Peedee belemnite) on a scale such that $13 \mathrm{C} / 12 \mathrm{C}$ of NBS-lime-stone (RM 8544) is 1.00195 times that of VPDB; and (3) 180/160 ratios of all substances be expressed relative to either VSMOW or VPDB an scales such that $180 / 160$ of SLAP is 0.9445 times that of VSMOW.

\section{1,059}

PB96-102025 Not available NTIS

National Inst of Standards and Technology (PL), Boulder, CO. Quantum Physics Div

Single Photon Laser Ionization as an In-situ Diagnostic for MBE growth.

Final rept.

A. L. Alstrin, A. K. Kunz, P. G. Strupp, and S. R Leone. 1994, 6p.

Contract ACS-PRF-27135-AC6

Sponsored by Petroleum Research Fund, Washington DC.

Pub. in Materials Research Society Symposia Proceedings, v324 p359-364 1994

Keywords: "Molecular beam epitaxy "Gallium arsenides, "Flux(Rate), Time-of-flight mass spectrometers, YAG lasers, Photons, Neodymium lasers, Diag nostic techniques, Scattering, Desorption, Laser radiation, lonization, Reprints.

Single photon laser ionization time-of-flight mass spec troscopy (SPI-TOFMS) is used to monitor the gaseous fluxes of $\mathrm{Ga}$ and Asn, during molecular beam epitaxy of GaAs. This noninvasive and real-time probe measures densities, and hence fluxes, of multiple chemical species impinging on or scattered from a substrate during conventional MBE. With single photon ionization a $118 \mathrm{~nm}$ (10.5 eV, ninth harmonic of Nd: YAG laser) the photon energy is large enough to ionize the species, but insufficient to both ionize and fragment. Results will be presented on the probing of scattering and desorption of III-V MBE species during GaAs growth.
This technique promises to be a valuable in-situ diagnostic for III-V and II-V| MBE.

01,060

PB96-102041 Not available NTIS

National Inst. of Standards and Technology (CSTL) Gaithersburg, MD. Thermophysics Div.

Ab initio Calculations for Helium: A Standard for Transport Property Measurements.

Final rept

R. A. Aziz, A. R. Janzen, and M. R. Moldover. 1995 , $4 p$.

Pub. in Physical Review Letters 74, n9 p1586-1589, 27 Feb 95.

Keywords: *Helium, "Transport properties, ${ }^{\star}$ Standards, Thermal conductivity, Virial equation, Coefficients, Viscosity, Potentials, Thermodynamics, Reprints, "Ab initio calculation.

For helium, the accuracy of calculated transport properties and virial coefficients based on accurate ab initio potential now exceeds that of the best measurements. The $a b$ initio results should be used to calibrate meas uring apparatus.

01,061

PB96-102298 Not available NTIS

National Inst. of Standards and Technology (PL), Boulder, CO. Quantum Physics Div.

High-Resolution Infrared Overtone Spectroscopy of N2-HF: Vibrational Red Shifts and Predissociation Rate as a Function of HF Stretching Quanta.

Final rept.

J. T. Farrell, O. Sneh, and D. J. Nesbitt. 1994, 7p.

Sponsored by National Science Foundation, Arlington, VA.

Pub. in Jnl. of Physical Chemistry 98, n24 p6068-6074 1994.

Keywords: "Nitrogen compounds, "Hydrofluoric acid "Infrared spectroscopy, Van der Waals forces, Infrared absorption, High resolution, Potentials, Difference frequency, Electrostatics, Red shift, Reprints.

The high-resolution infrared spectrum of the nu(sub $\mathrm{HF}$ ) $=2$ inversely maps to 0 stretch in N2-HF has been recorded using direct absorption of tunable infrared light in a slit jet spectrometer. The ban origin is located at $7657.40571 / \mathrm{cm}$ red-shifted $93.391 / \mathrm{cm}$ from the nu(sub HF) $=2$ inversely maps to 0 origin of the $\mathrm{HF}$ monomer. The changes in vibrational red shift with HF stretching quanta provide explicit information on the coupling between the high-frequency (i.e. intramolecular) and low-frequency (i.e., intermolecular) degrees of freedom, which can be understood with a simple electrostatic model. Additional evidence for coupling between the low- and high-frequency modes is provided through an analysis of the rotational constants.

01,062

PB96-102355 Not available NTIS

National Inst. of Standards and Technology (CSTL), Gaithersburg, MD. Biotechnology Div.

Non-Perturbative Relation between the Mutual Diffusion Coefficient, Suspension Viscosity, and Osmotic Compressibility: Application to Concentrated Protein Solutions.

Final rept

A. K. Gaigalas, V. Reipa, J. Hubbard, J. Edwards, and J. Douglas. 1995, $8 p$.

Pub. in Chemical Engineering Science, v50 n7 p1107 11141995.

Keywords: "Proteins, "Diffusion coefficient, "Viscosity "Osmosis, Concentration(Composition), Mixtures, Suspensions, Aqueous solutions, Compressibility, Reprints.

The authors test a proposed relation between mutual diffusion coefficient and viscosity in suspensions which is essentially identical to a dynamic mode-coupling the ory of diffusion in binary fluid mixtures near a critica mixing point. In contrast to conventional treatments of suspension hydrodynamics, this relation is nonperturbative in the sense that is not necessary to as sume that the volume fraction of suspended particles is a small parameter. The mutual diffusion coefficient was then related to the viscosity and osmotic compressibility of the concentrated suspension relative to the infinite dilution limit. The authors conclude that this non-perturbative relation provides a convenient estimate of protein diffusion coefficients as a function of protein concentration. 
01,063

PB96-102371 Not available NTIS

National Inst. of Standards and Technology (CSTL),

Gaithersburg. MD. Thermophysics Div.

Critical Lines for Type-lil Aqueous Mixtures by Generalized Corresponding-States Models.

Final rept.

J. S. Gallagher, D. G. Friend, J. A. Given, and J. M.

H. L. Sengers. $1994,8 p$

Sponsored by Department of Energy, Washington, DC. Pub. in International Jnl. of Thermophysics $15, \mathrm{n} 6$ p1271-1278 1994.

Keywords: "Aqueous solutions, "Binary mixtures, "Carbon dioxide, "Phase diagrams, Algorithms, Gases, Nitrogen, Chemical composition, Chemical equilibrium Critical point, Pressure, Reprints, "Critical lines, "TypeIII mixtures, Gas-gas equilibrium, ECS(Extended corresponding states)

An algorithm has been developed for calculating the gas-gas critical line of type-III binary fluid mixtures for extended corresponding-states (ECS) models. The algorithm searches for an extremum in pressure on the spinodal curve of an isothermal pressure-composition phase diagram of a binary mixture. The method has been applied to solutions of carbon dioxide and of $\mathrm{ni}$ trogen in water, starting at the water critical point. Two variants of ECS have been tested for their ability to represent reliable PVTx data in the nitrogen-water mix ture. It is demonstrated that in the latter system both ECS variants produce an artifact in the gas-gas critical line in the range of 0-0.2 mole fraction of nitrogen.

01,064
PB96-102769 Not available NTIS

National Inst. of Standards and Technology (PL), Gaithersburg, MD. Ionizing Radiation Div.

NBS/NIST Peltier-Effect Microcalorimeter: A FourDecade Review.

Final rept.

W. B. Mann, and M. P. Unterweger. 1995, 6p.

Pub. in Applied Radiation and Isotopes 46, n3 p1851901995.

Keywords: "Calorimeters, "Radioactivity, "Peltier effects, Radioactive isotopes, lonizing radiation, Nickel 63, Radium 226, Theromophysical properties, US NBS, Reprints.

Radioactivity measurements made with the National Bureau of Standards/National Institute of Standards microcalorimeter since January 1954 are reviewed.

\section{1,065}

PB96-102801 Not available NTIS

National Inst. of Standards and Technology (CSTL), Gaithersburg, MD. Thermophysics Div.

Length Scales for Fragile Glass-Forming Liquids. Final rept.

R. D. Mountain. 1995, 3p.

Pub. in Jnl. of Chemical Physics 102, n3 p5408-5410, 1 Apr 95.

Keywords: "Glass, "Supercooling, "Liquids "Computerized simulation, Molecular flow, Phase transformations, Secondary waves, Wavelengths, Clumps, Transverse waves, Mixtures, Reprints, Softsphere mixture, Molecular dynamic.

Molecular dynamics simulation results are used to demonstrate the existence of a growing length in supercooled, fragile glass-forming liquids. This length is the longest wavelength propagating shear wave the fluid can support. Explicit results are reported for an equimolar soft-sphere mixture. A possible connection between this length and the size of locally rigid clusters is discussed.

\section{1,066}

PB96-102835 Not available NTIS

National Inst. of Standards and Technology (PL), Boulder, CO. Quantum Physics Div.

Probing Potential-Energy Surfaces via High-ResoIution IR Laser Spectroscopy.

Final rept.

D. J. Nesbitt. 1994, 18p.

Grants NSF-PHY90-12244, NSF-CHE90-00641

Sponsored by National Science Foundation, Artington, VA. and Air Force Otfice of Scientific Research, Bolling AFB, DC

Pub. in Faraday Discussions 97, p1-18 1994.

Keywords: *IR laser spectroscopy, Foreign technology Infrared spectroscopy, Van der Waals, High resolution, Supersonic expansion, Energy transfer, Reprints.
The use of high-resolution IR lasers for spectroscopic detection and characterization of trace, weakly bound cluster species in low-density, jet-cooled environments has led to enormous program in the study of collision dynamics, intermolecular forces and intramolecular energy flow. As a particular focus of this talk, direct absorption methods in combination with slit supersonic expansions and crossed molecular beams offer an extremely general tool for probing unimolecular and biomolecular dynamics with full quantum-state resolution.

\section{1,067}

PB96-102884 Not available NTIS

National Inst. of Standards and Technology (PL), Gaithersburg, MD. Ionizing Radiation Div.

Total-Dielectric-Function Approach to Electron and Phonon Response in Solids.

Final rept.

D. R. Penn, S. P. Lewis, and M. L. Cohen. 1995,

$15 p$

Sponsored by National Science Foundation, Arlington, VA. and Department of Energy, Washington, DC. Pub. in Physical Review B 51, n10 p6500-6514, 1 Mar 95.

Keywords: "Electron scattering, "Phonon response, "Solids, Reprints, Superconductivity, Total dielectric function.

The interaction between two test charges, the response of a solid to an external field, and the normal modes of the solid can be determined from a total dielectric function that includes both electronic and lattice polarizabilities as well as local-field effects. In this paper we examine the relationship between superconductivity and the stability of a solid and derive sum rules for the electronic part of the dielectric function. It is also shown that there are negative eigenvalues of the total static dielectric function, implying the possibility of an attractive interaction between test charges. An attractive interaction is required for superconductivity.

\section{1,068}

PB96-102892 Not available NTIS

National Inst. of Standards and Technology (CSTL), Gaithersburg, MD. Thermophysics Div.

Principle of Congruence and Its Application to Compressible States.

Final rept.

C. J. Peters, L. J. Florusse, J. L. de Roos, J. de Swaan Arons, and J. M. H. Sengers. 1995, 27p. Pub. in Fluid Phase Equilibria, v105 p193-219 1995.

Keywords: "Congruence, *Chain molecules, Reprints, Corresponding states, Bubble points, Thermodynamic properties, Mixtures, Dew points

The principle of congruence claims that certain thermodynamic and transport properties of a mixture of $n$ alkanes are the same as those of the pure n-a'kane of the mole-fraction-averaged carbon number. We discuss the origin of the principle, and attempts at theoretical justification and validation with respect to experimental data. We demonstrate that the principle applies even in cases where no fundamental justification exists. Whenever the principle applies, prediction of thermodynamic behavior of a mixture of $n$-alkanes in inherently simpler and usually more accurate than that based on empirical equations of state, molecular interaction parameters, and mixing and combining rules. Our experimental verification serves to pinpoint those properties that can be safely and accurately predicted on the basis of the principle of congruence. The solubility of hydrogen in mixtures of long-chain n-alkanes is used as a prime example.

01,069

PB96-103171 Not available NTIS

National Inst. of Standards and Technology (PL), Boulder, CO. Quantum Physics Div.

Collisional Alignment of $\mathrm{CO} 2$ Rotational Angular Momentum States in a Supersonic Expansion. Final rept.

M. J. Weida, and D. J. Nesbitt. 1994, 14p.

Contract AFOSR-90-0055, Grant NSF-PHY90-12244

Sponsored by National Science Foundation, Arlington,

VA. and Air Force Office of Scientific Research, Bolling AFB, DC.

Pub. in Jnl. of Chemical Physics 100, n9 p6372-6385, 1 May 94

Keywords: "Alignment, "Carbon d!oxide, "Molecular collisions, "Angular momentum. Anisotropy, Infrared lasers, Polarization(Spin alignment), Supersonics, Expansion, Molecular rotation, Reprints.

The rotational alignment of $\mathrm{CO} 2$ seeded in a supersonic expansion is measured using a general, direct absorption method based on fast ( $75 \mathrm{kHz}$ ) polarization modulation and phase sensitive de tection with of a narrow band tunable IR laser. The anisotropic distribution of bar M(sub j)bar states is created by a directed velocity slip between the carrier gas and a nonspherical seed gas. Strong alignment signals are observed in a pinhole expansion that depend systematically on the carrier gas, stagnation pressure, and J state. In a slit expansion, however, no alignment is detected for comparable conditions. The observed effects are quite significant and occur at rather modest expansion conditions (e.g., $2.5 \% \mathrm{CO} 2$ in $\mathrm{He}$ at $1000 \mathrm{Torr}$ ).

\section{1,070}

PB96-103197 Not available NTIS National Inst. of Standards and Technology (MSEL), Gaithersburg, MD. Polymers Div.

Water Adsorption at a Polyimide/Silicon Wafer Interface.

Final rept.

W. L. Wu, W. J. Orts, C. J. Majkrzak, and D. L.

Hunston. 1995, 5p

Contract N00014-92-F-0036

Sponsored by Office of Naval Research, Arlington, VA. Pub. in Polymer Engineering and Science, v35 n12 p1000-1004 Jun 95

Keywords: "Interfaces, "Moisture, "Wafers, "Polyimides, Concentration(Composition), Adsorption, Water, Neutron scattering, Reflectivity, Polymers, Silicon, Reprints.

Neutron reflectivity (NR) was applied to measure the concentration of water at the buried interfaces between an amorphous polyimide and silicon single crystal wafers. Excess water was discovered within 30 Angstroms of the metal/polymer interface, where the water concentration reached $17 \%$ (by volume) for the samples without a coupling agent and $12 \%$ for the one with coupling agent. The above results demonstrate conclusively the unique power of NR in determining water concentration near a buried interface, and provide the first quantitative evidence for a water concentration profile which peaks in the interface region.

\section{1,071}

PB96-103205 Not available NTIS

National Inst. of Standards and Technology (PL), Boulder, CO. Quantum Physics Div.

Phase Shifts and intensity Dependence in Frequency-Modulation Spectroscopy.

Final rept.

H. R. Xia, J. I. Cirac, S. Swartz, J. L. Hall, P. Zoller, B. Kohler, and D. S. Elliott. 1994, 10p. Contract N00014-89-J-1227, Grant NSF-PHY9012244

Sponsored by Office of Naval Research, Arlington, VA Air Force Office of Scientific Research, Bolling AFB, DC. and National Science Foundation, Arlington, $\mathrm{VA}$.

Pub. in Jnl. of the Optical Society of America B 11, n4 p721-730 May 94

Keywords: "Phase shift, "Spectroscopy, "Intensity, Frequency modulation, Spectrum analysis, Optical heterodyning, Line spectra, Shape, Reprints, RF signal, Optical Block equations.

The authors discuss experimental observations of an induced phase shift of the if signal produced in frequency-modulated saturated-absorption measurements under the conditions of large probe-beam intensities. This phase shift can be understood in terms of the phase difference between the phase modulation of the optical driving field and the steady-state macroscopic polarization of the medium. The authors also present theoretically calculated spectra that are based on the optical Bloch equations and that are in excellent agreement with the experimental results.

01,072

PB96-111620 Not available NTIS

National Inst. of Standards and Technology (CSTL) Gaithersburg, MD. Thermophysics Div.

Comparison of a Fixed-Charge and a Polarizable Water Model.

Final rept.

R. D. Mountain. 1995, 7p

Pub. in Jnl. of Chemical Physics, v103 n8 p3084-3090, 22 Aug 95 
Keywords: "Hydrogen bonds, "Water, "Mathematical models, "Molecular structure, Chemical bonds, Pairing interactions, Distribution functions, Lifetime, Density, Temperature, Polarization, Reprints, "Molecular dynamics.

Molecular dynamics simulations are used to examine two models for water. The first model is the fixedcharge model introduced by Stillinger and Rahman and the second model is the polarizable model developed by Dang. The site-site, intermolecular pair distribution functions and the hydrogen bond lifetimes are determined for three fluid states for which neutron-diffraction determined pair function exist. The trends in the pair functions and the bond lifetimes with decreasing density and increasing temperature for both models are similar, with the fixed-charge model pair functions changing more slowly, and the bond lifetimes more rapidly than those for the polarizable model.

\section{1,073}

PB96-111760 Not available NTIS

National Inst. of Standards and Technology (MSEL), Gaithersburg, MD. Ceramics Div.

Surface Chemistry of Silicon Nitride Powder in the Presence of Dissolved lons.

Final rept.

V. A. Hackley, and S. G. Malghan. 1994, 11 .

Pub. in Jnl. of Materials Science, v29 p4420-4430 1994.

Keywords: "Silicon nitrides, "Surface chemistry, "Powders(Particles), Chemical reactions, Interfaces, nants, Adsorption, Ceramics, Reprints.

Colloidal processing of silicon nitride (Si3N4) powders depends largely on the control of reactions at the solidsolution interface. The role of dissolved ions in the surface chemistry of impurities, contaminants and additives in processing are discussed. The interaction of ions at the solid-solution interface was characterized by particle electrokinetic behavior determined for electroacoustic measurements in moderately concentrated suspensions. Ions were classified according to chemical similarity and surface specificity. Specific adsorption was inferred from the movement of the isoelectric point relative to the endemic native value.

\section{1,074}

PB96-111893 Not available NTIS

National Inst. of Standards and Technology (MSEL), Gaithersburg, MD. Polymers Div

Crystal Structure of Calcium Glutarate Monohydrate.

Final rept.

M. Mathew, and S. Takagi. 1995, 3p.

Sponsored by American Dental Association Health Foundation, Chicago, IL.

Pub. in Zeitschrift fuer Kristallographie, v210 p199-201 1995.

Keywords: *Crystal structure, Reprints, Monocrystals, $X$ ray diffraction, Hydrates, Chemical bonds, "Foreign technology, "Calcium gluta rate monohydrate.

The crystal structure of calcium glutarate monohydrate, $\mathrm{Ca}(\mathrm{C} 5 \mathrm{H} 6 \mathrm{O} 4) . \mathrm{H} 2 \mathrm{O}$, has been determined by single crystal $\mathrm{X}$-ray diffraction. The crystals are orthorhombic with $\mathrm{a}=6.805$ (1) Angstrom, $\mathrm{b}=$ 18.486(2) Angstrom, $c=5.884(1)$ Angstrom, space group P2(sub 1)2(sub 1)2(sub 2), $Z=4, V=740.1$ (2) Angstrom $(3), \mathrm{dm}=1.69$, and $\mathrm{dc}=1.688 \mathrm{mg} /$ cubic $\mathrm{m}$. The structure was refined by the full-matrix leastsquares techniques to $R=0.019, R w=0.026$, for 1198 reflections with I greater than or equal to 3 sigma (I). The absolute configuration was determined. $\mathrm{Ca}$ is $\mathrm{co}$ ordinated to seven oxygen atoms and the coordination polyhedron is best described as pentagonal bipyramid. One carboxylate group of the glutarate ion is bonded to three different $\mathrm{Ca}$ ions, forming a four-membered chelate ring with one $\mathrm{Ca}$ ion and unidentate bridge bonds to two other $\mathrm{Ca}$ ions. The other axis, with the hydrocarbon chains sandwich between the polar regions consisting of the $\mathrm{Ca}$ and carboxylate ions and water molecules.

01,075
PB96-112057 Not available NTIS

Notional Inst. of Standards and Technology (CSTL),
Natable Boulder, CO. Thermophysics Div.

Shear-Induced Melting of Two-Dimensional Solids.

Final rept.

T. Weider, M. A. Glaser, H. J. M. Hanley, and N. A.

Clark. 1993, 7p.

Pub. in Physical Review B, v47 n10 p5622-5628, 1 Mar 93.
Keywords: "Melting, "Solids, Reprints, Two dimensions, Shear, Molecular dynamics, Voroni construction.

We have carried out detailed nonequilibrium molecular-dynamics simulation studies of the shear-induced melting transition of a model two-dimensional solid. We find that the shear melting of the two-dimensional softdisk solid at temperture $T=1$ and density $p=1.03$ occurs in two stages: (1) a transition from elastic to plastic behavior takes place as soon as any finite shear rate is applied; (2) qualitativechanges in structural and dynamic behavior occur near a shear rate of gamma = 0.07 . For $y$ equal to or less than 0.07 , the system possesses very long-range bond-orientational correlations, and the instantaneous static structure factor ex hibits pronounced sixfold anisotropy, with a sixfold pattern that rotates uniformly with time in response to the applied shear. For y equal to or greater than 0.07 , the system behaves like an ordinary two-dimensional liquid under shear in that the ranges of translational and bond-orientational correlations are comparable and the instances static structure factor does not exhibit persistent sixfold anisotropy. We discuss our results in terms of the two competingpossibilities of a KosterlitzThouless- Halperin-Nelson-Young two-stage melting scenario or a single first-order melting transition.

\section{1,076}

PB96-112172 Not available NTIS

National Inst. of Standards and Technology (PL), Boulder, CO. Quantum Physics Div.

Laser Gas Ionization Technique Monitors MEB Crystal Growth.

Final rept.

S. R. Leone, A. L. Alstrin, P. G. Strupp, and R. V. Smilgys. 1993, 4p.

Pub. in Laser Focus World, 4p Jul 93.

Keywords: "Molecular beam epitaxy, "Crystal growth, Reprints, Laser radiation, Real time operations, Ionization.

Single-photon ionization technique simultaneously detects complete gaseous species to provide real-time feedback and control during molecular-beam-epitaxial crystal growth.

\section{1,077}

PB96-112255 Not available NTIS

National Inst. of Standards and Technology (MSEL) Gaithersburg, MD. Reactor Radiation Div.

Structure of a Triglyceride Microemulsion: A Small Angle Neutron Scattering Study.

Final rept.

S. F. Trevino, R. Joubran, N. Parris, and N. F. Berk 1994, $6 \mathrm{p}$

Pub. in Langmuir, v10 n8 p2547-2552 Aug 94.

Keywords: "Microemulsions, "Triglycerides, Reprints, Concentration(Composition), Temperature, Soybean oil, "Neutron scattering.

The microscopic structure of a microemulsion of soybean oil, water-ethanol (80/20 wt \%), and polyoxyethylene(40) sorbitol hexaoleats has been studied with small angle neutron scattering. The concentrations of the three components are varied such that the oil-surfactant ratio is constant and stable microemulsions are obtained in regions of the phase diagram corresponding to bicontinuous and $\mathrm{L} 2$ phases. Small angle neutron scattering is measured as a function of concentration and temperature in order to describe the structure of the microemulsions. The results are consistent with a biocontinuous phase in the cases of largest aqueous content. In the low aqueous concentration samples, a substantial correlation is found in the spatial distribution of this minority phase.

\section{1,078}

PB96-119375 Not available NTIS

National Inst. of Standards and Technology (CSTL), Gaithersburg, MD. Thermophysics Div.

Measurements of the Relative Permittivity of Liquid Water at Frequencies in the Range of 0.1 to $10 \mathrm{kHz}$ and at Temperatures between 273.1 and $373.2 \mathrm{~K}$ at Ambient Pressure.

Final rept.

D. P. Fernandez, A. R. H. Goodwin, and J. M. H.

Levelt Sengers. 1995, 25p.

Pub. in International Jnl. of Thermophysics, v16 n4 p929-955 1995

Keywords: "Water, "Dielectric properties, Electrical resistivity, Audio frequencies. Acoustic measurement, Capacitors, Electrodes, Polarization, Correction, Reprints.
The static relative permittivity (dielectric constant) of water has been determined from capacitance measurements at frequencies between 0.1 and $10 \mathrm{kHz}$, in the temperature range from 273.2 to $373.2 \mathrm{~K}$ at ambient pressure. The capacitor used for these measurements was formed from sapphire-insulated concentric cylinders. The specific conductance of the water used was maintained within $20 \%$ of the lowest value ever observed, which is better than in all previous experiments in this range. The new data shed some light on a discrepancy between sets of literature data in liquid water between the triple and boiling points.

\section{1,079}

PB96-119425 Not available NTIS

National Inst. of Standards and Technology (MSEL), Gaithersburg. MD. Ceramics Div.

Electroacoustic Characterization of Particle Size and Zeta Potential in Moderately Concentrated Suspensions.

Final rept.

V. A. Hackley, and S. G. Malghan. 1995, 8p.

Pub. in Ceramics Transactions, v56 p283-290 1995

Keywords: $\quad{ }^{*}$ Ceramics, $\quad{ }^{*}$ Powders (Particles) "Electroacoustic waves, Electrophoresis, Particle size, Size determination, Measuring instruments, Silicon dioxide, Suspensions, Zeta potential, Performance evaluation, Reprints.

A novel electroacoustic technique for the measurement of particle size and charge in opaque suspensions is reviewed, and a new commercial instrument based on this method is evaluated for applications to ceramic powder processing.

\section{1,080}

PB96-119730 Not available NTIS

National Inst. of Standards and Technology (EEEL) Gaithersburg, MD. Electricity Div.

Appearance Potentials of lons Produced by Electron-Impact Induced Dissociative Ionization of SF6, SF4, SF5Cl, S2F10, SO2, SO2F2, SOF2, and SOF4. Final rept.

K. L. Stricklett, J. M. Kassoff, J. K. Olthoff, and R. J. Van Brunt. 1995, 8p.

Pub. in International Symposium on Gaseous Dielectrics (7th), Knoxville, TN., April 24-28, 1994 p257-264

Keywords: "Appearance potential, Reprints, Sulfur fluorides, "Dissociative ionization, Sulfur oxyfluorides.

Appearance potential for ion produced by electron-impact induced dissociative ionization are measured. Appearance potentials are reported for SF5, SF4, SF5C $\mathrm{S} 2 \mathrm{~F} 10, \mathrm{SO} 2, \mathrm{SO} 2 \mathrm{~F} 2, \mathrm{SOF} 2, \mathrm{SOF} 4$

01,081

PB96-122098 (Order as PB96-117767, PC A08/ MF A02)

National Inst. of Standards and Technology Gaithersburg, MD

Low Electrolytic Conductivity Standards.

Y. C. Wu, and P. A. Berezansky. 1995, 7p.

Included in Jnl. of Research of the National Institute of Standards and Technology, v100 n5 p521-527 Sep/ Oct 95.

Keywords: "Conductivity, "Electrolytes, "Calibration standards, Reprints, Electric power plants, Water qual ity Quality assurance, Benzoic acids, Dielectric properties, Potassium chloride. NIST(National Institute of Standards and Technology).

Standards of low electrolytic conductivity were devel oped to satisfy the demands of the U.S. Navy and American industry for the measurement of high quality water. The criteria for the selection of appropriate solvent and solutes, based on the principles of equivalent conductivity and Onsager's limiting law, are described. Dilute solutions of potassium chloride and benzoic acid in $30 \%$ n-propanol-water have been chosen as standards. The electrolytic conductivity of both sets of these solutions as a function of molality was determined.

\section{1,082}

Not available NTIS

National Inst. of Standards and Technology (CSTL) Gaithersburg, MD. Thermophysics Div.

Hydrodynamic Similarity in an Oscillating-Body Viscometer.

Final rept.

R. F. Berg. 1995, 10p.

Pub. in International Jnl. of Thermophysics, v16 n5 p1257-1266 Sep 95. 
Keywords: "Viscometers, "Hydrodynamics, Calibration, Reprints, Oscillating bodies.

Hydrodynamic similarity can be used to calibrate simply and accurately an oscillating-body viscometer of arbitrarily complicated geometry. Usually, an explicit hydrodynamic model based on a simple geometry is re quired to deduce viscosity from the transfer function of an oscillating body such as a vibrating wire or a quartz torsion crystal. However, at low Reynolds numbers the transfer function of any immersed oscillator depends on the fluid's viscosity only through the viscous penetration depth. This hydrodynamic similarity can be exploited if the oscillator is over damped and thus is sensitive to viscosity in a broad frequency range. Even an osillator of poorly known geometry can be characterized over a range of penetration depths by measurements in a fluid of known $n$ and $p$ over the corresponding range of frequencies.

\section{1,083}

PB96-122551 Not available NTIS

National Inst. of Standards and Technology (CSTL), Gaithersburg, MD. Thermophysics Div.

Physical Limit to the Stability of Superheated and Stretched Water.

Final rept.

S. B. Kiselev, J. M. H. Levelt Sengers, and Q.

Zheng. 1995, 8p.

Pub. in Proceedings of the International Conference on the Properties of Water and Steam (12th), Orlando FL., September 9-16, 1994, p378-385.

Keywords: "Steam, "Spinodal, Molecular structure, Stability, Reprints, "Water structure, Equations of state.

Several highly accurate formulations of the thermodynamic properties of water and strearn are investigated in the regions of supercooled, superheated and stretched liquid water. It is shown that they give consid erably different locations for the spinodal, which diminishes the physical relevance of this curve. It is argued that nucleation theories based on local equilibrium fail long before the spinodal is reached. We calculate the physical limit of metastability for superheated and stretched water based on the theory of relaxation of metastable states of Patashinskii and Shumilo. For this application a crossover formalism is used to enable calculation of the critical radius of the nucleus for both positive and negative pressures. The calculated physical limit of metastability agrees with the extrema of superheat, realized in homogeneous states by Skripov et al., and of negative pressure, realized in homogeneous states by Zheng et al.

\section{1,084}

PB96-122619 Not available NTIS

National Inst. of Standards and Technology (EEEL) Gaithersburg, MD. Electricity Div.

Decomposition of SF6 and Production of S2F10 in Power Arcs.

Final rept.

H. D. Morrison, F. Y. Chu, M. Eygenraam, I. Sauers, and R. J. Van Brunt. 1995, 7p.

Pub. in International Symposium on Gaseous Dielectrics (7th), Knoxville, TN., April 24-28, 1994 p475-481 1995.

Keywords: "Sulfur hexafluoride, "Decomposition, "Arc discharges, "Electric arcs, Toxic substances, Dielectrics, Electrical insulation, Kinetics, Gas chromatography, Infrared spectroscopy, Gases, Reprints,
SF6, S2F10.

Decomposition of SF6 in electrical discharges produces many toxic solids and gases. S2F10 is the most toxic of the gaseous byproducts and has been found in arcs, sparks and corona. We have conducted a series of tests of a power arc discharge contained completely within a bus duct configuration. Among the many other gaseous byproducts, we have detected $\mathrm{S} 2 \mathrm{~F} 10$ at or below the part per million (ppm) by volume level, providing that S2F10 can be formed directly by a power arc within SF6-insulated equipment. The relative production rate of $S 2 F 10$ with respect to that of SOF2 and SF4, however, implies that S2F10 is not a significant contributor to the hazard of exposure to decomposed SF6.

01,085

PB96-122627 Not available NTIS

National Inst. of Standards and Technology (CSTL), Gaithersburg, MD. Thermophysics Div.
Simulation Studies of Supercooled and Glass Forming Liquids.

Final rept.

R. D. Mountain. 1995, 15p.

Pub. in Proceedings of the Winter Meeting on Statistical Physics (23rd), Cuernava, Mexico, January 9-12, 1994, p1-15 1995.

Keywords: "Computerized simulation, "Supercooling, "Glass, "Liquids, Molecular structure, Érgodic process, Convergence, Models, Time, Length, Relaxation time, Reprints, "Supercooled liquids, Molecular dynamics.

A brief review of the results of a number of molecular dynamics simulations of glass forming model systems is presented. A method for determining the ergodic convergence time is described. The results indicate that relaxation of dynamical correlations occurs over many time scales. The characterization of relevant length scales is not well understood. Some suggestions for further studies are presented.

\section{1,086}

\section{PB96-122734 Not available NTIS}

National Inst. of Standards and Technology (EEEL), Gaithersburg, MD. Electricity Div.

Modification of Cast Epoxy Resin Surfaces during Exposure to Partial Discharges.

Final rept.

H. Slowikowska, T. Las, J. Slowikowski, and R. J.

Van Brunt. 1995, 8p.

Pub. in International Symposium on Gaseous Dielectrics (7th), Knoxville, TN., April 24-28, 1994, p635-642 1995 .

Keywords: "Dielectrics, "Electrical insulation, "Gas discharges, "Epoxy resins, Electrical resistivity, Surface roughness, Morphology, Sulfur hexafluoride, Nitrogen, Oxygen, Gas mixtures, Reprints, PD(Partial discharges).

Cast epoxy resin materials are commonly used in insulating spacers of gas-insulated high-voltage power systems. It has been showed, that the discharge-induced decrease in surface resisitivity is responsible for dramatic changes in the stochastic behavior of the electrical discharge. In the present work, the nature of partial-discharge (PD) induced damage to cast epoxy resin surfaces has been investigated in more detail using various techniques to examine surface roughness and morphology, as well as resistivity. The effect of generating PD in gases other than air, such as SF6, $\mathrm{N} 2$, and N2/O2 mixtures, was also examined.

\section{1,087}

\section{PB96-122809 Not available NTIS}

National Inst. of Standards and Technology (EEEL), Gaithersburg, MD. Electricity Div.

Electron Attachment to Excited Molecules(1).

Final rept.

L. G. Christophorou. 1995, 1p.

See also DE94019124.

Pub. in International Symposium on Electron- and Photon-Molecule Collisions and Swarms, Berkley, CA., July 22-25, 1995, pE-1.

Keywords: "Eleciron attachment, "Molecular excitation, Molecular energy levels, Internal energy, Vibration, Rotation, Photodissociation, Cross sections, Lasers, Irradiation, Reprints, "Slow electrons.

The interactions of slow electrons with molecules-especially the processes of electron attachment-depend rather strongly on the internal energy content of the molecules themselves. As a rule, excited molecules interact with slow electrons with substantially larger cross section than do ground-state molecules. 1-5 Studies of electron attachment to vabrationally/ rotationally excited, 'hot', molecules $1,3,5-18$ and especially to electronically excited molecules 1,3,4,19-24 are rather recent, in contrast to the extensive studies on electron attachment to ground-state molecules which cover many decades $6,25,26$.

01,088

PB96-123252 Not available NTIS

National Inst. of Standards and Technology (CSTL), Gaithersburg, MD. Thermophysics Div.

Significant Contributions of IAPWS to the Power Industry, Science and Technology.

Final rept

J. M. H. Levelt Sengers. 1995, 12p

Pub. in Proceedings of the International Conference on the Properties of Water and Steam (12th), Orlando, FL., September 9-16, 1994, p1-121995.

Keywords: "Aqueous solutions, History, Steam tables, Water, Reprints, IAPWS(International Association for the Properties of Water and Steam), Supercritical states, Thermodynamic properties, Transport prop erties.

The lines are traced from the early international cooperational necessary for efficient design of and global trade in turbines, boilers and heat exchangers or power generation, to the past and current forms this cooperation assumes in the International Association for the Properties of Water and Steam (IAPWS). The mutually enriching interaction of IAPSW and the scientific world will be illustrated by several examples taken from the fields of critical phenomena, molecula simulation, and supercritical fluids. The acceptance of IAPWS formulations of steam and water properties of de facto world standards for many other applications besides power generation is a consequence of IAPW's firm commitment to excellence. IAPWS's branching out to impure water and steam, necessitated by the threa impurities pose to the components of power plants, has ed to fruitful interactions with aqueous physical chemistry and geochemistry, and may help reach a new synthesis in the science and engineering of reactive supercritical fluid mixtures.

\section{1,089}

PB96-123450 Not available NTIS

National Inst. of Standards and Technology (EEEL) Gaithersburg, MD. Electricity Div.

Fundamental Processes in Gas Discharges. Final rept.

L. G. Christophorou, R. J. Van Brunt, and J. K. Olthoff. 1995, 13p.

Pub. in Proceedings of the International Conference on Gas Discharges and Their Applications (11th), v1 pl$536-1-548$.

Keywords: "Gas discharges, "Discharges Dissociation, Electron, Excited atoms, Ionization, Negative ions, Reprints, "Foreign technology.

Recent aspects of fundamental processes in gas dis charges are discussed. These include the effect of internal energy of excitation of atoms and molecules on their interactions with slow electrons, the effect of tempearture on electron attachment and detachmen processes, photodissociation of molecules and photodetachment of anoins, and interactions involved in discharge byproduct formation and discharge diagnostics. Referecne is also made to fundamenta processes in gas discharge materials used in plasma processing.

01,090

PB96-123559 Not available NTIS National Inst. of Standards and Technology (CSTL), Gaithersburg, MD. Thermophysics Div.

Static Dielectric Constant of Water and Steam. Final rept.

D. P. Fernandez, A. R. H. Goodwin, R. C. Williams, S. G. Penoncello, Y. Mulev, and J. M. H. Levelt Sengers. $1995,8 p$.

Pub. in Proceedings of the International Conference on the Properties of Water and Steam (12th), Orlando FL., September 9-16, 1994, p109-116 1995

Keywords: "Dielectric properties, "Steam, "Water, Experimental data, Data correlation, Data bases, Reprints, Relative permittivity.

All relevant static dielectric constant data for water and steam have been collected and evaluated, and will be made available in print and in computenzed form. A new formulation of the static dielectric constant or relative permittivity of water and stream has been developed. It is based on the ITS-90 temperature scale, and uses a new fundamental equation for water and steam, likewise based on this scale. It also includes our recently obtained dielectric constant data in liquid water and in saturated steam. The new formulation ranges from -45 to +600 degrees $C$ in temperature, and from 0.1 to $1189 \mathrm{MPa}$ in pressure. It is based on the dipole correlation or g-factor of Alder and Harris, and formulates this factor with 10 adjustable coefficients and associated powers of reduced density and inverse re duced temperature.

01,091

PB96-123724 Not available NTIS

National Inst. of Standards and Technology (MSEL), Gaithersburg, MD. Reactor Radiation Div. 
Characterization of the Vibrational Dynamics in the Octahedral Sublattices of LaD2.25 and LaH2.25. Final rept.

T. J. Udovic, J. J. Rush, and I. S. Anderson. 1995 $10 \mathrm{p}$.

Pub. in Jnl. of Physics: Condensed Matter, v7 p7005. 70141995

Keywords: "Vibrational spectroscopy, "Denterides, Dynamics, Hybride, Reprints, Lanthanum, Neutron scattering, Octahedral site, "Tetrahedral site vibrations.

Incoherent inelastic neutron scattering spectroscopy was used to characterize the optic-vibrational density of states (DOS) of the octahedrally coordinated deuterium (Do) and hydrogen $(\mathrm{Ho})$ atoms in $\mathrm{LaD2.25}$ $\mathrm{LaH} 2.25$, and LaH2.03. The DOS exhibits a temperature- and concentration-dependent behavior consistent with that oserved previously for the analogous beta-TbH $2+x$ system. At low temperature, the Ho DOS for LaH2.03 is fairly sharp with minor spectral sidebands, indicating that the Ho atoms are predominantly isolated, with some atoms residing in shortrange-order domains. Increasing the Ho (or Do) concentration to LaH2.25 (or LaD2.25) yields a dispersionbroadened bimodal DOS characteristic of the Ho (or Do) $14 / \mathrm{mmm}$ long-range order that develops in the octahedral sublattice at low temperatures and these higher Ho (or Do) concentrations. For LaH2.25 at higher temperature $(340 \mathrm{~K})$, a broad, somewhat asymmetric DOS is suggestive of an Ho sublattice that is now largely disordered yet still possesses some degree of short-range order.

01,092

PB96-123732 Not available NTIS

National Inst. of Standards and Technology (EEEL), Gaithersburg, MD. Electricity Div.

Kinetic-Energy Distributions of lons Sampled from Radio-Frequency Discharges in Helium, Nitrogen, and Oxygen.

Final rept

R. J. Van Brunt, J. K. Olthoff, and S. B. Randovanov. 1995, 4p.

Pub. in Proceedings of the International Conference on Gas Discharges and Their Applications (11th), Tokyo, Japan, September 11-15, 1995, v1 pl-486-I-489.

Keywords: "Gas discharges, "Helium, Nitrogen, Oxygen, Reprints, "Foreign technology, Ion kinetic energies, Radio-frequency plasma.

Mass-resolved ion kinetic energy distributions are measured for radio-frequency (if) discharges sustained in helium, nitrogen, and oxygen in a parallelplate plasma reactor. The dominate ions for each of the gases are observed to be the parent ions $\mathrm{He}+$, $\mathrm{N} 2+$, and $\mathrm{O} 2+$, respectively over a wide range of pressures $(1.3$ to $67 \mathrm{~Pa}$ ) with an applied if voltage of 200 $\checkmark$. Ion kinetic-energy distributions at the grounded electrode were measured for these ions, as well as for less abundant ions, such as $\mathrm{He} 2+, \mathrm{N}+, \mathrm{N} 2 \mathrm{H}+, \mathrm{N} 3+$ $\mathrm{N} 4+, \mathrm{O}+$, and $3+$

01,093

PB96-123740 Not available NTIS

National Inst. of Standards and Technology (EEEL), Gaithersburg, MD. Electricity Div.

Measurement of S2OF10, and S2O2F10 Production

Rates from Spark and Negative Glow Corona Discharge in SF6/O2 Gas Mixtures.

Final rept.

R. J. Van Brunt, J. K. Olthoff, S. L. Firebaugh, and I. Sauers. 1995, 4p.

Sponsored by Electric Power Research Inst, Palo Alto, $C A$. and Department of Energy, Washington, DC

Pub. in Proceedings of the International Conference on Gas Discharges and Their Applications (11th), Tokyo, Japan, September 11-15, 1995, v1 pl-316-1-319.

Keywords: "Corona discharge, "Oxygen, Production rates, Spark discharge, Sulfur hexafluoride, Reprints, "Foreign technology, Disulfur decafluoride.

The rates for production of the compounds $\mathrm{S} 2 \mathrm{~F} 10$, and $\mathrm{S} 2 \mathrm{OF} 10$, and $\mathrm{S} 2 \mathrm{O} 2 \mathrm{~F} 10$ have been measured both in spark and continuous, constant-current (40 micro A) negative glow corona discharges generated using point-to-plane electrode gaps in 'pure' SF6 and SF6/ $\mathrm{O} 2$ gas mixtures containing different relative amounts of oxygen, up to 10 percent. The measurements were performed for the total gas pressures in the range of 100 to $200 \mathrm{kPa}$, and the SF6 discharge byproduct concentrations were mesured using a gas chromatographmass spectrometric technique and a cryogenic enrichment chromatographic technique, respectively, for the corona and spark experiments. When $\mathrm{O} 2$ is added to the gas, there is a dramatic drop in the S2F10 yield from the corna discharge. The results can be explained within the framework of a plasma-chemical model from considerations of the competition among reactions of $S F$, radicals produced by dissociation of SF6 in the discharge with SF5 itself as well as with $\mathrm{O} 2$ and $\mathrm{O}$, and the relative degree of $\mathrm{O} 2$ dissociation in the two types of discharges.

\section{1,094}

PB96-123765 Not available NTIS

National Inst. of Standards and Technology (MSEL), Gaithersburg, MD. Polymers Div.

Zimm Plot and Its Analogs as Indicators of Vesicle and Micelle Size Polydispersity.

Final rept.

J. H. van Zanten. 1995, 8p

Pub. in Jnl. of Chemical Physics, v102 n22 p9121. 9128 Jun 95.

Keywords: "Light scattering, "Liposomes, "Vesicles, Size distributions, Reprints.

The utility of using the Zimm plot and its analogs, the Debye and Berry plots, as a sensitive means of determining the degree of polydispersity present in solutions of surfactant aggregates such as vesicles or micelles is conclusively demonstrated. These methods of interpreting the excess scattered light intensity due to the surfactant aggregates lead to the determination of the weight-average molecular weight $(\mathrm{Mw})$ and $\mathrm{z}$-average mean square radius ( $(r 2 \mathrm{sub} g) \mathrm{z})$, or radius of gyration of the surfactant aggregates. These two parameters are very sensitive to any polydispersity which is present in the aggregate solution, since these two experimentally determined quantities represent differen moments of the aggregate size distribution. For a given geometric model, apparent surfactant aggregate structural parameters, such as the radius and wall thickness in the case of surfactant vesicles, can be calculated under the assumption of monodisperse aggregates from ( $r 2$ sub g) $\mathrm{z}$ and $\mathrm{Mw}$ determined from a Zimm or Debye analysis of static light scattering spectra.

01,095

PB96-135314 Not available NTIS

National Inst. of Standards and Technology (EEEL) Gaithersburg, MD. Electricity Div.

Decomposition of Sulfur Hexafluoride by X-rays.

Final rept.

J. K. Olthoff, and R. J. Van Brunt. 1995, 6p.

Pub. in International Symposium on Gaseous Dielectrics (7th), Knoxville, TN., April 24-28, 1994, p417-422.

Keywords: *Sulfur hexafluoride, "Physical radiation ef fects, "Decomposition reactions, "X-rays, Radiation doses, Chemical reaction kinetics, Electric discharges, Corona discharges, Byproducts, Electrical insulation, Gas pressure, Reprints.

In the paper, the authors present results of by-product formation in gaseous SF6 exposed to high-energy $X$ rays. The identity and concentration of the decomposition by-products are determined by gas chromotography/mass spectrometry techniques tha were developed to investigate the decomposition of SF6 exposed to corona discharges. The production curves of SOF2 and S2F10 are determined for a range of SF6 gas pressures, $\mathrm{X}$-ray energies, and X-ray fluxes. Evidence for the presence of other by-products, such as SOF4, SO2F2, and $\mathrm{S}_{2} \mathrm{O} 2 \mathrm{~F}_{10}$ is also pre sented. The decomposition data for the SF6 exposed to $X$-rays are compared with previously published data or SF6 exposed to corona discharges.

01,096

PB96-138516 Not available NTIS

National Inst. of Standards and Technology (MSEL),

Gaithersburg, MD. Reactor Radiation Div.

$q$ Dependence of Self-Energy Effects of the Plane Oxygen Vibration in YBa2Cu3O7.

D. Reznik, B. Keimer, F. Dogan, and I. A. Aksay.

1995, 4p.
Pub. in Physical Review Letters, v75 n12 p2396-2399 Sep 95.

Keywords: "Vibrations, Reprints, "YBCO, "Plane oxygen vibration, Brillouin, q-dependence, Temperature dependence.

The authors have measured the temperature dependence of the peak position and linewidth of the $42.5 \mathrm{meV}$ phonon branch in a twinned single crystal of
BYa2Cu2O- as a function of wave vector $q$ In the $(100) /(010)$ direction in the Brillouin zone, considerable softening and broadening occur below the superconducting transition temperature $\mathrm{Tc}$ at some values of $q$. The authors observe an order of magnitude smaller softening and no linewidth broadening for $q$ in the $(110) /(110)$ direction. Possible implications of these findings for the symmetry of the superconducting order parameter are discussed.

\section{1,097}

PB96-138565 Not available NTIS

National Inst. of Standards and Technology (CSTL), Gaithersburg, MD. Surface and Microanalysis Science Div.

Silicon Surface Chemistry by IR Spectroscopy in the Mid- to Far-IR Region: $\mathrm{H} 2 \mathrm{O}$ and Ethanol on Si(100).

Final rept.

L. M. Struck, B. E. Bent, Y. J. Chabal S. Christman,

E. E. Chaban, K. Raghavachari, G. W. Flynn, K.

Radermacher, S. Mantl, G. P. Williams, and A. E.

White. 1995, 6p

Pub. in Material Research Society Symposium Proceedings, v386 p395-400 1995.

Keywords: "Infrared spectroscopy, "Silicon, "Surface chemistry, Water, Ethanol, Reprints.

The technique of external reflection infrared (IR) spectroscopy is used to study silicon surface chemistry. External reflection is enhanced by implanting a buried cobalt silicide layer in silicon to act as an infrared reflector. The preparation of clean well-ordered surfaces from the ion implanted substrates is demonstrated. The reactions of water and ethanol with Si(100) are investigated.

01,098

PB96-140397 Not available NTIS

National Inst. of Standards and Technology (EEEL)

Boulder, CO. Optoelectronics Div.

Dielectric Spectroscopic Determination of Temperature Behavior of Electroclinic Parameters in the Liquid Crystal W317.

Final rept

P. A. Williams, and N. A. Clark. 1995, 5p.

Keywords: "Liquid crystals, Electric fields, Spectroscopy, Free energy, Reprints, "Electroclinic effect, W317 liquid crystal.

We report measurements of the temperature behavior of the electroclinic coupling coefficient $c(T)$ and the inverse tilt suscepitbility $A(T)$, the principal phenomenological parameters determining the magnitude of the electroclinic effect in the chiral smectic A phase of the liquid crystal W317, a material which exhibits an anomalously large electroclinic effect with unusual thermal behavior. We find that $c(T)$ decreases by approximately 30 percent in response to a 40 degrees $C$ increase in temperature. $A(T)$ exhibits a mean field behavior at high temperature, increasing by a factor of 3 as temperature is increased over the range 40 degrees $\mathrm{C}$ less than $\mathrm{T}$ less than 65 degrees $\mathrm{C}$. However below approximately 40 degrees $C, A(T)$ becomes nearly independent of temperature over a approximately 10 degrees $C$ range around temperature. This $A(T)$ behavior has not been previously found in electroclinic materials.

\section{1,099}

PB96-141064 Not available NTIS

National Inst. of Standards and Technology (MSEL), Gaithersburg, MD. Polymers Div.

Crystal Structure of Decacalcium Tetrapotassium Hexakis (Pyrophosphate) Nonahydrate.

Final rept.

M. Mathew, and H. L. Ammon. 1995, 4p.

Pub. in Jnl. of Chemical Crystallography, v25 n5 p2192221995

Keywords: "Crystal structure, "Calcium phosphates, "Pyrophosphates, Potassium phosphates, Layers, Reprints.

The crystal structure of $\mathrm{Ca} 10 \mathrm{~K} 4(\mathrm{P} 2 \mathrm{O} 7) 6.9 \mathrm{H} 2 \mathrm{O}$ has been determined by single crystal $\mathrm{X}$-ray diffraction. Crystal are hexagonal, space group $P 6($ sub 3$) \mathrm{cm}$ with $a=11.76(1), c=9.7701$ (1) Angstroms, and $Z=1$. The structure was refined to $R=0.028$ and $R w=0.037$ for 468 reflections with I greater than or equal to 3 sigma(l). The structure consists of a compact assembly of $\mathrm{Ca}$ and $\mathrm{P} 2 \mathrm{O} 7$ ions arranged in layers per- 
pendicular to the $\mathrm{c}$-axis in a hexagonal array large open channels along the $\mathrm{c}$-axis. The $\mathrm{K}$ ions and the water molecules are located in these open channels and are disordered.

01,100

PB96-141080 Not available NTIS

National Inst. of Standards and Technology (EEEL),

Boulder, CO. Electromagnetic Technology Div.

X-ray Observation of Electroclinic Layer Constriction and Rearrangement in a Chiral Smectic-A Liquid Crystal.

Final rept.

A. G. Rappaport, P. A. Williams, B. N. Thomas, D M. Walba, N. A. Clark, and M. Blanca Ros. 1995, 3p. Pub. in Applied Physics Letters, v67 n3 p362-364 Jul 95

Keywords: "Liquid crystals, $X$ ray, Reprints, *Electroclinic effect, "Layer constriction, Smectic.

An $x$-ray scattering study of electroclinic layer constric tion verifies the interpretation of the electroclinic effec as field-induced molecular tilt. The tilt angles deduced from the layer spacing changes are in close agreemen with those from optical measurements. Layer buckling a consequence of the layer constriction, is also observed and may be the cause of the loss of optical contrast observed in electroclinic devices.

01,101

PB96-145560 Not available NTIS

American Chemical Society, Washington, DC

Journal of Physical and Chemical Reference Data, Volume 24, No. 1, January/February 1995.

Bimonthly rept.

J. W. Gallagher. c1995, 669p.

See also PB96-145578 through PB96-145594, PB94 168556 and PB96-145818. Prepared in cooperation with American Inst. of Physics, New York. Sponsored by National Inst. of Standards and Technology Gaithersburg, MD

Available from American Chemical Society, 1155 Sixteenth St., NW, Washington, DC. 20036-9976.

Keywords: "Physical properties, "Chemical properties, Physical chemistry, Atomic properties, Chemical reac tions, Thermodynamics, Reaction kinetics, Vapor phases, Tables(Data), Reprints, "Reference materials. Contents:

The Millimeter- and Submillimeter-Wave Spectrum of trans-Ethyl Alcohol;

A Database for the Static Dielectric Constant of Water and Steam;

Theoretical Form Factor, Attenuation and Scattering Tabulation of $Z=1$-92 from $E=1$ $10 \mathrm{eV}$ to $\mathrm{E}=0.4-1.0 \mathrm{MeV}$.

01,102

PB96-145578 Not available NTIS

Ohio State Univ. Columbus. Dept. of Physics.

Millimeter- and Submillimeter-Wave Spectrum of trans-Ethyl Alcohol.

J. C. Pearson, K. V. L. N. Sastry, M. Winnewisser, E Herbst, and F. C. De Lucia. c1995, 32p.

Prepared in cooperation with New Brunswick Univ. Fredericton. Dept. of Physics. and Giessen Univ. (Germany, F.R.). Physikalisch-Chemisches Inst.

Included in Jnl. of Physical and Chemical Reference Data, v24 n1 p1-32 Jan/Feb 95. Availabale from American Chemical Society, 1155 Sixteenth St., NW, Washington, DC. 20036-9976

Keywords: "Ethanol, "Wave spectrums, Intensities, Microwave spectra, Molecular constants, Radio astronomy, Reprints, Interstellar molecules, Rotational spectrum, Internal rotation.

The rotational-torsional spectrum of the trans rotational isomer of ethyl alcohol was investigated in the 65-350 $\mathrm{GHz}$ frequency region. A total of 481 ground state transitions over a range of $\mathrm{J}$ and $\mathrm{Ka}$ values up to 33 and 10 , respectively, were measured and assigned. Doublets or triplets arising from the $A$ and $E$ torsional states of the $v=0$ torsional level of the three-fold-symmetric methyl internal rotation have been resolved in 168 of these transitions. Internal rotation theory predicts a significant number of c-type E-state transitions normally forbidden, but allowed when the rotational symmetry operators mix E-state rotational-torsional levels. Over 40 of these transitions have been observed. The newly measured transitions, along with the results of many previous measurements, have been analyzed using an IAM internal rotation Hamiltonia and a Watson A-reduced Hamiltonias to determine the ro- tational, centrifugal distortion, and torsional constants. (Copyright (c) 1995 American Institute of Physics and American Chemical Society.)

\section{1,103}

PB96-145586 Not available NTIS

National Inst. of Standards and Technology (CSTL), Gaithersburg, MD. Thermophysics Div.

Database for the Static Dielectric Constant of Water and Steam.

D. P. Fernandez, Y. Mulev, A. R. H. Goodwin, and J. M. H. Levelt Sengers. c1995, 36p.

Included in Jnl. of Physical and Chemical Reference Data, v24 n1 p33-69 Jan/Feb 95. Available from American Chemical Society, 1155 Sixteenth St., NW, Washington, DC. 20036-9976.

Keywords: "Databases, "Steam, "Water, Data, Compilation, Reprints, "Capacitance bridges, "Static dielectric constants, Electrode polarization, ITS-90, Resonant circuits.

All reliable sources of data for the static dielectric constant or relative permittivity of water and steam, many of them unpublished or inaccessible, have been collected, evaluated, corrected when required, and converted to the ITS-90 temperature scale. The data extend over a temperature range from 238 to $873 \mathrm{~K}$ and over a pressure range from 0.1 MPa up to $1189 \mathrm{MPa}$. The evaluative part of this work includes a review of the different types of measurement techniques, and the corrections for frequency dependence due to the impedance of circuit components, and to electrode polarization. It also includes a detailed assessment of the uncertainty of each particular data source, as compared to other sources in the same range of pressure and temperature. Both the raw and the corrected data have been tabulated, and are also available on diskette. A comprehensive list of references to the literature is included. (Copyright (c) 1995 American Institute of Physics and American Chemical Society.)

\section{1,104}

PB96-145594 Not available NTIS

Melbourne Univ., Parkville (Australia). School of Physics.

Theoretical Form Factor, Attenuation and Scattering Tabulation for $Z=1-92$ from $E=1-10 \mathrm{eV}$ to $E=0.4$ 1.0 MeV.

C. T. Chantler. c1995, 572p

Included in Jnl. of Physical and Chemical Reference Data, v24 n1 p71-643 Jan/Feb 95. Available from American Chemical Society, 1155 Sixteenth St., NW., Washington, DC. 20036-9976.

Keywords: "Anomalous dispersions, "Attenuation, Form factors, Reprints, Photoabsorption tabulation, Scattering cross-sections.

in the present study, the primary interactions of $x$-rays with isolated atoms from $Z=1$ (hydrogen) to $Z=92$ (uranium) are described and computed within a selfconsistent Dirac-Hartree-Fock framework. This has general application across the range of energy from 1 . $10 \mathrm{eV}$ to $400-1000 \mathrm{keV}$, with limitations (described below) as the low- and high-energy extremes are approached. Tabulations are provided for the $f 1$ and $f 2$ components of the form factors, together with the photoelectric attenuation coefficient for the atom, micro, and the value for the K-shell, microk, as functions of energy and wavelength. Also provided are estimated correction factors as described in the text, conversion factors, and a simple estimate for the sum of the scattering contributions (from an isolated atom). The method used herein is primarily theoretical and considers intermediate assumptions which limit the precision and applicability of previous theoretical tabulations. Particular concern involves the application of the dispersion relation to derive $\operatorname{Re}(f)$ from photoelectric absorption cross-sections.

\section{1,105}

PB96-145818 Not available NTIS

American Chemical Society, Washington, DC

Journal of Physical and Chemical Reference Data, Volume 24, No. 2, March/April 1995.

Bimonthly rept

J. W. Gallagher. c1995, 394p.

See also PB96-145826, PB96-145834, Volume 24, No 1 PB96-145560, and Volume 24 'No 3 PB96145842. Prepared in cooperation with American Inst. of Physics, New York. Sponsored by National Inst. of Standards and Technology, Gaithersburg, MD

Available from American Chemical Society, 1155 Sixteenth St., NW, Washington, DC. 20036-9976.
Keywords: "Physical properties, *Chemical properties, Physical chemistry Atomic properties, Chemical reac tions, Thermodynamics, Reaction kinetics, Vapo phases, Tables(Data), Reprints, "Reference materials Contents:

Rate Constants for the Decay and Reactions of the Lowest Electronically Excited Singlet State of Molecular Oxygen in Solution. An Expanded and Revised Compilation:

Thermodynamic Properties of the Aqueous Ba2+ Ion and the Key Compounds of Barium.

01,106

PB96-145826 Not available NTIS

Loughborough Univ. of Technology (England). Dept. of Chemistry.

Rate Constants for the Decay and Reactions of the Lowest Electronically Excited Singlet State of Molecular Oxygen in Solution. An Expanded and Revised Compilation.

F. Wilkinson, W. P. Helman, and A. B. Ross. c1995, $358 \mathrm{p}$

Prepared in cooperation with Notre Dame Univ., IN Radiation Chemistry Data Center.

Included in Jnl. of Physical and Chemical Reference Data, v24, n2 p663-1021 Mar/Apr 95. Available from American Chemical Society, 1155 Sixteenth St., NW, Washington, DC. 20036-9976.

Keywords: "Chemical kinetics, "Rate constants, Data compilation, Decay, Oxidation, Photochemistry, Photosensitization, Quenching, Reprints

An expanded and revised compilation on the reactivity of singlet oxygen, the lowest electronically excited singlet state of molecular oxygen, 10 (star)2(1deltag) in fluid solution is presented, which supersedes the publication of Wilkinson and Brummer, J. Phys. Chem Ref. Data 10,809 (1981). Rate constants for the chemical reaction and physical deactivation of singlet oxy gen available through 1993 have been critically compiled. Solvent deactivation rates (kd) are tabulated for 145 solvents or solvent mixtures and second-order rate constants for interaction of singlet oxygen with 1915 compounds are reports. (Copyright (c) 1995 American Institute of Physics and American Chemical Society.)

01,107

PB96-145834 Not available NTIS

National Inst. of Standards and Technology (CSTL) Gaithersburg, MD. Chemical Kinetics and Thermodynamics Div.

Thermodynamic Properties of the Aqueous Ba(sup $2+)$ lon and the Key Compounds of Barium.

V. B. Parker. c1995, 31p

Included in Jnl. of Physical and Chemical Reference Data, v24 n2 p1023-1054 Mar/Apr 95. Available from American Chemical Society, 1155 Sixteenth St., NW, Washington, DC. 20036-9976

Keywords: "Barium ions, Data evaluation, Enthalpy, Entropy, Key compounds, Reprints, CODATA, Gibbs energy, Thermochemical measurements.

Recornmended thermochemical property values, delta(f)H(deg), delta(f)G(deg) and $S($ deg) for the aqueous ion of barium, Ba2t, are given at $298.15 \mathrm{~K}$ in $\mathrm{S}$ units. The values are: delta(f) $\mathrm{H}(\mathrm{deg})=-534.64$ plus or minus $1.80 \mathrm{~kJ} . \mathrm{mol}(-1)$, delta(f) $\mathrm{G}(\mathrm{deg})=-557.60$ plus or minus $1.81 \mathrm{~kJ} . \mathrm{mol}(-1)$ and $\mathrm{S}(\mathrm{deg})=8.80$ plus or minus $0.50 \mathrm{~J}-\mathrm{K}(-1) \mathrm{mol}(-1)$. They are consistent with the CODATA Key Values for Thermodynamics. The evaluation involves the analysis of the enthalpy changes Gibbs energy changes, and the entropy measurements for all key substances in the key network. A consistent set of the rmochemical property values is given for $\mathrm{BaO}(\mathrm{cr}), \mathrm{BaH}_{2}(\mathrm{cr}), \mathrm{BaCl}_{2}(\mathrm{cr}), \mathrm{BaCl}_{2} 2 \mathrm{H} 2 \mathrm{O}(\mathrm{cr})$ $\mathrm{Ba}(\mathrm{NO} 3$ )2(cr), and $\mathrm{BaCO} 3(\mathrm{cr}$, witherite), as well as reconstituted recommended process values with uncertainties for reactions involving these substances (Copyright (c) 1995 American Institute of Physics and American Chemical Society.)

\section{1,108}

PB96-145842 Not available NTIS

American Chernical Society, Washington, DC Journal of Physical and Chemical Reference Data, Volume 24, No. 3, May/June 1995.

Bimonthly rept.

J. W. Gallagher. c1995, 347p

See also PB96-145859 through PB96-145875, Volume 24. No. 2, PB96-145818 and Volume 24, No. 4, PB96145883. Prepared in cooperation with American Inst. of Physics, New York. Sponsored by National Inst. of Standards and Technology, Gaithersburg, MD. 
Available from American Chemical Society, 1155 Sixteenth St., NW, Washington, DC. 20036-9976.

Keywords: "Physical properties, "Chemical properties, Physical chemistry, Atomic properties, Chemical reactions, Thermodynamics, Reaction kinetics, Vapor phases, Tables(Data), Reprints

Contents:

Critical Review of Rate Constants for Reactions of Transients from Metal lons and Metal Complexes in Aqueous Solution

Ideal Gas Thermodynamic Properties of Sulphur Heterocyclic Compounds;

Standard Reference Data for the Thermal Conductivity of Water.

01,109
PB96-145859 Not available NTIS

Cookridge Radiation Research Centre, Leeds (Engand).

Critical Review of Rate Constants for Reactions of Transients from Metal Ions and Metal Complexes in Aqueous Solution.

G. V. Buxton, Q. G. Mulazzani, and A. B. Ross.

c1995, 294p.

Prepared in cooperation with Consiglio Nazionale delle Ricerche, Bologna (Italy). Ist. di Fotochimica $\Theta$ Radiazioni d'Alta Energia, and Notre Dame Univ., IN Radiation Chemistry Data Center

Included in Jnl. of Physical and Chemical Reference Data, v24 n3 p1055-1349 May/Jun 95. Available from American Chemical Society, 1155 Sixteenth St., NW. Washington, DC. 20036-9976.

Keywords: "Aqueous solutions, "Chemical kinetics, Critical review, Data compilation, Pulse radiolysis, Rate constants, Transients, Reprints, Flash photolysis, Metal ions.

Kinetic data for transient metal species in aqueous solution have been critically reviewed. The compilation covers over 2000 measurements of rate constants involving 660 metal ions and metal complexes from Groups 4-15; lanthanides and actinides are not included. Most of the data have been obtained by the methods of pulse radiolysis or flash photolysis. Data have been collected from 500 publications through 1993. (Copyright (c) 1995 American Institute of Physics and American Chemical Society.)

\section{1,110}

PB96-145867 Not available NTIS

Akademiya Nauk SSSR, Moscow. Inst. Vysokikh Temperatur

Ideal Gas Thermodynamic Properties of Sulphur Heterocyclic Compounds.

. V. Dorofeeva, and L. V. Gurvich. c1995, 25p.

Included in Jnl. of Physical and Chemical Reference

Data, v24 n3 p1351-1376 May/Jun 95. Available from

American Chemical Society, 1155 Sixteenth St., NW,

Washington, DC. 20036-9976

Keywords: "Thermodynamic properties, Molecular structure, Reprints, *Sulphur heterocyclic compounds, Vibrational frequencies.

The available structural parameters, fundamental frequencies and enthalpies of formation for thiirane thiirene, thietane, 2H-thiete, 1,2-dithiete, tetrahydrothiophene, 2,3-dihydrothiophene, 2,5dihydrothiophene, thiophene, 1,2-dithiolane, 1,3dithiolane, 1,2,4-trithiolane, tetrahydro-2H-thiopyran 5,6-dihydro-2H-thiopyran, 2,4-dithiane, 1,4-dithiane, 1,4-dithiin, 1,3,5-trithiane, thiepane and 1,3,5,71,4-dithion, 1,3,5-trithiane, thiepane and 1,3,5,7ommended values were selected. Molecular constants and enthalpies of formation for some of the molecules were estimated, as experimental values for these compounds are not available.

01,111

PB96-145875 Not available NTIS

isbon Univ. (Portugal). Dept. de Quimica.

Standard Reference Data for the Thermal Conductivity of Water.

M. L. V. Ramires, C. A Nieto de Castro, $Y$.

Nagasaka, W. A. Wakeham, A. Nagashima, and M. J. Assael. c1995, 5p.

Prepared in cooperation with Keio Univ., Yokohama Japan). Dept. of Mechanical Engineering. Imperial Coll. of Science, Technology and Medicine, London (England). Dept. of Chemical Engineering. and Thessaloniki Univ., Salonika (Greece). Dept. of Chemical Engineering.

Included in Jnl. of Physical and Chemical Reference

Data, v24 n3 p1377-1381 May/Jun 95. Available from
American Chemical Society, 1155 Sixteenth St., NW, Washington, DC, 20036-9976

Keywords: "Water, "Thermal conductivity, Liquids, Reprints, Reference materials, Standard reference data.

New experimental data on the thermal conductivity of liquid water along the saturation line have been obtained recently, using the bare and coated transient ho wire technique, with high accuracy. The quality of the data is such that new standard reference values can be proposed with confidence limits of $0.7 \%$ at a $95 \%$ confidence level. These data and the correlation herein presented revise a previous correlation endorsed by IUPAC. (Copyright (c) 1995 American Institute of Phys ics and American Chemical Society.)

\section{1,112}

PB96-145883 Not available NTIS

American Chemical Society, Washington, DC

Journal of Physical and Chemical Reference Data, Volume 24, No. 4, July/August 1995.

Bimonthly rept

J. W. Gallagher. c1995, 249p

See also PB96-145891 through PB96-145917, Volume 24 No. 3, PB96-145842 and Volume 24, No. 5 , PB96145925. Errata sheets inserted. Prepared in cooperation with American Inst. of Physics, New York. Sponsored by National Inst. of Standards and Technology, Gaithersburg, MD.

Available from American Chemical Society, 1155 Sixteenth St., NW, Washington, DC. 20036-9976.

Keywords: "Physical properties, "Chemical properties, Physical chemistry, Atomic properties, Chemical reactions, Thermodynamics, Reaction kinetics, Vapo phases, Tables(Data), Reprints, "Reference materials. Contents:

Summary of the Apparent Standard Partial Molal Gibbs Free Energies of Formation of Aqueous Species, Minerals, and Gases at Pressure 1 to 5000 Bars and Temperatures 25 to 1000 degees C;

Atomic Weights of the Elements 1993

Spectral Data for Highly Ionized Krypton, $\mathrm{Kr} V$ through $\mathrm{Kr}$ XXXVI.

01,113

PB96-145891 Not available NTIS

Centre National de la Recherche Scientifique, Toulouse (France).

Summary of the Apparent Standard Partial Molal Gibbs Free Energies of Formation of Aqueous Species, Minerals, and Gases at Pressures 1 to 5000 Bars and Temperatures 25 to $1000 \mathrm{C}$.

E. H. Oelkers, H. C. Helgeson, E. L. Shock, V. A

Pokrovskii, D. A. Sverjensky, and J. W. Johnson. C1995, 159p.

Prepared in cooperation with California Univ., Berkeey. Dept. of Geology and Geophysics., Washington Univ, St. Louis, MO. Dept. of Earth and Planetary Sciences., Johns Hopkins Univ., Baltimore, MD. Dept. of Earth and Planetary Sciences. and Lawrence Livermore National Lab., CA. Earth Sciences Dept.

Included in Jnl. of Physical and Chemical Reference Data, v24 n4 p1401-1560 Jul/Aúg 95. Available from American Chemical Society, 1155 Sixteenth St. NW, Washington, DC. 20036-9976.

Keywords: "Aqeous species, "Electrolyte solutions, Gases, Water, Reprints, Water mineral interaction, Gibbs free energies of formation.

Accurate values of the apparent standard partial molal Gibbs free energies of formation (delta $\mathrm{G} \mathrm{deg}$ ) of aqueous species, minerals, and gases at high temperatures and pressures are a requisite for characterizing a variety of industrial and natural processes including corrosion of metals, solvent extraction, crystal growth, metamorphism, and the formation of hydrothermal ore deposits. Revision of the HKF equations of state for aqueous species other than $\mathrm{H} 2 \mathrm{O}$ (Helgeson, Kirkham and Flowers, 1981) by Tanger and Helgeson (1988) and Shock et al. (1992) permits calculation of delta $G$ deg for these species at temperatures to $1000 \mathrm{deg} C$ and pressures to 5000 bars.

\section{1,114}

PB96-145909 Not available NTIS

Commission on Atomic Weights and Isotopic Abundances, Reston, VA

Atomic Weights of the Elements, 1993

c1995, 15p

Included in Jnl. of Physical and Chemical Reference Data, v24 n4 p1561-1576 Jul/Aug 95. Available from
American Chemical Society, 1155 Sixteenth St., NW, Washington, DC 20036-9976

Keywords: "Atomic weights, Critical evaluation, Elements, Reprints, *Isotopic compositions, "IUPAC Commission on Atomic Weights.

The biennial review of atomic weight, $\operatorname{Ar}(\mathrm{E})$, determinations and other cognate data has resulted in changes for the standard atomic weight of titanium from 47.88 plus or minues 0.03 to 47.867 plus or minus 0.001 , of iron from 55.847 plus or minus 0.003 to 55.845 plus or minus 0.002 of antimony from 121.757 plus or minus 0.003 to 121.760 plus or minus 0.001 and of iridium from 192.22 plus or minus 0.03 to 192.217 plus or minus 0.003 . Recent investigations on chlorine and bromine confirmed the presently accepted values of $\mathrm{Ar}(\mathrm{Cl})$ and $\mathrm{Ar}(\mathrm{Br})$. To emphasize the fact that the atomic weight of lithium commonly available in laboratory reagents can vary significantly, the value of lithium, $\operatorname{Ar}(\mathrm{Li})$, was enclosed in brackets and a footnote was added. As a result of several changes, the Table of Standard Atomic Weights Abridged to Five Significant Figures has been updated. Because relative isotoperatio data for stable hydrogen, carbon, and oxygen are commonly being expressed on non-corresponding scales, the Commission recommends that such isotopic data be expreseed only relative to the references VSMOW and VPDB. (Copyright (c) 1995 American Institute of Physics and American Chemical Society.)

01,115

PB'96-145917 Not available NTIS

Japan Atomic Energy Research Inst., Ibaraki.

Spectral Data for Highly Ionized Krypton, Kr V through Kr XXXVI.

T. Shirai, K. Okazaki, and J Sugar c1995, 31p.

Prepared in cooperation with Institute of Physical and Chemical Research, Wako (Japan). and National Inst. of Standards and Technology, Gaithersburg, MD.

Included in Jnl. of Physical and Chemical Reference Data, v24 n4 p1577-1608 Jul/Aug 95. Available from American Chemical Society, 1155 Sixteenth St. NW, Washington, DC. 20036-9976.

Keywords: "Atomic data, "Krypton, Energy levels, Ions, Spectra, Wavelengths, Reprints, Grotrian diagrams Transition probabilities

Wavelengths, energy levels, ionization energies, line classifications, intensities and transition probabilities for $\mathrm{Kr} V$ through $\mathrm{Kr} X X X V I$, with the exception of $\mathrm{Kr}$ $\mathrm{XI}$ through $\mathrm{Kr}$ XVII, are tabulated. No data have been published for $\mathrm{Kr} X I$ through $\mathrm{Kr} X X \mathrm{VII}$. These data are based on the energy levels compilation of Sugar and Musgrove (13). Transition probabilities for selected M1 lines have been reported and are quoted here. A short review of the line identifications and wavelength measurements is given for each stage of ionization. The literature has been surveyed through February 1995. (Copyright (c) 1995 American Institute of Physics and American Chemical Society.)

01,116

PB96-145925 Not available NTIS

American Chemical Society, Washington, DC.

Journal of Physical and Chemical Reference Data,

Volume 24, No. 5, September/October 1995.

Bimonthly rept.

J. W. Gallagher. c1995, 117p

See also PB96-145933 through PB96-145958, Volume 24, No. 4, PB96-145883 and Volume 24, No. 6, PB96145966 Prepared in cooperation with American Inst of Physics, New York. Sponsored by National Inst. of Standards and Technology, Gaithersburg, MD Available from American Chemical Society, 1155 Sixteenth St., NW, Washington, DC. 20036-9976.

Keywords: "Physical properties, "Chemical properties, Physical chemistry, Atomic properties, Chemical reactions, Thermodynamics, Reaction kinetics, Vapor phases, Tables(Data), Reprints, "Reference materials.

Contents:

The Viscosity of Ammonia

Thermodynamics of Enzyme-Catalyzed Reactions:

Part 4. Lyases

Thermodynamic Properties of the Aqueous lons $(2+$ and $3+)$ of Iron and the Key Compounds of Iron.

01,117

PB'96-145933 Not available NTIS

Imperial Coll. of Science and Technology, London (England). 
Viscosity of Ammonia.

A. Fenghour, W. A. Wakeham, V. Vesovic, E. Vogel, J. T. R. Watson, and J. Millat. c1995, 18p.

Prepared in cooperation with Rostock Univ. (German D.R.). Fachbereich Chemie., National Engineering Nordum Inst. fuer Umwelt und Analytik G.m.b.H., Rostock (Germany). Gewerbepark am Weidenbruch. Included in Jnl. of Physical and Chemical Reference Data, v24 n5 p1649-1667 Sep/Oct 95. Available from American Chemical Society, 1155 Sixteenth St., NW, Washington, DC. 20036-9976.

Keywords: "Ammonia, "Viscosity, Correlation, Critical assessment, Liquids, Representation, Vapors, Reprints.

A new representation of the viscosity of ammonia is presented. The representative equations are based on a set of experimental data selected as a result of a critical assessment of the available information. The validity of the representation extends from $196 \mathrm{~K}$ to the critical temperature for both liquid and vapor phases. In the supercritical region the temperature range extends to $680 \mathrm{~K}$ for pressures at or below ambient and to 600 $\mathrm{K}$ for pressure up to $50 \mathrm{MPa}$. The accuracy of the representation varies from $0.5 \%$ for the viscosity of the dilute gas phase at moderate temperatures to about $5 \%$ for the viscosity at high pressures and temperatures. Tables of the viscosity generated by the correlating equation at selected temperatures and pressures and along the saturation line are presented to provide easy reference as well as for the validation of computer codes. (Copyright (c) 1995 American Institute of Physics and American Chemical Society.)

01,118

PB96-145941 Not available NTIS

National Inst. of Standards and Technology (CSTL), Gaithersburg, MD. Biotechnology Div.

Thermodynamics of Enzyme-Catalyzed Reactions. Part 4. Lyases.

R. N. Goldberg, and Y. B. Tewari. c1995, 29p.

Included in Jnl. of Physical and Chemical Reference Data, v24 n5 p1669-1698 Sep/Oct 95. Available from American Chemical Society, 1155 Sixteenth St., NW, Washington, DC. 20036-9976.

Keywords: "Thermodynamic properties, "Lyases, "Enzyme-catalyzed reactions, Data evaluation, Reprints, Apparent equilibrium constants, Reaction enthalpies.

Equilibrium constants and enthalpy changes for reactions catalyzed by the lyase class of enzymes have been compiled. For each reaction the following information is given: the reference for the data; the reaction studied; the name of the enzyme used and its Enzyme Commission number; the method of measurement; the conditions of measurement (temperature, $\mathrm{pH}_{\text {, ionic }}$ strength, and the buffer(s) and cofactor(s) used); the data and an evaluation of it; and, sometimes, commentary on the data and on any corrections which have been applied to it or any calculations for which the data have been used. The data from 106 references have been examined and evaluated. Chemical Abstract Service registry numbers are given for the substances involved in these various reactions. There is a cross reference between the substances and the Enzyme Commission numbers of the enzymes used to catalyze the reactions in which the substances participate. (Copyright (c) 1995 American Institute of Physics and American Chemical Society.)

01,119

PB96-145958 Not available NTIS

National Inst. of Standards and Technology (CSTL) Gaithersburg, MD. Chemical Kinetics and Thermodynamics Div.

Thermodynamic Properties of the Aqueous lons $(2+$ and $3+)$ of Iron and the Key Compounds of Iron. V. B. Parker, and I. L. Khodakovskii. c1995, 46p. Prepared in cooperation with Akademiya Nauk SSSR, Moscow. Inst. Geokhimii i Analiticheskoi Khimii.

Included in Jnl. of Physical and Chemical Reference Data, v24 n5 p1699-1745 Sep/Oct 95. Available from American Chemical Society, 1155 Sixteenth St., NW, Washington, DC. 20036-9976.

Keywords: "Thermodynamic properties, "Aqueous iron, "Iron compounds, Data evaluation, Enthalpy, Entropy, Key values, Reprints, CODATA, Gibbs energy, Reaction catalogs.

The evaluation involves the analysis of the enthalpy changes, Gibbs energy changes, and the entropy measurements for all key substances in the key network. A consistent set of thermochemical property values is given for $\mathrm{FeOOH}(\mathrm{Cr}, \mathrm{Goethite)}, \mathrm{FeCl} 2(\mathrm{Cr})$, $\mathrm{FeCl} 3(\mathrm{cr}), \mathrm{FeBr} 2(\mathrm{cr}), \mathrm{FeBr} 3(\mathrm{cr}) \mathrm{Fel} 2(\mathrm{cr})$, and $\mathrm{FeSO} 4.7 \mathrm{H} 2 \mathrm{O}(\mathrm{cr})$, as well as 'reconstituted' rec ommended process values with uncertainties involving these substances. All recommnded values are also given for a standard state of $P(d e g)=1 \mathrm{~atm}$. A computer based reaction catalog of measurements accompanies the text analysis.

\section{1,120}

\section{PB96-145966 Not available NTIS}

American Chemical Society, Washington, DC.

Journal of Physical and Chemical Reference Data, Volume 24, No. 6, November/December 1995.

Bimonthly rept.

J. W. Gallagher. c1995, 143p.

See also PB96-145974, PB96-145982 and Volume 24 No. 5, PB96-145925. Prepared in cooperation with American Inst. of Physics, New York. Sponsored by National Inst. of Standards and Technology, Gaithersburg, MD

Available from American Chemical Society, 1155 Sixteenth St., NW, Washington, DC. 20036-9976.

Keywords: "Physical properties, "Chemical properties, Physical chemistry, Atomic properties, Chemical reac tions, Thermodynamics, Reaction kinetics, Vapor phases, Tables(Data), Reprints, "Reference materials.

\section{Contents:}

Thermodynamics of Enzyme-Catalyzed Reactions:

Part 5. Isomerases and Ligases;

Energy Levels of Zinc, Zn I through Zn XXX.

\section{1,121}

PB96-145974 Not available NTIS

National Inst. of Standards and Technology (CSTL), Gaithersburg, MD. Biotechnology Div.

Thermodynamics of Enzyme-Catalyzed Reactions.

Part 5. Isomerases and Ligases.

R. N. Goldberg, and Y. B. Tewari. c1995, 36p.

Included in Jnl. of Physical and Chemical Reference Data, v24, n6 p1765-1801 Nov/Dec 95. Available from American Chemical Sociely, 1155 Sixteenth St., NW, Washington, DC. 20036-9976.

Keywords: "Isomerases, "Ligases, Data, Reprints, Equilibrium constants, Reaction enthalpies, Enzymecatalyzed reactions, Thermodynamic properties.

Equilibrium constants and enthalpy changes for reactions catalyzed by the isomerase and ligase classes of enzymes have been compiled. For each reaction the following information is given: the reference for the data; the reaction studies; the name of the enzyme used and its Enzyme Commission number; the method of measurement; the conditions of measurement (temperature, $\mathrm{pH}$, ionic strength, and the buffer(s) and cofactor(s) used); the data and an evaluation of it; and sometimes, commentary on the data and on any corrections which have been applied to it or any calculations for which the data have been used. The data from 176 references have been examined and evaluated. Chemical Abstract Service registry numbers are given for the substances involved in these various reactions. There is a cross reference between the substances and the Enzyne Commission numbers of the enzymes used to catalyze the reactions in which the substances participate. (Copyright (c) 1995 American Institute of Physics and American Chemical Society.)

\section{1,122}

PB96-145982 Not available NTIS

National Inst. of Standards and Technology (PL), Gaithersburg, MD. Atomic Physics Div.

Energy Levels of Zinc, Zn I through Zn XXX.

J. Sugar, and A. Musgrove. c1995, 69p.

Included in Jnl. of Physical and Chemical Reference Data, v24 n6 p1803-1872 Nov/Dec 95. Available from American Chemical Society, 1155 Sixteenth St., NW, Washington, DC. 20036-9976.

Keywords: "Zinc, "Energy levels, Atomic, Ions, Spectra, Reprints.

Atomic energy levels of zinc have been compiled for all stages of ionization for which experimental data are available. No data have yet been published for $Z n I X$, $Z n X, Z n X X V I$, and ZN XXVIII, and only several resonance lines of $Z n X X I X$ and $Z N X X X$. Very accurate calculated values are compiled for $Z n X X \mid X$ and $Z N$ $X X X$. Experimental $g$-factors and leading percentages from calculated eigenvectors are given. $A$ value for the ionization energy, either experimental when available or theoretical, is included for the neutral atom and each ion. A review of the published literature is given. (Copyright (c) 1995 American Institute of Physics and American Chemical Society.)

01,123

PB96-146618 Not available NTIS

National Inst. of Standards and Technology (CSTL), Gaithersburg, MD. Thermophysics Div.

High-Temperature High-Pressure Oscillating Tube Densimeter.

Final rept.

R. F. Chang, and M. R. Moldover, 1996, 6p.

Pub. in Review of Scientific Instruments, v67 n1 p251256 Jan 96

Keywords: "Densitometers, "Oscillations, Density, High pressure, High temperature, Tubes, Calibrating, Toluene, Thermophysical properties, Reprints.

We describe an oscillating tube densimeter for use at temperatures up to at least $575 \mathrm{~K}$ and at pressures up to $20 \mathrm{MPa}$. After the densimeter was calibrated under vaccum and filled with water, it was used to measure the density of toluene from 298 to $575 \mathrm{k}$ at $13.8 \mathrm{MPa}$. The results agree $(0.1$ percent rms deviation) with those obtained using other techniques. In the present densimeter, an alternating current is passed through the fube containing the sample to force the tube to oscillate in the field of a permanent magnet. This design avoids the use of electromagnets (with their attendant polymer or ceramic insolations) and does not require attachment of appendages to the oscillating tube.

01,124

PB96-146733 Not available NTIS

National Insi. of Standards and Technology (PL), Gaithersburg, MD. Ionizing Radiation Div.

Radiation-Chemical Reaction of 2,3,5-TriphenylTetrazolium Chloride in Liquid and Solid State.

Final rept.

A. Kovac, L. Wojnarovits, W. L. McLaughlin, S. E. Ebrahim Eid, and A. Miller. 1996, 4p.

Pub. in Radiation Physics and Chemistry, v47 n3 p483 4861996.

Keywords: "Radiolysis, "Reaction kinetics, "Pulses, Dosimetry, Oxidation, Reduction(Chemistry), Dosage, Liquids, Solids, Chemical reactions, Radiation chemistry, Reprints, TTC(Triphenyl-tetrazolium chloride),

In pulse radiation of 2,3,5-triphenyl-tetrazolium chloride (TTC) at around $360 \mathrm{~nm}$ fast formation of intermediate tetrazolium radical was observed under both oxidizing and reducing conditions. In the latter case biomolecular formation of formazan, absorbing at around $480 \mathrm{~nm}$, was observed. This reaction is accompanied by combination to the diformazan dimer, absorbing over the spectral range $500-550 \mathrm{~nm}$. A polyvinyl-alcohol-based TTC film was produced and tested for dosimetry purposes: it gave a measureable response in the 1-100kGy dose range by evaluating the 50 micrometers thick TTC films at the absorption maximum of $493 \mathrm{~nm}$

01,125

PB96-147079 Not available NTIS

National Inst. of Standards and Technology (EEEL) Boulder, CO. Electromagnetic Technology Div.

Pinch Effect in Commensurate Vortex-Pin Lattices. Final rept.

L. D. Cooley, and A. M. Grishin. 1995, 4p.

Pub. in Physical Review Letters, v74 n14 p2788-2791 Apr 95.

Keywords: "Commensurate lattices, "Critical state, Magnetization, Superconductors, Reprints, Flux pinning.

The critical state in a superconductor with periodic pins has properties similar to the pinch effect, known in plasma physics. It forms a terrace structure around the average flux density gradient, causing stratification of the transport current into the terrace edges where the flux density gradient is large. Regions of extremely high current thus interlace with regions of near zero current. The appearance of each new terrace inside the superconductor causes the magnetization jump, a new quantum effect in superconductors, corresponds to the addition of one flux quantum threading the pin lattice unit cell.

01,126
PB96-147152 Not available NTIS 
National Inst. of Standards and Technology (MSEL), Gaithersburg, MD. Reactor Radiation Div.

Neutron Scattering Study of the Lattice Modes of Solid Cubane.

Final rept.

P. M. Gehring, D. A. Neuman, W. A. Kamitakahara,

D. P. VanMeurs, J. J. Rush, and P. E. Eaton. 1995,

$6 \dot{p}$.

Pub. in Jnl. of Physical Chemistry, v99 p4429-4434 1995

Keywords: "Molecular structure, "Chemical bonds, "Neutron scattering, Lattices, Reprints, "Cubanes.

The authors use neutron time-of-flight scattering techniques to measure the low-frequency vibrational density of states of solid cubane at 100 and $300 \mathrm{~K}$. Debyelike behavior is observed up to approximately $60 \mathrm{~cm}$ 1) at both temperatures. At $100 \mathrm{~K}$, the density of states exhibits three distinct peaks at 78, 94, and $114 \mathrm{~cm}(-$ 1) before cropping to zero around $150 \mathrm{~cm}(-1)$. The authors identify the $114 \mathrm{~cm}(-1)$ peak with the $\mathrm{Eg}$ librational mode of cubane based on the marked temperature dependence of its frequency and a comparison to Raman measurements. In similar fashion they identify the $94 \mathrm{~cm}(-1)$ peak with the Ag librational mode. At $300 \mathrm{~K}$, the librational modes have softened to the extent that the density of states exhibits a plateau-like feature between 60 and $100 \mathrm{~cm}(-1)$.

01,127

PB96-147889 PC A03/MF A01

National Inst. of Standards and Technology (CSTL), Gaithersburg, MD. Thermophysics Div.

Collection of Results for the SPC/E Water Model. R. D. Mountain, and A. Wallqvist. Jan 96, 15p,

NISTIR-5778.

Prepared in cooperation with National Cancer Inst., Frederick, MD. Frederick Cancer Research and Development Center. and Science Applications International Corp., Frederick, MD.

Keywords: "Water, "Molecular properties, "Simulation, Constants, Dielectric constant, Diffusion, Potential energy, Sample cubic lattices, Tables(Data), SPC/ E(Simple Point Charge Extended), Simple point charge.

The report contains a compilation of molecular dynamics computer simulation results for the simple point charge extended model (SPC/E) for water. In addition to published results, this compilation contains unpublished results developed by the author. The properties included are the static dielectric constant, the self-diffusion coefficient, the internal energy, and the pressure. The simulation values of the dielectric constant are compared with a new correlation based on available experimental values.

01,128

PB96-156054 Not available NTIS

National Inst. of Standards and Technology (CSTL), Gaithersburg, MD. Thermophysics Div.

Virial Coefficients of Five Binary Mixtures of Fluorinated Methanes and Ethanes.

Final rept.

L. A. Weber, and D. R. Defibaugh. 1994, 18p.

Pub. in Internaitonal Jnl. of Thermophysics, v15 n5 p863-880 1994

Keywords: "Hydroflurorocarbons, Refrigerants, Viral coefficients, Reprints, "Pressure vapor temperature, *Polar gases.

The authors have made new measurements of the gas-phase PVT surface of five binary mixtures of hydrofluorocarbons (HFCs) in a Bumett/isochoric apparatus. The components chosen all have moderate to large reduced dipole moments. The authors present PVT data, de rived mixture virial coefficients, cross second virial coefficients, and binary interaction parameters for these systems, and the authors compare the results with a recently published model for calculating second and third viral coefficients of polar gases and their mixtures. That model accounts for the polar nature of the molecules with a term containing the reduced dipole moment and it contains mixing rules for the substance-specific parameters needed to calculate the second and third cross virial coefficients. The model and data are in satisfactory agreement, and the model can be used to greatly extend the useful range of the limited set of data.

01,129

PB96-160510 Not available NTIS

National Inst. of Standards and Technology (MSEL),

Gaithersburg, MD. Reactor Radiation Div.
Tetrahedral-Framework Lithium Zinc Phosphate Phases: Location of Light-Atom Positions in LiZnPO4 $\mathrm{H} 2 \mathrm{O}$ by Powder Neutron Diffraction and Structure Determination of $\mathrm{LiZnPOA}$ by ab Initio Methods.

Final rept.

W. T. A. Harrison, T. E. Gier, J. M. Nicol, and G. D. Stucky. 1995, 9p.

Pub. in Jnl. of Solid State Chemistry, v114 p249-257 1995

Keywords: "Ion exchange resins, "Cations, "Crysta structure, Powder(Particles), Neutron diffraction, Synthesis(Chemistry), Phase transformations, Lithium inorganic compounds, Zinc phosphates, Reprints, Powder neutron diffraction, Zeolite A phases.

The syntheses, crystal structures, and certain properties of two lithium zinc phosphate phases, LiZnPO4.H2O and LiZnPO4, are reported. $\mathrm{LiZnPO4.H2O}$ is an isostructure of the Li-A-type zeolite LiAlSiO4.H2O and consists of a fully ordered three-dimensional network of vertex-sharing $\mathrm{ZnO} 4$ and $\mathrm{PO} 4$ tetrahedral units surrounding 8-, 6-, and 4-ring windows. The extraframework lithium cation and water molecular are located in this cavity system. The structure of LiZnPO4 was solved ab initio using synchrontron X-ray powder data and consists of a new semicondensed tetrahedral-framework structure incorporating the guest lithium cations in squashed 6 ring channels

01,130

PB96-160528 Not available NTIS

National Inst. of Standards and Technology (MSEL), Gaithersburg, MD. Reactor Radiation Div.

Characterization of the Structure of TbD2.25 at 70 $K$ by Neutron Powder Diffraction.

Final rept.

Q. Huang, T. J. Udovic, J. J. Rush, J. Schefer, and I. S. Anderson. 1995, 4p.

Pub. in Jnl. of Alloys and Compounds, v231 p95-98 1995.

Keywords: "Terbium compounds, "Crystal structure, Powder(Particles), Neutron diffraction, Lattice parameters, Rare earth compounds, Chemical bonds, Reprints, Terbium deuteride, Long-range order, Octahedral site occupation.

A neutron-powder-diffraction pattern of the superstoichiometric rare-earth dideuteride TbD2.25 was measured at $70 \mathrm{~K}$. Profile refinements indicated that the TbD2.25 structure possesses $14 / \mathrm{mmm}$ symmetry with deuterium fully occupying the tetrahedral $(t)$ in erstices of the nominally fcc Tb lattice and the excess deuterium occupying one-fourth of the octahedral $(0)$ interstices with long-range order. Ideal order corresponds to the occupation of only those 0 sites within every fourth (042)cubic plane, in agreement with the results of a previous TbD $2+x$ study.

\section{1,131}

PB96-160585 Not available NTIS

Gaithersburg MD Precision Engineering Div.

Fabrication Issues for the Prototype National Institute of Standards and Technology SRM 2090A Scanning Electron Microscope Magnification Calibration Standard.

Final rept.

B. L. Newell, M. T. Postek, and J. P. van der Ziel.

$1995,5 p$

Pub. in Jnl. of Vacuum Science and Technology, Chapter 2671, vB 13 n6 p2671-2675 Nov/Dec 95

Keywords: "Electron microscopes, "Scanning Calibration standards, Prototypes, Lithography, Electron beams, Fabrication, Design, Reprints.

A new National Institute of Standards and Technology scanning electron microscope magnification calibration standard has been fabricated and distributed in production prototype form. The SRM 2090A samples contain structures ranging in pitch from 3000 to 0.2 microm and are useful at both high- and low-accelerating voltages. The design and fabrication of the samples has incorporated many of the improvements suggested through two previous prototype series. This article discusses optimization of the lithographic techniques in the fabrication process. We used electron beam lithography for fabrication because of the 0.1 microm features required on the samples.

01,132

PB96-160643 Not available NTIS
National Inst of Standards and Technology (CSTL), Gaithersburg, MD. Process Measurements Div. $Q$ Branch Lineshape Functions for CARS Thermometry.

G. J. Rosasco, and W. S. Hurst 1992, 6p.

Pub in Temperature, Its Measurements and Control in Science and Industry, v6 pt2 p655-660 Feb 92.

Keywords: "Raman spectroscopy, "Temperature measurement, Line spectra, Spectra analysis, Temperature dependence, Pressure, Collision parameters, Reprints, CARS(Coherent Anti-Stokes Raman Spectroscopy), Collisional narrowing, Dicke narrowing.

Knowledge of the pressure and temperature dependence of the spectra distribution is required to allow determination of the temperature from a coherent antiStokes Raman spectroscopy (CARS) spectrum. We review our understanding of the physics of line formation for the Raman vibrational Q branch of diatomic molecules. While we can demonstrate that the role of velocity, phase, and state changing collisions in line formation is reasonably well understood, there are relatively few first principle calculations which allow prediction of the molecular parameters which determine the spectra distribution. We show how quasi-empirical models, which are consistent with our physical understanding, can provide a reliable basis for prediction.

01,133

PB96-160734 Not available NTIS

National Inst. of Standards and Technology (MSEL) Gaithersburg, MD. Reactor Radiation Div.

Low-Energy Vibrations and Octahedral Site Occupation in $\mathrm{Nb} 95 \mathrm{~V} 5 \mathrm{H}(\mathrm{D}) \mathrm{y}$.

Final rept.

T. J. Udovic, J. J. Rush, R. Hempelmann, and D.

Richter. 1995, 3p.

Pub. in Jnl. of Alloys and Compounds, v231 p144-146 1995

Keywords: , Niobium compounds, "Neutron scattening, "Microstructure, Inelastic scattering, Alloys, Spectroscopic analysis, Vibration spectra, Lattice parameters, Hydrides, Reprints, Octahedral site occupation, Metal hydrides.

$\mathrm{Nb}(100-\mathrm{x}) \mathrm{VxHy}$ is a random b.c.c. alloy system with an $\mathrm{H}$ sublattice disordered with respect to site energies. Previous incoherent inelastic neutron scattering (IINS) studies ascertained both octahedral (o) and tetrahedra (t) site occupation by $\mathrm{H}$ for $\mathrm{x}=5,10$ and 50. On the basis of spectroscopic and statistical arguments, the low-temperature $\mathrm{H}$ vibrational peaks in the $\mathrm{Nb}$-rich, low-H-concentration $\mathrm{Nb} 95 \mathrm{~V} 5 \mathrm{H} 1$ alloy were assigned to $\mathrm{Nb} 4 \mathrm{~V} 2 \mathrm{o}$ sites and $\mathrm{NbV}_{3} \mathrm{t}$ sites. To probe the regime of lower $\mathrm{H}$ concentration and the effects of $\mathrm{D}$ substitution, further IINS spectra were measured for Nb95 $\mathrm{V} 5 \mathrm{Hy}(\mathrm{y}=0.5$ and 0.8$)$ and $\mathrm{Nb95V5D2.7}$

01,134

PB96-160742 Not available NTIS

National Inst. of Standards and Technology (MSEL) Gaithersburg, MD. Reactor Radiation Div.

Neutron Spectroscopic Comparison of beta-Phase Rare Earth Hydrides.

Final rept.

T. J. Udovic, J. J. Rush, and I. S. Anderson. 1995,

$6 p$. 1995.

Keywords: "Rare earth elements, "Hydrides, "Neutron scattering, "Microstructure, Inelastic scattering, Spectroscopic analysis, Vibrational spectra, Lattice pa rameters, Phase transformations, Reprints.

The Beta-phase hydrides Beta- $\mathrm{RH} 2+x$ of a variety of rare metals $(R=T b, Y, L a, C e$ and $D y)$ were inves tigated by incoherent inelastic neutron scattering Specroscopy The broad, complex vibrational density of states (DOS) of the tetrahedrally coordinated (t-site) Hydrogen $(\mathrm{Ht})$ typically found for these systems is manifestation of the dynamic coupling within the $H$ sublattice due to significant $\mathrm{Ht}$-Ht interactions. A break down of this dynamic coupling via isotopic dilution of the $\mathrm{Ht}$ atoms with larger mass deuterium atoms is observed spectroscopically by a collapse of the complex DOS into a single sharp feature.

01,135

PB96-160999 Not available NTIS

National Inst. of Standards and Technology (CSTL) Gaithersburg, MD. Process Measurements Div. 
Effects of Pipe Elbows and Tube Bundles on Selected Types of Flowmeters.

Final rept.

G. E. Mattingly, and T. T. Yeh. 1991, 10p.

Pub. in Flow Measurement and Instrumentation Jnl. v2 p4-13 Jan 91

Keywords: "Pipe elbows, "Measurement accuracy, *Flowmeters, Swirl, Reprints, "Foreign technology, Tube bundles.

The paper presents experimental results for the decay of pipe elbow-produced swirl in pipeflows and its ef fects on flowmeter measurement accuracy. Experiments include the decay of swirl produced by single and double elbow configurations for pipe diameter Reynolds number of 10 to the 4 th power to 10 to the 5 th power using water in a $50 \mathrm{~mm}$ diameter facility a NIST in Gaithersburg, MD. Results show that differen types of swirl are produced by the different piping configurations. The swirl decay is found to be dependent on the type of swirl and the pipe Reynolds number. A high Reynolds number very long lengths of straight constant diameter pipe are required to dissipate the single eddy type swirl that is produced by the two elbows out-of-plane configuration. Without flow conditioning, it is concluded that the specifications of upstream pipe lengths in the current flowmetering stand ards may not be sifficient to achieve the desired flow metering accuracy. Experimental results are also presented for the effects produced by tube bundle-type flow conditioners. These results show shifts in orifice meter discharge coefficients that are both positive and negative depending upon pertinent conditions. A range of orifice geometries, Reynolds numbers and meter locations are studied and explanations are put forth to explain these shifts.

\section{1,136}

PB96-161005 Not available NTIS

National Inst. of Standards and Technology (CSTL), Gaithersburg, MD. Process Measurements Div.

Pressure Measurements with the Mercury Melting Line Referred to ITS-90.

Final rept.

G. F. Molinar, V. E. Bean, J. Houck, and B. Welch.

$1991,2 \mathrm{p}$.

Pub. in Metrologia, v28 p353-354 Feb 91.

Keywords: "Melting line, "Mercury, Pressure, Standards, Reprints, "Foreign technology.

In this letter the authors recalculate the mercury melting line, for use as an accurate pressure transfer standard up to $1,200 \mathrm{MPa}$, with a reference to the new International Temperature Scale of 1990 (ITS-90). In previous work the authors obtained fifty-two experimental temperature and absolute pressure points, each poin representing a melting point of mercury under specified temperature and pressure conditions. The temperatures were referenced to the International Practical Temperature Scale of 1968 (IPTS-68).

\subsection{7}

PB96-161625 Not available NTIS

National Inst. of Standards and Technology (CSTL) Gaithersburg, MD. Biotechnology Div.

Imposed Oscillations of Kinetic Barriers Can Cause an Enzyme to Drive a Chemical Reaction Away from Equilibrium.

Final rept.

R. D. Astumian, and B. Robertson. 1993, 6 p.

Pub. in Jnl. of the American Chemical Society, v115 n24 p11063-11068 Dec 93

Keywords: "Chemical affinity, "Enzyme kinetics, Reprints, Thermodynamics, "Free energy transduction.

The overall Gibbs free energy change (delta G) of a chemical reaction is often termed the driving force of the reaction. The sign of delta $G$ defines the direction of spontaneous reaction, and the condition delta $\mathrm{G}=$ 0 defines the point of chemical equilibrium. This is strictly true for elementary reactions--reactions that pass through only one local maximum (the transition state) along the reaction coordinate connecting reactant and product states. However, under many circumstances it is also true for reactions that involve one or more intermediates, particularly if the steady state intermediate concentrations are very small. Here the authors show that externally imposed oscillations or fluctuations can drive a net chemical reaction away from equilibrium so long as the rate constants of a least one elementary step of the overall reaction depend on the fluctuating parameter. This is true even if the overall delta $G$ is independent of the perturbation and it is also true even if the concentrations of the intermediate states are very, very small (i.e., experimental undetectable).

\section{1,138}

PB96-161633 Not available NTIS

National Inst. of Standards and Technology (CSTL), Gaithersburg, MD. Biotechnology Div.

Quadratic Response of a Chemical Reaction to External Oscillations.

Final rept.

R. D. Astumian, B. Robertson, R. S. Li, and J. Ross. 1992, 7p.

Pub. in Jnl. of Chemical Physics, v96 n9 p6536-6542 May 92 .

Keywords: "Chemical reactions, *Oscillations, Reprints, *Quadratic response, External periodic perturbations.

The authors develop a second-order response theory to investigate the effects of external periodic perturbations on a chemical reaction at a stable steady state in an open reactor. The authors apply the theory to the quadratic Schlogl model, a single-variable nonlinear reaction. In the presence of oscillating reactant or product concentrations or oscillating rate coefficients, the average intermediate concentration, the fluxes, and the dissipation are each a Lorentzian function of frequency with midpoint at the inverse relaxation time of the system. Thus even very short relaxation times can be determined by measuring average rates as a function of frequency of the perturbation. The amplitude of the Lorentzian depends on the chemical mechanism of the reaction and is proportional to the square of the amplitude of the applied perturbation. The authors also show that energy from the perturbation can be used to drive the reaction in a direction opposite of that predicted by the Gibb's free energy difference of the reactants and products, even under circumstances where the overall affinity is independent of the perturbation.

\section{1,139}

PB96-161856 Not available NTIS

National Inst. of Standards and Technology (CSTL), Gaithersburg, MD. Thermophysics Div.

Vapor-Liquid Equilibria of Ternary Mixtures in the Critical Region on Paths of Constant Temperature and Overall Composition.

Final rept

L. J. Van Poolen, and J. C. Rainwater, 1995, 9p Pub. in International Jnl. of Thermophysics, v16 n2 p473-481 Mar 95

Keywords: "Critical region, *Ethane, Reprints, Ternary mixtures, Phase rule, "Vapor liquid equilibrium, Leung Griffiths model, N-butane, N-pentain.

High-pressure vapor-liquid equilibrium (VLE) data for the ternary mixture ethane $+n$-butane $+n$-pentane due to Thodos and co-workers have been correlated by a ternary version of the modified Leung-Griffiths model. Data were taken along paths of constant temperature and approximately constant overall composition, and a separate test was made for such constancy. Seventeen different specified overall compositions at four different temperatures were correlated. In general, agreement between the correlation and data is very good, particularly for those curves that satisfy the test for constant overall composition. The ternary model has been constructed from correlations of each of the three constituent binaries without any further adjustable parameters.

\section{1,140}

PB96-161898 Not available NTIS

National Inst. of Standards and Technology (CSTL), Boulder, CO. Thermophysics Div

Density Dependence of Fluid Properties and NonNewtonian Flows: The Weisenberg Effect.

Final rept.

J. C. Rainwater, H. J. M. Hanley, and A. Narayan. 1995, 17p.

Pub. in Jni. of Rheology, v39 n6 p1343-1359 Nov/Dec 95.

Keywords: "Rotating cylinders, Compressibility, Couette flow, Reprints, "Weissenberg effect, "NonNewtonian fluids, Rivlin-Ericksen tensors, Shear dilatancy.

Two approaches which describe the Weissenberg effect (height profile of a non-Newtonian fluid between rotating vertical concentric cylinders) are discussed. The first is based on an earlier calculation with rheological properties of a simple liquid from nonequilibrium molecular dynamics (NEMD). The calculation is redone here using new results on the density dependence of the normal pressure differences. The NEMD calculations are restricted to Couette flow, but describe specifically, in a consistent manner, the effects of finite compressibility. The pressure, viscosity, and normal pressure differences are all found from NEMD to be sensitive functions of density, which requires that the equations of motion be solved iteratively and self-consistently, and a sample calculation is presented for the soft sphere fluid. The second approach is that of Joseph and Fosdick. Their assumptions and techniques are examined and compared with the NEMD calculations.

01,141

PB96-161906 Not available NTIS

National Inst. of Standards and Technology (CSTL), Boulder, CO. Thermophysics Div.

Nonlinear Correlation of High-Pressure Vapor-Liquid Equilibrium Data for Ethylene + n-Butane Showing Inconsistencies in Experimental Compositions.

J. C. Rainwater, and J. J. Lynch. 1994, 9p.

Pub. in International Jnl. of Thermophysics, v15 n6 p1231-1239 Nov 94

Keywords: "Critical region, "Ethylene, High pressure Reprints, "Mole fraction errors, N-butane, Simplex optimization, Vapor-liquid equilibrium.

The modified Leung-Griffiths model is applied to the previously unpublished data, tabulated here, of Witliams for high-pressure vapor-liquid equilibria of ethylene $+n$-butane. It is not possible to obtain a hingly accurate correlation with the experimentally stated compositions, but evidence is given that those composition measurements may be suspect, although pressure temperature, and density data are accurate. A simplex optimization method was used for the parameters of the model, and the compositions were also treated as adjustible parameters. With this method a much more accurate correlation is obtained, but the optimized compositions differ in two of four cases by more than $3 \%$ from the stated compositions.

01,142

PB96-161914 Not available NTIS

National Inst. of Standards and Technology (CSTL), Gaithersburg, MD. Thermophysics Div.

Quantum Collisional Transfer Contributions to the Density Dependence of Gaseous Viscosity.

Final rept.

J. C. Rainwater. 1995, 7p.

Pub. in Proceedings of the Conference on Rarefied Gas Dynamics (19th), Oxford, England, July 25-29, 1994, p114-1201995.

Keywords: "Boltzmann equation, "Classical limit, *Density correction, Reprints, Kinetic theory, Quantum theory, Viscosity, Weyl transform.

The linearly extended Waldmann-Snider equation of Thomas and Snider is solved by a Chapman-Enskog method to derive the quantum collisional transfer contributions to the viscosity of a gas. These contributions, which are linear in density, are then reduced by Weyl correspondence methods to their classical limits, and are shown to agree with the known classical results.

01.143

PB96-161930 Not available NTIS National Inst. of Standards and Technology (CSTL), Boulder, CO. Process Measurements Div. Cryogenic Flow Calibration in NIST.

Final rept.

J. L. Scott, and M. A. Lewis. 1995, 5p

Pub. in International Jnl. of Metrology, v2 n5 p12-16 Sep/Oct 95.

Keywords: *Cryogenics, *Flow calibration, Data acquisition, Load cell, Reprints, "Mass flow measurement. A unique flow calibration facility for cryogenic liquids is located at the National Institute of Standards and Technology (NIST) in Boulder Colorado. A dynamic weighing system is used to measure totalized mass flow and, with the use of NIST thermodynamic property data for density, volumetric flow. Calibrations are typically performed with liquid nitrogen in a flow of 0.95 to $9.5 \mathrm{~kg} / \mathrm{s}$, pressure range of
temperature range of 80 to $90 \mathrm{k}$.

01,144

PB96-163670 Not available NTIS 
National Inst. of Standards and Technology (CSTL), Gaithersburg, MD. Process Measurements Div. Effect of Finite Beam Width on Elastic Light Scattering from Droplets.

Final rept.

J. T. Hodges, and C. Presser. 1993, 5p

Pub. in Annual Conference on Liquid Atomization and Spray Systems (6th), Worcester, MA., May 17-19, 1993, Volume of Extended Abstracts, p152-156.

Keywords: "Droplets, "Elastic scattering, "Gaussian beam, Reprints, Lorenz-Mie.

A number of experimental techniques based on elastic light scattering are used to measure the size of micronsized spherical particles and droplets. Some examples include, phase Doppler anemometry, laser diffraction methods, light intensity deconvolution as well as ensemble scattering measurements from axi-symmetric Gaussian and Gaussian sheet beams. In the interpretation of these measurements, the classical LorenzMie theory (LMT) of elastic light scattering or principles of diffraction and ray optics are invoked to predict the phase, amplitude and intensity of the scattered radiation as a function of particie size, refractive index and scattering angle. The reliability and accuracy of these measurements, thus depend on the degree to which the invoked theory predicts the observed features of the scattered field. In this context, it is therefore important to consider realistic situations for which departures from the LMT are significant enough to warrant the use of more exact theories of light scattering.

\section{1,145}

PB96-163803 Not available NTIS

National Inst. of Standards and Technology (CSTL), Boulder, CO. Thermophysics Div.

$\mathrm{CO} 2 / \mathrm{CH}_{4}$ Transport in Polyperfluorosulfonate Ionomers: Effects of Polar Solvents on Permeation and Solubility.

Final rept.

J. Pellegrino, and Y. S. Kang. 1995, 12p.

Pub. in Jnl. of Membrane Science, v99 p163-174 1995.

Keywords: "Permeation, *Solubility, Membranes, Reprints, Modeling, Alcohols, "Polyperfluorosulfonate ionomer, PFSI.

The authors measured $\mathrm{CO} 2$ and $\mathrm{CH} 4$ gas permeation through polyperfluorosulfonate ionomer (PFSI) that was solvated with water, methanol, ethanol, or 1-propanol. These measurements were compared with predictions based on a two-phase, effective-media, membrane model that considered a number of possible geometries for the distributed phase (the ionic clusters). The transport parameters (solubility and diffusivity) for each of the phases and the volume fraction of dispersed phase were either measured independently or estimated from prior literature data. The authors were unable to match all the experimental observations with this two-phase model. The authors conclude that different polar solvents an incorporate themselves into the fluorocarbon 'continuous phase' in ways that will alter the effective diffusion coefficient of that phase to gas permeation.

\section{1,146}

\section{PB 96-164199 Not available NTIS}

National Inst. of Standards and Technology (EEEL), Gaithersburg, MD. Semiconductor Electronics Div.

Electrical Characterization of Narrow Gap n-Type Bulk HgCdTe Single Crystals by Variable-Magnetic-Field Hall Measurements and Reduced-Conductivity-Tensor Analyses.

Final rept.

J. S. Kim, D. G. Seiler, R. A. Lancaster, and M. B.

Reine. 1996, 2p

Pub. in Extended Abstracts of the 1995 U.S. Workshop on the Physics and Chemistry of Mercury Cadmium Telluride and Other IR Materials, Baltimore, MD. October 10-12, 1995, p31-32 1996

Keywords: "Hall effect, "Resistance, "Supercondictivity, Reprints, Electron mobility, Electron transport, Gapless state, HgCdTe, Magnetotransport, MCT, Narrow gap.

We have investigated, for the first time, magnetotransport properties of very narrow gap $\mathrm{HgCdTe}$ single crystals in the light of the reduced-conductivity-tensor (RCT) scheme to determine the variations of the carrier density and mobility as a function of temperature in the semiconductor-to-semimetal transition region. In this paper, we report the results of variable-magnetic-field and variable-temperature Hall measurements ( 0 to $1.5 \mathrm{~T}$ and $10 \mathrm{~K}$ to $300 \mathrm{~K}$ ) on three n-type bulk $\mathrm{HgCdTe}$ single crystals, labeled MCT-1 $(x=0.190)$, MCT-2 $(x=0.175)$, and MCT-3 $(x=0.160)$. The experimental data are analyzed in the context of the RCT scheme of multicarrier characterization, which we have previously used to electrically characterize liquid-phase-epitaxially (LPE) grown $\mathrm{HgCdTe}$ films. The latter work deals with type classification if LPE samples according to the degree of anomalous electrical behavior present, from normal to severely anomalous.

\section{1,147}

PB96-164215 Not available NTIS

National Inst. of Standards and Technology (CSTL), Boulder, CO. Thermophysics Div.

Solubilities of Copper(II) and Chromium(III) betaDiketonates in Supercritical Carbon Dioxide.

Final rept.

A. F. Lagalante, B. N. Hansen, T. J. Bruno, and R. E Sievers. 1995, 5p

Pub. in Inorganic Chemistry, v34 p5781-5785 1995.

Keywords: "Carbon dioxide, "Copper, "Solubility, Parameter, Reprints, Beta-diketonates, Supercritical fluids.

The mole fraction solubilities of ten copper(II) and five chromium(III) beta-diketonates were measured in supercritical carbon dioxide with a spectroscopic technique and found to vary over 4 orders of magnitude. Observed trends indicate that the solubility on supercritical carbon dioxide is strongly dictated by the character of the hydrocarbon or fluorocarbon shell surrounding the central metal atom. A group-contribution approach was used to calculate the solubility parameter of the anion of the uncomplexed beta-diketone that was correlated to the experimentally measured solubility. A regular solutions approach was used for Cr(acac) 3 to quantitatively attempt to predict the solubility in supercritical carbon dioxide. Solubility data, solubility parameter trends, and limitations of regular solutions theory applied to supercritical fluids are discussed.

\section{1,148}

\section{PB96-167135 Not available NTIS}

National Inst. of Standards and Technology (CSTL), Boulder, CO. Thermophysics Div.

Molar Heat Capacity at Constant Volume for ( $x \mathrm{CO}$ $+(1-x) \mathrm{C} 2 \mathrm{H} 6)$ from 220 to $340 \mathrm{~K}$ at Pressures to 35 $\mathrm{MPa}$

Final rept.

J.W. Magee. 1995, 5p

Pub. in Jnl. of Chemical and Engineering Data, v40 n2 p438-442 1995

Keywords: "Heat capacity, "Calorimeters, "High pressure, Ethane, Carbon dioxide, Reprints, Isochoric Measurement, Mixtures.

Measurements of the molar heat capacity at constant volume $(\mathrm{Cv})$ for $(\mathrm{xCO} 2+(1-\mathrm{x}) \mathrm{C} 2 \mathrm{H} 6), \mathrm{x}=0.25,0.49$, 0.74 , were conducted. Tempertures ranged from about 220 to $340 \mathrm{~K}$, and pressures were as high as $35 \mathrm{MPA}$ Meaurements were conducted on samples in compressed gas and liquid states. The primary sources of uncertainty are the estimated temperature rise and the estimated quantity of substance in the calorimeter Overall, the uncertainty of the $\mathrm{C} v$ values is estimated to be less than plus or minus $2.0 \%$ for vapor and plus or minus $0.5 \%$ for liquid.

\section{1,149}

PB96-167150 Not available NTIS

National Inst. of Standards and Technology (CSTL), Boulder, CO. Thermophysics Div

REFPROP Refrigerant Properties Database: Capabilities, Limitations, and Future Directions.

Final rept

M. O. McLinden, J. G. Gallagher, M. L. Huber, and G. Morrison. 1993, 13p

Pub. in ASHRAE/NIST Refrigerants Conference, Gaithersburg, MD., August 19-20, 1993, p59-71.

Keywords: "Refrigerants, "Chlorofluorocarbons, Databases, Mixtures, Reprints, Equation of state Hydrochiorofluorocarbons, Hydrofiuorocarbons.

The REFPROP computer database has been developed to meet the needs of the refrigeration industry in their evaluation of alternative refrigerants. In this paper, we highlight the capabilities and discuss the limations of the database through examples of severa representative pure refrigerants and refrigerant blends. The database employs the Carnahan-StarlingDesantis (CSD), modified Bennedict-Webb-Rubin
(MBWR), and Haar-Gallagher equations of state for thermodynamic properties, and an extended corresponding states (ECS) model for thermodynamic and transport properties; the most appropriate model for specific applications is recommended.

\section{1,150}

PB96-167176 Not available NTIS

National Inst. of Standards and Technology (CSTL), Boulder, CO. Thermophysics Div.

Simulation and SANS Studies of Gelation Under Shear.

Final rept

C. D. Muzny, D. Hansen, G. C. Straty, D. J. Evans,

and H. J. M. Hanley. 1995, 10p.

Pub. in International Jnl. of Thermophysics, v16 n2 p337-346 Mar 95.

Keywords: "Gelation, "Shear, Reprints, "Foreign technology, "Small angle neutron scattering, "Nonequilibrium molecular dynamics, Colloidal silica, Lennard Jones fluid, Spinodal decomposition.

Computer simulations of a two-dimensional LennardJones fluid undergoing spinodal decomposition are reported for the system subjected to planar Couette flow. Key results are the images of the atomic structure and plots of the corresponding pair correlation functions. $A$ companion small-angle neutron scattering (SANS) study of shear-influenced gelation on colloidal silica suspensions at volume fractions phi $=0.1,0.12,0.18$ and 0.24 is discussed. It is found that the scattered in tensity-wave vector curves from the unsheared gels obey a power law for phi less than 0.24. At higher volume fractions, the power law does not seem to be followed Shear, however induces an apparent fractal structure in the gel at phi $=0.24$. Results from the computer and the SANS experiments indicate that the spinodal decomposition process and the gelation mechanism have features in common.

01,151

PB96-167184 Not available NTIS

National Inst. of Standards and Technology (CSTL) Boulder, CO. Thermophysics Div.

Small-Angle Neutron-Scattering Study of Dense Sheared Silica Gels.

Final rept.

C. D. Muzny, G. C. Straty, and H. J. M. Hanley. 1994, 4p.

Pub. in Physical Review E, v50 n2 pR675-R678 Aug 94.

Keywords: "Gels, "Shear, Fractal, Reprints, "Smal angle neutron scattering, Colloidal silica.

Small-angle neutron-scattering (SANS) shearing experiments on dense colloidal silica suspensions in H2O-D2O medium are reported for the wave vector range 0.03 less than $\mathrm{Q}$ (nm to the minus 1 power) less than 0.8 at volume fractions phi $=0.1,0.12,0.18,0.24$ and 0.3 . For the unsheared gels, apparent fractal struc tures were observed at the lower volume fractions, and a small maximum at a $Q$ corresponding to a contact particle morphology was apparent at all volume fractions. A fractional dimension $d-1.60$ plus or minus 0.05 was estimated for phi $=0.10$ and 0.12 by fitting the power law behavior of the structure factor. Shear induces an apparent fractal domain into the dense gels and enhances the contact particle structure.

01,152

PB96-167218 Not available NTIS

National Inst. of Standards and Technology (CSTL) Boulder, CO. Thermophysics Div.

Status of the Round Robin on the Transport Properties of R134a.

M.J. Assael, Y Nagasaka, C. A Nieto de Castro, E. Vogel, W. A. Wakeham, R. A. Perkins, and K. Strom. $1995,16 p$.

Pub. in International Jnl. of Thermophysics, v16 n1 Jan 95.

Keywords: "Refrigerants, "Thermal conductivity, Satu ration properties, Transport properties, Viscosity, Reprints, R134a, Dilute gas.

The paper contains a status report on an international project coordinated by the Subcommittee on Transport Properties of commission 12 of the International Union of Pure and Applied Chemistry. The project has been conducted to investigate the large discrepancies be tween the results reported by various authors for the transport properties of $\mathrm{R} 134 \mathrm{a}$. The project has involved the remeasurement of the transport properties of a sin- 
gle sample of $\mathrm{R} 134 \mathrm{a}$ in nine laboratories throughout the world in order to test the hypothesis that at least part of the discrepancy could be attributed to the purity of the sample. This paper provides an intercomparison of the new experimental results obtained to data in this project for the viscosity and the thermal conductivity in both gaseous and liquid phases. The agreement be tween the viscosity data from the laboratories contributing to the project was improved with several techniques, now producing consistent results. This suggests that the purity of the samples of R134a used in previous work was at least partly responsible for the discrepancies observed. For the thermal conductivity in the liquid phase the results of the measurements are also more consistant than before, although not for all experimental techniques. Not all of the previous measurements suffered from significant sample impurities, so the present measurements on a consistent high-purity sample can be used to detect data sets which are outliners, possibly because of impurities. Identification of laboratories and techniques with systematic differences may require the examination of data for several fluids. The implications for future measurements of the transport properties of other refrigerants are significant.

\section{1,153}

PB96-167358 Not available NTIS

National Inst. of Standards and Technology (CSTL), Boulder, CO. Thermophysics Div

Thermophysical Property Standard Reference Data from NIST.

Final rept.

D. G. Friend, and M. L. Huber. 1994, 10p.

Pub. in International Jnl. of Thermophysics, v15 n6 p1279-1288 Nov 94

Keywords: "Thermodynamic properties, "Refrigerants, Databases, Mixtures, Predictive models, Standards, Reprints, *Foreign technology, "Transport properties.

The National Institute of Standards and Technology (NIST) has a primary function to develop and disseminate standard reference data for the thermophysical properties of fluids and fluid mixtures of interest to the industrial and scientific communities. In this paper we discuss five computerized databases distributed by the Standard Reference Data (SRD) Program of NIST. The databases provide national standards for the properties of pure fluids, an accurate evaluated mixture program focusing on the properties of natural gas mixtures, a predictive package emphasizing hydrocarbon systems up to $\mathrm{C20}$, a database for refrigerant and prospective alternative refrigerant fluids, and the current scientifc thermophysical property surfaces for the pure water and steam.

\section{1,154}

PB96-167390 Not available NTIS

National Inst. of Standards and Technology (CSTL) Boulder, CO. Thermophysics Div.

Isochoric $(p-p-T)$ Measurements on Liquid and Gaseous Air from 67 to $400 \mathrm{~K}$ at Pressures to 35 $\mathrm{MPa}$.

Final rept.

J. B. Howley, J. W. Magee, and W. M. Haynes.

1994, $12 p$.

Pub. in International Jnl. of Thermophysics, v15 n5 p881-902 Sep 94.

Keywords: "Isochoric, "High pressure, Air, Density, Reprints, Mixtures, Vapor.

Comprehensive isochronic $p$ - $p$-T measurements have been carried out on liquid and gaseous air along 16 isochores at densities ranging from 2 to $32 \mathrm{~mol}-\mathrm{dm}$ to the minus 3 rd power. The air mixture has a nomina composition of $0.7812 \mathrm{~N} 2+0.2096 \mathrm{O} 2+0.0092 \mathrm{Ar}$ The $p-p-T$ data cover a temperature range from 67 to $400 \mathrm{~K}$ at pressures up to $35 \mathrm{MPa}$. Comparisions with experimental results from independent sources are presented using a fundamental equation of state based, in part, on the $p-p-T$ data from this study.

\section{1,155}

PB96-176508 Not available NTIS

National Inst. of Standards and Technology (PL), Boulder, CO. Time and Frequency Div.

Potential Surfaces and Dynamics of Weakly Bound Trimers: Perspectives from High Resolution IR Spectroscopy.

Final rept.

M. A. Suhm, and D. J. Nesbitt. 1995, 10p. Sponsored by National Science Foundation, Arlington,
VA.
Pub. in Chemical Society Reviews, p45-54 1995.

Keywords: “Clusters, "Trimers, Reprints, "High resolution, IVR, Hydrogen bonding, Predissociation, Potential energy surfaces, Three-body forces.

If we want to understand the properties of condensed molecular matter in detail, we have to study the interactions between molecules, which are often summarized under the name 'van der Waals interactions.' These intermolecular interactions are of a profoundly subtle, multidimensional, long-range, and coupled nature. This is in contrast to intramolecular interactions, where the concepts of chemical bond and harmonic force-field usually form a reasonably localized zeroorder reference point at sufficiently low excitation levels. Infrared spectroscopy provides a powerful general tool to study both inter- and intramolecular interactions, since it can cover the full frequency range of fundamental and few-quantum excitation of the associated degrees of freedom. It is in the 'floppy molecule', intermolecular regime where the inversion of high resoIution spectroscopic data to interaction potentials is particularly difficult, and thus the demand for interaction between theory and experiment is particularly intense. As one consequence is this difficulty, initial spectroscopic research efforts have concentrated on pairs of molecules, the obvious prototypes of any interaction study. These binary complexes or dimers have revealed an unprecedented richness of structural properties and dynamical behavior, when viewed through the eyes of high resolution microwave, far- and nearIR spectroscopy, 2-5 and modern theoretical tools. Indeed, the astonishing range of challenges and insights provided by dimer spectroscopy deflected the original thrust of a spectroscopy-based understanding of bulk matter toward an extensive, albeit very fruitful, series of diversions.

\section{1,156}

\section{PB96-176524 Not available NTIS}

National Inst. of Standards and Technology (MSEL), Gaithersburg, MD. Ceramics Div.

Magnetic Dielectric Oxides: Subsolidus Phase Relations in the BaO: Fe2O3: TiO2 System.

Final rept.

T. A. Vanderah, J. M. Loezos, and R. S. Roth. 1996, $13 p$.

Pub. in Jnl. of Solid State Chemistry, Article No. 0006 , v121 p38-50 1996.

Keywords: "Phase diagrams, "Solid solutions, "Banium oxides, Reprints, $\mathrm{BaO}, \mathrm{Fe} 2 \mathrm{O} 3, \mathrm{TiO} 2$.

The $\mathrm{BaO}-\mathrm{Fe} 2 \mathrm{O} 3-\mathrm{TiO} 2$ ternary phase diagram has been investigated at 1250-1270 degrees $C$ in air. $X$ ray diffraction studies of approximately 150 ray diffraction studies of approximately 150 firmed the existence of sixteen ternary compounds. Two of these compounds, BaFe4Ti2O11 and Ba12Fe28Ti15084, were previously reported, four were found to be isostructural with known chemically similar compounds, and ten apparently adopt new structure-types. The crystal structures of five of the new ternary phases are briefly described. The oxidation state of iron found in this study is similar to that reported for a study of the $\mathrm{BaO}-\mathrm{Fe} 2 \mathrm{O} 3-\mathrm{SnO} 2$ system in air at 1200 degrees $C$.

\section{1,157}

PB96-176607 Not available NTIS

National Inst. of Standards and Technology (PL), Boulder, CO. Quantum Physics Div

State-Resolved Rotational Energy Transfer in Open Shell Collisions: $\mathrm{Cl}((2) \mathrm{P} 3 / 2)+\mathrm{HCl}$.

Z. Q. Zhao, W. B. Chapman, and D. J. Nesbitt. 1995, $13 p$

Contract AFOSR93-NC-231

Sponsored by Air Force Office of Scientific Research, Bolling AFB, DC

Pub. in Jnl. of Chemical Physics, v102 n18 p70467058 May 95.

Keywords: "Hydrogen bonds, "Energy transfer, "Rotation, "Collisions, "Chlorine, Preparation, Photochemical reactions, Chemical dissociation, Interactions, Chemicals, Dynamics, Quantum theory, Elastic properties, Reactivities, Reprints, Open shells.

Time- and frequency-resolved infrared (IR) laser absorption methods are used to probe hot atom energy transfer in open shell interactions of $\mathrm{Cl}(2 \mathrm{P} 3 / 2)+\mathrm{HCl}(\mathrm{J})$ in the single collision regime. The $\mathrm{Cl}(2 \mathrm{P} 3 / 2)$ atoms are prepared by $308 \mathrm{~nm}$ laser photolysis of $\mathrm{Cl} 2$, and suffer collisions at $\mathrm{E}$ rel approx. $3500 \mathrm{~cm}$ to the minus one power with a room temperature $\mathrm{HCl}$ distribution in a ast flow cell. Selective collisional excitation of final $\mathrm{HCl}(\mathrm{Jf})$ states is monitored by transient IR absorption on $R(J$ equal to or greater than 4$)$ branch lines in the $\mathrm{HCl}(\mathrm{v}=1$ greater than 0$)$ band, while depletion of the initial $\mathrm{HCl}(\mathrm{Ji})$ states is monitored by transient bleaching of the room temperature Doppler profiles. Analysis of the $\mathrm{J}$ dependent Doppler profiles permits extraction of rotational loss cross sections, as a function of initial and final $\mathrm{J}$ states, respectively. Absolute transient concentrations of the $\mathrm{HCl}(\mathrm{Ji})$ and $\mathrm{HCl}(\mathrm{Jf})$ are measured directly from absorbances via Beer's Law, and used to extract absolute collisional cross sections.

\section{1,158}

PB96-176771 Not available NTIS

National Inst. of Standards and Technology (CSTL), Gaithersburg, MD. Process Measurements Div.

Surface Plasmon Microscopy of BiotinPhotopatterned Alkanethiol Self-Assembled Monolayers.

Final rept.

D. Piscevic, W. Knoll, and M. J. Tarlov. 1995, 8p.

Pub. in Supermolecular Science, v2 p99-106 1995.

Keywords: "Surface plasmon resonance, Alkanethiols Reprints, "Foreign technology, "Self assembled monolayers, Photopatterning, Biotin streptavidin.

The authors report surface plasmon imaging of streptavidin binding to photopatterned biotinylated alkanethiol self-assembled monolayers (SMAs) on gold. Micrometer-scale patterns of a mixed biotin- and hydroxyl-terminated monolayer were formed in an inert, hydroxy-terminated alkanethiol monolayer using a UV-photopatterning procedure. Using surface plasmon microscopy, contrast is readily observed between the mixed biotin- and hydroxy-terminated SAM region after specific binding of streptavidin has OCcurred and the pure hydroxy-terminated region where nonspecific binding of streptavidin is negligible. Surface plasmon microscopy was also to monitor in situ and in real time the binding of streptavidin to the patterned SAMs. The ability of surface plasmon microscopy to detect and spatially resolve 2-dimensional monolayer binding events may prove useful in diagnostic applications involving the parallel interrogation at surface biomolecular arrays.

\section{1,159}

PB96-180161 Not available NTIS

National Inst. of Standards and Technology (MSEL), Gaithersburg, MD. Polymers Div.

Adsorption of Potassium $\mathrm{N}$-phenylglycinate on Hydroxyapatite: Role of Solvents and lonic Charge. Final rept.

D. N. Misra. $1996,9 p$

Pub. in Colloids and Surfaces, A: Physiocochemical and Engineering Aspects, v108 p277-285 1996

Keywords: "Adsorption, "Hydroxyapatite, Ionic charge Reprints, Solvent role, "Foreign technology, Potassium salts, N-phenylglycinate.

The adsorption of the potassium salt of $\mathrm{N}$ phenylglycine (KNPG) on synthetic hydroxyapatite from aqueous and ethanol solutions was studied at 22 degrees C. The adsorption isotherm of KNPG from aqueous solutions is Langmuirian in shape, and the analysis showed that one glycinate ion was adsorbed per about two (100) faces of the unit cell of hydroxyapatite. For each glycinate ion adsorbed, 3.5 phosphate ions were released to the aqueous solution while the calcium concentration decreased slightly. The adsorption from ethanol (99.8\%) was total and irreversible from dilute solutions up to a threshold concentration, and reversible and Langmuirian thereafte from concentrated solutions, while the amounts of phosphate and calcium ions in solution are negligible. At maximum coverage from ethanol solution, the surface is fully occupied by reversibly adsorbed molecules which are perched on a one-to-one basis on the top of irreversibly adsorbed molecules totally covering the substrate. The amount of irreversibly adsorbed solute is thus equal to the reversibly adsorbed solute and about twice the maximum amount adsorbed from aqueous solutions

\section{1,160}

PB'96-186143 Not available NTIS

National Inst. of Standards and Technology (CSTL), Boulder, CO. Biotechnology Div. 
Thermodynamics of the Hydrolysis of 3,4,5Trihydroxybenzoic Acid Propyl Ester (nPropylgallate) to 3,4,5-Trihydroxybenzoic Acid in Toluene.

Final rept.

Y. B. Tewari, M. M. Schantz, M. V. Rekharsky, and R. N. Goldberg. 1996, 15p

Pub. in Jnl. of Chemical Thermodynamics, v28 p171185 May 96.

Keywords: "Calorimetry, "Enthalpy, "Equilibrium constants, Gallic acid, Reprints, High performance liquid chromatography, 2-( $\mathrm{N}$-morpholino) ethanesulfonic acid

Equilibrium measurements at several temperatures between $293 \mathrm{~K}$ and $309 \mathrm{~K}$ have been performed on the tannase catalyzed reaction; 3,4,5trihydroxybenzoic acid propyl ester(sin) $+\mathrm{H} 2 \mathrm{O}(\sin )=$ 2.4,5-trihydroxybenzoic acid(sin) + propan-1-01(sin) where $\sin =$ aqueous phosphate buffer, aqueous acetate buffer, and toluene. The change in binding of the hydrogen ion delta(r) $\mathrm{N}(\mathrm{H}+)$ for this biochemical reaction in aqueous solution was calculated both from an equilibrium model for the biochemical reaction and from the dependence of the apparent equilibrium constant on $\mathrm{pH}$. Calorimetric measurements were also performed for this biochemical reaction in aqueous phosphate and 2-( $\mathrm{N}$-morpholino)ethanesulfonic acid (MES) buffers. Standard transformed thermodynamic quantities for the overall biochemical reaction as well as standard thermodynamic quantities for chemical reference reactions that involve specific chemical species have been calculated from the experimental results.

\section{1,161}

PB96-186150 Not available NTIS

National Inst. of Standards and Technology (MSEL) Gaithersburg, MD. Reactor Radiation Div.

Characterization of the Structure of YD3 by Neutron Powder Diffraction.

Final rept.

T. J. Udovic, Q. Huang, and J. J. Rush. 1996, 13p.

Pub. in Jnl. of Physics and Chem Solids, v57 n4 p4234351996.

Keywords: "Alloys, "Inorganic compounds, "Metals, Neutron scattering, Crystal structure, Reprints, "Foreign technology.

Neutron-powder-diffraction (NPD) measurements of the hexagonal rare-earth trideuteride gamma-YD3 were undertaken between 10 and $400 \mathrm{~K}$. Rietveld refinements indicated that the YD3 structure possesses P3cl(D(sup 4)(sub 3d)) symmetry, in agreement with previous NPD studies of YD3 and HoD3. The unit cel is a (sq. root of $3 \times$ sq. root of 3 ) R30 degrees expansion of the conventional hcp unit cell in the ab plane. To accomodate the D atoms, the $c$ axis for YD3 is elongated by approx $15 \%$ (co/ao $=1.8019(1)$, ao $=$ 3.6627(1) Angstrom, at 295K) compared with that for $Y$. The D atoms occupy unusual interstitial positions of a slightly distored, hcp metal lattice, instead of the ideal octahedral (o) and tetrahedral (t) sites. In particular, the hypothetical o-site $\mathrm{D}$ atoms are displaced vertcally toward the $Y$-defined basal planes, with correlated placements at either near-plane or in-plane threefold metal $(m)$ sites. The $t-s i t e ~ D(D t)$ atoms are displaced horizontally, also in a correlated fashion, with respect to the $\mathrm{m}$-site $\mathrm{D}(\mathrm{Dm})$ atoms.

01,162

PB96-186176 Not available NTIS

National Inst. of Standards and Technology (MSEL), Gaithersburg, MD. Ceramics Div.

Preparation, Crystal Structure, Dielectric Properties, and Magnetic Behavior of Ba2Fe2Ti4O13. Final rept.

T. A. Vanderah, Q. Huang, W. Wong-Ng, R. G. Geyer, J. Baker-Jarvis, R. S. Roth, A. Santoro, B. C Chakoumakos, and R. B. Goldfarb. 1995, 7p Pub, in Jnl. of Solid State Chemistry, v120 p121-127 1995

Keywords: "Magnetic properties, "Dielectric constant, Barium iron titanate, Neutron diffraction, Reprints Single crystal structure determination.

The preparation, crystal structure, dielectric properties and magnetic behavior of the new compound Ba2Fe2Ti4O13 are reported. Structural studies carried out by single-crystal $X$-ray diffraction and neutron powder diffraction show that this phase is isostructural with K2Ti6O13 and Ba2ZnTi5O13 (C2/m(No. 12); $a=$ 15.216(1), $b=3.8979(3), c=9.1350$ (6) Angstrom, Beta
$=98.460(7)$ degrees; $V=535.90(8)$ Angstrom; $Z=2$ ) The cations Fe(sup $3+$ ) and Ti(sup 4+) are partially ordered among distorted octahedral sites with $\mathrm{Ba}$ (sup 2+) occupying eleven-coordinated polyhedra. with a dielectric constant of approx. 28 and a dielectric loss tangent of $2 \times 10$ to the minus 3 . The compound displays complex paramagnetic behavior with marked field dependence; the magnetization at $80 \mathrm{kA} / \mathrm{m}$ is sev eral orders of magnitude smaller than that of most ferrites.

01,163

PB96-200274 Not available NTIS

National Inst. of Standards and Technology (PL), Boulder, CO. Quantum Physics Div.

Excitation Transfer in Barium by Collisions with Noble Gases.

Final rept.

J. Brust, and A. C. Gallagher. 1995, 12p.

Grant NSF-PHY90-12244

Sponsored by National Science Foundation, Arlington

Pub. in Physical Review A, v52 p2120-2131 Sep 95

Keywords: "Barium, "Energy transfer, "Noble gases, Reprints, "Molecule-molecule collisions, Transition probabilities.

We present a time-resolved study of excitation transfer processes between the low-lying excited states of barum induced by collisions with noble gases. The transient excited-state populations have been measured by means of fluorescence and absorption spectroscopy. Cross sections for spin-changing collisions from $3 P 1$ to $1 D 2$ and from $1 D 2$ to $3 D 3$ as well as for fine structure mixing within the 3D multiplet were obtained by fitting the numerical solution of a seven-state system of rate equations including diffusion to the experimental data. We compare our results to experimenta findings for other alkaline earth metals and report an improved value of the lifetime of the 6s6p3P1 state of barium.

01,164

PB96-201082 Not available NTIS

National Inst. of Standards and Technology (MEL)

Gaithersburg, MD. Precision Engineering Div.

Scanning Electron Microscope Magnification Calibration Interlaboratory Study.

Final rept.

M. T. Postek, A. E. Vladar, S. N. Jones, and W. J.

Keery. 1993, 2p.

Pub. in Proceedings of the Annual Meeting of the Microscopy Society of America (51st), p1-2 1993.

Keywords: "Electron microscopes, "Scanning, "Calibration standards, Prototypes, Lithography, Electron beams, Fabrication, Design, Reprints.

The National Institute of Standards and Technology (NIST) is in the process of developing a new scanning electron microscope (SEM) magnification calibration reference standard useful at both high and low accelerating voltages. The standard will be useful for all applications to which the SEM is currently being used but it has been specifically tailored to meet many of the particular needs of the semiconductor industry. A small number of test samples with the pattern were prepared on silicon substrates using electron beam lithography at the National Nanofabrication Facility at Cornell University. The structures were patterned in titanium/palladium with maximum nominal pitch of approximately 3000 micro $\mathrm{m}$ scaling down to structures with minimum nominal pitch of 0.4 micro $\mathrm{m}$. Several of these samples were sent out to a number of university, research, semiconductor and their industrial laboratories in an interlaboratory study. The purpose of the study was to test the SEM instrumentation and to review the suitability of the sample design.

\section{1,165}

PB96-201181 Not available NTIS

National Inst. of Standards and Technology (MSEL), Gaithersburg, MD. Ceramics Div.

NMR Characterization of Injection-Moulded Alumina Green Compacts. Part 2. T2-Weighted Proton Imaging.

Final rept.

P. S. Wang, S. G. Malghan, S. J. Dapkunas, K. F.

Hens, and R. Raman. 1995, 6p.

Pub. in Jnl. of Materials Science, v30 p1069-1074

Keywords: "Nuclear magnetic resonance, "Ceramic slurries, "Proton imaging, Injection moulding, Alumina, Reprints, "Water distribution.
Injection-moulded alumina green compacts containing polypropylene, max, and stearic acid were studied for binder distribution by proton nuclear magnetic resonance (1H NMR) imaging. The solid imaging technique of nuclear spin-spin relaxation time, T2, weighted imaging at $400 \mathrm{MHz}$ was used. This imaging technique utilizes a multiple pulse sequence of DO-(phi/2)plus or minus $x$-tau-D10-D7-(phi)x-D7-D10-AQ-D0 for echo detection and phase encoding. Two- and three-dimensional images were constructed from the intensities of these nuclear echo signals. Spatially resolved two-dimensional images obtained by the application of this technique indicated that the green compacts fabricated from the same nominal binder composition did not contain the same amounts of binder. This observation agrees well with our previous conclusion drawn from nuclear spin echo studies by Hahn's pulse sequence. A $64 \times 64 \times 64$ three-dimensional imaging revealed that the inhomogeneity of binder distribution and internal inperfection do exist at certain locations of the samof these green compacts.

\section{1,166}

PB96-204011 Not available NTIS

National Inst. of Standards and Technology (CSTL), Gaithersburg, MD. Chernical Kinetics and Thermodynamics Div.

Enthalpy Increment Measurements from 4.5 to 318 $\mathrm{K}$ for Bismuth(cr). Thermodynamic Properties from $0 \mathrm{~K}$ to the Melting Point.

Final rept.

D. G. Archer. 1995, 10p.

Pub. in Jnl. of Chemical and Engineering Data, v40 p1015-10241995.

Keywords: "Bismuth, "Thermodynamic properties, Reprints, Enthalpy, Temperature scales, Measurements.

Enthalpy increments for bismuth(cr) were measured from 4.5 to $318 \mathrm{~K}$ with an adiabatic calorimeter. The calorimeter's performance was demonstrated through comparison of measured enthalpy increments for copper and aluminum oxide to literature values. The effect that different temperature scales had on these comparisons for copper at low temperatures was discussed. The new enthalpy increments for bismuth(cr) were combined with previously measured thermodynamic properties for temperatures below $4 \mathrm{~K}$ and about $300 \mathrm{~K}$ in order to generate the thermodynamic properties of bismuth $(\mathrm{cr})$ from $0 \mathrm{~K}$ to the melting point.

01,167

PB96-204151 Not available NTIS

National Inst. of Standards and Technology (CSTL), Gaithersburg, MD. Chemical Kinetics and Thermodynamics Div

Deuterium Isotope Effect in Vinyl Radical Com. bination/Dispropor tionationReactions.

Final rept.

A. Fahr, and A. H. Laufer. 1995, 3p.

Pub. in Jnl. of Physical Chemistry, v99 p262-264 1995.

Keywords: "Isotope effect, "Vinyl radical, Reprints, Combination reactions, Disproportionation reactions.

The deuterium isotope effect for the vinyl radical combination and disproportionation reactions have been investigated. Protonated or deuterated vinyl and methyl radicals are produced from the $193 \mathrm{~nm}$ photolysis of protonated or perdeuterated methyl vinyl ketone. On the basis of product yield measurements, no isotope effect for the combination reactions of either vinyl-vinyl or vinyl-methyl has been observed. From the relative yields of ethylene, an isotope effect of $\mathrm{kh} / \mathrm{kd}=1.20$ is determined for the vinyl-vinyl disproportionation reaction.

\section{1,168}

PB96-204169 Not available NTIS

National Inst. of Standards and Technology (CSTL), Gaithersburg, MD. Chemical Kinetics and Thermodynamics Div.

Temperature Dependence of the Ultraviolet $A b$ sorption Cross Section of CF3l.

Final rept.

A. Fahr, A. K. Nayak, and R. E. Huie. 1995, 10p

Pub. in Chemical Physics, v199 p275-284 1995.

Keywords: "Ultraviolet spectra, "Temperature dependence, Reprints, Temperatures, Absorption, Cross sections, "CF3l.

The ultraviolet absorption cross section of CF3I has been measured in the gas phase over the wavelength range $160-240 \mathrm{~nm}$ for temperatures $240,295,355 \mathrm{~K}$ 
and over the wavelength range $240-350 \mathrm{~nm}$ for temperatures ranging from 218 to $333 \mathrm{~K}$. Two intense and sharp absorption bands centered at about 160 and 171 $\mathrm{nm}$ and a broad and relatively strong band centered at about $267.5 \mathrm{~nm}$ were observed. The absorption band centered at $171 \mathrm{~nm}$ shows significant vibrational structure. The cross sections, at absorption peaks, between 160 and $175 \mathrm{~nm}$, increased by increasing temperature. At the near-UV maximum of $267.5 \mathrm{~nm}$, the absorption increased with decreasing temperature, but decreased at the lowest temperature. At wavelengths greater than about $280 \mathrm{~nm}$, the absorption cross section decreased with decreasing temperature. The absorption cross sections were found to all fit a triple Gaussian expression for lambda greater than $250 \mathrm{~nm}$.

\section{1,169}

\section{PB96-204177 Not available NTIS}

National Inst. of Standards and Technology (CSTL), Gaithersburg, MD. Chemical Kinetics and Thermodynamics Div

Temperature Dependent Ultraviolet Absorption Cross Sections of Propylene, Methylacetylene and Vinylacetylene.

Final rept.

A. Fahr, and A. Nayak. 1996, 8p.

Pub. in Chemical Physics, v203 p351-358 1996.

Keywords: "Temperature dependence, "Ultraviolet, "Absorption cross sections, Reprints, Propylene, Methylacetylene, Vinylacetylene, Planetary atmosphere.

The temperature dependence of the UV absorption cross sections of propylene $\left(\mathrm{CH}_{3} \mathrm{CH}=\mathrm{CH} 2\right)$ and the methylacetylene $(\mathrm{CH} 3 \mathrm{C}$ identical with $\mathrm{CH}$ ) from 160 to $200 \mathrm{~nm}$ and vinylacetylene $(\mathrm{CH} 2=\mathrm{CHC}$ identical with $\mathrm{CH}$ ) from 160 to $240 \mathrm{~nm}$ have been measured in the gas phase, for temperatures ranging from about 220 to $330 \mathrm{~K}$. The propylene spectrum exhibits a broad absorption band with a maximum at $172.0 \mathrm{~nm}$ with some structure to the short wavelength side of the broad band. At the absorption peaks a small increase (about $5 \%$ ) in the cross sections has been observed with decreasing temperature from 333 to $233 \mathrm{~K}$. At the long wavelength tail of the absorptionn band the cross sections are found to decrease with decreasing temperature. The absorption spectrum of methylacetylene in the region of 160 to $200 \mathrm{~nm}$ consists of a broad continuum with a maximum at $172.4 \mathrm{~nm}$. The cross section values, for methylacetylene, near the absorption peak are found to remain nearly independent of temperture. However, at the long wavelength side of the absorption band the cross sections increase with increasing temperature. The spectrum of vinylacetylene consists of a very strong and structured absorption in the 160 to $170 \mathrm{~nm}$ region and strong diffuse bands between around 190 and $230 \mathrm{~nm}$. The cross section values at the absorption peaks, determined at low temperatures, are significantly higher than those at higher temperatures.

\section{1,170}

\section{PB96-210711 PC A04/MF A01}

National Inst. of Standards and Technology (CAML), Gaithersburg, MD. Applied and Computational Mathematics Div

Diffuse-Interface Description of Fluid Systems.

D. M. Anderson, and G. B. McFadden. Aug 96, 37p, NISTIR-5887.

Figures in this document may not be legible in microfiche.

Keywords: "Critical point, "Diffuse interface, Hydrodynamics, Surface tension, Internal waves, Models, Fluid-fluid systems.

The authors consider a diffuse-interface model for fluid-fluid systems. In classical models, an iterface between two fluids is treated as infinitely thin, or sharp, and is endowed with properties such as surface tension. Diffuse-interface theories replace this sharp interface with continuous variation of an order parameter such as density in a way consistent with microscopic theories of the interface. Surface tension effects, for example, are incorporated into the model through a modified stress tensor in the classical Navier-Stokes equations. The authors relate the diffuse-interface model to classical, sharp interface models by deriving asymptotically the governing equations and the associated boundary conditions used in the sharp-interface formulation. The authors illustrate the diffuse-interface approach by modeling internal gravity waves, which have been observed experimentally be Berg et al. in xenon near its critical point. The authors obtain static density profiles, compute internal wave frequencies and compare with their experimental data and theoretical (classical) results both above and below the critical temperature. The results reveal a singularity in the diffuse-interface model in the limit of incompressible perturbations.

\section{1,171}

PB97-1091:6 (Order as PB97-109011, PC A11/

MF A03)

National Irist. of Standards and Technology, Gaithersburg, MD.

Biological Macromolecule Crystallization Database and NASA Protein Crystal Growth Archive.

G. L. Gilliland, M. Tung, and J. Ladner. 1996, $12 p$.

Prepared in cooperation with Maryland Univ., Rockville. Center for Advanced Research in Biotechnology. Included in Jnl. of Research of the National Institute of Standards and Technology, v101 n3 p309-320 May/ Jun 96

Keywords: "Crystallography, "X ray diffraction, Data bases, Information systems, Crystal structure, Three dimensional models, Microgravity, Nucleic acids, Pesticides, Proteins, "Biological macromolecules.

The NIST/CARB Biological Macromolecule Crystallization Datbase (BMCD), NIST Standard Reference Database 21 , contains crystal data and crystallization conditions for biological macromolecules. The database entries include data abstracted from published crystallographic reports. Each entry consists of information describing the biological macromolecule crystallization conditions for each crystal form. The BMCD serves as the NASA Protein Crystal Growth Archive in that it conains protocols and results of crystallization experiments undertaken in microgravity (space). These database entries report the results, whether successful or not from NASA-sponsored protein crystal growth experiments in microgravity and from microgravity crystallization studies sponsored by other international organizations. The BMCD was designed as a tool to assist $x$-ray crystallographers in the development of protocols to crystallize biological macromolecules, those that have previously been crystallized, and those that have not been crystallized.

\section{1,172}

PB97-109151 (Order as PB97-109011, PC A11/ MF A03)

Du Pont de Nemours (E.I.) and Co., Wilmington, DE Central Research and Development Dept.

Troublesome Crystal Structures: Prevention, Detection, and Resolution.

R. L. Harlow. 1996, 13p.

Included in Jnl. of Research of the National Institute of Standards and Technology, v101 n3 p327-339 May/ Jun 96.

Keywords: "Crystallography, *Error analysis, "Information systems, Data bases, Performance evaluation, Crystal structure.

A large number of incorrect crystal structures is being published today. These structures are proving to be a particular problem to those of us who are interested in comparing structural moieties found in the databases in order to develop structure-property relationships. Problems can reside in the input data, e.g. wrong unit cell or low quality intensity data, or in the structural model, e.g., wrong space group or atom types. Many of the common mistakes are, however relatively easy to detect and thus should be preventable; at the very least, suspicious structures can be flagged, it not by the authors then by the references and, ultimately, the crystallographic databases. This article describes some of the more common mistakes and their effects on the resulting structures, lists a series of tests that can be used to detect incorrect structures, and makes a strong plea for the publication of higher quality structures.

\section{1,173}

PB97-110118 Not available NTIS

National Inst. of Standards and Technology (CSTL), Gaithersburg, MD. Physical and Chemical Properties Div.

Compressed Liquid Densities, Saturated Liquid Densities, and Vapor Pressures of 1,1Difluoroethane.

Final rept.

D. R. Defibaugh, and G. Morrison. 1996, 6p.

Pub. in Jnl. of Chemical and Engineering Data, v41 n3 p376-381 1996.

Keywords: "Vapor pressures, "Liquid densities, Compression, Saturation, Measurements, Correlations, Comparisons, Reprints, "Difluoroethane.
The compressed liquid densities and vapor pressures of 1,1,-difluoroethane (HFC-152a) have been measured, correlated, and compared with other data. The liquid densities were measured with a combined standard uncertainty of plus or minus $0.05 \%$ using a vibrating tube densimeter over a temperature range of 243 $\mathrm{K}$ to $371 \mathrm{~K}$ and at pressures from near the saturated vapor pressure to $6500 \mathrm{kPa}$; thus the data extend nearly to the critical point ( $T C=386.41 \mathrm{~K}$ and $\mathrm{PC}=4514.7$ $\mathrm{kPa}$ ). The vapor pressures were measured with a combined standard uncertainty of plus or minus $0.02 \%$ using a stainless steel ebulliometer in the temperature range from $280 \mathrm{~K}$ to $335 \mathrm{~K}$. Saturated liquid densities were calculated by extrapolating the compressed liquid isotherms to the saturation pressure.

\section{1,174}

PB97-110241 Not available NTIS

National Inst. of Standards and Technology (CSTL), Gaithersburg, MD. Biotechnology Div.

Electrolytes Constrained on Fractal Structures: Debye-Huckel Theory.

Final rept.

S. H. Lee, and J. B. Hubbard. 1993, 5p

Pub. in Jnl. of Chemical Physics, v98 n2 p1504-1508 Jan 93.

Keywords: "Debye-Huckel theory, "Fractal structures, Electrolytes, Ionic screening. Thermodynamic properties, Reprints.

The authors calculate, via the Debye-Huckel theory, the effect of ionic screening on the thermodynamic properties of an electrolyte confined topologically to an isotropic random fractal structure embedded in three dimensiors. It is found that screening effects are generally imp ortant, even for low fractal dimensions. In many instances crossover effects occur, whereby with increasing ionic strength, a low dimension fractal is more stabilized by screening than a fractal of higher dimension.

\section{1,175}

PB97-110365 Not available NTIS

National Inst. of Standards and Technology (CSTL) Gaithersburg. MD. Chemical Kinetics and Thermodynamics Div.

Isopiestic Investigation of the Osmotic and Activity Coefficients of Aqueous $\mathrm{NaBr}$ and the Solubility of $\mathrm{NaBr} 2 \mathrm{H} 2 \mathrm{O}(\mathrm{cr})$ at 298.15 K: Thermodynamic Properties of the $\mathrm{NaBr}+\mathrm{H} 2 \mathrm{O}$ System over Wide Ranges of Temperature and Pressure.

Final rept.

J. A. Rard, and D. G. Archer. 1995, 16p.

Pub. in Jnl. of Chemical and Engineering Data, n40 p170-185 1995.

Keywords: "Sodium bromide, "Thermodynamics, Isopiestic, Activity, Solubility, Reprints, CODATA key values.

Isopiestic vapor-pressure measurements have been performed for aqueous solutions of well-characterized high-purity $\mathrm{NaBr}$ from 1.9551 to $9.4778 \mathrm{~mol}-\mathrm{kg}(-1)$ at $298.15 \mathrm{~K}$; the highest five molatities correspond to supersaturated concentrations. Solubilities have also been determined by the methods. A few equilibrations were made between solutions of $\mathrm{NaCl}$ and $\mathrm{H} 2 \mathrm{SO} 4$ to refine the osmotic coefficients of $\mathrm{H}_{2} \mathrm{So} 4$ at high molalities. These isopiestic results for $\mathrm{NaBr}$ have been combined with other experimental thermodynamic quantities (vapor pressures, activity coefficients, solubilities, freezing temperatures, and volumetric and calorimetric measurements) to yield revised parameters for an extended form a Pitzer's equation applicable over wide ranges of molality, temperature, and pressure. It was not possible to obtain a complete consistency between the experimental results for either the $\mathrm{NaBr}+\mathrm{H} 2 \mathrm{O}$ system or the $\mathrm{NaCl}+\mathrm{H} 2 \mathrm{O}$ system with entropies from the CODATA Key Values for Thermodynamics.

01,176

PB97-111223 Not available NTIS

National Inst. of Standards and Technology (CSTL), Gaithersburg, MD. Chemical Kinetics and Thermodynamics Div.

Resonance Enhanced Multiphoton Ionization Spectroscopy of the SnF Radical.

Final rept.

J. Pearson, R. N. Dixon, J. W. Hudgens, and R. D.

Johnson. 1996, $5 p$

See also PB90-170028.

Pub. in Jnl. of Chemical Physics, v104 n12 p440644101996. 
Keywords: *Chemical radicals, "Tin monofluoride, Visible spectrum, Vibrational spectra, Reprints,
$*$ Multiphoton ionization, "Multi-photon processes, Rydberg states.

The resonance enhanced multiphoton ionization spectrum of the free radical $\mathrm{SnF}$ has been recorded using excitation wavelengths in the range 540 to $360 \mathrm{~nm}$. The previously documented C2(delta), D, E(2)II, and $F(2)$ sigma states and two hitherto unidentified excited states, the $H$ and I(2)phi states, were all observed as two-photon resonances. Band contour simulations yielded spectroscopic constants for the E, F, and I states.

\section{1,177}

\section{PB97-111314 Not available NTIS}

National Inst. of Standards and Technology (CSTL), Gaithersburg, MD. Chemical Kinetics and Thermodynamics Div.

Fluoride Elimination Upon Reaction of Pentafluoroaniline with e (sub eq')(sup -), $\mathrm{H}$, and $\mathrm{OH}$ Radicals in Aqueous Solution.

Final rept.

L. C. T. Shoute, J. P. Mittal, and P. Neta. 1996, 5p. Pub. in Jnl. of Physical Chemistry, v100 n27 p11355 113591996.

Keywords: "Pentafluoroaniline, *Pulse radiolysis, Absorption spectra, Reduction, Oxidation, Reprints.

Reduction of pentafluoroaniline (PFA) leads to rapid fluoride elimination to form the aminotetrafluorophenyl radical. This radical undergoes rapid intramolecular electron transfer from the amino group to the phenyl radical site and protonates at the latter site to form the tetrafluoroaniline radical cation or its deprotonated form $(\mathrm{pKa}=2.3$ ). Oxidizing radicals such as $\mathrm{SO} 4-\mathrm{N} 3$ and $\mathrm{Cl}$ - oxidize PFA to the pentafluoroaniline radical cation. The $\mathrm{pKa}=1.9$ of the pentafluoroaniline radical cation is 3.6 units lower than that expected from the substituent effects of fluorine atoms. Addition of $\mathrm{OH}$ to PFA is followed by rapid HF elimination to yield the aminotetrafluorophenoxyl radical. In acidic solution, however, the reaction of $\mathrm{OH}$ leads to formation of the pentafluoroaniline radical cation. Fluo ride elimination upon reduction and upon $\mathrm{OH}$ addition to PFA was confirmed by determination of fluoride ion yield.

\section{1,178}

\section{PB97-111538 Not available NTIS}

National Inst. of Standards and Technology (CSTL) Gaithersburg, MD. Chemical Kinetics and Thermodynamics Div.

Ionization Energies, Appearance Energies and Thermochemistry of CF2O and FCO.

Final rept.

T. J. Buckley, R. D. Johnson, R. E. Huie, R. B

Klemm, Z. Zhang, and S. C. Kuo. 1995, $7 p$

Pub. in Jnl. of Physical Chemistry, v99 n14 p487948851995.

Keywords: "Ionization energy, "Photoionization, Discharge flow, Mass spectrometry, Formation heat, Bond strength, Appearance energy, Reprints.

With the discharge flow-photoionization mass spectrometer (DF-PIMS) coupled to the U-11 beamline a the National Synchrotron Light Source, the authors have measured the ionization energies of $\mathrm{C} 2 \mathrm{~F} 4, \mathrm{CF} 4$ and $\mathrm{CF} 2 \mathrm{O}$ and appearance energy of FCO+ from CF2O. The PIMS results corroborate those determined by other techniques. With high-level ab initio calculations that utilize a large basis set and isogyric corrections, they have determined an ionization energy of 9.3 plus or minus $0.1 \mathrm{eV}$ for FCO. At $298 \mathrm{~K}$ the heats of formation of FCO $(-152$ plus or minus $12 \mathrm{~kJ} / \mathrm{mol})$ and $\mathrm{FCO}+(745.3$ plus or minus $9.6 \mathrm{~kJ} / \mathrm{mol}$ ) (relative to (delta)fH $298\left(C_{2} 2 \mathrm{O}\right)=-607.9$ plus or minus $7.1 \mathrm{~kJ} / \mathrm{mol}$ ) were determined and agree to within the uncertainties of other measurements but with much higher precision. The authors have evaluated the bond strengths $D$ 298 (F-CFO) and D 298 (F-CO) to be 535 plus or minus 12 and 121 plus or minus $12 \mathrm{~kJ} / \mathrm{mol}$, respectively.

\section{1,179}

PB97-111967 Not available NTIS

National Inst. of Standards and Technology (CSTL) Gaithersburg, MD. Chemical Kinetics and Thermodynamics Div.

One-Electron Oxidation of Metalloporphycenes as Studied by Radiolytic Methods.

Final rept

D. M. Guldi, J. Field, J. Grodkowski, P. Neta, and E.

Vogel. 1996, 6p.

Pub. in Jnl. of Physical Chemistry, v100 n32 p13609 136141996.
Keywords: "Metalloporphycenes, "Radiolysis, Oxidation, Porphycenes, Pulse radiolysis, Absorption spectra, Reprints.

One-electron and two-electron oxidation of 2,7,12,17tetrapropylporphycene ( $\mathrm{H} 2 \mathrm{TPrPC}$ ) and its $\mathrm{Fe}, \mathrm{Co}, \mathrm{Ni}$, $\mathrm{Cu}$, and $\mathrm{Sn}$ complexes in $\mathrm{Ch} 2 \mathrm{Cl} 2, \mathrm{CCl} 4$, and 2-PrOH solutions have been studied by radiolytic techniques. Formation and decay of intermediates formed upon one-electron oxidation have been followed by kinetic spectrophotometric pulse radiolysis, and the absorption spectra of stable oxidation products have been recorded following gamma-radiolysis. H2TPrPc is oxidized to the pi-radical cation and then to the dication, which is stable in aprotic solvents but is transformed to a different product in 2-PrOH. Similar oxidation to the pi-radical cation and then to the dication was observed for the Cull, SnIV, CollI, and Fell porphycenes. Coll and Nill porphycenes underwent radiolytic oxidation to form stable Colll and Nill products. The stability of the latter is in contrast with previous electrochemical observations, the difference is ascribed tc the effect of the axial ligand.

01,180

PB97-112361 Not available NTIS

National Inst. of Standards and Technology (CSTL), Gaithersburg, MD. Chemical Kinetics and Thermodynamics Div.

Absorption Cross Sections, Kinetics of Formation, and Self-Reaction of the IO Radical Produced via the Laser Photolysis of N2O//2/N2 Mixtures.

Final rept.

1995, 7p.

Sponsored by Department of the Air Force, Washington, DC. and Department of the Army, Washington, DC

Pub. in Jnl. of Physical Chemistry, v99 p11701-11707 1995.

Keywords: "Iodine monoxide, "Laser photolysis, Atmosphere chemistry, Kinetics, Ozone, Spectra, Reprints.

The laser photolytic production of the 10 radical from $\mathrm{N} 2 \mathrm{O} / 12 / \mathrm{N} 2$ mixtures was used to measure the radical absorption spectrum from 340 to $450 \mathrm{~nm}$. Two new absorption peaks at 403 and $411 \mathrm{~nm}$ were observed along with an underlying continuum starting at about $420 \mathrm{~nm}$ and extended to about $350 \mathrm{~nm}$. An absorption cross section (2.8 plus or minus 0.5$) \times 10(-17) \mathrm{cm}(2)$, in good agreement with previous measurements, was determiend for the (4-0) band head at $427.2 \mathrm{~nm}$. From the rate of formation of 10 and the rate of loss of 12 , rate constants for the reactions $\mathrm{O}=12 \rightarrow 10+1$ and $\mathrm{O}+\mathrm{Ol} \rightarrow \mathrm{O} 2+\mathrm{I}$ of $(1.4$ plus or minus 0.4$) \times 10(-10)$ and (1.2 plus or minus 0.5$) \times 10$ to the minus 10 power $\mathrm{cm}(3) \mathrm{s}(-1)$, respectively, were derived. Finally, from the rate of decay of 10 , a rate constant for the reaction $10+10 \rightarrow$ products of (8.0 plus or minus 1.8$) \times 10(-$ 11) $\mathrm{cm}(3) \mathrm{s}(-1)$ was obtained. All of the uncertainties expressed are 2 standard deviations derived from the statistical analysis and do not include estimates for possible systematic errors.

01,181

PB97-112528 Not available NTIS

National Inst. of Standards and Technology (CSTL), Gaithersburg, MD. Chemical Kinetics and Thermodynamics Div.

Thermochemical Studies of Inorganic Chalocogenides by Fluorine-Combustion Calorimetry: Binary Compounds of Germanium Final rept.

P. A. G. O'Hare. 1995, 13p.

Pub. in Thermochimica Acta, v267 p 1-13 1995.

Keywords: "Chalcogenides, "Thermochemistry, "Enthalpy, Dissociation, Germanium silicides, Germanium selenides, Silicon compounds, Tellurides, Selenium, Reprints, "Fluorine-combustion calorimetry.

A general outline is given of the technique of fluorinebomb calorimetry, including equipment, experimental procedures, sample purity requirements, post-combustion analyses, and interpretation of the results. Some recent measurements are described on $\mathrm{Si} 2 \mathrm{Te} 3$, SiSe2, $\mathrm{GeS}$, and GeS2. How the thermochemistry-based results for the dissociation enthalpies D(sup o)(sub $\mathrm{m}$ ) of the gaseous diatomic molecules SiTe, SiSe, GeS, and GeSe correlate with the $\mathrm{D}$ (sup o)(sub $\mathrm{m}$ ) derived from spectroscopy is also discussed.

01,182

PB97-112536 Not available NTIS National Inst. of Standards and Technology (CSTL),
Gaithersburg, MD. Chemical Kinetics and Thermodynamics Div.

Thermodynamics of (Germanium + Selenium): A Review and Critical Assessment

Final rept.

P. A. G. O'Hare, A. Zywocinski, and L. A. Curtiss.

$1996,22 p$.

Pub. in Jnl. of Chemical Thermodynamics, v28 p4594801996.

Keywords: "Thermodynamic properties, "Germanium, "Selenium, Thermochemistry. Molecular structure, Heat capacity, Combustion kinetics, Heat of formation, Enthalpy, Dissociation, Gibbs free energy Wavenumbers, Reprints, Bond energy.

The review deals with the critical assessment of the thermodynamic properties of several germanium selenides: GeSe(cr), GeSe(g), GeSe2(cr), GeSe2(g) and $\mathrm{Ge} 2 \mathrm{Se} 2(\mathrm{~g})$. In the absence of experimental intormation, the structures, internuclear distances, and vibrational wavenumbers were calculated for GeSe2(g) and $\mathrm{Ge} 2 \mathrm{Se} 2(\mathrm{~g})$ by molecular orbital methods.

01,183

PB97-113112 Not available NTIS

National Inst. of Standards and Technology (MSEL) Gaithersburg, MD. Reactor Radiation Div.

Molecular Dynamics Investigation of the Surface Bulk Equilibrium in an Ethanol-Water Solution. Final rept

M. Tarek, D. J. Tobias, and M. L. Klein. 1996, 5p. Pub. in Jnl. of the Chemical Society, Faraday Trans. v92 n4 p559-563 1996.

Keywords: "Ethanol water solution, *Molecular dynam ics, "Surface tension, Surface excess, Hydrogen bonding, Density profiles, Air liquid interface, Reprints *Foreign technology.

Molecular dynamics simulations of the vapor/solution interface, initiated from an ethanol-water mixture at a mole traction of ethanol, $X_{C}=0.1$, have been performed. Rapid redistribution of ethanol molecules to the free surface was observed during simulations two different sized systems. Inspection of the calculated surface structure reveals that after redistributing the ethanol molecules are preferentially oriented such that the alkyl group points out the solution. A 'depletion layer' of enhanced water density beneath the ethanol surface excess was revealed by the simulation. Analysis of the structure involving molecules close to the surface shows that water molecules are arranged to maximize the hydrogen bonding between the oriented ethanol and the adjacent water molecules. The enhanced water density beneath the interface re sults from this optimization. The surface tension calculated from the simulation is ca, $50 \%$ higher than the results of macroscopic experimental measurements. This overestimation is attributed to shortcomings to both the potential function and the simulation methodology. The number of density profiles of the ethanol surface excess were compared with results from neutron reflectivity measurements. The calculated profiles agree better with the experimental measurements at the calculated surface tension (i.e. $X_{c}=0.022$ ) than those reported for $X_{c}=0.1$ mixtures.

01,184

PB97-118343 Not available NTIS

National Inst. of Standards and Technology (PL), Gaithersburg, MD. Molecular Physics Div.

Excitons in Complex Quantum Nanostructures. Final rept.

G. W. Bryant, P. S. Julienne, and Y. B. Band. 1996, $4 p$.

Pub. in Surface Science, n361/362 p801-804 Oct 96

Keywords: "Excitons, "Quantum wells, Reprints, Nanostructures, Semiconductor-semiconductor heterostructures, Semi-empirical models, Model calculations.

A theory for excitons in complex quantum nanostructures with complicated geometries and strong coupling between nanostructures is presented. Effective mass models with screened Coulomb interaction give reasonable ground-state energies for excitons in T-shaped quantum wires, coupled T-quantum wires and quantum dot quantum wells, provided the effects of complex geometry are included. A siz able shift between exciton states in quantum wells and T-shaped wires occurs due to a $40 \%$ enhancement in binding energy, confirnment in the wire, and significant interwire coupling. 


\section{1,185}

PB97-118350 Not available NTIS

National Inst. of Standards and Technology (PL),

Gaithersburg, MD. Atomic Physics Div.

Quantum Dots in Quantum Well Structures.

Final rept.

G. W. Bryant. 1996, $12 p$.

Pub. in Jnl. of Luminescence, v70 p108-119 Nov 96.

Keywords: *Quantum dots, "Energy levels, Quantum wells, Strain, Reprints.

Recent progress toward fabricating and characterizing quantum dots in III-V quantum well structures is reviewed. Quantum dots made by use of lithography and etching, including deep-etched, barrier-modulated strain-induced and interdiffused quantum dots, are described. Quantum dots fabricated by growth, including natural quantum dots, dots on patterned substrates and self-assembled dots, are discussed. Dot sizes and uniformity, energy-level splittings, and luminescence efficiencies that are now being achieved are discussed. The status of key issues, such as the energy relaxation in quantum dots, is mentioned.

\section{1,186}

PB97-119127 Not available NTIS

National Inst. of Standards and Technology (CSTL), Gaithersburg, MD. Chemical Kinetics and Thermodynamics Div.

Kinetics of the Reaction of the Sulfate Radical with the Oxalate Anion.

Final rept.

R. E. Huie, and C. L. Clifton. 1996, 5p

Pub. in International Jnl. of Chemical Kinetics, v28 p195-199 1996.

Keywords: "Atmospheric chemistry, ${ }^{\star}$ Chemical kinetics, *Sulfate radicals, Oxalate anions, Reprints.

The kinetics of the reaction of the sulfate radical, $\mathrm{SO} 4$, with the oxalate anion, $\mathrm{C} 2 \mathrm{O} 4(2-)$, was studied in aqueous solution and second-order rate constants, corrected for the effects of ionic strength, derived. Measurements were carried out over the temperature range 24 - 60 degrees $C$ resulting in the expression $\mathrm{k}(0)=$ 2.10 plus or minus $0.96 \times 10$ to the 8th power $\exp$ 1080 plus or minus $140 / \mathrm{T}$ ) L mol to the minus 1 power $s$ to the minus 1 power.

01,187

PB97-119143 Not available NTIS

National Inst. of Standards and Technology (PL),

Gaithersburg, MD. Molecular Physics Div.

Matrix Isolation Study of the Interaction of Excited Neon Atoms with BCI3: Infrared Spectra of BCl(sub 3 , sup + ), $\mathrm{BCl}($ sub 2 , sup + ), and $\mathrm{BCl}$ (sub 3 , sup -).

M. E. Jacox, K. K. Irikura, and W. E. Thompson.

$1996,8 p$.

See also PB97-112403.

Pub. in Jnl. of Chemical Physics, v104 n22 p88718878 Jun 96.

Keywords: "Neon, "Molecular ions, "Vibrational spectra, Boron chlorides, Interactions, Infrared spectra, Reprints, "Matrix isolation.

When a $\mathrm{Ne}: \mathrm{BCl} 3$ sample is codeposited at approximately $5 \mathrm{~K}$ with a beam of neon atoms that have been excited in a microwave discharge, the infrared spectrum of the resulting solid deposit shows a weak to moderately intense absorption of $\mathrm{BCl} 2$ and more prominent absorptions which are assigned to the v3 fundamentals of $\mathrm{BCl} 3$ (D3h) and of linear, centrosymmetric $\mathrm{BCl} 2$. The boron- and chlorine-isotopic structure of the spectrum is consistent with both of these assignments. Ab initio calculations support the $\mathrm{BCl} 2$ assignment. An absorption is also tentatively as signed to $\mathrm{V} 3(\mathrm{e})$ of $\mathrm{BCl}$. Ab initio calculations for $\mathrm{BCl} 3$ are consistent with that assignment. The processes which occur when the solid deposit is exposed to visible and ultraviolet radiation are considered.

01,188

PB97-119234 Not available NTIS

National Inst. of Standards and Technology (PL), Gaithersburg, MD. Optical Technology Div.

Vibrational Spectra of Molecular lons Isolated in Solid Neon. 13. Ions Derived from $\mathrm{HBr}$ and $\mathrm{HI}$.

Final rept.

C. L. Lugez, M. E. Jacox, and W. E. Thompson.

1996, 10p

See also PB95-125688.
Pub. in Jnl. of Chemical Physics, v105 n10 p390139101996.

Keywords: "Molecular ions, "Vibrational spectra, Infrared absorption, Infrared spectra, Electron capture, Matrix isolation, Reprints, *Solid neon.

When a $\mathrm{Ne}: \mathrm{HBr}$ or a $\mathrm{Ne}: \mathrm{HI}$ sample is codeposited at approximately $5 \mathrm{~K}$ with discharge-excited neon atoms, the infrared spectrum of the resulting solid includes not only the absorptions of the $\mathrm{HX}$ molecule and its multimers but also a prominent absorption of $\mathrm{HBr}+$ or $\mathrm{HI}+$. The absorption of each of the two cations lies close to its gas-phase band center. The vibrational fundamentals of $\mathrm{DBr}+$ and $\mathrm{Dl}+$ are also identified in experiments on deuterium-enriched samples. Other infrared absorptions are assigned to normal and deuteriumsubstituted (HBr)2+ and ( $\mathrm{HI} 2+$, as well as to $\mathrm{BrHBr}$ and IHI-. Studies of changes in the absorption spectrum which result from exposure of the deposit to filtered visible and near ultraviolet radiation support these assignments and provide further information on photodissociation and photodetachment processes which occur in these systems.

01,189

PB97-119341 Not available NTIS

National Inst. of Standards and Technology (PL),

Gaithersburg, MD. Optical Technology Div.

Self-Consistent ' $G W$ ' and Higher-Order Calculations of Electron States in Metals.

Final rept.

E. L. Shirley. 1996, 7p

96.

Keywords: "Electron states, "Metals, Self-consistent, Vertex correction, Bandwidths, Reprints

Past work, treating simple metals in the GW approximation, has largely neglected effects of self-consistency and higher-order vertex corrections on occupied bandwidths. The work presents self-consistent GW results, plus nearly self-consistent higher-order results, for jellium, iliustrating that both effects are large, yet largely canceling (e.g., $0.65-\mathrm{eV}$ effects on the sodium bandwidth, but a combined effect of only $0.13 \mathrm{eV}$ ). This supports findings that many-body effects substantially reduce such bandwidths.

\section{1,190}

PB97-119358 Not available NTIS

National Inst. of Standards and Technology (CSTL), Gaithersburg, MD. Chemical Kinetics and Thermodynamics Div.

Thermodynamic Properties of Silicides. 5. Standard Molar Enthalpy of Formation at the Temperature $298.15 \mathrm{~K}$ of Trimolybdenum Monosilicide Mo3si Determined by Fluorine-Combustion Calorimetry.

Final rept

I. Tomaszkiewicz, G. A. Hope, C. M. Beck, and P. A. G. O'Hare. 1996, 14p.

Pub. in Jnl. of Chemical Thermodynamics, v28 p29421996

Keywords: "Thermochemistry, "Heat capacities, *Enthalpy increments, Silicides, Temperatures, Calorimetry, Reprints, Trimolybdenum monosilicide.

The massic (formerly called specific) energies of combustion in fluorine of two different specimens of trimolybdenum monosilicide have been measured in a bomb calorimeter and the standard molar enthalpy of formation delta(f) $\mathrm{H}(\mathrm{m})$ (Mo3Si, $\mathrm{Cr}, 298.15 \mathrm{~K}$ ) determined to be $-(125.2$ plus or minus 5.8$) \mathrm{kJ}$-mol to the minus one power. A critical evaluation of the thermodynamic properties of $\mathrm{Mo3Si}$ is also presented, and recommended values for the standard molar enthalpy increments, standard molar heat capacities, standard molar enthalpies of formation, and standard molar Gibbs free energeis of formation have been tabulated to $\mathrm{T}=2300 \mathrm{~K}$

01,191

PB97-122402 Not available NTIS

National Inst. of Standards and Technology (CSTL), Gaithersburg, MD.

Oxidation of Ferrous and Ferrocyanide lons by Peroxyl Radicals.

Final rept.

G. I. Khaikin, Z. B. Alfassi, R. E. Huie, and P. Neta.

1996, 6p

Pub. in Jnl, of Physical Chemistry, v100 n17 p707270771996.

Keywords: "Oxidation, "Iron ions, "Peroxyl radicals, Ferrous ions, Ferrocyanide ions, Reprints.
Alkylperoxyl and arylperoxyl radicals were produced by pulse radiolysis in aqueous solutions, and their reactions with ferrous and ferrocyanide ions were studied by kinetic spectrophotometry. Oxidation of Fe(CN)6(4) took place with rate constants that varied from less than $1 \times 10$ to the 5 th power to $5 \times 10$ to the 7 th power $L$ mol(-1)s(-1), depending on the electron-withdrawing effects of the substituents on the peroxyl radical and presumably reflecting variations in reduction potentia of the peroxyl radical, as expected for outer-sphere electron transfer. Oxidation of $\mathrm{Fe}(\mathrm{aq})(2+)$, on the othe hand, took place by an inner-sphere mechanism controlled by the rate of dissociative interchange of the water ligand. The rate constants were nearly the same for all the peroxyl radicals examined $(k=(0.5-1.1) x$ 10 to the 6th power $L$ mol(-1)s(-1)) and involved the formation of a transient intermediate, $\mathrm{RO} 2(-) \mathrm{Fe}(3+)$, which later decomposed to yield $\mathrm{Fe}(\mathrm{aq})(3+)$. The decomposition was accelerated by $\mathrm{H}(+)$ and by $\mathrm{Fe}(2+)$. The proposed mechanism is a modification of a previously suggested reaction scheme.

\section{1,192}

PB97-122451 Not available NTIS

National Inst. of Standards and Technology (PL), Gaithersburg, MD. Molecular Physics Div.

Perpendicular C-H Stretching Band nug/nu13 and the Torsional Potential of Dimethylacetylene.

Final rept.

J. Pliva, A. S. Pine, and S. Civis. 1996, $11 p$

Pub. in Jnl. of Molecular Spectroscopy, Article No. 0220, v180 p15-25 1996

Keywords: *Free rotors, *Infrared spectrums, Internal rotation, Torsional potential, Tunable laser, Reprints, *Dimethylacetylene.

The perpendicular band of the fourfold degenerate $\mathrm{C}$ $\mathrm{H}$ stretching vibration of dimethylacetylene (2-butyne) occurring in the region 2955-3065 $\mathrm{cm}(-1)$ has been measured with Doppler-limited resolution at a temperature of approximately $195 \mathrm{~K}$ on a difference-frequency laser spectrometer, and on a high-resolution Fourier transform instrument at room temperature as well as in a molecular jet at approximately $20 \mathrm{~K}$. From a detailed rotational analysis of this complex band, based on an extension of the mode of the rotational-torsiona interactions described by $P$. R. Bunker and C. di Lauro (Chem. Phys, 190, 159-169, 1995) accurate spectroscopic constants were determined for the upper $\mathrm{CH}$ stretching state, along with values for the first three components of the Fourier expansion for the torsional potential in the ground state, and for six components of this potential in the upper state. Over 700 lines assigned to 37 well-resolved subbands plus 25 additional unresolved line-like $Q$-branches were fitted with an overall standard deviation of $0.0024 \mathrm{~cm}(-1)$. the results yield a value of 6.316 plus or minus $0.034 \mathrm{~cm}(-1)$ for the barrier to internal rotation in the ground state, and 6.643 plus or minus $0.006 \mathrm{~cm}(-1)$ for the $\mathrm{C}-\mathrm{H}$ stretching state. This value for the ground state barrier is somewhat higher than that recently determined from an analysis of the $\mathrm{CH} 3$ rocking band by $P$. R. Bunker et al.

01,193

PB97-122493 Not available NTIS

National Inst. of Standards and Technology (PL) Gaithersburg, MD. Radiometric Physics Div.

Measuring Nondipolar Asymmetries of Photoelectron Angular Distributions.

P. S. Shaw, U. Arp, and S. H. Southworth. 1996 , $10 \mathrm{p}$.

Pub. in Physical Review A, v54 n2 p1463-1472 Aug 96

Keywords: "Angular distributions, "X rays, *Photoelectrons, Non-dipolar effects, Synchrotrons, Reprints

In theories of photoelectron angular distributions at soft $x$-ray energies from $1 \mathrm{keV}$ to $10 \mathrm{keV}$, first-order corrections to the dipole approximation give rise to two nondipolar asymmetry parameters in addition to the wellknown dipolar asymmetry parameter, beta. The nondipolar parameters characterize the forward/backward asymmetry with respect to the propagation vector of the photon beam. Experimentally, the measurement of the non-dipolar effect has been hampered because of the complications resulting from the three-parameter angular distribution. In the paper, the authors suggest various experimental approaches to the measurement of the three asymmetry parameters using partially linearly polarized $x$-rays, corresponding to measurements on synchrotron radiation beamlines. They 
decribe methods to extract the asymmetry parameters using such approaches.

\section{1,194}

PB97-122543 Not available NTIS

National Inst. of Standards and Technology (CSTL), Gaithersburg, MD. Physical and Chemical Properties Div.

Absence of Quantum-Mechanical Effects on the Mobility of Argon lons in Helium Gas at $4.35 \mathrm{~K}$.

Final rept.

L. A. Viehland, and J. J. Hurly. 1996, 4p

Pub. in Jnl. of Chemical Physics, v105 n24 p1-4 1996.

Keywords: "Transport cross sections, *Argon, "Helium, Mobilities, Potential, Spectroscopy, Reprints.

Quantum-mechanical transport cross sections are accurately computed from recent spectroscopic potentials for the three lowest energy levels of the molecular ion $\mathrm{HeAr}+$. Statistical and ground state combinations of the cross sections are used to compute the transport coefficients describing the motion of $\mathrm{Ar}+$ ions through $\mathrm{He}$ gas at $4.35 \mathrm{~K}$. The calculated mobilities do not show the rapid decrease of the experimental values as the ratio of the electric field strength to the gas number density becomes very small. The experimental observations therefore are not due to the effects of orbiting resonance.

\section{Polymer Chemistry}

01,195

PB94-172293 Not available NTIS

National Inst. of Standards and Technology (MSEL), Gaithersburg, MD. Polymers Div.

Thermal Pulse Study of the Polarization Distributions Produced in Polyvinylidene Fluoride by Corona Poling at Constant Current.

Final rept.

J. A. Giacometti, and A. S. DeReggi. 1 Sep 93, 9p

Pub. in Jnl. of Applied Physics 74, n5 p3357-3365, 1 Sep 93

Keywords: "Vinyl resins, "Polarization, "Profiles, Electric corona, Pulse heating, Reprints, "Polyvinylidene fluoride, "Polarization profiles, Corona poling.

A thermal pulse study of the polarization profiles in samples of 12-micrometer-thick, biaxially oriented polyvinylidene fluoride after corona poling under approximately constant-current conditions, using a modified corona triode in atmospheric air, is reported. An electrical characterization of the corona triode is also reported to show how it may be operated in the constant-current mode. Samples poled without electrode on the corona-exposed surface show polarization distributions sensitive to the corona polarity, with polarization depletion on the corona side of the samples when the corona is positive. Polarization-reversal experiments show switching inhomogeneities with a pronounced dependence on the initial corona polarity. The above observations are consistent with a simple model in which positive charges from the positive corona partially penetrate the sample during poling and cause an inhomogeneous reduction of the poling field.

01,196

PB94-172392 Not available NTIS

National Inst. of Standards and Technology (MSEL), Gaithersburg, MD. Polymers Div.

Shear-Excited Morphological States in a Triblock Copolymer.

Final rept.

C. L. Jackson, M. Muthukumar, K. A. Barnes, A. I.

Nakatani, C. C. Han, F. A. Morrison, and J. W. Mays.

1994, 2p

Pub. in Polymer Preprints 35, p624-625 1994.

Keywords: "Shear rate, "Block copolymers, "Morphology, "Polystyrene, "Polybutadiene, Molecular structure, Small angle scattering, Transmission electron microscopy, Excitation, Neutron scattering, Reprints, Molecular configuration.

The effect of shear on the morphology of a triblock copolymer of polystyrene-d8/polybutadiene/polystyrened8 (SBS) (23 wt. \% styrene-d8, cylindrical morphology) was studied by small angle neutron scattering (SANS) and transmission electron microscopy (TEM). The copolymer was sheared $(0.585 / \mathrm{s}$ at $100 \mathrm{C})$ in a cone-and- plate rheometer and quenched to room temperature. The morphology consisted of two distinct structures, including a primary cylindrical spacing of $22 \mathrm{~nm}$, as ex pected for the equilibrium state, and a secondary spac ing of $12 \mathrm{~nm}$, which is hypothesized to be a shear-ex cited state for this material. A mechanism for producing such a unique morphology is proposed to be the conversion of a bridging configuration between cylinders to a looping configuration.

\section{1,197}

PB94-172624 Not available NTIS

National Inst. of Standards and Technology (MSEL), Gaithersburg, MD. Polymers Div.

Neutron Scattering Study of Shear Induced Turbidity in Polystyrene/Dioctyl Phthalate Solutions at High Shear Rates.

Final rept.

A. I. Nakatani, J. F. Douglas, Y. B. Ban, and C. C.

Han. 1993, 2p.

Pub. in Jnl. of American Chemical Society Polymer Preprints 34, p554-555 1993.

Keywords: "Polystyrene, *Neutron scattering, *Turbidity, "Solutions, "Mixing, Small angle scattering, Shear rate, Polymers, Binary systems(Materials), NonNewionian systems, Reprints, "Phthalic acid/(dioctylester), Polymer blends.

The phenomenon of shear induced 'mixing' and 'demixing' in polymer solutions has attracted theoretical and experimental attention since the 1950's. Helfand and Fredrickson (HF) developed a theory which is appropriate for describing the distorted scattering in polymer solutions at low shear rates, but the HF theory is not applicable to fluids in the non-Newtonian flow regime. The range of shear rates where PS DOP solutions becomes turbid to the eye is typically $>100 / \mathrm{s}$ in our experiments where the HF theory is no applicable. We have conducted small angle neutron scattering (SANS) investigations of these PS/DOP solutions at high shear rates in order to examine the scattering in this 'strong shear' regime.

\section{1,198}

PB94-172632 Not available NTIS

National Inst. of Standards and Technology (MSEL), Gaithersburg, MD. Polymers Div.

Time Dependent Small Angle Neutron Scattering Behavior in Triblock Copolymers Under Steady Shear.

Final rept

A. I. Nakatani, F. A. Morrison, J. W. Mays, M.

Muthukumar, and C. C. Han. 1993, 2p

Pub. in American Chemical Society Polymer Preprints 34, p630-631 1993.

Keywords: "Neutron scattering, "Block copolymers, "Temperature dependence, "Polystyrene, "Polybutadiene, Shear rate, Small angle scattering, Shear flow, Isotropy, Anisotropy, Reprints.

We examine a styrene-butadiene-styrene triblock copolymer (23 wt. \% styrene, cylindrical morphology, $M($ sub $w)=8,000 / 54,000 / 8,000$ ) in two ways: (a) the shear rate and temperature dependence of the steady shear small angle neutron scattering (SANS) patterns; (b) the transient SANS behavior during steady shear as a function of temperature and shear rate. At low shear rates, above and below the triplet optical density (ODT), an isotropic scattering pattern is observed. Below the quiescent ODT, at somewhat higher shear rates, the sample orients in the flow direction and the scattering becomes anisotropic. Below ODT, at even higher shear rates, a second pair of diffraction spots are observed at higher $\mathrm{q}$. In the third regime, the intensity of the low q peak passes through two distinct maxima and a minimum. The intensity of the higher $q$ peak appears to pass through a maximum at the same time the low q peak is at a minimum.

\section{1,199}

PB94-172756 Not available NTIS

National Inst. of Standards and Technology (IMSE), Gaithersburg, MD. Reactor Radiation Div.

Temperature Dependence of the Morphology of Thin Diblock Copolymer Films as Revealed by Neutron Reflectivity.

Final rept.

T. P. Russell, S. H. Anastasiadis, S. K. Satija, and C. F. Majkrzak. 1990, 6p

Pub. in Proceedings on Neutron Scattering for Materials Science Symposium, Boston, MA., November 2730, 1989, p145-150 1990. See also PB92-170620.

Keywords: "Temperature dependence, "Morphology, "Block copolymers, "Thin films, "Surface properties,
Neutron radiography, Reflectivity, Phase trans formations, Annealing. Transition temperature, Interfaces, Reprints.

In the report neutron reflectivity studies on the temperature dependence of the morphology of diblock $\mathrm{co}$ polymers in the vicinity of the microphase separation transition temperature (MST) is discussed. At temperatures below the MST the ordered multilayered lamellar morphology is found to penetrate through the entire specimen. At temperatures above the MST the periodic variation in the composition of the components is maintained but a clear dissipation of the order is found with increasing distance from either the air copolymer or copolymer/substrate interfaces. An exponentially damped squared cosine function from both interfaces is found to well describe the reflectivity results. The characteristic decay length is found to decrease with increasing temperature in accordance with mean field arguments. Results from two different copolymers with two different molecular weights and, consequently, two different MSTs are found to be identical on a reduced temperature scale.

01,200

PB94-185055 Not available NTIS

National Inst. of Standards and Technology (MSEL), Gaithersburg, MD. Polymers Div.

Grafted Interpenetrating Polymer Networks.

Final rept.

B. J. Bauer, R. M. Briber, and B. Dickens. 1994, 17p. Pub. in Interpenetrating Polymer Networks, Chapter 7 p179-195 1994

Keywords: *Graft polymerization, *Polymethyl meth acrylate, Thermal analysis, Neutron scattering, $X$-ray diffraction, Phase transformation, Reprints, *Interpenetrating polymer networks.

A new class of interpenetrating polymer network (IPN) has been studied in which grafting reactions between the two components are varied. Small-angle neutron scattering of grafted and nongrafted IPNs shows that grafting greatly enhances the miscibility of the components. Five nonfunctionalized poly(methyl methacrylates) (PMMAs) with alkacrylate, methacrylate, acrylate, and alpha-methylstyrene end groups were dissolved in styrene-divinylbenzene and polymerized. Small-angle $X$-ray scattering was used to characterize the extent of phase separation. The uniformity of the IPNs is strongly dependent on the grafting efficiency. Grafted and nongrafted IPNs were also made from the PMMAs and polyethylene glycol diacrylates. Thermal studies showed one transition in the grafted samples and two distinct transitions in a nongrafted sample.

01,201

PB94-185105 Not available NTIS

National Inst. of Standards and Technology (MSEL) Gaithersburg, MD. Polymers Div.

In-Line Optical Monitoring of Injection Molding.

Final rept.

A. J. Bur, F. W. Wang, C. L. Thomas, and J. L.

Rose. Apr 94, 9p.

Pub. in Polymer Engineering and Science 34, n8 p671679 Apr 94

Keywords: "Solidification, "Injection molding, "Sensors, "Polymers, Fiber optics, Polyethylene, Polystyrene, Crystallization, Fluorescence, In-situ processing, Monitoring, Plastics processing, Reprints.

An optical sensor, consisting of optical fibers to transmit light to and from the mold cavity, was constructed for the purpose of measuring the onset of polymer solidification during injection molding. The sensor was used to detect characteristic fluorescence radiation from a dye which had been doped into the resin at very low concentration. By measuring changes in fluorescence intensity, it was possible to detect whether the state of the resin was liquid or solid. We observed that, as the resin cooled in the mold, the onset of solidification was indicated by highly characteristic and distinct changes in the fluorescence intensity/time profile. Application of the method involved the use of a calibration relationship between the fluorescence intensity and temperature of the doped polymer in order to determine the distinct features which characterize the onse of solidification. Injection molding of a glass forming polymer (polystyrene) and a crystallizable polyme (polyethylene) was monitored by this technique.

01,202

PB94-185188 Not available NTIS 


\section{Polymer Chemistry}

National Inst. of Standards and Technology (MSEL), Gaithersburg, MD. Polymers Div.

Phase Behavior of a Hydrogen Bonding Molecular Composite.

Final rept.

M. D. Dadmun, and C. C. Han. 1993, 6p

Pub. in Materials Research Society Symposium Proceedings, v305 p171-176 1993

Keywords: "Composite materials, "Phase transformations, "Hydrogen bonds, Microscopy, Glass transition temperature, Polypeptides, Liquid crystals, Copolymers, Reprints, "Molecular composites, "Liquid crystalline polymers, Poly(hydroxypropyl-L-glutamine).

The phase behavior of a blend containing poly(hydroxypropyl-L-glutamine), a rod-like polypeptide, and a coil-like random copolymer with $1.5 \%$ hydrogen bonding monomer is studied using light scattering, DSC, and optical microscopy. The results show that this system has an LCST. The phase behavior of this binary system is complicated by a glass transition and an ordering transition. Evidence is also observed of a possible change in phase demixing process, from nucleation and growth to spinodal decor sition, for a $50 / 50$ blend with a change in temperature.

\section{1,203}

PB94-185469 Not available NTIS

National Inst. of Standards and Technology (MSEL) Gaithersburg. MD. Polymers Div

Influence of Surface Interaction and Chain Stiffness on Polymer-Induced Entropic Forces and the Dimensions of Confined Polymers.

Final rept.

A. L. Kholodenko, D. W. Bearden, and J. F. Douglas. 1994, $19 p$.

Pub. in Physical Review E 49, n3 p2206-2224 Mar 94.

Keywords: "Polymers, "Entropy, "Stiffness, "Surfaces, Mathematical models, Critical temperature, Reprints, "Confined semiflexible polymers, "Entropic Casimin forces

The theories of Dolan and Edwards and Eisenriegler et al. for Gaussian chains confined between parallel plates and to a half-space are generalized to chains having arbitrary stiffness. The generalized theory exploits a recently discovered relation between semiflexible polymers and Euclidean-type Dirac fermions in which 'flexible' and 'stiff' polymers correspond to the nonrelativistic (massive) and relativistic (massless) limits of the Dirac propagator, respectively. We show that half-space and parallel-plate problems are interrelated and this allows for a simplified and unified treatment of confined semiflexible polymers. The properties of confined semiflexible chains exhibit a complicated dependence on the polymer-surface interaction and chain stiffness. Results for polymer dimensions and entropic Casimir-like forces between plates are consistent with those obtained previously for flexible chains and corresponding results are obtained for semiflexible polymers. The new results for the forces between plates, having a semiflexible polymer in the gap, exhibit quafitative agreement with experimental data on confined chains at nonvanishing concentrations.

01,204

PB94-185543 Not available NTIS

National Inst. of Standards and Technology (MSEL),

Gaithersburg, MD. Polymers Div.

Fluorescence Monitoring of Polarity Change and Gelation during Epoxy Cure.

Final rept.

K. F. Lin, and F. W. Wang. 1994, 5p.

Pub. in Polymer 35, n4 p687-691 1994

Keywords: "Curing. "Fluorescence, "Polymerization, *Epoxy resins, Temperature, Gelation, Polarity, Monitoring, Molecular structure, Reprints, Hexatriene/ (dimethylaminophenyl)-phenyl,

Bisphenol A, Diethylene triamine.

Ether/diglycidyl

The fluorescence spectrum of 1-(4(DMA-DPH) dissolved in a stoichiometric mixture of diglycidyl ether of bisphenol A and diethylene triamine was measured as a function of cure time at various cure temperatures. The frequency of the fluorescence maximum for DMA-DPH increased during the curing reactions because of the change in the polarity of the epoxy resin. In an isothermal cure, the fluorescence frequency increased linearly with the cure time until the gelation occurred. The total change in fluorescence frequency that occurred from the beginning of the iso- thermal cure to the gelation time was $1,000 / \mathrm{cm}$ and was independent of the cure temperature, implying that the chemical structure of the infinite network at the gelation time was independent of the cure temperature. The rate constant, $\mathrm{K}$ (sub $\mathrm{T}$ ), for the polarity change during an isothermal cure of the epoxy resin, defined as the rate constant for the linear increase in fluorescence frequency, was determined. The activation energy of K(sub T) was estimated to be $60 \mathrm{~kJ} / \mathrm{mol}$.

\section{1,205}

PB94-185550 Not available NTIS

National Inst. of Standards and Technology (MSEL), Gaithersburg, MD. Polymers Div.

Electron Beam Crosslinking of Poly(vinylmethyl ether).

X. Liu, R. M. Briber, and B. J. Baver. 1994, 5p Pub. in Jnl. of Polymer Science B 32, p811-815 1994

Keywords: *Vinyl ether resins, "Crossfinking, Molecular structure, Electron beams, Solutions, Alcohols, Solvation, Chromatographic analysis, Reprints.

The electron beam induced branching of poly(vinylmethyl ether) (PVME) in bulk and in isopropanol solutions has been studied by gel permeation chromatography. The branching probability of bulk PVME induced by high-energy electrons can be characterized by gel permeation chromatography and a simple probability constant obtained. In isopropano solutions this branching probability is not constant as a function of dose and is found to decrease with de creasing concentration. These results indicate the importance of solvent effects on the crosslinking of PVME in isopropanol solution by electron beam radiation.

\section{1,206}

PB94-185758 Not available NTIS

National Inst. of Standards and Technology (MSEL), Gaithersburg, MD. Reactor Radiation Div.

Neutron Reflectivity of End-Grafted Polymers: Concentration and Solvent Quality Dependence in Equilibrium Conditions.

Final rept.

D. Perahia, D. G. Wiesler, S. K. Satija, S. T. Milner, L. J. Fetters, and S. K. Sinha. 1994, 4p.

Pub. in Physical Review Letters 72, n1 p100-103, 3 Jan 94

Keywords: "Polymers, "Density(Mass/volume) "Monomers, Concentration(Composition), Neutron scattering, Polystyrene, Temperature dependence, Neutron reflectometry is used to obtain a direct measurement of the monomer distribution of a weakly bound end-grafted polystyrene layer in equilibrium with bufk solution, as a function of the polymer concentration and temperature. We report for the first time two new regions in the density profile of the polymers, an excess polymer near the surface and a wetting layer on top of the brush. The physical properties of the polymer layer are strongly affected by the equilibrium between the bound and free polymer molecules.

\section{1,207}

PB94-198389 Not available NTIS

National Inst. of Standards and Technology (MSEL), Gaithersburg, MD. Polymers Div.

Facile Synthesis of Novel Fluorinated Multifunctional Acrylates.

Final rept.

J. M. Antonucci, J. W. Stansbury, and G. W. Cheng

1990, $1 p$

Pub. in Abstracts of Papers of the American Chemical Society 199, p111 Apr 90

Keywords: "Acrylates, "Fluorine organic compounds, *Chemical reactions, Reprints, Chemical preparation, Chemical analysis, Dienes, "Fluorinated acrylates, Oligomers.

Previously it was shown that the bulk reaction alkyl acrylates with paraformaldehyde catalyzed by triethylenediamine yields bis(acrylate) monomers having a 1,6 diene structure which favors free radical cyclopolymerization via 6-membered ring formation. In this study the feasibility of extending this reaction to convert fluoro-acrylates and-diacrylates to difunctiona and oligomeric fluorinated monomers was explored. The reaction of 2,2,3,3,4,4,4-heptafluorobutyl acrylate (7FBA) under the usual ambient, bulk conditions yield ed only trace amounts of products. Elevated temperature $(80 \mathrm{C})$ and the use of dimethyl sulfoxide resulted in an improved yield of two types of bis products, a 1,4-diene as well as the expected 1,6 diene (proton NMR). The analogous reaction with $2,2,3,3,4,4-$ hexafluoropentane-1,5-diacrylate yields a soluble, highly fluorinated, multifunctional oligomer. This type of aldehyde insertion reaction offers an attractive means of preparing otherwise not readily available highly fluorinated multifunctional monomers.

01,208

PB94-198496 Not available NTIS

National Inst. of Standards and Technology (MSEL), Gaithersburg, MD. Polymers Div.

Thermodynamic Interactions in Model Polyolefin Blends Obtained by Small-Angle Neutron Scattering.

Final rept.

N. P. Balsara, L. J. Fetters, N. Hadjichristidis, W. W. Graessley, R. Krishnamoorti, D. J. Lohse, and C. C. Han. 1992, 11p.

Pub. in Macromolecules 25, n23 p6137-6147 1992.

Keywords: *Polymer blends, "Polyolefins, Small angle scattering, Random phase approximation, Amorphous materials, Neutron scattering, Temperature dependence, Phase studies, Deuterium compounds, Polybutadiene, Reprints, Polyolefin blends.

The dependence of Flory-Huggins interaction parameter chi on temperature, composition, and chain length was investigated for binary blends of amorphous model polyolefins, materials which are structurally analogous to copolymers of ethylene and butene-1. The components were prepared by saturating the double bonds of nearly monodisperse polybutadienes $(78 \%, 88 \%$, and $97 \%$ vinyl content) with $\mathrm{H} 2$ and $\mathrm{D} 2$, the latter to provide contrast for small-angle neutron scattening (SANS) experiments. Values of chi were extracted from SANS data in the single-phase region for two series of blends, $\mathrm{H} 97 / \mathrm{D} 88$ and $\mathrm{H} 88 / \mathrm{D} 78$, using the random phase approximation and the Flory-Hugnins expression for free energy of mixing. These vi jes were found to be insensitive to chain length (one test only) and to the component volume fractions for phi $=0.25,0.50$, and 0.75 . Their temperature dependence $(27-170 \mathrm{C})$ obeys the form $\mathrm{chi}(T)=A T+B$ with coefficients that connote upper critical solution behavior.

01,209

PB94-199296 Not available NTIS

National Inst. of Standards and Technology (MSEL), Gaithersburg, MD. Polymers Div.

Fluorescence Anisotropy Measurements on a Stress.

Final rept.

A. J. Bur, R. E. Lowry, S. C. Roth, C. L. Thomas,

and F. W. Wang. 1992, 8p.

Pub. in Macromolecules 25, p3503-3510 1992.

Keywords: "Polymer chemistry, "Shear stress, "Molecular structure, "Orientation, "Melts(Crystal growth), "Anistropy, Reprints, Polybutadiene, Anthracene, Marking, Tracer techniques, Probes, Crosslinking, Relaxation time, Extensions, Crystal structure.

Polybutadiene tagged with anthracene was synthesized and used as a fluorescent molecular probe to study shear-induced orientation in a matrix polymer melt. With the tagged polybutadiene doped into a polybutadiene matrix at $0.1 \%$ concentration, steadystate fluorescence anisotropy measurements were carried out under zero shear and under finite shear conditions using an optically instrumented cone and plate rheometer. Measurements were made over a shear rate range for which the specimen displayed non-Newtonian behavior, .00264 to 5.3/S. Anisotropy was observed to decrease with increasing applied shear stress. The magnitude of the effect is small and is attributed to shear-induced onentation of the probe molecule which is engaged in the entanglement network of the host polymer. Diluting the entanglement network using plasticizer produced a smaller effect. For polybutadiene plasticized with $50 \%$ cetane, the authors observed that anisotropy was independent of the applied shear stress, indicating that the probe molecule was not participating in the orientation of the matrix entanglement network. A relationship between anisotropy, chromophore relaxation time, and orientation factors was derived and used to deduce an orientation distribution of fluorescent absorption dipoles and to illustrate the difference between shear and extension stress observations. Extension experiments, carried out using a cross-linked polybutadiene specimen, showed that anisotropy increased as a function of applied extensional stress. 
01,210

PB94-199304 Not available NTIS

National Inst. of Standards and Technology (MSEL), Gaithersburg, MD. Polymers Div.

Observations of Shear Induced Molecular Orientation in a Polymer Melt Using Fluorescence Anisotropy Measurements.

Final rept.

A. J. Bur, R. E. Lowry, S. C. Roth, C. L. Thomas,

and F. W. Wang. 1991, 3p.

Pub. in Macromolecuies 24, n12 p3715-3717 1991

Keywords: "Polymer chemistry, "Shear stress, *Molecular structure, *Orientation, "Melts(Crystal growth), Polybutadiene, Crystal structure, Anisotropy, Molecular weight, Fluorescence, Marking, Tracer techniques, Anthracene, Reprints.

Polybutadiene tagged with anthracene was synthesized and used as a fluorescent molecular probe to study shear induced orientation in a matrix polymer melt. With the tagged polybutadiene doped into a polybutadiene matrix at $0.1 \%$ concentration, steady state fluorescence anisotropy measurements were carried out under zero shear and under finite shear conditions using an optically instrumented cone and plate rheometer. For polybutadiene plasticized with $50 \%$ cetane, we observed that anisotropy of the tagged polybutadiene was independent of the applied shear stress indicating that the probe molecule was not participating in the orientation of the matrix entanglement network.

01,211

PB94-199312 Not available NTIS

National Inst. of Standards and Technology (MSEL),

Gaithersburg, MD. Polymers Div.

Observations of Shear Stress and Molecular Orientation Using Fluorescence Anisotropy Measurements.

Final rept.

A. J. Bur, R. E. Lowry, S. C. Roth, C. L. Thomas, and F. W. Wang. 1991, 4p.

Pub. in Proceedings of Annual Technical Conference (49th), Montreal, Quebec, Canada, May 5-9, 1991 , p842-845

Keywords: "Polymer chemistry, "Shear stress, *Molecular structure, "Orientation, "Melts(Crystal growth), Polybutadiene, Crystal structure, Anisotropy, Molecular weight, Fluorescence, Marking, Tracer techniques, Anthracene, Reprints.

Polybutadiene tagged with anthracene was synthesized and used as a fluorescent molecular probe to study shear induced orientation in a matrix polymer melt. With the tagged polybutadiene doped into a polybutadiene matrix at $0.1 \%$ concentration, steady state fluorescence anisotropy measurements were carried out under zero shear and under finite shear conditions using an optically instrumented cone and plate rheometer. For polybutadiene plasticized with $50 \%$ cetane, we observed that anisotropy of the tagged polybutadiene was independent of the applied shear stress indicating that the probe molecule was not participating in the orientation of the matrix entanglement network.

01,212

PB94-199387 Not available NTIS

National Inst. of Standards and Technology (NML),

Boulder, CO. Chemical Engineering Science Div.

Statistical Thermodynamics of Phase Separation and lon Partitioning in Aqueous Two-Phase Systems.

Final rept.

H. Cabezas, M. Kabiri-Badr, and D. C. Szlag. 1990, $7 p$.

Pub. in Bioseparation 1, n3/4 p227-233 1990.

Keywords: "Binary system(Materials), "Polymer blends, "Phase diagrams, "Thermodynamics, "Mathematical models, Aqueous solutions, Statistical mechanics, Separation, Molecular weight, Dextran, Polyoxyethylene, Reprints.

A general model for the phase behavior of polymerpolymer aqueous two-phase systems containing small amounts of added inorganic salts has been developed from statistical thermodynamics. The model is based on the solution theory of Hill and new electrolyte model based on Fluctuation Solution Theory. It includes the effect of polymer molecular weight with scaling expres. sions from the Renormalization Group theory of polymer solutions. The model has been used to calculate the phase diagram and the partitioning of salt for an aqueous two-phase system containing polyethylene glycol (MW=8000) and dextran $(M W=28700)$ with Na2SO4. The calculations have been compared to experiment with good agreement.

\section{1,213}

PB94-199809 Not available NTIS

National Inst. of Standards and Technology (MSEL), Gaithersburg, MD. Polymers Div.

Peeling a Polymer from a Surface or from a Line. Final repi.

E. A. DiMarzio, and C. M. Guttman. 1991, 9p.

Pub. in Jnl. of Chemical Physics 95, n2 p1189-1197 1991

Keywords: "Polymers, *Adhesion, *Surface chemistry, Equations of state, Rubbers, Elasticity, Molecular structure, Chemical bonds, Gels, Crosslinking, Reprints, Polymeric chains, Zippering.

The authors calculate the force on a long linear polymer molecule whose one end is zippered down onto a surface or onto a line and whose other end is at a perpendicular distance $\mathrm{A}$ from the surface of line. Random coil statistics are used for the unattached portion of the chain. The method is extended to the case when the bonds within the zippered portion are breaking and reforming. They also consider the case where the attached portion is in the form of loops and trains. Applications are discussed briefly. They include (1) Selfhealing systems of gels and rubbers where the cross links may be hydrogen bonds, (2) Adhesion, (3) The degree of crystallinity in crystal-amorphous lamella system, (4) The packing of DNA into the head of a bacieriophage virus, and pulling apart of double stranded DNA, (5) An insight into the theory of rubber elasticity, (6) Understanding the critical force for flow in thixotropic systems.

01,214

PB94-200052 Not available NTIS

National Inst. of Standards and Technology (MSEL) Gaithersburg, MD. Polymers Div.

Etfect of Curing History on Ultimate Glass Transition Temperature and Network Structure of Crosslinking Polymers.

Final rept.

S. S. Chang. 1992,11p.

Pub. in Polymer 33, n22 p4768-4778 1992.

Keywords: "Glass iransition temperature, "Curing, "Polymerization, "Crosslinking, Differential therma analysis, Phase transiormations, Reprints, *Glycidyl ethers, "Bisphenol-A, *Crosslinking network.

For a particular crosslinking polymer, it is often considered that the final state of crosslinking may be reached by post-curing at temperatures above its ultimate glass ransition iemperature. T(sub g,u) regardless of previous curing histories. Although this appears to be true over a certain range of variation in curing history the $\mathrm{T}($ sub $\mathrm{g}, \mathrm{u}$ ) and the network structure depend strongly on the curing history over a wide range of conditions for the homopolymerization of diglycidyl ether of bisphenol-A (DGEBA) catalyzed by 2-ethyl-4-methyl imidazole. As degrees of cure and crosslink increase, the glass transition temperature, $T$ (sub g), of a sample may increase from the monomeric DGEBA value of -22 $\mathrm{C}$ to the highest value near $180 \mathrm{C}$. Depending on the curing thermal history, some samples may only attain a $T($ sub $g, u)$ of approximately $100 \mathrm{C}$, even after postcuring at $200 \mathrm{C}$ for $16 \mathrm{~h}$. Although the influence of thermal history on $\mathrm{T}$ (sub g) may rank second after the influence of the degree of cure, it is the most important factor on T(sub g,u) for a fixed resin and catalyst composiion. The reversible physical aging process appears to be the least influential on $\mathrm{T}(\mathrm{sub} \mathrm{g}$ ). As all crosslinking reactions involve competing reactions with different $\mathrm{ki}$ netic parameters, we believe that these phenomena are universally observable to a greater or lesser degree in all crosslinking reactions.

01,215

PB94-211026 Not available NTIS

National Inst of Standards and Technology (MSEL) Gaithersburg, MD. Polymers Div.

Hypercubic Lattice SAW Exponents nu and gamma 3.99 Dimensions Revisited.

Final rept

J. F. Douglas, T. Ishinabe, A. Nemirovsky, and K. F. Freed. 1993, 11p.

Pub. in Jnl. of Physics A: Mathematical and General 26. p1835-1845 1993
Keywords: "Polymers, Partition functions, Computation, Exponents, Reprints, Self-avoiding walks, Spatial dimensions, Lattice models.

The self-avoiding walk (SAW) exponents nu and gamma are computed over a range of dimensions ( 1 = or $<d<$ infinity) from exact expressions for the meansquare end-to-end distance $<R$ (sub n, sup 2) > and the partition function $Q$ (sub $n$ ) of SAWs having a lim ited number of steps, $n=$ or $<11$. SAW exponents (nu, gamma) for arbitrary dimension d are estimated by applying standard extrapolation iechniques to our direct enumeration data which has been analytically continued to variable dimension. Exponent estimates obtained from continuum theories of self-avoiding paths are compared with the SAW calculations.

01,216

PB94-211034 Not available NTIS

National Inst. of Standards and Technology (MSEL)

Gaithersburg, MD. Polymers Div.

Effect of Swelling on the Elasticity of Rubber: Localization Model Description.

Final rept.

J. F. Douglas, and G. B. McKenna. 1993, 7p

Pub. in Macromolecules 26, n13 p3282-3288 1993

Keywords: "Elasiomers, "Spatial distribution,

"Mathematical models, Elastic properties,

"Localization model, *Mooney-Rivlin equation.

The success of the localization model in describing the elasticity of unswollen natural rubber is reviewed and the model is extended to describe the elasticity of swollen networks. In contrast to the Frenkel-Flory-Rehner modeling of network elasticity, the localization mode predicts that the mechanical response of swollen rubbers changes qualitatively in going from low to high cross-link densities. The implications of these predictions are discussed.

01,217

PB94-211141 Not available NTIS

National Inst. of Standards and Technology (MSEL) Gaithersburg, MD. Polymers Div.

How Far is Far from Critical Point in Polyme Blends. Lattice Cluster Theory Computations for Structured Monomer, Compressible Systems.

Final rept.

J. Dudowicz, M. Lifschitz, K. Freed, and J. Douglas.

1993, 17p

Pub. in Jnl. of Chemical Physics 99, n6 p4804-4820 15 Sep 93.

Keywords: *Polymer blends, Critical point, Compress ibility, Renormalization, Monomers, Computation, $\mathrm{Re}$ prints, Lattice cluster theory.

Although the lattice cluster theory (LCT) incorporates many features which are essential in describing real polymer blends, such as compressibility, monomer structures, local correlations, chain connectivity, and polymer-polymer interactions, it still remains a mean field theory and is therefore not applicable in the vicinity of the critical point where critical fluctuations become large. The LCT, however, permits formulating the Ginzburg criterion, which roughly specifies the temperature range in which mean field applies. The present treatment abandons the conventional assumptions of incompressibility and of composition and the molecular weight independent effective interaction parameter upon which all prior analyses of the Ginzburg criterion are based. Blend compressibility, monomer structure, and local correlations are found to exert profound influences on the blend phase diagram and othe critical properties and, thus, exhibit a significant impac on the estimate of the size of the nonclassical region

01,218

PB94-211422 Not available NTIS

National Inst. of Standards and Technology (MSEL), Gaithersburg, MD. Polymers Div.

Monitoring Polymer Cure by Fluorescence Recovery After Photobleaching.

Final rept.

Coni. 1991, 8p.

Pub in Rev. Prog. Quant. Nondestr. Eval. 10A, p112711341991

Keywords: "Curing, "Polymers, "Monitors, *Tracer techniques, Fiber optics, Fluorescence, Diffusion coefficient, Dyes, Laser applications, Reprints, *Fluorescence Recovery After Photobleaching technique.

Fluorescence recovery after photobleaching (FRAP) is a technique to measure the translational diffusion coef- 


\section{CHEMISTRY}

\section{Polymer Chemistry}

ficient of dye molecules in polymer matrices. The sensitivity of the diffusion coefficient to the degree of cure of the polymer matrix provides a means to monitor cure. The aim of the present work is to examine the potential of optic fibers as a means probing the interior of thick specimens by FRAP. The temporal response of the fluorescence intensity has been calculated for an evanescent wave type fiber optic probe. With this probe, the evanescent wave of the photobleaching laser creates a region near the fiber surface devoid of fluorescent molecules. The diffusion coefficient of the dye molecule is determined from the temporal response of the fluorescence. With fiber optics it should be possible to measure translational diffusion coefficients an order of magnitude smaller than those detected by the conventional microscope-based method.

\section{1,219}

PB94-211547 Not available NTIS

National Inst. of Standards and Technology (MSEL), Gaithersburg, MD. Polymers Div.

Neutron Scattering by Multiblock Copolymers of Structure $(A-B) N-A$.

Final rept.

G. Hadziioannou, H. Benoit, W. Tang, K. Shull, and

C. C. Han. 1992 5p.

Pub. in Polymer 33, n22 p4677-4681 1992.

Keywords: "Block copolymers, "Neutron scattering, "Small angle scattering, *Molecular structure, Styrene resins, Polymerization, Deuterium compounds, Monomers, Reprints.

Multiblock copolymers were prepared by anionic copolymerization of deuterated and classical styrene monomers. The use of bifunctional initiators gives copolymers with an odd number of sequences. Neutron scattering experiments were performed on these sam. ples in order to check the existing theories which are extended in the first part to multiblock copolymers with an odd number of blocks. The agreement between theory and experiment is excellent and, surprisingly, the height of the maximum of the curves:scattered intensity versus scattering angle, does not depend on the number of blocks.

\section{1,220}

\section{PB94-211612 Not available NTIS}

National Inst. of Standards and Technology (MSEL), Gaithersburg, MD. Polymers Div.

Shear Dependence of Critical Fluctuations in Binary Polymer Mixtures by Small Angle Neutron Scattering.

Final rept.

C. C. Han, and A. I. Nakatani. 1990, 1 .

Pub. in Abstracts of Papers of the American Chemical Society 200, p105 Aug 90.

Keywords: "Binary systems(Materials), "Shear rate, -Small angle scattering, "Polystyrene, "Concentration(Composition), Polybutadiene, Neutron scattering, Thermoplastic resins, Deuterium compounds, Polymer blends, Vinyl ether resins, Labeled substances, Temperature dependence, Phase diagrams, Reprints, Poly(ether/vinylmethyl)

Small angle neutron scattering (SANS) has been used to study the concentration fluctuations of binary polymer mixtures under shear. Two different polymer systems: deuterated polystyrene/poly(vinylmethylether) and deuterated polystyrene/polybutadiene have been studied as a function of temperature and shear rate.

\section{1,221 \\ PB94-211729 Not available NTIS}

National Inst. of Standards and Technology (MSEL), Gaithersburg, MD. Polymers Div

Small-Angle X-Ray and Neutron Scattering Study of Block Copolymer/Homopolymer Mixtures.

Final rept.

H. Hasegawa, H. Tanaka, T. Hashimoto, and C. C. Han. 1990, 1p.

Pub. in Abstracts of Papers of the American Chemical Society 200, p279 Aug 90.

Keywords: "Small angle scattering, "X-ray scattering, "Neutron scattering, "Block copolymers, "Binary systems(Materials), "Polystyrene, Polymer blends, Molecular weight, Thermoplastic resins, Polybutadiene, Deformation, Molecular chains, Reprints, Molecular conformations.

We investigated lateral and vertical one-dimensional components of radius of gyration for a single block copolymer chain (Study 1) and those of a single homopolymer chain (Study II) in a microdomain space formed by a mixture of diblock copolymers and homopolymers by means of small-angle neutron and $\mathrm{X}$-ray scattering techniques. The molecular weights of the homopolymers were much smaller than those of the corresponding block copolymer chains. The results imply that both the block copolymer and the homopolymer chains in the microdomain space are compressed in the direction parallel to the intertace and stretched in the direction perpendicular to the interface. The block copolymer chains undergo an isochoric affine deformation on addition of the homopolymers.

\section{1,222}

PB94-211976 Not available NTIS

National Inst. of Standards and Technology (MSEL) Gaithersburg, MD. Polymers Div.

Crossover to Strong Shear in a Low-MolecularWeight Critical Polymer Blend.

Final rept.

E. K. Hobbie, D. W. Hair, A. I. Nakatani, and C. C.

Han. 1992, 4p.

Pub. in Physical Review Letters 69, n13 p1951-1954 28 Sep 92

Keywords: "Small angle scattering, "Neutron scattering, "Polymer blends, "Shear flow, Molecular weight, Critical point, Light scattering, Reprints.

Small-angle neutron scattering has been used to measure the influence of shear flow on a low-molecular-weight polymer blend near the critical point. When combined with light scattering measurements of the equilibrium (gamma $=0$ ) critical dynamics, these meas urements reveal that the long-range critical fluctuations begin to break apart when the shear rate becomes comparable to the characteristic relaxation rate $1 /$ tau(sub c), where tau(sub c) is the equilibrium lifetime of the critical fluctuations. This effect is directly related to the decrease in the critical temperature caused by the flow, and the data are found to be in very good agreement with the theoretical predictions of Onuki and Kawasaki.

\section{1,223}

PB94-212321 Not available NTIS

National Inst. of Standards and Technology (MSEL), Gaithersburg, MD. Polymers Div.

Anomalous Freezing and Melting of Solvent Crysals in Swollen Gels of Natural Rubber.

Final rept.

C. L. Jackson, and G. B. McKenna. 1991, 9p.

Pub. in Rubber Chemistry and Technology $64, n 5$ p760-768 1991.

Keywords: "Elastomers, "Freezing, "Cryoscopy, Gels, Polymers, Crosslinking, Calorimetry, Solvents, Swelling, Reprints, "Freezing point depression.

The anomalously large solvent freezing point depression (fpd), observed in crosslinked rubbers swollen in solvent has been a subject of study for over thirty years without clear resolution. While a sizeable fpd is accounted for by the lowering of the thermodynamic potential of solvent molecules in a polymer solution described by Flory-Huggins theory, the additional fpd observed for crosslinked rubbers has been attributed to various physical effects such as restriction of solven crystals to small size by the network mesh or difficulty in nucleation of the solvent crystals. In this paper, we identify two points of misunderstanding in the literature on this problem, and attempt to clarify the analysis of pd for solvent swollen rubbers. The first point relates to the use of Flory-Huggins theory for solvent freezing where nucleation is a concern, rather than for solven melting, for which it was intended. We present new ex perimental calorimetric data on both the freezing and melting of solvent crystals in crosslinked and uncrosslinked natural rubber swollen in benzene to illustrate this point. The second point relates to a reconsideration of the hypothesis of Kuhn, et al., that the anomalous fpd can be accounted for by small crystallite size.

\section{1,224}

PB94-212339 Not available NTIS

National Inst. of Standards and Technology (MSEL) Gaithersburg, MD. Polymers Div.

Polymer Liquid Crystalline Materials.

Final rept.

C. L. Jackson, and M. T. Shaw. 1991, 22p.

Pub. in International Materials Reviews 36, n5 p165 1861991.

Keywords: "Liquid crystals, "Polymers, Molecular structure, Physical properties, Liquid phases, Molecular chains, Rheology, Melts, Solutions, Reprints
A review of the chemical structures, physical properties and current and potential applications of liquid crystalline polymers (LCPS) is presented. Comparisons are made between LCPS, low molecular weight liquid crystals (LCS) and conventional polymers for readers unfamiliar with the field. The ordered liquid phases are classified and molecular requirements for the formation of LC phases in both LCP melts (thermotropics) and LCP solutions (lyotropics) are discussed. The theoretical predictions of the formation of ordered phases given by Flory and Onsager are compared to experimental data, and other aspects of the phase behavior of LCPS in solution are considered, including physical gelation and crystal-solvate complexes. A wide variety of polymer structures which exhibit LC behavior are presented. These include main-chain (rigid-rod), semiflexible, main-chain with flexible spacers, side-chain LCPs and various new combinations which have been recently devised. An emphasis is placed on the relationship between chemical and physical structure of LCPS, rather than on the details of the synthesis.

\section{1,225}

PB94-212438 Not available NTIS

National Inst. of Standards and Technology (MSEL),

Gaithersburg, MD. Polymers Div.

Effect of Cross-Links on the Miscibility of a Deuterated Polybutadiene and Protonated Polybutadiene Blend.

Final rept.

H. Jinnai, H. Hasegawa, T. Hashimoto, R. Birber,

and C. C. Han. 1993, 7p.

Pub. in Macromolecules 26, n1 p182-188 1993.

Keywords: "Polymer blends, "Polybutadiene, "Crosslinking, Elastomers, Deuterium compounds, Hydrogen, Labeled substances, Small angle scattering, Neutron scattering, Binary systems(Materials), Temperature, Phase separation(Materials), Reprints

The effect of peroxide crosslinking on the phase diagram and the scattering function for a critical mixture of perdeuterated polybutadiene (DPB) and protonated polybutadiene (HPB) has been examined by smallangle neutron scattering as a function of temperature. The scattering curves for the cross-linked blends were essentially temperature independent. A comparison of the scattering for the cross-linked blend with that of the linear blend at the cross-linking temperature $(150 \mathrm{C})$ showed a suppression in the scattering due to the presence of the crosslinks. However, it was experimentally found that the concentration fluctuations present at the temperature of crosslinking dominate the scattering, which made it rather difficult to verify the prediction on the scattering function made by de Gennes.

\section{1,226}

PB94-212446 Not available NTIS

National Inst. of Standards and Technology (MSEL), Gaithersburg, MD. Polymers Div.

Inversion of the Phase Diagram from UCST to LCST in Deuterated Polybutadiene and Protonated Polybutadiene Blends.

Final rept

H. Jinnaj, H. Hasegawa, T. Hashimoto, and C. C.

Han. 1992, 3p.

Pub. in Macromolecules 25, n22 p6078-6080 1992.

Keywords: "Phase diagrams, "Polymer blends, "Polybutadiene, Microstructure, Elastomers, Hydrogen, Labeled substances, Deuterium compounds, Temperature dependence, Small angle scattering, Neutron scattering, Reprints.

Miscibility of elastomer blends with various microstructures have been extensively studied in recent years. The separation of the isotope labeling effect from the microstructure effect has been successfully carried out by Sakurai et al. by using mean-field approximation. One of the important predictions is the possibility of phase diagram inversion from UCST to LST or vice versa for these random copolymer blends. In this study, such phenomena have been found for a deuterated polybutadiene and hydrogenated polybutadiene pair by studies from small angle neutron scattering (SANS) experiments.

01,227

PB94-212479 Not available NTIS

National Inst. of Standards and Technology (MSEL),

Gaithersburg, MD. Polymers Div. 
Topological Influences on Polymer Adsorption and Desorption Dynamics.

Final rept.

H. E. Johnson, J. F. Douglas, and S. Granick. 1993, $4 \mathrm{p}$.

Pub. in Physical Review Letters 70, n21 p3267-3270, 24 May 93.

Keywords: "Polymers, "Adsorption, *Desorption, Companson, Molecular weight, Surface chemistry, Transport properties, Reprints.

The desorption of polymer chains through an overlayer of strongly adsorbed chains was studied to determine the influence of topological constraints on the polymer desorption process. The desorption time of linear chains fits a power law, $M$ (sup a), where $M$ is molecular weight and $\mathrm{a}=2.3+$ or -0.2 . A comparison of linear and star-branched chains shows that desorption of star-branched chains was greatly suppressed. These findings are reminiscent of entanglement effects in bulk systems. They suggest a unifying perspective from which to analyze polymer mobility at surfaces.

01,228

PB94-212610 Not available NTIS

National Inst. of Standards and Technology (NEL) Gaithersburg, MD. Fire Science and Engineering Div. Effects of Molecular Weight and Thermal Stability on Polymer Gasification.

Final rept.

T. Kashiwagi, and A. Omori. $1988,4 p$.

Pub. in Chem. Phys. Processes Combust., p68/1-68/ 41988

Keywords: "Polymers, "Gasification, "Molecular weight, "Thermal stability, Polystyrene, Polymethyl methacrylate, Plastics, Heat rate, Heat of vaporization, Reprints.

Effects of initial molecular weight and thermal stability on the gasification process of polymeric materials were studied by determining differences in gasification rate, global heat of vaporization and rate of heat release rate between two polystyrene (PS) samples and two polymethylmethacrylate (PMMA) samples.

01,229

PB94-212941 Not available NTIS

National Inst. of Standards and Technology (MSEL) Gaithersburg, MD. Polymers Div.

Effects of Variable Excluded Volume on the Dimensions of Off-Lattice Polymer Chains.

Final rept.

D. E. Kranbuehl, and P. H. Verdier. 1993, 6p

Pub. in Macromolecules 26, n15 p3986-3991 1993.

Keywords: "Molecular models, "Polymers, "Monte Carlo method, Statistical mechanics, Mathematical models, Volume, Reprints, "Off lattice polymer chains, "Bend stick models, Excluded model.

The expansion of bead-stick models of polymer chains by excluded volume has been obtained by Monte Carlo methods for chains with ratios d of hard-sphere bead diameter to stick length between zero (no excluded volume) and unity (connected beads touching) for chains of from 9 to 99 beads. We report values of mean square end-to-end length (I(sup 2)) and apparent power law exponents $2 \mathrm{nu}=\mathrm{d}(\ln (1$ sup 2$)) / \mathrm{d}(\ln (\mathrm{N}-1))$ for chains of $N$ beads, for eight values of $d$ from 0.30 to 0.93 . For the range of chain lengths reported here, the apparent power-law exponent is not independent of $d$ but rather shows a smooth, gradual transition from the well-known result for $d=0$ to the previously reported value for $d=1$. The variation of 2 nu with bead size is remarkably similar to its variation with shortrange attractive energy in other models. The results reported here are compared with those obtained by other workers on and off lattices, for hard-sphere and Lennard-Jones potentials, and with predictions of twoparameter and scaling theories.

01,230

PB94-217031 PC A03/MF A01

National Inst. of Standards and Technology (MSEL), Gaithersburg, MD. Polymers Div.

Determination of the Weight Average Molecular Weight of Two Poly(Ethylene Oxides), SRM 1923 and SRM 1924.

C. M. Guttman, and J. R. Maurey. Feb 94, 48p, NISTIR-5286.

Keywords: "Polyoxyethylene, "Molecular weight, *Calibration standards, US NBS, Organic polymers Chemical analysis, Quality assurance, Measurement,
Uncertainty, Precision, Accuracy, Chromatographic analysis, Size determination, "Standard reference materials, Statistical control, National Institute of Standards and Technology, Size exclusion chromatography, SRM 1923, SRM 1924.

The characterization of two narrow molecular weigh distribution poly(ethylene oxide) standard reference materials, SRM 1923 and SRM 1924, is described. The weight-average molecular weight of SRM 1923 by light scattering was determined to be $26.9 \times 1000 \mathrm{~g} / \mathrm{mol}$ with a sample standard deviation of $0.87 \times 1000 \mathrm{~g} /$ mole, based on 3 degrees of freedom. A combined expanded uncertainty of $2 \times 1000 \mathrm{~g} / \mathrm{mole}$ is estimated for this determination. The weight-average molecula weight of SRM 1924 by light scattering was determined to be $120.9 \times 1000 \mathrm{~g} / \mathrm{mole}$ with a sample standard deviation of $1.0 \times 1000 \mathrm{~g} / \mathrm{mole}$, based on 3 degrees of freedom. A combined expanded uncertainty of $9.0 \times 1000$ $\mathrm{g} /$ mole is estimated for this determination.

\section{1,231}

PB95-107090 Not available NTIS

National inst. of Standards and Technology (MSEL), Gaithersburg, MD. Polymers Div.

SANS and LS Studies of Polymer Mixtures Under Shear Flow.

Final rept.

A. I. Nakatani. 1993, 4p.

Pub. in Polymer Preprints, Japan 42, n1 p134-137 1993.

Keywords: *Polymers, "Mixtures, "Light scattering, "Shear flow, Neutron scattering, Phase transformations, Shear rate, Kinetics, Rheology. Reprints, SANS(Small angle neutron scattering), LS(Light scattering), Polymer blends.

Small angle neutron scattering (SANS) and light scattering (LS) techniques have been used recently for in situ observations of the behavior of polymer mixtures under the influence of an applied shear field. Many prior studies examine the phase behavior of polymer mixtures under shear by turbidimetric or light scattering methods. The smaller size scales accessible with SANS make a combination of LS and SANS desirable. A couette geometry shear cell for SANS and a cone and plate geometry cell for LS have been constructed at the National Institute of Standards and Technology. Details of the two instruments will be described. Various examples of the results from the two instruments will be presented.

\section{1,232}

PB95-107389 Not available NTIS

National Inst. of Standards and Technology (MSEL) Gaithersburg, MD. Polymers Div.

Polyethylene Crystallized from an Entangled Solution Observed by Scanning Tunneling Microscopy. Final rept.

D. H. Reneker, J. Schneir, B. Howell, and H. Harary. 1990, 3p.

Pub. in Polymer Communications 31, n5 p167-169 1990.

Keywords: "Polyethylene, "Crystallization, "Surfaces, Molecular weight, Scanning electron microscopy, Reprints, “Scanning tunneling microscopy.

Rapid cooling of a thin layer of an entangled solution of ultra-high molecular weight polyethylene on a mica sheet resulted in a structure composed of elongated patches of polymer a few nanometers thick, aligned over distances of several micrometers. Some rows of side-by-side patches were thicker and formed a structure with a moss-like appearance at lower magnification. The scanning tunneling microscope was shown to be useful for the examination, at high magnification, of polymer surfaces. The top surface of a thin, conducting layer of evaporated platinum and carbon on a polymer reveals much the same information as could be obtained by removing the platinum and carbon layer and examining it with a transmission electron microscope. The often troublesome step of removing the platinum/carbon replica is not needed for the scanning tunneling microscope.

\section{1,233}

PB95-108668 Not available NTIS

National Inst. of Standards and Technology (MSEL), Gaithersburg, MD. Polymers Div.

Response of a Terminally Anchored Polymer Chain to Simple Shear Flow.

Final rept.

R. S. Parnas, and Y. Cohen. 1991, 11p.

Pub. in Macromolecules 24, n16 p4646-4656 1991
Keywords: "Polymers, "Shear flow, "Dynamic response, Plastic flow, Molecular structure, Brownian movement, Fluid dynamics, Hydrodynamics, Reprints.

The dynamic behavior of a terminally anchored freelyjointed bead rod chain, subjected to uniform solvent shear flow, was investigated via Brownian dynamics simulations. Computed segment density distributions and other statistical expressions of the chain conformation demonstrate a strong effect of shear rate on chain behavior. Values of the effective hydrodynamic thickness computed from the numerical similations were also found to be a strong function of the shear rate. Stationary statistical results obtained for the chain extension show two regimes in the configuration space of the model chain, a Brownian regime at low shear rates and a hydrodynamic regime at high shear rates.

01,234

PB95-140075 Not available NTIS

National inst. of Standards and Technology (MSEL), Gaithersburg, MD. Reactor Radiation Div.

Morphology of Symmetric Diblock Copolymers as Revealed by Neutron Reflectivity.

Final rep

S. K. Satija, C. F. Majkrzak, S. H. Anastasiadis, and T. P. Russell. 1990,6p.

Pub. in Materials Research Society Symposia Proceedings 166, p139-144 1990

Keywords: "Block copolymers, "Morphology, "Thin films, "Polystyrene, "Polymethyl methacrylate, Surface properties, Substrates, Neutron radiography, Reflectivity, Annealing, Interfaces, Laminates, Phase transformations, Reprints.

Recently, it has been shown that symmetric diblock copolymers of polystyrene (PS), and polymethylmethacrylat thin films (5000A or less) on silicon substrates, exhibit a strong orientation of the lamellar microdomains parallel to the surface of the substrate. This orientation occurs when the copolymer films are annealed at temperatures above the glass transition temperatures of the PS and PMMA blocks and results from the interactions of the two blocks with the air and substrate interfaces. PMMA, the more polar species, preferentially resides at the silicon (silicon oxide)/copolymer interfaces, whereas PS, the lower surface energy component, preferentially segregates to the air/copolymer interface. These interactions, coupled with the chemical connectivity of the PS and PMMA blocks, result in the observed multilayered structure.

01,235

PB95-140125 Not available NTIS

National Inst. of Standards and Technology (MSEL), Gaithersburg, MD. Polymers Div.

Novel Polydiacetylenes Derived from Liquid Crystalline Monomers.

Final rept.

M. A. Schen. 1988, 10p

Pub. in Proceedings of International Conference on Electrical, Optical and Acoustic Properties of Polymers (1st), Canterbury, UK., September 5-7, 1988, p12/1$12 / 40$

Keywords: "Liquid crystals, "Acetylene, Small angle scattering, X-ray scattering, Reaction kinetics, Polymerization, Monomers, Reprints, "Polydiacetylenes.

The liquid crystal phase polymerization of a liquid crystalline diacetylene monomer, 1,6 bis-(4oxybenzylidene 4 '-n-octylaniline) 2,4 -hexadiyne is reported and characterized. Within the crystal and liquid crystal phases, first-order disappearance of monomer is seen by differential scanning calorimetry with a thermal activation energy of $155 \mathrm{~kJ} / \mathrm{mol}$. The lamellar structure characteristic of the monomer smectic phase is conserved with polymerization as shown by $\mathrm{X}$-ray scattering. The topochemical polymerization of diacetylenes is therefore extended to include polymerization in the liquid crystal phase.

\section{1,236}

PB95-140190 Not available NTIS

National Inst. of Standards and Technology (MSEL), Gaithersburg, MD. Polymers Div.

Glass Temperature of Polymer Blends: Comparison of Both the Free Volume and the Entropy Predictions with Data.

Finai rept.

H. A. Schneider, and E. A. Di Marzio. 1992, 9p.

Pub. in Polymer 33, n16 p3453-3461 1992.

Keywords: "Blends, *Polymers, "Glass transition temperature, "Volume, "Entropy, Mathematical models 
Phase transformations, Monomers, Experimental data, Reprints, "Polymer blends.

An equation derived from the hypothesis that the glass temperature is determined by entropy considerations and an equation derived from the hypothesis that the glass temperature is determined from free volume considerations are each compared to 17 polymer blend systems. The curves mimic experiment in about half (the same half) of the cases. The (roughly) equivalent predictions of the two equations suggests that the glass temperature of an infinite molecular weight polymer is proportional to the mass of a monomer unit divided by the number of flexible bonds of a monomer unit. A slightly modified treatment suggests proportionality to monomer volume per flexible bond. It is pointed out each of the two equations are zeroth order treatments and that they can each be improved. The imparameters.

01,237

PB95-141156 Not available NTIS

National Inst. of Standards and Technology (MSEL) Gaithersburg, MD. Polymers Div.

Effect of Hydrodynamic Interactions on a Terminally Anchored Bead-Rod Model Chain.

Final rept.

R. S. Parnas, and Y. Cohen, 1993, 5p.

Pub. in Proceedings of Symposium on Dynamics in Small Confining Systems, Boston, MA., November 30December 4, 1990, 5p 1993.

Keywords: "Hydrodynamics, "Polymers, "Chains, Brownian movement, Simulation, Shear flow, Molecu lar structure, Statistical distributions, Radius of gyration, Reprints, "Hydrodynamic interactions, "Bead-rod model.

Brownian dynamics simulations of a terminally anchored, freely-jointed bead-rod chain were used to reveal the effects of Yamakawa hydrodynamic interactions on the statistical properties of the chain. Properties such as the RMS and effective hydrodynamic thicknesses were computed with and without hydrodynamic interactions. Although hydrodynamic interactions significantly influenced the magnitude of the statistical properties of the terminally anchored beadrod chain, the general trends seen in such properties were not altered. Additionally, the results obtained with the freely-jointed bead-rod model are in better agreement with experimental results than those obtained with bead-spring models, for surface bound polymer layers.

01,238

PB95-150033 Not available NTIS

National Inst. of Standards and Technology (MSEL) Gaithersburg, MD. Reactor Radiation Div.

Observed Frustration in Confined Block Copoly-

mers.

P. Lambooy, T. P. Russell, G. J. Kellog, S. K. Satija, A. M. Mayes, and P. D. Gallagher. 1994, 4p.

Contract DE-FG03-88ER45375

Sponsored by Department of Energy, Washington, DC Pub. in Physical Review Letters 72, n18 p2899-2902 2 May 94.

Keywords: "Block copolymers, "Solids, "Surface properties, Neutron radiography, Reflectivity, Interfaces, Films, Separation, Reprints.

Symmetric, diblock copolymers confined between two solid surfaces were studied by neutron reflectivity. A multilayered morphology with an integral number of layers oriented parallel to the solid interfaces was found in all cases. The period of the confined multilayers deviated from the bulk period in a cyclic manner as a function of the confined film thickness. A first-order transition occurred between the expanded and contracted states of the copolymer chains. The data suggest that the deviation of the period from the bulk value decreases with increasing separation distance.

01,239
PB95-150454 Not available NTIS

National Inst. of Standards and Technology (MSEL), Gaithersburg, MD. Polymers Div

Structural Stabilization of Phase Separating PC/ Polyester Blends through Interfacial Modification by Transesterification Reaction.

Final rept.

H. Yoon, Y. Feng, Y. Qiu, and C. C. Han. 1994, 8p. Pub. in Jnl. of Polymer Science: Part B. Polymer Phys ics 32, p1485-1492 1994
Keywords: "Molecular structure, "Phase separation(Materials), "Polymer blends, "Interfaces "Esterification, Copolymers, Polycarbonate resins, Time dependence, Temperature dependence, Small angle scattering, Neutron scattering, Melting, Heat measurement, Reprints, Differential scanning calorimetry.

Competition between phase separation and transesterification in immiscible polymer blends of polycarbonate (PC) and a copolyester (PET) is studied as a function of time and temperature by differential scanning calorimetry (DSC) and small-angle neutron scattering (SANS). We found that (1) Global structure coarsens at $T$ greater than or equal to $200 \mathrm{C}$ due to the dominance of phase separation over transesterification and melts at $\mathrm{T}$ greater than or equal to $200 \mathrm{C}$ due to the dominance of transesterification at the domain interface. However, transesterification is slow but still significant even at $\mathrm{T}$ greater than or equal to $200 \mathrm{C}$. (2) An intricate balance of transesterification and phase separation rates controls global and interfacial structures. (3) Interfacial structures become measureable under certain conditions, and the interfacial thickness between PC or PET and the copolymers generated by transesterification increases with time. (4) DSC results are consistent with results obtained by SANS, but the latter is more sensitive than the former and differentiates the structural change at different length scales caused by phase separation and transesterification

01.240

PB95-151072 Not available NTIS

National Inst. of Standards and Technology (MSEL), Gaithersburg, MD. Polymers Div.

Thermal Stability of Internal Electric Field and Polarization Distribution in Blend of Polyvinylidene Fluoride and Polymethylmethacrylate.

Final rept.

N. Tsutsumi, Y. Ueda, T. Kiyotsukuri, A. S. DeReggi, and G. T. Davis. 1993, 7p.

Pub. in Jnl. of Applied Physics 74, n5 p3366-3372, 1 Sep 93

Keywords: "Thermal stability, "Polymer blends, "PMMA, "Polarization(Charge separation) Polyvinylidenes, Ferroelectric materials, Dyes, Electric fjelds, Reprints, "Polyvinylidene fluorides, "Internal electric fields.

It is confirmed that a melt-quenched and annealed blend of $80 \mathrm{wt} \%$ polyvinylidene fluoride and $20 \mathrm{wt} \%$ polymethylmethacrylate has the beta-crystal form of PVDF with optical clarity and other properties that are desirable for a host material in a guest-host system where the guest is an optically nonlinear dye that is orientationally stabilized by the strong internal electric field of a poled ferroelectric. Combined measurements in such a blend, of the internal electric field $E$ (sub i), the pyroelectric coefficient $C$ (sub pyro), and the polarization distribution after electrically poling and subsequently thermally aging for $2 \mathrm{~h}$ intervals at temperatures up to $120 \mathrm{C}$ are reported.

\subsection{1}

PB95-151247 Not available NTIS

National Inst. of Standards and Technology (MSEL) Gaithersburg, MD. Polymers Div.

Anisotropic Phase Separation Kinetics in a Polymer Blend Solution Following Cessation of Shear Studied by Light Scattering.

Final rept.

D. A. Waldow, A. I. Nakatani, and C. C. Han. 1992,

Pub. in Polymer 33, n21 p4635-4638 1992.

Keywords: "Polymer blends, "Phase separation(Materials), "Kinetics, "Anisotropy, "Laboratory equipment, "Shear flow, Light scattering, Solutions, Polystyrene, Polybutadiene, Monitoring, Reprints.

A light scattering instrument capable of monitoring the scattering from samples under the influence of a simple shear field has been constructed. The apparatus consists of transparent cone-and-plate fixtures and a two-dimensional charge coupled device array detector. The detector unit is also capable of measuring the scattering patterns as a function of time. The phase separation kinetics of an $8 \%$ solution of a polystyrene/ polybutadiene (50:50) blend in dioctyl phthalate following cessation of a steady shear is monitored with this instrument. The sample is two-phase in the quiescent state and the applied shear is sufficient to suppress all scattering observed from the quiescent sample (shear-induced mixing). The evolution of the scattering profiles following cessation of shear is quite differen parallel and perpendicular to the original flow direction. In the normal direction, a spinodal growth and coarsening mechanism similar to that observed in temperature quench experiments is observed. In the parallel direction, a different mechanism is followed. We believe this is the first report of this type of anisotropic behavior in the phase separation kinetics of a polymer blend.

01,242

PB95-151841 Not available NTIS

National Inst. of Standards and Technology (MSEL), Gaithersburg, MD. Polymers Div.

SANS Study of the Plastic Deformation Mechanism in Polyethylene.

Final rept.

W. Wu, G. D. Wignall, and L. Mandelkern. 1993, 4p. Pub. in Polymer 33, n19 p4137-4140 1992.

Keywords: "Polyethylene, "Neutron scattering, "Polymer blends, "Plastic deformation, Small angle scattering, Thermoplastic resins, Deuterium compounds, Protons, Separation, Melting, Reprints, SANS(Small Angle Neutron Scattering).

Small angle neutron scattering was used to investigate the role of partial melting and recrystallization in the solid state deformation of polyethylene. Blends containing 4 vol\% deuteropolyethylene in protonated polyethylene were deformed in a pure shear mode. The scattering cross section of the undeformed blends was well in excess of that expected for randomly (statistically) dispersed molecules, due to isotopic segregation effects between the deuterated and the protonated species. A significant reduction in the excess scattering was observed as the blends were deformed beyond the yield point. Upon further deformation the cross section underwent a further modest decrease. Such a reduction is known to occur on melting. The neutron scattering results, hence, support the notion of local melting during the yielding and the subsequent deformation processes.

01,243

PB95-151866 Not available NTIS

National Inst. of Standards and Technology (MSEL), Gaithersburg, MD. Polymers Div.

Structural Heterogeneity in Epoxies.

Final rept.

W. L. Wu, J. T. Hu, and D. L. Hunston. 1990, 6p. Pub. in Polymer

Keywords: "Molecular structure, "Curing, "Epoxy resins, "Heterogeneity, Small angle scattering, $X$-ray scattering, Mechanical properties, Fracture properties, Toughness, Modulus of elasticity, Impact strength, Glass transition temperature, Reprints.

The effects of different cure procedures on the structure and properties of epoxy samples made from diglycidyl ether of bisphenol A (DGEBA) and mixtures of two linear aliphatic diamines were studied. The elastic modulus, fracture toughness, impact resistance and glass transition temperature were determined for various cure schemes. The morphologies of the cured res ins were characterized with small angle $x$-ray scattering. The results show that samples with the same average morphology (molecular network structure) have similar elastic moduli and glass transition temperatures. If some heterogeneity is introduced in the molecular network structure without changing the average structure, however, the experiments indicate that the toughness can be increased without significantly sacrificing other properties.

01,244

PB95-151882 Not available NTIS National Inst. of Standards and Technology (MSEL), Gaithersburg, MD. Polymers Div.

Characterization of Polyquinoline Block Copolymer Using Small Angle Scattering.

Final rept.
W. L. Wu, J. K. Stille, J. W. Tsang, and A. J. Parker. 1989, 4p.

Pub. in Materials Research Society Symposia Proceedings, v134 p493-4961989.

Keywords: "Block copolymers, "Small angle scattering, Neutron scattering, X-ray scattering, Deuterium compounds, Separation, Rigidity, Flexibility, Reprints, *Polyquinolines.

To determine the compatibility between the rigid rod and the flexible chain polyquinolines, both small angle 
$\mathrm{x}$-ray and neutron scattering measurements were conducted on a sample containing deuterated flexible components. The scattering intensities from both $\mathrm{x}$-ray and neutron were reduced to the absolute scale in order to remove the scattering contribution from microvoids which tended to overshadow the signal from the molecular origin. Quantitative information regarding the extent of segregation of this rigid rod containing blends can be obtained from the scattering data free of microvoid contribution.

01,245
PB95-151890 Not available NTIS

National Inst. of Standards and Technology (MSEL), Gaithersburg, MD. Polymers Div.

Dynamic Light-Scattering Study of a Diluted Polymer Blend Near Its Critical Point.

Final rept.

H. Yajima, D. Hair, A. Nakatani, J. Douglas, and C.

Han. 1993, 4p

Pub. in Physical Review B 47, n18 p12 268-12 271, 1 May 93

Keywords: *Polymer blends, "Light scattering, "Critical point, "Dynamics, "Polystyrene, "Polybutadiene, Experimental design, Reprints, Phthalate/dioctyl.

The critical dynamics of a moderately-high-molecularweight polymer blend of polystyrene and polybutadiene diluted in dioctyl phthalate is studied near the consolute point by dynamic light scattering. Fast and slow modes are observed as in previous experiments on a low-molecular-weight binary-polymerblend melt. The light-scattering data are consistent with a Fisher renormalization of the correlation-length exponent.

\section{1,246}

PB95-152237 Not available NTIS

National Inst. of Standards and Technology (MSEL), Gaithersburg, MD. Polymers Div.

Thermal Behaviour of Methyl Methacrylate and $\mathrm{N}$ Phenyl Maleimide Copolymers.

Final rept.

L. Choudhary, D. S. Varma, I. K. Varma, and F. W.

Wang. 1993, 10p.

Pub. in Jnl. of Thermal Analysis 39, p633-642 1993

Keywords: "Copolymers, "Methyl methacrylate, "Synthesis(Chemistry), "Thermal stability, PMMA, Reprints, "Maleimide/N-phenyl.

The paper describes the synthesis and characterization of six copolymers of methyl methacrylate (MMA) and N-phenyl maleimide (NPM). Thermal stability of PMMA was markedly improved by incorporation of even a very low mole fraction of NPM in the backbone. An increase in mole fraction of NPM from 0.0979 to 0.4766 only marginally affected thermal stability.

\section{1,247}

PB95-152872 Not available NTIS

National Inst. of Standards and Technology (MSEL), Gaithersburg, MD. Polymers Div.

Thermodynamic interactions and Correlations in Mixtures of Two Homopolymers and a Block Copolymers by Small Angle Neutron Scattering. Final rept.

N. P. Balsara, S. V. Jonnalagadda, C. C. Lin, C. C. Han, and R. Krishnamoorti. 1993, 10p.

Pub. in Jnl. of Chemical Physics 99, n12 p1001110020, 15 Dec 93

Keywords: "Polymer blends, "Thermodynamics, "Copolymers, "Neutron scattering, "Polyolefins, Small angle scattering. Random phase approximation, Hydrogen, Deuterium, Synthesis(Chemistry), Phase studies, Reprints.

Thermodynamic interactions in mixtures of two homopolymers and a block copolymer were obtained from small-angle neutron scattering (SANS) measurements. Experimental SANS profiles from homogeneous, ternary mixtures of model polyolefins-poly(ethyl butylene)/poly(methyl butylene)/poly(methyl butylene)-b-poly(ethyl butylene)--were compared with theoretical predictions based on the multicomponent random phase approximation (RPA). The polymers were nearly monodisperse and were synthesized by saturating the double bonds in anionically synthesized polydienes with $\mathrm{H} 2$ and $\mathrm{D} 2$, thus yielding polyolefins with neutron scattering contrast.
National Inst. of Standards and Technology (MSEL), Gaithersburg, MD. Polymers Div.

Copolymerization of N-Phenyl Maleimide and gamma-Methacryloxypropyl Trimethoxysilane.

Final rept.

L. Choudhary, D. S. Varma, F. W. Wang, V.

Choudhary, and I. K. Varma. 1993, 5p.

Pub. in Jnl. of Applied Polymer Science 49, p91-95 1993

Keywords: "Copolymerization, Reaction kinetics, Decomposition reactions, Endothermic reactions, Re prints, "Maleimide/N-phenyl, "Silane/ methacryloxyprophyl trimethoxy.

Copolymerization of N-phenyl maleimide (NPM) and gamma-methacryloxypropyl trimethoxysilane (MTS) in dioxan solution using benzoyl peroxide as an initiator is described. The rate of copolymerization depended on the mole fraction of NPM in the initial feed and decreased with an increase in NPM content. Copolymers having a mole fraction of NPM varying from 0.29 to 0.55 exhibited similar thermal behavior in terms of initial decomposition temperature of maximum rate of weight loss. However, char yield at $600 \mathrm{C}$ depended on NPM content, showing a tendency to increase with the increase in NPM content.

\section{1,249}

PB95-153151 Not available NTIS

National inst. of Standards and Technology (MSEL), Gaithersburg, MD. Polymers Div.

Thermal Behavior of 4-Maleimidophenyl Glycidyl Ether Resins.

Final rept.

L. Choudhary, D. S. Varma, F. W. Wang, V

Choudhary, and I. K. Varma. 1993, 10p.

Pub. in Thermochimica Acta 220, p261-270 1993

Keywords: "Thermodynamic properties, "Polymerization, "Thermochemistry, Infrared spectroscopy, Nuclear magnetic resonance, Curing, Heat of reaction, Molecular structure, Epoxy resins, Reprints, "Ether/maleimidophenyl glycidyl.

A novel epoxy-maleimide resin, 4-maleimidophenyl glycidyl ether (MGE), was prepared from 4 aminophenol. Characterization was carried out by estimation of the epoxy equivalent and by IR and (1) HNMR spectroscopy. The MGE resin was cured by heating above $250 \mathrm{C}$. A decrease in the curing temperature was observed after the addition of a stoichiometric or non-stoichiometric amount of an aromatic diamine. The values of the curing temperature and the heat of the polymerization reaction (Delta $\mathrm{H}$ ) were independent of diamine concentration but depended on the structure of the diamine. The char yield of cured resins at $800 \mathrm{C}$ in a nitrogen atmosphere ranged from $25 \%$ to $40 \%$, which is much higher than the values reported for epoxy resins.

01,250

PB95-153367 Not available NTIS

National Inst. of Standards and Technology (MSEL), Gaithersburg, MD. Polymers Div.

Piezoelectric and Pyroelectric Polymers.

Final rept.

G. T. Davis. 1993, 31p

Pub. in Polymers for Electronic and Photonic Applica. tions, p435-465 1993.

Keywords: "Vinyl copolymers, "Piezoelectricity, "Pyroelectricity, "Polarization(Charge separation),
Nylon fibers, Ferromagnetic materials, Hysteresis, Thermal stresses, Reprints.

Piezoelectric and pyroelectric response from polymers arises from changes in polarization resulting from stress or temperature-induced changes in volume. Factors which determine the polarization that can be induced by poling are outlined for amorphous polymers and for semicrystalline polymers. Definitions of piezoelectric and pyroelectric coefficients and the conventions of nomenclature are presented. Typical coefficients for polymers are compared with those for other organic or inorganic materials which exhibit such properties. A brief description of the properties of specific polymers is presented with frequent reference to more detailed review articles.

\section{1,251}

PB95-153789 Not available NTIS

National Inst. of Standards and Technology (MSEL) Gaithersburg, MD. Polymers Div.
SANS Studies of Space-Time Organization of Structure in Polymer Blends.

Final rept.

T. Hashimoto, H. Jinnai, H. Hasegawa, and C. C. Han. 1993, 11p.

Pub. in Proceedings of International Symposium on Advanced Nuclear Energy Research (5th) - Neutrons as Microscopic Probes, Ibaraki, Japan, March 10-12, 1993, p73-83

Keywords: "Molecular structure, "Polymer blends, "Neutron scattering, "Polybutadiene, "Polyisoprene, Small angle scattering, Light scattering, Deuterium compounds, Synthetic elastomers, Spinodal decomposition, Time resolution, Protons, Reprints, SANS(Small Angle Neutron Scattering).

Self-organization of structure in polymer blends via spinodal decomposition (SD) was studied over a wide spatial-scale and time-scale (both being covered ove 4 orders of magnitude) by using a combined time-resolved small-angle neutron scattering and light scattering method. The system studied was a binary critical fluid of deuterated polybutadiene and proton polyisoprene having a narrow molecular weight dis tribution in a weak segregation limit. The scattering analyses made it possible to study space-time organization of various elements of the structures: (1) globa structure, (2) interfacial structure such as mean curvature of the tangled interface, interfacial area density and interfacial thickness, (3) 'interphase', i.e. interfacial region with characteristic thermal concentration fluctuations, and (4) the local structure characterized by the thermal concentration fluctuations within each domain. In the late stage SD, the form factor from the 'interphase' was found to be time-independent, having the q-dependence of $q(\sup -2.5)$

01,252

PB95-161196 Not available NTIS

National Inst. of Standards and Technology (MSEL), Gaithersburg, MD. Polymers Div.

Time-Resolved Small-Angle Neutron Scattering Study of Spinodal Decomposition in Deuterated and Protonated Polybutadiene Blends. 1. Effect of Initial Thermal Fluctuations.

Final rept.

$H$ Jinnai, $H$ Hasegawa, T Hashimoto, and C. C.

Han. 1993, 327p

Pub. in Jnl. of Chemical Physics 99, n6 p4845-4854, 15 Sep 93

Keywords: "Polymer blends, "Spinodal decomposition, "Polybutadiene, "Neutron scattering, Synthetic elastomers, Small angle scattering, Deuterium com pounds, Protons, Time resolution, Temperature ef fects, Binary systems(Materials), Phase diagrams, $R e$ prints.

Time-resolved small-angle neutron scattering (SANS) experiments have been performed on the self-assembling process of a binary mixture of deuterated polybutadiene and protonated polybutadiene at the critical composition. This mixture has an upper critical solution temperature type of phase diagram with the spinodal temperature at $99.2 \mathrm{C}$. Specimens held in the single-phase state at an initial temperature (T(sub i)) were quenched to a point inside the spinodal phase boundary at a final temperature (T(sub f)) to induce phase separation via spinodal decomposition (SD). In order to examine the effect that thermal concentration fluctuations have on SD, three different initial temperatures, $T$ (sub $i)=102.3 \mathrm{C}, 123.9 \mathrm{C}$, and $171.6 \mathrm{C}$, were chosen while $T$ (sub $f$ ) was fixed at $-7.5 \mathrm{C}$. A critica test of the linearized Cahn-Hilliard-Cook theory led to the conclusion that this theory can describe satisfac torily the early stage SD in the deep-quench region.

\section{1,253}

Not available NTIS

National Inst. of Standards and Technology (MSEL), Gaithersburg, MD. Polymers Div.

Localization of a Homopolymer Dissolved in a Lamellar Structure of a Block Copolymer Studied by Small-Angle Neutron Scattering.

Final rept.

Y. Matsushita, N. Torikai, Y. Mogi, I. Noda, and C. C. Han. 1993, 4p.

Pub. in Macromolecules 26, n24 p6346-6349 1993

Keywords: "Block copolymers, "Neutron scattering, * Molecular structure, "Polymers, Small angle scattering, Lamellar structure, Deuterium compounds, Molecular chains, Phase separation(Materials), Reprints.

The localization of deuterated styrene homopolymer dissolved in a lamellar structure of a styrene-2 


\section{Polymer Chemistry}

vinylpyridine diblock copolymer was studied in comparison with that of the end part of the block copolymer by observing diffraction from the styrene and 2vinylpyridine microdomains of deuterated styrene homopolymer/styrene-2-vinylpyridine diblock copolymer blends by small-angle neutron scattering (SANS). Even when the average scattering lengths of both domains were equal, that is, 'phase contrast matching' was theoretically achieved, diffraction was definitely observed. From the diffraction profiles in SANS and the dependence of domain spacing on the volume fraction of the styrene homopolymer, it was found that the homopolymers are isolated in the middle of the polystyrene domain, although they are concentrated less than the end parts of the block chains.

\section{1,254}

PB95-161691 Not available NTIS

National Inst. of Standards and Technology (MSEL) Gaithersburg, MD. Polymers Div.

Molecular Weight Dependence of the Lamellar Domain Spacing of $A B C$ Triblock Copolymers and Their Chain Conformation in Lamellar Domains.

Final rept.

Y. Mogi, K. Mori, H. Kotsuji, C. Han, Y. Matsushita, and I. Noda 1993, 5p.

Pub. in Macromolecules 26, n19 p5169-5173 1993.

Keywords: "Molecular weight, "Block copolymers, *Molecular chains, "X-ray scattering, Lamellar structure, Small angle scattering, Phase tion, Styrene vinylpyridine.

The molecular weight dependence of the lamellar domain spacing of isopren-styrene-2-vinylpyridine triblock copolymers in bulk was studied over the molecular weight range from $40 \mathrm{~K}$ to $280 \mathrm{~K}$ by using a smallangle $X$-ray scattering (SAXS) method. It was found that the lamellar domain spacings of the triblock copolymers are larger than those of styrene-2vinylpyridine diblock copolymers with the same molecular volumes. This result can be interpreted by theories of microphase separation at the strong segregation limit, taking into account the fact that the number of boundaries in the repeating structure of triblock copolymers is twice as large as that of diblock copolymers. Moreover, small-angle neutron scattering (SANS) studies imply that the middle block chain of triblock copolymers is contracted along the direction parallel to lamellae in almost the same manner as the block chain of the diblock copolymers.

\section{1,255}

PB95-161816 Not available NTIS

National Inst. of Standards and Technology (MSEL), Gaithersburg, MD. Polymers Div.

Elastic Scattering of Polymer Networks.

Final rept.

W. L. Wu. 1991, 4p.

Pub. in Macromolecules 24, n5 p1145-1148 1991

Keywords: "Neutron scattering, "Crosslinking, "Molecular structure, "Spatial distribution, "Polymers, Random phase approximation, Reprints.

The theoretical aspect of the scattering of crosslinked polymers was investigated with emphasis on the fact that the number of spatial neighbors for any given network is finite. A random phase approximation scheme was used; however the interaction terms was truncated to account for this finiteness. The result indicates that the expression based on infinite neighbors hold true in all the cases.

\section{1,256}

PB95-161865 Not available NTIS

National Inst. of Standards and Technology (MSEL), Gaithersburg, MD. Polymers Div.

Neutron Scattering Study of Shear Induced Turbidity in Polystyrene Dissolved in Dioctyl Phthalate. Final rept.

A. I. Nakatani, J. F. Douglas, Y. B. Ban, and C. C. Han. 1994, 9p.

Pub. in Jnl. of Chemical Physics 100, n4 p3224-3232, 15 Feb 94.

Keywords: "Neutron scattering, "Polystyrene, "Turbidity, "Solutions, Shear rate, Small angle scattering, Deuterium compounds, Hydrogenation, Temperature dependence, Specific heat, Critical temperature, Reprints, Phthalate/dioctyl.

The influence of shear on the small angle neutron scattering from a semidilute solution of polystyrene (PS) dissolved in dioctyl phthalate (DOP) is examined in the limit of strong shear (gamma greater than or $=$ to 100/ s). These experiments are restricted to room temperature, which is close to the theta point of PS/DOP. The fraction of deuterated PS chains is varied, while the total polymer weight fraction is fixed at $3 \%$. This is nea the critical composition of hydrogenated PS in DOP. The increased scattering at high shear rates ("shear induced turbidity') is interpreted in terms of a critical temperature shift. Scattering along the flow direction is distorted and the scattering data normal to flow is uninfluenced by shear above a wave vector cutoff $q^{*}$ which is independent of shear rate. This cutoff is found to correspond to the Debye length, characterizing the average intermolecular potential range.

01,257

PB95-163044 Not available NTIS

National Inst. of Standards and Technology (MSEL) Gaithersburg, MD. Polymers Div.

Synthesis and Polymerization of Difunctional and Multifunctional Monomers Capable of Cyclopolymerization.

Final rept.

J. W. Stansbury. 1990, 2p.

Pub. in Polymer Preprints 31, n1 p503-504 1990.

Keywords: "Synthesis(Chemistry), *Polymerization, "Monomers, "Cyclic compounds, Esters, Crosslinking, Vitreous state, Ethers, Intramolecular forces, Reprints.

The reaction of an acrylate ester with paraformaldehyde in the presence of DABCO has given access to a novel class of ether-fused pseudodimethacrylate monomers. The 1,6-diene unit incorporated in the monomers can be polymerized through an inter-intramolecular cyclopolymerization process which yields a soluble, ester-substituted, cyclic ether polymer structure A series of monomers was prepared to evaluate the effect of the substituents on the synthesis and cyclopolymerization. A method was developed which efficiently provided even monomers with very bulky ester groups. Solution polymerization studes indicated that both monomer concentration and ester structure exert significant influence on the mode of polymerization. Apparently, the more hindered monomers are more effective at cyclopolymerization The bulk polymerization of these monomers resulted in crosslinked, glassy polymers with little or no residual unsaturation. Multifunctional oligomers based on this same chemistry were also prepared and polymerized in this study.

\section{1,258}

PB95-163770 Not available NTIS

National Inst. of Standards and Technology (MSEL) Gaithersburg MD Polymers Div.

Applications of Fluorescence Spectroscopy in Polymer Science and Technology.

Final rept

F. W. Wang. 1991, 6p

Pub. in Polymer Science 2, p546-551 1991

Keywords: "Polymers, "Epoxy resins, "Curing, "Spectrum analysis, Marking, Labeled substances, Viscosity, Diffusion coefficient, Polystyrene, Bleaching Photochemical reactions, Transport properties, Reprints, "Fluorescent dyes.

Two approaches using fluorescent dyes dissolved in epoxy resins were used to determine the viscosity changes during the curing process. First, we used a dye whose fluorescence intensity increases with the in crease in the local viscosity, and a second dye whose fluorescence intensity is insensitive to the local viscos ity. The ratio of the fluorescence intensities of the two dyes was measured to monitor the cure of epoxy res ins. In another approach, we measured the diffusion coefficient of a fluorescent dye dissolved in epoxy resins by fluorescence recovery after photobleaching to monitor the cure of epoxy resins. Finally, self-diffusion in concentrated polystyrene solutions was measured by the method of fluorescence recovery after photobleaching.

\section{1,259}

PB95-164109 Not available NTIS

National Inst of Standards and Technology (MSEL) Gaithersburg MD. Polymers Div.

Characterization of Molecular Network of Thermosets Using Neutron Scattering.

Final rept.

W. L. Wu. 1990, 4p.

Pub. in Materials Research Society Symposia Proceedings, v166 p475-478 1990.
Keywords: "Molecular structure, "Epoxy resins, "Thermosetting resins, "Neutron scattering, Curing, Polymerization, Reprints.

Neutron scattering measurements were conducted to investigate the conformation of the molecular networks in epoxies. Emphasis was placed on the changes in the scattering intensity resulting from curing. Experimental results on homopolymerized epoxy as well as a theoretical interpretation of the results are presented.

\section{1,260}

PB95-164117 Not available NTIS

National Inst. of Standards and Technology (MSEL), Gaithersburg. MD. Polymers Div.

Small-Angle Neutron Scattering of Poly(vinyl alcohol) Gels.

Final rept.

W. Wu, H. Kurokawa, S. Roy, and R. S. Stein. 1991,

Pub. in Macromolecules 24, n15 p4328-4333 1991.

Keywords: "Neutron scattering, “Gels, "Polyvinyl alcohol, "Molecular structure, Small angle scattering, Temperature, Transition temperature, Sols, Reprints.

The structure of aqueous poly(vinyl alcohol) (PVA) gels with and without borate ions, hereafter referred to as alkaline gels and hydrogels, respectively, was investigated using small-angle neutron scattering (SANS). The correlation length Xi, a measure of the wavelength of concentration fluctuation, was estimated from the SANS results. The value of $\mathrm{Xi}$ showed a modest decrease with increasing temperature. Even at the high est temperature of observation, $95 \mathrm{C}$, Xi was still a few times greater than the radius of gyration of individual PVA molecules, indicating the presence of PVA clusters in the sol state. The scattered intensities for both the hydrogels and the alkaline gels over a wide temperature range could be superimposed onto one master curve by employing the reduced variables $1(q) /$ $X i(\exp 3)$ and $(X i)(q)$, where $q$ is the magnitude of the scattering vector. This indicates that the concentration fluctuation can be described adequately by a single characteristic length over a wide temperature range embracing the sol-gel transition temperature.

\section{1,261}

PB95-164125 Not available NTIS

National Inst. of Standards and Technology (MSEL), Gaithersburg, MD. Polymers Div.

Characterization of Polyquinoline Blends Using Small Angle Scattering.

Final rept.

W. L. Wu, J. K. Stille, J. W. Tsang, and A. J. Parker. $1990,5 p$

Sponsored by Defense Advanced Research Projects Agency, Arlington, VA and Air Force Office of Scientific Research, Bolling AFB, DC

Pub. in Materials Research Society Symposia Proceedings, v171 p189-1931990.

Keywords: "Polymer blends, "Neutron scattering, " $X$ ray scattering, Small angle scattering, Molecular chains, Deuterium compounds, Reprints, "Polyquinolines, Rigid rod polymers, Flexible chain polymers.

To determine the compatibility between the rigid rod and the flexible chain polyquinolines, both small angle $x$-ray and neutron scattering measurements were conducted on blends containing deuterated flexible chains. The scattering intensities from both $x$-ray and neutron were reduced to their absolute scales in order to remove the scattering contribution from microvoids which tended to overshadow the signal of molecular origin. Quantitative information regarding the molecular dispersion in a 50/50 rigid rod and flexible chain blend was obtained. The result indicated that this material was partially segregated but not to the point of single component phases.

01,262

PB95-164372 Not available NTIS

National Inst. of Standards and Technology (MSEL), Gaithersburg, MD. Polymers Div.

Small Angle Neutron Scattering Studies on Chain Asymmetry of Coextruded Poly(Vinyl Alcohol) Film.

Final rept

M. Shibayama, H. Kurokawa, S. Nomura, W. L. Wu, S. Roy, and R. S. Stein. 1990, 6p.

Pub. in Macromolecules 23, n5 p 1438-1443 1990.

Keywords: "Neutron scattering, "Polyvinyl alcohol, "Molecular chains, "Coextruding, Small angle scatter- 
ing. Deformation, Birefringence, Least squares method, Regression analysis, Radius of gyration, Reprints. Molecular conformation.

Chain asymmetry of solid state co-extruded poly(vinyl alcohol) film was studied using small-angle neutron scattering. The radius of gyration $R(s u b g)$ was estimated by a nonlinear least square regression analysis with a theoretical scattered intensity function which is based on a model of deformed Gaussian chains. The $R$ (sub g) values for deformed PVA indicate that the poly(vinyl alcohol) chains are deformed affinely at least up to the extension draw ratio of ca. 5 , which is in good accordance with the results of birefringence measurement.

01,263

PB95-164380 Not available NTIS

National Inst. of Standards and Technology (MSEL), Gaithersburg, MD. Polymers Div.

Small Angle Neutron Scattering Study on Poly(NIsopropyl Acrylamide) Gels Near Their VolumePhase Transition Temperature.

Final rept.

M. Shibayama, T. Tanaka, and C. C. Han. 1992,

$13 p$

Pub. in Jnl. of Chemical Physics 97, n9 p6829-2841 1 Nov 92.

Keywords: "Neutron scattering, "Polymers, "Gels, "Phase transformations, "Temperature dependence, Small angle scattering, Heavy water, Solutions, Lorentz transformations, Gauss equation, Ising model, Reprints, "Poly(amide/N-propylacryl).

The small angle neutron scattering experiments were conducted on $\mathrm{N}$-isopropyl acrylamide (NIPA) gels in D2O and on the corresponding NIPA solutions. The NIPA gels underwent a sharp, but a continuous volume phase transition at $34.6 \mathrm{C}$ from a swollen state to a shrunken state with increasing temperature. In the case of the gels, an excess scattering due to the presence of crosslinks was observed at low q region ( $q<$ $0.02 / A)$, where $q$ is the magnitude of the scattering vecor. The scattered intensity function for the gel was we described with a combination of Gauss and Lorentztype functions. The Gaussian part results from solidlike inhomogeneity, having a characteristic size of $\mathrm{Xi}_{\text {, }}$ which is due to the introduction of crosslinks into the system. The Lorentzian part is originated from the liquid nature of the local concentration fluctuations of the gel characterized with a thermal blob of dimension X The critical exponents for the gels support that the volume-phase transition of gels is classified to the three dimensional Ising model reported by $\mathrm{Li}$ and Tanaka.

\section{1,264}

PB95-164398 Not available NTIS

National Inst. of Standards and Technology (MSEL), Gaithersburg, MD. Polymers Div

Small-Angle Neutron Scattering Study on Weakly Charged Temperature Sensitive Polymer Gels.

Final rept.

M. Shibayama, T. Tanaka, and C. C. Han. 1992,

M. Sp.

Pub. in Jnl. of Chemical Physics 97, n9 p6842-6854, 1 Nov 92.

Keywords: "Neutron scattering, "Temperature dependence, "Polymers, "Gels, "Statics, Small angle scattering, Electrolytes, Phase transformations, Scattering coefficients, Phase separation(Materials), Heavy water, Reprints, "Poly(amide/N-propylacryl), "Polyacrylic acid.

The static structure factor for poly $(\mathrm{N}$ isopropylacrylamide-co-acrylic acid) (NIPAAAC) gels was investigated in terms of small-angle neutron scattering (SANS). The NIPAVAAC gels underwent a discrete volume phase transition at $50.8 \mathrm{C}$ from swollen to shrunken states as temperature increased. The static structure factors for NIPANAAC gels were well described by a Lorentz-type scattered intensity function at temperatures below $34 \mathrm{C}$ which was near the so called Theta temperature of NIPA in D2O. At highe temperatures, the static structure factor had a distinct scattering maximum although the gel was still in the highly swollen state. The static structure factors were analyzed quantitatively with the theory of Borue and Erukhimovich for polyelectrolyte solutions in a poor solvent. It is found that the concentration fluctuations lead to a microphase separation between polymer rich and poor domains before the system undergoes the volume-phase transition.
National Inst. of Standards and Technology (MSEL), Gaithersburg, MD. Polymers Div.

Competition between Hydrodynamic Screening ('Draining') and Excluded Volume Interactions in an Isolated Polymer Chain.

Final rept.

J. F. Douglas, and K. F. Freed. 1994, 12p.

Pub. in Macromolecules 27, n21 p6088-6099 1994.

Keywords: "Polymers, "Molecular structure, *Hydrodynamics, "Solutions, Solvents, Dynamics, Coefficient of friction, Intermolecular forces, Configuration interaction, Reprints, Excluded volume, Preaveraging approximation.

A remaining challenge in the theory of polymer solution dynamics is associated with establishing a relation between the macroscopic hydrodynamic properties of polymer solutions and the molecular structure of the polymer and solvent. This problem is unsolved even for the simplest case of polymer solutions at 'infinite dilution'. Recent studies have focused on technical problems such as the 'preaveraging' approximation, epsilon-expansion truncation errors, and the influence of ternary excluded volume interactions which limit the accuracy of analytic calculations. The present paper examines the role of polymer excluded volume in altering intramolecular hydrodynamic interactions and the possible significance of dynamic chain flexibility on hydrodynamic polymer solution properties. This investigation is aided by analysis of our previous renormalization group (RG) computations and simple exactly solvable models. We also examine experimental trends for the variation of the polymer hydrodynamic interaction with solvent and the variation of the translational friction of small molecules with molec ular size.

\section{1,266}

PB95-175733 Not available NTIS

National Inst. of Standards and Technology (MSEL), Gaithersburg, MD. Polymers Div.

Examination of the $\mathrm{V} / \mathrm{d}$ Expansion Method from Exact Enumeration for a Self-Interacting SelfAvoiding Walk.

Final rept.

T. Ishinabe, J. F. Douglas, A. M. Nemirovsky, and K F. Freed. 1994, 11p.

Pub, in Jnl. of Physics A: Mathematical and General 27, p1099-11091994.

Keywords: "Polymers, "Series expansion, "Enumeration, Comparison, Thermodynamics, Molecular structure, Reprints, "Self-avoiding walks

The 1/d expansion method of polymer chains is examined by comparing these expansions for several thermodynamic and structural quantities with the results of standard series analysis of exact enumeration data. The comparisons cover a wide range of spatial dimensions d, including non-integer ones, and are performed for particular values of interaction energy. Good agreement is generally found for $d>4$, whereas discrepancies become conspicuous as $d$ decreases to $d=2$. Reasonable values are obtained for the exponents nu and gamma in $d=2-4$ by applying the coherent-anomaly method of Suzuki to our $1 / d$ expansions through fifth order in $1 / d$.

\section{1,267}

PB95-175998 Not available NTIS

National Inst of Standards and Technology (CSTL), Boulder, CO. Thermophysics Div.

Gas Transport Properties of Solution-Cast Perfluorosulfonic Acid lonomer Films Containing lonic Surfactants.

Final rept.

J. Pellegrino, D. Wang, R. Rabago, R. Noble, and C Koval. 1993, 9p

Pub. in Jnl. of Membrane Science 84, p161-169 1993.

Keywords: "Polymeric films, "Gas transport, Membranes, Casting, Ions, Separation, Carbon dioxide, Methane, Permeability, Diffusion, Fluorine organic compounds, Surfactants, Reprints, "Poly(sulfonic acid/ tetrafluoro)

We have made solution-cast films of perfluorosulfonic acid ionomer (PFSA) that included ionic surfactants in the casting solution. Gas transport measurements were made, using mixtures of $\mathrm{CO} 2$ and $\mathrm{CH}_{4}$, through both solution-cast and commercial PFSA films that contained either $\mathrm{Na}(+)$ or HEDA(+) (monoprotonated ethylenediamine) counterions. The HEDA(t) provides facilitated transport of $\mathrm{CO} 2$ at low partial pressures. For all the films with the $\mathrm{Na}(+)$ counterion the dif- ference in permeability was greater at lower feed tota pressures than at the higher pressures (approximately $11 \mathrm{MPa}$ ). We interpret this result as a mechanical pressure effect on the free volume around the ionic clusters. Additional observations include a 'hindering' ef fect of the HEDA(+) counterion on both $\mathrm{CO} 2$ and $\mathrm{CH} 4$ permeation rates but more so for the $\mathrm{CO} 2$. We discuss these results in the context of separate factors that are economically attractive at all the pressures studied. However, the permeability of the membranes was below economic targets by a factor of 100 considering the fact that they were 30 micrometers thick

01,268

PB95-176145 Not available NTIS

National Inst. of Standards and Technology (MSEL) Gaithersburg, MD. Polymers Div.

Ring-Opening Polymerication of a 2-Methylene Spiro Orthocarbonate Bearing a Pendant Methacrylate Group.

Final rept.

J. W. Stansbury. 1994, 13p.

Sponsored by National Inst. of Dental Research, Bethesda, MD.

Pub. in Polymers of Biological and Biomedical Significance, Chapter 14, p171-1831994.

Keywords: "Monomers, "Synthesis(Chemistry) "Polymerization, "Methacrylates, Dental materials, Mo ecular structure, Medical supplies, Composite mate nals, Adhesives, Coatings, Chemical bonds, Crosslinking, Polycarbonate resins, Methylene radicals, Reprints, Chemical reaction mechanisms.

A methacrylate-substituted spiro orthocarbonate monomer was synthesized and evaluated in polymeriza tions using radical and/or cationic initiators. The monomer contains an exocyclic double bond on the spiro group for radical addition and ring opening independent of the remote methacrylate functionality. Crosslinked polymers were obtained by all modes of nitiation with mixed radical and cationic giving optimum conversions and ring opening. The incomoration of the pendant methacrylate group minimizes concerns of leachable products generated by polymerization mechanisms involving single ring opening with elimination of a cyclic carbonate. The spiro vinyl ether-type double bond appears to activate the monomer toward cationic polymerization. The ring-opening polymerization of spiro orthocarbonate monomers can yield ex pansion in volume and may improve a variety of dental and medical materials such as composites, adhesives, and coatings.

01,269

PB95-180014 Not available NTIS

National Inst. of Standards and Technology (PL) Gaithersburg, MD. Ionizing Radiation Div.

Polymerization Initiation by $\mathrm{N}$-p-Tolylglycine: FreeRadical Reactions Studied by Pulse and Steady State Radiolysis.

Final rept.

M. Al-Sheikhly, M. Farahani, and R. L. Bowen. 1994, 10p.

Sponsored by American Dental Association Health Foundation, Chicago, IL

Pub. in Jnl. of Applied Polymer Science 54, p1049 10581994

Keywords: "Polymerization, "Free radicals, "Adhesive bonding, Hydroxyl radicals, Oxidation, Reduction(Chemistry), Cations, Anions, Reprints, "Chemical reaction mechanisms, Peroxyl radicals, Gly cine/ $\mathrm{N}$-tolyl.

The extensive use of N-p-tolylglycine (NTG) and analogous compounds in adhesive bonding technologies requires a better understanding of their role in initiating ree-radical polymerization. The fast oxidation and reduction reactions of NTG proceed via the formation of various free radicals and radical cation and anion intermediates. These intermediates were identified and their reactivity with oxygen, to produce the correspond ing peroxyl radicals, was measured. Hydroxyl radicals $\mathrm{OH}$ ) were used to initiate oxidation reactions of NTG, while the reduction reactions were initiated with hydrated electrons (e(sub aq)(sup -)).

01,270

PB95-180196 Not available NTIS

National Inst. of Standards and Technology (MSEL) Gaithersburg, MD. Polymers Div.

Neutron Scattering Study of the Orientation of a Liquid Crystalline Polymer by Shear Flow.

Final rept.

M. D. Dadmun, and C. C. Han. 1994, 11p.

Pub. in Macromolecules 27, n26 p7522-7532 1994 
Keywords: "Neutron scattering, "Shear flow, "Polymers, "Orientation, Solutions, Shear rate, Dynamics, Rheology, Anistropy, Reprints, "Liquid crystalline polymers, Poly (glutamate/benzyl).

The orientational response of a liquid crystalline polymer (LCP) solution, specifically poly-(benzyl L-glutamate) (PBLG) in deuterated benzyl alcohol, to the application of a shear field has been determined by insitu neutron scattering. By analyzing the anisotropic two-dimensional scattering pattern, the orientation of the LCP at different shear rates (dot-gamma) in the flow-vorticity plane was determined. It was found that the LCP response has three regimes. At low shea rates, dot-gamma < dot-gamma 1, the orientation of the LCP molecule increases with dot-gamma; at intermediate flow rates, dot-gamma $1<$ dot-gamma < dotgamma 2, there is not much change in the orientation of the PBLG molecule with dot-gamma; and at high dot-gamma, dot-gamma > dot-gamma 2, there is again an increase in the orientation of the LCP with shear rate. The crossover shear rates, dot-gamma 1 , and dot-gamma 2, have been shown to correlate well to the first two relaxation times of a rodlike polymer in concentrated solution. The results are also compared and contrasted to some recent studies of the rheology of LCP in solution.

\section{1}

PB95-180683 Not available NTIS

National Inst. of Standards and Technology (PL) Gaithersburg, MD. Ionizing Radiation Div.

Radiochromic Solid-State Polymerization Reaction.

Final rept.

W. L. McLaughlin, M. Al-Sheikhly, D. F. Lewis, A Kovacs, and L. Wojnarovits. $1994,2 \mathrm{p}$.

Pub. in Polymer Preprints 35, n2 p920-921 Aug 94

Keywords: "Polymerization, Reaction kinetics, Electron beams, Dispersions, Dosimetry, Radiolysis, Uses, Reprints, “Radiochromic films.

The GafChromic radiochromic film is a colorless microcrystalline dispersion of an active monomer in gel binder. It has been developed for direct radiographic imaging and dosimetry (no chemical, optical, or thermal development). This relatively sensitive sensor may be used as a thin-coating on a transparent or reflecting substrate for broad-range dosimetry (510 (exp 4) Gy) and for registering permanent, high-resolution, high-contrast blue images. Pulse radiolysis studies show that the primary radiation-initiated imag formation (lambda $(\max )=675 \mathrm{~nm})$ and propagation of the polymerization are terminated within $2 \mathrm{~ms}$ with a first-order rate constant of $900 / \mathrm{s}$. Subsequently there is a much slower exponential hypsochromic shift of the absorption maximum from $675 \mathrm{~nm}$ to $665 \mathrm{~nm}$. The devices are currently finding application in clinical radiog raphy, stereotactic radiosurgery, food irradiation, blood irradiation, insect population control, and industrial radiation processing (including medical sterilization qualification and quality control).

\section{1,272}

PB95-181061 Not available NTIS

National Inst. of Standards and Technology (MSEL) Gaithersburg, MD. Polymers Div.

Terminally Anchored Chain Interphases: Their Chromatographic Properties.

Final rept.

J. H. van Zanten. 1994, 11p

See also PB95-181079

Pub. in Macromolecules 27, n23 p6797-6807, 7 Nov 94.

Keywords: "Polymers, "Chromatographic analysis, "Interfaces, "Solvents, Dispersions, Molecular structure, Phase studies, Block copolymers, Graft polymerization, Solutes, Thermodynamics, Separation processes, Reprints, "Polymer brushes.

A previously developed Flory-type mean-field analysis of the mixing of a multicomponent, polydisperse solvent with an interphase of terminally anchored chains of finite extensibility is utilized in the determination of the chromatographic properties of the interphase. These interphases could be surface-grafted polymer layers or block copolymers at interfaces. The partitioning and retention of solute molecules in the interphase depend on the chain configurations, the entropy of mixing, and the contact interactions among the species present. The theory allows for the calculation of average or global properties such as the polymer, solvent, and solute volume fractions in the interphase, the interphase thickness, and solute partition coefficients and retention factors. Size exclusion and enhancement, affinity, and gradient chromatography are considered.

\section{1,273}

PB95-181079 Not available NTIS

National Inst. of Standards and Technology (MSEL), Gaithersburg, MD. Polymers Div.

Terminally Anchored Chain Interphases: The Effect of Multicomponent, Polydisperse Solvents on Their Equilibrium Properties.

Final rept.

J. H. van Zanten. 1994, 8p.

See also PB95-18106

Pub. in Macromolecules 27, n18 p5052-5059, 29 Aug 94.

Keywords: "Polymers, "Interfaces, "Solvents, "Equilibrium, Qualitative analysis, Thermodynamics, Molecular structure, Numerical analysis, Surface properties, Dispersions, Phase studies, Reprints, "Polymer brushes.

A theoretical description of terminally anchored chain interphases is presented for the case of an interphase in contact with a multicomponent solvent composed of species of varying quality and size. This work is an extension of a model proposed by Lai and Halperin to calculate the global properties of a terminally anchored chain interphase, or polymer brush, in contact with a binary solvent in which each component is of monomeric size. Here it is shown that the solvent size, in addition to the solvent quality and chain surface density, has a profound influence on the equilibrium properties of the terminally anchored chain interphase. The approach outlined here yields a simple calculational procedure for qualitatively examining the various global properties of these interphases which are inherent to each potential application as a function of chain surface density and solvent quality and size.

\section{1,274}

PB95-202735 Not available NTIS

National Inst. of Standards and Technology (MSEL) Gaithersburg, MD. Reactor Radiation Div

Neutron Reflectivity Study of the Density Profile of a Model End-Grafted Polymer Brush: Influence of Solvent Quality.

Final rept.

A. Karim, S. K. Satija, J. F. Douglas, J. F. Ankner, and L. J. Fetters. 1994, 4p.

Pub. in Physical Review Letters 73, n25 p3407-3410, 19 Dec 94.

Keywords: ‘Density(Mass/volume), Polymers, Neutron scattering, Temperature dependence, Solvents, Reprints, "End grafted polymers, "Neutron reflectivity, "Polymer brush.

Neutron reflectivity measurements are made on a chemically end-grafted polymer brush swollen over a range of temperatures above and below the theta point. Good agreement between the brush profiles and recent self-consistent field calculations and numerical simulations is obtained for temperatures in the vicinity of the theta point and in a good solvent. The expansion of the main body of the brush resembles a swelling gel, while the brush tail expands similarly to a polymer in solution.

01,275

PB95-209896 PC A07/MF A02

National Inst. of Standards and Technology (MSEL), Gaithersburg, MD. Polymers Div.

Polymers Technical Activities 1994. NAC-NRC Assessment Panel, April 6-7, 1995.

L. E. Smith, and B. M. Fanconi. 1995, 142p, NISTIR5581

See also PB92-116284.

Keywords: “Polymers, “Composite materials, "Medical supplies, "Dental materials, Organic polymers, Standards, Technology transfer, Viscoelasticity, Rheologica properties, Computerized simulation, Transport properties, Mechanical properties, Durability, Models, Ceramics, "Polymer blends, Annual report.

The annual report of the Polymers Division, Materials Science and Engineering Laboratory, describes technical activities for the fiscal 1994 year and summarizes technical publications, industrial interactions, patents, and invited talks.

01,276
PB96-102231 Not available NTIS
National Inst. of Standards and Technology (MSEL), Gaithersburg, MD. Polymers Div.

Self-Avoiding-Walk Contacts and Random-Walk Self-Intersections in Variable Dimensionality. Final rept. J. F. Douglas, and T. Ishinabe. 1995, 27p Pub. in Physical Review E 51, n3 p1791-1817 Mar 95. Keywords: "Polymers, "Series expansion, "Enumeration, Lattices, Molecular structure, Critical temperature, Percolation, Integral equations, Exponents, Interactions, Probability theory, Reprints, "Selfavoiding walks, "Random walks, NN(Nearest-neighbor), Hamilton walks, Wiener sausage.

The average number of nearest-neighbor (NN) contacts $(\mathrm{m})$ of self-avoiding walks (SAW's) on a hypercubic lattice is calculated using direct enumeration and $1 / d$ expansion methods, where $d$ is the spatial dimension. These calculations are compared with exact analytic determinations for the asymptotic number of random-walk (RW) self-intersections in the limit of long chains $n$ maps to infinity. the number of RW (binary, ternary, etc.) self-intersections is a function of the probability $\mathrm{Cd}$ that a RW escapes from the origin to infinity and an accurate tabulation of $\mathrm{Cd}$ is given in the dimension range $2<d<10$.

01,277

PB96-119508 Not available NTIS

National Inst. of Standards and Technology (MSEL), Gaithersburg, MD. Polymers Div.

Shear-Induced Martensitic-Like Transformation in a Block Copolymer Melt.

Final rept.

C. L. Jackson, K. A. Barnes, F. A. Morrison, C. C. Han, J. W. Mays, and A. I. Nakatani. 1995, 10p Pub. in Macromolecules, n28 p713-722 1995

Keywords: "Martensitic transformations, "Shear, "Copolymers, *Melts(Crystal growth), Microstructure Styrenes, Polystyrene, Electron microscopy, Neutron scattering, Deformation, Reprints, SBS block copolymer, Polybutadiene.

The coexistence of two cylindrical microstructures of different symmetries has been observed in a sheared and quenched poly(styrene-d8)/polybut adiene/ poly(styrene-d8) (SBS) block copolymer (23 wt \% styrene-d8) by small-angle neutron scattering and transmission electron microscopy. Near the order-disorder transition (ODT) temperature, the equilibrium cylindrical microstructure with $\mathrm{d}(100)=21$ plus or minus 2 $\mathrm{nm}$ and cylinder diameter of about $12 \mathrm{~nm}$ orients in the shear field as expected based on previous reports. Above a critical shear rate and at an appropriate strain, a new shear-induced cylindricalmicrostructure forms.

\section{1,278}

PB96-122478 Not available NTIS

National Inst. of Standards and Technology (CAML), Gaithersburg, MD. Applied and Computational Mathematics Div.

Nonlinear Dynamics of Stiff Polymers.

Final rept.

R. E. Goldstein, and S. A. Langer, 1995, 4p.

Pub. in Physical Review Letters, v75 n6 p1094-1097 Aug 95.

Keywords: *Polymers, "Curve motion, "Nonlinear dynamics, Models, Formalism, Reprints.

A formalism is presented for the nonlinear dynamics of inextensible stiff polymers within the model of local viscous dissipation. By casting the internal elastic forces in an intrinsic representation enforcing the constraint of local inextensibility through a Lagrange multiplier function and utilizing techniques from the differential geometry of curve motion, the dynamics of configurations of arbitrary complexity is reduced to a scalar partical differential equation amenable to analytical and efficient numerical study. As an example, the formalism is applied to the 'folding' dynamics of stiff polymers with pairwise self-interactions and intrinsic curvature.

01,279

PB96-123245 Not available NTIS

National Inst. of Standards and Technology (BFRL), Gaithersburg, MD. Fire Science Div.

Polymer Combustion and Flammability: Role of the Condensed Phase.

Final rept.

T. Kashiwagi. 1994, 15p.

Pub. in International Symposium on Combustion (25th), Irvine, CA., July 31-August 5, 1994, p14231437. 
Keywords: "Polymers, "Combustion, "Flammability, Combustion products, Reprints, "Condensed phase.

The combustion process of polymers is a complex coupling of energy feedback from a flame to the polymer surface with gasification of the polymer surface with gasification of the polymer to generate combustible degradation products. Although there are extensive studies of the effects of wind velocity, gas phase oxygen concentration, external thermal radiation, and gravity on the combustion of polymers, the effects of polymer characteristics on combustion and flammability are not nearly as well understood as those in the gas phase. At present, detailed goveming equations for continuity, momentum, energy, and chemical species concentration in the gas phase can readily be written with appropriate boundary conditions, and their solutions can be derived for various cases. However, even those governing equations cannot be derived for the condensed phase without understanding of the governing chemical and physical processes that control the gasification of polymers. This paper concentrates on describing various observed phenomena in polymers (which have been often ignored or neglected) during their combustion, some or all of which might have significant effects on the burning rate and flammability properties. Because of a lack of understanding of the basic combustion mechanisms of poly. mers, theoretical models able to predict combustion phenomena and flammability properties are not available.

01,280

PB96-123526 Not available NTIS

National Inst. of Standards and Technology (MSEL), Gaithersburg, MD. Polymers Div.

Book Review: Statistical Physics of Macromolecules.

Final rept.

J. Douglas. 1995, 2p.

Pub. in American Scientist, v83 p479-480 1995.

Keywords: "Polymer physics, "Macromolecules, "Statistical analysis, Polymers, Reviews, Reprints.

A review of 'Statistical Physics of Macromolecules' is given. Basically, this text is well written and provides a good introduction to polymer science.

01,281

PB96-123591 Not available NTIS

National Inst. of Standards and Technology (MSEL),

Gaithersburg, MD. Polymers Div.

Slow Dynamics of Segregation in Hydrogen-Bonded Polymer Blends.

Final rept.

E. K. Hobbie, G. Merkle, B. J. Bauer, and C. C. Han 1995, 4p.

Pub. in Physical Review E, v52 n3 p3256-3259 Sep 95.

Keywords: "Molecular structure, "Neutron scattering, *Polymer blends, Phase separation, Hydrogen bonding, Reprints, Spinodal decomposition, Fractal dimensions.

Time-resolved small-angle neutron scattering has been used to study the dynamics of phase separation in hydrogen-bonded polymer blends under a variety of shallow-quench conditions. Above a well defined cutoff wave vector $\mathrm{qC}(\mathrm{star})$, the structure factor $\mathrm{S}(\mathrm{q}, \mathrm{t})$ is described by an exponential or a stretched exponential decay. The early-stage growth rate $R(q)$ appears to change sign precipitously at the cutoff, however, which is not consistent with a linear theory. Below the cutoff, $S(q, t)$ exhibits nonlinear growth with a characteristic time scale $\operatorname{tm}(q)$, and scales as $q(-D) f(t / t$ sup $m)$ with a scaling exponent $D$ that is often different from the universal value $d=3$.

\section{1,282}

PB96-128251 PC A03/MF A01

National Inst. of Standards and Technology (MSEL), Gaithersburg, MD. Polymers Div

Certification of the Standard Reference Material 1473a, a Low Density Polyethylene Resin.

J. R. Maurey, W. R. Blair, and C. M. Guttman. Oct

95, 24p, NISTIR-5639.

Keywords: "Certification, "Calibration standards, * Polyethlenes, Temperature, US NBS, Thermoplastic resins, Quality assurance, Organic polymers, Error analysis, Viscosisty, Test methods, "Standard reference material 1473a, NIST(National Institute of Standards and Technology), Plastometer, Melt viscosity.
The melt flow rate of Standard Reference Materia (SRM) 1473a, a polyethylene resin, was determined to be $1.17 \mathrm{~g} / 10 \mathrm{~min}$ at $190 \mathrm{deg} \mathrm{C}$ under the load of 2.16 $\mathrm{kg}$ using the ASTM Method D 1238-90b. The average results from 66 determinations on samples with a standard deviation of a single measurement of 0.015 $\mathrm{g} / 10 \mathrm{~min}$. A small but measureable drift from the firs timed extrudate to the third timed extrudate was observed.

01,283

PB96-146667 Not available NTIS

National Inst. of Standards and Technology (MSEL) Gaithersburg, MD. Polymers Div.

Response to 'Draining in Dilute Polymer Solutions and Renormalization'.

Final rept.

K. F. Freed, and J. F. Douglas. 1995, $2 p$

Pub. in Macromolecules, n28 p8460-8461 1995.

Keywords: "Polymers, *Hydrodynamics, "Mathematical models, Solutions, Fluid dynamics, Normality, Reprints, Renormalization group.

This paper discusses a dispute regarding the correc method of modeling the hydrodynamics of polymer solutions and the correct mathematical procedure for perorming renormalization group calculations for polymer solution hydrodynamic properties.

\section{1,284}

PB96-146808 Not available NTIS

National Inst. of Standards and Technology (MSEL), Gaithersburg, MD. Polymers Div.

Thermodynamic Properties of Dilute and Semidilute Solutions of Regular Star Polymers.

Final rept.

J. Roovers, P. M. Toporowski, and J. Douglas. 1995, $7 p$.

Pub. in Macromolecules, p7064-7070 Oct 95

Keywords: "Virial coefficients, "Polymers, "Thermodynamic properties, Solutions, Polybutadiene, Light scattering, Osmosis, Reprints, "Star ploymers, Dilute solutions, Semidilute solutions, Osmotic modulus.

The osmotic modulus of solutions of polybutadiene stars with $\mathrm{f}=32,64$ and 128 arms have been measured from the dilute to the semidilute regime in the good solvent cyclohexane by static light scattering. Some complementry results on low molecular weight stars have been obtained using SANS. Values of the dimensionless viral ratio $g$ A3/A2(exp2) M is approximately 0.62 for these many-arm stars accord well with the hard-sphere value (5/8). The concentration dependence of the osmotic modulus near the polyme coil overlap concentration, A2Mc is approximately 1 is characterized by a steep increase. In the semidilute regime the osmotic modulus becomes identical with that of a homogeneous linear polymer solution. A linear polybutadiene sample was also considered for comparison with the star polybutadiene measurements.

\section{1,285}

PB96-146840 Not available NTIS

National Inst. of Standards and Technology (MSEL) Gaithersburg, MD. Polymers Div.

Preparation and Characterization of Cyclopolymerizable Resin Formulations.

Final rept.

J. W. Stansbury, B. Dickens, and D. W. Liu. 1995

See also PB90-242181

Pub. in Jnl. of Dental Research, v74 n5 p1110-1115 1995.

Keywords: "Acrylic resins, "Dental materials, "Polymers, Polymerization, Chemical reactions, Viscosity, Molecualr weight, Acrylates, Formaldehyde, Reprints.

An amine-catalyzed reaction between acrylates and ormaldehyde has been used to convert monoacrylates to difunctional monomers and di-acrylates to multifunctional oligomers by linking the acrylic double bonds together in 1,6 -diene pairs. In this study, a convenient single-step process was used with mixtures of mono- and di-acrylate starting materials to produce a series of resins with potential for effective cyclopolymerization. Incremental changes in the ethyl acrylate (EA) to ethoxylated bisphenol A diacrylate (EBPAD) ratio directly supplied cyclopolymerizable resins with a broad range of viscosities and product distributions. Those resins produced from reaction mixtures rich in EA have low viscosities because of high diluent monomer contents and limited oligomerization of EBPAD due to end-cap formation. Resin viscosity and average molecular weight of the oligometric component of the resin were inversely related to the amount of EA used in the reaction. Through the choice of reactants and their ratio, this simple technique has the potential to provide cyclopolymerzable resins for use in a variety of dental polymer applications.

01,286

PB96-146865 Not available NTIS

National Inst. of Standards and Technology (MSEL) Gaithersburg, MD Polymers Div.

Light-Scattering Studies on Phase Separation in a Binary Blend with Addition of Diblock Copolymers. Final rept.

L. Sung, and C. C. Han. 1995, 8p

Pub. in Jnl. of Polymer Science. Pant B: Polymer Physics, v33 p2405-2412 1995.

Keywords: "Polymer blends, ${ }^{*}$ Copolymers, "Phase separation, Critical temperature, Diblock, Kinetics, Light scattering, Reprints, Spinodal decomposition

Time-resolved light-scattering measurements have been conducted to investigate the influence of diblock copolymer additive on the phase boundaries and the kinetics of the phase separation of a polyme blend. The blend studied was a polystyrene-d8/ polybutadiene (PSD/PB) mixture with a diblock copolymer composed of the same homopolymers. It was observed that the critical temperature of the blend, which has an upper critical solution temperature (UCST), decreased with increasing copolymer content and the kinetics of the phase separation via a spinodal decomposition mechanism slowed down in the presence of the copolymer. The features of the spinodal peak position and intensity as a function of time with and without $\mathrm{co}-$ polymer additive were analyzed for near and off-critical compositions in various temperature jumps. The intermediate and late-stage growth rates do not follow a universal scaling function with the addition of diblock copolymers.

01,287

PB96-148085 Not available NTIS

National Inst. of Standards and Technology (MSEL)

Gaithersburg, MD. Polymers Div.

Shear-Induced Mixing in Polymer Blends.

Final rept

E. K. Hobbie, A. I. Nakatani, and C. C. Han. 1994,

$19 p$.

Pub. in Modern Physics Letters B, v8 n19 p1143-1161

Keywords: "Polymers, "Shear flow, Neutron scattering, Critical temperature, Phases, Separation, Molecular weight Stratified flow Reprints, Critical polymer blends, SANS(Small angle neutron scattering).

The effect of shear flow on phase separation in critica polymer blends is reviewed. For a low-molecularweight blend, the response is in good agreement with the theoretical predictions of Onuki and Kawasaki. The break-up of large-scale critical fluctuations by the flow leads to a drop in the temperature Tc at which phase separation begins. For a high-molecular-weight blend, the data suggest that the mode-coupling contribution to the decay rate of composition fluctuations is significant in both the miscible and immiscible phases.

01,288

PB96-163753 Not available NTIS

National Inst. of Standards and Technology (MSEL) Gaithersburg. MD. Polymers Div.

Influence of Shear on the Ordering Temperature of a Triblock Copolymer Melt.

Final rept.

A. I. Nakatani, F. A. Morrison, J. F. Douglas, M.

Muthukumar, C. C. Han, J. W. Mays, and C. L.

Jackson. 1996, 11p.

Pub. in Jnl. of Chemical Physics, v104 n4 p1589-1599 Jan 96.

Keywords: "Block copolymers, "Melts, "Neutron scattering, Copolymers, Reprints, Temperature, Shear Polystyrene polybutadiene polystyrene.

The effect of shear on the ordering temperature of a triblock copolymer melt of polystyrene-polybut adientpolystyrene (SBS) is examined by in situ small angle neutron scattering (SANS). Results obtained by SANS arecompared to the rheologically determining orderdisorder transition temperature, TRODT $==115$ plus or minus $\mathrm{C}$. The SANS measurements from a Couette geometry shear cell are then used to construct a 'dy- 
namical phase diagram' based on characteristic changes in the scattering with temperature and shear rate, gamma. A shear rate dependent ordering temperature, Tord(gamma), is identified as the system is sheared isothermally from the disordered state. The scattering behavior is shownto be highly strain dependent. The authors compare their findings on theshear rate dependence of the ordering transition in triblock materials with previous observations on diblock copolymer materials and theoretical expectations for the shear rate dependence of the order-disorder transition temperature. A simple scaling argument leads to a good description of the shear rate dependence of $T$ ord(gamma) in both diblock and triblock copolyme measurements over the range of shearrates examined.

\subsection{9}

PB96-176532 Not available NTIS

National Inst. of Standards and Technology (MSEL) Gaithersburg, MD. Polymers Div.

Solid State (13)C NMR and Raman Studies of Cellulose Triacetate: Oligomers, Polymorphism, and Inferences about Chain Polarity.

Final rept.

D. L. VanderHart, J. A. Hyatt, R. H. Atalla, and V. C. Tirumalai. 1996, 10p

Pub. in Macromolecules, v29 p730-739 1996.

Keywords: "Cellulose triacetate, *Oligomers, Crystal Structure, Polymorphy, Reprints, "Chain polarity, Raman

13 C CPMAS NMR and Raman spectra have been taken to oligomers (DP $=2-9$ ) and several preparations of cellulose triacetate (CTA). CTA exhibits poly morphism and the NMR spectra of the CTA(I) and noncrystalline CTA have been isolated Magnetic inequivalence within the unit cell of CTA(II) is greate than for CTA(I), indicating a lower symmetry in the CTA(II) unit cell. An attempt is made to correlate some of the NMR resonances with proposed X-ray crystal structures for each of these allomorphs. From both the Raman and the NMR spectra of the oligomers, in comparison with the different CTA preparations, it is determined that the pentamer and higher oligomers crys tallize into the CTA(I) lattice. On the basis of the Raman spectra, it is argued that CTA(I) and CTA(II) are distinguished by backbone conformational differences. In a single attempt to explore the role of the solvent in determining the crystal habit of the oligomers, the pentamer persisted in the CTA(I) lattice even when crystallization took place in dibenzyl ether the solvent out of which the CTA polymer forms CTA(II) crystalitites. Based on these findings, an approach of obtaining a definitive crystal structure of CTA(I) would be to grow and perform a structural determination on a single crystal of an oligomer of DP greater than 4 .

\section{1,290}

PB96-180146 Not available NTIS

National Inst. of Standards and Technology (PL) Gaithersburg, MD. Ionizing Radiation Div.

Radiochromic Solid-State Polymerization Reaction.

Final rept

W. L. McLaughlin M. Al-Sheikhly D. F Lewis, A

Kovacs, and L. Wojnarovits. 1996, 15p.

See also PB95-180683.

Pub. in Irradiation of Polymers: Fundamental and Technological Applications, American Chemical Socety Symposium Series 620, Chapter 11, p152-166 1996.

Keywords: "Diacetylenes, "Film dosimeters, Dosimetry, Reprints, GafChromic films, Polydiacetylenes Pulse radiolysis, Radiation processing, Radiographic imaging.

Radiochromic films (GafChromic DM 1260 and MD55), consisting of thin, colorless transparent coatings of a polycrystalline, substituted-diacetylene sensor layer on a clear polyester base, are studied by pulse radiolysis and flash photolysis, in terms of the kinetics of their response to ionizing radiation and ultraviolet light These films have recently been established for broad applications in radiographic imaging, nuclear medicine and dosimetry for radiotherapy, blood irradiation, insect population control, food irradiation, and industrial radiation processing. The radiochromic reaction is a solid-state polymerization, whereby the films turn deep blue proportionately to radiation dose, due to progressive 1,4-trans additions as polyconjugations along the ladder-like polymer chains. The pulsed-electron-induced propagation of polymerization has an observed first-order rate constant of the order of 10 to the 3rd power s to the minus 1 power, depending on the irradiation temperature. The UV-induced polymerization is faster by about one order of magnitude in the case of the electron beam effect, the radiation-induced $a b$ sorption spectrum exhibits a much slower blue-sift of the primary absorption band on the 10 to the minus 3 power - 10 to the minus 1 power second time scale. This effect is attributed to crystalline strain rearrangements of the stacked polymer strand units.

\section{1,291}

PB96-193719 PC A10/MF A02

National Inst. of Standards and Technology (MSEL), Gaithersburg, MD. Polymers Div.

Polymers Technical Activities, 1995.

L. E. Smith, and B. M. Fanconi. 1995, 177p, NISTIR5749

See also PB95-209896.

Keywords: "Polymers, "Composite materials, "Medical supplies, "Dental materials, Organic polymers, Standards, Technology transfer, Viscoelasticity, Electronic packaging, Rheological properties, Transport properties, Computerized simulation, Mechanical properties, Durability, Models, Ceramics, Polymer blends, Annual report.

Technical activities of the Polymers Division, 854 , for the 1995 fiscal year are described. The report is orga nized by programs: Electronic Packaging and Interconnections, Polymer Blends, Polymer Matrix Composites, Viscoelastic Behavior of Polymers, Polymer Characterization, Dental and Medical Materials, Theory and Modeling and Other. The objectives, accomplishments and outputs of each project included in the Programs are given, as well as summaries of publications, patents, industrial and academic interactions, invited talks and participation by Division staff in technical and professional committees.

\section{1,292}

PB96-200753 Not available NTIS

National Inst. of Standards and Technology (MSEL), Boulder. CO. Materials Reliability Div.

Thermal Conductivity of Polypyromellitimide Film with Alumina Filler Particles from 4.2 to $300 \mathrm{~K}$.

Final rept.

D. L. Rule, D. R. Smith, and L. L. Sparks. 1996, 8p. Pub. in Cryogenics, v36 n4 p283-290 1996.

Keywords: "Polymeric films, "Thermal conductivity, "Contact resistance, Alumina, Reprints, "Foreign technology, Polypyromellitimide film, Kapton.

The thermal conductivities of several types of a commercial polyimide (namely polypyromellitimide, PPMI) film were measured over a range of temperatures from 4.2 to $300 \mathrm{~K}$ with an unguarded steady state parallelplate apparatus. Specimens were made by stacking multiple layers of film together. Conductive grease was used between the layers of film to reduce thermal contact resistance. Two specimens were made from two different types of neat film with a thickness of 76 micro $\mathrm{m}$, and three specimens were made from films that contained two different amounts of alumina filler and had thicknesses of 25 or 76 micro m. The conductivity of PPMI film increases with the amount of alumina fille present. The thermal conductivity of specimens made from film of the same type but of different thickness is independent of film thickness, within the limits of ex perimental uncertainty. The conductivity of a specimen subjected to a simulated curing process by being held at a temperature of 150 degrees $\mathrm{C}$ for 90 minutes was indistinguishable from that of a similar, control specimen not subjected to such treatment.

\section{1,293}

\section{PB96-204078 Not available NTIS}

National Inst. of Standards and Technology (BFRL), Gaithersburg, MD. Fire Science Div.

Gas Phase Oxygen Effect on Chain Scission and Monomer Content in Bulk Poly(methyl methacrylate) Degraded by External Thermal Radiation. Final rept.

J. E. Brown, and T. Kashiwagi. 1996, 10p.

Pub. in Polymer Degradation and Stability, v52 p1-10 1996.

Keywords: "Chain scission, "Polymers, "Thermal degradation, Reprints, Decomposition, Fire research, Gasification, Molecular weight, Chromatography, "Foreign technology, Poly(methyl methacrylate).

The effect of atmospheric oxygen on the thermal decomposition of poly(methyl methacrylate), PMMA, in a slab-like configuration was investigated. Blackbody ir- radiation of $12 \mathrm{~mm}$ thick PMMA slabs on one side was used to simulate the thermal decomposition and gasification of the polymer in a fire environment. Results are reported for chain scission number obtained from molecular weight measurements and for residual monomer content at various levels below the slab surfaces irradiated at 17 and $30 \mathrm{k} \mathrm{W} / \mathrm{sq}$. $\mathrm{m}$. in atmospheres containint $0,10,21$ and $41 \%$ oxygen in nitrogen. The scission number and polydispersity of surface layers, about $0.1 \mathrm{~mm}$ thick, were found to increase linearly with the mole fraction of oxygen in nitrogen. Over this range ( 0 to $41 \%$ O2) the scission number increased from 1.5 to 5.0 and the polydispersity increased from 3.6 yo 11.3 when the PMMA was degraded at the lowe flux, while at the higher flux.

\section{1,294}

PB96-204482 Not available NTIS

National Inst. of Standards and Technology (MSEL) Gaithersburg, MD. Polymers Div.

Phase Separation in Thin Film Polymer Blends With and Without Block Copolymer Additives.

Final rept.

L. Sung, A. Karim, J. F. Douglas, and C. C. Han.

1996, $2 \mathrm{p}$.

Pub. in Proceedings of the American Chemical Society Division of Polymeric Materials: Science and Engineering, New

Keywords: "Meetings, "Polymer blends, "Thin films, Reprints, "Phase separation kinetics, Symmetric diblock copolymer, Surface pattern formation, Optical microscopy.

Studies of finite size effects on the equilibrium properties and kinetic processes of materials confined to thin films provide important insight into thin film coating applications. Many recent experimental and theoretica investigations have focused on the influence of film thickness on phase stability. Modification of surface interactions near the boundaries of confined materials can lead to essentially new phenomena which should provide the basis for technological applications. Surfactant materials, such as block copolymers, are known to be effective in modifying interfacial properties so that these additives are expected to have a large effect in thin films.

01,295

PB97-111900 Not available NTIS

National Inst. of Standards and Technology (MSEL), Gaithersburg, MD. Polymers Div.

Compatibilization of Polymer Blends by Complexation. 2. Kinetics of Interfacial Mixing.

Final rept.

Y. Feng, R. A. Weiss, A. Karim, D. G. Peiffer, C. C.

Han, and J. F. Ankner. 1996, $7 p$

Pub. in Macromolecules, v29 n11 p3918-3924 1996 Keywords: "Neutron reflectivity, "Polymer interface, Complexation, lonomer, Diffusion, Reprints.

The interfacial composition and kinetics of interfacia mixing of two polymers that exhibit strong exothermic interactions, poly $(N, N$ '-dimethyle thylenesebacamide) (mPA) and the lithium salt of a lightly sulfonated poly styrene ionomer containing $7.4 \mathrm{~mol} \%$ sulfonate groups (Li-SPS), were characterized by neutron reflectivity measurements. Blends of Li-SPS and MPA are miscible below ca. 150 degrees $C$ as a result of the formation of an ion-dipole complex between the $\mathrm{Li}+$ sulfonate and amide groups. Neutron reflectivity meas urements were carried out on spun-coated bilayer films of the polyamide and a deuterated sample of the ionomer. The films were annealed at 96 degrees $C$ which is above the melting temperature of the MPA $(T M=75$ degrees $C)$ but below the glass transition temperature of the ionomer $(\mathrm{Tg}=120$ degrees $\mathrm{C})$. The interface between the two films exhibited an asymmetric concentration profile due to the large viscosity mismatch between the two polymer, and the interfacial mixing followed neither Fickian nor Case II diffusion A kinetic model that combined Fickian diffusion with a suppression of mobility due to intermolecular crosslinking adequatelyrepresented the experimental data.

01,296

PB97-113146 Not available NTIS

National Inst. of Standards and Technology (MSEL), Gaithersburg, MD. Polymers Div.

Microstructure Effect on the Phase Behavior of Blends of Deuterated Polybutadiene and Protonated Polyisopene.

Final rep

R. N. Thudium, and C. C. Han. 1996, 7p.

Pub. in Macromolecules, v29 n6 p2143-2149 1996 
Keywords: "Elastomer blends, "Interaction parameters, "Microstructure effects, Phase behavior, Neutron scattering, Reprints.

Small-angle neutron scattering (SANS) was used to measure the miscibility of blends of protonated polyisoprene (HPI) with 3,4 microstructure in the range of $7-44 \%$, and of deuterated polybutadiene (DPB) with 1,2 microstructure in the range of $9-70 \%$. These data suggest that, for deuterated polybutadiene/protonated polyisoprene blends, overall miscibility is controlled by the 3,4 content of the polyisoprene. This isomeric group is strongly immiscible with the 1,4 -butadiene group and the immiscibility decreases with temperature, while the 3,4 group is weakly miscible with the 1,4 isoprene group and miscibility increases with temperature. Conversely, the 3,4 group is strongly miscible with the 1,2 butadiene group and miscibility decreases with increasing temperature. The differing trends of the interaction parameter with temperature suggest that shifts in the LCST phase diagram can be controlled by judicious changes in microstructure. These theoretical predications were confirmed with experimental smallangle light scattering (SALS) results on all protonated blends. The statistical segment length, $b$, appears to be independent of temperature, but is dependent on total vinyl level. For the blends used in the study, b has a minimum value at approximately $30-35 \%$ total vinyl isomeric groups.

01,297

PB97-119135 Not available NTIS

National Inst. of Standards and Technology (MSEL) Gaithersburg, MD. Polymers Div.

Morphology and Phase Separation Kinetics of a Compatibilized Blend.

Final rept.

C. L. Jackson, L. Sung, and C. C. Han. 1996, 4p.

Pub. in Society of Plastics Engineers Annual Technical Meeting, Indianapolis, IN., May 5-9, 1996, p15991602

Keywords: "Polymer blends, "Block copolymers, *Microscopy, Light scattering, Reprints.

The phase diagram for a low molecular weight blend of deuterated polystyrene (PSD, $M(W)=1000 \mathrm{~g} / \mathrm{mol})$ and polybutadiene (PB, M $=5100 \mathrm{~g} / \mathrm{mol}$ ) was determined by light scattering measurements. The critical temperature of the blend was 51 degrees $C$ and the critical composition was approximately $0.75 \mathrm{~g} / \mathrm{g}$ polysytrene (75\% PSD by weight). Upon addition of the PSD-PB diblock copolymer (total $M(W)=10,600 \mathrm{~g} /$ mol) linearly to a lower temperature and the rate of phase separation was greatly retarded. The size scale of the phase separated microstructure was also greatly reduced as block copolymer was added, indicating compatibilization of the blend.

\section{CIVIL ENGINEERING}

\section{General}

01,298

PB95-151825 Not available NTIS

National Inst. of Standards and Technology (BFRL), Gaithersburg, MD. Building and Fire Research Lab. Office.

Think Metric.

Final rept

R. N. Wright. 1992, 2p.

Pub. in Jnl. of Structural Engineering 118, n12 p32533254 Dec 92.

Keywords: "Metric system, "Structural engineering, "Standardization, Structural design, Metrication, si units, Metrology, International system of units, Standards, Conversion, Reprints.

The modern metric, International System (SI) of measurement is coming to structural engineering practice. For instance, Federal agencies are required by law to implement SI procurements, specifications, grants, etc., by September 30, 1992. Perhaps the most important challenge this brings to structural engineers is to learn to think quantitatively and exert good engineering judgment in metric dimensions.
01,299

PB95-216891 PC A08/MF A02

National Inst. of Standards and Technology (BFRL) Gaithersburg, MD.

White Papers Prepared for the White House: Construction Industry Workshop on National Construction Goals. Held on December 14-16, 1994. A. J. Fowell. 6 Mar 95, 155p, NISTIR-5610.

Keywords: "Construction industry, "Goals, "National interests, Research management, Competition, Meetings, Proceedings, Commercial buildings, Government buildings.

The report contains seven white papers prepared for a December 1994 White House--Construction Industry Workshop organized by the Civil Engineering Re search Foundation on behalf of the National Science and Technology Council's Subcommittee on Construction and Building.

\section{Civil Engineering}

01,300

PB94-217163 PC A03/MF A0

George Mason Univ., Fairfax, VA.

Human Factors Considerations for the Potential Use of Elevators for Fire Evacuation of FAA Air Traffic Control Towers.

B. M. Levin, and N. E. Groner. Aug 94, 25p, NIST/ GCR-94/656.

Contract NIST-50SBNBIC6678

See also PB92-187129 and PB92-238641. Sponsored by National Inst. of Standards and Technology (BFRL) Gaithersburg, MD

Keywords: "Fire safety, "Elevators(Lifts), "Evacuation "Airport towers, "Human factors, Fire protection, Emergencies, Escape systems, Fires, Human factors engineering, Air traffic control.

The Federal Aviation Administration (FAA) is interested in the possibility of using elevators for evacuation of air traffic control towers during fire emergencies. Assuming that the FAA could design, install, and maintain elevators that could safely be used by tower occupants during fire evacuations, it would be important to study a number of human factor considerations. This repor which is partly based on interviews of occupants in thirteen FAA lowers, discusses these issues. Given the fact that there has been a 20 -year campaign to discourage elevator use during fire emergencies, mos interviewees indicated a willingness to use such elevators as a backup mode of escape with some reluctance. The controls in the elevator would not need any major modification but a special communication system would be needed. Fire emergency plans and train ing are important to assure proper use of the proposed system and confidence in the safety it provides.

\section{1,301}

PB96-167978 PC A04/MF A01

Virginia Transportation Research Council, Charlottesville.

Application of Electromagnetic-Acoustic Trans ducers for Nondestructive Evaluation of Stresses in Steel Bridge Structures.

Final rept.

M. G. Lozev, A. V. Clark, and P. A. Fuchs. cApr 96

43p, VTRC-96-R30

Prepared in cooperation with National Inst. of Stand ards and Technology, Boulder, CO. and West Virginia Univ., Morgantown. Constructed Facilities Center. Sponsored by Virginia Dept. of Transportation, Richmond.

Keywords: "Bridges(Structures), "Nondestructive tests, Bridge foundations, Steel structures, Transducers, Electromagnetic testing, Virginia, Uitrasonic tests, Dynamic loads.

The report presents the results of a study to (1) assess the applicability of electromagnetic-acoustic transducers for nondestructive evaluation of stresses in bridge structures and (2) evaluate the new ultrasonic instruments as an effective technique for stress sur veys in bridge structures. Field tests were performed on two bndges, one a simply supported design and the other an integral backwall bridge. Residual stress measurements were made on a vertical scanline in the web at midspan of a simply-supported bridge. Live load measurements were made by determining the normalized change in arrival times of surface waves propagating between two tranducers mounted on the bottom flange. Good agreement between strain gage and ultrasonic data was obtained, both for the time-history of strain and also for the equivalent stress range.

01,302

PB97-115794 PC A16/MF A03

National Inst. of Standards and Technology (BFRL), Gaithersburg, MD

Proceedings of a Workshop on Developing and Adopting Seismic Design and Construction Standards for Lifelines. Held in Denver, Colorado on September 25-27, 1991

Rept. for 1991-92

R. D. Dikkers, R. M. Chung, B. Mohraz, H S. Lew, and R. N. Wright. Oct 96, 346p, NISTIR-5907. Sponsored by Federal Emergency Management Agency, Washington, DC.

Keywords: "Earthquake resistance, "Seismic design, "Meetings, Earthquake engineering, Construction, Design standards, Vulnerability, Public utilities, Electric power, Natural gas distribution systems, Liquid fuels, Telecommunication, Transportation sector, Water systems, Sewer systems, Government agencies, Federal government, Soil-structure interactions, Government policies, Economic analysis, Regulations, Guidelines, "Lifelines.

These recommendations for developing and adopting seismic design and construction standards for lifelines describe the properties intended for lifeline systems, equipment, and materials. They provide both the mechanism for communication between buyers and sellers of lifeline products and services and the basis for regulations protecting the public health, safety and welfare.

\section{Construction Equipment, Materials, \& Supplies}

\section{1,303}

PB94-145661 PC A08/MF A02

National Inst. of Standards and Technology (BFRL), Gaithersburg, MD.

System for Calibration of the Marshall Compaction Hammer.

H. W. Shenton, M. M. Cassidy, P. A. Spellerberg, and D. A. Savage. Jan 94, 152p, NISTIR-5338, FHWA/RD-94/002

See also PB92-134683. Prepared in cooperation with American Association of State Highway and Transportation Officials, Gaithersburg. MD. Materials Reference Lab. Sponsored by Federal Highway Administration, Washington, DC

Keywords: "Marshall method, "Hot mix paving mixtures, "Flexible pavements, Test methods, Bituminous concretes, Road materials, Specifications, Asphalts Calibration, Compacting.

The Marshall method is used by many state and local highway agencies for the design of hot-mix asphalt pavement. Although the procedure is specified by several industry standards, round-robin programs have confirmed wide variability in Marshall test results. Much of the scatter in the data is attributed to compaction hammer variables, such as: variation in drop weight, drop height, friction, hammer alignment, pedestal support and foundation. With the objective of reducing the variability of Marshall test results, a robust, easy to use and relatively inexpensive test apparatus, described in the report, has been developed for calibration of mechanical Marshall compaction hammers.

01,304

PB94-172236 Not available NTIS

National Inst. of Standards and Technology (BFRL), Gaithersburg MD Building Materials Div.

Digitized Simulation of Mercury Intrusion Porosimetry

Final rept

E. Garboczi, and D. Bentz. 1990, 16p

Pub. in Proceedings of a Conference on Advances in Cementitious Matenals, Gaithersburg, MD. July 22. 26,1990, p365-380.

Keywords: "Cements, "Porous materlals, "Microstructure, "Computerized simulation, 
Porosimeters, Mercury, Pore structure, Permeability, Porosity, Percolation, Reprints, "Mercury intrusion porosimetry.

Mercury intrusion porosimetry is a widely used method for measuring the pore-size distribution of porous materials. The paper describes an algorithm that has been developed that simulates mercury intrusion into a twodimensional porous material. The simulation can be implemented on model structures or on micrographs of real porous materials. Using a burning algorithm, the critical pore diameter for mercury connectivity can be directly computed and studied as a function of microstructure. The point at which the intruding mercury becomes continuous is found to occur at the inflection point of the cumulative intrusion curve, in agreement with recent experimental results.

\section{1,305}

PB94-172640 Not available NTIS

National Inst. of Standards and Technology (NEL), Gaithersburg, MD. Building Materials Div.

Serial Sectioning of Hardened Cement Paste for Scanning Electron Microscopy.

Final rept.

P. E. Stutzman. 1990, 13p.

Pub. in Proceedings of Conference on Advances in Cementitious Materials, Gaithersburg, MD., July 2226, 1990, p237-2491991.

Keywords: "Cements, Scanning electron microscopy, Sequential sampling, Surface preparation, Microstructure, Polishing, Procedures, Indentation tioning.

Serial sectioning is a technique of making and examining thin sections of material to obtain information on three-dimensional structures from a series of two-dimensional images. Procedures were developed to make serial sections of hardened cement paste by the removal of thin layers by polishing. Backscattered electron imaging of the remaining paste block was used to record the paste microstructure after each section was removed. Procedures developed cover the polishing practice, the removal of thin layers by polishing, the estimation of layer thickness, and the 10 cation and alignment of specific regions for imaging.

\section{1,306}

PB94-185121 Not available NTIS

National Inst. of Standards and Technology (NEL) Gaithersburg, MD. Structures Div.

Detection of Voids in Grouted Ducts Using the Impact-Echo Method.

Final rept.

N. J. Carino, and M. Sansalone. 1990, 15p.

Pub in Proceedings of RILEM Workshop on Testing during Concrete Construction, Mainz, Germany, March 5-7, 1990, p369-383.

Keywords: "Nondestructive tests, "Voids, "Concrete structures, Stress waves, Ducts, Grout, Wave propagation, Spectrum analysis, Structural members, Defects, Reprints, "Impact-echo method.

The impact-echo method was used to detect simulated voids in grouted post-tensioning tendon ducts cast in a $1 \cdot m$ thick concrete wall specimen. The study was part of a program to evaluate nondestructive test methods based on stress wave propagation. The locations of the voids in the ducts were not known by the authors until after their results had been reported to the principal investigator of the project. The impact-echo method was successful in locating the voids. However, the study showed that additional research is needed to gain a complete understanding of the interaction of stress waves with voids in cylindrical ducts.

01,307

PB94-185618 Not available NTIS

National Inst. of Standards and Technology (BFRL),

Gaithersburg, MD. Building Materials Div.

Corrosion Resistant Epoxy-Coated Reinforcing

Steel.

Final rept.

R. G. Mathey, and J. R. Clifton. 1994, 7p.

Pub. in Proceedings of the ASCE Structures Congress (12th), Atlanta, GA., April 24-28, 1994, v1 p109-115.

Keywords: "Epoxy coatings, "Corrosion resistance, "Reinforced concrete, "Bridge decks, Concrete structures, Steel structures, Case studies, Structural engineering, Construction materials, Corrosion inhibition, Reprints, "Reinforcing steels.

Research conducted in the 1970 s to evaluate and de velop criteria for nonmetallic coatings to protect steel reinforcing bars embedded in concrete bridge decks rom the corrosive action of chlorides is reviewed to provide a case study for successes and disappointments in structural research. The objectives of the re search, the technical challenges faced, the conduct of the research, and how the results were implemented for improvement of structural engineering practice are discussed. Also, reasons for the successful transition from research to field application are explored.

\section{1,308}

PB94-196557 PC A06/MF A02

National Inst. of Standards and Technology (BFRL). Gaithersburg, MD Building Materials Div.

Graphical Analysis of the CCRL Portland Cement Proficiency Sample Database (Samples 1-72). (Part 1. Univariant Analysis of Portland Cement)

C. B. Spring J. H Pielent S. Leigh, and N. A.

Heckert. Mar 94, 1210, NISTIR-5387.

Prepared in cooperation with American Society for Testing and Materials, Philadelphia, PA.

Keywords: "Portland cements, "Construction mate rials, "Concrete construction, "Standards, "Test methods, Production, Statistical analysis, Graphic methods, Histograms, Physical properties, Chemical properties, Compression tests, Sampling. Turbidimeters, Fineness, Performance evaluation, Air entrainment, Tables(Data), "Building technology, "National Institute of Standards and Technology, CCRL(Cement and Concrete Reference Laboratory)

A large database of Portland Cement and Portland Cement Concrete interlaboratory test results has been generated at the Cement and Concrete Reference Laboratory (CCRL) since 1965. The database rep resents a rich resource inasmuch as the cements tested were produced by many different production facilities, from raw materials obtained from different geological areas, and over a long period of time. Participation varies from 120 to over 200 laboratories, located throughout the United States and in several other countries. Computer and statistical techniques are used to present the data from the first 72 samples in graphical forms to better understand cement and cement testing. Data for each of 10 physical test properties and 11 chemical 'compounds' of Portland cement are represented graphically through use of box plots. For each property and compound, the box plots of data from multiple samples are plotted sequentially as distributed by CCRL and also sorted on median val ues. In several instances, box plots for one test prop erty are ordered by the median values of a nother property to analyze possible relationships. Also, the characteristics of each of 72 cements are profiled (graphically) in relation to the population of results of other cements.

\section{1,309}

PB94-198777 Not available NTIS

National Inst. of Standards and Technology (NEL) Gaithersburg, MD. Building Materials Div.

Digitized Direct Simulation Model of the Microstructural Development of Cement Paste. Final rept.

D. P. Bentz, and E. J. Garboczi. 1990, $8 p$. Pub in Mater. Res. Soc. Symp. Proc. Phys. Phenom Granular Mater., v195 p523-530 1990. Sponsored by Northwestern Univ., Evanston, IL. Center for Advanced Cement-Based Materials.

Keywords: "Cements, "Curing, "Computerized simula tion, "Hydration, Diffusivity, Algorithms, Percolation, Transport properties, Microstructure, Models, Reprints

The complex microstructure of hardened cement paste is produced by hydration reactions between cement particles and the water in which they are suspended. In recent years, algorithms like the diffusion-limited aggregation (DLA) and Eden models have demonstrated that simple growth rules can result in complex aggregated structures. The model described in the pape simulates, via simplified growth rules, the microstructural development of hydrating cement paste. The model has simularities to DLA, but with the additional novel features of dissolution of solid particles, and a free-space nucleation probability. The percolation aspects and transport properties of the model's pore space are computed and discussed.

\section{1,310}

PB94-198785 Not available NTIS

National Inst of Standards and Technology (NEL), Gaithersburg, MD. Building Materials Div.
Digitized Simulation Model for Microstructural Development.

Final rept.

D. P. Bentz, and E. J. Garboczi. 1991, 16p.

Pub. in Ceram. Trans. Adv. Cem. Mater., v16 p211. 2261991.

Keywords: "Cements, "Curing, "Computerized simulation, "Hydration, Diffusivity, Algorithms, Porosity, Transport properties, Microstructure, Models, Reprints.

Characterization of the microstructure of hydrating cement is difficult due to its complexity. Models of the hydration process provide one mears of characterization as they attempt to duplicate features of rea microstructures. This paper presents a simple model for hydration consisting of random dissolution, diffusion, nucleation, and surface reaction. The model has been developed in a digitized format. Space is rep. resented as an array of pixels, each of which is classified as a given material: reactant, dissolved species, hydrate, or pore. Having the model in digitized format allows for the visualization of the hydration as it occurs and for rapid subsequent quantification of the resultant microstructure. In addition, real images or cement particles can be digitized and used as the starting material for model execution. Algorithms for, and results of, the model are presented.

\section{1}

PB94-198793 Not available NTIS

National Inst. of Standards and Technology (BFRL), Gaithersburg, MD. Building Materials Div

Modelling the Leaching of Calcium Hydroxide from Cement Paste: Effects on Pore Space Percolation and Diffusivity.

Final rept.

D. P. Bentz, and E. J. Garboczi. 1992, 11 p.

Pub. in Materials and Structures 25, p523-533 1992. Sponsored by National Science Foundation, Arlington,

Keywords: "Cements, "Leaching, "Hydration, "Models, Porosity, Percolation, Microstructure, Diffusivity, Calcium hydrides, Durability, Reprints.

As concrete is exposed to the elements, its underlying microstructure can be attacked by a variety of aggressive agents. For example, rainwater and groundwater can degrade the concrete by dissolving soluble constituents such as calcium hydroxide. Using computer simulation, the paper examines the effects of calcium hydroxide dissolution on two material properties: the percolation properties or connectivity of the capillary pore space, and the relative ionic diffusivity. A microstructural model for cement paste is used to produce a hydrated specimen which is subsequently subjected to the leaching process. Pore space percolation characteristics and relative ionic diffusivity are computed throughout the leaching process as a function of total capillary porosity. Material variables examined are water:solids ratio and silica fume content. Percolation theory is used to develop the concept of a critical volume fraction of calcium hydroxide plus capillary pore space. It is shown that this critical combined volume fraction determines the magnitude of the effect of leaching on relative ionic diffusivity.

01,312

PB94-198801 Not available NTIS

National Inst. of Standards and Technology (NEL) Gaithersburg, MD. Building Materials Div.

Diffusion Studies in a Digital-Image-Based Cement Paste Microstructural Model.

Final rept.

D. P. Bentz, E. J. Garboczi, D. Gingold, C. J. Lobb, and $\mathrm{H}$. M. Jennings. 1991, 9p.

Pub. in Ceram. Trans. Adv. Cem. Mater., v16 p227235 1991. Sponsored by Northwestern Univ., Evanston, IL. Center for Advanced Cement-Based Materials. Keywords: "Cements, "Curing, "Computerized simulation, "Hydration, Diffusivity, Algorithms, Percolation, Transport properties, Microstructure, Models, Reprints.

A digital-image-based microstructural model of cement paste, along with a new, fast algorithm for computing and conductance of random conductor lattices, are used to study the diffusivity of cement paste as a function of degree of hydration and water:cement ratio. The computational methods and some preliminary data are presented, and comparison with experimental results is discussed. 
National Inst. of Standards and Technology (NEL), Gaithersburg, MD. Structures Div.

Maturity Method.

Final rept.

N. J. Carino. 1991, 46p

Pub. in CRC Handbook on Nondestructive Testing of Concrete, Chapter 5, p101-146 1991.

Keywords: ${ }^{*}$ Concretes, ${ }^{*}$ Curing, "Compressive strength, Temperature effects, Mechanical properties, Activation energy, Arthenius equation, Standards, Aging(Materials), Concrete durability, Reprints, *Maturity method, ASTM standards.

The chapter reviews the history and technical basis of the maturity method, a technique for estimating the strength gain of concrete based upon the measured temperature history during curing. The combined eflects of time and temperature on strength gain are quantified by means of a maturity function. The widely used maturity functions are reviewed critically. It is shown that the traditional maturity function is inadequate compared with the function based upon the Arrhenius equation. The concept of equivalent age, which is the most convenient measure of maturity, is explained. The strength gain of a specific concrete mixture is estimated using the measured maturity and the strength vs. maturity relationship for that mixture. Varous proposed strength-maturity relationships are reviewed. It is also explained why the maturity method can only be used reliably to estimate relative strength. Examples are presented to illustrate how this technique can be used in combination with other in-place tests of concrete strength. The ASTM standard dealing with the method is also summarized.

\section{1,314}

PB94-199502 Not available NTIS

National Inst. of Standards and Technology (BFRL), Gaithersburg, MD. Structures Div.

Maturity Functions for Concrete Made with Various Cements and Admixtures.

Final rept.

N. J. Canino, and R. C. Tank. 1991, 15p

Pub. in Proceedings of International Workshop on Testing during Concrete Construction (RILEM), Mainz, Germany, March 5-7, 1990, p192-206 1991.

Keywords: "Concretes, "Curing, "Compressive strength, Temperature effects, Mechanical properties, Arrhenius equation, Aging(Materials), Cements, Models, Concrete durability, Reprints, "Maturity functions.

A model is proposed for estimating relative strength gain of concrete under isothermal curing conditions. The key feature of the model is the relationship between curing temperature and the rate constant for relative strength development. The strength gain of seven concrete and mortar mixtures under three curing temperatures was studied. It was found that a simple exponential function may be used to describe the observed variations of the rate constant with curing temperature. It is shown that the relative strength development of concrete can be estimated from its temperature history using parameters determined experimentally from tests of isothermally-cured mortar specimens.

\section{1,315}

PB94-199759 Not available NTIS

National Inst. of Standards and Technology (NEL), Gaithersburg, MD. Building Materials Div.

Interaction between Naphthalene Sulfonate and Silica Fume in Portland Cement Pastes.

Final rept.

S. Diamond, and L. Struble. 1988, 10p.

Contract AFOSR-155A86-0057

Pub. in Materials Research Society Symposia Proceedings, v114 p117-126 1988. Sponsored by Air Force Office of Scientific Research, Bolling AFB, DC.

Keywords: "Portland cement, "Admixtures, "Curing, Hardening(Materials), Concentration(Composition), Superplasticity, Compressive strength, Reprints, Silica fume, Naphthalene sulfonate, Superplasticizers

Portland cement pastes were mixed with predissolved naphthalene sulfonate superplasticizer at normal water:cement ratios. Solutions were separated from the fresh pastes at intervals and the residual concentration of the superplasticizer determined by UV spectrophotometry. At low dosage levels essentially all of the superplasticizer was found to be removed from solution within a few minutes; at high dosage levels a substantial concentration was maintained in solution a least to approximately the time of set. In pastes in which silica fume replaced $10 \%$ by weight of the ce- ment, it was found that the incorporation of silica fume significantly increased the uptake of superplasticizer. In separate trials it was found that the silica fume by itself absorbed little superplasticizer, even from high $\mathrm{pH}$ solution simulating that of cement paste.

\section{1,316}

PB94-215670 PC A08/MF A02

National Inst. of Standards and Technology (BFRL), Gaithersburg, MD.

Highway Concrete (HWYCON) Expert System User Reference and Enhancement Guide.

L. J. Kaetzel, J. R. Clifton, P. Klieger, and K. Snyder. May $93,170 p$, NISTIR-5184

See also PB93-198885 and PB94-182995.

Keywords: *Concretes, "Expert systems, "Highway maintenance "Information systems Concrete durability, Road materials, Pavement condition, Bridge decks, Maintenance management, Concrete pavements, Pavement damage.

The report discusses the expert system HWYCON (Highway Concrete). HWYCON is designed to assis state highway departments in three areas: (1) diagnosing distresses in highway pavements and structures; (2) selecting materials for construction and reconstruction; and (3) obtaining recommendations on materials and procedures for repair and rehabilitation methods. HWYCON is an operational system and will be distributed to state DOT's through SHRP. This document is intended to provide a reference for users of the system who need information that is not covered in the overview and installation document provided with the sys tem.

01,317

PB95-143079 PC A03/MF A01

National Inst. of Standards and Technology (BFRL) Gaithersburg, MD.

Quantitative X-Ray Powder Diffraction Methods for Clinker and Cement.

P. E. Stutzman. May 94, 26p, NISTIR-5403.

See also PB92-183664.

Keywords: "Portland cements, "Clinker, $X$-ray diffraction, Quantitative analysis, Particle size distribution, Chemical composition, Construction materials, *Reference materials, Phase abundance, Sample preparation.

Performance of concrete is influenced by the portland cement phase composition. Phase abundance determination has traditionally been accomplished using two different methods; optical microscopy, and a Bogue calculation based on a chemical analysis. $X$-ray powder diffraction is a direct, bulk analytical method for phase analysis of fine-grained materials including clinker and cements. Each phase produces a unique diffraction independently of the others with each pattern intensity being proportional to phase concentration. Difficulties in X-ray powder diffraction analysis include correction for sample absorption, selection of ref erence standards, and determination of individual pattern intensity. These problems are minimized by use of an internal standard, profile fitting, and careful reference standard selection.

01,318

PB95-143095 PC A03/MF A01

National Inst. of Standards and Technology (BFRL), Gaithersburg, MD. Building Materials Div.

Diagnosis of Causes of Concrete Deterioration in the MLP-7A Parking Garage.

P. E. Stutzman, and J. R. Clifton. Jun 94, 26p,

NISTIR-5492.

Keywords: *Parking garages, *Concrete durability *Deterioration, Alkali aggregate reactions Cracking(Fracturing), Efflorescence, Petrography Water repellants.

Parking garage MLP-7A at the National Institutes of Health $(\mathrm{NIH})$ is a four-level, cast-in-place concrete structure, approximately seventeen years of age which is exhibiting expansion and cracking of the parape walls. An accelerated expansion test of aggregate extracted from the structure indicates that the aggregate is reactive and that there is a potential for further expansion. The performance of water repellant treat ments were evaluated in field studies on the garage structure. Each repellant treatment reduced water infiltration relative to an untreated test region however some treatments performed better in regions with visible, fine surface cracks
01.319

PB95-155552 PC A05/MF A01

National Inst. of Standards and Technology (TS), Gaithersburg, MD. National Voluntary Lab. Accreditation Program

National Voluntary Laboratory Accreditation Program: Construction Materials Testing.

.

P. R. Martin. Sep 94, 79p, NIST/HB-150/5

Also available from Supt. of Docs as SN003-003

03301-4. See also PB94-178225 and PB95-128658

Keywords: *Highway construction, "Construction materials, *Laboratories, "Certification, Admixtures, Concretes, Aggregates, Cements, Rocks, Soils, Road ma terials, Stainless steels, Geotechnical fabrics, Geotextiles, Procedures, Requirements, Calibration standards, Standardization, Licensure, Methodology, Accuracy, Performance evaluation, National Voluntary Laboratory Accreditation Program, US NIST.

NIST Handbook 150-5 presents the technical requirements of the National Voluntary Laboratory Accredita. tion Program (NVLAP) for the Construction Materials Testing program. It is intended for information and use by staff of accredited laboratories, those laboratories seeking accreditation, other laboratory accreditation systems, users of laboratory services, and others needing information on the requirements for accreditation under the Construction Materials Testing program. This publication supplements NIST Handbook 150 (PB94-178225), NVLAP Procedures and General Requirements, which contains Part 285 of Title 15 of the U.S. Code of Federal Regulations (CFR) plus all general NVL AP procedures, criteria, and policies. Handbook $150-5$ contains information that is specific to the Construction Materials Testing program and does not duplicate information contained in the Procedures and General Requirements. It is organized to cross-reference with Handbook 150

01,320

PB95-175055 Not available NTIS

National Inst. of Standards and Technology (BFRL) Gaithersburg, MD. Building Materials Div.

Cellular Automaton Simulations of Cement Hydration and Microstructure Development.

Final rept

D. P. Bentz, P. V. Coveney, E. J. Garboczi, M. F Kleyn, and P. E. Stutzman. 1994, 26p.

Sponsored by Northwestern Univ., Evanston, IL

Pub. in Modelling and Simulation in Materials Science and Engineering 2, p783-808 1994

Keywords: "Hydration, "Cement, "Microstructure, *Simulation, Models, Slurries, Percolation, Image analysis, Algorithms, Reprints.

Cellular automation algorithms, which operate on a starting digital image of a water-cement suspension, are described. The algorithms simulate the microstructure development process due to hydration reactions that occur between cement and water. This paper describes the evolution of the cement model from a simple model, which treated the cement particles as a single-phase materials, with a greatly simplified hydration chemistry, into a model which has many more chemical species and includes numerous reactions which eventually convert the viscous watercement suspension into a rigid porous solid. Methods are presented for generating two- and three-dimensional images representing suspension initial conditions; these are derived from both micrographs of real cements and computer-based algorithms. A convenient measure of the point at which the initial paste turns into a solid material is the percolation threshold of the solids. Consideration of these models has already led to the prediction and subsequent experimental observation of a sharply defined onsel of shear wave propagation, from ultrasonic measurements through hydrating cement slurries. The amount of hydration needed to reach the percolation threshold can be determined in the present simulations, and our results are compared with time of shear wave onset in actual cement slurries.

\section{1,321}

PB95-175063 Not available NTIS

National Inst. of Standards and Technology (BFRL), Gaithersburg, MD. Building Materials Div Evolution of Porosity and Calcium Hydroxide in Laboratory Concretes Containing Silica Fume. Final rept.

D. P. Bentz, and P. E. Stutzman. 1994, 7p.

Pub. in Cement and Concrete Research 24, n6 p104410501994 


\section{Construction Equipment, Materials, \& Supplies}

Keywords: "Durability, "Concrete, "Calcium hydroxides, Image analysis, Scanning electron micros. copy, Interfaces, Microstructure, Hydration, Construction materials, Aggregates, Reprints, "Silica fume.

Laboratory concretes containing only coarse aggregates and 0,10 , or $20 \%$ silica fume as a cement replacement were prepared at a constant water-to-solids ratio and sampled at 1,7 , and 28 days. Scanning electron microscopy was utilized to monitor the progress of the hydration reactions both in the bulk paste and in regions near an aggregate surface. Phase volume fractions were determined by quantitative image analysis of the backscattered electron images. In addition, the size of 'individual' two-dimensional cross sections of calcium hydroxide crystals and capillary pores were assessed. Silica fume additions are seen to affect both the amount and size of these microstructural features. Differences are observed between interfacial zone and bulk areas which support previous mercury intrusion measurements of mortar specimens. This analysis lends support to the hypothesis that when silica fume is present, calcium hydroxide crystals form and then dissolve away, contributing to a connected pathway of capillary porosity in the interfacial zone regions.

01,322

PB95-180147 Not available NTIS

National Inst. of Standards and Technology (BFRL)

Gaithersburg, MD. Structures Div

High-Performance Concrete: Research Needs to Enhance Its Use.

Final rept.

N. J. Carino, and J. R. Clifton. 1991, 7p.

Pub. in Concrete International 13, n9 p70-76 Sep 91

Keywords: *Concretes, Research, Durability, Mechanical properties, Construction materials, Workshops, Reprints, "High-performance concretes.

The National Institute of Standard and Technology and the American Concrete Institute cosponsored a workshop on high-performance concrete (HPC). The objectives were to identify technical and institutional barriers which impede the widespread use of HPC and to recommend actions to overcome these barriers. The recommended research, summanized in this paper, is proposed as the basis for developing a national program to exploit the potential of HPC and assure U.S. competence in concrete technology.

\section{1,323
PB95-190674 PC A05/MF A01}

National Inst. of Standards and Technology (BFRL) Gaithersburg, MD. American Association of State Highway and Transportation Officials, Gaithersburg MD. Materials Reference Lab.

Field Evaluation of the System for Calibration of the Marshall Compaction Hammer.

H. W. Shenton, and M. M. Cassidy. Feb 95, 80p,

H.W. Shenton, and M. M. Cassidy.
NISTIR-5553, FHWA/RD-95/063.

Prepared in cooperation with American Association of State Highway and Transportation Officials, Gaithersburg, MD. Materials Reference Lab. Sponsored by Federal Highway Administration, Washington, DC.

Keywords: "Calibrating, "Compactors, "Hot mix paving mixtures, Bituminous materials, Asphalts, Road materials, Test methods, Highway construction, Mechanical properties, "Marshall compaction hammer.

A system for calibrating the Marshall compaction hammer has recently been developed at the National Institute of Standards and Technology, in collaboration with the American Association of State Highway Transportation Officials (AASHTO), Materials Reference Laboratory (AMRL). The calibration system consists of a spring-mass device with an integra! force transducer and a high-speed data acquisition system. The proposed calibration procedure is based on adjusting the number of hammer blows delivered to a specimen, such that a standard compactive effort is supplied during the compaction process, regardless of slight variations in the Marshall hammer. In an earlier laboratory evaluation program, the calibration system and procedure proved to be effective in reducing the variability of Marshall test results. Presented in the report is a summary of a field evaluation program of the calibration system and procedure. In this study, Marshall specimens were prepared in bituminous laboratories using production Marshall hammers: twelve labora tories, or field sites, participated in the study. Sixteen Marshall specimens were prepared at each site.
National Inst. of Standards and Technology (BFRL), Gaithersburg, MD. Structures Div.

Prediction of Potential Concrete Strength at Later Ages.

Final rept.

N. J. Carino. 1994, 13p

Pub. in Significance of Tests and Properties of Concrete and Concrete-Making Materials, ASTM STP 169C, Chapter 15, p140-152 1994.

Keywords: "Concrete, "Reinforced concrete, "Compressive strength, Reprints, Strength(Mechanics), Construction materials, Failure(Mechanics), Curing, Standards.

This chapter deals with methods for estimating the potential, later-age strength of a concrete mixture based upon the compressive strength measured on cylindrical specimens at early ages. The results of the cooperative research program leading to the development of ASTM Test Method for Making. Accelerated Curing, and Testing Concrete Compression Test Specimens (C 684) are reviewed. This review is based on Chapter 13 of ASTM STP $169 \mathrm{~B}$, which was written by M.H. Wills, Jr., and was titled 'Accelerated Strength Tests.' The earlier text has been augmented by the addition of information on the high-temperature and pressure accelerated test method that was added to ASTM C 684 in 1989. In addition, the chapter has been expanded by including the basis of ASTM Test Method for Developing Early-Age Compression Test Values and Projecting Later-Age Strengths (C 918).

01,325

PB96-122445 Not available NTIS

National Inst. of Standards and Technology (BFRL), Gaithersburg, MD. Structures Div.

Recent Development in Nondestructive Testing of Concrete.

Final rept.

N. J. Carino. 1992, 57p

Pub. in Advances in Concrete Technology, International Symposium on Advances in Concrete Technology, Athens, Greece, May 11-13, 1992, p281-337.

Keywords: *Concrete, "Nondestructive testing, Flaw detection, Impulse response, Reprints, Break-off test, Impact-echo, Torgue test.

Advances in nondestructive testing of concrete are summarized. Test methods are classified into those used to assess in-place strength and those used to locate hidden defects. In the first category, recent developments are presented on the pullout test, the breakoff test, the torque test, a review is presented of infrared thermography, ground penetrating radar, and several methods based upon stress wave propagation. The principles of the methods, their advantages and their inherent limitations are discussed. Where appropriate, requirements of relevant ASTM standards are discussed. Future directions for research in the area of nondestructive testing of concrete are presented.

\section{1,326}

\section{PB96-146816 Not available NTIS}

National Inst. of Standards and Technology (BFRL), Gaithersburg, MD. Building Materials Div.

Interfacial Transport in Porous Media: Application to dc Electrical Conductivity of Mortars.

Final rept.

L. M. Schwartz, E. J. Garboczi, and D. P. Bentz.

1995, 11 p.

Pub. in Jnl. of Applied Physics, v78 n10 p5898-5908 Nov 95.

Keywords: "Electrical resistivity, "Direct current, "Mortars(Materials), Concretes, Transport properties, Percolation, Durability, Construction materials, Reprints.

A mortar is a composite of inert sand grains surrounded by a porous cement paste matrix. We investigate the electrical conductivity of model mortars that include enhanced electrical conduction in the matrixsand grain interfacial region. The electrical conductivity is evaluated by a combination of finite element, finite difference, and random walk methods for periodic and disorderd models of motar. Since the effective conductivity within the interfacial zone is often much higher than the bulk matrix conductivity, the qualitative features of transport in these systems is often controlled by the connectivity if the interfacial zone. Special attention is thus given to the geometrical percolation of this zone.
National Inst. of Standards and Technology (BFRL), Gaithersburg, MD. Building Materials Div.

Guide to a Format for Data on Chemical Admixtures in a Materials Property Database.

C. F. Ferraris. Apr 96, 31p, NISTIR-5796.

Keywords: "Admixtures, "Properties, "Data bases, *Format, Concretes, Construction materials, Standards, NIST(National Institute of Standards and Technology).

This guide is the second in a series of related documents that present recommended formats for use in computerization of concrete materials property data. It addresses the problem of distinguishing one chemical admixture from another by providing a logical scheme for systematically organizing and subdividing matenaal characteristics and parameter to create a unique chemical admixture material identifier. The organiza. tion and structure presented in this guide provide a framework for cross-referencing chemical admixture properties, data, and other information which is consistent with reporting recommendations contained in the other $\mathrm{ACl}$ Committee 126 guides. ACl Committee 126 guides are consistent with the principles laid down in the standard guides that have been prepared by ASTM Committee E-49. This guide is intended for use by those responsible for entering data into a concrete materials property database or preparing tables of concrete properties and information for use by others.

\section{1,328}

PB96-186192 PC A04/MF AO1

National Inst. of Standards and Technology (BFRL), Gaithersburg, MD. Building Materials Div.

Guide to a Format for Data on Chemical Admixtures in a Materials Property Database. (Reannouncement with new abstract).

C. F. Ferraris. Apr 96, 31p, NISTIR-5796.

Supersedes PB96-165394. Reannouncement with new abstract.

Keywords: "Admixtures, "Properties, "Data bases, *Format, Concretes, Construction materials, Standards, NIST(National Institute of Standards and Technology).

The formats for data on chemical admixtures that are described in the report are intended to aid the creation of a coherent system of concrete materials property databases. The preliminary document is a guide that presents a recommended format for use in computerization of concrete materials property data. It addresses the problem of distinguishing one chemical admixture from another by providing a logical scheme for organizing and subdividing material characteristics and parameters to create a unique chemical admixture material identifier. The organization and structure presented in the guide provide a framework for cross-referencing chemical admixture properties, data, and other information which is consistent with the principles laid down in the standard guides that have been prepared by ASTM Committee E-49 and which are due to be adopted by American Concrete Institute (ACI) Committee 126. The preliminary working document is intended to assist the work of $\mathrm{ACl}$ committee 126 by providing a draft for use by committee members and others who may wish to offer suggestions for its development. The preliminary document will be superseded by an official $\mathrm{ACl}$ document in the series on concrete materials property database formats that is being prepared by $\mathrm{ACl} 126$.

\section{1,329}

PB96-202296 PC A09/MF A02

National Inst. of Standards and Technology (BFRL), Gaithersburg, MD. Office of Applied Economics. Development of a Method for Measuring WaterStripping Resistance of Asphalt/Siliceous Aggregate Mixtures.

T. Nguyen, E. Byrd, D. Bentz, and J. Seiler. Jul 96, 152p, NISTIR-5865.

See also PB96-197249. Sponsored by National Research Council, Washington, DC. Transport Research Board.

Keywords: "Asphalt pavements, "Water effects, Spectroscopic analysis, Interfacial tension, Test methods.

The main objective of this study was to develop a sensitive, spectroscopic technique to nondestructively evaluate the water stripping resistance at the molecular level of an asphalt on a siliceous aggregate. This study consisted of three phases. Phase 1 involved the 
development of a nondestructive, spectroscopic technique to measure the amount and thickness of the water layer at the interface between an asphalt and a siliceous aggregate. Phase 2 was to develop a technique to measure the adhesion loss of the asphall/ag gregate mixture exposed to water. And Phase 3 aimed to relate the quantity of the interfacial water layer obtained in Phase 1 with the adhesion loss data gen erated in Phase 2.

01,330

PB96-214713 PC A11/MF AO3

National Inst. of Standards and Technology (BFRL), Gaithersburg, MD. Structures Div.

Shear Design of High-Strength Concrete Beams: A Review of the State-of-the-Art.

D. Duthinh, and N. J. Carino. Aug 96, 205p, NISTIR5870 .

Keywords: "Concrete structures, "Beams(Supports), Reinforced concrete, Shear strength, State of the art.

This state-of-the-art review of the shear design of highstrength concrete beams consists of four parts. In the first part, various analysis methods are presented: (1) The plastic solution assumes that both steel reinforce ment and concrete, modeled as a modified Mohr-Coulomb material, are at yield. Under shear loading, the concrete web develops an inclined compression field which satisfies both upper and lower bound theorems. A plastic solution of shear friction is also discussed. (2) Both the compression field theory and the modified compression field theory (MCFT) are exact theories in the sense that they satisfy equilibrium, compatibility of displacements and stress-strain relationships. The MCFT accounts for the contribution of the tensile strength of concrete to shear resistance. (3) Other exact solutions are also discussed, that do not assume that the principal stress and principal strain directions are aligned with the shear cracks in the web. (4) The $45 \mathrm{deg}$ truss, the variable angle truss (VAT) and strutand-tie models (STM) belong to a class of solutions that only satisfy equilibrium.

\section{Highway Engineering}

\section{1,331}

PB94-161940 PC A06/MF A01

National Inst. of Standards and Technology (BFRL) Gaithersburg, MD. Structures Div.

Draft Guidelines for Pre-Qualification and Prototype Testing of Seismic Isolation Systems.

H. W. Shenton. Mar 94, 104p, NISTIR-5359.

Keywords: "Vibration isolators, "Bridges(Structures) "Prototypes, "Test methods, "Earthquake resistant structures, Building codes, Displacement, Structural vibration, Dynamic structural analysis, Earthquake engineering, Dynamic response, Standards, Stiffness, Vibration damping, Loads(Forces), Test facilities.

At the present time, at least in the United States, seismic isolation systems are custom designed and built on a per project basis. As a result, testing has become an essential element in the design and construction of isolated structures. Prototype and quality control tests are required by the 1991 Uniform Building Code and the American Association of State Highway and Transportation Officials, 1991 Guide Specifications for Seismic Isolation Design. Currently, however, standards do not exist for conducting these tests. The Building and Fire Research Laboratory of the National Institute of Standards and Technology has developed draft guidelines for testing and evaluation of seismic isolation systems. Presented in the report are guidelines for conducting pre-qualification and prototype tests. Pre-qualification tests are currently not required by the codes but are generally conducted during development of a new system and are aimed at evaluating fundamental properties and characteristics of the isolation system. The guidelines include general requirements of the test facility, instrumentation, calibration, data acquisition, data analysis and reporting of result. A total of twenty three tests are included in the guidelines. Performance criteria have been established for all tests, systems that do not meet or exceed these criteria may not function adequately in service. The guidelines are to serve as a resource document for voluntary standard/specification writing organizations, and for practitioners and researchers involved in the design, manufacture and testing of seismic isolation systems.

\section{1,332}

PB94-161957 PC A03/MF A01

National Inst. of Standards and Technology (BFRL), Gaithersburg, MD. Structures Div.

Draft Guidelines for Quality Control Testing of Sliding Seismic Isolation Systems.

H. W. Shenton. Mar 94, 36p, NISTIR-5371.

Keywords: "Vibration isolators, "Bridges(Structures), "Quality control, "Test methods, "Earthquake resistant structures, Standards, Earthquake engineering, Displacement, Building codes, Vibration damping, Energy dissipation, Structural vibration, Dynamic response, Dynamic structural analysis.

Seismic isolation systems designed according to the 1991 Uniform Building Code, or the 1991 AASHTO Guide Specification for Seismic Isolation Design are required to undergo a series of prototype and quality control tests before being installed in the structure. At the present time, however, standards do not exist for conducting these tests, consequently, results are subject to unknown variability. The document represents the initiation of the process to develop standards for quality control testing of seismic isolation systems built in the U.S. The guidelines are devoted specifically to quality control testing of sliding systems (another report is devoted to quality control testing of elastomeric isolation systems). The guidelines address component part and material tests to be conducted during production, and tests on completed isolation units or components. Production tests are outlined in broad terms for a generic pure sliding device. Two completed isolation unit tests are outlined: sustained compression, and effective stiffness and energy dissipation. Complete details of the test set-up, test procedure, data acquisition, analysis and reporting of results are given in the guidelines. Performance criteria are established for all tests; systems that do not meet these criteria may not perform satisfactorily in service and should be set aside for disposition by the engineer of record.

01,333

\section{PB94-182995 PC A13/MF A03}

Construction Technology Labs., Skokie, IL.

Optimization of Highway Concrete Technology. Final rept.

D. Whiting, M. Nagi, P. Okamoto, K. Smith, M.

Darter, J. Clifton, L. Kaetzel, T. Yu, and D. Peshkin. cJan 94, 2910, SHRP-C-373, isBN-0-309-05751-5. Contraci SHRP-C-206

See also PB88-244330, PB90-113440 and PB91100537. Prepared in cooperation with ERES Consultants, Inc., Savoy, IL. and National Inst. of Standards and Technology, Gaithersburg, MD. Sponsored by Strategic Highway Research Program, Washington,

Keywords: *Concrete pavements, *Portland cements, *Bridge maintenance, Pavement damage, Permeability, Temperature effects, Density, Pavement overlays, Highway maintenance, Compressive strength, Aggregates, Mechanical properties, Admixtures.

The report provides (1) state-of-the-art information on new developments in portland cement concrete matenals and applications to users in the highway agencies, especially young engineers just beginning their careers in the field; and (2) new materials and testing methodologies for a variety of important highway and bridge rehabilitation applications. The report includes a condensation of the state-of-the-art synthesis of concrete highway technology prepared as part of this research project. Descriptions of evaluations of test methods for in-place concrete density with the use of nuclear methods and determination of water content of fresh concrete using microwave drying are also incorporated in the report. The report gives summaries of guidelines for avoiding thermal effects in concrete pavement slab placements and packing-based aggregate proportioning for concrete mixtures. The report contains a detailed description of field evaluations of a variety of concrete mixes used for early opening of full depth concrete pavement repairs and bridge deck overlays. Opening times ranged from 2 to 24 hours. Brief descriptions of the methodology and content of the HWYCON expert system, available from SHRP, are also included. Finally, a description of the contents of audiovisual implementation packages for highway personnel dealing with materials testing and pavement and bridge rehabilitation is included. (Copyright (c) 1994 National Academy of Sciences.)

\section{1,334}

PB94-193471 PC A08/MF A02
Montana State Univ., Bozeman. Dept. of Chemistry Binder Characterization and Evaluation by Nuclear Magnetic Resonance Spectroscopy.

Final rept.

P. W. Jennings, J. A. Pribanic, B. Franconi, and D. L. VanderHart. cMay 93, 155p, ISBN-0-309-05252-1 SHRP-A-335.

Contract SHRP-A-002C

Prepared in cooperation with National Inst. of Standards and Technology, Gaithersburg, MD. Sponsored by Strategic Highway Research Program, Washington, DC.

Keywords: "Binders, "Nuclear magnetic resonance, *Magnetic spectroscopy, "Bituminous concretes "Highways, Asphalt, Molecular diffusion, Chemical properties, Molecular structure, Aggregates, Road materials.

Nuclear magnetic resonance (NMR) spectroscopy was used in solution-state and solid-state experiments on eight asphalt cements. Using a variety of chemical and solution-state NMR spectroscopic techniques, data were obtained about the amounts of aromatic carbon in an average molecule of the asphalt sample, how that carbon is arranged in terms of the size of the average aromatic system and the extent of substitution. The arrangement of the aliphatic portion has also been described in terms of the average number of alicyclic rings, of aliphatic chain length and extent of branching Concentrations of carboxylic acids and phenols were measured both before and after laboratory oxidative aging. All these data show both surprising similarities and significant differences among the asphalts studied. By using solid-state NMR spectroscopic techniques the structure of asphaltic cements was probed on a small-distance scale of a few angstroms to 30 micrometers. Differences in molecular mobility were found among the asphalts studied. Changes in molecula mobility after laboratory oxidative aging were found to be small. The implications of these data with regard to the micellar model of asphalt are discussed. The research results' potential impact on understanding asphalt cement properties is explored. (Copyright (c) 1993 National Academy of Sciences.)

01,335

PB95-163259 Not available NTIS

National Inst. of Standards and Technology (BFRL), Gaithersburg, MD. Structures Div.

Evaluating the Seismic Performance of Lightly-Reinforced Circular Concrete Bridge Columns.

Final rept.

A. W. Taylor, and W. C. Stone. 1993, 10p

Sponsored by Federal Highway Administration McLean, VA. Office of Research, Development, and Technology.

Pub. in Proceedings of National Earthquake Conference, Memphis, TN., May 2-5, 1993, p543-552.

Keywords: "Failure modes, "Concrete structures, "Bridges(Structures), Earthquake damage, "Cyclic loads, Dynamic response, Earthquake engineering, Reinforced concrete, Loads(Forces), Displacement, Hysteresis, Bridge design, Structural analysis, Earthquake engineering, Structural failure, Reprints, "Spirally-reinforced bridge columns, "Hysteretic failure model.

The ability to analytically predict the inelastic dynamic behavior of reinforced concrete bridge structures during earthquakes is dependent on the availability of ap propriate hysteretic failure models. To date, severa such models have been implemented in research computer codes However little information is available concerning the selection of appropriate parameters for the solution of specific types of reinforced concrete structures. As part of an effort to develop an integrated seismic design procedure (ISDP) for reinforced concrete bridge piers, the National Institute of Standards and Technology (NIST) is conducting extensive stud es of hysteretic failure models and laboratory data from tests of bridge columns subjected to cyclic latera loading. A digital database of load-displacement histories has been established for circular, spirally-reinforced bridge columns. A system identification analysis was performed to determine optimal values of three hysteretic failure model parameters for each test specimen. Subsequent regression analyses were conducted to develop equations correlating the hysteretic failure model parameters to the material and geometric properties of the specimens.

01,336

PB95-163267 available NTIS

National Inst. of Standards and Technology (BFRL), Gaithersburg, MD. Structures Div. 
Jacket Thickness Requirements for Seismic Retrofitting of Circular Bridge Columns.

Final rept.

A. W. Taylor, and W. C. Stone. 1993, 10p.

Pub. in Proceedings of Symposium on Practical Solutions for Bridge Strengthening and Rehabilitation, Des Moines, IA., April 5-6, 1993, p249-258.

Keywords: "Earthquake resistant structures, "Bridges(Structures), "Reinforcement(Structures), "Thickness, Bridge design, Design criteria, Reinforcing materials, Reinforced concrete, Earthquake engineering. Hysteresis, Failure modes, Earthquake damage, Structural failure, Columns(Supports), Reprints, "Steel jacketing, "Circular bridge columns, "Seismic retrofitting, Cumulative damage model.

Steel jacketing is a common seismic retrofit strategy for circular, spirally-reinforced concrete bridge piers. Although jacketing is recognized as an effective and relatively inexpensive method for improving column ductility, little guidance is available for determining the required thickness of the jacket. In this study jacket thickness requirements were investigated using a timestep inelastic analysis algorithm which incorporates a hysteretic failure rule and a cumulative damage model. A variety of column designs were subjected to a range of real earthquake acceleration histories, and the resulting damage levels, or damage indices were calculated. These same columns were then re-analyzed with steel jackets of varying thicknesses. It was determined that a relatively thin steel jacket--on the order of a few millimeters in thickness--can dramatically improve the seismic performance of a column. Guidelines are presented for calculating required jacket thicknesses, and practical considerations regarding the installation and performance of jacisets are discussed.

01,337

PB95-170551 PC A06/MF A02

National Inst. of Standards and Technology, Gaithersburg, MD.

Robotics Application to Highway Transportation. Volume 2. Literature Search.

Final rept. Sep 92-Nov 93.

E. W. Kent, and R. Finkelstein. Jan 95, 114p, FHWA RD-94/054.

Contract DTFH61-92-Y-00160

See also PB95-171633. Prepared in cooperation with Robotic Technology, Inc., Potomac, MD. Sponsored by Federal Highway Administration, McLean, VA. Office of Advanced Research.

Keywords: "Robotics, "Highway transportation, "Literature surveys, "Exoskeleton, "Technology assessment, Human factors engineering. Highway design, Highway construction, Highway maintenance, Man machine systems, Robots, Manipulators, Data bases, Bibliographies, Automation.

The National Institute of Standards and Technology, at the request of the Federal Highway Administration, has conducted a study of potential applications of automation and robotics technology in construction, maintenance, and operation of highway systems. The study included a workshop exploring industry perceptions of needs and barriers to adoption, a workshop and a literature search to assess current state of the art practices and trends, and site visits by automation experts to typical highway worksites. Potential technology opportunities were highlighted for short, medium, and long-term efforts in a matrix of intersections between common highway jobs and areas of current technological thrust. From among the opportunities identified, six potential research a reas were developed as specific proposals, and subjected to life-cycle cost-benefits analysis. Four were projected to return significant savings by comparison with current practice. Of these, two were identified as also likely to return benefits of significant impact on total highway expenditures and the national economy due to their ability to leverage savings across large numbers of jobs or their effect on a large percentage of highway traffic. This is Volume II of IV.

\section{1,338}

PB95-171633 PC A20/MF AO National Inst. of Standards and Technology,

Robotics Application to Highway Transportation. Volume 3. Proposed Research Topics and Cost/ Benefit Evaluations by CERF.

Final rept. Sep 92-Nov 93.

E. W. Kent. Jan 95, 468p, FHWARD-94/094

Contract DTFH61-92-Y-00160

See also Volume 2, PB95-170551. Prepared in cooperation with Civil Engineering Research Foundation,
Washington, DC. Sponsored by Federal Highway Administration, McLean, VA. Office of Advanced Research.

Keywords: "Robotics, "Cost benefit analysis, "Highway transportation, "Technology assessment, Highway design, Highway maintenance, Highway construction, Bridge inspection, Man machine systems, Automation, Surveys, Research programs, Nondestructive tests Trenching.

The National Institute of Standards and Technology, at the request of the Federal Highway Administration, has conducted a study of potential applications of automation and robotics technology in construction, maintenance, and operation of highway systems. The study included a workshop exploring industry perceptions of needs and barriers to adoption, a workshop and a literature search to assess current state of the art practices and trends, and site visits by automation experts to typical highway worksites. Potential technology op portunities were highlighted for short, medium, and ong-term efforts in a matrix of intersections between common highway jobs and areas of current technoogical thrust. From among the opportunities identified six potential research areas were developed as specific proposals, and subjected to life-cycle cost-benefits analysis. Four were projected to return significant savings by comparison with current practice. Of these, two were identified as also likely to return benefits of significant impact on total highway expenditures and the na tional economy due to their ability to leverage savings across large numbers of jobs or their effect on a large percentage of highway traffic. This is Volume III of IV.

\section{1,339}

PB95-193173 PC A07/MF A02

National Inst. of Standards and Technology, Gaithersburg, MD.

Robotics Application to Highway Transportation. Volume 4. Proposals for Potential Research.

Final rept. Sep 92-Nov 93.

E. W. Kent Jan 95, 132p, FHWA/RD-94/095.

Contract DTFH61-92-Y-00160

See also PB95-171633. Sponsored by Federal Highway Administration, McLean, VA. Office of Advanced Research.

Keywords: "Robotics, "Highway construction, "Highway maintenance, "Highway management, "Technology assessment, Telemetry, Life cycle costs Cost benefit analysis, Robots, Automation, Bridge inspection, Real time.

The National Institute of Standards and Technology at the request of the Federal Highway Administration, has conducted a study of potential applications of automation and robotics technology in construction, maintenance, and operation of highway systems. The study included a workshop exploring industry perceptions of needs and barriers to adoption, a workshop and a literature search to assess current state of the art practices and trends, and site visits by automation experts to typical highway worksites. Potential technology opportunities were highlighted for short, medium, and long-term efforts in a matrix of intersections between common highway jobs and areas of current technological thrust. From among the opportunities identified six potential research areas were developed as specific proposals, and subjected to life-cycle cost-benefits analysis. Four were projected to return significant sav ings by comparison with current practice.

\section{1,340}

\section{PB95-251641 PC A06/MF A02}

National Inst. of Standards and Technology (MEL) Gaithersburg, MD. Intelligent Systems Div.

Study of Potential Applications of Automation and Robotics Technology in Construction, Maintenance and Operation of Highway Systems: A Final Report. Volume 4.

E. W. Kent. Jun 95, 118p, NISTIR-5667-V4.

See also PB95-251690. Sponsored by Federal Highway Administration, McLean, VA.

Keywords: "Highway construction, "Highway maintenance, "Robotics, "Automation, Research and development, Proposals, Computer aided design, Sites, System integration, Pavements, Image analysis, Cracking(Fracturing).

The National Institute of Standards and Technology (NIST) at the request of the Federal Highway Administration, has conducted a study of potential applications of automation and robotics technology in construction, maintenance, and operation of highway systems. The study included a workshop exploring industry perceptions of needs and barriers to adoption, a workshop and a literature search to assess current state of the art practices and trends, and site visits by automation experts to typical highway worksites. The final volume contains the set of final research proposals setting out specific studies with proposed deliverables, support levels, and timetables.

01,341

\section{PB95-251682 PC A06/MF AO2}

National Inst. of Standards and Technology (MEL), Gaithersburg, MD. Intelligent Systems Div.

Study of Potential Applications of Automation and Robotics Technology in Construction, Maintenance and Operation of Highway Systems: A Final Report. Volume 1.

E. Kent. Jun 95, 105p, NISTIR-5667-V1.

See also Volume 3, PB95-251690. Sponsored by Federal Highway Administration, McLean, VA.

Keywords: "Highway construction, "Highway maintenance, "Robotics, "Automation, Highway bridges, Technology assessment, Computer applications, Sys
tems integration, Bridge inspection, Bibliographies.

The National Institute of Standards and Technology (NIST) at the request of the Federal Highway Administration, has conducted a study of potential applications of automation and robotics technology in construction, maintenance, and operation of highway systems. The study included a workshop exploring industry perceptions of needs and barriers to adoption, a workshop and a literature search to assess current state of the art practices and trends, and site visits by automation experts to typical highway worksites. The first volume of the report contains an overview of the study and the methods employed, a summary of the principal results, several white papers examining selected topics of interest, and a bibliographic study.

\section{1,342}

\section{PB95-251690 PC A15/MF A03}

National Inst. of Standards and Technology (MEL), Gaithersburg, MD. Intelligent Systems Div.

Study of Potential Applications of Automation and Robotics Technology in Construction, Maintenance and Operation of Highway Systems: A Final Report. Volume 3 .

E. Kent. Jun 95, 336p, NISTIR-5667-V3.

See also PB95-251682. Sponsored by Federal Highway Administration, McLean, VA.

Keywords: "Highway construction, "Highway maintenance, "Robotics, "Automation, "Meetings, Technology innovation, Technology assessment, Requirements, Control systems, Highway design, Teleoperators, Man machine systems, Robot sensors, Inspection.

The National Institute of Standards and Technology (NIST) at the request of the Federal Highway Administration, has conducted a study of potential applications of automation and robotics technology in construction, maintenance, and operation of highway systems. The study included a workshop exploring industry perceptions of needs and barriers to adoption, a workshop and a literature search to assess current state of the art practices and trends, and site visits by automation experts to typical highway worksites. The third volume contains proceedings of workshops organized to educate the study panel with respect to the highway industry, and with respect to current state of the art.

\section{1,343}

PB95-255865 PC A19/MF A04

National Inst. of Standards and Technology (MEL), Gaithersburg, MD. Intelligent Systems Div.

Study of Potential Applications of Automation and Robotics Technology in Construction, Maintenance and Operation of Highway Systems: A Final Report. Volume 2

E. Kent. Jun 95, 428p, NISTIR-5667-V2 See also Volume 1, PB95-251682, Volume 3 PB95251690 and Volume 4, PB95-251641. Sponsored by Federal Highway Administration, McLean, VA.

Keywords: "Highway construction, "Highway maintenance, "Robotics, "Automation, Research and development, Proposals, Benefit cost analysis, Life cycle costs, Technology innovation, Sites, Systems integration, Highway bridges, Inspection, Pavements, Trenching.

The National Institute of Standards and Technology (NIST), at the request of the Federal Highway Adminis- 
tration, has conducted a study of potential applications of automation and robotics technology in construction, maintenance, and operation of highway systems. The study included a workshop exploring industry perceptions of needs and barriers to adoption, a workshop and a literature search to assess current state of the art practices and trends, and site visits by automation experts to typical highway worksites. The second volume presents proposals for particular research possibilities developed by the study panel, and a life-cycle cost-benefit analysis of the proposed research under the assumption the expected technological goals could be achieved.

\section{1,344}

PB96-101050 PC A03/MF A01

National Inst. of Standards and Technology (BFRL), Gaithersburg, MD. Building Materials Div.

Degradation of Powder Epoxy Coated Panels Immersed in a Saturated Calcium Hydroxide Solution Containing Sodium Chloride.

Final rept. Aug 92-Jul 94

J. W. Martin, T. Nguyen, D. Alsheh, E. Byrd, J. Seiler, J. A. Lechner, and E. Embree. Oct 95, 46p, FHWA/RD-94/174

Contract DTFH61-92-Y-30115

Sponsored by Federal Highway Administration, McLean, VA. Office of Engineering and Highway Operations Research and Development

Keywords: *Reinforcing steels, "Bars, "Epoxy coatings, "Immersion test(Corrosion), *Corrosion, Manine environments, Chemical attack, Degradation, Protective coatings, Peeling, Exfoliation corrosion, Blistering, Calcium hydroxides, Sodium chloride, Thermography, Anodic blisters, Cathodic disbondment, Wet adhesion, Infrared thermography.

Blasted-steel panels were coated with two commercial powder epoxy coatings. Approximately half $(80)$ of the coated panels were scribed; while the other half remained defect-free. All of the panels were immersed in a saturated calcium hydroxide solution containing 3.5 percent sodium chloride maintained at either 35 or $50 \mathrm{deg} . \mathrm{C}$. None of the unscribed panels degraded after $3074 \mathrm{~h}$ of immersion at $35 \mathrm{deg} \mathrm{C}$; whereas, all of the scribed panels degraded within $24 \mathrm{~h}$ after immersion, regardless of the immersion solution temperature. Scribed panels degraded in three ways: (1) anodic corrosion, (2) Cathodic disbondment, and (3) wetadhesion loss.

\section{1,345}

\section{PB96-131537 PC A03/MF A01}

National Inst. of Standards and Technology (BFRL), Gaithersburg, MD. Building Materials Div. Alkali-Silica Reaction and High Performance Concrete.

C. F. Ferraris. Aug 95, 20p, NISTIR-5742.

Sponsored by Nuclear Regulatory Commission, Washington, DC.

Keywords: "Alkali aggregate reactions, "Silicon dioxide, "Concretes, Air, Cement aggregate reactions, Concrete durability, Standards, Tests, Chemical reactions, Performance, Construction materials, ASR(Alkali-silica reaction), HPC(High performance concrete), National Institute of Standards and Technology.

Damage due to alkali-silica reaction (ASR) in concrete is a phenomenon that was first recognized in the U.S. since 1940 and has since been observed in many countries. Despite numerous studies published, the machanism is not yet clearly understood. Nevertheless, the three major factors in concrete have been identified, i.e., the alkalies contained in the pore solution, reactive amorphous or poorly crystallized silica present in certain aggregates, and water. In this study, we attempted to address the question of whether highperformance concrete (HPC) is susceptible to ASR. It was found that air content is the most important variable (other than the three majors factors cited above) that increase expansion of concretes affected by ASR. This study seems to indicate that even HPC is susceptible to $A S R$ if reactive aggregates are used.

\section{1,346}

PB96-146352 PC A07/MF A02

National Inst. of Standards and Technology (BFRL) Gaithersburg, MD. Building and Fire Research Lab.
Seismic Performance of Circular Bridge Columns Designed in Accordance with AASHTO/CALTRANS Standards.

Building science series (Final).

W. C. Stone, and A. W. Taylor. Feb 93, 132p, NIST/ BSS-170

Also available from Supt. of Docs. as SN003-003 03195-0. Sponsored by Federal Highway Administration, McLean, VA.

Keywords: "Bridges(Structures), "Earthquake resistant structures, "Dynamic structural analysis, Seismic design, Bridge design, Columns(Supports), Earthquake damage, Failure modes, Cyclic loads, Dynamic loads, Lateral pressure, Hysteresis, Reinforcement(Structures), Reinforced concrete, Earthquake engineering, Standards, Circular bridge columns, Spiral reinforcement.

Limitations of present procedures for the design of bridge columns to withstand seismic loads are discussed. Techniques for achieving the earthquake resistant capabilities are described and new design criteria based on acceptable damage indices as functions of earthquake magnitude, distance, and structural importance, are proposed. Using the proposed procedure and criteria the performance of 72 representative bridge columns designed in accordance with 1992 CALTRANS specifications is analyzed. Analysis parameters included earthquake magnitude, distance from epicenter, subsurface soil characteristics, column aspect ratio, and normalized column axial load. Design charts, based on allowable damage index versus earthquake magnitude, are developed and retrofit strategies are discussed for those designs which do not meet the proposed design criteria.

\section{1,347}

PB96-193685 PC A08/MF A02

National Inst. of Standards and Technology (BFRL), Gaithersburg, MD

Guidelines for Pre-Qualification, Prototype and Quality Control Testing of Seismic Isolation Sys-

Final rept.

H. W. Shenton. Jan 96, 137p, NISTIR-5800

See also PB94-161940.

Keywords: "Vibration isolators, "Test methods, *Earthquake resistant structures, Building codes, Displacement, Structural vibration, Dynamic structural analysis, Earthquake engineering, Dynamic response, Vibration dampling, Sliding, Hybrid systems, Prototypes, Loads(Forces), Bridges(Structures), Standards, Requirements, Quality control, Elastomeric systems, Qualification.

The Building and Fire Research laboratory of the National Institute of Standards and Technology has developed guidelines for testing and evaluation of seismic isolation systems. Included in the report are comprehensive guidelines for conducting pre-qualification, prototype and quality control tests of the isolation system. The guidelines are independent of the type of isolation system and application. Thus, they can be used to test elastomeric, sliding or hybrid isolation systems, for applications that involve buildings, bridges, facilities and other special structures. The guidelines include general requirements of the test facilities and other special structures. The guidelines include general requirements of the test facility, instrumentation, calibration, data acquisition, data analysis and reporting of results. All tests are presented in a standard format that includes test designation, purpose, sequence, procedure, performance criteria, special requirements and exceptions.

\section{1,348}

PB96-197249 PC A04/MF A01

Transportation Research Board, Washington, DC. IDEA Program

Development of a Method for Measuring WaterStripping Resistance of Asphalt/Siliceous Aggregate Mixtures.

Rept. of investigation (Final) Apr 93-May 96

T. Nguyen, E. Byrd, D. Bentz, and J. Seiler. 6 May 96, 37p, TRB/NCHRP-ID002

Prepared in cooperation with National Inst. of Standards and Technology, Gaithersburg, MD. Sponsored by Federal Highway Administration, Washington, DC and American Association of State Highway and Transportation Officials, Washington, DC

Keywords: "Bituminous concretes, "Flexible pavements, "Water resistance, "Nondestructive tests, Aggregates, Mechanical proerties, Infrared spectroscopy.
Adhesion, Concrete durability, Pavement bases, Stripping.

The objective of the study was to develop a nondestructive spectroscopic method for measuring water stripping resistance of aspha!tsiliceous aggregates ex posed to water. Phase 1 of the study developed a technique based on Fourier transform infrared spectroscopy/multiple reflection mode to quantify water layer at the asphalt-siliceous aggregate interface. In Phase 2 a technique to measure adhesion loss of the asphaltaggregate system exposed to water environment was developed. Phase 3 correlated the quantity of interfacial water layer with the adhesion loss data. In addition, results on the use of spectroscopic technique to evaluate the effectiveness of asphalt antistripping agents are presented. Based on interfacial wate inforamtion, mechanisms of stripping asphalt from siliceous aggregates and of transport of water from the environment to asphalt-aggregate interface are presented.

\section{1,349}

PB96-202353 PC A06/MF AO1

National Inst. of Standards and Technology (BFRL) Gaithersburg, MD. Office of Applied Economics.

Economics of New-Technology Materials: A Case Study of FRP Bridge Decking.

Final rept.

M. A. Ehlen, and H. E. Marshall. Jul 96, 76p,

NISTIR-5864.

Keywords: "Bridge decks, "Cost analysis, "Composite materials, Life cycle costs, Highway bridges, Economic analysis, Technology innovation, Construction materials, Advanced materials, FRP(Fiber reinforced polymers).

The purpose of this report is to provide a general method for evaluating the life-cycle cost effectiveness of new-technology materials in relation to conventional materials. The methods provides users with a tool that helps them choose that material among competing al ternative materials that performs the required function at minimum life-cycle cost. To illustrate an application of the economic method, the authors prepare a case study of highway bridge decks. The authors evaluate the use of FRP (Fiber reinforced polymers) materials as an alternative to conventionally used concrete. The rapidly increasing research on FRPs suggest that it will be a major construction material in the future. The authors choose sbridge decks for two reasons: first this application of FRPs appears technically promising, and second, there is a large number of bridges in the United States that will need to be replaced in the next 10 15 years, suggesting that there will be considerable interest in a case study of this application.

\section{Soil \& Rock Mechanics}

01,350

PB96-128111 PC A05/MF A01

National Inst. of Standards and Technology (BFRL) Gaithersburg, MD. Structures Div.

Ground Improvement Techniques for Liquefaction Remediation Near Existing Lifelines.

R. D. Andrus, and R. M. Chung. Oct 95, 89p, NISTIR-5714

Keywords: "Liquefaction, "Soil stabilization, "Earthquake resistance, Grouting, Soil dynamics, Mix ing, Drainage, Conduits, Pipelines, Buildings, Storage tanks, Bridges(Structures), Dams, Soil structure interactions, Deformation, Earth movements, Ground motion, Displacement, Ground subsidence, Seismic effects, Soil mechanics, Earthquake engineering, Cost estimates, "Lifeline systems.

This report reviews five low vibration ground improvement techniques suitable for remedial work near existing structures. The five techniques are: compaction grouting, permeation grouting, jet grouting, in situ soil mixing and drain pile. The factors which can influence the effectiveness of each technique are identified. Cost estimates are given for each technique, except the drain pile technique which is not yet available in the United States. Nineteen case studies of liquefaction remediation and remedial work near existing lifelines are reviewed Advantages and constraints of the five techniques are compared. A combination of techniques may provide the most cost-effective ground improve ment solution for preventing damage to existing life- 


\section{Soil \& Rock Mechanics}

lines resulting from liquefaction-induced horizontal ground displacement, subsidence, and uplift.

\section{1,351}

\section{PB96-214697 PC A10/MF A03}

Colorado School of Mines, Golden. Div. of Engineering. Estimation of System Damping at the Lotung Site by Application of System Identification.

S. D. Glaser, and A. L. Leeds. Aug 96, 197p, NIST/ GCR $-96 / 700$

Contract NIST-60NANB5D0074

See also PB96-165972. Sponsored by National Inst. of Standards and Technology (BFRL), Gaithersburg, MD. and National Science Foundation, Arlington, VA.

Keywords: "Nuclear power plants, "Soil mechanics, "Excitation "Seismic waves, Soil structure inter. actions, Earthquake resistant structures, Liquefaction, Displacement, Structure response, Vibration damping, Stiffness, Earthquake magnitude, Seismic effects, Earthquakes, Ground motion, Soil profiles, Site characterization, Nonlinearity, Earthquake engineering. Graphs(Charts), Lotung(Taiwan), System identification.

This report presents detailed system identification (SI) analyses of the full suite of seismic events at the Lotung site. Estimates of resonant frequencies and damping, and the dependence of these quantities on earthquake intensity is presented. The Lotung site is introduced in Chapter 2 through geological, seismological, and geotechnical descriptions. The SI precedures are discussed in detail in Chapter 3. Chapter 4 presents results of the system identification of the Lotung site data, with a discussion and conclusions given in Chapter 5. Appendix A describes the signal processing used on the raw data, with the integration procedure given in Appendix B. The pore water pressure time histories are shown in Appendix C. A literature review of damping values measured by other researchers is compiled in Appendix D. Finally, comparisons of actual interval outputs and modeled outputs for all events and intervals is given in Appendix

\section{COMBUSTION, ENGINES, \& PROPELLANTS}

\section{General}

01,352

PB97-112270 Not available NTIS

National Inst. of Standards and Technology (CSTL),

Gaithersburg, MD. Process Measurements Div.

Analysis of Droplet Arrival Statistics in a PressureAtomized Spray Flame.

Final rept

J. T. Hodges, C. Presser, A. K. Gupta, and C. T. Avedisian $1994,9 \mathrm{p}$

Pub. in International Symposium on Combustion (25th), Irvine, CA., July 31-August 5, 1994, p353-361.

Keywords: "Combustion, "Fuel sprays, "Drops, Flow visualization, Image analysis, Flames, Kerosene, Reprints.

The statistical behavior of droplets in a kerosene pressure-atomized spray was investigated under swirling and burning conditions. This case was studied in order to better understand individual droplet transport processes downstream of the fuel spray flame. A two-component phase-Doppler particle sizing system was employed to provide information on the droplet size and velocity distributions as well as interarrival-time statistics. Time-resolved information is presented on the instantaneous values of diameter, and the respective axial and radial velocity components. The results provide some evidence of nonsteady interarrival-time statistics (associated with droplet clustering) immediately downstream of the fuel nozzle and at radial coordinates near the center of the spray. The unsteady statis tical behavior was most prominent within the shear layer formed near the inner spray boundary. Decomposition of the results into size classes indicated that clustering (attributed to entrainment by recirculating gases) occurred only for the smallest droplets, 0-20 micrometers in diameter. For larger droplets, analysis on the random nature of the spray process revealed that droplet transport generally follows steady Poisson statis tics. Furthermore, the information provided here shows that size-class decomposition is important in the analy sis of spray behavior.

\section{Combustion \& Ignition}

\section{1,353}

AD-A274 609/7 PC A03/MF A01

National Inst. of Standards and Technology, Gaithersburg, MD.

Gordon Research Conference on the Physics and Chemistry of Laser Diagnostics in Combustion Held in Plymouth, New Hampshire on 12-16 July 1993.

Final rept.

K. C. Smyth. 16 Jul 93, 24p.

Keywords: "Combustion, Diagnostic equipment, Lase applications, Symposia, Quantitative analysis, Phase siudies, Multiphase flow, Flow fields, Laser diagnostics, In situ analysis, Sectroscopy.

The 1993 Gordon Research Conference on the Phys ics and Chemistry of Laser Diagnostics in Combustion was held at Plymouth State College (South) in Plym outh, New Hampshire, July 12-16, 1993. This conference is primarily concerned with the fundamenta physics and chemistry underlying the wide variety of laser-based optical spectroscopic diagnostic techniques which are used for studying combustion proc esses. The focus is on in-situ and nonperturbing optical methods for one- two- and three-dimensional measurements of species concentrations, temperature, and velocity. The development of such quantitative methods using linear and nonlinear optical interactions en compasses a broad scope of interdisciplinary research including basic and applied physics and chemistry.

01,354

N96-15569/2 (Order as N96-15552, PC A20/MF

A04)

National Inst. of Standards and Technology, Gaithersburg, MD.

Combustion of a Polymer (PMMA) Sphere in Micro gravity.

J. C. Yang, and A. Hamins. Aug 95, 6p.

In NASA. Lewis Research Center, the 3RD International Microgravity Combustion Workshop $p 115$ 120.

Keywords: "Aerospace vehicles, "Combustion, "Liquid fuels, "Reduced gravity, "Polymers, "Polymethyl methacrylate, "Propulsion, Aerospace safety, Burning rate Chemical reactions, Flammability, Gravitational efChemical reactions,
fects, *Microgravity.

Polymer combustion is a highly complicated process where chemical reactions may occur not only in the gas phase, but also in the condensed phase as well as at the solid-gas interphase. The chemistry depends strongly on the coupling between the condensed phase and gas phase phenomena. For some polymers, additional complications arise due to the formation of char layers. For others, the behavior of the condensed phase involves swelling, bubbling, melting sputtering, and multi-stage combustion. Some of these features bear resemblance to the phenomena observed in coal particle combustion. In addition to its relevance to spacecraft fire safety, the combustion of polymeric materials is related to many applications including solid and hybrid rocket propulsion, and of recent interest, waste incineration. The burning rate is one of the most important parameters used to characterize the combustion of polymers. It has been used to rank the polymer flammability under the same experimenta conditions and to evaluate various modes of inhibiting polymer flammability. The main objective of this work is to measure the burning rates of a polymeric material in low gravity. Because of inherent logistical difficulties involved in microgravity experiments, it is impossible to examine a wide spectrum of polymeric materials. It is desirable to investigate a polymer whose combustion is less complicated, and yet will lead to a better understanding of the burning characteristics of other more complicated materials. Therefore, a typical noncharring polymer is selected for use in this experimental study. PMMA (polymethylmethacrylate) has been chosen because its thermo-physical properties are well characterized. Although the combustion of PMMA has been extensively studied in $1 \mathrm{G}$ experiments, only a limited amount of work has been conducted in low gravity. A spherical sample geometry is chosen in this study because it is the simplest configuration in terms of the microgravity hardware design requirements. Furthermore, a burning PMMA sphere in microgravity represents a one-dimensional flame with overall combustion characteristics expected to be analogous to the combustion of a liquid fuel droplet, a field with many well-developed theories and models. However, differences can also be expected such as the flame-front standoff ratios and the condensed phase processes occurring during combustion.

01,355

PB94-142973 PC A04/MF A01

National Inst. of Standards and Technology (BFRL), Gaithersburg, MD.

In situ Burning of Oil Spills: Mesoscale Experiments.

W. D. Walton. Nov 93, 63p, NISTIR-5266.

See also PB94-101839. Sponsored by Minerals Management Service, Herndon, VA. Technology Assessment and Research Branch.

Keywords: "Oil spills, "In-situ combustion, "Fire tests, Crude oil, Burning rate, Plumes, Smoke, Particulates, Water pollution control, Mitigation, Oil-water interfaces, Mesoscale tests.

In 1991 a series of 14 mesoscale fire experiments were performed to measure the burning characteristics of crude oil on salt water. These oil bums in a pan ranged in size from $6 \mathrm{~m}$ square to $15 \mathrm{~m}$ square. Results of the measurements for burning rate, oil temperature, water temperature, smoke particle size distribution, smoke plume trajectory, and smoke particulate yield are provided. The burning rate as indicated by the regression rate of the oil surface was found to be 0.055 ( or -) $0.005 \mathrm{~mm} / \mathrm{s}$ and smoke particulate yields were found to be approximately 0.13 of the oil burned on a mass basis.

\section{1,356}

PB94-156866 PC A08/MF AO2

California Univ., Berkeley. Dept. of Mechanical Engineering.

Concurrent Flow Flame Spread Study

H. T. Loh. Mar 92, 161p, NIST/GCR-92/603.

Grant NIST-NANB7D737

See also N93-24946. Sponsored by National Inst. of Standards and Technology (BFRL), Gaithersburg, MD.

Keywords: "Flame propagation, "Gas flow, Combustion, Temperature distribution, Reaction kinetics, Pyrolysis, Mathematical models, Boundary layer, Computer programs, Diffusion flames, Computational fluid dynamics.

An experimental study has been performed of the spread of flames over the surface of thick PMMA and thin filter paper sheets in a forced gaseous flow of varied oxygen concentration moving in the direction of flame spread. It is found that the rate of spread of the PMMA pyrolysis front is time independent, linearly dependent on the gas flow velocity and approximately square power dependent on the oxygen concentration of the gas. The experimental data with thin filter paper sheets shows that the flame spread rate is independent of the flow velocity for forced flow conditions and linearly dependent on the oxygen concentration of the flow. In both experiments, it was found that the flame spread rate data can be correlated in terms of parameters deduced from heat transfer considerations only. This indicates that heat transfer from the flame to the condensed fuel is the primary mechanism controlling the spread of flame. Finite rate chemical kinetic effects have apparently a small influence on the flame spread process itself. Analytical and numerical methods were also employed to study theoretically the flame spread process over thermally thick fuel and in the influence on the flow field behavior in the presence of a flame. 
Numerical Analysis Support for Compartment Fire Modeling and Incorporation of Heat Conduction into a Zone Fire Model.

Final rept. 15 Aug 88-31 Mar 91

W. F. Moss. Mar 92, 51p, NIST/GCR-92/605.

Grant NANB-8D0857

See also PB90-250192 and PB92-172790. Sponsored by National Inst. of Standards and Technology (BFRL) Gaithersburg, MD.

Keywords: "Mathematical models, "Conduction, "Heat transmission, "Fires, Numerical analysis, Prototypes, Temperatures, Fortran, Floors, Walls, Ceilings, Differential equations, Zone fire model.

The research goal for the first year of the grant was to determine the best available numerical technology for use in zone fire modeling. The goal for the second year was to incorporate heat conduction into a zone fire model in a numerically robust and efficient manner. Three prototype zone fire models named MCCFM CONRAD1 and CONRAD2 were constructed to test the numerical technology used to realize these goals. These zone fire models and their implementations as Fortran codes are presented. The code MCCFM, de veloped during the first year of the grant, demonstrates the advantages of using mass as a solution variable instead of density. CONRAD1 and CONRAD2 examine two strategies for coupling the heat conduction equation (a one dimensional partial differential equation) with the zone fire modeling ordinary differential equations. CONRAD2 reduces the heat conduction problem to a set of implicitly defined functional equations, a strategy never before used in zone fire modeling.

\section{1,358}

PB94-164019 PC A05/MF A01

National Inst. of Standards and Technology (BFRL) Gaithersburg, MD.

Fire Data Management System, FDMS 2.0, Technical Documentation.

Technical note.

R. W. Portier. Feb 94, 86p, NIST/TN-1407.

Also available from Supt. of Docs.as $\mathrm{SNOO}^{-003-}$ 03251-4. See also PB93-182038.

Keywords: "Data bases, "User manuals, "Managemen information systems, "Fire tests, Information retrieval, Information systems, Data management Systems en gineering, Data storage systems, Data processing, "FDMS Computer data base, "Fire Data Managemen System.

Fire Data Management System, FDMS, is a computer database specifically designed to store and retrieve fire test results. A version, FDMS 1.0, of the database is currently available. This quide provides detailed descriptions of the physical file implementations planned for the next generation of the database, FDMS 2.0.

01,359

PB94-165941 PC A12/MF A03

California Inst. of Tech., Pasadena.

Experimental and Numerical Studies on Two-Dimensional Gravity Currents in a Horizontal Channel.

W. R. Chan, E. E. Zukoski, and T. Kubota. Jul 93, 264p, NIST/GCR-93/630

Grant NIST-NANB9D0958

Sponsored by National Inst. of Standards and Technology (NEL), Gaithersburg, MD. Center for Fire Research.

Keywords: "Fire tests, "Computational fluid dynamics, Smoke, Channel flow, Currents.

The objective of this investigation is to examine the be havior of two-dimensional gravity currents, especially as applied to the spreading of smoke, generated from a room fire, along a long corridor. Both experimental and numerical techniques were used to provide a model that can adequately explain and predict the behavior of a gravity current under certain boundary conditions. A series of experiments was carried out to study the effects of Reynolds number on gravity currents in a horizontal water channel. Measurements of the time varying front position, velocity profile of the following current, and the depth of a gravity current were made using either dyed liquids or hydrogen bubble technique. Quantitative results were shown to agree with previously published results.

01,360

PB94-165974 PC A04/MF A01

Michigan Univ., Ann Arbor.
Radiation and Mixing Properties of Buoyant Turbulent Diffusion Flames.

U. U. Koylu, Z. Dai, L. K. Tseng, and G. M. Faeth. Jul 93, 61p, NIST/GCR-93/631.

Grant NANB1D1175

See also PB84-155829. Sponsored by National Inst. of Standards and Technology, Gaithersburg, MD.

Keywords: "Fires, "Flame propagation, Diffusion flames, Plumes, Optical properties, Raleigh scattering, Soot, Turbulent flow.

Two aspects of unwanted fires were considered: (1) the optical properties of soot in the fuel-lean region of buoyant turbulent diffusion flames, and (2) the structure and mixing properties of buoyant turbulent plumes. The scattering, absorption and extinction properties of soot were measured for conditions where soot structure was known from earlier transmission electron microscopy measurements. The plume study involved laser-induced iodine fluorescence measurements of mean and fluctuating mixture fractions. The results indicated that past measurements of plume properties represent transitional plumes and that selfpreserving turbulent plumes are somewhat narrower, with higher levels of mean and fluctuating mixture fractions near the axis.

\section{1,361}

PB94-172988 Not available NTIS

National Inst. of Standards and Technology (BFRL), Gaithersburg, MD. Fire Science Div.

Greatly Enhanced Soot Scattering in Flickering Ch4/Air Diffusion Flames.

Final rept.

K. C. Smyth, J. E. Harrington, E. L. Johnsson, and W. M. Pitts. 1993, 11p.

Pub. in Combustion and Flame 95, p229-239 1993.

Keywords: "Soot, "Laser induced fluorescence, "Diffusion flames, "Optical images, Combustion, Hydroxyl radicals, Scattering, Strain rate, Particles, Methane, Sampling, Reprints.

Planar images of laser-induced fluorescence from $\mathrm{OH}$ radicals and elastic scattering from soot particles are presented in time-varying, laminar $\mathrm{CH} 4 /$ air diffusion flames burning in a co-flowing, axisymmetric configuration at atmospheric pressure. Acoustic forcing is used to phase lock the periodic flame flicker to the pulsed laser system operating at $10.13 \mathrm{~Hz}$. For conditions where the tip of the flame is clipped, the intensity of the light scattered by the soot particles increases dramatically (by more than a factor of 7 for the maximum signals at a point) compared to a steady-state, laminar flame with the same mean fuel flow velocity. Comparison of the scattering signals integrated along the flame radius is carried out in the steady-state and time-varying flames as a function of height above the burner. Time-varying flames exhibit a larger range of combustion conditions than observed in corresponding steady. state flames, including different residence times, temperature histories, local stoichiometries, and strain and scalar dissipation rates. Thus, their investigation promises to yield new insights into a wide variety of chemistry-flowfield interactions which are prominent in turbulent combustion.

\section{1,362}

PB94-185113 Not available NTIS

National Inst. of Standards and Technology (CSTL), Gaithersburg, MD. Process Measurements Div.

Fluorinated Hydrocarbon Flame Suppression Chemistry

Final rept.

D. Burgess, W. Tsang, M. R. Zachariah, and P. R

Westmoreland. 1994, 6p.

Preprints of papers presented at the American Chemical Society National Meeting (207th), San Diego, CA., March 13-17, 1994, p141-146.

Keywords: "Combustion kinetics, "Thermochemistry, "Fluorohydrocarbons, "Fire suppression, "Flames, Combustion chemistry, Fuels, Transport properties, Combustion efficiency, Inhibitors, Reprints, "Chemical reaction mechanisms, $A b$ initio molecular orbital theory

A comprehensive, detailed chemical kinetic mechanism was developed for fluorinated hydrocarbon destruction and flame suppression. Existing fluorinated hydrocarbon thermochemistry and kinetics were compiled and evaluated. For species where no or incomplete thermochemistry was available, these data were calculated through application of ab initio molecular orbital theory. Group additivity values were determined consistent with experimental and ab initio data. For re actions where no or limited kinetics was available these data were estimated by analogy to hydrocarbon reactions, by using empirical relationships from other fluorinated hydrocarbon reactions by ab initio transition state calculations, and by application of RRKM and QRRK methods. The chemistry was modeled considering different transport conditions (plug flow, premixed flame, opposed flow diffusion flame) and using different fuels (methane, ethylene), equivalence ratios agents (fluoromethanes, fluoroethanes) and agen concentrations. An overview of this work is presented.

\section{1,363}

PB94-185287 Not available NTIS

National Inst. of Standards and Technology (BFRL), Gaithersburg, MD. Fire Science Div.

Flame Retardants - Overview.

Final rept.

R. G. Gann. 1994, 7p.

Pub. in Kirk-Othmer Encyclopedia of Chemical Technology, v10 p930-936 1994

Keywords: "Flame retardants, Fire tests, Fire resistant coatings, Fire prevention, Polymers, Marketing, Performance evaluation, Reprints.

This article provides an introduction to succeeding chapters on fire retardants in the "Encyclopedia of Chemical Technology.' The text describes why fire retardants are useful, general mechanisms for their ef fectiveness, how the performance of polymer/retardant composites is measured, a snapshot of the market for these chemicals, and forces that might change that market.

01,364

PB94-185352 Not available NTIS National Inst. of Standards and Technology (BFRL) Gaithersburg, MD. Fire Science Div.

Laser Imaging of Chemistry-Flowfield Interactions: Enhanced Soot Formation in Time-Varying Diffusion Flames.

Final rept

J. E. Harrington, C. R. Shaddix, and K. C. Smyth 1994, 14p

Pub. in Proceedings of the Society of Photo-Optical Instrumentation Engineers Laser Techniques for StateSelected and State-to-State Chemistry II, Los Angeles, CA., January 27-29, 1994, v2124 14p.

Keywords: "Diffusion flames, "Soot, "Laser induced flurescence, "Flow fields, Reprints, i ight scattering, Luminosity, Methane, Combustion products, Image processing, Unsteady flow.

Models of detailed flame chemistry and soot formation are based upon experimental results obtained in steady, laminar flames. For successful application of these descriptions to turbulent combustion, it is instruc tive to test predictions against measurements in timevarying flowfields. This paper reports the use of optical methods to examine soot production and oxidation processes in a co-flowing axisymmetric $\mathrm{CH}$ 4/air diffusion flame in which the fuel flow rate is acoustically forced to create a time-varying flowfield. For a particular forcing condition in which tip clipping occurs $(0.75$ $\checkmark$ loudspeaker excitation), elastic scattering of vertically polarized light from the soot particles increases by nearly an order of magnitude with respect to that observed for a steady flame with the same mean fue flow rate. The visible flame luminosity and laser-induced fluorescence attributed to polycyclic aromatic hydrocarbons (PAH) are also enhanced. Peak soo volume fractions, as measured by time-resolved laser extinction/tomography at 632.8 and $454.5 \mathrm{~nm}$ and calibrated laser-induced incandescence (LII), show a factor of 4-5 enhancement in this flickering flame. The LII method is found to track the soot volume fraction closely and to give better signal-to-noise than the extinction measurements in both the steady and time-varying flowfields. A Mie analysis suggests that most of the enhanced soot production results from the formation of larger particles in the time-varying flowfield.

\section{1,365}

PB94-193844 PC A04/MF AO

California Univ., Berkeley. Dept. of Mechanical Engineering.

Propagation in Concurrent Flows.

Final progress rept. 1 Sep 92-31 Aug 93.
A. C. Fernandez-Pello. Jun 94, 68p, NIST/GCR-94/

A. C.

Grant NIST-60NANB1D1174

See also PB91-157206. Sponsored by National Inst of Standards and Technology (BFRL), Gaithersburg. MD. 


\section{Combustion \& Ignition}

Keywords: "Flame propagation, "Turbulence, *Fire tests, Velocity distribution, Buoyancy, Soot, Burning rate, Test facilities, Heat flux, Combustion products, Mathematical models, Heat transfer, "Concurrent flow. A research program is being conducted to study the mechanisms controlling the spread of flames in an oxidizing gas flow moving in the direction of flame propagation. During this reporting period research has been conducted to study the effect of the oxidizer flow characteristics on the concurrent flame spread over thick PMMA sheets. The parameters varied in the experiments are the oxidizer flow velocity, turbulence intensity and oxygen concentration, and the geometrical orientation (floor and ceiling). Their effect on the flame spread process is studied by measuring the rate of flame spread, flame length, surface heat flux, products of combustion and soot. The results of the experiments show that the combined effect of flow velocity, turbulence intensity, and oxygen concentration has a complex influence on the flame spread process. At low flow velocity, the flame spread rate increases monotonically with turbulence intensity. At high flow velocity, however, the flame spread rate increases with flow turbulence at low turbulence intensities, but it decreases at high turbulence intensity values. The effect is more pronounced at high oxygen concentration. These trends appear to be due to a strong influence of the turbulence intensity on the flame temperature and length, and on that heat flux from the flame to the solid fuel. Turbulence enhances mixing, which increases the flame temperature and then the heat flux.

\section{1,366}

PB94-193927 PC A11/MF A03

California Univ., Berkeley

Backdraft Phenomena.

Doctoral thesis 1990-92 (Final).

C. M. Fleischmann. cJun 94,237 p, NIST/GCR-94/

646.

Grant NIST-60NANBOD 1042

Sponsored by National Inst. of Standards and Technology (BFRL), Gaithersburg, MD.

Keywords: "Flame propagation, "Flow velocity, Combustion physics, Fires, Mixing layers(Fluids), Compart ments, Ignition, Theses, Mathematical models, Froude number, Convective flow, Flow visualization, "Backdraft phenomena, "Compartment fires.

The purpose of this project was to develop a fundamental physical understanding of backdraft phenomena. The research was divided into three phases exploratory simulations, gravity current modeling, and quantitative backdraft experiments. The primary goa of the first phase was to safely simulate a backdraft in the laboratory. A half-residential-scale compartment was built to conduct exploratory experiments. The initial experiments concluded with a scenario describing the fundamental physics of backdrafts. The importance of the gravity current which enters the compartment after opening was identified. In the second phase, the gravity current speed and the extent of its mixed region was investigated in a series of scaled salt water experiments. The scaled compartment $(0.3 \mathrm{~m} \times 0.15 \mathrm{~m}$ $0.15 \mathrm{~m}$ ) was fitted with a variety of end openings; full slot, door, and window. Video and photo data indicate that the mixing layer which rides on the gravity curren in the full opening case, expands to occupy nearly the entire current in the partial opening cases. The Froude number and nondimensional head height are independent of beta and are in good agreement with numerical simulations and special limits from the literature. In the final phase, 28 backdraft experiments were conducted in a $1.2 \mathrm{~m}$ by $1.2 \mathrm{~m}$ by $2.4 \mathrm{~m}$ compartment. (Copyright (c) 1993 Charles Martin Fleischmann.)

01,367

PB94-195856 PC A03/MF A01

Maryland Univ., College Park. Dept. of Fire Protection Engineering.

Fire Growth Models for Materials.

Final rept. Jun 92-Dec 93

J. G. Quintiere, and B. Rhodes. Jun 94, 49p, NIST/ GCR-94/647.

Grant NIST-6ONANB2D1266

Sponsored by National Inst. of Standards and Technology (BFRL), Gaithersburg, MD.

Keywords: “Burning rate, "Materials, "PMMA, Fire hazards, Calorimeters, Flame propagation, Heat flux, Ignition, Mathematical models, Test f́acilities, Combustion, *Fire models.

Ignition and burning rate data have been developed for thick (25 mm.) black Polycast PMMA in a Cone Calo- rimeter heating assembly. The objective was to establish a testing protocol that would lead to the prediction of ignition and burning rate from Cone data. This has been done for a thermoplastic like PMMA. For black PMMA we measured ignition temperatures of 250 to $350 \mathrm{C}$ and vaporization temperatures of approximately 325 to $380 \mathrm{C}$ over irradiance levels of 15 to $60 \mathrm{~kW}$ $\mathrm{sq} \mathrm{m}$. The incident flame heat flux, for irradiation levels of 0 to $75 \mathrm{~kW} / \mathrm{sq} \mathrm{m}$, was found to be approximately $37 \mathrm{~kW} / \mathrm{sq} \mathrm{m}$ for black PMMA. Its constancy has been shown due to the geometry of the Cone flame. Also, this flame can be shown to be nearly transparent for Cone irradiance (greater than 90 percent). The heat of gasification of the black PMMA used was found to be approximately $2.8 \mathrm{~kJ} / \mathrm{g}$; higher than other values reported for PMMA. This is believed to be due to dif ferences in molecular structure or pigmentation effects and the types of PMMA tested. A burning rate mode was demonstrated to yield good accuracy (greater than 80 percent) in comparison to measured transient values.

\section{1,368}

PB94-196045 PC A99/MF A06

Brown Univ., Providence, RI. Div. of Engineering. Behavior of Charring Materials in Simulated Fire Environments.

Final rept. 1990-92

E. M. Suuberg, I. Milosavljevic, and W. D. Lilly. Jun 94, 625p, NIST/GCR-94/645.

Grant NIST-6ONANBOD 1042

Sponsored by National Inst. of Standards and Technology (BFRL), Gaithersburg, MD.

Keywords: "Fire models, "Pyrolysis, "Cellulose, *Charring, Fire chemistry, Reaction kinetics, Polymers, Heat transfer, Laboratories, Thermodynamics, Combustion products, Fire research, Solids, Flammable gases, US NBS, Carbon monoxide, Char, Char depth, NIST(National Institute of Standards and Technology).

The focus of this study was the behavior of thick charring solids in fire situations. Clearly one of the most important parameters governing the fire phenomenon is the rate of release of combustible volatiles into the gas phase, in which they actually burn. Over the years, fire researchers have learned how to model the processes in the gas phase, so that the rate of heat feedback to the solid surface can be reasonably well predicted. Likewise, there exists the ability to model the heat transfer processes at the solid surface and within the solid itself. This study was concerned with the possibility that the inability to come to complete closure on the charring polymer fire problem might derive from difficulties in applying laboratory scale kinetics to actual fire conditions. Specifically, we were concerned about how well small scale laboratory experiments used to derive the kinetics of pyrolysis could be used to predict the behavior of charring solids in fire situations.

\section{1,369}

PB94-198439 Not available NTIS

National Inst. of Standards and Technology (BFRL) Gaithersburg, MD. Fire Measurement and Research Div.

Effective Measurement Techniques for Heat, Smoke, and Toxic Fire Gases.

Final rept.

V. Babrauskas. 1991, 14p.

Pub. in Fire Safety Jnl. 17, p13-26 1991.

Keywords: "Fires, "Smoke, *Heat, "Toxicity, "Test methods, Combustion products, Measuring instruments, Bench tests, Test facilities, Measurement, Reprints, "Fire gases

The latest techniques which have been developed for the measurement of heat, smoke, and toxic gas emissions from fires are reviewed. The current objective of minimizing apparatus dependence of the data is emphasized. Forms of data and their units are outlined. Differences between data obtained in large-scale and in bench-scale tests are discussed, and areas where further research is needed are indicated.

\section{1,370}

PB94-198447 Not available NTIS

National Inst. of Standards and Technology (NEL), Gaithersburg, MD. Fire Measurement and Research Div.

Modern Test Methods for Flammability.

Final rept.

V. Babrauskas. $1990,20 p$

Pub. in Proceedings of a Conference on Recent Adv.

Flame Retard. Polym. Mater., Stanford, CT., May 1990, p50-69.
Keywords: "Flammability, "Test methods, "Bench tests, "Fire hazards, Burning rate, Combustion, Toxicity, Smoke, Ignition, Test facilities, Reprints.

During the last decade, significant improvements have become available in flammability testing. Rationallybased new methods, derived from fundamental engineering principles, are replacing the previously used empirical tests. The major emphasis in this development work has been to provide a basic set of benchscale methods which can be used to predict full-scale product performance. Reference methods for conducting full-scale tests will continue to be needed to handle products or situations where the bench-scale methods are not applicable. The bulk of the testing needs, however, can now be fulfilled by use of bench-scale tests which are not only simple to run, but are known to accurately predict the full-scale performance.

\section{1,371}

PB94-198462 Not available NTIS

National Inst. of Standards and Technology (NEL) Gaithersburg, MD. Fire Measurement and Research Div.

Standardization of Formats and Presentation of Fire Data - The FDMS.

Final rept.

V. Babrauskas, R. D. Peacock, and M. Janssens. 1991, 8p

Pub. in Fire and Materials 15, n2 p85-92 Apr-Jun 91.

Keywords: "Standardization, "Fires, "Data transfer(Computers), "Data base management systems, Test methods, Data processing, Standards, Computer programs, Data integration, Reprints, *Fire test data, "Fire Data Management System.

Effective exchange of fire test data, even within a single laboratory, has been difficult due to the multiple incompatible data formats and hardware. This issue has been addressed by a careful study of user needs, leading to the development of a series of standard formats whereby fire test data could easily be exchanged among laboratories and design professionals. Both scalar and vector data are included. These formats have been made practical by the development of a computer program, the Fire Data Management System (FDMS), and pertinent hardware standards. The system includes the most commonly used of modern day fire test methods, but also has provisions for future extension to other tests of interest.

\section{1,372}

PB94-200292 Not available NTIS

National Inst. of Standards and Technology (NEL) Gaithersburg, MD. Fire Science and Engineering Div. Generation and Characterization of Acetylene Smokes.

Final rept.

T. G. Cleary, R. A. Fletcher, G. W. Mulholland, L. K. Ives, and J.W. Gentry. 1990, 4p.

Pub. in Chemical Physics Processes Combustion, p128-1-128-4 1990

Keywords: *Particles, *Diffusion flames, "Smoke generators, Acetylene, Combustion products, Mass spectroscopy, Aerosol generators, Soot, Particle size, Lam inar flow, Reprints.

Acetylene smokes from a laminar diffusion flame are characterized. The smoke generator is capable of producing mono-sized $7 \mathrm{~nm}$ particles and soot agglomerates containing $20-30 \mathrm{~nm}$ in diameter primary particles. Size distributions and structural information were obtained by electron and optical microscopy. Laser microprobe mass spectroscopy was used to measure the chemical composition of the smokes.

\section{1,373}

PB94-200300 Not available NTIS

National Inst. of Standards and Technology (NEL), Gaithersburg, MD. Fire Science and Engineering Div. Ultrafine Combustion Aerosol Generator.

Final rept.

T. G. Cleary, G. W. Mulholland, L. K. Ives, R. A

Fletcher, and J. W. Gentry. 1992, 5p.

Pub. in Aerosol Science and Technology 16, n3 p1661701992

Keywords: "Diffusion flames, "Aerosol generators, "Smoke, Smoke generators, Particles, Combustion products, Particle size, Laminar flow, Soot, Reprints.

A laminar diffusion flame system capable of producing a narrowly distributed ultrafine aerosol is described. Diffusion battery and transmission electron microscopy 
indicate a mean particle diameter on the order of 10 to $7 \mathrm{~nm}$ respectively. Laser microprobe mass analysis indicates that the particles are composed of phosphates and sulfates in addition to carbonaceous material.

\section{1,374}

PB94-211679 Not available NTIS

National Inst. of Standards and Technology (BFRL), Gaithersburg, MD. Fire Science Div.

Laser-Induced Fluorescence Measurements of Formaldehyde in a Methane/Air Diffusion Flame.

Final rept.

J. E. Harrington, and D. C. Smyth. 1993, 7p

Pub. in Chemical Physics Letters 202, n3,4 p196-202, 22 Jan 93.

Keywords: "Diffusion flames, "Laser induced fluorescence, "Formaldehyde Combustion, Test methods, Methane, Excitation, Extinction, Burning rate, Reprints.

Laser-induced fluorescence has been observed from the formaldehyde electronic transition in a well-characterized, laminar methane/air diffusion flame burning at atmospheric pressure. This represents the first optica measurement in flames of naturally occurring form aldehyde, an important intermediate in the oxidation of hydrocarbons. Both $355 \mathrm{~nm}$ and tunable dye laser excitation of fluorescence are demonstrated. The observed fluorescence signals a re corrected for partition function effects and for estimated collisional quenching rates to obtain relative concentration profiles.

\section{1,375}

PB94-212693 Not available NTIS

National Inst. of Standards and Technology (NEL) Gaithersburg, MD. Fire Science and Engineering Div. Application of Boundary Element Methods to a Transient Axis-Symmetric Heat Conduction Problem.

Final rept.

F. Kavoosi, M. di Marzo, H. R. Baum, and D. D.

Evans. $1989,7 p$ Pub. in Proceedings of National Heat Transfer Con-
ference, Philadelphia, PA., August 6-9, 1989, p79-85.

Keywords: "Conductive heat transfer, "Sprinklers, "Boundary element method, Heat transfer coefficients, Cooling, Drops(Liquids), Evaporation, Fire fighting, Heat flux, Reprints.

A long term study of the phenomena controlling the extinguishment of solid fuel fires is based on the modeling of the interactions of sprinkler generated droplets mpinging hot solid surfaces. The thermal interactions during the evaporation of a liquid droplet deposited on low conductivity semi-infinite solid are complex because the evaporative process is coupled to intense local cooling of the solid. Sharp temperature gradients in the proximity of the droplet edge cause instabilities in finite difference solutions using reasonable time steps due to the explicit coupling of the liquid-solid region. A boundary element method is proposed in order to overcome these difficulties. The methodology and ts application to this specific problem are described in detail. Formal solutions are obtained in the form of surace integrals over the solid and the droplet surfaces. These surface integrals are discretized into summations, which lead to matrix equations for the discretized surface temperatures and heat fluxes. Results for some preliminary tests are discussed to provide an insight on the various aspects of this solution procedure

\section{1,376}

\section{PB95-104964 PC A09/MF A02}

National Inst. of Standards and Technology (BFRL), Gaithersburg, MD. Fire Science Div.

Annual Conference on Fire Research: Book of Ab. stracts, October 17-20, 1994

S. B. Smith. Oct $94,181 p$, NISTIR-5499.

See also report for 1993, PB94-121324.

Keywords: "Fire suppression, "Meetings, "Fire hazards, "Flame propagation, "Abstracts, Fires, Research, Fire extinguishing agents, Combustion, Soot, Smoke, Flow distribution, Plumes, Pool fires.

Contents: Suppression Using Halocarbons - Labora. tory Studies; Fire Hazard, Risk, and Data: Suppression Using Halocarbons - Large-Scale Studies; Suppression Using Water; Pool Fires; Fire-Induced Flows; Chemistry and Physics of Material and Product Combustion; Soot; Fire Signatures; Flame Spread; Fire Plumes.

01,377

PB95-107405 Not available NTIS
National Inst. of Standards and Technology (MSEL), Gaithersburg, MD. Metallurgy Div.

Optimization of Inert Gas Atomization.

Final rept.

S. D. Ridder, F. I. Espina, and F. S. Biancaniello. 1989, $11 p$.

Pub. in Proceedings of International Symposium on Physical Chemistry of Powder Metals Production and Processing. St. Marys, PA., October 16-18, 1989 , p163-173.

Keywords: "Atomization, "Rare gases, Particle size distribution, Powder metallurgy, Size determination, Powder metals, Expert systems, Feedback control, Hypersonic flow, Reprints.

Inert gas atomization via close-coupled hypersonic jets (Mach number $>7$ ) has promise in providing meta powder with mean size < 30 micrometers in large quantities for even difficult materials such as 304 SS Recent studies at the National Institute of Standards and Technology (formerly NBS) have been focused on the implementation of an in-situ particle size measurement sensor for feed-back control of powder size during atomization. This work involves a detailed analysis of the compressible supersonic jets, liquid disruption studies using high-speed cinematography $(10,000 \mathrm{fps})$ and holography ( 25 ns exposure time), development of the particle sizing sensor, and incorporation of this data into an intelligent process control system.

01,378

PB95-128674 PC A03/MF A01

National Inst. of Standards and Technology (BFRL),

Gaithersburg, MD. Fire Safety Engineering Div.

FIREDOC Users Manual, 3rd Edition.

N. H. Jason. Dec 93, 50p, NISTIR-5305

Errata sheet inserted. See also 2nd edition, PB91178830.

Keywords: "User manuals(Computer programs), "Information systems, "Fire safety, "Information retrieval, Information transfer, Online systems, US NIST, Bibliographies, Data bases, "FIREDOC computer program, "Fire Research Information Sevices

FIREDOC is the on-line bibliographic database which reflects the holdings (published reports, journal articles, conference proceedings, books, and audiovisual items) of the Fire Research Information Services (FRIS) at the Building and Fire Research Laboratory (BFRL), National Institute of Standards and Technology (NIST). This manual provides step-by-step procedures for entering and exiting the database via telecommunication lines, as well as a number of techniques for searching the database and processing the results of the searches. This Third Edition is necessitated by the change to a UNIX platform. The new computer allows for faster response time if searching via a modem and, in addition, offers internet accessibility. FIREDOC may be used with personal computers, using DOS or Windows, or with Macintosh computers and workstations A new section on how to access Internet is included, and one on how to obtain the references of interest to you. Appendix F: Quick Guide to Getting Started will be useful to both modem and Internet users.

\section{1,379}

\section{PB95-140919 Not available NTIS}

National Inst. of Standards and Technology (BFRL), Gaithersburg, MD. Fire Science Div.

Comparison of Experimental and Computed Species Concentration and Temperature Profiles in Laminar, Two-Dimensional Methane/Air Diffusion Flames.

Final rept.

T. S. Norton, K. C. Smyth, J. H. Miller, and M. D.

Smooke. 1993, 34p.

Pub. in Combustion Science and Technology 90, p1341993

Keywords:

"Concentration(Composition), "Temperature distribution, Methane, Laminar flow, Scalars, Radicals, Dis sipation, Combustion, Reprints.

Experimental concentration measurements of the major stable species and five radical species $(\mathrm{OH}, \mathrm{H}$ atom, $\mathrm{O}$ atom, $\mathrm{CH}$, and $\mathrm{CH} 3$ ) obtained on a rectangular Wolfhard-Parker slot burner are compared with a detailed computation of the chemical structure of an axisymmetric laminar, $\mathrm{CH} 4$ /air diffusion flame burning at atmospheric pressure. In order to examine these $\mathrm{CH} 4$ /air flames with different geometries and different sizes, the species profiles are plotted as functions of the local mixture fraction, and the scalar dissipation rate has been matched in a region around the stoichiometric surface. The overall agreement in the absolute concentrations, the shape of the profiles, and their location in terms of the local mixture fraction is good to excellent for the stable species (except for O2) and for the most abundant radicals $\mathrm{OH}, \mathrm{H}$ atom, and $\mathrm{O}$ atom. For example, the calculated $\mathrm{OH}$ maximum concentration is in much better agreement with the experimental results than are full equilibrium and partial equilibrium estimates. Less satisfactory agreement is found for the $\mathrm{CH}$ and $\mathrm{CH} 3$ radicals. In addition, significant discrepancies are observed in the temperature field ano in the degree of $\mathrm{O} 2$ penetration into rich flame regions.

01,380

\section{PB95.143160 PC A08/MF A02}

Pennsylvania State Univ., University Park. Dept. of Mechanical Engineering.

Fund

Final rept.

R. J. Santoro. Nov 94, 174p, NIST/GCR-94/661 Grant NIST-6ONANBOD 1035

See also PB88-173976 and AD-A192 733. Sponsored by National Inst. of Standards and Technology (BFRL) Gaithersburg, MD.

Keywords: "Diffusion flames, "Carbon monoxide, "Soot, Combustion products, Laminar flow, Concentration(Composition), Measuring instruments, Mathematical models.

Studies investigating the oxidation of soot and carbon monoxide (CO) have been conducled in a series of laminar diffusion flames. Both overventilated and underventilated conditions have been examined. For the overventilated studies, the production and destruction of CO has been found to be influenced by the amount of soot present in the flame Measurements of the hydroxyl radical $\mathrm{OH}(\cdot)$ have demonstrated that soot can compete for $\mathrm{OH}(-)$ when undergoing oxidation and thus, impede the oxidation of $\mathrm{CO}$ to $\mathrm{CO} 2$. Absolute concentration measurements for $\mathrm{OH}(-)$ have shown that superequilibrium values of $\mathrm{OH}(-)$ are achieved in the upper region of these diffusion flames. The results clearly demonstrate that soot particles are far from passive species in flames and can directly affect the chemical pathways involved in the oxidation process through radiative effects on temperature and soot particle reactivity effects on radical concentrations. The chemical makeup and structure of the smoke produced at high equivalence ratio is qualitatively different from smoke produced under overventilated conditions; the smoke is mainly organic rather than graphitic and it has an agglutinated structure rather than an agglomerate structure with distinct primary spheres usually observed in overventilated burning

01,381

PB95-150041 Not available NTIS

National Inst. of Standards and Technology (BFRL)

Gaithersburg, MD. Fire Science Div.

Airborne Smoke Sampling Package for Field Meas. urements of Fires.

Final rept.

J. R. Lawson, G. W. Mulholland, and H. Koseki.

1994, $18 p$

Pub. in Fire Technology 30, n1 p155-172 1994.

Keywords: "Airborne equipment, "Measuring instruments, "Combustion products, "Plumes, Crude oil, Petroleum products, Polycyclic aromatic hydrocarbons, Particulates, Sampling, Field tests, Reprints, "Smoke yield, Pool fires.

A unique airborne smoke sample package (ASSP) for determining the smoke yield of large fires has been de veloped. The ASSP, which weighs less than $4 \mathrm{~kg}$, is light enough to be flown suspended below a tethered helium-filled balloon or attached to a small radio-controlled aircraft. Measurements are made by flying the sampling equipment into a fire's smoke plume. Additional smoke plume measurements that can be made with the ASSP include particle size distribution using a cascade impactor, smoke agglomerate structure using transmission electron microscope (TEM) grids and polycyclic aromatic hydrocarbons (PAHs) analysis using various sorbent tubes. The application of the ASSP in measuring laboratory and large outdoors petroleum pool fires is discussed.

01,382

PB95-150215 Not available NTIS

National Inst. of Standards and Technology (BFRL), Gaithersburg, MD. Fire Science Div. 


\section{Combustion \& Ignition}

Oxidation of Soot and Carbon Monoxide in Hydrocarbon Diffusion Flames.

Final rept.

R. Puri, R. J. Santoro, and K. C. Smyth. 1994, 20p.

Pub. in Combustion and Flame 97, p125-144 1994.

Keywords: "Diffusion flames, "Oxidation, "Soot, "Carbon monoxide, Hydrocarbons, Combustion products, Laser induced fluorescence, Hydroxyl radicals, Methane, Collision rates, Concentration(Composition), Reprints.

Ouantitative $\mathrm{OH}(-)$ concentrations and primary soot particle sizes have been determined in the soot oxidation regions of axisymmetric diffusion flames burning methane, methane/butane, and methane/1-butene in air at atmospheric pressure. The total carbon flow rate was held constant in these flames while the maximum amount of soot varied by a factor of seven along the centerline. Laser-induced fluorescence measurements of $\mathrm{OH}(-)$ were placed on an absolute basis by calibration against earlier absorption results. The primary size measurements of the soot particles were made using thermophoretic sampling and transmission electron microscopy. $\mathrm{OH}(-)$ concentrations are greatly reduced in the presence of soot particles. Whereas large superequilibrium ratios are observed in the high-temperature reaction zones in the absence of soot, the $\mathrm{OH}(-)$ concentrations approach equilibrium values when the soot loading is high. The diminished $\mathrm{OH}(-)$ concentrations are found to arise from reactions with the soot particles and only to a minor degree from lower temperatures due to soot radiation losses. Analysis of the soot oxidation rates computed from the primary particle size profiles as a function of time along the flame centerlines shows that $\mathrm{OH}(-)$ is the dominant oxidizer of soot, with O2 making only a small contribution. Higher collision efficiencies of $\mathrm{OH}(-)$ reactions with soot particles are found for the flames containing larger soot concentrations at lower temperatures. A comparison of the soot and $\mathrm{CO}$ oxidation rates shows that although $\mathrm{CO}$ is inherently more reactive than soot, the soot successfully competes with $\mathrm{CO}$ for $\mathrm{OH}(-)$ and hence suppresses CO oxidation for large soot concentrations

\section{1,383}

PB95-150264 Not available NTIS

National Inst. of Standards and Technology (BFRL),

Gaithersburg, MD. Fire Safety Engineering Div.

Fire Service and Fire Sciences: A Winning Combination.

Final rept.

R. E. Sanders, and D. Madrzykowski. 1994, 6 p.

Pub. in NFPA Jnl. 88, n2 p55-60 Mar/Apr 94

Keywords: "Fire fighting, "Risk assessment, "Fire hazards, Fire tests, Fire safety, Fire departments, Fire suppression, Sprinklers, Fire damage, Computerized simulation, Public relations, Reprints, Louisville(Kentucky).

The NIST study provided the information that was needed to predict conditions firefighters would face on the fire floor, elevator lobby, and floor above, without placing firefighters at extraordinary risk, providing for a meaningful and safe training exercise for high rise fire fighting. Also by using the test data and HAZARD I to transfer fire science technology to the media, legis lators, and the public in an understandable context, this exercise provided the catalyst that was needed to pass the sprinkler retrofit ordinance in Louisville, Kentucky.

\section{PB95-151056 Not available NTIS}

National Inst. of Standards and Technology (NML), Gaithersburg, MD. Chemical Kinetics Div.

Progress in the Development of a Chemical Kinetic Database for Combustion Chemistry.

Final rept.

W. Tsang, G. Mallard, and J. T. Herron. 1988, 5p.

Pub. in Scientific and Technical Data in a New Era, p167-171 1988.

Keywords: "Combustion chemistry, "Chemical reaction kinetics, "Thermodynamic properties, "Databases, Combustion products, Combustion kinetics, Thermochemistry, Hydrocarbons, Alkanes, Combustion properties, Free radicals, Data compilation, Information storage, Information systems, Reprints.

A data base of chemical kinetic information for use in the modeling of the combustion of organic fuels has been developed at the National Institute of Standards and Technology. The authors have now extended the data base to cover light alkanes with up to four carbon atoms and are now carrying out work involving C3 and
C4 unsaturates. They have also carried out extensive compilations of chemical kinetic data pertinent to combustion. They begin with a brief description of the rationale for the effort and their approach to the problem. This will be followed by a summary of their most recent work. They will conclude with a discussion of their approach to solving a number of the special problems that have been encountered.

\section{1,385}

PB95-153748 Not available NTIS

National Inst. of Standards and Technology (BFRL) Gaithersburg, MD. Fire Science Div.

Turbulent Spray Burner for Assessing Halon Alternative Fire Suppressants.

Final rept.

W. Grosshandler, D. Lowe, W. Rinkinen, and C.

Presser. 1993, 8p.

Sponsored by Department of the Air Force, Wright-Patterson AFB, OH.

Pub. in Proceedings of American Society of Mechanical Engineers Winter Annual Meeting, New Orleans, LA., November 28-December 3, 1993, p1-8.

Keywords: "Sprays, "Atomization, "Fire suppression, "Aircraft fires, Flame propagation, Turbulent flow, Liquid fuels, Aircraft fuels, Combustion kinetics, Combustion properties, Thermodynamic properties, Halon al ternatives, Nozzles, Fire research, Reprints.

A research program to characterize candidate compounds for replacing halon 1301 for in-flight aircraft fire protection is described in the paper. The tion properties are examined, and a number of fuels and flame arrangements are investigated in an attemp to develop a general test protocol which will reliably predict the relative fire suppression efficiency of new agents being considered for a variety of applications. A coaxial turbulent spray burner was built to evaluate the relative effectiveness of agents for suppressing high intensity fuel fires such as one might encounter in a jet engine nacelle. A key element of the facility is the agent delivery system, which is designed to inject the desired amount of material into the air upstream of the fuel nozzle. The influence of air velocity, fue flow, and injection period on the amount of a N(sub 2) required to extinguish the turbulent spray flame is discussed, and the effectiveness of twelve gaseous agents is compared.

\section{1,386}

PB95-161790 Not available NTIS

National Inst. of Standards and Technology (BFRL), Gaithersburg, MD. Fire Science Div.

Reactivity of Product Gases Generated in Idealized Enclosure Fire Environments.

Final rept.

W. M. Pitts. 1992, $10 p$

Pub. in Proceedings of International Symposium on Combustion (24th), Sydney, Australia, July 5-10, 1992 p1737-1746.

Keywords: "Combustion products, "Reactivity, High temperature gases, Combustion chemistry, Carbo monoxide, Chemical reactions. Flue gases, Reaction products, Reprints, "Combustion gases, "Enclosure fires.

Previous experiments have demonstrated that the mole fractions of major product gases trapped in a hood located above a fire can be correlated in terms of the global equivalence ratio. Full-kinetic calculations are employed to characterize the reactivity and reaction behavior for the product gases observed in a hood experiment burning natural gas as fuel. A range of temperatures $(700-1300 \mathrm{~K})$ typical of enclosure fires is considered. Mixing is assumed to be infinitely fast (per fectly-stirred reactor) or infinitely slow (plug-flow reactor). Both isothermal and adiabatic cases are treated. Calculations are reported for a range of residence times (0-20 s) and global equivalence ratios $(0.5-2.83)$ The dominant variable for reaction behavior is found to be temperature. Effects due to mixing and heat transfer assumptions are less important. The results in dicate that the hood product gases are reactive for temperatures greater than $800 \mathrm{~K}$. For rich mixtures, reaction generates primarily carbon monoxide as opposed to carbon dioxide. At higher temperatures the formation of hydrogen is favored over water while water is favored in the $800-1000 \mathrm{~K}$ range. In the lowe temperature range $\mathrm{HO} 2$ is the dominant free radical Uncertainties in rates for reactions involving this species introduce considerable uncertainty into the calculated behaviors. At high temperatures (1100-1300 K) the important free radicals are $\mathrm{H}$ atom and $\mathrm{OH}$. The findings suggest that the results of the hood experiments cannot be used directly for the modeling of species production in enclosure fires.

\section{1,387}

PB95-162160 Not available NTIS

National Inst. of Standards and Technology (BFRL), Gaithersburg, MD. Fire Science Div.

Laser-Induced Fluorescence Measurements of $\mathrm{OH}$. Concentrations in the Oxidation Region of Laminar, Hydrocarbon Diffusion Flames.

Final rept.

R. Puri, M. Moser, R. J. Santoro, and K. C. Smyth. 1992, 8p.

Pub. in Proceedings of International Symposium on Combustion (24th), Sydney, Australia, July 1992, p1015-1022.

Keywords: "Diffusion flames, "Concentration(Composition), "Laser induced fluorescence, "Soot, "Oxidation, Combustion, Laminar flow, Hydroxyl radicals, Ethene, Methane, Flow rate, Combustion products, Reprints.

Ouantitative $\mathrm{OH}(-)$ concentrations have been measured in four coannular diffusion flames, burning methane at a single fuel flow rate and ethene at fuel flow rates which produce non-smoking, incipient smoking conditions. Laser-induced fluorescence has been used to make point profile, line image, and two-dimensional image measurements. These data have been placed on an absolute basis by reference to earlier absorption results. Particular emphasis has been placed on the investigation of the soot oxidation region in these laminar flames, since current soot models must utilize ejther assumed or estimated $\mathrm{OH}(-)$ concentrations. As the soot concentration increases, both the $\mathrm{OH}(-)$ concentration and the flame temperature are found to decrease. Estimates of the superequilibrium levels of $\mathrm{OH}(-)$ increase as the measured concentrations decrease, which makes accurate a priori prediction of $\mathrm{OH}(-)$ concentrations problematic in diffusion flames containing significant soot concentrations.

01,388

PB95-163457 Not available NTIS

National Inst. of Standards and Technology (NML),

Gaithersburg, MD. Chemical Kinetics Div.

Incinerability of Perchloroethylene and Chlorobenzene.

Final rept.

W. Tsang, and D. Burgess. 1992, 17p.

Pub. in Combu

6 p31-47 1992

Keywords: "Chlorobenzenes, "Tetrachloroethylene, *Incinerators, "Computerized simulation, Combustion kinetics, Hydroxyl radicals, Methane, Data bases, Reprints, "Perchloroethylene.

The paper reports on the results of computer simulations of the destruction of trace quantities of perchloroethylene and chlorobenzene in organic combustion systems. These results are derived from a data base dealing with methane combustion kinetics, recent measurements on hydrogen atom attack on the chlorinated compounds, and estimates based on literature values of the rate constants for a variety of destruction processes. The roles of $\mathrm{OH}$-radicals and $\mathrm{H}$ atoms as the key agents for destruction are dem. onstrated. At higher temperatures, unimolecular decompositions begin to become important. The juxtaposition of the various processes makes difficult the construction of a simple algorithm that will readily de. fine the relative ease of destruction of any two compounds. Additional complications arise from the dependence on fuel composition and at higher hazardous waste concentrations. The advantages of using computer simulations in conjunction with laboratory and field results and in deriving a better understanding of incinerator behavior are discussed.

01.389

PB95-176020 Not available NTIS

National Inst. of Standards and Technology (CSTL), Gaithersburg, MD. Process Measurements Div.

Effect of Dodecanol Content on the Combustion of Methanol Spray Flames.

Final rept.

C. Presser, A. K. Gupta, C. T. Avedisian, and H. G.

Semerijan. 1994, 16p.

Sponsored by Department of Energy, Washington, DC. Pub. in Atomization and Sprays 4, p207-222 1994.

Keywords: "Methyl alcohol, "Combustion, Droplets, Sprays, Flames, Binary mixtures, Reprints, ־Dodecanol, "Dodecanoic acid, Lauric acid. 
The structure of a swirl-stabilized spray flame, fueled by a 75/25 mixture (by volume) of methanol and dodecanol has been examined. Spatially resolved information on droplet size and velocity distributions was obtained under burning conditions using a phase Doppler interferometry system. The effect of system gain (i.e., voltage setting of the photomultiplier tube detectors and laser power) on interpretation of the results was also assessed. The relatively large volatility difference between methanol and dodecanol provided an opportunity to examine the occurrence of microexplosions within spray flames. Evidence of microexploded droplets was revealed by a sudden decrease in droplet size and velocity, and an increase in number density at different spatial positions within the flame. On this basis, results were obtained that indicated the occurrence of microexplosions in the 75/25 mixture flame, but at a reduced extent as compared to prior results reported for methanol flames containing a larger fraction of dodecanol.

01,390

PB95-180576 Not available NTIS

National Inst. of Standards and Technology (BFRL),

Gaithersburg, MD. Fire Science Div.

Acid Gas Production in Inhibited Diffusion Flames.

Final rept.

G. Linteris, M. D. King, A. Liu, C. Womeldorf, and Y. E. Hsin. 1994, 15p

Sponsored by Department of the Air Force, Wright-Patterson AFB, OH.

Pub. in Proceedings of Halon Options Technical Working Conference, Albuquerque, NM., May 3-5, 1994, p177-191

Keywords: "Diffusion flames, "Combustion products, "Fire extinguishing agents. Fire suppression, Inhibitors, Combustion, Transport properties, Fluorinated hydrocarbons, Acids, Concentration(Composition), Production rate, Hydrogen fluoride, Hydrogen chloride, Reprints, “Acid gas production.

The proposed replacements to halon 1301, mainly fluoninated and chlorinated hydrocarbons, are expected to be required in significantly higher concentrations than $\mathrm{CF} 3 \mathrm{Br}$ to extinguish fires. At these higher concentrations the by-products of the inhibited flames may include correspondingly higher portions of corrosive gases, including $\mathrm{HF}$ and $\mathrm{HCl}$. To examine the chemical and transport-releated mechanisms important in producing these acid gases, a series of inhibited flame tests have been performed with several types of laboratory-scale burners, varying agent type and concentration, and fuel type. Production rates were meas ured for co-flow laminar and turbulent diffusion flames. Systematic selection of the agent concentrations, burner type, and air flow rates allowed an assessment of the relative importance of agent transport and chemical kinetics on the acid gas production rates. These experimental results were then compared to a model which estimates the maximum $\mathrm{HF}$ and $\mathrm{HCl}$ production rates based on stoichiometric reaction to the most stable products. The results demonstrate the relative significance of $\mathrm{F}, \mathrm{Cl}$, and $\mathrm{H}$ in the inhibitor and fuel, as well as the effect of different burner configurations.

01,391

PB95-180584 Not available NTIS

National Inst. of Standards and Technology (BFRL), Gaithersburg, MD. Fire Science Div.

Burning Rate of Premixed Methane-Air Flames Inhibited by Fluorinated Hydrocarbons.

Final rept

G. T. Linteris, and L. Truett. 1994, 12p.

See also PB95-180592. Sponsored by Department of the Air Force, Wright-Patterson AFB, OH.

Pub. in Proceedings of Halon Options Technical Working Conference, Albuquerque, NM., May 3-5, 1994, $12 \mathrm{p}$.

Keywords: "Burning rate, *Diffusion flames, "Fire extinguishing agents, "Fluorinated hydrocarbons, Fire suppression, Fire protection, Combustion, Oxidation, Flame propagation, Inhibitors, Concentration(Composition), Reprints.

This paper presents the first measurements of the burning rate of premixed flames inhibited by three fluorinated hydrocarbons that have oxidation chemistry which is similar to agents which may be used as replacements for CF3Br. The burning rate of premixed methane-air flames stabilized on a Mache-Hebra nozzle burner was determined using the total area method from schlieren images of the flame. The inhibitors were tested over a range of concentrations and fuel-air equivalence ratios. The measured burning rate reduc- tions are compared with those predicted by numerical solution of the species and energy conservation equations employing a detailed chemical kinetic mechanism recently developed at the National Institute of Standards and Technology (NIST). This paper presents initial efforts at testing and validation of the mechanism using buming rate data. The mode of inhibition of these chemicals is inferred through interpretation of the numerical results.

\section{1,392}

PB95-180592 Not available NTIS

National Inst. of Standards and Technology (BFRL), Gaithersburg, MD. Fire Science Div.

Experimental and Numerical Burning Rates of Premixed Methane-Air Inhibited by Fluoromethanes.

Final rept

G. T. Linteris, and L. Truett. 1994, 4p.

See also PB95-180584. Sponsored by Department of the Air Force, Wright-Patterson AFB, OH.

Pub. in Proceedings of Combustion Institute/Eastern State Section Meeting, Clearwater Beach, FL., December $5-7,1994,4 p$.

Keywords: "Burning rate, "Fire extinguishing agents, "Diffusion flames, Fire suppression, Flame propagation, Combustion, Methane, Fire protection, Inhibitors Mass transfer, Heat transfer, "Fluoromethanes.

The agents which are currently being considered as a replacements for fire suppressant agent $\mathrm{CF} 3 \mathrm{Br}$ are mostly fluorinated hydrocarbons and perfluorinated alkanes. This abstract describes measurements of the reduction in burning rate of premixed methane-air flames by the single carbon inhibitors $\mathrm{CF} 4, \mathrm{CF} 3 \mathrm{H}$, and $\mathrm{CF} 2 \mathrm{H} 2$. Early studies of the inhibitory effects of halogenated hydrocarbons on flames were conducted in premixed systems. The premixed laminar burning rate is a fundamental parameter describing the overall reaction rate, heat release, and heat and mass transpor in a flame. In addition, the reduction in the premixed flame burning rate is useful for understanding the mechanism of chemical inhibition of fires since diffusion flames often have a stabilization region which is premixed, and good correlation has been found between the reduction in burning rate and the concentration of inhibitors found to extinguish diffusion flames. The present burning rate measurements allow an early assessment of the performance of the National Institute of Standards and Technology fluorinated species chemical kinetic mechanism in premixed flames and are considered to be an initial step in the validation and refinement of the mechanism.

\section{1,393}

PB95-203246 Not available NTIS

National Inst. of Standards and Technology (BFRL) Gaithersburg, MD Fire Science Div.

Quantitative Measurements of Enhanced Soot Production in a Flickering Methane/Air Diffusion Flame.

Final rept.

C. R. Shaddix, J. E. Harrington, and K. C. Smyth. 1994, 10p.

Pub. in Combustion and Flame 99, p723-732 1994

Keywords: "Diffusion flames, "Soot, Flow fields, Oxidation, Methane, Flicker, Air, Reprints, Laser induced incandescence, Time varying systems.

Integrated models of soot production and oxidation are based upon experimental results obtained in steady, laminar flames. For successful application of these de scriptions to turbulent combustion, it is instructive to est predictions of soot concentrations against experimental measurements obtained in time-varying flowfields. This paper reports quantitative measurements of the local soot volume fraction in a co-flowing, flickering $\mathrm{CH}$ 4/air diffusion flame burning at atmospheric pressure. Acoustic forcing of the fuel flow rate is used to phase lock the periodic flame flicker close to the natural flicker frequency. Our measurements show that soot production is four times greater for a forcing condition in which flame tip clipping occurs, compared with a steady flame burning with the same mean fuel flow velocity. The soot field in the flickering flame has been characterized using tomographic reconstruction of extinction data obtained at $632.8 \mathrm{~nm}$, laser-induced incandescence (LII) images calibrated against steady $\mathrm{CH} 4 /$ air extinction results, and vertically polarized scattering data.

\section{1,394}

PB95-231700 PC A10/MF A03

National Inst. of Standards and Technology (BFRL) Gaithersburg, MD. Fire Science Div.

Carbon Monoxide Production in Compartmen Fires: Reduced-Scale Enclosure Test Facility. N. P. Bryner, E. L. Johnsson, and W. M. Pitts. Nov 94, 218p, NISTIR-5568. See also PB93-146702

Keywords: "Fire research, "Carbon monoxide, "Fire tests, "Combustion products, Solid fuels, Reaction kinetics, Flashover, Fire chemistry, Prototypes, Oxidation, "Compartment fires, Fuel/air ratio, Scale models.

Carbon monoxide production during room fires has been investigated using natural-gas fires within a reduced-scale enclosure (RSE), an $0.98 \mathrm{~m} \times 0.98 \mathrm{~m} \times$ $1.46 \mathrm{~m}(w \times h \times d)$ room with a single door opening centered in the front wall. This series of 125 fires with hea release rates (HRR) from 7 to $650 \mathrm{~kW}$ and global equivalence ratio phi sub g from 0.2 to 4.2 , respec tively, has demonstrated that the upper layer is nonuniform in temperature and gas species, and that upper-layer oxygen is depleted for underventilated fires with high-temperature upper layers. For fires hav ing HRR exceeding $400 \mathrm{~kW}$ (phi sub g > 2), carbon monoxide concentrations of up to 3.5 percent have been observed in the front portion of the upper layer. Carbon monoxide concentrations in the rear were consistently lower being on the order of 2.0 percent for phi sub $g>2$.

\section{1,395}

PB95-231817 PC A04/MF A01

National Inst. of Standards and Technology (BFRL) Gaithersburg, MD

Suppression of High Speed Turbulent Flames in a Detonation/Deflagration Tube.

G. Gmurczyk, and W. L. Grosshandler. Jan 95, 62p, NISTIR-5642.

Sponsored by Wright Lab., Wright-Patterson AFB, OH and Naval Air Systems Command, Washington, DC

Keywords: "Fire extinguishing agents, "Aviation safety Aircraft fires, Fire fighting, Fire tests, Performance evaluation, Chemical properties, Detonation, Military air facilities, Halon 130

Live-fire, full-scale testing has been conducted at Wright-Patterson Air Force Base to identify an agen to replace CF3Br (halon 1301) for suppressing fires in military aircraft dry bays. The three chemicals being considered (C2HF5, HFC-125; C3F8, FC-218; and CF31, halon 13001) had been evaluated in a previous laboratory study, in which unique properties of each chemical were identified in small-scale experiments. The FC-218 provided the most consistent performance in this new series of experiments which examined lean stoichiometric and rich initial conditions. Large pres sure build ups were not observed during suppression of the propane/air mixtures under the current set of conditions. None of the agents could be ruled out for dry bay applications based upon the results of this study

01,396

PB95-242327 PC A05/MF A02

Michigan Univ., Ann Arbor. Dept. of Aerospace Engineering.

Mixing and Radiation Properties of Buoyant Turbulent Diffusion Flames.

Z. Dai, L. K. Tseng, U. O. Koeylue, and G. M. Faeth. Jun $95,100 p$, NIST/GCR-95/67

Contract NIST-60NANB1D1175

Also pub. as Michigan Univ., Ann Arbor. Gas Dynamics Labs rept no GDL/GMF-94-01. See also PB94 165974. Sponsored by National inst. of Standards and Technology (BFRL), Gaithersburg, MD

Keywords: "Turbulence, "Diffusion flames, Models, Mixing, Plumes, Soot, Optical properties, Velocity, Combustion products, Fuels.

An investigation of the mixing and radiation properties of buoyant turbulent diffusion flames is described. The study was divided into two phases: (1) the structure and mixing properties of turbulent plumes, which must be understood in order to resolve effects of turbulence radiation interactions and to benchmark models of buoyant turbulent flows; and (2) the fractal and structure properties of soot aggregates, which must be understood in order to develop nonintrusive methods for measuring soot properties of soot in flame environments. Measurements of the turbulent mixing prop- 


\section{Combustion \& Ignition}

erties of buoyant turbulent plumes involved laser induced fluorescence (LIF), laser velocimetry (LV) and combined LIF/LV to find mixture fraction and velocity statistics in buoyant turbulent plumes.

\section{1,397}

PB96-102132 Not available NTIS

National Inst. of Standards and Technology (BFRL) Gaithersburg, MD. Fire Science Div.

Simultaneous Optical Measurement of Soot Volume Fraction, Temperature, and $\mathrm{CO} 2$ in Heptane Pool Fire.

M. Choi, A Hamins, $H$. Rushmeier, and T.

Kashiwagi. 1994, 10p

Pub. in Proceedings of the International Symposium on Combustion (25th), Irvine, CA., July 31-August 5, 1994, p1471-1480 Keywords: "Optical $\begin{aligned} & \text { properties, "Soot, "Fires, } \\ & \text { Carbon }\end{aligned}$
dioxide, Concentration(Composition), Heat transfer, Temperature, Surfaces, Heat flux, Reprints, Soot volume fraction, Pool fires.

Detailed measurements of the temperature, soot volume fraction and $\mathrm{CO} 2$ concentrations have been performed for a $10 \mathrm{~cm}$ diameter heptane fire. In addition, the concentrations of $\mathrm{H} 2 \mathrm{O}$ and $\mathrm{CO}$ were inferred from generalized state relationships. The heat feedback to the surface was calculated by using a reverse Monte Carlo method in conjunction with RADCAL. The use of average and instantaneous values of the temperature and species concentrations results in a $21 \%$ difference in the heat flux to the surface. Simultaneous optical measurements using two probes were used to investigate the importance of temperal correlations on the heat transfer calculations. Measurements made throughout the fire indicate that non-simultaneous data sets can be used to accurately predict the heat transfer to the surface.

\section{1,398}

\section{PB96-102173 Not available NTIS}

National Inst. of Standards and Technology (BFRL), Gaithersburg, MD. Fire Safety Engineering Div.

Generation Rate and Distribution of Products of Combustion in Two-Layer Fire Environments: A Model and Applications.

Final rept.

L. Y. Cooper. 1994, 26p

See also PB91-107151.

Pub. in Fire Safety Jnl., v23 p245-270 1994

Keywords: "Two phase flow, "Flame propagation, "Thermodynamics, Combustion products, Fires, Com partment analysis, Time dependence, Stoichiometry, Methane, Algorithms, Mathematical models, Reprints, "Fire models, GGERM(Generalized Global Equivalence Ratio Model).

A model is developed for predicting the generation rates of oxygen, fuel, and other products of combustion in rooms containing fires and time-dependent fire environments. The model is called the Generalized Global Equivalence Ratio Model (GGERM) It extends the steady state global equivalence ratio model established previously from data of several steady state ex perimental studies. After describing the GGERM, a concise algorithm is outlined for implementing it in twolayer zone-type compartment fire models. With the algorithm in place, such models could be used to simulate the distribution of combustion products in single or multi-room fire environments under conditions of arbitrary ventilation.

01,399

PB96-102306 Not available NTIS

National Inst. of Standards and Technology (BFRL)

Gaithersburg, MD. Fire Safety Engineering Div.

Computing Radiative Heat Transfer Occurring in a Zone Fire Model.

Final rept.

G. P. Forney. 1994, 16p

See also PB92-156777.

Pub. in Fire Science and Technology, v14 n1-2 p31. 471994.

Keywords: "Radiative heat transfer, "Compartment analysis, "Algorithms, Fires, Smoke, Gases, Walls, Heat transmission, Conduction, Convection, Numerical analysis, Computerized simulation, Mathematical models, Reprints, "Fire models, Zone models.

This paper presents algorithms for efficiency computing the radiative heat exchange between four wall sur- faces, several fire, and two interior gases. A two-wall and a ten-wall radiation model is also discussed. The structure of this radiation model is exploited to show that only a few configuration factors need to be calculated directly (two rather than 16 for the four-wall model and eight rather than 100 for the ten-wall model) and matrices needed to solve for the net radiative flux striking each surface are shown, after the appropriate transformation is taken, to be diagonally dominant. Iterative methods may then be used to solve the linea equations more efficiently than direct methods such as Gussian elimination.

\section{1,400}

PB96-102314 Not available NTIS

National Inst. of Standards and Technology (BFRL), Gaithersburg, MD. Fire Safety Engineering Div. Analyzing and Exploiting Numerical Characteristics of Zone Fire Models.

Final rept.

G. P. Forney, and W. F. Moss. 1994, 12p

See also PB92-172790.

Pub. in Fire Science and Technology, v14 n1-2 p49601994.

Keywords: *Algorithms, "Compartment analysis, Mathematical models, Computerized simulation, Fires, Conduction Pressure dependence, Differential equations, Numerical analysis, Reprints, "Fire models.

In order to design robust and stable zone fire modeling algorithms, the numerical properties of the fire model ing differential equations must be understood. This paper examines some of these properties. Many sets of differential equations for zone fire modeling can be derived using the conservation of mass and energy. A comparison between various possible formulations is made in terms of numerical properties. One property that many formulations possess is the presence of mul tiple time scales. Pressures equilibrate much faster than other quantities, such as density and temperature. Numerically, this property is known as stiffness. Stiffness, in the context of fire modeling, and numerical methods for handling it are discussed.

\section{1,401}

PB96-102454 Not available NTIS

National Inst. of Standards and Technology (BFRL), Gaithersburg, MD. Fire Science Div.

Assessing Halon Alternatives for Aircraft Engine Nacelle Fire Suppression.

Final rept.

W. Grosshandler, C. Presser, D. Lowe, and W.

Rinkinen. 1995, 6p.

Pub. in Jnl. of Heat Transfer, v117 p489-494 May 95

Keywords: "Fire extinguishing agents, *Aircraft fires "Environmental chemical substitutes, Nacelles, Fire suppression, Thermodynamic properties, Effectiveness, Performance evaluation, Fire fighting. Alter natives, Fluorocarbons, Halohydrocarbons, Fuel sprays, Jet engine fuels, Reprints, Hydrofluorocarbons, Hydrochlorofluorocarbons.

A coaxial turbulent spray burner was built to evaluate the relative effectiveness of different chemicals for suppressing fires in a jet engine nacelle. An agent delivery system was designed to inject the desired amount of material into the air upstream of a fuel nozzle and to control the agent injection rate through variation of the storage pressure and the duration of time that a solenoid valve remains open. The influence of air velocity, fuel flow, and injection period on the amount of nitrogen required to extinguish a jet fuel spray flame is dis cussed. The effectiveness of eleven different fluorocarbons, hydrofluorocarbons, and hydrochlorofiuorocarbons is compared to that of halon 1301.

01,402

PB96-109574 PC A03/MF A01

National Inst. of Standards and Technology (BFRL) Gaithersburg, MD. Fire Science Div.

Effect of Suppressants on Metal Fires.

T. J. Ohlemiller, and J. R. Shields. Aug 95, 28p, NISTIR-5710.

Keywords: "Fire suppressants, "Titanium, "Magnesium, *Combustion kinetics, Fire extinguishing agents, Halons, Environmental chemical substitutes, Alternatives, Burning rate, Heat transmission, Reaction kinetics, Vapors, Air flow, Fire tests.

The present study is limited to examining the effect of the vapors of various halogen-containing suppressants on the burning of pure magnesium and titanium rods in a slow flow of oxygen-containing gas. The test configuration was instrumented to allow quantitation of the effect of the vapors on the rate of burning. The agents examined in the presence of burning magnesium included halon 1301 (the reference case), HFC-125, HFC-227ea, FC-218 and CF3I. For titanium, time limitations narrowed the study to halon 1301, HFC227ea(sup 2) and HFC-125.

\section{1,403}

PB96-114764 PC A08/MF A02

Kentucky Univ., Lexington. Dept. of Mechanical Engineering.

Turbulent Flame Spread on Vertical Corner Walls. Doctoral thesis.

C. Qian. Apr 95, 163p, NIST/GCR-95/669.

Grant NIST-60NANB3D1443

Sponsored by National Inst. of Standards and Technology (BFRL), Gaithersburg, MD

Keywords: "Walls, "Burning rate, Flame propagation, Turbulent flow, Heat flux, Pyrolysis, Thermodynamics, Combustion kinetics, Heat trasnfer, Infared imagery, Fire tests, Fire research, Theses, "Turbulent flames, *Flame spreading.

In this study, attention is given to the corner fire spread mechanism and the flame spread behavior. Infrared (IR) radiometry and image analysis techniques have been developed in this study to measure flame spread rate on large areas with high resolution and frequency. In addition to the flame spread measurement, the fireinduced flow was studied by flow visualization, and the total incident heat flux to the wall surface from the flame was measured by Gardon-type heat flux meters. Based on these experimental studies, a thermal mode for corner fire spread has been successfully developed.

01,404

PB96-119342 Not available NTIS

National Inst. of Standards and Technology (BFRL)

Gaithersburg, MD. Fire Safety Engineering Div.

Flame Heights and Heat Release Rates of $1991 \mathrm{Ku}$ wait Oil Field Fires.

Final rept.

D. D. Evans, D. Madrzykowski, and G. A. Haynes

1994, 11p.

Pub, in Fire Safety Science Proceedings of the International Symposium (4th), Ottawa, Ontario, Canada, June 13-17, 1994, p1279-1289.

Keywords: "Oil wells, "Flame propagation, "Radiative heat transfer, "Fires, Kuwait, Burning rate, Heat flux, Heat of combustion, Diffusion flames, Heat release rate, Dissipation, Smoke, Turbulent diffusion, Jet flow, Reprints, Flame height

A series of measurements were made in the Al Mawqa/ Al Ahmadi oil field region of Kuwait to explore the feasibility of assessing the heat release rate of individual well fires through flame height and thermal radiation measurements. The heat release rate of the crude oil well fires was correlated with the flame height. The 12 Kuwait oil field fires measured ranged in calculated heat relaese rate from $90 \mathrm{MW}$ to $2 \mathrm{GW}$ which correspond to flow rates of $0.003 \mathrm{cu} \mathrm{m} / \mathrm{s}$ (1500 bbls/day) to $0.059 \mathrm{cu} \mathrm{m} / \mathrm{s}$ (30000 bbls/day) which is only $20 \mathrm{per}$ cent greater than published National Oceanic and Atmospheric Administration (NOAA) estimates based on information from the Kuwait Oil Company.

\subsection{5}

PB96-122676 Not available NTIS

National Inst. of Standards and Technology (BFRL), Gaithersburg, MD. Fire Science Div

Experimental Study of the Stabilization Region of Lifted Turbulent-Jet Diffusion Flames.

Final rept.

C. D. Richards, and W. M. Pitts. 1991, 4p.

See also PB92-117191.

Pub. in Combustion Institute/Eastern States Section Fall Technical Meeting (24th), Ithaca, NY., October 14$16,1991, p 1-4$

Keywords: *Diffusion flames, "Turbulent flow, "Lift, *Jet flow, Fuel combustion, Flame stability, Rayleigh scattering, Image analysis, Concentrations(Composition), Spatial resolution, Light scattering, Reprints, Fuel jets, Temporal resolution

The stabilization mechanism of a lifted turbulent-jet diffusion flame remains an active area of research despite numerous studies. The early work of Vanquickenbourne and Van Tiggelen provided experimental documentation of lift-off heights for a variety of 
fuels. Although this time-averaged view provides a means of predicting lift-off height, more physical insight is required to develop a model of stabilization which includes current knowledge on the structure of turbulent jets and mixing. The present study is in many ways an update of the Vanquickenborne and Van Tiggelen experiments. The anchoring position of a lifted flame is obtained through image analysis of video images and concentration profiles are independently acquired in the corresponding isothermal fuel jets with Rayleigh light scattering (RLS). These diagnostics provide both temporal and spatial resolution.

01,406
PB96-122866 Not available NTIS

National Inst. of Standards and Technology (BFRL), Gaithersburg: MD. Fire Safety Engineering Div.

Field Modeling: Simulating the Effect of Sloped Beamed Ceilings on Detector and Sprinkler Response.

Final rept.

W. D. Davis, G. P. Forney, and R. W. Bukowski.

1994, 42p.

Pub. in National Fire Protection Research Foundation, Batterymarch Park, Quincy, MA., 42p.

Keywords: "Fire detectors, "Sprinkler systems, "Ceilings, "Mathematical models, Smoke detectors, Fire suppression systems, Fire detection systems, Beams, Ventilation, Fire safety, Reprints.

The report describes the results of the second year of the project. During the second year, numerical simulations of smoke movement in response to sloped, beam ceilings were studied. Slopes of 10,25 and 50 degrees were studied with beams running along or across the slope. It was found that channeling of smoke flow was more prevalent as ceiling slope increased for parallel beam cases. For beams perpendicular to the slope, increasing the ceiling slope decreased the effectiveness of the beams in preventing smoke flow up the ceiling. Based on the predicted smoke movement, recommendations on sensor selection and placement are made for the slope beamed ceilings.

\section{1,407}

PB96-122890 Not available NTIS

National Inst. of Standards and Technology (BFRL) Gaithersburg, MD. Fire Science Div.

Smoke Emission from Burning Crude Oil.

Final rept.

D. Evans, W. Walton, H. Baum, H. Koseki, A.

Ghoniem, G. Mulholland, and J. Lawson. 1991, 29p. Pub. in Proceedings of the Arctic and Marine Oil Spill Conference (14th), Vancouver, B.C., June 12-14, 1991, p421-449.

Keywords: "Oil spills, "In situ combustion, "Burning rate, "Combustion kinetics, Crude oil, Plumes, Smoke Soot, Combustion products, Combustion properties, Fir tests, Oil-water interfaces, Reprints.

Research has shown that burning can be an effective means to remove oil from the surface of the water after a spill. The paper describes instrument packages developed to determine the amount of various combustion products emitted from large $(15 \mathrm{~m} \times 15 \mathrm{~m})$ crude oil pool fires. The increase in burning rate and smoke production with increasing fire size is discussed. Progress is reported on new calculation methods for smoke dispersion and downwind deposition of particulate. Results of example calculations are presented.

\section{1,408}

PB96-122965 Not available NTIS

National Inst. of Standards and Technology (BFRL), Gaithersburg, MD. Fire Science Div.

Development of Hazard Assessment and Suppression Technology for Oil and Gas Well Blowout and Diverter Fires.

Final rept.

J.P. Gore, and D. D. Evans. 1991, 6p.

Pub. in OCS Study MMS 91-0057 (ALSO) Biennial Report of the Technology Assessment and Research Program (7th), Reston, VA., p27-32 1991.

Keywords: "Blowouts, "Oil wells, "Natural gas wells, "Fire suppression, Fires, Flame propagation, Radiative heat transfer, Jet flow, Burning rate, Diffusion flames, Fire fighting, Fire extinguishing agents, Reprints.

The report summarizes progress during the past two years on understanding the radiation production from both vertical and horizontal jet-flames. These two cases represent two forms of blowout fire events on offshore platforms. The laminar flamelet representation of a turbulent flame is used successfully to predict radiation from vertical methane jet-flames in the range of 1 to $7 \mathrm{MW}$. Peak temperature in the vertical methane jet-flame with water addition were over predicted by about $20 \%$ using the model. The trajectory of horizontal jet-flames, which turn under the influence of buoyancy are predicted using a parabolic finite difference scheme developed in this research program. References are given to other technical reports and publications for details of the work summarized in this paper.

\section{1,409}

PB'96-123120 Not available NTIS

National Inst. of Standards and Technology (BFRL), Gaithersburg, MD. Fire Science Div.

Laser-Induced Fluorescence Measurements of $\mathrm{OH}$ in Laminar Diffusion Flames in the Presence of Soot Particles.

Final rept.

R. Puri, M. Moser, R. J. Santoro, and K. C. Smyth. 1991, 4p

Pub. in Eastern States Section the Combustion Institute (24th) Fall Technical Meeting, Ithaca, NY, October 14-16, 1991, p34-1-34-4.

Keywords: "Diffusion flames, "Soot, Combustion products, Laminar flow, Reprints, "Hydroxyl radicals.

Combustion in a simple view is the process of oxidation to form stable products, usually $\mathrm{CO} 2$ and $\mathrm{H} 2 \mathrm{O}$, accompanied by the release of energy. Although we often view this as a simple case of oxygen reacting with a fuel to exothermically form product species, it is well known that radicals such as $\mathrm{OH}, \mathrm{H}$ and $\mathrm{O}$ have a significant role in the reaction chemistry. It is the specifics of these reactions which determine the rates at which they proceed and the routes by which minor products such as NO, soot and CO result. The radical species participate in numerous reactions, and there exists a competition among several chemical steps for each reactive species. Important to determining the dominant set of reactions are the local conditions involving temperature, pressure and species concentrations. As these conditions vary, the dominant reactive pathways will also change. It is this dynamic situation which challenges researchers as they attempt to understand the variety of processes which characterize combustion. In the present study, measurements of hydroxyl radicals obtained in laminar diffusion flames containing soot particles are described.

\section{1,410}

PB96-123385 Not available NTIS

National Inst. of Standards and Technology (BFRL) Gaithersburg, MD. Fire Science Div.

Chemical Stability of Upper-Layer Fire Gases.

Final rept.

W. M. Pitts. 1991, 4p

Pub. in Fall Technical Meeting of the Eastern Section (24th): Combustion Institute, Cornell University, Ithaca, NY., October 14-16, 1991, 4p.

Keywords: "Carvon monoxide, "Fire gases, Kinetics, Reaction models, Toxic products, Reprints.

The chemical stability of upper-layer fire gases generated during burning in an enclosure is investigate by the use of a full-kinetic model. Initial concentrations of flame gases are those observed in experiments where fire gases are trapped in a hood. Calculations are preformed as a fucntion of layer temperature $(700$ 1300 ), global equivalence ratio (0.5-2.8), mixing mode (perfectly stirred and plug-flow reactors), and heat loss conditions (adiabatic or isothermal). The calculations indicate the flame gases become reactive for temperatures above $800 \mathrm{~K}$.

\section{1,411}

PB96-123625 Not available NTIS

National Inst. of Standards and Technology (BFRL) Gaithersburg, MD. Fire Science Div.

New Approach for Reducing the Toxicity of the Combustion Products from Flexible Polyurethane Foam.

Final rept

B. C. Levin, M. Paabo, E. Braun, and R. H. Harris.

1991, 6p.

Pub. in Annual Conference on Flame Retardancy of Polymeric Materials, Applications, Industry Developments, Markets, Stamford, CT., May 14-16, 1991, p16.

Keywords: "Polyurethane foam, "Hydrogen cyanide, "Combustion chemistry, "Thermodynamic properties
Combustion products, Copper compounds, Flammability, Ignition, Burning rate, Toxicity, Inhalation, Fire hazards, Fire tests, Reprints.

Hydrogen cyanide ( $\mathrm{HCN}$ ) is one of the gases which is produced during the thermal decomposition of flexible polyurethane foams and which (in combination with carbon monoxide and other fire gases) contributes to the toxicity of the smoke. Flexible polyurethane foams treated with copper or various copper compounds produced significantly less HCN when thermally decomposed that the identical but untreated control foams. The decreased atmospheric concentrations of HCN resulted in the reduction of the acute inhalation toxicity (as measured by lethality in Fischer 344 rats) produced from exposure to this smoke. This reduction of HCN and toxicity occurred regardless of whether the copper or copper compound was added to the foam during its formulation (prior to the foaming process) or as a posttreatment (after formulation).

01,412

PB96-131479 PC A11/MF A03

National Inst. of Standards and Technology (BFRL), Gaithersburg, MD

Solid Propellant Gas Generators: Proceedings of the 1995 Workshop. Held in Gaithersburg, Maryland on June 28-29, 1995.

J. C. Yang, and W. L. Grosshandler. Nov 95, 226p, NISTIR-5766.

Keywords: *Solid propellant combustion, "Gas generators, "Fire extinguishing agents, "Meetings, Fire suppression, Fire fighting, Halons, Aircraft fires, Aircraft safety, Solid propellants, Nacelles, Test methods, Certification, Effectiveness, Performance evaluation, Halon 1301, Fire protection systems.

The intent of the workshop was to bring together gas generator manufacturers, researchers, and potential users to discuss various critical issues related to the evaluation and performance of the gas generators as a fire fighting tool and the search for new propellants. The specific objectives of the workshop, which reflected the need for such an apparatus, were: Identification of certification procedure(s) for gas generators in fire suppression applications; determination of critical parameters for evaluating the fire suppression efficiency of various gas generators; development of a standard methodology to facilitate testing of gas generators; identification of possible applications other than protection of engine nacelles and dry bays; and identification of a new generation of propellants.

01,413

PB96-135322 Not available NTIS

National Inst. of Standards and Technology (BFRL), Gaithersburg, MD. Fire Science Div.

Application of Thermodynamic and Detailed Chemical Kinetic Modeling to Understanding Combustion Product Generation in Enclosure Fires.

Final rept.

W. M. Pitts. 1994, 33p

Pub. in Fire Safety Jnl., v23 n3 p271-303 1994.

Keywords: "Combustion products, "Thermodynamics, "Rooms, "Fire tests, Combustion chemistry, Gases, Enclosures, Carbon monoxide, Chemical reaction kinetics, Chemical equilibrium, Models, Reprints.

Experiments in idealized two-layer fire environments have demonstrated that concentrations of carbon monoxide and other gaseous combustion products can be correlated in terms of the global equivalence ratio. In this paper, the results of detailed chemical kinetic modeling and equilibrium calculations are used to gain insight into the chemical stability of the gases observed within the upper layers of such fires. It is demonstrated that the production of upper-layers gases is kinetically controlled and that for rich conditions concentrations of the upper-layer gas components are far from those expected for thermo-dynamic equilibrium at the layer temperatures. Criteria are provided for determining whether or not the correlations can be employed to predict the generation of combustion products in enclosure fires.

01,414

PB96-146741 Not available NTIS

National Inst. of Standards and Technology (BFRL), Gaithersburg, MD. Fire Science Div. 
Inhibition of Premixed Methane-Air Flames by Halon Alternatives.

Final rept.

G. T. Linteris, and L. Truett. 1995, 6p.

Pub. in Proceedings of the International Conference of Fire Research and Engineering, Orlando, FL., September 10-15, 1995, p153-158.

Keywords: "Fire extinguishing agents, "Alternatives, "Combustion kinetics, Inhibitors, Fire suppression, Fire fighting, Burning rate, Fluorinated hydrocarbons, Methane, Chemical reaction kinetics, Flame propagation, Energy transfer, Heat transfer, Mass transfer, Environmental chemical substitutes, Halons, Reprints

This article describes the first measurements of the reduction in burning rate of premixed methane-air flames inhibited by the two-carbon fluorinated species C2F6 $\mathrm{C} 2 \mathrm{HF} 5, \mathrm{C}_{2} \mathrm{H}_{2} \mathrm{~F} 4$ and the three-carbon species C3F8 and C3HF7 all of which are being considered as replacements to $\mathrm{CF} 3 \mathrm{Br}$. The burning rate of premixed methane-air flames stabilized on a Mache-Hebra nozzle burner is determined using the total area method from a schlieren image of the flame. The inhibitors are tested over a range of concentration and fuel-air equivalence ratio. The measured burning rate reduction caused by addition of the inhibitor is compared (for the two-carbon species) with that predicted by numerical solution of the mass, species, and energy con servation equations employing a detailed chemical kinetic mechanism recently developed at the National Institute of Standards and Technology (NIST).

\section{1,415}

PB96-147046 Not available NTIS

National Inst. of Standards and Technology (BFRL), Gaithersburg, MD. Fire Safety Engineering Div. Gravity-Current Transport in Building Fires.

Final rept.

H. R. Baum, K. W. Cassel, K. B. McGrattan, and R. G. Rehm. 1995, 6p.

Pub. in Proceedings of the International Conference on Fire Research and Engineering, Orlando, FL., Septem ber 10-15, 1995, p27-32 1995.

Keywords: "Fires, "Numerical simulation, Smoke, Gas flow, Convective flow, Dispersion, Heat transfer coefficients, Buildings, Buoyancy, Navier-Stokes equations, Reprints, "Gravity currents.

Gravity currents (GC) are also of interest in the movement of gases in buildings. A GC produced by a fire can transport smoke, toxic material and hot gases, and when the building has long corridors, the current often is one of the most important mechanisms for large scale mass and energy transport. It is now possible to compute the structure of GCs in detail and to compare features of GCs with available experimental and analytical results. In the next section, the authors describe GCs by solving the Navier-Stokes equations in two dimensions. The authors make comparisons between results from salt-water, fresh-water experiments carried out by Zukoski and coworkers, and the results obtained from the authors' computational simulations.

\section{1,416}

PB96-147897 PC A04/MF A01

California Inst. of Tech., Pasadena. Dept. of Mechanical Engineering.

Review of Flows Driven By Natural Convection in Adiabatic Shafts.

E. E. Zukoski. Oct $95,48 p$, NIST/GCR-95/679.

Grant NIST-60NANB6D1444

Sponsored by National Inst. of Standards and Technology (BFRL), Gaithersburg, MD.

Keywords: "Adiabatic conditions, "Natural convection, "Fire tests, "Turbulent flow, Shafts, Vertical orientation, Hot gases, Air flow. Convective heat transfer, Turbulent mixing, Buoyancy, Burning rate, Combustion, Flame propagation, Computational fluid dynamics, Gas dynamics, Mathematical models, Stack effect.

An experimental study of the motion of hot gas through vertical shafts and passages under the influence of buoyancy forces is being made with the support of the Building and Fire Research Laboratory of the Natioan Institute of Science and Technology, the Department of Commerce. The aim of this work is to derive the information required for the preparation of models which can be used to describe these flows in computer-based models of fire spread through structures.

\section{1,417}

PB96-148200 Not available NTIS

National Inst. of Standards and Technology (BFRL), Gaithersburg, MD. Fire Science Div.
Examination of the Correlation between Cone Calorimeter Data and Full-Scale Furniture Mock-Up Fires.

Final rept.

T. J. Ohlemiller. 1995, $6 p$.

Sponsored by Society of Fire Protection Engineers, Boston, MA

Pub. in Proceedings of the International Conference of Fire Research and Engineering, Orlando, FL., September 10-15, 1995, p217-222 1995.

Keywords: *Calorimeters, *Furniture, *Fires, Heat transfer, Barriers, Rates(Per time), Fire research. Fire tests, Reprints, "Cone calorimeters, California Technical Bulletin 133, Heat release rate.

As part of an on-going study of the factors which affect heat release rate performance of furniture in the California Technical Bulletin 133, the authors have focused most recently on the role played by interliners or barriers. A barrier in this context is a layer of very low flammability material placed between the fabric and the polyurethane foam to minimize the participation of the latter in a fire. In CB 133 that fire is initiated on the seating area of an upholstered furniture item by an 18 kW gas burner that sprays flames on the seat, seatback and inner arm surfaces. The response of the chair is required to be at most an $80 \mathrm{~kW}$ peak in heat release rate. The test applies to furniture in public occupancies; it has been adopted in four states and is under consideration in several others.

01,418

PB96-154794 PC A10/MF A03

Maryland Univ., College Park. Dept. of Fire Protection Engineering.

Predicting the Ignition Time and Burning Rate of Thermoplastics in the Cone Calorimeter.

Master's thesis.

D. Hopkins. Sep 95, 193p, NIST/GCR-95/677

Grant NIST-60NANB2D1266

Sponsored by National Inst. of Standards and Technology (BFRL), Gaithersburg, MD.

Keywords: *Thermoplastic resins, *Ignition time, *Burning rate, "Flammability testing, Polyethylene, Polypropylene, Nylon 66, Fire tests, Ignition temperature, Flame propagation, Heat flux, Heat measurement, Calorimetry, Theses, Cone calorimeter, Mass loss.

Ignition and burning rate data are developed for Nylon $6 / 6$, Polyethylene, and Polypropylene in a Cone Calorimeter heating assembly. The objective is to examine a testing protocol that leads to the prediction of ignition and burning rate for thermoplastics from Cone data. The flame heat flux is not measured, but is inferred from Cone data. The flame heat flux is not measured, but is inferred from Cone data. The constancy of the flame heat flux for thermoplastics in the Cone calorimeter is due to the geometry of the flame. The burning rate model is shown to yield good accuracy in companson to measured transient values. Ignition and burning rate data are developed for Redwood and Red Oak in a Cone Calorimeter heating assembly. Measements of the flame plus external heat flux are presented. The data is intended to be used for future work to develop a testing protocol and burning rate model for charring materials.

01,419

PB96-160593 Not available NTIS

National Inst. of Standards and Technology (BFRL), Gaithersburg, MD. Fire Science Div.

New Generation of Fire Resistant Polymers. Part 1. Computer-Aided Molecular Design.

Final rept.

M. R. Nyden, and J. E. Brown. 1993, 7p.

Pub. in UJNR on Fire Research, Tsukuba, Japan, October 1992, p257-263.

Keywords: "Computer-aided design, "Fire resistant materials, "Molecular structure, Nonflammable materials, Flammability, Thermal degradation, Charring, Combustion products, Mathematical models, Polymers, Reprints.

Molecular dynamics modeling and experimental measurements are used to identify factors which reduce flammability by promoting the formation of heat resistant chars during the thermal degradation of polymers. Computer movies of the calculated trajectories reveal that cross-linked model polymers tend to undergo further cross-linking when bumed. The presence of strong potential energy interactions with a surface or filler further facilities the formaton of high molecular weight, thermally stable chars.
01,420

PB96-161831 Not available NTIS

National Inst. of Standards and Technology (BFRL) Gaithersburg, MD. Fire Science Div.

Effect of $\mathrm{CF} 3 \mathrm{H}$ and $\mathrm{CF} 3 \mathrm{Br}$ on Laminar Diffusion Flames in Normal and Microgravity.

Final rept.

B. A. VanDerWege, M. T. Bush, S. Hochgreb, and G. T. Linteris. 1995, $6 \mathrm{p}$.

See also N96-15609 and PB96-161849.

Pub. in International Microgravity Combustion Conference (3rd), Cleveland, OH., April 11-13, 1995, 6p. Keywords: "Chemical inhibition, "Flame chemistry, Reprints, Flame models, Flame retardants, Diffusion flame.

Chemical inhibition of diffusion flames through addition of halogenated inhibitors is a problem of significant practical and scientific interested. Extension studies on diffusion flames in microgravity have shown that these flames have significantly different characteristics than those under normal gravity $(1,2)$. However, the mechanisms through which inhibitors reach the reactions zone to suppress combustion in diffusion flames and the effectiveness of these compounds under reduced gravity have yet to be investigated. This study reports preliminary results of investigations on the behavior of laminar jet diffusion flames upon the addition of bromotrifluoromethane (CF3Br) and trifluoromethane (CF3H) to the surroundings under normal and microgravity conditions. The results show that the flame structure in microgravity is significantly different from that under normal gravity conditions, and more importantly, that conditions for flame stability are less stringent under microgravity.

01,421

PB96-161849 Not available NTIS

National Inst. of Standards and Technology (BFRL), Gaithersburg, MD. Fire Science Div.

Effect of $\mathrm{CF} 3 \mathrm{H}$ and $\mathrm{CF} 3 \mathrm{Br}$ on Laminar Diffusion Flames in Normal and Microgravity.

Final rept.

B. A. VanDerWege, M. T. Bush, S. Hochgreb, and G T. Linteris. 1995, 4p.

See also PB96-161831.

Pub. in Eastern States Section Meeting/The Combus tion Institute, Worcester, MA., October 16-18, 1995 p443-446.

Keywords: *Chemical inhibition, "Flame chemistry, Reprints, Flame models, Flame retardants, Diffusion flame.

Due to the ban on production of bromotrifluoromethane (CF2Br) because of its high ozone destruction potential, there has been recent interest in finding a replacement for it for fire extinguishing applications. While a variety of potential replacement are being considered, halogenated hydrocarbons may be a viable alternative for some applications. Consequently, an improved understanding of their action in flames will aid in their effective use. In addition, CF $3 \mathrm{Br}$ is used as a fire suppressant on the space shuttle, and its action in microgravity has not been tested in diffusion flames. The present study investigates the effects of $\mathrm{CF} 3 \mathrm{Br}$ and trifluoromethane (CF3H), the simplest compound representative of the fluorocarbons, in laminar diffusion flames. The primary experiments are laminar gas-jet diffusion flames buming in a quiescent environment containing the inhibitor in normal and microgravity. Experiments were conducted with CF2Br mole fractions in the oxidizer gas of $0.5 \%$ to $3 \%, \mathrm{CF} 3 \mathrm{H}$ mole fractions of $4 \%$ to $12 \%$, oxygen mole fractions from $18 \%$ to $30 \%$, and ambient pressures of $101 \mathrm{kPa}$ and $25 \mathrm{kPa}$. Additional opposed-jet counterflow diffusion flame experiments were used to investigate flame structures observed in the microgravity flames.

01,422

PB96-164256 Not available NTIS

National Inst. of Standards and Technology (BFRL), Gaithersburg, MD. Fire Science Div.

Asymptotic and Numerical Analysis of a Premixed Laminar Nitrogen Dioxide-Hydrogen Flame.

Final rept.

G. T. Linteris, and F. A. Williams. 1995, 13p.

Pub. in Combustion Science and Technology, v105 p165-182 1995.

Keywords: "Nitrogen dioxide, "Hydrogen, "Combustion chemistry, Laminar flames, Premixed flames, Combustion kinetics, Reduction(Chemistry), Burning rate, Thermodynamics, Thermochemistry, Stoichiometry, Numerical analysis, Reprints. 
A kinetic mechanism of eight-some reactions for flames in mixtures of hydrogen and nitrogen dioxide is systematically reduced to twenty-four-, elevenseven-, two-, and one-step mechanisms. The numerically predicted burning rates for the full mechanism describing a near-stocichiometric burner-stablized flame at a pressure of 25 torr, and final temperature of 2000 $\mathrm{K}$ are compared with the results using the reduced mechanisms, and the sources of inaccuracies are identified.

01,423

PB96-183108 PC A04/MF AO1

National Inst. of Standards and Technology (BFRL), Gaithersburg, MD.

NASA Fire Detector Study.

W. D. Davis, and K. A. Notarianni. Mar 96, 40p, NISTIR-5798.

Color illustrations reproduced in black and white.

Keywords: "Fire detectors, "Air flow, "Ventilation, Fire detection systems, Clean rooms, Hangars, Ceilings, Size, Warning systems, Smoke detectors, Radiation detection, Infrared detectors, Ultraviolet detectors, Activation, Mathematical models, Fire protection, NASA Computational fluid dynamics, Heat detectors, Fire models.

A study of fire detection methods for use by NASA in protecting their high bay structures is presented. A high bay structure is defined in this study as a structure hav ing a ceiling height in excess of $18 \mathrm{~m}(60 \mathrm{ft})$. The analysis conducted in this study dealt with prediction of the expected performance of heat, smoke, and radiation detection systems for simulated fires of the type ex pected in NASA high bay facilities. The expected performance of smoke, heat, and radiation detectors in simulated fires is calculated using computer modeling The results of this analysis will aid the development of fire detection strategies for high bay spaces.

\section{1,424}

PB96-183132 PC A03/MF A01

National Inst. of Standards and Technology (BFRL), Gaithersburg, MD. Fire Safety Engineering Div.

Numerical Simulation of Rapid Combustion in an Underground Enclosure.

K. B. McGrattan, H. R. Baum, and S. P. Deal. Apr 96, 19p, NISTIR-5809.

See also PB93-174902.

Keywords: "Underground space, "Combustion, "Thermodynamic properties, Ignition, Mass transport Energy transport, Convective flow, Thermal absorption, Accelerating agents, Energy dissipation, Momentum, Equations of motion, Mach number, Equations of state, High temperature, Conservation equations, Conservation laws, Mathematical models, Computational fluid dynamics, "Fire models, Zone models, Field models.

The scenario of interest is a 2-second firing of a rocket in an underground enclosure intended to mimic the effect of burning a high temperature accelerant (HTA) Because of the unusual nature of the problem, at least in the context of typical fire scenarios, two types of numerical models have been applied to the problem. The first, a zone model, divides each room in the enclosure into one or two control volumes, and the transport o mass and energy from the burn room is estimated from the basic consenvation laws. The second model, a field model designed for relatively low Mach number flows, solves the conservation equations of mass, momentum and energy discretized over hundreds of thousands of cells.

01,425

PB96-183181 PC A05/MF A0

National Inst. of Standards and Technology (BFRL), Gaithersburg, MD. Fire Science Div.

Minimum Mass Flux Requirements to Suppress Burning Surfaces with Water Sprays.

J. C. Yang, C. I. Boyer, and W. L. Grosshandler. Apr 96, 52p, NISTIR-5795.

Sponsored by Fire Administration, Emmitsburg, MD.

Keywords: "Solid fuels, "Burning rate, "Fire estinguishing agents, Wood, Methy!methacrylates, Polystyrene, Flames, Flow rates, Heat flux, Flow velocity, Water flow, Drop size, Spraying, Mist, Nozzles, Atomization, Low pressure. Mass flow, Fire fighting, Fire suppression, Piezoelectric gages, Requirements, Micronozzles.

Experimental measurements of extinghișhment times of burning solid fuels using water were conducted using a prototype micronozzle array and a piezoelectric droplet generator. Solid fuels considered included solid white pine, polymethyl methacrylate, and polystyrene foam. External heat flux was applied to the sample surface during burning. The effects of drop size, sample orientation with respect the the nozzle, and nozzle distance from the sample surface on extinguishment time were examined. The extinguishment time was found to decrease with increasing water flow rate. For a given water flow rate, significant reduction in extinguishment time decreased when the nozzle was positioned further from the sample surface. At high flow rates, the extinghishment was independent of the nozzle-to-sample distance.

\section{1,426}

PB96-190012 Not available NTIS

National Inst. of Standards and Technology (BFRL), Gaithersburg, MD. Fire Safety Engineering Div.

Large Eddy Simulations of Smoke Movement in Three Dimensions.

Final rept.

H. R. Baum, K. B. McGrattan, and R. G. Rehm. 1996, 10p.

Pub. in INTERFLAM: Proceedings of the International Interflam Conference (7th), Cambridge, England, March 26-28, 1996, p189-198.

Keywords: "Smoke, "Convection, "Transport, "Computerized simulation, Fire, Gases, Mathematical models, Computation, Navier-stokes equations, Fluid flow, Reprints.

This paper describes a methdology for simulating the transport of smoke and hot gases in enclosures The approach is based on the use of efficient CFD techniques and high performance computers to solve a form of the Navier Stokes equations specialized to the smoke movement problem. The fire is prescribed in a manner consistent with a mixture fraction based approach to combustion but the combustion phenomena themselves are not simulated. The mixing and transport of smoke and hot gases is calculated directly from an approximate for of the Navier Stokes equations. The next two sections give a brief description of the mathematical and computational aspects of the model, while the final section illustrates its capability with sample results and a comparison with experiment.

01,427

PB96-190079 Not available NTIS

National Inst. of Standards and Technology (BFRL). Gaithersburg, MD. Fire Safety Engineering Div.

Large Fire Experiments for Fire Model Evaluations. Final rept

D. D. Evans. 1996, 6p

Pub. in Proceedings of the International Interflam Conference (7th), Cambridge, England, March 26-28, 1996, p329-334.

Keywords: "Fire research, "Hydrocarbons, "Fuels, Oilspills, Smoke, Yield, Models, Fire safety, Building codes, Reprints.

Recent movement towards performance based evaluation of building safety has placed a premium on demonstrating the accuracy of engineering methods and increased the demand for fire performance data from large scale experiments. Data from large scale experiments are generally the basis for development and evaluation of fire models. Verification of engineering methods for prediction of fire related performance of structures, contents, and fire protection systems has become a priority need to support the development of performance based codes and standards. Generally a great impediment to model verification is the lack of means to quantify the degree of agreement between experiments and predictions or repeated experiments. The results have shown that modeling of building fire flows at a resolution of several centimeters is feasible. The advent of high resolution calculations for use in fire safety analysis has increased the demand for high resolution measurements of fire.

01,428

PB96-190178 Not available NTIS

National Inst. of Standards and Technology (BFRL).

Gaithersburg, MD. Fire Safety Engineering Div.

Office Work Station Heat Release Rate Study: Full Scale versus Bench Scale.

Final rept

D. Madrzykowski. 1996, 9p.

Pub. in INTERFLAM '96, Proceedings of the International Interflam Conference (7th), March 26-28, 1996, Cambridge, England, 247-55 1996.
Keywords: "Fire research, "Calorimeters, "Office equipment, Furniture, Fire tests, Heat flux, Reprints, Cone calorimeter, Radiation heat flux.

The National Institute of Standards and Technology (NIST) has conducted a study with office work stations to examine their heat release rates and to determine if the peak heat release rate for a work station can be predicted accurately from cone calorimeter results. Fifteen full scale fire experiments were conducted. Three types of work station panel construction and three work station configurations were examined. Preliminary results for the most common panel construction, fabric over fiberglass batting with a $6 \mathrm{~mm}$ thick hardboard core, are presented here. A method utilizing the peak heat release rate from the cone calorimeter experiments has been used successfuily to predict peak heat release rates for the most common construction work station.

\section{1,429}

PB'96-190210 Not available NTIS

National Inst. of Standards and Technology (BFRL), Gaithersburg, MD. Fire Science Div.

Analysis of High Bay Hangar Facilities for Detector Sensitivity and Placement.

Final rept.

K. A. Notarianni, W. Davis, D. Lowe, S. Laramee,

and J. E. Gott. 1996, 10p.

Pub. in INTERFLAM '96: Proceedings of the International Interflam Conference, Cambridge, England, March 26-28, 1996, p487-496.

Keywords: "Hangars, "Sprinkler systems, "Fire suppression "Smoke detectors, Fire protection, Fire detectors, Activation, Ventilation, Air flow, Ceilings, Thermodynamic properties, Burning rate, Combustion kinetics, Heat flux, Flame propagation, Fire detection systems, Bays(Structural units), Reprints.

The study was conducted to investigate the response of various fire detectors and automatic sprinklers in high bay aircraft hangars. Laboratory and full-scale experiments as well as computer modeling were conducted to better understand the movement of heat and products of combustion in high bay spaces. Temperature distribution across the ceiling was measured along with the response of various types of fire protection devices as a function of fire size, fuel type, and ventilation conditions. Key findings are presented relating to detector spacing, threshold fire sizes, sprinkler type and temperature ratings, burn rates, heat release rates, and the effect of draft curtains.

\section{1,430}

PB96-193701 PC A04/MF A01

Kansas State Univ., Manhaltan. Dept. of Physics. Post-Flame Soot

Final rept. Sep 94-Dec 95

C. M. Sorensen. Jun $96,36 p$, NIST/GCR-96/694 Grant NIST-NANB4H1652

Sponsored by National Inst. of Standards and Technology (BFRL), Gaithersburg, MD.

Keywords: "Soot, "Combustion products, Diffusion flames, Acetylene, Agglomerates, Light scattering, Standards.

The smoke agglomerates produced by a co-annular diffusion flame with acetylene fuel were characterized by sampling/microscopy and by light scattering measurements. Particles were sampled at various heights above the flame using both thermophoretic sampling and impaction. Transmission electron microscopy was used for the smaller agglomerates obtained by thermophoretic sampling and optical microscopy was used for analysis of particles as large as .4 mm in diameter collected by impaction. The number of primary spheres was estimated from the projected area of the agglomerate and the primary sphere size. The fractal analysis extended over four orders of magnitude in the radius of gyration - the widest range studied for smokes.

01,431

PB96-195532 PC A04/MF AO

California Univ., San Diego, La Jolla. Dept. of Applied Mechanics and Engineering Sciences.

Chemical Inhibition of Methane-Air Diffusion Flame.

Final rept. 15 Sep 93-15 Sep 95

K. Seshadri. Jun 96, 43p, NIST/GCR-95/685.

Grant NANB3D1435

Sponsored by National Inst. of Standards and Technology, Gaithersburg, MD. 


\section{Combustion \& Ignition}

Keywords: "Halons, "Diffusion flames, "Methane, "Combustion kinetics, "Thermochemistry, Fire extinguishing agents, Oxygen, Burning rate, Flame propagation, Inhibition, Asymptotic series, Numerical analysis, Reprints, Halon 1301.

The principal objective of the research is to clarify the mechanisms of chemical inhibition of methane-air diffusion flames by $\mathrm{CF} 3 \mathrm{Br}$ and $\mathrm{CF} 3 \mathrm{H}$. An experimental, numerical, and analytical study is conducted. An asymptotic analysis is performed to characterize the structure and critical conditions of extinction of uninhibited methane-air diffusion flames. This analyses is extended to methane-air diffusion flames inhibited with CF3Br. Critical conditions of extinction of the flame are measured over a wide range with agents added to the air stream and to the fuel stream. Numerical calculations with detailed chemistry are performed to calculate the structure and critical conditions of flame extinction. The numerical results are compared with the measurements.

\section{1,432}

\section{PB96-202254 PC A06/MF A01}

Michigan Univ., Ann Arbor. Dept. of Aerospace Engineering.

Mixing and Radiation Properties of Buoyant Luminous Flame Environments.

Z. Dai, S. K. Krishnan, R. Sangras, J. S. Wu, and G. $M$. Faeth. Jun 96, 91p, GDL/GMF-95-02, NIST/GCR96/691.

Grant NIST-60NANB4D1696

See also PB95-242327. Sponsored by National Inst. of Standards and Technology (BFRL), Gaithersburg, MD.

Keywords: "Diffusion flames, "Fire tests, "Optical properties, Soot, Combustion products, Acetylene, Propylene, Ethylene, Propane, Standards, Turbulent flow.

An investigation of the radiation and mixing properties of buoyant turbulent diffusion flames is described. The study was divided into two phases: (1) the optical and radiative properties of soot, which must be understood in order to develop nonintrusive methods for measuring soot properties and to estimate the continuum radiation properties of soot in flame environments, and (2) the structure and mixing properties of buoyant turbulent plumes, which must be understood in order to resolve effects of turbulence/radiation interactions and to benchmark computationally tractable models of buoyant turbulent flows.

\section{1,433}

\section{PB96-202304 PC A13/MF A03}

Maryland Univ., College Park. Dept. of Mechanical Engineering.

Sparse Water Sprays in Fire Protection.

M. di Marzo. Jun 96, 254p, NIST/GCR-96/687.

Grant NIST-NANB5D0136

Sponsored by National Inst. of Standards and Technology (BFRL), Gaithersburg, MD.

Keywords: "Fire tests, „Evaporative cooling, Heat flux, Spraying, Fire protection, Water.

Selected reports: Dropwise Evaporative Cooling; Infrared Thermography of Dropwise Evaporative Cooling; Evaporative Cooling Due to a Gently Deposited Droplet; Infrared Thermography of Dropwise Evaporative Cooling of a Semi-Infinite Solid Subjected to Radiant Heat Input; Modeling of Dropwise Evaporative Cooling on a Semi-Infinite Solid Subjected to Radiant Heat Input; Multi-Droplet Evaporative Cooling: Experimental Results; Effect of Liquid-Solid Contact Angle on Droplet Evaporation; Effect of Dissolved Gasses on Spray Evaporative Cooling With Water; and Flooding Criterion for Evaporative Cooling on Horizontal Semi-Infinite Solids

\section{1,434}

\section{PB96-204433 Not available NTIS}

National Inst. of Standards and Technology (CSTL), Gaithersburg, MD. Process Measurements Div.

Role of Combustion on Droplet Transport in Pressure-Atomized Spray Flames.

Final rept.

A. K. Gupta, C. Presser, J. T. Hodges, and C. T.

Avedisian. 1996, $11 \mathrm{p}$.

Pub. in Jnl. of Propulsion and Power, v12 n3 p543-553 Jun 96.

Keywords: *Spray combustion, *Flames, Reprints, Droplets, Kerosene, Velocity.

The transport of droplets in a pressure-atomized kerosene spray flame was examined using a two-compo- nent phase Doppler system to measure the drople size and velocity distributions at several locations within the spray. The effect of combustion on droplet trans port was examined by comparing the results to a nonburning spray under similar flow conditions. Directions of motion of droplets are calculated from the measured droplet velocity components to provide information on trajectories and dispersion of droplets. Results show that combustion reduces the strength of gas recirculation as evience by significantly fewer droplets being transported upstream toward the nozzle along the centerline for the burning spray as compared to the nonburning spray. Combustion enhances droplet vaporization and results in reduced number density and larger droplet mean diameters and velocities when compared to the nonburning spray. At the spray cen terline there is a wide range of droplet trajectories that are associated with recirculated droplets and those originating from the nozzle There is some correlation between droplet velocity and diameter. Some large size droplets are also found to be entrained into the recirculation pattern. At the spray boundary, few droplets deviate from the mean direation. Also, droplet velocity is well correlated with increasing diameter but appears to approach an asymptotic value in which droplet velocity becomes insensitive to diameter.

\section{1,435}

PB97-110050 Not available NTIS

National Inst. of Standards and Technology (BFRL) Gaithersburg, MD. Fire Safety Engineering Div.

Flammability Characterization with the Lift Apparatus and the Cone Calorimeter.

Final rept.

T. G. Cleary. 1992, 17p.

Pub. in Proceedings of the Fire Retardant Chemicals Association Spring Conference, Orlando, FL., March 29-April 1, 1992, 17p.

Keywords: "Flammability, "Calorimeters, Heat measurement, Characterization, Ignitability, Flame spread, Reprints, "Life apparatus, "Cone calorimeters.

Two small-scale test apparatuses, the LIFT apparatus and the Cone Calorimeter provide ignitability, flame spread, and heat release rate data for combustible solid materials. Data gathered with these apparatuses can be reduced to a limited number of key flammability or fire properties for a particular material. These key properties, in conjunction with the appropriate modelling equations, characterize the time to ignition, flame spread rate, and heat release rate over the range of applicable heating conditions and surface temperatures likely in full-scale fires. These key properties may be used as input parameters in a fire model that pre dicts flame spread and heat release rates for certain full-scale fire scenarios.

01,436
PB97-110068 Not available NTIS

National Inst. of Standards and Technology (BFRL), Gaithersburg, MD. Fire Science Div.

Letter Report on Flame Spread Testlng of a Composite Material.

Final rept.

T. G. Cleary. 1993, 16p.

Sponsored by Naval Surface Warfare Center Carderock Div., Annapolis, MD.

Pub. in a Letter Report on Flame Spread Testing of a Composite Material, p1-16 Feb 93.

Keywords: "Composites, "Flame spread, Ignition, Reprints, LIFT apparatus, ASTM E 1321

Ignition delay time, lateral flame spread and upward flame spread data were gathered on a composite ma terial (glass fiber/organic resin type). These tests were performed at the request of the Naval Surface Warfare Center. The Navy desires some assessment of the flame spread characteristics of the selected composite the data gathered here are part of that flame spread characterization. The ignition delay time and latera spread results were obtained with the LIFT apparatus in accordance with ASTM E 1321. The upward flame spread tests were performed using an experimental electrical radiant panel apparatus.

\section{1,437}

PB97-110076 Not available NTIS

National Inst. of Standards and Technology (BFRL), Gaithersburg, MD. Fire Safety Engineering Div. Interaction of an Isolated Sprinkler Spray and a Two-Layer Compartment Fire Environment. Phenomena and Model Simulations.

Final rept.

L. Y. Cooper. 1995, 19p.

See also PB91-216804
Pub. in Fire Safety Jnl., v25 n2 p89-107 Sep 95.

Keywords: "Fire tests, "Sprinkler systems, Spraying, Mathematical models, Nozzles Computerized simulation, Water, Combustion, Buildings, Reprints, *Foreign technology.

A general description of the interaction of sprinklers and compartment-fire-generated smoke layers is presented. Various possible aspects of the interaction phenomena (upper-layer smoke entrainment into the sprinkler spray, momentum and mass exchange between droplets and entrained gas, gas cooling by evaporation, buoyancy effects, and others) are discussed in the context of a two-layer-type description of the fire environment. The inputs and outputs for a mathematical submodel which simulates the phenom. ena are discussed. The submodel is suitable for general use in any two-layer, zone-type compartment fire model. Results from exercising the submodel are presented. These example calculations simulate the interaction between the spray of a real sprinkler device and a range of two-layer fire environments. The calculations reveal an important generic interaction phenomenon, namely, an abrupt and large change in the growth rate of the upper layer that would accompany an increase in upper-layer thickness beyond a critical thickness (for a given upper-layer temperature) or an increase in upper-layer temperature beyond a critical temperature (for a given upper-layer thickness). Exceeding these critical values would lead to a very large rate of growh of upper layer thickness, a growth that could lead to rapid and complete smoke filling of even the largest compartments of fire origin.

\section{1,438}

PB97-110357 Not available NTIS

National Inst. of Standards and Technology (BFRL) Gaithersburg, MD. Fire Safety Engineening Div.

Heat Flux from Flames to Vertical Surfaces.

Final rept.

J. G. Quintiere, and T. G. Cleary. 1992, 10p.

Pub. in American Society of Mechanical Engineers Winter Annual Meeting, Anaheim, CA., November 1992, 10p.

Keywords: *Heat flux, "Flames, "Vertical surfaces, Walls, Corners, Dimensional analysis, Reprints.

Dimensional analysis is used to examine heat transfer from flames to vertical surfaces. Configurations include a line fire against a wall, a square burner flame against a wall and in a corner, and window flames impinging on a wall. Dimensionless parameters that affect flame heat flux include $x /(f), y / D, I(f) / D$, and $k D$ where $x$ is vertical distance, $y$ is horizontal distance, $l(f)$ if flame length, $D$ is burner dimension, and $k$ is the flame $a b$ sorption coefficient. Only the effect of these variables is shown; no general correlation is developed, and more data are needed.

\section{1,439}

PB97-118632 Not available NTIS

National Inst. of Standards and Technology (BFRL), Gaithersburg, MD. Fire Science Div.

NIST Research on Less-Flammable Materials.

Final rept.

R. G. Gann. 1996, 5p.

Pub. in Society for the Advancement of Material and Process Engineers Jnl., v32 n3 p16-20 May/Jun 96.

Keywords: "Building materials, "Furniture, *Flammability, "Fire tests, Polymers, Composite materials, Service life, Fire safety, Fire research, Research programs, Reprints, US NIST.

A principal objective of the NIST Fire Research Program is supporting the development by U.S. manufacturers of a new generation of buiding and furnishing materials and products that contribute less to a fire, maintain their fire safety performance over the product life, and are environmentally friendly. The paper describes the NIST roles in fire safety science and engineering, as well as research projects currently underway on less fire-prone materials and products.

\section{1,440}

Not available NTIS

National Inst. of Standards and Technology (BFRL), Gaithersburg, MD. Fire Science Div.

Computations of Enhanced Soot Production in Time-Varying CH4/Air Diffusion Flames.

Final rept.

C. R. Kaplan, C. R. Shaddix, and K. C. Smyth. 1996, $11 \mathrm{p}$.

See also PB95-203246. 
Pub. in Combustion and Flame, v106 n4 p392-405 Sep 96.

Keywords: "Soot, *Diffusion flames, "Methane, TurbuIent flow, Heat transmission, Flicker, Instability, Temporal variations, Navier-Stokes equation, Computations. Two dimensional models, Mathematical models. Reprints.

The paper presents time-dependent numerical simulations of both steady and time-varying $\mathrm{CH} 4$ /air diffusion flames to examine the differences in combustion conditions which lead to the observed enhancement in soo production in the flickering flames. The numerical model solves the two-dimensional, time-dependent reactive-flow Navier-Stokes equations coupled with submodels for soot formation and radiation transport.

\section{1,441}

PB97-119325 Not available NTIS

National Inst. of Standards and Technology (CAML) Gaithersburg, MD.

Transport by Gravity Currents in Building Fires.

Final rept.

R. G. Rehm, K. B. McGrattan, H. R. Baum, and K.

W. Cassel. 1996, 12p.

Pub. in Proceedings of the Internationa! Symposium on Fire Safety Science (5th), Melbourne, Australia, 12p 1996.

Keywords: "Fires, "Computational fluid dynamics, *Boundary conditions, Buildings, Walls, Convection, Smoke, Gas flow. Heat transfer, Buoyancy, Convective flow, Eddies(Fluid mechanics), Navier-Stokes equation, Two-dimensional models, Three-dimensional models, Computerized simulation, Reprints, "Gravity currents.

Gravity currents (GC) are important physical phenomena which transport smoke and hot gases in corridors of buildings. In the paper, they are studied using large eddy simulations (LES). The transient Navier-Stokes $(\mathrm{N}-\mathrm{S})$ equations are numerically integrated with very high resolution, in two dimensions and with high resolution in three dimensions. The LES computations require no adjustable parameters, and are found to agree well with available experimental results in the absence of heat transfer.

01,442

PB97-122311 Not available NTIS

National Inst. of Standards and Technology (BFRL), Gaithersburg, MD. Fire Science Div.

Materials and Fire Threat.

Final rept.

R. G. Gann, R. Lyon, U. Sorathia, and L. Gritzo.

1996, 8p.

Pub. in Society for the Advancement of Material and Process Engineers Jnl., v32 n3 p8-15 May/Jun 96.

Keywords: "Aircraft fire, *Ship fires, "Fire resistant materials, "Polymers, Fiber reinforced composites, Composite materials, Materials replacement, Flammability Military applications, Naval vessels, Ship structura components, Barriers, Fire protection, Fire research, Fire safety, Reprints.

Fiber-reinforced polymer composites offer the U.S military the potential for significant reductions in weight and signatures. Current seaborne applications of composite materials in the U.S. Navy include sonar bow domes and windows, and coastal minehunter MHC-51 hulls. The U.S. navy is also evaluating composite materials of both primary and secondary load-bearing structures such as foundations, deckhouses, and hulls; machinery components such as composite piping valves, centrifugal pumps, and heat exchangers; and auxiliary or support items such as gratings, stanchions, ventilation ducts, and screens. This new interest in composite materials is due to increased need for a corrosion-free, lightweight, and affordable low-cost alternative to metallic components. The U.S. Army is evaluating composite combat vehicles and the U.S. Air Force has taken the lead in transitioning composite technology to military advantage as evidenced by superior performance of the Stealth Fighter.

\section{1,443}

PB97-122519 Not available NTIS

National Inst. of Standards and Technology (BFRL), Gaithersburg, MD. Fire Science Div.

NO Production and Destruction in a Methane/Air Diffusion Flame.

Final rept.

K. C. Smyth. $1995,35 p$.

Pub. in Combustion Science and Technology, v115 p151-176 1996.
Keywords: "Diffusion flames, "Methane, "Nitrogen oxides, "Combustion chemistry, Combustion products, Laminar flow. Thermodynamic properties, Laser induced fluorescence, Boltz mann equation, Reprints, Collisional quenching.

Concentration profiles have been measured for naturally occurring $\mathrm{NO}$ in a laminar $\mathrm{CH} 4$ /air diffusion flame burning on a rectilinear Wolfhard-Parker slot burner at atmospheric pressure. The observed fluorescence signals have been corrected for (1) the Boltzmann population in the $R$ (sub 1) rotational level of the ground vibronic state and (2) collisional quenching rates as a function of the local temperature and collider concentrations. A reaction path analysis and determination of NO fluxes strongly indicate that prompt NO production outweights the thermal route, but uncertainties in determining the relative contributions to instantaneous NO production are large.

\section{Fuel \& Propellant Tanks}

01,444

PB97-110043 Not available NTIS

National Inst. of Standards and Technology (BFRL), Gaithersburg, MD. Fire Science Div.

Effect of Fuel Tank Rupture Mode on the Ignitability of Expelled Fuel.

Final rept.

T. G. Cleary, and R. G. Gann. 1993, 7p.

Sponsored by National Highway Traffic Safety Administration, Washington, DC

Pub. in Effect of Fuel Tank Rupture Mode on the Ignitability of Expelled Fuel, p1-7 Mar 93

Keywords: "Fuel tanks, "Crash tests, Fuel work rupture, Trucks, Automotive fuels, Reprints

At the request of the Office of Defects Investigation (ODI) of the National Highway Traffic Safety Administration (NHTSA), the Building and Fire Research Laboratory (BFRL) of the National Institute of Standards and Technology (NIST) analyzed data from vehicle collision tests and tank crush tests to provide an assessment of the effect of tank rupture mode on the ignitability of expelled fuel for two different ignition scenarios: compression of a tank, resulting in increased internal pressure and rapid, violent expulsion of the gasoline as a result of a puncture or rupture; and simple puncture of a vehicle fuel tank, resulting in gasoline drainage. For the work, ODI/NHTSA provided NIST with: video tapes of the tank crush tesis and video tapes and high-speed film of two high speed vehicle collision tests (one of the latter resulted in violent expulsion of fluid: the other in fuel tank puncture and fluid drainage) estimates of the fuel dispersion from the tank crush tests and the vehicle collision tests; and a list of potential ignition sources and their locations on the vehicle of interest.

\section{Jet \& Gas Turbine Engines}

\section{1,445}

AD-A278 138/3 PC A04/MF A01

National Bureau of Standards, Boulder, $\mathrm{CO}$

Bibliography of Books and Published Reports on Gas Turbines, Jet Propulsion, and Rocket Power Plants.

E. F. Fiock, and C. Halpern. 1 Jun 51, 68p, NBSCIRCULAR-509

Keywords: "Jet engines, "Gas turbines, "Bibliographies, Jet propulsion, Rockets, Auxiliary power plants, Reports, "Rocket engines, N-10173. No abstract available.

\section{1,446}

AD-A278 213/4 PC A06/MF A02

National Bureau of Standards, Gaithersburg, MD.

Bibliography of Books and Published Reports on

Gas Turbines, Jet Propulsion, and Rocket Power Plants, January 1950 through December 1953. E. F. Fiock, and C. Halpern. Dec 53, 115p, NBS CIRC-509-SUPPL

Keywords: "Gas turbines, "Bibliographies, Jet propulsion, Rockets, Reports, "Rocket engines, "Jet engines.
No abstract available.

01,447

N95-26123/6 (Order as N95-26119, PC A03/MF

A01)

National Inst of Standards and Technology Gaithersburg, MD

Measurement Methods and Standards for Process ing and Application of Thermal Barrier Coatings. Abstract Only.

S. J. Dapkunas. Mar 95, 1p.

in NASA. Lewis Research Center, Thermal Barrier Coating Workshop $p 9$

Keywords: "Spraying, "Thermal control coatings, "Vapor deposition, Aircraft engines, Diesel engines, Gas turbine engines, Stationary engines.

Application of thermal barrier coatings deposited by thermal spray, physical vapor and possibly other methods is expected to be extended from aircraft gas turbines to industrial and utility gas turbines as well as diesel engines. This increased usage implies the participation of greater numbers of processors and users, making the availability of standards for process control and property measurement more important. Available standards for processing and evaluation of therma barrier coatings are identified as well as those needed in the future but currently unavailable.

01,448

PB95-175956 Not available NTIS

National Inst. of Standards and Technology (CSTL), Gaithersburg, MD. Inorganic Analytical Research Div. Determination of Hydrogen in Titanium Alloy Je Engine Compressor Blades by Cold Neutron Capture Prompt Gamma-ray Activation Analysis.

Final rept

R. L. Paul, and R. M. Lindstrom, 1994, 6p.

Pub. in Review of Progress in Quantitative Nondestructive Evaluation, v13 p1619-1624 1994

Keywords: "Jet engines, "Titanium alloys, "Hydrogen embrittlement, "Concentration(Composition) "Compressor blades, "Nondestructive tests, Test methods, Structural failure, Hydrogen, Gamma ray scattering, Compressors, Defect analysis, Reprints, "Cold neutron capture prompt gamma-ray activation analysis

Titanium, an important structural metal, readily absorbs hydrogen. Since the presence of trace amounts of hydrogen causes embrittlement in metals, a reliable nondestructive method of measuring hydrogen in tita nium is much needed. We have found cold neutron capture prompt gamma-ray activation analysis (CNPGAA) to be ideal for the measurement of trace amounts of hydrogen. The technique is nondestructive, gives bulk analyses, the results are independent of the chemical form of the element being measured, and the hydrogen peak is interference free. The detection limit for hydrogen in titanium and its alloys is near $100 \mathrm{mg}$ $\mathrm{kg}$. We have used CNPGAA to measure hydrogen in two titanium alloy jet engine compressor blades from an engine in which a blade had failed during service. While $\mathrm{H}$ concentrations in one blade were nearly uniform (approximately $200 \mathrm{mg} / \mathrm{kg} \mathrm{Ti}$ ) at all points meas ured, $\mathrm{H}$ concentrations in the second blade ranged from 140 to $750 \mathrm{mg} / \mathrm{kg} \mathrm{Ti}$, with greater $\mathrm{H}$ concentrations toward the edges of the blade than in the interior. Analysis of three cross sections, cut from different regions of another blade, showed a similar concentration gradient.

\section{Reciprocation \& Rotating Combustion Engines}

\section{1,449}

PB́94-172103 Not available NTIS

National Inst. of Standards and Technology (NEL) Gaithersburg, MD. Chemical Process Metrology Div. Thin Film Thermocouples for Measurement of Wal Temperatures in Internal Combustion Engines. Final rept.

D. Burgess, M. Yust, and K. Kreider. 1990, 6p. Pub. in Proceedings of American Institute of Aeronautics and Astronautics/American Society of Mechanical Engineers Thermophysics and Heat Transfer Conference, Seattle, WA., June 18-20, 1990, p43-48.

Keywords: "Thermocouples, "Thermal conductions, *Internal combustion engines, Ceramics, Heat flux 


\section{Reciprocation \& Rotating Combustion Engines}

Thin films, Lasers, Temperature measuring instruments, Zirconium oxides, Aluminum oxide, Thermal diffusion, Reprints, Zirconia, Alumina.

The transient temperature response of thin film thermocouples (TFTC) was measured using a pulsed laser heating technique. Pt/Pt-10\% Rh, Pt/Au, and In2O3/In2O3-SnO2 (ITO) TFTC's with thicknesses ranging from 0.4 to 28 microns were fabricated by sputter deposition on alumina, plasma-sprayed zirconia, and Min-K 2000 substrates. The zirconia TFTC's are used in plugs for determination of the wall heat flux in internal combustion engines. The thermocouple output of the TFTC's was measured as a function of time following irradiation-heating of the thermocouple junction by a 12 ns laser pulse. The magnitude of the temperature jump (thermocouple output) and the temporal response of the TFTCs were found to be consistent with those predicted from a solution to the heat conduction equation. This model assumes that the heat flow is one-dimensional and that the film is thick compared to the thermal diffusion length during the laser heating pulse. For example, it was found that TFTCs with nominal 4 micron thick junctions has temperature decay imes of about 5 microseconds, 60 microseconds, and $20 \mathrm{~ms}$ for alumina, zirconia, and Min-K 2000 substrates, respectively. These results are related to similar work using thin film resistance sensors and to measurements of the frequency response of TFTCS using an intensity modulated laser heating method. The fabrication of the TFTCs and the utility of heat flux sensors in internal combustion engines are discussed.

01,450

PB'95-104915 PC A04/MF A01

National Inst. of Standards and Technology (MSEL), Gaithersburg, MD. Ceramics Div

Abrasive Wear by Diesel Engine Coal-Fuel and Related Particles.

L. K. Ives. Sep 94, 66p, NISTIR-5461, ORNL/SUB/ 83-21322/03

Contracts DE-A105-830R21322, DE-ACO5-

$840 R 21400$

See also DE93000834 and DE87008697. Sponsored by Oak Ridge National Lab., TN. and Department of Energy, Washington, DC.

Keywords: "Diesel engines, "Abrasion, "Coal, "Wear tests, Piston rings, Fuel slurries, Fuel substitution, Pulverized fuels, Scanning electron microscopy, Engine cylinders, Wear resistance.

The purpose of the work summarized in this report was o obtain a basic understanding of the factors which are responsible for wear of the piston ring and cylinder wall surfaces in diesel engines utilizing coal-fuel. The approach included analytical studies using scanning electron microscopy and energy dispersive $x$-ray analyses to characterize coal-fuel and various combustion particles, and two different wear tests. The wear tests were a modified pin-on-disk test and a block-on-ring test capable of either unidirectional or reciprocating-rotational sliding. The wear tests in general were con ducted with mixtures of the particles and lubricating oil The particles studied included coal-fuel, particles resulting from the combustion of coal fuel, mineral matte extracted during the processing of coal, and severa other common abrasive particle types among which quartz was the most extensively examined. The variables studied included those associated with the par ticles, such as particle type, size, and hardness; variables related to contact conditions and the surrounding environment; and variables related to the type and properties of the test specimen materials.

01,451

PB́95-152120 Not available NTIS

National Inst. of Standards and Technology (MSEL) Gaithersburg, MD. Ceramics Div.

New Method to Evaluate Deposit Forming Tendencies of Liquid Lubricants by Differential Scanning Calorimetry.

Final rept.

Y. Zhang, P. Pei, J. M. Perez, and S. M. Hsu. 1992, $7 p$.

Sponsored by Department of Energy, Washington, DC. Energy Conversion and Utilization Technologies Pro-

Pram. in Lubrication Engineering 48, n3 p189-195 1992.

Keywords: “Lubricating oils, ”Deposits, ”Engine tests, Lubricants, Calorimeters, Lubrication, Contamination, Test methods, Oxidation, Reprints, "Two-peak method, Pressurized differential scanning calorimetry.

A new laboratory bench test, using the technique of pressurized differential scanning calorimetry (PDSC) has been developed to evaluate deposit forming tendency for liquid lubricants. This method, called 'PDSC two-peak method', attempts to simulate deposit forma tion mechanism in an engine by heating thin films of oil on steel discs in an oxidative atmosphere. In this paper, the development and optimization of the twopeak test procedure are described. The test was evaluated by characterizing the deposit forming tendencies of five liquid lubricants. The deposit performance ranking for the five oils obtained from this new test method was consistent with engine test ranking.

\section{1,452}

PB95-152138 Not available NTIS

National Inst. of Standards and Technology (IMSE), Gaithersburg, MD. Ceramics Div.

Deposit Forming Tendencies of Diesel Engine Oils. Correlation between the Two-Peak Method and Engine Tests.

Final rept.

Y. Zhang, J. M. Perez, P. Pei, and S. M. Hsu. 1992 $6 p$.

Sponsored by Department of Energy, Washington, DC Energy Conversion and Utilization Technologies Program.

Pिub. in Lubrication Engineering 48, n3 p221-226 1992.

Keywords: "Diesel engines, "Lubricating oils, "Deposits, "Engine tests, Lubricants, Colorimeters, Sampling, Contamination, Test methods, Lubrication, Reprints, "Two-peak method, Pressurized differential scanning calorimetry.

The ability to determine the deposit forming tendencies of lubricants utilizing a newly developed laboratory bench method was established using three individua sets of lubricants. The 'Two-Peak Method' was developed at NIST using a pressurized differential scanning calorimeter and a unique sample pan. The method is described and repeatability between operators is demonstrated. The oils used in the study were evaluated in engine oil performance tests, or field tests, by the cooperating laboratories. For each set of oils highly correlatable results between the two-peak method and the engine performance tests were obtained. Several reference oils were included in each set of samples to show agreement between the three sets of samples. The method can be used to predict top land deposits that result increased oil consumption in different types of diesel engines.

\section{Rocket Engines \& Motors}

01,453

\section{PB95-169215 Not available NTIS}

National Inst. of Standards and Technology (CSTL),

Boulder, CO. Process Measurements Div.

Vortex Shedding Flowmeters for SSME Ducts.

Final rept.

J. D. Siegwarth, and M. A. Lewis. 1992, 9p.

Pub. in Proceedings of Advanced Earth-to-Orbit Propulsion Technology Conference, Huntsville, AL., May 19-21, 1992, NASA Conference Publication 3174, v1 p217-2251992.

Keywords: "Space shuttle main engine, "Liquid oxygen, "Ducted rocket engines, Vortex shedding, Flow meters, Gas flow, Flow measurement, Ducted flow, meters, Gas flow, Flow measurement,

Previous work has shown that vortex shedding flowmeters can measure flow in the Space Shuttle Main Engines (SSME) liquid oxygen ducts under the extreme conditions found there and without any upstream flow conditioning. Though measurement of liquid flow in the $28 \mathrm{~mm}$ (1.1 in.) duct is more difficult, recent tests have shown vortex shedders can make this measurement also. An encapsulated sensor design is presented and vortex shedding flowmeters for selected fuel ducts are discussed. A hydrogen gas test facility is needed to complete development of gas meters because of the high velocities found in the fuel ducts.

\section{Rocket Propellants}

01,454

AD-A280 398/9 PC A05/MF A01
National Bureau of Standards, Boulder, CO Cryogenic Research and Development (Quarterly Report Number 2 for Period Ending December 31 , 1960).

Quarterly rept. no. 2 for period ending $31 \mathrm{Dec} 60$

31 Dec 61, 88p, NBS-6736.

Keywords: *Cryogenics, *Liquid hydrogen, Temperature control, Thermodynamics, Vapor pressure, Electric batteries, Thermophysical properties.

No abstract available.

\section{1,455}

\section{AD-A280 399/7 PC A04/MF A01}

National Bureau of Standards, Boulder, $C O$

Cryogenic Research and Development (Progress Report Number 4 for Period Ending December 31, 1961).

Progress rept. no. 4 for period ending 31 Dec 61

31 Dec $61,74 p$, NBS-7219.

Keywords: "Cryogenics, "Liquid hydrogen, Thermodynamic properties, Gravity(Artificial), Density, Specific heat.

No abstract available.

\section{1,456}

AD-A280 401/1 PC A04/MF A01

National Bureau of Standards, Boulder, $C O$

Cryogenic Research and Development (Quarterly Report Number 1 for Period Ending September 30, 1960).

Quarterly rept. no. 1 for period ending 30 Sep 60.

30 Sep $60,71 p$, NBS-6728.

Keywords: "Cryogenics, "Cryogenic propellants, Liquid hydrogen, Thermal properties, Thermal conductivity, Gravity, Densitometers, Gravity(Artificial), Gas dynamics, Pressure transducers, Zero gravity, Compressibility.

No abstract available.

\section{1,457}

AD-A280 679/2 PC A04/MF A01

National Bureau of Standards, Gaithersburg, MD. Cryogenic Research and Development (June 30, 1961)

30 Jun $61,73 p$, NBS-6785.

Keywords: "Cryogenics, Thermophysical properties, Isotherms, Flanges, Rings, Temperature, Pressure, Densitometers, Ball bearings, Hydrogen, "Cryogenic propellants, Liquid hydrogen.

No abstract available.

\section{1,458}

AD-A286 612/7 PC A05/MF A01

National Bureau of Standards, Gaithersburg, MD

Progress Report to National Aeronautics and Space Administration on Cryogenic Research and Development.

Progress rept. no. 4, for period ending 31 Dec 61.

31 Dec 61, 77p, NBS-7219.

Keywords: "Cryogenics, "Liquid hydrogen, Instrumentation, Hydrogen. Physical properties, Specific heat, Fluids, Saturation, Density, Vapor pressure, Heat of vaporization, Thermal conductivity, Dielectrics, Temperature, Detectors, Pressure transducers, Vibration Thermodynamics.

No abstract available

\section{COMMUNICATION}

\section{Common Carrier \& Satellite}

\section{1,459}

AD-A286 619/2 PC A10/MF A03

National Bureau of Standards, Gaithersburg, MD lonospheric Radio Propagation. 25 Jun $48,214 p$, NBS-462.

Keywords: "Ionospheric propagation, *Radio waves, Signal to noise ratio, Solar disturbances, Field inten- 
sity, Wave propagation, Noise(Radio), High frequency, Magnetic fields, Radio transmission, Antenna radiation pattems, Sky waves, Range(Distance).

No abstract available.

\section{1,460}

FIPS PUB $182 \quad$ PC E03

National Inst. of Standards and Technology (CSL), Gaithersburg, MD

Integrated Services Digital Network (ISDN); Category: Telecommunications Standard; Subcategory: Integrated Services Digital Network.

\section{Oct $93,35 \mathrm{p}$}

Three ning vinyl binder also available; North American Continent price $\$ 7.00$; all others write for quote.

Keywords: *Communication networks, "Digital communications, Communications management, Data transmission, Protocols, Data links, Packet switching, Interoperability, Signal transmission, Telecommunication, *Integrated senvices digital network, Telecommunications standards, Primary rate interface, Basic rate interface.

The publication defines the generic protocols necessary to establish transparent Integrated Services Digital Network (ISDN) connections among government networks and between government and conformant common carrier networks. This FIPS provides a minimal set of bearer services, and is based on national standards, international standards, and implementation agreements developed by the North American ISDN Users' Forum (NIUF). Future versions of this FIPS will provide protocols for additional services, teleservices and applications. This standard supports a range of integrated services including voice, data, image, and video services.

01,461

FIPS PUB 187 PC A02/MF A01

National Inst. of Standards and Technology (CSL), Gaithersburg, MD

Administration Standard for the Telecommunications Infrastructure of Federal Buildings. Category: Telecommunications Standard; Subcategory: Telecommunications Administration.

11 Aug $94,9 p$

Prepared in cooperation with National Communications System, Arlington, VA

Three ring vinyl binder also available; North American Continent price $\$ 7.00$; all others write for quote.

Keywords: "Telecommunication, "Public buildings, "Federal information processing standards, Passageways, Communication cables, Electrical grounding, Communication equipment, Wire, Administration, $\mathrm{Na}$ tional government, Specifications.

This standard, by adoption of ANSI/TIANEIA-606-1993, Administration Standard for the Telecommunications Infrastructure of Commercial Buildings, specifies the administrative requirements of the telecommunications infrastructure within a new, existing, or renovated office building or campus. Telecommunications infrastructure cal, be thought of as the collection of those components (telecommunications equipment spaces, cable pathways, grounding, wiring, and termination hardware) that provide the basic support for the distribution of all information within a building or campus. Administration of telecommunications includes documentation (record-keeping, drawings, labeling, etc.) of telecommunications outlet boxes, connectors, cables, termination hardware, patching and cross-connect facilities, conduits, other cable pathways, telecommunications closets, and other spaces.

\section{1,462}

PB94-123080 PC A03/MF A01

National Inst. of Standards and Technology (CSL), Gaithersburg, MD.

Technology Trends in Telecommunications: An Overview.

Oct 93, 36p, NISTIR-5282.

Keywords: "Telecommunication, "Trends, "Technological innovations, Economic surveys, Computer networks, User requirements, Data transmission, Wireless communication, Signal processing, Global aspects, Competition, Economic impact, Computer information security, Local area networks, Fiber optics, Information systems, "Diversification.

The article describes the technology trends for telecommunications and their impact on the services provided to the user. The impact of divestiture, together with the advancement of some enabling technologies, is leading to a rapid diversification of the telecommunication industry. The diversification is the subject of the study that summarizes trends in a historic perspective.

01,463

PB94-139045 PC A04/MF A01

National Inst. of Standards and Technology (CSL), Gaithersburg, MD.

Good Security Practices for Electronic Commerce, Including Electronic Data Interchange.

Special pub. (Final).

R. G. Saltman. Dec 93, 68p, NIST/SP-800/9.

Also available from Supt. of Docs. as SN003-00303243-3. See also PB90-147489 and PB90-148784. Sponsored by Farmers Home Administration, Washington, $\mathrm{DC}$.

Keywords: "Electronic mail, "Data processing security Computer security, Computer communications, Commerce, Risk assessment, Documents, Data management, Access control, Cryptography, Authentication Computer privacy, EC(Electronic Commerce), EDI(Electronic Data Interchange).

Electronic commerce (EC) is the use of documents in electronic form, rather than paper, for carrying out functions of business or government that require interchange of information, obligations, or monetary value between organizations. Electronic data interchange (EDI) is the computer-to-computer transmission of strictly formatted messages that can represent documents; EDI is an essential component of EC. With EC human participation in routine transaction processing is limited or non-existent. Transactions are processed and decisions are made more rapidly, leaving much less time to detect and correct errors. This report presents security procedures and techniques (which encompass internal controls and checks) that constitute good practice in the design, development, testing and operation of EC systems. Principles of risk management and definition of parameters for quantitative risk assessments are provided. The content of the trading partner agreement is discussed, and the components of $\mathrm{EC}$, including the network(s) connecting the partners, are described. Some security techniques considered include audit trails, contingency planning, use of acknowledgments, electronic document management activities of supporting networks, user access controls to systems and networks, and cryptographic techniques for authentication and confidentiality.

\section{1,464}

PB94-142494 PC A03/MF A01

National Inst. of Standards and Technology, Gaithersburg, MD.

Introduction to Traffic Management for Broadband ISDN.

Y. Chang, D. Su, and S. Wakid. Dec 93, 25p,

NISTIR-5214

See also PB93-149433

Keywords: "Communications networks, "Traffic management, Communications management Computer networks, Traffic control, Telecommunication, Standards, Protocols, Congestion, "Broadband Integrated Services Digital Network, *Integrated Services Digita Network, Asynchronous transfer mode.

This paper presents an overview of issues related to the traffic management for the Broadband Integrated Services Digital Networks (B-ISDN). B-ISDN is an emerging high bandwidth telecommunications infrastructure with transmission speeds in the range of mega-bit to giga-bits per second. This paper first analyzes traffic profiles in a B-ISDN environment and their impact on traffic management. It then presents an overview of traffic management for existing communications protocols, and discusses, in depth, how it can be applied to the B-ISDN environment. Finally, the paper gives a brief review of the current work on BISDN traffic management by the National Accredited Standards Committee T1 and the International Telegraph and Telephone Consultative Committee (CCITT).

\section{1,465}

PB94-142528 PC A05/MF A01

National Inst. of Standards and Technology (CSL), Gaithersburg, MD.
Context Analysis of the Network Management Domain. Conducted as Part of the Domain Analysis Case Study.

C. Dabrowski, and S. B. Katz. Dec 93, 84p, NISTIR5309

Keywords: *Communication networks, "Network control, Software engineering, Protocols, Fault detection, "Soltware reuse, "Domain analysis, Context analysis, FODA(Feature Oriented Domain Analysis), Network management.

A key to increasing software producibility in the development of large, reliable software applications is the systematic reuse of existing software products. Domain analysis is a pivotal technique for developing reusable products that can be used to engineer software systems. The Domain Analysis Case Study was created to investigate domain analysis methods. This report is a product of the Domain Analysis Case Study. This report describes the application of the first phase of a domain analysis effort to the domain of network management systems--software systems that manage communications networks. The first phase of the domain analysis process is called the context analysis phase.

\section{1,466}

PB94-162559 PC A16/MF A03

National Inst. of Standards and Technology (CSL), Gaithersburg, MD. Advanced Systems Div.

North American ISDN Users' Forum Agreements on Integrated Services Digital Network.

Special pub. (Final).

D. P. Stokesberry, and T. A. Antonishek. Mar 94 $354 \mathrm{p}, \mathrm{NIST} / \mathrm{SP}-823 / 5$.

Also available from Supt. of Docs. as SN003-00303248-4. See also PB93-173391.

Keywords: Communication networks, Digital communications, Telephone systems, Computer networks, North America, Workstations, Agreements, "Integrated Services Digital Network, Basic rate interface, Primary rate interface, Application profiles, Conformance tests, NIUF forum, Voice mail.

This document compiles the existing North American Integrated Services Digital Network (ISDN) Users Forum (NIUF) agreements for an ISDN developeu and approved in the NIUF as of October 1992 . New agreements superseded or added during 1992 cover Layer $1 \mathrm{BRI}$ at the $\mathrm{U}$, and $\mathrm{S} / \mathrm{T}$ reference points. In addition, this document references the Conformance tests which have been completed by the NIUF. These include: Layer 3 BRI, Layer 2 BRI, PRI at the U reference point, and PRI at the U/S $/ T$ reference points. Finally, this document contains the Application Profiles for: Secure Voice Mail; Data Conferencing--Multi-Point; ISDN Telephone Workstation Integration; Engineering Workstation Interface; and the Remote Agent Application Profile.

\section{1,467}

PB94-164035 PC A06/MF A02

National Inst. of Standards and Technology, Gaithersburg, MD.

Supplement to Stable Implementation Agreements for Open Systems Interconnection Protocols. Version 3, September 1990. Change Page Index, Version 3, June 1990 (Stable) Change Pages Issued December 1990; Output from September 1990 OSI Workshop (NIST Special Publication 500-177).

Dec $90,108 \mathrm{p}$.

See also PB90-269556.

Keywords: *Computer networks, "Communication networks, Standards, Agreements, Implementation, Protocols, "Open systems interconnections.

This document records, in replacement page format, all changes to stable material current (according to Version and Edition number) as of the end of the June Workshop. In this case, that would be NIST SP 500177, Version 3, Edition 1 with previous Change Pages incorporated. By following the instructions and replacing or inserting the indicated pages, text will be created which reflects the current status of relevant stable material as of September 14, 1990.

\section{1,468}

PB94-166006 PC A99/MF A06

North American ISDN Users' Forum, Gaithersburg, MD

Catalog of National ISDN Solutions for Selected NIUF Applications.

Feb 94, 679p.

Supersedes PB93-162881. 
Keywords: "Telecommunication, "Technology utilization, Decision making, Telephone systems, Work stations, Local area networks, Supercomputers, Facsimile communication, Voice communication, Screens(Displays), Computer networks. Computer software, Data transmission, Bibliographies, Dictionaries, Real property, Computer files, "ISDN(Integrated Services Digital Network), LAN(Local area networks) Videoconferencing systems, Teleconferencing, Telecommuting.

The NIUF is pleased to present the Second Edition of its ISDN Solutions Catalog. The purpose of the Second Edition remains the same as the first, to make it easier for vendors to develop and support ISDN applications and for users to understand and install them. In February of 1993, the NIUF authorized work on this Second Edition. It provides a more robust set of solutions in Section 3 , and adds Section 2 for the decision maker', who must decide whether to use ISDN. Many other refinements were made. The scope is essentially the same as the First Edition, with additional coverage of National ISDN-2. New solutions in the Catalog cover National ISDN-2, especially Primary Rate. Of course changes in products and services are tracked through an updated chapter of product information.

\section{1,469}

PB94-214756 PC A08/MF A02 National Inst. of Standards and Technology,

Information Infrastructure: Reaching Society's

Goals. Report of the Information Infrastructure Task Force Committee on Applications and Technology.

Special pub.

Sep $94,160 p$, NIST/SP-868.

Also available from Supt. of Docs. as $\mathrm{SNOO3}^{-003}$ 03283-2.

Keywords: "Sectoral analysis, ${ }^{*}$ Computer networks "Information services, Information systems, National interests, Information dissemination, Delivery systems Citizen participation, Transportation sector, Electric power industry, Social services, Handicapped persons, Emergency preparedness, Public safety, Arts, Humanities, Telecommunications, "NII(National Information Infrastructure), "National Information Infrastructure, Government services, Information superhighways, Information networks. Telecommuting, Intelligent Vehicle Highway System.

Table of Contents:

Introduction;
NII:

An Investment in People with Disabilities;

Supply and Demand of Electric Power and the NII;

Improving Transportation:

The NII and Intelligent Transportation Systems;

Promoting Telecommuting

An Application of the NII:

The Effect of the NII on Local, State, and Federal Emergency Management;

Public Empowerment with Environmental Information:

Arts, Humanities, and Culture on the NII;

and Public Safety and the NII:

Supporting Law Enforcement and Criminal Justice.

\section{1,470}

PB94-217023 PC A04/MF AO

National Inst. of Standards and Technology (CSL), Gaithersburg, MD.

Videoconferencing Procurement and Usage Guide. Rept. for Oct 93-Jul 94.

M. A. Wallace, and D. E. Rorrer. Aug 94, 54p, NISTIR-5485.

Sponsored by Internal Revenue Service, Washington,

Keywords: "Teleconferencing, "Video communication, "Procurement, Interactive systems, Systems analysis, Integrated systems, Requirements, Personnel development, Training, Sites, Videoconferencing

A brief history and technical discussion of videoconferencing is presented along with the processes that are required in order to evaluate the need for a videoconferencing system. Guidance is provided for the evaluation, selection, purchase, installation, and use of various options and system types. Communication and physical requirements are discussed with respect to each of the most common systems that are currently available. Personnel training and site preparation requirements are also developed.
01,471

PB94-219094 PC A03/MF A01

National Inst. of Standards and Technology (CSL), Gaithersburg, MD. Advanced Systems Div.

ISDN Conformance Testing Guidelines: Guidelines for Implementors of ISDN Customer Premises Equipment to Conform to Both National ISDN-1 and North American ISDN Users' Forum Layer 3 Basic Rate Interface Basic Call Control Abstract Test Suites. Special pub.

SP-823/6.

Also available from Supt. of Docs as $\mathrm{SNOOH}^{-003-}$ 03278-6.

Keywords: "Tests, "Protocols, "Telecommunication, Interfaces, Guidelines, Communication networks, Communications management, Communication equipment, "ISDN(Integrated Services Digital Network), *Integrated Services Digital Network.

The document is intended to provide information, as a supplement to the abstract test suites, to allow conformance to both the Bellcore National ISDN-1 (NI-1) and North American ISDN Users' Forum (NIUF) NIU. 301 specifications for the ISDN Layer 3 Basic Rate Interface for Basic Call Control (user-side). It was developed to guide implementors in the design of customer premises equipment in a manner which would allow them to pass both the NI-1 and NIUF conformance tests. It may also be useful to vendors of test equipment, government procurement agents, and testing laboratories.

\section{1,472}

PB95-103677 PC A09/MF A03

National Inst. of Standards and Technology (EEEL), Gaithersburg, MD. Electricity Div.

Report on the Workshop on Advanced Digital Video in the National Information Infrastructure. Held in Washington, D.C. on May 10-11, 1994.

C. Fenimore, B. Field, H. Frank, G. Reitmeier, W.

Stackhouse, C. Van Degrift, E. Georg, and M.

Papillo. Jul 94, 195p, NISTIR-5457.

Keywords: "Meetings, "Information systems, "Digital data, "Video data, Communication networks, Information services, Television systems, Telecommunication, Telemedicine, High definition television, Video compression, Standards, Interactive systems, "NII(National Information Infrastructure), "National Information Infrastructure, "ADV(Advanced Digital Video), "Advanced Digital Video, ATM(Asynchronous Transfer Mode), ATV(Advanced Television System).

\section{Contents:}

Summary Report by the Program Committee:

Workshop Agenda

Breakout Group Reports:

Visions and Services of the National Information Infrastructure;

Production and Distribution of Entertainment in the NII;

Medical Applications:

Putting Advanced Digital Video on the Technology Curve;

National Information Infrastructure:

An Administration Perspective:

Interoperability Aspects of the Grand Alliance HDTV System;

Video Compression Technology:

Advanced Digital Video over ATM Networks;

Digital Video in the Internet:

Status, Technology, and Issues;

Standards Panel

Old Game, New Rules:

Advancing the National Interactive

Communications Systems;

The National Information Infrastructure and the Grand Alliance ATV System:

A Commentary on Some Aspects of Interoperation;

A Commentary on Requirements for the Interoperation of Advanced Television with the National Information Infrastructure;

Arguments in Support of Embedded

Multiresolution Signaling Strategies for HD

Video Transmission.

\section{1,473}

\section{PB95-105383 PC A13/MF A03}

National Inst. of Standards and Technology (CSL), Gaithersburg, MD.
Security in Open Systems.

Special pub.

R. Bagwill, J. Barkley, L. Carnahan, P. Markovitz, A Nakassis, K. Olsen, M. Ransom, J. Wack, S. Chang, and R. Kuhn. Jul 94, 290p. NIST/SP-800/7.

Also available from Supt. of Docs. as SNO03-00303276-0. Sponsored by National Communications System, Arlington, VA.

Keywords: "Telecommunication, "Applications programs(Computers), "Computer security, Standards, Software engineering, Operating systems(Computers), UNIX(Operating system), Specifications, Cryptography, Man computer interface, Computer networks, Open systems, US NIST, PSN(Public Switched Network), POSIX(Portable Operating System Interface)

The report is intended to provide information for the practicing programmer involved in development of telecommunications application software. It provides information on building security into software based on open system platforms. It is not intended to be tutorial in nature and assumes some knowledge of open systems and UNIX. It covers the following topics: Portable Operating System Interface (POSIX) Open System Environment; Functional Requirements Specifications for Computer Security; POSIX Security Interfaces and Mechanisms; Standard Cryptographic Service Calls; Human/Computer Interaction Services Security; the $X$ Window System; SQL; Network Security Threats; Improving Security in a Network Environment; X.400 Message Handling Services; X.500 Directory Services.

\section{1,474}

PB95-143145 PC A03/MF A01

National Inst. of Standards and Technology (CSL) Gaithersburg, MD. Systems and Software Technology Div.

Information Technology Engineering and Meas rement Model: Adding Lane Markings to the Information Superhighway.

M. Zelkowitz, and B. Cuthill. Nov 94, 46p, NISTIR5522.

See also PB94-163383. Prepared in cooperation with Maryland Univ., College Park. Dept. of Computer Science.

Keywords: "Information systems, "Systems engineering, "Software engineering, "Computer systems design, Distributed data processing, Database management. Information flow, Information dissemination, Computer networks, Information services, Information technology, Telecommunications, "National Information Infrastructure, Information superhighways, Information networks, ITEM(Information Technology Engineering and Measurement)

Development of the National Information Infrastructure (NII) will depend upon building vast networks on the interconnected computers communicating over highspeed digital lines. This paper describes the growth of the NII concept and proposes a model-the Information Technology Engineering and Measurement (ITEM) model-that may be useful in describing the set of services an operational NII may contain.

01,475

PB́95-143178 PC A03/MF A01

National Inst. of Standards and Technology (CSL) Gaithersburg, MD. Advanced Systems Div.

Channel Coding for Code Excited Linear Prediction (CELP) Encoded Speech in Mobile Radio Applications.

E. Bracha, N. Farvardin, and Y. Yesha. Aug 94, 40p, NISTIR-5503.

See also PB93-173938.

Keywords: "Mobile communication systems, "Channels(Data transmission) "Coding, Radio communication, Error correction codes, Computerized simulation, Algorithms, "CELP(Code Excited Linear Prediction), "Code Excited Linear Prediction.

The National Wireless Performance Benchmarking Program seeks to provide tools, performance metrics, methodologies and testbed facilities to the wireless industry and users, so as to allow consistent and impartial performance measurement of wireless communication systems. As a part of this program, a research program is currently being undertaken, in which the mobile radio channel effects on Code Excited Linear Prediction (CELP) encodes speech data are studied. This research uses software simulations to test the various algorithms and evaluate their performance. This repor provides a detailed description of the tests performed 
in this study, including bit error and bit protection sensitivity tests as well as various error correction code tests. It also evaluates different error correction codes for use for channel coding of wireless communication systems.

01,476

PB95-151270 Not available NTIS

National Inst. of Standards and Technology (PL), Boulder, CO. Quantum Physics Div.

Slant Path Atmospheric Refraction Calibrator: An Instrument to Measure the Microwave Propagation Delays Induced by Atmospheric Water Vapor.

Final rept.

S. Walter, and P. Bender. 1992, 10p.

Pub. in Institute of Electrical and Electronics Engineers Transactions on Geoscience and Remote Sensing 30, n3 p462-471 May 92.

Keywords: "Microwave transmission, "Water vapor, Tropospheric propagation, Light transmission, Radio transmission, Atmospheric composition, Atmospheric refraction, Microwave radiometers, Performance evaluation, Remote sensing, Travel time, Time delay, Measuring instruments, Reprints, "SPARC calibrator, Atmospheric transmissivity

The Slant Path Atmospheric Refraction Calibrator, SPARC, has been developed to measure the water vapor induced propagation delay experienced by radio signal traversing the atmosphere. SPARC measures the difference in the travel times between an optical and a microwave signal propagating along the same atmospheric path. Since the refractivity of dry air is similar for both the microwave and optical signals, the difference in arrival times is a sensitive measure of the water vapor induced microwave delay. The in strument is capable of measuring the difference in the optical and microwave travel times with an accuracy of 15 ps or better. SPARC was developed to provide an accurate, independent method for evaluating other techniques used to determine the atmospheric wate vapor induced delay. The theoretical and experimental issues involved in making measurements of the delay induced by water vapor are discussed. Measurements along a 13.35-km ground-based path are presented which will illustrate the instrument's stability, precision and accuracy.

01,477

PB95-154696 PC A03/MF A01

National Inst. of Standards and Technology (CSL) Gaithersburg, MD.

ISDN LAN Bridging.

T. Boland. Nov 94, 26p, NISTIR-5532.

Also available from Supt. of Docs. Sponsored by Internal Revenue Service, Washington, DC.

Keywords: "Local area networks, Computer networks Communication networks, Routing, Narrowband, Multimedia, Procurement, Personal computer, Protocols, Data processing security, Data integrity "ISDN(Integrated Services Digital Network) "Integrated Services Digital Network, "Bridging.

This paper describes the interconnection of a user to a local area network (LAN) across a wide area using an Integrated Services Digital Network (ISDN). This interconnection enables users to access remote applications and services as though they were local to the user.

\section{1,478}

PB95-163176 Not available NTIS

National Inst. of Standards and Technology (ICST) Gaithersburg, MD. Advanced Systems Div.

ISDN Conformance Testing.

Final rept.

D. H. Su, and L. A. Collica. 1991, 9p

Pub. in Proceedings of the Institute of Electrical and Electronics Engineers 79, n2 p190-198 1991

Keywords: Protocols, Telecommunications, Communication networks, Digital communications, Interoperability, Interfaces, Tests, Standards, Reprints, "ISDN(Integrated Services Digital Networks) "Integrated Services Digital Networks, "Conformance esting.

Advances in communication networks have increased the complexity of communications protocols and interfaces significantly, and correct interpretation of stand ards has become a critical issue in ensuring interoperability of equipments from different vendors. The chal lenge to the telephony industry in providing high quality Integrated Services Digital Network (ISDN) service and interoperable ISDN products is unprecedented, as the ISDN protocols and interfaces are very complex and more open in terms of how services are to be provided. Conformance testing is a fundamental task in meeting this challenge.

01,479

PB95-163689 Not available NTIS

National Inst. of Standards and Technology (ICST), Gaithersburg, MD Advanced Systems Div.

Application Profile for ISDN.

Final rept.

S. Wakid, and K. Roberts. 1991, 6p.

Pub. in Proceedings of the Institute of Electrical and Electronics Engineers 79, n2 p199-204 Feb 91.

Keywords: "Data processing, Standards, File management systems, Data transfer(Computers), Facsimile communication, Information retrieval, Electronic mail, Reprints, "ISDN(Integrated Services Digital Network), *Integrated Services Digital Network, EDI(Electronic Data Interchange).

This paper emphasizes some key data processing applications to be used in conjunction with the voice and facsimile services as a platform for deploying Integrated Services Digital Network (ISDN) in North America. Special emphasis is placed on the emerging standards of both ANSI T1 and $X_{3}$ in an attempt to harmonize the computer and telecommunication industries towards providing the user with an integrated platform that is vendor transparent and ubiquitous. This generic platform, which spans a large sector of businesses, is only presented as a base context for defining user specific needs and incubating the applications that directly relate to businesses. Distributed Transaction Processing. Electronic Data Interchange, Information Retrieval, Electronic Mail, and File Transfer together with seven supplementary services for voice and G4 facsimile are proposed for ISDN deployment in the marketplace. This paper, therefore, describes how the relevant standards may be exercised via the various ISDN channels and emphasizes the value of the North American ISDN Users' Forum for commercializing this platform

\section{1,480}

PB95-168563 Not available NTIS

National Inst. of Standards and Technology (EEEL),

Boulder, CO. Electromagnetic Technology Div

Lightwave Standards Development at NIST.

Final rept

D. L. Franzen. 1994, 310p.

Pub. in Proceedings of Department of Defense Fiber Optics ' 94 'Optical Networks in the Concept of a Global Grid', McLean, VA., March 22-24, 1994, p443-445.

Keywords: "Optical communication, "Optical fibers, "Optical detectors, "Standards, Infrared detectors, Wave dispersion, Frequency response, Fiber optics, Wavelengths, Reprints, Optical power, US NIST

Standards being developed at the National Institute of Standards and Technology support the following parameters of interest to lightwave communications: optical fiber geometry, optical fiber chromatic dispersion, absolute optical power, high speed detector frequency response, and wavelength.

01,481

PB95-174967 PC A03/MF A01

National Inst. of Standards and Technology (CSL), Gaithersburg, MD.

Asynchronous Transfer Mode Procurement and Usage Guide.

T. Boland. Dec 94, 43p. NISTIR-5561

Sponsored by Internal Revenue Service, Washington, DC.

Keywords: "Procurement, "Utilization, Telecommunication, Data transfer(Computers), Computer networks, Data transmission, Access control, Data processing security, Local area networks, Management, "ATM(Asynchronous Transfer Mode), "Asynchronous Transfer Mode, B-ISDN(Broadband Integrated Services Digital Network)

This paper gives complete procurement and usage guidance to acquisition of asynchronous transfer mode (ATM) technology. ATM uses a high-speed cell switching technology to move data rapidly between distinct end points.

\section{1,482}

PB95-178604 PC $\$ 9.00$
National Inst. of Standards and Technology (NML), Gaithersburg, MD. National Telecommunications and Information Administration, Washington, DC. Office of Management and Budget, Washington, DC.

Global Information Infrastructure: Agenda for Cooperation.

Also available from Supt. of Docs. See also PB93 231272 Prepared in communications and Information Administration, Washington, DC and Office of Management and Budget, Washington, DC. Office of Information and Regulatory Affairs.

Keywords: "Information systems, "Access to information "Online systems, Information dissemination, Information retrieval, Information technology Information services, Computer networks, Benefits, Investments, Policies, International cooperation, Global aspects, "Gll(Global Information Infrastructure), "Global Intormation Infrastructure, Information networks, Information superhighways.

Table of Contents:

Introduction;

Building a Foundation for the Global Information Infrastructure (GII)-- Five Basic Principles;

Encouraging the Use of the Gll:

Implementing The Gll:

Conclusion

Appendix A - Summary of Recommendations;

Appendix B - Gll Hearing Testimony;

Appendix C - Gll Key Contacts.

\section{1,483}

PB95-189445 PC A03/MF A01

National Inst. of Standards and Technology (CSL) Gaithersburg, MD.

Impact of the FCC's Open Network Architecture on NS/NP Telecommunications Security.

Special pub.

K. Olsen, and J. Tebbutt. Feb 95, 50p, NIST/SP-800/

11

Also available from Supt. of Docs. as SN003-003 03318-9. See also PB95-105383.

Keywords: "National security, "Emergency preparedness, "Telecommunication, "Security, Access control Data processing security, Threats, Vulnerability, Computer networks, Communication networks, FCC(Federal Communications Commission) ONA(Open Network Architecture), PSN(Public Switched Network), NS/EP(National Security/Emergency Preparedness).

The Public Switched Network (PSN) provides National Security and Emergency Preparedness (NS/EP) telecommunications. Service vendors, equipment manufacturers, and the federal government are concerned that vulnerabilities in the PSN could be exploited and result in disruptions or degradation of service. The $\mathrm{Na}$ tional Institute of Standards and Technology (NIST) is investigating the vulnerabilities and related security is sues that result from use of the Federal Communica tions Commission's (FCC's) Open Network Architecture (ONA). This report provides an overview of ONA describes NS/EP telecommunications security concerns, and describes NS/EP telecommunications security concerns that the FCC's ONA requirement introduces into the PSN.

01,484

PB95-210936 PC A03/MF A01

National Inst. of Standards and Technology (CSL), Gaithersburg, MD. Systems and Software Technology Div.

Electronic Implementors' Workshop.

A. T. Landberg, R. H. Bagwill, and B. J. Gray. Mar

95, 19p, NISTIR-5623

Presented at the RWS-CC Meeting (13th), Osaka, Japan, March 7-8, 1995.

Keywords: "Telecommunication, *Interoperability *Information technology, Computer networks, Digita communications, Communication networks, Systems integration, Data access, User needs, Information dissemination, Standards, Protocols, "Electronic workshops, Internet.

The report has been prepared for the 13th RWS-CC Meeting held in Osaka, Japan, March 7-8, 1995. It de scribes the various elements of an electronic workshop and provides a list of capabilities needed to operate one. The primary elements of an electronic workshop include use of the Internet for basic communications such as electronic mail and public forums and publica- 
tion and distribution of publications using computer based media such as Info Servers and CD ROM technology. The necessary prerequisites for establishing an electronic workshop are discussed along with policy and procedures that must be put into plane. Going electronic in the operation of the OSE Implementors Workshop has meant substantial cost savings while improving the timeliness and accessibility of information to the public and members.

\section{1,485}

PB95-231882 PC A2O/MF A04

National Inst. of Standards and Technology (CSL), Gaithersburg, MD.

Standards Policy and Information Infrastructure.

May 95, 454p, NIST/GCR-95/670.

Keywords: "Standards, "Policy making, "Intellectual property, Telecommunication, Government policies, Federal government, Communication networks, Information systems, Information technology, Information flow, Information dissemination, Standarization, Interoperability, "National Information Infrastructure, Open systems.

The purpose of this book is not to think about how formal de jure standards might best be developed, but to consider how standards work as a dynamic, living process--a dialog about technology and how limited common implementations of technology may be useful to enable interoperability and spur market development. The test lies in the market response to the standards process and its expressions--reference models architectures, draft specifications, or standards--and in the further response of the standards process to the market.

\section{1,486}

PB95-267993 PC A06/MF A02

National Inst. of Standards and Technology (TS), Gaithersburg, MD. National Voluntary Lab. Accreditation Program.

National Voluntary Laboratory Accreditation Program. GOSIP: Government Open Systems Interconnection Profile.

Handbook

J. Horlick, S. Nightingale, and J. P. Favreau. Feb 95 , 120p. NIST/HB-150/12.

Also available from Supt. of Docs. as SN003-003 03353-7. See also FIPS PUB 146-1.

Keywords: "Computer networks, *Communication networks, "Message processing, Protocols, File management systems, Data transmission systems, Computer architecture, Requirements, Certification, Procedures, Calibration standards, Standardization, Equipment, Management, Auditing Test methods, Personnel, "GOSIP(Government Open Systems Interconnection Profile), "Government Open Systems Interconnection Profile, NVLAP(National Voluntary Laboratory Accreditation Program).

NIST Handbook 150-12 presents the technical requirements of the National Voluntary Laboratory Accreditation Program (NVLAP) for the GOSIP - Government Open Systems Interconnection Profile program. It is intended for information and use by staff of accredited laboratories, those laboratories seeking accreditation, other laboratory accreditation systems, of laboratory services, and others needing information on the requirements for accreditation under the GOSIP program

01,487

PB96-106851 PC A04/MF A01

National Inst. of Standards and Technology (CSL), Gaithersburg, MD. Advanced Systems Div.

NIST ATM Network Simulator: Operation and Programming, Version 1.0

N. Golmie, A. Koenig, and D. Su. Aug 95, 63p NISTIR-5703.

Keywords: "Communication networks, "Systems simulation, "Data transmission systems, "Manuals, Telecommunication, Computer graphics, Technology assessment, Models, Information systems, "ATM(Asynchronous Transfer Mode), "Asynchronous "ATM(Asynchronous Transfer Mode), "Asynchronous pology.

An Asynchronous Transfer Mode (ATM) network simulator has been developed to provide a means for researchers and network planners to analyze the behavior of ATM networks without the expense of building a real network. The simulator is a tool that gives the user an interactive modeling environment with a graph- ical user interface. With this tool, the user may create different network topologies, control computer parameters, measure network activity, and log data from simulation runs. Part 1 of this document is the user's manual for the simulator; it includes instructions for creating network configurations, specifying component parameters, manipulating the display, logging and saving measurements, and post-processing of data. Part 2 has been prepared as a guide for the user who wishes to modify the simulator software to accommodate net work components not previously defined or to change the behavior of components already defined.

\section{1,488}

PB96-119367 Not available NTIS

National Inst. of Standards and Technology (EEEL), Gaithersburg, MD. Electricity Div.

National Information Infrastructure and Advanced Digital Video.

Final rept.

C. Fenimore. $1994,17 p$

See also PB95-103677

Pub. in International Symposium of Digital Imagery ' 94 'Digital Image and Interactive Services', Tokyo, Japan, October 17-24, 1994, p1-17.

Keywords: "Digital data, "Video data, "Information services, "Image processing, Video compression, Television systems, Telecommunication, Interactive systems, Communication networks, Information systems, Standards, Reprints, National Information Infrastructure.

The U.S. Administration regards the development of a National Information Infrastructure (NII) as a way of putting vast amounts of information at the fingertips of users in America and around the world. Digital video services are likely to be the most technically demanding NII service. Recognizing this, the National Institute of Standards and Technology (NIST), the Technology Policy Working Group of the Administration's Information Infrastructure Task Force and several industrial or ganizations sponsored a recent Workshop to: (1) define a vision of the role of digital video in the NII: (2) identify the architectural, scaling, and performance is sues in realizing this vision; and (3) recommend the research, experiments, and other steps to taken to re solve these issues.

01,489

PB96-119433 Not available NTIS

National Inst. of Standards and Technology (EEEL). Gaithersburg, MD. Electricity Div.

Perception of Clamp Noise in Television Receivers. Final rept.

S. Herman, B. F. Field, P. Boynton, and G. de Haan. 1994, 4p

Pub. in International Display Research Conference Monterey, CA., October 10-13, 1994, p317-320.

Keywords: "Television systems, "Clamping circuits, *Signal-to-noise ratio Clamps, Signal processing, Video signais, Scintillation, Time constants, Reprints. Clamp circuits in television systems adjust the black level of each scan line to a reference voltage derived from the 'back porch' of the T.V signal. If the TV signa is noisy, then the derived black level can vary from scan line, resulting in a displayed streaking effect called 'clamp noise.' This paper reports on clamp nois research performed on a video processing supercomputer at the National Institute of Standards and Technology (NIST). This research measured the average input video signal-to-noise ratio (SNR) at which human observers can just begin to perceive clamp noise against a background of moving color pictures. This threshold was measured as a function of two parameters: two-dimensional scintillation nois due to broadband video noise, and the time constant of the clamp circuitry. These results may give TV system designers guidance in choosing tradeoffs between scintillation noise processing and clamp noise reduction.

\section{1,490}

PB96-122411 Not available NTIS

National Inst. of Standards and Technology (EEEL) Gaithersburg, MD. Electronics and Electrical Engineering Lab. Office.

Making Displays Deliver a Full Measure.

Final rept.

H. S. Bennett, C. Fenimore, B. F. Field, and E. F

Kelley $1995,8 p$

Pub. in Official Monthly Publication of the Society for Information Display, 'Information Display', v11 n1 p2027 Jan 95.
Keywords: "Display devices, "High resolution, * Standards, Video data, Information systems, Image processing, Measurement, Performance evaluation, Technology innovation, Reprints.

High-Resolution Displays are essential for market acceptance of advanced video systems and for sophisticated exploitation of 'the information age'. Developing and manufacturing such displays will require advanced measurement capabilities. This is a subject that interests us greatly at the National Institute of Standards and Technology (NIST), where the development of measurement standards and their application to industrial competitiveness have long been a central part of the authors' mission.

01491

PB96-122452 Not available NTIS

National Inst. of Standards and Technology (CSL), Gaithersburg, MD. Advanced Systems Div.

Error Protecting Characteristics of CDMA and Impacts on Speech.

Final rept.

D. Cypher, S. Wakid, J. Bose, and D. Vaman. 1995,

$6 \mathrm{p}$

Pub. in Institute of Electrical and Electronics Engineers Workshop (3rd) on the Architecture and Implementation of High Performance Communication Subsystems (HPCS '95), Mystic, CT., August 23-25, 1995, p141146.

Keywords: "Code division multiple access, "Mobile communication systems "Satellite communication, "Speech, Synchronism, Feedback control, Automatic control, Errors, Reprints.

A Code Division Multiple Access (CDMA) wireless network is simulated to analyze errors, determine impact on services, and provide an upper bound on the number of active channels. The simulation emphasizes the encoder, decoder/correlator, and a threshold detector: but assumes a resultant Gaussian Function for the 'ai interface' noise. Since the characteristics of a wireless enviroment vary significantly, we examine, as a whole, the errors on the received signal without accounting for specific error contributions from power, fading, propagation, interference from other users, and other factors in such environment. The CDMA encoder uses the 64 Walsh codes that are specified in the draft Wideband CDMA Air Interface Compatibility Standard for 1.85 to $1.99 \mathrm{GHz}$ PCS Applications (1). We use a digitized actual speech database to test for the intelligibility of the voice senvice over such a CDMA air interface. Therefore we are able to learn about the nature of the errors inherent in such a frequency spreading technique and also determine an upper bound on the Simultaneous active channels that would preserve the quality of serv ice (QOS).

\section{1,492}

PB96-131487 PC A03/MF A01

National Inst. of Standards and Technology, Gaithersburg, MD

Application Software Interface: ISDN Services for an Open Systems Environment

D. P. Stokesberry. Feb 95, 36p, NISTIR-5595.

Keywords: "Communication networks, "Specifications, Integrated systems, Digital communications, Computer program portability, Interoperability, Computer networks, Telecommunication, Information technology, Criteria, Evaluation, Standards, Interfaces, *ISDN(Integrated Services Digital Network), *Integrated Services Digital Network, Open Systern Environment, ATM(Asynchronous Transfer Mode), APP(Application Portability Profile), ASI(Application Software Interface), National Information Infrastructure.

This report provides the specific information required to evaluate the Application Software Interface (ASI) as one component of an Open System Environment (OSE) which might include POSIX, GOSIP, and other specifications to provide the functionality necessary to address a broad range of federal information technology requirements.

\section{1,493}

PB96-131511 PC A03/MF A01

National Inst. of Standards and Technology (CSL), Gaithersburg, MD. Distributed Systems Engineering.
Sharing Information via the Internet: An Infoserver Case Study.

R. H. Bagwill. Nov 95, 28p, NISTIR-5757.

Keywords: "Information dissemination, "Data access, Computer networks, On-line systems, Computer com- 
munications, Application programs(Computers), Electronic mail, Specifications, Standards, Requirements, Case studies, *Internet, World wide web, Gopher, Data formats, Software protocols.

A variety of proprietary systems are available from commercial vendors, but the most rapid deployment of electronic document distribution has been by systems on the Internet using software based on open standards. This report describes some of the experiences of the Distributed Systems Engineering Group has had employing this software.

\section{1,494}

PB96-131578 PC A03/MF AO1

National Inst. of Standards and Technology (CSL), Gaithersburg, MD. Information Access and User Interface Div.

Electronic Access to Standards on the Information Highway.

S. J. Laskowski, and V. V. Ramayya. Aug 95, 18p,

NISTIR-5708

See also PB95-103719. Prepared in cooperation with National Standards Systems Network Project, Arlington, $V A$.

Keywords: "Accessibility, "On-line systems, *Standards, Information systems, Computer networks, Technical assistance, User needs, Information needs, Information retrieval, Information technology, Telecommunication, "National Information Infrastructure, *Information highways, NSSN(National Standards Systems Network), Internet, World wide web, Virtual libraries, System architecture.

We discuss how the information highay can be used to support electronic access standards and standards information. We then show how a network of collections of standards and standards information based on an Internet architecture can be viewed as a 'virtual library of standards' by the users, while allowing standards providers to control and maintain the ownership of these collections on a set of heterogeneous databases. Next, we focus on how the needs of users and developers can be supported through search for standards via a common user interface. We conclude with some thoughts on support for standards development on the information highway and other related issues.

\section{1,495}

PB96-138607 Not available NTIS

National Inst. of Standards and Technology (PL), Boulder, CO. Time and Frequency Div.

High Resolution Time Interval Counter.

Final rept.

V. S. Zhang, D. D. Davis, and M. A. Lombardi. 1994 $10 \mathrm{p}$.

See also DE95009972, and N95-32331 (available as N95-32319)

Pub. in Annual Precise Time and Time Interval (PTTI) Applications and Planning Meeting (26th), Reston, VA., December 6-8, 1995, p191-200 1994.

Keywords: "Time interval analyzers, "Modems, Time log, Frequency measurement, Frequency analysis High resolution, Counters, Reprints.

In recent years, we have developed two types or high resolution, multi-channel time interval counters. In the NIST two-way time transfer MODEM application, the counter is designed for operating primarily in the interrupt-driven mode, with 3 start channels and 3 stop channels. The intended start and stop signals are 1 PPS, although other frequencies can also be applied to start and stop the count. The time interval counters used in the NIST Frequency Measurement and Analysis System are implemented with 7 start channels and 7 stop channels. Four of the 7 start channels are devoted to the frequencies of $1 \mathrm{MHz}, 5 \mathrm{MHz}$ or $10 \mathrm{MHz}$ while triggering signals to all other start and stop channels can arrange from 1 PPS to $100 \mathrm{kHz}$. Time interval interpolation plays a key role in achieving the high resolution time interval measurements for both counters.

\section{1,496}

PB96-139415 PC A03/MF A01

National Inst. of Standards and Technology (NCSL) Gaithersburg, MD. Systems and Network Architecture Div.
Telecommunications Security Guidelines for Telecommunications Management Network. Computer Security

Special pub.

J. Kimmins, C. Dinkel, and D. Walters. Oct 95, 46p, $\mathrm{NIST} / \mathrm{SP}-800 / 13$

Also available from Supt. of Docs. as SN003-00303376-6. See also PB95-189445.

Keywords: "Secure communications, "Voice communications, "Guidelines, Communication networks, Threats, Access control, Confidentiality, Vulnerability, Life cycles, Operation and maintenance, Integrity, Requirements, Telecommunication, "PSN(Public Switched Network), "Public Switched Network, Open systems architecture.

The Public Switched Network (PSN) provides critical commercial telecommunications services and National Security and Emergency Preparedness (NSEP). Telecommunication Service providers, equipment manufacturers, users, and the Federal Government are concerned that vulnerabilities in the PSN could be exploited and result in disruptions or degradation of service. This guideline focuses on two specific components of a Telecommunications Management Network (TMN) Network Elements (NEs) and Mediation Devices (MDs) - with emphasis on the security features needed to protect the Operations, Administration, Maintenance, and Provisioning (OAM\&P) of these components. This document is intended to provide a security baseline for NEs and MDs that is based on commercial security needs. In addition, some National Security and Emergency Preparedness (NS/EP) security requirements will be integrated into the baseline to address specific network security needs. The guideline should assist telecommunications vendors in developing systems and service providers in implementing systems with appropriate security for integration into the PSN.

\section{1,497}

PB96-141320 Not available NTIS

National Inst. of Standards and Technology (EEEL), Gaithersburg, MD. Electricity Div.

Summary Report on the Workshop on Advanced Digital Video in the National Information Infrastructure.

Final rept.

C. Fenimore, B. Field, H. Frank, G. Reitmeier, W.

Stackhouse, C. Van Degrift, E. Georg, and M.

Papillo. 1995, 5p.

See also PB95-103677 and PB96-119367.

Pub. in Society of Motion Picture and Television Engineers, Inc., v104 n3 p148-152 Mar 95.

Keywords: "Digital data, "Video data, "Standards, "Telecommunication, Communication systems, High definition, Television systems, Image processing Video compression, Interactive systems, Information services, Reprints, National Information Infrastructure.

The development of a National Information Infrastructure (NII) is a way of putting vast amounts of information at the fingertips of users in America and around the world. Digital video is likely to be the most technically demanding NII service. Recognizing this, several industrial and governmental organizations sponsored a recent workshop to define a vision of the role of digital video in the NII; identify the architectural, scaling, and performance issues in realizing this vision; and recommend the research, experiments, and other steps to be taken to resolve these issues. It was the sense of the participant in the workshop that the Grand Alliance proposal for HDTV is the best available alternative for terrestrial broadcast of HDTV in the U.S

\section{1,498}

PB96-160452 Not available NTIS

National Inst. of Standards and Technology (CSL), Gaithersburg, MD. Computer Security Div.

Comparison of FDDI Asynchronous Mode and DQDB Queue Arbitrated Mode Data Transmission for Metropolitan Area Network Applications.

Final rept.

W. E. Burr, S. Wakid, D. Vaman, and X. Qian. 1994 $12 \mathrm{p}$

Pub. in Institute of Electrical and Electronics Engineers Workshop on Metropolitan Area Networks (5th), Taormina, Italy, May 12, 1992, $12 \mathrm{p}$ Apr 94.

Keywords: "Wide area networks, "Computer networks, * Data transmission, Packet transmission, Network Data transmission, Packet transmission, Network ance evaluation, Communication networks, Protocols, Mathematical models, Reprints, DQDB(Distributed Queve Dual Bus), FDDI(Fiber Distributed Data Interface)
The performance of the FDDI token ring and IEEE 802.6 DQDB protocols are compared using discrete event simulation models. A Metropolitan Aera Network (MAN) of $100 \mathrm{~km}$ and with 50 stations was moldeled. A 100 Mbps channel is used for both networks, with a traffic model with large (1 kbyte) low priority packets and smaller (100 byte) high priority packets. The delay and fairness characteristics of both networks are analyzed. The simulations show that FDDI has advantages in fairness and maximum capacity, while DQDB offers lower delay at all except very heavy loads and has a stronger priority mechanism.

\section{1,499}

PB96-160460 Not available NTIS

National Inst. of Standards and Technology (CSL), Gaithersburg, MD. Advanced Systems Div.

Standardization for ATM and Related B-ISDN Technologies.

Final rept.

D. Cypher, and S. Wakid. 1993, 14p.

Pub. in Association for Computing Machinery (ACM) Standard Review, pt-14 Sep 93.

Keywords: "Computer networks *Interfaces, *Standards, Communication networks, Protocols, User needs, Wide area networks, Local area networks, Operation and maintenance, Compatibility, Telecommunication, Reprints, ATM(Asychronous Trasfer Mode), BISDN(Broad Integrated Services Digital Network), Interconnections.

The Computer and Telecommunication industries are finally converging towards one single communication technology that permits transparency between the Local and Wide Area networks. This Asynchronous Transfer Mode (ATM) enables the remote interconnection of Local Area Networks (LANs) with compatibly switched telecommunication services rather than expensive private lines. ATM provides scalable high bandwidths, therefore promoting applications in imagery, visualization, video, and others; however, its key value is in shielding the dependency of protocols on media thus providing a seamless shell for interfacing and upgrading current communication gear. In, addition, users are able to dynamically allocate bandwidth on demand, use one unified addressing scheme, and obtain a spectrum of integrated services.

01,500

PB96-160551 Not available NTIS

National Inst. of Standards and Technology (CSL),

Gaithersburg, MD. Advanced Systems Div.

Hardware Measurement Techniques for HighSpeed Networks.

Final rept.

A. Mink, Y. Fouquet, and S. Wakid. 1994, 21p.

Pub. in Institute of Electrical and Electronics Engineers Summer Topical Meeting, Santa Barbara, CA., July 2830, 1993, Jnl. of High-Speed Networks, v3 p187-207 1994.

Keywords: "Computer performance evaluation, "Very large scale integration, "Data flow analysis, "Network analysis, Computer networks, Computer systems hardware, Computer architecture, High speed, Applications programs(Computers), Bandwidth, Standardization, Protocols, Kernel functions, Reprints, Ethernet.

The utilization of the inherent bandwidth in high-speed networks is often obstructed by implementation of the protocols. NIST and ARPA developed VLSI measurement components that permit researchers and product developers to better understand computing and communication bottlenecks by accurate measurement techniques. To illustrate and promote the use of such public domain components, this paper uses image transfer as an example application via a commercial UNIX (BSD 4.3.1 variant) implementation of the TCP IP protocol over an Ethernet (10 Mbits/s), and a HIPPI ( $800 \mathrm{Mbits} / \mathrm{s}$ ) medium. The only modification done to this commercially available kernel was to add the simple probe code necessary to interface to the NIST Multikron measurement chip. The accurate data obtained for the various aspects of this protocol implementation clearly illustrate the major bottlenecks, provide insight with regard to scaling, and determine upper bounds on performance.

01,501

PB96-160635 Not available NTIS

National Inst. of Standards and Technology (CSL), Gaithersburg, MD. Advanced Systems Div. 


\section{Provision of Isochronous Service on IEEE 802.6.} Final rept.

X. Qian, S. Kumar, D. Vaman, S. Wakid, and D.

Cypher. 1994, 7p

Pub. in Institute of Electrical and Electronics Engineers Transactions on Communications, $7 p$ Apr 94

Keywords: "Circuit interconnections, "Communication networks, "Data links, Data transmission, Message processing, Channel capacity, Communications traffic, Signal transmission, Packet switching, Bandwidth, Reprints, Integrated Services Digital Network.

Ths paper concentrates on the provision of isochronous services to users via the existing IEEE 802.6 framework using $\mathrm{q} .931$ as the signalling protocol for providing call control functions. We present possible scenarios of isochronous service provisioning via the IEEE 802.6 network. Real time performance for cal setup procedures is simulated and analyzed with different priority schemes for transferring signalling messages. Call setup delays are determined using both Poisson-type and bursty type data traffic models.

01,502
PB96-160767 Not available NTIS

National Inst. of Standards and Technology (CSL), Gaithersburg, MD. Advanced Systems Div.

ISDN in North America.

Final rept.

S. Wakid, and D. Stokesberry. 1993, 13p.

Pub. in Institute of Electrical and Electronic Engineers Communications Magazine, 13p May 93.

Keywords: "Communication networks, "Standards, Digital communications, Agreements, Telecommunication, North America, Reprints, "ISDN(Integrated Services Digital Network), "Integrated Services Digital Network, FIPS(Federal Processing Standards).

This paper is an overview of the progression of Integrated Services Digital Networks (ISDN) in North America with a focus on the activities of the North American ISDN Users' Forum (NIUF), a consortium of users, service providers, network equipment manufac turers, customer premises equipment manufacturer, and applications software developers. This article discusses the role of the NIUF in the product developmen cycle, provides an overview of its activities and briefly discusses other important and complementary ISDN work. It outlines the beginning deployment of a natioan ISDN and a major demonstration of ISDN capability within North America. Finally, it offers the perspective of ISDN as an enabling technology and not as a network that meets all communications requirements for all users.

\section{1,503}

PB96-160775 Not available NTIS

National Inst. of Standards and Technology (CSL) Gaithersburg, MD. Advanced Systems Div.

North American Agreements on ISDN.

Final rept.

S. A. Wakid, and K. Roberts. 1992, $6 p$.

See also PB94-162559 and PB96-160767.

Pub. in Institute of Electrical and Electronics Engineers Communications Magazine, Special Issue, p42-47 Aug 92.

Keywords: "Communication networks, "Standards, Digital communications, Agreements, Telecommunication, North America Reprints, *ISDN(Integrated Services Digital Network), "Integrated Services Digital Network, FIPS(Federal Processing Standards).

This article presents the work of the NIUF and discusses its relationship to the Accredited Standards Committee T1 and other standrards bodies. The important role of the NIUF within the ISDN arena is eviden when considering the Federal Information Processing Standard (FIPS) for ISDN and the Transcontinental ISDN Project (TRIP), both of which are briefly described. Brief statements on the relationship of the ISDN FIPS to the Government Open System Interconnection Profile (GOSIP) and the military standrad on ISDN are included.

\section{1,504}

PB96-161229 Not available NTIS

National Inst. of Standards and Technology (CSL), Gaithersburg, MD

GOSIP Testing Program.

Final rept.

S. M. Radack. $1991,4 \mathrm{p}$.

See also PB94-211455 and PB96-119359.

Pub. in Computer Systems Laboratory Bulletin, $\mathrm{p}-4$ May 91.
Keywords: "Computer communications, "Standards, *Testing, Communication networks, Conformance, Interoperability, Protocols, Specifications, Reprints, "GOSIP(Government Open Systems Interconnection Profile), " Government Open Systems Interconnection Profile, OSI(Open Systems Interconnection), Federal Information Processing Standards.

This bulletin discusses the testing program recently established by the Computer Systems Laboratory for Federal Information Processing Standard (FIPS) 146, Government Open Systems Interconnection Profile (GOSIP).

\section{1,505}

PB96-161245 Not available NTIS

National inst. of Standards and Technology (CSL), Gaithersburg, MD.

Industry/Government Open Systems Specification: The Development of GOSIP Version 3.

Final rept.

S. M. Radack. 1993, 2p

See also PB94-219110.

Pub. in Federation of Facts, Quarterly Publication, p3,6 1993.

Keywords: *Specifications, *Government/industry relations, Computer networks, Cornmunication networks, Data transmission, Interoperability, Standards, Protocols Testing, Reprints, *GOSIP(Government Open Systems Interconnection Profile), "Government Open Systems Interconnection Profile, OSI(Open Systems Interconnection), IGOSS(Industry/Government Open Systems Specification).

Open Systems Interconnection (OSI) standards describe in a general way how data communications should take place in an open network, and include many options and undefined conditions. The development of products that implement OSI standards has been slow. It has been necessary to define the options, subsets, parameters and other conditions that are precisely specified in the standards, and to get agreements among the implementors to implement in a compatible way. Conformance and interoperability testing are needed to assure that different implementations work together, Different profiles, or groupings, of the OSI standards have been developed by different organizations to carry out similar functions. This condition also may be slowing down the development of OSIcompliant products.

\section{1,506}

\section{PB96-172325 PC A05/MF A01}

National Inst. of Standards and Technology (NCSL), Gaithersburg, MD. Systems and Network Architecture

Guidelines for the Evaluation of Electronic Data Interchange Products.

Special pub.

J. J. Garguilo, and P. Markovitz. Feb 96, 63p, NIST/ SP-500/231.

Also available from Supt. of Docs. as SN003-00303382-1.

Keywords: *Information systems, *Telecommunication, "Commerce, Standards, Procurement, Computer networks, Information exchange, Performance evaluation, Industries, "EDI(Electronic Data Interchange), "Electronic Data Exchange, Electronic commerce.

Electronic Data Interchange (EDI) is defined as the computer-to-computer exchange of standardized business information. As with most software products, EDI products can differ greatly. The host of options potentially present in an EDI product can make purchasing the 'right' one a difficult task. This document assists the reader in determining which EDI product, among many candidate products, best meets the reader's requirements. Specifically, this document addresses: (1) EDI product fuctionality, (2) EDI product performance and (3) the integration of EDI products into the business process.

\section{1,507}

PB96-176672 Not available NTIS

National Inst. of Standards and Technology (CSL), Gaithersburg, MD. Advanced Network Technologies Div.

Strategy to Support Multipoint Communication Service Over Native ATM Service.

Final rept.

S. Y. Kim. 1996, 4p.

Pub. in Institute of Electrical and Electronics Engineers Southeastcon '96, Tampa, FL., April 11-14, 1996, p14.
Keywords: "Protocols, Communication networks, Telecommunication, Standards, Reprints, "Multipoint communication, "ATM(Asynchronous Transfer Mode), *Asynchronous Transfer Mode, Adaptation layer, Functionality.

This paper presents the simplified Multipoint Communication Service Protocol on the adaptation layer with multicast functionality over ATM. This protocol structure makes the adaptation layer take over the domain management function of the MCS protocol as the adaptation layer can do this function without difficulty by its own multicast functionality to support multimedia teleconferencing. As both the multicast functionality and the domain management function of the MCS layer combine into the adaptation layer over ATM, the authors can get more efficient multipoint communication without duplication of functions.

01,508

PB96-183165 PC A05/MF A0

National Inst. of Standards and Technology (CSL), Gaithersburg, MD.

Distributed Communication Methods and RoleBased Access Control for Use in Health Care Applications.

J. Poole, J. Barkley, K. Brady, A. Cincotta, and W. Salamon. Apr 96, 66p, NISTIR-5820.

Keywords: "Software tools, "Access control, "Health care, Distributed, Security, CORBA, OLE, RBAC, Role-based.

The use of software in the health care industry is becoming of increasing importance. One of the major roadblocks to efficient health care is the fact that important information is distributed across many sites. These sites can be located across a significant area. The problem is to provide a uniform mechanism to integrate this information. This paper documents the results of an investigation into the suitability of several different distributed access mechanisms. Five methods were examined; the Common Object Request Broke (CORBA), Object Linking and Embedding (OLE), remote procedure call (RPC), remote database access (SQLRDA) and Protocol independent Interfaces (PII, the authors specifically examined sockets). These mechanisms were compared with regard for use in health care applications. In particular, the following capabilities were compared: Ease of use by the developer. Class of applications for which the technology is particularly effective in developing, Security capabilities.

\section{1,509}

PB96-190111 Not available NTIS

National Inst. of Standards and Technology (PL), Boulder, CO. Time and Frequency Div.

Wavelet Variance, Allan Variance, and Leakage. Final rept

D. A. Howe, and D. B. Percival. 1995, 4p.

Pub in Proceedings of the Institute of Electrical and Electronics Engineers Transactions on Instrumentation, v44 n2 p94-97 Apr 95.

Keywords: *Clock, "Waves, "Time series analysis, Synchronism, Time measurement, Frequencies, Standards, Phase meters, Variance(Statistics), Reprints, Wavelet transform.

Wavelets have recently been a subject of great interest in geophysics, mathernatics and signal processing The discrete wavelet transform can be used to decompose a time series with respect to a set of basis functions, each one which is associated with a particular scale. The properties of a time series at different scales can then be summarized by the wavelet variance, which decomposes the variance corresponding to some of the recently discovered wavelets can provide a more accurate conversion between the time and frequency domains than can be accomplished using the Allan variance. This increase in accuracy is due to the fact that these wavelet variances give better protection against leakage than does the Allan variance.

01,510

PB96-190202 Not available NTIS

National Inst. of Standards and Technology (PL), Boulder, CO. Time and Frequency Div.

New 5 and $10 \mathrm{MHz}$ High Isolation Distribution Amplifier.

Final rept.

C. W. Nelson, F. L. Walls, M. Sicarrdi, and A. De Marchi. 1994, 5p.

Pub. in Proceedings of the Institute of Electrical and Electronics Engineers Intemational Frequency Control Symposium, Boston, MA., June 1-3, 1994, p567-571. 
Keywords: *Distributed amplifiers, "Amplifier design, Noise, Phase control, Frequency control, Temperature, Coefficients, Reprints, "Isolation amplifiers,
Phase noise.

Increasing performance demands made by precision timing have made NIST's present $5 \mathrm{MHz}$ distribution amplifier system obsolete. A new design providing improved phase stability with temperature, harmonic punity, and phase noise is presented. By building on previous designs, a modified cascode amplifier was created with performance increases of more than 10 fold in phase noise, temperature coefficient and isolation.

\section{1,511}

PB96-201124 Not available NTIS

National inst. of Standards and Technology (EEEL), Boulder, CO. Optoelectronics Div.

Precise Laser-Based Measurements of Zero-Dispersion Wavelength in Single-Mode Fibers.

Final rept.

J. B. Schlager, S. E. Mechels, and D. L. Franzen.

1996, $2 \mathrm{p}$.

Pub. in Conference on Optical Fiber Communication (OFC '96), San Jose, CA., February 25-March 1, 1996, Technical Digest Series, v2 p293-294.

Keywords: "Optical communication, "Optical fibers, *Optical detectors, "Standards, Infrared detectors, Wave dispersion, Frequency response, Fiber optics, Wavelengths, Tunable lasers, Reprints.

The transmission performance of ultralong-distance optical communication systems critically depends on fiber chromatic dispersion. To achieve desired performance, system designers require knowledge of the zero-dispersion wavelength, lambda sub 0 to within 0.1 $\mathrm{nm}$. The authors have developed two laser-based systems to measure lambda sub 0 of long optical fibers. Both systems are based on tunable narrow-line lase sources with output wavelengths that can be accurately measured with an interferometric wavemeter. The first system uses the phase-shift method to measure group delay in the fiber sample as a function of wavelength. The second system directly measures the wavelength-averaged differential group delay or fiber chromatic dispersion by dithering the laser wavelength in a new implementation of the differential phase-shift technique. Both systems give lambda sub zero with a precision (repeatability) of $0.022 \mathrm{~nm}$ or less at the $99 \%$ confidence level.

\section{1,512}

\section{PB97-110498 Not available NTIS}

National Inst. of Standards and Technology (CSL), Gaithersburg, MD.

Introduction to Secure Telephone Terminals.

Final rept.

D. Branstad. 1992, 6p.

Pub. in CSL Bulletin, p1-6 Mar 92.

Keywords: "Telephone systems, *Data encryption, "Secure communications, Telecommunications, Computer security, Cryptography, Data processing security, Standards, Reprints.

The bulletin addresses several frequently asked questions about secure telephone terminals, discusses practical security issues from a federal user's viewpoint, and provides sources for additional information. A secure telephone terminal is a device that connects to a telephone line or a cellular telephone system and provides a variety of security services to the conversation of information being transmitted.

\section{1,513}

PB97-112395 Not available NTIS

National Inst. of Standards and Technology (CSL), Gaithersburg, MD.

Using Technology to Manage and Protect Intellectual Property.

Final rept.

R. J. Linn. 1996, 17p.

Pub. in Proceedings of the IMA/Copyright Office Forum on Technology-Based Intellectual Property Management, Reston, VA., March 7, 1996, p1-17.

Keywords: "Intellectual property, "Property rights, *Protection, Computer networks, Inforamtion technology, Internet, Digital systems, Information services, Encryption, Access control, Authentication, Copyrights, Systems management, Legal aspects, Reprints, Information infrastructure, Digital libranies, Digital signature.

Digital libraries and related information technologies are the fruits of recent research and development and are enabling the management, dissemination, and protection of intellectual property by means of a networkbased information infrastructure. Presented is a dis cussion of several technologies whose convergence enables new applications which can serve as the foundations for a digital information infrastructure and enable new modes of electronic commerce. The goal of the report is to help those unfamiliar with information technologies better understand the potential of technology to manage, disseminate, and protect informa tion and intellectual property in a new, networked world.

\section{1,514}

PB97-122287 Not available NTIS

National Inst. of Standards and Technology (CSL) Gaithersburg, MD. Information Access and User Inter face Div.

Usability Engineering: Industry-Government Collaboration for System Effectiveness and Efficiency.

Final rept.

L. L. Downey, S. J. Laskowski, E. A. Buie, and H. R. Hartson. 1996, 2p.

Pub. in SIGCHI Bulletin, v28 n4 p66-67 Oct 96.

Keywords: *Systems engineering, *System effectiveness, "Efficiency, "Usability, Information systems, Computer networks, User needs, User requirements, Government/industry relations, Reprints.

The report summarizes the activities of Usability Engineering: Industry-Government Collaboration for System Effectiveness and Efficiency, a symposium held February 26, 1996, at the National Institute of Standards and Technology (NIST) in Gaithersburg, Maryland.

\section{Communication \& Information Theory}

01,515

PB95-125753 Not available NTIS

National Inst. of Standards and Technology (NEL) Gaithersburg, MD. Statistical Engineering Div

Capacity of the Lp Norm-Constrained Poisson Channel.

Final rept.

M. R. Frey. 1992, 6p.

Pub. in Institute of Electrical and Electronics Engineers Transactions on information Theory 38, n2 p445-450 Mar 92.

Keywords: *Information capacity, "Channel capacity, Norms, Reprints, "Communication channels, Poisson channels, Coding capacity, Channels(Data trans mission).

The information and coding capacities of the Poisson channel are derived with a constraint imposed on the L(sub p) norm (Norm chi (sub i))(sub p) of the encode intensity chi; that is, we fix $1=$ or $<p=$ or $<$ infinity and suppose (Norm chi (sub i))(sub p) $=$ or $<\mathrm{Q}$ for all $t$ (a member of) (in brackets:0,T) and some $Q=$ or > 0 . Formulae are given for the capacity which establish previously known results for $p=1, p=2$, and $p=$ infinity to be part of a system of capacity problems with common form and solution. These formulae apply with or without causal feedback and whether or not the encoder intensity is OOK-constrained.

\section{Graphics}

\section{1,516}

FIPS PUB 148 PC E99

Electronic Industries Association, Washington, DC. Engineering Dept

Procedures for Document Facsimile Transmission ssued by General Services Administration, Apri 14, 1982. Federal Standard 1063.

14 Apr 82, 30p, ElA-RS-466.

Sponsored by General Services Administration, Washington, DC

Keywords: "Facsimile transmission, "Signals, *Interoperability, Facsimile communications, Data transmission, Signal processing, Pitch, Audio tones, Binary codes, Interfaces, Connectors, Terminals, Pro- cedures, Documents, Federal Information Processing Standards, Calls.

This Standard is concerned with the procedures which are necessary for document transmission between two facsimile stations operating on voice band analog circuits. These procedures essentially comprise: call estabishment and call release; compatibility checking, status and control command; checking and supervision of line conditions; control function and facsimile operator recall; and both recognized optional functions as well as other (non-standard) options. In this Standard two separate signalling systems are described; first a simple tonal system using single frequency tones, and second a binary-coded system offering a much wider range of signals for more complex procedures.

\section{Policies, Regulations, \& Studies}

\section{7}

AD-A297 905/2 PC A02/MF A01

National Inst. of Standards and Technology (NML) Washington, DC. Central Radio Propagation Lab. Standard of Attenuation for Microwave Measure-

R. E. Grantham, and J. J. Freeman. 4 Oct $48,8 p$,

CRPL-9-8.

Pub. in Transactions of the American Institute of Electrical Engineers, V67 p1-7 1948.

Keywords: "Microwave equipment, "Standards, Re prints, Measurement, Specifications, Accuracy, Electrical properties, Consistency, Radiation attenuation, Calibration, Direct current, Attenuators.

In line with the program of the National Bureau of Standards for extending the standards of electrical quantities up through microwaves, the establishment of a standard of attenuation was undertaken. For such a standard to be useful, it must be readily adaptable to the calibration of commercial attenuators over the entire frequency range. Also, for self consistency, such a standard must be capable of being checked by $\mathrm{d}$ $c$ measurements, thus relating the radiofrequency ( $r$ f) standard to the more accurate primary standards. A standard of any physical quantity consists not merely in the unique specification of that particular quantity but equally important, in the specification of the operations, or procedures, through which an unknown quantity is measured in terms of it. The purpose of this paper is to describe such a standard, and the preliminary experiments employed in its evaluation and development.

\section{8}

AD-P009 114/0 PC A03/MF A01

National Inst. of Standards and Technology (NML) Boulder, CO. Time and Frequency Div.

Comparison of GPS Broadcast and DMA Precise Ephemerides.

M. A. Weiss, G. Petit, and S. Shattil. 2 Dec $93,14 \mathrm{p}$. This article is from 'Proceedings of the Annual Precise Time and Time Interval (PTTI) Applications and Planning Meeting (25th) Held in Marina Del Rey, California on 29 November - 2 December 1993', AD-A280 955 p293-306.

Keywords: *Ephemerides, Estimates, Perturbations, Phase modulation, Residuals, Time, Global positioning system, Navigation satellites, Kalman filtering, Component Reports, Satellite orbits

We compare the broadcast ephemerides from Global Positioning Satellites (GPS) to the postprocessed ephemerides from the Defense Mapping Agency (DMA). We find significant energy in the spectrum of the residuals at $1 \mathrm{cycle} / \mathrm{day}$ and higher multiples. We estimate the time variance of the residuals and show that the short term residuals, from $15 \mathrm{~min}$, exhibit power law processes with greater low frequency perturbations than white phase modulation. We discuss the significance of these results for the performance of the GPS Kalman filter which estimates the broadcast orbits.

01,519

AD-P009 132/2 PC A02/MF A01

National Inst. of Standards and Technology, Boulder, CO.

NIST Internet Time Service.

J. Levine. 2 Dec 93, $7 p$.

This article is from 'Proceedings of the Annual Precise

Time and Time Interval (PTTI) Applications and Plan- 
ning Meeting (25th) Held in Marina Del Rey, California on 29 November - 2 December 1993', AD-A280 955, p505-511.

Keywords: "Time standards, Calibration, Clocks, Daylight, Formats, Communications networks, Uncertainty, Radio links, Synchronism, Component Reports, internet, US NIST.

We will-describe the NIST Network Time Service which provides time and frequency information over the internet. Our first time server is located in Boulder, Colorado, a second backup server is under construction there, and we plan to install a third server on the Eas Coast later this year. The servers are synchronized to UTC(Universal Coordinated Time)(NIST) with an uncertainty of about $0.8 \mathrm{~ms}$ RMS and they will respond to time requests from any client on the internet in several different formats including the DAYTIME, TIME and NTP protocols. The DAYTIME and TIME protocols are the easiest to use and are suitable for providing time to PCs and other small computers. In addition to UTC(NIST), the DAYTIME message provides advance notice of leap seconds and of the transitions to and from Daylight Saving Time. The Daylight Saving Time notice is based on the US transition dates of the first Sunday in April and the last one in October. The NTP is a more complex protocol that is suitable for large machines; it is normally run as a demon process in the background and can keep the time of the client to within a few milliseconds of UTC (NIST). We will describe the operating principles of various kinds of client software ranging from a simple program that queries the server once and sets the local clock to more complex demon processes (such as NTP) that continuously correct the time of the local clock based on periodic calibrations.

\section{1,520}

\section{PC A02/MF A01}

National Inst. of Standards and Technology, Boulder Co

Future of Time and Frequency Dissemination.

J. Levine. 2 Dec 93, 6p.

This article is from 'Proceedings of the Annual Precise Time and Time Interval (PTTI) Applications and Planning Meeting (25th) Held in Marina Del Rey, California on 29 November - 2 December 1993', AD-A280 955 p573-578.

Keywords: "Frequency standards, "Time standards Bandwidth, Computer networks, Technology forecasting, Communications traffic, "Time transfer, Component Reports.

I will try to extrapolate the changes in the dissemination of time and frequency information that have taken place during the last 25 years to predict the future developments both in the methods of disseminating time and frequency and in the kinds of customers we will be asked to serve. Two important developments are likely to play pivotal roles in driving the evolution of dissemination. The first is the commercial availability of very high quality clocks - devices whose performance may eventually rival that of the current generation of primary frequency standards. The widespread use of these devices may blur the traditional distinction between client and server, and may replace it with a more symmetrical interchange of data among peers. The second is the increasing demand for digital time and frequency information driven by the increasing sophistication of everything from traffic lights to electric power meters. The needs of these individual users may not tax the state of the art of primary frequency standards in principle, but their large numbers and wide geo graphical distribution present a technological challenge that is difficult to meet at a reasonable price using existing methods. Some of these problems may be solved (or at least addressed) using developments in communications and consumer electronics such as the increasing use of fiber-optic telephone circuits and the increasing bandwidth and sophistication of the cable network used to transmit television pictures. To be useful, these advances in hardware must stimulate paralle advances in software algorithms and methods. These advances are more difficult to predict with great confidence, but the developments of the last few years will be examined to provide some indications of the future.

\section{1,521}

\section{N19960042622 (Order as N19960042616, PC}

A20/MF AC

National Inst. of Standards and Technology, Boulder CO.
Utc Dissemination to the Real-Time User.

J. Levine. 1 May 96, 10p.

In National Inst. Of Standards and Technology, 27TH Annual Precise Time and Time Interval (Ptti) Applications and Planning Meeting p 103-112.

Keywords: "Accuracy, "Atomic clocks, *Frequency standards, "International cooperation, "Real time operation, "Statistical analysis, "Time measurement, "Time signals, "Transit time, "Universal time, Annual variations, Cesium, Hyperfine structure, Synchronism.

The current definition of Coordinated Universal Time (UTC) dates from 1972. The duration of a UTC second is defined in terms of the frequency of a hyperfine transition in the ground state of cesium. This standard frequency is realized in a number of different laboratories using ensembles of commercial cesium clocks and a few primary frequency standards. The data from all of these devices are transmitted periodically to the $\mathrm{Bu}$ reau International des Poids et Mesures (BIPM) in Sevres, France, where they are combined in a statistical procedure to produce International Atomic Time (TAI). The time of this scale is adjusted as needed (' $\mathrm{CO}$ ordinated') by adding or dropping integer seconds so as to keep it within plus or minus $0.9 \mathrm{~s}$ of UT1, a time scale based on the observation of the transit times of stars and corrected for the predicted seasonal variations in these observations. When the leap seconds are included into TAI, the result is called UTC. The difference between TAI and UTC is therefore an exact integer number of seconds. This difference is currently $29 \mathrm{~s}$ and will become $30 \mathrm{~s}$ at O UTC on 1 January 1996.

\section{1,522}

N94-30641/2 (Order as N94-30639/6, PC A25/

MF A06)

National Inst. of Standards and Technology, Boulder, CO.

Time and Frequency Technology at NIST.

D. B. Sullivan. May $94,6 \mathrm{p}$.

In NASA Goddard Space Flight Center the 25TH Annual Precise Time and Time Interval (Ptti) Applications and Planning Meeting p 33-38.

Keywords: "Clocks, "Frequency standards, "Standards, "Time measurement, "Timing devices, Laser pumping, Oscillators, Semiconductor lasers, Temporal distribution, Computer networks, Digital systems, Standardization, Telephones, Cesium frequency standards, Rubidium frequency standards, Time transfer.

The state of development of advanced timing systems at NIST is described. The work on cesium and rubidium frequency standards, stored-ion frequency standards, diode lasers used to pump such standards, time transfer, and methods for characterizing clocks, oscillators, and time distribution systems is presented. The emphasis is on NIST-developed technology rather than the general state of the art in this field.

\section{1,523}

N94-30660/2 (Order as N94-30639/6, PC A25 MF A06)

National Inst. of Standards and Technology, Boulder $\mathrm{CO}$

Comparison of GPS Broadcast and DMA Precise Ephemerides.

M. A. Weiss, G. Petit, and S. Shattil. May 94, 14p. In NASA. Goddard Space Flight Center, the 25TH Annual Precise Time and Time Interval (Ptti) Applications and Planning Meeting p 293-306.

Keywords: "Ephemerides, "Global positioning system, "Kalman filters, "Satellite tracking, Autonomous spacecraft clocks, Ground stations, Position (Location), Tracking stations.

We compare the broadcast ephemerides from Global Positioning Satellites (GPS) to the postprocessed ephemerides from the Defense Mapping Agency (DMA). We find significant energy in the spectrum of the residuals at 1 cycle/day and higher multiples. We estimate the time variance of the residuals and show that the short term residuals, from $15 \mathrm{~min}$, exhibit power law processes with greater low frequency perturbations than white phase modulation. We discuss the significance of these results for the perormance of the GPS Kalman filter which estimates the broadcast orbits.

01,524

N94-30684/2 (Order as N94-30639/6, PC A25/

MF A06)

National Inst. of Standards and Technology, Boulder, $\mathrm{CO}$
Future of Time and Frequency Dissemination. J. Levine. May 94, 6p.

In NASA. Goddard Space Flight Center, the 25TH Annual Precise Time and Time Interval (Ptti) Applications and Planning Meeting p 573-578.

Keywords: "Clocks, "Frequency standards, "Time measurement, Technology assessment, Accuracy, Computer networks, Information dissemination, Telecommunication, Trends

I will try to extrapolate the changes in the dissemination of time and frequency information that have taken place during the last 25 years to predict the future developments both in the methods of disseminating time and frequency and in the kinds of customers we will be asked to serve. Two important developments are likely to play pivotal roles in driving the evolution of dissemination. The first is the commercial availability of very high quality clocks -- devices whose performance may eventually rival that of the current generation of primary frequency standards. The widespread use of these devices may blur the traditional distinction between client and server, and may replace it with a more symmetrical interchange of data among peers. The second is the increasing demand for digital time and frequency information driven by the increasing sophistication of everything from traffic lights to electric power meters. The needs of these individual users may not tax the state of the art of primary frequency standards in principle, but their large numbers and wide geographical distribution present a technological challenge that is difficult to meet at a reasonable price using existing methods. Some of these problems may be solved (or at least addressed) using developments in communications and consumer electronics such as the increasing use of fiber-optic telephone circuits and the increasing bandwidth and sophistication of the cable network used to transmit television pictures. To be useful, these advances in hardware must stimulate parallel advances in software algorithms and methods. These advances are more difficult to predict with great confidence, but the developments of the last few years will be examined to provide some indications of the future.

01,525

PB94-172772 Not available NTIS

National Inst. of Standards and Technology (NML) Boulder, CO. Time and Frequency Div.

Time Scale Algorithm for Post-Processing: AT1 Plus Frequency Variance.

Final rept

M. A. Weiss, and T. Weissert. 1990, 1 p.

Pub. in Proceedings of Conference on Precision ElecPromagnetic Measurements, Ottawa, Can

Keywords: "Time measurement, Kalman filters, Reprints, Frequency step detection, Time scale algorithms, Postprocessing.

There are some applications where the output of a time scale is not needed in real time. Such applications can make use of a post-processed time scale for improving long term performance. A post-processed scale can take advantage of improved characterizations of clocks in the scale and improved capability of frequency step detection. The authors study a particular time scale al gorithm run in post-processed mode being studied a the National Institute of Standards and Technology. Results are reported based on both simulation and real data.

01,526

PB94-216017 Not available NTIS

National Inst. of Standards and Technology (PL), Boulder, CO. Time and Frequency Div.

Using LORAN-C Broadcasts for Automated Frequency Calibrations.

Final rept.

M. Lombardi. 1992, $13 p$.

Pub. in Proceedings of National Conference of Stand ards Laboratories Workshop and Symposium Managing Worldwide Measurements, Washington, DC. August 2-6, 1992, p361-373.

Keywords: "Frequency measurement, "LORAN C, "Calibration, Ground wave propagation, Oscillators, Accuracy, Reprints, Path noise

The LORAN-C (LOng RAnge Navigation) system is a radio navigation system maintained by the United States Coast Guard. LORAN-C signals are widely used as a navigation aid. Since the signals are extremely stable, they can also serve as an excellent reference for frequency calibrations. Any quartz, rubid- 
ium, or cesium oscillator can be calibrated with LORAN-C. This paper tells how to use LORAN-C signals for frequency calibrations. It describes the LORAN-C system, lists the equipment that you need, shows the data you can obtain, and discusses the accuracy you can expect. It also discusses the NIST Frequency Measurement System, an automated calibration system that uses LORAN-C signals

01,527
PB94-216066 Not available NTIS

National Inst. of Standards and Technology (PL), Boulder, $\mathrm{CO}$. Time and Frequency Div.

Ultra-High Stability Synthesizer for Diode Laser Pumped Rubidium.

Final rept.

J. Lowe, F. L. Walls, and R. E. Drullinger. 1992, 5p. Pub. in Proceedings of Institute of Electrical and Electronics Engineers Frequency Control Symposium, Hershey, PA., May 27-29, 1992, p183-187.

Keywords: "Rubidium frequency standards, "Frequency standards, "Local oscillators, Frequency stability, Optical pumping, Room temperature, Design, Reprints, Laser diodes.

We describe the design of a synthesized local oscillaor for a rubidium (Rb) passive frequency standard pumped by radiation from a diode laser. The design goals for this new oscillator are: (1) To operate at room temperature, (2) To have the ability to step quickly to different frequencies for measuring parameters such as magnetic fields, (3) To contribute less than $2 \times$ 10 (sup -14)/tau(sup 1/2) to the frequency stability of the $\mathrm{Rb}$ standard for measurement times between 30 and 200 seconds. Potential limits for obtaining these goals, as well as solutions to such problems, are discussed

\section{1,528}

PB́95-108585 Not available NTIS

National Inst. of Standards and Technology (NML), Gaithersburg, MD. Time and Frequency Div.

High-Order Harmonic Mixing with GaAs Schottky Diodes.

Final rept

M. Prevedelli, F. L. Walls, and S. P. Beaton. 1990,

4p.

Pub. in Proceedings of the Annual Symposium on Frenquency Control (44th), Baltimore, MD., May 23 25, 1990, p555-558.

Keywords: "Frequency multipliers, Frequency synthesizers, Frequency control, Laser radiation, Schottky diodes, Planar structures, Extremely high frequency, Far infrared radiation, Gallium arsenides, Klystrons, Reprints, "Harmonic mixing.

In many areas of precision frequency metrology, it is useful to use a single nonlinear element to both mi,ltiply a source frequency and mix this harmonic with higher frequency source. This process is commonly referred to as harmonic mixing. When the frequency span is great, it is advantageous to use large harmonic numbers to reduce the complexity, the number of loca oscillators, and ultimately the cost. Planar GaAs Schottky diodes are good candidates for high order harmonic mixing because of their high cut-off frequency. Their robust construction should lead to excellent lifetime, reliability, and reproducibility. The authors have investigated the performance of one type of fast planar Schottky diode for harmonic mixing with harmonic numbers from 8 to 201. Their measurements in dicate that, with optimum biasing and power, harmonic mixing with harmonic numbers of up to approximately 80 can be achieved with good signal-to-noise ratios. Detectable signals have been observed for harmonic numbers of 201. From the nonlinear characteristics of the diode and the signal-to-noise ratio obtained at lower frequencies, it looks as though we can multiply from $70 \mathrm{GHz}$ to about $4.5 \mathrm{THz}$ in one step.

01,529
PB95-151098 Not available NTIS

National Inst. of Standards and Technology (PL), Boulder, $\mathrm{CO}$. Time and Frequency Div.

Preliminary Comparison of Time Transfers via LASSO, GPS and Two-Way Satellite.

Final rept.

P. Uhrich, R. Tourde, M. Granveaud, C. Veillet, D. Feraudy, J. M. Torre, J. F. Mangin, J. Gaignebet, J. $L$. Hatat, W. Hanson, A. Clements, J. Jespersen, M Lombardi, P. Grudler, and F. Baumont. 1991, 9p. Pub. in Proceedings of European Frequency and Time Forum (5th), Besancon, France, March 12-14, 1992 p96-104.
Keywords: *Global positioning system, "Navigation satellites, "Laser range finders, "Signal transmission "Time signals, Position indicators, Satellite ground support, Time measuring instruments, Timing devices, Rangefinding, Ground stations, Calibration, Accuracy, Synchronism, Precision, Reprints, "Time transfer.

Presently, the three primary techniques for achieving nanosecond time transfer are Common-View GPS. Two-Way Satellite Time Transfer and the Laser Ranging Technique of the LASSO Experiment. Through the cooperative efforts of several international laboratories, all the three methods were implemented at two stations within Europe, CERGA and TUG. At each of these two stations, a common reference clock to al three systems allows the direct intercomparison of these three state-of-the-art techniques. The paper will discuss the operations at these two stations, present the available data and an intercompanison of it.

\section{1,530}

PB95-151445 Not available NTIS

National Inst. of Standards and Technology (PL), Boulder, CO. Time and Frequency Div.

Smart Clock: A New Time.

Final rept.

M. A. Weiss, D. W. Allan, D. D. Davis, and J. Levine. 1992, $4 p$

Pub. in Institute of Electrical and Electronics Engineers Transactions on Instrumentation and Measurement 41, n6 p915-918 Dec 92

Keywords: "Clocks, "Synchronism, *Calibration, Time measuring instruments, Reference standards, Time standards, Time signals, Random walk, Frequency stability, Accuracy, Reprints, "Smart Clock, "Time stability,

The Smart Clock, opens up the possibility for any clock to be automatically synchronized to an external standard with a minimum of measurements. The Smart Clock enhances the accuracy or stability of a clock or oscillator by characterizing it against an external standard. The Smart Clock algorithm uses optimal estimation and prediction to apply a correction to the output of the oscillator, maintaining any combination of time or frequency accuracy or stability within specified limits. The algorithm decides when external measurements are necessary to maintain the desired accuracy or stability. The authors present here a tutorial description of the Smart Clock system and an example of how it could be used to maintain a simple clock to $1 \mathrm{~s}$ using measurements over the telephone lines.

\section{1,531}

PB95-151452 Not available NTIS

National Inst. of Standards and Technology (PL), Boulder, CO. Time and Frequency Div.

Calibration of GPS Equipment in Japan.

Final rept.

M. A. Weiss, and D. D. Davis. 1988, 9p

Pub. in Proceedings of Annual Precise Time and Time Interval (PTTI) Applications and Planning Meeting (20th), Tysons CornerNienna, VA., November 29-December 1, 1988, p101-109.

Keywords: "Japan, "Global positioning system *Calibration "Time measuring instruments, Ground stations, Satellite tracking, Tracking stations, Interlaboratory comparisons, Time standards, Standardization, Time measurement, Precision, Clocks, Diurnal variations, Reprints, "Time transfer.

With the development of common view time comparisons using GPS satellites the Japanese time and frequency standards laboratories have been able to contribute with more weight to the international unification of time under the coordination of the Bureau of International de Poids et Measures (BIPM). During the period from June 1 through June 11, 1988, the differential delays of time transfer receivers of the global Poisitioning System (GPS) were calibrated at three different laboratories in Japan, linking them for absolute time transfer with previously calibrated labs of Europe and North America. The authors report here the results of the calibration trip, along with some interesting problems that developed conceming this technique.

01,532

PB95-151460 Not available NTIS

National Inst. of Standards and Technology (PL), Boulder, CO. Time and Frequency Div.
Confidence on the Second Difference Estimation of Frequency Drift.

Final rept.

M. A. Weiss, D. W. Allan, and D. Howe. 1992, 6p.

Pub. in Proceedings of Institute of Electrical and Elec tronics Engineers Frequency Control Symposium, May 27-29, 1992, p300-305.

Keywords: *Frequency standards, *Frequency stability, Rubidium frequency standards, Frequency modulation, White noise, Simulation, Estimates, Reprints, Frequency drift.

We use simulation to compare the confidence in estimating frequency drift in the presence of stochastic noise between two different estimators: (1) a mean second difference using neighboring data, and (2) a single second difference over the entire data set. In each case we simulate $100 \mathrm{~s}$ data sampling and 10,000 samples ( 11 and $1 / 2$ days of data). The two estimators show a similar confidence when the noise is purely random walk frequency modulation, but when there is also white frequency modulation, as is common in oscillators, method (2) is more efficient. An ad vantage of (1) is that it provides an internal confidence to an estimate on a single data set. As a practical example, when we simulate a typical rubidium gas cell frequency standard the confidence of the drift estimate was ten times better for method (2) over method (1)

01,533

PB95-151478 Not available NTIS

National Inst. of Standards and Technology (PL), Boulder, CO. Time and Frequency Div.

Promise into Practice: Implementing TA2 on Real Clocks at NIST.

Final rept.

M. A. Weiss, and T. Weissert. 1991, 7p.

Pub. in Proceedings of European Frequency and Time

Forum (5th), March 12-14, 1991, p442-448.

Keywords: Global positioning system, Kalman filters Random walk, Stability, Clocks, Reprints, "Time scale algorithms, "TA2 algorithm, Postprocessors, US NIST

TA2 is a time scale algorithm designed to run as a post-processor by smoothing, i.e. combining estimates from runs in both forward and backward temporal directions. It has been tested using the time-scale data at NIST from 1989 through the first half of 1990 . We review the characterization parameters for clocks with white FM, and random-walk FM, and the dependence of these estimates on integration time. We also discuss the transformation of these parameters from their use as time deviations to frequency variances. We com pare the scale generated by TA2 over $541 \mathrm{~d}$ starting at the beginning of 1989 , with the two other scales at NIST, AT 1 and TA.

01,534

PB95-153458 Not available NTIS

National Inst. of Standards and Technology (PL), Boulder, CO. Time and Frequency Div.

NIST-7, the New US Primary Frequency Standard. Final rept.

R. E. Drullinger, J. P. Lowe, D. J. Glaze, and J. Shirley. 1993, 3p.

Pub. in Proceedings of European Frequency and Time Forum (7th), Neuchatel, Switzerland, March 16-18 1993, p549-551 and Proceedings of the Institute for Electrical and Electronics Engineers International Frequency Control Symposium, Salt Lake City, UT., June $2-4,1993$ p $71-74$

Keywords: "Cesium frequency standards, "Frequency standards, "Primary standards, Optical pumping, Frequency stability, Reprints, "NIST-7 standard.

NIST-7, an optically pumped, cesium-beam frequency standard has replaced NBS-6 as the official US primary frequency standard. The present short-term stability of the standard, measured with respect to an ac tive hydrogen maser, is characterized by sigma(sub y)(tau) approx $=8 \times 10(\exp -13$ ) tau(sup -1/2). Our first evaluation has resulted in an uncertainty of $4 \times 10$ (exp -14). An improved servo-electronic system is being developed and this should improve stability and allow for more precise evaluation of the various systematic errors.

01,535

PB95-153482 Not available NTIS

National Inst. of Standards and Technology (PL), Boulder, CO. Time and Frequency Div. 
Error Analysis of the NIST Optically Pumped Primary Frequency Standard.

Final rept.

R. E. Drullinger, J. H. Shirley, J. L. Lowe, and D. J.

Glaze. 1992, 2p.

Pub. in Proceedings of Conference on Precision Electromagnetic Measurements, Paris, France, June 9-12, 1992, p198-199.

Keywords: "Frequency standards, "Primary standards, Optical pumping, Error analysis, Reprints, US NIST.

An error analysis of the NIST optically pumped, primary frequency standard is presented. Where possible, direct, in-situ measurements have been made. Alternatively, electrical measurements have been made on subassemblies during construction. Where measurements are not yet available, model calculations have been applied to the design. The analysis indicates that in the present configuration of the servo, the error budget is dominated by microwave power in stability entering through the second-order Doppler shitt at the level of a few parts in 10(exp 14).

\section{1,536}

PB95-161089 Not available NTIS

National Inst. of Standards and Technology (PL), Boul-

der, CO. Time and Frequency Div.

Satellite Two-Way Time Transfer: Fundamentals and Recent Progress.

Final rept.

D. A. Howe, D. W. Hanson, J. L. Jespersen, and M.

A. Lombardi. $1988,12 p$.

Pub. in Proceedings of Precise Time and Time Interval Conference, Redondo Beach, CA., November 28-30, 1988, p117-128.

Keywords: "Synchronous satellites, "Ground stations, "Calibration, "Signal processing, Synchronism, Signal reception, Signal transmission, Time signals, Synchronous platforms, Time measurement, Time standards, Accuracy, Reprints, "Time transfer.

The authors discuss fundamental aspects of two-way timing and show an implementation of a satellite twoway time transfer system which has been used for twoyears between USNO, Washington, D.C. and NIST. Boulder, $\mathrm{CO}$. The raw data collection procedure will be discussed. The authors also outline the rationale for the choice of satellite uplink/downlink frequencies, signal structure, and reduction of data. Short-term noise in the time transfer limits the precision to about 300 ps in a $300 \mathrm{~s}$ average. Uncertainty in accuracy is due to uncertainty in the non-reciprocity of the two-way signal path. Accuracy limits due to the atmosphere, earthsatellite rotating system (Sagnac effect), and the equipment are discussed. The goal is to achieve an accuracy level of $1 \mathrm{~ns}$ after a suitable calibration of earth-station (differential) equipment delays.

\section{O1,537
PB95-161410 Not available NTIS}

National Inst. of Standards and Technology (PL), Boulder, CO. Time and Frequency Div.

Keeping Time on Your PC.

Final rept.

M. A. Lombardi. 1993, 4p.

Pub. in Byte Magazine, p57-62 Oct 93

Keywords: "Clocks, "Personal computers, "Calibration, Time signals, Time measurement, Timing devices, Synchronism, Accuracy, ACTS, GOES satellites, Computer networks, Reprints, Smart Clock, World Wide Web.

A summary of the differential methods that can be used to set computer clocks on time. It describes how the clock inside a PC works, how computer clocks can be set by modem or through the Internet, and discusses the NIST Smart Clock Technology. It also discusses how computer time can be obtained by radio or through the use of an add-on clock board.

\section{1,538}

\section{PB95-163218 Not available NTIS}

National Inst. of Standards and Technology (PL), Boulder, CO. Time and Frequency Div.

Diode-Laser Pumped, Rubidium Cell Frequency Siandards.

Final rept.

C. Szekely, R. E. Drullinger, F. L. Walls, J. P. Lowe,

and A. Novick. 1993, 5 .

$\mathrm{Pub}$. in Proceedings of European Frequency and Time Forum (7th), Neuchatel, Switzerland, March 16-18, 1993, p593-597.

Keywords: "Rubidium frequency standards, *Frequency standards, Semiconductor lasers, Fre- quency stability, Optical pumping, Reprints, Phase noise.

We report on our project to experimentally investigate the limits to the short-term stability achievable in optically pumped, rubidium cell frequency standards. Theory indicates that the atomic limited stability is several orders of magnitude better than what has actually been achieved. The difference is related to the complex spectral output of the lamps used in conventional standards as well as phase noise in the interrogating microwave radiation. Using a novel filter technique, we have built a microwave synthesis chain with phase noise capable of supporting a standard with (sigma(sub y))(tau) $=$ or $<4 \times 10(\exp -14)$ tau(sup -1/ 2). With extended-cavity, diode lasers we have demonstrated measured line $Q$ and signal-to-noise ratios commensurate with (sigma(sub y)) (tau) $=4 \times 10(\exp$ -13) tau(sup -1/2) in a commercial, buffer gas type standard and (sigma(sub y))(tau) $=2 \times 10(\exp -13)$ tau(sup -1/2) in an evacuated, wall-coated cell. Both of these numbers can be improved significantly in a fully engineered and optimized standard.

\section{1,539}

PB95-163838 Not available NTIS

National Inst. of Standards and Technology (PL), Boulder, CO. Time and Frequency Div.

Confidence on the Three-Point Estimator of Frequency Drift.

Final rept

M. A. Weiss, and C. Hackman. 1993, 8p.

Pub. in Proceedings of Annual Precise Time and Time Interval (PTTI) Applications and Planning Meeting (24th), McLean, VA., December 1-3, 1992, p451-458 1993.

Keywords: "Frequency standards, "Atomic clocks, Estimators, Uncertainty, Reprints, "Frequency drift, Allan variance.

It has been shown that a three-point second difference estimator is nearly optimal for estimating frequency drift in many common atomic oscillators. We derive a formula for the uncertainty of this estimate as a function of the integration time and of the Allan variance associated with this integration time.

\section{1,540}

PB95-163853 Not available NTIS

National Inst. of Standards and Technology (NML) Gaithersburg, MD. Time and Frequency Div. Use of lonospheric Data in GPS Time Transfer. Final rept.

M. Weiss, T. Weissert, C. Thomas, M. Imae, and K. Davies. $1990,7 p$

Pub. in Proceedings of European Frequency and Time Forum (4th), Neuchatel, March 13-15, 1990, p327-333.

Keywords: *Ionospherics, "Global positioning system, "Error analysis, "Delay time, Synchronism, Data smoothing, Periodic variations, Electromagnetic noise, Time standards, Frequency standards, Time measuring instruments, Interlaboratory comparisons, Calibrating, Accuracy, Reprints, "Time transfer, Ionospheric delay.

With solar activity near maximum, the largest error in the use of the Global Positioning System (GPS) for common-view time transfer can be the correction for ionospheric delay. The paper describes the use of data from two different codeless ionospheric measurement systems to correct common-view time transfer measurements. The authors compare the measured ionospheric values with those from the ionospheric values with those from the ionspheric model parameters broadcast from the GPS satellites, computer time transfer values among three time standards laboratories: Observatoir de Paris, Paris, France; National Institute of Standards and Technology, Boulder. Colorado, USA; and Communications Research Laboratory, Tokyo, Japan. Combining these time transfers the authors can obtain closure around the world. The authors make another comparison between these results and ionospheric measurements using a Faraday rotation system.

\section{1,541}

PB95-180501

Not available NTIS

National Inst. of Standards and Technology (PL), Boulder, CO. Time and Frequency Div.
Microwave Leakage as a Source of Frequency Error and Long-Term Instability in Cesium AtomicBeam Frequency Standards.

Final rept.

W. D. Lee, J. P. Lowe, J. H. Shirley, and R. E

Drullinger. $1994,4 p$

Pub. in Proceedings of European Frequency and Time Forum (8th), Weihenstephan, Germany, March 9-11, 1994, p513-516.

Keywords: "Cesium frequency standards, "Frequency standards, Frequency stability, Microwave radiation, Instability, Reprints, Microwave leakage, Systematic errors.

We identify leakage from the microwave electronics in cesium atomic-beam frequency standards as a potential source of frequency error and instability in these standards. The problem arises when radiation that is phase coherent with the interrogating radiation finds its way to the atoms in regions outside the Ramsey cavity. We discuss techniques for evaluating and reducing the problem.

01,542

PB95-180519 Not available NTIS

National Inst. of Standards and Technology (PL), Boulder, CO. Time and Frequency Div.

Velocity Distribution of Atomic Beams by Gated Optical Pumping.

Final rept.

W. D. Lee, J. H. Shirley, and R. E. Drullinger. 1994,

Pub. in Proceedings of Institute of Electrical and Electronics Engineers International Frequency Control Symposium, Boston, MA., June 1-3, 1994, p658-661.

Keywords: "Cesium frequency standards, "Frequency standards, "Atomic beams, "Velocity distribution, Velocity measurement, Optical pumping, Doppler effect, Accuracy, Reprints, "NIST-7 frequency standard, US NIST.

In evaluating the accuracy of a cesium-beam frequency standard, accurate measurement of the atomic velocity distribution is important. In frequency stand ards which employ atoms with thermal velocities, the measured atomic resonance frequency differs from the true resonance by several parts in $10(\exp 13)$ due to the second-order Doppler shift. To achieve the frequency accuracy goal for NIST-7 of 1 . 10(exp-14), an upper bound on the uncertainty of the mean-square atomic velocity of about 1 percent is needed. We present the results of experiments designed to measure the velocity distribution of NIST-7 using two independent techniques: gated optical pumping, and Ramsey fringe inversion. We show that these techniques yield velocity distributions and corresponding second-order Doppler shifts that agree within the stated tolerances.

01,543

PB95-180535 Not available NTIS

National Inst. of Standards and Technology (PL), Boulder, CO. Time and Frequency Div.

Cross-Correlation Analysis Improves Time Domain Measurements.

Final rept.

A. Lepek, and F. L. Walls. 1993, 9p.

Pub. in Proceedings of Institute of Electrical and Electronics Engineers International Frequency Control Symposium, Salt Lake City, UT., June 2-4, 1993, p312320.

Keywords: Frequency stability, Cross correlation, Time domain, Reprints, "Allan variance.

We introduce cross correlation-based variances to estimate the Allan variance, the modified Allan variance, and the time variance in the presence of the measurement system noise. These variances substantially lower the short-term measurement noise floor: however, they require significantly more data. The cross correlation based variances are also used as a tool to analyze and improve the measurement system noise. The cross correlation based variances bear a precise relation to the 3-cornered-hat sigma. We have reduced the short-term noise floor for sigma(sub y) (1 s) by factor of 100 relative to our initial hardware and software configuration

01,544

PB95-180618 Not available NTIS

National Inst. of Standards and Technology (PL), Boulder, CO. Time and Frequency Div. 
Hybrid Digital/Analog Servo for the NIST-7 Frequency Standard.

Final rept.

J. P. Lowe, W. D. Lee, F. L. Walls, and R. E.

Drullinger. 1994, 4p.

Pub. in Proceedings of Institute of Electrical and Electronics Engineers International Frequency Control Symposium, Boston, MA., June 1-3, 1994, p662-665.

Keywords: " $\mathrm{Cesium}$ frequency standards, "Frequency standards, Hybrid systems, Servomechanisms, Digital systems, Analog systems, Design, Stability, Reprints, "NIST-7 frequency standard, US NIST.

This paper describes a hybrid (analog/digital) approach to the servo electronics for the NIST-7 cesiumbeam, frequency standard. A digital servo system has been added to the existing analog system to enhance the capabilities of both servos. The analog servo allows a $5 \mathrm{MHz}$ oscillator to be frequency locked directly to the atomic transition frequency. The digital servo reduces the frequency perturbations due to changes in the ambient magnetic field. Also, the existence of separate servo systems allows independent measurements of systematic errors. Design specifications are given and stability measurement results are presented.

01,545

PB95-180972 Not available NTIS

National Inst. of Standards and Technology (PL), Boulder, CO. Time and Frequency Div.

Reducing the Effect of Local Oscillator Phase Noise on the Frequency Stability of Passive Frequency Standards.

Final rept.

C. Szekely, F. L. Walls, J. P. Lowe, R. E. Drullinger, and A. Novick. 1993, 6p.

Pub. in Proceedings of Institute of Electrical and Electronics Engineers International Frequency Control Symposium, Salt Lake City, UT., June 2-4, 1993, p8186.

Keywords: "Frequency standards, "Frequency stability, Rubidium, Frequency standards, Notch filters, Local oscillators, Performance tests, Reduction, Reprints, Phase noise.

We report on the experimental test of a new concept for reducing limitation on short-term frequency stability of passive frequency standards due to local oscillator phase noise. Systems that use sinewave modulation to interrogate a stable resonance are limited in shortterm frequency stability by phase noise at the second harmonic of the modulation, $f(m)$. This effect limits the fractional frequency stability. This new concept uses notch filters at + or $-2 f(m)$ from the carrier to reduce this effect. Tests on a modified passive rubidium standard demonstrate an improvement of approximately 18 in fractional frequency stability. The dual notch filters proved to be feasible and were obtained commercially.

\section{1,546}

PB95-181129 Not available NTIS

National Inst. of Standards and Technology (PL), Boulder, CO. Time and Frequency Div.

Practical Standards for PM and AM Noise at 5, 10 and $100 \mathrm{MHz}$

Final rept

F. L. Walls. 1993, 10p

Pub. in Proceedings of European Frequency and Time Forum (7th), Neuchatel, Switzerland, March 16-18, 1993, p189-198.

Keywords: *Amplitude modulation, "Phase modulation, "Standards, Very high frequency, High frequency, Carnerfrequencies, Reprints, "Noise standards.

This paper describes a practical implementation of secondary standards for phase modulation noise (PM) and amplitude modulation noise (AM) at carrier frequencies of $5 \mathrm{MHz}$, and $100 \mathrm{MHz}$. In each case the standard simultaneously produces output signals at approximately $+15+17 \mathrm{dBm}$. The PM and AM noise between the two signals can be set to a calibrated level, which is constant to better than $0.2 \mathrm{~dB}$ for Fourier frequency offsets from nearly dc to $5 \%$ of the carrier. or set ot a minimum value. The calibrated level of PM and AM noise is used to evaluate the accuracy versus Fourier frequency.

\section{1,547}

PB95-181137 Not available NTIS

National Inst. of Standards and Technology (PL), Boulder, CO. Time and Frequency Div.
Sifting Through Nine Years of NIST Clock Data with TA2.

Final rept.

M. A. Weiss, and T. P. Weissert. 1993, 12p.

Pub. in Proceedings of the European Frequency and Time Forum (7th), Neuchatel, Switzerland, March 1619, 1993, p199-210.

Keywords: *Clocks, Optimization, Anomalies, Reprints, TA2 algorithm, Time scales, Stochastic noise, US NIST.

We have extended the new TA2 post-process time scale at NIST beyond our previous reports to include all of the period from January of 1984 to the present. Derived from the ensemble of clocks at NIST, this time scale includes the benefits of several recent refinements to the algorithm. By iteratively running the algorithm on the ensemble clock data and characterizing anomalous behavior in the dominant individual clocks of the ensemble between iterations, we obtain an optimized scale which benefits from the informed anticipation of that anomalous behavior and demonstrates an overall decrease in scale disruption. Herein we discuss: changes to the TA2 algorithm that we made while processing the eight-year run, our method of characterizing anomalous behavior in individual clocks, the way unanticipated anomalous behavior is dealt with by the algorithm, and our resulting nine-year time scale.

\section{1,548}

PB95-220463 PC A06/MF A02

National Inst. of Standards and Technology (PL), Boulder, CO. Time and Frequency Div.

Time and Frequency: Bibliography of NIST Publications, March 1995.

G. E. Bennett, and D. B. Sullivan. Mar 95, 110p, NISTIR-5035.

Keywords: "Atomic clocks, "Time standards, "Frequency standards, Lasers, Time, Ion storage, Time measurement, Frequency measurement, Time transfer, US NIST

This bibliography includes most NIST publications in this field dating from the development of the first atomic clock. The publications are sorted into 23 categories. While the majority cover topics that are central to time and frequency measurements and standards, the bibliography does include other publications of the Time and Frequency Division that are only peripherally relat ed to the field.

\section{1,549}

PB96-102652 Not available NTIS

National Inst. of Standards and Technology (PL), Boulder, CO. Time and Frequency Div.

Measurement Methods and Algorithms for Comparison of Local and Remote Clocks.

Final rept.

J. Levine. 1993, 9p.

Pub. in Proceedings of Annual Precise Time and Time Interval Applications and Planning Meeting (PTTI) (24th), McLean, VA., December 1-3, 1992, p277-285 1993.

Keywords: "Time measuring instruments, "Synchronism, *Algorithms, Time measurements, Calibration, Comparison, Clocks. Time standards, Time domain, Frequency domain, Computers, Global positioning system, Accuracy, Reprints, Time transfer.

We will discuss several methods for characterizing the performance of clocks with special emphasis on using calibration information that is acquired via an unreliable of noisy channel. We will discuss time-domain variance estimators and frequency-domain techniques such as cross-spectral analysis. Each of these methods has advantages and limitations that we will illustrate using data obtained via GPS, ACTS and, other methods. We will also discuss the inverse problem of communicating frequency and time corrections to a real-time steered clock.

\section{1,550}

\section{PB96-103049 Not available NTIS}

National Inst. of Standards and Technology (PL), Boulder, CO. Time and Frequency Div.

Time Generation and Distribution.

Final rept.

D. B. Sullivan, and J. Levine. 1991, 9p.

Pub. in Proceedings of the Institute of Electrical and Electronics Engineers, v79 p906-914 Jul 91.

Keywords: "Time standards, "Frequency standards, "Synchronism, Atomic clocks, Time signals, Frequency measurements, Calibration, Reprints.
This paper presents a broad overview of time and frequency technology, particularly those trends relating to the generation and distribution of time and frequency signals. The characterization of components and systems is also addressed.

\section{1}

PB96-112230 Not available NTIS

National Inst. of Standards and Technology (PL), Boulder, CO. Time and Frequency Div.

Local Oscillator Requirements and Strategies for the Next Generation of High-Stability Frequency Standards.

Final rept.

F. L. Walls. 1992, 4p

Pub. in Proceedings of the Institute of Electrical and Electronics Engineers Frequency Control Symposium Hershey, PA., May 27-29, 1992, p2-5.

Keywords: "Oscillators, "Frequency stability, *Standards, Reprints, Meetings, Requirements, Strategies, "Foreign technology, Frequency synthesis.

This paper provides a brief introduction to the special session on high-stability frequency synthesis from if to the visible. The special session is motivated by a number of proposals that have been made for developing frequency standards with frequency stabilities in the 10 to the minus 16 th power to 10 to the minus 18th powe range. Currently available local oscillators, frequency synthesis techniques, and the measurement methods are not, however, sufficient to support this level of stability. Some general approaches to solve these problems, such as the effect of interrogation type on local oscillator induced white frequency noise, are briefly in troduced. The details are to be found in the individual presentations.

01,552

PB96-119482 Not available NTIS

National Inst. of Standards and Technology (PL), Boulder, CO. Time and Frequency Div.

Accurate Measurement of Time.

Final rept.

W. M. Itano, and N. F. Ramsey. 1993, 10p

Pub. in Scientific American, v269 n1 p56-65 Jul 93

Keywords: "Atomic clocks, "Time measurements, "Frequency measurements, Time standards, Trapped particles, lon storage, Hydrogen masers, Cesium frequency standards, Rubidium frequency standards, Atomic beams, Reprints, Atomic fountains.

The current status of atomic frequency standards and clocks is reviewed. The operating principles of fre quency standards based on cesium atomic beams, hydrogen masers, rubidium cells, trapped ions, and atomic fountains are discussed. Applications of such frequency standards are discussed.

\section{1,553}

PB96-119490 Not available NTIS

National Inst. of Standards and Technology (PL), Boul der, CO. Time and Frequency Div.

Atomic Clock.

Final rept.

W. M. Itano. 1992, 3p

Pub. in McGraw-Hill Encyclopedia of Science and Technology, 7th Edition, p240-242 1992

Keywords: "Atomic clocks, "Time measurements "Frequency measurement, Time standards, Atomic beams, Rubidium frequency standards, Gas cells, Cesium frequency standards, Hydrogen masers, Reprints.

Atomic clocks measure the passage of time using an internal resonance frequency of an atom. The most commonly used types of atomic clocks are the cesium atomic beam, the hydrogen maser, and the rubidium gas cell. In addition, there are many other kinds of atomic clocks under development which may prove to have even greater accuracy and stability. Atomic clocks are used in international timekeeping, navigation, communications, radio astronomy, space exploration, and fundamental science.

\section{1,554}

PB96-123187 Not available NTIS

National Inst. of Standards and Technology (PL), Boulder, CO. Time and Frequency Div. 


\section{Policies, Regulations, \& Studies}

Secondary Standard for PM and AM Noise at 5, 10 , and $100 \mathrm{MHz}$.

Final rept.

F. L. Walls, 1992, 10p

Pub. in Special Issue of the Institute of Electrical and Electronics Engineers Instrumentation and Measurement Transactions of CPEM '92, p290-299.

Keywords: *Amplitude modulation, *Phase modulation, "Standards, "Electromagnetic noise, Frequency domain, Time domain, High frequency, Electrical measurement, Reprints, Phase noise.

This paper describes a practical implementation of a portable secondary standard for phase modulation (PM) and amplitude modulation (AM) noise at 5,10 , and $100 \mathrm{MHz}$. The accuracy of the standard for both $\mathrm{PM}$ and $\mathrm{AM}$ noise is + or $-0.14 \mathrm{~dB}$, and the temperature coefficient is less than $0.02 \mathrm{~dB} / \mathrm{K}$. The noise floor of the standard for $\mathrm{PM}$ noise measurements, is less than $-190 \mathrm{dBC}$ rel, to $1 \mathrm{sq} \mathrm{rad} / \mathrm{Hz}$ at 5,10 and 100 $\mathrm{MHz}$. The noise floor for AM measurements depends on the configuration. A calibrated level of PM and AM noise of approximately $-130+$ or $-0.2 \mathrm{~dB}$ rel to 1 square rad $/ \mathrm{Hz}$ (for Fourier frequencies from approximately $1 \mathrm{~Hz}$ to $10 \%$ of the carrier frequency) is used to evaluate the accuracy versus Fourier frequency. Similar PM/AM measurements. Some types of timedomain measurement equipment can also be calibrated.

\section{5}

PB96-176581 Not available NTIS

National Inst. of Standards and Technology (PL), Boulder, CO. Time and Frequency Div.

Implementation of a Standard Format for GPS Common View Data.

Final rept.

M. A. Weiss, and C. Thomas. 1995, 10p

Also available as N95-32323; order as N95-32319.

Pub. in Annual Precise Time and Time Interval (PTT)

Applications and Planning Meeting (26th), Reston,

VA., December 6-8, 1994, p75-84 1995.

Keywords: "Global positioning system, *Standardization, Calibration, Time signals, Precision, Implementation, Data processing, Ionospherics, Implementation, Data processing, Ionospherics, ability.

A new format for standardizing common view time transfer data, recommended by the Consultative Committee for the Definition of the Second, is being implemented in receivers commonly used for contributing data for the generation of International Atomic Time. We discuss three aspects of this new format that potentially improve GPS common-view time transfer: (1) the standard specifies the method for treating short term data, (2) it presents data in consistent formats including needed terms not previously available, and (3) the standard includes a header of parameters important for the GPS common-view process.

\section{1,556}

PB96-190319 Not available NTIS

National Inst. of Standards and Technology (PL), Boulder, CO. Time and Frequency Div.

Time and Frequency Metrology.

Final rept.

D. B. Sullivan, and J. Levine. 1996, 10p.

Pub. in Advances in Metrology and Its Role in Quality Improvement and Global Trade, New Delhi, India, February $20-23,1996$, p $287-296$

Keywords: "Time standards, "Frequency standards, ${ }^{\star}$ Calibration, Time signals, Time measurement, Frequency measurement, Atomic clocks, Oscillators, Ion storage, Hydrogen masers, Cesium frequency standards, Rubidium frequency standards, Reprints, Time transfer.

The paper presents an overview of some of the trends which are shaping the directions of development in time and frequency metrology. The paper focuses on the characteristics of frequency sources, methods for comparing separated clocks/oscillators, and techniques for distributing time and frequency signals.

\section{1,557}

PB96-190376 Not available NTIS

National Inst. of Standards and Technology (PL), Boulder, CO. Time and Frequency Div.
Confidence on the Modified Allan Variance and the Time Variance.

Final rept.

M. A. Weiss, F. L. Walls, C. A. Greenhall, and T.

Walter. 1995, 14p.

Pub. in Proceedings of the European Frequency and Time Forum, Besancon, France, March 8-10, 1995, $14 p$.

Keywords: "Degrees of freedom, "Confidence limits, Time domain, Overlap, White noise, Electromagnetic noise, Signal to poise ratio, Frequency stability, Time stability, Reprints, *Allan variance, Chi square distribution, Time variance.

The paper presents tabulated factors for calculating confidence intervals for the square root of the Modified Allan Variance and the related time Variance when the analysis uses full overlapping of the data. Confidence intervals are determined by multiplying factors from the appropriate table times sample deviations. These factors have been calculated from the equivalent degrees of freedom (EDF) for the five common power law noise types. Closed form expressions for calculating the EDF for these variances and noise types have only recently become available for fully overlapped data analysis. We compute the EDF using two different closed form expressions as well as simulations to ensure correctness of the results. We also show the relative advantage of using the modified Allan variance instead of the Allan variance to evaluate frequency or time stability in the presence of white phase modulated noise.

01,558

PB96-200381 Not available NTIS

National Inst. of Standards and Technology (PL), Boulder, CO. Time and Frequency Div.

Wavelet Analysis for Synchronization and Timekeeping.

Final rept.

D. A. Howe, and D. B. Percival. 1994, 5p.

Pub. in Institute of Electrical and Electronics Engineers International Frequency Control Symposium, Boston, MA., June 1-3, 1994, p567-571

Keywords: "Wavelet analysis, "Synchronism, Fre quency standards, Time functions, Frequency synchronization, Time measurement, Phase melet transform, Wavelet variance.

We discuss the concept of the wavelet variance as a generalized formalism for representing variations in a time series on a scale by scale basis. In particular, we note that the wavelet variance corresponding to some of the recently discovered wavelets can provide a more accurate conversion between the time and frequency domains that can be accomplished using the Allan variance. The increase in accuracy is due to the fact that these wavelet variances give better protection against leakage than does the Allan variance.

\section{1,559}

PB96-200647 Not available NTIS

National Inst. of Standards and Technology (PL), Boulder, CO. Time and Frequency Div.

How to Get NIST-Traceable Time on Your Computer.

Final rept

M. A. Lombardi. 1994, 15p.

Pub. in Proceedings of the Workshop and Symposium of the Role of Metrology in a Changing World, Chicago, IL., July 31-August 4, 1994, p299-313.

Keywords: *Computers, "Time signals, ${ }^{*}$ Calibration standards, Computer networks, Time measurement, Time standards, Accuracy, Synchronism, ACTS GOES satellites, Global positioning system, Reprints, *Time keeping, Smart clock.

The paper describes a number of different ways that you can use to get accurate time that is traceable to NIST on your computer system. This includes receiving time signals by radio, by telephone, or through the internet. The cost and performance of each technique is also described.

\section{1,560}

PB96-200654 Not available NTIS

National Inst. of Standards and Technology (PL), Boulder, CO. Time and Frequency Div. Introduction to Frequency Calibration. Part 1. Final rept.

M. Lombardi. 1996, 12p.

Pub. in Cal Lab: The International Jnl. of Metrology v3 n1 p17-28 Feb 96
Keywords: "Frequency measurement, "Calibration standards, Frequency synchronization, Frequency stability, Phase modulation, Oscillators, Specifications, Reprints, Traceability, Transfer standards.

The article is part I of a two-part series on frequency calibrations. Part 1 introduces the topic of frequency calibrations, discusses the specifications involved, and describes the different types of oscillators.

\section{1,561}

PB96-200662 Not available NTIS

National Inst. of Standards and Technology (PL), Boulder, CO. Time and Frequency Div.

NIST Frequency Measurement Service.

Final rept.

M. A. Lombardi. $1995,7 p$

See also PB92-165851.

Pub. in Cal Lab: The International Jnl. of Metrology, v2 n3 p11-17 Jun 95

Keywords: "Frequency measurement, "Calibration, *Accuracy, Frequency stability, Frequency synchronization, Interlaboratory comparisons, Validatio

The NIST Frequency Measurement Service (FMS) began operation in 1984 to assist users who needed to make high-level frequency calibrations traceable to NIST. The FMS provides a complete solution to all frequency calibration problems by providing a frequency measurement system that can be installed in the customer's lab. The system includes all hardware, software, and documentation needed to automate the frequency measurement process. NIST provides training and phone support and validates customer data through a modem hookup. Each lab receives a monthly report that certifies traceability to NIST.

\section{1,562}

PB96-200688 Not available NTIS

National Inst. of Standards and Technology (EEEL), Boulder, CO. Electromagnetic Fields Div.

General Order ' $N$ ' Analytic Correction of Probe-Position Errors in Planar Near-Field Measurements.

Final rept.

A. Muth $1995,10 p$

Pub. in Annual Meeting and Symposium of 1995 Antenna Measurement Techniques Association (17th), Williamsburg, VA., November 13-17, 1995, p331-340.

Keywords: "Probes(Electromagnetic), "Antennas, Near field, Extremely high frequency, Reprints, Error correction.

An analytic technique recently developed at NIST (1)(2) to correct for probe position errors in planar nearfield measurements has been implemented to arbitrary accuracy. The nth-order correction scheme is composed of an mth-order ordered expansion and an $n$ $\mathrm{m}$ higher-order approximation, where both $\mathbf{n}$ and $\mathrm{m}$ are arbitrary. The technique successfully removes very large probe position errors in the near-field, so the residual near-field probe position errors are substantially below levels that can be measured on a near-field range. Only the error-contaminated near-field measurements and an accurate probe position errors are substantially below levels that can be measured on a near-field range. Only the error-contaminated nearfield measurements and an accurate probe position error function are needed for implementation of the correction technique. The method also requires the ability to obtain derivatives of the error-contaminated near field defined on an error-free regular grid with respect to the coordinates. In planar geometry the derivatives are obtained using FFTs (1), giving an approximate operation count of $(3.2(m-1)-1+(n-m)) \mathrm{N}$ log $\mathrm{N}$, where $\mathrm{N}$ is the number of data points. Efficient computer codes have been developed to demonstrate the technique. The results of simulations are more accurate than those obtained using the well-known k correction (3), which can correct for position errors in some direction in $\mathrm{k}$ space, but further contaminates the sidelobe levels.

\section{1,563}

PB96-200779 Not available NTIS

National Inst. of Standards and Technology (PL), Boulder, CO. Time and Frequency Div.

Effect of Harmonic Distortion on Phase Errors in Frequency Distribution and Synthesis.

Final rept.

F. L. Walls, and F. G. Ascarrunz. 1995, 8p

Pub. in Proceedings of the European Frequency and

Time Forum (9th), Besancon, France, March 8-10, 1995, 8p. 
Keywords: "Phase error, "Frequency distribution, "Frequency synthesizers, Frequency measurement, Frequency stability, Phase detectors, Radio frequencies, Roots of equations, Harmonics, Reprints, "Harmonic distortion, Timing errors.

The paper explores the effect of harmonic distortion on phase errors in frequency distribution and synthesis. Harmonic distortion gives rise to large timing errors in phase or zero crossing detectors. These large timing errors may cause unacceptable sensitivity to environmental conditions or signal fluctuations. Two types of phase detectors are considered in the paper. The timing errors in an ideal zero crossing detector is calculated as a function of harmonic number and the phase of the harmonic relative to the fundamental. Experimental data from several mixers at $5 \mathrm{MHz}$ and different drive levels show that this model is a first-order approximation to real devices. The low sensitivity to the second harmonic is an important advantage over true zero-crossing detectors because it is difficult to significantly reduce the second harmonic without introducing large phase shifts due to filtering networks.

\section{Radio \& Television Equipment}

\section{1,564}

PB94-172020 Not available NTIS

National Inst. of Standards and Technology (EEEL), Boulder, CO. Electromagnetic Fields Div.

Electric-Field Strengths Measured Near Personal Transceivers.

Final rept.

J. Adams. 1993, 4p.

Pub. in Proceedings of International Symposium on Electromagnetic Compatibility, Dallas, TX., August 9$13,1993, \mathrm{p} 42-45$

Keywords: "Transmitter receivers, "Electric fields, "Electrical measurement, Very high frequencies, Ultrahigh frequencies, Portable equipment, Walkie talkies Exposure, Standards, Reprints.

Electric-field strengths were measured at a number of points near $5-W$ personal transceivers. The points were located on cylinders of revolution with radii of 7 and $12 \mathrm{~cm}$ around the antenna. The transceivers operated on four authorized frequencies of 40.27, 162.475, 464 , and $823 \mathrm{MHz}$, and radiated powers of $5,5,5$, and $3 W$, respectively. In some cases, these measured values exceeded the exposure limits suggested in ANSI Standard C95.1-1982.

01,565

PB96-147202 Not available NTIS

National Inst. of Standards and Technology (EEEL), Boulder, CO. Electromagnetic Technology Div.

Heterodyne Mixing and Direct Detection in High Temperature Josephson Junctions.

Final rept.

E. N. Grossman, and L. R. Vale. 1994, 20p.

Pub. in International Symposium on Space Terahertz

Technology (5th), Ann Arbor, MI., May 10-12, 1994 p244-263.

Keywords: "Lasers, "Heterodyne detection, Reprints, "Josephson junctions, Microwave noise temperature.

The authors have examined various properties of high characteristic frequency YBCO superconductor- normal-superconductor (SNS) Josephson junctions that are important to their performance as low-noise $\mathrm{THz}$ frequency mixers. Without far-infrared laser illumination, the microwavefrequency noise temperature to our lowest noise device shows good agreement with the predictions of the resistivity shunted Josephson model in applicable regions of bias. It has a maximum noise temperature of 36 plus or minus $K$ at a physical temperature of $4 \mathrm{~K}$. When illuminatedwith a $404 \mathrm{GHz}$ farIR laser local oscillator (LO) and a chopped $77 \mathrm{~K}$ blackbody signal, strong modulation of the $1 \mathrm{GHz}$ IF noise power is observed. However, certain features of the modulated IF power signal strongly suggest that a larger fraction of it is not true heterodyne detection. The spurious component is probably due to direct detection ofthe broadband hot load/cold load signal. The authors believe that reliable measurement of heterodyne performance will require narrowband signal sources.

\section{Verbal}

01,566

PB94-211539 Not available NTIS

National Inst. of Standards and Technology (NCSL), Gaithersburg, MD. Advanced Systems Div.

Benchmarks for the Evaluation of Speech Recognizers.

Final rept.

W. M. Fisher. 1991, 2p.

Pub. in Digital Signal Processing 1, n2 p64-65 Apr 91. Keywords: "Speech recognition, "Computer applications, "Technology assessment, CD-ROM, Data bases, Computer software, Evaluation, Reprints, US NIST.

Materials produced by the National Institute of Standards and Technology (NIST) for the standardized evaluation of computer speech recognition systems are described.

\section{COMPUTERS, CONTROL \& INFORMATION THEORY}

\section{General}

01,567

FIPS PUB 140-1 PC E05

National Inst. of Standards and Technology (CSL),

Gaithersburg, MD.

Security Requirements for Cryptographic Modules; Category: Computer Security; Subcategory: Cryptography.

11 Jan 94, 44p.

Supersedes FIPS PUB 140

Three ring vinyl binder also available; North American Continent price $\$ 7.00$; all others write for quote.

Keywords: "Computer security, "Cryptography, "Federal information processing standards, Telecommunication, Data processing security, Algorithms, Software engineering, Requirements.

The selective application of technological and related procedural safeguards is an important responsibility of every Federal organization in providing adequate security in its computer and telecommunications systems. The publication provides a standard to be used by Federal organizations when these organizations specify that cryptographic-based security systems are to be used to provide protection for sensitive or valuable data. Protection of accryptographic module within a security system is necessary to maintain the confidentiality and integrity of the information protected by the module. This standard specifies the security requirements that are to be satisfied by a cryptographic module. The standard provides four increasing, qualitative levels of security intended to cover a wide range of potential applications and environments. The security requirements cover a reas related to the secure design and implementation of a cryptographic module. These areas include basic design and documentation, module interfaces, authorized roles and services, physical security, software security, operating system security, key management, cryptographic algorithms, electromagnetic interference/electromagnetic compatibility (EMI/EMC), and self-testing. This revision supersedes FIPS 140 in its entirety.

\section{1,568 \\ FIPS PUB 180-1 PC E04}

National Inst. of Standards and Technology (CSL), Gaithersburg, MD.

Secure Hash Standard. Category: Computer Security.

17 Apr 95, 25p.

Supersedes FIPS PUB 180

Three ring vinyl binder available; North American Continent price $\$ 7.00$; all others write for quote.

Keywords: "Computer security, "Federal Information Processing Standards, "Algorithms, Message processing, Data processing, Cryptology, Data encryption,
"FIPS PUB 180, "Hash algorithm, Digital signatures.

This standard specifies a Secure Hash Algorithm (SHA-1) which can be used to generate a condensed representation of a message called a message digest. The SHA-1 is required for use with the Digital Signature Algorithm (DSA) as specified in the Digital Signature Standard (DSS) and whenever a secure hash al gorithm is required for Federal applications. The SHA1 is used by both the transmitter and intended receiver of a message in computing and verifying a digital signature.

\section{1,569}

FIPS PUB 185 PC E03

National Inst. of Standards and Technology (CSL), Gaithersburg, MD

Escrowed Encryption Standard (EES); Category: Computer Security; Subcategory: Cryptography. 9 Feb $94,11 \mathrm{p}$

Three ring vinyl binder also available; North American Continent price $\$ 7.00$; all others write for quote.

Keywords: "Data encryption, "Federal information processing standards, Cryptography, Computer secu rity, Telecommunication, Algorithms, Data processing security, LEAF(Law Enforcement Access Field).

The standard specifies an encryption/decryption algorithm and a Law Enforcement Access Field (LEAF) creation method which may be implemented in electronic devices and used for protecting government telecommunications when such protection is desired. The algorithm and the LEAF creation method are classified and are referenced, but not specified, in the standard. Electronic devices implementing the standard may be designed into cryptographic modules which are integrated into data security products and systems for use in data security applications. The LEAF is used in a key escrow system that provides for decryption of telecommunications when access to the telecommunications is lawfully authorized.

\section{1,570}

FIPS PUB 186 PC E04

National Inst. of Standards and Technology (NCSL), Gaithersburg, MD.

Digital Signature Standard (DSS). Category: Computer Security; Subcategory: Cryptography.

19 May $94,24 p$.

Three ring vinyl binder also available; North American price $\$ 7.00$; all others write for quote.

Keywords: "Cryptography, "Computer security, Algorithms, Access control, Data processing security, Data integrity, Data encryption, Message processing, Information security, Random number-generators, Authentication, Verification, "Digital signatures, Federal Information Processing Standard, ADP(Automated Data Processing)

This standard specifies a Digital Signature Algorithm (DSA) which can be used to generate a digital signature. Digital signatures are used to detect unauthorized modifications to data and to authenticate the identity of the signatory. In addition, the recipient of signed data can use a digital signature in proving to a third party that the signature was in fact generated by the signatory. This is known as nonrepudiation since the signatory cannot, at a later time, repudiate the signature.

\section{1,571}

FIPS PUB 188 PC E04

National Inst. of Standards and Technology (CSL) Gaithersburg, MD.

Standard Security Label for Information Transfer; Category: Computer Security; Subcategory: Security Labels.

6 Sep $94,28 p$

Also available from Supt. of Docs.

Three ring vinyl binder also available; North American Continent price $\$ 7.00$; all others write for quote.

Keywords: "Computer information security, "Federal information processing standards, Computer security, Access control, Protocols, Computer communications, Syntax, Computer networks, Data processing securlty, "Security labels.

Information Transfer security labels convey information used by protocol entities to determine how to handle data communicated between open systems. Informa. tion on a security label can be used to control access, specify protective measures, and determlne handling 


\section{General}

restrictions required by a communications security policy. This standard defines a security label syntax for information exchanged over data networks and provides encodings of that syntax for use at the Application and Network Layers.

\section{1,572}

FIPS PUB 46-2 PC E04

National Inst. of Standards and Technology (CSL) Gaithersburg, MD

Data Encryption Standard (DES); Category: Computer Security; Subcategory: Cryptography.

30 Dec 93, 23p

Supersedes FIPS PUB 46-1.

Three ring vinyl binder available; North American Continent price \$7.00; all others write for quote.

Keywords: "Data encryption, "Federal information processing standards, Computer security, Algorithms Cryptography, Data integrity, Data processing security

The selective application of technological and related procedural safeguards is an important responsibility of every Federal organization in providing adequate security to its electronic data systems. The publication specifies a crypotographic algorithm which may be used by Federal organizations to protect sensitive data. Protection of data during transmission or while in storage may be necessary to maintain the confidentiality and integrity of the information represented by the data. The algorithm uniquely defines the mathematical steps required to transform data into crypotographic cipher and also to transform the ciphe back to the original form. The Data Encryption Standard is being made available for use by Federal agencies within the context of a total security program consisting of physical security procedures, good informa tion management practices, and computer system/net work access controls. The revision supersedes FIPS 46-1 in its entirety.

01,573

PB94-134897 PC A03/MF A01

National Inst. of Standards and Technology (CSL)

Gaithersburg, MD

General Procedures for Registering Computer Security Objects.

N. A. Nazario. Dec 93, 23p, NISTIR-5308.

Keywords: "Computer security, "Standards, Services Fees, Requirements, Labels, "CSOR(Computer Secu rity Objects Register), Registration.

The document defines a Computer Security Objects Register (CSOR). It outlines the information required for the assignment of unique identifiers to the specifications of Computer Security Objects (CSOS). A CSO can be defined as a resource, tool, or mechanism used to maintain a condition of security in computerized environments. This broad definition covers many elements that are referred to in standards but that are either selected or defined by separate user communities (e.g., network security labels, cryptographic algorithm operation modes, authentication techniques). The CSOR services organizations and individuals seeking to further interoperability by using a common set of tools and techniques in the area of computer security.

01,574

PB94-135001 PC A09/MF A02

National Inst. of Standards and Technology (CSL), Gaithersburg, MD.

Report of the NIST Workshop on Digital Signature Certificate Management. Held on December 10-11, 1992.

D. K. Branstad. Oct 93, 187p, NISTIR-5234.

Keywords: "Computer security, "Cryptography, "Meetings, Certification, Standards, Data processing Standards and Technology.

This report summarizes the major topics of discussion held during a Workshop on Ditigal Signature Certificate Management held at the National Institute of Standards and Technology (NIST) on December 10-11, 1992. The purpose of the workshop was to review ex sting and required technologies for digital signature certification authorities and to develop recommendations for certificate contents, formats, generation, distribution and storage. The results of the workshop have been provided as input to a federally sponsored study of support infrastructures of a set of international and national signature certification authorities. A summary of the major issues discussed and the recommendations on several of them are presented.
01,575

PB94-162583 PC A04/MF A01

National Inst. of Standards and Technology (CSL) Gaithersburg, MD. Computer Security Div

Proceedings of the Workshop on the Federal Criteria for Information Technology Security. Held in Ellicot City, Maryland on June 2-3,1993.

J. A. Cugini, P. Toth, G. Troy, T. Mayfield, M.

Abrams, L. Fraime, V. Gligor, L. M. Ambuel, and $F$. Mayer. Mar 94, 74p, NISTIR-5386.

Prepared in cooperation with National Security Agency/Central Security Service, Fort George G. Meade, $\mathrm{MD}$

Keywords: "Meetings, "Computer security, Access control, Data processing security, Computer privacy Standards, North America, Europe, "Federal Criteria, National Institute of Standards and Technology, $\mathrm{Na}$ tional Security Agency, Common Criteria Editorial Board, ISO(International Standards Organization).

The first draft of the Federal Criteria was made public in Januany 1993. Several thousand copies of the Fed eral Criteria were distributed and comments on this first draft were received. The purpose of the Federal Criteria Workshop was to address these comments. The purpose of these proceedings is to inform the Federal Criteria commentators and the workshop attendees of the outcome of the workshop. Also, these proceedings will be used as a blueprint for updating the Federal Criteria. These proceedings, and the revised portions of the draft Federal Criteria contents, will be the two principal National Institute of Standards and Technology (NIST)/National Security Agency (NSA) inputs to the Common Criteria Editorial Board (CCEB). Preliminary plans for dealing with the Federal Criteria comments are included in this document, and all revisions will be performed under the direction of NIST and the NSA.

\section{6}

PB94-164373 PC A03/MF A01

National Inst. of Standards and Technology (CSL), Gaithersburg, MD.

Conformance Ass zssment of Transport Layer Security Implementatıons.

W. A. Jansen. Dec 93, 23p, NISTIR-5325.

See also AD-A207 905 and AD-A255 422

Keywords: "Computer networks, "Protocols, "Computer security, Tests, Data integrity, Computer information security, Authentication, Access control, OSI(Open System Interconnection), TLSP(Transport Layer Security Protocol), SP4(Security Protocol at layer 4), SDNS(Secure Data Network System)

The paper presents a tramework for evaluating conformance of a protocol implementation to the Security Protocol at layer 4 (SP4) standard. SP4 is one element of the Secure Data Network System (SDNS) architec. ture, used to provide security services at the Transpor layer of the Open System Interconnection (OSI) reference model. SP4 also forms the basis of the International Organization for Standardization (ISO) standard for the OSI Transport Layer Security Protocol (TLSP). Therefore, with few exceptions, the findings of the paper are applicable to TLSP. The paper explores the relationship between conformance assessment, interoperability assessment, and security evaluation of security protocols. The OSI conformance testing methodology and framework is reviewed, and a strategy is given for applying this methodology to SP4 implementations.

\section{7}

PB94-185402 Not available NTIS

National Inst. of Standards and Technology (CSL), Gaithersburg, MD. Computer Security Div.

Conformance Testing of a Lower Layer Security Protocol.

Final rep

W. A. Jansen. 1993, 13p

Pub. in Proceedings of Symposium on Military Communications Network, The Hague, The Netherlands, June 8-11, 1993, p843-855.

Keywords: "Computer networks, "Computer security, "Protocols, "Conformity Tests, Communication neiworks, Reprints, Conformance testing, OSI(Open System Interconnection), SDNS(Secure Data Network System), ISO(International Organization for Standardization), TLSP(Transport Layer Security Protocol)

This paper presents a framework for evaluating conformance of a protocol implementation to the Security Prolocol at layer 4 (SP4) standard. SP4 is one element of the Secure Data Network System (SDNS) architecture, used to provide security services at the transport layer of the Open System Interconnection (OSI) reference model. SP4 also forms the basis of the recently completed ISO standard for the Transport Layer Security Protocol (TLSP). The paper explores the relationship between conformance testing and security evaluations of security protocols in general, and SP4 in particular. The OSI conformance testing methodology is reviewed, and a strategy is given for applying the methodology to SP4 implementations.

\section{1,578}

PB94-191202 PC A21/MF A04

Independent Monitoring, Cambridge, MA

Federal Certification Authority Liability and Policy: Law and Policy of Certificate-Based Public Key and Digital Signatures.

M. S. Baum. Jun 94, 485p, NIST/GCR-94/654. Sponsored by National Inst. of Standards and Technology, Gaithersburg, MD.

Keywords: "Computer security, "Cryptography, "Law(Jurisprudence), Computer information security, Government policies, Legal aspects, Data processing security, Electronic mail, Recommendations, Digital signatures, EDI(Electronic Data Interchange), National Institute of Standards and Technology, FCA(Federal Certification Authority), Infrastructure, PEM(Privacy Enhanced Mail)

The report identifies diverse technical, legal and policy issues affecting a certificate-based public key cryptographic infrastructure utilizing digital signatures supported by 'trusted entities.' It examines potential legal implications, surveys existing legal paradigms and the structures and roles of relevant governmental agencies and presents various institutional approaches to controlling liability. It considers the underpinnings of a lega and policy framework which might serve as a foundation for security policies and their implementation and concludes with a series of recommendations, botl general and specific.

\section{1,579}

PB94-193653 PC A07/MF A02

National Inst. of Standards and Technology (CSL),

Gaithersburg, MD. Computer Security Div.

Study of Federal Agency Needs for Information Technology Security.

Rept. for Feb-Aug 92

D. M. Gilbert. May 94, 126p, NISTIR-5424.

See also PB93-138956.

Keywords: "Data processing security, "Federal agencies, Requirements, Standards, Computer security, Computer information security, Surveys, Tables(Data) "IT(Information Technology), *Information Technology, "National Institute of Standards and Technology.

The report presents the results of a National Institute of Standards and Technology (NIST) study to determine and document what federal agencies need to meet their information technology (IT) security requirements. A meeting of the NIST IT Security Needs Study Working Group was held at NIST in September 1992 to review and comment on the study results. This re port reflects the working group input. The results of the study contribute to a sound basis for planning future NIST IT security standards, guidance, and related activities.

\section{1,580}

PBg4-198256 Not available NTIS

National Inst. of Standards and Technology (NEL), Gaithersburg, MD. Robot Systems Div.

Hierarchical Interaction between Sensory Processing and World Modeling in Intelligent Systems.

Final rept.

S Albus. $1990,7 p$

Pub. in Proceedings of the Institute of Electrical and Electronics Engineers International Symposium on Intelligent Control (5th), Philadelphia, PA., September 5 7, 1990, v1 p53-59.

Keywords: "Artificial intelligence, "Machine vision, Learning machines, Robotics, Sensory perception, Models, Image processing, Pattern recognition, Reprints

The role of the sensory processing system is to transform sensory maps into world model maps and to extract entity attributes and states. The role of the world modeling system is to store best estimates of the extracted values, and to generate from them, predicted inputs. There is a tight coupling between sensory proc 
essing and world modeling. The world model generates predicted sensory input from stored state estimates. The sensory processing system compares predictions with observations, and returns the difference signals to the world model for updating state estimators. The sensory processing system also performs spatial and temporal integration on various combinations of observed and predicted data, and compares the resulting correlation function against thresholds of recognition and detection.

01,581

PB94-198264 Not available NTIS

National Inst. of Standards and Technology (NEL). Gaithersburg, MD. Robot Systems Div.

Role of World Modeling and Value Judgment in Perception.

Final rept.

J. S. Albus. $1990,10 p$

Pub. in Proceedings of the Institute of Electrical and Electronics Engineers International Symposium on intelligent Control (5th), Philadelphia, PA., September 5 7, 1990, v1 p154-163.

Keywords: "Artificial intelligence, "Models, Machine learning, Knowledge representation, Robotics, Task planning(Robotics), Sensory perception, Interfaces Reprints, "World models.

The world model is the intelligent system's best estimate of the external world. It provides an interface be tween sensory processing and task decomposition. A world model architecture is proposed that represents knowledge in terms of maps, entity frames, and state variables. The structure is hierarchically organized so as to provide multiple levels of resolution in space and time. Three types of map coordinates are important: egospheres, object coordinates, and world coordinates. Value judgments provided an evaluation of hypothesized plans, and perceived objects, events, and situations. Evaluations produce value state-variables that indicate cost, benefit, risk, priority, desirability, attractiveness, and uncertainty.

\section{1,582}

PB94-198272 PC AO2

National Inst. of Standards and Technology (NEL), Gaithersburg, MD. Robot Systems Div.

Theory of Intelligent Systems.

Final rept.

J. S. Albus. 1990, 10p

Pub. in Proceedings of the Institute of Electrical and Electronics Engineers International Symposium on Intelligent Control (5th), Philadelphia, PA., September 5 7, 1990, p866-875.

Keywords: "Artificial intelligence, Robotics, Learning machines, Machine vision, Feedback control, Models, Theories, Reprints.

A theoretical model is proposed consisting of seven basic elements: actuators, sensors, sensory processing, world modeling, task decomposition, value judgment, and global memory/communications. These elements are integrated into a hierarchical system architecture wherein: (1) control bandwidth decreases about an order of magnitude at each higher level, (2) perceptual resolution of spatial and temporal patterns contracts about an order-of-magnitude at each higher level, (3) goals expand in scope and planning horizons expand in space and time about an order-of-magnitude at each higher level, and (4) models of the world and memories of events expand in space and time by abou an order-of-magnitude at each higher level. At each level, tightly coupled functional modules perform task decomposition, world modeling, sensory processing, and value judgments. Feedback control loops are closed at every level.

01,583

PB94-199247 Not available NTIS

National Inst. of Standards and Technology (NCSL), Gaithersburg, MD. Systems and Network Architecture Div.

Integrated Network Management.

Final rept

P. Brusil, and D. P. Stokesberry. 1989, 7p.

Pub. in Proceedings of International Federation for In formation Processing TC 6NG 6.6 Symposium on Integrated Network Management, Boston, MA., May 1617, 1989, p3-9.

Keywords: "Computer networks, "Integrated systems, Reprints, Communication networks, Vendors, "Network management, OSI(Open Systems Interconnection)
The document contains the introductory remarks to the First International Symposium on Integrated Network Management.

\section{1,584}

\section{PB94-209459 PC A08/MF A02}

lowa State Univ. Ames. Dept of Computer Science. Report of the NIST Workshop on Key Escrow Encryption. Held in Gaithersburg, Maryland on June 8-10, 1994.

A. E. Oldehoeft, and D. K. Branstad. Jun 94, 164p, NISTIR-5468

See also FIPS PUB 185. Sponsored by National Inst. of Standards and Technology (CSL), Gaithersburg, MD. Computer Security Div.

Keywords: "Data encryption, "Data processing security, Federal information processing standards, Algorithms, Computer software, Computer program integrity, Requirements, Industries, Computer security, "Key escrow encryption, EES(Escrowed Encryption Standard), US NIST.

The purpose of this workshop was to initiate a dialogue among representatives from industry, government and academia regarding issues of key escrow encryption. Workshop presentations and discussions included current progress in the planned usage of CAPSTONE/ CLIPPER chips, the alternative of software key escrowing, multipurpose key escrowing methods, private key escrow management, integrity requirements of application systems, and international implications. The proposed plan of action, arising from this workshop, is to establish a joint industry/government working group to critically evaluate all known key escrowing proposals; examine the adequacy of existing (and possibly suggesting new) vehicles for collaborative industry/government research and development; identify and evaluate suitable algorithms for use in conjunction with key escrow along with criteria for determining acceptability; identify and address the issues of intellectual property which are inherent in any public royaltyfree usage of algorithms, key escrow methodology, and supporting infrastructure; and create a key escrowing task force to manage and expedite the search for key escrow alternatives.

\section{1,585}

PB94-213030 Not available NTIS

National Inst. of Standards and Technology (NCSL), Gaithersburg, MD. Systems and Software Technology Div.

Formal Specification and Verification of Control Software for Cryptographic Equipment.

Final rept.

D. R. Kuhn, and J. F. Dray, 1990, 11p.

Pub. in Proceedings of Annual Computer Security Applications Conference (6th), Tucson, AZ., December 37, 1990, p32-43.

Keywords: "Cryptography, "Computer program verification, "Specifications, Access control, Software engineering Computer security, Microprocessors, Workstations, Reprints, Smart cards, Formal methods, VDM(Vienna Development Method).

This paper describes the application of formal specification and verification methods to two microprocessor-based devices: a 'smart-token' system that controls access to a network of workstations, and a message authentication device implementing the ANSI X9.9 message authentication standard. A statebased specification for the smart token system was prepared and verified using the Unisys Formal Development Methodology. The message authentication device specification used the Vienna Development Method, which is based on denotational semantics. Formal specification and verification were found to be practical, cost-effective tools for detecting potential security weaknesses. The security of the access control system was strengthened as a result of the analysis. An unexpected result was the discovery of ambiguities in a published standard.

\section{1,586}

PB94-215746 PC A05/MF A01

National Inst. of Standards and Technology (NCSL), Gaithersburg, MD. Computer Security Div.
Head Start on Assurance: Proceedings of an Invitational Workshop on Information Technology (IT) Assurance and Trustworthiness. Held in Williams burg, Virginia on March 21-23, 1994.

Special pub.

M. D. Abrams, and P. R. Toth. Aug 94, 92p, NISTIR5472

See also PB91-235978. Sponsored by Aerospace Computer Security Associates, Silver Spring, MD

Keywords: "Meetings, "Computer security, Data processing security, Computer privacy, Computer information security, Data integrity, Government policies, Distributed computer systems, Operating systems(Computers).

The purpose of the Invitational Workshop on Informa tion Technology (IT) Assurance and Trustworthiness was to identify crucial issues on assurance in IT systems and to provide input into the development of policy guidance on determining the type and level of assurance appropriate in a given environment. The readers of these proceedings include those who handle sensitive information involving national security, privacy, commercial value, integrity, and availability. Ex isting IT security policy guidance is based on compute and communications architectures of the early $1980 \mathrm{~s}$. Technological changes since that time mandate a review and revision of policy guidance on assurance and trustworthiness, especially since the changes encompass such technologies as distributed systems, local area networks, the worldwide Internet, policy-enforcing applications, and public key cryptography.

\section{1,587}

Not available NTIS

National Inst. of Standards and Technology (CSL), Gaithersburg, MD. Computer Security Div. Bibliography of Computer Security Glossaries. Final rept.

S. P. McCrea. $1990,2 p$

Pub. in National Computer Systems Laboratory Bulletin, $\mathrm{p} 1-2$ Sep 90

Keywords: "Computer security, "Dictionaries, "Bibliographies, Federal agencies, Data processing security, Reprints, NCSL(National Computer Systems Laboratory), US NIST, ANSI(American National Standards Institute)

The National Computer Systems Laboratory (NCSL) developed a bibliography that identifies eight glos sa ries that contain computer security terms and definitions. These glossaries were developed by Federal Government agencies, the American National Stand ards Institute (ANSI) Accredited Standards Committee $\times 3 \mathrm{~K} 5$, and private sector organizations. The purpose of the bibliography is to provide a guide to compute security glossaries that can be used by Federal Gov ernment agencies to promote common understanding of computer security terms, concepts, and procedures.

\section{1,588}

PB94-218575 PC A03/MF A01

National Inst. of Standards and Technology (MEL) Gaithersburg, MD. Intelligent Systems Div.

Adaptive, Predictive 2-D Feature Tracking Algorithm for Finding the Focus of Expansion. S. Krishnan, and D. Raviv. Jun 94,30p, NISTIR 5460.

Grant NSF-IRI-9115939

Prepared in cooperation with Florida Atlantic Univ. Boca Raton. Robotics Center. Sponsored by National Science Foundation, Arlington, VA.

Keywords: "Motion, *Optical tracking, "Computer vi sion, "Two dimensional flow, Feature extraction, Pat ern recognition, Image processing, Algorithms, Pixels, FOE(Focus of Expansion)

This paper describes a robust, pixel-based adaptive al gorithm to track image features, both spatially and tem porally, over a sequence of mcnocular images. The algorithm assumes no a priori knowledge about the feaures to be tracked, or the motion of the camera or the objects, in the 3-D scene. The features to be tracked are selected by the algorithm and these correspond to the peaks of a 'matching surface' constructed from the first image of the sequence to be analyzed. Any kind of motion can be tolerated keeping in mind the pixelsper-frame motion limitations. In our experiments, we have used the algorithm for tracking up to 3 pixels motion between frames with absolutely no subpixel calculations being necessary. Taking into account constraints of temporal continuity, the algorithm uses simple and efficient predictive tracking over multiple 


\section{General}

frames. The algorithm accepts a slow, continuous change of brightness D.C. level in the pixels of the feature. Trajectories of features on multiple objects can be computed. As an application of the algorithm we show how the algorithm can be used to find the Focus of Expansion (FOE) using a sequence of real images.

\section{1,589}

PB95-130985 PC A09/MF A02

National Inst. of Standards and Technology (CSL),

Gaithersburg, MD

Computer Security Training and Awareness Course Compendium.

K. Everhart. Sep 94, 192p, NISTIR-5495

See also PB92-205442 and PB90-780172.

Keywords: "Computer security, "Training, "Curricula, Directories, Government employees, Governmen agencies, Personnel development, Vendors, Auditing, Computer viruses, Local area networks, Data process ing security, Computer information security, Information systems, Telecommunication.

The training and awareness courses in this compendium correspond to the matrix in NIST Special Publication 500-172, Computer Security Training Guidelines (PB90-780172). Special Publication 500-172 is used as reference under the OPM regulation that implements Public Law 100-235, the Computer Security Act of 1987 , which requires training for all employees responsible for the management and use of federal computer systems that process sensitive information. Under the regulation, agencies will be responsible for identifying the employee to be trained and providing appropriate training. In addition to the table of contents which lists the courses, there are four appendices: (1) lists the course by training areas within audience categories as defined in NIST Special Publication 500 172: (2) lists the vendors that participated in this effort (3) lists the specific products for which training is available; and (4) lists the product specific courses.

\section{1,590}

\section{PB95-150983 Not available NTIS}

National Inst. of Standards and Technology (NEL), Gaithersburg, MD. Electricity Div.

Compensation of Markov Estimator Errors in TimeJittered Sampling of Nonmonotonic Signals.

Final rept.

G. Tong, and T. M. Souders. 1993, 5p.

Pub. in Institute of Electrical and Electronics Engineers Transactions on Instrumentation and Measurement 42 n5 p931-935 Oct 93 .

Keywords: Probability density functions, Sampling, Errors, Reprints, "Signal estimation, "Markov estimators, Sampling voltage trackers, Bias compensation, Time jitter.

The so-called Markov estimator is sometimes used to estimate signals from their time-jittered samples. The estimates are unbiased for monotonic signals, but exhibit errors in regions of nonmonotonicity. A method of compensation is presented to reduce this error. It requires a knowledge of the probability density functions (PDF) of the time jitter, and a proposed method for de termining the PDF based on the generalized Markov estimator has been verified through simulations. The performance of the compensation approach is presented for four different nonmonotonic waveforms.

\section{1,591}

\section{PB95-161030 Not available NTIS}

National Inst. of Standards and Technology (MEL), Gaithersburg, MD. Robot Systems Div.

Visual Road Following without 3-D Reconstruction. Final rept.

M. Herman, D. Raviv, H. Schneiderman, and M

Nashman. 1993,11p.

Pub in Proceedings of Society of Photo-Optical Instrumentation Engineers Applied Imagery Pattern Recognition Workshop (22nd), Washington, DC., October 1993, v2103 p1-11.

Keywords: "Robots, "Autonomous navigation, *Computer vision, Robotics, Robot dynamics, Ground vehicles, Two dimensional models, Optical flow(Image analysis), Cues, Algorithms, Real time, Reprints, "Road following.

The paper discusses an alternative approach to visua road following in which a minimal road model is generated. The model contains only task-relevant information and a minimum of vision processing is performed to extract this information. This approach leads to rapid and continuous update of the road model from the vis- ual data. Road following is achieved by servoing on the visual cues in the 2-D model. In this paper, two specific examples of road following that use this approach are presented. In the first example, we show that road following commands can be generated from visual cues consisting of the projection into the image of the tangent point on the edge of the road, along with the optical flow of this point. Using this cue, the resulting servo loop is very simple and fast. In the second example, we show that lane markings can be robustly tracked in real time while confining all processing to the 2-D image plane. Neither knowledge of vehicle motion nor a calibrated camera is required.

\section{1,592}

\section{PB95-161170 Not available NTIS}

National inst. of Standards and Technology (CSL), Gaithersburg, MD. Computer Security Div.

SDNS Security Management.

Final rept.

W. A. Jansen. 1992, $10 p$.

Pub. in Proceedings of National Computer Security Conference (15th), Baltimore, MD., October 13-16, 1992, p574-583

Keywords: "Computer networks, "Computer security, Standards, Protocols, Access control, Systems management, Reprints, *SDNS(Secure Data Network System), "Secure Data Network System, OSI(Open Systems Interconnection)

The Secure Data Network System (SDNS) program has developed a security architecture based on the International Organization for Standardization (ISO) Basic Reference Model for Open Systems Interconnection (OSI). The SDNS standards include lower layer security protocols, a key management protocol, and a framework for access control. This paper provides an overview of SDNS security management and its relationship to OSI systems management. A conceptual data model of SDNS security management information is presented. The overview also includes the naming hierarchy for the object classes defined for SDNS management and the registration scheme proposed for identifying elements of SDNS management information.

\section{1,593}

\section{PB95-162236 Not available NTIS}

National Inst. of Standards and Technology (NEL), Gaithersburg, MD. Robot Systems Div.

Reconstruction during Camera Fixation.

Final rept

D. Raviv. $1991,8 p$

Pub. in Proceedings of Society of Photo-Optical Instrumentation Engineers: Intelligent Robots and Computer Vision IX: Neural, Biological and 3-D Methods, Boston, MA., November 7-9, 1990, v1382 p312-319 1991.

Keywords: "Autonomous navigation, "Robots, "Computer vision, Robot dynamics, Optical flow(Image analysis), Cameras, Reprints, "Camera fixation.

A fixation point is a point in 3-D space that projects to zero optical flow in an image over some period of time while the camera is moving. This paper deals with quantitative aspects of camera fixation for a static scene. In general, when the camera undergoes translation and rotation, there is an infinite number of points that produce equal optical flow for any instantaneous point in time. Using a camera-centered spherical coordinate system, it is shown how to find these points in space. If the elevation component of the optical flow is set to zero, then these points form a circle (called the Equal Flow Circle (EFC)) and a line. A special case of the EFCs is the Zero Flow Circle (ZFC) where both components of the optical flow are equal to zero. In a set of experiments using simulated as well as real data we show how the concept of the EFC and ZFC can be used to explain the optical flow produced by points near the fixation point, and to explicitly map the space while fixating. It is also shown experimentally that points near the fixation point may change the sign of their optical flow as the camera moves.

\section{1,594 \\ PB95-162244 Not available NTIS}

National Inst. of Standards and Technology (MEL), Gaithersburg, MD. Robot Systems Div.

Unified Approach to Camera Fixation and VisionBased Road Following.

Final rept.

D. Raviv, and M. Herman. 1994, 53p.

Pub. in Institute of Electrical and Electronics Engineers Transactions on Systems, Man, and Cybernetics 24, n8 53p Aug 94
Keywords: *Autonomous navigation, "Robotics, *Computer vision, Robots, Robot dynamics, Optical
flow(Image analysis), Cameras, Reprints, "Camera fixation, "Road following

Both camera fixation and vision-based road following are problems that involve tracking or fixating on 3-D points and features. These problems also require an analysis of depth and motion. We present a theoretical approach to analyzing optical flow and show the application of this unified approach to both problems. In general, when a camera undergoes translation and rotation, there is an infinite number of points in 3-D space that produce equal optical flow for any point in time. Using a camera-centered spherical coordinate system, we show how to find these points in space. If the elevation component of the optical flow is set to zero, then these points form a circle (called the Equal Flow Circle (EFC)) and a line. A special case of the EFCs is the Zero Flow Circle (ZFC) where both components of the optical flow are equal to zero. The fixation point is the intersection of all the ZFCs. Points inside and outside of the ZFC can be quantitatively mapped using the EFCs. Using the ZFCs approach, we suggest a new, quantitative, vision-based approach to road following We show that motion commands can be generated from a visual feature, or cue, consisting of the projection into the image of the tangent point on the edge of the road, along with the optical flow of this point. Using this cue, we suggest a vision-based control approach.

\section{1,595}

PB95-162376 Not available NTIS

National Inst. of Sta

Data Encryption Standard.

Final rept.

E. Roback. $1990,6 p$.

letin, p1-6 Jun 90

Keywords: "Data encryption, "Cryptology, "Secure communications, Cryptography, Coding, Message processing, Information security, Data integrity, Computer security, Electronic security, Reprints, Data security.

This NCSL Bulletin provides an overview of the most frequently asked questions regarding Federal Information Processing Standard 46-1 (FIPS PUB 46-1), Data Encryption Standard (DES), and associated topics in cluding: its background, technical overview, applicability, waivers and procedures, DES crytographic keys, endorsement of DES devices, exportability issues, and validation of DES implementations. The document does not establish new policy. The Bulletin also provides a list of reference documents regarding the DES.

01,596

PB95-163432 Not available NTIS

National Inst of Standards and Technology (NCSL) Gaithersburg, MD. Computer Security Div.

Computer Security Management and Planning in the U.S. Federal Government.

Final rept.

E. F. Troy. 1990, 519p

Pub. in Proceedings of Comsec International 1990 London, United Kingdom, October 10-12, 1990, 519p.

Keywords: "Federal government, "Computer information security, "Selective dissemination of information, "Data base administrators, "Access control, Data processing security, Electronic security, Classified information Classified materials, Vulnerability, Privacy, Confidentiality, Risk assessment, Computer networks, Operating systems(Computers), Computer program in tegrity, Data management systems, Policies, Reprints, Computer security management, Computer security planning, Trusted systems, Computer Security Act.

The paper describes a number of activities sponsored by NIST to improve computer security management in the U.S. Federal government. The paper presents the results of the computer Security Act-mandated security plan review process and describes a number of procedural initiatives NIST has taken to help Federal computer security managers meet their computer security responsibilities. The paper also discusses new NIST technical initiatives in applying trusted systems technology concepts to sensitive systems, as a powerful new aid to assure better security protection. The paper asserts that a balanced combination of technical and non-technical security controls is needed to provide adequate computer security for Federal systems. 
01,597

PB95-163440 Not available NTIS

National Inst. of Standards and Technology (NCSL).

Gaithersburg, MD. Computer Security Div.

Guidance to Federal Agencies on the Use of Trusted Systems.

Final rept:

E. F. Troy, and E. E. Flahavin. 1990, 4p.

Pub. in National Computer Systems Laboratory Bulletin, $\mathrm{p} 1-4$ Jul 90

Keywords: "Federal agencies, "Computer information security, "Selective dissemination of information, "Access control, Data processing security, Electronic security, Privacy, Confidentiality, Computer networks, Data management systems, Operating systems(Computers), Policies, Standards, Guidelines, Reprints, "Trusted systems, "Unclassified sensitive information, Computer security management.

The document provides initial guidance to Federal departments and agencies on the use of trusted systems technology in computer systems which handle unclassified sensitive information.

\section{1,598}

PB95-163895 Not available NTIS National Inst. of Standards and Technology (NEL),
Gaithersburg, MD. Robot Systems Div.

Configuration and Performance Evaluation of a Real-Time Robot Control System: A Skeleton Approach.

Final rept.

T. Wheatley, and J. Michaloski. 1990, 4p.

Pub. in Proceedings of Institute of Electrical and Electronics Engineers International Conference on Systems Engineering, Pittsburgh, PA., August 9-11, 1990, p268-271.

Keywords: "Robot control, "Systems analysis, "Performance evaluation, Real time, Computer system hardware, Operating systems (Computers), Multiprocessors, Concurrent processing. Hierarchies Control systems, Reprints, Skeleton systems.

The use of a skeleton system to model a multi-processor robol control architecture offers the system designer a powerful tool to configure and evaluate system parameters. This paper describes the skeleton approach as applied to the NASREM robot control architecture. The skeleton approach creates the shell of a functioning real-time control system utilizing the actual hardware and operating system code without using actual application code. This is done by replacing the processing part of the application code with time delays. Parameterization of time delays, communication paths, message buffer lengths, and process allocation provides for rapid prototyping of alternative system architectures. Actual system performance is measured to provide realistic data on computation and communication loads. The skeleton reporting facility provides quantitative assessments of system activity. To illustrate the use of this technique, the servo level of the NASREM hierarchy will be modeled using a 5.0 msec cycle time on a multiprocessor system, and compared with the actual system.

\section{1,599}

\section{PB95-169108 Not available NTIS}

National Inst. of Standards and Technology (CSL), Gaithersburg, MD. Systems and Software Technology Div.

Approaches Using Virtual Environments with Mosaic.

Final rept.

S. Ressler. 1994, 8p

Pub. in Proceedings of International WWW Conference ' 94 Mosaic and the Web (2nd), Chicago, IL., October 17-20, 1994, p853-860.

Keywords: "Virtual reality, "Environment simulation, "Interactive graphics, Computer graphics, Interactive control, Computerized simulation, Man computer interfaces, Data transmission, Information technology, Reprints, "Mosaic, "Computer generated environments, User interfaces, World Wide Web, National Information Infrastructure.

The paper describes several ways to use a computer generated environment with Mosaic. The first is to allow the user to interact with an environment displayed as part of the Mosaic document, an in-line image. The second is to create an independent process with which the user can have high bandwidth interaction, i.e. real time manipulations, which can drive
Mosaic remotely. These techniques demonstrate that the use and interaction with spatially oniented information spaces are practical. Both techniques have advantages and disadvantages which are discussed.

\section{1,600}

PB95-171435 PC A03/MF A01

Flonida Atlantic Univ., Boca Raton. Robotics Center.

New Method to Calculate Looming for Autonomous Obstacle Avoldance.

K. Joarder, and D. Raviv. Nov 94, 32p, NISTIR-5512. Grant NSF-IRI-9115939

Sponsored by National Inst. of Standards and Technology (MEL), Gaithersburg, MD. Intelligent Systems Div, and National Science Foundation, Arlington, VA Div. of Information, Robotics and Intelligent Systems.

Keywords: "Autonomous navigation, "Obstacle avoidance, Computer vision, Cameras, Ocular fixation, Textures, Motion, Image processing, "Visual looming.

The idea of visual looming, i.e., the expansion of an object's size on the retina due to its decreasing distance from the observer, can be used as a powerful visual cue for autonomous obstacle avoidance. In this paper we describe a method of measuring looming quantitatively by fixating a camera at a point on the surface of an object and studying the texture near this point. A reduced distance of the camera from the surface results in a decline in the density of the texture primitives in the image. We show analytically how looming can be calculated from the relative change in this density and from the local orientation of the surface. The method is applicable for both stationary and moving obstacles and does not require calculation of range. Results of looming are presented for various textural surfaces, in different orientations.

\section{1,601}

PB95-171955 PC A05/MF A01

National Inst. of Standards and Technology (CSL), Gaithersburg, MD. Computer Security Div.

Multi-Agency Certification and Accreditation (C and A) Process: A Worked Example.

E. Flahavin, A. Lee, and D. Wolcott. Dec 94, 86p, NISTIR-5540.

Contract DEA-50SBNB1C6732

Sponsored by Drug Enforcement Administration, Washington, DC

Keywords: "Federal agencies, "Computer security Data processing security. Systems engineening, Federal information processing standards, Risk assessment DEA(Drug Enforcement Administration) C\&A(Certification and Accreditation), Mountain Pass project.

This document describes a worked example of a multiagency certification and accreditation (C\&A) process implemented by several Federal agencies. The objective of the document is to provide a practical example of how to perform multi-agency C\&A. The example is based on a project implemented for the Drug Enforcement Administration (DEA), called Mountain Pass. The project was implemented to improve the EI Paso Intelligence Center (EPIC) information system and related communications to satisfy EPIC's current and anticipated system needs.

\section{1,602}

PB95-180386 Not available NTIS

National Inst. of Standards and Technology (CSL), Gaithersburg, MD.

Taxonomy for Security Standards.

Final rept.

W. A. Jansen. 1994, 10p.

Pub. in Proceedings of National Computer Security Conference (17th), Baltimore, MD., October 11-14 1994, p165-174.

Keywords: "Computer security, "Standards, "Taxonomy, US DOD, Standardization, Classifications, Reprints.

This paper presents a taxonomy of security standards developed to ensure a systematic review of security standardization areas appropriate to the Department of Defense (DoD) Goal Security Architecture. The taxonomy relies on a simple paradigm based on the notions of uniformity and quality. This approach attempts to provide full coverage of all relevant security standards, yet be simple to understand and apply. The taxonomy is also open to further refinements and adjustments. Because of the flexibility and simplicity of the taxonomy, other initiatives involving the classification of standards may benefit from its use.
01,603

PB95-203089 Not available NTIS

National Inst. of Standards and Technology (EEEL), Gaithersburg, MD. Electricity Div.

Casual Regularizing Deconvolution Filter for Optimal Waveform Reconstruction.

Final rept.

N. G. Paulter. 1994, 8p.

Pub. in Institute of Electrical and Electronics Engineers Transactions on Instrumentation and Measurement 43 n5 p740-747 Oct 94.

Keywords: Iterative methods, Optimization, Convergence, Reprints, "Signal reconstruction, "Waveform reconstruction, Deconvolution filters.

A causal regularizing filter is described for selecting an optimal reconstruction of a signal from a deconvolution of its measured data and the measurement instrument's impulse response. Measurement noise and uncertainties in the instrument's response can cause the deconvolution (or inverse problem) to be ill-posed, thereby precluding accurate signal restoration. Nevertheless, close approximations to the signal may be obtained by using reconstruction techniques that alter the problem so that it becomes numerically solvable. A regularizing reconstruction technique is implemented that automatically selects the optimal reconstruction via an adjustable parameter and a specific stopping criterion, which is also described. Waveforms reconstructed using this filter do not exhibit large oscillations near transients as observed in other regularized reconstructions. Furthermore, convergence to the optimal solution is rapid.

\section{1,604}

PB95-210522 PC A03/MF A01

National Inst. of Standards and Technology (MEL), Gaithersburg, MD. Intelligent Systems Div.

Calculating Time-to-Contact Using Real-Time Quantized Optical Flow.

T. A. Camus. Mar 95, 18p, NISTIR-5609.

Keywords: "Computer vision, "Optical flow(Image analysis), Robotics, Autonomous navigation, Collision avoidance, Real time, Algorithms, Correlation, Measurement, "Time-to-contact, Time-to-collision.

Recently it has been demonstrated that robust, realtime optical flow is possible using standard computing hardware. One limitation of this correlation-based algorithm is that it does not give truly real-valued image velocity measurements. Therefore, it is not obvious that it can be used for a wide range of robotic vision tasks. One particular application for optical flow is time to-contact: based on the equations for the expansion of the optical flow field it is possible to compute the number of frames remaining before contact with an observed object. Although the individual motion measurements of this algorithm are of limited precision, they can be combined to produce remarkably accurate time-to-contact measurements, which can be produced in real-time on standard computing hardware.

\section{1,605}

PB96-122833 Not available NTIS

National Inst. of Standards and Technology (CSL) Gaithersburg, MD. Computer Security Div.

EDI and EFT' Security Standards.

Final rept.

E. Barker. 1994, 17p.

Pub. in Handbook of Information Security Management, 1994-95 Yearbook, Chapter IV-9, pS-397-S-413 1994.

Keywords: "Electronic security, "Data encryption "Cryptography, "Standards, Electronic funds transfer Data processing security Computer security, Risk management, Authentication, Confidentiality, Specifications, Reprints, Electronic data interchange.

The use of electronic rather than paper-based trans actions has reduced costs and improved efficiency for many organizations. However, the use of this technology also exposes the business community and its customers to potential/severe risks from the accidental or deliberate alteration of data. In response to this increased risk, several standards-setting bodies have developed or are developing standards to protect information transferred electronically. This chapter dis cusses two computer-based technologies--electronic funds transfer (EFT) and electronic data interchange (EDI)--and several of the standards that have been developed specifically for them including message authentication, encryption, and key management 


\section{General}

\section{1,606}

PB96-123484 Not available NTIS

National Inst. of Standards and Technology (CSL), Gaithersburg, MD. Computer Security Div.

Common Criteria: On the Road to International Harmonization.

Final rept.

J. Cugini. 1995, 6p.

Pub. in Computer Standards and Interfaces, v17 n4 p315-320 Sep 95.

Keywords: "Information technology, "Data processing security, "Systems management, Information systems, Information security, Computer security, Electronic security, Access control, Safeguards, Computer networks, Operating systems(Computers), Reprints Common criteria.

The Common Criteria is an effort of the United States, Europe, and Canada to align their existing Information Technology (IT) security criteria into one Common Criteria. The goals of the Common Criteria are to enhance existing IT product security development and evalua tion criteria, to protect previous investments in IT product security, and to facilitate international harmonization of IT product security development and evaluation criteria. The Common Criteria has both functional and assurance requirements. These requirements can be combined to form Protection Profiles and Security Targets.

\section{1,607}

\section{PB96-123492 Not available NTIS}

National inst. of Standards and Technology (CSL) Gaithersburg, MD. Computer Security Div.

Functional Security Criteria for Distributed Sys tems.

J. Cugini, R. Dobry, V. Gligor, and T. Mayfield. 1995 Pub. in National Information Systems Security Conference (18th), Baltimore, MD., October 10-13, 1995 p310-321.

Keywords: "Information technology, "Data processing security, "Systems management, "Access control, $R e$ prints, Computer security, Computer privacy, Data encryption, Cryptography, Data integrity, Authentication, Auditing, Distributed systems, Information systems, Computer networks, Requirements, Common Criteria.

The National Security Agency (NSA) with the cooperation of the National institute of Standards and Technology (NIST) formed a technical group to create security requirements for distributed systems. These in clude requirements for data confidentiality, data integrity, cryptography, distributed identification and authentication, as well as for access control, auditing, system management, trusted path, and trusted recovery for distributed systems. These requirements are being reviewed for incorporation within the Common Criteria which is a joint effort of the United States (NSA and NIST), Canada, France, Great Britain, and Germany to come up with a single criteria for security requirements.

\section{1,608}

PB96-131610 PC A13/MF A03

National Inst. of Standards and Technology (NCSL) Gaithersburg, MD. Computer Security Div.

Computer Security: An Introduction to Computer Security. The NIST Handbook.

Special pub.

B. Guttman, and E. A. Roback. Oct 95, 290p, NIST/ SP-800/12.

Also available from Supt, of Docs, as SNO03-00303374-0.

Keywords: "Computer security, "Electronic security, "Handbooks, Data processing security, Data integrity, Computer information security, Cryptography, Secure communications, Authentication, Computer networks, Access control, Risk management, Contingency planning, Disasters, Program management, Training, Guidelines, Management controls, Operational controls, Technical controls, Life cycle analysis.

The Handbook provides a broad overview of computer security to help readers understand their computer security needs and develop a sound approach to the selection of appropriate security controls. The Handbook includes chapters on each of the major types of com puter security controls and an example of how they could be applied within a hypothetical organization.
The Handbook contains references to more detailed how-to books and articles.

01,609

PB96-141304 Not available NTIS

National Inst. of Standards and Technology (EEEL), Gaithersburg, MD. Electricity Div.

Bounds on Least-Squares Four-Parameter Sine-Fit Errors Due to Harmonic Distortion and Noise. Final rept.

J. P. Deyst, T. M. Souders, and O. M. Solomon. $1995,6 p$

See aiso DE94007859.

Pub. in Institute of Electrical and Electronics Engineers Transactions on Instrumentation and Measurement, v44 n3 p637-642 Jun 95.

Keywords: "Least square fit, "Signal conditioning, Algorithms, Reprints, Errors, Harmonics, Noise.

Least-squares sine-fit algorithms are used extensively in signal-processing applications. The parameter estimates produced by such algorithms are subject to both random and systematic errors when the record of input samples consists of a fundamental sine wave corrupted by harmonic distortion or noise. The errors occur because, in general, such sine-fits will incorporate a portion of the harmonic distortion or noise into their estimate of the fundamental. Bounds are developed for these errors for least-squares four-parameter (amplitude, frequently, phase, and offset) sine-fit algorithms. The errors are functions of the number of periods in the records, the number of samples in the record, the number of samples in the record, the harmonic order, and fundamental and harmonic amplitudes and phases. The bound do not apply to cases in which harmonic components become aliased.

\section{1,610}

PB96-156112 Not available NTIS

National inst. of Standards and Technology (CSL), Gaithersburg, MD. Computer Secunty Div. Security Program Management.

Final rept.

B. Guttman, and E. Roback. 1993, 5p.

Pub. in CSL Bulletin, p1-5 Aug 93.

Keywords: "Computer security, "Information systems, * Federal agencies, Information resources, Information management, Electronic security, Access control, Computer networks, Policies, Systems analysis, Reprints.

This bulletin discusses the establishment and operation of a security program as a management function and describes some of the features and issues common to most organizations.

\section{1,611}

\section{PB96-160577 Not available NTIS}

National inst. of Standards and Technology (CSL), Gaithersburg, MD. Computer Security Div.

Addressing U.S. Government Security Requirements for OS!

Final rept.

N. A. Nazario. $1992,4 p$

Pub in Panel Session Overview for the National Computer Security Conference (15th), Baltimore, MD., October $13-16,1992, \mathrm{p} 740-743$.

Keywords: "Computer information security, "Data transmission, "Requirements, "Federal government, Computer networks, Data processing security, Electronic security, Secure communications, Protocols, Standards, Reprints, OSI(Open Systems Interconnection), GOSIP(Government Open Systems Interconnection Profile), Security labels.

This session focuses on security requirements that need to be addressed within the U.S. Government Open Systems interconnection Profile (FIPS 146-1) and current efforts to meet them. The session examines Government requirements, discusses emerging security protocols, and describes a labeling infrastructure necessary to support security services and protocols.

\section{1,612}

PB96-160817 Not available NTIS

National Inst. of Standards and Technology (CSL), Gaithersburg, MD. Systems and Software Technology Div.
Point Probe Decision Trees for Geometric Concept Classes.

Final rept

E. M. Arkin, M. T. Goodrich, J. S. B. Mitchell, S. S. Skiena, D. Mount, and C. D. Piatko. 1993, 12p.

Pub. in Workshop on Algorithms and Data Structures (WADS '93) (3rd), Montreal, Canada, Spring 1993, v709 p95-106.

Keywords: "Computer vision, "Decision tree analysis, Image resolution, Geometric accuracy, Computational geometry, Reprints, Probing.

A fundamental problem in model-based computer vision is that of identifying to which of a given set of concept classes of geometric models an observed model belongs. Considering a 'probe' to be an oracle that tells whether or not the observed model present at a given point in an image, we study the problem of computing efficient strategies ('decision trees') for probing an image, with the goal to minimize the number of probes necessary (in the worst case) to determine in which class the observed model belongs. We prove a hardness result and give strategies that obtain decision trees whose height is within a log factor of optimal.

01,613

PB96-160825 Not available NTIS

National Inst. of Standards and Technology (CSL), Gaithersburg, MD. Computer Security Div.

Precise Identification of Computer Viruses.

Final rept.

L. E. Bassham, and W. T. Polk. 1992, 9p.

Pub. in National Computer Security Conference Proceedings (15th), p1-9 1992

Keywords: "Computer viruses, "Personal computers, "Identification, Debugging(Computers), Data integrity, Data encryption, Error detection codes, Computer security, Electronic security, Vulnerability, Reprints.

The number of personal computer viruses continues to grow at an alarming rate. Many of these viruses are variants (i.e., close relatives) of 'old' viruses. This often resuits in less than accurate identification of viruses. A public domain technique for precise identification of viruses if needed, This paper explores various alternatives.

\section{1,614}

PB96-160882 Not available NTIS

National Inst. of Standards and Technology (CSL), Gaithersburg, MD. Computer Security Div.

Concept Paper: An Overview of the Proposed Trust Technology Assessment Program.

Final rept.

E. E. Flahavin, and P. R. Toth. 1992, 9p.

Pub. in National Computer Security Conference Pro. ceedings (15th), 9p 1992.

Keywords: "Computer information security, "Information technology, Electronic security, Data processing security, Classified information, Systems engineering, Performance tests, Assessments, Evaluation, Quality assurance, Reprints, "Trusted systems, National Voluntary Laboratory Accreditation Program. This paper provides an overview of the philosophy, objectives, and methodology of a proposed new program for the evaluation of trusted information technology products. The program will focus on products with the features and assurances characterized by the TCSEC (Trusted Computer System Evaluaton Criteria, or Orange Book) B1 and lower levels. The program is intended to be fully compatible with the Federal Criteria Version 1. The program will continue to emphasize the credibility and fairness of the evaluation process. The program will allow a sealless transition from the current process in which National Security Agency (NSA) alone evaluates products and populates the Evaluated Products List (EPL). The new program is to be called the Trust Technology Assesment Program (TTAP).

\section{1,615}

PB96-161815 Not available NTIS

National Inst. of Standards and Technology (CSL), Gaithersburg, MD. Computer Security Div.

Response to Comments on the NIST Proposed Digital Signature Standard.

Final rept.

M. E. Smid, and D. K. Branstad. 1992, 14p.

See also PB94-135001.

Pub. in Proceedings of Crypto '92, Santa Barbara, CA. August 16-20, 1992, p1-14.

Keywords: "Computer security, "Cryptography, "Meetings, Reprints, Certification, Standards, Data 
processing security, *Digital signatures, National Institute of Standards and Technology.

NIST received comments from 109 separate government agencies, companies, and private individuals concerning the proposed Digital Signature Standard. Both positive and negative comments were received. However, the number of negative comments was significantly larger than normally received for a proposed Federal Information Processing Standard (FIPS). This paper summarizes the major comments, both positive and negative and provides responses where appropriate. The paper highlights the anticipated significant modifications to the proposed standard and concludes by discussing the future milestones that need to be accomplished before the proposed DSS becomes a FIPS.

\section{1,616}

PB96-166004 PC A08/MF A02

National Inst. of Standards and Technology (CSL), Gaithersburg, MD. Computer Security Div.

Public Key Infrastructure Invitational Workshop. Held in McLean, Virginia on September 28, 1995 W. E. Burr. Nov $95,146 \mathrm{p}$, NISTIR-5788.

Sponsored by MITRE Corp., McLean, VA. Program Management Office.

Keywords: "Computer security, "Meetings, Computer networks, Cryptography, Data encryption, Access control, Data integrity, Authentication, Verification, Confidentiality, Data processing security, Electronic security, Infrastructure, Costs, "Public key certificates, *Digital signatures.

The document is the report of the Invitational Workshop on Public Key Infrastructure. A public key infrastructure provides a means for issuing and managing public key certificates, which may be used to provide security services, such as authentication, integrity, confidentiality and non-repudiation, between strangers who have no previous knowledge of each other. $\mathrm{Pa}$ pers were presented on the current state of technology and standards for a Public Key Infrastructure, management and technical issues, escrowing keys used for confidentiality exchanges, and cost models.

01,617

PB96-179155 (Order as PB96-177381, PC A07/ MF A02)

Cray Research, Inc., Calverton, MD.

MasPar MP-1 as a Computer Arithmetic Laboratory.

M. A. Anuta, D. W. Lozier, and P. R. Turner. 1996,

10p.

Prepared in cooperation with National Inst. of Standards and Technology, Gaithersburg, MD. and Naval Academy, Annapolis, MD.

Included in Jnl. of Research of the National Institute of Standards and Technology, v101 n2 p165-174 Mar/ Apr 96.

Keywords: *Computer arithmetics, Fixed-point, Floating-point, Logarithmic, Level-index, Reprints, Residue number system, Serial simulation, Parallel simulation.

The paper is a blueprint for the use of a massively parallel SIMD computer architecture for the simulation of various forms of computer arithmetic. The particular system used is a DEC/MasPar MP-1 with 4096 processors in a square array. This architecture has many advantages for such simulations due largely to the simplicity of the individual processors. Arithmetic operations can be spread across the processor array to simulate a hardware chip. Alternatively they may be performed on individual processors to allow simulation of a massively parallel implementation of the arithmetic.

01,618

PB96-195318 PC A03/MF A01

National Inst. of Standards and Technology (CSL), Gaithe rsburg, MD. Computer Security Div.

TMACH Experiment Phase 1. Preliminary Developmental Evaluation.

E. C. Flahavin. Jun 96, 14p, NISTIR-5810.

Sponsored by Defense Advanced Research Projects Agency, Arlington, $V A$.

Keywords: "Federal agencies, *Computer security, *Access control, "European communities, Data processing security, Electronic security, Privacy, Confideniality, Computer networks, Data management systems, Operating systems(Computers), Policies, Standards, Performance evaluation, Criteria, ${ }^{*}$ Trusted systems, Commercial Licensed Evaluation Facilities.
In November 1990, James Burrows, then Director of the Computer Systems Laboratory of the National Institute of Standards and Technology (NIST), met with European officials and Steve Walker of Trusted Information Systems to discuss initiating a preliminary developmental evaluation of the trusted Mach (Tmach) system against the European developed Inforamtion Security Evaluation Criteria (ITSEC). As a result, NIST finalized an agreement with the Advanced Research Projects Agency (ARPA) to coordinate and oversee the TMach evaluation work to be done by Germany and the United Kingdom. The document describes the multi-national evaluation experiment of the Trusted Mach system. The report focuses on Phase I - The Development Evaluation Phase. The objective is to provide a historical journal discussing the experiment, and providing insight into what has been learned and accomplished thus far. Discussed are the objectives of the effort, the participants, how the evaluation has proceeded, and the benefit. It is in the interest of the U.S. Government to understand how these two criteria differ in practice and how evaluations done under each may be compared.

\section{1,619}

PB97-110811 PC A05/MF A01

National Inst. of Standards and Technology (CSL), Gaithersburg, MD.

Computer Security: Generally Accepted Principles and Practices for Securing Information Technology Systems.

Special pub.

M. Swanson, and B. Guttman. Sep 96, 63p, NIST/ SP-800/14.

Keywords: "Computer security, Information systems, Federal agencies, Local area networks, Risk, Computer viruses, Computer prevacy, Data integrity, Management, Personal computers, Principles, Computer networks, Data encryption, "Generally Accepted Principles and Practices.

As more organizations share information electronically. a common understanding of what is needed and expected in securing information technology (IT) resources is required. This document provides a baseline that organizations can use to establish and review their IT security programs. The document gives foundation that organizations can reference when conducting multi-organizational business as well as internal business. Management, internal auditors, users, system developers and security practioners can use the guideline to gain an understanding of the basic security requirements most IT systems should contain. The foundation begins with generally accepted system security principles and continues with common practices that are used in securing IT systems.

\section{Computer Hardware}

01,620

N95-24130/3 (Order as N95-24108, PC A17/MF

A04)

National Inst. of Standards and Technology, Gaithersburg, MD.

Optical Storage Media Data Integrity Studies.

F. L. Podio. Mar 94, 12p.

In NASA. Goddard Space Flight Center, Fourth NASA Goddard Conference on Mass Storage Systems and Technologies p 265-276.

Keywords: *Data storage, "Error correcting codes, "Optical disks, "Optical memory (Data storage), "Quality control, Degradation, Error analysis, Information systems, On-line systems.

Optical disk-based information systems are being used in private industry and many Federal Government agencies for on-line and long-term storage of large quantities of data. The storage devices that are part of these systems are designed with powerful, but not unlimited, media error correction capacities. The integrity of data stored on optical disks does not only depend on the life expectancy specifications for the medium. Different factors, including handling and storage conditions, may result in an increase of medium errors in size and frequency. Monitoring the potential data degradation is crucial, especially for long term applications. Efforts are being made by the Association for Information and Image Management Technical Committee C21, Storage Devices and Applications, to specify methods for monitoring and reporting to the user medium errors detected by the storage device while writing, reading or verifying the data stored in that medium. The Computer Systems Laboratory (CSL) of the $\mathrm{Na}$ tional Institute of Standard and Technology (NIST) has a leadership role in the development of these standard techniques. In addition, CSL is researching other data integrity issues, including the investigation of error-resilient compression algorithms. NIST has conducted care and handling experiments on optical disk media with the objective of identifying possible causes of degradation. NIST work in data integrity and related stand. ards activities is described.

\section{1,621}

PB94-135761 PC A06/MF A02

National Inst. of Standards and Technology (CSL), Gaithersburg, MD.

Planning for the Fiber Distributed Data Interface (FDDI)

W. E. Burr. Oct 93, 110p, NIST/SP-500/212

Also available from Supt. of Docs. as SN003-003 03239-5. Sponsored by Internal Revenue Service, Washington, DC

Keywords: *Local area networks, *Standards, Fiber op tics, Workstations, Protocols, Wiring, "FDDI, "Fiber Distributed Data Interface, GOSIP(Government Open Systems Interconnection Profile), Token rings

The Fiber Distributed Data Interface (FDDI) is an emerging Standard fiber optic Local Area Network (LAN) technology suitable for backbone and high performance workstation applications. FDDI will be widely used by federal agencies in the 1990's. This report describes FDDI standards and the media that FDDI uses and provides information about writing for FDDI LANs and about effectively configuring FDDI LANs. It also describes the relationship of FDDI to the Government Open Systems Interconnection Profile (GOSIP) and discusses connecting FDDI to other networks.

\section{1,622}

PB94-162518 PC A05/MF A02

National Inst. of Standards and Technology (CSL), Gaithersburg, MD.

Computer Systems Laboratory Annual Report, 1993.

E. B. Lennon, S. M. Radack, and R. K. Roach. Feb 94, 100p, NISTIR-5342.

See also PB93-181873.

Keywords: "Computers, *Computer software, Organizational structure, Information systems, Systems engineering, Computer security, Telecommunication, Federal information processing standards, Computer networks, Computer architecture, Technology transfer "Computer Systems Laboratory, National Institute of Standards and Technology

The Computer Systems Laboratory (CSL) Annual Re port - 1993 describes the annual computer and related telecommiunications activities and accomplishments of the laboratory. Following the Director's Foreword, a CSL overview is presented, followed by overviews of the five technical divisions. The Technology Transfer section describes the vehicles used by CSL to disseminate research and technical information to the public A list of Federal Information Processing Standards (FIPS) and FIPS order information conclude the annual report.

01,623

PB94-216470 Not available NTIS

National Inst. of Standards and Technology (NEL) Gaithersburg, MD. Electricity Div.

Guarding Against Transients.

Final rept

F. D. Martzloff. 1990, 1p.

Pub. in Institute of Electrical and Electronics Engineers Spectrum, p16 Dec 90.

Keywords: "Computers, "Surges, "Circuit protection, Reliability, Data processing, Electromagnetic interference, Quality, Overvoltage, Overcurrent, Reprints.

This article is a response to a reader's comment on the IEEE Spectrum article (April 1990) by Martzloff entitled Protecting Computer Systems Against Power Transients.

\section{1,624}

PB95-108486 Not available NTIS

National Inst. of Standards and Technology (NCSL), Gaithersburg, MD. Advanced Systems Div. 
Standardization of Testing Methods for Optical Disk Media Characteristics and Related Activities at NIST.

Final rept.

F. L. Podio, R. Onyshczak, and E. S. Villagran. 1990, $5 \mathrm{p}$.

Pub. in Optical Information Systems 10, n4 p174-178 Jul/Aug 90.

Keywords: "Optical disks, "Tests, "Standardization, Media, Standards, Optical storage materials, Service life, Reprints, US NIST.

Standard testing methods for measuring the characteristics of optical disks are needed to verify conformance to the related industry media interchange standards. Technical Committee (TC) X3B11 is addressing the standardization of those testing methods. Through its optical disk computer storage media research program, the National Institute of Standards and Technology, National Computer Systems Laboratory (NIST/ NCSL) is involved in the research of testing methods for media characteristics and is participating in the development of optical media standards in TC X3B11 and other standard committees. Standard testing methods for predicting the life expectancy of the media are also needed. NIST is designed a methodology for predicting life expectancy of optical media and is also contributing to those standardization efforts and data permanence studies.

\section{1,625}

PB95-136388 PC A11/MF A03

National Inst. of Standards and Technology (CSL) Gaithersburg, MD. Advanced Systems Div.

NIST Workshop on the Computer Interface to Flat Panel Displays. Held in San Jose, California on January 13-14, 1994.

Special pub.

M. P. Williamson, W. E. Burr, and J. W. Roberts. Aug 94, 231p, NIST/SP-500/219.

Also available from Supt. of Docs. as SN003-00303289-1.

Keywords: "Display devices, "Standards, "Meetings, Liquid crystal display systems, Cathode ray tubes, Electric connectors, Personal computers, Flat surfaces, Workstations, "Flat panel displays, "Compute interface, Interface standards, US NIST.

On January 13 and 14, 1994, the NIST Workshop on the Computer Interface to Flat Panel Displays was held at the San Jose Hilton and Towers in San Jose, California. The meeting was attended by representatives from computer, flat panel display, and graphics controller companies. The objectives of this workshop were: to determine the need for a standard or a series of standards for the computer interface to flat panel displays: to identify what types of standards are needed; to identify approaches for developing flat panel display interface standards; and to obtain a consensus on a coordinated plan for standards development. The workshop attendees agreed that a standard flat panel display interface for integrated devices is needed. A special interest group was formed under the Video Electronics Standards Association (VESA) to undertake the development of a standard or a series of standards for the interface between a flat panel display and its controller. This interface standard will address both active and passive flat panel displays in integrated devices. It will cover both the electrical and the mechanical (connector) specifications. The interface for remote flat panel displays requires additional technical discussions before a standard can be written. Additional workshops on display interfaces and/or technical sessions at the SID (Society for Information Display) Symposium should be explored. In addition, the VESA Monito Committee invited interested parties to participate in their committee which will be considering interfaces and connectors to desktop and remote flat panel displays in addition to their CRT interface work.

\section{1,626}

PB95-169496 PC A08/MF A02

National Inst. of Standards and Technology (CSL) Gaithersburg, MD. Systems and Software Technology Div.

Guide on Open System Environment (OSE) Procurements.

Special pub.

G. E. Fisher. Oct $94,161 \mathrm{p}$, NIST/SP-500/220.

Also available from Supt. of Docs. as $\mathrm{SN}-003-003$ 03302-2. See also PB95-166260

Keywords: "Government procurement, Requirements, Guidelines, Specifications, Operating
systems(Computers), Man computer interface, Software engineering, Data management, Data transfer(Computers), Computer graphics, Computer networks, Management, Computer systems hardware, ${ }^{*} \mathrm{OSE}\left(\right.$ Open System Environment), ${ }^{*}$ Open System Environment, RFPs(Requests for proposals)

The guide provides program managers and senior system engineers with much-needed information for developing plans and requirements to establish an Open System Environment (OSE). U.S. Government procurements that have already taken place have provided numerous lessons-learned which have been in corporated in this report. A decision model is defined in the introductory material to provide a mechansim for making choices concerning the applicability of standards. Side-bars in the text provide associated information about the use of specific standards in order to more clearly describe the effects of relationships among different standards. Additional information in cludes text that can be included in requests for proposals (RFPs), a sample statement of work based on the guidance contained in the report, a glossary, and methods for evaluating individual proposals made in response to OSE requirements.

\section{1,627}

PB95-189437 PC A03/MF A01

National Inst. of Standards and Technology (CAML), Gaithersburg, MD. Applied and Computational Mathematics Div.

MasPar MP-1 as a Computer Arithmetic Laboratory.

M. A. Anuta, D. W. Lozier, and P. R. Turner. Jan 95, 24p, NISTIR-5569.

Prepared in cooperation with Cray Research, Inc. Calverton, MD. and Naval Academy, Annapolis, MD. Dept. of Mathematics.

Keywords: "Arithmetic units, " SIMD(Computers), Algorithms, Computer architecture, Computer systems hardware, Massively parallel processors, Very large scale integration, Computer software, Array processors, "DEC/MasPar MP-1 computer systems, LI(Level-index) arithmetic, SLI(Symmetric level-index) arithmetic.

This paper describes the use of a massively parallel SIMD computer architecture for the simulation of various forms of computer arithmetic. The particular system used is a DEC/MasPar MP-1 with 4096 processors in a square array. This architecture has many advantages for such simulations due largely to the simplicity of the individual processors. Arithmetic operations can be spread across the processor array to simulate a hardware chip. Alternatively, they may be performed on individual processors to allow simulation of a massively parallel implementation of the arithmetic. Compromises between these extremes permit speed-area trade-offs to be examined. The paper includes a description of the architecture and its features. It then summarizes some of the arithmetic systems which have been, or are to be implemented. The implementation of the level-index (LI) and symmetric level-index (SLI) systems is described in some detail. An extensive bibliography is included.

\section{1,628}

PB95-189486 PC A03/MF A01

National Inst. of Standards and Technology (CSL), Gaithersburg, MD. Advanced Systems Div.

Operating Principles of MultiKron II Performance Instrumentation for MIMD Computers.

A. Mink. Dec 94, 32p, NISTIR-5571.

See also PB92-181072. Sponsored by Advanced Research Projects Agency, Arlington, VA

Keywords: "MIMD(Computers), "Very large scale integration, Chips(Electronics), Multiprocessors, Computer performance evaluation, Parallel processors, "Multikron II.

The Multikron II design is an enhanced version of our earlier Multikron performance instrumentation chip. They are both 179 pin chips, and although pin compatibility is close they are not $100 \%$ compatible. The MultiKron II has a longer TRACE sample, 20 bytes vs 16 , to allow a larger user data and Timestamp fields. The 16 counters for resource utilization measurements are now writable, to allow for 'virtual' use, and their programming interface has changed to allow more options. Although designed for a 64-bit processor bus interface, an internal holding register provides for 32 bit mode operation.
National Inst. of Standards and Technology (CSL), Gaithersburg, MD.

Computer Systems Laboratory Annual Report 1994.

E. B. Lennon, S. M. Radack, and R. K. Roach. 26 Mar 92, 126p, NISTIR-5576.

See also PB94-162518.

Keywords: "Computers, "Information technology, *Information systems, Systems engineering, Computer security, Organizational structures, Computer software, Telecommunication, Federal Information Processing Standards, Computer networks, Computer architectures, Technology transfer, US NIST, *Computer Systems Laboratory.

The annual report describes the annual computer and related telecommunications activities and accomplishment of the Laboratory. Following the Director's Foreword, an overview of the Laboratory is presented, including a current CSL Organization Chart. Overviews of CSL's major technical units are featured next, followed by a section on Interactions and Accomplishments which details the vehicles CSL uses to disseminate research and inforamtion to the public and technical communities. A list of Federal Information Proc essing Standards (FIPS) concludes the annual report.

01,630

PB95-231783 PC A03/MF A01

National Inst. of Standards and Technology (CSL), Gaithersburg, MD

Operating Principles of the SBus MultiKron Interface Board.

A. Mink, G. G. Nacht, and J. K. Antonishek. May 95 , $14 p$, NISTIR-5652.

See also PB92-181072 PB93-234730 and PB95189486. Sponsored by Defense Advanced Research Projects Agency, Arlington, VA.

Keywords: "Computer systems hardware, "Circuit boards, "Very large scale integration, Printed circuits, MIMD(Computers), Multiprocessors, Computer architecture, Data acquisition, SIB(SBus Multikron Interface Board), US NIST

The MultiKron Experimenter's Toolkit contains an SBus Multikron interface board (SIB) or alternatively a VME MultiKron interface board (MIB), installation software, data logging software, and analysis software all of the software supplied is written in C. The Toolkit allows users to take advantage of the National Institute of Standards and Technology (NIST) Multikron performance measurement chip in systems that do not al ready have a MultiKron designed into them. The SIB is applicable to both multiprocessor systems and single-processor systems. The Experimenter's Toolkit allows researchers to obtain hands-on experience with the MultiKron performance measurement chip, without the engineering effort required to design and build a hardware interface between the Multikron and their computer. Over 800,000 Trace Samples can be collected during an experiment to the SIB on-board memory; a practically-unlimited number of Samples can be collected if an optional external data-collection computer is used.

01,631

PB96-102686 Not available NTIS

National Inst. of Standards and Technology (CSL) Gaithersburg, MD.

Conformance Testing for OSI Protocols.

Final rept.

R. J. Linn. 1990, 17p.

Pub. in Computer Networks and ISDN Systems 18 , p203-219 1989/90.

Keywords: "Computer communications, "ISO, *Protocols, Computer architecture, Communication networks, Conformity, Tests, Test methods, Specifications, Standards, Reprints, "OSI(Open Systems Interconnection), "Open Systems Interconnection, Test systems, ANC1(Abstract Syntax Notation 1), TICN(Tree and Tabular Combined Notation), Estelle.

In the early 1980's, research and development was initiated on methods to test the developing Internationa Standards Organinzation's Open Systems Interconnection (ISO/OSI) communications protocols. A tutorial overview of the test methods and test notation med TTCN which were developed within ISO are presented. Issues regarding multilayer test methods are still not completely resolved. These issues are identified and alternatives to ISO's methods are explored. Application of the formal description techniques named ASN.1 and Estelle to multi-layer test systems 
is illustrated. Concluding remarks summarize the status of current practice in conformance testing and status of evolving OSI testing standards.

01,632

PB96-131529 PC A03/MF A01

National Inst. of Standards and Technology (CSL), Gaithersburg, MD. Advanced Systems Div.

Operating Principles of Multikron Virtual Counter Performance Instrumentation for MIMD Computers.

A. Mink. Nov 95, 24p, NISTIR-5743

See also PB93-234730, PB95-189486 and PB95231783. Sponsored by Defense Advanced Research Projects Agency, Arlington, VA.

Keywords: "Computer systems hardware, *Multiprocessors, *Circuit boards, Very large scale integration, MIMD(Computers), Printed circuits, Computer architecture, Pertormance tests, Multikron Performance Instrumentation.

The MultiKron* and MultiKron II performance instrumentation provided both Trace sampling and Resource Counters, but required a separate measurement data collection facility for collecting sample data. Although providing a large amount of measurement detail, trace sampling has the disadvantage of requiring additional investment in logic, wires and space to provide for the collection facilities. An alternative measurement approach that would eliminate the need for a collection tacility, and its associated cost, is to eliminate Trace sampling and only provide for a very large number of Resource Counters, at the cost of some loss of measurement detail. The Multikron virtual counter (Multikron vc) performance instrumentation chip provides such a feature. Similar in concept to virtual memory, thousands of virtual counters are available but only a small number are real counters that can be active at any one time. Unlike virtual memory, where swapping is transparent to the programmer, due to extra hardware and kernel software support, swapping of counter blocks must currently be handled by the programmer.

\section{1,633}

PB96-160304 Not available NTIS

National Inst. of Standards and Technology (CSL), Gaithersburg, MD. Advanced Systems Div.

Research on Methods for Determining Optical Disk Media Life Expectancy Estimates.

Final rept.

F. L. Podio. 1992, 9p.

Pub. in Sociefy of Photo-Optical Instrumentation Engineers, v1663 p447-455 Feb 92

Keywords: "Optical data storage materials, "Life(Durability), "Aging tests(Materials), Optical disks, Service life, Stress testing. Test methods, Error analysis, Quality control, Standards, Reprints, Arrhenius model, Byte error rate.

Methodologies for determining extrapolated life ex pectancy values for optical disk media were inves tigated at the National Institute of Standards and Technology (NIST). These investigations showed that the life expectancy values of optical disk media vary greatly due to several factors, including the method used to derive the value of the quality parameter (the byte error rate), the areas measured in the disks, the written patterns used, and the criteria for data analysis. Vendor's life expectancy claims can be properly assessed by prospective users if standard methods for calculating and reporting extrapolated life expectancy values are implemented.

01,634

PB96-160619 Not available NTIS

National Inst. of Standards and Technology (CSL), Gaithersburg, MD. Advanced Systems Div.

Status of Emerging Standards for Removable Computer Storage Media and Related Contributions of NIST.

Final rept

F. L. Podio. 1991, 25p

Aiso available as N93-14778 (Order as N93-14771). Pub. in National Space Science Data Center Conference on Mass Storage Systems and Technologies for Space and Earth Science Applications, Greenbelt, MD., July 23-25, 1991, NASA Conference Publication 3165, v3 p163-187.

Keywords: "Computer storage devices, "Data storage, "Digital data, Magnetic tapes, Optical disks, Optical memory(Data storage), Optical data (Storage mate- rials), Interchangeability, Error detection codes, Life Durability), Service life, Error analysis, Standards, Reprints, Siandard reference materials.

Standards for removable computer storage media are needed so that users may reliably interchange data both within and among various computer installations. Industry standards for digital magnetic tapes require the use of Standard Reference Materials (SRMs) developed and maintained by NIST In addition, NIST has been studying Care and handling procedures for optical digital data disks and is involved in a program to investigate error reporting capabilities of optical disk drives. NIST has developed a methodology for determining the life expectancy of optical disks. NIST is developing care and handling procedures for optical digial data disks and is involved in a program to inves tigate error reporting capabilities of optical disk drives.

\section{1,635}

PB96-160627 Not available NTIS

National Inst. of Standards and Technology (CSL), Gaithersburg, MD. Advanced Systems Div.

NIST Program for Investigating Error Reporting Capabilities of Optical Disk Drives.

Final rept.

F. L. Podio. 1991, 8p.

Pub. in Proceedings of a Workshop held in Colorado Springs, CO., August 5, 1991, p1-8.

Keywords: "Error detection codes, "Optical disks, *Research programs, Computer storage devices, Optical data storage materials, Optical memory(Data storage), Quality control, Error correcting codes, Life(Durability), Service life, Error analysis, Reprints.

Optical disk drives are designed with sirong but unlimted error corrections capabilities. If the level of errors overcome the error detection and corrections mechanisms implemented in the optical disk drive controllers, incorrectable errors may occur. Federal Government data managers are interested in being able to monito error rate activity in optical disk drive systems. This could be achieved through the interface, if the drives would provide information on correctable error rate activity, such as maximum number of correctable errors, maximum number of errors per interleave, and the location of those errors. Reports on errors encountered while reading header information is also necessary. This information would provide data managers with a better understanding of the status of their data and would allow them to design more efficient recopying policies to copy the data to similar or different media in a timely and economic fashion.

\section{1,636}

PB96-161252 Not available NTIS

National Inst. of Standards and Technology (CSL), Gaithersburg, MD.

Information Technology Standards in Federal Acquisitions.

Final rept.

S. M. Radack. $1992,8 p$

See also PB96-161260

Pub. in Federal Data Center Issues 1992. A View to the Future Council of Federal Data Center Directors, p1-8.

Keywords: "Information technology, "Government procurement, "Electronic security, "Standards, Federal agencies, Acquisition, Information resources, Computer program portability, Interoperability, Computer information security, Reprints, GOSIP(Government Open Systems Interconnection Profile), POSIX(Portable Operating Sytems Interface for Com puter Environments), Conformance testing.

Information technology (IT) standards are an importan part of Federal Government strategies for managing information resources. Standards help to promote the interoperability of different manufactures' systems, the portability of applications, and the sharing of ideas data, and training. But while the abstract concept of standards is acceptable and easily understood, it is no always easy for managers and users to put standards to work for their organizations. This paper discusses some of the activities of the National Institute of Standards and Technology (NIST) that support the implementation of standards in products that organizations can buy.

01,637

PB96-161260 Not available NTIS

National Inst. of Standards and Technology (CSL) Gaithersburg, MD.
Using Information Technology Standards in Federal Acquisitions.

Final rept.

S. M. Radack. 1992,6p

See also PB96-161252

Pub. in Computer Systems Laboratory Bulletin, p1-6 Dec 92.

Keywords: "Information technology, "Government procurement, "Electronic security, "Standards, Federal agencies, Acquisition, Information resources, Computer program portability, Interoperability, Computer information security, Reprints, GOSIP(Government Open Systems Interconnection Profile), POSIX(Portable Operating Sytems Interface for Computer Environments), Conformance testing.

Information technology (IT) standards are an important part of federal government strategies for managing information resources. Standards help to promote the interoperability of different manufactures' systems, the portability of applications, and the sharing of ideas, data, and training.

01,638

PB96-193768 PC A05/MF A01

National Inst. of Standards and Technology (CSL), Gaithersburg, MD. Software Diagnostics and Conform ance Testing Div

Computer Systems Laboratory Computing and Applied Mathematics Laboratory Technical Accomplishments, October 1994-March 1996.

E. B. Lennon. Jun $96,67 p$, NISTIR-5854.

Keywords: "Computers, "Information technology, *Information systems, "Applied mathematics, Systems engineering, Computer security, Organizational structures, Computer software, Telecommunications, Computer networks, Computer architecture, Technology transter, Research programs, "Computer Systems Laboratory, National Institute of Standards and Technology.

The document describes the technical accomplishments and activities of National Institute of Standards and Technology (NIST's) Computer Systems Laboratory/Computing and Applied Mathematics Laboratory during the period October 1994 through March 1996.

\section{Computer Software}

01.639

AD-A274 872/1 PC A04/MF A01

National Inst. of Standards and Technology,

Ada Compiler Validation Summary Report: Certificate Number: 931029S1.11330, Digital Equipment Corporation, DEC Ada for DEC OSF/1 AXP Systems, Version 3.1, DEC 3000 Model 400 AXP Workstation, DEC 3000 Model 400 AXP Workstation.

26 Oct 93, 70p

Keywords: "Compilers, "Ada programming language, Standards, Test and evaluation, "Validation summary reports.

The Ada implementation described above was tested according to the Ada Validation Procedures (Pro92) against the Ada Standard (Ada83) using the current Ada Compiler Validation Capability (ACVC). This Validation Summary Report (VSR) gives an account of the iesting of this Ada implementation.

\section{1,640}

AD-A275 977/7 PC A03/MF A01

National Inst. of Standards and Technology Gaithersburg, MD.

Ada Compiler Validation Summary Report: Certificate Number: 931217S1.11336 Control Data Systems, Inc. NOS NE Ada, Version 1.4 Cyber 180-930 $31 \Rightarrow$ Cyber 180-930-31.

17 Dec 93, 48p.

Keywords: "Compilers, "Ada programming language Validation, Data processing, Standards, "Validation summary reports, Computer program verification.

This Valldation Summary Report describes the extent to which a specific Ada compiler conforms to the Ada Standard, ANSI/MIL-STD-1815A. This report explains all technical terms used within it and thoroughly reports 


\section{Computer Software}

the results of testing this compiler using the Ada Compiler Validation Capability. An Ada compiler must be implemented according to the Ada Standard, and any implementation-dependent features must conform to the requirements of the Ada Standard. The Ada Stand ard must be implemented in its entirety, and nothing can be implemented that is not in the Standard. Even though all validated Ada compilers conform to the Ada Standard, it must be understood that some differences do exist between implementations. The Ada Standard permits some implementation dependencies--for example, the maximum length of identifiers or the maximum values of integer types. Other differences between compilers result from the characteristics of particular operating systems, hardware, or implementation strategies. All the dependencies observed during the process of testing this compiler are given in this report. The information in this report is derived from the test results produced during validation testing. The validation process includes submitting a suite of standard ized tests, the ACVC, as inputs to an Ada compiler and evaluating the results.

01,641

AD-A276 181/5 PC A04/MF A01 National Inst. of Standards and Technology,

Ada Compiler Validation Summary Report. Certificate Number: 931119S1.11332, DDC-1, Inc. DACS MIPS R3000 Bare Ada Cross Compiler System, Version 4.7.1 Sun SPARCstation IPX $\Rightarrow$ DACS Sun SPARC/SunOS to MIPS R3000 Bare Instruction Set Architecture Simulator, Version 4.7.1.

10 Dec 93, 55p, NIST92DDI510-5-1.11.

Keywords: "Compilers, "Ada programming language, Test and evaluation, Standards, "Validation summary reports, Computer program verification.

This Validation Summary Report describes the extent to which a specific Ada compiler conforms to the Ada Standard, ANSI/MIL-STD-1815A. This report explains all technical terms used within it and thoroughly reports the results of testing this compiler using the Ada Compiler Validation Capability. An Ada compiler must be implemented according to the Ada Standard, and any implementation-dependent features must conform to the requirements of the Ada Standard. The Ada Standard must be implemented in its entirety, and nothing can be implemented that is not in the Standard. Even though all validated Ada compilers conform to the Ada Standard, it must be understood that some differences do exist between implementations. The Ada Standard permits some implementation dependencies--for ex ample, the maximum length of identifiers or the maximum values of integer types. Other differences be tween compilers, result from the characteristics of particular operating systems, hardware, or implementation strategies. All the dependencies observed during the process of testing this compiler are given in this report. The information in this report is derived from the tes results produced during validation testing. The validation process includes submitting a suite of standardized tests, the ACVC, as inputs to an Ada compiler and evaluating the results.

\section{1,642}

AD-A276 283/9 PC A08/MF A02

National Inst. of Standards and Technology,

Ada Compiler Validation Summary Report: Certificate Number: 931119S1.11331 DDC-I, Inc. DACS Sun SPARC/SunOS to 80386 PM Bare Ada Cross Sun SPARC/SunOS to 80386 PM Bare Ada Cross
Compiler System, Version 4.6.4 Sun Sparcstation $1+=>$ Bare Board iSBC $386 / 116$.

19 Nov $93,158 p$

Keywords: "Compilers, "Ada programming language, Standards, "Validation summary reports, Computer program verification, Runtime.

This Validation Summary Report describes the extent to which a specific Ada compiler conforms to the Ada Standard, ANSI/MIL-STD-1815A. This report explains all technical terms used within it and thoroughly reports the results of testing this compiler using the Ada Compiler Validation Capability. An Ada compiler must be implemented according to the Ada Standard, and any implementation-dependent features must conform to the requirements of the Ada Standard. The Ada Standard must be implemented in its entirety, and nothing can be implemented that is not in the Standard. Even though all validated Ada compilers conform to the Ada Standard, it must be understood that some differences do exist between implementations. The Ada Standard permits some implementation dependencies--for ex- ample, the maximum length of identifiers or maximum values of integer types Other differences between compilers result from the characteristics of particular operating systems, hardware, or implementation strategies. All the dependencies observed during the process of testing this compiler are given in this report. The information in this report is derived from the test results produced during validation testing. The validation process includes submitting a suite of standardized tests the ACVC, as inputs to an Ada compiler and evaluating the results.

\section{1,643}

\section{AD-A277 981/7 PC A03/MF A01}

National Inst. of Standards and Technology (NCSL) Gaithersburg, MD. Software Standards Validation Group.

Ada Compiler Validation Summary Report. Certificate Number: 930927S1.11328 Green Hills Software C Ada, Version 1.1 ZENY 386 => ZENY 386 30 Sep $93,324 p$.

Keywords: "Compilers, "Ada programming language, Standards, "Validation summary reports, Computer program verification.

The Ada implementation described was tested according to the Ada Validation Procedures (Pro92) against the Ada Standard (Ada83) using the current Ada Compiler Validation Capability (ACVC). This Validation Summary Report (VSR) gives an account of the testing of this Ada implementation.

\section{1,644}

AD-A279 642/3 PC A06/MF A02

National Inst. of Standards and Technology, Gaithersburg, MD

Ada Compiler Validation Summary Report: Certificate Number: 940325S1.11348 DDC-I, DACS Sun SPARC/Solaris to 80386 PM Bare Ada Cross Com piler System, Version 4.6.4 Sun SPARCclassic => Intel iSBC 386/116 (Bare Machine).

25 Mar 94, 118p.

Keywords: "Compilers, "Ada programming language, Standards, Validation.

This Validation Summary Report describes the extent to which a specific Ada compiler conforms to the Ada Standard, ANSI/MIL-STD-1815A. This report explains all technical terms used within it and thoroughly reports the results of testing this compiler using the Ada Compiler Validation Capability. An Ada compiler must be implemented according to the Ada Standard, and any implementation-dependent features must conform to the requirements of the Ada Standard. The Ada Standard must be implemented in its entirety, and nothing can be implemented that is not in the Standard. Even though all validated Ada compilers conform to the Ada Standard, it must be understood that some differences do exist between implementations. The Ada Standard permits some implementation dependencies--for example, the maximum length of identifiers or maximum values of integer types. Other differences between compilers result from the characteristics of particular operating systems, hardware, or implementation strategies. All the dependencies observed during the process of testing this compiler are given in this report. The information in this report is derived from the test results produced during validation testing. The validation process includes submitting a suite of standardized tests, the ACVC, as inputs to an Ada compiler and evaluating the results

\section{1,645}

AD-A279 $643 / 1$ PC A06/MF A02
National Inst. of Standards and Technology, Gaithersburg, MD

Ada Compiler Validation Summary Report: Certificate Number: 940325S1.11341 DDC-I, DACS Sun SPARC/SunOS to 80186 Bare Ada Cross Compiler System, Version 4.6.4 Sun SPARCstation IPX $=>$ Intel iSBC 186/100 (Bare Machine).

25 Mar 94, 118p.

Keywords: "Compilers, "Ada programming language Standards, "Validation summary reports, Computer program verification.

This Validation Summary Report describes the extent to which a specific Ada compiler conforms to the Ada Standard, ANSI/MIL-STD-1815A. This report explains all technical terms used within it and thoroughly reports the results of testing this compiler using the Ada Compiler Validation Capability. An Ada compiler must be implemented according to the Ada Standard, and any implementation-dependent features must conform to the requirements of the Ada Standard. The Ada Standard must be implemented in its entirety, and nothing can be implemented that is not in the Standard. Even though all validated Ada compilers conform to the Ada Standard, it must be understood that some differences do exist between implementations. The Ada Standard permits some implementation dependencies--for example, the maximum length of identifiers or maximum values of integer types. Other differences between compilers result from the characteristics of particular operating systems, hardware, or implementation strategies. All the dependencies observed during the process of testing this compiler are given in this report. The information in this report is derived from the test results produced during validation testing. The validation process includes submitting a suite of standardized tests, the ACVC, as inputs to an Ada compiler and evaluating the results.

01,646

AD-A279 644/9 PC A06/MF A02

National Inst. of Standards and Technology,

Ada Compiler Validation Summary Report: CertifiAda Compiler Validation Summary Report: Certificate Number: 94032551.11349 DDC-1, DACS Sun
SPARC/Solaris to 80386 PM Bare Ada Cross Compiler System with Rate Monotonic Scheduling, Version 4.6.4 Sun SPARCclassic $=>$ Intel iSBC $386 / 116$ (Bare Machine).

25 Mar 94, 119p.

Keywords: "Compilers, "Ada programming language, Standards, "Validation summary reports, Computer program verification.

This Validation Summary Report describes the extent to which a specific Ada compiler conforms to the Ada Standard, ANSI/MIL-STD-1815A. This report explains all technical terms used within it and thoroughly reports the results of testing this compiler using the Ada Compiler Validation Capability. An Ada compiler must be implemented according to the Ada Standard, and any implementation-dependent features must conform to the requirements of the Ada Standard. The Ada Standard must be implemented in its entirety, and nothing can be implemented that is not in the Standard. Even though all validated Ada compilers conform to the Ada Standard, it must be understood that some differences do exist between implementations. The Ada Standard permits some implementation dependencies--for example, the maximum length of identifiers or maximum values of integer types. Other differences between compilers result from the characteristics of particular operating systems, hardware, or implementation strategies. All the dependencies observed during the process of testing this compiler are given in this report. The information in this report is derived from the test results produced during validation testing. The validation process includes submitting a suite of standardized tests, the ACVC, as inputs to an Ada compiler and evaluating the results.

\section{1,647}

AD-A279 645/6 PC A04/MF A01

National Inst. of Standards and Technology, Gaithersburg, MD.

Ada Compiler Validation Summary Report: Certificate Number: 940325S1.11354 DDC-1, DACS Sun SPARC/Solaris Native Ada Compiler System, Version 4.6.2 Sun SPARCclassic $\Rightarrow$ Sun SPARCclassic.

25 Mar 94, 57p.

Keywords: "Compilers, "Ada programming language, Standards, "Validation summary reports, Computer program verification.

This Validation Summary Report describes the extent to which a specific Ada compiler conforms to the Ada Standard, ANSI/MIL-STD-1815A. This report explains all technical terms used within it and thoroughly reports the results of testing this compiler using the Ada Compiler Validation Capability. An Ada compiler must be implemented according to the Ada Standard, and any implementation-dependent features must conform to the requirements of the Ada Standard. The Ada Standard must be implemented in its entirety, and nothing can be implemented that is not in the Standard. Even though all validated Ada compilers conform to the Ada Standard, it must be understood that some differences do exist between implementations. The Ada Standard permits some implementation dependencies--for example, the maximum length of identifiers or maximum values of integer types. Other differences between compilers result from the characteristics of particular 
operating systems, hardware, or implementation strategies. All the dependencies observed during the process of testing this compiler are given in this report. The information in this report is derived from the test results produced during validation testing. The validation process includes submitting a suite of standardized tests, the ACVC, as inputs to an Ada compiler and evaluating the results.

\section{1,648}

AD-A279 646/4 PC A05/MF A02 National Inst. of Standards and Technology,

Ada Compiler Validation Summary Report: Certificate Number: 940325S1.11346 DDC-1, DACS Sun SPARC/Sunos to $680 \times 0$ Bare Ada Cross Compiler System (BASIC MODE), Version 4.6.9 Sun SPARCstation IPX $=>$ Lynwood j435TU (68030) (Bare Machine).

25 Mar 94, 100p.

Keywords: "Compilers, *Ada programming language, Standards, "Validation summary reports, Computer program verification.

This Validation Summary Report describes the extent to which a specific Ada compiler conforms to the Ada Standard, ANSI/MIL-STD-1815A. This report explains all technical terms used within it and thoroughly reports the results of testing this compiler using the Ada Compiler Validation Capability. An Ada compiler must be implemented according to the Ada Standard, and any implementation-dependent features must conform to the requirements of the Ada Standard. The Ada Standard must be implemented in its entirety, and nothing can be implemented that is not in the Standard. Even though all validated Ada compilers conform to the Ada Standard, it must be understood that some differences do exist between implementations. The Ada Standard permits some implementation dependencies--for example, the maximum length of identifiers or maximum values of integer types. Other differences between compilers result from the characteristics of particular operating systems, hardware, or implementation strategies. All the dependencies observed during the process of testing this compiler are given in this report. The information in this report is derived from the test results produced during validation testing. The validation process includes submitting a suite of standardized tests, the ACVC, as inputs to an Ada compiler and evaluating the results.

\section{1,649}

AD-A279 757/9 PC A06/MF A02

National Inst. of Standards and Technology, Gaithersburg, MD.

Ada Compiler Validation Summary Report: Certificate Number: 940325 S1.11343 DDC-I, DACS Sun SPARC/Solaris to 80186 Bare Ada Cross Compile System, Version 4.6.4 Sun SPARCclassic $=>$ Intel iSBC 186/100 (Bare Machine).

25 Mar 94, 118p.

Keywords: "Compilers, "Ada programming language Standards, Validation, "Validation summary reports Computer program verification.

This Validation Summary Report describes the exten to which a specific Ada compiler conforms to the Ada Standard, ANSI/MIL-STD-1815A. This report explains all technical terms used within it and thoroughly reports the results of testing this compiler using the Ada Compiler Validation Capability. An Ada compiler must be implemented according to the Ada Standard, and any implementation-dependent features must conform to the requirements of the Ada Standard. The Ada Standard must be implemented in its entirety, and nothing can be implemented that is not in the Standard. Even though all validated Ada compilers conform to the Ada Standard, it must be understood that some differences do exist between implementations. The Ada Standard permits some implementation dependencies--for example, the maximum length of identifiers or maximum values of integer types. Other differences between compilers result from the characteristics of particular operating systems, hardware, or implementation strategies. All the dependencies observed during the process of testing this compiler are given in this report. The information in this report is derived from the test results produced during validation testing. The validation process includes submitting a suite of standardized tests, the ACVC, as inputs to an Ada compiler and evaluating the results.

National Inst. of Standards and Technology, Gaithersburg, MD.

Ada Compiler Validation Summary Report: Certificate Number: 940325S1.11344 DDC-I, DACS Sun SPARC/Solaris to 80186 Bare Ada Cross Compiler System with Rate Monotonic Scheduling, Version 4.6.4 Sun SPARCclassic $\Rightarrow>$ Intel iSBC $186 / 100$ (Bare Machine).

25 Mar 94, 117p.

Keywords: "Compilers, "Ada programming language, Standards, "Validation summary reports, Computer program verification

This Validation Summary Report describes the extent to which a specific Ada compiler conforms to the Ada Standard, ANSI/MIL-STD-1815A. This report explains all technical terms used within it and thoroughly reports the results of testing this compiler using the Ada Compiler Validation Capability. An Ada compiler must be implemented according to the Ada Standard, and any implementation-dependent features must conform to the requirements of the Ada Standard. The Ada Standard must be implemented in its entirety, and nothing can be implemented that is not in the Standard. Even though all validated Ada compilers conform to the Ada Standard, it must be understood that some differences do exist between implementations. The Ada Standard permits some implementation dependencies--for example, the maximum length of identifiers or maximum values of integer types. Other differences between compilers result from the characteristics of particular operating systems, hardware, or implementation strategies. All the dependencies observed during the process of testing this compiler are given in this report. The information in this report is derived from the test results produced during validation testing. The validation process includes submitting a suite of standardized tests, the ACVC, as inputs to an Ada compiler and evaluating the results.

\section{1,651}

\section{AD-A279 778/5 PC A06/MF A02}

National Inst. of Standards and Technology, Gaithersburg, MD.

Ada Compiler Validation Summary Report: Certificate Number: 940325S1.11347 DDC-I, DACS Sun SPARC/SunOS to $680 \times 0$ Bare Ada Cross Compiler System (SECURE MODE), Version 4.6.9 Sun SPARCstation IPX $\Rightarrow$ Lynwood j435TU (68030) (Bare Machine).

25 Mar 94, 101p

Keywords: "Compilers, "Ada programming language, Standards, "Validation summary reports, Computer program verification.

This Validation Summary Report describes the extent to which a specific Ada compiler conforms to the Ada Standard, ANSI/MIL-STD-1815A. This report explains all technical terms used within it and thoroughly reports the results of testing this compiler using the Ada Compiler Validation Capability. An Ada compiler must be implemented according to the Ada Standard, and any implementation-dependent features must conform to the requirements of the Ada Standard. The Ada Standard must be implemented in its entirety, and nothing can be implemented that is not in the Standard. Even though all validated Ada compilers conform to the Ada Standard, it must be understood that some differences do exist between implementations. The Ada Standard permits some implementation dependencies-for example, the maximum length of identifiers or maximum values of integer types. Other differences between compilers result from the characteristics of particular operating systems, hardware, or implementation strategies. All the dependencies observed during the process of testing this compiler are given in this report. The information in this report is derived from the test results produced during validation testing. The validation process includes submitting a suite of standardized tests, the ACVC, as inputs to an Ada compiler and evaluating the results.

01,652

AD-A279 779/3 PC A06/MF A02

National Inst. of Standards and Technology, Gaithersburg, MD.
Ada Compiler Validation Summary Report: Certificate Number: $940325 S 1.11342$ DDC-I, DACS Sun SPARC/SunOS to 80186 Bare Ada Cross Compiler System with Rate Monotonic Scheduling Version 4.6.4 Sun SPARCstation IPX $=>$ Intel iSBC $186 / 100$ (Bare Machine).

25 Mar 94, 117p.

Keywords: "Compilers, "Ada programming language, Standards, "Validation summary reports, Compute program verification.

This Validation Summary Report describes the extent to which a specific Ada compiler conforms to the Ada Standard, ANSI/MIL-STD-1815A. This report explains all technical terms used within it and thoroughly reports the results of testing this compiler using the Ada Compiler Validation Capability. An Ada compiler must be implemented according to the Ada Standard, and any implementation-dependent features must conform to the requirements of the Ada Standard. The Ada Stand ard must be implemented in its entirety, and nothing can be implemented that is not in the Standard. Even though all validated Ada compilers conform to the Ada Standard, it must be understood that some differences do exist between implementations. The Ada Standard permits some implementation dependencies--for example, the maximum length of identifiers or maximum values of integer types. Other differences between compilers result from the characteristics of particula operating systems, hardware, or implementation strategies. All the dependencies observed during the process of testing this compiler are given in this report. The information in this report is derived from the test results produced during validation testing. The validation process includes submitting a suite of standardized tests, the ACVC, as inputs to an Ada compiler and evaluating the results.

\section{1,653}

AD-A279 804/9 PC A06/MF A02

National Inst. of Standards and Technology, Gaithersburg, MD.

Ada Compiler Validation Summary Report: Certificate Number: 940325S1.11351 DDC-I, DACS Sun SPARC/SunOS to Pentium PM Bare Ada Cross Compiler System with Rate Monotonic Scheduling, Version 4.6.4 Sun SPARCstation IPX $\Rightarrow$ Inte Pentium (operated as Bare Machine) based in Xpress Desktop (Intel product number: XBASE6E4F-B).

11 Apr 94, 118p.

Keywords: "Compilers, "Ada programming language, Standards, Validation, "Validation summary reports, Computer program verification.

This Validation Summary Report describes the exten to which a specific Ada compiler conforms to the Ada Standard, ANSI/MIL-STD-1815A. This report explains all technical terms used within it and thoroughly reports the results of testing this compiler using the Ada Compiler Validation Capability. An Ada compiler must be implemented according to the Ada Standard, and any mplementation-dependent features must conform to the requirements of the Ada Standard. The Ada Standard must be implemented in its entirety, and nothing can be implemented that is not in the standard. Even though all validated Ada compilers conform to the Ada Standard, it must be understood that some differences do exist between implementations. The Ada Standard permits some implementation dependencies--for ex ample, the maximum length of identifiers or the maximum values of integer types. Other differences beween compilers result from the characteristics of particular operating systems, hardware, or implementation strategies. All the dependencies observed during the process of testing this compiler are given in this report. The information in this report is derived from the test results produced during validation testing. The validation process includes submitting a suite of standard ized tests, the ACVC, as inputs to an Ada compiler and evaluating the results.

\section{1,654}

AD-A279 805/6 PC A06/MF A02

National Inst. of Standards and Technology, Gaithersburg, MD. 


\section{Computer Software}

Ada Compiler Validation Summary Report: Certificate Number: 940325S1.11353 DDC-I, DACS Sun SPARC/Solaris to Pentium PM Bare Ada Cross Compiler System with Rate Monotonic Scheduling, Version 4.6.4 Sun SPARCclassic $\Rightarrow$ Intel Pentium (operated as Bare Machine) based in Xpress Desktop (Intel product number: XBASE6E4F-B).

11 Apr 94, 123p.

Keywords: *Compilers, *Ada programming language, Standards, Validation.

This Validation Summary Report describes the extent to which a specific Ada compiler conforms to the Ada Standard, ANSI/MIL-STD-1815A. This report explains all technical terms used within it and thoroughly reports the results of testing this compiler using the Ada Compiler Validation Capability. An Ada compiler must be implemented according to the Ada Standard, and any implementation-dependent features must conform to the requirements of the Ada Standard. The Ada Stand ard must be implemented in its entirety, and nothing can be implemented that is not in the Standard. Even though all validated Ada compilers conform to the Ada Standard, it must be understood that some differences do exist between implementations. The Ada Standard permits some implementation dependencies--for example, the maximum length of identifiers or the maximum values of integer types. Other differences between compilers, result from the characteristics of particular operating systems, hardware, or implementation strategies. All the dependencies observed during the process of testing this compiler are given in this report. The information in this report is derived from the test results produced during validation testing. The validation process includes submitting a suite of standardized tests, the ACVC, as inputs to an Ada compiler and evaluating the results.

\section{1,655}

AD-A279 864/3 PC A06/MF A02

National Inst. of Standards and Technology, Gaithersburg, MD.

Ada Compiler Validation Summary Report: Certificate Number: 940325S1.11350 DDC-I, DACS Sun SPARC/SunOS to Pentium PM Bare Ada Cross Compiler System, Version 4.6.4 Sun SPARCstation IPX $\Rightarrow>$ Intel Pentium (Operated as Bare Machine) Based in Xpress Desktop (Intel Product Number XBASE6E4F-B)

11 Apr 94, 118p.

Keywords: "Compilers, "Ada programming language, Standards, "Validation summary reports, Computer program verification.

This Validation Summary Report describes the extent to which a specific Ada compiler conforms to the Ada Standard, ANSI/MIL-STD-1815A. This reports explains all technical terms used within it and thoroughly reports the results of testing this compiler using the Ada Compiler Validation Capability. An Ada compiler must be implemented according to the Ada Standard, and any implementation-dependent features must conform to the requirements of the Ada Standard. The Ada Standard must be implemented in its entirety, and nothing can be implemented that is not in the Standard. Even though all validated Ada compilers conform to the Ada Standard, it must be understood that some differences do exist between implementations. The Ada Standard permits some implementation dependencies--for example, the maximum length of identifiers or the maximum values of integer types. Other differences between compilers, result from the characteristics of particular operating systems, hardware, or implementation strategies. All the dependencies observed during the process of testing this compiler are given in this report The information in this report is derived from the tes results produced during validation testing. The validation process includes submitting a suite of standardized tests, the ACVC, as inputs to an Ada compiler and evaluating the results.

\section{1,656}

\section{AD-A280 145/4 PC A05/MF A02}

National Inst. of Standards and Technology, Gaithersburg, MD.

Ada Compiler Validation Summary Report: Certificate Number 940325S1.11345 DDC-I. DACS Sun SPARC/SunOS to $680 \times 0$ Bare Ada Cross Compiler System, Version 4.6.9 Sun SPARCstation IPX $=>$ Motorola MVME143 68030/68882 (Bare Machine).

11 Apr 94, 100p.

Keywords: "Ada programming language, "Compilers, Computer program verification, Functional analysis,
Optimization, Software engineering, Test and evaluation, Standards, "Validation summary reports, ACVC(Ada Compiler Validation Capability).

No abstract available.

01,657

AD-A280 295/7 PC A06/MF A02 National Inst. of Standards and Technology

Ada Compiler Validation Summary Report: Certificate Number: 940325 S1.11352 DDC-I DACS Sun SPARC/Solaries to Pentium PM Bare Ada Cross Compiler System, Version 4.6.4 Sun SPARCclassic $\Rightarrow$ Intel Pentium (Operated as Bare Machine) Based in Xpress Desktop (Intel Product Number: XBASE6E4F-B).

25 Mar 94, 117p.

Keywords: "Ada programming language, "Compilers, Operating systems(Computers), Software engineering, "Validation summary reports, Standards, Computer program verification.

The ADA implementation described above was tested according to the Ada Validation Procedures Pro92 against the Ada Standard Ada83 using the current Ada Compiler Validation Capability (ACVC). This Validation Summary Report (VSR) gives an account of the testing of this ADA implementation. For any technical terms used in this report, the reader is referred to Pro92. A detailed description of the ACVC may be found in the current ACVC User's Guide UG89.

01,658

AD-A288 571/3 PC A03/MF A01

National Inst. of Standards and Technology, Ada Compiler Validation Summary Report: CertifiAda Number 94090251.11377 UNISYS Corporation. IntegrAda for Windows NT, Version 1.0. Inte Deskside Server with Intel 80486DX266 $\Rightarrow$ Intel Deskside Server with Intel 80486DX266.

D. K Jefferson, L. A. Johnson, and D. J. Peifer. 14 Sep 94, 36p.

Keywords: "Validation, "Compilers, "Ada programming language, Standards.

The Ada implementation described above was tested according to the Ada Validation Procedures Pro92 against the Ada Standard Ada83 using the current Ada Compiler Validation Capability (ACVC). This Validation Summary Report (VSR) gives an account of the testing of this Ada implementation. For any technical terms used in this report, the reader is referred to Pro92.

01,659

AD-A288 572/1 PC A03/MF A01

National Inst. of Standards and Technology,

Ada Compiler Validation Summary Report: Certificate Number 940902S1.11376. UNISYS Corporation IntegrAda for Windows NT, Version 1.0. Intel Deskside Server for Intel Pentium $60 \mathrm{MHz}=>$. Intel Deskside Server with Intel Pentium $60 \mathrm{MHz}$.

K Jefferson, L. A Johnson, and D. J. Reifer 14 Sep $94,36 p$.

Keywords: *Validation, *Compilers, "Ada programming language, Standards.

The Ada implementation described above was tested according to the Ada Validation Procedures Pro92 against the Ada Standard Ada83 using the current Ada Compiler Validation Capability (ACVC). This Validation Summary Report (VSR) gives an account of the testing of this Ada implementation. For any technical terms used in this report, the reader is referred to Pro 92.

01,660

AD-A288 573/9 PC A03/MF A01

National Inst. of Standards and Technology, Gaithersburg, MD.

Ada Compiler Validation Summary Report: Certificate Number 94101251.11379 TISOFT, Inc. Green HIlls Optimizing Ada Compileer, Version 1.8.7 with PATCK ID 1 COMPAQ ProLiiant 2000 Model 55/66 => COMPAQ ProLiant 2000 Model 5/66.

Oct $94,34 p$

Keywords: "Validation, "Compilers, *Ada programming language, Standards.

The Ada implementation described above was tested according to the Ada Validation Procedures Pro92 against the Ada Standard Ada83 using the current Ada Compiler Validation Capability (ACVC). This Validation Summary Report (VSR) gives an account of the testing of this Ada implementation. For any technical terms used in this report, the reader is referred to Prog2.

\section{1,661}

AD-A288 574/7 PC A05/MF A01

National Inst. of Standards and Technology, Gaithersburg, MD

Ada Complier Validation Summary Report: Certificate Number: 940929S1.11378. Digital Equipment Corporation DEC Ada for DEC OSF/1 AXP Systems, Version 3.2; DEC 3000 Model 400 AXP Workstation $\Rightarrow$ DEC 3000 Model 400 AXP Workstation.

4 Oct $94,77 \mathrm{p}$

Keywords: "Validation, "Compilers, "Ada programming language, Facilities, Standards.

The Ada implementation described above was tested according to the Ada Validation Procedures Pro92 against the Ada Standard Ada83 using the current Ada Compiler Validation Capability (ACVC). This Validation Summary Report (VSR) gives an account of the testing of this Ada implementation. For any technical terms used in this report, the reader is referred to Pro92.

\section{1,662}

AD-A289 895/5 PC A05/MF A01

National Inst. of Standards and Technology (NCSL), Gaithersburg, MD. Software Standards Validation Group.

Ada Compiler Validation Summary Report. Certificate Number 941117 S1.11380. Electronic Data Sys tems Corp. Compiler: OC Systems Legacy Ada/ 370 , Release 1.4.1 (without optimization).

2 Dec 94, 94p.

Keywords: "Compilers, "Ada programming language Optimization, Information systems, Validation, Electronic equipment, Spn-19950119011.

The following Ada implementation was tested and determined to pass ACVC I. II.: Compiler Name and Version - OC Systems Legacy Ada/370, Release 1.4.1 Host Computer System - AMDAHL 5990 under VM/ ESA, Release 2.1, and Target Computer System AMDAHL 5990 under VM/ESA, Release 2.1. This Validation Summary Report (VSR) gives an account of the testing of this Ada implementation. For any technica terms used in this report, the reader is referred to (Pro92). A detailed description of the Ada Compiler Validation Capability (ACVC) may be found in the current ACVC User's Guide (UG89).

01,663

AD-A293 709/2 PC A05/MF A01

National Inst. of Standards and Technology, Gaithersburg, MD, ADA Compiler Validation Summary Report, VC pumber 95030351.11381 . Digital Equipment CorAlpha Systems, Version 3.2

28 Mar 95, 78p.

Keywords: "Validation, "Compilers, "Ada programming language, Test and evaluation, Digital systems, Host computers, Standards, Work stations, Implementation(Computers).

This Ada implementation was tested and determined to pass ACVC 1.11. Testing was completed on March 3, 1995. Host Computer System: DEC 3000 Model 400 AXP Workstation under OpenVMS Alpha Operating System, Version 6.1 Target Computer System: DEC 3000 Model 400 AXP Workstation under OpenVMS Alpha Operating System, Version 6.1.

01,664

AD-A296 794/1 PC A05/MF A01

National Inst. of Standards and Technology, Gaithersburg, MD

Ada Compiler Validation Summary Report, vC No. 950609S1.11390 Digital Equipment Corporation. Compiler Name: DEC Ada Version 3.2 for OpenVMS VAX Systems.

Final rept.

12 Jun 95, 80p.

Keywords: "Compilers, "Ada programming language, Validation, Standards, Computer program verification.

This Ada implementation was tested and determined to pass ACVC I.II. Testing was completed on 9 June 
1995. Host Computer System: VAXstation 3100 Model 76 under OpenVMS VAX Operating System, Version 6.2 Target Computer System: VAXstation 3100 Model 76 under OpenVMS VAX Operating System, Version 6.2 (KAR) P. 1.

\section{1,665}

DE94014586 PC A03/MF A01

National Inst. of Standards and Technology (NEL) Gaithersburg, MD. Center for Computing and Applied Mathematics.

Measuring Performance of Parallel Computers. Final Report.

F. Sullivan. 1994, 23p, DOE/ER/25046-T1

Contract AI05-87ER25046

Sponsored by Department of Energy, Washington, DC.

Keywords: "Parallel Processing, Algorithms, Benchmarks, Performance Testing, EDB/990200.

Performance Measurement - the authors have develped a taxonomy of parallel algorithms based on data motion and example applications have been coded for each class of the taxonomy. Computational benchmark kemels have been extracted for several applications, and detailed measurements have been performed. Algorithms for Massively Parallel SIMD machines measurement results and computational experiences indicate that top performance will be achieved by 'iteration' type algorithms running on massively paralle SIMD machines. Reformulation as iteration may entail unorthodox approaches based on probabilistic methods. The authors have developed such methods for some applications. Here they discuss their approach to performance measurement, describe the taxonomy and measurements which have been made, and report on some general conclusions which can be drawn from the results of the measurements.

\section{1,666}

\section{DE94014587 PC A03/MF A01}

National Inst. of Standards and Technology (NEL) Gaithersburg, MD. Center for Computing and Applied Mathematics.

Measuring Performance of Paraliel Computers. Progress Report, 1989.

F. Sullivan. 1994, 23p, DOE/ER/25046-T2.

Contract A105-87ER25046

Sponsored by Department of Energy, Washington, DC. Keywords: "Parallel Processing, Algorithms, Benchmarks, Performance Testing, Progress Report, EDB/ 990200.

Performance Measurement - the authors have developed a taxonomy of parallel algorithms based on data motion and example applications have been coded for each class of the taxonomy. Computational benchmark kemels have been extracted for several applications and detailed measurements have been performed. Algorithms for Massively Parallel SIMD machines measurement results and computational experiences indicate that top performance will be achieved by iteration' type algorithms running on massively paralle SIMD machines. Reformulation as iteration may entail unorthodox approaches based on probabilistic methods. The authors have developed such methods for some applications. Here they discuss their approach to performance measurement, describe the taxonomy and measurements which have been made, and repor on some general conclusions which can be drawn from the results of the measurements.

01,667

FIPS PUB 119-1 PC $\$ 278.00$

National Inst. of Standards and Technology (CSL), Gaithersburg, MD.

ADA; Category: Software Standard; Subcategory: Programming Language.

13 Mar 95, 546p, ANSI/ISO/IEC-8652-1995.

Supersedes FIPS PUB 119. Prepared in cooperation with Ame rican National Standards Inst., New York.

Three ring binder also available. North American Continent price $\$ 6.25$; all others write for quote.

Keywords: "Ada programming language, "Standards, "Data processing, Computer program portability, Compilers, Decoding, Semantics, Syntax, High level languages, Conformance, Software standards, Programming languages, Federal Information Processing Standards.

The purpose of the standard is to promote portability of Ada programs for use on a variety of data processing systems. The standard is for use by implementors as the reference authority in developing compilers, in- terpreters, or other forms of high level language processors; and by other computer professionals who need to now the precise syntactic and semantic rules of the standard.

\section{1,668}

FIPS PUB 153-1 PC E99

National Inst. of Standards and Technology (CSL), Gaithersburg, MD.

Programmer's Hierarchical Interactive Graphics System (PHIGS). Category: Software Standard; Subcategory: Graphics.

27 Jan 95, 199p.

Supersedes FIPS PUB 153

Three ring vinyl binder available; North American Continent price $\$ 7.00$; all others write for quote.

Keywords: "Interactive graphics, "Computer software, * Federal information processing standards, Computer program portability, Computer graphics, Computer animation, Computer aided design, Data structures Specifications, PHIGS(Programmers Hierarchical Interactive Graphics Systems), PLUS(Plus Lumiere and Surfaces).

The publication is a revision of FIPS PUB 153 and supersedes that document in its entirety. This revision provides a substantial, upward-compatible enhancement of the basic Programmer's Hierarchical Interactive Graphics System (PHIGS) functionality known as Plus Lumiere and Surfaces, (PHIGS PLUS) (ANS ISO 9592.1a, 2a, 3a 4:1992). PHIGS PLUS adds facilities for the specification of curved lines, curved and facetted surfaces, lighting and shading, and adds mechanism for color specification to allow non-indexed color specification. Amendments to each part of the PHIGS specification details revisions required by PHIGS PLUS. Also, each language binding of PHIGS has been amended as a result of PHIGS PLUS. The specifications and amendments that comprise the complete PHIGS standard as a result of this revision are detailed in the Specification section of this document.

01,669

FIPS PUB $194 \quad$ PC EO4

National Inst. of Standards and Technology (CSL) Gaithersburg, MD.

Open Document Architecture (ODA) Raster Document Application Profile (DAP). Category: Software Standard; Subcategory: Graphics.

13 Mar 95, 54p

Three ring vinyl binder available; North American Continent price $\$ 7.00$; all others write for quote.

Keywords: "Federal information processing standards "Computer graphics, "Documents, Raster scanning, Format, Data transfer(Computers), Image processing, Text processing, DAP(Documents Application Profile) ISP(International Standard
Document Architecture).

This Federal Information Processing Standard (FIPS) adopts the International Organization for Standardization (ISO)/International Electrotechnical Commission (IEC) 12064-1 International Standard Profile (ISP) FOD 112, which specifies the use of subset of the Open Document Architecture (ODA) standard. It facilitates the interchange of raster documents among differen raster graphics applications by specifying the constraints on document structure and content according to the rules of the ODA standard. The documents supported by this standard are based on a paradigm of an electronic engineering drawing, illustration, or other electronic image. The FIPS PUB specifies the structure and parameters for describing and interchanging bilevel untiled compressed images as well as tiled raster images.

\section{1,670}

\section{FIPS PUB 21-4 PC E99}

National Inst. of Standards and Technology (CSL), Gaithersburg, MD.

COBOL. Category: Software Standard; Subcategory: Programming Language. Includes 23 Jan $95,975 p$

Supersedes FIPS PUB 21-3. Also available from Supt. of Docs. Also pub. as American National Standards Committee, New York rept no. ANSI-X3.23-1985, ANSI-X3.23A-1989 and ANSI-X3.23B-1993. Prepared in cooperation with American National Standards Committee, New York.

Three ring vinyl binder also available; North American Continent price $\$ 7.00$, all others write for quote.
Keywords: "COBOL, "Standards, "Programming languages, Memory(Computers), ANSI(American National Standards Institute).

This standard is a revision of American National Standard for Programming Language COBOL, ANSI X3.231974. The language specifications contained in this standard were drawn from both ANSI X3.23-1974 and the CODASYL COBOL Journal of Development. Like its prodecessors, this document provides specifications for both the form and interpretation of programs expressed in COBOL. It is intended to provide a high degree of machine independence in such programs in order to permit their use on a variety of automatic data processing systems.

01,671

FIPS PUB 21.4A PC E99

National Inst. of Standards and Technology (CSL), Gaithersburg, MD.

COBOL. Category: Software Standard; Subcategory: Programming Language. Part $A$.

23 Jan $95,94 p$

Supersedes FIPS PUB 21-3A. Also available from Supt. of Docs. Also pub as American National Standards Committee, New York rept no. ANSI-X3.23A1989. Prepared in cooperation with American National Standards Committee, New York.

Three ring vinyl binder also available; North American Continent price $\$ 7.00$, all others write for quote.

Keywords: "COBOL, "Standards, "Programming languages, Memory(Computers), ANSI(American National Standards Institute)

This supplement, the first to the document entitled 'American National Standard for Information Systems - Programming Language - COBOL, ANSI X3.23-1985, ISO 1989-1985,' presents a new COBOL module, the Intrinsic Function module. This module provides the capability of referencing a data item whose value is derived automatically during the execution of a program.

\section{2}

FIPS PUB 21-4B PC E99

National Inst. of Standards and Technology (CSL), Gaithersburg, MD.

COBOL. Category: Software Standard; Subcategory: Programming Language. Part $B$.

23 Jan $95,80 p$

Supersedes FIPS PUB 21-3. Also available from Supt. of Docs. Also pub. as American National Standards Committee, New York rept. no. ANSI-X3.23B-1993. Prepared in cooperation with American National Standards Committee, New York

Three ring vinyl binder also available; North American Continent price $\$ 7.00$, all others write for quote.

Keywords: "COBOL, "Standards, "Programming languages, Memory(Computers), ANSI(American $\mathrm{Na}$ tional Standards Institute)

This amendment, the second to the document entitled American National Standard for Information Systems - Programming Language - COBOL, ANSI X3.23-1985, ISO 1989:1985, presents correction of errors and clarification of ambiguities in Programming Language COBOL.

\section{1,673}

FIPSPUB184 PC E10

National Inst. of Standards and Technology (CSL), Gaithersburg, MD

Integration Definition for Information Modeling (IDEF1X); Category: Software Standard; Subcategory: Modeling Techniques.

21 Dec 93, 163p.

Also available from Supt. of Docs. See also AD-A266 176 .

Three ring vinyl binder also available; North American Continent price \$7.00; aii others write for quote.

Keywords: "Data models, "Systems engineering, Data base management systems, Information systems, Data management, Semantics, Syntax, Computer models, "IDEF1X(Information Definition for Information Modeling), Software standards, Modeling techniques, Graphical languages, Data definition.

This standard is based on the Integration Information Support System (IISS), Volume V - Common Data Model Subsystem, Part 4 - Information Modeling Manual - IDEF1 Extended, 1 (IDEF1X) November 1985 (AD-A181 952). This standard describes the IDEF1X modeling language (semantics and syntax) and associated rules and techniques, for developing a logical 


\section{Computer Software}

model of data. IDEF $1 \mathrm{X}$ is used to produce a graphical information model which represents the structure and semantics of information within an environment or system. Use of this standard permits the construction of semantic data models which may serve to support the management of data as a resource, the integration of information systems, and the building of computer databases.

01,674

N94-36857/8 (Order as N94-36853/7, PC A10/

MF A03)

National Inst. of Standards and Technology,

Gaithersburg, MD.

Open System Environments.

F. Schulz. Aug 94, 31p.

In National Aeronautics and Space Administration, NASA Sti Program Coordinating Council Tweltth Meeting: Standards p 85-115

Keywords: "Distributed processing, "Information systems, "Standards, "Applications programs (Computers), Computer networks, Internets, Protocol (Computers), Systems compatibility, Systems engineering, User requirements.

An open system is defined as a system that implements open specifications for interfaces, services, and supporting formats to enable properly engineered applications software to be ported with minimal changes across a wide range of systems; and to interact with users in a style which facilitates user portability. Based on that definition, a reference model for an open system environment is presented.

\section{1,675}

PB94-139623 PC A03/MF A01

National Inst. of Standards and Technology (CSL), Gaithersburg, MD

Computing Effects and Error for Large Synthetic Perturbation Screenings.

N. Drouin, R. Kacker, and G. Lyon. Dec 93, 15p

NISTIR-5296.

See also PB93-161339, PB93-178572 and PB93189835. Sponsored by Defense Advanced Research Projects Agency, Arlington, VA.

Keywords: "Parallel programming, *Statistical analysis, Analysis of variance Standard error, Experimental design, Computation, "Synthetic Perturbation Screening, Hadamard matrices.

Synthetic Perturbation Screening (SPS) is a powerful, statistically-based method of performance improvement for parallel computer programs. SPS uses statistically designed experiments to identify segments of code that consume significant computing resources or otherwise impede a parallel application. Analysis typically starts with a preliminary screening of a large number of code segments; SPS does this via fractional factorial designs based upon Hadamard matrices. Since Hadamard matrix designs are not commonly discussed in introductory texts, there is a need to survey briefly the calculation of their effects and especially, their standard error. The result is a practical, supplementary sketch that should help users of SPS automatic tools understand how investigations and analyses are being performed.

\section{1,676}

PB94-140167 PC A04/MF A01

National Inst. of Standards and Technology, Gaithersburg, MD.

Guide to Software Engineering Environment Assessment and Evaluation.

B. B. Cuthill. Nov 93,69p, NISTIR-5295.

Sponsored by Defense Information Systems Agency, Arlington, VA.

Keywords: "Software engineering, "Evaluation, Software tools, Methodology, Requirements, User needs, "SEE(Software Engineering Environment), CASE(Computer Aided Software Engineering).

This guide outlines general approaches to software engineering environment (SEE) assessment and evaluation. This guide defines SEE assessment as the process of accurately describing the capabilities of a software engineering environment to support and integrate a range of computer aided software engineering (CASE) tools. The quide defines SEE evaluation as the process of determining how well a SEE matches the customer's requirements. The assessment and evaluation approaches presented here focus on accurately defining the integration and functional capabilities of candidate SEEs and the requirements of the customer.
01,677

PB94-143401 PC A06/MF A02

National Inst. of Standards and Technology (CSL), Gaithersburg, MD

Next Generation Computer Resources: Reference Model for Project Support Environments (Version 2.0)

Special pub.

A. Brown, D. Carney, P. Oberndorf, and $M$

Zelkowitz. Nov 93, 123p, NIST/SP-500/213.

Also available from Supt. of Docs. as SN003-00303244-1. Also pub. as Carnegie-Mellon Univ., Pitts burgh, PA. Software Engineering Inst. rept. no. CMU/ SEl/93-TR-23. See also AD-A255 575. Prepared in cooperation with Carnegie-Mellon Univ., Pittsburgh, PA Software Engineering Inst. Sponsored by Space and Naval Warfare Systems Command, Washington, DC.

Keywords: "Software engineering, Systems engineering, User needs, Support services, Software tools, Computer software management, Project management, "Reference models, "PSEs(Project Support Environments), Frameworks.

Software engineering environments (SEEs) are typically built on hardware and operating system plat forms. Current SEE architectures distinguish between a set of relatively fixed number of services called the framework, and application-specific tools called enduser services. This report extends the concept of SEE frameworks with appropriate end-user services for defining project support environments.

01,678

PB94-150919 PC A03/MF A01

National Inst. of Standards and Technology, Gaithersburg, MD.

Guide to Configuration Management and the Revision Control System for Testbed Users.

S. Bodarky. Aug 91, 25p, NISTIR-4646.

See also PB91-107615. Sponsored by Assistant Secretary of Defense (Production and Logistics), Washington, DC. Computer-aided Acquisition and Logistic Support Program.

Keywords: "Configuration management, "Computer software, Software tools, Standards, File managemen systems, Documents, Software engineering. Systems analysis, ${ }^{*} \mathrm{RCS}$ (Revision Control System) *PDES(Product Data Exchange using STEP), STEP(Standard for the Exchange of Product Model Data).

Product Data Exchange using STEP (PDES) refers to the United States organizational activities in support of the development of the Standard for the Exchange of Product Model Data (STEP). These activities have resulted in the creation of large amounts of information and software, which reside in the PDES File System on the computer systems at the National institute of Standards and Technology. The PDES software, documents, and data have been placed under configuration management using the Revision Control System (RCS), in order to ensure that change to these items occurs in a controlled manner, and that any services provided by them are done so as reliably as possible. This document provides instructions for anyone need ing to access this material.

\section{1,679}

PB94-160793 PC A03/MF A01

National Inst. of Standards and Technology (CAML), Gaithersburg, MD. Applied and Computational Mathematics Div.

Review of Mathematical Function Library for Microsoft-FORTRAN, John Wiley and Sons, 1989. Final rept.

D. W. Lozier, and F. W. J. Olver. Dec 90, 16p,

NiSTIR-4490,

Prepared in cooperation with Maryland Univ., College Park. Inst. for Physical Science and Technology.

Keywords: "FORTRAN, "Subroutine libraries, Computer programming, Software, Algorithms, US NIST, Calculators, Tables(Data), Mathematical tables, Manuals.

The loose-leaf manual and accompanying diskettes under review, which we shall refer to as the 'UL Library', may be regarded as an attempt to replace the numerical tables of the higher transcendental functions supplied in the NBS Handbook by a comprehensive software package. The functions treated include Bessel and related functions, hypergeometric and confiuent hypergeometric functions, elliptic functions and integrals, exponential integral and related functions, error function and related functions, Gamma and incomplete Gamma functions, orthogonal polynomials, probability functions and random number generators.

01,680

PB94-163086 PC A03/MF A01

National Inst. of Standards and Technology (NCSL), Gaithersburg, MD. Systems and Software Technology Div.

Distributed Supercomputing Software: Experiences with the Parallel Virtual Machine - PVM.

R. D. Schneeman. Mar 94, 25p, NISTIR-5381. See also DE91014961.

Keywords: "Supercomputers, "Distributed computer systems, "Parallel processing, ${ }^{*}$ Computer software, Computer program portability, Requirements, Paralle programming Graphic methods, Man computer interface, Models, Heterogeneity, US NIST, Graphical user interface

The Parallel Virtual Machine (PVM) is a general purpose distributed system developed by researchers at the Oak Ridge Nationai Laboratory and Emory University. The PVM system consists of a portabie suite of software specifically designed for use by parallel and supercomputing application engineers. The Nationa institute of Standards and Technology (NIST) researchers are studying PVM to assist them in defining the system service requirements needed to support parallel programming and supercomputing activities in the general purpose distributed setting. The report focuses on defining the profile requirements culminating from our PVM assessment; therefore, the document will also provide reference material for those involved in evaluating distributed system software for the supercomputing domain.

\section{1,681}

PB94-164399 PC A03/MF A01

National Inst. of Standards and Technology (CSL), Gaithersburg, MD

Time-Perturbation Tuning of MIMD Programs. G. Lyon, R. Snelick, and R. Kacker. Jun 82, 22p, NISTIR-4859.

See also PB93-161339. Sponsored by Defense Advanced Research Projects Agency, Arlington, VA

Keywords: "Parallel programming, Experimental design, Distributed computer systems, Optimization, Performance evaluation, Response time(Computers), "Time-perturbation tuning, "MIMD, " Multiple-instruction Multiple-data, Code perturbation, DEX(Design experiments)

Time-perturbation tuning (TPT) is a novel technique for assaying and improving the performance of programs on Multiple-instruction Multiple-data (MIMD) systems. Small, synthetic program delays are combined with statistically designed experiments (DEX). Claims for TPT are two: (1) Conceptually, it brings the powerful, mathematical perspective of experiment design to interdependent, sometimes refractory aspects of MIMD program tuning; (2) Practically, it provides a needed speedup mechanism, synthetic time delays for what otherwise would be ad-hoc, hand tailored program setups for DEX. Overall, the technique identifies bottlenecks in programs directly, as quantitative effects upon response time. TPT works on programs for both shared and distributed-memory, and it scales well with increasing system size.

\section{1,682}

PB94-165206 PC A08/MF A02

National inst. of Standards and Technology (CSL), Gaithersburg, MD. Information Systems Engineering Div.

User's Guide for the PHIGS Validation Tests (Version 2.1).

K. G. Brady, J. V. Cugini, and Q. Wang. Apr 94, $174 \mathrm{p}$, NISTIR-5398.

Supersedes PB93-126365 and PB93-228617. See also PB90-269580.

Keywords: *interactive graphics, *Standards, ${ }^{*}$ Computer program verification, Computer graphics, Hierarchies, Tests, Data structures, Specifications, Subroutine libraries, Computer software, PHIGS(Programmers Hierarchical Interactive Graphics System), PVT(PHIGS Validation Tests), NIST(National Institute of Standards and Technology).

The Programmers Hierarchical Interactive Graphics System (PHIGS) Validation Tests (PVT), developed by the National Institute of Standards and Technology 
(NIST), consist of a large set of Fortran and C programs which may be used to test how well implementations of PHIGS conform to the standard. The tests are organized into a hierarchical structure of modules which corresponds to the conceptual overview of the standard. The tests are associated with the standard via a set of semantic requirements which are derived directly from the standard. Cross-reference tables allow the user to find tests relating to specific PHIGS functions and data structures. Directions for installation and operation of the tests are included.

01,683

PB94-172301 Not available NTIS

National Inst. of Standards and Technology (NCSL), Gaithersburg, MD. Systems and Network Architecture Div.

RDI-SIM ECMA Inter-Domain Routing Protocol Simulation Tool.

Final rept.

K. R. Glenn. 1990, 5p.

Pub. in Proceedings of Institute of Electrical and Electronics Engineers Global Telecommunications Conference and Exhibition, San Diego, CA., December 25, 1990, p579-583.

Keywords: "Routing, *Protocols, "Computerized simulation, "Software tools, Performance evaluation, Reprints, ECMA(European Computer Manufacturers Association), OSI(Open Systems Interconnection), ISIS(Intermediate System to Intermediate System), Interdomain.

Three classes of Open Systems Interconnection (OSI) routing protocols have been defined by the international Organization for Standardization (ISO): End System to Intermediate System (ES-IS), intra-domain Intermediate System to Intermediate System (IS-IS), and inter-domain IS-IS. The paper addresses a simulation tool developed at the National Institute of Standards and Technology (NIST) to aid in the design and performance evaluation of the European Computer Manufacturers Association (ECMA) inter-domain IS-IS protocol proposal. The paper discusses the innerworkings of the simulation tool and how it models the ECMA protocol. Finally, a set of experiments are defined that test the performance of the routing algorithm defined by ECMA.

01,684

PB94-172566 Not available NTIS

National Inst. of Standards and Technology (CSL), Gaithersburg, MD. Advanced Systems Div. Time-Perturbation Tuning of MIMD Programs.

Final rept.

G. Lyon, R. Snelick, and R. Kacker. 1992, 14p.

Pub. in Proceedings of International Conference on Modelling Techniques and Tools for Computer Performance Evaluation (6th), Edinburgh, Scotland, September 16-18, 1992, p211-224. Sponsored by Defense Advanced Research Projects Agency, Arlington, VA.

Keywords: *Software engineering, Response time(Computers), Computer systems performance, Multiprocessors, Reprints, “TPT *Time Perturbation Tuning, "MIMD, "Multiple Instruction Multiple Data, DEX(Designed experiments).

Time-perturbation tuning (TPT) is a novel technique for assaying and improving the performance of programs on Multiple Instruction Multiple Data (MIMD) systems. Conceptually, TPT brings the powerful, mathematical perspective of statistically designed experiments (DEX) to the interdependent, sometimes refractory aspects of MIMD program tuning. Practically, TPT provides a needed speedup mechanism of synthetic time delays for what otherwise would be ad hoc, hand-tailored program setups for DEX. Overall, the technique dentifies bottlenecks in programs directly as quantitative effects upon response time. TPT works on programs for both shared and distributed-memory, and it scales well with increasing system size.

\section{1,685}

PB94-172657 Not available NTIS

National Inst. of Standards and Technology (CSL), Gaithersburg, MD. Advanced Systems Div.

Using Synthetic-Perturbation Techniques for Tuning Shared Memory Programs (Extended Abstract). Final rept.

R. Snelick, J. JaJa, R. Kacker, and G. Lyon. 1993

Pub. in Proceedings of 1993 Int. Conf. on Paralle

Processing, St. Charles, IL, Aug. 16-20, 1993, pplI-2II-10. Sponsored by Defense Advanced Research Projects Agency, Arlington, VA.
Keywords: *Parallel programming, Software engineering, Computer program portability, Memory(Computers), Multiprocessors, Case studies, image processing, Sorting routines, Parallel processing, Reprints, "SPI, "Synthetic Perturbation Tuning, "MIMD, *Multiple Instruction Multiple Data.

The Synthetic-Perturbation Tuning (SPT) methodology is based on an empirical approach that introduces artificial delays into the Multiple Instruction Multiple Data (MIMD) program and captures the effects of such delays by using the modern branch of statistics called design of experiments. SPT provides the basis of a powerful tool for tuning MIMD programs that is portable across machines and architectures. The purpose of this paper is to explain the general approach and to extend it to address specific features that are the main source of poor performance on the shared memory programming model. These include performance deg radation due to load imbalance and insufficient parallelism, overhead introduced by synchronizations and by accessing shared data structures, and compute time bottlenecks. The authors illustrate the practicality of SPT by demonstrating its use on two very differen case studies: a large image processing benchmark and a parallel quicksort.

\section{1,686}

PB94-178407 PC A21/MF A04

National Inst. of Standards and Technology (CSL), Gaithersburg, MD. Advanced Systems Div.

Second Text REtrieval Conference (TREC-2). Held in Gaithersburg, Maryland on August 31-September 2, 1993.

Special pub.

D. K. Harman. Mar 94, 497p, NIST/SP-500-215.

Also available from Supt. of Docs. as SNO03-003 03255-7. See also PB93-191641.

Keywords: "Meetings, "Information retrieval, "Tex processing, Thesauri, Automation, Natural language processing, Pattern recognition, Routing, Probability theory, Documents, Approximation, Search structuring, Knowledge bases(Artificial intelligence), Machine learning, Algorithms, Semantics, Vector processing.

The second Text REtrieval Conference (TREC-2) was held in August 1993 and was attended by about 150 people involved in the 31 participating groups. The goal of the Conference was to bring research groups together to discuss their work on a new large test collection. There was a large variation of retrieval techniques reported on, including methods using automatic thesauri, sophisticated term weighting, natural language techniques, relevance feedback, and advanced pattern matching. As results had been run through a common evaluation package, groups were able to compare the effectiveness of different techniques, and discuss how differences between the systems affected pertormance.

\section{1,687}

PB94-185568 Not available NTIS

National Inst. of Standards and Technology (CSL) Gaithersburg, MD. Advanced Systems Div.

Synthetic-Perturbation Tuning of MIMD Programs. Final rept.

G. Lyon, R. Snelick, and R. Kacker. 1994, 24p.

Pub. in Jnl. of Supercomputing 8, p5-28 1994

Keywords: *Parallel programming, Computer program verification, Distributed computer systems, Performance evaluation, Response time(Computers), Concurrent processing, Reprints, ${ }^{*}$ SPT(Synthetic perturbation tuning), *Synthetic perturbation tuning, *MIMD(Multiple instruction Multiple data), *Multiple instruction Multiple data, DEX(Designed experiments).

Synthetic-perturbation tuning (SPT) is a novel technique for assaying and improving the performance of programs on the Multiple instruction Multiple data (MIMD) systems. Conceptually, SPT brings the powerful, mathematical perspective of statistically designed experiments to the interdependent, sometimes refractory aspects of MIMD program tuning. Practically, synthetic perturbations provide a much needed quickchange mechanism for what otherwise would be ad hoc, hand-configured experiment setups. Overall, the technique identifies bottlenecks in programs directly as quantitative effects upon a measured response. SPT works on programs for both shared and distributed memory, and it scales well with increasing system size.

01,688

PB94-193638 PC A03/MF A01
National Inst. of Standards and Technology (CSL), Gaithersburg, MD. Advanced Systems Div.

Simple Scalability Test for MIMD Code.

G. Lyon, and R. Kacker. Jun 94, 21p, NISTIR-5417. Sponsored by Defense Advanced Research Projects Agency, Arlington, VA.

Keywords: "Parallel programming, *Scaling, "Computer program portability, Parallel processors, Computer performance evaluation, Tests, Computer program verification, Coding, Taylors series, *MIMD(Multiple Instruction Multiple Data), Code scalability, DEX(Designed experiments)

Code scalability, crucial on any parallel system, determines how well parallel code avoids becoming a bottleneck as its host computer is made larger. Scalability of computer code can be estimated by statistically designed experiments that empirically approximate a multivariate Taylor expansion of the code's execution response function. Each suspected code bottleneck corresponds to a first-order term in the expansion, the coefficient for that term indicating how sensitive execution is to changes in the suspect location. However, it is the coefficients for second-order interactions between code segments and the number of processors that are fundamental in discovering which program elements limit parallel speedup. Extending an earlier formulation, a new unified view via these second-order terms yields an informal scaling test of high utility in code development.

\section{1,689}

PB94-198967 Not available NTIS

National Inst. of Standards and Technology (CAML), Gaithersburg, MD. Applied and Computational Mathematics Div

Software Libraries, Numerical and Statistical.

Final rept.

R. F. Boisvert. 1993, 4p.

Pub. in Encyclopedia of Computer Science, p122912321993

Keywords: *Subroutine libraries, Computer software, Software reuse, Computation, Computer calculations, Reprints, "Mathematical software, Statistical software. A program library is a collection of computer programs for a particular application. This article surveys program libraries for general-purpose numerical computation and statistical analysis.

\section{1,690}

PB94-198975 Not available NTIS

National Inst. of Standards and Technology (CAML), Gaithersburg, MD. Applied and Computational Mathematics Div

Portable Vectorized Software for Bessel Function Evaluation.

Final rept.

R. F. Boisvert, and B. V. Saunders. 1992, 14p.

Pub. in Association for Computing Machinery Transactions on Mathematical Software 18, n4 p456-469 Dec 92. See also PB91-216598.

Keywords: "Bessel functions, *Computation, "Computer software, Computer calculations, Hyperbolic functions, Hypergeometric functions, Vector processing, Computer program portability, Reprints, *Mathematical software.

A suite of computer programs for the evaluation of Bessel functions and modified Bessel functions of orders zero and one for a vector of real arguments is described. Distinguishing characteristics of these programs are that (a) they are portable across a wide range of machines, and (b) they are vectorized in the case when multiple function evaluations are to be performed. The performance of the new programs are compared with software from the FNLIB collection of Fullerton on which the new software is based.

\section{1,691}

PB94-198983 Not available NTIS

National Inst. of Standards and Technology (CAML), Gaithersburg, MD. Applied and Computational Mathematics Div.

Virtual Software Repository System.

Final rept.

R. F. Boisvert, J. L. Springmann, and M. L.

Strawbridge. 1992, 5p

Pub. in Proceedings of Semi-Annual Cray User Group Meeting (13th), Washington, DC., September 14-18, 1992, p68-72.

Keywords: "Computer software, Software reuse, Computation, Computer calculations, Distributed computer 


\section{Computer Software}

systems, Computer networks, Catalogs, On-line systems, Reprints, "Mathematical software, Statistical software, GAMS(Guide to Available Mathematical Software).

Much reusable software is available for solving routine mathematical and statistical problems. Unfortunately, locating this software is often quite difficult for the average user in current distributed computing environments. The Guide to Available Mathematical Software (GAMS) virtual software repository seeks to remedy this situation by providing users with convenient access to some 8000 software modules from more than 50 packages physically distributed among several Internet repositories, including netlib. In this paper we illustrate the use of GAMS and outline its implementation.

\section{1,692}

PB94-200573 Not available NTIS

National Inst. of Standards and Technology (NCSL), Gaithersburg, MD. Information Systems Engineering Div.

Graphical Conceptual Navigation as a Presentation Technique for a Graphics Standard.

Final rept.

J. V. Cugini. $1989,10 p$

Pub. in Proceedings of Annual Conference and Exposition Dedicated to Computer Graphics (10th). Philadelphia, PA., April 17-20, 1989, v1 p87-96.

Keywords: "Computer graphics, "Standards, Knowledge representation, Knowledge bases(Artificial intelligence), Object-oriented programming, Reprints, "Conceptual navigation, COOL(COncept Oriented Language), Prolog programming language, Conceptual networks

Conceptual navigation can serve as a powerful tool to help users understand and explore a given domain. COOL (COncept Oriented Language) is a system, implemented with Prolog and GKS, which gives the knowledge engineer a high level language in which to express the conceptual relations among objects within a domain. The facilities for inspecting the Knowledge base (KB) support the metaphore of a complex graph of related concepts. Thus, a close conceptual relationship among objects is expressed as spatial proximity. Although not mandatory, the system is set up to allow the conceptual graph to be grounded in some authoritative reference text explaining or defining the domain. The system also has the ability to express relations among the concepts of the domain intensionally, as well as extensionally. The GKS standard is a suitable domain for the COOL system.

\section{1,693}

PB94-203437 PC A03/MF A01

National Inst. of Standards and Technology (CSL), Gaithersburg, MD. Systems and Software Technology Div.

Quality Characteristics and Metrics for Reusable Software (Preliminary Report).

Rept. for Oct 92-Jun 94.

W. J. Salamon, and D. R. Wallace. May $94,43 p$,

NISTIR-5459.

Contracts BMDO-92-A-012, BMDO-OA-005

Sponsored by Ballistic Missile Defense Organization, Washington, DC

Keywords: "Software reuse, "Software reliability, Quality, Characteristics, Computer program reliability, Computer software, Software metrics.

This report identifies a set of quality characteristics of software and provides a summary of software metrics that are useful in measuring these quality characteristics for software products. The metrics are useful in assessing the reusability oi software products.

\section{1,694}

PB94-212016 Not available NTIS

National Inst. of Standards and Technology (NCSL), Gaithersburg, MD. Advanced Systems Div.

Associated Object Model for Distributed Systems. Final rept.

Z. Hong, and W. McCoy. 1990, 18p.

Pub. in Operating Systems Review 24, n4 p34-51 Oct 90.

Keywords: "Distributed computer systems "Applications programs(Computers), "Object programs, Models, Distributed processing, Operating systems(Computers), Reprints.

A model for supporting distributed applications, called the Associated Object Model, is described. In this model, multi-objects (different distributed application objects) can join in an association, making it possible for application objects to cooperate in wider degree than possible under other models. A mechanism supporting associations is introduced in this paper. By the mechanism, many associations between cooperating application objects can be established and maintained. The paper also includes some discussions about the description and implementation of the model and the mechanism.

\section{1,695}

PB94-213303 Not available NTIS

National Inst. of Standards and Technology (MEL) Gaithersburg, MD. Factory Automation Systems Div. Debugger for Tcl Applications.

Final rept

D. Libes. 1993, 16p.

Pub. in Proceedings of $T c l / T k$ Workshop, Berkeley, CA., June 10-11, 1993, p3-18.

Keywords: "Debugging(Computers), Applications programs(Computers), Interpreters, Software tools, Reprints, "Tcl(Tool Command Language) programming language, "Tool Command Language, "Tcl programming language, $C$ programming language.

This paper describes an implementation of a debugger for Tcl applications. The debugger has a typical frontend but with some extremely unusual commands, in part because of the features and limitations of Tcl. The debugger is modeless, allowing users to issue $\mathrm{Tcl}$ and application commands along with debugger commands. Each type of command may invoke the other, allowing debugging to be programmed, dynamically or in advance. The debugger is written in C and is very fast. When linked in but not used, it does not slow applications at all. The debugger requires no modifications to the $\mathrm{TCl}$ core, and can be plugged into applications with little effort

\section{1,696}

PB94-213311 Not available NTIS

National Inst. of Standards and Technology (MEL) Gaithersburg, MD. Factory Automation Systems Div. Kibitz-Connecting Multiple Interactive Programs Together.

Final rept.

D. Libes. 1993, 11p.

475 May 93

Keywords: "Interactive systems, "Computer software, * Interprocessor communication, Automation, Software reuse, Computer program portability, Debugging(Computers), Reprints, "Expert programming language, Kibitz.

Expect is a programming language for automating interactive programs. Recently, people have begun using Expect to connect multiple interactive programs together, allowing for new classes of applications. With some basic building blocks and a little scripting, it is possible to build such applications quickly. This paper discusses the general technique, while focusing on a particular example: Kibitz. Kibitz connects multiple sessions and applications together, providing a means for consulting, group editing or other cooperative tasks. Kibitz in turn, can be used as a module in building additional programs of this type. Using Kibitz, we demonstrate how to enable cron or background processes to call upon and interact with users, e.g. for guidance or debugging. Owing to program reuse, our approach avoids many portability issues already addressed and solved by existing programs. Kibitz has no special coding for byte swapping, structure encoding, or job control, and requires neither kernel modifications nor setuid or other permissions even though it runs over a network and deals with multiple users, job control, and sophisticated programs such as shells and full screen editors.

\section{1,697}

PB94-213329 Not available NTIS

National Inst. of Standards and Technology (NEL), Gaithersburg, MD. Factory Automation Systems Div. Using Expect to Automate System Administration Tasks.

Final rept

D. Libes. $1990,8 p$.

Pub. in Proceedings of Large Installation System Administrator's Conference (4th), Colorado Springs, CO., October 17-19, 1990, p107-114.

Keywords: "UNIX(Operating system), "Computer systems programs, "Systems management, Interactive systems, Interprocessor communication, Computer software management, Automation, Reprints, Systems administration.

UNIX system administration often involves programs designed only for interactive use. 'Expect' is a program which can 'talk' to interactive programs. A script is used to guide the dialogue. Scripts are written in a high-level language and provide flexibility for arbitrarily complex dialogues. By writing an 'Expect' script, one can run interactive programs non-interactively. Shell scripts are incapable of managing these system administration tasks, but 'Expect' scripts can control them and many others. 'Expect' is similar in style to the shell, and can easily be mastered by any system administrator who can program in the shell already. This paper presents real examples of using 'Expect' to automate system administration tasks such as passwd and fsck. Also discussed are a number of other systems administration tasks that can be automated.

01,698

\section{PB94-500691 CP T99}

National Inst. of Standards and Technology (CSL), Gaithersburg, MD. Software Standards Validation Group.

FORTRAN Compiler Validation System, Version 2.1.

Software.

1 Jan 94, mag tape, NIST/SW/MT-93/008.

Language: FORTRAN. Supersedes PB85-226736.

Available in 9-track, ASCII character set tape, 1600 bpi, 6250 bpi, or 3480 cartridge. Documentation in cluded; may be ordered separately as PB94-114055.

Keywords: "Software, "Compilers, "Computer program verification, Federal information processing standards, Error detection codes, Validation, Magnetic tapes, "Fortran programming language, Conformance testing.

This Version of the Fortran Compiler Validation System (FCVS) has been revised to include all of the Temporary Program Files (TPFs) that were used with Version 2.0. The tests are progressively complex exercises of statement combinations representing typical language usage. They can be used to evaluate compiler usability and conformance to the 1978 FORTRAN Standard, and can aid in precise identification of compiler flaws. The test case outputs are tailored to each of the tests involved in order to aid in the specific diagnosis of instances of non-conformance or of compiler errors. For each test, the output indicates whether the test passes; if a test fails, the correct results and the compller produced results are displayed on the output report. The test cases are so designed that the use can quickly and easily locate the source code which caused the failure for further analysis. The FCVS architecture supports thorough quality testing of the compiler; at the same time, with no loss of efficiency or in crease in complexity, the system also provides for the auditability of the testing operation. The system was produced to be used in support of the procurement of 1978 FORTRAN Standard compilers, but it is also designed for the general user.

\section{1,699}

\section{PB94-502077 Diskette $\$ 250.00$}

National Inst. of Standards and Technology, Gaithersburg, MD

M (also known as MUMPS) Validation Test Suite, Version $\mathbf{8 . 3}$ (for Microcomputers).

Software.

c1 Jul 94, diskette.

System: MS DOS 3.0 or higher operating system. Language: MUMPS. Supersedes PB91-507699, PB91507707 , PB91-507715, PB91-507723

The software is on one $31 / 2$ inch DOS diskette, $1.4 \mathrm{M}$ high density. The diskette is in ASCIl format. Documentation included; may be ordered separately as PB94-180486.

Keywords: "Software, "Compilers, "Tests, Federal information processing standards, Precompilers, Validation summary reports, Interpreters, Computer systems programs, Computer program verification, Microcomputers, Diskettes, "M programming language, *MUMPS programming language.

The M (also known as MUMPS) Validation Test Suite, Version 8.3 was developed to assess conformance to the FIPS PUB 125-1. This test suite contains 3,592 tests and incorporates features such as (1) pass/fail conditions for individual tests, (2) imbedded instructions and warnings, and (3) fully automated tabulation of the test results. This test suite can be used by Federal Agencies, M programming language developers, 
physicians, hospital administrators, large group medical practices, and other industries which have requirements for $M$ to assess the technical correctness of one or more $M$ programming language processors. This test suite is approximately $99 \%$ self driven.

01,700

PB94-937300 Contact NTIS for subscription information and price.

National Inst. of Standards and Technology, Gaithersburg, MD.

Validated Products List (Cobol, Fortran, ADA, Pascal, MUMPS, SQL).

$1994,1 p$.

Supersedes PB93-937300.

Paper copy available on Standing Order, deposit account required (U.S., Canada, and Mexico \$100; all others $\$ 200$ ). Single copies also available in paper copy only.

Keywords: "Cobol programming language, "Fortran programming language, Language programming, Vederal Information Processing Standards, summary reports, "Pascal programming language, "Ada programming language, SQL programming language.

The Validated Processor List identifies those COBOL, Fortran, Ada and Pascal programming language processors that have a current validation certificate and those SQL language processors that have a registered test report, referencing the applicable Federal Information processing Standard (FIPS) as of the date of the publication. The list also includes GOSIP Conformance Testing Registers. The testing of language processors to determine the degree to which they conform to the Federal Standards is required by Government agencies in accordance with Federal Information Resources Management regulation (FIRMR) Parts 201.13 and 201.39, and the associated Federal ADP and Telecommunications Standards Index. The list is updated and published quarterly.

01,701

PB95-105037 PC A03/MF A01

National Inst. of Standards and Technology (CSL) Gaithersburg, MD. Systems and Software Technology Div.

Making Sense of Software Engineering Environment Framework Standards.

B. Cuthill. May 94, 35p, NISTIR-5487.

Sponsored by Assistant Secretary of Defense, Washington, DC. Director of Defense Information.

Keywords: "Softwear engineering, "Standards, Specifications, Operating systems(Computers), Software tools, Data base management systems, Man computer interface, "SEEs(Software Engineering Environments), "Software Engineering Environments, POSIX(Portable Operating System Interface), PCTE(Portable Common Tool Environment), CORBA(Common Object Reques Broker Architecture), ODBMS(Object Database Management Standard), CDIF(Computer Aided Software Engineering (CASE) Data Interchange Format), $\times 3 \mathrm{H} 6$ Draft Messaging Standard, X Windows, Motif.

The purpose of software environment framework standards and specifications is to enhance tool portability, interoperability, and integration by creating public interfaces to functionality incorporated into the framework. If tools can access framework services in predictable ways, tool vendors can take advantage of these services to avoid duplicating services and concentrate on the unique functionality of the tool. This describes the functionality and integration support supplied by a selected set of software environment framework standards and specifications with respect to common models.

01,702

PB95-105045 PC A03/MF A0

National Inst. of Standards and Technology (CAML) Gaithersburg, MD. Applied and Computational Mathematics Div.

Software Needs in Special Functions.

D. W. Lozier. Aug 94, 19p, NISTIR-5490.

Keywords: "Computer software, "Computation, Algorithms, Subroutine libranies, Interactive systems, Requirements, Tests, Computer applications, Computer program verification, "Special functions.

Currently available software for special functions exhibits gaps and defects in comparison to the needs of modern high-performance scientific computing and also, surprisingly, in comparison to what could be con- structed from current algorithms. In this paper, we expose some of these deficiencies and identify the related need for user-oriented testing software.

\section{1,703}

PB95-125894 Not available NTIS

National Inst. of Standards and Technology (CSL), Gaithersburg, MD. Systems and Software Technology Div.

Software Safety and Program Slicing.

Final rept.

K. B. Gallagher, and J. R. Lyle. 1993, 10p.

Pub. in Proceedings of the Annual Conference on Computer Assurance (8th), COMPASS '93, Gaithersburg, MD., June 14-17, 1993, p71-80.

Keywords: "Computer program reliability, "Safety, *Quality assurance, Software engineering, Computer program verification, Functional integration, Prototypes, Reprints, "Program slicing, US NIST.

Software quality assurance auditors are faced with a myriad of difficulties. One particular problem is the localization of safety critical code that may be interleaved throughout the entire system. Moreover, once this code is located, its effects throughout the system are difficult to ascertain. We present a method that uses program slicing to mitigate these difficulties in two ways. First, we show how program slicing can be used to locate all code that contributes to the value of variables that might be part of a safety critical component. Second we show how slicing-based techniques can be used to validate functional diversity, i.e, that there are no interactions of one critical component with another critical component and that there are no interactions of non critical components with the safety critjcal components.

\section{1,704}

\section{Not available NTIS}

National Inst. of Standards and Technology (CSL), Gaithersburg, MD. Information Systems Engineering Div.

Object SQL: Language Extensions for Object Data Management.

Final rept.

L. J. Gallagher. 1992, 10p

Pub. in Proceedings of ISMM International Conference on Information and Knowledge Management, Baltimore, MD., November 8-11, 1992, p17-26.

Keywords: *Query languages, "Object-oriented programming, "Data management, Data bases, Data base management systems, Standards, Specifications, Standardization, Reprints, *SQL database language, ISO(International Organization for Standardization), RDA(Remote Database Access).

Database Language SQL is enjoying success as an effective International Standard for the definition and management of relationally structured data. National and international SQL standardization committees are now focusing on development of future extensions for meeting the stated requirements of managing complex objects in engineering and multimedia environments. These extensions include object identifiers, abstract data types, inheritance hierarchies, and all of the other features normally associated with object data management. This paper presents the object management features contained in the draft specification for the next SQL standard, discusses problems and alternatives, and addresses planned future standardization activities.

\section{1,705}

\section{PB95-136339 PC A10/MF A03}

National Inst. of Standards and Technology (CSL), Gaithersburg, MD. Information Systems Engineering Div.

Domain Analysis of the Alarm Surveillance Domain. Version 1.0. Conducted as Part of the Domain Analysis Case Study Project.

C. Dabrowski, and J. Watkins. Sep 94, 210p NISTIR-5494

Keywords: "Software reuse, "Communications networks, "Management, Fault detection, Information systerns, Models, Domain analysis, Alarm surveillance, FODA(Feature Oriented Domain Analysis)

The Domain Analysis Case Study was created to examine the potential use of domain analysis methods in supporting software reuse. To this end, the Domain Analysis Case Study has investigated the use of a particular domain analysis method, called the Feature Oriented Domain Analysis (FODA) method. This report describes the results of the application of the second phase of the FODA method-the domain modeling phase--to the alarm surveillance domain. During this phase, a domain model of alarm surveillance systems was created. This model captured commonalities and variabilities of the alarm surveillance domain with respect to the features of systems in the domain, the functions system perform, and the underlying structure of data used by systems. The domain model is intended to be reused to derive functional requirements for new software systems in this domain.

\section{1,706}

PB95-136370 PC A03/MF A01

National Inst. of Standards and Technology (CAML) Gaithersburg, MD. Applied and Computational Mathematics Div.

Faster BKL Monte Carlo Simulations.

J. L. Blue, I. Beichl, and F. Sullivan. 20 Sep 94, 11p, NISTIR-5489

Keywords: "Computerized simulation, "Monte Carlo method, "Run time(Computers), Algorithms, Crysta growth.

For Monte Carlo simulations of systems of size $M$ that use the method of Bortz, Kalos, and Liebowitz (BKL) the best computer time per event has been $\mathrm{O}(\mathrm{M}$ to the $1 / 2$ power). We present two new methods whose computer time per event is $\mathrm{O}(\mathrm{M}$ to the $1 / \mathrm{K}$ power) or $\mathrm{O}$ (log M). In practice, for typical simulation sizes, $K=4$ or $\mathrm{K}=5$ is fastest, requiring even less computer time than the $\mathrm{O}(\log \mathrm{M})$ method. For typical simulation sizes, we are able to achieve speedup factors of 5 to 7 .

\section{1,707}

PB95-136610 PC A03/MF AO

National Inst. of Standards and Technology (CSL), Gaithersburg, MD. Systems and Software Technology Div.

Teport on the Advanced Software

D. R. Wallace, D. R. Kuhn, and T. R. Rhodes. 25 Aug 94, 15p, NISTIR-5500.

Keywords: "Computer software, "Software engineering, "Technology assessment, Computer program reliability, Software reuse, Computer security, Standards, Businesses, Computer program portability, Computer applications, Requirements, US NIST.

On February 1, 1994, the Director of the Compute Systems Laboratory of the National Institute of Standards and Technology (NIST) convened an invitational workshop attended by eleven executives for whom software is critical to business. The purpose of the workshop was to identify advanced software technology requirements for U.S. business and to identify opportunities that NIST might pursue in the area of ad vanced software technology. This report summarizes the deliberations of the workshop.

01,708

PB95-151262 Not available NTIS

National Inst. of Standards and Technology (CSL) Gaithersburg, MD. Systems and Software Technology

Analysis of Selected Software Safety Standards. Final rept.

D. R. Wallace, D. R. Kuhn, and L. M. Ippolito. 1992 $14 \mathrm{p}$.

Pub in Proceedings of COMPASS '92 Conference, Gaithersburg, MD., June 15-18, 1992, p1-14.

Keywords: "Computer software, "Software reliability "Data processing security, "Standards, Computer program integrity, Software engineering, Computer security, Quality assurance, Requirements, Guidelines, Reprints, Software safety.

The study examines standards, draft standards, and guidelines that provide requirements for the assurance of high integrity software. The study focuses on identifying the attributes necessary in a document for providing reasonable assurance for high integrity software, and on identifying the relative strengths and weak nesses of the documents. The documents vary widely in their requirements and the precision with which the requirements are expressed. Overall there is little relationship between the degree of risk and the rigor of applicable standards. Recommendations are provided for a base standard for the assurance of high integrity software.

01,709

PB'95-152062 Not available NTIS 


\section{Computer Software}

National Inst. of Standards and Technology (CSL), Gaithersburg, MD. Systems and Software Technology Div.

Use of an Environment Classification Model.

Final rept.

M. V. Zelkowitz. 1993, 10p

Pub. in Proceedings of International Conference on Software Engineering (15th), Baltimore, MD., May 17$21,1993, p 348-357$

Keywords: "Software engineering, "Environments, Environment models, Integrated systems, Mapping, Reprints.

Various reference models have been proposed for the classification of features present in an integrated software engineering environment. In this paper, two such models are studied and a target is mapped to the set of services present in these models. The results of this mapping and comments on the effectiveness of the models are given.

01,710

PB95-153029 Not available NTIS

National Inst. of Standards and Technology (NCSL) Gaithersburg, MD. Systems and Network Architecture Div.

Formal Methods in Conformance Testing: Result and Perspectives.

Final rept.

A. R. Cavalli, J. P. Favreau, and M. Phalippou. 1993 $16 \mathrm{p}$.

Pub. in Proceedings of the International Workshop on Protocol Test Systems (6th), Pau, France, September 1993, p1-16.

Keywords: Tests, Standardization, Protocols, Reprints, "Conformance testing, "Formal methods.

The application of formal methods to conformance testing becomes a more and more active research area. This paper presents the results and perspectives of the application of these languages for tests and test generation methods.

\section{1,711}

PB95-154670 PC A06/MF A02

National Inst. of Standards and Technology (CSL) Gaithersburg, MD. Information Systems Engineering Div.

Mapping Integration Definition for Information Modeling (IDEF $1 X$ ) Model into CASE Data Interchange Format (CDIF) Transfer File.

I. Simakhodskiy. Nov 94, 103p, NISTIR-5530.

Also available from Supt. of Docs. See also FIPS PUB 184

Keywords: "Data transfer(Computers), "Standards, Models, Software tools, "IDEF1X(Integration Definition for Information Modeling), "Integration Definition for Information Modeling, "CDIF(CASE Data interchange Format), "CASE Data Interchange Format, Meta data

This document describes a mapping of an Integration Definition for Information Modeling (IDEF $1 X$ ) mode into a CASE Data Interchange Format (CDIF) transfer file. This work will demonstrate that it is possible to use the CDIF standard exchange for moving information between different IDEF tools. It will also show how CDIF can provide a common definition of model data for communicating between different CASE tools.

\section{1,712}

PB95-161444 Not available NTIS

National inst. of Standards and Technology (CAML) Gaithersburg, MD. Applied and Computational Mathematics Div.

Underflow-Induced Graphics Failure Solved by SLI Arithmetic.

Final rept.

D. W. Lozier. $1993,8 p$

Pub. in Proceedings of the Institute of Electrical and Electronics Engineers Symposium on Computer Arithmetic (11th), Windsor, Ontario, Canada, June 29-July 2, 1993, p10-17

Keywords: "Computer graphics, "Floating point arithmetic, Computation, Algorithms, Errors, Scaling, Accuracy, Reprints, SLI(Symmetric level-index) arithmetic.

Floating-point underflow is often regarded as either harmless or as an indication that the computational al gorithm is in need of scaling. A counter example to this view is given of a function for which contour plotting is difficult due to floating-point underflow. The function arose as an asymptotic solution to a model problem in turbulent combustion in which two chemical species (fuel and oxidizer) mix and react in a vortex field. Scaling is not a viable option because of extreme sensitivity to a small physical parameter. Standard graphics software packages produce erroneous contours without any indication of difficulty. This example provides support for considering symmetric level-index (SLI) arithmetic, a new form of computer arithmetic which is immune to underflow and overflow.

\section{1,713}

PB95-161881 Not available NTIS

National Inst. of Standards and Technology (CSL), Gaithersburg, MD. Information Systems Engineering Div.

Standards and Linkages: What Data Sharing Needs.

Final rept.

J. J. Newton, and D. C. Wahl. 1994, 5p.

Pub. in Data Base Newsletter 22, n1 p3-7 Jan/Feb 94

Keywords: "Data structures, "Information transfer, *Linkages, Data links, Data integration, Data management, Information processing, Data elements, Standardization, Information systems, Reprints, "Data sharing, Legacy systems, Data administration, Data representation.

Legacy systems data must be integrated into newlydeveloped standardized system data in order to achieve fully interchangeable information. Standards for data element representation and legacy data linkages are two components of a successful program for fully-realized data sharing. A data hierarchy reference model provides a framework for integration of data standards. Chained, coupled, and multi-purpose linkages supply characteristic associations to existing elements.

\section{1,714}

\section{PB95-163309 Not available NTIS}

National Inst of Standards and Technology (CAML) Gaithersburg, MD. Applied and Computational Mathematics Div.

Data-Parallel Algorithm for Three-Dimensional Delaunay Triangulation and Its Implementation.

Final rept.

Y. A. Teng, F. Sullivan, I. Beichl, and E. Puppo.

1993, 10p.

Pub. in Proceedings of Supercomputing '93, Portland, OR., November 15-19, 1993, p112-121.

Keywords: "Triangulation, "Computational geometry, "Three dimensional models, Parallel processing, Algorithms, Run time(Computers), Computer program reliability, Connection machine, Reprints.

In this paper, we present a parallel algorithm for constructing the Delaunay triangulation of a set of vertices in three-dimensional space. The algorithm achieves a high degree of parallelism by starting the construction from every vertex and expanding over all open faces thereafter. In the expansion of open faces, the search is made faster by using a bucketing technique. The algorithm is designed under a data-parallel paradigm. It uses segmented list structures and virtual processing for load-balancing. As a result, the algorithm achieves a fast running time and good scalability over a wide range of problem sizes and machine sizes. We also incorporate a topological check to eliminate inconsistencies due to degeneracies and numerical errors. The algorithm is implemented on Connection Machines $\mathrm{CM}-2$ and $\mathrm{CM}-5$, and experimental results are presented.

\section{1,715}

PB95-163655 Not available NTIS

National Inst. of Standards and Technology (NCSL), Gaithersburg, MD. Computer Security Div

Computer Virus Attacks.

Final rept.

J. P. Wack, and S. A. Kurzban. 1990, 4p.

Pub. in National Computer Systems Laboratory Bulletin, p1-4 Aug 90.

Keywords: "Computer viruses, "Electronic security, "Threat evaluation, "Risk analysis, Computer program integrity, Vulnerability, Computer information security, Data processing security, Personal computers, Reprints, Worms(Computers), Logic bombs, Time bombs(Computers), Trojan horses.

The popular media contain many stories about computer viruses and similar phenomena. There are many things users and organizations can do to protect themselves from the damage that viruses can do, but some protective measures remain to be fully developed. The solution to the virus problem involves a broad range of issues, and requires us to regard viruses and computer crime in a more serious light.

01,716

PB95-173084 PC A05/MF A01

National Inst. of Standards and Technology (CSL), Gaithersburg, MD. Systems and Software Technology Div.

Framework for the Development and Assurance of High Integrity Software.

Special pub.

D. R. Wallace, and L. M. Ippolito. Dec 94, 92p, NIST/ SP-500/223

Also available from Supt. of Docs. as SN003-00303312-0. See also PB90-111691.

Keywords: "Software engineering, "Project management, Computer software management, Computer program reliability, Computer program verification, Configuration management, Quality assurance, Software tools, Fault tolerant computing, Error detection codes

The purpose of this document is to recommend a framework for the development and assurance of high integrity software. The framework addresses the fact that these processes must take into account properties and requirements of a high integrity system and the processes and standards used in developing other system components. This framework provides guidance to developers, assurers, and buyers of software, researchers for high integrity software systems, and vendors of Computer Aided Software Engineering tools and integrated environments.

\section{1,717}

PB95-174959 PC A03/MF A01

National Inst. of Standards and Technology (CSL) Gaithersburg, MD. Systems and Software Technology Div.

\section{SGML Parser Validation Procedures.}

R. B. Wilson. Jan 95,33 , NISTIR-5538.

Sponsored by Assistant Secretary of Defense (Production and Logistics), Falls Church, VA. Computer-aided Acquisition and Logistic Support Program.

Keywords: "Parsers, "Computer program verification, Federal informatlon processing standards, Tests, "SGML(Standard Generalized Markup Language), "Standard Generalized Markup Language, Conformance testing, US NIST.

This document establishes operating policy and procedures for the Computer Systems Laboratory's (CSL) validation program for Federal Information Processing Standards (FIPS) 152, Standard Generalized Markup Language (SGML), parsers. The testing methodology is based on ANSI X3.190-1992, Text and Office Systems - Conformance Testing for Standard Generalized Markup Language Systems.

01,718

PB95-175550 Not available NTIS

National Inst. of Standards and Technology (CSL), Gaithersburg, MD. Systems and Software Technology Div.

Testers Open Dialogue at Inaugural NIST Workshop.

Final rept.

M. M. Gray, and K. Liburdy. 1994, 2p.

Pub. in Institute of Electrical and Electronics Engineers Software 11, n5 p120-121 Sep 94.

Keywords: "Computer program verification, "Tests, *Automation, Standards, Computer software, Technology assessment, Costs, Reprints, Conformance testing.

This paper is a summary report of a recent invitationa workshop on automated testing. One of the goals of the workshop was to propose an agenda to support and accelerate efforts in automated testing. Attendees were urged to identify 'areas of synergy' and a common focus that could lead to increased participation and funding for automated testing and reduce the "exorbitant' cost of building test suites. Participants also reviewed existing and emerging technologies and explored the relationship between automated testing and standards development.

01719

PB95-175741 Not available NTIS

National Inst. of Standards and Technology (PL), Boulder, CO. Time and Frequency Div. 
Obtaining and Installing a Public Domain TEX.

Final rept.

W. Itano. 1991, 2p.

Pub. in Optics and Photonics News 2, n10 p41, 61, Oct 91.

Keywords: *Text processing, "Computer software, Microcomputers, Reprints, *Public domain, TEX computer program, Typesetting

Instructions are given for obtaining a public-domain version of the TEX computer program for MS-DOS computers. The installation and operation procedures are outlined.

\section{1,720}

PB95-178992 PC A03/MF AO1

National inst. of Standards and Technology (CSL), Gaithersburg, MD. Information Systems Engineering Div.

Glossary of Software Reuse Terms.

S. Katz, C. Dabrowski, K. Miles, and M. Law. Dec $94,37 \mathrm{p}, \mathrm{NIST} / \mathrm{SP}-500 / 222$.

Also available from Supt. of Docs.

Keywords: "Software reuse, "Dictionaries, Software engineering, Computer programming, Software tools. One method proposed for increasing the efficiency of software production in the development of large, reliable software applications is the systematic reuse of existing software products. Effective software reuse will require new techniques to supplement traditional software engineering practices. Preliminary research has already produced new methods and reports. As a result, new terminology has emerged. This repor provides a baseline set of recommended definitions for terms commonly used in the software reuse community. The glossary will be expanded as further research results become available and are evaluated for use in software reuse programs.

01,721

PB $95-180360 \quad$ Not available NTIS

National Inst. of Standards and Technology (PL), Boulder, CO. Time and Frequency Div.

Getting Started on Mosaic.

Final rept.

W. M. Itano. 1994, 2p.

Pub. in Optics and Photonics News 5, n6 p48-49 Jun 94.

Keywords: *Computer software, Super computers, Information retrieval, Operating systems(Computers), Applications programs(Computers), Reprints, "Mosaic computer program, World Wide Web, Internet.

Mosaic, a free computer program developed by the National Center for Supercomputing Applications, is described. Mosaic works with the World Wide Web and Internet-based information retrieval system. Information on obtaining, installing, and using the software is given. Mosaic operates on computers running the $x$ Window, Microsoft Windows, and Macintosh operating systems.

01,722

PB95-180550 Not available NTIS

National Inst. of Standards and Technology (MEL) Gaithersburg. MD. Factory Automation Systems Div. Handling Passwords with Security and Reliability in Background Processes.

Final rept.

D. Libes. 1994, 8p

Pub. in Proceedings of Systems Administration Conference (8th), (LISA VIII), San Diego, CA., September 19-23, 1994, 8p.

Keywords: *Interactive systems, "Computer security, Data processing security, Computer program reliability, Automation, Access control, Reprints, Passwords, Expect computer program, Background processes.

Traditionally, background automation of interactive processes meant giving up security and reliability. With the advent of software such as Expect for controlling interactive processes, it has become possible to improve reliability and security with relative ease. This paper reviews the reliability aspects but focuses primarily on the security aspects, presenting several nonobvious techniques for dealing with passwords and other sensitive information in background processes. These techniques require no changes to existing programs and no new security systems are necessary. With the appropriate tools and examples, these techniques can be applied with surprisingly little effort to a wide variety of problems.
01,723

PB95-180832 Not available NTIS

National Inst. of Standards and Technology (CAML), Gaithersburg, MD. Applied and Computational Mathe matics Div.

Performance Characteristics of Fast Elliptic Solvers on Parallel Platforms.

Final rept.

R. Pozo. 1994, 5 p.

Pub. in Proceedings of the European Parallel Virtual Machine Users Group Meeting (1st), Rome, Italy, October 9-11, 1994, p1-5.

Keywords: "Elliptic differential equations, "Computation, Connection machine, Computer calculations, Computer software, Computer networks, Partial differential equations, Interprocessor communication, Run time(Computers), Performance evaluation, Reprints, "PVM(Parallel Virtual Machine), "Paraliel Virtual Machine, OSC(Orthogonal spline collocation).

We present performance analyses of a fast three-dimensional elliptic orthogonal spline collocation (OSC) method on the Connection Machine CM-5, and various network clusters using the Parallel Virtual Machine (PVM) software package. While the OSC method is an efficient approach for solving Poisson, Helmholtz, and other separable partial differential equation (PDE) problems, it poses serious challenges for parallel architectures because of its high communication to computation ratio: $\mathrm{O}(\mathrm{N}$ cubed) data movement to $\mathrm{O}(\mathrm{N}$ cubed $\log N$ ) computation. Furthermore, the communication pattern is one of the most expensive: personalized all-to-all. Results show that communication time occupies roughly $5 \%$ of total execution time in native message-passing systems (such as NX and CMMD), $10 \%$ with PVM layer on MPP's and roughly $50 \%$ for Ethernet networks.

01,724

PB95-194205 PC A03/MF A01

National Inst. of Standards and Technology (CSL), Gaithersburg, MD. Systems and Software Technology Div.

Comparing Remote Procedure Calls: Open Network Computing, Distributed Computing Environment and International Organizakion for Standardization.

J. F. Barkley. Oct 93, 23p, NISTIR-5277.

Sponsored by Defense Information Systems Agency, Arlington, VA. and Department of the Army, Fort Belvoir, VA. Sustaining Base Information Services.

Keywords: *Computer networks, ${ }^{*}$ Computer communications, $\quad$ "Protocols, Applications programs(Computers), Compilers, Semantics, Programming languages, Computer program portability, Run time(Computers), *RPC(Remote procedure calls), "Remote procedure calls, ONC(Open Network Computing). DCE(Distributed Computing Environment), ISO(International Organization for Standardization).

Almost all computer systems are connected to a network supporting data communications. As a result, many techniques have evolved to support the development of applications which require processes on different systems to communicate and coordinate their activities. One such technique is remote procedure call (RPC). RPC is a mature method with several specifications and implementations. Among these are: Open Network Computing (ONC) RPC, Distributed Computing Environment (DCE) RPC, and the RPC specification from the International Organization for Standardization (ISO). This report describes the RPC concept, how this concept is commonly implemented, and compares the features and capabilities of these three RPCs. The RPC language, semantics, and protocol of ONC RPC, DCE RPC, and ISO RPC are compared. since ONC RPC and DCE RPC have implementations, the output of their RPC language compiler and the support provided by their runtime libraries are also compared.

\section{1,725}

PB95-198727 PC A04/MF A01

National Inst. of Standards and Technology (CSL), Gaithersburg, MD. Systems and Software Technology Div.

Study on Hazard Analysis in High Integrity Software Standards and Guidelines.

L. M. Ippolito, and D. R. Wallace. Jan 95, 65p NISTIR-5589.

See also PB92-112267.

Keywords: *Computer software, "Hazards, "Computer program integrity, Standards, Guidelines, Safety, Qual- ity assurance, Software engineering, Computer program reliability, Systems analysis.

This report presents the results of a study on hazard analysis, especially software hazard analysis, in high integrity software standards and guidelines. It describes types of system hazard analysis (that influence software), types of software hazard analysis, techniques for conducting hazard analysis (along with some of their advantages and disadvantages), and other practices and processes that should be employed in order to ensure the safety of software.

\section{1,726}

PB95-199329 PC A03/MF A01

National Inst. of Standards and Technology (CSL), Gaithersburg, MD. Systems and Software Technology Div.

Object-Oriented Technology Research Areas. W. J. Salamon, and D. R. Wallace. 21 Feb 95, 29p NISTIR-5600.

Keywords: *Object-oniented programming, "Technology assessment, Distributed computer systems, Distributed processing, Software engineering, Computer program verification, Technology innovation, Research projects, *Object-oriented technology, US NIST.

This paper discusses some of the issues surrounding object technology. The topics which are discussed are object-oriented (OO) development methodologies, measuring the quality of $\mathrm{OO}$ software, testing, the use of $\mathrm{OO}$ technology in high-integrity systems, and distributed object computing. The purpose of this report is to identify research topics in $\mathrm{OO}$ technology for the $\mathrm{Na}$ tional Institute of Standards and Technology (NIST) Computer Systems Laboratory. A bibliography is included to assist the reader in selecting material for further reading.

\section{1,727}

PB95-203253 Not available NTIS

National Inst. of Standards and Technology (NCSL), Gaithersburg, MD. Systems and Network Architecture Div.

PET and DINGO Tools for Deriving Distributed Implementations from Estelle.

Final rept.

R. Sijelmassi, and B. Strausser. 1993, 18p. Pub. in Computer Networks and ISDN Systems 25 p841-8511993.

Keywords: "Software tools, *Distributed computer systems, Operating systems(Computers), Compilers, Finite state machines, Specifications, Translators, Computer networks, Reprints, *Estelle translator, Osi(Open systems interconnection)

The combination of the Portable Estelle Translato (PET) and the Distributed ImplementatioN GeneratOr (DINGO) tools produces distributed implementations from Estelle specifications. The resulting implementations run as one or more operating system processes distributed over several sites of a target distributed system. In addition, the tools generate elements of an XWindow interface which allows centralized or distributed monitoring of some or all of the running modules.

\section{1,728}

PB95-216883 PC A25/MF A06

National Inst. of Standards and Technology (CSL), Gaithersburg, MD. Advanced Systems Div.

Overview of the Text REtrieval Conference (3rd) (TREC-3). Held in Gaithersburg, Maryland on November $2-4,1994$.

Special pub.

D. K. Harman Apr 95 595p, NIST/SP-500/225.

Also available from Supt. of Docs. as SN003-003 03328-6. Sponsored by Advanced Research Projects Agency, Arlington, VA

Keywords: "Meetings, "Information retrieval, "Text processing, Thesaun, Automation, Natural language processing, Pattern recognition, Routing, Probability theory, Documents, Search structuring, Knowledge bases(Artificial intelligence), Vector processing, Semantics, Evaluation.

The third Text REtrieval Conference (TREC-3) was held in Gaithersburg. Maryland, November 2-4, 1994 and was attended by 150 people involved in the 33 participating groups. The goal of the Conference was to bring research groups together to discuss their work on a new large text collection. There was a large variation of retrieval techniques reported on, including 


\section{Computer Software}

methods using automatic thesauri, sophisticated term weighting, natural language techniques, relevance feedback, and advanced pattern matching. As results were run through a common evaluation package, groups were able to compare the effectiveness of different techniques, and discuss how differences between the systems affected performance.

\section{1,729}

\section{PB95-220554 PC A03/MF A01}

National Inst. of Standards and Technology (NCSL), Gaithersburg, MD. Systems and Network Architecture Div.

Analysis of ANSI ASC X12 and UN/EDIFACT Electronic Data Interchange (EDI) Standards.

R. Aronoff, and K. Hsing. Apr 95, 35p, NISTIR-5631.

Keywords: "Information systems, "Message processing, "Software engineering, *Standards, Data processing, Electronic commerce, Health insurance, EDI(Electronic Data Interchange), EDI, American National Standards Institute ASC X12, UN/ EDIFACT(United Nations Electronic Data Exchange for Information), UN/EDIFACT.

The report discusses the two Electronic Data Interchange (EDI) standards that are being developed by the ANSI ASC X12 and the UN/EDIFACT committees respectively. Discussion is focused on the basic ED data structures, syntax rules, and functionality. In order to illustrate the concepts and issues involved, a par ticular application, health care claim submission, is examined. An example scenario is presented which demonstrates how EDI can be used to support this traditional business process. An analysis is then presented which compares these two standards in detail. This analysis look at functionality, efficiency, and existing levels of standards development. To investigate issues of relative efficiency, a comparison is made of record lengths for the basic control/service elements for both standards.

\section{1,730}

\section{PB95-220588 PC A05/MF A01}

National Inst. of Standards and Technology (CSL), Gaithersburg, MD. Information Systems Engineering Div.

Persistent Object Base System Testing and Evaluation.

E. N. Fong. Apr 95, 84p, NISTIR-5636

Sponsored by Defense Advanced Research Projects Agency, Arlington, VA.

Keywords: "Software engineering, "Object database management, Database management systems, Performance evaluation, Performance tests, "POB(Persistent Object Based Systems), "POB.

The report summarizes the role of the Computer Systems Laboratory (CSL) of the National Institute of Standards and Technology (NIST) in support of the Advanced Research Projects Agency (ARPA) in the testing and evaluation of persistent object base (POB) systems. The actual evaluation consists of designing a testing suite for exercising the POB system for the features supported. The goal of actual testing is to reveal how well each feature is being supported. The testing methodology, the abstract test suite, and the actual test results on the ARPA funded prototype POB system called Open OODB developed by Texas Instruments are described.

01,731

PB95-231726 PC A03/MF A01

National Inst. of Standards and Technology (CSL), Gaithersburg, MD. Systems and Software Technology Div.

Introduction to the P1003.1g and CPI-C Network Application Programming Interfaces.

K. OIsen. 25 May 95, 50p, NISTIR-5657.

Keywords: "Computer networks, "Computer programming, "Distributed processing, "Programming environments, Applications programs(Computers) Interprocessor communication, Protocols, Data transfer(Computers), Specifications, APIs(Application programming Interfaces)

Numerous network application programming interfaces (APIS) have been developed to assist programmers in developing distributed applications. Both IEEE P1003.1g and Common Programming Interface for Communications (CPI-C) are examples of network APIs. The report provides an overview of the P1003.1g and $\mathrm{CPI}-\mathrm{C}$ calls is given, along with a simple programming example for each API. The report does not con- tain sufficient detail needed to develop distributed applications using these network APIs, however, a list of references is provided.

\section{1,732}

\section{PB95-242335 PC A03/MF A01}

National Inst. of Standards and Technology (CAML), Gaithersburg, MD. Applied and Computational Mathematics Div.

Parallel and Serial Implementations of SLI Arithmetic.

D. W. Lozier, and P. R. Turner. Jun 95, 28p, NISTIR5660

See also PB93-153476 and PB95-161444.

Keywords: *Floating point arithmetic, Computation, Parallel processing, Vector processing, Algorithms, Accuracy. Reprints, "Foreign technology, SLI(Symmetric Level Index).

The paper describes the various algorithms and software implementations of the Level-Index LI and Symmetric Level-Index SLI arithmetic schemes After a brief introduction to the number representations and the arithmetic algorithms, the authors describe the original precompiler for including $\mathrm{LI}$ and $\mathrm{SLI}$ variables and their arithmetic in a Fortran 77 program.

\section{1,733}

\section{PC A05/MF A01}

Reliable Software Technology Corp., Reston, VA.

Testability of Object-Oriented Systems.

Final rept. on Phase 1

Jun 95, 83p, NIST/GCR-95/675

Contract NIST-50DKNA-Y-00119

Sponsored by National Inst. of Standards and Technology (CSL), Gaithersburg, MD.

Keywords: "Object-oriented programming, "Tests, *Improvements, Software reuse, Fault tolerant computing, Computer software.

We have studied the feasibility of building an object oriented software testability improvement tool that can be sued in conjuction with RST Corporation's C++ Softwre Testability Analysis (TM) or as a standalone assertion injection tool. We have also produced a se of recommended validation and design techniques that will improve testability, and as a side benefit, the tes ing of OO systems. If awarded a Phase II effort, RST will provide NIST with a means of improving the testability of $\mathrm{C}++$ systems; to do so within the Phase II cost limit, RST will leverage knowledge gained from building next-generation software assessment tools. Here, RST will rely on experience gained during the building of RST's C and Fortran-77 testability tools, as well as the experimental results from the Phase I effect (which required our building a prototype $\mathrm{C}++$ front-end for PiSCES Software Analysis Toolkit(TM)). Such a Phase II innovation can also be used by NIST or othe US Government agencies to empirically research the fault masking ability provided between procedural and object-oriented languages.

\section{4}

PB95-251674 PC A03/MF AO

National Inst. of Standards and Technology (CSL) Gaithersburg, MD. Systems and Software Technology Div.

Center for High Integrity Software System Assurance: Initial Goals and Activities.

D. R. Wallace, and M. Zelkowitz. Jun 95, 28p NISTIR-5677

Keywords: "Computer program reliability, Quality as surance, Technology transfer, Industries, National government, Universities, Software engineering, Measurement, Goals, Software tools, Prototypes, "Center fo High Integrity Software System Assurance, ${ }^{*}$ CHISSA(Center for High Integrity Software System Assurance), US NIST, Formal methods.

To enable the U.S. software industry to build high in egrity software and to provide U.S. industries and gov ernment confidence in the software systems on which they are dependent, the National Institute of Standards and Technology (NIST) created the Center for High Integrity Software System Assurance (CHISSA) to es tablish measurements and software methods for software assurance for use by those who build these systems. The measurements and associated methods will be embodied in a software development and assurance framework that will enable CHISSA to guide research in development, analysis, and testing techniques, to promote research in measurement and experimentation in the software engineering domain to be able to measure the impact of new high-integrity techniques, to accelerate use of effective technology into industry, and to develop standards and guidelines in cooperation with industry, other Federal agencies, and the research community.

\section{1,735}

PB95-267829 PC A03/MF A01

National Inst. of Standards and Technology (CAML) Gaithersburg, MD. Applied and Computational Mathematics Div.

\section{Expression Formatter for MACSYMA.}

B. R. Miller. Jul 95, 26p, NISTIR-5618.

Keywords: "Format, Computer software, Formulas(Mathematics), Coefficients, Semantics, ${ }^{\star} \mathrm{CAS}$ (Computer Algebra System), *Computer Algebra System, MACSYM computer program.

A package for formatting algebraic expressions in MACSYMA is described. It provides facilities for userdirected heirarchical structuring of expressions, as well as for directing simplifications to selected subexpressions. It emphasizes a semantic rather than syntactic description of the desired form. The package also provides utilities for obtaining efficiently the coefficients of polynomials, trigonometric sums and power senies.

01,736

PB95-267886 PC A05/MF A01

National Inst. of Standards and Technology (CSL) Gaithersburg, MD. Systems and Software Technology Div.

Unravel: A CASE Tool to Assist Evaluation of High Integrity Software. Volume 1. Requirements and Design.

J. R. Lyle, D. R. Wallace, J. R. Graham, D. W Binkley, K. B. Gallagher, and J. P. Poole. Aug 95, $94 p$, NISTIR-5691-V1.

See also Volume 2, PB95-267894

Keywords: "Software engineering, "Computer program reliability, "Software tools, Computer program verification, Computer software maintenance, Debugging(Computers), Safety, Algorithms, Requirements, "Program slicing, CASE(Computer Aided Software Engineering), ANSI C programming language.

This report describes a Computer Aided Software Engineering (CASE) tool, unravel, that can assist evaluation of high integrity software by using program slices to extract computations for examinations. The tool can currently be used to evaluate software written ANSI C and is designed such that other languages can be added. Program slicing is a static analysis technique that extracts all statements relevant to the computation of a given variable. Program slicing is useful in program debugging, software maintenance and program understanding. Application of program slicing is evaluation high integrity software reduces the effort in examining software by allowing a software reviewer to focus attention on one computation at a time. Once a software reviewer has identified a variable for furthe investigation, the reviewer directs unravel to compute a program slice on the variable. Instead of examining the entire program, only the statements in the slice need to be examined by the reviewer. By speeding up the process of locating relevant code for examination by the reviewer, a larger sample of the software can be inspected with greater confidence that some relevant section of source code has not been missed. Volume 1 of this report describes the requirements, design and evaluation of unravel.

\section{1,737}

PB95-267894 PC A04/MF AO1

National Inst. of Standards and Technology (CSL), Gaithersburg, MD. Systems and Software Technology

Unravel: A CASE Tool to Assist Evaluation of High Integrity Software. Volume 2. User Manual.

J. R. Lyle, D. R. Wallace, J. R. Graham, D. W

Binkley, K. B. Gallagher, and J. P. Poole. Aug 95,

$56 \mathrm{p}$, NISTIR-5691-V2.

See also Volume 1, PB95-267886

Keywords: * Software engineering, *Computer program reliability, "Software tools, Computer program verifica. tion, Computer software maintenance, Debugging(Computers), Uafety, User manuals(Computer programs), "Program slicing, CASE(Computer Aided Software Engineering), ANSi $\mathrm{C}$ programming language.

This is the second volume of a two volume report on unravel, a Computer Aided Software Engineering 
(CASE) tool for software written in ANSI C, that assist evaluation of high integrity soltware by using program slices to extract computations for examination. In this volume, we provide a user manual for unravel. This manual is intended to provide the user with enough information to use unravel without any other reference. To this end, a brief simplified description of program slicing is provided in additon to a tutorial example and a detailed description of unravel operation. This user manual also discusses limitations of unravel and how to deal with code containing extensions to ANSI C that would inhibit the correct operation of unravel.

\section{1,738}

PB95-937300 Contact NTIS for subscription

information and price.

National Inst. of Standards and Technology, Gaithersburg, MD.

Validated Products List (Cobol, Fortran, ADA, Pascal, MUMPS, SQL).

1995, $1 p$

Supersedes PB94-937300

Paper copy available on Standing Qrder, deposit account required (U.S., Canada, and Mexico \$100; all others \$200). Single copies also available in paper copy only.

Keywords: "Cobol programming language, "Fortran programming language, Language programming, *Federal Information Processing Standards, *Validation summary reports, *Pascal programming language, "Ada programming language, SQL programming language.

The Validated Processor List identifies those CQBQL, Fortran, Ada and Pascal programming language processors that have a current validation certificate and those SQL language processors that have a registered test report, referencing the applicable Federal Information processing Standard (FIPS) as of the date of the publication. The list also includes GQSIP Conformance Testing Registers. The testing of language processors to determine the degree to which they conform to the Federal Standards is required by Government agencies in accordance with Federal Information Resources Management regulation (FIRMR) Parts 201.13 and 201.39, and the associated Federal ADP and Telecommunications Standards Index. The list is updated and published quarterly.

\section{1,739}

\section{PB96-102678 Not available NTIS}

National Inst. of Standards and Technology (MEL), Gaithersburg, MD. Manufacturing Systems Integration Div.

Ouch Those Programs are Painful.

Final rept.

D. Libes. $1995,3 p$

Pub. in Q'Reilly and Associates, Inc., p9-11 1994.

Keywords: *Interactive systems, "Computer communications, Interprocessor communication, Applications programs(Computers), UNIX(Qperating system), Systems management, Software tools, Reprints, "Expect computer program, Shelis(Computers), TCL computer language, Programmed dialogue.

UNIX programs used to be designed so that they could be connected with pipes created by a shell. This paradigm is insufficient when dealing with many modern interactive problems. Expect is a program designed to control interactive programs. Expect reads a script that resembles the dialogue itself but which may include multiple paths through it. The scripting language used by Expect is usually $\mathrm{Tcl}$ but may be other languages. In addition, Expect may be compiled into $\mathrm{C}, \mathrm{C}++$, or other complied languages.

01,740

PB96-103031 Not available NTIS

National Inst. of Standards and Technology (CSL), Gaithersburg, MD. Advanced Systems Div.

Using Synthetic Perturbations and Statistical Screening to Assay Shared-Memory Programs. Final rept.

R. Snelick, J. JaJa, R. Kacker, and G. Lyon. 1995

$7 p$.

Pub. in Information Processing Letters, v54 p147-153 1995

Keywords: "Parallel processing, MIMD(Computers), Response time(Computers), Computer performance evaluation, Computer systems performance, Distributed computer systems, Statistical analysis, Reprints, "Shared memory programs, "Synthetic perturbation screening.
Synthetic-perturbation screening (SPS, hereafter, fol brevity) is a diagnostic technique employing artifical code-in discussion to follow, delays-placed within segments of an MIMD program. These insertions simulate code changes in suspected program bottlenecks Screening techniques based upon statistical experimental design then flag those program segments that are most sensitive to perturbation (delay). A subset of program segments so flagged can be candidates for improvement. The results are sensitivity analyses o specimen programs in terms of their questionable sections of code.

\section{1,741}

PB96-109533 PC A07/MF A02

National Inst. of Standards and Technology (CSL) Gaithersburg, MD. Information Systems Engineering Div.

Mapping Integration Definition for Function Modeling (IDEFO) Model into CASE Data Interchange Format (CDIF) Transfer File.

I. V. Simakhodskiy. Sep 95, 128p, NISTIR-5719 See also PB95-154670.

Keywords: "Data transfer(Computers), "Standards, *Models, Software tools, "IDEFQ(Integration Definition for Function Modeling), *Integration Definition for Function Modeling, "CDIF(Case Data Interchange Format), *Case Data Interchange Format, CASE(Computer Aided Software Engineering), Computer Aided Software Engineering.

The Integration Definition for Function Modeling (IDEFO) is a standard modeling technique used in the ogical definition of an organization's data resources. Qne serious limitation to the standard is the lack of a formal standard mechanism for transferring information between different IDEF tools and between IDEF tools and other tools supporting different modeling echniques. This leaves the users of IDEF tools at a great disadvantage. A user is restricted to using a particular tool provided by a particular vendor thus seriously hindering the reusability of the information represented in a model. Qne solution is the use of a stand ard method to exchange data between tools. The CASE Data Interchange Format (CDIF) Family of Standards is primarily designed to be used as a description of a mechanism for transferring information between CASE tools. This document will describe a conceptual mapping of an IDEFO model into the CDIF transfer tile. The document will cover both syntax and semantics of the model. This work will demonstrate that it is possible to use the standard exchange for moving information between different tools.

\section{1,742}

PB96-109582 PC A02/MF A01

National Inst. of Standards and Technology (CAML) Gaithersburg, MD. Applied and Computational Mathematics Div.

Error-Bounding in Level-Index Computer Arithmetic.

D. W. Lozier, and P. R. Turner. Qct 95, 10p, NISTIR5724.

Presented at the International IMACS-GAMM Symposium on Numerical Methods and Error-Bounds, Oldenburg, Germany, July 9-12, 1995. Prepared in cooperation with Naval Academy, Annapolis, MD. Dept of Mathematics

Keywords: "Computer programs, "Computation, *Accuracy, Algorithms, Computer systems perform ance, Level Index, SLI(Symmetric Level Index).

This paper proposes the use of level-index (LI) and symmetric level-index (SLI) computer arithmetric for practical computation with error bounds. Comparisons are made with floating-point and several advantages are identified.

\section{1,743}

PB96-111844 Not available NTIS

National Inst. of Standards and Technology (CSL), Gaithersburg, MD. Systems and Software Technology Div.

SQA Standards and Total Quality Management.

Final rept.

C. E. Wardle, D. R. Wallace, R. Khorramshahgol, E G. McGuire, and B. Kaplan. 1991, 19p.

Pub. in Annual Pacific Northwest Software Quality Conference (9th), Portland, QR., Qctober 7-8, 1991, v9 p1-19.

Keywords: "Total quality management, "Computer program integrity, Computer software, Software engineering, Software reliability, Program verification(Computers), Computer architecture, Quality control, Reprints, *SQA(Software Quality Assurance), "Software Quality Assurance, Software stand ards, Process improvement.

Total Quality Management (TQM) programs, which focus on continuous process improvement, are becom ing increasingly popular in corporations today and may, in fact, be superceding quality assurance activities. Quality assurance has been used by corporations to ensure the quality of released products. What we are finding now is the thrust of management attention is on process improvement rather than on quality assurance. This paper examines some major standards and programs in SQA and TQM. Because few companies are using SQA standards, the difficulties in implementing SQA standards are addressed. Results from an on-going research project studying SQA standards and the interrelationships between TQM and SQA are presented.

01,744

PB96-112214 Not available NTIS

National Inst. of Standards and Technology (CSL) Gaithersburg, MD. Systems and Software Technology Div.

High Integrity Software Standards Activities at NIST.

Final rept

D. R. Kuhn, D. R. Wallace, and J. C. Cherniavsky. 1991, 15p.

Pub. in Annual Pacific Northwest Software Quality Conference (9th), Portland, QR., Qctober 7-8, 1991, v9 p47-61.

Keywords: *Computer software, "Standards, *Software reliability, Computer program integrity, Computer program performance, Standardization, Quality assurance, Software engineering, Research and develop ment, Technology transfer, Guidelines, Research programs, Reprints, "High integrity.

This paper provides information about the National Institute of Standards and Technology (NIST) effort to produce a comprehensive set of standards and guidelines for the assurance of high integrity software. In particular, the paper presents the results of a Workshop on the Assurance of High Integrity Software held at NIST on January 22-23, 1991 and activities at NIST in support of assuring high integrity software.

01,745

PB96-122122 (Order as PB96-117767, PC A08/

MF A02)

National Inst. of Standards and Technology, Gaithersburg, MD.

Performance Measures for Geometric Fitting in the NIST Algorithm Testing and Evaluation Program for Coordinate Measurement Systems

T. H. Hopp, and M. S. Levenson. 1995, 12p.

Included in Jnl. of Research of the National Institute of Standards and Technology, v100 n5 p563-574 Sep/ Qct 95.

Keywords: "Algorithms, "Computer software, "Calibration standards, Reprints, Metrology, Geometry, Performance tests, Orthogonal functions, Quality control

The Algorithm Testing and Evaluation Program for Coordinate Measurement Systems (ATEP-CMS) is a Special Test Service offered under the NIST Calibration Program. ATEP-CMS evaluates the performance of geometric fitting software used in coordinate measurement systems. It is a Special Test because it is a new type of NIST service, experimental in nature and unsupported by historical data. This report documents and explains the rationale of the performance measures used in ATEP-CMS and analyzes the uncertainties of those measures.

01,746

PB96-128202 PC A03/MF A01

National Inst. of Standards and Technology (CSL), Gaithersburg, MD. Distributed Systems Engineering. Distributed Systems: Survey of Open Management Approaches.

J. Hungate, and G. Fernandes. 21 Sep 95, 34p, NISTIR-5735

Keywords: "Distributed computer systems, *Management, System engineering, Computer networks, Computer communications, Integrated systems, Interoperability, Models.

Networks and distributed systems are becoming critical for the working of many enterprises. Traditionally, 


\section{Computer Software}

tools necessary to perform effective system management were inherent to proprietary operating systems and dealt with user and resource allocation and administration. With the introduction of local area networks (LANs), distributed computing environments started to develop. System management tools were enhanced with network management facilities, but rarely in an integrated fashion. Management services, providing mechanisms to monitor and control a great diversity of components and user interactions with these components, and an integrated approach to assure consistency are just now being addressed by standard development organizations and user consortia. The report gives an overview of some existing approaches proposed by differnet organizations: IEEE POSIX Working Group P1003.0, X/Open, ISO/IEC, and the Network Management Forum (NMF)

\section{1,747}

PB96-131503 PC A03/MF A01

National Inst. of Standards and Technology (CSL), Gaithersburg, MD. Systems and Software Technology Div.

Method to Determine a Basis Set of Paths to Perform Program Testing.

J. Poole. Nov 95, 18p, NISTIR-5737.

Keywords: "Computer program verification, "Software tools, Algorithms, Computer software, "Debugging(Computers), "Flowgraphs, Software testing.

A major problem in unit testing of programs is to determine which test cases to use. One technique that is in widespread use is to take the control flowgraph from each of the program functions and calculate a basis set of test paths. A basis set is a set of paths through the functions that are linearly independent and the paths in the basis set can be used to construct any path through the program flowgraph. Path construction is defined as adding or subtracting the number of times each edge is traversed. While this is not a total solution for test case generation, it does provide a good starting set of test cases. This paper gives an algorithm for taking a function's flowgraph and determining a basis set of paths. Proofs that the algorithm generates a set of paths that fulfill the above requirements are provided. A prototype tool, STest, is also discussed. Some general ideas for further improvement are also provided.

01,748

PB96-141296 Not available NTIS

National Inst. of Standards and Technology (CAML), Gaiv

Experience with MPI: 'Converting Pvmmake to Mpimake under LAM' and 'MPI and Parallel Genetic Programming'.

Final rept.

J. E. Devaney. 1995, 13p.

Pub. in MPI Developers Conference, Notre Dame, IN., June 22-23, 1995.

Keywords: "Parallel processing, "Coniputer codes, Personal computers, Computer programming, Utility routines, Software engineering, Systems approach, Algorithms, Reprints, *MPI(Message-Passing Interface), "Message-Passing Interface, PVM(Parallel Virtual Machine).

The paper looks at issues which arose in porting the pvmmake utility from PVM to MPI. Pvmmake application which allows a user to send files, execute commands, and receive results from a single machine on any machine in the virtual machine. Its actions are controlled by the contents of a configuraton file. A utility with the same features, mpimake, was coded up to run under LAM. The implementation under MPI requires the transfer of dynamic data structures such as lists and trees. This paper discusses the match between the requirements of this algorithm and the datatype feature on MPI.

\section{1,749}

PB96-146758 Not available NTIS

National Inst. of Standards and Technology (CSL), Gaithersburg, MD. Advanced Systems Div.

Scalability Test for Parallel Code.

Final rept.

G. Lyon, R. Kacker, and A. Linz. 1995, 17p

See also PB94-193638.

Pub. in Institute of Electrical and Electronics Engineers International Software Metrics Symposium (2nd), Lond, United Kingdom, October 24-26, 1994, Software: Practice and Experience, v25 n12 p1299-1314 Dec 95.
Keywords: "Parallel processing(Computers), "MIMD(Computers), "Response time(Computers) Computer performance evaluation, Computer systems performance, Computer program verification, Coding, Distributed computer systems, Sensitivity analysis, Optimization, Experimental design, Reprints, *Code scalability.

Code scalability, crucial on any parallel system, determines how well parallel code avoids becoming a bottleneck as its hosi computer is made larger. Each suspected code bottleneck corresponds to a first-orde erm in the expansion, the coefficient for that term indicating how sensitive execution is to changes in the suspect location. However, it is the expansion coefficients for second-order interactions between code segments and the number of processors that are fun damental to discovering which program elements impede parallel speedup. A new, unified view of these second-order coefficients yields an informal relative scalability test of high utility in code development. Dis cussion proceeds through actual examples, including a straightforward illustration of the test applied to SLA LOM, a complex, multiphase benchmark.

\section{1,750}

\section{PB96-154588 P04/MF A01}

National Inst. of Standards and Technology (CSL) Gaithersburg, MD. Systems and Software Technology Div.

C++ in Safety Critical Systems.

D. W. Binkley. Nov 95, 34p, NISTIR-5769.

Keywords: "Systems engineering, "Safety engineering, Reliability, Computer programs, Verification, Validation, Classification, Guidelines, Software, Nuclear reactor safety, Fault tolerant systems, Aviation, Motor ve hicle transportation, "High integrity software, ${ }^{*} \mathrm{C}++$ (Programming language).

The safety of software is influenced by the choice of implementation language and the choice of program ming idioms. The $\mathrm{C}++$ language is gaining popularity as the implementation of choice for large software projects because of its promise to reduce the complexity and cost of their construction. But is $\mathrm{C}++$ an appropriate choice for such systems. An assessment of how well $\mathrm{C}++$ fits into recent software guidelines for safety critical systems is presented along with a collection of techniques and idioms for constructing safer $\mathrm{C}++$ code.

\section{1,751}

\section{PB96-154992 PC A03/MF A01}

National Inst. of Standards and Technology (CSL) Gaithersburg, MD. Systems and Software Technology Div.

Standard Generalized Markup Language Test Suite Evaluation Report.

C. S. Russell. Nov 95, 28p, NISTIR-5762

Keywords: "Parsers, "Computer program verification, Federal information processing standards, Tests, Doc uments, Publishing, Automation, "SGML(Standard Generalized Markup Language), Standard General ized Markup Language, Conformance Testing, CALS(Computer Acquisition and Life Cycle Support).

NIST has been tasked by the CALS Project Office to organize an SGML Conformance Testing Service. The first step in producing a conformance testing service was to produce a thorough test suite. The goal of this project was to evaluate existing test suites for use as the basis within an SGML conformance testing service. This document describes the methodology used in analyzing Exoterica Corporation's SGML Test Suite and the different types of tests run with the various SGML products at NIST's disposal.

\section{1,752}

PB96-155791 Not available NTIS

National Inst. of Standards and Technology (CSL) Gaithersburg, MD. Advanced Systems Div.

Findings and Recommendations from a Software Reengineering Case Study.

Final rept.

M. K. Ruhl. 1991, 5p.

Pub. in Proceedings of Annual Systems Reengineering Workshop (2nd), Silver Spring, MD., March 25-27, 1991, p1-5.

Keywords: "Software engineering, "Computer software maintenance, "Data base management, Relationa data bases, Feasibility studies, Cost effectiveness, US IRS, Case studies, Reprints, CASE(Computer-Aided Software Engineering), Open system environment, Network data bases, US NIST.
Our objective was to examine the effort needed and issues by attempting to reengineer the system for use on a more open environment and convert the network database to a relational database.

\section{1,753}

\section{PB96-158712 PC A07/MF A02}

National Inst. of Standards and Technology (CSL), Gaithersburg, MD. Systems and Software Technology

Application Portability Profile (APP): The U.S. Government's Open System Environment Profile Version 3.0.

Special pub.

G. E. Fisher. Feb 96, 112p, NIST/SP-500/230.

Supersedes PB93-216943. Also available from Supt. of Docs. as SN003-003-03389-8.

Keywords: "Computer program portability, "Interoperability, "Distributed processing, Computer program transferability, Federal agencies, Computer networks, Computer communications, Software engineering, Operating systems(Computers), Applications programs(Computers), Network analysis, Data transfer(Computers), Data flow, Data management, Computer graphics, Graphical user interface, Humancomputer interface, Standardization, Specifications, Recommendations, "Open System Environment, *Application Portability Profile, Federal Information Processing Standards.

The report is designed to provide recommendations on a variety of specifications that will generally fit the requirements of U.S. government information systems. As the U.S Government's Open System Environment (OSE) profile, the guidance is provided to assist Federal agencies in making informed choices regarding the selection and use of OSE specifications, and in the development of more selective application profiles based on the APP. It is directed toward managers ind project leaders who have the responsibilities of ac uiring, developing, and maintaining information systems supported by heterogeneous application platform environments.

\section{1,754}

PB96-160791 Not available NTIS

National Inst. of Standards and Technology (CSL), Gaithersburg, MD. Systems and Software Technology Div.

SQA and TQM in Software Quality Improvement. Final rept.

C. E. Wardle, and D. Wallace. 1993, 2p.

Pub. in Software Engineering Research Forum (SERF '93), Orlando, FL., November 1993, 2p.

Keywords: "Total quality management, Software engineering, Software reliability, Computer program integrity, Program verification(Computers), program reliability, Research and development, $\mathrm{Re}$ prints, "SQA(Software Quality Assurance), "Software quality assurance, Software development, Process improvement

Software Quality Assurance (SQA) has traditionally been used to ensure the quality of released software products. The authors suggest that a combination of techniques drawn from both SQA and TQM will be more effective in producing the desired level of software quality, than will either SQA or TQM separately.

01,755

PB96-160858 Not available NTIS

National Inst. of Standards and Technology (CSL), Gaithersburg, MD. Systems and Software Technology Div.

Slicing in the Presence of Parameter Aliasing.

Final rept.

D. Binkley. 1993, 8p.

Pub. in Proceedings of the Software Engineering $\mathrm{Re}$ p261-268.

Keywords: "Software engineering, "Computer program integrity, Algorithms, Debugging(Computers), Computer software maintenance, Software reliability, Computer programming, Parameterization, Functional integration, Reprints, “Program slicing, Aliasing, Data dependence, Program dependence graph.

The notion of a program slice, originally introduced by Mark Weiser, is useful in program debugging, automatic parallelization, program integration, and software maintenance. A slice of a program is taken with respect to a point $p$ and a variable $x$; the slice consists of all 
statements of the program that might affect the value of $x$ at point $p$. This paper presents an algorithm for interprocedural slicing in the presence of parameter aliases: two formal parameters that refer to the same memory location. The algorithm is parameterized by a set of parameter aliasing information.

01,756

PB96-160908 Not available NTIS

National Inst. of Standards and Technology (CSL) Gaithersburg, MD. Information Systems Engineering Div.

Impact of Computer-Aided Acquisition and Logistic Support (CALS) in the Application of Standards. Final rept.

D. K. Jefferson. 1992, $11 p$.

Pub. in International Conference on the Application of Standards for Open Systems (7th), Paris, France, November 4, 1992, p1-11.

Keywords: "Concurrent engineering, "Standards, Information systems, Weapon systems, Logistics management, Systems engineering, Distributed processing Application program(Computers), Protocols, Reprints, *CALS(Computer-aided Acquisition and Logistic Support), *Computer-aided Acquisition and Logistic Support, Conformance testing, CALS(Continuous Acquisition and Life-cycle Support), Open systems.

Computer-aided Acquisition and Logistic Support (CALS) is a major initiative by the U.S. Department of Defense to enable the use of shared digital technica information in support of weapon systems. CALS is reducing the use of paper-based technical information, improving the information systems supporting weapon systems, and improving work processes. CALS is based on broad support nationally and internationally particularly from the CALS/Concurrent Engineering Industry Steering Group and the Natioanl Institute of Standards and Technology within the U.S. Department of Commerce. CALS is based on Open Systems and has actively supported the development of standards, Application Protocols/Profiles, and conformance tests and test services.

01,757

PB96-160924 Not available NTIS

National Inst. of Standards and Technology (CSL) Gaithersburg, MD. Systems and Software Technology Div.

IEEE's POSIX: Making Progress.

Final rept.

D. R. Kuhn. 1991, 4p.

Pub. in Institute of Electrical and Electronics Engineers Spectrum, p36-39 Dec 91.

Keywords: "Software development, "Computer program verification, Operating systems(Computers) Computer program portability, Object-oriented programming, Interfaces, Interoperability, Computeraided design, UNIX(Operating system) $\mathrm{C}++$ (Programming language), Standards, Reprints, *POSIX(Portable Operating Systems Interface for Computer Environments), "Portable Operating Systems Interface for Computer Environments.

As the circuit design drew near, the pace became more frantic by the hour, elevating the project leader's anxiety level and his blood pressure. And for good reason - all the users of DEC workstations, where the computer-aided design software resided, were out at a seminar. To be sure, IBM, Sun, and other workstations were available that would have allowed other engineers to complete the job on time. Only a DEC version was obtainable in house; in no way could it be ported to the IBM or Sun on time to beat the deadline - even though the project leader had received assurances from the software vendor that such versions 'were forthcoming.'

01,758

PB96-160932 Not available NTIS

National Inst. of Standards and Technology (CSL), Gaithersburg, MD. Systems and Software Technology Div.

Open Systems Software Standards in Concurrent Engineering.

Final rept.

D. R. Kuhn, W. J. Majurski, W. McCoy, and F.

Schulz. 1994, 30p.

Pub. in Concurrent Engineering Techniques and Applications, Control and Dynamics Systems, v62 p59-88 Feb 94.

Keywords: *Operating systems(Computers) *Computer program portability, *Concurrent engineer- ing, Software development, Software tools, Platforms, Interoperability, Interfaces, Application programs(Computers), Standards, Reprints, programs(Computers), $\quad$ Standards, Reprints,
POSIX(Portable Operating Systems Interface for Computer Environments), OST(Open Systems Interconnection).

The movement toward concurrent engineering comes at a time when the computer industry is undergoing a fundamental change in the way computers and software are developed and used. Products based on 'open systems' standards - particularly the ISO Open System Interconnection (OSI), and IEEE POSIX - are beginning to replace reliance on proprietary computing platforms. Engineers who develop products that incorporate embedded computer and software are thus faced with the challenge of understanding two technological developments that will change the way they do their jobs: concurrent engineering and open systems.

01,759

PB96-160940 Not available NTIS

National Inst. of Standards and Technology (CSL), Gaithersburg, MD. Systems and Software Technology Div.

Predicate Differences and the Analysis of Dependencies in Formal Specifications.

Final rept.

D. R. Kuhn. 1991, 10p.

Pub. in National Computer Security Conference (14th), Washington, DC., October 1-4, 1991, Information Systems Security: Requirements and Practices, v2 p436445.

Keywords: "Predicate logic, "Computer software, *Specifications, "Electronic security, Mathematical logic, Boolean functions, Software engineering, Software reliability, Computer program integrity, Computer program verification, Standards, Computer information security, Reprints.

This paper defines the notion of predicate differences in predicate calculus. The paper shows how predicate differences may be used to analyze the effect of changes to formal specifications; to investigate the conditions under which invalid assumptions will render a system non-secure; and in some cases may help to simplify re-verification of a modified formal specification.

\section{1,760}

PB96-160957 Not available NTIS

National Inst. of Standards and Technology (CSL) Gaithersburg, MD. Systems and Software Technology

Technique for Analyzing the Effects of Changes in Formal Specifications.

Final rept.

D. R. Kuhn. 1992, 5p.

Pub. in the Computer Jnl., v35 n6 p574-578 1992.

Keywords: "Predicate logic, *Computer software, *Specifications, "Electronic security, Mathematical logic, Boolean functions, Software engineering, Software reliability, Computer program integrity, Computer program verification, Standards, Computer information security, Reprints.

This paper defines the notion of predicate differences and shows how predicate differences may be used to analyze the effects of changes in formal specifications. Predicate differences have both theoretical and practical applications. As a theoretical tool, predicate differences may be used to define a meaning for the 'size' of a change to a formal specification. Practical applications include analyzing the effect of design changes on a previously verified design; defining an affinity function for reusable software components; computing slices of formal specifications, similar to program slices; investigating the conditions under which invalid assumptions will render a system non-secure; and formalizing the database inference problem.

\section{1,761}

PB96-160981 Not available NTIS

National Inst. of Standards and Technology (CSL), Gaithersburg, MD. Systems and Software Technology Div.

Program Slicing.

Final rept.

J. R. Lyle. 1994, 6p

See also PB95-125894.

Pub. in Encyclopedia of Software Engineering, v2 p873-878 Feb 94.

Keywords: "Debugging(Computers), "Software engineering, "Computer software maintenance, Computer programming, Computer program vertification, Computer program reliability, Computer program integrity, Software reliability, Mathematical calculations, Algorithms, Functional integration, Reprints, *Program slicing.

Program slicing is a family of program decomposition techniques based on extracting statements relevant to a computation in a program. This is advantageous because the slice can colfect an algorithm for a given calculation that may be scattered throughout a program excluding irrelevant statements. It should be easier for a programmer interested in a subset of the program's behavior to understand the corresponding slice than to deal with the entire program. The utility and power of program slicing comes from the potential automation of tedious and error-prone tasks. Program slicing has applications in program debugging, program testing program integration, parallel program execution, and software maintenance. Several variations on this theme have been developed, including program dicing, dynamic slicing, and decomposition slicing.

\section{1,762}

PB96-161211 Not available NTIS

National Inst. of Standards and Technology (CSL), Gaithersburg, MD.

Conference Report: International Conference on the Application of Standards for Open Systems (6th).

Final rept.

S. M. Radack. 1991, 3p.

Pub. in International Conference on the Application of Standards for Open Systems (6th), Gaithersburg, MD. October 2-4, 1990, Jnl. of Research of the National Institute of Standards and Technology, v96 n6 p767-769 Nov/Dec 91

Keywords: *Standards, Application programs(Computers), Computer program portability, Distributed processing, Interoperability, Interfaces, Policy making, International cooperation, Computer security, Reprints, "Open systems, OSI(Open Systems Interconnection), Conformance testing.

Worldwide interest in advancing open computing systems was highlighted at the Sixth International conference on the application of Standards for Open Systems. The conference featured papers and discussion on key issues affecting the implementation of open systems including policy development, international collaboration, trade issue, implementation, conformance, and security.

\section{1,763}

PB96-161377 Not available NTIS

National Inst. of Standards and Technology (CSL), Gaithersburg, MD. Systems and Software Technology Div.

Report of a Workshop on the Assurance of High Integrity Software.

Final rept.

D. R. Wallace, D. R. Kuhn, and J. C. Cherniavsky

1991, 5p.

Pub. in Proceedings of the Annual Conference on Computer Assurance (COMPASS '91) (6th) Gaithersburg, MD., June 24-27, 1991, p151-155. ance evaluation, "Standards, Software engineering, Computer program integrity, Electronic security, Computer information security, Quality assurance, Specifications, Reprints, "High integrity, Critical systems.

This paper provides information about the National Institute of Standards and Technology (NIST) effort to produce a comprehensive set of standards and guidelines for the assurance of high integrity software. In particular, the paper presents the results of a Workshop on the Assurance of High Integrity Software held at NIST on January 22-23, 1991.

01,764

PB96-161385 Not available NTIS

National Inst. of Standards and Technology (CSL), Gaithersburg, MD. Systems and Software Technology Div.

Standards for High Integrity Software.

Final rept.

D. R. Wallace, and L. Beltracchi. 1992, 2p.

Pub. in Transactions of the Water Reactor Safety Information Meeting (20th), Bethesda, MD., October 21-23 1992, p9-3-9-4.

Keywords: "Software reliability, "Computer performance evaluation, *Standards, Software engineering 


\section{Computer Software}

Computer program integrity, Electronic security, Computer information security, Ouality assurance, Specifications, Reprints, "High integrity, Critical systems.

High integrity software is software that must be trusted to work dependably in some critical function, and whose failure to do so may have catastrophic results, such as serious injury, loss of life or property, business failure or breach of security. Examples include software used in safety systems of nuclear power plants, medical devices, electronic baking, air traffic control, automated manufacturing, and military systems.

\section{1,765}

\section{PB96-161393 Not available NTIS}

National Inst. of Standards and Technology (CSL), Gaithersburg, MD. Systems and Software Technology Div

Verification and Validation.

Final rept.

D. R. Wallace. 1994, 24p.

See also PB90-111691 and PB96-161401.

Pub. in Encyclopedia of Software Engineering, p1409. 1434 Feb 94

Keywords: "Software engineering, " Computer program verfication, "Validation, Software development, Software reliability, Computer program integrity, Computer performance evaluation, Error detection codes, Project management, Quality assurance, Standards, Reprints.

This chapter describes how the software verification and validation $(\mathrm{V} \& \mathrm{~V})$ methodology and software provide a strong framework for developing quality software. First, the chapter describes software $V \& V$, its objectives, recommended tasks, and quidance for selecting V\&V techniques to perform V\&V. An analysis of two studies of V\&V's cost-effectiveness concludes that cost benefits of $V \& V$ early error detection outweigh the cost of performing V\&V. The chapter describes several software engineering standards for $V \& V$, project management, and quality assurance. The chapter describes each V\&V standard according to its V\&V requirements and techniques. The chapter provides insights on how to use management, quality, and V\&V techniques to structure a quality software development.

\section{1,766 Not available NTIS}

National Inst. of Standards and Technology (CSL), Gaithersburg, MD. Systems and Software Technology Div.

Verification and Validation of Reengineered Software.

Final rept.

D. R. Wallace. 1993, 1p.

See also PB96-161393.

Pub. in Business Reengineering: The Competitive Edge Proceedings of the Annual DAMA-NCR Symposium (6th), Gaithersburg, MD., May 11-12, 1993, p80.

Keywords: *Computer program verification, *Validation, Software engineering, Software reuse Software reliability, Computer program integrity, Ouality assurance, Federal agencies, Reprints, "Software reengineering.

Many Federal agencies are currently examining their inventories of software systems to identify those which are suitable for reuse Reuse of software may range from conversion of code from one language to another adaption of the system for another platform, or the use of an existing system in a new system with additional requirements. To accomplish these and other forms of reuse, the agencies are finding that reengineeringing is often necessary.

\section{1,767}

PB96-165964 PC A05/MF A01

National Inst. of Standards and Technology (CSL),

Gaithersburg, MD. Advanced Systems Div.

Using S-Check, Alpha Release 1.0.

R. D. Snelick, N. V. Drouin, and J. K. Antonishek.

Feb 96, 54p, NISTIR-5789.

Sponsored by Defense Advanced Research Projects Agency, Arlington, VA.

Keywords: "Parallel processing, "Computer performance evaluation, "Debugging(Computers), Multiprocessers, Software rools, Distributed computer systems, Sensitivity analysis, Users guide, Synthetic Perturbation Screening.

S-Check is a tool for detecting performance bottlenecks in programs on computer systems with multiple processors. S-Check automates the techniques of Synthetic Perturbation Screnning. Synthetic Perturbation Screening systematically perturbs selected program code segments and determines performance sensitivities of these selected segments by using the statistical techniques of Design of Experiments. The resulting sensitivity analysis serves as a basis for performance evaluations. This document serves as a user's guide for S-Check.

\subsection{8}

\section{PB96-167838 PC A03/MF A01}

National Inst. of Standards and Technology (CSL), Gaithersburg, MD. Information Systems Architecture Div.

Application of the Pointer State Subgraph to Static Program Slicing.

D. W. Binkley, and J. R. Lyle. Mar 96, 22p, NISTIR5799.

Contract DISA-DNR046115

Sponsored by Defense Information Systems Agency, Arlington, $V A$.

Keywords: "Static analysis, "Computer programs, Software engineering, Data flow analysis, Interface slicing, Pointers.

A new technique for performing static analysis of programs that contain unconstrained pointers is presented. The technique is based on the pointer state subgraph: a reduced control flow graph that takes advantage of the fact that in any program there exists a smaller program that computes only the values of pointer variables. The pointer state subgraph is useful in building static analysis tools. As an example the application of the pointer state subgraph to program slicing is considered. Finally, some experimental results, obtained using the ANSI-C slicer Unravel, are reported. These results show a clear reduction in the time taken to compute data-flow information from programs that contain pointers. They also show a substantial reduction in the space needed to store this information.

\section{1,769}

\section{PB96-169099 PC E99}

Massachusetts Inst. of Tech., Cambridge.

$X$ Window System, Version 11, Release 5 .

R. W. Scheifler. c1988, 897p.

Keywords: *Protocols, "Computer programming, ${ }^{*} X$ Window System, Programming languages, Software tools, Operating systems(Computers), Personal computers, Computer networks, Computer graphics, Computer systems programs, Format, C programming language, User interfaces.

Table of Contents:

Acknowledgments;

Protocol Formats:

Syntactic Conventions;

Common Types:

Errors;

Keyboards

Pointers;

Predefined Atoms;

Connection Setup

Requests;

Connection Close;

Events;

Flow Control and Concurrency;

Appendix A - KEYSYM Encoding;

and Appendix B - Protocol Encoding.

\section{1,770}

\section{PB96-179411 Not available NTIS}

National Inst. of Standards and Technology (CAML). Gaithersburg, MD. Applied and Computational Mathematics Div.

Tree-Lookup for Partial Sums Or: How Can I Find This Stuff Quickly.

Final rept.

I. Beichl, and F. Sullivan. 1996, 3p.

Pub. in Institute of Electrical and Electronics Engineers Computational Science and Engineering, v3 n1 p1315 Spring 96.

Keywords: *Parallel processing, "Searching, "Monte Carlo method, "Data structures, Trees(Mathematics), Data processing, Data storage, Data management, Structured programming, Computer systems performance, Run time(Computers), Simulation, Reprints.

Suppose that you need to maintain a large list of data that is to be searched and modified frequently in the course of a computation. Although there are several elegant ways to do this, the authors present one, a simple tree that doesn't require a lot of thinking to implement. It can speed up execution tremendously because instead of searching through a list item after item for each update, possibly examining ' $n$ ' items per operation.

\section{1,771}

PB96-180187 Not available NTIS

National Inst. of Standards and Technology (CSL), Gaithersburg, MD. Information Systems Architecture Div.

Application of Metadata Standards.

Final rept.

J. Newton. 1996, 14p.

Pub. in Institute of Electrical and Electronics Engineers Metadata Conference Proceedings (1st), Silver Spring, MD., April 16-18, 1996, p1-14.

Keywords: "Standards, Data transfer(Computers), Data management, Interoperability, Formats, Data bases, Standardization, Specifications, Procedures, Reprints, "Metadata, "Data elements, Attributes.

Application of metadata standards in the development of data elements assures a consistent, sharable set of data described and named in a predictable way. $\mathrm{Na}$ tional and international efforts are underway to produce this metadata standardization. Classification of the components of data elements into a taxonomic structure facilitates the selection of appropriate components for particular elements. In this way, sets of complete and discrete data elements can be developed in to coherent descriptions of subject areas. A set of standardized attributes, some with sample values, prescribe the base set of information to be recorded about each data element. Finally, procedures for registration of standard data elements provide a facility for sharing the benefits of standardization among organizations.

01,772

PB96-183140 PC A04/MF A01

National Inst. of Standards and Technology (CSL), Gaithersburg, MD. Information Systems Architecture Div.

Interoperability Experiments with CORBA and Persistent Object Base Systems.

E. Fong, and D. Yang. Apr 96, 38p, NISTIR-5824. See also PB95-220588.

Keywords: "Software engineering, "Object database management, "Computer programming, Distributed data processing, Models, Standards, Operating systems(Computers), Software tools, "POB(Persistent Object Based Systems), "Platform interoperability, Persistent Object Based Systems, CORBA(Common Object Request Broker Architecture), Common Object Request Broker Architecture.

This report describes the design and development of interoperability experiments using the common Object Request Broker Architecture (CORBA) products. The experiments will focus on establishing practical experiences for how to do 'plug and play' using CORBA products with Persistent Object Base (POB) systems such as Texas instruments' Open OODB and commercially available object database systems (ODBMS) such as MATISSE. The experiments will also investigate methodologies for integration of new or legacy distributed applications through the CORBA middleware infrastructure.

01,773

\section{PB96-188164 PC A06/MF A01}

National Inst. of Standards and Technology (CSL), Gaithersburg, MD. Information Systems Architecture Div.

Reference Information for the Software Verification and Validation Process.

Special pub.

D. R. Wallace, L. M. Ippolito, and B. B. Cuthill. Apr 96, 96p, NIST/SP-500-234.

Also available from Supt. of Docs. as SN003-00303410-0.

Keywords: "Computer program verification, "Software engineering, "Computer program management, Standards, Guidelines, Computer software maintenance, Knowledge based systems, Performance evaluation, Tests, Health care, Software validation.

Computing systems may be employed in the health care environment in efforts to increase reliability of care and reduce costs. Software verification and validation (V\&V) is an aid in determining that the software requirements are implemented correctly and com- 
pletely and are traceable to system requirements. It heips to ensure that those system functions controlled by software are secure, reliable, and maintainable. Software $V \& V$ is conducted throughout the planning, development and maintenance of software systems including knowledge-based systems, and may assist in assuring appropriate reuse of software.

01,774

PB96-190053 Not available NTIS

National Inst. of Standards and Technology (MEL) Gaithersburg, MD. Manufacturing Systems Integration Div.

Dynamic Objects and Meta-Level Programming of an EXPRESS Language Environment

Final rept.

P. Denno. 1996, 6p

Pub. in Dynamic Objects Workshop, Object World, 1996, p 1-6.

Keywords: "Programming languages, LISP programming languages, Object programs, Reprints, "EXPRESS language environment.

This paper describes design and programming techniques employed in the development of a language environment for the EXPRESS information modeling language. A fundamental concern in the development of language environments for object flavored languages is the degree to which the object model of the implementation language matches that of the language being modeled. This paper describes how object model mismatch was eliminated and a responsive, incremental EXPRESS language environment is being developed using the Common Lisp Object System (CLOS) metaobject protocol (MOP) and dynamic object techniques.

\section{1,775}

PB96-193636 PC A03/MF A01

National Inst. of Standards and Technology (CAML) Gaithersburg, MD. Applied and Computational Mathematics Div.

Sparselib++ v. 1.5 Sparse Matrix Class Library. Reference Guide.

R. Pozo, K. A. Remington, and A. Lumsdaine. Jun $96,25 p$, NISTIR-5861.

Prepared in cooperation with Notre Dame Univ., IN.

Keywords: *Linear systems, *Matrices(Mathematics), Algebra, Distributed data processing, Array processors, Parallel processing, Computer architecture, Object oriented programming, Computer codes, Subroutine libraries, Data storage systems, Computatuion, Iterations, Sparse matrix, SparseLib plus plus, C plus plus programming language, Kernel functions.

SparseLib++ is a $\mathrm{C}++$ class library for efficient sparse matrix computations across various computational platforms. The software package consists of matrix objects representing several sparse storage format currently in use (in the release: compressed row, compressed column and coordinate formats), providing basic functionality for managing sparse matrices, together with efficient kernel mathematical operations (e.g. sparse matrix-vector multiply). The Sparse BLAS Toolkit(1) is used to enhance portability and performance across a wide range of computer architectures. Included in the package are various preconditioners commonly used in iterative solvers for linear systems of equations. The focus here is on computational support for iterative methods (see IML $++(5)$ ), but the sparse matrix objects presented here can be used in their own right.

\section{1,776}

\section{PB96-195219 PC A04/MF A01}

National Inst. of Standards and Technology (CAML) Gaithersburg, MD. Applied and Computational Mathematics Div.

IML++ v.1.2 Iterative Methods Library Reference Guide.

J. Dongarra, A. Lumsdaine, R. Pozo, and K. A.

Remington. Jun $96,43 p$, NISTIR-5860.

Prepared in cooperation with Notre Dame Univ., IN. and Oak Ridge National Lab., TN.

Keywords: "Algorithms, "Computer program documentation, Iterative methods, Object oriented computing, Linear algebra, Matrices(Mathematics), Vector fields.

The Iterative Methods Library, $\mid \mathrm{ML++}$, is a collection of algorithms implemented in $\mathrm{C}++$ for solving both symmetric and nonsymmetric linear systems of equations by using iterative techniques. The goal of the package is to provide working code which separates the numerical algorithm from the details of the matnx/vector implementation. The separation allows the same algorithm to be used without modification, regardless of the specific data representation.

01,777

PB96-195326 PC A03/MF AO1

National Inst. of Standards and Technology (CAML) Gaithersburg, MD. Applied and Computational Mathematics Div.

MV++ V. 1.5a Matrix/Vector Class Reference Guide. R. Pozo. 27 Jun 96, 18p, NISTIR-5859.

See also DE95017458.

Keywords: "Matrix algebra, "Vector fields, "User guide Algorithms, Compute program documentation, MV++ $\checkmark 1.5$ a computer program.

$M V++$ is a small, efficient set of concrete vector and matrix classes specifically designed for high performance numerical computing. The $\mathrm{MV}++$ package in cludes interfaces to the computational kernels found in the Basic Linear Algebra Subprograms (BLAS), such as scalar updates, vector sums, and dot products. The idea behind $\mathrm{MV}++$ is to leverage vendor-supplied or optimized BLAS routines that are fine-tuned for particular plantforms. The various MV++ classes form the building blocks of larger user-level libraries such as SparseLib++(2) and LAPACK $++(1)$. The MV ++ library was built to supply simple, concrete, numerical vector and column oriented dense matrix classes.

\section{1,778}

PB96-200977 Not available NTIS

National Inst. of Standards and Technology (CAML) Gaithersburg, MD. Applied and Computational Mathematics Div.

Software Needs in Special Functions.

Final rept.

D. W. Lozier. $1995,17 \mathrm{p}$

See also PB95-105045.

Pub. in Jnl. of Computational and Applied Mathematics 66, p344-360 Jun 96.

Keywords: *Special functions(Mathematics) "Computer software, "Faults, "Error analysis, Computer programming, Computations, Algorithms, Diag-
nosis, User needs, User requirements, Reprints, Functional testing.

Currently available software for special functions ex hibits gaps and defects in comparison to the needs of modern high-performance scientific computing and also, surprisingly, in comparison to what could be constructed from current algorithms. In the paper we expose some of these deficiencies and identify the related need for user-oriented testing software.

\section{1,779}

PB96-201009 Not available NTIS

National Inst. of Standards and Technology (NCSL), Gaithersburg, MD. Systems and Network Architecture Div.

Experimental Evaluation of Specification Techniques for Improving Functional Testing.

Final rept.

K. L. Mills. 1996, 13p.

Pub. in Jnl. of Software and Systems Engineering, n32 p83-95 Jan 96.

Keywords: "Computer programming, "Specifications, * Man-computer interface, Computer software, Natura language(Computers), Performance tests, Graphica user interface, Real time, Reprints, *Functional testing Structured analysis, Program execution.

The article reports results from one experiment to compare the effectiveness of functional testing given three different sets of software specifications. Three groups of experienced programmers independently developed functional test cases for the same automated cruise control software. One group worked solely from a natural language description, one was given a graphica real-time structured analysis (RTSA) specification to augment the natural langauge description, and a third was given, in addition to the English language description and the RTSA specification, an executable specification. Two measures of performance were evaluated: the degree of statement coverage achieved by the test cases created by each group, and the amount of time taken to create the test cases. No significan difference in performance was found among any of the groups for either of the measures.

\section{1,780}

PB97-112502 Not available NTIS
National Inst. of Standards and Technology (CSL), Gaithersburg, MD. Advanced Network Technologies Div.

Knowledge-Based Approach for Automating a Design Method for Concurrent and Real-Time Systems.

Final rept

K. L. Mills, and H. Gomaa. 1996, 8p.

Pub. in International Conference on Software Engineering and Knowledge Engineering, SEKE '96 (8th) Lake Tahoe, NV., June 10-12, 1996, p529-536.

Keywords: "Concurrent engineering, "Real time oper ation, "Computer-aided design, Knowledge bases(Artificial intelligence), Design analysis, Software engineering, Automation, Software tools, Specifications, Semantics, Reprints.

The paper describes a knowledge-based approach to automate CODARTS, a software design method fo concurrent and real-time systems. The approach uses multiple paradigms to represent knowledge embedded within CODARTS. Semantic data modeling provides the means to model specifications and related designs. Production rules form the basis for modeling a set of heuristics that can generate concurrent designs based upon semantic concepts from the specification and design meta-models. Together, the semantic data models and production rules, encoded using an expert-sys tem shell, compose CODA, an automated designer's assistant.

01,781

PB97-113138 Not available NTIS

National Inst. of Standards and Technology (CSL), Gaithersburg, MD. Information Systems Architecture Div.

Measurement of Process Complexity.

Final rept.

R. Tesoriero, and M. V. Zelkowitz. 1996, 12p.

Pub. in Proceedings of the European Control and Metrics Conference (7th), Wilmslow, United Kingdom, May 15-17, 1996, p304-315.

Keywords: "Software engineering, "Technology transfer, "Complexity, Computer program reliability, Computer programming, Design analysis, Scheduling, Specifications, Measurement, Requirements, Reprints, Process models.

The ability to quantitatively assess a given project has meant one of two different approaches: (1) a study of the complexity of the software being produced, (2) or a study of the process being used to develop that software. In the apper, the authors try to indicate that neither approach is sufficient. They are developing a model that evolved from an earlier study on software engineering technology transfer. In it, the authors look at the evolving nature of the software development to develop a process model that keeps up with these changes. The paper presents an initial overview of the process model.

01,782

PB97-113799 PC A03/MF A01

National Inst. of Standards and Technology (MEL), Gaithersburg, MD. Manufacturing Systems Integration Div.

Representation of Axes for Geometric Fitting.

T. H. Hopp. Sep 96, 18p, NISTIR-5897.

Keywords: "Dimensional measurement, "Algorithms, Metrology, Geometry, Estimates, *Geometric fitting software, Coordinates measuring systems.

The authors review methods for representing geometric axes in three dimensions and discuss why these methods have undesirable properties for geometric fitting applications. The authors then describe a new representation that seems better suited for use in problems of fitting geometric elements to data points. This representation was developed in the course of the authors' work on testing of data analysis software in $\mathrm{co}$ ordinate measurement systems. The new representation has allowed us to lower significantly the estimated uncertainty of the authors' test results. Details and properties of the representation are discussed.

\section{1,783}

PB97-113906 PC A04/MF A01

National Inst. of Standards and Technology (CSL), Gaithersburg, MD.

Experimental Models for Software Diagnosis. M. V. Zelkowitz, and D. R. Wallace. Aug 96, 39p, NISTIR-5889.

Prepared in cooperation with Maryland Univ., College Park. Dept. of Computer Science. 


\section{Computer Software}

Keywords: "Software engineering, "Information systems, "Computer program validation, Computer systems design, Distributed data processing, Data collection, Experimental data, Models.

Experimentation and data collection are becoming accepted practices within the software engineering community to determine the effectiveness of various software development practices. However, there is wide disagreement as to exactly what the term 'experimentation' means in this domain. It is important that we be able to understand this concept and identify how we can best collect data needed to validate software methods that seem to be effective. This understanding wil provide a basis for improved technical exchange of information between scientists and engineers within the software community.

\subsection{4}

PB97-114169 PC A07/MF A02

National Inst. of Standards and Technology (CSL), Gaithersburg, MD. Software Diagnostics and Conformance Testing Div.

Structured Testing: A Testing Methodology Using the Cyclomatic Complexity Metric.

Special pub.

D. R. Wallace, A. H. Watson, and T. J. McCabe. Aug 96, 124p, NIST/SP-500/235.

Portions of this document are not fully legible. Also available from Supt. of Docs. as SN003-003-03426-6.

Keywords: "Computer programs, "Test methods, Integration, Object oriented programs, Life cycles, Quality assurance, Measurement, Software engineering, Cyclomatic complexity.

The purpose of this document is to describe the struc tured testing methodology for software testing, also known as basis path testing. Based on the cyclomatic measure of McCabe structured testing uses the control flow structure of software to establish path coverage criteria. The resultant test sets provide more through testing than statement and branch coverage. Extensions of the fundamental structured testing tech niques for integration testing and object-oriented systems are also presented. Several related software complexity metrics are described. Summaries of technical papers, case studies, and empirical results are presented in the appendices.

01,785

PB97-119044 Not available NTIS

National Inst. of Standards and Technology (CAML), Gaithersburg, MD. Applied and Computational Mathematics Div.

Making Connections.

Final rept.

I. Beichl, and F. Sullivan. 1996, 4p.

Pub. in Institute of Electrical and Electronics Engineers Computational Science and Engineering, v3 n3 p9-12 Fall 96.

Keywords: "Data structures, "Connection machine, *Parallel processing, Algorithms, Sequences(Mathematics), Computations, Computational geometry, Reprints, Depth first search.

The paper is part of a series of expository articles on scientific computing with non-numeric methods. The depth first search method of graph traversal is used to find connectivity information.

\section{1,786}

PB97-121636 PC A99/MF EO8

National Inst. of Standards and Technology (CSL) Gaithersburg, MD. Information Access and User Interface Div.

Text REtrieval Conference (4th) (TREC-4). Held in Gaithersburg, Maryland on November 1-3, 1995.

Special pub.

D. K. Harman. Oct 96, 776p, NIST/SP-500-236

Also available from Supt. of Docs. as SN003-00303430-4.

Keywords: "Meetings, "Information retrieval, "Text processing, Thesauri, Automation, Natural language processing, Pattern recognition, Routing, Probability theory, Documents, Search structuring, Knowledge bases(Artificial intellegience), Vector processing, Semantics, Evaluation.

This report constitutes the proceedings of the fourth Text REtrival Conference (TREC-4) held in Gaithersburg, Maryland, November 1-3, 1995. The conference was co-sponsored by the National Institute of Standards and Technology (NIST) and the Defense
Advanced Research Agency (DARPA), and was attached by 140 people involved in the 36 participating groups. The goal of the conference was to bring research groups together to discuss their work on a large test collection. There was a wide variation of retrieval techniques reported on, including methods using automatic thesaurii, sophisticated term weighting, natural language techniques, relevance feedback, and advanced pattern matching. As results had been run through a common evaluation package, groups were able to compare the effectiveness of different techniques, and discuss how differences between the systems affected performance. In addition to the main evaluation, 5 more focussed evaluations, called 'tracks' were run. The conference included paper sessions and discussion groups. This proceeding includes papers from most of the participants (several poster groups did not submit papers), tables of the system results, and brief system descriptions including timing and storage information.

\section{1,787}

PB97-122303 Not available NTIS

National Inst. of Standards and Technology (CSL), Gaithersburg, MD. Information Systems Architecture Div.

CSL View of Applications Portability, Scalability, and Interoperability.

Final rept.

G. E. Fisher. 1996, 1p

Pub. in ACM StandardView, v4 n2 p1 Jun 96.

Keywords: "Computer program portability, "Interoperability, "Software engineering, Distributed processing, Computer program transferability, Applications programs (Computers), Integrated systems, Computer networks, Standardization, Specifications, Standards, Recommendations, Reprints, Application Portability Profile, Scalability, US NIS, Computer Systems Laboratory.

The document presents an overview summary of the Computer Systems Laboratory position on portability, scalability, and interoperability in the computer software arena.

\section{Control Systems \& Control Theory}

01,788

PB95-107108 Not available NTIS

National Inst. of Standards and Technology (NEL), Gaithersburg, MD. Robot Systems Div.

Three Dimensional Position Determination from Motion.

Final rept

M. Nashman, and K. Chaconas. 1991, 10p.

Pub. in Proceedings of Society of Photo-Optical Instru mentation Engineers - Sensor Fusion III: 3-D Perception and Recognition, Boston, MA., November 5-8, 1990, v1383 p166-175 1991

Keywords: "Manipulators, "Control systems, *Position finding, "Motion, Algorithms, Image processing, Three dimensional motion, Real time, Sensory feedback, Trajectory planning, Reprints.

The analysis of sequences of images over time provides a means of extracting meaningful information which is used to compute and track the three-dimensional position of a moving object. This paper describes an application in which sensory feedback based on time-varying camera images is used to provide position information to a manipulator control system. The system operates in a real-time environment and provides updated information at a rate which permits intelligent trajectory planning by the control system.

\section{1,789}

PB95-143137 PC A03/MF A01

National Inst. of Standards and Technology (MEL), Gaithersburg, MD. Robot Systems Div.

Reference Model Architecture for Intelligent Systems Design.

J. S. Albus. Sep 94, 48p, NISTIR-5502.

Keywords: "Control systems design, "Real time systems, "Artificial intelligence, Autonomy, Self adaptive control systems, Hierarchies, Task planning(Robotics) Canonical forms, Data bases, Robots, Manufacturing Workstations, Models, RCS(Real-time Control System)
A reference model architecture based on the Real-time Control System (RCS) is proposed for real-time intelligent control systems. RCS partitions the control probem into four basic elements: behavior generation (or task decomposition), world modeling, sensory processing, and value judgment. It clusters these elements into computational nodes that have responsibility for specific subsystems, and arranges these nodes in hierarchical layers such that each layer has characteristic functionality and timing. The RCS reference model architecture has a systematic regularity, and recursive structure that suggests a canonical form. Control systems based on the RCS have been built for a numbe of applications. Examples of a control system architecture and a world model database for an intelligent robot in a manufacturing work-station are described. Each level of the control architecture performs real-time planning and sensory interactive execution. Each leve of the world model knowledge database contains state variables, system parameters, entity frames, and maps.

\section{1,790}

PB95-198859 PC A03/MF A01

National Inst. of Standards and Technology (MEL) Gaithersburg, MD. Factory Automation Systems Div. Expert Control System Shell Version 1.0 User's Guide.

S. A. Osella. Feb 95, 44p, NISTIR-5601.

Keywords: "Control systems design, "Expert systems, * Software tools, Prototypes, Systems engineering, Real time systems, Controllers, Graphical user interface, Control equipment, Software engineering, User manuals(Computer programs).

The Expert Control System Shell (ECSS) is a software tool for rapidly prototyping and deploying control systems of arbitrary complexity. With the ECSS, a contro system designer creates a controller which consists of a graphical user interface (called the operator fron panel) and, optionally, a collection of expert system ule-based programs. A controller can be operated in manual mode where an operator interacts directly with the system to be controlled without using the exper system, in automatic mode where the expert system is used to perform most, if not all, system interactions, or in a combination of the two modes. The User's Guide is discussed in the following order: (1) a tutoria on how to create a controller, (2) creating controllers, (3) creating and importing device interface functions 4) creating rule-based programs, (5) importing expert system functions, and (6) operating controllers.

\section{Information Processing Standards}

01,791

DE94015308 PC A03/MF A01

National Inst. of Standards and Technology, Gaithersburg, MD.

NIST Cooperative Laboratory for OSI Routing Technology.

D. Montgomery. 23 May 94, 40p, DOE/ER/25114-1. Contract Al05-92ER25114, Grant NCR-9120054 Sponsored by Department of Energy, Washington, DC. Keywords: "Computer Networks, "Equipment Interfaces, "Standards, Data Transmission, Information Dissemination, On-Line Systems, Performance Testing, EDB/990300.

This document is one of two reports on the Integrated ISIS protocol. Required by the IAB/IESG in order for an Internet routing protocol to advance to Draft Standard Status. Integrated ISIS is an Interior Gateway Protocol and is designed to carry both IP and ISO CLNP routing information. Integrated ISIS is currently designated as a Proposed Standard. The protocol was first published in RFC 1195. Internet Draft was published subsequently to RFC 1195 and documents the current version of the protocol. This report documents experience with Integrated isIS. This includes reports on interoperability testing, field experience and the current state of Integrated ISIS implementations. It also presents a summary of the Integrated ISIS Management Information Base (MIB), and a summary of the Integrated ISIS authentication mechanism.

\section{1,792}

FIPS PUB 120-1C PC A06/MF A02

National Inst. of
Gaithersburg. MD. 
Graphical Kernel System (GKS). Category: Software Standard. Subcategory: Graphics. International Standard: Information Technology; Computer Graphics; Graphical Kernel System (GKS) Language Bindings. Part 4: $\mathrm{C}$

8 Jan $91,113 p$

Also available from Supt. of Docs. Also pub. as Amercan National Standards Inst., New York rept. no. ISO/ IEC-8651-4.1991E. Supersedes FIPS PUB 120. Prepared in cooperation with American National Standards Inst., New York.

Keywords: *Federal information processing standards, "Computer graphics, *Computer software, Applications programs(Computers), Computer program portability. GKS(Graphical Kernel System), ANSI(American National Standard Institute), ISO(Intemational Organization for Standardization), C programming language.

This revision supersedes Federal Information Processing Standard (FIPS) PUB 120 and modifies the standard by adding a requirement for validation of GKS implementations that are acquired by Federal agencies. FIPS 120-1 adopts American National Standard Graphical Kernel System (ANS GKS), ANSI X3.1241985. Functional Description, which consists of four parts (X3.124.1-1985 FORTRAN Binding, X3.124.21988 Pascal Binding, X3.124.3-1989 Ada Binding), as a FIPS.ANS GKS specifies a library (or toolbox package) of subroutines for an application programmer to incorporate within a program in order to produce and manipulate two-dimensional pictures. The purpose of the standard is to promote portability of graphics application programs between different installations. The standard is for use by implementors as the reference authority in developing graphics software systems; and by other computer professionals who need to know the precise syntactic and semantic rules of the standard.

01,793

FIPS PUB 158-1 PC E01

National Inst. of Standards and Technology (NCSL), Gaithersburg, MD.

User Interface Component of the Applications Portability Profile Category: Software Standard; Subcategory: Application Program Interface.

Federal information processing standards.

c8 Oct 93, 5p.

Supersedes FIPS PUB 158. Also available from Supt. of Docs.

Three ring binder also available. North American Continent price $\$ 7.00$; all other write for quote.

Keywords: "Standards, "Windowing, Interfaces, Com. puter graphics, "Federal information processing standards, "Man computer interface, "Application programs(Computers), "Software engineening, Portable Operating System Interface (POSIX), Compute program portability, Application program interface, Open systems interconnections.

This publication announces the adoption of the $X$ Protocol, Xlib Interface, $X t$ Intrinsics and Bitmap Distribution Format specifications of the $X$ Window System, Version 11, Release 5 ( $X$ Window System is a trademark of the Massachusetts Institute of Technology (MIT) as a Federal Information Processing Standard). This FIPS is identical to FIPS PUB 158, except that the specification is updated from Release 3 to Release 5 of the $X$ Window System, Version 11. The standard is for use by computing professionals involved in system and application software development and implementation. The standard is part of a series of specifications needed for application portability. The standard covers the Data Stream Encoding, Data Stream Interface, and Subroutine Foundation layers of the reference model. It is the intention of NIST to provide standards for other layers of the reference model as consensus develops within industry. This standard addresses the user interface functional area of the Applications Portability Profile (NIST SP 500-87).

01,794

FIPS PUB 173-1 PC A04/MF A01

National Inst. of Standards and Technology (CSL), Gaithersburg, MD

Spatial Data Transfer Standard (SDTS): Category: Software Standard; Subcategory: Information Interchange.

10 Jun $94,51 p$

Supersedes FIPS PUB 173

Keywords: "Data transfer(Computers), "Federal information processing standards, Digital data, Mapping, Data structures, Geographic information systems, Topology, Specifications, Requirements, Data dictionaries, TVP(Topological Vector Profile).
This standard provides specifications (developed through the U.S. Department of the Interior (DOI), United States Geological Survey (USGS), National Mapping Division) for the organization and structure of digital spatial data transfer, definition of spatial features and attributes, and data transfer encoding. The purpose of the standard is to promote and facilitate the transfer of digital spatial data between dissimilar computer systems. This revision adds Part 4, Topological Vector Profile (TVP), to the specifications adopted by the Spatial Data Transfer Standard (SDTS) and makes minor changes and clarifications to the Parts 1 and 3 of the SDTS.

\section{1,795}

FIPS PUB 173-1A PC $\$ 44.50$

National Inst. of Standards and Technology (CSL) Gaithersburg, MD.

Spatial Data Transfer Standard (SDTS). Category: Software Standard; Subcategory: Information Interchange. (FIPS PUB 173-1A).

10 Jun 94, 348p.

Supersedes FIPS PUB 173

Three ring vinyl binder also available; North American Continent price $\$ 7.00$; all other write for quote.

Keywords: *Data transfer(Computers), "Federal information processing standards, "Position(Location), Mapping, Data structures, Format, Data integrity, Geo graphic information systems, Topology, Rasters.

This standard provides specifications (developed through the Department of the Interior) for the organization and structure of digital spatial data transfer, definition of spatial features and attributes, and data transfer encoding. The purpose of the standard is to promote and facilitate the transfer of digital spatial data between dissimilar computer systems.

\section{1,796}

FIPS PUB 173-1B PC E99

National Inst. of Standards and Technology (CSL) Gaithersburg, MD.

Spatial Data Transfer Standard (SDTS). Category: Software Standard; Subcategory: Information Interchange. (FIPS PUB 173-1B).

10 Jun 94, 54p.

Supersedes FIPS PUB 173

Three ring vinyl binder also available; North American Continent price $\$ 7.00$; all other write for quote.

Keywords: "Data transfer(Computers), "Federal information processing standards, "Position(Location), Mapping, Data structures, Format, Data integrity, Geographic information systems, Topology, Rasters.

This standard provides specifications (developed through the Department of the Interior) for the organization and structure of digital spatial data transfer, definition of spatial features and attributes, and data transfer encoding. The purpose of the standard is to promote and facilitate the transfer of digital spatial data between dissimilar computer systems.

\section{1,797}

FIPS PUB $189 \quad$ PC A03/MF A01

National Inst. of Standards and Technology (CSL). Gaithersburg, MD.

Portable Operating System Interface (POSIX). Part 2. Shell and Utilities. Category: Software Standard Subcategory: Operating Systems.

11 Oct $94,12 \mathrm{p}$

Also available from Supt. of Docs.

Three ring vinyl binder also available; North American Continent price $\$ 7.00$; all others write for quote.

Keywords: "Federal information processing standards, "Operating systems(Computers), Data processing, Application programs(Computers), Computer program portability, Utility routines, Computer software, Software engineening, Specifications, Open systems.

This publication announces the adoption of International Standard ISO/IEC 9945-2:1993, Information Technology--Portable Operating System Interface (POSIX)--Part 2: Shell and Utilities as a Federal Information Processing Standard (FIPS). ISO/IEC 9945 2:1993 defines a command language interpreter (shell) and a set of utility programs. This standard is for use by computing professionals involved in system and application software development and implementation and is part of a series of specifications needed for ap plication portability. This standard addresses the Applcations Portability Profile functional area that deals with methods by which a person interacts with the operating system.
01,798

FIPS PUB $190 \quad$ PC A04/MF A01

National Inst. of Standards and Technology (CSL),

Gaithersburg, $M D$.

Guideline for the Use of Advanced Authentication Technology Alternatives. Category: Computer Security. Subcategory: Access Control.

Final rept.

J. F. Dray. 28 Sep 94, 66p.

Also available from Supt of Docs.

Three ring vinyl binder also available; North American Continent price \$7.00; all others write for quote.

Keywords: "Computer security, "Authentication, "Federal information processing standards; Cryptography, Telecommunication, Data processing security, Data encryption, Biometrics, Access control, Passwords.

This Guideline describes the primary alternative methods for verifying the identities of computer system users, and provides recommendations to Federal agencies and departments for the acquisition and use of technology which supports these methods. Although the traditional approach to authentication relies primarily on passwords, it is clear that password-only authentication often fails to provide an adequate level of protection. Stronger authentication techniques become increasingly more important as information processing evolves toward an open systems environment. Modern technology has produced authentication tokens and biometric devices which are reliable, practical, and costeffective. Passwords, tokens, and biometrics can be used in various combinations to provide far greater assurance in the authentication process than can be attained with password alone.

01,799

FIPS PUB 191 PC A04/MF A01

National Inst. of Standards and Technology (CSL). Gaithersburg, MD

Guideline for the Analysis of Local Area Network Security. Category: Computer Security; Subcategory: Risk Analysis and Contingency Planning.

Final rept.

L. J. Carnahan. 9 Nov $94,62 \mathrm{p}$.

Also available from Supt. of Docs.

Three ring vinyl binder also available; North American Continent price $\$ 7.00$; all others write for quote.

Keywords: "Local area networks, "Computer security, "Federal information processing standards, Risk assessment, Vulnerability, Distributed computer systems, Data processing security, Protocols, Message processing, Access control, Authentication, Data integrity, Personal computers, Training.

Local area networks (LANs) that are used to store, process or transmit sensitive information must provide appropriate protection to prevent undesirable events such as compromise of information or denial of service This document can be used as a tool to help improve the security of a local area network. A LAN security architecture is described that discusses threats and vulnerabilities that should be examined, as well as security services and mechanisms that should be explored. A five step process is defined that helps the reader to determine LAN assets, environment-specific threats and vulnerabilities, the risk of those threats to the LAN, and possible security services and mechanisms that can be used to help reduce the risk to the LAN. A discussion concerning LAN security policy, contingency planning, and training issues is also included.

\section{1,800}

FIPS PUB $192 \quad$ PC A03/MF A01

National Inst. of Standards and Technology (CSL), Gaithersburg, MD

Application Profile for the Government Information Locator Service (GILS). Category: Software Standard; Subcategory: Information Interchange.

7 Dec 94, 34p.

Three ring vinyl binder also available; North American Continent price $\$ 7.00$; all others write for quote.

Keywords: "Federal information processing standards, Information retrieval, Government, Specifications, Information systems, Internets, Protocols, Records Services, "GILS(Government Information Locato Service), "Government Information Locator Service.

This standard describes an application profile for the Government Information Locator Service (GILS). This 


\section{Information Processing Standards}

application profile is based primarily on the American National Standard for Information Retrieval Application Service Definition and Protocol Specification for Open Systems Interconnection (ANSI/NISO Z39.50-1992), developed by the National Information Standards Organization (NISO). The Government Information Locator Service (GILS) is a decentralized collection of servers and associated information services that will be used by the public either directly or through intermediaries to find public information throughout the Federal government.

\section{1,801}

FIPS PUB $193 \quad$ PC A04/MF A01

National Inst. of Standards and Technology (CSL), Gaithersburg, MD

SQL Environments. Category: Software Standard; Subcategory: Database.

3 Feb $95,72 p$

Also available from Supt. of Docs.

Three ring vinyl binder also available; North American Continent price \$7.00; all others write for quote.

Keywords: *Federal information processing standards, *Programming environments, "Data processing, Data bases, Multimedia, Integrated systems, Tests, Procurement, Specifications, `SQL(Structured Query Language), "Structured Query Language, Internet, ERI(External repository interface), CLI(Call Level Interface).

AN SQL environment is an integrated data processing environment in which heterogeneous products, all supporting some aspect of the FIPS SQL standard (FIPS PUB 127), are able to communicate with one another and provide shared access to data and data operations and methods under approprate security, integrity, and access control mechanisms. This Federal Information
Processing Standards Publication (FIPS PUB) is the beginning of a continuing effort to define appropriate conformance profiles that can be used by both vendors and users to specify exact requirements for how various products fit into an SQL environment. The emphasis in this first publication is to specify general purpose, SQL extemal repository interface (SQLERI) server profiles for non-SQL data repositories. Two major SQL ERI Server Profiles are specified: read-only and readwrite. To make it easier to specify integration among heterogeneous, non-SQL data models, this specification defines a new minimal level of the SQL language that can be supported by various non-SQL implementations.

\section{1,802}

FIPS PUB $195 \quad$ PC E18

National Inst. of Standards and Technology (CSL), Gaithersburg, MD

Federal Building Grounding and Bonding Requirements for Telecommunications. Category: Teleing and Bonding.

15 Aug $95,46 \mathrm{p}$

Also pub. as Telecommunications Industry Association, Washington, DC. Standards and Technology Dept. rept. no. ANSI/TIAVEIA-607-1994. Prepared in cooperation with Telecommunications Industry Association, Washington, DC. Standards and Technology Dept.

Three ring vinyl binder also available; North American Continent price $\$ 7.00$, all others write for quote.

Keywords: "Telecommunication, "Public buildings, Communication cables, Electrical grounding, Wires, Communication equipment, National government, Passageways, Electrical circuits, Safety engineening, Fire prevention, "Federal information processing Fire preven
standards.

This standard, by adoption of ANSI/TIAVEIA-607-1994, Commercial Building Grounding and Bonding Requirements for Telecommunications, specifies the requirements for a uniform telecommunications grounding and bonding infrastructure for Federal buildings where telecommunications equipment is installed. The standard provides the requirements for a ground reference for telecommunications systerns within the telecommunications entrance facility, the telecommunications closet, and equipment room; it also provides the requirements for bonding and connecting pathways, cable shields, conductors, and hardware at telecommunications closets, equipment rooms, and entrance facilities. The grounding and bonding approach described in this standard is consistent with the cabling topology specified in FIPS PUB 174, Federal Building Telecommunications Wining Standard (ANSI/EIATIA568-1991), and installed in accordance with FIPS PUB
175, Federal Building Standard for Telecommunications Pathways and Spaces (ANSI/EIATIA-5691990).

\section{1,803}

PB94-142536 PC A07/MF A02

National Inst. of Standards and Technology (CSL) Gaithersburg, MD.

Report on Application Integration Architectures (AIA) Workshop. Held in Dallas, Texas on February 8-12, 1993.

R. Hodges, C. Thompson, and E. Fong. Jan 94,

138p, NISTIR-5326.

Also available from Supt. of Docs. Prepared in cooperation with Texas Instruments, Inc., Dallas.

Keywords: "Meetings, "Standards, Interoperability, Computer software, Information management, "IT(Information technology), "Information technology, Open systems.

This report provides a proceedings of the workshop on Application Integration Architectures (AIA) held on February 8-12, 1993, in Dallas, Texas. The workshop addressed various means of coordinating and improving information technology (IT) standards to achieve open systems interoperability. The purpose of this workshop was to provide a forum where individuals active in one or more standards efforts or industrial consortia in the information technologies software area could meet to discuss how their efforts relate and work to formulate a roadmap to insure convergence of de jure or de facto standards in software information technology.

\section{1,804}

PB94-161809 PC A03/MF A01

National Inst. of Standards and Technology (CSL), Gaithersburg, MD. Information Systems Engineering Div.

Computer Graphics Metafile (CGM): Procedures for NIST CGM Validation Test Service.

L. S. Rosenthal, and J. A. Schneider. Feb 94, 29p, NISTIR-5372

See also FIPS PUB 128-1E and PB93-198273.

Keywords: "Computer graphics, "Standards, Federal information processing standards, Conformity, Tests, Verifying, Interpreters, "Computer Graphics Metafile, CALS, Continuous Ácquisition and Life-Cycle Support, Computer-aided Acquisition and Logistics Support, Application Protocols, National Institute of Standards and Technology.

This document provides general procedures for the National Institute of Standards and Technology's (NIST) Computer Graphics Metafile (CGM) Validation Test Service. The NIST CGM Validation Test Service provides a way of determining the degree to which an implementation conforms to the CGM standard (Federal Information Processing Standard 128-1, Computer Graphics Metafile, and the Continuous Acquisition and Life-Cycle Support Application Profile, MIL-D-28003A) The goal of the NIST Test Service is to maximize the robability of successful interchange of CGMs beween conforming systems. The document is divided into three testing programs: metafile testing, generator testing, and interpreter testing. In order to take into ac count the differences among the three testing programs, many procedures have been tailored to the specific testing program. The policies and procedures presented in this document are organized into five sections: an introduction, general procedures, and specific procedures for each of the three testing programs.

\section{1,805}

PB94-163474 PC A06/MF A02

National Inst. of Standards and Technology (CSL), Gaithersburg, MD

Compatibility Analysis of the ANSI and ISO IRDS Services Interfaces.

J. Berube, and A. Goldfine. Sep 92, 104p, NISTIR4904.

See also PB88-163779.

Keywords: "Data management, "Dictionaries, -Standards, Compatibility, IRDS (Information Resource Dictionary System), ANSI(American National Standards Institute), ISO(International Organization for Standardization).

The primary purpose of the report is to compare: the ANSI IRDS standards (ANSI 1992), (IRDS 1988); and the ISO IRDS standards (FRAMEWORK 1990), (ISO 1992). The following are also part of the context of the report and will be surveyed: the IBM Repository Man- ager Interface (RM 1990); the ATIS IRDS proposal (ATIS 1990); and the PCTE standard (PCTE 1988) The principal objective of the report is to establish the level of compatibility and interoperability possible between an ANSI IRDS environment and an ISO IRDS environment. This is done by attempting to establish if these environments can be reconciled: in definition. That is, can they share data. in operation. That is could a single product offer the two types of services.

\section{1,806}

\section{PB94-207453 PC A07/MF A02}

National Inst. of Standards and Technology (NCSL) Gaiv IGOSS-Industry/Government Open Systems Specification.

Special pub.

G. Mulvenna. May 94, 131p, NIST/SP-500/217

Also available from Supt. of Docs as SNO03-00303269-7. See also FIPS PUB-107. FIPS PUB 100-1 and PB93-166809.

Keywords: *Computer networks, "Standards, Protocols, Government/industry relations, Computer architecture, File management systems, Message processing, Communication networks, "OSI(Open Systems Interconnection), "Open Systems interconnection, GOSIP(Government Open Systems Interconnection Profile), IGOSS(Industry/Government Open Systems Specification).

The IGOSS is a standard reference to use when acquiring and operating computer networking products and services intended to conform to the Open Systems Interconnection (OSI) protocols. The IGOSS is jointly authored by the U.S. Government, the Canadian Government, Manufacturing Automation Protocol (MAP) group, the Technical and Office Protocol (TOP) group, and the electric power group. Each of these organizations has previously issued their own procurement profiles and are now consolidating their procurement requirements into a single domain.

01,807

PB94-211455 Not available NTIS

National Inst. of Standards and Technology (NCSL) Gaithersburg, MD. Systems and Network Architecture Div.

U.S. GOSIP Testing Program.

Final rept.

J. P. Favreau, K. L. Mills, and J. S. Nightingale.

1990, 5p

Pub. in Proceedings of International Conference on the Applications of Standards for Open Systems (6th), Falls Church, VA., October 2-4, 1990, p125-129.

Keywords: "Tests, Protocols, Federal information processing standards, Federal agencies, Government procurement, Reprints, "GOSIP(Government Open Systems Interconnection Profile), "Govemment Open Systems Interconnection Profile, OSI(Open Systems Interconnection)

This paper discusses progress to date in the development and deployment of a Testing Policy for U.S. Government Open Systems Interconnection Profile (GOSIP) protocol implementations. The policy itself is fully discussed in the Federal Information Processing Standards (FIPS): 'GOSIP Conformance and Interoperation Testing and Registration.' Realization of the policy has included solicitation and assessment of abstract test suites, means of testing, conformance test laboratories and interoperability test suites and facilities. Public registers of all of these items are compiled and published so that Federal Agencies may make informed procurement decisions for GOSIP products.

\section{1,808}

PB94-216645 Not available NTIS

National Inst. of Standards and Technology (CSL), Gaithersburg, MD. Advanced Systerns Div.

NIST-Coordinated Standard for Fingerprint Data Interchange.

Final rept.

R. M. McCabe. 1993, 5p.

Pub. in Jnl. of Forensic Identification 43, n4 p344-348 1993.

Keywords: "Data transfer(Computers), "Standards, Image processing, Data compression, Format, Quality assurance, Reprints, "US NIST, "Fingerprints, ANSI(American National Standards Institute).

This editorial is a clarification of the National Institute of Standards and Technology's (NIST's) role in the de- 
velopment of the American National Standards Institute (ANSI) standard ANSI/NIST-CSL 1-1993 entitled 'Data Format for the Interchange of Fingerprint Information.' Several misrepresentations of the NIST role appeared in an editorial published in the May/June 1993 issue of the Journal of Forensic Identification.

\section{1,809}

PB94-219110 PC A06/MF A02

National Inst. of Standards and Technology (CSL), Gaithersburg, MD.

Industry/Government Open Systems Specification

Testing Framework. Version 1.0.

J. P. Favreau. Jun 94, 124p, NISTIR-5438.

Supersedes PB92-110105.

Keywords: "Tests, Quality control, Data transmission, Data transfer(Computers), Protocols, Computer networks, Message processing, Commodities, Procurement, Government/industry relations, Standards, *IGOSS(Industry/Government Open Systems Specification), "Industry/Government Open Systems Specification, US NIST, OSI(Open Systems Interconnection).

The development of National Institute of Standards and Technology (NIST) Special Publication 500-217 which specifies the Industry/Government Open Systems Specification (IGOSS), resulted in the need to establish policy and procedures aimed at ensuring that the IGOSS Partners' data communications products adhere to the technical documents referenced by IGOSS and that they interoperate. The goal of this document is to aid an Industry/Government Acquisition Authority in procurement of IGOSS products by employing publicly accessible registers verifying supplie claims of conformance and documenting instances of interoperability of IGOSS CONFORMANT PRODUCTS. This document supersedes NISTIR 4594.

01,810

PB95-126207 Not available NTIS

National Inst. of Standards and Technology (NCSL) Gaithersburg, MD. Information Systems Engineering Div.

Information Resource Dictionary System (IRDS): A Status Report.

Final rept.

A. Goldfine. $1990,2 p$

Pub. in AFFIRMation 12, n8 p1-2 Oct 90.

Keywords: "Data dictionaries, "Standards, Federal information processing standards, Standardization, Information systems, Systems engineering, Reprints, *IRDS(Information Resource Dictionary System) *Information Resource Dictionary System, ANSI(American National Standards Institute).

This article discusses the status of the Information $\mathrm{Re}$ source Dictionary System (IRDS). It provides brief background information, reviews the current standardization situation, and discusses extensions to the IRDS that are under development.

01,811

PB95-171377 PC A03/MF A01

National Inst. of Standards and Technology (CSL), Gaithersburg, MD

Perspective on Software Engineering Standards.

D. R. Wallace, and R. J. Martin. Dec 94, 12p,

NisTIR-5546.

See also PB95-136610 and NUREG/CP-0136.

Keywords: *Software engineering, "Standards, Federal information processing standards, Computer software, Organizations, Industries, US NIST.

This paper provides information about the National Institute of Standards and Technology's (NIST) Federal Information Processing Standards (FIPS) and other standards organizations and presents a perspective on software engineering standards.

\section{1,812}

PB95-180840 Not available NTIS

National Inst. of Standards and Technology (CSL), Gaithersburg, MD

Federal Government and Information Technology Standards: Building the National Information Infrastructure.

Final rept.

S. M. Radack. 1994, 13p

Pub. in Government Information Quarterly 11, n4 p373-385 1994.

Keywords: *Standards, "Information processing, Government policies, National government, Federal infor- mation processing standards, Computers, Information systems, Telecommunication, Reprints, NII(Nationa Information Infrastructure), NPR(National Performance Review), US NIST.

For many years, standards have been important considerations in the Federal government's policies for the use of information technology. The Computer Systems Laboratory (CSL) at the National Institute of Standards and Technology develops and issues technical standards that are used by the Federal government in its information technology systems. The new Federal initiative for the National Information Infrastructure (NII) and the National Performance Review (NPR) make information technology an agent for change and empha size standards as a means for achieving connectivity of computer and telecommunications technologies and for easy access to information. The Federal govern ment will be challenged to address the technical, organizational and policy issues that affect the develop ment of the standards needed for future information systems.

\section{1,813}

PB96-111190 PC A03/MF A01

National Inst. of Standards and Technology (CSL), Gaithersburg, MD

Federal Implementation Guideline for Electronic Data Interchange: ASC X12 003040 Transaction Set 838 Trading Partner Profile (Confirmation of Vendor Registration). Implementation Convention. J. P. Favreau. Aug 95, 25p, NIST/SP-881/2

Also available from Supt. of Docs, as SNOO3-00303364-2. See also PB96-112651.

Keywords: "Computer programs, *Information ex change, "Logistics management, Procurement, Standards, Acquisition, Databases, Telecommunication, Businesses, Corporations, Purchasing, Vendors, Electronic Data Interchange ASC X12 003040 Transaction Set 838 .

The ASC $\times 12003040$ Transaction Set 838, Trading Partner Profile (Confirmation of Vendor Registration), Implementation Convention defines the Federal Government interpretation of the use of ASC X12 003040 standards. This implementation convention provides the information necessary for the user to be able to develop an interface program between the compute application and the ASC $\times 12$ translator.

\section{1,814}

\section{PB96-114913 PC A11/MF A03}

National Inst. of Standards and Technology (NCSL)

Gaithersburg, MD. Systems and Network Architecture Div

Federal Implementation Guideline for Electronic Data Interchange. ASC X12 003050 Transaction Set 850 Award Instrument. Implementation Convention.

Special pub.

J. P. Favreau. Aug 95, 228p, NIST/SP-881/3

Also available from Supt. of Docs as SN003-003 03365-1. See also PB96-114921.

Keywords: "Information systems, "Standards, "Procurement, Commerce, Computer networks, Information exchange, Computer software, United States government, Contract administration, Data processing, *Electronic Data Interchange, "EDI(Electronic Data Interchange), Standard ASC 12003050 Transaction Set 860 , Draft standard.

The ASC $\times 12003050$ Transaction Set 850, Award Instrument, Implementation Convention defines the Federal Government interpretation of the use of ASC $\times 12$ 003050 standards. This implementation convention provides the information necessary for the user to be able to develop an interface program between the computer application and the ASC X12 translator.

01,815

PB96-114921 PC A11/MF A03

National Inst. of Standards and Technology (NCSL) Gaithersburg, MD. Systems and Network Architecture Div.

Federal Implementation Guideline for Electronic Data Interchange. ASC X12 003050 Transaction Set 860 Modifications to Award Instrument. Implemen tation Convention.

Special pub.

J.P. Favreau. Aug 95, 230p, NIST/SP-881-4.

Also available from Supt. of Docs as SN003-003 03366-9. See also PB96-114913.

Keywords: *Information systems, "Standards, *Procurement, Commerce, Computer networks, Infor- mation exchange, Computer software, United States government, Contract administration, Data processing *Electronic Data Interchange, "EDI(Electronic Data Interchange), Standard ASC X12 003050 Transaction Set 860 , Draft standards.

This Draft Standard for Trial Use contains the format and establishes the data contents of the Purchase Order Change Request - Buyer Initiated Transaction Set (860) for use within the context of an Electronic Data Interchange (EDI) environment. The transaction set can be used to provide the information required for the customary and established business and industry practice relative to a purchase order charge. This transaction can be used: (1) by a buyer to request a change to a previously submitted purchase order or (2) by a a buyer to confirm acceptance of a purchase order change initiated by the seller or by mutual agreement of the two parties.

01,816

PB96-114939 PC A07/MF A02

National Inst. of Standards and Technology (CSL), Gaithersburg, MD.

Z39.50 Implementation Experiences.

Special pub.

P. Over, W. E. Moen, R. Denenberg, and L. Stovel. Sep 95, 129p, NIST/SP-500/229.

Also available from Supt. of Docs. Prepared in cooperation with University of North Texas, Denton School of Library and Information Sciences., Library of Congress, Washington, DC. and Research Libraries Group, Inc., Mountain View, CA.

Keywords: "Computer networks, "Computer software *Information retrieval, Implementation, Z39.50 ANSIINISO protocol, Open systems interconnections. Contents:

Z39.50 for Full-Text Search and Retrieval

Basic Z39.50 Server Concepts and Creation;

Building a Z39.50 Client;

Implementing Explain;

Implementing Z39.50 in a multi-national and multilingual environment;

Use of Z39.50 for Search and Retrieval of Scientific and Technical Information;

Structural Components of the Isite Information System;

Z39.50 - implications and implementation at the AT\&T library network:

The Implementation of Z39.50 in the National Library of Canada's AMICUS System

Developing a Multi-Platform Z39.50 Service;

and Use of Z39.50 for the Delivery of Current Awareness Products.

01,817

PB96-119359 Not available NTIS

National Inst. of Standards and Technology (NCSL), Gaithersburg, MD. Systems and Network Architecture Div.

Lessons from the Establishment of the U.S. GOSIP Testing Program.

Final rept.

J. P. Favreau, and J. S. Nightingale. 1991, 16p.

See also PB94-211455.

Pub. in Proceedings of the International Workshop on Protocol Test Systems (4th), Leidschendam, The Netherlands, October 15-17, 1991, 16p.

Keywords: "Computer communications, "Protocols, Computer networks, Communication networks, Interfaces, Interoperability, Message processing, Computer architecture, Systems engineering, Standardization, Specifications, Requirements, Reprints, *OSI(Open Systems Interconnection), *Open Systems Interconnection, "GOSIP(Government Open Systems Interconnection Profile), "Government Open Systems Interconnection Profile, Conformance.

Currently, the U.S. Government mandates use of Open Systems Interconnection (OSI) protocols in procurement of new networks and major upgrades to existing networks. Vendors strive to meet the challenge of making OSI products available. Users are confused by this new technology. In order to respond to this problem the National Institute of Standards and Technology has set up a comprehensive testing program. An overview of the U.S. GOSIP Testing Program is presented with some of the issues in OSI protocol conformance and interoperability testing raised during its design and implementation.

PB96-122908 Not available NTIS 
National Inst. of Standards and Technology (NCSL), Gaithersburg, MD. Systems and Network Architecture Div.

Open Issues in OSI Protocol Development and Conformance Testing.

Final rept.

J. P. Favreau, G. V. Bochmann, and P. Mondain-

Monval. 1991, $19 p$ Pub. in Computer Networks ' 91 , Wroclaw, Poland, $19 p$
1991 .

Keywords: *Protocols, "Conformance, "Interoperability, Standards, Testing, Computer networks, Computer software, Computer equipment, Procurement, Communications, Reprints, "OSI(Open Systems Interconnection), "Open Systems Interconnection.

As of August 15, 1990 the U.S. Government mandated use of Open Systems Interconnection (OSI) in procurement of new networks and major upgrades to existing networks. The goal of OSI is to allow interworking of separately developed pieces of software and equipment. However, it is not easy to allow interworking of separately developed pieces of software and equipment. However, it is not easy to insure that OSI products provided by different vendors will properly interoperate. This paper presents some unresolved issues in OSI protocol development and conformance testing, and proposes an approach for the protocol development and testing cycle that would alleviate some of the present problems.

\section{1,819}

PB96-122924 Not available NTIS

National Inst. of Standards and Technology (CSL), Gaithersburg, MD. Information Systems Engineering Div.

Database Management Standards: Status and Applicability.

L. Gallagher. 1991, 11p.

Pub. in Computers and Standards, 11p, 18 Nov 91.

Keywords: "Data base management, "Standards, Computer languages, Query languages, Conformance, Applicability, Reprints, ISO(International Organization for Standards), RDA(Remote Database Access), NDL computer language, SQL computer language.

This article presents the current status of existing and emerging ISO, ANSI, and FIPS standards for database management, specifically Database Languages NDL and SQL and Remote Database Access (RDA). It describes the general content of each standard and discusses its applicability, availability, completeness, maturity, stability, existing usage, and known limitations. Where appropriate, it also addresses the availability of conformance test suites and future plans for enhancements and follow-on standardization efforts.

\section{1,820}

PB96-131495 PC A03/MF A01

National Inst. of Standards and Technology (CSL), Gaithersburg, MD. Distributed Systems Engineering. Comparison of POSIX Open System Environment (OSE) and Open Distributed Processing (ODP) Reference Modeis.

G. Fernandes, and J. Hungate. 13 Nov 95, 26p, NISTIR-5736.

Keywords: "Models, "Standardization, Computer program portability, Interfaces, Distributed processing, Interoperability, Data flow analysis, Computer networks, Information systems, Information centers, Informaton services, Operating systems(Computers), Information dissemination, Systems management, Specifications, "POSIX(Portable Operating System Interface for Computing Environments), "Portable Operating System Interface for Computing Environments, "OSE(Open System Environment), "Open System Environment, "ODP(Open Distributed Processing), "Open Distributed Processing, Distributed systems.

This report presents two existing reference models for describing distributed systems. These models are the Open Distributed Processing reference model and the POSIX OSE reference model. They both address distributed open systems, but from different perspectives, with different objectives and methodology. After a brief description of the two models, a comparison is made referring in particular to the extent distributed systems management is accommodated in both models.
National Inst. of Standards and Technology (CSL), Gaithersburg, MD NIST POSIX Testing Program.

\section{Final rept.}

E. Lennon. 1991, 4p.

Pub. in Computer Systems Laboratory Bulletin, p1-4 Oct 91.

Keywords: "Operating systems(Computers), *Computer program portability, *Interfaces, Compute program verification, Application programs(Computers), Computer systems programs, Interoperability, Distributed processing, Reprints, *POSIX(Portable Operating Systems Interface for Computer Environments), "Conformance testing *Portable Operating Systems Interface for Computer Environments.

This CSL Bulletin describes NIST's program to test POSIX products for conformance to Federal Information Processing Standard (FIPS) 151-1, Portable Operating System Interface for Computer Environments (POSIX). The bulletin discusses the NIST POSIX Conformance Test Suite, POSIX testing procedures, accredited POSIX testing laboratories, POSIX validated products, and the availability of POSIX information.

\section{1,822}

PB96-168984 PC A09/MF A02

National Inst. of Standards and Technology (CSL), Gaithersburg, MD. Electronic Commerce Acquisition Program.

Federal Implementation Guideline for Electronic Data Interchange. ASC X12 003050 Transaction Sel 843 Response to Request for Quotation. Impiementation Convention.

Special pub.

J. P. Favreau. 1996, 154p, NIST/SP-881-7.

Keywords: "Information systems, "Standards, "Procurement, Commerce, Computer networks, Tele communication, Information exchange, Computer software, United States Government, Contract administration, Data processing, Purchasing, "EDI(Electronic Data Interchange), "Electronic Data Interchange, Standard ASC X12 003050 Transaction Set 843, Draft Standard, Electronic commerce.

This Draft Standard for Tidal Use contains the format and establishes the data contents of the Response to Request for Quotation Trasaction Set (843) for use within the context of an Electronic Data Interchange (EDI) environment. The transaction set can be used to provide potential buyers with price, delivery schedule, and other terms from potential sellers of goods and services, in response to a request for such information.

\section{1,823}

\section{PB96-172374 PC A04/MF AO}

National Inst. of Standards and Technology (NCSL), Gaithersburg, MD. Systems and Network Architecture Div.

Federal Implementation Guideline for Electronic Data Interchange. ASC X12 003050 Transaction Set 855 Purchase Order Acknowledgment: Implementation Convention.

Special pub.

J. P. Favreau. Feb 96, 41p, NIST/SP-881/6.

Also available from Supt. of Docs. as SN003-003 03398-7. See also PB96-114921.

Keywords: "Information systems, "Standards, "Procurement, Commerce, Computer networks, Telecommunication, Information exchange, Computer software, United States Government, Contract administration, Data processing, Purchasing, "EDI(Electronic Data Interchange). "Electronic Data Interchange, Standard ASC X12 003050 Transaction Set 855, Draft standard, Electronic commerce.

This Draft Standard for Tidal Use contains the format and establishes the data contents of the Purchase Order Acknowledgement Transaction Set (855) for use within the context of an Electronic Data Interchange (EDI) environment. The transaction set can be used to provide for customary and established business and industry practice relative to a seller's acknowledgment of a buyer's purchase order. This transaction set can also be used as notification of a vendor generated order. The usage advises a buyer that a vendor has or will ship merchandise as prearranged in their partnership.

\section{1,824}

PB96-172531 PC A10/MF A03
National Inst. of Standards and Technology (NCSL), Gaithersburg, MD. Systems and Network Architecture Div.

Federal Implementation Guideline for Electronic Data interchange. ASC $\times 12003050$ Transaction Set 840 Request for Quotation. implementation Convention.

Special pub.

J.P. Favreau. Feb 96, 196p, NIST/SP-881-8

Also available from Supt. of Docs. as SNO03-00303402-9.

Keywords: "Data processing, "Computer networks, "Standards, Information systems, Government procurement Information exchange, Contract administration, Computer software, "Electronic data interchange, ASC X12003050 Transaction Set 840.

This Draft Standard for Tidal Use contains the format and establishes the data contents of the Request for Quotation Transaction Set (840) for use within the context of an Electronic Data Interchange (EDI) environment. The transaction set can be used to provide buyers with the ability to solict price, delivery schedule and other items from potential sellers of goods and services.

\section{1,825}

PB'96-172549 PC A09/MF A02

National Inst. of Standards and Technology (NCSL), Gaithersburg, MD. Systems and Network Architecture Div.

Federal Implementaion Guideline for Eletronic Data interchange. ASC X12 003050 Transaction Set 865 Purchase Order Change Acknowledgement Request - Seller Initiated. Implementation Convention.

Special pub.

J.P. Favreau. Feb 96, 162p, NIST/SP-881-5.

Also available from Supt. of Docs. as SNO03-00303397-9. See also PB96-114921.

Keywords: "Information systems, "Standards, "Computer networks, "Procurement, Commerce, Information exchange, Computer software, United States Government, Contract administration, Purchasing, Telecommunications, Data processing, "EDI(Electronic Data Interchange), Electronic Data Interchange, Standard ASC X 12003050 Transaction Set 865, Implementation Convention, Electric Commerce, Draft standard.

The ASC X12 003050 Transaction Set 865, Purchase Order Change Acknowledgement/Request - Sell Initiated Implementation Convention defines the Federal Government interpretation of the use of ASC X12 003050 standards. This implementation convention provides the information necessary for the user to be able to develop an interface program between the computer application and the ASC X12 translator.

\section{1,826}

PB96-175716 Not available NTIS

National Inst. of Standards and Technology (NCSL), Gaithersburg, MD. Systems and Network Architecture Div.

Five Q's (Cues) of the U.S. GOSIP Testing Program. Final rept.

J. P. Favreau. 1992, 14p.

Pub. in Government Open Systems Interconnection Profite Procurement Symposium, Gaithersburg, MD., December 7, 1992, p1-14.

Keywords: "Computer networks, "Interoperability, Conformance, Systems engineering, File management systems, Data transmission systems, Product development, Procedures, Requirements, Specifications, Standards, Testing, Procurement, Quality assurance, Computer communications, Reprints, "GOSIP(Government Open Systems Interconnection Profile), "Government Open Systems Interconnection Profile.

The National Institute of Standards and Technology (NIST) has developed a comprehensive test policy and procedures that must demonstrate technical credibility, acceptability to both vendors and users, assurance of interoperability and provide a basis for international recognition of national testing. The U.S. GOSIP Testing Program includes the identification of abstract test suites, the development of method to assess means of testing, the setting up a test laboratory accreditation program, a definition for the role of interoperability testing and the creation of publicly available registers.

01,827

PB96-178892 PC A04/MF A01 
National Inst. of Standards and Technology (NCSL), Gaithersburg, MD. Systems and Network Architecture Div

Federal Implementation Guideline for Electronic Data Interchange: ASC X12 003050 Transaction Set 836 Procurement Notices. Implementation Convention.

Special pub

J. P. Favreau. Feb 96, 43p, NIST/SP-881/9.

Also available from Supt. of Docs. as $\mathrm{SNOO}^{-003-}$ 03400-2. See also PB96-172374.

Keywords: "Telecommunication, "Contract management, "Government procurement, "Federal government, Standards, Computer networks, Information systems, Data processing systems, Purchasing, Acquisition, Contract administration, Implementation, Computer communications, "Electronic Data Interchange, Electric commerce, Standard ASC X12 003050 Transaction Set 836.

This implementation convention provides the information necessary for the user to be able to develop an interface program between the computer application and the ASC X12 translator.

\section{1,828}

PB96-180047 Not available NTIS

National Inst. of Standards and Technology (CSL), Gaithersburg, MD. Information Systems Architecture Div.

Open System Environment Implementors Workshop (OIW); Standardization Role Defined.

Final rept.

J. Hungate. 1996, 2p

Pub. in Open Systems Standards Tracking Report, v5 n2 p2-3, 2 May 96

Keywords: "Computer networks, "Interoperability, "Standardization, Distributed computer systems, Systems management, Integrated systems, Systems engineering, Interfaces, Specifications, Data flow analysis, Reprints, "Open Systems Environment.

The Open System Environment Implementors' Workshop (OIW), cosponsored by NIST and the IEEE, offers a neutral, inexpensive, public-consensus forum that is recognized by national and international standards organizations. The output of this technical work is primarily information technology (IT) profiles, also known as implementors' agreements or functional standards.

\section{Pattern Recognition \& Image Processing}

01,829

PB94-145620 PC A03/MF A01

National Inst. of Standards and Technology (MEL), Gaithersburg, MD. Robot Systems Div.

Reliable Optical Flow Algorithm Using 3-D Hermite Polynomials.

H. Liu, T. H. Hong, M. Herman, and R. Chellappa.

Dec 93, 35p, NISTIR-5333.

Prepared in cooperation with Maryland Univ. Coll., College Park.

Keywords: "Image analysis, Image reconstruction, Scene analysis, Computer vision, Hermite polynomials, Three dimensional, Matrices, Algorithms Theorems, "Optical flow.

Algorithms that attempt to accurately compute optica flow must cope with occlusion, brightness changes, ir regular motion, and the aperture problem. Most optical flow algorithms have tried to overcome one or more of the problems mentioned above. Yet claims abou the reliability of computed flow are still based on ad hoc evaluation schemes. While a perfect algorithm that is tree of all the problems is not yet available, we present a reliable algorithm, which despite all the difficulties, generates the output flow field wherever possible, and associates with the output evaluation metrics that reflect the reliability of the output. The evaluation metrics are complete in the sense that they are theoretically related to the physical phenomena that cause the inherent problems noted above. Our approach to computing optical flow expands the spatio-tempora image in terms of Hermite polynomials and then derives multiple Gaussian smoothed gradient constraint equations, which constitute an overdetermined linea system that can be solved for image flow with a least square method.

\section{1,830}

PB94-168044 PC A06/MF A02

National Inst. of Standards and Technology (NCSL), Gaithersburg, MD. Advanced Systems Div.

Evaluating Form Designs for Optical Character Recognition. M. D. Garris, and D. L. Dimmick. Feb 94, 117p,
NiSTIR-5364.

Keywords: "Optical character recognition, "Human factors engineering, "Forms(Paper), "Taxes, Errors, Performance evaluation, Data bases, NIST(National Institute of Standards and Technology), IRS(Internal Revenue Service)

The National Institute of Standards and Technology (NIST), under the sponsorship of the Internal Revenue Service, has conducted an extensive study of three different redesigned tax forms. The NIST Model Recognition System was used in conjunction with the NIST Scoring Package to generate performance measures at the form, field, and character levels. The analyses of these measures conclude that factors introduced onto forms by the writer are the primary cause of segmentation errors, which are the major source of errors within the recognition system. Analysis shows that $97 \%$ of these segmentation errors can be attributed to factors introduced by the writer. The paper cites three ways in which these types of human factors can be handled so as to increase recognition performance. First, the algorithms and techniques deployed within the system can be improved. Second, the instances of human factors leading to system errors can be detected. Third, writers can be influenced by the design of the form including the layout and structure of the fields. By applying a combination of these three ap proaches, human factors can be dealt with, and the errors made by a form processing system can be effectively reduced to classification errors.

\section{1}

PB94-168051 PC A03/MF A01

National Inst. of Standards and Technology (CSL), Gaithersburg, MD. Advanced Systems Div.

Unconstrained Handprint Recognition Using a Limited Lexicon.

M. D. Garris. Dec 93, 15p, NISTIR-5310.

See also PB94-118213.

Keywords: "Word recognition, "Handwriting, Character recognition, Pattern recognition, Neural nets, Automation, Classification, Lexicography, NIST(National Institute of Standards and Technology).

A word recognition system has been developed at the National Institute of Standards and Technology (NIST) to read free-formatted text paragraphs containin handprinted characters. The system has been developed and tested using samples of handprint from NIST Special Database 1. This database of binary images contains 2,100 different writers' printings of the Preamble to the U.S. Constitution. Each writer was asked to print these sentences in an empty $70 \mathrm{~mm}$ by $175 \mathrm{~mm}$ box. The Constitution box contains no guidelines fo the placement and spacing of the handprinted text, no are there guidelines to instruct the writer where to stop printing one line and to begin the next. This paper discusses the word recognition system in detail.

\section{1,832}

PB94-188711 PC A12/MF AO3

National Inst. of Standards and Technology (CSL), Gaithersburg, MD. Advanced Systems Div.

Second Census Optical Character Recognition Systems Conference.

Final rept. 1993-94.

J. Geist, R. A. Wilkinson, S. Janet, N. W. Larsen, R. M. Klear, M. J. Matsko, C. J. C. Burges, R. Creecy, J. J. Hull, T. P. Vogl, C. L. Wilson, P. J. Grother, and B. Hammond. May $94,266 \mathrm{p}$, NISTIR-5452.

See also PB92-238542.

Keywords: "Meetings, "Optical character recognition *Handwriting, Character recognition, Image processing, Dictionaries, Scoring, Voting, Tests, US Bureau of the Census, NIST(National Institute of Standards and Technology).

The results of the Second Census Optical Characte Recognition (OCR) Systems Conference are described There were two conference tests, one scanned from microfilm, the other from paper. Each test consisted of three thousand miniforms with three answer fields per form. The answers consisted of phases associated with the respondant's occupation.
Twenty-five organizations participated in the conference, but only ten submitted results for scoring by the National Institute of Standards and Technology (NIST). The results from one organization were good enough to suggest that OCR may now be sufficiently well developed to be used in a future Census.

\section{3}

PB94-199106 Not available NTIS

National Inst. of Standards and Technology (NML), Gaithersburg, MD. Gas and Particulate Science Div. Object Finder for Digital Images Based on Multiple Thresholds, Connectivity, and Internal Structures. Final rept.

D. S. Bright. 1989, 120p

Pub. in Jnl. of Computer Assisted Microscopy 1, n4 p307-329 Dec 89.

Keywords: "Image processing, Transmission electron microscopy, Ion microscopy, Electron diffraction, Reprints, "Feature extraction, Blob Splitting Algorithm, Image segmentation, Digital images.

The Blob Splitting Algorithm (BSA), developed for segmenting digital images (Bright 1987a) finds simple objects in micrographs that have troublesome backgrounds. Locating objects can be difficult when they have a wide range of sizes or shapes, when they have fuzzy edges, when they are not clearly separated, or when they do not stand out well from the background. The algorithm works without any preprocessing, filtering, or transforming of the images. It is not sensitive to object width or even to object size or shape, but rather to intensities relative to a local surround. Although computationally expensive, it is robust and is used routinely in our laboratory for locating particles in TEM and ion microscope images, locating spots in electron diffraction patterns, and finding regions of interest in complexparticles.

\section{1,834}

\section{PB94-200029 Not available NTIS}

National Inst. of Standards and Technology (NCSL), Gaithersburg, MD. Advanced Systems Div.

VLSI Architectures for Template Matching and Block Matching.

Final rept.

C. Chakrabarti, and J. JaJa. 1991, $24 \mathrm{p}$.

Grants NSF-DCR-86-00378, NSF-OIR-85-00108

Pub. in Parallel Architectures and Algorithms for Image Understanding, p3-26 1991. Sponsored by National Science Foundation, Arlington, $V A$

Keywords: "Image processing, "Templates, "Blocks, "Computer architecture, Pattern recognition, Parallel processing, Very large scale integration, Systolic arrays, Chips (Electronics), Input output processing, $R e-$ prints.

Image template matching and block matching are representative of many window based tasks in image processing. While the existing architectures for template matching and block matching consist of a systolic array of processors, most of them either ignore the i/ $O$ issues or employ schemes involving frequent offchip memory accesses. We propose a systolic architecture consisting of a linear array of processors which handles the $1 / O$ band width problem efficiently.

\section{1,835}

PB94-203510 PC A03/MF A01

National Inst. of Standards and Technology (MEL),

Gaithersburg, MD. Robot Systems Div.

Certainty Grid to Object Boundary Algorithm.

J. A. Horst, H. M. Huang, and T. M. Tsai. Jun 94 , 16p, NISTIR-5447.

Keywords: "Image processing, "Robotics, Algorithms, Edge detection, Curve fitting, Approximation, Mapping, ${ }^{*} \mathrm{CGOB}\left(\mathrm{C}\right.$ rtainty grid to object boundary), ${ }^{*}$ Certainty grid to object boundary.

An edge linking algorithm is modified for a mobile robot map representation application. Certainty grid 'images' are transformed to an appropriate set of object boundary curves. The latter are expressed as oriented piecewise linear segments. Image processing techniques, such as edge detection, thinning, curve tracing, and linear approximation are employed with various modifications. The most significant modification is a new method for linear curve approximation that is simple accurate, and efficient. The method monitors chord and arc length, and its excellent performance is demonstrated against similar algorithms. The certainty grid to object boundary algorithm is tested against simulated noisy certainty grid maps. 


\section{1,836}

PB94-206281 PC A03/MF A01

National Inst. of Standards and Technology (MEL),

Gaithersburg, MD. Robot Systems Div.

Visual-Motion Fixation Invariant.

D. Raviv, and N. Ozery. Jun 94, 11p, NISTIR-5442.

Grant NSF-IRI-9115939

See also PB92-133016. Prepared in cooperation with Florida Atlantic Univ., Boca Raton. Sponsored by $\mathrm{Na}$ tional Science Foundation, Washington, DC. Directorate for Engineering.

Keywords: *Computer vision, "Three dimensional motion, "Optical flow(Image analysis), Invariance, Three dimensional bodies, Cameras, Rotation, Fixation.

The paper deals with a visual-motion fixation invariant. The authors show that during fixation there is a measurable nonlinear function of optical flow that produces the same value for all points of a stationary environment regardless of the 3-D shape of the environment. During fixated camera motion relative to a rigid object, e.g., a stationary environment, the projection of the fixated point remains (by definition) at the same location in the image, and all other points located on the 3-D rigid object can only rotate relative to the 3-D fixation point. This rotation rate of the points is invariant for all points that lie on the particular environment, and it is measurable from a sequence of images. This new invariant is obtained from a set of monocular images, and is expressed explicitly as a closed form solution. In the paper the authors show how to extract this invariant analytically from a sequence of images using optical flow information, and they present results obtained from real data experiments.

\section{1,837}

\section{PB94-207768 PC A05/MF A01}

National Inst. of Standards and Technology (CSL), Gaithersburg, MD. Advanced Systems Div.

Face Recognition Technology for Law Enforcement Applications.

C. L. Wilson, C. S. Barnes, R. Chellappa, and S. A. Sirohey. Jul 94, 77p, NISTIR-5465.

Keywords: "Face(Anatomy), "Law enforcement, "Pattem recognition, Neural networks, Character recognition, Image processing, Computer aided analysis, tion, Segmented elements, Eigenvectors. Technoc transfer, "Forensic sciences, "Face recognition, National Institute of Standards and Technology.

The goal of the report is to relate existing face recognition technology to law enforcement applications. These applications range from static matching of controlled photographs as in mugshot matching to surveillance video images and have different constraints in terms of complexity of processing requirements and thus present a wide range of different technical challenges. The ongoing research activities have been given a renewed emphasis over the last five years. The existing techniques and systems have been tested on different sets of images of varying complexities. Also, very little synergism exists between studies in psychophysics and the engineering literature. Most importantly, there exist no evaluation or benchmarking studies using large databases with the image quality that anses in law enforcement applications.

\section{1,838}

PB94-217106 PC A04/MF A01

National Inst. of Standards and Technology (CSL), Gaithersburg, MD. Advanced Systems Div.

NIST Form-Based Handprint Recognition System. M. D. Garnis, J. L. Blue, G. T. Candela, P. J. Grother, S. A. Janet, C. L. Wilson, D. L. Dimmick, and J. Geist. Jul 94, 72p, NISTIR-5469.

See also PB94-168044 and PB94-188711

Keywords: "Optical character recognition, "Handwriting, Data structures, Utility routines, Computer software, Feature extraction, UNIX(Operating system), "Handprint, US NIST, CD-ROM, C programming language.

The National Institute of Standards and Technology (NIST) has developed a standard reference formbased handprint recognition system for evaluating optical character recognition. NIST is making this recognition system freely available to the general public on CD-ROM. This is a source code distribution written primarily in $\mathrm{C}$ with two additional utilities having FORTRAN components. Library utilities are provided with the recognition system for conducting form reg- istration, form removal, field isolation, field segmentation, character normalization, feature extraction, character classification, and dictionary-based postprocessing. A host of data structures and low-level utilities are also provided. The recognition system has been successfully compiled and tested on a host of UNIX workstations. This report documents the system in terms of its installation, organization, and functionality.

\section{1,839}

Not available NTIS

National Inst. of Standards and Technology (NEL) Gaithersburg, MD. Robot Systems Div

Real-Time Implementation of a Differential Range Finder.

Final rept.

R. Rangachar, T. Hong, M. Herman, and J. Lupo. 1990, 12p.

Pub. in Proceedings of Society of Photo-Optical Instrumentation Engineers - Real Time Image Processing II, Orlando, FL., April 16-18, 1990, v1295 p211-222.

Keywords: "Optical range finders, "Optical flow(Image analysis), "Real time, Edge detection, Spatial distribution, Temporal distribution, Algorithms, Gradients, Cameras, Motion, Reprints, "Differential range PIPE(Pipelined Image Processing Engine)

A scheme to estimate differential range using optical flow along a known direction is described. The factors affecting the accuracy of results, and various spatial and temporal smoothing algorithms used to increase the accuracy of the method are described. The effect of using edge detectors and a prior knowledge of the environment is considered next. While the former reduces the noise, the latter improves the range discriminability of the method. The authors have implemented the method on a real time, high speed Pipelined Image Processing Engine (PIPE), which processes sixty frames per second. For the PIPE implementation, a horizontally moving camera is used which produces optical flow along a scan line.

\section{1,840}

PC A03/MF A01

National Inst. of Standards and Technology (CSL) Gaithersburg, MD. Advanced Systems Div.

Comparison of FFT Fingerprint Filtering Methods for Neural Network Classification.

C. I. Watson, G. T. Candela, and P. J. Grother. Sep $94,38 p$, NISTIR-5493.

See also PB92-213339 and PB93-184273. Sponsored by Federal Bureau of Investigation, Washington, DC

Keywords: "Fast Fourier transformations, "Neural nets, Data bases, Image enhancement, Pattern recognition, Karhunen-Loeve expansion, Errors, Feature extraction, *Fingerprint classification.

Two types of Fourier Transform based filters are presented and used to enhance fingerprint images for use with a neural network fingerprint classification system developed at the National Institute of Standards and Technology (NIST). With image enhancement the sysem is capable of achieving classification error rates of $8.65 \%$ with $10 \%$ rejects, a 2 percentage point improvement in error rate versus using no fingerprint enhancement. Speed of the filters range from 2 to $9 \mathrm{sec}-$ onds. Classification tests were performed using ridgevalley based feature extraction, Karhunen Loeve transform, and a Probabilistic Neural Network (PNN) classifier. The testing method used differs from past reports because no rolling of the same print is allowed to appear in both the training and testing set used by the Neural Network classifier.

01,841

PB95-143285 PC A03/MF A01

National Inst. of Standards and Technology (MEL). Gaithersburg, MD. Intelligent Systems Div.

Visual Pursuit Systems.

J. C. Fiala, and A. J. Wavering. Oct 94, 21p, NISTIR5513.

Also available from Supt. of Docs. Prepared in cooperation with Boston Univ., MA. Dept. of Cognitive and Neural Systems.

Keywords: "Computer vision, "Optical tracking, Systems analysis, Predictions, Bandwidth, Image processing, Algorithms, Kinematics, "Visual pursuit systems, TRICLOPS system.

Visual pursuit systems are reviewed using a generalzed form with the principal delay in the visual feedback pathway. The performance of several different control methods are compared through analysis of the tracking error. A high-performance active vision system, called TRICLOPS, is used to obtain experimental data on the tracking performance of the various methods, some of which had previously only been tested through simulation. Image processing delay is shown to be the primary limiting factor in performing high-speed tracking. Prediction is necessary to achieve high performance tracking, and the effectiveness of prediction depends on several factors, including the coordinate system used for motion modeling and the noise content of the target motion signal. It is demonstrated that, using relatively simple tracking algorithms which incorporate prediction with an advanced robotic device like in the domain of visual pursuit of simple targets.

01,842

PB95-151932 Not available NTIS

National Inst. of Standards and Technology (NML), Gaithersburg, MD. Ionizing Radiation Div.

Tomographic Decoding Algorithm for a Nonoverlapping Redundant Array.

Final rept.

L. I. Yin, and S. M. Seltzer. 1993, 10p.

Sponsored by Department of Energy, Washington, DC

Pub. in Applied Optics 32, n20 p3726-3735, 10 Jul 93.

Keywords: "Image reconstruction, "Tomography, X-ray imagery, Computerized simulation, Pinhole cameras, Three dimensional, Algonithms, Reprints, "Image decoding.

A tomographic reconstruction algorithm is developed for the nonoverlapping redundant array $x$-ray imaging system whereby the background contributions from out-of-focus planes can be eliminated. The algorithm makes use of two constraints derived from the physical characteristics of the nonoverlapping redundant array system in tandem with the correlation decoding process. It is simple, direct, and noniterative. Tomographic images of computer-generated planar and three-dimensional objects are provided to illustrat $\theta$ the effectiveness of the algorithm.

01,843

PB95-153003 Not available NTIS

National Inst. of Standards and Technology (CAML), Gaithersburg, MD. Applied and Computational Mathematics Div.

Image Restoration and Diffusion Processes.

Final rept.

A. S. Carasso. 1993, 12p.

Pub. in Proceedings of Society of Photo-Optical Instrumentation Engineers: Mathematical Methods in Medical Imaging, San Diego, CA., July 15-16, 1993, v2035 p255-266.

Keywords: "Image restoration, "Image reconstruction, Noise reduction, Diffusion theory, Reprints, TikhonovMiller restoration, SEB restoration, III posed problems, Image deblurring.

A new supplementary a priori constraint, the slow evolution from the boundary constraint, (SEB) sharply reduces noise contamination in a large class of spaceinvariant image deblurring problems that occur in medical, industrial, surveillance, environmental, and astronomical applications. The noise suppressing properties of SEB restoration can be proved mathematically, on the basis of rigorous error bounds for the reconstruction, as a function of the noise level in the blurred image data. This analysis proceeds by reformulating the image deblurring problem into an equivalent ill-posed problem for a time-reversed diffusion equation. The SEB constraint does not require smoothness of the image. An effective, fast, non-iterative procedure, based on FFT algorithms, may be used to compute SEB restorations. For a $512 \times 512$ image, the procedure requires about 45 seconds of cpu time on a Sun/sparc2. A documented deblurring experiment, on an image with significant high frequency content, illustrates the computational significance of the SEB constraint by comparing SEB and Tikhonov-Miller reparameters.

01844

PB95-161808 Not available NTIS

National Inst. of Standards and Technology (NEL), Gaithersburg, MD. Robot Systems Div. 
Real Time Differential Range Estimation Based on Time-Space Imagery Using PIPE.

Final rept.

R. Rangachar, T. H. Hong, M. Herman, R. Luck, and J. Lupo. 1990, 10p.

Pub. in Proceedings of Society of Photo-Optical Instrumentation Engineers: Real-Time Image Processing II, Orlando, FL., April 16-18, 1990, v1295 p247-256.

Keywords: "Optical flow(Image analysis), "Image processing, Spatial resolution, Temporal resolution, Pipelining(Computers), Edge detection, Gradients, Reprints, PIPE(Pipelined Image Processing Engine).

The paper examines spatio-temporal methods of image flow and shows how differential range can be estimated from time-space imagery. We consider one scan line of the image obtained from a camera moving in the horizontal direction. At the next instant of time, we shift the previous line up by one pixel, and obtain another line from the image. We continue the procedure to obtain a time-space image, where each horizontal line represents the spatial relationship of the pixels, and each vertical line the temporal relationship. Each feature along the horizontal scan line will generate an edge in the time-space image, the slope of which depends upon the distance of the feature from the camera. We apply two mutually perpendicular edge operators to the time-space image, and determine the slope of each edge. We show that this corresponds to optical flow. We use the result to obtain the differential range, and show how this can be implemented on the Pipelined Image Processing Engine (PIPE). We discuss several kinds of edge operators, show how using the zero crossings reduces the noise, and demonstrate how better discrimination can be achieved by knowing the range we are interested in.

01,845

PB95-163994 Not available NTIS

National Bureau of Standards (ICST), Gaithersburg, MD. Advanced Systems Div.

Self-Organizing Neural Network Character Recognition on a Massively Parallel Computer.

Final rept.

C. L. Wilson, R. A. Wilkinson, and M. D. Garris.

1990, $5 p$.

Pub. in Proceedings of International Joint Conference on Neural Networks, San Diego, CA., June 17-21, 1990, p325-329.

Keywords: "Neural nets, "Character recognition, "Massively parallel processors, Array processors, Image processing, Self organizing systems, Algorithms, Accuracy, Reprints.

Two neural network based methods are combined to develop font independent character recognition on a distributed array processor. Feature localization and noise reduction are achieved using least squares optimized Gabor filtering. The filtered images are then presented to an ART-1 based learning algorithm which produces self-organizing sets of neural network weights used for character recognition. Implementation of these algorithms on a highly parallel computer with 1,024 processors allows high speed character recognition to be achieved in $8 \mathrm{~ms} / \mathrm{image}$ with greater than $99 \%$ accuracy on machine print and $80 \%$ accuracy on unconstrained hand printed characters.

01,846

PB95-164075 Not available NTIS

National Inst. of Standards and Technology (EEEL), Gaithersburg, MD. Electronics and Electrical Engineering Lab. Office.

Physics-Based Vision: Principles and Practice, Shape Recovery (Book Review).

Final rept.

J. A. Worthey. 1993, 3p

Pub. in Color Research and Application 18, n3 p221223 Jun 93.

Keywords: "Computer vision, Stereoscopic vision, Multisensor fusion, Specular reflection, Photometry, Shapes, Reprints, "Shape recovery, "Machine vision.

Shape Recovery is a useful compilation of recent papers in the machine vision specialty of 'shape from shading'.

01,847

PB95-171096 PC A03/MF A01

National Inst. of Standards and Technology (MEL),

Gaithersburg, MD. Intelligent Systems Div.
General Motion Model and Spatio-Temporal Filters for Computing Optical Flow.

$H$. Liu, T. H. Hong, M. Herman, and R. Chellappa. Nov $94,50 p$, NISTIR-5539.

Prepared in cooperation with Maryland Univ., College Park.

Keywords: Image motion compensation, Image filters, Image analysis, Mathematical models, Three dimen*Image motion analysis, "Optical flow.

Traditional optical flow algorithms assume local image translational motion and apply simple image filtering Recent studies have taken two separate approaches toward improving the accuracy of computed flow: the application of spatio-temporal filtering schemes and the use of generalized motion models such as the affine model. Each has achieved some improvement over traditional algorithms in specialized situations. In this paper, we analyze the interdependency between them and propose a unifed approach. The general motion model we adopt characterizes arbitrary 3-D steady motion. Under perspective projection, we derive an mage motion equation that describes the spatio-temporal relation of gray-scale intensity in an image sequence, thus making the utilization of 3-D filtering possible. However, to accommodate this complex motion we need to extend the filter design to derive additional motion constraint equations. Using Hermite polynominals, we design differentiation filters, whose orthogonality and Gaussian derivative properties insure numerical stability. The resulting algorithm produces accurate optical flow and other useful motion parameters. It is evaluated quantitatively using the scheme established by Barron, et al. and qualitatively with real images.

\section{1,848}

PB95-171971 PC A03/MF A01

National Inst. of Standards and Technology (CSL), Gaithersburg, MD. Advanced Systems Div.

Binary Decision Clustering for Neural Network Based Optical Character Recognition.

C. L. Wilson, P. J. Grother, and C. S. Barnes. Dec 94, 23p, NISTIR-5542.

See also PB92-238542 and PB94-217106.

Keywords: *Optical character recognition, "Neural nets, "Handwriting, Pattern recognition, Dynamical systems, Image processing, Karhunen-Loeve expansion, Errors, BDMs(Binary Decision Machines).

A multiple neural network system for handprinted character recognition is presented. It consists of a set of input networks which discriminate between all two class pairs, and an output network which takes the signals from the input networks and yields a digit recognition decision. For a ten digit classification problem this requires forty-five binary decision machines in the input network. The output stage is typically a single trained network. The neural network paradigms adopted in these input and output networks are the multi-laye perceptron, the radial basis function network, and the probabilistic neural network. A simple majority vote rule was also tested in place of the output network.

\section{1,849}

PB A03-189502 A01

National Inst. of Standards and Technology (MEL), Gaithersburg, MD. Intelligent Systems Div.

Motion-Model-Based Boundary Extraction.

H. Liu, T. H. Hong, M. Herman, and R. Chellappa. Jan $95,29 p$, NISTIR-5587.

Presented at International Conference on Computer Vision, Cambridge, MA., June 20-23, 1995. Prepared in cooperation with Maryland Univ., College Park.

Keywords: "Computer vision, "Motion, "Edge detection, Algorithms, Flow, Iteration, Mathematical models, Pattern recognition, Three dimensional models, Segmentation.

Motion boundary extraction and optical flow computation are two subproblems of the motion recovery problem that cannot be solved independently of each other They represent the most common dilemma in motion research. A popular approach uses an iterative scheme that consists of motion boundary extraction and optical flow computation components and refines each result through iteration. This approach is typically time-consuming and sometimes does not converge. We present a local, noniterative algorithm that extracts motion boundaries and computes optical flow simultaneously. This is achieved by modeling a 3-D image intensity block with a general motion model that pre- sumes locally coherent motion. Local motion coherence, which is measured by the fitness of the motion model, is the criterion we use to determine whether motion should be estimated or otherwise motion boundaries should be located. The motion boundary extraction algorithm is evaluated quantitatively and qualitatively against other existing algorithms in a scheme originally developed for edge detection. The results show

01,850

PB95-251724 PC A03/MF A01

National Inst. of Standards and Technology (CSL), Gaithersburg, MD. Advanced Systems Div.

Method and Evaluation of Character Stroke Preservation on Handprint Recognition.

M. D. Garris. Jul 95, 32p, NISTIR-5687

Keywords: *Optical character recognition "Handwriting, Artificial intelligence, Image processing Pertormance evaluation, Statistical analysis, Algorithms, Edge detection, Automation

A new technique for intelligent form removal has been developed along with a new method for evaluating its impact on optical character recognition (OCR). The form removal technique automatically detects the dominant lines in an image and erases them while preserving as much of the overlapping character strokes as possible. This method of form removal relaxes the recognition systems dependence on rigid form design, printing, and reproduction by automatically detecting and removing some of the physical structures (lines) on the form. The line detection and removal technique operates on loosely defined zones in which no image deskewing is pertormed. The technique was tested on a large number of randomly-ordered handprinted lowercase alphabet fields, as these letters (especially those with descenders) frequently touch and extend through the line along which they are written. It is shown that intelligent form removal can improve lowercase recognition by as much as $3 \%$, but this net increase in performance is insufficient to understand the inpact on the recognition. There is expected to be trade-offs with the introduction of any new technique into a complex recognition system. A new statistical analysis was designed to evaluate the impact of intelligent line removal on OCR. The evaluation method compares the statistical distributions of individual confusion pairs between two systems and automatically determines the significant improvements and the significant losses in performance. In order for system developers to continue to reduce error rates, sophisticated analyses like this become necessary to understand the real impact a modification has on recognition performance. The statistical analysis presented in the paper was used to evaluate the new line removal technique, and the results are reported.

01,851

PB95-267803 PC A03/MF A01

National Inst. of Standards and Technology (CSL). Gaithersburg, MD. Advanced Systems Div.

Improving Neural Network Performance for Character and Fingerprint Classification by Altering Network Dynamics.

C. L. Wilson, J. L. Blue, and O. M. Omidvar. Aug 95 12p, NISTIR-5695.

See also PB95-171971

Keywords: "Optical character recognition, "Neura nets, Self organizing systems, Optimization, Conjugate gradient method, Iteration, Handwriting. Periormance evaluation, Machine learning, Image processing, Pattern recognition, Reprints, "Fingerprint classification, PNN(Probabilistic Neural Network), MLPS(Multilaye Perceptrons)

The paper shows that performance equal to or bette than the Probabilistic Neural Network (PNN) can be achieved with a single three-layer Multilayer Perceptron (MLP) by making fundamental changes in the network optimization strategy. These changes are: (1) Neuron activation functions are used which reduce the probability of singular Jacobians; (2) Successive regularization is used to constrain volume of the weigh space being minimized; (3) Boltzmann pruning is used to constrain the dimension of the weight space; and (4) Prior class probabilities are used to normallze al error calculations so that statistically significant sam ples of rare but important classes can be Included without distortion of the error surface. All four of these changes are made in the inner loop of a con ugate gra dient optimization iteration and are Intended to simplify 


\section{Pattern Recognition \& Image Processing} the training dynamics of the optimization. On
handprinted digits and fingerprint classification problems these modifications improve error-reject performance by factors between 2 and 4 and reduce network size by $40 \%$ to $60 \%$.

\section{1,852}

PB95-267845 PC A03/MF A01

National Inst. of Standards and Technology (CSL), Gaithersburg, MD. Advanced Systems Div.

Effect of Training Dynamics on Neural Network Performance.

C. L. Wilson, J. L. Blue, and O. M. Omidvar. Aug 95, 32p, NISTIR-5696.

Prepared in cooperation with District of Columbia Univ., Washington. Dept. of Computer Science.

Keywords: "Neural nets, "Machine learning, "Optical character recognition, Self organizing systems, Optimization, Dynamical systems, Conjugate gradient method, Iteration, Handwriting, Pattern recognition, Image processing, Mathematical models, Fingerprint classiPerceptrons) PNN(Probabilistic Neural Network).

In this paper, analysis of a simple model of recurrent network dynamics is used to gain qualitative insights into the training dynamics of multilayer perceptrons (MLPs). These insights allow the training methods used by MLPS to be modified to significantly improve network performance. We will show that performance equal to or better than the Probabilistic Neural Network (PNN) can be achieved with a single three-layer MPL by making fundamental changes in the network optimization strategy. These changes are: (1) Neuron activation functions are used which reduce the probability of singular Jacobians; (2) Successive regularization is used to constrain the volume of the minimized weight space; (3) Boltzmann pruning is used to constrain the dimension of the weight space; and (4) Prior class probabilities are used to normalize all error calculations so that statistically significant samples of rare but important classes can be included without distorting the error surface. All four of these changes are made in the inner loop of a conjugate gradient optimization iteration and are intended to simplify the training dynamics of the optimization. On handprinted digits and fingerprint classification problems, these modifications improve error-reject performace by factors between 2 and 4 and reduce network size by $40 \%$ to $60 \%$.

01,853

PB95-267936 PC A03/MF A01

National Inst. of Standards and Technology (CSL)

Gaithersburg, MD. Advanced Systems Div.

PCASYS: A Pattern-Level Classification Automation System for Fingerprints.

G. T. Candela, P. J. Grother, C. I. Watson, R. A.

Wilkinson, and C. L. Wilson. Aug 95, 46p, NISTIR

5647.

Keywords: *Pattern recognition, *Automation, Image processing, Data base management, Image classification, Image enhancement, Feature extraction, Neural nets, Algorithms, Machine learning, Accuracy, "Fingerprint classification, PNN(Probabilistic Neutral Network), AFIS(Automated Fingerprint Identification System)

This report describes a system we have developed that automatically classifies images of fingerprints into six pattern-level classes. Our program takes gray-level images of fingerprints as input, and for each fingerprint it produces a hypothesized classification as arch, left loop, right loop, scar, tented arch, or whorl, as well as a number indicating how much confidence should be assigned to its classification decision. The system performs these processing steps: image segmentation image enhancement; feature extraction; registration; application of a linear transform that both applies a pattern of regional weights and reduces dimensionality; running of a main classifier, which is a Probabilistic Neural Net, and of an auxiliary whorl-detecting classi fier that traces and analyzes pseudoridges (approximate trajectories through the ridge flow); and finally, the combining of the outputs of the main and auxiliary classifiers so as to decide on a hypothesized class and a confidence level.

\section{1,854}

PB96-111687 Not available NTIS

National Inst. of Standards and Technology (CSL) Gaithersburg, MD. Advanced Systems Div.
Human and Machine Recognition of Faces: A Survey.

Final rept.

R. Chellappa, C. L. Wilson, and S. Sirohey. 1995

$36 \mathrm{p}$

Pub. in Proceedings of Institute of Electrical and Electronics Engineers, v83 n5 p705-740 May 95.

Keywords: "Computer vision, "Visual perception, *Humans, "Pattern recognition, "Photographic images, Face(Anatomy), Optical images, Feature extraction, Image processing, Computer aided analysis, Identification systems, Psychophysics, Reprints.

The goal of this paper is to present a critical survey of existing literature on human and machine recognition of faces. Machine recognition of faces has several applications, ranging from static matching of controlled photographs, as in mug shots matching and credit card verification to surveillance video images. Over the las 20 years researchers in psychophysics, neural sciences and engineering, image processing, analysis and computer vision have investigated a number of is sues related to face recognition by humans and machines. Ongoing research activities hvae been given renewed emphasis over the last five years. Existing techniques and systems have been tested on different sets of images of varing complexities.

\section{1,855}

PB96-119797 Not available NTIS

National Inst. of Standards and Technology (CSL), Gaithersburg, MD. Advanced Systems Div.

Self-Organizing Neural Network Character Recognition Using Adaptive Filtering and Feature Extraction.

Final rept

C. L. Wilson, R. A. Wilkinson, and M. D. Garris

$1995,24 p$

Pub, in Progress in Neural Networks, Chapter 10, v3 p295-318 1995.

Keywords: "Feature extraction, "Character recognition, "Neural networks, Image processing, Pattern recogntion, Adaptive features, Machine learning, Compute architecture, Massively parallel processors, Optical filters, Data management, Algorithms, Accuracy, Reprints.

Neural network methods show great promise for providing highly accurate, noise resistant, parallel algo viding highly accurate, noise resistant, parallel algoOne specific area of image recognition, the conversion of images of hand written and machine print characters to computer representation, has been studied in detail in the past. Both special purpose hardware and software approaches have been used on the character recognition problem with promising results. The present work addresses the problem of using a specific class of computer architecture, an array of 1024 processors arranged in a $32 \times 32$ grid and operated in a parallel mode, as a neural network character recognition device.

01,856

PB96-123195 Not available NTIS

National Inst. of Standards and Technology (CSL), Gaithersburg, MD. Advanced Systems Div.

Improving Neural Network Performance for Character and Fingerprint Classification by Altering Network Dynamics.

Final rept.

C. L. Wilson, J. L. Blue, and O. M. Omidvar. 1995

Sp. also PB95-267803.

Pub. in World Congress on Neural Networks Proceed ings II, Washington, DC., July 20, 1995, p151-158.

Keywords: "Optical character recognition, "Neural nets, Self organizing systems, Optimization, Conjugate gradient method, Heration, Handwriting, Performance evaluation, Machine learning, Image processing, Pattern recognition, Reprints, "Fingerprint classification, PNN(Probabilistic Neural Network), MLPs(Multilayer Perceptions).

In this paper, the authors will show that performance equal to or better than PNN can be achieved with a single three-layer MLP by making fundamental changes in the network optimization strategy. These changes are: (1) Neuron activation functions are used which reduce the probability of singular Jacobians; (2) Successive regularization is used to constrain volume of the weight space being minimized; (3) Boltzmann pruning is used (4) to constrain the dimension of the weight space; and (4) Prior class probabilities are used to normalize all error calculations so that statistically significant samples of rare but important classes can be included without distortion of the error surface. All four of these changes are made in the inner loop of a conjugate gradient optimization iteration (5) and are intended to simplify the training dynamics of the optimization.

01,857

PB96-186184 Not available NTIS

National Inst. of Standards and Technology (CSL), Gaithersburg, MD. Advanced Systems Div.

Binary Decision Clustering for Neural-Network Based Optical Character Recognition.

Final rept.

C. L. Wilson, P. J. Grother, and C. S. Barnes. 1995 $13 p$.

See also PB95-171971

Pub. in Pattern Recognition, v29 n3 p425-437 Jul 96.

Keywords: "Optical character recognition, "Neura nets, "Handwriting, Pattern recognition, Dynamica systems, Image processing, Karhunen-Loeve expansion, Errors, Reprints, BDMs(Binary Decision Machines).

A multiple neural network system for handprinted cha racter recognition is presented. It consists of a set of input networks which discriminate between all-twoclass pairs, for example ' 1 ' from ' 7 ' and an output network which takes the signals from the input networks and yields a digit recognition decision. For a ten-digit classification problem this requires 45 binary decision machines in the input network. The output stage is typically a single trained network. The neural network paradigms adopted in these input and output networks are the multi-layer perceptron, the radial-bias function network and the probabilistic neural network. A simple majority vote rule was also tested in place of the output network The various resulting digit classifiers were trained on 7480 isolated images and tested on a disjoint set of size 32140 . The Karhunen-Loeve transforms of the images of each pair of two classes formed the training set for each BDM. Several different combinations of neural network input and output structures gave similar classification performance. The minimum error rate achieved was $2.5 \%$ with no rejection obtained by comining a PNN input array with an RBF output stage. This combined network has an error rate of $0.7 \%$ with $10 \%$ rejection.

01,858

\section{PB96-191374 PC A03/MF A01}

National Inst. of Standards and Technology (CSL), Gaithersburg, MD. Advanced Systems Div. Generalized Form Registration Using StructureBased Techniques.

M. D. Garris, and P. J. Grother. Apr 96, 16p, NISTIR5726

See also PB94-217106.

Keywords: "Forms(Paper), "Optica! character recognition, "Handwriting, Data structures, Utility routines Computer software, Databases, Image processing, Automation, Registration, Feature extraction, US NIST.

A new method for registering forms has been develA ned the National Institute of Standards and Technology. This method automatically estimates the amount of rotation and translation in the image withou any detailed knowledge of the form. This is accomplished through the automatic detection of dominant vertical and horizontal structures (lines) commonly found in forms. A general method for rotation estimation and a robust method for translation estimation are presented. Results demonstrate that this technique is extremely tolerant to spurious annotations on the form and scanner noise in the image, and the computational requirements of the utility can be tuned by optionally choosing to process and analyze downsampled versions of the image. All 3,669 Handwriting Sample Forms distributed with NIST Special Database 19 were successfully registered with the new technique, and using the same code, 255 uniformly laid out IRS tax forms and 500 Census miniforms were also tested and registered. Every type of form contained in the numerous NIST (public) form databases can be registered using this technique. These results also demonstrate how easy it is to set up the computer to register new types of forms, introducing a set-up interface that is much more automated and less tedious than what is currently required to specify new forms for the NIST public domain Form-Based Handprint Recognition System. 
01,859

PB96-193669 PC A03/MF A01

National Inst. of Standards and Technology (CSL), Gaithersburg, MD. Information Access and User Interface Div.

Component-Based Handprint Segmentation Using Adaptive Writing Style Model.

M. D. Garris. Jun 96, 29p, NISTIR-5843.

Keywords: "Optical character recognition, *Handwriting, "Data structures, Feature extraction, Image processing, Forms(Paper), Statistical analysis, Classifying, Databases, Expert systems, *Handprint, Character segmentation.

Building upon the utility of connected components, NIST has designed a new character segmentor based on statistically modeling the style of a person's handwriting. Simple spatial features (the thickness of the pen stroke and the height of the handwriting) capture the characteristics of a particular writer's style of handprint, enabling the new method to maintain a traditional character-level segmentation philosophy without the integration of recognition or the use of oversegmentation and linguistic postprocessing. Estimates for stroke width and character height are used to compute aspect ratio and standard stroke count features that adapt to the writer's style at the field level. The new method has been developed with a predetermined set of fuzzy rules making the segmentor much less fragile and much more adaptive, and the new method successfully reconstructs fragmented characters as well as splits touching characters. The new segmentor was integrated into the NIST public domain Form-Based Handprint Recognition System and then tested on a set of 490 Handwriting Sample Forms found in NIST Special Database 19. When compared to a simple component-based segmentor, the new adaptable method improved the overall recognition of handprinted digits by $3.4 \%$ and field level recognition by $6.9 \%$, while effectively reducing deletion errors by $82 \%$. The same program code and set of parameters successfully segments sequences of uppercase and lowercase characters without any context-based tuning. While not as dramatic as digits, the recognition of uppercase and lowercase characters improved by $1.7 \%$ and $1.3 \%$ respectively. The segmentor maintains a relatively straightforward and logical process flow avoiding convolutions of encoded exceptions as is common in expert systems. As a result, the new segmentor operates very efficiently, and throughout as high as 362 characters per second can be achieved. Letters and numbers are constructed from a predetermined configuration of a relatively small number of strokes. Results in the paper show that capitalizing on this knowledge through the use of simple adaptable features can significantly improve segmentation, whereas recognition-based and oversegmentation methods fail to take advantage of these intrinsic quality of handprinted characters.

01,860

PB96-195524 PC A04/MF A01

National Inst. of Standards and Technology (EEEL), Gaithersburg, MD.

Specification for Interoperability between Ballistic Imaging Systems. Part 1. Cartridge Cases.

B. F. Field, E. F. Kelley, and R. M. McCabe. Jun 96, $44 \mathrm{p}$, NISTIR-5855.

Keywords: "Cartridges(Explosives), *Guns, "Identification, *Image processing, Ammunition, Crimes, Pattern recognition, Computer equipment Interoperability, Databases, Specifications, Optical equipment, "Forensics.

To facilitate interoperability between existing ballistic imaging systems, the Office of National Drug Control Policy (ONDCP), the Federal Bureau of Investigation (FBI), and the Bureau of Alcohol, Tobacco, and Firearms (ATF) executed a Memorandum of Understanding that recognized, in part, that it is imperative that the Drugfire and IBIS Ballistic Imaging Systems are interoperable. Further, the National Institute of Standards and Technology (NIST) was asked to provide technical assistance in this effort. This document is the first of a series of planned documents that will specify the hardware and software requirements to permit interoperability between the Drugfire and IBIS systems as specified in the MOU. This specific document provides a complete specification of hardware requirements for capture of cartridge image and ancillary data for each system, and the data exchange formats required for transmitting and processing information requests.
01,861

PB96-210703 PC A07/MF AO2

Maryland Univ., College Park. Dept. of Electrical Engineering.

General Motion Model and Spatio-Temporal Filters for 3-D Motion Interpretations.

$H$. Liu. Nov $95,122 p$, NISTIR- 5763 .

Sponsored by National Inst. of Standards and Technology (MEL), Gaithersburg, MD. Intelligent Systems Div.

Keywords: "Image motion compensation, Image filters, Image analysis, Algorithms, Three dimensional, Hermite polynomials, Mathematical models, Matrices(Mathematics), Theorems, "Image motion analysis, ${ }^{*}$ Optical flow.

Motion cues are a rich source of visual information. Obect boundaries due to motion parallax, perception of collision, and transparency provide crucial information to any mobile vision system, biological or robotic. This report presents the formulation, design, evaluation and implementation of a motion algonithm which accurately and efficiently interprets the above motion cues.

\section{Magnetic Detection}

01,862

PB95-175667 Not available NTIS

National Inst. of Standards and Technology (EEEL), Boulder, CO. Electromagnetic Fields Div.

Gradiometer Antennas for Detection of Long Subsurface Conductors.

Final rept.

D. A. Hill. 1994, 12p.

Sponsored by Army Belvoir Research Development and Engineering Center, Fort Belvoir, VA.

Pub. in Jnl. of Electromagnetic Waves and Applications 8, n2 p237-248 1994.

Keywords: "Gradiometers, "Magnetic detection, *Electromagnetic measurement, Electrical impedance, Antennas, Boreholes, Magnetic dipoles, Magnetic fields, Tunnels, Electromagnetic wave transmission, Reprints, *Subsurface conductors.

The use of gradiometer antennas for detection of long conductors in tunnels is analyzed. For reception in vertical boreholes, the gradiometer consists of two vertica magnetic dipoles with a vertical separation. The source is a vertical magnetic dipole located in an adjacent vertical borehole. Both sum and difference responses are useful, but the difference response has the potential advantage of suppressing the primary field and making the scattered field easier to detect. The difference response is most effective in suppressing the primary field for a parallel scan where the transmitting antenna and receiving gradiometer are always at the same height.

\section{Optical Detection}

\section{1,863}

PB96-177381 PC A07/MF A02

National Inst. of Standards and Technology, Gaithersburg, MD.

Journal of Research of the National Institute of Standards and Technology, March/April 1996. Volume 101, Number 2.

1996, 105p.

See also PB96-179114 through PB96-179163 and PB96-175666. Also available form Supt. of Docs. as SN703-027-00069-5.

Keywords: "Calibration, "Cryogenic radiometers, "Quartz-halogen lamps, "Gravimetry, "Computer arithmetic, "Quantized dissipation, Absolute spectral re- sponse, Color temperature, Lamp orientation, Human serum, Definitive method, Candela, Breakdown, Re prints.

Contents:

The NIST Detector-Based Luminous Intensity Scale;

The NIST High Accuracy Scale for Absolute Spectral Response from $406 \mathrm{~nm}$ to $920 \mathrm{~nm}$;

Irradiance of Horizontal Quartz-Halogen Standard Lamps;

Development of the Ion Exchange-Gravimetric Method for Sodium in Serum as a Definitive Method;

The MasPar MP-1 as a Computer Arithmetic Laboratory;

Evidence That Voltage Rather Than Resistance is Quantized in Breakdown of the Quantum Hall Effect.

01,864

PB96-179114 (Order as PB96-177381, PC A07)

MF A02)

National Inst. of Standards and Technology, Gaithersburg, MD.

NIST Detector-Based Luminous Intensity Scale.

C. L. Cromer, G. Eppeldauer, J. E. Hardis, A. C.

Parr, T. C. Larason, and Y. Ohno. 1996, 24p.

Included in Jnl. of Research of the National Institute of Standards and Technology, v101 n2 p109-132 Mar/ Apr 96.

Keywords: "Calibration, "Candela, Illuminance, Lumen, Luminous intensity, Lux, Measurement, Photometers, Scale, Standards, Units, Reprints.

The Systeme International des Unites (SI) base unit for photometry, the candela, has been realized by using absolute detectors rather than absolute sources This change in method permits luminous intensity calibrations of standard lamps to be carried out with a relative expanded uncertainty (coverage factor $\mathrm{k}=2$, and thus a 2 standard deviation estimate) of $0.46 \%$ almost a factor-of-two improvement. A group of eight reference photometers has been constructed with silicon photodiodes, matched with filters to mimic the spectral luminous efficiency function for photopic vision. The wide dynamic range of the photometers aid in their calibration. The components of the photometers were carefully measured and selected to reduce the sources of error and to provide baseline data for aging studies Periodic remeasurement of the photometers indicate that a yearly recalibration is required. The design, characterization, calibration, evaluation, and application of the photometers are discussed.

01,865

PB96-179122 (Order as PB96-177381, PC A07/ MF A02)

National Inst. of Standards and Technology, Gaithersburg MD.

NIST High Accuracy Scale for Absolute Spectral Response from $406 \mathrm{~nm}$ to $920 \mathrm{~nm}$.

T. C. Larason, S. S. Bruce, and C. L. Cromer. 1996, 8p.

Included in Jnl. of Research of the National Institute of Standards and Technology, v101 n2 p133-140 Mar/ Apr 96.

Keywords: *Absolute spectral response, *Cryogenic radiometers, Light-trapping detectors, Measurements Optical power, Scale, Silicon photodiode, Quantum efficiency, Quality system, Reprints.

The authors describe how the National Institute of Standards and Technology obtains a scale of absolute spectral response from $406 \mathrm{~nm}$ to $920 \mathrm{~nm}$. This scale of absolute spectral response is based solely on detector measurements traceable to the NIST High Accuracy Cryogenic Radiometer (HACR). Silicon photodiode light-trapping detectors are used to transfer optical power measurements from the HACR to a monochromator-based facility where routine mea surements are performed. The transfer also involves modeling the quantum efficiency (QE) of the silicon photodiode light-trapping detectors. The authors describe their planned quality system for these measurements that follows ANSI/NCSL Z540-1-1994. A summary of current NIST capabilities based on these measurements is also given.

01,866

PB96-179130

(Order as PB96-177381, PC A07)

MF A02)

National Inst. of Standards and Technology, Gaithersburg, MD. 


\section{Optical Detection}

Irradiance of Horizontal Quartz-Halogen Standard Lamps.

E. A. Early, and A. Thompson. 1996, 13p.

Included in Jnl. of Research of the National Institute of Standards and Technology, v101 n2 p141-153 Mar' Apr 96.

Keywords: "Color temperature, "Lamp orientation, "Quartz-halogen lamps, Spectral irradiance, Standard lamps, Ultraviolet, Reprints.

Spectral irradiance calibration often require that irradiance standard lamps be oriented differently than the normal calibration orientation used at the National Institute of Standards and Technology and at other standards laboratories. For example, in solar measurements the instruments are generally upward viewing requiring horizontal working standards for minimization of irradiance calibration uncertainties. To develop a working standard for use in a solar ultraviolet intercomparison. NIST determined the irradiance of quartz-halogen lamps operating in the horizontal position, rather than in the customary vertical position. An experimental technique was developed which relied upon equivalent lamps with independent mounts for each orientation and a spectroradiometer with an integrating sphere whose entrance port could be rotated 90 degrees to view either lamp position.

\section{1,867}

PB96-179148 (Order as PB96-177381, PC A07/

National Inst. of Standards and Technology, Gaithersburg, MD.

Development of the Ion Exchange-Gravimetric Method for Sodium in Serum as a Definitive Method.

J.R. Moody, and T. W. Vetter. 1996, 10p.

Included in Jnl. of Research of the National Institute of Standards and Technology, v101 n2 p155-164 Mar/ Apr 96.

Keywords: "Human serum, "Gravimetry, "Definitive method, Accuracy, Instrumental determination, Ion-exchange, Repeatability, Sodium, Uncertainty, Reprints

An ion exchange-gravirnetric method, previously developed as a National Committee for Clinical Labora tory Committee for Clinical Laboratory Standards (NCCLS) reference method for the determination of sodium in human serum, has been re-evaluated and improved. Sources of analytical error in this method have been examined more critically and the overall uncertainties decreased. Additionally, greater accuracy and repeatability have been achieved by the application of this definitive method to a sodium chloride reference material.

01,868

PB96-179163 (Order as PB96-177381, PC A07) MF A02)

National Inst. of Standards and Technology, Gaithersburg, MD.

Evidence That Voltage Rather Than Resistance is Quantized in Breakdown of the Quantum Hall Effect.

M. E. Cage. 1996, 6p.

Included in Jnl. of Research of the National Institute of Standards and Technology, v101 n2 p175-180 Mar/ Apr 96.

Keywords: "Breakdown, *Quantized dissipation, Resistance states, Voltage states, Hall effect, Level scattering, Electron gas, Reprints.

Quantized longitudinal voltage drops are observed along a length of a GaAs/AIGaAs heterostructure quantum Hall effect device at applied currents large enough for the device to be in the breakdown regime. The range of currents is extensive enough to demonstrate that it is the longitudinal voltage that is quantized, rather than the longitudinal resistance. A blackbox and a quasi-elastic inter-Landau level scattering (QUILLS) model are then employed to calculate the fraction of electrons making transitions into higher Landau levels, the transition rates, and the maximum electric field across the device.
Developing Quality System Documentation Based on ANSI/NCSL Z540-1-1994: The Optical Technology Division's Effort.

S. S. Bruce, and T. C. Larason. Aug 96, 58p,

NISTIR-5866.

See also PB95-103461.

Keywords: "Quality assurance, "Absolute spectral response, "Calibration, Optical measuning instruments, Photometers, Spectroradiometers, Optical detectors, Optical properties, Radiance, Test facilities, ISO(International Standards Organization), International Standards Organization 4000, ANSI/NCSL Z540-1-1994.

The Optical Technology Division, formerly known as the Radiometric Physics Division, at the National Institute of Standards and Technology (NIST) began a project in 1993 to develop a quality system that is based on the American National Standard for Calibra tion - Calibration Laboratories and Measuring and Test Equipment, ANSI/NCSL Z540-1-1994. This document is intended to complement the NCSL 'Handbook for the Interpretation and Application of ANSI/NCSL Z540-1 1994 ' and to provide guidance to other calibration serv ices at NIST, particularly those starting to develop their quality systems. The appendix of this publication contains a reprint of a paper entitled, 'Building a Quality System Based on ANSI/NCSL Z540-1-1994 An Effor by the Radiometric Physics Division at NIST'.

\section{Radiofrequency Detection}

\section{1,870}

PB94-218591 PC A03/MF A01

National Inst. of Standards and Technology (EEEL), Boulder, CO. Electromagnetic Fields Div. RangeCAD and the NIST RCS Uncertainty Analy sis.

R. L. Lewis, L. A. Muth, and R. C. Wittmann. Aug 94, 18p, NISTIR-5022.

Keywords: "Uncertainty, "Error analysis, *Radar cross sections, Calibration, Radar signals, Noise(Radar), Amplitude, Estimation, Mathematical models, Range CAD computer program.

We discuss the salient features of a computer program, RangeCAD, and then translate the program's output into a catalogue of radar cross section (RCS) uncertainties. This specific catalogue was developed by the National Institute of Standards and Technology (NIST) to standardize RCS uncertainty computations at the various RCS measurement sites. We check uncertainty estimates generated by RangeCAD against alternative formulations that approximate equivalent uncertainty specifications. Based on this comparison we conclude that the uncertainty estimates generated by RangeCAD provide realistic values for the NIST RCS uncertainty analysis.

\section{1,871}

PB95-203568 Not available NTIS

National Inst. of Standards and Technology (EEEL) Boulder, CO. Electromagnetic Fields Div.

Proposed Analysis of RCS Measurement Uncertainty.

Final rept.

R. C. Wittmann, M. H. Francis, L. A. Muth and R. L. Lewis. 1994, 7p.

Pub. in Proceedings of the Antenna Measurement Techniques Association Symposium, Long Beach, CA., October 3-7, 1994, p51-57.

Keywords: "Radar cross sections, Measurement, Uncertainty, Errors, Reprints.

From a study of several radar cross section (RCS) measurement facilities, we identify significant sources of uncertainty and develop methods for estimating their effect. Our goal is to provide a 'reasonable' and uniform formalism for evaluating RCS measurements which can be used on a variety of test ranges to produce comparable estimates of uncertainty.

01,872

PB95-216925 PC A03/MF A01

National Inst. of Standards and Technology (EEEL), Boulder, CO. Electromagnetic Fields Div.
Polarimetric Calibration of Reciprocal-Antenna Radars.

R. L. Lewis, L. A. Muth, and R. C. Wittmann. Mar 95, 24p, NISTIR-5033.

Keywords: "Radar cross sections, Radar antennas, Radar targets, Error analysis, Depolarization, Calibration, "Radar polarimetry, Measurement uncertainty, Reciprocity.

We discussed how radar target depolanization enhances a radar's cross polarization contamination, and we dramatize the effect by presenting a graphical study of radar cross section (RCS) measurement error due to depolarization by an inclined dihedral reflector. Since error mitigation requires full polarimetric RCS measurements, we recommend upgrading singly polarized radars to full polarimetric capability. We present a novel polarimetric calibration technique that is applicable to reciprocal antenna radars, saves calibration time by requiring a single calibration target, uses simple mathematical expressions, and smoothes measured calibration data to reduce clutter.

\section{ELECTROTECHNOLOGY}

\section{General}

\section{1,873}

PB94-126901 PC A09/MF A02

National Inst. of Standards and Technology (EEEL), Gaithersburg, MD.

Electronics and Electrical Engineering Laboratory 1994 Program Plan: Supporting Technology for U.S. Competitiveness in Electronics.

J. M. Surette. Dec 93, 184p, NISTIR-5337.

See also PB93-228625 and PB94-161320.

Keywords: "Electrical measurement, *Electronics industry, Semiconductor devices, Electric equipment Optoelectronic devices, Microwave equipment, Optical fibers, Electromagnetic compatibility, Superconductors, Metrology, Competitiveness, US NIST.

The Electronics and Electrical Engineering Laboratory (EEEL), working in concert with other NIST Laboratories, is providing measurement and other generic technology critical to the competitiveness of the U.S. electronics industry and the U.S. electrical-equipment industry. This 1994 Program Plan describes the projected metrological support that EEEL intends to provide to U.S. industry.

\section{1,874}

PB94-161320 PC A04/MF AO1

National Inst. of Standards and Technology (EEEL), Gaithersburg, MD

Electronics and Electrical Engineering Laboratory: 1994 Strategic Plan. Supporting Technology for U.S. Competitiveness in Electronics.

R. M. Powell. Apr 94, 70p, NISTIR-5409.

See also PB92-123082.

Keywords: "Electrical measurement, Electronics indus try Optical communication, Semiconductor devices, Microwave equipment, Video equipment, Optical processing, Signal processing, Optical storage, Magnetic storage, Electric power, Integrated optics, Optical fibers, Electromagnetic compatibility, Metrology, Standards, Superconductors, Electronics and Electrical Engineering Laboratory, Competitiveness, US NIST, Fiber optic sensors.

The U.S. electronics and electrical-equipment industries are outstripping available measurement capability with adverse effects on their international competitiveness. Improved measurement support is an essential part of any successful strategy for improving their competitiveness. Among U.S. manufacturing industries the electronics industry is the largest employer with 1.8 million employees and is virtually tied with the chemica industry for largest shipments of nearly $\$ 300$ billion (1992). The electrical-equipment industry is also quite large, with shipments of nearly $\$ 50$ billion (1990). U.S competitiveness in many fields of electronic and elec- 
trical products has been declining. Improved competitiveness will require outstanding performance from manufacturers in every step required to realize a competitive product in the marketplace: research and development, manufacturing marketplace exchange, and after-sales support. All of these steps are highly measurement intensive. The Electronics and Electrical Engineering Laboratory (EEEL), within the National Institute of Standards and Technology, has identified the principal needs for improved measurement capability and other supporting technology in several important fields: semiconductors, magnetics, superconductors, low frequency, microwaves, lightwaves, power, video, electromagnetic compatibility, electronic data exchange, and national electrical standards. The document describes EEEL's strategic plan for a response to these needs. The plan was developed in consultation with U.S. industry and other NIST Laboratories.

01,875

PB94-165990 PC AO6/MF AO2

National Inst. of Standards and Technology (EEEL), Boulder, CO. Electromagnetic Fields Div.

Bibliography of the NIST Electromagnetic Fields Division Publications.

R. M. Lyons, and K. A. Gibson. Aug 92, 108p,

NISTIR-3993.

Supersedes PB94-112547.

Keywords: "Electromagnetic fields, "Bibliographies, "US NIST, Electrical measurement, Dielectric properties, Electromagnetic interference, Electromagnetic noise, Remote sensing, Time domain, Radiation, Impedance, Attenuation, Radiation hazards, Antennas, Near fields, Metrology, Waveforms, Microwaves, Standards, "Nonionizing radiation.

The bibliography lists the publications by the staff of the Electromagnetic Fields Division of the National Institute of Standards and Technology for the period January 1970 through July 1992. It supersedes NISTIR 3973 which listed the publications of the Electromagnetic Fields Division from January 1970 through July 1991. Selected earlier publications from the Division's predecessor organizations are included.

\section{1,876}

PB94-172186 Not available NTIS

National Inst. of Standards and Technology (IMSE), Gaithersburg, MD. Ceramics Div.

Dielectric Properties Measurements and Data.

Final rept.

J. A. Carpenter. 1991, $11 \mathrm{p}$.

Pub. in Materials Research Society Symposia Proceedings, v189 p477-487 1991.

Keywords: Dielectric properties, Minerals, Polymers, Solids, Ceramics, Microwave frequencies, Reprints, "Dielectric measurements.

This paper reviews measurement techniques and sources of data for the dielectric constant and loss factor of solid materials. Accurate values of such properties are basic to intelligent design of materials processing schemes using electromagnetic energy. Emphasis is on techniques and data for these properties in the 'microwave' range of frequencies of roughly 10(sup 8) to 10 (sup 11) $\mathrm{Hz}$ and at elevated temperatures.

\section{1,877}

PB94-185410 Not available NTIS

National Inst. of Standards and Technology (EEEL), Boulder, CO. Electromagnetic Fields Div.

Time-Domain Measurements of the Electromagnetic Backscatter of Pyramidal Absorbers and Metallic Plates.

Final rept.

R. T. Johnk, A. Ondrejka, S. Tofani, and M. Kanda.

1993, 5p.

Pub. in Institute of Electrical and Electronics Engineers Transactions on Electromagnetic Compatibility $35, n 4$ p429-433 Nov 93.

Keywords: "Electromagnetic scattering, Electromagnetic measurement, Very high frequency, Ultrahigh frequency, $\mathrm{MHz}$ range $01-100, \mathrm{MHz}$ range $100-$ 1, 000, Absorbers(Materials), Time domain, Metal plates, Backscattering, Microwave scattering, Broadband, Pyramids, Reprints.

A wideband time-domain measurement system has been developed for the evaluation of the backscatter performance of dissipative macrostructures. Backscatter measurements have been performed in an ordinary room environment on metal plates as well as samples of pyramidal absorbing material. The backscattering performance of pyramidal absorbers is evaluated in the $50-$ to $1,000 \mathrm{MHz}$ frequency rang with a varying incident field angle of incidence. In the case of rectangular metal plates, numerically generated results are compared with measured data in order to gauge the accuracy of the system.

\section{1,878}

PB94-185634 Not available NTIS

National Inst. of Standards and Technology (EEEL), Gaithersburg, MD. Electricity Div.

Approach to Setting Performance Requirements for Automated Evaluation of the Parameters of High-Voltage Impulses.

Final rept.

T. R. McComb, C. Cherbaucich, L. Coffen, E. Hanique, K. Lehmann, J. McBride, J.J. Ribot, G

Rizzi, P. Vaessen, W. Zaengl, M. F. Deschamps, and G. J. FitzPatrick. 1993, 4p.

Pub. in Proceedings of the International Symposium on High Voltage Engineering (8th), Yokohama, Japan, August 23-27, 1993, p309-312.

Keywords: "High voltage, "Waveforms, "Signal processing, Wave analyzers, Pulse analyzers, Automation, Performance evaluation, Computer applications, Data bases, Reprints.

This paper reports the present status of an ongoing study of digital signal processing applied to various impulse waveforms. In a round-robin study, twelve lab oratories are using their own software to evaluate the parameters of impulse waveforms in a data base of thirty-one waveforms with the objective of establishing minimum performance requirements. This paper presents the results obtained for smooth full impulses and some examples of results on more complex waveforms.

\section{1,879}

PB94-185931 Not available NTIS

National Inst. of Standards and Technology (EEEL), Gaithersburg, MD. Semiconductor Electronics Div. Reproducibility of JEDEC Standard Current and Voltage Ramp Test Procedures for Thin-Dielectric Breakdown Characterization.

Final rept.

J. S. Suehle. 1993, 13p.

Pub. in Proceedings of the International Integrated $\mathrm{Re}$ liability Workshop, Lake Tahoe, CA., October 24-27, 1993, p22-34.

Keywords: *Dielectric breakdown, *Electrical measurement, Interlaboratory comparisons, Integrated circuits Electric fields, Charge density, Reproducibility, Tes methods, Reprints, JEDEC.

Six laboratories and a reference laboratory participated in an interlaboratory experiment that was conducted to determine the reproducibility of breakdown electric field, $E(B R)$, and breakdown charge density, $q(\mathrm{bd})$ measurements using the JEDEC standard voltage and current ramp dielectric test procedures. The results indicate that the measurement of $E(B R)$ is much more reproducible than $q(b d)$. Much of the variability in the $\mathrm{q}$ (bd) measurements was found to be due to an allowed range of values that could be chosen for a parameter in the current ramp procedure. When this source of variability is accounted for, the results indicate that the standard test procedures can be implemented to obtain critical dielectric integrity parameters with good reproducibility for a large variety of tes equipment.

\section{1,880}

\section{PB94-193786 PC A04/MF A01}

National Inst. of Standards and Technology (EEEL). Boulder, CO. Electromagnetic Fields Div.

Coaxial Reference Standard for Microwave Power. Technical note.

F. R. Clague, and P. G. Voris. Apr 93, 54p, NIST/TN 1357.

Also available from Supt. of Docs. as SNOO3-00303213-1.

Keywords: "Power measurement, "Microwaves, *Standards, Coaxial cables, Calibration, Performance, Design, "Reference standards, *Bolometer mounts, *Thermistor mounts.

Design and construction details are given for the bolometer (thermistor) mounts used by NIST as working reference standards for microwave power calibration in coaxial transmission line. The effective efficiency of these reference standards can be measured directly in the NIST coaxial microcalorimeters. The standards are then used to calibrate other microwa ve power sensors. Two versions are described: one with a Type $\mathrm{N}$ connector and one with an APC-7 connector. The operating frequency range is 0.05 to $18 \mathrm{GHz}$ with either connector. Detailed drawings and performance measurements are included.

01,881

PB94-193984 PC A04/MF A01

National Inst. of Standards and Technology (EEEL) Gaithersburg, MD. Semiconductor Electronics Div.

Realizing Suspended Structures on Chips Fabricated by CMOS Foundry Processes Through the MOSIS Service.

J. Marshall, M. Gaitan, M. Zaghloul, J. I. Pi, C. Pina W. Hansiord, D. Novotny, and V. Tyree. Jun 94,60p, NISTIR-5402.

Prepared in cooperation with George Washington Univ., Washington, DC. School of Engineering and Applied Science. and University of Southern California Marina del Rey. Information Sciences Inst.

Keywords: Chips(Electronics), Etching, Wafers, Tiles, Design, Silicon, CMOS, Hazards, "Micromachining, Microelectromechanics, MOSIS.

Chips can be inexpensively fabricated at Complementary Metal-Oxide Semiconductor (CMOS) foundries through the MOSIS (MOSIS is an acronym for 'MOS Implementation System') Service at the University of Southern California's Information Sciences Institute. MOSIS now supports CMOS-compatible micromachining to realize microelectromechanical systems (MEMS) and devices such as suspended corners, cantilevers, and pixels. The MEMS designs are fabricated through MOSIS on a multi-project wafer, and the user performs a post-processing maskless anisotropic etch. Two new design tiles called 'open' and 'pstop' have been added to support these designs.

\section{1,882}

PB94-200409 Not available NTIS

National Inst. of Standards and Technology (EEEL) Gaithersburg, MD. Electricity Div.

President's Column for Dielectrics and Electrical Insulation Society Newsletter.

Final rept

A. H. Cookson. 1993, 1p.

Pub. in EE Electrical Insulation Magazine 9, n3 and 5 May/Jun 93

Keywords: Planning, Reprints, "Dielectrics and Electrical Insulation Society.

A summary of plans for the coming year, for the Dielectrics and Electrical Insulation Society, is given.

\section{1,883}

PB94-213774 PC A03/MF A01

National Inst. of Standards and Technology (EEEL) Gaithersburg, MD. Semiconductor Electronics Div. Electronics and Electrical Engineering Laboratory Technical Publication Announcements Covering Laboratory Programs, January to March 1994 with 1994/1995 EEEL Events Calendar.

J. M. Rohrbaugh. Aug 94, 33p, NISTIR-5471. See also PB94-193752

Keywords: "Microelectronics, "Metrology, "Bibliographies, Electrical measurement, Integrated circuits, Millimeter waves, Microwaves, Optical fibers, Integrated optics, Semiconductor devices, Electric power, High temperature superconductors, Electromagnetic interference, Magnetic measurement, Abstracts.

This is the fortieth issue of a quarterly publication pro viding information on the technical work of the Nationa Institute of Standards and Technology Electronics and Electrical Engineering Laboratory (EEEL). This issue of the EEEL Technical Publication Announcements covers the first quarter of calendar year 1994. This issue contains citations and abstracts for Laboratory publications published in the quarter. Main topic areas include: Fundamental Electrical Measurements; Semiconductor Microelectronics: Signal Acquisition, Processing, and Transmission; Electrical Systems; Electromagnetic Interference; Additional Information.

01,884

PB94-219334 (Order as PB94-219326, PC A05/

MF A02)

National Inst. of Standards and Technology, Gaithersburg, MD. 
Sources of Uncertainty in a DVM-Based Measurement System for a Quantized Hall Resistance Standard.

K. C. Lee, M. E. Cage, and P. S. Rowe. 1994, 14p Included in Jnl. of Research of the National Institute of Standards and Technology, v99 n3 p227-240 May/ Jun 94.

Keywords: "Resistors, "Standards, "Calibrating, Voltmeters, Electrical resistance meters, Hall effect, Drift(Instrumentation)

The paper describes a simple automated measurement system that uses a single, high accuracy, commercially available digital voltmeter (DVM) to compare the voltages developed across a $10 \mathrm{kilohm}$ standard resistor and a quantized Hall resistor when the same current is passed through the two devices. From the se measurements, the value of the $10 \mathrm{kilohm}$ standard resistor is determined. The sources of uncertainty in this system are analyzed in detail and it is shown that i is possible to perform calibrations with relative combined standard uncertainties less than $0.1 \mathrm{ppm}$.

01,885

PB94-219359 (Order as PB94-219326, PC A05/ MF A02)

National Inst. of Standards and Technology, Gaithersburg, MD.

Three-Axis Coil Probe Dimensions and Uncertainties during Measurement of Magnetic Fields from Appliances.

M. Misakian, and C. Fenimore. 1994, 7p.

Included in Jnl. of Research of the National Institute of Standards and Technology, v99 n3 p247-253 May/ Jun 94.

Keywords: "Electric appliances, "Magnetic fields, "Probability theory, Coils, Computation.

Comparisons are made between the average magnetic flux density for a three-axis circular coil probe and the flux density at the center of the probe. The results, which are determined assuming a dipole magnetic field, provide information on the uncertainty associated with measurements of magnetic fields from some electrical appliances and other electrical equipment. The present investigation extends an earlier treatment of the problem, which did not consider all orientations of the probe. A more comprehensive examination of the problem leaves unchanged the conclusions reached previously.

01,886

PB95-135562 PC A06/MF A02

National Inst. of Standards and Technology (EEEL),

Boulder, CO. Electromagnetic Fields Div.

Bibliography of the NIST Electromagnetic Fields

Division Publications.

R. M. Lyons, and K. A. Gibson. Sep 94, 120p

NISTIR-5028.

Supersedes PB94-165990.

Keywords: "Electromagnetic fields, "Bibliographies, Electrical measurement, Dielectric properties, Electromagnetic interference, Electromagnetic noise, Radiation hazards, Remote sensing Time domain Near field, Microwaves, Metrology, Attenuation, Waveforms, Standards, Impedance, Antennas, Nonionizing radiation, US NIST.

This bibliography lists the publications by the staff of the Electromagnetic Fields Division of the National Institute of Standards and Technology for the period Jan wary 1970 through July 1994. It supersedes NISTIR 5009 which listed the publications of the Electromagnetic Fields Division from January 1970 through July 1993. Selected earlier publications from the Division's predecessor organizations are included.

\section{1,887}

PB95-144309 PC A06/MF A02

National Inst. of Standards and Technology (EEEL), Gaithersburg, MD.

Electronics and Electrical Engineering Laboratory 1994 Technical Accomplishments Supporting Technology for U.S. Competitiveness in Electronics.

Special pub.

Dec 94, 102p, NISTIR-5551.

See also PB94-136777.

Keywords: "Electronics industry, "Metrology, Tech nology innovation, Competition, United States, Measurement, Semiconductor devices, Technology transfer, Research projects, Commercial development, Organi- zational structure, US NIST, EEEL(Electronics and Electrical Engineering Laboratory)

The Electronics and Electrical Engineering Laboratory (EEEL) working in concert with other National Institute of Standards and Technology (NIST) Laboratories, is providing measurements capability and other generic technology critical to the competitiveness of the U.S electronics industry and the U.S. electrical-equipment industry. This report summarizes selected technical accomplishments and describes activities conducted by the Laboratory in Fiscal Year 1994. Also included is a profile of EEEL's organization, its customers, and the Laboratory's long-term goals.

\section{1,888}

PB95-150389 Not available NTIS

National Inst. of Standards and Technology (EEEL), Gaithersburg, MD. Electricity Div.

Performance Evaluation of a New Digital Partial Discharge Recording and Analysis System.

Final rept.

P. von Glahn, and R. J. VanBrunt. 1994, 5p.

Pub. in Proceedings of Conference Record of the 1994 Institute of Electrical and Electronics Engineers International Symposium on Electrical Insulation, Pittsburgh, PA., June 5-8, 1994, p12-16.

Keywords: *Digital recording systems, "Electrical insulation, Epoxy matrix composites, Performance evaluation, Personal computers, Stochastic processes, Real time, Digitizers, Reprints, "Partial discharges.

We describe the design and performance evaluation of a new digital partial discharge (PD) recording system capable of real-time recording of PD pulse trains for later off-line computerized stochastic analysis. By way of illustration, measurements were made of the time-varying stochastic behavior of ac-generated PDs in point-to-dielectric gaps in air where the insulation material was cast epoxy with aluminum oxide filler extending the work reported previously. Sample analysis results are presented, demonstrating that the new system provides analysis results comparable with the results achieved by the existing NIST analog PD stochastic analysis system. Sample stochastic analysis results are presented demonstrating the additional insights possible with the new system.

01,889

PB95-150579 Not available NTIS

National Inst. of Standards and Technology (EEEL) Gaithersburg, MD. Electricity Div.

Electrical Breakdown in Transformer Oil in Large Gaps.

Final rept.

K. L. Stricklett, D. M. Weidenheimer, N. R. Pereira, and D. C. Judy. 1992, 7p

Sponsored by Defense Nuclear Agency, Washington, DC. and Department of Energy, Washington, DC.

Pub. in Proceedings of Annual Report Conference on Electrical Insulation and Dielectric Phenomena, Victoria, British Columbia, Canada, October 18-21, 1992 p248-254.

Keywords: "Electric discharges, "Dielectric breakdown, "Pulse transformers, "Spark gaps, Shadowgraph photography, Electric fields, High speed photography, Puises, Switches, Reprints.

The Aurora accelerator uses four parallel Blumlein pulse-forming lines to provide an intense flash $x$-ray pulse. Proper timing of the pulses generated by each Blumiein is important to the quality of the radiation. The pulse on each Blumlein, and the synchronization between the Blumleins are affected by the closure of high-voltage triggered oil switches in each line. The triggered oil switch utilizes a uniform field geometry with a gap spacing between 40 and $50 \mathrm{~cm}$, a unique environment for observation of arc development in transformer oil. High-speed photography of switch closure shows timing to be influenced by the initiation and spatial development of prebreakdown streamers.

01,890

PB95-150777 Not available NTIS

National Inst. of Standards and Technology (NML) Gaithersburg, MD. Center for Atomic, Molecular and Optical Physics.

New International Representations of the Volt and Ohm Effective January 1, 1990.

Final rept.

B. N. Taylor. 1990, 4p

Pub. in Institute of Electrical and Electronics Engineers

Transactions on Instrumentation and Measurement 39 $n 1,2-5,1990$.
Keywords: "Standards, International agreements, Josephson effect, Reprints, "Resistance standards, "Voltage standards, "Volt, "Ohm, Consultative Committee on Electricity, Quantum Hall effect.

Starting on January 1, 1990, new representations of the volt and ohm based on the Josephson and quantum Hall effects, respectively, are to come into effect worldwide. Implementation of the new representations in the U.S. requires that on this date, the value of the present national representation of the volt maintained at the National Institute of Standards and Technology (NIST) be increased by 9.264 parts per million (ppm) and that the value of the national representation of the ohm maintained at NIST be increased by $1.69 \mathrm{ppm}$. The resulting increases in the U.S. representations of the ampere and watt will be about $7.57 \mathrm{ppm}$ and 16.84 $\mathrm{ppm}$, respectively.

01,891

PB95-150934 Not available NTIS

National Inst. of Standards and Technology (EEEL),

Boulder, $\mathrm{CO}$. Electromagnetic Fields Div.

Bistatic Scattering of Absorbing Materials from 30 to $1000 \mathrm{MHz}$

Final rept.

S. Tofani, A. R. Ondrejka, M. Kanda, and D. A. Hill. 1992, 4p.

Pub. in Institute of Electrical and Electronics Engineers Transactions of Electromagnetic Compatibility 34, n3 p304-307 Aug 92

Keywords: "Absorbers(Materials), Electromagnetic scattering, Electromagnetic measurement, Very high frequency, Ultrahigh frequency, Scattering coefficients, $\mathrm{MHz}$ range 01-100, $\mathrm{MHz}$ range 100-1000, Anechoic chambers, Specular reflection, Time domain, Broadband, Reprints, "Microwave absorbers, "Bistatic scattering.

A wide-band time-domain reflectometer has been used to evaluate the bistatic performance of the scattering coefficient of $\mathrm{r} /$ /microwave absorbers. The scattering coefficient has been measured inside an anechoic chamber in the $30-1000 \mathrm{MHz}$ frequency range in the case of specular reflection. The scattering coefficien increases with incidence angle, and the measurement accuracy is + or $-2 \mathrm{~dB}$.

01,892

PB95-153227 Not available NTIS

National Inst. of Standards and Technology (EEEL) Boulder, CO. Electromagnetic Fields Div.

New Coaxial Microwave Microcalorimeter Evaluation Technique.

Final rept.

F. R. Clague. $1992,2 p$

Pub. in Proceedings of Conference on Precision Electromagnetic Measurements, Paris, France, June 9-12, 1992, p387-388

Keywords: "Power measurement, "Calorimeters, "Standards, Coaxial cables, Microcalorimetry, Corrections, Reprints, "Microwave power standards, "Microcalorimeters.

A technique for improving the evaluation of the microcalorimeter portion of a coaxial microwave power standard is described. The evaluation results in a factor that corrects for varying thermal paths and losses. This technique allows major components of the correction factor to be determined by direct measurement rather than estimated indirectly.

\section{1,893}

PB95-154662 PC A03/MF A01

National Inst. of Standards and Technology (EEEL)

Boulder, CO. Electromagnetic Fields Div.

Effective Medium Theory for Ferrite-Loaded Materials.

Technical note.

R. G. Geyer, J. Mantese, and J. Baker-Jarvis. Oct 94, 26p, NIST/TN-1371.

Also available from Supt. of Docs. as SN003-00303304-9. See also PB92-205376. Prepared in COoperation with General Motors Research Labs., Warren, MI.

Keywords: "Electromagnetic theory, Binary systems(Materials), Composite materials, Barium titanates, Very high frequency, Ultrahigh frequency, Dielectric properties, Magnetic properties, Permeability, Permittivity, Inclusions, Integral equations, Spheres, Maxwell-Garnett formulae, Magnesium copper zinc ferrites, Two phase systems.

A ferrite-loaded composite medium is modeled by spherical inclusions spaced equally on a cubic lattice 
within a host matrix. Both the inclusions and host matrix may be magnetically permeable and possess dielectric and magnetic loss. The ferrite-loaded medium may be considered to consist of excited Hertzian electric and magnetic dipole sources. Effective medium rules of a modified Maxwell-Garnett form can be denived by analysis of plane-wave propagation through the composite. These rules do not yield symmetric characterization of two-phase media. They are compared with other effective medium theories (LorentzLorenz. Maxwell-Garnett, and Bruggeman) and broadband coaxial transmission line data measured on ferrite-loaded titanates of known composition. The modified Maxwell-Garnett rules give both lower and upper bounds for the effective permittivities and permeabilities of the composite and yield accurate estimates of bulk electric and magnetic properties for low volumetric inclusion loading. The Bruggeman formalism yields the best predicitive permittivity and permeability values when volumetric percentages of the inclusions and host matrix are approximately equal. Generally, maximal magnetic loss factors occur at a frequency where the static initial permeability decreases by a factor of one-half, and the relaxation fequency for ferrite composites increases with decreasing static initial permeability.

\section{1,894}

\section{PB95-159885 PC A09/MF A03}

National Inst. of Standards and Technology (EEEL), Gaithersburg, MD.

Electronics and Electrical Engineering Laboratory 1995 Program Plan. Supporting Technology for U.S. Competitiveness in Electronics.

Jan 95, 200p, NISTIR-5563.

Keywords: "Electronics industry, "Electrical measurement, "Metrology, "Technology transfer, Electric equipment, International trade, Competition, Microelectronics, Law enforcement, Standards, Electric utilities, Electric power generation, Semiconductor devices, Optoelectronic devices, US NIST.

The Electronics and Electrical Engineering Laboratory (EEEL), working in concert with other National Institute of Standards and Technology (NIST) laboratories, is providing measurement and other generic technology critical to the competitiveness of the U.S. electronics industry and the U.S. electrical-equipment industry. This 1995 Program Plan describes the projected metrological support that EEEL intends to provide to U.S. industry.

\section{1,895}

PB95-161253 Not available NTIS

National Inst. of Standards and Technology (EEEL), Gaithersburg, MD. Electricity Div.

Frequency Extension of the NIST AC-DC Difference Calibration Service for Current.

Final rept.

J. R. Kinard, T. E. Lipe, and C. B. Childers. 1993,

$12 p$.

Pub. in Proceedings of the National Computer Systems Laboratory Workshop and Symposium, Albuquerque, NM., July 25-29, 1993, 12p.

Keywords: "Electrical measurement, "Electric current, "Standards, Frequencies, Reprints, Thermal converters, US NIST.

This paper describes the NIST standards of the ac-dc difference for current and the results of a study underway to extend the frequency range down to $10 \mathrm{~Hz}$ and up to $100 \mathrm{kHz}$.

\section{1,896}

PB95-161287 Not available NTIS

National Inst. of Standards and Technology (EEEL), Gaithersburg, MD. Electricity Div.

Modeling and Test Point Selection for a Thermal Transfer Standard.

Final rept.

A. D. Koffman, and H. L. Stott. 1993, 12p.

Pub. in Proceedings of National Computer Systems Laboratory Workshop and Symposium, Albuquerque, NM., July 25-29, 1993, p299-310.

Keywords: Case studies, Calibration, Selection, Frequency, Voltage, Reprints, "Thermal transfer standards, "Multirange instruments, Test points.

Full calibration support for multirange instruments can be costly and time consuming. This paper presents a case study in which a new empirical-model-based approach was used to substantially reduce the number of tests required to fully characterize an instrument. The Fluke 792A Thermal Transfer Standard was the subject instrument for the study. Test results showed that measurements made at 50 test points were sufficient to allow accurate predictions of the instrument's performance at all 255 test points specified by the manufacturer. An accurate model relating ac/dc difference to voltage and frequency for the instrument was formulated using complete test data for many devices collected by the manufacturer over several production runs. An empirical test point selection procedure was used to select an optimal set of test points and subsequently to predict the ac/dc differences of other 792As based on the limited set of measurements taken at the selected test points.

\section{1,897}

PB95-161485 Not available NTIS

National Inst. of Standards and Technology (EEEL) Boulder, CO. Electromagnetic Fields Div.

Characterization of Unknown Linear Systems Based on Measured CW Amplitude.

Final rept.

and J. W. Adams. 1993, 5p.

Pub. in Proceedings of International Symposium on Electromagnetic Compatibility, Dallas, TX., August 913, 1993, p78-82.

Keywords: "Linear systems, Electromagnetic compatibility, Continuous radiation, Laplace transformation, Transfer functions, Approximation, Characteristics, Reprints, Impulse response.

An approximate squared-magnitude function is derived from a given measured cw amplitude response to characterize an unknown linear system. Various possible system transfer functions (both amplitude and phase) and the corresponding impulse responses are then deduced. These transfer functions may or may not be minimum phase. The first impulse maximum and accumulated energy content are the greatest when the transfer function is at minimum phase.

\section{1,898}

\section{PB95-161568 Not available NTIS}

National Inst of Standards and Technology (EEEL),

Boulder, CO. Electromagnetic Fields Div.

Comments on 'Protecting EFIE-Based Scattering Computations from Effects of Internal Resonances'.

Final rept.

R. B. Marks. 1993, 3p

Pub. in Institute of Electrical and Electronics Engineers Transactions on Antennas and Propagation 41, n3 p387-389 Mar 93.

Keywords: "Electromagnetic scattering, Electric fields, Magnetic fields, Integral equations, Computation, Reprints.

This letter discusses a recently-published paper which presents an approach to the problem of interna resonances of the electric field integral equation. This method, which requires the determination of the smallest singular value of the associated integral operator may fail due to the accumulation of singular values at the origin. In contrast, another method, already in the literature, applies the magnetic field integral equation, which does not suffer from this problem.

\section{1,899}

PB95-162210 Not available NTIS

National Inst. of Standards and Technology (EEEL). Boulder, CO. Electromagnetic Fields Div.

Condensed Catalogue of Electromagnetic Environment Measurements, $30-300 \mathrm{~Hz}$.

Final rept.

J. Randa, D. Gilliland, W. Gjertson, W. Lauber, and M. Mclnerney. 1993,6p.

Pub. in Proceedings of International Symposium on Electromagnetic Compatibility, Dallas, TX., August 9 13, 1993, p126-131.

Keywords: *Electromagnetic environments, *Extremely low frequency, "Catalogs(Publications), Electromagnetic measurement, Bibliographies, Reprints.

The IEEE Electromagnetic Compatibility Society's Technical Committee on Electromagnetic Environments (TC-3) has undertaken a long-term project to compile an inventory or catalog of published measurements of electromagnetic environments. The frequency spectrum has been divided into tractable bands which will be considered one at a time. We have now completed the $30-300 \mathrm{~Hz}$ band. We present here an abridged version of the resulting bibliography, along with a brief summary of what has been measured.
01,900

PB95-163127 Not available NTIS

National Inst. of Standards and Technology (EEEL) Gaithersburg, MD. Electricity Div.

Observations of Partial Discharges in Hexane Under High Magnification.

Final rept.

K. L. Stricklett, E. F. Kelley, H. Yamashita, T. V Blalock, A. L. Wintenburg, I. Alexeff, C. Fenimore, and M. O. Pace. $1990,6 p$.

Proceedings of International Conference on Conduction and Breakdown in Dielectric Liquids (10th), Grenoble, France, September 10-14, 1990, p381-386.

Keywords: "Electric discharges, “Dielectric breakdown "Hexanes, Shadowgraph photography, Photographic techniques, Direct current, Liquids, Reprints, "Partial discharges, Streamer initiation, Point cathodes.

Partial discharges are observed in $n$-hexane by shadow photography under the application of dc voltages. A non-uniform field geometry is employed and the growth of low-density streamers at a point cathode is recorded. Photographs of the partial discharge streamers are obtained at 200x magnification. The use of an image-preserving optical delay allows a record of the conditions which exist in the liquid prior to the initiation of low density streamer to be obtained.

01,901

PB95-163572 Not available NTIS

National Inst. of Standards and Technology (EEEL), Gaithersburg, MD. Electricity Div.

Importance of Unraveling Memory Propagation Effects in Interpreting Data on Partial Discharge Statistics.

Final rept.

R. J. Van Brunt, E. W. Cernyar, and P. von Glahn. 1993, 12p.

Sponsored by Department of Energy, Washington, DC Office of Energy Storage and Distribution.

Pub. in Institute of Electrical and Electronics Engineers Transactions on Electrical Insulation 28, n6 p905-916 Dec 93.

Keywords: "Electric discharges, "Electrical insulation, Monte Carlo method, Static electricity, Pulse amplitude, Stochastic processes, Pattern recognition, Dielectrics, Simulation, Reprints, "Partial discharges.

The significance of memory propagation in controlling the stochastic behavior of partial-discharge phenomena is demonstrated by determination of various conditional amplitude and phase-of-occurrence distributions for both measured and simulated discharge pulses. A system that can be used to measure directly a set of both conditional and unconditional pulse amplitude and phase distributions needed to reveal memory effects and quantify the phase-resolved stochastic properties of partial-discharge pulses, is briefly described. It is argued that not only is an unraveling of memory effects essential in any attempt to understand the physica basis for the observed stochastic behavior of partialdischarge phenomena, but also that the data on conditional distributions provide additional statistical information that may be needed to optimize the reliability of partial-discharge pattern recognition schemes now being considered for use in insulation testing.

01,902

PB95-163580 Not available NTIS

National Inst. of Standards and Technology (EEEL) Gaithersburg, MD. Electricity Div.

Nonstationary Behavior of Partial Discharge during Insulation Aging.

Final rept.

R. J. Van Brunt, P. von Glahn, and T. Las. 1993, 2p. See also PB95-163598. Sponsored by Nuclear Regulatory Commission, Washington, DC. Div. of Engineer-

Pub. in Proceedings of the International Conference on Partial Discharges, Canterbury, United Kingdom, September 28-30, 1993, p29-30.

Keywords: "Electrical insulation, "Aging(Materials), "Electric discharges, Surface resistivity, Alternating current, Aluminum oxides, Epoxy resins, Stochastic processes, Dielectrics, Fillers, Reprints, "Partial discharges

The statistical properties of pulsating partial discharges (PD) generated by applying an alternating voltage to a point-dielectric gap were measured with a stochastic analyzer under conditions where the PD induced changes in the dielectric surface conductivity. The 


\section{General}

epoxy materials with and without $\mathrm{Al} 203$ filler were considered in this investigation. In the case of epoxy with filler, dramatic changes in the statistical behavior of the PD were observed to be correlated with changes in surface conductivity. These results illustrate the difficulties to be encountered in defining meaningful PD pulse patterns that can be used for reliable defect site identification. An analysis of the positive and negative integrated-charge distribution also reveal problems of relating PD pulse-height data to average current measured by standard techniques.

\section{1,903}

PB95-163598 Not available NTIS

National Inst. of Standards and Technology (EEEL), Gaithersburg, MD. Electricity Div.

Partial Discharge: Induced Aging of Cast Epoxies and Related Nonstationary Behavior of the Discharge Statistics.

Final rept.

R. J. Van Brunt, P. von Glahn, and T. Las. 1993, 7p. See also PB95-163580. Sponsored by Nuclear Regulatory Commission, Washington, DC. Div. of Engineering.

Pub. in 1993 Annual Report, Conference on Electrical Insulation and Electric Phenomena, Pocono Manor PA., October 17-20, 1993, p455-461.

Keywords: "Electric discharges, "Electrical insulation, "Epoxy resins, "Aging(Materials), Time dependence, Alternating current, Stochastic processes, Monte Carlo method Electrical measurement, Aluminum oxides, Dielectrics, Simulation, Surface resistivity, Fillers, Reprints, ${ }^{*}$ Partial discharges.

Measurements were made of the time dependences of positive and negative integrated-charge distributions for ac-generated pulsating partial discharge $(P D)$ in point-to-dielectric gaps where the dielectric materia was cast epoxy either with or without an aluminum oxide filler. Other statistical characteristics of the PD were measured such as pulse-phase distributions. The dielectric surface resistivity was measured before and after exposure to PD. For epoxy with filler, the PD statistical characteristics changed significantly during times when there was a corresponding decrease in local surface resistivity. For example, the positive PD pulses were observed to cease after a time that is inversely proportional to the frequency of the applied voltage. Partial discharge from epoxy without filler exhibited a much more stationary behavior. The connection between changes in surface resistivity and changes in stochastic behavior are explained using a Monte Carlo simulation.

\section{1,904}

\section{PB95-163960 Not available NTIS}

National Inst. of Standards and Technology (EEEL)

Boulder, CO. Electromagnetic Fields Div.

Verification of Scattering Parameter Measurements.

Final rept.

D. F. Williams, and R. B. Marks. 1992, 2p.

Pub. in Proceedings of Conference on Precision Electromagnetic Measurements, Paris, France, June 9-12, 1992, p371-372.

Keywords: "Electrical measurement, "Calibration, Electrical impedance, Verification, Comparison, Accuracy, Reprints, "Scattering parameters, Automatic network analyzers, On wafer calibration.

A powerful new technique enables the verification of the measurement accuracy of scattering paramete calibrations. This technique determines the relative reference impedance, the reference plane offset, and the worst-case measurement deviations of any calibration in comparison to a standard calibration. Experimental results for on-wafer measurements are presented.

\section{1,905}

\section{PB95-164612 Not available NTIS}

National Inst. of Standards and Technology (CSTL), Gaithersburg, MD. Process Measurements Div.

Electrical Characterization of Radio-Frequency Discharges in the Gaseous Electronics Conference Reference Call.

Final rept.

M. A. Sobolewski. 1993, 13p

Pub. in Jnl. of Vacuum Science and Technology A 10, n6 p3550-3562 Nov/Dec 92

Keywords: "Radio frequency discharge, "Gas discharges, "Glow discharges, "Argon plasma, Electrical measurement, Electrical impedance, Error analysis, Reprints, Reference cells, Plasma processing.
Measurements of the electrical characteristics of radiofrequency $(r)$ discharges can be subject to large errors due to limitations in the measurement instruments and the stray impedance of the discharge cell. This study reports electrical measurements of argon discharges in the GEC Reference Cell in which special care has been taken to identify and minimize these sources of error. These techniques should improve the utility of electrical measurements for gauging the reproducibility of plasma conditions among if discharge cells, for testing theoretical results, and for monitoring plasma processing.

\section{1,906}

PB95-164695 Not available NTIS

National Inst. of Standards and Technology (EEEL), Gaithersburg, MD. Electricity Div.

Volitage Ratio Measurements of a Zener Reference Using a Digital Voltmeter.

Final rept

R. L. Steiner, E. A. Early, and C. Kiser. 1992, 2p

Pub. in Conference Record for Conference on Precision Electromagnetic Measurements, Paris, France, June 9-12, 1992, p300-301.

Keywords: "Electrical measurement, Calibration, Voltmeters, Accuracy, Reprints, "Voltage measurement, Voltage ratio, Voltage standards, Josephson arrays.

A high precision digital voltmeter can be used to measure the ratio of $1 \mathrm{~V}$ to $10 \mathrm{~V}$ very accurately. Preliminary tests of calibrating $10-\mathrm{V}$ Zener references from a $1-\mathrm{V}$ Josephson array standard indicate that an accuracy with an uncertainty of several parts in 10(exp 8) is possible.

\section{1,907}

PB95-168761 Not available NTIS

National Inst. of Standards and Technology (EEEL),

Boulder, CO. Electromagnetic Fields Div.

Revised Uncertainty Analysis for the NIST 30-MHz Attenuation Calibration System.

Final rept.

J. A. Jargon. 1994, 6p

Pub. in Proceedings of Measurement Science Conference Symposium and Workshop, Pasadena, CA., January 27-28, 1994, 6p.

Keywords: Very high frequency, Electrical measurement, Phase shift, Modification, Revisions, Attenuators, Uncertainty, Reprints, *Attenuation Calibration System, US NIST.

Although the 30-MHz Attenuation Calibration System has been in operation for many years at the National Institute of Standards and Technology, several modifications have been made to the system since the last published uncertainty analysis. The linear displacement of the standard attenuator's receiving coil is now measured with a laser interferometer instead of a steel ruled scale and optical projector, and a new comparison receiver has been installed in the system. The expanded uncertainty is on the order of + or $-0.003 \mathrm{~dB}$ per $10 \mathrm{~dB}$ step. Type $\mathrm{A}$ uncertainties depend upon the repeatability and resettability of the system and the device under test. Type $B$ uncertainties are due to the standard waveguide below-cutoff (WBCO) attenuator, the resolution of the comparison receiver, the change in level of the precision phase shift standard, the level set attenuator, if leakage, and mismatch uncertainty.

\section{1,908}

\section{PB95-180329 Not available NTIS}

National Inst. of Standards and Technology (EEEL),

Boulder, CO. Electromagnetic Fields Div.

Electronic Mode Stirring for Reverberation Chambers.

Final rep

D. A. Hill. 1994, 6p.

Pub. in Institute of Electrical and Electronics Engineers Transactions on Electromagnetic Compatibility 36, n4 p294-199 Nov 94.

Keywords: "Reverberation chambers, Electromagnetic theory, Rectangular configuration, Two dimensional, Superhigh frequency, Modal response, Numerical solution, Bandwidth, Reprints.

A modal analysis and a uniform-field approximation are presented for the fields in an idealized two-dimensional, rectangular cavity excited by an electric line source. The model is used to evaluate the effectiveness of frequency stirring, an alternative to mechanical stirring in reverberation chamber immunity measurements. Numerical results indicate that good field uni- formity (standard deviation less than $1 \mathrm{~dB}$ ) can be obtained with a bandwidth of $10 \mathrm{MHz}$ at a center frequency of $4 \mathrm{GHz}$. The bandwidth requirement is determined primarily by the number of modes excited, and higher frequencies can achieve the same field uniformity with a smaller bandwidth because of the higher mode density. Cavity excitation by two single-frequency sources is also analyzed.

\section{1,909}

PB95-180444 Not available NTIS

National Inst. of Standards and Technology (EEEL), Gaithersburg, MD. Electricity Div.

NIST Strategies for Reducing Testing Requirements.

Final rept.

A. D. Koffman, T. M. Souders, and G. N

Stenbakken. 1994, 7p.

Pub. in Proceedings of Conference Test and Calibration Symposium, Arlington, VA., November 30-December 2, 1994, p267-273.

Keywords: "Electronic equipment, "Test methods, Mathematical models, Standard deviation, Analog systems, Calibration, Optimation, Reduction, Strategy, Reprints, Singular value decomposition, US NIST.

For the past several years, research has been carried out in the Electricity Division at the National Institute of Standards and Technology (NIST) to reduce the testing requirements for analog and mixed-signal devices. The most significant testing technique to result has been a model-based approach to the testing and calibration of such devices. The model is developed from empirical data, physical information a priori information, or a combination of the three. Algebraic operations are performed on these data to create a model. The model approximately spans the vector space within which the device behavior can be described. With this model, the device can be characterized using significantly fewer measurements than is possible with traditional methods. A brief description of the techniques will be presented, along with a summary of the results achieved in testing analog and mixed-signal devices.

\section{1,910}

PB95-181046 Not available NTIS

National Inst. of Standards and Technology (EEEL), Gaithersburg, MD. Electricity Div.

Physics and Chemistry of Partial Discharge and Corona: Recent Advances and Future Challenges. Final rept.

R. J. Van Brunt. 1994, 24p.

Sponsored by Nuclear Regulatory Commission, Washington, DC. Div. of Engineering

Pub. in Institute of Electrical and Electronics Engineers Transactions on Dielectrics and Electrical Insulation 1 n5 p761-784 Oct 94

Keywords: "Electric discharges, Electrical insulation, Dielectric breakdown, Electric corona, Aging(Materials), Stochastic processes, Reprints, * Partial discharges.

Results of recent research on physical and chemica processes in partial discharge (PD) phenomena are re viewed. The terminology used to specify different types or modes of PD are discussed in light of a general theory of electrical discharges. The limitations and assumptions inherent to present theoretical models are examined. The influence of memory propagation ef fects in controlling the stochastic behavior of PD is shown. Examples of experimental results are presented that demonstrate the nonstationary characteristics of PD which can be related to permanent or quasipermanent discharge-induced modifications (aging) of the site where the PD occur. Recommendations for future research are proposed.

\section{1,911}

PB95-198917 PC A03/MF A01

National Inst of Standards and Technology (EEEL) Boulder, CO. Electromagnetic Fields Div.

Electromagnetic Shielding Characterization of Gaskets.

D. A. Hill. Feb 95, 29p, NISTIR-5032.

Keywords: "Electromagnetic shielding, "Gaskets, Two dimensional models, Mathematical models, Reverberation chambers, Transfer impedance, Time domain, Slots, Shielding effectiveness, Coaxial fixtures

Numerous measurement methods are used for determining the shielding performance of $r$ gaskets, but different methods give significantly different results for 
the same gasket. Various measurement methods and the reasons for the discrepancies are reviewed. Simple models and theories are used to explain the meaning of transfer impedance and shielding effectiveness for gaskets and to determine the frequency range of validity. Transfer impedance should be a valid characterization at low frequencies, and shielding effectiveness is more appropriate at high frequencies. The precise frequency limitations of these characterizations and current measurement methods are not well known, but a time-domain method is proposed for determining gasket properties over a broad frequency range.

\section{1,912}

PB95-198925 PC A03/MF AO1

National Inst. of Standards and Technology (EEEL), Gaithersburg, MD. Semiconductor Electronics Div.

Electronics and Electrical Engineering Laboratory Technical Publication Announcements Covering Laboratory Programs, July to September 1994 with 1995 EEEL Events Calendar.

J. M. Rohrbaugh. Mar 95, 25p, NISTIR-5607.

See also PB94-213774.

Keywords: "Microelectronics, "Metrology, "Bibliographies, Electrical measurement, Integrated circuits, Integrated optics, High temperature superconductors, Superconducting films, YBCO superconductors, Superconducting devices, Electromagnetic interference, Magnetic measurement, Microwave equipment, Video equipment, Electric power, Antennas, Silicon, Abstracts, SOI(Semiconductors), Partial discharges, SIMOX.

This is the forty-second issue of a quarterly publication providing information on the technical work of the $\mathrm{Na}$ tional Institute of Standards and Technology, Electronics and Electrical Engineering Laboratory (EEEL). The issue of the EEEL Technical Publication Announcements covers the third quarter of calendar year 1994. Abstracts are provided by technical areas for papers published. Main topic areas include: Fundamenta Electrical Measurements; Semiconductor Microelectronics; Signal Acquisition, Processing, and Transmission; Electrical Systems; Electromagnetic Interference; Product Data Systems (includes net information tools); Video Technology; Additional Information.

01,913

PB95-202404 Not available NTIS

National Inst. of Standards and Technology (EEEL) Boulder, CO. Electromagnetic Fields Div.

Method to Determine the Calorimetric Equivalence Correction for a Coaxial Microwave Microcalorimeter.

Final rept.

F. R. Clague. 1994, 5p.

Pub. in Institute of Electrical and Electronics Engineers Transactions on Instrumentation and Measurement 43, n3 p421-425 Jun 94.

Keywords: "Power measurement, "Calorimeters, "Standards, Coaxial cables, Microcalorimetry, Reprints, "Microwave power standards, "Microcalorimeters, Reference standards, Thermistor mounts.

A way is presented to obtain the microcalorimeter correction factor by direct measurement rather than by an indirect estimate or modeling. The microcalorimeter is used to measure the effective efficiency of a reference standard thermistor mount. The correction factor accounts for the different thermal paths and losses in the microcalorimeter reference standard combination. The uncertainty in the measurement depends primarily on an accurate determination of the correction factor. This has been an especially difficult problem in the coaxial case because of the center conductor. The method requires the fabrication of components that duplicate the thermal and RF loss in the microcalorimeter and reference standard. Using the technique with the new $\mathrm{Na}$ tional Institute of Standards and Technology (NIST) type $\mathrm{N}$ coaxial microcalorimeter has substantially reduced the systematic uncertainty. The total uncertainty is about one-half the uncertainty of the prior NIST standard at frequencies above $1 \mathrm{GHz}$.

\section{1,914}

PB95-202412 Not available NTIS

National Inst. of Standards and Technology (EEEL). Boulder, CO. Electromagnetic Fields Div.
Developing a NIST Coaxial Microwave Power Standard at $1 \mathrm{~mW}$.

Final rept.

F. R. Clague, and J. D. Splett. 1994, $8 p$.

Pub. in Proceedings of National Conference of Standards Laboratories 1994 Workshop and Symposium, Chicago, IL., July 31-August 4, 1994, p291-298.

Keywords: "Power measurement, "Standards, Coaxial configurations, Calibration, Thermistors, Uncertainty, Error analysis, Reprints, *Microwave power standards, Reference standards, Thermistor mounts, US NIST.

Some customers of the NIST microwave power calibration sevvice report that they are using their calibrated reference standard at a power of about $1 \mathrm{~mW}$, rather than $10 \mathrm{~mW}$ where the NIST measurement is made. Since the coaxial reference standards accepted by NIST for calibration are dual-element thermistor mounts, they are subject to a dual-element substitution error if not used at the calibration power level. The error differs for each mount. The error is not easily measured, nor is it possible to readily estimate an additional uncertainty for using the mount at a different power. Initial measurements indicate the error can be up to 50 percent of the quoted calibration uncertainty. If the calibration uncertainty does not increase too much, a reasonable solution is to extend the NIST calibration service to powers under $10 \mathrm{~mW}$. This paper briefly describes the present standard and the ap proach being taken to add the $1 \mathrm{~mW}$ capability. Preliminary uncertainty estimates are included.

\section{1,915}

PB95-203519 Not available NTIS

National Inst. of Standards and Technology (EEEL), Boulder, CO. Electromagnetic Fields Div.

Compensation for Substrate Permittivity in ProbeTip Calibration.

Final rept.

D. F. Williams, and R. B. Marks. 1994, 11p.

Pub. in Proceedings of Automatic Radio Frequency Techniques Group Conference Digest (44th), Boulder, CO., December 1-2, 1994, p20-30.

Keywords: Capacitance, Compensation, Permittivity Substrates, Reprints, "Probe tip calibration, Multiline TRL method, On wafer measurement, Coplanar waveguides, Scattering parameters.

We demonstrate a method of compensation for the effect of substrate permittivity on coplanar waveguide probe-tip scattering parameter calibrations, modeling the effect as a capacitance at the probe tip. Comparison to on-wafer multiline TRL (through-reflect-line) calibration verifies its accuracy. The method allows calibration to the probe tip using generic off-wafe standards with accuracy comparable to that of onwafer calibration.

01,916

PB95-209821 PC A03/MF A01

National Inst. of Standards and Technology (EEEL), Gaithersburg, MD. Semiconductor Electronics Div.

Electronics and Electrical Engineering Laboratory Technical Progress Bulletin Covering Laboratory Programs, April to June 1991, with 1992 EEEL Events Calendar.

Oct $91,24 p$, NISTIR-4670

See also PB92-164672 and PB94-145968.

Keywords: "Microelectronics, "US NIST, "Metrology, Standards, Research projects, Electrical engineering, Semiconductor devices, Signal processing Electrooptics.

Contents includes research summaries on Fundamental Electrical Measurements; Semiconductor Microelectronics; Signal Acquisition, Processing and Transmission; Electrical Systems; and Electromagnetic Interference.

\section{1,917}

PB95-210480 PC A03/MF A01

National Inst. of Standards and Technology (EEEL), Gaithersburg, MD. Semiconductor Electronics Div. Electronics and Electrical Engineering Laboratory Technical Progress Bulletin Covering Laboratory Programs, January to March 1992, with 1992/1993 EEEL Events Calendar.

Rept. for Jan-Mar 92

J. A. Gonzalez. Sep 92 , 34p, NISTIR-4901.

See also PB92-164672 and PB94-165958.

Keywords: "Electrical engineering, "Electronics *Metrology, "US NIST, Semiconductor devices, Signa processing, Detectors, Electrical measurement, Electrooptics, Research projects.

Contents includes research summanies of: Semiconductor Microelectronics; Signal Acquisition, Processing, and Transmission; Electrical Systems; and Electromagnetic Interference.

\section{1,918}

PB95-231841 PC A03/MF A01

National Inst. of Standards and Technology (EEEL), Gaithersburg, MD. Semiconductor Electronics Div. Electronics and Electrical Engineering Laboratory Technical Publication Announcements Covering Laboratory Programs, October to December 1994 with 1995 EEEL Events Calendar.

J. M. Rohrbaugh. May 95, 34p, NISTIR-5649. See also PB95-198925.

Keywords: "Semiconductors, "Electrical engineering, "Signals, "Electrical systems, Electronics, Optical fibers, Microwaves, Semiconductors, Magnet measurement, "Electromagnetic interference.

This is the forty-third issue of a quarterly publication providing information on the technical work of the $\mathrm{Na}$ tional Institute of Standards and Technology, Electronics and Electrical Engineering Laboratory. This issue of the EEEL Technical Publication Announcements covers the fourth quarter of calendar year 1994. Abstracts are provided by technical areas for papers published.

01,919

PB95-261954 (Order as PB95-261897, PC A07)

MF A02)

National Inst. of Standards and Technology, Gaithersburg, MD.

High Accuracy Measurement of Aperture Area Relative to a Standard Known Aperture.

J. B. Fowler, and G. Dezsi. 1995, 7p.

Prepared in cooperation with National Office of Measures, Budapest (Hungary)

Included in $\mathrm{Jnl}$ of Research of the National Institute of Standards and Technology, v100 n3 p277-283 May/ Jun 95

Keywords: "Apertures, "Area, "Metrology, Accuracy, Calibration standards, Precision, Radiometers, Uncertainty, National Institute of Standards and Technology.

Precise knowledge of the area of apertures used in high precision radiometry is extremely important. A method is presented here for the determination of the area of round and irregularly shaped apertures by comparison to a standard aperture which has been measured by other means to high accuracy. The method presented here is quick and has no physical contact with the fragile edge of the aperture opening.

\section{1,920}

PB96-102496 Not available NTIS

National Inst. of Standards and Technology (EEEL), Boulder, CO. Electromagnetic Technology Div.

Voltage-Standard Devices.

Final rept.

C. A. Hamilton. 1992, 4p.

Pub. in Concise Encyclopedia of Magnetic and Superconducting Materials, p621-624 1992

Keywords: "Electrical measurement, "Standards, Measuring instruments, Volt-ampere characteristics, Electric current, Ohms Law, Electric equipment, Reprints.

The International Systems of Units, abbreviated SI, has been developed to meet the need for a uniform and consistent set of units. One of the most important and widely used of these units is the volt. The SI definition of the volt is that electromotive force between two points on a conductor carrying a constant current of 1 A when the power dissipated between the two points is $1 \mathrm{~W}$. The realization of the SI volt, therefore depends on experiment that relate the primary electrical units to mechanical units of length, force and power. Precise modem instrumentation requires voltage measurements with a reproducibility exceeding the accuracy with which the SI volt can be realized. To meet this need, realizations of the volt that are stable and reproducible at a level near $0.01 \mathrm{ppm}$ have been developed even though their value relative to the SI volt is uncertain within about $0.4 \mathrm{ppm}$.

01,921

PB96-102637 Not available NTIS 


\section{General}

National Inst. of Standards and Technology (EEEL), Gaithersburg, MD. Electricity Div.

Bonding Wires to Quantized Hall Resistors.

Final rept.

K. C. Lee. $1995,4 p$

Pub. in Institute of Electrical and Electronics Engineers Transactions on Instrumentation and Measurement, v44 n2 p249-252 Apr 95.

Keywords: "Resistors, "Wire bonding, Resistance meters, Quality, Reprints, "Bonding.

Three different techniques for attaching wires on quantized Hall resistors with gold-germanium-nickel (AuGe/ Ni) alloyed contacts were evaluated. The best quality and most robust samples were made by evaporating bonding pads that overlapped the alloyed contacts and the substrate, so that bonds could be made over the substrate rather than over the heterostructure.

\section{1,922}

PB96-103114 Not available NTIS

National Inst. of Standards and Technology (EEEL), Gaithersburg, MD. Electricity Div.

Nonstationary Behaviour of Partial Discharge during Discharge Induced Ageing of Dielectrics.

Final rept.

R. J. Van Brunt, P. Von Glahn, and T. Las. 1995, 9p. Sponsored by Nuclear Regulatory Commission, Washington, DC. Div. of Engineering.

Pub. in Institute of Electrical and Electronics Engineers Proceedings: Science, Measurement and Technology, v142 n1 p37-45 Jan 95.

Keywords: "Dielectric materials, "Electric discharges, *Aging(Materials), Monte Carlo method, Simulation, Pattern recognition, Stochastic processes, Surface properties, Electric conductivity, Reprints, Nonstationary behavior, Partial discharges, Point-dielectric gaps.

Changes in the stochastic behavior of pulsating partial discharge with time have been observed when an alternative voltage is applied to point-dielectric gaps in which the dielectric is a cast epoxy resin either with, or without, $\mathrm{Al} 2 \mathrm{O} 3$ filler. The changes in discharge behavior are shown, with the help of a Monte-Carlo simulation, to be consistent with discharge induced increases in the surface conductivity of the epoxy. This 'ageing' effect is shown to be accelerated as the frequency of applied voltage is increased from 50 to 800 $\mathrm{Hz}$. The implications of the results on accelerated aging tests and definition of partial discharge 'signatures' for possible pattern recognition are discussed.

\section{1,923}

PB96-106455 PC A03/MF A01

National Inst. of Standards and Technology (EEEL), Gaithersburg, MD. Semiconductor Electronics Div.

Electronics and Electrical Engineering Laboratory Technical Progress Bulletin Covering Laboratory Programs, April to June 1995 with 1995 EEEL Events Calendar.

J. M. Rohrbaugh. Sep 95, 36p, NISTIR-5709.

See also PB95-143186.

Keywords: "Electrical engineering, "Research projects, Semiconductor devices, Metrology, Laboratories, Abstracts, US NIST, Antennas, Lasers, Optical fibers, Superconductors.

This is the fifty-first issue of a quarterly publication providing information on the technical work of the National Institute of Standards and Technology, Electronics and Electrical Engineering Laboratory. This issue of the EEEL Technical Progress Bulletin covers the second quarter of calendar year 1995. Abstracts are provided by technical area for both published papers and papers approved by NIST for publication.

\section{1,924}

PB96-111851 Not available NTIS

National Inst. of Standards and Technology (EEEL),

Boulder, CO. Electromagnetic Fields Div.

Relative Accuracy of Isolated and Unisolated Noise Comparison Radiometers.

Final rept.

D. F. Wait. $1994,2 p$

Pub. in Conference on Precision Electromagnetic Measurements Digest, Boulder, CO., June 27-July 1 , 1994, p256-257.

Keywords: "Radiometers, "Noise measurement, Accuracy, Isolators, Noise reduction, Comparison, Estimates, Radiometric correction, Equations, Reprints.

$A$ rigorous radiometer equation is derived and used to develop better corrections and uncertainty estimates.
Isolated and well corrected unisolated radiometers have similar accuracies for low reflection coefficien sources. To ignore corrections for the finite isolation of a radiometer, about $38 \mathrm{~dB}$ isolation is needed.

01,925

PB96-112156 Not available NTIS

National Inst. of Standards and Technology (EEEL), Gaithersburg, MD. Electricity Div.

Continuous Recording and Stochastlc Analysis of PD.

Final rept.

P. von Glahn, and R. J. Van Brunt. 1995, 12p.

Pub. in Institute of Electrical and Electronics Engineers Transactions on Dielectrics and Electrical Insulation, v2 n4 p590-601 Aug 95.

Keywords: "Digital recording systems, "Stochastic analysis, Reprints, Design, Personal computers, Digitizers, Real time, "Partial discharges.

We describe the design and use of a digital partial discharge (PD) data recording system capable of continuous real-time recording of PD pulse trains. The recording system consists of a custom two-channel PD digitizer coupled to a personal computer via a 16-bit parallel interface. The digitizer is under software contro with the resulting data being stored in binary files on the computer's hard disk. The stored data subsequently are subjected to stochastic analysis using ap propriate computer software. Because all data are retained and the computer provides the desired stochastic analysis of data files, the new system is well suited to investigate non-stationary PD behavior such as encountered in aging studies. The results confirm and extend previous measurements made with an analog stochastic analyzer. With these sample results, we demonstrate how the system allows detailed stochastic analyzes not possible with data obtained from existing conventional PD measurement systems.

\section{1,926}

PB96-113568 (Order as PB96-113535, PC A05/ MF A01

National Institute of Standards and Technology, Boulder, CO.

Screened-Room Measurements on the NIST Spherical-Dipole Standard Radiator.

G. Koepke, and J. Randa. 1994, 13p.

Sponsored by Naval Air Systems Command, Washington, DC.

Included in Jnl. of Research of the National Institute of Standards and Technology, v99 n6 p737-749 Nov/ Dec 94.

Keywords: "Radiators, *Electromagnetic interference "Standards, Interlaboratory comparisons, Electromagnetic compatibility, Test chambers, Radiation sources, Field strength, Spherical configuration, Dipoles, Graphs(Charts) "Standard radiators, Screened-rooms, NIST(National Institute of Standards and Technology).

We report the results of a study of measurements of radiated emissions from the NIST spherical-dipole standard radiator in several screened rooms. The study serves as a demonstration of possible applications of the standard radiator as well as an investigation of radiated-emissions measurements in screened rooms. The screened-room measurements were performed in accordance with MIL-STD 462 (1967). Large differences occurred in the field intensity measured a different days at the same laboratory. There was a systematic difference at low frequencies between the screened-room results obtain in a transverse electromagnetic (TEM) cell, open-area test site (OATS), and anechoic chamber.

\section{1,927}

PB96-117767 PC A08/MF A02

National Inst. of Standards and Technology, Gaithersburg, MD.

Journal of Research of the National Institute of Standards and Technology, September/October 1995. Volume 100, Number 5 .

1995, $160 \mathrm{p}$

See also PB96-122098 through PB96-122148 and PB96-113311. Also available from Supt. of Docs. as SN703-027-00066-1.

Keywords: *Research and development, "Calibration standards, "Metrology, Reprints, Conductivity, Electric measuring instruments, Rockwell hardness, Diamond pyramid hardness tests, Performance tests, Plastic concrete, Blackbody radiation, NIST(National Institute of Standards and Technology).
Table of Contents:

Articles:

Low Electrolytic Conductivity Standards:

Potential and Current Distributions Calculated Across a Quantum Hall Effect Sample at Low and High Currents;

Microform Calibration Uncertainties of Rockwell Diamond Indenters:

Performance Measures for Geometric Fitting in the NIST Algorithm Testing and Evaluation Program for Coordinate Measurement Systems;

A Study on the Reuse of Plastic Concrete Using Extended Set-Retarding Admixtures:

A Third Generation Water Bath Based Blackbody Source.

\section{1,928}

\section{PB96-118039 PC A19/MF A04}

National Inst. of Standards and Technology, Gaithersburg, MD.

Opportunities for Innovation: Optoelectronics. D. E. Edgerly. May 95, 426p, NIST/GCR-95/672.

Keywords: "Electrooptics, "Technology utilization, *Fiber optics, Communication networks, Telecommun cations, Technology innovation, Local area networks, Cable television, Phased array antennas, Sensors, Holography, Automotive industry, Air pollution monitors, Optical equipment, Transmitters, Receivers, Electronics industry, "Optoelectronics.

Contents:

Preface:

Optoelectronic Technology Applications in Data Processing and Data Communications Networks;

Optoelectronic Technologies for Advanced Optical Telecommunications Networks;

The Evolving Cable Television Architecture; Intra-machine Optoelectronic Interconnection

Optoelectronics for Phased Array Antennas;

Fiber Optic Sensors:

Optoelectronic Technology for Optical Information Processing;

Opportunities for Optoelectronics in Holographic Data Storage Systems and Neural Networks;

Automotive Industry Applications of Optoelectronics;

and Electro-optical Instrumentation for Pollution Monitoring.

\section{1,929}

PB96-119235 Not available NTIS

National Inst. of Standards and Technology (TS), Gaithersburg, MD. Standards Code and Information Program.

Electrical Product Requirements (Especially Quality Requirements) in the United States.

\section{Final rept.}

M. Breitenberg. 1995, 10p.

Pub. in Proceedings of Seminar in Microelectronics and It's Applications, Campinas, Brazil, June 20-23, 1995, p1-10.

Keywords: "Electrical equipment, "Quality assurance, "Standards, "Certification, *United States, Testing, Quality control, Requirements, Specifications, Inspection, Assessments, Reliability, Reprints.

The US standardization, testing, certification, and quality system registration system is an intricate one, based on principles of openness and transparency. Responsibility for the saftey of elecctrical products is shared between the public and private sectors, with opportunity for consumers, government agencies at all levels, retailers, manufactors, and third party testing and inspection bodies to participate in the decisions that affect them. This paper attempts to answer some frequently raised questions on the U.S. system.

\section{1,930}

PB96-122486 Not available NTIS

National Inst. of Standards and Technology (EEEL) Gaithersburg, MD. Electricity Div.

Standardised Computer Data File Format for Storage, Transport, and Off-Line Processing of Partial Discharge Data.

Final rept.

T. Hucker, P. von Glahn, H. G. Kranz, and T.

Okamoto. $1995,4 p$

Pub. in International Symposium on High Voltage Engineering (9th). Subject 5: Dielectrics Diagnostics and Expert Systems, Graz, Austria, August 28-September $1,1995, \mathrm{p} 5613-\uparrow-5613-4$ 
Keywords: "Data files, "Data storage, "Formats, Electrical discharges, Data exchange, Data retrieval, Data processing, Data analysis, Reprints, "Partial discharges.

The authors present an overview of a proposed data file format for digitized partial discharge (PD) data. The proposed format will permit investigators at different institutions to exchange PD data and collaborate on the analysis and understanding of the PD phenomenon. The authors include an example in which investigators at all three institutions evaluated the same data record and report their analysis results.

01,931

PB96-122767 Not available NTIS

National Inst. of Standards and Technology (EEEL) Gaithersburg, MD. Electricity Div.

Influence of Surface Charge on the Stochastic Behavior of Partial Discharge in Dielectrics.

Final rept.

R. J. van Brunt, P. von Glahn, and T. Las. 1995, 8p. Pub. in Proceedings of the International Conference on Space Charge in Solid Dielectrics (2nd), Antibes Juan-las-Pins, France, April 2-7, 1995, p439-446.

Keywords: *Surface properties, "Charge distribution, "Dielectrics, "Stochastic processes, Pulse duration Electrical pulses, Aluminum oxides, Impurities, Statistical analysis, Electric discharges, Reprints, $\mathrm{PD}$ (Partial discharge), Surface charge.

Experimental techniques have been developed in recent years that enable a complete stochastic characterization of pulsating partial-discharge (PD) phenomena in terms of a set of conditional and unconditiona discharge pulse phase (or time), amplitude, and integrated-charge distributions and related correlation coefficients. Investigations using these techniques have shown the importance of pulse-to-pulse memory propagation on the statistical behavior of PD. It has been shown that the observed stochastic behavior of PD can be simulated using a theoretical model that invokes surface Charging and considers the effect of surface charge on the probability of discharge pulse initiation

01,932

PB96-122775 Not available NTIS

National Inst. of Standards and Technology (EEEL), Gaithersburg, MD. Electricity Div.

Comment and Discussion on Digital Processing of PD Pulses.

Final rept.

P. von Glahn. 1995, 15p

Pub. in Institute of Electrical and Electronics Engineers Transactions on Dielectrics and Electrical Insulation, v2 n4 p685-699 Aug 95.

Keywords: "Digital systems, "Signal processing "Pulses, Dielectrics, Pulse amplitude, Pattern recognition, Errors, Identification, Reprints, PD(Partial discharge), PD pulses.

Some of the more salient aspects of the digital processing technology of PD signals are examined. Most of the efforts in this field are concentrated on the application of digital analyzers for pulse height analysis pattern recognition and identification of the physical phenomena. It is demonstrated that errors in the signal processing unit can lead to dominant mistakes in the interpretation of the test results.

01,933

PB́96-122791 Not available NTIS

National Inst. of Standards and Technology (EEEL)

Boulder, CO. Electromagnetic Fields Div.

Electromagnetic Properties of Materials: The NIST Metrology Program.

Final rept.

C. M. Weil. 1993, 8p

Pub. in Proceedings of the DOD Electromagnetic Win dows Symposium (5th), Boulder, CO., October 19-21, 1993, v1 p175-182.

Keywords: "Electromagnetic properties, "Metrology, -Standards, Magnetic materials, Dielectrics, Electrical measurement, Microwave frequencies, Research programs, Reprints, US NIST.

The Electromagnetic Properties of Materials program at the National Institute of Standards and Technology (NIST) is described, including an outline of the current goals of the project and details of measurement techniques being used at NIST for characterizing dielectric and magnetic materials at RF and microwave frequencies.
01,934

PB96-123153 Not available NTIS

National Inst. of Standards and Technology (EEEL) Gaithersburg, MD. Electricity Div

Methods for Aligning the NIST Watt-Balance.

Final rept.

G. N. Stenbakken, R. Steiner, P. T. Olsen, and E. R. Williams. 1995, 5p.

Pub. in Proceedings of Instrumentation and Measurement Technology Conference, Waltham, MA., April 24 $26,1995, \mathrm{p} 247-251$

Keywords: "Magnetic forces, Electrical measurement, Simulation, Reprints, "Watt measurement, Induced voltage, Mass stability.

The NIST watt-balance has been developed to explore the possibility of monitoring the stability of the mass standard by means of electrical quantum standards. The mass standard is the last basic standard that is kept as an artifact. The watt-balance uses a movable coil in a radial magnetic field to compare the mechanical energy required to lift a kilogram mass in earth's gravity with the electrical energy required to move the coil the same distance in a magnetic field. The electrical energy is monitored in terms of quantized Hall resistance and Josephson's junction voltage standards. The accuracy of this experiment depends on a large number of factors. Among them are the ability to align the apparatus so that the movable coil and magnet are coaxial and aligned to the local vertical. Misalignment of the coil and magnet results in forces and torques on the coil. The coil is suspended like a pendulum, so responds easily to these torques and horizontal forces. This paper describes a computer program that was written to calculate the shape of the magnetic field and the torques and forces on the movable coil that result from any misalignments. This information is being used to develop an alignment procedures that minimizes misalignments and the errors they cause. This program has enhanced our understanding of the cause of torques about the vertical axis on the coil and the dependence of this torques on the magnetic field gradient.

01,935

PB96-123179 Not available NTIS

National Inst of Standards and Technology (EEEL), Gaithersburg, MD. Electricity Div.

Influence of Electrode Material on Measured Ion Kinetic-Energy Distributions in Radio-Frequency Discharges.

Final rept.

R. J. Van Brunt, J. K. Olthoff, and S. B. Radovanov.

$1995,2 p$

Pub. in Proceedings of the International Conference on Phenomena in Ionized Gases (22nd), Hoboken, NJ., July 31-August 4, 1995.

Keywords: "Gas discharges, "Radio trequency discharges, Mass spectrometry, Plasma, Surface charging, Reprints, Ion kinetic energy, Ion sampling.

Evidence is presented for a significant influence of electrode surface material and condition on the measurement of the kinetic energies of ions samples from discharges through an orifice in the electrode. Significant differences in ion energy shifts and/or discrimination of low-energy ions are found using aluminum and stainless-steel electrodes in a radio-frequency (if) discharge cell. It is argued that the observed differences in energy shifts may be attributable in part to differences in charging of oxide layers on the electrode surface around the sampling orifice.

\section{1,936}

PB96-123757 Not available NTIS

National Inst. of Standards and Technology (EEEL), Gaithersburg, MD. Electricity Div.

Physics and Chemistry of Partial Discharge and Corona - Recent Advances and Future Challenges. Final rept.

R. J. Van Brunt. 1995, 42p.

Pub. in 1994 Annual Report - Conference on Electrical Insulation and Dielectric Phenomena, Arlington, TX., October 23-26, 1994, p29-70 1995

Keywords: "Electric discharges, Electrical insulation, Dielectric breakdown, Electric corona, Aging(Materials), Stochastic processes, Reprints, *Partial discharges.

Results of recent research on physical and chemical processes on partial-discharge (PD) phenomena are reviewed. The terminology used to specify different types of modes of PD are discussed in the light of the general theory of discharges. The limitations and assumptions inherent to present theoretical models of PD are examined. The influence of memory propagation effects in controlling the stochastic behavior of PD is shown. Examples of experimental results are presented which demonstrate the nonstationary characteristics of PD which can be related to PD-induced aging of insulating materials. Recommendations for future research are proposed.

01,937

PB96-123781 Not available NTIS

National Inst. of Standards and Technology (EEEL), Gaithersburg, MD. Electricity Div.

Behavior of Surface Partial Discharge on Aluminum Oxide Dielectrics.

Final rept.

P. von Glahn, and R. J. Van Brunt. 1995, 7p

Pub. in 1995 Annual Report: Conference on Electrica Insulation and Dielectric Phenomena, Virginia Beach, VA., October 22-25, 1995, p365-371.

Keywords: "Dielectrics, "Partial discharge, Aluminum xide, Data recording, Behavior, Phase distributions, Purity effect, Statistical parameters, Reprints.

Partial discharge (PD) was generated with alternating voltage applied to a point electrode touching the surfaces of aluminum oxide (Al2O3) dielectrics of different purity in air. The amplitudes and phases of all pulsating PD events that occured during voltage application periods up to 35 minutes were recorded and the results were analyzed to reveal details of the characteristics of $P D$ exhibit rapid changes with time that depend significantly on voltage and the purity of the Al2O3 sam ple. Example are shown of statistical data for individual positive and negative pulses in a voltage cycle that are much more refined than data that can be obtained from conventional Pd measurment systems. The results presented here illustrate the advantages of using coninuous data records in the investigation and characterzation of pulsating PD phenomena.

01,938

PB96-128269 PC A05/MF A01

National Inst. of Standards and Technology (EEEL)

Boulder, CO. Electromagnetic Technology Div.

Metrology for Electromagnetic Technology: A Bibliography of NIST Publications, September 1995

A. G. Bradford. Sep 95, 81p, NISTIR-5040.

See also PB95-135588 and PB96-128210.

Keywords: "Metrology, "Bibliographies Superconductor devices, Magnetic measurements, Cryogenics, Josephson junctions, High temperature superconductors, US NIST, Cryoelectronics, Electromagnetic metrology

This bibliography lists the publications of the personne of the Electromagnetic Technology Division of NIST during the period from January 1970 through the publication of this report. A few earlier references that are directly related to the present work of the Division are also included. This edition of the bibliography is the first since the Electromagnetic Technology Division split into two Divisions, and it includes publications in cryoelectronic metrology and superconductor and magnetic measurements. The optical electronic metrology section found in earlier editions is now being produced seperately by the new Optoelectronics Division of NIST. That companion bibliography to this publication is NISTIR 5041.

01,939

PB96-128277 PC A08/MF A02

National Inst of Standards and Technology (PL) Gaithersburg, MD. Radiometric Physics Div.

Metrology Issues in Terahertz Physics and Technology.

R. U. Datla, E. Grossman, and M. K. Hobish. $13 \mathrm{Dec}$ 94, 164p, NISTIR-5701.

Proceedings of a workshop held in Gaithersburg, MD on December 13, 1994.

Keywords: "Metrology, "Physics, "Meetings, Technology utilization, Infrared radiation, Research and development, Radiation measuring instruments, Accuracy, Calibrating, Standards, Infrared equipment, "Terahertz physics, THz technology.

Tremendous progress has been made in recent years in the development of new sources, detectors, antennas, and materials for the terahertz (THz) spectral region, i.e., from $100 \mathrm{GHz}$ ( $3 \mathrm{~mm}$ wavelength) to $10 \mathrm{THz}$ (30 micro $m$ wavelength). However, very little attention 
has been paid to questions of accurate measurement techniques, calibration, and standards at $\mathrm{THz}$ frequencies. In order to discuss metrology issues and the role NIST might play in the rapidly advancing field of THz technology, the staff in various divisions of NIST'S Physics Laboratory, in collaboration with the staff in the Electronics and Electrical Engineering Laboratory (EEEL), convened a Workshop on Metrology Issues in $\mathrm{THz}$ Physics and Technology. The workshop was held at NIST on December 13,1994 , and was attended by
50 representatives from industry, academia, and federal agencies.

\section{1,940}

PB96-135116 Not available NTIS

National Inst. of Standards and Technology (EEEL),

Boulder, CO. Electromagnetic Fields Div.

Analysis of an Open-Ended Coaxial Probe with LiftOff for Nondestructive Testing.

Final rept

J. Baker-Jarvis, M. D. Janezic, P. D. Domich, and R. G. Geyer. 1994, 8p.

Pub. in Institute of Electrical and Electronics Engineers Transactions on Instrumentation and Measurement, v43 n5 p711-718 Oct 94

Keywords: *Calibration, *Dielectric constants, Reprints, Loss factor, Microwaves, Nondestructive, Permeability, Measurement, ${ }^{\star}$ Coaxial probes, Open ended probe.

The open-ended coaxial probe with lift-off is studied using a full-wave analysis, and an uncertainty analysis is presented. The field equations for the following terminations are worked out: (1) the sample extends to infinity in the positive axial direction, (2) the sample is backed by a well-characterized material, and (3) the sample is backed by a short-circuit termination. The equations are valid for both dielectric and magnetic materials. The model allows the study of the openended coaxial probe as a nondestructive testing tool. The analysis allows a study of the effects of air gaps on the probe measurements. The reflection coefficient and phase are studied as a function of lift-off, coaxial line size, permittivity, permeability, and frequency. Numerical results indicate the probe is very sensitive to lift-off. For medium to high permittivity values and electrically small probes, gaps on the order of fractions of a millimeter strongly influence the reflection coefficient.

\section{1,941}

\section{PB96-137187 PC A03/MF A01}

National Inst. of Standards and Technology (EEEL), Gaithersburg, MD. Semiconductor Electronics Div. Electronics and Electrical Engineering Laboratory Technical Publication Announcements Covering Laboratory Programs, April to June 1995 with 1995 EEEL Events Calendar.

J. M. Rohrbaugh. Dec 95, 23p, NISTIR-5773.

See also PB95-242277.

Keywords: "Electrical engineering, "Electronics, "Research management, "Electrical measurement, Semiconductor devices, Metrology, Signal processing, Electromagnetism, Fiber optics, Abstracts, Catalogs(Publications), Bibliographies, Superconductors, Antennas, Electric power, Lasers, *National Institute of Standards and Technology.

This is the forty-fifth issue of quarterly publication providing information on the technical work of the Nationa Institute of Standards and Technology, Electronics and Electrical Engineering Laboratory. This issue of the EEEL Technical Publication Announcements covers the second quarter of calendar year 1995. Abstracts are provided by technical areas for papers published.

\section{1,942}

\section{PB96-147905 PC A04/MF A01}

National Inst. of Standards and Technology (EEEL), Gaithersburg, MD. Semiconductor Electronics Div.

Electronics and Electrical Engineering Laboratory Technical Progress Bulletin Covering Laboratory Programs, July to September 1995 with 1996 EEEL Events Calendar.

J. M. Rohrbaugh. Jan 96, 37p, NISTIR-5774.

See also PB96-106455.

Keywords: "Electrical engineering, "Research projects, Semiconductors, Electromagnetic interference, Signals, Electrical systems, Metrology, Abstracts.

Contents:

Introduction;

Fundamental Electrical Measurements;

Semiconductor Microelectronics:
Signal Acquisition, Processing, and Transmission; Electrical Systems;

Electromagnetic Interference:

Additional Information.

\section{1,943}

PB96-155452 Not available NTIS

National Inst. of Standards and Technology (EEEL), Boulder, CO. Electromagnetic Fields Div.

Catalogue of Electromagnetic Environment Measurements, $30-300 \mathrm{~Hz}$.

Final rept.

J. Randa, D. Gilliland, W. Gjertson, W. Lauber, and W. Mclnerney. $1995,8 p$.

See also PB95-162210.

Pub. in Institute of Electrical and Electronics Engineers Transactions on Electromagnetic Compatability, v37 n1 p26-33 Feb 95

Keywords: *Electromagnetic environments, " Extremely low frequency, "Bibliographies, Electromagnetic fields, Electromagnetic measurements, Bandwidth, Reprints.

The IEEE Electromagnetic Compatibility Society's Technical Committee on Electromagnetic Environments (TC-3) has undertaken a long-term project to compile an inventory or catalogue of published meas urements of electromagnetic envionments. The au thors have now completed the $30-300 \mathrm{~Hz}$ band. The paper presents the resulting bibliography, along with a brief overview of what has been measured.

\section{1,944}

PB96-156062 Not available NTIS

National Inst. of Standards and Technology (EEEL), Boulder, CO. Electromagnetic Fields Div.

NIST Metrology Program on Electromagnetic Characterization of Materials.

Final rept.

C. M. Weil. 1994, 14p.

See also PB96-122791.

Pub. in Proceedings of the Symposium on Materials and Processes for Wireless Communication, Boston, MA., November 15-16, 1994, p35-48.

Keywords: "Electromagnetic properties, ${ }^{\star}$ Ceramics, "Polymers, "Microwave communities, Dielectric materials, Magnetic materials, Electrical measurement, Wireless communications, Substrates, Metrology, Research programs, Reference standards, Reprints.

The Electromagnetic Properties of Materials (EPM) Program at the National Institute of Standards and Technology (NIST) is described, including an outline of the current goals of the project and details of meas urement techniques being used at NIST for characterizing dielectric and magnetic materials of importance on wireless communications in the RF spectrum of interest.

\section{1,945}

PB96-157854 Not available NTIS National Inst. of Standards and Technology (EEEL) Boulder, CO. Electromagnetic Fields Div.

Applicability of Effective Medium Theory to Ferroelectric/Ferrimagnetic Composites with Composition and Frequency-Dependent Complex Permittivities and Permeabilities.

Final rept.

R. G. Geyer, J. V. Mantese, A. L. Micheli, J.

Grosvenor, D. F. Dungan, and J. Baker-Jarvis. 1996,

Pub. in Jnl. of Applied Physics, v79 n3 p1655-1660 Feb 96.

Keywords: "Composites, "Ferrites, Microwaves, Permeability, Permittivity, Reprints, Effective medium, Mixing rules.

High-frequency (1 $\mathrm{MHz}-1 \mathrm{GHz}$ ) transmission line measurements were used to determine the composition and frequency-dependent complex permitivities and complex permeabilities of ferroelectric ferrimagnetic (barium titanate and a magnesium-copper-zinc ferrite) composites. The effective medium rules of Maxwell-Garnett give both lower and upper bounds for the effective permittivities and permeabilities and yield accurate estimates of the bulk electric and magnetic properties at low volume fill fraction of either component provided the proper host matrix is chosen. Bruggeman theory yielded the best predictive values for the permittivity and permeability ove the entire composition range. In all cases these complex quantities were shown to be constrained by Bergman-Milton bounds.
01,946

PB96-157862 Not available NTIS

National Inst. of Standards and Technology (EEEL) Boulder, CO. Electromagnetic Fields Div.

Influence of Films' Thickness and Air Gaps in Sur. face Impedance Measurements of High Temperature Superconductors Using the Dielectric Resonator Technique.

Final rept.

R. G. Geyer, J. Ceremuga, J. Krupka, and J. Modelski. 1995, 5p

Pub. in Institute of Electronics, Information and Communications Engineers Transactions on Electronics, Japan, vE78-C n8 p1106-1110 Aug 95.

Keywords: `Dielectric resonators, *Surface resistance, Reprints, "Foreign technology, "High temperature superconductors.

The dielectric resonator technique is commonly used for microwave surface resistance measurements of High Temperature superconducting (HTS) films. Thickness of superconductors and its impact on measurement results has not been taken into consideration so far. A theoretical mode-match solution analysis of a TE011 $10 \mathrm{GHz}$ sapphire resonator was performed. The results of this analysis demonstrate that the thickness of the films under test can significantly affect the resonant frequencies (Fres) and quality factor $Q$ of the resonant system, particularly when the thickness is less than three times the penetration depth lambda of the films at the operating temperature.

01,947

PB96-157888 Not available NTIS

National Inst. of Standards and Technology (EEEL), Boulder, CO. Electromagnetic Fields Div

Two-Tier Multiline TRL for Calibration of Low-Cost Network Analyzers.

Final rept.

J. A. Jargon, and R. B. Marks. 1995, 8p.

Pub. in Automatic RF Techniques Group Meeting (ARFTG), Scottsdale, AZ., November 30-December 1, 1995, p1-8.

Keywords: *Network analyzers, *Scattering parameters, Wireless, Calibration, Measurement, Multiline, Reprints, TRL, Two-tier.

We compare calibrations for use on three-sampler vector network analyzers (VNAs) which do not allow the direct application of some advanced error-correction schemes such as TRL (thru-reflect-line). Here we compare various alternatives, including an approximate version of TRL that has been introduced commercially and two-tier multiline TRL using external software. We consider both coaxial and coplanar open-short-loadthru (OSLT) calibrations for the first tier, showing that the latter can lead to inaccuracies. Finally, we investigate the stability of the load reflection terms to show that the first tier calibration need not be frequently repeated.

\section{1,948}

PB96-158688 PC A06/MF A01

National Inst. of Standards and Technology (MSEL), Gaithersburg, MD. Ceramics Div.

Conference Proceedings: International Workshop on Instrumented Indentation. Held in San Diego, California on April 22-23, 1995.

Special pub.

D. T. Smith. Feb 96, 78p, NIST/SP-896.

Also available from Supt. of Docs. as SN003-00303386-3.

Keywords: *Instruments, "Indentation hardness tests, *Meetings, Calibration standards, Thin films, Metal coatings, Mechanical properties, Nanoindentation, NIST(National Institute of Standards and Technology).

An international workshop was held on April 22-23, 1995, at the Town and Country Hotel in Sand Diego, to discuss the scientific and standardization issues as. sociated with instrumented identation, also known as dynamic hardness testing or depth-sensing, ultra-lowload, or nano indentation. The workshop was sponsored jointly by the NIST Standard Reference Materials Program and the Institute for Mechanics and $\mathrm{Ma}$ terials in San Diego, with additional support for student travel from Nano Instruments, Inc., Oak Ridge, TN, and Instron Corporation, Canton, MA, and was part of the program of the 1995 International Conference on Metallurgical Coatings and Thin Films (ICMCTF95).

01,949

PB96-159215 PC A08/MF A02 
National Inst. of Standards and Technology, Gaithersburg, MD.

Journal of Research of the National Institute of Standards and Technology, November/December 1995. Volume 100, Number 6 .

1995, 144p.

See also PB96-159223 through PB96-159264 and PB96-117767. Also available from Supt. of Docs. as SN-703-027-00067-9.

Keywords: "Research and development, "Calibration standards, "Metrology Performance tests, NIST(National Institute of Standards and Technology).

Contents:

Calibration of Electret-Based Integral Radon Monitors Using NIST PolyethyleneEncapsulated 226Ra/222Rn Emanation (PERE) Standards;

Microstructural Characterization of CobaltTungsten Coated Graphite Fibers;

On Using Collocation in Three Dimensions and Solving a Model Semiconductor Problem;

Precision Tests of a Quantum Hall Effect Device

DC Equivalent Circuit Using Double-Series and Triple-Series Connections;

Analysis of the $(5 d 2+5 d 6 s)-5 d 6 p$ Transition Arrays of OS VII and Ir VIII, and the 6s 2S6p2P Transitions of Ir IX.

01,950

PB96-159223 (Order as PB96-159215, PC A07)

MF A02)

National Inst. of Standards and Technology,

Gaithersburg, MD.

Calibration of Electret-Based Integral Radon Monitors Using NIST Polyethylene-Encapsulated (226)Ra/(222)Rn Emanation (PERE) Standards.

R. Colle, P. Kotrappa, and J. M. R. Hutchinson.

1995, 10p.

Prepared in cooperation with Rad Elec Inc., Frederick, MD.

Included in Jnl. of Research of the National Institute of Standards and Technology, v100 n6 p629-639 Nov/ Dec 95

Keywords: "Calibration standards, "Metrology, "Electret, Emanation, Environment, Ionization chamber, Measurement, Radium-226, Radon-222.

The recently developed $222 \mathrm{Rn}$ emanation standards that are based on polythylene-encapsulated 226Ra solutions were employed for a first field-measurement application test to demonstrate their efficacy in calibrating passive integral radon monitors. The performance of the capsules was evaluated with respect to the calibration needs of electret ionization chambers (E-PERM, Rad Elec Inc.). The encapsulated standards emanate well-characterized and known quanties of $222 \mathrm{Rn}$, and were used in two different-sized, relatively-small, accumulation vessels (about $3.6 \mathrm{~L}$ and $10 \mathrm{~L}$ ) which also contained the deployed electret monitors under test.

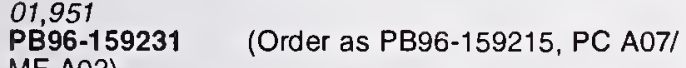

MF A02)

National Inst. of Standards and Technology, Gaithersburg, MD.

Microstructural Characterization of Cobalt-Tungsten Coated Graphite Fibers.

N. S. Wheeler. 1995, 18p.

Prepared in cooperation with Virginia Univ., Charlottes ville. Dept. of Materials Science and Engineering.

Included in Jnl. of Research of the National Institute

of Standards and Technology, v100 n6 p641-659 Nov' Dec 95

Keywords: "Carbon, "Cobalt, "Tungsten, Composite, Graphite, TEM, XRD.

The research concerns an electrodeposited cobalttungsten alloy coating on graphite fibers. Annealed and unannealed coated fibers were analyzed by scanning electron microscopy/energy dispersive $x$-ray spectroscopy (SEM/EDS), x-ray diffraction (XRD), and transmission electron microscopy (TEM). The mole fraction of tungsten in the as-deposited cobalt-tungsten coating was found to be ( 7.10 plus or minus 0.82$) \%$, and the crystalline lattice was determined to be hexagonal closepacked. Note: The uncertainties reported here are expanded uncertainties (i.e., 2 standard deviation estimates).

01,952

PB96-159249

(Order as PB96-159215, PC A07/

MF A02)
National Inst. of Standards and Technology, Gaithersburg, MD.

Using Collocation in Three Dimensions and Solving a Model Semiconductor Problem.

J. F. Marchiando. 1995, 15p

Included in Jnl. of Research of the National Institute of Standards and Technology, v100 n6 p661-676 Nov/ Dec 95.

Keywords: "Boundary, "Collocation, "Semiconductors, Poisson's equation, Three dimensional.

A research code has been written to solve an elliptic system if coupled nonlinear partial differential equa tions of conservation form on a rectangulary shaped three-dimensional domain. The code uses the method of collation of Gauss points with tricubic Hermite piecewise continuous polynomial basis functions. The system of equations is solved by iteration. The system of nonlinear equations is linearized, and the sysem of linear equations is solved by iterative methods.

01,953

PB96-159256 (Order as PB96-159215, PC A07/

MF A02)

National Inst. of Standards and Technology, Gaithersburg, MD.

Precision Tests of a Quantum Hall Effect Device DC Equivalent Circuit Using Double-Series and Triple-Series Connections.

A. Jeffery, R. E. Elmquist, and M. E. Cage. 1995, 8p. Included in Jnl. of Research of the National Institute of Standards and Technology, v100 n6 p677-685 Nov/ Dec 95.

Keywords: "Electrical circuits, "Circuits, "Quantum Hall effect, Cryogenic current comparator, Two dimensional electron gas.

Precision tests verify the dc equivalent circuit used by Ricketts and Kemeny to describe a quantum Hall effect device in terms of electrical circuit elements. The terms employ the use of cryogenic current comparators and the double-series and triple-series connection techniques of Delahaye. Verification of the dc equivalent circuit in double-series and triple-series connections is a necessary step in developing the acquantum Hall effect as an intrinsic standard of resistance.

01,954

(Order as PB96-159215, PC A07/

National Inst. of Standards and Technology, National Inst.

Analysis of the $(5 d(2)+5 d 6 s)-5 d 6 p$ Transition Arrays of Os VII and Ir VIII, and the 6s (2)S-6p (2)P Transitions of Ir IX.

G. J. van het Hof, Y. N. Joshi, J. F. Wyart, and J.

Sugar. 1995, 10p

Prepared in cooperation with Centre National de la Recherche Scientifique, Orsay (France). Lab. Aime Coton. and Saint Francis Xavier Univ., Antigonish (Nova Scotia). Dept. Physics.

Included in Jnl. of Research of the National Institute of Standards and Technology, v100 n6 p687-697 Nov/ Dec 95.

Keywords: "Energy levels, "Iridium, "Osmium, Spectra.

The spectra of osmium and iridium were photographed in the 300 Angstroms to 1600 Angstroms region on a $3 \mathrm{~m}$ normal incidence spectrograph using a triggered spark source. The $(5 d 2+5 d 6 s)-5 d 6 p$ transition arrays of Os VII and Ir VIII were analyzed. All levels of these configurations in both spectra have been established. There are 77 lines in Os VII and 71 lines in Ir VIII classified. The parametric least squares fitting calculations are used to interpret both spectra. The $6 s 251 / 2$ $6 \mathrm{p} 2 \mathrm{P} 1 / 2,3 / 2$ transitions in Ir IX have also been identified.

\section{1,955}

\section{Not available NTIS}

National Inst. of Standards and Technology (CSTL), Gaithersburg, MD. Inorganic Analytical Research Div. Faraday Constant.

Final rept.

W. F. Koch. 1991, 6p

Pub. in Units and Fundamental Constants, Subvolume b: Fundamental Constants, Chapter 3.2.13, p3-213-3218.

Keywords: "Faraday constant, "Electrochemistry, Cou lomb, Reprints, Coulometry, Electricity, Silver, "Foreign technology, Fundamental physical constant.

The discovery of the quantitative relationship among electric current, time, and the amount of chemical change effected by the electric current was the first announced by Michael Faraday in the 1830's (1839 F) Because of religious convictions, Faraday, himself, never denived a mathematical expression for these laws of nature. However, these laws may be simply expressed in summary as: mole $=(1 / F) f l d t$, where mole is the amount of substance, $I$ is the current in amperes and $t$ is the time in seconds. The proportionality factor $F$ has been appropriately named the Faraday constant (or just Faraday) and has units of coulombs per mole of univalent substance, $\mathrm{C} / \mathrm{mol}$. (A mole of univalent substance is synonymous with the term 'equivalent'.)

\section{1,956}

PB96-160320 Not available NTIS

National Inst. of Standards and Technology (EEEL) Gaithersburg, MD. Semiconductor Electronics Div.

Electron and Hole Trapping in Irradiated SIMOX, ZMR and BESOI Buried Oxides.

Final rept

R. E. Stahlbush, G. J. Campisi, J. B. McKitterick, G. A. Brown, W. P. Maszara, and P. Roitman. 1992, $12 \mathrm{p}$

Pub. in Institute of Electrical and Electronics Engineers Transactions on Nuclear Science, v39 n6 p2086-2097 Dec 92.

Keywords: "Buried oxides, "Electron traps, Reprints, "RESOI, Silicon on insulator, SIMOX, ZMR

Shallow electron and deep hole trapping in the buried oxides of SIMOX, ZMR and BESOI material are examined. By irradiating the oxides with $x$-rays at cryogenic temperatures, 40 - $50 \mathrm{~K}$, hole motion is frozen and electrons are trapped. The oxide charge is determined by $\mathrm{C}-\mathrm{V}$ measurements. Following the cryogenic irradiation, the electrons are detrapped by field stressing (tun neling) or by annealing (thermal excitation). Hole trap ping is examined by annealing after the trapped electrons are removed by field stressing. Substantial shallow electron and deep hole trapping distributed uniformly through the oxide is observed for all buried oxides that are processed above about 1100 degrees $C$. A comparison to thermal oxides grown at 850 degress $C$ and annealed at 1300 degress $C$ with and without a polysilicon capping layer shows that the top silicon layer significantly increases trap formation.

01,957

PB96-160353 Not available NTIS

National Inst. of Standards and Technology (EEEL) Gaithersburg, MD. Semiconductor Electronics Div.

Effect of Single versus Multiple Implant Processing on Defect Types and Densities in SIMOX.

Final rept.

D. Venables, S. J. Krause, J. C. Park, J. D. Lee, and

P. Roitman. 1993, 2p.

Pub. in Proceedings of the Institute of Electrical and Electronics Engineers SOI Conference, Palm Springs, CA., October 1993, p48-49.

Keywords: "Silicon, Defects, Reprints, "SIMOX, TEM, Silicon on insulator, Stacking faults.

Silicon-on-insulator material fabricated by high-dose oxygen implantation (SIMOX) has applications for high speed, radiation hard and low power devices. However, a continuing problem has been crystalline defects in the top silicon layer. SIMOX is fabricated by two dis tinct methods: a single oxygen implant to a dose of $1.8 \times 10$ to the 18 th power $\mathrm{cm}$ to the minus 2 power followed by a high-temperature anneal (equal to or greater than 1300 degress $C, 4-6 \mathrm{hr}$ ) or by multiple lowe dose implants (approx. $6 \times 10$ to the 17 th power $\mathrm{cm}$ to the minus 2 power) with high-temperature anneals after each implant. To data, there has been no system. atic companison of the defect structures produced by these two fabrication methods. Therefore, we have compared the defect structure and densities on multiple vs. single implant wafers produced on a high current implanter. In this paper we describe the origin and characteristics of the defect structures in contemporary SIMOX and show how their densities are controlled by the processing method and conditions.

01,958

PC A07/MF A02

National Inst. of Standards and Technology (EEEL), Boulder, CO. Electromagnetic Fields Div.

Calibration Service for Coaxlal Reference Standards for Microwave Power.

Technical note.

F. R. Clague. May 95, 116p, NIST/TN-1374

Also available from Supt. of Docs. as SNO03-003 03348-1. 
Keywords: "Microwaves, "Power measurement, "Coaxial configurations, "Calibration standards, Calorimeters, Thermistors, Error analysis, Uncertainty, Frequencies, NIST(National Institute of Standards and Technology), Microwave power standards, Thermistor mounts.

A calibration service at the National Institute of Standards and Technology (NIST) for coaxial microwave power reference standards is described. The service provides measurements of the reference standard effective efficiency from $50 \mathrm{MHz}$ to $18 \mathrm{GHz}$ at a power level of $10 \mathrm{~mW}$. In the Report of Calibration, the effective efficiency is reported in percent. The NIST microwave power standards consist of both a microcalorimeter and an associated reference standard. The reference standard is a bolometric type power detector (a thermistor mount). The only thermistor mounts accepted for measurement are those constructed to NIST specifications which are compatible with the coaxial microcalorimeter. These thermistor mounts and the automated microcalorimeter are described. A detailed error analysis with an estimate of the calibration uncertainties and their sources is included.

\section{1,959}

PB96-164520 PC A05/MF A01

National Inst. of Standards and Technology (EEEL) Gaithersburg, MD

Electronics and Electrical Engineering Laboratory 1995 Technical Accomplishments: Advancing Metrology for Electrotechnology to Support the U.S. Economy.

J. Surette. Dec 95, 72p, NISTIR-5818.

See also PB96-147905.

Keywords: *Electrical engineering, "Research and de velopment, "Metrology, Standards, US NIST, Semiconductor devices, Superconductors, Magnetic properties, Microwaves, Display devices, Electromagnetic compatibility, Research management.

Table of Contents:

Laboratory Director's Message;

EEEL and its Customer Interactions;

Fiscal Year 1995 Activities;

Selected FY 1995 Technical Accomplishments

Programs Matrix-Managed by EEEL;

EEEL Awards and Recognition;

FY 1995 CRADAs;

and EEEL Organization.

01,960
PB96-165410 PC A07/MF A02

National Inst. of Standards and Technology (EEEL), Boulder, CO. Electromagnetic Fields Div.

Band-Limited, White Gaussian Noise Excitation for Reverberation Chambers and Applications to Radiated Susceptibility Testing.

Technical note.

M. L. Crawford, T. A. Loughry, M. O. Hatfield, and G. J. Freyer. Jan 96, 112p, NIST/TN-1375.

Also avallable from Supt. of Docs. as $\mathrm{SNO03-003-}$ 03392-8.

Keywords:

${ }^{\star}$ Reverberation

chambers,

"Electromagnetic shielding, "Electromagnetic susceptibility, Bandwidth, Shielding effectiveness, Mode stirred chambers, Frequency stirring.

The report gives the results of demonstration tests conducted to (1) evaluate the electromagnetic environment (EME) produced by band-limited, white gaussian noise (BLWGN) excitation of a reverberation chamber and to verify its applications to susceptibility and shielding effectiveness (SE) testing. Data were collected to compare the EME produced in a reverberation chamber by $\mathrm{CW}$ and swept frequency excitation using both mechanical stirring and BLWGN to excite the cavity mode structure. The feasibility of using the BLWGN technique for radiated susceptibility testing was evaluated by comparison with mechanical stirring in a reverberation chamber and with anechoic chamber results. Within normal measurement uncertainties, the response of both types of systems were the same fo mechanical stirring and for BLWGN excitation and were consistent with the results obtained in the anechoic chamber.

\section{1,961}

PB96-165998 PC A05/MF A01

National Inst. of Standards and Technology (MSEL), Gaithersburg, MD. Polymers Div.
Beyond the Technology Roadmaps: An Assessment of Electronic Materials Research and Development.

M. A. Schen, T. J. Russell, R. F. Leheny, G. Borsuk, A. Yang, H. Simon, and V. Hess. Mar 96, 60p, NISTIR-5777.

Prepared in cooperation with Defense Advanced Research Projects Agency, Arlington, VA., Department of Commerce, Washington, DC. Technology Administration., National Science Foundation, Arlington, VA. and Naval Research Lab., Washington, DC

Keywords: "Electronic equipment, "Microelectronics, *Materials, Photonics, Research management, Microwaves, Radiofrequency, Electronic industry, Modules(Electronics), Mass storage.

This report, produced by the National Science and Technology Council, Electronic Materials Working Group, captures the findings of the December 6-7. 1994 industry-government-university Workshop on Electronic Materials held in Dallas, TX. It provides summary of the dominant electronics materials issues facing U.S. industry and contains recommendations critical to the advancement and competitiveness of the U.S. electronics and materials industries. The technologies encompassed within this report are microelectronics, radio frequency and microwave electronics, photonics, mass storage, and module interconnection. In addition, materials characterization and materials research, two areas essential to the understanding, discovery and utilization of materials, are included.

01,962

PB96-175237 PC A10/MF A03

National Inst. of Standards and Technology (EEEL), Gaithersburg, MD.

Electronics and Electrical Engineering Laboratory: 1996 Program Plan. Supporting Technology for U.S. Competitiveness in Electronics.

L. L. Sacchet. 1996, 198p, NISTIR-5832.

See also PB95-159885.

Keywords: "Electronic industry, "Electrical measurement, "Metrology, "Technology transfer, "Electronics, Electric equipment, Intemational trade, Competition, Microelectronics, Law enforcement, Standards, Electric utilities, Semiconductor devices, Electromagnetic compatibility, Electromagnetic fields, Optoelectronics devices, Commercialization, Abstracts, Research projects

The Electronics and Electrical Engineering Laboratory (EEEL), working in concert with other NIST Laboratories, is providing measurement capability and other generic technology critical to the competitiveness of the U.S. electronics industry and the U.S. electricalequipment industry. This 1996 Program Plan describes the technical projects that EEEL plans to conduct in fiscal year 1996 to provide metrological support to U.S. industry.

01,963

PB96-176573 Not available NTIS

National Inst. of Standards and Technology (MSEL), Gaithersburg, MD. Ceramics Div.

Electromagnetic Coupling Character of (001) Twist Boundaries in sintered Bi2Sr2CaCu2O8 $+x$ Bicrystals.

J. L. Wang, X. Y. Cai, R. J. Kelley, D. C

Larbalestier, M. D. Vaudin, and S. E. Babcock. 1994 $10 \mathrm{p}$.

Pub. in Physica C, v230 p189-198 1994.

Keywords: “Bicrystals, "Electromagnetic coupling, Reprints, "Critical current density, Grain boundaries, Weak coupling, Weak links.

The electromagnetic characteristics of (001) twist grain boundaries in Bi2Sr2CaCu2O8+x (BSCCO-2212) have been deduced from measurements of the resistive transitions, voltage-current (V-I) characteristics, and field-dependent transport critical current densities $(\operatorname{Jct}(\mathrm{H}))$ of single- and bicrystalline samples. The bicrystals were prepared by solid-state sintering of (001) faces of freshly-cleaved, bulk-scale single crystals placed at a pre-determined misorientation angle, $O$. A low-angle 2 degrees bicrystal was strongly coupled with $\mathrm{V}-\mathrm{I}$ and $\mathrm{Jct}(\mathrm{H})$ characteristics that were indistinguishable from those of the single crystals, indicating that the sintering process itself does not produce weak coupling. 30 degrees and 36 degrees (001) bicrystals were weakly coupled. 23 degrees and 88 degrees twist bicrystals were strongly coupled, even though their boundary Tc values were reduced. This behavior contrast starkly with that of (001) tilt boundaries in YBa2Cu3O7-, where a reduced boundary Tc is always associated with weak coupling.

\section{1,964}

PB96-180070 Not available NTIS

National Inst. of Standards and Technology (EEEL), Gaithersburg, MD. Semiconductor Electronics Div. Scanning Capacitance Microscopy Measurements and Modeling: Progress Towards Dopant Profiling of Silicon.

Final rept.

J. J. Kopanski, J. F. Marchiando, and J. R. Lowney. 1996, 6p.

See also PB96-148150 and PB96-164207.

Pub. in Jnl. of Vacuum Science and Technology B, v14 n1 p242-247 Jan/Feb 96.

Keywords: *Capacitance imaging, Modeling, Collocation, Reprints, "Scanning capacitance microscopy, ${ }^{\star}$ Poisson's equation.

A scanning capacitance microscope (SCM) has been implemented by interfacing a commercial contactmode atomic force microscope with a high-sensitivity capacitance sensor. The SCM has promise as a nextgeneration dopant-profiling technique because the measurement in inherently two dimensional, has a potential spatial resolution limited by tip diameter of at least $20 \mathrm{~nm}$, and requires no current carrying metalsemiconductor contact. Differential capacitance images have been made with the SCM of a variety of bulk-doped samples and in the vicinity of pn junctions and homojunctions. Also, a computer code has been written that can numericaliy solve Poisson's equation for a model SCM geometry by using the method of collocation of Gaussian points. Measured data and model output for similar structures are presented. How data and model output can be combined to achieve an experimental determination of dopant profile is discussed.

\section{1,965}

PB96-183066 PC A03/MF A01

National Inst. of Standards and Technology (EEEL), Gaithersburg, MD. Semiconductor Electronics Div.

Electronics and Electrical Engineering Laboratory Technical Publication Announcements Covering Laboratory Programs, July to September 1995 with 1996 EEEL Events Calendar.

J. M. Rohrbaugh. Apr 96, 26p, NISTIR-5816. See also PB96-137187.

Keywords: *Electrical engineering, "Research projects, Semiconductor devices, Metrology, Laboratories, Abstracts, Electromagnetic interference, Optical fibers, Lasers, Antennas, Electrical measurement.

This is the forty-sixth issue of a quarterly publication providing information on the technical work of the $\mathrm{Na}$ tional Institute of Standards and Technology, Electronics and Electrical Engineering Laboratory. This issue of the EEEL Technical Publication Announcements covers the third quarter of calendar year 1995 Abstracts are provided by technical areas for papers published.

\section{1,966}

\section{PB96-183116 PC A04/MF A0}

National Inst. of Standards and Technology (EEEL) Gaithersburg, MD. Semiconductor Electronics Div.

Electronics and Electrical Engineering Laboratory Technical Progress Bulletin Covering Laboratory Programs, October to December 1995 with 1996 EEEL Events Calendar.

J. M. Rohrbaugh. Apr 96, 40p, NISTIR-5815. See also PB96-147905.

Keywords: *Electrical engineering, ${ }^{*}$ Research projects, Semiconductor devices, Metrology, Microelectronics, Laboratories, Abstracts, Signal processing, Optical fibers, Electromagnetic interference, Standards, US NIST, Dielectrics, Superconductors, Electrical measurement, Antennas, Lasers, Electronics, Electrical insulation, Microwave equipment.

This is the fifty-third issue of a quarterly publication providing information on the technical work of the National Instititute of Standards and Technology, Electronics and Electrical Engineering Laboratory. This issue of the EEEL Technical Progress Bulletin covers the fourth quarter of calendar year 1995. Abstracts are provided by technical area for both published papers and papers approved by NIST for publication. 
01,967

PB96-186010 Not available NTIS

National Inst. of Standards and Technology (PL), Boulder, CO. Time and Frequency Div.

Surface Transverse Wave Oscillators with Extremely Low Thermal Noise Floors.

Final rept.

I. D. Avramov, F. L. Walls, T. E. Parker, and G. K.

Montress. 1994, 16p.

Pub. in Proceedings of the Institute of Electrical and Electronics Engineers International Frequency Control Symposium, Boston, MA., June 1-3, 1994, p379-394.

Keywords: "Phase noise, "Power oscillator, Reprints, "Surface transverse wave resonators, Line discriminator, A-B class power amplifier.

This paper presents state-of-the-art results on $1 \mathrm{Ghz}$ surface transverse wave (STW) power oscillators running at extremely high loop power levels. High-O single-mode STW resonators used in these designs have an insertion loss of $3.6 \mathrm{~dB}$, an unloaded $\mathrm{O}$ of 8000 , a residual phase noise of $-142 \mathrm{dBc} / \mathrm{Hz}$ at $1 \mathrm{~Hz}$ intercept and operate at an incident power of up to $31 \mathrm{dBm}$ in the loop. Other low-Q STW resonators and coupled resonator filters (CRF) with an insertion loss in the 5$9 \mathrm{~dB}$ range can conveniently handle power level in escess of $2 \mathrm{~W}$. These devices were implemented in voltage controlled oscillators (VCO's) running from a $9.6 \mathrm{~V}$ source at an output power of $23 \mathrm{dBm}$ and a RF/ dc efficiency of $28 \%$. Their tuning range was $750 \mathrm{kHz}$ and the noise floor - $180 \mathrm{dBc} / \mathrm{Hz}$. The oscillators, stabilized with the high-O devices, use specially designed AB-class power amplifiers, deliver an output power of $29 \mathrm{dBm}$ and demonstrate a noise floor of $-184 \mathrm{dBc} /$ $\mathrm{Hz}$ and a $1 \mathrm{~Hz}$ intercept of $-17 \mathrm{dBc} / \mathrm{Hz}$. The $1 \mathrm{~Hz}$ intercept was improved to $-33 \mathrm{dBc} / \mathrm{Hz}$ using the UTO-1023 as a loop amplifier. In this case the output power was $22 \mathrm{dBm}$. In all cases the loop amplifier was the limiting factor for the close-to-carrier oscillator phase noise performance.

\section{1,968}

PB96-190228 Not available NTIS

National Inst. of Standards and Technology (EEEL), Boulder, CO. Electromagnetic Technology Div.

Models of Granualr Giant Magnetoresistance Multilayer Thin Films.

Final rept.

J. O. Oti, S. E. Russek, S. C. Sanders, and R. W.

Cross. 1996, 9p.

Pub. in Institute of Electrical and Electronics Engineers Transactions on Magnetics, v32 n2 p590-598 Mar 96.

Keywords: "Thin films, "Magnetoresistance, Models, Anisotropy, Exchange, Multilayers, Spin-valves, Micromagnetic, Reprints

Phenomenological micromagnetic and large-scale magnetization-dependent models of resistivity that produce giant magnetoresistance in granular multilayer magnetic thin films are described. Included in the models are intralayer and interlayer scattering components formulated explicitly in terms of the microstructural properties and characteristic transport lengths of the medium. The micromagnetic model provides insight into the influence of the magnetization distribution on the giant magnetoresistance response of the medium. The large-scale model which is derived from the micromagnetic model, is useful for obtaining media transport parameters from experimental data. Both models are used to study a set of annealed NiFe/ Ag multilayer films.

01,969

\section{PB96-191390 PC A04/MF A01}

National Inst. of Standards and Technology (EEEL), Gaithersburg, MD. Semiconductor Electronics Div.

Electronics and Electrical Engineering Laboratory Technical Progress Bulletin Covering Laboratory Programs, January to March 1996, with 1996 EEEL. Events Calendar.

J. M. Rohrbaugh. Jun 96, 40p, NISTIR-5853.

See also PB96-183116.

Keywords: "Electrical engineering, "Research projects, Semiconductor devices, Metrology, Laboratories, Abstracts, US NIST, Antennas, Lasers, Optical fibers, Superconductors, Electronics, Microwaves, Electrical measurement, Microelectronics.

This is the fifty-fourth issue of a quarterly publication providing information on the technical work of the $\mathrm{Na}$ tional Institute of Standards and Technology, Electronics and Electrical Engineering Laboratory. This issue of the EEEL Technical Progress Bulletin covers the first quarter of calendar year 1996. Abstracts are provided by technical area for both published papers and papers approved by NIST for publication.

01,970

PB96-200282 Not available NTIS

National Inst. of Standards and Technology (EEEL), Boulder, CO. Electromagnetic Fields Div.

Data Evaluation of a Linear System by a SecondOrder Transfer Function.

Final rept.

D. G. Camell, and M. T. Ma. 1995, 5p

See also PB95-182390.

Pub in Institute of Electrical and Electronics Engineers EMC Symposium Conference Record, August 14-18, 1995, p511-515.

Keywords: "Electromagnetic interference, "Convolution integrals, Electromagnetic pulses, Transfer functions, Continuous radiation, Resonant frequency, Linear systems, Magnitude, Reprints, Impulse response.

A recently developed technique for predicting the response of a linear system to an electromagnetic pulse, based only on the measured sontinuous-wave magnitude, is applied to a particular system as a case study. The measured magnitude representing the sys tem's transfer function is deduced first from the meas ured response to a known cw source. The authors next derive an analytic expression for the magnitude square of the transfer function to approximate the measured data and obtain a system transfer function in terms of the complex frequency. Finally, the authors predict the system's cw phase characteristics and its multiple solutions due to a given impulse source.

\section{1,971}

PB96-200332 Not available NTIS

National Inst. of Standards and Technology (EEEL), Boulder, CO. Electromagnetic Technology Div. Observation of the Transverse Second Harmonic Magneto-Optic Kerr Effect from Ni81Fe19 Thin Film Structures.

Final rept.

T. M. Crawford, C. T. Rogers, T. J. Silva, and Y. K. Kim. 1996, 3p.

Pub. in Applied Physics Letters, v68 n11 p1573-1575 Mar 96.

Keywords: "Thin films, "Multiple reflection theory, Magnetic interfaces, Second harmonics, Reprints, "Kerr ef fect, MOKE, Non-linear optics, Second order susceptibility, SH conversion efficiency.

We report second-harmonic magneto-optic Kerr measurements on air-exposed, polycrystalline Ni81Fe19 thin films, ranging in thickness from $1 \mathrm{~nm}$ to 2 micro $\mathrm{m}$, on $\mathrm{Al} 2 \mathrm{O} 3$ coated $\mathrm{Si}(001)$. For samples thicker than $20 \mathrm{~nm}$, in the tranverse Kerr geometry, we observe a factor of 4 change in second-harmonic intensity upon magnetization reversal. For thin samples, we observe interference between second-harmonic fields from the various interfaces and deterioration of ferromagnetism in the 1 and $2 \mathrm{~nm}$ films. Modeling suggests that the Ni81Fe19/Al2O3 interface has a larger second-order susceptibility than the air/Ni81Fe19 surface.

\section{1,972}

PB96-200407 Not available NTIS

National Inst. of Standards and Technology (EEEL), Boulder, CO. Electromagnetic Fields Div.

$30 \mathrm{MHz}$ Comparis on Receiver.

Final rept.

J. A. Jargon. 1995, 3p.

Pub. in Asia-Pacific Microwave Conference Proceedings, Taejon, Korea, October 10-13, 1995, v1 p94-96. Keywords: "Electrical measurements, "Microwave circuits, Phase shift, Reprints, "Attenuation Calibration System.

The National Institute of Standards and Technology has developed a highly sensitive $30 \mathrm{MHz}$ comparison receiver for detecting low-level if signals in the nanvolt region. The purpose of this instrument is to detect a null between two signals that can be adjusted in phase and magnitude. This paper contains a description of the receiver, specifications, and theory of operation.

PB96-200837 available NTIS

National Inst. of Standards and Technology (MSEL). Gaithersburg, MD. Ceramics Div.
Measurements of Properties of Materials in Electronic Packaging.

Final rept.

J. A. Carpenter. 1991, 13p.

Pub. in Proceedings of a Symposium Metal-Ceramic Joining, Detroit, MI., October 8-9, 1990, p205-217 1991.

Keywords: "Electronic packaging, "Measurement, "Reliability analysis, Manufacturing, Performance Physical properties, Mechanical properties, Electrical properties, Thermal properties, Costs, Reprints.

Designers require reliable values of properties of the materials in electronic packaging in order to accurately predict the performance of their products. This chapte looks at techniques for measuring the important elec trical, thermal, mechanical, and physical properties of electronic packaging and three other properties which assess the ease, efficiency, degree of success, or costs of processing the materials' manufacturability properties.

\section{1,974}

PB96-200852 Not available NTIS

National Inst of Standards and Technology (EEEL) Boulder, CO. Electromagnetic Technology Div.

Self-Reciprocal Fourier Functions.

Final rept.

M. W. Coffey. 1994, 3p.

Pub. in Jnl. of Optical Society of America A, v11 n9 p2453-2455 Sep 94

Keywords: "Fourier transformation, "Self reciprocal function, Optical applications, Diffraction theory, Inverse transform, Reprints, Mellin transform, Laplace transform.

By definition, a self-reciprocal (SR) function is its own Fourier or Hankel transform. Areas of application of SR functions, including fourier optics, are noted. Integral representations for SR functions are obtained and are illustrated with the exponential Fourier transformation on the half-line. It is pointed out that there are a large number of classes of SR functions, and examples of these functions are given.

\section{1,975}

PB96-200878 Not available NTIS

National Inst. of Standards and Technology (EEEL) Boulder, CO. Electromagnetic Technology Div.

Wideband Current and Magnetic Field Sensors Based on Iron Garnets.

Final rept.

M. N. Deeter, K. B. Rochford, A. H. Rose, and G. W. Day. 1993, 6p.

Pub. in Proceedings of the Annual Defense Advanced Research Projects Agency Symposium on Photonics Systems for Antenna Applications (3rd), Monterey, CA., January 20-22, 1993, p92-97.

Keywords: "Magnetic field sensors, "Iron garnets, Faraday effect, Flux concentration, Waveguides, Mag neto-optics, Reprints, Electric current sensors, Radiation-induced darkening.

Sensors based on the Faraday effect can be config ured to measure either magnetic fields or electric currents. Such sensors can be constructed without introducing conducting materials which are often undesirable. Presently, the most sensitive Faraday effect sensors employ ferrimagnetic iron garnets as the sensing elements. With these materials, we have demonstrated minimum detectable fields as low as $1 \mathrm{pT} / \mathrm{Hz} 1 / 2$ and currents as low as $200 \mathrm{nA} / \mathrm{Hz} 1 / 2$. The paper reviews these and other recent research results obtained at NIST and considers prospects for still further improvements in sensitivity and frequency response.

\section{6}

PB96-200902 Not available NTIS

National Inst. of Standards and Technology (EEEL)

Boulder, CO. Electromagnetic Technology Div.

Performance of the Electron Pump with Stray Capacitances.

Final rept.

H. D. Jensen, and J. M. Martinis. 1994, 2 p.

Pub. in Physica B, v194-196 p1255-1256 1994.

Keywords: "Electron pumps, "Coulomn blockage, *Metrology, Reprints, Single electron tunneling.

We calculate the effect of stray capacitance on the performance of the electron pump for a simplified and experimentally appropriate circuit model. We show that to first order the effect of the stray capacitances can 


\section{General}

be accounted for by replacing the junction capacitance $C$ with $C+A C$ (stray), where a equals 1 and $C$ (stray) is the average total stray capacitance of the metal islands between the junctions.

\section{1,977}

PB96-201025 Not available NTIS

National Inst. of Standards and Technology (EEEL),

Boulder, CO. Electromagnetic Technology Div.

Novel Hot-Electron Microbolometer.

Final rept.

M. Nahum, and J. M. Martinis. 1994, 2p.

See also DE93002542

Pub. in Physica B, v194-196 p109-110 1994

Keywords: *Infrared detectors, *Bolometers, *Antennas, Superconducting junctions, Electron tunneling, Microbolometers, Hot electrons.

The authors present measurements of a novel power detector which can be used as an ultra-sensitive detector of millimeter and sub-millimeter radiation. The absorbing element consists of a thin film resistor with submicrometer dimensions, which is connected to superconducting electrodes. This device exploits the Andreev reflection of electrons, and the weak interaction between electrons and phonons at low temperatures, in order to produce a large temperature rise for a small input power (approximately $10 \mathrm{mK} / \mathrm{fW}$ ). The temperature rise of the electrons is measured from the temperature dependence of the current-voltage characteristic of a superconductor-insulator-normal metal Iunnel junction, where part of the resistor strip forms the normal electrode. The authors have measured a voltage responsitivity approximately 10 to the ninth power VN, and an amplifier-limited electrical NEP approximately $3 \times 10$ to the minus 18 th power $\mathrm{WHz}$ to the minus one half power at an operating temperature of $100 \mathrm{mK}$. If infrared radiation were coupled to the absorbing element by using superconducting planar anntennas, then the sensitivity of this detector would be at least a factor of 10 higher than the best available direct detectors and thus may become the detector of choice for important astrophysics investigations.

\section{1,978}

PB96-202346 PC A04/MF A01

National Inst. of Standards and Technology (EEEL), Gaithersburg, MD. Semiconductor Electronics Div.

Electronics and Electrical Engineering Laboratory Technical Publication Announcements Covering Laboratory Programs, October to December 1995, with 1996 EEEL Events Calendar.

J. M. Rohrbaugh. Jul 96, 31p, NISTIR-5872.

See also PB96-183066.

Keywords: "Electrical engineering, "Research projects, Electrical measurement, Semiconductor devices, Metrology, Abstracts, Electromagnetic interference, Laboratories, Optical fibers, Signals

This is the forty-seventh issue of a quarterly publication providing information on the technical work of the $\mathrm{Na}$ tional Institute of Standards and Technology, Electronics and Electrical Engineering Laboratory. This issue of the EEEL Technical Publication Announcements covers the fourth quarter of calendar year 1995. Abstracts are provided by technical areas for papers published.

\section{1,979}

\section{PB96-210026 PC A04/MF A01}

National Inst. of Standards and Technology (EEEL), Boulder, CO. Electromagnetic Fields Div.

Rapid Evaluation of Mode-Stirred Chambers Using Impulsive Waveforms.

Technical note.

J. M. Ladbury, R. T. Johnk, and A. R. Ondreika. Jun

96, 39p, NIST/TN-1381.

Also available from Supt. of Docs. as SN003-00303417-7.

Keywords: "Reverberation, "Electromagnetic compatibility, Electromagnetic fields, Electromagnetic noise.

In this paper, the authors present an experimental technique for the rapid evaluation of mode-stirred (or reverberation) chambers. The measurement provides an estimate of the average chamber quality factor $(Q)$ by measuring the chamber impulse response and observing the decay of the spectral components of the response. The results show good agreement with those obtained using conventional $\mathrm{CW}$ techniques. The measurement is well suited for low-frequency analysis of a chamber, where costly and time consuming mode-tuned approaches are generally employed.
Since this technique does not use paddle stirnng, the time savings can be considered.

\section{1,980}

PB96-210778 PC A08/MF A02

National Inst. of Standards and Technology (EEEL), Boulder, CO. Electromagnetic Fields Div.

Bibliography of the NIST Electromagnetic Fields Division Publications.

R. M. Lyons. Aug 96, 134p, NISTIR-5050.

See also PB95-135562.

Keywords: "Electromagnetic fields, "Bibliographies, Antennas, Dielectric properties, Electrical measurement, Electromagnetic interference, Microwaves, Metrology, Electromagnetic noise, Remote sensing, Time domain, Waveforms, Attenuation, Electrical imped ance, Impedance measurement, Near fields, Antenna radiation patterns, Radiation hazards, Standards, "US NIST, Nonionizing radiation.

This bibliography lists the publications by the staff of the Flectromagnetic Fields Division of the National Institute of Standards and Technology for the period January 1970 through July 1996 . It supersedes NISTIP 5039 , which listed the publications of the Electro magnetic Fields Division from January 1970 through July 1995. Selected earlier publications from the division's predecessor organizations are included.

\section{1,981}

PB96-214622 PC A03/MF A01

National Inst. of Standards and Technology (EEEL), Gaithersburg, MD. Semiconductor Electronics Div.

Electronics and Electrical Engineering Laboratory Technical Publication Announcements Covering Laboratory Programs, January to March 1996, with 1996 EEEL Events Calendar.

J. M. Rohrbaugh. Aug 96, 25p, NiSTIR-5892. See also PB96-202346.

Keywords: "Electrical engineering, "Research projects, Semiconductor devices, Metrology, Laboratories, AD stracts, US NIST, Antennas, Electromagnetic interference, Optical fibers, Lasers, Microelectronics, Electrical measurements, Standards, Electronics, Microwaves.

This is the forty-eight issue of a quarterly publication providing information on the technical work of the $\mathrm{Na}$ tional Institute of Standards and Technology. Electronics and Electrical Engineering Laboratory. This issue of the EEL Technical Publication Announcements covers the first quarter of calendar year 1996. Abstracts are provided by technical areas for both published papers and papers approved by NIST for publication.

\section{1,982}

PB97-111454 Not available NTIS

National Inst. of Standards and Technology (EEEL) Gaithersburg, MD. Electricity Div.

Proposed Tests to Evaluate the Frequency-Dependent Capacitor Ratio for Single Electron Tunneling Experiment.

Final rept.

S. Avramov-Zamurovic, N. M. Zimmerman, A. F

Clark, and A. Jeffery. 1996, 2p.

Pub. in Conference on Precision Electromagnetic Measurements, Braunschweig, Germany, June 17-20, 1996, p265-266.

Keywords: "Capacitor ratio, "Single electron tunneling, Voltage divider, Frequency dependence, Capacitance, Reprints, "Foreign technology.

A precise measurement of the ratio of two cryogenic capacitors is needed for a capacitor charging experiment using Single Electron Tunneling (SET) phenomena. To support the capacitor charging metrology a frequency characterization of the capacitors is required. To cover the frequency range from $1 \mathrm{~Hz}$ to $1 \mathrm{kHz}$, resistive and inductive voltage divider bridges are proposed. Preliminary tests suggest that the uncertainty with which the capacitor ratio can be evaluated is less than one part per 10 to the sixth power.

\section{1,983}

PB97-111512 Not available NTIS

National Inst. of Standards and Technology (EEEL), Gaithersburg, MD. Electricity Div.

Transient Errors in a Precision Resistive Divider. Final rept.

S. A. Boggs, G. J. FitzPatrick, and J. Kuang. 1996 , 4p.

Pub. in Proceedings of the Institute of Electrical and Electronics Engineers International Symposium on
Electrical Insulation, Montreal, Quebec, Canada, June 16-19, 1996, p482-485.

Keywords: "Resistive dividers, "Transient errors, Heating effects, High voltage, Impulse measurements, Reprints.

Resistive dividers have the advantages of dc response and stability. However unlike capacitive dividers, they inevitably involve power dissipation and also generally involve an appreciable inductance. These aspects of a resistive divider result in transient errors, i.e., errors which are a function of the applied waveform. The paper discusses transient measurement errors of precision high voltage resistive dividers such as the one recently developed by NIST.

\section{1,984}

PB97-111843 Not available NTIS

National Inst. of Standards and Technology (EEEL), Gaithersburg, MD. Electricity Div.

Uncertainties of Frequency Response Estimates Derived from Responses to Uncertain Step-Like Inputs.

Final rept.

J. P. Deyst, T. M. Souders, and J. J. Blair. 1996, 4p. Pub. in Institute of Electrical and Electronics Engineers Instrumentation and Measurement Technology Conference, Brussels, Belgium, June 4-6, 1996, p151-154. Keywords: "Frequency response, "Step response, Electrical measurement, Signal conditioning, Algorithms, Errors, Test methods, Mathematical models, Estimates, Reprints.

The frequency response of a linear time-invariant system can be estimated from the measurement of its response to an ideal step input. However, an ideal step is unrealizable, and various other error sources affect the accuracy of such estimates. The paper investigates the effect of using an uncertain (inexactly known), steplike test signal. An approach is developed here for determining the systematic uncertainties of the frequency response estimate of a device under test (DUT), when it is estimated from the response, are converted to the frequency domain and processed, resulting in uncertainties for the frequency response of the DUT. Also, a mathematical proof is provided for the 'envelopemodulation' method of calculating the systematic uncertainties of a frequency response estimate of a device, as derived from the uncertain response of the device to an ideal step.

\section{1,985}

PB97-111918 Not available NTIS

National Inst. of Standards and Technology (EEEL), Gaithersburg, MD. Electricity Div.

Optical and Mass Spectrometric Investigations of Ions and Neutral Species in SF6 Radio-Frequency Discharges.

Final rept.

R. Foest, J. K. Olthoff, R. J. Van Brunt, E. C. Benck, and J. R. Roberts. 1996, 12p.

Pub. in Physical Review E, v54 n2 p1876-1887 Aug 96.

Keywords: "Radio frequency discharges, "Electrode surface effects, Ion kinetic energy, Laser induced fluorescence, Electric characteristics, Degree of dissociation, Reprints.

Radio-frequency ( $\mathrm{rf}$ ) discharges at $13.56 \mathrm{MHz}$ were generated in pure SF6 using a capacitively coupled, parallel-plate GEC reference cell for gas pressures in the range of 4-33.3 Pa (30-250 mTorr) and for peakto-peak applied if voltages in the range of 100-300 V. The following measurements were made during operation of the discharge: (1) electrical characteristics which included power dissipation, voltage-current phase angle, and the dc self-bias; (2) time-averaged vertical and horizontal profiles of the optical emissions from neutral atomic fluorine for the $2 p(4) 3 p^{\prime} 2 F(0) 7 / 2$ $\rightarrow 2 p(4) 3 s^{\prime} 2 D 5 / 2$ and $2 p(4) 3 p(2) p(0) 3.2 \rightarrow$ $2 p(4) 3 s(2) P 3 / 2$ transitions; (3) spatially resolved, laserinduced fluorescence (LIF) utilizing the $2 p(4) 3 s(4) p 5 /$ 2 metastable level of atomic fluorine; (4) mass spectra of neutral species in the plasma; and (5) kinetic-energy distributions and relative fluxes of mass-selected positive ions extracted from the plasma through a $0.1 \mathrm{~mm}$ diameter onfice in the grounded electrode. The dependence of the electrical characteristics on gas pressure confirms previous observations and model predictions which indicate, for example, that the plasma becomes more resistive as pressure increases.

\section{1,986}

PB97-111926 Not available NTIS 
National Inst. of Standards and Technology (EEEL), Gaithersburg, MD

New Refractometer by Combining a Variable Length Vacuum Cell and a Double-Pass Michelson Interferometer.

Kinal rept. Kujii, E. R. Williams, and R. L. Steiner. 1996, 2p.

Pub. in Conference on Precision Electromagnetic Measurements, Braunschweig, Germany, June 17-20, 1996, p128-129.

Keywords: "Interferometers, "Refractometers, "Polarized interferometer, Air index, Watt determination, Reprints, "Foreign technology, "Michelson interferometers, Variable length vacuum.

A new refractometer with a variable length vacuum cell has been developed, where the refractive index of air is determined by measuring the changes in the optical path difference between the air of interest and a vacuum as a function of the changes in the cell length. An accuracy of $4 \times 10$ to the minus 9 th power has been achieved.

01,987

PB97-111959 Not available NTIS

National Inst. of Standards and Technology (EEEL) Gaithersburg, MD. Electricity Div.

Measurement and Reduction of Alignment Errors of the NIST Watt Experiment.

Final rept.

A. Gillespie, K. Fujii, D. Newell, R. Steiner, G.

Stenbakken, E. Williams, P. T. Olsen, and A. Picard.

1996, 2p.

Pub. in Conference on Precision Electromagnetic Measurements, Braunschweig, Germany, June 17-20, 1996, p614-615.

Keywords: "Magnetic fields, "Electrical measurement, Monitoring, Standards, Fundamental constants, Superconducting magnets, Reprints, "Watt balance method, Induced voltage, Mass stability, Kilogram.

The effects of uncertainties in the alignment of the NIST watt balance with local gravity and the magnetic field of the balance have been analyzed, and techniques for measuring all misalignment parameters have been developed. The systematic uncertainty in the watt measurement due to alignment has been reduced to 0.04 micrometers W/W.

01,988

PB97-112007 Not available NTIS

National Inst. of Standards and Technology (EEEL), Boulder, CO. Electromagnetic Technology Div.

Observation of Hot-Electron Shot Noise in a Metallic Resistor.

Final rept.

A. H. Steinbach, J. M. Martinis, and M. H. Devoret. 1996, $4 p$.

Pub. in Physical Review Letters, v76 n20 p3806-3809 May 96.

Keywords: "Mesoscopics, "Noise, "Shot noise, "Resistors, Reprints.

The authors have measured the current noise of silver thin-film resistors as a function of current and temperature and for resistor lengths of 7000,100,30 and 1 micrometers. As the resistor becomes shorter than the electron-phonon interaction length, the current noise of large current increases from a nearly current independent value to the interacting hot-electron value (square root 3/4)2el. However, further reduction in length below the electron-electron interaction length decreases the noise to a value approaching the independent hot-electron value (1/3)2el first predicted for mesoscopic resistors.

\section{1,989}

PB97-112379 Not available NTIS

National Inst. of Standards and Technology (EEEL), Gaithersburg, MD. Electricity Div.

High-Current Thin Film Multijunction Thermal Converters and Multi-Converter Modules.

Final rept.

J. R. Kinard, T. E. Lipe, C. B. Childers, and D. B.

Novotny. 1996, 2p.

Pub. in Conference on Precision Electromagnetic Measurements, Braunschweig, Germany, June 17-20 1996, p594-595.

Keywords: *Current converters, *Thin films, "Thermal converters, Thermal sensors, Multilayers, Ac-dc difference, Multijunctions, Reprints, "Foreign technology. High-current, thin-film multijunction thermal converters (HCFMJTCs) have been fabricated at NIST with heater ranges from a new milliamperes to $1 \mathrm{~A}$. Multi-converter modules containing HCFMJTCs have also been constructed to measure currents up to several amperes.

01,990

PB97-113039 Not available NTIS

National Inst. of Standards and Technology (EEEL),

Gaithersburg, MD. Electricity Div.

Wideband Sampling Voltmeter.

Final rept.

T. M. Souders, B. C. Waltrip, O. B. Laug, and J. P.

Deyst. 1996, 6p.

Pub. in Institute of Electrical and Electronics Engineers Instrumentation and Measurement Technology Conference, Brussels, Belgium, June 4-6, 1996, p11391144 Jun 96.

Keywords: "Sampling, "Wideband, "Voltmeters, Error sources, RMS voltage, Time-base, Reprints, "Foreign technology, Quasi-equivalent-time.

A high accuracy sampling voltmeter, designed to span the frequency range of $10 \mathrm{~Hz}$ to $200 \mathrm{MHz}$ is described. The instrument operates autonomously, at a measurement update rate of at least one per second. A novel quasi-equivalent time sampling process is sued, with a custom strobed comparator as the sampling device and decision element. The architecture and control are presented, along with the time-base design principles. Major error sources associated with the time-base are also discussed.

01,991

PB97-113062 Not available NTIS

National Inst. of Standards and Technology (EEEL) Gaithersburg, MD. Electricity Div.

NIST Watt Balance: Progress Toward Monitoring the Kilogram.

Final rept.

R. Steiner, A. Gillespie, K. Fujii, A. Picard, G.

Stenbakken, P. T. Olsen, E. Williams, and D. Newell. $1996,2 p$

See also PB96-123153.

Pub. in Conference on Precision Electromagnetic Measurements, Braunschweig, Germany, June 17-20, 1996, p6-7.

Keywords: "Magnetic forces, Electrical measurement Simulation, Reprints, "Watt measurement, "Foreign technology, Induced voltage, Mass stability.

Random uncertainty of 0.08 microW/W in the NIST watt balance has been achieved by inmprovements in a velocity measurement using three laser interferometers and by the elimination of filter delays and electrical noise. The latest results of this experiment are presented.

01,992

PB97-113211 Not available NTIS

National Inst. of Standards and Technology (EEEL), Gaithersburg, MD. Flectricity Div.

DC-MHz Wattmeter Based on RMS Voltage Measurements.

Final rept.

B. C. Waltrip, and N. M. Oldham. 1996, 2p

Pub. in Institute of Electrical and Electronics Engineers Instrumentation and Measurement Technology Conference, Brussels, Belgium, June 4-6, 1996, p214-215.

Keywords: "Wattmeters, "Power, Buffer, RMS voltage, Reprints, "Foreign technology, 3 DVM method.

A wideband wattmeter for measuring active power over a frequency range of dc to $1 \mathrm{MHz}$ is described. The wattmeter is based on the three voltmeter method in which three rms voltage measurements are used to calculate power.

\section{1,993}

PB97-113252 Not available NTIS

National Inst. of Standards and Technology (EEEL) Gaithersburg, MD. Electricity Div.

Magnetometer Calibration Services.

Final rept

E. R. Williams, and D. W. Matson. 1995, 5p.

Pub. in Proceedings of the National Conference on Standards Laboratories, The Impact of Metrology on Global Trade, July 16-20, 1995, Dallas, TX., p495-499.

Keywords: "Magnetometers, "Calibrations, Gauss meters, Magnetic standards, Reprints, Hall effect meters, Nuclear magnetic resonance.

A new facility to calibrate magnetometers in San Diego has recently been completed as a cooperative effor between the Naval Primary Standards Laboratory and the National Institute of Standards and Technology. All measurements are NIST traceable through a nuclear magnetic resonance-based measurement. Magnetic fields from the 0.1 microT to $1.4 \mathrm{~T}$ can be calibrated by comparisons with fields generated by a series of coils or an electromagnet.

01,994

PB97-113880 PC A04/MF A01

National Inst. of Standards and Technology (EEEL), Gaithersburg, MD. Semiconductor Electronics Div.

Electronics and Electrical Engineering Laboratory Technical Progress Bulletin Covering Laboratory Programs, April to June 1996 with 1996-1998 EEEL Events Calendar.

J. M. Rohrbaugh. Oct 96, 37p, NISTIR-5895.

See also report dated Sep 95, PB96-106455.

Keywords: "Electrical engineering, "Research projects, Semiconductor devices, Metrology, Laboratories, Abstracts, US NIST, Antennas, Superconductors, Electronics, Microwaves, Electromagnetic interference, Microelectronics, Optical fibers, Electrical measurement, Lasers, Lightwave technology.

This is the fifty-fifth issue of a quarterly publication providing information on the technical work of the National Institute of Standards and Technology, Electronics and Electrical Engineering Laboratory. This issue of the EEEL Technical Progress Bulletin covers the second quarter of calendar year 1996. Abstracts are provided by technical area for both published papers and papers approved by NIST for publication.

01,995

PB97-118616 Not available NTIS

National Inst. of Standards and Technology (MSEL) Gaithersburg, MD. Ceramics Div.

Effect of Beam Voltage on the Properties of Aluminum Nitride Prepared by Ion Beam Assisted Deposition.

Final rept.

J. H. Edgar, C. A. Carosella, C. R. Eddy, and D. T.

Smith. 1996, $7 p$

Pub. in Jnl. of Materials Science: Materials in Electronics, v7 p247-253 1996.

Keywords: "Aluminum nitride, "Beam voltage, Mechanical properties, Reprints, "Ion beam assisted deposition.

The effects of nitrogen-beam voltage on the structure, stress, energy band gap and hardness of AIN thin films deposited on Si(111), Si(100) and sapphire (0001) by ion beam assisted deposition (IBAD) are reported As the nitrogen-bean voltage was increased from 50 to $200 \mathrm{~V}$, the stress and disorder in the AIN films increased as determined by X-ray diffraction, FTIR and Raman spectroscopy. The preferred orientation of the film's c-axis changed from completely normal to the film at $100 \mathrm{~V}$, to a mixture of normal and in the plane of the film at $200 \mathrm{~V}$. For AIN films deposited under the same conditions, the films were more highly oriented on sapphire (0001) than in SI(111). The hardness of the films increased from 18.2 to $23.7 \mathrm{GPa}$ with the nitrogen-beam voltage, and possible reasons for this change in hardness are considered.

01,996

PB97-122253 Not available NTIS

National Inst. of Standards and Technology (EEEL), Gaithersburg, MD. Electricity Div.

Dependence of the Thermal Electron Attachment Rate Constant in Gases and Liquids on the Energy Position of the Electron Attaching State.

Final rept.

L. G. Christophorou. 1996, 21 p.

Pub. in Zeitschritt fur Physikalische Chemie, Bd., v95 S p195-215 1995

Keywords: *Electron attachment, *Thermal electrons, *Energy positions, Gases, Liquids, Constants, Reprints, "Foreign technology.

Thermal electron attachment rate constants for halocarbons in the gaseous phase, (ka)th, and thermal electron attachment rate constants for a number of molecules in liquid cyclohexane, (ka) L, are summarized in an effort to discern the dependence of the thermal electron attachment rate constant for molecules embedded in gases and in liquids on the energy position, E(NIS), of the electron attaching state. For these molecules the (ka)th varies only slightly with $E(N I S)$ as long as $\mathrm{E}$ (NIS) is less than $0.0 \mathrm{eV}$ (i.e., as long as the electron affinity of the molecule is positive), but it de- 


\section{General}

creases precipitously with increasing $E(N I S)$ when $\mathrm{E}$ (NIS) greater than $0.0 \mathrm{eV}$ (i.e., when the electron affinity of the molecule becomes negative). The (ka) L exhibits a similar behavior except that it remains virtually constant well above $0.0 \mathrm{eV}$, up to $\mathrm{E}$ (NIS) approximately $0.9 \mathrm{eV}$, and thereafter decreases precipitously This energy shift between the gaseous and the liquid phase correlations is consistent with the magnitude of the polarization energy of the anions in liquid cyclohexane and its effect on $\mathrm{E}$ (NIS).

\section{1,997}

\section{PB97-122329 Not available NTIS}

National Inst. of Standards and Technology (EEEL) Gaithersburg, MD. Electricity Div.

NIST Watt Experiment: Monitoring the Kilogram.

Final rept.

A. Gillespie. 1996, $1 p$

See also PB97-111959.

Pub. in Bulletin/Conference Abstract Program of the 1996 March Meeting, St. Louis, MI., March 18-22, 1996, v41 n1 p506.

Keywords: "Calibration standards, "Magnetic field, "Voltage, "Mass, Interlaboratory comparisons, Electrical measurement, Weight measurement, Fundamental constants, Mechanical measurement, Metrology Precision, Monitoring, Reprints, "Kilograms, Current loops.

An apparatus has been constructed which measures both the force on a current loop in a magnetic field and the voltage induced around that loop when it moves at some velocity through that same magnetic field. By comparing the force times the velocity to the curren times the voltage, mechanical power is compared to electrical power, and a value for the watt which is consistent in both electrical mechanical units can be derived. The kilogram is defined in terms of a physical artifact, and intercomparisons among various kilogram masses suggest that the kilogram mass standard changes over time. Present research on the watt experiment aims to improve the precision of the measurement so that a defined value of the watt can be used to monitor the drift of the kilogram mass standard in terms of fundamental natural constant.

\section{Antennas}

\section{1,998}

PB94-172491 Not available NTIS

National Inst. of Standards and Technology (EEEL) Boulder, CO. Electromagnetic Fields Div.

Planar Near-Field Alignment.

Final rept.

D. Kremer, A. Newell, A. Repjar, M. Pinkasi, C.

Rose, and A. Trabelsi. 1993, 7p.

Pub. in Proceedings of the Annual Meeting and Sym posium of Antenna Measurement Techniques Association (15th), Dallas, TX., October 4-8, 1993, p198-204.

Keywords: Alignment, Reprints, "Antenna measurements. Planar near field technique.

This paper discusses one method of characterizing the scan plane for planar near-field measurements. The method uses a theodolite autocollimator, a laser interferometer, an electronic level and an optical square. The data obtained using these techniques are firs used to make alignment corrections to the scan plane; then new data are used to determine the best fit for the realigned scan plane. The normal to this plane is referenced using a permanently placed mirror. In addition, the final data obtained can be used in probe position-correction techniques, developed for planar nearfield measurements.

\section{1,999}

PB94-185436 Not available NTIS

National Inst. of Standards and Technology (EEEL) Boulder, CO. Electromagnetic Fields Div.

Standard Probes for Electromagnetic Field Measurements.

Final rept.

M. Kanda. 1993, 16p.

Pub. in Institute of Electrical and Electronics Engineers Transactions on Antennas and Propagation 41, n10 p1349-1364 Oct 93.

Keywords: "Probes(Electromagnetic), "Electric probes "Antennas, Electrical measurement, Electric fields,

Magnetic fields, Radio frequencies, Near field, Sensors, Reprints.

This tutorial paper discusses various standard antennas for measuring radio-frequency electric and magnetic fields. A theoretical analysis of each antenna's receiving characteristics is summarized and referenced. The standard probes described are an electrically short dipole, a resistively-loaded dipole, a hallwave dipole, an electrically small loop, and a resistively-loaded loop. A single-tum loop designed for simultaneous measurement of the electric and magnetic components of near-fields and other complex electromagnetic environments is also described. Each type of antenna demonstrates a different compromise between broadband frequency response and sensitivity.

\section{2,000}

PB94-219219 PC A06/MF A02

National Inst. of Standards and Technology, Gaithersburg, MD.

Journal of Research of the National Institute of Standards and Technology. March/April 1994. Volume 99, Number 2.

B. N. Taylor, and D. R. Harris. 1994, 118p.

See also PB94-219227 through PB94-219268, PB94169737 and PB94-219326. Also available from Supt. of Docs. as SN703-027-00057-1.

Keywords: "Calorimeters, "Radiation doses, "Water, Dissolved gases, Calorimetric dosemeters, Measuring instruments, Temperature effects, Thermistors, Exothermic reactions, Endothermic reactions, Heat defect, Convective barrier, National Institute of Standards and Technology.

Contents:

Articles--A Sealed Water Calorimeter for Measuring Absorbed Dose;

Planar Near-Field Measurements of Low-Sidelobe Antennas;

On the Physics Required for Prediction of Long Term Performance of Polymers and Their Composites;

The Measurement and Uncertainty of a Calibration Standard for the Scanning Electron Microscope

New Values for Silicon Reference Materials, Certified for Isotope Abundance Ratios:

News Briefs.

02,001

PB94-219235

MF A02)

National Inst. of Standards and Technology, Gaithersburg, MD.

Planar Near-Field Measurements of Low-Sidelobe Antennas.

M. H. Francis, A. C. Newell, K. R. Grimm, J.

Hoffman, and H. E. Schrank. 1994, 25p.

Prepared in cooperation with Nichols Research Corp. Vienna, VA. and System Engineering Corp., Columbia, MD.

Included in JnI. of Research of the National Institute of Standards and Technology, v99 n2 p143-167 Mar/ Apr 94.

Keywords: "Antennas, "Microwaves power transmission, "Sidelobes, Microwave probes, Waveguides, Antenna radiation patterns, Near fields, Far field, Antenna arrays, Measuring instruments, Uncertainty, Planar near field, National Institute of Standards and Technology.

The planar near-field measurement technique is a proven technology for measuring ordinary antennas operating in the microwave region. The development of very low-sidelobe antennas raises the question whether this technique can be used to accurately measure these antennas. The authors show that data taken with an open-end waveguide probe and processed with the planar near-field methodology, including probe correction, can be used to accurately measure the sidelobes of very low-side-lobe antennas to levels of $-55 \mathrm{~dB}$ to $-60 \mathrm{~dB}$ relative to the main beam peak. A special probe with a null in the direction of the main beam was also used for some of these measurements. This special probe reduced some of the measurement uncertainties but increased the uncertainties due to probe-antenna interactions. The authors discuss the major sources of uncertainty and show that the probeantenna interaction is one of the limiting factors in making accurate measurements. The test antenna for this study was a slotted-waveguide array whose low sidelobes were known.
02,002

PB95-108643 Not available NTIS

National Inst. of Standards and Technology (EEEL), Boulder, CO. Electromagnetic Fields Div

Correction Factor for Nonplanar Incident Field in Monopole Calibrations.

Final rept.

J. Randa. 1993, 3p.

Pub. in Institute of Electrical and Electronics Engineers Transactions on Electromagnetic Compatibility $35, \mathrm{n} 1$ p94-96, 1 Feb 93.

Keywords: "Antenna radiation patterns, "Calibration, Monopole antennas, Antenna design, Electromagnetic properties, Monopoles, Incidence, Electromagnetic fields, Electromagnetic compatibility, Reprints.

In calibrating monopole antennas, the length of the antenna can be comparable to the separation distance. In that case, there is a significant variation in both the magnitude and the phase of the incident field along the length of the antenna under test. This paper presents an expression for a correction factor to account for this effect. We evaluate the correction factor for some representative cases and present some guidelines for when this factor should be taken into account. The efect can exceed $1 \mathrm{~dB}$ in some practical cases.

02,003

PB95-152781 Not available NTIS

National Inst. of Standards and Technology (EEEL) Boulder, CO. Electromagnetic Fields Div. Time-Domain Antenna Characterizations. Final rept.

O. E. Allen, D. A. Hill, and A. R. Ondrejka. 1993, 8p. Pub. in Institute of Electrical and Electronics Engineers Transactions on Electromagnetic Compatibility 35, n3 p339-346 Aug 93

Keywords: "Broadband antennas, Hom antennas, Time domain, Frequency domain, Characteristics, Directivity, Comparison, Reprints.

A set of time-domain characterizations that can efficiently describe wide bank antennas is proposed in this paper. The experimentally measured responses of ransverse electromagnetic horn antennas are used to evaluate the utility of these characterizations. Comparisons are made between the antennas' frequency-domain response and their time-domain characterizations. The comparisons show that the time-domain characterizations can provide significant insight into an antenna's behavior as well as providing a means to accurately compare two or more different antennas.

02,004

PB95-153755 Not available NTIS

National Inst. of Standards and Technology (EEEL)

Boulder, CO. Electromagnetic Fields Div.

New Extrapolation/Spherical/Cylindrical Measurement Facility at the National Institute of Standards and Technology.

Final rept.

J. R. Guerrien, D. P. Kremer, and T. Rusyn. 1993 10p.

Pub. in Proceedings of Annual Meeting and Symposium on Antenna Measurement Techniques Association (15th), Dallas, TX., October 4-8, 1993, p343-352.

Keywords: "Test facilities, Antenna radiation patterns, Polarization(Waves), Cylindrical configuration, Spherical configuration, Probes(Electromagnetic), Near field, Extrapolation, Gain, Reprints, "Antenna measurements, US NIST.

A new multi-purpose antenna measurement facility was put into operation at the National Institute of Standards and Technology (NIST) in 1993. This facility is currently used to perform gain, pattern, and polarization measurements on probes and standard gain horns. The facility can also provide spherical and cylindrical near-field measurements. The frequency range is typically from 1 to $75 \mathrm{GHz}$. This paper discusses the capabilities of this new facility in detail

\section{2,005}

PB95-161915 Not available NTIS

National Inst. of Standards and Technology (EEEL), Boulder, CO. Electromagnetic Fields Div. 
Optically Linked Three-Loop Antenna System for Determining the Radiation Characteristics of an Electrically Small Source.

Final rept.

D. R. Novotny, K. D. Materson, and M. Kanda. 1993, Pub. in Proceedings of International Symposium on Electromagnetic Compatibility, Dallas, TX., August 9 13, 1993, p300-304.

Keywords: *Antenna arrays, "Radio emission, Electromagnetic compatibility, Poynting theorem, Loop antennas, Near field, Dipole moments, Electric moments Magnetic moments, Reprints, 'Electromagnetic emissions, "Photonic probes.

This paper presents the experimental results of an an tenna system for determining the radiation characteristics of an electrically small source. Three orthogonal loop antennas, each terminated at diametrically opposite points with identical loads, encircle the source and characterize its equivalent electric and magnetic dipole moments. The total radiated power can be determined from this near-field measurement of the device under test. The test system operates from $3 \mathrm{kHz}$ to over 100 $\mathrm{MHz}$ with up to $90 \mathrm{~dB}$ of dynamic range.

02,006

PB95-180378 Not available NTIS

National Inst. of Standards and Technology (EEEL)

Boulder, CO. Electromagnetic Fields Div.

Dual Frequency mm-Wave Radiometer Antenna for Airborne Remote Sensing of Atmosphere and Ocean.

Final rept.

M. D. Jacobson, L. S. Fedor, D. A. Hazen, D. P

Kremer, W. B. Madsen, and M. H. Francis. 1994, 8p. Contract NIPR-N93051

Sponsored by Department of Defense, Washington, DC.

Pub. in Microwave Jnl. 27, n9 p24-38 Sep 94.

Keywords: "Radiometers, "Antennas, Extremely high frequency, Superhigh frequency, Millimeter waves, Remote sensors, Remote sensing, Airborne equipment, Atmospheres, Oceans, Reprints.

Accurate multiwavelength radiometric remote sensing of the ocean and the atmosphere from an aircraft requires antennas with the same beamwidth at the varlous frequencies of operation. An offset antenna was designed with a pressure-compensating corrugated feed horn to meet this criterion. A specially designed fairing was incorporated into the antenna to optimize the aerodynamics and minimize the liquid build up on the antenna surfaces. The antenna has two positions, the zenith (up) position and the nadir (down) position. The far-field pattern results show that the antenna beamwidths at 23.87 and $31.65 \mathrm{GHz}$ agree well with design criterion. This antenna was recently used in an ocean remote-sensing experiment and performed according to expectations.

02,007

PB96-102561 Not available NTIS

National Inst. of Standards and Technology (EEEL), Boulder, CO. Electromagnetic Fields Div.

Standard Antennas for Electromagnetic Interference Measurements and Methods to Calibrate Them.

Final rept.

M. Kanda. 1994, $13 p$

Pub. in Institute of Electrical and Electronics Engineers Transactions on Electromagnetic Compatibility, v36 n4 p261-273 Nov 94

Keywords: *Antennas, "Electromagnetic interference, Radiofrequency interference, Electric fields, Magnetic fields, Calibrating, Radio reception, Reprints.

This tutorial paper discusses various standard antennas for measuring radio-frequency electric and magnetic fields. A theoretical analysis of each antenna's receiving characteristics is summarized and referenced. The antennas described are an electrically short dipole, a resistively loaded short dipole and halfwave dipole, an electrically small loop, a resistively loaded loop, photonic probes, and a single-turn loop designed for simultaneous measurement of the electric and magnetic components of near-fields and other complex electromagnetic (EM) environments. Each type demonstrates a different compromise between broadband responses and sensitivity. This paper also discusses the calibration techniques for these probes using standard EM fields established in transmission electron microscopy (TEM) cells, waveguide cells, anechoic chambers, and open-field sites.
02,008

PB96-111166 PC A08/MF A02

National Inst. of Standards and Technology (EEEL), Boulder, CO. Electromagnetic Fields Div.

Spherical-Wave Source-Scattering Matrix Analysis of Antennas and Antenna-Antenna Interactions.

Technical note.

R. L. Lewis. Jul 95, 159p, NIST/TN-1373.

Also available from Supt. of Docs. as SN003-00303358-8.

Keywords: "Autennas, "Near field, Spherical waves, Algorithms, Antenna coupling, Antenna measurements, Probes(Electromagnetic)

Expressions are presented for describing incoming and outgoing fields about an antenna in terms of a se ries of exciting and emergent vector spherical-wave functions. The exciting and emergent fields around the antenna's exterior are related to the field in the antenna feed via a source-scattering matrix representation. A series of so called spherical-wave source-scattering matrix coelficients are then related to more conventional parameters such as antenna gain and receiving cross section. An overview of rotation and translation theorems for transforming vector spherical-wave functions between two distinct coordinate systems is given, followed by a general solution to the problem of ex pressing the coupling between two coupled antennas in terms of each antenna's spherical-wave sourcescattering matrix representation. The authors go on to consider special results to substantiate their formulation, such as showing equivalence between the coupling equations for transmission in opposite directions when the antennas are reciprocal, showing uniform convergence of some series representations for an tenna coupling and simultaneously obtaining a coordinate-system translation theorem for the dyadic Green's function, and lastly showing that the authors' two-antenna coupling equations reduce to expressions for the incident and emergent fields about a single antenna when the other antenna is an elementary dipole. Efficient probe-corrected spherical and hemispherical scanning algorithms are then developed for processin measured near-field data to obtain antenna's far-field pattern. Finally, the authors describe a number of selfconsistency tests and theoretical-data simulations that were developed to validate their spherical-scanning algorithm, and they describe an independent experimental verification.

\section{2,009}

PB96-112289 Not available NTIS

National Inst. of Standards and Technology (EEEL) Boulder, CO. Electromagnetic Fields Div.

Dual-Frequency Millimeter-Wave Radiometer Antenna for Airborne Remote Sensing of Atmosphere and Ocean.

Final rep

M. H. Francis, D. P. Kremer, M. D. Jacobson, W. B. Madsen, L. S. Fedor, and D. A. Hazen. 1995, 7p. Pub. in Proceedings of the Antenna Measuremen Techniques Association Symposium, Long Beach, CA., October 3-7, 1994, p3-9.

Keywords: "Antennas, "Radiometers, "Millimeter wave equipment, "Airborne equipment, "Remote sensors, Extremely high frequencies, Remote sensing, Design criteria, Far fields, Oceans, Earth atmosphere, Reprints, Planar near field.

Accurate multiwavelength radiometric remote sensing of the ocean and the atmosphere from an aircraft re quires antennas with the same beamwidth at the various frequencies of operation. Scientists at the National Oceanic and Atmospheric Administration designed an offset antenna with a pressure-compensating corrugated feed hom to meet this criterion. A specially designed fairing was incorporated into the antenna to op timize the aerodynamics and minimize the liquid buildup on the antenna surfaces. The antenna has two postions: the zenith (up) position and the nadir (down) position. The planar near-field facility at the National insitute of Standards and Technology was used to determine the far-field pattern of the antenna. The results show that the antenna beamwidths at 23.87 and 31.65 Ghz are nearly the same as expected from the design criterion. This antenna was recently used in an ocean remote-sensing experiment and performed according to expectations.

\section{2,010}

PB96-135082 Not available NTIS

National Inst. of Standards and Technology (EEEL)

Boulder, CO. Electromagnetic Fields Div.
Development of Near-Field Test Procedures for Communication Satellite Antennas.

Final rept.

C. F. Stubenrauch. 1994, 19p.

See also PB86-164357 and PB89-156152.

Pub. in Antenna Measurement Techniques Association Workshop on Communication Satellite Antenna Measurements, Ann Arbor, Ml., July 2, 1993, p1-19.

Keywords: *Satellite antennas, "Tests, "Specifications, *Near fields. Antenna design, Multibeam antennas, Communication satellites, Simulation, Procedures, Reprints.

The presentation defines and discusses the capabilities of near-field antenna test techniques, specifically for the requirements associated with the development and verification testing of reconfigurable, multibeam frequency reuse, commercial satellite antennas. This discussion focuses on the planar near-field measurement method and covers the determination of sampling criteria and scan limits, development of diagnostic and design assist methods, development of beam alignment technqiues, and specification of hardware requirements for the measurement system.

\section{2,011}

PB96-141361 Not available NTIS

National Inst. of Standards and Technology (EEEL), Boulder, CO. Electromagnetic Fields Div.

Alternative Contour Technique for the Efficient Computation of the Effective Length of an Antenna.

Final rep

R. T. Johnk, and M. Kanda. 1994, 3p.

Pub. in Institute of Electrical and Electronics Engineers Transactions on Antennas and Propagation, v42 n5 p747-749 May 94.

Keywords: *Dipole antennas, *Length, "Galerkin method, Convergence, Current density, Electric potential, trostatics, Reprints.

The scattering of a plane wave broadside incident to a dipole antenna with a symmetrically placed, opencircuited gap is treated. The induced currents on the antenna are found by solving the electric-field integral equation by means of a Galerkin moment method. The resulting current distribution is then used to compute line integrals of the scattered electric field along appropriate paths. The line integral is first evaluated directly across the dipole gap in order to compute the effective length, but severe convergence problems are encountered. This problem is due to the presence of charge singularities at the gap edges. Instead of incorporating the singular charge behavior into the basis functions, a line integral is evaluated along a path that alleviates the convergence difficulties. This remedy is developed first for an electrostatic case and then for the dynamic scattering problem.

02,012

PB96-147137 Not available NTIS

National Inst. of Standards and Technology (EEEL),

Boulder, CO. Electromagnetic Fields Div.

Comparison of k-Correction and Taylor-Series Correction for Probe-Position Errors in Planar NearField Scanning.

Final rept.

M. H. Francis. 1995, 7p

Pub. in Annual Meeting and Symposium Antenna Measurement Techniques 1995 Assoc. (17th), Williamsburg, VA., November 13-17, 1995, p341-347.

Keywords: "Antennas, "Extremely high frequencies, Antenna arrays, Phased arrays, Near fields, Far fields, Planar structures, Antenna radiation patterns, Response functions, Error analysis, Taylor series, Reprints, "Probe-position errors.

The authors investigated two methods of probe-position error correction to determine how well the corrected results compare to the uncorrupted far field: the k-correction method and the Taylor-series method. For this investigation, the authors measured a $1.2 \mathrm{~m}$ dish at $4 \mathrm{GHz}$ and a $1.2 \mathrm{~m}$ by $0.9 \mathrm{~m}$ phased array at 2.2 $\mathrm{GHz}$. Measurements were made first without position errors and then with deliberate z-position errors. The authors performed probe-position error correction using both methods and compared the results to the error-free far field.

02,013

PB96-183157 PC A04/MF A01

National Inst. of Standards and Technology (EEEL),

Boulder, CO. Electromagnetic Fields Div. 


\section{Antennas}

Standard Source Method for Reducing Antenna Factor Errors in Shielded Room Measurements. Technical note.

D. Camell, G. Koepke, R. Smith, and B. Rakoski.

Mar 96, 43p, NIST/TN-1382.

Also available from Supt. of Docs. as SNO03-00303405-3.

Keywords: "Antennas, "Electromagnetic fields, "Calibration, *Shields, Test facilities, Errors.

In this report the authors will examine the use of a well characterized standard source of electromagnetic radiation as a means to calibrate the effects of the shielded room on a receiving antenna used for MILSTD 461/ 462 RE102 emissions measurement. The goal was to compensate for the shielded room environment so that radiated emissions measurements can be more accurately compared from one room to another. This was accomplished by using a characterized spherica dipole source to calibrate an antenna's response in the location that it was used. An interlaboratory comparison was made of the detected emissions from a simulated equipment-under-test at three sites to see how this in-situ calibration of the receive antenna helped the shielded room test repeatability.

\section{2,014}

PB96-200126 Not available NTIS

National Inst. of Standards and Technology (EEEL), Boulder, CO. Electromagnetic Fields Div.

Methodology for Electromagnetic Interference Measurements.

Final rept.

M. Kanda. $1995,27 p$.

See also PB96-102561

Pub. in Handbook of Electromagnetic Compatibility, Chapter 15, p599-625 1995.

Keywords: "Electromagnetic interference, "Antennas, * Measurement, Standards, Radio frequency interference, Radio reception, Electric fields, Magnetic fields, Radio receptor, Reprints.

Establishing standards for electromagnetic (EM) field measurements is a multifacted endeavor which re quires measurements made (1) in anechoic chambers, (2) at open sites, and (3) within guided-wave structures and the means of transferring these measurements from one situation to another. The underlying principles of these standard measurements and transfer standards fall into one of two categories: measurements and theoretical modeling. That is, either a parameter or a set of parameters is measured, or a parameter is calculated by established physical and mathematical principles. In the following, the three measurement topics and field transfer standards will be discussed, with the guided-wave structures restricted to the transverse electromagnetic (TEM) cell and the waveguide chamber. Throughout the discussion the interplay between measured quantitites and predicted (modeled) quantities will be seen. The frequencies considered here range from $10 \mathrm{kHz}$ to $40 \mathrm{Ghz}$ (and upward) and are determined by our ability to made an actual measurement and the restrictions imposed by rigorous theoretical analysis of a given model.

\section{2,015}

PB96-200373 Not available NTIS

National Inst. of Standards and Technology (EEEL), Boulder, CO. Electromagnetic Fields Div.

Comparison of Ultralow-Sidelobe-Antenna FarField Patterns Using the Planar-Near-Field Method and the Far-Field Method.

Final rept.

M. H. Francis, A. C. Newell, K. R. Grimm, J

Hoffman, and $H$. E. Schrank. 1995, 9p.

Pub. in Institute of Electrical and Electronics Engineers Antennas and Propagation Magazine, v37 n6 p7-15 Dec 95

Keywords: "Antennas, "Sidelobes, "Measurement, Far fields, Near fields, Measuring instruments, Reprints, Planar near field.

The development of very-low-sidelobe antennas raises the question of whether or not the planar-near-field method can be used to accurately measure these antennas. Recently, scientists at several organizations showed that data taken and processed with the planarnear-field methodology, including probe correction can be used to accurately measure the sidelobes of ver-low-sidelobe antennas. This can be done to levels of $-55 \mathrm{~dB}$ to $-60 \mathrm{~dB}$, relative to the mainbeam peak (1). This paper highlights these results, including a comparison of the far field, from the planar-near-field meth- od with the far field, found on a far-field range. The test antenna for this study was slotted-waveguide array, the low sidelobes for which were known. The near-field measurements were conducted on the NIST planar-near-field facility.

\section{2,016}

PB96-200696 Not available NTIS

National Inst. of Standards and Technology (EEEL), Boulder, CO. Electromagnetic Fields Div. Polarimetric Calibration of Reciprocal-Antenna Radars.

Final rept.

L. A. Muth, R. L. Lewis, and R. C. Wittmann. 1995

$6 \mathrm{p}$.

See also PB95-216925.

Pub. in Annual Meeting and Symposium of the 1995 Antenna Measurement Techniques Association (17th). Williamsburg, VA., November 13-17, 1995, p3-8.

Keywords: "Radar cross sections, "Depolarization, "Polarimetry, Radar antennas, Radar targets, Cross polarization, Error analysis, Calibration standards, Reprints, Reciprocal antenna radars.

We discuss how RCS targer depolanization enhances cross-polanization contamination, and we present a graphical study of measurement error due to depolarization by an inclined dihedral reflector. Error correction requires complete polarimetric RCS measurements. We present a simple polarimetric calibration scheme that is applicable to reciprocal antenna radars. This method uses a dihedral calibration target mounted on a rotator. Because the calibration standard can be rotated, there is no need to mount and align multiple separate standards, and clutter and noise may be rejected by averaging over rotation angle.

\section{2,017}

PB97-110555 Not available NTIS

National Inst. of Standards and Technology (MEL), Gaithersburg, MD. Precision Engineering Div.

Numerical Evaluation of Hypersingular Integrals for Scattering by a Dielectric Wedge.

Final rept

E. Marx. $1993,4 p$

Pub. in Institute of Electrical and Electronics Engineers Antennas and Propagation Society of International Symposium, Ann Arbor, MI., June 28-July 2, 1993 , 1993 Digest, v2 p906-909.

Keywords: "Dielectric wedges, "Electromagnetic scattering, Hypersingular integral, Numerical integration, Reprints, Neighboring-patch contributions.

Some components of the electromagnetic field near the edge of a dielectric wedge diverge. The computation of the fields scattered by the wedge can be done by solving a singular integral equation (SIE) or a hypersingular integral equation (HIE) (2) - (4). The unknown boundary function diverges near the edge for the SIE and tends to a constant for the HIE. Thus, the method using the HIE does not require the computa. tion of the boundary function closer to the edge that the closest field point, since this function can be approximated by a constant and the remainder of the integral can be evaluated numerically. Nevertheless, the integrand varies rapidly, like l/R2, over the patch and a number of special precautions have to be taken. The authors need to verify the expected behavior of fields near sharp edges because the theory is not rigorous. If the unknown function is constant near the edge of the wedge, the behavior of the fields is determined by the remainder of the integrand. The neighboring-patch contributions were previously examined under the assumption taht the small-argument approximation of the Hankel functions could be used. Here the authors relax this assumption. Similar corrections are required in the computation of the field components when the distance between the field point and the patch is small compared to the wavelength. Although the field integrals are finite, the behavior of the integrand is the same as that for the neighboring-patch contributions.

\section{Circuits}

\section{2,018}

PB94-135803 PC A03/MF AO

National Inst. of Standards and Technology (EEEL), Boulder, CO. Electromagnetic Fields Div.
NIST Model PM2 Power Measurement System for $1 \mathrm{~mW}$ at $1 \mathrm{GHz}$ F. R. Clague. Dec 93, 46p, NISTIR-5016.

Keywords: "Microwave equipment, "Power measurement, Computerized control systems, Electric power meters, GHz range 01-100, Ultrahigh frequency, Computer programs, Automation, Uncertainty.

The design and operation of an automated measurement system designed to measure power accurately at the level of $1 \mathrm{~mW}$ and at the frequency of $1 \mathrm{GHz}$ are described. The system consists of commercial IEEE Std-488 bus-controlled instruments, a computer controller, and software. The results of a series of measurements are output to the computer display and, optionally, to a printer. The results are the mean of the measurement series and an estimate of the Type A (here random) and Type B (here systematic) uncertainty. The estimated total expanded uncertainty for the average of six consecutive measurements of a nominal $1 \mathrm{~mW}, 1 \mathrm{GHz}$ source is typically less than 1 percent. The system can measure any power for 0.1 to $10 \mathrm{~mW}$ at any microwave frequency by making appropriate changes to the software and, possibly, the hardware.

\section{2,019}

PB94-169737 PC A07/MF A02

National Inst. of Standards and Technology, Gaithersburg, MD.

Journal of Research of the National Institute of Standards and Technology. January/February 1994. Volume 99 , Number 1

1994, $131 \mathrm{p}$.

See also PB94-169745 through PB94-169802 and PB94-140555. Also available from Supt. of Docs. as SN703-027-00056-3.

Keywords: Lattice parameters, X-ray diffraction, Blackbody radiation, Electric measuring instruments, Dimensional measurement, Thermal noise, Silicon, Length, Calibration, "Noise calibration system, "NCS1 system.

\section{Contents:}

Precision Comparison of the Lattice Parameters of Silicon Monocrystals:

The NIST $30 \mathrm{MHz}$ Linear Measurement System; Uncertainties in Dimensional Measurements Made at Nonstandard Temperatures:

A Null-Balanced Total-Power Radiometer System NCS1;

Derivation of the System Equation for NullBalanced Total-Power Radiometer System NCS1;

Evaluation of Uncertainties of the Null-Balanced Total-Power Radiometer System NCS1;

Cryogenic Blackbody Calibrations at the National Institute of Standards and Technology Low Background Infrared Calibration Facility.

\section{2,020}

\section{PB94-169752 Not available NTIS}

National Inst. of Standards and Technology, Boulder, CO.

NIST $30 \mathrm{MHz}$ Linear Measurement System.

J. A. Jargon, R. A. Ginley, and D. D. Sutton. 1994, $12 \mathrm{p}$.

Included in Jnl. of Research of the National Institute of Standards and Technology, v99 n1 p19-30 Jan/Feb 94.

Keywords: "Electric measuring instruments, Power measurement, High frequency, Linear systems, Nonlinearity, Automation, Calibration, Thermistors, Attenuators, Uncertainty, Reprints, Reference standards, US NIST.

There has been a recent interest in and demand for a calibration service at NIST to support if attenuators and voltage doublers that operate specifically at 30 $\mathrm{MHz}$. The first step required to offer such a service is to develop a reference standard. For the best possible accuracy, a tuned single-element thermistor mount was chosen. A linear measurement system was designed and constructed at NIST to calibrate the nonlinearity of this mount. The paper contains a description of the LMS, an explanation of the measurement scheme, calibration results, and an uncertainty analysis.

02,021

PB94-169778 Not available NTIS

National Inst. of Standards and Technology, Boulder, $\mathrm{CO}$ 
Null-Balanced Total-Power Radiometer System NCS1.

S. P. Pucic. 1994, 9p

Included in Jnl. of Research of the National Institute of Standards and Technology, v99 n1 p45-53 Jan/Feb 94.

Keywords: "Thermal noise, "Calibration, Noise temperature, $\mathrm{GHz}$ range 01-100, Cryogenic temperature, Radiometers, Reprints, "Noise calibration system, "NCS1 system, Noise standards.

A recently developed radiometer system NCS1 is used to calibrate thermal noise temperature at any frequency between $1.0 \mathrm{GHz}$ and $12.0 \mathrm{GHz}$. Any cryogenic noise source can be measured; the upper limit of noise temperatures measured without a loss of accuracy is estimated to be about $100,000 \mathrm{~K}$. For a typical hot noise source with the noise temperature of $8400 \mathrm{~K}$ and a reflection coefficient magnitude of 0.1 , the expanded uncertainty is about $1.8 \%$, and the system sensitivity about $2 \mathrm{~K}$. Implemented in Type $\mathrm{N}$ connector, it can be easily modified to calibrate noise sources with other coaxial connectors or waveguide flanges.

\section{2,022}

PB94-169786 Not available NTIS

National Inst. of Standards and Technology, Boulder, CO

Derivation of the System Equation for Null-Balanced Total-Power Radiometer System NCS1.

S. P. Pucic. 1994, 9p.

Included in Jnl. of Research of the National Institute of Standards and Technology, v99 n1 p55-63 Jan/Feb 94.

Keywords: Noise temperature, Mathernatical models, Calibration, Broadband, Radiometers, Reprints, "Noise calibration system, "NCS1 system.

A system equation of a recently developed null-balanced, total-power radiometer system is rigorously de rived. Delivered noise power and temperature is related to available power (temperature) through an extension of the mismatch factor to broadband systems. The available power ratio, the available gain, and the deliv ered power ratio (efficiency) are defined. Properties of idealized, but in principal realizable components such as an infinitely directive isolator and a lossless matched waveguide-below-cutoff attenuator are used. A cascading technique is repeatedly applied to the fundamental noise equation. Mathematically modeling the experimental procedure of sequentially attaching the wo noise standards and the unknown source to the system input, the authors obtain the system of three equations that can be solved for the noise temperature of the unknown noise source.

\section{2,023}

PB94-169794 Not available NTIS

National Inst. of Standards and Technology, Boulder, CO.

Evaluation of Uncertainties of the Null-Balanced Total-Power Radiometer System NCS1.

S. P. Pucic. 1994, $11 p$

Included in Jnl. of Research of the National Institute of Standards and Technology, v99 n1 p65-75 Jan/Feb 94.

Keywords: Noise temperature, $\mathrm{GHz}$ range 01-100 Thermal noise, Radiometers, Uncertainty, Calibration, Reprints, "Noise calibration system, "NCS1 system, Noise standards

Standard uncertainties are evaluated for the null-balanced, total-power, heterodyned radiometer syster with a switched input that was recently developed at NIST to calibrate thermal noise sources. Eight significant sources of uncertainty due to systematic effects are identified, two, attributable to the two noise stand ards, and one each to connectors, the input mismatch the input switch asymmetry, the isolator, the broadband mismatch and the attenuator. The combined standard uncertainty of a typical coaxial noise source calibration at the representative frequency of 2 $\mathrm{GHz}$ is about $1 \%$. A strategy for reducing uncertainties is discussed.

02,024

PB94-172228 Not available NTIS

National Inst. of Standards and Technology (EEEL) Gaithersburg, MD. Electricity Div.
Leakage Current Detection in Cryogenic Current Comparator Bridges.

Final rept.

R. E. Elmquist. 1993, 3p

Pub. in Institute of Electrical and Electronics Engineers Transactions on Instrumentation and Measurement 42 n2 p167-169 Apr 93

Keywords: "Resistance bridges, "Comparator circuits, Leakage current, Electrical measurement, Error analysis, Cryogenic equipment, Ratios, Tests, Reprints, Cryogenic current comparators, Quantum Hall effect.

Several tests have been developed to locate leakage currents in cryogenic current comparator (CCC) resistance ratio bridges used at NIST to measure ratios of $1,000 \mathrm{ohms} / 100 \mathrm{ohms}, 6,453.2 \mathrm{ohms} / 100 \mathrm{ohms}$, and $10 \mathrm{k}$ (ohms)/100 ohms. The major advantage of the tests is that they can be performed in situ using the sensitivity of the CCC bridge. These test procedures have been used to reduce the leakage error uncertainty of CCC ratio measurements linking working standards to the quantized Hall resistance (QHR) and to the NIST calculable capacitor experiment. CCC bridges require that the current which passes through a standard resistor must equal the current through the appropriate CCC winding to very high precision. This can be difficult to verify at or below 1 pA because a large number of possible leakage paths exist. Errors due to six important leakage current paths are given.

\section{2,025}

\section{PB94-172384 Not available NTIS}

National Inst. of Standards and Technology (EEEL), Gaithersburg, MD. Electricity Div.

Investigation of the Effects of Aging on the Calibration of a Kerr-Cell Measuring System for High Voltage Impulses.

Final rept.

G. J. FitzPatrick, and T. R. McComb. 1993, 4p.

Pub. in Proceedings of International Symposium on High Voltage Engineering (8th), Yokohama, Japan, August 23-27, 1993, p387-390.

Keywords: "High voltage, "Kerr cells, "Calibration, Aging(Materials), Electrical measurement, Electric pulses, Precision, Impulses, Reprints, Reference standards, Transfer standards.

Kerr-cell measuring systems can be used for high-voltage measurements from direct voltage up to impulses with a few nanoseconds risetime. In principle, this allows a measuring system for impulses to be calibrated at high-voltage using direct or alternating voltage which can be measured with a smaller uncertainty than is needed for impulse measurements. Unfortunately, the liquid normally used in such systems, nitrobenzene, degrades with time. This paper reports on an investigation into methods of calibrating an aged Kerr-cell so that a measuring system based on a Kerr-cell could be used as a Reference Measuring System. The repeatability of measurements using a fixed geometry to determine the voltage scale factor was also investigated.

\section{2,026}

PB94-172442 Not available NTIS

National Inst. of Standards and Technology (EEEL), Gaithersburg MD Electricity Div.

Intercomparison of NIST, NPL, PTB, and VSL Thermal Voltage Converters from $100 \mathrm{kHz}$ to $1 \mathrm{MHz}$

Final rept.

J. R. Kinard, R. B. D. Knight, P. Martin, J. Dessens, M. Klonz, and J. P. M. de Vreede. 1993, 3p.

Pub. in Institute of Electrical and Electronics Engineers Transactions on Instrumentation and Measurement 42 n2 p615-617 Apr 93. See also PB93-129328, PB93151181 and PB94-172459.

Keywords: Electrical measurement, $\mathrm{kHz}$ range 1001000, Interlaboratory comparisons, Reprints, "Thermal voltage converters, "Thermal converters, Intercomparison.

Coaxial, thermal voltage converters were hand-carried among NIST, NPL, PTB, and VSL for intercomparison of ac-dc difference from $100 \mathrm{kHz}$ to $1 \mathrm{MHz}$. This paper briefly describes the highly varied methods and underlying principles on which ac-dc difference determinations a re based in each laboratory, the transport standards used and the results of the intercomparisons. The ac-dc differences reported by the participating laboratories are in very good agreement. It has been concluded that representative values of ac-dc difference in the $100 \mathrm{kHz}$ to $1 \mathrm{MHz}$ frequency range have been established with 2(Sigma) uncertainties of 4-8 ppm.
02,027

PB94-172459 Not available NTIS

National Inst. of Standards and Technology (EEEL), Gaithersburg, MD. Electricity Div.

Intercomparison of Thermal Converters at NIM, NIST, PTB, SIRI and VSL from 10 to $100 \mathrm{MHz}$.

Final rept

J. R. Kinard, Z. Zhen, D. Huang, J. de Vreede, G Rebuldela, and D. Janik. 1993, 4p.

Pub. in Institute of Electrical and Electronics Engineers Transactions on Instrumentation and Measurement 42 , n2 p618-621 Apr 93

Keywords: Electrical measurement, $\mathrm{MHz}$ range 01 100, Interlaboratory comparisons, Reprints, “Therma voltage converters, "Thermal converters, intercomparison.

Coaxial thermal voltage converters have been intercompared among NIM, NIST, PTB, SIRI, and VSL in the frequency range from $10 \mathrm{MHz}$ to $100 \mathrm{MHz}$. The intercomparisons were made from 1988 through 1990 This paper briefly describes the highly varied methods and underlying principles on which RF-dc difference determinations a re based in each laboratory, the transport standards used, and the results of the intercomparisons. The results from the participating laboratories are in very good agreement; therefore, we believe that the determinations of RF-dc difference in this frequency range are very well established.

02.028

PB94-172582 Not available NTIS

National Inst. of Standards and Technology (EEEL) Gaithersburg, MD. Electricity Div.

Comparative Measurements of High-Voltage Im pulses Using a Kerr Cell and a Resistor Divider. Final rept.

T. R. McComb, and G. J. FitzPatrick. 1993, 4p.

Pub. in Proceedings of International Symposium on High Voltage Engineering (8th), Yokohama, Japan, August 23-27, 1993, p383-386.

Keywords: "High voltage, "Electric pulses, Electrica measurement, comparative evaluations, Kerr cells, Voltage dividers, Lightning, Impulses, Tests, Reprints, Reference standards, Transfer standards.

Recent proposals in committee drafts of IEC TC42 have placed greater emphasis on the use of comparative measurements as a method of qualifying impulse measuring systems. This paper describes some further nvestigations of comparative measurements where a Kerr-cell system is compared against a system based on a resistor divider. The paper describes the experimental techniques used to make comparative meas urements, and the results of tests on the linearity of the systems are presented. Finally, recommendations are made for the use of comparative measurements in qualifying impulse measuring systems.

\section{2,029}

PB94-172954 Not available NTIS

National Inst. of Standards and Technology (MSEL), Boulder, CO. Materials Reliability Div.

Evaluation and Qualification Standards for an $X$ Ray Laminography System.

Final rept.

T. A. Siewert, M. W. Austin, G. Lucey, and M. Plott. Sep 92, 9p

Pub. in Materials Evaluation, p1027-1035 Sep 92 Sponsored by Harry Diamond Labs., Adelphi, MD

Keywords: "Circuit boards, "X-ray inspection, Calibration standards, Qualifications, Resolution, Reprints, *X-ray laminography, "Laminography, Image quality.

$X$-ray laminography, a tomographic technique that can examine individual planes of focus within a three-dimensional structure, promises to be an excellent method of inspecting complicated circuit boards. The system has accuracy and reproducibility that are appropriate for circuit board inspection, however its application has been limited by a lack of physical standards to verify machine performance. The report describes the development of devices to measure the resolution of a laminography system and reports the system resolution measured with these devices. The system which the authors evaluated uses a scanned beam technique to select a plane of focus (slice) within a three-dimensional structure. The scanned beam technique provides very good z-dimension resolution. It is able to minimize the contribution from out-of-plane objects to the image. However the authors noted one example of an incorrect height (measurement artifact due to ge- 


\section{ELECTROTECHNOLOGY}

\section{Circuits}

ometry), when used with a hollow sphere image quality indicator design (not representative of solder joint shapes).

\section{2,030}

\section{PB94-185451 Not available NTIS}

National Inst. of Standards and Technology (NEL), Boulder, CO. Electromagnetic Technology Div.

Design and Operation of Series-Array Josephson Voltage Standards.

Final rept.

R. L. Kautz, 1992, 38p.

Pub. in Proceedings of Int. School of Physics Enrico Fermi, Villa Marigola, Italy, June 27-July 7, 1989, p2592961992

Keywords: "Standards, Phase locked systems, Josephson junctions, Josephson effect, Operation, Arrays, Design, Chaos, Electrochemical cells, Calibration, Reprints, "Voltage standards.

Series arrays typically including 1,500 Josephson junctions driven at $90 \mathrm{GHz}$ have been used to generate quantized reference voltages in excess of $10 \mathrm{~V}$. Such standards simplify the procedure and reduce the measurement uncertainties in the calibration of electrochemical cells.

\section{2,031}

\section{PB94-198306 PC A03}

National Inst. of Standards and Technology (PL), Boulder, CO. Time and Frequency Div.

Precision Oscillators: Dependence of Frequency on Temperature, Humidity and Pressure.

Final rept.

D. W. Allan, J. Barnes, F. Cordara, R. Kinsman, J.

Kusters, R. Smythe, F. L. Walls, M. Garvey, and W.

Hanson. 1992, 12p.

Keywords: *Oscillators, "Frequency stability, Temperature dependence, Pressure dependence, Test methods, Specifications, Guidelines, Precision, Humidity, Reprints.

The purpose of the research is to arrive at tractable (non-burdensome) guidelines, standards, and precautions for test methods used in determining the dependence of the output frequency of precision oscillators on temperature, humidity, and pressure. Over specification, under specification, or the lack of proper specification will miscommunicate. We offer a perspective for the manufacturer and the designer, as well as the user so that clear understanding and communication can occur. The guidelines, standards and precautions encourage consistency and repeatability for measurement and specification of environmental sensitivities. We believe very large cost savings will be appreciated if these guidelines, standards and precautions are followed.

\section{2,032}

PB94-198413 Not available NTIS

National Inst. of Standards and Technology (EEEL), Gaithersburg, MD. Electricity Div

Automatic Inductive Voltage Divider Bridge Operates from $10 \mathrm{~Hz}$ to $100 \mathrm{kHz}$

Final rept.

S. Avramov, N. M. Oldham, D. G. Jarrett, and B. C.

Waltrip. $1993,5 p$.

Pub. in Institute of Electrical and Electronics Engineers Transactions on Instrumentation and Measu rement 42, n2 p131-135 Apr 93. See also PB93-135572.

Keywords: "Voltage dividers, "Impedance bridges, Calibrating, Instrument compensation, Automation, Computer applications, Alternating current, Inductance, Reprints, IVD(Inductive voltage dividers).

A bridge to calibrate programmable and manual inductive voltage dividers is described. The bridge is based on a programmable $30 \mathrm{~b}$ binary inductive voltage divider with terminal linearity of + or $-0.1 \mathrm{ppm}$ in phase and + or $-2 \mathrm{ppm}$ quadrature at $400 \mathrm{~Hz}$. Measurements of programmable test dividers can be automated using software developed to align the bridge components and perform an automatic balance.

\section{3}

PB94-211588 Not available NTIS

National Inst. of Standards and Technology (NEL),

Boulder, CO. Electromagnetic Technology Div.
$24 \mathrm{GHz}$ Josephson Array Voltage Standard. Final rept.

C. A. Hamilton, R. L. Kautz, M. Stieg, M. B Simmonds, K. Chieh, and W. F. Avrin. 1991, 4p. Pub. in Institute of Electrical and Electronics Engineers Transactions on Instrumentation and Measurement 40 , n2 p301-304 1991

Keywords: *Standards, Superhigh frequency, Reprints, "Voltage standards, Josephson arrays.

A Josephson array voltage standard that operates at $24 \mathrm{GHz}$ has been designed and fabricated. Optimum selection of junction parameters makes possible step stability times of 20 minutes, which is more than adequate for calibrations at the $1-\mathrm{V}$ level. Designs using both probe and finline microwave couplers were test ed. The probe coupler was about $3 \mathrm{~dB}$ more efficient and allowed a smaller chip and a less expensive mount. The reduced operating frequency allows substantial advantages in cost and reliability over more conventional devices that operate at 70-100 GHz.

\section{2,034}

PB94-212669 Not available NTIS

National Inst. of Standards and Technology (EEEL) Boulder, CO. Electromagnetic Technology Div.

Proposed High-Accuracy Superconducting Power Meter for Millimeter Waves.

Final rept.

R. L. Kautz, D. G. McDonald, D. K. Walker, and D. Williams. 1993, 4p.

Contract N66001-92MP00017

Sponsored by Naval Ocean Systems Center, San Diego, CA

Pub. in Institute of Electrical and Electronics Engineers Transactions on Applied Superconductivity $3, n$ p2152-2155 Mar 93

Keywords: "Power meters, "Millimeter waves, Ex tremely high frequency, Superconducting devices, Microwave equipment, Bolometers, Niobium, Reprints.

The accuracy of conventional microwave power meters is limited by the fact that some of the power dissipated in the meter is not sensed by the bolometric detector. In a proposed power meter, superconducting materials are used to virtually eliminate this source of error. Our goal is to measure a power of $10 \mathrm{~mW}$ at frequencies in the WR-22 band ( 33 to $50 \mathrm{GHz}$ ) with an accuracy of $0.02 \%$.

\section{2,035}

PB94-216702 Not available NTIS

National Inst. of Standards and Technology (EEEL) Gaithersburg, MD. Electricity Div.

Digital Techniques in HV Tests - Summary of 1989 Panel Session.

Final rept.

T. R McComb C. Fenimore, E. Gockenbach, K.

Schon, L. Van der Sluis, B. Ward, Y. X. Zhang, J.

Kuffel, and R. Malewski. 1992, 5p.

Pub. in Institute of Electrical and Electronics Engineers Transactions on Power Delivery 7, n4 p1800-1804 Oct 92

Keywords: "Digital techniques, "High voltage, "Tests, Signal processing, Analog to digital converters, Industries, Reprints.

A panel session on digital techniques in high voltage (HV) tests was held at the IEEE PES Summer Meeting in Long Beach, CA (July 1989). This panel addressed the question of how signal processing can be used to enhance HV tests and extract more information from them. Part 1 dealt with the evaluation of digitizers and records, and Part 2 dealt with the application of digitizers to industrial testing. This paper presents an outline of the Panel Session and lists pertinent reference material.

\section{2,036}

PB95-126371 Not available NTIS

National Inst. of Standards and Technology (NEL), Gaithersburg, MD. Electricity Div.

Wide Band Active Current Transformer and Shunt. Final rept.

T. Guang-qiu, and X. Xiu-ye. 1991, 5p.

Pub in Institute of Electrical and Electronics Engineers Transactions on Instrumentation and Measu rement 40 n6 p908-912 1991.

Keywords: "Bypasses, "Circuits, "Transformers, Voltage converters (AC to $A C)$, Alternating current, Inductance, Frequency ranges, Audio frequencies, Resistors, Transducers, Electromagnetic measurement, Reprints.
An active inductive shunt with high accuracy and wide frequency range is introduced in this paper. The new shunt can be used in the audio frequency band. It has a wide current ratio $(0.01-1)$ and good loading capacity. If the external burden of the inductive shunt is an ac standard resistor, it will become an ac current to voltage transducer that can be used in many electromagnetic measurements. The relative uncertainty in the current ratio of the new shunt is $(1+j 1) \mathrm{ppm}$ to $(8+j 8) \mathrm{ppm}$ over the frequency range from $40 \mathrm{~Hz}$ to 10 $\mathrm{kHz}$.

\section{2,037}

PB95-150520 Not available NTIS

National Inst. of Standards and Technology (EEEL)

Gaithersburg, MD. Electricity Div.

Developing Linear Error Models for Analog Devices.

Final rept.

G. N. Stenbakken, and T. M. Souders. 1993, 6p.

Pub. in Proceedings of Instrumentation and Measurement Technology Conference '93, Irvine, CA., May 1820, 1993, p280-285.

Keywords: "Analog to digital converters, "Analog circuits, "Tests, Mathematical models, Error analysis, Simulation, Optimization, Reprints.

Techniques are presented for developing linear error models for analog and mixed-signal devices. A simulation program developed to understand the modeling process is described and results of simulations are presented Methods for optimizing the size of empinical error models based on simulated error analyses are included. Once established, the models can be used in a comprehensive approach for optimizing the testing of the subject devices. Models are developed using data from a group of 13-bit AVD converters and compared with the simulation results.

\section{2,038}

PB95-150538 Not available NTIS

National Inst. of Standards and Technology (EEEL) Gaithersburg, MD. Electricity Div.

Diakoptic and Large Change Sensitivity Analysis. Final rept.

G. N. Stenbakken, and J. A. Starzyk. 1992, 5p.

$P$ ub. in IEE Proceedings-G 139, n1 p114-118 Feb 92. Keywords: "Electrical networks, "Analog circuits, *Circuit analysis, Sensitivity analysis, Nonlinear systems, Reprints.

An approach to the analysis of large circuits based on the use of the large change sensitivity technique applied to decomposed networks is presented. As a result of this approach, a simple, compact notation for the solution vector is derived. The method is applicable to nonlinear analogue networks with hierarchical decomposition simulated by inserted ideal switches. A simple illustrative example is given.

\section{2,039}

PB95-150785 Not available NTIS

National Inst. of Standards and Technology (EEEL), Gaithersburg, MD. Electricity Div.

Flux-Locked Current Source Reference.

Final rept.

W. L. Tew, and E. R. Williams. 1993, 5p.

Pub. in Institute of Electrical and Electronics Engineers Transactions on Instrumentation and Measurement 42, n2 p186-190 Apr 93.

Keywords: Temperature range 0000-0013 K, SQUID devices, Quantization, Stability, Reprints, "Current sources, Flux transformers, Voltage references.

The quantization of flux in a closed superconducting circuit is used to provide a stable reference current. A $10 \mathrm{~mA}$ current source is coupled through a toroidal transformer to a dc SQUID input, and the resulting signal is fed back as an error current. The result is a net flux linkage that exhibits short-term stability of 1 part in $10(\exp 9) / \mathrm{h}$. The net current is quantized with a step size of $59.4 \mathrm{nA}$, and it will exhibit the same stability as the flux provided the mutual inductance of the transformer remains constant. This current is passed through a precise $100 \mathrm{ohm}$ resistor and compared against Zener diode references. The observed temperature coefficient for the flux transformer is $28.5+$ or $-3 \mathrm{ppm} / \mathrm{K}$ at $4.2 \mathrm{~K}$. Possible sources for the temperature dependence are discussed.

02,040

PB95-152310 Not available NTIS 
National Inst. of Standards and Technology (EEEL), Gaithersburg, MD. Electricity Div.

Cryogenic Precision Capacitance Bridge Using a Single Electron Tunneling Electrometer.

Final rept.

R. N. Ghosh, E. R. Williams, A. F. Clark, and R. J.

Soulen. 1994, 2p.

Pub. in Physica B 194-196, p1007-1008 1994.

Keywords: *Capacitance bridges, Cryogenic temperature, Cryogenic equipment, Electrometers, Precision, Reprints, Single electron tunneling, Electron charge, Dilution refrigerators.

The electronic charge can be determined by placing a known number of electrons on a calibrated capacitor and measuring the resulting voltage. Single electron tunneling (SET) electrometers with sufficient sensitivity for this application have been fabricated. We report on the design and preliminary results of a capacitance bridge experiment using an SET electrometer to measure two capacitors in a dilution refrigerator. This includes discussion of issues such as the leakage rate of a capacitor at cryogenic temperatures and the optimum coupling of the electrometer to the bridge circuit.

02,041

PB95-152849 Not available NTIS

National Inst. of Standards and Technology (EEEL), Gaithersburg, MD. Electricity Div.

Automatic Calibration of Inductive Voltage Dividers for the NASA Zeno Experiment.

Final rept.

S. Avramov, and N. M. Oldham. 1993, 3p.

Pub. in Review of Scientific Instruments 64, n9 p26762678 Sep 93.

Keywords: "Voltage dividers, "Calibration, Temperature control, Temperature measurement, Automation, Linearity, $\mathrm{Hz}$ range, Reprints, Inductive voltage dividers, Zeno experiment.

Two inductive voltage dividers (IVDs) used for temperature measurements in NASA's Zeno experiment were tested. In order to obtain the required resolution of 10 parts-per-billion, a 30-bit binary inductive voltage divider developed at the National Institute of Standards and Technology was used to measure the differential linearity of the Zeno IVDs. Automatic measurements were performed on the dividers in the Zeno engineering model at frequencies of 266 and $351 \mathrm{~Hz}$ over a ratio range of $0.55-0.56$. The measured differential linearity limits of temperature resolution of 5 microk.

02,042

PB95-152856 Not available NTIS

National Inst. of Standards and Technology (EEEL), Gaithersburg, MD. Electricity Div.

Inductive Voltage Divider Calibration for the NASA Flight Experiment.

Final rept.

S. Avramov, N. M. Oldham, and R. W. Gammon.

1993, 8p.

Contract NASA-NAS-3-25370

Sponsored by National Aeronautics and Space Administration, Washington, DC

Pub. in Proceedings of National Computer Systems Laboratory Workshop and Symposium, Albuquerque, NM., July 25-29, 1993, p225-232.

Keywords: "Voltage dividers, *Calibration, Temperature control, Thermostats, Automation, Impedance bridges, $\mathrm{Hz}$ range, Linearity, Tests, Reprints, Inductive voltage dividers, Zeno experiment.

The inductive voltage dividers (IVDs) used in the thermostat of NASA's Zeno experiment were tested using an automatic IVD bridge developed at the National Institute of Standards and Technology (NIST). To achieve + or - 10 microK temperature control, the thermostat must be able to measure resistance ratios with a differential linearity of + or -0.1 parts-per-million (ppm). The test results show that within a ratio range of 0.5 to 0.6 at frequencies between $200 \mathrm{~Hz}$ and 400 $\mathrm{Hz}$, the thermostat IVDs were linear to + or $-0.07 \mathrm{ppm}$.

02,043

PB95-153573 Not available NTIS

National Inst. of Standards and Technology (EEEL), Boulder, CO. Electromagnetic Fields Div.

Terminal Invariant Description of Amplifier Noise. Final rept.

G. F. Engen, and D. F. Wait. 1992, 2p

Pub. in Proceedings of Conference on Precision Electromagnetic Measurements, Paris, France, June 9-12, 1992 p250-251.
Keywords: *Electromagnetic noise, "Electric terminals, Models, Electric connectors, Electrical impedance, Reprints, "Amplifier noise.

An alternative treatment of amplifier noise which, in contrast to some of the earlier treatments, is 'terminal invariant' at the output as well as input terminals is presented. As a practical consequence, the model provides a reduced sensitivity to connector imperfections, and the problems in defining characteristic impedance.

\section{2,044}

PB95-162061 Not available NTIS

National Inst. of Standards and Technology (EEEL), Boulder, CO. Electromagnetic Fields Div.

Broadband Mismatch Error in Noise Measurement Systems.

Final rept.

S. Perera. 1992, $2 p$

Pub. in Proceedings of Conference on Precision Elecromagnetic Measurements, Paris, France, June 9-12, 1992, p256-257.

Keywords: "Electromagnetic noise measurement, Microwave radiometers, Radiofrequency filters, Thermal noise, Error analysis, Heterodyning, Reprints, Mismatch(Electrical).

Microwave noise measurement systems of a doublesideband heterodyne design, with a wide bandwidth and an electrically long transmission line at the input, may suffer from a large error; systems with a high IF are especially vulnerable. An IF filter may be employed to reduce the error to a negligible level.

\section{2,045}

PB95-163713 Not available NTIS

National Inst. of Standards and Technology (NML) Boulder, CO. Time and Frequency Div.

High Spectral Purity X-Band Source.

Final rept.

F. L. Walls, and C. M. Felton. 1990, 7p.

Pub. in Proceedings of the Annual Symposium on Frequency Control (44th), Baltimore, MD., May 23-25, 1990, p542-548

Keywords: "Frequency synthesizers, " $X$ band, Frequency discriminators, Microwave oscillators,

We have developed a $X$-band frequency source that has very high spectral purity and is suitable for frequency synthesis and many kinds of high-resolution spectroscopy. A commercial dielectric resonator oscillator (DRO) is frequency locked to a high-Q cavity used as a frequency discriminator. Many systems have been developed in the past for locking a source to a reference cavity. The distinguishing features of our approach are given. We describe the design of the discriminator cavity, the phase compensated frequencylocked loop, and the phase and amplitude noise performance. We also discuss vibration sensitivity, techniques for automatically locking to the low frequency reference, and the reduction of $60 \mathrm{~Hz}$ sidebands.

\section{2,046}

PB95-163861 Not available NTIS

National Inst. of Standards and Technology (NEL), Boulder, CO. Electromagnetic Technology Div.

Series Array of DC SQUIDs.

Final rept.

R. P. Welty, and J. M. Martinis. 1991, 3p.

Pub. in Institute of Electrical and Electronic Engineers Transactions on Magnetics 27, n2 p2924-2926 Mar 91

Keywords: "SQUID devices, Direct current, Amplifiers, Arrays, Aluminum oxides, Niobium, Reprints, SIS(Superconductors), Multilayers.

We have fabricated series arrays of $100 \mathrm{dc}$ SQUIDs using a $\mathrm{Nb}$-AIO $(\mathrm{x})$-Nb trilayer junction process. The SQUIDs are modulated with a common flux bias line and produce an output voltage swing of severa millivolts across the array. The large output voltage allows direct connection to room temperature electronics without the transformer coupling and resulting frequency limitations commonly associated with dc SQUID amplifiers. We have measured a bandwidth of $\mathrm{dc}$ to at least $175 \mathrm{MHz}$ for a 100 -SQUID array.

\section{2,047}

PB95-164687 Not available NTIS

National Inst. of Standards and Technology (EEEL), Gaithersburg, MD. Electricity Div.
Accuracy Comparisons of Josephson Array Systems.

Final rept.

R. L. Steiner, A. F. Clark, C. Kiser, T. J. Witt, and D. Reymann. 1993, 4p.

Pub. in Institute of Electrical and Electronics Engineers Transactions on Applied Superconductivity 3, n1 p1874-1877 Mar 93

Keywords: "Electrical measurement, "Standards, Comparative evaluations, Josephson junctions, Accuracy, Reprints, *Josephson arrays, "Voltage standards.

Five Josephson-array voltage standard systems were compared using several different methods. All of the tests were performed on site at a 1.018-V level, eithe by direct connection or through successive measurements of independent voltage sources. The resulting agreement between different systems measuring the same source were generally better than 10.0 parts in 10(exp -9), limited by source noise and detector resolution. Direct array-to-array comparisons for independent systems achieved agreement to within random uncertainties of 0.2 parts in 10 (exp -9)

\section{2,048}

PB95-168423 Not available NTIS

National Inst. of Standards and Technology (EEEL)

Boulder, CO. Electromagnetic Technology Div.

Tunable High Temperature Superconductor Microstrip Resonators.

Final rept.

J. A. Beall, R. H. Ono, D. Galt, and J. C. Price. 1993, 3p.

Sponsored by Office of Naval Research, Arlington, VA Pub. in Institute of Electrical and Electronics Engineers Atlanta, GA., June 14-18, 1993, p1421-1423.

Keywords: "Resonators, High temperature superconductors, Ferroelectric materials, Superconducting devices, Microstrip devices, Tuning devices, Microwave equipment, YBCO superconductors, Strontium titanates, Thin films, Reprints, Yttrium barium cuprates.

We have fabricated and characterized electrically tunable high temperature superconductor microstrip resonators incorporating $\mathrm{YBa} 2 \mathrm{Cu} 3 \mathrm{O}(7-\mathrm{x})$ superconducto and $\mathrm{SrTiO} 3$ ferroelectric films. Early versions of these and similar devices were described previously. The resonators consist of two co-linear microstrip line-sections separated by a 5 micrometer gap. The capacitance of the gap influences the frequencies of the odd order coupled resonances. Inductively choked dc bias lines are attached to each line section so that a bias voltage can be applied to the gap. When the gap is filled with a ferroelectric material the odd resonances can be tuned. Frequency shifts of $300 \mathrm{MHz}$ have been observed with a bias voltage of $50 \mathrm{~V}$ for resonances at $5.6 \mathrm{GHz}$ and $11.6 \mathrm{GHz}$. The tunability is independent of temperature from $4 \mathrm{~K}$ to $80 \mathrm{~K}$

\section{2,049}

\section{PB95-168530 Not available NTIS}

National Inst. of Standards and Technology (EEEL), Boulder, CO. Electromagnetic Fields Div.

Multi-State Two-Port: An Alternative Transfer Standard.

G. F. Eugen, R. M. Judish, and J. Juroshek. 1994,

G. F.

Pub. in Proceedings of Automatic Radio Frequency Techniques Group Conference Digest (43rd), San Diego, CA., May 27, 1994, p11-18.

Keywords: "Network analyzers, Solid state devices, Electric connectors, Performance evaluation, Reproducibility, Calibration, Automation, Tests, Reprints, *Transfer standards, *Impedance generators.

In a companion paper, the proposed use of a 'stable solid programmable impedance generator' as a calibration transfer and verification standard for vector network analyzers (VNA) has been suggested. An obvious requirement is that the multistate device provide a high degree of stability and repeatability. This paper describes a series of preliminary tests, using the NIST six-port systems, to evaluate the parameters of interest. The application of this device in connector evaluation is also reported.

02,050

PB95-168811 Not available NTIS

National Inst. of Standards and Technology (EEEL) Boulder, CO. Electromagnetic Technology Div. 


\section{Circuits}

Observation of Vortex Dynamics in Two-Dimensional Josephson-Junction Arrays.

Final rept.

S. G. Lachenmann, T. Doderer, D. Hoffmann, S. P. Benz, R. P. Huebener, and P. A. A. Booi. 1994, 7p Pub. in Physical Review B 50, n5 p3158-3164, 1 Aug 94.

Keywords: "Josephson junctions, Scanning electron microscopy, Two dimensional, Aluminum oxides, Niobium, Reprints, Josephson arrays, Vortex dynamics.

Spatially resolved images of the dynamic states of current-biased two-dimensional arrays of $\mathrm{Nb} / \mathrm{AlO}(\mathrm{x}) / \mathrm{Nb}$ Josephson junctions were obtained using low-temperature scanning electron microscopy. The arrays were square or rectangular and the maximum size was 20 junctions $X 20$ junctions. In overdamped arrays, our images at zero or small applied perpendicular magnetic field, together with model calculations, confirm the nucleation of a vortex at one sample edge (or an antivortex at the opposite edge) and its subsequen motion into the array interior. Vortex annihilation due to vortex-antivortex collision was observed to tak place in the middle of the array or at the edge opposite to the nucleation edge. These dynamics and the underlying model considerations are similar to that for Abrikosov vortices in the current-induced resistive state of thin film type-II superconductors. The phenomenon of 'row switching' is directly confirmed in images of underdamped arrays. The specific rows experiencing this process change randomly when the same bias point on the current-voltage characteristic is established many times, starting each time from zero current.

\section{2,051 \\ PB95-169165 Not available NTIS}

National Inst. of Standards and Technology (EEEL), Boulder, CO. Electromagnetic Technology Div Optimization of ECR-Based PECVD Oxide Films fo Superconducting Integrated Circuit Fabrication. Final rept.

J. E. Sauvageau, C. J. Burroughts, M. W. Cromar, and J. A. Koch. 1994, 6p.

Pub. in Proceedings of Annual Technical Conference of Society of Vacuum Coaters (37th), Boston, MA. May 8-13, 1994, 6p.

Keywords: "Chemical vapor deposition, "Chemical reactors, "Oxide films, "Silicon dioxide, Electron cyclotron resonance, Dielectric films, Superconducting devices, Integrated circuits, Microwave equipment, Optimization, Reprints, Plasma deposition.

A commercial microwave electron cyclotron resonance (ECR) reactor has been optimized for plasma en hanced chemical vapor deposition of silicon dioxide films at deposition temperatures lower than $150 \mathrm{C}$. A spool piece was added to the system resulting in improved deposition uniformity and lower deposition temperatures. Response surface methodology was used in the ECR system optimization before and after the modification. The response variables were deposition rate uniformity index of refraction film stress, and wet-etch rate. As a result of the studies, several oxide deposition processes have been developed at ambient temperatures. These processes are presently being tion facility at NIST. The optimization process and results are discussed.

\section{2,052}

PB95-169314 Not available NTIS

National Inst. of Standards and Technology (EEEL) Boulder, CO. Electromagnetic Technology Div.

Phase-Locked Oscillator Optimization for Arrays of Josephson Junctions.

Final rept.

K. Wiesenfeld, S. P. Benz, and P. A. A. Booi. 1994

\section{$12 p$.}

Sponsored by Office of Naval Research, Arlington, VA Pub. in Jnl. of Applied Physics 76, n6 p3835-3846, 15 Sep 94.

Keywords: "Josephson junctions, Phase locked systems, Two dimensional, Frequency standards, Design criteria, Stability, Reprints, "Josephson oscillators, Josephson arrays.

An overview of phase locking in two-dimensional (2D) arrays of identical Josephson junctions is presented General design criteria are discussed for optimization of power and linewidth. A harmonic balance technique is used to derive an analytic expression for the fundamental power as a function of bias voltage for a sin- gle shunted tunnel junction with an external shunt resistor having parasitic inductance. A linear stability analysis is performed on the in-phase state of $2 \mathrm{D}$ arrays in the absence of any external load. Most excitation modes in the 2D array are damped, leading to stable phase locking between parallel junctions within each row: however, within the theoretical model no mechanisms intrinsic to the array were found to induce phase locking between rows of junctions. The results of these calculations and their impact on and relevance to the design of phase-locked Josephson oscillators are discussed.

\section{2,053}

PB95-175071 Not available NTIS

National Inst. of Standards and Technology (EEEL), Boulder, CO. Electromagnetic Technology Div. Experimental Results on Single Flux Quantum Logic.

S. P. Benz, C. J. Burroughs, and C. A. Hamilton

1993, 4p

Sponsored by Defense Advanced Research Projects Agency, Arlington, VA

Pub. in Institute of Electrical and Electronics Engineers Transactions on Applied Superconductivity 3, n1 p2582-2585 Mar 93

Keywords: "Superconducting devices, "Logic devices, Josephson junctions, SQUID devices, Design, Reprints, Single flux quantum.

We have optimized the design and calculated the margins for a number of single flux quantum (SFQ) logic elements including AND, OR, XOR, Splitter, DC-toSFQ converter, and SFQ-to-DC converter. These are the fundamental building blocks necessary to construct more complex logic functions such as the half adder, and full adder. Experimental tests of the primary gates, the AND, OR, XOR, and splitter, were made by imbedding each test gate between DC-to-SFQ converters at the inputs and SFQ-to-DC converters at the outputs. Automated testing of each circuit was used to determine functionality, optimum bias levels, and margins. The experimental bias current margins for each gate are consistent with the simulations. This is the first experimental functional confirmation of these SFQ logic gates.

02,054

PB95-175121 Not available NTIS

National Inst. of Standards and Technology (EEEL),

Boulder, CO. Electromagnetic Technology Div.

Characterization of the Emission from 2D Array Josephson Oscillators.

Final rept.

P. A. A. Booi, and S. P. Benz. 1994, 10 p.

Pub. in Proceedings of International Symposium on Space Terahertz Technology (5th), Ann Arbor, MI., May 10-12, 1994, p234-243.

Keywords: "Josephson junctions, Phase locked systems, Experimental data, Two dimensional, Noise temperature, Line width, Emission, Reprints, "Josephson oscillators, Josephson arrays.

We present experimental results on the emission from phase-locked two-dimensional arrays of Josephson junctions. We have coupled the emission from $10 \times 10$ arrays to a room-temperature mixer through a fin-line antenna and WR-12 waveguide. A single voltage-tunable peak was detected up to $230 \mathrm{GHz}$. A stripline resonance in the antenna reduced the array's dynamic resistance and thereby the emission linewidth to as low as $10 \mathrm{kHz}$. We extract an effective noise temperature of $14 \mathrm{~K}$ from the linewidth data. When the array's emission was coupled to an on-chip detector junction through a dc blocking capacitor, we detected voltagetunable emission from $75 \mathrm{GHz}$ up to $300 \mathrm{GHz}$, and in some circuits emission above $400 \mathrm{GHz}$. The coherent power spectrum depends primarily on internal resonances.

\section{2,055}

PB95-175139 Not available NTIS

National Inst. of Standards and Technology (EEEL), Boulder, CO. Electromagnetic Technology Div.

Emission Linewidth Measurements of Two-Dimensional Array Josephson Oscillators.

Final rept.

P. A. A. Booi, and S. P. Benz. 1994, 3p.

Pub. in Applied Physics Letters 64, n16 p2163-2165, 18 Apr 94.

Keywords: "Josephson junctions, Noise temperature, Two dimensional, Line width, Emission, Reprints, "Josephson oscillators, Josephson arrays.
We have coupled emission from $10 \times 10$ arrays of Josephson junctions at $4 \mathrm{~K}$ to a room-temperature mixer through a fin-line antenna and a WR-12 waveguide. A single voltage-tunable peak was detected in the frequency range from 53 to $230 \mathrm{GHz}$. A stripline resonance in the antenna reduced the array's dynamic resistance and thereby the emission linewidth to as low as $10 \mathrm{kHz}$. We extract an effective noise temperature of $14 \mathrm{~K}$ from the linewidth data.

\section{2,056}

PB95-175147 Not available NTIS

National Inst. of Standards and Technology (EEEL). Boulder, CO. Electromagnetic Technology Div

Frequency Dependence of the Emission from 2D Array Josephson Oscillators.

Final rept.

P. A. A. Booi, S. P. Benz, T. Doderer, S.

Lachenmann, R. P. Huebener, D. Hoffmann, and J. Schmidt. 1993, 3p.

Contract ONR-N00014-92-F-00040

Sponsored by Office of Naval Research, Arlington, VA. Pub. in Institute of Electrical and Electronics Engineers Transactions on Applied Superconductivity $3, n 1$ p2493-2495 Mar 93.

Keywords: "Josephson junctions, Phase locked systems, Frequency dependence, Two dimensional, Coherent radiation, Standing waves, Emission, Reprints, *Josephson oscillators, Josephson arrays.

Coherent emission from two-dimensional arrays of Josephson junctions, coupled to a detector junction through a dc blocking stripline capacitor, was detected over a frequency range from 50 to $210 \mathrm{GHz}$. A power of 0.26 (micro) W which is larger than the 0.1 (micro)W expected from the RSJ model was detected in a range from 140 to $150 \mathrm{GHz}$. Frequencies where no emission was detected correspond to standing waves in the capacitor when multiples of the half-wavelength match the capacitor length. Low temperature scanning electron microscopy confirmed the presence of standing waves at these frequencies, but also revealed standing waves at other frequencies indicating an impedance mismatch and a possible extension of the standing waves into the array.

\section{2,057}

PB95-175246 Not available NTIS

National Inst. of Standards and Technology (EEEL), Boulder, CO. Electromagnetic Technology Div. Automated Josephson Integrated Circuit Test Sys. tem.

Final rept.

C. J. Burroughs, and C. A. Hamilton. 1993, 3p. Pub. in Institute of Electrical and Electronics Engineers Transactions on Appl. Supercond. 3, p2687-2689 Mar 93.

Keywords: "Circuit testers, "Integrated circuits, Test equipment, Josephson junctions, Automation, Reprints, "Superconducting networks.

We have developed an automated test system for complex superconductive integrated circuits. Its low speed capability consists of 96 identical I/O channels which are controlled by a PC-486 computer. Each channel is capable of driving currents and reading voltages at frequencies up to $40 \mathrm{kHz}$. Integrating this low speed $1 / O$ capability with high speed test equipment controlled over the IEEE 488 bus allows meas urements at frequencies up to the limits of the test equipment. The system can automatically set biases, display I-V curves, measure parameter margins, plot threshold curves, extract experimental circuit values, and collect statistical data on parameter spreads and error rates. Issues of noise suppression, ground loop handling, and auto-calibration are discussed.

\section{2,058}

PB95-175600 Not available NTIS

National Inst. of Standards and Technology (EEEL) Boulder, CO. Electromagnetic Technology Div. Josephson Voltage Standard Based on SingleFlux-Quantum Voltage Multipliers.

Final rept.

C. A. Hamilton. 1992, 4p.

Pub. in Institute of Electrical and Electronics Engineers Transactions on Applied Superconductivity 2, n3 p 139142 Sep 92.

Keywords: "Josephson junctions, Frequency multipliers, Design, Reprints, "Voltage standards, Single flux quantum.

A Josephson single-flux-quantum voltage/frequency multiplier circuit is proposed as the basic building block 
for a new generation of voltage standards. The circuit uses magnetic coupling to synchronize a series array of independent junction oscillators to the flux flow in a Josephson transmission line. A cascade of these circuits can multiply an arbitrarily low input frequency up to the frequency limit of the circuit (approx. $250 \mathrm{GHz}$ ) and then add the voltages across approximately 30,000 oscillators to generate precise voltages up to $10 \mathrm{~V}$. Because the oscillators can be switched on and off with a bias current, the output voltage is rapidly programmable. A complete design for a voltage standard programmable in 1.2 (micro) $\mathrm{V}$ increments to a maximum of $10 \mathrm{~V}$ is proposed. Using existing fabrication technology, the circuit would cover a substrate area of about $1 \mathrm{sq} \mathrm{cm}$ and use 67,210 junctions.

02,059

PB95-175857 Not available NTIS

National Inst. of Standards and Technology (EEEL),

Boulder, CO. Electromagnetic Technology Div.

Fabrication Issues in Optimizing YBa2Cu307-x

Flux Transformers for Low I/f Noise.

Final rept.

F. Ludwig, E. Dantsker, D. T. Nemeth, J. Clarke, S.

Knappe, H. Koch, R. E. Thomson, D. Koelle, and A

H. Miklich, 1994, 4p

Contract DE-AC03-76SF00098

Sponsored by Department of Energy, Washington, DC. Pub. in Superconducting Science and Technology 7 , p273-276 1994.

Keywords: "Transformers, Superconducting devices, High temperature superconductors, YBCO superconductors, Strontium titanates, Magnetometers, Fabrication, Reprints, "Flux transformers, Yttrium barium cuprates, Multilayers.

We describe an improved interconnect technology for the fabrication of multiturn flux transformers from YBa2Cu3O (7-x)-SrTiO3-YBa2Cu3O(7-x) multilayers. The essential improvements are reductions in the thicknesses of the trilayer films, typically to $100 \mathrm{~nm}$, $250 \mathrm{~nm}$, and $250 \mathrm{~nm}$ respectively, and in the deposition rate, to $0.07 \mathrm{~nm} /$ laser pulse. This process yields crossovers in which the critical current density in the upper $\mathrm{YBa} 2 \mathrm{Cu} 3 \mathrm{O}(7-\mathrm{x})$ film at $77 \mathrm{~K}$ is $(2-3) \times 10$ (sup 6) $\mathrm{A} / \mathrm{sq}$ $\mathrm{cm}$. In situ trilayers exhibited $1 / f$ flux noise levels at 1 $\mathrm{Hz}$ below the measurement sensitivity of 15 (mu)(Phi)(sub 0) $\mathrm{Hz}$ (sup -1/2), where Phi(sub 0) is the flux quantum. However, the flux noise of trilayers in which each layer had been patterned was significantly higher. The best flip-chip magnetometer had a white noise of $40 \mathrm{fT} \mathrm{Hz}$ (sup -1/2), increasing to $340 \mathrm{IT}$ $\mathrm{Hz}$ (sup -1/2) at $1 \mathrm{~Hz}$; the corresponding flux noise levels were 9 (mu)(Phi)(sub 0 ) $\mathrm{Hz}$ (sup -1/2) and 75 (mu)(Phi)(sub 0) Hz(sup -1/2).

02,060

PB95-176053 Not available NTIS

National Inst. of Standards and Technology (EEEL),

Boulder, CO. Electromagnetic Technology Div.

Phase Locking in Two-Junction Systems of High-

Temperature Superconductor-Normal MetalSuperconductor Junctions.

Final rept.

C. D. Reintsema, R. H. Ono, T. E. Harvey, N.

Missen, and L. R. Vale. 1994, 3p.

Pub. in Applied Physics Letters 64, n5 p637-639, 31 Jan 94

Keywords: "Phase locked systems, "Josephson junctions, High temperature superconductors, Temperature range 0000-0013 $\mathrm{K}$, Temperature range 0013 $0065 \mathrm{~K}$ Temperature dependence, YBCO superconductors, Planar structures, Reprints, Yttrium barium cuprates, Josephson arrays, Multilayers.

Mutual phase locking between two high-temperature step-edge superconducting-normal metalsuperconducting junctions has been investigated using a two-junction circuit with a nonsuperconducting feedback path. The strength of the phase-locked state has been characterized as a function of locking frequency and temperature. Results are presented for a plana circuit as well as for a multilayer circuit incorporating a superconducting ground plane. The observed behavior was significantly enhanced for the circuit over a ground plane. Characterization of the phase locked state at $4 \mathrm{~K}$ yielded locking strengths as large as (absolute value of $\mathrm{I}($ sub $L)) /(\mathrm{I}$ bar $)(\mathrm{sub} \mathrm{c})=9 \%$, and maximum locking frequencies to $1.06 \mathrm{THz}$. The magnitude of the locking strength decreased rapidly with increasing temperature with complete loss of coherence oc curring at temperatures greater than $35 \mathrm{~K}$.
02,061

PB95-176277 Not available NTIS

National Inst. of Standards and Technology (EEEL) Boulder, CO. Electromagnetic Technology Div.

Two-Stage Integrated SQUID Amplifier with Series Array Output.

Final rept.

R. P. Welty, and J. M. Martinis. 1993, 4p.

Contract ONR-N00014-92-F-0003

Sponsored by Office of Naval Research, Arlington, VA Pub. in Institute of Electrical and Electronics Engineers Transactions on Applied Superconductivity $3, \mathrm{n} 1$ p2605-2608 Mar 93

Keywords: "SQUID devices, Integrated circuits, Low noise, Reprints, "SQUID amplifiers, SQUID arrays.

We have fabricated a 2 -stage integrated dc SQUID amplifier which uses a compact series array of $100 \mathrm{dc}$ SQUIDs as the readout device for a low-noise single SQUID. The results suggest the general utility of series SQUID arrays as readout devices for SQUIDS.

\section{2,062}

PB95-180022 Not available NTIS

National Inst. of Standards and Technology (PL), Boulder, CO. Time and Frequency Div.

Investigations of $A M$ and PM Noise in X-Band Devices.

Final rept.

F. G. Ascarunz, E. S. Ferre, and F. L. Walls. 1993,

Pub. in Proceedings of Institute of Electrical and Electronics Engineers International Frequency Control Symposium, Salt Lake City, UT., June 2-4, 1993, p303 311

Keywords: "Microwave amplifiers, *Microwave oscillators, "Mixers(Electronics), "Electromagnetic noise, "Amplitude modulation, "Phase modulation, Electrica measurement, Superhigh frequency, $X$ band, White noise, Reprints.

In this paper we report on measurements of phase modulation (PM) and amplitude modulation (AM) noise in a variety of amplifiers, dielectric resonator oscillato (DRO) sources and mixers at $10.6 \mathrm{GHz}$. There is little information on AM noise and only limited information on PM noise in microwave devices. Two channel measurement systems and cross-correlation analysis were used in these noise measurements. In amplifiers there are at least two random noise processes that generate equal levels of PM and AM noise when the amplifier is in the linear operating range. The noise processes correspond to a flicker noise process and a white noise process. In the DRO sources we show that these noise mechanisms are present in the active components and determine the AM and PM character istics of the oscillator. We report on noise residuals in mixers from three different companies.

\section{2,063}

PB95-198685 PC A04/MF A01

National Inst. of Standards and Technology (EEEL), Gaithersburg, MD. Electricity Div.

10 kV DC Resistive Divider Calibration.

R. S. Turgel. Jan 95, 57p, NISTIR-5566.

Keywords: "Voltage dividers, "Calibration, Electrica networks, Direct current, Temperature control, High voltage, Computer programs, Voltmeters, Resistors, Kilovolts.

A calibration method for a $10 \mathrm{kV}$ dc ratio divider was developed. The method uses a high-precision digita voltmeter, at the $10 \mathrm{~V}$ level, to establish a 10:1 voltage ratio for each decade of the resistive divider and combines these results to obtain an overall voltage ratio of 10,000:1. The linearity of the voltmeter was checked using a Josephson-array voltage standard. The overall ratio agreed with measurements made at $10 \mathrm{kV}$ using a Park-type divider to within the uncertainty limits.

\section{2,064}

PB95-202644 Not available NTIS

National Inst. of Standards and Technology (EEEL) Boulder, CO. Electromagnetic Fields Div.

Accuracy and Repeatability in Time Domain Network Analysis.

Final rept.

L. A. Hayden, R. B. Marks, and J. B. Rettig. 1994,

Pub. in Proceedings of Automatic Radio Frequency Techniques Group Conference Digest, Boulder, CO. December 1-2, 1994, p39-46
Keywords: "Network analysis, "Electrical measurement, Electrical networks, Electromagnetic noise, Time domain, Reproducibility, Reflectometers, Attenuation, Accuracy, Reprints

This paper examines the importance of measurement repeatability in time domain network analysis and includes an analysis of the limitations imposed on theoretical accuracy by measurement noise. A closed-loop correction algorithm implemented in a fast, equivalenttime sampling, time domain reflectometer improves source timing accuracy, the dominant cause of nonrepeatability. An example measurement of attenuation in a $3.5 \mathrm{~mm}$ coaxial air-line demonstrates performance approaching this theoretical noise limit and the limits imposed by connector repeatability.

\section{2,065}

PB95-202925 Not available NTIS

National Inst. of Standards and Technology (EEEL), Boulder, CO. Electromagnetic Fields Div.

Time Domain Network Analysis Using the Multiline TRL Calibration.

Final rept.

R. B. Marks, L. A. Hayden, J. A. Jargon, and F.

Williams. 1994, 9p.

Pub. in Proceedings of Automatic Radio Frequency Techniques Group Conference Digest (44th), Boulder, CO., December 1-2, 1994, p47-55

Keywords: "Network analyzers, "Calibration, Time domain, Reprints, Multiline TRL method, On wafer measurement, Scattering parameters.

We apply the multiline TRL (through-reflect-line) meth od to the calibration of a time domain network analyze (TDNA). The calibration removes the effects of cables and connectors, nonideal source and sampler re sponses, source and sampler mismatch, and frequency-dependent characteristic impedance of the transmission lines. Multiline TRL is especially well suited to TDNA and provides not only a complete calibration but also a full characterization of the transmission lines, information useful in the study of interconnections.

\section{2,066}

PB96-102082 Not available NTIS

National Inst of Standards and Technology (EEEL), Boulder, CO. Electromagnetic Technology Div

Resonances in Two-Dimensional Array Oscillator Circuits.

P. A A Booi, and S. P. Benz. 1995, 4p

Sponsored by Office of Naval Research, Arlington, VA. Pub. in Institute of Electrical and Electronics Engineers Transactions on Applied Superconductivity, v5 n2 p2899-2902 Jun 95.

Keywords: "Josephson junctions, "Resonance, *Emission, Antennas, Waveguides, Resonance lines, Spectra line width, Oscillators, Circuits, Reprints

The authors present experimental results on the emission from phase-locked two-dimensional arrays of Josephson junctions. The authors have coupled the emission from $10 \times 10$ arrays to a room-temperature mixer through a fin-line antenna and WR-12 waveguide. A single voltage-tunable peak was detected up to $230 \mathrm{GHz}$. A stripline resonance in the antenna reduced the array's dynamic resistance and thereby the emission linewidth to as low as $10 \mathrm{kHz}$. The authors extract an effective noise temperature of $14 \mathrm{~K}$ from the linewidth data.

\section{2,067}

PB96-102223 Not available NTIS

National Inst. of Standards and Technology (EEEL), Boulder, CO. Electromagnetic Technology Div. Direct Observation of Vortex Dynamics in Two-Dimensional Josephson-Junction Arrays.

Final rept

T. Doderer, S. G. Lachenmann, R. P. Huebener, P. A. A. Booi, and S. P. Benz. 1995, 4p.

Pub. in Institute of Electrical and Electronics Engineers Transactions on Applied Superconductivity, v5 n2 p2723-2726 Jun 95.

Keywords: "Josephson junctions, "Arrays, "Vortices, Absolute zero, Magnetic fields, Electric current, Nucleation, Bias, Reprints, Two dimensional array, SEM(Scanning electron microscopy), Vortex dynamics, LTSEM.

Spatially resolved images of the dynamic states of current-biased overdamped two-dimensional arrays of 


\section{Circuits}

$\mathrm{Nb} / \mathrm{AlO} \times \mathrm{Nb}$ Josephson junctions were obtained using low-temperature scanning electron microscopy. The authors present two-dimensional imaging results describing various vortex dynamic regimes in zero applied magnetic field. The nucleation of current-induced vortices at the array boundaries and their subsequent motion into the array interior are observed for bias currents slightly above the array critical current. With increasing bias current, vortex-vortex interaction be comes important. Discussions on the coherent microwave radiation emission are presented.

\section{2,068}

PB96-102629 Not available NTIS

National Inst. of Standards and Technology (EEEL) Gaithersburg, MD. Electricity Div.

100 Ampere, $100 \mathrm{kHZ}$ Transconductance Amplifier. Final rept.

O. B. Laug. 1995, 6p

Pub. in Institute of Electrical and Electronics Engineers Instrumentation and Measurement Technology Con ference (IMTC), Waltham, MA., April 24-26, 1995 p506-511 1995.

Keywords: "Power amplifiers, "Voltage amplifiers, Reprints, Transconductance, Electric power meters.

A high-current, wide-band transconductance amplifier is described that provides an unprecedented level of output current at high frequencies with exceptional stability. It is capable of converting a signal voltage ap plied to its input into a ground-referenced output current up to 100 amperes rms over a frequency range from dc to $100 \mathrm{kHz}$ with a useable frequency extending to $1 \mathrm{MHz}$. The amplifier has a 1000 watt output capability, + or - 10 volts of compliance, and can deliver up to 400 amperes peak-to-peak of pulsed current. The amplifier design is based on the principle of paralleling a number of precision bipolar voltage-to-current converters. The design incorporates a unique ranging system controlled by opto-isolated switches, which permit a full-scale range from 5 to 100 amperes. The design considerations for maintaining wide bandwidth, high output impedance, and unconditional stability for all loads are discussed.

\section{2,069}

PB96-102785 Not available NTIS

National Inst. of Standards and Technology (EEEL), Boulder, CO. Electromagnetic Fields Div.

Comments on 'Conversions between S, Z, Y, h, $A B C D$, and $T$ Parameters Which Are Valid for Complex Source and Load Impedances'.

Final rept.

R. B. Marks, and D. F. Williams. 1995, 2p

Pub. in Institute of Electrical and Electronics Engineers Transactions on Microwave Theory and Techniques, v43 n4 p914-914 Apr 95

Keywords: "Characteristic impedance, "Electric converters, "Microwave circuits, Electrical impedance, Electric networks, Matrices(Circuits), Wave scattering, Travelling waves, Transmission circuits, Reprints, * Scattering parameters.

A recently published paper presents formulas for converting between various network parameters. However, these parameters are defined using an unconventional definition of the waves and therefore yield un expected results.

\section{2,070}

PB96-111984 Not available NTIS

National Inst. of Standards and Technology (EEEL), Boulder, CO. Electromagnetic Fields Div.

Uncertainties of the NIST Coaxial Noise Calibration System.

Final rept.

S. P. Pucic. 1994, 2p

Pub. in Proceedings of Conference on Precision Electromagnetic Measurements Digest, Boulder, CO., June 27-July 1, 1994, p254-255.

Keywords: "Calibration, Radiometers, Broadband, Cryogenic temperature, Standards, Reprints, *Noise calibration systems, National Institutes of Standards and Technology.

Uncertainties of the NIST broadband coaxial calibration system are analyzed. The expanded relative uncertainty for a source with the ENR of approximately $15 \mathrm{~dB}$ is typically $1.6 \%$

02,071

PB96-112263 Not available NTIS
National Inst. of Standards and Technology (EEEL), Gaithersburg, MD. Electricity Div.

Binary versus Decade Inductive Voltage Divider Comparison and Error Decomposition.

Final rept.

S. Avramov-Zamurovic, G. N. Stenbakken, A. D.

Koffman, N. M. Oldham, and R. W. Gammon. 1995

$5 p$.

Pub. in Institute of Electrical and Electronics Transactions on Instrumentation and Measurement, v44 n4 p904-908, 28 Aug 95.

Keywords: "Voltage dividers, Reprints, Electrical networks, Linearity, Structural models, Inductive voltage dividers

An automatic Inductive Voltage Divider (IVD) characterization method that can measure linearity by comparing IVD's with different structures is suggested. Structural models are employed to decompose an error vector into components that represent each divider. Initial tests at $400 \mathrm{~Hz}$ show that it is possible to assign independent errors due to the binary and decade structures with a 2-sigma uncertainty of 0.05 parts per million (ppm) at the measured ratio values.

\section{2,072}

PB96-112271 Not available NTIS

National Inst. of Standards and Technology (EEEL), Gaithersburg, MD. Electricity Div.

Cryogenic Precision Capacitance Bridge Using a Single Electron Tunneling Electrometer.

Final rept.

R. N. Ghosh, A. F. Clark, B. A. Sanborn, and E. R.

Williams. 1994, 6p.

See also PB95-126074 and PB95-152310.

Pub. in Coulomb and Interference Effects in Small Electronic Structures, p311-316 1994.

Keywords: “Capacitance bridges, “Electrometers, Reprints, Cryogenic temperature, Cryogenic equipment, Precision, Single electron tunneling, Electron charge.

The value of the electronic charge can be determined by placing a known number of electrons on a calibrated capacitor and measuring the resulting voltage, which can lead to a measure of the fine structure constant. alpha. Single electron tunneling (SET) electrometers with sufficient sensitivity for this application have been fabricated. We report on the design and preliminary results of a capacitance bridge experiment using an SET electrometer as a detector to measure two capacitors in a dilution refrigerator. AC measurements of the capacitance ratio have a precision of one part in 10 to the 4th power and DC measurements provide information on the leakage rate of the standard capacitors.

\section{2,073}

PB96-112362 Not available NTIS

National Inst. of Standards and Technology (MSEL),

Gaithersburg, MD. Reactor Radiation Div.

Vortex Dynamics and Melting in Niobium.

Final rept

J. W. Lynn, N. Rosov, and T. E. Grigereit. 1995, 2p. Pub. in Jnl. of Magnetism and Magnetic Materials, v140-144 p2067-2068 1995.

Keywords: "Niobium, "Neutron scattering, Reprints, Melting, "Foreign technology, Vortex dynamics.

Small angle neutron scattering has been used to investigate the vortex scattering in a single of crystal niobium. Below the irreversibility line resolution-limited Bragg peaks are observed, indicating that a crystalline vortex lattice with long range order exists. Above the irreversibility line intrinsic transverse widths develop, while close to $\mathrm{Hc} 2$ intrinsic radial widths also develop. Nevertheless the basic six-fold dymmetry of scattering is observed throughout the vortex phase, indicating that a correlated flux fluid exists in the reversible regime.

\section{2,074}

PB96-119334 Not available NTIS

National Inst. of Standards and Technology (EEEL), Gaithersburg, MD. Electricity Div.

High-Temperature Superconductor Cryogenic Current Comparator.

Final rept.

R. E. Elmquist, and R. F. Dziuba. 1995, 3p.

Pub in Institute of Electrical and Electronics Engineers Transactions on Instrumentation and Measurement, v44 n2 p262-264 Apr 95.

Keywords: "Computer circuits, "High temperature, "Superconductors, "Cryogenics, Magnetic shielding,
Diamagnetism, Superconductivity, Resistance, SOVID detectors, Reprints, "Cryogenic current comparators HTS(High temperature superconductor).

The National Institute of Standards and Technology (NIST) is developing a cryogenic current comparator (CCC) to operate at $77 \mathrm{~K}$, using high-temperature superconductor (HTS) ceramic shielding material and an HTS-based superconducting quantum interference device (SQUID) detector. The shielding properties of at least two polycrystalline oxide HTS materials appear sufficient for use in a high-accuracy CCC. A measurement of current-linkage error, a figure of merit for CCC devices, is made for one type of HTS CCC. The design of a second HTS CCC which uses improved magnetic shielding is described.

\subsection{5}

PB96-119771 Not available NTIS

National Inst. of Standards and Technology (PL), Boulder, CO. Time and Frequency Div.

Reducing Errors, Complexity, and Measurement Time of PM Noise Measurements.

Final rept.

F. L. Walls. $1993,9 p$

Pub. in Proceedings of the Institute of Electrical and Electronics Engineers International Frequency Contro Symposium, Salt Lake City, UT., June 2-4, 1993, p289297.

Keywords: "Signal generators, *Frequency synthesizers, “Oscillators, Reprints, Standards, "Phase noise.

This paper shows that a new measurement technique based on the two-oscillator technique and the addition of a noise source in series with the reference oscillator can significantly reduce calibration time for accurate PM measurements in oscillators and other components as compared to the traditional two-oscillator technique. This technique also significantly reduces the measurement time and improves the accuracy of 3-corner-hat measurements. Measurement complexity is greatly reduced. The noise source is used to generate a known level of PM noise (PMCAL) for calibrating the product of mixer sensitivity and amplifier gain with Fourier frequency. This can be used to correct for PLL effects whem PMCAL is larger than the residual phase noise in the oscillator under test. PMCAL is typically constant to equal to or greater than $0.1 \mathrm{~dB}$ for Fourier frequencies from 0 to $5 \%$ of the carrier (maximum width typically less than $500 \mathrm{MHz}$ ). When the PMCAL is off, the noise added to the reference signal is typically less than $-150 \mathrm{dBc} / \mathrm{Hz}$ at $1 \mathrm{~Hz}$ and $-190 \mathrm{dBc} /$ $\mathrm{Hz}$ at $10 \mathrm{kHz}$ for carrier frequencies of 5 to $100 \mathrm{MHz}$ A similar system also works in the microwave range.

02,076

PB96-122502 Not available NTIS

National Inst. of Standards and Technology (EEEL), Boulder, CO. Electromagnetic Technology Div.

Optical Sampling Using Nondegenerate Four-Wave Mixing in a Semiconductor Laser Amplifier.

Final rept.

M. Jinno, J. B. Schlager, and D. L. Franzen. 1994

$3 p$.

Pub. in Tech. Digest, OSA, Optical Amplifers and Their Applications, Topical Meeting, Breckennidge, CO., August 3-5, 1994, v4 p147-149 1994

Keywords: "Laser amplifiers, "Optical sampling, Semiconductors, Reprints, Four wave mixing, Fiber laser. Picosecond optical sampling using nondegenerate four-wave mixing in a serniconductor amplifier (SLA) is demonstrated for the first time. High-peak-power probe pulses and electrical gating of the SLA produce an optical sampling signal with high signal-to-noise ratio.

\section{2,077}

PB96-122585 Not available NTIS

National Inst. of Standards and Technology (EEEL), Boulder, CO. Electromagnetic Fields Div.

Microwave Characterization of Printed Circuit Transmission Lines.

Final rept.

R. B. Marks, and D. F. Williams. 1994, 8p

Pub. in National Electronics Packaging and Production

Conference (NEPCON) East '94, Proceedings of the Technical Program, Boston, MA., June 13-16, 1994, p520-527.

Keywords: "Microwaves, *Printed circuit boards, Electrical characterization, Reprints, Electronic packaging, Impedance.

This paper reviews basic methodology for the microwave characterization of printed circuit transmission 
lines in terms of scattering parameters, impedances, and frequency-dependent transmission lines parameters. The focus is on a suite of methods developed at the National Institute of Standards and Technology for the characterization of high-performance electronic packaging and interconnections as well as monolithic microwave integrated circuits.

\section{2,078}

PB96-122825 Not available NTIS

National Inst. of Standards and Technology (EEEL), Boulder, CO. Electromagnetic Fields Div.

Open-Ended Coaxial Probes for Nondestructive Testing of Substrates and Circuit Boards.

Final rept.

J. R. Baker-Jarvis, and M. D. Janezic. 1994, 6p.

Pub. in Proceedings of Materials Research Society, April 4-8, 1994, San Francisco, CA., v347 p215-220.

Keywords: "Circuit boards, "Coaxial cables, "Nondestructive tests, Substrates, Probes, Dielectrics, Wave equations, Mathematical models, Numerical analysis, Sensitivity, Low frequencies, Reprints, "Open-ended probes.

The results of the full-wave model theory for the openended coaxial probe with lift-off are presented and are applied to mesurements of thin materials. The model allows the study of the open-ended coaxial prode as a nondestructive testing tool. The equations presented are valid for both dielectric and magnetic materials. The analysis yields insight into the effects of air gaps on probe measurements. Numerical results indicate that the probe is very sensitive to lift-off at low frequencies. This sensitivity decreases somewhat as frequency increases.

\section{2,079}

PB96-123583 Not available NTIS

National Inst. of Standards and Technology (EEEL), Gaithersburg, MD. Semiconductor Electronics Div.

Wire Bonding to Multichip Modules and Other Soft Substrates.

Final rept.

G. G. Harman. 1995, 10p.

Pub. in Proceedings of the International Conference on Multichip Modules, Denver, CO., April 19-21, 1995, p292-301.

Keywords: *Multichip modules, *Microwave substrates, "Material modules, Skin(Anatomy), Reprints, "Wire bonding, Soft substrates.

Several classes of 'soft substrates' can be difficult to wire bond. These include MCM-Ds, MCM-Ls, flex substrates, some complex IC chips with multilevel polymer-insulated metallization, and microwave hybrids made on PTFE substrates. The bonding solutions in. clude: increasing the bond-pad metal thickness and area and applying a hard metal under-layer, a hard metal top-layer, or some combination of these, -capped with a highly-bondable metal. A summary is given of the bond-pad metallurgy and bonding machine parameters that have produced successful wire bonding to a wide variety of MCMs and other 'soft substrates.' Wire-bonding yield is also generally correlated to the elastic modulus and $\mathrm{Tg}$ of the polymer or laminate, and to the yield strength of the metal pads. Thus, the choice of material properties for the substrate and bond pads is at least as important as the actual bonding machine setup in achieving high-yield wire bonding. Other considerations, such as the possible use of high-frequency ultrasonic bonding and high-clock-rate skin-effect losses on the multilayer bond pads, are discussed.

\section{2,080}

PB96-135157 Not available NTIS

National Inst. of Standards and Technology (EEEL), Boulder, CO. Electromagnetic Technology Div.

High-Frequency Oscillators Using Phase-Locked Arrays of Josephson Junctions.

Final rept.

S. P. Benz, and P. A. A. Booi. 1994, 4p.

Pub. in Proceedings of the Institute of Electrical and Electronics Engineers International Frequency Control Symposium, Boston, MA., June 1-3, 1994, p666-669. Keywords: "Josephson junctions, Phase locked systems, Emission, Linewidths, Reprints, Power, " $2 \mathrm{D}$ arrays.

The authors present a basic description of Josephson junctions and discuss their use as $\mathrm{GHz}$ and $\mathrm{THz}$ Oscillators. The resistively shunted junction model is used to calculate the available power, linewidth and operat- ing frequency of the oscillators. The authors discuss how phase-locked arrays of junctions are used to achieve higher power and narrower linewidth. Two experimental examples of phase-locked emission are shown: one from on-chip detection circuits at $150 \mathrm{GHz}$ and one detected off-chip showing a $13 \mathrm{kHz}$ linewidth at $88.8 \mathrm{GHz}$

02,081

PB96-135165 Not available NTIS

National Inst. of Standards and Technology (EEEL), Boulder, CO. Electromagnetic Technology Div.

Noise Reduction in Low-Frequency SQUID Measurements with Laser-Driven Switching.

Final rept.

C. E. Cunningham, G. S. Park, B. Cabrera, and M. E. Huber. 1993, 3p

See also AD-A253821

Pub. in Applied Physics Letters, v63 n8 p1152-1154 Aug 93.

Keywords: "SQUID devices, "Noise reduction, "Low frequency, "Switches, Optical switching, Niobium, Laser radiation, Superconducting devices, Circuits, Modulation, Frequency dependence, Reprints, SQUID(Superconducting Quantum Interference Device), Microbridges.

The authors have developed a technique to modulate the input to a superconducting quantum interference device (SQUID) using a very low noise double-pole, double-throw switching zetwork. This network is composed of four niobium microbridges that are driven normal in pairs by pulses of laser light. An input signa is modulated at a frequency above the $1 /$ frequency noise regime, amplified by the SQUID, and subsequently demodulated to obtain an output in which the excess low-frequency SQUID noise has been removed. With this technique, the authors have reduced the low-frequency noise by an order of magnitude in energy over measurements made without this technique.

\section{2,082}

PB96-135207 Not available NTIS

National Inst. of Standards and Technology (EEEL), Gaithersburg, MD. Semiconductor Electronics Div.

Wire Bonding to Multichip Modules and Other Soft Substrates.

Final rept

G. G. Harman. 1995, 9p.

See also PB96-123583.

Pub. in Proceedings of the International Conference and Exhibition on Multichip Modules Compendium of Papers 1992-1995, Denver, CO., April 19-21, 1995, p629-637.

Keywords: "Multichip modules, "Microwave substrates, "Material modules, Skin(Anatomy), Reprints, "Wire bonding, Soft substrates.

Several classes of 'soft substrates' can be difficult to wire bond. These include MCM-Ds, MCM-Ls, flex substrates, some complex IC chips with multilevel poly mer-insulated metallization, and microwave hybrids made on PTFE substrates. The bonding solutions include: increasing the bond-pad metal thickness and area and applying a hard metal under-layer, a hard metal top-layer, or some combination of these, capped with a highly-bondable metal. A summary is given of the bond-pad metallurgy and bonding machine parameters that have produced successful wire bonding to a wide variety of MCMs and other 'soft substrates.' Wire-bonding yield is also generally correlated to the elastic modulus and $\mathrm{Tg}$ of the polymer or laminate, and to the yield strength of the metal pads. Thus, the choice of material properties for the substrate and bond pads is at least as important as the actual bonding machine setup in achieving high-yield wire bonding. Other considerations, such as the possible use of high-frequency ultrasonic bonding and high-clock-rate skin-effect losses on the multilayer bond pads, are discussed.

\section{2,083}

PB96-148093 Not available NTIS

National Inst. of Standards and Technology (EEEL), Gaithersburg. MD Electricity Div.

AC-DC Difference Characteristics of High-Voltage Thermal Converters.

Final rept.

D. X. Huang, T. E. Lipe, J. R. Kinard, and C. B.

Childers, 1995, 4 p.

Pub. in Institute of Electrical and Electronics Engineers Transactions on Instrumentation and Measurement, VIM-44 n2 p387-390 Apr 95
Keywords: "Alternating current, "Direct current, Converters, Dielectric loss, Measurement, Electric potential, Coefficients, Resistors, Reprints, Thermal voltage converters, Voltage coefficients, HVTCs(High Voltage Thermai Converters)

This paper describes a study of high-voltage therma converters (HVTC's) at voltages above $100 \mathrm{~V}$ at frequencies up to $100 \mathrm{kHz}$. Techniques for the construction of HVTC's are described, and the effects of aging and dielectric loss on the resistor, changes in the tim ing sequence of ac-dc difference tests, relay deadtimes, warmup times, and voltage level dependence are given

02,084

PB96-148135 Not available NTIS

National Inst. of Standards and Technology (EEEL) Gaithersburg, MD. Electricity Div

Performance of Multilayer Thin-Film Multijunction Thermal Converters.

Final rept.

R. Kinard, D. X. Huang, and D. B. Novotny. 1995

$4 p$.

See also PB95-196143

Pub. in Institute of Electrical and Electronics Engineers Transactions on Instrumentation and Measurement v44 n2 p383-386 Apr 95.

Keywords: "Thin films, "Converters, Fabrication, Alternating current, Direct current, Performance, Thermodynamic properties, Physical properties, Silicon, Ma chining, Microminiaturization, Reprints, MJTCS(Multijunction thermal converters), Multilayer TVCs, Multijunction TVCs, Micromachining.

New multilayer, thin-film multijunction thermal convert ers (MJTC's) suitable as high performance ac-dc transfer standards have been fabricated and studied at $\mathrm{Na}$ tional Institute of Standards and Technology (NIST). This paper describes their thermal and physical features and the materials chosen to improve performance. Performance data are given over a wide range of frequencies and conditions.

02,085

PB96-157821 Not available NTIS

National Inst. of Standards and Technology (EEEL) Boulder, CO. Electromagnetic Fields Div.

Optimizing Time-Domain Network Analysis.

Final rept.

D. C. DeGroot, and R. B. Marks. 1995, 10p.

Pub in Automatic RF Techniques Group Meeting (ARFTG), Scottsdale, AZ., November 30-December 1 1995, p19-28.

Keywords: Automation, Calibration, Reprints, "Time domain network analysis, "Instrument settings.

In this work, we demonstrate how changes in sample density, time-window size, and waveform averaging af fect the accuracy and acquisition time of calibrated time-domain network analysis. One of the key results from this study is that accuracy can be enhanced by eliminating the incident step-edge signal from the timedomain reflection waveform before maximizing the instrument's vertical scale. This study identifies the trad offs between accuracy and measurement speed and examines other trands to provide general guidance in establishing reliable and efficient time-domain network analysis measurements for a variety of if and microwave applications.

02,086

PB96-158654 PC A03/MF A01

National Inst. of Standards and Technology (EEEL) Boulder, CO. Electromagnetic Fields Div.

Direct Comparison Transfer of Microwave Power Sensor Calibrations.

Technical note.

M. P. Weidman. Jan 96, 20p, NIST/TN-1379.

Also available from Supt. of Docs. as SN003-003 03385-5.

Keywords: "Power measurement, "Microwave equip ment, "Calibration, Electrical measurement, *Microwave power standards.

This report describes a basic, but potentially accurate transfer technique for comparing microwave powe sensors. The technique is not new, but the specific ap plications are. This report is written to supplement the existing literature. The method transfer the effective efficiency of a standard power sensor to an unknown (uncalibrated) power sensor. The power sensors may be bolometric (thermistor mounts), thermoelectric, or 


\section{ELECTROTECHNOLOGY}

\section{Circuits}

diode types, and each type will have inherent limitations. The technique can be implemented with a variety of commercial coaxial and rectangular waveguide components. Measurement uncertainty is discussed in this report so that a potential user can quantify transfer uncertainties.

\section{2,087}

PB96-164017 Not available NTIS

National Inst. of Standards and Technology (EEEL), Gaithersburg, MD. Semiconductor Electronics Div.

Natural Convection from an Array of Electronic Packages Mounted on a Horizontal Board in a Narrow Aspect Ratio Enclosure.

Final rept.
V. H. Adams, Y. Joshi, and D. L. Blackburn. 1995,

$7 p$.

Pub. in Proceedings of the National Heat and Mass Transfer Conference, Surathkal, India, December 28 30,1995 , p911-917.

Keywords: "Electronic packaging, "Natural convection, "Heat transfer, Chips(Electronics), Circuit boards, Computational fluid dynamics. Temperature distribution, Temperature measurement, Air cooling, Space(Room), Reprints, Electronic cooling.

Three-dimensional natural convection flow and heat transfer were numerically studied for a 3 by 3 array of discretely heated electronic packages mounted on a horizontal circuit board in an air-filled, narrow aspect ratio rectangular enclosure with length, width, and height ration of $6: 6: 1$. It was found that conduction-only analysis under-predicts heat transfer from the top surfaces of the electronic packages by a factor of 1.5 to 4.4 , with a resultant over-prediction of the maximum chip to ambient temperature difference of up to $235 \%$.

\section{2,088}

PB96-176441 Not available NTIS

National Inst. of Standards and Technology (EEEL), Boulder, CO. Electromagnetic Fields Div.

Single-Port Technique for Adaptor Efficiency Evaluation.

Final rept

S. P. Pucic, and W. C. Daywitt 1995, 6p.

Pub. in Proceedings of the Automatic Radio Frequency Techniques Group Conference (45th), Orlando, FL., May 19, 1995, p113-118.

Keywords: "Adapters, "Electrical measurement, "Radiometers, Electric connectors, Broadband, Reflectance, Efficiency, Dissipation, Insertion Ioss, Losses, Reprints, Swept frequency measurements.

The single-port adaptor efficiency evaluation' (SPAEE) technique uses swept-frequency measurements to evaluate broadband efficiency of low-loss, reciprocal 2ports, including noninsertable devices such as adaptors. The 2-por is terminated in two reflective terminations, a shielded open and a short. The frequency range is limited only by the availability of the two reflective terminations. The major advantages of the SPAEE technique are its simplicity, speed, and accuracy.

\section{2,089}

\section{PB96-200134 Not available NTIS}

National Inst. of Standards and Technology (EEEL), Boulder, CO. Electromagnetic Technology Div Metallic-Barrier Junctions for Programmable Josephson Voltage Standards.

Final rept

R. L. Kautz, and S. P. Benz. 1995, 4p

Pub. in Applied Superconductivity Conference, Edinburgh, Scotland, July 3-6, 1995, p1407-1410.

Keywords: "Josephson junctions, "Niobium, dium, Gold, Reprints, SNS junctions.

The current amplitudes of Shapiro steps are studied in large-area metallic-barrier Josephson junctions by simulation and experiment. In the absence of a ground plane, simulations show tht junctions larger than about 4 times the Josephson penetration depth are of limited utility because the microwave power required to induce Shapiro steps increases rapidly with junction size. Experimentally, step amplitudes as large as $7 \mathrm{~mA}$ are observed in Nb-PdAu-Nb sandwich junctions.

\section{2,090}

PB96-200142 Not available NTIS

National Inst. of Standards and Technology (EEEL),

Boulder, CO. Electromagnetic Technology Div.
Shapiro Steps in Large-Area Metallic-Barrier Josephson Junctions.

Final rept.

R. L. Kautz. $1995,9 p$

Pub. in Jnl. of Applied Physics, v78 n9 p5811-5819 Nov 95.

Keywords: "Josephson junctions, "Superconductivity Phase locking, Voltage standards, Reprints, Josephson effect, Shapiro steps.

The current amplitudes of Shapiro steps in large-area metallic-barrier Josephson junctions, both with and without a ground plane, are investigated with the goa of optimizing junction parameters for programmable voltage standards. Using the resistively shunted junction model without capacitance, the authors calculate maximum step amplitudes as a function of reduced frequency and junction dimension for both one- and twodimensional junctions. For junctions without a ground plane, the authors conclude that step amplitudes of order $10 \mathrm{~mA}$ are practical, but significantly larger amplitudes require excessive microwave power.

\section{2,091}

PB96-200167 Not available NTIS

National Inst. of Standards and Technology (EEEL) Boulder, CO. Electromagnetic Technology Div.

Novel Vortex Dynamics in Two-Dimensional Josephson Arrays.

Final rept.

S. G. Lachenmann, T. Doderer, R. P. Huebener, P A. A. Booi, and S. P. Benz. 1994, 8p.

Pub. in Proceedings of the International Conference on Nonlinear Superconducting Devices and High-Tc Materials, Capri, Italy, October 8-13, 1994, p365-372.

Keywords: "Josephson junctions, "Arrays, "Vortices, Superconductivity, Reprints, Two dimensional array, Vortex dynamics.

The authors present spatially resolved studies of vortex dynamics in two-dimensional Josephson-junction arrays. For bias currents smaller than the array critica current, a small local thermal perturbation at the array boundaries lowers the vortex entry barrier and the array switches to the resistive state. For bias currents slightly above the array critical current vortices and antivortices are nucleated at opposite edges of the array. An alternating crossing vortex motion (ACVM) is observed experimentally.

\section{2,092}

PB96-200233 Not available NTIS

National Inst. of Standards and Technology (EEEL), Boulder, CO. Electromagnetic Technology Div.

Superconductor- Normal-Superconductor Junctions for Digital/Analog Converters.

Final rept.

S. P. Benz. 1995, 3p

Pub. in International Superconductive Electronics Conference (5th), Nagoya, Japan, September 18-21, 1995 p216-218.

Keywords: "Josephson junctions, "Niobium, "Superconductors, Palladium, Gold, Arrays, Optica wave guides, Analog to digital converters, Optical circuits, Voltage standards, Reprints, SNS junctions.

Series arrays of $\mathrm{Nb}-\mathrm{PdAu}-\mathrm{Nb}$ Josephson junctions have been fabricated with characteristics ideally suited for application in programmable voltage standards and digital/analog convertors. Large arrays of junction with applied microwave power showed constant voltage steps with current amplitudes as large as $7 \mathrm{~mA}$. A novel coplanar waveguide design has enabled uniform microwave power coupling to a 5-segment array of 8192 total junctions so that each array segment has constant voltage steps over the same bias current range. The 8192-junction device generated $1.1 \mathrm{~mA}$ steps at $186 \mathrm{mV}$ with $11 \mathrm{GHz}$ power and a maximum constant voltage step of $260 \mathrm{mV}$ at $15.34 \mathrm{GHz}$.

\section{2,093}

\section{PB96-200241 Not available NTIS}

National Inst. of Standards and Technology (EEEL) Boulder, CO. Electromagnetic Technology Div.

Superconductor- Normal-Superconductor Junctions for Programmable Voltage Standards. Final rept.

S. P. Benz. 1995, 3p

Pub. in Applied Physics Letters, v67 n18 p2714-2716 Oct 95

Keywords: "Josephson junctions, "Niobium, "Arrays, Palladium, Gold, Optical waveguides, Analog to digital conventers, Optical circuits, Voltage standards, Reprints, SNS junction.

Series arrays of $\mathrm{Nb}-\mathrm{PdAu}-\mathrm{Nb}$ Josephson junctions were fabricated with characteristics ideally suited for application in programmable voltage standards and D/ A converters with fundamental accuracy. Large arrays of junctions with applied microwave power showed constant voltage steps with current amplitudes as large as $7 \mathrm{~mA}$. A novel coplanar waveguide design enabled uniform microwave power coupling to a five-segment array of 8192 junctions, so each segment had constant voltage steps over the same bias range. The $8192-$ unction device generated $1.1 \mathrm{~mA}$ steps at $186 \mathrm{mV}$ with $11 \mathrm{GHz}$ power and a maximum constant voltage step of $260 \mathrm{mV}$ at $15.34 \mathrm{GHz}$

02,094

PB96-200258 Not available NTIS

National Inst. of Standards and Technology (EEEL)

Boulder, CO. Electromagnetic Technology Div.

Design of High-Frequency, High-Power Oscillators Using Josephson-Junction Arrays.

Final rept.

P. A. A. Booi, and S. P. Benz. 1995, 4p.

See also PB96-200266

Pub. in Proceedings of the Applied Superconductivity Conference, Edinburgh, Scotland, July 3-6, 1995, Inst. Phys. Conf. Series No. 148, p1479-1482

Keywords: "Josephson junctions, "Arrays, Niobium, Aluminum oxide, Frequency standards, Pertormance tests, Aluminum, Reprints, "Josephson oscillators.

The authors analyze the limitations imposed by junction capacitance and the parasitic inductance associated with shunt resistors on the performance of $\mathrm{Nb} / \mathrm{Al}$ AlOx/Nb-junction-array oscillators. The authors use wide junctions that are in situ deposited on top of PdAu resistor films (to minimize inductance) and are situated about Nb ground planes to ensure uniform current inection. From the measured parasitics, the authors infer that maximum power and frequency that can be obtained for critical-current densities Jc less than or equal to $100 \mathrm{kA} / \mathrm{cm}$ squared. The authors illustrate these findings with experimental results of a 1.968 unction array have $\mathrm{Jc}=10 \mathrm{Ka} / \mathrm{cm}$ squarted that was ound to couple $0.1-0.8 \mathrm{~mW}$ to a 56 -ohm load in the range $100-300 \mathrm{GHz}$

\section{2,095}

PB96-200266 Not available NTIS

National Inst. of Standards and Technology (EEEL), Boulder, CO. Electromagnetic Technology Div.

High-Power, High-Frequency Oscillators Using Distributed Josephson-Junction Arrays.

Final rept

P. A. A. Booi, and S. P. Benz. 1995, 3p

See also PB96-200258.

Pub. in International Superconductive Electronics Conference (5th) (ISEC '95), September 18-21, 1995, Nagoya, Japan, p513-515.

Keywords: "Josephson junctions, "Arrays, Phase locked systems, Reprints, "Josephson oscillators.

The authors present experimental results showing emission that is coupled from distributed series arrays of wide, resistively shunted tunnel junctions to on-chip $50 \mathrm{ohm}$ loads. The authors have detected poweer output of $0.85 \mathrm{~mW}$ at $240 \mathrm{GHz}$ and power greater than 100 microW at most frequencies in the range of 100 $300 \mathrm{GHz}$.

\section{2,096}

P896-200787 Not available NTIS

National Inst. of Standards and Technology (PL), Boulder, CO. Time and Frequency Div.

Origin of $\mathrm{I} / \mathrm{P} \mathrm{PM}$ and AM Noise in Bipolar Junction Transistor Amplifiers.

Final rept

F. L. Walls, E. S. Ferre-Pikal, and S. R. Jefferts. 1995, 11p.

Pub. in Proceedings of the International Institute of Electrical and Electronics Engineers Frequency Control Symposium, San Francisco, CA., May 31-June 2, 1995, p294-304.

Keywords: "Microwave amplifiers, "Amplitude modulation, *Phase modulation, "Electromagnetic noise, Frequency domain, Standards, Electrical measurement, Very high frequency, High frequency, Reprints.

In the paper, the authors report the results of extensive research on phase modulation (PM) and amplitude modulation (AM) noise in linear bipolar junction transis- 
tor (BUT) amplifiers. BJT amplifiers exhibit 1/f PM and AM noise about a carrier signal that is much larger than the amplifier's thermal noise at those frequencies in the absence of the carrier signal. The authors work whows that the 1/f PM noise of a BJT based amplifier is accompanied by $1 / f$ AM nose which can be higher, lower or nearly equal depending on the circuit implementation. The 1/fAM and PM noise in BJT's is primarily the result of the $1 / f$ fluctuations in transistor, current, transistor capacitance, circuit supply voltages, circuit impedances, and circuit configuration. The author discuss the theory and present experimental data in reference to common emitter emplifiers, but the analysis can be applied to other configurations as well. The study provides the functional dependence of $1 / \mathrm{f} A M$ and PM noise on transistor parameters, circuit parameters, and signal frequency, thereby laying the groundwork for a comprehensive theory of $1 / f$ AM and PM noise in BJT amplifiers. The authors show that in many cases the 1/f PM and AM noise can be reduced below the thermal noise of the amplifier.

PB,097-200795 Not available NTIS

National Inst. of Standards and Technology (PL), Boulder, CO. Time and Frequency Div.

Quest to Understand and Reduce I/f Noise in Amplifiers and Baw Quartz Oscillators.

Final rept.

F. L. Walls. $1995,18 \mathrm{p}$

Pub. in Proceedings of the European Frequency and Time Forum (9th), Besancon, France, March 8-10, 1995, p227-244.

Keywords: "Microwave amplifiers, "Microwave oscillators, "Quartz resonators, Amplitude modulation, Phase modulation, Electromagnetic noise, Frequency meas urement, Reprints, Phase noise.

The paper attempts to trace the highlights in the development of precision oscillators from a historical poin of view and speculates on further improvements in their $1 / f$ performance. Using Leeson's model the closed loop phase modulation (PM) noise and frequency stability can be estimated from the stability of the quartz resonator, the resonator quality factor, and the noise of the sustaining amplifier coupled with the stability of the oven and various environmental param eters. The inherent frequency stability of the oscillato is primarily limited by the $1 / f$ PM noise of the BAW quartz resonator and the sustaining amplifier. The paper, therefore, focuses on describing the quest to characterize, understand, and reduce the 1/f PM noise of amplifiers and BAW resonators.

\section{2,098}

PB96-200936 Not available NTIS

National Inst. of Standards and Technology (EEEL). Gaithersburg, MD. Electricity Div.

$100 \mathrm{~A}, 100 \mathrm{kHz}$ Transconductance Amplifier. Final rept.

O. B. Laug. 1996, 5p.

See also PB96-102629

Pub. in Institute of Electrical and Electronics Engineers Transacations on Instrumentation and Measurement v45 n3 p440-444 Jun 96.

Keywords: "Power amplifiers, "Voltage amplifiers, Transconductance, Electric power meters, Reprints.

A high-current, wide-band transconductance amplifier is described that provides an unprecedented level of output current at high frequencies with exceptional stability. It is capable of converting a signal voltage applied to its input into a ground-referenced output rms current up to $100 \mathrm{~A}$ over a frequency range from dc to $100 \mathrm{kHz}$ with a useable frquency extending to 1 $\mathrm{MHz}$. The amplifier has a $1000-\mathrm{W}$ output capability plus or minus $10 \mathrm{~V}$ of compliance, and can deliver up to $400 \mathrm{~A}$ of pulsed peak-to-peak current. The amplifier design is based on the principle of paralleling a number of precision bipolar voltage-to-current converters. The design incorporates a unique ranging system controlled by optoisolated switches, which permit a fullscale range from $5 \mathrm{~A}$ to $100 \mathrm{~A}$. The design considerations for maintaining wide bandwidth, high output inpedance, and unconditional stability for all loads are discussed.
High Power Generation with Distributed Josephson-Junction Arrays.

Final rept.

A. A. P. Booi, and S. P. Benz. 1996, 3p.

See also PB96-200266.

Pub. in Applied Physics Letters, v68 n26 p3799-3801 Jun 96

Keywords: "Josephson junctions, "Arrays, Phase locked systems, Current, Power, Reprints, ”Josephson oscillators.

The authors have experimentally coupled emission from a distributed series array of 1968 wide Josephson junctions to an on-chip 10.8 omega load and detected $0.16 \mathrm{~mW}$ at $240 \mathrm{HGz}$. The result is achieved by reducing the parasitic inductance associated with shunt resistors so that functions with critical currents of $23 \mathrm{~mA}$ are effectively shunted at the operating frequency. The power is less than the $1.3 \mathrm{~mW}$ expected from theory due to the presence of a large impedance mismatch. Optimization of the load design will allow the detection of $\mathrm{mW}$ power.

\section{2,100}

PB97-112569 Not available NTIS

National Inst. of Standards and Technology (EEEL), Gaithersburg, MD. Electricity Div.

Low Voltage Standards in the $10 \mathrm{~Hz}$ to $1 \mathrm{MHz}$ Range.

Final rept.

N. M. Oldham, M. E. Parker, B. C. Waltrip, and S.

Avramov-Zamurovic. 1996, $2 p$

Pub. in Conference on Precision Electromagnetic Measurements, CPEM '96 Conference Digest, Braunschweig, Germany, June 17-20, 1996, p570571.

Keywords: "Electrical measurement, Standards, Potentiometers(Resistors), Reprints, "Voltage standards, "Thermal voltage converters, Thermal converters, Low voltage.

A step down procedure is described for establishing voltage standards in the $1 \mathrm{mV}$ to $100 \mathrm{mV}$ range at frequencies between $10 \mathrm{~Hz}$ and $1 \mathrm{MHz}$. The step down employs low-voltage thermal voltage converters and micropotentiometers. Techniques are given for meas. uring input inpedance and calculating loading errors.

\section{2,101}

PB97-113187 Not available NTIS

National Inst. of Standards and Technology (PL), Boulder, CO. Time and Frequency Div.

Frequency Synthesis and Metrology at 10(-17) and Beyond.

Final rept.

F. L. Walls, F. Ascarrunz, C. W. Nelson, and L. M. Nelson. 1996, 3p

Pub. in Proceedings of the Symposium on Frequency Standards and Metrology (5th), Woods Hole, MA., October 15-19, 1995, p468-470 1996. Keywords: $\quad$ "Frequency synthesizers,
*Mixers(Electronics), "Amplifiers, Spectrum analysis, Stability, Signal generators, Superhigh frequency, Phase modulation, Amplitude modulation, Electro magnetic noise, Transmission lines, Reprints, "Timing errors.

The paper investigates the systematic timing errors due to variations in temperature and signal amplitude in distribution amplifiers, cables, mixers, frequency $\mathrm{di}-$ viders and multipliers. The effect of harmonic distortion in the reference signals on timing errors in phase detectors is also considered. The authors outline the basic configurations necessary to synthesize and distribute the reference frequencies and measure the performance of potential new clocks having a frequency stability of approximately $3 \times 10$ to the -15 th powe tau(sup 1/2) and 10 to the -17 th power in the long term.

\section{2,102}

PB97-113195 Not available NTIS

National Inst. of Standards and Technology (PL), Boulder, CO. Time and Frequency Div.

Reducing the 1/f $\mathrm{AM}$ and PM Noise in Electronics for Precision Frequency Metrology.

Final rept

F. L. Walls, E. S. Ferre-Pikal, and S. R. Jefferts.

1996, 2p

Pub. in Proceedings of the Symposium on Frequency Standards and Metrology (5th). Woods Hole, MA., October 15-19, 1995, p480-481 1996.

Keywords: "Phase modulation, "Amplitude modulation, "Electromagnetic noise, "Frequency synthesizers,
Electrical

measurement Amplifiers, Mixers(Electronics), Signal generators, Transfer functions, Reprints.

The authors report on a fundamental breakthrough in understanding how $1 / f \mathrm{PM}$ and amplitude modulation (AM) noise is generated. They show how one can analyze the transfer function of a two port device to calculate the dependence of added AM and PM noise on circuit and active element parameters. Using this new approach, the authors have developed sample silicon BJT amplifiers with exceptionally low $1 / f$ AM and PM noise.

02,103

PB97-119176 Not available NTIS

National Inst. of Standards and Technology (EEEL), Gaithersburg, MD. Electricity Div.

Conversion of a 2-Terminal-Pair Bridge to a 4-Terminal-Pair Bridge for Increased Range and Precision in Impedance Measurements.

Final rept.

A. Jeffery, J. Q. Shields, and L. H. Lee. 1996, 2p. Pub. in Conference on Precision Electromagnetic Measurements, Braunschweig, Germany, June 17-20, 1996, p358-359.

Keywords: "Bridges, "Electrical measurements, Ratio transformers, Measurements, Impedance bridges, Alternating current, Precision measurements, Reprints, "Foreign technology, Four-terminal-pair.

A new 4-terminal-pair bridge, capable of a relative uncertainty of 1 in 10 to the 9 th power, has been constructed at NIST by converting a 2-terminal pair bridge. The conversion requirees only addition of components which are easily removed if 2-pair measurements are to be made. The design and testing of this bridge will be described.

02,104

PB97-119317 Not available NTIS

National Inst. of Standards and Technology (EEEL), Gaithersburg, MD. Electricity Div.

Active High Voltage Divider with 20-PPM Uncertainty.

Final rept.

O. Petersons, E. Simmon, and G. J. FitzPatrick.

1996, 2p

Pub. in Conference on Precision Electromagnetic Measurement Digest (CPEM '96), Braunschweig, Germany, June 17-20, 1996, p486-487.

Keywords: "Voltage dividers, Electrical networks, Capacitors, Circuitry, Feedback amplifiers, Reprints.

A voltage divider has been designed which consists of a group of solid dielectric capacitors maintained in a temperature-controlled environment, an external compressed-gas capacitor, and special electronic circuitry. The prototype divider has been constructed and preliminary results obtained to validate the operating principle and accuracy target. The principal innovative part is a feedback amplifier, complemented with an 'openloop' voltage source controlled from the high voltage. This enables achievement of a voltage ratio that is equal to the reciprocal of the capacitance ratio to well within one ppm without encountering dynamic instability problems.

\section{Electromechanical Devices}

02,105

PB95-150488 Not available NTIS

National Inst. of Standards and Technology (EEEL),

Gaithersburg, MD. Semiconductor Electronics Div.

Test Structures for Determining Design Rules for Microelectromechanical-Based Sensors and Actuators.

Final rept.

C. Zincke, M. Gaitan, M. E. Zaghloul, and L. W.

Linholm. 1994, 7p.

Pub. in Proceedings of Institute of Electrical and Electronics Engineers International Conference on Microelectronic Test Structures, San Diego, CA., March 22 24, 1994, v7 p44-50.

Keywords: "Electromechanical devices, Microminiaturization, Design criteria, Etching, Actuators, Sensors, CMOS, Reprints, *Microactuators, *Microsensors, "Test structures, Wafer alignment, MOSIS service 


\section{ELECTROTECHNOLOGY}

\section{Electromechanical Devices}

We present two test structures for establishing design rules for minimum spacing of a new class of microelectromechanical-based sensors and actuators fabricated through commercial complementary metaloxide-semiconductor (CMOS) foundries. The microelectromechanical devices are suspended membranes of passivation glass that encapsulates polysilicon and aluminum layers in the CMOS process. The membranes are suspended by anisotropically etching the silicon substrate through openings in the passivation glass. These test structures measure the lateral undercutting and the rotational misalignment of openings in passivation oxide that are used to make the microelectromechanical devices, and give information for the layout, and proximity to circuits of the microelectromechanical devices. Two test structures are discussed, one optical and one electrical, and results for a 2 micrometer $n$ - and $p$-well CMOS process run are presented.

\section{2,106}

\section{PB95-153797 Not available NTIS}

National Inst. of Standards and Technology (EEEL), Gaithersburg, MD. Electronics and Electrical Engineering Lab. Office.

Directions in MEMS Research Application Development.

Final rept

R. E. Hebner. 1993, 19p.

Pub. in Proceedings of Microsensors and cember 6-7, 1993, p1-19.

Keywords: "Microminiaturization, Materials science, Thin films, Metrology, Uses, Reprints, "Microelectromechanics, "MEMS systems, Microheating elements, Thermal converters.

NIST's laboratory-based metrology programs are planned and implemented in cooperation with industry and focus on measurements, standards, evaluated data, and test methods. It is as part of this program that NIST is developing microelectromechanical systems (MEMS). The NIST program has three primary thrusts: the development of MEMS-based devices for metrology applications; materials characterization, and standards.

\section{2,107}

\section{PB96-111174 PC A05/MF A01}

National Inst. of Standards and Technology (TS) Gaithersburg, MD. National Voluntary Lab. Accreditation Program.

Efficiency of Electric Motors. National Voluntary Lab. Accreditation Program (NVLAP).

Handbook.

L. S. Galowin, W. J. Rossiter, and W. A. Hall. Aug

95, 85p, NIST/HB-150/10

Also available from Supt. of Docs. as SN003-00303356-1.

Keywords: "Electric motors, "Testing laboratories, *Standards, US NIST, Calibrating, Tests, Accreditation.

NIST Handbook 150-10 presents the technical requirements of the National Voluntary Laboratory Accreditation Program (NVLAP) for the Efficiency of Electric Motors (EEM) field of accreditation. The same document is published for use by the Standards Council of Canada (SCC); selected differences may be specified for implementation in both countries. This handbook is intended for information and use by staff of accredited laboratories, those laboratories seeking accreditation, other laboratory accreditation systems, users of laboratory services, and others needing information on the requirements for accreditation under the EEM program.

\section{2,108}

PB96-122544 Not available NTIS

National Inst. of Standards and Technology (EEEL), Gaithersburg, MD. Electricity Div.

Programmable Guarded Coaxial Connector Panel. Final rept.

L. L. Kile. $1995,5 p$

Pub. in National Conference of Standards Laboratories, Dallas, TX., July 16-20, 1995, p285-289.

Keywords: *Coaxial cables, *Connectors, "Automated systems, Programmable switch, Resistance measurements, Reprints.

A programmable guarded connector panel using coaxial connectors has been specifically designed for an automated measurement system for the comparison of four-terminal resistors. A computer controlled XYZ positioning system is used to move a 4 -connector $Z$ arm over a panel of 72 coaxial connectors mounted in the $X Y$ plane. This provides for 30 four-terminal channels. The outer shields of the connectors are electrically isolated from one another to allow the shields to be driven at guard voltages to supress errors caused by leakage currents. The resistance repeatability of the plug-socket connections including resistance variations of $12 \mathrm{~m}$ of AWG 12 connecting cable is typically 10 microohms. Variations of thermoelectric voltages over a 10-minute measurement period of the plug-socket connections are typically less than $10 \mathrm{nV}$. This automatic switching system may be useful for other types of precision measurements where guarding, contact resistances, and thermoelectric voltages a re critical factors.

\section{2,109}

PB96-146709 Not available NTIS

National Inst. of Standards and Technology (EEEL). Gaithersburg, MD. Electricity Div.

Integrated Thin-Film Micropotentiometers. Final rept.

J. R. Kinard, D. X. Huang, and D. B. Novotny. 1995, $3 p$.

Pub. in Institute of Electrical and Electronics Engineers Transactions on Instrumentation and Measurement, vIM-44 n2 p374-376 Apr 95

Keywords: *Potentiometers, *Thin films, *Microinstrumentation, Machining. Silicon, Microminiaturization, Alternating current, Frequencie
Reprints, Thermal converters, Microvolts, Millivolts.

Integrated micropotentiometers, new devices fabricated with thin-film technology and the micromachining of silicon, have been developed for the accurate determination of ac voltage from 1 to $100 \mathrm{mV}$ at frequencies from audio to $1 \mathrm{MHz}$ and with the potential for higher frequencies.

\section{2,110}

PB96-180153 Not available NTIS

National Inst. of Standards and Technology (EEEL), Gaithersburg, MD. Electricity Div.

Distributions of Measurement Error for Three-Axis Magnetic Field Meters during Measurements Near Appliances.

Final rept.

M. Misakian, and C. Fenimore. 1996, 6p

Pub. in Institute of Electrical and Electronics Engineers Transactions on Instrumentation and Measurement, v45 n1 p244-249 Feb 96.

Keywords: "Electric appliances, "Magnetic fields, *Magnetic measurement, "Residential buildings, Coils, Magnetic dipoles, Magnetic flux, Magnetic probes, Fre quency measurement, Measuring methods, Error anal ysis, Uncertainty, Probability distribution functions, Reprints, Power frequency.

Comparisons are made between the average magnetic flux density as would be measured with a three-axis coil probe and the flux density at the center of the probe. Probability distributions of the differences between the two quantities are calculated assuming dipole magnetic field and are found to be asymmetric. The distributions allow estimates of uncertainty for resultant magnetic field measurements made near some electrical appliances and other electrical equipment.

\section{Electron Tubes}

02,111

AD-A285 495/8 PC A06/MF A02

National Bureau of Standards, Gaithersburg, MD. Tabulation of Data on Receiving Tubes. Handbook 68. $1960,111 \mathrm{p}$

Keywords: “Tabulation processes, “Data acquisition Transmit receive tubes, Electrodes, Handbooks, ${ }^{*}$ Receiving tubes

A tabulation of Receiving-Type Electron Tubes with some characteristics of each type has been prepared in the form of two major listings, a Numerical Listing in which the tubes are arranged by type number, and a Characteristic Listing in which the tubes are arranged by tube type and further ordered on the basis of one or two important parameters. The tabulation is accom- panied by a listing of similar tube types and basing diagrams for the listed tubes.

02,112

PB96-103064 Not available NTIS

National Inst. of Standards and Technology (PL), Gaithersburg, MD. Quantum Metrology Div.

Planar Lenses for Field-Emitter Arrays.

Final rept.

C. M. Tang, T. A. Swyden, and A. C. Ting. 1995, 5p. Pub. in Jnl. of Vacuum Science and Technology B, v13 n2 p571-575 Mar/Apr 95

Keywords: "Electron beams, *Field emission, "Planar structures, "Lenses, Collimation, Focusing, Electrodes, Gates(Circuits), Experimental data, Cathodes, Reprints, "Field emitter arrays, "Spindt cathodes

Experimental data is analyzed for a method of collimating and focusing electron beams emitted from fieldemitter arrays (FEAs) where the focusing electrode is co-planar of nearly co-planar or nearly co-planar with the gate electrode. The focusing mechanism is provided by the fringe field formed along the edge of the gate electrode due to the potential difference between grid and the substrate or the gate and another lens electrode. The concept is verified by EGUN2 simulations. Experiments performed to verify the theory and focusing of electron beams emitted from FEAs were observed. Elongated emission patterns were consistently observed, indicative of one-dimensional focusing.

\section{Optoelectronic Devices \& Systems}

02,113

PB94-145968 PC A03/MF A01

National Inst. of Standards and Technology (EEEL), Gaithersburg, MD. Semiconductor Electronics Div.

Electronics and Electrical Engineering Laboratory Technical Progress Bulletin Covering Laboratory Programs, January to March 1991, with 1991 EEEL Events Calendar.

J. A. Gonzalez. Jul 91, 31p, NISTIR-4621.

See also PB92-112309.

Keywords: "Microelectronics, "Metrology, Electrical measurement, Semiconductors, Bipolar transistors, Signal processing. High temperature superconductors, Laser radiation, Optical waveguides, Microwaves, Silicon, Photodiodes, Antennas, Magnetic materials, Electromagnetic interference, Millimeter waves, Progress report, Abstracts, Fiber optic sensors, Erbium lasers.

This is the thirty-fourth issue of a quarterly publication providing information on the technical work of the $\mathrm{Na}$ tional Institute of Standards and Technology, Electronics and Electrical Engineering Laboratory. This issue of the EEEL Technical Progress Bulletin covers the first quarter of calendar year 1991. Abstracts are provided by technical area for both published papers and papers approved by NIST for publication. Topics covered include the following: Fundamental Electrical Measurements; Semiconductor Microelectronics; Signal Acquisition, Processing, and Transmission; Electrical Systems; Electromagnetic Interference.

02,114

PB94-152733 PC A05/MF A01

National Inst. of Standards and Technology (MSEL), Gaithersburg, MD. Ceramics Div.

Photonic Materials: A Report on the Results of a Workshop. Held in Gaithersburg, Maryland on August 26-27, 1992, Volume 1.

Internal rept.

J. A. Carpenter, and S. W. Freiman. Feb $94,94 p$, NISTIR-5299.

Keywords: "Optoelectronic devices, "Phonetics, *Meetings, Light emitting diodes, Semiconductor lasers, Silicon solar cells, Infrared detectors, Display devices, Optical disks, Holography, Lithium niobates, Aluminum gallium arsenides, Optical fibers, Optical storage, Packaging, Flat panel displays.

This is a report on the results of a workshop held August 26-27, 1992, on the title subject. Sponsored by NIST and the Optoelectronics Industry Development Association (OIDA), the major objectives were to identify and set priorities on R\&D needed to advance photonic materials in commercial products appearing
in the market place in the next $3-15$ years. Working 
group sessions were held in six areas: sources and detectors, display, storage, active devices (other than the four previously listed), packaging, and passive devices (other than packaging)

02,115

PB94-154341 PC A03/MF A01

National inst. of Standards and Technology (NEL), Gaithersburg, MD. Center for Electronics and Electrical Engineering

Electronics and Electrical Engineering Laboratory Technical Progress Bulletin Covering Programs, October to December 1993, with 1994/1995 EEEL Events Calendar.

J. M. Rohrbaugh. Feb 94, 37p, NISTIR-5357.

See also PB93-120723.

Keywords: *Optoelectronic devices, "Microelectronics, "Metrology, Integrated circuits, Dimensional measurement, Electromagnetic interference, Signal processing, Optical fibers, Magnetic materials, Millimeter waves, Photodetectors, Microwave equipment, Integrated optics, High temperature superconductors, Semiconductors, Progress report, Abstracts, Fiber optic sensors.

This is the forty-fifth issue of a quarterly publication providing information on the technical work of the National Institute of Standards and Technology, Electronics and Electrical Engineering Laboratory. This issue of EEEL Technical Progress Bulletin covers the fourth quarter of calendar year 1993. Abstracts are provided by technical area for both published papers and papers approved by NIST for publication. Major subject headings include the following: Fundamental Electrical Measurements; Semiconductor Microelectronics; Signal Acquisition, Processing, and Transmission; Electrical Systems; Electromagnetic Interference; Law Enforcement Standards; Video Technology.

\section{2,116}

PB94-159761 PC A04/MF A01

National Inst. of Standards and Technology (EEEL), Boulder, CO. Electromagnetic Technology Div.

Metrology for Electromagnetic Technology: A Bibliography of NIST Publications.

M. E. DeWeese, and S. Moynihan. Sep 92, 73p NISTIR-3994.

Keywords: "Metrology, "Bibliographies, Optical communication, Optical fibers, Fiber optics, Optoelectronic devices, Solid state lasers, Superconducting devices, High temperature superconductors, Josephson junctions, Electrooptics, Magnetic measurement, Cryogenics, "Electromagnetic metrology, Cryoelectronics, SQUID devices, US NIST.

This bibliography lists the publications of the personnel of the Electromagnetic Technology Division of NIST during the period from January 1970 through publication of this report. A few earlier references that are directly related to the present work of the Division are also included

02,117

PB94-169802 Not available NTIS

National Inst. of Standards and Technology,

Gaithersburg, MD

Cryogenic Blackbody Calibrations at the National Institute of Standards and Technology Low Background Infrared Calibration Facility.

R. U. Datla, M. C. Croarkin, and A. C. Parr. 1994, $43 \mathrm{p}$

Included in Jnl. of Research of the National Institute of Standards and Technology, v99 n1 p77-119 Jan/ Feb 94

Keywords: "Blackbody radiation, "Calibration, Intermediate infrared radiation, Near infrared radiation, Infrared radiometers, Cryogenic equipment, Light sources, Measurement, Uncertainty. Sensors, Reprints, Low Background Infrared Calibration Facility, LBIR facility, US NIST.

The Low Background Infrared Calibration Facility (LBIR) at the National Institute of Standards and Technology has been in operation for calibration measurements of the radiant power emitted from infrared radiation (IR) sources, such as cryogenic blackbodies, for more than 2 years. The $\mathbb{R}$ sources are sent to NIST by customers from industry government, and university laboratories. An absolute cryogenic radiometer is used as the standard detector to measure the total radiant power at its aperture. The low background is provided by a closed cycle helium refrigeration system that maintains the inner parts of the calibration chamber at $20 \mathrm{~K}$. The radiance temperature of the blackbody is deduced from the measured power and compared with the blackbody temperature sensor data. The calibration procedures and data analysis are illustrated using the measurements of a typical blackbody.

02,118

PB94-185154 Novailable NTIS

National Inst. of Standards and Technology (EEEL), Gaithersburg, MD. Semiconductor Electronics Div. Interface Roughness-Induced Changes in the Near-E(sub 0$)$ Spectroscopic Behavior of Short-Period AIAs/GaAs Superlattices.

Final rept.

D. Chandler-Horowitz, J. G. Pellegrino, N. V.

Nguyen, and S. B. Qadri. 1994, 5 p

Pub. in Materials Research Society Symposium Proceedings, v326 p145-1491994.

Keywords: "Superlattices, Aluminum gallium arsenides, Aluminum arsenides, Optical properties, Perturbations, Ellipsometry, Reprints, "Interface roughness, Photoreflectance.

The perturbations on the optical properties introduced by increased interface roughness in $3 \times 3$ short-period AlAs/GaAs superlattices (SLs) were investigated hrough an examination of the position and line shape of the $E(0)$ (direct gap) feature in photoreflectance (PR) and spectroscopic ellipsometry (SE). The degree of interface roughness in the SLS was controlled by a choice of the growth conditions and the buffer layer thickness. The structural behavior was characterized by X-ray diffraction. The measured spectra from PR and SE were compared to those from an $\mathrm{Al}(x) \mathrm{Ga}(1$ $x)$ As $(x=0.5)$ alloy reference specimen grown under nearly identical conditions.

02,119

PB94-185485 Not available NTIS

National Inst. of Standards and Technology (PL) Gaithersburg, MD. Electron and Optical Physics Div. One Gigarad Passivating Nitrided Oxides for $100 \%$ Internal Quantum Efficiency Silicon Photodiodes. Final rept.

R. Korde, J. S. Cable, and L. R. Canfield. 1993, 5p Pub. in Institute of Electrical and Electronics Engineers Transactions on Nuclear Science 40, n6 p1655-1659 Dec 93.

Keywords: "Ultraviolet detectors, "Silicon diodes, Photodiodes, Vacuum ultraviolet radiation, Radiation resistance, Quantum efficiency, Silicon dioxide, Reprints.

$100 \%$ internal quantum efficiency silicon photodiodes with 4 to $8 \mathrm{~nm}$ passivating silicon dioxide have been fabricated by rapid thermal nitridation in nitrous oxide and ammonia ambients with the aim of increasing their radiation hardness. The fabricated diodes were exposed to $10.2 \mathrm{eV}$ photons using a hydrogen plasma light source and a normal incidence monochromator The measured quantum efficiency degradation indicates that the interface trap area density increases sublinearly with dose up to a measured dose of one Gigarad. No noticeable change in quantum efficiency Over the range of 50 to $250 \mathrm{~nm}$ was observed after exposure to $100 \%$ relative humidity. This suggests that the nitrided $\mathrm{SiSiO} 2$ interface is practically insensitive to moisture.

\section{2,120}

PB94-185824 Not available NTIS

National Inst. of Standards and Technology (EEEL),

Boulder, CO. Electromagnetic Technology Div.

Modified Airy Function Method for the Analysis of Tunneling Problems in Optical Waveguides and Quantum Well Structures.

Final rept.

R. Sukhdev, A. K. Ghatak, I. C. Goyal, and R. L.

Gallawa. 1993, 6p.

Pub. in Institute of Electrical and Electronics Engineers Jnl. of Quantum Electronics 29, n2 p340-345 Feb 93

Keywords: "Optical waveguides, "Quantum wells, Electron tunneling, One dimensional, Airy function, WKB approximation, Potentials, Reprints.

We present a simple method for the analysis of tunneling through an arbitrary one-dimensional potential barrier, based on the modified Airy function approach. We have considered truncated step-linear step-exponential, parabolic, and quartic potential barriers. The results have been compared with those obtained by the conventional WKBJ, modified WKBJ, and the matrix method. The effect of the truncation level on the tunneling coefficient has also been investigated. The tunneling coefficient is sensitive to the truncation level For the step-linear potential, the tunneling coefficien is a monotonically decreasing function of the truncation level, while for the parabolic potential, it oscillates before saturating to a constant value.

\section{1}

PB94-185907 Not available NTIS

National Inst. of Standards and Technology (EEEL) Gaithersburg, MD. Electricity Div.

Optical Current Transducer for Calibration Studies. Final rept

E. D. Simmon, A. H. Rose, and G. J. Fitzpatrick.

1993, 4p.

Pub. in Proceedings of International Symposium on High Voltage Engineering (8th), Yokohama, Japan, August 23-27, 1993, p399-402.

Keywords: "Electric current, "Electrical measurement, Faraday effect, High voltage, Electric power, Magnetooptics, Error analysis, Operation, Design, Tests, Reprints, "Optical current transducers, "Current sensors

Optical current transducers (OCTs) are well-suited for current measurements in high voltage applications because they offer advantages over conventional oil-filled current transformers such as greater immunity from electromagnetic interference (EMI), intrinsic safety and wide dynamic range. This paper describes an OCT designed and built at the National Institute of Standards and Technology (NIST) for in the development of calibration methods for OCTs for power system applications. The design and operating characteristics of the NIST OCT are described, and the results of tests for sensitivity, linearity, and dynamic range are reported. Some of the sources of measurement error are discussed.

\section{2,122}

PB94-188810 PC A09/MF A02

National Inst. of Standards and Technology (EEEL), Gaithersburg, MD. Semiconductor Electronics Div.

Semiconductor Measurement Technology: Im proved Characterization and Evaluation Measurements for $\mathrm{HgCdTe}$ Detector Materials, Processes, and Devices Used on the GOES and TIROS Satellites.

Special pub.

D. G. Seiler, J. R. Lowney, W. R. Thurber, J. J.

Kopanski, and G. G. Harman. Apr 94, 188p, NIST/ SP-400-94

Also available from Supt of Docs as SN003-00303263-8. See also PB92-112382.

Keywords: "Satellite instruments, "Infrared detectors, "TIROS satellites *GOES satellites, Mercury cadmium ellurides, Intermediate infrared radiation Shubnikovde Haas effect, Meteorological satellites, Photoconductive cells, Magnetoresistivity, Metallizing, Packaging, Bonding, Calibration, Scanning capacitance microscopy, Test structures.

An extensive study was carried out to improve the characterization and evaluation methods used for HgCdTe (mercury cadmium telluride) photoconductive infrared detectors used in GOES and TIROS satellites. High-field magnetotransport techniques were used to determine the electrical properties of the detector accumulation layers, which partially control their detectivities. Assessments were made of the quality of the bonding and packaging used in detector fabrication, and a list of recommended practices was produced. The applicability of scanning capacitance microscopy and test structures to detector-array evaluation is discussed and finally recommendations are made for standardized detector calibration. The results of this work have provided new and more refined measurement methods that can be adapted by the detector manufacturers to improve performance and yield.

02,123

PB94-198223 Not available NTIS

National Inst. of Standards and Technology (NEL) Gaithersburg, MD. Chemical Process Metrology Div. Electro-Optical Sensor for Surface Displacement Measurements of Compliant Coatings.

Final rept.

D. Adair, W. M. Madigosky, and N. E. Mease. 1991, $2 p$.

Pub. in Review of Scientific Instruments 62, n6 p16521653 1991. Sponsored by Admiralty Research Establishment, Portland (England). and Naval Research Lab., Washington, DC. 
Keywords: "Displacement measurement, "Vibration measurement, Light emitting diodes, Phototransistors, Hydroelasticity, Hydrodynamics, Gallium arsenides Silicon, Sensors, Reprints, Compliant coatings.

A method has been developed primarily to quantify the characteristics of hydroelastic instability waves on an elastic surface but which may also be applied to vibration measurements in general. The instability waves to be investigated result from the reaction of a compliant coating to the hydrodynamic forces of the boundary layer flowing over it. The present displacement sensor comprises a gallium arsenide infrared emitting diode in conjunction with a spectrally matched silicon phototransistor. It was found that this conbination could be designed to give an analog linear response in the region $4.5 \mathrm{~mm}<d<6.5 \mathrm{~mm}$, where is the distance from the reflecting surface to the phototransistor. Variation in voltage to change in d was found to be approximately $1.5 \mathrm{mV} / \mathrm{m}$ when using polished aluminum as the reflecting surface and $0.5 \mathrm{mV} /(\mathrm{mu}) \mathrm{m}$ when using silicon gel impregnated with calcium carbonate.

\section{2,124}

\section{PB94-199478 Not available NTIS}

National Inst. of Standards and Technology (NML) Gaithersburg. MD. Electron and Optical Physics Div. Silicon Photodiodes Optimized for EUV and Soft XRay Regions.

Final rept

L. R. Canfield, J. Kerner, and R. Korde. 1990, 6p.

Pub. in Proceedings of Society of Photo-Optical Instru-

mentation Engineers - Extreme Ultraviolet, X-ray, and

Gamma-ray Instrumentation for Astronomy, San

Diego, CA., July 11-13, 1990, v1344 p372-377.

Keywords: "X-ray detection, "Ultraviolet detectors, "Silicon diodes, "Photodiodes, Extreme ultraviolet radiation, Soft x rays, Radiometry, Reprints

Silicon photodiode detectors are now being routinely fabricated with almost no internal loss mechanisms. Such detectors have also been optimized in a windowless configuration for use in the extreme ultravioleUs soft $X$-ray region. These photodiodes have stable efficiencies (electrons per incident photon) which are essentially linear with photon energy (in this region), and which exceed 1 electron per incident photon for photon energies of about $10 \mathrm{eV}$ and beyond. In addi. tion to reporting the most recent performance parameters of devices with active areas of $1 \mathrm{sq} \mathrm{cm}$ and 3 $\mathrm{sq} \mathrm{cm}$, the temporal stability of devices with measurements spanning more than a year will be shown to be quite acceptable for photon energies $>25 \mathrm{eV}$. Silicon photodiodes of this type have numerous applications in the extreme ultraviolet and soft $X$-ray regions where their relative ease of use, low cost, stability, extremely high efficiency, and ability to operate in the presence of gases can be profitably exploited. Although silicon detectors are by nature very wide band detectors, narrow bandpass characteristics may be easily developed by directly overcoating the silicon dioxide outer surface of the basic photodiode with one or more thin films of appropriate materials. Examples of photodiodes whose responses have been thus tailored for use in the extreme ultraviolet are presented.

\section{2,125}

PB94-199551 Not available NTIS

National Inst. of Standards and Technology (EEEL),

Boulder, CO. Electromagnetic Technology Div.

Magneto-Optic Magnetic Field Sensor with $1.4 \mathrm{pT} /$

square root of $1(\mathrm{~Hz})$ Minimum Detectable Field at

$1 \mathrm{kHz}$.

Final rept.

M. N. Deeter, G. W. Day, T. J. Beahn, and M.

Manheimer. 1993, 2p.

Pub. in Electronics Letters 29, n11 p993-994, 27 May 93. Sponsored by Laboratory for Physical Sciences, College Park, MD.

Keywords: Yttrium iron garnets, Faraday effect, Magnetooptics, Substitutes, Sensitivity, Cylinders, Gallium, Reprints, *Magnetic field sensors, Fiber optic sensors, Nickel zinc ferrites.

The Lefter demonstrates that the sensitivity of magneto-optic magnetic field sensors employing iron garnets can be increased by approximately two orders of magnitude by applying flux concentration. A minimum detectable field of $1.4 \mathrm{pT} /(\mathrm{Hz})$ (sup $1 / 2)$ was measured when a cylindrical gallium-substituted yttrium iron garnet crystal was combined with two conically tapered nickel-zinc ferrite cylinders.
National Inst. of Standards and Technology (EEEL), Boulder, CO. Electromagnetic Technology Div. Characterization of Vertical-Cavity Semiconductor Structures.

Final rept.

D. H. Christensen, J. G. Pellegrino, R. K. Hickernell, R. S. Rai, S. M. Crochiere, and C. A. Parsons. 1992 $8 p$.

Pub. in Jnl. of Applied Physics 72, n12 p5982-5989, 15 Dec 92

Keywords: "Surface emitting lasers, Electron microscopy, Photoluminescence, Gallium arsenides, Metrology, Wafers, Reprints, Double crystal x-ray diffractometry, Photoreflectance.

Several analytical tools are applied to characterize vertical-cavity surface-emitting laser structures grown on GaAs wafers. These epitaxial structures are amenable to $x$-ray, electron-beam, and optical metrologies. Cross-sectional scanning electron microscopy and transmission electron microscopy were used to measure layer thicknesses and uniformity. Photoluminescence wafer mapping was used to determine alloy composition uniformity across the wafer. Photoreflectance was also used to determine alloy composition. Cross-sectional microphotoluminescence was used to measure average alloy compositions in the top and bottom mirrors. Reflectance spectroscopy was used to characterize the cavity resonances and mirror layers. Double-crystal $x$-ray diffractometry (DCXRD) was used to characterize mirror layer dimensions, uniformity, and average alloy composition. Excellent agreement was found among these measure ment techniques and between simulations and meas urements. The results demonstrate the accuracy of the device simulation tools and the applicability of DCXRD in analyzing these structures.

\section{2,127}

PB94-200698 Not available NTIS

National Inst. of Standards and Technology (EEEL), Boulder, CO. Electromagnetic Technology Div

Faraday Effect Current Sensors.

Final rept.

G. W. Day, M. N. Deeter, and A. H. Rose. 1992, 8p. Pub. in Proceedings of Australian Conference on Optical Fibre Technology (17th), Hobart, Tasmania, Aus tralia, November 29-December 2, 1992, p20-27.

Keywords: "Electric current, Faraday effect, Optical fibers, Thermal stability, Sagnac effect, Birefringence, Reviews, Reprints, "Fiber optic sensors, "Current sensors, Sagnac interferometers.

This paper contains a review of Faraday effect current sensors, including both those based on bulk materials and on single mode optical fiber. Among the topics dis cussed are new materials for bulkbased sensors leading to the best reported sensitivities, methods of dealing with fiber birefringence, temperature stability, speed limitations, and new configurations, including the Sagnac Interferometer.

02,128

PB94-200706 Not available NTIS

National Inst. of Standards and Technology (EEEL), Boulder, CO. Electromagnetic Technology Div.

Faraday Effect Sensors: A Review of Recent Progress.

Final rept.

G. W. Day, M. N. Deeter, and A. H. Rose. 1991, 16p. Pub. in Proceedings of International Conference on Optical Fiber Sensors, Wuhan, China, October 9-11, 1991, p11-26.

Keywords: "Electric current, "Magnetic fields, "Magnetometers, Reprints, Magnetic measurement, Faraday effect, Thermal stability, Optical fibers, Birefringence, Reviews, "Fiber optic sensors, "Current sensors.

The last few years have seen dramatic progress in the development of Faraday effect sensors for measuring both magnetic fields and electric current. In the case of magnetic field sensors, the most significant ad vances have resulted from an investigation of new materials, especially the ferrimagnetic iron garnets. Minimum detectable magnetic fields have been reduced by several orders of magnitude to about $100 \mathrm{pT} / \mathrm{Hz}$ (sup 1/2) at $500 \mathrm{~Hz}$ and bandwidths of hundreds of megahertz have been obtained. In the case of electric current sensors, new methods of avoiding the effects of linear birefringence in optical fiber have resulted in much smaller and more stable sensors. One of those approaches, annealing of the fiber to reduce birefringence, has yielded sensors with temperature stabilities near that of the material limit, < 10 (sup -4) $K$. These topics and other developments that have occurred since a previous summary (Proc. SPIE 985138 . $150(1988))$ are discussed in the review.

02,129

PB94-200714 Not available NTIS

National Inst. of Standards and Technology (IMSE) Gaithersburg, MD. Ceramics Div.

Correlation of HgCdTe Epilayer Defects with Underlying Substrate Defects by Synchrotron X-Ray Topography.

B. E. Dean, C. J. Johnson, S. C. McDevitt, R. C.

Dobbyn, M. Kuriyama, J. Ellsworth, H. R. Vydyanath, J. J. Kennedy, G. T. Neugebauer, and J. L. Sepich. 1991, 7p

Pub. in Jnl. of Vacuum Science and Technology B 9 , n3 p1840-1846 May/Jun 91.

Keywords: "Mercury cadmium tellurides, "Crystal defects, Focal plane devices, Infrared detectors, Synchrotron radiation, X-ray sources, Substrates, Reprints, Diffraction imaging, Epilayers

Synchrotron $X$-ray topography studies have been conducted at the National Synchrotron Light Source (NSLS) at Brookhaven National Laboratories to correlate defects in $\mathrm{HgCdTe}$ epilayers with those in underlying CdTe family substrates. IR detectors are being fabricated on these epilayers to investigate the performance impact of specific defects. This paper describes synchrotron $X$-ray facilities and methods. Images of substrates and epilayers are discussed and mapping of epilayer/substrate defects, such as microtwins, subgrain boundaries and slip lines, is demonstrated. Efforts to map detector array performance to epilayer and substrate topographs are described.

02,130

PB94-212594 Not available NTIS

National Inst. of Standards and Technology (NEL), Boulder, CO. Electromagnetic Fields Div.

Optically Sensed EM-Field Probes for Pulsed Fields.

Final rept.

M. Kanda, and K. D. Masterson. 1992, 7p.

Pub. in Proceedings of the Institute of Electrical and Electronics Engineers 80, n1 p209-215 Jan 92.

Keywords: "Probes(Electromagnetic), "Photonics, Electromagnetic fields, Electromagnetic pulses, Transfer functions, Electrooptics, Modulators, Reprints.

We discuss the characteristics of photonic probes that give them the potential to measure pulsed electromagnetic fields more accurately than has been possible in the past. An overview of system design is presented with particular emphasis placed on the transfer functions of appropriate antennas and electro-optic modulators. Noise sources which limit the sensitivity to low level signals are discussed and used to determine the noise equivalent field (NEF). The characteristics of some sensors developed by NIST are presented, and their performances relative to estimated practical limits for the NEF are indicated. Optical guided wave technology appears to hold considerable promise for fabricating modulators with a wide variety of operating parameters that can improve probe performance and extend their applications to a wider region of the electromagnetic spectrum.

\section{2,131}

PB94-212917 Not available NTIS

National Inst. of Standards and Technology (EEEL),

Gaithersburg, MD. Semiconductor Electronics Div.

High-Spatial-Resolution Resistivity Mapping Applied to Mercury Cadmium Telluride.

Final rept.

J. J. Kopanski, J. R. Lowney, D. B. Novotny, J.

Ramsey, D. G. Seiler, and A. Simmons. 1992, 7p

Sponsored by Night Vision and Electro-Optics Lab., Fort Belvoir, VA. and Defense Advanced Research Projects Agency, Arlington, $V A$.

Pub. in Jnl. of Vacuum Science and Technology B 10, n4 p1553-1559, Jul/Aug 92.

Keywords: "Mercury cadmium tellunides, "Electrical resistivity, Liquid phase epitaxy, High resolution, Bulk semiconductors, Room temperature, Electric contacts, Recrystallization, Thin films, Reprints, "Resistivity mapping, Contact resistivity.

The fine-scale resistivity variations of slices of bulk, $n$ type $\mathrm{Hg}(1-\mathrm{x}) \mathrm{Cd}(\mathrm{x}) \mathrm{Te}(\mathrm{HgCdTe})$ grown by the solid- 
state recrystallization (SSR) process and thin-film epitaxial layers of $\mathrm{HgCdTe}$ grown by liquid phase epitaxy (LPE) on SSR substrates were mapped at room temperature $(297 \mathrm{~K})$. An automatic probe station was used to make four-probe resistance measurements, spaced 80 micrometers apart, on lithographically defined metal-to-HgCdTe contacts. Most slices of SSR $\mathrm{HgCdTe}$ were found to have resistivities that increased from the center to the outside edge, and some SSR material also showed small inclusions with resistivity either higher or lower than the surrounding material. LPE material was found to have a more random variation in resistivity than SSR $\mathrm{HgCdTe}$ material. Also, the metal-semiconductor contact resistivity of In/ $\mathrm{Pb} / \mathrm{In} / \mathrm{Ni}$ contacts to n-type $\mathrm{HgCdTe}$ was measured to be in the range of 0.3 to $20 \times 10(-5)$ ohm $\mathrm{cm}$ for $\mathrm{HgCdTe}$ resistivities varying from 0.01 to $0.1 \mathrm{ohm} \mathrm{cm}$.

\section{2,132}

PB94-212925 Not available NTIS

National Inst. of Standards and Technology (EEEL), Gaithersburg, MD. Semiconductor Electronics Div.

Review of Semiconductor Microelectronic Test Structures with Applications to Infrared Detector Materials and Processes.

Final rept.

J. J. Kopanski, and C. E. Schuster. 1993, 23p.

Pub. in Semicond. Sci. Technol. 8, p888-910 1993.

Keywords: "Mercury cadmium tellurides, "Infrared detectors, Integrated circuits, Process control, Gallium arsenides, Microelectronics, Silicon, Reviews, Reprints, *Test structures.

The impact of microelectronic test structures, as they have been applied to silicon integrated circuits (ICS) and gallium arsenide monolithic microwave integrated circuits (MMICS), is reviewed. General principles for the use of test structures with possible applications to infrared (IR) detector technology based on HgCdTe and other materials are emphasized. The uses of test structures for $\mathrm{Si}$ and GaAs, test chip design methodology and some examples of how test structures have been applied for process control and to increase yield are discussed. Specific test structures and techniques that have been applied to IR detectors are also reviewed. The basic design considerations and measurements possible with each class of test structure are discussed. The important experience of the $\mathrm{Si}$ and $\mathrm{GaAs}$ industries, applicable to IR detectors, is that significant yield improvement is possible with improved process control using test structures. Increased research efforts to expand the applications of test structures to IR detector manufacture are indicated.

\section{2,133}

PB94-213485 Not available NTIS

National Inst. of Standards and Technology (EEEL), Gaithersburg, MD. Semiconductor Electronics Div. Investigation of Mercury Interstitials in $\mathrm{Hg}(\mathbf{1 -}$ x)CdxTe Alloys Using Resonant Impact-Ionization Spectroscopy.

Final rept.

C. L. Littler, E. Maldonado, X. N. Song, D. G. Seiler J. R. Lowney, Z. Yu, and J. L. Elkind. 1992, 5p Pub. in Jnl. of Vacuum Science and Technology B 10, n4 p1466-1470 Jul/Aug 92

Keywords: *Mercury cadmium tellurides, Activation energy, Electron traps, Hole traps, Interstitials, Impurities, Reprints, Resonance ionization spectroscopy.

A technique for studying low concentrations of trap levels in narrow-gap $\mathrm{Hg}(1-x) \mathrm{Cd}(x) \mathrm{Te}$ has been combined with the deliberate introduction of defects to determine the activation energies of these impurities in this ternary material. In this investigation, mercury $(\mathrm{Hg})$ interstitials, believed to be responsible for dark current in metal-insulator-semiconductor devices, were deliberately introduced into samples with $x$ approx $=0.22$ and $x$ approx $=0.24$. Each sample was divided into two parts with the second part of each slice used as a control. The results from these samples provide direct evidence that $\mathrm{Hg}$ interstitials create trap levels near 45 and $60 \mathrm{meV}$ above the valence-band edge for these $x$-value samples.

02,134

PB94-216074 Not available NTIS

National Inst. of Standards and Technology (EEEL),

Gaithersburg, MD. Semiconductor Electronics Div.
Heavily Accumulated Surfaces of Mercury Cadmium Telluride Detectors: Theory and Experiment. Final rept.

J. R. Lowney, D. G. Seiler, W. R. Thurber, C. L.

Littler, Z. Yu, and X. N. Song. 1993, 7p.

Pub. in Jnl. of Electronic Materials 22, n8 p985-991 1993.

Keywords: "Mercury cadmium tellurides, "Infrared detectors, "Passivation, Shubnikov-de Haas effect, Photoconductive cells, Two dimensional, Electron gas, Surfaces, Reprints.

Some processes used to passivate n-type mercury cadmium telluride photoconductive infrared detectors produce electron accumulation layers at the surfaces, which result in 2D electron gases. The dispersion relations for the electric subbands that occur in these layers have been calculated from first principles. Poisson's equation for the built-in potential and Schroedinger's equation for the eigenstates have been solved self-consistently. The cyclotron effective masses and Fermi energies have been computed for each subband density for 12 total densities between 0.1 to $5.0 \times 10$ (sup 12)/sq cm. Results are discussed. These results provide a basis for characterizing the passivation processes, which greatly affect device performance.

\section{2,135}

PB94-216280 Not available NTIS

National Inst. of Standards and Technology (EEEL) Boulder, CO. Electromagnetic Technology Div

Integrated Optic Laser Emitting at 906, 1057, and $1358 \mathrm{~nm}$.

Final rept

K. J. Malone, N. A. Sanford, and J. S. Hayden. 1993, 3p.

Pub. in Electronics Letters 29, n8 p691-693, 15 Apr 93. Keywords: "Neodymium lasers, "Glass lasers, Infrared lasers, Near infrared radiation, Integrated optics, Phosphate glass, Doped materials, Optical waveguides, Reprints

Laser oscillation at 906,1057 , and $1358 \mathrm{~nm}$ has been achieved in the same neodymium-doped-glass integrated optic laser. This is believed to be the first report of a $906 \mathrm{~nm}$ integrated optic laser. High slope efficiency and high output power were observed at 1057 $\mathrm{nm}$. The laser was fabricated by silver jon exchange in a phosphate glass.

\section{2,136}

\section{PB94-216298 Not available NTIS}

National Inst. of Standards and Technology (EEEL), Boulder, CO. Electromagnetic Technology Div. Integrated Optic Laser Emitting at 905, 1057, 1356 $\mathrm{nm}$.

Final rept.

K. J. Malone, N. A. Sanford, J. S. Hayden, and D. L. Sapak. 1993, 3p.

Pub. in Advanced Solid-State Lasers, New Orleans LA., February 1-3, 1993, 3p.

Keywords: "Neodymium lasers, "Glass lasers, Infrared lasers, Near infrared radiation, Integrated optics, Phosphate glass, Doped materials, Optical waveguides, Reprints.

We have achieved laser oscillation at 1057, 1356, and $905 \mathrm{~nm}$ in the same neodymium-doped-glass integrated optic device. We believe this is the first report of a $905 \mathrm{~nm}$ integrated optic laser. The laser was fabnicated by solid silver ion exchange in a phosphate glass.

02,137

PB95-107215 Not available NTIS

National Inst. of Standards and Technology (EEEL) Gaithersburg, MD. Semiconductor Electronics Div. Interface Roughness of Short-Period AIAs/GaAs Superlattices Studied by Spectroscopic Ellipsometry.

Final rept.

N. V. Nguyen, J. G. Pellegrino, P. M. Amintharaj, D. G. Seiler, and S. B. Qadri. 1993, 8p.

Pub. in Jnl. of Applied Physics 73, n11 p7739-7746. 1 Jun 93

Keywords: * Superlattices, "Interfaces, Molecular beam epitaxy, Gallium arsenides, Aluminum arsenides, Thin films, Optical properties, Ellipsometry, Roughness, Reprints.

Spectroscopic ellipsometry (SE) has been used to study the effects of interface roughness on the optical properties of ultrathin short-period $3 \times 3$ GaAs/AIAs superlattices grown by molecular-beam epitaxy (MBE). The complex dielectric function and thickness of the whole superlattice and the thickness of the native oxide overlayer were simultaneously determined by an inversion technique from data in the $1.5-5.0 \mathrm{eV}$ region. The main optical critical points $E(0), E(0)+\operatorname{Delta}(0), E(1)$, $E(1)+\operatorname{Delta}(1)$, and $E(2)$ were deduced by line-shape fitting of the second derivative of the complex dielectric function of the superlattice to the analytical line-shape expression. The interface roughness is found to shift the optical transitions, except $E(2)$, to higher energy and broaden their line shapes. A simple interpretation of the shift and broadening is given. The interface roughness and layer thicknesses obtained by SE are found to be consistent with the results of x-ray diffraction and Raman scattering studies previously reported. The results in the study demonstrate the capability of the post-growth nondestructive characterization by SE to provide useful information about the interface quality of superlattice structures, and consequently to optimize the MBE growth conditions in order to achieve the desired structural parameters.

02,138

PB95-108775 Not available NTIS

National Inst. of Standards and Technology (EEEL)

Gaithersburg, MD. Semiconductor Electronics Div.

Interface Sharpness in Low-Order III-V Superlattices.

Final rept

J. Pellegrino, S. B. Oadri, P. M. Amirtharaj, N. V

Nguyen, and J. Comas, 1992, 8p.

Pub. in Thin Solid Films 220, p176-183 1992

Keywords: "Superlattices, "Interfaces, Molecular beam epitaxy, Surface emitting lasers, Aluminum arsenides, Gallium arsenides, Thin films, $X$-ray diffraction, Roughness, Reprints, Edge emitting lasers.

Superlattices composed of aluminum, gallium and in dium are currently employed in a variety of device-related applications. Among these are edge-emitting GRINSCH lasers and vertical cavity surface emitting laser diodes. As the individual layer thickness is reduced, the role of interface sharpness becomes more critical in ensuring good two-dimensional growth. This work addresses the relationship between interface roughness and superlattice crystallinity for short-period AlAs/GaAs superlattices. Thin short-period superlattices with active layer thicknesses of $30 \mathrm{~nm}$ or less were also investigated to help determine the interface sharpness in the initial stages of growth. X-ray diffraction was used to assess interface roughness and to calculate superlattice periodicity. These results are compared with those obtained by reflection high en ergy electron diffraction (RHEED), Raman spectroscopy, and spectroscopic ellipsometry. The results indicate that interface roughness is promoted by a reduced arsenic flux growth condition at normal growth temperatures for short-period superlattices. The results also suggest that, for thin superlattices, a $10 \mathrm{~nm}$ buffer layer enhances interface roughness in the initial stages of growth and compromises the subsequent epilayer crystallinity. An analysis of these results in the light of structural, dynamical, and optical data is presented.

02,139

PB95-108783 Not available NTIS

National Inst. of Standards and Technology (EEEL)

Gaithersburg, MD. Semiconductor Electronics Div.

Interface Sharpness during the Initial Stages of Growth of Thin, Short-Period III-V Superlattices. Final rept.

J. G. Pellegrino, S. B. Oadri, C. M. Cotell, J. Comas, P. M. Amirtharaj, and N. V. Nguyen. 1993, 6p. Pub. in Jnl. of Vacuum Science and Technology A 11 n4 p917-922, Jul/Aug 93

Keywords: "Superlattices, "Interfaces, Molecular beam epitaxy, X-ray diffraction, Gallium arsenides, Roughness, Thin films, Reprints, Heterostructures.

Superlattices composed of III-V heterostructures have established applications in high-speed electronic and optoelectronic devices. As layer thicknesses are reduced, the role of heterostructure interface sharpness becomes more critical to ensuring high quality two-dimensional growth. In this work, short-period (less then $1 \mathrm{~nm}$ ) superlattices with active layer thicknesses of 31 $\mathrm{nm}$ were investigated to assess interface roughness in the initial stages of growth. X-ray diffraction was used to evaluate interface roughness and to calculate superlattice periodicity. Results suggest that surface roughening by islanding may be promoted by GaAs buffer layers that are $10-100 \mathrm{~nm}$ thick. Smoother inter- 
faces were obtained in samples with buffer layers 250 $\mathrm{nm}$ and greater.

02,140

PB95-125928 Not available NTIS

National Inst. of Standards and Technology (EEEL), Boulder, CO. Electromagnetic Technology Div.
International Intercomparison of Detector Responsivity at 1300 and $1550 \mathrm{~nm}$.

Final rept.

R. L. Gallawa, J. L. Gardener, D. H. Nettleton, and

K. D. Stock. 1992, 2p.

Pub. in Proceedings of a Conference on Precision Electromagnetic Measurements, Paris, France, June 9-12, 1992, p268-269.

Keywords: "Ge semiconductor detectors, "Infrared detectors, Near infrared radiation, Interlaboratory comparisons, International cooperation, Optical communication, Spectral response, Optical measurement, Power meters, Calibration, Standards, Reprints, Intercomparison.

An international intercomparison of spectral responsivity measurements at wavelengths of interest to optical communications was recently completed. Thirteen countries participated in the test, which was conducted in the course of a year. Agreement is within about $1 \%$.

02,141

PB95-126017 Not available NTIS

National Inst. of Standards and Technology (EEEL), Boulder, CO. Electromagnetic Technology Div. International Intercomparison of Detector Responsivity at 1300 and $1550 \mathrm{~nm}$.

Final rept

J. L. Gardener, R. L. Gallawa, K. D. Stock, and D. H. Nettleton. 1992, 6p.

Pub. in Applied Optics 31, n34 p7226-7231, 1 Dec 92

Keywords: "Ge semiconductor detectors, "Infrared detectors, "Photodiodes, Interlaboratory comparisons, Near infrared radiation, Optical communication Radiometry, Reprints, Intercomparison.

An intercomparison of spectral responsivity measurements has been carried out among the national laboratories of 13 countries. Measurements were made at wavelengths of 1300 and $1550 \mathrm{~nm}$, which are important in optical communication systems. Three germa nium photodiodes were circulated in each of three sep. arate paths, beginning and ending at the National Institute of Standards and Technology as the coordinating laboratory. The results show agreement within $1 \%$.

\section{2,142 \\ PB95-126348 Not available NTIS}

National Inst. of Standards and Technology (NEL),

Boulder, CO. Electromagnetic Technology Div.

Far-Infrared Kinetic Inductance Detector.

Final rept.

E. N. Grossman, D. G. McDonald, and J. E.

Sauvageau. 1991, 4p.

Sponsored by Strategic Defense Initiative Organization, Washington, DC. Innovative Science and Tech-

Pub. in Institute of Electrical and Electronics Engineers Transactions on Magnetics 27, n2 p2677-2680 Mar 91

Keywords: "Superconducting devices, "Infrared bolometers, "Infrared detectors, Superconducting films, Far infrared radiation, SQUID devices. Mixers(Electronics), Temperature range 0000-0013 K, Thin films, Inductance, Inductors, Heterodyning, Reprints, Niobium titanium, Photoinductors.

Extremely sensitive far-infrared detectors suitable for both direct detection and heterodyne applications are possible, based on micrometer-sized thin films with thickness less than a superconducting penetration depth. The penetration depth of such a film, and therefore its inductance, varies with temperature and with quasiparticle population (described by an effective temperature $\left.T^{*}\right)$, resulting in both bolometric and nonequilibrium 'photoinductive' responses. Common magnet alloys such as $\mathrm{NbTi}$ are the natural choice for liquid-He temperature operation. A detailed analysis predicts a (phonon-limited) NEP of about $4 \times 10(\exp -17)$ $\mathrm{W} / \mathrm{Hz}$ (sup 1/2) for a bolometer with an iridium kinetic inductor operated at $0.1 \mathrm{~K}$. A heterodyne noise temperature of $2250 \mathrm{~K}$ (SSB) at $3 \mathrm{THz}$, with $200 \mathrm{MHz}$ bandwidth is predicted for a $\mathrm{NbTi}$ mixer operated at 4
National Inst. of Standards and Technology (EEEL). Boulder, CO. Electromagnetic Technology Div. Metrology for Electromagnetic Technology: A Bibliography of NIST Publications. A. J. Smith. Sep 94, 93p, NISTIR-5029. Supersedes PB94-108776.

Keywords: "Optoelectronic devices, "Superconducto devices, "Magnetic measurement, "Metrology, "Bibliographies, Optical communication, Optical fibers, Optical waveguides, Fiber optics, Solid state lasers, YBCO superconductors, High temperature superconductors, Josephson junctions, SQUID devices, Cryogenics, "Electromagnetic metrology Cryoelectronics, US NIST.

This bibliography lists the publications of the personne of the Electromagnetic Technology Division of the $\mathrm{Na}$ tional Institute of Standards and Technology (NIST) during the period from January 1970 through publication of this report. A few earlier references that are directly related to the present work of the Division are also included. Keywords include cryoelectronics, electromagnetic metrology lasers, optical fibers, and superconducting materials. This bibliography is expected to be the last from the Electromagnetic Technology Division since the Division is in the process of being split into two Divisions. Each will produce its own bibliography in future editions.

\section{2,144}

PB95-140083 Not available NTIS

National Inst. of Standards and Technology (NEL) Boulder, CO. Electromagnetic Technology Div.

Superconducting Kinetic Inductance Radiometer.

Final rept.

J. E. Sauvageau, D. G. McDonald, and E. N.

Grossman. 1991, 4p.

Pub. in Institute of Electrical and Electronics Engineers Transactions on Magnetics 27, n2 p2757-2760 1991

Keywords: "Superconducting devices, "Infrared radiometers, "Infrared bolometers, Superconducting films, Impedance bridges, Integrated circuits, Temperature measurement, SQUID devices, Thermometers, Inductors, Prototypes, Silicon, Reprints, Inductance bridges, Photoinductors.

We are developing a bolometer based on a differentia thermometer that senses temperature changes through changes in the kinetic inductance of a superconducting thin film. The composite bolometer is the sensor for a prototype radiometer that will provide an absolute measure IR power. The radiometer is in tended to measure the spectrally dispersed power of a $300 \mathrm{~K}$ black body. This absolute radiometer is being developed for use at the Low Background Infrared (LBIR) Facility at NIST, Gaithersburg. The noise floo of the temperature transducer for the radiometer has been measured to be $0.75 \mathrm{pW}$ for a $100 \mathrm{sec}$. integration time. This is 150 times lower noise level than that of the commercial absolute radiometer currently used at the LBIR Facility in Gaithersburg.

\section{2,145}

\section{PB95-141115 Not available NTIS}

National Inst. of Standards and Technology (EEEL), Boulder, CO. Electromagnetic Technology Div.

LP11-Mode Leakage Loss in Coated Depressed Clad Fibers.

B. P. Pal, R. L. Gallawa, and I. C. Goyal. 1992, 3p.

Pub. in Institute of Electrical and Electronics Engineers Photonics Technology Letters 4, n4 p376-378 Apr 92

Keywords: "Optical fibers, "Fiber optics, "Transmission loss, Fiber optics transmission lines, Leakage, Reprints, Cutoff wavelength.

A quantitative investigation of the leakage loss spectrum of the LP(11)-mode in coated depressed index clad fibers is made using the matrix method. The study reveals oscillations similar to those seen in the measurement of cutoff wavelength. Our results do not agree with recently published results and a plausible explanation of the discrepancies is given.

\section{2,146}

PB95-150397 Not available NTIS

National Inst. of Standards and Technology (CSTL) Gaithersburg, MD. Biotechnology Div.

Retinal-Protein Complexes as Optoelectronic Components.

Final rept.

N. N. Vsevolodov, and T. V. Dyukova. 1994, 8p.

Pub. in Trends in Biotechnology 12, n3 p81-88 Mar 94.
Keywords: "Eye pigments, "Eye proteins, "Optical storage, "Rhodopsin, "Biosensors, Photosensitivity(Biological), Biotechnology, Complexes, Reprints, "Bacteriorhodopsin.

Naturally occurring retinal-protein complexes (RPCS) have recently received much attention with regard to their potential use as light-sensitive elements for optical recording. The best-known RPC is bacteriorhodopsin (BR), a photosensitive protein from the membrane of extreme halophilic bacteria, which has been studied in great detail. The remarkably robust nature of BR, coupled with its ability to reversibly change color upon illumination and its high cyclicity of ground-to-photoinduced state transitions, makes BR a promising material for optical information processing.

\section{2,147}

PB95-151114 Not available NTIS

National Inst. of Standards and Technology (EEEL), Gaithersburg, MD. Electricity Div

Recent Developments at NIST on Optical Current Sensors and Partial Discharge Diagnostics.

Final rept

R. J. Van Brunt. 1993, 15p.

Pub. in Proceedings of Workshop on Advanced Diagnostics for Substation Equipment, Palo Alto, CA., November 9-11, 1992, p6-159 - 6-173 1993.

Keywords: "Electric current meters, "Electric discharges, "Fault detection, Optical measurement, Pattern recognition, Faraday effect, Electric substations, Electrical insulation, Stochastic processes, Electric power, Diagnostic techniques, Reprints, *Fiber optic sensors, "Partial discharges, Optical fiber sensors, US NIST.

The purpose of this presentation is to draw attention to the NIST projects and discuss their relevance to the improvement of measurements applied to electricpower systems, especially those under the substations. The first activity discussed here is concerned with the use of optical fiber techniques to measure current. The second is concerned with a new approach to analyzing partial-discharge data that should prove useful in improving the reliability of pattern recognition schemes that are under development to identify the characteristics of defect sites in the insulation at which the discharges occur.

\section{2,148}

PB95-153177 Not available NTIS

National Inst. of Standards and Technology (EEEL) Boulder, CO. Electromagnetic Technology Div.

Vertical-Cavity Optoelectronic Structures: CAD, Growth, and Structural Characterization.

Final rept.

D. H. Christensen, S. M. Crochiere, J. G. Pellegrino,

W. F. Tseng, R. K.

Parsons. 1993, 6p.

Pub. in Materials Research Society Symposia Proceedings, v281 p307-312 1993.

Keywords: "Semiconductor lasers, Scanning electron microscopy, Transmission electron microscopy, Computer aided design, Aluminum gallium arsenides, Moecular beam epitaxy, X-ray diffraction, Photoluminescence, Laser cavities, Superlattices, $R e-$ flectance, Mirrors, Reprints, Double crystal x-ray diffractometry, Heterostructures.

Simulations of reflectance spectra and electric field distributions for vertical-cavity structures were used in the computer aided design of epitaxial mirrors and lasers. The binary GaAs/AlAs superlattice alloys and $\mathrm{Al}(\mathrm{x}) \mathrm{Ga}(1-\mathrm{x})$ As random alloys that compose these structures were grown by molecular beam epitaxy. Photoluminescence, photoreflectance, reflectance spectroscopy, scanning electron microscopy, transmission electron microscopy, and double crystal $x$-ray diffractometry were applied to characterize cavity and Bragg mirror layer thicknesses and alloy composition.

\section{2,149}

\section{PB95-153185 Not available NTIS}

National Inst. of Standards and Technology (EEEL), Boulder, CO. Electromagnetic Technology Div.

Vertical-Cavity Semiconductor Structures: Materials Characterization.

Final rept.

D. H. Christensen, J. R. Hill, D. T. Schaafsma, W. F Tseng, R. K. Hickernell, and J. G. Pellegrino. 1993, $2 \mathrm{p}$.

Pub. in Proceedings of Conference on Lasers and Electro-Optics, Baltimore, MD., May 2-7, 1993, v11 p234-235 
Keywords: "Semiconductor lasers, "Laser materials, $\mathrm{X}$ ray diffraction, Photoluminescence, Reflectance, Characteristics, Laser cavities, Reprints, Double crystal xray diffractometry, Microca vities, Microlasers.

Materials characterization of a variety of vertical-cavity semiconductor structures is reported. Double crystal $\mathrm{x}$ ray diffractometry, reflectance spectroscopy, and photoluminescence measurements are correlated and used to determine cavity and Bragg mirror layer thicknesses.

02,150

National Inst. of Standards and Technology (EEEL), Boulder, CO. Electromagnetic Technology Div. Vertical-Cavity Semiconductor Lasers: Structural Characterization, CAD, and DFB Structures.

Final rept.

D. H. Christensen, C. A. Parsons, J. G. Pellegrino, S. M. Crochiere, R. K. Hickernell, D. T. Schaafsma, J. R. Hill, and R. S. Rai. 1993, $7 p$

Pub. in Proceedings of Society of Photo-Optical Instrumentation Engineers: Laser Diode Technology and Applications V, Los Angeles, CA., January 18-20, 1993, v1850 p115-121.

Keywords: "Distributed feedback lasers, "Semiconductor lasers, "Computer aided design, Scanning electron microscopy, Transmission electron microscopy, Near infrared radiation, Laser materials, Materials tests, $X$-ray diffraction, Computerized simulation, Software tools, Laser arrays, Photoluminescence, Characteristics, Reflectance, Reprints, Nondestructive evaluation, Double crystal $x$-ray diffractometry.

One of the key technologies required for manufacturing vertical-cavity laser arrays at a production scale is rapid and nondestructive evaluation of the laser matenial. A brief review of methods for materials characterization of vertical-cavity semiconductor lasers is presented. Techniques based on reflectance spectroscopy, photoluminescence, photoreflectance, double crystal $\mathrm{x}$-ray diffractometry, scanning electron micros copy, and transmission electron microscopy are used to determine alloy composition, cavity spacer thickness, and Bragg mirror layer thickness. Critical aspects of data gathering, analysis, interpretation, and simulation are highlighted. The optical simulation software used for computer aided device design and simulation of reflectance spectra is also briefly discussed. The usefulness of the software is demonstrated by applying it to the design of a novel distributed-feedback verticalcavity laser with a vertically integrated pump reflector. The characterization techniques are applicable to al types of vertical-cavity devices: lasers, detectors, light emitting diodes, Bragg mirrors, modulators, micro-cavities, and vertically integrated devices.

\section{2,151}

PB95-153391 Not available NTIS

National Inst. of Standards and Technology (EEEL), Boulder, CO. Electromagnetic Technology Div.

High Speed, High Sensitivity Magnetic Field Sensors Based on the Faraday Effect in Iron Garnets. Final rept.

M. N. Deeter. 1993, 6p.

Pub. in Proceedings of Optical Fiber Sensors Conference (9th), Firenze, Italy, May 4-6, 1993, p409-414.

Keywords: Faraday effect, Optical measurement, Magnetic measurement, Magnetic anisotropy, Yttrium iron garnets, Optical waveguides, High sensitivity, High speed, Reprints, "Magnetic field sensors, "Fiber optic sensors, Garnet films.

Recent research demonstrating greater performance of Faraday effect sensors based on iron garnet crystals is described. Bulk crystals are coupled to high-permeability flux concentrators, resulting in an increase in sensitivity of at least two orders of magnitude. Iron garnet films with perpendicular magnetic anisotropy and exploited in an optical waveguide geometry exhibit greater frequency response than bulk crystals. These films also exhibit larger values of saturation Faraday rotation and ideally should exhibit no hysteresis.

\section{2,152}

\section{PB95-153409 Not available NTIS}

National Inst. of Standards and Technology (EEEL), Boulder, CO. Electromagnetic Technology Div.
Magneto-Optic Magnetic Field Sensors Based on Uniaxial Iron Garnet Films in Optical Waveguide Geometry.

Final rept.

M. N. Deeter, G. W. Day, R. Wolfe, and V. Fratello. 1993, $1 p$.

Pub. in Digests of International Magnetics Conference INTERMAG'93, Stockholm, Sweden, April 13-16 1993, pEF-09.

Keywords: Yttrium iron garnets, Magnetic anisotropy Magnetic hysteresis, Magnetic measurement, Optica waveguides, Optical measurement, Magnetooptics, Barkhausen effect, Faraday effect, Ferrimagnetism, Reprints, "Magnetic field sensors, Uniaxial anisotropy Garnet films.

The properties of iron garnet films exhibiting uniaxia (perpendicular) magnetic anisotropy are investigated as they relate to magneto-optic magnetic field sensors. When configured in an optical waveguide geometry, such films are expected to magnetize by the mechanism of domain rotation rather than domain wall motion. This distinction should result in magnetic field sen sors which exhibit improved linearity and frequency response in comparison to garnet-based magneto-optic magnetic field sensors that rely on domain wall motion. We present data which show the predicted purely linear response function as well as data which exhibit hysteresis at higher driving fields.

\section{2,153}

PB95-153763 Not available NTIS

National Inst. of Standards and Technology (EEEL) Boulder, CO. Electromagnetic Technology Div,

Accurate Characterization of High Speed Photodectors.

Final rept.

P. D. Hale, and D. L. Franzen. 1993, 10 p.

Pub. in Proceedings of Society of Photo-Optical Instrumentation Engineers: Photodetectors and Power $\mathrm{Me}$ ters, San Diego, CA., July 15-16, 1993, v2022 p218227.

Keywords: "Photodetectors, "Photodiodes, Frequency domain, Characteristics, Heterodyning, Metrology, Uncertainty, Reprints.

We designed a simple heterodyne system for fre quency domain photodetector characterization which is intended to avoid many of the disadvantages of earlier characterization systems and to minimize errors due to intensity fluctuation, frequency calibration, and source impedance mismatch without using vector network analysis. A detailed uncertainty analysis for the system indicates $95 \%$ confidence intervals between + or -0.25 and + or $-0.6 \mathrm{~dB}$ at $25 \mathrm{GHz}$, depending on the photodetector output impedance.

\section{2,154}

\section{PB95-162426 Not available NTIS}

National Inst. of Standards and Technology (EEEL). Boulder, CO. Electromagnetic Technology Div.

Polarization Dependence of Response Functions in $3 \times 3$ Sagnac Optical Fiber Current Sensors.

Final rept.

K. B. Rochford, G. W. Day, and P. R. Forman. 1993,

Sponsored by Los Alamos National Lab., NM.

Pub. in Proceedings of Optical Fiber Sensors Conference (9th), Firenze, Italy, May 4-6, 1993, 4p.

Keywords: Response functions, Optical coupling, Polarized light, Sagnac effect, Complexity, Reprints "Electric current sensors, "Fiber optic sensors, "Optical fiber sensors, Polarization dependence, Optical couplers, Sagnac interferometers.

The response functions of an ideal Sagnac optical fibe current sensor, based on a $3 \times 3$ coupler, fundamentally depend on the polarization state of light entering the coupler, even for a zero birefringence system. The desired response functions, sinusoids separated by 120 deg phase shifts, are obtained only for circularly polarized light. The response functions for linearly polarized and depolarized inputs are sinusoids separated by 180 deg, and yield only zero-slope small-signal responses. in addition, two outputs are degenerate, so the re sponse is similar to that observed in $2 \times 2$ systems. Thus, $3 \times 3$ couplers offer no advantage over $2 \times 2$ systems for linearly polarized light. This result increases system complexity in that polarization control optics are required to supply the proper polarization, even for an ideal, zero-birefringence system.

\section{2,155}

PB95-162467 Not available NTIS
National Inst. of Standards and Technology (EEEL), Boulder, CO. Electromagnetic Technology Div Submicroampere-Per-Root-Hertz Current Sensor Based on the Faraday Effect in Ga: YIG.

Final rept.

A. H. Rose, M. N. Deeter, and G. W. Day. 1993, 3p. Pub. in Optics Letters 18, n17 p1471-1473, 1 Sep 93. Keywords: "Yttrium iron garnets, "Faraday effect, Optical measurement, Materials replacement, Gallium additions, Sensitivity, Bandwidth, Substitutes, Reprints, "Electric current sensors.

We demonstrate an optical current sensor that is based on the Faraday effect in gallium-substituted yttrium iron garnet and has a measured sensitivity of about $3 \mathrm{deg} / \mathrm{A}$ a noise-equivalent current of about 220 $n \mathrm{~A} / \mathrm{Hz}$ (sup 1/2) and a $-3-\mathrm{dB}$ bandwidth of about 2.6 $\mathrm{MHz}$. The bandwidth-sensitivity product is a factor of about 10 greater than that of an all-silica-fiber current sensor with the same diameter.

\section{2,156}

\section{PB95-164232 Not available NTIS}

National Inst. of Standards and Technology (PL)

Gaithersburg, MD. Radiometric Physics Div.

Thermal and Nonequilibrium Responses of Superconductors for Radiation Detectors.

Final rept.

Z. M. Zhang, and A. Frenkel. 1993, 14p.

Pub. in ASME Winter Annual Meeting, New Orleans, LA., November 28-December 3, 1993, p1-14.

Keywords: "Superconducting devices, "Radiation detectors, "Infrared detectors, "High temperature superconductors "Bolometers, Optical properties, Thermal properties, Nonequilibrium conditions, HighTc superconductors, Reprints.

This work summarizes the progress in the study of the superconductor response to optical radiation and in the development of infrared detectors. The recent advances in the design of high-temperature (high-T(c)) superconducting radiation detectors using silicon microfabrication technology are emphasized. Thermal and optical properties important for the detector performance are discussed. The mechanism of the nonequilibrium optical response and its potential use to build fast and sensitive radiation detectors are described. Future challenges and opportunities in the development of high-T(c) superconducting radiation detectors are highlighted.

02,157

PB95-164299 Not available NTIS

National Inst. of Standards and Technology (EEEL), Gaithersburg, MD. Semiconductor Electronics Div. Hg1-xCdxTe Characterization Measurements: Current Practice and Future Needs.

Final rept.

D. G. Seiler, S. Mayo, and J. R. Lowney. 1993, 24p Pub. in Semicond. Sci. Technol. 8, p753-776 1993

Keywords: "Mercury cadmium tellurides, *Infrared detectors, Semiconductor materials, Electrical measurement, Optical measurement, Characteristics, Reprints. An extensive industrial survey of the importance and use of characterization measurements for $\mathrm{HgCdTe}$ materials, processes and devices has been completed. Seventy-two characterization/measurement techniques were considered and thirty-five responses were received. This information was sought for a study on materials characterization and measurement techniques of parameters and properties necessary to improve the manufacturing capabilities of HgCdTe infrared detectors. The nature of materials characterization is defined and an overview is given of how it is related to improving IR detector manufacturing. Finally, we present a description of the characterization survey and a summary of the survey results.

\section{2,158}

PB95-168480 Not available NTIS

National Inst. of Standards and Technology (EEEL), Boulder, CO. Electromagnetic Technology Div.

Low-Coherence Interferometric Measurement of Group Transit Times in Precision Optical Fibre Delay Lines.

Final rept.

B. L. Danielson. 1993, 4p

Pub. in Proceedings of Optical Fibre Measurement Conference (2nd), Torino, Italy, September 21-22, 1993, p159-162.

Keywords: "Optical fibers, "Delay lines, Optical measurement, Transit time Interferometry, Calibration Reprints, Optical time domain reflectometry, Group delay. 
We describe a low-coherence interferometric method for measuring the transit time in optical fiber delay lines as long as $1.5 \mathrm{~km}$. Group delays in $100 \mathrm{~m}$ standard reference fibers can be determined with an expanded uncertainty of about 4 ps (1 $\mathrm{mm})$ and a resolution of $0.15 \mathrm{ps}(0.03 \mathrm{~mm})$. The principal limitations of this approach are identified and discussed.

\section{P2,159 168498 Not available NTIS}

National Inst. of Standards and Technology (EEEL), Boulder, CO. Electromagnetic Technology Div Magneto-Optic Magnetic Field Sensors Based on Uniaxial Iron Garnet Films in Optical Waveguide Geometry.

Final rept.

M. N. Deeter, G. W. Day, R. Wolfe, and V. J

Fratello. 1993, 3p.

Pub. in Institute of Electrical and Electronics Engineers Transactions on Magnetics 29, n6 p3402-3404 Nov 93.

Keywords: *Ferrite garnets, Ferrimagnetic resonance, Magnetic hysteresis, Magnetic anisotropy, Magnetic films, Yttrium iron garnets, Domain walls, Optical waveguides, Faraday effect, Magnetooptics, Reprints, *Magnetic field sensors, Uniaxial anisotropy, Garnet films.

Iron garnet films which exhibit perpendicular uniaxial magnetic anisotropy are promising materials for magneto-optic magnetic field sensing. In an optical waveguide geometry, these materials exhibit large values of saturation Faraday rotation which in turn produce high sensitivity. The domain structure of these films favors magnetization rotation as the primary magnetization process. This process is significantly faster than domain wall motion, which is the primary magnetization process in bulk iron garnet crystals. We present data which confirm the high sensitivity and wideband frequency response to these materials. One film exhibits a virtually flat frequency response from dc to at least $1 \mathrm{GHz}$. Potential problems with waveguide sensors, such as birefringence and optical coupling efficiency appear to be soluble.

\section{2,160}

\section{PB95-168647 Not available NTIS}

National Inst. of Standards and Technology (EEEL),

Boulder, CO. Electromagnetic Technology Div.

Terahertz Detectors Based on Superconducting Kinetic Inductance.

Final rept.

E. N. Grossman, D. G. McDonald, and J. E.

Sauvageau. $1991,16 p$.

Pub. in Proceedings of International Symposium on Space Terahertz Technology, Pasadena, CA., February 1991, p407-422.

Keywords: "Infrared radiometers, "Infrared detectors, "Bolometers, "Superconducting devices, Optical heterodyning, Antenna couplers, Far infrared radiation, Reprints, "Heterodyne mixers, SIS(Superconductors), Mixers(Optics), Kinetic inductance, Photoinductive detectors.

The inductance of a superconducting stripline varies with the concentration of Cooper pairs in the superconductor. This inductance variation may be used as the basis for highly sensitive radiometers bolometers, and heterodyne mixers. We describe re: cent progress on three kinetic inductance devices: a large-area, absolute radiometer intended for use in the NIST Low-Background Infrared Calibration Facility, and small, antenna-coupled devices used either in a bolometric mode as direct detectors, or in a non-equilibrium, 'photoinductive' mode as heterodyne mixtures. The photoinductive mixers of a particular interest because their frequency coverage starts at approximately the energy gap, 2Delta, and extends upward. The impedance matching concerns which make extension of SIS mixers to high frequencies so difficult in practice are greatly relaxed for photoinductors because they lack the large parasitic capacitances inherent in a junction-like geometry.

\section{2,161}

\section{PB95-168662 Not available NTIS}

National Inst. of Standards and Technology (EEEL),

Boulder, CO. Electromagnetic Technology Div

Optical Performance of Photoinductive Mixers at Terahertz Frequencies.

Final rept.

E. N. Grossman, J. E. Sauvageau, and D. G.

McDonald. 1993 17p

Pub. in Proceedings of International Symposium on

Space Terahertz Technology (4th), Los Angeles, CA., February 1993, p588-604.
Keywords: "Infrared detectors, "Bolometers *Superconducting devices, Superconducting films, Fa infrared radiation, Temperature dependence, Electrical properties, Optical properties, SQUID devices, Niobium, Reprints, Kinetic inductance, Photoinductive detectors, Heterodyne mixers, Mixers(Optics)

We have investigated the electrical and optical properties of detectors based on the change in kinetic in ductance of a superconducting film with inciden terahertz-frequency radiation. Two different geometric configurations, stripline and slotline, of these photoinductive detectors have been explored. Both in clude a loop of thin niobium coupled to the incident radiation through a lithographic antenna; the loop inductance is read out via an integrated D.C. SQUID. The slotline geometry is substantially simpler to fabricate but electrically, the two geometries have very simila properties. The loop inductance varies with temperalure in good agreement with the 2-fluid model, while the critical current varies with temperature in agreement with Ginzburg-Landau theory.

\section{2,162}

PB95-168845 Not available NTIS

National Inst. of Standards and Technology (EEEL), Boulder, CO. Electromagnetic Technology Div.

Spatial Uniformity of Optical Detector Responsivity.

Final rept.

D. Livigni, and X. Li. 1994, 16p.

Pub. in Proceedings of National Computer Systems aboratory Workshop and Symposium: The Role of Metrology in a Changing World, Chicago, IL., July 31 August 4, 1994, p337-352.

Keywords: "Optical detectors, "Infrared detectors, Optical measurement, Indium gallium arsenides, Spatia dependencies, Laser radiation, Coherent radiation, $\mathrm{Ge}$ semiconductor detectors, Si semiconductor detectors Germanium, Silicon, Variability, Accuracy, Reprints.

A scanning system for measuring the spatial uniformity of the responsivity of optical detectors and methods of quantifying the degree of uniformity are described. Surface plots and contour maps of the measured responsivity are presented, along with a statistica treatment. Factors which can affect the accuracy of the uniformity measurement are described, including sampling theorem restrictions and interference artifacts produced when coherent light is used. Examples of these artifacts are presented, along with scans of ac tual $\mathrm{Si}, \mathrm{Ge}$, and In GaAs detectors.

\section{2,163}

PB95-168985 Not available NTIS

National Inst. of Standards and Technology (EEEL) Boulder, CO. Electromagnetic Technology Div.

Ultrasensitive-Hot-Electron Microbolometer.

Final rept.

M. Nahum, and J. M. Martinis. 1993, 3p.

Pub. in Applied Physics Letters 63, n22 p3075-3077, 29 Nov 93

Keywords: "Infrared detectors, "Bolometers, Superconducting devices Submillimeter waves, Millimeter waves, Far infrared radiation, Electron-phonon coupling, Superconducting junctions, Tunnel junctions, Hot electrons, Reprints, Microbolometers, Millikelvin temperature.

We present measurements on a novel power detector which can be used as an ultrasensitive detector of millimeter and submillimeter radiation. The absorbing element consists of a thin film resistor strip which is connected to superconducting electrodes. This device ex ploits the Andreev reflection of electrons and the weak electron-phonon coupling at low temperatures to produce a large temperature rise for a small input power (approx $=10 \mathrm{mK} / \mathrm{fW}$ ). The temperature rise of the electrons is detected by a tunnel junction where part of the metal strip forms the normal electrode. We have measured a voltage responsivity of approximately 10(exp 9) V/W and an amplifier-limited electrical noise equivalent approx $=3 \times 10(\exp -18) \mathrm{W} / \mathrm{s}$ root of $\mathrm{Hz}$ at an operating temperature of $100 \mathrm{mK}$. If infrared radiation were efficiently coupled to the absorbing element with an antenna or a waveguide, then the sensitivity of this detector would be at least a factor of 10 better than the best available direct detector op erating at the same temperature.

02,164

PB95-169066 Not available NTIS

National Inst. of Standards and Technology (EEEL), Boulder, CO. Electromagnetic Technology Div.
Electrically Calibrated Pyroelectric Detector-Refinements for Improved Optical Power Measurements.

Final rept.

R. J. Phelan, J. H. Lehman, and D. R. Larson. 1993,

Pub. in Proceedings of Society of Photo-Optical Instrumentation Engineers: Photodetectors and Power Meters, San Diego, CA., July 15-16, 1993, v2022 p160164.

Keywords: "Pyroelectric detectors, "Photodetectors, "Optical measurement, Power measurement, Variability, Accuracy, Reprints, *Optical power.

This paper describes the present efforts at NIST to improve the accuracy of the electrically calibrated pyroelectric detector for measuning optical power by an order of magnitude. The principal limitation, the uniformity of the responsivity over the receiving aperature, has been significantly improved.

\section{2,165}

PB95-169116 Not available NTIS

National Inst. of Standards and Technology (EEEL),

Boulder, CO. Electromagnetic Technology Div.

Kinetic-Inductance Infrared Detector Based on an Antenna-Coupled High-Tc SQUID.

Final rept.

J. P. Rice. 1993, 2p.

Pub. in Proceedings of International Superconductive (nics Conference

Keywords: "Infrared detectors, "SQUID devices, Superconducting devices, High-Tc Superconductors, Antenna couplers, Performance, Design, Reprints, Kinetic inductance.

We describe the design and estimate the performance of a high-T(c) kinetic-inductance infrared detector.

02,166

PB95-169124 Not available NTIS

National Inst. of Standards and Technology (EEEL) Boulder, CO. Electromagnetic Technology Div.

High-Tc Superconducting Antenna-Coupled Microbolometer on Silicon.

Final rept.

J. P. Rice, Grossman, L. J. Borcherdt, and D. A

Rudman. 1994, 12p.

Pub. in Society of Photo-Optical Instrumentation Engineers 2159, p98-109 1994

Keywords: "Bolometers, High temperature superconductors, Superconducting devices, Yttrium oxides, Zirconium oxides, YBCO superconductors, Silicon, Epitaxial growth, Micromachining, Fabrication, Substrates, Reprints, "Microbolometers, Yttrium barium cuprates.

A process is described for fabricating antenna-coupled resistive-edge microbolometers based on the high $\mathrm{T}(\mathrm{c})$ superconductor YBa2Cu307 (YBCO) on silicon. The $Y B C O$ and a buffer layer of yttria-stabilized zirconia (YSZ) were grown epitaxially on silicon to minimize excess electrical noise. A silicon-micromachined $\mathrm{YBCO} /$ $Y S Z$ air-bridge was incorporated to minimize the thermal conductance and the heat capacity. The thermal conductance of the air-bridge was measured to be 3 $X 10(\exp -6) \mathrm{W} / \mathrm{K}$ at a temperature of $100 \mathrm{~K}$. At an operating temperature of $89 \mathrm{~K}$, the detector is estimated to have a response time of 2 microseconds, a responsivity in the $1000 \mathrm{~V} / \mathrm{W}$ range, and a noise-equivalent power (NEP) in the 10(exp -12) $\mathrm{W} / \mathrm{Hz}(\operatorname{sup~1/2)}$ range at $1000 \mathrm{~Hz}$

\section{2,167}

PB95-169132 Not available NTIS

National Inst. of Standards and Technology (EEEL), Boulder, CO. Electromagnetic Technology Div. Effect of Semiconductor Laser Characteristics on Optical Fiber Sensor Performance.

Final rept

K. B. Rochford, A. H. Rose, I. Clarke, and G. W.

Day. 1994, 11p.

Pub. in Proceedings of Society of Photo-Optical Instrumentation Engineers: Laser Diode Technology and Applications VI, Los Angeles, CA., January 25-27, 1994 , v2148 p269-279.

Keywords: "Semiconductor lasers, Frequency stability, Noise spectra, Error analysis, Calibration, Performance, Reprints, *Optical fiber sensors, *Fiber optic sensors.

Optical sensor systems have source requirements that can be significantly different from those of optical com- 
munications and other technologies that have generally driven the development of semiconducto sources. In this paper, we examine basic interferometric, polarimetric, and other sensors. Relevant semiconductor source data is reviewed to illustrate the impact of source characteristics on sensor performance. The effect of low-frequency amplitude and frequency noise on sensor precision is described. Errors in sensor calibration due to amplitude and wavelength drifts are discussed. Examples of sensor performance using typical source data illustrate these issues.

P2,168

National Inst. of Standards and Technology (EEEL), Boulder, CO. Electromagnetic Technology Div

Terahertz Shapiro Steps in High Temperature SNS Josephson Junctions.

Final rept.

P. A. Rosenthal, and E. N. Grossman. 1994, 8p. Sponsored by National Aeronautics and Space Administration, Washington, DC.

Pub. in Institute of Electrical and Electronics Engineers Transactions on Microwave Theory and Techniques 42, n4 p707-714 Apr 94

Keywords: "Josephson junctions, Far infrared radiation, High temperature superconductors, Temperature dependence, Laser radiation, Infrared lasers, Reprints, "Shapiro steps, Microfabrication, Microbridges, Josephson oscillators.

We have studied the far infrared behavior of high-T(c) superconductor-normal metal-superconductor (SNS) microbridges with $T(c)>85 \mathrm{~K}$ and critical current-resistance products (I(C)R(N)) as high as $10 \mathrm{mV}$ at $4 \mathrm{~K}$. These are the highest I(C)R(N) products reported to date for microfabricated Josephson junctions of any material. The junctions were integrated at the feeds of planar log-periodic antennas made from Au thin films. The junctions had dc normal state resistances $R(N)$ between 6 and 38 ohms, reasonably well matched to the antenna's estimated RF impedance of $53 \mathrm{ohms}$. Far infrared laser radiation at 404,760 , and $992 \mathrm{GHz}$ in duced distinct Shapiro steps (i.e. constant voltage steps at voltages $n(h t / 2 e), n=1,2, \ldots)$ in the current voltage characteristics as well as modulation of the crit ical current. Steps were observed at voltages up to 17 $\mathrm{mV}$ and $6 \mathrm{mV}$, at temperatures of $9 \mathrm{~K}$ and $57 \mathrm{~K}$, respectively. This corresponds to maximum Josephson oscillation frequencies of 8 and $3 \mathrm{THz}$ at these temperatures.

02,169

PB95-169173 Not available NTIS

National Inst. of Standards and Technology (EEEL) Boulder, CO. Electromagnetic Technology Div.

Comparative Photoluminescence Measuremen and Simulation of Vertical-Cavity Semiconductor Laser Structures.

Final rept.

D. T. Schaafsma, D. H. Christensen, R. K

Hickernell, and J. G. Pellegrino. 1994, 6p

Pub. in Materials Research Society Symposia Proceedings, v326 p483-488 1994

Keywords: "Quantum well lasers, "Surface emitting lasers, "Photoluminescence, Optical measurement, Dielectric properties, Epitaxy, Simulation, Comparison Reprints, Distributed systems.

We present comparisons of photoluminescence (PL) data for various vertical-cavity surface-emitting lase (VCSEL) and distributed quantum well structures taken with the pump beam (and the collection path) in two different configurations: normal to the surface of the sample; and perpendicular to a cross-section of the epitaxial layers. We demonstrate that the cross-sectional PL (XPL) technique can resolve individual features in the structures, and that the surface-normal PL (NPL) spectra are perturbed by the multilayer mirrors in the VCSELs. We elucidate a potential method fo transforming between the NPL spectra and the nonperturbed XPL spectra and evaluate the sensitivity of this method to various measurement as well as material parameters. This simulation technique is well-suited to wide parametric variations of the dispersion curves for the complex dielectric constant of the matenals, the pump field distribution, and the depth profile of the gain medium.

02,170

PB95-169181 Not available NTIS

National Inst. of Standards and Technology (EEEL), Boulder, CO. Electromagnetic Technology Div.
Measurement and Simulation of Photoluminescence Spectra fr
Quantum-Well Laser Structures. Quantum

D. T. Schaafsma, R. K. Hickemell, and D. H.

Christensen. 1994, 11p.

Pub. in Proceedings of Society of Photo-Optical Instrumentation Engineers: Quantum Well and Superlattice Physics V, Los Angeles, CA., January 24-25, 1994 v2139 p92-102.

Keywords: "Quantum well lasers, "Surface emitting lasers, "Photoluminescence, Optical measurement, Dielectric properties, Spatial resolution, Quantum electrodynamics, Superlattices, Simulation, Epitaxy, Reprints, Distributed systems, Multilayers.

We compare photoluminescence data collected in either a surface-normal configuration (NPL) or with the pump and collection paths perpendicular to a crosssection of the epitaxial layers (XPL) for various verticalcavity surface-emitting lasers and distributed quantum well structures. We report the spatial resolution of the XPL technique, particularly as it applies to distinguishing features in complex multilayer structures. We assess a potential simulation method for transforming the perturbed NPL spectra into the unperturbed XPL spectra, taking into account a number of experimental and material parameters which may influence the lineshape. These factors include the pump field distribution and its iniliuence on the weighting of the emitters, the collection optics, and the changes in the dispersive complex dielectric constant of the quantum wells. This information is of import not only to optimizing device manufacture, but to basic physical and materials research as well Thereas the XPL technique is a relatively simple but destructive characterization tool, a complete understanding of NPL emission could be made to yield the same information via rapid, nondestructive means.

\section{2,171}

PB95-169264 Not available NTIS

National Inst. of Standards and Technology (EEEL), Boulder, CO. Electromagnetic Technology Div.

Waveguide Polarizers Processed by Localized Plasma Etching.

Final rept.

D. L. Veasey, D. R. Larson, and I. Veigl. 1994, 3p. Pub. in Applied Optics 33, n7 p1242-1244, 1 Mar 94.

Keywords: "Optical waveguides, "Plasma etching, "Polarizers, Chemical vapor deposition, Reprints, "Waveguide polarizers, In situ monitoring, Claddings.

We develop a downstream localized plasma-etching process that permits in situ monitoring of light throughput in a semiconductor-clad channel waveguide as the semiconductor thickness is trimmed. Hydrogenated amorphous silicon films are deposited on ion-exchanged channel waveguides by plasma-enhanced chemical vapor deposition. We then employ the localized plasma-etching process to maximize accurately the extinction ratio between TE and TM polarizations propagating in the clad waveguide. We achieve polarzation extinction ratios of greater than $30 \mathrm{~dB}$ for both TE-pass and TM-pass polarizers

\section{2,172}

PB95-169298 Not available NTIS

National Inst. of Standards and Technology (EEEL), Boulder, CO. Electromagnetic Technology Div.

Self-Calibrating Fiber Optic Sensors: Potential Design Methods.

Final rept.

H. K. Whitesel, G. W. Day, A. H. Rose, and C. A

Miller. 1993, 42p.

Pub. in Naval Surface Warfare Center Technical Report CARDIVNSWC-TR-80-92/15, 42p May 93

Keywords: "Self tests, "Calibration, Self repairing devices, Redundant components, Temperature sensors, Military applications, Naval equipment, Reprints, "Optical fiber sensors, "Fiber optic sensors, "Self calibration.

Potential applications of optical fiber sensors in the Navy and elsewhere involve networks of hundreds of thousands of sensors. Routine maintenance and calibration of these sensor networks, if undertaken manually, would involve unacceptable commitments of ship personnel and time. This has led to the present investigation into methods that can be used to produce sensor systems that can be self testing and/or self calibrat- ing. A self testing sensor is one in which one or more internal tests are used to verify the performance of the sensor. Self calibration extends the self testing concept to the point where when defects are identified, calibration corrections can be made automatically. Three design concepts can be considered for optical fiber sensors: substitution, redundancy, and internal diagnostics. Examples of how diagnostics of these types can be used to test and recalibrate various types of sensors are described in this report; a more detailed study of potential designs for a self calibration polarimetric temperature sensor is also included.

\section{2,173}

PB95-169306 Not available NTIS

National Inst. of Standards and Technology (EEEL) Boulder, CO. Electromagnetic Technology Div. Self Calibrating Fiber Optic Sensors: Potential De sign Methods.

Final rept.

H. K. Whitesel, C. A. Miller, G. W. Day, A. H. Rose, and W. F. Hamann. 1993, 18p.

Pub. in Proceedings of International Symposium on Spectral Sensing Research, Maui, HI., November 15 20, 1992, v1 p324-341 1993.

Keywords: *Self tests, "Calibration, Self repairing devices, Redundant components, Military applications, Naval equipment, Reprints, "Optical fiber sensors, "Fiber optic sensors, "Self calibration.

Potential applications of optical fiber sensor networks in the Navy include environmental and equipment monitoring and control. Such systems may involve hundreds if not thousands of sensors. Conventional methods of performing routine maintenance and calibration would require unacceptable amounts of manpower and time. This has led to the present investigation into methods that can be utilized to produce sensor systems that can be self-testing and/or self-calibrating. A self testing sensor is one in which internal tests are used to verify the performance of the sensor. Self calibration extends the self testing concept to a poin where, when changes in system operational characteristics are measured, calibration corrections can be made automatically. Three general design concepts were considered: substitution, redundancy, and internal diagnostics. Substitution involves applying a measurand of known value to the sensor in a manne similar to most laboratory calibrations. Redundancy in volves using multiple sensors in such a way that the failure of a single sensor can be detected: Correct values are then attributed to sensors giving consistent re sults. Internal diagnostics can take many forms, but in volves the use of various optical tests to insure the proper performance of the sensor.

\section{4}

PB95-175279 Not available NTIS

National Inst. of Standards and Technology (EEEL) Boulder, CO. Electromagnetic Technology Div.

Correlatlon of Optical, X-ray, and Electron Micros copy Measurements of Semiconductor Multilayer Structures.

Final rept.

D. H. Christensen, R. K. Hickernell, D. T.

Schaafsma, J. R. Hill, R. S. Rai, J. G. Pellegrino, and M. J. McCollum. 1994, 12p.

Pub. in Proceedings of Society of Photo-Optical Instrumentation Engineers: Spectroscopic Characterization Techniques for Semiconductor Technology V, Los Angeles, CA., January 25-26, 1994, v2141 p177-188.

Keywords: "Semiconductor devices, Electron microscopy, Optical microscopy, $X$ ray microscopy, Aluminum
gallium arsenides, Photoluminescence, Refractive index, Quantum wells, Reflectance, Correlation, Simulation, Epitaxy, Reprints, Bragg mirrors, Multilayers.

Techniques based on optical, $\mathrm{X}$-ray, and electron microscopy measurements are applied to characterize a wide variety of semiconductor multilayer structures. Bragg mirrors serve as valuable test structures for evaluating the epitaxial uniformity of crystal growth systems. Careful characterization of half-wave spaced single quantum wells provides a method for determining their complex refractive indices using reflectance spectroscopy. Comparison of cross-sectional microphotoluminescence to surface-norma photoluminescence, combined with these character zation techniques, allows studies of spontaneous emission in microcavities and elucidates the difficulties with using surface-normal photoluminescence to determine the alloy composition of the mirror layers. The ap plication of these characterization methods to visible wavelength AlGaAs mirrors, 485-720nm, enables the 


\section{Optoelectronic Devices \& Systems}

development of these mirrors for uses such as optically tailored substrates and visible surface-emitter or detector arrays.

\subsection{5}

PB95-175592 Not available NTIS

National Inst. of Standards and Technology (EEEL), Boulder, CO. Electromagnetic Technology Div.

Photodetector Frequency Response Measurements at NIST, US, and NPL, UK: Preliminary Results of a Standards Laboratory Comparison. Final rept.

P. D. Hale, D. A. Humphreys, and A. Gifford. 1994,

$12 \mathrm{p}$.

Pub. in Proceedings of Society of Photo-Optical Instrumentation Engineers: Technologies for Optical Fiber Communications, Los Angeles, CA., January 25, 1994, v2149 p345-356.

Keywords: "Photodiodes, "Photodetectors, *Frequency response, Near infrared radiation, Fre. quency measurement, Interlaboratory comparisons, Infrared detectors, Heterodyning, Neodymium lasers, YAG lasers, Reprints, Mach-Zehnder modulators, Intercomparison.

We report the first comparison of high speed photodiode frequency response measurements up to $40 \mathrm{GHz}$ between NIST and NPL in the 1.3 and $1.5 \mathrm{mi}-$ crometer wavelength regions. This comparison is an important step in establishing international agreement on photodiode response measurements, with traceability to international microwave power and DC current standards. Measurements at NIST used a Nd:YAG heterodyne system. NPL used DFB heterodyne and integrated-optical modulator-based techniques. Measurements of a photodiode with nominally $20 \mathrm{GHz}$ optical bandwidth show good agreement with average scatter of + or $-0.15 \mathrm{~dB}$ (2 sigma) below 20 $\mathrm{GHz}$, and + or $-0.30 \mathrm{~dB}$ from 20 to $33 \mathrm{GHz}$. The results diverge systematically above $33 \mathrm{GHz}$, due to calibra. tion of the RF power sensors. Scatter in the data is well represented by the combined uncertainties of the measurement systems up to $33 \mathrm{GHz}$.

\section{PB, 176 Not available NTIS}

National Inst. of Standards and Technology (EEEL), Boulder, CO. Electromagnetic Technology Div

Determination of the Complex Refractive Index of Individual Quantum Wells from Distributed Reflectance.

Final rept.

R. K. Hickernell, D. H. Christensen, J. G. Pellegrino, J. Wang, and J. P. Leburton. 1994, $4 p$.

Pub. in Applied Physics Letters 75, n6 p3056-3059, 15 Mar 94.

Keywords: "Quantum wells, "Refractive index, Aluminum gallium arsenides, Optical measurement, Reflectance, Absorption, Reprints, Bragg reflectors, Distributed systems.

We investigate the measurement of the complex refractive index of individual quantum wells by reflectance spectroscopy. Placing the wells at half-wavelength spacing to cause resonant feedback produces an order-of-magnitude increase in measurement sensitivity over that of nonresonant structures. Ouantum well dispersive and absorptive effects on reflectance can be differentiated in certain spectral regions. Experimental data confirm a theoretical model of refractive index and absomtion for quantum wells of GaAs in $\mathrm{Al}(0.2) \mathrm{Ga}(0.8) \mathrm{As}$ in the region of the well band gap.

\section{2,177}

\section{PB95-175782 Not available NTIS}

National Inst. of Standards and Technology (EEEL), Gaithersburg, MD. Semiconductor Electronics Div. Electrical Characterization of Liquid-Phase Epitaxially Grown Single-Crystal Films of Mercury Cadmium Telluride by Variable-Magnetic-Field Hall Measurements.

Final rept.

J. S. Kim, D. G. Seiler, L. Colombo, and M. C. Chen. 1994, $10 p$.

Pub. in Semiconductor Science and Technology 9 , p1696-1705 1994.

Keywords: "Mercury cadmium tellurides, Liquid phase epitaxy, Temperature dependence, Semiconducting films, Infrared detectors, Magnetic fields, Single crystals, Hall effect, Magnetoresistivity, Classification, Reprints.

We report a method for a new classification procedure for liquid-phase epitaxially grown mercury cadmium telluride single crystals. Variable-magnetic-field hall measurements are performed on nine liquid-phase epitaxially grown $\mathrm{Hg}(0.78) \mathrm{Cd}(0.22) \mathrm{Te}$ single-crystal films for magnetic fields from 0 to $1.4 \mathrm{~T}$ and in the temperature range from $10 \mathrm{~K}$ to $300 \mathrm{~K}$. The data from these measurements are analyzed in the context of the reduced-conductivity-tensor scheme proposed by Kim and co-workers. Based on the degree of deviation from an ideal one-carrier behavior, these experimental samples are classified into several types to emphasize the transition in the behavior of the normal to anomalous n-type samples, finally leading to p-type samples. Our classification is also based on a general trend in the temperature dependence of the mobility and density of the majority carriers, which were extracted from the magnetoresistivity data. The classification provides a useful benchmark for materials characterization in the infrared detector industry.

\section{2,178}

PB95-202826 Not available NTIS

National Inst. of Standards and Technology (PL), Boulder, CO. Quantum Physics Div.

Low-Noise High-Speed Diode Laser Current Controller.

Final rept.

K. G. Libbrecht, and J. L. Hall. 1993, 3p. Contract No0014-89-J-1227, Grant NSF-PHY9012244

Sponsored by Office of Naval Research, Arlington, VA. Air Force Office of Scientific Research, Bolling $A F B, D C$. and National Science Foundation, Washington, DC

Pub. in Review of Scientific Instruments 64, n8 p2133. 2135 Aug 93.

Keywords: "Semiconductor lasers, Current regulations, Direct current, High speed, Low noise, Reprints, Current controllers.

We describe a new diode laser current controller which features low current noise, excellent dc stability, and the capacity for high-speed modulation. While it is simple and inexpensive to construct, the controller compares favorably with the best presently available commercial diode laser current controllers.

\section{2,179}

PB95-202909 Not available NTIS

National Inst. of Standards and Technology (EEEL) Boulder, CO. Electromagnetic Technology Div. Integrated-Optical Devices in Rare-Earth-Doped Glass.

Final rept.

K. J. Malone. 1994, 25p

Pub. in Proceedings of Society of Photo-Optical Instrumentation Engineers: Glass Integrated Optics and Optical Fiber Devices, San Diego, CA., July 24-25, 1994 vCR53 p132-156.

Keywords: "Optoelectronic devices, "Integrated optics Solid state lasers, Optical waveguides, Neodymium, Erbium additions, Doped materials, Fabrication, Glass Reviews, Reprints.

A short overview is given of integrated-optical devices in rare-earth-doped glasses. Achievements in device performance are discussed. Fabrication of these components as well as analytical and diagnostic techniques that can improve performance are considered. The article concludes with a discussion of some current topics in this field.

\section{2,180}

PB95-203154 Not available NTIS

National Inst. of Standards and Technology (EEEL),

Boulder, CO. Electromagnetic Technology Div.

Faraday Effect Current Sensor with Improved Sensitivity-Bandwidth Product.

Final rept.

K. B. Rochtord, A. H. Rose, M. N. Deeter, and G. W Day. 1994, 3p

\section{CRO-93-806}

See also PB94-200698. Sponsored by Defense Nuclear Agency, Washington, DC

Pub. in Optics Letters 19, n22 p1903-1905, 15 Nov 94 Keywords: Yttrium iron garnets, Faraday effect, Sensitivity, Bandwidth, Design, Reprints, "Electric curren sensors.

We report a new design for a Faraday effect current sensor based on yttrium iron garnet that has substantially greater bandwidth than previous designs and is much easier to fabricate. The measured sensitivity is $0.7 \mathrm{deg} / \mathrm{A}$, with a $-3-\mathrm{dB}$ bandwidth of $500 \mathrm{MHz}$, which gives an improvement in sensitivity-bandwidth product of approximately 45. A noise-equivalent current of 840 $n A / H z(s u p ~ 1 / 2)$ was measured at $1.8 \mathrm{kHz}$ by difference-over-sum processing. The use of turning prisms with phase-preserving coatings greatly simplifies construction, improves electrical isolation, and increases sensitivity through proximity effects.

\section{2,181}

PB95-203576 Not available NTIS

National Inst. of Standards and Technology (EEEL), Boulder, CO. Electromagnetic Technology Div.

Accurate Measurement of Optical Detector Nonlinearity.

Final rept.

S. Yang, I. Vayshenker, X. Li, and T. R. Scott. 1994, $10 p$.

Pub. in Proceedings of National Computer Systems Laboratory Workshop and Symposium 'Role of Metrology in a Changing World', Chicago, IL., July 31-August 4, 1994, p353-362

Keywords: "Optical detectors, Optical measurement, Comparative evaluations, Computerized simulation, Nonlinearity, Accuracy, Reprints

In this paper we describe the results of our efforts to analyze and compare by computer simulation three common methods of measuring detector nonlinearity:

(1) superposition method, (2) attenuation method, and (3) differential or ac-dc method. We describe a definition and expressions which we used to intercompare the data analyses of these methods. Issues that are common to these methods or specific to one individual method and have an impact on the measurement accuracy were studied. We conclude that superposition and differential methods are better choices than the attenuation method. Suggestions about the choice of polynomial order of the fitting curve with regard to the data accuracy and detector nonlinearity are also given.

02,182

PB95-261921 (Order as PB95-261897, PC A07/

MF A02)

National Inst. of Standards and Technology, Gaithersburg, MD.

Determination of the Transmittance Uniformity of Optical Filter Standard Reference Materials.

J. C. Travis, N. K. Winchester, and M. V. Smith.

1995, $16 p$.

Included in Jnl. of Research of the National Institute of Standards and Technology, v100 n3 p241-256 May/ Jun 95.

Keywords: "Optical filters, "Calibration standards, *Transmittance, Spectrophotometry, Homogeneity, Certification, Charge coupled devices, Cameras, National Institute of Standards and Technology.

An instrument based on a scientific grade charge-coupled device (CCD) camera system is performancequalified to evaluate the transmittance homogeneity of solid optical filter standard reference materials. Measurement results are presented for the new instrument, and compared, where appropriate, with an older, scanning instrument, for a variety of filters spanning transmittances down to 0.01 . The new instrument is found to give comparable results with the older instrument, with reduced random uncertainty and improved information content.

\section{2,183}

PB96-102793 Not available NTIS

National Inst. of Standards and Technology (EEEL), Gaithersburg, MD. Semiconductor Electronics Div. Scaling of the Nonlinear Optical Cross Sections of GaAs-AlGaAs Multiple Quantum-Well Hetero n-i-pi's.

Final rept.

D. S. McCallum, A. N. Cartwright, A. L. Smirl, J. Comas, W. F. Tseng, and J. G. Pellegrino. 1994, 8p. Pub. in Institute of Electrical and Electronics Engineers Jnl. of Ouantum Electronics, v30 n12 p2790-2797 Dec 94.

Keywords: "Absorption cross sections, "Nonlinear optics, "Ouantum wells, "Heterojunctions, Gallium arsenides, Aluminum gallium arsenides, Carrier density(Solid state), Semiconducters(Materials), Reprints.

The authors study the dependence of the Stark shift optical nonlinearity of GaAs-AIGaAs multiple quantumwell hetero $n-i-p-i$ 's on the number of quantum wells per intrinsic region in otherwise identical hetero $n-i-p-$ i's. The authors determine that sigma (sub eh), the 
nonlinear absorption cross section, is proportional to the number of quantum wells per intrinsic region. A study of the fluence dependence of sigma(sub eh) shows that the saturation carrier density is inversely proportional to the number of wells per intrinsic region. The authors find that the turn-on time of the nonlinear abosrption change in their samples is independent of the number of quantum wells per intrinsic region. All of these results are consistent with the absence of retrapping of photogenerated carriers.

\section{2,184}

PB96-111729 Not available NTIS

National Inst. of Standards and Technology (EEEL), Boulder, CO. Electromagnetic Technology Div. Niobium Microbolometers for Far-Infrared Detection.

Final rept.

M. E. MacDonald, and E. N. Grossman. 1995, 4p.

Pub. in Institute of Electrical and Electronics Engineers Transactions on Microwave Theory and Techniques, v43n4 p893-896 Apr 95

Keywords: "Bolometers, "Far infrared radiation, *Niobium, Thin films, Temperature measurement, Room temperature, Bias, Noise, Detectors, Reprints, "Microbolometer.

Microbolometers have been fabricated using a thin niobium film as the detector element. These detectors operate at room temperature, are impedance matched to planar antennas, and are suitable for broadband use at far-infrared wavelengths. The authors have achieved responsitivities of up to $21 \mathrm{~V} / \mathrm{W}$ at a bias of $6.4 \mathrm{~mA}$, and electrical noise equivalent powers (NEP) of as low as $1.1 \times 10(\exp -10) \mathrm{W} /$ (square root of $\mathrm{H} 2$ ) at $1 \mathrm{kHz}$ at a bias of $3.6 \mathrm{~mA}$. At this bias, the detectors are $1 / f$-noise limited below $1 \mathrm{kHz}$ and are Johnson noise limited above $10 \mathrm{kHz}$. The $1 / \mathrm{f}$ noise in $\mathrm{nV} /$ (square root of $\mathrm{Hz}$ ) increases approximately linearly with bias with a typical level of $0.39 \mathrm{l}(\mathrm{MA}) /($ square root of $\mathrm{f}(\mathrm{kHz})$ ). This level of $1 / f$ noise is approximately a factor of 7 below the best reported for bismuth microbolometers.

02,185

PB96-111935 Not available NTIS

National Inst. of Standards and Technology (EEEL), Gaithersburg, MD. Electronics and Electrical Engineering Lab. Office.

Can Displays Deliver a Full Measure: Manufacturing.

Final rept.

H. S. Bennett, C. Fenimore, B. F. Field, and E. F. Kelley. 1994, 2p.

Pub. in Proceedings of the Annual Display Manufacturing Technology Conference (1st) 'Digest of Technical Papers', San Francisco, CA., January 11-13, 1994 p45-46.

Keywords: *Display devices, *Manufacturing, "Design criteria, "Standardization, High resolution, Research and development, Technology transfer, Standards, Measurement, Computation, Research programs, Reprints.

The National Institute of Standards and Technology (NIST) recently initiated a new program on measurements for displays. As part of this new program, NIST completed a preliminary assessment of the needs for measurements, standards, and computations to assist in the development of high-resolution displays. In this paper, we summarize the major results of this assessment and describe briefly NIST's ongoing intramural and extramural programs on displays.

\section{2,186}

PB96-119227 Not available NTIS

National Inst. of Standards and Technology (EEEL), Gaithersburg, MD. Electricity Div.

Display-Measurement Round-Robin.

Final rept

D. J. Bechis, M. D. Grote, D. P. Bortfeld, E. F.

Kelley, G. R. Jones, P. A. Boynton, L. H. Hammer,

and M. J. Polak. 1995, 4p.

Pub. in Society for Information Display, International Symposium, Digest of Technical Papers, Orlando, FL. May 23-25, 1995, v26 p641-644, 15 May 95.

Keywords: "Cathode ray tube screens, *Display devices, Electronic equipment, Test and evaluation, Visual perception, Man-computer interface, Graphics, Performance evaluation, Standards, Procedures, Specifications, Reporting, Reprints, "Performance

Display measurement procedures intended for use by other laboratories and by industry for measuring, ana- lyzing, and reporting the performance of display mon itors are tested through the round-robin process in preparation for acceptance of the procedures as a standard. The National Information Display Laboratory NIDL) and National Institute of Standards and Technololgy (NIST) results presented here show much quantitative agreement in support of the measurement procedures.

02,187

PB96-119276 Not available NTIS

National Inst. of Standards and Technology (EEEL), Boulder, CO. Optoelectronics Div.

Polarization Insensitive $3 \times 3$ Sagnac Current Sensor Using Polarizing Spun High-Birefringence Fiber.

Final rept.

I. G. Clarke, K. B. Rochford, A. H. Rose, and G. W.

Day. 1994, 4p

Pub. in International Conference on Optical Fibre Sensors (10th), Glasgow, Scotland, UK, October 11-13, 1994, Postdeadline paper, v2360 4p

Keywords: *Electric current, *Sensors, *Birefringence, "Optical fibers, Fiber optics, Polarization(Waves), Response functions, Sensitivity analysis, Reprints, `Sagnac current sensor, Optical fiber sensor, Polarization dependence.

The response functions of previously reported $3 \times 3$ Sagnac current sensors are strongly dependent on input polarization so systems require careful control of the input polarization state. In this paper, the authors describe a simple method for eliminating this polarization dependence so that stable response functions are obtained. The authors demonstrate the existence of a wavelength range over which spun high-birefringence fiber guides only one elliptically polarized mode, thus preserving a single polarization state. Using this effect, the authors show that $3 \times 3$ Sagnac current sensor response functions can exhibit current sensing without the need for polarization control.

\section{2,188}

PB96-122528 Not available NTIS

National Inst. of Standards and Technology (EEEL), Gaithersburg, MD. Electricity Div.

Survey of the Components of Display-Measurement Standards.

Final rept.

E. F. Kelley, G. R. Jones, P. A. Boynton, M. D.

Grote, and D. J. Bechis. 1995, 4p

Pub. in Society for Information Display International Symposium, Digest of Technical Papers, Orlando, FL. May 23-25, 1995, p637-640.

Keywords: "Display devices, "Standards, *Measurement, Cathod ray tubes, Liquid crystal dis play systems, Reprints, *Flat panel displays.

Several display standards a re reviewed and distinctive elements are compared. With flat panel displays becoming more common and the CRT displays being so well established, the associated standards activities can be somewhat bewildering, even overwhelming. This paper attempts to identify complementary and inconsistent elements of related display standards.

\section{2,189}

PB96-122684 Not available NTIS

National Inst. of Standards and Technology (EEEL), Boulder, CO. Electromagnetic Technology Div Polarization Dependence of Response Functions in $3 \times 3$ Sagnac Optical Fiber Current Sensors.

Final rept.

K. B. Rochford, G. W. Day, and P. R. Forman. 1994 $6 p$.

See also PB95-162426.

ub. in Jni. of Lightwave Technology, v12 n8 p15041509 Aug 94

Keywords: "Fiber optics, *Sagnac effect, *Functions(Mathematics) Interferometers, Birefringence, Electric current, Polarization(Waves), Phase shift, Optical coupling, Reprints, *Electric current sensors, "Optical fiber sensors, Sagnac interferometer.

We show theoretically that the response functions of a lossless Sagnac optical fiber current sensor based on a $3 \times 3$ coupler fundamentally depend on the polarization state of light entering the coupler, even for systems with no linear birefringence. Thus, $3 \times 3$ Sagnac systems offer no advantage over $2 \times 2$ systems for linearly polarized input light. The predicted polarization dependence of $3 \times 3$ Sagnac response functions is ex- perimentally confirmed. This result establishes the need for increased system complexity in $3 \times 3$ Sagnac current sensors since polarization control optics are required to provide the proper input polarization.

\section{2,190}

PB96-122700 Not available NTIS

National Inst. of Standards and Technology (EEEL), Boulder, CO. Electromagnetic Technology Div.

Millimeter-Resolution Optical Time-Domain Reflectometry Using a Four-Wave Mixing Sampling Gate. Final rept.

J. B. Schlager, M. Jinno, and D. L. Franzen. 1995

Pub. in Institute of Electrical and Electronics Engineers Photonics Technology Letters, v7 n2 p206-208 Feb 95.

Keywords: *Semiconductor devices, *Laser mode locking, "Light amplifiers, Optical coupling, Switches, Spatial resolution, Waves, Mixing, Reprints, "Optical sampling, Optical reflectometry, Wave mixing.

We report on optical time-domain reflectometer that employs an ultrafast optical switch based on nondegenerate four-wave mixing in a semiconductor laser amplifier. Two-point spatial resolution on the order of $1 \mathrm{~mm}$ over $2 \mathrm{~m}$ is demonstrated; Fresnel reflections with optical return losses greater than $53 \mathrm{~dB}$ are detected. Submillimeter-resoluton optical time-domain reflectometery over a $100-m$ range should be possible with modifications to the pulse sources.

02,191

PB96-123021 Not available NTIS

National Inst. of Standards and Technology (EEEL) Gaithersburg, MD. Electricity Div.

Nonlinear Color Transformations in Real Time Using a Video Supercomputer.

Final rept.

E. F. Kelley, B. F. Field, and C. Fenimore. 1993, 4p Pub. in Proceedings of the Society for Imaging Science and Technology/Society for Information Display Color Imaging Conterence: Transtorms and Transportability of Color, Scottsdale, AZ., November 7-11, 1993, p122125.

Keywords: "Color coding, "Video compression, *Supercomputers, "Visual discrimination, Nonlinea systems,' Flat panel displays, Display devices, Colors Electroluminescence, Video data, Image convertors, Computerized simulation, Reprints, Color image processing.

Investigations of the effects of color transformations used for video displays are often hampered by the in ability to see the effects of these changes in real time for a variety of input signals. Using a video supercomputer, the Princeton Engine, the effects of a parametric nonlinear color transformation can be shown in real time.

\section{2}

PB96-123716 Not available NTIS

National inst. of Standards and Technology (EEEL) Gaithersburg, MD. Electricity Div.

Refraction of Light by Graded Birefringent Media. Final rept.

K. L. Stricklett, and C. Sununu. 1994, 5p.

Pub. in Conference of Electrical Insulation and Dielectric Phenomena, Pocono Manor, PA., October 17-20, 1993 Institute of Electrical and Electronics Engineers 1993 Annual Report, p444-448 1994.

Keywords: "Light refraction, *Birefringent media, Reprints, Kerr effect, Electric field mapping, Graded refractive index

To date, proposals to use the Kerr effect to map divergent electric fields have not considered refraction of light in the graded potential. Spatial variation of the strength or direction of the field will produce non-uniform refractive indices, and significant refraction of light in regions of high divergence may disort the final image and introduce an error in the field measurement. Experimental evidence for this effect is presented and its implications to optical field mapping are discussed.

02,193

PB96-128210 PC A04/MF A01

National Inst. of Standards and Technology (EEEL) Boulder, CO. Optoelectronics Div.

Bibliography of the NIST Optoelectronics Division. A. J. Smith, and L. S. Derr. Sep 95, 67p, NISTIR-

5041

See also PB96-128269. 
Keywords: "Metrology, "Bibliographies, Optical communications, Optoelectronic devices, Optical fibers, Fiber optics, Electrooptics, Solid state lasers, US NIST.

The Optoelectronics Division was established in 1994 to provide the optoelectronics industry and its suppliers and customers with comprehensive and technically advanced measurement capabilities, standards, and traceability to those standards. The Division is organized into four groups: the Sources and Detectors Group, the Fiber and Integrated Optics Group, the Optical Components Group, and the Optoelectronic Manufacturing Group, with work in six project areas.

\section{2,194}

PB96-138490 Not available NTIS

National Inst. of Standards and Technology (EEEL), Gaithersburg, MD. Electricity Div.

Electro-Optic-Based RMS Voltage Measurement Technique.

Final rept.

N. G. Paulter. 1995, 8p.

Pub. in Review of Scientific Instruments, v66 n6 p3583-3690 Jun 95.

Keywords: "Electromagnetic fields, "Voltage measurement, Reprints, "Electro-optic crystals, "RMS voltage.

A new electro-optic-based technique for measuring the rms value of an applied voltage is presented. The technique incorporates a ratiometric method whereby the requirement for absolute laser power information is unnecessary. Description, analysis, and experimental results of the prototype measurement system are presented.

\section{2,195}

PB96-140363 Not available NTIS

National Inst. of Standards and Technology (EEEL), Boulder, CO. Electromagnetic Fields Div.

Radiometer Equation for Noise Comparison Radiometers.

Final rept.

D. F. Wait. 1995, 4p.

Pub. in Institute of Electrical and Electronics Engineers Transactions on Instrumentation and Measurement, v44 n2 p336-339 Apr 95

Keywords: "Electromagnetic noise measurement, "Radiometers, "Calibrating, Accuracy, Error analysis, High temperature, Uncertainty, Reprints

A complete radiometer equation is derived and used to develop better corrections and uncertainty estimates. We show that a corrected unisolated radiometer can have an accuracy similar to that of a well-isolated radiometer. The radiometer error is insignificant for radiometers with $40-\mathrm{dB}$ isolation. However, for hightemperature applications (such as sources and standards above $3000 \mathrm{~K}$ ), isolation is usually unnecessary.

\section{2,196}

PB96-156088 Not available NTIS

National Inst. of Standards and Technology (EEEL)

Gaithersburg, MD. Semiconductor Electronics Div.

CMOS Circuit Design for Controlling Temperature in Micromachined Devices.

Final rept.

C. Zincke, M. Gaitan, and M. E. Zagloul. 1995, 4p

Pub. in Proceedings of the Midwest Symposium on Circuits and Systems (37th), Lafayette, LA., August 3-5, 1995, p183-186.

Keywords: "Circuit design, "Temperature, Pixel, Reprints, "Constant power, CMOS, EDP etch, Micrmachining, MOSIS, Open area.

The authors present a CMOS circuit used for controlling the temperature of a CMOS compatible microheating element, known as a thermal pixel. The circuit uses nonlinear compensation to maintain constant power over large variation in the resistance. An external analog voltage controls the power delivered to the thermal pixel. This circuit was designed and fabricated through the MOSIS service foundry. Measurements are presented that verify the design and performance of the circuit.

\section{2,197}

PB96-157961 Not available NTIS

National Inst. of Standards and Technology (EEEL), Gaithersburg, MD. Semiconductor Electronics Div.
Characterization of LPE HgCdTe Film by Magnetoresistance.

Final rept.

J. S. Kim, D. G. Seiler, L. Colombo, and M. C. Chen. 1994, 2p.

Pub. in Extended Abstracts of the 1994 U.S. Workshop on the Physics and Chemistry of Mercury Cadmium Telluride and Other IR Materials, San Antonio, TX., October 4-6, 1994, p167-168

Keywords: "Mercury cadmium tellurides, "Electrical resistivity, Liquid phase epitaxy, High resolution, Reprints, "Reduced conductivity tensor.

Magnetoresistance has been shown in the past to be a valuable tool for studying complex energy bands of semiconductors. In this paper, we demonstrate that magnetoresistance can be used as an extremely useful physical quantity to electrically characterize $\mathrm{HgCdTe}$ materials, structures, or devices which are of a multicarrier conduction nature. We take advantage of simple relationships between the magnetoresistance. The applied magnetic field, the lattice temperature, and relevant physical quantities in the reduced conductivity tensor (RCT) scheme. We have performed variable-magnetic-field and vanabletemperature Hall measurements $(0$ to $1.5 \mathrm{~T}$ and $10 \mathrm{~K}$ $300 \mathrm{~K}$ ) on LPE HgCdTe films and a GaAs-based HEMT structure. Magnetoresistance data obtained from these measurements are analyzed in the context of the RCT scheme.

\section{2,198}

\section{PB96-158704 PC A03/MF A01}

National Inst. of Standards and Technology (PL), Gaithersburg, MD. Radiometric Physics Div.

Liquid-Nitrogen-Cooled High Tc Electrical Substitution Radiometer as a Broadband IR Transfer Standard.

Technical note

J. P. Rice, and Z. M. Zhang. Jan 96, 18p, NIST/TN1414 .

Also available from Supt. of Docs. as SN003-00303375-8.

Keywords: "Infrared radiometers, "Calibration standards, Temperature control, Superconductors, High temperature, Infrared detectors, Electrical-substitution, NIST(National Institute of Standards and Technology).

A requirement exists for broadband radiometers that can be used to transfer NIST infrared radiometric scales to calibration laboratories elsewhere. The authors discuss the design of a liquid-nitrogen-cooled transfer standard radiometer that represents a practical tradeoff between room-temperature and liquid-helium-cooled radiometers. The detector utilizes high-Tc superconductors as temperature sensors for increased sensitivity. Such sensors have already been shown to enable temperature regulation of 100 microK peak-topeak at $85 \mathrm{~K}$, and have the potential for further improvements by three orders of magnitude. Electrical substitution is used to linearize the response over the dynamic range and to remove susceptibility to sensor aging effects

\section{2,199}

\section{PB96-165378 PC A04/MF A01}

National Inst. of Standards and Technology (EEEL), Boulder, CO. Optoelectronics Div.

Optical Detector Nonlinearity: Simulation.

Technical note.

S. Yang, I. Vayshenker, X. Li, T. R. Scott, and M. Zander. May 95, 42p, NIST/TN-1376.

Also available from Supt. of Docs. as SN003-00303338-3. See also PB95-169355 and PB95-203576.

Keywords: "Optical detectors, "Infrared detectors, "Power meters, Optical measurement, Mathematical models, Computerized simulation, Nonlinearity.

The authors developed a unified mathematical treatment for five commonly used measurement methods of optical detector nonlinearity and conducted computer simulation to compare these methods for different measurement conditions and data processing options. They found that the triplet and differential methods will give the overall best results, and third and fourth order polynomial representations of the measurement result will yield least total error for a common practical measurement system.

\section{2,200}

PB96-176649 Not available NTIS

National Inst. of Standards and Technology (MSEL), Gaithersburg, MD. Reactor Radiation Div.
Simulations of Neutron Focusing with Curved Mirrors.

Final rept.

J. R. D. Copley, 1996, 5p.

Pub. in Review of Scientific Instruments, v67 n1 p190194 Jan 96.

Keywords: "Mirrors, "Neutron reflection, Gravitational effects, Ray tracing, Reprints, "Ellipsoidal mirror, Toroidal mirror.

The authors have developed a ray-tracing program to calculate the imaging properties of ellipsoidal and toroidal mirrors in glancing angle configurations. It has been proposed that such mirrors be used in small angle neutron scattering instruments, in order to achieve increased count rates without sacrificing angular resolution. The authors describe the method of calculation, including effects due to gravity, and they present a number of instructive examples of calculations for both types of mirrors. The authors also illustrate a practical method of reducing image degradation associated with gravitational effects

\section{2,201}

PB96-200308 Not available NTIS

National Inst. of Standards and Technology (EEEL) Boulder, CO. Optoelectronics Div.

Fiber-Optic Faraday-Effect Magnetic-Field Sensor Based on Flux Concentrators.

Final rept.

M. N. Deeter. 1996, $4 p$

Pub. in Applied Optics, v35 n1 p154-157 Jan 96

Keywords: "Faraday effect, "Magneto optics, Magnetic field sensors, Optical fiber sensors, Yttrium iron garnets, Ferrimagnetism, Sensitivity, Reprints, Electric current sensors.

The principles and performance of a fiber-optic Faraday effect magnetic-field sensor designed around an yttrium-iron-granet (YIG) sensing element and two flux concentrations are described. The system design exploits the technique of polarization-rotated reflection in which a single polarization-maintaining optical fibe links the sensor head to the optical source and detection system. In the sensing head, ferrite flux concentrators are magnetically coupled to the YIG sensing element to achieve maximum sensitivity.

\section{2,202}

\section{PB96-200860 Not available NTIS}

National Inst. of Standards and Technology (EEEL) Boulder, CO. Optoelectronics Div.

Optoelectronics at NIST.

Final rept.

G. W. Day. 1995, 2p.

See also PB96-128210.

Pub in Proceedings of the Annual Institute of Electrical and Electronics Engineers Laser and Electro-Optics Society Meeting (8th), San Francisco, CA., October 30-November 2, 1995, v2 p73-74.

Keywords: "Lasers, "Optoelectronic devices, "Research programs, Optical fibers, Wavelengths, Frequency stability, Frequency measurement, Calibration standards, Metrology, Reprints, "US NIST.

The Optics Division, one of the first divisions formed after NBS was established in 1901, was initially charged with work on radiometry, spectroscopy, and polarimetry. Over the next five or six decades optics remained a significant topic of research. Within a year of Maiman's first demonstration of the ruby laser in 1960, a similar laser was constructed at the NBS-Boulder Laboratories. Demands for assistance in measuring the power or energy produced by such lasers led to the establishment of laser calibration services beginning in 1967. Another early program was aimed at developing techniques for laser frequency stabilization and absolute frequency and wavelength measurements. That effort culminated in 1972 with the determination of a much improved value for the speed of light, which led ultimately to a redefinition of the meter.

\section{2,203}

PB96-204441 Not available NTIS

National Inst. of Standards and Technology (PL), Gaithersburg, MD. Radiometric Physics Div. Improving Color Measurements of Displays.

Final rept.

J. E. Hardis, 1996, 10p.

Pub. in Color Imaging: Device-Independent Color, Color Hard Copy, and Graphic Arts, San Jose, CA., January 29-February 1, 1996, v2658 p182-191. 
ELECTROTECHNOLOGY

Power \& Signal Transmission Devices

Keywords: "Calibration, "Cathode ray tubes, "Matrix transformation, Colorimeters, Reprints, Measurement, Standards, Tristimulus, Chromaticity.

It is generally believed that the most accurate means of measuring the CIE tristimulus values $X, Y, Z$ or chromaticity coordinates $x, y$ from a display is by using a spectroradiometer. Nevertheless, tristimulus colorimeters employing three or four colored filters find wide use because of their simplicity and lower cost. These devices cannot be calibrated to give accurate results in all situations because the spectral responsivities of their filtered detectors are not exactly the CIE color-matching functions. However, for a display that produces a linear superposition of three primary colored lights of fixed spectra, a tristimulus colorimeter can be correctly calibrated to measure all colors on that display. Signals from all of the filtered detectors are used to compute each of the $X, Y, Z$ values. The calibration matrix is computed by data fitting to a reference colorimeter.. An improvement to the previously published method is reported, and a numerical example is shown. This technique is more tractable with today's digital instrumentation than it was when it was discovered, yet it remains underused. The American Society for Testing and Materials (ASTM), through its Committee on Color and Appearance, is revising its standard on display measurements using tristimulus colorimeters to encourage the adoption of the technique.

\section{2,204}

\section{PB97-104186 PC A11/MF A03}

National Inst. of Standards and Technology, Gaithersburg, MD. Advanced Technology Program. Optoelectronics and Optomechanics Manufacturing: An ATP Focused Program Development. Workshop Proceedings. Held in Gaithersburg, Maryland on February 15, 1995

T. Lettieri, V. R. McCrary, and J. C. Boudreaux. Feb 96, 210p, NISTIR-5715.

Keywords: "Electrooptics, *Computer aided manufacluring, "Research programs, Photonics, Economics, Product development, Display systems, Communication networks, Fiber otpics, Planning, Semiconductor devices, Packaging, Optical sensors, Modules(Electronics), Computer aided design Chips(Electronics), Circuit interconnections, Industrial research, Workshops, Technology transfer, Precision engineering, "Optoelectronics, Optomechanics.

The ATP Workshop on Optoelectronics and Optomechanics Manufacturing was held at the $\mathrm{Na}$ tional Institute of Standards and Technology in Gaithersburg, MD on February 15, 1995. The purpose of the Workshop was to respond to industry interest and provide an open forum for the exchange of ideas among interested members of the optics/photonics community concerning the strategic importance of optoelectronics (OE) and optomechanics (OM) technologies to U.S. economic growth. The Workshop brought together over 160 experts in OE/OM for the purpose of addressing the issues and requirements of U.S. industry in the area of OE/OM manufacturing. Input gained from the morning presentations and the breakout sessions will be used to help formulate the scope and range of a proposed ATP Focused Program in $\mathrm{OE} / \mathrm{OM}$ manufacturing.

02,205

PB97-111835 Not available NTIS

National Inst. of Standards and Technology (EEEL), Boulder, CO. Optoelectronics Div.

Fundamentals and Problems of Fiber Current Sensors.

Final rept.

G. W. Day, K. B. Rochford, and A. H. Rose. 1996,

$6 p$.

Pub. in Proceedings of the International Conference on Optical Fiber Sensors, Advanced Sensing Photonics (11th), Sapporo, Hokkaido, Japan, May 21-24, 1996, p124-129 May 96.

Keywords: *Detectors, "Faraday effect, Metrology, Reprints, "Electric current sensors, Fiber optic sensors, Optical fiber sensors.

The paper briefly reviews the history and present commercial status of optical fiber sensors, and then summarizes recent research aimed at improved performance, lower cost, and wider areas of applications.

02,206

PB97-113179 Not available NTIS
National Inst. of Standards and Technology (EEEL). Boulder, CO. Optoelectronics Div Integrated Optical Polarization-Discriminating Receiver In Glass.

Final rept.

D. L. Veasey, and D. R. Larson. 1995, 6p.

Pub. in Jnl. of Lightwave Technology, v13 n11 p22442249 Nov 95.

Keywords: "Receivers, "Glass, "Polarization, Waveguides, Cladding, Hybrid, Integrated, Optical, Polarizers, Semiconductors, Splitter, Reprints.

The authors have successfully demonstrated an integrated optical TE-TM mode discriminator using waveguide polarizers and guided-wave photodetectors for use in polarimetric optical sensor and positioning systems. The photonic integrated circuit consists of a Y-branch waveguide splitter formed by potassium-sodium ion exchange in silicate glass. Hydrogenated amorphous silicon claddings were deposited on each branch of the splitter to act as polarizers. One output cladding was trimmed to a thickness which attenuated the TE polarization, while the other cladding was trimmed to attenuate light having TM polarization. The thickness trimming is accomplished using a process of localized plasma etching which allows in situ extinction optimization by monitoring transmitted light. Optical extinction ratios of up to $27 \mathrm{~dB}$ were demonstrated on Y-branch waveguides for polarizers with claddings 1.2 $\mathrm{mm}$ in length. The integrated receiver was completed with the deposition of metal-semiconductor-metal photodetectors on each of the output waveguide branches following the polarizers. Amorphous silicon claddings were contacted with chrome-gold interdigitated Schotky contacts to form the waveguide detectors.

\section{2,207}

PB97-116040 PC A06/MF AO1

National Inst. of Standards and Technology (EEEL), Boulder, CO. Optoelectronics Div.

Bibliography of the NIST Optoelectronics Division. A. J. Smith. Sep 96, 78p, NISTIR-5052.

See also report for 1995, PB96-128210.

Keywords: "Electrical engineering, *Electrooptics, "Bibliographies, Research projects, Metrology, Optical communications, Optical fibers, Fiber optics, Optoelectronic devices, Laboratories, Electronics, Electrical measurement, Semiconductor devices, Superconduciors, Lasers, "Optoelectronics.

This bibliography lists publications of the staft of the Optoelectronics Divison and its predescessor organizational units, from about 1970 through the data of this report.

\section{2,208}

PB97-119200 Not available NTIS

National Inst. of Standards and Technology (EEEL), Gaithersburg, MD. Electricity Div.

Specular and Diffuse Reflection Measurements of Electronic Displays.

Final rept.

G. R. Jones, E. F. Kelley, and T. A. Germer. 1996, $4 \mathrm{p}$

Pub. in International Symposium Digest of Technical Papers 1996, San Diego, CA., May 12-17, 1996, p203206.

Keywords: "Display devices, "Specular reflection, "Diffuse reflection, "Measurement, Electronic equipment, Bidirectional reflection, Distribution functions, Coefficients, Electroluminescence, High resolution, Estimation, Reprints.

Display standards describe measurements of the diffuse and specular reflection coefficients. The adequacy of such procedures is compared with the bidirectional reflection distribution function (BRDF) measurements. Alternative methods are examined and their estimates of the specular and diffuse reflection coefficients are compared to the results of the BRDF measurement.

\section{2,209}

PB97-122394 Not available NTIS

National Inst. of Standards and Technology (EEEL), Gaithersburg, MD. Electricity Div.

Survey of the Components of Display-Measurement Standards.

Final rept.

E. F. Kelley, G. R. Jones, P. A. Boynton, M. D.

Grote, and D. J. Bechis. 1996, 4p.

See also PB96-122528.
Pub. in Jnl. of the Society for Information Display, v3 n4 p219-222 Dec 95.

Keywords: "Display devices, "Measurement, "Performance evaluation, Flat panel displays, Liquid crystal display systems, Cathode ray tube screens, Electronic equipment, Logical elements, Visual perception, Components, Standards, Reprints

Several display standards are reviewed and distinctive elements are compared. The paper attempts to identify complementary and inconsistent elements of related display standards.

\section{Power \& Signal Transmission Devices}

\section{2,210}

PB94-135639 PC A03/MF AO

National Inst. of Standards and Technology (EEEL) Boulder, CO. Electromagnetic Fields Div.

Crosstalk between Microstrip Transmission Lines. D. A. Hill, K. H. Cavcey, and R. T. Johnk. Dec 93, 38p, NISTIR-5015.

Keywords: "Microstrip transmission lines, *Crosstalk Characteristic impedance, Mutual inductance, Scale models, Permittivity, Predictions, Mutual capacitance, Scalar polentials, Vector potentials.

Methods for prediction of crosstalk between microstrip transmission lines are reviewed and simplified for the weak coupling case. Classical coupled transmission line theory is used for uniform lines, and potential and induced EMF methods are used for crosstalk between nonuniform lines. It is shown that the potential method is equivalent to classical coupled transmission line theory for the case of uniform lines. An experiment was performed for uniform coupled microstrip lines for frequencies from $50 \mathrm{MHz} 105 \mathrm{GHz}$, and good agreement between theory and measurement was obtained for both near-end and far-end crosstalk.

02,211

PB94-165537 PC A06/MF A02

National Inst. of Standards and Technology (EEEL) Boulder, CO. Electromagnetic Fields Div.

Transmission/Reflection and Short-Circuit Line Methods for Measuring Permittivity and Permeability.

Technical note

J. Baker-Jarvis, M. D. Janezic, J. H. Grosvenor, and R. G. Geyer. Dec 93, 121p, NIST/TN-1355-R

Also available from Supt. of Docs. as SN003-00303258-1. See also PB92-205376.

Keywords: "Transmission lines, "Magnetic permeability, "Permittivity, "Electrical measurement, Coaxial cables, Waveguides, Dielectric properties, Calibrating, Short circuits, Reflection, Algorithms, Optimization, Mathematical models, Frequency stability, Uncertainty analysis.

The transimission/reflection and short-circuit line methods for measuring complex permittivity and permeability of materials in waveguides and coaxial lines are examined. Equations for complex permittivity and permeability are developed from first principles. In addition, new formulations for the determination of complex permittivity and permeability independent of reference plane position are derived. For the one-sample transmission/reflection method and two-position short-circuit line measurements, the solutions are unstable at requencies corresponding to integral multiples of onehalf wavelength in the sample. For two-sample methods the solutions are unstable for frequencies where both samples resonate simultaneously. Criteria are given for sample lengths to maintain stability. An optimized solution is also presented for the scattering parameters. This solution is stable over all frequencies and is capable of reducing scattering parameter data on materials with higher dielectric constant. An uncertainty analysis for the various techniques is developed and the results are compared. The errors incurred due to the uncertainty in scattering parameters, length measurement, and reference plane position are used as in puts to the uncertainty models.

\section{2,212}

PB94-172350 Not available NTIS

National Inst. of Standards and Technology (MSEL), Gaithersburg, MD. Polymers Div. 
Effect of DC Tests on Induced Space Charge.

Final rept.

N. Hozumi, J. Tanaka, A. DeReggi, B. Dickens, and

N. Nagusrinivas. $1990,3 p$.

Pub. in Proceedings Conference Record of the 1990

Institute of Electrical and Electronics Engineers International Symposium on Electrical Insulation, Toronto, Canada, June 3-6, 1990, p332-334.

Keywords: "Electric cables, "Space charge, Aging(Materials), Direct current, Polyethylenes, Tests, Reprints.

Poling-induced space charges in peeled samples of ac aged/dc tested cables were studied using the thermal pulse technique. Samples were poled at about 0.12 $\mathrm{MV} / \mathrm{cm}$ for 3.5 hours at $70 \mathrm{C}$ using the same polarity as that used for the dc tests. Previous work seemed to indicate that dc testing could have permanent effects which could be detected by the amount of poling-induced charge. An attempt was made to examine this by using three samples with about the same length of ac aging but with different amounts of $\mathrm{dc}$ testing. The sample with one $d c$ test and the sample with $23 d c$ tests were appreciably oxidized whereas the sample with $11 \mathrm{dc}$ tests was not oxidized. Other interesting observations were made.

\section{2,213}

PB94-172814 Not available NTIS

National Inst. of Standards and Technology (EEEL), Boulder, CO. Electromagnetic Fields Div.

Reciprocity Relations in Waveguide Junctions.

Final rept.

D. F. Williams, and R. B. Marks. 1993, 6p.

Pub. in Institute of Electrical and Electronics Engineers Transactions on Microwave Theory and Techniques $41, n 6 / 7$ p1105-1110 Jun/Jul 93.

Keywords: Characteristic impedance, Reprints, "Waveguide junctions, Microwave metrology, Reference impedance, Scattering parameters, Reciprocity.

The Lorentz reciprocity condition is applied to junctions composed of reciprocal media which connect uniform but otherwise arbitrary waveguides. An expression relating the forward and reverse transmission coefficients is derived and factored into two terms: the first involving the phase of the reference impedance in the guide, and the second a new reciprocity factor. The usual condition equating the forward and reverse transmission coefficients is shown not to hold in the general case. Experimental evidence supporting the theoretical results is presented.

\section{2,214}

PB94-199130 Not available NTIS

National Inst. of Standards and Technology (MSEL), Gaithersburg, MD. Polymers Div.

Spatial Dependence of Electrical Fields Due to space Charges in Films of Organic Dielectrics Used for Insulation of Power Cables.

Final rept.

M. G. Broadhurst, A. S. DeReggi, B. Dickens, and R. M. Eichhorn. 1990, 5p

Pub. in Conference of Record of the Institute of Electrical and Electronics Engineers International Symposium on Electrical Insulation, Toronto, Canada, June 3-6, 1990, p405-409.

Keywords: "Electrical insulation, "Dielectrics, "Electric wire, "Space charge, "Electric fields, Reprints, Polyethylenes, Electrical properties, Electric charge, Electrical measurement.

The authors measured the electric fields due to space charge within film samples of several commercial polyolefins used as medium voltage power cable insulation. They charged the films at 60 deg. $C$ with $D C$ voltages applied for several hours. For dry charged films, vacuum deposited gold or aluminum provided the electrodes. For wet charged films, one or both surfaces of the film were bare and in contact with an aqueous 0.05 molar solution of $\mathrm{NaCl}$. They measured the fields using the thermal pulse (TP) method. To use this method with the wet charged films, they painted colloidal graphite electrodes onto the films after they were charged. The results show that thermoplastic polyethylene (PE) charges negatively for both wet and dry charging. Crosslinked polyethylene (XLPE) charges negatively when dry charged, but small amounts of either positive or negative charge were found after wet charging.
National Inst. of Standards and Technology (EEEL), Boulder, CO. Electromagnetic Fields Div.

Currents Induced on Multiconductor Transmission LInes by Radiation and Injection.

Final rept.

D. A. Hill. 1992, $6 p$.

Pub. in Institute of Electrical and Electronics Engineers Transactions on Electromagnetic Compatibility 34, n4 p445-450 Nov 92

Keywords: "Transmission lines, Excitation, Tests, Reprints, *Induced current, Wire assemblies.

Multiconductor transmission line theory is used to compare currents induced on individual wires within a bundle by injection and radiated excitation. Calculations show that the two types of excitation generally induce significantly different current distributions, but the differences are much smaller for electrically short lines. The results are relevant to the validity of using current injection testing to replace radiated immunity testing.

\section{2,216}

PB94-211968 Not available NTIS

National Inst. of Standards and Technology (EEEL),

Boulder, CO. Electromagnetic Fields Div.

Aperture Coupling to a Coaxial Air Line: Theory and Experiment.

Final rept.

D. A. Hill, M. L. Crawtord, M. Kanda, and D. I. Wu.

1993, 6p.

Pub. in Institute of Electrical and Electronics Engineers Transactions on Electromagnetic Compatibility $35, \mathrm{n} 1$ p69-74, 1 Feb 93

Keywords: "Electromagnetic shielding, Reverberation chambers, Polarization(Waves), Circular configuration, Dipole moments, Apertures, Reprints, "Coaxial air lines.

Coupling through a circular aperture in the shield of a coaxial air line is studied theoretically and experimentally. Polarizability theory is used to compute the effective dipole moments that excite the coaxial line in the internal region. Measurements of shielding effectiveness were made in a reverberation chamber over wide-frequency ranges. Agreement between theory and measurements is generally within + or - $10 \mathrm{~dB}$. Recommendations for improvements in the measurements and theory are made for achieving closer agreement that would be desirable for an artifact standard for shielding effectiveness measurements.

02,217
PB94-216389 Not available NTIS

National Inst. of Standards and Technology (EEEL), Boulder, CO. Electromagnetic Fields Div.

Accurate Experimental Characterization of Interconnects: A Discussion of 'Experimental Electrical Characterization of Interconnects and Discontinuities in High-Speed Digital Systems'.

Final rept

R. B. Marks, and D. F. Williams. 1992, 4p.

Pub. in Institute of Electrical and Electronics Engineers Transactions on Components. Hybrids, Manufacturing Technology 15, n4 p601-604 Aug 92.

Keywords: "Circuit interconnections, *Transmission lines, Characteristic impedance, Electrical measurement, Digital circuits, Permittivity, Capacitance, High speed, Accuracy, Reprints.

This paper discusses two issues concerning the accuracy of electrical characterizations of interconnect transmission lines, particularly in regard to a recently published paper. The error in the characteristic impedance may be reduced through an alternative approximation to the capacitance of the transmission line. Furthermore, measurements of both the propagation constant and characteristic impedance, which are the two primary parameters characterizing the line, may be improved by the use of a well conditioned algorithm.

\section{2,218}

\section{PB94-216397 Not available NTIS}

National Inst. of Standards and Technology (EEEL), Boulder, CO. Electromagnetic Fields Div.

Interconnection Transmission Line Parameter Characterization.

Final rept.

R. B. Marks, and D. F. Williams. 1992, 8p.

Pub. in Proceedings of the ARFTG Conterence (40th), Orlando, FL., December 3-4, 1992, p88-95 1992.

Keywords: *Circuit interconnections, *Transmission lines, Characteristic impedance, Frequency depend- ence, Dielectric properties, Substrates, Silicon, Reprints, Coplanar waveguides.

This paper introduces a new method for the characterization of transmission lines fabricated on lossy or dispersive dielectrics. The method, which is more accurate than conventional techniques, is used to examine the resistance, inductance, capacitance, and conductance per unit length of coplanar waveguide transmission lines fabricated on lossy silicon substrates.

\section{2,219}

PB94-216504 Not available NTIS

National Inst. of Standards and Technology (EEEL) Gaithersburg, MD. Electricity Div.

Important Link in Entire-House Protection: Surge Reference Equalizers.

Final rept.

F. D. Martzloff, and M. Samotyj. 1993, 4p.

Pub. in Proceedings of Zurich EMC Symposium, Zurich, Switzerland, March 9-12, 1993, p395-398.

Keywords: "Electronic equipment, "Circuit protection, "Surges, Households, Residential buildings, Cable television, Telephones, Communication equipment, Voltage regulators, Reprints.

The increasing use of electronics in residential applications has been paralleled by a realization that surge protection may be necessary for this type of equipment. Installing a surge-protective device on the power-line port as well as on the communications-line port of a piece of equipment might appear sufficient to ensure this protection. However, the normal operation of one of the protective devices during a surge event can create differences in the voltages of the references of the two ports. This difference in voltages, applied across the equipment or across a communication link between two pieces of equipment, can result in permanent damage as well as upset. Equalizing these voltages can be achieved by proper routing of the two lines through a single device, called Surge Reference Equalizer, and thus avoid the risk of damage.

\section{2,220}

\section{PB95-151593 Not available NTIS}

National Inst. of Standards and Technolo

Boulder, CO. Electromagnetic Fields Div.

Accurate Transmission Line Characterization.

Final rept

D. F. Williams, and R. B. Marks. 1993, 3p.

Pub. in Institute of Electrical and Electronics Engineers 249 Aug 93 .

Keywords: "Transmission lines, Characteristic impedance, Frequency dependence, Dielectric properties, Calibration, Substrates, Silicon, Reprints, Coplanar waveguides.

This letter introduces a new method for the characterization of transmission lines fabricated on lossy or dispersive dielectrics. The method, which is more accurate than conventional techniques is used to examine the resistance, inductance, capacitance, and conductance per unit length of coplanar waveguide transmission lines fabricated on lossy silicon substrates.

\section{2,221}

\section{PB95-168787 Not available NTIS}

National Inst. of Standards and Technology (EEEL) Boulder, CO. Electromagnetic Fields Div.

Measurements of the Characteristic Impedance of Coaxial Air Line Standards.

Final rept.

J. R. Juroshek, and G. M. Free. 1994, 6p

Pub. in Institute of Electrical and Electronics Engineers Transactions on Microwave Theory and Techniques 42, n2 p 186-191 Feb 94

Keywords: "Characteristic impedance, "Standards, *Impedance measurement, Electrical measurement, Network analyzers, Transmission lines, Capacitance, Electric connectors, Reprints, ${ }^{\star}$ Coaxial air lines, Scattering parameters.

A method for electrically measuring the characteristic impedance of coaxial air line standards is described. This method, called the gamma method, determines the characteristic impedance of a coaxial air line from measurements of its propagation constant and capacitance per unit length. The propagation constant is measured on a network analyzer, and the capacitance per unit length. The propagation constant is measured on a network analyzer, and the capacitance per unit length is measured on a capacitance bridge at $1 \mathrm{kHz}$. 
The measurements of characteristic impedance with the gamma method are independent of any dimensional measurements. Measurements of the characteristic impedance using the gamma method are compared to theoretical predictions from dimensional measurements. Test results are shown for $14 \mathrm{~mm}, 7$ $\mathrm{mm}$, and $3.5 \mathrm{~mm}$ coaxial air lines.

\section{2,222}

\section{PB95-169538 PC A05/MF A02}

National Inst. of Standards and Technology (EEEL), Boulder, CO. Electromagnetic Technology Div.

Superconductor Critical Current Standards for Fusion Applications. Final Progress Report, October 1993-July 1994.

L. F. Goodrich, J. A. Wiejaczka, A. N. Srivastava, and T. C. Stauffer. Nov 94, $100 p$, NISTIR-5027. Sponsored by Department of Energy, Washington, DC Office of Fusion Energy. and Massachusetts Inst. of Tech., Cambridge. Plasma Fusion Center.

Keywords: "Thermonuclear reactor materials, "Superconducting wires, "Niobium stannides, ${ }^{*}$ Critical current, "Electrical measurement, Interlaboratory comparisons, Fusion reactors, Homogeneity, Standards, Mandrels, Niobium intermetallics, Titanium intermetallics, ITER tokamak, Tables(Data), Graphs(Charts), Titanium alloy 6Al 4V, Reference materials, Reference wires, Intercomparison, Niobium titanides.

This report describes research conducted to help es tablish a standard critical current measurement technique for Nb3Sn wires that may be used in fusion applications. The main part of this report is a detailed presentation of results of the first ITER international interlaboratory comparison of $\mathrm{Nb} 3 \mathrm{Sn}$ critical curren measurements. A common procedure and a common reaction and measurement mandrel was used by US laboratories in this comparison, whereas there was no common procedure followed by other international lab. oratories. The largest difference in I(c) measurements of two laboratories that did not use a common procedure was $23 \%$. The largest difference in I(c) measurements of two laboratories that did use a common procedure was $6.5 \%$. There may still be room for improvement, but this indicates the strong need for a common detailed procedure. Results on the homogeneity of one of the Nb3Sn wires used in this study and a commentary on creating a Nb3Sn Reference Wire are also presented.

\section{2,223}

PB95-175535 Not available NTIS

National Inst. of Standards and Technology (EEEL), Boulder, CO. Electromagnetic Technology Div.

Reduction of Interfilament Contact Loss in Nb3Sn Superconductor Wires.

Final rept.

R. B. Goldfarb, and K. Itoh. 1994, 4p.

Sponsored by Department of Energy, Washington, DC Pub. in Jnl. of Applied Physics 75, n4 p2115-2118, 15 Feb 94.

Keywords: "Superconducting wires, "Niobium stannides, Proximity effect, Eddy currents, AC losses, Magnetization, Reduction, Reprints, Hysteresis losses, Twisted filaments, Length dependence.

Interfilament contact in Nb3Sn wires made by the internal-tin-diffusion process causes excess hysteresis loss beyond the intrinsic magnetic hysteresis loss of the filaments. In analogy with eddy-current and proximity-effect coupling losses, the excess contact loss can be reduced by decreasing the twist-pitch length of the filaments in the wire. One consequence of interfilament contact is that volume magnetization measurements are strongly dependent on sample length below about one twist pitch. We define a characteristic length whose reciprocal is equal to the sum of the reciprocals of the sample length and the twist pitch. Hysteresis loss is a universal function of characteristic length for different sample lengths and twist pitches. We discuss several experimental parameters for the magnetic determination of hysteresis loss.

02,224

PB95-176178 Not available NTIS

National Inst. of Standards and Technology (EEEL),

Boulder, CO. Electromagnetic Technology Div.

Micromagnetic Scanning Microprobe System.

Final rept.
C. A. Thompson, R. W. Cross, and A. B. Kos. 1994,

$7 p$. p383-389 Feb 94 .
Keywords: "Magnetoresistance, "Thin films, "Scanning electron microscopy, "Microanalysis, Magnetic tapes Magnetic recordings, Instrumentation, Data acquisition, Domains, Apparatus, Barkhausen noise, Reprints, Read heads.

The authors describe the apparatus, instrumentation and data acquisition techniques which make up the micromagnetic scanning microprobe system (MSMS) The system was developed to study magnetoresistive (MR) thin films used in magnetic recording read heads. It uses a dc, four-probe resistance measurement cou pled with two pairs of orthogonal field sources. Results from magnetoresistance measurements of the dy. namic response of a MR read head film are shown to demonstrate system operation and performance. Significant variations in MR responses were seen across the width of the device because of local domain formation. The MSMS is an effective tool for characterizing the effects of domain formation on the output of a MR read head.

\section{2,225}

PB95-180337 Not available NTIS

National Inst. of Standards and Technology (EEEL). Boulder, CO. Electromagnetic Fields Div.

Crosstalk between Microstrip Transmission Lines. (NIST Reprint).

Final rept.

D. A. Hill, K. H. Cavcey, and R. Johnk. 1994, 8p. See also PB94-135639.

Pub. in Institute of Electrical and Electronics Engineers Transactions on Electromagnetic Compatibility $36, n 4$ p314-321 Nov 94

Keywords: "Microstrip transmission lines, "Crosstalk, Characteristic impedance, Mutual inductance, Permittivity, Reprints, Mutual capacitance, Scalar potentials, Vector potentials.

Methods for prediction of crosstalk between microstrip transmission lines are reviewed and simplified for the weak-coupling case. Classical coupled transmission line theory is used for uniform lines, and potential and induced EMF methods are used for crosstalk between nonuniform lines. It is shown that the potential method is equivalent to classical coupled transmission line theory for the case of uniform lines. An experiment was performed for uniform coupled microstrip lines for frequencies from $50 \mathrm{MHz}$ to $5 \mathrm{GHz}$, and good agreement between theory and measurement was obtained for both near- and far-end crosstalk.

\section{2,226}

PB95-202784 Not available NTIS

National Inst. of Standards and Technology (EEEL), Boulder, CO. Electromagnetic Technology Div

Critical-Current Degradation in Nb3 Al Wires Due to Axial and Transverse Stress.

Final rept.

T. Kuroda, H. Wada, S. L. Bray, and J. W. Ekin.

1993, 5p.

See also PB93-153211

Keywords: "Superconducting wires, "Critical current, "Stress analysis, Axial stress, Microstructure, Strains, Reprints, Transverse stress, Niobium aluminides, Multifilaments.

Effects of axial and transverse stress on the critical cur rent of Nb-tube processed multifilamentary Nb3Al wires have been studied. The degradations of the critical current due to axial or transverse stress for these $\mathrm{Nb3Al}$ wires are much smaller than those for Nb3Sn wires. The microstructures and stress states of the Nb3Al wires have been investigated to understand the stress dependences of the critical current through the microscopic observations and the elastic theory. From an engineering standpoint, the importance of mechanical strength of the matrix material where superconducting filaments are embedded has been found especially for transverse stress effects.

\section{2,227}

PB96-119219 Not available NTIS

National Inst. of Standards and Technology (EEEL), Boulder, CO. Electromagnetic Technology Div.

Comparing the Accuracy of Critical-Current Measurements Using the Voltage-Current Simulator. Final rept.

D. Aized, J. W. Haddad, C. H. Joshi, L. F. Goodrich, and A. N. Srivastava. 1994, $4 \mathrm{p}$

Pub. in Institute of Electrical and Electronics Engineers Transactions on Magnetics, v30 n4 p2014-2017 Jul 94. Keywords: *Critical current, "Superconductors, Simulations, Reprints, Electric circuit, Data acquisition, Comparison, "Voltage current simulation.
A passive voltage-current simulator developed by the National Institute of Standards and Technology (NIST) is used to compare the accuracy of critical current measurements and the power-law behavior of high temperature superconductors (HTS). In this study, critical current measurements made from four data acquisition and analysis systems are compared with those carried out at NIST. This paper also discusses various measurement techniques, methods of calculating critical current, and n-values. The V-I simulator is believed to be an advancement towards defining the standards for critical current measurements and ensuring the traceability of results at different test facilities.

\section{2,228}

PB96-119268 Not available NTIS

National Inst. of Standards and Technology (EEEL), Gaithersburg, MD. Electricity Div.

Gaseous Dielectrics Research: Possible SF6 Substitutes.

Final rept

L. G. Christophorou, and R. J. Van Brunt. 1995, 28p. Pub. in Electrical Transmission and Distribution Systems. Sulfur Hexafluoride and Atmospheric Effects of Greenhouse Gas Emissions Conference, August 9-10, 1995, Washington, DC., p148-175

Keywords: "Electrical insulation, "Environimental chemical substitutes, "Sulfur hexafluoride, Gases, Dielectrics, Mixtures, Greenhouse effect, Global air pollution, Nitrogen, Reprints.

Sulfur hexafluoride (SF6) is currently the most common insulating gas used in enclosed electrical systems. It is, however a potent greenhouse gas and, thus, its use could impart fears of environmental costs, regulations, and restrictions, in spite of its current low levels in the environment. An aspect of this potential problem, namely the search for alternative high-voltage insulants, such as high-pressure gaseous dielectrics (e.g., N2 and N2/SF6 mixtures), is addressed in this paper.

\section{2,229}

PB96-119409 Not available NTIS

National Inst. of Standards and Technology (EEEL), Boulder, CO. Electromagnetic Technology Div.

Simple and Repeatable Technique for Measuring the Critical Current of Nb3Sn Wires.

Final rept.

L. F. Goodrich, and A. N. Srivastava. 1994, 4p.

Pub. in Proceedings of the International Workshop on Critical Currents in Superconductors (7th), Alpbach, Austria, January 24-27, 1994, p609-612.

Keywords: "Critical current, "Niobium stannides, "Superconductors, "Wire, Interlaboratory comparisons, Mandrels, Strains, Titanium alloys, Variations, Reproducibility, Reprints.

We evaluated an alternative approach for measuring the critical current (IC) of Nb3Sn wire which uses a standard mandrel geometry and apparatus interface. Preliminary data indicate that the tension in the conductor before reaction and measurement may affect the repeatability. We show preliminary summary statistics for measurements of conductors performed by five US laboratories. The reaction and measurement mandrel used was fabricated using a Ti-6 Al-4V alloy. This high temperature alloy was used to avoid transferring the specimen between mandrels, thus reducing the likelihood of inadvertent mechanical damage of the specimen. The U.S. International Thermonuclear Ex. perimental Reactor (US ITER) Home Team adopled this approach in a recent test because it was expected to be easily implemented and yield consistent results.

\section{2,230}

PB96-123468 Not available NTIS

National Inst. of Standards and Technology (EEEL), Gaithersburg, MD. Electricity Div.

SF6/N2 Mixtures: Basic and High-Voltage-Insulation Properties.

Final rep

L. G. Christophorou, and R. J. Van Brunt. 1995, 52p. Pub. in Institute of Electrical and Electronics Engineers Transaction on Dielectrics and Electrical Insulation, Virginia Beach, VA., October 23-26, 1995, p952-1003.

Keywords: *Dielectrics, "Electrical insulation, Collision cross sections, Electron transport, Gas mixtures, Global warming, Reprints, High-voltage insulation.

The widespreed use of SF6 by the electric power and other industries has led to increased concentrations of SF6 in the atmosphere. This causes concern as to pos- 


\section{ELECTROTECHNOLOGY}

\section{Power \& Signal Transmission Devices}

sible effects on global warming, because SF6 is a potent greenhouse gas. This paper first touches on this issue and then documents the behavior of high pressure gases such as N2 and SF6/N2 mixtures that can be realistically considered as acceptable intermediate or long-term replacements for pure SF6 in some HV applications. The possible use of dilute SF6/N2 mixtures as an alternative to pure SF6 for some of industry's insulation needs (albeit at higher pressure) is documented, and existing knowledge on these mixtures and on the individual components (N2 and SF6), both basic and applied, is compiled. A guide to existing literature is provided.

\section{2,231}

\section{PB96-123633 Not available NTIS}

National Inst. of Standards and Technology (EEEL), Gaithersburg, MD. Electricity Div.

Keeping Up with the Reality of Today's Surge Environment.

Final rept

F. D. Martzloff. 1995, $7 \mathrm{p}$

Pub. in Proceedings of the Power Quality Solutions, Long Beach, CA., September 9-15, 1995, p246-252.

Keywords: "Surges, "Electric faults, "Overvoltage, Electrical equipment, Electric power distribution, Electric power failures, Overcurrent, Power measurement, Electromagnetic compatibility, Circuit protection, Voltage regulators, Reprints.

The paper proposes to establish a program for characterizing surge events by the capability of a surge event to deliver a surge current through the power system in end-user facilities. This characterization would complement or even supersede the conventional monitoring of surge voltages. Two approaches are suggested: (1) using a metal-oxide varistor with the lowest possible voltage 10 'attract' surges away from other SPDS connected in the facility, and then recording the surge current waveform in the varitor; (2) gathering data on field failure attributable to surges or swells for all types of electrical appliances, then attempting to replicate the failure mode in the laboratory.

\section{2,232 \\ PB96-131586 PC A05/MF A01}

National Inst. of Standards and Technology (EEEL), Gaithersburg, MD. Electricity Div

Classified Bibliography: Insulation Condition Monitoring Methods, 1989-1995.

F. D. Martzloff. Nov 95, 96p, NISTIR-5760.

See also PB91-222687

Keywords: "Bibliographies, "Electrical insulation, "Cables "Nondestructive testing. Defects, Aging, Monitoring, Test methods, Experimental data, Measurements, Polymers, Spectroscopy, Time domain, Nuclear power plants, Detection, Wire, Simulation, Data reduction, Dielectric properties, Electrical testing, Damage assessment, Partial discharges.

This Bibliography is a complement to the Annotated Bibliography that was initially compiled for identifying promising test methods to detect incipient defects due to aging of cables. That compilation led to the selection of two methods for a demonstration at the National Institute of Standards and Technology (NIST), Partial Discharge Analysis and Time-Domain Spectroscopy. The literature search covers the period 1989-1995, with emphasis on partial discharge measurements, data reduction and simulation, on measurement of dielectric properties, and to some degree, on insulation aging.

\section{2,233}

\section{PB96-137781 Not available NTIS}

National Inst. of Standards and Technology (EEEL),

Boulder, CO. Electromagnetic Technology Div

Anomalous Switching Phenomenon in CriticalCurrent Measurements When Using Conductive Mandrels.

Final rept

L. F. Goodrich, J. A. Wiejaczka, and A. N.

Srivastava. 1995, 3p

Pub. in Institute of Electrical and Electronics Engineers Transactions on Applied Superconductivity, v5 n3 p3442-3444 Sep 95.

Keywords: "Critical current, "Superconducting wires, Switiching, Voltage-current, Reprints, Bypass current, Conductive mandrel, Multivalued, $\mathrm{Nb}-\mathrm{Ti}$.

NIST and other laboratories have observred an anomalous switching phenomenon that can occur in criticalcurrent measurement of coiled $\mathrm{Nb}-\mathrm{Ti}$ and $\mathrm{Nb} 3 \mathrm{Sn}$ superconductors when mounted on an electrically-conductive measurement mandrel. During aquistion of the voltage-current $(\mathrm{V}-\mathrm{I})$ characteristic, large voltage discontinuities are observed. This switching phenomenon results in a multivalued V-I curve, and apparently multiple 'critical-current' values. An explanation of this phenomenon, some necessary conditions for switching to occur, as well as methods of detecting the phenome non are given.

\section{2,234}

PB96-140389 Not available NTIS

National Inst. of Standards and Technology (EEEL) Boulder, CO. Optoelectronics Div.

Fiber Coating Diameter: Toward a Glass Artifact Standard.

Final rept.

D. H. Williams, M. Young, and L. A. Tietz. 1995, 3p. Pub. in Proceedings of the Optical Fibre Measurement Conference (3rd), Liege, Belgium, September 25-26, 1995, 3p.

Keywords: “Fiber optics, "Calibration, Coatings, Reprints, Standards, Micrometers, "Foreign technology, Optical fibers, Standard Reference Materials.

This paper proposes a glass fiber, 245 micrometers in diameter, with an appropriate index of refraction as a standard for the calibration of outer primary coating diameter measurements. Fibers will be manufactured by either a melt process or a draw process. The index of refraction will be measured by the Becke line method and the diameter with a contact micrometer.

\section{2,235}

\section{PB96-141262 Not available NTIS}

National Inst. of Standards and Technology (EEEL), Boulder, CO. Electromagnetic Technology Div

Microwave Properties of Voltage-Tunable YBa2Cu307-delta/SrTiO3 Coplanar Waveguide Transmission Lines.

Final rept.

D. C. DeGroot, J. A. Beall, R. B. Marks, and D. A. Rudman. 1995, 4p

Pub. in Institute of Electrical and Electronics Engineers Transactions on Applied Superconductivity, v5 n2 p2272-2275 Jun 95

Keywords: "Waveguides, Integrated circuits, Reprints, Microwaves, Electromagnetic shielding, Superconductors, Ferroelectric, "Coplanar waveguides

To explore the electrical characteristics of monolithic microwave circuits with integrated high-temperature superconductor and ferroelectric materials, the authors fabricated a series of coplanar waveguide transmission lines in laser-deposited $\mathrm{YBa}$ Cu3O708 and $\mathrm{SrTiO}$ thin films. The authors characterized the voltage-tunable two-port microwave response of the transmission lines at cryogenic temperatures using a calibrated network analyzer system. Total phase shifts and phase tuning in these devices increased for increasing ferroelectric film thickness with only moderate increases in transmission loss.

\section{2,236}

PB96-147038 Not available NTIS

National Inst. of Standards and Technology (EEEL), Boulder, CO. Electromagnetic Fields Div.

Dielectric Measurements on Printed-Wiring and Circuit Boards, Thin Films, and Substrates: An Overview.

Final rept.

J. R. Baker-Jarvis, and C. A. Jones. $1995,12 \mathrm{p}$

Pub. in Proceedings of the Material Research Society Symposium, San Francisco, CA., April 18, 1995, p153 164.

Keywords: "Calibration, "Coaxial cables, Dielectric constants, Loss factor, Microwaves, Permeability, Permittivity, Reflection method, Resonance, Resonator, Reprints, Open-ended probe.

A review of the most common methods of permittivity measurements on thin films, printed-wiring and circuit boards, and substrates is presented. Transmission-line lechniques, coaxial apertures, open resonators, surface-wave modes, and dielectric resonators methods are examined. The frequency range of applicability and typical uncertainties associated with the methods are summarized.

\section{2,237 \\ PB96-155510 Not available NTIS}

National Inst. of Standards and Technology (EEEL), Boulder, CO. Electromagnetic Technology Div Size Effects in Submicron NiFe/Ag GMR Devices. Final rept

S. E. Russek, R. W. Cross, S. C. Sanders, and J. O Oti. $1995,4 p$.

Pub. in Instifute of Electrical and Electronics Engineers Transactions on Magnetics, v31 n6 p3939-3942 Nov 95.

Keywords: "Magnetoresistance, Barkhausen noise, Reprints, Submicrons, "GMR.

The authors measured the magnetoresistive response of submicron NIFe/Ag giant magnetoresistive (GMR) devices as a function of current density and field angle. In addition to magnetostatic broadening, the authors observe large jumps in the magnetoresistive response (Barkhausen jumps) due to domain switching. These effects lead to irregular device-specific magnetoresistive response curves. The large Barkhausen jumps are more pronounced at low current density while at high current densities the response is smoother due to self field stabilization. The detailed structure of the Barkhausen jumps is very sensitive 10 the angle of the applied magnetic field. These effects are general properties of wide class of GMR materials that rely on incoherent reversal of many small magnetic domains.

\section{2,238}

\section{PB96-162649 PC A04/MF A01}

National Inst. of Standards and Technology (EEEL), Boulder, CO. Electromagnetic Fields Div.

Radiated Emissions and Immunity of Microstrip Transmission Lines: Theory and Measurements.

Technical note.

D. A. Hill, D. G. Camell, K. H. Cavcey, and G. H. Koepke. Jul 95, 37p, NIST/TN-1377.

Also available from Supt. of Docs. as SN003-00303360-0.

Keywords: "Microstrip transmission lines "Irradiation, -Directivity Emission, Immunity, Measurement, Power, Efficiency, Reverberation, Test chambers, NIST(National Institute of Standards and Technology), Mode stirring, Radiated power, Radia efficiency.

The authors analyze radiation from a microstrip transmission line and calculate total radiated power by numerical integration. Reverberation chamber methods for measuring radiated emissions and immunity are reviewed and applied to three microstrip configurations. Measurements from 200 to $2000 \mathrm{MHz}$ are compared with theory, and excellent agreement is obtained for two configurations that minimize feed cable and finite ground plane effects. Emissions measurements are found to be more accurate than immunity measurements because the impedance mismatch of the receiving antenna cancels when the ratio of the microstrip and reference radiated power measurements is taken.

\section{2,239}

PB96-164090 Not available NTIS

National Inst. of Standards and Technology (EEEL), Gaithersburg, MD. Electronics and Electrical Engineering Lab. Office.

President's Column 'Editorial'.

Final rept

A. H. Cookson. 1994, 1p.

Pub. in Institute of Electrical and Electronics Engineers Electrical Insulation Magazine, v10 n5 p3 Sep/Oct 94. Keywords: "Dielectrics, "Electrical insulation, "Semiconductors, Reprints, Electronics, Medicine, IEEE

As departing President of IEEE's Dielectrics and Electrical Insulation Sociely after iwo years, there are changes that have occured and highlights that have been noticed. The Society now has stronger conferences on a global scale with greater student representation. Membership size has grown and financially the Society is in sound condition. Work for the future will include broadening the scope of the Society to increase interest in the areas of semiconductors, electronic and medicine related to unique insulation and dielectrics challenges.

\section{2,240}

PB96-167143 Not available NTIS National Inst. of Standards and Technology (EEEL) Boulder, CO. Electromagnetic Fields Div. Accurate Electrical Characterization of High-Speed Interconnections.

Final rept

R. B. Marks, and D. F. Williams. 1994, 6p. See also PB94-216389. 
Pub. in Proceedings of International Symposium on Microelectronics, Boston, MA., November 14-17, 1994 p96-101.

Keywords: "Circuit interconnections, "Transmission lines, Characteristic impedance, Electrical measurement, Digital circuits, Permittivity, Capacitance, High speed, Accuracy, Reprints.

A program at the National Institute of Standards and technology supports the electrical characterization of electronic packaging and interconnections in terms of scattering parameters, impedances, and transmission line. This paper reviews the basic methodology, including its orgins in the characterization of monolithic microwave integrated circuits, and describes the resulting calibration and measurement methods that have been developed.

02,241

PB96-176482 Not available NTIS

National Inst. of Standards and Technology (EEEL), Boulder, CO. Electromagnetic Fields Div.

Dimensional Characterization of Precision Coaxial Transmission Line Standards.

Final rept.

G. V. Sherwood. 1993, 10p.

Pub. in Proceedings of the Measurement Science Conference, Annaheim, CA., January 21-22, 1993, Session 5-B p 1-10.

Keywords: "Coaxial cables, "Dimensional measurement, "Transmission lines, Waveguides, Standards, Calibration, Mechanical measurement, Pneumatic instruments, Surface roughness, Microwave equipment, Metrology, Reprints, Coordinate measuring machines, Air gauging, Gauging methods.

Precision, air-dielectric coaxial transmission line standards are commonly used with automatic network analyzers for impedance measurements. This report summarizes recent efforts at NIST to attain improved dimensional and geometric characterization of coaxial lines. The use of computer-sided methods that augment traditional air gaging, roundness testing, and coordinate measuring machine analysis will also be discussed.

\section{2,242}

PB96-176599 Not available NTIS

National Inst. of Standards and Technology (EEEL), Boulder, CO. Electromagnetic Fields Div.

Line-Reflect-Match Calibrations with Nonideal Microstrip Standards.

Final rept.

D. F. Williams, and J. B. Schappacher. 1995, 4p.

Pub. in Automatic RF Techniques Group Conference Digest (46th), Scottsdale, AZ., November 30-December 1, 1995, p35-38.

Keywords: "Microstrips, "Reference impedance, Reprints, "Line reflect match calibrations, "Scattering-parameter measurement, Thru-reflect-line calibration.

We apply a previously developed Line-Reflect-Match (LRM) calibration that compensates for the nonideal electrical behavior of the match standard to microstrip transmission lines and investigate impedance definitions, standard parasitics, and calibration accuracy.

02,243

PB96-180138 Not available NTIS

National Inst. of Standards and Technology (EEEL), Gaithersburg, MD. Electricity Div.

Surging the Upside-Down House: Measurements and Modeling Results.

Final rept.

F. D. Martzloff, A. Mansoor, K. O. Phipps, and W. M. Grady. 1996, 15p

See also PB96-112313.

Pub. in PQA '95, International Conference on Power Quality (4th): Applications and Perspectives, New York, NY., May 9-11, 1995, 17p Aug 95.

Keywords: "Electrical equipment, "Residential buildings, "Surges, "Overcurrent, "Electromagnetic compatibility, Electromagnetic equipment, Circuit protection, Voltage regulation, Surge suppressors, Communication equipment, Wiring, Voltage regulators, Cascaded elements, Reprints.

To demonstrate real-world scenarios, a replica of the wiring system in a typical residence was installed in the laboratory. This paper reports selected results from many measurements, and presents the corresponding numerical modeling, thereby leading to mutual valida- tion of the two processes. Two exposure scenarios for producing differences of voltages between the power and data ports of appliances are illustrated. Additional measurements and parametric variations are reported here to characterize the impedance of the various components of the wiring system and the source impendance of the resulting overvoltages appearing between the ports.

02,244

PB96-186119 Not available NTIS

National Inst. of Standards and Technology (EEEL) Gaithersburg, MD. Electricity Div.

Electrohydrodynamic Instability and Electrical Discharge Initiation in Hexane.

Final rept.

K. L. Stricklett, and R. A. C. Altafim. 1995, 4p Pub. in Annual Report, Conference on Electrical Insulation and Dielectric Phenomena, Virginia Beach, VA., October 22-25, 1995, p163-165.

Keywords: "Electrohydrodynamics, "Hexanes, Dielectric fluids, Surface instability, Reprints, "Laser triggered breakdown, Prebreakdown.

An experimental technique is described that tests the hydrodynamic stability of the fluid boundary in a fluid insulated system: a quasi-uniform field configuration is used and a pulsed, Nd:YAG laser is employed to create a micro-bubble at the surface of one electrode. The gap is pulse-charged and the laser is synchronized with the time-of-application of the voltage pulse. Under appropriate experimental conditions of voltage and laser pulse energy, the bubble evolves to produce full electrical breakdown by the onset and propagation of instabilities in the bubble surface. Experimental data obtained in hexane are presented.

\section{2,245}

\section{PB96-191382 PC A03/MF A01}

National Inst. of Standards and Technology (EEEL), Boulder, CO. Electromagnetic Fields Div.

Dielectric and Magnetic Measurements from $-50 \mathrm{C}$ to $200 \mathrm{C}$ and in the Frequency $\mathrm{B}$ and $50 \mathrm{MHz}$ to 2 $\mathrm{GHz}$.

J. Baker-Jarvis, and J. H. Grosvenor. Mar 96, 20p, NISTIR-5045.

Keywords: "Dielectric properties, "Magnetic properties, "Electrical measurement, "Transmission lines, Cavity resonators, Coaxial cables, Calibrating, Waveguides, High temperatures, Microwave measurements.

This is an overview of techniques for dielectric and magnetic measurements of low-loss through high-loss materials in the frequency range from $50 \mathrm{MHz}$ to $2 \mathrm{GHz}$ and over a temperature range of $-50 \mathrm{deg} C$ to $200 \mathrm{deg}$ C. The authors conclude that a single fixture is not adequate to satisfy the measurement objectives. The necessary measurements can be met using a combination of reentrant cavity, coaxial line, and dielectric resonato fixtures. In order to minimize heat loss, the coaxial line fixture should be milled from stainless steel stock and then gold plated. The reentrant cavity and split post resonator fixtures should be fitted with high temperature coaxial cables and temperature control obtained from an environmental furnace.

\section{2,246}

PB96-200118 Not available NTIS

National Inst. of Standards and Technology (EEEL), Boulder, CO. Electromagnetic Fields Div.

Coaxial Line-Reflect-Match Calibration.

Final rept.

J. A. Jargon, R. B. Marks, and D. F. Williams. 1995, $4 \mathrm{p}$.

Pub. in Asia-Pacific Microwave Conference Proceedings, Taejon, Korea, October 10-13, 1995, v1 p86-89.

Keywords: "Electrical measurements, "Coaxial cables, "Calibration standards, Transmission lines, Microwave equipment, Metrology, Reflection method, Test methods, Impedance matching, Reprints.

The authors describe a coaxial line-reflect-match calibration that corrects for imperfections in the load used as a match standard. The method provides a practical means of obtaining accurate, wideband calibrations with compact coaxial standard sets. When the load model is valid, the load may be characterized using an additional line of moderate length.

\section{2,247}

PB96-200324 Not available NTIS

National Inst. of Standards and Technology (EEEL) Boulder, CO. Electromagnetic Fields Div.
Alternative EMC Compliance Test Facilities.

Final rept.

M. L. Crawford. 1995, 30p.

Pub. in Handbook of Electromagnetic Compatibility, Chapter 18, p681-7101995.

Keywords: "Electromagnetic capability, "Test facilities Electrical measurement, Performance, Electromagnetic shielding. Transmission lines, Reprints, Mode stirred method.

Chapter 15 described conventional electromagnetic compatibility compliance test facilities which consist of open-field or shielded enclosures. Shielded enclosures have low residual noise levels and electrical isolation or shielding. Presumably, open-field sites provide both a reference and a perturbation-free environment in which actual operating conditions of the equipment can be simulated. However, open-field sites do not provide isolation from the environment and hence they can be used only under special circumstances.

\section{2,248}

PB96-204490 Not available NTIS

National Inst. of Standards and Technology (EEEL), Gaithersburg, MD. Electricity Div.

Correlations between Electrical and Acoustic Detection of Partial Discharge in Liquids and Implications for Continuous Data Recording.

Final rept.

P. von Glahn, K. L. Stricklett, R J. Van Brunt, and L. A. V. Cheim. 1996, 6p.

Pub. in Conference Record of the Institute of Electrical and Electronics Engineers International Symposium on Electrical Insulation, Montreal, Quebec, Canada, June 16-19, 1996, v1 p67-74.

Keywords: "Acoustic detection, "Continuous recording, "Electrical detection, Reprints, Partial discharge, Signal analysis, Statistical properties, Correlation analysis

Simultaneous measurements were made of electrical and acoustic signals from partial discharge (PD) produced by applying an alternative voltage at a frequency of $70 \mathrm{~Hz}$ to a point-plane electrode gap immersed in transformer oil. Both internally and externally mounted acoustic sensors were tested, and in all cases the intensity of the acoustic PD signal was found, on average, to increase with the amplitude of the electrical PD signal. The correlation between acoustic and electrical PD signal was found to be consistent with results reported from previous investigations. It is shown here that, because of this strong correlation, it is possible to extract statistical information from continuously recorded acoustic PD data, such as pulse phase (time) and amplitude distributions that are in agreement with those obtained from electrical data. It is demonstrated that by having continuous records of all PD events that occur during a test, it is possible to uncover new statistical information that is useful in attempts to understand the physical basis for the phenomenon.

\section{Resistive, Capacitive, \& Inductive Components}

\section{2,249}

AD-A284 623/6 PC A03/MF A01

National Bureau of Standards, Gaithersburg, MD.

Precision Resistors and Their Measurement.

J. L. Thomas. 8 Oct 48, 35p, NBS-470.

Keywords: "Wirewound resistors, Precision, Calibration, Circular, Construction, Electrical resistance, Limitations, Measurement, Resistance, Test and evaluation.

This circular contains information on the construction and characteristics of wire-wound resistors of the precision type. There are also included descriptions of the methods used at this Bureau for the test of precision resistors and the calibration of precision resistance measuring apparatus. Although the presentation is nontechnical, there is a considerable amount of information on the characteristics and limitations of apparatus of this type that should be of interest to any one making accurate measurements of electrical resistance. 


\title{
ELECTROTECHNOLOGY
}

\author{
Resistive, Capacitive, \& Inductive Components
}

National Inst. of Standards and Technology (EEEL), Boulder, CO. Electromagnetic Technology Div.

Electromechanical Properties of Superconductors for DOE Fusion Applications.

J. W. Ekin, S. L. Bray, C. L. Lutgen, and W. L. Bahn Jan 94, 116p, NISTIR-5013.

Keywords: "Thermonuclear reactor materials, "Superconducting magnets, "Superconductors,
*Niobium stannides, Superconducting wires, Stress analysis, Axial stress, Critical current, Fusion reactors, US DOE, Niobium aluminides, Vanadium gallides.

The electrical performance of many superconducting materials is strongly dependent on mechanical load. This report presents electromechanical data on a broad range of high-magnetic-field superconductors. The conductors that were studied fall into three general categories: candidate conductors, experimental conductors, and reference conductors. Research on candidate conductors for fusion applications provides screening data for superconductor selection as well as engineering data for magnet design and performance analysis. The effect of axial tensile strain on criticalcurrent density was measured for several Nb3Sn candidate conductors including the US-DPC (United States Demonstration Poloidal Coil) cable strand and an ITER (International Thermonuclear Experimental Reactor) candidate conductor. Also, data are presented on promising experimental superconductors that have strong potential for fusion applications. Axial strain measurements were made on a V3Ga tape conductor that has good performance at magnetic fields up to $20 \mathrm{~T}$. Axial strain data is also presented for three experimental Nb3Sn conductors that contain dispersion hardened copper reinforcement for increased tensile strength. Finally, electromechanical characteristics were measured for three different $\mathrm{Nb} 3 \mathrm{Sn}$ reference conductors from the first and second VAMAS (Versailles Project on Advanced Materials and Standards) international Nb3Sn critical-current round robins. Published papers containing key results, including the first measurement of the transverse stress effect in Nb3Sn, the effect of stress concentration at cable-strand crossovers, and electromechanical characteristics of $\mathrm{Nb} 3 \mathrm{Al}$, are included throughout the report.

02,251
PB94-151776 PC A11/MF A03

National Inst. of Standards and Technology (EEEL), Boulder, CO. Electromagnetic Fields Div.

NIST 60-Millimeter Diameter Cylindrical Cavity Resonator: Performance Evaluation for Permittivity Measurements.

Technical note.

E. J. Vanzura, R. G. Geyer, and M. D. Janezic. Aug

93, 241p, NIST/TN-1354

Also available from Supt of Docs as SN003-003. 03226-3.

Keywords: "Cavity resonators, Cylindrical configuration, Dielectric materials, Performance evaluation, Computer programs, $X$ band, Uncertainty, Superhigh frequency, "Permittivity measurements, Dielectric measurements, MEAS-RES computer program, CAVITYPROG computer program.

Uncertainty estimates are developed for dielectric permittivity calculations made using the NIST $60-\mathrm{mm}$ diameter cylindrical resonator. A mode-filtering helical waveguide makes up the cavity's cylindrical wall, which permits the generation of high-purity $T E(01 p)$ resonant modes for high accuracy permittivity measurements. The cavity's length can be varied from 408 to $433 \mathrm{~mm}$. Fixed-length and fixed-frequency measurements in the $X$-band frequency range are evaluated with particular emphasis on $10 \mathrm{GHz}$. Resonator theory and design, measurement tolerances, and software are included.

\section{2,252}

\section{PB94-185501 Not available NTIS}

National Inst. of Standards and Technology (CSTL),

Gaithersburg, MD. Process Measurements Div.
High Temperature Silicide Thin-Film Thermocouples.

Final rept

K. G. Kreider. 1994, 6p.

Pub. in Materials Research Society Symposia Proceedings, v322 p285-290 1994 .

Keywords: *Thermocouples, Temperature measuring instruments, High temperature, Seebeck effect, Thin films, Molybdenum silicides, Rhenium silicides, TantaIum silicides, Titanium silicides, Tungsten silicides, Sputtering, Reprints.
High-temperature silicides have been used in mechanical structures, heating elements, and electronic, CMOS applications. Their stability in high temperature oxidizing environments and excellent electrical conductivity may also make them useful as high temperature thin-film sensors in harsh environments. We have investigated sputter deposited MoSi2, ReSi2, TaSi2 TiSi2, and WSi2 thin films and characterized their performance as thermoelements and stability up to 1200 C. A multilayer technique was developed to ensure constant silicide stoichiometry during oxidation thereby maintaining a constant Seebeck coefficient. In addition, techniques were developed to suppress the formation of metal oxides from the silicides. The results indicated excellent stability of Seebeck coefficient up to $1200 \mathrm{C}$. These results are compared with the problems of thin film instability in the Seebeck coefficient found in noble metal thermocouples. Potential applications for temperature and heat transfer measurements will be discussed.

\subsection{3}

PB94-185519 Not available NTIS

National Inst. of Standards and Technology (CSTL), Gaithersburg, MD. Process Measurements Div

Thin Film Transparent Thermocouples.

Final rept.

K. G. Kreider. 1992, 5p.

Pub. in Sensors and Actuators A 34, p95-99 1992.

Keywords: "Thermocouples, Temperature measuring instruments, Antimony oxides, Indium oxides, Seebeck Antimony tin oxides, Indium tin oxides.

Transparent thin-film electrical conductors have been sputter deposited to form transparent thermocouples. Indium-tin oxide (ITO) and antimony-tin oxide (ATO) have demonstrated Seebeck coefficients of 7-77 micro V/C, depending on their conductivity and fabrication parameters. In addition to results for in-house-fabricated thermocouples, thermoelectric measurements are reported on commercial ATO, ITO, and fluorinedoped tin oxide transparent films.

\section{2,254}

PB94-200060 Not available NTIS

National Inst. of Standards and Technology (EEEL) Gaithersburg, MD. Electricity Div.

NIST Capacitance Measurement Assurance Program (MAP).

Final rept.

Y. M. Chang. 1993, 10p

Pub. in Proceedings of Measurement Science Conference, Anaheim, CA., January 21-22, 1993, p1-10.

Keywords: "Capacitance, Calibration, Reprints, Measurement Assurance Program, MAP program, Reference standards, US NIST.

This paper describes the recently developed capacitance Measurement Assurance Program (MAP) service at the National Institute of Standards and Technology (NIST). Using a commercial digital capacitance meter as the transport standard two separate pilot programs for the capacitance MAP have been carried out for standards at both the $1,000 \mathrm{pF}$ and $100 \mathrm{pF}$ levels. The first was carried out as a single transfer with a gov ernment standards laboratory, and the second was de signed for round-robin measurements as a multiple transfer by three industrial standards laboratories. In contrast to the normal MAP, where the transport stand ards are measured by the client laboratory, the capaciance MAP involves measurements performed on 'dummy' standards by both the meter (transport standard) and the laboratory capacitance measuring sys tems. Results from these two pilot programs are pre sented. Also included are requirements and procedures for laboratories interested in participating in the capacitance MAP service.

\section{2,255}

PB94-211521 Not available NTIS

National Inst. of Standards and Technology (NEL), Boulder, CO. Electromagnetic Technology Div. Roles of Copper in Applied Superconductivity. Final rept.

F. R. Fickett. 1990, 8p.

Pub. in Proceedings of Conference on Copper '90: Refining, Fabrication, Markets, Vasteras Congress Centre, Sweden, October 1-3, 1990, p310-317

Keywords:

$$
\text { "Superconducting }
$$

devices, *Superconductivity, "Copper alloys, "Copper, High
temperature superconductors, Superconducting magnets, Historical aspects, Cryogenics, Cuprates, Uses, Reprints.

Copper plays many roles in applied superconductivity, but its contribution is seldom acknowledged. In this paper, we review the uses of copper in each of many categories, both historically and in the latest applications. A brief description of the desired properties of the copper used for each application will be given, along with some suggestions as to how to achieve these properties in commercial copper products.

\section{2,256}

PB95-151288 Not available NTIS

National Inst. of Standards and Technology (EEEL), Gaithersburg, MD. Electricity Div.

Electron Scattering and Dissociative Attachment by SF6 and Its Electrical-Discharge By-Products.

Final rept.

H. X. Wan, J. H. Moore, J. K. Olthoff, and R. J. Van

Brunt. 1993, 16p

Grant NSF-CHE91-20504

Sponsored by National Science Foundation, Washing-

ton, DC and Department of Energy, Washington, DC Pub. in Plasma Chemistry and Plasma Processing 13, n1 p1-16 1993

Keywords: "Electron-molecule collisions, "Electron scattering, "Sulfur fluorides, "Electric discharges, Scattering cross sections, Chemical dissociation, Electron attachment, Sulfur hexafluoride, Sulfur dioxide, Milli eV range, EV range 1-10, EV range 10-100, Plasma chemistry, Plasma etching, Negative ions, Reprints.

Discrete electron-molecule processes relevant to SF6 etching plasmas are examined. Absolute, total scattering cross sections for $0.2-12$-eV electrons on SF6 SO2, SOF2, SO2F2, SOF4, and SF 4 , as well as cross sections for negative-ion formation by attachment of electrons, have been measured. These are used to calculate dissociative-attachment rate coefficients as a function of E/N for SF6 by-products in SF6.

\section{2,257}

PB95-153516 Not available NTIS

National Inst. of Standards and Technology (EEEL), Gaithersburg, MD. Electricity Div.

Pressure Dependencies of Standard Resistors.

Final rept.

R. F. Dziuba. 1993, 7p.

Pub. in Proceedings of National Computer Systems Laboratory Workshop and Symposium, Albuquerque, NM., July 25-29, 1993, p363-369.

Keywords: "Resistors, "Pressure, "Electrical resistance, Electrical measurement, Standards, Reprints.

The U.S. representation of the ohm is based on the quantized Hall resistance of $6453.20175 \mathrm{ohms}$ with a combined standard uncertainty of 0.02 ppm. To maintain the ohm at this resistance or other resistances at this uncertainty level requires well characterized standard resistors. A sometimes overlooked parameter affecting standard resistors is pressure. A variety of standard resistors are examined for pressure dependencies over the range 250 hectopascals (hPa) above and below a standard atmosphere. Results indicate that the pressure coefficients of resistance for some standards are significant.

\section{2,258}

PB95-153557 Not available NTIS

National Inst. of Standards and Technology (EEEL), Boulder, CO. Electromagnetic Technology Div.

Preparation of Low Resistivity Contacts for HighTc Superconductors.

Final rept.

J. W. Ekin. 1993, 37p

Pub. in Processing and Properties of High-Tc Superconductors, Chapter 9, v1 p371-407 1993

Keywords: *High temperature superconductors, "HighTC superconductors, "Electric contacts, YBCO superconductors, Soldering, Joining, Reprints, Yttrium barium cuprates.

Methods for preparing practical contacts to high-T(c) superconductors are described, including quick, easy techniques for attachment of voltage-taps, and detailed descriptions of how to fabricate high-quality contacts for current connections. Methods for soldering and lead attachment that preserve the low-resistivity of high-quality contacts are also presented. 
National Inst. of Standards and Technology (CSTL), Gaithersburg, MD. Process Measurements Div. Sputtered High Temperature Thin Film Thermocouples.

Final rept.

K. G. Kreider. $1993,5 p$

Pub. in Jnl. of Vacuum Science and Technology A 11, n4 p1401-1405 Jul/Aug 93

Keywords: "Thermocouples, Temperature measuring instruments, Temperature range 0400-1000 K, Rhodium containing alloys, Rhodium oxides, Platinum alloys, Thin films, Seebeck effect, Aluminum oxides, Annealing, Reprints.

Thin film thermocouples have the advantage of extremely fast response $(<1 \mathrm{~ms})$, low cost, and very fine spatial resolution. These capabilities have permitted measurements never before possible, such as the instantaneous temperature measurements of the inside surfaces of a diesel engine cylinder during operation. Although these type $S$ platinum plus platinum $10 \%$ rhodium thermocouples are stable at the 900-1100 C range, oxidation of rhodium can take place in the 600$800 \mathrm{C}$ range in air which lessens the thermoelectric potential of the positive element. We evaluated the effect of air exposures to high temperature thin film thermocouples and attempted to find solutions to the problem. We found that after $50 \mathrm{~h}$ at $750 \mathrm{C}$ the Seebeck coefficient of a platinum $10 \%$ rhodium element was reduced $50 \%$ compared to the as-sputtered condition. The reduction of Seeback coefficient at 700 and $800 \mathrm{C}$ was also substantial. This reduction in Seeback coefficient could be recovered by annealing the thin film at $900 \mathrm{C}$. The degradation after $750 \mathrm{C}$ could be suppressed using a 1 micrometer sputtered aluminum oxide coating. The reversible behavior suggests the formation of Rh2O3 at $700-800 \mathrm{C}$ and its subsequent reduction at temperatures at which the free energy of formation is zero or positive. Microstructural evidence confirm these conclusions.

\section{2,260}

PB95-161386 Not available NTIS

National Inst. of Standards and Technology (EEEL), Gaithersburg, MD. Electricity Div.

Lithium-Drift Technique for Making Submicron Thick Silicon Membranes.

Final rept

K. C. Lee. $1992,8 p$

Pub. in Proceedings of Symposium on Electrochemical Microfabrication, Phoenix, AZ., October 16, 1992, p18.

Keywords: "Electrochemistry, Pressure sensors, Cantilever beams, Room temperature, Wafers, Reprints, "Silicon membranes, Anodic etching, Lithium diffusion, Microfabrication.

An electrochemical method for preparing silicon membranes that relies on the use of room temperature lithium diffusion to create a compensated, high resistivity layer with very low etch rate near one surface of a $p$ type Si wafer is described. The bulk wafer can be dissolved away, leaving behind a membrane that can be between several tenths of a micrometer and several micrometers in thickness. The various parameters that affect the thickness of the membrane are described. As an example of this technique, the preparation of a $670 \mathrm{~nm}$ thick silicon membrane is described.

02,261

PB95-168852 Not available NTIS

National Inst. of Standards and Technology (PL), Boulder, CO. Time and Frequency Div.

Ultralinear Small-Angle Phase Modulator.

Final rept.

J. Lowe, and F. L. Walls. 1991, 4p.

Pub. in Proceedings of European Frequency and Time Forum, Besancon, France, March 12-14, 1991, p461464

Keywords: "Quartz resonators, Phase modulation, Frequency response, Electric fields, Direct current, Linear systems, Reprints, "Phase modulators, Polarizing effect.

A dc electric field applied to a quartz plate resonator causes changes in the elastic constants which can lead to a change in the frequency of the resonator. This effect, known as the polarizing effect, has been shown to be extremely linear. We have used this effect to build a phase modulator with 2 nd-harmonic distortion that is at least $117 \mathrm{~dB}$ below the fundamental modulation and low added phase noise A description of the modulato as well as methods of measurement are discussed.
02,262

PB95-169009 Not available NTIS

National Inst. of Standards and Technology (PL). Boulder, CO. Time and Frequency Div.

Relationship of AM to PM Noise in Selected RF Oscillators

Final rept.

L. M. Nelson, C. W. Nelson, and F. L. Walls. 1994,

Pub. in Institute of Electrical and Electronics Engineers Transactions on Uitrasonics. Ferroelectrics, and Frequency Control 41, n5 p680-684 Sep 94

Keywords: "Radiofrequency oscillators, *Quartz resonators, *Electromagnetic noise, Amplitude modulation Phase modulation, Cross correlation, Very high frequency, High frequency, Reprints, Flicker noise, Phase noise.

We have studied the amplitude modulation (AM) and phase modulation (PM) noise in a number of $5 \mathrm{MHz}$ and $100 \mathrm{MHz}$ oscillators to provide a basis for developing models of the origin of AM noise. To adequately characterize the AM noise in high performance quartz oscillators, we found it necessary to use two-channe cross-correlation AM detection. In the quartz oscillators studied, the power spectral density (PSD) of the f(sup 1) and $f($ sup 0$)$ regions of $A M$ noise is closely related to that of the PM noise. The major difference between different oscillators of the same design depends on the flicker noise performance of the resonator. We therefore propose that the $f($ sup -1$)$ and $f($ sup 0$)$ regions of $\mathrm{AM}$ and PM noise arise from the same physical processes, probably originating in the sustaining amplifier.

\section{2,263}

PB95-171419 PC A03/MF A01

National Inst. of Standards and Te

Gaithersburg, MD. Electricity Div.

Design Challenges in a Commerci Effect-Based Resistance Standard.

K. C. Lee, and M. E. Cage. Nov 94, 42p, NISTIR5533.

Keywords: "Electrical resistance, "Primary standards Standards, Temperature range 0000-0013 K, Cryogenic temperature, Cryogenic cooling. Temperature dependence, Electrical measurement, Commercial equipment, Wire wound resistors, Calibration. Closedcycle cooling systems, Design criteria, "Resistance standards, "Quantum Hall effect.

Of the electrical measurements that underpin the broad spectrum of modern industry, measurements of voltage, resistance, and impedance are among the most important. National Standards laboratories have developed intrinsic standards based on quantum ef fects, such as Josephson's Effect and the quantum Hall effect that can be used to realize standards of voltage and resistance. The equipment necessary to observe these phenomena is very complex and expensive, and these standards have not yet found wide application in industrial laboratories. This paper assesses the possibility of developing a commercially viable quantum Hall effect-based resistance standards system. The capabilities of resistance calibration systems currently in use are summarized, and specifications for competitive quantum Hall effect-based system are described. The realization of such a system presents numerous challenges, from making suitable samples, to developing high accuracy, automated measurement systems, to developing low cost, reliable cryogenic systems. These technical challenges are discussed in detail, and several possible commercial systems are described

\section{2,264}

PB95-175527 Not available NTIS

National Inst. of Standards and Technology (EEEL) Boulder, CO. Electromagnetic Technology Div.

Characterization of a Tunable Thin Film Microwave YBa2Cu3O7-x/SrTiO3 Coplanar Capacitor.

Final rept.

D. Galt, J. C. Price, J. A. Beall, and R. H. Ono. 1993, Pub. in Applied Physics Letters 63, n22 p3078-3080 Nov 93

Keywords: "Superconducting devices, "Resonators High temperature superconductors, YBCO superconductors, Strontium titanates, Microwave equipment, Microstrip devices, Variable capacitors, Thin films, Coplanarity, Tuning, Reprints, Yttrium barium cuprates, Ferroelectric capacitors

We have fabricated and characterized electrically tunable high temperature superconductor coplanar microstrip resonators incorporating tunable $\mathrm{SrTiO} 3$ ferroelectric thin films. The low frequency capacitance of the $\mathrm{SrTiO}_{3}$ capacitor is measured directly. High frequency capacitance and loss information are extracted low frequency data. Hysteresis loops display an onset low frequency data. Hysteresis loops display an onset and coercive voltage (at $10 \mathrm{kHz}$ ) as a function of temperature are extracted from these loops.

02,265

PB95-180451 Not available NTIS

National Inst. of Standards and Technology (EEEL)

Boulder, CO. Electromagnetic Technology Div.

Improved Eddy-Current Decay Method for Resistivity Characterization.

Final rept

A. B. Kos, and F. R. Fickett. 1994, 3p

Sponsored by International Copper Research Association, Inc New York

Pub. in Institute of Ëlectrical and Electronics Engineers Transactions on Magnetics 30, n6 p4560-4562 Nov 94 Keywords: "Electrical resistivity, "Electrical measure ment, "Eddy currents, Low temperature, Curve fitting Metals, Copper, Reprints

Eddy-current decay is a unique, nondestructive method for determining the low-temperature resistivity of large samples of pure metal.. Furthermore, it is the only means available for measurement of the residual resistivity ratio (RRR), rho( $273 \mathrm{~K}) / \mathrm{rho}(4 \mathrm{~K})$, of samples with shapes that do not lend themselves to conventional four-wire resistance measurement techniques. An im provement to an earlier implementation of the eddy current decay method of resistivity characterization is presented. It involves modernizing the earlier appara tus by the use of a digitizing oscilloscope, commercial curve-fitting software, digital averaging techniques and modern electronics. Data are shown for high-purity copper cylinders.

02,266

PB95-180477 Not available NTIS

National Inst of Standards and Technology (EEEL)

Boulder CO. Electromagnetic Fields Div.

Dielectric Properties of Single Crystals of $\mathrm{Al} 2 \mathrm{O} 3$ LaAlO3, SrTiO3, and MgO at Cryogenic Temperatures.

Final rept

Krupka, $\mathrm{R}$

1994, 190p

Pub. in Institute of Electrical and Electronics Engineers Transactions on Microwave Theory and Techniques 42, n10 p $1886-1890$ Oct 94

Keywords: "Aluminum oxide, "Strontium titanates, "Magnesium oxides, "Dielectric properties, Temperature range 0013-0065 $\mathrm{K}$, Temperature range 0065 $0273 \mathrm{~K}$, Temperature range 0273-0400 K Superconducting films, Microwave frequencies, Permittivity, Substrates, Reprints, "Lanthanum alu minates, "Neodymium gailates.

A dielectric resonator technique has been used for measurements of the permittivity and dielectric loss tangent of single-crystal dielectric substrates in the temperature range 20-300 $\mathrm{K}$ at microwave frequencies. Application of superconducting films made it possible to determine dielectric loss tangents of about $5 \times 10(\exp -7)$ at $20 \mathrm{~K}$. Two permittivity tensor components for uniaxially anisotropic samples were measured. Generally, single-crystal samples made of the same material by different manufacturers or by different processes have significantly different losses, al though they have essentially the same permittivities The permittivity of one crystalline ferroelectric substrate, $\mathrm{SrTiO} 3$, strongly depends on temperature. This temperature dependence can affect the performance of ferroelectric thin-film microwave devices, such as electronically tunable phase shifters, mixers, delay lines and filters.

02,267

PB95-181160 Not available NTIS

National Inst. of Standards and Technology (EEEL)

Boulder, CO. Electromagnetic Technology Div.

Dielectric Properties of Thin Film SrTiO3 Grown on LaAlO3 with YBa2Cu307-x Electodes.

Final rept.

H. Wu, F. Barnes, D. Galt, J. Price, and J. Beall.

1994, 10p.

Pub. in Proceedings of Society of Photo-Optical Instrumentation Engineers: High Tc Microwave Superconductors and Applications, Los Angeles, CA January 25-27, 1994, v2156 p131-140 


\section{ELECTROTECHNOLOGY}

\section{Resistive, Capacitive, \& Inductive Components}

Keywords: "Strontium titanates, "Dielectric properties, High-Tc superconductors, Temperature dependence, Microwave frequencies, Microstrip devices, YBCO superconductors, AC losses, Thin films, Capacitance, Capacitors, Substrates, Reprints, Yttrium barium cuprates, Lanthanum aluminates, Microstrip resonators.

We have fabricated and characterized YBCO (YBa2Cu3O(7-x)) microstrip resonators on $\mathrm{LAO}(\mathrm{LaAlO} 3)$ substrates that include thin film STO(SrTiO3) coplanar capacitors to study the dielectric properties of thin film STO. The low frequency capacitance of the STO/LAO capacitor is measured as a function of temperature and dc bias. We use the observed resonant frequencies to extract the microwave frequency capacitance of the structure and the Q's to determine the microwave losses. A conformal map is developed and used to transform the observed capacitances into dielectric constant values for the thin film STO.

\section{2,268}

PB95-202461 Not available NTIS

National Inst. of Standards and Technology (EEEL), Boulder, CO. Electromagnetic Technology Div.

Domain Effects in Faraday Effect Sensors Based on Iron Garnets.

Final rept.

M. N. Deeter, 1995, 4p.

See also PB92-197482.

Pub. in Applied Optics 34, n4 p655-658, 1 Feb 95.

Keywords: "Magnetic measurement, "Ferrite garnets, Yttrium iron garnets, Magnetic anisotropy, Magnetic domains, Magnetic films, Faraday effect, Thick films, Comparative evaluations, Magnetooptics, Diffraction, Reprints, *Magnetic field sensors.

Domain-induced diffraction effects produced by two iron garnet thick films and two bulk crystals are compared. The thick films, characterized by a serpentine magnetic domain structure, produced nonlinear response functions; this is in qualitative agreement with a one-dimensional diffraction model. Bulk iron garne crystals, which exhibited a complex three-dimensional domain structure, produced qualitatively similar effects that diminished with increasing crystal length. Differential signal processing resulted in a linear signal for the thick films and a primarily sinusoidal response for the bulk crystals.

\section{2,269}

PB95-251625 PC A03/MF A01

National Inst. of Standards and Technology (EEEL), Gaithersburg, MD. Electricity Div.

SF6 Insulation: Possible Greenhouse Problems and Solutions.

L. G. Christophorou, and R. J. Van Brunt. Jul 95,

28p, NISTIR-5685.

Keywords: "Sulfur hexafluoride, "Electrical insulation, Dielectric materials, Gas-insulated substations, Gas insulated cables, Gas-insulated transformers, Physica properties, Chemical properties, Dielectric properties, Recycling, Alternatives, Environmental chemical substitutes, Nitrogen, Mixtures, Greenhouse gases, Ozone depletion, Air pollution abatement, "Insulating gas.

Sulfur hexafluoride (SF6) is the most commonly used insulating gas in electrical systems to date. It is however, a potent greenhouse gas and, thus, its use entails fears of environmental costs, regulations, and restrictions, in spite of its current low levels in the environment. This potential problem and current efforts in the search for short-term and long-term solutions are briefly outlines and discussed in this report. Limiting the release of SF6 in the environment, recycling SF6, and using it where it is needed the most, are among the elements of an emerging consensus effort for the short run. A long-term solution may include the search for alternative high-voltage insulants such as highpressure gaseous dielectrics (e.g., N2 and N2/SF6 mixtures).

\section{2,270}

PB96-102389 Not available NTIS

National Inst. of Standards and Technology (EEEL)

Boulder, CO. Electromagnetic Technology Div.
Ferroelectric Thin Film Characterization Using Superconducting Microstrip Resonators.

Final rept.

D. Galt, J. C. Price, J. A. Beall, and T. E. Harvey $1995,4 p$

Pub. in Institute of Electrical and Electronics Engineers Transactions on Applied Superconductivity, v5 n2 p2575-2578 Jun 95.

Keywords: "Ferroelectricity, "Thin films, "Resonators, "Microwave frequencies, Superconductors, Dielectric properties, Capacitors, Bias, Solid state devices, Reprints, YBCO film, Microstrip devices.

The authors describe a novel technique for characterizing the dielectric response of ferroelectric thin films at microwave frequencies. The method involves a microstrip resonator which incorporates a ferroelectric capacitor at its center. To demonstrate this method the authors have fabricated a superconducting microstrip resonator from a laser ablated Yba2Cu3O(7-delta) (YBCO) film on a LaAIO3(LAO) subsrate with a SrTiO3(STO) capacitor at its center. The authors report the observed dielectric behavior of the STO laser ablated film as a function of bias at liquid $\mathrm{He}$ and N2 temperatures and at high and low frequencies. It is observed that the electrically tunable dielectric constant of the STO film is roughly independent of frequency up to $20 \mathrm{GHz}$ (especially at high bias). The loss tangent of the STO/LAO capacitor decreases with increasing bias and is apparently independent of frequency between 6 and $20 \mathrm{GHz}$

02,271

PB96-103148 Not available NTIS

National Inst. of Standards and Technology (PL), Boulder, CO. Time and Frequency Div.

Environmental Sensitivities of Quartz Oscillators. Final rept.

F. L. Walls, and J. J. Gagnepain. 1991, 9p.

See also N91-25797.

Pub. in Institute of Electrical and Electronics Engineers Transactions on Ultrasonics, Ferroelectrics, and Frequency Control, v39 n2 p241-249 Mar 92.

Keywords: "Oscillators, "Quartz, "Environmental effects, "Sensitivity, Frequency analysis, Amplitudes, Mechanical vibrations, Noise, Magnetic fields, Electric fields, Irradiation, Temperature, Humidity, Pressure, Acceleration, Reprints.

The frequency, amplitude, and noise of the output signal of a quartz oscillator are affected by a large number of environmental effects. The paper examines the physical bases for the sensitivity of precision oscillators to temperature, humidity, pressure, acceleration and vibration, magnetic field, electric field, load, and radjation. The sensitivity of quartz oscillators to radiaton is a very complex topic and poorly understood. Therefore only a few general results are mentioned. The senstivity to most external influences often varies significantly both from one oscillator type to another and from one unit of a given type to another. For a given unit, the sensitivity to one parameter often depends on the value of other parameters and on history.

\section{2,272}

PB96-103155 Not available NTIS

National Inst. of Standards and Technology (EEEL), Gaithersburg, MD. Electricity Div.

Digital Impedance Bridge.

Final rept.

B. C. Waltrip, and N. M. Oldham. 1995, 4p.

See also PB91-101196.

Pub. in Institute of Electrical and Electronics Engineers Transactions on Instrumentation and Measurement, v44 n2 p436-439 Apr 95.

Keywords: "Impedence measurement, "Digital systems, "Electric bridges, Electrical impedance, Circuits, Standards, Comparative evaluations, Inductance, Resistors, Reprints.

An impedance bridge that compares two-terminal standard inductors to characterized ac resistors in the frequency range of $10 \mathrm{~Hz}$ to $100 \mathrm{kHz}$ is described. A dual-channel, digitally-synthesized source and sampling digital multimeter are used to generate and measure relevant bridge signals. A linear interpolation algorithm is used to autocalibrate the bridge to a $1 \mathrm{nF}$ gas dielectric capacitor. An intercomparison of the new bridge with existing measurement standards conducted in the low audio frequency range shows agreement of 50 to 200 parts in 10 (exp 6) for inductors for $1 \mathrm{mH}$ to $10 \mathrm{H}$
02,273

PB96-111869 Not available NTIS

National Inst. of Standards and Technology (EEEL), Boulder, CO. Electromagnetic Fields Div.

Measurements of Permittivity and the Dielectric Loss Tangent of Low Loss Dielectric Materials with a Dielectrlc Resonator Operating on the Higher Order Te(sub 0 gamma delta) Modes.

Final rept.

J. Krupka, R. G. Geyer, and D. Cros. 1994, 6p.

Pub. in International Microwave Conference (10th), MIKON - 94, Ksiaz Castle, Poland, May 30-June 2, 1994, v2 p567-572.

Keywords: "Resonators, "Dielectric resonators, Reprints, Microwaves, Permittivity, Metrology, Modes, Foreign technology.

Dielectric resonator techniques are commonly used for permittivity and the dielectric loss tangent measurements of low-loss materials. Usually, the TE mode (12) or TE mode (3-5) is used. In this case, if dielectric characterization is required at various frequencies, more than one sample of the material under test must be provided. These samples must have differing physical dimensions. In order to perform dielectric measurements on a single sample at several contiguous frequency subbands, higher-order TE modes may be utilized. These higher-order modes are tuned by changing the distance between the conductor ground planes of the dielectric resonator. Identification of higher-order TE resonant modes in several cylindrical samples of the material under test allows properties to be determined over a broad frequency spectrum.

02,274

PB96-119326 Not available NTIS

National Inst. of Standards and Technology (EEEL) Gaithersburg, MD. Electricity Div.

Automated Resistance Measurements at NIST.

Final rept

R. F. Dziuba. 1995, 7p

Pub. in National Conference of Standards Laboratories (NCSL) Workshop and Symposium, Dallas, TX., July 16-20, 1995, p189-195.

Keywords: "Resistors, "Automation, "Calibration, "US NBS, Measurements, Quality assurance, Standards Reprints, Standard resistors, NIST(National Institute of Standards and Technology).

The National Institute of Standards and Technology (NIST) provides a calibration service for dc standard resistors over 17 decades of resistance from 10(exp -4) Ohms to 10(exp 12) Ohms using seven independ ent measurement systems. Four measurement systems a re completely automated for calibrating resistors from 1 Ohms to $1 \mathrm{M}$ Ohms. A fifth system for high resistance measurements is semiautomated. Plans are underway to fully automate this system, along with the remaining two measurements is semiautomated. Plans are underway to fully automate this system, along with the remaining two measurement systems. The primary consideration to automate a measurement system is to improve the quality of measurements, and not simply to relieve the operator from tedious repetitive meas urements. This paper describes the extent and future plans of resistance measurement automation at NIST.

\section{2,275}

PB96-122494 Not available NTIS

National Inst. of Standards and Technology (EEEL) Gaithersburg, MD. Electricity Div.

Constant Temperature and Humidity Chamber for Standard Resistors.

Final rept.

D. G. Jarrett. $1995,5 p$

Pub. in National Conference of Standards Labora tories, Dallas, TX., July 16-20, 1995, p501-505.

Keywords: "Test chambers, "Laboratory equipment, Resistors, Environmental control, Calibration, Relative humidity, Air temperature, Heating systems, Air conditioning, Insulation, Reprints.

An environmental chamber has been developed for housing standard resistors under controlled temperature and humidity conditions during calibration in air. The design allows sufficient space for standard resistors to be housed in a volume of 0.25 cubic meters with sixteen pairs of coaxial connectors feeding through the chamber wall to the lab environment. The box is of double-walled construction with the inner box electrically and thermally insulated from the outer box Improved insulation and control circuitry along with 
added volume are some of the improvements this new air chamber has over other chambers previously used for calibrating standard resistors at NIST.

\subsection{6}

PB96-172333 PC A03/MF A01

National Inst. of Standards and Technology (EEEL), Gaithersburg, MD. Electricity Div

NIST Measurement Assurance Program for Capacitance Standards at $1 \mathrm{kHz}$.

Technical note.

Y. M. Chang. Mar 96, 27p, NIST/TN-1417

Also available from Supt. of Docs. as SN003-00303393-6. See also PB94-200060

Keywords: "Capacitance, "Calibration, Measuring instruments, "Measurement Assurance Program, MAP program, Reference Standards, US NIST.

The document describes the capacitance Measurement Assurance Program (MAP) service at the Naional Institute of Standards and Technology (NIST) This service, which uses a commercial digital capacitance meter as the transport standard, provides calibration for capacitance standards at both the $1000 \mathrm{pF}$ and $100 \mathrm{pF}$ levels, at a frequency of $1 \mathrm{kHz}$. In contras to the normal MAP, where the transport standards are measured by the client laboratory, the capacitance MAP involves measurements performed on 'dummy' standards by both the Meter (transport standard) and the laboratory capacitance measuring system. Measurement procedures and requirements for client laboratories are included. Also presented are error analy sis, assigned values, and equations to estimate the combined uncertainties of the assigned values.

\section{2,277 \\ PB96-176565 Not available NTIS}

National Inst. of Standards and Technology (PL), Boulder, CO. Time and Frequency Div

Fundamental Limits on the Frequency Stabilities of Crystal Oscillators.

Final rept.

F. L. Walls, and J. R. Vig. 1995, 14p.

Pub. in Institute of Electrical and Electronics Engineers Transactions on Ultrasonics, Ferroelectrics, and Frequency Control, v42 n4 p576-589, 13 Jul 95

Keywords: "Frequency stability, "Crystal oscillators, Environmental effects, Ouartz oscillators, Ouartz reso nators, Reprints, BAW resonators, Flicker FM.

The frequency instabilities of precision bulk acoustic wave (BAW) quartz crystal oscillators are reviewed. The fundamental limits on the achievable frequency stabilities, and the degree to which the fundamental limits have been approached to data are examined. Included are the instabilities as a function of time, temperature, acceleration, ionizing radiation, electromagnetic fields, humidity, atmospheric pressure, power supply, and load impedance. Most of the fundamental limits are zero or negligibly small, a few are finite. We speculate about progress which may be achievable in the future with respect to approaching the fundamental limits. Suggestions are provided about the paths that may lead to significant stability improvements.

\section{2,278}

PB96-180179 Not available NTIS

National Inst. of Standards and Technology (MEL), Gaithersburg, MD. Automated Production Technology Div.

Complicated Cases and Shielded Rooms: Audiometric Booths Shielded to Attenuate Electromagnetic Interference.

Final rept.

V. Nedzelnitsky. 1996, 2p

Pub. in Jnl. of the Acoustical Society of America, v97 n5 pt2 p3321-3322 May 95

Keywords: "Electromagnetic

shielding,

Electroacoustics, *Test chambers, Electromagnetic

interference, Radiofrequency, Hearing aids, Electromagnetic compatibility, Standards, Reprints, Audiometric equipment.

Electromagnetic compatibility (EMC) issues involving acoustical instruments, especially their susceptibility and immunity to electromagnetic interference (EMI), are increasing in importance. Of particular significance is the impact on commercial practice and international trade of two Councils of the European Communities directives regarding generic apparatus and medical devices. Directive $93 / 42 / E E D$ is being interpreted to include hearing aids and audiometric instruments. In the
U.S.A., at least, existing standards for measuring such shielded-room performance were not specifically de veloped for audiometric booths. Methods in some of these standards have been adapted and applied to that purpose Selected standards, methods, and some considerations regarding their application to measuring the shielding performance of audiometric booths in hospital/clinical environments are discussed.

\section{2,279}

PB96-186036 Not available NTIS

National Inst. of Standards and Technology (MSEL),

Boulder, CO. Materials Reliability Div.

Determination of Sheet Steel Formability Using Wide Band Electromagnetic-Acoustic Trans. ducers.

Final rept

A. V. Clark, C. M. Fortunko, M. G. Lozev, S. R

Schaps, and M. C. Renken. 1992, 18p.

Pub. in Research in Nondestructive Evaluation, v4 n3 p165-182 1992

Keywords: "Formability, "Acoustics, "Sheet metal, Resonance, Reprints, "Electromagnetic acoustic trans ducers, R-value.

An electromagnetic-acoustic transducer (EMAT) system was used in conjunction with a 'sample' CW signal-processing method to generate, receive, and process longitudinal and shear waves in thin steel sheets. Using the system, swept-frequency measurements were made up to $7.5 \mathrm{MHz}$, To relate the measure ments to sheet steel formability, a dimensionless fre quency ratio, $\mathrm{K}$, was computed from the resonant frequencies. From theoretical considerations, $K$ should be related to a measure of steel sheet formability, $r$. This parameter is traditionally measured by plastically deforming uniaxial tension specimens. Good correlation was found between $K$ and $r$ for a set of steel shee represenative of those typically used to produce auto mobile body parts.

\section{2,280}

PB96-186044 Not available NTIS

National Inst. of Standards and Technology (MSEL) Boulder, CO. Materials Reliability Div.

Effect of Liftoff on Accuracu of Phase Velocity Measurements Made with Electromagnetic-Acoustic Transducers.

Final rept.

A. V. Clark, and Y. Berlinsky. 1992, 41p.

Pub. in Research in Nondestructive Evaluation, $41 \mathrm{p}$ 1992.

Keywords: "Acoustics, Liftoff, Reprints, "Electromagnetic acoustic transducers, "Phase velocity.

Electromagnetic-acoustic transducers (EMATs) work on transduction principles which allow them to operate with a clearance (liftoff) between them and a conducting specimen. They have the potential for on-line ultrasonic measurements of rapidly moving materials. Liftoff causes changes in the effective inductance and re sistance of the EMAT. Consequently, it causes a phase shift in the output voltage of a receiving EMAT. This can cause errors when the EMAT is used for velocity measurements. In this paper, the authors develop a model for the effect of liftoff. The model gives good agreement with measured liftoff-induced arrival ime changes. The model can be extended to the case of an EMAT used with a voltage stepup transformer. The maximum signal is obtained for EMATs operating at resonance; however, the maximum sensitivity to liftoff also occurs then. Thus, a tradeoff must be made between optimum signal and supressing liftoff artifacts. The authors' model, and experimental results, can be used to make these tradeoffs.

\section{2,281}

PB96-186051 Not available NTIS

National Inst. of Standards and Technology (MSEL), Boulder, CO. Materials Reliability Div.

Methods to Improve the Accuracy of On-Line Ultra sonic Measurement of Steel Sheet Formability.

Final rept.

A. V. Clark, Y. Berlinsky, N. Izworski, S. R. Schaps, Y. Cohen, and D. V. Mitrakovic. 1992, 36p. Pub. in Jnl. of Nondestructive Evaluation, 36p 1992 Keywords: “Formability, "Steel sheet, Nondestructive tests, Reprints, "Electromangetic acoustic transducers, Ultrasonic velocity, $R$ value.

Ultrasonics offers a potential means to measure sheet steel formability nondestructively. The formability can be characterized by the plastic strain ratio, or r-value To be practical, an ultrasonic system should resolve r-value accurately, on rapidly moving material. The authors report here on research which quantifies factors which could degrade this resolution. The authors also present means to suppress these artifacts. They have developed a moving sheet device (MSD) to be used as a test bed to demonstrate the feasibility of on-line measurement of r-value in a steel mill. The device can move specimens at speeds comparable to those in industrial practice. An automated velocity measurement system has also been developed and integrated with the MSD. This allows ultrasonic measurements to be made with an array of transducers. Measurements were made in both static and dynamic mode. Artifacts due to sheet motion were found to be small, and should not significantly degrade 4-value resolution.

\section{2,282}

PB96-190384 Not available NTIS

National Inst. of Standards and Technology (EEEL), Boulder, CO. Electromagnetic Technology Div.

High Frequency Magnetic Field Sensors Based on the Faraday Effect in Garnet Thick Films.

Final rept.

R. Wolfe, E. M. Gyorgy, R. A. Lieberman, M. N. Deeter, G. W. Day, V. J. Fratello, and S. J. Licht 1992, 3p.

See also PB92-198167.

Pub. in Applied Physics Letters, v60 n17 p2048-2050 Apr 92

Keywords: "Magnetic measurement, Magnetic anistropy, Magnetooptics, Faraday effect, Epitaxial films, Reprints, "Fiber optic sensors, "Magnetic field sensors, Stripe domains.

The Faraday effect in the thick epitaxial films of magnetic garnets of the type used in magnetooptic isolators can be used as the basis for a fiber-optic magnetic field sensor. These films have uniaxial anisotropy perpendicular to the surface and they contain bismuth to enhance the Faraday rotation. The typical magnetic domain pattern of meandering stripes changes in response to an applied field perpendicular to the film and this changes the polarization of infrared light propagating perpendicular to the film. Theory and experiment show that the speed of operation is limited by relaxation or resonance effects to upper frequencies between $10(6)$ and $10(9) \mathrm{Hz}$. Maximum sensitivity requires low magnetic moment and large thickness, in conflict with the requirements for high speed.

02,283

PB97-110233 Not available NTIS

National Inst. of Standards and Technology (CSTL), Gaithersburg, MD. Process Measurements Div.

Thin Film Thermocouple Research at NIST.

Final rept.

K. G. Kreider. 1991, 18p

See also PB91-190116.

Pub. in ISA Conference Proceedings of the International Instrumentation Symposium (37th), Anaheim, CA., October 29-31, 1991, p13-30.

Keywords: "Thin films, "Thermocouples, "Indium oxides, "Tin oxides, Sputtering, Ceramics, Transparence, Platinum, Calibrating, Reprints.

Thin Film thermocouples have unique capabilities for measuring surface temperatures at high temperatures (above 800K) under harsh conditions. Their low mass, approximately $2 \times$ ten to the minus 5 power $\mathrm{g} / \mathrm{mm}$ permits very rapid response and very little disturbance of heat transfer to the surface being measured. This has led to applications inside gas turbine blades and vanes and ceramic liners in diesel cylinders. The most successful high temperature (up to $1300 / \mathrm{K}$ ) thin film thermocouples are sputter deposited from platinum and platinum $10 \%$ rhodium targets although results using base metal alloys, gold, and platinel will also be presented. The paper reviews the fabrication techniques used to form the thermocouples, approaches used to solve the high temperature insulation and adherence problems, current applications, and test results using the thin film thermocouples. In addition, a discussion will be presented on the recent research on thin film transparent thermocouples.

02,284

PB97-111876 Not available NTIS

National Inst. of Standards and Technology (EEEL), Gaithersburg, MD. Electricity Div. 


\section{ELECTROTECHNOLOGY}

Resistive, Capacitive, \& Inductive Components

\section{Resistors.}

Final rept

R. F. Dziuba. 1996, 13p

Pub. in Encyclopedia of Applied Physics, v16 p423-

435 Jul 96.

Keywords: "Resistors, "Automation, "Calibration, Measurements, Quality assurance, Standards, Hall effect, Reprints.

Resistors are inportant components in electrical and electronic circuits. The article begins with a brief historical account of the evolution of the different types of resistors starting with wirewounds and proceeding to carbon composition and the various film-type resistors. The concept of resistance is briefly described along with Ohm's Law and the unit of resistance. By international agreement, since, January 1, 1990, the unit of resistance has been expressed in terms of the quantum Hall effect which occurs in suitable semiconductors operating at high magnetic fields and low temperatures and is based on fundamental constants. The importance of resistors in the measurement of current, voltage ratios, temperature, and displacement is also discussed. The article describes, in more detail, resistor fabrication including the main constructural elements of base material, resistive element, terminations, and protective enclosure. Then separate cat egories of carbon composition, wirewound, metal foil, thin film, and thick film resistors are characterized. The article next discusses the key characteristics of the different types of resistors, including accuracy, stability, power rating, temperature coefficient, load coefficient, voltage coefficient, humidity effects, pressure effects, and freqency effects. Finally, the common classification of resistors, according to their intended use and inherent performence, is described. The classification includes standard resistors, resistors in electronic circuits, integrated circuit resistors, high current resistors, and high voltage resistors.

\section{2,285}

PB97-111884 Not available NTIS

National Inst. of Standards and Technology (EEEL) Gaithersburg, MD. Electricity Div.

Loading Effects in Resistance Scaling.

Final rept.

R. E. Elmquist, and R. F. Dziuba. 1996, 2p.

Pub. in Conference on Precision Electromagnetic Measurements 1996 Digest. (CPEM '96), Braunschweig, Germany, June 17-20, 1996, p334335.

Keywords: "Electrical resistance, "Electrical measurement, Standards, Loading, Reprints

Power loading effects in dc resistance references are not well understood even for the most commonly used high precision standards. The paper will examine loading effects and their contribution to the uncertainty o recent NIST comparisons of the quantum Hall effect and calculable capacitor.

02,286

PB97-112338 Not available NTIS

National Inst. of Standards and Technology (EEEL), Gaithersburg, MD. Electricity Div.

Development of Thin-Film Multijunction Thermal Converters at NIST.

Final rept.

J. R. Kinard, D. B. Novotny, T. E. Lipe, and D. X

Huang. 1996, 2p.

See also PB96-148135.

Pub. in Conference on Precision Electromagnetic Measurements 1996 Digest, (CPEM '96), 494.

Keywords: "CMOS, *Thin films, Microinstrumentation, Machining, Alternating current, Direct current, Photolithography, Transfer characteristics, Standards, Reprints, "Multijunction thermal converters.

The paper gives an overview of the development of thin-film multijunction thermal converters (FMJTCs) a the National Institute of Standards and Technology (NIST) A historical perspective of film thermal converters is presented, followed by descriptions of the motivation, fabrication processes, physical characteristics and the electrical properties of the FMJTCs produced at NIST. Integrated micropotentiometers which incorporate FMJTCS and thermal converters, produced by an alternative fabrication technology using a CMOS foundry, are also described. The paper concludes with a report on the current status of the FMJTC project and future directions.
02,287

PB97-112353 Not available NTIS

National Inst. of Standards and Technology (EEEL), Gaithersburg, MD. Electricity Div.

Empirical Linear Prediction Applied to a NIST Calibration Service.

Final rept.

A. D. Koffman, T. M. Souders, G. N. Stenbakken, T. E. Lipe, and J. R. Kinard. 1996, $6 p$.

Pub. in Proceedings of the National Conference of Standards Laboratories Workshop and Symposium, Monterey, CA., August 25-29, 1996, p207-212.

Keywords: "Calibration, "Electronic equipment, Test methods, Mathematical models, Linear systems, Predictions, Reprints, Thermal transfer standards.

Empirical linear prediction, developed at NIST, has recently been applied to the NIST calibration of a commercial multi-range ac-dc thermal transfer standard. This approach reduced the number of required test points by 62 percent, resulting in significant cost savings. The calibration model was developed using extensive test data obtained from the instrument manufacturer. Calibration measurements for the instrument under lest were made at the reduced set of test points enabling subsequent predictions of the response at all unmeasured points using the model. Uncertainties for the unmeasured points were developed by testing the goodness of fit of the calibration measurements to the model. These uncertainty intervals depend on the quality of the model as well as on the number of points actually measured. The ability of the model to characterize the instrument under test is key to achieving low uncertainties. A brief mathematical description of the modeling and prediction process is presented along with measurement results.

\section{2,288}

PB97-112452 Not available NTIS

National Inst. of Standards and Technology (EEEL), Gaithersburg, MD. Electricity Div.

Low Thermal Guarded Scanner for High Resistance Measurement Systems.

Final rept.

J. A. Marshall, T. A. Marshall, D. G. Jarrett, and R. F. Dziuba. 1996, 2p.

Pub. in Conference on Precision Electromagnetic Measurements, CPEM '96 Conference Digest, Braunschweig, Germany, June 17-20, 1996, p20-21.

Keywords: "Electrical resistance, "Electrical measurement Resistance bridges, Standards, Test equipment, Reprints, Current comparators, Resistance standards. The design and testing of a low thermal guarded scanner developed to provide completely guarded switching when used with guarded resisted bridge networks is described.

\section{2,289}

PB97-119150 Not available NTIS

National Inst. of Standards and Technology (EEEL), Gaithersburg, MD. Electricity Div.

Automated Guarded Bridge for Calibration of Multimegohm Standard Resistors.

Final rept.

D. G. Jarrett. 1996, $2 \mathrm{p}$.

Pub. in Conference on Precision Electromagnetic Measurements, June 17-20, 1996, Braunschweig, Germany, p336-337, 1996.

Keywords: *Resistors, "Standard resistors, "Graphical user interface, "Automated bridge, Calibrators, Electrometers, High resistance, Multimegohm, Reprints, "Foreign technology, Wheatstone bridge.

The implementation of an automated guarded bridge for calibrating multimegohm standard resistors is described. A guarded Wheatstone bridge has been assembled with programmable dc calibrators in two of the arms allowing multiple ratios and test voltages to be remotely selected. Preliminary measurements will be reported along with the balancing algorithm.

\section{2,290}

PB97-119168 Not available NTIS

National Inst. of Standards and Technology (EEEL), Gaithersburg. MD. Electricity Div.

Resistance Measurements from $10 \mathrm{M}$ Omega to 1 TOmega at NIST.

Final rept.

D. G. Jarrett. 1996, 8p

Pub. in National Conference of Standards Laboratories, Monterey, CA., August 25-29, 1996, p291-298.
Keywords: "Resistance measurements, "Standard resistors, "Resistors, Temperature, Chambers, Environments, Humidity, Multimegohm, Reprints, National Institutes of Standards and Technology.

Described are the measurement systems and methods used for calibrating standard resistors from $10 \mathrm{M}$ ohms to $1 \mathrm{~T}$ ohms at the National Institute of Standards and Technology (NIST). Presently four systems are used for the calibration of standard resistors at and above $10 \mathrm{M}$ ohms. An automated guarded multimegohm bridge has recently been developed to augment a manual guarded Wheatstone bridge and a semiautomated teraohmmeter system. An automated resistance ratio bridge is used during the scaling process Scaling from one decade to the next is done by using guarded Hamon boxes and the high resistance bridges.

02,291

PB97-119184 Not available NTIS

National Inst. of Standards and Technology (EEEL), Gaithersburg, MD. Electricity Div.

NIST Comparison of the Quantized Hall Resistance and the Realization of the SI Ohm Through the Calculable Capacitor.

Final rept.

A. Jeffery, R. E. Elmquist, L. H. Lee, J. Q. Shields,

and R. F. Dziuba. 1996, 2p.

See also PB97-119192

Pub. in Institute of Electrical and Electronics Engineers Transactions on Instrumentation and Measurement, p10-11 1996.

Keywords: *Calculable capacitors, "Comparators, Fundamental constants, Cryogenic currents, Fine structure constants, Reprints, "Four-terminal-pair-bridge, National Institutes of Standards and Technolology.

The latest NIST results from the comparison of the quantized Hall resistance (QHR) with the realization of the SI ohm obtained from the calculable capacitor measurement will be reported. Various systematic checks have been performed.

\section{2,292}

PB97-119192 Not available NTIS

National Inst. of Standards and Technology (EEEL) Gaithersburg, MD. Electricity Div

NIST Comparison of the Quantized Hall Resistance and the Realization of the Si Ohm Through the Calculable Capacitor. Conference Proceedings, June 17-20, 1996.

Final rept.

A. Jeffery, R. E. Elmquist, L. H. Lee, J. Q. Shields, and R. F. Dziuba. 1996, $2 p$

See also PB97-119184

Pub. in Proceedings of the Conference Precision Electromagnetic Measurements, Braunschweig Germany, June 17-20, 1996, CPEM '96 Conference Digest, p10$11,1996$.

Keywords: "Calculable capacitors, "Comparators, Fundamental constants, Cryogenic currents, Fine structure constants, Reprints, Four-terminal-pair-bridge, National Institutes of Standards and Technology

The latest NIST results from the comparison of the quantized Hall resistance (QHR), with the realization of the Sl ohm obtained from the calculable capacito measurement will be reported. Various systematic checks have been performed.

\section{2,293}

PB97-122600 Not available NTIS

National Inst. of Standards and Tech Gaithersburg, MD. Electricity Div. um-Gap Capacitors.

Final rept.

N. M. Zimmerman 1996, 6p.

Pub. in Institute of Electrical and Electronics Engineers Transactions on Instrumentation and Measurement. v45 n5 p841-846 Oct 96 .

Keywords: "Capacitors, Measurements, Resistance, Reprints, "Vacuum-gap capacitors, "Low-loss capacitors.

The authors report on measurements of capacitors with about $1 \mathrm{pF}$ of capacitance, which have unmeasurably small leakage at very low frequencies, placing a lower bound of about 10 to the 19 th power omega on the parallel resistance at an effective frequency of $1 \mathrm{mHz}$. These measurements are made possible by two themes: the use of vacuum-gap capacitors 
(i.e., no dielectric material, operated in vacuum), and detection of leakage using single electron tunneling (SET) electrometers, which have very high input impedance. The authors also report on good achieved results in time stability and lack of frequency and voltage dependence.

\section{Semiconductor Devices}

\section{2,294}

DE95009287 PC A03/MF AO

National Renewable Energy Lab., Golden, CO.

Atomic-scale characterization of hydrogenated amorphous-silic on films and devices. Annual subcontract report, 14 February 1994--14 April 1995. PROGRESS REPT.

A. Gallagher, D. Tanenbaum, A. Laracuente, and B. Jelenkovic. Aug 95, 18p, NRELTP-411-8246. Contract AC36-83CH10093

Sponsored by Department of Energy, Washington, DC

Keywords: *Silicon Solar Cells, Amorphous State, Electrical Properties, Hydrogenation, Progress Report, Thin Films, EDB/140501.

Properties of the hydrogenated amorphous silicon (a$\mathrm{Si}$ H) films used in photovoltaic (PV) panels are reported. The atomic-scale topology of the surface of intrinsic a-Si:H films, measured by scanning tunneling microscopy (STM) as a function of film thickness, are reported and diagnosed. For 1-500-nm-thick films deposited under normal device-quality conditions from silane discharges, most portions of these surfaces are uniformly hilly without indications of void regions. However, the STM images indicate that 2-6-nm silicon particulates are continuously deposited into the growing film from the discharge and fill approximately $0.01 \%$ of the film volume. Although the STM data are not sensitive to the local electronic properties near these particulates, it is very likely that the void regions grow around them and have a deleterious effect on a-Si:H photovoltaics. Preliminary observations of particulates in the discharge, based on light scattering, confirm that particulates are present in the discharge and that many collect and agglomerate immediately downstream of the electrodes. Progress toward STM measurements of the electronic properties of cross-sectioned a-Si: $\mathrm{H}$ $\mathrm{PV}$ cells is also reported.

\section{2,295}

\section{PB94-155579 PC A03/MF A01}

National Inst. of Standards and Technology (EEEL)

Gaithersburg, MD. Semiconductor Electronics Div.

Development and Characterization of Insulating Layers on Silicon Carbide: Annual Report for February 14,1988 to February 14, 1989.

J. J. Kopanski, and D. B. Novotny. Sep 89, 48p, NISTIR-89-4157.

Sponsored by National A ronautics and Space Administration, Cleveland, $\mathrm{OH}$. Lewis Research Center

Keywords: "Silicon carbides, "Capacitors, *Semiconductor devices, "Vapor deposition, Cubic lat tices, Electrical properties, Semiconductor traps MIS(Semiconductors), Capacitance-voltage character istics, Metal oxide semiconductors, Multilayer insulation, Oxide coatings, Silicon inorganic compounds "MIS capacitors, "MOS capacitors, "Compound semiconductors.

Processes to fabricate metal-insulator-semiconductor (MIS) capacitors on the cubic (3C) form of silicon car bide (SiC) were studied. The insulating layers were formed from either thermally grown oxide or chemicalvapor-deposited (CVD) silicon dioxide. The effects of wet or dry oxygen and of oxidation temperatures be tween 1050 and $1200 \mathrm{C}$ on the electrical properties o devices with thermal oxides were determined. Elec trical characterization techniques appropriate for these devices on $\mathrm{SiC}$ were developed and applied to the fab ricated capacitors. The capacitors were characterize from multiple-frequency capacitance-voltage ( C-V) measurements as a function of temperature from room temperature to $300 \mathrm{C}$. The apparent interface trap level densities were estimated from the high-frequency $C$ $\checkmark$ curves.

\section{2,296}

\section{PB94-159787 PC A03/MF A01}

National Inst. of Standards and Technology (EEEL) Gaithersburg, MD. Semiconductor Electronics Div.
Center for Electronics and Electrical Engineering Technical Progress Bulletin Covering Center Programs, October to December, with 1991 CEEE Events Calendar.

J. A. Gonzalez. May 91, 45p, NISTIR-4548.

See also PB91-159749 and PB93-205524.

Keywords: "Electrical measurement, "Metrology, Semiconductor materials, Bipolar transistors, Signal processing, Josephson junctions, Electromagnetic interference, Millimeter waves, Optical fibers, High temperature superconductors, Microwaves, Photodiodes, MOSFET Lasers, Antennas, Magnetic materials, Electric power, Progress report, Abstracts, Fiber optic sensors, US NIST, SIMOX.

This is the thirty-third issue of a quarterly publication providing information on the technical work of the $\mathrm{Na}$ tional Institute of Standards and Technology (formerly the National Bureau of Standards) Center for Electronics and Electrical Engineering. This issue of the CEEE Technical Progress Bulletin covers the fourth quarter of calendar year 1990. Abstracts are provided by technical area for both published papers and papers approved by NIST for publication. Main headings from the Table of Contents include the following: Semiconductor Technology Program; Signals and Systems Metrology Program; Fast Signal Acquisition, Processing, and Transmission; Electrical Systems; Electromagnetic Interference; Additional Information: 1991 CEEE Calendar; Sponsor List; Key Contacts in Center, Center Organization.

\section{2,297}

\section{PB94-163458 PC A05/MF A01}

National Inst. of Standards and Technology (EEEL), Gaithersburg, MD. Semiconductor Electronics Div. Design Guide for CMOS-On-SIMOX. Test Chips NIST3 and NIST4.

J. C. Marshall, M. W. Cresswell, C. H. Ellenwood, M. E. Zaghloul, L. W. Linholm, and P. Roitman. Jan 93, 93p. NISTIR-4889

See also PB93-152106. Prepared in cooperation with George Washington Univ., Washington, DC. School of Engineering and Applied Science.

Keywords: "Very large scale integration, "Integrated circuits, *Computer aided design, Chips(Electronics). Computer programs, Gallium arsenides, Silicon, Transistors, Guidelines, Manuals, MOSFET, CMOS, Test structures, Test chips, Sol(Semiconductors), SIMOX. The design guidelines for test chips NIST3 and NIST4 are specified in this manual. These chips were designed for process monitoring and device parameter extraction for a CMOS (Complementary Metal-OxideSemiconductor)-on-SOI (Silicon-On-Insulator) process. The chips contain structures which are common to a standard CMOS process as well as structures specifically designed for a SIMOX (Separation by the IMplantation of OXygen) process. In order to facilitate the CAD process, a unique 'technology file' was created for the Magic VLSI layout editor used on a Sun-3/280 system running Sun Version 3.5. This SIMOX technology file is very general and can be used to build CMOS as well as SIMOX chips. NIST3 is $6380 \mathrm{mi}$ crometers $\times 4780$ micrometers and contains several large-geometry MOSFETs, resistors, and capacitors. NIST 4 is $1 \mathrm{~cm} \times 1 \mathrm{~cm}$ and contains approximately 300 small-geometry test structures. The SIMOX specific structures found on these chips include MOSFETs, capacitors, interconnects, and pads to be discussed in more detail. The test guide for the test structures on NIST3 and NIST4 is included in a separate manual (PB93-152106).

\section{2,298}

PB94-164316 PC A03/MF A01

National Inst. of Standards and Technology (EEEL), Gaithersburg, MD. Semiconductor Electronics Div. Color Supplement to NIST Special Publication 400 . 93: Semiconductor Measurement Technology: Design and Testing Guides for the CMOS and Lateral Bipolar-on-SOI Test Library.

J. C. Marshall, and M. E. Zaghloul. Mar 94, 40p NISTIR-5324

Color illustrations reproduced in black and white. Prepared in cooperation with George Washington Univ., Washington, DC. School of Engineering and Applied Science.

Keywords: "Very large scale integration, "Integrated circuits, Computer aided design, Field effect transisIors, Bipolar transistors, Chips(Electronics), Silicon, MOSFET, CMOS, Test chips, Test structures, SOI(Semiconductors), SIMOX
This report is the supplement to the NIST Special Publication entitled, 'Semiconductor Measurement Technology: Design and Testing Guides for the CMOS and Lateral Bipolar-on-SOI Test Library.' This supplement contains the complete set of figures from the above mentioned document with the test structures provided in color for easier interpretation.

02,299

PB94-165958 PC A03/MF A0

National Inst. of Standards and Technology (EEEL) Gaithersburg, MD. Semiconductor Electronics Div.

Electronics and Electrical Engineering Laboratory Technical Progress Bulletin Covering Laboratory Programs, October to December 1992, with 1992 1993 EEEL Events Calendar.

J. M. Rohrbaugh. Mar 93, 31p, NISTIR-5145.

See also PB93-158632.

Keywords: "Electrical engineering, "Electronics, "Metrology, "US NIST, Semiconductors, Semiconductor devices, Electrical measurement, Microelectronics Signal processing, Integrated circuits, Dimensiona measurement, Electromagnetic interference, Lasers, Optical fibers, Magnetic materials, Microwaves, Antennas, Electric power, Superconductors, Abstracts.

This is the forty-first issue of a quarterly publication providing information on the technical work of the $\mathrm{Na}$ tional Institute of Standards and Technology, Electronics and Electrical Engineering Laboratory. This issue of the EEEL Technical Progress Bulletin covers the fourth quarter of calendar year $\$ 992$. Abstracts are provided by technical area for both published papers and papers approved by NIST for publication.

02,300

PB94-172483 Not available NTIS

National Inst. of Standards and Technology (EEEL) Gaithersburg, MD. Semiconductor Electronics Div.

Efficient Method to Compute the Maximum Transient Drain Current Overshoot in Silicon on Insulator Devices.

Final rept.

C. Korman, I. Mayergoyz, M. Gaitan, and G. Tai.

$1993,6 p$

Pub. in Jnl. of Applied Physics 73, n6 p2611-2626, 15

Mar 93.

Keywords: *Field effect transistors, Transport theory Poisson equation, Computation, Silicon, MOSFET, Reprints, "SOl(Semiconductors), Drain current overshoot.

The authors present an efficient method to compute the maximum transient drain current overshoot in silicon-on-insulator metal-oxide-silicon field effect transistors. The method is based on the physical idea that the number of majority carriers remains unchanged immediately after a change in the applied gate bias. The maximum overshoot is computed by solving the Poisson and the stationary minority carrier transpor equations under the constraint that the number of majority carriers is conserved. Hence, the novel aspect of the method is that it allows one to compute the maximum drain current overshoot without resorting to a computationally costly transient simulation. The accuracy of the method is verified by comparing the value of the drain current computed by the method with the maximum value of the drain current computed by transient simulations. The comparisons show that, with this method, the maximum transient drain current overshoot can be computed quite accurately for fast changes in the gate bias.

02,301

PB94-178019 PC A07/MF A02

National Inst. of Standards and Technology (EEEL), Gaithersburg, MD. Semiconductor Electronics Div. Semiconductor Measurement Technology: Design and Testing Guides for the CMOS and Lateral Bipolar-on-SOl Test Library.

J. C. Marshall, and M. E. Zaghloul. Mar 94, 142p, NIST/SP-400-93.

Also available from Supt. of Docs. as $\mathrm{SNOO}^{-003}$ 03262-0. See also PB93-152106 and PB94-163458. Prepared in cooperation with George Washington Univ., Washington, DC. School of Engineering and APplied Science.

Keywords: "Very large scale integration, "Computer aided design, "Integrated circuits, Chips(Electronics), Bipolar transistors, Electronic modules, Computer pro-
grams, Wafers, MOSFET, CMOS, Tests, Sol(Semiconductors), Test structures, Test chips, SIMOX, NIST8, NIST9. 


\section{ELECTROTECHNOLOGY}

\section{Semiconductor Devices}

Design and testing guides have been developed for the test library from which test chip NIST8 and test wafer NIST9 were derived. They were designed for use in process monitoring and device parameter extraction to evaluate and compare CMOS (Complementary MetalOxide-Semiconductor) test structures, including devices and circuits, fabricated on both bulk silicon and SOI (Silicon-on-Insulator), specifically SIMOX (Separation by the IMplantation of OXygen), wafers. The test library consists of both CMOS-on-SOI and lateral bipolar-on-SOI modules. From it, 20 modules were assembled to create CMOS test chip NIST8 that was fabricated using a standard bulk CMOS foundry through the MOSIS service. SOI/SIMOX test wafer NIST9 contains approximately 1000 modules and was also assembled from modules in this test library. Fourteen processing masks are used to fabricate depletionmode MOSFETs, lateral bipolar devices, and CMOS MOSFETs with source-to-channel ties. The SOI/ SIMOX technology file used with the Magic VLSI layout editor was modified to include the layers necessary to generate these 14 processing masks. This modification is discussed, and unique test structure designs are presented.

\section{2,302}

\section{PB94-181997 Not available NTIS}

National Inst. of Standards and Technology (EEEL), Gaithersburg, MD. Semiconductor Electronics Div. Application of the Modified Voltage-Dividing Potentiometer to Overlay Metrology in a CMOS/Bulk Process.

Final rept.

R. A. Allen, M. W. Cresswell, L. W. Linholm, T. A. Hill, J. D. Benecke, S. R. Volk, H. D. Stewart, J. C. Owen, and C. H. Ellenwood. Mar 94, 6p.

Contract DE-AC04-94AL85000

Pub. in Proceedings of the Institute of Electrical and Electronics Engineers International Conference on Microelectronic Test Structures, San Diego, CA., March 22-24, 1994, v7 p51-56. Sponsored by Department of Energy, Washington, DC.

Keywords: "Very large scale integration, "Integrated circuits, "Overlays, Positioning, Alignment, Metrology, Wafers, CMOS, Lithography, Reprints, Test structures, Registration.

The measurement of layer-to-layer feature overlay will, in the foreseeable future, continue to be a critical metrological requirement for the semiconductor industry. Meeting the image placement metrology demands of accuracy, precision, and measurement speed favors the use of electrical test structures. In this paper, a twodimensional, modified voltage-dividing potentiometer is applied to a short-loop VLSI process to measure image placement. The contributions of feature placement on the reticle and registration on the wafer to the overall measurement are analyzed and separated. Additional sources of uncertainty are identified, and methods developed to monitor and reduce them are described.

\section{2,303}

\section{PB94-185592 Not available NTIS}

National Inst. of Standards and Technology (EEEL), Gaithersburg, MD. Semiconductor Electronics Div. Electro-Thermal Simulation of an IGBT PWM Inverter.

Final rept.

H. A Mantooth, and A. R. Hefner 1993, 10p Pub. in Proceedings of the Power Electronics Specialists Conference, Seattle, WA., June 20-24, 1993, p7584.

Keywords: "Inverters, Chips(Electronics), Computerized simulation, Thermal analysis, Silicon, Reprints, Insulated gate bipolar transistors, Circuit simulators.

A recently developed electro-thermal network simulation methodology is used to analyze the behavior of a full-bridge, pulse-width-modulated, voltage-source inverter which uses IGBTs (Insulated Gate Bipolar Transistors) as the switching devices. The electro-thermal simulations are performed using the Saber Circuit simulator and include the control logic circuitry, the IGBT gate drivers, the physics-based IGBT electrothermal model, and the thermal network component models for the power device silicon chips, packages, and heat sinks. It is shown that the thermal response of the silicon chip determines the IGBT temperature rise during the device switching cycle. It is also shown that the full electro-thermal analysis is required to accurately describe the power losses and circuit efficiency.
02,304

PB94-185949 Not available NTIS

National Inst. of Standards and Technology (EEEL), Gaithersburg, MD. Semiconductor Electronics Div. Experimental Investigation of the Validity of TDDB Voltage Acceleration Models.

Final rept.

J. S. Suehle, P. Chaparala, C. Messick, W. M. Miller, and K. C. Boyko. 1993, 9p.

Pub. in Proceedings of the International Integrated Reliability Workshop, Lake Tahoe, CA., October 24-27. 1993, p59-67.

Keywords: “Dielectric breakdown, "Silicon dioxide, Temperature dependence, High temperature, Integrated circuits, Electric fields, Reprints, Field acceleration models.

Time-Dependent Dielectric Breakdown (TDDB) data are presented for 15- and 22.5-nm oxides collected over a wide range of electric fields and temperatures. The results indicate that it is necessary to obtain data over this range to distinguish between the two field ac celeration models and to quantify the electric field and temperature dependencies of the thermal activation energy and the field acceleration factor respectively. We also demonstrate the use of temperatures as high as $400 \mathrm{C}$ to accelerate time-dependent dielectric breakdown.

\section{2,305}

PB94-185956 Not available NTIS

National Inst. of Standards and Technology (EEEL) Gaithersburg, MD. Semiconductor Electronics Div

Field and Temperature Acceleration of Time-Dependent Dielectric Breakdown in Intrinsic Thin SiO2.

Final rept.

J S Suehle, P. Chaparala, C. Messick, W. M. Miller, and K. C. Boyko. 1994, 6p

Pub. in Proceedings of the Institute of Electrical and Electronics Engineers International Reliability Physics Workshop, San Jose, CA., April 12-14, 1994, p120 125.

Keywords: "Dielectric breakdown, "Silicon dioxide, Temperature dependence, High temperature, Integrated circuits, Electric fields, Dielectric films, Thin films, Reprints, Field acceleration models.

Time-Dependent Dielectric Breakdown (TDDB) data are presented for $15-$ and $22.5-\mathrm{nm}$ oxides collected over a wide range of electric fields and temperatures. The results indicate that it is necessary to obtain data over this range to distinguish between the two field ac celeration models and to quantify the electric field and temperature dependencies of the thermal activation energy and the field acceleration factor, respectively. We also report on the TDDB characteristics of thin $\mathrm{SiO} 2$ films at temperatures as high as $400 \mathrm{C}$ and demonstrate the use of these temperatures to accelerate TDDB

\section{2,306}

PB94-185964 Not available NTIS

National Inst. of Standards and Technology (EEEL) Gaithersburg, MD Semiconductor Electronics Div. Junction Locations by Scanning Tunneling Microscopy: In-Air-Ambient Investigation of Passivated GaAs pn Junctions.

Final rept.

W. F. Tseng, J. A. Dagata, R. M. Silver, J. Fu, and J. R. Lowney. 1994, 5p.

Pub. in Jnl. of Vacuum Science and Technology B 12 n1 p373-377 Jan/Feb 94. Sponsored by Office of Naval Research, Arlington, VA.

Keywords: "Semiconductor junctions, "P-n junctions, Gallium arsenides, Molecular beam epitaxy, Scanning tunneling microscopy, Aluminum arsenides, Passivation, Cleavage, Reprints, Atomic force microscopy

Scanning tunneling microscopy (STM) and atomic orce microscopy operating in air have been used to investigate locations of molecular-beam epitaxially grown GaAs multiple pn junctions cleaved and passivated with P2S5. Symmetrically and asymmetrically doped junctions were prepared within topographically delineated AlAs/GaAs marker regions for this in-air study of electronic junction contrast. Our results indicate that the STM-delineated junction locations do not coincide with the electrical junction locations, but rather shift into the p-type regions.

\section{2,307 \\ PB94-193752 PC A03/MF AO}

National Inst. of Standards and Technology (EEEL), Gaithersburg, MD. Semiconductor Electronics Div. Electronics and Electrical Engineering Laboratory Technical Publication Announcements Covering Laboratory Programs, October to December 1993 with 1994/1995 EEEL. Events Calendar.

J. M. Rohrbaugh. Jun 94, 23p, NISTIR-5435

See also PB94-118403 and PB94-154341.

Keywords: "Electrical measurement, "Microelectronics, "Metrology, "Bibliographies, Dimensional measurement, Integrated circuits, Integrated optics, High temperature superconductors, YBCO superconductors, Electromagnetic interference, Electric contacts, Electronic packaging. Photodetectors, Abstracts, Magnetic force microscopy.

This is the thirty-ninth issue of a quarterly publication providing information on the technical work of the $\mathrm{Na}$ tional Institute of Standards and Technology, Electronics and Electrical Engineering Laboratory (EEEL). This issue of the "EEEL Technical Publication Announcements' covers the fourth quarter of calendar year 1993. Abstracts are provided by technical areas for papers published. Major subject headings include the following: Semiconductor Microelectronics; Signal Acquisition, Processing, and Transmission; Electrical Systems; Electromagnetic Interference.

02,308

PB94-193810 PC A03/MF A01

National Inst. of Standards and Technology (EEEL), Gaithersburg, MD. Semiconductor Electronics Div.

Electronics and Electrical Engineering Laboratory Technical Progress Bulletin Covering Laboratory Programs, January to March 1994 with 1994/1995 EEEL Events Calendar.

J. M. Rohrbaugh. Jun 94, 50p. NISTIR-5434

See also PB93-234698, PB94-118403 and PB94154341

Keywords: "Electrical measurement, *Microelectronics, "Metrology, "Bibliographies, Integrated circuits, Dimensional measurement, Dielectric breakdown, Josephson junctions, High temperature superconductors, Microwave equipment, Sulfur hexafluoride, Antennas, Integrated optics, Optical fibers, Photodetectors, Radiometers, Electric power, Electromagnetic interference, Abstracts, Fiber optic sensors, Test structures, Microfabrication, Magnetic force microscopy, Flat panel displays.

This is the forty-sixth issue of a quarterly publication providing intormation on the technical work of the $\mathrm{Na}$ tional Institute of Standards and Technology, Electronics and Electrical Engineering Laboratory (EEEL). This issue of the 'EEEL Technical Progress Bulletin' covers the first quarter of calendar year 1994. Abstracts are provided by technical area for both published papers and papers approved by NIST for publication. Major subject headings include the following: Fundamental Electrical Measurements; Semiconductor Microelectronics; Signal Acquisition, Processing, magnetic Interference; Video Technology.

\section{2,309}

PB94-194354 PC A03/MF A01

National Inst. of Standards and Technology (EEEL). Gaithersburg, MD. Semiconductor Electronics Div. Electronics and Electrical Engineering Laboratory Technical Progress Bulletin Covering Laboratory Programs, July to September 1993 with 1994 EEEL Events Calendar.

J. M. Rohrbaugh. Nov 93, 49p, NISTIR-5288.

Keywords: ${ }^{\star}$ Electrica! measurement, "Microelectronics, "Metrology, "Bibliographies, Josephson junctions, High temperature superconductors, Integrated circuits, Electromagnetic interference, Dimensional measurement, YBCO superconductors, Millimeter waves, Microwave equipment, Semiconductors, Magnetic materials, Optical fibers, Antennas, Abstracts, Quantum Hall effect, Microfabrication.

This is the forty-fourth issue of a quarterly publication providing information on the technical work of the $\mathrm{Na}$ tional Institute of Standards and Technology, Electronics and Electrical Engineering Laboratory (EEEL). This issue of the 'EEEL Technical Progress Bulletin' covers the third quarter of calendar year 1993. Abstracts are provided by technical area for both published papers and papers approved by NIST for publication. Major subject headings include the following: Fundamental Electrical Measurements; Semiconductor Microelectronics; Signal Acquisition, Processing, 
and Transmission; Electrical Systems; Electromagnetic Interference; Law Enforcernent Standards: Product Data Systems; Video Technology.

02,310

PB94-198561 Not available NTIS

National Inst. of Standards and Technology (MSEL), Gaithersburg, MD. Metallurgy Div.

Numerical Simulation of Submicron Photolithographic Processing.

Final rept.

E. Barouch, J. W. Cahn, U. Hollerbach, and S. A.

Orszag. Sep $91,22 p$

Pub. in Jn!. of Scientific Computing 6, n3 p229-250 Sep 91. Sponsored by Defense Advanced Research Projects Agency, Arlington, VA. Technology Reinvestment Project. and Air Force Office of Scientific Re search, Bolling AFB, DC.

Keywords: "Photolithography, "MOSFET, Field effect transistors, Chips(Electronics), Computerized simulation, Photomasks, Reprints.

A complete numerical simulation package for submicron photolithography is described in depth. Four of the computational steps are analyzed: aerial image generation, exposure, postexposure bake, and disgeneration, exposure, postexposure bake, and disgate is described. In addition, the utility of phase-shif masks is described, and the effects of aberrations are explored.

\section{2,311}

PB94-198728 Not available NTIS

National Inst. of Standards and Technology (EEEL), Gaithersburg, MD

Majority and Minority Mobilities in Heavily Doped Silicon for Device Simulations.

Final rept.

H. S. Bennett, and J. R. Lowney. 1992, 6p.

Pub. in Proceedings of International Workshop on $\mathrm{Nu}$ merical Modeling of Processes and Devices for Integrated Circuits, Seattle, WA., May 31-June 1, 1992 , p123-128.

Keywords: "Electron mobility, "Hole mobility, Carrie mobility, Doped materials, Simulation, Silicon, Reprints.

As silicon devices approach 0.2 micrometer in size, it will be essential to have accurate values for the majority and minority mobilities of electrons and holes. ity and minority mobilities of electrons and holes. donor and acceptor densities between 10(sup 17)/cc and 10 (sup 20)/cc. All the important scattering mechanisms have been included. The ionized impurity scattering has been treated with a quantum-mechanical phase-shift analysis. The results are in good agreement with experiment. In addition, the ionized impurity cattering rates calculated from the quantum-mechanical phase shifts and those rates calculated from the Born approximation are shown to differ by more than actors of three. The commonly used Born approximation is not valid for low energy carriers near band extrema.

\section{2,312}

PB94-200391 Not available NTIS

National Inst. of Standards and Technology (EEEL) Gaithersburg, MD. Semiconductor Electronics Div.

Electron Traps, Structural Change, and Hydrogen Related SIMOX Defects.

Final rept.

J. F. Conley, P. M. Lenahan, and P. Roitman. 1992 $2 p$.

Contract DNA-IACRO-88-800

Pub. in Extended Abstract, Proceedings of Institute of Electrical and Electronics Engineers International SO Conference, Ponte Vedra Beach, FL. October 6-8, 1992, p26-27. Sponsored by Office of Naval Research Arlington, VA. and Defense Nuclear Agency, Washington, DC

Keywords: Electron spin resonance, Vacuum ultraviolet radiation, Crystal defects, Electron traps, Hydrogen, Reprints, "SOI(Semiconductors), "SIMOX, Buried oxides.

Evidence for structural changes in SIMOX buried ox ides is presented. We show that deep electron traps may be created in SIMOX buried oxides; these electron traps could compensate for some positively charged E' centers. In addition, we report observation of relatively high densities of hydrogen-related defects in VUV illuminated oxides. We suggest that this short range disorder may be responsible for the differences between the response of ordinary thermal oxide and SIMOX buried oxides.

\section{2,313}

PB94-200565 Not available NTIS

National Inst. of Standards and Technology (EEEL) Gaithersburg, MD. Semiconductor Electronics Div. Test Structures for the In-Plane Locations of Projected Features with Nanometer-Level Accuracy Traceable to a Coordinate Measurement System. Final rept.

M. W. Cresswell, R. A. Allen, L. W. Linholm, E. C. Teague, C. H. Ellenwood, and W. B. Penzes. 1993 $7 p$.

Pub in Proceedings of Institute of Electrical and Electronics Engineers International Conference on Microelectronic Test Structures, Sitges, Barcelona, Spain March 22-25, 1993, v6 p255-261.

Keywords: "Microelectronics, "Lithography, Metrology Substrates, Masks, Reprints, Test structures, Coordinate measurement systems, Traceability.

A new test structure has been designed to measure the positions of the images of an array of fiducial marks projected from a mask into a resist film on a substrate. The resist film on the substrate covers a nominally matching array of partially formed versions of the tes structure prepatterned in a conducting film. Instances of the finished structure are formed on the substrate by further selective removal of conducting material from the partially formed test structures where they are overlaid by images of the fiducial marks on the mask. At each array point, the feature of the completed test structure that is defined by the overlay of the image of the fiducial marks on the mask is called the pointer. The part of the partially formed test structure that is unaffected by the overlay of the images of the fiducial marks on the mask serves as a ruler. Electrical testing accurately provides the precise location of the pointer relative to the ruler within each test structure. The paper describes the test structure and provides examples of measurements that indicate precision to within less than $2 \mathrm{~nm}$ and accuracy of better than $7 \mathrm{~nm}$.

\section{2,314}

PB94-211653 Not available NTIS

National Inst. of Standards and Technology (NEL) Gaithersburg, MD. Semiconductor Electronics Div.

Wire Bond Testing.

Final rept.

G. G. Harman. 1989, 48p.

Pub. in Reliability and Yield Problems of Wire Bonding in Microelectronics: The Application of Materials and Interface Science, Chapter 1, p1-48 1989.

Keywords: "Semiconductor devices, "Microelectronics, Fracture mechanics, Test methods, Gallium arsenides Silicon, Reviews, Reprints, "Wire bonds.

The methods of testing wire bonds are reviewed. These include the bond pull test, the nondestructive bond pull test, and ball shear test, the 'pluck' test, and the thermal stress test. In the process of discussing the tests, their usage in production is emphasized and their reliability implications are discussed.

\section{2,315}

\section{PB94-211711 Not available NTIS}

National Inst. of Standards and Technology (EEEL), Boulder, CO. Electromagnetic Technology Div. YBa2Cu307-x to Si Interconnection for Hybrid Superconductor/Semiconductor Integration.

Final rept.

T. E. Harvey, J. Moreland, B. Jeanneret, R. H. Ono, and D. A. Rudman. 1992, 3p.

Pub. in Applied Physics Letters 61, n18 p2225-2227, 2 Nov 92 . Sponsored by Department of Energy, Wash ington, DC.

Keywords: "High temperature superconductors, "Superconducting films, "Integrated circuits, "Circuit interconnections *Electric contacts, Metal oxide semiconductors, YBCO superconductors, Hybrid circuits, Laser ablation, Single crystals, Thin films, Substrates, Silicon, Silver, Gold, Reprints, Yttrium barium cuprates, Ohmic contacts.

We have successfully made low resistance contacts between high-quality films of $\mathrm{YBa} 2 \mathrm{Cu} 3 \mathrm{O}(7-\mathrm{x})$ (YBCO) and single-crystal Si substrates through $\mathrm{Ag}$-Au interconnections. The YBCO films were deposited by lase ablation on an epitaxial yttria-stabilized zirconia buffer layer on Si and had zero-resistance critical temperatures of 83-85 $\mathrm{K}$ after patterning into lines. Specific contact resistivities (resistance-area products) of the
YBCO to $\mathrm{Si}$ interconnection, limited by the $\mathrm{Au}$ to $\mathrm{Si}$ interface of 10(sup -6) ohm (cm squared) were achieved on heavily doped Si after deposition and patterning of the YBCO film. This demonstrates the use of high-temperature superconduclors as a wiring layer compatible with conventional Si metal-oxide semiconductor processing.

\section{2,316}

PB94-211794 Not available NTIS

National Inst. of Standards and Technology (EEEL) Gaithersburg, MD. Semiconductor Electronics Div. Investigation of the Drive Circuit Requirements for the Power Insulated Gate Bipolar Transistor (IGBT).

Final rept.

A. R. Hefner $1991,12 p$.

Pub. in Institute of Électrical and Electronics Engineers Transactions on Power Electronics 6, n2 p208-219 Apr

Keywords: "Bipolar transistors, Requirements, Reprints, "Insulated gate bipolar transistors, Drive circuits, Power transistors.

The drive circuit requirements of the insulated gate bipolar transistor (IGBT) are explained with the aid of an analytical model It is shown that non-quasi-static effects limit the influence of the drive circuit on the time rate-of-change of anode voltage. Model results are compared with measured turn-on and turn-off waveforms for different drive, load, and feedback circuits and for different IGBT base lifetimes.

\section{2,317}

PB94-212776 Not available NTIS

National Inst. of Standards and Technology (EEEL), Gaithersburg, MD. Semiconductor Electronics Div. Multicarrier Characterization Method for Extracting Mobilities and Carrier Densities of Semiconductors from Variable Magnetic Field Measurements.

Final rep

J. S. Kim, D. G. Seiler, and W. F. Tseng. 1993, 12p Pub. in Jnl. of Applied Physics 73, n12 p8324-8335, 15 Jun 93.

Keywords: "High electron mobility transistors, "Carrier mobility, "Carrier density, Indium gallium arsenides, Electrical conductivity, Magnetic fields, Tensors, Reprints, Multilayers.

A simple, practical method is described to extract the carrier concentration and mobility of each component of a multicarrier semiconductor system (which may be either a homogeneous or multilayered structure) from variable magnetic field measurements. Advantages of the present method are mainly due to the inclusion of both the longitudinal and transverse components of the conductivity tensor and normalization of these quantities with respect to the zero-field longitudinal component of the conductivity tensor. This method also provides a simple, direct criterion by which one can easily determine whether the material under test is associated with a one-carrier or multicarrier conduction. The method is demonstrated for a simple one-carrier system (GaAs single-channel high-electron-mobility-transistor (HEMT) structure) and two multicarrier systems (an InGaAs-GaAs double-channel HEMT-structure and two types of carriers present in an InGaAs singlechannel HEMT structure). The analysis of the experimental data obtained on these samples demonstrates the utility of the method presented here for extracting carrier concentrations and mobilities in advanced semiconductor structures.

02,318

PB94-212966 Not available NTIS

National Inst. of Standards and Technology (EEEL), Gaithersburg, MD. Semiconductor Electronics Div. Defect of Thermal Ramping and Annealing Conditions on Defect Formation in Oxygen Implanted Silicon-On-Insulator Material.

Final rept.

S. J. Krause, J. C. Park, J. D. Lee, M. El-Ghor, and P. Roitman. 1992, 2p.

Sponsored by Defense Nuclear Agency, Washington, DC

Pub in Extended Abstract, Proceedings of Institute of Electrical and Electronics Engineers International SOI Conference, Ponte Vedra Beach, FL., October 6-8, 1992, p80-81.

Keywords: "Crystal defects, ${ }^{*}$ CMOS, Integrated circuits, Ion implantation, Stacking faults, Annealing, Silicon, Oxides, Reprints, "SOl(Semiconductors), Thermal ramping, SIMOX. 


\section{ELECTROTECHNOLOGY}

\section{Semiconductor Devices}

SIMOX (Separation by IMplantation of OXygen) is a leading technology for providing SOI material for complementary metal-oxide-semiconductor (CMOS) circuits which have increased radiation hardness and higher operating speed. Defects in the top Si layer af fect CMOS device yield, operation, and reliability. Defects in annealed SIMOX form from oxide precipitation and dissolution during thermal ramping and annealing. These defects include dislocations, stacking faults, stacking fault pairs, and stacking fault tetrahedra. Multiple cycles of implantation and annealing and higher temperature implantation, above $600 \mathrm{C}$, have been used to reduce the defect density. Thermal ramping rate and annealing ambient can significantly affect precipitation processes and defect formation during thermal ramping. In this work, the effect of thermal ramping conditions, including oxide capping, on defect formation was studied.

\section{2,319}

PB94-213105 Not available NTIS

National Inst. of Standards and Technology (MEL), Gaithersburg, MD. Precision Engineering Div.

Precision, Accuracy, Uncertainty and Traceability and Their Application to Submicrometer Dimensional Metrology.

Final rept.

R. D. Larrabee, and M. T. Postek. 1993, 12p.

Pub. in Solid-State Electronics 36, n5 p673-684 1993.

Keywords: "Dimensional measurement, "Semiconductor devices, "Integrated circuits, "Metrology, Reproducibility, Calibration, Precision, UnCertainty, Accuracy,
Nanotechnology.

The terms in the title of this paper are often used to characterize the quality of any measurement result. However, these terms (particularly accuracy and traceability) are very often confused (and abused) in practice. They often do not have the same meaning to the seller, buyer, and user of metrology instruments. Each of these terms has a very specific meaning and definition and each should be fully understood and quantified properly before being used to convey metrological information for any purpose. This paper summarizes the generally-accepted generic metrological meaning and significance of these terms for the purpose of clarifying any misunderstanding that might otherwise arise between the metrologist and the user of metrological data. These meanings are illustrated by discussing their application to dimensional standards presently available to the semiconductor industry from NIST.

\section{2,320}

PB94-213147 Not available NTIS

National Inst. of Standards and Technology (EEEL) Gaithersburg, MD. Electricity Div.

Custom Integrated Circuit Comparator for HighPerformance Sampling Applications.

Final rept.

O. B. Laug, T. M. Souders, and D. R. Flach. 1992,

Pub. in Proceedings of Institute of Electrical and ElecPub, in Proceedings of Institute of Electrical and Elec-
tronics Engineers Instrumentation and Measurement Technology Conference, Secaucus, NJ., May 12-14, 1992, p437-441

Keywords: "Application specific integrated circuits, "Comparator circuits, Frequency domain, Time domain, Electrical measurement, Electric pulses, Voltage, Performance, Design, Silicon, Reprints, Waveform sampling.

This paper reports on the design and performance of an application specific integrated circuit (ASIC) comparator that has been optimized for equivalent-time waveform sampling applications. The comparator which has been fabricated with an $8.5 \mathrm{GHz} f$ (sub tau) bipolar silicon process, features a bandwidth of $>2$ $\mathrm{GHz}$, a settling-time accuracy of $0.1 \%$ in $2 \mathrm{~ns}$, and al most total elimination of 'thermal tails' in the settling response. Several novel design features that have been used to achieve this level of performance are presented. The comparator can be used in a sampling system for both frequency domain measurements, e.g. wideband rms voltage measurements, and high accuracy time domain pulse measurements.

\section{2,32}

PB94-216413 Not available NTIS

National Inst. of Standards and Technology (EEEL), Gaithersburg, MD. Semiconductor Electronics Div.
High-Level CAD Melds Micromachined Devices with Foundries.

Final rept.

J. C. Marshall, M. Parameswaran, M. Zaghloul, and M. Gaitan. 1992, $8 p$.

Sponsored by Navy Advance Test EquipmenuMetrology Project, San Diego, CA and Army Test Measurements and Diagnostic Equipment Activity, Redstone Arsenal, AL

Pub. in Institute of Electrical and Electronics Engineers Circuits and Devices 8, n6 p10-17 Nov 92.

Keywords: "Computer aided design, "CMOS, Chips(Electronics), Etching, Silicon, Reprints,
"Micromachining, MOSIS service.

The methodology for implementing the design of silicon micromachined devices in a standard CMOS foundry process is discussed, and a modified Magic technology file is introduced. The modified technology file is used to design silicon micromachined devices that are fabricated using a standard CMOS foundry through the MOSIS service. An additional maskless etch in EDP is required to realize the micromechanical structures once devices are delivered The modified technology file implements a layer that we call 'open' that consists of a combination of vias and contact cuts. This open area exposes the silicon surface for an anisotropic etch procedure that creates suspended bridges of polysilicon or metal encapsulated in $\mathrm{SiO}_{2}$. Results from fabricated chips are included.

\section{2,322}

PB94-216629 Not available NTIS

National Inst. of Standards and Technology (EEEL), Gaithersburg, MD. Semiconductor Electronics Div.

Characterization of Interface Defects in OxygenImplanted Silicon Films.

Final rept.

S. Mayo, J. R. Lowney, and P. Roitman. 1993, 8p. Pub. in Jnl. of Electronic Materials 22, n2 p207-214 1993.

Keywords: "Silicon films, "Film resistors, "Photoresistors, "Crystal defects, Ion implantation, Photoconductivity, Annealing, Interfaces, Oxygen, Wafers, Reprints, Photoinduced transient spectroscopy, SOI(Semiconductors), SIMOX

Defects in ungated $\mathrm{n}$ - or $\mathrm{p}$-type and gated $\mathrm{p}$-type resistors have been characterized by photoinduced transient spectroscopy (PITS). These resistors were fabricated with p-type separation by implanted oxygen (SIMOX) wafers with a single-energy 200-keV oxygen implant to a total fluence of $1.8 \times 10($ sup 18$) / \mathrm{sq} \mathrm{cm}$. One wafer, used for gated resistor fabrication was implanted at $595 \mathrm{C}$ and sequentially annealed at 1,325 $\mathrm{C}$ for $4 \mathrm{~h}$ in argon (plus $0.5 \%$ oxygen) followed by 4 $h$ in nitrogen (plus $0.5 \%$ oxygen). Another wafer, used for ungated resistor fabrication, was implanted at 650 $\mathrm{C}$ and annealed at $1,275 \mathrm{C}$ for $2 \mathrm{~h}$ in nitrogen (plus $0.5 \%$ oxygen). Our results indicate that more damage is present in the wafer annealed at $1,275 \mathrm{C}$ than in the one annealed at $1,325 \mathrm{C}$. We estimate the average trap density at the back interface to be in the 10(sup $11) / \mathrm{sq} \mathrm{cm}$ range.

02,323

PB94-216637 Not available NTIS

National Inst. of Standards and Technology (EEEL) Gaithersburg, MD. Semiconductor Electronics Div.

Charge Trapping and Breakdown Mechanism in SIMOX

Final rep

S. Mayo, J. S. Suehle, and P. Roitman. 1992, 2p. Contract DNA-IACRO-88-800

Sponsored by Defense Nuclear Agency, Washington, DC

Pub. in Extended Abstract, Proceedings of 1992 Institute of Electrical and Electronics Engineers International SOl Conference, Ponte Vedra Beach, FL., October 6-8, 1992, p28-29.

Keywords: "Breakdown(Electronic threshold), "Dielectric breakdown, Electron traps, Hole traps, Capacitors, Reprints, "SIMOX, SIMOX(Separation by IMplanted Oxygen), Buried oxides, SOI(Semiconductors).

We have studied the charge build-up mechanism in SIMOX (Separation by IMplanted OXygen) capacitors injected with electrons from the gate or substrate. The breakdown voltage corresponds to electric fields of 10 (sup 7) $\mathrm{V} / \mathrm{cm}$ for electron injection from the gate, or $7.5 \times 10$ (sup 6 ) $\mathrm{V} / \mathrm{cm}$ for electron injection from the substrate. These field values, comparable to breakdown fields in capacitors fabricated with thermally grown ox ides, are reported here for the first time in SIMOX burexides. The breakdown voltage asymmety in the SIMOX structure is determined by differences in interface morphology at the gate or substrate. Injection from the substrate yields higher current under equal bias and results in lower breakdown fields.

02,324

PB95-108510 Not available NTIS National Inst. of Standards and Technology (NEL) Gaithersburg, MD. Precision Engineering Div. Practical Photomask Linewidth Measurements. Final rept.

J. Potzick. 1990 9p.

Pub in Proceedings of Society of Photo-Optical Instrumentation Engineers - Integrated Circuit Metrology, In spection and Process Control IV, San Jose, CA. March 5-6, 1990, v1261, p114-122.

Keywords: "Dimensional measurement, "Integrated circuits, "Line width, "Photomasks, "Lithography, Process control, Comparison, Accuracy, Reprints, Microlithography, Micrometrology

The measurement cycle for practical accurate photomask linewidth measurement is analyzed as a differential measurement--the linewidth to be measured is compared to a known linewidth on a standard photomask. The linewidth measuring instrument is thus a comparator. The conditions necessary for a valid measurement are discussed with regard to both the instrument and the comparison process. The prin ciples discussed here apply to many other types of measurement as well.

02,325

PB95-111522 PC A03/MF A01

National Inst. of Standards and Technology (EEEL) Gaithersburg, MD. Semiconductor Electronics Div User's Manual for the Program MONSEL-1: Munte Carlo Simulation of SEM Signals for Linewidth Metrology.

Special pub.

J. R. Lowney, and E. Marx. Aug 94, 46p, NIST/SP. 400-95.

Also available from Supt. of Docs. as SNOO3-00303282-4.

Keywords: "Integrated circuits, "Dimensional measurement, "Line width, Scanning electron microscopy, Monte Carlo method, Electron scattering, Computerized simulation Programming manuals, Backscattering, Metrology, MONSEL-I computer code, Fortran 77 programming language, $X$-ray lithography, Silicon substrates, Multilayers.

This user's manual is a guide to the FORTRAN code MONSEL-I which is a Monte Carlo simulation of the transmitted and backscattered electron signals in a scanning electron microscope (SEM) associated with line specimen with a trapezoidal cross section. The line is deposited on a multi-layer substrate. The primary purpose of the code is to determine the actual inewidth from measured SEM signals. However, it can be used for many other purposes such as transmission electron microscopy. Future extensions to model secondary electron signals and multiple lines are planned.

\section{6}

PB95-125076 PC A05/MF AO1

National Inst. of Standards and Technology (EEEL), Gaithersburg, MD. Semiconductor Electronics Div.

Standard Reference Materials: Certification of a Standard Reference Material for the Determination of Interstitial Oxygen Concentration in Semiconductor Silicon by Infrared Spectrophotometry. Special pub.

B. Rennex. Aug 94, 85p, NIST/SP-260/121.

Also avaiłable from Supt. of Docs. as $\mathrm{SNOO}^{-003-}$ $03281-6$

Keywords: *Silicon, Concentration(Composition), Infrared spectrophotometers, Certification, Calibration, Interstitials, Semiconductors, Uncertainty, Tables(Data), Graphs(Charts), "Standard reference materials, "Oxygen concentration, Infrared spectrophotometry, Number fraction.

A Standard Reference Material, SRM-2551, has been A fion of interstitial oxygen number fraction (commonly referred to as the oxygen concentration) in semiconductor silicon. This SRM is intended for calibration of infrared spectrophotometers used to measure the $1107 / \mathrm{cm}$ interstitial oxygen peak in silicon. Its purpose 
is to enable its users to improve their measurement agreement. The expanded SRM uncertainty is $0.17 \%$ for the low-oxygen specimens, $0.13 \%$ for the mediumoxygen specimens, and $0.12 \%$ for the high-oxygen specimens. The certifying instrument was a Fouriertransform infrared spectrophotometer which measured the oxygen peak height. Specimens from an earlier international Grand Round Robin (GRR) were used to convert these infrared values to oxygen number fraction (concentration) values. A major source of uncertainty had been measurement drift; this was largely compensated using a control specimen. The remaining sources of uncertainty were instrument reproducibility. nonuniformity in oxygen concentration and thickness over the specimen area, and variation in residual oxygen in the SRM float-zone specimens, each of which float-zone specimens served as the zero-oxygen reference for a measurement. These sources were combined in quadrature to arrive at the above-quoted 2 sigma estimate of expanded SRM uncertainty.

02,327

PB95-141172 Not available NTIS

National Inst. of Standards and Technology (EEEL), Gaithersburg, MD. Semiconductor Electronics Div.

Physics for Device Simulations and Its Verification by Measurements.

Final rept.

H. S. Bennett, and J. R. Lowney. 1994, 41 p.

Pub. in Semiconductors, v59 pt2 p33-73 1994

Keywords: *Semiconductor devices, ${ }^{*}$ Computerized simulation, Ouantum mechanics, Carrier mobility, Bipolar transistors, Space charge, Doped materials, Band theory, Gallium arsenides, Silicon, Plasmons, Phonons, Reprints.

The motivations for using computers to simulate the electrical characteristics of transistors are discussed. Our work and that of others in the area of device physics and modeling are described. We compare conventional device physics with an alternative approach to device physics that is more directly traceable to quantum-mechanical concepts. We then apply this new approach to quasi-neutral regions, space-charge regions, and regions with high levels of carrier injection. Examples of applying quantum-mechanically-based device physics to energy band diagrams for bipolar transistors are given. The limits for using theoretical results from uniform media in numerical simulations of devices with large concentration gradients are discussed. We conclude with a discussion of the requirements for verifying and calibrating device simulators for the submicrometer domain.

\section{2,328}

\section{PB95-143111 PC A05/MF A01}

National Inst. of Standards and Technology (MSEL), Gaithersburg, MD. Polymers Div.

Metrology and Data for Microelectronic Packaging and Interconnection: Results of a Joint Workshop on Materials Metrology and Data for Commercial Electrical and Optical Packaging and Interconnection Technologies. Held in Gaithersburg, Maryland on May 5-6, 1994. Volume 1. Results.

M. A. Schen. Nov $94,90 p$, NISTIR-5520.

See also PB94-152733. Sponsored by Optoelectronics Industry Development Association, Mountain View, CA. and Semiconductor Research Corp., Research Triangle Park, NC.

Keywords: "Circuit interconnections, " Electronic packaging, "Optoelectronic devices, "Metrology, *Microelectronics, "Meetings, Printed circuit boards, Integrated circuits, Chips(Electronics), urement Ceramics, Polymers connections, Flat panel displays, US NIST

This NISTIR documents the results of a joint Industry - University - Government workshop on 'Materials Metrology and Data for Commercial Electrical and Optical Packaging and Interconnection Technologies' conducted on May 5-6, 1994 at the Hilton Hotel in D and was cosponsored by the $\mathrm{Na}$ of Standards and Technology, the Institute for Interconnecting and Packaging Electronic Circuits, the Optoelectronics Industry Development Association, and the Semiconductor Research Corporation. The workshop consisted of eight separate working groups. Four technology-focused working groups met to address the challenges and priorities in materials measurement and data to support the design, manufacture, and reliability assessment of critical electrical and optical packaging and interconnection materials structures, and processes. Four performance-focused working groups also met to address the status, challenges, and gaps in metrology and data to describe the properties of materials utilized in the critical packaging and interconnection materials, structures, and processes identified earlier.

\section{2,329}

PB95-143186 PC A03/MF A01

National Inst. of Standards and Technology (EEEL) Gaithersburg, MD. Semiconductor Electronics Div.

Electronics and Electrical Engineering Laboratory Technical Progress Bulletin Covering Laboratory Events Calendar.

J. M. Rohrbaugh. Sep 94, 44p, NISTIR-5483.

See also PB94-193810.

Keywords: "Electrical measurement,

*Microelectronics, *Optoelectronic devices, "Metrology, "Bibliographies, Integrated circuits, Dimensional measurement, Dielectric breakdown, Josephson junctions, High temperature superconductors, Microwave equipment, Sulfur hexafluoride, Optical fibers, Electric power, YBCO superconductors, Antennas, Body armor, Abstracts, Fiber optic sensors, Test structures, Microfabrication, Cryoelectronics, Magnetic force microscopy, Scanning capacitance microscopy US NIST.

This is the forty-seventh issue of a quarterly publication providing information on the technical work of the $\mathrm{Na}$ tional Institute of Standards and Technology, Electronics and Electrical Engineering Laboratory (EEEL). This issue of the EEEL Technical Progress Bulletin covers the second quarter of calendar year 1994. Abstracts are provided by technical area for both published papers and papers approved by NIST for publication. Major subject headings include the following Fundamental Electrical Measurements: Semiconductor Microelectronics; Signal Acquisition, Processing, and Transmission; Electrical Systems; Law-Enforcement Standards; Product Data Systems (includes ne information tools); Video Technology.

\section{2,330}

PB95-143327 PC A08/MF A02

National Inst. of Standards and Technology, Gaithersburg, MD.

Metrology and Data for Microelectronic Packaging and Interconnection: Results of a Joint Workshop on Materials Metrology and Data for Commercial Electrical and Optical Packaging and Interconnec tion Technologies. Held in Gaithersburg, Maryland on May 5-6, 1994. Volume 2. Presentation Material. M. A. Schen Nov 94,156 p NISTIR-5520-V2.

See also Volume 1, PB95-143111. Sponsored by Optoelectronics Industry Development Association Mountain View, CA. and Semiconductor Research Corp., Research Triangle Park, NC

Keywords: "Circuit interconnections, "Electronic packaging, "Optoelectronic devices, "Integrated circuits, *Meetings, Very large scale integration, Governmen policies, Chips(Electronics), Optical communication, Optical storage, Memory devices, Market surveys Microelectronics, Metrology, Hardware, Optical interconnections.

This volume 2 consists of Appendix $C$, which contains presentations by representatives from Motorola, GTE Laboratories, Cornell University, SRC, IPC, ARPA ITRI, and the National Institute of Standards and Technology (NIST)

02,331

PB95-150058 Not available NTIS

National Inst. of Standards and Technology (EEEL), Gaithersburg, MD. Semiconductor Electronics Div. Monte Carlo Model for SEM Linewidth Metrology. Final rept.

J. R. Lowney, M. T. Postek, and A. E. Vladar. 1994,

Pub. in Proceedings of Society of Photo-Optical Instrumentation Engineers: Integrated Circuit Metrology, Inspection, and Process Control VIII, San Jose, CA February 28-March 2, 1994, v2196 p85-96

Keywords: "Scanning electron microscopy, *Dimensional measurement, "Integrated circuits, * Line width " Edge detection Mathematical models Monte Carlo method, Computerized simulation, Electron transport, Electron scattering, Backscattering, Metrology, Substrates, Silicon, Gold, Reprints, X-ray lithography, Microlithography.

A scanning electron microscope (SEM) can be used to measure the dimensions of the microlithographic features of integrated circuits. However without a good model of the electron-beam/specimen interaction, accurate edge location cannot be obtained. A Monte Carlo code has been developed to model the interaction of an electron beam with a line lithographically produced on a multi-layer substrate. The purpose of the code is to enable one to extract the edge position of a line from SEM measurements. It is based on prior codes developed at NIST, but with a new formulation for the atomic scattering cross sections and the inclusion of a method to simulate edge roughness or rounding. The code is currently able to model the transmitted and backscattered electrons, and the results from the code have been applied to the analysis of electron transmission through a gold line on a thin silicon substrate, such as used in an X-ray lithographic mask. By comparing the predictions of the code with measured data, it is possible to obtain edge positions to the order of + or - $10 \mathrm{~nm}$, which is needed for the advanced lithography projected for the year 2000 . The uncertainty of this measurement is limited by the sample geometry and surface roughness and not by the measurement process.

\section{2,332}

PB95-150066 Not available NTIS

National Inst. of Standards and Technology (EEEL), Gaithersburg, MD. Semiconductor Electronics Div. Transverse Magnetoresistance: A Novel Two-Terminal Method for Measuring the Carrier Density and Mobility of a Semiconductor Layer.

Final rept.

J. R. Lowney, W. R. Thurber, and D. G. Seiler. 1994 3p. 30 May 94

Keywords: "Carrier density, "Carrier mobility, "Magnetoresistance, Semiconductor devices, tellurides, Infrared detectors, Electrical measurement, Cryogenic temperature, Reprints, Magnetic field dependence, Transverse magnetoresistance.

The magnetic-field dependence of the two-terminal magnetoresistance that occurs in rectangularly shaped samples can be used to determine both the free-carrier density and the mobility of a semiconductor layer. An approximate equation for the magnetoresistance was derived for variable length-to-width ratio. This technique was used to determine the electron density and mobility of accumulation layers in n-type at 6 and $77 \mathrm{~K}$. It should be applicable to a wide variety of fabricated devices and allow significant improvements in processing methods and quality control.

\section{2,333}

PB95-150348 Not available NTIS

National Inst. of Standards and Technology (EEEL), Gaithersburg, MD. Semiconductor Electronics Div.

High Temperature Reliability of Thin Film SiO2. Final rept. J. S. Suehle, P. Chaparala, and C. Messick. 1994

Pub in Proceedings of International High Temperature Electronics Conference (2nd), Charlotte, NC., June 5 10, 1994, pVIII 15-VIII 23

Keywords: "Silicon dioxide, "Dielectric breakdown, Temperature range 0400-1000 K, Time dependence, Reliability(Electronics), High temperature, Dielectric films, Reprints.

We present Time-Dependent Dielectric Breakdown (TDDB) test results for thin $\mathrm{SiO} 2$ gate oxides at stress temperatures up to $400 \mathrm{C}$. The data were collected at the wafer level using a specially designed probe station that uses a water-cooled probe card and text fixture. It is demonstrated that these oxides exhibit extrapolated lifetimes in excess of $6.3 \times 10(\exp 10) \mathrm{s}(2000$ years) at $2.0 \mathrm{MV} / \mathrm{cm}$ for a stress temperature of 350 $C$ under positive or negative bias. Our results indicate that the physical mechanism of TDDB does not change significantly up to stress temperatures of $400 \mathrm{C}$. It is necessary to obtain data over a wide range of electric fields and temperatures to distinguish between field acceleration models and to quantify the electric field and emperature dependencies of the thermal activation energy and the field acceleration factor, respectively.

02,334

PB95-150975 Not available NTIS

National Inst. of Standards and Technology (EEEL), Gaithersburg, MD. Semiconductor Electronics Div. 


\section{Semiconductor Devices}

Effects of Heavy Doping on Numerical Simulations of Gallium Arsenide Bipolar Transistors.

Final rept.

M. Tomizawa, T. Ishibashi, H. S. Bennett, and J. R. Lowney. 1992, 10p. epitaxy, Mathematical models, Numerical solution, Sensitivity analysis, Doped materials, Carrier mobility, Carrier density, Carrier lifetime, Gain, Reprints, "Gallium arsenide transistors.

Using the best available physical models is essential for predictive numerical simulations of advanced, high performance GaAs transistors. Among the many input parameters for numerical simulations, the effective intrinsic carrier concentrations, $\mathrm{n}(\mathrm{ie})$ minority carrier mobilities, mu, and recombination lifetimes, tau, are very critical parameters. The results from recent theoretical calculations for $n$ (ie) were implemented into a two-dimensional, drift-diffusion simulator for GaAs transis tors. In order to compare predicted and measured d.c. common emitter gains, NPN GaAs homojunction bipolar transistors with different but heavily doped bases and similarly doped emitters that have widths between 0.1 and 0.45 micrometer were fabricated by molecula beam epitaxy. The predicted gains of $8,25,46,72$ for these transistors agreed very well with their measured gains of $9,22,42$ and 70 at high current, respectively. Without using the new theoretical data for n(ie) but setting $n(i e)$ equal everywhere to the intrinsic carrier concentration, $n(i)$, the predicted gains became 4, 14, 27 and 35 , respectively. Sensitivity analyses on mobilities, lifetimes, and $\mathrm{n}$ (ie) showed that physically correct $\mathrm{n}$ (ie) values are quantitatively very important for predictive simulations of GaAs bipolar transistors.

\section{2,335}

PB95-151155 Not available NTIS

National Inst. of Standards and Technology (CSTL),

Gaithersburg, MD. Thermophysics Div.

Effect of Electrode-Polymer Interf́acial Layers on Polymer Conduction. Part 2. Device Summary.

Final rept.

A. van Roggen, and P. H. E. Meijer. 1988, $11 p$

Pub. in Proceedings of International Symposium on Molecular Electronic Devices (3rd), Arlington, VA., October 6-8, 1986, p427-437 1988.

Keywords: *Electrodes, "Crystals, "Polymers, "Electric devices, Electron tunneling, Experimental design, Interfaces, Conductivity, Molecular electronics, Or ganic semiconductors, Reprints.

In this paper, chain-folded crystals are investigated as possible electronic devices. Active devices, showing negative resistance in the IV curve, have been demonstrated experimentally on single crystals of polymers. The operating principle of these devices is tunneling, which was confirmed by theory. The current paper describes the experimental evidence, the theoretical background with explicit calculations of the expected current-voltage profile, the model for such devices, and points to several possible device types and applications

02,336

PB95-152278 Not available NTIS

National Inst. of Standards and Technology (EEEL) Gaithersburg, MD. Semiconductor Electronics Div.

Electrical Test Structure for Overlay Metrology Referenced to Absolute Length Standards.

Final rept.

M. W. Cresswell, W. B. Penzes, R. A. Allen, E. C.

Teague, L. W. Linholm, and C. H. Ellenwood. 1994

$10 \mathrm{p}$.

Pub. in Proceedings of Society of Photo-Optical Instrumentation Engineers: Integrated Circuit Metrology, Inspection, and Process Control VIII, San Jose, CA. February 28-March 2, 1994, p512-520.

Keywords: "Very large scale integration, "Integrated circuits, "Overlays, Potentiometers(Resistors), Microelectronics, Positioning, Alignment, Metrology, Lithography, Reprints, "Test structures, Coordinate measurement systems, Length standards, Traceability.

This test structure is based on the voltage-dividing potentiometer principle and was originally replicated in a single lithography cycle to evaluate feature placement by a primary pattern generator. A new test structure has now been developed from the single-cycle version and has been used for measuring the overlay of fea tures defined by two different exposures with a stepping projection aligner. The as-measured overlay val- ues are processed by an algorithm which minimizes the effects of nominal random pattern imperfections. The algorithm further partitions measurements of overlay into contributions which derive, respectively, from misregisteration of the image fields projected by the two masks and from the drawn misplacement of features on the masks. The numerical estimates of these contributions so obtained from the electrical measurements were compared with those extracted from the same features by the NIST Line Scale Interferometer. providing traceability to absolute length standards. The two sets of measurements were found to agree to within the several-nanometer uncertainty cited for the line scale interferometer's readings alone. The motivation for this work was to compare the nanometer-level distortions, produced by alternative chucking arrangements, of proximity $X$-ray masks having various support-ring architectures. However, the techniques may also be used to evaluate optical aligner tools and to determine image placement quality on optical reticles with traceability to the International Standard of length, the meter.

\section{2,337}

\section{PB95-152773 Not available NTIS}

National Inst. of Standards and Technology (EEEL), Gaithersburg, MD. Semiconductor Electronics Div.

Exact Solution of the Steady-State Surface Temperature for a General Multilayer Structure.

Final rept.

J. Albers. 1994, 9p.

Pub. in Proceedings of Annual Institute of Electrical and Electronics Engineers Semiconductor Thermal Measurement and Management Symposium (10th), San Jose, CA., February 1-3, 1994, p129-137

Keywords: "Semiconductor devices, "Surface temperature, Thermal conductivity, Fourier analysis, Steady state, Heat flow, Microelectronics, Silicon, Reprints, SOI(Semiconductors), Multilayers, SIMOX.

A recursive technique is developed and is shown to provide the surface temperature of the multilayer steady-state heat flow equation. This recursive technique can be used with any number of layers while incurring only a small increase in computation time for each added layer. For the case of complete, uniform top surface coverage by a heat source, the technique gives rise to the generalized one-dimensional thermal resistance result. An example of the use of the new recursive method is provided by the preliminary calculations of the surface temperature of a buried oxide (SOI, SIMOX) structure coritaining several thicknesses of the surface silicon layers. This new technique should prove useful in the investigation and understanding of the steady-state thermal response of modern multilayer microelectronic structures.

\section{2,338}

PB95-152807 Not available NTIS

National Inst. of Standards and Technology (EEEL), Gaithersburg, MD. Semiconductor Electronics Div.

Comparisons of Measured Linewidths of Sub-Micrometer Lines Using Optical, Electrical, and SEM Metrologies.

Final rept

R. A. Allen, P. Troccolo, J. C. Owen, J. Potzick, and L. W. Linholm. 1993, 10p.

Pub. in Proceeding of Society of Photo-Optical Instrumentation Engineers: Integrated Circuit Metrology, In spection, and Process Control VII, San Jose, CA March 2-4, 1993, v1926 p34-43.

Keywords: "Very large scale integration, "Integrated circuits, "Dimensional measurement, "Line width, Scanning electron microscopy, Optical microscopy, Comparative evaluations, Metal films, Lithography, Substrates, Metrology, Titanium, Quartz, Wafers, Reprints, Test structures.

An investigation is being carried out to determine the ability of three methods of linewidth metrology to meas ure the dimensions of features to less than 0.5 micrometer. The three methods are transmitted-light optical microscopy, electrical test structure, and scanning electron microscopy (SEM). Electrical, SEM, and reflected-light microscopy techniques are widely used for linewidth metrology in VLSI fabrication. However, none of these widely-used techniques currently permits traceability to international standards of length. Transmitted-light optical microscopy allows traceability; however, this technique is applicable only to transparent substrates. To permit the inclusion of transmitted-light optical microscopy in this investigation, $100-\mathrm{nm}$ thick Ti films were patterned using normal VLSI processing techniques on a $150-\mathrm{mm}$ diameter quartz wafer. The cross-bridge resistor test structure was used as this structure has been widely used in industry and it allows the results from all three metrological techniques to be compared. The design bridge widths of the test structures range from 0.4 micrometer to $f 0$ micrometer. The results of these measurements show systematic and uniform offsets between the different techniques. In this paper we discuss the different techniques and describe the observed results.

02,339

PB 95-152914 Not available NTIS

National Inst. of Standards and Technology (EEEL) Gaithersburg, MD. Semiconductor Electronics Div. Physics for Device Simulations and Its Verification by Measurements.

Final rept.

H. S. Bennett, and J. R. Lowney. 1994, $41 \mathrm{p}$ Pub. in Semiconductors, v59 pt2 p33-73 1994.

Keywords: "Computerized simulation, "Transistors, Silicon transistors, Bipolar transistors, Quantum mechanics, Energy bands, Gallium arsenides, Carrier mobility, Calibration, Phonons, Plasmons, Reprints.

The motivations for using computers to simulate the electrical characteristics of transistors are discussed. Our work and that of others in the area of device physics and modeling are described. We compare conventional device physics with an alternative approach to device physics that is more directly traceable to quantum-mechanical concepts. We then apply this new approach to quasi-neutral regions, space-charge regions, and regions with high levels of carrier injection. Examples of applying quantum-mechanically-based device physics to energy band diagrams for bipolar transistors are given. The limits for using theoretical results from uniform media in numerical simulations of devices with large concentration gradients are discussed. Calculations of the effective intrinsic carrier concentrations for gallium arsenide and silicon are also given, along with published data. In addition, calculations of the mobilities for GaAs that are based in part on quantum-mechanical phase shifts are compared with published data. We then conclude with a discussion of the requirements for verifying and calibrating device simulators for the submicrometer domain.

\section{2,340}

PB95-152997 Not available NTIS

National Inst. of Standards and Technology (EEEL), Gaithersburg, MD. Semiconductor Electronics Div.

Experimental Study of Reverse-Bias Failure Mechanisms in Bipolar Mode JFET (BMFET).

Final rept.

G. Busatto. 1993, 7p.

Pub. in Proceedings of Power Electronics Specialists Conference, Seattle, WA., June 20-24, 1993, p482488 .

Keywords: "Field effect transistors, "Bipolar transistors, "Breakdown(Electronic threshold) "Avalanche breakdown, Nondestructive tests, Failure(Electronics), Integrated circuits, Layout, Reprints, Power transistors Second breakdown, Reverse bias.

A systematic, non-destructive, experimental study of the bipolar mode FET (BMFET) behavior during its failure is presented. The variation of the reverse bias safe operating area (RBSOA) for an inductive load with different bias conditions is described. It is shown that the device breakdown is strongly dependent on the reverse current gain. On the basis of the experimental results, insight into the physics of the failure mechanisms is given, and it is shown that most of the RBSOA limitations appear to result from device lay-out problems.

02,341

PB95-153359 Not available NTIS

National Inst. of Standards and Technology (MEL) Gaithersburg, MD. Precision Engineering Div.

Integration of Scanning Tunneling Microscope Nanolithography and Electronics Device Processing.

Final rept.

J. A. Dagata, W. Tseng, J. Bennett, H. Harary, E. A Dobisz, and J. Schneir. 1992, 9p.

Pub. in Jnl. of Vacuum Science and Technology A 10 n4 p2105-2113 Jul/Aug 92

Keywords: "Integrated circuits, Scanning tunneling microscopy, Molecular beam epitaxy, Quantum electronics, Semiconductor devices, Fabrication, Reprints, "Nanoelectronics, "Nanolithography, Reactive ion etching. 
The emerging field of nanoelectronics demands innovative methods to fabricate nanometer-scale structures. Such structures will play a critical role in the quantum-effect device physics of future highly integrated circuit architectures. An integrated approach to compound semiconductor nanostructure fabrication based on scanning tunneling microscope (STM) nanolithography, molecular-beam epitaxy, and reactive ion etching techniques is described. The critical elements of this approach, which have been demonstrated recently, are reviewed. Prospects for the coevolutionary development of nanoelectronics and STM-based fabrication and characterization are considered.

02,342

P895-153656 Not available NTIS

National Inst. of Standards and Technology (EEEL), Gaithersburg, MD. Semiconductor Electronics Div.

Performance of Commercial CMOS Foundry-Compatible Multijunction Thermal Converters.

Final rept.

M. Gaitan, J. Kinard, and D. X. Huang. 1993, 3p.

See also PB95-153664.

Pub. in Proceedings of International Conference on Solid-State Sensors and Actuators (7th), Yokohama Japan, June 7-10, 1993, p1012-1014.

Keywords: "Microwave sensors, "Voltage measuring instruments, Electric current meters, Electric powe meters, Computer aided design, Alternating current Very large scale integration, Low costs, Integrated circuits, Performance, Electrical measurement, CMOS, Reprints, "Thermal converters, Multijunction thermal converters, MOSIS service.

We report on the periormance of multijunction thermal converters (MJTCs) fabricated using commercial CMOS integrated circuit (IC) foundries through MOSIS. Calibration testing shows that the devices are suitable for the measurement of ac voltage, current and power for frequencies above audio using conventional thermal transfer techniques. These devices show promise for applications as low-cost, high-precision RF and microwave power sensors with integrated electronics. The fabrication methodology allows easy integration with VLSI microcircuits using standard design libraries leading to low-cost, foundry-independent products.

02,343

PB95-153664 Not available NTIS

National Inst. of Standards and Technology (EEEL), Gaithersburg, MD. Semiconductor Electronics Div.

Multijunction Thermal Converters by Commercial CMOS Fabrication.

Final rept.

M. Gaitan, J. Suehle, J. R. Kinard, and D. X. Huang 1993, 2p.

See also PB95-153656

Pub. in Proceedings of Institute of Electrical and Electronics Engineers Instrumentation/Measurement Technology Conference, Irvine, CA., May 17-20, 1993, p243-244.

Keywords: Electrical measurement, Commercial sector, Fabrication, Transfer characteristics, Alternating current, Direct current, CMOS, Reprints, "Thermal converters, Multijunction thermal converters.

New multijunction thermal converters fabricated in a commercial CMOS foundry are described and the results of measurements of their ac-dc transfer characteristics are given.

02,344

PB95-153805 Not available NTIS

National Inst. of Standards and Technology (EEEL), Gaithersburo, MD. Semiconductor Electronics Div. Modeling Buffer Layer IGBTs for Circuit Simula. tion.

Final rept.

A. R. Hefner. 1993, 10p

Pub. in Proceedings of Power Electronics Specialists Conference, Seattle, WA., June 20-24, 1993, p60-69.

Keywords: "Bipolar transistors, Application specific integrated circuits, Mathematical models, Dynamic propenties, Reprints, "Insulated gate bipolar transistors, Circuit simulators, Buffer layers.

The dynamic behavior of commercially available buffer layer IGBTs is described. It is shown that buffer laye IGBTs become much faster at high voltages than nonbuffer layer IGBTs with similar low voltage characteristics. Because the fall times specified in manufac- turers' data sheets do not reflect the voltage dependence of switching speed, a new method of selecting devices for different circuit applications is suggested. A buffer layer IGBT model is developed and implemented into the Saber circuit simulator, and a procedure is developed to extract the model parameters for buffer layer IGBTs. It is shown that the new buffer layer IGBT model can be used to describe the dynamic be. havior and power dissipation of buffer layer IGBTs in user-defined application circuits.

\section{2,345}

PB95-161014 Not available NTIS

National Inst. of Standards and Technology (EEEL) Gaithersburg, MD. Semiconductor Electronics Div. Simulating the Dynamic Electro Thermal Behavior of Power Electronic Circuits and Systems.

Final rept.

A. R. Hefner, and D. L. Blackburn. 1993, 10p. Pub. in Institute of Electrical and Electronics Engineers Transactions on Power Electronics 8, n4 p376-385 Oct 93.

Keywords: *Electronic circuits, "Electrical networks, *Thermal analysis, Temperature distribution, Mathematical models, Semiconductor devices, Chips(Electronics), Heat sinks, Integrated circuits, Electric current, Voltage, Reprints, "Circuit simulators, Thermal networks.

A methodology is presented for simulating the dynamic electrothermal behavior of power electronic circuits and systems. In the approach described the simulator solves for the temperature distribution within the semiconductor devices, packages, and heat sinks (thermal network) as well as the currents and voltages within the electrical network. The thermal network is coupled to the electrical network through the electrothermal models for the semiconductor devices. The electrothermal semiconductor device models calculate the electrical characteristics based upon the instantaneous value of the device silicon chip surface temperature and calculate the instantaneous power dissipated as heat within the device. The thermal network describes the flow of heat from the chip surface through the package and heat sink and thus determines the evolution of the chip surface temperature used by the semiconductor device models.

\section{2,346}

P895-161022 Not available NTIS

National Inst. of Standards and Technology (EEEL), Gaithersburg, MD. Semiconductor Electronics Div.

Thermal Component Models for Electro-Thermal Network Simulation.

Final rept.

A. R. Hefner, and D. L. Blackburn. 1993, 11p.

Pub. in Proceedings of Institute of Electrical and Electronics Engineers SEMI-THERM Symposium (9th) Austin, TX., February 2-4, 1993, p88-98.

Keywords: "Electronic circuits, *Electrical networks, "Thermal analysis, Temperature distribution, Temperature measurement, Semiconductor devices, Integrated circuits, Heat sinks, Electric current, Voltage, Reprints, "Circuit simulators, Thermal networks.

A procedure is given for developing thermal compo nent models for electro-thermal network simulation. in the new electro-thermal network simulation methodology, the simulator solves for the temperature distribution within the semiconductor devices, packages, and heat sinks (thermal network) as well as the currents and voltages within the electrical network. Examples of electro-thermal network simulations are given, and the temperature measurement methods used to validate the thermal component models are described.

\section{2,347}

P895-161576 Not available NTIS

National Inst. of Standards and Technology (EEEL), Boulder, CO. Electromagnetic Technology Div.

Verification of Commercial Probe-Tip Calibrations. Final rept.

R. B. Marks, and D. F. Williams. 1993, 5p.

Pub. in Automatic Radio Frequency Techniques Group (ARFTG) Conference Digest (42nd), San Jose, CA. December 2-3, 1993, p37-41.

Keywords: "Integrated circuits, "Electric probes, "Calibration, Electrical measurement, Electronics lab. oratories, Verification, Accuracy, Industry, Reprints, "Probe tip calibration, "Wafer probe stations, Scattering parameters, On wafer calibration, On wafer probes.

We present results of a verification procedure useful in evaluating the accuracy of probe-tip scattering pa- rameter measurements. The procedure was applied to calibrations and measurements performed in industrial aboratories. Actual measurement discrepancies, due primarily to calibration errors, are directly compared to bounds determined by the comparison method. The results demonstrate the utility of the verification technique as well as serious flaws, particularly at high frequencies, in some conventional calibrations.

\section{2,348}

PB95-162129 Not available NTIS

National Inst. of Standards and Technology (MEL) Gaithersburg, MD. Precision Engineering Div.

$X$-ray Mask Metrology: The Development of Linewidth Standards for X-ray Lithography.

Final rept.

M. T. Postek, J. R. Lowney, A. E. Viadar, R. D.

Larrabee, W. J. Keery, and E. Marx. 1993, 15p.

Pub. in Proceedings of Society of Photo-Optical Instrumentation Engineers: Electron-Beam, X-ray, and IonBeam Submicrometer Lithographies for Manufacturing III, San Jose, CA., March 1-2, 1993, v1924 p435-449.

Keywords: "Integrated circuits, "Dimensional measurement, "Line width, "Lithography, "Standards, Scanning electron microscopy, Monte Carlo method, Electron beams, Metrology, Reprints, "X ray lithography, "X ray masks, Transmitted electrons, Secondary electrons.

The calibration of masks used in X-ray lithography has been successfully accomplished in the scanning electron microscope (SEM) by using the transmitted scanning electron detection technique. This has been made possible because these masks present a measurement subject different from most (if not all) other objects used in semiconductor processing because the support membrane is, by design, X-ray transparent. This characteristic can be used as an advantage in electron beam-based mask metrology because, de pending upon the incident electron beam energies, substrate composition and substrate thickness, the membrane can also be essentially electron transparent. The areas of the mask where the absorbe structures are located are essentially X-ray opaque as well as electron opaque. Viewing the sample from a perspective below an X-ray mask (by placing the detector beneath the mask) provides excellent electron signal contrast between the absorber structure and the base membrane. Thus, the mask can be viewed in the transmitted electron detection mode of the SEM and precise, potentially accurate dimensional measurements can be made. Monte Carlo modeling of the transmitted electron signal was used to support this work in order to determine the optimum electron detector position and characteristics. This work represents the potential for the first accurate linewidth measure ment standard measured at NIST in the SEM as well as the potential for linewidth standards for the X-ray lithography community.

\section{9}

PB95-163663 Not available NTIS

National Inst. of Standards and Technology (EEEL), Boulder, Co. Electromagnetic Fields Div.

Comparison of Three Techniques for the Precision Measurement of Amplifier Noise.

Final rept.

D. F Wait $1992,2 p$

Pub. in Proceedings of Conference on Precision Electromagnetic Measurements, Paris, France, June 9-12, 1992, p252-253

Keywords: "Microwave amplifiers, "Electromagnetic noise measurement, Electrical measurement, Superhigh frequency, $\mathrm{X}$ band, Precision, Radiometers, Comparison, Reprints, *Amplifier noise.

This paper discusses three new measurement techniques and the experimental results for precision fourparameter amplifier noise measurements. Two different measurement systems were used with two different types of low-noise, X-band amplifiers. The current measurement accuracy is about + or $-0.2 \mathrm{~dB}$.

02,350

PB95-163671 Not available NTIS

National Inst. of Standards and Technology (EEEL) Boulder, CO. Electromagnetic Fields Div.

Measurement Accuracies for Various Techniques for Measuring Amplifier Noise.

Final rept.

D. F. Wait. 1992, 10p

Pub. in Proceedings of the ARFTG Conference Digest (39th), Albuquerque, NM., June 5, 1992, p43-52.

Keywords: "Microwave amplifiers, "Electromagnetic noise measurement, Electrical measurement, 


\section{ELECTROTECHNOLOGY}

\section{Semiconductor Devices}

Superhigh frequency, $\mathrm{X}$ band, Microwave radiometers, Accuracy, Reprints, "Amplifier noise, Automatic network analyzers

The National Institute of Standards and Technology (NIST) has a program to develop an amplifier noise calibration service. Extensive measurements of the noise for different types of low-noise, X-band (8 - 12 $\mathrm{GHz}$ ) amplifiers were made. This paper concentrates on the accuracy of measuring generalized noise figure for various techniques using the NIST $8-12 \mathrm{GHz}$ noise calibration radiometer and a commercial automatic network analyzer.

\section{2,351}

PB95-163697 Not available NTIS

National Inst. of Standards and Technology (EEEL), Boulder, CO. Electromagnetic Fields Div. Planar Resistors for Probe Station Calibration. Final rept.

D. K. Walker, D. F. Williams, and N. Morgan. 1992, 9p.

Pub. in ARFTG Conference Digest (40th), Orlando, FL. , December 3-4, 1992, p1-9.

Keywords: "Film resistors, Electrical impedance, Planar structures, Equivalent circuits, Photoresist coatings, Nickel chromium alloys, Integrated circuits, Residues, Reprints, "Wafer probe stations, On water probes, On wafer calibration.

This paper investigates the effects of variations in sheet resistance, geometry, distance from the probe tip, and fabrication processes on the impedance of planar nickel-chromium resistors. Resistor reactance is a strong function of film resistance, but depends only weakly on geometry and distance from the probe tip. Photoresist contamination in the resistive film induces more complicated impedance behavior, even at low frequencies. The impact on circuit design and timeand frequency-domain calibrations is considered in light of these results.

\section{2}

\section{PB95-163945 Not available NTIS}

National Inst. of Standards and Technology (EEEL), Boulder, CO. Electromagnetic Fields Div. Calibrating On-Wafer Probes to the Probe Tips. Final rept

D. F. Williams, and R. B. Marks. 1992, 8p.

Pub in Proceedings of the ARFTG Conference Digest (40th), Orlando, FL., December 3-4, 1992, p136-143.

Keywords: "Integrated circuits, "Electric probes, "Calibration, Electrical measurement, Microwave circuits, Accuracy, Reprints, "Probe tip calibration, wafer probes, On wafer calibration.

This paper investigates the accuracy of on-wafer scattering-parameter calibrations at the probe tips. Data show the extent to which certain probe-tip calibrations are consistent with one another and applicable to the characterization of devices or circuits fabricated on different wafers or embedded in different transmissionline media. Calibrations to the probe tips are especially well suited to lower-frequency microwave measurements. Further results demonstrate conditions under which probe-tip calibrations fail.

02,353

PB95-163952 Not available NTIS

National Inst. of Standards and Technology (EEEL), Boulder, CO. Electromagnetic Technology Div,

LRM Probe-Tip Calibrations with Imperfect Resistors and Lossy Lines.

Final rept

D. F. Williams, and R. B. Marks. 1993, 4p.

Pub. in Proceedings of ARFTG Conference Digest (42nd), San Jose, CA., December 2-3, 1993, p32-36.

Keywords: "Integrated circuits, "Electric probes, "Calibration, Electrical measurement, Microwave circuits, Broadband, Reprints, *Probe tip calibration, "Wafer probe stations, Scattering parameters, On wafer calibration, On wafer probes.

The line-reflect-match calibration is extended, without significant loss of measurement accuracy, to accommodate imperfect match standards and lossy lines typical of monolithic microwave integrated circuits. We characterize the match and line standards using an additional line standard of moderate length. The new method provides a practical means of obtaining accurate, wideband calibrations with compact standard sets.

\section{2,354}

PB95-164174 Not available NTIS

National Inst. of Standards and Technology (EEEL), Gaithersburg, MD. Electricity Div.

Anomalous Behavior of a Quantized Hall Plateau in a High-Mobility Si Metal-Oxide-Semiconductor Field-Effect Transistor.

Final rept.

K. Yoshihiro, C. T. Van Degrift, M. E. Cage, and D

Yu. 1992, 11p.

Pub. in Physical Review B 45, n24 p14 204-14 214 15 Jun 92

Keywords: "Field effect transistors, *MOSFET, "Electrical resistance, High electron mobility transis tors, Silicon transistors, Metastable state, Electrica measurement, Anomalies, Reprints, "Quantum Hall effect, Millikelvin temperature.

Measurements at $14 \mathrm{~T}$ and $340 \mathrm{mK}$ of the quantized Hall resistance of the $\mathrm{i}=4$ plateau of a Si metal-oxidesemiconductor field-effect transistor (Si-MOSFET) made with a precision of 0.005 ppm and an accuracy of $0.015 \mathrm{ppm}$ revealed unexpected irregularities. Smooth variations of + or $-0.04 \mathrm{ppm}$ were observed across the plateau even though the Si-MOSFET had a mobility of $1.2 \mathrm{~m}$ (squared) $\mathrm{N} \mathrm{s}$ and a diagonal resistivity less than 0.002 ppm of the plateau resistivity. Furthermore, measurements over a period of severa months indicated that the plateau shape is metastable. A variety of possible causes for these phenomena are discussed, but none provides a satisfactory explanation.

\section{2,355}

PB95-164273 Not available NTIS

National Inst. of Standards and Technology (EEEL) Gaithersburg, MD. Semiconductor Electronics Div.

Electrical Test Structure for Improved Measurement of Feature Placement and Overlay in Integrated Circuit Fabrication Processes.

Final rept.

R. A. Allen, and C. E. Schuster. 1993, 3p.

Pub. in Proceedings of the Government Microcircuit Applications Conference (GOMAC), New Orleans, LA. November 1-5, 1993, p159-161.

Keywords: "Integrated circuits, "Semiconductor devices, *Alignment, Potentiometers(Resistors), Microwave circuits, Millimeter waves, Process control Gallium arsenides, Metrology, Overlays, CMOS, Reprints, *Test structures.

The modified voltage-dividing potentiometer has previously been demonstrated to have a resolution of under $10 \mathrm{~nm}$ when applied to short-loop, single-leve processes. This test structure has recently been ap plied to several full cycle processes, which we are re porting here for the first time. In this paper we describe the successful demonstration of test vehicle implemenations and test results obtained from applying the new design to a GaAs Microwave/Millimeter Wave Mono lithic Integrated Circuits (MIMIC) process and a CMOS Bulk process. The demonstrated success in these sub stantially different processes indicates that these results should apply to a wide range of semiconductor fabrication environments.

02,356

PB95-164356 Not available NTIS

National Inst. of Standards and Technology (EEEL), Gaithersburg, MD. Semiconductor Electronics Div.

Effect of Annealing Ambient on the Removal of Oxide Precipitates in High-Dose Oxygen Implanted Silicon.

Final rept.

S. Seraphin, S. J. Krause, P. Roitman, D. S. Simons, and B. F. Cordts. 1991, 3p.

Sponsored by National Science Foundation, Washington DC.

Pub. in Applied Physics Letters 59, n23 p3003-3005 2 Dec 91.

Keywords: Ion implantation, Oxygen additions, Oxynitrides, Annealing, Ambience, Reprints, "Oxide removal, "SIMOX, Oxide precipitates.

The effect of annealing ambient on the precipitate removal processes in high-dose oxygen implanted silicon (separation by implantation of oxygen (SIMOX)) has been studied with transmission electron microscopy, electron energy-loss spectroscopy, and secondary ion mass spectroscopy. The rate of removal of oxide precipitates from the top silicon layer in SIMOX is higher during annealing in argon than in nitrogen.
The removal is reduced in nitrogen due to the formation of an oxynitride complex at the precipitate surfaces which inhibits oxygen diffusion across the interfaces. Similar effects have been observed for oxide precipitation during nitrogen ambient annealing in bulk silicon.

\section{2,357}

PB95-164679 Not available NTIS

National Inst. of Standards and Technology (MSEL),

Gaithersburg, MD. Ceramics Div.
Influence of Lattice Mismatch on Indium Phosphide Based High Electron Mobility Transistor (HEMT) Structures Observed in High Resolution Monochromatic Synchrotron X-Radiation Diffraction Imaging.

Final rept.

B. Steiner, J. Comas, W. Tseng, and U. Laor. 1993 $6 p$. Sponsored by National Aeronautics and Space Administration, Washington, DC.

Pub. in Materials Research Society Symposia Proceedings, v281 p127-1321993

Keywords: "High electron mobility transistors, *Crystal defects, Molecular beam epitaxy, Synchrotron radiation, Monochromatic radiation, $X$-ray diffraction, High resolution, Indium phosphides, Substrates, Reprints, "Lattice mismatch.

The formation of mismatch dislocations in layered semiconductor structures was found recently in high resolution monochromatic synchrotron $x$-radiation difraction images to be correlated with characteristics of the substrate as well as with the layer thickness and degree of lattice mismatch of nonpseudomorphic layers. We have now extended these studies to examine the accommodation to strain as a function of lattice mismatch in a series of high electron mobility transistor (HEMT) structures grown by molecular beam epitaxy (MBE) on indium phosphide substrates. Five distinct types of irregularity are observed: (1) lattice warping, (2) the formation of a nonpseudomorphic layer, (3) the formation of extended arrays of linear mismatch dislocations at the interface between the substrates and nonpseudomorphic layer, (4) the formation of oval regions of tweed-like local lattice variation imbedded among these arrays, (5) extended tweed-like local lattice variation over large peripheral areas in which the formation of straight mismatch dislocation arrays is not observed.

\section{2,358}

PB95-169397 (Order as PB95-169371, PC A07) MF A02)

National Inst. of Standards and Technology, Gaithersburg, MD.

Optical Characterization in Microelectronics Manufacturing.

S. Perkowitz, D. G. Seiler, and W. M. Duncan. 1994 $36 \mathrm{p}$.

Prepared in cooperation with Texas Instruments, Inc., Dallas.

Included in Jnl. of Research of the National Institute of Standards and Technology, v99 n5 p605-640 Sep/ Oct 94.

Keywords: "Semiconductor devices, "Manufacturing *Quality control, Polarimetry, Infrared spectroscopy Raman Spectra, Photoluminescence.

The six techniques described in the paper (ellipsometry, infrared spectroscopy, microscopy, modulation spectroscopy, photoluminesence, and Raman scattering) were chosen because they are currently or potentially widely used in the industry; they measure a broad array of semiconductor parameters; and they operate in different regions of the electromagnetic spectrum. The discussion of each technique indicates the basic semiconductor quantities measured, gives the scientific basis of the lechnique, and indicates how the measurement is made.

02,359

PB95-169405 (Order as PB95-169371, PC A07) MF A02)

National Inst. of Standards and Technology, Gaithersburg, MD.

Critical Issues in Scanning Electron Microscope Metrology.

M. T. Postek. 1994, 32p

ncluded in Jnl. of Research of the National Institute of Standards and Technology, v99 n5 p641-672 Sep/ Oct 94.

Keywords: "Integrated circuits, "Precision, "Manufacturing, Electron microscopy, Secondary emission, Metrology. 
During the manufacturing of present-day integrated circuits, certain measurements must be made of the
submicrometer structures composing the device with a high degree of repeatability. Optical microscopy, scanning electron miscroscopy, and the various forms of scanning probe microscopies are major microscopical techniques used for this submicrometer metrology. New techniques applied to scanning electron microscopy have improved some of the limitations of this technique and time will permit even further improvements. The paper reviews the current state of scanning electron microscope (SEM) metrology in light of many of these recent improvements.

\section{2,360}

PB95-170395 PC A03/MF A01

National Inst. of Standards and Technology (EEEL), Gaithersburg, MD. Semiconductor Electronics Div.

Electronics and Electrical Engineering Laboratory Technical Progress Bulletin Covering Laboratory Programs, July to September 1994 with 1994/1995 EEEL Events Calendar.

J. M. Rohrbaugh. Nov $94,37 p$, NISTIR-5529. See also PB95-143186.

Keywords: "Electrical

measurement, * Superconducting devices, "Microelectronics, superconductors, Electromagnetic interference, Dimensional measurement, YBCO superconductors, Josephson junctions, Integrated circuits, Microwave equipment, Integrated optics, Video equipment, Mangetic materials, Optical fibers, Photodetectors, Semiconductors, Antennas, Abstracts, SOI(Semiconductors), SIMOX.

This is the forty-eighth issue of a quarterly publication providing information on the technical work of the $\mathrm{Na}$ tional Institute of Standards and Technology, Electronics and Electrical Engineering Laboratory. This issue of the EEEL Technical Progress Bulletin covers the third quarter of calendar year 1994. Abstracts are provided by technical area for both published papers and papers approved by NIST for publication. Major subject headings include the following: Fundemental Electrical Measurements; Semiconductor Microelectronics; Signal Acquisition, Processing, and Transmission; Electrical Systems; Electromagnetic Interference; Product Data Systems; Video Technology.

\section{2,361}

PB95-175097 Not available NTIS

National Inst. of Standards and Technology (EEEL), Gaithersburg, MD. Semiconductor Electronics Div. Status and Trends in Power Semiconductor Devices.

Final rept.

D. L. Blackburn. 1993, 7p

Pub. in Proceedings of IECON ' 93 Institute of Electrical and Electronics Engineers International Conference on Industrial Electronics, Lahaina, Maui, Hawaii, November 15-19, 1993, p619-625.

Keywords: "Semiconductor devices, Electronic packaging, Chips(Electronics), Semiconductor materials, Bipolar transistors, Integrated circuits, Silicon carbides, Electric power, Simulation, Wafers, Trends, Reprints.

A brief description of some recent developments that affect the application of power semiconductor devices is given. Developments in 'chips', packages, simulation, and new materials are included.

\section{2,362}

PB95-175337 Not available NTIS

National Inst. of Standards and Technology (EEEL), Gaithersburg, MD. Semiconductor Electronics Div.

Evidence for a Deep Electron Trap and Charge Compensation in Separation by Implanted Oxygen Oxides.

Final rept.

J. F. Conley, P. M. Lenahan, and P. Roitman. 1992, $7 p$.

Sponsored by Office of Naval Research, Arlington, VA. and Defense Nuclear Agency, Washington, DC

Pub. in Institute of Electrical and Electronics Engineers

Transactions on Nuclear Science 39, n6 p2114-2120

Dec 92.

Keywords: "Electron traps, Electron spin resonance, Ion implantation, Oxygen additions, Reprints, "SOI(Semiconductors), "SIMOX.

We present direct evidence for the creation of deep electron traps in Separation by IMplantation of OXygen buried oxides. In addition, we present combined elec- trical and electron spin resonance evidence which demonstrate that at least some positively charged paramagnetic $E^{\prime}$ centers are compensated by negatively charged centers. Finally, we present evidence which strongly suggests that a substantial fraction of the deep electron traps are coupled to E' centers.

\section{2,363 \\ PB95-175345 Not available NTIS}

National Inst. of Standards and Technology (EEEL), Gaithersburg, MD. Semiconductor Electronics Div. New Test Structure for Nanometer-Level Overlay and Feature-Placement Metrology.

Final rept.

M. W. Cresswell, R. A. Allen, L. W. Linholm, E. C. Teague, C. H. Ellenwood, and W. B. Penzes. 1994 $6 p$.

Pub. in Institute of Electrical and Electronics Engineers Transactlons on Semiconductor Manufacturing 7, n3 p266-271 Aug 94

Keywords: "Microelectronics, "Overlays, "Lithography, Semiconductor devices, Integrated circuits, Process control, Measurement, Photomasks, Metrology, Reprints, Test structures.

A new electrical test structure for overlay measurement has been evaluated by replicating arrays of its complementary components from two different photomasks into a conducting film on a quartz substrate. The features resulting from images projected from the first mask were used as a reference grid which was calibrated by the NIST line-scale interferometer. A first subset of the relative placements of the images projected from the second mask, which were derived from the electrical overlay measurements and the ref erence grid, agreed to within $13 \mathrm{~nm}$ with corresponding measurements made directly by the line-scale interferometer over distances up to $13.5 \mathrm{~mm}$. A second comparison made at another substrate location indicated that gradients of projected feature linewidths across the exposure site may need to be measured and corrected for, in the electrical extraction of overlay.

\section{2,364}

PB95-175824 Not available NTIS

National Inst. of Standards and Technology (EEEL), Gaithersburg, MD. Semiconductor Electronics Div.

Defect Pair Formation by Implantation-Induced Stresses in High-Dose Oxygen Implanted Siliconon-Insulator Material.

Final rept.

J. D. Lee, J. C. Park, D. Venables, S. J. Krause, and P. Roitman. 1994, 6p.

Pub. in Materials Research Society Symposia Proceedings, v316 p753-758 1994

Keywords: *Crystal dislocations, Transmission electron microscopy, $X$ ray diffraction, Crystal dislocations, lon implantation, Temperature dependence, Integrated circuits, Oxygen additions, Doped materials, Microstructure, Annealing, Strains, Silicon, Stresses, Reprints, "SOI(semiconductors), "SIMOX.

Defect microstructure and the near-surface strain of high-dose oxygen implanted silicon-on-insulator material (SIMOX) were investigated as a function of dose implant temperature, and annealing temperature by transmission electron microscopy and high resolution $x$-ray diffraction. Dislocation half loops (DHLs) begin to form by stress assisted climb at a critical stress leve due to implantation-induced damage. DHLs evolve into through-thickness defect (TTD) pairs by expansion during annealing. Both DHL and TTD-pair density increase with higher implant dose and lower implant temperature. Possible methods for defect density reduc tion are suggested based on the results of this study.

\section{2,365}

PB95-176285 Not available NTIS

National Inst of Standards and Technology (EEEL), Boulder, CO. Electromagnetic Fields Div.

On-Wafer Impedance Measurement on Lossy Substrates.

Final rept

D. F. Williams, and R. B Marks. 1994, 2 p.

Pub. in Institute of Electrical and Electronics Engineers Microwave and Guided Wave Letters 4, n6 p175-176 Jun 94

Keywords: "Electrical impedance, "Impedance measurement, Characteristic impedance, Transmission lines, Planar structures, Resistors, Wafers, Reprints Silicon substrates, Scattering parameters.

This paper introduces a new method for measuring impedance parameters in transmission lines fabricated on lossy or dispersive dielectrics. The method, which uses an independent calibration to provide an impedance reference, compares well with conventional techniques when applied to lossless substrates. The effectiveness of the technique is illustrated for resistors fabricated on lossy silicon substrates.

02,366

PB95-176301 Not available NTIS

National Inst. of Standards and Technology (EEEL), Gaithersburg, MD. Semiconductor Electronics Div.

Interaction of Stoichiometry, Mechanical Stress, and Interface Trap Density in LPCVD Si-rich SiNxSi Structures.

Final rept.

S. C Witczak M Gaitan J S Suehle, M. C.

Peckerar, and D. I. Ma. 1994, 10p.

Pub, in Solid-State Electronics 37, n10 p1695-1704 1994.

Keywords: "Silicon nitrides, Chemical vapor deposition, $X$ ray diffraction, Rutherford scattering, Dielectric films, Electron traps, Hole traps, Backscattering, Stoichiometry, Interfaces, Capacitors, Stresses, Wafers, Reprints.

Mechanical and electrical properties were correlated in LPCVD SiN(x)-Si structures through the characterization of six wafers patterned with MNS capacitors whose insulator films were deposited rich in Si under various processing conditions. The samples were measured for mechanical stress at the Si-SiN(x) interface with X-ray diffraction. The deposited $\operatorname{SiN}(x)$ films were measured for stoichiometry by Rutherford backscattering spectroscopy. Low-temperature C-V measurements were used for the first time to estimate $\mathrm{Si}-\mathrm{SiN}(\mathrm{x})$ interface trap densities in the capacitors. The interface trap densities were confirmed with the aid of a model based on a numerical analysis of the capacitor small-single response. The measurement results indicate that an increase in the $\mathrm{Si} / \mathrm{N}$ ratio in the insulating films was accompanied by a decrease in the film tensile stress. Those SiN $(x)$ films made sufficiently rich in Si were successfully deposited under compressive stress. Furthermore, a decrease in the magnitude of the stress was accompanied by a decrease in interface trap densities, suggesting that interfacial mechanical stress may be influential in the formation of $\operatorname{Si}-\operatorname{SiN}(x)$ interface traps. Interface trap densities were lowest in those structures whose insulating films were deposited under compression.

02,367

PB95-180568 Not available NTIS

National Inst. of Standards and Technology (EEEL) Gaithersburg, MD. Semiconductor Electronics Div. Microelectronic Test Structures for Feature Placement and Electrical Linewidth Metrology.

Final rep

L. W. Linholm, R. A. Allen, and M. W. Cresswell. 1994, 28p.

Pub. in Handbook of Critical Dimension Metrology and Process Control, vCR52 p91-118 1994

Keywords: *Integrated circuits, "Microelectronics, "Line width, "Lithography, Overlays, Reviews, Reprints, *Test structures, Registration.

This paper presents a critical review of electrical test methods for determining feature placement with total measurement uncertainties below $10 \mathrm{~nm}$ and electrical linewidth for sub-half micrometer design linewidths with measurement precision below $1 \mathrm{~nm}$. Microelectronic test structures are electrical devices that are used to determine selected tool, process, device, material, or circuit parameters by means of electrical tests. They are supported by a variety of commercial test equipment often found in semiconductor manufacturing facilities. They provide low-cost, post-patterning metrology for determining both feature placement and electrical linewidth. Properly characterized test structures and measurement methods provide an economic means of determining the critical parameters needed to develop, control, and operate the next generation of patterning tools.

\section{2,368}

PB95-180808 Not available NTIS

National Inst. of Standards and Technology (MEL), Gaithersburg, MD. Precision Engineering Div.

Accuracy in Integrated Circuit Dimensional Measurements.

Final rept.

J. E. Potzick. 1993, 10p

Pub. in Proceedings of Society of Photo-Optical Instrumentation Engineers: Critical Review of IC Metrology 


\section{Semiconductor Devices}

and Process Control, Monterey, CA., September 28 29, 1993, p1-10.

Keywords: "Integrated circuits, "Photomasks, *Dimensional measurement, Calibration, Metrology, Length, Accuracy, Reprints.

The measurement of critical dimensions of features on integrated circuits and photomasks is modeled as the comparison of the images of the test object and of a standard object in a measuring device. A length meas uring instrument is then a comparator. The calibration of the standard and the conditions necessary for a valid comparison are discussed. The principles discussed here apply to many other types of measurement as well.

\section{2,369}

\section{PB95-202610 Not available NTIS}

National Inst. of Standards and Technology (EEEL), Boulder, CO. Electromagnetic Fields Div.

Dielectric Properties of Materials at Cryogenic Temperatures and Microwave Frequencies.

Final rept.

R. G. Geyer. 1994, 2p.

Pub. in Proceedings of Conterence on Precision Electromagnetic Measurements Digest, Boulder, CO., June 27-July 1, 1994, p350-351.

Keywords: "Tetrafluoroethylene resins, *Polystyrene, *Quartz, "Teflon, Dielectric properties, Dielectric materials, Microwave frequencies, Temperature range 0065-0273 K, Temperature range 0273-0400 K, Room temperature, Single crystals, Permittivity, Microelectronics, Substrates, Reprints, Dielectric measuretronics, Substrate
ments, Rexolite.

The permittivity and dielectric loss tangent of singlecrystal quartz, cross-linked polystyrene (Rexolite), and polytetrafluoroethylene (Teflon) were measured at microwave frequencies and at temperatures of $77 \mathrm{~K}$ and $300 \mathrm{~K}$ using a dielectric resonator technique. Application of high-temperature superconducting (HTS) films at the endplates of the dielectric resonator made it possible to determine dielectric loss tangents of about $7 \times 10(\exp -6)$ at $77 \mathrm{~K}$. Two permittivity tensor components for uniaxially anisotropic crystalline quartz were measured. Although the permittivities at $77 \mathrm{~K}$ changed very little from their room temperature values at $300 \mathrm{~K}$, large changes in dielectric losses were observed. The decreased loss characteristics of these microelectronic substrates can markedly improve the performance of many microwave devices at cryogenic temperatures.

\subsection{0}

PB95-203170 Not available NTIS

National Inst. of Standards and Technology (EEEL), Gaithersburg, MD. Semiconductor Electronics Div.

Electrical Method for Determining the Thickness of Metal Films and the Cross-Sectional Area of Metal Lines.

Final rept.

H. A. Schafft, S. Mayo, S. N. Jones, and J. S.

Suehle. 1994, 7p.

Pub. in Proceedings of the Integrated Reliability Workshop, Lake Tahoe, CA., October 16-19, 1994, p5-11.

Keywords: "Integrated circuits, "Metal films, "Film thickness, *Dimensional measurement, Circuit interconnections, Reliability(Electronics), Aluminum alloys, Test methods, Microelectronics, Electromigration, Metallizing, Reprints, Thickness measurement, Resistance measurement, Test structures.

The electrical thickness of an aluminum-alloy metallization can be determined from resistance meas urements of a van der Pauw cross structure at two temperatures, with corrections for the deviation from Matthiessen's rule and for thermal expansion. Thickness determinations, made in this way, agree with those made with a calibrated scanning electron microscope (SEM) to within the uncertainty of the instrument. The electrical cross-sectional area of metal lines can be determined by making resistance measurements at two temperatures.

\section{2,371}

PB95-203188 Not available NTIS

National Inst. of Standards and Technology (EEEL) Gaithersburg, MD. Semiconductor Electronics Div.
JEDEC 'TCR' Interlaboratory Experiment: Lessons Learned.

H. A. Schafft, J. S. Suehle, and J. Albers. 1994, 8p. Pub. in Proceedings of the Integrated Circuit Reliability Workshop, Lake Tahoe, CA., October 16-19, 1994, p12-19.

Keywords: "Integrated circuits, Interlaboratory comparisons, Thermal resistance, Joule heating, Line width, Measurement, Precision, Wafers, Standards, Reprints, "Temperature coefficient of resistance, Silicon substrates.

Described are the results of an interlaboratory experiment involving wafer-level measurements intended to do the following: (1) to determine the precision and bias of both the JEDEC Standard Test Method (JESD33) for determining the temperature coefficient of resistance (TCR) and joule heating of a metal line and the ASTM standard (F1261) for measuring the electrical width of a metal line; (2) to assess the reproducibility of measuring the temperature drop across the interface between the silicon substrate and the hot chuck; and (3) to obtain a temperature calibration of the hot chucks used by the participating laboratories.

02,372

PB95-208724 PC A04/MF A01

National Inst. of Standards and Technology (EEEL),

Gaithersburg, MD. Semiconductor Electronics Div.

Electronics and Electrical Engineering Laboratory Technical Progress Bulletin Covering Laboratory Programs, October to December 1994 with 1995 EEEL Events Calendar.

J. M. Rohrbaugh. Mar 95, 56p, NISTIR-5608.

See also PB95-170395.

Keywords: *Electrical engineering, "Semiconductor devices, "Metrology, Signal processing, Electrooptics, Reliability.

This is the forty-ninth issue of a quarterly publication providing information on the technical work of the National Institute of Standards and Technology, Electronics and Electrical Engineering Laboratory. This issue of the EEEL Technical Progress Bulletin covers the fourth quarter of calendar year 1994. Abstracts are provided by technical area for both published papers and papers approved by NIST for publication.

\section{2,373}

PB95-242277 PC A03/MF A01

National Inst. of Standards and Technology (EEEL),

Gaithersburg, MD. Semiconductor Electronics Div.

Electronics and Electrical Engineering Laboratory Technical Publication Announcements Covering Laboratory Programs, January to March 1995 with 1995 EEEI Events Calendar.

J. M. Rohrbaugh. Jun 95, 17p, NISTIR-5669.

See also PB95-231841

"Electrical

*Metrology, "Bibliographies, Antennas, Video equip-

ment, Optical fibers, Semiconductors, Superconductors, Magnetic materials, Electromagnetic interference, Electrical engineering, Lasers, Microwave equipment, Electronics, Abstracts.

This is the forty-fourth issue of a quarterly publication providing information on the technical work of the $\mathrm{Na}$ tional Institute of Standards and Technology, Electronics and Electrical Engineering Laboratory. This issue of the EEEL Technical Publication Announcement covers the first quarter of calendar year 1995. Abstracts are provided by technical areas for papers pub lished. Major subject headings include the following: Fundamental Electrical Measurements: Semiconductor Microelectronics; Signal Acquisition, Processing, and Transmission; Electrical Systems; Electromagnetic Interference; Law Enforcement Standards; Video Technology.

\section{2,374}

PB95-260766 PC A05/MF A01

National Inst. of Standards and Technology (EEEL), Gaithersburg, MD. Semiconductor Electronics Div

Semiconductor Measurement Technology:

HOTPAC. Programs for Thermal Analysis Including Version 3.0 of the TXYZ Program, TXYZ30, and the Thermal MultiLayer Program, TML. Special pub.

J. Albers. Aug 95, 96p, NIST/SP-400-96 Also available from Supt. of Docs. as SN003-003. 03351-1.
Keywords: "Semiconductor devices, "Integrated circuits, "Thermal analysis, "Computer programs, Models, Heat transmission, Steady flow, Steady state, Thermal conductivity, Temperature, Area, Surface temperature, Average, Semiconductor(Materials), TXYZ3.0, TXYZ computer program, HOTPAC software package, Line averaging, Area averaging.

This report presents and discusses a number of recent developments in the steady-state thermal analysis of multiple-layer structures. These include: (1) analytical evaluation of line and area average temperatures, and (2) a recursion relation technique for calculating the steady-state surface temperature of a multilayer structure with an arbitran number of layers. The application of the analytic averaging to the TXYZ code is incorporated in the updated code, TXYZ30, while the multilayer recursion relation solution along with analytic averaging are included in the Thermal Multilayer code TML. Both of these are contained in the HOTPAC software package. The first part of this report contains a discussion of the general elements of the multiple-layer thermal model. This is presented in some detail for the case of the Kokkas three-layer problem and the associated TXYZ code. The previous update of the code TXYZ20, is also discussed.

\section{2,375}

PB95-261947 (Order as PB95-261897, PC A07/

MF A02

National Inst. of Standards and Technology, Gaithersburg, MD.

Using Quantized Breakdown Voltage Signals to Determine the Maximum Electric Fields in a Quantum Hall Effect Sample.

M. E. Cage, and C. F. Lavine. 1995, 8p.

Included in Jnl. of Research of the National Institute of Standards and Technology, v100 n3 p269-276 May/ Jun 95

Keywords: "Quantum electronics, "Hall effect, *Electrical faults, *Electric fields, Electrical measure ment, Electron plasma, Landau damping, Two dimensional flow, Dissipation, Quantum Hall Effect, Nationa Institute of Standards and Technology, Quantized voltage states, Quasi-elastic inter-Landau level scattering.

We estimate the maximum values of the electric field across the width of a GaAs/AlGaAs heterostructure quantum Hall effect sample at several currents when the sample is in the breakdown regime. This estimate is accomplished by measuring the quantized longitudinal voltage drops along a length of the sample and then employing a quasi-elastic inter-Landau level scattering (QUILLS) model to calculate the electric field. We also present a pictorial description of how QUILLS transitions occuring between states distributed across the sample width can be detected as voltage signals along the sample length.

\section{2,376}

PB96-102017 Not available NTIS

National Inst. of Standards and Technology (EEEL), Gaithersburg, MD. Semiconductor Electronics Div.

Exact Recursion Relation Solution for the SteadyState Surface Temperature of a General Multilayer Structure.

Final rept.

J. Albers. 1995, 8p.

Pub. in Institute of Electrical and Electronics Engineers Transactions on Components, Packaging, and Manufacturing Technology, ptA18 n1 p31-38 Mar 95.

Keywords: *Semiconductor devices, "Fourier analysis, * Laplace equation, Electric conductivity, Surfaces Thermal conductivity, Temperature, Steady state, Heat flow, Layers, Reprints, Multiple layers.

A recursion relation technique has been used in the past to determine the surface potential from the multilayer electrical Laplace equation. This has provided for a vastly simplified evaluation of the electrical spreading resistance and four-probe resistance. The isomorphism of the multilayer Laplace equation and the multilayer steady-state heat flow equation suggests the possibility of developing a recursion relation applicable to the multilayer thermal problem. This recursive technique is developed and is shown to provide the surface temperature of the multilayer steady-state heat flow equation.

02,377
PB96-102363 Not available NTIS

National Inst. of Standards and Technology (EEEL), Gaithersburg, MD. Semiconductor Electronics Div. 
MEMS in Standard CMOS VLSI Technology. Final rept.

M. Gaitan. 1995, 5p.

Pub. in Proceedings of Annual Conference on Information Sciences and Systems (29th), Baltimore, MD., March 22-24, 1995, p169-173

Keywords: "Fabrication, "Very large scale integration, "Integrated circuits, "CMOS, Microelectronics, Micromechanics, Metal oxide semiconductors, Thermoelectric materials, Sensors, Computer aided design, Reprints, "Microelectromechanics, MEMS, CAD, IC.

The methodology for the design and fabrication of a class of thermo-electro-mechanical devices and systems that can be fabricated in a commercial CMOS foundry environment is presented. The fabrication technique allows the monolithic integration of the micromechanical devices with CMOS VLSI circuits using standard design methods. Microheating elements and thermoelectric sensors are presented as examples of the application of this technique.

\section{2,378}

\section{PB96-102702 Not available NTIS}

National Inst. of Standards and Technology (EEEL), Gaithersburg, MD. Semiconductor Electronics Div. Model for Determining the Density and Mobility of Carriers in Thin Semiconducting Layers with Only Two Contacts.

Final rept.

J. R. Lowney. 1995, 5p.

Pub. in Jnl. of Applied Physics 78, n2 p1008-1012, 15 Jul 95.

Keywords: "Carriers, "Magnetoresistance, Reprints, Density, Mobility, Semiconductors, Electrical measurement, Coallocation, Hall effect.

A new method for determining the carnier densities and mobilities in a thin semiconducting layer was recently described. It is based on fitting the transverse magnetoresistance of the layers as a function of magnetic field, and it requires only two contacts. To improve the accuracy and generalize the procedure to multicarrier systems, a computer code was written to solve for the magnetoresistance as a function of magnetic field and the length-to-width ratio of a rectangular sample. A nonlinear-least-squares fit was made to the results of the computer model for a single-carrier system. The results for multicarrier systems are discussed. This method is especially useful as a monitor for improving the quality control of the electrical characteristics of thin conducting layers in finished devices. The code is also useful for interpreting standard fourterminal measurements as well.

\section{2,379}

PB96-102710 Not available NTIS

National Inst. of Standards and Technology (EEEL), Gaithersburg, MD. Semiconductor Electronics Div. nals for Linewidth Metrology.

Final rept.

J. R. Lowney. 1995, 6p.

See also PB95-111522

Pub. in Microbeam Analysis 4, p131-136 1995.

Keywords: "Integrated circuits, "Dimensional measurement, "Line width, Reprints, Scanning electron microscopy, Monte Carlo method, Electron scattering, Computerized simulation, "MONSEL-I computer code.

This is a guide to the FORTRAN code MONSEL-II, which is a Monte Carlo simulation of the transmitted, backscattered, and secondary electron signals in a scanning electron microscopy (SEM) associated with lines with a trapezoidal cross section. The lines are deposited on a multilayer substrate. The primary purpose of the code is to interpret the actual linewidths from measured SEM signals. However, it can be used for many other purposes such as corresponding image analysis in transmission electron microscopy.

\section{2,380}

PB96-102876 Not available NTIS

National Inst. of Standards and Technology (EEEL), Gaithersburg, MD. Semiconductor Electronics Div. Buffer Layer Modulation-Doped Field-Effect-Transistor Interactions in the Al0.33Ga0.67As/GaAs Superlattice System.

Final rept.

J. G. Pellegrino, C. A. Richter, J. A. Dura, B.

Roughani, P. M. Amirtharaj, and S. B. Qadri. 1995,

$5 p$.

Pub. in Jnl. of Vacuum Science and Technology A 13, n3 p787-791 May/Jun 95
Keywords: *Electron transport, Superlattices, $X$ ray diffraction, Mobility, Reprints, "Field effect transistors, "Molecular beam epitaxy, MODFET(Modulation Doped Field Effect Transistors)

The correlation between the structural and transport properties for a series of high-quality modulationdoped field-effect transistor (MODFET) structures was made for various growth temperatures. X-ray reflectivity, x-ray diffraction, and magnetotransport measures were used to assess structural quality and transport parameters. Four samples with growth temperatures in the range 500-630 degrees $C$ were examined. The results show a correlation exists between the measured electron mobility and the quality of the interface width, as measured from satellite peaks of the buffer layer In addition, these results show, for the first time to the best of our knowledge, that a direct correlation can be made between $x$-ray reflectivity structural measurements and measured electron mobility of high-quality gallium-arsenide-based MODFETs. Both $x$-ray and transport results suggest a higher-quality structure was obtained at higher growth temperatures.

02,381

PB96-102926 Not available NTIS

National Inst. of Standards and Technology (EEEL), Gaithersburg, MD. Semiconductor Electronics Div. TDDB Characterization of Thin SiO2 Films with Bimodal Failure Populations.

Final rept.

J. Prendergast, J. S. Suehle, P. Chaparala, E.

Murphy, and M. Stephenson. 1995, 7p.

Pub. in Proceedings of the International Reliability Physics Symposium, Las Vegas, NV., April 4-6, 1995. p124-130.

Keywords: "Silicon dioxide, "Dielectric breakdown, Reprints, Time dependence, Reliability(Electronics), Dielectric films, Field acceleration models.

For many years Time Dependent Dielectric Breakdown (TDDB) has been the subject of much controversy Two different field dependencies have been observed and several acceleration models have been suggested. Of the two most popular models one predicts that the $\log 150 \%$ is proportional to the electric field $(E)$ $(1-3)$ while the other predicts that it is proportional to the reciprocal of the electrical field (1/E) (4-6). This paper will help to explain the discrepancies in the electric field dependence observed and demonstrate distinct differences in the TDDB behavior between extrinsic dielectric breakdown

\section{2,382}

PB96-112206 Not available NTIS

National Inst. of Standards and Technology (EEEL), Gaithersburg, MD. Semiconductor Electronics Div.

Effect of Anneal Temperature on Si/Buried Oxide Interface Roughness on SIMOX.

Final rept.

G. Jacobs, A. Genis, and L. P. Allen. 1995, 2p.

Pub. in Proceedings of the Institute of Electrical and Electronics Engineers SOI Conference, Nantucket Island, MA., October 3-6, 1994, p49-50.

Keywords: "Temperature dependence, "Silicon, Reprints, Roughness, *SIMOX, Atomic force microscopy, SOI(Semiconductors)

Fully depleted, thin film silicon-on-insulator SIMOX devices are attractive for their short channel characteristics and high speed compared with bulk silicon. Their potential for applications in low power devices and circuitry places extreme demands upon the starting SIMOX substrate material. Threshold voltage contro for fully depleted SOI devices has been recognized as a key issue for realization of fully depleted SOI technology. To that end examination of the interface roughness between the device silicon layer and the buried oxide of the SOl structure is an active area of materials research and development. In this work, we have used atomic force microscopy (AFM) to measure the interface roughness of SIMOX SOl substrates as function of anneal parameters. This study has shown that the silicon/buried oxide interface roughness is a strong function of the post implant annealing temperature.

02,383

PB96-112248 Not available NTIS

National Inst. of Standards and Technology (PL), Boulder, $\mathrm{CO}$. Time and Frequency Div.
New Model of 1/F Noise in Baw Quartz Resonators. Final rep

F. L. Walls, P. H. Handel, R. Besson, and J. J. Gagnepain. 1992, 7p.

Pub. in Proceedings of the Institute of Electrical and Electronics Engineers Frequency Control Symposium, Hershey, PA., May 27-29, 1992, p327-333

Keywords: "Quartz resonators, "Resonators, "Noise Reprints, Models, Bulk acoustic wave devices, $1 / /$ noise, Electronic noise, Quantum 1/f effect.

The paper presents a new model for predicting the 1 $f$ (flicker) frequency noise in quartz resonators as a function of the unloaded resonator quality factor $Q$ and volume under the electrodes for bulk acoustic wave (BAW) resonators. The functional form of this mode originates from a quantum $1 / f$ theory for scattering of phonons from the primary oscillator mode. Using this new model, we are able to match the $1 / f$ frequency noise observed in the best quartz controlled oscillators and resonators. Quite unexpectedly, this model indicates that the amplitude of $1 / \mathrm{f}$ frequency noise migh be improved by making resonators with smaller electrodes. BVA resonators show approximately a factor of 3 improvements in $1 / f$ frequency noise (Sy(f)) over electroded resonators with the same unloaded $Q$-factor and electrode volume.

02,384

PB96-112297 Not available NTIS

National Inst. of Standards and Technology (EEEL),

Boulder, CO. Electromagnetic Fields Div.

Substrate and Thin Film Measurements.

Final rept.

C. A. Jones. 1994, 13p.

Pub. in Proceedings of Wireless Workshop, Phoenix Scottsdale/Chandler, AZ., October 16-19, 1994, p1-3.

Keywords: "Substrates, "Thin films, "Electromagnetic properties, Reprints, Microwaves, Measurements, Dielectric constants, Loss tangents

This paper describes the use of cavity resonators, coaxial probes, capacitive measurements and dielectric resonators for characterizing the electromagnetic properties of substrates and thin films.

02,385

PB96-112313 Not available NTIS

National Inst. of Standards and Technology (EEEL). Gaithersburg, MD. Electricity Div.

Surging the Upside-Down House: Looking into Upsetting Reference Voltages.

Final rept.

T. S. Key, and F. D. Martzloff. 1994, 6p.

Pub. in Proceedings of the International Conference on Power Quality (3rd): End-Use Applications and Perspectives, Amsterdam, The Netherlands, October 24 27, 1994, p1-6.

Keywords: "Surges, "Electronic equipment, "Circuit protection, "Voltage regulations, "Residential buildings, Telephones, Cable television, Communication equipment, Electric fuses, Power supply circuits, Wiring, Overvoltage, Voltage regulation, Retrofitting, Reprints

Electronic equipment with two input ports-power and communications-can be exposed to damaging differences of voltage across the two ports during surge events. Two exposure scenarios of producing such differences of voltages are explained and illustrated by measurements performed in a replica of a residential or light commercial installation of power, telephone and cable TV wiring. Severa! mitigation methods are described, and one possible retrofit solution is shown.

02,386

PB96-113311 PC A09/MF A03

National Inst. of Standards and Technology, Gaithersburg, MD.

Journal of Research of the National Institute of Standards and Technology, July/August 1995. Volume 100, Number 4. Special Issue: The Gaseous Electronics Conference Radio-Frequency Reference Cell.

1995, 196p.

See also PB96-113329 through PB96-113436 and PB95-261897. Color illustrations reproduced in black and white. Also available from Supt. of Docs. as SN703-027-00065-2.

Keywords: "Research and development, "Metrology, "Standards, "Meetings, RF systems, Microelectronics, Gases, Kinetics, Spectroscopy, Plasmas(Physics), Di- 


\section{Semiconductor Devices}

agnostic techniques, Cells, "Gaseous electronics, NIST(National Institute of Standards and Technology). No abstract available.

02,387

PB96-113329

MF A03)

National Inst. of Standards and Technology, Gaithersburg, MD.

Gaseous Electronics Conference RF Reference Cell: An Introduction.

J. K. Olthoff, and K. E. Greenberg. 1995, 13p.

Prepared in cooperation with New Mexico Univ., Albu querque.

Included in Jnl. of Research of the National Institute of Standards and Technology, v100 n4 p327-339 Jul Aug 95.

Keywords: "Microelectronics, "Gases, "Standards, "Cells, Plasma etching, Radio frequencies, Reactors Research and development, Diagnostic techniques *Gaseous electronics, Reference cell, NIST(National Institute of Standards and Technology).

This paper provides an introduction to the Gaseous Electronics Conference (GEC) RF Reference Cell, and to the articles published in this Special Issue of the Journal of Research of the National Institute of Standards and Technology. A brief summary of the history and purpose of the Reference Cell concept is presented, and recent changes to the GEC Cell design are documented. The paper concludes with highlights of research performed on GEC Cells, and with an appendix of all known publications that present research performed on GEC Cells.

\section{2,388}

PB96-113337

MF A03)

National Inst. of Standards and Technology Gaithersburg, MD.

Current and Voltage Measurements in the Gaseous Electronics Conference RF Reference Cell.

M. A. Sobolewski. 1995,11p.

Included in Jnl. of Research of the National Institute of Standards and Technology, v100 n4 p341-351 Jul/ Aug 95.

Keywords: "Microelectronics, "Gases, "Standards, "Cells, "Meetings, Electric current, Diagnostic techniques, Electric discharges, Plasmas(Physics), Radio frequencies, Electrical impedance, Electrical measurement, Comparison, "Gaseous electronics, Reference cell, NIST(National Institute of Standards and Technology).

Measurements of the electrical characteristics of discharges in the Gaseous Electronics Conference Radio-Frequency Reference Cell are reviewed here. Topics include: common sources of error in the measurements; companisons of current and voltage data among GEC cells; the effects of gas impurities, surface conditions and the external circuitry on the reproducibility of the electrical characteristics; and comparisons of current and voltage data with results of other measurements.

\section{2,389}

PB96-113345

MF A03)

National Inst. of Standards and Technology

Gaithersburg, MD

Optical Emission Spectroscopy on the Gaseous Electronics Conference RF Reference Cell.

J. R. Roberts. 1995, 19p.

included in Jnl. of Research of the National Institute of Standards and Technology, v100 n4 p353-371 Jul Aug 95.

Keywords: "Microelectronics, "Gases, "Standards "Cells, "Meetings, Light emission, Plasmas(Physics) Radio frequency discharge, Spectroscopy, Calibrating, "Gaseous electronics, Reference cell, NIST(National institute of Standards and Technology).

A summary of the experimental observations of the op tical emission from the Gaseous Electronics Con ference (GEC) if Reference Cell plasma will be discussed. Spatially and temporally resolved results are provided for various reference and nonreference conditions, including etching type plasmas. These meas urements provide a detailed description of the temporal evolution of optical emission from different excited atomic states within different atomic and ionic species as well as their radial and axial distributions. Some of the measurements have been placed on a absolute radiometric scale to provide comparisons to model calculations.

\section{2,390}

PB96-113352 (Order as PB96-113311, PC A09/ MF A03)

Sandia National Labs., Albuquerque, NM.

Optical Diagnostics in the Gaseous Electronics Conference Reference Cell.

G. A. Hebner, and K. E. Greenberg. 1995, $10 \mathrm{p}$.

Contracts KC-03-01-03, DE-AC04-94AL85000

Prepared in cooperation with New Mexico Univ., Albuquerque. Dept. of Chemical and Nuclear Engineering. Sponsored by Department of Energy, Washington, DC. Office of Basic Energy Sciences.

Included in Jnl. of Research of the National Institute of Standards and Technology, v100 n4 p373-382 Jul/ Aug 95

Keywords: "Microelectronics, "Gases, "Standards, "Cells, "Meetings, Plasmas(Physics), Absorption, Argon, Electric discharges, Radio frequencies, Electric fields, Helium, Fluorescence, Metastable state, Diagnostic techniques, Plasma processing, NIST(National Institute of Standards and Technology).

A number of laser-induced fluorescence and absorption spectroscopy studies have been conducted using Gaseous Electronics Conference Reference Cells. Laser-induced fluorescence has been used to measure hydrogen atom densities, to measure argon metastable spatial profiles, to determine the sheath electric field, and to infer the electron density and temperature. Absorption spectroscopy, using lamp sources and diode lasers, has been used to measure metastable atom densities in helium and argon discharges and fluorocarbon densities in siticon etching discharges. The experimental techniques and sample results of these investigations are reviewed.

02,391

PB96-113360 (Order as PB96-113311. PC A09/

MF A03)

National Inst. of Standards and Technology, Gaithersburg, MD

Studies of lon Kinetic-Energy Distributions in the Gaseous Electronics Conference RF Reference Cell.

J. K. Oltho

1995, 18p. Included in Jnl. of Research of the National Institute of Standards and Technology, v100 n4 p383-400 Jul/ Aug 95.

Keywords: "Microelectronics, "Gases, "Standards, "Cells, "Meetings, Argon plasma, Oxygen plasma, Irradiation, Kinetic energy, Mass spectroscopy, Radio frequencies, Electric discharges, "Gaseous, Reference cell, Argon-oxygen plasmas, Ion energy analyzers, NIST(National Institute of Standards and Technology). A review is presented of kinetic-energy distribution measurements for ions striking grounded surfaces in a gaseous Electronics Conference (GEC) if Reference Cell. Two experimental arrangements that have been used to measure ion energies in the GEC Cell are described, and a comparison of their performance under different operating conditions is presented. Significant results from ion-energy analysis in the Reference Cell are highlighted, including evidence of effects due to surface conditions on ion sampling, verification of electrical behavior of the cell, inferences about ion-molecule reactions indicated by the shapes of measured ion kinetic-energy distributions (IEDs), and the use of measured IEDs for the validation of theoretical models. The paper concludes with a detailed study of IEDs measured for if plasmas generated in mixtures of argon and oxygen, using both experimental arrangements.

\section{2,392}

PB96-113378 (Order as PB96-113311, PC A09/ MF A03)

Texas Univ. at Dallas, Richardson.

Microwave Diagnostic Results from the Gaseous Electronics Conference RF Reference Cell.

L. J. Overzet. 1995, 14p.

Grant NSF-ECS-9257383

Sponsored by National Science Foundation, Arlington, $\checkmark A$

Included in Jnl. of Research of the National Institute of Standards and Technology, v100 n4 p401-414 Jul Aug 95

Keywords:

"Microwave

transmission,

"Microelectronics, "Gases, "Cells, "Meetings, Electron density, Electric discharges, Microwave interferometers, Reactors, Diagnostic techniques, Plasmas(Physics), Time dependence, "Gaseous electronics, Reference cell, NIST(National Institute of Standards and Technology).

Three groups have published electron density data taken in the Gaseous Electronics Conference (GEC) reference reactor using microwave interferometry. The agreement in the data from these groups at higher pressures is excellent especially when one considers that the GEC reactors involved have some key differences. The electron densities compare favorably in argon, helium, and nitrogen above $33.3 \mathrm{~Pa}(250$ mTorr); but, the measurements tend to diverge some at $13.3 \mathrm{~Pa}$ (100 mTorr) and in $133 \mathrm{~Pa}$ helium above approximately $200 \mathrm{~mA}$. It is speculated that the latter difference occurs as the discharges change from a bulk ionization or a-mode to a secondary electron emission or y-mode, and that this transition occurs at lower voltages and currents for reactors with aluminum electrodes than it does for those with stainless steel electrodes. In addition, time resolved electron densities are presented.

02,393

PB96-113386

MF A03)

Dublin City Univ. (Ireland). Dept. of Physics.

Langmuir Probe Measurements in the Gaseous Electronics Conference RF Reference Cell.

M. B. Hopkins. 1995, 11p.

Included in Jnl. of Research of the National Institute of Standards and Technology, v100 n4 p415-425 Jul/ Aug 95.

Keywords: "Microelectronics, "Gases, "Standards, "Cells, "Meetings, Electrostatic probes, Plasma diagnostics, Reactors, Radio frequencies, "Gaseous electronics, Reference cell, NIST(National Institute of Standards and Technology).

The use of a Langmuir probe system in two GEC cells is reviewed. The major problems associated with probe diagnostics and GEC cell are outlined and discussed. While the data base is still insufficient to give definitive values for many parametes, a number of standard measurements are put forward. The plasma density in argon is $9 \times 10(\exp 9) /$ cubic cm (plus or minus 20\%) at an applied rf voltage of $250 \mathrm{~V}$ ( $500 \mathrm{~V}$ peak to peak) and a gas pressure of $13.3 \mathrm{~Pa}$ (100 mTorr). The electron density scales linearly with applied voltage. The plasma to ground sheath resistance is shown to be very important with a value of 810 Ohms in argon at a pressure of $13.3 \mathrm{~Pa}$ (100 mTorr) and discharge current of $0.1 \mathrm{~A}$. The value of plasma to ground resistance scales inversely with discharge current and sublinear with pressure.

02,394

PB96-113394 (Order as PB96-113311, PC AO9/

MF A03)

Sandia National Labs., Albuquerque, NM.

Inductively Coupled Plasma Source for the Gaseous Electronics Conference RF Reference Cell. P. A. Miller, G. A. Hebner, K. E. Greenberg, P. D. Pochan, and B. P. Aragon. 1995, 13p.

Contract DE-AC04-94AL85000

Prepared in cooperation with New Mexico Univ., Albuquerque. Dept. of Chemical and Nuclear Engineering. and Applied Physics, Inc., Albuquerque, NM. Sponsored by Department of Energy, Washington, DC. and SEMATECH, Austin, TX.

Included in Jnl. of Research of the National Institute of Standards and Technology, v100 n4 p427-439 Jul/ Aug 95.

Keywords: "Microelectronics, "Gases, "Standards, "Cells, "Meetings, Plasma density, Electrostatic probes, Coupling, Plasmas(Physics), Plasma potenNIST(National Institute of Standards and Technology). In order to extend the operating range of the GEC RF Reference Cell, we developed an inductively coupled plasma source that replaced the standard parallelplate upper-electrode assembly. Voltage and current probes, Langmuir probes, and an $80 \mathrm{GHz}$ interferometer provided information on plasmas formed in argon, chlorine, and nitrogen at pressures from $0.1 \mathrm{~Pa}$ to 3 $\mathrm{Pa}$. For powers deposited in the plasma from $20 \mathrm{~W}$ to $300 \mathrm{~W}$, the source produced peak electron densities between $10(\exp 10) /$ cubic $\mathrm{cm}$ and $10(\exp 12) / \mathrm{cubic} \mathrm{cm}$ and electron temperatures near $4 \mathrm{eV}$. The electron density peaked on axis with typical full-width at half maximum of $7 \mathrm{~cm}$ to $9 \mathrm{~cm}$. Discharges in chlorine and nitro- 
gen had biomodal operation that was clearly evident rom optical emission intensity. A dim mode occurred at low power and a bright mode at high power. The transition between modes had hysteresis.

02,395

(Order as PB96-113311, PC A09/ MF A03)

Michigan Univ., Ann Arbor. Dept. of Nuclear Engineering.

Reactive Ion Etching in the Gaseous Electronics Conference RF Reference Cell.

M. L. Brake, J. T. P. Pender, M. J. Buie, P. D.

Pochan, P. A. Miller, A. Ricci, and J. Soniker. 1995, $8 p$.

Contracts DE-AC04-94AL85000, DE-FI04-

89AL588772

Prepared in cooperation with Sandia National Labs. Albuquerque, NM. Sponsored by Department of Energy, Washington, DC. and SEMATECH, Austin, TX. Included in Jnl. of Research of the National Institute of Standards and Technology, v100 n4 p441-448 Jul/ Aug 95.

Keywords: "Microelectronics, "Gases, "Standards, "Cells, *Meetings, Electric discharges, Plasma etching, Wafers, Rates(Per time), Depth, Radio frequencies, "Gaseous electronics, Reference cell, Reactive ion etching. NIST(National Institute of Standards and Technology)

This paper describes the results of using the GEC reference cell as a reactive ion etcher. Silicon wafers with layers of polysilicon and silicon dioxide on crystalline silicon patterns with photoresist have been invesfigated with fluorine and chlorine chemistries. Scanning electron microscopy (SEM), profilometry, and refraction techniques were used to determine the etch parameters such as etch rate, uniformity and selectivity. The discharges are in general monitored by measuring the optical emission spectroscopy and the bias voltages. Depending upon the discharge and chemistry conditions, similar etch rates and etch patterns of different GEC cells were obtained.

02,396

(Order as PB96-113311, PC A09/

$\mathrm{MFA03}$

New Mexico Univ., Albuquerque. Dept. of Chemical and Nuclear Engineering.

Dusty Plasma Studies in the Gaseous Electronics

Conference Reference Cell.

H. M. Anderson, and S. B. Radovanov. 1995, 14p Contract DE-AC04-76DP00789

Sponsored by Department of Energy, Washington, DC. and Sandia National Labs., Albuquerque, NM

Included in Jnl. of Research of the National Institute of Standards and Technology, v100 n4 p449-462 Jul/ Aug 95

Keywords: "Microelectronics, "Gases, "Standards, "Cells, "Meetings, Radio frequencies, Electric discharges, Plasmas(Physics), Dust, Particles, Plasma clouds, Lasers, Light scattering, Trapping, Statistical analysis, "Gaseous electronics, Reference cells LS(Laser light scattering), DLLS(Dynamic laser ligh scattering), NIST(National Institute of Standards and Technology)

A number of important investigations into the formation, growth, charging, transport and consequences of particulate due in plasmas have been made using the Gaseous Electronics Conference Reference Cell as the reactor test-bed. The greatest amount of work to data has been directed toward a better understanding of the role that electrostatic, ion drag, neutral fluid drag and gravitational forces play in governing the dynamic behavior of particles cloud motion. Also statistical correlation's in the fluctuation of scattered laser light intensity (dynamic laser light scattering (DLLS)) can be used to determine information about particle size, motion, and growth dynamics. New results from DLSS experiments performed in the Reference Cell are presented that show process-induced dust particles conined in an electrostatic trap exhibit low-frequency oscillatory motion consistent with charge density wave CDW) motion predicted for strongly coupled Coulomb liquids.

\section{2,397}

PB96-113428

(Order as PB96-113311, PC A09/

MF A03)

Scientific Research Associates, Inc., Glastonbury, CT.
One-Dimensional Modeling Studies of the Gaseous Electronics Conference RF Reference Cell.

T. R. Govindan, and M. Meyyappan. 1995, 10p. Contract DOC-50-DKNA-3-00143

Sponsored by Department of Commerce, Washington, DC.

Def in Jnl of Research of the National Institute Standards and Technology, v100 n4 p463-472 Jul/ Aug 95

Keywords: "Microelectronics, "Gases, "Standards, "Cells, "Mathematical models, "Meetings, Plasmas(Physics). Fluid mechanics, Transport properties, Kinetics, Radio frequencies, Electric discharges, One dimensional, "Gaseous electronics, Ref erence cell, Hybrid model, NIST(National Institute of Standards and Technology).

A review of the one-dimensional modeling studies in the literature of the Gaseous Electronics Conference GEC) reference plasma reactor is presented. Most o the studies are based on the fluid model description of the discharge and some utilize hybrid fluid-kinetic schemes. Both models are discussed here briefly. The models provide a basic understanding of the discharge mechanisms and reproduce several critical discharge features observed experimentally.

\section{2,398}

PB96-113436 (Order as PB96-113311, PC A09/ MF A03)

Houston Univ., TX. Dept. of Chemical-Petroleum Engi-

Two-Dimensional Self-Consistent Radio Frequency Plasma Simulations Relevant to the Gase ous Electronics Conference RF Reference Cell.

D. P. Lymberopoulos, and D. J. Economou. 1995

22p.

Grant NSF-CTS-9216023

Sponsored by National Science Foundation, Arlington, VA., Robert A. Welch Foundation, Houston, TX. and SEMATECH, Austin TX.

Included in Jnl. of Research of the National Institute of Standards and Technology, v100 n4 p473-494 Jul/ Aug 95

Keywords: "Plasmas(Physics), "Simulation "Microelectronics, "Gases, "Standards, "Cells, "Meetings, Argon plasma, Electric discharges, Mathe matical models, Radio frequencies, Two dimensional *Gaseous electronics, Reference cell, RF discharges, NIST(National Institute of Standards and Technology)

Over the past few years multidimensional self-consistent plasma simulations including complex chemistry have been developed which are promising tools for fur thering our understanding of reactive gas plasmas and for reactor design and optimization. These simulations must be benchmarked against experimental data obtained in well-characterized systems such as the Gaseous Electronics Conference (GEC) reference cell. Two-dimensional simulations relevant to the GEC Cel are reviewed in this paper with emphasis on fluid simulation.

\section{2,399}

PB96-117692 PC A05/MF A01

National Inst. of Standards and Technology (EEEL) Gaithersburg, MD. Semiconductor Electronics Div. Semiconductor Measurement Technology: Test Structure Implementation Document: DC Parametric Test Structures and Test Methods for Monolithic Microwave Integrated Circuits (MMICs). Special pub.

C. E. Schuster. Sep 95,96p, NIST/SP-400-97.

Also available from Supt. of Docs. as $\mathrm{SNO03-003-}$ 03368-5. See also PB92-154640. Sponsored by Defense Advanced Research Projects Agency, Arlington, VA. and Air Force Wright Aeronautical Labs., WrightPatterson AFB, OH.

Keywords: "Integrated circuits, "Semiconductor devices, "Microwave circuits, "Metrology, Gallium arsenides, Microelectronics, Fabrication, Expert systems, Microwave frequency converters, Communication equipment Millimeter waves, Test methods, Test structures, MMIC, Monolithic Microwave Integrated circuits.

This document describes a set of microelectronic test structure designs for manufacturers of GaAs MMIC devices. These designs enable the dc measurement of process and device parameters that can be used to diagnose, monitor, compare, and predict the performance of the fabrication process or the devices pro duced. The test structure designs are embodied in a computer-aided design library known as NISTGAAS, which contains 8 types of test structures, implemented in 125 combinations of process layer and size and based on a 2 × 6 probe-pad array. Any design, once fabricated on a wafer, can be probed using commonly available commercial parametric test system equipment. This document specifies how to implement and test each type of test structure and how to analyze the results. It also provides guidance on how to apply the set of test structures at the wafer level. Although NISTGAAS was designed for the process described in this document, it was also designed and demonstrated to be adaptable for other MMIC processes. Since NISTGAAS contains cell designs rather than a chip design, it provides a flexible test structure methodology that also provides the MMIC community with a common reference point for assessing process and device performance.

02,400

PB96-119300 Not available NTIS

National Inst. of Standards and Technology (EEEL) Gaithersburg, MD. Semiconductor Electronics Div. Characterization of Two-Dimensional Dopant Profiles: Status and Review.

Final rept.

A. C. Diebold M. Kump J J Kopanski, and D. G.

Seiler 1994, 20p.

Pub. in Proceedings of the Electrochemical Society, Miami, FL., October 10-14, 1994, v94-33 p78-97.

Keywords: "Transistors, "Computer-aided design, *Models, Verification, Calibrating, Two dimensional, Dopes, Simulation, Microscopy, Spatial resolution, Spectroscopic analysis, Reprints, "Scanning probe methods.

Process and device simulators are being used for the technology computer-aided design (TCAD) of 0.25-micrometers gate-length transistors predicted to begin manufacture in 1998. This paper compares and reviews the status of inverse modeling and direct physical analysis methods to obtain two-dimensional dopant profiles for verification and calibration of these TCAD simulators. Spatial resolution, dopant accuracy, and concentration range requirements are discussed. Direct characterization methods include scanning probe microscopies (scanning capacitance microscopy, scanning tunneling microscopy combined with 'spectroscopy,' Mu-spreading resistance probe, scanning Kelvin probe microscopy, and scanning potential microscopy), electron holography, and tomographic secondary ion mass tomographic spectroscopy.

\section{2,401}

PB96-119516 Not available NTIS

National Inst. of Standards and Technology (EEEL), Gaithersburg, MD. Semiconductor Electronics Div.

Oxidation of $\mathrm{SiC}$.

Final rept.

J. J. Kopanski. 1992, 9p.

Pub. in Properties of Silicon Carbide, Book Chapter $5.2, p 121-129$

Keywords: "Oxidation, "Silicon carbides, Metal oxide semiconductors, Mis(Semiconductors), Chemical vapor deposition, Epitaxy, Wafers, Vacuum deposition, Sublimation, Electrical properties, Semiconductors(Materials), Ceramics, Reprints, Thermal oxidation.

Thermal oxidation of the two most common forms of single-crystal silicon carbide with potential for semiconductor electronics applications is discussed: 3C$\mathrm{SiC}$ formed by heteroepitaxial growth by chemical vapor deposition on silicon, and $6 \mathrm{H}$-SiC wafers grown in bulk by vacuum sublimation or the Lely method. SiC is also an important ceramic and abrasive that exists in many different forms. Its oxidation has been studied under a wide variety of conditions. Thermal oxidation of $\mathrm{SiC}$ for semiconductor electronic applications is discussed in the following section. Insulating layers on $\mathrm{SiC}$, other than thermal oxide, are discussed in Section $C$, and the electrical properties of the thermal oxide and metal-oxide-semiconductor capacitors formed on $\mathrm{SiC}$ are discussed in Section D.

\section{2,402}

PB96-119524 Not available NTIS

National Inst. of Standards and Technology (EEEL), Gaithersburg, MD. Semiconductor Electronics Div. 


\section{Semiconductor Devices}

Defect Formation Mechanism Causing Increasing Defect Density during Decreasing Implant Dose in Low-Dose Simox.

Final rept.

J. D. Lee, J. C. Park, S. Krause, and P. Roitman

1994, 2p.

Pub. in Proceedings of the International IEEE SOI Conference, Nantucket Island, Massachusetts, October 3-6, 1994, p69-70.

Keywords: "Large scale integration, "Defects, "Quality control, Oxygen, Ion implantation, Dosage, Crystal dislocations, Production costs, Wafers, Reprints, SIMOX wafers, Defect density, Defect formation mechanisms.

Silicon-on-insulator material synthesized by oxygen implantation (SIMOX) is a leading candidate for advanced large scale integrated circuit applications due to thickness uniformity and moderate defect density. In the past few years, there has been a significant reduction of the defect density by optimizing processing conditions. Today, commercial SIMOX wafers are available by single implant at a high dose of $1.8 \times$ 10(exp 18)/square $\mathrm{cm}$ (defect density about 10(exp 6)/ square $\mathrm{cm}$ ), single implant at a low-dose of $-0.5 \times$ 10(exp 18)/square $\mathrm{cm}$ (defect density less than 10(exp 3)/square $\mathrm{cm}$ ), and multiple implants/anneals (defect density less than $10(\exp 4)$ /square $\mathrm{cm})$. Studies on defect formation mechanisns may suggest further modification of the processing conditions for both production cost and material quality.

\section{2,403}

PB96-122460 Not available NTIS

National Inst. of Standards and Technology (EEEL), Gaithersburg, MD. Electricity Div.

Progress on the Quantized Hall Resistance Recommended Intrinsic/Derived Standards Practice. Final rept.

R. E. Elmquist. 1995, 7p.

Pub. in National Conference on Standards Laboratories, Dallas, TX., July 16-20, 1995, p647-653.

Keywords: "Electrical resistance, "Device characterization, Fundamental constant, Electron gas, Reprints *Quantum Hall effect.

The quantized Hall resistance (QHR) standard re quires characterization test which determine if and how a particular QHR device should be used as an intrinsic standard. The initial characterization at a qualified QHR laboratory would provide the following: (1) Verification that the device resistance was approximately equal to that of other QHR devices under prescribed conditions; (2) Assurance that the QHR device meets recognized quality construction standards; (3) Determination of the effect of temperature in the range below $1.5 \mathrm{k}$; (4) Determination of the approximate magnetic flux density which must be applied to measure the QHR standard. The device could than be used as a standard in another laboratory, which would be expected to characterize the device using procedures which are given in the Recommended Instrinsic/Denived Standards Practice. Some of the laboratory procedures are described.

\section{2,404}

PB96-122668 Not available NTIS

National Inst. of Standards and Technology (EEEL)

Development of a Standard Reference Material for

Measurement of Interstitial Oxygen Concentration

in Semiconductor Silicon by Infrared Absorption. Final rept.

B. G. Rennex, J. R. Ehrstein, and R. I. Scace. 1994

$1 \mathrm{p}$

See also PB95-125076.

Pub. in Extended Abstract of the Meeting of the Electrochemical Society (186th), Miami, FL., October 9-14, 1994, Abstract No. 469, p738.

$\begin{array}{ll}\text { Keywords: } & \text { "Calibration standards, } \\ \text { *Semiconductors(Materials). } & \text { "Oxygen, }\end{array}$ *Concentrations(Composition), Silicon, Interstices Infrared spectrophotometers, Certification, Reprints, Standard Reference Material, SRM-2551, FTIR(Fourier Transform Infrared Radiation).

A Standard Reference Material, SRM-2551, has been prepared, measured, and certified for the determination of institial oxygen concentration in semiconductor silicon. Its purpose is to assist users in the calibration of infrared spectrophotometers used to measure the $1107 \mathrm{~cm} \mathrm{-1}$ oxygen peak. The SRM uncertainty is $0.17 \%$ for the low-oxygen specimens, $0.13 \%$ for the medium-oxygen specimens, and $0.12 \%$ for the highoxygen specimens.
02,405

PB96-122692 Not available NTIS

National Inst. of Standards and Technology (MSEL), Gaithersburg, MD. Polymers Div.

Electronics Packaging Materials Research at NIST. Final rept.

M. A. Schen, G. T. Davis, F. I. Mopsik, J. R

Manning, C. A. Handwerker, D. T. Read, W. L. Wu, and W. E. Wallace. 1995, 13p

Pub. in Materials Research Society Symposium Proceedings, San Francisco, CA., April 17-21, 1995, v390 p19-31.

Keywords: "Electronic packaging, "Metrology, "Research programs, Electronic components, Microelectronics, Circuit interconnections, Technology innovation, Research management, Reprints, US NIST, coefficient of thermal expansion.

The Materials Science and Engineering Laboratory at NIST has augmented its laboratory-based research in support of the U.S. commercial microelectronics industry by expanding its efforts in electronics packaging interconnection, and assembly $(\mathrm{P} / \mathrm{I} / \mathrm{A})$ materials technologies. In conjunction with industry, university, and other government agency partners, these new NIST efforts target materials technology issues that underlie the priorities contained within the various electronics industry technology roadmaps. A dominant aspect of the laboratory P///A program focuses on the in-situ metrology and data needs associated with the materials and complex material assembles which comprise today's microelectronic components and circuits.

02,406

PB96-122841 Not available NTIS

National Inst. of Standards and Technology (CAML) Gaithersburg, MD. Applied and Computational Mathematics Div.

Parallel Monte Carlo Simulation of MBE Growth. Final rept.

I. M. Beichl, Y. A. Teng, and J. L. Blue. 1995, 7p

Pub. in International Parallel Processing Symposium (9th), Santa Barbara, CA., April 25-28, 1994, p46-52.

Keywords: "Monte Carlo method, “Parallel processing, "Molecular beam epitaxy, Algorithms, Crystal growth, Computerized simulation, Computer software, Sequences(Mathematics), Reprints, MBE(Molecular beam epitaxy).

We present a parallel algorithm for Monte Carlo simulation of molecular beam epitaxial growth (MBE) focusing on the software we have developed and the experiences gained. An efficient sequential method to do MBE simulation was developed that uses a single binary tree to store the rates of occurence of all possible events at a given time. The challenge was to find a way to adapt this method to a parallel machine.

\subsection{7}

PB96-123674 Not available NTIS

National Inst. of Standards and Technology (EEEL), Gaithersburg, MD. Semiconductor Electronics Div. Nano-Defects in Commercial Bonded SOI and SIMOX.

Final rept.

D. K. Sadana, J. Lasky, H. J. Hovel, K. Petrillo, and P. Roitman. 1994, 2p.

Pub. in Proceedings of the International Institute of Electrical and Electronics Engineers SOI Conference, Nantucket Island, MA., October 3-6, 1994, p111-112.

Keywords: "Crystal defects, "Defects, Reprints, *SIMOX, "SOI(Semiconductors), Nano defects.

Two new classes of defects have been identified in commercial SIMOX, plasma thinned BSOI and BESOI materials. The first class of defects are revealed when the materials are treated in concentrated HF, and their density is in the range 10 to the 2nd power - 10 to the third power $\mathrm{cm}-2$. The second class of defects densities of 10 to the 4 th power -10 to the 5 th power $\mathrm{cm}$ -2 are present in both plasma thinned BSOI and BESOI after Secco etching. The defect densities in SIMOX after the secco etching were 10 to the 6th power - 10 to the 7 th power $\mathrm{cm}-2$ which was expected.

\section{2,408}

\section{PB96-135017 Not available NTIS}

National Inst. of Standards and Technology (EEEL), Boulder, CO. Electromagnetic Technology Div.
Thermal Isolation of High-Temperature Superconducting Thin Films Using Silicon Wafer Bonding and Micromachining.

Final rept.

C. A. Bang, J. P. Rice, M. I. Flik, D. A. Rudman, and M. A. Schmidt. 1993, 5p.

Pub. in Jnl. of Microelectromechanical Systems, v2 n4 p160-164 Dec 93.

Keywords: "High temperature superconducting, "Superconductors, "Wafer bonding, "Micromachining, Thin films, Silicon, Reprints, Thermal isolation, Micromachining.

Using a new micromachining technology, thermally isolated thin films of high-temperature superconductor have been microfabricated. The intended application for these structures is in infrared bolometers. A silicon wafer bonding process produces a low thermal mass island of single-crystal silicon on a silicon nitride membrane which provides thermal isolation. The silicon can act as a seed for the epitaxial growth of $\mathrm{YBa} 2 \mathrm{Cu} 3 \mathrm{O} 7$ on a ytria-stabilized zirconia buffer layer. This paper describes the overall concept of the thermally isolated device, and demonstrates that the micromachined structure can be fabricated with high-quality superconducting films.

\section{2,409}

PB96-135181 Not available NTIS

National Inst. of Standards and Technology (EEEL), Boulder, CO. Electromagnetic Technology Div.

\section{Growth of Epitaxial KNbO3 Thin Films.}

Final rept.

T. M. Graettinger, P. A. Morris, A. Roshko, D. J

Lichtenwalner, A. F. Chow, A. I. Kingon, and $O$.

Auciello. 1994, 12p.

Pub. in Materials Research Society Symposium Prop265-276.

Keywords: "Epitaxial growth, "lon beams, "Thin films, Reprints, Morphology, Sputtering, MOCVD.

$\mathrm{KNbO3}$ possesses high nonlinear optical coefficients making it a promising material for frequency conversion of infrared light into the visible wavelength range using integrated optical devices. While epitaxial thin films of $\mathrm{KNbO} 3$ have previously been grown using ion beam sputtering, defects (i.e grain boundaries, domains, surface roughness) in these films resulted in high optical losses and no measurable in-plane birefringence. Previous films were grown on $\mathrm{MgO}$ substrates, which have a greater than $4 \%$ lattice mismatch with $\mathrm{KNbO3}$. In the work reported here, the authors have grown films on $\mathrm{MgO}, \mathrm{MgAl} 2 \mathrm{O} 4, \mathrm{NdGaO} 3$, and $\mathrm{KTaO} 3$ to investigate the role of lattice mismatch on the resulting film quality. Films have also been grown with and without oxygen ion assistance. The orientations, morphologies, and defects in the films were examined using $x$-ray diffraction and AFM to determine their relationships to the growth conditions and substrate lattice mismatch.

02,410

PB96-135355 Not available NTIS

National Inst. of Standards and Technology (EEEL)

Boulder, CO. Electromagnetic Technology Div.

Effects of Etching on the Morphology and Surface Resistance of YBa2Cu3O7-delta Films.

Final rept.

A. Roshko, S. E. Russek, K. A. Trott, J. S. Martens, D. Zhang, S. C. Sanders, and M. E. Johansson.

1995, 4p

Pub. in Institute of Electrical and Electronics Engineers Transactions on Applied Superconductivity, v5 n2 p1733-1736 Jun 95.

Keywords: "Yttrium oxides, "Thin films, Reprints, RHEED, HTS.

The changes in surface morphology and surface resistance of sputtered and laser ablated $\mathrm{YBa} 2 \mathrm{Cu} 3 \mathrm{O} 7$ sigma films both before and after etching have been examined. Six different etchants were used: citric acid, nitric acid, Br-methanol, EDTA, disodium EDTA, and ion milling. The surface morphologies of the films were examined by reflection high energy electron diffraction (RHEED) and atomic force microscopy (AFM), both before and after etching. The surface resistance (Rs) was measured at $94 \mathrm{GHz}$ using a confocal resonator. An amorphous layer was found on the film surfaces after exposure to air. A few of the etches restored some of the surface crystallinity, but most caused increases in the overall surface roughness. Several of 
the wet etches attacked dislocations. Ion milling caused the largest degradation of surface crystallinity and a corresponding increase in (Rs). Some of the chemical etches increased $R$, by less than $15 \%$

02,411

PB96-135389 Not available NTIS

National Inst. of Standards and Technology (EEEL) Boulder, CO. Electromagnetic Fields Div.

LRM Probe-Tip Calibrations Using Nonideal Standards.

Final rept

D. F. Williams, and R. B. Marks. 1995, 4p

See also PB95-163952.

Pub. in Institute of Electrical and Electronics Engineers Transactions on Microwave Theory and Techniques, v43 n2 p466-469 Feb 95.

Keywords: *Integrated circuits, "Electric probes, "Calibration, Electrical measurement, Reprints Microwave circuits, Broadband, "Probe tip calibration, "Wafer probe stations.

The line-reflect-match calibration is enhanced to accommodate imperfect match standards and lossy line typical of monolithic microwave integrated circuits. The authors characterize the match and line standards using an additional line standard of moderate length The new method provides a practical means of obtain ing accurate, wideband calibrations with compact standard sets. Without the enhancement, calibration errors due to imperfection in typical standards can be severe.

02,412

PB96-137765 Not available NTIS

National Inst. of Standards and Technology (EEEL), Boulder, CO. Electromagnetic Fields Div

Microwave Dielectric Properties of Anisotropic Materials at Cryogenic Temperatures.

Final rept.

R. G. Geyer, and J. Krupka. 1995, 3p.

Pub. in Institute of Electrical and Electronics Engineers Transactions on Instrumentation and Measurement v44 n2 p329-331 Apr 95

Keywords: "Cryogenics, Dielectric properties, Micro wave frequencies, Permittivity, Reprints, Crystals, Superconductors, "Anisotropic materials, Dielectric resonators.

The permittivity and dielectric loss tangent of crosslinked polystyrene (Rexolite), polytetrafluoroethylene (Teflon), and single-crystal quartz were measured at microwave frequencies and at tempera tures of $77 \mathrm{~K}$ and $300 \mathrm{~K}$ using a dielectric resonato technique. Dielectric loss tangents as low as $7 \times 10$ to the minus 6th power at $77 \mathrm{~K}$ were determined by applying high-temperature superconducting (HTS) films as the endplates of the dielectric resonator. Two permittivity tensor components for uniaxially aniso tropic crystalline quartz were measured. Although the permittivities at $77 \mathrm{~K}$ changed very little from their room temperature values at $300 \mathrm{~K}$ large changes in dielec tric losses were observed. The decreased losses of these microelectronic substrates can markedly improve the performance of many microwave devices a cryogenic temperatures.

02,413

PB96-137807 Not available NTIS

National Inst. of Standards and Technology (EEEL) Gaithersburg, MD. Semiconductor Electronics Div. Use of Monte Carlo Modeling for Interpreting Scanning Electron Microscope Linewidth Measurements.

Final rept.

J. R. Lowney. $1995,6 p$

Pub. in Scanning, v17 p281-286 1995

Keywords: "Line width, "Lithography, Monte Carlo method, Electron beams, Metrology, Reprints, "X ray lithography, *Scanning electron microscopy.

A scanning electron microscope (SEM) can be used o measure the dimensions of the microlithographic features of integrated circuits. However, without a good model of the electron-beam/specimen interaction, accurate edge location cannot be obtained. A Monte Carlo code has been developed to model the inter action of an electron beam with one or two lines lithographically produced on a multilayer substrate. The purpose of the code is to enable one to extract the edge position of a line from SEM measurements. It is based on prior codes developed at the National institute of Standards and Technology, but with a new for- mulation for the atomic scattering cross sections and the inclusion of a method to simulate edge roughness or rounding. The code is currently able to model the transmitted and backscattered electrons, and the results from the code have been applied to the analysis of electron transmission through gold lines on a thin silicon subtrate, such as is used in an $x$-ray lithographic mask. Significant reductions in backscattering occur because of the proximity of a neighboring line.

\section{2,414}

PB96-138433 Not available NTIS

National Inst. of Standards and Technology (EEEL), Gaithersburg, MD. Semiconductor Electronics Div

Electrical Characterization of Integrated Circuit Metal Line Thickness.

Final rept.

S. Mayo, and H. A. Schafft. 1995, 8p

Pub. in Solid-State Electronics, v38 n12 p1993-2000 1995.

Keywords: "Aluminum, "Film thickness, "Integrated circuits, Interconnect lines, Metallization, Reprints, Matthiessen's rule.

Resistance measurements of thin aluminum-silicon alloy lines, 10 and 30 microm wide were made at various temperatures in the $9.2-295.5 \mathrm{~K}$ range. Deviations from Matthiessen's rule were observed over the whole temperature range. At temperatures near room ambient data for these lines are in good agreement with those reported for aluminum-copper alloy wires. A formalism was developed to calculate line thickness and cross-sectional area from electrical resistance data. Line thickness calculations are in good agreement with thickness data measured via scanning electron microscopy. The stress distributions in these lines were modeled by using finite element stress analysis. The results show large stress gradients localized at the line edge region, whereas at the central part of the line there is a high stress value and a low stress gradient. In submicrometer lines the whole line body is under large stress gradients.

\section{2,415}

PB96-141106 Not available NTIS

National Inst. of Standards and Technology (EEEL), Boulder, CO. Optoelectronics Div.

Cross-Sectional Photoluminescence and Its Application to Buried-Layer Semiconductor Structures. Final rept.

D. T. Schaafsma, and D. H. Christensen. 1995, 6p Pub. in Jnl. of Applied Physics, v78 n2 p694-699 Jul 95.

Keywords: "Semiconductors, "Photoluminescence, Quantum wells, Lasers, Emission spectra, Reprints, Burried-layers, VCSEL.

We present an overview of a cross-sectional scanning microphotoluminescence technique that is used to examine various buried-layer semiconductor structures for which traditional surface-normal techniques cannot yield sufficient information or must be coupled with time-consuming and painstaking processes such as wet etching. This technique has a wide range of application; two-- defect-driven interdiffusion in quantum wells and the modificaton of spontaneous emission from quantum wells in vertical-cavity surface-emitting lasers (VCCSELs)-are discussed here. The data obtained using this method can be used to distinguish emission spectra from quantum wells as little as one micrometer apart in depth and a few nanometers different in wavelength. The comparison of normal incidence with cross-sectional data from VCSELs can be used to more effectively optimize the match between cavity resonance and quantum well emission in high$Q$ devices.

\section{2,416}

PB96-146634 Not available NTIS

National Inst. of Standards and Technology (MSEL), Gaithersburg, MD. Reactor Radiation Div.

In-situ Neutron Reflectivity of MBE Grown and Chemically Processed Surfaces and Interfaces.

Final rept.

J. A. Dura, and C. F. Majkrzak. 1996, 6p

Pub. in Proceedings of International Workshop on Semiconductor Characterization: Present Status and Future Needs, Gaithersburg, MD., January 30-February 2, 1995, p549-554 1996.

Keywords: "Molecular beam epitaxy, "Neutron sources, "Reflectivity, Crystal growth, Semiconductor devices, Quantitative analysis, Isotopic labeling,
Composition(Property), Xray analysis, Comparative evaluations, Reprints, MBE(Molecular beam epitaxy) In situ neutron reflectivity.

Several properties make neutron reflectivity particuarly suited to investigations of semiconductor strucures produced by both molecular beam epitaxy (MBE) and chemical means. Reflectivity can provide quantitative information about composition, layer thickness and interface width. Since the scattering length for neutons depends upon nuclear interactions, in contrast to $x$-rays, a large difference in scattering length can occur between systems of similar atomic number and one can employ isotope tagging at a particular fabrication step to determine its effect in the finished structure. In this paper we describe a new facility for in-situ neutron reflectivity and related measurements within a MBE chamber, review the principles underlying reflectivity and grazing angle diffraction, discuss the unique capabilities of neutrons in comparison with $\mathrm{X}$-rays, and give some examples of applications in which neutron reflec tivity can be uniquely utilized.

02,417

PB96-146774 Not available NTIS

National inst. of Standards and Technology (MEL)

Gaithersburg, MD. Precision Engineering Div.

Progress Toward Accurate Metrology Using Atomic Force Microscopy.

Final rept.

T. McWaid, J. Schneir, J. S. Villarrubia, R. Dixson,

and V. W. Tsai. 1996, 5p.

Pub. in Semiconductor Characterization Present Status and Future Needs, p313-317 1996.

Keywords: "Metrology, "Accuracy, "Microscopy, Microstructure Calibration standards, Reprints, AFM(Atomic force microscopy), Critical dimension.

Accurate metrology using atomic force microscopy (AFM) requires accurate control of the tip position, an estimate of the tip geometry and an understanding of the tip-surface interaction forces. We describe recen progress at National Institute of Standards and Technology (NIST) towards accurate AFM metrology. We begin with a brief introduction to the potential applications of accurate AFM metrology within the semiconductor industry. Next, we describe the technological infrastructure required before industry personnel can successfully use AFM for repeatable, reproducible, and accurate measurements. NIST's programmatic response to the identified needs is then outlined. We give particular attention to the developmen of a calibrated atomic force microscope (C-AFM), since the development of this instrument is essential to the successful completion of most of our specific projects.

\section{2,418}

PB96-148044 Not available NTIS

National Inst. of Standards and Technology (EEEL)

Boulder, CO. Electromagnetic Technology Div.

Josephson D/A Converter with Fundamental Accuracy.

Final rept.

C. A. Hamilton, C. J. Burroughs, and R. L. Kautz.

1995, 3p.

See also AD-A110 081/7

Pub. in Institute of Electrical and Electronics Engineers Transactions on Instrumentation and Measurement, v44 n2 p223-225 Apr 95

Keywords: "Josephson junctions, "Converters, Electric potential, Standards, Accuracy, Arrays, Reprints, Voltage standard.

A binary sequence of series arrays of shunted Josephson junctions is used to make a 14-b D/A converter With 13 bias lines, any step number in the range -8192 to $+8192-1.2 \mathrm{~V}$ to $+1.2 \mathrm{~V}$ can be selected in the time required to stabilize the bias current (a few microseconds). The circuit is a fast accurate dc reference, and it makes possible the digital synthesis of ac waveforms whose amplitudes derive directly from the internationally accepted definition of the volt

02,419

PB96-148051 Not available NTIS

National Inst. of Standards and Technology (EEEL) Boulder, CO. Electromagnetic Technology Div. Performance and Reliability of NIST $10-\mathrm{V}$ Joseph. son Arrays.

Final rept.

C. A. Hamilton, and C. J. Burroughs. 1995, 3p.

Pub. in Institute of Electrical and Electronics Engineors Transactions on Instrumentation and Measurement, v44 n2 p238-240 Apr 95 


\section{ELECTROTECHNOLOGY}

\section{Semiconductor Devices}

Keywords: "Josephson junctions, "Reliability, "Arrays, Superconductivity, Electric potential, Standards, Fabrication, Niobium, Lead(Metal), Aluminum oxides, Niobium oxides, Reprints.

This paper reviews eight years of fabrication of $10-\mathrm{V}$ Josephson array chips at National Institute of Standards and Technology (NIST), and the performance and reliability of these chips at 22 different standards laboratories. Failure mechanisms and statistical data on failure rates are presented for devices made with both $\mathrm{Nb} / \mathrm{Nb} 2 \mathrm{O} 5 / \mathrm{Pb}$ and $\mathrm{Nb} / \mathrm{Al} 2 \mathrm{O} 3 / \mathrm{Nb}$ junctions.

\section{2,420}

\section{PB96-148168 Not available NTIS}

National Inst. of Standards and Technology (EEEL), Gaithersburg, MD. Semiconductor Electronics Div. Measurement of Patterned Film Linewidth for Interconnect Characterization.

Final rept.

L. W. Linholm, R. A. Allen, M. W. Cresswell, H. A.

Schafft, J. A. Kramar, E. C. Teague, R. N.

Ghoshtagore, and S. Mayo. 1995, 4p.

Pub. in Proceedings of the Institute of Electrical and Electronics Engineers Conference on Microelectronic Test Structures, Nara, Japan, March 22-25, 1995, v8 p23-26 Mar 95

Keywords: "Integrated circuits, "Microelectronics, Semiconductors(Materials), Electrical measurement, Resisters, Test equipment, Conductors, Line width, Reprints.

Test results from high-quality electrical and physical measurements on the same cross-bridge resistor test structure with approximately vertical sidewalls have shown differences in linewidth as great as $90 \mathrm{~nm}$ for selected conductive films. These differences were independent of design linewidth. As dimensions become smaller, the accurate measurement of the patterned conductor width is necessary to assure predictable timing performance of the interconnect system as well as control of critical device parameters.

\section{2,421}

PB96-155478 Not available NTIS

National Inst. of Standards and Technology (EEEL), Gaithersburg, MD. Semiconductor Electronics Div.

Methodology for the Certification of Reference

Specimens for Determination of Oxygen Concentration in Semiconductor Silicon by Infrared Spectrophotometry.

Final rept.

B. G. Rennex, J. R. Ehrstein, and R. I. Scace. 1996

$6 p$

Pub. in Jnl. of the Electrochemical Society, v143 n1 p258-263 1996.

Keywords: "Silicon, "Semiconductors, Fourier transform spectrometers, Interstitial, Oxygen, Reprints, Standards, FTIR.

The methodology and experiment for certification of reference specimens for determining interstitial oxygen concentration in semiconductor silicon are reported. These reference specimens are intended for calibration of infrared spectrophotometers which measure the $1107 \mathrm{~cm}$ to the minus 1 power oxygen peak in silicon to enable users to improve their measurement agreement. Based on an earlier international Grand Round Robin study, this measurement agreement is at best $5.4 \%$ (2(sigma)). Industry requirements for measure. ment comparison are much more demanding, and a methodology to satisfy those requirements is described. The most important aspect of this methodology is to reduce interlaboratory variation by the use of a single infrared instrument for certification. The certification uncertainty depends primarily on the improved repeatability of this instrument. Other sources of uncertainty were nonuniformity in both oxygen concentration and thickness over the specimen area, and variations in residual oxygen among the float-zone specimens which provided zero-oxygen reference for the reference sets. These various sources were combined in quadrature to arrive at 2 (sigma) estimates of uncertainty under $0.2 \%$ at three oxygen levels.

\section{2,422}

PB96-156070 Not available NTIS

National Inst. of Standards and Technology (EEEL)

Gaithersburg, MD. Semiconductor Electronics Div.
Micromachined Display Output for a Cellular Neural Network.

Final rept

R. Yentis, C. Zincke, M. E. Zaghloul, and M. Gaitan. $1995,4 p$

Pub. in Proceedings of the Institute of Electrical and Electronics Engineers International Symposium on Circuits and Systems, Seattle, Washington, April 30-May 3, 1995, p660-663.

Keywords: "Neural networks, "Display devices, "Computer-aided design, Integrated circuits, Micro electronics, Chips(Electronics), CMOS, Input/output processing, Very large scale integration, Sensors, Design analysis, Reprints, "Micromachining, MOSIS system, MEMS(Microelectromechanical System).

A major problem with current Cellular Neural Networks is that the size of the network is partially limited by the number of input/output pins. This paper address this issue by combining a thermal pixel with the output of each cell. The output of all of the cells can then be seen in parallel with the aid of a thermal camera of other device located off-chip. THe layout for the cell is given and discussed. The chip was fabricated through the MOSIS service.

02,423

PB96-160668 Not available NTIS

National Inst. of Standards and Technology (PL) Gaithersburg. MD. Physics Lab. Office.

Interfaces in Mo/Si Multilayers.

Final rept.

J. M. Slaughter, P. A. Kearney, D. W. Schulze, E. B. Saloman, R. N. Watts, C. M. Falco, and C. R. Hills 1990, 10p

Pub. in Society of Photo-Optical Instrumentation Engineers X-ray/EUV Optics for Astronomy, Microscopy, Polarimetry, and Projection Lithography, San Diego, CA., July $9-13,1990, v 1343$ p73-82

Keywords: "Interfaces, "Reflective coatings, "Molybdenum, "Silicon, Epitaxy, Surface properties, ray diffraction, Synchrotron radiation, Spectroscopic analysis, Chemical analysis, Reprints.

$\mathrm{Mo} / \mathrm{Si}$ multilayer mirrors with high peak reflectivities have been fabricated in many laboratories. This mate rial pair works very well for wavelengths between 125 Angstroms adn 250 Angstroms, and is therefore very useful in optics for astronomy and microscopy. How ever, complete understanding of the properties of these structures is presently limited by lack of under standing of the details of the interfaces. We report re sults from a study of $\mathrm{Mo} / \mathrm{Si}$ interfaces performed with state-of-the-art surface science instruments and electron microscpoy. Low-angle $x$-ray diffraction and synchrotron radiation characterization results for multilayers prepared under a variety of conditions are reported.

\section{2,424}

PB96-160718 Not available NTIS

National Inst. of Standards and Technology (MEL) Gaithersburg, MD. Precision Engineering Div.

Metrology Standards for Advanced Semiconducto Lithography Referenced to Atomic Spacings and Geometry.

Final rept.

E. C. Teague, L. W. Linholm, M. W. Cresswell, F. E Scire, J. S. Villarrubia, J. S. Jun, W. B. Penzes, and J. A. Kramar. 1993, 5p.

Pub. in Proceedings of the Institute of Electrical and Electronics Engineers International Conference on Microelectronic Test Structures, v6 p213-217 Mar 93. Keywords: "Metrology, "Images, Semiconductors(Materials), Lithography, Masks, Heterodyning, Microelectronics, Reprints, CD metrology, Image placement, Mask metrology, Overlay error, Nanometrology.

This paper outlines how the needs for calibrating the positional accuracy of features on an $\mathrm{X}$-ray mask membrane or an opticalreticle can be addressed by application of a high-accuracy coordinate metrology system known as the NIST Molecular Measuring Machine (MCubed). Based on scanning tunneling microscopy and state-of-the-art heterodyne optical interferometry, the measurements of $M$-cubed are referenced to fundamental standards of length and angle and with the damental standards of length and angle and with the probe will be validated against the interatomic spacings and geometry of single crystal surfaces.

02,425

PB96-160726 Not available NTIS
National Inst. of Standards and Technology (MEL), Gaithersburg, MD. Precision Engineering Div. Nanometrology.

Final rept.

E. C. Teague. 1991, 37p.

Pub. in AIP Conference Proceedings 241, Scanned Probe Microscopy, Santa Barbara, CA., January 1-11, 1991, p1.37 Feb 91.

Keywords: "Metrology, Heterodyning, Transducers, Flexing, Bearings, Microscopy, Interferometry, Reprints, Nanometrology, Probe geometry, Scanning tunneling microscopy.

Nanometrology is defined here as the science of measuring the dimensions of objects or object features to uncertainties of one nanometer or less. Four major tasks of nanometrology are examined: realizing a metric in the measurement space; establishing the metric in a coordinate reference frame; generating and measuring repeatable motion relative to the coordinate reference frame; and linking the testpiece to the coordinate reference frame/metric by a probe. Optical scales, $x$-ray interferometry, heterodyne interferometry, FabryPerot etalons, impedance-based transducers, and STM scales are evaluated as possible means to realize a metric.

\section{2,426}

PB96-160783 Not available NTIS

National Inst. of Standards and Technology (MEL), Gaithersburg, MD. Precision Engineering Div.

Scanned Probe Microscopies: Opportunities and Issues in Metrology.

Final rept.

E. C. Teague. 1991, $4 p$

Pub. in Scanning Probe Microscopies: STM and Beyond, Proceedings 241, Santa Barbara, CA., January $1-11,1991,4$ p Feb 91

Keywords: "Metrology, "Integrated circuits, "Trends, Electronics industry, Microelectronics, Surfaces, Geometry, Microscopy, Scanning, Requirements, Reprints, Frequency metrology, Atomically-flat surfaces, Scanned probe microscopy.

Projections about manufacturing trends in the integrated circuit industry are made which indicate that by 2001 uncertainty requirements for dimensional metrology will be less than 1 nanometer. Brief arguments are presented that new scanning probe microscopes offer many opportunities to meet this need because they have the potential of reducing the uncertainty of locating a feature on a testpiece and because they provide new access to the highly ordered atomic structure of crystal surfaces. Such access has much potential for giving greatly increased accuracy for generating reference lines and geometry against which motion of machine elements can be measured and characterized. The need for large-area, atomically-flat surfaces is discussed.

02,427

PB96-163787 Not available NTIS

National Inst. of Standards and Technology (EEEL), Gaithersburg, MD. Semiconductor Electronics Div. High-Accuracy Principal-Angle Scanning
Spectroscopic Ellipsometry of Semiconductor Interfaces.

Final rept.

N. V. Nguyen, D. Chandler-Horowitz, J. G.

Pellegrino, and P. M. Amirtharaj. 1996, 5p

Pub. in Proceedings of the International Workshop on Semiconductor Characterization: Present Status and Future Needs, Gaithersburg, MD., January 30-February 2, 1995, p438-4421996.

Keywords: "Semiconductor interfaces, "Principal angle, Reprints, Interfaces, "Spectroscopic ellipsometry, Strain roughness.

A high-performance spectroscopic ellipsometer has been custom built, in house at NIST, based on the commonly used rotating-analyzer configuration. The data accuracy was highly enhanced by using the principal-angle scanning technique. This technique requires an accurate setting of the angle of incidence which is accomplished by an interferomter and highprecision goniometers for the sample stage and the polarizer. For each wavelength, the principal angle of incidence was automatically searched to obtain a 90 s phase shift of the polarized light upon reflection, i.e., delta $=90$ degrees, and the polarizer azimuth was set to psi. At this condition, the ac component of detected intensity is near a null. With zone averaging, systematic errors such as the detector nonlinearity, and the 
analyzer and polarizer calibration constants, are minimized. To illustrate the use and capability of this system, the authors use the results of a recent study of the optical properties of $\mathrm{SiO} 2 / \mathrm{Si}$ system and, in particlar, the transitional region, defined as an interlayer between the thermally grown $\mathrm{SiO} / \mathrm{Si}$ system and, in particular, the transitional region, defined as an interlayer between the thermally grown $\mathrm{SiO}_{2}$ film and the Si substrate. In this study, the complex dielectric function and the thickness of the interlayer were determined.

\section{2,428}

\section{PB96-164025 Not available NTIS}

National Inst. of Standards and Technology (EEEL), Gaithersburg, MD. Semiconductor Electronics Div. Enhanced Voltage-Dividing Potentiometer for High-Precision Feature Placement Metrology.

Final rept.

R. A. Allen, M. W. Cresswell, C. H. Ellenwood, and

L. W. Linholm. 1996, 6p.

Pub. in Institute of Electrical and Electronics Engineers Transactions on Instrumentation and Measurement, v45 n1 p136-141 Feb 96.

Keywords: "Metrology, "Potentiometer, Reprints, Voltage dividing, Spatial separations, Voltage dividing.

Enhancements to the voltage-dividing potentiometer, an electrical test structure for measuring the spatial separations of pairs of conducting features, are presented and discussed. These enhancements reduce or eliminate systematic errors which can otherwise lead to uncertainties as large as several hundred nanometers. These systematic errors, attributed by modeling to asymmetries at certain intersections of conducting features in the test structure, are eliminated by modifications to the test structure and test procedures.

\section{2,429}

PB96-164173 Not available NTIS

National Inst. of Standards and Technology (EEEL),

Gaithersburg, MD. Semiconductor Electronics Div.

Modeling Buffer Layer IGBT's for Circuit Simula-

tion.

Final rept.

A. R. Hefner. 1995, 13p.

See also PB95-153805.

Pub. in Institute of Electrical and Electronics Engineers Transactions on Power Electronics, v10 n2 p111-123 Mar 95

Keywords: "Bipolar transistors, Application specific integrated circuits, Mathematical models, Dynamic propenties, Reprints, *Insulated gate bipolar transistors, Circuit simulators, Buffer layers.

The dynamic behavior of commercially available buffer layer IGBT's described. It is shown that buffer layer IGBT's become much faster at high voltages than nonbuffer layer IGBT's with similar low voltage characteristics. Because the fall times specified in manufactures' data sheets do not reflect the voltage dependence of switching speed, a new method of selecting devices for different circuit applications is suggested. A buffer layer IGBT model is developed and implemented into the Saber circuit simulator, and a procedure is developed to extract the model parameters for buffer layer IGBT's. It is shown that the new buffer layer IGBT model can be used to describe the dynamic behavior and power dissipation of buffer layer IGBT's in user-defined application circuits. The results of the buffer layer IGBT model are verified using commercially available IGBT's.

02,430

PB96-164249 Not available NTIS

National Inst. of Standards and Technology (EEEL), Gaithersburg, MD. Semiconductor Electronics Div.

Microelectronic Test Structures for Overlay Metrol-

ogy.

L. W. Linholm, R. A. Allen, and M. W. Cresswell.

1995, 3p.

See also PB95-180568.

Pub. in Nikkei Microdevices, p63-65 1995.

Keywords: "Microelectronics, "Overlays, "Positioning, "Alignment, Integrated circuits, Process control, Registration, Line width, Measurement, Lithography, Very large scale integration, Requirements, Metrology, Reprints, "Test structures, Test chips.

Control of image placement has been and is expected to remain one of the most important challenges re- quired in the manufacturing of advanced microelecronic devices. The metrology to monitor and evaluate the performance of lithographic tools with those capabilities is lagging. Electrical test structures provide lowcost, post-patterning metrology for overlay that is routinely available during the advanced stages of process development and during manufacturing

\section{2,431}

PB96-167192 Not available NTIS

National Inst. of Standards and Technology (EEEL), Gaithersburg, MD. Semiconductor Electronics Div. Optical Characterization of Materials and Devices for the Semiconductor Industry: Trends and Needs.

Final rept.

S. Perkowitz, D. G. Seiler, and W. M. Bullis. 1996,

3p.

Pub. in Proceedings of the International Workshop on Semiconductor Characterization: Present Status and Future Needs, Gaithersburg, MD., January 30-February 2, 1995, p422-424 1996 .

Keywords: "Semiconductors, *Optical characterization Surveys, Processes, Devices, Reprints, Contactless, Nondestructive methods.

Contactless, nondestructive optical methods are used to characterize materials, process, and devices in the semiconductor industry. In response to industria needs, the Semiconductor Electronics Division of the National Institute of Standards and Technology conducted a survey of the need for use of optical characterization methods within the semiconductor industry. Data from forty-two firms were analyzed to show the mpact of the methods, what they measure, their range and precision, and their cost. A significant finding of the study is the need expressed by many industrial users for imporved standards and test methods for optical characterization.

\section{2,432}

PB96-175856 PC A07/MF AO2

National Inst. of Standards and Technology (EEEL) Gaithersburg, MD. Semiconductor Electronics Div. NIST List of Publications, LP 103, March 1996. National Semiconductor Metrology Program. J. Walters. Mar $96,102 p$

Keywords: "US NIST, "Documents, Bibliographies Semiconductors(Materials), Semiconductor industry Metrology, Software

This List of Publication includes all paper relevant to semiconductor technology published by NIST staff, including work of the National Semiconductor Metrology Program the Semiconductor Electronics Division, and other parts of NIST having independent interests in semiconductor metrology. Bibliographic information is provided for publications from 1990 through 1995 Within each year, citations of published papers are listed alphabetically by first author. Indexes are provided by topic area and by author. Publications are referred to in the Topic and Author Indexes according to publication year and citation number (i.e., 95-3 refers to the third publication in the year 1995). A' listing of software available from the Semiconductor Electronics Division is given on page 57 , along with contacts for additional information and for copies of the computer programs.

\section{2,433}

PB96-176458 Not available NTIS

National Inst. of Standards and Technology (EEEL), Gaithersburg, MD. Semiconductor Electronics Div.

Novel Magnetic Field Characterization Techniques for Compound Semiconductor Materials and Devices.

Final rept

C. A. Richter, D. G. Seiler, J. G. Pellegrino, W. F

Tseng, and W. R. Thurber. 1996, 5 p.

Pub. in Proceedings of the International Workshop on Semiconductor Characterization: Present Status and Future Needs, Gaithersburg, MD., January 30-February 2, 1995, p673-677 1996

Keywords: "Semiconductors, "Magnetic fields, Reprints, "Foreign technology, "Magnetoresistance, 2D electron gas, Magnetophonon effect, Universal conductance fluctuations, Shubnikov-de Haas oscillations.

Quantum-mechanical effects observed in the magnetoresistance of semiconductor devices and materials give important information such as carrier density, scattering rates, and band structure parameters. However, the small size of these effects often limits their observation and subsequently their practical use.
The purpose of this paper is to show how these quantum mechanical effects can be more easily observed and used as characterization tools by modulating the applied magnetic field, which can increase the sensitivity of magnetotransport measurements.

\section{2,434}

PB96-176722 Not available NTIS

National Inst. of Standards and Technology (EEEL), Boulder, CO. Electromagnetic Fields Div.

Microwave Characterization of Flip-Chip MMIC Components.

Final rept.

R. B. Marks, J. A. Jargon, C. K. Pao, and C. P. Wen $1995,8 p$

See also PB96-176730 and PB96-176748.

Pub. in Electronic Components and Technology Conference (45th), Las Vegas, NV., May 21-24, 1995 p343-350.

Keywords: "Microwave circuits, MIM(Semiconductors) Transmission lines, Capacitors, Calibration, Stand ards, Computer-aided design, Data bases, Reprints, "MMIC(Monolithic Microwave Integrated Circuits) "Monolithic Microwave Integrated Circuits, "Flip-chip technology, Coplanar waveguide, Circuit models, On wafer measurement, Spiral inductors.

The authors apply custom calibration standards and software to the accurate on-wafer measurement of MIM capacitors and spiral inductors on flip-chip coplanar-waveguide MMICs. The authors suggest equivalent circuit models and document their deficiencies. The results are applicable to the development of an accurate CAD database.

02,435

PB96-176730 Not available NTIS

National Inst. of Standards and Technology (EEEL), Boulder, CO. Electromagnetic Fields Div.

Microwave Characterization of Flip-Chip MMIC Interconnections.

Final rept

R. B. Marks, J. A. Jargon, C. K. Pao, and C. P. Wen

1995, 4p

See also PB96-176722 and PB96-176748.

Pub. in International Microwave Symposium, Orlando, FL., May 14-19, 1995, v3 p 1463-1466.

Keywords: "Microwave circuits, MIM(Semiconductors) Transmission lines, Capacitors, Calibration, Standards, Computer-aided design, Data bases, Reprints, "MMIC(Monolithic Microwave Integrated Circuits) *Monolithic Microwave Integrated Circuits, "Flip-chip technology, Coplanar waveguide, Circuit models, On wafer measurement, Spiral inductors.

The report accurate on-wafer measurements of transmission lines on flip-chip coplanar-wave-guide MMICs. The results are applicable to the development of an accurate CAD database. The authors also report and apply a new technique for the measurement of trans mission line capacitance.

02,436

PB96-176748 Not available NTIS

National Inst. of Standards and Technology (EEEL),

Boulder, CO. Electromagnetic Fields Div.

Electrical Measurements of Microwave Flip-Chip Interconnections.

Final rept

R. B. Marks, J. A. Jargon, C. K. Pao, and C. P. Wen $1995,6 p$

See also PB96-176722 and PB96-176730.

Pub. in ISHM '95 Proceedings, International Symposium on Microelectronics, Los Angeles, CA., Octobe 24-26, 1995, p424-429.

Keywords: "Microwave circuits, MIM(Semiconductors) Circuit interconnections, Electrical measuremen Transmission lines, Capacitors, Calibration, Standards. Computer-aided design, Data bases, Reprints, *MMIC(Monolithic Microwave Integrated Circuits), *Monolithic Microwave Integrated Circuits, "Flip-chip technology, Coplanar waveguide, Circuit models, On wafer measurement, Spiral inductors.

The authors apply custom calibration standards and software to the accurate on-wafer measurement of components on flip-chip coplanar-waveguide MMICs. The authors characterize transmission lines, MIM capacitors, and spiral inductors and develop equivalent circuit models. The results are applicable to the development of an accurate CAD database.

02,437

PB96-179445 Not available NTIS 
National Inst. of Standards and Technology (PL), Gaithersburg, MD. Molecular Physics Div.

Theory for Quantum-Dot Quantum Welis: Palr Correlation and Internal Quantum Conflnement in Nanoheterostructures.

Final rept.

G. W. Bryant. 1995, 4p.

Pub. in Physical Review B, v52 n24 pR16997-17000 15 Dec 95.

Keywords: "Binding energy, "Quantum wells, "Oscillator sirength, Quantum dots, Correlation, Exciton, Hgs, Reprinis.

Spherical quantum-dot-quantum-well nanocrystallites, with internal radial quantum-well band profiles, can now be fabricated. Pair states in these nanoheterostructures are determined by electron-hole attraction, global confinement in the dot, and local confinement within the well. Configuration-interaction calculations using effective-mass models with screened pair interaction give accurate exciton energies for CDS/ $\mathrm{HgS}$ quantum-dot quantum wells. Binding energy and pair correlation are suppressed but pair-oscillator strengths are enhanced by adding a HgS quanium well to a CDs quantum dot.

\section{2,438}

PB96-179478 Not available NTIS

National Inst. of Standards and Technology (EEEL) Gaithersburg, MD. Semiconductor Electronics Div.

Time-Dependent Dielectric Breakdown of Intrinsic SiO2 Films under Dynamic Stress.

Final rept.

P. Chaparala, J. S. Suehle, C. Messick, and M.

Roush. 1995, $9 \mathrm{p}$.

See also PB94-185956

Pub. in Proceedings of the Institute of Electrical and Electronics Engineers International Integrated Reliability Workshop, Lake Tahoe, CA., October 22-25, 1995, p104-112.

Keywords: "Dielectric breakdown, "Silicon dioxide Temperature dependence, High lemperature, Integrated circuits, Reprints, Electric fields, Dielectric files, Thin films, Field acceleration models.

The authors present time-dependet dielectric breakdown (TDDB) characteristics for 9, 15, and $22 \mathrm{~nm}$ silicon dioxide films stressed under dc, unipolar and bipolar pulsed bias conditions. The authors' results indicate that the increased lifetime observed under pulsed stress conditions diminishes as the stress electric field and oxide thickness are reduced. TDDB data under pulse bias conditions exhibit similar field and temperature dependencies as under static stress. $C-V$ measurements indicate that lifetime enhancement only occurs for electric fields and thickness where charge trapping is significant.

\section{2,439}

PB96-179510 Not available NTIS

National Inst. of Standards and Technology (PL), Gaithersburg, MD. Electron and Optical Physics Div. Improved Dose Metrology in Optical Lithography. Final rept.

C. L. Cromer, T. B. Lucatorto, T. R. O'Brian, and M. Walhout. 1996, $6 p$.

Pub. in Solid State Technology, v39 n4 p75-80 Apr 96.

Keywords: "Metrology, "Laser annealing, "Ultraviolet radiation, Optical properties, Photoresistors, Radiometers, Semiconductors, Microelectronics, Calibration standards, Standardization, Reprints, "Optica lithography, Critical dimensions, Irradiance measurement, Laser etching, Microlithography.

Maintaining critical dimensions (CDs) in optical lithography requires careful control over the UVIDUV dose applied to a photoresist, especially as CDs become smaller. To meet the growing need for a UV-measurement infrastructure in the semiconductor industry, the National Institute of Standards and Technology (NIST) is improving its primary radiometric standards by an order of magnitude, reducing the absolute uncertainty in irradiance measurements to about $0.5 \%$ in the 100 $400 \mathrm{~nm}$ range. Parallel improvements in transfer standards and source calibrations will allow the dissemination of highly accurate radiometric scales directly to in dustry.

P2,440

National Inst. of Standards and Technology (EEEL), Gaithersburg, MD. Semiconductor Electronics Div.
Now Physics-Based Model for TIme-Dependent- Dielectric-Breakdown.

B. Schlund, J. Suehle, C. Messick, and P.

Chaparala. 1995, 9p.

Pub. in Proceedings of the Institute of Electrical and Electronics Engineers International Integrated Reliability Workshop Final Report, Lake Tahoe, CA., October 22-25, 1995, p72-80.

Keywords: "Silcon dioxide, Reliability(Electronics), Reprints, Dielectric films, Time dependence, "Time-Dependent Dielectric Breakdown, "Thin dielectric reliability.

A new, physics based model for time dependent dielectric breakdown has been developed and is presented along with test data obtained by NIST on oxides provided by National Semiconductor. Testing included Fields from $5.6 \mathrm{MV} / \mathrm{cm}$ to $12.7 \mathrm{MV} / \mathrm{cm}$, and temperatures ranging from 60 degrees $C$ to 400 degrees $C$. The physics, mathematical model and test data, all confirm a linear, rather than an inverse field dependence. The primary influence on oxide breakdown was determined to be due to the dipole interaction energy of the field with the orientation of the molecular dipoles in the dielectric. The resultant failure mechanism is shown to be the formation and coalescence of vacancy defects, similar to that proposed by Dumin et al.

02,441

PB96-190038 Not available NTIS

National Inst. of Standards and Technology (PL), Gaithersburg, MD. Alomic Physics Div.

Microlithography by Using Neutral Metastable Atoms and Self-Assembled Monolayers.

Final rept.

K. K. Berggren, A. Bard, J. L. Wilbur, J. J.

McClelland, S. L. Rolston, W. D. Phillips, M. Prentiss, G. M. Whitesides, J. D. Gillaspy, and A. G. Helg. 1995, 3p

Pub. in Science, v269 p1255-1257 Sep 95.

Keywords: "Lithography, "Microstructures, "Metastable atoms, Gold, Etching, Surfaces, Aqueous solutions, Argon, Reprints, "Atom lithography, Microlithography, SAMS(Self-assembled monolayers).

Lithography can be performed with beams of neutral atoms in metastable excited states to pattern self-assembled monolayers (SAMs) of alkanethiolates on gold. An estimated exposure of a SAM of dodecanethiolate (DDT) to 15 to 20 metastable argon per DDT molecule damaged the SAM sufficiently to allow penetration of an aqueous solution of ferricyanide to the surface of the gold. This solution etched the gold and transformed the patterns in the SAMS into structures of gold; these structures had edge resolution of less than 100 nanometers. Regions of SAMS as large as 2 square centimeters were patterned by exposure to a beam of metastable argon atoms. These observations suggest that this system may be useful in new forms of micro- and nanolithography.

\section{2,442}

PB96-200365 Not available NTIS

National Inst. of Standards and Technology (PL), Boulder, CO. Time and Frequency Div.

Design Criteria for BJT Amplifiers with Low I/f AM and PM Noise.

Final rept.

E. S. Ferre-Pikal, F. L. Walls, and C. W. Nelson

1995, 10p.

Pub. in Institute of Electrical and Electronics Engineers International Frequency Control Symposium, San Francisco, CA., May 31-June 2, 1995, p305-314.

Keywords: "Bipolar transistors, "Amplifiers, Design criteria, Junction transistors, Electric circuits, Noise, Reprints.

In this paper, the authors discuss guidelines for designing linear bipolar junction transistor (BJT) amplifiers with low $1 / \mathrm{f}$ amplitude modulation (AM) and phase modulation (PM) noise. These guidelines are derived from a new theory that relates $A M$ and PM noise to transconductance fluctuations, junction capacitance fluctuations, and circuit architecture. The authors analyze the noise equations of each process for a common emitter (CE) amplifier and use the results to suggest amplifler designs that minimlze the $1 / f$ noise whlle providing other required attributes such as high gain. Although the authors use a CE amplifier as an example. the procedure applies to other configurations as well. Experimental noise results for several amplifier configurations are presented.
02,443

PB96-200845 Not available NTIS

National Inst. of Standards and Technology (MSEL) Gaithersburg, MD. Ceramics Div.

Overview of U.S. Government Advanced Packaging Programs.

Final rept.

J. A. Carpenter, J. Evans, E. B. Hakim, C. Pagel, D. Palmer, J. Lyke, and N. J. Naclerio. 1993, 6p.

Pub. in Proceedings of the International Conference and Exhibition on Multichip Modules, Denver, CO. April 14-16, 1993, p495-500.

Keywords: "Electronic packaging, "Interagency coordination, Photonics, Circuit interconnections, US DOD, US DOE, US DOC, NASA, Government/industry relations, Reprints, Multichip module.

An overview is provided of non-classified advanced electronics packaging programs, particularly those involving multichip module (MCM) technologies, sponsored and performed by the U.S. Departments of Defense, Energy, and Commerce and the National Aeronautics and Space Administration (NASA), and how those programs are coordinated among one another and with U.S. industry.

02,444

PB96-200969 Not available NTIS

National Inst. of Standards and Technology (EEEL), Gaithersburg, MD. Semiconductor Electronics Div. Monte Carlo Simulation of Scanning Electron Microscope Signals.

Final rept

J. R. Lowney. 1996, 6p.

Pub. in Scanning, v18 p301-306 1996.

Keywords: "Scanning electron microscopes, "Monte Carlo, "Metrology, Linewidth, Lithography, Reprints.

Two computer codes for simulating the backscattered, transmitted, and secondary-electron signals for targets in a scanning electron microscope are described. The frist code, MONSEL-II, has a model target consisting of three parallel lines on a three-layer substrate, while the second, MONSEL-III, has a model target consisting of a two-by-two array of finite lines on a three-layer substrate. Elastic electron scattering is determined by published fits to the Mott cross section. Both plasmongenerated electrons and ionized valence electrons are included in the secondary production. An adjustable quantity, called the residual energy loss rate, is added to the formula of Joy and Luo to obtain the measured secondary yield. The codes show the effects of signal enhancement due to edge transmission, known as blooming, as well as signal reduction due to neighboring lines, known as the 'black-hole' effect.

\section{2,445}

PB96-201074 Not available NTIS

National Inst. of Standards and Technology (MEL), Gaithersburg, MD. Precision Engineering Div. Report on the NIST Low Accelerating Voltage SEM Magnification Standard Interlaboratory Study. Final rept.

M. T. Postek, A. E. Vladar, S. Jones, and W. J. Keery. 1993, 18p.

Pub. in Integrated Circuit Metrology, Inspection and Process Control VII, San Jose, CA., March 2-4, 1993 p268-285.

Keywords: "Dimensional measurements, "Scanning electron microscopy "Standards, Line widths, Lithography, Reprints, "Magnification standards, Nanofabrication, Standard reference materials.

NIST is in the process of developing a new scanning electron microscope (SEM) magnification calibration reference standard useful at both high and low accelerating voltages. This standard will be useful for all applications to which the SEM is currently being used but it has been specifically tailored to meet many of the particular needs of the semiconductor industry. A small number of test samples with the pattern were prepared on silicon substrates using electron beam lithography at the National Nanofabrication Facility at Cornell University. The structures were patterned in titanium/palladium with maximum nominal pitch struciures of approximately 3000 micrometers scaling down to structures with minimum nominal pitch of $0.4 \mathrm{mi}$ crometer. Eighteen of these samples were sent out to a total of 35 university, research, semiconductor and other industrial laboratories in an interlaboratory study. The purpose of the study was to test the SEM instrumentation and to review the suitability of the sample 
design. The laboratories were asked to take a series of micrographs at various magnifications and accelerating voltages designed to test several of the aspects of instrument performance related to general SEM operation and metrology. If the instrument in the laboratory was used for metrology, the laboratory was also asked to make specific measurements of the sample. In the first round of the study (representing 18 laboratories), data from 35 instruments from several manufacturers were obtained and the second round yielded information from 14 more instruments. The results of the analysis of the data obtained in the study are presented in the paper.

\section{2,446}

PB96-201090 Not available NTIS

National Inst. of Standards and Technology (MEL),

Gaithersburg, MD. Precision Engineering Div.

Scanning Electron Microscope Metrology.

Final rept.

M. T. Postek. 1993, 45p

See also PB95-169405

Pub. in Handbook of Critical Dimension Metrology and Process Control, Monterey, CA., September 28-29, 1993, p46-90.

Keywords: "Integrated circuits, "Precision *Manufacturing, Electron microscopy, Secondary emission, Metrology, Reprints.

During the manufacturing of present-day integrated circuits, certain meausrements must be made of the submicrometer structures composing the device with a high degree of precision. Optical microscopy, scanning electron microscopy and the various forms of scanning probe microscopies are major microscopical echniques used for submicrometer metrology. New techniques applied to scanning electron microscopy have improved some of the limitations of this technique and time will permit even further improvements. This presentation will review the current state of scanning electron microscope (SEM) metrology in light of many of these recent improvements.

\section{2,447}

PB96-201108 Not available NTIS

National Inst. of Standards and Technology (MEL), Gaithersburg, MD. Precision Engineering Div.

SEM Linewidth Metrology of $X$-ray Lithography Masks.

Final rept

M. T. Postek, J. R. Lowney, A. E. Vladar, R. D.

Larrabee, W. J. Keery, and E. Marx. 1993, $5 p$.

Pub. in American Society of Mechanical Engineers, v8 p78-82 1993.

Keywords: "Scanning electron microscopy, "Metrology, "Lithography masks, $X$ rays, Measurements, Reprints.

Accurate dimensional metrology of the submicrometer gold absorber structures of $x$-ray masks can be accomplished in the scanning electron microscope (SEM). This is possible because the $x$-ray masks present a measurement object that is unique from most other objects viewed in the SEM. This is because the silicon support membrane is, by design, $x$-ray transparent The characteristic can be used as a distinct advantage in electron beam-based mask metrology since, de pending upon the incident electron beam energies, substrate composition and substrate thickness, the membrane can also be essentially electron transparent. The area of the mask where the absorber structures are located are essentially $\mathrm{x}$-ray opaque, as well as, electron opaque. Viewing the sample from a perspective below the mask, by placing an electron detector beneath the mask, provides excellent electron signal contrast between the absorber structure and the base membrane.

\section{2,448}

PB96-201132 Not available NTIS

National Inst. of Standards and Technology (EEEL) Gaithersburg, MD. Semiconductor Electronics Div.

New Physics-Based Model for Time-Dependent- Dielectric-Breakdown.

Final rept.

B. Schlund, J. S. Suehle, C. Messick, and P.

Chaparala. $1996,8 p$

See also PB96-186093.

Pub. in Proceedings of the Intemational Reliability Physics Conference, Dallas, TX., April 30-May 2, 1996 p84-92.

Keywords: "Dielectric breakdown, "Oxides, Time dependence, Mathematical models, Linear systems, Crystal defects, Reprints.
A new physics-based model for time dependent dielectric breakdown has been developed, and is presented along with tesI data obtained by NIST on oxides provided by National Semiconductor. Testing included fields from $5.4 \mathrm{MV} / \mathrm{cm}$ to $12.7 \mathrm{MV} / \mathrm{cm}$, and temperatures ranging from $60 \mathrm{C}$ to $400 \mathrm{C}$. The physics, mathematical model, and test data, all confirm a linear, rather than an inverse field dependence. The primary influence on oxide breakdown was determined to be due to the dipole interaction energy of the field with the orientation of the molecular dipoles in the dielectric. The resultant failure mechanism is shown to be the formation and coalescence of vacancy defects, similar to that proposed by Dumin et al.

\section{2,449}

PB96-204102 Not available NTIS

National Inst. of Standards and Technology (EEEL), Gaithersburg, MD. Semiconductor Electronics Div. Electric Field Dependent Dielectric Breakdown of Intrinsic SiO2 Films Under Dynamic Stress.

Final rept.

P. Chaparala, J. S. Suehle, C. Messick, and M.

Roush. 1996, 6p.

See also PB96-179478.

Pub. in Proceedings of the Institute of Electrical and Electronics Engineers International Reliability Physics Conference, Dallas, TX., April 30-May 2, 1996, p6166.

Keywords: "Dielectric breakdown, "Silicon dioxide, Temperature dependence, Reprints, High temperature, Integrated circuits, Electric fields. Thin films, Oxides, Time-dependent.

Time-dependent dielectric breakdown (TDDB) characteristics are reported for $6.5 \mathrm{~nm}, 9 \mathrm{~nm}, 15 \mathrm{~nm}$, and 22 $\mathrm{nm}$ intrinsic silicon dioxide films stressed under $\mathrm{dc}$ and bipolar pulsed stress conditions for a wide range of electric fields and temperatures. The authors results show that the increased ifetime observed under bipolar pulsed stress conditions diminishes as the stress electric field and oxide thickness are reduced. Similar electric field and temperature dependencies of TDDB are observed under both static and dynamic stress conditions. It is observed that lifetime enhancement only occurs for electric fields and thicknesses where charge trapping is significant. Contradictory to the convention notion, TDDB tests on intrinsic thin oxides indicate that static stress testing cannot be considered as a conservative test of bipolar stressing for estimating oxide reliability. These results also confirm the existence of two separate failure mechanisms for TDDB that are function of electric field and oxide thickness.

\section{2,450}

\section{PB96-204136 Not available NTIS}

National Inst. of Standards and Technology (EEEL), Gaithersburg, MD. Semiconductor Electronics Div. Hybrid Optical-Electrical Overlay Test Structure. Final rept.

M. W. Cresswell, R. A. Allen, L. W. Linholm, W. F Guthrie, and A. W. Gurnell. 1996, 4p.

Pub. in Proceedings of the Institute of Electrical and Electronics Engineers International Conference on Microelectronic Test Structures, Trento, Italy, March 26-28, 1996, v9 p9-12.

Keywords: "Semiconductors, "Test structure, Reprints, Lithography, Metrology, Overlay.

The purpose of this work is to explore the use of electrical test structures for calibrating optical overlay instruments with respect to certain application-dependent errors which are otherwise expected to become a serious impediment to realization of the goals of the National Technology Roadmap for Semiconductors over the next decade. A new hybrid test structure, from which overlay measurements can be extracted electricallty, as well as by optical instruments used for inspecting production wafers, has been designed and fabricated with built-in overlay values ranging from -60 to $+60 \mathrm{~nm}$. Structures patterned in a single conducting film and having critical-dimension (CD) design-rules ranging from 1.0 to 2.0 micrometers have been tested. Electrical overlay parameters, derived from multiple step-and-repeat exposure-site measurements, generally match the corresponding optical parameters to within several nanometers, subject to nominal quality of the pattern-replication process. The paper focuses on the analysis of electrical measurements, their dependence on $C D$ design rules, and their comparison with the corresponding measurements made both by a commercial optical-overlay instrument and by a coordinate-measurement system having measurements traceable to absolute dimensional standards.
02,451

PB97-110134 Not available NTIS

National Inst. of Standards and Technology (EEEL), Gaithersburg. MD Semiconductor Electronics Div. Characterization of Two-Dimensional Dopant Profiles: Status and Review.

Final rept.

A. C. Diebold, M. R. Kump, J. J. Kopanski, and D. G. Seiler. 1996, 6p.

See also PB96-119300.

Pub. in Jnl. of Vacuum Science and Technology B, v14 1 p196-201 Jan/Feb 96.

Keywords: "Transistors, ${ }^{*}$ Computer aided design, *Models, Verification, Calibrating. Two dimensional Dopes, Simulation, Microscopy, Spatial resolution, Spectroscopic analysis, Scanning probe methods, $\mathrm{Re}$ prints.

The National Technology Roadmap for Semiconductors calls for development of two- and three-dimensional dopant profiling methods for calibration of technology computer-aided design process simulators. The authors have previously reviewed 2D dopant profiling methods. In the particle, the authors briefly review methods used to characterize etched transistor cross sections by expanding the previous discussion of scanned probe microscopy methods. They also mention the need to participate in the ongoing comparison of analysis results for test structures that the authors have provided the community.

\section{2,452}

PB97-111439 Not available NTIS

National Inst. of Standards and Technology (EEEL) Gaithersburg, MD. Semiconductor Electronics Div. Double Modulation and Selective Excitation Photoreflectance for Characterizing Highly Luminescent Semiconductor Structures and Samples with Poor Surface Morphology.

Final rept.

P. M. Amirtharaj, D. Chandler-Horowitz, and D. P Bour. 1996, 12p

Pub. in Proceedings of the Material Research Society Fall Meeting, Boston, MA., November 25-29, 1995 v406 p229-240 1996.

Keywords: $\quad$ *Double-modulation,

"Lasers, "Photoreflectance, "Semiconductors, Quantum-well, Selective-excitation, Reprints.

Photoreflectance (PR) is a powerful, contactless, and nondestructive technique capable of probing interband electronic transitions and built-in electric-fields at the surface and in interface regions in semiconductor materials and microstructures. It has been widely used as a characterization too. Howevr, its application to highly luminscent systems, such as quantum-well (QW) la sers and samples with poor surface morphology has been limited because of the difficulty in minimizing the interference from the luminescence and the pump beam that is scattered from the surface. The authors present a double modulation procedure where both the probe and pump beams are modulated which allows the PR component to be completely separated from the luminescence and scattered contributions. The separation is achieved through the appropriate choice of modulation frequencies and specially designed tuned amplifiers. A complete PR system, along with the necessary circuits, is presented. In addition, the au thors have also exploited the freedom provided by the system to choose any pump wavelength to selectively modulate specific regions of the multilayer structure (QW, barrier, and cladding layer) and extract detailed information regarding the properties of each layer.

02,453

Not available NTIS

National Inst of Standards and Technology (EFEL) Gaithersburg, MD. Semiconductor Electronics Div. Mechanism of Defect Formation in Low-Dose Oxy gen Implanted Silicon-on-Insulator Material.

Final rept.

S. Bagchi, J. D. Lee, S. J. Krause, and P. Roitman $1996,6 p$.

Pub. in Jnl. of Electronic Materials, v25 n1 p7-12 1996

Keywords: *Silicon islands, *Defect formation, Dislocation half-loop, Stacking fault pyramide. Threading dis ocation, Reprints, SIMOX

The defects and microsructure of low-dose (less than $0.7 \times 10$ to the 18 th power $\mathrm{cm}$ to the minus 2 power) rial were investigated as a function of implant dose and 


\section{Semiconductor Devices}

annealing temperature by plan-view and cross-sectional transmission electron microscopy. The threading-dislocations in low-dose $(0.2$ approximately $0.3 \times$ 10 to the 18 power $\mathrm{cm}$ to the minus 2 power), annealed SIMOX originate from unfaulting of long (approximately 10 micrometer), shallow ( 0.3 micrometer), extrinsic stacking faults generated durng the ramping stage of annealing. As dose increases, the defect density is reduced and the structure of the buried oxide layer evolves dramatically. It was found that there is a dose window which gives a lower defect density and a continuous buried oxide with a reduced density of $\mathrm{Si}$ islands and the buried oxide.

\section{2,454}

PB97-111827 Not available NTIS

National Inst. of Standards and Technology (EEEL),

Gaithersburg, MD. Semiconductor Electronics Div.

Electrical Test Structures Replicated in Silicon-onInsulator Material.

Final rept.

M. W. Cresswell, J. J. Sniegowski, R. N

Ghoshtagore, J. S. Villarrubia, R. A. Allen, and L. W.

Linholm. 1996, 18p.

Pub. in Proceedings of Society of Photo-Optical Instrumentation Engineers Metrology, Inspection and Process Control for Microlithography X, Santa Clara, CA. March 10-15, 1996, v2725 p659-676.

Keywords: "Silicon, "Electrical measurement *Calibration, Semiconductors(Materials), Integrated circuits, Line width, Test equipment, Standards, Microelectronics, Reprints, MEMS(Microelectromechanical systems), Microelectromechanical systems.

Measurements of the linewidths of subm icrometer features made by different metrology techniques have frequently been characterized by differences of up to 90 $\mathrm{nm}$. The purpose of the work reported here is to address the special difficulties that this phenomenon pre sents to the certification of reference materials for the calibration of linewidth-measurement instruments. Accordingly, a new test structure has been designed and fabricated, and has undergone preliminary tests. Its distinguishing characteristics are assured cross-sectional profile geometries with known side-wall slopes, surface planarity, and compositional uniformity when it is formed in mono-crystalline material at selected orientations to the crystal lattice. To allow the extraction of electrical linewidth, the structure is replicated in a silicon film of uniform conductivity which is separated from the silicon substrate by a buried oxide layer. The utilization of a silicon-on-insulator (SOI) substrate further allows the selective removal of substrate material from local regions below the reference features, thus facilitating measurements by optical and electronbeam transmission microscopy. The combination of planar feature surfaces having known side-wall slopes is anticipated to eliminate factors which are believed to be responsible for methods divergence in linewidth measurements, a capability which is a prerequisite fo reliable certification of the linewidths of features on reference materials.

\section{2,455}

PB97-112296 Not available NTIS

National Inst. of Standards and Technology (EEEL), Gaithersburg, MD. Semiconductor Electronics Div. Interdigitated Stacked P-I-N Multiple Quantum Well Modulator.

Final rept.

X. R. Huang, S. K. Cheung, A. N. Cartwright, A. L.

Smirl, and W. F. Tseng. 1996, 3p.

Pub. in Institute of Electrical and Electronics Engineers Photonics Technology Letters, v8 n9 p1172-1174 Sep 96

Keywords: "Quantum wells, "Optical absorption, "Modulators, Lasers, Interdigitated, Hetero, Reprints.

The authors demonstrate low-voltage operation of a strained InGaAs-GaAs interdigitated hetero n-i-p-i modulator (or stacked SEED) that is grown and fabricated using a shadow-mask growth technique fo making the metal contacts to the $n$ - and $p$-layers separately. An absorption change of $6 \times 10$ to the $3 r d$ power $\mathrm{cm}$ to the minus 1 with an applied bias as low as approximately $1 \mathrm{~V}$ is observed in an unoptimized structure. Optical switching of the unbiased structure is also demonstrated.

\section{2,456}

PB97-119283 Not available NTIS

National Inst. of Standards and Technology (EEEL), Gaithersburg, MD. Semiconductor Electronics Div.
Micromachined Coplanar Waveguides in CMOS Technology.

Final rept

V. Milanovic, M. Gaitan, E. D. Bowen, and M. E.

Zaghloul. 1996, 3p

Pub. in Institute of Electrical and Electronics Engineers Microwave and Guided Wave Letters, v6 n10 p3803820 ct 96

Keywords: "CMOS, "Micromachining, "Coplanarity "Waveguides, Lossy media, Transmission loss, Phase velocity, Magnetic dispersion, Substrates, Silicon, Reprints.

Coplanar waveguides were fabricated in standard complimentary metal-oxide semiconductor (CMOS) with post-processing micromachining. IC's were designed with commercial CAD tools, fabricated through the MOSIS service, and subsequently suspended by maskless top-side etching. Absence of the lossy silicon substrate after etching results in significantly improved insertion loss characteristics, dispersion characteristics, ad phase velocity. Measurement were performed at frequencies from 1 to $40 \mathrm{GHz}$, before and after micromachining. These show improvement in loss characteristics of orders of magnitude.

\section{2,457 \\ PB97.122428 Not available NTIS}

National Inst. of Standards and Technology (EEEL), Gaithersburg, MD. Semiconductor Electronics Div. Application of the Collocation Method in Three Dimensions to a Model Semiconductor Problem.

Final rept.

J. F. Marchiando. 1996, 12p

Pub. in International Jnl. for Numerical Methods in En gineering, v39 p1029-1040 1996.

Keywords: "Semiconductors, "Collocation, Mathematical models, Three dimensional, Boundary value problems, Reprints, Poisson's equation.

A research code has been written to solve an elliptic system of coupled non-linear partial differential equations of conservation form on a rectangularly shaped three-dimensional domain. The code uses the method three-dimensional domain. The code uses the method piecewise continuous polynomial basis functions. The system of equations is solved by iteration. The system of non-linear equations is linearized, and the system of linear equations is solved by iterative methods. When the matrix of the collocation equations is duly modified by using a scaled block-limited partial pivoting procedure of Gauss elimination, it is found that the rate of convergence of the iterative method is significantly improved and that a solution becomes possible. The code is used to solve Poisson's equation for a model semiconductor problem. The electric potential distribution is calculated in a metal-oxide-semiconductor structure that is important to the fabrication of electron devices.

\section{2,458}

PB97-122527 Not available NTIS

National Inst. of Standards and Technology (EEEL), Gaithersburg, MD. Semiconductor Electronics Div.

Characterization of Time-Dependent Dielectric Breakdown in Intrinsic Thin SiO2.

Final rept.

J. S. Suehle, and P. Chaparala. 1996, 9p

See also PB96-179478.

Pub. in Microelectronics Jnl., v27 p657-665 1996.

Keywords: "Dielectric breakdown, "Silicon dioxide, Temperature dependence, High temperature, Integrated circuits, Electric fields, Dielectric files, Thin films, Reprints, "Foreign technology.

Time-dependent dielectric breakdown data collected from 6.5-, 9-, 15-, 20-, and 22.5-nm-thick SiO2 films are presented. The failure distributions are of single mode with no apparent extrinsic population. The logarithm of the median-test-time-to-failure, $\log (\mathrm{t} 50)$ is described by a linear electric-field dependence. Contrary to reports in earlier studies, the field-acceleration parameter is observed to be insensitive to temperature and has a value to approximately 1.0 decade/ $/ \mathrm{Mv} / \mathrm{cm}$ for the range of oxide thicknesses studied. Capacitance-voltage studies indicate that there is no strong correlation between oxide trapped charges and timeto-failure under constant voltage stress conditions.

\section{Batteries \& Components}

02,459

PB96-164231 Not available NTIS

National Inst. of Standards and Technology (EEEL) Gaithersburg, MD. Electronics and Electrical Engineering Lab. Office.

Rechargeable Batteries for Personal/Portable. Final rept.

A. G. Lieberman, L. Eliason, and K. Higgins. 1995, $16 \mathrm{p}$.

Sponsored by National Inst. of Justice, Washington, DC.

Pub. in Rechargeable Batteries for Personal/Portable Transceivers, NIJ Standard-0221.01, 16p Sep 95.

Keywords: "Test methods, "Requirements, "Nickelcadmium batteries, "Radio equipment, Storage batteries, Performance tests, Transmitter receivers, Radio communication, Walkie talkies, Portable equipment Reprints, "Rechargeable batteries.

The purpose of this document is to establish performance requirements and test methods for rechargeable (secondary) batteries used in personal/portable transceivers by law enforcement agencies. The scope of this standard is limited to nickel-cadmium secondary batteries.

\section{Electric Power Production}

\section{2,460}

PB94-161817 PC A03/MF A01

National Inst. of Standards and Technology (NEL), Gaithersburg, MD. Center for Building Technology. Earthquake Resistant Construction of Electric Transmission and Telecommunication Facilities Serving the Federal Government Report.

F. Y. Yokel. Feb 90, 48p, NISTIR-89/4213, FEMA

202.

Sponsored by Federal Emergency Management Agency, Washington, DC

Keywords: "Telecommunication, "Electric power generation, *Earthquake resistant structures, Seismic design, Design standards, Building codes, Telephone systems, Television systems, Radio communication, Computer networks, Transmission lines, Power transmission, Soil-structure interactions, Earthquake engineering, Lifeline systems.

The vulnerability of electrical transmission and telecommunication facilities to damage in past earthquakes, as well as available standards and technologies to protect these facilities against earthquake damage are reviewed. An overview is presented of measures taken by various Federal agencies to protect electrical transmission and telecommunication facilities against earthquake hazards. It is concluded that while most new facilities which are owned and operated by Federal agencies are presently designed to provide some, though not necessarily adequate, earthquake resistance, there generally is no effort to retrofit existing facilities. No evidence was found of requirements to protect electrical transmission and communication facilities which have major contractual obligations to serve the Federal Government and only limited seismic design requirements are stipulated for electrical transmission systems constructed with Federal funding.

\section{2,461}

PB95-188850 PC A03/MF A01

National Inst. of Standards and Technology (EEEL), Gaithersburg, MD. Electricity Div.

Evaluation of the Economic Impacts Associated with the NIST Power and Energy Calibration Services.

A. N. Link. Jan 95, 27p, NISTIR-5565.

See also DE94010428. Prepared in cooperation with North Carolina Univ. at Greensboro. Dept. of Economics. 
Keywords: "Electric power, "Calibration, "Economic impacts, Benefit cost analysis, Government policies, Watt hour meters, Electric utilities, Evaluation, Power and Energy Calibration Project, Federal laboratories, US NIST.

It is well established in the economics literature that investments in measurement-related technology research by Federal laboratonies represents an importan resource commitment to the innovation process, and that these investments have a significant impact on economic growth. The purpose of this study is to identify, and quantify where possible, the net economic benefits associated with the NIST Power and Energy Calibration Project. The NIST services from the project that are considered in this report are: (1) maintenance of the national standard for the watthour; (2) research to lower the level of uncertainty associated with watthour revenue meters; (3) general technical support to industry associated with measurement activities. For this particular study, the calculation of a benefit-to-cost ratio is believed to be the most reliable evaluation metric. This ratio is computed as the ratio of benefits (received by economic units directly dependent on the activities of the NIST power and energy calibration services) to the costs to society to generate those benefits, namely the cost to operate the Power and Energy Calibration Project. An alternative metric, an intemal rate of return measure, is also presented in this report in order to facilitate a comparison of the benefit-to-cost result to the results from similar studies.

\section{2,462}

\section{PB96-147913 PC A05/MF A01}

National Inst. of Standards and Technology (EEEL), Gaithersburg, MD. Electricity Div.

Technical Impact of the NIST Calibration Service for Electrical Power and Energy.

J. D. Ramboz, and F. D. Martzloff. Dec 95, 68p,

NISTIR-5564.

See also PB95-188850.

Keywords: "Electric power meters, "Electrical measurement, "Calibration, Economic analysis, Watt hour meters, Standards, Power measurement, Electric utilities, NIST(National Institute of Standards and Technology).

A survey was conducted to assess the economic impact of the NIST Power and Energy Calibration service. Information was sought from electric utilities, manufacturers, testing laboratories, and Public Utilities Commissions on the structure of their operations and the economic aspects of the existence of traceability to National Standards by NIST. The information returned by the respondents was not sufficiently comprehensive and consistent to allow broad generalization leading to reliable assessment of the economic impact of the NIST services. However, the technical impact of these services was sufficiently documented to present this report describing the infrastructure and the relationships among the stakeholders.

\section{Electric Power Transmission}

02,463

PB94-172061 Not available NTIS

National Inst. of Standards and Technology (EEEL), Gaithersburg, MD. Electricity Div.

Coordinating Cascaded Surge Protection Devices: High-Low versus Low-High.

Final rept.

J. S. Lai, and F. D. Martzloff. 1993, 8p.

Pub. in Institute of Electrical and Electronics Engineers Transactions on Industry Applications 29, n4 p680-687 Jul/Aug 93. See also PB92-159664.

Keywords: "Cascaded elements, "Circuit protection, "Surges, Reliability(Electronics), Varistors, Standards, Electric fuses, Coordination, Reprints.

Cascading surge protection devices located at the service entrance of a building and near the sensitive equipment is intended to ensure that each device shares the surge stress in an optimum manner to achieve reliable protection of equipment against surges impinging from the utility supply. However, depending on the relative clamping voltages of the two devices, their separation distance, and the waveform of the impinging surges, the coordination may or may not be effective. The paper provides computations with experimental venification of the energy deposited in the devices for a matrix of combinations of these three parameters. Results show coordination to be effective for some combinations and ineffective for some others, which is a finding that should reconcile contradictor conclusions reported by different authors making different assumptions. From these results, improved coordination can be developed by application standards writers and system designers.

\section{2,464}

PB94-216488 Not available NTIS

National Inst. of Standards and Technology (EEEL), Gaithersburg, MD. Electricity Div.

Cascading Surge-Protective Devices: Options for Effective Implementation.

Final rept.

F. D. Martzloff, and J. S. Lai. 1992, $8 \mathrm{p}$

Sponsored by Electric Power Research Inst., Palo Alto $\mathrm{CA}$.

Pub. in Proceedings of International Conference on Power Quality (2nd): End-Use Applications and Perspectives, Atlanta, GA., September 28-30, 1992, pC $11: 1-\mathrm{C}-11: 8$

Keywords: "Cascaded elements, "Circuit protection, *Surges, Arresters, Computerized simulation, Standards, Overvoltage, Reprints

The basic and critical parameters for a successful coordination of cascaded surge-protective devices include the relative voltage clamping of the two devices, their electrical separation through wining inductance, and the actual waveform of the impinging surge. The authors examine in detail the implications of the situation resulting from the present uncoordinated application of devices with low clamping voltage at the end of branch circuits and devices with higher clamping voltage at the service entrance. As an alternative, several options are offered for discussion, that might result in effective, reliable implementation of the cascaded protection concept.

\section{2,465}

PB94-216496 Not available NTIS

National Inst. of Standards and

Coordinatig, MD. Electricity Div An Elusive Goal.

Final rept.

F. D. Martzloff, and J. S. Lai. 1992, 4p.

Pub. in Electrical Business, p1-4 Sep 92.

Keywords: "Cascaded elements, "Circuit protection, *Surges, Reliability(Electronics), Overvoltage, Lightning protection, Standards, Reprints.

Cascading two or more surge-protective devices lo cated at the service entrance of a building and near sensitive load equipment is done to ensure that each device shares the surge stress in a manner commensurate with its rating. The final purpose is to achieve reliable protection of equipment against surges impinging from the utility supply, as well as internally generated surges. Coordination may or may not be effective, depending upon the relative clamping voltages of the two devices, their separation distance, and the waveform of the impinging surge. The article presents the results of computations, confirmed by measurements of the energy deposited in the devices for combinations of these three parameters. From these re sults, a suggestion is made on a possible approach to a coordinated scheme. However, without consensus among interested parties, some questions remain on the likelihood of ensuring coordination for all possible circumstances.

\section{Engine Studies (Energy Related)}

02,466

PB95-200218 PC A07/MF A02

Southwest Research Inst., San Antonio, TX. Engine, Fuel, and Vehicle Research Div.

Evaluation of Wear Resistant Ceramic Valve Seats in Gas-Fueled Power Generation Engines. Topical Report, December 1991-April 1994

R. W. Burrahm, R. J. Branecky, P. C. Sui, M. C.

Shen, J. P. Latusek, and S. M. Hsu. Dec 94, 127p

SWRI-4770, GRI-94/0411.

Contract GRI-5091-288-2319

Color illustrations reproduced in black and white. Prepared in cooperation with National Inst. of Standards and Technology (MSEL), Gaithersburg, MD. Ceramics Div. Sponsored by Gas Research Inst., Chicago, IL.

Keywords: "Ceramics, "Wear resistance, "Engine wear, "Engine valves, "Diesel engines, "Electric gen erators, Life cycle costs, Engine failures, Economic analysis, Maintenance costs, Structural analysis, Finite element method, Natural gas.

This project is directed at the reduction of valve reces sion in natural gas-fueled engines. Ceramic valve seat inserts have been procured, installed in a Caterpillar G3516 natural gas generator set, and tested for 1000 hours. Two different silicon nitride materials are being utilized for the valve seats in addition to stock Eatonite metallic inserts. Three valve face materials are being tested. These include stock Caterpillar stellite 1 faced, stellite 6 faced, and unfaced valves. A test matrix was used to allow comparison of all three valve face materials in combination with all three insert materials. The testing is scheduled to continue for an additional 7000 hours. No problems have been encountered with the test materials. In general, it has been shown that two types of silicon nitride materials have at least short term durability in engine operation. Neither material has exhibited any deficiencies thus far. An economic analysis spreadsheet has been created to calculate potential cost savings potential using ceramic valve seat inserts. Valve recession data for the first 1000 hours shows expected trends. Exhaust valve positions are wearing more than intake valve positions. If the intake positions and all positions with unfaced valve are ignored, then ceramic inserts paired with Stellite 1 valves show the most wear.

\section{Environmental Studies}

02,467

PB94-212388 Not available NTIS

National Inst. of Standards and Technology (EEEL), Gaithersburg, MD. Electricity Div.

Investigation of S2F10 Production and Mitigation in Compressed SF6-Insulated Power Systems. Final rept.

D. R. James, I. Sauers, G. D. Griffin, K. L. Stricklett, F. Y. Chu, J.R. Robins, H. D. Morrison, R. J. Van Brunt, and J. K. Olthoff. 1993, 13p.

Pub. in Institute of Electrical and Electronics Engineers Electrical Insulation Magazine 9, n3 p29-40, 51, Mayl Jun 93.

Keywords: "Sulfur fluorides, "Electric power distribution, *Insulation, "Toxic substances, "Circuit breakers, Air pollution, Electrical insulation, Byproducts, Operation, Maintenance, Electric discharges, Reprints.

A Cooperative Research and Development Agreement (CRADA) has been established to study the production and mitigation of S2F10 (disulfur decafluoride), one of a number of toxic by-products formed in electrical dis charges in the insulating gas SF6. The particular concern for S2F10 is due to its highly toxic nature, the Ceiling Limit Value being 10 parts per billion (ppb, or 1 part in 10 to the 8th power) and the need for development of sensitive detection techniques down to this level. There are a large number of SF6 gas-insulated electric power systems already in service in North America, particularly circuit breakers. SF6 is extensively used as an insulation and current interruption medium in power circuit breakers, compressed gas transmission lines and various components in substations. Ensuring the safe operation and maintenance of this equipment is an important issue for utilities, government agencies, and manufacturers.

02,468

PB96-155528 Not available NTIS

National Inst. of Standards and Technology (EEEL), Gaithersburg, MD. Electricity Div.

Investigation of S2F10 Production and Mitigation in Compressed SF6-Insulated Power Systems.

Final rept.

I. Sauers, G. D. Griffin, D. R. James, K. L. Stricklett, H. D. Morrison, F. Y. Chu, M. F. Frechette, R. J. Van Brunt, and J. K. Olthoff. 1995, 27 p.

See also PB94-212388. Sponsored by Electric Power Research Inst., Palo Alto, CA. and Department of Energy, Washington, DC

Pub. in Oak Ridge National Laboratory, Oak Ridge, Tennessee Report No. ORNL/M-4314, v1 27p Oct 95. 
Keywords: "Sulfur fluorides, "Electric power distribution, "Insulation, Toxic substances, Circuit breakers, Reprints, Air pollution, Electrical insulation.

A Cooperative Research and Development Agreement (CRADA) was established in 1991 to study the production and mitigation of S2F10 (disulfur decafluoride), one of a number of toxic by-products formed by elec trical discharges in the insulating gas SF6. Since com pressed SF6 is extensively used as an insulation and current interruption medium in electric power equipment, ensuring the safe operation and maintenance of this equipment is an important issue for utilities, government agencies, and manufacturers. The particula concern for S2F is due to (1) its highly toxic nature: the Threshold Limit Value - Ceiling (TLV-C) is $10 \mathrm{ppbv}$ ( 1 ppbv = 1 part in 10 to the 9 th power by volume $=$ $1 \mathrm{~nL} / \mathrm{L})$, the level which can not be exceeded during any part of the working exposure; and (2) the lack of sensitive detection techniques down to the TLV-C.

\section{Fuels}

\section{2,469}

AD-A279 952/6 PC A09/MF A02

National Bureau of Standards, Gaithersburg, MD.

National Standard Petroleum Oil Tables.

4 Mar 36, 185p.

Keywords: "Oils, "Standards, Petroleum industry, Scale, Specific gravity, Densimeters, Tables(Data), Weight, Temperature.

The tables given are based on an investigation of American petroleum oils carried out by the National Bureau of Standards, and the arrangement is largely according to the recommendations of a committee appointed by the American Petroleum Institute to represent the petroleum industry. In order to overcome the confusion that has existed in the petroleum-oil industry by reason of the use of two so-called Baume scales for light liquids, the American Petroleum Institute, the U. S. Bureau of Mines, and the National Bureau of Standards, in December 1921, agreed to recommend that in the future only the scale based on the modulus 141.5 be used in the petroleum-oil industry, and that it be known as the A.P. I. scale. The relation of degrees A. P. I. to specific gravity is expressed by the formula.

\section{2,470}

DE94004399 PC A02/MF A01

National Inst. of Standards and Technology (CSTL). Gaithersburg, MD.

Development of Measurement Capabilities for the Thermophysical Properties of Energy-Related Fluids. Annual Report, December 1, 1990--November 30, 1991.

Progress rept.

1991, 10p, DÖE/ER/13823-T2

Contract Al05-88ER13823

Sponsored by Department of Energy, Washington, DC. Keywords: "Liquid Fuels, "Lubricants, "Measuring Instruments, "Refrigerants, "Working Fluids, Aqueous Solutions, Calorimetry, Densimeters, Density, Design, Dielectric Properties, Enthalpy, Phase Studies, Physical Properties, Progress Report, Thermal Conductivity, Thermodynamic Properties, Viscosimeters, Viscosity, "Thermophysical properties, EDB/400102, EDB/ 440800.

The objectives of this project are to develop state-ofthe-art experimental apparatus that can be used to measure the thermophysical properties of a wide range of fluids and fluid mixtures important to the energy, chemical, and energy-related industries and to carry out carefully selected benchmark measurements on key systems. The measurement capabilities to be developed include new apparatus for transport properties, thermodynamic properties, phase equilibria properties, and dielectric properties. The specific measurement capabilities to be developed are: thermal conductivity apparatus; vibrating wire viscometer; dualsinker densimeter; high-temperature vibrating tube densimeter; dynamic phase equilibria apparatus; apparatus for dilute solutions; total-enthalpy flow calorimeter; and dielectric constant apparatus. The research also includes benchmark experimental measurements on pure and mixed alternative refrigerants and their mixtures with lubricants, on aqueous solutions, and on carefully selected systems consisting of species of diverse size (methane + neopentane) and polarity (methane + ammonia) that are important for the development of predictive models for energy-related fluids.

\section{2,471}

DE94008991 PC A02/MF A01

National Inst. of Standards and Technology, Boulder, CO. Thermodynamics Div

Low Temperature $H($ sub 2)S Separation Using Membrane Reactor with Redox Catalyst.

J. J. Pellegrino, M. Ko, and L. Watts. 1993, 9p,

CONF-931156-41.

Contract Al21-86MC23120

Fuels Technology Contractors' Review Meeting, Morgantown, WV (United States), 16-18 Nov 1993. Sponsored by Department of Energy, Washington, DC.

Keywords: "Chemical Reactors, "Desulfurization, "Natural Gas, "Separation Processes, Hydrogen sulfides, Catalysts, Design, Membranes, Oxidation, Sodium Hydroxides, "Gas pipelines, EDB/030300.

A low temperature redox catalyst is combined with an $H$ (sub 2)S selective membrane to create a compositecatalytic-membrane-reactor (CCMR) to help reach pipeline-quality natural gas $\mathrm{H}$ (sub 2)S levels of below $4 \mathrm{ppM}$. The goal of this program is to identify the apparent kinetic and mass transfer parameters for the CCMR. Using these kinetic and mass transfer parameters a preliminary process design can be made to ascertain the potential of this technology for replacement of conventional amine scrubbing and Claus sulfur recovery processes. In pursuit of this goal the project contains the following activities: construction of reactor and mass transfer equipment suitable for accurate measurements and safe handling of pure $H$ (sub 2) $S$; initial proof-of-concept experiments with a prototype CCMR; obtain literature data and develop thermodynamic models for multicomponent natural gas components' solubility in electrolyte solutions; make physical and chemical measurements on the catalyst; obtain kinetic data using a 3-phase slurry reactor; obtain kinetic data for a catalytic membrane under varying mass transfer conditions; develop mathematical models to guide experimental work and for interpretation of resuits; optimize the permselective membrane part of the CCMR using commercially available materials; further proof-of-concept experiments with an optimized CCMR; and develop preliminary process design and economic analysis for the use of a CCMR in gas cleanup

\section{2,472}

PB94-146677 PC A03/MF A01

National Inst. of Standards and Technology (CSTL), Boulder, CO. Thermophysics Div.

Thermophysical Properties of Fluids for the Gas Industry. Final Report, February 1, 1988-August 31, 1993.

T. J. Bruno, and W. M. Haynes. Nov 93, 20p, GRI93/0396.

Contract GRI-5088-260-1700

See also PB93-207470. Sponsored by Gas Research Inst., Chicago, IL.

Keywords: "Fluids, "Thermophysical properties, *Natural gas, Gas industry, Equations of state, Experimental design, Mathematical models, Hydrocarbons, Detectors

The U.S gas industry standard for computing thermophysical properties is the A.G.A. Transmission Measurement Committee Report No. 8 equation of state (AGA 8). The report summarized the results from several experimental, theoretical, and modeling programs directed at the extensive evaluation of the accuracy with which various types of natural gas physical properties can be calculated using AGA 8 and related methods. The most important results were the assembly of benchmark data sets for speed of sound, viscosity, fugacity, heat capacity, critical region PVT, mixture compressibilities, and vapor pressure measurements for natural gas fluids.

\section{2,473}

PB94-193802 PC A08/MF AO2

Purdue Univ., Lafayette, IN. Thermal Sciences and Propulsion Center.

Structure and Radiation Properties of Pool Fires. Final rept. 1 Sep 91-31 Aug 92 .

M. Klassen, and J. P. Gore. Aug 92, 156p, NIST/ GCR-94/651.

Grant NIST-60NANB1D1169

Sponsored by National Inst. of Standards and Tech-

nology (BFRL), Gaithersburg. MD.
Keywords: "Radiative heat transfer, "Fuels, Burning rate, Combustion, Data analysis, Spectral emission, Burners, Heat flux, Soot, Heat measurement, "Pool fires.

An experimental and theoretical study of radiative feedback, burning rates, radiative heat loss fractions, and flame heights for pool fires with diameters ranging from $4.6 \mathrm{~cm}$ to $100 \mathrm{~cm}$ was completed. Transient measurements of soot and temperature distributions were obtained in $7.1 \mathrm{~cm}$ and $30 \mathrm{~cm}$ heptane and toluene fires using a three-wavelength emission/absorption probe. The heat release rates of the fires varied from $0.6 \mathrm{~kW}$ to $2,166 \mathrm{~kW}$ allowing a study over a wide range. A variety of fuels were tried but most of the measurements were restricted to methanol, heptane, and toluene as representatives of the alcohols, paraffins and aromatics. Radiative feedback was measured using a new in situ purged optical probe in serted at the level of the fuel surface. Measurements of reflection of energy from the fuel surface were also obtained.

\section{2,474}

PB94-199254 Not available NTIS

National Inst. of Standards and Technology (NML), Gaithersburg, MD. Chemical Thermodynamics Div.

Calculation of Higher Heating Values of Biomass Materials and Waste Components from Elemental Analyses.

Final rept.

T. J. Buckley. 1991, 13p

Pub. in Resources, Conservation and Recycling 5, n4 p329-341 Aug 91

Keywords: "Biomass, "Waste utilization, "Calorific value, "Hazardous materials, Comparison, Performance evaluation, Chemical analysis, Numerical analysis, Experimental design, Fuels, Reprints, Standard reference materials, Vondracek formula

Higher heating values on a dry basis (HHV2) measured and those calculated from elemental analyses of over 140 biomass and waste component materials have been compared. The data was evaluated by six higher heating value formulas. The Vondracek formula was found to give the best agreement between calculated and measured heating values over a wide range (10 to $47 \mathrm{MJ} / \mathrm{kg}$ ). This was evident by the good linear correlation between measured and calculated HHV2's. Standard reference materials also have been evaluated and shown generally to give good agreement. The calculation of higher heating values from elemental analyses is a good check for the reliability of a material's composition and heating value measurements in most cases. Materials with differences between calculated and measured higher heating values in excess of 6 percent should be considered suspect and examined closely.

\section{5}

PB94-206646 PC A05/MF A02

Southwest Research Inst., San Antonio, TX. Nondestructive Evaluation Science and Technology Div. Assessment of Technology for Detection of Stress Corrosion Cracking in Gas Pipelines. Final Report, July 1993-March 1994

A. E. Crouch, C. M. Teller, J. L. Fisher, G. M. Light, and C. M. Fortunko. Apr 94, 99p, GRI-94/0145. Contract GRI-5093-271-2639

Prepared in cooperation with National Inst. of Stand ards and Technology, Boulder, CO. Sponsored by Gas Research Inst., Chicago, IL.

Keywords: "Nondestructive tests, "Natural gas pipelines, "Stress corrosion, Cracking(Fracturing), Wipers, Inspection, Technology assessment, Electromagnetic waves, ILI(In-line inspection), Smart pigs.

The objective of the project was to assess the nondestructive evaluation (NDE) technology that may be applied to natural gas steel pipelines for the detection of stress corrosion cracking (SCC). In particular, the application of such technology would be from the inside of the pipe while the pipeline is in service, and inspection commonly called in-line inspection (ILI). The assessment focused on two basic NDE methods, ultrasonics and electromagnetics (including magnetic flux leakage). A third technology was data acquisition analysis systems. Based on the experience of British Gas (BG) and others doing SCC inspections in different industries, crack discrimination is the important challenge for any such inspection system. 
National Inst. of Standards and Technology (NEL), Gaithersburg. MD. Fire Science and Engineening Div. Estimate of Flame Radiance via a Single Location Measurement in Liquid Pool Flames.

Final rept.

A. Hamins, M. Klassen, J. Gore, and T. Kashiwagi.

1991, 6p.

Pub. in Combustion and Flame 86, n3 p223-228 1991

Keywords: "Radiative heat transfer, "Fuels, "Flames, "Heat loss, Radiative transfer, Heat transmission, Radiant heating, Combustion, Heat flux, Reprints, "Pool fires.

A method for estimating the radiative heat loss fraction is developed for non-premixed flames in a poor fire configuration. An experimental investigation of small to intermediate pool flames shows that a particular location can be determined such that a single radiance measurement yields an adequate estimate of the radiative heat loss fraction. The procedure is tested on a variety of fuels which yield flames over a wide range of luminosities and values of radiative heat loss fraction.

\section{2,477}

PB94-211604 Not available NTIS

National Inst. of Standards and Technology (NEL), Gaithersburg, MD. Fire Science and Engineering Div. Measurement of Radiative Feedback to the Fuel Surface of a Pool Fire.

Final rept.

A. Hamins, M. Klassen, J. Gore, and T. Kashiwagi. 1990, 4p.

Pub. in Chem. Phys. Processes Combust., p58/1-58/ 41990.

Keywords: "Radiative heat transfer, "Fuels, "Surfaces, Radiant heating, Heat flux, Radiative transfer, Heat transmission, Methanol, Combustion, Reprints, "Pool fires, "Heat feedback.

The measurement of the radiative heat feedback to the fuel surface of fires buming in a pool fire configuration is discussed. Radiative feedback in a $30 \mathrm{~cm}$ methanol pool is compared to total heat feedback. Measurements show that the radiative flux decreases from pool center towards the pool edge.

\section{2,478}

PB95-108544 Not available NTIS

National Inst. of Standards and Technology (NML), Gaithersburg, MD. Chemical Process Metrology Div.

Combustion of Methanol and Methanol/Dodecanol Spray Flames.

Final rept.

C. Presser, A. K. Gupta, C. T. Avedisian, and H. G. Semeriian. 1992, 7p.

Pub. in Jnl. of Propulsion and Power 8, n3 p553-559 May/Jun 92.

Keywords: "Methanol, "Combustion, "Spraying, "Flame propagation, Lasers, Velocity distribution, Drops(Liquids), Interferometry, Mixtures, Size determination, Reprints.

The structure of methanol and methanol/dodecanol mixture spray flames has been examined. The mixture was studied in order to obtain evidence for the occurrence of microexplosions. Droplet size and velocity measurements were carried out under burning conditions using phase/Doppler interferometry Laser sheet beam photography was used to observe the global features of the spray within the nonluminous portion of the flames. Droplet size and velocity distributions near the nozzle exit were found to be similar and monomodal for both fuels. Further downstream in the mixture flame the velocity distribution became bimodal near the spray boundary along with a change in size distribution to smaller droplets and an increase in number density. These results suggest that microexplosions may occur in $50 / 50$ mixtures of methanol/dodecanol spray flames.

02,479

PB95-108551 Not available NTIS

National Inst. of Standards and Technology (NML),

Gaithersburg, MD. Chemical Process Metrology Div.

Study of Droplet Transport in Alcohol-Based Spray

Flames Using Phase/Doppler Interferometry.

Final rept.

C. Presser, A. K. Gupta, C. T. Avedisian, and H. G. Semeriian. $1990,5 p$.

Pub. in Proceedings of Annual Conference Extended Abstracts ILASS-Americas (4th), Hartford, CT., May 21-23, 1990, p243-247 1990

Keywords: "Alcohol fuels, "Atomization, "Spraying, "Transport, "Flame propagation, Vaporization, Com- bustion, Combustion products, Particulates, Inteferometry, Reprints.

Studies with alternative fuels (i.e., methanol and ethanol) are of particular interest because of the current initiative to gradually replace the limited supply of conventional fossil fuels (i.e., gasoline, heating oils, etc.) Alcohol-based fuels are attractive, since they bum more cleanly and represent a potential to reduce the environmental impact of combustion processes. Investigation of the effect of alcohol fuels on spray and flame characteristics (i.e., droplet size and velocity) is important since chemical and physical properties of the fue have a significant influence upon droplet atomization vaporization, transport, combustion, and pollutant and particulate formation processes. The paper examines the effects of physical and chemical properties of two alcohol fuels on droplet transport processes of swirling spray flames. Experiments were carried out with methanol and a miscible mixture of methanol/1-dodecanol. The mixture fuel was selected to investigate the possibility of microexplosions in spray flames. Droplet size and velocity distributions were obtained using a phase/ Doppler system. Laser sheet beam photography was also used to observe the internal features of pressureatomized spray flames.

\section{2,480}

PB95-108569 Not available NTIS

National Inst. of Standards and Technology (NML), Gaithersburg, MiD. Chemical Process Metrology Div. Structure of a Swirl-Stabilized Kerosene Spray Flame.

Final rept.

C. Presser, A. K. Gupta, and H. G. Semeriian. 1990, $4 p$.

Sponsored by Department of Energy, Washington, DC Pub. in Proceedings of Fall Technical Meeting Chemical and Physical Processes in Combustion, Orlando FL., December 3-5, 1990, p81-1-81-4.

Keywords: "Kerosene, "Swirling, "Spraying, "Flames, Velocity distribution, Atomization, Drops(Liquids) Laser doppler velocimeters, Combustion, Reprints.

The interaction of the fuel spray with the surrounding combustion air, in particular near the spray boundary affects its combustion and emission characteristics. Near the spray boundary, droplets of many different sizes, traveling at different velocities, are expected to be found. In addition, combustion air swirl affects fuel/ air mixing and modifies the entire spray pattern. Therefore, spatially and temporally resolved information about the effect of swirl on droplet properties, especially near the spray boundary, is important for understanding the structure of sprays and spray flames. The results presented demonstrate the strong interaction between the spray droplets and the combustion air flow, which significantly modifies the flame structure. They also demonstrate the need to assess the effect of instrument characteristics, such as dynamic range and system gain, on the measurements for determination of accurate mean flow properties.

\section{2,481}

PB95-126314 Not available NTIS

National Inst. of Standards and Technology (NEL), Gaithersburg, MD. Structures Div.

Ashland Tank Collapse Investigation.

Final rept.

J. L. Gross, J. H. Smith, and R. N. Wright. 1989

$19 p$.

See also PB95-126322.

Pub. in Jnl. of Performance of Constructed Facilities 3, n3 p144-162 Aug 89.

Keywords: "Storage tanks, "Structural failure, "Steel structurers, "Fractures(Materials), "Steels, "Brittleness, *Accident investigations, Crack propagation, Structural analysis, Welded joints, Fracture strength, Toughness, Pennsylvania, Cracking(Fracturing), Fuel tanks, Construction matenals, Fuel oils, Collapse, Reprints, "Welded stee tanks, "Floreffe Terminal, Ashland Petroleum Company, Reconstruction.

On January 2, 1988, a four million gallon capacity oil storage tank at the Ashland Petroleum Company Floreffe Terminal near West Elizabeth, Pennsylvania, collapsed as it was being filled to capacity for the first tlme since its reconstruction. The tank had been dismantled in Cleveland, Ohio, after more than 40 years of service and reconstructed at the Floreffe site in 1986. The National Institute of Standards and Technology (NIST) conducted an independent investigation into the physical cause of the Ashland tank collapse.
It was determined that the failure was caused by a britthe fracture of the tank shell that initiated from a defect which existed prior to reconstruction of the tank. Complete rupture of the tank shell occurred because the steel did not possess adequate toughness at the operating temperature to prevent brittle fracture propagation. The collapse shows the importance of using steel with sufficient fracture toughness to prevent propagation of a brittle fracture in tanks whose sudden failure would mean unacceptable human, environmental or economic losses.

\section{2,482}

\section{PB95-126322 Not available NTIS}

National Inst. of Standards and Technology (NEL) Gaithersburg, MD. Structures Div.

Ashland Tank-Collapse Investigation: Closure by Authors.

Final rept

J. L. Gross, J. H. Smith, and R. N. Wright. 1991, 2p. See also PB95-126314.

Pub. in Jnl. of Performance of Constructed Facilities 5, n2 p150-151 May 91.

Keywords: "Storage tanks, "Structural failure "Steel structures, "Fractures(Materials), "Steels, "Brittleness, "Accident investlgations, Crack propagation, Structural analysis, Welded joints, Fracture strength, Toughness Pennsylvania, Cracking(Fracturing), Fuel tanks, Construction materials, Fuel oils, Collapse, Reprints, "Welded steel tanks, "Floreffe Terminal, Ashland Petroleum Company, Reconstruction.

This Closure addresses several issues raised in a discussion of the paper titled, 'Ashland Tank Collapse Investigation," (PB95-126314) which appeared in the Joumal of Performance of Constructed Facilities ASCE. That article related to the structural failure of a four million gallon capacity oil storage tank at the Ashland Petroleum Company Floreffe Terminal as it was being fueled to capacity for the first time since its reconstruction.

\section{2,483}

PB95-136644 PC A03/MF A01

National Inst. of Standards and Technology (CSTL). Boulder, CO. Thermophysics Div.

Constituents and Physical Properties of the C6+ Fraction of Natural Gas. Topical Report, April-June 1994.

T. J. Bruno. Aug 94, 40p, NISTIR-5212, GRI-94/

Contract GRI-5093-260-2720

Sponsored by Gas Research Inst., Chicago, IL.

Keywords: "Natural gas, "Chemical composition, * Physical properties, Data bases, Gas chromatography, Chemical analysis, Quality assurance, Thermophysical properties, Hydrocarbons, Thermodynamics, Odorants, Treatment compounds.

The U.S. gas industry relies upon gas chromatographic analysis to obtain detailed gas compositional information needed for determining phase equilibrium behavior, thermophysical properties, and compliance with gas quality and safety engineering specifications. Analysis of the $C(6+)$ fraction constituents of natural gas is technically complex and expensive. The assembly of a physical properties database for known $\mathrm{C}(6+)$ fraction constituents is needed to facilitate the development of improved chromatographic separation procedures for analyzing raw and processed natural gas streams for compliance with gas quality and end use specifications. The report provides a critically reviewed compilation of physical properties data for 132 hydrocarbon compounds identified as being native constituents of natural gas, 21 gas treatment compounds, and 23 gas odorant compounds.

\section{2,484}

PB95-140950 Not available NTIS

National Inst. of Standards and Technology (MSEL)

Gaithersburg, MD Polymers Div.

Chemical and Microbiological Problems Associated with Research on the Biodesulfurization of Coal.

Final rept.

G. J. Kelly. 1991, 11 . Pub. in Resources,

Keywords: "Coal, "Desulfurization, "Biological treatment *Air pollution abatement "Sulfur inorganic compounds, "Sulfur organic compounds, Microorganisms, Microbiology, Biochemistry, Chemistry, Removal, Thermophiles, Reprints. 


\section{Fuels}

The study of microbial processes for the removal of organic and inorganic sulfur from coals is complicated by the lack of direct methods of measurement for organic sulfur content and the related incomplete understanding of the specific forms of organic sulfur in coal. In addition, the accessibility of specific chemical groups in the coal matrix to microorganisms and their enzymes is uncertain, raising questions about the nature and validity of model compound studies. Thus, interpretation of data from numerous efforts focused on the microbial removal of inorganic and organic sulfur from coals remains controversial. The discussion here reviews recent developments in the chemical characterization of coal sulfur related to bioprocessing research and describes some of our recent efforts in involving sulfur transformations by hyperthermophilic archaebacteria.

\section{2,485}

PB95-141099 Not available NTIS

National Inst. of Standards and Technology (CSTL), Boulder, CO. Thermophysics Div.

Process Gas Chromatography Detector for Hydrocarbons Based on Catalytic Cracking.

Final rept.

S. L. Outcalt, H. Ingham, and T. J. Bruno. 1992, 13p. Sponsored by Gas Research Inst., Chicago, IL.

Pub. in Process Control and Quality 2, p357-369 1992. Keywords: "Thermopiles, "Gas chromatography, *Catalytic cracking, *Hydrocarbons, "Detectors, Fuels, Temperature measurement, Thermocouples, Catalysis, Reprints, "Hydrocarbon detectors.

A detector has been designed and built for the gas chromatographic detection of hydrocarbon species, especially in plant or on-line applications. Detection of the temperature change that occurs as a result of the catalytic cracking of hydrocarbons is the functional basis of the detector. The main component of this detector is a thermopile bundle constructed with type-K thermocouple wire. The sensing junctions of the thermopile are coated with a catalyst made up of a silicon dioxide-aluminum oxide-zeolite mixture. The temperature change associated with catalytic cracking occurs predominantly on the catalyst surface and is measured by the thermopile. The thermopile is directly connected to an electronic integrator, and provides a signal as a result of the temperature change. The tests that were performed to characterize the performance capabilities of the detector are reponted. In addition, variable parameters were studied to determine the optimal conditions for obtaining the greatest sensitivity of the detector. The catalytic cracking detector is a very simple, inexpensive, and rugged device and is proposed as a potential alternative to other detectors for use in field or plant environments.

\section{2,486}

PB95-150447 Not available NTIS

National Inst. of Standards and Technology (BFRL), Gaithersburg, MD. Fire Safety Engineering Div.

Estimate of the Effect of Scale on Radiative Heat Loss Fraction and Combustion Efficiency.

Final rept.

J. C. Yang, A. Hamins, and T. Kashiwagi. 1994, 6p. Pub. in Combustion Science and Technology 96, p183-188 1994

Keywords: "Burning rate, "Thermal radiation, "Heat loss, *Scaling factor, "Fuel combustion, "Combustion efficiency, Flames, Radiative heat transfer, Heat transmission, Diameters, Combustion control, Reprints, "Pool fires, "Flame height, Fuel fires.

The effect of fire size on radiative heat loss fraction kappa(sub r) and combustion efficiency kappa(sub a) was examined by an analysis of scale for pool flames with varying diameter (D). Correlations between $D$ and kappa(sub r) or kappa(sub a) were obtained. For $0.1 \mathrm{~m}$ $<D<1 m$, kappa(sub r) and kappa(sub a) are relatively constant and independent of $\mathrm{D}$. For larger pool diameters, kappa(sub r) decreases with increasing $D$.

\section{2,487}

PB95-150900 Not available NTIS

National Inst. of Standards and Technology (NEL), Gaithersburg, MD. Fire Measurement and Research Div.

Optical Measurements of Atomic Hydrogen, Hydroxyl, and Carbon Monoxide in Hydrocarbon Diffusion Flames.

Final rept.

P. J. H. Tjossem, and K. C. Smyth. 1988, 4p.

Pub. in Chem. Phys. Processes Combust., p8/1-8/4 1988
Keywords: "Diffusion flames, "Laser induced fluorescence, "Hydrogen, "Hydroxyl radicals, "Hydrocarbons, Carbon monoxide, Methane, Ionization, Concentration(Composition), Multiphoton absorption, Polarization, Reprints.

Recent quantitative $\mathrm{OH}(-)$ measurements and relative data for $\mathrm{H}$ atoms and $\mathrm{CO}$ are presented for a $\mathrm{CH}_{4}$ air diffusion flame. These results have been obtained using absorption, laser-induced fluorescence, and multiphoton ionization technique.

\section{2,488}

PB95-163374 Not available NTIS

National Bureau of Standards (IMSE), Gaithersburg, MD. Polymers Div.

Microbial Degradation of Polysulfides and Insights into Their Possible Occurrence in Coal.

Final rept.

L. Tilstra, G. J. Olson, G. Eng, and R. M. Kelly. 1990, $16 \mathrm{p}$

$\mathrm{Pub}$. in Proceedings of International Symposium on the Biological Processing of Coal (1st), Orlando, FL., May
$1-3,1990$, p3.27-3.42.

Keywords: "Microbial degradation, "Biodeterioration, "Polysulfides, "Coal, "Air pollution abatement, Desulfurization, Sulfur-oxidizing bacteria, Thiobacillus oxidans, Reduction(Chemistry), Oxidation, Biological treatment, Microorganisms, Reprints, Sulfur reducing bacteria.

Sulfur-oxidizing mesophilic (Thiobacillus thiooxidans) and sulfur-reducing hyperthermophilic (pyrococcus, Pyrodictium and others) bacteria are used as probe of specific forms of sulfur coal. When these bacteria are grown in medium with elemental sulfur or simple polysulfides (e.g., methyl trisulfide, cystine), easily measured products which are diagnostic of desulfurization are produced. As part of this research we have synthesized model compounds which migh more closely approximate some of the forms in which sulfur occurs in coal. These include: (1) organic sulfur model compounds which have bulky chemical groups surrounding the sulfur atoms and (2) polymeric polysulfides. Tests on these polysulfides will clarify the ability of the above bacteria to oxidize or reduce varous forms of sulfur. The studies are also providing information as to the potential for these microorganisms to serve as probes of specific functional groups in coal and for the microbiological removal of sulfur from coal. Our approach could be extended to studies of large model compounds containing sulfur in other types of linkages such as heterocyclic rings.

\section{2,489}

PB95-168449 Not available NTIS

National Inst. of Standards and Technology (TS) Gaithersburg, MD. Office of Standards Services.

Method of Sale for CNG Paves Way to Greater Public Acceptance.

Final rept.

C. S. Brickenkamp. 1994, 1 p.

Pub. in Natural Gas Fuels Magazine, p47 Sep 94

Keywords: "Compressed gases, “Sales, “Marketing Gas fuels, Liquid fuels, Comparisons, Selling, Cos analysis, Market research, Alternative fuels, Reprints, "Gasoline gallon equivalent, Compressed natural gas. The article describes the National Conference on Weights and Measures adoption of the Gasoline GalIon Equivalent (GGE) as the method of sale for compressed natural gas (CNG) when sold at reatil as an engine fuel. The GGE is defined as 5.660 Pounds $\mathrm{CNG}$. This amount of $\mathrm{CNG}$ approximates the equivalent energy of a gallon of gasoline.

\section{2,490}

PB95-175188 Not available NTIS

National Inst. of Standards and Technology (CSTL), Boulder, CO. Thermophysics Div

Measurement of Diffusion in Fluid Systems: Applications to the Supercritical Fluid Region.

Final rept.

T. J. Bruno. 1994, 5p.

Pub. in Jnl. of Thermophysics and Heat Transfer 8, n2 p329-3331994.

Keywords: "Diffusion coefficient, "Aviation fuels, "Supercritical fluids, ${ }^{*}$ Chromatography, Thermophysical properties, Diffusion
Concentration(Composition), Solubility, Binary mix tures, Chemical analysis, Nuclear magnetic resonance, Light scattering, Reprints.

In this article, the experimental procedures that are applicable to the measurement of diffusion in supercritica fluid solutions will be reviewed. This topic is of great importance to the proper design of advanced aircraft and turbine fuels, since the fuels on these aircraft may sometimes operate under supercritical fluid conditions. More specifically, we will consider measurements of the binary interaction diffusion coefficient $D(s u b 12)$ of a solute (species 1) and the solvent (species 2). In this discussion the supercritical fluid will be species 2 , and the solute, species 1 , will be at a relatively low concentration, sometimes approaching infinite dilution. After a brief introduction to the concept of diffusion, we will discuss in detail the use of chromatographic methods, and then briefly treat light scattering, nuclear magnetic resonance spectra, and physical methods.

02,491

PB95-175212 Not available NTIS

National Inst. of Standards and Technology (CSTL),

Boulder, CO. Thermophysics Div.

Fugacity Coefficients of Hydrogen in (Hydrogen + Butane).

Final rept.

T. J. Bruno, and S. L. Outcalt. 1993, 10p

Sponsored by Gas Research inst., Chicago, IL

Pub. in Jnl. of Chemical Thermodynamics 25, p106110701993.

Keywords: "Evanescence, "Hydrogen, "Butanes, *Fugacity, Thermodynamic properties, Permeability, Membranes, Gas flow, Transpiration, Binary mixtures, Reprints, "Fugacity coefficients.

The fugacity coefficients of hydrogen in (hydrogen + butane) were measured as a function of composition with a physical-equilibrium technique at (temperature, pressure) pairs of (433.15 K, 3.39 MPa), (473.15 K, 3.38 MPa), (473.15 K, 22.65 MPa), and (523.15 K, $3.42 \mathrm{MPa}$ ). The physical-equilibrium technique involved the use of an experimental chamber that was divided into two separate regions by a semipermeable membrane through which hydrogen, but not butane, could permeate. Measurement of the gas pressures on each side of the membrane, in addition to a measurement of the composition and the system temperature, allowed the calculation of the fugacity and fugacity coefficient of hydrogen in the mixture. The qualitative features of the measurements are discussed, and comparisons are made with predictions obtained from the Redlich-Kwong and Peng-Robinson models.

\section{2,492}

PB95-253571 PC A03/MF A01

National Inst. of Standards and Technology (TS), Gaithersburg, MD. Weights and Measures Program. Advanced Mass Calibration and Measurement Assurance Program for State Calibration Laboratories.

K. L. Fraley, and G. L. Harris. Jun 95, 28p, NISTIR5672

Keywords: "Mass, "Calibrating, "Design, "Standards, Metrology, Laboratories, State government, Surveillance, Precision, Measurement, "Mass standards, US NIST, Surveillance tests, Measurement assurance.

This publication provides guidelines for evaluating data from the advanced mass calibration and for establishing measurement assurance programs in state metrology laboratories. The NIST Office of Weights and Measures (OWM) will use these guidelines when evaluating data for laboratories requesting technical support or accreditation at this level.

\section{2,493}

\section{PB95-256335 PC A04/MF A01}

National Inst. of Standards and Technology Gaithersburg, MD.

Sliding Vane Flow Conditioner Tests in a 100 Diameter Long 10 inch Natural Gas Orifice Meter at Pa. cific Gas and Electric. Topical Report, 1990-1992. J. W. Stuart. Oct $92,58 p$, GRI-92/0576.

Contract GRI-5088-271-1680

Prepared in cooperation with Pacific Gas and Electric Co., San Francisco, CA. Sponsored by Gas Research Inst., Chicago, IL

Keywords: "Natural gas, "Orifice meters, "Flow measurement, Field tests, Gas flow, Vanes, Gas pipelines, High pressure, Technical assistance, Standards, Orifice flow, "Sliding vanes.

Sliding vane tests in a 10-inch, high pressure, natural gas test loop conducted at the Pacific Gas and Electric company in January 1991 are documented in this volume. The work is part of cooperative research in flow conditioning aimed offer technical support for revisions of industry standards in gas metering. 
02,494

PB96-122437 Not available NTIS

National Inst. of Standards and Technology (CSTL),

Boulder, CO. Thermophysics Div.

Thermophysical Properties of Fluids for the Gas Industry.

Final rept.

T. J. Bruno, and W. M. Haynes. 1992, 12p.

Contract GRI-5088-260-1700

See also PB94-146677. Sponsored by Gas Research Inst., Chicago, IL.

Pub. in Gas Research Institute Annual Report, JanDec $92,12 p$.

Keywords: "Fluids, "Thermophysical properties, *Natural gas, Gas industry, Equations of state, Experimental design, Mathematical models, Hydrocarbons, Detectors, Reprints.

The U.S. gas industry standard for computing thermophysical properties is the A.G.A. Transmisson Measurement Committee Report No. 8 equation of state (AGA 8). This report summarized the results from several experimental, theoretical, and modeling programs directed at the extensive evaluation of the accuracy with which various types of natural gas physical properties can be calculated using AGA 8 and related methods. The most important results were the assembly of benchmark data sets for speed of sound, viscocity, fugacity, heat capacity, critical region PVT, mixture compressibilities, and vapor pressure meas urements for natural gas fluids. When tested against these benchmark properties data, the AGA 8 equation of state model was found to be generally accurate with in plus or minus $0.1 \%$ for sound speeds (and densities) and within plus or minus $0.03 \%$ for compressibilities over the ranges of pressure, temperature, and com position that encompass the major region of custody transfer or natural gas. Work was also completed on the fabrication and testing of a prototype catalytic cracking detector for the selective detection of hydrocarbons; a U.S. patent was awarded for this invention with the assignment to GRI

\section{2,495}

PB96-141379 Not available NTIS

National Inst. of Standards and Technology (CSTL) Gaithersburg, MD. Inorganic Analytical Research Div. Determination of Sulfur in Fossil Fuels by Isotope Dilution Thermal lonization Mass Spectrometry.

Final rept

W. R. Kelly, P. J. Paulsen, K. E. Murphy, R. D.

Vocke, and L. T. Chen. 1994, 9p.

Pub. in Analytical Chemistry, v66 n15 p2505-2513 Aug

Keywords: "Fossil fuels, "Sulfur, "Chemical composi tion, Coal, Petroleum products, Kerosene, Chemical analysis, Mass spectroscopy, Isotope dilution, Ioniza tion potential, Reprints, Standard reference materials

Total sulfur has been measured in 13 petroleum and 14 coal Standard Reference Materials (SRMs) by isotope dilution thermal ionization mass spectrometry. Samples were spiked with enriched stable S-34 and conbusted in sealed Carius tubes using nitric and hydrochloric acids. The oxidized sulfur was reduced to $\mathrm{H} 2 \mathrm{~S}$, precipitated as As2S3, and then dissolved in an ammoniacal solution of As2O3. A portion of this solution, equivalent to 1.5 micrograms of sulfur, was added to a single Re filament coated wth silica gel. The amount of sulfur in the samples was determined from the S-32/S-34 ratio by mesuring the $A s(75) S(32)$ As(75)S(34) molecular ions in a Faraday detector. The total uncertainty (95 percent confidence interval) for homogeneous materials such as oils is about 0.5 percent, and for less homogeneous materials such as coals is 1 - 4 percent.

\section{2,496}

\section{PB96-155486 Not available NTIS}

National Inst. of Standards and Technology (CSTL),

Gaithersburg, MD. Organic Analytical Research Div.

Development of Gas Standards from Solid 1,4dichlorobenzene.

Final rept.

G. C. Rhoderick. 1995, 7p

Pub. in Jnl. of Chromatography A, v710 p229-235 1995.

Keywords: "Natural gas, Liquified, Recrystalize, Response factor, Solid, Reprints, "Foreign technology, "Gas standards.

For over fifteen years the National Institute of Stand ards and Technology (NIST) has been preparing gas standards containing volatile organic compounds at sub micro $\mathrm{mol} / \mathrm{mol}(\mathrm{ppm}$ ) concentrations. These stand ards have been prepared using organic compounds that are either yases or liquids at room temperature. A microgravimetric technique was developed previously to prepare standards containing these compounds in treated aluminum gas cylinders using a one step dilution. Requests were received to prepare gas standards containing the compound 1.4 dichlorobenzene. These requests posed a major problem in that 1.4-dichlorobenzene is a solid at room temperature Research was undertaken using the microgravimetric procedure to determine if it was feasible to prepare gas standards from solid phase compounds. In the first stage of research the liquid phas compound 1.2-dichlorobenzene previously studied at NIST in gas mixtures was used as an internal standard. Results from analyses of a pepared gas standard showed that the response factor on a gas chromatograph flame-ionization detector for 1.3dichlorobenzene was $3 \%$ less than that for 1.2 dichlorobenzene.

\section{2,497}

PB96-155494 Not available NTIS

National Inst. of Standards and Technology (CSTL) Gaithersburg, MD. Organic Analytical Research Div.

Measurement of Atmospheric Methyl Bromide Using Gravimetric Gas Standards.

Final rep

G. C. Rhoderick. 1995, 4p.

Pub. in Environmental Science and Technology, v29 n11 p2797-2800 Nov 95.

Keywords: "Methyl bromide, Samples, Calibration, Regression analysis, Reprints, "Gravimetric standards, Ozone layer.

Methyl bromide $(\mathrm{CH} 3 \mathrm{Br})$ is the largest source of gaseous bromine in the atmosphere. Methyl bromide is, therefore, environmentally important due to its potential to contribute to the loss of the stratospheric ozone layer through catalytic destruction. Several sets of at mospheric bethyl bromide measurements have been reported, but little has been reported on the calibration standards used to determine those values. For years, the National Institute of Standards and Technology (NIST) has prepared gravimetric gas standards conlaining methyl bromide at the low parts per billion (ppb 10 to the minus 9 th power $\mathrm{mol} / \mathrm{mol}$ ) level. These stand ards have been used by federal and state governments in urban air monitoring programs.

\section{2,498}

PB96-167234 Not available NTIS

National Inst. of Standards and Technology (CSTL), Boulder, CO. Thermophysics Div.

Viscosity of Defined and Undefined Hydrocarbon Liquids Calculated Using an Extended Corresponding-States Model.

Final rept.

M. E. Baltatu, R. A. Chong, and M. L. Huber. 1996

9p.

Pub. in International Jnl. of Thermophysics, v17 n1 p213-221 1996.

Keywords: "Petroleum fractions, Reprints, "Foreign technoloogy, "Viscosity prediction, "Corresponding states.

We predict the viscosity of petroleum fractions using extended corresponding states. Our model builds upon the TRAPP procedure, which is the most advanced ap proach to predict transport properties of straight-chain nonpolar hydrocarbons and their mixtures. We perform comparisons with experimental viscosity data for pure hydrocarbons, treating them as nonstandard components; we find deviations of $10-15 \%$. We also extend the model to predict the transport properties of petroleum fractions and compare with an experimental database of more than 80 crude oils, including highly aromatic petroleum fractions. The model predicts the viscosity of the crude oil fractions within experimental uncertainty

\section{2,499}

PB96-176664 Not available NTIS

National Inst. of Standards and Technology (CSTL),

Boulder, CO. Thermophysics Div.

New Data and Correlations for the Custody Transfer of Natural Gas Liquids.

Final rept.

J. F. Ely, W. M. Haynes, C. D. Holcomb, and J. W.

Magee. 1995, 19p

Pub. in Proceedings of the Gas Processors Association Annual Convention (74th), San Antonio, TX. March $13-15,1995$, p35-53.
Keywords: "Natural gas liquids, "Thermophysical properties, "Liquid phases, "Phase transformations, Compressed natural gas, Phase studies, Phase equilibria, Vapor phases, Equations of state, Volume, Mixtures, Density(Mass/Volume), Reprints, Coexisting density, Corresponding states, Vapor liquid equilibria.

This report presents new experimental density measurements and density prediction models for natural gas liquids. Experimentally, vibrating tube densimeters have been used to measure coexisting densities for ethane, propane, n-butane, isopentane, n-pentane, and isohexane and for binary mixtures of ethane+propane, propane $+n$-butane, and $n$ butaine $+n$-pentane. The density measurements were combined with simultaneous vapor-liquid equilibrium measurements using a vapor and liquid recirculation phase-equilibrium apparatus at temperatures from 240 $\mathrm{K}$ to either $410 \mathrm{~K}$ or the critical temperature of the system, whichever is less. Isothermal data were obtained for the binary mixtures over the entire composition range for each of the systems. Also, an isochoric gas expansion technique has been used to carry out $(p, p, T)$ measurements on two binary mixtures of ethane + propane with mol fractions of 0.35 and 0.65 and one binary mixture of propane $+n$-butane with a propane mol fraction of 0.61 .

02,500

PB97-119267 Not available NTIS

National Inst. of Standards and Technology (CSTL),

Gaithersburg, MD. Process Measurements Div.

Internal Droplet Circulation Induced by SurfaceDriven Rotation.

Final rept.

C. M. Megaridis, J. T. Hodges, J. Xin, J. M. Day, and C. Presser. 1994, 14p.

Pub in International Jnl. of Heat and Fluid Flow, v15 n5 p364-377 Oct 94

Keywords: "Droplets, "Rotation, "Liquid circulation, Circulation, Numerical models, Fluid transport, Spherical liquid volume, Rotation, Reprints.

The paper presents a combined theoretical/experimental study of internal liquid circulation induced by droplet surface rotation. A numerical model is presented first, examining the fluid transport within a spherical liquid volume whose surface is subjected to rotation about a central axis. The model predicts that the steady-state motion established from spatially nonuniform surface rotation has a helical character and bears little resemblance to the toroidal internal flows developed within droplets under axisymmetric conditions. Similar internal flow patterns are predicted for temporally varying surface rotation occuring during droplet spin-up or spin-down. Planar laser-induced fluorescence is employed to provide high-resolution images of fluid flow developed within millimeter-sized suspended droplets that are exposed to steady laminar air streams to induce repeatable surface rotation. The predicted spiral flow patterns are corroborated by the pendant droplet visualization experiments, and suggest that nonuniform rotation or transient spinning may significantly alter internal droplet dynamics.

\section{Heating \& Cooling Systems}

\section{2,501}

\section{PB94-140407 PC A08/MF A02}

National Inst. of Standards and Technology (BFRL), Gaithersburg, MD

Computer Programs for Simulation of Lighting/ HVAC Interactions.

G. N. Walton. Dec $93,156 p$, NISTIR-5322.

See also PB91-144386. Sponsored by Department of Energy, Washington, DC., and Electric Power Research Inst., Palo Alto, CA

Keywords: "Space HVAC systems, "Lighting systems, "Computerized simulation, "Buildings, Coefficients, Algorithms, Interactions, Heat transfer, Cooling load, Energy audits, Thermal measurements, HLITE computer program, VLITE computer program, National Institute of Standards and Technology.

The report describes two computer programs developed for the analysis of lighting/HVAC interactions: HLITE and VLITE. VLITE is used to compute the coefficients (view factors) describing radiation interchange between surfaces. These coefficients are used in a thermal network model which is solved by HLITE for 


\section{Heating \& Cooling Systems}

transient temperatures and cooling loads. These programs are research tools. HLITE is based on a simple finite volume model for heat transfer combined with sufficiently short time steps to permit explicit time integration in most of the simulation. Controls are modeled in a manner emulating the operation of controls in real buildings. The accuracy of the mathematical solution is appropriate to the models used and data available resulting in a fast, flexible simulation tool.

\section{2,502}

\section{PB94-143344 PC A04/MF A01}

National Inst. of Standards and Technology (BFRL), Gaithersburg, MD. Building Environment Div.

Evaluation of GSA Maintenance Practices of Large Centrifugal Chillers and Review of GSA Refrigerant Management Practices.

J. Y. Kao. Jan 94, 51p, NISTIR-5336.

DC.

Keywords: *Centrifugal pumps, "Refrigerating machinery, "Refrigerants, US GSA, Maintenance, Inspection, Nondestructive tests, Air pollution control, CFC(Chlorofluorocarbons).

The study contains two major subjects involving maintenance of large centrifugal chillers in the General Services Administration (GSA) facilities. The first part is to use nondestructive testing (NDT) techniques for chiller testing and maintenance. NDT techniques investigated are visual inspection, leak testing, vibration analysis, infrared thermal testing, eddy current testing, oil analysis, and acoustic emission testing. With the exception of acoustic emission testing, all other techniques are recommended for GSA chiller maintenance. The second part of the study is about refrigerant management.

\section{2,503}

PB94-218559 PC A04/MF A01

National Inst. of Standards and Technology (BFRL), Gaithersburg, MD. Building Environment Div.

Study of Heat Pump Performance Using Mixtures of R32/R134a and R32/R125/R134a as 'Drop-In' Working Fluids for R22 with and Without a LiquidSuction Heat Exchanger.

P. I. Rothfleisch, and D. A. Didion. Dec 93, 65p, NisTIR-5321.

Keywords: "Heat pumps, "Refrigerants, *Working fluids, Heat exchangers, Performance(Engineering), Heat transfer, Uncertainty, Mathematical models, Thermodynamic cycles, Residential buildings.

A ductless mini-split residential heat pump with a modified indoor coil was utilized to compare the performance of R22 and a mixture of $34 \%$ R $32 / 66 \%$ R $134 a$ by weight. This test was intended to serve as an indicator of 'drop-in' performance so the system was optimized for each refrigerant by varying only the charge mass and expansion valve setting. At the $27.8 \mathrm{C}(82$ mixture were $94 \%$ and $90 \%$ of the values for R22, spectively. Additional tests were conducted with a liquid-suction intracycle heat exchanger. The modified system was operated with both single-phase and twophase refrigerant entering the low pressure side of the liquid-suction heat exchanger. The addition of the liquid-suction heat exchanger showed a minimal performance improvement with the performance of the twophase variation being slightly higher. The best performing liquid-suction heat exchanger variant (two-phase refrigerant on the low pressure side) was also run with a ternary mixture of $30 \% \mathrm{R} 32 / 10 \% \mathrm{R} 125 / 60 \% \mathrm{R} 134 \mathrm{a}$, by weight. The results for this mixture were similar to the binary mixture.

\section{2,504}

PB95-105524 PC A03/MF A01

National Inst. of Standards and Technology (BFRL), Gaithersburg, MD. Building Environment Div.

Predicting the Energy Performance Ratings of a Family of Type I Combination Appliances.

S. T. Liu, and G. E. Kelly. Aug 93, 30p, NISTIR 5250

Sponsored by Department of Energy, Washington, DC Office of Codes and Standards.

Keywords: "Housing(Dwellings), "Space heaters, "Water heaters, "Energy efficiency, Water heating, Comuterized simulation, "ANSI/ASHRAE Standard 124-1991, "Combination appliances.

ANSI/ASHRAE Standard 124-1991 specifies the laboratory tests and the calculation procedure for estimat- ing seasonal and annual performance of combination appliances which are designed to provide both space heating and water heating. A boiler that includes a tankless coil for water heating is covered by those sections in ASHRAE Standard 124 that pertain to Type I combination appliances. In an effort to minimize the test burdens on manufacturers, a computer simulation study was conducted to determine if a subset of a family series of Type I combination appliances could be tested and used to predict the performance of the rest of the appliances in the family. Computer simulation was conducted on a family of five different size boilers with an identical tankless coil to calculate their Combined Annual Efficiency (CAE) as specified in ASHRAE Standard 124. To this end, the Energy Factor (EF) for water heating and the Annual Fuel Utilization Efficiency (AFUE) for space heating were calculated. For the water heating test that was simulated, daily hot water draw volumes of 243.4 liters (64.3 gal.) and 454.2 liters (120 gal.) were used. The results showed that for the five boilers, the AFUE for space heating differed by less than 1 percent.

\section{2,505}

PB95-220521 PC A03/MF A01

National Inst. of Standards and Technology (BFRL), Gaithersburg, MD. Building Environment Div.

Performance Testing of a Family of Type I Combination Appliance.

S. T. Liu, G. E. Kelly, and C. P. Terlizzi. Apr 95, 34p, NISTIR.5626.

Sponsored by Department of Energy, Washington, DC.

Keywords: *Space HVAC systems, *Performance evaluation, Boilers, Tests, Hot water heating, Energy effi ciency.

In an effort to minimize the test burdens on manufac turers, a family series of gas-fired hot water boilers were tested to determine if a subset of a family series of Type I combination appliances could be tested and used to predict the performance of the rest of the appliances in the family series. Tests were conducted on a family series of three different size boilers with an identical indirectly heated storage tank to determine their Combined Annual Efficiency (CAE) as specified in ASHRAE Standard 124

\section{2,506}

PB96-135066 Not available NTIS

National Inst. of Standards and Technology (CSTL), Gaithersburg, MD. Thermophysics Div.

Shape of the Temperature-Entropy Saturation Boundary.

Final rept.

G. Morrison. 1994, 10p.

Pub. in International Jnl. of Refrigeration, v17 n7 p4945031994

Keywords: "Compression, *Diagrams, "Entropy, Reprints, Refrigerants, Saturation, Substitute, Temperature.

The phase diagram in temperature-entropy space plays an important role in the visualization design and analysis of operating cycles in refrigerators and heat pumps. The shape of the phase boundary in this space varies dramatically from one material to another. The origin of these differences is shown to be a consequence of molecular structure. Relationships of varying accuracy are developed to estimate the slope of the vapour branch of the T-S diagram.

\section{Miscellaneous Energy Conversion \& Storage}

02,507

PB95-155586 PC A99/MF A06

National Inst. of Standards and Technology (MSEL), Gaithersburg. MD. Materials Reliability Div.

Composite Struts for SMES Plants.

Rept. for May 91-Nov 92

R. P. Reed, and J. D. McColskey. Oct 94, 624p, NISTIR-5024

Contract DNA-IACRO-91-854

Prepared in cooperation with Cryogenic Materials, Inc., Boulder, CO. Sponsored by Defense Nuclear Agency, Washington, DC

Keywords: "Epoxy matrix composites, "Glass fiber reinforced plastics, "Struts, "Composite structures,
* Magnetic energy storage equipment, Structural components, Stress analysis, Strains, Tensile properties, Modulus of elasticity, Composite materials, Displacement, Fatigue tests, Mechanical properties, Thermal conductivity, "Superconducting Magnetic Energy Storage Plants.

This report summarizes NIST research efforts in the development of composite struts for Superconducting Magnetic Energy Storage plants (SMES). Large SMES plants require radial structural support from the superconducting coils to the outer walls to assist in restraining the coil cool-down and Lorentz forces. The struts proposed for this structural support must have good axial compressive strength and low axial thermal conductivity, as well as low cost. A variety of E-glass fibers, resins, lay-up angles and manufacturing methods were evaluated in the course of this program, at temperatures ranging from 295 to $76 \mathrm{~K}$. Analysis meth ods are presented that describe the response of fiber reinforced cylinders and end fitting designs to axially varying mechanical and thermal loads. Finally, failuremode and tube design studies were conducted in order to optimize support tube-design based on minimum cross-sectional area with allowable strain values input as design constraints.

\section{Policies, Regulations \& Studies}

02,508

PB94-206018 PC A04/MF A01

National Inst. of Standards and Technology (CAML) Gaithersburg, MD. Office of Applied Economics.

Energy Prices and Discount Factors for Life-Cycle Cost Analysis 1994. Annual Supplement to NIST Handbook 135 and NBS Special Publication 709. S. R. Petersen. Oct 93, 64p, NISTIR-85-3273-8. See also PB87-180253, PB88-138227, PB94-500055 and report for 1993, PB92-238633. Sponsored by Department of Energy, Washington, DC. Federal Energy Management Program Staff.

Keywords: "Energy conservation, "Prices, *Life cycle costs, "Cost analysis, Federal buildings, Residentia buildings, Economic analysis, Present worth, Fuels, Price index, Electric appliances, Energy supplies, Tables(Data).

This is the 1994 annual edition of energy prices and discount factors for performing life-cycle cost analyses of energy conservation and renewable energy projects. It supports the Federal life-cycle costing methodology by updating the energy price projections and discoun factors that are described, explained, and illustrated in NIST Handbook 135 (HB I35). It supports private-sector life-cycle costs analysis by updating the energy price indices that are described, explained, and illustrated in NBS Special Publication 709 (SP 709). It also supports the Energy Conservation Mandatory Perform. ance Standards for New Federal Residential Buildings (10 CFR 435) by providing a table of factors for updating appliance label values.

\section{2,509}

PB95-105011 PC A04/MF A01

National Inst. of Standards and Technology (CAML), Gaithersburg, MD. Office of Applied Economics.

Energy Price Indices and Discount Factors for LifeCycle Cost Analysis 1995. Annual Supplement to NIST Handbook 135 and NBS Special Publication 709. (Revised).

S. R. Petersen. Oct $94,56 p$, NISTIR-85/3273-9

See also PB94-206018. Sponsored by Federal Energy Management Program, Washington, DC.

Keywords: "Life cycle costs, "Cost analysis, "Prices, *Energy conservation, Federal buildings, Residential buildings, Fuels, Economic analysis, Electric appliances, Present worth, Price index, Energy supplies, Tables(Data).

This is the 1995 annual edition of energy price indices and discount factors for performing life-cycle cost analyses of energy conservation and renewable energy projects. It supports the Federal life-cycle costing methodology by updating the energy price projections and discount factors that a re described, explained, and illustrated in the National Institute of Standards and Technology (NIST) Handbook 135 (HB 135). It supports private-sector life-cycle cost analysis by updating the energy price indices that are described, explained, and illustrated in NBS Special Publication 709 (SP 
709). It also supports the Energy Conservation Mandatory Performance Standards for New Federal Residential Buildings (10 CFR 435) by providing a table of factors for updating appliance label values.

\section{2,510}

PB96-162441 PC A05/MF A01

National Inst. of Standards and Technology (BFRL), Gaithersburg, MD. Office of Applied Economics.

Energy Price Indices and Dlscount Factors for LifeCycle Cost Analysis 1996. Annual Supplement to NIST Handbook 135 and NBS Special Publication 709. (Revised).

S. R. Petersen. Oct 95, 69p, NISTIR-85/3273-10. See also PB95-105011. Sponsored by Federal Energy Management Program, Washington, DC.

Keywords: "Life cycle costs, "Cost analysis, "Prices, "Energy conservation, Federal buildings, Residential buildings, Fuels, Economic analysis, Electric appliances, Present worth, Price index, Energy supplies, Tables(Data)

This is the 1996 annual edition of energy price indices and discount factors for performing life-cycle cost analyses of energy and water conservation and renewable energy projects. It supports the federal life-cycle costing methodology by updating the energy price projections and discount factors that are described, explained, and illustrated in NIST Handbook 135 (HB 135). It supports private-sector life-cycle cost analysis by updating the energy price indices that are delication 709 (SP 709). It also supports the Energy Conservation Mandatory Performance Standards for New Federal Residential Buildings (10 CFR 435) by providing a table of factors for updating appliance label values.

\section{2,511}

\section{PB96-172317 PC A10/MF A02}

National Inst. of Standards and Technology (BFRL) Gaithersburg, MD. Office of Applied Economics.

Life-Cycle Costing Manual for the Federal Energy Management Program. 1995 Edition.

Handbook.

S. K. Fuller, and S. R. Petersen. Feb 96, 176p, NIST/HB-135.

Also available from Supt. of Docs. as SN003-00303373-1. Supersedes PB88-138227. Sponsored by Federal Energy Management Program, Washington, DC.

Keywords: "Federal buildings, "Life cycle costs, "Energy management, Energy conservation, Economic analysis, Cost benefit analysis, Cost effectiveness, Water conservation, Investments, Capitalized costs, Renewable energy sources, Public buildings, Manuals, Energy economics.

Handbook 135 is a guide to understanding the lifecycle cost (LCC) methodology and criteria established by the Federal Energy Management Program (FEMP) for the economic evaluation of energy and water conservation projects and renewable energy projects in all federal buildings. The purpose of this handbook is to facilitate the implementation of the FEMP rules by explaining the LCC method, defining the measures of economic performance used, describing the assumptions and procedures to follow in performing evaluations, giving examples, and noting NIST computer software available for computation and reporting purposes.

\section{2,512}

\section{PB96-210745 PC A05/MF A01}

National Inst. of Standards and Technology (BFRL), Gaithersburg, MD. Office of Applied Economics.

Energy Price Indices and Discount Factors for LifeCycle Cost Analysis 1997. Annual Supplement to NIST Handbook 135 and NBS Special Publication 709. (Revised).

S. R. Petersen. Jul 96, 68p, NISTIR-85-3273-11.

See also PB96-162441. Sponsored by Federal Energy Management Program, Washington, DC.

Keywords: "Life cycle costs, "Cost analysis, "Prices, "Energy conservation, Federal buildings, Residential buildings, Fuels, Electric appliances, Energy supplies, Economic analysis, Present worth, Price index, Tables(Data)

This report is the FY 1997 edition of energy price indices and discount factors for performing life-cycle cost analyses of energy and water conservation and renewable energy projects in federal facilities. It supports the federal life-cycle costing methodology by updating the energy price projections and discount factors that are described, explained and illustrated in NIST Handbook 135 (HB 135, Life-Cycle Cosilng Manual for the Federal Energy Management Program (PB96172317)). It supports private-sector life-cycle cost analysis by updating the energy price indices that are described, explained, and illustrated in NBS Special Publication 709 (SP 709 (PB87-180253)). It also supports the Energy Consenvation Mandatory Performance Standards for New Federal Residential Building (10 CFR 435) by providing a table of factors for updating appliance label values.

\section{Reserves}

02,513

PB96-119755 Not available NTIS

National Inst. of Standards and Technology (EEEL), Gaithersburg, MD. Electricity Div.

Procedure for Measuring Trace Quantities of S2F10, S2OF10, and S2O2F10 in SF6 Using a Gas Chromatograph-Mass Spectrometer.

Final rept.

R. J. Van Brunt, J. K. Olthoff, K. L. Stricklett, and D. J. Wheeler. 1995, 8p.

Pub. in Proceedings of the Intemational Symposium on Gaseous Dielectrics (7th), Knoxville, TN., April 24-28, 1994, p441-448 1995.

Keywords: "Gas chromotography, "Mass spectrometers, "Trace quantities, Reprints, Procedures.

The compounds S2F10, S2OF10, and S2O2F10 are formed by decomposition of gaseous sulfur hexafluoride (SF6) in electrical discharges. The species S2F10 is known to be highly toxic to humans, and there is recent evidence that S2O2F10 may also be very toxic. There is, therefore, an interest in having analytical methods to detect these compounds in compressed SF6 at trace levels down to 10 parts in 10 to the 9th power by volume (10 ppbv). Two chromatographic methods have been used to direct these compounds at the $10 \mathrm{ppbv}$ level or lower. The first method developed by Sauers and coworkers is based on a cryogenic enrichment procedure first proposed by Janssen, and uses a gas chromatograph with an electron-capture detector. The second method, which is the focus of the present work, utilizes a gas chromatograph-mass spectrometer (GC/MS) with a thermal-chemical converter. The purposes of the present work are: (1) to define a procedure for using the CC/MS method to make fast, reliable measurement of trace $\mathrm{S} 2 \mathrm{~F} 10, \mathrm{~S} 2 \mathrm{OF} 10$, and $\mathrm{S} 2 \mathrm{O} 2 \mathrm{~F} 10$ in pressurized SF6; (2) to discuss the major sources of error that can be encountered in using the method; and (3) to discuss the factors that limit the sensitlvity such as interference from other compounds.

\section{Solar Energy}

\section{2,514}

PB94-211075 Not available NTIS

National Inst. of Standards and Technology (PL), Boulder, CO. Quantum Physics Div.

Plasma Chemistry in Disilane Dlscharges.

Final rept.

J. R. Doyle, D. A. Doughty, and A. Gallagher. 1992,

Contract SERI-XG-1-1216

Pub. in Jnl. of Applied Physics 71, n10 p4771-4780, 15 May 92 . Sponsored by Solar Energy Research Inst., Golden, $\mathrm{CO}$.

Keywords: "Silanes, "Radio frequency discharge, "Plasma chemistry, "Amorphous silicon, Silicon solar cells, Semiconducting films, Reprints, Disilanes.

We have measured the initial silane and polysilane product yields from disilane decomposition in rf and dc discharges, at 25 and $250 \mathrm{C}$ and $20 \mathrm{~Pa}$ (0.15 Torr) pressure as typically used for alpha-Si:H film deposition. From analyses of these yields we conclude that the initial $\mathrm{Si} 2 \mathrm{H} 6$ fragmentation pattern is $\mathrm{SiH} 3+\mathrm{SiH} 2$ $+\mathrm{H}(91+$ or $-9 \%)$ and $\mathrm{H} 3 \mathrm{SiSiH}+2 \mathrm{H}(9+$ or $-9 \%)$, that the primary product of the $\mathrm{H}+\mathrm{Si2H} 6$ reaction is $\mathrm{SiH} 4+\mathrm{SiH} 3$, and that $\mathrm{SiH} 3$ is the dominant radical causing film growth. We have measured a radical-surface reaction probability of $0.34+$ or -0.03 , very similar to that observed for $\mathrm{SiH} 3$ in $\mathrm{SiH} 4$ discharges. We report a spatial distribution of emission indicative of a gamma-regime discharge. From deposition on glass fibers strung between the electrodes, we find that highly strained alpha-Si:H film is produced everywhere except on or near the electrodes, suggesting that energetic ion impact is necessary to yield useful films in disilane discharges.

ENVIRONMENTAL POLLUTION \& CONTROL

\section{General}

\section{2,515}

PB94-172533 Not available NTIS

National Inst. of Standards and Technology (CAML) Gaithersburg, MD. Statistical Engineering Div.

Replicate Measurements for Data Quality and Environmental Modeling.

Final rept.

W. Liggett. 1994, 32p.

Pub. in Handbook of Siatistics, v12 p71-102 1994

Keywords: "Environmental research, "Mathematical models, "Replicating, Statistical analysis, Multivariate analysis, Experimental design, Measurement, Reprints, "Data quality.

Replicate measurements can be used to improve sampling and measurement procedures and thereby data quality and to select an environmental model that agrees reasonably with the data. The implications for study design of these uses of replicates and corresponding statistical analysis methods are presented. The statislical analysis methods presented for quality improvement and model selection are especially appropriate for environmental studies. The quality im provement method discussed is a multivariate method generalized to include estimation of a power transformation for each constituent measurement. The model selection method discussed employs a bootstrap based on replicates that is applicable to models fit by robust estimation.

\section{2,516}

PB94-172806 Not available NTIS

National Inst. of Standards and Technology (CSTL) Gaithersburg, MD.

Current Activities Within the National Biomonitoring Specimen Bank.

Final rept.

S. A. Wise, B. Koster, J. Langland, and R. Zeisler. $1993,12 p$

Pub. in The Science of the Total Environment 139/140, p1-12 1993 Sponsored by Environmental Protection Agency, Washington, DC., National Oceanic and Atmospheric Administration, Rockville, MD., and Department of the Interior, Washington, DC.

Keywords: "Tissue banks, "Bioaccumulation, "Marine mammals, "Water pollution effects(Animals), Pollution monitoring, Biological accumulation, Data collection, Environmental monitoring, Terrestrial ecosystems, Specimens, Organic compounds, Contamination, Quality assurance, Reprints.

The National Institute of Standards and Technology (NIST) has been involved in biological environmental specimen banking activities since 1979. These activities, which are known collectively as the National Biomonitoring Specimen Bank (NBSB), include the banking of a variety of specimens (human liver, sediment, mussels/oysters, fish tissue and marine mammal tissues) from several different projects supported by different government agencies. The two most recent projects, the Alaska Marine Mammal Tissue Archival Project (AMMTAP) and the National Marine Mammal Tissue Bank (NMMTB), focus on the collection, banking and analysis of marine mammal tissues and they 


\section{General}

are part of a comprehensive plan to address manine mammal monitoring, specimen banking and quality assurance of analytical measurements associated with contaminant analyses in manine mammals.

\section{2,517}

PB94-185527 Not available NTIS

National Inst. of Standards and Technology (CAML) Gaithersburg, MD. Statistical Engineering Div.

Scientific Protocols in Statistical Standards for Environmental Studies.

Final rept.

W. Liggett. 1993, 6p.

Pub. in Proceedings of American Statistical Association Conference on Quality and Product, San Francisco, CA., p38-43, 1993

Keywords: "Environmental surveys, "Mathematica models, "Statistical inference, "Protocols, Statistica analysis, Randomization, Measurement, Errors, Ex perimental design, Reprints.

In environmental studies, the validity of the probability model that underlies statistical inference often depends on the operation of physical mechanisms. Deliberate randomization, although important, often cannot remove this dependence. Traditionally, statisticians have considered the impact of physical mechanisms on study conclusions to be the responsibility of $\mathrm{sci}$ entific collaborators. Because scientists are guided by values other than the validity of probability models, statisticians must take responsibility themselves. Thus, statisticians must understand the relevant physical mechanisms so that they can develop scientific protocols that result in statistically-tractable probability models. As a practical approach, statisticians can become involved in the design and execution of pilot studies with the aim of resolving probability-model issues. Pilo studies adequate in this regard are necessary as a basis for environmental statistical standards.

\section{2,518}

PB94-198330 Not available NTIS

National Inst. of Standards and Technology (TS) Gaithersburg, MD. Standard Reference Materials Program.

Standard Reference Materials for Dioxins and Other Environmental Pollutants.

Final rept.

R. Alvarez. 1991, 7p

Pub. in Science of the Total Environment 104, n1-2 p1 71991.

Keywords: "Dioxins, ${ }^{*}$ Chemical analysis, "Environmental pollution, "Pollutants, Chemical composition, Certification, Concentration(Composition) Calibrating, Analytical techniques, Pesticides, Isotopic labeling, Physical properties, Chlorine organic compounds, Reprints, "Standard reference materials, National Intitute of Standards and Technology.

The National Institute of Standards and Technology is sues approximately 1100 Standard Reference Matenals (SRMs) certified for chemical composition or physical properties. A number of these SRMs have been developed to assist chemists in analyzing environmental samples more reliably for chloninated dioxins and other organic pollutants. Certification of the pollutant concentration in a natural matrix SRM is based on concordant analyses by the NIST Organic Analytical Research Division using at least two inde pendent methods. For a calibration solution, such as SRM 1614, Dioxin (2,3,7,8-TCDD) in Isooctane, certification is based on agreement of the calculated concentration based on the gravimetric preparation and the concentration as determined experimentally. SRM 1614 also includes a (13)C-labeled 2,3,7,8-TCDD for use as an intemal standard in methods based on gas chromatography/mass spectrometry. The certified concentrations, in $n g / g$, are $98.3+3.3$ for the unlabeled dioxin and $95.6+$ or -1.5 for the labeled dioxin. The certificates for SRM 1588, Organics in Cod Liver Oil and SRM 1589, Polychlorinated Biphenyls (As Aroclo 1260 ) in Human Serum, provide noncertified concentrations of dioxins. Concentrations of chlorinated dioxins in two urban particulate SRMs have been reported in the literature.

\section{2,519}

PB94-206364 PC A08/MF A02

National Inst. of Standards and Technology (BFRL) Gaithersburg, MD.
U.S. Green Building Conference, 1994.

Special pub.

A. H. Fanney, K. M. Whitter, A. E. Traugott, and L. N. Simon. Jun $94,153 p$, NIST/SP-863

Also available from Supt. of Docs. as SN003-003-

03274-3. Proceedings of a conference held in

Gaithersburg, MD. on February 16-17, 1994.

Keywords: "Buildings, "Environmental engineering "Meetings, Construction materials, Indoor air pollution, Carbon dioxide, Technology transfer.

The report constitutes the proceedings of the Green Building Conference held in Gaithersburg, Maryland, February 16-17, 1994. The conference was co-sponsored by the National Institute of Standards and Technology (NIST) and the US. Green Building Council (USGBC). Over 450 individuals attended the conference representing building product manufacturers, building owners and managers, environmental groups, utilities, contractors, builders, architects, engineers, and the local, state, and the federal governments. The conference provided an opportunity to acquire practical, useful information on green buildings, resources, and guidelines.

\section{2,520}

PB95-147146 PC A09/MF A02

National Inst. of Standards and Technology (TS), Gaithersburg, MD.

Opportunities for Innovation: Pollution Prevention. D. E. Edgerly. Aug 94, 181p, NIST/GCR-93/659. Grant 50SBNB3C7626

Keywords: "Pollution abatement, "Waste recycling *Small businesses, "Waste disposal, "Industrial wastes, Automotive industry, Chemical industry, Paper industry, Textile industry, Paints, Coatings, Degreasing, Dyes, Dyeing, Printing, Office equipment, * Medium-sized businesses, Opportunities for innova. tion, Waste minimization, Pollution prevention, Waste reduction.

The objective was to identify technological opportunities within a number of selected industries and/or manufacturing/finishing processes, to reduce pollution. These industries/processes were selected as representative of and applicable to the broad range of U.S manufacturing businesses. These include: metals coating (i.e., painting) which is widely done in a number of major industries, including the automotive industry metals degreasing; recyclability of office equipment; chemical manufacturing; printing; textiles dye and dyeing; and the pulp and paper industry. Additionally, the promulgation of new regulations, requining companies to change their historical manufacturing and waste generating/disposal practices, will continue to create technological needs and opportunities for savy businesses to develop solutions.

\section{2,521}

\section{PB95-161394 Not available NTIS}

National Inst. of Standards and Technology (TS), Gaithersburg, MD. Office of Technology Evaluation and Assessment.

Encouraging Environmentally-Aware Inventions.

Final rept.

G. P. Lewett. 1993, 17p.

Sponsored by International Federation of Inventors Associations, Geneva (Switzerland).

Pub. in Proceedings of Conference Invention and Protection of the Environment, Tunis, Tunisia, September 20-25, 1993, p1-17.

Keywords: "Environmental protection, *Research and development, "Technology transfer, "Inventions, Pollution abatement, Environmental research, Technology innovation, Commercialization, Appropriate technology, Research programs, Research management, Reprints, "Energy Related Inventions Program, Environmental aspects, Environmental considerations.

The paper provides a review of the Energy-Related Inventions Program to indicate how its experience can be utilized to stimulate invention of environmentally-oriented inventions. It also briefly describes federal programs which generate new environmental technologies.

02,522

PB95-164026 Not available NTIS

National Inst. of Standards and Technology (CSTL) Gaithersburg, MD. Organic Analytical Research Div.
Standard Reference Materials for the Determination of Trace Organic Constituents in Environmental Samples.

Final rept.

S. A. Wise. $1993,44 p$.

Pub. in Environmental Analysis: Techniques, Applications and Quality Assurance, Chapter 12, p403-446 1993.

Keywords: "Organic compounds, "Chemical analysis "Reference standards, "Certification, Polychlorinated biphenyls, Polycyclic aromatic hydrocarbons, Pesticides, Trace amounts, Test methods, Analytical techniques, Reprints, "Environmental samples, Standard reference materials.

Since 1980 , NIST has issued a number of Standard Reference Materials (SRMs) for the determination of trace level organic contaminants such as polycyclic aromatic hydrocarbons (PAHs), polychlorinated biphenyls (PCBs), and chlorinated pesticides. This chapter describes these SRMs and the analytical techniques used for the certification measurements for these materials. The certified and noncertified concentrations for the PAHs, PCBs, and pesticides are summarized for the most recent natural matrix SRMs.

02,523

PB95-164042 Not available NTIS

National Inst. of Standards and Technology (CSTL) Gaithersburg, MD. Organic Analytical Research Div.

Liquid Chromatographic Determination of Polycyclic Aromatic Hydrocarbon Isomers of Molecular Weight 278 and 302 in Environmental Standard Reference Materials.

Final rept.

S. A. Wise, A. Deissler, and L. C. Sander. 1993, 16p. Pub. in Polycyclic Aromatic Compounds 3, p169-184 1993.

Keywords: "Liquid column chromatography, "Polycyclic aromatic hydrocarbons, "Reference standards, "Quantitative chemical analysis, Isomers, Chromatographic analysis, Fluorescence spectroscopy, Extraction columns, Pollution sampling, Particulates, Sediments, Coal tar, Reprints, "Environmenta samples, Standard reference materials, Dibenzopyrenes, Dibenzofluoranthenes.

Dibenzoanthracenes,

Polycyclic aromatic hydrocarbons (PAHs) of molecular weight (MW) 278 (dibenzanthracene isomers) and 302 (dibenzopyrene/dibenzofluoranthene isomers) are seldom measured in environmental samples. A multidimensional liquid chromatography (LC) procedure was developed for the quantification of isomers of MW 278 and 302 . Using the multidimensional LC procedure, six isomers of MW 278 and nine isomers of MW 302 were quantified in four environmental Standard Reference Materials (SRMS): coal.tar (SRM 1597), air particulate matter (SRMs 1648 and 1649), and marine sediment (SRM 1941).

02,524

PB95-220513 PC A03/MF A01

National Inst. of Standards and Technology (TS), Gaithersburg, MD. Office of Standards Services. ISO Environmental Management Standardization Efforts.

M. H. Saunders. Apr 95, 18p, NISTIR-5638

Keywords: "ISO, "Standardization, "Environmenta protection, Environment management, Manufacturing, Product development, Process control(Industry). Design criteria, Design analysis, Life cycle costs, Labeling, Auditing, Performance evaluation, Recommendations, "Life cycle analysis, Process management, Product standards.

The Intemational Organization for Standardization (ISO) is currently developing a family of environmenta management standards which address management systems and the environmental aspects of products in the areas of life cycle assessment and labeling. These standards have the potential to exert a significant influence on the design, manufacture, and marketing of products. They are also likely to affect the type of environmental data gathered by businesses and how those data are communicated intemally and externally.

\section{2,525}

PB95-253605 PC A04/MF A01

National Inst. of Standards and Technology (BFRL) Gaithersburg, MD. 
International Green Building Conference and Exposition (2nd). Held in Big Sky, Montana on August $13-15,1995$.

Special pub.

A. H. Fanney, K. M. Whitter, and T. B. Cohn. Aug 95, 63p, NIST/SP-888.

Also available from Supt. of Docs. as SNO03-00303349-9. See also PB94-206364.

Keywords: "Buildings, "Pollution abatement, *Meetings, Design analysis, Construction materials, Operation and maintenance, Building materials, Energy efficiency, Environmental engineering, Space HVAC systems, Landscaping, Demolition, Economic analysis, Technology innovation, Green buildings, Life cycle assessment, Building design.

The report constitutes the proceedings of the Green Building Conference held in Big Sky, Montana, August $13-15,1995$. The conference focused on the design, construction, operation, maintenance, and demolition of buildings in an environmental and cost-efficient manner.

\section{2,526}

PB96-112354 Not available NTIS

National Inst. of Standards and Technology (CSTL), Gaithersburg, MD. Inorganic Analytical Research Div. Application of a Novel Slurry Furnace AAS Protocol for Rapid Assessment of Lead Environmental Contamination.

Final rept.

M. S. Epstein, S. M. Smith, and J. J. Breen. 1995

Pub. in American Chemical Society Book, Chapter 30 , p265-270 1995.

Keywords: "Lead(Metal), "Absorption spectroscopy, "Analytical methods, "Chemical analysis, Furnaces Slurries, Atomization, Sampling, Sample preparation, Environmental pollution, Environmental monitoring, Public health, Health hazards, Reprints, "Atomic absorption.

Threats to public health must be identified and quickly eliminated by officials who have the proper information to make educated decisions. Instances of environmental injustice, where minority and low-income populations are subject to disproportionately high and adverse human health or environmental nisks, need to be identified and corrected. Analytical science plays the pivotal role in providing data for environmental decision-making, from site hazard assessment, through evaluation of remediation efforts, and finally in the appraisal of pollution prevention technology.

\section{2,527}

PB96-112370 Not available NTIS

National Inst. of Standards and Technology (CSTL), Gaithersburg, MD. Analytical Chemistry Div.

Considerations in the Design of an Environmental

Specimen Bank: Experiences of the National Biomonitoring Specimen Bank Program.

Final rept.

S. A. Wise, and B. J. Koster. 1995, 7p

Pub. in Environmental Health Perspectives, v103 sup3 p61-67 Apr 95.

Keywords: "Tissue banks, "Reference standards, "Environmental monitoring, "Bioassay, Chemical analysis, Inorganic chemistry, Organic chemistry, Sampling, Bioaccumulation, Marine mammals, Fishes, Animal tissues, Humans, Biological effects, Cryogenic storage, Quality assurance, Reprints, National Biomonitoring Specimen Bank.

Since 1979 the National Institute of Standards and Technology (NIST) have been involved in environmental specimen banking activities as part of the $\mathrm{Na}$ tional Biomonitoning Specimen Bank (NBSB). These activities have focused on the development of procedures for the collection, processing, analysis, and longterm storage of a variety of environmental specimens including: human liver, mussels and oysters, fish tissue (liver and muscle), marine mammal tissues (blubber, liver, and kidney), and marine sediments. The experiences of the NBSB, the issues that should be addressed in the design and operation of a valid specimen bank program are presented.

\section{2,528}

\section{PB96-158662 PC A03/MF A01}

National Inst. of Standards and Technology (TS),

Gaithersburg, MD. Office of Standards Services.
ISO Environmental Management Standardization Efforts.

Technical note.

M. H. Saunders. Feb 96, 25p, NISTIR-5638-1.

Supersedes PB95-220513.

Keywords: "ISO, "Standardization, "Environmental protection, Environment management, Product development, Process control(Industry), Design criteria, Design analysis, Life cycle costs, Labeling, Auditing, Performance evaluation, Recommendations, "Life cycle analysis, Process management, Product standards.

The Intemational Ogranization for Standardization (ISO) is currently developing a family of 'environmental management' standards which address management systems and the environmental aspects of products in the areas of life cycle assessment and labelling. These standards have the potential to exert a significant inflyence on the design manufacture and marketing of products. They are also likely to affect the type of environmental data gathered by businesses and how those data are communicated internally and externally. The report outlines the current state of development of these planned ISO standards and also covers developments relating to third party certification of environmental management systems.

\section{2,529}

\section{PB97-114359 PC A05/MF AO}

Trade Promotion Coordinating Committee, Washington, DC. Environmental Trade Working Group.

India: Environmental Technologies Export Market Plan.

Final rept.

Oct 96, 64p

Keywords: "India, "Pollution control equipment, Environmental protection, "Market research, Water treatment, Waste water treatment, Air pollution control, Hazardous materials Solid waste management Sanitary engineering. Pollution monitoring, Pollution sam pling, Infrastructure, Energy efficiency, Renewable energy sources, Exports, Financing, Market analysis, analysis, Export trade information.

Table of Contents:

Introduction and Objectives:

Market Overview;

Environmental Market Segments;

Routes to the Market;

Financing;

Financing Agencies and U.S. Government Programs;

Appendix A - Environmental Management in India;

Appendix B - U.S. Contracts for India;

Appendix C - Key Contacts in India;

Appendix D - Selected Publications:

Appendix $\mathrm{E}$ - Key Internet Web Sites:

and Appendix F - Environmental Trade Working Group Key Contacts.

02,530

PB97-118715 Not available NTIS

National Inst. of Standards and Technology (CAML) Gaithersburg, MD. Statistical Engineering Div.

Pilot Studies for Improving Sampling Protocols.

Final rept.

W. S. Liggett, and K. G. W. Inn. 1996, 18p.

Pub. in Principles of Environmental Sampling, p1852021996.

Keywords: "Environmental research, "Sampling, "Measurement, Experimental design, Protocols, Selectivity, Optimization, Sensitivity, Specificity, Statistical analysis, Statistical inference, Reprints.

Development of an environmental sampling and measurement protocol by means of experiments is feasible. The experiments require dissimilar plots, each of which is large enough for execution of a sel of alternative protocols, but the experiments do not require that the property of interest be known for the plots. This chapter discusses the statistical framework for such experiments: what constitutes a proper set of alternative protocols, how the protocol responses can be used to estimate relative performance, and how the best protocol can be determined.

\section{2,531}

PB97-121826 PC A12/MF A03

National Inst. of Standards and Technology (BFRL) Gaithersburg, MD.
International Green Building Conference and Exposition (3rd). Held in San Diego, California on November $17-19,1996$. (Reannouncement with new abstract).

Special pub

A. H. Fanney, and P. R. Svincek. Nov 96, 230p,

NIST/SP-908,

Also available from Supt. of Docs. as SNO03-000303433-9. See also report on 2nd conference, PB95253605. Sponsored by American Inst. of Architects, Washington, DC. San Diego Gas and Electric Co. CA. and American Society of Landscape Architects, Washington, DC.

Keywords: "Buildings, "Pollution abatement, "Energy efficiency, "Meetings, Environmental engineering, Design analysis, Construction materials, Building matenals, Energy conservation, Operation and maintenance, Space HVAC systems, Pholovoltaic cells, Solar cells, Indoor air quality, Technology innovation, Economic analysis, Real estate, Insurance, Financial management, Green buildings, Life cycle assessment, Building design, Sustainability.

Sessions within the conference and corresponding papers within these proceedings focused on the role of cheif officers in bringing green products and services to market: A review of the U.S. Green Building Council's Leadership in Energy and Environmental Design Building Rating System; A review of the latest international green building projects including examples of commercial, industrial, historical, retail, office, academic, and residential projects; The selection of sustainable building materials: An overview of the latest practices in energy conservation; A world overview of solar photovoltaic components and systems; Commercial, residential, and legal indoor air quality issues; Design tools and resources that are applicable to green buildings including the Internet, 3D design visualization, computer simulations, and the Department of Energy's energy simulation programs; and The implications of green buildings on the real estate, financial, and insurance industries. (Reannouncement with new abstract).

\section{Air Pollution \& Control}

\section{2,532}

PB94-143542 PC A05/MF A01

National Inst. of Standards and Technology, Gaithersburg, MD.

Ground-Based Smoke Sampling Techniques Training Course and Collaborative Local Smoke Sampling in Saudi Arabia.

Final rept. Aug 92-Aug 93

N. P. Bryner, and B. A. Benner. Jan 94, 94p, NISTIR-5306.

Keywords: "Air pollution sampling, "Smoke, "Oil wells, "Fires, Particulates, Saudi Arabia, Health, Kuwait.

Smoke and gaseous emissions such as those generated by multiple oil-well fires can significantly impact the health of the local population. To assess the immediate risk to public health, the chemical and physical properties of the smoke and gaseous emissions must be quickly characterized. Local sampling via portable gas analyzers, filters and pumps, and particle sizing instrumentation provides real-time characterization of pollutant levels. These analyses provide a snapshot of the physical and chemical properties of the aerosols sampled in Saudi Arabia, but due to the very limited number of samples, they do not provide a reasonable basis for estimating the short- or long-term health risk due to exposure to smoke and gaseous emissions.

\section{2,533}

\section{PB94-146636 PC A04/MF A01}

National Inst. of Standards and Technology (CAML), Gaithersburg, MD. Applied and Computational Mathematics Div.

Modulation of Fossil Fuel Production by Global Temperature Variations, 2.

B. W. Rust, and F. J. Crosby. Jan 94,64p, NISTIR5332

See also PB83-182592.

Keywords: "Fossil fuels, "Atmospheric temperature, "Northern Hemisphere, "Air pollution, Production, Climatic changes, Global warming, Variations, Mathematical models, Graphs(Charts), Gaia hypothesis, Rust-Kirk model. 


\section{Air Pollution \& Control}

The report includes the inverse modulation of global fossil production by variations in Northern Hemispheric temperatures. The present study incorporates recent revisions and extensions of the fuel production record and uses a much improved temperature record. The authors show that the new data are consistent with the predictions of the original Rust-Kirk model which they then extend to allow for time lag between variations in the temperature and the corresponding responses in fuel production. The modulation enters the new model through the convolution of a lagged averaging function with the temperature time-series. The authors also include explicit terms to account for the perturbations caused by the Great Depression and World War II. The final model accounts for $99.84 \%$ of the total variance in the production record. This modulation represents a feedback which is consistent with the carbon dioxide problem; climate change; fossil fuel production global warming Gaia hypothesis; temperature variations.

\subsection{4}

PB94-155587 PC A04/MF AO1

National Inst. of Standards and Technology (NML), Gaithersburg, MD. Center for Chemical Technology. Sulfur Dioxide Capture in the Combustion of Mixtures of Lime, Refuse-Derived Fuel, and Coal.

Final rept.

K. L. Churney, and T. J. Buckley. Nov 90, 59p,

NISTIR-4443.

Sponsored by Department of Energy, Washington, DC. Biofuels and Municipal Waste Technology Div.

Keywords: "Refuse derived fuels, "Sulfur dioxide, "High sulfur coal, "Cocombustion, "Air pollution control Calcium oxides, Fly ash, Chlorine, Manganese oxides, Sorbents, Tests, Combustion chambers.

Chlorine and sulfur mass balance studies have been carried out in the combustion of mixtures of lime, refuse-derived fuel, and coal in the NIST multikilogram capacity batch combustor. The catalytic effect of manganese dioxide on the trapping of sulfur dioxide by lime was examined. Under the authors conditions, only $4 \%$ of the chlorine was trapped in the ash and no effect of manganese dioxide was observed. Between 42 and $14 \%$ of the total sulfur was trapped in the ash, depending upon the lime concentration. The effect of manganese dioxide on sulfur capture was not detectable. The temperature of the ash was estimated to be near 1200 deg C, which was in agreement with that calculated from sulfur dioxide capture thermodynamics.

02,535

\section{PB94-161635 PC A03/MF A01}

Maryland Univ., College Park

Rare-Earth Isotopes as Tracers of Particulate Emissions: An Urban Scale Test.

Final rept.

J. M. Ondov, W. R. Kelly, J. Z. Holland, P. J

Paulsen, Z. C. Lin, and S. A. Wight. Jan 91, 43p,

CBRM-TR-91-2

See also PB91-195347. Prepared in cooperation with National Inst. of Standards and Technology (NML), Gaithersburg, MD. Center for Analytical Chemistry. Sponsored by Maryland Dept. of Natural Resources, Annapolis. Chesapeake Bay Research and Monitoring Div. and Maryland Power Plant Research Program Annapolis.

Keywords: "Tracer techniques, "Isotopic labeling, "Particulates, "Rare earth elements, "Air pollution, Urban areas, Feasibility studies, Coal fired power $\begin{array}{lll}\text { plants, Fly ash, Flue } & \text { gases, } \\ \text { Concentration(Composition), Neodymium 148, Field }\end{array}$ tests.

The particulate emissions of a $100 \mathrm{MW}(\mathrm{e})$ coal-fired power plant were successfully tagged with enriched rare-earth isotopes on three occasions during the month of August, 1988. On 28 August, an 8-hour release was made using $94 \%$ isotopically pure $148 \mathrm{Nd}$ injected at a rate of $61 \mathrm{mg} / \mathrm{hr}$ and 14 ambient aerosol samples collected along a 72 degree arc $20-\mathrm{km}$ distant from the plant were analyzed by pulse-counting thermal-ionization mass spectrometry for excess $148 \mathrm{Nd}$ and naturally-occurring (total) Nd background. The results were in good agreement with Gaussian plume model predictions. We conclude that the experiment and preliminary results successfully demonstrate the feasibility of using an enriched rare-earth isotopes as tracers of emissions from coal-fired power plants. The large signal-to-noise ratios achieved here are sufficient for tracing particles over much larger distances.
02,536

PB94-164381 PC A05/MF A01

National Inst. of Standards and Technology (BFRL), Gaithersburg, MD

CONTAM93 User Manual.

G. N. Walton. Mar 94, 80p, NISTIR-5385.

Keywords: "User manuals (Computer programs), "Indoor air pollution, "Air flow, "Buildings, Environmental transport, Spatial distribution, Ventilation, Contaminants, Algorithms, Requirements, Air quality, Environmental engineering, "CONTAM93 computer program, CONTAM computer program, CONTAMX computer program, Multizone analysis.

This manual describes the use of two computer programs for analyzing the air movement and indoor air quality in multizone buildings. The first program CONTAM is used to create and edit the building description including data for all features relating to airflow or to the generation and removal of contaminants. It uses a graphic interface to establish the spatial relationship of these features. These data along with weather data are used by the program CONTAMX to calculate the airflows and dynamic levels of indoor contaminants. The results of the calculation may be reviewed graphically in CONTAM and printable files may be generated. Together these two programs are called CONTAM93.

\section{2,537}

PB94-194388 PC A04/MF A01

National Inst. of Standards and Technology (BFRL), Gaithersburg, MD.

CONTAM88 Building Input Files for Multi-Zone Airflow and Contaminant Dispersal Modeling.

J. B. Fang, and A. K. Persily. Jun 94, 53p, NISTIR-

5440

Keywords: "Indoor air pollution, "Air flow, "Buildings, Computerized simulation, Algorithms, Ventilation, Contaminants, Computer files, Dispersants, Data bases, Infiltration(Fluids), "CONTAM88 computer program, "Multi-zone air flow, "Contaminant dispersal modeling.

Input files for the multi-zone airflow and contaminant dispersal computer program CONTAM88 are described for four large buildings. The files were developed for a twelve-story multi-family residential building, a five-story mechanically-ventilated office building with an atrium, a seven-story mechanically-ventilated office building with an underground parking garage, and a one-story school building. The physical characteristics of each building and its idealization as a multi-zone airflow system are described. These input files enable a user to employ CONTAM88 to study airflow and contaminant dispersal in these large buildings without developing building idealizations and inputting them into CONTAM88. Results of selected computer simulations are presented to demonstrate the effects of wind speed, indoor-outdoor temperature difference, and the percentage of outdoor air intake in the supply air on air change rates and interzonal airflows for these four buildings. Appendices are also included that contain a building component leakage database useful in multizone airflow modeling and updated information on the use of CONTAM88.

\section{2,538}

PB94-199874 Not available NTIS

National Inst. of Standards and Technology (BFRL), Gaithersburg, MD. Building Environment Div.

Environmental Evaluation of a New Federal Office Building.

W. S. Dols, A. K. Persily, and S. J. Nabinger. 1993,

9p.

Pub. in Proceedings of IAQ 92 Environments for People Conference, San Francisco, CA., October 19-21, 1992, p85-93. Sponsored by General Services Administration, Washington, DC.

Keywords: "Indoor air pollution, "Office buildings, "Air pollution monitoring, "Federal buildings, "Air quality, Environmental engineering, Ventilation, Concentration(Composition), Carbon dioxide, Carbon monoxide, Radon, Formaldehyde, Volatile organic compounds, Performance evaluation, Reprints, Overland(Missouri)

A study of the environmental performance of three new office buildings is being conducted, involving long-term monitoring of building ventilation rates and indoor levels of selected pollutants, both before occupancy and during early occupancy. This paper describes the effort in a seven-story office building in Overland, Missouri. The measurement results discussed in this paper include tracer gas decay measurements of ventilation rates and the concentrations of carbon dioxide, carbon monoxide, radon, formaldehyde, and volatile organic compounds (VOCs)

02,539

PB95-108825 Not available NTIS

National Inst. of Standards and Technology (NEL), Gaithersburg, MD. Building Environment Div.

Ventilation Rates in Office Buildings.

Final rept.

A. Persily. $1989,9 p$

Sponsored by Geological Survey, Reston, VA., General Services Administration, Washington, DC., and Department of Energy. Washington, DC

Pub. in Proceedings of the ASHRAE/SOEH Conference on IAQ 89 The Human Equation: Health and Comfort, San Diego, CA., April 17-20, 1989, p128-136.

Keywords: "Ventilation, "Office buildings, "Air quality, "Thermal confort, "Indoor air pollution, "Flow measurement, Design criteria, Air flow, Performance evalua tion, Standards, Tracer techniques, Reprints.

The ventilation rate of an office building impacts the building air quality in terms of both thermal comfort and pollutant levels. The National Institute of Standards and Technology has been studing ventilation in office buildings for several years. The research has included the measurement of ventilation rates in 14 office build ings for periods on the order of one year, and has produced a data set of about 3000 ventilation measurements under a range of weather and building operation conditions. These data are a unique source of actua ventilation rate measurements in office buildings, and are the subject of the paper. The data provides an indication of current practice. Although the ventilation rates vary considerably among the buildings and for each individual structure, many of the buildings have ventilation rates below recommended levels and/or design values during significant portions of the year.

\section{2,540}

PB95-126066 Not available NTIS

National Inst. of Standards and Technology (BFRL), Gaithersburg, MD. Fire Safety Engineering Div.

Dispersion and Deposition of Smoke Plumes Generated in Massive Fires.

Final rept.

A. F. Ghoniem, X. Zhang, O. M. Knio, H. Baum, and R. Rehm. 1993, 19p.

don, VA Pub. in Jnl. of Hazardous Materials 33, p275-293 1993. Keywords: *Plumes, "Fires, “Smoke, "Environment effects, "Trajectories, "Computational fluid dynamics, Deposition, Buoyancy, Environmental pollution, Dispersion, Pollution transport, Equations of motion, Wind direction, Air pollution, Reprints, *Buoyant smoke plumes.

Massive fires resulting from the uncontrolled burning of crude oil from spills or industrial accidents produce large smoke-laden buoyant plumes which rise in the wind direction before they equilibrate within a stably stratified atmosphere. Beyond this point, the plume material cools by entrainment and the plume becomes negatively buoyant due to the heavy smoke loading. The trajectory of the descending plume, which determines the ground distribution of smoke, is the subject of this paper. A computational model for the simulation of large-scale smoke plumes resulting from such fires is developed and applied to investigate the effects of the plume initial properties on its trajectory and smoke deposition patterns. Attention is focused on the descent and dispersion of wind-driven plumes in a homogeneous atmosphere, and the smoke deposition on flat terrain. Results show that the plume dynamics in the cross-wind direction are dominated by two buoyantly generated, coherent, streamwise vortices which distor the plume cross section into a kidney-shaped structure. The strength of the two vortices and their separation increases as the plume falls. The plume width grows under the action of these vortices at a rate which increases as the plume settles on the ground, leading to a smoke footprint which does not resemble the prediction of Gaussian dispersion models.

\section{2,541}

PB95-138129 PC A04/MF A01

National Inst. of Standards and Technology (TS), Gaithersburg, MD. National Voluntary Lab. Accreditation Program. 
National Voluntary Laboratory Accreditation Program: Bulk Asbestos Analysis.

Handbook.

E. B. Steel, J. Verkouteren, and D. F. Alderman. Aug 94, 72p, NIST/HB-150/3

Also available from Supt. of Docs. as SN003-00303288-3. See also PB94-178225.

Keywords: "Asbestos, "Laboratories, "Certification, *Polarized light, "Microscopy, Requirements, Licensure, Methodology, Standardization, Performance evaluation, Quality assurance, Administrative procedures, National Voluntary Laboratory Accreditation Program, US NIST

The handbook presents the specific requirements that laboratories must comply with in order to be accredite under the National Voluntary Laboratory Accreditation Program (NVLAP) for Bulk Asbestos Analysis. Details are provided regarding the on-site assessment and proficiency testing requirements for laboratories analyzing bulk samples by means of polarized light microscopy.

\section{2,542}

PB95-150140 Not available NTIS

National Inst. of Standards and Technology (BFRL), Gaithersburg, MD. Building Environment Div.

Study of Ventilation and Carbon Dioxide in an Of. fice Building.

Final rept.

S. J. Nabinger, A. K. Persily, and W. S. Dols. 1994,

Sponsored by Public Buildings Service, Washington, DC.

Pub. in American Society of Heating, Refrigerating and Air-Conditioning Engineers Transactions 100, pt2 10p 1994.

Keywords: "Office buildings, "Ventilation, *Carbon dioxide "Indoor air quality, Air flow rates, Air circulation, Air infiltration, Flow measurement, Tracer techniques, Air pollution monitoring, Indoor air pollution, Space HVAC systems, Reprints, Air exchange rates.

Ventilation rates and indoor carbon dioxide levels were monitored for two years in a new office building nea St. Louis, Missouri. These measurements were made to assess the operation and performance of the ventilation system in this building and to investigate the relationship between indoor carbon dioxide levels and ai change rates. Ventilation rates were measured with the tracer gas decay technique using an automated meas uring system. Indoor carbon dioxide concentrations were also measured with an automated system. The relationship between the indoor carbon dioxide levels and the building air change rates was similar to that seen in other office buildings. This discussion points out limitations in the use of equilibrium analysis of carbon dioxide concentrations in office buildings.

\section{2,543}

PB95-151189 Not available NTIS

National Inst. of Standards and Technology (NML), Gaithersburg, MD. Gas and Particulate Science Div. Guidelines for Refractive Index Measurements of Asbestos.

Final rept

J. R. Verkouteren, J. M. Phelps, and E. B. Steel.

$1989,5 p$.

Pub. in Microbeam Anal. 24, p429-433 1989.

Keywords: "Asbestos, "Refractive index, "Optical measurement, "Guidelines, Chrysotile, Amosite, Crocidolite, Polarized light, Microscopy, Calibration standards, Reference standards, Reprints, Standard reference materials, Focal screening, Dispersion staining, Becke line technique.

Refractive index is one of the primary optical properties used to identify minerals using polarized light microscopy. Through the association with the Bulk Asbestos Laboratory Accreditation Program, the authors have received requests from hundreds of Laboratories fo technical assistance in the area of bulk asbestos analysis. Many of these requests are for information on the measurement of refractive index, specifically using the focal screening (dispersion staining) and Becke line techniques. The authors have developed Standard Reference Materials (SRMs) of chrysotile, amosite and crocidolite (SRM 1866) to serve laboratories as primary calibration standards for the measurement of the optical properties of asbestos, including refractive index. These materials will be discussed and used as examples to explain the application of focal screening and the Becke line technique to refractive index measurement.

\section{2,544}

PB95-162095 Not available NTIS

National Inst. of Standards and Technology (BFRL), Gaithersburg, MD. Building Environment Div.

Ventilation, Carbon Dioxide and ASHRAE Standard 62-1989.

Final rept.

A. K. Persily. 1993, $4 p$

Pub. in American Society of Heating, Refrigeration and Air-Conditioning Engineers Jnl. 35, n7 p40-44 Jul 93

Keywords: "Ventilation, "Carbon dioxide, "Air quality standards, "Indoor air quality, Commercial buildings, Office buildings, Indoor air pollution. Air flow, Flow rates, Flow measurement, Air infiltration, Air circulation, Air pollution monitoring. Space HVAC systems, Reprints, "ASHRAE Standard 62-1989.

The article is an attempt to reduce some of the confusion concerning the issues of ventilation, carbon dioxide and ASHRAE Standard 62-1989 that has been evident in numerous discussions of indoor air quality. The article presents a brief discussion of how indoor carbon dioxide levels are related to ventilation rates, including the potential for using these indoor concentrations to determine outdoor airflow rates. The role of carbon dioxide is Standard 62, specifically the significance of the $1000 \mathrm{ppm}$ quideline is also discussed. Much of the discussion in the article is specific to mechanically-ventilated office buildings, but many of the conclusions are relevant to other building types.

\section{2,545}

PB95-162277 Not available NTIS

National Inst. of Standards and Technology (NML), Gaithersburg, MD. Gas and Particulate Science Div. Development of a Gas Standard Reference Material Containing Eighteen Volatile Organic Compounds. Final rept.

G. C. Rhoderick. 1991, 8p

Pub. in Fresenius' Jnl. of Analytical Chemistry $341, n 9$ p524-531 Nov 91.

Keywords: "Reference standards, "Gas analysis, "Quantitative chemical analysis, Gas chromatography, Gravimetric analysis, Microanalysis, Flame ionization, Electron-capture detectors, Interlaboratory comparisons, Calibrating, Certification, Reprints, "Volatile organic compounds, Standard reference materials.

A procedure to prepare primary gas cylinder standards for eighteen volatile organic compounds (VOC's) at the $1-15 \mathrm{nmol} / \mathrm{mol}$ (ppb) level was developed. These gas standards were evaluated over a period of 2 years and were determined stable and accurate. This research resulted in the development of Standard Reference Material (SRM) 1804 containing these 18 volatile organic compounds in nitrogen at a nominal concentration of $5 \mathrm{ppb}$ for each compound

\section{2,546}

\section{PB95-162285 Not available NTIS}

National Inst. of Standards and Technology (NML), Gaithersburg, MD. Gas and Particulate Science Div. Stability/Instability of Gas Mixtures Containing 1,3Butadiene in Treated Aluminum Gas Cylinders.

Final rept.

G. C. Rhoderick. 1990, 9p

Pub. in Proceedings of 1990 EPA/A\&WMA International Symposium on Measurement of Toxic and Related Air Pollutants, Raleigh, NC., May 1990, p709717.

Keywords: "Butadienes, "Stability, "Gas cylinders, "Binary mixtures, "Calibration standards, Nitrogen, Air pollution, Chemical analysis, Quantity ratio, Concentration(Composition), Reprints, "Aluminum gas cylinders, "1,3-Butadiene.

The Gas Metrology Research Group of the National Institute of Standards and Technology (NIST), has been involved in research and development of gas standards of volatile toxic organic compounds for many years. Over thirty toxic organic compounds have been studied in gas mixtures contained in high pressure alu. minum gas cylinders with specially treated interior surfaces. Almost all compounds studied to date have shown very good long term stability at the parts-perbillion (ppb, nanomole/mole) to parts-per-million (ppm, micromole/mole) range. One exception, 1,3-butadiene, is a compound that many scientists and policy makers are very interested in measuring in the environment. The author discusses data obtained over several years or gas mixtures of 1,3-butadiene in nitrogen, at concentrations of $2 \mathrm{ppm}, 100 \mathrm{ppb}$, and $10 \mathrm{ppb}$. The data demonstrate that mixtures of 13 -butadiene at the 2 $\mathrm{ppm}$ level have remained stable for over three years. However, gas mixtures of 1,3-butadiene at the $10 \mathrm{ppb}$ level have shown decreases in concentration of more than $70 \%$ over a two-year period. Decreases in the concentration of 1,3-butadiene have been observed immediately after the preparation of the gas mixture in several cylinders. This indicates that gas mixtures of 1,3-butadiene are not stable at the ppb levels and therefore are not reliable as accurate calibration standards.

\section{2,547}

PB95-163622 Not available NTIS

National Inst. of Standards and Technology (NML) Gaithersburg, MD. Gas and Particulate Science Div. Standards for Atmospheric Measurements.

Final rept

R. A. Velapoldi, and W. D. Dorko. 1990, $7 p$.

Pub. in Fresenius Jnl. of Analytical Chemistry 338, n4 p479-485 1990.

Keywords: "Air pollution sampling, "Chemical analysis, Reference standards, "Quality control, Air pollutants, Gas analysis, Atmospheric chemistry, Interlaboratory comparisons, Quality assurance, Accuracy, Standardization, Reprints, Standard reference materials.

The effects of global climate change and air pollutants on the earth and human population, have made the measurement of atmospheric gaseous and particulate species as well as kinetic rate constants for modeling of utmost importance. The paper reviews: (1) the philosophy and process of the development, preparation and certification of gas Standard Reference Materials (SRM's) at the National Institute of Standards and Technology (2) some of the SRM's currently available, with a listing of their components, concentrations, and specific uses; (3) the results of a recent laboratory intercomparison; (4) a brief discussion of some of the measurements and SRM research currently in progress; and finally (5) some ideas for new standards that might be needed in the future.

\section{2,548}

PB95-163705 Not available NTIS

National Inst. of Standards and Technology (NML), Gaithersburg, MD. Chemical Kinetics Div.

Atmospheric Reactivity of alpha-Methyl-Tetrahydrofuran.

Final rept.

T. J. Wallington, W. O. Siegal, R. Liu, M. J. Kurylo,

Z. Zhang, and R. E. Huie. 1990, $4 p$

Pub. in Environmental Science and Technology 24 n10 p1596-1599 1990

Keywords: "Atmospheric chemistry, "Air pollution, "Fuel additives, Biomass, Volatile organic compounds, Environmental impact, Hydroxyl radicals, Reaction kinetics, Exhaust emissions, Reprints, "Furan/methyltetrahydro

Biomass derived alpha-methyl-tetrahydrofuran, MTHF, has been proposed as an automotive fuel additive Since MTHF is a volatile organic compound, the environmental impact of evaporation to the atmosphere needs to be considered if this compound is to be used in substantial quantities in such applications. The major loss process of MTHF in the atmosphere is expected to occur via reaction with hydroxyl radicals $(\mathrm{OH})$, hence we have conducted a study of the kinetics of the reaction of $\mathrm{OH}$ radicals with MTHF using both absolute (flash photolysis resonance fluorescence) and relative rate techniques. The absolute rate experiments were performed over the temperature range 240-400 K at total pressures of 35 torr of argon; the relative rate experiments were conducted at $295 \mathrm{~K}$ in 740 torr of synthetic air. The results from both techniques were in good agreement.

02,549

PB95-163754 Not available NTIS

National Inst. of Standards and Technology (NML) Gaithersburg, MD. Gas and Particulate Science Div. Measurement of the Uniformity of Particle Deposition of Filter Cassette Sampling in a Low Velocity Wind Tunnel.

Final rept.

C. C. Wang, R. A. Fletcher, E. Steel, and J. W.

Gentry. 1990, $4 p$

Pub. in Jnl. of Aerosol Science 21, nS1 pS621-S624 1990.

Keywords: "Air pollution sampling, "Aerosols, "Wind tunnels, Indoor air pollution, Asbestos, Wind velocity, Bias, Error analysis, Reprints. 
Microscopic particle counting is often employed for determining airborne fiber concentrations. Wind tunnel testing was used to determine whether there is a systematic sampling bias for anisokinetic and non-isoaxial conditions. Low wind velocities $(0.2-0.5 \mathrm{~m} / \mathrm{s})$ were employed to simulate indoor air environments.

02,550

PB95-169041 Not available NTIS

National Inst. of Standards and Technology (CSTL), Boulder, CO. Thermophysics Div

Membrane Gas Separation for a Fluidized-Bed Incinerator.

Final rept.

J. J. Pellegrino, D. M. Stull, and B. W. Logsdon.

1993, 5p.

Pub. in Proceedings of the American Filtration Society Meeting, Chicago, IL., May 3-6, 1993, p390-394.

Keywords: "Hazardous materials, "Low level radioactive wastes, "Incinerators, "Air pollution control, Fluidized beds, Membranes, Separation processes, Permeability, Combustion products, Carbon dioxide, Oxygen, Nitrogen, Water, Reprints, Gas permeation.

For their proposed fluidized-bed incineration process, EG\&G Rocky Flats' technical staff are considering the possible scenario of placing a 'molecular filter' (a gas separation membrane) system on their stack gases. The permeate through the membrane would principally be the products of combustion: $\mathrm{CO} 2$ and $\mathrm{H} 2 \mathrm{O}$, and the retentate is circulated back to the incinerator as the fluidizing gas. Data were obtained for cellulose acetate and polyamide membranes using a $\mathrm{CO} 2$-rich stream with $\mathrm{N} 2$ and $\mathrm{O} 2$. Additional measurements were made to provide some basis for estimating the fate and effect of contaminants. Gas permeation measurements, with both types of membranes, were made on streams that contained trace $\mathrm{C}($ sub 1$)-\mathrm{C}$ (sub 3) contaminants. These latter measurements were also made with cellulose acetate samples which had been exposed to saturated organic solvent vapors for extended periods.

\section{2,551}

PB95-175659 Not available NTIS

National Inst. of Standards and Technology (CSTL) Gaithersburg, MD. Surface and Microanalysis Science Div.

Sources of Urban Contemporary Carbon Aerosol. Final rept.

L. M. Hildemann, D. Klinedinst, G. A. Klouda, L. A

Currie, and G. R. Cass. 1994, 12p.

Pub. in Environmental Science and Technology 28, n9 p1565-1576 1994

Keywords: "Aerosols, "Carbonaceous materials, "Air pollution sources, "Urban areas, Carbon, Carbon 12, Carbon 14, Fossil fuels, Particulates, Exhaust emissions, Anthropogenic sources, Air pollution sampling, Air pollutants, Los Angeles, Reprints, Emission inventories.

Emissions from the major sources of fine carbonaceous aerosol in the Los Angeles basin atmosphere have been analyzed to determine the amounts of the $\mathrm{C}-12$ and $\mathrm{C}-14$ isotopes present. From these measurements, an inventory of the fossil carbon and contemporary carbon particle emissions to the Los Angeles atmosphere has been created. Using a mathematical model for atmospheric transport, predictions are made of the atmospheric fine particulate fossil carbon and contemporary carbon concentrations expected due to primary source emissions. It is concluded that the high fraction of contemporary carbon measured historically in Los Angeles is not due to local emission sources of biogenic material; rather, it is due to a combination of local anthropogenic pollution sources and background marine aerosol advected into the city.

\section{2,552}

PB95-176004 Not available NTIS

National Inst. of Standards and Technology (BFRL), Gaithersburg, MD. Building Environment Div.

Limits of $\mathrm{CO}_{2}$ Monitoring in Determining Ventilation Rates.

Final rept.

A. K. Persily. 1994, 4p.

Pub. in TAB Jnl., p19-22 1994

Keywords: "Carbon dioxide, "Ventilation, *Indoor air pollution, "Air pollution monitoring, Air infiltration, Air flow, Flow rates, Concentration(Composition). Concentration rates, Buildings, Reprints, ASHRAE Standard 62-1989.

The article is an attempt to reduce some of the confu sion concerning the issues of ventilation, carbon diox- ide (CO2), and ASHRAE Standard 62-1989 that has been evident in recent discussions of indoor air quality. The article presents a brief discussion of how indoor $\mathrm{CO} 2$ levels are related to ventilation rates, including the use of indoor concentration measurements to determine outdoor air flow rates. The role of carbon dioxide in Standard 62, specifically the significance of the $1,000 \mathrm{ppm}$ guideline, is also discussed. The article primarily addresses mechanically ventilated office buildings, but many of the statements are also relevant to other building types

\section{2,553}

PB95-176012 Not available NTIS

National Inst. of Standards and Technology (BFRL), Gaithersburg, MD. Building Environment Div.

Ventilation Effectiveness Measurements in Two Modern Office Buildings.

Final rept.

A. K. Persily, W. S. Dols, and S. J. Nabinger. 1993,

$6 \mathrm{p}$

Contract DE-Al01-91CE21042

Sponsored by Department of Energy, Washington, DC Pub. in Proceedings of Indoor Air '93, Helsinki, Finland, July 4-8, 1993, p195-200.

Keywords: "Office buildings, *Ventilation, "Indoor air pollution, "Air infiltration, Air flow, Flow rates, Flow measurement, Air quality, Air intakes, Space HVAC systems, Reprints, Air change effectiveness.

Ventilation effectiveness measurements were made in two office buildings employing the age-of-air approach. The objective of these tests was to study the applicability of the associated tracer gas procedures in mechanically ventilated office buildings. Experimental issues were investigated including the ability to establish a uniform tracer concentration at the start of the test and the relationship of ventilation system configuration and system operation on the test procedure. The test results indicate good mixing of the ventilation air within the occupied space. In addition, experience was obtained with the measurement procedures that will assist the development of a standardized approach to measuring ventilation effectiveness in the field.

\section{2,554}

PB95-178893 PC A05/MF A01

National Inst. of Standards and Technology (BFRL), Gaithersburg, MD. Building Environment Div.

Indoor Air Quality Impacts of Residential HVAC Systems. Phase 2.A Report: Baseline and Preliminary Simulations.

S. J. Emmerich, and A. K. Persily. Jan 95, 80p, NISTIR-5559.

See also Phase 1 Report, PB95-135596. Sponsored by Consumer Product Safety Commission, Washington, DC.

Keywords: *Air pollution monitoring, "Indoor air pollution, "Space HVAC systems, "Baseline measurements, Residential buildings, Air pollutants, Air intakes Air infiltration, Air flow, Flow measurement, Ventilation, Air pollution control, Mathematical models, Computerized simulation

NIST is performing whole building airflow and contaminant dispersal computer simulations with the program CONTAM93 to assess the ability of modifications of central forced-air heating and cooling systems to control pollutant sources relevant to the residential environment. The report summarizes the results of Phase II.A of this project, which consisted of three major efforts: baseline simulations of contaminant levels with out indoor air quality (IAQ) controls, design of the IAQ control retrofits, and preliminary simulations of contaminant levels with the IAQ control retrofits. In Phase II.B of the study, all of the baseline cases will be modified to incorporate the IAQ control retrofits. The retrofit results will then be compared to the baseline results to evaluate the effectiveness of the retrofits.

\section{2,555}

PB95-180238 Not available NTIS

National Inst. of Standards and Technology (BFRL), Gaithersburg, MD. Building Environment Div. Application of a Multizone Airflow and Contaminant Dispersal Model to Indoor Air Quality Control in Residential Buildings.

Final rept.

S. J. Emmerich, A. K. Persily, and G. N. Walton. 1994, 15p

Pub. in Proceedings of the AIVC Conference (15th): The Role of Ventilation, Buxton, Great Britian, September 27-30, 1994, p493-507 1994.
Keywords: "Air flow, *Indoor air polfution, *Residential buildings, "Air quality, "Computerized simulation, Ventilation, HVAC systems, Air circulation, Air pollution control, Tracer studies, Contaminants, Reprints, "CONTAM93 model, Contaminant dispersal models, Multi-zone air flow.

A new multizone airflow and contaminant dispersal model CONTAM93 is described, along with a demonstration of its application in a study of ventilation and contaminant control in single-family residential buildings. While CONTAM93 is based on existing theory of network airflow analysis and contaminant dispersal the model employs a unique graphic interface for data input and display. The interface uses a sketchpad to describe the connections between zones and icons to represent zones, openings, ventilation system components, and contaminant sources and sinks. The model, its graphic interface and plans for its further development are described. As a demonstration of the capabilities of CONTAM93, the paper describes a study of ventilation and contaminant control in eight single-family residential buildings. The overall objective of the effort is to study the impact of residential HVAC systems on indoor air quality (IAQ). This paper describes the study and the use of CONTAM93 to calculate whole building air change rates for a range of weather conditions and to simulate fan pressurization and tracer gas decay tests in the houses.

02,556

\section{PB95-198735 PC A04/MF A01}

National Inst. of Standards and Technology (BFRL) Gaithersburg, MD. Building Environment Div.

Measurements of Indoor Pollutant Emissions from EPA Phase II Wood Stoves.

S. J. Nabinger, A. K. Persily, K. S. Sharpless, and S. A. Wise. Feb 95, 68p, NISTIR-5575.

Sponsored by Consumer Product Safety Commission Washington, DC. Directorate for Health Sciences.

Keywords: "Stoves, *Air pollution sampling, *Indoor air pollution, *Residential buildings, Polycyclic aromatic hydrocarbons, Particulates, Emission factors, Waste gases, Particle size, Air pollution monitoring, "Wood burning appliances.

Measurements of indoor pollutant emissions were made for four wood stoves meeting the U.S. Environmental Protection Agency (EPA) Phase II emission requirements in a $37 \mathrm{sq}$. $\mathrm{m}$ (400 sq. ft) test house at $\mathrm{Na}$ tional Institute of Standards and Technology (NIST). The stoves were operated in a manner consistent with typical residential use and in accordance with the manufacturers' instructions. Three tests were conducted for each stove, with each test lasting approximately ten hours. During the tests some of the following quantities monitored included: combined gaseous and particulate phase concentrations of individual polycyclic aromatic hydrocarbons (PAH) averaged over the test period, in cluding benzo(a)pyrene (BAP); and, indoor and outdoor air temperature and relative humidity. Based on these measurements, emission rates of the individual PAH compounds were determined for each test.

\section{2,557}

\section{PB95-220422 PC A03/MF A01}

National Inst. of Standards and Technology (BFRL), Gaithersburg, MD. Building Environment Div.

Computer Simulations of Aiflow and Radon Transport in Four Large Buildings.

J. B. Fang, and A. K. Persily. Apr 95, 50p, NISTIR5611

Sponsored by Environmental Protection Agency, Washington, DC. Office of Air and Radiation.

Keywords: "Air flow, "Radon, "Indoor air quality, *Compulerized simulation, Ventilation, Air infiltration, Air circulation, Radionuclide migration, Leakage, Flow rates, Air intakes, Space HVAC systems, Temperature measurement, Spatial distribution, Flow models.

Computer simulations of aifflow and radon transport in four large buildings are performed using the multi-zone aiflow and pollutant transport model CONTAM88. These buildings include a twelve-story multi-family residential building, a five-story mechanically ventilated office building with an atrium, a seven-story mechanically ventilated office building with an underground parking garage and a one-story mechanically ventilated school building. Interzone airflow rates and radon concentrations are predicted in these buildings as a function of wind speed and direction, indoor-outdoor temperature difference, and ventilation system operation. Ventilation system factors that are studied include the operation of exhaust fans in the apartment 
building and variations in the percent outdoor air intake in the office buildings. Simulations in the office buildings are also made with the ventilation systems off and with variations in the balance of the supply and return airflow rates.

\section{2,558}

PB95-255899 PC A05/MF AO1

Yale Univ. New Haven, CT. School of Architecture. New Mass Transport Elements and Components for the NIST IAQ Model.

J. W. Axley. Jul 95, 90p, NIST/GCR-95/676.

Sponsored by National Inst. of Standards and Technology (BFRL), Gaithersburg, MD. Building Environment Div.

Keywords: "Indoor air pollution, "Air flow, "Air pollution dispersion, "Mathernatical models, Aerosols, Particles, Air circulation, Environmental transport, Mass transport, Flow measurement, Contaminants, Filtration Ventilation, Computerized simulation, Contaminant dispersal models.

This report presents new mass transport elements for the next generation of the NIST IAQ Model that may be used to model (1) homogenous (buik-air) chemistry within well-mixed chamber, (2) aerosol mass transpor within well-mixed chambers and fractional particle fil ration in building filtration devices, and (3) hetero geneous (surface-related) physical processes and chemical transformations including those governing the behavior of gas-phase air cleaning devices. In an effort to maintain rigor, generality, and flexibility, each ransport process is formulated in terms of the elemental mass transport step that together govern the overall process. In this way, the more complex processes may be represented as component equations that are assembled from fundamental element equations.

\section{2,559}

\section{PB96-106877 PC A05/MF A01}

National Inst. of Standards and Technology (BFRL) Gaithersburo, MD. Building Environment Div.

Indoor Air Quality Impacts of Residential HVAC Systems Phase II.B Report: IAQ Control Retrofit Simulations and Analysis.

S. J. Emmerich, and A. K. Persily. Sep 95, 94p NISTIR-5712

Sponsored by Consumer Product Safety Commission, Bethesda, MD.

Keywords: "Indoor air pollution, "Air pollution monitoring, "Air flow, "Space HVAC systems, Air infiltration, Air intakes, Flow measurement, Retrofitting, Residen tial buildings, Vents, Ambient air quality, Air pollutants Ventilation systems, Heating systems, Computerized simulation, Graphs(Charts)

The National institute of Standards and Technology (NIST) performed a preliminary study of the potentia for using central forced-air heating and cooling system modifications to control indoor air quality (IAQ) in residential buildings. The objective of this effort was to pro vide insight into the use of state-of-the-art IAQ models to evaluate such modifications, the potential of these modifications to mitigate residential IAQ problems, the pollutant sources they are most likely to impact, and their potential limitations. This study was not intended to determine definitively whether the IAQ control options studied are reliable and cost-effective. The report summarizes the results on Phase II.B of this project, which consisted of three main efforts: computer simulations of contaminant levels with IAQ control retrofits evaluation of the effectiveness of the IAQ control retroits, and development of recommendations for future research.

\section{2,560}

PB96-109566 PC A03/MF A01

National Inst. of Standards and Technology (BFRL), Gaithersburg, MD. Office of Applied Economics.

EMISS: A Program for Estimating Local Air Pollution Emission Factors Related to Energy Use in Buildings: User's Guide and Reference Manual. Final rept.

S. R. Petersen. Oct 95, 36p, NISTIR-5704.

Sponsored by Federal Energy Management Program, Washington, DC.

Keywords: "Emission factors, "Energy use, "Air pollution monitoring, Buildings, Energy consumption, Energy conservation, Heating systems, Regional analysis, Life cycle costs, Nitrous oxide, Sulfur dioxide, Carbon dioxide, Electric power, Distillates, Natural gas,
Coal, Liquified natural gas, Residual fuels, Fossil fuels Combustion, Sulfur content, User quides, EMISS computer program, End users.

EMISS is a computer program used to generate data files with regional or local air pollution emission factors for use with the Nist BLCC (Building Life-Cycle Cost) program. BLCC uses these emission factors to cal culate air pollution emissions associated with energy use in buildings and reductions in those emissions at tributable to energy conservation measures. Emission actors for fossil fuels can be regionalized or localized by specifying the percentage of sulfur in the fuel, the heating content of the fuel, and the end-use combustion process at the building site. A data base with statespecific electricity emission factors and U.S. averag sulfur content of fossil fuels provides default data for use in setting up a regional emission factors file.

\section{2,561}

B96-117221 Not available NTIS

National Inst. of Standards and Technology (CSTL), Gaithersbirg, MD. Analytical Chemistry Div

Analysis by a Combination of Gas Chromatography and Tandem Mass Spectrometry: Development of Quantitative Tandem-in-Time Ion Trap Mass Spectrometry: Isotope Dilution Quantification of 11-Nor-Delta-9-Tetrahydro cannabinol-9 Carboxylic Acid.

Final rept.

C. S. Phinney, and M. J. Welch. 1995, 5p. Pub. in Rapid Communications in Mass Spectrometry, v9 p1056-1060 1995.

Keywords: "Toxic substances, "Mass spectrometers Reprints, Sampling, Organic compounds, Quantitative analysis, "Drug analysis, Tandem Mass Spectrometry.

Collision-induced dissociation (CID) was performed in an ion-trap mass spectrometer to investigate quanlification by isotope dilution and gas chromatography coupled with tandem mass spectrometry (GC/MS/MS) The operating system software was modified to allow simultaneous CID of two ions differing only slightly in mass/charge ratios. Simultaneous excitation of the base peaks of the parent compound and its deuterated analog in a co-eluting GC peak leads to quantitative and reproducible formation of daughter ions. Changing conditions in the mass analyzer as the GC peak elutes required broadening of the $\mathrm{CID}$ excitation frequency pattern. This had to be done so that relatively uniform dissociation energy would be imparted to both parent ions during the concentration changes associated with the elution of a GC peak. A commonly measured human metabolite of marijuana, 11-nor-delta-9tetrahydro cannabinol-9-carboxylic acid, was analyzed in freeze-dried urine. The mean value, 14.0 equal to or greater than 0.61 ( 1 standard deviation) $\mathrm{ng} / \mathrm{mL}$, agrees very well with the GC/MS mean measured value of 14.1 equal to or greater than $0.45 \mathrm{ng} / \mathrm{mL}$. The overall coefficient of variation for the individual ion-trap MS/MS sample measurements is $4.3 \%$.

\section{2,562}

Not available NTIS

National Inst. of Standards and Technology (BFRL), Gaithersburg, MD. Building Environment Div.

Few Caveats on Carbon Dioxide Monitoring.

Final rept.

A. P. Persily. 1994, 4p

Pub. in Indoor Air Quality Jnl., v1 n4 p22-25 1994.

Keywords: *Carbon dioxide, "Air pollution monitoring. "Indoor air pollution, Commercial buildings, Ecological concentration, Air flow, Air circulation, Ventilation systems, Space HVAC systems. Tracer techniques, Er rors, Reprints.

While techniques exist to relate indoor carbon dioxide concentrations to building ventilation, they are not as simple as sometimes suggested. Some of the techniques can yield reliable results when used correctly, while others can result in serious errors. Using any of these approaches requires that one understands the technique and the following factors are considered: the outdoor carbon dioxide concentration; the number of people in the building and the rate at which they generate carbon dioxide; variations in the number of occupants within the building and over time; variations in the carbon dioxide concentration within the building; the measurement uncertainty of the carbon dioxide monitor being used vertilation system operation prior to and during the measurements; and, indoor sources of carbon dioxide other than the occupants

02,563
PB96-135124 Not available NTIS
National Inst. of Standards and Technology (CSTL), Gaithersburg, MD. Organic Analytical Research Div. Distinguishing the Contributions of Residential Wood Combustion and Mobile Source Emissions Using Relative Concentrations of Dimethylphenanthrene Isomers

Final rept.

B. A. Benner, S. A. Wise, L. A. Currie, R. B. Zweidinger, R. K Stevens, C. W Lewis, G. A

Klouda, and D. B. Klinedinst. 1995, 8p.

Pub. in Environmental Science and Technology, v29 n9 p2382-2389 Sep 95.

Keywords: “Air pollution sources, *Combustion products, "Emissions, Particulates, Polycylic aromatic hydrocarbons, Emission factors Mass spectroscopy, Carbon 13 , Carbon 1 concentration, Vehicle air pollution, Wood burning ap-
pliances, Air pollution monitoring, Reprints, pliances, Air pollution
*Dimethylphenanthrenes.

As part of the United States Environmental Protection Agency's Integrated Air Cancer Project, air particulate matter samples collected in Boise, ID, were analyzed by gas chromatography with mass spectrometric detection (GC-MS) and apportioned between their two main sources: residential wood combustion (RWC) and motor vehicle (MV) emissions. The technique used for distinguishing the source contributions involved dimethylphenanthrene (1,7-DMP), a polycyclic aromatic hydrocarbon (PAH) emitted primarily by burning soft woods (e.g., pines), with that of a PAH emitted in modest concentrations by both RWC and MV sources, 2,6-dimethylphenanthrene (2,6-DMP). These results ratio of 48 samples collected in a roadway tunnel, with any enrichment in the Boise sample ratios over the mean tunnel ratio attributable to the RWC source These resulting RWC contributios were compared with fraction RWC results obtained by radiocarbon measurements (C-14/C-13) of the same extracts from Boise, with generally good correlations between the two techniques observed, suggesting that the methods are comparable when used to distinguish emissions of MVs from RWC of soft woods.

\section{2,564}

PB96-146642 Not available NTIS

National Inst. of Standards and Technology (BFRL) Gaithersburg, MD. Building Environment Div.

Effectiveness of a Heat Recovery Ventilator, an Outdoor Air Intake Damper and an Electrostatic Particulate Filter at Controlling Indoor Air Quality in Residential Buildings.

Final rept.

S. J. Emmerich, and A. K. Persily. 1995, 13p.

Pub. in Air Infiltration and Ventilation Centre Conference (16th), Palm Springs, CA., September 19-22, 1995. p263-275 1995.

Keywords: "Residential buildings, "Ventilation systems, *Indoor air pollution, Space HVAC systems, Ventilators, Heat recovery Air intakes, Dampers, Electrostatic precipitators, Filters, Air flow, Air infiltration, Particulates, Air pollution control, Reprints.

A preliminary study of the potential for using central forced-air heating and cooling system modifications to control indoor air quality (IAQ) in residential buildings was performed. The main objective was to provide insight into the potential of three IAO control options to mitigate residential IAQ problems, the pollutant sources the controls are most likely to impact, and the potential limitations of the controls. Another important objective was to identify key issues related to the use of multizone models to study residential IAQ and to identify areas for follow-up work

\section{5}

PB96-146659 Not available NTIS

National Inst. of Standards and Technology (BFRL), Gaithersburg, MD. Building Environment Div.

Multizone Modeling of Three Residential Indoor Air Quality Control Options.

Final rept.

S. J. Emmerich, and A. K. Persily. 1995, 8p. Pub. in Building Simulation '95, International IBPSA Conference (4th), Madison. WI., August 14-16, 1995 p213-220.

Keywords: "Indoor air pollution, "Air pollution control * Space HVAC systems, Computerized simulation, Heating systems, Cooling systems, Ventilation sys tems, Residential buildings, Environmental transport Ecological concentration, Air flow, Electrostatic 


\section{Air Pollution \& Control}

precipitators, Filters, Heat recovery, Ventilators, Air intakes, Dampers, Reprints.

The impact of central forced-air heating and cooling system modifications on the levels of selected pollutants in single-family houses was evaluated by simulat ing pollutant concentrations due to a variety of sources in eight houses with typical HVAC systems. Simulations were performed with a multizone airflow and pollutant transport model and were repeated with the systems modified to include an electrostatic particulate filter, a heat recovery ventilator, and an outdoor air intake damper. The system modification reduced the pollutant levels in the houses for some cases; however, the results also demonstrated potential limitations in both the simulation method and the performance of the devices.

\section{2,566}

\section{PB96-147392 PC A05/MF A01}

National Inst. of Standards and Technology (TS), Gaithersburg, MD. National Voluntary Lab. Accreditation Program.

Airborne Asbestos Analysis: National Voluntary Laboratory Accreditation Program.

Handbook.
S. Turner, E. B. Steel, and D. F. Alderman. Oct 95 ,

83p, NIST/HB-150-13

Also available from Supt. of Docs.

Keywords: "Asbestos, "Air pollution, “Chemical analysis, "Handbooks, Electron microscopy, Airboric dures, Requirements, Site suney, Laboratories, "National Voluntary Laboratory Accreditation Program, NIST(Nationa! Institute of Standards and Technology), TEM(Transmission electron microscopy).

Any laboratory (including commercial, manufactor, university, or federal, state, or local government laboratory) that performs the test method that comprises the Airborne Asbestos Analysis Program many apply for National Voluntary Laboratory Accreditation Program (NVLAP) accreditation. Accreditation will be granted to a laboratory that satisfactorily fulfills the conditions for accreditation defined in NIST Handbook 150, NVLAP Procedures and General Requirements, which contain Title 15, Part 285 of the Code of Federal Regulations. These conditions include satisfactory performance in selected proficiency testing as required, and fulfilling the on-site assessment requirements, including resolu tion of identified deficiencies. The names of NVLAPaccredited laboratories are published in the NVLAP annual directory and other media to which information is regularly provided.

\section{2,567}

PB96-165782 PC A08/MF A02

National Inst. of Standards and Technology (BFRL), Gaithersburg, MD. Building Environment Div.

Multizone Modeling of Three Residential Indoor Air Quality Control Options.

S. J. Emmerich, and A. K. Persily. Mar 96, 145p, NISTIR-5801.

Supersedes PB95-135596, PB95-178893 and PB96106877. Sponsored by Consumer Product Safety Commission, Washington, DC. Directorate of Engineering Science.

Keywords: "Indoor air quality, "Heating systems, "Cooling systems, "Residential buildings, Space HVAC systems, Indoor air pollution, Central systems, Ventilation systems, Air flow, Air infiltration, Flow measurement, Particulates, Microorganisms, Nitrogen dioxide, Carbon monoxide, Filtration, Electrostatic precipitators, Dampers, Heat recovery equipment, Computerized simulation, "Multizone analysis, Forced air, Volatile organic compounds.

The National Institute of Standards and Technology (NIST) performed a preliminary study of the use of central forced-air heating and cooling system modifications to control indoor air quality (IAW) in residential buildings. The objective of the effort was to provide insight into the use of state-of-the-art multizone airflow and IAQ models to evaluate such modifications, the potential of these modifications to mitigate residential IAW problems, the pollutant sources they are most likely to impact, and their potential limitations. The multizone airflow and pollutant transport program CONTAM93 was used to simulate the pollutant concentrations due to a variety of sources in eight buildings with typical HVAC systems under different weather conditions. Three indoor air quality control technologies were incorporated into the house models to determine their effectiveness in controlling the mod- eled pollutant sources. The technologies include the following: electrostatic particulate filtration, heat recovery ventilation, and an outdoor air intake damper on the forced-air system return.

\section{2,568}

\section{PB96-167812 PC A04/MF A01}

National Inst. of Standards and Technology (BFRL), Gaithersburg, MD. Building Environment Div.

Study to Determine the ExIstence of an Azeotropic R-22 'Drop-In' Substitute.

M. S. Kim, G. Morrison, W. J. Mulroy, and D. A. Didion. Mar 96, 50p, NISTIR-5784.

See also PB92-149814. Sponsored by Electric Power Research Inst., Palo Alto, CA. and Korea Science and Engineering Foundation.

Keywords: "Refrigerants, "Environmental chemical substitutes, "Azeotropes, Cooling systems, Mixtures, Cyclopropane, Butanes, Propane, Refrigeration, Computerized simulation, Heat pumps, HFC-134a, HCFC(Hydrochlorofluorocarbon), CFC(Chlorofluorocarbon).

The reduction in chlorofluorocarbon (CFC) and hydrochlorofluorocarbon (HCFC) production and the scheduled phase-out of these ozone depleting refrigerants requires the development and determination of environmentally safe refrigerants for use in heat pumps, water chillers, air conditioners, and refrigerators. Azeotropic mixtures are attractive as alternative refrigerants because they behave very nearly as pure materials. A simple correlative scheme that allows one to judge whether or not an azeotrope is likely in a binary refrigerant mixture is discussed. This paper presents laboratory and computer simulation model evaluation of two of the azeotropic refrigerant mixtures which are identified, HFC-134a (1,1,1,2134 a with R-600a (Isobutane), in a generic heat pump apparatus. A third azeotropes mixture, HFC-134a with apparatus. A third azeotropes mixture, HFC-134a with
$\mathrm{R}-\mathrm{C} 290$ (Cyclopropane) is examined by computer simulation only

\section{2,569}

\section{PB97-111249 Not available NTIS}

National Inst. of Standards and Technology (BFRL), Gaithersburg, MD. Building Environment Div.

Relationship between Indoor Air Quality and Carbon Dioxide.

Final rept.

A. K. Persily. $1996,6 p$

Pub. in Indoor Air' 96 , the International Conference on Indoor Air Quality and Climate (7th), Nagoya, Japan, July 21-26, 1996, v2 p961-966.

Keywords: "Carbon monoxide, "Indoor air pollution, "Air pollution monitoring, Commercial buildings, Air flow, Flow rates, Air circulation, Air infiltration, Odors, Ventilation, Ecological concentration, Reprints, Air exchange rates.

The paper describes the relationship of indoor $\mathrm{CO} 2$ concentrations to building air quality and ventilation, with a focus on how $\mathrm{CO} 2$ can be used to evaluate air quality and ventilation performance. While $\mathrm{CO} 2$ concentrations do not provide a comprehensive indication of indoor air quality, they can be used to assess the acceptability of a space in terms of human body odor. Also, under some circumstances, $\mathrm{CO} 2$ can be used to evaluate building ventilation, specifically air change rates and percent outdoor air intake.

\section{2,570}

\section{PB97-113203 Not available NTIS}

National Inst. of Standards and Technology (BFRL), Gaithersburg, MD. Building Environment Div.

CONTAM94: A Multizone Airflow and Contaminant Dispersal Model with a Graphic User Interface.

Final rept.

G. N. Walton. 1995, 6p.

Pub. in Building Simulation '95: Proceedings of the Conference of International Building Performance Simulation Association (4th), Madison, WI., August 14-16, 1995, p674-679.

Keywords: "Air flow, "Contaminants, "Indoor air pollution, Air circulation, Air pollution monitoring, Environmental transport, Graphical user interface, Computerized simulation, Algorithms, Computer models, Reprints, CONTAM94 model, Multizone buildings.

CONTAM94 is an airflow and contaminant migration analysis program combining algonthms for modeling airflow and contaminant dispersal in multizone buildings. It employs a simplified graphic description of the building for both data entry and the presentation of simulation results. It can handle buildings containing a large number of zones.

02,571

PB97-114227 PC A05/MF A01

National Inst. of Standards and Technology (BFRL) Gaithersburg, MD. Building Environment Div.

Carbon Monoxide Dispersion in Residential Buildings: Literature Review and Technical Analysis A. K. Persily. Oct 96, 71p, NISTIR-5906.

Sponsored by National Fire Protection Association, Quincy, MA

Keywords: "Carbon monoxide, "Air pollution dispersion, "Residential building, "Indoor air pollution, Air circulation, Ventilation, Air flow, Flow rates, Diffusion models, Rooms, Air pollution monitoring, Tracer techniques, Detection, Exposure, Environmental engineering, Computerized simulation, Literature surveys.

While the use of $\mathrm{CO}$ detectors can decrease the likelihood of exposure to such CO levels, questions exist concerning the installation of these devices in residential buildings, primarily with regards to the location and number of detectors. A literature review and technical analysis was conducted to assess information on CO dispersion in residential buildings that could support the development of guidance on detector installation. The review covered a number of issues including $\mathrm{CO}$ concentration measurements in residential buildings, sources of indoor $\mathrm{CO}$, mixing within and between rooms, tracer gas techniques for assessing building airflow, and computer models of air movement and contaminant dispersal in buildings. The material obtained in the literature review is discussed, and a technical analysis of the issues related to $\mathrm{CO}$ dispersion in residential buildings is presented.

\section{2,572}

PB97-118749 Not available NTIS

National Inst. of Standards and Technology (BFRL), Gaithersburg, MD. Building Materials Div. Materials-Science Based Approach to Pheno Emissions from a Flooring Material in an Office Building.

J. W. Martin, A. K. Persily, F. R. Guenther, E. Byrd Lakley, T. Nguyen, and W. S. Liggett. 1996, 6p. Pub. in Indoor Air' 96 , International Conference on Indoor Air Quality and Climate (7th), Nagoya, Japan, July 21-26, 1996, v2 p109-114.

Keywords: "Office buildings, "Phenols, "Floors, "Indoor air pollution, Epoxy resins, Emissions, Building matenals, Chemical analysis, Physical properties, Reprints, Volatile organic compounds.

It was hypothesized that phenol emissions from an epoxy floor-leveling material were the source of the complaints. A materials-science based study was performed to ascertain whether phenol, or any other volatile organic compounds, was being emitted from the floor-leveling material. The chemical composition and physical properties of the leveling material were determined using a variety of analytical procedures typica of a materials-science based approach. It was concluded that the floor-leveling material contained phenol, and that the measured concentrations ranged from $0.25 \%$ to $0.52 \%$ of the material's mass.

\section{2,573}

PB97-118806 Not available NTIS

National Inst. of Standards and Technology (BFRL) Gaithersburg, MD. Building Environment Div.

Issues in the Field Measurement of VOC Emission Rates.

Final rept.

A. K. Persily. $1996,6 p$.

Pub. in Indoor Air '96, International Conference on Indoor Air Quality and Climate (7th), Nagoya, Japan, July 21-26, 1996, v2 p49-54.

Keywords: "Emissions, "Indoor air pollution, "Air pollution monitoring, Ecological concentration, Mass balance, Absorption, Desorption, Design analysis, Computerized simulation, Reprints, "Volatile organic compounds.

The measurement of VOC emission rates in the field can be valuable in indoor air quality research, in evaluations of the indoor air quality impacts of design, and in the diagnosis of indoor air quality problems. The paper reviews the mass balance theory employed in field measurements of VOC emission rates. Some concems associated with these measurements are dis- 
cussed including the assumption of equilibrium VOC concentrations and the neglect of adsorption and desorption of VOC on building surfaces. Computer simulations are described that provide an order-ofmagnitude assessment of the impacts of these issues.

\section{2,574}

PB97-122352 Not available NTIS

National Inst. of Standards and Technology (CSTL), Gaithersburg, MD. Surface and Microanalysis Science Div.

Radiocarbon Measurements of Atmospheric Volatile Organic Compounds: Quantifying the Biogenic Contribution.

Final rept.

G. A. Klouda, C. W. Lewis, R. A. Rasmussen, R. K. Stevens, L. A. Currie, D. J. Donahue, A. J. T. Jull, R L. Seila, G. C. Rhoderick, and R. L. Sams. 1996, 8p. Pub. in Environmental Science and Technology, v30 n4 p1098-1105 Jun 96.

Keywords: "Carbon 14, "Natural emissions, *Air pollution sampling, "Biomass, Air pollution sources, Ozone, Precursors, Ecological concentration, Atmospheric chemistry, Photochemistry, Urban areas, Photochemical reactions, Data quality, Quality control, Reprints, “Volatile organic compounds, Attanta(Georgia).

The radiocarbon C-14 abundance of atmospheric volatile organic compounds (VOC) gives a quantitative estimate of contributions from biomass and fossil-mass sources, important information for effective regulation of ozone precursors. The authors report the details of a methodology to perform such measurements and the first exploratory $\mathrm{C}-14$ results on VOC fractions separated from two composited urban tropospheric air samples, collected during the summer (1992) in Atlanta, GA. The results of these experiments, designed to (1) evaluate the entire C-14 VOC measurement process and (2) obtain reliable estimates of biogenic contributions to atmospheric VOC, emphasize how important controls are throughout this multi-step chemical process to ensure quality data.

\section{Environmental Health \& Safety}

\section{2,575}

PB95-140117 Not available NTIS

National Inst. of Standards and Technology (PL), Gaithersburg, MD. Ionizing Radiation Div.

Radiation Accident at an industrial Accelerator Facility.

Final rept.

D. A. Schauer, B. M. Coursey, C. E. Dick, M. F

Desrosiers, A. D. Jacobson, W. L. McLaughlin, and

J. M. Puhl. 1993, 10p.

Sponsored by Navy Health Sciences Education and Training Command, Bethesda, MD.

Pub. in Health Physics 65, n2 p131-140 1993.

Keywords: *Radiation accidents, "Occupational safety and health, "Radiation injuries, "Industrial medicine, *Accident investigations, Industrial accidents, Occupational exposure, Radiation dosage, Partial body irradiation, Accelerators, Biological radiation effects, Radiation heating, Materials processing reactors, Reprints.

On 11 December 1991, a radiation overexposure occurred at an industrial radiation facility in Maryland. The radiation source was a 3-MV potential drop accelerator designed to produce high electron beam currents for materials-processing applications. During maintenance on the lower window pressure plate, an operator placed his hands, head, and feet in the beam. This was done with the filament voltage of the electron source turned 'off,' but with the full accelerating potential on the high voltage terminal. The operator's body, especially his extremities and head, were exposed to electron dark current. In an attempt to reconstruct the accident, radiochromic film and alanine measurements were made with the accelerator operated at two beam currents. Electron paramagnetic resonance spectrometry, which measures the concentration of radiation-induced paramagnetic centers in calcified tissues, was used to estimate the dose to the victim's extremities.

\section{Radiation Pollution \& Control}

\section{2,576}

PB94-165602 PC A09/MF A03

National Inst. of Standards and Technology (BFRL), Gaithersburg, MD.

Measurement and Determination of Radon Source Potential: A Literature Review.

A. B. Tanner. Apr 94, 199p, NISTIR-5399.

Keywords: *Radon, *Air pollution sources, "Indoor air pollution, Natural radioactivity, Radium, Soil gases, Soil-structure interactions, Radionuclide migration, Mapping Buildings, Diffusion Permeability, Flow rates, Site characterization, Foundations, Construction materials, Regional analysis, Soil tests, Activity levels.

Radon source potential may be estimated for areas of a nation, state, county, housing development, or build ing lot. The critical characteristics of the soil are its radium concentration, emanation coefficient, permeability to gas, and diffusion coefficient for radon under typical conditions. This report summarizes and evaluates available information on radon potential mapping and site-specific characterization. More than 100 reports have been found that bear on radon potential mapping. and indicate fair to good agreement with indoor radon results where correlations have been possible.

02,577

PB94-185782 Not available NTIS

National Inst. of Standards and Technology (BFRL) Gaithersburg, MD. Building Materials Div.

Expansion of Cementitious Materials Exposed to Sulfate Solutions.

Final rept.

J. R. Pommersheim, and J. R. Clifton. 1994, $6 p$

Pub. in Materials Research Society Symposia Proceedings, v333 p363-368 1994. Sponsored by Nuclear Regulatory Commission, Washington, DC.

Keywords: *Cements, *Concretes, *Radioactive waste disposal, *Environmental effects, *Sulfates, *Low-level radioactive wastes, Underground storage, Construction materials, Environmental protection, Degradation, Exposure, Diffusion, Crack propagation, Reprints.

Disposal of low-level radioactive waste in concrete vaults buried underground is being considered in the United States and a potentially significant degradation process is sulfate attack. The results of experimental studies and of modeling of the kinetics of sulfate attack of cement-based materials are given. It was concluded that the expansion process involves a two-stage mechanism. The first stage is likely to be diffusion controlled and the second stage appears to be acceleratory due to the development and spread of cracks.

\section{2,578}

PB96-190103 Not available NTIS

National Inst. of Standards and Technology, Gaithersburg, MD. Occupational Health and Safety Div.

Response Comparison of Electret Ion Chambers, LiF TLD, and HPIC

Final rept.

T. Hobbs, P. Kotrappa, J. Tracy, and B. Biss. 1996 , 8p.

Pub. in Radiation Protection Dosimetry, v63 n3 p181. 1881996.

Keywords: "Dosimeters, "Electrets, *Environmental monitoring, Ionization chambers, Radiation measuring instruments, Dielectrics, Thermoluminescent, Reprints.

During each quarter of 1991 electret ion chambers, (nai) LiF(6) LiF and (7) LiF thermoluminescence dosimeters, all integrating ionizing radiation monitors were placed at a location in the grounds of the National Institute of Standards and Technology. One group was exposed to the total ambient field, while a second group was sealed for testing a method of protection against radon gas instrusion. A high-pressure ion chamber at the same location provided another measure of the field. Simultaneously with the environmental response test, other sealed and unsealed groups of electret ion chambers and thermoluminescence dosemeters were exposed during each quarter to discrete exposures from calibrated sources of (60) Co and $(137) \mathrm{Cs}$. All responses were evaluated at the end of each quarter. The results indicated that the electret ion chamber gave results comparable to thermoluminescence dosemeters for routine environmental monitoring. No significant difference was found for any monitor type when sealed results were compared with unsealed results.

\section{Solid Wastes Pollution \& Control}

02,579

AD-A279 133/3 PC A03/MF A0

National Bureau of Standards, Gaithersburg, MD.

Recommendations for the Disposal of Carbon-14

Wastes.

1952, 21p

Keywords: "Carbon, "Hazardous wastes, Biological agents, Chemical compounds, "Waste disposal.

No abstract available.

02580

AD-A301 258/0 PC A04/MF A01

National Bureau of Standards, Gaithersburg, MD.

Performance of Plastic Packaging for Hazardous Materials Transportation. Part 1. Mechanical Properties.

Final rept. Jun 75-Jun 76

J. M. Crissman, C. M. Guttman, and L. J. Zapas. Oct 76, 53p, DOT/MTB/OHMO-76/4

Contract DOT-AS-50074

Keywords: "Packaging, "Hazardous materials *Polyethylene plastics, Test and evaluation, Density, Vibration, Transportation, Static loads, Cracking(Fracturing), Damage assessment, Brittleness, Failure(Mechanics), Compressive strength, Regulations. Thermal stresses, Shipping containers.

This report, prepared for the U. S. Department of Transportation, contains background information useful in evaluating the performance of plastic packagings for hazardous materials transportation, insofar as mechanical properties are concerned. Current DoT regulations and test methods are reviewed as well as testing procedures from other organizations and countries. Also included are recommendations to modify curren DoT regulations to make test methods more quantitative Finally experimental data are presented which represent the initial stage of a study it is hoped will ultimately lead to the establishment of criteria upon which the long time behavior of plastic containers can be predicted based on short time tests. (AN).

\section{2,581}

\section{PB94-199882 Not available NTIS}

National Inst. of Standards and Technology (NML) Gaithersburg, MD. Chemical Thermodynamics Div. Assessing the Credibility of the Calorific Content of Muncipal Solid Waste.

Final rept.

E. S. Domalski, K. L. Churney, A. E. Ledford, T. J.

Buckley, R. C. Paule, M. L. Reilly, J. C. Colbert, and S. S. Bruce. 1991, 4p.

Pub. in Pure and Applied Chemistry 63, n10 p1415 14181991

Keywords: "Municipal wastes, ${ }^{*}$ Calorific value, "Hea of combustion, "Heat measurement, Solid wastes, Calorimeters, Performance evaluation, Sample preparation, Reprints

A study has been carried out to establish the limits of reliability of the calorific content of municipal solid waste (MSW) by the bomb calorimetric procedure currently used in commercial test laboratories. This procedure involves using gram-size samples derived from MSW that have been processed down to a particle size of $2 \mathrm{~mm}$ or less. Critics argue that one cannot sample a multi-ton quantity of MSW and extract gram-size samples which are representative of the entire gross sample. They argue further that processing down to 2 $\mathrm{mm}$ or less significantly alters the composition of the sample. In order to test the bomb calorimetric procedure, a multi-kg capacity combustion flow calorimete was designed and constructed for the determination of the enthalpies of combustion of kilogram-size samples of MSW in flowing oxygen near atmospheric pressure.

\section{2,582}

PB95-140968 Not available NTIS

National Inst. of Standards and Technology (MSEL), Gaithersburg, MD. Polymers Div. 
Bioleaching of Cobalt from Smelter Wastes by 'Thiobacillus ferrooxidans'.

Final rept.

G. J. Olson, C. K. Sakai, E. J. Parks, and F. E

Brinckman. 1990, 4p.

Pub. in Jnl. of Industrial Microbiology 6, n1 p49-52 Sep 90.

Keywords: "Waste treatment, "Materials recovery, "Cobalt, "Biological treatment, "Leaching, Microorganisms, Smelting, Mattes, Solubility, Reprints Thiobacillus ferrooxidans.

The bioleaching of cobalt from domestic, industrial smelter wastes was studied. Thiobacillus ferrooxidans solubilized Co from sulfidic dross furnace mattes. At pulp densities of $4 \%$, up to $600 \mathrm{mg}$ of Co per liter of leaching solution was released from nickel matte, corresponding to removal of about two-thirds of the original amount of $\mathrm{Co}$ in the matte. Bioleaching methods may be useful as a component of a process for solubilization and recovery of Co from sulfidic smelter mattes.

\section{Water Pollution \& Control}

02,583

PB95-104907 PC A06/MF A02

National Inst. of Standards and Technology (BFRL),

Gaithersburg, MD. Fire Safety Engineering Div.

In Situ Burning Oil Spill Workshop Proceedings. Held in Orlando, Florida on January 26-28, 1994.

Special pub.

N. H. Jason. Aug 94, 112p, NIST/SP-867

Also available from Supt. of Docs, as SN003-00303290-5. See also PB94-101839. Sponsored by Minerals Management Service, Herndon, VA.

Keywords: "Meetings, "Oil spills, "In-situ combustion, "Fire tests, "Burning rate, "Water pollution control, Crude oil, Regulations, State government, Legal as pects, Louisiana, Decision making. Environmental research, Public health, Smoke.

This workshop is part of the U.S. Minerals Management Service's continuing effort to ensure the re evance of their research program to the needs of the user community. Specific emphasis was given to environmental and operational implications of in situ burn response technology. The goals of the In Situ Oil Burning workshop were: to present the state of knowledge to representatives of industry, government, and research organizations; to review the present status guidelines for use of in situ burning as an oil spill response method; and to prioritize research and information needs to support decisions on the use of in situ burning of oil spills.

\section{2,584}

\section{PB95-140091 Not available NTIS}

National Inst. of Standards and Technology (CSTL) Gaithersburg. MD. Organic Analytical Research Div. Comparison of Methods for Gas Chromatographic Determination of PCBs and Chlorinated Pesticides in Marine Reference Materials.

Final rept.

M. Schantz, R. Parris, J. Kurz, K. Ballschmiter, and S. Wise. 1993, 13p.

Pub. in Fresenius J. Anal. Chem. 346, p766-778 1993

Keywords: "Gas chromatography, "Water pollution detection, "Marine environments, "Polychlorinated biphenyls, "Pesticides, "Chemical analysis, Chlorine organic compounds, Comparison, Marine biology, Water pollution effects, Sediments, Reprints, *Standard reference materials, "Certified reference materials, SRM 1588, SRM 1941, SRM 1974, CRM 349, CRM 350

Three gas-chromatographic (GC) columns with different selectivity (DB-5, DB-1701, and C-18) and two different GC detectors (electron-capture and massspectrometric) were used to analyze three Standard Reference Materials (SRMs), which are available from the National Institute of Standards and Technolog (NIST), and two Certified Reference Materials (CRMs) which are available from the Community Bureau of Reference (CBR), for polychlorinated biphenyl (PCB) congeners and chlorinated pesticides. The materials analyzed were: SRM 1588, Organics in Cod Liver Oil SRM 1941, Organics in Marine Sediment; SRM 1974 Organics in Mussel Tissue (Mytilus edulis); CRM 349
Chlorobiphenyls in Cod Liver Oil; and CRM 350, Chlorobiphenyls in Mackerel Oil. Results are reported.

02,585

PB95-140109 Not available NTIS

National Inst. of Standards and Technology (CSTL), Gaithersburg, MD. Organic Analytical Research Div.

NIST Standard Reference Materials (SRMs) for Polychlorinated Biphenyl (PCB) Determinations and Their Applicability to Toxaphene Measurements. Final rept.

M. M. Schantz, R. M. Parris, and S. A. Wise. 1993,

Pub. in Chemosphere 27, n10 p1915-1922 1993.

Keywords: "Polychlorinated biphenyls, "Pesticides, "Environmental surveys, "Chemical analysis, *Toxaphene "Water pollution detection, Chlorine organic compounds, Water pollution effects, Marine environments, Rivers, Sediments, Air pollution detection, Dust, Interlaboratory comparisons, Marine biology, Reprints, "Standard reference materials, SRM 1588, SRM 1939, SRM 1941, SRM 1974, SRM 1945, SRM 1649.

In the past five years the National Institute of Standards and Technology (NIST) has developed several Standard Reference Materials (SRMs) to assist in the validation of measurements of polychlorinated biphenyl (PCB) congeners and chlorinated pesticides in environmental samples. The following natural matrix SRMs have been issued: SRM 1588, Organics in Code Liver Oil; SRM 1939, PCB Congeners in River Sediment; SRM 1941, Organics in Marine Sediment; SRM 1974, Organics in Mussel Tissue (Mytilus edulis); and SRM 1945, Whale Blubber. Recently, SRM 1649 has been analyzed for selected PCB congeners and chlorinated pesticides. Toxaphene measurements have not been made at NIST for these SRMs; however, these SRMs represent the environmental matrices that are typically analyzed for toxaphene. Recommended values for toxaphene concentrations could be established on any of these materials through interlaboratory comparison exercises involving laboratories experienced in making toxaphene measurements. In this paper we describe these SRMs and discuss their suitability as potential reference materials for toxaphene measurements.

\section{2,586}

\section{PB95-161402 Not available NTIS}

National Inst. of Standards and Technology (CSTL) Gaithersburg, MD. Organic Analytical Research Div. Development of the National Marine Mammal Tissue Bank.

T. Lillestolen, N. Foster, and S. Wise. 1993, 11p.

Pub. in Science of the Total Environment 139/140 p97-107 1993.

Keywords: "Marine mammals *Animal tissues, "Tissue banks, "Bioaccumulation, Bioassay, Water pollution effects(Animals), Biological accumulation, Chemical analysis, Contaminants, Water pollutants, Environmental monitoring, Quality assurance, Reference standards, Reprints, "National Marine Mammal Tissue Bank, Standard reference materials.

The National Manine Mammal Tissue Bank (NMMTB) has been established as part of a comprehensive effort to obtain reliable information on contaminant levels in marine mammal tissues. A four component program consisting of the NMMTB, stranding networks, monitoring and quality assurance has been developed. The development and current status of the NMMTB and its relationship to the other three components will be described.

02,587

PB95-163747 Not available NTIS

National Inst. of Standards and Technology (BFRL), Gaithersburg, MD. Fire Safety Engineening Div.

In situ Burning of Oil Spills: Mesoscale Experiments and Analysis.

Final rept.

W. D. Walton, D. D. Evans, K. B. McGrattan, D. Madrzykowski, A. D. Putorti, R. G. Rehm, H. Koseki, E. J. Tennyson, H. R. Baum, and W. H. Twilley. $1993,56 p$.

Sponsored by Minerals Management Service, Herndon, VA.

Pub. in Proceedings of the Arctic and Marine Oil Spill Program Technical Seminar (16th), Calgary, Alberta, Canada, June 7-9, 1993, p679-734.

Keywords: "Oil spills, "Fire tests, "In situ combustion, "Burning rate, Crude oil, Combustion properties, Com- bustion kinetics, Oil-water interfaces, Plumes, Particulates, Oil pollution control, Water pollution control, Reprints, Smoke yield.

A series of six mesoscale and one large laboratory fire experiments were performed to measure the buming characteristics of Louisiana crude oil on water in a pan. Results of the measurements for burning rate and smoke emissions are compared to those from previous burns of various scales. Predictions of smoke plume trajectory and particulate deposition at ground level from the Large Eddy Simulation (LES) model developed as part of this research effort are presented. LES is a steady-state three-dimensional calculation of smoke plume trajectory and smoke particulate deposition based on a mixed finite difference and Langrangian particle tracking method.

\section{2,588}

PB95-164034 Not available NTIS

National Inst. of Standards and Technology (CSTL) Gaithersburg, MD. Organic Analytical Research Div.

Quality Assurance of Contaminant Measurements in Marine Mammal Tissues.

Final rept.

S. A. Wise, $1993,11 p$

Pub. in Proceedings of Symposium on Coastal and Ocean Management (8th): Coastal Zone '93, New Orleans, LA., July 19-23, 1993, p2531-2541.

Keywords: "Marine mammals, "Animal tissues, *Bioaccumulation, *Chemical analysis, "Quality assurance, Water pollution effects(Animals), Biological accumulation, Bioassay, Standardization, Interlaboratory comparisons, Tissue banks, Reference standards, Reprints, Standard reference materials.

The National Institute of Standards and technc.ugy (NIST), in conjunction with the National Oceanic and Atmospheric Administration (NOAA) is developing a program to improve the quality and comparability of analytical measurements for contaminants in marine mammal tissues. This quality assurance program consists of the (1) preparation of marine mammal tissue control materials, (2) interlaboratory companison exercises, and (3) development of standard reference materials. The development and status of the program is described.

02,589

PB95-171344 PC A05/MF A01

National Inst. of Standards and Technology (CSTL) Gaithersburg, MD. Organic Analytical Research Div. National Manine Fisheries Service, Silver Spring, MD. Office of Protected Resources.

Alaska Marine Mammal Tissue Archival Project: Specimen Inventory.

B. J. Koster, S. A. Wise, and P. R. Becker. Nov 94 , $78 p$, NISTIR-5462

See also PB91-184796 and PB92-143718. Prepared in cooperation with National Marine Fisheries Service, Silver Spring, MD. Office of Protected Resources.

Keywords: "Mañne mammals, "Animal tissues, "Tissue banks, "Bioassay "Reference standards "Water pollution effects(Animals), Whales, Seals(Mammals) Bioaccumulation, Biological accumulation, Chemical analysis, Environmental monitoring, Alaska, *Standard reference materials, Sea lions.

The Alaska Marine Mammal Tissue Archival Project (AMMTAP) was initiated in 1987 to aid in the monitoring of contaminants in the Alaskan environment. The tissue samples are then archived in the National Biomonitoring Specimen Bank (NBSB) at the Nationa Institute of Standards and Technology (NIST). In the NBSB, the tissues are stored in vapor phase liquid nitrogen freezers until required for analysis. This document contains the current inventory of the AMMTAP archive. Included in the inventory is information such as: species, location collected, morphometries, tissue collected and dispositions of the tissue, i.e., archived or homogenized for analysis. This document contains the records for the collection of tissue from 94 animals samples between July 87 - October 93 .

02,590

PB95-209870 PC A06/MF A02

National Inst. of Standards and Technology (CSTL), Gaithersburg, MD 
Concentrations of Chlorinated Hydrocarbons, Heavy Metals and Other Elements in Tissues Banked by the Alaska Marine Mammal Tissue Archival Project.

P. R. Becker, E. A. Mackey, M. M. Schantz, B. J. Koster, S. A. Wise, D. C. G. Muir, R. Demiralp, and R. R. Greenberg. Mar $95,124 \mathrm{p}$ NISTIR-5620.

See also PB92-143718 and PB95-171344. Prepared in cooperation with Department of Fisheries and Oceans, Winnipeg (Manitoba). Freshwater Inst.

Keywords: "Marine mammals, "Animal tissues, *Chemical analysis, "Biological accumulation, "Bioassay, Bioaccumulation, Water pollution effects(Animals), Whales, Seals(Mammals), Heavy metals, Polychlorinated biphenyls, Trace elements, Chlorinated hydrocarbons, Pesticides, Muscles, Kidney, Liver, Neutron activation analysis, Tissue banks, Environmental monitoring, Alaska, Bioconcentration, Blubber.

The National Oceanic and Atmospheric Administration maintains two marine mammals tissue banks within NIST's National Biomonitoring Specimen Bank. The document is the second report in the NIST Interagency
Report Series in which analytical data on samples collecled and banked as part of the Alaska Marine Mammal Tissue Archival Project are summarized. Results for inorganic constituents in liver tissues from 15 bulukha whales, 14 ringed seals, 3 bowhead whales, 3 beared seals, and 1 spotted seal, and for organic contaminants in blubber tissues from 12 belukha whales are presented and discussed.

02,591

PB96-111737 Not available NTIS

National Inst. of Standards and Technology (CSTL), Gaithersburg, MD.

Quantitative Analysis of Selected PCB Congeners in Marine Matrix Reference Materials Using a Novel Cyanobiphenyl Stationary Phase.

Final rept.

B. R. Hillery, J. E. Girard, M. M. Schantz, and S. A.

Wise. 1995, 8p.

Pub. in Jnl. of High Resolution Chromatography, v18 p89-96 Feb 95

Keywords: "Polychlorinated biphenyls, "Quantitative chemical analysis, "Marine mammals, "Reference standards, Water pollution effects(Animals). Bioaccumulation, Bioassay, Environmental monitoring, Gas chromatography, Fish oils, Cod liver oil, Fishes, Whales, Reprints, Cyanobiphenyls, Stationary phase, Blubber, Mackerel.

A novel $p, p$-cyanobiphenyl stationary phase ( $p$ cyanobiphenyl, $p$-allyloxy methlpolysiloxane) has been evaluated for the GC investigation of polychloninated biphenyls (PCBs). Several PCB congeners which coelute on the phases typically used for PCB analysis (e.g. $5 \%$ phenyl methylpolysiloxane) are separated on the p,p-cyanobiphenyl phase, including the hexachlorobiphenyl congers PCB 163, PCB 164, and PCB 138. In this work, a p,p-cyanobiphenyl stationary phase was used to measure selected PCB congeners in two Standard Reference Materials (SRMS) available from the National Institute of Standards and Technology (NIST), and two Certified Reference Materials (CRMs) available from the Community Bureau of Reference (BCR). The materials analyzed were SRM 1588, Organics in Cod Liver Oil; SRM 1945, Organics in Whale Blubber; CRM 349, Chlorobiphenyls in Cod Liver Oil; and CRM 350, Chlorobiphenyls in Macherel Oil. Concentrations are reported for several PCB congeners which coelute on the $5 \%$ phenyl methylpolysiloxane, including PCB 163 and PCB 164.

\section{2,592}

\section{PB96-111778 Not available NTIS}

National Inst. of Standards and Technology (CSTL), Gaithersburg, MD. Analytical Chemistry Div.

Certification of Polycyclic Aromatic Hydrocarbons in a Marine Sediment Standard Reference Material. Final rept.

S. A. Wise, M. M. Schantz, B. A. Benner, M. J. Hays, and S. B. Schiller. 1995, $8 \mathrm{p}$

Pub. in Analytical Chemistry, v67 n7 p1171-1178, 1 Apr 95

Keywords: "Polycyclic aromatic hydrocarbons, "Environmental monitoring, "Reference standards, *Chemical analysis, "Analytical methods, Isomers, Water pollution effects, Test methods, Gas chromatography, Mass spectroscopy, Separation techniques, Fluorescence spectroscopy, Liquid chromatography, Sampling, Certification, Reprints, "Marine sediments, Standard reference materials.
Four different analytical techniques were used for the determination of polycyclic aromatic hydrocarbons (PAHs) in a new marine sediment reference material, SRM 1941 a, Organics in Marine Sediment. These procedures were based on reversed-phase liquid chromatography (LC) with fluorescence detection, a multidimensional LC procedure, and gas chromatography/mass spectrometry on two stationary phases with different selectivity for the separation of PAH isomers. The results from these four aproaches were in good agreement and were combined to provide cerified concentrations for $23 \mathrm{PAHs}$, which represents the largest number of certified concentrations for PAHs in any natural matrix SRM.

\section{2,593}

PB96-123690 Not available NTIS

National Inst. of Standards and Technology (CSTL), Gaithersburg, MD. Organic Analytical Research Div. Certlfication of Standard Reference Material (SRM) 1941a, Organics in Marine Sediment.

Final rept.

M. M. Schantz, B. A. Benner, M. J. Hays, R Demiralp, R. R. Greenberg, S. B. Schiller, G. G. Lauenstein, S. A. Wise, W. R. Kelly, and R. D. Vocke. 1995, 8p.

Pub. in Analytical Chemistry, v352 p166-173 1995.

Keywords: "Bottom sediments, "Water pollution effects, "Chemical analysis, "Reference standards, Environmental monitoring, Certification Marine environments, Pesticides, Polychlorinated biphenyls, Polycylic aromatic hydrocarbons, Aliphatic hydrocarbons, Organic carbon, Trace elements, Sulfur, Gas chromatography, Mass spectroscopy, Reprints, Marine sediments, Standard reference materials.

SRM 1941a, Organics in Marine Sediment, has been recently issued with certified concentrations 23 polycyclic aromatic hydrocarbons, 21 polychlorinated biphenyl congeners, 6 chlorinated pesticides, and sufur. Noncertified concentrations have been also reported for additional PAHs, PCB congeners, and chlorinated pesticides and for percent total organic carbon (TOC), aliphatic hydrocarbons, and trace elements. SRM 1941a is the most extensively characterized natural matrix SRM issued by the National Institute of Standards and Technology (NIST).

\section{2,594}

B96-131560 PC A03/MF A01

National Inst. of Standards and Technology (BFRL), Gaithersburg, MD.

Smoke Plume Trajectory from In situ Burning of Crude Oil in Alaska: Field Experiments.

K. B. McGrattan, W. D. Walton, A. D. Putorti, D. D.

Evans, W. H. Twilley, and J. McElroy. Nov 95, 42p NISTIR-5764

See also PB94-114519. Sponsored by Alaska State Dept. of Environmental Conservation, Juneau. Div. of Spill Prevention and Response.

Keywords: "Oil spills, "In situ combustion, "Smoke, "Plumes, Atmospheric diffusion, Burning rate, Emulsions, Particulates, Combustion kinetics, Combustion properties, Crude oil, Oil pollution control, Water pollution control, Field tests, Fire tests, Comparison, LES(Large Eddy Simulation).

In the present report, experimental data collected at two sets of mesoscale burns are compared with the results of the LES model run using the recorded meteorological and physical conditions. The two experiments are the Newfoundland Offshore Burn Experiment (NOBE), Aug. 1993, and the Alaska Clean Seas Burning of Emulsions, Sept. 1994. Each series o bums was conducted under different conditions, and different data collection techniques were employed a each. The results show that the predictions of the LES model are in good agreement with the experimental measurements, given the uncertainty if the input parameters.

\section{2,595}

PB96-167127 Not available NTIS

National Inst. of Standards and Technology (CSTL), Gaithersburg, MD. Analytical Chemistry Div.

Trace Element Concentrations in Cetacean Liver Tissues Archived in the National Marine Mammal Tissue Bank.

Final rept.

E. A. Mackey, R. Demiralp, P. R. Becker, S. A. Wise, R. R. Greenberg, and B. J. Koster. 1995, 17p

See also PB95-161402.

Pub. in Science of the Total Environment, v175 p25411995.
Keywords: "Bioaccumulation, "Marine mammals, "Trace elements, Dolphins, Reprints, "Foreign technology, Harbor porpoise, Neutron activation analysis, Pilot whales, Specimen banking.

The National Biomonitoring Specimen Bank (NBSB), a collaborative project of several U.S. government agencies, includes manine mammal tissues collected for the Alaska Marine Mammal Tissue Archival Projec (AMMTAP) and the National Marine Mammal Tissue Bank (NMMTB). Tissues were collected from 139 animals representing 13 species of marine mammals from around the U.S. Recently, concentrations for up to 30 elements in liver tissues of nine long-finned pilot whales (Globicephala melas), six harbors porpoises (Phocoena phocoena), and four white-sided dolphins (Lagenorhyunchus acutus) from the NMMTB were measured using instrumental neutron activation analysis. Results from analysis of these tissues are presented, compared with results for liver tissues from other marine mammals from the AMMTAP, and compared with published values.

\section{2,596}

PB96-167275 Not available NTIS

National Inst. of Standards and Technology (CSTL), Gaithersburg, MD. Inorganic Analytical Research Div. Relationship of Silver with Selenium and Mercury in the Liver of Two Species of Toothed Whales (Odontocetes)

Final rept.

P. R. Becker, E. A. Mackey, R. Demiralp, B. J.

Koster, S. A. Wise, R. Suydam, and G. Early. 1995,

$10 \mathrm{p}$ 1995.

Keywords: "Bioaccumulation, "Marine mammals, "Water pollution effects(Animals), Liver, Mercury, Selenium, Silver, Reprints, "Foreign technology, "Instrumental neutron activation analysis, Pilot whale, Beluga whale.

Liver specimens archived in the National Biomonitoring Specimen Bank from beluga whales, Delphinapterus leucas, and from Alaska and pilot whales, Globicephala melas, from the North Atlantic were analysed for silver, selenium and total mercury. Silver concentrations in beluga whales were one to three orders of magnitude higher than the concentrations in pilot whales and those reported elsewhere for the other marine mammals. The concentrations of silver in the livers of beluga whales were the same or in some instances higher than the concentrations of selenium or mercury, Like mercury, silver was positively correlated with selenium in both pilot and beluga whales. This suggests a possible role for selenium in the accumulation and storage of silver in both species of whales, and raises questions about the potential for silver a such high concentrations to affect radical-scavenging enzyme systems in these manine mammals.

\section{2,597}

PB96-200993 Not available NTIS

National Inst. of Standards and Technology (BFRL), Gaithersburg, MD. Fire Safety Engineering Div.

Smoke Plume Trajectory from In situ Burning of Crude Oil: Field Experiments.

Final rept

K. B. McGrattan, R. J. Ferek, and E. E. Uthe. 1995, $6 p$.

See also PB96-131560

Pub. in International Conference on Fire Research and Engineering, Orlando, FL., September 10-15, 1995 p47-52.

Keywords: *Oil spills, *In situ combustion, *Atmospheric diffusion, "Combustion products, Smoke, Plumes, Particulates, Atmospheric transport Burning rate, Combustion kinetics, Oil pollution control, Fire tests, Reprints.

There is growing interest in the environmental consequences of large fires, since the transport of combustion products by a windblown fire plume can distribute potentially hazardous materials over a wide area. Pools of burning oil and other petroleum products are of particular concern due to the vast flow of these materials through the global economy and because of the fragility of the environment in many regions where oil is extracted or transported. The present work is part of a larger study of a closely related issue, the feasibility of cleaning up oil spills on water through buming

02,598

PB97-119226 Not available NTIS 
National Inst. of Standards and Technology (CSTL), Gaithersburg, MD. Analytical Chemistry Div.

National Status and Trends Program Specimen Bank: Sampling Protocols, Analytical Methods, Results, and Archive Samples.

Final rept.

G. Lauenstein, A. Cantillo, B. Koster, R. Zeisler, S.

Wise, M. Schantz, and S. Stone. 1996, 168p.

See also PB89-175855.

Pub. in NOAA Technical Memorandum NOS ORCA 98, v98 168p 1996.

Keywords: "Sediments, "Tissue banks, "Environmental monitoring, "Reference standards, "Animal tissues, Aquatic animals, Fish, Oysters, Mussels, Marine mammals, Biological effects, Bioaccumulation, Bioassay, Chemical analysis, Sampling, Quality assurance, Reprints, National Status and Trends Program.

The National Oceanic and Atmospheric Administration's (NOAA) National Status and Trends (NS\&T) Program monitoring components, the National Benthic Surveillance and Mussel Watch Projects, have archived tissues and sediments in a specimen bank since 1985 and 1986, respectively. Additional samples were archived after the Exxon Valdez oil spill in Prince Williams Sound, Alaska. Samples from the Environmental Protection Agency's Mussel Watch Program, collected from 1976 to 1978 , were added to the NS\&T Specimen Bank in 1992. Contaminant analyses were performed to provide additional data on environmenta quality and to ensure that data existed for sample stability studies. Samples in the NS\&T Specimen Bank are available for retrospective analyses should a contaminant or group of contaminants be identified that was introduced into the environment during the years of the NS\&T Program or during the EPA's Mussel Watch Program but that were not previously measured.

\section{HEALTH CARE}

\section{Health Care Technology}

\section{2,599}

PB96-160544 Not available NTIS

National Inst. of Standards and Technology (CSL), Gaithersburg, MD. Advanced Systems Div.

Prototyping a Graphical User Interface for DHCP. Final rept.

W. J. Majurski, R. E. Dayhoff, and D. L. Maloney.

1992, 11p.

Pub. in MUMPS Computing: MUG Proceedings Issue, Phoenix, AZ., June 15-19, 1992, 11p.

Keywords: "Biomedical measurement, "Graphical user interface, "Computer programs, Computer graphics, Image processing, Software engineering, Operating systems(Computers), Reprints, DHCP(Decentralized Hospital Computing Program), Medical imaging, $X$ windows, MIMPS(Massachusetts General Hosptial Utility Multi-Programming System).

We have started looking at how a large end user application, the Department of Veterans Affairs' DHCP, can adapt to this changing environment. We describe a minimal prototype $X$ window binding that we have constructed and modifications to several DHCP subsystems to adapt to it. We then discuss the types of changes that may be needed to adapt DHCP to a graphical user interface environment.

\section{INDUSTRIAL \& MECHANICAL ENGINEERING}

\section{General}

\section{2,600}

PB94-196029 PC A03/MF A01

National Inst. of Standards and Technology (TS),

Gaithersburg, MD. Metric Program.

Federal Metric Progress in 1993.

G. P. Carver. Apr 94, 15p, NISTIR-5413.

See also PB89-114490.

Keywords: "Metric systems, Handbooks, Units of measurement, Government agencies, Interagency cooperation, Natlonal govemment.

During 1993, cooperation among the federal agencies on interagency issues led to more uniform metric transition approaches Individually, agencies made significant progress in implementing metric use in their programs. However, the amount of progress by different agencies also showed significant variation. Cooperative progress included; Acquisition Guidelines; Functional subcommittees; Strategic Approach; Public Affairs; Metric Transition Plan Revision; Expanded Agency-Industry Cooperation; and Federal Standard 376.

02,601

PB94-206307 PC A02/MF A01

National Inst. of Standards and Technology (TS), Gaithersburg, MD. Metric Program

Metric Path to Global Markets and New Jobs: A Question-and-Answer and Thematic Discussion. G. P. Carver. Jun 94, 9p, NISTIR-5463.

Keywords: "Metric system, "Job creation, Global aspects, Govemment policies, Exports, Competitiveness, International trade.

The logic for use of the metric system in the United States is strongest when metric use is viewed as an element of our national economic infrastructure, as an investment in efficiency, and as a necessity for internatioal competitiveness.

\section{2,602}

PB95-226916 PC A05/MF A01

National Inst. of Standards and Technology (CSTL), Gaithersburg, MD. Process Measurements Div

Laser Doppler Velocimeter Studies of the Pipeflow Produced by a Generic Header.

Technical note.

T. T. Yeh, and G. E. Mattingly. Apr 95, 79p, NIST/ TN-1409.

Also available from Supt. of Docs.

Keywords: "Flow measurement, "Flow meters, Fluid flow, Installation effects, LDV, Header flow.

The report presents recent results obtained in a consortium-sponsored research program on flowmeter installation effects being conducted at NISTGaithersburg, MD. The piping element tested and reported here is generic header which has a specific arrangement of one inlet and two outlets. LDV velocity measurements are reported for the pipeflows produced downstream of the header with and without a conventional 19-tube concentric tube bundle flow conditioner. The results indicate that the secondary flow produced by the generic header is very similar to that produced by the closely-coupled double elbows out-of-plane piping configuration. The axial velocities are slower than the ideal flow near the pipe center and higher near the walls. Transverse velocity distributions show a very strong swirl eddy flow pattem produced by this header. This swirl eddy is relatively stable and organized and its dissipation is slower than that observed for the double elbows out-of-plane. The 19-tube tube bundle is quite effective in reducing the transverse velocities associated with the energetic, swirl flow from this header. Several profile indexes are introduced and presented for the header flow to characterize its swirt, profile peakness and flow displacements. These indexes can also be used to develop criteria for improving flow meter performance in the outlet piping from this header.

\section{2,603}

PB95-255824 PC A03/MF A01

National Inst. of Standards and Technology (BFRL), Gaithersburg, MD. Building Environment Div.

Simple Method of Composition Shifting with a DlstIllation Column for a Heat Pump Employing a Zeotropic Refrigerant Mlxture.

P. I. Rothfleisch. Jul 95, 30p, NISTIR-5689.

Sponsored by Department of Energy, Washington, DC Building Equipment Div. and Environmental Protection Agency, Research Triangle Park, NC. Air and Energy Engineering Research Lab.

Keywords: "Refrigerants, "Air conditioning equipment, "Heat pumps, Refrigerating, Chemical composition, Distillation, Columns(Process engineering), Zeotropic mixtures.

The work presents a simplified method of controlling heat pump capacity by shifting the composition of a zeotropic refrigerant mixture with a distillation column. Simplicity is achieved by incorporating the distillation column into the typical suction accumulator used by residential heat pumps. A U.S. patent has been applied for under the title Accumulator Distillation Insert for Zeotropic Mixtures. An experimental system employing this distillation concept has been evaluated in the laboratory for zeotropic mixtures of R32/134a (30/70) and $\mathrm{A} 32 / 125 / 134 \mathrm{a}(23 / 25 / 52)$. For the binary mixture the circulating refrigerant composition was shifted to $\mathrm{R} 32$ $134 a(54 / 46)$. For the ternary mixture the circulating refrigerant composition was shifted to R32/125/134a (36/ $36 / 28$ ).

02,604

PB96-123146 Not available NTIS National Inst. of Standards and Technology (EEEL) Gaithersburg, MD. Electricity Div.

Effects of Nonmodel Errors on Model-Based Testing.

Final rept

G. N. Stenbakken. 1995, 5p.

Pub. in Proceedings of Instrumentation and Measure. ment Technology Conference, Waltham, MA., April 24. $26,1995, \mathrm{p} 38-42$.

Keywords: "Confidence intervals, Simulation, Testing, Empirical modeling, Reprints, "Nonmodel errors, Prediction intervals.

In previous work, methods have been developed for efficient testing of components and instruments that are based on models of these units. These methods allow for the full behavior of these units to be predicted from a small but efficient set of test measurements. Such methods can significantly reduce the testing cost of such units by reducing the amount of testing required. But these methods are valid only as long as the model accurately represents the behavior of the unit. Previous papers on this subject described many methods for developing accurate models and using them to develop efficient test methods. However, they gave little consideration to the problem of testing units which change their behavior after the model has been developed, for example, as a result of changes in the manufacturing process. Such changed behavior is referred to as nonmodel behavior or nonmodel error. When units with this new behavior are tested with these more efficient methods, their predicted behavior can show significant deviations from their true behavior. This paper describes how to analyze the data taken at the reduced set of measurements to estimate the uncertainty in the model predictions, even when the device has significant nonmodel error. Results of simulation are used to verify the accuracy of the estimates and to show the expected.

\section{2,605}

PB96-165956 PC A04/MF A01

National Inst. of Standards and Technology (BFRL), Gaithersburg, MD. Building Environment Div. Enhancement of R123 Pool Boiling by the Additlon of N-Hexane.

M. A. Kedzierski. Mar 96, 45p, NISTIR-5780 See also PB96-128129. Sponsored by Department of Energy, Washington, DC.

Keywords: "Pool boiling, Hexanes, Heat transfer, Thermal conductivity, Surface treatment, Heat flux, Mixtures, Natural convection, Thermodynamic properties, Performance tests, " $\mathrm{N}$-hexane, ${ }^{\text {* }} \mathrm{R}-123$ refrigerant. 
The paper presents the heat transfer data used to file international patent WO 94/18282. The data consisted of pool boiling performance of a GEWA-T(trademark) surface for three fluids: (1) pure R123, (2) R123/nhexane (99/1), and (3) R123/n-hexane (98/2). The heat flux and the wall superheat were measured for each fluid at $277.6 \mathrm{~K}$. The observations were used to describe various boiling modes on the GEWA$T$ (trademark) surface. The addition of hexane to pure R123 caused a simultaneous reduction in the bubble diameter and increase in the site density. The increase in site density enhanced the boiling despite the reduction in bubble size. Presumably, the site density enhancement was caused by a layer enriched in hexane at the heat transfer surface. The addition of hexane to R123 also improved natural convection.

\section{2,606}

\section{PB96-210893 PC A07/MF A02}

National Inst. of Standards and Technology (CSTL), Gaithersburg, MD. Process Measurements Div.

Flowmeter installation Effects Due to a Generic Header.

Technical note

T. T. Yeh, and G. E. Mattingly. Jul 96, 111p, NIST/ TN-1419.

Also available from Supt. of Docs. as SN003-00303415-1.

Keywords: "Flowmeters, *Orifice flow, *Installation, Orifice meters, Turbines, Orifices, Flow measurement, Inlets, Outlets, Pipe flow, Fluid flow, Discharge coefficient, Performance evaluation, Graphs(Charts), Tables(Data), "Headers.

This report presents recent results obtained in a consortium-sponsored research program on flowmeter installation effects being conducted at NISTGaithersburg, MD. The piping element tested and reported here is a generic header which has a specific arrangement of one inlet and two outlets. The results reported here are for the outlet nearest the inlet operated fully open with the other outlet fully closed. The meter performance measured in this outlet are reported with and without the installation of a conventional 19-tube concentric tube bundle flow conditioner.

\section{Environmental Engineering}

\section{2,607}

PB95-216917 PC A03/MF A01

National inst. of Standards and Technology (BFRL),

Gaithersburg, MD. Building Environment Div.

Theoretical Evaluation of the Vapor Compression

Cycle with a Liquid-Line/Suction-Line Heat Exchanger, Economizer, and Ejector.

P. A. Domanski. Mar 95, 41p, NISTIR-5606.

Keywords: *Air conditioning, *Ejectors, "Heat exchang ers, Refrigeration, Heat transfer, Feedwater heaters, Efficiency, "Vapor compression cycle, Liquid-line/suction-line heat exchange.

The report presents a theoretical analysis of three vapor compression cycles which are derived from the Rankine cycle by incorporating a liquid-line/suction-line heat exchanger, economizer, or ejector. These addendums to the basic cycle reduce throttling losses using different principles, and they require different mechanical hardware of different complexity and cost. The theoretical merits of the three modified cycles were evaluated in relation to the reversed Carnot and Rankine cycle. Thirty-eight fluids were included in the study using the Carnahan-Starling-DeSantis equation of state. In general, the benefit of these addendurns increases with the amount of the throttling losses realized by the refrigerant in the Rankine cycle.

\section{Hydraulic \& Pneumatic Equipment}

02,608
PB95-128906 PC A04/MF A01

National Inst. of Standards and Technology (CSTL), Boulder, CO. Process Measurements Div.
Uncertainty Analysis of the NIST Nitrogen Flow Facility.

Technical note.

J. L. Scott, and M. A. Lewis. Mar 94, 56p, NIST/TN1364

Supersedes COM-71-150325. Also available from Supt. of Docs. as SN003-003-03294-8. See also PB93-159465

Keywords: *Test facilities, Cryogenic equipment, Cryogenic fluids, Liquid nitrogen, Gas flow, Mass flow, Error analysis, Statistical analysis, Orifice meters, Flow meters, Sensitivity, Buoyancy, Density, "Nitrogen flow facility, "Measurement uncertainty, Discharge coefficients, US NIST

An uncertainty analysis of the National Institute of Standards and Technology nitrogen flow facility was performed. The facility functions as a cryogenic flow calibration laboratory and as an applied research laboratory for high pressure nitrogen gas flow measurement. The report includes the analysis of uncertainty in instrumentation, liquid nitrogen mass flow, gaseous nitrogen mass flow determined by a system turbine meter, and the uncertainty in orifice meter discharge coefficient calculation using a defined propagation of errors technique. Uncertainties determined by statistical and non-statistical means are presented separately.

\section{2,609}

PB95-175832 Not available NTIS

National Inst. of Standards and Technology (CSTL), Gaithersburg, MD. Thermophysics Div.

PC-Based Spinning Rotor Gage Controller.

Final rept.

J. P. Looney, F. G. Long, D. F. Browning, and C. R.

Tilford. 1994, 8p.

Pub. in Review of Scientific Instruments 65, n9 p30123019 Sep 94.

Keywords: "Pressure measurement, "Vacuum gages, "Rotor dynamics, Vacuum apparatus, Measuring instruments, Rotors, Signal processing, Data analysis Controllers, Reprints, "Spinning rotor gage controllers.

The spinning rotor gage (SRG) is a molecular drag vacuum operate between about $10(\exp -4)$ and $1 \mathrm{~Pa}$. In this paper we describe the design, and present performance data for a new SRG controller that uses a plugin peripheral board and a personal computer $(\mathrm{PC})$ to perform all control, signal processing, and data analysis functions. This controller offers several advantages over those presently available, including the simultaneous operation of multiple SRGs (at least four SRGs with a single PC), complete sof tware control of all operating parameters, and significantly improved low-pressure performance.

\section{Industrial Safety Engineering}

\section{2,610}

PB95-174975 PC A04/MF A01

National Inst. of Standards and Technology (BFRL), Gaithersburg, MD. Fire Science Div.

Protection of Data Processing Equipment with Fine Water Sprays.

Annual rept. Sep 93-Sep 94.

W. Grosshandler, D. Lowe, K. Notarianni, and W

Rinkinen. Oct $94,60 \mathrm{p}$, NISTIR-55†4.

See also PB93-219780. Sponsored by Fire Administration, Emmitsburg, MD.

Keywords: *Data processing equipment, *Fire protection, "Sprinkling, Fire fighting, Drops(Liquids), Size determination, Spraying, Nozzle geometry, Concentration(Composition), Velocity distribution, Fire extinguishing agents, Electronic equipment, "Fine water sprays, Halon 1301.

The major objective of the work presented here has been to determine how a fine water spray compares to a gaseous agent in extinguishing fires in data processing equipment, an environment typically protected by halon 1301. A scaled-down, generic electronics package was designed and a chamber built to contain the water spray to emulate the physical system of interest. The mock electronics cabinet is $0.5 \mathrm{~m}$ wide, 0 ? $\mathrm{m}$ deep and $0.4 \mathrm{~m}$ high. The fuel is a $3 \mathrm{~mm}$ thick plate of poly(methyl methacrylate), placed vertically in an aluminum frame centered among a number of alu- minum circuit boards. The limitations imposed by the different transport phenomena associated with droplet versus gas dispersion have been investigated. The influence on extinguishing efficiency of the nozzles geometry, the location relative to the fire, the water application rate, and the amount of shielding surrounding the fire within the simulated cabinet are all parameters which have been examined. A gaseous agent, CF3H is used for comparison. A phase-Doppler particle analyzer measured the droplet size distribution and velocity. The water pressure has a significant effect on the size of the region in which a fire can be effectively suppressed. By contrast, similar fires in all geometric configurations can be successfully extinguished with $\mathrm{CF} 3 \mathrm{H}$ as long as the concentrations in the chamber are close to those recommended in NFPA 2001.

\section{2,611}

PB95-270062 PC A03/MF A01

National Inst. of Standards and Technology (BFRL), Gaithersburg, MD.

Proceedings of the 1995 Workshop on Fire Detector Research. Held on February 6-7, 1995.

W. L. Grosshandler. Jun 95, 46p, NISTIR-5700. See also PB95-189452.

Keywords: "Fire detection systems, "Research and development, "Meetings, Technology assessment, Smoke, Fire safety.

A workshop was convened February 6 and 7, 1995 to indentify the needs of users and specifiers of fire detection systems which are not currently being met by the U.S. fire protection industry. A series of experts from industry, government, certifying organizations and academia were invited to review the various applications for fire detection systems and to discuss recen development that could impact the future of the industry. The speakers were divided into focused panels of users and specifiers, systems and components manufactureres, regulators and certifiers, and researchers. Small working groups were convened after the panel discussions to identify critical research issues, concentrating on sensors, signal processing, systems integration and regulations.

\section{Job Environment}

\section{2,612}

PB97-122485 Not available NTIS

National Inst. of Standards and Technology (PL) Gaithersburg, MD. Ionizing Radiation Div.

Overview of a Radiation Accident at an Industrial Accelerator Facility.

Final rept.

D. A. Schauer, B. M. Coursey, C. E. Dick, M. F Desrosiers, A. D. Jacobson, W. L. McLaughlin, ano J. M. Puhl. 1994, 4p.

See also PB95-140117. Sponsored by Armed Forces Radiobiology Research Inst., Bethesda, MD.

Pub. in Advances in the Biosciences, v94 p321-324 1994.

Keywords: "Accelerator facilities, *Accident investiga. tions, "Occupational safety and health, Industrial acci dents, Occupational exposure, Radiation dosage, Ra diation injuries, Partial body irradiation, Dosimetry, Biological radiation effects, Radiation accidents, Reprints, Dose reconstruction, EPR(Electron Paramagnetic Resonance).

On 11 December 1991, a radiation overexposure occurred at an industrial radiation facility in Gaithersburg Maryland. During maintenance on the lower window pressure plate, an operator placed his hands, head and feet in the beam. The operator's body, especially his extremities and head, were exposed to electron dark current. After prolonged periods of inactivity, particularly after the vacuum in the accelerator tube is lost an electron bearn current can be present even if the electron filament is cold or dark.

\section{Laboratory \& Test Facility Design \& Operation}

02,613

AD-A279 240/6 PC A03/MF A0 
National Bureau of Standards, Gaithersburg, MD. Standard Samples and Reference Standards Issued by the National Bureau of Standards.

31 Aug 54, 28p, NBS-CIRC-552.

Keywords: "Standards, "Scientific literature, Sampling, Circular, Periodicals, Weight, Procurement, Tables(Data), Chemical composition, Physical properties, Metals, Ceramic materials, Chemicals, Spectroscopy, Ph factor, Viscosity, Melting point, Density, Refractive index, Heat of combustion, "Reference standards, "Standard samples, Fees, Ores.

No abstract available.

\section{2,614}

AD-A279 948/4 PC A05/MF A01

National Bureau of Standards, Gaithersburg, MD

Reference Tables for Thermocouples.

H. Shenker, J. I. Lauritzen, R. J. Corruccini, and S.

T. Lonberger. 27 Apr 55, 89p.

Keywords: *Thermocouples, Tables(Data), Platinum, Rhodium Copper, Electrical measurement, Calibration, Reference tables, Constantan, Chromel, Temperature measuring instruments.

No abstract available.

\section{2,615}

AD-A279 949/2 PC A05/MF A01

National Bureau of Standards, Gaithersburg, MD.

Corrected Optical Pyrometer Readings.

D. E. Poland, J. W. Green, and J. L. Margrave. 21

Apr 61, 77p.

Keywords: "Pyrometers, Optical properties, Conversion, Emissivity, Tables(Data), "Temperature measurement.

The table of corrected optical pyrometer readings enables optical pyrometer users to convert observed temperature immediately to the true temperature, if the ef fective emissivity of the material being observed is known. The table gives observed temperatures from 1,000 to $3,000 \mathrm{~K}$ in increments of 5 degrees, from 3,000 to $5,000 \mathrm{~K}$ in increments of 10 degrees, from 5,000 to $10,000 \mathrm{~K}$ in increments of 50 degrees, and from 10,000 to $39,900 \mathrm{~K}$ in increments of 100 degrees. For these, true temperatures are tabulated for 49 emissivities ranging from 0.02 to 0.98 in increments of 0.02 . These calculations were made on a 650 electronic computer using Planck's law, and the value c sub $2=1,438 \mathrm{~cm}$ deg for the radiation constant.

\section{2,616 \\ AD-A280 086/0 PC A03/MF A01}

National Bureau of Standards, Gaithersburg, MD.

Federal Basis for Weights and Measures: A Historical Review of Federal Legislative Effort, Statutes, and Administrative Action in the Field of Weights and Measures in the United States.

R. W. Smith. 5 Jun $58,27 p$

Keywords: "Measurement, Weight, Federal law, Standardization, Standards, Length, Mass, Capacity(Quantity), "Weights and measures, Government policies.

No abstract available.

02,617

AD-A280 278/3 PC A08/MF A02

National Bureau of Standards, Gaithersburg, MD.

Guide to Instrumentation Literature.

W. G. Brombacker, J. F. Smith, and L. M. Van der

Pyl. 14 Dec 55, 162p, NBS-567.

Keywords: "Instrumentation, "Literature surveys, *Measuring instruments, Standards, Abstracts, Bibliographies, Scientific literature, Biology, Medicine, Engineering, Electronics, Physics, Chemistry, "Guides, *Source list.

No abstract available.

\section{2,618}

\section{AD-A280 562/0 PC A03/MF A01}

National Bureau of Standards, Gaithersburg, MD. Precision Laboratory Standards of Mass and Laboratory Weights.

T. W. Lashof, and L. B. Macurdy. 20 Aug 54, 28p.

Keywords: "Laboratory equipment, "Weight, "Standards, "Mass, "Balances, Precision, Calibration.

No abstract available.
02,619

AD-A286 618/4 PC A03/MF A01

National Bureau of Standards, Boulder, $\mathrm{CO}$.

Design and Construction of a Liquid Hydrogen

Temperature Refrigeration System.

D. B. Chelton, J. W. Dean, and B. W. Birmingham. 12 Jan $60,42 p, T N-38$.

Keywords: "Refrigeration systems, "Liquid hydrogen, "Low temperature, "Bubble chambers, Design criteria Construction, Circuits, Control systems, Heat exchangers, Physics, Nuclear instrumentation.

No abstract available.

02,620

DE93019682 PC A02/MF A01

National Inst. of Standards and Technology, Boulder, CO. Thermodynamics Div.

Development of a Dual-Sinker Densimeter for HighAccuracy Fluid P-V-T Measurements. Appendix A. M. O. McLinden, and N. V. Frederick. 1993, 8p,

CONF-9305134-3-APP.A.

Contract Al05-88ER13823

Symposium on Energy Engineering Sciences (11th) Argonne, IL (United States), 3-5 May 1993. Sponsored by Department of Energy, Washington, DC.

Keywords: *Densimeters, Design, Pressure Measurement, Temperature Measurement, Thermostats, Volume, EDB/440500.

A dual-sinker densimeter to very accurately measure the pressure-volume-temperature $(\mathrm{P}-\mathrm{V}-\mathrm{T})$ properties of fluids over a temperature range of $80 \mathrm{~K}$ to $520 \mathrm{~K}$ and at pressures up to $35 \mathrm{MPa}$ is in the final stages of development at NIST. The density of a fluid is determined by measuring the difference in the buoyancy forces experienced by two sinkers of identical mass, surface area, and surface material, but very different volumes. The buoyancy forces on the sinkers are transmitted to a semi-microbalance by means of a magnetic suspension coupling. This paper reviews the principle of the measurement and describes the overall design of the system.

02,621

\section{DE95005780 PC A03/MF A01}

National Inst. of Standards and Technology (NEL) Gaithersburg, MD. Automated Production Technology Div.

Minutes of the CAALS Workshop on modularity and communications standards.

G. W. Kramer. 1991, 11p, DOE/EW/50009-T2

Contract Al01-90EW5000'9

Sponsored by Department of Energy, Washington, DC

Keywords: "Measuring Instruments, Communications Equipment Interfaces, Meetings, Standards, EDB/ 426000.

This Workshop was organized similarly to the previous meeting. After a presentation on Instrument Interfacing, three of the four Working Groups met individually. The Strategic Planning Committee did not meet at this Workshop, but it held a meeting in April; the minutes of that meeting are included. The Physical link and Transport Committee has defined the requirements for the link connecting modules to their controller, examined a new ASTM standard for low-level communication, and is determining what fits into to the various layers of the ISO model. The Module Requirements Group has proposed a process control model, has defined the core functionality, and has listed the requirements for a Standard Laboratory Module. The Software Engineering Group proposed that a document detailing the CAALS Modularity Architectural Specification be prepared and presented a working outline. The future structure and charters of the committees and the decision making criteria of the Workshop itself were questioned. These items will be examined in greater detail at the next Workshop.

02,622

N95-15937/2 (Order as N95-15919/0, PC A11/ MF A03)

National Inst. of Standards and Technology, Gaithersburg, MD

Numeric Data Distribution: The Vital Role of Data Exchange in Today's World.

M. W. Chase. 14 Sep $94,2 p$

In NASA, Washington, Fourth Annual International AC quisitions Workshop: Access to Multiple Media Worldwide $2 p$

Keywords: "Accuracy, "Dimensional measurement, "Information dissemination, "Measure and integration,
"Numerical data bases, "Reference systems, "Reliability, "Standards, Chemical properties, Feedback, On-line systems, Physical properties.

The major aim of the NIST Standard Reference Data Program (SRD) is to provide critically evaluated numeric data to the scientific and technical community in a convenient and accessible form. A second aim of the program is to provide feedback into the experimental and theoretical programs to help raise the general standards of measurement. By communicating the experience gained in evaluating the world output of data in the physical sciences, NIST/SRD helps to advance the level of experimental techniques and improve the reliability of physical measurements

02,623

PB94-138989 PC A18/MF A04

National Inst. of Standards and Technology,

Report of the National Conference on Weight and Measures (78th). Held in Kansas City, MO. on July 18-22, 1993 .

Special pub.

C. S. Brickenkamp, and A. H. Turner. Oct 93, 425p, NIST/SP-854.

Also available from Supt. of Docs. as $\mathrm{SNO03}^{-003-}$ 03245-0. Library of Congress catalog card no. 2627766

Keywords: "Weight measurement, "Metrology, "Meetings, US NIST, Regulations, Laws(Legislation) Standards, Quality control, Kansas City(Missouri), Legal metrology.

The 78th Annual Meeting of the National Conference on Weights and Measures (NCWM) was held July 18 through 22, 1993, at the Ritz-Carlton Hotel, Kansas City, Missouri. The theme of the Meeting was 'Excellence Through Standards'. Reports by the standing and annual committees of the Conference comprise the major portion of the publication, along with the addresses delivered by Conference officials and other authorities from government and industry. Special meetings included those of the Metrologists, the Associate Membership Committee, the Retired Officials Committee, the Scale Manufacturers' Association, the American Petroleum Institute, the Industry Committee on Packaging and Labeling, the regional weights and measures associations, the National Association of State Departments of Agriculture Weights and Measures Division and the National Council on State Metrication.

02,624

PB94-142478 PC A03/MF A01

National Inst. of Standards and Technology (CSTL), Gaithersburg, MD. Process Measurements Div.

Assessment of Uncertainties of Calibration of Resistance Thermometers at the National Institute of Standards and Technology.

G. F. Strouse, and W. L. Tew. Jan 94, 19p, NISTIR. 5319

See also PB93-159465.

Keywords: "Resistance thermometers, "Calibration, International agreements, Temperature scales, Platinum, Estimates, "Measurement uncertainty, ITS-90, US NIST

The National Institute of Standards and Technology (NIST) has adopted a new policy for the expression of measurement uncertainty, effective 1 January 1994 that is consistent with recommendations from the Comite International des Poids et Mesures. Using this new method of expressing uncertainties, this paper gives the expanded uncertainties for NIST-calibrated resistance thermometers (from $0.65 \mathrm{~K}$ to $1234.93 \mathrm{~K}$ ) that are suitable as either defining interpolating devices of the International Temperature Scale of 1990 (ITS90 ) or as scale transfer thermometers.

02,625

PB94-142510 PC A03/MF A01

National Inst. of Standards and Technology (CSTL),

Gaithersburg, MD. Process Measurements Div.

Assessment of Uncertainties of Liquid-in-Glass Thermometer Calibrations at the National Institute of Standards and Technology.

$J$. A. Wise. Jan $94,23 p$, NISTIR-5341. See also PB89-128888 and PB94-142478.

Keywords: "Temperature measurement, "Thermometers, "Calibration, Calibration standards, Uncertainty, "Liquid-in-glass thermometers, US NIST. By January 1, 1994, the method recommended by the International Committee of Weights and Measures in 
1981 for expressing uncertainties for calibrations will be implemented at the National Institute of Standards and Technology. This paper explains how the Type A and Type B standard uncertainties and the combined uncertainty for liquid-in-glass thermometer calibrations are computed.

\section{2,626}

PB94-160785 PC A03/MF A01

National Inst. of Standards and Technology (MEL), Gaithersburg, MD

Comparison of Finite Element and Analytic Calculations of the Resonant Modes and Frequencies of a Thick Shell Sphere.

D. E. Gilsinn, H. J. Hansen, Y. XU, M. Huerta-

Garnica, and E. C. Teague. May $91,24 p$, NISTIR4568.

Prepared in cooperation with Tianin Univ. (China). and Centro de Investigacion y de Estudios Avanzados, Mexico City.

Keywords: High resolution, Dimensional measurement, Boundary value problems, Finite element analysis, Spherical shells, Resonant frequency, Simulation Vibration, Comparison, "Coordinate measuring machines, "Molecular Measuring Machine, Normal mode analysis.

An ultra-high accuracy planar coordinate measuring machine, called the Molecular Measuring Machine (M(sup 3)) is being built at the National Institute of Standards and Technology (NIST). The design goal is to achieve a spatial resolution of $0.1 \mathrm{~nm}$ over an area of $50 \mathrm{~mm} \times 50 \mathrm{~mm}$. This goal requires that gravity loading and vibration response of a complicated mechanical structure be modeled to a high accuracy. Finite element analysis (FEA) was applied in the design of $M($ sup 3 ) as analytical modeling of the complicated structure was precluded. In order to achieve confidence in a specific FEA package, normal mode analysis results for a thick spherical shell that represented a first approximation to the $M($ sup 3$)$ structure were compared to those predicted by another FEA package and to analytic results of the thick shell problem. The analytic model predicted degenerate modes due to the structural symmetry of the sphere. These were confirmed subsequently by both of the FEA packages. The FEA and analytic results differ by less than $5 \%$.

\section{2,627}

PB94-160850 PC A03/MF A01

National Inst. of Standards and Technology (TS), Gaithersburg, MD. National Voluntary Lab. Accreditation Program.

NVLAP Procedures U.S. Code of Federal Regulations. Title 15, Subtitle A, Chapter 2, Part 7. (Effective December 1984; Amended September 1990). J. Horlick, and H. M. Richmond. Nov $90,22 p$, NISTIR-4493.

Keywords: "Laboratories, "Accreditation, Administrative procedures, Requirements, Quality assurance, "National Voluntary Laboratory Accreditation Program, "NVLAP system.

The NVLAP Procedures are a part of the U.S. Code of Federal Regulations and set forth the conditions under which the National Voluntary Laboratory Accreditation Program (NVLAP) will function. The Procedures contain general information about NVLAP requirements for establishing a program in a specific field of testing, the process for accrediting a laboratory, and conditions and criteria for accreditation. NVLAP is a system for accrediting testing laboratories found competent to perform specific tests and types of tests. Competence is defined as the ability of a laboratory to meet conditions and conform to criteria specified in the Procedures as tailored and interpreted for the test methods, types of test methods, products, services, or standards for which the laboratory seeks accreditation.

\section{2,628}

PB94-161932 PC A03/MF AO1

National Inst. of Standards and Technology (MSEL), Gaithersburg, MD. Polymers Div.

Recertification of the Standard Reference Material 1475 A, a Linear Polyethylene Resin.

C. M. Guttman, and J. R. Maurey. Oct 93, 36p

NISTIR-5199.

See also PB93-159465.

Keywords: "Certification, "Calibration standards, "Polyethylenes, "References(Standards), US NIST, Quality assurance, Performance testing, Melt viscosity, Chromatographic analysis, Size determination, Or- ganic polymers, Molecular weight, "Standard reference material 1475A, Measurement uncertainty.

The size exclusion chromatography (SEC) and the melt flow rate of Standard Reference Material (SRM) $1475 \mathrm{a}$, a polyethylene resin, were determined and compared with measurements on the samples of SRM 1475. Within the errors of the measurements by SEC and melt flow rate, the measured characteristics of the SRM 1475 a are identical to those of SRM 1475 .

\section{2,629}

PB94-173754 PC A03/MF A01

National Inst. of Standards and Technology (MEL) Gaithersburg, MD. Factory Automation Systems Div. Process for Selecting Standard Reference Algorithms for Evaluating Coordinate Measurement Software.

M. E. A. Algeo, and C. Diaz. Jan 94, 15p, NISTIR5374

See also PB93-149623 and PB94-163029.

Keywords: "Metrology, "Computer program verification, Computer applications, Computer software Measuring instruments, Dimensional measurement, Calibrating, Coordinates. Data analysis, US NIST, Calibrating, Coordinates, Data analysis, US NIST, measuring systems, "SRA, *Standard reference algonithm, ATEP(Algorithm Testing and Evaluation Program), ATS(Algorithm Testing System).

The report is directed to vards the dimensional metrology community interested in standardizing geometric fitting algorithms used in coordinate measuring systems (CMSs) and is intended to stimulate suggestions for establishing a standard reference algorithm (SRA) selection process. An acceptance procedure for evaluating and selecting the SRAs is identified. The SRA selection process provides control of variation among national reference algorithms and ensures addressing the needs of industry. It is envisioned as a two-stage process: an alpha phase conducted by the National Institute of Standards and Technology (NIST) and a beta phase of public review. The report describes the details of both phases and provides a summary of related activities.

\section{2,630}

PB94-178225 PC A03/MF AO

National Inst. of Standards and Technology (TS) Gaithersburg, MD. National Voluntary Lab. Accreditation Program.

National Voluntary Laboratory Accreditation Program: Procedures and General Requirements. Handbook

J. L. Cigler, and V. R. White. Mar 94, 44p, NIST/HB150

Also available from Supt. of Docs. as SN003-00303260-3.

Keywords: *US NBS, *Laboratories, Certification, ProCedures, Requirements, Calibration standards, Standardization, Licensure, Methodology, Accuracy, Performance evaluation, Government policies, "National Voluntary Laboratory Accreditation Program, NVLAP, NIST(National Institute of Standards and Technology)

NIST Handbook 150 is intended for information and use by staff of accredited laboratories, those seeking accreditation, other laboratory accreditation systems, users of laboratory services, and others needing information on the requirements for accreditation under the National Voluntary Laboratory Accreditation Program (NVLAP). It presents the basic procedures and genera accreditation requirements of NVLAP for use in accrediting calibration and testing laboratories. This handbook contains Part 285 of Title 15 of the U.S. Code of Federal Regulations (CFR) plus all general procedures, criteria, and policies formerly contained in the individual NVLAP technical handbooks and separately published NVLAP policies.

02,631

PB94-185147 Not available NTIS

National Inst. of Standards and Technology (MSEL), Gaithersburg, MD. Metallurgy Div.

High-Temperature Laser-Pulse Thermal Diffusivity Apparatus.

Final rept.

A. Cezairliyan, T. Baba, and R. Taylor. Mar 94, 25p. Pub. in International Jnl. of Thermophysics $15, \mathrm{n} 2$ p317-341 Mar 94

Keywords: "Thermal diffusivity, "High temperature tests, "Temperature measuring instruments, Radiative heat transfer, Thermophysical properties. Thermal properties, Boundary conditions, Transport properties Pulsed lasers, Uncertainity, Reprints, "Laser pulse method.

A high-temperature laser-pulse apparatus for the measurement of thermal diffusivity in the temperature range from 1,500 to $2,500 \mathrm{~K}$ has been designed, constructed, and tested at the National Institute of Standards and Technology. A curve-fitting method is introduced by which the entire experimental temperature history curve is fitted with the theoretical curve under the boundary condition of radiative heat losses. The new apparatus and the curve-fitting method permit thermal diffusivity measurements with an uncertainty of not more than $3 \%$

\section{2,632}

PB94-186848 PC A06/MF A02

National Inst. of Standards and Technology (EEEL) Gaithersburg, MD.

Optical Fiber Sensors: Accelerating Applications in Navy Ships.

W. Day, P. S. Lovely, H. K. Whitesel, and R. K.

Hickernell. May 94, 105p, NISTIR-5018.

Prepared in cooperation with Naval Surface Warfare Center, Annapolis, MD

Keywords: "Naval vessels, "Fiber optics transmission lines, Pressure gages, Temperature measuring instruments, Cost analysis, Multiplexing, Military technology, Control systems, Specifications, Economic analysis Signal processing, Damage assessment, Commercialization, Market research, *Optical fiber sensors.

The Navy needs new sensors for shipboard machinery monitoring and control, condition-based maintenance, and damage assessment Optical fiber sensors are strongly preferred because of their immunity to elec trical disturbances, as well as potential size, weight and performance advantages. But despite well over a decade of development and promise, relatively few optical fiber sensors available today can meet the Navy's needs with acceptable performance and cost. This re port examines the reasons and recommends strategies to help the Navy achieve its goals. Some of the recommendations confirm approaches that the Navy is already implementing. Optical fiber sensors have very valuable potential advantages, but those that the Navy can use may remain too expensive to be deployed if the Navy uses traditional methods of writing specifica tions and soliciting development and procurement bids. For this reason, the study focuses on cooperation with industry and promoting commercial off-the-shelf and dual-use technology.

\section{2,633}

PB94-187630 PC A03/MF A01

National Inst. of Standards and Technology (TS) Gaithersburg, MD. Metric Program.

Metric for Success.

G. P. Carver. May 94, 18p, NISTIR-5425.

Keywords: "Metric system, *Metrication, International system of units, Standards, United States, Federal agencies, Metrology, Units of measurement, Governmentindustry relations, National government History, NIST(National Institute of Standards and Technology).

The federal agencies are working with industry to ease adoption of the metric system. The goal is to help U. industry to compete more successfully in the global marketplace, increase exports, and create new jobs. The strategy is to use federal procurement financial assistance, and other business-related activities to encourage voluntary conversion. Based upon the positive experiences of firms and industries that have converted, federal agencies have concluded that metric use will yield long-term benefits that are beyond any one-time costs or inconveniences. It may be time for additional steps to move the Nation out of its dual-system comfort zone and to continue the progress toward metrication. The report includes Metric Highlights in U.S. History.

02,634

PB94-198819 Not available NTIS

National Inst. of Standards and Technology (NML), Gaithersburg, MD. Thermophysics Div.

Milliwatt

Final rept.

R. F. Berg, and N. P. De Luca. 1991, 3p.

Pub. in Review of Scientific Instruments 62, n2 p527 5291991

Keywords: "Mixers, Milliwatt power range, Low power, Thermal stability, Fluoroform, Methanol, Mixing, Samples, Reprints, "Fluid mixers. 
Mixing without excessive heating is sometimes needed for temperature-sensitive fluids. We modified an inexpensive low-frequency electronic filter based on a mechanical resonator to produce a compact $(16 \mathrm{~mm})$ and low-power (1-3 mW) mixer for low viscosity fluids. Tests made in air and methanol at room temperature and in fluoroform near its critical point are compared against the predictions of an electromechanical model.

\section{2,635}

PB94-199999 Not available NTIS

National Inst. of Standards and Technology (CSTL), Gaithersburg, MD. Thermophysics Div.

Millisecond-Resolution Pulse Heating System for Specific-Heat Measurements at High Temperatures.

Final rept.

A. Cezairliyan. 1992, 35p.

Pub. in Compendium of Thermophysical Property Measurements Methods, Chapter 17, v2 p483-517 1992.

Keywords: "Specific heat, "High temperature tests, "Test methods, "Pulse heating, Experiment design, Errors, Temperature measurement, Thermophysical properties, Test facilities, Reprints.

In the first volume of this Compendium, a general description and a brief survey of the pulse techniques, utilizing resistive self-heating of the specimen, for the measurement of specific heat at high temperatures were given. The presentation covered techniques over a wide regime of time response: from nearly a second to submicrosecond resolution. Most of the techniques were of an exploratory nature and were developed for specific immediate applications. However, as a result of extensive research performed during the last 25 years, the millisecond-resolution pulse heating technique for specific-heat measurements has reached a mature stage. The objective of the present chapter is to give details of the millisecond-resolution technique developed in the Dynamic Measurements Laboratory of the U.S. National Institute of Standards and Technology (NIST), formerly the U.S. National Bureau of Standards (NBS). The presentation includes a description of the measurement system, measurement procedure, consideration of various phenomena that affect the design and operation of the system, estimate of errors, and discussion summarizing recent improvements and additions to the overall system.

\section{2,636}

PB94-200003 Not available NTIS

National Inst. of Standards and Technology (CSTL), Gaithersburg, MD. Thermophysics Div.

High-Speed Spatial Scanning Pyrometer.

uinal rept.

A. Cezairliyan, R. F. Chang, G. M. Foley, and A. P.

Miiller. 1993, 9p.

Pub. in Review of Scientific Instruments 64, n6 p15841592 Jun 93.

Keywords: "Pyrometers, "Spectral emission, "Temperature measurement, Linear arrays, Line spectra, Temperature measuring instruments, Errors, Tungsten, Thermophysical properties, Reprints.

A high-speed spatial scanning pyrometer has been designed and developed to measure spectral radiance temperatures at multiple target points along the length of a rapidly heating/cooling specimen in dynamic thermophysical experiments at high temperatures (above about $1800 \mathrm{~K}$ ). The design, which is based on a self-scanning linear silicon array containing 1024 elements, enables the pyrometer to measure spectral radiance temperatures (nominally at $650 \mathrm{~nm}$ ) at 1024 equally spaced points along a $25-\mathrm{mm}$ target length. The elements of the array a re sampled consecutively every 1 microsecond thereby permitting one cycle of measurements to be completed in approximately $1 \mathrm{~ms}$. The pyrometer output is recorded digitally with a fullscale resolution of $0.025 \%$ every 1 microsecond. Procedures for calibration and temperature measurement as well as the characteristics and performance of the pyrometer are described. The details of sources and estimated magnitudes of possible errors are given. An example of measurements of radiance temperatures along the length of a tungsten rod, during its cooling following rapid resistive pulse heating, is presented.

\section{2,637}

PB94-211364 Not available NTIS

National Inst. of Standards and Technology (MEL), Gaithersburg, MD. Precision Engineering Div.
Advanced Angle Metrology System.

Final rept.

W. T. Estler, and Y. H. Queen. 1993, 4p.

Pub. in Annals of the CIRP 42, n1 p573-576 1993.

Keywords: "Metrology, Calibration, Resolution, Accuracy, Reprints, "Angle measurement, "AAMACS system, US NIST.

We describe the new Advanced Automated Master Angle Calibration System (AAMACS) at the U. S. National Institute of Standards and Technology. The heart of this system is a set of three stacked concentric indexing tables with 832, 729, and 625 teeth respectively. The system is fully error corrected and achieves a full-circle positioning accuracy of + or -0.02 arc-seconds with an angular positioning resolution of 0.0034 arc-seconds. System accuracy is limited by autocollimator noise. We will describe the error mapping process and the use of the system for high-accuracy autocollimator calibrations.

\section{2,638}

PB94-211687 Not available NTIS

National Inst. of Standards and Technology (TS) Gaithersburg, MD. Weights and Measures Program.

Ensuring Accuracy and Traceability of Weighing Instruments.

Final rept.

G. L. Harris. 1993, 80.

Pub. in American Society for Testing and Materials Standa rdization News, p44-51 Apr 93.

Keywords: "Weight indicators, Weight measurement, Calibration, Metrology, Balances, Accuracy, Reprints, Mass standards, ISO 9000 , Accreditation, Traceability.

The paper reviews available standards, guides, recommended practices, and publications to provide both a general overview of available reference material and recommendations related to ensuring accuracy and traceability of weighing instruments. References are promulgated by the following organizations: (1) $\mathrm{Na}$ tional Institute of Standards and Technology; (2) National Conference on Weights and Measures; (3) International Organization for Legal Metrology; (4) International Standards Organization; (5) Instrument Society of America for Testing and Materials.

\section{2,639}

PB94-211695 Not available NTIS

National Inst. of Standards and Technology (NEL) Gaithersburg, MD. Precislon Engineering Div.

Particle Size Standards and Their Certification at NIST.

Final rept.

A. W. Hartman, 1991, 9p.

Pub. in Proceedings of Measurement Science Conference, Anaheim, CA., January 31-February 1, 1991, $9 \mathrm{p}$

Keywords: "Particle size, Particle size distribution, Electron microscopy, Certification, Microspheres, $\mathrm{Re}$ prints, Standard reference materials, US NIST.

An overview is given of the National Institute of Standards and Technology's (NIST's) Standard Reference Materials (SRMs) for particle size and of the techniques used in their certification.

\section{2,640}

PB94-212370 Not available NTIS

National Inst. of Standards and Technology (CSTL), Gaithersburg, MD. Thermophysics Div.

Intercomparison of the Effective Areas of a Pneumatic Piston Gauge Determined by Different Techniques.

Final rept.

K. K. Jain, C. D. Ehrlich, J. C. Houck, and J. K. N.

Sharma. 1993, 9p.

Pub. in Meas. Sci. Technol. 4, p249-257 1993.

Keywords: "Pneumatic equipment, "Pressure gages, *Manometers, Calibration, Measuning instruments, Test methods, Pressure measurement, Uncertainty, Reprints, "Piston gages.

A variety of primary measurement techniques is now available for the measurement of pressure to $1 \mathrm{MPa}$ and above. To ascertain the systematic uncertainty, i any, which exists in the measured pressure using the individual techniques, it is best to perform direct intercomparisons of primary instruments. However, when direct intercomparison is not possible, the next best alternative is to use a highly stable, reproducible transfer artifact such as a simple piston gage. Such intercomparisons are described here, utilizing a piston gage calibrated by a mercury manometer (with 0.1 MPa full-scale pressure), four large diameter 'dimensional' piston gages from two different manufacturers (all with $1 \mathrm{MPa}$ full-scale pressure), and a controlled clearance piston gage (with $7 \mathrm{MPa}$ full-scale pressure). The area ratio derived from dimensional measurements on two of the large diameter gages, when compared with the ratio obtained from measurements traceable to a manometer agrees within 1 part per million $(\mathrm{ppm})$. For one of the large diameter piston gages, the area value obtained from the manometer agrees within 3 ppm with its dimensional area, and within 10 ppm with the value obtained by its direct calibration against the controlled clearance piston gage.

\subsection{1}

PB94-216306 Not available NTIS

National Inst. of Standards and Technology (NML), Gaithersburg, MD.

Models and Interactions.

Final rept.

J. Mandel. 1991, 5p.

Pub. in Jnl. of Testing and Evaluation 19, n5 p398-402 1991.

Keywords: "Experimental data, Interlaboratory comparisons, Analysis of variance, Data analysis, Graphic methods, Test methods, Models, Reprints, Two way tables.

It is shown that the classical analysis of variance for two-way tables, with mean squares for rows, columns and interaction, is often a hindrance to understanding. For interlaboratory data resulting from the study of a test method, a row-linear model is generally more appropriate. However, in practice, a material-by-material analysis of such data, involving minimal prior assumptions, is the most appropriate way of dealing with interlaboratory test data. The importance of graphics is illustrated.

\section{2,642}

\section{PB94-219060 PC A04/MF A01}

National Inst. of Standards and Technology (TS), Gaithersburg. MD. National Voluntary Lab. Accreditation Program.

National Voluntary Laboratory Accreditation Program: Energy Efficient Lighting Products.

Handbook.

L. S. Galowin, W. Hall, and W. J. Rossiter. Jul 94

$74 \mathrm{p}, \mathrm{NIST} / \mathrm{HB}-150 / 1$

Also available from Supt. of Docs as SN003-00303279-4. See also PB94-178225.

Keywords: "Energy efficiency, "Laboratories, *Accreditation, "Tests, Lighting equipment, Procedu res, Methodology, Requirements, Calibration standards, Quality, Energy policy, "EEL program, *Energy Efficient Lighting program, "NVLAP(National Voluntary Laboratory Accreditation Program), "National Voluntary Laboratory Accreditation Program, US NIST.

The purpose of this handbook is to set out procedures and technical requirements for the National Voluntary Laboratory Accreditation Program (NVLAP) accreditation of laboratories which perform test methods covered by the Energy Efficient Lighting (EEL) Products program. It complements and supplements the NVLAP programmatic procedures and general requirements found in NIST Handbook 150 (PB94-178225). The interpretive comments and additional requirements contained in this handbook make the general NVLAP criteria specifically applicable to the EEL program.

\section{2,643}

PB94-219326 PC A05/MF A02

National Inst. of Standards and Technology, Gaithersburg, MD.

Journal of Research of the National Institute of Standards and Technology. May/June 1994. Volume 99, Number 3.

B. N. Taylor, and D. R. Harris. 1994, 100p.

See also PB94-219334 through PB94-219409 and PB94-219219. Also available from Supt. of Docs. as SN703-027-00058-0.

Keywords: "Resistors, "Standards, "Calibrating, Voltmeters, Electrical resistance meters, Hall effect, Drift(Instrumentation)

Contents:

Articles:

Sources of Uncertainty in a DVM-Based Measurement System for a Quantized Hall Resistance Standard; 
A dc Method for the Absolute Determination of Conductivities of the Primary Standard $\mathrm{KCl}$ Solutions from 0 deg $C$ to 50 deg $C$;

Three-Axis Coil Probe Dimensions and Uncertainties During Measurement of Magnetic Fields from Appliances;

Improved Automated Current Control for Standard Lamps;

Refractive Indices of Fluids Related to Alternative Refrigerants;

A Theoretical Analysis of the Coherenece-Induced Spectral Shift Experiments of Kandpal, Vaishya, and Joshi;

Comments on the paper 'Wolf Shifts and Their Physical Interpretation Under Laboratory Conditions' by K. D. Mielenz;

Reply to Professor Wolf's comments on my paper on Wolf shifts;

Errata--Erratum:

Precision Comparison of the Lattice Parameters of Silicon Monocrystals;

Conference Reports--Workshop on Characterizing Briefs. Diamond Films III

02,644
PB94-219342

(Order as PB94-219326, PC A05/

MF A02)

National Inst. of Standards and Technology, Gaithersburg, MD.

dc Method for the Absolute Determination of Conductivities of the Primary Standard $\mathrm{KCl}$ Solutions from $0 \mathrm{C}$ to $50 \mathrm{C}$.

Y. C. Wu, W. F. Koch, D. Feng, E. Arvay, A. Tomek, L. A. Holland, and E. Juhasz. 1994, $6 p$

Prepared in cooperation with National Office of Measures, Budapest (Hungary).

Included in Jnl. of Research of the National Institute of Standards and Technology, v99 n3 p241-246 Mayl Jun 94.

Keywords: "Standards, "Conductivity, "Potassium chloride, Electrical resistivity, Tests, Aqueous electrolytes.

A new method for the absolute determination of electrolytic conductivity based on direct current and potentio-metric measurements is described. The unique design of the cell uses a removable center section whose length and cross-sectional area are accurately known. Two pairs of matched $\mathrm{Ag}, \mathrm{AgCl}$ electrodes are used in a four terminal mode of resistance measurement. Measurements of the electrolytic conductivity of primary standard potassium chloride solution using this novel dc conductance cell are compared with the currently adopted IUPAC and OIML recommendations. In addition, measurements have been made of the electrolytic conductivity of a solution of potassium chloride having a molality of $1 \mathrm{~mol} / \mathrm{kg}$ (mole $\mathrm{KCl}$ per kilogram $\mathrm{H} 2 \mathrm{O}$ ). The values so obtained ove the temperature range of $0 \mathrm{deg} C$ to $50 \mathrm{deg} C$ are recommended as the new primary standards for electroIytic conductivity.

02,645

PB95-103826 PC A02/MF A01

National Inst. of Standards and Technology (CSTL), Gaithersburg, MD. Process Measurements Div.

New Expressions of Uncertainties for Humidity Calibrations at the National Institute of Standards and Technology.

P. H. Huang, and J. R. Whetstone. Jun 94, 9p, NISTIR-5455.

Keywords: "Humidity measurement, "Calibration, "Uncertainty, Error analysis, Mixing ratios, Dew point, Tables(Data), US NIST.

Beginning January 1, 1994, the National Institute of Standards and Technology (NIST) requires all calibration reports to state the uncertainties according to the new policy. Reference is to be made to some published document. Uncertainties of calibration results are expressed using the method described in the 'Guide to the Expression of Uncertainty in Measurement' (NIST Technical Note 1297). This report explains how each component of the combined standard uncertainty was determined for providing the expanded uncertainties of humidity parameters

02,646
PB95-107280 Not available NTIS

National Inst. of Standards and Technology (TS),

Gaithersburg, MD. Office of Measurement Services.
Should NIST Accredit U.S. Calibration Laboratories.

Final rept.

S. D. Rasberry, and J. D. Simmons. 1991, 11p

Pub. in Proceedings of Measurement Science Conference, Anaheim, CA., January 31-February 1, 1991, $11 \mathrm{p}$.

Keywords: Measurement, Standards, Reprints, "Calibration laboratories, "Laboratory accreditation, *Accreditation, Reference materials, Traceability, US NIST.

For nine decades the National Institute of Standards and Technology (NIST), and under its former name the National Bureau of Standards, has followed one model of providing traceability to National standards of measurement. In that model NIST offers calibration and reference material services, and leaves entirely to users of those services the burden of maintaining and demonstrating traceability. In this talk the authors explore the possibility of a new model. The NIST services mentioned above would be continued as would the feature of user responsibility for traceability. What would be added is accreditation by NIST of laboratory calibration competence, conformance to quality laboratory standards, and proficiency in providing calibration services. A NIST 'home' for such a system already exists in the National Voluntary Laboratory Accreditation Program (NVLAP) which currently accredits about 1000 testing laboratories. At least four pressures are building to move in this direction. They include (1) company quality programs aimed at the highest level of attainment (2) customer requirements (including those of Federal executive branch departments); (3) requirements for accreditation as mandated in new laws; and (4) international trade requirements resulting from EC-92 developments.

02,647

PB95-108502 Not available NTIS

National Inst. of Standards and Technology (MEL),

Gaithersburg, MD. Precision Engineering Div.

Metrology Model for Submicrometer Dimensional Measurements.

Final rept.

J. Potzick. 1993, 10p

Pub. in Proceedings of Measurement Science Conference, Anaheim, CA., January 20-22, 1993, p1-10.

Keywords: "Dimensional measurement, "Length, Planar structures, Calibration, Metrology, Standards, Comparison, Precision, Accuracy, Models, Reprints, Nanostructures, Traceability.

A model for the accurate dimensional measurement of small planar objects is developed in terms of the comparison of the images of the test object and a standard object in a measuring device. A length measuring instrument is thus a comparator. The calibration of the standard and the conditions necessary for a valid comparison are discussed. The principles discussed here apply to many other types of measurement as well.

\section{2,648}

PB95-108700 Not available NTIS

National Inst. of Standards and Technology (NEL), Gaithersburg, MD. Automated Production Technology Div.

Vibration Laboratory Automation at NIST with Personal Computers.

Final rept.

B. F. Payne. 1990, 15p

Pub. in Proceedings of National Computer Systems Laboratory Workshop and Symposium, Washington, DC., August 19-23, 1990, p41-55.

Keywords: "Vibration measurement, "Automation, * Personal computers Accelerometers, Calibration, Standards, Laboratories, Measuring instruments, Vibration simulators, Shakers, High level languages, Reprints, US NIST.

The National Institute of Standards and Technology (NIST) provides calibration services for vibration pickups over a wide frequency range. Two experimental test measurement setups have been automated, each using a personal computer. One measurement system is for calibration of vibration shakers that uses an absolute reciprocity method, and the shaker is calibrated by measuring the changes in the electrical admittance of the shaker as a function of the mass attached to the shaker table. The second measurement system is for accelerometer calibration by comparison of a test accelerometer to a standard shaker which has been calibrated by an absolute method.
These automated measurement systems make use of modern high level languages, one in Pascal and one in ' $C$ '. The paper also gives some comparisons of the use of personal computers and technical laboratory computers.

\section{2,649}

PB95-143087 PC A03/MF A01

National Inst. of Standards and Technology (PL), Gaithersburg, MD

Guidelines for Evaluating and Expressing the Uncertainty of NIST Measurement Results. 1994 Edition.

Technical note.

B. N. Taylor, and C. E. Kuyatt. Sep 94, 34p, NIST/

TN-1297.

Supersedes PB93-159465. Also available from Supt. of Docs. as SN003-003-03292-1.

Keywords: Confidence level, Error analysis, Guide lines, Uncertainty, Standards, Accuracy, Precision *Measurement uncertainty, ISO Guide, US NIST.

This Technical Note succinctly presents, in the context of the NIST Policy on Statements of Uncertainty Associated with Measurement Results, those aspects of the ISO Guide to the Expression of Uncertainty in Measurement that will be of most use to the NIST staff and others in implementing that policy, which is included as Appendix C. In this second (1994) edition of Technical Note (TN) TN 1297. Appendix D has been added to clarify and provide additional guidance on a number of topics in response to questions asked since the publication of the first (1993) edition.

02,650

PB95-150884 Not available NTIS

National Inst. of Standards and Technology (CSTL) Gaithersburg, MD. Thermophysics Div.

Vacuum Gauges and Partial Pressure Analyzers. Final rept.

C. R. Tilford. $1993,4 p$

Pub. in Jnl. of Physics: Condens. Matter 5, pA81-A84 1993.

Keywords: "Vacuum gages, "Gas analysis, Partial pressure, Measuring instruments, Pressure measurement, Vacuum apparatus, Ionization gages, Reprints, Capacitance diaphragm gages, Partial pressure ana lyzers, Residual gas analyzers.

This paper briefly reviews the characteristics of severa important vacuum instruments; capacitance diaphragm gauges, ionization gauges, spinning rotor gauges, and partial pressure or residual gas analyzers.

\section{2,651}

PB95-162202 Not available NTIS

National Inst. of Standards and Technology (NEL), Gaithersburg, MD. Precision Engineering Div. Dimensional Characterization of Small Bores: A Survey.

Final rept.

J. Raja, and R. Veale. 1990,10p

Pub. in Proceedings of Measurement Science Conference, Anaheim, CA., February 8-9, 1990, 10p.

Keywords: "Holes, *Cavities, " Dimensional measure ment, Methodology, Precision, Diameters, Waveguides, Reprints, CMM(Coordinate measuring machines).

The measurement of small bores is now and has always been a difficult measurement problem. The problem is especially difficult when the length to diamete ratio of the bore is large. This paper presents a survey of the various techniques now in use as well as othe methods which have been developed in the past. It lists the advantages, disadvantages, and expected accu racy which can be achieved using the various methods. Current work using coordinate measuring ma chines to characterize the internal dimensions of waveguides is also described.

02,652

PB95-162434 Not available NTIS

National Inst. of Standards and Technology (MSEL), Gaithersburg, MD. Ceramics Div.

Loading Device for Fracture Testing of Compac Tension Specimens in the Scanning Electron Microscope.

Final rept

J. Rodel, J. F. Kelly, M. R. Stoudt, and S. J.

Bennison. 1991, 7p

Pub. in Scanning Microscopy 5, n1 p29-35 Mar 91. 
Keywords: "Loading rate, "Fractography, "Scanning electron microscopy, Loads(Forces), Crack propagation, Aluminum oxide, Ceramics, Performance evaluation, Design criteria, Case studies, Reprints, Piezoelectric translators.

A loading device for compact tension specimens allowing fracture experiments in the scanning electron microscopy (SEM) has been designer. Its key elements are a piezoelectric translator for applying controlled displacements to the loading points on the specimen transmission of displacement from the piezoelectric driver to the specimen was found to be the major mechanical design problem. The peripheral equipment include a function generator and a high voltage amplifier to drive the piezoelectric translator as well as a video overlay and standard video equipment to record the image continuously during the course of the experiment. A case study on alumina describes qualitative observations on the toughening mechanism, crackinterface bridging, operating in this material. Quantitative information pertaining to the closure stresses associated with this toughening mode can be obtained by measuring the crack profile.

02,653

PB95-163093 Not available NTIS

National Inst. of Standards and Technology (MEL), Gaithersburg, MD. Precision Engineering Div.

Two New Probes for a Coordinate Measuring Machine.

Final rept.

J. Stone, L. Carroll, G. Caskey, J. Rose, K. Lau, S.

Phillips, and A. Resnick. 1993, 4p.

Pub. in Proceedings of the ASPE Annual Meeting (8th), Seattle, WA., November 7-12, 1993, p82-85

Keywords: "Measuring instruments, "Probes, Dimensional measurement, Accuracy, Reprints, *CMM(Coordinate measuring machines), "Coordinate measuring machines, Analog probes, Touch-trigger probes.

We have performed tests of the measuring capabilities of two new analog probes used with coordinate measuring machines. Both probes are capable of sub-micrometer accuracies. The first is an LVDT-based threedimensional analog probe with a range of $0.1 \mathrm{~mm}$ and a resolution of 0.025 micrometers. We found that the repeatability of the probe is 0.04 micrometers ( 1 standard deviation) and three-dimensional errors are less than 0.5 micrometers at small deflections. The second probe is a non-contact capacitance probe. The probe stylus is a precision ball mounted on the end of a hypodermic needle; the probe detects the position of a nearby surface through measuring the capacitance between the surface and the ball. The probe can be used as a true analog probe or it can simulate the operation of a touch-trigger probe, generating a trigger pulse when the reading exceeds a threshold value. The repeatability in analog mode is 0.05 micrometers and the repeatability as a touch-trigger probe is $0.1 \mathrm{mi}-$ crometers. Used as a touch-trigger probe in three dimensions, the probe accuracy is about 1.3 micrometers.

\section{2,654}

PB95-164703 Not available NTIS

National Inst. of Standards and Technology (NML), Boulder, CO. Chemical Engineering Science Div. Cryogenics.

Final rept.

L. L. Sparks. 1993, 20p

Sponsored by American Society for Testing and Materials, Philadelphia, PA.

Pub. in Manual on the Use of Thermocouples in Temperature Measurement, Chapter 11, p214-233 1993.

$\begin{array}{ll}\text { Keywords: } & \text { "Temperature dependence, } \\ \text { "Thermocouples, "Cryogenic temperature, Magnetic }\end{array}$ fields. Thermoelectricity, Reprints, Reference tables, ITS-90.

Successful use of thermocouples for absolute or differential temperature measurement at low temperatures requires that particular attention be paid to thermocouple sensitivity and to the relative importance of thermoelectric effects such as dilute impurities, strain, and inhomogeneity. An important subsel of cryogenic temperature measurement involves magnetic-field environments. Thermocouple types and the magneticfield effect on the thermoelectric sensitivity are discussed. Reference tables based on ITS-90 are given for zero-field applications.

\section{2,655}

PB95-168506 Not available NTIS

National Inst. of Standards and Technology (CSTL), Gaithersburg, MD. Thermophysics Div.

Look at Uncertainties over Twenty Decades of Pressure Measurement.

FInal rept.

C. D. Ehrlich. 1994, 10p.

Pub. in Proceedings of VIII IMEKO World Congress, Torino, Italy, September 5-9, 1994, p1941-1950.

Keywords: "Pressure measurement, "Uncertainity, Transducers, Pressure gages, Accuracy, Test facilities, Test methods, Reprints.

A wide variety of instrumentation is required to perform state-of-the-art pressure measurements over the almost 20 decade range of pressure that is used in science and industry today. The uncertainties associated with such measurements similarly span a wide range, from several parts in a million near atmospheric pressure to around $10 \%$ or more at both pressure ex tremes. This paper provides an overview of the techniques, instrumentation and uncertainties associated with the most common primary measurements of pressure practiced today. A discussion of some of the more prominent types of pressure gages and transducers in the various pressure regimes is also included.

\section{2,656}

\section{PB95-169819 PC A20/MF AO4}

National Inst. of Standards and Technology (TS), Gaithersburg, MD. Weights and Measures Program.

Report of the National Conference on Weights and Measures (79th). Held in San Diego, California on July 17-21, 1994 .

Special pub.

C. S. Brickenkamp, and A. H. Turner. Nov 94, 458p, NIST/SP-870.

Also available from Supt. of Docs. as SN003-00303306-5. Library of Congress catalog card no. 2627766. See also PB94-138989.

Keywords: "Weight measurement, *Metrology, *Meetings, Regulations, Laws(Jurisprudence), Standards, Quality control, US NBS, Safety, Scale(Ratio), Training. Mass flowmeters, San Diego(California), Legal metrology, Grain moisture.

The 79th Annual Meeting of the National Conference on Weights and Measures (NCWM) was held July 17 through 21, 1994, at the Doubletree Hotel at Horton Plaza, San Diego, California. The theme of the meeting was 'Consensus Through Communication.' Reports by the standing and annual committees of the Conference comprise the major portion of this publication, along with the addresses delivered by Conference officials and other authorities from government and industry. Special meetings included those of the Metrologists, the Associate Membership Committee, the Retired Officials Committee, the Scale Manufacturers' Association, the American Petroleum Institute, the Industry Committee on Packaging and Labeling, and the regional weights and measures associations.

\section{2,657}

PB95-175014 Not available NTIS

National Inst. of Standards and Technology (CSTL). Gaithersburg, MD. Thermophysics Div.

Influence of the Filament Potential Wave Form on the Sensitivity of Glass-Envelope Bayard-Alpert Gages.

Final rept.

P. J. Abbott, and J. P. Looney. 1994, 6p.

Pub. in JnI. of Vacuum Science and Technology A 12, n5 p2911-2916 Sep/Oct 94.

Keywords: "Bayard-Alpert ionization gages, "Waveforms, "Sensitivity, "Filaments, Controllers, Vacuum gages, Nonlinearity, Glass, Pressure gages, Reprints.

Nonlinearities of about $10 \%-15 \%$ in the sensitivity of glass envelope Bayard-Alpert gages (BA gages) have been observed in the pressure range 10 (exp -7) to $10(\exp -2) \mathrm{Pa}$. These nonlinearities were studied in modified BA gage tubes with platinum coatings on thei inner glass surfaces by measuring the equilibrium potential of the platinum coating as a function of pressure. The sensitivities of the gage systems (gage tube plus controller) were found to depend on the inner surface potential, and this potential was found to depend on pressure and on the details of the filament potentia waveform provided by the gage controller. It was found that the nonlinearities could be minimized by holding the inner surface to a fixed direct-current (dc) potential, by modifying the alternating-current filament potential wave form, or by using a controller that provides a noise-free dc filament-heating current.

\section{2,658}

PB95-180428 Not available NTIS

National Inst. of Standards and Technology (BFRL), Gaithersburg, MD. Building Environment Div.

Design and Machining of Copper Specimens with Micro Holes for Accurate Heat Transfer Measurements.

Final rept

M. A. Kedzierski, and J. L. Worthington. 1993, 16p Pub. in Experimental Heat Transfer 6, p329-344 1993

Keywords: "Drilling, "Micromachining, "Temperature measurement, "Copper, Thermocouples, Experiment design, Temperature distribution, Uncertainty, Errors, Reprints, Micro holes

This article presents a technique that has been developed specifically for drilling 0.5334 -mm-diameter, 19$\mathrm{mm}$-deep holes in copper for use in temperature measurement. The holes accept thermocouples, which are used for the measurement of the temperature gradient and the wall temperature of the specimen. Errors due to the intrusion of the probe, and the finite size and mass of the probe, are reduced as the diameter of the probes is reduced. A machining procedure for drilling deep micro holes in copper cannot be found in conventional texts; this article advocates holes that are deeper than those traditionally recommended. This article is written for both machinists and experimentalists. Both heat transfer and machining criteria are considered. The necessary equipment and their specifications are discussed. Special attention is given to specifying drilling speeds, feed rates, and lubricants. Step-by-step drilling instructions are given. An analysis is performed to reveal the important parameters for reducing the errors associated with the uncertainty in the location, the relative position of the thermocouples, and the individual temperature measurements.

\section{2,659}

PB95-180998 Not available NTIS

National Inst. of Standards and Technology (CSTL), Gaithersburg, MD. Thermophysics Div.

Measurement of Very-Low Partial Pressures.

Final rept

C. R. Tilford. 1994, $6 p$

Pub. in Jnl. of the Vacuum Society of Japan 37, n9 p667-672 1994.

Keywords: "Pressure measurement, "Ultrahigh vacuum, "Partial pressure, "Residual gas, Measuring instruments, Sensitivity, Mass spectrometers, Gas analysis, Vacuum apparatus, Vapor pressure, Ionization gages, Reprints.

The measurement of partial pressures in the ultra high and extreme high vacuum ranges (below about $10(\exp$ -6)) is important for residual gas analysis and the measurement of low-pressure constituents (both contaminants and deliberate additions) in higher-pressure process gases. Mass-spectrometer type instruments-partial pressure or residual gas analyzers (PPAs and RGAs)--are generally used for this purpose. Typically, these instruments corisist of an electron-impact ionizer, a quadrupole mass filter, and an ion detector. This paper discusses these elements and other factors that determine the very-low-pressure performance of these instruments--detection limits, mass resolution, and stability and pressure-dependence of the sensitivity. Particular attention is paid to ion-source design and operating parameters, the coupling of ions between the ionizer and the mass analyzer, and the influence of the total pressure on partial pressure sensitivities.

02,660

PB95-181004 Not available NTIS

National Inst. of Standards and Technology (CSTL), Gaithersburg, MD. Thermophysics Div.

Process Monitoring with Residual Gas Analyzers (RGAs): Limiting Factors.

Final rept.

C. R. Tilford. 1994, 5p

Pub. in Surface and Coatings Technology 68/69, p7087121994.

Keywords: „Pressure measurement, "Partial pressure, "Gas analysis, "Residual gas, Mass spectrometers, Calibration, Metrology, Vacuum apparatus, Sensitivity, Reprints, "Residual gas analyzers.

Many vacuum processes are limited by low-level impurities introduced with process gases or generated with- 
in the process chamber. Specific impurities are often monitored using residual gas analyzers (RGAs) or partial pressure analyzers (PPAs), most commonly, mass spectrometers of the quadrupole type. Unfortunately, the performance of these instruments can be affected significantly by a number of instrument and vacuum environment variables, so that the sensitivities of even calibrated instruments can differ significantly from expected values. These variables include ion source parameters, total pressure and prior exposure to active gases. The magnitude of the deviations in sensitivity varies for different instruments, but can reach orders of magnitude in extreme cases. This paper reviews the important factors affecting instrument performance, illustrates the magnitude of the effects for different instruments, recommends instrument test procedures, and suggests operating parameters and procedures that can minimize these effects.

\section{2,661}

PB95-189478 PC A05/MF A01

National Inst. of Standards and Technology (TS), Gaithersburg, MD. National Voluntary Lab. Accreditation Program.

National Voluntary Laboratory Accreditation Program: POSIX. Portable Operating System Interface. Handbook

J. Horlick, and M. M. Gray. Jan 95, 76p, NIST/HB

$150 / 7$.

Also available from Supt. of Docs as SN003-00303317-1. See also PB94-178225 and FIPS PUB 151-

Keywords: *Laboratories, "Requirements, Certification, Procedures, Calibration standards, Standardization, Management, Auditing, Personnel, Equipment, Tests, "POSIX(Portable Operating System Interface), "Portable Operating System Interface, *NVLAP(National Voluntary Laboratory Accreditation Program), "National Voluntary Laboratory Accreditation Program, US NIST.

The handbook presents the technical requirements of the National Voluntary Laboratory Accreditation Program (NVLAP) for the Portable Operating System Interface (POSIX program). It is intended for information and use by staff of accredited laboratories, those laboratories seeking accreditation, other laboratory accreditation systems, users of laboratory services, and others needing information on the requirements for accreditation under the POSIX program. This publication supplements NIST Handbook 150, 'NVLAP Procedures and General Requirements' (PB94-178225) which contains Part 285 of Title 15 of the U.S. Code of Federal Regulations (CFR) plus all general NVLAP procedures, criteria, and policies.

\section{2,662}

PB95-203444 Not available NTIS

National Inst. of Standards and Technology (MEL), Gaithersburg, MD. Precision Engineering Div.

Morphological Estimation of Tip Geometry for Scanned Probe Microscopy.

Final rept.

J. S. Villarnubia. 1994, 14p.

Pub. in Surtace Science 321, p287-300 1994.

Keywords: "Tips, "Scanning tunneling microscopy, "Morphology, "Image processing, Geometry, Surface properties, Mathematical models, Image analysis, Reprints, ${ }^{*}$ Atomic force microscopy.

Morphological constraints inherent in the imaging process limit the possible shapes of the tip with which any given tunneling microscope or atomic force microscope image could have been taken. Broad tips do not produce narrow image protrusions. Therefore, feature sizes within the image may be used to place an upper bound on the size of the tip. In this paper, mathematical morphology is used to derive, for each point on an image, a corresponding bounding surface for the tip. The actual tip must be equal to or smaller than the largest tip which satisfies all of the constraints. Example calculations are performed, demonstrating that if the imaged specimen contains sharp features and high relief, the tip shape deduced by this method will be a good estimate of the actual one. Once known, the tip geometry can be deconvoluted from images to recover parts of the actual surface which were accessible to the tip.

02,663

PB95-242293 PC A04/MF A01

National Inst. of Standards and Technology (MEL), Gaithersburg, MD. Manufacturing Systems Integration Div.
ISO TC 184/SC4 Reference Manual.

J. D. Wellington, and B. M. Smith. Jun 95, 61p, NISTIR-5665.

Keywords: "Standardization, "Manuals, Procedures, Research projects, Administration, Manufacturing, Digital systems, Technology transfer, International cooperation, Intemational standards.

The reference manual contains background information on the International Organization for Standardization (ISO) and its Technical Committee 184. It gives a detailed explanation of the technical work of TC 184's Subcommittee 4 (TC 184/SC4) and information on the working groups and advisory committees that carry out that technical work.

\section{2,664}

PB95-242376 PC A06/MF A02

National Inst. of Standards and Technology (TS) Gaithersburg, MD. National Voluntary Lab. Accreditation Program.

National Voluntary Laboratory Accreditation Program: Electromagnetic Compatibility and Telecommunications. FCC Methods.

E. R. Lindstrom, and J. Horlick. Apr 95, 118p, NIST/ HB-150-11.

Also available from Supt. of Docs. as SN003-00303339-1. See also PB94-178225.

Keywords: "Laboratories, "Certification, "Handbooks, Requirements, Electronics laboratories, Telecommunication, Standards, Test methods, Electric devices, Measurements, "Federal Communications Commission, NVLAP(National Voluntary Laboratory Accreditation Program), ECT(Electromagnetic Compatibility and Telecommunications), ECT LAP, US NIST.

NIST Handbook 150-11 presents the technical requirements of the National Voluntary Laboratory Accreditation Program (NVLAP) for accreditation under the Electromagnetic Compatibility and Telecommunications (ECT) LAP. There are two program handbooks for he ECT LAP. The handbook covers test methods used to demonstrate compliance with FCC requirements given in Title 47 of the U.S. Code of Federal Regulations (CFR), Telecommunication, Part 15, Digitial Devices, and FCC Part 68, Analog and Digital. A second program handbook, NIST Handbook 150-14, describes the requirements for test methods in Military Standards 461/462, Electromagnetic Compatibility. Program handbooks are intended for information and use by staff of accredited laboratories and laboratories seeking accreditation, other laboratory accreditation systems, users of laboratory services, and others needing information on the requirements for accreditation.

\section{2,665}

PB95-242400 PC A03/MF A01

National Inst. of Standards and Technology (MEL) Gaithersburg, MD. Precision Engineering Div.

Measuring Long Gage Blocks with the NIST Line Scale Interferometer.

Technical note.

J. S. Beers. May 95, 24p, NIST/TN-1410.

Also available from Supt. of Docs. as SN003-00303341-3. See also PB93-146645.

Keywords: "Interferometers, Length, Silicon dioxide, Measuring instruments, Uncertainty, Dimensions, Manufacturing, US NIST, Long gage blocks, Line scale interferometer.

An improved technique is described for temporarily converting long gage blocks into line scales so they can be measured by fringe counting (dynamic) interferometry in the National Institute of Standards and Technology (NIST) line scale interferometer. The new process employs fuzed silica rather than the previously used steel conversion gage blocks. Conversion blocks are pairs of small $(26 \mathrm{~mm})$ gage blocks with graduation lines on their side faces. The new conversion blocks have a number of advantages as shown by experimental evidence.

\section{2,666 \\ PB95-251658 PC A03/MF A01}

National Inst. of Standards and Technology (MEL), Gaithersburg, MD. Manufacturing Systems Integration Div.

Algorithm Testing and Evaluation Program for $\mathrm{Co}$ ordinate Measuring Systems: Testing Methods.

C. Diaz. Jul 95, 34p, NISTIR-5686.

See also PB95-231833.

Keywords: "Tests, "Metrology, "Computer program verification, Computer applications, Computer soft- ware, Measuring instruments, Data analysis, Calibrat ing, Coordinate measuring systems, Algorithm testing and evaluation program, Standard reference algorithm. The Algorithm Testing and Evaluation Program for $\mathrm{Co}$ ordinate Measuring Systems (ATEP-CMS) is a NIST Special Test service offered through the Office of Measurement Services Calibration Program. ATEPCMS evaluates the performance of data analysis software used in coordinate measuring systems (CMSs) The report details the CMS testing and evaluating methods that ATEP-CMS uses to test and evaluate the performance of CMS software. The report is intended as a guide to procedures for ATEP-CMS testing personnel.

\section{2,667}

PB95-251716 PC A08/MF A02

National Inst. of Standards and Technology (MEL), Gaithersburg, MD. Precision Engineering Div.

Gage Block Hiandbook.

Monograph.

T. Doiron and J. S. Beers. Jun 95, 160p, NIST/

MONO-180.

Also available from Supt. of Docs.

Keywords: "Calibrating, "Measuring instruments, *Metrology, Interferometers, Standards, Length, Statistical quality control, Comparison, "Gage blocks.

Calibration of gage blocks is one of the oldest and most sophisticated measurements in dimensional metrology. This work explains gage blocks are calibrated by both interferometry and mechanical comparison at the National Institute of Standards and Technology. The basic physical and geometrical properties, and the limitations on calibration caused by these properties are explained. All known sources of uncertainty are discussed along with the methods used to estimate these uncertainties. Finally, the measurement assurance program used to control the calibrations processes are explained with estimates of the process parameters.

\section{2,668}

PB95-254439 PC A03/MF A01

National Inst. of Standards and Technology (MSEL), Gaithersburg, MD.

Materials Science and Engineering Laboratory AnDec 93, 30p.

Keywords: "US NIST, "Laboratories, Metallurgy, Ceramics, Polymers, Reliability, Standards, Radiation.

Contents

An overview of NIST's Materials Science and Engineering Laboratory, including the

Metallurgy Division

Ceramics Division;

Polymers Division;

Materials Reliability Division;

Reactor Radiation Division

and Office of Intelligent Processing of Materials

\subsection{9}

PB95-255840 PC A03/MF A01

National Inst. of Standards and Technology (MEL) Gaithersburg, MD. Precision Engineering Div.

NIST SRM 9983 High-Rigidity Ball-Bar Stand. User Manual.

D. Sawyer, S. D. Phillips, G. Caskey, P. Snoots, B. Borchardt, and D. Ward. Jun 95, 16p, NISTIR-5659.

Keywords: "Performance evaluation, Rigidity, Assembly, Manuals, "CMMs(Coordinate Measuring Machines), "Coordinate Measuring Machines, "Ball-bar stands, US NIST, SRM 2083, SRM 9983

The National Institute of Standards and Technology (NIST) SRM 9983 high rigidity ball bar stand is designed to support a ball bar for coordinate measuring machine (CMM) performance evaluation. This document is a user manual for NIST SRM 9983. The manual contains the complete instructions for assembling and using the stand.

\section{2,670}

PB95-261897 PC A07/MF A02

National Inst. of Standards and Technology, Gaithersburg MD.

Journal of Research of the National Institute of Standards and Technology, May/June 1995. Volume 100, Number 3 .

$1995,127 p$

See also PB95-261905 through PB95-261962 and PB95-169371. Also available from Supt. of Docs. as SN-703-027-00064-4 
Keywords: "Standards, "Metrology, "Research projects, US NBS, Magnetic properties, Electric properties, Interlaboratory comparisons, Calibration standards, Optical filters, Electromagnetic absorption, Radio frequencies, Isotope ratio, National Institute of Standards and Technology

No abstract available.

\section{2,671}

\section{PB95-267944 PC A02/MF A01}

National Inst. of Standards and Technology (TS), Gaithersburg, MD. National Voluntary Lab. Accreditation Program.

National Voluntary Laboratory Accreditation Program (NVLAP): Commercial Products Testing.

Handbook

L. I. Knab. Jul 95, 9p, NIST/HB-150/16

Also available from Supt. of Docs. as SNO03-00303355-3. See also PB94-178225.

Keywords: "Laboratories, "Accreditation, "Test facilities, Paints, Plastics, Papers, Plumbing, NVLAP, Commercial products.

National Institute of Standards and Technology (NIST) Handbook 150-16 presents the technical requirements of the National Voluntary Laboratory Accreditation Program (NVLAP) for Commercial Products Testing. It is intended for information and use by staff of accredited laboratories, those laboratories seeking accreditation, other laboratory accreditation systems, users of laboratory services, and others needing information on the requirements for accreditation under the Commercial Products Testing program.

\section{2,672}

\section{PB95-270039 PC A22/MF A04}

National Inst. of Standards and Technology (MSEL), Gaithersburg, MD. Ceramics Div.

Ceramic Powders Characterization: Results of an International Laboratory Study.

Special pub.

S. G. Malghan, S. M. Hsu, R. G. Munro, and L. S. H Lum. Jul 95, 514p. NIST/SP-879.

Also available from Supt. of Docs. as SN003-00303337-5. See also PB92-171446.

Keywords: "Ceramics, "Powder(Particles), "Interlaboratory comparisons, *Germany (Unified), "Sweden, "United States, Morphology, Physical properties, Chemical properties, Test methods, Comparative evaluations, Statistical quality control, Phase, Chemical composition, Chemical analysis, Industries, Universities, Federal agencies, Laboratories.

This report contains the results of an international interlaboratory comparison program (round-robin) of powder characterization under the auspices of international Energy Agency (IEA). The results include the contributions of 25 industrial, university and governmental laboratories in the Federal Republic of Germany, Sweden and the United States. These laboratories surveyed a variety of methods for the characterization of physical properties and chemical characteristics of ceramic starting powders. For the first time, data from various measurement methods are available for critical comparison. Results of the same method from different laboratories are also provided. Recommendations for future studies are drawn from results of the present round-robin.

\section{2,673}

\section{PB96-103080 Not available NTIS}

National Inst. of Standards and Technology (CSTL), Gaithersburg, MD. Thermophysics Div.

Comments on the Stability of Bayard-Alpert Ionization Gages.

Final rept.

C. R. Tilford, A. R. Filippelli, and P. J. Abbott. 1995 ,

3p.

Pub. in Jnl. of Vacuum Science and Technology, v13 n2 p485-487 Mar/Apr 95.

Keywords: "Bayard-Alpert gages, "Stability, Ionization gages, Measuring instruments, Tests, Experimental data, Reprints

A recent group of three articles presents stability testing results for ionization gages, discusses the causes of gage instability, and describes a new type of BayardAlpert (BA) ionization gage. Although the authors are in general agreement with the analysis of gage instability presented by Bills, they are concerned that the test results of Arnold and Borichevsky and the discussion in all three references imply instabilities for existing types of BA gages that are much larger than they find to be the case. Here the authors briefly describe their experience with BA gage stability, indicate why they think their results are different from those of Arnold and Borichevsky, and describe the operating conditions that they believe will result in improved stability.

\section{2,674}

PB96-1135

MF A01)

National Physical Lab., Teddington (England)

Intercomparison of the ITS-90 Radiance Temperature Scales of the National Physical Laboratory (U.K.) and the National Institute of Standards and Technology.

G. Machin, B. C. Johnson, C. Gibson, and R. L.

Rusby. 1994, 6p.

Prepared in cooperation with National Inst. of Standards and Technology, Gaithersburg MD.

Included in Jnl. of Research of the National Institute of Standards and Technology, v99 n6 p731-736 Nov/ Dec 94.

Keywords: "Temperature scales, *Radiance, *Interlaboratory comparisons, Calibration standards, Pyrometers, Quality assurance, Temperature measurement, United States, United Kingdom, NIST(National Institute of Standards and Technology), ITS-90.

An intercomparison of radiance temperature scales has been performed by the National Physical Laboratory (NPL) and the National Institute of Standards and Technology (NIST) using a standard transfer pyrometer operating at a wavelength of approximatelly 1000 $\mathrm{nm}$. It was found that the radiance temperature scales estabfished by the two laboratories were in agreement to $0.1 \%$ or better of the temperature over the range 1000 degrees $\mathrm{C}$ to 2500 degrees $\mathrm{C}$.

\section{2,675}

PB96-123138 Not available NTIS

National Inst. of Standards and Technology (MEL), Gaithersburg, MD. Precision Engineering Div.

Stylus Flight in Surface Profiling.

Final rept.

J. F. Song, and T. V. Vorburger. 1994, 14p.

Pub. in International Mechanical Engineering Congress and Exposition, Chicago, IL., June 6-11, 1994, Manufacturing Science and Engineering, v68 n1 p161 174.

Keywords: "Surface properties, "Flight, "Profile, Roughness, Slope, Specimen, Stylus, Surface, Waveform, Reprints.

In this paper, theoretical and experimental work on stylus flight is described. Experiments on the surfaces of different roughness specimens with sinusoidal, rectangular, triangular and random waveforms support the theoretical model, which predict stylus flight from the traversing speed, stylus force, stylus radius and parameters of the surface.

\section{2,676}

PB96-123567 Not available NTIS

National Inst. of Standards and Technology (CSTL), Gaithersburg, MD. Thermophysics Div.

Long-Term Stability of Bayard-Alpert Gauge Performance: Results Obtained from Repeated Calibrations against the National Institute of Standards and Technology Primary Vacuum Standard.

Final rept.

A. R. Fillippelli, and P. J. Abbott. 1995, 5p.

Pub. in Jnl. of Vacuum Science and Technology, vA 13 n5 p2582-2586 Sep/Oct 95.

Keywords: "Bayard-Alpert ionization gages, "Calibration, "Reference standards, Vacuum gages, Vacuum apparatus, Ultrahigh vacuum, Interlaboratory comparisons, Laboratory tests, Stability, Time, Utilization, Reprints.

This article presents and briefly discusses information on the long-term stability of the sensitivity of BayardAlpert ionization gages with time and use, derived from an analysis of data for Bayard-Alpert gauge calibrations performed at the National Institute of Standards and Technology over a ten year period.

\section{2,677}

PB96-135025 Not available NTIS

National Inst. of Standards and Technology (MSEL), Gaithersburg, MD. Metallurgy Div.
Radiance Temperatures (in the Wavelength Range 523-907 nm) of Group IVB Transition Metals Titanium, Zirconium, and Hafnium at Their Melting Points by a Pulse-Heating Technique.

Final rept.

A. Cezairliyan, J. L. McClure, and A. P. Miiller. 1994 $17 p$.

Pub. in International Jnl. of Thermophysics, v15 n5 p993-1009 1994.

Keywords: "Metals, "Melting points, "Temperature measurement, Reprints, "Radiance temperatures, *Pulse heating

The melting-point radiance temperatures (at six wavelengths in the range 523-907 $\mathrm{nm}$ ) of the Group IVB transition metals titanium, zirconium, and hafnium were measured by a pulse-heating technique. The method is based on rapid resistive self-heating of the specimen from room temperature to its melting point in less than $1 \mathrm{~s}$ and on simultaneously measuring the specimen radiance temperatures every $0.5 \mathrm{~ms}$ with a high-speed six-wavelength pyrometer. Melting was manifested by a plateau in the radiance temperatureversus-time function for each wavelength. The meltingpoint radiance temperatures for a given specimen were determined by averaging the measured temperatures along the plateau at each wavelength.

\section{2,678}

\section{PB96-160239 Not available NTIS}

National Inst. of Standards and Technology (TS), Gaithersburg, MD. Office of Standards Services.

U.S. Government Accreditation and Conformity Assessment System Evaluation.

Final rept.

W. G. Leight. 1993, 20p

Pub. in Conformity Assessment Conference, Gaithersburg, MD., July 14, 1993, 20p.

Keywords: "Laboratories, "Certification, "Standards, Conformity, Accreditation, Testing, Requirements, International trade, Federal government, United States, Reprints, US NIST.

The National Institute of Standards and Technology has since its earliest days been concerned with standards and conformity assessment, with regard to both the physical standards of measurement and documentary standards covering products and testing. We perform calibration services, issue standard reference materials and standard reference data, and conduct many other standards-related activities.

\section{2,679}

PB96-160841 Not available NTIS

National Inst. of Standards and Technology (CSTL), Gaithersburg, MD. Process Measurements Div.

Executive Summary: Proceedings of the Workshop on the Measurement of Transient Pressure and Temperature.

Final rept.

V. E. Bean, and G. J. Rosasco. 1992, 8p.

See also PB92-183680.

Pub. in Proceedings of the Workshop on the Measurement of Transient Pressure and Temperature, Gaithersburg, MD., April 23-24, 1991, p1-8 Apr 92.

Keywords: "Pressure measurement, "Meetings, Temperature measurement, Military requirements, Explosion effects, Pressure transducers, Shock waves, High pressure, Calibration, Transients, Standards, Reprints.

The Workshop on the Measurement of Transient Pressure and Temperature was held at the National Institute of Standards and Technology (NIST), Gaithersburg, MD, on 23-24 April 1991. There were 77 attendees, $55 \%$ from government and $45 \%$ from the private sector Aerospace, automotive and instrumentation manufacturers were present along with engineers from primate sector and government (Army, Navy, Air Force, and NASA) research organizations. The Army, Navy and NASA primary standards laboratories also were represented. The workshop drew international attention with participants from Taiwan, Sweden, Austria, and France. Only France currently offers transient pressure calibrations as part of their national measurement system. The purpose of the workshop was to identify current and emerging measurement problems and needs for transient pressure and temperature measurements, to explore scientific and technical barriers, and opportunities for achieving measurement accuracy goals, and to assist in the formulation of an appropriate role for NIST. 
National Inst. of Standards and Technology (MEL), Gaithersburg, MD. Precision Engineening Div.

Veriflcation of Revised Water Vapour Correction to the Index of Refractlon of Air.

Final rept.

J. Beers, and T. Doiron. 1992, $2 p$

Pub. in Metrologia, v29 p315-316 1992.

Keywords: "Environmental compensation, "Refractive indexes, Reprints, Humidity, "Foreign technology, "Edlen equation, Wavelength correction.

The Edlen equation has become the standard method of relating the index of refraction of air to the air temperature, pressure and humidity. The recent suggestion for improvements in the humidity dependence by Birch and Downs has been verified at the $0.633 \mathrm{mi}-$ crometers $\mathrm{He}-\mathrm{Ne}$ laser wavelength using the line scale interferometer at the National Institute of Standards and Technology (NIST).

02,681

PB96-165840 PC A15/MF A03

National Inst. of Standards and Technology (TS), Gaithersburg, MD. Weights and Measures Program.

Report of the National Conference on Weights and Measures (80th) as Adopted by the 80th National Conference on Weights and Measures, 1995. Held In Portland, Maine on July 16-20, 1995.

Special pub.

G. M. Ugiansky, and A. H. Tumer. Feb 96, 307p, NIST/SP-894.

Also available from Supt. of Docs. as $\mathrm{SNOON}^{-003}$ $03379-1$. See also PB96-166616.

Keywords: "Weight measurement, "Metrology *Meetings, Regulations, Laws(Jurisprudence), Standards, Quality control, US NBS, Safety, Beale(Ratio) Portland(Maine), Legal metrology, Grain moisture.

The 80th Annual Meeting of the National Conference on Weights and Measures (NCWM) was held July 16 through 20,1995, at the Holiday Inn By the Bay, Portland, Maine. The theme of the meeeting was 'Quality Partnerships and Programs through Education.' Reports by the standing and annual committees of the Conference comprise the major portion of this publication, along with the addresses delivered by Conference officials and other authorities from government and industry. Special meetings included those of the Metrologists, the Associate Membership Committee, the Retired Officials Committee, the Scale Manufacturers' Association, the American petroleum Institute, the Industry Committee on Packaging and Labeling, the regional weights and measures associations, and the National Association of State Departments of Agriculture Weights and measures associations, and the National Association of State Departments of Agriculture Weights and Measures Division.

\section{2,682}

PB96-178926 PC A03/MF A01

National Inst. of Standards and Technology (TS) Gaithersburg, MD. Weights and Measures Program.

Specifications and Tolerances for Reference Standards and Field Standard Weights and Measures. 2. Specifications and Tolerances for Field Standard Measuring Flasks.

Handbook.

G. L. Harris. 1996, 18p, NIST/HB-105/2

Supersedes COM-71-50065. Also available from Supt. of Docs. as SN003-003-03406-1. See also PB9O232752 .

Keywords: "Flasks, "Standards, Specifications, Tolerances(Mechanics), Measurement, Handbooks, Weight measurement, Volumetric analysis.

Field standard volumetric flasks and graduated cylinders as described herein are intended to be used by weights and measures officials, manufacturers and distributors of liquid products, research and testing laboratories, and others concerned with accurate measurements of the volume of liquids. This handbook volumetric flasks with graduated necks and graduated cylinders for legal metrology applications as 'field standards.'

\section{2,683}

PB96-179544 Not available NTIS

National Inst. of Standards and Technology (MEL), Gaithersburg, MD. Automated Production Technology Div.
Testing the Sensitivity of Accelerometers Using Mechanlcal Shock Pulses Under NIST Special Publlcatlon 250 Test No. 24040 S.

Final rept.

D. J. Evans. 1995, 15p

Pub: in American Society of Mechanical Engineers International Mechanical Engineering Congress and Exposition, San Francisco, CA., November 12-17, 1995, p1-15.

Keywords: "Accelerometers, "Shock tests, "Mechanical vibration, Piezoelectric transducers, Calibration standards, Sensitivity, Reference standards, Acceleration, Inteferometry, Shock(Mechanics), Shock absorbers, Frequency analysis, Test and evaluation, Reprints, Drop-ball apparalus.

The complex sensitivity of a laboratory reference $\mathrm{p}$ ezoelectric accelerometer in combination with a charge amplifier is obtained by comparison against two NIST standards which are calibrated periodically by reciprocity and by interferometric techniques. For each condition of test, a dynamic signal (FFT) analyzer is used to obtain estimates of the complex frequency response functlon (complex voltage output of the system under test relative to that of the reference system) and the coherence function from rms trispectral averages, as well as to obtain time averages of the complex linear spectra of the voltage outpuis of the system under test and the reference system. Together with the calibrated complex sensitivity of the reference system, these spectra may then be used to estimate the magnilude of the sensitivity of the system under test as a function of frequency, and to obtain an estimate of the magnitude of the peak output voltage of the system under test relative to the magnitude of the peak acceleration for each condition of test.

\section{2,684}

PB96-179569 Not available NTIS

National Inst. of Standards and Technology (MEL). Gaithersburg, MD. Automated Production Technology

Div.

Ultrasound Power Measurement Techniques at NIST.

Final rept.

S. E. Fick. $1995,6 p$

Pub. in American Society of Mechanical Engineers Congress and Exposition, San Francisco, CA., November 12-17, 1995, p1-6.

Keywords: *Ultrasonic wave transducers, "Power measurement, "Calibration, Ultrasonic radiation, tronic switching, Reprints, US NIST.

This paper tells how ultrasound power levels between 100 microwatts and 25 watts at frequencies between $0.5 \mathrm{MHz}$ and $200 \mathrm{HMz}$ are measured at NIST Ultrasonic radiation pressure, the phenomenon most commonly used by ultrasound power meters, is described in the context of an instrument devised at NIST to minimize all known associated components of uncertainty. Also, described are an electronic switching technique for high precision pulse generation, and a calibration transfer technique which bases replicated ultrasound power levels on measurements of dc voltage.

\section{2,685}

PB96-201116 Not available NTIS

National Inst. of Standards and Technology (BFRL), Gaithersburg, MD. Fire Science Div.

Low Heat-Flux Measurements: Some Precautions. Final rept.

A. F. Robertson, and T. J. Ohlemiller. 1995, 16p.

Pub. in Fire Safety Jnl., v25 n2 p109-124 1995.

Keywords: "Heat fluxes, "Measurement errors, Radiation, Gages, Sensors, Reprints, "Foreign technology.

Simple experiments are described for the purpose of illustrating measurement errors and their avoidance during use of Gardon or Schmidt-Boelter total heat flux sensors. These errors can assume serious proportions of the observed signal at flux levels below about 15 $\mathrm{kW} / \mathrm{sq}$. $\mathrm{m}$. They result from two sources, both a consequence of the flux gage's temperature relative to its surrounding: firstly, convective heating of the gage by the boundary layer from hot surfaces surrounding it and second, heat exchange with the ambient environment by radiation and convection. Some proposals are made for standardizing measurement methods, but it seems unlikely that errors in measurement can be completely eliminated. It thus becomes important for users of experimentally derived data to understand the limitations which may exist in the data reported.

\section{2,686}

PB96-210141 PC A10/MF A03

National Inst. of Standards and Technology (TS) Gaithersburg, MD. Office of Standards Services.

Proceedings of the Open Forum on Laboratory Accreditation at the National Institute of Standards and Technology, October 13, 1995.

Special pub.

W. Leight, and L. Galowin. 1996, 195p, NIST/SP

902.

Also available from Supt. of Docs. as $\mathrm{SNOO3}^{-003-}$ 03418-5.

Keywords: "Accreditation, United States, Meetings, Trade associations, Organization, Tests, "Laboratory accreditation, NIST'National Institute of Standards and Technology).

The American National Standards Institute (ANSI) ACIL (formerly the American Council of Independen Laboratories), and NIST co-sponsor an informal Laboratory Accreditation Working Group (LAWG) composed of representatives of laboratories, their governmental and private sector customers, accreditation bodies, and those public and private sector institutions that require laboratory accreditation. At a Forum on October 13, 1995, reports were presented by sectoral interests, along with a Vision and Principles proposed by the LAWG Steering Committee, aimed at developing a consensus for the formation of an integrated, cost effective infrasturcture for laboratory accreditation in the United States, mutual recognition of qualified bod ies, and facilitation of trade based on test results from competent bodies. These Proceedings are a transcript of papers presented and ensuing floor discussions at the Forum.

\section{2,687}

PB96-214705 PC A07/MF A02

National Inst. of Standards and Technology (TS) Gaithersburg, MD. Weights and Measures Program. State Weights and Measures Laboratories: Program Handbook.

G. L. Harnis. Jul 96, 113p, NIST/HB-143.

Supersedes PB85-183358. Also available from Supt. of Docs. as SNO03-003-03420-7.

Keywords: "Laboratories, "States(United States), "Accreditation, "Calibration standards, Metrology, Units of measurement, Weight, Tolerances(Mechanics), Standardization, Accuracy Requirements, Procedures, Tests, Criteria, Handbooks, State services, State Standards Program.

As part of its program to encourage a high degree of technical and professional competence in such activities, the National Institute of Standards and Technology (NIST) Office of Weights and Measures (OWM) has developed performance standards and formalized procedures for voluntary accreditation of State lega metrology laboratories. This Handbook describes the procedures followed in accrediting State (and a few other jurisdictional) legal metrology laboratories fo competence.

\section{2,688}

\section{PB96-215074 PC A2O/MF A04}

National Inst. of Standards and Technology, Gaithersburg, MD. Office of Standards Services. Directory of U.S. Private Sector Product Certification Programs.

Special pub. (Final)

C. W. Hyer. Jul 96, 446p, NIST/SP-903

Supersedes PB90-161712. Also available from Supt. of Docs. as SNOO3-003-03414-2.

Keywords: "Consumer products, "Product inspection, "Directories, "United States, Inspection, Certification, Specifications, Quality assurance, Accreditation, Tests, Registration, Standards, Private sector, Listings, Conformity assessment.

This document presents information on 178 private sector groups in the United States which engage in product certification activities. Entries describe the type and purpose of each organization, the nature of the activity, a pictorial representation of the organization's mark (if available), products certified, standards used certification requirements, any accreditation or recognition by a U.S. or foreign private sector or government agency, availability of services, methods of cost determination, and other relevant detalls.

\section{2,689}

PB97-108575 PC A04/MF A01 
National Inst. of Standards and Technology (EEEL), Gaithersburg, MD. Electricity Div. NIST High-Accuracy Sampling Wattmeter.

Technical note.

G. N. Stenbakken, and A. Dolev. Aug 96, 50p, NIST/ TN -1420 .

Portions of this document are illegible in microfiche products. Also available from Supt. of Docs. as SN003$003-03422 \cdot 3$

Keywords: "Wattmeters, Ammeters, Voltmeters, Power measurement, Product development, Engineering drawings, US NIST

A high-accuracy sampling wattmeter was developed at the National Institute of Standards and Technology (NIST) to investigate the feasibility of using waveform sampling techniques for making very accurate power measurements at frequencies from $50 \mathrm{~Hz}$ to $1000 \mathrm{~Hz}$ The goal of this effort was to develop an instrument having a full scale measurement uncertainty over these frequencies of less than plus or minus 50 micro WN. The prototype instrument that came out of the development was used to demonstrate the accuracy achievable with the digital sampling method. The new high-accuracy sampling wattmeter was built around a wideband instrument developed earlier at NIST.

\section{2,690}

PB97-110217 Not available NTIS

National Inst. of Standards and Technology (TS),

Gaithersburg, MD

Examination Procedure Outlines: Keys to Solving the Handbook 44 Puzzle.

Final rept.

J. Koenig. 1993, 5p

Pub. in Weighing and Measurement, p26-30 Aug 93

Keywords: "Standards, *Weight measurement, Meters, Procedures, Safety notes, Scales, Reprints,
"Examination Procedure Outlines(EPOs), Handbook 44.

The article covers the development, organization, and use of the Examination Procedure Outlines (EPO's) published by the National Conference on Weights and Measures in cooperation with the National Institute of Standards and Technology (NIST). EPO's are outlines of requirements from the General Code and specific device codes of NIST Handbook 44. Specifications Tolerances, and Other Technical Requirements for Weighing and measuring Devices, that apply to a certain category of commerical devices such as vehicle scales or motor-fuel dispensers. They are used by State and local weights and measures officials as well as device manufacturers and service companies to check a devices compliance with legal requirements.

\section{2,691}

\section{PB97-110266 Not available NTIS}

National Inst. of Standards and Technology (CSTL) Gaithersburg, MD. Process Measurements Div.

Current Status and Trends in Temperature Measurements at NIST, Cooperative Projects and New Mutual Agreement between NIST and IMGC

Final rept.

B. W. Mangum. 1992, 11p.

Pub. in Proceedings of the Italy/USA Metrology Semjnar, Torino, Italy, May 5-8, 1992, p1-11.

Keywords: "Temperature measurements, "Standards, Thermometry, Reprints, "Foreign technology, "International Temperature Scale of 1990, National Institute of Standards and Technology.

In the brief paper, the authors will discuss the status of the current realization of the International Temperature Scale of 1990 (ITS-90)I over its entire range associated with contact thermometry, various development projects, and the current research. Then, they will address the trends in temperature measurements at the National Institute of Standards and Technology (NIST), discuss some cooperative projects between NIST and the Istituto de Metrologia ' $G$. Colonnetti' (IMGC), and conclude with a brief discussion of the Mutual Agreement between NIST and IMGC on Equivalence of National Scales of Temperature.

\section{2,692}

PB97-111850 Not available NTIS

National Inst. of Standards and Technology (TS), Gaithersburg, MD. Calibration Program.

Measurement Comparability, Traceability, and Measurement Assurance Programs.

Final rept.

S. Dittmann 1996, 6 p.

Pub. in Metrology for the Americas Symposium, Miami, FL., November 6-10, 1995, p1-61996.
Keywords: "Reference standards, "Tracer techniques "Interlaboratory comparisons, Metrology, Measure ment, Accuracy, Standardization, Quality assurance, Reprints.

The paper presents three parts of a national metrology system - a system linked to other nations (e.g., trading partners) and to the local industries, and one in which industries having common metrology needs are strongly linked. The three parts of this system are measurement comparability, traceability, and assurance.

\section{2,693}

PB97-118368 Not available NTIS

National Inst. of Standards and Technology (MSEL)

Gaithersburg, MD. Metallurgy Div.

Issues in High-Speed Pyrometry.

Final rept.

A. Cezairliyan, and F. Righini. 1996, 8p

Pub in Merrologia, v33 p299-306 1996.

Keywords: "Temperature measurements, "Pyrometry Radiation thermometry, High temperatures, Optica, Reprints, "Foreign technology, "High-speed techniques.

A brief summary is given of the developments in optical techniques for rapid temperature measurement during the last three decades in two national laboratories, the National Institute of Standards and Technology in the USA and the Instituto di Metrologia 'G. Colonnetti' in Italy. Results of research conducted in originating and advancing the state-of-the-art in high-speed (millisecond- and microsecond-resolution) pyrometry for measurement of temperatures in the range 1000 $\mathrm{K}$ to $5000 \mathrm{~K}$ are discussed. The main emphasis is on several key issues related to high-speed pyrometry including: high-speed operation, calibration of pyrometers, determination of true temperature and high-temperature reference points. Anticipated future directions in research, developments and applications in high-speed pyrometry are briefly discussed.

\section{2,694}

PB97-118376 Not available NTIS

National Inst of Standards and Technology (MSEL) Gaithersburg, MD. Metallurgy Div.

Simultaneous Measurement of Normal Spectral Emissivity by Spectral Radiometry and Laser Polarimetry at High Temperatures in Pulse-Heating Experiments: Application to Molybdenum and

Tungsten.

A. Cezairliyan, S. Krishnan, and J. L. McClure. 1996, $19 p$

Pub. in International Jnl. of Thermophysics, v17 n6 p1455-1473 Nov 96

Keywords: "High-speed measurements, "Spectral radiometry, "Laser polarimetry, High temperatures, Molybdenum, Pulse heating, Pyrometry, Reprints.

Spectral radiometry and laser polarimetry are two independent methods for measurement of spectral emissivity of materials. In the paper, a high-speed system is described for the rapid measurements of normal spectral emissivity of a specimen based on the simultaneous utilization of the two techniques. One of the goals of the work is to ascertain the reliability of the laser polarimetry technique in measurement of normal spectral emissivity at high temperatures. To accomplish this, normal spectral emissivity, in the vicinity of $0.633 \mathrm{~m}$, of molybdenum and tungsten were measured by the two techniques in the temperature range 2000$2800 \mathrm{~K}$. The results obtained by the iwo techniques are in agreement with $1 \%$. The total uncertainty (two standard-deviation level) in measurement of emissivity by either spectral radiometry or laser polarimetry technique is estimated to be not more than plus or minus $2 \%$

\section{2,695}

PB97-119101 Not available NTIS

National Inst. of Standards and Technology, Gaithersburg, MD. Office of the Director. Design of Technically Complex Facilities. Final rept.

S. R. Hogan. 1996, 50p

Pub in American Institute of Architects Symposium: Building Performance, Defining the Architect's Role Washington, DC., April 12-13, 1996, 50p.

Keywords: *Advanced technology laboratory *Design criteria, Laboratories, Research facilities, Environmental control, Temperature control, Product manage ment, Metrology, Reprints, US NIST.
NIST's new Advanced Technology Laboratory (ATL) has a program goal to create the most environmentally stable laboratory in the world, in support of NIST's mission in basic research and measurement standards. The presentation will outline NIST and its place in the global measurement community, an overview of the new ATL design, a focus on several areas of research for which the new ATL is critical, and a presentation on the research project itself.

02,696

PB97-121610 PC A08/MF A02

National Inst. of Standards and Technology (TS), Gaithersburg, MD. Office of Information Services Science, Technology, and Competitiveness: Retrospective on a Symposium in Celebration of NIST's 90th Anniversary and the 25th Anniversary of the Gaithersburg Laboratories, November 14-15, 1991. Special pub.

G. W. Hixenbaugh. Oct $96,138 p$, NIST/SP-837.

Color illustrations reproduced in black and white. Also available from Supt. of Docs. as SN003-003-3431-2.

Keywords: "Meetings, "Federal agencies, "Research and development, Government agencies, Standards, Technoogy, Robotics, Fiber optics, Tomography, Fire protection, Competition, "US NIST. Gaithersburg(Maryland)

This symposium held in observance of the 90th anniversary of the National Institute of Standards and Technology and the 25th anniversary of NIST's Gaithersburg, MD, laboratories. The first day's program included sessions on emerging technologies, proprietary vs, non-proprietary research, educating the workforce, and the view from the U.S. Congress. On the second day NIST directors and researchers described some of the latest research at the Institute. Topics included advanced technology; competitiveness: computational geometry; computer performance fire research; artificial intelligence and robotics; the history of NIST and the future of NIST; optical technology; science education; and surface science.

\section{Manufacturing Processes \& Materials Handling}

02,697

PB94-176666 PC A09/MF A02 Northwestern Univ., Evanston, IL. BIRL Industrial Reearch Lab.

Opportunities for Innovation: Advanced Surface Engineering.

W. D. Sproul, and K. O. Legg. May 94, 191p, NIST/ GCR-94/640-

Grant NIST-60NANB32D1223

See also PB94-100278. Sponsored by National Inst. of Standards and Technology, Gaithersburg, MD.

Keywords: "Surface finishing, "Technology innovation, "Coating processes, State of the art, Quality control, Deposition, Sputtering, Plating, Spray coating, Hea treatment, Ion implantation, Cathode sputtering Chemical vapor deposition, Laser applications, Diamonds, Nitriding, "Advanced technology, Diamond films, Plasma enhanced chemical vapor deposition.

Over the last 20 years more advanced surface treatments have been developed. While some have been widely deployed (physical vapor deposition, chemica vapor deposition, thermal spray, and plasma nitriding for example), others are less commonly used, or are just beginning to appear commercially (including ion implantation, diamond coating, and laser coating). The purpose of this volume is to provide information on the basics of advanced surface engineering processes to small businesses, especially to those not currently directly involved with the technology, in order to make them aware of the current state of the art. Each chapter has been written by an industrial expert in the particular area, and following the basic description of the technology are sections outlining the needs of the surface treatment (such as better controls, improved materials, or better knowledge of fundamental processes) and the future markets that can be expected to open. Meeting these needs and exploiting these new markets is likely to be best done by the combined efforts of present providers with companies whose experience lies in entirely different areas, and it is this type of cross-fertilization that this volume seeks to promote. 
02,698

Not available NTIS

National Inst. of Standards and Technology (MSEL), Gaithersburg, MD. Polymers Div.

Comparison of the Unidirectional and Radial InPlane Flow of Fluids Through Woven Composite Reinforcements.

Final rept.

R. S. Parnas, and A. J. Salem. 1993, 12p.

Pub. in Polymer Composites 14, n5 p383-394 Oct 93.

Keywords: *Flow distribution, "Woven composites, Resin transfer molding, "Fluid flow, Fiber composites, Comparison, Glass fiber reinforced plastics, Newtonian fluids, Preforms, Reinforcing fibers, Reprints.

The in-plane flow of fluids through dense fibrous woven reinforcements was studied to aid the development of constitutive models for use in simulations of composite fabrication by resin transfer molding. As a first part of his effort, both one-dimensional and radial flow experiments were conducted with Newtonian fluids in severa woven glass fabrics. Analysis of the one-dimensional flow experiments shows that the two experimenta techniques are often, but not always, consistent, and both techniques suggest a relationship between the physical structure of the reinforcement and the mathe matical structure of the permeability tensor. Preform features at the laminar and interlaminar scales were hypothesized to influence the experimental results.

02,699

PB96-122981 Not available NTIS

National Inst. of Standards and Technology (MEL) Gaithersburg, MD. Automated Production Technology Div.

Effects of Spindle Dynamic Characteristics on Hard Turning.

Final rept.

K. Harper, M. Davies, and A. Donmez. 1995, 4p.

Pub. in International Precision Engineering Seminar Proceeding (8th), Compiegne, France, May 15-19, $1995,4 p$

Keywords: *Machining, "Lathes, "Precipitation hardening. Manufacturing, Mechanical properties, Spindles, Reprints, "Foreign technology.

Error motions between the tool and workpiece during cutting operations affect the surface finish of the fina products. These error motions arise from a number of different sources including asynchronous axial machine and process vibrations, tool wear, and tool edge build up. In this work, the error motions and dynamic characteristics of a tapered-roller bearing spindle with adjustable bearing preload were measured, and their effect on hard turned surfaces was assessed.

02,700

\section{PB96-160494 Not available NTIS}

National Inst. of Standards and Technology (MEL) Gaithersburg, MD. Automated Production Technology Div.

Evaluation of a Tapered Roller Bearing Spindle for High-Precision Hard Turning Applications.

Final rept.

M. A. Donmez, K. Harper, L. Greenspan, Y

Matsumoto, and L. Keller. 1993, 6p.

Pub. in Annual Meeting of the American Society for Precision Engineering (8th), Seattle, WA., November $7-12,1993,6 \mathrm{p}$

Keywords: *Spindles, "Maching, "Axes of rotation, Precision, Defects, Roller bearings, Metrology, Reprints "Hard turning.

In this study, we are to evaluate a spindle which consisted of a pair of high precision tapered roller bearing including a HYDRA-RIB tapered roller bearing. Rolle bearing spindles are used for applications that require high bearing stiffness, and can be good candidates for applications such as precision hard turning. For evaluation purposes, we have classified our measurements into four categories: axis of rotation errors, ther mal growth, static stiffness, and dynamic stiffness and damping. In this presentation, we will focus on the axis of rotation error measurements. In addition to evaluating the performance of the spindle, we will show that the axis of rotation error measurements can also be used to help diagnose, analyze and improve the spindle design.

\section{Nondestructive Testing}

\section{2,701}

PB94-172111 Not available NTIS

National Inst. of Standards and Technology (CAML) Gaithersburg, MD. Applied and Computational Mathe matics Div.

Infinite Divisibility and the Identification of Singular Waveforms.

Final rept.

A. S. Carasso. 1992, 14p.

Pub. in Proceedings of Conference on Probabilistic and Stochastic Methods in Analysis, with Applications, Ciocco, Italy, July 14-27, 1991, p273-286 1992

Keywords: "Ultrasonic tests, Nondestructive tests, System identification, Wave propagation, Elastic waves, Volterra equations, Stochastic processes, Waveforms, Reprints, Flaw detection, Impulse response, Deconvolution, Divisibility.

Infinitely divisible probability density functions on the half-line $\mathrm{t}=$ or $>0$ form a convolution semigroup on $t=o r>0$, as they describe stochastic processes with stationary, non-negative, independent increments. A subclass D of such densities are C(sup infinity) functions on the whole t-line when extended by zero for $t<0$. Such functions may be viewed as physically re alizable, causal, C(sup infinity) approximations to the Dirac delta-function, with further positivity properties. The use of such probe waveforms for system identification is particularly advantageous in transient wave propagation problems, where the system's impulse re sponse is typically highly singular. An ill-posed deconvolution problem must be solved to recover the system's response; the semigroup and positivity properties of the input probe enable the deconvolution problem to be implemented as a Cauchy problem or a diffusion equation. The approach allows the analys to monitor the gradual and systematic development of sharp singularities in the presence of noise. One important context where this theory applies is ultrasonic flaw detection in nondestructive evaluation.

\section{2,702}

PB94-191640 PC A03/MF A01

National Inst. of Standards and Technology (MEL), Gaithersburg, MD. Ultrasonics Group.

NIST Callbratlon of ASTM E127-Type Ultrasonic Reference Blocks.

J. A. Slotwinski, and G. V. Blessing. May 94, 49p, NISTIR-5430

See also PB-258 105

Keywords: *Calibrating, ${ }^{\star}$ Standards, Ultrasonic wave transducers, Underwater acoustics, Ultrasonic tests, Nondestructive tests, "Ultrasonic reference blocks, Aluminum alloy 7075 , US NIST.

This document describes the procedures and instrumentation used by the National Institute of Standards and Technology (NIST) in calibrating aluminum ultrasonic reference blocks. The Ultrasonics Group performs this calibration service for technological and industrial customers. The calibration is based on the American Society of Testing and Materials (ASTM) recognized practice for calibrating aluminum reference blocks designated E127-92a. Instructions for requesting calibrations of ultrasonic reference blocks are contained in Appendix I of this document. The NIST ultrasonic reference block calibration service was originated in the late 1970's, and is described in the NBS Technical Note 924 (PB258105). At that time the application of one master block for use as a reference standard was considered to be a temporary or interim measure. Today NIST uses this master block to set the ultrasonic system sensitivity in all calibrations of ultrasonic reference block sets. In addition there are several newly implemented practices employed for checking the calibration system characteristics, both over a period of years and in preparation for a particular calibration. This current document updates the NBS Technical Note 924 in describing these practices for our calibration procedure.

\section{2,703}

PB96-103098 Not available NTIS

National Inst. of Standards and Technology (EEEL), Gaithersburg, MD. Semiconductor Electronics Div.
Comparison of Techniques for Nondestructive Composition Measurements in CdZnTe Substrates.

Final rept.

S. P. Tobin, J. P. Tower, P. W. Norton, V. C. Lopes, W. M. Duncan, A. J. Syllaios, C. K. Ard, N. C. Giles R. Balasubramanian, A. B. Bollong, T. W. Steiner, D. K. Bowen, B. K. Tanner, D. Chandler-Horowitz, and P. M. Amirtharaj. 1995, 10p

Pub, in Jnl. of Electronic Materials, v24 n5 p697-705 1995.

Keywords: "Nondestructive tests, "Alloys, *Photoluminescence, Semiconductors(Materials), Optical measurement, Chemical composition, Lattice parameters, Cadmium alloys, $X$ ray diffraction, Precision, Accuracy, Comparative evaluations, Variations, Epitaxy, Reprints, Photoreflectance.

The authors report an overview and a comparison of nondestructive optical techniques for determining alloy composition $\mathrm{x}$ in $\mathrm{Cd}, \mathrm{Zn}$, Te substrates for $\mathrm{HgCdTe}$ epitaxy. The methods for single-point measurements include a new $x$-ray diffraction technique for precision lattice parameter measurements using a standard high-resolution diffractometer, room-temperature photoreflectance, and low-termperature photoluminescence. The authors compare measurements on the same set of samples by all three techniques. Comparisons of precision and accuracy, with a discussion of the strengths and weaknesses of different techniques, are presented.

02,704

PB96-111786 Not available NTIS

National Inst of Standards and Technology (MSEL) Boulder, CO. Materials Reliability Div.

Contributions of Out-of-Plane Material to a Scanned-Beam Laminography Image.

Final rept.

T. A. Siewert, and M. A. Austin. 1994, 6p.

Sponsored by Harry Diamond Labs., Adelphi, MD.

Pub. in Materials Evaluation, v52 n10 p1193-1198 Oct 94.

Keywords: "X ray inspection, "Nondestructive tests, *Image resolution, Blurring, Focusing, Gray scale, "Laminography, "X ray laminography, "Out-of-plane.

The authors studied the edge of a thin step wedge with a scanned-beam laminography system to learn how material above and below the plane of focus contributes to the image. The edge of a planar, $0.5 \mathrm{~mm}(0.02$ in.) Thick lead sheet with large $x$ and $y$ dimensions was centered in the field of view. Images were captured as the lead sheet was moved in $0.075 \mathrm{~mm}(0.003 \mathrm{in}$.) Steps above and below the plane of focus of the sys tem. The blurring (measured as its width on the image) was no more than $0.1 \mathrm{~mm}(0.004 \mathrm{in}$., or the system resolution) when the sheet was in focus and increased linearly with the deviation from focus. Blurring of out of-plane objects is an essential feature of this laminography system, and is used to minimize the contribution to the image from material above and below the plane of focus. The authors also imaged various three-dimensional structures to show the ability of laminography to resolve different planes of focus.

\subsection{5}

PB96-141023 Not available NTIS

National Inst. of Standards and Technology (MSEL), Boulder, CO. Materials Reliability Div.

Void Shape in Sintered Titanium.

Final rept.

H. Ledbetter, M. Dunn, S. Kim, and R. Fields. 1995 $7 p$

Pub. in Review of Progress in Quantitative Non destructive Evaluation, v14 p1633-1639 1995

Keywords: "Sound velocities, "Titanium, Ultrasonics, Deformation, Reprints, *Void shapes.

Void shape in sintered materials is important because the shape affects both physical and plastic-deformation problem. For example, the mechanical stress distribution around a disc-shaped void differs drastically from that around a spherical void. Thompson, Spitzig. and coworkers made ultrasonic studies on compacted iron. Haynes reviewed the general topic of mechanical properties of sintered materials. Because voids are small, three-dimensional, and essentially empty space between grains, their geometry determination presents formidable problems. Here, we take an indirect approach to void shape: measure longitudinal-wave and transverse-wave sound velocities, use a solid-mechan- 


\section{Nondestructive Testing}

ics model that predicts effective ('composite') sound velocities from matrix sound velocities and occlusion phase geometry, use model inversely to estimate void shape.

\section{2,706}

PB96-154596 PC A05/MF A01

National Inst. of Standards and Technology (EEEL) Gaithersburg, MD. Semiconductor Electronics Div.

Semiconductor Meas urement Technology: Survey of Optical Characterization Methods for Materials Processing, and Manufacturing in the Semiconductor Industry.

Special pub.

W. M. Bullis, S. Perkowitz, and D. G. Seiler. Dec 95, 54p, NIST/SP-400-98

Also available from Supt. of Docs, as SNO03-00303381-2. Prepared in cooperation with Emory Univ., Atlanta, GA.

Keywords: "Semiconductor devices, "Optical measurement, "Silicon, Ellipsometry, Infrared spectroscopy, Interferometry, Photoluminescance, Optical micros copy, Raman spectroscopy, Scutterometry, Microelectronics, Test methods, Surveys.

Contactless, nondestructive optical methods are used to characterize many critical properties of materials, processes, and devices in the semiconductor industry. To determine the extent of use and the relative importance of various opticals methods in the industry, the Smeiconductor Electronics Division of the National Institute of Standards and Technology conducted a survey of this field. The survey also sought to identify both advantages and limitations of these techniques as well as future requirements for and anticipated use of optical characterization methods within the semiconductor industry. Data from 42 firms were analyzed to show the impact of the methods, what they measure, their range and precision, and their cost. A significant finding of the study is the need expressed by many industrial users for improved standards and test methods for optical characterization, especially in the area of film thickness and composition.

\section{2,707}

PB96-190327 Not available NTIS

National Inst. of Standards and Technology (MSEL), Boulder, CO. Materials Reliability Div.

Ultrasonic Methods.

Final rept.

R. B. Thompson, W. Y. Lu, and A. V. Clark. 1996

30p.

Pub. in Handbook of Measurement of Residual Stresses, Chapter 7, p149-178 1996.

Keywords: "Ultrasonic tests, "Residual stress, "Stress measurement, Nondestructive tests, Acoustic properties, Elastic properties, Reprints, Acoustoelastic measurement, Through-thickness.

The average stress is determined in the region through which the waves propagate, as indicated by the crosshatching: (1) through-thickness pulse-echo; (2) through-thickness pitch-catch; and (3) surface pitchcatch.

\section{Quality Control \& Reliability}

\section{2,708}

PB94-199064 Not available NTIS

National Inst. of Standards and Technology (NEL) Gaithersburg, MD. Statistical Engineering Div. Statistical Quality Control Technology in Japan.

Final rept.
K. O. Bowen, T. H. Hopp, R. N. Kacker, and R. J. Lundegard. 1991, 7p.

Pub. in Chance 4, p15-21 1991

Keywords: "Quality control, "Japan, "Technology assessment, Industries, Laboratories, Government agencies, Reprints, "Foreign technology, "Statistical quality control, US NIST, TQC(Total Quality Control).

From May 17 to June 1, 1989, a survey team organized by the National Institute of Standards and Technology visited Japan to assess research and application of statistical quality control technology. The team explored the philosophy and conduct of total quality control (TQC) in Japanese industries, government laboratories, and national agencies. The philosophy and practice of TQC in Japan is quite different from that of the U.S. industries.

\section{Tooling, Machinery, \& Tools}

\section{2,709}

PB94-206109 PC A03/MF A01

National Inst. of Standards and Technology (MEL), Gaithersburg, MD. Precision Engineering Div.

User's Guide to NIST SRM 2084: CMM Probe Performance Standard.

Special pub.

G. W. Caskey, S. D. Phillips, B. R. Borchardt, D. E. Ward, and D. S. Sawyer. Jun 94, 32p. NIST-SP-260120.

Also available from Supt. of Docs. as SN003-00303275-1.

Keywords: "Dimensional measurement, "Standards, "Probes, Machine tools, User manuals, Performance, Uncertainty, Metrology, Calibration, Spheres, Mounts, Design, "Standard reference materials, "Coordinate measuring machines.

National and international published standards on the performance evaluation of coordinate measuring machines (CMMs) require various artifacts to facilitate testing of these machines. Standard Reference Material (SRM) 2084, developed by the National Institute of Standards and Technology, is a calibrated $10 \mathrm{~mm}$ diameter sphere and mount designed to aid in CMM, and specifically CMM probe, performance assessment. This publication serves as a users' manual, with general instructions on the care and use of SRM 2084. Reference to relevant tests in the ASME B89.1.12M1990 CMM performance standard as well as several performance issues not addressed in the Standard are presented.

\section{2,710}

\section{PB95-203303 Not available NTIS}

National Inst. of Standards and Technology (PL), Boulder, CO. Quantum Physics Div.

Low-Frequency, Active Vibration Isolation System. Final rept.

R. Stebbins, D. Newell, S. Richman, J. Mason, P. Bender, and J. Faller. 1994, 11p.

Sponsored by National Science Foundation, Arlington, VA.

Pub. in Proceedings of Society of Photo-Optical Instrumentation Engineers: Vibration Monitoring and Control, San Diego, CA., July 28-29, 1994, v2264 p27-37.

Keywords: "Vibration isolators, Extremely low frequency, Infrasonic frequencies, Seismic noise, Ground motion, Control systems, Active control, Performance, Design, Reprints.

We are developing an active vibration isolation system for reducing the disturbing effects of ground motion about six orders of magnitude between 1 and $30 \mathrm{~Hz}$. The system consists of three cascaded stages, each comprising a suspended platform on which are located seismometers and actuators for six degrees of freedom. Our goal is to reduce the vibration noise to (1 $\times 10(\exp -13) \times(1 \mathrm{~Hz} / \mathrm{f})(\sup 2.5)+3 \times 10(\exp -15)) \mathrm{m}$ $\mathrm{Hz}($ sup $1 / 2)$ on the third stage. We have built and tested the initial stage, which is intended to reduce our laboratory noise level to that of a quiet site. The design, control system models, measured performance and current limitations for this stage are presented. The two main stages require a vacuum environment and interferometric readout. The design and models for the main stages are also presented.

\section{General}

02,711

PB94-198488 Not available NTIS

National Inst. of Standards and Technology (CSL), Gaithersburg, MD. Advanced Systems Div.

Dilemma-Preservation versus Access.

Final rept.

T. C. Bagg. 1993, 5p

Pub. in Preservation Research and Development Round Table Proceedings, Washington, DC., Septem ber 28-29, 1992, p57-61 1993. Sponsored by Library of Congress, Washington, DC.

Keywords: "Data storage, "Information management "Media, Preserving, Access, Indexes (Documentation) Information retrieval, Digital data, Papers, Microfilm, Reprints.

The dilemma is to find suitable techniques and media to preserve information now stored on media with a short life expectancy. A stable recording media such as an acid free buffered paper on microfilm, where the information is in human readable form and has access suitable to its needs, is the best solution today. Information requiring frequent access should be indexed and stored in a digital form for rapid automated retrieval with a back-up on a permanent medium. Today's digital systems, which are essential for rapid ac cess, are not suitable for preservation because of the continuous changes in computer technology.

02,712

PB95-125837 Not available NTIS

National Inst. of Standards and Technology (CSL) Gaithersburg, MD. Systems and Software Technology Div.

Important Papers in the History of Document Preparation Systems: Basic Sources.

Final rept.

R. Furuta. 1992, 26p.

Pub. in Electronic Publishing 5, n1 p19-44 Mar 92.

Keywords: *Documents, "Text processing, "Computer applications, Format, Data processing, Text editors, Reprints.

This report provides a narrative description of influential papers that discuss computer-based document preparation systems. The report's focus is on the systems actually used to prepare documents--editors and formatters, and the goal is to provide an introduction to the papers that have been influential on the community of researchers who investigate such systems.

02,713

PB96-157896 Not available NTIS

National Inst. of Standards and Technology (BFRL) Gaithersburg, MD. Fire Safety Engineering Div.

Evolution of a United States Information System. Final rept.

N. H. Jason. 1993, 10p

Pub. in Proceedings of the inFIRE Annual Conference, Norwood, MA., April 28-30, 1993, p89-98.

Keywords: "Bibliographic databases, "Computer networks, "Fire research, Information dissemination, Reprints, Internet.

As part of the current information activities in the United States, the Conference for Exploration of a Nationa and Engineering Information Service is discussed, in addition to the Internet and the proposed NREN ( $\mathrm{Na}$ tional Research Education Network). Challenges to the fire community to bridge the information gap are presented.

02,714

PB96-157904 Not available NTIS

National Inst. of Standards and Technology (BFRL) Gaithersburg, MD. Fire Safety Engineering Div. 
Information Transfer in the 21st Century.

Final rept.

N. H. Jason. 1993, 10p

Pub. in Proceedings of the International Fire Information Conference (1st), Moreton-in-Marsh, 1421993.

Keywords: "Fire research, "Information transfer, Politics, Libraries, Reprints, "Foreign technology.

The impact of technological advances and some political events are discussed in the context of current information activities. Several examples of new and exciting projects in information technology in print and nonprint activities are presented. The role of the information professional and specifically the future role of fire libraries concludes the discussion.

\section{Information Systems}

\section{2,715}

N94-31228/7 PC A06/MF AO2

National Inst. of Standards and Technology, Gaithersburg, MD.

Putting the Information Infrastructure to Work: Report of the Information Infrastructure Task Force Committee on Applications and Technology.

May 94, 116p, NIST/SP-857, ISBN-0-16-043188-3.

Keywords: "Communication networks, "Computer networks, "Information systems, Technology utilization, Commerce, Computers, Education, Environmental monitoring, Libraries, Manufacturing, Public health, Technology assessment.

The Committee on Applications and Technology of the Information Infrastructure Task Force is charged with coordinating efforts to develop, demonstrate, and promote applications of information technology in several diverse areas and to develop and recommend technology strategies and policy to accelerate the implementation of the National Information Infrastructure (NII). The goal of this document is to focus the public debate on the uses of the NII and the benefits of advanced computing and communications technologies. Seven applications papers are presented which address manufacturing, electronic commerce, health care, education and lifelong learning, environmenta monitoring, libraries, and government service delivery. In each of these papers a description of the application arena is given along with a summary of the current information technology uses, short and long term goals, and issues related to how these goals could be achieved.

\section{2,716}

N94-36858/6 (Order as N94-36853/7, PC A10/

MF A03)

National Inst. of Standards and Technology, Gaithersburg, MD

Open System Environment Procurement.

G. Fisher. Aug 94, 43p.

In National Aeronautics and Space Administration, NASA Sti Program Coordinating Council Twelfth Meeting: Standards p 116-158.

Keywords: "Computer networks, "Government procurement, "Information systems, "Standards, Data bases, Data transfer (Computers), Proposals.

Relationships between the request for procurement (RFP) process and open system environment (OSE) standards are described. A guide was prepared to help Federal agency personnel overcome problems in writing an adequate statement of work and developing realistic evaluation criteria when transitioning to an OSE. The guide contains appropriate decision points and transition strategies for developing applications that are affordable, scalable and interoperable across a broad range of computing environments. While useful, the guide does not eliminate the requirement that agencies possess in-depth expertise in software development, communications, and database technology in order to evaluate open systems.

N2,717 $\quad$ (Order as N95-15919/0, PC A11/

MF A03)

National Inst. of Standards and Technology, Gaithersburg, MD

Slandards and Technology

National Center for Standards and Certification Information: Service and Programs.

J. Overman. 14 Sep $94,7 p$

In NASA, Washington, Fourth Annual International ACquisitions Workshop: Access to Multiple Media Worldwide $7 p$.

Keywords: "Certification, "Information dissemination "Information systems, "International cooperation, *Libraries, "Regulations, "Standards, Commerce Competition, Directories, Documentation.

The National Center for Standards and Certification Information ( $\mathrm{NCSCl}$ ) provides information on U.S., foreign and international voluntary standards, government regulations, and conformity assessment procedures for non-agricultural products. The Center serves as a referral service and focal point in the United States for information on standards and standards-related information. $\mathrm{NCSCl}$ staff respond to inquiries, maintain a reference collection of standards and standards-re lated documents, and serve as the U.S. inquiry point for information to and from foreign countries.

\section{2,718}

PB94-172194 Not available NTIS

National Inst. of Standards and Technology (NCSL), Gaithersburg, MD. Systems and Network Architecture Div.

Formal Multi-Layer Test Methodology and Its Application to OSI.

Final rept.

J. P. Favreau, R. J. Linn, and S. Nightingale. 1989,

$18 p$.

Pub. in Proceedings of International Conference on Formal Description Techniques for Distributed Sys tems and Communications Protocols (2nd), Van couver, Canada, December 5-8, 1989, p375-392 1990.

Keywords: "Tests, "Specifications, File management systems, Protocols, Reprints, "OSI, "Open Systems Interconnection, FTAM(File Transfer and Management), Estelle specification language, ASN1(Abstrac Syntax Notation One), FTP(File Transfer Protocol)

A multi-layer test methodology is introduced which takes advantage of the emergence of formal specification methods. Protocols are specified using Estelle and Abstract Syntax Notation One. Then, the Estelle specifications are augmented in with the logic necessary for lesting. In this methodology, test cases are stimuli which are injected into the augmented protocol machines. This methodology has been applied success fully to the design and implementation of a test system for FTAM/FTP gateways. Results are presented fo FTAM/FTP gateway testing, FTAM interoperation testing, and evaluation of a commerical FTAM test system which is under development.

\section{2,719}

PB94-172202 Not available NTIS

National Inst. of Standards and Technology (NCSL), Gaithersburg, MD. Systems and Network Architecture Div.

ISO/NEC Workshop on Worldwide Recognition of OSI Test Results Regional Progress - North America.

Final rept.

J. P. Favreau. 1993, 13p.

Pub. in Proceedings of ISO/IEC Worldwide Recognition of Open Systems Interconnection Test Results Workshop, Brussels, Belgium, November 2-4, 1993 $13 p$

Keywords: "Tests, "North America, Certification, Ac creditation, Standardization, Information systems, Re prints, "OSI, "Open Systems Interconnection GOSIP(Government OSI Profile), IGOSS(Industry Government Open Systems Specification).

This paper presents a summary of the developments in North America towards harmonization of testing and certification/registration in the information technology field. The current status follows an intense period of activity to develop North American regional strategies favorable to future multilateral recognition of national regional certificates/registers on a global basis. Specia emphasis is given to the consolidation of profiles via the design the Industry/Government Open Systems Specification (IGOSS), the quality Improvement of the Government Open System Interconnection Profile (GOSIP) Testing Program and the creation of the North American Open Systems Testing and Certification Policy Council as the basic steps towards achieving world wide recognition of test results.

\section{2,720 Not available NTIS}

Not available NTIS
National Inst. of Standards and Technology (CSL) Gaithersburg, MD. Advanced Systems Div.

Bringing Natural Language Information Retrieval Out of the Closet.

Final rept.

D. K. Harman, and G. T. Candela. Jul 90,7p.

Pub. in SIGCHI Bulletin 22, n1 p42-48 Jul 90.

Keywords: "Information retrieval, "Natural language processing, Information systems, Prototypes, Man computer interface, Human factors engineering, ACcess, Tests, Response time(Computers), Reprints

A prototype information retrieval system was developed that gives users fast and easy access to textual information. The system uses a statistical ranking methodology that allows a user to input a query using only natural language, such as a sentence or a noun phrase, with no special syntax required. The system returns a set of text titles or descriptions, ranked in order of likely relevance to the query. The user can then select one or more titles for further examination of the corresponding text. The prototype was tested by over forty users.

\section{2,721}

PB94-172921 Not available NTIS

National Inst. of Standards and Technology (CSL), Gaithersburg, MD. Systems and Software Technology Div.

Porting Multimedia Applications to the Open System Environment.

Final rept.

R. D. Schneeman. 1992, 9p.

Pub. in Institute of Electrical and Electronics Engineers Software 9, n6 p39-47 Nov 92

Keywords: "Computer program portability, Computer program transferability, Standards, US NIST, Reprints, "OSE, "Open System Environment, "Multimedia

Organizations in both industry and government are rapidly moving toward the Open System Environment (OSE), which is based on standards for information technology. Part of that transition means migrating existing software to that environment. Those overseeing OSE migration projects need implementation strategies that use available standards to initiate such transitions. My colleagues and I at the National Institute of Standards and Technology's Computer Systems Laboratory have been researching software approaches to such strategies.

\section{2,722}

PB94-207354 PC A03/MF A01

National Inst. of Standards and Technology (MEL) Gaithersburg, MD. Factory Automation Systems Div. World Wide Web and Mosaic: User's Guide.

C. I. Schlenoff. Jun 94, 16p, NISTIR-5453.

Keywords: "Information systems, "Information re trieval, Computer networks, Documents, Protocols, User manuals, "WWW(World Wide Web), "World Wide Web, "Mosaic software package, US NIST, Internet, Wide area networks.

The National Institute of Standards and Technology (NIST) is moving forward in providing and accessing information through the World Wide Web (WWW) by using the Mosaic software package. The WWW is a seamless world in which all information, from any source, can be accessed in a simple and consistent manner. Mosaic is a networked information discovery, retrieval, and collaboration tool used to view documents available through the WWW. This document was created for the following purposes: to help new users become more familiar with the WWW concept; to demonstrate how to use Mosaic and its capabilities: and to provide a pointer to other documents further describing the WWW and Mosaic.

\section{2,723}

PB95-103719 PC A07/MF A02

National Inst. of Standards and Technology (CSL), Gaithersburg, MD. Advanced Systems Div.

Framework for National Information Infrastructure Services.

O. G. Farah, W. Majurski, W. McCoy, R.

Schneeman, D. Cypher, J. Pottmeyer, and W. Jansen. Jul 94, 148p, NISTIR-5478.

Prepared in cooperation with Defense Information Sys tems Agency, Arlington, VA.

Keywords: "Computer networks, "Information technology, Information systems, Telecommunication, On- 
line systems, Information management, Information services, Information flow, Information transfer, Information dissemination, Information processing, Information retrieval, Information needs, User needs, Delivery systems, "National Information Infrastructure, Information superhighways, Electronic delivery.

The document provides an initial description of a framework for National Information Infrastructure (NII) Services (hereafter referred to as the Services Framework), motivated by emerging information technologies. The original goal of the document was to define an architecture for NII services. Instead, the document represents a less ambitious attempt to define a framework within which architectures of NII services can be discussed. The Services Framework as presented herein is intended to serve as a point of departure for discussing the definition, scope, and alignment of NII services.

\section{2,724}

PB95-128641 PC A08/MF A01

National Inst. of Standards and Technology, Gaithersburg, MD. Office of Information Services.

Databases Available in the Research Information Center of the National Institute of Standards and Technology.

Special pub.

D. Cunningham. Sep $94,158 \mathrm{p}$, NIST/SP-869

Supersedes PB94-114568. Also available from Supt. of Docs. as SNO03-003-03284-1.

Keywords: "Data bases, *Information services, Information systems, Indexes(Documentation), Subject indexing, Directories, Vendors, CD-ROM, Tables(Data), *US NIST.

Databases available online in the Research Information Center of the National Institute of Standards and Technology (NIST) are listed by acronym and by full title. In addition, descriptions of the databases, dates covered, producers, hard copy counterpart, principal sources and vendors are listed. A list of CD-ROM databases, a general subject index, a cross reference index, and a full text database list are also supplied.

\section{P2,725 $205-154738 \quad$ PC A06/MF A02}

National Inst. of Standards and Technology (CSL), Gaithersburg, MD. Systems and Software Technology Div.

User Study: Informational Needs of Remote National Archives and Records Administration Customers.

Special pub.

J. Moline, and S. Otto. Nov 94, 123p, NIST/SP-500/ 221

Also available from Supt. of Docs. as SN003-00303305-7.

Keywords: "Data acquisition, "User needs, *Information services, Customer service, Access, Information needs, Delivery systems, Records management, Information retrieval, Information systems, "National Archives and Records Administration, User surveys, Government services.

The National Archives and Records Administration (NARA) possesses a vast wealth of information and records holdings from the Federal Government, which documents the history of the American people. The purpose of this particular study was to conduct a survey to investigate the information and records needs of citizens who lack direct access to NARA's facilities and determine how to best meet those needs. The basic question answered is what remote users of the National Archives want in the way of information and materials and how they want to receive it.

\section{2,726}

PB95-168555 Not available NTIS

National Inst. of Standards and Technology (CSL), Gaithersburg, MD. Information Systems Engineering Div.

Coping with Different Retrieval Methods in Next Generation Networks.

Final rept.

D. Flater, and Y. Yesha. 1994, 7p

Pub. in Proceedings of the International Conference on Parallel and Distributed Computer Systems (7th), Las Vegas, NV., October 6-8, 1994, p549-555.

Keywords: "Query languages, "Information retrieval, "Search profiles, "Computer networks, Natura! language processing, Information systems, Data storage systems, Heuristic methods, Systems engineering, Man computer interfaces, Statistical analysis, Reprints.
Methods of information retrieval that rely on automated indexing, automated classification, and/or analysis of natural language queries are often categorically condemned by proponents of traditional retrieval methods. With statistical retrieval, a powerful and expressive query language is processed with limited success; with traditional retrieval, a less expressive query language is processed with $100 \%$ success. Some feel that a battle must be fought to decide what sort of information retrieval will serve the needs of the world. The challenge is to support both kinds of retrieval in an information space that is too large for global indexing.

\section{2,727}

PB95-168605 Not available NTIS

National Inst. of Standards and Technology (PL), Boulder, CO. Time and Frequency Div.

Using Archie to Find Files on the INTERNET.

Final rept.

J. M. Gilligan. 1992, 2p

Pub. in Optics and Photonics News, p61, 64, Dec 92

Keywords: "Data transfer(Computers), Data retrieval, Data transmission, Computer networks, Data links, Protocol(Computers), Reprints, "Archie, "FTP(File Transfer Protocol), "File transfer protocol, INTERNET. The document provides a beginner's guide to usy anonymous FTP on the Internet.

\section{2,728}

PB95-168720 Not available NTIS

National Inst. of Standards and Technology (PL), Boulder, CO. Time and Frequency Div.

Retrieving Articles from the Internet (without a UNIX Workstation). Part 1. File Formats and Software Tools.

Final rept.

W. M. Itano. 1994, 2p

Contract ONR-N00014-89-J-1467

See also Part 2, PB95-168738. Sponsored by Office of Naval Research, Arlington, VA

Pub. in Optics and Photonics News 5, n9 p36, 43, Sep 94.

Keywords: "Information retrieval, "Data retrieval, "Software tools, "Computer networks, Information transfer, Data transmission, Man computer interfaces, Data storage, Document storage, Reprints, "File format, "INTERNET.

File formats commonly used for electronic storage of Internet-accessible documents (TEX and PostScript) are discussed. File archiving, compression, and encoding formats used to reduce storage requirements and to facilitate transmission by electronic mail are dis cussed also. Sources for non-commercial software for processing electronic documents having these formats on IBM-compatible computers are listed.

\section{2,729}

PB95-168738 Not available NTIS

National Inst. of Standards and Technology (PL), Boulder, CO. Time and Frequency Div.

Retrieving Articles from the Internet (without a UNIX Workstation). Part 2. An Example.

Final rept.

W. M. Itano. 1994, 2 p.

See also Part 1, PB95-168720.

Pub. in Optics and Photonics News $5, n 10$ p61, 67 Oct 94

Keywords: *Information sources, "Information retrieval, "Data retrieval, "Computer networks, Information transfer, Data transmission, Man computer interfaces, Data storage, Document storage, Software tools, Reprints, *INTERNET, File format.

Some Internet sources for scientific and technical doc uments are described. A detailed example of retrieving a document from an Internet host computer and converting it to printed format on an IBM-compatible computer is given.

\section{2,730}

PB95-182275

\section{PC A05/MF A0}

National Inst. of Standards and Technology (CSL), Gaithersburg, MD. Computer Security Div.

Keeping Your Site Comfortably Secure: An Introduction to Internet Firewalls.

Special pub.

J. P. Wack, and L. J. Carnahan. Dec 94, 92p, NIST/ SP-800/10.

Also available from Supt. of Docs. as SN003-00303313-8. See also PB94-139045.
Keywords: "Computer security, "Data processing security, Information systems, Computer networks, Local area networks, Access control, Computer privacy, $\mathrm{Au}$ thentication, Telecommunication, "Internet, TCP/ IP(Transmission Control Protocol//nternet Protocol).

This document provides an overview of the Internet and security-related problems. It then provides an overview of firewall components and the general reasoning behind firewall usage. Several types of network access policies are described, as well as technical implementations of these policies. Lastly, the document contains pointers and references for more detailed information. The document is designed to assist users in understanding the nature of Internet-related problems and what types of firewalls will solve or alleviate specific problems. Users can then use this document to assist in purchasing or planning a firewall.

\section{2,731}

PB95-219218 PC A04/MF A01

National Inst. of Standards and Technology (CSL), Gaithersburg, MD. Systems and Software Technology Div.

Electronic Access: Blueprint for the National Archives and Records Administration.

Special pub.

J. Moline, and S. Otto. Apr 95, 66p, NIST/SP-500/

227

Also available from Supt. of Docs. as SNOO3-00303330-8. See also PB95-217014.

Keywords: *Information storage, *Information retrieval, *Records management, *Stralegic planning, Access to information, Information dissemination, Preservation, Primary sources, Computer system design, Information networks, Optical scanning, "Electronic Access Project, "National Archives and Records.

The Electronic Access Project of the National Archives and Records Administration (NARA) had three main tasks. They were to design a methodology for exploring the informational needs of remote customers, to survey the targeted population, and to develop a blueprint for NARA's long-term information delivery systems that would enable NARA to meet their customers' needs. This report covers the last task. It discusses the findings of the user study as they influence the design of an electronic access system for the National Archives and Records Administration and presents a blueprint for meeting the users' needs.

\section{2,732}

\section{PB95-231908 PC A04/MF A01}

National Inst. of Standards and Technology (NCSL), Gaithersburg, MD. Systems and Network Architecture Div.

Guidelines for the Evaluation of X.500 Directory Products.

Special pub.

J Tebbutt May 95,74p, NIST/SP-500/228.

Also available from Supt. of Docs. Sponsored by Department of Agriculture, Washington, DC

Keywords: "Distributed data bases, "Information systems, Performance evaluation, Access control, Data processing security, Computer networks, Data storage, Relational data bases, Data base management systems, "X500 Directory.

The document provides readers with the means to evaluate various $X 500$ products and make informed choices as to which available products best match the requirements of their organizations. The document is aimed at procurement officials, systems administration staff and others involved in the process of obtaining or recommending $X .500$ products for use in their organizations.

\section{2,733}

\section{PB96-131545 PC A03/MF A01}

National Inst. of Standards and Technology (CSL), Gaithersburg, MD. Systems and Software Technology Div.

Defining Environment Integration Requirements. B. Cuthill, and M. Zelkowitz. May 95, 34p, NISTIR5654

See also PB94-143401 and PB95-143145.

Keywords: "Software engineering, "Information systems, "Requirements, Automation, Computer networks, Information flow, Systems management, Software tools, Systems engineering, Corporate information management, Integrated systems, Distributed computer systems, Interfaces, Data management, Organizational structure, "Enterprise integration, Enter- 
prise modeling, ITEM(Information Technology Engineering and Measurement).

This paper discusses the use of enterprise and process modeling to classify the features of the enterprise process, its automation and the external stimuli on the enterprise that effect enterprise choices for tool and environment integration. This report focuses on the use of metadata and message types as mechanisms for integrating environments. This report includes an example of generating metadata and messaging requirements in the software development domain.

02,734

PB96-139407 PC A07/MF A02

National Inst. of Standards and Technology (TS),

Gaithersburg, MD. Office of Information Services.

Databases Available in the Research Information Center of the National Institute of Standards and Technology (December 1995).

Special pub.

D. Cunningham. Dec $95,150 p$, NIST/SP-895.

Supersedes PB95-128641. Also available from Supt. of Docs. as SN003-003-03383-9.

Keywords: "Data bases, *Information centers, Information services, Directories, Subject indexing, Indexes(Documentation), CD-ROMs, Vendors, Tables(Data), "US NIST.

Databases available online in the Research Information Center of the National Institute of Standards and Technology (NIST) are listed by acronym and by full title. In addition, descriptions of the databases, dates covered, producers, hard copy counterpart, principal sources and vendors are listed. A list of CD-ROM databases, a general subject index, a cross reference index, and a full text database sources and vendors are listed. $A$ list of CD-ROM databases, a general subject index, a cross reference index, and a full text database list are also supplied.

02,735

PB96-147053 Not available NTIS

National Inst. of Standards and Technology (TS), Gaithersburg, MD. Office of Information Services.

General Types of Information Services.

Final rept.

P. W. Berger. 1992, 13p

Sponsored by Engineering Foundation, New York. and Council on Library Resources, Inc., Washington, DC. Pub. in Proceedings of the Conference on a National Engineering Information Service (NENGIS), Palm Coast, FL., June 14-19, 1992, p351-363.

Keywords: *Information services, Information systems, Library services, Data systems, Reprints, National Engineering Information System, Data services, Analytic services

The report provides a description and evaluation of four types of information services: Library Services, Specialized Information Services, Data Services, and Analytic Services.

\section{2,736}

PB96-160536 Not available NTIS

National Inst. of Standards and Technology (CSL), Gaithersburg, MD.

Copyright and Information Services in the Context of the National Research and Education Network. Final rept.

R. J. Linn. 1994, $12 p$.
Pub. in Intellectual Property Project Proceedings, v1 n1 p9-20 Jan 94.

Keywords: "Copyrights, "Property rights, ${ }^{*}$ Requirements, Information services, Information dissemination, Computer networks, Legal aspects, Authentication, Systems management, Reprints, National Resource and Education Networks, High Performance Computing Act of 1991, Public Law 102-194, Digital signatures.

The High Performance Computing Act (HPCA) of 1991 (P.L. 102-194) places unenforcable requirements to protect copyrights and intellectual property rights on the National Research and Education Network (NREN). This paper discusses the roles and responsibilities of the NREN and associated information services; technical approaches to authentication, redistribution and authorization of use of electronic documents over the NREN; and an amendment to the high Performance Computing Act.
National Inst. of Standards and Technology (CSL), Gaithersburg, MD.

Information Technology Standards in a Changing World: The Rose of the Users.

Final rept.

J. H. Burrows. 1993, 8p

Pub. in Computer Standards and Interfaces, v15 p49. 561993.

Keywords: *Information technology, *Interoperability *User requirements, Information systems, Integrated systems, Standards, Interfaces, Reprints, Open Systems Interconnection.

Standards for information technology (IT) systems are important to users in effectively applying IT and carrying out the business of their organizations. Users need standards to interconnect products developed by different vendors and to move software, data ad applications from one system to another. However, the formal standards development process does not always respond to user needs in a timely way. The cost and complexity of the process often inhibits direct user participation over the long period of time needed to develop standards. Users also have difficulty applying the standards when they are developed in a fragmented and non-integrated fashion.

\section{2,738}

PB96-167846 PC A05/MF A0

National Inst. of Standards and Technology, Gaithersburg, MD.

Data Communications Strategy

J. Mulvenna, and T. Boland. Jan 96, 62p, NISTIR-

5793.

Sponsored by Internal Revenue Service, Arlington, VA

Keywords: *Interoperability, "Computer networks, Network management, Computer architecture, Distributed computer systems, Data transfer(Computers), Data transmission, File management systems, Procurement, Protocols, Standards, Interfaces, Information retrieval, Data processing security, *Open systems interconnection, "Data communication systems, Internet.

The absence of a single solution for providing worldwide computer interoperability places more responsibility on procurement authorities to make the right choice. In order to make intelligent procurement decisions procurement authorities need to be informed about the provided services and marketplace acceptance of computer networking products. The document provides technical, managerial, and procurement personnel with some of the factors that need to be considered when making procurment decisions.

\section{2,739}

PB96-183041 PC A03/MF A01

National Inst. of Standards and Technology (CSL), Gaithersburg, MD.

X.500 Directory Schema Design Handbook.

C. A. Warnar, and J. Tebbutt. Apr 96, 25p, NISTIR5819.

See also PB95-231908

Keywords: "Distributed data bases, "Information systems, "Directories, Performance evaluation, Data processing security, Computer networks, Design, Relational databases, Database management systems ×500 Directory.

This document contains a high level schema description including a description of the schema components, the storage of schema information in the Directory Information Tree (DIT), and the tailoring of the schema components to meet an organization's needs. Pilo projects and other work in the area of schema design are reviewed and summarized.

\section{Operations \& Planning}

\section{2,740}

PB95-181152 Not available NTIS

National Inst. of Standards and Technology (CSL), Gaithersburg, MD. Advanced Systems Div.

Prototype Information Retrieval System to Perform a Best-Match Search for Names.

Final rept.

N. E. Willman. 1994, 11p.

Sponsored by Social Security Administration, Washington, DC.
Pub in Proceedings of RIAO 94' Conference on Intelligent Multimedia Information Retrieval Systems and Management, New York, NY., October 11-13, 1994, p751-761.

Keywords: "Information retrieval, "Information systems, Performance evaluation, Pattern recognition, Errors, Prototypes, Ranking, Reprints, "Proper names.

Personal names are often used as keywords in searches to retrieve information. The presence of misspellings and other errors in names can make an exact match of a keyword and a search query impossible. In these cases, it is necessary to be able to retrieve personal names that are similar to that of the personal name given in the search query. In addition, i an interactive search is required, it is necessary to retrieve matches quickly and to rank them in terms of their similarity to the search query. This paper discusses a prototype information retrieval system which was developed to test the feasibility of such a search, and the experimental results obtained using this sys tem

\section{2,741}

PB95-232633 PC A03/MF A01

National Inst. of Standards and Technology (TS), Gaithersburg, MD. Office of Information Services.

Abstract and Index Collection in the Research Information Center of the National Institute of Stand ards and Technology.

Special pub.

D. Cunningham. May 95, 40p, NIST/SP-884

Supersedes PB94-152204. Also available from Supt of Docs. as SN003-003-03340-5.

Keywords: "Abstracts, *Indexes(Documentation) Technical reports, Information centers, Subject index ing. Data bases, Sources, Publishing, "National Institute of Standards and Technology.

An alphabetical arrangement of abstracts and indexes available in the Research Information Center (RIC) of the National Institute of Standards and Technology (NIST) is listed by most current title of the publication Other information includes description of the abstract or index, RIC holdings, principal sources, publisher or association, corresponding RIC database and $C D$ ROM availability and the classitication number. A general subject and former title/database name index follow the main text of the report.

02,742

PB97-118665 Not available NTIS

National Inst. of Standards and Technology (CSL), Gaithersburg, MD. Information Access and User Inter face Div.

Lab Report Special Section: Natural Language Processing and Information Retrieval Group Information Access and User Interfaces Division, $\mathrm{Na}$ tional Institute of Standards and Technology.

Final rept.

D. Harman. $1995,5 p$

Pub. in SIGIR Forum, v29 n2 p6-10 Oct 95

Keywords: "Natural language processing, *Text processing, "Information retrieval, Knowledge-based systems, Search structuring, Access, User needs, Automation, Human-computer interface, Reprints, US NIST, Electronic text.

The Natural Language Processing and Information $\mathrm{Re}$ trieval Group was formed in 1994 at the National Insttute of Standards and Technology (NIST) in recognition of the importance of managing the ever-increasing amount of electronically available text. The formal objective of the group is 'to work with industry, academia and other government agencies to promote the use of more effective and efficient techniques for manipulating (largely) unstructured textual information, especially the browsing, searching, and presentation of the information'

\section{2,743}

PB97-118673 Not available NTIS

National Inst. of Standards and Technology (CSL), Gaithersburg, MD. Information Access and User Interface Div

Panel: Building and Using Test Collections.

Final rept.

D. Harman. 1996 , 3p.

Pub. in Proceedings of the Annual International ACM SIGIR Conference on Research and Development in Information Retrieval (19th), SIGIR '96, Zurich, Switzerland, August 18-22, 1996, p335-337.

Keywords: *Information retrieval, "Text processing, "Documents, "Data collection, Knowledge-based sys- 


\section{Operations \& Planning}

tems, Search structuring, Multimedia, Computer languages, Semantics, Syntax, Reprints.

The panel, emphasizing audience participation, focuses on issues in building and using test collections for information retrieval. The panel presents an opportunity to share experiences gained from past test collection building and usage to help guide the development of these new test collections.

\section{Reference Materials}

\section{2,744}

PB94-152204 PC A03/MF A01

National Inst. of Standards and Technology, Gaithersburg, MD.

Abstract and Index Collection in the Research Information Center of the National Institute of Standards and Technology.

Special pub.

D. Cunningham. Jan 94, 41p, NIST-SP-859

Also available from Supt. of Docs. as SNO03-00303250-6.

Keywords: "Abstracts, "Indexes(Documentation), Technical reports, Information centers, Subject indexing, Data bases, Sources, Publishing, "National Institute of Standards and Technology

An alphabetical arrangement of abstracts and indexes available in the Research Information Center (RIC) o the National Institute of Standards and Technology (NIST) is listed by most current title of the publication. Other information includes description of the abstract or index, RIC holdings, principal sources, publisher or association, corresponding RIC database and CD ROM availability and the classification number. A general subject and former title/database name index follow the main text of the report.

\section{2,745}

PB94-178068 PC A12/MF A03

National Inst. of Standards and Technology (TS), Gaithersburg, MD. Office of Information Services

NIST Serial Holdings, 1994.

Special pub. (Final).

S. A. Sanders. Apr 94, 270p, NIST/SP-777-94-ED

Supersedes PB94-120847. Also available from Supt. of Docs. as SN003-003-03261-1.

Keywords: "Periodicals, "Catalogs(Documentation), "Collection, "Information centers, "Bibliographies, Standards, Libraries, "National Institute of Standards and Technology, "NIST.

This publication contains bibliographic information on approximately 5,000 titles held in the NIST Research Information Center, representing current and noncurrent journals, periodicals, annuals, memoirs, proceedings and transactions.

\section{2,746}

\section{PB95-188926 PC A12/MF A03}

National Inst. of Standards and Technology (TS) Gaithersburg, MD. Office of Information Services.

NIST Serial Holdings, 1995.

Special pub. (Final).

S. A. Sanders. Feb 95, 271p, NIST/SP-777-95.

Supersedes PB94-178068. Also available from Supt of Docs. as SNO03-003-03319-7.

Keywords: "US NIST, "Periodicals, "Catalogs(Documentation), "Bibliographies, Standards, Libraries.

The publication contains bibliographic information on approximately 5,000 titles held in the NIST Research Information Center, representing current and noncurrent journals, periodicals, annuals, memoirs, proceedings and transactions.

\section{2,747}

PB95-226692 PC A05/MF A01

National Inst. of Standards and Technology (PL), Gaithersburg, MD

Guide for the Use of the International System of Units (SI).

Special pub.

B. N. Taylor. Apr 95, 87p, NIST-SP-811

Supersedes PB92-116383. Also available from Supt. of Docs. as SN003-003-03329-4.
Keywords: "International system of units, "Metric system, "Conversion tables, Units of measurement

The guide is a practical tool for the use of NIST authors in the preparation of manuscripts in conformance with the NIST policy that requires the use of the International System of Units for all publications.

\section{2,748}

\section{PB96-172523 PC A13/MF A03}

National Inst. of Standards and Technology (TS), Gaithersburg, MD. Office of Information Services.

NIST Serial Holdings, 1996.

Special pub.

S. A. Sanders. Feb 96, 270p.

Supersedes PB95-188926. Also available from Supt. of Docs. as SNO03-003-03394-4

\section{Keywords: "US NIST, "Periodicals, Documents.}

This publication contains bibliographic information on approximately 5,000 titles held in the NIST Research Information Center, representing current and noncurrent journals, periodicals, annuals, memoirs, proceedings and transactions.

\section{General}

\section{2,749}

\section{AD-A286 683/8 PC A03/MF A01}

National Bureau of Standards, Boulder, $\mathrm{CO}$

Helium Refrigeration and Liquefactlon Using a Liq. uid Hydrogen Refrigerator for Precooling.

Technical note.

D. B. Shelton, J. W. Dean, T. R. Strobridge, B. W.

Birmingham, and D. B. Mann. 27 Jan 60, 36p.

Keywords: "Helium, "Refrigeration systems, "Liquefaction, "Liquid hydrogen, Circuits, Evaporators, Heat exchangers, Hydrogen, Production, Requirements, Temperature, Low temperature, Gases, "Precooling, Refrigerators.

Consideration is given to the use of a hydrogen refrigerator to assist in the production of temperatures below those obtained with hydrogen. A hydrogen refrigerator is used to maintain a precooling evaporator at or near $21 \mathrm{deg} K$ in a helium gas circuit. The helium circuit may be arranged to produce liquid for external use or to produce refrigeration between $21 \mathrm{deg} K$ and 4.2 deg $K$ in a closed system. Charts have been developed that show the requirements of the composite helium - hydrogen system and the effect of heat exchanger performance. The relative quantities of refrigeration (hydrogen and helium) at various temperature levels have been determined.

\section{2,750}

PB94-165966 PC A06/MF A02

National Inst. of Standards and Technology (MEL), Gaithersburg, MD.

Publications of the Manufacturing Engineering Laboratory Covering the Period January 1989-September 1992.

C. Albus. Nov 92, 115p, NISTIR-4974.

See also PB93-123041.

Keywords: "Bibliographies, "US NIST, Dimensional measurement, Robotics, Artificial intelligence, Production englneering, Information systems, Metrology, Primary standards, Calibrating, Precision, Sensors, Automation, "Manufacturing Engineering Laboratory.

The mission of the Manufacturing Engineering Laboratory (MEL) is to bring the resources of the National Institute of Standards and Technology to bear on the standards and measurement problems associated with America's discrete parts manufacturing. To fulfill this mission MEL conducts active programs of research in the areas of: High-precision dimensional measurement and precision engineering; Robotics and intelligent machines; Manufacturing data description, data adminis. tration, and information processing; and Sensors for manufacturing processes. This list of publications reflects the diversity of scientific and technical problems which have been attacked over the past fourteen years in fulfillment of the Laboratory's mission.

02,751

PB95-140943 Not available NTIS

National Inst. of Standards and Technology (CSTL), Gaithersburg, MD. Process Measurements Div.

Heat Transfer in Thin, Compact Heat Exchangers with Circular, Rectangular, or Pin-Fin Flow Passages.

Final rept.

D. A. Olson. 1992, 10p.

Contract NLRC-L7400C

Sponsored by National Aeronautics and Space Administration, Hampton, VA. Langley Research Center.

Pub. in Jnl. of Heat Transfer 114, p373-382 May 92

Keywords: "Heat transfer, "Pressure gradients, "Channel flow, "Flow geometry, Heat flux, Friction factor, Reynolds number, Nusselt number, Helium, Gas flow, Viscosity, Thermal conductivity, Reprints, "Compact heat exchangers

We have measured heat transfer and pressure drop of three thin, compact heat exchangers in helium gas at $3.5 \mathrm{MPa}$ and higher, with Reynolds numbers of 450 to 36,000 . The flow geometries for the three heat exchanger specimens were: circular tube, rectangular channel, and staggered pin fin with tapered pins. The specimens were heated radiatively at heat fluxes up to $77 \mathrm{~W} / \mathrm{sq} \mathrm{cm}$. Correlations were developed for the isothermal friction factor as a function of Reynolds number, and for the Nusselt number as a function of Reynolds number and the ratio of wall temperature to fluid temperature. The specimen with the pin internal geometry had significantly better heat transfer than the other specimens, but it also had higher pressure drop. For certain conditions of helium flow and heating, the temperature more than doubled from the inlet to the outlet of the specimens, producing large changes in gas velocity, density, viscosity, and thermal conductivity. These changes in properties did not affect the correlations for friction factor and Nusselt number in turbulent flow.

02,752

PB95-169082 Not available NTIS

National Inst. of Standards and Technology (CSTL), Gaithersburg, MD. Process Measurements Div. Energy Flows in an Orifice Pulse Tube Refrigerator

Final rept.

W. Rawlins, R. Radebaugh, P. Bradley, and K.

Timmerhaus. 1993, 8p.

Pub. in Advances in Cryogenic Engineering, v39 ptB p1449-1456 1993

Keywords: "Mass flow, "Flow measurement, Enthalpy, Refrigerators, Entropy, Heat flux, Refrigerating machinery, Reprints, "Orifice pulse tube refnigerators, "Energy flow, "Enthalpy flow.

A technique which allows for the instantaneous measurements of mass flow rate and temperature in an orifice pulse tube refrigerator (OPTR) during actual operation has been developed recently. This paper pre sents the values of enthalpy, entropy, and work fluxes at the cold end of the pulse tube evaluated from these measurements. They are thermodynamically self consistent within $1 \%$. An analytical model describing the operation of an OPTR was developed at the National Institute of Standards and Technology (NIST) in the late 1980's. This model assumes adiabatic performance of the pulse tube and purely sinusoidal mass flow rates, temperature, and pressure oscillations in the OPTR. The experimentally measured enthalpy flux varies from $60 \%$ to $85 \%$ of that predicted by the adiabatic model. The experimental work reported here also gives values for various phase relationships that are needed for some calculations with the analytical model.

\section{2,753}

PB95-169090 Not available NTIS

National Inst. of Standards and Technology (CSTL), Gaithersburg, MD. Process Measurements Div. Graded and Nongraded Regenerator Performance. Final rept.

W. Rawlins, K. D. Timmerhaus, R. Radebaugh, J Gary, and P. Bradley. 1993, 13p. Sponsored by National Aeronautics and Space Administration, Moffett Field, CA. Ames Research Center. 
Pub. in Proceedings of International Cryocooler Conference (7th), Santa Fe, NM., November 17-19, 1992 p471-483 1993.

Keywords: "Regenerators, "Cooling systems, "Performance testing, Regeneration(Engineering), Refrigerating machinery, Cryogenic cooling, Temperature measurement, Heat loss, Mass flow, Effectiveness, Performance evaluation, Reprints, "Pulse tube refrigerators.

A method to measure regenerator performance, in situ, in an rifice pulse tube refrigerator has been successfully developed. This was accomplished by inserting two, $2 \mathrm{~mm}$ long, tungsten wires with diameters of $4 \mathrm{mi}$ crometers perpendicular to the fluid flow at the cold end of the regenerator. The paper compares the performance between two different regenerators constructed from stainless steel mesh: one with a graded mesh (where three different mesh sizes are used for the matrix material) and another with a single mesh size throughout. Both expeirmental and numerica model results are presented. The numerical model indicated that there was only a very slight advantage to using a graded mesh in a generator, whereas experiments showed the graded mesh regenerator to have a slightly higher ineffectiveness.

\section{2,754}

PB95-188835 PC A11/MF A02

National Inst. of Standards and Technology (MEL) Gaithersburg, MD.

Program of the Manufacturing Engineering Laboratory, 1995. Infrastructural Technology, Measurements, and Standards for the U.S. Manufacturing Industries.

G. P. Carver. Feb 95, 241p, NISTIR-5599.

See also PB94-165966.

Keywords: "Metrology, Dimensional measurement, Production engineering, Artificial intelligence, Automation, Robotics, Standards, "Manufacturing Engineering Laboratory, US NIST.

The National Institute of Standards and Technology (NIST) Manufacturing Engineering Laboratory works with the U.S. manufacturing industries to develop and apply infrastructural technology measurements, and standards to meet their needs. This report summarizes the resources, objectives, needs addressed, accomplishments, and plans for the projects carried out by the Manufacturing Engineering Laboratory in support of U.S. industry.

\section{2,755}

\section{PB95-198750 PC A04/MF A01}

National Inst. of Standards and Technology (MEL), Gaithersburg, MD. Factory Automation Systems Div. Apparel Manufacturing Glossary for Application Protocol Development.

M. E. Read. Feb 95, 60p, NISTIR-5572.

Sponsored by Defense Logistics Agency, Alexandria, VA.

Keywords: "Clothing industry, "Manufacturing, "Dictionaries, Pattern making, Standards, Specifications, APS(Application Protocols), APDES(Appare Product Data Exchange Standard), STEP(Standard for the Exchange of Product Model Data).

A glossary of terms used in apparel manufacturing, specifically those used in the pattern-making process, are listed verbatim from six primary sources. These terms are needed in the development of new, International Organization for Standardization (ISO) Standard for the Exchange of Product Model Data (STEP) application protocols for the apparel industry. The purpose of this report is to present to the apparel industry a working set of terms and meanings from the published literature, and to act as a catalyst in the development of a set of consensus terms and meanings.

\section{2,756}

\section{PB96-136973 PC A03/MF A01}

National Inst. of Standards and Technology (BFRL), Gaithersburg, MD.

Effect of Inclination on the Performance of a Compact Brazed Plate Condenser and Evaporator.

M. A. Kedzierski. Nov 95, 33p, NISTIR-5767.

Sponsored by Trane Co., La Crosse, WI.

Keywords: "Heat exchanges, "Refrigerating machinery, Schematic diagrams, Refrigerants.

The origins of the compact brazed plate heat exchanger (CBE) began in the 1920's with the first com- mercially successful gasketed-plate heat exchangers (Saunders, 1988). Milk producers and other food and drink processors satisfied hygiene requirements by periodically disassembling and cleaning the gasketedplates. Applications for the compact brazed plate hea exchangers (CBEs) have increased in recent years. Currently, the compactness of the CBE drives its use as refrigerant evaporators and condensers. The purpose of this study was to identify the performance deg radation associated with tilting a CBE from the designed vertical position.

\section{2,757}

PB96-163621 Not available NTIS

National Inst. of Standards and Technology (MEL), Gaithersburg, MD. Precision Engineering Div.

Gage Block Standards, Measurement Capabilities and Laboratory Accreditation.

Final rept.

T. Doiron. 1992, 12p

Pub. in NCSL Workshop and Symposium, August 1992, p183-194

Keywords: "Gage blocks, "Interlaboratory tests, Reprints, Accuracy, Error budget, Standards, “Laboratory accreditation.

As the United States begins to seriously consider laboratory accreditation for calibration labs, the calibration of gage blocks (one of the most accurate and sophisticated of industrial measurements) has been suggested as the prototype dimension laboratory accreditation. One of the technical problems in implementing laboraory accreditation is determining the laboratory accuracy which is adequate for calibration systems. The author will discuss some of the problems inherent in the classifcation schemes of both the US and ISO stand ards and their relation to the current state of the art for gage block calibration.

\section{2,758}

PB96-190293 Not available NTIS

National Inst. of Standards and Technology (MEL), Gaithersburg, MD. Precision Engineering Div.

Increasing the Value of Atomic Force Microscopy Process Metrology Using a High-Accuracy Scanner, Tip Characterization, and Morphologica Image Analysis.

Final rept.

J. Schneir, J. S Villarrubia, T. H. McWaid, V. W.

Tsai, and R. Dixson. 1996, 7p.

Pub. in Jnl. of Vacuum Science and Technology, v14 n2 p1540-1546 Mar/Apr 96

Keywords: "Metrology, Tip, Manufacturing, Scanners, Image analysis, Reprints, "Atomic force microscopes

Atomic force microscopes are being used increasingly for process metrology. As a case study, the measurement by atomic force microscope of a soda lime glas optical disk patterned using optical lithography and reactive plasma etching is examined. The atomic force microscope used for this measurement has a highly accurate scanner system. The $X, Y$, and $Z$ axes are calibrated using laser interferometry. To determine the shape of the tip used a commercially available tip calibration artifact was imaged both before and after the measurement. The image was corrected for the tip shape using mathematical morphology. The value of the atomic force microscope measurement is defined to be the impacts of the metrology on the product or process. It is shown that the value of atomic force microscopy process metrology on an optical disk is increased by using an accurate scanner, tip characterization, and morphological image analysis; however, the cost per measurement in increased as well. In general, the characteristics of the metrology required de pends on the secific manufacturing process being supported.

02,759

PB96-190368 Not available NTIS

National Inst. of Standards and Technology (MEL) Gaithersburg, MD. Precision Engineering Div.

Scanned Probe Microscope Tip Characterization Without Calibrated Tip Characterizers.

Final rept.

J. S. Villarrubia. $1996,4 p$

Pub. in Jnl. of Vacuum Science and Technology B, v14 n2 p1518-1521 Mar/Apr 96

Keywords: *Metrology, Morphology, Reprints, "Alomic force microscopes, "Blind reconstruction, Scanned probe microscopy, Scanning tunneling microscopy.

In scanned probe microscopy the image is a combination of information from the sample and the tip. In order to reconstruct the true surface geometry, it is necessary to know the actual tip shape. It has been proposed that this shape may be constructed from images of 'tip characterizer' artifacts of independently known shape. The requirements for this strategy-dimensiona uncertainty and instability of the characterizer small compared to the tip size-are not trivial. An alternative is 'blind reconstruction', which requires no information about the characterizer geometry apart from that contained within its image, yet produces an outer bound on the tip shape which for appropriately chosen characterizers is a good approximation. With blind reconstruction dimensional instability of characterizers is less problematical, and characterizer measureability is no longer a constraint, so more complex distributed characterizer has a known shape, blind reconstruction and the known-characterizer method may be combined.

\section{2,760}

PB96-195276 PC A11/MF A03

National Inst. of Standards and Technology (MEL) Gaithersburg, MD

Program of the Manufacturing Engineering Laboratory, 1996. Infrastructural Technology, Measurements, and Standards for the U.S. Manufacturing Industries.

May $96,218 p$, NISTIR-5845.

See also report for 1995, PB95-188835.

Keywords: "Metrology, Dimensional measurement, Production engineering, Standards, Calibration, Robotics, Automation

The NIST Manufacturing Engineering Laboratory works with the U.S. manufacturing industries to develop and apply infrastructural technology, measurements, and standards to meet their needs. This report summarizes the Manufacturing Engineering laboratory in support of U.S. industry.

\section{2,761}

PB96-201157 Not available NTIS

National Inst. of Standards and Technology (MEL) Gaithersburg, MD. Automated Production Technology

\section{Ultrasonic NDE of Sprayed Ceramic Coatings.}

Final rept.

J. A. Slotwinski, and G. V. Blessing. 1996, 8p.

Pub. in Review of Progress in Quantitative Nondestructive Evaluation, v15 p 1613-1620 1996.

Keywords: "Ceramic coatings, "Plasma spray *Nondestructive evaluation, Alumina, Elastic moduli, Ultrasonics, Zirconia, Reprints.

Thermal spraying of protective coatings has been in use since 1917 when the initial application was the spraying of zinc layers onto steel structures to prevent corrosion. In the 1970's plasma-spray technology was first used with the introduction of vacuum plasmaspraying. Today, gases such as argon and nitrogen sometimes with an additional gas such as helium or hydrogen) are often used in plasma-spray guns. In plasma-spraying an electric-arc discharge heats the gas stream to high temperature (equal to or greater than $10,000 \mathrm{~K})$, turning it into a plasma. The gas exits the spray-gun at high speed (approx. $200 \mathrm{~m} / \mathrm{s}$ to 600 $\mathrm{m} / \mathrm{s}$ ) towards the material to be coated. Material powder (often carried by a second gas stream) is injected into the plasma stream, where it melts into liquid droplets. These droplets are carried onto the target surface, where they rapidly cool (approx. 10 to the sixth power $\mathrm{K} / \mathrm{s}$ ) into solid, flat splats. A layer of material can be built up by repeated spraying of the same surface area.

\section{2,762}

PB97-116107 PC A03MF A01

National Inst. of Standards and Technology (BFRL), Gaithersburg, MD.

Intracycle Evaporative Cooling in a Vapor Compression Cycle.

B. S. Kim, and P. A. Domanski. Sep 96, 28p, NISTIR-5873

Sponsored by Department of Energy, Washington, DC Office of Building Technology.

Keywords: "Evaporative cooling, "Vapor compression refrigeration cycle, Heat pumps, Refrigerating, Buildings, Air conditioning.

The temperature glide of zeotropic mixtures during phase change provides the opportunity to limit throttling losses of the refrigeration cycle by intracycle evaporative cooling of the refrigerant leaving the condenser. Intracycle evaporative cooling is similar to the 


\section{General}

use of the liquid-line/suction-line heat exchanger with the difference that a two-phase low-pressure refrigerant, instead of superheated vapor, is used to subcool the high-pressure liquid leaving the condenser Intracycle evaporative cooling was evaluated by semi-theoretical simulation model and in the NIST's Small Breadboard Heat Pump at the cooling mode operating condition typical for a water-to-water residentia heat pump. The capacity, coefficient of performance (COP), pressures, and temperature profiles of refrig erant and heat-transfer fluid in the heat exchangers are reported. The laboratory measured improvement of the COP was $4.0 \%$ for R-32/152a, 3.6\% for R-407C and 1.8 percent for $R-23 / 152 a$

02,763

PB97-118681 Not available NTIS

National Inst. of Standards and Technology (MEL) Gaithersburg, MD. Automated Production Technology Div.

Transient Analysis of a Line-Focus Transducer Probing a Liquid/Solid Interface.

Final rept.

N. N. Hsu, D. Xiang, S. E. Fink, and G. V. Blessing. 1996, 8p.

Pub. in Review of Progress in Quantitative Nondestructive Evaluation, Seattle, WA., July 30-August 4 , 1995, p995-1002 1996.

Keywords: "Line-focus transducer, "Transient ultrasonic wave, Materials, Reprints, Time-domain Green's function Transducer response function, Ultrasonic tests, Liquid-solid interfaces.

The use of a line-focus ultrasonic transducer in a vertical scanning reflection acoustic microscope system is well known for quantitative materials characterization. The technique relies on the measurement of the reflected radio frequency tone burst echo emplitude, $V$ as a function of amount of defocus, $z$, and analysis of the interference minima of the $V(z)$ curve to obtain varous interface wave speeds. The technique uses we developed theory representing fixed frequency ultrasound generated and detected by a cylindrical lens in the frequency domain. The authors have developed a large aperture lensless line-focus transducer which is highly efficient and has a bandwidth wide enough to allow the generation and detection of narrow ransient pulses. From this transducer placed in water near a solid sample, the resulting echo waveforms have multiple features which can be interpreted as the arrival of specularly reflected axial ray and leaky surface waves. Using this transducer, the authors have developed a time-resolved and polarization-sensitive testing tehcnique for materials characterization. The objective of the paper is to provide a theoretical basis for interpretation and analysis of these time domain waveforms.

\section{2,764}

PB97-122576 Not available NTIS

National Inst. of Standards and Technology (MEL) Gaithersburg, MD. Automated Production Technology Div.

Material Characterization By a Time-Resolved and Polarization-Sensitive Ultrasonic Technique.

Final rept.

D. Xiang, N. N. Hsu, and G. V. Blessing. 1996, 8p. Pub. in Review of Progress in Quantitative Non destructive Evaluation, Seattle, WA., July 30-August 4 1995, v15 p1431-1438 1996.

Keywords: *Ultrasonic microscopy, "Transducers Leaky waves, Reprints, "Line-focus transducer, PVDF transducers.

Acoustic microscopy provides materials characterization by measuring the leaky surface wave speed and attenuation in sample materials. These measurements may be interpreted by analyzing a $V(z)$ curve, which is a record of the voltage of the transducer as a function of the defocus distance $z$ between the focal poin and sample surface. Since the $V(z)$ curve results from the interference between the leaky surface waves and the direct-reflected wave in a tone-burst mode of operation, the fast Fourier transform (FFT) technique is commonly used to analyze the $V(z)$ curve in the frequency domain. While this manner of acoustic microscopy utilizes a small aperture lens and high frequency tone bursts in order to obtain high spatial resolution, the system cost is greater than the alternative shortpulse broadband systems. Furthermore, high spatia resolution is not necessary for many practical meas urements, for example in the case of composite materials.

\section{2,765}

PBg7-122584 Not available NTIS

National Inst. of Standards and Technology (MEL) Gaithersburg, MD. Automated Production Technology Div.

Design, Construction and Application of a Large Aperture Lens-Less Line-Focus PVDF Transducer. Final rept.

D. Xiang, N. N. Hsu, and G. V. Blessing. 1996, 7p. Pub. in Uitrasonics, v34 p341-347 1996.

Keywords: "Transducers, "Materials characterization, Reprints, *Green's function, "PVDF transducers, "Linefocus transducers.

A large aperture lens-less line-focus transducer for materials characterization is described. The transduce design is based on a time-domain Green's function formalism, which also yields good theoretical corroboration of the experimental results. The transducer construction is readily achieved by conforming commercial polyvinylidene fluoride (PVDF) film to a cylindrical (convex) surface, followed by casting a tungsten-powder-loaded epoxy resin backing material into an at tached housing. This transducer can be used to characterize sample material properties by simultaneously measuring surface wave speeds and bulk wave transit times, from which the thickness and anisotropy may be deduced.

\section{Computer Aided Design (CAD)}

\section{2,766}

PB94-139649 PC A03/MF A01

National Inst. of Standards and Technology, Gaithersburg, MD

Security Considerations for SQL-Based Implementations of STEP.

L. E. Bassham, and W. T. Polk. Oct 93, 26p, NISTIR5283

See also PB89-168009, PB93-113637 and PB94 104585.

Keywords: *Computer security, Computer aided design, Computer aided manufacturing, Distributed processing, Data management, Standards, Access control Data integrity, *PDES(Product Data Exchange using STEP), STEP(Standard for the Exchange of Product Model Data). SQL database language, RDA(Remote Database Access)

The Database Language SQL (SQL) is a standard interface for accessing and manipulating relational databases. As such, SQL can be used in many different operational environments, with correspondingly different needs for security. One specific application of this standard is in the field of Product Data Exchange using STEP (PDES). This paper examines the security implications of the versions of the SQL standard as used to implement STEP. STEP does not imply any particular security policy, so a variety of security policies are examined. The paper has been written as a companion document to the National Institute of Standards and Technology's (NTIS's) general SQL security document, 'Security Issues in the Database Language SQL', and references that document frequently.

02,767

PB94-154325 PC A03/MF A01

National Inst. of Standards and Technology, Gaithersburg, MD

APDE Demonstration System Architecture. $\mathrm{Na}$ tional PDES Testbed Report Series.

S. N. Clark, and A. B. Feeney. Jan 93, 21p, NISTIR-

5318

See also PB93-208114. Sponsored by Assistant Secretary of Defense (Production and Logistics), Washing ton, DC. Computer-aided Acquisition and Logistic Support Program.

Keywords: "Computer aided design, *Computer aided manufacturing, Standards, Prototypes, Systems engineering *APDE(Application Protocol Development Environment), "Application protocols, STEP(Standard fo the Exchange of Product Model Data), PDES(Product Data Exchange using STEP), ARM(Application Reference Model), AIM(Application Interpreted Model).

The National Product Data Exchange using STEP (PDES) Testbed project at the National Institute of Standards and Technology (NIST) is focused on the development and implementation of the emerging international Standard for the Exchange of Product model data (STEP). One sub-project within the Testbed is the effort to establish an Application Protocol Development Environment (APDE). The report describes the architecture of a prototype system which will be built at NIST to demonstrate some of the features needed in an APDE and some approaches to providing these features. The demonstration system will focus on a specific step in the AP developmen process, namely Application Reference Model (ARM) interpretation. The functionality demonstrated is based on specifications documented in an earlier report

\section{2,768}

PB94-154358 PC A03/MF A01

National Inst. of Standards and Technology (MEL) Gaithersburg, MD. Factory Automation Systems Div. Variant Design for Mechanical Artifacts-A State of the Art Survey.

Internal rept.

J. E. Fowler. Feb 94, 28p, NISTIR-5356

Keywords: *Computer aided design, Reasoning, Specifications, Feature extraction, Mathematical models Standards, "Variant design, STEP(Standard for the Exchange of Product Model Data)

Variant design refers to the technique of adapting existing design specifications to satisfy new design goals and constraints. Specific support of variant design techniques in current computer aided design systems would help to realize a rapid response manufacturing environment. A survey of approaches supporting variant design is presented. Capabilities used in current commercial computer aided design systems are discussed along with approaches used in recent research efforts. Information standards applicable to variant design are identified as well. Barriers to variant design in current systems are identified and ideas are presented for augmentation of current systems to support variant design.

\section{2,769}

PB94-159795 PC AO4/MF A01

National Inst. of Standards and Technology (MEL) Gaithersburg, MD. Factory Automation Systems Div. Structural EXPRESS Editor.

T. R. Kramer, K. C. Morris, and D. A. Sauder. Aug

92, 73p, NISTIR-4903

See also PB90-269507. Prepared in cooperation with Catholic Univ. of America, Washington, DC. Dept. of Mechanical Engineering.

Keywords: *Editing routines, Computer aided design, Computer aided manufacturing, Standards, Interactive systems, Data structures, Algorithms, "EXPRESS modeling language, "STEP (Standard for the Exchange of Product Model Data), PDES(Product Data Exchange using STEP), SEXE(Structural EXPRESS Editor).

EXPRESS is the official information modeling language of the next generation of product information standards, commonly referred to as STEP or Standard for the Exchange of Product Model Data. This pape presents a Structural EXPRESS Editor (SEXE), which has two parts: an Interactive Editor, and a Translation Module. The Interactive Editor was built using a too called the Data Probe Editor (DPE) Builder and is an interactive, friendly editor with which a user defines the structure of an EXPRESS schema. The output of the Interactive Editor is a STEP exchange file that is trans lated into EXPRESS by the Translation Module, a software system written in LISP. The paper describes the DPE Builder and both parts of SEXE. The editor is as sessed and EXPRESS editors, generally, are discussed. Alternative representations of EXPRESS are presented. A few issues in information modeling highlighted in the course of building the editor are discussed.

\section{2,770}

PB94-160868 PC A05/MF A01

Catholic Univ. of America, Washington, DC.

Issues and Recommendations for a STEP Applica tion Protocol Framework. National PDES Testbed. T. R. Kramer, M. E. Palmer, and A. B. Feeney. 17 Jan 92, 90p, NISTIR-4755.

Contract NANB9H0923

See also PB92-112374. Sponsored by National Inst of Standards and Technology, Gaithersburg, MD. and Assistant Secretary of Defense (Production and Logistics), Washington, DC. Computer-aided Acquisition and Logistic Support Program. 
Keywords: Product development, Standards, Protocols, Computer aided design, Computer aided manufacturing, Tests, Standardization, "STEP(Standard for the Exchange of Product Model Data), change Using STEP), AIM(Application Interpreted Model).

Technical experts are developing the next generation product information exchange standards, called the Standard for the Exchange of Product Model Data (STEP). The STEP project is using the application protocol (AP) methodology for defining the information requirements of industry and for specifying standards for representing these requirements. Several APs have already been drafted. Before the AP methodology is ex panded to address more complex problems, and before any more AP projects are initiated, the fundamental methods for developing and testing APs must be completed and proven. This report provides a description of application protocols, a summary of AP develop ment issues (supplemented by discussions and case studies in the appendices), a listing of the relevant documentation and issues papers, and recommendations for resolving the identified issues. This includes recommendations on functional requirements for an AP framework, elements of AP structure, coordination of AP development, and AP classification. This report provides a baseline study for subsequent work on an AP Framework by members of the STEP community.

02,771

PB94-163482 PC A03/MF A01

National Inst. of Standards and Technology, Gaithersburg, MD

Validation Testing System Requirements. National PDES Testbed Report Series.

K. C. Morris, M. McLay, and P. J. Carr. Sep 91, 32p, NISTIR-4676.

See also PB88-235452 and PB92-123090. Sponsored by Assistant Secretary of Defense (Production and Logistics), Washington, DC. Computer-aided Acquisition and Logistic Support Program.

Keywords: "Software engineering, "Requirements, Product development, Models, Tests, Computer software, Software tools, Data flow analysis, Interfaces, "VTS'(Validation Testing System), PDES(Product Data Exchange using STEP), STEP(Standard for the Exchange of Product Model Data), ISO(International Standards Organization).

This document describes requirements for software to support the testing of information models for validity and correctness. The requirements provide a basis for the software development to support the Validation Testing System (VTS) within the National PDES Testbed. This document describes the soffware needs as currently known for a complete validation testing system. It identifies the scope of the VTS software, presents functional requirements for the proposed system describes the data flow environment, external interfaces, performance, and documentation requirements of the system, and prioritizes the requirements for the purpose of implementing the proposed system.

\section{2,772}

PB94-219086 PC A04/MF A01

Information Assets, Inc , Houston, TX.

Framework for Information Technology Integration in Process Plant and Related Industries.

W. G. Beazley, and J. B. Chapman. Jul 94, 74p

NIST/GCR-94/657

See also PB87-209052. Sponsored by National Inst. of Standards and Technology (BFRL), Gaithersburg, MD. Building Environment Div.

Keywords: "Industrial plants, "Automation "Petrochemistry, Design, Construction, Operation, Engineering, Constraints, Data processing, Digital data Benefit cost analysis, Computer aided design, Computer aided manufactu ring.

Initially, the report presents a constraint framework that can identify all the root constraints and their associated activities for the engineering, construction, and operation of a petrochemical plant. Second, it demonstrates how the constraint framework leads to functional modIs of design that can be traced directly to the constraints they address. This avoids some of the probems in basing models on design tasks assigned within phases. Third, it demonstrates how external constraints do, in fact, influence design and how to analyze their influence. Fourth, it discusses some of the data hat is produced to document that the constraints have been satisfied. It provides a list of some of the tools that support the creation and maintenance of these data sets. Last it discusses the business case for 3D design and for digital Piping and Instrumentation Drawings (P\&ID) delivery.

\section{2,773}

\section{PC A03/MF A01}

National Inst of Standards and Technology (MEL) Gaithersburg, MD. Factory Automation Systems Div. Formulation of Position on U.S. Standards Role in Enterprise Integration

H. M. Bloom. Aug 94, 43p, NISTIR-5484.

Keywords: *Standards, "Product development, United States, Government policies, Technology innovation, Computer aided design, Computer aided manufacturing, "Enterprise integration, PDES(Product Data Exchange using STEP), STEP(Standard for the Exchange of Product Model Data), US NIST, IGES(Initial Graphics Exchange Specification), US PRO.

This paper gives a brief overview of the concepts of enterprise integration, defines a national strategy for enterprise integration (EI) that involves not only standards bodies but also the government and industry groups with an interest in El technology and standards, and proposes that one specific standards organization, the US PRO that oversees the Initial Graphics Exchange Specification (IGES) Product Data Exchange using STEP (PDES) Organization, develop a new extended scope that will tackle the El standards issues. Finally, a business case for US PRO involvement in $E l$ is discussed, first through the identification of key issues requiring resolution, and then through listing the pros and cons of extending the scope to enterprise inlegration framework standards. As reference information to support the paper, the key government and in dustrial groups that are involved in El-related technology development programs are described along with the organizations that have a stake in the standards process.

\section{2,774}

PB95-107314 Not available NTIS

National Inst. of Standards and Technology (NEL), Gaithersburg, MD. Factory Automation Systems Div. Summary and Notes of the Joint ISO/IGES/PDES Organization Technical Committee Meeting. Held in Albuquerque, New Mexico on October 15-20, 1989.

Final rep

S. R. Ray, and G. R. Rinaudot. 1989, 85p

Pub. in Joint IGES/PDES/ISO Meeting Minutes, Albuquerque, NM., October 15-20, 1989, p1-85.

Keywords: "Computer aided design, "Computer aided manufacturing, "Standards, Automation, Reprints, "IGES(Initial Graphics Exchange Specification) *Initial Graphics Exchange Specification, "PDES(Product Data Exchange using STEP), "Product Data Exchange using STEP, STEP(Standard for the Exchange of Product Model Data), ISO(International Organization for Standardization).

The document is a summary of the Initial Graphics Exchange Specification/Product Data Exchange using STEP (IGES/PDES) Organization General Assembly meeting and the IGES/PDES technical committee meetings held in Albuquerque, New Mexico, on Octo ber 15 th - 20th, 1989

\subsection{5}

PB95-125829 Not available NTIS

National Inst. of Standards and Technology (NEL) Gaithersburg, MD. Factory Automation Systems Div. National PDES Testbed: An Overview.

Final rept

C. M. Furlani. 1989, $8 \mathrm{p}$

Pub. in Proceedings of Annual Conference on University Programs in Computer-Aided Engineering, Design, and Manufacturing (7th), Laramie, WY., July 23-26, 1989, p1-8.

Keywords: "Computer aided design, ${ }^{~}$ Computer aided manufacturing, "Data transfer(Computers), change Specification), Product Data Exchange using STEP STEP(Standard for the Exchange of Product Model Data), AMRF(Automated Manufacturing Research Facility), US NIST.

A description of the National PDES Testbed which has recently been established at the National Institute of Standards and Technology (NIST) to provide support for industry and government projects in developing and testing the Product Data Exchange Specification
(PDES), the next generation of data interchange standards for automated manufacturing An overview of the Automated Manufacturing Research Facility (AMRF), which provides the foundation for the Testbed, is in cluded.

\section{2,776}

PB95-136347 PC A03/MF A01

National Inst. of Standards and Technology, Gaithersburg, MD

Challenges to the National Information Infrastructure: The Barriers to Product Data Sharing. National PDES Testbed Report Series.

D. A. Sauder, M. J. Mitchell, and A. B. Feeney, 15 Sep 94, 13p, NISTIR-5498.

See also PB93-208114. Sponsored by CALS Evaluation and Integration Office, Washington, DC

Keywords: "Computer aided design, "Computer aided manufacturing, "Standards, Data processing, Product development, Data management, Specifications, STEP(Standard for the Exchange of Product Model Data), "Standard for the Exchange of Product Model Data, NII(National Information Infrastructure) SGML(Standard Generalized Markup Language), US NIST.

Industry has a need to share data among functions within its own enterprise and among its suppliers and partners. A standard specification for manufacturing data is needed that can support the entire product development and support life-cycle. The emerging international standard called the Standard for the Exchange of Product Model Data (STEP) is addressing this need by specifying what data is needed within industrial application areas, how it should be represented, and how it should be exchanged. The Application Protocol Development Environment (APDE) is a software project at the National Institute of Standards and Technology (NIST) designed to facilitate the development, acceptance, and use of STEP. The paper discusses the status of STEP barriers to the development, acceptance, and use of STEP, and the benefits of using STEP in industry. Aspects of the APDE project are also discussed including current status, lans for a STEP information base use of the Standard Generalized Markup Language (SGML) for facilitating the information base, barriers to the project, and expected benefits for STEP.

\section{7}

PB95-137790 PC A02/MF AO

National Inst. of Standards and Technology (MEL) Gaithersburg, MD. Factory Automation Systems Div. STEP On-Line Information Service (SOLIS). The IGES/PDES Organization.

G. R. Rinaudot. Oct 94, 9p, NISTIR-5511.

See also PB91-159756.

Keywords: "Information services, "On-line systems, Standards, Product development, Computer aided de sign, Computer aided manufacturing, "STEP(Standard for the Exchange of Product Model Data), "Standard for the Exchange of Product Model Data, US NIST, SOLIS(STEP On-Line Information Service), IGES(Initial Graphics Exchange Specification), PDES(Product Data Exchange using STEP).

This document contains an overview and describes the objectives of the STEP (STandard for the Exchange of Product model Data) On-Line Information Service (SOLIS). This report is also intended to provide a general explanation of this service and its capabilities. The service is provided and maintained by the National Institute of Standards and Technology (NIST), Manufacturing Engineering Laboratory (MEL), IGES/PDES (Initial Graphics Exchange Specification/Product Data Exchange using STEP) Office in the Factory Automation Systems Division. SOLIS enhances the ability to gain consensus on STEP by expanding the availability of the draft standards, supporting documents, and software used by the community of experts who are contributing to this standard.

\section{2,778}

PB95-143103 PC A03/MF A01

National Inst. of Standards and Technology (MEL), Gaithersburg, MD. Factory Automation Systems Div. SGML Environment for STEP.

L. Phillips, and J. Lubell. 22 Jun 94, 36p, NISTIR-

5515

See also PB94-114501 and PB94-150919. Sponsored by Assistant Secretary of Defense (Production and Logistics), Washington, DC. Computer-aided Acquisition and Logistic Support Program.

Keywords: "Documents, "Standards, Parsers, Tex processing, "STEP(Standard for the Exchange of 


\section{Computer Aided Design (CAD)}

Product Model Data), "Standard for the Exchange of Product Model Data, "SGML(Standard Generalized Markup Language), "Standard Generalized Markup Language, US NIST, APDE(Application Protocol Development), DTD(Document Type Definition).

The paper provides an in-depth discussion of the components of a proposed Standard Generalized Markup Language (SGML) environment for the Standard for the Exchange of Product Model Data (STEP). Section one introduces the SGML environment for STEP and provides a definition of SGML and its applicability to STEP. Section two provides a background discussion of the Application Protocol Development Environmental (APDE) project and its relationship to the SGML environment for STEP. Section three provides additional basic concepts in SGML. Section four provides further discussion on the benefits of using SGML and the rationale for choosing SGML for STEP. Section five provides a detailed overview of the components of an SGML environment for STEP. Finally, section six concludes with an overview of the lessons learned, current status, and the near-term and long-term future plans for the STEP SGML environment.

\section{2,779
PB95-154688 PC A03/MF AO}

National Inst. of Standards and Technology (MEL), Gaithersburg, MD. Factory Automation Systems Div. Initial NIST Testing Policy for STEP: Beta Testing Program for AP 203 Implementations. Nationa PDES Testbed Report Series.

M. J. Mitchell. 3 Nov $94,25 p$, NISTIR-5535.

Also available from Supt. of Docs. Sponsored by CALS Evaluation and Integration Office, Washington, DC.

Keywords: "Tests, Policies, Protocols, Programs, Laboratories, Methodology, Standards, Computer aided design, Product development, "STEP(STandard for the Exchange of Product model data), "STandard for the Exchange of Product model data, "US NIST, Con formance testing, ITI(Industrial Technology Institute) Application protocols.

The standard for Product Data Representation and Exchange, ISO 10303, defines a neutral, computer-inter pretable representation for describing product data in a manner that is independent from any particular system. This standard is more commonly known as STEP STandard for the Exchange of Product model data. This document describes the policy and procedures for a 'beta' conformance testing program for one of the application protocols: Configuration Controlled 3D Design for Mechanical Parts and Assemblies (ISO 10303 203). However, the concepts described are applicable to ISO 10303 in general, and the characteristics of the longer term testing program are described. This document describes the policy and procedures used by the National Institute of Standards and Technology (NIST) and the Industrial Technology Institute (ITI) for the Beta STEP Conformance Testing Program. The objective of this beta program is to develop an acceptable degree of confidence in the test methods and procedures.

\section{2,780}

\section{PB95-171427 PC A03/MF A01}

National Inst. of Standards and Technology (CSL) Gaithersburg, MD. Information Systems Engineering Div.

Initial Graphics Exchange Specification (IGES): Procedures for the NIST IGES Validation Test Service.

J. A. Schneider, and L. S. Rosenthal. Dec 94, 36p NISTIR-5541.

Keywords: "Tests, Computer aided design, Computer aided manufacturing, Data transfer(Computers) Preprocessors, Federal information processing standards, "CALS, "IGES(Initial Graphics Exchange Speci fication), "Initial Graphics Exchange Specification Continuous Acquisition and Life-Cycle Support, Computer-aided Acquisition and Logistics Support, US NIST, Postprocessors, Conformance testing.

This document provides general procedures for the National Institute of Standards and Technology's (NIST) Initial Graphics Exchange Specification (IGES) Validation Test Service. The NIST IGES Validation Test Service provides a way of determining the degree to which an implementation conforms to the FIPS PUB 177, which adopts the ANSI standard (ASME/ANSI Y14.26M-1989) in its entirety. The NIST IGES Validation Test Service also provides a way of determining the degree to which an implementation confirms to the Continuous Acquisition and Life-Cycle Support (CALS) specification, MIL-D-28000, Class II subset. The goal of the NIST Validation Test Service is to maxımize the probability of successful data exchange between dissimilar CAD/CAM systems. The document is divided into two testing programs: preprocessor and postprocessor testing. The procedures are organized into four sections: an introduction, general procedures, and specific procedures for the two testing programs.

\section{2,781}

PB95-180543 Not available NTIS

National Inst. of Standards and Technology (MEL), Gaithersburg, MD. Factory Automation Systems Div. Concepts of the NIST EXPRESS Server.

Final rept.

D. Libes. 1994, 6p

Pub. in Proceedings of International Workshop on Services in Distributed and Networked Environments (SDNE) (1st), Prague, Czech Republic, June 27-28, 1994, 6p.

Keywords: "Remote consoles, Computer aided design, Computer aided manufacturing, Compilers, Electronic mail, File structures, Software tools, Reprints, *EXPRESS, *US NIST, PDES(Product Data Exchange using STEP), STEP(Standard for the Exchange of Product Model Data)

The National Institute of Standards and Technology (NIST) has built numerous software toolkits and applications for manipulating STEP and EXPRESS data. The NIST EXPRESS Server is a computational facility at NIST, which provides the ability to run these toolkitbased applications remotely without installing them locally. Users e-mail EXPRESS Schemas and other data files to the Server. The Server runs the requested applications on the files and returns any diagnostics or output, also by e-mail. Applications requiring interaction can either be returned via e-mail so that they can be run locally, or run remotely by telnet or rlogin across the Internet

\section{2,782}

PB95-242285 PC A04/MF A01

National Inst. of Standards and Technology (EEEL), Gaithersburg, MD. Electricity Div.

Operating Procedures and Life Cycle Documentation for the Initial Graphics Exchange Specification.

G. F. Morea, E. Reid, and C. Parks. 22 Jun 95, 62p, NISTIR-5666.

Keywords: "Changes, "Editing, "Standards, Administrative procedures Documentation Record keeping Standardization, Computer aided design, Data processing, Data transfer(Computers), Concurrent engineering, "IGES(Initial Graphics Exchange Specification), "Initial Graphics Exchange Specification.

The purpose of this document is to provide a record of the underlying rationale and the detailed procedures by which the Initial Graphics Exchange Specification (IGES) is changed, new versions are approved, and the document itself is edited, maintained, and published by the IGES Project of the IGES/PDES Organization (IPO). The document also defines the life cycle documentation supporting these activities and provides guidance for its use, including record keeping requirements, to provide evidence of compliance with these procedures. Procedures given in this document are intended to be supplementary to and consistent with the documented procedures of the General Assembly of the IPO for the IGES Project. In general, procedures in this document are at a more detailed, working level.

\section{2,783}

\section{PB95-253563 PC A03/MF A01}

National Inst. of Standards and Technology (MEL) Gaithersburg, MD. Manufacturing Systems Integration Div.

Product Models and Virtual Prototypes in Mechanical Engineering.

M. J. Pratt. May 95, 22p, NISTIR-5650

Presented at the IFIP Working Group 5.10 Workshop on Virtual Prototyping, Providence, RI., September 21 . 23, 1994.

Keywords: "Product development, "Models, "Prototypes, "Mechanical engineering, Virtual reality, Computerized simulation, Computer graphics, Computer aided design, Computer aided manufacturing, edge based systems, Feature extraction.

The paper gives an overview of some of the modeling and virtual prototyping techniques used in product real- ization, with emphasis on the mechanical engineering field. It is pointed out that virtual prototypes, in the commonly accepted sense of computer models permitting realistic graphical simulation, represent only one class amongst the many types of computer models used in design and planning for manufacture. Each such model is usually created for some comparatively narrow purpose, and one of the major problems faced by developers of product realization systems concerns the transmutation of one type of model into another. A related problem is that of interpretation by any model of information generated by interrogations of another model. These difficulties are compounded by the increasing presence in such models of semantic information concerning different aspects of the intended functionality or manufacturing requirements of the modelled artifact.

\section{2,784}

PB95-267860 PC A03/MF A01

National Inst of Standards and Technology (MEL) Gaithersburg, MD. Factory Automation Systems Div. Design Engineering Research at NIST.

K. W. Lyons, and P. F. Brown. May 94, 22p, NISTIR5464 .

Keywords: "Design, *US NIST, "Production engineering, Research management, Technology transfer, Research and development.

The National Institute of Standards and Technology (NIST) has established an Engineering Design Laboratory (EDL) to assist in determining the best practices and methods to design new products and processes This paper includes a description of issues facing designers and outlines research activities that are currently being done, or are planned, at NIST.

\section{2,785}

PB96-141049 Not available NTIS

National Inst. of Standards and Technology (MEL) Gaithersburg, MD. Manufacturing Systems Integration Div.

Object-Oriented Tel/Tk Binding for Interpreted Control of the NIST EXPRESS Toolkit in the NIST STEP Application Protocol Development Environment.

Final rept

D. Libes, and S. N. Clark. 1995, 14p.

Pub. in Proceedings of the EXPRESS User Group Workshop (EUG '95), Grenoble, France, October 21 $22,1995, p 1-14$

Keywords: "Software tools, "Object-oriented programming "Standards, Computer aided design, Computer aided manufacturing, Systems engineering, Protocols, Reprints, PDES(Product Data Exchange using STEP) National PDES Testbed, STEP(Standard for the EX change of Product model data), APDE(Application Protocol Development Environment), TCL(Tool Command Language), TCL programming language, TK(Task knowledge)

The National Institute of Standards and Technology (NIST) has built numerous software toolkits and applications for manipulating STEP and EXPRESS data. These toolkits are traditionally used as compiled librar ies which are linked to other complied modules. This paper describes a binding allowing the toolkit interfaces to be called from interpreted scripts. This significantly reduces the time required to construct and compile new applications. An $\times 11$ extension allows the construction of graphic elements, providing easy creation and integration of existing applications into $X$ graphic user interfaces. We describe how the combination of bindings has been used to construct a STEP Application Protocol Development Environment.

\section{2,786}

PB96-154539 PC A03/MF A01

National Inst. of Standards and Technology (MEL) Gaithersburg, MD. Manufacturing Systems integration Div.

Guidelines for the Development of Mapping Tables. A. B. Feeney, and D. Craig. 16 Nov 95, 24p, NISTIR5716.

Prepared in cooperation with Product Data Integration Technology, Long Beach, CA

Keywords: "Computer aided language, "Computer aided manufacturing, "Standards, Data processing, Product development, Data management "STEP(Standard for the Exchange of Product Mode Data), APDE(Application Protocol Development Environment). 
The mapping table is a pivotal component of an application protocol (AP). The mapping table documents the traceability of the application information requirements between the specification of these requirements in clause 4 of the AP and the application interpreted model (AIM) that documents how standardized constructs are applied to satisfy these requirements in clause 5. The document is intended to provide guidance to application protocol development teams on the creation of mapping tables. Additionally, the document may aid reviewers and implementors of APs in understanding mapping tables. The document describes the methodology for producing a complete mapping table, focusing on the development of the mapping table content. Specifics on style, format, boiler plate text, and other presentation issues are provided in the Supplementary directives for the drafting and presentation of ISO 10303 (Supplementary directives). Additional guidance on other areas of AP development is found in the Guidelines for development and approval of STEP application protocols.

\section{2,787}

PB96-154976 PC A03/MF A01

National Inst. of Standards and Technology (CSL), Gaithersburg, MD

STandard for the Exchange of Product Model Data (STEP): Procedures for NIST STEP Validation.

L. S. Rosenthal. Nov 95, 19p, NISTIR-5771. See also PB95-154688.

Keywords: “Tests, Policies, Protocols, Programs, Laboratories, Standards, Computer aided design, Product development, "STEP(Standard for the Exchange of Product Model Data), Standard for the Exchange of Product Model Data, Conformance testing. Application protocols, ITI(Industrial Technology Institute), ISO 10303.

The standard for Product Data Representation and Exchange, ISO 10303, defines a neutral, computer-interpretable representation for describing product data in a manner that is independent from any particular system. This standard is more commonly known as STEP, Standard for the Exchange of Product model data and it is designed to support a wide range of design, engineering, and product support applications. STEP was adopted verbatim as an American National Standard (ANS) titled Product Data Exchange using Step, ANSI USPRO 200. Support for specific applications is provided through an Application Protocol. This document provides general procedures for the National Institute of Standards and Technology's (NIST) STEP FIPS Validation Service. The Validation Service provides a way of determining the degree to which an implementation conforms to the proposed STEP FIPS. The goal of the Validation Service is to maximize the probability of successful interchange among systems which implement the same STEP applications protocol.

\section{2,788}

\section{PB96-160965 Not available NTIS}

National Inst. of Standards and Technology (CSL), Gaithersburg, MD. Information Systems Engineering Div.

Template-Driven Systems Development with IDEF: Enterprise Standards for Reuse.

Final rept.

M. H. Law. 1992, 10p.

Pub. in IDEF Users Group Business Process Design: A Strategy for Corporate Information Management, Washington, DC., October 19-22, 1992, p1-10.

Keywords: "Software reuse, "Systems approach, "Integrated systems, Computer-aided design, Computer-aided manufacturing, Software engineering, Software reliability, Object-oriented programming, Parameterization, Standards, Reprints, IDEF(Integrated Definition), CASE(Counter-Aided Software Engineering), System development, Domain analysis, Life cycles(Software).

This paper addresses the use of reusable templatedriven architectures to improve system and software development methods with an extended IDEF. Extended IDEF is proposed as a family of modeling techniques that has been integrated with the proposed Federal Information Processing Standards (FIPS) for IDEFO and IDEFIX, and extended to offer more support for the system and software development lifecycle Integrated through a metamodel, Extended IDEF will present a number of distinct views to users working in different aspects of the lifecycle, such as activity modeling, data modeling, object modeling, state/event modeling, etc. Integration through the metamodel is intended to maintain the critical relationships among the
IDEF techniques; extension through the lifecycle is intended to expand the utility of IDEF.

\section{2,789}

\section{PB96-165402 PC A11/MF A03}

National Inst. of Standards and Technology (BFRL), Gaithersburg, MD. Building Environment Div

Group 1 for the Plant Spatial Configuration STEP Application Protocol.

. W. Kline, M. E. Palmer, W. Burkett, and J. Skeels. Jun 95, 202p, NISTIR-5675.

Prepared in cooperation with Product Data Integration Technology, Long Beach, CA.

Keywords: *Computer aided manufacturing, "Industrial plants, *Computer aided design, *Standards, Standard tools, Pipes tubes, Models, *STEP(Standard for the Exchange of Product Model Data), ISO 1303, Standard for the the Exchange of Product Model Data, Application protocols, Spatial distribution.

The document specifies the scope and information re quirements of the STEP application protocol (AP) for the exchange of spatial configuration information of plant systems with a central emphasis on piping sys tems. It specifies the information required to construct a piping system, including the shape, material, and arrangements of the system components. It also specifies requirements for the physical aspects of other plant systems (e.g., structure) needed to design and layout the piping system. The document includes the application activity model (AAM) and application ref erence model (ARM) for this AP. The document was submitted to ISO TC184/SC4 and to international ex perts for review and comment on the scope and requirements of the AP. Additional clauses and annexes and refinements will be added to the AP after the completion of the ISO Committee Draft for Comment review.

\section{2,790}

PB96-183231 PC A03/MF A01

National Inst. of Standards and Technology (MEL), Gaithersburg, MD. Manufacturing Systems Integration Div.

Aspects of a Product Model Supporting Apparel Virtual Enterprises.

P. O. Denno. 16 Apr 96, 15p, NISTIR-5821.

See also PB96-128194.

Keywords: *Clothing industry, *Computer aided de sign, Computer aided manufacturing, Data transfer Patterns, Tests, Models, Data structures, Data exchange, Standard, Product data, Textile industry, STEP(Standard for the Exchange of Product Model Data).

Emerging computer technology, including an infra structure for virtual enterprises and more capable apparel product development software, is providing an opportunity to reinvent how apparel is produced. The development of an information-rich and unambiguous garment product model would help realize this opportunity. This product model would improve the effectiveness of product development software and enhance the industry's ability to distribute work in virtual enterprises. This paper suggests what such a product mode should contain, how it can be developed and how it would be utilized throughout the product development life cycle to enable apparel virtual enterprises.

\section{2,791}

\section{PB96-202320 PC A03/MF A01}

National Inst. of Standards and Technology (MEL) Gaithersburg, MD. Manufacturing Systems Integration

Application Protocol Information Base World Wide Web Gateway.

Technical repi

J. Lubell. $31 \mathrm{Jul} 96,27 p$, NISTIR-5868

Keywords: “Documents, "Standards, Parsers, Tex processing, Information retrieval, *STEP(Standard for the Exchange of Product Model Data), Standard for the Exchange of Product Model Data, SGML(Standard Generalized Markup Language), Standard Generalized Markup Language, APID(Application Protocol Information Base). Application Protocol Information Base, Internet, World Wide Web, HTML, Browsers.

The Application Protocol Information Base (APIB) is an on-line repository of documents for the Standard for the Exchange of Product Model Data (STEP, officially ISO 10303-Product Data Representation and Exchange). STEP Application Protocols are standards that are intended to be implemented in software sys- tems, and Integrated Resources are used by them as building blocks. Application Protocols and Integrated Resources are represented in the Standard Generalized Markup Language (SGML) in the APIB in order to facilitate efficient information search and retrieval. This paper describes a World Wide Web gateway to the APIB, implemented using the Common Gateway Interface(CGI) standard. The APIB gateway allows STEP developers to efficiently search for ISO 10303 standards and supporting information. The only client software required to use the APIB gateway is a third party web browser.

\section{2,792}

PB97-111561 Not available NTIS

National Inst. of Standards and Technology (MEL) Gaithersburg, MD. Manufacturing Systems Integration Div.

Representing Designs with Logic Formulations of Spatial Relations.

Final rept.

S. C. Chase. 1996, 9p.

Pub. in International Conference on Artificial Intel ligence in Design (AID) (4th): Workshop Notes, Visua Representation, Reasoning and Interaction in Design Stanford University, CA., June 22-27, 1996, p1-9.

Keywords: *Logic programming, "Computer-aided design, "Representations, Computer graphics, Feature extraction, Pattern recognition, Shape, Geometry, Parameterization, Formalism, Computer models, Specifications, Reprints, "Spatial relations.

A new method of describing designs by combining the paradigms of shape algebras and predicate logic representations is presented. Representing shapes and spatial relations in first order predicate logic provides a natural, intuitive method of developing complete computer systems for reasoning about designs. Shape algebraic representations provide several advantages over more traditional geometric representations. The method described involves the definition of a large set of high level design relations from a small set of simple structures and spatial relations.

\section{2,793}

PB97-111579 Not available NTIS

National Inst. of Standards and Technology (MEL), Gaithersburg, MD. Manufacturing Systems Integration Div

Using Logic to Specify Shapes and Spatial Relations in Design Grammars.

Final rept.

S. C. Chase. 1996, $4 p$

Pub. in International Conference on Artificial Intelligence in Design (AID) (4th): Workshop Notes, Grammatical Design, Stanford University, CA., June 22-27, 1996, p1-4.

Keywords: "Computer-aided design, "Pattern recognition, "Shape, "Geometry, Computer graphics, Feature extraction, Representations, Parameterization, Logic programming, Formalism, Computer models, Specifications, Reprints, Spatial relations.

The use of predicate logic formulations of shape and spatial relations provides a natural, intuitive way to extend shape representations of the shape grammar formalism to provide generalized, parametric grammars.

\section{2,794}

PB97-112320 Not available NTIS

National Inst. of Standards and Technology (MEL) Gaithersburg, MD. Precision Engineering Div.

Combining Interactive Exploration and Optimization for Assembly Design.

Final rept.

G. J. Kim, and S. Szykman. 1996, 12p.

Pub. in American Society of Mechanical Engineers De sign Engineering Technical Conference and Computers in Engineering Conference, Irvine, CA., August 18$22,1996, \mathrm{p} 1-12$

Keywords: *Computer aided design, *Tools, Optimization, Standards, Quality assurance, Reprints.

The paper presents an integrated framework for assembly design. The framework allows the designer to represent knowledge about the design process and constraints, as well as information about the artifac being designed, design history and rationale. Because the complexity of assembly design leads to extremely large design spaces, adequately supporting design space exploration is a key issue that must be addressed. This is achieved in part by allowing the designer to use both top-down and bottom-up ap 
proaches to assembly design. Exploration of the design space is further enabled by incorporating a simulated annealing-based optimization tool that allows the designer to rapidly complete partial designs, refine complete designs, and generate multiple design alternatives.

\section{2,795}

PB97-113096 Not available NTIS

National Inst. of Standards and Technology (MEL) Gaithersburg, MD. Manufacturing Systems Integration Div.

Improving the Design Process by Predicting Downstream Values of Design Attributes.

Final rept.

S. Szykman. 1996, 9p.

Pub. in Proceedings of the American Society of Mechanical Engineers Design Engineering Technical Conference and Computers in Engineering Conference, inine, CA., August 18-22, 1996, p1-9.

Keywords: "Computer-aided design, *Product development, Design analysis, Design criteria, Feature extraction, Attributes, Specifications, Automation, Compute tools, Computer models, Reprints, " Design process.

The paper presents a computational approach to developing design space models that are utilized to improve the design process by predicting values of downstream design attributes based on information available at early stages, such as preliminary design speci fications. The predictive models are similar in function, though not in form, to the internal (mental) models created by experienced designers; however, the advantages of these models are that it may be possible to construct them in the absence of a designer's internal models, and that they can be passed on to and used by less experienced designers. Once created, the computational models aid designers in exploration of design alternatives and to reduce design costs and product development time.

\section{2,796}

\section{PB97-114268 PC A03/MF A01}

National Inst. of Standards and Technology (MEL) Gaithersburg, MD. Manufacturing Systems Integration Div.

Interoperability Requirements for CAD Data Transfer in the AutoSTEP Project.

S. P. Frechette. Mar 96, 28p, NISTIR-5844.

Keywords: *Computer aided design, *Computer aided manufacturing, “Automotive engineering, Production planning, Questionnaires, Standards, Networks, Development, Communications, Packaging, Information exchange, Motor vehicles, Interoperability, *STEP(Standard for the Exchange of Product Model Data), "Standard for the Exchange of Product Mode Data, iso 10303

This document describes user requirements for implementing STEP in a production environment for packaging applications used in the auto industry. The main purpose of the document is to report the results of a requirements survey of AutoSTEP project participants concerning the implementation of STEP in production practice.

\section{2,797}

PB97-116073 PC A99/MF A06

National Inst. of Standards and Technology (BFRL) Gaithersburg, MD

Group 1 for the Process Engineering Data STEP Application Protocol.

S. W. Kline, M. E. Palmer, N. Appel, and M. Gilbert. Oct 96, 656p, NISTIR-5909.

Prepared in cooperation with Product Data Integration Technology, Long Beach, CA.

Keywords: *Computer aided design, "Computer aided manufacturing, Product development, Automation Standards, Production planning, Specifications, Process engineering, Systems engineering, Protocols, rent engineering. STEP(Standard for the Exchange of Product Model Data), ISO(International Standards Organization), ISO10303.

The document specifies the scope and information requirements of the STEP application protocol (AP) for the exchange of process engineering data with a central emphasis on process design and major equipment. It specifies the information required to exchange process engineering and conceptual design information for process plants. The information forms the conceptual basis for the specification, selection, and oper- ation of process plant equipment over the plant life cycle. The AP supports continuous and batch processes, process simulations, stream data, unit operations, conceptual design requirements for major process equipment, and conceptual process control strategies. The document includes the application activity model (AAM) and application reference model (ARM) for this AP. The document was submitted to ISO TC184/SC4 and to international experts for review and comment on the scope and requirements of the AP. The document follows the format and clause numbering scheme prescribed for this type of International organization for Standardization (ISO) standard. Additional clauses and annexes and refinements will be added to the Ap after the completion of the ISO Group 1 review.

\section{2,798}

PB97-118764 Not available NTIS

National Inst. of Standards and Technology (MEL), Gaithersburg, MD. Manufacturing Systems Integration Div.

Capabilities for Product Data Exchange.

Final rept.

M. J. Mitchell. 1996, 7p.

Pub. in Proceedings of an International Multidisciplinary Conference Intelligent Systems: A Semiotic Perspective, Capabilities for Product Data Exchange, 7p 1996.

Keywords: "Computer-aided design, "Computer-aided manufacturing, Reprints, Product development, Standards, Specifications, Data management, Communication, Integrated systems, ISO, *STEP(Standard for the Exchange of Product Model Data), *Standard for the Exchange of Product Model Data, "PDES(Product Data Exchange Specification), "Product Data Exchange Specifications.

The paper describes the need for standardized representations of product information which are suitable for electronic communication between engineering and manufacturing functions. It then describes how a single international standard, ISO 10303 - Product data representation and exchange, has been designed to meet this industrial need. The benefits of STFP and the scope of the standard are presented. A high level technical description is given; this includes a description of the segments of the standard that can be used by readers to determine if the standard satisfies any of their company's needs for product data exchange. Key players in the development and implementation of STEP along with sources of additional information are provided

\section{2,799}

Not available NTIS

National Inst. of Standards and Technology (MEL), Gaithersburg, MD. Manufacturing Systems Integration Div.

SC4 Short Names Registry.

Final rept.

J. Lubell. 1996, 15p.

See also PB95-242293.

Pub. in Express User Group International Conference, Toronto, Canada, October 5-6, 1996, p1-15.

Keywords: "Registries, *Standardization, *Software tools, Computer-aided manufacturing, Computer-aided design, Digital systems, Procedures, Standards, Reprints, Data types, Product Data Exchange, SQL(Structured Query Language), STEP(Standard for the Exchange of Product Model Data), EXPRESS programming language

The paper describes a software environment recently implemented for maintaining a registry of unique short names for each of the entity data types within each of the EXPRESS schemas in the ISO TC184/SC4 standards. The new environment replaces an inefficient application and has already provided time savings for both the registry's administrator and editors of SC4 standards. It is also improving the quality of SC4 standards.

\section{Computer Aided Manufacturing (CAM)}

\section{2,800}

FIPSPUB183 PC E07

National Inst. of Standards and Technology (CSL), Gaithersburg, MD.
Integration Definition for Function Modeling (IDEF0); Category: Software Standard; Subcategory: Modeling Techniques.

21 Dec 93, 94p.

Also available from Supt. of Docs. See also AD-B062 465 and $A D-A 181952$

Three ring vinyl binder also available; North American Continent price \$7.00; all others write for quote.

Keywords: "Computer aided manufacturing, *Management information systems, Scheduling, Shops(Work areas), Integrated systems, Computer ap plications, Graphical languages, Functions, *IDEFO(Integration Definition for Function Modeling) Software standards, Modeling techniques, Business process improvement, Activity modeling.

The standard is based on the Air Force Wright Aeronautical Laboratories integrated Computer-Aided Manufacturing (ICAM) Architecture, Part II, Volume IV Function Modeling Manual (IDEFO), June 1981 (ADB062 457). The standard describes the IDEFO model ing language (semantics and syntax), and associated rules and techniques, for developing structured graphical representations of a system or enterprise. Use of this standard permits the construction of models comprising system functions (activities, actions, processes, operations), functional relationships, and data (information or objects) that support systems integration. The standard is the reference authority for use by system or enterprise modelers required to utilize the IDEFO modeling technique by implementors in developing tools for implementing the technique, and by other computer professionals in understanding the precise syntactic and semantic rules of the standard.

\section{2,801}

PB94-100278 PC A07/MF A02

Rensselaer Polytechnic Inst., Troy, NY.

Opportunities for Innovation: Advanced Manufacturing Technology.

C. F. Rancourt, and G. R. Simons. Jul 92, 136p,

NiST/GCR-92/610-1.

Grant NANBOD1071

Sponsored by National Inst. of Standards and Technology, Gaithersburg, MD.

Keywords: "Manufacturing, "Technology assessment, "Technology utilization, Computer aided manufacturing Computer aided design Competition Numerical control, Machining, Automation, Assembling, Total quality management, Statistical analysis, Data acquisition, Data processing, Integrated systems, Systems engineering, Process planning, EDI(Electronic Data Interchange), CNC(Computer Numerical Control).

Contents:

The Fit and Interaction of Competitiveness and Manufacturing Technology:

Computer-Aided Design:

Computer-Aided Process Planning and Group Technology;

Electronic Data Interchange;

CNC Machining

Automated Assembly

Statistical Quality Methods:

Quality Measurement Techniques:

Data Acquisition and Processing;

Systems Integration.

\section{2,802}

PB94-142460 PC A03/MF AO

National Inst. of Standards and Technology (MEL) Gaithersburg. MD. Robot Systems Div.

Enhanced Machine Controller Architecture Overview.

F. M. Proctor, and J. Michaloski. Dec 93, 26p, NISTIR-5331.

Keywords: "Computer aided manufacturing, *Controllers, Numerical control, Machine tools, Systems engineering, Computer architecture, Workstations, EMC(Enhanced Machine Controller) RCS(Real-time Control System), National Institute of Standards and Technology.

The National Institute of Standards and Technology (NIST) Enhanced Machine Controller program has defined an architecture for controllers, with an emphasis on machine tools. The architecture defines a set of components and their interfaces. The objective is to re duce the cost of controller development, installation, maintenance, and integration by defining a framework for building controllers from commercial off-the-shelf components.

02,803

PB94-142502 PC A03/MF A01 
National Inst. of Standards and Technology (CSL), Gaithersburg, MD. Systems and Software Technology Div.

Applying Virtual Environments to Manufacturing. S. Ressler. Jan 94, 28p, NISTIR-5343.

Keywords: "Manufacturing, Computer graphics, Man computer interface, Technology assessment, Computer applications, Case studies, "Virtual environments, Synthetic environments, Virtual reality.

This document presents a high level overview on the use of Virtual Environments for manufacturing applications. A brief introduction of the many component technologies used to create 'virtual environments' gives the reader enough background for discussion. The terms virtual environment, virtual reality and synthetic environments all refer to the concepts of placing a human inside a synthetic or virtual environment and allowing interaction with the environment in a number of ways. In recent years applications of virtual environments are beginning to appear. In addition to the basics of the various technologies, information on where to go to get more information on these technologies is also included. A series of case studies illustrating the use of virtual environments for a variety of manufacturing applications is presented

\section{2,804}

PB94-142791 PC A12/MF A03

National Inst. of Standards and Technology,

Gaithersburg, MD.

Feasibility Study: Reference Architecture for Machine Control Systems integration.

T. R. Kramer, and M. K. Senehi. Nov 93, 255p NISTIR-5297.

See also PB91-240796 and PB92-112242. Prepared in cooperation with Catholic Univ. of America, Washington, DC.

Keywords: *Computer aided manufacturing, *Control systerns, "Integrated systems, Systems engineering, Industrial plants, Computer architecture, Hierarchies, Models, Feasibility studies, National Institute of Standards and Technology, RCS(Real-time Control System), MSI(Manufacturing System Integration).

The Manufacturing Engineering Laboratory (MEL) at the National Institute of Standards and Technology (NIST), has been conducting research on control of mechanical systems for more than sixteen years. The Robot Systems Division has developed an architecture, the Real-time Control System (RCS) which focuses on providing real-time control of equipment. The Factory Automated Systems Division has developed the Manufacturing System Integration (MSI) architecture which focuses on providing information integration with factory production systems. While the architectures share some common features such as the use of hierarchical control and task decomposition, there are also differences. This report documents the work performed in assessing the feasibility of combining the RCS and MSI architectures into a single reference architecture. The report is written primarily for the team of researchers charged with developing the joint architecture. It includes a literature survey a framework for developing the joint architecture, a detailed set of issues about architectures and control architectures, and a preliminary sketch of the joint architecture.

\section{2,805}

\section{PB 94-160819 PC A03/MF A01}

National Inst. of Standards and Technology (MEL), Gaithersburg, MD. Factory Automation Systems Div. Technical Program of the Factory Automation Systems Division 1993.

H. M. Bloom, and L. W. Masters. Jul 93, 49p

NISTIR-5225.

See also report for 1992, PB92-205392.

Keywords: *Automation, "Industrial plants, *Data base management systems, "Standards, Interfaces, Research and development, Computer aided manufacturing, Industries, National Institute of Standards and Technology, Concurrent engineering, STEP(Standard for the Exchange of Product Model Data), PDES(Product Data Exchange using STEP).

The report describes the 1993 technical program of the Factory Automation Systems Division (FASD), one of five technical divisions in the Manufacturing Engineering Laboratory (MEL), within the National Institute of Standards and Technology (NIST). MEL supports the U.S. mechanical manufacturing industry through research and measurement services that are oriented toward a modern automated environment. The mission of FASD is to provide a focus for national research and standards efforts related to information systems for manufacturing. In carrying out its mission, the Division contributes to the strength of manufacturing in the United States and to the ability of the United States to remain competitive in world markets. The Division provides leadership in the development of national and international standards relating to information technology and manufacturing systems to meet U.S. industry needs for the twenty-first century. The work is organized around five key technologies: Enterprise Integration, Product Data Exchange, FCIM Processes, Communications and Networking, and Database and Database Management. Typical outputs for the Division include: draft specifications for future standards, journal papers describing research results, prototype software systems that demonstrate proof-of-concept, test methodologies for supporting the implementation of standards and testbeds for use by NIST, industry, and academia.

\section{2,806}

PB94-161924 PC A03/MF A01

National Inst. of Standards and Technology (MEL), Gaithersburg, MD. Factory Automation Systems Div. Bibliography on Apparel Sizing and Related issues.

Y. T. Lee. Feb 94, 25p, NISTIR-5365

Contract DLA-A708

See also PB93-158665. Sponsored by Defense Logistics Agency, Alexandria, VA. Mfg. Engineering Branch.

Keywords: "Clothing, "Anthropometry, "Size determination, "Bibliographies, Measurement, Clothing industry, Standards, APDES(Apparel Product Data Exchange Specification)

Anthropometric data and sizing systems is an important component of apparel quality. Apparel can not be top quality unless it fits satisfactorily the potential wearers. Much research has been conducted on this topic area. Some of these research results are documented but scattered. The purpose of the report is to collec these documentation/references for the quick use of apparel researchers who are studying sizing and its related issues. The report presents an annotated bibliography for apparel sizing and related issues. The report lists full bibliographic reference data and a brief abstract or summary for each technical paper or book. The literature collection for the bibliography includes documents from books, journals, reports, and national and international standards.

\section{2,807}

\section{PB94-162831 PC A04/MF A01}

National Inst. of Standards and Technology, Gaithersburg, MD. Automated Mfg. Research Facility. U.S. Navy Coordinate Measuring Machines: A Study of Needs.

D. C. Stieren, R. C. Veale, H. H. Harary, and S. C.

Feng. Dec 93, 55p, NISTIR-5379.

Contract N00014-92-F-0082

Sponsored by Naval Research Lab., Washington, DC Navy Manufacturing Technology Program.

Keywords: "Metrology, "Computer aided manufacturing, "Dimensional measurement, "Weapon systems, Inspection, Navy, Measuring instruments, Quality as surance, Surveys, Production engineering,
*Coordinate Measuring Machines, Integrated manufacturing.

This report presents the results of a one year study of Coordinate Measuring Machine (CMM) technology at US Navy manufacturing facilities, both in-house and contractor. The study was sponsored by the Navy Manufacturing Technology (ManTech) program through the NIST Automated Manufacturing Research Facility (AMRF) and was conducted by technical staf members of the NIST Manufacturing Engineering Laboratory (MEL). The results of the study are an analysis of the state-of-the-practice in the Navy regarding CMM utilization specifically with respect to weapon system manufacture. The results of the study are also an analysis of the Navy's needs in CMM technologies.

\section{2,808}

\section{PB94-163490 PC A09/MF A02}

National Inst. of Standards and Technology (MEL), Gaithersburg, MD. Robot Systems Div.
NIST Support to the Next Generation Controller Program: 1991 Final Technical Report

J. S. Albus, R. Quintero, F. Proctor, N. Tarnoff, T.

Kramer, J. Michaloski, and N. Dagalakis. Jul 92

188p, NISTIR-4888

Keywords: "Process control, "Computer aided manufacturing, *Controllers, Technology assessment, Air force, Control systems, Real time systems, Deburring Chamfering, Data structures, Knowledge bases(Artificial intelligence), Robots, Software engineering, Automation, Machine tools, "National Institute of Standards and Technology, PDES(Product Data Exchange using STEP). STEP(Standard for the Exchange of Product Model Data).

This report covers work performed by the National Institute of Standards and Technology (NIST) Robo Systems Division between October 1, 1990 and December 31, 1991 in continuation of work previously undertaken by NIST during FY90 for the U.S. Air Force Next Generation Controller Program (NGC)

02,809

PB94-164183 PC A04/MF AO

National Inst. of Standards and Technology (MSEL), Gaithersburg, MD.

Intelligent Processing of Materials, Technical Activities 1993 (NAS-NRC Assessment Panel, April 21-22, 1994)

1994, 74p, NISTIR-5312.

See also report for 1992, PB94-112430.

Keywords: "Materials, "Process control, "Artificial intelligence, Metals, Alloys, Ceramics, Polymers, Monitor ing, Sensors, Nondestructive tests, Arc welding, Aerospace environment, Eddy current tests, Metal sheets, Research projects.

The 1993 report of the Office of Intelligent Processing of Materials continued to focus on cooperative programs with industry and includes projects on metals and alloys, ceramics, polymers and polymer blends. The report also summarizes research on sensors for potential applications to process monitoring.

\section{2,810}

PB94-172681 Not available NTIS

National Inst. of Standards and Technology (NEL) Gaithersburg, MD. Robot Systems Div.

AMRF Composite Fabrication Workstation.

Final rept.

R. J. Norcross, W. R. Bunch, and R. D. Kilmer. 1990 , $6 p$.

Pub. in Proceedings of International Symposium on Robotics and Manufacturing (3rd): Research, Education, and Applications, British Columbia, Canada July 18-20, 1990, p655-660.

Keywords: "Thermosetting plastics, "Workstations "Robots, "Computer aided manufacturing, "Fiber reinforced composites, Manipulators, Robotics, Composite materials, Real time, Matrix materials, Control systems, Reprints.

The Robot Systems Division of the National Institute of Standards and Technology (NIST) has initiated development of an advanced manufacturing workstation for the fabrication of composite parts. The focus of this project will be the application of sensor-based hierarchically controlled robot systems to automated fiber placement of thermoplastic composite materials. In such a system, continuous fiber reinforced thermoplastic polymer is fed from a spool and applied onto a mandrel or part form. The orientation of the fiber is based on the part's geometry and structural design. As the composite material is applied it is heated to melt the polymer matrix, and then consolidates in place as it cools. The intention of this project is to demonstrate that the use of fiber placement via two cooperating robot manipulators and in-situ consolidation, can provide the capability to efficiently produce complex shaped composite parts.

\section{2,811}

PB94-172780 Not available NTIS

National Inst. of Standards and Technology (IMSE) Gaithersburg, MD. Office of Nondestructive Evaluation.

Intelligent Processing of Materials.

Final rept.

H. T. Yolken. 1992, $10 p$

Pub. in Flight-Vehicle Materials, Structures, and Dynamics Technologies - Assessment and Future Direc-

tions, Chapter 15, p375-384 1992. See also PB94112430 
Keywords: "Process control, "Computer aided manufacluring, "Nondestructive lesting, "Composite materials, Microstructure, Artificial intelligence, Sensors, Signal processing, Control systems, Reprints, "Intelligent processing.

Advanced materials are capable of providing outstanding or specialized properties, or combinations of properties, that cannot be obtained in conventional materials. These unique properties are the result of the so. phisticated microstructure that is designed and built into the materials. However, advanced materials generally require unusual and difficult to control processing operations in order to achieve their unique microstructures. A promising direction towards overcoming these difficulties involves intelligent processing of materials. This approach controls the microstructure in contrast to conventional materials processing where process variables such as temperafure and pressure are automatically controlled to preselected values. In an intelligent processing system advanced nondestructive evaluation (NDE) sensors are utilized to characterize the evolution microstructure of the matenal in real time. Moreover, these data and data from conventional process variable sensors are transmitted to a computerized decision-making system. This computer system transmits control signals based on the sensor data, a process model, and process data.

\section{2,812}

\section{PB94-187655 PC A03/MF A01}

National Inst. of Standards and Technology (MEL), Gaithersburg, MD. Factory Automation Systems Div. State-of-the-Art Survey of Methodologies for Rep-

resenting Manufacturing Process Capabilities.
M. E. A. Algeo. Mar 94, 30p, NISTIR-5391.

Keywords: "Computer aided manufacturing, sources, Standards, Mathematical models, Data bases, State of the art, Methodologies.

Represertations of manufacturing process capabilities are essential for the integration of manufacturing applications as well as the dynamic management of factory data. The survey presents methodologies for describing and using such information within the domain of discrete parts manufacturing. Current practices in industry are identified. Standards and research efforts related to representing manufacturing process capabilities also are described. Finally, a course of action for advancing current practices is presented.

\section{2,813}

\section{PB94-187739 PC A03/MF A0}

National Inst. of Standards and Technology (MEL) Gaithersburg, MD. Factory Automation Systems Div. Body Dimensions for Apparel.

Y. T. T. Lee. Apr 94, 30p, NISTIR-5411.

See also PB93-158665. Sponsored by Defense Logistics Agency, Alexandria, VA.

Keywords: "Clothing industry, "Anthropometry, "Body measurement(Biology), Body size(Biology). Computer aided manufacturing, Patterns, Pattern making, Fitting, Sizing, Size determination, APDES(Apparel Product Data Exchange Standard).

This report represents a compilation of body dimensions that are used in the manufacturing and fitting of apparel. It is the result of a comparison of five body measurements reports, including documentations of national and intemational apparel sizing standards. The information in this report will provide the basis for the development of the information model of made-10measure pattern making. It will also contribute to the conducting of future body measurements surveys and the development of new or improved sizing standards for apparel.

02,814

PB94-187788 PC A03/MF A01

Catholic Univ. of America, Washington, DC. Dept. of Mechanical Engineering.

NIST RS274/NGC Interpreter Version 1.

T. R. Kramer, F. M. Proctor, and J. L. Michaloski. Apr 94, 32p, NISTIR- 5416

See also PB94-163490. Sponsored by National Inst. of Standards and Technology (MEL), Gaithersburg. MD. Robol Systems Div.

Keywords: "Computer aided manufacturing, "Numerical control, "Interpreters, Machining, Input out put processing, Process control, Models, NGC(Next Generation Controller), NIST(National Institute of Standards and Technology).
An interpreter has been written which reads numerical control code and produces calls to a set of canonical machining functions. The output of the interpreter can be used to drive a 3 -axis machining center. This report describes version 1 of the interpreter.

\section{2,815}

PB94-191715 PC A06/MF A02

National Inst. of Standards and Technology (MEL), Gaithersburg, MD.

Control Entity Interface Speciflcatlon.

S. Wallace, M. K. Senehi, E. Barkmeyer, S. Ray, and E. K. Wallace. Sep 93, 116p, NISTIR-5272.

See also PB91-240796.

Keywords: "Computer aided manufacturing, "Control systems, "Interfaces, "Specifications, Integrated systems, Herarchies, Productlon planning, Models, Production control, NIST(National Institute of Standards and Technology), MSI(Manufacturing Systems Integration), Job control.

This document is concemed with defining the details of the interfaces for control entities which are incorporated into an integrated system that conforms to the National Institute of Standards and Technology (NIST) Manufacturing Systems Integration (MSI) architectural model as revised in 1992. The purpose of this document is twofold: to document the progress and current status of the MSI architecture's control entity interfaces and to provide designers and implementors with specifications for an MSI architectural compliant control entity. Section 2 provides an overview of the MSI project. Section 3 discusses production plans in the context of the interaction between planners and job controllers. Section 4 discusses generic data objects which are common to several of the 5 control entity interfaces. Sections 5-9 detail the 5 different control entity interfaces: the planning interface, the job control interface. the planning-10-job-control interface, the guardian planning interface and the guardian job control interface. Section 10 gives an example scenario of how the different messages from the 5 Interfaces work together.

\section{2,816}

PB94-194552 PC A03/MF A01

National Inst. of Standards and Technology (MEL), Gaithersburg, MD. Factory Automation Systems Div. Visualization Appllcations for Manufacturing: A State-of-the-Art Survey. Final Report.

H. T. Moncarz. May 94, 35p, NISTIR-5427.

Keywords: "Computer aided manufacturing, "Computer graphics, "Technology assessment, "Scientific visualization, Standards, Production engineering, Computerized simulation, Data transmission, Algonithms, Software tools, State of the art, Surveys, Product development, "Rapid response manufacturing.

A state-of-the-art (SOTA) survey was pertormed for visualization software applications (VAs) in the context of a rapid response manufactuning (RRM) environment. VAs are standalone, commercially-available products, that have sophisticated capabilities for interactively visualizing data. The data includes geometry, image, and scientific data. VAs' capabilities to visualize data can be advantageously applied to manufacturing if VAs are integrated within RRM. In general, integration enables any RRM process to access data from other RRM processes in the product life cycle. However, having access to data isn't necessarily useful if it is too overwhelming for a human to use it. VAS offer the potential for users to cognitively integrate the data from multiple RRM processes to make sense of it and to intelligently use it. This SOTA describes the capabilities of VAs and methods for integrating them within RRM to ultimately enable the full potential of VAs to be realized.

\section{2,817}

PB94-199932 Not available NTIS

National Inst. of Standards and Technology (NEL), Gaithersburg, MD. Factory Automation Systems Div. Implementing a Transitton Manager In the AMRF Cell Controller.

Final rept.

B. A. Catron. $1993,5 p$

Pub. in the Proceedings of Intemational Conference CAD/CAM Robotics and Factories of the Future (3rd), Southfield, MI., August 14-17, 1988 , v3 p42-46.

Keywords: "Computer aided manufacturing, "Controllers, Control systems, Synchronism, Process control, Integrated systems, Automation, Remote control, Reprints, "Transition management, US NIST.

The Transition Manager is one of several managers which are responsible for providing functionality to the cell controller in the Automated Manufacturing Research Facility (AMRF) at the National Institute of Standards and Technology. The cell controller implementation of the Transition Manager is based on a model developed at the University of Virginia (UVA). This model provides identical functionality at each level of the AMRF control hierarchy. The paper outlines the changes and additions which were needed in order to integrate the Transition Manager into the AMRF cell controller. An extension to the model is also proposed to allow more flexibility in the automated remote control capabilities. The extension to the UVA model will allow the cell controller the flexibility of controlling resources and then releasing the resource when finished.

02,818

PB94-199940 Not available NTIS

National Inst. of Standards and Technology (NEL) Gaithersburg, MD. Factory Automation Systems Div. Generic Manufacturlng Controllers.

Final rept.

B. A. Catron, and B. H. Thomas. 1989, 3p.

Pub. in Proceedings of Institute of Electrical and Electronics Engineers International Symposium on Intelligent Control, Arlington, VA., August 24-26, 1988, p742-744.

Keywords: "Computer aided manufacturing, "Controllers, "Software reuse, Integrated systems, Process control, Computer program portability, Reprints, AMRF(Automated Manufacturing Research Facility), US NIST.

The cost of developing software for the factory is continuing to increase due in part to the problems of integrating equipment and controllers from multiple vendors. A consistent philosophy is needed for designing reusable code for integrating manufacturing control systems. Development of a 'generic controller' will minimize the problem of redundant software. An outline of a generic controller architecture is discussed and key concepts are identified. A generic controller is unde development at the Automated Manufacturing $\mathrm{Re}$ search Facility at the National Institute of Standards and Technology. The implementation is outlined and future work identified.

\section{2,819}

PB94-213758 PC A05/MF A01

National Inst. of Standards and Technology (MEL), Gaithersburg, MD

Technical Program Description Systems Integration for Manufacturing (SIMA)

H. M. Bloom. Jul 94, 94p, NISTIR-5476.

See also PB91-193367 and PB93-189801.

Keywords: "Computer aided manufacturing, *Integrated systems, "Standards, Data transler(Computers), Interfaces, Computer aided design, Product development, Prototypes, Specifications, Systems engineering, "SIMA(Systems Integration for Manufacturing Applications), "Systems Integration for Manufacturing Applications, STEP(Standard for the Exchange of Product Model Data), US NIST, AMSANT(Advanced Manufacturing System and Networking Testbed).

The National Institute of Standards and Technology (NIST) has established an Advanced Manufacturing System and Networking Testbed (AMSANT) which supports research and development (R\&D) in high-performance manufacturing systems and testing high-performance computer and networking hardware and software in a manufacluring environment. A standardsbased data exchange effor for computer integrated manufacturing will focus on improving data exchange among computer aided design, process and manufacturing activities. This new program, Systems Integration for Manufacturing Applications (SIMA), is part of the govermment's High Performance Computing and Communications Initiative. Applications may include enterprise integration for manufacturing applications integrated product/process design, simulation and agile manufacturing. Prototype systems, and interface specifications will be communicated to appropriate standards organizations. The report describes the objectives of the program and highlights the projects to be initiated in FY94 and proposed for FY95. 
02,820

PB95-144549 PC A06/MF A02

National Inst. of Standards and Technology (MEL),

Gaithersburg, MD. Factory Automation Systems Div. Reference Architecture for Machine Control Systems Integration: Interim Report.

M. K. Senehi, T. R. Kramer, J. Michaloski, W. G. Rippey, S. Wallace, R. Quintero, and S. R. Ray. Oct 94, 111p, NISTIR-5517.

See also PB94-142791. Prepared in cooperation with Catholic Univ. of America, Washington, DC.

Keywords: "Computer aided manufacturing, "Control systems, "Integrated systems, Automation, Systems engineering, Industrial plants, Hierarchies, Models, Protocols, US NIST.

The Manufacturing Engineering Laboratory (MEL) at the National Instifute of Standards and Technology (NIST) has been conducting research on control of mechanical systems for more than sixteen years. The Robot Systems Division and Factory Automation Systems Division have been working jointly for over a yea to develop a reference architecture for computer integrated control of mechanical systems, drawing on the best of past work in both divisions. The architecture is not yet complete, and work on the architecture is continuing. This report describes the architecture in its current, incomplete state. The architecture is a hierarchical control architecture aimed at the domain of discrete parts manufacturing, including considerations of data systems, communications systems, planning, resource allocation, real-time control, and error recovery.

02,821

PB95-150827 Not available NTIS

National Inst. of Standards and Technology (NEL), Gaithersburg, MD. Factory Automation Systems Div. Using Grafcet to Design Generic Controllers.

Final rept.

B. H. Thomas, and C. R. McLean. 1988, 10p.

Pub. in Proceedings of International Conference on Computer Integrated Manufacturing, Troy, NY., May 23-25, 1988, p110-119.

Keywords: "Computer aided manufacturing, "Controllers, "Systems engineering, Control systems, Software engineering, Parallel processing, Process control, Software reuse, Reprints, Grafcet programming language.

The purpose of this paper is to outline some ways Grafcet is being used to help design generic controllers within the Automated Manufacturing Research Facility. Grafcet is a powerful graphical language for expressing control flow and allows the expression of both parallel and sequential control logic. The paper describes the rationale for using Grafcet as a design tool for ex pressing software for a generic controller. Grafcet helps the designer determine: modularization of the code, functions that can be performed in parallel, communication flow, and problems in control flow.

\section{2,822}

\section{PB95-151908 Not available NTIS}

National Inst. of Standards and Technology (NEL) Gaithersburg, MD. Automated Production Technology Div.

Integration of Real-Time Process Planning for Small-Batch Flexible Manufacturing

Final rept.

C. Yang, and K. Lee. 1989 5p.

Pub. in Proceedings of IASTED International Symposium on Robotics and Manufacturing (12th), Santa Barbara, CA., November 13-18, 1989, p230-234.

Keywords: "Computer aided manufacturing, *Process control, "Planning, Automation, Control systems, Hierarchies, Task complexity, Task planning(Robotics), Workstations, Machine tools, Reprints.

Automation of just-in-time, small-lot, mixed-batch manufacturing at the workcell level is a topic of interest in Flexible Manufacturing Systems (FMS) research today. The control processes involved in producing diferent parts in small batches can be very complex. This paper describes a method of generating the process plan in real time based on part manufacturing attributes and execution of the plan by a three-level hierarchical controller. This method is currently implemented in the workstation controller for the Mare Island Flexible Manufacturing Workstation (MIFMW), a small-batch workcell designed for just-in-time, untended operation.
02,823

PB95-162335 Not available NTIS

National Inst. of Standards and Technology (MSEL) Gaithersburg, MD. Metallurgy Div.

Development of Adaptive Control Strategies for Inert Gas Atomization.

Final rept.

S. D. Ridder, S. A. Osella, P. I. Espina, and F. S.

Biancaniello. 1990, 10p.

Pub. in MD (Intell. Process. Mater.) 21, p79-88 1990.

Keywords: "Atomizers, "Particle size, "Process control "Metal powders, Real time operations, Artificial intelligence, Expert systems, Supersonic flow, Cinematog raphy, Gas flow, Rare gases, Adaptive control, Particulates, Controllers, High speed, Monitors, Reprints, SiGMA method.

The Supersonic inert Gas Metal Atomization (SiGMA) technique produces ultra-fine $(<45$ micrometer median particle diameter), rapidly solidified metal powder via high energy gas atomization. Research at the National Institute of Standards and Technology (NIST, formerly NBS) on SiGMA has focused on providing this system the ability to monitor and control particle size during atomization. The process controller currently under development for SiGMA includes a novel real-time particle size measurement sensor (incorporating an adaptive pattern recognition scheme) into a multiple level 1/O command interface. The modular nature of both the hardware and software design will be adaptable to other particulate producing equipment. Gas and liquid flow imaging, gas flow modeling, real-time particle size measurement and process control will be discussed.

\section{2,824}

PB95-162343 Not available NTIS

National Inst. of Standards and Technology (IMSE). Gaithersburg, MD. Metallurgy Div.

Process Modeling and Control of Inert Gas Atomization.

Final rep

S. D. Ridder, S. A. Osella, P. I. Espina, and F. S.

Biancaniello. 1990, 18p.

Pub. in Proceedings of Thermal Structures Conference, Charlottesville, VA., November 13-15, 1990 p383.400.

Keywords: "Atomizing, "Particle size, "Process control Real time operations, Artificial intelligence, Expert sys tems, Rare gases, Gas flow, Particulates, Controllers Reprints.

Research at the National Institute of Standards and Technology (NIST, formerly NBS) on gas atomization has focused on providing this process the ability to monitor and control particle size during atomization. Studies included gas and liquid flow imaging, gas flow modeling, real-time particle size measurement and process control. The process controller incorporates a multi-level expert system shell and a novel real-time particle size measurement sensor (incorporating an adaptive pattern recognition scheme). The modular nature of both the hardware and software design is adapt able to other particulate producing equipment.

\section{2,825}

PB95-173555 PC A11/MF A03

National Inst. of Standards and Technology (MEL), Gaithersburg, MD. Intelligent Systems Div.

Workshop on the Application of Virtual Reality to Manufacturing. Final Report. Held in Gaithersburg, Maryland on August $9,1994$.

E. W. Kent. Dec 94, 227p, NISTIR-5543.

Keywords: "Computer aided manufacturing, "Virtual reality, "Human factors engineering, Man computer interface, Protocols, Interactive systems, Distributed processing, Surveys, Graphs(Charts), US NIST.

The Intelligent Systems Division (ISD) of the Manufacluring Engineering Laboratory (MEL) at the National Institute of Standards and Technology (NIST) is committed to the standardization of protocols for the application of information and interface technology for the virtual and distributed manufacturing of discrete parts. A part of this effort, ISD is developing an initiative in human interfaces in manufacturing emphasizing the integration of human factors, virtual reality, and manufacturing systems technologies. A one-day workshop was held on 9 August 1994 to examine the applicatio of information and interface technology for the virtual and distributed manufacturing of discrete parts. The purpose of this report is to document the workshop including presentations, participant discussions, and the results of working group deliberations.

02,826

PB95-181228 Not available NTIS

National Inst. of Standards and Technology (CAML),

Gaithersburg, MD. Statistical Engineering Div

Analysis of Autocorrelations in Dynamic Processes.

Final rept.

N. F. Zhang, and J. F. Polland. 1994, 15p.

Pub. in Technometrics 36, n4 p354-368 Nov 94

Keywords: "Autoregressive processes, "Process control, "Data analysis, Error detection codes, Mass balance, Material balance, Autocorrelation, Time series analysis, Mathematical models, Reprints.

Data collected by process information and control sys tems are almost always correlated due to process dynamics combined with short sampling times. The traditional time series approach for dealing with autocorrelated data has been to model the autocorrelation. This modeling effort is substantially more difficult than simply treating the time series as a sequence of independent data. In this article, we develop and demonstrate a methodology that yields insight into the error introduced by not modeling the autocorrelation of the process data when performing material balances around process equipment. This work shows that in many cases data analysis (e.g. gross-error detection) can be done using the simplified models.

02,827

PB95-217097 PC A03/MF A01

National Inst. of

Gaithersburg, MD.

Japan Technology Program Assessment. Simulation: State-of the-Art in Japan.

A. Jones. Mar 95, 37p, NISTIR-5614

See also PB95-171112. Sponsored by Assistant Secretary for Technology Policy (Commerce), Washington, DC. Japan Technology Program.

Keywords: *United States, *Japan, *Technology transfer Computerized simulation, Computerized manufacturing, Industries, Government policies, "Foreign technology, Translations.

The report summarizes the current state-of-the-art of simulation in Japan, and provides some insight into the future directions the Japanese are likely to pursue. It is divided into three parts. In part one, the authors provide a translation of a paper that was published in Journal of the Operations Research Society of Japan by Shigeki Umeda and Susumu Morito. In part two, they provide a copy of the paper by Shigeki Umeda which is contained in the proceedings of the recently held conference on New Directions in Simulation for Manufacturing and Communications. These authors have graciously agreed to allow their papers to be included in the report. In part three, the authors provide comments from five US researchers who attended the conference on New Directions in Simulation for Manufacturing and Communications.

\subsection{8}

PB95-231866 PC A05/MF A01

National Inst. of Standards and Technology (MEL), Gaithersburg, MD

Conceptual Design Plan for the National Advanced Manufacturing Testbed.

Special pub

M. M. Hessel. Apr 95, 87p, NIST/SP-882

Also available from Supt. of Docs. as SN003-00303336-7.

Keywords: "Manufacturing, "Government/industry relations, Computer aided manufacturing. Demonstration projects, US NIST, Distributed manufacturing, Testbeds, Advanced manufacturing.

The National Advanced Manufacturing Testbed (NAMT) Program provides a collaborative context for helping U.S. manufacturers achieve a competitive advantage. It is a national industry-government teaming program designed to leverage private sector and federal investments, expertise and facilities to accelerate the development and implementation of technologies for distributed and virtual manufacturing (DVM). The objective of the NAMT program is to establish and operate a distributed, multinode, multi-project testbed among industry, government agencies and universities. This testbed will facilitate coordination between individual projects and development and demonstra- 


\section{Computer Aided Manufacturing (CAM)}

tion of industry-defined scenarios which advance DVM technologies to support integration and interoperability among suppliers, vendors and manufacturers.

\section{2,829}

PB 95-251708 PC A03/MF A01

National Inst. of Standards and Technology (MEL), Gaithersburg, MD. Manufacturing Systems Integration Div.

Information Technologies Make Business Sense for the Custom Therapeutic Footwear Industry.

H. T. Moncarz. Jun 95, 12p, NISTIR-5673.

Presented at a workshop held in Charleston, SC. in March 1994.

Keywords: "Footwear industry, *Information technology, "Computer aided manufacturing, Shoes, Orthopedic equipment, Therapeutic uses, Design analysis, Process control, Production engineering, Produc development, Standards, Requirements, Data transmission, Technology utifization, Life cycle analysis, STEP(Standard for the Exchange of Product Model Data)

The commercialization and integration of emerging technologies for custom therapeutic footwear (CTF) manufacturing will provide economic and health-care benefits to the nation and will improve responsiveness to consumer desires. Using STEP Application Protocols (APs), complex product information can be unambiguously transferred throughout the virtual and distributed, multi-enterprise marketplace that is anticipated for the future CTF industry. In that marketplace, infor mation representing foot shapes, shoe lasts (forms fo ootwear manufacturing), orthopedic insets, and the pattems that comprise the footwear pieces must be transferred among different organizations. This paper will describe that marketplace, and type of STEP APS required, and possible business scenarios.

\section{2,830}

PB96-112008 Not available NTIS

National Inst. of Standards and Technology (MEL) Gaithersburg, MD. Factory Automation Systems Div. Production Management Information Model for Discrete Manufacturing.

Final rept.

S. R. Ray, and S. Wallace. 1995, 15p.

Pub. in Production Planning and Control, v6 n1 p65 791995.

Keywords: "Computer aided manufacturing, Production management, "Management information systems, Automation, Integrated systems, Control sys tems, Systems design, Production planning, Production control, Resource allocation, Process control Scheduling, Specifications, Hierarchies, Mathematical models, Reprints, Manufacturing Systems Integration, NIAM(Nijssens Information Analysis Model), Systems architecture

The Manufacturing Systems Integration (MSI) project at the National Institute of Standards and Technolgy is developing a system architecture that incorporates an integrated production planning and control environment. This paper presents the production management information model within the MSI project. The main focus of the model is to identify and characterize the relationships between orders and workpieces, to identify the information necessary to achieve workpieces, to identify the information necessary to achieve workpiece tracking and to identify the information necessary to achieve resource requirements specifications for process plans.

\section{2,831}

PB96-112685 PC A07/MF A02

National Inst. of Standards and Technology, Gaithersburg, MD.

Requisite Elements, Rationale, and Technology Overview for the Systems Intergration for Manufacturing Applications (SIMA) Program. Background Study.

E. J. Barkmeyer, T. H. Hopp, M. J. Pratt, and G. R

Rinaudot. Sep 95, 133p, NISTIR-5662.

See also PB94-213758.

Keywords: "Computer aided manufacturing, "Integrated systems, Product development, Systems engineering, Software, Cost estimates, Standards, -SIMA(Systems Integration for Manufacturing Applications), "Systems Integration for Manufacturing Applications.

The report--which documents the findings of a back ground study of industry needs in the area of manufac- turing systems integration--defines the initial MSE project focus in detail and sets the technical direction for project efforts. The report also describes the principal types of product realization software applications now in use, reviews efforts within manufacturing industry towards developing integration solutions, discusses relevant research trends in the field of product realization, and surveys standards which may be applicable to the work of the MSE project. A brief review is also given of some potentially relevant supporting technologies from the realm of information technology.

\section{2,832}

Not available NTIS

National Inst. of Standards and Technology (MEL) Gaithersburg, MD. Manufacturing Systerns Integration

Roadmap for the Computer Integrated Manufacturing (CIM) Application Framework.

Final rept.

S. L. Stewart and J. A. St. Pierre. 1995, 21p.

Pub. in SEMATECH Roadmap for the Computer Integrated Manufacturing (CIM) Application Framework, p1-21 May 95.

Keywords: "Object oriented systems, "Systems engineering "Production management, computer software, Computer aided manufacturing, Integrated systems, Data management, Interfaces, Frameworks, Specifications, Technology transfer, Reprints, "CIM(Computer Integrated Manufacturing), "Computer Integrated Manufacturing.

This technology transfer reports on the first year of a joint project between the National Institute of Standards and Technology (NIST) and SEMATECH. It includes a roadmap for promoting adoption of the SEMATECH Computer Integrated Manufacturing (CIM) Framework as an industry standard. The CIM Framework is an object-oriented software infrastructure that creates a common environment for integrating applications and sharing information in production environments. The roadmap consists of the following stages: specifications; consensus; standardization; and testing/certification. Each stage is described in detail in the document.

\section{2,833}

PB96-128228 PC A03/MF A01

National Inst. of Standards and Technology (MEL), Gaithersburg, MD. Manufacturing Systems Integration

Overview of the Manufacturing Engineering Toolkit Prototype.

M. J. luliano. Oct 95, 12p, NISTIR-5730.

Keywords: "Software tools, "Computer aided manufacturing, "Prototypes, Standards, Interfaces, Validation, Systems integration, Systems approach, Systems engineering, Software engineering, Life(Durability), Applications programs(Computers), METK(Manufacturing Engineering Toolkit)

A computer-aided Manufacturing Engineering Toolkit (METK) prototype is currently under development at the National Institute of Standards and Technology (NIST). The toolkit is being used to identify the integration standards and issues which must be addressed to implement plug-compatible environments in the future. The toolkit consists of commercial-off-the-shelf (COTS) manufacturing software applications housed together on a high speed computer workstation. The purpose of the CAME project at NIST is to provide an integrated framework, operating environment, common databases, and interface standards for manufacturing engineering software applications. This paper describes an initial METK prototype.

\section{2,834}

PB96-136965 PC A10/MF A03

National Inst. of Standards and Technology (MEL) Gaithersburg, MD. Manufacturing Systems Integration Div.

Computer-Aided Manufacturing Engineering Forum (1st). Technical Meeting Proceedings. Held in Gaithersburg, Maryland on March 21-22, 1995.

M. Smith, and S. Leong. Mar 95, 211 , NISTIR-5699. Sponsored by Naval Research Lab., Washington, DC Navy Manufacturing Technology Program.

Keywords: "Computer aided manufacturing, "Meetings, "Planning, Data processing, Process control, Validation, Integrated systems, Systems engineering, Metals, Machines, Materials handling, Models, Concurrent engineering.
Although many software tools are currently available or manufacturing engineers, they do not work together and cannot be readily integrated. This lack of integration significantly diminishes the productivity of manulacturing engineers, reduces the quality of their work, and increases the time it takes to plan the production of a part. The Manufacturing Engineering Tool Kit project is attempting to develop an integrated tool kit This tool kit will be used to generate a process plan for production and to validate the engineering data package and process plan prior to release to production. The application software tools are based on commercially available off-the shelf products. This paper describes the validation requirements for the engineering data package and the categories and types of data errors compiled from a need analysis from the industry end users.

\section{2,835}

PB 96-147954 PC A05/MF A01

Catholic Univ. of America, Washington, DC. Dept. of Mechanical Engineering.

NIST RS274KT Interpreter.

T. R. Kramer, and F. Proctor. 26 Oct 95, 58p, NISTIR-5738.

Grant NIST-7ONANB2H1213

See also PB94-187788. Sponsored by National Inst of Standards and Technology (MEL), Gaithersburg MD. Intelligent Systems Div.

Keywords: "Computer aided manufacturing, Numerical control, "Interpreters, Machining, Process control, Models, Computer programs, Software engineering, NGC(Next Generation Controller), Nationa Institute of Standards and Technology, RS274KT interpreter.

The NIST 'RS274KT interpreter' is a software system which reads numerical control code in the "KT dialect of the RS274 numerical control language and produces calls to a set of canonical machining functions. The output of the interpreter can be used to drive a Keamey and Trecker 8004 -axis machining center. The report describes the RS274KT interpreter.

\section{2,836}

PB96-147962 PC A04/MF AO1

National Inst. of Standards and Technology (MEL) Gaithersburg, MD. Manufacturing Systems Integration Div.

Product Realization Process Modeling: A Study of Requlrements, Methods and Research Issues. K. W. Lyons, M. R. Duffey, and R. C. Anderson. Jun 95, 48p, NISTIR-5745.

Prepared in cooperation with George Washington Univ., Washington, DC.

Keywords: *Computer aided manufacturing, "Process models, "Product development, Computerized simulation, Industrial plants, Electromechanical devices, Materials handling, Artificial intelligence, Automation.

The purpose of the document is to identify and document key requirements, industry practices, and research questions which should drive new methods and computer tools for process modeling of product realiza tion. It addresses a wide range of industry-relevant modeling issues to help focus discussion on future re search directions and its intended audience are modeling researchers and practitioners in industry, universities, and other federal agencies. Although process modeling methods have been applied to many types of development efforts (e.g., software engineering, VL$\mathrm{SI}$ ), our sole focus in the report is realization of discrete electro-mechanical products.

\section{2,837}

PC A02/MF A01

National Inst. of Standards and Technology (MEL), Gaithersburg, MD.

Machine Performance Standard Provides Opportunity to Improve Quality and Productivity.

. D. Lovett. Dec 95, 8p, NISTIR-5775.

Sponsored by American Society of Mechanical Engineers, Washington, DC.

Keywords: "Process control *Machine tools, "Computer aided manufacturing, Measurement, Performance evaluation, Standards, Quality control.

Evaluating manufacturing capability and monitoring any decrease in performance of CNC machines may provide some trends of manufacturing process variables which can be used for estimating better contro over the manufacturing process. This in turn could lead to improved quality and a reduction in manufacturing 
lead time. A performance standard for CNC machining centers and a draft standard for turning centers provide methods for evaluating performance of machines comparative analysis as well as ongoing assessment of machine tool performance.

\section{2,838}

\section{PB96-154877 PC A08/MF A02}

National Inst. of Standards and Technology (MEL) Gaithersburg, MD. Manufacturing Systems Integration Div.

NIST SIMA Interactive Management Workshop. Held in Fort Belvoir, Virginia on November 14-16, 1994.

C. Johnson, S. Frechette, and M. Luce. Sep 95,

127p, NISTIR-5717.

Keywords: "Computer aided manufacturing *Integrated systems, "Workshops, Product development Systems engineering Software, Standards, "SIMA(Systems Integrated for Manufacturing Applications), "Systems Integrated for Manufacturing Applications.

The Manufacturing Systems Integration Divison (MSID) hosted a workshop November 14-16, 1994, on Systems Integrations for Manufacturing Application (SIMA). The workshop was held at the Defense Sys tems Mangement College (DSMC) in Fort Belvoir, Virginia. A total of 27 people participated in the workshop including representatives from industry, industry consortia, other government manufacturing programs, and the Natioanl Institute of Standards and Technology (NIST). The focus of the workshop was to identify critical problems and actions that can be taken to solve these problems in the context of advancing information technology for manufacturing systems and improving the effectiveness of related programs. This documen contains diagrams that illustrate relationships between problems and actions related to removing barriers to manufacturing systems integration. In addition, it con tains a description of the Interactive Management process that was used to facilitate the NIST SIMA work shop at Fort Belvoir, Virginia.

\section{2,839}

\section{PB96-160437 Not available NTIS}

National Inst. of Standards and Technology (MEL), Gaithersburg, MD. Automated Production Technology Div

Quality in Automated Manufacturing.

Final rept.

D. S. Blomquist. $1991,34 p$.

Pub. in Control and Dynamic Systems, v45 p163-196 Aug 91.

Keywords: "Computer-aided manufacturing, "Dimensional measurement, "Automation, Process control, Computerized control systems, Machine tools, Real-time systems, Integrated systems, Quality control, Error correcting codes, Reprints, Coordinate measuring machines, Initial Graphics Exchange Specifications.

The Quality in Automation (QIA) program is described at the Manufacturing Engineering Laboratory of the National Institute of Standards and Technology (NIST). The purpose of the QIA program is to develop a quality control and quality assurance system that exploits deterministic metrology principles in an automated manufacturing environment, producing small batches with commercially available and affordable equipment. This 'deterministic manufacturing' is based on the premise that most errors occuring in the manufacturing process are repeatable, thus predictable. The QIA program combines statistical process control methods with onmachine sensing and gauging, real-time error compensation and distributed processing to produce parts of consistently high quality.

02,840

PB96-160486 Not available NTIS

National Inst. of Standards and Technology (MEL) Gaithersburg, MD. Automated Production Technology Div.

Development of a New Quality Control Strategy for Automated Manufacturing.

Final rept.

M. A. Donmez. 1992, $12 p$

Pub in Proceedings of Manufacturing International 1992, MI92 Technologies, Economics, Information for Global Partnership Realignments, Dallas, TX., March 19-April 1, 1992, 12p.

Keywords: "Computer-aided manufacturing, "Machine tools, "Quality control, "Automation, Process control,
Computerized control systems, Real time systems, Inspection, Error corection, Accuracy, Reprints.

A new quality control strategy is being developed at the Manufacturing Engineering Laboratory of the $\mathrm{Na}$ tional Institute of Standards and Technology (NIST) with a project called Quality in Automation (QIA). The objective of this strategy is to demonstrate that the likelihood of producing defective parts can be dramatically reduced through better control of the manufacturing process. The QIA project is an effort to develop and implement a real-time, on-line quality control/quality assurance system to improve the quality of the parts produced in a small-batch, automated manufacturing environment using commercially available and affordable equipment. In order to obtain high-quality parts consistently, we are building a three-layered control strategy around the metal cutting process. These three control layers are: real-time, process-intermittent, and post-process.

\section{2,841}

PB96-165428 PC A03/MF A01

National Inst. of Standards and Technology (MEL), Gaithersburg, MD. Manufacturing Systems Integration Div.

Machining Process Planning Activity Model for Systems Integration.

S. C. Feng. Mar 96, 26p, NISTIR-5808

See also PB96-112685.

Keywords: "Machining, "Planning, "Models, Computer aided manulacturing, Systems analysis, Software engineering, "STEP(Standard for the Exchange of Product Model Data), "Standard for the Exchange of Product Model Data.

A key issue of integrating process planning systems with design systems and production planning systems is how to overcome barriers in data exchange and sharing amongst software systems. A machining process planning activity model was developed at NIST to address some of the barriers. The model represents functional components and data requirements in process planning systems. The purpose of the model is to create the context in which data requirements and data flow for NC machining process planning are defined. The model was developed as a unification of many previously developed process planning activity models.

\section{2,842}

PB96-165980 PC A08/MF A02

National Inst. of Standards and Technology (MEL) Gaithersburg, MD. Automated Production Technology Div.

PIECS: A Software Program for Machine Tool Process-Intermittent Error Compensation.

H. T. Bandy, and D. E. Gilsinn. Mar 96, 140p,

NISTIR-5797.

Keywords: "Machine tools, "Automation, "Error correc tion codes, Computer aided manufacturing, Numerical control, Feedback control, Coding, Cutting tools, Quality control, Algorithms, Real time, PIECS computer program, C programming language.

This report documents software, called PIECS, that performs process-intermittent error compensation for a turning center. The program is a part of a larger three loop control architecture that includes a real-time geometric-thermal error compensation, loop and a postprocess loop. In process-intermittent error compensation, a part is measured by on-machine gauging after a semifinish cut which uses the same cutting parameters (speed, feed, and depth of cut) as are used in the finish cut, to reproduce process-dependent errors such as cutting-force induced tool or part deflection. During gauging a touch-trigger probe signal indicates that the part surface has been contacted. The coordinates of the points are then transformed to the part coordinate system and compared to the corresponding nominal coordinates so that errors may be determined. The error vector is defined as having its head at the measured coordinates of the gauged point and its tail at the nominal coordinate for that point. Since the philosophy chosen in this program is to compensate proc. ess-intermittent errors by changing the position and orientation of features, least squares curve fitting through the ends of the error vectors is used to determine the adjusted tool cirve. The compensation curve becomes the tool path for the corresponding feature for the finish cut. The report includes a description of the program algorithm, the input and output data sets as well as descriptions of each of the C-programming language functions that compose PIECS. A listing of the program is included in the appendix.
02,843

PB96-183058 PC A04/MF A01

National Inst. of Standards and Technology (MEL) Gaithersburg, MD. Manufacturing Systems Integration

Procedure for Product Data Exchange Using STEP Developed in the AutoSTEP Pilot.

D. A. Rosenfeld, and S. Dhar. Apr 96, 33p, NISTIR

5833

Prepared in cooperation with Industrial Technology Inst., Ann Arbor, MI. Center for Electronic Commerce. Keywords: "Automotive industry, "Product models, "Information exchange, "Computer aided manufacturing, Standards, Computer aided design, Interoperability, Production planning, Information systems Motor vehicles, "STEP(Standard for the Exchange of Product Model data), "Standard for the Exchange of Product Model data, ISO 10303, STEP AP203.

The AutoSTEP Pilot Project, directed by the Automotive Industry Action Group (AIAG), is an effor whose goal is to promote the use of STEP (STandard for the Exchange of Product model data) as a neutra mechanism for product data exchange within the automotive industry. To explore and demonstrate the capabilities of STEP. AutoSTEP organized a number of trading pairs to exchange product model data represented in STEP Application Protocol 203, configuration controlled design conformance class six, or data represented with advanced boundary representation (b-rep). Model data would be transferred from one company's CAD system to its partner company's sys tem via STEP. AutoSTEP Phase One organized pairs of automotive companies and their first-tier suppliers and involved exchanges relating to packaging applications. Later phases will include exchanges of product model data relating to other applications, and will extend the information exchange further down the supply chain. Exchange results are logged in a database, and reports assessing the results will be produced. This document provides a complete description of the product model data exchange scenario envisioned for transactions using STEP, both in general and in the context of the AutoSTEP Pilot. It outlines the entire process that model data undergoes in STEP exchanges, and it identifies the significant metrics throughout the process. It also provides forms and tables for the benefit of the AutoSTEP participants to as sist them in performing exchanges and logging metrics.

\section{2,844}

PB96-193735 PC A05/MF A0

National Inst. of Standards and Technology (MEL) Gaithersburg, MD. Manufacturing Systems Integration

Systems Integration for Manufacturing Applications Program 1995 Annual Report.

J. E. Fowler, and M. E. Luce. May $96,56 p$, NISTIR 5839.

See also PB94-213758 and PB96-154877.

Keywords: "Computer aided manufacturing *Integrated systems, "Standards, Data transfer(Computers), Systems engineering, Technology transfer, Interfaces, Computer aided design, Product development, Prototypes, Specifications, "SIMA(Systems Integration for Manufacturing Applications), "Systems Integration for Manufacturing Applications(SIMA).

The Systems Integration for Manufacturing Applications (SIMA) Program is NIST's coordinating focus for manufacturing activities supporting the High Performance Computing and Communications/Information Infrastructure Technology and Applications initiative. The document summarizes the organizational structure of the program, provides descriptions of the technical projects comprising the program, and highlights accomplishments for those projects for FY94 and FY95

02,845

PB96-195334 PCA11/MF A03

National Inst. of Standards and Technology (MEL) Gaithersburg, MD. Manufacturing Systems Integration Div.

Computer-Aided Manufacturing Engineering Forum (2nd). Technical Meeting Proceedings. Held in Gaithersburg, Maryland on August 22-23, 1995. M. Smith, and S. Leong. May 96, 202p, NISTIR5846.

See also PB96-136965. Prepared in cooperation with Naval Research Lab., Washington, DC. Navy Manufacturing Technology Program. 
Keywords: "Computer aided manufacturing, "Meetings, Planning, Data processing, Process control, Integrated systems, Systems engineering, Materials handling, Concurrent engineering, Computerized simulation.

The paper reports results of the Computer-Aided Manufacturing Engineering (CAME) Forum Second Technical Meetings held in August 1995. This meeting built on work completed since the first technical meeting held in March 1995. The meeting brought together software developers, manufacturing technology managers and engineers, and university researchers to address important issues related to developing a Manufacturing Engineering Tool Kit (METK). The meeting was organized around three major segments. During the first segment, NIST computer scientists and engineers updated participants on the status of ongoing METK development work. Presentations were given on issues regarding the manufacturing data validation methodology, business case and implementation plan, and system architecture and tools integration. An overview was given on the Systems Integration for Manufacturing Applications (SIMA) Production System Engineering project. The METK baseline pilot system was demonstrated to the Forum attendees. The second segment used breakout groups to solicit ideas and feedback from meeting attendees on the issues discussed in the presentation topics. In the third segment, breakout groups prepared outbriefs and presented results to all meeting participants.

\section{2,846}

PB97-110472 Not available NTIS

National Inst. of Standards and Technology (MEL), Gaithersburg, MD. Automated Production Technology Div.

Compensation of Errors Detected by ProcessIntermittent Gauging.

Final rept.

H. T. Bandy, and D. E. Gilsinn. 1995, 4p.

Pub. in Proceedings of the American Society for Precision Engineering Annual Meeting, October 15-20, 1995, v12 p440-443

Keywords: "Machine tools, "Automation, "Error correction codes, Computer aided manufacturing, Numerical control, Feedback control, Cutting tools, Quality control, Algonithms, Real time, Metals, Reprints.

The paper describes a software design for implementing compensation of errors detected by process-intermittent gauging. This is the function of one control loop in the three-loop Quality In Automation (QIA) architecture that is under development at the National Institute of Stadards and Technology (NIST) (Donmez, 1992). Process-intermittent error compensation is useful for improving the accuracy of metal cutting processes on turning or machining centers (Brandy, 1991). Duning machining processes, errors due to tool wear, tool deflection, part deflection, etc., are independent of the accuracy of the machine tool itself, and can, therefore, be measured using the machine tool. The approach discussed here is to reduce those process errors. The term process-intermittent refers to inspection occurring during an interval between the semifinish and finish machining processes. Although in-process measurement techniques may allow uninterrupted machining (Fan, 1991), process intermittent inspection has advantages. For instance, a simple measurement device such as a tough-trigger probe may be used instead of the more intrusive apparatus required for measurement during active machining. Also, since a complete profile of the error pattern may be obtained in advance of finish machining, computational filters may be applied to the compensating tool path to minimize the effects of measurement irregularities.

\section{2,847}

PB97-110480 Not available NTIS

National Inst. of Standards and Technology (MEL), Gaithersburg, MD. Automated Production Technology Div

Data Management for Error Compensation and Process Control.

Final rept

H. T. Bandy, and D. E. Gilsinn. 1995, 10p

Pub. in Proceedings Reprint, Modeling, Simulation, and Control Technologies for Manufacturing, Philadelphia, PA., October 25-26, 1995, v2596 p114-123.

Keywords: "Machine tools, "Automation, "Error correction codes, Computer aided manufacturing, Numerical control, Feedback control, Data management, Cutting tools, Metals, Quality control, Reprints.
Strategies for managing data flow are described for a system which controls processes to compensate machine tool and process errors. A CAD representation of the part is the basis for generating the data used in the processes and analyses. A process-oriented view of part features is used for tracking data regarding design, part programming, manufacture, error compensation, and inspection. Part ID and file name nomenclature have been developed to facilitate data identification. All data are stored in a database, enabling error trends over time to be studied so that error compensation algorithms may be refined.

\section{2,848}

PB97-112551 Not available NTIS

National Inst. of Standards and Technology (EEEL), Gaithersburg, MD. Electricity Div.

Exploring the Low-Frequency Performance of Thermal Converters Using Circuit Models and a Digitally Synthesized Source.

Final rept.

N. Oldham, M. E. Parker, B. Bell, and S. Avramov-

Zamurovic. 1996, 2p.

Pub. in Conference on Precision Electromagnetic Measurements, CPEM '96 Conference Digest Braunschweig, Germany, June 17-20, 1996, p495496.

Keywords: "Electrical measurement, "Low frequency Standards, Electrical networks, Temperature control, Tests, Reprints, "Thermal voltage converters, Voltage standards, Thermal converters.

Low-frequency tracking errors of thermal voltage converters are described and estimated using circuit mod els. A digitally synthesized source is used to confirm ac-dc differences in the $0.001 \mathrm{~Hz}$ to $40 \mathrm{~Hz}$ range.

\section{2,849}

PB97-113781 PC A03/MF A01

National Inst. of Standards and Technology (EEEL), Gaithersburg, MD

Conformance Testing and Specification Management.

Rept. for Jun 95-Jun 96

J. A. St. Pierre, K. G. Brady, and S. L. Stewart. Sep

96, $21 p$, NISTIR-5879.

Contract SEMATECH-95101037

Sponsored by SEMATECH, Austin, TX.

Keywords: "Conformance testing, "Object oriented programs, "Production management, Computer software, Computer aided manufacturing. Specifications, CIM(Computer Integrated Manufacturing), Compute Integrated Manufacturing, JAVA programming language, CORBA(Common Object Request Broker Architecture), Common Object Request Broker Architec ture, Electronic.

This is the final report for the second year of a joint project between the National Institue of Standards and Technology (NIST) and SEMATECH. It presents the results from investigation in two areas that were proposed in the first year report: conformance testing and specification management. In conformance testing, a number of technologies are reviewed with special emphasis on using Java and Corba to develop a conformance testing environment. For specification manage ment, technologies were reviewed for ways to use a single format with multiple uses and multiple distribu tion formats. This task looked at pragmatic solutions to the problem of maintaining complex documents with multiple uses through combinations of radily available software.

\section{2,850}

PB97-116123 PC A06/MF AO

National Inst. of Standards and Technology (MEL) Gaithersburg, MD. Manufacturing Systems Integration Div.

Unified Process Specification Language: Requirements for Modeling Process.

C. Schlenoff, A. Knutilla, and S. Ray. Sep 96, 78p, NISTIR-5910.

Keywords: "Computer aided manufacturing, "Process control, "Software tools, Production management, Scheduling, Systems integration, Industrial plants, Automation, Life cycles, PSL(Process Specification Language), Process Specification Language.

A wide range of applications deal with the manipulation and expression of collections of activities. Examples in clude project management, workflow management, business process reengineering, product realization process modeling, process planning, production scheduling, simulation, and Computer Aided Software Engineering, each of which is supported by some combination of graphical programming and control languages, Petri nets, PERT charts or other representation methodology. Each of these applications serves a specific audience and need, and focuses on particular aspects of a process. Nevertheless, much could be gained by sharing information among applications. One of the primary obstacles to such integration is the lack of any common representation of what is really the common underlying concept of process. The objective of the work described here is an investigation of the feasibility of a unifying specification of process which is applicable to all of the above applications, yet powerful and robust enough to meet each set of requirements. The document represents the results of the first phase of the work - that of researching the process representational requirements for design/ manufacturing process life-cycle applications. These requirements are categonzed into four categories: core, outer core, plug-ins, and application, which aided in describing the role of the requirements in the overall challenge of process representation.

\section{Computer Software}

02,851

PB95-155578 PC A09/MF A03
National Inst. of Standards and Technology (TS), National Inst. of

Opportunities for Innovation: Software for Manufacturing.

D. E. Edgerly. Aug 94, 194p, NIST/GCR-94/658.

Keywords: "Manufacturing, "Computer software, Technology innovation, Computer applications, Models, Data management, Information management, Knowledge bases(Artificial intelligence), Computer aided design, Computer aided manufacturing, Numerical control, Total quality management, Software engineering.

The purpose of this Monograph is to survey the existing software applicable to the manufacturing enterprises, to identify the gaps and the opportunities for software developers to close these gaps. Information technology is constantly growing and adapting. This Monograph proposes directions for this growth and is a snapshot of current conditions, to be used as a starting guide.

\section{Engineering Materials}

\section{2,852}

PB95-162889 Not available NTIS

National Inst. of Standards and Technology (MSEL), Gaithersburg, MD. Materials Reliability Div.

Welding for Cryogenic Service.

Final rept.

T. A. Siewert, and C. N. McCowan. 1993, 4p.

Pub. in ASM Materials Handbook, v6 p1016-1019 Dec 93.

Keywords: "Cryogenics, "Welding, Mechanical properties, Aluminum, Nickel, Stainless steels, Materials, Reprints.

The report summarizes the factors that affect weld metal strength and toughness for cryogenic service. It provides advice on how to produce welds that match the properties of the wrought structural material.

\section{Job Environment}

\section{2,853}

PB95-161907 Not available NTIS

National Inst. of Standards and Technology (BFRL), Gaithersburg, MD. Fire Science Div.

Water Mist Fire Suppression Workshop Summary. Final rept.

K. A. Notarianni. $1993,2 p$

Pub. in Society of Fire Protection Engineers Bulletin, p8-9 Summer 1993. 
Keywords: "Sprays, "Fire suppression, "Commercialization, "Fire extinguishing agents, Workshops, Water vapor, Spraying, Fire fighting, Drop size, Technology assessment, Reprints, "Water mist.

The water mist fire suppression workshop was organized to facilitate the commercialization of water mist technology. The imminent lack of availability of halon fire suppressants has sparked worldwide efforts in developing other fire fighting agents. Water mist systems are potential replacements in many industrial uses, as well as in new markets, such as commercial passenge aircraft. Speakers presented state-of-the-art papers on the incentives of using misting sprays, the advances in spray drop size measurement and the engineering criteria for water mist fire suppression systems. Three papers discussed projects demonstrating the use of water mist systems in aircraft, marine, and telecommunications applications. The speakers and attendees were divided into three panels: Research Needs; End-Use Criteria; Marketing. The proceedings brings together the recommendations of each panel and the individual technical papers.

\section{Joining}

02,854

AD-A279 121/8 PC A04/MF A01

National Bureau of Standards, Gaithersburg, MD. Screw-Thread Standards for Federal Services, 1957. Part 3.

$1957,75 p$

Keywords: "Screw threads, "Standards, Keywords: Nuts(Fastenings),
Thew threads, Handbooks, Tolerances(Mechanics), Sizes(Dimensions).

No abstract available.

\section{2,855}

\section{AD-A279 290/1 PC A10/MF A03}

National Bureau of Standards, Gaithersburg, MD.

Screw-Thread Standards for Federal Services, 1957. Part 1.

Nov 60, 215p, NBS-HB-H28-PT-1.

Keywords: *Screw threads, "Standards, Gages, Handbooks, Screws, Fasteners, Couplers, Joints, Threaded products, Federal services.

No abstract available.

\section{2,856}

PB94-185873 Not available NTIS

National Inst. of Standards and Technology (MSEL), Boulder, $\mathrm{CO}$. Materials Reliability Div.

International Institute of Welding: Report on 1992 Actions.

Final rept

T. Siewert. Dec 92 3p.

Pub. in Materials Evaluation 50, n12 p1389-1392 Dec 92. See also report for 1993, PB94-185881.

Keywords: "Welding, "Meetings, "Nondestructive tests "Quality assurance, "Standardization, Inspection, Standards, Weldments, Defects, Test methods, Quality control, Reprints, International Institute of Welding.

Commission V on Quality Control and Quality Assurance of Welded Products handles inspection and quality issues for the International Institute of Welding. The activities of this commission are important to the nondestructive evaluation industry because the annual meetings serve as an international review of research activities and many of its draft standards are forward to the International Organization for Standards (ISO) for international approval. Thus, this summary of the meeting provides an up-to-date review of research in other countries and advance notice of standardization activities. This commission has activities in many areas, with subcommissions that cover major techniques for nondestructive inspection ( $x$-ray, ultrasonic electrical, magnetic, and optical techniques) and groups with interests in inspection of offshore construction, in significance of defects, and in quality assurance in welding technology. This year, Commission $\mathrm{V}$ met for three days and sponsored a seminar on the fourth. Thirty delegates and experts from sixteen countries at tended the meetings. Following are reports of the subcommissions and working groups, in the order of presentation.
02,857

PB94-185881 Not available NTIS

National Inst. of Standards and Technology (MSEL), Boulder, CO. Materials Reliability Div.

International Institute of Welding: Report on 1993

Actions.

Final rept.

T. A. Siewert. 1994, 4p.

Pub. in Materials Evaluation 52, n1 p44, 46, 47, and 49 Jan 94. See also report for 1992, PB94-185873.

Keywords: "Welding, "Meetings, "Nondestructive tests, "Quality assurance, "Standardization, Inspection, Standards, Weldments, Defects, Test methods, Quality control, Reprints, International Institute of Welding. Commission V on Quality Control and Quality Assurance of Welded Products handles inspection and quality issues for the International Institute of Welding (IIW). The activities of this commission are important to the nondestructive evaluation industry because the annual meetings serve as an international review of research activities. Many of its draft standards are forwarded to the International Organization for Standards (ISO) for international approval. Thus, this summary of the meeting provides an up-to-date review of research in other countries and advance notice of standardization activities.

\section{2,858}

PB94-185899 Not available NTIS

National Inst. of Standards and Technology (MSEL), Boulder, CO. Materials Reliability Div.

Through-the-Arc Sensing for Monitoring Arc Welding.

Final rept.

T. A. Siewert, R. B. Madigan, T. P. Quinn, and M. A. Mornis. 1993, 4p.

Pub. in Proceedings of International Conference on Trends in Welding Research (3rd), Gatlinburg, TN., June 1-5, 1992, p1037-1040 1993. Sponsored by Department of the Navy, Washington, DC

Keywords: *Monitoring, *Quality control, Real time operation, Signal processing, Video signals, Welding machines, Wear, Algorithms, Process control(Industry), Reprints, "Through-the-arc sensing, "Pulsed gas metal arc welding.

Current and voltage records for pulsed gas metal arc welding were captured at data collection rates up to $9 \mathrm{kHz}$, then correlated with a high-speed video image of the arc. Loss of shielding gas coverage and contact tube wear caused characteristic changes in the current and voltage records. Change in contact-tube-to-work distance or change in the droplet transfer mode, also affected the current and voltage records. Algorithms were developed which use the current and voltage signals to automatically detect these undesirable welding conditions or variations in the welding procedure. The algorithms were incorporated in a computerized data collection and analysis system for real-time monitoring of the weld.

\section{2,859}

PB94-210143 PC A03/MF A01

National Inst. of Standards and Technology (TS), Gaithersburg, MD. Office of Standards Services.

Program Handbook: Requirements for Obtaining NIST Approval/Recognition of a Laboratory Accreditation Body Under P.L. 101-592. The Fastener Quality Act.

R. L. Gladhill. Aug 94, 49p, NISTIR-5428.

Keywords: "Fasteners, "Quality control, "Laboratories, "Accreditation, Requirements, Product inspection, Methodology, 'Tests, Costs, Foreign government, Private organizations, "US NIST, Fastener Quality Act, Public Law 101-592.

On November 16, 1990 the United States Congress enacted the Fastener Quality Act, P.L. 101-592 (The Act) The Act requires the establishment of procedures and conditions for the accreditation of laboratories engaged in the inspection and testing of certain fasteners entered into commerce within the United States. This document describes the National Institute of Standards and Technology (NIST) process to evaluate accreditation bodies as required by section 6 of the Act. The text of this handbook provides specific NIST interpretations of the general requirements. It explains the procedures, conditions, and requirements for gaining NIST approval/recognition.

\section{2,860}

PB95-147922
National Inst. of Standards and Technology (MEL) Gaithersburg, MD. Automated Production Technology Div.

Portsmouth Fastener Manufacturing Workstation. User's Manual.

V. J. Lee, L. J. Fronczek, R. J. Gavin, and K. B. Lee. Feb 93, 116p, NISTIR-5507.

See also PB94-118221. Sponsored by Naval Research Lab., Washington, DC. Navy Manufacturing Technology Program.

Keywords: "Fasteners, "Computer aided manufacturing, "Workstations, Control systems, UNIX(Operating system), Man machine systems, Computer software Operators(Personnel), User manuals(Computer programs), US NIST, AMRF(Automated Manufacturing Research Facility).

A user manual is written for the integrated fastener manufacturing workstation which was jointly developed in the Automated Manufacturing Research Facility at the National Institute of Standards and Technology (NIST) by the Automated Production Technology Division and Portsmouth Naval Shipyard. The manual is intended to be used to train the operators or machinists to run the computer-controlled workstation. The workstation is controlled by a complex UNIX-based control system and requires operator interactions with the computer, machine and gaging devices. The workstation software is developed with ease of operation in mind and provides an operator-friendly environment for the machinists who usually have very little experience with computers, particularly a UNIX multitasking, multi-window system. The manual describes the workstation, the software, and operation procedures and leads the operator to order part batches, examine status and perform diagnostics.

\section{2,861}

PB95-162855 Not available NTIS

National Inst. of Standards and Technology (MSEL) Gaithersburg, MD. Materials Reliability Div.

Status Report: AWS Standards for Identifying Arc Welds (A91.1) and Recording Weld Data (A9.2).

Final rept.

T. A. Siewert. 1993, 4p

Pub. in Proceedings of International Conference on Computerization of Welding Information IV, Orlando, FL., November 3-6, 1992, p3-6 1993.

Keywords: "Arc welding, "Data bases, Data processing. Standards, Materials specifications, Physical properties, Reprints, PQR, NDE data, Property data, Standard formats.

Standard formats are being developed for a wide range of data associated with materials and their properties. Two committees have cooperated in the development of two standards for welds, one covering the identification of welds (unique identification of a specific weld as well as details on the preparation of the weld) and the other covering the properties and inspection of welds. Separation of the data into these two categories makes the standards compatible with the formats de veloped by E49 for other materials. The report provides more details on these standards.

\section{2,862}

PB95-162863 Not available NTIS

National Inst. of Standards and Technology (MSEL) Gaithersburg, MD. Materials Reliability Div.

Computers in Welding: A Primer.

Final rept.

T. A. Siewert, R. B. Madigan, and T. P. Quinn. 1994,

Pub. in Welding Jnl., p39-43 Feb 94

Keywords: "Welding, "Computers, "Computer applications, Computer components, Computer hardware Computer software, Reprints.

The article is a basic summary of computer concepts and components. If you haven't started to use computers yet, use this article to gain a basic understanding. If you feel somewhat comfortable with computers, you can use this article as a review, but it also may help you develop a plan to upgrade your skills and knowledge. We have added recommendations for further reading and a section on terms and definitions at the end to help you learn the jargon.

02,863

PB95-162871 Not available NTIS

National Inst. of Standards and Technology (MSEL) Gaithersburg, MD. Materials Reliability Div. 
Through-the-Arc Sensing for Real-Time Measurement of Gas Metal Arc Weld Quality.

Final rept.

T. A. Siewert, R. B. Madigan, T. P. Quinn, and M. A.

Mornis. 1992, 9p.

Sponsored by Department of the Navy, Washington, $\mathrm{DC}$.

Pub. in Proceedings of International Conference on Computerization of Welding Information IV, Orlando, FL., November 3-6, 1992, p198-206.

Keywords: "Gas metal arc welding, "Quality control, "Real time operations, Computer systems programs, Electric potential, Electric current, Records, Detection, Reprints, Droplet transfer, Voltage records, Current records.

The voltage drop across the arc in gas metal arc welding is affected by droplet transfer, spatter formation and many other arc characteristics. The current is affected in a similar manner. The authors collected voltage and current records with a high-speed data acquisition system, and correlated features in the records to various welding problems. Interruption in the shield ing gas coverage, wear of the contact tube, change in the contact-tube-to-work distance, and change in the droplet transfer mode were detected through this sensing strategy. These effects were developed into general correlations and incorporated into a computerized data collection and analysis system.

\section{2,864}

PB95-163614 Not available NTIS

National Inst. of Standards and Technology (MEL) Gaithersburg, MD. Precision Engineering Div.

Variations in Size Measurements by Indicating Gaging Systems.

Final rept.

R. Veale, A. Strang, and C. Hsiao-Yu. 1994, 13p.

Pub. in Proceedings of the 1994 Measurement Science Conference, Pasadena, CA., January 27-28, 1994, p1-13.

Keywords: *Size determination, "Dimensional measurement, "Screw threads, Measuring instruments, Pitch(Inclination), Gages, Tests, Reprints. Pitch diameters.

The National Institute of Standards and Technology has investigated the efficacy of indicating gaging systems used to measure pitch diameter and functional size of threaded fasteners. Three external systems and four internal systems, representing four manufacturers, were used in the test. Twenty-seven external threads and eleven internal threads were measured. Some of the samples were purposely produced with form errors near and beyond product tolerances. The study shows that when the form errors are outside the product tolerances, indicating gages from different manufacturers give different results and do not always match the values obtained by alternate methods. Results of the tests are presented in tabular form.

\section{2,865}

PB95-189460 PC A03/MF A01

National Inst. of Standards and Technology (CAML) Gaithersburg, MD. Applied and Computational Mathematics Div.

Lubrication Theory for Reactive Spreading of a Thin Drop.

R. J. Braun, B. T. Murray, W. J. Boettinger, and G. B. McFadden. Dec 94, 50p, NISTIR-5557.

Keywords: "Soldering, "Lubrication, "Capillary flow, "Drops, Mass transfer, Transport properties, Solder Concentration(Composition). Marangoni convection, Heat transfer, Temperature gradients.

Solder drops spreading on metallic substrates are a reactive form of the wetting problem. A metallic component may diffuse into the metal substrate and form intermetallics. This reaction with the substrate may af fect the bulk transport in the spreading drop via coupling with the flow field via the Marangoni effect. Motivated by this situation, we extend a lubrication theory for the spreading of thin drops in the presence of gravity and thermocapillarity to include mass transport and solutocapillarity. We use an approximate solute profile in the drop to derive coupled evolution equations for the free surface shape and concentration field. Numerical solutions for the non-reactive (single component) drop agree well with previous theory. Including reactive effects in the model affects the flow patterns and spreading rates at relatively early times; but by the end of the spreading, solutal effects have died out in the model. We compare our results with some experimental results.
02,866

PB95-198743 PC A03/MF A01

National Inst. of Standards and Technology (MSEL),

Boulder, CO. Materials Reliability Div.

IIW Commission V Quality Control and Quality Assurance of Welded Products Annual Report 1994/ 95.

T. A. Siewert. Mar 95, 35p, NISTIR-5034, IIW-V1046-95.

Sponsored by International Inst. of Welding.

Keywords: "Quality assurance, *Quality control, "Weld tests, "Welded joints, Standards, Radiography, Welding, Inspection, Nondestructive tests, Standards, Test methods, Standardization, "International Institute of Welding.

The Annual Report 1994/1995 for Commission V, Quality Control and Quality Assurance of Welded Products, of the International Institute of Welding is presented. It includes (a) minutes, resolutions, and the future program adopted at its Annual Assembly in September 1994, (b) the organization, officials, and delegates, (c) schedules of meetings, and (d) the status of documents published by Commission V. It reviews current research and work on standardization.

\section{2,867}

\section{PB95-209300 PC A04/MF A01}

National Inst. of Standards and Technology (MSEL),

Boulder, CO. Materials Reliability Div.

Droplet Transfer Modes for a MIL 100S-1 GMAW Electrode.

P. R. Heald, R. B. Madigan, T. A. Siewert, and S.

Liu. Oct $91,54 \mathrm{p}$, NISTIR-3976.

See also PB91-174250. Prepared in cooperation with Colorado School of Mines, Golden. and Phillips Co., Borger, TX. Sponsored by David Taylor Research Center, Annapolis, MD. Programmable Automated Welding System Program.

Keywords: "Gas metal arc welding, "Welding electrodes, "Drops, "Mass transfer, Weldments, Standard deviation, Weld metal, Data acquisition, Computer applications, Computer aided manufacturing, MIL 100S1 electrode.

Welds were made with a $1.2-\mathrm{mm}$-diameter MIL $100 \mathrm{~S}$ 1 electrode using Ar-2\% O2 shielding gas to investigate the effects of contact-tube-to-work distance (13, 19 , and $25 \mathrm{~mm}$ ) on metal transfer. The transfer modes were identified by the sound of the arc, images from a laser back-lit high-speed video system, and digital records of the voltage and current fluctuations. The spray transfer region was mapped on a current-voltage plot, with a range that included the boundaries of adjacent transfer modes. The metal transfer mode boundaries shifted with an increase in contact-tube-to-work distance. Increasing the contact-tube-to-work distance from $13 \mathrm{~mm}$ to $19 \mathrm{~mm}$ required a $15 \mathrm{~mm} / \mathrm{s}$ increase in the wire feed rate for the globular-to-drop-spray transition.

PC A03/MF A01

National Inst. of Standards and Technology (MSEL), Gaithersburg, MD. Reactor Radiation Div.

Determination of the Residual Stresses Near the Ends of Skip Welds Using Neutron Diffraction and $X$-ray Diffraction Procedures.

P. C. Brand, G. E. Hicho, and H. J. Prask. Jun 95 40p, NISTIR-5671.

Also pub. as National Inst. of Standards and Technology (MSEL), Gaithersburg, MD. Metallurgy Div. rept. no. REPT-29.

Keywords: "Residual stress, "X ray diffraction, "Neutron diffraction, "Metal plates, Spot welds, Welding, Stress analysis, Stress distribution, Nondestructive tests, Stress measurement.

Welding is known to be a significant factor in the formation of residual stresses. Residual stresses are suspected as the reason for the occurrence of leaks in some railroad tank cars that had stiffeners welded to them. Residual stresses at the surface and in the bulk of metals can be measured non-destructively by $x$-ray and neutron diffraction respectively. In this report the results of such non-destructive residual stress measurements on one-pass, skip, bead-on plate welds are presented. The plate specimens used for this investigation contained two beads that represent the skip weld process. Of the full weld bead only positions close to the end of the first and the start of the second bead are investigated. The results show longitudinal tensile stresses and compressive transverse and perpendicular residual stresses in the region close to both the weld end and the weld start.

\section{2,869}

PB96-131461 PC A06/MF A02

National Inst. of Standards and Technology (MSEL), Gaithersburg, MD. Materials Reliability Div.

Control of Gas-Metal-Arc Welding Using Arc-Light Sensing.

R. B. Madigan, T. P. Quinn, and T. A. Siewert. Nov 95, 120p, NISTIR-5037.

See also AD-A177 893.

Keywords: "Gas metal arc welding, "Feedback control, Automation, Models, Process control, Radiation, Sensors, Arc length, Droplet-transfer frequency, National Institute of Standards and Technology.

In this development of closed-loop control of the gasmetal-arc welding process using light emitted by the arc, two process parameters were controlled in real time: arc length and droplet-transfer frequency. An arclength sensor and controllers to regulate arc length and to actuate welding current were developed. The transfer frequency depended on current, wire-feed speed, and electrode extension, these results led to the design of a transfer-frequency controller.

\section{0}

PB96-135058 Not available NTIS

National Inst. of Standards and Technology (MSEL) Boulder, CO. Materials Reliability Div.

Mathematical Models of Transport Phenomena Associated with Arc-Welding Processes: A Survey.

Final rept.

P. G. Jonsson, J. Szekely, R. T. C. Choo, and T. P. Quinn. 1994, 22p.

Pub. in Modelling Simulation Materials Science and Engineering, v2 p995-1016, 1994.

Keywords: "Arc models, "Welding, Surveys, Reprints, Mathematical models, Process control, GNAW, GTAW.

A comprehensive survey is presented of research on transport phenomena, that is, heat flow, fluid flow, mass transfer, and electrodynamics, associated with arc welding. Both gas-metal arc-welding (GMAW) and gas-tungsten arc welding (GTAW) systems are considered, and appropriate emphasis is placed on the intrinsic differences between these systems. The topics discussed include the behavior of the arc, and its interaction with both the electrode and the surrounding medium, on the one hand, and the behavior of the weld pool on the other. The limited work done on the coupling between arcs, electrodes, and weld pools is also discussed, as are some process-control issues, but the problems of thermal stress evolution and stress-in duced deformation in the solid state are not treated. A critical discussion of the comparison between mode predictions and experimental measurements is a key feature of the presentation, which concludes with a discussion of the critical problems that still need to be addressed.

02,871

PB96-135074 Not available NTIS

National Inst. of Standards and Technology (MSEL), Boulder, CO. Materials Reliability Div. Electrode Extension Model for Gas Metal Arc Welding.

Final rept.

T. P. Quinn, R. B. Madigan, and T. A. Siewert. 1994,

Pub. in Welding Research Supplement, p241s-248s Oct 94

Keywords: "Welding, *Electrode extensions, Reprints, Transfer functions, Temperature profiles, Spray transfer mode, "Describing functions, GMAW, Electrode feed speed.

The electrode extension during gas metal arc welding is predicted using a one-dimensional model of the melting electrode. Jouile heating in the electrode, heat directly applied to the end of the electrode from the condensing electrons, and heat transferred from the droplet, together with conduction along the electrode are considered. The thermal conductivity, the thermal diffusivity, and the electrical resistivity of the electrode material are allowed to bary with temperature. The steady-state electrode extension is predicted to an accuracy of $1.9 \mathrm{~mm}(0.074 \mathrm{in}$.). The onset of shortcircuiting as the current is decreased for a given electrode feed speed is predicted within $9 \%$. Dynamic anal- 
ysis shows that the gas metal arc welding process acts as a low-pass filter for electrode extension with respect to the square of the current (proportional to power) and with respect to electrode feed speed. As the mean welding current is increased the electrode extension (or arc length if the contact-tube-to-work distance is constant) has a smaller response to perturbations in the current or electrode feed speed. The quasi-linear transfer functions between electrode extension and current squared and between electrode extension and electrode feed speed can be described by one zero, two pole parametric fits. The transfer functions are linear in the amplitude of the excitation up to $10 \%$ of the mean excitation. The model transfer functions were verified with experiments.

\section{2,872}

\section{PB96-135330 Not available NTIS}

National Inst. of Standards and Technology (MSEL), Boulder, CO. Materials Reliability Div.

Contact Tube Wear Detection in Gas Metal Arc

Welding.

T. P. Quinn, R. B. Madigan, M. A. Mornis, and T. A. Siewert. 1995, 7p

Pub. in Welding Research Supplement, p115s-121s Apr 95.

Keywords: "Wear tests, "Gas metal arc welding "Welding current, "Sensors, Reprints, Weldments Welding electrodes, Wear, Robots, Sliding contact, Tube wear detection.

A real-time algorithm has been developed to detect contact tube wear during gas metal arc welding. The integral from 0.3 to $4 \mathrm{~Hz}$ of the power spectral density of voltage (constant current and pulsed current power sources) or current (constant voltage power sources) is called the wear parameter and is used to measure wear. The contact tube is predicted to be worn when the parameter grows nonlinearly or exceeds a threshold. For 11 contact tubes worn by welding on a rotating pipe, the wear parameter predicted the observed increase in the area of the contact tube's exit bore of $140 \%$ of the original area within a standard deviation of $20 \%$. Welds were made with a constant voltage power source and with a pulsed current power source at two different voltages. The pulsed current welds were made with and without automatic voltage control by wire feed speed and with and without weaving in a $V$-groove. No significant differences were detected between the wear parameters for all test welds, indicating that the algorithm can be used under these welding conditions.

\section{2,873}

PB96-138540 Not available NTIS

National Inst. of Standards and Technology (MSEL), Boulder, CO. Materials Reliability Div.

Report on 1994 Actions of the International Institute of Welding.

Final rept.

T. A. Siewert. $1995,4 p$.

See also PB94-185881.

Pub. in Materials Evaluation, p396-399 Mar 95

Keywords: "Welding, "Meetings, "Nondestructive tests, "Quality assurance, *Standardization, Inspection, Standards, Weldments, Defects, Test methods, Reprints, International Institute of Welding.

Commission $V$ covers issues of weld inspection and quality control for the International Institute of Welding. The report summarizes the information presented at the 1994 Annual Assembly: descriptions of both research and draft ISO standards being developed from the research data. The information comes from the various multinational subcommissions, working groups, and task groups within Commission $V$. Thus, this summary provides an up-to-date review of research activities in other countries than the US and advanced notice of standardization activities.

02,874

\section{PB96-158076 Not available NTIS}

National Inst. of Standards and Technology (MSEL), Gaithersburg, MD. Materials Reliability Div.

International Institute of Welding: Report on 1995

Actions.

T. Siewert. 1995, 4p

See also PB96-138540.

Pub. in Materials Evaluation, v53 n12 p1380-1383 Dec 95.

Keywords: "Welding, "Inspection, "Nondestructive tests, "Stadards, Weldments, Test methods, Defects,
Quality assurance, Quality control, Radiology, Ultrasonic tests, Standardization, Reprints, International Institute of Welding, Radioscopy.

This report summarizes the information presented at the 1995 Annual Assembly descriptions of both re search and draft ISO standards having been developed from the research data. The information comes from the various multinational sub-commissions, work ing groups, and task groups within Commission V. This summary provides an up-to-date review of research activities in other countries and advance notice of standardization activities.

\section{2,875}

PB96-158084 Not available NTIS

National Inst. of Standards and Technology (MSEL),

Gaithersburg MD. Materials Reliability Div.

What's Available in Welding Software.

Final rept.

T. A. Siewert. $1995,5 p$

Pub. in Welding Jnl., p39-43 Nov 95

Keywords: "Welding, "Computer software, Weldments, Computerized simulation, Expert systems, Reference materials, Mathematical calculations, Standards, Reprints.

Welding software is making large strides in sophistication and power, as the developers take advantage of the latest hardware technology and new software concepts. The list includes current software, a short summary of its capabilities, and where to buy it.

\section{2,876}

\section{PB96-160676 Not available NTIS}

National Inst. of Standards and Technology (TS), Gaithersburg, MD. Lab. Accreditation Program.

Implementation of the Fastener Quality Act.

Final rept.

S. W. Stiefel. $1993,7 p$.

See also PB94-210143.

Pub. in National Board of Boiler and Pressure Vesse Inspectors Proceedings, General Meeting (62nd), San Diego, CA., May 3-7, 1993, p63-69.

Keywords: *Implementation, "Quality control, Fasteners, Product inspection, Laboratory procedures, Test methods, Requirements, Accreditation, Certification, Reprints, "Fastner Quality Act, Pubic Law 101-592.

This presentation describes: the objective of Public Law 101-592, (the fastener Quality Act), various requirements and responsibilities under that act, the status of the procedures required for implementation, and the process being used at the National Institute of Standards and Technology (NIST) to develop the laboratory accreditation program for those laboratories that will be doing the testing under the act

\section{2,877}

\section{PB96-183124 PC A04/MF A01}

National Inst. of Standards and Technology (MEL), Gaithersburg, MD. Intelligent Systems Div.

Proceedings of NIST Workshop: Industry Needs in Welding Research and Standards Development. Held on August 15-16, 1995

W. G. Rippey. Apr 96, 32p, NISTIR-5822.

Keywords: "Welding, "Standards, "Meetings, US NIST, Research and development, Gaithersburg(Maryland).

Thirty-two attendees representing technology users, technology providers, private and university research, and government gathered for this two-day workshop on welding technology. The attendees defined twelve sub-topics. The top four ranked, indicating where Industry has the strongest needs, were: Interface standards for data exchange, Electrical and mechanical interface standards, Predictive process models and knowledge base, Simulation and off-line programming.

\section{2,878}

PB96-186028 Not available NTIS

National Inst. of Standards and Technology (MSEL),

Boulder, CO. Materials Reliability Div.

Uitrasonic Sensing of GMAW: Laser/EMAT Defect Detection System.

Final rept.

N. M. Carlson, J. A. Johnson, E. D. Larsen, and A

Van Clark. 1992, 13p.

See also DE92017916.

Pub. in International Conference on Welding Research Trends in Welding Technology (3rd), ASM International, Metals Park, OH., p2-14 1992.
Keywords: "Defects, "Gas Metal-Arc Welding, Transducers, Computerized control systems, Reprints, Data acquisition systems, Detection, Feedback, Monitoring, Nondestructive analysis, Personal computers, *Ultrasonography, Welded joints.

In-process ultrasonic sensing of welding allows detection of weld defects in real-time (1). For a sensing effort to be practical in a production environment, noncontracting ultrasonic system is developed. The system components are a pulsed laser for ultrasound generation and an electromagnetic acoustic transducer (EMAT) for ultrasound reception. A personal computer (PC)-based system acquires and analyzes data to determine the quality of the welding process on a pass-by-pass basis. The laser/EMAT system interrogates an area in the weld volume where defect conditions are most likely to occur. This area of interest is computer calculated on a pass-by-pass basis using weld planning information provided by the off-line programmer (OLP). The absence of a signal level above threshold in the computer calculated time interval indicates a disruption of the sound path to the EMAT due to the presence of a defect condition. The ultrasonic sensor system then provides an input signal to the weld controller about the defect condition.

\section{2,879}

PB96-186077 Not available NTIS

National Inst. of Standards and Technology (MSEL) Boulder, CO. Materials Reliability Div

Well-Shielded EMAT for On-Line Ultrasonic Monitoring of GMA Welding.

Final rept.

A. V. Clark, S. R. Schaps, and C. M. Fortunko. 1992 $3 p$.

Pub, in Proceedings of Institute of Electrical and Electronics Engineers Ultrasonics Symposium, Orlando, FL., 1991, 3p 1992.

Keywords: "Acoustics, "Ultrasonics, "Welding, Reprints, "EMATs.

A hybrid laser/EMAT system is currently being developed for pass-by-pass inspection of weldment defects. The laser source is inherently broadband, whereas the EMAT is a narrow-band detector. The authors review some of the theory used to develop an optimized EMAT system for use with a laser source. The EMAT will be used close to a welding torch. The welding system has a $40 \mathrm{kHz}$ (fundamental) switching power supply. This gives rise to severe electromagnetic inter ference (EMI). The authors review the means of temperature- and EMI-hardening used to develop a practical EMAT system for this environment. They developed a balanced system of counter-wound coil and differential preamplifier which greatly suppressed EMI. Other measures, such as multiple shielding, bandpass filtering, and attention to grounds, were used. The system gives about $32 \mathrm{~dB}$ signal-to-noise ratio, when operated $125 \mathrm{~mm}$ from a welding torch.

\section{2,880}

PB96-191366 PC A04/MF A01

National Inst. of Standards and Technology (MSEL), Boulder, CO. Materials Reliability Div.

IIW Commission V Quality Control and Quality Assurance of Welded Products, Annual Report 1995

T6. A. Siewert. Apr 96, 36p, NISTIR-5044, IIW-V $1059-96$

See also report for 1994/95, PB95-198743. Sponsored by Intemational Inst. of Welding

Keywords: "Welding, *Quality control, Nondestructive tests, Inspection, Eddy current tests, Ultrasonic tests Meetings, US NIST.

The Annual Report 1995-96 for Commission V, Quality Control and Quality Assurance of Welded Products, of the International Institute of Welding includes (1) minutes, resolutions, and the future program adopted at its Annual Assembly in June 1995, (2) the organization, officials, and delegates, (3) schedules of meetings, and (4) the status of documents published by Commission $v$. It reviews current research and work on standardization.

02,881

PB97-114185 PC A06/MF A01

National Inst. of Standards and Technology (TS), Gaithersburg, MD. National Voluntary Lab. Accreditation Program 
National Voluntary Laboratory Accreditation Program (NVLAP): Fasteners and Metals.

Handbook

D. F. Alderman. Aug 96, 76p, NIST/HB-150-18.

Also available from Supt. of Docs. as SNO03-00303421-5.

Keywords: "Fasteners, "Quality control, Standards, Tests, Laboratories, Accreditation, Chemical analysis, Metallography, Corrosion resistance, US NIST.

NIST Handbook $150-18$ presents the technical requirements of the National Voluntary Laboratory Accreditation Program (NVLAP) for Fasteners and Metals. It is intended for information and use by staff of accredited laboratories, those laboratories seeking accreditation, other laboratory accreditation systems, users of laboratory services, and others needing information on the requirements for accreditation under the Fastners and Metals program. This publication supplements the Fasteners Quality Act (PL 101-592) as amended by the National Technology Transfer and Advancement Act (PL 104-113) and its implementing regulations as described in Title 15, Part 280 of the U.S. Code of Federal Regulations, and NIST Handbook 150, NVLAP Procedures and General Requirements (which contains all general NVLAP procedures, criteria, and policies).

\section{Manufacturing, Planning, Processing \& Control}

\section{2,882}

PB94-185493 Not available NTIS

National Inst. of Standards and Technology (MEL), Gaithersburg, MD. Robot Systems Div.

Issues Concerning Material Removal Shape Element Volumes (MRSEVs).

Final rept.

T. R. Kramer. 1994, 13p.

Pub. in International Jnl. of Computer Integrated Manufacturing 7, n3 p139-151 1994.

Keywords: "Machining, Numerical control, Metal cutting, Computer aided manufacturing, Automation, Shape, Subroutine libraries, Reprints,
"MRSEVS(Material removal shape element volumes), "MRSEVs(Material removal shape element volumes), PDES(Product Data Exchange using STEP), STEP(Standard for the Exchange of Product Model Data).

In machining discrete parts, material removal shape element volumes (MRSEVs) may be used to convey shape information from process planning to NC-programming. This paper discusses 16 issues regarding MRSEVs and presents, in summary form, a proposed library of MRSEVs for three-axis machining.

\section{2,883}

\section{PB95-147906 PC A04/MF A01}

National Inst. of Standards and Technology (MEL), Gaithersburg, MD. Factory Automation Systems Div. Program Requirements to Advance the Technology of Custom Footwear Manufacturing.

H. T. Moncarz. Oct 94, 56p, NISTIR-5521.

Keywords: "Footwear, "Orthopedic equipment, "Manufacturing. "Research and development "Meetings, Shoes, Technology innovation, Prototypes, Cost effectiveness, US NIST.

The National Institute of Standards and Technology (NIST) and the South Carolina Research Authority (SCRA) co-hosted the first Custom Footwear Manufacturing Workshop in Charleston, SC on March 10-11. 1994. At the workshop we discussed the potential creation of a nationwide, collaborative research and development (R\&D) effort to benefit the American footwear industry. We discussed applicable technologies for custom and therapeutic footwear manufacturing, related business requirements, national impacts on the economy and health care, and potential strategies to launch a nationwide R\&D effort. We decided that our best strategy will be to concentrate initial efforts on the technology requirements of therapeutic footwear. We believe that the proper development, commercialization, and particularly, the integration of the advanced technologies discussed will enable the cost-effective design, manufacture, and distribution of therapeutic footwear. This report documents the workshop and is intended as a starting point to initiate the $R \& D$ program proposed
02,884

PB95-171112 PC A03/MF A01

National Inst. of Standards and Technology (MEL), Gaithersburg, MD. Precision Engineering Div.

Japan Technology Program Assessment: Precision Engineering/Precision Optics in Japan.

C. J. Evans. Dec 94, 18p, NISTIR-5491.

Keywords: "Precision, "Machining, "Optics, "Japan, Technology assessment, Manufaciuring, Lithography, Research and development, Technology innovation Metrology, Precision engineering, National Institute of Standards and Technology.

The report, based on two visits to Japan, discusses the structure of precision engineering research and development in Japan, and current directives in precision machining, precision optics and advanced lithography.

\section{2,885}

\section{PB95-210167 PC A03/MF A01}

Catholic Univ. of America, Washington, DC. Dept. of Mechanical Engineering.

Issues Concerning Material Removal Shape Element Volumes (MRSEVs).

T. R. Kramer. 26 Mar 92, 32p, NISTIR-4804.

Contract NIST-70NANB9H0923

See also PB94-185493. Sponsored by National Inst. of Standards and Technology (MEL), Gaithersburg MD. Intelligent Systems Div.

Keywords: "Machining, *Numerical control, Metal cutting, Computer aided manufacturing, Automation, Subroutine libraries, Shape, Production control, Material forming, "Material removal shape element volumes.

In machining discrete parts, Material Removal Shape Element Volumes (MRSEVs) may be used to convey shape information from process planning to numerically controlled (NC) programming. This paper discusses sixteen issues regarding MRSEVs and presents, in summary form, a proposed library of MRSEVS for 3-axis machining

\section{2,886}

PB96-109525 PC A03/MF A01

National Inst. of Standards and Technology (CAML) Gaithersburg, MD. Statistical Engineering Div.

Agile Manufacturing from a Statistical Perspective. R. G. Easterling. Oct 95, 44p, NISTIR-5573.

Also pub as Sandia National Labs., Albuquerque, NM. New Initiatives Dept. rept. no. SAND95-1552. Prepared in cooperation with Sandia National Labs., Albuquerque, NM. New Initiatives Dept.

Keywords: "Manufacturing, "Industrial management, "Systems analysis, Industrial plants, Product development, Industrial plants, Statistics, Research programs, Agile manufacturing.

The objective of agile manufacturing is to provide the ability to quickly realize high-quality, highly-custuring, or any other endeavor, is defined as changeproficiency--the ability to thrive in an evironment of unpredictable change. This report discusses the general direction of the agile manufacturing initiative, including research programs at the National Institute of Standards and Technology (NIST), the Department of Energy, and other government agencies, but focuses on agile manufacturing from a statistical perspective. The role of statistics can be important because agile manufacturing requires the collection and communication of process characterization and capability information, much of which will be data-based. The statistical community should initiate collaborative work in this important area.

\section{2,887}

\section{PB96-112693 PC A99/MF E08}

National Inst. of Standards and Technology (MEL), Gaithersburg, MD

Proceedings of the Annual Manufacturing Technology Conference (2nd): Toward a Common
Agenda. Held in Gaithersburg, Maryland on April 18-20, 1995

Special pub.

C. Albus. Aug 95, 913p, NIST/SP-886.

Also available from Supt. of Docs as $\mathrm{SNOOH}^{-003}$. 03361-8. See also report for 1994, PB95-206181.

Keywords: "Manufacturing, "Meetings, "Proceedings, US NIST, State of the art, Market research, Aerospace industry, Electronics industry, Automotive industry,
Construction industry, Food industry, Textile industry, Gaithersburg(Maryland).

This conference helped to define the major elements of a common manufacturing agenda that involves the efforts of government, industry, academia and workforce organizations. The conference built on the progress made at last year's priority-setting conference on manufacturing technology, which attracted more than 600 participants.

\section{Quality Control \& Reliability}

\section{2,888}

PB94-136009 PC A11/MF A03

National Inst. of Standards and Technology, Gaithersburg, MD.

NIST Handbook 44, 1994: Specifications, Tolerances and Other Technical Requirements for Weighing and Measuring Devices as Adopted by the 78th National Conference on Weights and Measures 1993.

Handbook.

H. V. Oppermann, and T. G. Butcher. Oct 93, 230p,

NIST/HB-44.

Supersedes PB93-213106. Also available from Supt. of Docs. as SN003-003-03242-5.

Keywords: "Weight measurement, "Tolerances(Mechanics), "Measuring instruments, "Metrology, "Handbooks, Liquid level indicators, Water meters, Weight indicators, Grains(Food), Dimensional measurement, Hydrocarbons, Fuel tanks, Specifications, Liquefied petroleum gases, Length, Grain moisture, Taximeters.

Handbook 44 was first published in 1949, having been preceeded by similar handbooks of various designations and in several forms beginning in 1918. This 1994 edition was developed by the Committee on Specifications and Tolerances of the National Conference on Weights and Measures, with the assistance of the Office of Weights and Measures of the National Institute of Standards and Technology. It includes amendments adopted by the 78th annual meeting of the National Conference on Weights and Measures, 1993. Handbook 44 is published in its entirety each year following the annual meeting of the National Conference on Weights and Measures.

\section{2,889}

\section{PB94-160835 PC A05/MF A01}

National Inst. of Standards and Technology, Gaithersburg, MD.

National Type Evaluation Program: Index of Device Evaluations by Company. NCWM Publication 5 Part A (Second Edition)

K. Newell. 8 May $90,86 p$, NISTIR-4333.

Keywords: "Metrology, Indexes(Documentation), Performance evaluation, "National Type Evaluation Program.

The publication contains the listing of all devices that have been evaluated under the National Type Evaluation Program (NTEP) since January 1, 1986 to May 8 , 1990. It replaces the first Edition and Supplements 1, 2 , and 3.

\section{2,890}

\section{PB94-163029 PC A03/MF A01}

National Inst. of Standards and Technology (MEL) Gaithersburg, MD. Factory Automation Systems Div. Concept for an Algorithm Testing and Evaluation Program at NIST.

C. Diaz. Jan 94, 14p, NISTIR-5366.

See also PB90-148362.

Keywords: "Dimensional measurement, "Data analysis, *Computer program integrity, Metrology, Coordinates, Computer software, Algonithms, Computer program verification, Standards, National Institute of Standards and Technology.

This report proposes a National Institute of Standards and Technology (NIST) service for testing and evaluating coordinate measuring systems (CMS) software. This program addresses industry's need for a formal mechanism that tesis data analysis software used in CMSs. The NIST Algorithm Testing and Evaluation Program (ATEP) combines the NIST Algorithm Testing System (ATS), test procedures based on emerging na- 
tional standards, and control over the ATS reference algorithms. This report presents a concept for this for$\mathrm{mal}$ mechanism to provide testing methods and tools to test and evaluate CMS data analysis software.

\section{2,891}

\section{PB94-163466 PC A03/MF A01}

National Inst. of Standards and Technology (MEL). Gaithersburg, MD. Factory Automation Systems Div. Models, Managing Models, Quality Models: An Example of Quality Management.

J. E. Tyler. Dec 91, 20p, NISTIR-4738.

See also PB91-193367.

Keywords: "Product development, "Quality, *Management, "Standards, Models, Data bases, Technology innovation, Design standards, Production models, Commerce, Competition, International trade, STEP(Standard for the Exchange of Product Model Data), PDES(Product Data Exchange using STEP) Malcolm Baldrige National Quality Award, IGES(Initial Graphics Exchange Specification), ISO(International Organization for Standardization), Quality levels, Model assessment, Information models, Data models. Quality is an increasingly important element in competitiveness. This paper will focus on strategies used by an international standards committee for improving the development of its complex standard. The paper outlines an approach for use within the standards-making community. Aside from the standards-making community, any organization interested in establishing quality metrics and quality thresholds to assess technical quality should find this paper useful. An overview of a methodology for evaluating the technical quality of information and data models is also included. Quality management techniques for models are described along with management's role in ensuring that high quality models are produced. Many basic concepts of the Malcolm Baldrige National Quality Award were used in building the foundation for this new approach to standards development.

\section{2,892}

PB94-164043 PC A06/MF A02

National Inst. of Standards and Technology (CSTL), Gaithersburg, MD. Thermophysics Div.

NIST Measurement Services: NIST Pressure Calibration Service.

Special pub.

V. E. Bean. Feb 94, 102p, NIST-SP-250-39

Also available from Supt. of Docs. as SN003-00303249-2.

Keywords: "Pressure measurement, "Primary standards, "Calibration, Quality control, Uncertainty, NIST.

This report is the documentation for the NIST pressure calibration service. Topics discussed include design philosophy and theory of pressure primary standard piston gages, uncertainties of the primary standards, uncertainties of NIST transfer standard piston gages, calibration quality control, methods of piston gage calibration, and international intercomparisons of pressure measurement. A sample calibration report is included as an appendix.

\section{2,893}

PB94-169760 Not available NTIS

National Inst. of Standards and Technology,

Uncertainties in Dimensional Measurements Made at Nonstandard Temperatures.

D. A. Swyt. 1994, $14 p$.

Included in Jnl. of Research of the National Institute of Standards and Technology, v99 n1 p31-44 Jan/Feb 94.

Keywords: "Dimensional measurement, "Length, Temperature effects, Tolerances(Mechanics), Thermal expansion, Uncertainty, Metrology, Reprints, Reference temperature.

The report examines the effects of uncertainties in temperature and coefficient of thermal expansion on the
expanded uncertainty of length dimensional measureexpanded uncertainty of length dimensional measurements made away from the international standard reference temperature of $20 \mathrm{C}$ for artifact standards and workpieces of various materials. Specific cases examined deal with (1) uncertainties of thermal-expansion coefficients associated with values given in engineering references, standard reference data, standard reference materials and direct measurements; and (2) uncertainties of part temperature measurements associ- ated with realizing the International Temperature Scale of 1990 (ITS-90) and determining part temperatures relative to ITS- 90 with the principal types of thermometry and achievable levels of temperature control.

\section{2,894}

PB94-185683 Not available NTIS

National Inst. of Standards and Technology (MEL) Gaithersburg, MD. Precision Engineering Div.

Development of a Calibrated Atomic Force Microscope.

Final rept

T. H. McWaid, and J. Schneir. 1994, 6p.

Pub. in Proceedings of the ASPE Conference, Tucson, AZ., 1994, 6p.

Keywords: "Microscopes, Process control, Calibration Metrology, Operation, Height, Pitch(Inclination), Design, Reprints, "Atomic force microscopes, Atomic force microscopy.

Advances in the manufacture of integrated circuits, $x$ ray optics, magnetic read-write heads, optical data storage media, razor blades, etc. require advances in ultraprecision metrology. Each of these industries is currently investigating the use of Atomic Force Microscopy (AFM) to improve the precision and accuracy of heir manufacturing process control measurements. To facilitate the use of AFM for manufacturing, we are de veloping a specially designed AFM system that we call the Calibrated AFM (C-AFM). The C-AFM will be used to calibrate artifacts which, in turn, can be used to calibrate commercial AFMs. The system design goals are first presented. The critical electro-mechanical and merology issues involved in the design, construction, and operation of the C-AFM are then summarized. The current status and performance of the instrument is then presented. The effects of stage stiffness on system performance are discussed in depth. Finally, plans for the future development of the instrument are summarized.

\section{2,895}

PC A07/MF A02

National Inst. of Standards and Technology, Gaithersburg, MD. Office of Weights and Measures

State Weights and Measures Laboratories: State Standards Program Description and Directory. 1994 Edition.

Special pub.

G. L. Harris. Jun $94,132 p$, NIST/SP-791.

Supersedes PB93-217529. Also available from Supt of Docs. as SN003-003-03272-7.

Keywords: "Laboratories, "Standards, "Directories, Units of measurement, States(United States), Puerto Rico, Virgin Islands, Tolerances(Mechanics), Calibration, Tests, "State services, "Weights and measures, National Type Evaluation Program, State Standards Program, Accreditation.

The support of its mission to promote uniform standards of measurement throughout the United States, the National Institute of Standards and Technology (NIST) received funding in 1965 to provide new standards of mass, length, and volume to State weights and meas ures laboratories. This program, called the (New) State Standards Program, also provided the equipment needed to perform calibration in these measuremen areas. Part I describes the accreditation program whereby NIST accredits State weights and measures laboratories. Part II is the directory of State weights and measures laboratories and lists the services they provide to State and local weights and measures agencies as well as to industry. The directory is intended to assist potential users of the laboratory senices in ocating and obtaining needed measurement services.

\section{2,896}

PB94-216132 Not available NTIS

National Inst. of Standards and Technology, Gaithersburg, MD. Office of Product Standards Policy. Performance Standards: The Pro's and Con's.

Final rept.

D. R. Mackay. 1985, 3p.

Pub. in Proceedings of American Water Works Association Annual Conference, Washington, DC., June 23 27, 1985, p403-405

Keywords: "Performance tests, "Standards, Requirements, Reprints.

This paper defines the characteristics of performance standards, describes the promotion of the use of such standards and discusses the positive and negative as pects of performance standards. The purpose of this paper is to acquaint more people with the subject of performance standards.

02897

PB95-108809 Not available NTIS

National Inst. of Standards and Technology (MEL)

Gaithersburg, MD. Precision Engineering Div.

Evolution of Automatic Line Scale Measurement at the National Institute of Standards and Technology.

Final rept.

W. B. Penzes, and J. S. Beers. 1990, 18p.

Pub. in IMEKO Proceedings of the Symposium on Measurement and Inspection in Industry by Computer Aided Laser Metrology, Balatonfured, Hungary, September 24-27, 1990, p1-18.

Keywords: "Dimensional measurement, "Length Laser interferometry, Historical aspects, Data acquisition, Automation, Uncertainty, Accuracy, Stability, Cor rection, Reprints, Laser metrology, US NIST.

The paper describes automatic length scale measure ment by laser interferometry at NIST. It will show the continuous progress in measurement technique, instrumentation, and, consequently, measurement accuracy since 1965 to the present time. The discussion will include the latest improvement in stability, and the new yaw and pitch correction of the subcarriage. Changes in the measurement process including the automatic environmental data acquisition and the interferometer deadpath correction are also described.

\section{2,898}

PB95-108858 Not available NTIS

National Inst. of Standards and Technology (NEL) Gaithersburg, MD. Precision Engineering Div.

Some Considerations for Interim Testing of Coordinate Measuring Machine Performance Using a Specific Artifact.

Final rept.

S. D. Phillips, and B. Borchardt. 1991, 13p

Pub. in Proceedings of the Measurement Science Conference, Anaheim, CA., January 31-February 1, 1991 $13 p$

Keywords: *Tests, Dimensional measurement, Metrology, Errors, Process control, Quality assurance, Reprints, "CMMs(Coordinate Measuring Machines) "Coordinate Measuring Machines, Machine Checking Gage.

The use of a specific artifact, known as the Machine Checking Gage, is examined for use as an interim test of Coordinate Measuring Machine (CMM) perform ance. The results of several tests using this gage on two different CMMs is reported. Suggestions for mak ing the test more robust and informative are described.

\section{2,899}

PB95-108866 Not available NTIS

National Inst. of Standards and Technology (NEL) Gaithersburg, MD. Precision Engineering Div.

Development of an Automated Part Inspection Sys tem Using the DMIS Standard.

Final rept.

S. D. Phillips, D. C. Stieren, and J. G. Salsbury

1990, 10p.

Pub. in Proceedings of the Conference on Precision Metrology with Coordinate Measurement Systems, San Jose, CA., September 18-19, 1990, 10p.

Keywords: *Dimensional measurement, *Inspection, *Automation, Computer aided design, Standards Measuring instruments, Reprints, DMIS(Dimensiona Measuring Interface Specification), CMMs(Coordinate Measuring Machines), US NIST.

The recently approved dimensional measuring interface specification (DMIS) standard is briefly reviewed. An inspection system which incorporates this standard is being developed at the National Institute of Stand ards and Technology (NIST), and the implementation of DMIS is outlined It closes with a discussion of the benefits and limitations of such a system and a summary of the inspection process

\section{2,900}

PB95-125704 Not available NTIS

National Inst. of Standards and Technology (MSEL) Boulder, CO. Materials Reliability Div. 


\section{Quality Control \& Reliability}

High-Sensitivity Acoustic Emission Sensor/Preamplifier Subsystems.

Final rept.

C. M. Fortunko, M. A. Hamstad, and D. W. Fitting.

$1993,17 p$.

Pub. in Review of Progress in Quantitative Nondestructive Evaluation, v12 17p 1993.

Keywords: "Acoustic emission, "Piezoelectric ceramics, "Ultrasonic wave transducers, Failure analysis, Nondestructive tests, Ultrasonics, Acoustic measurement, Preamplifiers, Uiltrasonic tests, Reprints.

An experimental study was conducted to evaluate the suitability of five piezoelectric materials for use in highsensitivity, broadband (10 kHz-2 MHz) acoustic-emission (AE) 'point' sensors. The scope of the study included effects of the material parameters and preamplifier input-impedance characteristics. Computermodel predictions were compared with results of measurements. The NIST Standard Reference Material (SRM) 'conical' transducer was used as a reference high-performance sensor. The experimental study was carried out using 'pinducers' made of: PZT. $5 \mathrm{~A}$ lead metaniobate $X$-cut quartz, 36 deg $Y$-cut LiNbO3, and PVDF it is shown that materials exhibiting the highest dielectric constant are best suited for high-performance AE sensor applications.

\section{2,901}

PB95-136354 PC A03/MF A01

National Inst. of Standards and Technology (MEL), Gaithersburg, MD. Factory Automation Systems Div. Sensitivity of Three-Point Circle Fitting.

T. H. Hopp. Sep 94, 14p, NISTIR-5501.

Keywords: "Inspection, "Metrology, "Curve fitting, Dimensional measurement, Circles (Geometry), Coordinates, Estimating, ${ }^{~} \mathrm{CMMs}$ (Coordinate Measuring $\mathrm{Ma}$ chines), "Coordinate Measuning Machines.

This paper establishes to first order the sensitivity of the center coordinates and radius of a circle through three points in terms of small, random perturbations of those points. This problem arises in the estimation of measurement errors from coordinate measurement systems. Formulas developed herein express the uncertainty of circle parameters as functions of point measurement uncertainty and the arc angle between points. We show that for practical measurement procedures, task uncertainties depend only on the mean and variance of the point measurement errors and are essentially independent of their statistical distribution.

02,902

PB95-140455 Not available NTIS

National Inst. of Standards and Technology (MSEL), Gaithersburg. MD.

Nondestructive Evaluation and Materials Process. ing

Final rept

H. Schwartz. 1991,16p.

Pub. in Review of Progress in Quantitative Nondestructive Evaluation, v10A p35-50 1991.

Keywords: "Nondestructive tests, "Test methods, Ultrasonic tests, Polymers, Process control, Heat treatment, Research, Eddy currents, Fluorescence, Aluminum alloys, Reprints, *Intelligent processing of materials, US NIST.

A brief introduction to this paper will be given describing the relationship of materials science to the industrial challenge facing the United States and the role of the National Institute of Standards and Technology (NIST) in helping to meet this challenge. The main portion of the talk will deal with the important role of Nondestructive Evaluation (NDE) in the processing of materials. NDE research and engineering at NIST aimed at developing sensors for such applications will be dis. cussed and illustrated by the following examples: fluorescent sensors for polymer processing, ultrasonic sensors for high Tc superconductor heat treatment, and eddy current probes for aluminum processing. The focus on the intelligent processing of materials will be illustrated by examples involving high pressure gas atomization and hot isostatic processing of metal powders. Future directions of the NIST program will be presented.

\section{2,903}

PB95-146379 PC A11/MF A03

National Inst. of Standards and Technology (TS), Gaithersburg, MD. Office of Standards Services.
NIST Handbook 44, 1995: Specifications, Tolerances and Other Technical Requirements for Weighing and Measuring Devices as Adopted by the 79th National Conference on Weights and Measures 1994

H. V. Oppermann, and T. G. Butcher. Oct 94, 240p, NIST/HB-44.

Supersedes PB94-136009. Also available from Supt. of Docs. as SN003-003-03298-1.

Keywords: "Weight measurement, "Measuring instruments, "Handbooks, Liquid level indicators, Time measuring instruments, Weight indicators, Dimensional measurement, Tolerances(Mechanics), Liquefied petroleum gases, Water meters, Grains(Food) Cryogenic fluids, Hydrocarbons, Specifications, Containers, Odometers, Flowmeters, Ammonia, Length, Volume, Fabrics, Rope, Wire, Milk, Grain moisture, Taximeters.

Handbook 44 was first published in 1949, having been receded by similar handbooks of various designations and in several forms beginning in 1918. This 1995 edition was developed by the Committee on Specifications and Tolerances of the National Conference on Weights and Measures with the assistance of the of fice of Weights and Measures of the National Institute of Standards and Technology. It includes amendments adopted by the 79th annual meeting of the National Conference on Weights and Measures in 1994. Hand book 44 is published in its entirety each year following the annual meeting of the National Conference on Weights and Measures

\section{2,904}

PB95-151916 Not available NTIS

National Inst. of Standards and Technology (MSEL), Gaithersburg, MD. Matenials Reliability Div.

Ultrasonic Technique for Sizing Voids Using Area Functions.

Final rept.

J. Yang, and L. J. Bond. 1992, 6p.

Sponsored by British Council, London (England).

Pub. in IEE Proceedings-A 139, n2 p45-50 Mar 92.

Keywords: *Voids, "Ultrasonic tests, "Size determination, Dimensional measurement, Nondestructive tests, Approximation, Size(Dimensions), Backscattening, Reprints.

A technique is reported for determining the sizes of voids in structural materials by the inversion of backscattered ultrasonic signals using the area function formula. The formulation of this method is based on the Born approximation which is a weak scattering approximation, but it is shown to work well for voids. Results for extracting the radii of spherical voids, or the tangent plane distances of spheroidal voids are presented, using theoretical and experimental scattering data.

\section{2,905}

PB95-152179 Not available NTIS

National Inst of Standards and Technology (NEL), Gaithersburg. MD. Precision Engineering Div. Automated Optical Roughness Inspection. Final rept.

J. H. Zimmerman, T. V. Vorburger, and H. T. Moncarz. 1989, 13p.

Pub. in Proceedings of Society of Photo-Optical Instrumentation Engineers: Optical Testing and Metrology II, v954 p252-265 1989.

Keywords: "Surface roughness, "Inspection, *Computer aided manufacturing, "Optical measuring instruments, Light scattering, Robots, Robotics, Surface properties, Process control, Controllers, Quality control, Reprints, "Surface Roughness Instrument Controllers.

This paper describes the theory and implementation of automated optical roughness inspection. Our work has been accomplished at the Inspection Workstation IWS) in the Automated Manufacturing Research Facilty (AMRF) at the National Bureau of Standards. The Surface Roughness Instrument Controller (SRIC) supervises the automated optical roughness inspection of parts. This controller is an integrated, data-driven, hierarchical control software system. The SRIC controls two pieces of equipment--the surface roughness instrument (SRI) and the automatic dial indicator (ADI). The SRI is a photo-optical surface roughness inspection device which monitors surface roughness by measuring the angular distribution of light scattered from the surface of a part. Its tasks are coordinated with those of the IWS inspection robot. Using the SRI optical signals as sensory input, the robot properly aligns the part in front of the SR! so that a valid optical scattering reading is obtained. The ADI is used to help the robot to position the part in front of the SRI for its initial reading. The SRIC uses the optical data obtained to estimate values of roughness average and root mean square roughness for parts machined in the AMRF.

02,906

PB95-163077 Not available NTIS

National Inst. of Standards and Technology (NEL), Gaithersburg, MD. Precision Engineering Div.

Automated Inspection: The Integration of National Standards and Commercial Products at NIST.

Final rept.

D. C. Stieren, and S. Phillips. 1991, 7p

Sponsored by Miami Univ., Coral Gables, FL. Dept. of Industrial Engineering.

Pub. in Productivity and Quality Management Frontiers-III, p330-336 1991.

Keywords: "Automated inspection, "Integrated systems, "Dimensional measurement Computer aided design, Computer aided manufacturing, Measuring instruments, Standards, Interfaces, Reprints, "US NIST, Manufactured parts, Coordinate measuring machines, IGES(Initial Graphics Exchange Specification), Postprocessing.

An automated inspection system for manufactured parts is being implemented within the Quality in Automation program at the National Institute of Standards and Technology. The project is a cooperative research effort among NIST and six U.S. industrial firms to correct the traditional problems associated with post-process gauging procedures, and closes the loop for these procedures from the production of a CAD representation of a part to the analysis of inspection results for that part.

02,907

PB95-164455 Not available NTIS

National Inst. of Standards and Technology (MSEL), Gaithersburg, MD. Materials Reliability Div.

X-Ray Image Quality Indicator Designed for Easy Alignment.

Final rept.

A. Siewert, and M. W. Austin. 1992,4p.

Pub. in Matenals Evaluation, p1069-1072 Sep 92.

Keywords: "X-ray inspection, "Weldsd joints, "Image processing *X-ray imager, Image analysis, Quality control, Radiography, Imaging techniques, Nondestructive tests, Reprints, "Image quality indicator.

A new image quality indicator (IQI) design is proposed that is particularly suitable for fundamental measurements of image unsharpness. The design is that of a thin strip, with its larger cross-sectional dimension aligned parallel to the beam at some location along the strip. One end of the strip is given a half twist so some section near the center of the strip is perfectly aligned with the beam, without requining the careful alignment as with some $|Q|$ designs. A strip configuration is apt because it is available for many materials at low cost and in thin dimensions. Our report describes preliminary evaluation of the concept using a steel strip with cross-sectional dimensions of 0.07 by $3 \mathrm{~mm}$.

\section{2,908}

PB95-164463 Not available NTIS

National Inst. of Standards and Technology (MSEL), Gaithersburg, MD. Materials Reliability Div.

Through-the-Arc Sensing for Measuring Gas Metal Arc Weld Quality in Real Time.

Final rept.

T. Siewert, B. Madigan, T. Quinn, and M. Mornis

1992, 4p.

Pub. in Materials Evaluation, p314-318 Nov 92.

Keywords: "Arc welding, "Electrical measurement, "Real time, "Quality control, Drop transfer, Shielding, Wear, Electric current, Voltage, Data acquisition, Monitoring, Inspection, Reprints, "Through-the-arc sensing * Gas metal arc welding.

The voltage drop across the arc in gas metal arc welding is affected by droplet transfer, spatter formation, and many other arc characteristics, and the current is affected in a similar manner. We collected voltage and current records with a high-speed data acquisition system, and correlated features in the records to various welding problems. Interruption in the shielding gas coverage, wear of the contact tube, change in the contacttube-to-work, distance, and change in the droplet 
transfer mode were detected through this sensing strategy. These effects were developed into general correlations and incorporated into a computerized data collection and analysis system.

\section{2,909}

PB95-174470 PC A09/MF AO2

National Inst. of Standards and Technology (TS) Gaithersburg, MD. Weights and Measures Program.

Uniform Laws and Regulations in the Areas of Legal Metrology and Motor Fuel Quality, 1994 as Adopted by the 79th National Conference on Weights and Measures 1994.

Handbook

J. A. Koenig, and K. S. Butcher. Dec 94, 183p, NIST/ HB-130-1995.

Supersedes PB93-125086. Also available from Supt. of Docs. as SN003-003-03308-1.

Keywords: "Weight measurement, "Regulations, *Handbooks, Packaging, Standardization, Revisions Automotive fuels, Consumer affairs, Commodities, La bels, Prices, Food, Sales, Metrology,

The handbook compiles the latest Uniform Laws and Regulations and related interpretations and guidelines adopted by the National Conference on Weights and Measures (NCWM). At the 1983 annual meeting, the NCWM voted to change the title of Handbook 130 and the titles of the Laws and Regulations compiled in the handbook. The edition includes amendments adopted at the annual meetings in 1993 and 1994. The Conference recommends adoption and promulgation by weights and measures jurisdictions of these Uniform Laws and Regulations as updated in the handbook.

02,910

PB95-175451 Not available NTIS

National Inst. of Standards and Technology (MEL) Gaithersburg, MD. Factory Automation Systems Div. Dimensional Inspection Planning Based on Product Data Standards.

Final rept.

S. C. Feng. 1994, 10p

Pub. in Proceedings of International Conference on Concurrent Engineering (1st): Research and Applications, Pittsburgh, PA., August 29-31, 1994, p333-342.

Keywords: "Dimensional measurement, "Inspection, * Standards, Computer aided design, Models, Information processing, Planning, Computer aided manufacturing, Reprints, "DMIS(Dimensional Measuring Interface Standard), "Dimensional Measuring Interface Standard, STEP(Standard for the Exchange of Product Model Data), CMMs(Coordinate Measuring Machines).

This paper describes an activity model for dimensiona inspection planning that bridges the gap between product design and the dimensional measurement of manufactured products. Functionally, this model specifies requirements for developing a part of a product data exchange standard that enables design, inspection resource, and measurement data to be exchanged among computer aided design systems, computer aided process planning systems, and coordinate measuring systems. A set of diagrams has been generated to represent the activity and its sub-activities, inputs, outputs, controls, and mechanisms, when such inspection planning is based on technologies of product data exchange, process planning, and information modeling.

\section{2,911}

PB95-180816 Not available NTIS

National Inst. of Standards and Technology (MEL) Gaithersburg, MD. Precision Engineering Div.

Improving Photomask Linewidth Measurement Accuracy via Emulated Stepper Aerial Image Measurement.

Final rept.

J. E. Potzick. 1994, 8p

Pub. in Society of Photo-Optical Instrumentation Engineers Photomask Technology and Management 10 , n47 p1-8 Sep 94.

Keywords: "Photomasks, "Dimensional measurement, "Line width, Integrated circuits, Uncertainty, Chromium, Accuracy, Reprints.

The most significant contribution to uncertainty in the measurement of photomask linewidths is the rough shape of the edge of the etched chrome lines. This uncertainty can be greatly reduced if the emulated stepper aerial image of the feature is measured instead of its geometric linewidth. That is: measure what the photomask does, not what it is. Phase-shift and other kinds of mask can be measured in the same way.

02,912

PB95-189494 PC A03/MF A01

National Inst. of Standards and Technology (CSL), Gaithersburg, MD.

Proceedings Report of the International Invitation Workshop on Development Assurance. Held in Ellicott City, Maryland on June 16-17, 1994.

P. Toth. Jan 95, 35p, NISTIR-5590.

Keywords: "Quality assurance, "Product development Product inspection, Evaluation, Competition, Models, Methodology, "Developmental assurance, US NIST.

Ninety-eight participants from the US, EC, Canada and Japan representing government agencies, both defense and civil, private corporations, evaluators, users, and vendors of trusted products met to discuss the concept of developmental assurance. The majority of the participants felt that the concept of developmental assurance was valid. The participants recommended that all future developmental assurance work take place on the international level. The developmental assurance concept must first be proven at the lower levels of trust. Developmental assurance may provide a level of assurance that approaches the current $\mathrm{C} 2 / \mathrm{E} 2$. If this is possible, developmental assurance may then be extended to the higher levels of trust. Developmental assurance may not replace third party evaluations completely but may help to speed up the evaluation process. Developmental assurance in combination with third party evaluation may provide useful level of assurance in a more reasonable period of time.

02,913

PB95-209862 PC A03/MF A01

National Inst. of Standards and Technology (MEL). Gaithersburg, MD. Precision Engineering Div.

Design, Specification and Tolerancing of Microm. eter-Tolerance Assemblies.

D. A. Swyt. Mar 95, 27p, NISTIR-5615.

See also PB92-164680

Keywords: "US NIST, "Metrology, "Tolerances(Mechanics), Electronics industry, Auto mobile industry, Specifications, Engines.

Increasing numbers of economically important prod ucts manufactured by U.S. companies are comprise of assemblies of component parts which have macroscopic dimensions and microscopic tolerances. $M$ crometer-tolerance assemblies include not only integrated-circuit devices and mangetic-memory read write heads, but automobile-engine systems, including fuel injectors, hydraulic valve lifters and even piston and cylinder assemblies. Focusing on these, this paper: (1) reports examples of micrometer-tolerance assemblies; (2) discusses strategies in design, specification, and tolerancing (DS\&T) which some companies have adopted for the manufacture of such assemblies; and (3) identifies generic-technology, measure ment, and standards issues in DS\&T associated with that manufacture.

\section{2,914}

\section{PB95-210589 PC A04/MF A01}

National Inst. of Standards and Technology (MEL), Gaithersburg, MD. Precision Engineering Div.

Interim Testing Artifact (ITA): A Performance Eval uation System for Coordinate Measuring Machines (CMMs). User Manual.

A. T. Singer, J. L. Land, S. D. Phillips, G. Caskey, D. Ward, P. Snoots, B. Faust D. Sawyer, and B.

Borchardt. Feb 95, 74p, NISTIR-5602.

Keywords: *Dimensional measurement, *Measuring in struments, *Inspection, "Performance evaluation, Kinematics, Manufacturing, Tests, Pneumatic equipment Components, User manuals, CMMs(Coordinate Meas uring Machines), ITA(Interim Testing Artifact)

Coordinate Measuring Machines (CMMs) have emerged as the primary tool for manufactured part dimensional inspection. However, models which com pletely characterize CMM errors are not currently avail able. The Interim Testing Artifact (ITA) was developed at the National Institute of Standards and Technology (NIST) to help CMM users determine the performance of their CMMs. This document is a user manual which contains the complete instructions for the assembly, use and maintenance of the ITA. National Inst. of Standards and Technology (MEL),
Gaithersburg, MD. Manufacturing Systems Integration Algorithm Testing and Evaluation Program for $\mathrm{Co}$ ordinate Measuring Systems: Long Range PIan. C. Diaz. May 95, 16p, NISTIR-5651.

See also PB94-163029.

Keywords: "Data analysis, "Computer program, "Dimensional measurement, Algorithms, Metrology, Institute of Standards and Technology.

The report is a long range plan for the NIST Algorithm Testing and Evaluation Program (ATEP). ATEP is a Special Test Service provided at NIST through the Office of Measurements Services Calibrations Program. ATEP is a serivce for testing and evaluating data analysis software found in coordinate measuring systems. The purpose of the long range plan is to outline the projected evolution of the service so that the service will evolve systematically.

\section{6}

PB95-251666 PC A03/MF A01

National Inst. of Standards and Technology (MEL) Gaithersburg, MD. Manufacturing Systems Integration

User's Guide for the Algorithm Testing System Version 2.0 .

D. A. Rosenfeld. Jun 95, 44p, NISTIR-5674

See also PB93-175990.

Keywords: "Verification inspection, "Fitting, "Geometry, "Computer program verification, Algorithms, Computer software, Data analysis, Graphical user interface, Input output processing, Online systems, User manuals(Computer programs), ATEP(Algorithm Testing and Evaluation Program), CMS(Coordinate Measuring Systems), US NIST.

The Algorithm Testing System (ATS) is a software system which support the Algorithm Testing and Evaluation Program for Coordinate Measuring Systems Standards and Technology (NIST) special test service for evaluating the performance of CMS geometric fitting software. ATEP-CMS uses the ATS to analyze such software. The ATS typically performs such an analysis by generating data sets, applying its own fitting routines to fit geometries onto the datasets, and comparing its own fit results to the fit results of the software under test for the same data. The ATS provides the above capabilities, and extensive on-line help. The report provides a user's guide for the ATS. It introduces the overall design and functionality of the ATS and guides the user through a number of hands-on tutorials.

\section{2,917}

PB95-255832 PC A03/MF AO

National Inst. of Standards and Technology (MEL), Gaithersburg, MD. Intelligent Systems Div.

Integrated Vision Touch-Probe System for Dimensional Inspection Tasks.

M. Nashman, W. Rippey, T. H. Hong, and M

Herman. Jun 95, 18p, NISTIR-5678.

Keywords: "Verification inspection, "Dimensional measurement, "Computer vision, "Tactile sensors(Robotics), Control systems, Real time operation, Hierarchies, Image processing, CMMs(Coordinate Measuring Machines), NGIS(Next Generation Inspection System).

The paper discusses the integration of vision and touch sensors in a coordinate measuring machine (CMM) controller used for dimensional inspection tasks. A real-time hierarchical control system is presented in which a vision system extracts positions of features. The probe is tracked by the vision system as it scans surfaces so that its motion can be visually served. Minimalist sensor-derived representations, involving only task-specific information, are used in this process. Although the camera itself remains incalibrated, a realtime calibration of very limited scope is performed each processing cycle to transform the task-specific image informaiton into 3-D information as feedback to guide the probe. The ability to integrate vision and touch sensors for CMM tasks promises expanded capabilities for flexible inspection data acquisition.

\section{2,918}

PB95-267928 PC A03/MF AO1

National Inst. of Standards and Technology (MEL), Gaithersburg, MD. Precision Engineering Div. 
Estimation of Measurement Uncertainty of Small Circular Features Measured by CMMs.

S. D. Phillips, B. Borchardt, and W. T. Estler. Aug 95, 16p, NISTIR-5698.

Keywords: "Dimensional measurement, "Accuracy, "Estimating, Circular plates, Algorithms, Metrology, Geometry, Uncertainty, Graphs(Charts), "CMMs(Coordinate Measuring Machines), "Coordinate Measuring Machines.

This paper examines the measurement uncertaingy of small circular features as a function of the sampling strategy, i.e., the number and distribution of measurement points. Specifically, we examine measuring a circular feature using a three-point sampling strategy in which the angular distance between the points varies from widely spaced, 120 degrees, to closely grouped a few degrees. Both theoretical and experimental results show that the measurements uncertainty is a strong function of the sampling strategy. The uncertainty is shown to vary by four orders of magnitude as a function of the angular distribution of the measurement points. A conceptual framework for theoretically estimating the measuring uncertainty is described and a good agreement with experiment is obtained when the measurements are consistent with the assumptions of the theoretical model.

\section{2,919}

PB96-115019 PC A04/MF A01

National Inst. of Standards and Technology, Gaithersburg, MD. Standards Application and Assistance Program.

Standards Setting in the European Union: Standards Organization and Officials in EU Standards Activities.

Special pub

R. A. Rensberger, and R. van de Zande. Sep 95 ,

$74 \mathrm{p}, \mathrm{NIST} / \mathrm{SP}-891$

Also available from Supt. of Docs. as SN003-00303369-3. Prepared in cooperation with European Union, Brussels (Belgium)

Keywords: "European Union, "Standards, ${ }^{*}$ Requirements, Certification, Test facilities, Standardization, Organizations, Specifications, Telecommunication, Electrotechnology, Quality assurance, International trade.

The guide includes a brief history of the role of standards in the European Union and the lastest information on the EU's harmonization directives for implementing the 'New Approach' and the Global Approach for harmonizing technical regulations and standards to reduce barriers to trade. The standards guide also contains information on the three key European standards organizations that are mandated by the EU Commission to draft European technical standards; information on European testing and certification activities; and a list of EU officials with standards-related responsibilities.

\section{2,920}

\section{PB96-123401 Not available NTIS}

National Inst. of Standards and Technology (TS),

Gaithersburg, MD. Metric Program.

Federal Labs Have Key Role in Metrication.

Final rept.

R. Richter. 1995, 2p.

Pub. in NEWSLINK: A Monthly Publication of the Federal Laboratory Corsortium for Technology Transfer (FLC), v11 n5 p2-3 May 95.

Keywords: "Metrication, "Federal agencies, *International trade, Laboratories, Standards, Metric system, Metrology, SI units, International system of units, Exports, Reprints, Market competition.

As international trade becomes more competitive, the slightest disadvantage quickly is translated into lost opportunities. Many U.S. exporters may find themselves at a competitive disadvantage because of the incompatibility between the metric standards used in world markets and many U.S. standards which remain nonmetric.

\section{2,921}

\section{PB96-128236 PC A03/MF A01}

National Inst. of Standards and Technology (MEL), Gaithersburg, MD. Manufacturing Systems Integration Div.

User's Guide to 'SuperFit' Modeling Software for CMM Probe Lobing.

T. H. Hopp. Oct 95, 12p, NISTIR-5720

Keywords: "Dimensional measurement, "Computer programs, Data analysis, Inspection, User manuals,
*CMM(Coordinate Measuring Machines), "Coordinate Measuring Machines, Superfit Modeling Software.

Superfit is a software package developed by the $\mathrm{Na}$ tional Institute of Standards and Technology (NIST) that fits a model of probe lobing to coordinate data obtained by probing a calibration ball. It runs under the Microsoft Windows(TM) 3.1 operating system. This document describes how to use the Superfit software.

02,922

PB96-128244 PC A03/MF A01

National Inst. of Standards and Technology (MEL), Gaithersburg, MD. Manufacturing Systems Integration Div.

Reference Manual for the Algorithm Testing System Version 2.0

D. A. Rosenfeld. Oct 95, 44p, NISTIR-5722.

See also PB95-251666.

Keywords: "User manuals, "Dimensional measurement, "Data analysis, Computer program integrity, Metrology, Coordinates, Algorithms, Computer software tests, Computer program verification, Coordinate measuring systems, Geometric fitting software.

The Algorithm Testing System (ATS) is a software system which supports the Algorithm Testing and Evaluation Program for Coordinate Measuring Systems (ATEP-CMS). ATEP-CMS is a NIST Special Test Service for evaluating the performance of CMS geometric fitting software. The ATS typically performs an analysis by generating data sets, applying its own fitting routines to fit geometrics onto the data sets, and comparing its own fit results to the fit results of the software under test for the same data. This report is a reference manual for the ATS. It documents fully the usuage of the ATS, describes in detail the data generation and analysis capabilities of the ATS, and defines the naming conventions used by the ATS.

\subsection{3}

PB96-155429 Not available NTIS

National Inst. of Standards and Technology (TS), Gaithersburg, MD. Standards in Trade Program.

Metrology and Regional Trade Pacts.

Final rept.

H. Oppermann. 1995, 7p

Pub. in Metrology for the Americas Symposium, Miami, FL., November 6-8, 1995, p1-7.

Keywords: "Dimensional measurements, "Standards, "International trade, Specifications, Metrology, Regulations, Laboratories, Accreditation, Reprints, Trade agreements, Technical barriers.

International investment and trade are driving forces for national economies, building a sound industrial base, and developing jobs. However, as tariffs have decreased, technical barriers have become significant as potential impediments to trade. These technical barriers are often the result of technical regulations (mandatory standards). Some of the major technical barriers to trade stem from product standards, conformity assessment, laboratory accreditation, and demonstrating traceability to national laboratories

\section{2,924}

PB96-160684 Not available NTIS

National Inst. of Standards and Technology (MEL). Gaithersburg, MD. Precision Engineering Div.

New Concepts of Precision Dimensional Measurement for Modern Manufacturing.

Final rept.

D. A. Swyt. $1991,52 p$.

See also PB91-240812

Pub. in Control and Dynamic Systems, v45 p111-162.

Keywords: "Dimensional measurement, "Accuracy "Precision, "Machine tools, Manufacturing, Process control, Integrated systems, Measuring instruments, Tolerance(Mechanics), Specifications, Metrology, Error analysis, Reprints, Coordinate measuring machines.

A hallmark of modern products is the high precision of the dimensions of their functional parts - high, that is, compared to that of their less-modern contemporaries. Modern products of this century are video cassette recorders, the precision of whose parts allows read-write heads to be aerodynamically flown over the recording medium at misrcoscopic altitudes.

02,925

PB96-160759 Not available NTIS
National Inst. of Standards and Technology (TS), Gaithersburg, MD. Office of Technology Information. Metrology

J. J. Ulbrecht. 1991, 1p

Pub. in Academic Press Dictionary of Science, $1 p$ Fall 91.

Keywords: "Metrology, Units of measurement, Weight, Standardization, Reprints.

Metrology is the field of knowledge concerned with measurement. While all scientific disciplines impact on human life in one way or another, the interaction with weigts and measures runs like a continuous line through everybody's life.

02,926

PB96-166616 PC A13/MF A03

National Inst. of Standards and Technology Gaithersburg, MD. Office of Weights and Measures.

NIST Handbook 44, 1996: Specifications, Tolerances, and Other Technical Requirements for Weighing and Measuring Devices as Adopted by the 80th National Conference on Weights and Measures, 1995.

T. G. Butcher, C. Cotsoradis, and T. L. Grimes, Jan $96,252 p$

Supersedes PB95-146379.

Keywords: "Weight measurement, "Measuring instruments, "Handbooks, Metrology, Liquid level indicators Time measuring instruments, Weight indicators, Dimensional measurement, 'Tolerances(Mechanics) Liquified petroleum gases, Water meters, Grains(Food), Cryogenic fluids, Hydrocarbons, Fue tanks, Specifications, Containers, Odometers, Flowmeters, Ammonia, Length, Volume, Grain moisture, Taximeters.

Handbook 44 was first published in 1949, having preceded by similar handbooks of various designations and several forms, beginning in 1918. This 1996 edition was developed by the Committee on Specifications and Tolerances of the National Conference on Weights and Measures, with the assistance of the Office of Weights and Measures of the National Institute of Standards and Technology (NIST). It includes amendments adopted by the 80th annual meeting of the National Conference on Weights and Measures in 1995. Handbook 44 is published in its entirety each year following the annual meeting of the National Conference on Weights and Measures.

02,927

PB96-172309 PC A12/MF A03

National Inst. of Standards and Technology (TS) Gaithersburg, MD. Weights and Measures Program. Uniform Laws and Regulations in the Areas of Legal Metrology and Motor Fuel Quality as Adopted by the 80th National Conference on Weights and Measures 1995. 1996 Edition.

Handbook

J. A. Koenig, K. S. Butcher, and T. L. Grimes. Jan $96,231 p$, NIST/HB-130-1996.

Supersedes 1995 Edition, PB95-174470. Also available from Supt. of Docs. as SN003-003-03391-0.

Keywords: "US NIST, "Weight measurement, "Law(Jurisprudence), Regulations, Handbooks, Automotive fuels, Consumer affairs. Packaging, Standardization, Commodities, Price, Labels, Food, Sales.

This handbook compiles the latest Uniform Laws and Regulations and related interpretations and guidelines adopted by the National Conference on Weights and Measures (NCWM). At the 1983 Annual Meeting, the NCWM voted to change the title of Handbook 130 and the titles of the Laws and Regulations compiled in this handbook. The former title of the handbook was "Model State Laws and Regulations.' 'Model State' was changed to 'Uniform' in the titles to reflect that these Laws and Regulations are (1) intended to be standards rather than just guidelines, and (2) intended for adoption by political subdivisions other than States when deemed appropriate. The compilation itself was approved by the NCWM in 1979. This edition includes amendments adopted at the Annual Meeting in 1995.

02,928

PB97-110423 Not available NTIS

National Inst. of Standards and Technology (MEL), Gaithersburg, MD. Precision Engineering Div. 
Present and Future Standard Specimens for Surface Finish Metrology.

Final rept.

T. V. Vorburger, J. F. Song, E. Marx, B. R. Scace,

and T. R. Lettieri. 1992, 4p.

Pub. in International Symposium on Optical Fabrication Testing and Surface Evaluation, Society of PhotoOptical Instrumentation Engineers, v1720 p78-81 Feb 92.

Keywords: "Surface profiling, "Metrology, Bidirectional reflectance, Roughness average, Sinusoidal, Spatial wavelength, Reprints, "Standard reference materials.

The accurate measurement of surface finish requires standard specimens to calibrate and check the operation of the measuring instruments. This is true both for profiling techniques such as the stylus and area averaging techniques such as light scattering. For profiling stylus instruments, the international standard ISO 5436 has enumerated four types of standard specimens which may be classified as step height specimens, periodic roughness specimens, random roughness specimens, and specimens for checking probe tip resolution. A draft of a related U.S. standard uses the same taxonomy. For light scattering instruments, the use of standard specimens are discussed in the ASTM standard for total integrated scatter. In addition, several ad hoc standard specimens have been used in an interlaboratory comparison of instruments that measure the bidirectional reflectance distribution function (BRDF). The group has developed a series of specimens, known as Standard Reference Materials (SRMs), for calibrating stylus profiling instruments. The authors are also in the process of developing a series of profile specimens for calibrating the linearity of BRDF instruments. Both types of specimens have sinusoidal profiles and are discussed in turn.

\section{Research Program Administration \& Technology Transfer}

02,929

PB94-211844 Not available NTIS

National Inst. of Standards and Technology, Gaithersburg, MD. Office of the Director.

Importance of Measurement in Technology-Based Competition.

Final rept.

H. Hellwig. 1990, 4p.

Pub. in Institute of Electrical and Electronics Engineers Transactions on Instrumentation and Measurement 39 , n5 p685-688 1990.

Keywords: "Measurement, "Manufacturing "Technology innovation, "Quality assurance, Metrology, Measuring instruments, Production, Productivity Online systems, Reprints.

In this paper, measurement challenges are related to the changes taking place in emerging technologies, quality and production. The act of measurement is increasingly associated with the process rather than the product. As a result, the role of measurement is becoming proactive and anticipatory. Measurements are increasingly done on-line and at the place of need within the production process. Measurement problems must be addressed early in the research and development phase; and the purpose of measurement must be seen as assuring good product at all times as opposed to the traditional role of measurement which was focussed on eliminating bad products.

\section{2,930}

\section{PB95-206181 PC A22/MF A04}

National Inst. of Standards and Technology (MEL), Gaithersburg, MD.

Proceedings of the Manufacturing Technology Needs and Issues: Establishing National Priorities and Strategies Conference. Held in Gaithersburg, Maryland on April 26-28, 1994.

Special pub.

C. F. Albus, and J. D. Meyer. Feb 95, 510p, NIST/ SP-877.

Also available from Supt. of Docs. as $\mathrm{SNOOH}^{-003-}$ $03320-1$. Sponsored by Department of Defense, Washington, DC.

Keywords: "Meetings, "Manufacturing, "Technology assessment, *Government policies, Governmentin dustry relations, Priorities, User needs, Industries,
Competition, Govemment agencies, Programs, Technology innovation, Small businesses.

The conference addressed the many challenges and opportunities confronting manufacturers. Presentations were made by representatives from a host of manufacturing industries, each of which has been trying to chart its course for the future by developing a 'road map' of technology directions and needs. The conference was a unique opportunity to examine the spectrum of manufacturing industries, identify the commonalities and differences, and start assessing the value of road maps. The conference also provided a glimpse of the full spectrum of government programs in manufacturing technology. The ultimate goal was to develop a plan for moving forward to assure that the United States maintains a strong, dynamic manufacturing sector that is globally competitive and capable of meeting national challenges. Among other themes, the papers reflect growing government collaboration with industry, increasing cooperation within and among federal agencies, expanded technical agendas in government, and experimentation with innovative government programs.

\section{2,931}

PB96-109491 PC A11/MF A03

National Inst. of Standards and Technology (MEL), Gaithersburg, MD

Joint DoD/NIST Workshop on International Manufacturing Systems Research and Development. Held in Rockville, Maryland on November 3-5, 1992. Proceedings.

Special pub.

J. D. Meyer. Oct $95,244 p$, NIST/SP-873.

Also available from Supt of Docs as SN003-00303344-8. Sponsored by Assistant Secretary of Defense (Production and Logistics), Washington, DC

Keywords: "Manufacturing, "Research and development, "Meetings, US NIST, Computer aided manufacturing, Competition, North America, Europe, Asia, International cooperation, Rockville(Maryland), $\mathrm{CIM}$ (Computer Integrated Manufacturing)

An international workshop was held in Rockville, Maryland, on November 3-5, 1992 to discuss major re search and development programs in manufacturing systems technology. In addition to individual R\&D programs and technology sources in North America, Western Europe, Eastern Europe, and Asia, the workshop participants also discussed R\&D needs, priorities, underlying motivations, and opportunities for international collaboration. Approximately 25 leading experts attended the workshop, which was co-sponsored by the U.S. Department of Defense's Manufacturing Technology Program.

\section{Robotics/Robots}

\section{2,932}

PB94-172046 Not available NTIS

National Inst. of Standards and Technology (NEL), Gaithersburg, MD. Robot Systems Div.

Toward a Reference Model Architecture for RealTime Intelligent Control Systems (ARTICS)

Final rept.

J. S. Albus, and R. Quintero. 1990, $8 p$

Pub. in Proceedings of International Symposium on Robotics and Manufacturing (3rd): Research, Education, and Applications, British Columbia, Canada, July 18-20, 1990, p243-250.

Keywords: "Control systems, "Robotics, "Real time systems, Artificial intelligence, Automation, Technology innovation, Technology transfer, Government/ industry relations, Computer architecture, Models, Systems engineering, Reprints, ARTICS(Architecture for Real-Time Intelligent Control Systems).

The paper presents a concept for the development of a reference model open-system architecture for realtime, sensory interactive, intelligent machine systems. Central to the notion is a desire to accelerate technological development, technology transfer and commercialization of world class control system products in the field of robotics, intelligent machines, and automation. A plan is outlined whereby a reference model Architecture for Real-Time Intelligent Control Systems (ARTICS) can be defined through the cooperative efforts of industry, academia, and government. As envisioned, ARTICS would be a series of evolving guide- lines specifying an infrastructure of hardware components, software components, interfaces, communications protocols and application development tools. An ARTICS reference model would make it possible fo industry to develop and market a diverse line of control system components which could be interchangeable and realizable on many different vendors' intelligent machine systems platforms.

\section{3}

PB94-173010 Not available NTIS

National Inst. of Standards and Technology (MEL) Gaithersburg, MD. Robot Systems Div.

World Model Registration for Effective Off-Line Programming of Robots.

Final rept.

N. Tarnoff, A. Jacoff, and R. Lumia. 1990, 8p

Pub. in Proceedings of International Symposium on Robotics and Manufacturing (3rd): Research, Education, and Applications, British Columbia, Canada, July 18-20, 1990, p783-790.

Keywords: "Robots, "Computer programming "Manufacturing, Trajectories, Robot dynamics, Contro systems, Off line systems, Calibrating, US NIST, Reprints, "World models, AMRF(Automated Manufacturing Research Facility).

The paper discusses progress in world model calibration and environmental modeling of sensory information in the context of the Off-Line Programming (OLP) project at the National Institute of Standards and Technology's (NIST) Automated Manufacturing Research Facility (AMRF). The project began in 1988 to demonstrate an integrated OLP implementation. The baseline OLP system accepts IGES CAD data, enables graphical simulation of objects and devices and produces VAL II robot trajectories. The robot trajectories, however, are based on a world model that is inherently inaccurate. A basic OLP system and most existing commercial OLP systems, therefore, are more effective only as a prototyping tool. Consequently, the authors are now developing the new OLP system into a production tool for generating robust robot control programs. The authors recently completed work on world model calibration and environmental modeling of force and torque in an attempt to improve world model registration of the real world.

\section{2,934}

PB94-213162 Not available NTIS

National Inst. of Standards and Technology (NEL) Gaithersburg, MD. Robot Systems Div.

Hierarchical Ada Robot Programming System (HARPS): A Complete and Working Telerobot Control System Based on the NASREM Model.

Final rept.

$S$. Leake, T Green, S Cofer and T Sauenwein.

1989, $7 \mathrm{p}$.

Pub. in Proceedings of Institute of Electrical and Elec tronics Engineers International Conference on Robotics and Automation, Scottsdale, AZ., May 14-19, 1989 , p1022-1028.

Keywords: "Telerobotics, "Control systems, Real time Robots, Robot sensors, Teleoperators, Computer vision, Software engineering, Software tools, Reprints, "NASREM(NASA Standard Reference Model) "NASA Standard Reference Model, Ada programming language, HARPS (Hierarchical Ada Robot Programming System), US NIST.

The Hierarchical Ada Robot Programming System (HARPS) is a telerobot control system which can perform some simple but useful tasks: this capability is demonstrated by performing an ORU exchange demo. HARPS is based on the National Institute of Standards and Technology (NIST) NASA Standard Reference Model (NASREM) model. The primary programming language for all developed software is Ada, and the project incorporated a number of different Computer Aided Software Engineering (CASE) development tools. NASREM was found to be a valid and usefu model for building a telerobot control system: Its hierarchical and distributed structure creates a very natural and logical flow for developing and implementing large. complex and robust control systems. Similarly, the ability of Ada to create and enforce abstraction was found to enhance the implementation of such a control system. The CASE tools utilized showed some promise in helping to design a large system which processed a tremendous amount of data involving very complex computations and relationships. An overview of NASREM, the ORU exchange demo the HARPS system, and the development tools used in HARPS is given in this paper. 
02,935

PB94-217098 PC A05/MF A01

National Inst. of Standards and Technology (MEL),

Gaithersburg, MD. Intelligent Systems Div.

Publications of the Intelligent Systems Division (Previously Robot Systems Division) Covering the Period January 1971-April 1994.

D. L. F. Russell. Jul 94, 80p, NISTIR-5474

Keywords: "Automation, "Robotics, "Artificial intelligence, "Bibliographies, Computer aided manufacturing, Robot dynamics, Control systems, Real time Interactive systems, Software engineering, Construction, Mining, Documents, AMRF(Automated Manufacturing Research Facility), US NIST, MEL(Manufacturing Engineering Laboratory), Intelligent Systems Division.

This document consists of a list of publications by the staff of the Intelligent Systems Division, Manufacturing Engineering Laboratory (MEL), National Institute of Standards and Technology (NIST) for the period of January 1971 through April 1994. Publications cover research done by the division in the areas of intelligent machines and systems, robotics, real-time sensory interactive control, open-systems architectures, software development techniques, world modeling, construction, mining and automated manufacturing systems.

\section{2,936}

PB95-164133 Not available NTIS

National Inst. of Standards and Technology (NEL) Gaithersburg, MD. Robot Systems Div.

Integrated Mobile Robot System for Testing Vision Algorithms.

Final rept.

H. H. Yakali, D. Raviv, and M. Herman. 1990, 8p. Pub. in Proceedings of Conference on Recent Ad vances in Robotics (3rd), Boca Raton, FL., July 2-3, $1990,8 p$.

Keywords: "Robot dynamics, "Computer vision, Robots, Robotics, Algorithms, Robot sensors, Real time, Image processing, Workstations, Tests, Reprints.

We describe an integrated real-time vision-based closed-loop system which will serve as a platform for testing vision-based navigation algorithms. The system consists of several subsystems: a charge coupled device (CCD) camera, a Pipelined Image Processing Engine (PIPE) real-time image processor, a SUN3/160 workstation and a DENNING mobile robot. We detail each of the subsystems, and the communications between them. Finally, we describe an algorithm used to test the system and discuss future work.

\section{2,937}

\section{PB95-198677 PC A03/MF AO}

National Inst. of Standards and Technology (MEL), Gaithersburg, MD. Intelligent Systems Div.

Real-Time Obstacle Avoidance Using Central Flow Divergence and Peripheral Flow.

D. Coombs, M. Herman, T. Hong, and M. Nashman. Feb 95, 22p, NISTIR-5605.

Keywords: "Robot dynamics, "Obstacle avoidance, "Computer vision, Real time, Robotics, Navigation Image processing, Flow visualization, Control systems, PIPE(Pipelined Image Processing Engine).

The lure of using motion vision as a fundamental element in the perception of space drives this effort to use flow features as the sole cues for robot mobility. Realtime estimates of image flow and flow divergence provide the robot's sense of space. The robot steers down a conceptual corridor, comparing left and right peripheral flows. Large central flow divergence warns the robot of impending collisions at 'dead ends.' When this occurs, the robot turns around and resumes wandering. Behavior is generated by directly using flow-based information in the 2-D image sequence; no 3-D reconstruction is attempted. Active mechanical gaze stabilization simplifies the visual interpretation problems by reducing camera rotation. By combining corridor following and dead-end deflection, the robot has wandered around the lab at $30 \mathrm{~cm} / \mathrm{s}$ for as long as $20 \mathrm{~min}-$ utes without collision. The ability to support this behav ior in real-time with current equipment promises ex panded capabilities as computational power increases in the future.

\section{2,938}

PB95-242350 PC A09/MF A02

National Inst. of Standards and Technology (MEL) Gaithersburg, MD. Intelligent Systems Div.
Unified Telerobotic Architecture Project (UTAP) Standard Interface Environment (SIE), May 1995. J. Michaloski, R. Russell, T. E. Wheatley, S. Lee, R
D. Steele, R. Lumia, and P. G. Backes. May 95, 179p, NISTIR-5658.

Prepared in cooperation with Jet Propulsion Lab., Pasadena, CA. and New Mexico Univ., Albuquerque. Sponsored by Air Force Systems Command, Kelly AFB, TX. Robotics and Automation Center of Excellence.

Keywords: "Artificial intelligence, "Robot control, "Robotics, "Teleoperators, Computer architecture, Standards, Interfaces, Terminology, Definitions, Robot sensors, Draft standard.

The Unified Telerobotic Architecture Project (UTAP) open architecture is intended for operator-supervised, teleoperated and shared control robotic maintenance tasks. The basic premise is to augment, not replace, the human operator by blending the individual skills of each into a system - the human's superior cognition and pattern recognition, and the robot's tireless, precise, and repeatable, motion. Further, many of the tasks are unsafe and hazardous. This document describes the UTAP architecture and a standard environment and Application Programming Interface for the architecture. The document presents a discussion of the Interface Framework including the reasoning process applied to the interface definition. It also presents a UTAP configuration and classification strategy. It describes the UTAP information model used for representing the interface data. The UTAP interfaces, including the format, types, syntax, semantics and significant features of operation are presented. Appendix I describes the architecture and module functionality. Appendix II summarizes the UTAP interface messages, and Appendix III contains specific definitions for each of the UTAP interfaces. Appendix IV contains profiles for the Remote modules in the system. Appendix $V$ discusses a sample scenario for a refurbishing task, and then presents an overview of the message traffic.

\section{2,939}

PB96-154562 PC A03/MF A01

National Inst. of Standards and Technology (MEL), Gaithersburg, MD. Intelligent Systems Div.

Image Gradient Evolution: A Visual Cue for Danger.

H. Liu, T. H. Hong, M. Herman, and R. Chellappa.

Oct $95,19 p$, NISTIR-5728.

Prepared in cooperation with Maryland Univ., College Park. Center for Automation Research.

Keywords: "Computer vision, "Position sensing, *Collision avoidance, Autonomous navigation, Robot dynamics, Robot sensors, Motion perception. Three dimensional motion, Optical flow(Image analysis), Image processing, Divergence, Image analysis, Artificial intelligence, "Image gradient.

This paper is concerned with the task of visual motionbased navigation. A critical requirement of the task is the ability to estimate 3-D depth and motion from visual information. We present a new concept called image gradient evolution (IGE), which utilizes the change of image spatial gradients over time as a threat cue; an approaching object induces 2-D expanding motion and causes the image spatial structure to stretch so the image gradients decrease. Based on this idea, our method offers a one-step solution directly from image gradients, instead of from optical flow and its derived properties.

\section{2,940}

PB96-183173 PC A06/MF A01

National Inst. of Standards and Technology (MEL), Gaithersburg, MD. Intelligent Systems Div.

Scale-Space-Based Visual-Motion-Cue for Autonomous Navigation.

S. R. Kundur, D. Raviv, and E. Kent. Feb 96, 96p,

NISTIR-5790.

Grant NSF-IRI-9115939

Prepared in cooperation with Florida Atlantic Univ. Boca Raton. Sponsored by National Science Foundation, Arlington, VA. Div, of Information, Robotics and Intelligent Systems

Keywords: "Computer vision, "Autonomous navigation, "Robotics, Collision avoidance, Image analysis, Three dimensional bodies, Artificial intelligence, Image processing. Algorithms, Cameras, Visual perception, Computer motion.

This paper presents a new visual motion cue, the authors call the Hybrid Visual Threat Cue (HVCT) that provides some measure for a change in relative range as well as absolute clearances, between a 3D surface and a fixated observer in motion. The visual field associated with the HVTC can be used to demarcate the regions around a moving observer into safe and danger zones of varying degree, which may be suitable or autonomous navigation tasks, in particular collision avoidance and maintenance of clearance. The HVTC is independent of the 3D environment and needs almost no a-priori information about it. It is rotation independent, and is measured in (time(sup -1)) units. When there is a relative motion between a fixation point on a $3 \mathrm{D}$ surface and an observer, the perceived texture details the image vary. The rate at which the details vary provides an indication of the observer's relative motion with respect to the 3D surface. Scale space representation which is a multiscale approach provides a concrete way to analyze the variations of image details The authors derive a relation between the relative temporal variations of the image inner scale and the HVTC. A practical method to extract the HVTC from a sequence of images of a 3D textured surface obtained by a fixated, fixed-focus monocular camera in motion is also presented. A global dissimilarity meas ure is extracted directly from the raw data of the gray level of textured images from which the HVTC is obtained. This approach of extracting the HVTC is independent of the type of 3D surface texture and needs no optical flow information, 3D reconstruction, segmentation, feature tracking. It needs almost no camera calibration. This algorithm to extract the HVTC was applied to a set of twelve different texture patterns (of 3D scenes) from the Brodatz's album, where the authors observed a similar behavior for most of the textures.

\section{2,94}

PB96-193727 PC A05/MF A01

Florida Atlantic Univ., Boca Raton

Novel Active-Vision-Based Motion Cues for Local Navigation.

S. R. Kundur, and D. Raviv. Feb 96, 65p, NISTIR-

5791.

Grant NSF-IRI-9115939

Sponsored by National Inst. of Standards and Technology (MEL), Gaithersburg, MD. Intelligent Systems Div. and National Science Foundation, Arlington, VA Div. of Information, Robotics and Intelligent Systems. Keywords: "Robots, "Autonomous navigation, *Computer vision, *Collision avoidance, Sensors, Controllers, Cameras, Image processing, Visual perception, Real time operation.

In the absence of a-priori information about the information an autonomous mobile robot relies on sensory information to make local judgments about its surrounding. Generation of a local collision-free path based on sensory data plays an important role in the control of the robot's motion. The paper presents a novel active vision-based approach for generating local collision-free paths for mobile robot navigation in indoor as well as outdoor environments. Two measurable visual motion cues that provide some measure for a relative change in range as well clearance between 3D surface and fixated observed in motion are described. These visual cues are independent of the 3D environment and need no a-priori knowledge about it. For each visual motion cue, there is a visual field surrounding the moving observer. In other words, there are imaginary 3D surfaces attached to the observer that move with it, each of which correspond to a value of the cue. These visual fields can be used to demarcate regions around a moving observer into safe and danger zones of varying degree to make local decisions about the sterring as well as speed commands to the mobile robot. We describe a practical method to extract these cues from a sequence of images. This approach needs no feature tracking between images and almost no camera calibration.

\section{Tooling, Machinery, \& Tools}

\section{2,942}

PB94-135621 PC A03/MF A01

National Inst. of Standards and Technology, Gaithersburg, MD

Open Architectures for Machine Control.

F. M. Proctor, B. Damazo, C. Yang, and S. Frechette. Dec 93, 20p, NISTIR-5307.

Keywords: "Machine tools, "Controllers, "Standards, Interactive graphics, Man machine systems, Error cor- 
recting devices, Modular structures, Extensibility, Workstations, Computer architecture, National Institute of Standards and Technology, EMC(Enhanced Machine Controller), NURBS(Non-Uniform Rational BSplines).

A major impediment to improving the performance and functionality of machine tools is the limited access that users or third parties have to the internals of the machine controller. This limit has suppressed the emergence of a community of third-party vendors who could provide a wide range of applications at competitive prices. The result is that users are often faced with allor-nothing compromises when choosing controllers, and are restricted to the original controls vendors for everything from spare parts to software. Machine tool users would benefit from an open architecture that can serve as both a target for innovative third party product development, and as a specification which produces multiple competitive sources for interoperable products. The National Institute of Standards and Technology has initiated a project which will demonstrate the feasibility of open architectures for machine control. This project, the Enhanced Machine Controller (EMC), has selected several target applications which improve the accuracy and ease of use of machine tool controllers: selectable look-and-feel, tool management, alternate part programming languages, splinebased motion, on-machine inspection, and thermalgeometric error compensation.

\section{2,943}

PB94-186673 PC A07/MF A02

National Inst. of Standards and Technology, Gaithersburg, MD

Prediction of Geometric-Thermal Machine Tool Errors by Artificial Neural Networks.

D. E. Gilsinn, and M. A. Donmez. Apr 94, 149p,

NISTIR-5367.

Sponsored by Naval Research Lab., Washington, DC Navy Manufacturing Technology Program.

Keywords: "Machine tools, "Error correction codes, *Neural nets, Conjugate gradient method, Optimization, Cutting machines(Tools), Algorithms, Artificial neural networks, Fortran programming language, Gradient descent method.

In machining operations, the precision of the workpiece dimensions depends on the accuracy of the relative position of the cutting tool and the workpiece. Among the key factors that affect the accuracy of this relative position are the geometric errors of the machine tool and the thermal effects on these geometric errors. Recent work on developing models to predict volumetric errors on NC lathes has led to a synthesis technique that combines the modeling of individual axis related geometric-thermal components by way of a rigid body kinematic model to produce predicted errors in the work volume of the machine. An alternative method of modeling these component errors is described in this report. Neural network computing is shown to be a viable technique for developing mappings between ma. chine tool component error measurements and the vector consisting of both a component slide position and the temperature state of the machine as reported by the thermal sensors. The conjugate gradient algorithm, used to compute the optimum neural network weights for the machine tool error components, is described. A case study of the mapping results for one component error of an actual NC lathe is given. Finally, the source codes for the neural network algorithm and the conjugate gradient algorithm are given in FORTRAN.

\section{2,944 \\ PB94-199858 Not available NTIS}

National Inst. of Standards and Technology (NEL), Gaithersburg, MD. Precision Engineering Div.

Computer Vision Based Tool Setting Station.

Final rept.

T. D. Doiron. $1989,12 \mathrm{p}$.

Contract DE-Al05-85OR21584

Pub. in Proceedings of VISION '89 Conference, Chicago, IL., April 24-27, 1989, p8/61-8/72. Sponsored by Department of Energy, Washington, DC

Keywords: "Computer vision, "Tools, "Metrology, Calibrating, Edge detection, Cameras, Reprints.

In order to use a computer vision system for high accuracy gaging, the intensity array reported to the computer from the camera must correspond closely to the geometry of the part to be measured. To verify this correspondence for two different vision systems, a number of tests are reported and discussed. A number of effects due to the camera sensor geometry, the type of edge finder employed, the thermal properties of the camera, and the interface method used between the camera and computer are explored.

\section{2,945}

PB95-107231 Not available NTIS

National Inst. of Standards and Technology (NEL) Gaithersburg, MD. Automated Production Technology Div.

Real Time Compensation for Tool Form Errors in Turning Using Computer Vision.

Final rept.

G. Nobel, M. Alkan Donmez, and R. Burton. 1990,

Pub. in Proceedings of Society of Photo-Optical Instrumentation Engineers - Dimensional Stability, San Diego, CA., July 12-13, 1990, v1335 p186-193.

Keywords: "Turning(Machining), "Errors, "Compute vision, Real time, Cutting tools, Algorithms, Numerical control, Inspection, Reprints, CMMs(Coordinate Measuring Machines).

Deviations from the circular shape of the cutting edge of a single-point turning tool cause form errors in the workpiece during contour cutting. One can compensate for these tool-form errors by determining the size of the effective deviation at a particular instant during cutting, and then adjusting the position of the cutting tool accordingly. An algorithm for the compensation of tool-nose-radius errors in real time has been developed and implemented on a CNC turning center. A previously developed computer-vision-based tool-in spection system is used to determine the size of the deviations. Information from this system is fed to the error compensation computer which modifies the too path in real time. Workpieces were cut utilizing the compensation system and were inspected on a coordinate measuring machine. Significant improvements in workpiece form were obtained.

02,946

PB95-152088 Not available NTIS

National Inst. of Standards and Technology (NEL), Gaithersburg, MD. Precision Engineering Div.

Displacement Method for Machine Geometry Calibration.

Final rept.

G. Zhang. R. Ouyang, B. Lu, A. Donmez, B.

Borchardt, R. Capparelii, R. Hocken, and R. Veale.

1988, 17p.

Pub. in ClRP Annals 38, n1 17p 1988.

Keywords: "Displacement measurement, "Machine tools, "Calibration, Three dimensional, Error analysis, Metrology, Interferometry, Reprints, "Coordinate measuring machines.

It is shown that the volumetric errors of a three-axis machine tool or measuring machine can be determined by measuring the displacement error along 22 lines in the machine work zone. Pitch and yaw are derived from measuring the displacement error on two parallel ines for each axis. The squareness and straightness errors are determined by measuring two diagonals and one additional line Roll errors are derived from the straightness errors on two parallel planes. Formulae for calculating these components of error and also the three-dimensional positioning error at any particular point are given. Experiments done at both Tianjin and NBS show that this method simplifies machine calibration, reduces the equipment needed, and can improve accuracy.

\section{2,947}

PB95-154720 PC A07/MF A02

National Inst. of Standards and Technology (MSEL) Gaithersburg, MD. Metallurgy Div.

Review and Upgrading of Military Fastener Test Standard MIL-STD-1312.

S. R. Low. Sep 94, 147p, NISTIR-5524.

Contract MIPR-2-0117

Also available from Supt. of Docs. Sponsored by Defense Industrial Supply Center, Philadelphia, PA

Keywords: "Test methods, "Military standards, "Fasteners, Durability, Fastenings, Tensile strength Mechanical properties, Military equipment, Shea stresses, Tension, Fatigue tests(Mechanics), "MILSTD-1312.

The National Institute of Standards and Technology (NIST) has conducted a study of United States Military Standard MIL-STD-1312, Fastener Test Methods. Military Standard MIL-STD-1312 is a unified compilation of over 35 individual fastener test methods for determining the capability of fasteners to withstand various environmental and mechanical conditions encountered in service. Each of the individual fastener test methods is itself a fastener test standard, and is designated with its own document number. For this study, only selected test methods which prescribe certain mechanical tests for fasteners were reviewed. These test methods were evaluated and recommendations formulated for upgrading or replacing them to reflect the current stateof-the-art.

\section{2,948}

PB95-168902 Not available NTIS

National Inst of Standards and Technology (CSTL)

Boulder, CO. Process Measurements Div.

Design Equations and Scaling Laws for Linear Compressors with Flexure Springs.

Final rept.

22p.

Sponsored by National Aeronautics and Space Administration, Moffett Field, CA. Ames Research Center Pub. in Proceedings of International Cryocooler Conference (7th), Santa Fe, NM., November 17-19, 1992. p783-804 1993

Keywords: "Scaling laws, "Design criteria, "Refrigerant compressors, Seals(Stoppers), Electric motors, Mathematical models, Springs, Refrigerating machinery, Refrigerators, Reprints, "Flexural springs.

Linear-resonant compressors with flexure springs and clearance seals have recently been developed for use in long-life Stirling and pulse tube refrigerators. This paper describes a set of equations that are used to design the various components in the compressor given specified performance criteria. The components considered are the moving-coil linear motor, the mass of the moving components, the magnet assembly, the flexure springs, and the clearance seals. Both radially and axially magnetized magnets are analyzed and the criteria for selection are developed. Methods for reducing the compressor size and mass are discussed as well as the influence of the stroke-to-diameter ratio on the design of flexure springs. The design equations have allowed the development of scaling laws for linear motor compressors covering a wide range of compressor sizes from 3 watts to 4 kilowatts.

02,949

PB95-169207 Not available NTIS

National Inst. of Standards and Technology (CSTL)

Boulder, CO. Process Measurements Div.

Comparing NIST.B $50 \mathrm{~mm}$ Orifice Meter Gas Data to the ANSI Equation.

Final rept.

J. L. Scott. $1993,7 p$

Pub. in Proceedings of Fluid Engineering Conference Fluid Measurement and Instrumentation Forum, Washington, DC., June 20-24, 1993, p13-19.

Keywords: * $O$ rifice meters, "Flowmeters, "Flow distortion, Orifice flow, Flow measurement, Reynolds number, Discharge coefficient, Orifices, Reprints.

A revised equation for computing discharge coefficients in orifice meters has been adopted by the American National Standards Institute (ANSI). The empirical data base used to develop this equation did not include gaseous fluid measurements for the $50 \mathrm{~mm}$ orifice meter. New experimental data that extend the Reynolds number range of the data base have been generated using nitrogen gas in this meter size. These new data are compared to the discharge coefficients calculated from the equation and to the original, lower Reynolds number data. The comparisons are beta ratio dependent, but, in general, the comparisons between the new data and the original data are quite good for all the beta ratios tested. Work was sponsored by Gas Research Institute (GRI)

\section{2,950}

PB95-203287 Not available NTIS

National Inst. of Standards and Technology (MEL), Gaithersburg, MD. Precision Engineering Div.

Geometric Characterization of Rockwell Diamond Indenters.

Final rept.

J. F. Song, F. F. Rudder, T. V. Vorburger, J. H.

Smith A. W. Hartman, and B. R. Scace. 1994, 6p

Pub. in Proceedings of IMEKO World Congress (13th) 'From Measurement to Innovation', Torino, Italy, September 5-9, 1994, v1 p779-784. 
Keywords: "Calibration, Rockwell hardness, Uncertainty, Pens, Microform, Reprints, "Diamond indenters, Surface finish.

By using a stylus instrument, a series of calibration and check standards, and calibration and uncertainty calculation procedures, we have calibrated Rockwell diamond indenters with traceability to fundamental measurements. The combined measurement uncertainties $(95 \%)$ of the calibration are less than $1 / 10$ of the tolerance requirements of calibration grade Rockwell dia. mond indenters. Our combined measurement uncertainties are: + or -0.4 micrometer in least squares radius, + or $-0.01 \mathrm{deg}$ in cone angle, and + or -0.023 deg in holder axis alignment. The profile deviation from least squares radius and cone flank straightness is also calibrated. Engineering features of the diamond indenter's surface finish, such as surface roughness, and tlat or sharp-shaped spherical tip are explored and quantified.

\section{2,951 \\ PB95-203295 Not available NTIS}

National Inst. of Standards and Technology (MEL) Gaithersburg, MD. Precision Engineering Div. Microform Calibrations in Surface Metrology. Final rept.

J. F. Song, F. F. Rudder, T. V. Vorburger, J. H. Smith, A. W. Hartman, and B. R. Scace. 1995, 10p Pub. in Int. Jnl. Mach. Tools Manufact. 35, n2 p301 3101995.

Keywords: "Microform, "Calibration, Rockwell hardness, Measurement, Uncertainty, Pens, Reprints "Surface metrology, Diamond indenters, Surface texture.

Microform calibrations include the measurement of complex profile forms and position errors of micrometer scale in combination with the measurement of deviations from specified profile and surface texture of profile segments. Tolerances on the profile form are specified and may correspond geometrically to surface texture parameters. One example of microform calibration is the calibration of Rockwell diamond indenters used for hardness testing of materials. Previously reported measurement techniques do not meet the stringent microform calibration requirements for Rockwell diamond indenters. Inadequate microform calibration of hardness indenters may be one factor resulting in significant interlaboratory differences in results from Rockwell hardness tests. By using a stylus instrument in combination with a series of calibration and check standards and calibration and measurement uncertainty calculation procedures, we have calibrated Rockwell diamond indenters in accordance with the definitions specified in ISO and ASTM standards. Our procedures for conducting microform calibration yield total measurement uncertainties less than ten percent of the tolerance values specified in ISO and ASTM standards. In this paper, the general calibration requirements, calibration and check standards, and calibration and uncertainty procedures that we use in performing microform calibrations are introduced. Some general considerations on stylus radius correction, data fitting, calibration traceability, uncertainty and reproducibility are also discussed.

\section{2,952}

PB95-203451 Not available NTIS

National Inst. of Standards and Technology, Gaithersburg, MD. Program Office.

Post-Process Control of Machine Tools.

Final rept.

T. V. Vorburger, K. W. Yee, B. R. Scace, and F. F

Rudder. 1994, 15p

Pub. in Manufacturing Review 7, n3 p252-266 Sep 94

Keywords: "Machine tools, "Control, "Automation, Quality assurance, Real time, Error detection codes, Process control, Reprints, US NIST, QIA(Quality in Automation), Post-process.

The automated control of machine-tool accuracy is discussed based on a quality architecture that is being implemented at the National Institute of Standards and Technology (NIST) under the Quality in Automation (QIA) project. The quality architecture contains three control loops: real-time, process-intermittent, and postprocess. This paper highlights the post-process loop, although the OIA project emphasizes the measure. ment and control of process variables to control the quality of the product, post-process dimensional measurements of the finished product play a role in the sys tem and may be used for correction of certain variables. The strategy for the post-process loop includes classification schemes for the adjustable parameters in the system, for the error diagnostics, and for the error model of the machine tool.

\section{2,953}

PB95-242301 PC A04/MF A01

National Inst. of Standards and Technology (MEL), Gaithersburg, MD. Automated Production Technology Div.

Precision in Machining: Research Challenges.

H. A. Soons, and S. L. Yaniv. May 95, 57p, NISTIR5628

Keywords: "Machining, "Technology innovation, *Precision, Machine tools, Errors, Accuracy, Closed loop systems, Metrology, Production control, Manufacturing, Tolerances(Mechanics), Research and development.

This report looks at critical research needs for leadingedge technology developments in machining and precision that are important to the competitiveness of the US discrete-part industry. Key driving forces in manufacturing are identified. These include the increased demand for higher speed, greater flexibility, greater precision, automation, advanced materials, and the emergence of new processes. Infrastructural developments required for improvements in and control of manufacturing precision are discussed. Research challenges in machine tool characterization, machine tool performance enhancement, closed-loop precision manufacturing, process modeling, high speed machining and costs of precision are identified.

\section{2,954}

PB96-111695 Not available NTIS

National Inst. of Standards and Technology (MEL), Gaithersburg, MD. Precision Engineering Div. Fabrication of Optics by Diamond Turning.

Final rept.

R. L. Rhorer, and C. J. Evans. 1995, 13p.

Pub. in Handbook of Optics, Chapter 41, p41.1-41.13 1995.

Keywords: "Optics, "Fabrication, "Diamonds, "Tools, Wear, Technology utilizing, Cost effectiveness, Cutting tools, Reprints, "Diamond turning.

Single point diamond turning is a cost effective method of fabricating aspheric and specialty optics. Tool wear is a major limit for certain materials. The range of applications of diamond turning, and the factors to consider in selecting an optical fabrication strategy are reviewed.

\section{2,955}

PB96-135363 Not available NTIS

National Inst. of Standards and Technology (CAML), Gaithersburg, MD. Statistical Engineering Div.

Proposed Changes to Charpy V-Notch Machine Certification Requirements.

Final rept

J. D. Splett, and J. C. M. Wang. 1995, 13p.

Pub. in Symposium on Pendulum Impact Machines: Procedures and Specimens for Verification, Montreal, Quebec, Canada, May 18-19, 1994, p182-194 May 95.

Keywords: "Certification programs, Reprints, Impact testing, Requirements, "Charpy $V$ Notch machines, *Pendulum impact machines, Notched bar testing.

In 1989 the administration of the Charpy V-Notch Certification Program was assumed by the National Institute of Standards and Technology. The United States Army originated the program to insure the measurement integrity of Charpy $V$-notch machines across the country. The program has been operated for many years using candidate machine acceptance limits which can possibly be traced to a 1955 paper by Driscoll, however, the original statistical justification for using these acceptance criteria has been lost or never existed. A statistical analysis of recent certification program data indicates that the existing candidate machine acceptance limits should be modified. In this paper, the authors will discuss and justify potential changes to candidate machine acceptance limits.

\section{2,956}

PB96-141189 Not available NTIS

National Inst. of Standards and Technology (EEEL),

Boulder, CO. Electromagnetic Technology Div.
Effect of Magnetic Field Orientation on the Critical Current of HTS Conductor and Coils.

Final rept.

J. P. Voccio, A. J. Rodenbush, C. H. Joshi, J. W.

Ekin, and S. L. Bray. 1995, 4p

Pub. in Institute of Electrical and Electronics Engineers Transactions on Applied Superconductivity, v5 n2 p1822-1825 Jun 95

Keywords: "High-Tc superconductors, "Magnetic fields, Magnet coils, Reprints, Conductors, Silver, Temperature, "Critical current.

The critical current of short samples of HTS multifilamentary conductor and ring-shaped coils has been measured at helium temperatures with varying magnetic field orientation with respect to the conductor The samples and coil conductor consist of a multifilamentary composite of BSCCO-2223 filaments in a silver matrix. Short conductor samples were tested in a variable temperature system with up to $8 \mathrm{~T}$ background field using a sample rotational system. Ringshaped coils made from the sample type of conductor were exposed to a large background field at liquid helium temperatures and critical current was measured with the ring located at various axial positions within the bore.

\section{2,957}

PB96-155551 Not available NTIS

National Inst. of Standards and Technology (MEL), Gaithersburg, MD. Precision Engineering Div.

Metrology Approach to Unifying Rockwell C Hardness Scales.

Final rept.

J. F. Song, J. H. Smith, and T. V. Vorburger. 1995 ,

13p.

Pub. in Proceedings of the International Symposium on Hardness Testing in Theory and Practice (9th), Dusseldorf, Germany, November 23-24, 1995, p19-31.

Keywords: "Rockwell hardness, "Calibration, Microforms, Etching, Diamond pyramid hardness tests, Indentation hardness tests, Verification, Metrology, Uncertainty, Reference standards, Reprints, "Diamond indenters, Traceability.

Current Rockwell C hardness scales (HRC) are unified by performance comparisons. Unless a reliable metrology approach is used for the direct verification of standard hardness machines and diamond indenters, the unified hardness scale may exhibit a systematic offset from the true value of the harness scale. Based on the recently developed NIST microform calibration system, a metrology approach is suggested to unify Rockwell $C$ hardness scales. The true value and reference-value scales are defined. It is proposed to use the reference standards, including standard hardness machines and NIST standard-grade Rockwell diamond indenters, to create, maintain, and reproduce the reference-value scale for the metrology-based Rockwel hardness standard system, and to overcome the systematic offset in existing performance-based hardness scales.

\section{2,958}

PB96-155569 Not available NTIS

National Inst. of Standards and Technology (MEL), Gaithersburg, MD. Precision Engineering Div.

Stylus Technique for the

Final rept.

J. F. Song, F. F. Rudder, T. V. Vorburger, and J. H Smith. 1995, 10p.

Pub. in Proceedings of the International Symposium on Hardness Testing in Theory and Practice (9th), Dusseldorf, Germany, November 23-24, 1995, p129-138.

Keywords: "Rockwell hardness, "Calibration, "Pens, Etching, Microforms, Diamond pyramid hardness tests, Indentation hardness tests, Metrology, Verification Uncertainty, Reference standards, Reprints, "Diamond indenters.

Based on a stylus technique, a microform calibration system was developed at NIST for the direct verification of Rockwell diamond indenters. The least-squares radius and profile deviations, cone angle and cone flank straightness, and the holder axis alignment erro can be calibrated with traceability to fundamental measurements. The calibration requirements, instrument setup, calibration and check standards, calibration and uncertainty procedure, and the calibration results are described. Based on this calibration system, the use of the NIST standard-grade Rockwell diamond indenters is also suggested for the establishment of the 
reference-value scale for a metrology-based Rockwell hardness standard system and for the unification of Rockwell hardness standards.

\section{2,959}

\section{PB96-160569 Not available NTIS}

National Inst. of Standards and Technology (MEL), Gaithersburg, MD. Automated Production Technology Div.

Integrated Inspection System for Improved Machine Performance.

Final rept.

J. Mou, and M. A. Donmez. 1993, 10p.

Pub. in Proceedings of Society of Photo-Optical Instrumentation Engineers, Vision, Sensors, and Control for Automated Manufacturing Systems, Boston, MA., September 9-10, 1993, v2063 p22-31.

Keywords: "Machine tools, "Displacement measurement, *Inspection, "Precision, Computerized control systems, Process control, Accuracy, Quality control, Error analysis, Calibration, Data analysis, Algorithms, Reprints, CNC(Computer Numerical Control)

An integrated inspection system for improving the accuracy of CNC machine tools is proposed. The sysem described in this paper emphasizes the integration of the on-machine inspection and analysis techniques with the information coming from post-process inspection. The proposed system utilizes the information from both post-process and on-machine inspection to improve machine performance automatically. Algorithms are derived for analyzing the post-process and on-machine inspection data to identify residual systematic errors and relate them to the machine performance. Various data analysis algorithms and techniques are compared. A feature comparison approach is developed to relate the dimensional and form errors of a manufactured workpiece to the systematic machine tool errors. Inverse kinematics technique and statistical methods are used to identify and characterize the contribution of each geometric error component. A self tuning algorithm is also proposed to fine tune the geometric-thermal model.

02,960

PB97-113898 PC A05/MF A01

National Inst. of Standards and Technology (MEL), Gaithersburg, MD. Precision Engineering Div.

Static Structural Analysis of a Reconfigurable Rigid Platform Supported by Elastic Legs.

F. F. Rudder. Jun 96, 62p, NISTIR-5885.

Keywords: "Machine tools, "Foundations, Platforms, Joints(Junctions) Supports,

Positioning devices(Machinery), Metrology.

The report describes a static structural analysis of a rigid planar platform supported by elastic legs. The platform deformations are computed relative to a specified platform position and orientation. The analysis considers deformations due to static platform loads, non-uniform temperatures of the legs, and manufacturing errors of each leg length. The model was developed as a tool to assist in the interpretation of metrology data and the development of an error budget for the NIST Octahedral Hexapod Milling Machine.

02,961

PB97-116206 PC A03/MF A01

National Inst. of Standards and Technology (MEL), Gaithersburg, MD. Office of Manufacturing Programs. Standards Promote Credibility and Technology Transfer: The Need for

C. D. Lovett. Oct 96, 13p, NISTIR-5899.

Keywords: "Technology transfer, "Standards, "Machining, Machine tools, International trade, Competition, Technical organizations, International Standards Organization.

The intent of this paper is to call aftention to the need for greater industry participation in both national and intemational standards committees. This need is described by giving an overview of U.S. standardization by the voluntary consensus-based process and describing some of the benefits of participating in standards-developing committees. The U.S. National Machining Center Standards Committee is cited as an example of a voluntary consensus-based process, involving cooperation among industry, government and academia.

\section{Tribology}

02,962

PB94-172731 Not available NTIS

National Inst. of Standards and Technology (MSEL), Gaithersburg, MD. Ceramics Div.

Considerations on Data Requirements for Tribological Modeling.

Final rept.

A. W. Ruff. 1991, 16p.

Pub. in Tribological Modeling for Mechanical Designers, ASTM STP 1105, p127-142 1991

Keywords: "Tribology, "Data bases, Mechanical engineering, Lubrication, Test methods, Friction, Wear, Materials, Models, Design, Reprints.

An idealized database for tribology is described tha contains numenc data with characteristics needed for use in modeling approaches in tribology. Tribologica data on materials are considered in detail, and othe types of required data are identified. Testing methodologies for obtaining data are examined. Priority areas for future work in data gathering and data evaluation in tribology are suggested.

\section{2,963}

PB94-211018 Not available NTIS

National Inst. of Standards and Technology (MSEL). Gaithersburg, MD. Ceramics Div.

Tribological Characteristics of Alpha-Alumina at Elevated Temperatures.

Final rept.

X. Dong, S. Jahanmir, and S. M. Hsu. 1991, 9p. Pub. in Jnl. of American Ceramic Society $74, \mathrm{n} 5$ p1036-10441991.

Keywords: "Aluminum oxide, " Tribology, "Mechanica properties, High temperature tests, Friction, Performance evaluation, Wear, Ceramics, Reprints.

The tribological characteristics of alpha-alumina sliding on a similar material under dry sliding conditions are divided into four distinct regimes. At low temperatures, $\mathrm{T}<200 \mathrm{C}$, tribochemical reactions between alumina surface and water vapor in the environment control the tribological performance. At temperatures above 800 $\mathrm{C}$, formation of silicon oxide layers on the wear track, by viscous flow and diffusion of the grain boundary phase, reduces the coefficient of friction to 0.4 ; and the wear coefficient is reduced to a value less than .000001 . The results of the wear tests and observations of the fundamental mechanisms controlling the tribological behavior of this material are consolidated in a simple wear transition diagram. The wear transition diagram can be used for the identification of wear mechanisms and the transition boundaries.

\section{2,964}

PB94-212057 Not available NTIS

National Inst. of Standards and Technology (MSEL), Gaithersburg, MD. Ceramics Div.

Tribochemical Reaction of Stearic Acid on Copper Surface Studied by Surface Enhanced Raman Spectroscopy.

Final rept.

Z. S. Hu, S. M. Hsu, and P. S. Wang. $1992,6 \mathrm{p}$

Pub. in Tribology Transactions 35, n3 p417-422 1992

Keywords: "Tribology, "Lubricants, *Copper, "Stearic acid, "Surface chemistry, Chemical reactions, Raman spectroscopy, Chemisorption, Wear, Reprints, Cupric stearate, Hexadecane.

Micro-Raman, back-scattered by Ar ion laser, was used to study the tribochemical reaction of copper surface and hexadecane containing 0.4 weight percent stearic acid. The characteristic Raman signals of cupric stearate at $1,547,623,288$, and 243 wavenumbers were detected during the reaction and the formation of cupric stearate confirmed. The tribochemical reaction was initiated by the formation of a chemisorbed stearic acid on the native oxide film of the copper surface. This chemisorbed stearic acid is in the form of monomer and is approximately perpendicular to the copper surface. The native oxide film is necessary for this chemisorption to occur. Furthe wearing of the surface converts the chemisorbed stearic acid into cupric stearate.

\section{5}

PB94-212362 Not available NTIS

National Inst. of Standards and Technology (MSEL), Gaithersburg, MD. Ceramics Div.
Tribology Education: Present Status and Future Challenges.

Final rept.

S. Jahanmir, and F. E. Kennedy. 1991, 3p

Pub. in Jnl. of Tribology 113, n2 p229-231 1991.

Keywords: "Tribology, "Education, "Mechanical engineering, "Lubrication, Recommendations, Surveys, Forecasting, Friction, Wear, Reprints, *Research Committee on Tribology, American Society of Mechanical Engineering.

The ASME-Research Committee on Tribology has been concerned that some of today's engineering students may not be learning enough about fundamentals of tribology. A survey of engineering schools was conducted to assess the current status of tribology education at universities. The survey results were presented and discussed at a panel session on Tribology Education. The panel consisted of an invited group of distinguished tribologists representing academia, industry, and federal government. A set of recommendations was prepared based on the presentations and the discussions that followed. Since only a small fraction of engineering colleges offer any treatments on tribology in their curricula, it is recommended that tribology fundamentals be incorporated in the course offerings to prepare the engineering students for future advanced technical developments.

\section{2,966}

PB95-164158 Not available NTIS

National Inst. of Standards and Technology (MSEL) Gaithersburg, MD. Ceramics Div.

Asperity-Asperity Contact Mechanisms Simulated by a Two-Ball Collision Apparatus.

Final rept.

T. N. Ying, and S. M. Hsu. 1993, 9p.

Pub. in Wear 169, p33-41 1993.

Keywords: "Wear, "Test methods, "Friction, Wear resistance, Interfaces, Friction measurement, Wea tests, Tribology, Reprints, *Two-ball collision test apparatus, "Asperity.

Wear of materials is controlled by asperity-asperity contacts. Since the asperities are of uneven height, the stresses imposed by different pairs of asperities necessarily are unequal. When two surfaces come to gether, there is a distribution of the contact stresses. In a wear experiment, we measure wear by measuring the cumulative wear across the interface from the beginning of the experiment, and we measure friction by measuring the average friction of all the asperity con tacts across the interface at the particular instance in time. The fnction and wear characteristics at the asperity level have interested the tribology community for a long time. Data at that level, however, are difficult to obtain and the conditions that lead to a single asperity wear event are not understood. This paper describes the design and construction of a two-ball collision test apparatus to simulate asperity contacts. Materials used include steels, brass, and aluminum of different hardness and elasticity. Dynamic friction and wear of the collision were measured under dry, paraffin oil lu bricated conditions. The results suggested that at a coefficient of 0.4 , wear of the asperity contact occurred. A simple plain strain model confirmed the observations that under certain conditions, asperity wear should occur at a coefficient of 0.39 .

02,967

PB95-180949 Not available NTIS

National Inst. of Standards and Technology (MSEL)

Boulder, CO. Materials Reliability Div.

Tribometer for Measurements in Hostile Environ ments.

Final rep

A. J. Slifka, D. K. Chaudhuri, R. Compos, and J. D. Siegwarth. 1993, 6p.

Sponsored by National Aeronautics and Space Administration, Huntsville, AL. George C. Marshall Space Flight Center.

Pub. in Wear 170, p39-44 1993

Keywords: "Friction measurement, "Wear tests, Coefficient of friction, Friction, Tribology, Test facilities, Measuring instruments, Wear, Reprints, "Tribometers,

A tribometer at the National Institute of Standards and Technology in Boulder, Colorado is used to measure the coefficient of friction and the wear rate for various specimens in a controlled atmosphere of oxygen or non-corrosive gas. The wide range of demonstrated operating temperature of 80 to $1030 \mathrm{~K}$ is presently un avallable in any commercial apparatus. The machine 


\section{Tribology}

use ball-on-flat or ring-on-flat specimen geometries for comparison of conforming and non-conforming contacts. The apparatus is described and some test results are compared with known values.

\section{MATERIALS SCIENCES}

\section{General}

\section{2,968}

AD-A301 675/5 PC A14/MF A03

National Bureau of Standards, Boulder, $\mathrm{CO}$.

Weatherability of Plastic Materials.

M. R. Kamal. Feb 67, 306p

Proceedings on Weatherability of Plastic Materials, Applied Polymer Symposia, Number 4, Gaithersburg, MD 8-9 Feb 67

Keywords: "Polymers, "Plastics, "Weatherproofing, *Weathering *Meetings, Crystal structure, Ultraviolet radiation, Weather, Aging(Materials), Degradation, Exposure, Laminates, Polystyrene, Atmospheric temperature, Copolymers, Embrittlement, Glass, Light sources, Rain, Oxidation, Radiation absorption, Light transmission, Photochemical reactions, Pigments, ACcelerated testing, Elongation, Carbon black, Reinforcing materials, Humidity, Polyester plastics, Polyethylene plastics, Polyvinyl chloride, Sunlight, Plastic coatings, Radiant intensity, Durability, Photooxidation.

The growth of the plastics industry in recent years has been accompanied with a significant expansion in the outdoor application of plastic materials.

\section{9}

PB94-162534 PC A05/MF A01

National Inst. of Standards and Technology (MSEL) Gaithersburg, MD.

Materials Science and Engineering Laboratory Annual Report, 1993. NAS-NRC Assessment Panel, April 21-22, 1994.

Rept. for 1 Oct 92-30 Sep 93

L. H. Schwartz, and H. L. Rook. Apr 94, 86p,

NISTIR-5311

See also PB94-112430.

Keywords: *Technology innovation, Research and development, Personnel, Technology transfer Laboratories, Research management, Research projects, Reviews, "Materials Science and Engineering Laboratory, "MSEL, National Institute of Standards and Technology.

The Report contains background information on resources, activities, and representative highlights of the Materials Science and Engineering Laboratory (MSEL). The report includes a summary of the strategic plan of MSEL and the technical areas proposed for future emphasis.

\section{2,970}

PB94-172939 Not available NTIS

National Inst. of Standards and Technology (MSEL), Gaithersburg, MD

Industry and Government-Laboratory Cooperative R and D: An Idea Whose Time Has Come.

Final rept.

L. H. Schwartz. Sep 90, 2p.

Pub. in MRS Bulletin 15, n9 p4-5 Sep 90

Keywords: "Materials, "Research and development "Cooperation, Government/industry relations, Joint ventures, Technology innovation, Technology transfer Reprints, CRADAS(Cooperative Res earch and Development Agreements).

The article explores the role that Federal Laboratories can play in addressing the area of materials processing via joint technology development with industry. The discussion includes a brief description of the Technology Transfer Act of 1988 and the mechanism of Cooperative Research and Development Agreements (CRADAS) created therein. These CRADAS are an ideal vehicle with which to pursue such joint technology development.
02,971

PB94-198884 Not available NTIS

National Inst. of Standards and Technology (MSEL), Gaithersburg, MD. Polymers Div.

Diffraction Imaging of Polycrystalline Materials.

Final rept

D. R. Black, H. E. Burdette, M. Kuriyama, and R. D. Spal. $1991,8 p$

Pub. in Jnl. of Materials Research 6, n7 p1469-1476 1991.

Keywords: "X-ray diffraction, "Imaging techniques, Residual stress, Crystal defects, Grain size, Polycrystalline, Strains, Shape, Reprints, “Diffraction imaging, Industrial materials.

For the characterization of industrial materials, a new diffraction imaging technique is proposed and applied to obtain direct information about individual grains and their size and shape distributions and, in turn, strains in materials. Unlike traditional powder diffractometry, the highly parallel and monochromatic beam available from a synchrotron $x$-ray source is employed to observe and measure diffraction images from individual grains and component particles in consolidated materials prepared by various processes. Unlike traditional diffractometry, this new technique provides the ability to measure shape, size and strain without model based analyses. The spatial distribution of strain within individual grains, displayed as a diffraction image (topograph), indicates the presence of defects, such as dislocations, subgrain boundaries and precipitates, and sheds new light on the origins of residual strains (stresses) in industrial materials. The resolution of the imaging system used is limited to particles 1 micrometer or larger due to diffraction broadening and the resolution of the recording medium.

\section{2}

PB94-200227 Not available NTIS

National Inst. of Standards and Technology (MSEL), Gaithersburg, MD. Ceramics Div.

Crack Growth Resistance of Strain-Softening Materials under Flexural Loading.

Final rept.

T. J. Chuang. 1993, $4 p$

Pub. in Proceedings of American Society of Civil Eng neers Structure Congress, Indianapolis, IN., April 29, 1991, p466-469.

Keywords: "Crack propagation, "Flexural properties, "Strain(Mechanics) "Brittle materials, Loads(Forces) Flexural strength. Mechanical properties, Toughness, Deformation, Reprints, Subcritical crack growth.

The subcritical crack growth resistance of a bend bar is investigated for a group of brittle materials exhibiting strain-softening behavior. Based on a nonlinear constitutive law ascribed to the process zone ahead of the crack tip in a strain gradient field, the evolution of the process zone size, crack length and applied load is predicted for a given deformation history. The results showed that the crack growth resistance or toughness increases to a peak then decays precipitously to zero as the crack grows towards the compressive edge in qualitative agreement with experimental data. The fac that the $K$ value, in general, never reaches a plateau suggests that a bend bar geometry is not suitable for K(sub IC) measurement.

\section{2,973}

PB94-218567 PC A04/MF A01

National Inst. of Standards and Technology (MSEL), Gaithersburg, MD. Metallurgy Div.

NIST Workshop on Nanostructured Material (1st): Report of an Industrial Workshop Conducted by the National Institute of Standards and Technology. Held in Gaithersburg, Maryland on May 14 15,1992 .

Internal rept.

R. D. Shull. Mar 94, 56p, NISTIR-5456.

Keywords: "Meetings, Particle size, Composite materials, Information transfer, Synthesis(Chemistry), Industries, Phase studies, Physical properties, Thermodynamics, Mechanical properties, Corrosion, Processing, "Nanomaterials, "Nanostructures, "Advanced materials, Nanocomposites.

This workshop, the 'First NIST Workshop on Nanostructured Materials, 'was the first of a series of workshops on the synthesis, characterization, properties, and applications of nanostructured materials to be held every three years at NIST The workshop was organized to bring together people from industry, fed- eral agencies, and universities to: (1) expedite the flow of information in the field, (2) identify key synthesis and processing methods with scalability potential, (3) acquaint the various communities with the unique properties of nanostructured materials, (4) assist industry in identifying potential application areas, and (5) identify needed areas of study. The workshop also was planned because of the recognition that in order for the U.S. to take advantage of the promise these materials hold, a concerted effort in this area is needed early. The workshop could highlight this need and provide directions for a concerted effort. Finally, it was felt that the workshop would provide NIST with additional guidance in directing its own programmatic initiative in the area.

02,974

PB95-150108 Not available NTIS

National Inst. of Standards and Technology (MSEL) Gaithersburg, MD. Metallurgy Div.

Method for Determining Both Magnetostriction and Elastic Modulus by Ferromagnetic Resonance.

Final rept.

R. D. McMichael. 1994, 3p.

Pub. in Jnl. of Applied Physics 75, n10 p5650-5652, 15 May 94

Keywords: "Modulus of elasticity, "Ferromagnetic resonance, "Magnetostriction, Ferromagnetic materials, Elastic properties, Bending, Substrates, Ferromag netism, Strains, Anisotrophy, Nickel, Polymers, Magnetic properties, Reprints.

The magnetostriction constant lambda(sub s) includes a combination of both elastic constants C(sub ij) and magnetoelastic coupling constants $\mathrm{B}($ sub $\mathrm{i}$ ). To provide information on both C(sub ij) and B(sub i), a method for measurement of both lambda(sub s) and the elastic modulus $Y$ of thin films has been developed using ferromagnetic resonance (FMR). As in prior FMR schemes for measuring lambda(sub s), a strain is produced in the film by bending the substrate, and the resulting anisotropy field is measured. However, by using a triangular polymer beam as a substrate, three important advantages are obtained (1) the triangular beam shape has uniform strain along its surface, (2) the polymer material supports large strains $(>10(\exp -3)$ ) without breaking, and (3) the low elastic modulus of the beam allows measurement of the sample elastic modu'us through variation of the sample aspect ratio. Measurements on Ni foils yield values of lambda(sub s) and $Y$ which fall within the scatter of literature data.

02,975

PB95-162525 Not available NTIS

National Inst. of Standards and Technology (NML) Gaithersburg, MD. Standard Reference Data.

Access Paths for Materials Databases: Approaches for Large Databases and Systems.

Final rept.

J. Rumble. 1991, 10p.

Pub. in Computerization and Networking of Materials Databases: Second Volume, ASTM STP 1106, p1321411991.

Keywords: *Materials, *Databases, *Information retrieval, Information systems, Man computer interfaces Graphical user interface, User requirements, User needs, Query languages, Reprints, Access paths, Search strategies.

Materials databases can contain widely differing data records in terms of the size and number of data elements, depending on the types of data. Acceptance of materials databases depends on user interfaces containing search strategies that will find answers to queries as needed by engineers and scientists. The pape will outline typical access paths (search strategies) from three points of view: first, simple and complex matching; second, specification of multiple criteria; and third, search refinement.

02,976

PB95-196697 PC A06/MF A02

National Inst. of Standards and Technology (MSEL), Gaithersburg, MD.

Materials Science and Engineering Laboratory Annual Report, 1994. NAS-NRC Assessment Panel, April 6-7, 1995.

Rept. for 1 Oct 93-30 Sep 94

L. H. Schwartz. Apr 95, 103p, NISTIR-5577. See also PB94-162534.

Keywords: "Research and development, Research management, Technology innovation, Research 
projects, Organizational structure, Laboratonies, Resources, Technology transfer, "US NIST, tory), "Materials Science and Engineering Laboratory.

The report contains background information on the vision, mission, priority-setting process, implementation and organization of The National Institute of Standards and Technology's (NIST's) Materials Science and Engineering Laboratory (MSEL), as well as accomplishments and impacts, resources, activities, and technology transfer for the Laboratory.

02,977

PB95-267985 PC A05/MF A01

National Inst. of Standards and Technology (TS) Gaithersburg, MD. National Voluntary Lab. Accreditation Program.

National Voluntary Laboratory Accreditation Program: Thermal Insulation Materials.

Handbook.

L. I. Knab. May 95, 80p, NIST/HB-150-15.

Also available from Supt. of Docs. as $\mathrm{SNON3}^{-003-}$ 03354-5. See also PB88-152541.

Keywords: "Thermal insulation, "Insulating materials, "Laboratones, Materials testing, Test methods, Requirements, Certification, Procedures, Calibration standards, Standardization, Equipment, Management, Auditing, Personnel, NVLAP(National Voluntary Laboratory Accreditation Program).

NIST Handbook 150-15 presents the technical requirements of the National Voluntary Laboratory Accreditation Program (NVLAP) for Thermal Insulation Matenals. It is intended for information and use by staff of accredited laboratories, those laboratories seeking accreditation, other laboratory accreditation systems users of laboratory services, and others needing information on the requirements for accreditation under the Thermal Insulation Materials program.

\section{2,978}

PB96-112677 PC A11/MF A03

National Inst. of Standards and Technology (MSEL), Gaithersburg, MD.

Glimpse of Materials Research in China: A Report from an Interagency Study Team on Materials Visiting China from June 19, 1995 to June 30, 1995.

Special pub.

S. M. Hsu, and L. H. Schwartz. Sep 95, 233p, NIST/ SP-893.

Portions of text in Chinese. Also available from Supt of Docs. as SN003-003-03370-7.

Keywords: "Materials, "Research programs, "China International cooperation, Research and development, Electronics, Polymers, Electrooptics, Semiconductor devices, Ceramics, Tribology, Universities, "Foreign technology.

During the period June 16, 1995 to June 30, 1995, an interagency delegation of materials scientists from the Department of Commerce (DOC), Department of Energy (DOE), National Aeronautical and Space Administration (NASA), and the National Science Foundation (NSF) visited China and Hong Kong to explore areas for possible cooperation in materials research between the two countries under the general science and technology agreement. Recognizing the daunting task of assessing the state-of-the-art in a nation with so vas a scientific and academic structure as China, the study team's itinerary was organized to split into four smalle teams which began together in Beijing, spread out to visit separate locations and then met again in Shanghai. The visit ended at the Hong Kong University of Science and Technology (HKUST) which graciously provided facilities for the drafting of this report. The itineraries of these four teams (Appendix E) allowed for visits to 30 institutions in a period of a week and one half. Reports describing each of these visits are assembled in Appendix $F$, and should be referred to for detailed description.

\section{2,979}

PB96-119243 Not available NTIS

National Inst. of Standards and Technology (MSEL) Gaithersburg, MD.

Evolution Equations for Phase Separation and Ordering in Binary Alloys.

Final rept.

J. W. Cahn, and A. Novick-Cohen. 1994, 33p.

Pub. in Jnl. of Statistical Physics, v76 n3/4 p877-909 1994.
Keywords: "Alloys, "Phase separation, "Phase ordering, Reprints, Equations, Binary alloys, Allen-Cahn equations, Cahn-Hilliard equations.

The authors explore two phenomenological approaches leading to systems of coupled Cahn-Hilliard and Cahn-Allen equations for describing the dynamics of systems which can undergo first-order phase separation and order-disorder transitions simulateneously, starting from the same discrete lattice free energy function. In the first approach, a quasicontinuum limit is taken for this discrete energy and the evolution of the system is then assumed to be given by gradient flow. In the second approach, a discrete set of gradient flow evolution equations is derived for the lattice dynamics and a quasicontinuum limit is then taken. The authors demonstrate in the context of BCC Fe-Al binary alloys that it is important that variables be chosen that accommodate the variations in the average concentration as well as the underlying ordered structure of the possible coexistent phases. Only then will the two approaches lead to roughly the same continuum descriptions.

\section{2,980}

PB96-119698 Not available NTIS

National Inst. of Standards and Technology (MSEL), Gaithersburg, MD. Reactor Radiation Div.

Neutron Techniques in Materials Science and Related Disciplines.

Final rept.

J. M. Rowe, and H. J. Prask. 1995, 14p.

Pub. in Proceedings of Society of Photo-Optical Instrumentation Engineers: Neutrons and Their Applications, Crete, Greece, June 12-18, 1994, v2339 p304-317 1995.

Keywords: "Materials science, "Neutron activation analysis, Neutrons, Reprints, Depth profiling, Diffraction, Inelastic scattering, Reflectometry, *Foreign technology.

Thermal and cold neutron probes provide researchers in such fields as materials science, physics, chemistry, and biology information that often can be obtained by no other means. The main focus of this paper will be on recent studies at NIST which illustrate how thermal and cold neutrons are utilized for materials research.

\section{2,981}

PB96-136981 PC A08/MF A02

National Inst. of Standards and Technology (MSEL), Gaithersburg, MD. Metallurgy Div.

Metallurgy Technical Activities 1994 (NAS-NRC Assessment Panel, April 6-7, 1995)

E. N. Pugh, and S. C. Hardy. 1995, 151p, NISTIR5582

Keywords: "Metallurgy, "Properties, "Metrology, "Research projects, Government/industry relations, Metal matrix composites, Intermetallics, Superconductors, Corrosion, Electrodeposition, Mechanical properties, Magnetic materials, High temperature, Advanced materials, National Institute of Standards and Technology.

This report summarizes the FY 1994 activities of the Metallurgy Division, National Institute of Standards and Technology (NIST). These activities center on struclure-processing-properties relations of metals and alloys, on methods of measurement, and on the generation and evaluation of critical materials data. Efforts comprise studies of metals processing and process sensors; advanced materials - including metal matrix composites, intermetallic alloys, and superconductors; corrosion and electrodeposition; mechanical properties; magnetic materials; and high temperature reactions.

\section{2,982}

PB96-160213 Not available NTIS

National Inst. of Standards and Technology (EEEL) Gaithersburg, MD. Semiconductor Electronics Div.

Effect of Intermediate Thermal Processing on Microstructural Changes of Oxygen Implanted Silicon-on-Insulator Material.

Final rept.

J. D. Lee, J. C. Park, S. J. Krauss, P. Roitman, and M. K. El-Ghor. 1992, 6p.

Pub. in Proceedings of the Materials Research Society Symposium, Boston, MA., December 2-6, 1991, v235 p133-1381992.

Keywords: *Annealing, Defects, Reprints, "SIMOX, "Silicon on insulator, Transmission electron microscopy.

The microstructural changes in oxygen implanted silicon-on-insulator material (SIMOX) at intermediate an- nealing steps and the changes by rapid thermal annealing (RTA) were studied with transmission electron microscopy. Defects found in as-implanted SIMOX, including multiply faulted defects, short stacking faults, and (113) defects, were all removed in anneals from 900 degrees $C$ to 1100 degrees $C$. The threading dislocations in annealed materials from at these temperatures during thermal ramping. RTA shows that the microstructure is significantly influenced by the ramp rate. The very high ramp rate in RTA results in very flat interfaces and a buried oxide layer with no $\mathrm{Si}$ islands, but significantly increases the defect density. Overall, the results show that intermediate thermal processing steps strongly affect the final microstructure of SIMOX material.

\section{2,983}

\section{PB96-160262 Not available NTIS}

National Inst. of Standards and Technology (EEEL) Gaithersburg, MD. Semiconductor Electronics Div.

Interface Roughness, Composition, and Alloying of Low-Order AlAs/GaAs Superlattices Studies by $X$-ray Diffract
Final rept.

W. R. Miller, J. G. Pellegrino, and J. Comas. 1993

6p.

Pub. in Proceedings of the Materials Research Society Symposium, 1992, v280 p265-270 1993

Keywords: "Interface roughness, Superlattices, Reprints, "X ray diffractometry AIAs/GaAs, Heterostructures, Migration enhanced epitaxy.

Low-order, monolayer by monolayer (1x1) AIAs/GaAs superlattices grown by MBE using different growth techniques have been studied by $X$-ray diffractometry. High-resolution multiple-crystal diffractometry was used to study diffraction features near the (004) peaks due to the substrate and epilayers. Using this technique, we have investigated the effect of growth techniques on the strain and tilt of the superlattices. Highresolution $X$-ray diffraction (HRXRD) results suggest that the sample grown by migration-enhanced epitaxy (MEE) is more highly strained and has more tilt than the same superlattice sample grown using the interrupted growth (IG) technique.

\section{2,984}

PB96-160429 Not available NTIS

National Inst of Standards and Technology (MSEL) Gaithersburg, MD. Reactor Radiation Div.

Instrumental Smearing Effects in Radially Symmetric Small-Angle Neutron Scattering by Numerical and Analytical Methods.

Final rept.

J. G. Barker, and J. S. Pedersen. 1995, 9p.

Pub. in Jnl. of Applied Crystallography, v28 p105-114 1995.

Keywords: "Resolution functions, Reprints, "Foreign technology, "Small angle neutron scattering, Smearing shadowing, Beamstop shadowing.

A numerical calculation method for determining the resolution functions for radially symmetric collimation and scattering is described. In the present approach the total number of integrations is reduced to four by use of the radial symmetry of the geometry Futhermore, the beam-stop shadowing effect is included exactly. A typical calculation can be completed in minutes on current personal computers. An interactive computer program allows the user to enter the experimental parameters such as aperture size and wavelength spread, allowing smearing calculations to be handled rountinely as a 'black box' operation. The exact smearing treatment is compared both to an improved technique involving Gaussian resolution functions where corrections for the beam stop are included and to results from Monte Carlo simulations. In most experiments, the use of a Gaussian distribution to approximate the resolution is preferred on account of its ease of calculation. But in a few cases, such as Porod scattering, the present more extensive numerical calculation or inclusion of the developed beam-stop-shadowing correction factors into a Gaussian scheme are needed for an adequate prediction of the smeaning effects.

\section{2,985}

PB96-161823 Not available NTIS

National Inst. of Standards and Technology (MSEL) Gaithersburg, MD

Lattice Statics of Interfaces and Interfacial Cracks in Bimaterial Solids.

Final rept.

V. K. Tewary, and R. Thomson. 1991, 39p.

Pub. in Jnl. of Materials Research, p1-39 1991. 


\section{General}

Keywords: *Composites, "Films, "Fracture, Reprints Interfaces, Calculation, Green's function.

A method for calculating a lattice statics Green's function is described for a bimaterial lattice or a bicrysta containing a plane interface. The method involves creation of two half space lattices containing free surfaces and then joining them to form a bicrystal. The two half space lattices may have different structures as in a two phase bicrystal or may be of the same type but joined at different orientations to form a grain boundary interface. The method is quite general but, in this paper, has been applied only to a simple model bicrystal formed by two simple cubic lattices with nearest neigh bor interactions. The bimaterial Green's function is modified to account for an interfacial crack which is used to calculate the displacement field due to an applied external force. It is found that the displacement field, as predicted by the lattice theory, does not have the unphysical oscillations predicted by the continuum theory.

02,986

PB96-161922 Not available NTIS

National Inst. of Standards and Technology (MSEL), Gaithersburg, MD.

Correlations between Flaw Tolerance and Reliabil ity in Zirconia.

M. J. Readey, C. L. McCallen, P. D. McNamara, and B. R. Lawn 1993, 5p

Pub. in Jnl. of Materials Science, v28 p6748-6752 1993.

Keywords: "Flaw tolerance, "Zirconia, Reliability, Indentations, Reprints, "Weibull modulus, R-curve.

Interrelations between flaw tolerance and reliability in Y-TZP, Ce-TZP and Mg-PSZ ceramics are investigated. Indentation-strength tests indicate an enhanced flaw tolerance with increasing $R$-curve behav iour from tetragonal goes to martensite transformation. The Weibull modulus of unindented specimens in creases with the enhanced tolerance. However, even the most tolerant zirconias show persistent scatter in strength, implying that variability in materia microstructure may be as important a factor in reliability evaluation in these materials as variability in flaw size.

\section{2,987}

PB96-161997 Not available NTIS

National Inst. of Standards and Technology (MSEL) Gaithersburg, MD.

Whither Computational Materials Science. Some Thoughts from the Mechanical Properties Front.

Final rept.

R. Thomson. $1993,6 \mathrm{p}$

Pub. in Computational Materials Science, v2 p137-142 1994.

Keywords: "Elasticity theory, Mechanical properties, Reprints, Computations, Fractures, "Computationa materials science.

A claim is made that analysis will remain important and become a useful ally in helping computational materals science live up to its ultimate potential. Examples are given in the mechanical properties area where numerical simulations have been able to parameterize and mark out areas of validity for elasticity theory. The important role of developing asymptomic paths from one level or category of theory to another is discussed.

\section{2,988}

PB96-163613 Not available NTIS

National Inst. of Standards and Technology (MSEL) Gaithersburg, MD.

Fracture in Multilayers.

Final rept.

P. Anderson, I. H. Lin, and R. Thompson. 1992, 6p.

Pub. in Scripta Metallurgica, 6p 1992.

Keywords: "Fractures, "Multilayers, "Interfaces, Reprints, "Foreign technology, “Green's functions.

Fracture in multilayers is a subject about which very little is known. One of the only papers on the mechanisms of interfacial fracture of multilayers at the microstructural scale is that by Hirth and Evans. General references to the experimental literature are given in the paper by Lashmore and Foecke in this series. The main thrust of this paper, however, will be the dis cussion and interpretation of a single observation of an interfacial crack in a $\mathrm{Cu} / \mathrm{Ni}$ multilayer by Lashmore, and since the theory is so little developed, the authors will often be projecting what the authors know about interfacial cracking into the multilayer world. For presentation of the results by Lashmore, again, see the paper in this series by lashmore and Foecke. Some preliminary interpretation of this micrograph is reported in Reference, based on extrapolation from dislocation emission theory in homogeneous solids. It was suggested there that the fracture toughness of multilayers may be higher than in the corresponding homogeneous materials because emitted dislocations will shield the crack more forcefully due to the limited mobility of shielding glide dislocations in the multilayer.

\section{2,989}

\section{PB96-163696 Not available NTIS}

National Inst. of Standards and Technology (MSEL) Gaithersburg, MD.

Cracks and Dislocations in Face-Centered Cubic Metallic Multilayers.

Final rept.

D. S. Lashmore, and R. Thomson. 1992, 8p.

Pub. in Jnl. of Materials Science, v7 n9 p2379-2386 Sep 92

Keywords: *Coherent interfaces, Reprints, "Dislocation emission, * Interfacial fracture, Metallic multilayers.

In this paper, the authors have demonstrated that very perfect thin multilayers of the $\mathrm{Cu} / \mathrm{Ni}$ system can be prepared with coherent interfaces if the layer modulation wavelength is in the $10 \mathrm{~nm}$ range. At modulation thickness above about $60 \mathrm{~nm}$, the interfaces become incoherent. The authors have injected a crack into a coherent interface in the $10 \mathrm{~nm}$ case which has generated dislocations into the interface forming the crack plane as well as into the layers adjacent to the crack plane. The dislocations injected into the crack plane presumably from misfit dislocations on that interface and are grouped so close to the crack tip that individual dislocations are not completely imaged. The dislocations injected into the adjacent layers are distributed rather widely. The authors have analyzed the dislocation emission from a crack in the fcc geometry appropriate to the multilayers using a simplified elastic theory developed for cracks in homogeneous materials. The mixed mode loading which the misfit stresses are ex pected to produce lead one to expect these materials to be ductile and to have high toughness. Very high dislocation densities on the crack plane near the crack however, may lead to a brittle mode of failure, which is beyond the purview of the elastic theory. The dislocations are observed to have strong interactions with alternating interfaces in the multilayers, and this effect could be due to elastic bunching of the dislocations at alternating interfaces caused by the misfit stress.

\section{2,990}

PB96-164223 Not available NTIS

National Inst. of Standards and Technology (MSEL),

Boulder, CO. Materials Reliability Div.

Compressibility of Polycrystal and Monocrystal Copper: Acoustic-Resonance Spectroscopy. Final rept.

H. Ledbetter, S. Kim, C. Fortunko, and P. Heyliger

1996, 7p.

International Jnl. of Thermophysics, $v 17 \mathrm{n} 1$ p263-269 Jan 96

Keywords: "Bulk-modulus, "Compressibility, "Copper, Reprints, "Elastic constants, Ultrasonics.

Using a method used mainly by geophysicists for small specimens-acoustic-resonance spectroscopy (ARS)we measured the elastic-stiffness constants of centimeter-size copper specimens with rectangular-parallelepiped shapes. The polycrystal consisted of heavily twinned 75-micromoles crystallites. From the specimens' macroscopic resonance-vibration frequencies (midkilohertz to low-megahertz), we calculated the least-squares elastic-stiffness coefficients, two and three for the two cases. Using the same specimens, we augmented the ARS measurements with conventional pulse-echo-method measurements. Using rod specimens, we measured the Young modulus $E$ and torsional modulus $\mathrm{G}$, and we calculated the bulk modulus B. The less direct and less familiar ARS method gives the same results as a usual pulse-echo method and a rod-resonance method.

\section{2,991}

PB96-176516 Not available NTIS National Inst. of Standards and Technology (PL), Boulder, CO. Quantum Physics Div.
Growth and Nucleation of Hydrogenated Amorphous Silicon on Silicon (100) Surfaces.

Final rept.

D. M. Tanenbaum, A. Laracuente, and A. C.

Gallagher. 1995, 6p.

Pub. in Materials Research Society Symposium Proceedings, v377 p143-148 1995

Keywords: *Dynamic scaling, "Nucleation, Islanding, Reprints, "Nanoparticulates, A-SiiH.

A scanning tunneling microscope (STM) has been used to study the topology of the surfaces of a series of thin hydrogenated amomhous silicon (a-Si:H) films deposited by if discharge from pure silane. The substrates were atomically flat, oxide-free, single-crystal Si(100). Films were grown in our laboratory and transferred to the STM with no air exposure between growth and measurement. A series of thin films between 1 and $50 \mathrm{~nm}$ in thickness reveals the initial growth stage of a-Si:H on $\mathrm{Si}(100)$. Initial nucleation and islanding can be seen on these films. The surface has a distribution of island sizes. The rms roughness and a surface lateral correlation length were measured as functions of film thickness. The rms roughness grows sublinearly with thickness from $0.3-0.5 \mathrm{~nm}$ as the film thickness is raised from 1 to $50 \mathrm{~nm}$. The lateral size of the surface features also grows with film thickness. The growth of the roughness and correlation length can be compared with the dynamic scaling model In addition the topographs reveal occasional structures of larger size and low density on the film surface. These structures are nanoparticles of silicon deposited from the plasma during film growth. The frequency of these features scales with film thickness, and represents 10 to the minus 5th power - 10 to the minus 4 th power of the total film volume.

02,992

PB96-179577 Not available NTIS

National Inst. of Standards and Technology (MSEL), Gaithersburg, MD. Reactor Radiation Div.

Design of a High-Flux Backscattering Spectrometer for Ultra-High Resolution Inelastic Neutron Measurements.

Final rept.

P. M. Gehring, C. W. Brocker, and D. A. Neumann 1995, 6p.

Pub. in Materials Research Society Symposium Proceedings, v376p113-1181995.

Keywords: "Nuetron measurements, "Backscattering spectrometers, Design, Reprints, High-flux, Ultra-high resolution

The authors discuss the design of a new backscattering spectrometer to be installed at the Cold Neutron Research Facility at the National Institute of Standards and Technology. Si (111) crystals cover both monochromator and analyzer which are spherically bent to a radius of curvature of approxmately 2 $m$ to focus the incident and scattered neutron beams. The bending increases the intrinsic lattice gradient of Si beyond its Darwin limit, resulting in an energy resoIution of approximately 0.75 mueV FWHM. The monochromator is Doppler-driven, allowing users access to a dynamic range of plus or minus 60 mueV The elastic Q-range covers 0.15 to 1.8 Angstroms to the minus one power. The most novel aspect of this design lies in the incorporation of a phase-space transform chopper. This device rotates at $4700 \mathrm{~mm}$ while neutrons are Bragg-diffracted from sets of pyrolytic graphite crystals affixed to its periphery. The process enhances the neutron flux at the backscattering energy of $2.08 \mathrm{meV}$ but at the expense of a larger horizontal divergence. Computer simulations indicate a resultant flux increase of order 3 should be obtained.

02,993

PB96-180039 Not available NTIS

National Inst. of Standards and Technology (MSEL), Gaithersburg, MD

Diffusion of Water along 'Closed' Mica Interfaces. Final rept.

R. Horn, K. T. Wan, S. Courmont, and B. R. Lawn.

1996, 3p.

Pub. in Jnl. of Colloid and Interface Science, v159 p509-511 1993.

Keywords: “Adhesion, "Cracks, “Mica, Fracture, Humidity, Reprints, Interface diffusion.

The responses of internal cracks at recontacted mica interface to change in external humidity are reported and analyzed. Cracks on interfaces reformed in lattice registry remain effectively stationary; cracks formed a 
interfaces with a twist misorientation expand and contract reversibly with increasing and decreasing humidity. The results indicate that the internal cracks in the misoriented configuration are accessible to atmospheric water, which diffuses along the dilated interface and lowers the interface energy, but remain impervious to other constituent molecules of air.

02,994

PB96-180054 Not available NTIS

National Inst. of Standards and Technology (MSEL), Gaithersburg, MD. Polymers Div.

Synthesis of Hybrid Organic-Inorganic Materials from Interpenetrating Polymer Network Chemistry. Final rept.

C. L. Jackson, B. J. Bauer, A. I. Nakatani, and J. D.

Barnes. 1996, 7p.

Pub. in Chemistry of Materials, v8 n3 p727-733 1996.

Keywords: "Polymer networks, "Acrylate, Synthesis Sol-gel, Scattering, Reprints, "Interpenetrating polymer network, Transmission electron microscopy.

Organic-inorganic interpenetrating polymer networks were synthesized with an $\mathrm{SiO} 2$ phase made by sol-gel chemistry and the organic phase made from poly(2hydroxyethyl acrylate). The resultant morphologies were characterized by small-angle scattering and electron microscopy. When the vinyl polymerization is more rapid than the sol-gel reaction, gross phase separation occurs giving a heterogeneous structure. For comparable rates of the two polymerizations, the specimens have a dendritic morphology on the scale of 0.5 micro $m$. The more rapid formation of the silica phase relative to the organic network produces rigid and optically transparent materials, with a finely divided structure as seen by transmission electron microscopy. The glass content of these materials is about $0.15 \mathrm{~g} / \mathrm{g}$ and the sizes of the SiO2-rich domains are 100 Angstroms or less. Addition of tetrakis(2(acryloxy)ethoxy)silane was also studied and found to promote phase mixing between the organic and inorganic phases.

\section{2,995}

PB96-180104 Not available NTIS

National Inst. of Standards and Technology (MSEL), Gaithersburg, MD. Polymers Div.

Composition and Solubility Product of a Synthetic Calcium Hydroxyapatite.

Final rept.

M. Markovic, B. O. Fowler, M. S. Tung, and E. S.

Lagergren. 1996, 12p.

Pub. in Mineral Scale Formation and Inhibition, Washington, DC., August 21-26, 1995, p271-282.

Keywords: "Calcium hydroxyapatite, "Chemical analysis, "Solubility products, Composition, Reprints, Statistical analysis, Thermal analysis.

Synthetic calcium hydroxyapatites (HA's) have, in general, excellent biocompatibility with tooth and bone tissues, and they are frequently used as biomedical materials. The properties of synthetic HA's depend strong$y$ on preparative conditions. They are well known to be variable and the composition deviates from the stoichiometric $\mathrm{HA}$ formula $\mathrm{Ca} 10(\mathrm{POa}) 6(\mathrm{OH}) 2$, that comprises the unit-cell content. In order to properly characterize these HA's, accurate determinations of complete chemical composition, unit-cell parameters, solubility, surface area, crystallinity, etc., are needed. The focus of this paper is on the procedures and methods for accurate and precise determinations of two important parameters, the Ca/P molar ratio and solubility product of a HA that was prepared and characterized in the author's group for use as a HA reference material (HA RM).

02,996

PB96-180245 Not available NTIS

National Inst. of Standards and Technology (MSEL), Gaithersburg, MD.

Effect of Chemical Interaction on Barenblatt Crack Profiles in Brittle Solids.

Final rept.

K. T. Wan, and B. R. Lawn. 1992,7p.

Pub. in Acta Metallurgica Materials, v40 n12 p333133371992.

Keywords: "Brittle solids, "Chemical interactions, Cohesive zone, Reprints, Bareblatt profile, Crack-tip field, Molecular wedge.

A self-consistent solution for continuum-slit Barenblatt cracks with interactive chemistry is presented. Environmental species entering the crack mouth are limited in their transport along the ever-narrowing interface by molecular size restrictions. The ensuing cohesion zon behind the tip consists of three regions: an extended far region of weak solid-fluid-solid attraction; a small intermediate region of strong solid-fluid-solid repulsion and an exclusinary near-tip intrinsic region of strong solid-solid attraction. To facilitate an analytical solution of the equations for the crack-opening displacements the cohesion stresses are taken to be uniformly distributed within each of these zones. The magnitudes of these stresses are expressed in terms of the intersurface energies that define equilibrium crack states, for virgin and healed interfaces. Illustrative calculations of the crack profiles are given for the welldocumented mica-water system. it is shown that the penetrating species cause a significant local bulge in the repulsion region, consistent with Thomson's picture of a molecular wedge.

\section{2,997}

PB96-180252 Not available NTIS

National Inst. of Standards and Technology (MSEL) Gaithersburg, MD.

Pressurized Internal Lenticular Cracks at Healed Mica Interfaces.

Final rept.

K. T. Wan, R. G. Hom, S. Courmont, and B. R.

Lawn. 1993, 9p.

Pub. in Jnl. of Materials Research, v8 n5 p1128-1136 May 93.

Keywords: "Adhesion, "Fracture mechanics, "Interface cracks, Mica, Reprints, Lenticular flaws, Pressure difference.

The equilibrium states of intemal penny cracks at interfaces in thin-sheet bodies are investigated. Consideration is given to cracks held open by a center-loading force from an entrapped particle in combination with a uniform pressure from a fixed mass of entrapped gas. A fracture mechanics analysis indicates that under these conditions the crack are stable, but are amendable to growth from an enhancement in net pressure (increase in internal pressure or decrease in external pressure) or effective particle size. Essential details of the theory are confirmed by experiments on linticular crack at healed interfaces in muscovite mica The results are pertinent to flaw responses in brittle ceramic systems where structural integrity is an issue.

02,998

PB96-180286 Not available NTIS

National Inst. of Standards and Technology (PL), Gaithersburg, MD. Radiometric Physics Div.

Development of Neutral-Density Infrared Filters Using Metallic Thin Films.

Final rept

Z. M. Zhang, R. U. Datla, and L. M. Hanssen. 1995, $6 p$.

Pub. in Materials Research Society Symposium Proceedings, v374 p117-122 1995

Keywords: "Infrared filters, "Optical density, Optical properties, Spectrometers, Reprints.

Broadband infrared filters with uniform spectral transmittance are used for spectrometer calibration and other applications. However, commercially available neutral-density filters with optical density (OD) greater than 2 exhibit significant variations in $O D$ over the wavelength region from 2 micrometers to 25 micrometers. In this work, the authors found a single-layer alloy film that for appropriate thicknesses, yields a flat transmittance for $\mathrm{OD}$ near 3 and 4 . The transmittance and reflectance of the filters were measured using a Founer-transform infrared spectrometer. The optical constants of the alloy films were obtained from transmittance and reflectance measurements, which can be used for future design optimization.

\section{2,999}

PB96-183082 PC A07/MF A02

National Inst. of Standards and Technology (MSEL).

Gaithersburg, MD. Materials Reliability Div.

Materials Reliability. Technical Activities, 1995.

H. I. McHenry, and T. A. Siewert. Apr 96, 107p.

NISTIR-5748.

See also report for 1995, PB93-173466.

Keywords: "Research projects, "Materials, Quality, Reliability, Safety, Process control, Metrology, Welding, Casting. Thermomechanical treatment, Microstructure, Steels, Metals, Ultrasonic tests, Physical properties, Nondestructive tests.

Contents:
Division Organization:

Introduction:

Highlights

Research Staff;

Technical Activities:

Intelligent Processing of Materials;

Ultrasonic Charactenzation of Materials;

Micrometer-Scale Measurements for Materials Evaluation;

Other Projects

Outputs and Interactions:

Recent Publications:

Technical and Professional Committee

Leadership:

Industrial and Academic Interactions;

and Appendix:

Organizational Charts.

\section{3,000}

PB96-186135 Not available NTIS

National Inst. of Standards and Technology (MSEL), Gaithersburg, MD. Polymers Div.

What is a 'Standard Reference Material' - What Is Any Reference Material.

Final rept.

J. A. Tesk, and J. Colbert. 1996, 3p.

Pub. in Newsletter of the Society of Biomaterials, The Torch, v18n1 p6-7 and 17, Jan/Feb 96.

Keywords: "Standards, Reprints, "Standard Reference Material, "Certified Reference Material, "NIST Reference Materials, ISO Reference Materials.

One of the pressing topics of discussion at the 1995 meeting of the Biomaterials Society Standards Committee (SC) was the need for Reference Materials (RMs). As discussions proceeded, however, it became apparent that some members of the SC were unaware of the different kinds of RMs that can be obtained and that the significance of their differences lies in their intended applications. This situation is not unique to SC members; it appears to be prevalent to varying degrees throughout the scientific and engineering community, including the Biomaterials Society at large. The fact is that there are two classifications of reference matenals, readily distinguished by their definitions, which prescribe the kind of technical information that is supplied with them when they are acquired.

\section{3,001}

PB96-186168 Not available NTIS

National Inst. of Standards and Technology (MSEL), Gaithersburg, MD. Reactor Radiation Div.

Neutron and Raman Spectroscopies of 134 and 134a Hydrofluorocarbons Encaged in Na-X Zeolite. Final rept.

T. J. Udovic, J. M. Nicol, R. R. Cavanagh, C. P. Grey, D. R. Corbin, J. J. Rush, and M. K. Crawtord. 1995, 6p

Pub. in Materials Research Society Symposium Proceedings, v376 p751-756 1995.

Keywords: "Hydrofluorocarbon, "Neutron spectroscopy, "Raman spectroscopy, Refrigerants, Reprints, Inelastic neutron scattering, Nax zeolite.

Inelastic neutron scattering methods were used in conjunction with Raman spectroscopy to probe the vibrational density of states of the hydrofluorocarbons (HFCs) 134 (HF2C-CF2H) and 134a (F3C-CFH2) adsorbed in the cages of dehydrated $\mathrm{Na}-\mathrm{X}$ zeolite. A comparison of the vibrational spectra of the encaged HFC species with those of their gas-phase analogs in dicates that the HFCs adsorb nondissociatively at room temperature and are most likely associated with $\mathrm{Na}$ cations in the supercages at the Sm sites. Guesthose interactions are manifested by adsorption-induced perturbations of the gas-phase torsional and $C$ $H$ stretching vibrations and the presence of additional features presumably due to low-energy whole-molecule vibrations and adsorbate-coupled zeolite framework vibrations. Moreover, although the 134 trans conformer is favored by $5 \mathrm{~kJ} / \mathrm{mole}$ in the gas phase at 300 $\mathrm{K}$, the gauche conformer seems to be more prevalent in the zeolite at this temperature and below. This suggests that a sizeable fraction of the $\mathrm{Na}-\mathrm{X}$ adsorption sites provides a stabilizing configuration for the otherwise higher-energy gauche conformation, perhaps due to hydrogen-bonding interactions with the zeolite framework.

03,002

PB96-190335 Not available NTIS

National Inst. of Standards and Technology (MSEL), Boulder, CO. Materials Reliability Div. 


\section{General}

\section{Charpy Specimen Tests at $4 \mathrm{~K}$.}

Final rept.

R. L. Tobler, and A. Bussiba. 1992, $8 p$

Pub. in International Cryogenic Materials Conference

(9th), Huntsville, AL., June 11-14, 1991, Advances in

Cryogenic Engineering (Materials), v38 p217-224

1992.

Keywords: "Austenitic steels, "Fracture toughness, Impact tests, Mechanical properties, Materials, Standards, Reprints, ${ }^{*}$ Cryogenic test procedures.

The paper describes nonstandard methods of testing Charpy specimens at $4 \mathrm{~K}$. We show that the initial temperature can be achieved using a helium flow method with $U$-type as well as C-type machines. Unfortunately, heating during impact loading weakens the correlation with quasistatic fracture toughness parameters. Alternative test procedures using fatigue precracked specimens were not fully satisfactory, either: (1) Precracking reduces the impact energy by $35-54 \%$, but the specimen heating is still significant; (2) slow bending curtails the heating most effectively, but then the desired simplicity of a screening test is lost.

\section{3,003}

PB96-195284 PC A09/MF A02

National Inst. of Standards and Technology (MSEL),

Gaithersburg, MD. Metallurgy Div.

Metallurgy. Technical Activities, 1995.

Rept. for 1 Oct 94-30 Sep 95

E. N. Pugh, and S. C. Hardy. 1995, 163p, NISTIR-

5750 .

See also PB96-136981.

Keywords: "Metallurgy, "Metrology, "Research projects, Mechanical properties, Electronic packaging, Metallurgy, Electrochemistry, Alloys, Steels, Electrodeposition, Corrosion, Intermetallics, IPM(Intelligent processing of materials), Advanced materials, Nanomaterials.

The report describes the technical activities of the Metallurgy Division in 1995. The format this year is different from that in preceding years in that the descriptions of the Division's projects are grouped under majo Materials Science and Engineering Laboratory (MSEL) program rather than under specific Groups. Partial Table of Contents: Intelligent Processing of Materials High-Tc Superconductivity; Electronic Packaging and Interconnection; Nanostructured Materials; Dental and Medical Materials; MSEL Other; Metals Processing Metals Data and Characterization.

\section{3,004}

PB96-200159 Not available NTIS

National Inst. of Standards and Technology (MSEL), Boulder, CO. Materials Reliability Div.

Scanning Electron Microscopy Observations of Misfit Dislocations in Epitaxial In0.25Ga0.75As on GaAs(001).

Final rept.

R. R. Keller, and J. M. Phelps. 1996, 3p.

Pub. in Jnl. of Materials Research, v11 n3 p552-554 Mar 96.

Keywords: "Scanning electron microscopes, "Dislocations, "Epitaxial films, Thin films, Electron channeling, Reprints, Kikuchi patterns, Backscattered electrons.

Dislocations in the misfit epitaxial film system In0.25Ga0.75As on GaAs(001) were imaged using a modified electron channeling contrast technique in a LaB6 SEM. We obtained images at an incident beam energy of $30 \mathrm{KeV}$, a beam divergence of less than 1 mrad and a specimen tilt of 70 degrees in conjunction with a movable scintillator detector mounted at a takeoff angle of approximately 3 degrees to 5 degrees. We achieved a spatial resolution of approximately 80 to $100 \mathrm{~nm}$ with this technique. Such resolution allowed rapid imaging of clusters consisting of only a few closely spaced dislocations in a $55 \mathrm{~nm}$ thick film. At such small film thicknesses, we did not require accurate knowledge of the incident beam direction in order to obtain sufficiently strong channeling contrast for qualitative characterization. The observed defect arrangements included features that we believe represent clustered threading segments.

\section{3,005}

PB96-200761 Not available NTIS

National Inst. of Standards and Technology (MSEL), Boulder, CO Materials Reliability Div.
Elastic Constants and Microcracks in YBa2Cu3O7. Final rept.

Y. Shindo, H. Ledbetter, and H. Nozaki. 1995, $4 p$ Pub. in Jnl. of Materials Research, v10 n1 p7-10 Jan

Keywords: "Elastic constants, "Microcracks, "Superconductors, Polycrystalline, Crack density, Crack aspect ratio, Reprints, Oxide superconductors.

We analyze theoretically the effect of microcracks and voids on the apparent elastic constants of polycrystalline YBa2Cu307. Using measurements by Holcomb and Mayo, we calculate crack density and crack aspect ratio. We obtain reasonable intrinsic elastic constants. For the bulk modulus, for example, we predict values close to these obtained by neutron-diffraction studies: $123 \mathrm{GPa}$ for a polycrystal and 122 $\mathrm{GPa}$ for a monocrystal.

\section{3,006}

PB96-200910 Not available NTIS

National Inst. of Standards and Technology (BFRL), Gaithersburg, MD. Building Materials Div.

Precision of Marchall Stability and Flow Test Using 6-in. (152.4-mm) Diameter Specimens.

Final rept

P. S. Kandhal, Y. Wu, F. Parker, and P. A.

Spellerberg. 1996, 6p.

Pub. in Jnl. of Testing and Evaluation (JTEVA), v24 n1 p20-25 Jan 96.

Keywords: "Stones, "Marshall stability, Percent voids, Precision statement, Marshall flow, Reprints, Large stone mixes.

Earlier studies have shown that the repeatability of Marshall stability flow and air voids content measurements on 6 -in. $(152.5-\mathrm{mm})$ diameter specimens of large stone mixes is better than the repeatability on 4 in. (101.6- $\mathrm{mm})$ diameter specimens. A round robin study involving twelve participating laboratories was conducted to provide information for developing a precision statement for the ASTM Test Method for Resistance to Plastic Flow of Bituminous Mixtures Using Marshall Apparatus (6 inch-Diameter Specimen) (D 5581). Difference two-sigma (d2s) limits were developed to determine acceptable single and multilaboratory differences for bulk specific gravity, percent voids, Marshall stability, and Marshall flow measurements. Anal'y' sis of other data collected during the study indicated that stability and flow measurements are not sensitive to minor differences in various 6-in. (152.4- $\mathrm{mm}$ ) diameter breaking heads currently in use.

\section{3,007}

PB96-204045 Not available NTIS

National Inst. of Standards and Technology (MSEL), Gaithersburg, MD. Ceramics Div.

Guide to Locating and Accessing Computerized Numeric Materials Databases.

Final rept.

E. F. Begley, and S. J. Dapkunas. 1993, 6p

Pub. in Jnl. of Materials Engineering and Performance, v2 n6 p881-886 1993

Keywords: "Materials, "Data bases, "Statistical data, Access, Data retrieval, Information retrieval, Information dissemination, Reprints.

The article provides the materials engineer and component designer a guide to these sources including the data available and the points of contact for gaining access.

\section{3,008}

PB96-204086 Not available NTIS

National Inst. of Standards and Technology (MSEL), Gaithersburg, MD. Ceramics Div.

NIST/NCMS Program on Electronic Packaging: First Update.

Final rept.

J. A. Carpenter, B. Dickens, K. G. Kreider, G. J. Piermarini, D. T. Read, R. D. Evans, J. R. Manning, and R. L. Mattis. 1992, 16p.

Pub. in Proceedings of the Conference of the International Electronics Packaging Society (IEPS), Austin, TX., September 27-30, 1992, p449-464.

Keywords: *Electronic packaging, * Metrology, "Quality control, Manufacturing, Circuit interconnections, Printed circuit boards, Measurement, Reliability analysis, Reprints, Materials properties.

In 1992 the National Institute of Standards and Technology (NIST) expanded its intramural efforts on devel- opment of metrology for microelectronics packaging and interconnection. The new intramural projects concentrate on development or refinement of metrology for measuring the properties of the materials as they actually exist in modern packaging and interconnection, and not as measured on idealized or bulk specimens. Also, work begin in 1991 accelerated a major consortium effort led by the National Center for Manufacturing Species (NCMS) and jointly supported by the consortium members and the NIST Advanced Technology Program (ATP) to develop new materials and production technology for printed wiring boards. This is an update on the progress of those efforts.

03,009

PB96-214754 PC A05/MF A01

National Inst. of Standards and Technology (MSEL), Gaithersburg, MD.

Materials Science and Engineering Laboratory Annual Report, 1995. Technical Activities.

Rept. for 1 Oct 94-30 Sep 95.

L. H. Schwartz, and H. L. Rook. 1995, 68p, NISTIR5746 .

See also PB95-196697.

Keywords: "Research and development, Research management, Technology innovation, Research projects, Organizational structure, Laboratories, Resources, Technology transfer, "US NIST, "MSEL(Materials Science and Engineering Laboratory), "Materials Science and Engineering Laboratory(MSEL)

The 1995 Annual Report was organized to assist the NAS-NAE-NRC Board on Assessment and intended to be used as background information at the annual review meeting. Coupled with the more detailed technical reports of the divisions of MSEL, this report describes the activities, accomplishments, output and impacts of the MSEL during the period of Fiscal Year 1995 (October 1, 1994 to September 30,1995 )

\section{3,010}

PB97-111587 Not available NTIS

National Inst. of Standards and Technology (MSEL) Gaithersburg, MD. Reactor Radiation Div.

Neutron Diffraction Texture Study of Deformed Uranium Plates.

Final rept.

C. S. Choi, and M. Staker. 1996, 6p.

Pub. in Jnl. of Materials Science, v31 p3397-3402 1996.

Keywords: *Uranium plates, "Neutron diffraction, Orientational distribution functions, Deformation, Reprints.

The texture of two depleted uranium (DU) samples, labelied DUWR and DUWRs, were studied by neutron diffraction. DUWR was prepared by warm rolling of a cast ingot, and DUWR2 was prepared by adding $20 \%$ tensile strain to the warm-rolled DUWR. Complete three-dimensional orientation distribution functions were determined using four neutron pole figures for the DUWR, and using six neutron pole figures for the DUWR2 sample, by the WIMV method of the program popLA. The textures of the two samples were essentially identical to each other. They could be described by a twisted helical density tube spiralling continously along the psi-axis of the Euler space. The projection of the backbone of the density tube along the Bragg angle-axis cast a linear shadow running parallel to the diagonal of the integral-psi plane, which could be defined by a integral $=p s i+90$ degrees (and integral $=$ $\mathrm{psi}+270$ degrees) relation. The helical tube was confined within narrow Braggs angle-angle limits, from 14 degrees to 30 degrees with the peak orientation at (103) $(010)$

\section{Adhesives \& Sealants}

\section{3,011}

PB94-212222 Not available NTIS

National Inst. of Standards and Technology (MSEL), Gaithersburg, MD. Polymers Div. 
Micromechanics of Fracture in Rubber-Toughened Epoxies.

Final rept.

D. L. Hunston, H. Mizumachi, and W. McDonough

1990, $6 \mathrm{p}$

Pub. in Proceedings of International Conference on Adhesion '90 (4th), Cambridge, England, September 10-12, 1990, p37/1-37/6.

Keywords: "Crack propagation, "Adhesive bonding, "Epoxy resins, "Fractures(Materials), *Micromechanics, Adhesives, Deformation, Bonded joints, Failure, Fracture mechanics, Mechanical properties, Reprints.

Previous work has shown the importance of the cracktip deformation zone in determining the fracture energies for both samples of bulk adhesive and the corresponding adhesive joints. To gain a better understanding of deformation zone development, the cracktip events in compact tension specimens of bulk adhesive were monitored during loading and failure. The results show no clear onset point for crack growth but rather a gradual acceleration. Moreover, in regions of stable crack growth, the size of the deformation zone correlates with the crack velocity. This has important implications for failure criteria.

03,012

PB96-183249 PC A05/MF A01

National Inst. of Standards and Technology (BFRL),

Gaithersburg, MD. Building Materials Div.

Performance of Tape-Bonded Seams of EPDM Membranes: Comparison of the Peel Creep-Rupture Response of Tape-Bonded and Liquid-Adhesive-Bonded Seams.

Building science series.

W. J. Rossiter, M. G. Vangel, E. Embree, K. M. Kraft, and J. F. Seiler. May 96, 70p, NIST/BSS-175.

Also available from Supt. of Docs. as SNO03-00303411-8.

Keywords: "Adhesive tapes, "Roofs, Seams(Joints), Creep rupture tests, Construction materials, EPDM(Ethylene Propylene Diene Terpolymer).

A study was conducted to compare the creep-rupture response (i.e., time-to-failure or TTF) of tape-bonded and liquid-adhesive-bonded seams of EPDM (ethylene-propylene-diene terpolymer) roofing membranes. Two commercial tape systems (i.e., tape and primer) and one liquid adhesive were applied to well-cleaned EPDM rubber.

\section{Carbon \& Graphite}

\section{3,013}

PB94-185022 Not available NTIS

National Inst. of Standards and Technology (MSEL),

Gaithersburg, MD. Metallurgy Div.

Thermal Diffusivity of POCO AXM-5Q1 Graphite in the Range 1500 to $2500 \mathrm{~K}$ Measured by a LaserPulse Technique.

Final rept.

T. Baba, and A. Cezairliyan. 1994, 22p

Pub. in International Jnl. of Thermophysics 15, n2 p343-364 Mar 94.

Keywords: "Graphite, "Thermal diffusivity, "Temperature measuring instruments, Radiative heat transfer, Thermophysical propeities, Thermal properties, Boundary conditions, Transport properties, Pulsed lasers, Uncertainity, Reprints, "Laser pulse method.

The thermal diffusivity of $\mathrm{POCO} A X M-5 \mathrm{Q} 1$ graphite was measured in the temperature range 1,500 to 2,500 $\mathrm{K}$ utilizing the laser-pulse technique. The uncertainty of the values is estimated to be no more than $3 \%$. The measured values are compared with the results of other investigators.

\section{3,014}

PB94-211471 Not available NTIS

National inst. of Standards and Technology (MSEL), Gaithersburg, MD. Ceramics Div.

Analysis of Thermal Wave Propagation in Diamond

Films.

A. Feldman, H. P. R. Frederikse, and S. Norton.

1990, 110.

1990, $11 \mathrm{p}$.
See also PB94-211489.
Pub. in Proceedings of Society of Photo-Optical Instrumentation Engineers Diamond Opt. 3, v1325 p304-314 1990.

Keywords: "Diamond films, *Thermal diffusivity, Chemical vapor deposition. Thermal conductivity, Thick films, Thin films, Wave propagation, Heating, Reprints, Photothermal radiometry, Thermal waves.

The use of photothermal radiometry to obtain the thermal diffusivity of CVD diamond is analyzed. The finite size of the heating beam and the radiation detector are taken into account. Heating a finite circula rly symmetric region of the specimen surface is compared with uni form heating of the specimen surface. Thermal wave propagation in thick and thin diamond films in vacuum in air, and on several substrate materials is modelled.

\section{3,015}

PB94-211489 Not available NTIS

National Inst. of Standards and Technology (MSEL), Gaithersburg, MD. Ceramics Div.

Thermal Wave Propagation in Diamond Films.

Final rept.

A. Feldman, H. P. R. Frederikse, and S. Norton.

$1991,6 p$

See also PB94-211471.

Pub. in Proceedings of International Conference on New Diamond Science and Technology (2nd), Washington, DC., September 23-27, 1990, p881-886 1991.

Keywords: "Diamond films, "Thermal diffusivity, Chemical vapor deposition, Thermal conductivity, Wave propagation, Computation, Reprints, Photothermal radiometry, Thermal waves.

Photothermal radiometry, one of several methods based on the propagation of thermal waves, is a convenient noncontact technique to measure the therma diffusivities of diamond films. Results of calculations for thermal wave propagation in diamond films are presented. Taken into account are the thickness of the film, the substrate material, the presence of air, the presence of a black absorbing layer, the finite size of the heating beam, and the finite size of the infrared detector used to measure the thermal energy emitted by the specimen.

\section{3,016}

PB95-162392 Not available NT1S

National Inst. of Standards and Technology (MSEL), Gaithersburg, MD. Ceramics Div.

Lineshape Analysis of the Raman Spectrum of Diamond Films Grown by Hot-Filament and Microwave-Plasma Chemical-Vapor Deposition.

Final rept.

L. H. Robins, E. N. Farabaugh, and A. Feldman

$1990,13 p$

Pub. in Jnl. of Materials Research 5, n11 p2456-2468 1990.

Keywords: "Crystal growth, "Crystal defects, "Raman spectroscopy, "Chemical vapor deposition, Atomic energy levels, Photoluminescence, Thin films, Diamonds, Reprints, "Diamond films.

Raman spectra were measured in 48 different polycrystalline diamond forms grown by hot-filament and microwave-plasma chemical vapor deposition and one gem-quality diamond, and characterized by fitting the data to a model lineshape function. The lineshape function contains the following three components: a narrow symmetric line at $-1333 / \mathrm{cm}$, the Raman line of diamond; a broad symmetric band a $-1520 / \mathrm{cm}$ due to $\mathrm{s}$ doublet p-bonded carbon; and a slowly varying background due to photoluminescence $(\mathrm{PL})$. Four spectral parameters are observed to change in a correlated manner from one specimen to another (1) the linewidth of the diamond Raman line, which varies from 3 to $30 / \mathrm{cm}$; (2) the intensity in the tails of the diamond line, several halfwidths away from the peak (3) the intensity of the $s$ doublet p-bonded carbon band, relative to the diamond line, which varies from -0 to 1; and (4) the intensity of the PL background, relative to the diamond line, which varies from 0.03 to 9 The observed correlations suggest that these changes in the Raman spectrum are caused by a common set of defects.

\section{3,017}

PB95-162400 Not available NTIS

National Inst. of Standards and Technology (MSEL), Gaithersburg, MD. Ceramics Div.
Studies of Defects in Diamond Films and Particles by Raman and Luminescence Spectroscopies. Final rept.

L. H. Robins, E. N. Farabaugh, and A. Feldman.

$1990,12 p$.

Pub. in Proceedings of Society of Photo-Optical Instrumentation Engineers: Diamond Opt. 3, v1325 p1301411990

Keywords: *Crystal growth, "Crystal defects, "Raman spectroscopy "Chemical vapor deposition, Atomic energy levels, Diamonds, Cathodoluminescence, Luminescence, Photoluminescence. Thin films, Reprints "Diamond films.

Diamond films grown under a variety of deposition conditions in hot-filament or microwave-plasma chemical vapor deposition (CVD) reactors were characterized by Raman and cathodoluminescence (CL) spectroscopies. The magnitudes of the following four Raman spectral features were observed to vary from specimen to specimen in a correlated manner: (1) the linewidth of the diamond Raman line; (2) the intensity of the tails of the diamond Raman line at several half widths from the peak; (3) the intensity ratio of the $s$ doublet p-bonded carbon Raman band to the diamond Raman line; and (4) the intensity ratio of the broad photoluminescence (PL) background that underlies the Raman spectrum to the diamond Raman peak. We suggest that each of these features varies with the abundance of $s$ doublet p-bonded carbon. A model of competing recombination between two sets of defects is proposed to explain the inverse correlation.

\section{3,018}

PB95-162418 Not available NTIS

National Inst of Standards and Technology (MSEL) Gaithersburg, MD. Ceramics Div.

Surface Roughness Evaluation of Diamond Films Grown on Substrates with a High Density of Nucleation Sites.

Final rept.

L. H. Robins, E. P. Whitenton, E. N. Farabaugh, and A. Feldman. 1991, $6 p$

Pub. in Proceedings of International Conference New Diamond Science and Technology (2nd), Washington DC., September 23-27, 1990, p619-624 1991

Keywords: "Crystal growth, "Nucleation, "Chemical vapor deposition, "Surface roughness, Substrates, Diamonds, Scanning tunneling microscopy Wet methods, Dry methods, Spectrum analysis, Polishing, Scan ning electron microscopy, Profilometers, Thin films, Raman spectroscopy, Reprints, "Diamond films.

Diamond films were deposited on silicon substrates which were seeded with diamond by either of two techniques, abrasive dry polishing or wet polishing. Depositions were carried out in a microwave-plasma chemical vapor deposition (CVD) reactor. Films were characterized by scanning electron microscopy (SEM) imaging Raman spectroscopy, optical transmittance and reflec tance spectroscopy, stylus profilometry, and scanning tunneling microscope (STM) surface profilometry.

\section{3,019}

PB96-119714 Not available NTIS

National Inst. of Standards and Technology (MSEL), Boulder, CO. Materials Reliability Div.

Tribological Behavior of 440/Diamond-Like-Carbon Film Couples.

Final rept

A. J. Slifka, R. Compos, R. Wei, P. Wilbur, and D. K Chaudhuri. 1992, 6p

Pub. in Advanced Earth-to-Orbit Propulsion Tech nology, Huntsville, AL., May 19-21, 1992, p398-403 NASA CP-3174.

Keywords: "Coatings, "Diamond, "Tribology, Reprints Friction, Wear.

Diamond-Like-Carbon films were characterized for their tribological properties. Films were made on $440 \mathrm{C}$ substrates by first sputter-depositing an intermediate layer of $\mathrm{SiC}$, then extracting $450 \mathrm{eV}$ carbonacceous ions to produce the diamond-like-carbon film. The intermediate SiC layer provides adhesions between $440 \mathrm{C}$ and the diamend-like-carbon film, and also provides the necessary nucleation sites for diamond-likecarbon film formation. Films were prepared in three thicknesses, $0.5,1$, and 2 micrometer, and tested at two temperatures, liquid oxygen (-184 deg C) and room temperature (25 degrees $C$ ). A coefficient of friction of 0.03 was measured in an oxygen environment. Specific wear rates that are two orders of magnitude lower than for $440 \mathrm{C}$ on $440 \mathrm{C}$ were measured. Data are 
presented for $440 \mathrm{C}$ on diamond-like-carbon, $440 \mathrm{C}$ on $440 C$, and Si3N4 on $440 C$, for comparison.

\section{3,020}

PB96-119748 Not available NTIS

National Inst. of Standards and Technology (MSEL),

Gaithersburg, MD. Reactor Radiation Div.

Structural and Magnetic Properties of $\mathrm{CuCl}_{2}$ Graphite Intercalation Compounds.

Final rept.

M. Suzuki, I. S. Suzuki, C. R. Burr, K. Koga, D. G.

Wiesler, and N. Rosov. 1994, 12p.

Pub. in Physical Review B, v50 n13 p9188-9199, 1 Oct 94.

Keywords: "Electrochemical cells, "Copper chlorides, Reprints, Catalysts, Electric conductors, Cathodes, *Graphite intercalation compounds.

Structural and magnetic properties of stage-1, stage2 , and stage-3 CuCl2 graphite intercalation compounds (GIC's) were studied by means of x-ray, electron- and neutron-diffraction, dc magnetic susceptibility, and electron spin resonance (ESR) measurements. The Cu2+ ions form an isosceles triangular lattice with one short side and two longer sides. The inplate dc magnetic susceptibility shows Cure-Weiss behavior above $150 \mathrm{~K}$, a broad maximum around 62-65 $\mathrm{K}$, indicative of low-dimensional magnetic correlations, and a Curie-type behavior below $20 \mathrm{~K}$, attributable 10 paramagnetic inhomogeneities in the sample.

\section{3,021}

PB97-112601 Not available NTIS

National Inst. of Standards and Technology (MEL), Gaithersburg, MD. Precision Engineering Div.

Chemical Aspects of Tool Wear in Single Point Diamond Turning.

Final rept.

E. Paul, C. J. Evans, A. Mangamelli, M. L.

McGlauflin, and R. S. Polvani. 1996, 16p

Pub. in Precision Engineering, v18 p4-19 1996.

Keywords: "Nickel alloys, "Electro deposited coatings, "Diamonds, "Tools, "Wear, Cutting, Machining, Reprints.

A hypothesis is proposed that ascrbies chemical wear of diamond tools to the presence of unpaired $d$ electrons in the sample being machined. The hypothesis is used to explain a range of results for metals, alloys, and other materials including 'electroless' nickel. The hypothesis is further tested by experiments presented here on the machining of a range of high purity elements. The implications for diamond turnability of other materials are discussed.

\section{Ceramics, Refractories, \& Glass}

\section{3,022}

\section{AD-A244 582/3 PC A02/MF A01}

National Inst. of Standards and Technology, Gaithersburg, MD

Effect of Green Density and the Role of Magnesium Oxide Additive on the Densification of Alumina Measured by Small-Angle Neutron Scattering. (Reannouncement with New Availability Informa(Rean).

G. G. Long, S. Krueger, and R. A. Page. Jul 91, 9p, ARO-26123.3-MS

Contract ARO-MIPR-102-90

Pub. in Jnl. of the American Ceramic Society, v74 n7 p1578-1584 Jul 91

Keywords: "Aluminum oxides, Additives, Density, Green(Color), Magnesium oxides, Microstructure, Neutron scattering, Porous materials, Sintering, Reprints, Alumina, Small angle scattering, Densification.

Small angle neutron scattering measurements were used to examine the effect of green density and the role of $\mathrm{MgO}$ additive on the evolution of the porous microstructure of alumina during intermediate- and final-stage sintering. It was found that the initial connectivity in the green state plays a dominant role in establishing the channel diameters during the intermediate stage of sintering, and contributes also to determining the onset density at which the final stage of sintering begins. The role of $\mathrm{MgO}$ as a sintering aid lies, at least in part, in prolonging the stability of intermediate stage sintering such that the body achieves greater density before the transition to final-stage sintering after which isolated pores are formed.

03,023

AD-A249 178/5 PC A03/MF A01

National Inst. of Standards and Technology, Gaithersburg, MD.

Evolution of the Pore Size Distribution in FinalStage Sintering of Alumina Measured by SmallAngle $X$-ray Scattering. (Reannouncement with New Availability Information)

S. Krueger, G. G. Long, D. R. Black, D. Minor, and P. R. Jemian. 10 Oct $91,11 \mathrm{p}$, ARO-26123.4-MS. Contract MIPR-ARO-102-90

Pub. in Jnl. of the American Ceramic Society, v74 n10 p2538-2546 Oct 91 .

Keywords: "Aluminum oxides, "Sintering, " $X$ ray scattering, Magnesium oxides, Doping, Ceramic materials, High resolution, Density, Entropy, Neutron scaltering, Reprints, "Pore size distribution, Alumina, Magnesia, Small angle.

Small-angle $X$-ray scattering was used to follow the evolution of the pore size distribution during final-stage sintering of alumina and of alumina doped with 0.25 wt \% magnesia. The volume-weighted (Guinier) results indicate that the effective size of the largest pores in creases as the body goes from $97 \%$ to more than $99 \%$ dense. The surface-area-weighted (Porod) results show that the median size of the smallest pores decreases slightly over the same density range. Taken together, these data indicate that the pore size distribution becomes broader as final-stage densification proceeds. This was confirmed by a maximum entropy analysis, which was used to derive pore size distributions directly from the data. Finally, the evolution of the pore size distributions in alumina, with and without sintering aid, were compared.

\section{3,024}

AD-A249 179/3 PC A02/MF A01

National Inst. of Standards and Technology, Gaithersburg, MD.

Characterization of the Densification of Alumina by Multiple Small-Angle Neutron Scattering (Reannouncement with New Availability Informa. tion).

S. Krueger, G. G. Long, and R. A. Page. 1991, 10p, ARO-26123.2-MS

Contract MIPR-ARO-102-90

Pub. in Acta Crystallographica, vA47 p282-290 1991.

Keywords: Density, Microstructure, Neutron scattering, Sintering, Reprints, "Aluminum oxide, Small angle scattering.

Multiple small-angle neutron scattering was used to follow the evolution of the pore-size distribution in alpha $\mathrm{Al} 2 \mathrm{O} 3$ through the intermediate and final stages of sintering. This new technique enables the study of microstructure in the $0.08-10$ micro size regime, which is the size range of importance for many materials systems, without needing to increase the resolution of currently available small-angle scattering instruments. The microstructure evolution results indicate a nearly constant effective pore radius for the alumina throughout the intermediate sintering stage, ranging from 0 19 micro at $54 \%$ of theoretical density to $0-17$ micro at $79 \%$ dense. As the alumina densifies further, there is a transition region after which the effective pore radius grows rapidly to 0.6 micro at $97-5 \%$ dense.

\section{3,025}

AD-A249 510/9 PC A03/MF A01

National Inst. of Standards and Technology, Gaithersburg, MD.

Small-Angle Neutron Scattering Characterization of Processing/Microstructure Relationships in the Sintering of Crystalline and Glassy Ceramics. (Reannouncement with New Availability Information).

G. G. Long, S. Krueger, R. A. Gerhardt, and R. A

Page. Dec 91, 11p, ARO-26123.5-MS

Contract MIPR-ARO-102-90

Pub. in Jnl. Mater. Res., v6 n12 p2706-2715, Dec 91.

Keywords: *Neutron scattering, "Sintering, "Ceramic materials, Microstructure, Polycrystalline, Reprints, "Glassy silica, "Crystalline ceramics, Porosity, SANS(Small Angle Neutron Scattering)

Small-angle neutron scattering measurements were used to examine the pore microstructure evolution of glassy silica and polycrystalline alpha-alumina as function of sintering. It was shown that the two majo sintering mechanisms, viscous flow and surface and volume diffusion, lead to very different microstructure evolution signatures in terms of the average pore size as a function of density. However, with respect to topology, the evolution of the porosity per unit surface area as a function of density is remarkably similar in the two systems.

\section{3,026}

\section{DE94013170 PC A02/MF A01}

National Inst. of Standards and Technology, Gaithersburg, MD

Ceramic Characterization

M. D. Vaudin, E. R. Fuller, J. P. Cline, and A. L

Dragoo. 1987, 7p, CONF-8710535.

Contract Al05-85OR21569

Ceramic characterization for international standards meeting, Dearborn, MI (United States), 26 Oct - 1 Nov 1987. Sponsored by Department of Energy, Washington, DC.

Keywords: "Silicon Carbides, Electron Microscopy, Grain Boundaries, Microstructure, Standards, X-Ray Diffraction, EDB/360202.

Objective of this task is to measure and characterize the ceramic properties (crystalline phase composition grain boundary properties, residual stresses) of a series of standard ceramic specimens to assist in developing international standards for ceramic characterization. This report describes electron microscopy of the microstructure and grain boundaries of ESK silicon carbide; supporting $x$-ray diffraction data for the bulk phase composition is included.

\section{3,027}

\section{DE94013486 PC A03/MF A01}

National Inst. of Standards and Technology, Gaithersburg, MD

Densification of Nano-Size Powders. 1994 Report Progress rept.

W. Chen, S. G. Malghan, J. S. Dapkunas, G

Piermarini, and A. Pechenik. 1994,12p, DOE/OR

22041-2.

Contract Al05-920R22041

Sponsored by Department of Energy, Washington, DC. Keywords: "Aluminium Oxides, "Silicon Nitrides, Compacting, Density, Fabrication, Hot Pressing, Powders, Progress Report, Sintering, Vickers Hardness, EDB/ 360201.

Green compacts from a nano-size silicon nitride powder were fabricated having density up to $67 \%$ of theoretical at $2.8 \mathrm{GPa}$ pressure using liquid nitrogen and pentane as compaction lubricant media. Pressureless sintering of these transparent samples did not promote further densification beyond that obtained for the green state. To further increase the density of these samples, a hot-pressing device was designed. In a series of experiments, hot-pressing of these samples at.0.5 to 1.0 GPa and $800 \mathrm{C}$, followed by pressureless sintering at $1400 \mathrm{C}$ was studied. The resulting silicon nitride ceramic had a Vickers hardness of 9.0 GPa while transparency under visible light was maintained. Without the use of hot pressing, the hardness obtained was 5.8 GPa. In addition, the effect of compaction pressure on densification was studied for nano-size Al(sub 2)O(sub 3) to further understand factors contributing to achieving high green densities. The dense $\mathrm{Al}($ sub 2) O(sub 3) green samples were pressureless sintered to near full density at temperatures several hundred degrees lower than those needed for sintering low density green material.

03,028

DE94013593 PC A03/MF A01

National Inst. of Standards and Technology (IMSE), Gaithersburg, MD.

Equipment for Investigation of Cryogenic Compaction of Nanosize Silicon Nitride Powders. 1993 Report.

Progress rept.

W. Chen, G. J. Piermarini, S. J. Dapkunas, S. G Malghan, and A. Pechenik 1993, 11p, DOE/OR 22041-T1.

Contract Al05-920R22041

Sponsored by Department of Energy, Washington, DC.

Keywords: "Presses, "Silicon Nitrides, Calibration, Compacting, Computerized Control Systems, Design, Lubricants, Nitrogen, Particle Size, Progress Report, EDB/360201.

This paper describes a highly-specialized system for studies of time-dependent compaction of nanosize sili- 
MATERIALS SCIENCES

Ceramics, Refractories, \& Glass

con nitride powders under a variety of atmospheres and at temperatures ranging from 77 to $1,000 \mathrm{~K}$. The system incorporates a screw-driven press (10 ton capacity) with a piston-cylinder type die and can produce decylindrical powder compacts, $3 \mathrm{~mm}$ in diameter and approximately $1 \mathrm{~mm}$ in thickness, using pressures up to $3 \mathrm{GPa}$. The system is computer-controlled and permits accurate measurements of the sample volume, and, after appropriate calibration, can determine the rate and degree of densification of the compacting powder as pressure is applied. Frictional forces between the piston and the die are measured during the compaction process. For calibration of the system, powders with known volume-change accompanied by phase transition under pressure were studied, and good agreement with published results was demonstrated. Several Si(sub 3)N(sub 4) samples have been compacted and sintered at 1,300 to $1,600 \mathrm{C}$. A maximum random packing density of $64 \%$ has been obtained using liquid nitrogen as a lubricant medium at pressure lower than $2.5 \mathrm{GPa}$. Both green samples and sample sintered at temperatures to $1,500 \mathrm{C}$ exhibited visual transparency under visible light.

\section{3,029}

DE95013505 PC A05/MF A01

National Inst. of Standards and Technology (MSEL),

Gaithersburg, MD. Ceramics Div.

Low temperature fabrication from nano-size ceramic powders.

E. J. Gonzalez, G. J. Piermarini, and B. Hockey.

1995, 79p, DOE/OR/22041-T2.

Contract AlO5-92OR22041

Sponsored by Department of Energy, Washington, DC.

Keywords: "Aluminium Oxides, "Silicon Nitrides, Compacting, Equations, Mechanical Properties, Microstructure, Powder Metallurgy, Processing, Sintering, EDB/360201, EDB/360203, EDB/360202.

The objective of the compaction process is to produce a dense green-state compact from a nanosize powder that subsequently can be sintered at high temperatures to form a dense ceramic piece. High density in the green-state after pressing is of primary importance for achieving high densities after sintering. Investigation of the compaction behavior of ceramic powders, therefore, is an important part of characterization of raw ceramic powders and evaluation of their compaction behavior, analysis of interaction between particles, and the study of microstructure of green body (unsintered) during pressure-forming processes. The compaction of nanosize ceramic particles into high density green bodies is very difficult. For the nanosize materials used in this study (amorphous Si(sub 3)N(sub 4) and (gamma) Al(sub 2)O(sub 3)), there is no evidence by TEM of partial sintering after synthesis. Nevertheless, strong aggregation forces, such as the van der Waals surface forces of attraction, exist and result in moderate precursor particle agglomeration. More importantly, these attractive surface forces, which increase in magnitude with decreasing particle size, inhibit interparticle sliding necessary for particle rearrangement to denser bodies during subsequent compaction. Attempts to produce high density green body compacts of nanosize particles, therefore, generally have been focused on overcoming these surface forces of attraction by using either dispersive fluids or high pressures with or without lubricating liquids. In the present work, the use of high pressure has been employed as a means of compacting nanosize powders to relatively high green densities.

\section{3,030}

N94-35335/6 PC A07/MF AO2

National Inst. of Standards and Technology,

Classification of Advanced Technical Ceramics.

S. Schneider. Jul 93, 128p.

Keywords: "Ceramics, "Chemical composition, "Chemical properties, "Classifications, Data bases, Utilization, Ceramic coatings, Ceramic fibers, Ceramic matrix composites, Glass, information dissemination, Porous materials, Powder (Particles).

This document presents the basis and scheme for a unified classification system for advanced (technical) ceramics. The utility of the classification system is multi-fold. Its use is advantageous at the company level for purposes involving assembly of design and materials propenty databases, or tabulation of inventories, or invoicing. Industry can use the system for gathering and sorting trend data on market behavior or R\&D expenditures, or for literature categorization. At the government level, the system can be used for gathering national and international economic data, or ther vital statistics, or for determining demographics of the field. The classification system is based on defining advanced technical ceramics as 'a highly engineered, high performance, predominately non-metallic, inorganic, ceramic material having specific functional attributes.' This excludes commodity products, such as building materials and refractories. Five hundred diferent product types are identified as advanced technical ceramics in this classification. The classification is a non-hierarchical, matrix-type scheme accessible by a number of entry and retrieval routes to build relational databases. The classification provides a machine readable coding system built upon four independent descriptor fields (application, chemical character and product form, processing, and propenty data) and corresponding subdivisions that may be sequenced in order to match the users preference.

\section{3,03}

PB94-162591 PC A10/MF A03

National Inst. of Standards and Technology (MSEL), Gaithersburg, MD. Ceramics Div.

Ceramics Technical Activities, 1993 (NAS-NRC Assessment Panel April 21-22, 1994).

1994, 215p, NISTIR-5313.

See also PB93-173508.

Keywords: "Research projects, "Ceramics, Data bases, Mechanical properties, Standards, Composite materials, Powder(Particles), Optical materials, Electronics, Glass, Surface properties, Thin films, Standards and Technology, Advanced materials, Synchrotron radiation.

In 1993 the Ceramics Division continued to emphasize a technical program directly relevant to the needs of U.S. industry. The program is made up of tasks which involve standard materials development, construction of evaluated databases, and laboratory research focused on topics that address the dominant issues af fecting commercialization of advanced ceramics, namely, processing costs and reliability. In accord with the strategic plan laid out by the Materials Science and Engineering Laboratory, the Ceramics Division is continuing to expand its direct involvement with industrially related research issues. Standard materials and data activities continue to represent an important portion of the Division's program. Three new Standard Reference Materials were developed this year, and an evaluated database on high Tc superconducting materials was begun in collaboration with a Japanese lab oratory.

\section{3,032}

PB94-172434 Not available NTIS

National Inst. of Standards and

Gaithersburg, MD. Polymers Div.

Error Propagation Biases in the Calculation of Indentation Fracture Toughness for Ceramics.

Final rept.

J. R. Kelly, M. E. Cohen, and J. A. Tesk. 1993, 4p. Pub in Jni, of the American Ceramic Society $76, \mathrm{n} 10$ p2665-2668 Oct 93

Keywords: "Ceramics, "Bias, "Fracture(Mechanics) Error analysis, Cracks, Mechanical properties, Toughness, Regression analysis, Loads(Forces), Monte Carlo method, Reprints.

Indentation fracture toughness models generally share the derived parameter $P c(\exp -3 / 2)$, where $P$ is the indentation load and $\mathrm{c}$ the measured crack length. $\mathrm{Bi}$ ases, inherent to error propagation through this nonlinear transformation (c to c(exp 3/2)), can be introduced into calculated values for $\mathrm{K}$ (sub ic) depending upon the amount of averaging of crack length data performed prior to the transformation. The work utilizes Monte Carlo simulations to evaluate the bias in $\mathrm{K}$ (sub Ic) calculated using both mean and linear regression methods. Significant positive biases were demonstrated when using mean-based calculations where coefficients of variation (cv) in c exceeded $10 \%$. Regression methods produced significantly less bias. With $\mathrm{cv}<10 \%$ or when $\mathrm{c}$ is averaged per load, both methods produce essentially unbiased estimates for $\mathrm{K}$ (sub lc).

\section{3,033}

PB94.172970 Not available NTIS

National Inst. of Standards and Technology (MSEL) Gaithersburg, MD. Ceramics Div.
Surface Forces and Adhesion between Dissimilar Materials Measured in Various Environments.

Final rept.

D. T. Smith, and R. G. Horn. 1990, 7p.

Pub. in Materials Research Society Symposia Proceedings, v170 p3-9 1990. Sponsored by Office of Naval Research, Arlington, $V A$

Keywords: "Surface chemistry, "Adhesion, *Solids, *Dissimilar materials bonding, Materials science, Liquid phases, Vapor phases, Silicon dioxide, Mica, Ce

ramics, Reprints, Israelachvili surface force apparatus. used extensively over the past decade to make de tailed measurements of surface forces and adhesion between very smooth solids in various liquid and vapor environments. Most of those measurements have been made with mica surfaces, but the authors have recently developed a method of preparing smooth silica suraces for use in place of the micra. The silica surfaces adhere in dry and humid atmospheres, but do not adhere when immersed in water. The use of a second material not only broadens the scope of the Israelachvili technique, but also enables studies of forces and adhesion between dissimilar materials. In this work, the authors present the results of measurements of adhesion in air and forces in aqueous solution between two silica surfaces; they also report preliminary results of the adhesion between a mica surface and a silica surface.

\section{3,034}

PB94-185378 Not available NTIS

National Inst. of Standards and Technology (MSEL) Gaithersburg, MD. Ceramics Div.

Contact Electrification Induced by Monolaye Modification of a Surface and Relation to AcidBase Interactions.

Final rept.

R. G. Horn, D. T. Smith, and A. Grabbe. 1993, 2p.

Pub. in Nature 366, p442-443, 2 Dec 93.

Keywords: "Surface chemistry, "Electrification "Electric contacts, *Layers, “Acid-base equilibrium, - Silicon dioxide, Reprints, Adhesion, Coatings, Revisions, pH, Polarization(Charge separation), lon atom interactions, Surface force apparatus.

Electrical charge separation following contact between two materials (contact electrification or the triboelectric effect) is well known to occur between different materials as a consequence of their different electronic structures. Here the authors show that the phenomenon occurs between two surfaces of the same material if one is coated with a single chemisorbed monolayer. The authors use the surface force apparatus to study contact electrification and adhesion between two silica surfaces, one coated with an amino-silane. The presence of this monolayer results in significantly enhanced adhesion between the surfaces, owing to elecaccord with Derjaguin's electrostatic theory of adhesion. These observations demonstrate a link between acid-base interactions and contact electrification.

\section{3,035}

\section{PB94-198843 Not available NTIS}

National Inst. of Standards and Technology (MSEL)

Gaithersburg, MD. Reactor Radiation Div.

Analysis of SANS from Controlled Pore Glasses. Final rept.

N. F. Berk, C. J. Glinka, W. Haller, and L. C. Sander. 1990, 6p

Pub in Materials Research Society Symposia Proceedings Neutron Scattering Materials Science, v166 p409-4141990.

Keywords: *Porous materials, *Glass, Small angle scattering, Neutron scattering, Probability density func tions, Computerized simulation, Morphology, Silica Reprints

Small angle neutron scattering measurements have been performed on several samples of silica controlled pore glasses with pore sizes ranging from roughly 7 to $30 \mathrm{~nm}$. The scattering intensity is strongly peaked at small $Q$ and shows approximate Porod law behavio at large $Q$. Contrast variation measurements have shown that the pore space in these samples is entirely interconnected and thus forms a bicontinuous microstructure. The scattering data have been analyzed using the leveled wave method based on an early scheme for representing two-phase microstructures resulting from spinodal decomposition. The authors determined model probability density 
MATERIALS SCIENCES

\section{Ceramics, Refractories, \& Glass}

functions by fitting the SANS data with the leveled wave scattering function and then used these to construct leveled wave images of the corresponding porous structures.

\section{3,036}

PB94-200110 Not available NTIS

National Inst. of Standards and Technology (MSEL), Gaithersburg, MD.

Cavitation Damage During Flexural Creep of SiAION-YAG Ceramics.

Final rept

C. F. Chen, S. M. Wiederhorn, and T. J. Chuang.

1991, 5p.

Pub. in Jnl. of the American Ceramic Society $74, n 7$ p1658-1662 Jul 91 .

Keywords: "Ceramics, "Sialon, "Creep properties, "Cavitation, Flexing, High temperature tests, Tensile strength, Density measurement, Stress analysis, Reprints.

Cavitation damage in flexure bars crept at $1,170 \mathrm{C}$ was studied by a density measurement technique. The cavity density within the flexure beams could be approximated by a linear function of position from the tensile surface. A threshold stress for cavitation damage is suggested from the results of this study. Below the threshold tensile stress, cavitation ceases, whereas above the threshold, cavitation damage is in the form of wedge-shaped cracks at grain-boundary triple junctions. Cavitation is not observed in compression for the conditions used in this study. From a stress analysis
of the flexure bars, a cavitation threshold of $55 \mathrm{MPa}$ is estimated for this material.

\section{3,037}

\section{PB94-200268 Not available NTIS}

National Inst. of Standards and Technology (MSEL), Gaithersburg, MD. Ceramics Div

Analysis of Creep in a Si-SiC C-Ring by Finite Element Method.

Final rept.

T. J. Chuang, Z. D. Wang, and D. Wu. 1992,6p.

Contract GRI-TPSU-NBS-1302-37922

Pub. in Jnl. of Engineering Materials and Technology 114, p311-316 Jul 92. Sponsored by Gas Research Inst., Chicago, IL.

Keywords: "Creep buckling, "Finite element method, "Silicon carbides, Stress distribution, Stress relaxation, Creep properties, Creep tests, Displacement, Stress strain relations, Reprints.

The long-term creep deformation of a siliconized silicon carbide ceramic $\mathrm{C}$-ring subjected to a compression load at $1300 \mathrm{C}$ in air is studied by the finite element method. Based on asymmetric creep responses observed under uniaxial creep tests, multidirectional constitutive equations in power-law form are derived using the parameters of effective stress/strain. The elastic solutions are first solved and used as a guide to reach the final mesh design and as initial conditions. Solving the initial value problem in which equilibrium and constitutive equations are satisfied at all times, this model gives time-history of stresses and displacements. Fair agreements were obtained in load-point displacement rate, damage zone, neutral axis locations, stress relaxation and redistribution when compared with simple curved beam theory and experimental data. As common with other nonlinear problems, the convergence of the finite element solutions strongly depends on the time as well as the finess of the element size, particularly at the regions where principal stresses are close to the threshold stress for creep damage.

\section{3,038}

PB94-200656 Not available NTIS

National Inst. of Standards and Technology (MSEL), Gaithersburg, MD. Ceramics Div.

Transient Subcritical Crack-Growth Behavior in Transformation-Toughened Ceramics.

Final rept.

R. H. Dauskardt, W. C. Carter, D. K. Veirs, and R. O. Ritchie. 1990 10p

Pub. in Acta Metallurgica et Materialia 38, n11 p232723361990 . Sponsored by Department of Energy, Washington, DC.

Keywords: "Crack propagation, "Ceramics, "Fatigue(Mechanics), "Transients, Transformations, Loads(Forces), $\quad$ Fracture(Mechanics), Cracking(Fracturing), Mechanical properties, Reprints, "Subcritical crack growth.

Transient subcritical crack-growth behavior following abrupt changes in the applied load are studied in trans- formation-toughened ceramics. A mechanics analysis is developed to model the transient nature of transformation shielding of the crack tip, Ks, with subcritica crack extension following the applied load change. Conditions for continued crack growth, crack growth followed by arrest, and no crack growth after the load change, are considered and related to the magnitude and sign of the applied load change and to materials properties such as the critical transformation stress. Using experimentally derived steady-state subcritical crack-growth relationships for cyclic fatigue in a trans formation-toughened Mg-PSZ, the analysis is found to provide similar trends in $\mathrm{Ks}$ and accurate prediction of the post load-change transient crack-growth behavio compared to experimentally measured data.

Not available NTIS

National Inst. of Standards and Technology (MSEL), Gaithersburg, MD. Ceramics Div.

Imaging of Fine Porosity in a Colloidal Silica: Po tassium Silicate Gel by Defocus Contrast Micros copy.

Final rept.

H. Kerch, F. Cosandey, and R. Gerhardt. 1993, 14p. Pub. in Jnl. of Non-Crystalline Solids 152, p18-31 1993.

Keywords: "Silicon dioxide, "Porosity, "Potassium silicates, "Gels, Transmission electron microscopy, Image processing, Fines, Colloids, Reprints.

The fine porosity present in a 10:90 colloidal silica:potassium silicate gel was imaged in the trans mission electron microscope by the technique of defocus contrast microscopy. With this imaging method it is possible to detect very small pores ( $>$ or $=1$ $\mathrm{nm}$ ) and to obtain an accurate measure of their size and distribution. The technique allows independent measurement of the spatial location, morphology and size of the pores in the gel. Specifically, defocus contrast proves that the colloidal gel exhibits two pore populations in the micro- and mesopore range: a fine porous texture which is found uniformly across the gel particles, and larger pores with an interconnected globular morphology. This result, together with data from previous studies, showed that the gel composition possesses a polydisperse distribution of porosity which extends to many length scales. The influence of the gel structure on the resultant nitrogen sorption data is also discussed.

\section{3,040}

PB94-212958 Not available NTIS

National Inst. of Standards and Technology (MSEL) Gaithersburg, MD. Ceramics Div.

Observed and Theoretical Creep Rates for an Alumina Ceramic and a Silicon Nitride Ceramic in Flexure.

Final rept

R. F. Krause. 1992, 4p.

Pub. in Jnl. of the American Ceramic Society 75 , n5 p1307-1310 1992

Keywords: "Aluminum oxide, "Silicon nitrides, "Ceramics, "Creep rate, "Flexing, Comparison, Compressive properties, Tensile properties, Reprints.

Observed creep curvature rates are compared to theoretical rates for both an alumina ceramic at $1,000 \mathrm{C}$ and a silicon nitride ceramic at $1,200 \mathrm{C}$ in four-point flexure. The observed rates have been calculated from published rise-displacement rates, and the theoretical rates have been calculated from published power-law parameters for compressive and tensile creep, which differ appreciably for these ceramics. Although both compressive and tensile creep measurements are easier to analyze than flexural creep measurements the latter are usually less expensive and easier to conduct. The present work shows the usefulness of flexural creep tests to verify the accuracy of compressive and tensile creep tests.

\section{3,04}

PB94-216025 Not available NTIS

National Inst. of Standards and Technology (MSEL) Gaithersburg. MD. Ceramics Div

Structures of Vapor-Deposited Yttria and Zirconia Thin Films.

G. G. Long, D. R. Black, A. Feldman, D. K. Tanaka, G. G. Long, D. R. Black, A. Feldman, D. K. Tanaka,
Z. Zhang, E. N. Farabaugh, and R. D. Spal. 1992, Z. Zp.

Pub. in Thin Solid Films 217, p113-119 1992.

Keywords: "Zirconium oxides, "Yttrium oxides, "Crysta structure, $X$-ray absorption, Ceramic coatings, Bulk materials, Thin films, Porosity, Comparison, Reprints, Electron beam evaporation.

The structures of think films of zirconia and yttria, deposited by electron beam evaporation, have been examined by $X$-ray absorption fine structure (XAFS) analysis. It was found that the structure of the yttria film was similar to that of bulk yttria, which is a cubic oxide phase. The zirconia film, however, possessed a structure different from that of the bulk material. An analysis of the zirconia film data indicated a structure with a predominant tetragonal phase. Although lower coordination numbers were found in the films than in the standard powder specimens, it was not clear from the extended fine structure whether this deficit was due to film porosity observed in prior work or to the disorder that is generally observed in films. An analysis of the near-edge structure, however, suggested that porosity, rather than disorder, was present in the films. In addition, the K-edge positions observed for both the zirconia and the yttria films were higher in energy by approximately $3 \mathrm{eV}$ than the corresponding $\mathrm{K}$-edge in the powder specimens, indicating that the films may be more insulating than the standard bulk material.

03,042

PB94-216249 Not available NTIS

National Inst. of Standards and Technology (MSEL), Gaithersburg, MD. Ceramics Div.

Statistical Analysis of Parameters Affecting the Measurement of Particle-Size Distribution of Silicon Nitride Powders by Sedigraph (Trade Name). Final rept.

S. G. Malghan, L. S. H. Lum, E. Lagergren, J. Kelly, and R. N. Kacker. 1992,10p.

Pub. in Powder Technology 73, p275-284 1992.

Keywords: "Silicon nitrides, "Particle size distribution, "Powder(Particles), "Statistical analysis, Sedimentation, Experimental design, Surfactants, Regression analysis, Ultrasonic frequencies, Reprints, "Sedigraph method.

The need to achieve repeatability in the measurement of particle size distribution of submicron particles of silicon nitride powders, and its dependence on the appropriate application of deagglomeration forces and subsequent stabilization of particles from reforming into agglomerates are discussed. An analysis of parameters affecting the particle size distribution measurement by Sedigraph(Trademark), a gravity sedimentation method, is presented and major parameters are identified. Statistical factorial design of experiments was used to determine the influence of various parameters such as ultrasonic power, time and energy, type of surfactant, and concentration of surfactant in a suspension on particle size determination. The results were analyzed using statistical techniques such as graphical and least squares regression. This analysis led to the calculation of main and interaction effects of the major parameters mentioned above. Depending on the ultrasonic power level selected for a powder, the interaction effects between ultrasonic power, ultrasonic time, and surfactant concentration for the two types of surfactants were identified.

03,043

PB94-216264 Not available NTIS

National Inst. of Standards and Technology (MSEL), Gaithersburg, MD. Ceramics Div.

Characterization of Phase and Surface Composition of Silicon Carbide Platelets.

Final rept.

S. G. Malghan, M. Vaudin, J. P. Cline, M. K. Jain, P. S. Wang, and L. S. H. Lum. 1991, 9p.

Pub. in Ceram. Trans. Adv. Compos. Mater. 19, p2532611991.

Keywords: "Silicon carbides, "Surface properties, * Phase studies, Ceramics, Surface chemistry, X-ray diffraction, Carbon, Washing, Transmission electron microscopy, Photoelectron spectroscopy, X-ray spectroscopy, Electrokinetics,

Reprints, ${ }^{\star}$ Platelets(Materials)

Silicon carbide platelets manufactured by a carbothermic reduction process were characterized for bulk phase composition and surface properties by transmission electron microscopy (TEM), X-ray diffraction (XRD), X-ray photoelectron spectroscopy (XPS) and electrokinetic sonic amplitude (ESA). These studies indicated that the platelets consisted of $4 \mathrm{H}$ and $6 \mathrm{H}$ polytypes as major phases, and $39 \mathrm{H}, 15 \mathrm{R}$ and $7 \mathrm{H}$ as minor phases. The XRD established the percent weight ratio of $4 \mathrm{H}$ to $6 \mathrm{H}$ as 57 to 43 . The ESA and XPS techniques identified differences between the sur- 
face properties of two types of platelets--acid washed and unwashed. The surface of the platelets was found to contain several forms of carbon. The thickness of the oxygen layer on the two types of platelets was calculated using Si $2 p$ photoelectron data.

\section{3,044}

PB94-216272 Not available NTIS

National Inst. of Standards and Technology (MSEL), Gaithersburg, MD. Ceramics Div.

Deposition of Colloidal Sintering-Aid Particles on Silicon Nitride.

Final rept.

S. G. Malghan, P. S. Wang, A. Sivakumar, and P.

Somasundaran. $1993,18 \mathrm{p}$

Pub. in Composite Interfaces 1, n3 p 193-210 1993

Keywords: *Silicon nitrides, "Deposition, "Surface chemistry, "Colloids, Powder(Particles) Electrokinetics, Zirconium oxides, Yttrium oxides, Photoelectron spectroscopy, Sintering, $X$-ray spectroscopy, Substrates, Auger electron spectroscopy, Par ticle size, Reprints.

The deposition of alumina (Al2O3) and yttria (Y2O3) particles on silicon nitride (Si3N4) by electrostatic and electrosteric interactions was studied using acoustophoretic measurements. The characteristics of Si3N4, Al2O3, and Y2O3 powders in an aqueous environment were studied by measuring the electrokinetic sonic amplitude (ESA), and appropriate conditions for deposition by adsorption were identified. The deposition of $\mathrm{Al} 2 \mathrm{O} 3, \mathrm{Y} 2 \mathrm{O} 3$, and $\mathrm{Y}(\mathrm{NO} 3) 3$ on $\mathrm{Si} 3 \mathrm{~N} 4$ particles was conducted at different $\mathrm{pH}$ values where these chemically dissimilar particles exhibit electrical charges of opposite sign. The deposition studies indicated that the particle size has a strong effect on the amount of coating on Si3N4 particles. The presence of polyacrylic acid as an electrosteric stabilizer for Si3N4 had no measurable effect on $\mathrm{Al}_{2} \mathrm{O} 3$ adsorption. The shift of the isoelectric point of Si3N4 with $\mathrm{Al} 2 \mathrm{O} 3$ showed that the total quantity of $\mathrm{Al} 2 \mathrm{O} 3$ adsorbed on Si3N4 reached a maximum at approximately $75 \%$ cov erage of the total surface area of Si3N4. Brems strahlung-excited Auger electron spectroscopy (AES) results showed that the as-received Si3N4 powder has a native oxide film of $8.9 \mathrm{~A}$. The presence and relative differences in the surface composition of coated particles were established using $\mathrm{X}$-ray photoelectron spectroscopy (XPS)

\section{3,045}

PB94-216587 Not available NTIS

National Inst. of Standards and Technology (MSEL) Gaithersburg, MD. Ceramics Div.

Rapid Hot Pressing of Ultra-Fine PSZ Powders.

Final rept

M. D. Matthews, and A. Pechenik. 1991, 7p.

Pub. in Jnl. of the American Ceramic Society $74, n 7$ p1547-1553 1991.

Keywords: "Powder(Particles), "Zirconium oxides, "Yttrium oxides, "Compacting, "Densification, High temperature superconductors, Hot pressing, Deformation, Fines, Mechanical properties, Scanning electron microscopy, Transmission electron microscopy, X-ray diffraction, Reprints.

The process of compaction and densification of ultrafine powder of partially-stabilized zirconia with 3 mole $\%$ of $\mathrm{Y} 2 \mathrm{O} 3$ (Y3-PSZ) during rapid hot pressing was investigated. Special apparatus was designed to allow rapid application of $1.6 \mathrm{GPa}$ of quasi-isostatic pressure at temperatures of $1,100-1,300 \mathrm{C}$ to powder compacts encapsulated in glass under vacuum. Pressure was applied for 10 seconds, then the samples were rapidly cooled, removed from glass and characterized using scanning electron microscopy (SEM), transmission electron microscopy (TEM) and X-ray diffraction. Density and mechanical properties of the prepared mate rials were measured and compared with that of simila materials fabricated via conventional hot pressing.

\section{3,046}

PB95.125746 Not available NTIS

National Inst. of Standards and Technology (IMSE), Gaithersburg, MD. Ceramics Div.

Environmentally Enhanced Fracture of Ceramics.

Final rept.

S. W. Freiman. $1988,11 \mathrm{p}$

Sponsored by Office of Naval Research, Arlington, VA Pub. in Materials Research Society Symposia Proceedings, v125 p205-215 1988.

Keywords: "Ceramic materials, "Glass, "Single crystals, "Fracture(Mechanics), "Stress corrosion, Mechanical properties, Crack
Cracking(Fracturing), Polycrystalline, Bonding, Reprints.

This paper presents a review of our current understanding of environmentally induced crack growth in glasses, single crystals, and polycnystalline ceramics. It is shown that the rate of crack growth is controlled by the chemical activity of the active species in the environment as well as by the crack tip stress. A molecular model of a stress-induced chemical reaction between vitreous silica and water is described, and the implications of this model for predicting the effects of other chemical species on crack growth are discussed. Effects of chemical changes both in the bonding in the solid as well as in the reacting solutions on the crack growth mechanism are elucidated. Finally, the complicating effects of multigrain arrays on crack extension in polycrystalline ceramics are pointed out.

\section{3,047}

PB95-140208 Not available NTIS

National Inst. of Standards and Technology (MSEL), Gaithersburg, MD

New Materials, Advanced Ceramics and Standards. Final rept.

S. Schneider. 1991, 6p

Pub. in Key Engineering Materials 53-55, p480-485 1991.

Keywords: ${ }^{*}$ Ceramics, "Standards, ${ }^{*}$ Classifications, *Materials science, Materials tests, Comparison, Mechanical tests, Structural engineering, Marketing, Reprints, *Advanced materials.

Today's technological world might well be termed the age of new materials for industrial needs that now require engineered materials that perform totally new functions or old functions in much better ways. Unfortunately, companion standards development has not kept pace with technology advances and this lack rep. resents one of the more important technical unknowns in the commercial market equation. For advanced ceramics the problem is acute for currently there are no consensus standards that allow national or international comparisons. While the standards needs are diverse and product specific there are two areas that crosscut all advanced ceramic product lines. First, there is an international need for a unified classification system that uniquely categories an array of advanced products by a series of set apart features, like application and materials chemistry. Second, there is need for a set of mechanical test standards to assure the structural reliability of advanced ceramics. This paper presents a perspective on these issues and problems, in the context of the age of new materials.

\section{3,048}

PB95-140570 Not available NTIS

National Inst. of Standards and Technology (MSEL), Gaithersburg, MD. Ceramics Div.

Phase Equilibria in the Systems $\mathrm{CaO}-\mathrm{CuO}$ and $\mathrm{CaO}-\mathrm{Bi} 2 \mathrm{O} 3$.

Final rept.

R. S. Roth, N. M. Hwang, C. J. Rawn, B. P. Burton, and J. J. Ritter. 1991, 4p.

Pub. in Jnl. of the American Ceramic Society $74, n 9$ p2148-21511991.

Keywords: *Bismuth oxides, "Calcium oxides, "Copper oxides, Binary mixtures, Polymorphism, Superconductors, Reprints, "Calcium bismuthates, "Copper bismuthates, Phase equilibrium.

New data are presented on the phase equilibria of the binary systems $\mathrm{CaO}-\mathrm{CuO}$ and $\mathrm{CaO}-\mathrm{Bi} 2 \mathrm{O} 3$. Corrected compositions are reported for $\mathrm{Ca} 4 \mathrm{Bi6013}$ and $\mathrm{Ca} 2 \mathrm{Bi} 2 \mathrm{O} 5$ and a new high temperature polymorph is reported for $\mathrm{Ca} 2 \mathrm{Bi} 2 \mathrm{O} 5$. The composition and decomposition temperatures for $\mathrm{Ca}(1-\mathrm{x}) \mathrm{CuO} 2$ are given for both air and one atmosphere oxygen.

\section{3,049}

PB95-161303 Not available NTIS

National Inst. of Standards and Technology (MSEL), Gaithersburg, MD. Ceramics Div.

Tensile Creep of a Silicon Nitride Ceramic.

Final rept.

R. F. Krause, and S. M. Wiederhorn. 1994, 6p. Pub. in Key Engineering Materials 89-91, p619-624 1994

Keywords: "Silicon nitrides, "Ceramic materials, "Tensile creep, Failure, Least squares method, Strain rate, Fatigue(Materials), Tensile properties, Reprints.

The tensile creep behavior of a hot isostatically pressed silicon nitride ceramic containing 6 percent yttria was charactenzed at temperatures between 1250 and $1400 \mathrm{C}$ and applied stresses between 72 and 250 MPa. Creep extension of specimens with nominal 10 $\mathrm{mm}$ gage length was measured by laser extensometry showing final extensions between 20 and 200 micrometers. Secondary creep strain rates varied from 0.3 to 3000 Gs (exp -1). Some tests ended with specimen failure between 1 and $500 \mathrm{~h}$, but other tests were discontinued after 1000 up to $2500 \mathrm{~h}$ without failure. A leastsquares fit of the Norton and Arrhenius functions to 31 strain-rate tests yielded a stress exponent of $8.4+0$ - 0.9 and an activation energy of $1310+$ or - $140 \mathrm{~kJ} /$ mol. A Monkman-Grant function was fitted to represent strain rate versus failure time.

\section{3,050}

PB95-161501 Not available NTIS

National Inst. of Standards and Technology (MSEL), Gaithersburg, MD. Ceramics Div

Analysis of Physical Properties of Ceramic Powders in an International Interlaboratory Comparison Program.

Final rept.

S. G. Malghan, S. M. Hsu, A. L. Dragoo, H. Hausner and R. Pompe. 1991, 16p.

Sponsored by Department of Energy, Washington, DC Pub in Proceedings of International Symposium on Ceramic Materials and Components for Engines (4th) p920-935 1992.

Keywords: "Interlaboratory comparisons, "Physical properties, "Ceramics, "Powder(Particles), International cooperation, Particle size distribution, Surface properties, Area, Density(Mass/volume), Reprints.

Accuracy in the measurement of physical characteristics of ceramic starting powders is a critical factor in the control and reproducibility of powder processing unit operations. An international interlaboratory comparison program on powders characterization has been in progress under the auspices of the International Energy Agency. In this paper, the results of powder characterization effort on five powders by 25 industrial, university and governmental laboratories are presented. Selected results of density, specific surface area, and particle size distribution are presented and discussed in terms of factors affecting these measurements. The reasons for the discrepancies in the data are outlined.

\section{3,051}

PB95-162681 Not available NTIS

National Inst. of Standards and Technology (IMSE), Gaithersburg, MD

Recent VAMAS Activity in Ceramics.

Final rept.

L. H. Schwartz, and B. Steiner. $1990,3 p$.

Pub. in Ceramic Bulletin 69, n3 p312-314 1990

Keywords: "Ceramics, "Mechanical tests, "Standards, Fracture tests, Mechanical properties, Hardness tests, Classifying, Wear tests, International coordination, Reprints, "Advanced materials, VAMAS(Versailles Project on Advanced Materials and Standards)

Current activity dealing with ceramics in the Versailles Project on Advanced Materials and Standards (VAMAS) is reviewed. The activity includes enhanced environmental fracture testing, hardness testing, wea testing, and classification. The nature of VAMAS is described.

03,052

PB95-163069 Not available NTIS

National Inst. of Standards and Technology (MSEL), Gaithersburg, MD

Real-Time Small-Angle X-Ray Scattering Study of the Early Stage of Phase Separation in the SiO2BaO-K20 System.

Final rept.

G. B. Stephenson, W. K. Warburton, W. Haller, and A. Bienenstock. 1991, 21p.

Pub. in Physical Review B 43, n16 p13417-13437 1991.

Keywords: "X-ray scattering, *Phase separation(Materials), "Silicates, Small' angle scattering, Spinodal decomposition, Glass, Real time operations, Silicon dioxide, Barium oxides, Potassium oxides, Amorphous materials, Deformation, Reprints.

Time-resolved small-angle $x$-ray scattering was used to study the early stage of phase separation in a silicate system. Scattering patterns were acquired in real time as a sample was quenched in situ from a temperature $\mathrm{T} 1$ in the single-phase region directly to a tem- 
MATERIALS SCIENCES

\section{Ceramics, Refractories, \& Glass}

perature $\mathrm{T} 2$ in the two-phase region and held isothermally. This was made possible by using the high $x$-ray intensity from a synchrotron radiation source, a fast position-sensitive detector based on a photodiode array, a sample heater capable of rapid quenches, and an appropriate sample composition. The composition studied, 90-mole-\% $\mathrm{SiO} 2,5-\mathrm{mole}-\% \mathrm{BaO}$, and 5-mole$\% \mathrm{~K} 2 \mathrm{O}$, undergoes subliquidus phase separation at temperatures below $1,021 \mathrm{~K}$ to form alkali-rich and at kali-poor amorphous phases. This is the best agreement between experiment and spinodal decomposition theory yet demonstrated for any system. For amor phous systems in particular, these results provide direct evidence that deformation is the rate-limiting step for the interdiffusion of network-forming and network modifying species over the short length scales involved in the early stage of phase separation.

\section{3,053}

PB95-164570 Not available NTIS

National Inst. of Standards and Technology (MSEL), Gaithersburg, MD. Ceramics Div.

Measuring Contact Charge Transfer at Interfaces: A New Experimental Technique.

Final rept.

D. T. Smith. $1991,18 \mathrm{p}$

Contract N0014-88-F-0034

Sponsored by Office of Naval Research, Arlington, VA. Pub. in Jnl. of Electrostatics 26, p291-308 1991

Keywords: "Surface energy, "Electric charge, "Charge transfer, *Ceramics, *Solid-solid interfaces, Experimental design, Silicon dioxide, Mica, Materials science, Interfaces, Test chambers, Charge density, Reprints.

A new experimental technique has been developed for measuring the amount of electric charge transferred when two dissimilar surfaces are brought into contac and separated without lateral motion in a controlled environment. The primary surfaces studied, silica and mica, are prepared with very low surface roughness (average roughness less than $0.5 \mathrm{~nm}$ ); the macroscopic contact area, which can be measured directly, is therefore a better measure of microscopic contact area than is typically the case in contact electrification experiments, and the surface charge density can be more accurately determined. Observations reported include (1) the transfer of charge densities as high as $.01 \mathrm{C} / \mathrm{sq} \mathrm{m}$ after only a few contacts of a mica and silica surface in dry air or $\mathrm{N} 2$ gas at room temperature and (2) a reduction in transferred charge density in the silica-mica system as the relative humidity of the air in the environmental chamber is increased from $0 \%$ to $98 \%$. The technique also permits the study of the rate at which the surface charge decays under various environmental conditions.

\section{3,054}

PB95-175493 Not available NTIS

National Inst. of Standards and Technology (MSEL), Gaithersburg, MD. Ceramics Div.

Fabrication of Transparent gamma-Al2O3 from Nanosize Particles.

Final rept.

M. R. Gallas, B. Hockey, A. Pechenik, and G. J.

Piermarini. 1994, 6p.

Pub. in Jnl. of the American Ceramic Society $77, \mathrm{n} 8$ p2107-2112 Aug 94 .

Keywords: "Aluminum oxides, Transmission electron microscopy Temperature dependence, Pressure dependence, Heat treatment, Transparence, Hardness, Compacting, Temperature range 0065-0273 K, Room temperature, Reprints, Diamond anvils, Nanomaterials.

The compaction and heat-treatment behavior of nanosize gamma-Al2O3 powder (average diameter = $20 \mathrm{~nm}$ ) was studied. A diamond anvil high-pressure cell was used to compact the powder at pressures up to $3 \mathrm{GPa}$, both in air at room temperature and under liquid nitrogen, followed by pressureless heat treatment at $800 \mathrm{C}$. For all conditions studied, the fabricated compacts were optically transparent. X-ray diffraction confirmed retention of the gamma-phase. The compacts were also characterized before and after heat treatment by microhardness measurements and by transmission electron microscopy. For both ambient and cryogenic compaction, sample hardness increased with pressure, and heat treatment resulted in about $50 \%$ increase in hardness independent of the initial green-state value. Samples compacted in LN2 were significantly harder (up to $9.6 \mathrm{GPa}$ ) than those compacted in air
03,055

PB95-175568 Not available NTIS

National Inst. of Standards and Technology (MSEL), Gaithersburg, MD. Ceramics Div.

Polyelectrolytes as Dispersants in Colloidal Processing of Silicon Nitride Ceramics.

Final rept.

V. A. Hackley, and S. G. Malghan. 1993, 2p.

Pub. in Polymer Preprints 34, $n 1$ p1024-1025 Mar 93.

Keywords: "Silicon nitrides, "Ceramics, "Colloiding, "Dispersants, "Polyelectrolytes, Powder(Particles), Sintering, Carboxylates, High temperature tests, Rheology, Homogeneity, Reprints.

Colloidal processing of submicron powders has become increasingly important in the commercial production of structural ceramic shapes. Silicon nitride is one of the materials of choice for high temperature loadbearing applications such as turbines and bearings for heat engines. To obtain chemical and microstructural homogeneity in the final sintered product, the powders must be dispersed at high solid loadings (up to $60 \%$ by volume) and then consolidated and dried. Carboxylate polyelectrolytes are routinely added to aqueous slurries to act as dispersants and obtain suitable rheological properties. Recipes for slurry preparation are often determined by trial and error, without specific knowledge of the complex interactions between solution, polyelectrolyte and colloidal particles. We have examined the electrostatic component of these interactions for PAA and PMMA using an electroacoustic technique and adsorption isotherms. These results are correlated with polymer structure and molecular weight and with the fluidity of dense slurries.

\section{3,056}

PB95-175576 Not available NTIS

National Inst. of Standards and Technology (MSEL), Gaithersburg MD Ceramics Div.

Surface Chemical Interactions of Si3N4 with Polyelectrolyte Deflocculants.

Final rept.

V. A. Hackley, R. Premachandran, and S. G.

Malghan. 1994, 4p.

Pub. in Key Engineering Materials 89-91, p679-682 1994.

Keywords: "Silicon nitrides, ${ }^{*}$ Colloiding, "Surface chemistry, "Polyelectrolytes, "Deflocculating, Electroacoustics, Solid solutions, Interfaces, Particle size distribution, Cations, Anions, $\mathrm{pH}$, acnlic acids Reprints, Poly(diamine epoxychlorohydrin), Ammonium poly(methacrylate).

The interaction of organic polyelectrolyte deflocculants at the Si3N4 solid-solution interface was investigated using electroacoustic measurements, polymer adsorption and particle size distribution analysis. One cationic and two anionic polymers were studied: quaternized poly(diamine epoxychlorohydrin), ammonium poly(methacrylate) and poly(acrylic acid), respectively. Electrostatic interactions are emphasized as a function of $\mathrm{pH}$, concentration and molecular weight.

\section{3,057}

PB95-175584 Not available NTIS

National Inst. of Standards and Technology (MSEL) Gaithersburg, MD. Ceramics Div.

Effects of Soxhlet Extraction on the Surface Oxide Layer of Silicon Nitride Powders.

Final rept

V. A. Hackley, P. S. Wang, and S. G. Malghan. 1993, 7p.

Pub. in Materials Chemistry and Physics 36, p112-118 1993.

Keywords: "Silicon nitrides, *Powder(Particles), "Surface chemistry, "Cleaning, " ${ }^{*}$ Colloids, ${ }^{*}$ Oxide films, Electroacoustics, Isoelectronic sequence, $\mathrm{pH}, \mathrm{X}$ ray spectroscopy, Auger spectroscopy, Interfaces, Solid solutions, Reprints, *Soxhlet extraction.

An aqueous soxhlet extraction procedure was used to surface-clean five commercial silicon nitride powders. The solid-solution interface properties were characterized before and after extraction by electrokinetic sonic amplitude measurements. The isoelectric point $\mathrm{pH}$ (sub iep) was found to increase significantly for some powders after treatment. The powder surface was analyzed using $X$-ray photoelectron spectroscopy and $X$ ray induced Auger electron spectroscopy before and after extraction. The surface oxygen content and oxide layer thickness decrease after treatment. A linear correlation was found between oxide thickness and $\mathrm{pH}$ (sub iep) which yields a pristine $\mathrm{pH}$ (sub iep) of about 9.7 for the unoxidized Si3N4 particle.

03,058

PB95-180931 Not available NTIS

National Inst. of Standards and Technology (BFRL) Gaithersburg, MD. Building Materials Div.

Calculation of the Thermal Conductivity and Gas Permeability in a Uniaxial Bundle of Fibers.

Final rept.

D. J. Skamser, D. P. Bentz, R. T. Coverdale, H. Jennings, D. L. Johnson, M. S. Spotz, and N. Martys. 1994, 12p.

Contract N00014-90-J-4020

Sponsored by Defense Advanced Research Projects Agency, Arlington, VA and Office of Naval Research Arlington, VA.

Pub. in Jnl. of the American Ceramic Society 77, n10 p2669-26801994.

Keywords: "Ceramic fibers, *Permeability, "Thermal conductivity, "Transport properties, Gas flow, Bundles, Microstructure, Thermophysical properties, Conduction(Heat transfer), Algorithms, Ceramics, Stokes law(Fluid mechanics), Reprints.

A model of the local microstructure of a bundle of fibers is simulated and used as the basis for calculations of transport properties. This, in turn, can be used in a macroscopic model of the chemical vapor infiltration process An expanding/overlapping circle representation of the microstructure simulates the deposition of matrix in a uniaxial bundle of fibers. An iterative heat conduction algorithm is used to calculate the trans. verse thermal conductivity based on the thermal conductivities of the solid and gas phases. The permeability of gas through the microstructure is calculated for flow both parallel and transverse to overlapping cylinders using a Strokes equation and assuming a Darcy's law behavior. Both the simulations of the microstructure and associated calculations of the transport properties compare favorably with experimental data.

03,059

PB95-202750 Not available NTIS National Inst. of Standards and Technology (MSEL) Gaithersburg, MD.

Stability and Surface Energies of Wetted Grain Boundaries in Aluminum Oxide.

Final rept.

D. Y. Kim, S. M. Wiederhorn, B. J. Hockey, C. A.

Handwerker, and J. E. Blendell. 1994, 10p

Pub. in Jnl. of the American Ceramic Society $77, \mathrm{n} 2$ p444-453 Feb 94

Keywords: "Aluminum oxide, *Grain boundaries, "Free energy, "Stability, "Surfaces, Crystal lattices, Interfaces, Wettability, Microstructure, Reprints.

The stability of a calcium-aluminum-silicate liquid film between two near-basal plane surfaces of sapphire at $1650 \mathrm{C}$ was studied. Samples were prepared having an average basal misorientation across the interface of 6-7 deg about (10-10). Three types of interfaces were observed: faceted, solid-liquid interfaces; lowangle grain boundaries consisting of aligned arrays of dislocations; and boundaries consisting of alternating regions of dislocations and faceted solid-liquid interfaces. The type of interface observed depended on the orientation of the interface and could be predicted by using a construction based on Wulff shapes. Because the type of interface depends on crystal alignment and interface angle, these results suggest an absolute method of determining the surface free energy of wetted boundaries.

03,060

PB95-203758 PC A04/MF A01

Pennsylvania State Univ. University Park. Center for Advanced Materials.

Structural Ceramics Database. Topical Report, June 1989-May 1991

E. F. Begley, and R. G. Munro. May 91, 65p, CAM9201, GRI-92/0042

Contracts GRI-5084-238-1302, GRI-TPSU-NBS

1302-379

Prepared in cooperation with National Inst. of Standards and Technology (MSEL), Gaithersburg, MD. Ceramics Div. Sponsored by Gas Research Inst., Chicago, IL.

Keywords: "Ceramics, "Information systems, "Data bases, "Research projects, "Structural engineering, 
Physical properties, Chemical properties, Criteria, High temperature tests, Implementation, Heat resistant materials, "Structural Ceramics Database System, *Advanced materials.

The objective of the project was to strengthen the link between the development of new materials in research laboratories and the application of these materials by design engineers. The medium for improving this link is a user-friendly database on materials properties of advanced ceramics. Papers containing data appropriate for the structural ceramics database (SCD) were identified by a computerized search of the open technical literature. Acceptance criteria were applied during careful review of the papers. At the end of this process. approximately seventy-five papers remained. Material and property information were extracted and evaluated by experts within the Ceramics Division at NIST who were particularly concerned with assessing the material specifications, the measurement methods and procedures, and the reliability of the data. Concurrently, user-friendly database software was developed to manage the data on personal computers. With this software, users can easily find the properties of a given material or identify materials that meet specified property values. After completion of code development and data entry, test sites were selected to review the database system.

\section{3,061}

PB95-210498 PC A03/MF A01

National Inst. of Standards and Technology (MSEL), Gaithersburg, MD. Ceramics Div.

Room-Temperature Flexure Fixture for Advanced Ceramics.

G. D. Quinn. Aug 92, 22p, NISTIR-4877.

See also PB92-222959.

Keywords: "Ceramics, "Test facilities, "Fixtures, *Temperature effects, Flexural strength, Room temperature, Standards, Design criteria, Mechanical properties, Loads(Forces), "Advanced materials.

A test fixture for the measurement of the flexure strength of advanced ceramic specimens is presented. This report presents a four-point flexure fixture that is in accordance with the requirements of U.S. Army MIL STD 1942 and ASTM C 1161. These standards prescribe specific specimen and fixture sizes, tolerance, and alignment requirements. The standards specify that the fixture must have sufficient articulation to ensure even line-loading pins. The fixture is designed so that it can be set up and aligned easily and quickly (about 30 minutes). The fixture in this report is intended for use with flat and parallel specimens.

\section{3,062}

\section{PB95-220547 PC A05/MF A02}

National Inst. of Standards and Technology (MSEL), Gaithersburg. MD. Ceramics Div.

Evaluation of Thermal Wave Imaging for Detection of Machining Damage in Ceramics.

L. Wei, H. H. K. Xu, and S. Jahanmir. Mar 95, 100p, NISTIR-5645.

Contract DE-AC05-840R21400

Color illustrations reproduced in black and white. Sponsored by Department of Energy, Washington, DC Office of Transportation Technologies. and Martin Marietta Energy Systems, Inc., Oak Ridge, TN.

Keywords: *Ceramics, "Machining, "Damage assessment, "Imaging techniques, Grinding(Material removal), Cracks, Subsurface investigations, Nondestructive tests, Performance tests, Aluminum oxide, Silicon nitrides, "Thermal wave imaging.

The feasibility of a thermal wave imaging technique is evaluated for the detection and characterization of machining damage in ceramics. First, a well-defined crack system produced by Vickers indentation is used to examine the characteristic response of thermal wave signals to different forms of cracks (i.e., median/radial and lateral). The results clearly indicate that thermal wave imaging can be used for the detection of surface and subsurface cracks in ceramics. Thermal wave imaging is then conducted on the surfaces of ground alumina and the cross-section of scratched silicon nitride. Careful examinations of the thermal wave images confirm that this technique can be used to detect the grindinginduced subsurface microcracks. The limitations of the thermal wave imaging technique in the detection of small cracks and precautions regarding choosing ap propriate sets of imaging parameters are pointed out.
National Inst. of Standards and Technology (MSEL) Gaithersburg, MD. Ceramics Div.

Applications of Diamond Films and Related Materials: International Conference (3rd). Held in Gaithersburg, Maryland on August 21-24, 1995. Supplement to NIST Special Publication 885.

A. Feldman, W. A. Yarbrough, M. Murakawa, $Y$ Tzeng, and M. Yoshikawa. 1995, 52p, NISTIR-5692SUP

See also PB95-255204. Prepared in cooperation with Auburn Univ., AL., Pennsylvania State Univ., University Park. and Nippon Inst. of Tech., Saitama. Sponsored by Tokyo Inst. of Tech. (Japan)

Keywords: *Diamonds, *Thin films, "Technology utilization, "Meetings, Raman spectra, Spectroscopic analysis, Vapor deposition, Epitaxy, Crystal defects, Crystal lattices, Crystal growth, Polycrystals, Cutting tools, Degradation, Annealing, Adhesion, Electrical resistivity, CVD(Chemical vapor deposition).

This supplement contains ten papers which had not been included in the conference proceedings of the Applied Diamond Conference 1995 (ADC'95) due to late arrival. In addition, the supplement contains two errata that correct two papers that appear in the proceedings.

03,064

PB96-102975 Not available NTIS

National Inst. of Standards and Technology (MSEL) Gaithersburg, MD. Reactor Radiation Div.

$X$-ray Powder Diffraction from Carbon Nanotubes and Nanoparticles.

Final rept.

D. Reznik, C. H. Olk, D. A. Neumann, and J. R. D. Copley. 1995, 9p.

Pub. in Physical Review B, v52 n1 p116-124 Jul 95

Keywords: *Powders, *Diffraction, Reprints, $X$ ray diffraction, Interlayers, Cross section, "Nanomaterials.

We report detailed $x$-ray powder-diffraction measurements on a sample consisting of approximately 60 carbon nanotubes and approximately 40 carbon nanoparticles. These measurements demonstrate the existence of short-range interlayer stacking correlations. Our calculations show that such correlations should not be observable in idealized models of nanotubes. The observation of short-range interlayer correlations can be explained if many of the nanotubes and nanoparticles have polygonal cross sections, largely consisting of flat regions having graphitic interlayer correlations. This polygonization is almost cer tainly driven by the van der Waals interactions responsible for the $A B A B$ stacking in crystalline graphite.

\section{3,065}

PB96-102983 Not available NTIS

National Inst. of Standards and Technology (MSEL) Gaithersburg, MD. Ceramics Div.

Damage Processes in Ceramics Resulting from $\mathrm{Di}$ amond Tool Indentation and Scratching in Various Environments.

Final rept.

A. W. Ruff, H. Shin, and C. J. Evans. 1995, 12p.

Pub. in Wear Jnl. 181-183, p551-562 1995.

Keywords: *Ceramic materials, "Diamonds, Reprints Damage, Tools, Scratching, ${ }^{\star}$ Foreign technology.

Studies have been carried out to determine the influ ence of different chemical environments and too shapes on damage produced during diamond tool scratching and indenting of two advanced ceramics: chemical-vapor-deposited silicon carbide and a composite aluminum oxide-titanium carbide. A nanoindentation/scratching apparatus was used in a controlled-load mode for the studies. Two diamond too shapes were used: a wedge with -45 degrees rake and $0.5 \mathrm{~mm}$ radius curved edge, and a Vickers pyramid. The environments studied were: air, water, mineral oil, mineral oil + stearic acid and two commercial waterbased fluids. It was of interest to identify damage mechanisms, critical loads for initiation of severe damage, specific scratching energies and the effect on damage of the different tool shapes.

\section{3,066}

PB96-111646 Not available NTIS

National Inst. of Standards and Technology (CSTL) Gaithersburg, MD. Surface and Microanalysis Science Div.
Photodesorption Dynamics of $\mathrm{CO}$ from Si(111): The Role of Surface Defects.

Final rept.

P. M. Chu, S. A. Buntin, L. J. Richter, and R. R.

Cavanagh. 1994, 6p.

Pub. in Surface Science, v321 p127-1321994.

Keywords: “Silicon, “Carbon monoxide, “Desorption, "Surface defects, Etching, Ion beams, Annealing, Diagnostic techniques, Photochemical reactions, Reprints, "Photodesorption.

Laser-induced desorption of $\mathrm{CO}$ from an ion beam etched and annealed $\mathrm{Si}(111)$ surface is reported State-resolved measurements of the desorbed CO reveal very high translational and vibrational energy contents, with the rotational excitation being quite low. The results suggest that the $\mathrm{CO}$ photodesorption is derived from $\mathrm{CO}$ bound to ion beam etch-induced active defect site(s) on the $\mathrm{Si}(111) 7 \times 7$ surface that are only minimally influenced by either the ion beam etching conditions or the anneal. Comparison of the photoyields at 266 and $355 \mathrm{~nm}$ suggests that the desorption mechanism cannot be described using existing models for thermal- or carrier-mediated processes.

\section{3,067}

PB96-111703 Not available NTIS

National Inst. of Standards and Technology (MSEL), Gaithersburg, MD. Ceramics Div

Silicon Nitride Boundary Lubrication: Lubrication Mechanism of Alcohols.

Final rept.

R. S. Gates, and S. M. Hsu. 1995, 9p

See also PB95-213583 and PB96-111711.

Pub. in STLE Tribology Transactions, v38 n3 p645-653 1995

Keywords: "Silicon nitrides, "Lubrication, "Alcohols, *Wear tests, Chemical reactions, Chemical analysis, Boundaries, Friction, Tribology, Wear, Alkoxides, Reprints, "Boundary lubrication, GPC-GFAA analysis, Silicon alkoxides.

This paper describes the lubrication mechanism of alcohols with silicon nitride under boundary lubrication conditions. Dynamic wear tests and static chemical reaction studies were conducted to study the chemical interaction between alcohols and silicon nitride. Direct evidence of chemical reactions occurring between alcohols and silicon nitride was collected. Gel-permeation-chromat ography-graphite- furnace-atomic-absorption (GPC-GFAA) analysis detected the presence of high molecular weight (HMW), silicon-containing, metallo-organic compounds in the wearing contact. Secondary ion mass spectrometry (SIMS) analysis of the reactionproducts from wear tests revealed the formation of silicon alkoxides. These alkoxide subsequently reacted to form HMW products which had been independently verified as capable of lubricating silicon nitride surfaces.

\section{3,068}

PB96-111711 Not available NTIS

National Inst. of Standards and Technology (MSEL), Gaithersburg, MD. Ceramics Div.

Silicon Nitride Boundary Lubrication: Effect of Oxygenates.

Final rept.

R. S. Gates, and S. M. Hsu. 1995, 12p

See also PB96-111703.

Pub. in STLE Tribology Transactions, v38 n3 p607-617 1995.

Keywords: "Silicon nitrides, "Wear tests, "Lubrication Oxygen compounds, Boundaries, Friction, Tribology, Wear, Lubricating oils, Alcohols, Reprints, *Boundary lubrication, BTF(Ball-on-three-flat), Oxygenates.

A ball-on-three-flat (BTF) wear tester was used to investigate the boundary lubricating characteristics of oxygenates on a commercial silicon nitride. A wide variety of oxygen-containing compounds were tested neat and/or at $1 \%$ by weight in a paraffin oil. Compounds containing hydroxyl functional groups were more effective compared to a base case of neat paraffin oil. Decreases of up to $58 \%$ in friction coefficient, and $95 \%$ in wear were obtained. In most cases, films were observed in and around the wear scar, suggesting chemical reactions had taken place in the contact. Additional wear tests, conducted using neat shorterchain linear primary alcohols, i.e., 6-10 carbons, demonstrated boundary lubrication protection, with longer chain length providing better antiwear performance.

\section{3,069}

PB96-111919 Not available NTIS 


\section{Ceramics, Refractories, \& Glass}

National Inst. of Standards and Technology (MSEL), Gaithersburg, MD. Reactor Radiation Div.

Small Angle Neutron Scattering Study of the Struc. ture and Formation of Ordered Mesopores in Silica. Final rept.

C. J. Glinka, J. M. Nicol, G. D. Stucky, Q. Huo, E.

Ramli, and D. I. Margolese. 1995, 6p.

Pub. in Materials Research Society Symposia Proceedings, v371 p47-52 1995.

Keywords: "Porous materials, "Neutron scattering, *Silica, Reprints, Small angle scattering, Molecular sieves, Mesoporous materials.

The nanoscale structure and synthesis mechanisms of a recently developed class of inorganic mesoporous materials with ordered arrays of uniform mesopores have been investigated by small angle neutron scattering (SANS). SANS measurements with solvents imbibed into the pores to vary the scattering contrast demonstrate that the low angle diffraction peaks from these materials are entirely due to the pore structure and that the pores are fully accessible to both aqueous and organic solvents. SANS measurements on the concentrated cationic surfactant and silicate that the existence of preassembled supramolecular arrays that mimic the final pore structure is not essential for the synthesis of these materials.

\section{3,070}

PB96-112339 Not available NTIS

National Inst. of Standards and Technology (MSEL), Boulder, CO. Materials Reliability Div.

Torsion Modulus and Internal Friction of a FiberReinforced Composite.

Final rept.

H. Ledbetter, and M. Lei. 1994, 2p

Pub. in Jnl. of Applied Physics, v76 n5 p3212-3213 1994

Keywords: "Fiber composites, "Glass fiber reinforced plastics, "Epoxy matrix composites, Reprints, Torsional stress, Elastic properties, Ultrasonics, Internal friction.

By a kilohertz-frequency resonance method, we determined the torsion modulus and internal friction of a uniaxially fiber-reinforced composite. The composite was composed of glass fibers in an epoxy-resin matrix. We studied three fiber contents: 0.41 and 49 vol \%. The internal friction failed to fit a classical freedamped-oscillator model where one assumes a linear rule-of-mixture for three quantities: oscillator mass, force constant, and mechanical-resistance constant. The torsion modulus approximately fits a plane-wave scattering ensemble-average model. The microstructure showed strong fiber-distribution nonhomogeneity. Considering this nonhomogeneity yielded a better agreement between model and observation. Thus, torsion-modulus measurements provide a method to detect and quantify fiber-distribution nonhomogeneity.

03,071
PB96-122510 Not available NTIS

National Inst. of Standards and Technology (MSEL), Gaithersburg, MD. Ceramics Div.

Epitaxial Growth of BaTiO3 Thin Films at $600 \mathrm{C}$ by Metalorganic Chemical Vapor Deposition.

Final rept.

D. L. Kaiser, M. D. Vaudin, L. D. Rotter, C. S.

Hwang, R. B. Marinenko, J. G. Gillen, Z. L. Wang, and J. P. Cline. 1995, 3p.

Pub. in Applied Physics Letters, v66 n21 p2801-2803 May 95

Keywords: *Barium compounds, Chemical vapor deposition, Reprints, Thin films, Temperature, ${ }^{\star}$ Epitaxial growth.

$\mathrm{BaTiO} 3$ thin films were grown epitaxially on (100) $\mathrm{MgO}$ substrates by metalorganic chemical vapor deposition (MOCVD) at a temperature of 600 degrees C. This substrate temperature is the lowest reported temperature for the growth of epitaxial BaTiO3 films by an MOCVD process. The films had a cube-cube orientation relationship with the substrate and were oriented with an a-axis perpendicular to the substrate plane. Nanoscale energy dispersive $x$-ray spectrometry measurements showed no evidence of interdiffusion between the film and substrate.

\section{3,072}

PB96-122999 Not available NTIS

National Inst. of Standards and Technology (MSEL), Gaithersburg, MD. Ceramics Div.
Chemically Assisted Machining of Si3N4.

Final rept.

C. J. Hsy, J. C. Wang, and S. M. Hsu. 1995, 17p Pub. in Proceedings of the CIMTEC (8th): High Performance Materials in Engine Technology, Faenza, Italy, June 28-July 4, 1994, p497-513.

Keywords: "Silicon nitride, "Ceramics, Cutting, Hydration, Machining, Surface chemistry, Surfac films, Reprints, "Chemical assisted machining.

Ceramics are hard and brittle. Machining such materials is time-consuming, difficult, and expensive. Current machining technology requires ultra-stiff tools, diamond-bonded wheels, and low material removal rate to minimize surface damage. This study demonstrates the feasibility of a novel ceramic machining concept that utilizes tribo-chemical reactions at the toolworkpiece interface to reduce the stress and minimize the surface damage. A series of cutting tests using a diamond wheel on silicon nitride with different chemical compounds has been conducted. Results suggest that by using different chemistries, the material removal rate and the surface finish of the machined Si3N4 surface can be significantly altered. Halogenated hydrocarbons show significant improvement over some commercial machining fluids currently in use.

03,073

PB96-123575 Not available NTIS

National Inst. of Standards and Technology (MSEL), Gaithersburg, MD. Ceramics Div.

Electrokinetic Sonic Analysis of Silicon Nitride Suspensions.

Final rept.

V. A. Hackley, R. S. Premachandran, and S. G.

Malghan. 1992, 8p.

Pub. in American Chemical Society, p141-148 1992

Keywords: "Electroacoustics, "Silicon nitrides soelectric point, Electrokinetics, Reprints, Interfacial chemistry.

In colloidal processing of ceramic slips, reactions occurring at the solid-solution interface play a dominant role in the dispersion properties. Electrokinetic sonic amplitude (EsA) measurements have been used to characterize the aqueous interfacial chemistry of Si3N4 suspensions. The ESA signal is proportional to the dynamic mobility and depends on the colloid shearplane potential. Samples were subjected to acidic and alkaline soxhlet extraction, then titrated potentiometrically. Variations in the ESA signal and isoelectric $\mathrm{pH}$ indicate differences in the surface charge behavior of each powder. These differences may arise from surface contamination by specifically absorbed ionic species or modification of potential determining surface sites as a result of treatment history. This paper is intended to provide hands-on information regarding the electrokinetic sonic analysis of aqueous ceramic dispersions.

\subsection{4}

PB96-135132 Not available NTIS

National Inst. of Standards and Technology (BFRL) Gaithersburg, MD. Building Materials Div.

Modelling Drying Shrinkage of Cement Paste and Mortar. Part 1. Structural Models from Angstroms to Millimeters.

Final rept.

D. P. Bentz, D. A. Quenard, V. Baroghel-Bouny, E. J. Garboczi, and H. M. Jennings. 1995, 9p.

Pub. in Materials and Structures, v28 p450-458 1995

Keywords: *Calcium silicates, "Hydrates, *Drying, "Shrinkage, Cements, Pases, Mortars(Material), Concretes, Physical properties, Sorption, Sorptive properties, Microstructure, Models, Simulation, Reprints.

The nanostructure of calcium silicate hydrate (C-S-H) gel contributes to many physical properties of concrete, including the important engineering properties of creep and shrinkage. A set of structural models for this gel and computational techniques for their validation have been developed. The basic nanostructure of $C$. $\mathrm{S}-\mathrm{H}$ is conceived as a self-similar agglomeration of spherical particales at two levels (diameters of $5 \mathrm{~nm}$ and $40 \mathrm{~nm}$ ). Computational techniques are presented for simulating transmission electron microscopy images and computing sorption characteristics of the model nanostructures. Agreement with available experimental data is reasonable. The development of these nanostructural models is a first step in a multiscale approach to computing the drying shrinkage of model cement-based materials. Such an approach will provide a better understanding of the relationships between microstructure and the shrinkage behavior of these systems

03,075

PB96-160411 Not available NTIS

National Inst. of Standards and Technology (MSEL),

Gaithersburg, MD. Ceramics Div.

Analysis of Small-Angle Scattering Data Dominated by Multiple Scattering for Systems Containing Eccentrically Shaped Particles or Pores.

Final rept.

A. J. Allen, and N. F. Berk. 1994, 14p.

Pub. in Jnl. of Applied Crystallography, v27 p878-891 1994.

Keywords: "Neutron scattering, "Porous ceramics, Sintering, Microstructure, Reprints, "X ray scattering, Multiple small angle scattering.

A previously developed formalism to interpret the beam broading due to multiple small-angle scattering of a collimated beam of radiation in condensed matter is extended to treat the case of nonspherical scattering particles or pores. The new formalism concerns the situation where coherent single-particle scattering is incoherently, or stochastically, compounded by a random system of spheroidal particles, of any given mean aspect ratio, in a uniform matrix. By appropriate transformation of axes to reflect a spherroidal particle symmetry, Bethe's analysis of scattering when the sample thickness greatly exceeds the scattering mean free path is combined with the dynamical analysis of singleparticle scattering to model the beam broadening arising from a system containing nonspherical scattering objects. For the range of experimental parameters used in practical small-angle scattering studies of technological materials such as porous ceramics, it is shown that, while the previous formulation suffices for spheres, globules and even short capillary pores, the variation in beam broadening as a function of incident wavelength exhibits distinguishable signatures for systems in which a collapsed planar or extreme capillary scattering morphology predominates.

\section{3,076}

PB96-161765 Not available NTIS

National Inst. of Standards and Technology (MSEL), Gaithersburg, MD. Ceramics Div.

Friction Processes in Brittle Fracture.

Final rept.

B. Lawn. 1992, 29p

Pub. in Fundamentals of Friction: Macroscopic and $\mathrm{Mi}$ croscopic Processes, Braunlage, July-August 1991 p137-1651992.

Keywords: *Brittle materials, *Fiber reinforced composites, Reprints, Fracture, Friction, Strength, Toughness, "Foreign technology, "Contact cracks, Interface chemistry.

In this paper the authors consider the interrelations between friction and fracture in highly brittle materials. First, they examine frictional effects in the mechanics of crack formation at elastic and elastic-plastic contacts on brittle surfaces. Then they consider how fundamental intersurface forces manifest themselves as intemal friction' at crack interfaces in 'model' solids like mica and glass, with special reference to environmental chemistry. Finally, the authors examine the controlling role of frictional processes in the strength and toughness of modern ceramic systems.

03,077

PB96-163704 Not available NTIS

National Inst. of Standards and Technology (MSEL), Gaithersburg, MD.

Model for Microcrack Initiation and Propagation beneath Hertzian Contacts in Polycrystalline $\mathrm{Ce}$ ramics.

Final rept.

B. R. Lawn, N. P. Padture, F. Guiberteau, and H

Cai. 1994, 11p.

Pub. in Acta Metallurgica Materials, v42 n5 p168316931994.

Keywords: "Microcrack, "Crack propagation, "Ceramics, Fracture mechanics, Reprints, Initiation, "Foreign technology, Shear faults.

A fracture mechanics model of damage evolution within Hertzian stress fields in heterogeneous brittle ceramics is developed. Discrete microcracks generate from shear faults associated with the heterogeneous ceramic microstructure; e.g. in polycrystalline alumina, they initiate at the ends of intergrain twin lamellae and extend along intergrain boundaries. Unlike the well-de- 
fined classical cone fracture that occurs in the weakly tensile region outside the surface contact in homogeneous brittle solids, the fault-microcrack damage in polycrystalline ceramics is distributed within a subsurface shear-compression zone below the contact The shear faults are modelled as sliding interfaces with friction, in the manner of established rock mechanics descriptions but with provision for critical nucleation and matrix restraining stresses. This allows for constrained microcrack pop-in during the loading halfcycle. Ensuing stable microcrack extension is then analyzed in terms of a K-field formulation. For simplicity, only mode I extension is considered specifically here, although provision exists for including mode II. The compressive stresses in the subsurface field constrain microcrack growth during the loading half-cycle, such that enhanced extension occurs during unloading.

\section{3,078}

PB96-163795 Not available NTIS

National Inst. of Standards and Technology (MSEL), Gaithersburg, MD.

Model for Toughness Curves in Two-Phase Ceramics. 2. Microstructural Variables.

Final rept

N. P. Padture, J. L. Runyan, S. J. Bennison, L. M.

Braun, and B. R. Lawn. 1993, 7p.

Pub. in Jnl. of the American Ceramic Society, v76 n9 p2241-2247 Sep 93.

Keywords: "Damage accumulation, "Microstructural design, Reprints, Strength, "Fracture mechanics model, Toughness curve, Two-phase composites.

The fracture mechanics analysis of Part I is here ex tended to consider the effects of volume fraction and scale of second-phase particles on the toughnesscurve properties of ceramic-matrix composites. In creasing these variables enhances the flaw tolerance of the material, but only up to certain limits, beyond which bulk microcracking occurs. These limits define domains of damage accumulation and potential strength degradation by microcrack coalesence. In the familiar approximation of elliptical crack-wall profiles the authors show that the principal effects of increasing volume fraction (or expansion mismatch) and particle size is to enhance the slope and scale of the T-curve, respectively.

\section{3,079}

Not available NTIS

National Inst. of Standards and Technology (MSEL), Gaithersburg, MD. Ceramics Div.

Objective Evaluation of Short-Crack Toughness Curves Using Indentation Flaws: Case Study on Alumina-Based Ceramics.

Final rept.

L. M. Braun, S. J. Bennison, and B. R. Lawn. 1992, L. . Pub. in Jnl. of the American Ceramic Society, v75 n11 p3049-3057 Nov 92

Keywords: "Alumina, "Flaw tolerance, Reprints, "Indentation strength, Aluminum titanate, Toughness curves, Two phase composite.

An objective methodology is developed for evaluating toughness curves (T-curves) of ceramics using identation flaws. Two experimental routes are considered: (1) conventional measurement of inert strength as a function of indentation load; (2) in situ measurement of crack size as a function of applied stress. Central to the procedure is a proper calibration of the indentation coefficients that determine the K-field of indentation cracks in combined residual-contact and applied-stress loading, using data on an appropriate base material with single-valued toughness. Tests on a finegrain alumina serve to demonstrate the approach

03,080

PB96-179452 Not available NTIS

National Inst. of Standards and Technology (MSEL), Gaithersburg, MD.

Deformation and Fracture of Mica-Containing Glass-Ceramics in Hertzian Contacts.

Final rept.

H. Cai, M. A. Kalceff, and B. R. Lawn. 1994, 9p. Pub. in Jnl. of Materials Research, v9 n3 p762-770 Mar 94.

Keywords: "Hertzian contact, Deformation, Machinability, Microfracture, Reprints, Glass-ceramic, Shear fault.

The Hertzian indentation response of a machinable mica-containing glass-ceramic is studied. Relative to the highly brittle base glass from which it is formed, the glass-ceramic shows evidence of considerable 'ductility' in its indentation stress-strain response. Section views through the indentation sites reveal a transition from classical cone fracture outside the contact area in the bae glass to accumulated subsurface deformation-microfracture in the glass-ceramic. The deformation is attributed to shear-driven sliding at the weak interfaces between the mica flakes and glass matrix. Extensile microcracks initiate at the shear-fault interfaces and propagate into the matrix, ultimately coalescing with neighbors at adjacent mica flakes to effect easy material removal. The faults are subject to strong compressive stresses in the Hertzian field, suggesting that frictional tractions are an important element in the micromechanics. Bend-test measurements on indented specimens show that the glass-ceramic, although weaker than its base glass counterpart, has su perior resistance to strength degradation at high contact loads. Implications of the results in relation to microstructural design of glass-ceramics for optimal toughness, strength, and wear and fatigue properties are discussed.

\section{3,081}

PB96-179460 Not available NTIS

National Inst. of Standards and Technology (MSEL), Gaithersburg, MD.

Flaw Tolerance and Toughness Curves in TwoPhase Particulate Composites: SiC/Glass System. Final rept.

H. Cai, N. P. Padture, B. M. Hooks, and B. R. Lawn. 1994, 9p.

Pub. in Jnl. of the European Ceramic Society, v13 p149-157 1994.

Keywords: "Composites, "Flaw tolerances, Glass, Re prints, "Foreign technology, Indentation-strength, Silicon carbide, Toughness-curve.

Flaw-tolerance and associated toughness-curve (Tcurve) characteristics in Sic/glass particle/matrix composites are studied. Two glass compositions, chosen to produce composites at extremes of high $(\mathrm{H})$ and low (L) thermal expansion mismatch relative to the SiC par. ticles, are investigated. In-situ observations of crack extension from indentation flaws reveal widely different responses: in the L composite the path is relatively undistorted from the planar geometry, with trans-particle fractures: in the $\mathrm{H}$ composite the path deflects strongly around the particles, with consequent interfacial bridge formation and activity in the crack wake. Surface fracture patterns produced by spherical indenters confirm the implied transition from transparticle to inter-particle with increasing internal residual stress, and point to a potential degradation in short-crack properties like wear and fatigue. Indentation-strength measurements also show different characteristics in the two compos. ites: minor flaw tolerance in the L material, consistent with a single-valued, 'rule of mixtures' toughness: major tolerance in the $\mathrm{H}$ material, consistent with a pronounced T-curve. The T-curves themselves are each composite and analyzed.

\section{3,082}

PB96-179593 Not available NTIS

National Inst. of Standards and Technology (MSEL), Gaithersburg, MD. Polymers Div.

Wear of Enamel against Glass-Ceramic, Porcelain, and Amalgam.

Final rept.

L. A. George, and F. C. Eichmiller. 1996, 8p

Pub. in Ceramic Transactions Series: Challenges in Ceramic Development, Manufacture, and Commercialization, v66 p 181-188 1996 .

Keywords: "Amalgam, "Betz-quartz, "Glass-ceramic, "Wear, Enamel, Procelain, Reprints.

The wear of human enamel opposing certain dental materials is a problem in restorative dentistry. A wear study was carried out on enamel that was in contact with three dental restorative materials, a beta-quartz glass-ceramic, a fused porcelain (Ceramco, Inc., Burlington, $\mathrm{NJ}$ ), and a dispersed phase amalgam (Dispersalloy, Johnson \& Johnson, Skillman, NJ). Twenty-seven human teeth tooled to form enamel cones were randomly assigned to the three restorative materials. Three groups of 9 enamel cones each were abraded against sample disks of each material. The test was carried out on a two-body, unidirectional, rolary wear testing machine. The wear of the enamel was evaluated by measurement of the volume of enamel lost during testing. The average wear volumes lost $(\mathrm{cm}$ cubed) for the enamel were: glass-ceramic, $5.0 \times 10$ to the minus 5 power ( $\mathrm{s}=2.1 \times 10$ to the minus 5 power); porcelain, $5.2 \times 10$ to the minus 4 power (s $=5.4 \times 10$ to the minus 4 power); amalgam, $3.5 \times 10$ to the minus 5 power ( $\mathrm{s}=1.8 \times 10$ to the minus 5 power). Comparison of the mean worn volume of enamel lost for the three materials, by the Turkey method of multiple comparisons with a significance level of $5 \%$, indicated no significant difference between only the enamel worn against glass-ceramic and against amalgam. The results indicate that beta-quartz glass-ceramic material would produce less wear to opposing enamel than traditional fused porcelains. The wear of enamel opposing glass-ceramic should be equivalent to that resulting from contact with amalgam.

\section{3,083}

PB96-179601 Not available NTIS

National Inst. of Standards and Technology (MSEL) Gaithersburg, MD

Effect of Grain Size on Hertzian Contact Damage in Alumina.

Final rept.

F. Guiberteau, N. P. Padture, and B. R. Lawn. 1994

$7 p$.

Pub. in Jnl. of the American Ceramic Society, v77 n7 p1825-1831 1994

Keywords: "Alumina, "Damage accumulation, Deformation, Grain size, Microfracture, Reprints, *Hertzian contact.

The role of microstructural scale on deformationmicrofracture damage induced by contact with spheres is investigated in monophase alumina ceramics ove a range 3-48 um in grain size. Measurement of a universal indentation stress-strain curve indicates a critical contact pressure approximately $5 \mathrm{GPa}$, above which irreversible deformation occurs in alumina. A novel sectioning technique identifies the deformation elements as intragrajn shear faults, predominantly crystallographic twins within a confining subsurface zone of intense compression-shear stress. The twins concentrate the shear stresses at the grain boundaries and, above a threshold grain size, initiate tensile intergranular microcracks. Below this threshold size, classical Hertzian cone fractures initiate outside the contact circle.

\section{3,084}

PB96-180013 Not available NTIS

National Inst. of Standards and Technology (MSEL), Gaithersburg, MD

Indentation Fatigue: A Simple Cyclic Hertzian Test for Measuring Damage Accumulation in Polycrystalline Ceramics.

Final rept.

F. Guiberteau, N. P. Padture, H. Cai, and B. R

Lawn. 1996, 14p.

Pub. in Philosophical Magazine A, v68 n5 p1003-1916 1993.

Keywords: "Alumina, "Damage accumulation, "Ceramics, Fatigue, Internal stress, Microfracture, Reprints, Hertzian contact.

A simple Hertzian contact procedure for investigating cyclic fatigue damage in brittle polycrystalline ceramics is described. Repeat loading of a shperical indenter on a coarse alumina ceramic produces cumulative mechanical damage. The mode of damage is one of deformation-induced intergranular microfracture, leading ultimately at large numbers of cycles and high contact pressures to severe grain dislodgement. In contrast to the classical Hertzian cone cracks that form in more homogeneous materials in the regions of tensile stress outside the contact circle, the damage in the coarse grain alumina develops in a zone of high shear stress and hydrostatic compression beneath the contact circle. The fatigue damage is evident in inert environments, confirming the mechanical nature of the process, although exposure to moisture accelerates the ef fect. The relatively modest degradation in failure stress with number of repeat contacts for indented flexure specimens suggests that conventional strength and toughness testing procedures may not always provide sensitive indications of the extent of damage that can be incurred in concentrated loading

\section{3,085}

PB96-180088 Not available NTIS

National Inst. of Standards and Technology (MSEL), Gaithersburg, MD. 
Model for Toughness Curves in Two-Phase Ceramics. 1. Basic Fracture Mechanics.

Final rept.

B. R. Lawn, N. P. Padture, L. M. Braun, and S. J.

Bennison. 1996, 6p.

See also PB96-163795.

Pub. in Jnl. of the American Ceramic Society, v76 n9 p2235-2240 1993.

Keywords: "Crack bridging, Strength, Reprints, - Fracture mechanics model, Microstructural design, Toughness-curve, Two-phase composites.

A fracture mechanics model is presented for the toughening of ceramics by bridging from second-phase particles, resulting in toughness curve (T-curve) behavior. It is assumed that the second-phase particles are in a state of residual thermal expansion dilatational mismatch relative to the matrix. In the long-crack region, these stresses augment frictional sliding stresses at the interphase boundaries, enhancing the crack resistance; in the short-crack region, the same stresses drive the crack, diminishing the crack resistance. The principal manifestation of these countervailing influences is a reduced sensitivity of strength to initial flaw size, i.e., an increased flaw tolerance.

\section{3,086}

PB96-180278 Not available NTIS

National Inst. of Standards and Technology (MSEL), Gaithersburg, MD.

Transient Creep Behaviour of Hot Isostatically Pressed Silicon Nitride.

Final rept.

S. M. Wiederhom, B. J. Hockey, D. C. Cranmer, and R. Yeckley. 1993, 9p.

Pub. in Jnl. of Materials Science, v28 p445-453 1993. Keywords: "Creep rupture, "Silicon nitride, "Ceramics, Lifetime, Reprints.

Transient creep is shown to dominate the high-temperature behavior of a grade of hot isostatically pressed silicon nitride containing only $4 \mathrm{wt} \%$ Y2O3 as a sintering aid. Contributing factors to transient creep are discussed and it is concluded that the most likely cause of long-term transient creep in the present study is intergranular sliding and interlocking of silicon nitride grains. In early stages of creep, devitrification of the intergranular phase, and intergranular flow of that phase may also contribute to the transient creep process. The occurrence of transient creep precluded the determination of an activation energy on the as-received material. However, after creep in the temperature range $1330-1430$ degrees $C$ for times exceeding approximately $1100 \mathrm{~h}$, an apparent activation energy of approximately $1260 \mathrm{~kJ}$ mol to the minus 1 power was measured. It is suggested that the apparent activation energy for creep is determined by the mobility and concentration of diffusing species in the intergranular glassy phase. The time-to-rupture was found to be a power function of the minimum strain rate, independent of applied stress or temperature. Hence, creep-rupture behavior followed a MonkmanGrant relation. A strain rate exponent of -1.12 was determined.

03,087

PB96-193677 PC A11/MF A03

National Inst. of Standards and Technology (MSEL), Gaithersburg, MD. Ceramics Div.

Ceramics Technical Activities, 1995.

S. W. Freiman, and S. J. Dapkunas. 1995, 211p,

TIR-5747

See also PB94-162591.

Keywords: "Ceramics. "Research projects, Mechanical properties, Standards, Ceramic coatings, Microstructure, Superconductors, Powder(Particles), Surface properties.

Current programs of the Ceramics Division are reviewed.

\section{3,088}

PB96-200829 Not available NTIS

National Inst. of Standards and Technology (MSEL), Gaithersburg, MD. Ceramics Div.

Multimedia Tutorial on Phase Equilibria Diagrams. Final rept.

E. F. Begley, and C. G. Lindsay. 1993, 2p.

Pub. in Innovations in Ceramic Education, v72 n12 p103-104 Dec 93.

Keywords: "Phase diagrams, "Multimedia, Computer applications, Video tapes, Ceramics, Physical prop- erties, Phase studies, Chemical composition, Vapor phases, Crystal structure, Solid phases, Reprints, Phase equilibria, US NIST.

In materials research, the phase diagram is a critical processing tool describing the relationship between temperature, chemical composition, and gaseous environment to the crystalline phases that should appear. The Ceramics Div. at the National Institute of Stand ards and Technology has initiated the development of innovative distributable, multimedia software incorporating digital video interactive (DVI) technology to assist in the understanding and use of these diagrams.

\section{3,089}

PB96-204060 Not available NTIS

National Inst. of Standards and Technology (MSEL) Gaithersburg, MD. Ceramics Div.

Need for Advanced Characterization Techniques in Product Manufacturing: A Case Study on Ceramic Matrix Composites.

Final rept.

L. M. Braun. 1996, 5p.

Contract DE-AI05-920422014

Sponsored by Department of Energy, Washington, DC. Pub. in Ceramic Product Development, Manufacture. and Commercialization, p213-217 1996

Keywords: "Ceramics, "Composites, Reprints, Interfaces, Fiber-reinforced, Properties.

The capability of industry to manufacture reliable and cost-effective fiber-reinforced ceramic matrix compos ites requires detailed characterization and control of the composite properties. Advanced characterization techniques, including in situ characterization of material properties, offer the potential for process control, quality control, and lifetime monitoring. Advanced characterization techniques currently being developed to aid in the manufacture and scientific understanding of ceramic components are discussed.

\section{3,090}

PB96-204110 Not available NTIS

National Inst. of Standards and Technology (MSEL) Gaithersburg, MD. Ceramics Div.

Diffusive Crack Growth at a Bimaterial Interface.

Final rept.

T. J. Chuang, J. L. Chu, and S. Lee. 1995, 6p.

Contract NASA-C082000-R

Sponsored by National Aeronautics and Space Administration, Cleveland, $\mathrm{OH}$. Lewis Research Center.

Pub. in AIAAASME/ASCE/AHSIASC Structures, Structural Dynamics and Materials Conference (33rd) Dallas, TX., April 13-15, 1992, p2964-2969 1995.

Keywords: "Crack growth, "Ceramic composites, Reprints, Creep rupture, "Bimaterial interface, Energy release rate, High temperature fracture, Interfacial crack. The diffusional microcrack growth behavior in a bimaterial system is investigated with an aim at estimating service life of advanced ceramic composites under creep-rupture conditions. The crack is assumed to grow via a coupled surface and grain-boundary diffusion under steady state conditions. The tensile stress distribution along the interface ahead of the moving crack tip is solved, and it is found that a new length parameter exists as a scaling factor for which the solution becomes identical to the single phase case when plotted on the nondimensional physical plane. In contrast to the elastic stress solution which shows singularity at the tip, together with oscillatory character away from the tip, the creep stresses have a peak value away from the tip due to a wedging effect and interfacial sliding eliminates stress oscillation resulting in a decoupling between mode I and mode II loading. The solution ties the far-field loading parameter to the crack tip conditions in terms of the unknown crack velocity to give a specific $V-K$ relationship. It is shown that an exponent of 12 in the conventional crack growth power law emerges at the higher applied stress range.

\section{3,091}

PB96-204128 Not available NTIS

National Inst. of Standards and Technology (MSEL), Gaithersburg, MD. Ceramics Div.

Life Prediction of a Continuous Fiber Reinforced Ceramic Composite Under Creep Conditions.

Final rept.

T. J. Chuang. 1993, $12 p$.

Contract NASA-C-82000-R

Sponsored by National Aeronautics and Space Administration, Cleveland, $\mathrm{OH}$. Lewis Research Center.

Pub. in Fatigue and Fracture of Aerospace Structural

Materials, ASME 1993, v36 p73-84 1993.
Keywords: "Ceramics, "Composites, "Creep rupture, Fiber-reinforced, Cavity growth, Damage mechanics, Engine materials, Life prediction, Reprints.

The paper is concerned with development of a lifetime prediction methodology for a unidirectional fiber reinforced ceramic composite subjected to creep conditions. A continuum damage mechanics approach is adopted in which constitutive creep laws incorporating damage are constructed based on micromechanical modeling. A unit cell model is established to take advantage of the periodic feature of the material. The model which entails two elements (one representing the fiber phase and the other the matrix phase) connected in parallel is subjected to a constant stress applied in the fiber direction. From the requirements of equilibrium and compatibility, a system of simultaneous differential equations was derived for the dependent variables: stress, strain and damage as functions of time, with the initial conditions given by the elastic state of the material. The algorithm for solving this time-dependent problem was given in a flow chart from which the lifetime limited by creep can be computed. The results suggested that creep life is strongly dependent on applied stress, temperature and volume fraction of the fibers.

\section{3,092}

PB96-204185 Not available NTIS

National Inst. of Standards and Technology (MSEL), Gaithersburg, MD. Ceramics Div.

Bulk Modulus and Young's Modulus of Nanocrystalline gamma-Alumina.

Final rept.

M. R. Gallas, and G. J. Piermanini. 1994, 4p.

Pub. in Jnl. of the American Ceramic Society, v77 n11 p2917-2920 Nov 94

Keywords: "Nanocrystalline ceramic, "Bulk modulus, 'Young's modulus, Reprints, Compression, Gammaalumina, Diamond anvil cell, Energy dispersive $x$-ray.

Compression measurements were performed for the first time on nanocrystalline gamma-alumina utilizing a diamond anvil cell (DAC) and the energy dispersive $X$ ray diffraction method. The cubic unit cell (alpha $=0.7924 \mathrm{~nm}$ ) for gamma-alumina was found to have a volume compression of about $2.4 \%$ over the pressure range from ambient to $3.8 \mathrm{GPa}$ at room temperature under both hydrostatic and nonhydrostatic conditions. Using the first-order Bridgman equation and the Birch equation of state, the isothermal bulk modulus $(B O)$ was determined to be 162 plus or minus $14 \mathrm{GPa}$ and Young's modulus (E) was estimated to be 253 plus or minus $22 \mathrm{GPa}$ assuming a Poisson's ratio for gamma-alumina of 0.24 plus or minus 0.2 .

\section{3,093}

PB96-204524 Not available NTIS

National Inst. of Standards and Technology (MSEL), Gaithersburg, MD.

Creep and Creep Rupture of Structural Ceramics. Final rept

S. M. Wiederhom, B. J. Hockey, and T. J. Chuang.

1991, $22 p$.

Pub. in NATO Advanced Research Workshop on 'Toughening Mechanisms in Quasi-Brittle Materials' Evanston, IL., July 1990, p555-576 1991.

Keywords: "Ceramics, "Crack growth, "Creep, Reprints, Rupture, Strength cavitation, "Foreign technology.

Structural ceramics are often two phase materials, in which rigid refractory grains, fibers, or whiskers are bonded by a less refractory matrix. At elevated temperatures, creep occurs by deformation of the matrix, resulting in the localization of stresses along grain boundaries, followed by cavitation and eventually be structural failure of the ceramic. The sequence of events prior to failure depends on the grain size and the amount of bonding matrix within the solid. Large amounts of matrix phase reduce constraints at grain boundaries, and thus the stresses that cause cavita. tion. Large grains, fibers or whiskers provie easy paths for crack growth, so that, once initiated, cracks readily propagate to failure. In structural ceramics, failure time can be expressed as a power function of the creep rate; the coefficient and exponent of the power function are determined by the failure mechanism. In the paper, models for creep rupture of ceramics are presented for cavity coalescence and for crack growth as primary failure processes. The model for cavity coalescence. in particular, is used to rationalize creep rupture data for a two phase ceramic that fails by cavity coalescence. 
03,094

PB96-204532 Not available NTIS

National Inst. of Standards and Technology (MSEL), Gaithersburg, MD.

Fracture Mechanism Maps: Their Applicability to Silicon Nitride.

Final rept.

S. M. Wiederhorn, G. D. Quinn, and R. Krause.

1994, 26p.

See also AD-A220 428

Pub. in Life Prediction Methodologies and Data for Ceramic Materials, ASTM STP 1201, p36-61 1994.

Keywords: *Ceramic materials, "Fracture(Mechanics), "Hot pressing, "Silicon nitrides, Reprints, Crack propagation, Creep, Failure, Fatigue(Materials), Flexual properties, Magnesium oxides.

Fracture mechanism maps provide means of assessing the structural reliability of ceramics at elevated temperatures. They can be used to summarize large quantities of data dealing with effects of load, temperature and environment on component lifetime. they also can be used to generate a design envelope that defines stress allowables for a given application. In this paper, the authors review the history and philosophy behind fracture mechanism maps and then discuss methods of obtaining such maps in an efficient manner. Based on data obtained in simple tensile tests, these methods are illustrated for one of the newer grades of silicon nitride. The map is then used to compare this material with a high temperature structural alloy, and another, older grade of silicon nitride. Finally, the authors discuss the use of fracture mechanism maps for design.

\section{3,095}

PB97-110027 Not available NTIS

National Inst. of Standards and Technology (MSEL), Gaithersburg, MD

Flaw-Insensitive Ceramics.

Final rept.

S. J. Bennison, N. P. Padture, J. L. Runyan, and B.

R. Lawn. 1991, 5p.

Pub. in Philosophical Magazine Letters, v64 n4 p1911951991.

Keywords: "Ceramics, "Flaw insensitivity, Bridging, Microstructures, Processing, Reliability, Reprints.

Ceramics are notorious for their 'brittleness', i.e. the sensitivity of mechanical strength to flaws on the microstructural scale. The association notion of the 'critical flaw' has dominated design considerations concerning structural reliability and materials processing of ceramic components. The accounts for the trend over the last decade towards a processing strategy of elimination strength-degrading flaws at source. Here the authors propose a fundamentally different approach, that of processing ceramics with crack-impeding elements integrated into the indigenous microstructures, such that any pre-existing or serviceinduced flaws are effectively stabilized. Strength data on a tailored alumina/aluminium-titanate material demonstrate the capacity of the approach to produce simple ceramics with unique flaw insensitivity.

PB,096 $110258 \quad$ Not available NTIS

National Inst. of Standards and Technology (MSEL), Gaithersburg, MD.

Tension/Compression Creep Asymmetry in Si3N4. Final rept.

W. E. Luecke, and S. M. Wiederhorn. 1994, 6p Pub. in Key Engineering Materials, v89-91 p587-592 1994

Keywords: "Cavitation, "Creep, "Silicon nitride, Tension, Compression, Reprints, "Foreign technology.

The authors have deformed a commercial HIP-ed silicon nitride in both tension and compression at 1430 degrees $\mathrm{C}$ at stresses between 40 and $300 \mathrm{MPa}$. The material creeps up to 100 times faster in tension than in compression. The stress dependence of the tensile strain rate is not characterized by a single stress exponent, rising from about two at low stresses to about five at higher stresses. When stressed in tension, the material responds by cavitating, with the cavitation contributing signifciantly to the tensile strain. In compression, however, the material exhibits a stress exponent of unity, and cavitation is almost completely sup. pressed. The asymmetry in creep behavior between tension and compression is due to the dilatation of the material.

03,097
PB97-110282 Not available NTIS
National Inst. of Standards and Technology (MSEL), Gaithersburg, MD. Ceramics Div.

Characterizing Materials Properties for Ceramic Matrix Composites.

Final rept.

R. G. Munro. 1991, 8p

Pub. in Proceedings of the Advanced Materials and Processing Technology Workshop on Ceramic Matrix Composites, Alexandria, VA., August 1-2, 1991, Part 2, p2-153-2-160.

Keywords: "Ceramics, "Composites, Data bases, Material properties, Matrix materials, Reprints.

The requirements for the effective reporting of materials property data for ceramic matrix composites are reviewed. The discussion focuses on bicomponent materials consisting of a reinforcement that is distributed in a ceramic matrix. The reinforcement may be short, whisker-like crystallites or long fibers with large lengthto-diameter ratios. Examples are given to illustrate the sometimes dramatic and the sometimes subtle significance of the information requirements.

\section{3,098}

PB97-110290 Not available NTIS

National Inst. of Standards and Technology (MSEL) Gaithersburg, MD. Ceramics Div.

Database Development and Management (Project A.2.2): The Annual Report for 1992-1993.

Final rept.

R. G. Munro, and E. F. Begley, 1993,6p.

Pub. in Projects within the Center for Advanced Materials: 1992-1993 Annual Report to the Gas Research Institute, Center for Advanced Materials, 6p 1993.

Keywords: “Data bases, "Ceramics, "Structural ceramics, High temperature, Development, Management, Reprints.

The Database Development and Management Project is directed towards the production and dissemination of a computerized database of materials properties for advanced, high-temperature, structural ceramics. The principle output of the project is the Structural Ceramics Database which is provided in a user-friendly software package that operates on DOS-based personal computers. The database is distributed worldwide via the NIST Standard Reference Data Program. The report discusses how the scope of the database has expanded in the current year, from the thermal and mechanical properties of silicon carbides and silicon nitrides, to a more extensive coverage of the thermal mechanical, and chemical corrosion properties of structural ceramic oxides, carbides, and nitrides in general.

\section{3,099}

PB97-110308 Not available NTIS

National Inst. of Standards and Technology (MSEL), Gaithersburg, MD. Ceramics Div.

Role of Corrosion in a Material Selector Expert System for Advanced Structural Ceramics.

Final rept.

R. G. Munro. 1995, 9p.

Pub. in American Society for Testing and Materials Special Publication, p127-1351995.

Keywords: "Ceramics, "Structural ceramics, *Corrosion, Expert systems, Heat exchangers, High edge base, Reprints.

The primary goal in the development of advanced structural ceramics has been to provide high-strength, corrosion-resistant materials for use in high-tempera. ture, aggressive environments. Much of the developmental effort has focused on heat exchangers for use in industrial gas- or coal-fueled systems with temperatures on the order of 1200 to 1400 degrees C. While ceramics are often popularly described as inert to chemical degradation, the corrosion resistance of these materials actually ranges from excellent to very poor, depending especially on the compositions of the environment and the materials selector expert system for advanced ceramics. The present paper examines the development of a set of rules for a material selector expert system based on relations among composition microstructure, transport processes, operating conditions, and corrosion. The concurrent information re quirements that must be provided in a computerized materials property database are discussed also.

\section{3,100}

PB97-110316 Not available NTIS

National Inst. of Standards and Technology (MSEL), Gaithersburg, MD. Ceramics Div.
Variances in the Measurement of Ceramic Powder Properties.

Final rept.

R. G. Munro, S. G. Malghan, and S. M. Hsu. 1995

$10 \mathrm{p}$.

Pub. in Jnl. of Research of the National Institute of Standards and Technology, v100 n1 p51-60 Jan/Feb 95.

Keywords: "Ceramic powder, "Variances, Characterization, Composition, Density, Particle size, Surface areas, Round robin, Reprints

Variances in the measurement of properties used to characterize ceramic powders are discussed in the context of the International Energy Agency's siudy Annex II, Subtask 2, which includes chemical and physical measurements for five powders: two grades of silicon nitride, and one grade each of silicone carbide, silicon, and ziconia. The analysis presented here includes results for 39 properties reported by 25 laboratories using approximately 700 samples of the powders. Measurement uncertainties are cliscussed in the contexts of measurement variations within given laboratories (within-laboratory variance, sometimes called repeatability), among different laboratories (betweenlaboratory variance, also called reproducibility), and among different measurement techniques (between methods variance). The analysis shows that the be tween-laboratory variance tends to be significantly greater than either the within-laboratory or the between-methods variances. The implication of this re sults is that the most important improvements in pow der characterization measurements may be achieved through the standardization of the measurement methodologies.

\section{3,101}

PB97-110324 Not available NTIS

National Inst. of Standards and Technology (MSEL), Gaithersburg, MD.

Flaw-Tolerance and Crack-Resistance Properties of Alumina-Aluminum Titanate Composites with Tailored Microstructures.

Final rept.

N. P. Padture, S. J. Bennison, and H. M. Chan

1993, 9p

Pub. in Jnl. of the American Ceramic Society, v76 n9 p2312-2320 Sep 93.

Keywords: "Alumina, "Aluminum titanate, Mechanical properties, Microstructure, Processing, Reprints, * Flaw tolerance, "Crack resistance.

The microstructures of alumina-aluminum titanate (A AT) composites have been tailored with the intent of altering their crack-resistance ( $R$ - or T-curve) behavio and resulting flaw tolerance. Specifically, two microstructural parameters which influence grain-localized crack bridging, viz (1) internal residual stresses and (2) microstructural titanate was added to alumina to induce intense internal residual stresses from ex treme thermal expansion mismatch. It was found tha A-AT composites with uniformly showed significantly improved flaw tolerance over single-phase alumina. Coarsening of the duplex microstructure via grain growth scaling was relatively ineffective in improving the flaw tolerance further. Onset of spontaneous microcracking precluded further exploitation of this scaling approach. Therefore, an alternative approach to coarsening was developed, in which a uniform distribution of large alumina grains was incorporated within a fine-grain A-AT matrix ('duplex-bimodal'), via a powder processing route. The duplex-bimodal com posites yielded excellent flaw tolerance with steady state toughness of approximately $8 \mathrm{MPa}-\mathrm{m}(1 / 2)$. A qualitative model for microstructure development in these duplex-bimodal composites is presented.

03,102

PB97-110332 Not available NTIS

National Inst. of Standards and Technology (MSEL) Gaithersburg, MD.

In situ-Toughened Silicon Carbide.

Final rept.

N. P. Padture. 1994, 5p.

Pub. in Jnl. of the American Ceramic Society, v77 n2 p519-523 Feb 94

Keywords: "Silicon carbide, Sintering, Microstructure, Reprints, Yttrium aluminum garnet, Crack-wake bridg ing, Fracture toughness.

A new processing strategy based on atmospheric pres sure sintering is presented for obtaining dense SiC based materials with microstructures consisting of (1) 
uniformly distributed elongate-shape alpha-SiC grains and (2) relatively high amounts $(20 \mathrm{vol} \%)$ of secondphase yttrium aluminum garnet (YAG). This strategy entails the sintering of beta-SiC powder doped with alpha-SiC, $\mathrm{Al} 2 \mathrm{O} 3$, and $\mathrm{Y}_{2} \mathrm{O} 3$. The $\mathrm{Al} 2 \mathrm{O} 3$ and $\mathrm{Y} 2 \mathrm{O} 3$ aid in the liquid-phase sintering of $\mathrm{SiC}$ and form in situ YAG, which has a significant thermal expansion mismatch with $\mathrm{SiC}$. During a subsequent grain-growth heat treatment, it is postulated that the alpha-SiC 'seeds' assist in controlling in situ growth of the elongated alpha-SiC grains.

03,103

PB97-110340 Not available NTIS

National Inst. of Standards and Technology (MSEL), Gaithersburg, MD.

Postfailure Subsidiary Cracking from Indentation Flaws in Brittle Materials.

Final rept.

N. P. Padture. 1993, 7p.

Pub. in Jnl. of Materials Research, v8 n6 p1411-1417 Jun 93.

Keywords: "Ceramics, "Cracking, "Indentation, Fracture, Lateral-cracks, Glass, Reprints.

Vickers indentation sites in ceramics have been examined after specimen failure from medial-radial indentation cracks. Evolution of a newcracking pattern of 'ortho-lateral' cracks, originating at the intact corners of the Vickers indentation and running orthogonal to the classic-lateral cracks and parallel to the new fracture surface, has been observed. In some instances postfailure extension of the classic-lateral cracks toward the surface was also observed. Enhanced residual tensile stress from relaxation of constraints on the indentation-plastic cavity by the generation of a fracture surface is postulated to drive the subsidiary cracking. A simple qualitative model to explain this phenomenon is presented. Possible implications of such postfailure subsidiary cracking on residual-stress-driv en flaws, postmortem fractography, and wear in ce ramics are discussed.

\section{3,104}

PB97-110456 Not available NTIS

National Inst. of Standards and Technology (MSEL), Gaithersburg, MD

High Temperature Structural Reliability of Silicon Nitride.

Final rept.

S. M. Wiederhorn, G. D. Quinn, and R. Krause

1994, 6p.

Pub. in Key Engineering Materials, v89-91 p575-580 1994.

Keywords: "Ceramics, "Silicon nitride, "Reliability, Strength, Creep, Fracture maps, Reprints, "Foreign technology.

Structural reliability of ceramics at elevated temperatures is often determined by crack growth, or by creep induced cavitation. Ceramics that fail in this manner fit a Monkman-Grant relation, in which the time to failure is represented as a power las function of the creep rate. In the paper the authors show that the Monkman Grant approach can be used to obtain fracture maps for characterizing creep and creep rupture behavior at high temperatures. Maps are presented for ceramics currently being considered for turbine or heat exchanger applications. Attributes of current grades of silicon nitride are compared.

\section{3,105}

PB97-110464 Not available NTIS

National Inst. of Standards and Technology (MSEL), Gaithersburg, MD.

Tensile Creep Testing of Structural Ceramics.

Final rept.

S. M. Wiederhorn, R. Krause, and D. C. Cranmer.

$1991,8 p$.

See also PB93-166619.

Pub. in Annual Automotive Technology Development Contractors Coordination Meeting, Dearborn, MI., October 28-31, 1991, p1-8.

Keywords: "Ceramics, "Creep tests, Loads(Forces) Mechanical properties, Tension tests, Test methods, Deformation, Strains, Fractures(Materials), Mechanical tests, Reprints.

In order to use structural ceramics in heat engines, their high temperature properties must be characterized. Temperatures as high as 1400 degrees $C$ are expected, hence, test equipment capable of this temperature will be needed. Furthermore, as the dominant wear-out mechanism for high temperature applications is creep rupture, test equipment must not only be capable of high temperatures and stresses, but must be stable and must be able to sustain applied stresses for extended periods of time. In the paper, the authors dis cuss an inexpensive tensile tests apparatus that can be used to collect creep data for the prediction of component lifetime. They use this equipment to collect creep and creep rupture data on two grades of silicon nitride. They also demonstrate the general tendency of silicon nitride ceramics to follow a Monkman-Grant type of stress rupture behavior. The tendency permits a simple design methodology to be used for lifetime prediction.

\section{3,106}

\section{PB97-112247 Not available NTIS}

National Inst. of Standards and Technology (MEL) Gaithersburg, MD. Precision Engineering Div

Chip Morphology, Tool Wear and Cutting Mechanics in Finish Hard Turning.

Final rept.

M. A. Davies, Y. Chou, and C. J. Evans. 1996, 6p. Pub. in Annals of the CIRP, v45 n1 p77-82 Jan 96.

Keywords: "Machining, "Boron nitrides, "Wear, Cutting tools, Models, Reprints, Cubic baron nitrides.

Topography of surfaces produced in finish hard turning using cubic boron nitride (CBN) tools is affected by a large number of factors including tool wear and the mechanics of the chip formation process. The paper shows first that tool wear rates are affected by interactions between the world material and binder phase of the CBN tool. For finish hard turning, Iow CBN content, ceramic binder tools give longer lives and bette finish than high CBN content metallic binder tools. For low CBN tools, wear rate is directly related to the microstructure of the work material and to the CBN grain size. SEM studies suggest that chip morphology is independent of work material microstructure, but varies with tool wear. Orthogonal cutting tests show that above a critical speed, segmented chips are formed by catastrophic localized shear and that chip segmentation spacing may be reflected in a mdoulation of the machined surface. Segment spacing is a function of depth of cut, rake angle, and surface speed, approaching a limiting value with speed. Specific cutting energies decrease with speed, also approaching an asymptote. A simple mechanical model gives reasonable predictions of segment spacing along the original surface, although a full thermo-plastic model will be required to account for other aspects of the chip formation process.

03,107

PB97-115802 PC A05/MF A01

National Inst. of Standards and Technology (BFRL), Gaithersburg, MD. Building Materials Div.

Multi-Scale Picture of Concrete and Its Transport Properties: Introduction for Non-Cement Researchers.

E. J. Garboczi, and D. P. Bentz. Oct 96, 53p,

NISTIR-5900.

Color illustrations reproduced in black and white.

Keywords: "Concretes, "Microstructure, Computerized simulation, Cements, Mortars(Material), Digital techniques, Silicate cements, Porosity, Hydration, Composite materials, US NIST.

Concrete is a multi-length scale composite material. From the nanometer to the millimeter scale, it is a random composite, and a different random composite at each length scale. Percolation processes play a key role in the microstructure of concrete, and help to describe the overall dependence of transport properties like ionic diffusivity on the microstructure. Computer models have been developed to describe the microstructure and transport properties, as the randomness of the material precludes most (but not all) analytical formulations. The overall description of concrete, over six orders of magnitude of length scales, in terms of computer models, precolation theory, and composite ideas is of interest to those studying other random materials as well, like ceramics and rocks. This report is written to present the ideas for concrete in such a way so as to be accessible to the non-cement researcher. It is hoped that these ideas will prove to be useful in other materials.

03,108

PB97-118731 Not available NTIS

National Inst. of Standards and Technology (MSEL), Gaithersburg, MD.
Sources of Strain-Measurement Error in FlagBased Extensometry.

Final rept.

W. E. Luecke, and J. D. French. 1996, 10p.

Pub. in Jnl. of the American Ceramic Society, v79 n6 p1617-1626 Jun 96

Keywords: "Strain measurement, "Extensometry, Advanced ceramics, Creep errors, Flag-based, Reprints

The paper examines the sources of error in strain measurement using flag-based extensometry that uses either scanning laser or electroptical extensometers. These errors fall into two groups: errors in measuring the true gauge length of the specimen, which arise from the method of attachment of the flags, and errors arising from unanticipated disortions of the specimen during testing. The sources of errors of the first type include gauge-length errors from nonparallel flags and uncertainties in the true attachment point of the flag. During the test, strain-measurement errors of the second type can arise from horizontal translation of nonparallel flags, flag rotation that is induced by slippage, and flag motion from bending of the gauge length.

\section{3,109}

PB97-122220 Not available NTIS

National Inst. of Standards and Technology (MSEL), Gaithersburg, MD. Reactor Radiation Div.

Antiferromagnetic Interlayer Correlations in Annealed Ni80Fe20/Ag Multilayers.

Final rept.

J. A. Brochers, P. M. Gehring, R. W. Erwin, T. L. Hylton, K. R. Coffey, M. A. Parker, J. K. Howard, J. F. Ankner, and C. F. Majkrzak. 1996, 13p. Pub. in Physical Review B, v54 n14 p9870-9882 Oct 96.

Keywords: *Annealing, "Antiferromagnetics, *Diffraction, Interlayers, Multilayers, $X$ ray diffraction, Cross section, Reprints.

Sputtered Ni80Fe20/Ag multilayers, annealed postgrowth, exhibit giant magnetoresistance (GMR) with pronounced field sensitivity. The authors have characterized a series of Ni8OFe2O(20 Angstroms/Ag(40 Angstroms)) multilayers annealed at temperatures ranging from 305 to 335 degrees $C$ using $x$-ray and polarized neutron reflectivity techniques. For all of the samples, specular $x$-ray measurements reveal that the laterally averaged interfaces between the Ni80Fe20 and $\mathrm{Ag}$ layers are not well defined. The growth-plane morphology of the multilayers, determined from offspecular x-ray diffraction, shows a dependence on annealing temperature. Specular and off-specular polarized neutron reflectivity data indicate that the GMR in the annealed samples does not arise from long-range antiferromagnetic alignment of coherent ferromagnetic sheets, as generally observed in related materials. Instead, annealing promotes the formation of planar ferromagnetic domains of micrometer size within each Ni80Fe20 layer that are antiferromagnetically correlated along the growth axis. The length scales of these domains are consistent with a model in which weak dipolar forces dominate the interactions between them.

03,110

PB97-122337 Not available NTIS

National Inst. of Standards and Technology (MSEL) Gaithersburg, MD. Reactor Radiation Div.

Small Angle Neutron Scattering Study of the Structure and Formation of MCM-41 Mesoporous Molecular Sieves.

Final rept.

C. J. Glinka, J. M. Nicol, G. D. Stucky, Q. Huo, J. B.

Higgins, M. E. Leonowicz, E. Ramli, and D.

Margolese. 1996, 6p.

Pub. in Jnl. of Porous Materials, v3 p93-98 1996.

Keywords: "Porous materials, "Neutron scattering * Molecular sieves, Mesopores, Liquid crystals, Re prints, Mesoporous sieves.

The nanoscale structure and synthesis mechanisms of the MCM-41 class of inorganic mesoporous materials have been investigated by small angle neutron scattering (SANS). SANS measurements with solvents imbibed in the pores to vary the scattering contrast demonstrate that the low angle diffraction peaks from these materials are entirely due to the pore structure and that the pores are fully accessible to both aqueous and organic solvents. Static and shear flow SANS measure. ments on the concentrated cationic surfactant and silicate precursor solutions typically used in the synthesis 
of the mesopore materials indicate that the existence of preassembled supramolecular arrays that mimic the final pore structure is not essential for the synthesis of these materials.

\section{3,111}

Not available NTIS

National Inst. of Standards and Technology (MSEL), Gaithersburg, MD. Ceramics Div.

Nanoindentation and Instrumented Scratching Measurements on Hard Coatings.

Final rept.

A. W. Ruff. 1996, 3p.

Pub. in American Society for Testing and Materials Publication - Effect of Surface Coatings and Treatments on Wear, vSTP1278 p124-126 1996.

Keywords: "Ceramics, "Coatings, "Elastic modulus, Hardness, Indentation, Surface treatments, Scratching, Reprints, Plasma sprayed coatings.

Elastic, plastic, and cracking properties of ZrO2, a ZrO2-metal composite, and a Ni-NiO composite, all plasma-sprayed as coatings on metallic substrates have been studied. Data comparisons were made with bulk ZrO2, bulk nickel, and single-crystal silicon as reference materials. A nanoindentation and scratching apparatus was used for the measurements. Three different indenter and scratching tool shapes were used: 200 micrometers radius sphere, Vickers four-sided pyramid, and a 45 degree wedge with a $0.5-\mathrm{mm}$ radius curved edge. During indentation, over a load range of $10 \mathrm{mN}$ to $1 \mathrm{~N}$, continuous load versus depth data were obtained in each case to permit analysis for material hardness and elastic modulus. The loading curve data are used to calculate hardness, and the unloading curve data are used to calculate elastic modulus. The analytical models used are described and compared. An alternate method for deriving elastic modulus from the initial portion of the loading curve is described. Comparison among the results for different tool shapes will be discussed.

\section{3,112}

PBं97-122501 Not available NTIS

National Inst. of Standards and Technology (MSEL), Gaithersburg, MD. Ceramics Div.

Wear Modeling of Si-Based Ceramics.

Final rept.

M. C. Shen, and S. M. Hsu. 1996, 6p.

Pub. in Proceedings of the International Tribology Conference, Yokohama, Japan, October 29-November 2 , 1995, p403-408 1996.

Keywords: "Ceramics, "Wear mechanics, "Silicon compounds, Fracture, Models, Carbides, Nitrides, Reprints, "Foreign technology.

Existing ceramic wear models have been evaluated against a large set of wear data of a Si3N4 and a SiC under dry sliding condition. The wear data cover a wide range of speeds and loads. The data include various wear mechanisms: deformation, fracture, and severe fracture corresponding to milk wear, severe, and ultrasevere wear regimes. Transition zones separate individual wear regimes. The wear models are assessed for their ability to predict both the level of wear and the locations of the wear transitions. The fracture models predict well in the severe wear regime but deviate from the data in the milk and ultra-severe wear regimes. In predicting the locations of wear transitions, the mildto-severe wear transitions can be predicted reasonably well. The severe-to-ultra-severe wear transition in both materials can be successfully described by a critica velocity model. This suggests that thermal-shock-induced fracture damages are responsible for the existence of the ultra-severe wear regime. Consequently, a modified model was developed to include the thermal shock stresses.

03,113

PB97-122592 Not available NTIS

National Inst. of Standards and Technology (MSEL),

Gaithersburg, MD. Ceramics Div.

Chemical Effect in Ceramics Grinding.

Final rept.

T. N. Ying, and S. M. Hsu. 1996, 6p.

Pub. in Proceedings of the International Tribology Conference, Yokohama, Japan, October 29-November 2, 1995, p1725-1730 1996

Keywords: "Ceramics, "Coolants, "Machining rate, Particles, Diamond wear, Silicon nitride, Reprints, *Foreign technology.

Ceramics are tough and hard and require diamond tooling to machine or cut. The chemical effects of cool- ants in ceramic grinding have received relatively little attention. The paper describes various chemical ef fects of coolants on machining rates, surface finish and explains why some chemistries can improve grind ing rates of tough ceramics several order of magnitudes.

\section{Coatings, Colorants, \& Finishes}

03,114

AD-A310 087/2 PC A03/MF A0

National Bureau of Standards, Gaithersburg, MD.

Volatile Corrosion Inhibitors.

Dec 64, 15p.

Availability: Document partially illegible.

Keywords: "Corrosion, "Corrosion inhibition, "Volatility, Packing materials, Test and evaluation, Materials, Steel, Coatings, External, Storage, Oils, Paper, Water vapor.

Significant reports covering the period since 1962 were selected for this study, with reference to relevant earlier works. Recent government RD on volatile corrosion inhibitors ( $\mathrm{VCl}$ ) has generally proceeded along three lines: extension of tests defining the protective value of these materials for longer storage periods to steel equipment, tolerances of various nonferrous materials and finishes toward tarnishing or corrosion by these materials, and effectiveness of exterior packaging materials in extending use of these inhibitors to more se vere exposures of water vapor and liquid. Volatile corrosion inhibitors in the form of salts, dissolved in oils, or coated on paper within enclosed shipment parcels or storage spaces offer improved corrosion protection to hare steel while it is in a ready condition for withdrawal and immediate use.

03,115

PB94-160801 PC A03/MF A01

National Inst. of Standards and Technology (MSEL) Gaithersburg, MD. Polymers Div.

Cylinder Wipe Air-Drying Intaglio ink Vehicles for U.S. Currency Inks.

Annual rept

B. Dickens, B. J. Bauer, and W. R. Blair. Jan 91,

29p. NISTIR-4498.

See also PB90-112343 and PB91-144345. Sponsored by Department of the Treasury, Washington, DC. Office of Research and Technical Services.

Keywords: "Alkyd resins, "Inks, "Synthesis(Chemistry) Polymers, Drying oils, Pentaerythritol esters, Linseed oil, Sebacic acid, Succinic acid, Performance tests, Thermosetting resins, Curing, Drying, "Intaglio ink resins.

A family of air-dry intaglio resin alkyds has been developed. The alkyds are based on pentaerythritol, sebacic, acid, linseed oil fatty acids and succinic anhy dride. In laboratory tests, intaglio inks made from these resins washed well and cured to satisfactorily resistan films. Industrial quantities for press trials have been synthesized by a third party. A second series of resins has also been developed. These resins are based on tripentaerythritol, linseed oil fatty acids and succinic anhydride. Satisfactory washing and solvent resist ance are obtained in laboratory tests. A third series of resins based on poly(vinyl alcohol) was synthesized but did not appear to be satisfactory for industrial de velopment. The temperature program is important in the synthesis of alkyds. The influence of temperature has been studied so that satisfactory resins can be made industrially in minimum time.

03,116

PB94-199338 Not available NTIS

National Inst. of Standards and Technology (NEL) Gaithersburg, MD. Chemical Process Metrology Div. Gas Phase Reactions Relevant to Chemical Vapor Deposition: Optical Diagnostics.

Final rept.

D. B. Burgess. 1990, 6p.

Pub. in Materials Research Society Symposia Proceedings, v168 p137-142 1990.

Keywords: "Chemical vapor deposition, *Vapor phases, "Reaction kinetics, "Optical tests, Silanes, Silicon, Silicon nitrades, Laser applications, Photolysis, Fabrication, Emission, Decomposition reactions, Nu cleation, Ammonia, Silicon hydrides, Reprints, Laser induced fluorescence.
Laser photolysis, optical emission, and laser-induced fluorescence (LIF) were used to study laser driven decomposition processes in the gas phase pertaining to (s). These processes are important to silicon/siliconnitride chemical vapor deposition, flame-driven gas phase silicon-particle nucleation, and laser-induced processes for materials fabrication. UV laser photolysis was used to generate $\mathrm{SiHx}$ and $\mathrm{NH}$ x species from silane and ammonia. A number of photo-fragments were identified by emission from excited states. The rate of reaction of $\mathrm{NH} 2$ with silane was measured using LIF to detect $\mathrm{NH} 2$ as a function of time following photolysis of ammonia.

\subsection{7}

PB94-199346 Not available NTIS

National Inst. of Standards and Technology (NEL), Gaithersburg, MD. Chemical Process Metrology Div.

Gas Phase Reactions Relevant to Chemical Vapor Deposition: Numerical Modeling

Final rept.

D. Burgess, and M. R. Zachariah. 1990, 6p.

Pub. in Materials Research Society Symposia Proceedings, v169 p31-36 1990

Keywords: *Chemical vapor deposition, "Mathematical models, "Vapor phases, "Reaction kinetics, Laser applications, Particles, Nucleation, Concentration(Composition), Fabrication, Temperature, Silicon, Silicon nitrides, Decomposition, Silanes, Reprints, Chemical reation mechanisms

Numerical modeling of gas phase chemical kinetic was used to investigate reactions following silane decomposition to suggest experimental conditions and to interpret concentration measurements. The effects of concentration and temperature gradients on kinetic pathways and reactive intermediates were studied. These processes are relevant to silicon and siliconnitride chemical vapor deposition (CVD), to flame-driven gas phase particle nucleation, and to laser-induced processes for materials fabrication.

03,118

PB94-200540 Not available NTIS

National Inst. of Standards and Technology (IMSE),

Gaithersburg, MD. Ceramics Div.

Perspective on Fiber Coating Technology.

Final rept.

D. C. Cranmer. $1988,4 p$.

Contract N00014-86-F-0096

Pub. in Ceram. Eng. Sci. Proc. 9, n9-10 p1121-1124 1988. Sponsored by Strategic Defense Initiative Organization. Washington, DC. Innovative Science and Technology.

Keywords: "Coating processes, "Fibers, "Deposition, Ceramic fibers, Carbon fibers, Composite materials, Reprints.

A variety of techniques exist for depositing coatings on ceramic and carbon fibers. The paper reviews several of these techniques and their advantages and disadvantages and points out several deficiencies in uniformly and reproducibly coating the fibers.

\section{9}

PB94-215704 PC A03/MF A01

National Inst. of Standards and Technology (BFRL), Gaithersburg, MD.

Diffusion of Cations Beneath Organic Coatings on Steel Substrate

J. Pommersheim, T. Nguyen, Z. Zhang, and C. Lin. Apr 93,42 p, NISTIR-5 102 .

Prepared in cooperation with Bucknell Univ., Lewisburg, PA. Dept. of Chemical Engineering. and Xiamen Univ. (China). Dept. of Chemistry.

Keywords: "Polymeric films, "Steels, "Corrosion mechanisms, Cations, Diffusion, Transport properties, Organic coatings, Substrates, Sodium, Mathematical models, Computer codes, US NBS, National Institute of Standards and Technology.

Theoretical and experimental studies were carried out for the transport of cations in the channel between a polymer coating and a metal substrate from a defect in the absence of an electrical applied potential. The model consists of two stages: an initial period during which ions diffuse in the coating/metal interfacial "channel' and adsorb on the coating surface and a propagation period during which ions also diffuse into the coating. The mathematical models were solved to predict the cation concentration and flux under the coating and the relative rate of diffusion between initial and propa- 


\section{Coatings, Colorants, \& Finishes}

gation periods. Model parameter values were derived from the results of an experiment conducted in a specially designed diffusion cell. The results also indicated that during the propagation period, the rate limiting step was the lateral diffusion along the coating $/ m$ met
interface rather than diffusion through the coating.

\section{3,120}

PB94-216447 Not available NTIS

National Inst. of Standards and Technology (NEL), Gaithersburg, MD. Building Materials Div.

Non-Osmotic, Defect-Controlled Cathodic Disbondment of a Coating from a Steel Substrate. Final rept.

J. Martin, E. Embree, and W. Tsao. 1990, 9p.

Sponsored by Naval Civil Engineering Lab., Port Hueneme, CA.

Pub. in Jnl. of Coatings Technology 62, n790 p25-33 1990.

Keywords: "Coatings, "Blistering, "Surface defects, Steels, Substrates, Cracking(Fracturing), Stress corrosion tests, Protective coatings, Barrier coatings, Reprints, "Cathodic disbondment model.

A non-osmotic, defect-controlled cathodic disbondment model is proposed for explaining experimental results which were inconsistent with an osmotically controlled disbondment process. The proposed model attempts to integrate the physics of blister initiation and growth (that is, the development of internal stresses and the fracture of the coating from the substrate as the blister grows in size) with the better elucidated chemistry of blister growth. The proposed mode has many features which are analogous to the buckling and stress corrosion cracking models proposed for other materials. It also provides an alternative explanation for the well-known barrier-effect in coatings.

\section{3,121}

PB95-108734 Not available NTIS

National Inst. of Standards and Technology (MSEL), Gaithersburg, MD. Ceramics Div.

Interface Modification and Characterization of Silicon Carbide Platelets Coated with Alumina Particles.

Final rept

P. T. Pei, J. F. Kelly, and S. G. Malghan. 1993, 11p. Pub. in Colloids and Surfaces A: Physicochemical and Engineering Aspects 70, p277-287 1993.

Keywords: "Coating processes, "Surface chemistry, face coating Particle size, Colloids, Flocculation, Scanning electron microscopy, Additives, Electrodynamics, Aluminum coatings, Reprints.

A colloidal-chemistry-based technique was developed for interface modification of silicon carbide platelets (SiCp) by coating with alumina particles. The coating process utilizes electrostatic and/or electrosteric interactions between the particles to promote heterocoagulation. Since an understanding of the chemical interface between the SiCp and alumina is important for improving the coating process, the powders were characterized by the measurement of the electrokinetic sonic amplitude (ESA). The major factors studied were particle size, type of alumina, presence of polyacrylate surfactant, and concentration of alumina in the coating slip. The surface coverage and morphology of the alumina coating on SiCp was determined quantitatively by scanning electron microscopy (SEM). The results indicate that around $\mathrm{pH} \mathrm{5.8,} \mathrm{the}$ surface charge difference between $\mathrm{SiCp}$ and alumina was the largest and the coating of alumina on SiCp was feasible. The SEM data showed that surface coverage was uniform, and the highest surface coverage on SiCp was $66 \%$ when the surface area ratio of aly mina to SiCp was $250 \%$ in the suspension. No significant improvement of the coating rate was observed by the addition of a polyacrylate for dispersion of SiCp. The concentration of alumina in the slip exhibited a strong influence on the amount of surface coverage on SiCp.

\section{3,122}

PB95-140893 Not available NTIS

National Inst. of Standards and Technology (NEL), Gaithersburg, MD. Building Materials Div.

In situ Measurements of Chloride Ion and Corrosion Potential at the Coating/Metal Interface.

Final rept.

T. Nguyen, and C. Lin. 1990, 4p.

Pub. in Proceedings of the Annual Meeting of the Adhesion Society (13th), (Extended Abstract), Savannah, GA., February 18-21, 1990, 4p
Keywords: *Metal coatings, *Chlorides, *Corrosion, *In situ measurement, "Steels, Chemical attack, Chemical reactions, Interfaces, Metal-metal bonding, Corrosion tests, Reprints, "Corrosion potential.

One of the main reasons for the lack of a complete understanding of corrosion and adhesion failures of a coated metal is the lack of analytical instrumentation to probe the behaviors of corrosive agents at the coating/steel interface. We have developed a procedure based on microelectrodes for studying in situ the behaviors of potential and chloride ions at a coating/metal interface. The procedure requires an attachment of a $\mathrm{Cl}(-)$ selective microelectrode at the coating/metal interface, thus allowing direct measurements of $\mathrm{Cl}(-)$ concentration and corrosion potential changes at localized areas under a coating. Although it is intricate to prepare these microelectrodes, the procedure provided very useful information for mechanistic studies of corrosion under coatings, as well as for transport studies of $\mathrm{Cl}(-)$ ions through a coating on metal. The procedure should also be very useful for studying the roles of $\mathrm{Cl}(-)$ in localized corrosion.

03,123

PB95-140927 Not available NTIS

National Inst. of Standards and Technology (MSEL) Gaithersburg, MD. Metallurgy Div.

Aging Effects on XRF Measurements of Solder Coatings.

F. Ogburn, and $\mathrm{H}$. J. Brown. 1988, 2p.

Pub. in Plating and Surface Finishing, p58-59 Nov 88

Keywords: "Aging(Materials), "X ray fluorescence, "Solders, "Metal coatings, Metastable state, Thickness, Degradation, Nondestructive tests, Errors, Lead alloys, Tin alloys, Reprints.

The use of fluorescent $x$-ray methods to measure the thickness and composition of electroplated solder coatings is subject to measurable errors associated with the spontaneous decomposition of the metastable state of the freshly deposited coating. The rate of change at room temperature drops off with time, but is evident for at least 90 days.

\section{3,124}

PB95-146387 PC A05/MF A01

National Inst. of Standards and Technology (BFRL), Gaithersburg, MD.

Methodologies for Predicting the Service Lives of Coating Systems.

\section{Building science series.}

J. W. Martin, S. C. Saunders, F. L. Floyd, and J. P. Wineburg. Oct $94,78 p$, NIST/BSS-172

Also available from Supt. of Docs. as SN003-00303291-3. See also PB88-175542. Prepared in COoperation with Washington State Univ., Pullman. Dept. of Pure and Applied Mathematics, and Duron Paints and Wallcoverings, Beltsville, MD. Sponsored by Federal Highway Administration, Washington, DC. and Du Pont de Nemours (E.I.) and Co., Philadelphia, PA Marshall Labs

Keywords: "Organic coatings, "Service life, *Environmental effects, "Life tests, "Coating processes, Reliability, Durability, Exposure, Life(Durability), Criteria, Fault trees, Time series analysis, Aging tests(Materials), Pollution abatement.

Over the last two decades, the organic coatings industry has undergone rapid technological and structural changes. These changes have been induced by legislative actions such as restrictions pertaining to hazardous chemicals, toxic effluents, and volatile organic compounds. The consequence of these changes has been the displacement of almost all commercially-important, well-established coatings (largely high-solvent coatings) by newer systems, the formulation and application of which are based on different chemistries and technologies. This report reviews the attributes of the service life prediction problem which are common to all materials, components, and systems in an effort to establish a set of criteria for assessing the adequacy of existing or proposed service life prediction meth odology for coating systems. The current durability methodology and the reliability-based methodology are then evaluated against these criteria. The proposed criteria include the ability to (1) handle high variability in the time-to-failure data for nominally identical coated panels exposed in the same service environment, (2) analyze multivariate and censored time-to-failure data (3) establish a connection between laboratory and field exposure results, and (4) quantitatively predict the service life of a coating system exposed in its intended service environment
03, 125

PB95-176087 Not available NTIS

National Inst. of Standards and Technology (MSEL), Gaithersburg, MD. Ceramics Div

Modified Surface Layers and Coatings.

Final rept.

A. W. Ruff. 1994, 11p.

Pub. in Tribology in the USA and the Former Soviet Union: Studies and Applications, Chapter 3.5, p1972071994.

Keywords: "Surface properties, "Ceramic coatings, "Layers, "Tribology, Lubrication, Finishes, Ceramic materials, Self lubricating materials, Surface finishing, Residual stress, Adhesion, Reprints.

The use of advanced surface modification and coating methods to improve tribological characteristics of materials is an important development in recent years. Considerable effort is being expended on development of new coating materials, both hard and soft, on new techniques of alteration of near-surface material, and on important questions of coating adhesion, and system residual stresses. This Chapter will briefly mention some of the current areas of research and development in the U.S., although space limitations and lack of information on proprietary methods will not permit a thorough discussion. In addition, examples of two techniques of surface improvement will be described.

03,126

PB96-123211 Not available NTIS

National Inst. of Standards and Technology (MSEL) Gaithersburg, MD. Ceramics Div.

Anisotropy of the Surfaces of Pores in Plasma Sprayed Alumina Deposits.

Final rept.

J. Ilavsky, A. J. Allen, G. G. Long, C. C. Berndt, A

N. Goland, S. Krueger, and H. Herman. 1995, 6p.

Contract DE-AC02-76CH00016

Sponsored by Department of Energy, Washington, DC Pub. in Proceedings of Thermal Spraying (ITSC '95) - Current Status and Future Trends, Kobe, Japan, May 22-26, 1995, p483-488.

Keywords: "Plasma sprayed alumina, "Pore surface area, "Neutron scattering, Porosity, Reprints.

Porosity is an important factor in determining the phys cal properties of plasma sprayed deposits. A significant fraction $(2-15 \%)$ of the deposit volume consists of voids. These voids are in the form of pore between the splats and around any inclusions (unmelted particles) that may be present, and in the form of cracks within the splats. Neither the pores nor the cracks are spherical in shape, and the splat structure is circularly symmetric only when viewed from the spray direction. The microstructure of a plasma sprayed deposit contains significant anisotropy in the void shapes and there may be significant alignment of some of these shapes along particular directions. In this research, the voids in plasma sprayed deposits were investigated by measuring the specific surface areas of the voids from the terminal slope of small-angle neutron scattering, i.e., Porod scattering, by the deposits, and by means of mercury intrusion porosimetry. The results indicate that much of the void surface area is found perpendicular to the substrate and is associated with cracks. The second population of voids is found mostly parallel with the substrate and is associated with interlamellar pores. The surface areas measured by means of porosimetry were smaller than the surface areas derived from scattering, and were probabily influenced by the presence of closed porosity, by the void model and by the low precision for measuring small voids.

03,127

PB96-123237 Not available NTIS

National Inst. of Standards and Technology (MSEL) Gaithersburg, MD. Polymers Div

Flow-Induced Structure in Polymers: Chapter 16. Shear-Induced Changes in the Order-Disorder Transition Temperature and the Morphology of a Triblock Copolymer.

Final rept.

C. L. Jackson, F. A. Morrison, A. I. Nakatani, K. A. Barnes, C. C. Han, J. W. Mays, and M. Muthukumar 1994, 13p.

Pub. in Symposium of the National Meeting of the American Chemical Society (208th), Washington, DC. August 21-25, 1994, p233-245.

Keywords: “Copolymers, Reprints, "Triblock copolymer, Transition temperature, Steady shear flow.

An overview of ongoing research on a triblock copolymer subjected to steady-shear flow is presented. The 
copolymer is composed of polystyrene-polybut adienepolystyrene ( $23 \mathrm{st} \%$ polystyrene-degrees) with cylindisorder transition (ODT) was studied by in-situ SANS in a couette geometry as a function of temperature and shear rate. We observed that the ODT temperature shifted upward by 20 degrees $C$ as the shear rate was increased from - 0.1 to $25 \mathrm{~s}$ to the minus 1 . Sheared samples were also quenched and studied by transmission electron microscopy. A unique shear-induced morphology was found and tentatively identified as a transformation from the original p31m space group to a $\mathrm{p} 3 \mathrm{~m} 1$ space group of lower symmetry, similar to a martensitic transformation in metals.

\section{3,128}

PB96-154984 PC A04/MF A01

National Inst. of Standards and Technology (BFRL), Gaithersburg, MD. Building Materials Div.

Development of a Test Method for Leaching of Lead from Lead-Based Paints Through Encapsulants.

T. Nguyen, M. E. McKnight, and E. Byrd. Feb 96 40p, NISTIR-5783.

Sponsored by Department of Housing and Urban Development, Washington, DC. Office of Lead-Based Paint Abatement and Poisoning Prevention.

Keywords: "Lead(Metal), "Leaching, "Encapsulation, *Test methods, "Lead coatings, Chemical analysis, Paints, Acetic acid, Diffusion, Polymers, Standards, Pollution abatement, Toxic substances

Lead in paints is one of the major sources of lead poisoning in children. Polymeric encapsulant is considered a viable approach for controlling the hazards associated with lead-based paints. Encapsulant is considered a viable approach for controlling the hazards associated with lead-based paints. This study aimed to provide a technical basis for the development of a standard test method for lead leaching through polymeric encapsulants. The effects of pigment type and concentration in the film, chemical types and $\mathrm{pH}$ of leaching solutions, and encapsulants of different generic resins on the leaching of lead from a lead-based film with and without an encapsulant covering it were investigated.

\section{3,129}

PB97-110431 Not available NTIS

National Inst. of Standards and Technology (MEL),

Gaithersburg, MD. Precision Engineering Div.

Proposed Coating Technology Consortium. (National Coil Coaters Association Fall Conference). Held in Rosemont, Illinois in September 1992. Final rept.

T. Vorburger. 1993, 4p

Pub. in Coil Lines, v1 n3 4p Mar 93.

Keywords: "Coatings, Appearance, Consortium, Defects, Film thickness, Inspection, Reprints, "National Institute of Standards and Technology.

At NCCA's Fall Conference in Rosemont, IL, this past September, attendees heard about a proposed consortium to improve coating technology. The consortium is being organized at the National Institute of Standards and Technology (NIST) in Gaithersburg, MD, but will be directed by a board composed mainly of industrial members.

\section{3,130}

\section{PB97-118772 Not available NTIS}

National Inst. of Standards and Technology (MSEL), Gaithersburg, MD. Polymers Div.

Shear-Induced Changes in the Order-Disorder Transition Temperature and the Morphology of a Triblock Copolymer.

Final rept.

A. I. Nakatani, F. A. Morrison, C. L. Jackson, M Muthukumar, C. C. Han, J. F. Douglas, and J. W.

Mays. $1996,15 p$.

See also PB96-123237

Pub. in Jnl. of Macromolecular Science: Chemistry Physics Reviews in Macromolecular Chemistry and Physics, nB35 p489-503 1996.

Keywords: "Copolymers, Reprints, "Triblock copolymer, Transition temperature, Steady shear flow.

A summary of the work on a triblock copolymer under steady shear is presented. The experiments were conducted using small-angle neutron scattering (SANS), as a function of shear rate and temperature, and transmission electron microscopy (TEM) on quenched specimens. The triblock copolymer is composed of pol- ystyrene $d 8 /$ polybutadiene/polystyrene- $d 8$, and the ordered microstructure normally consists of hexagonally packed cylinders. Two temperature-dependent, characteristic shear rates, gamma(c1) and gamma(c2), are identified from the scattering results. The first characteristic shear rate identifies the shear rate required to go from a disordered state to an ordered state, while the second characteristic shear rate is interpreted as the shear rate necessary to produce a different ordered morphology.

\section{Composite Materials}

03,131

PB94-160066 PC A13/MF A03

National Inst. of Standards and Technology (MSEL), Gaithersburg, MD. Polymers Div.

Report on the Workshop on Manufacturing Polymer Composites by Liquid Molding. Held in Gaithersburg, Maryland on September 20-22, 1993. R. S. Parnas, A. J. Salem, K. N. Kendall, and M. V. Bruschke. Feb 94, 284p, NISTIR-5373.

Prepared in cooperation with General Electric Corporate Research and Development, Schenectady, NY and Ford Motor Co., Dearborn, MI.

Keywords: "Meetings, "Composite materials, "Molding techniques, "Composite fabrication, Permeability, Binders(Materials), Molding materials, Design, Mathematical models, Validation, Fiber composites, Reinforcing materials, Heat transfer, Rheology, Kinetics, "Liquid composite molding, Standard reference materials.

The report documents the workshop on manufacturing composites by liquid molding, held at NIST September $20-22,1993$. The workshop was jointly sponsored by NIST, Ford, and General Electric, and attracted a diverse international group of participants from Industry, University and government. Eight invited lectures were presented, and then the workshop divided into five discussion groups to focus on particular issues. A smal number of critical issues were identified, including preform deformation inside and outside of the mold high speed processing, and the establishment of standard reference materials, data, and test methods.

\section{3,132}

PB94-172848 Not available NTIS

National Inst. of Standards and Technology (IMSE), Gaithersburg, MD

High Temperature Degradation of Structural Composites.

Final rept.

S. M. Wiederhorn, and B. J. Hockey. 1991, 16p.

Contract DE-AI05-850R21569

Pub. in Advanced Structural Inorganic Composites, p365-380 1991. Sponsored by Department of Energy, Washington, DC

Keywords: "Ceramic matrix composites, "Creep properties, ${ }^{*}$ Thermal degradation, *Structural components, Toughness, Failure(Mechanics), Whisker composites, Cavitation, Fracture(Mechanics), Mechanical properties, Reprints.

Ceramic matrix composites are being developed to improve the toughness of ceramics in structural applications. Modest success has been experienced in manufacturing ceramic matrix composites for which it is possible to double the room temperature toughness while still retaining the high strength of the untoughened material. As many of these new composites are expected to be used at high temperatures, their mechanical behavior at both high and low temperature must be characterized. In the paper, creep and creep rupture of both particulate and whisker reinforced ceramic matrix composites are discussed. Principal findings of experimental investigations on these materials indicate: (1) resistance to creep deformation is greater in compression than in tension; (2) the creep rupture lifetime follows a Monkman-Grant behavior in which the lifetime can be expressed as a power function of the steady state creep rate; (3) the principal mechanism of failure in creep is interfacial cavitation. Cavities nucleate at interfaces and then grow into full facet cavities. Failure occurs either by the growth or the linkage of these cavities to form critical size cracks in the composite.

03,133

PB94-185279 Not available NTIS
National Inst. of Standards and Technology (MSEL), Boulder, CO. Materials Reliability Div.

Selection of Appropriate Ultrasonic System Components for NDE of Thick Polymer-Composites.

Final rept.

C. M. Fortunko, and D. W. Fitting. 1990, 8 p.

Pub. in Review of Progress in Quantitative Nondestructive Evaluation, v10B 8p 1990

Keywords: *Polymer matrix composites, *Ultrasonic flaw detection, "Test methods, Ultrasonic tests, Bandwidth, Construction materials, Transducers, Fibers, Polymers, Nondestructive tests, Pulse generators, Reprints.

In certain marine applications, thick polymer-composte materials may have to endure different operatin environments than those experienced in traditiona aerospace applications. In particular, structures made of such materials may experience very large compressive and bending forces. To prevent in-service failure appropriate NDE methods and instrumentation are needed to characterize the state of the material. Specifically, in addition to detecting high-contrast anomalies (cracks and delaminations) it may be of interest to determine the pore content measure the fiber volume, assess the severity of fiber waviness, and the like. The NDE requirements of thick marine composites cannot be met by using traditional ultrasonic instrumentation, particularly in the pulse-echo mode. For example, conventional ultrasonic instruments often lack features that permit effective pulse-echo operation below $1 \mathrm{MHz}$ and have limited dynamic range. In this paper, we describe specific approaches that overcome the above limitations. Specifically, we describe a particularly effective transmitreceive circuit configuration that offers large dynamic range greater than $60 \mathrm{~dB}$, wide bandwidth ( $100 \mathrm{kHz}-60 \mathrm{MHz}$ ), and fast recovery. We also describe the design of a very-low-noise, highimpedance input preamplifier. We found that this preamplifier is particularly useful for conditioning signals generated by very small ('phase insensitive') receiver transducers.

\section{3,134}

PB94-198645 Not available NTIS

National Inst. of Standards and Technology (MSEL) Gaithersburg, MD. Ceramics Div.

Matrix Grain Bridging Contribution to the Toughness of Whisker Reinforced Ceramics.

Final rept.

P. F. Becher, E. R. Fuller, and P. Angelini. 1991, 5p. Pub. in Jnl. of the American Ceramic Society $74, n 9$ p2131-21351991.

Keywords: "Ceramic matrix composites, "Whisker composites, "Fracture strength, Composite materials, Reinforcing fibers, Silicon carbides, Aluminum oxide, Matrix materials, Grain size, Mechanical properties, Reprints.

The fracture toughness of whisker reinforced noncubic ceramic matrices increases with increase in matrix grain size, d, as well as increased whisker content. An analytical description for a grain bridging toughening contribution is derived which is combined with the whisker bridging contribution to describe the toughness of such composites. Results for SiC whisker reinforced alumina composites confirm the predicted $d$ 1 2 dependence of the fracture toughness.

03,135

PB94-199361 Not available NTIS

National Inst of Standards and Technology (MSEL), Gaithersburg, MD. Ceramics Div.

Interface Properties for Ceramic Composites from a Single Fiber Pull-Out Test.

Final rept.

E. P. Butler, E. R. Fuller, and H. M. Chan. 1990, 8p. Pub. in Materials Research Society Symposia Proceedings, v170 p17-24 1990

Keywords: "Ceramic matrix composites, "Interfaces, Surface properties, "Fiber composites, Silicon carbides, Borosilicates, Friction measurement, Shear strength, Fracture properties, Thermal expansion, Reprints, "Pull-Out test.

An experimental approach has been developed using a single fiber pull-out test to measure the intrinsic materials properties of a ceramic composite interface. These properties are determined from the force-displacement curve, which is directly related to reinforcement toughening of composites via a fiber-debonding and frictional pull-out process. These interface properties were evaluated for a model composite system 
of continuous $\mathrm{SiC}$ fibers with various surface treatments in a borosilicate glass matrix. For the processing conditions used the interface fracture toughness and the interface frictional shear resistance, were found to be $1.0+$ or $-0.5 \mathrm{~J} / \mathrm{sq} \mathrm{m}$ and $3.3+$ or $-0.6 \mathrm{MPa}$ respectivley, for as-received fibers. Experiments con ducted with long embedded fiber lengths to be deconvolved into an interface friction coefficient of acid washed fibers has an increased interface toughness, $3.6+$ or $-0.1 \mathrm{~J} / \mathrm{sq} \mathrm{m}$, and friction coefficient, $0.08+$ or -0.02 , but approximately the same initial clamping matrix thermal expansion mismatch and from measurements of the stress birefringence of glass were in general agreement with this value.

\section{3,136}

PB94-199536 Not available NTIS

National Inst. of Standards and Technology (MSEL) Gaithersburg, MD. Ceramics Div.

Micro-Mechanical Aspects of Asperity-Controlled Friction in Fiber-Toughened Ceramic Composites. Final rept.

W. C. Carter, E. P. Butler, and E. R. Fuller. 1991, 6p. Pub. in Scripta Metallurgica et Materialia 25, n3 p579 584 1991. Sponsored by Department of Energy, Washington, DC

Keywords: "Ceramic matrix composites, "Reinforcing fibers, "Mechanical properties, "Friction, Cracking(Fracturing), Ceramic bonding, Interfaces Matrix materials, Fiber composites, Ceramic fibers, Reprints.

Evidence for the presence of asperities at the sliding interface of a ceramic-fiber composite is presented The origins of different types of asperities are discussed. A micro-mechanical model for the role of the interfacial asperities is developed and related to measured quantities. Asperities play a direct role in the behavior of the debonded tip, but only indirectly enhance the dominate fracture mechanism which is strain-energy-release by friction along the mode II crack flank.

\section{3,137}

PB94-200235 Not available NTIS

National Inst. of Standards and Technology (MSEL) Gaithersburg, MD. Ceramics Div.

Generic Model for Creep Rupture Lifetime Estimation on Fibrous Ceramic Composites.

Final rept.

T. J. Chuang. 1992, 17p.

Contract NASA-C-82000-R

Pub. in Fracture Mechanics of Ceramics, v10 p441 457 1992. Sponsored by National Aeronautics and Space Administration, Cleveland, $\mathrm{OH}$. Lewis Research Center.

Keywords: "Ceramic matrix composites, "Fiber composites, "Fracture mechanics, "Creep rapture strength, "Fatigue life, Crack initiation, Life(Durability), Ceramic fibers, Creep properties, Mechanical properties, Toughness, Reprints.

Because of their high strength and toughness at elevated temperatures, fiber reinforced ceramic composites have become potential candidates for the nex generation of turbine engine materials for aerospace applications. A generic model is proposed for assess ing lifetime of this class of materials when subjected to long-term creep rupture conditions. This two dimensional model consists of interfacial cracks growing beween square grains and rectangular fibers in the direction normal to the principal tensile stress axis. Neglecting transient effects, the total lifetime is derived based on the criterion that rupture is due to coalescence of adjacent cracks. It is found that lifetime is in versely proportional to crack growth rate, volume fraction, and aspect ratio of the fibers; but extremely sensitive to the applied stress owing to the high power of the $\mathrm{V}$-K(sub I) law. This lifetime estimation seems to be in fair agreement with the creep rupture data of SiCw/Si3N 4 composite with 0 and 30 vo $1 \%$ reinforcement tested at $1250 \mathrm{C}$ in air. Furthermore, TEM performed on the post crept specimens revealed that creep damage is predominantly in the form of microcracks at matrix/matrix as well as fiber/matrix interfaces, approximately in accord with the model simulation.

\section{3,138}

PB94-200243 Not available NTIS

National Inst. of Standards and Technology (MSEL) Gaithersburg, MD. Ceramics Div.
Asymmetric Tip Morphology of Creep Microcracks Growing Along Bimaterial Interfaces.

Final rept.

T. J. Chuang, J. L. Chu, and S. Lee. 1992, 9p.

Pub. in Acta Metall. Mater. 40, n10 p2683-2691 1992.

Keywords: "Microcracking, "Interfaces, "Crack propagation, "Composite materials, Elastic properties, Deformation, Diffusivity, Laminates, Morphology, Dihedral angle, Reprints, "Bimaterials.

The asymmetric tip morphology of a creep microcrack propagating along a bimaterial interface is presented based on the assumptions that the near-tip shapes are developed from surface diffusion controlled crack growth and that a steady state prevails. Following Chuang and Rice, a single master curve still can adequately describe the near-tip shapes to within $6 \%$ accuracy, but four cases of crack-tip morophology emerge depending on the ratio of surface to interfacial free energy and diffusivity of the adjoining phases. For fixed ratios of the two surface diffusivities, crack tip morphology maps in the space of specific surface energies are constructed with which areas associated with each individual case are indicated. Predicted cases on a set of bimaterial systems are tabulated and discussed. TEM photos of creep crack tips in alumina and silicon nitride are presented for illustration. The information given here is essential to a coupled analysis diffusion and elastic deformation at a composite interface which may ultimately yield predictions of the delamination rate between the reinforcing phase and the matrix phase as a function of applied stress, temperature and other relevant materials' constants.

\section{3,139}

PB94-200532 Not available NTIS

National Inst. of Standards and Technology (MSEL) Gaithersburg, MD. Ceramics Div.

Assessment of Testing Methodology for Ceramic Matrix Composites.

Final rept.

D. C. Cranmer. 1991, 8p.

Pub. in Ceram. Trans. Adv. Compos. Mater., v19 p1003-10101991.

Keywords: "Ceramic matrix composites, "Materials testing. "Mechanical properties, Assessments Sample preparation, Interlaboratory comparisons, Strength, Creep tests, Fatigue tests, Whisker composites, Ceramics, Reprints.

An assessment of strength, creep, and fatigue measurement techniques for ceramic matrix composites is made. Many of the techniques available for composites testing are reviewed. Tensile tesis, not flexure, should be used for experimental measurements, and different tests are needed for material development and system design. Careful attention must be paid to specimen preparation. Tests used for monolithic ceramics can be used for whisker-reinforced materials. Interlaboratory comparisons are also needed to determine the reproducibility and limitations of the methodology as well as material behavior.

\section{3,140}

PB94-200649 Not available NTIS

National Inst. of Standards and Technology (MSEL), Boulder, CO. Materials Reliability Div.

Elastic Properties of Uniaxial-Fiber Reinforced Composites: General Features.

Final rept.

S. Datta, H. Ledbetter, and M. Lei. 1991, 6p.

Pub. in Nondestructive Evaluation and Material Properties of Advanced Materials, p23-28 1991.

Keywords: "Fiber composites, "Elastic properties, *Reinforcing fibers, Modulus of elasticity, Poisson ratio, Matrix materials, Fiber volume fraction, Reinforcing materials, Mechanical properties, Reprints, Scatteredplane-wave model, Ensemble-average model, Elastic constants.

The authors consider the complete set of elastic constants of composites comprising isotropic uniaxial fibers in an isotropic matrix. Such materials exhibit transverse-isotropic symmetry and five independent elastic constants, and the authors calculate and display all these constants over the entire fiber-volumefraction range, 0.0-1.0. They also consider some practical elastic constants such as the principal Young moduli and the principal Poisson ratios. Except for one principal Poisson ratio, all other elastic constants show negative departures from a linear rule-of-mixture. Because one often uses sound velocities to study such materials, the authors give resulis for the four principal sound velocities.
03,141

PB94-211778 Not available NTIS

National Inst. of Standards and Technology (MSEL) Gaithersburg, MD. Ceramics Div.

Effect of Microstructure on the Wear Transition of Zirconia-Toughened Alumina.

Final rept.

C. He, Y. S. Wang, J. S. Wallace, and S. M. Hsu. $1993,8 p$.

Pub. in Wear 162-164, p314-321 1993.

Keywords: "Ceramic matrix composites, "Microstructure, "Wear tests, "Aluminum oxide, "Zirconium oxides, Mechanical properties, Lubrication, Particle size distribution, Environmental effects, Fractures(Materials), Reprints.

The mechanical properties of alumina ceramics can be improved by the addition of pure or partially stabilized zirconia particles. In the present study, the wear characteristics of zirconia-toughened alumina (ZTA) composites under a non-reactive fluid (paraffin oil) lubricated condition were investigated. The wear transition load (i.e. the load at which a rapid increase in wear occurs) increased with increasing zirconia content up to $20 \mathrm{vol} . \%$. The transition from mild to fracture-controlled wear of ZTA depends on the material properties (e.g. hardness, elastic modules), the contact conditions (e.g. hertzian stress, coefficients of friction) and the microstructure of the material. The effect of the microstructure on wear was demonstrated and a HallPetch-type relationship between the microfracture stresses and the grain size was found. The effect of the grain size distribution on the wear transition load was also shown.

03,142

PB94-211984 Not available NTIS

National Inst. of Standards and Technology (MS $=L$ ), Gaithersburg, MD. Ceramics Div.

Tensile Creep of Whisker Reinforced Silicon Nitride.

Final rept.

B. J. Hockey, S. M. Wiederhom, W. Liu, J. G.

Baldoni, and S. T. Buljan. 1991, 9p.

Pub. in Jnl. of Materials Science 26, n14 p3931-3939, 15 Jul 91.

Keywords: "Whisker composites, "Silicon nitrides, *Tensile creep. Tensile properties, Reinforcing fibers, Ceramics, Creep properties, Mechanical properties, Tensile deformation, Cavitation, Reprints.

In this paper we discuss the creep and creep rupture behavior of a grade of hot pressed silicon nitride reinforced with $30 \mathrm{v} / \mathrm{O} \mathrm{SiC}$ whiskers. The material was tested in both tension and compression at temperatures ranging from $1100 \mathrm{C}$ to $1250 \mathrm{C}$ for periods as long as 1000 hr. Principal findings were: (1) transient creep due to devitrification of the intergranular phase dominates high temperature creep behavior; (2) at high temperatures and stresses, cavitation at the whisker/ silicon nitride interface enhances the creep rate and reduces the lifetime of the silicon nitride composite; (3) resistance to creep deformation is greater in compression than in tension; (4) the time to rupture is a power function of the creep rate, so that the temperature and stress dependence of the failure time is determined solely by the temperature and stress dependence of the creep rate.

03,143

PB94-212453 Not available NTIS

National Inst. of Standards and Technology (MSEL), Gaithersburg, MD. Metallurgy Div.

Status of Electrocomposites.

Final rept.

C. E. Johnson, and M. Browning. 1990, 24p.

Pub. in Proceedings of the American Electroplaters and Surface Finishers Annual Technical Conference, Boston, MA., July 9-12, 1990, p1203-1226.

Keywords: "Electrochemistry "Metal matnix composites, "Intermetallics, Coatings, Electrodeposition, Electroforming, Matrix materials, Hot pressing, Mechanical properties, Reprints.

This presentation is intended to discuss the current state of the art in the use of electrochemical techniques for the fabrication of metal and intermetallic matrix composites. Electroforming techniques are being evaluated for fabrication of near net-shape composite structures, for example, reusable rocket thrust chambers. Copper and nickel coated fibers are presently being used in polymer matrix composites for the pur- 
pose of EMI shielding. If sufficiently thick, the coated fibers can be consolidated into metal matrix composites by hot pressing without the need for infusion of additional metal. Electrocoatings are also used as diffusion barriers to protect carbon fibers from degradation at high temperatures due to carbide formation and dissolution of carbon into the matrix alloy. Another use of electroplating is to provide improved fiber/matric interfacial adhesion. Multilayer fiber coatings (e.g. Fe/ $\mathrm{Cu} / \mathrm{Al}$ ) can provide gradual transitions in the coefficien of thermal expansion and/or Young's modulus. These techniques can greatly improve the mechanical and thermal properties of the composites. Metal matrix composites which combine high stiffness and strength with low specific gravity are of particular interest in applications such as transportation (e.g. air and space craft, automobiles) and high speed machinery (e.g textile machinery, drive shafts).

03,144

PB94-213204 Not available NTIS

National Inst. of Standards and Technology (MSEL)

Boulder, CO. Materials Reliability Div. Aluminum Composite.

Final rept.

H. Ledbetter, and M. Austin. 1991, 9p.

Pub. in International Jnl. of Thermophysics $12, \mathrm{n} 4$ p731-739 Jul 91.

Keywords: "Metal matrix composites, "Aluminum graphite composites, "Thermal expansion, Extensometers, Silicon carbides, Elastic properties, Microstructure, Single crystals, Thermophysical properties, Neutron diffraction, Reprints

Using a push-rod dilatometer, we measured between 76 and $390 \mathrm{~K}$ the thermal expansion of a particle-reinforced-composite wrought plate obtained by powdermetallurgy methods. The particles, $30 \%$ by volume consisted of monocrystals of alpha-SiC with sizes near 10 micrometers. The matrix consisted of a 6061 aluminum alloy with original particle sizes near 20 micrometers. We found the perpendicular thermal expansivity, alpha 3 , higher by $26 \%$ than the in-plane thermal expansivity, alpha 1 approximately equal to alpha 2. These values differ from a rule-of-mixture prediction by -3 and $-23 \%$, respectively. All three alpha components lie outside the Rosen-Hashin bounds. Levin's isotropic model agrees within $10 \%$ with the alpha 1-alpha 2-alpha 3 average. Both the anisotropy and the bounds violation result from microstructura nonhomogeneity arising from processing. Rosen and Hashin's transverse-isotropic-symmetry relationships account approximately for these effects by introducing the anisotropic elastic constants. Using neutron diffraction, we determined that the SiC particles are textureless.

03,145

PB94-216728 Not available NTIS

National Inst. of Standards and Technology (MSEL), Gaithersburg, MD Polymers Div.

Review of Cure Monitoring Techniques for On-Line Process Control.

Final rept.

W. G. McDonough, B. M. Fanconi, F. I. Mopsik, and D. L. Hunston. 1990, $8 p$

Pub. in Proceedings of Annual ASM/ESD Advanced Composites Conference (6th), Materials Park, $\mathrm{OH}$. October 8-11, 1990, p637-644.

Keywords: *Process control(Industry), *Polymer matrix composites, "Curing, "Monitoring, "Nondestructive tests, Spectroscopy, Quality control, Real time operations. Inspection, Fluorescence, Ultrasonics, Reprints.

A driving force for implementation of on-line process control is the need to improve the efficiency and reliability of polymer composite processing. A critical element in process control is on-line process monitoring. This paper reviews and compares the most important techniques currently under development, i.e. dielectric ultrasonic, optical and spectroscopic monitoring meth ods. A number of technical and scientific issues that must be addressed for these techniques to reach their full potential are identified. The chief technical barrier is to adapt the methods to operate in the harsh environment of a factory with industrial grade resin systems. The major scientific barrier is a need to improve ou ability both to interpret the information provided by the sensor and translate this data into process control information. Although these issues are challenging, many programs are currently underway to address them so the future for on-line process control of composites looks very bright.

\section{3,146}

PB94-2192

MF A02)

National Inst. of Standards and Technology, Gaithersburg, MD.

Physics Required for Prediction of Long Term Performance of Polymers and Their Composites.

G. B. McKenna. 1994, 21p.

Included in Jnl. of Research of the National Institute of Standards and Technology, v99 n2 p169-189 Mar/ Apr 94.

Keywords: "Polymers, "Glass transition temperature "Composite materials, "Viscoelasticity Stress relaxation Mechanical properties, Mathematical models Predictions, Performance tests, Temperature, Volume Constitutive equations, Material clocks, Nonlinearity, National Institute of Standards and Technology.

The long term performance of polymers and their composites is an important aspect of their increasing use in engineering applications. Temporal, thermal, and mechanical stresses can all contribute to the deterioration of performance. Here we examine the concepts of the physics of glassy polymers and how they are important in developing constitutive equations that describe their volume/temperature/stress time response. The understanding of such response forms the basis of the prediction of long term performance.

\section{3,147}

\section{PB94-780129 AV E99}

Minnesota Technology, Inc., Minneapolis, MN

Polymer Composites Workshop. Held in Winona, Minnesota on April 29-30, 1992 (Video).

Audio-Visual.

J. Wright. 4 Jun 92,5 VHS videos

See also PB92-182658. Sponsored by National Inst of Standards and Technology (TS), Gaithersburg, MD. This video package contains 5 VHS videos with a tota viewing time of approximately $8 \mathrm{hrs}, 45$ minutes.

Keywords: "Polymer matrix composites, ${ }^{*}$ Composite materials, "Meetings, Technology innovation, Filament winding, Financing, Small businesses, Contact molding, Manufacturers, Maintenance, Compression molding, Injection molding. Pultrusion process, Video tapes, Minnesota Technology, National Institute of Standards and Technology.

The set of five video tapes covers the Polymer Com posites Workshop held in Winona, Minnesota on April 29-30, 1992. Presentations include: Minnesota Technology, Inc.; The NIST Perspective; The Regional Perspective; Filament Winding; Regional and Nationa Funding Sources for Small Businesses; Contact Molding: Opportunities for Interaction Between Primary Manufacturing Companies and Small Business; Composite Repair; Compression Molding/Class A Resin Transfer; Injection Molding; Reaction Injection Mold ing; Structural Resin Transfer Molding; Pultrusion.

\section{3,148}

\section{PB95-125696 Not available NTIS}

National Inst. of Standards and Technology (IMSE)

Boulder, CO. Fracture and Deformation Div.

Appropriate Ultrasonic System Components fo NDE of Thick Polymer Composites.

Final rept

C. M. Fortunko, and D. W. Fitting. 1991, 8p

Pub. in Review of Progress in Quantitative Non destructive Evaluation, v10B p2105-2112 1991

Keywords: *Ultrasonic tests, *Polymer matrix compos ites, "Ultrasonic flow detection, Acoustic measure ment, Nondestructive tests, Signal processing. Pre amplifiers, Ultrasonic wave transducers, Fiber volume fraction, Cracks, Fiber composites, Delaminating, $R e$ prints

In certain marine applications, thick polymer-compos ite materials may have to endure different operating environments than those experienced in traditiona aerospace applications. In particular, structures made of such materials may experience very large compres sive and bending forces. To prevent in-service failure, appropriate NDE methods and instrumentation are needed to characterize the state of the material. Spe cifically, in addition to detecting high-contrast anomalies (cracks and delamination), it may be of interest to determine the pore content, measure the fiber volume, assess the severity of fiber waviness, and the like in this paper we describe specific approaches that over come the limitations of conventional ultrasonic instru mentation. Specifically, we describe a particularly ef fective transmit/receive circuit configuration that offers large dynamic range (greater than $60 \mathrm{~dB}$ ), wide bandwidth $(100 \mathrm{kHz}-60 \mathrm{MHz})$, and fast recovery. We also describe the design of a very-low-noise, high impedance input preamplifier. We found that this preamplifier is particularly useful for conditioning signals generated by very small ('phase insensitive') receiver transducers.

03,149

PB95-125811 Not available NTIS

National Inst. of Standards and Technology (MSEL),

Gaithersburg, MD. Ceramics Div.

Determination of Fiber-Matrix Interfacial Properties of Importance to Ceramic Composite Toughening. Final rept.

E. R. Fuller, E. P. Butler, and W. C. Carter. 1991,

$19 p$

Sponsored by Department of Energy, Washington, DC. $P u b$. in Toughening Mechanisms in Quasi-Brittle Materials, p385-4031991.

Keywords: "Ceramic composites, "Fiber composites, Mechanical properties, Interfaces Silicon carbides, Borosilicate glass, Toughness, Shear strength, Reprints, "Debonding, "Single fiber pull-out test

The fiber-matrix interface is investigated for its role during fiber debonding and pull-out and its effect on the toughening of ceramic composites. Possible interface debonding criteria (critical interface shear strength; interface toughness or critical energy-release rate and asperity interlock) are considered. The interfacial and mechanical properties of a model SiC-fiber, borosilicate-glass-matrix composite system are measured by a single fiber pull-out test. Initial stresses are determined by photoelastic methods.

\section{3,150}

PB95-140828 Not available NTIS

National Inst. of Standards and Technology (MSEL), Gaithersburg, MD. Metallurgy Div.

Surface Energy Reduction in Fibrous Monotectic Structures.

Final rept.

A.C. Sandlin, and R. J. Schaefer $1991,6 \mathrm{p}$.

Pub. in Metallurgical Transactions A 22, n8 p188118861991.

Keywords: "Surface energy, "Directional solidification(Crystals), "Monotectic alloys, "Metal matrix composites, Grain boundaries, Morphology, Lead alloys, Aluminum alloys, Copper alloys, Microstructure, Metal fibers, Reprints.

A study has been made of the morphology of directionally solidified $\mathrm{Cu}-\mathrm{Al}-\mathrm{Pb}$ monotectic alloys. The structure consisted of a hexagonal array of $\mathrm{Pb}$ rods in a Cu-based matrix. In addition, highly-curved grain boundaries in the Cu-based matrix with lens-shaped $\mathrm{Pb}$ fibers on the boundary and a 'denuded zone', depleted of $\mathrm{Pb}$ rods, were observed. Existence of these boundaries is shown to reduce the overall surface energy of the system leading to the formation of the highly-curved grain boundaries.

\section{3,151}

PB95-140935 Not available NTIS

National Inst. of Standards and Technology (BFRL), Gaithersburg, MD. Fire Safety Engineering Div

One- and Two-Sided Burning of Thermally Thin Materials.

Final rept.

T. Ohlemiller, and J. Shields. 1993, 8p.

Contract NLRC-C-32003-R

Sponsored by National Aeronautics and Space Administration, Cleveland, $\mathrm{OH}$. Lewis Research Center. Pub. in Fire and Materials 17, p103-110 1993.

Keywords: "Burning rate, *Composite materials, *Fire tests, *Flammability, Flame propagation, Temperature dependence, Heat flux, Fire safety, Combustion, Reprints.

The rate of heat release from a thermally thin material burning on both sides will be more than twice the value seen when only one side is burning. Two simplified models demonstrate that this is a consequence of the Arrhenius temperature dependence of the gasification rate of the solid. Experiments carried out on three composite materials over a range of incident heat fluxes confirm this effect it is inferred that a further consequence of this heat release enhancement is an increased tendency for concurrent flame spread in the two-sided burning case. Materials whose application could lead to two-sided burning should thus be as- 


\section{Composite Materials}

sessed in this mode to obtain a true picture of their flammability potential.

03,152

PB95-143293 PC A03/MF AO1

National Inst. of Standards and Technology (BFRL), Gaithersburg, MD. Fire Science Div.

Investigation into the Flammability Properties of Honeycomb Composites.

Special pub.

M. R. Nyden, and J. E. Brown. Oct 94, 23p, NISTIR5509 .

Keywords: "Polymers, "Composite materials, "Aircraft construction materials, "Flammability, "Aircraft fires, Fire resistant materials, Ignition, Burning rate, Honeycomb structures, Formaldehyde, Sandwich structures Absorption spectra, Combustion, "Honeycomb composites.

The study, which is the subject of this report, was carried out in two stages. The objective of the first phase was to investigate the effect of electron beam irradiation and grafting on polymer flammability. The time to ignition and the rate-of-heat-release were measured for the combustion of a series of samples in which a fire resistant polymer was used to protect the surface of a more flammable polymer. The flammability properties of honeycomb composite materials, which are currently used in the interior cabin compartments of commercial aircraft, were examined in the second phase of this project. Analyses of the gases evolved during the thermal degradation of the components indicated that the phenol-formaldehyde resin makes a significant contribution to the flammability of these composites. The possibility that a more fire resistant formulation could be developed was examined by testing a series of resins which differed in the relative amounts of phenol and formaldehyde used in the reaction mix tures. The flammabilities of resins synthesized in excess phenol were measurably less than those synthesized in excess formaldehyde.

03,153

PB95-151528 Not available NTIS

National Inst. of Standards and Technology (MSEL), Gaithersburg, MD. Ceramics Div.

Measuring Matching Wear Scars on Balls and Flats.

Final rept.

E. P. Whitenton, and D. E. Deckman. 1989, 18p.

Pub. in Surface Topography 2, p205-222 1989.

Keywords: "Wear tests, "Aluminum oxides, *Profilometers, Profiles, Topography, Lubrication, Surface properties, Reprints, *Wear scars, Ball-on-three flat wear tests, Four-ball wear tests.

Four-ball and ball-on-three-flat wear tests are often performed to assess the tribological performance of lubricants and materials. Test results are described by the worn volume and or topography parameters of the balls or flats. As a general rule, relatively little effor goes into quantifying the wear scar of the top ball even though there is often useful information there. A computer-controlled profiling machine is described to measure the topography of the top ball and the bottom balls or flats. Several techniques are examined to turn this topographic information into useful parameters and aids for assessing the interaction between the balls or flats.

\section{3,154}

PB95-152294 Not avaiłable NTIS National Inst. of Standards and Technology (MSEL)
Gaithersburg, MD.

Creep Rupture of MoSi2/SiCp Composites.

Final rept.

J. D. French, S. M. Wiederhorn, and J. J. Petrovic. 1994, 6p.

Pub. in Materials Research Society Symposia Proceedings, v322 p203-208 1994

Keywords: "Creep rupture strength, "Ceramic matrix composites, "Silicon carbides, Composite materials Creep properties, Creep tests, Ductility, Molybdenum compounds, Reprints, *Molybdenum disilicide.

We studied the creep rupture of a series of MoSi2 materials reinforced with $\mathrm{SiC}$ particles. Particulate contents were in the range of $O$ to 40 volume percent Temperature and stress ranges were $1050 \mathrm{C}$ to 1200 $\mathrm{C}$ and $10 \mathrm{MPa}$ to $50 \mathrm{MPa}$, which gave failures ranging from 1 hour to 1500 hours. The creep curves show an extensive tertiary regime, accounting for $25-95 \%$ of the total lifetime. Tertiary creep increases with increasing stress and temperature. Cavitation occurs throughout the creep life, and tertiary creep is associated with the linkage of cavities into large cracks. The creep life improves with increasing SiC particle content, with a concurrent loss of creep ductility. Significant improvement occurs only when the particle content is greater than 30 volume percent. Our studies suggest that the creep and creep rupture behavior of MOSi2 can be further improved by increasing the content of SiC particles.

\section{3,155}

PB95-152880 Not available NTIS

National Inst. of Standards and Technology (MSEL), Boulder, CO Materials Reliability Div.

Residual Stresses in Aluminum-Mullite (alpha-Alumina) Composites.

Final rept

D. Balzar, and H. Ledbetter. 1993, 9p.

Pub. in Advances in X-ray Analysis, p489-497 1993.

Keywords: "X-ray diffraction, "Metal matrix composites, "Residual stress, "Aluminum alloys, "Particulate reinforced composites, "Aluminum oxides, Strains, Matrix materials, Reinforcing materials, Anisotropy, Tensile properties, Reprints, Rietveld refinement.

We studied particle-reinforced 6061-aluminum-alloy composites with particle volume fractions ranging from 0 to 0.25 . The mullite particles are approximately sphericai and contain embedded alpha-alumina phase. We obtained lattice parameters of all three phases in the composites and starting materials by using Rietveld refinement of $x$-ray diffraction patterns. In all three phases stresses are tensile and approximately of the same magnitude, contradicting a requirement for mechanical equilibrium. Stresses increase with both increasing particle size and volume fraction. Measurements of extracted-from-composites particles showed no evidence of a possible chemical reaction at the matrix-particle interface. The matrix is (111) and (100) textured, but measurements of elastic constants reveal only small anisotropy. Thus, explanation of the mechanical-equilibrium violation remains uncertain.

\section{3,156}

PB95-161295 Not available NTIS

National Inst. of Standards and Technology (MSEL), Gaithersburg, MD. Ceramics Div.

Flat and Rising R-Curves for Elliptical Surface Cracks from Indentation and Superposed Flexure.

Final rept.
R. F. Krause, $1994,7 p$.

Pub. in Jnl. of the American Ceramic Society 77, n1 p172-178 1994

Keywords: "Whisker composites, "Aluminum oxides, "Silicon carbides, "Surface cracks, "Flexing, *Indentation, Fracture strength, Deformation, Stress intensity factors, Surface properties, Loads(Forces), Cracking(Fracturing), Defects, Reprints, "R-curves, *Superposed flexure.

Flat and rising R-curves, fracture resistance versus crack extension were determined for a sintered $99 \%$ alpha-silicon carbide and for a hot-pressed composite of $25 \mathrm{wt} \%$ silicon carbide whiskers and alumina, respectively. The R-curves were evaluated from a combination of measured crack lengths, which were produced over a range of Vickers indentation loading, and of measured strengths, which were correlated either with the indentation flaws or with the most severe natural flaws or flexure specimens. A published analysis of the stress-intensity factor for a surface crack in flexure was interpreted to show that the crack front takes the form of a semiellipse where both the ratio of the minor to major radii and the configuration coefficient itself decrease with increasing crack extension. A power-law function of the indentation load was fitted to the product of an effective configuration coefficient and the flexural strength to evaluate the R-curves. When the configuration coefficient is assumed constant, a customary practice, the R-curves appear to have steeper rises. The assumed constancy of the coefficient of the indentation driving force may also have an effect on R-curves, but the effect would be much less.

03,157

PB95-161378 Not available NTIS

National Inst. of Standards and Technology (MSEL), Boulder, CO. Materials Reliability Div.

Elastic Properties of Al2O3/Al Composites: Measurements and Modeling.

Final rept.

H. Ledbetter, and S. Datta. 1991, 6p

Pub. in Damage and Oxidation Protection in High Temperature Composites AD, v25-2 p83-88 1991.
Keywords: "Metal matrix composites, "Elastic properties, "Particulate reinforced composites, Reinforcing materials, Composite materials, Aluminum oxides, Aluminum, Modulus of elasticity, Shear properties, Poisson ratio, Acoustic velocity, Reprints.

We considered seven Al2O3-particle-reinforced Al-matrix composites with particle volume fractions ranging from 0 to 0.24 . At ambient temperature, we measured the elastic constants by two dynamic methods: $\mathrm{kHz}$ resonance and $\mathrm{MHz}$ pulse-echo-superposition. We report the usual elastic-constant set: bulk, shear, Young moduli, and Poisson ratio. The microstructures show a random distribution of spherical particles, from specimen to specimen ranging from 30 to 100 micrometers. The highest observed Young-modulus anisotropy was 1.04. To model this system, we used the long-wavelength limit of a plane-wave ensemble-average method. Measurements and modeling agree well: the average disagreement in the Young modulus is about one percent. As expected, except for the Poisson ratio, all measurements fall below a linear rule-of-mixture.

\section{3,158}

PB95-161824 Not available NTIS

National Inst. of Standards and Technology (MSEL), Gaithersburg, MD. Polymers Div.

Thermoacoustic Technique for Determining the Interface and/or Interply Strength in Polymeric Composites.

Final rept.

W. L. Wu. $1990,5 p$

Pub. in Society for the Advancement of Material and Process Engineers Jnl. 26, n2 p11-15 1990.

Keywords: "Polymer matrix composites, "Graphite composites, "Thermal analysis, "Acoustic emission, Fiber composites, Laser heating, Interfacial energy, Debonding(Materials), Mechanical properties, Reprints.

Local heating was applied on polymeric composites reinforced with graphite fibers to induce interfacia debonding events and the acoustic emissions associated with the debonding events were monitored. Correlations among some of the acoustic parameters and the interfacial strength were observed. The possibility of adopting this scheme to determine interfacial strength in composites is delineated.

\section{3,159}

PB95-162012 Not available NTIS

National Inst. of Standards and Technology (MSEL) Gaithersburg, MD. Polymers Div.

Interaction between Micro and Macroscopic Flow in RTM Preforms.

Final rept.

R. S. Parnas, A. J. Salem, T. A. K. Sadiq, H. P

Wang, and S. G. Advani. 1994, 15p.

Pub. in Composite Structures 27, p93-107 1994.

Keywords: "Porous materials, "Composite materials, *Resin transfer molding, "Flow distribution, "Preforms, Resin matrix composites, Fiber composites, Microparticles, Macromolecules, Permeability, Reprints

Both experimental and modeling results are presented to convey the wide span of length scales over which flow in porous media can occur in a single material. Flow in such heterogeneous porous media are shown to be important in composites processing because the structure of fibrous reinforcements contains multiple length scales. Flow phenomena that arise due to material heterogeneity have been shown to include void formation and to explain the differences observed between measurements of the 'wet' permeability and the 'dry' permeability. The work presented indicates that the heterogeneous reinforcement structure may also contribute to the differences observed in permeability measurements carried out by the radial flow and the one-dimensional flow methods. The concept of heterogeneous porous media is also extended to the molecular level by considering the flow problem in a reinforcement sized with grafted macromolecules.

03,160

PB95-162194 Not available NTIS

National Inst. of Standards and Technology (MSEL), Gaithersburg, MD. Ceramics Div.

Fracture Toughness of Advanced Ceramics at Room Temperature: A Vamas Round-Robin.

Final rept.

G. Quinn. 1993, 9p.

Sponsored by Department of Energy, Washington, DC

Office of Transportation Systems Utilization. 
Pub. in Ceramic Engineering and Science Proceedings 14, n7-8 p92-100 1993.

Keywords: "Ceramic matrix composites, "Fracture strength, Mechanical properties, Ceramic materials, Test methods, Standards, Silicon nitrides, Toughness, Fracture mechanics, Reprints.

The VAMAS project is an international collaboration for prestandardization research. The participating countries are Canada, France, Germany, Italy, Japan, the United Kingdom, the United States, and the Commission of European Communities. Technical Working Area No. 3, Ceramics, has the objective of undertaking research on the reliability and reproducibility of test procedures for advanced technical ceramics. The Japan Fine Ceramics Center (JFCC) in 1988 organized a VAMAS round-robin to evaluate fracture toughness by three methods on two advanced ceramics The three methods are schematically illustrated. Preliminary results from thirteen of the participating twenty-two laboratories have been previously reported. A comprehensive, more recent report with the results of the five United States laboratories has been prepared. The five USA laboratories were: NIST, NASA-Lewis Worcester Polytechnic Institute, Allied-Signal, NortonSt. Gobain. The present paper highlights the principal results of the project.

03,161

PB95-162533 Not available NTIS

National Inst. of Standards and Technology (MSEL) Gaithersburg, MD. Ceramics Div.

Fabrication of Flaw-Tolerant Aluminum-TitanateReinforced Alumina.

Final rept.

J. L. Runyan, and S. J. Bennison. 1991, 7p.

Pub. in Jnl. of the European Ceramic Society $7, \mathrm{n} 2$ p93-99 1991.

Keywords: "Particulate reinforced composites "Aluminum oxide, "Fabrication, "Defects, "Ceramic composites, Composite materials, Ceramics, Crack propagation, Crack arrest, Grain structure Microstructure, Reprints, "Aluminum titanates.

Fabrication and flaw tolerance properties of particulate aluminum-titanate-reinforced alumina composites have been studied. High-density $(-99 \%$ theoretical $)$ composites with controlled microstructures are readily produced via a conventional ceramics processing scheme utilizing starting powders of alpha-alumina and beta-aluminum titanate. Indentation-strength measurements demonstrate certain composites exhibit unusu ally pronounced flaw tolerance behavior. Direct observations of crack evolution from Vickers indentations during loading reveal strong stabilization of cracks with sizes in the millimeter range. It is concluded that this stabilization gives rise to the flaw tolerance properties and results from pronounced crack-resistance (Rcurve) behavior. Grain-localized crack bridging is strongly active in this system and is believed to be a major contributor to the R-curve properties.

03,162

PB95-163325 Not available NTIS

National Inst. of Standards and Technology (IMSE), Boulder, CO. Fracture and Deformation Div

Elastic Green's Function for a Bimaterial Compos ite Solid Containing a Free Surface Normal to the Interface.

Final rept.

V. K. Tewary. 1991, 17p

Pub. in Jnl. of Materials Research 6, n12 p2592-2608 1991.

Keywords: "Composite materials, "Greens function, Fracture(Mechanics), Stress analysis, Series(Mathematics), Numerical solution, Singularity, Anisotropy, Integrals, Cracks, Reprints, Hilbert problems, Free surfaces.

Elastic plane strain Green's function is calculated for an anisotropic bimaterial composite solid containing a free surface normal to the interface. An exact integra representation is obtained for the Green's function which is evaluated numerically. The integral is also evaluated analytically which gives a series representation for the Green's function. The singularities in the stress field associated with the presence of the free surface are identified and discussed. For the purpose of illustration, the formalism is applied to a cubic solid containing a Sigma-5 grain boundary.
National Inst. of Standards and Technology (IMSE), Boulder, CO. Fracture and Deformation Div. Green's Function for Generalized Hilbert Problem for Cracks and Free Surfaces in Composite Mate-

rials.

Final rept.

V. K. Tewary. 1991, 7p.

Pub. in Jnl. of Materials Research 6, n12 p2585-2591 1991.

Keywords: "Composite materials, "Greens function, Vectors(Mathematics), Fracture mechanics, Stress analysis, Eigenfunctions, Cracks, Reprints, "Hilbert problems, Free surfaces.

Green's function for a generalized vector Hilbert problem is calculated which can be used to solve the Hilbert problem with any integrable inhomogeneity. The Green's function is obtained by using a complex transform defined by eigenfunctions of the homogeneous Hilbert problem. This method should be particularly convenient for the stress analysis of anisotropic composite materials containing cracks and free surfaces.

03,164

PB95-163341 Not available NTIS

National Inst. of Standards and Technology (IMSE), Boulder, CO. Fracture and Deformation Div

Generalized Plane Strain Analysis of a Bimaterial Composite Containing a Free Surface Normal to the Interface.

Final rept.

V. K. Tewary, and R. D. Kriz. 1991, 14p.

Pub. in Jnl. of Materials Research 6, n12 p2609-2622 1991

Keywords: "Composite materials, "Stress analysis, "Binary systems(Materials), "Solid-solid interfaces, Fracture(Mechanics), Laminates, Grain boundaries, Strain distribution, Green's functions, Plane waves, Elastic properties, Reprints.

The elastic plane strain Green's function calculated in earlier papers is modified to account for the generalized plane strain and applied to calculate the stress and the displacement field in a bimaterial composite containing a free surface normal to the interface which is subjected to an out of plane load. The result is obtained in terms of a closed integral representation which is evaluated numerically as well as analytically. The method is applied to a cubic solid containing a Sigma-5 grain boundary and to fiber reinforced laminated composites. The singularities in the stress are identified and discussed.

\section{3,165}

PB95-163929 Not available NTIS

National Inst. of Standards and Technology (IMSE), Gaithersburg, MD.

Creep and Creep Rupture of Ceramic Matrix Composites.

Final rept

S. M. Wiederhorn. 1992, 26p.

Pub. in Flight-Vehicle Materials, Structures, and Dynamics: Ass

Keywords: "Creep rupture strength "Ceramic matrix composites, Matrix materials, Whisker composites, composites, Matrix materials, Whisker composites,
Creep properties, Aluminum oxides, Asymmetry, Ceramics, Silicon carbides, Silicon nitrides, Reprints.

Ceramic matrix composites are being developed to improve the toughness of ceramics in structural applications. As many of these new materials are expected to be used at high temperatures, their mechanical behavior at both high and low temperature must be characterized. In this paper, creep and creep rupture of both particulate and whisker reinforced ceramic matrix composites are discussed. Creep in these materials tends to be asymmetric, creep occurring faster in tension than in compression. Also, extended transient creep occurs for those materials that react with the air or undergo phase transformations. The creep rupture lifetime of ceramic matrix composites may be expressed as a power function of the creep rate regardless of load or test temperature, which implies that improvement in lifetime can be achieved solely by reducing the rate of creep in these materials. Structural use of these materials seems to be limited to about 1300 $\mathrm{C}$ at the present time.

\section{3,166}

PB95-203220 Not available NTIS

National Inst. of Standards and Technology (MSEL), Gaithersburg, MD. Polymers Div.
Environmental Durability of Glass-Fiber Composites.

Final rept.

C. L. Schutte. 1994, 59p.

Pub. in Materials Science and Engineering R13, $n 7$ p265-323, 15 Nov 94

Keywords: "Environmental effects, "Fiber composites, "Polymer matrix composites, *Durability, Degradation, Polymer chemistry, Stress analysis, Moisture, Tem perature, Reprints.

Durability of glass-fiber/polymer composites is dictated by the du rability of the components: glass fiber, matrix and the interface. Environmental attack by moisture, for example, can degrade the strength of the glass fiber; plasticize, swell, or microcrack the resin; and degrade the fiber/matrix interface by either chemical o mechanical attack. The relative rates of these degradation processes are a function of the chemistry of the resin, temperature, length of time of exposure, degree of stress (whether cyclic or static), chemistry and morphology of coating of coupling agent on the glass fiber, and type of glass fiber. Several examples illustrate how the chemistry and morphology of the coatings of cou pling agents that are on the glass fiber influence the strength and durability of the interfacial region.

03,167

PB96-102397 Not available NTIS

National Inst. of Standards and Technology (BFRL) Gaithersburg, MD. Building Materials Div.

Geometrical Percolation Threshold of Overlapping Ellipsoids.

Final rept.

E. J. Garboczi, K. A. Snyder, J. F. Douglas, and M.

R. Thorpe. 1995, 10p.

Pub. in Physical Review E, v52 n1 p819-828 Jul 95

Keywords: "Percolation, "Composite materials, Porous materials, Ellipsoids, Shapes, Thresholds, Microstructure, Reprints.

A recurrent problem in material science is the prediction of the percolation threshold of suspensions and composites containing complex-shaped constituents. The authors consider an idealized material built up from freely overlapping objects randomly placed in a matrix, and numerically compute the geometrical percolation threshold $p$ (sub c) where the objects first form a continuous phase. Ellipsoids of revolution, ranging from the extreme oblate limit of platelike particles to the extreme prolate limit of needlelike particles, are used to study the influence of object shape on the value of $p($ sub $c)$. The reciprocal threshold $1 / p$ (sub c) ( $p$ (sub $c$ ) equals the critical volume fraction occupied by the overlapping ellipsoids) is found to scale linearly with the ratio of the larger ellipsoid dimension to the smaller dimension in both the needle and plate limits.

03,168

PB96-103163 Not available NTIS

National Inst. of Standards and Technology (MSEL) Gaithersburg, MD. Ceramics Div.

Wear Transitions in Monolithic Alumina and Zirconia-Alumina Composites.

Final rept.

Y. S. Wang, C. He, B. J. Hockey, P. I. Lacey, and S. M. Hsu. 1994, 9p.

Pub. in Wear 181-183, p156-1641995.

Keywords: "Aluminum oxide, "Zirconium oxides, "Composite materials, "Tribology, Wear, Microstructure, Grain size, Phase transformations, Residual stress, Ceramics, Toughness, Reprints.

The tribological properties of composite materials can be improved by its specially designed microstructure. Four zirconia-alumina composites (ZrO2-Al2O3) and a monolithic alumina were compared under paraffin oil lubricated wear test to investigate the effect of grain size, the residual stress and the phase transformation on the wear transitions. The tensile stress on the contact surface has been identified as the dominant stress which initiates the fracture wear of brittle materials. Wear transition occurs in the brittle materials when the tensile stress at the contact exceeds the critical value. The effect of zirconia content on the mean grain size of alumina and the wear transition resistance of the materials are presented.

03,169

PB96-109509 PC A17/MF A04

National Inst. of Standards and Technology (MSEL), Boulder, CO. Materials Reliability Div. 
Composite Materials for Offshore Operations: Proccedings of the International Workshop (1st). Held in Houston, Texas on October 26-28, 1993.

Special pub.

S. S. Wang, and D. W. Fitting. Aug 95, 400p, NIST/ SP-887.

Also available from Supt. of Docs as SN003-00303357-0. Prepared in cooperation with Houston Univ. TX. Sponsored by Minerals Management Service, Washington, DC

Keywords: "Composite materials, "Offshore drilling "Meetings. Petroleum industry, Fiberglass reinforced plastics, Fabrication, Construction, Safety, Tubes, Technology assessment, Research and development, Government policies.

The eight working group sessions focused on topics selected by the international steering committee of the workshop: (1) fabrication, construction, maintenance, and repair; (2) material performance, damage toler ance, durability, and environmental degradation; (3) structural design, optimization, testing, and reliability; (4) nondestructive evaluation; condition monitoring and inspection; (5) flammability and fire retardation; (6) facilities and secondary structural applications; (7) ad vanced applications; and (8) certification issues and policy concerns. In each group, leading experts, invited by the steering committee, made presentations and guided and stimulated the discussions. This proceedings describes the scope, the organization, and the program of the workshop. It contains a summary, rec ommendations reported by the steering committee representatives, invited lectures, keynote addresses, and most of the papers presented in the working-group sessions. Also included are a list of working-group chairs and panelists and their discussion summaries A list of registered workshop participants is given at the end of this volume.

\section{3,170}

PB96-119318 Not available NTIS

National Inst. of Standards and Technology (MSEL) Gaithersburg, MD. Polymers Div.

Intrinsic Viscosity and the Polarizability of Particles Having a Wide Range of Shapes.

Final rept.

J.F. Douglas, and E. J. Garboczi. 1995, 69p.

Pub, in Advances in Chemical Physics, vXCl p85-153 1995.

Keywords: "Mixtures, "Composite materials, "Solids, "Liquids, Shapes, Visocity, Polarization, Particles, Virial coefficients, Refractivity, Wave scattering, Reprints.

The intrinstic viscosity (Nu) and the electric alpha(sub e) and magnetic alpha(sub $\mathrm{m}$ ) polarizabilities of objects having general shape are required in the calculation of some of the most basic properties (viscosity, refracture index, dielectric constant, magnetic permeability, thermal and electrical conductivity, and others) can often be expressed in terms of these functionals of object shape. These virial coefficients also provide basic input into effective medium theories describing higher concentration mixtures. We present an argument that the ratio of (Nu) and alpha(sub e) the average electric polarizability tensor trace) is an invariant to a good approximation.

\section{3,171}

PB96-122577 Not available NTIS

National Inst. of Standards and Technology (MSEL) Gaithersburg, MD.

Cavitation Contributes Substantially to Tensile Creep in Silicon Nitride.

Final rept.

W. E. Luecke, S. M. Wiederhorn, B. J. Hockey, R. F. Krause, and G. G. Long. 1995, 12p.

Pub. in Jnl. of the American Chemical Society, v78 n8 p2085-2096 Mar 95

Keywords: "Silicon nitride, "Tensile creep, "Cavitation, Dilation, Reprints.

During tensile creep of a hot isostatically pressed (HIPed) silicon nitride, the volume fraction of cavities increases linearly with strain; these cavities produce nearly all of the measured strain. In contrast, compres sive creep in the same stress and temperature range produces very little cavitation. A stress exponent that increases with stress characterizes the tensile creep response, while the compressive creep response exhibits a stress dependence of unity. Futhermore, under the same stress and temperature, the material creeps nearly 100 times faster in tension than in compression. Transmission electron microscopy (TEM) indicates that the cavities formed during tensile creep occur in pockets of residual crystalline silicate phase located at silicon nitride multigrain junctions. Small-angle $X$-ray scattering (SAXS) from crept material quantifies the size distribution of cavities observed in TEM and demonstrates that cavity addition, rather than cavity growth, dominates the cavitation process. These observations are in accord with a model for creep based on the deformation of granular materials in which the microstructure must dilate for individual grains to slide past one another. During tensile creep the silicon nitride grains remain rigid; cavitation in the multigrain junctions allows nitride grain boundary sliding accommodates this expansion and leads to extension of the specimen. In compression, where cavitation is suppressed, deformation occurs by solutionreprecipitation of silicon nitride.

03,172

PB96-123542 Not available NTIS

National Inst. of Standards and Technology (MSEL), Gaithersburg, MD. Polymers Div.

Modification of the Phase Stability of Polymer Blends by Diblock Copolymer Additivies.

Final rept.

J. Dudowicz, K. F. Freed, and J. F. Douglas. 1995,

Pub. in Macromolecules, n28 p2276-2287 1995

Keywords: "Composite materials, "Polymer blends, Phase separation(Materials), Reprints, “Diblock copolymer additives.

The addition of a third component to a binary mixture usually alters the critical temperature and critical composition for phase seperation In practice, these shifts can be large, leading to both increasing and decreasing of the phase stability of the mixture. Lattice cluster theory calculations show that the addition of a small amount of $A-b-B$ dibock copolymer to an $A / B$ polyme blend shifts the phase boundary as 'impurities' do in other fluid mixtures. The novel feature associated with the dilution of the blend by a diblock copolymer additive is that both stabilization and destabilization can be achieved for certain conditions merely by 'tuning' the asymmetry of the block lengths in the diblock copolymer. More substantial shifts are found in the phase diagram of a blend (polystyrene/poly (vinyl menthyl ether)) exhibiting a lower critical solution temperature. We also examine the relation between the shifts in the critical temperature and in the critical composition shift when the block copolymer is nearly symmetric, but no simple relation seems to exist for asymmetric diblock copolymers.

03,173

PB96-126206 (Order as PB96-126180, PC A05/ MF A01)

National Inst. of Standards and Technology, Gaithersburg, MD

Mixing Plate-Like and Rod-Like Molecules with Solvent: A Test of Flory-Huggins Lattice Statistics. E. A. Di Marzio, A. J. M. Yang, and S. C. Glotzer. $1995,14 p$

Prepared in cooperation with Armstrong World Industries, Lancaster, PA

Included in Jnl. of Research of the National Institute of Standards and Technology, v100 n2 p173-186 Marl Apr 95.

Keywords: "Solvents, Lattice dynamics, Layered silicates, Liquid crystals, Flory Huggins theory, Discotic phase, Plate-rod transition, Rod-rod transition.

Boehm and Martire have shown that the Flory-Huggins (FH) lattice model applied to mixtures of squares and rigid rods in solvent on a two dimensional lattice gives different results depending on whether rods or squares are placed first onto the lattice. This correct derivation places the validity of the $\mathrm{FH}$ model itself into question since the final result should be independent of the order of placement. An analysis of the FH model in terms of Poisson statistics suggests an alternative formula for the probability of successfully placing a rectangle into an area partially filled with other rectangles, which when incorporated into the FH counting procedure gives the exact thermodynamic result for the tiling of squares (i.e., no solvent and no rods).

03,174

PB96-141270 Not available NTIS

National Inst. of Standards and Technology (BFRL), Gaithersburg, MD. Building Materials Div.
Unreacted Cement Content in Macro-Defect-Free Composites: Impact on Processing-StructureProperty Relations.

Final rept.

P. G. Desai, J. A. Lewis, and D. P. Bentz. 1994, 8p. Pub. in Jnl. of Materials Science, v29 n24 p6445-6452 1994.

Keywords: "Cements, "Counstruction materials, Fracture(Mechanics), Reprints, Ceramic materials, Moisture, Physical properties, Defect analysis, Flexural properties, MDF(Macro defect-free) cements.

The effect of unreacted cement content on the processing, structure and properties of macro-defect-free (MDF) composites fabricated from calcium aluminate cement (CAC), alpha-alumina ( $\mathrm{Al} 2 \mathrm{O} 3)$, and polyvinyl alcohol-acetate (PVAA) has been investigated. Thre systems were formed with inital CAC:Al2O3 ratios of $50: 50,35: 65$, and $25: 75$ by volume in their respective formulations. The amount of unreacted cement was reduced from 68.1 vol\% which is present in standard (100\% CAC) MDF cement, to 14.9 vol\% for composites with an initial CAC: $\mathrm{Al} 2 \mathrm{O} 3$ ratio of $25: 75$, while the hy. dration product content was reduced from 18.1 vol\% to $11.4 \mathrm{vol} \%$ for these respective systems. A hard corel soft shell continuum percolation model was used to determine that alumina substitution did not significantly affect the percolative nature of the interphase and bulk polymer regions. However, experiments showed that the reduction in unreacted cement content through $\mathrm{Al} 2 \mathrm{O} 3$ substitution affected both the processing and microstructural features of these composites.

03,175

PB96-146873 Not available NTIS

National Inst. of Standards and Technology (MSEL), Gaithersburg, MD. Polymers Div.

Influence of an Impenetrable Interface on a Polymer Glass-Transition Temperature.

Final rept.

W. E. Wallace, J. H. van Zanten, and W. L. Wu.

1995, 4p.

Pub. in Physical Review E, v52 n4 pR3329-R3332 Oct

Keywords: "Glass transition temperature, "Polymers, Substrate effects, Thermal expansion, Thin films, Reprints.

The thermal expansion of polystyrene thin films, supported on hydrogen-terminated silicon substrates, is measured by $x$-ray reflectivity. Films on the order of 400 Angstroms and thinner show no glass transition up to at least 60 degrees $C$ above the bulk glass-transition temperature, while a break in the thickness versus temperature curve, signaling the glass transition and the onset of bulk behavior, is observed for thicker films. This increase in the glass-transition temperature is in contrast to similar studies on the silicon native-oxide surface where a decrease in the glass-transition temperature is observed. This illustrates the importance of the character of the substrate surface in determining thin film behavior.

03,176

PB96-146881 Not available NTIS

National Inst. of Standards and Technology (MSEL), Gaithersburg, MD. Metallurgy Div.

Electrodeposited Cobalt-Tungsten as a Diffusion Barrier between Graphite Fibers and Nickel.

Final rept.

N. S. Wheeler, and D. S. Lashmore. 1995, 7p.

Pub. in Jnl. of Composites Technology and Research v17 n4 p301-307 Oct 95.

Keywords: "Cobalt base alloys, "Composites "Diffusion, Tungsten, Nickel, Coatings, Reprints.

An electrodeposited cobalt alloy coating have a composition of 7.1 plus or minus 0.8 at \% tungsten was developed to serve as a diffusion barrier between graphite fibers and nickel. A nickel matrix was electrodeposited around the fibers, and the results composite was annealed in vacuum under various time/temperature conditions. The coating was shown to inhibit interdiffusion for up to $24 \mathrm{~h}$ at 800 degrees C. This represents an improvement of 200 degrees over the uncoated fibers.

03,177

PB96-167200 Not available NTIS

National Inst. of Standards and Technology (MSEL),

Gaithersburg, MD. Polymers Div. 
Analysis of Transverse Flow in Aligned Fibrous Porous Media.

Final rept.

F. R. Phelan, and G. Wise. 1996, 10p.

Pub. in Composites Part A, v27A n1 p25-34 1996.

Keywords: "Fibrous porous media, "Porous media, Reprints, "Foreign technology, Darcy's law, Lubrication theory, Permeability prediction, Structure property relationships.

The permeability of preform materials used in liquid moulding proceses such as resin transfer moulding and structural reaction injection moulding is a complex function of weave pattern, packing characteristics, tow structure and intra-tow properties. The development of tools for predicting permeability as a function of these parameters is of great practical importance because such capability would speed process design and optimization, and provide a step towards establishing processing-performance relations. In this study, transverse flow in aligned fibrous porous media has been investigated. A semi-analytical model based on lubrication analysis is derived to predict the effect of tow shape and intra-tow permeability on the overall bed permeability for flow through rectangular arrays of porous elliptical cylinders. The Brinkman equation is used to model flow inside the porous structures, and the model flow inside the porous structures, and the
Stokes equation to model flow in the open media between the structures. The model predictions are verified by comparing with rigorous finite element calculations. The model shows that the influence of the intratow permeability on the overall bed permeability increases with inter-tow packing, and increasing degree of tow ellipticity. The influence is particularly critical for 'stacked' geometers for which previous models predict a zero permeability. A method for predicting intra-tow permeability is also proposed and investigated by comparing the model predications for overall bed permeability with some experimental data for flow in a model porous medium. The comparison shows that use of the method enables the experimentally measured permeability value to be properly bounded.

\section{3,178}

\section{PB96-176656 Not available NTIS}

National Inst. of Standards and Technology (MSEL), Boulder, CO. Materials Reliability Div.

Estimation of the Orientation Distribution of ShortFiber Composites Using UItrasonic Velocities.

Final rept.

M. L. Dunn, and H. Ledbetter. 1996, 9p.

Pub. in Jnl. of the Acoustical Society of America, v99 n1 p283-291 Jan 96.

Keywords: "Composites, "Fiber reinforced composites, *Ultrasonic velocities, Reprints, Orientation distribution function, SiC/AL composite.

A method for estimating the orientation distribution function (ODF) of short-fiber composites using measured ultrasonic velocities is presented. The method is based on the coupling of (1) a forward micromechanics model of the effective ultrasonic velocities of the textured composite in terms of the coefficients in an expansion of the ODF in generalized spherical harmonics, (2) an inversion algorithm to obtain the coefficients of the ODF from the measured velocities and the forward model. The approach is used to determine the ODF coefficients for a transversely isotropic shortfiber SiC/Al metal-matrix composite. The ODF coefficients obtained by inversion of ultrasonic velocities agree reasonably with those obtained from neutron-dif fraction pole figures. Corresponding elastic constants predicted with these coefficients also agree well with independently measured elastic constants.

\section{3,179
PB96-180260 Not available NTIS}

National Inst. of Standards and Technology (MSEL), Gaithersburg, MD

Fracture of Silicon Nitride and Silicon Carbide at Elevated Temperatures.

Final rept.

S. M. Wiederhorn, A. B. Fields, and B. J. Hockey.

1994, $10 p$.

Pub. in Materials Science and Engineering, vA176 p51-60 1994.

Keywords: "Ceramic matrix composites, "Statistics, *Fracture(Mechanics), Silicon nitride, Silicon carbide, High temperature, Damage, Failure(Mechanics), Tension, Stresses, Strain(Mechanics), Reprints, "Foreign technology

High temperature fracture of structural ceramics often results from creep and creep-related processes. Creep cavitation in particular plays an important role in the failure process, resulting in crack nucleation and crack growth prior to failure. Equations modeling cavitation and crack growth mechanisms are consistent with the Monkman-Grant behavior observed for structural ceramics. Both the mechanism of fracture and the mode of crack growth are affected by the test geometry. In flexure, cracks tend to remain stable because of stress relaxation near the tensile surface of test specimens. In tensile specimens, stress relaxation due to cavity formation occurs in the vicinity of crack tips. Intermittent crack growth results, in which the density of cavities at the crack tip has to reach a critical level for the occurrence of crack motion. Crack growth occurrs by microcrack nucleation and linkage with the dominant crack

03,180

PB96-183207 PC A04/MF A01

National Inst. of Standards and Technology (MSEL), Gaithersburg, MD. Polymers Div.

Hygrothermal Effects on the Performance of Polymers and Polymeric Composites: A Workshop Report. Held in Gaithersburg, Maryland on September 21-22, 1995

M. Y. M. Chiang, and G. B. McKenna. Apr 96, 42p, NISTIR-5826.

Keywords: "Polymers, "Composite materials, *Meetings, Moisture Performance, Models, Osmosis, Microelectronics, Packaging, Films, Automotive industry. *Hygrothermal effect

This report contains the highlights of the presentations and discussions at the NIST/Industry/Academe Workshop: Hygrothermal Effects on the Performance of Polymers and Polymeric Composites that took place on 21 and 22 September 1995 at the Hilton Hotel Gaithersburg, Maryland. The workshop was organized by the Polymers Division of the Materials Science and Engineering Laboratory (MSEL) at the National Institute of Standards and Technology (NIST). Sponsorshop was provided by the Center for Theoretical and Computational Materials Science in MSEL. This workshop brought together 46 attendees; including (10) invited speakers, fifteen (15) representatives from the academic community, eighteen (18) companies and several Divisions within NIST, to define the state-of-the art in hygrothermal issues in polymers and polymeric composites.

\section{3,181}

PB96-190087 Not available NTIS

National Inst. of Standards and Technology (MSEL), Boulder, CO. Materials Reliability Div.

Hemispherical Test Fixture for Measuring the Wavefields Generated in an Anisotropic Solid. Final rept.

D. W. Fitting, and C. M. Fortunko. 1991, 6p.

Pub. in Ultrasonics Symposium, Lake Buena Vista, Florida, December 8-11, 1991, p831-836.

Keywords: "Wave propagation, "Anistropy, "Solids, Directivity, Composite materials, Radiation, Patterns, rectivity, Composite materials, Radiation, Patterns,
Ultransonic tests, Reprints, Wavefields, Christoffel equation.

Recently, a closed-form representation for the exact solution of the Chirstoffel equation for wave propagation in an anisotropic solid has been derived. This development and the need to know the wavefield generated during ultrasonic inspection of composites has prompted the design and fabrication of an experimental system for measurement of wavefields in such materials. In the test fixture, a source transducer is affixed to the flat portion of a hemisphere of the anisotropic solid. A receiving transducer having a large field of view (FOV) is scanned over the surface of the hemisphere by a motorized $\mathrm{C}$-arm, mapping out the wavefield generated in the specimen. The hemispherical shape of the test specimen permits scanning the wavefield over nearly a full $2 \mathrm{pi}$ solid angle, an improvement over the limited angular range afforded by a flat panel of the anisotropic solid. In this paper, the authors report the result of preliminary experiments designed to evaluate the fixture.

\section{3,182}

PB96-200175 Not available NTIS

National Inst. of Standards and Technology (MSEL) Boulder, CO. Materials Reliability Div.
Orthotropic Elastic Constants of a Boron-Aluminum Fiber-Reinforced Composite: An AcousticResonance-Spectroscopy Study.

Final rept.

H. Ledbetter, C. Fortunko, and P. Heyliger. 1995, 5p. Pub. in Jnl. of Applied Physics, Chapter 78, v3 p1542 1546 Aug 95.

Keywords: "Composites, "Elastic constants, Model calculations, Reprints, Fiber reinforcements, Ultrasonic resonance spectroscopy

Acoustic-resonance spectroscopy was used to determine the complete elastic constants of a uniaxial-fiberreinforced metal-matrix composite: boron-aluminum This material exhibits orthotropic macroscopic symme try and, therefore, nine indepedent elastic stiffnesses Cii. The off-diagonal elastic constants (C12, C13 C23), which contain large errors when measured by conventional methods, were especially focused on. Good agreement emerged among present results, a previous pulse-echo study, and theoretical predictions using a plane-scattered-wave ensemble-average model. Attempts to measure the internal-friction "tensor' failed.

03,183

PB96-200803 Not available NTIS

National Inst. of Standards and Technology (MSEL) Gaithersburg, MD

Tensile Creep of Silicide Composites.

Final rept.

S. M. Wiederhorn, R. J. Gettings, D. E. Roberts, C Ostertag, and J. J. Petrovic. 1992, 7p. Pub. in Materials Science and Engineering, vA155 p209-215 1992

Keywords: "Creep, "Silicides, "Composites, High temperatures, Tensile creep, Reprints, Molybdenum disilicide

A study has been conducted on the creep behavior of a silicide composite consisting of $50 \mathrm{~mol} \% \mathrm{MoSi} 2$ and WSi2 reinforced with $\mathrm{SiC}$ whiskers. Activation energies for creep in compression and tension were $312 \mathrm{~kJ} \mathrm{~mol}$ to the minus 1 power and $557 \mathrm{~kJ}$ mol to the minus power respectively. Stress exponents for creep were 3.2 in tension and 23 in compression. These values lie within the range of values obtained by other inves tigators. The creep resistance of the composite is considerably better than that of current alloys used in heat engines. Most of this improvement comes from the addition of $\mathrm{SiC}$ whiskers to the base alloy. It is suggested that the addition of higher concentrations of whiskers or particles would result in additional improvement of the creep behavior of silicide composites.

03,184

PB96-210695 PC A04/MF A01

National Inst. of Standards and Technology (BFRL) Gaithersburg, MD. Building Materials Div.

Materials Aspects of Fiber-Reinforced Polymer Composites in Infrastructure.

J. W. Chin. Aug 96, 47p, NISTIR-5888

Keywords: "Polymer composites, "Civil engineering Retrofitting, Composite materials, Highway bridges, Corrosion, Beams(Supports), Maintenance, Reviews.

This paper provides a review of the technical literature pertaining to materials aspects of fiber-reinforced poly mer (FRP) composites in infrastructural and other civil engineering applications. The main focus is placed upon the durability, chemical and mechanical aspects of structures reinforced with or constructed from FRP materials. Categories which are addressed include marine applications, structural shapes, joining/fastening, reinforced concrete and rehabilitation/retrofitting of structures. Effects of moisture, salt water, alkalinity and mechanical loading on the performance of FRP components are emphasized.

\section{3,185}

PB97-112478 Not available NTIS

National Inst. of Standards and Technology (CSTL), Gaithersburg, MD. Process Measurements Div. in situ Characterization of Vapor Phase Growth of Iron Oxide-Silica Nanocomposites: Part 1. 2-D Planar Laser-Induced Fluorescence and Mie Imaging.

Final rept.

B. K. McMillin, P. Biswas, and M. R. Zachariah.

1996, 10p

Pub. in Jnl. of Materials Research, v11 n6 p1552-1561 Jun 96 


\section{Composite Materials}

Keywords: "Fluorescence, "Laser scattening, "Nanocomposites, "Vapor phase, I"

Planar laser-based imaging measurements of fluorescence and particle scattering have been obtained during flame synthesis of iron-oxide/silica application of laser-induced fluorescence, the spectroscopy of $\mathrm{FeO}(\mathrm{g})$, and the experimental approach for measurement of gas phase precursors to particle formation are discussed. The results show that the vapo phase $\mathrm{FeO}$ concentration rapidly rises at the primary reaction front of the flame and is very sensitive to the amount of precursor added, suggesting nucleationcontrolled particle growth. The FeO vapor concentration in the main nucleation zone was found to be insensitive to the amount of silicon precursor injected, indicating that nucleation occurred independently for the iron and silicon components. Light scattering measurements indicate that nanocomposite particles sinter faster than single component silica, in agreement with TEM measurements.

\section{Corrosion \& Corrosion Inhibition}

\section{3,186}

DE94017332 PC A02/MF A01

National Inst. of Standards and Technology (MSEL), Gaithersburg, MD.

Comparison of the Corrosion Rates of FeAl, Fe(sub 3)Al and Steel in Distilled Water and 0.5 M Sodium Chloride. Technical Report Number 2, January-March 1991.

Progress rept.

R. E. Ricker, and J. L. Fink. Aug 91, 9p, DOE/OR/ 21941-T2

Contract Al05-90OR21941

Sponsored by Department of Energy, Washington, DC

Keywords: "Aluminium Alloys, "Carbon Steels, "Iron Base Alloys, "Sodium Chlorides, "Corrosive Effects, Water, Comparative Evaluations, Corrosion, Experimental Data, Intermetallic Compounds, Progress Report, Tables(data), "Corrosion tests, EDB/360105.

The corrosion rate of an $\mathrm{Fe}$ (sub 3) Al alloy (FA117) and an $\mathrm{FeAl}$ (FA362) alloy in distilled water and $0.5 \mathrm{M} \mathrm{NaCl}$ was determined and compared to two low carbon steels. The results demonstrate that the corrosion rate of these two intermetallic compounds were more than an order of magnitude less than that of the two steels in both of the environments. The corrosion rates of the two iron aluminides were not significantly different.

03,187

PB94-198348 Not available NTIS

National Inst. of Standards and Technology (MSEL), Gaithersburg, MD. Metallurgy Div.

Evaluation of Corrosion Data: A Review.

Final rept.

D. B. Anderson. 1992, 12p.

Pub. in Computer Modeling in Corrosion, American Society for Testing and Materials Special Technical Publication 1154, p162-173 1992.

Keywords: "Corrosion, "Materials, "Environmental effects, *Industrial plants, Exposure, Field tests, Atmos pheric corrosion, Electrochemical corrosion, Pitting, Concentration cell corrosion, Cracks, Reprints.

The needs, objectives, and techniques for evaluation of material property data are reviewed with respect to their applicability to certain types of corrosion data. Examples of approaches that have been taken for data generated from exposures in natural environments and industrial processes are described to illustrate methods used for characterizing the materials, the environment and exposure conditions, the measurement techniques used to describe these components, and the delineation of controlling factors with respect to specific forms of corrosion.

\section{3,188}

PB94-198876 Not available NTIS

National Inst. of Standards and Technology (MSEL), Gaithersburg, MD. Metallurgy Div.
Modeling Polarization Curves and Impedance Spectra for Simple Electrode Systems. Final rept.

U. Bertocci, and R. E. Ricker. 1992, 19p

Pub. in Proceedings of Symposium on Computer Modeling for Corrosion, San Antonio, TX., November 12 13, 1990, n1154 p143-1611992.

Keywords: "Electrochemical corrosion, "Electrodes *Impedance, "Electrolytic polarization, "Spectrum analysis, Models, Steady states, Impedance measurement, Reprints.

A method for modeling the behavior of electrochemical systems based on electrode kinetics instead of equivalent circuits is developed and examples of the applica tion of this modeling method to three electrochemical systems are presented. It is shown that the behavior of simple, as well as relatively complex, electrochemical systems can be modeled on desktop computers yielding impedance spectra as a function of input quantities that describe steady state electrode kinetics and the electrode potential. This approach, since it is based on quantities that describe the kinetics of the actual processes that occur at the electrode, has the advantage over other approaches of having a built-in physical interpretation. The impedance plots produced in this way can give a feeling of what to expect experimentally, and can be useful in testing hypotheses, in establishing reasonable ranges for quantities not easily accessible to measurement, and in suggesting experimental strategies.

03,189

PB94-213345 Not available NTIS

National Inst. of Standards and Technology (NEL), Gaithersburg, MD. Building Materials Div.

Relation between AC Impedance Data and Deg-

radation of Coated Steel. 1. Effects of Surface Roughness and Contamination on the Corrosion Behavior of Epoxy Coated Steel.

Final rept.

C. Lin, T. Nguyen, and M. McKnight. 1992, $18 \mathrm{p}$.

Pub. in Progress in Organic Coatings 20, n2 p169-186 1992

Keywords: "Surface roughness, "Degradation, "Corrosion, "Steels, "Epoxy resins, "Contamination, Substrates, Protective coatings, Environment effects, Organic compounds, Reprints, AC impedance spectroscopy.

The effects of surface roughness and contamination on the corrosion behaviors of epoxy coated steel substrate were investigated using AC impedance spectroscopy. The results of the $A C$ impedance spectroscopy were analyzed using equivalent circuit model to discern the different steps of the corrosion processes of coated steel. Two kinds of corrosion of coated steel, which can be discerned by $\mathrm{AC}$ impedance spectroscopy, were observed depending on surface treatment. The area of coating deficiency that causes rapid corrosion of coated metal could be calculated accurately from the $\mathrm{AC}$ impedance data as verified by scanning electron microscopy (SEM) and computer image analyses. Interpretations for the experimental results were suggested and the factors dominating the protective properties of an organic coating were discussed.

03,190

PB95-152096 Not available NTIS

National Inst. of Standards and Technology (MSEL), Gaithersburg, MD.

Interaction between Dislocations and Intergranular Cracks.

Final rept.

H. Zhang, A. H. King, and R. Thomson. 1991, 10p Pub. in Jnl. of Materials Research 6, n2 p314-323 1991.

Keywords: "Dislocations(Materials) *Intergranular corrosion, "Emission, Isotropic media, Crack propagation, Cracking(Fracturing), Surface properties, Crack tips, Reprints.

The elastic interactions of dislocations and intergranular cracks in isotropic materials have been studied. In the first part of the paper, a model based on Rice-Thomson theory is established under which the conditions for dislocation emission and crack propagation can be described in terms of an emission surface, cleavage surface and loading line in the local kspace associated with a mixed mode intergranular crack. For a given crack, the local k-field changes with the emission of dislocations from the crack tip, which alters the balance of cleavage and emission. In the second part, we present experimental results of in situ TEM observation of intergranular cracks in molybdenum. Alternating brittle crack propagation and dislocation emission is observed. The number of emitted dislocations corresponding to each crack propagation is in good agreement with the calculated values.

03,191

PB95-162327 Not available NTIS

National Inst. of Standards and Technology (MSEL) Gaithersburg, MD. Metallurgy Div.

Evidence of Film-Induced Cleavage by Electrodeposited Rhodium.

Final rept.

R. E. Ricker, J. L. Fink, and E. Escalante. 1993, 7p Pub. in Corrosion-Deformation Interactions, p734-740 1993.

Keywords: "Stress corrosion cracking, "Cleavage "Electrodeposition, "Rhodium, Crack tips Fracture(Mechanics), Brittleness, Crack propagation, Metal powder, Cracking(Fracturing), Reprints.

Transgranular stress corrosion cracking (T-SCC) has been the subject of numerous investigations, but there is no universally accepted mechanism for this form of crack propagation. One of the mechanisms that has been proposed for T-SCC is film induced cleavage. ACcording to this mechanism, T-SCC cracks propagate by discontinuous micro-cleavage initiated by the corrosion films that form at the crack tip. A critical hypothesis in this postulated mechanism is that when the crack in the surface layer reaches the matrix, it will continue to propagate in a brittle manner in the normally ductile matrix for some distance before it arrests. The objective of this work was to unambiguously evaluate this hypothesis by electrodepositing Rh, a metal which normally cleaves at room temperature, on samples of pure $\mathrm{Ni}$, which does not normally cleave, and then deform these samples and examine the resulting cracks and the morphology of the cracks at the $\mathrm{Rh} / \mathrm{Ni}$ interface.

03,192

PB95-162475 Not available NTIS

National Inst. of Standards and Technology (NEL), Gaithersburg. MD. Building Materials Div.

Sorption of Moisture on Epoxy and Alkyd Free Films and Coated Steel Panels.

Final rept.

H. N. Rosen, and J. W. Martin. 1991, 9p.

Pub. in Jnl. of Coatings Technology $63, n 792$ p85-93 Jan 91.

Keywords: "Sorption, "Moisture, "Protective coatings "Steels, "Corrosion, Degradation, Corrosion inhibition, Diffusion, Moisture content, Isotherms, Humidity, Corrosion prevention, Epoxy compounds, Reprints.

Adsorption isotherm curves at $23 \mathrm{C}$ were determined for alkyd and epoxy free films and coated steel panels as well as for alkyd 'pocket' panels, consisting of an unadhered film surrounding steel panels. The isotherms consisted of a linear portion to about $75 \%$ relative humidity $(\mathrm{RH})$ followed by a rapidly rising portion to $100 \% \mathrm{RH}$. A model assuming weak bonding forces between paint film and water with microvoid condensation at high $\mathrm{RH}$ gave an excellent fit to the data. Transient adsorption and desorption data on the approach to equilibrium conditions showed differences between alkyd and epoxy coatings. Surface boundary layer resistance was significant for alkyd films and sorption followed Fickian models. For the epoxy coating, surface resistance was not significant and moisture movement was non-Fickian.

03,193

PB96-148036 Not available NTIS

National Inst. of Standards and Technology (BFRL), Gaithersburg, MD. Building Materials Div.

Water Permeability and Chloride Ion Diffusion in Portland Cement Mortars: Relationship to Sand Content and Critical Pore Diameter.

Final rept.

P. Halamickova, R. J. Detweiler, D. P. Bentz, and E J. Garboczi. 1995, 13p

Pub. in Cement and Concrete Research, v25 n4 p790 8021995.

Keywords: "Porosity, "Mortars(Material), "Portland cements, Interfaces, Construction materials, Diffusion, lons, Chlorides, Percolation, Permeability, Cements, Sands, Reprints, Mercury intrusion porosimetry, Pore structure.

The pore structure of hydrated cement in mortar and concrete is quite different from that of neat cement 
paste. The porous transition zones formed at the aggregate-paste interfaces affect the pore size distribu-
tion. The effect of the sand content on the development of pore structure, the permeability to water, and the diffusivity of chloride ions was studied on portland cement mortars. Mortars of two water-to-cement ratios and three sand volume fractions were cast together with pastes and tested at degrees of hydration ranging from 45 to $70 \%$. An electrically-accelerated concentration cell test was used to determine the coefficient of chloride ion diffusion while a high pressure permeability cell was employed to assess liquid permeability. The data set provides an opportunity to directly examine the application of the Katz-Thompson relationship to cement-based materials.

\section{Elastomers}

\section{3,194}

\section{AD-A307 789/8 PC A03/MF A01}

National Bureau of Standards, Washington, DC. Inst. for Applied Technology.

Synthesis of Thermally Stable Elastomers. Apr 66, 21p.

Keywords: "Elastomers, "Thermal stability, Polymers, High temperature, Synthesis(Chemistry), Anhydrides, Nitrogen, Siloxanes, Chemical bonds, Silicon, Ethers, Inhibitors, Phenyl radicals, Silicates, Silicarbanes, Polyphenylether, Metallosiloxane, Silazanes.

Significant reports covering the period since 1960 were selected for this study with reference to relevant earlier works. Recent Government-sponsored research and development (RD) in high temperature elastomers has been directed along two lines: (1) new polymer synthesis and (2) evaluation of compounds to improve the thermal stability of current elastomers. Progress has been made in each of these areas. Large groups of inorganic and semi-inorganic polymer systems were evaluated. Systems included silazanes, siloxanes, and fluorocarbons.

\section{3,195}

PB95-107033 Not available NTIS

National Inst. of Standards and Technology (MSEL), Gaithersburg, MD. Polymers Div.

Localization Model of Rubber Elasticity: Comparison with Torsional Data for Natural Rubber Networks in the Dry State.

Final rept.

G. B. McKenna, J. F. Douglas, K. M. Flynn, and Y

Chen. 1991, 7p.

Pub. in Polymer 32, n12 p2128-2134 1991.

Keywords: "Natural rubber, "Elastic properties, "Crosslinking, Strain energy methods, Vulcanizing, Elastomers, Thermodynamics, Free energy, Plastic analysis, Torsion, Networks, Reprints, Gaylord-Douglas Localization Model, Free energy density function.

Previously reported results from determinations of the first derivative of the elastic contribution to the free energy density function for dicumyl peroxide crosslinked natural rubber were analyzed within the context of the Gaylord-Douglas 'localization model' of rubber elasticity. It was found that the dry state properties of the rubbers are well described by the localization model and that the non-classical contribution to rubber elasticity arising from the confinement of network chains by surrounding chains varies according to the theory. The only other parameter in the model, the prefactor to a classical term, was set to the classical phantom value assuming a tetrafunctional network and that each dicumyl peroxide molecule decomposed to form one crosslink

\section{Fibers \& Textiles}

\section{3,196}

PB94-216256 Not available NTIS

National Inst. of Standards and Technology (MSEL), Gaithersburg, MD. Ceramics Div.
Coating of Fibers by Colloidal Techniques in $\mathrm{Ce}$ ramic Composites.

Final rept.

S. G. Malghan, D. B. Minor, P. S. Wang, and C. P. Ostertag. 1990, 11p.

Pub. in Ceramic Engineering and Science Proceedings 11, n9-10 p1674-1684 1990 .

Keywords: "Ceramic fibers, "Silicon carbides, "Ceramic composites, "Deposition, Composite materials, Colloids, Surface chemistry, Aluminum oxide Fiber composites, Powder(Particles), Reprints.

A process for coating silicon carbide tibers with alu mina particulates for control of interface characteristics in the sintering of fiber reinforced composites is described. The process is based on colloidal slurry processing techniques, in which the fibers are allowed to take up a positive surface charge, while the alumina particles are imparted a negative surface charge. When the oppositely charged fibers and particles are brought together, the particles deposit on the surface of fibers Some unique features of the process, as well as characteristics of the resultant coating are described. Process variables of the coating technique that control the coating morphology, thickness and adherence to the fiber are examined.

03,197

PB95-108676 Not available NTIS

National Inst. of Standards and Technology (MSEL), Gaithersburg, MD. Polymers Div.

Effect of Heterogeneous Porous Media on Mold Filling in Resin Transfer Molding.

Final rept.

R. S. Parnas, and F. R. Phelan. 1991, 8p.

Pub. in Society for the Advancement of Material and Process Engineers Quarterly 22, n2 p53-60 Jan 91.

Keywords: "Fluid flow, "Resin transler molding, "Molding techniques, "Flow characteristics, "Fibers Preforms, Porous media, Permeability, Curing, Polymers, Heterogeneity, Bundles, Reprints.

A model for fluid flow in the resin transfer molding (RTM) process is developed. The model takes into account two competing flow processes which occur simultaneously: the macroscopic flow of the resin through the preform and the impregnation of the individual fiber bundles which make up the preform. The model predicts the entrapment of air in the fiber bundles and the subsequent formation of voids in the cured part In addition, the competition between the two flow processes leads to transient phenomena in which mold inlet pressure continues to rise even after the flow front has reached the outlet of the mold. This result agrees qualitatively with experimental data and has implications regarding the use of D'Arcy's law to model transient mold filling processes with heterogeneous preforms.

03,198

PB96-128194 PC A03/MF AO

National Inst. of Standards and Technology (MEL) Gaithersburg, MD. Manufacturing Systems Integration

Extensions of the Prototype Application Protocol of Ready-to-Wear Apparel Pattern Making.

Y. T. Lee. Oct 95, 44p, NISTIR-5727.

See also PB93-158665. Sponsored by Defense Logistics Agency, Alexandria, VA.

Keywords: "Clothing industry, "Computer aided design, Computer aided manufacturing, Data transfer(Computers), Patterns, Prototypes, Pattern making, Tests, Models, Data structures, Data ex change, Specifications, Standards, Product data, Information modeling, "Application protocols, "Standard for the Exchange of Product Model Data(STEP) "STEP(Standard for the Exchange of Product Model Data), "EXPRESS language, EXPRESS-G computer program, Apparel industry.

A prototype application protocol for ready-to-wear pattern making was developed for the communication of pattern pieces and related information between various computer aided design (CAD) systems. The basic methodology for the application protocol is the Standard for the Exchange of Product Model Data (STEP) STEP is an emerging international standard, the result of an effort to develop a mechanism for digitally representing the physical and functional characteristics of a product throughout the product's life cycle. The STEP application protocol addresses a specific application area and contains an information model written in the conceptual modeling language EXPRESS. The experience gained through the implementation of the prototype application protocol and recommendations received from apparel researchers provided us useful input to improve the prototype application protocol. The report presents a revised version of the information model using both the EXPRESS language and the EXPRESS-G graphical notation. An application activity model that defines the scope and information require ments of the prototype application protocol is also presented in this report. Neither the EXPRESS-G diagrams nor the application activity model was shown in the original prototype application protocol report.

\section{3,199}

\section{PB96-193792 PC A06/MF A01}

National Inst. of Standards and Technology (MEL) Gaithersburg, MD. Manufacturing Systems Integration Div.

Survey of Standards for the U.S. Fiber/Textile/Apparel Industry

C. G. Pawlak. Apr 96, 94p, NISTIR-5823.

See also PB93-139095.

Keywords: "Textile industry, "Clothing industry, "Surveys, "Technology utilization, Design standards, Fibers, Textiles, Test methods, Industries, Integration systems, Standards, Specifications, "Apparel clothing.

The report documents a survey of standards relevan to the U.S. Fiber/Textile/Apparel (FTA) industry. The standards are discussed in four main groups--integration standards, test methods, quality standards, and standard reference data and materials. The Appendix of the report lists the titles of all standards found grouped together by the organization responsible for them. Those organizations are also listed along with contact information for them. The report attempts to bring together useful information concerning FTA standards as a starting point to support the industry in intelligently planning future standards' development efforts.

\section{Iron \& Iron Alloys}

03,200

AD-A279 759/5 PC A04/MF AO

National Inst. of Standards and Technology,

Characterization of the Hydrogen Induced Cold Cracking Susceptibility at Simulated Weld Zones in HSLA-100 Steel.

M. R. Stoudt, and R. E. Ricker. Apr 94, 73p, NISTIR5408

Keywords: "Welding, *Cracks, "Low alloy steels, Simulation, Hydrogen, Navy, Microstructure, Martensite, Carbon, Precipitation, Strength(Mechanics), Toughness, Hardening, Mechanical properties, Strain(Mechanics), Sea water, Corrosion, Electrochemistry, "HY-100 Steel, "HSLA-100 Steel, Characterization, Fugacities, HY(High Yield), HSLA(High Strength Low Alloy), Susceptibility.

The relative susceptibilities to hydrogen induced cold cracking were evaluated for $\mathrm{HY}-100$ a steel presently in service in naval applications and for HSLA-100. The martensitic microstructure of the $\mathrm{HY}-100$ undergoes wide variations in the heat affected zone during weld ing which strongly influence the resistance of that alloy to cold cracking. The HSLA-100, a low carbon precipitation hardened steel with similar strength and toughness to that of the HY-100, possesses a significantly lower degree of hardenability which results in minimal microstructural variations in the heat affected zone under the same simulated welding conditions. The mechanical properties of the base metal and the heat af fected zone created during a simulated, single welding pass were characterized by the slow strain rate technique for both steels in an inert environment and in artificial seawater under free corrosion and controlled hydrogen fugacities. The electrochemical behaviors of both steels were also evaluated in artificial seawater.

\section{1}

PB94-172087 Not available NTIS

National Inst. of Standards and Technology (MSEL) Gaithersburg, MD. Metallurgy Div. 
Monte Carlo and Mean-Field Calculations of the Magnetocaloric Effect of Ferromagnetically Interacting Clusters.

Final rept.

L. H. Bennett, R. D. McMichael, L. J.

Swartzendruber, R. D. Shull, and R. E. Watson.

1992, 2p.

Pub. in Jnl. of Magnetism and Magnetic Materials 104107, p1094-1095 1992. See also DE91014792.

Keywords: "Ferromagnetic materials, "Monte Carlo method, "Curie temperature, "Magnetic properties, Magnetic moments, Entropy, Solid clusters, Ferromagnetism, Mean-field theory, Reprints, "Magnetocaloric effect.

The magnetic entropy change of a ferromagnet induced by an application of a magnetic field is greatest in the temperature region near the Curie point, and the magnitude of the effect is expected to rise monotonically with the size of the individual moments which make up the material. The authors explore the case of nanocomposite materials with ferromagnetically interacting clusters having large cluster magnetic moments as a function of cluster size. As cluster size increases, both Monte Carlo and meanfield calculations show a decrease in the entropy change at $T(c)$ for a given applied field and constant total magnetic moment, and an increase in the entropy change well above $T(c)$. In addition, for the first time the authors present a comparison of the results of mean-field and Monte Carlo calculations of the magnetocaloric effect in classical Heisenberg magnetocaloric effect have taken the mean-field approach, which is known to underestimate the spontaneous magnetization below $T(c)$. These issues are relevant to devices employing magnetic refrigeration.

\section{3,202}

\section{PB94-172251 Not available NTIS}

National Inst. of Standards and Technology (CAML). Gaithersburg, MD. Applied and Computational Mathematics Div

PC-Based Prototype Expert System for Data Management and Analysis of Creep and Fatigue of Selected Materials at Elevated Temperatures.

Final rept.

J. T. Fong, and B. Bernstein. 1990, 4p

Pub. in Proceedings of International Conference on Computer Applications to Materials Science and EngiSponsored by Illinois Inst. of Tech., Chicago

Keywords: "Creep properties, "Expert systems, "Data management, "Fatigue(Mechanics) "Stainless steel, *High temperature, Data bases, Mechanical properties, Algorithms, Stresses, Strains, Information systems, Viscoelasticity, Computer programs, Materials, Fatigue life, Reprints, LPA PROLOG Professional Computer Program, CFD Expert System.

An engineering expert system named CFD for storing, analyzing, and assessing experimental data of the creep and fatigue properties of a class of structural materials at elevated temperatures is presented. The system is coded in micro-PROLOG, a PC version (1.4) o a commercially-available sottware named LAP PROLOG Professional and is implemented on an IBM-PCXT or compatible with DOS 211 or up The system is menu-driven and is designed to assist a computerunsophisticated user in managing creep and fatigue data for a variety of materials at various temperature, stress or strain levels. To illustrate the feasibility of this prototype system, selected data of AISI 304 stainless steel (fatigue life vs. hold time at $593 \mathrm{C}$ ) and AISI 316 stainless steel (stress relaxation test at $700 \mathrm{C}$ ) are examined and plotted. Based on the linear theory of viscoelasticity, the stress relaxation data are used to predict creep properties using a numerical algorithm due to Hopkins and Hamming (1957).

\section{3,203}

\section{PB94-172541 Not available NTIS}

National Inst. of Standards and Technology (MSEL), Boulder, CO. Materials Reliability Div.

Nitrogen Effect on Elastic Constants of f.c.c. Fe$18 \mathrm{Cr}-19 \mathrm{Mn}$ Alloys.

Final rept.

S. Lin, and $H$. Ledbetter. 1993, 5p

Pub. in Materials Science and Engineering A167, p81. 85 1993. Sponsored by Department of Energy, Washington, DC. Office of Fusion Energy.

Keywords: Iron base alloys, Chromium alloys, Manganese alloys, Bulk modulus, Elastic properties, FCC lattices, Interstitials, Nitrogen, Reprints, "Iron alloy $18 \mathrm{Cr} 19 \mathrm{Mn}$.

Previously, the authors studied the effects of interstitial carbon-plus-nitrogen $(\mathrm{C}+\mathrm{N})$ on the elastic constants of f.c.C. Fe-18Cr-10Ni-1Mn alloys. Consistent with a volume increase, all the elastic stiffnesses decrease with increasing $\mathrm{C}+\mathrm{N}$. The present alloys show dif ferent behavior: although volume increases, interstitial nitrogen atoms increase the bulk modulus. The peculiar bulk-modulus-electron-concentration behavior ( $B$ vs. $n$ (sub e) of $3 d$ electron elements is described. A first $B$ increases with increasing $n$ (sub e); beyond a critical concentration, $\mathrm{B}$ decreases rapidly. Application of Ducastelle's model (bandstructure and repulsion energies) shows that interstitial nitrogen increases the bandstructure contribution to the bulk modulus.

\section{3,204}

PB94-172715 Not available NTIS

National Inst. of Standards and Technology (MSEL), Boulder, CO. Materials Reliability Div.

Microstructure and Tensile Properties of Microalloyed Steel Forgings.

Final rept.

P. T. Purtscher, and Y. W. Cheng. 1993, 10p.

Pub. in Proceedings of Iron and Steel Society's Mechanical Working Conterence, Montreal, Canada, October 1992, p331-340 1993.

Keywords: "Steels, "Tensile properties, "Microstructure, "Forging, Austenite, Ferrites, Defor mation, Metalworking, Strain rate, Grain size, Hard ness, Mechanical properties, Simulation, Cooling Pearlite, Automotive components, Reprints, *Microalloying.

Forging is studied by thermomechanical simulation of the process variables on the microstructure, hardness, and tensile properties of medium-C, ferrite plus pearlite steel with $\mathrm{V}$ or $\mathrm{Nb}$ additions, steels used for forged automotive components. Experiments were carried ou on a test machine with a load capacity of $500 \mathrm{kN}$ and capable of high strain rates. The prior austenite grain size increases as the reheat temperature goes up from 950 to $1210 \mathrm{C}$ and decreases with applied strain up $50 \%$. The prior austenite grain size of the $\mathrm{Nb}-$ and $\mathrm{V}$ treated steels are nearly equal. As the cooling rate after deformation decreases from 1.9 to $0.8 \mathrm{C} / \mathrm{s}$, the ferrite content increases. Deformation reduces the prior austenite grain size and increases the ferrite content. The hardness, yield, ultimate, and fracture stresses are not a strong function of reheating and de formation temperature or strain, but are lowered by about $10 \%$ when the cooling rate is reduced.

\section{3,205}

PB94-185394 Not available NTIS

National Inst. of Standards and Technology (MSEL) Gaithersburg, MD. Metallurgy Div.

Effect of Backfill and Atomizing Gas on the Powde Porosity and Mechanical Properties of 304L Stain less Steel.

Final rept.

G. M. Janowski, F. S. Biancaniello, G. E. Hicho, R. J. Fields, and S. D. Ridder. 1993, 6p.

Pub. in $\mathrm{P} / \mathrm{M}$ in Aerospace, Defense and Demanding Applications, p363-370 1993.

Keywords: "Stainless steel-304L, "Powder metallurgy, *Porosity, "Mechanical properties, Austenitic steels, Atomization, Argon, Nitrogen, Particle size distribution, Reprints, Backfill gas.

A series of nitrogenated austenitic stainless steels was produced by gas atomization and HIP consolidation using various combinations of nitrogen and argon as the melt chamber backfill gas and as the atomizing gas. Analysis of the gas-only flow patterns and the physical properties of nitrogen and argon suggest that their atomization behavior should be quite comparable. The similar particle size distributions support this hy pothesis and indicate that the liquid metal ligament and sheet break-up were unaffected by gas type. It was found that the nitrogen content of the powder was largely controlled by the backfill gas and that the fraction of hollow particles was determined by the atomization gas. The nitrogen content of the standard 304L alloy was $0.15 \mathrm{wt} \%$ when processed using nitrogen for both backfilling and atomizing, and a modified version of $304 \mathrm{~L}$ with 23 wt $\%$ Cr contained 0.21 wt \% nitrogen when processed in the same manner. Hardness, yield strength, and the ultimate tensile strength of the HIP consolidated powders were improved with the ad dition of nitrogen while retaining significant tensile elongation (about 40\%)
03,206

PB94-185675 Not available NTIS

National Inst. of Standards and Technology (MSEL), Gaithersburg, MD. Metallurgy Div.

Langevin Approach to Hysteresis and Barkhausen Modeling in Steel.

Final rept.

R. D. McMichael, L. J. Swartzendruber, and L. H.

Bennett. 15 May 93, 3p.

Pub. in Jnl. of Applied Physics 73, n10 p5848-5850, 15 May 93.

Keywords: "Carbon steels, "Hysteresis, "Barkhausen effect, Stochostic processes, Electromagnetism, Rolling(Metallurgy), Ferromagnetic materials, Magnetic properties, Metastable state, Reprints.

To represent the multiplicity of metastable states in a bulk ferromagnetic material with domain-wall pinning effects, fluctuations in the domain-wall energies are described in terms of an ensemble of stochastic Langevin functions. The model parameters used are a correlation length, a rms value for the amplitude of the fluctuations in the domain-wall energy gradient, and a 'demagnetizing factor.' The model generates both hysteresis loops and Barkhausen effect (BE) jump size distributions. Jump size distributions were determined experimentally for low-carbon rolled sheet steel with the field applied both parallel and perpendicular to the rolling direction. Both the model and the experimental BE jump size distributions show a power-law behavior for small jumps and a rapid cutoff at large jump sizes

\section{3,207}

PB94-200136 Not available NTIS

National Inst. of Standards and Technology (MSEL), Boulder, CO. Materials Reliability Div.

Hot-Deformation Apparatus for Thermomechanical Processing Simulation.

Final rept.

Y. W. Cheng, and H. I. McHenry. 1988, 8 p.

Pub. in Proceedings of International Symposium on Physical Simulation of Welding, Hot Forming, and Continuous Casting, Canada, p1-81988

Keywords: "Hot working, "Quenching(Cooling), *Thermomechanical treatments, "Simulation, Reprints, Hardening(Materials), Heat treatment, High strength steels, Microstructure, Deformation, *Thermomechanical processing, *Steel-ASTM-A710.

A hot-deformation apparatus designed to simulate thermomechanical processing of steel plates is described, and some initial results for a study on controlled rolling followed by direct quenching (CR-DQ) of ASTM A710 steel are presented. The apparatus has the following features: a servohydraulic load frame with a $250 \mathrm{kN}$ capacity in tension or compression; a variable-actuator traveling speed up to $500 \mathrm{~mm} / \mathrm{s}$; a multiple-strike capability with controllable displacements and strain rates: a maximum heating rate of $150 \mathrm{deg}$. $\mathrm{C} / \mathrm{s}$ with a $10 \mathrm{~kW}$ induction heater (for a cylindrical steel specimen $\mathrm{mm}$ in diameter and $18 \mathrm{~mm}$ in height); a maximum cooling rate of $25 \mathrm{deg} \mathrm{C} / \mathrm{s}$ with helium-gas cooling; and vacuum to $1.33 \times 10(\exp -3) \mathrm{Pa}(1 \times 10$ (exp-5) torr) within $25 \mathrm{~min}$. Experimental results indicated that the hardenability of the steel increased with a simplified CR/DQ processing. The CR/DQ processed specimens showed variation of microstructure within the specimen due to nonuniform deformation during compression. Mechanisms that cont crease of hardenability are discussed.

\section{3,208}

PB94-211927 Not available NTIS

National Inst. of Standards and Technology (MSEL), Gaithersburg, MD. Metallurgy Div.

Determination of the Prior-Austenitic Grain Size of Selected Steels Using a Molten Glass Etch.

Final rept.

G. E. Hicho, L. C. Smith, C. A. Handwerker, and D. A. Kauffman. 1991, 11p.

Pub. in Jnl. of Heat Treating 9, n1 p37-47 1991.

Keywords: "Austenitic stainless steels, "Grain size, "Heat treatment, Microstructure, Austenite, Precipitates, Etching, Glass, Grain boundaries, Reprints

A hot etching procedure was used to reveal the prioraustenitic grain size of several steels. Etching experiments, using molten glass, were conducted on a HSLA precipitation hardened steel, two alloy steels, and a control-rolled steel. Experiments were also conducted 
MATERIALS SCIENCES

Iron \& Iron Alloys

on the HSLA precipitation hardenable steel in order to determine the effects of prolonged heating on the austenite grain size. For comparison purposes, the austenite grain size of the HSLA precipitation hardenable steel, as a function of temperature, was determined using a hot-stage microscope. Photomicrographs of the various steels showing the microstructures obtained using chemical etchants are also presented. A comparison of prior-austenitic grain sizes which were determined using the molten glass etch and chemical etches is shown.

03,209

PB94-212396 Not available NTIS

National Inst. of Standards and Technology (MSEL),

Gaithersburg, MD. Metallurgy Div.

Beneficial Effects of Nitrogen Atomization on an Austenitic Stainless Steel.

Final rept.

G. M. Janowski, F. S. Biancaniello, and S. D. Ridder. 1992, 10p.

Pub. in Metallurgical Transactions A 23A, p3263-3272

Dec 92.

Keywords: "Austenitic stainless steels, *Atomization, -Nitrogen, Copper alloys, Hardness, Powder metallurgy, Porosity, Hot pressing, Nickel alloys, Reprints. Fully dense nitrogenated austenitic stainless steels were produced by gas atomization and HIP consolidation. The base alloy, 304L, contained about 0.15 weight percent nitrogen when melted under a nitrogen atmosphere, and a modified version of $304 \mathrm{~L}$ with 23 weight percent $\mathrm{Cr}$ contained 0.21 weight percent nitrogen. A series of experiments using various combinations of N2 and Ar as the melt chamber backfill gas and atomizing gas demonstrated that the nitrogen content of the powder was largely controlled by the backfill gas and that the fraction of hollow particles was determined by the atomization gas. The hollow powder particles, which are common in inert-gas atomized materials, were virtually eliminated in the nitrogen atomized powders. Additional atomizing experiments using copper and a nickel-base superalloy indicate that low gas solubility in the metal leads to gas entrapment. Hardness and compression behavior (yield strength and flow stress) are substantially improved with the addition of nitrogen. The results of this study suggest that the properties of nitrogenated stainless steels fabricated in this manner are comparable to other high nitrogen austenitic alloys.

\section{3,210}

PB94-213188 Not available NTIS

National Inst. of Standards and Technology (MSEL), Boulder, CO. Materials Reliability Div.

Dynamics vs. Static Young's Moduli: A Case Study.

Final rept.

H. Ledbetter. 1993, 2p.

Pub. in Materials Science and Engineering A165, pL9L10 1993

Keywords: "Austenitic stainless steels, "Dynamic modulus of elasticity, "Modulus of elasticity, Static tests Dynamic tests, Mechanical properties, Uncertainty Elastic properties, Case studies, Reprints.

For one material, a $316 \mathrm{LN}$ austenitic steel, a comparison is made between the Young's modulus measured statically with usual commercial equipment and that measured dynamically at mega-hertz frequencies. The first gives a + or - $6 \%$ uncertainty; the second $a+$ or $-0.5 \%$ uncertainty. The two Young's moduli, $207 \mathrm{GPa}$ (static) and $202 \mathrm{GPa}$ (dynamic), agree within the statistical bounds of the static measurement, $195 \mathrm{GPa}$ and $219 \mathrm{GPa}$, the extreme static values being $178 \mathrm{GPa}$ and $229 \mathrm{GPa}$. Simple thermodynamics that shows the difference between dynamic and static elastic constants is also presented.

03,211

PB94-213212 Not available NTIS

National Inst. of Standards and Technology (MSEL), Boulder, CO. Materials Reliability Div.

Cast-Iron Elastic Constants: Effect of Graphite Aspect Ratio.

Final rept.

H. Ledbetter, and S. Datta. 1992, 4p.

Pub. in Zeitschrift fuer Metallkunde 83, n3 p195-198 1992.

Keywords: "Cast iron, "Elastic properties, Bulk modulus, Shear modulus, Young modulus, Aspect ratio, Poisson ratio, Graphite, Reprints, Scattered plane wave method.
Using a scattered-plane-wave ensemble-average model developed for composite materials, we calculated the effective elastic constants of cast iron. We focused on the effect of graphite-particle aspect ratio on the Young modulus. Between model and observation, we found good agreement. Oblate spheroidal graphite flakes lower the elastic stiffness much more than do spheres. To obtain good model-measurement agreement, one must use graphite's lower third-orderbound (Kroner-bound) elastic constants. Besides the Young modulus, we give calculated results for all the other usual quasi-isotropic elastic constants: shear modulus, Poisson ratio, and bulk modulus.

03,212

PB95-108759 Not available NTIS

National Inst. of Standards and Technology (NML).

Gaithersburg, MD. Gas and Particulate Science Div. Apparent Bias in the X-Ray Fluorescence Determination of Titanium in Selected NIST SRM Low Alloy Steels.

Final rept.

P. A. Pella, R. B. Marinenko, J. A. Norris, and A.

Marlow. 1991, 4p.

Pub. in Applied Spectroscopy 45, n2 p242-245 1991.

Keywords: *X-ray fluorescence, " $T$ Titanium, "Low alloy steels, "Chemical analysis, Standards, Calibrating, Emission spectroscopy, Metallurgy, Comparison, Electron microprobe analysis, Reprints, "SRM 1165, "SRM 1264, Standard reference materials

$\mathrm{X}$-ray fluorescence (XRF) results of analysis of titanium in National Institute of Standards and Technology (NIST) SRM 1165 and 1264 low alloy steels were found to be significantly different from the certified values. These differences appear to be unique to the XRF results since results from other methods such as emission spectroscopy did not show any discrepancies. The microhomogeneity of selected samples was examined by electron probe microanalysis and revealed the presence of titanium inclusions containing varying amounts of zirconium, niobium, and tantalum. Differences in metallurgical uniformity is believed to be XRF analysis of the new 1760 series alloys indicates that these are more accurate for purposes of calibration than the 1160 series and 1260 series for such elements as titanium.

03,213

PB95-153235 Not available NTIS

National Inst. of Standards and Technology (MSEL), Boulder, CO. Materials Reliability Div.

Ultrasonic Measurement of Sheet Anisotropy and Formability.

Final rept.

A. V. Clark, R. B. Thompson, R. C. Reno, G. V

Blessing, and D. Matlock. 1989, 7p.

Pub. in Proceedings of International Congress and Exposition, Detroit, MI., February 27-March 3, 1989, p89. 95.

Keywords: "Metal sheets, "Anisotrophy, "Modules of elasticity, "Steels, "Ultrasonic tests, Resonant frequencies, Textures, Nondestructive tests, Process control, Mechanical properties, Tensile properties, Reprints, "Formability, Electromagnetic-acoustic transducers.

The anisotropy of Young's modulus in steel sheet is closely related to the r-value. Commercially available instrumentation is often used to predict formability by measuring the resonant frequencies of coupons cut at 0,45 , and 90 degrees to the rolling direction. This paper describes recent advances in instrumentation to acquire equivalent information nondestructively, with possibility of on-line process control. The technique uses electromagnetic-acoustic transducers (EMAT's) to excite and detect ultrasonic guided waves propagating in the plane of the sheet. Theories of wave propagation relate the velocities of these waves to Young's modulus anisotropy or to the orientation distribution coefficients (ODC's) describing the texture. The paper reviews the principles of the measurement and then presents correlations of the elastic anisotropy, as deduced by the ultrasonic instrumentation, with tensile measurements of r. Measurements of Young's modulus anisotropy and neutron diffraction measurements of ODC's are also included.

03,214

PB95-161261 Not available NTIS

National Inst. of Standards and Technology (MSEL), Gaithersburg, MD. Materials Reliability Div.
Cryogenic Toughness of Austenitic Stainless Stee! Weld Metals: Effect of Inclusions.

Final rept.

A. O. Kluken, C. N. McCowan, and T. A. Siewert 1992, 19p

Pub. in Microstructural Science, v20 p45-63 1992

Keywords: "Cryogenic equipment, "Welded joints, *Austenitic stainless steels, "Toughness, "Inclusions, Voids, Fractures(Materials), Fractography, Mechanica properties, Microstructure, Image analysis, Impurities, Crack initiation, Reprints.

The present investigation studies the effect of the volume fraction inclusion and size distribution of inclu sions on the cryogenic ( $4 \mathrm{~K}$ ) toughness of austenitic stainless steel weld metals. Two weld series with systematic variations in the volume fraction of inclusions were made by varying the oxygen potential of the shielding gas at a constant heat input. To obtain differences in the size distribution of inclusions, at a given volume fraction of inclusions, the heat input was varied. The subsequent examinations of the welds involved both optical microscopy and scanning electron microscopy. Automatic image analysis was used to de termine the inclusion volume fraction and size distribution. The results from these examinations revealed that the arithmetic mean three-dimensional inclusion diameter increased with increasing volume fraction of inclusions. Moreover, for one of the weld series, a shift in the chemical composition of inclusions from spherical manganese silicates to predominantly angular chromium oxides was also observed with increasing content of weld-metal oxygen. Fracture toughness testing of the welds showed that the energy required to initiate and propagate cracks decreased as inclusion volume fraction increased. Fractographic examinations revealed that all specimens failed in a ductile manner with fracture surfaces exhibiting typical ductile dimple morphologies.

\section{3,215}

PB95-175253 Not available NTIS

National Inst. of Standards and Technology (MSEL) Boulder, CO. Materials Reliability Div.

Friction and Oxidative Wear of $440 \mathrm{C}$ Bal! Bearing Steels Under High Load and Extreme Bulk Temperatures.

Final rept

D. K. Chauduri, A. J. Slifka, and J. D. Siegwarth 1993, 14p.

Contract NASA-H-01026D

Sponsored by National Aeronautics and Space Administration. Washington, $\mathrm{DC}$

Pub. in Wear 160, p37-50 1993

Keywords: *Steels, *Sliding friction, *Wear, Temperature range 0065-0273 K Temperature range 0273 $0400 \mathrm{~K}$, Temperature range 0400-1000 K, Tempera ture dependence, Coefficient of friction, Ball bearings, Oxidation, Reprints, Steel $440 \mathrm{C}$

Unlubricated sliding friction and wear of $440 \mathrm{C}$ steels in an oxygen environment have been studied under a variety of load, speed and temperature ranging from approximately -185 to $675 \mathrm{C}$. A specially designed test apparatus with a ball-on-flat geometry has been used for this purpose. The observed dependencies of the initial coefficient of friction, the average dynamic coefficient of friction, and the wear rate on load, speed, and test temperatures have been examined from the standpoint of existing theories of friction and wear. High contact temperatures are generated during the sliding friction causing rapid oxidation and localized surface melting. A combination of fatigue, delamination, and loss of hardness due to tempering of the martensitic structure is responsible for the high wear rate observed and the coefficient of friction.

\section{3,216}

PB96-123393 Not available NTIS

National Inst. of Standards and Technology (MSEL), Boulder, CO. Materials Reliability Div.

Prediction of the Strength Properties for Plain-Carbon and Vanadium Micro-Alloyed Ferrite-Pearlite Steel.

Final rept.

P. T. Purtscher, Y. W. Cheng, and R. P. Foley. 1995, $10 \mathrm{p}$.

Pub. in Mechanical Working and Steel Processing Conference Proceedings (36th), ISS-AIME, Baltimore, MD., October 1994, v32 p279-288 1995.

Keywords: "Steels, "Alloying, "Fracture mechanics, Microstructure, Carbon steels, Toughness, Strength, Regression analysis, Reprints, Composite deformation theory. 


\section{Iron \& Iron Alloys}

Composite deformation theory is examined and applied to the case of ferrite and pearlite microstructures. The goal of this paper is to use the basic microstructural features (grain size and volume fraction of ferrite, and interlamellar spacing of pearlite) to predict as much of the stress-strain curve as possible, and from the stress-strain curve, to calculate strength. Two different deformation schemes are examined for the prediction of stress-strain curves: first, strain partitioning between ferrite and pearlite, and second, equal strain in ferrite and pearlite. The strain partitioning case can be used to predict the yield strength when the ferrite volume fraction is greater than about $33 \%$. When the volume fraction of ferrite is less than about $15 \%$, an equal-strain deformation scheme can be used to predict the flow curve up the uniform strain (both yield and ultimate strengths). Strengthening from microalloy precipitation can be incorporated by adding the assumed additional strengthening to the flow curves for the two phases.

\section{3,217}

PB96-135140 Not available NTIS

National Inst. of Standards and Technology (MSEL), Gaithersburg, MD

Catastrophic Failures Propagate Field of Fracture Mechanics.

Final rept

R. L. Blumberg. 1995, 2p.

Pub. in Materials Research Society Bulletin, v20 n8 p74-75 Aug 95.

Keywords: "Fracture mechanics, "Stresses, "Failure(Mechanics), Wires, Stress-strain relations, Failure analysis, Mechanical properties, Reprints, Size effect, Fracture analysis, Flaws.

The development of fracture mechanics dates back five centuries to Leonardo da Vinci, who carried out experiments on the fracture of iron wire. He demonstrated that the strength of a piece of wire varies inversely with its length; long wires are weaker, on average, while short wires are stronger. This behavior, better known today as the size effect, supported the theory that wire fracture is initiated by rare flaws. A longer piece of wire is more likely to contain a larger flaw that causes fracture at a lower critical stress.

\section{3,218}

PB96-161971 Not available NTIS

National Inst. of Standards and Technology (MSEL), Gaithersburg, MD.

Fundamentals of Fracture: A 1993 Prologue, and Other Comments.

Final rept.

R. Thomson. 1994, 7p

See also PB86-189131.

Pub. in Materials Science and Engineering, vA176 p1. 71994.

\section{Keywords:}

Dislocations(Materials), Reprints, Cracks, Fracture(Mechanics), "Foreign technology.

In the first of this paper, I discuss the trends in fracture science as it is reflected in the papers given at this and the past three conferences on the fundamentals of fracture. In the second part of the paper, I present some results carried out by myself and callaborators on certain atomic aspects of fracture in a simple twodimensional hexagonal lattice, i.e. the dislocation emission criterion, the non-local shift in the emission criterion caused by the shielding of dislocations already present, and the structure and some properties of the interfacial crack.

\section{3,219}

PB96-190194 Not available NTIS

National Inst. of Standards and Technology (MSEL), Gaithersburg, MD. Materials Reliability Div.

Charpy Impact Test as an Evaluation of $4 \mathrm{~K}$ Fracture Toughness.

Final rept.

H. Nakajima, K. Yoshida, H. Tsuji, M. M. Morra, R

G. Ballinger, R. L. Tobler, and I. S. Hwang. 1992, 9p. Pub. in International Cryogenic Materials Conference Publication, Huntsville, AL., June 11-14, 1991, Advances in Cryogenic Engineering (Materials), v38 p207-215 1992 .

Keywords: "Cryogenics, "Austenite, "Alloys, "Impact tests, "Fracture properties, Toughness, Mechanical properties, Standards, Tests methods, Low temperature tests, Reprints, Austenitic structural alloys, Charpy test.
A $4 \mathrm{~K}$ Charpy test is defined as a Charpy test in which the initial temperature of a specimen is $4 \mathrm{~K}$. Two methods, a glass Dewar method and a flow method, are compared in this study to demonstrate that both provide consistent results as long as an initial specimen temperature is the same. An improved correlation between the Charpy absorbed energy and the fracture toughness at $4 \mathrm{~K}$ is also examined to clarify the limitation of Charpy test applications at cryogenic temperatures.

\section{3,220}

PB96-190251 Not available NTIS

National Inst. of Standards and Technology (MSEL), Boulder, CO. Materials Reliability Div.

Prediction of Strengthening Due to $\mathrm{V}$ Additions in Direct-Cooled Ferrite-Pearlite Forging Steels.

\section{Final rept.}

P. T. Purtscher, Y. Cheng, R. Kuziak, and R. P. Foley. 1996, 11p

Pub. in Mechanical Working and Steel Processing Conference (37th), Hamilton, Ontario, Canada, October 1995, p405-415 1996.

Keywords: "Forging steels, "Thermodynamic modeling, Vanadium, Yield strength, Microstructure, Solubility products, Reprints.

Two different approaches predict the yield-strength increment (deltaYS) due to $\mathrm{V}$ additions in ferrite-pearlite forging steels. The first is based on the cooling rate and on the $\mathrm{V}$ and $\mathrm{N}$ contents dissolved in austenite before transformation to ferrite. The second is based on the $V$ available for precipitations strengthening in ferrite. Based on comparisons between the prediction schemes and measured results in the literautre for a range of compositions and forging variables, both approaches predict similar trends. There is no consistently better agreement with the measured deltaYS for one approach than for the other. Considering the reheat/deformation temperatures typically used in indus trial practice, the first approach based on $\mathrm{V}$ and $\mathrm{N}$ dissolved in austenite is preferred.

\section{3,221}

PB96-190269 Not available NTIS

National Inst. of Standards and Technology (MSEL), Boulder, CO. Materials Reliability Div. Temperature-Induced Transition in Ductile Frac ture Appearance of a Nitrogen-Strengthened Aus tenitic Stainless Steel.

Final rept.

P. T. Purtscher, G. Krauss, and D. K. Matlock. 1993, Pub. in Metallurgical Transactions A, v24A p2521. 2529 Nov 93.

Keywords: *Stainless steel, "Cryogenic properties, Reprints, Ductile fracture, Shear fracture, Sigma phase, Void nucleation.

A nitrogen-strengthened autenitic stainless steel was tested in uniaxial tension at room temperature (295 K) and in liquid nitrogen $(76 \mathrm{~K}$ ). A transition in ductile fracture appearance from a cup-cone fracture at room temperature to shear fracture at cryogenic temperature is observed and correlated to deformation behavior and micromechanisms (void nucleation and strain localization) of fracture. The flow stresses, fracture stresses, and strain hardening rates are all higher at liquid nitrogen temperature compared to those at room temperature, and the significant increases in platic flow stresses are accompanied by planar deformation mechanisms. At both temperatures, primary void nucleation is observed mainly at scattered, large patches of sigma phase, and initial primary void growth is associated with tensile instability (necking) in the specimen. Postuniform elongation at $195 \mathrm{~K}$ leads to secondary void growth and coalescence is highly localized an does not contribute to macroscopic elongation. At 76 $\mathrm{K}$, uniform strain increases, total strain decreases, and strain location into shear bands between the primary voids and the surface of the neck leads directly to failure. Secondary void nucleation, growth, and coalescence are limited to shear bands and also do not contribute to the macroscopic elongation. The observations of void nucleation are characterized in terms of a continuum analysis for the interfacial stress at voidnucleating particles. The critical interfacial stress for void nucleation at the lower temperature correlates with the increased flow properties of the matrix.

\section{3,222}

PB96-190285

Not available NTIS National Inst. of Standards and Technology (MSEL),
Boulder, CO. Materials Reliability Div.
Ductile Fracture and Tempered Martensite Embrittlement of 4140 Steel.

Final rept.

J. A. Sanders, P. T. Purtscher, D. K. Matlock, and G. Krauss. 1992, 10p.

Pub. in Speich Symposium Proceedings, Iron and Steel Society Meeting, Montreal, Canada, October 1992, p67-76.

Keywords: "Ductile fracture, "Fractography, "Steel, Strain hardening, Tensile properties, Void nucleation, Reprints, Tempered martensite embrittlement.

Specimens of martensitic 4140 steel were tempered at 200,300 , and 400 degrees $C$ and subjected to uniaxial tensile testing and fractographic examination. Ductility does not increase as flow stresses decrease with increasing tempering temperature. This manifestation of tempered martensite embrittlement is related to the introduction of new void-nucleating carbide particles by high temperature tempering and the effect of the particle distributions on ductile fracture. Calculations of void-nucleating stresses at the interfaces of carbide particles at fracture, incorporating the effects of macroscopic changes during necking, were made. The effect of changing particle distributions on these calculations are discussed.

\section{3,223}

PB96-190343 Not available NTIS

National Inst. of Standards and Technology (MSEL), Boulder, CO. Materials Reliability Div.

Fatigue Crack Thresholds of a Nickel-Iron Alloy for Superconductor Sheaths at $4 \mathrm{~K}$.

Final rept.

R. L. Tobler, and I. S. Hwang. 1994, 8p.

Pub. in Advances in Cryogenic Engineering, v40 p1315-1322 1994.

Keywords: "Fracture mechanics, "Nickel base superalloy, Liquid helium, Fatigue tests, Cryogenic properties, Reprints, Superconductor conduit, Austenitic alloys.

Short crack simulation (SCS) tests were used to characterize the fatigue crack growth rates da/dN and thresholds deltaK(Th) of a $\mathrm{Ni}$-Fe superalloy proposed for conduit sheath applications in superconducting magnets at $4 \mathrm{~K}$. Experiments described demonstrate the reproducibility of the SCS data and confirm that this alloy has a relatively high fatigue crack growth rate threshold at $4 \mathrm{~K}$. The effects of plate thickness and thermal treatment (650 degrees $C, 180 \mathrm{~h}$ ) on nearthreshold fatigue properties are minor compared with a strong cryogenic temperature effect that increases the fatigue threshold from about $2 \mathrm{MPa}$.m(to the one half power) at room temperature to 9 or $10 \mathrm{MPa} \cdot \mathrm{m}$ (to the one half power) at $4 \mathrm{~K}$.

\subsection{4}

PB96-190350 Not available NTIS

National Inst. of Standards and Technology (MSEL), Boulder, CO. Materials Reliability Div.

Mechanical Properties and Warm Prestress of Ultra-Low Carbon Steel at $4 \mathrm{~K}$.

Final rept.

R. L. Tobler, L. M. Ma, and R. P. Reed. 1991, 8p. Pub. in Supercollider III, Proceedings of the Supercollider Symposium (3rd), p139-146 1991.

Keywords: "Steel, "Fracture toughness, Ferritis steel, Cryogenic properties, Prestress effect, Tension tests, Warm prestress, Reprints, Low carbon steel.

The yield strength of ultra-low carbon steel increases from $137 \mathrm{MPa}$ at $195 \mathrm{~K}$ to $705 \mathrm{MPa}$ at $4 \mathrm{~K}$. In liquid helium the material is brittle and cleaves in tension. Consequently it is difficult or impossible to properly fatigue-precrack toughness specimens for test at $4 \mathrm{~K}$. We precracked a series of compact specimens at room temperature and then fractured them at $4 \mathrm{~K}$. The toughness measurements at $4 \mathrm{~K}$ are affected by precracking at $295 \mathrm{~K}$, and they provide only an estimate of $\mathrm{K}(\mathrm{l} \mathrm{c})$ for this steel: $15 \mathrm{MPa} \mathrm{M}$ (to the one half power). The tensile properties of the steel at 295 and $4 \mathrm{~K}$ are reported, and the effects of prestress on the tensile and fracture properties at $4 \mathrm{~K}$ are discussed.

\section{Lubricants \& Hydraulic Fluids}

O3,225
PB95-125969 Not available NTIS 
MATERIALS SCIENCES

Materials Degradation \& Fouling

National Inst. of Standards and Technology (MSEL) Gaithersburg, MD. Ceramics Div.

Control of Friction and Wear of Alpha-Alumina with a Composite Solid-Lubricant Coating.

Final rept.

A. Gangopadhyay, and S. Jahanmir. 1990, 8p.

Pub. in Proceedings of a Conference on Tribology of Composite Materials, Oak Ridge, TN., May 1-3, 1990 p337-344.

Keywords: "Aluminum oxide, "Solid lubricants, "Ceramics, "Friction reduction, "Wear resistance, High temperature lubricants, Tribology, Alternatives, Performance evaluation, Coatings, Coefficient of friction Reprints, *Advanced materials.

The successful utilization of advanced structural ce ramic materials as tribological components in high temperature engine applications requires friction coefficients in the range of 0.1 to 0.2 . The low values of friction coefficients are needed for energy conservation and control of the wear rate, since it has been shown that the wear of ceramics is adversely affected by friction. Presently, liquid lubricants are not available for the high temperature applications. Solid-lubricant coatings appear as a viable alternative to achieve a low friction coefficient. In the present study, the tribologica characteristics of a multicomponent solid-lubrican coating deposited on alumina is investigated up to a temperature of $800 \mathrm{C}$, and compared with that of uncoated alumina.

\section{3,226}

PB95-213583 PC A18/MF A04

National Inst. of Standards and Technology (MSEL) Gaithersburg, MD. Ceramics Div.

Boundary Lubrication of Silicon Nitride.

Special pub.

R. S. Gates, and S. M. Hsu. Feb 95, 408p, NIST/SP. 876.

Also available from Supt. of Docs. as SN003-003 03316-2. See also PB91-236711. Sponsored by Department of Energy, Washington, DC

Keywords: "Tribology, "Boundary lubrication, "Ceramics, "Silicon nitrides, Friction, Wear tests, Alcohols, Silicon compounds, Alkoxides, Fluid-solid inter actions, Surface chemistry, Organic compounds, Oxy gen, Phosphorus, Sulfur, Nitrogen, Halogens.

Successful use of advanced ceramics in many tribological applications requires an understanding of the physical, chemical, and mechanical properties of the material. This report describes a systematic study to investigate the effects of selected organic chemical compounds on the friction and wear of silicon nitride under boundary lubrication conditions. A broad range of chemistries were investigated. Results are grouped according to predominant chemical compound class as oxygen, phosphorus, halogen, sulfur, and nitrogencontaining chemistries.

03,227

PB96-119466 Not available NTIS

National Inst. of Standards and Technology (MSEL) Gaithersburg, MD. Ceramics Div.

Mechano-Chemical Model: Reaction Temperatures in a Concentrated Contact.

Final rept.

S. M. Hsu, M. C. Shen, E. E. Klaus, H. S. Cheng

and P. I. Lacey. 1994, 10p.

Pub. in Wear, v175 p209-218 1994

Keywords: "Boundary lubrication, "Models, "Wea tests, "Tribology, "Surface chemistry, Chemical reactions, Fluid mechanics, Hydrodynamics, Kinetics, Degradation, Temperature, Wear, Reprints, "Contact mechanics.

Successful boundary lubrication is essential in the design and operation of many components. The lubrication process is complex and it involves contact mechanics, fluid mechanics, tribochemistry and material deformation and fracture. Two schools of thought have emerged over the years in examining the mechanisms and modeling of boundary lubrication. The chemical school believes that chemical reactions at the rubbing surfaces control the efficacy of the lubrication process. The mechanical school believes that while chemistry is a factor, hydrodynamics, elastohydrodynamics (EHD), and micro-EHD can account for most of the load-bearing mechanisms, so at least in design, they are the principal issues. This paper attempts to bring the two schools together to examine a common set of experimental data. The experiments involved running wear tests on a four-ball wear tester using microliter of lubricant until seizure. Lubricant degradation and breakdown are therefore a factor in the wear test. The key to both models is the temperatures in the contest. This paper describes the two models and focuses on the temperatures in the contact. The temperatures calculated from the two models differ significantly.

\section{Materials Degradation \& Fouling}

\section{3,228}

DE93041307 PC A06/MF A02

National Inst. of Standards and Technology (MSEL), Gaithersburg, MD. Ceramics Div.

Review of Corrosion Behavior of Ceramic Heat Ex. changer Materals: Corrosion Characteristics of Silicon Carbide and Silicon Nitride. Final Report, September 11, 1992--March 11, 1993.

Progress rept.

R. G. Munro, and S. J. Dapkunas. 1993, 103p, DOE/ PC/92179-T1

Contract Al22-92PC92179

Sponsored by Department of Energy, Washington, DC.

Keywords: "Heat Exchangers, "Silicon Carbides, *Silicon Nitrides *Corrosion Resistance, Ashes, Flexural Strength, Gases, Liquid Metals, Materials, Molten Salts, Oxidation, Progress Report, Protective Coatings, Reviews, Slags, Temperature Range 1000-4000 $\mathrm{K}, \mathrm{EDB} / 360205$.

The present work is a review of the substantial effort that has been made to measure and understand the effects of corrosion with respect to the properties, performance, and durability of various forms of silicon carbide and silicon nitride. The review encompasses corrosion in diverse environments, usually at temperatures of $1000 \mathrm{C}$ or higher. The environments include dry and moist oxygen, mixtures of hot gaseous vapors, molten salts, molten metals, and complex environments pertaining to coal ashes and slags.

\section{3,229}

PB94-172368 Not available NTIS

National Inst. of Standards and Technology (MSEL), Gaithersburg, MD. Ceramics Div.

Wear Mechanism Maps of Ceramics.

Final rept.

S. M. Hsu, P. I. Lacey, Y. S. Wang, and S. W. Lee. $1990,10 p$

Pub. in Advances in Engineering Tribology, SP-31, p123-132 1990. Sponsored by Gas Research Inst., Chicago, IL.

Keywords: "Wear tests, "Ceramic fibers, "Tribology, *Metal matrix composites, Lubrication, Comparison, Polycrystalline, Composite materials, Reinforcing fibers, Plastic deformation, Fractures(Materials), Reprints.

The wear mechanisms of polycrystalline ceramics have been studied using wear map methodology. Wear of ceramic materials was examined under a wide range of speed and load to identify different wear regimes, and to establish the critical operating limits. The methodology also facilitates comparison among different materials under various systems parameters such as dry sliding, non-reactive fluids, and reactive fluids. The present study compares the wear mechanisms of alumina, silicon nitride, partially stabilized zirconia, and silicon carbide whisker reinforced alumina. Particular attention is given to the location of the transition zone from mild plastic deformation to rapid fracture controlled wear and the concomitant increase in wear rate.

\section{3,230}

PB94-172400 Not available NTIS

National Inst. of Standards and Technology (MSEL), Gaithersburg, MD. Ceramics Div

Fracture Mechanics Analysis of Near-Surface Cracks.

Final rept.

S. Jahanmir, J. W. Dally, and Y. M. Chen. 1990, $6 p$. Pub. in Proceedings of Japan International Tribology Conference, Nagoya, Japan, October 29-November 1 , 1990, p581-586.

Keywords: "Ceramics, "Wear tests, "Fracture mechanics, "Crack propagation, Tribology, Photoelasticity, Particles, Stress intensity factors, Surfaces, Materials testing, Reprints.
The purpose of the analysis is to develop a qualitative understanding on the propagation of cracks relevant to the formation of wear particles in an elastically deforming stress field. A shallow subsurface crack ori ented parallel to the boundary and a vertical surfacebreaking crack were analyzed. Three different series of photoelastic experiments were conducted on each crack type and results for the stress intensity factors for both the opening mode and the shearing mode were determined. The relative signs of $K(I)$ and $K(I I)$ which depend on the location of crack tip with respec to the point of load application, were used to predic the crack trajectories. The implications of these results on the process of wear particle formation in elastically deforming materials, such as ceramics, are discussed.

03,231

PB94-172749 Not available NTIS

National Inst. of Standards and Technology (MSEL) Gaithersburg, MD. Ceramics Div.

Wear of Selected Materials and Composites Sliding against MoS2 Films.

Final rept.

A. W. Ruff, and M. B. Peterson. 1993,6p

Pub. in Wear 162-164, p492-497 1993. See also PB93-138949. Sponsored by Research and Development Lab. Wright-Patterson, $\mathrm{OH}$

Keywords: "Wear tests, "Molybdenum disulfide, "Composite materials, "Films, *Tribology, "Vacuum coating, Friction tests, Coatings, Solid lubricants, Roller bearings, Space environments, Physical vapor dep osition, Reprints.

Improved vacuum deposition methods are now available to produce dense, suitability oriented, durable films of molybdenum disulfide on substrates appropriate for tribological applications. It is of interest to examine materials in sliding contact with such films in order to identify optimum combinations, and to improve further tribological performance of the system. Results of wear and friction measurements are presented to a number of materials including self-lubricating composites sliding against four different types of vacuumdeposited MoS2 films. The testing program utilized a controlled environment, pin-on-ring tribometer, with load and speed conditions appropriate to a possible application. Differences in wear over four orders of magnitude, and friction up to a factor of seven times, were measured among the materials. One application area of interest for these material combinations would be as ball retainers in rolling element bearings for space satellite systems.

03,232

PB94-187317 PC A03/MF A01

National Inst. of Standards and Technology (BFRL) Gaithersburg, MD.

Sulfate Attack of Cementitious Materials: Volumetric Relations and Expansions

J. R. Clifton, and J. M. Pommersheim. Apr 94, 24p, NISTIR-5390.

Prepared in cooperation with Bucknell Univ. Lewisburg, PA

Keywords: "Cements, "Concretes, "Sulfates, *Environmental effects, "Mathematical models, Hydration, Expansion, Volume, Chemical composition, Density(Mass/volume), Gypsum, Construction mate rials, Tricalcium aluminate, Chemical reaction mecha nisms, Ettringite.

A model was developed which predicts the conditions Ander which volumetric expansion of cementitious materials are most likely and the amount of expansion expected. Model parameters include concrete composition, phase densities, water-cement ratio, degree of hy dration, extent of expansive reaction and reaction sto chiometry. The model was applied to several differen reactions involving sulfate attack of concrete, including gypsum and ettringite formation reactions. The mode predicted that ettringite formation from monosulfate would not cause general expansion. However, loca expansion could occur if ettringite occupied the same space vacated by reacting monosulfate. The model predicted that if sufficient unhydrated tricalcium aluminate (C3A) was available in mature cement paste and it reacted with gypsum to form ettringite, the reac tion could cause expansion at low water-to-cement ratios.

03,233

Not available NTIS

National Inst. of Standards and Technology (MSEL) Gaithersburg, MD. Ceramles Dlv. 


\section{Materials Degradation \& Fouling}

Mechanism of Mild to Severe Wear Transition in Alpha-Alumina.

Final rept.

S. Jahanmir, and X. Dong. 1992, 9p.

Pub. in Jnl. of Tribology 114, n3 p403-411 1992.

Keywords: "Aluminum oxide, "Wear, "Friction factor, "Coefficient of friction, Temperature, Ceramics, Tribology, Fractures(Materials), Reprints.

Friction and wear experiments were conducted on high purity alpha-alumina sliding agains! a similar material in air under different contact loads and at temperatures ranging from $23 \mathrm{C}$ to $900 \mathrm{C}$. Experimental results indicate that tribochemical reactions between water vapor and alpha-alumina at room temperature produce aluminum hydroxide which result in relatively low coefficients of friction and low wear rates. Both the coefficient of friction and the wear rate of alumina were low at intermediate temperatures $(200 \mathrm{C}$ to $800 \mathrm{C}$ ), if the contact stress was below a threshold value. Above this load, wear occurred by fracture, the wear coefficient exceeded a value of .0001 , and the coefficient of friction increased to 0.90 . At $900 \mathrm{C}$, the coefficient of fric tion was 0.40 and the wear coefficient was reduced to 0001, because of the formation of a silicon-rich layer on the wear track. A contact mechanics model based on linear elastic fracture mechanics indicated that the propagation of cracks from pre-existing flaws controls the onset of catastrophic wear in the intermediate temperature range.

\section{3,234}

\section{PB94-213014 Not available NTIS}

National Inst. of Standards and Technology (CSTL), Gaithersburg, MD. Process Measurements Div.

Iridium Oxide Thin-Film Stability in High-Temperature Corrosive Solutions.

Final rept.

K. Kreider. 1991, 5p

Pub. in Sensors and Actuators B 5, p165-169 1991

Keywords: "Iridium oxides, Temperature range 0400 1000. Radioactive waste facilities, Underground facilities, High temperature, Aluminum oxide, Thin films, $\mathrm{pH}$ meters, Substrates, Stability, Reprints, Sputtered films.

The stability of sputtered iridium oxide films is inves tigated after exposure to $\mathrm{pH} \mathrm{4,7,} \mathrm{and} 10$ solutions at $200 \mathrm{C}$ (15 bar) and $245 \mathrm{C}$ (40 bar). These reactively sputtered films are being considered as $\mathrm{pH}$-sensing electrodes in high-temperature high-pressure saline solutions such as those found underground in potential nuclear repositories. The sputtered iridium oxide films (SIROF) have a linear response of approximately 53$58 \mathrm{mV}$ per $\mathrm{pH}$ at room temperature under a wide range of solution conditions. Other advantages include their ruggedness, small size, high voltage/low impedance output, and the low cost of fabrication. To evaluate their stability, the films are exposed in a Teflon-lined bomb for up to $70 \mathrm{~h}$ at high temperature. The $\mathrm{pH}$ testing of 50 samples includes cycling between $\mathrm{pH} 2$ and $\mathrm{pH} 12$ before and after exposure. Changes of the potential intercept and the slope are most severe after acidic conditions at $250 \mathrm{C}$. It is found that ion-assisted deposition helps to maintain film adherence and continuity on the alumina substrates during exposure to $200 \mathrm{C}$ solutions.

\section{3,235}

PB95-107041 Not available NTIS

National Inst. of Standards and Technology (MSEL),

Gaithersburg, MD. Polymers Div.

Aging in Glasses Subjected to Large Stresses and Deformations.

Final rept.

G. B. McKenna, M. M. Santore, A. Lee, and R. S.

Duran. 1991, 8p

Pub. in Jnl. of Non-Crystalline Solids 131, p497-504 1991.

Keywords: "Glass, "Aging(Materials), "Dilatometry, Viscoelasticity, Torsion, Deformation, Creep, Glass transition temperature, Kinetics, Thermodynamics, Reprints, Erasure, Rejuvenation, Volume recovery.

Physical aging studies near to the conventional glass transition temperature Tg were made using a model epoxy glass. Nonlinear viscoelastic responses were measured after quenching the samples from above Tg to below it. The physical aging response in creep in simple extension was studied as a function of stress magnitude and in stress relaxation in torsion at a function of deformation magnitude. In each test the time t(star) for the aging time shift factor to approach a constant value was determined and found to be independ- ent of the stress or deformation magnitude. These results are interpreted to mean that large mechanical stimuli do not alter the underlying therodynamic state of the glass and aging is not 'erased' by the large stresses or deformations.

\section{3,236}

PB95-140588 Not available NTIS

National Inst. of Standards and Technology (MSEL) Gaithersburg, MD. Ceramics Div.

Critical Factors in Non-Lubricated, Non-Abrasive Wear Testing.

Final rept.

A. W. Ruff. 1992, $6 \mathrm{p}$.

Pub. in Jnl. of Friction and Wear 13, n1 p48-53 1992.

Keywords: "Wear tests, "Test methods, *Nondestructive tests, "Standardization, Tribology, Morphology, Wear, Friction, Wear resistance, Reprints, "Non-abrasive wear testing.

Some wear testing approaches used in research and in standardized testing are discussed along with data from several interlaboratory studies of wear. Four critical factors are suggested to explain the relatively high variation in measured wear properties from three prominent test geometries and methods. The factors are: uniform wear scar morphology, wear debris retention in the contact, test system dynamic stiffness, and specimen shape. Further research efforts are needed to better identify and understand these factors, so that the test methods can be improved and the wear data obtained using them are more accurate and useful.

\section{3,237}

PB95-140596 Not available NTIS

National Inst. of Standards and Technology (IMSE), Gaithersburg, MD. Polymers Div.

Tribological Data: Needs and Opportunities.

Final rept.

A. W. Ruff. $1989,6 p$.

Sponsored by Department of Energy, Washington, DC Pub. in Proceedings of the International Congress on Tribology (5th), Helsinki, Finland, June 12-15, 1989 p26-31

Keywords: "Tribology, "Data bases, "Information systems, Information retrieval, Wear, Data retrieval, Information transfer, Data processing, Friction, Information management, Reprints, "ACTIS data base.

Many opportunities exist today for evaluated tribology data and information to influence material selection component design, predictive model development, and planning of research and development programs for new materials and understanding. In order for tribology databases to have a significant impact, there is need for improved standards of tribological measurement, and for new standards of data systems themselves. Basic considerations applying to tribology data and information are presented and discussed in this paper. Current developments in the U.S. in a joint government-private sector activity concerned with a comprehensive data and information system for tribology ACTIS, are also described.

\section{3,238}

PB95-162517 Not available NTIS

National Inst. of Standards and Technology (MSEL) Gaithersburg, MD. Ceramics Div.

Introduction to ASTM 1199 'Wear Test Selection for Design and Application'.

Final rept.

1993, 2p

Pub. in Tribology: Wear Test Selection for Design and Application, ASTM STP 1199, 2p 1993.

Keywords: "Wear tests, "Experimental design, "Test methods, "Standards, Design analysis, Test facilities, Erosion, Wear, Tribology, Reprints, "ASTM STP 1199. ASTM'S Committee on Wear and Erosion has sponsored numerous symposia in subject areas such as wear, solid particle erosion, and wear modeling. The focus of those symposia and the resulting Specia Technical Publications (STPS) involved numerous scientific and engineering subjects, as well as the area of test methodology. One important area that has not been covered so far is the connection between laboratory testing and actual operating performance of systems or components. The connection between laboratory testing, test results, and final design and performance is a crucial one in the practical application of tribology science and engineering.

\section{3,239}

PB95-163796 Not available NTIS
National Inst. of Standards and Technology (MSEL). Gaithersburg, MD. Ceramics Div.

Wear Model for Alumina Sliding Wear.

Final rept.

Y. S. Wang, S. M. Hsu, and R. G. Munro. 1990, 6p. Pub. in Proceedings of Japan International Tribology Conference, Nagoya, October 29-November 1, 1990 , p1225-1230.

Keywords: "Aluminum oxide, "Wear tests, "Mathematical models, "Sliding friction, "Microstructure, Ceramics, Tribology, Lubrication, Tensile stress, Loads(Forces), Velocity, Reprints.

A wear model incorporating microstructural effects has been developed to understand the wear data of alumina sliding on alumina under dry, paraffin oil lubricated, and water lubricated conditions. The model accounts for the wear transitions which were observed under different speeds and loads. A normalized tensile stress delta(sub max)/delta(sub D) is proposed as a critical parameter to explain wear transitions.

\section{3,240}

PB95-164067 Not available NTIS

National Inst. of Standards and Technology (MSEL), Gaithersburg, MD. Ceramics Div.

Molecular Órbital Study of Water Enhanced Crack Growth Process.

Final rept.

W. Wong-Ng, G. S. White, and S. W. Freiman. 1990 ,

Pub. in Materials Research Society Extended Abstract (EA-23), p11-14 1990.

Keywords: "Silicon dioxide, "Environmental effects, "Molecular orbitals, "Crack propagation, "Water immersion, Covalent bonds, Self consistent field, Chemical bonds, Atomic properties, Ions, Strains, Reprints.

To further our understanding of the mechanism of environmentally enhanced crack growth, molecular orbital calculations (MO) using the ab initio self-consistent field (SCF) technique have been employed to investigate the effect of applied strain on the atomic charges, bond overlap population and ionic character of the Si-O bond at the crack tip of silica. Pyrosilicic acid $\mathrm{H} 6 \mathrm{Si} 2 \mathrm{O} 7$ with eclipsed conformation has been employed as the model molecule. Strain effects up to $30 \%$ were simulated in terms of bond stretching and angle distortion.

\section{3,241}

PB96-141395 Not available NTIS

National Inst. of Standards and Technology (MSEL). Boulder, CO. Materials Reliability Div.

Artificial Crack in Steel: An Ultrasonic-ResonanceSpectroscopy and Modeling Study.

Final rept

H. Ledbetter, P. Heyliger, K. Pei, S. Kim, and C. Fortunko. 1995, 7p

Pub. in Review of Progress in Quantitative Nondestructive Evaluation, v14 p2019-2025 1995.

Keywords: "Cracks, "Steels, "Spectroscopy, Ultrasonics, Reprints, Defect detection, Finite element method, Ritz method, Natural-vibration frequencies.

The problem of detecting and characterizing cracks in solids is a major research area involving contributions from mathematics, physics, mechanics, and materials science. In the present study the authors approached this problem from both measurement and modeling viewpoints. In the measurements, the authors used ultrasonic-resonance spectropscopy to determine the macroscopic-vibration-frequency shifts caused by an artificial (electromachined) crack. The modeling used a finite-element method to approximate the macroscopic vibration frequencies of a cracked solid. As a check, the authors used the Ritz method to calculate the frequencies of the noncracked solid.

\section{Miscellaneous Materials}

03,242

AD-A302 669/7 PC A04/MF A01

Optical Materials, Inc., Cambridge, MA

Preliminary Subject and Authors Index to Compilations of Data on Properties of Materials.

J. G. Beckerley. 1966, 47p, NBS-9058.

Contract CST-1321

ADA302670. 
Keywords: "Materials, "Subject indexing, Commerce, Technology transfer, Chemistry, Engineering, Physics, Space sciences, Standards, Mathematics, Telecommunications, Industrial research, Physical sciences, Scientists, Applied mechanics.

This report is intended for use in conjunction with NBS Report 9057, Preliminary List of References Containing Compilations of Data on Properties of Materials. I contains two parts: (1) a preliminary subject index; and (2) a name index of authors, editors, translators, and corporate sponsors.

03,243

AD-A302 670/5 PC A05/MF A01

Optical Materials, Inc., Cambridge, MA.

Preliminary List of References Containing Compilations of Data on Properties of Materials.

J. G. Beckerley. 1966, 66p, NBS-9057

Contract CST-1321

ADA302669.

Keywords: "Materials, "Bibliographies, "Scientific lit erature, Commerce, Technology transfer, Chemistry, Engineering, Physics, Space sciences, Standards, Mathematics, Telecommunications, Industrial research, Physical sciences, Applied mechanics.

This preliminary list contains 1,000 references in alphabetical order by author or editor. The references contain compilations of numerical data on the physica properties of materials.

\section{3,244}

DE93019683 PC A02/MF A01

National Inst. of Standards and Technology (NEL) Gaithersburg, MD. Thermophysics Div.

Dielectric Studies of Fluids with Reentrant Resonators. Appendix B.

A. R. H. Goodwin, and M. R. Moldover. 1993, 10p, CONF-9305134-4-APP.B

Contract Al05-88ER13823

Symposium on Energy Engineering Sciences (11th) Argonne, IL (United States), 3-5 May 1993. Sponsored by Department of Energy, Washington, DC

Keywords: "Dielectric Properties, Mixtures, Calibration Cavity Resonators, Fluids, Fluorine Compounds, Measuring Methods, Phase Transformations, RF Systems, Temperature Dependence, "Refrigerants, EDB/ 420400.

We have used a reentrant radio-frequency $(i f)$ cavity as a resonator operating near $375 \mathrm{MHz}$ to measure changes in the dielectric constant of fluids within it. The utility of these measurements was demonstrated by determining the dipole moment of $1,1,1,2,5,3$ hexafluoropropane, a candidate replacement refrigerant (denoted R236ea) and by detecting the phase boundaries in the mixture $((1-x) \mathrm{C}$ (sub 2) $\mathrm{H}$ (sub 6) + $x C O($ sub 2)), for the mole fraction $x=0.492$. The densities of the coexisting phases of the mixture, were deermined using the Clausius-Mossotti relation which has errors on the order of $0.5 \%$ in this application. To test the accuracy of the present techniques, the if resonator was calibrated with helium and then used to redetermine the molar polarizability $A($ sub $c$ ) of argon. The results were in excellent agreement with published values. Our design of the recent resonator makes it suitable for use with corrosive fluids at temperatures up to 400 (degrees)C.

\section{3,245}

DE94004236 PC A03/MF A01

National Inst. of Standards and Technology, Boulder CO. Thermodynamics Div.

Thermophysical Properties of HFC-143a and HFC 152a. Quarterly Report, 1 July 1993-30 September 1993.

Progress rept.

W. M. Haynes. Oct 93, 27p, DOE/CE/23810-22A

Contract FG02-91CE23810

Sponsored by Department of Energy, Washington, DC

Keywords: "Freons, "Refrigerants, Compiled Data Data Compilation, Density, Equations of State, Physical Properties, Progress Report, Specific Heat, Thermodynamic Properties, Vapor Pressure, Volume Tables(data), *Thermophysical properties, EDB 360606 .

Numerous fluids have been identified as promising alernative refrigerants, but much of the information needed to predict their behavior as pure fluids and as components in mixtures does not exist. In particular, reliable thermophysical properties data and models are needed to predict the performance of the new refrigerants in heating and cooling equipment and to design and optimize equipment to be reliable and energy efficient. Objective of this project is to provide highly accurate, selected thermophysical properties data for refrigerants HFC-143a (CH(sub 3) CF(sub 3)) and HFC152a ( $\mathrm{CH}$ (sub 3) CHF(sub 2)) and to use these data to fit complex equations of state and detailed transport property models. The new data will fill gaps in the existing data sets and resolve problems and uncertainties that exist in and between the data sets.

03,246

DE94017738 PC A03/MF A01

National Inst. of Standards and Technology (NEL),

Gaithersburg, MD. Thermophysics Div.

Development of Measurement Capabilities for the Thermophysical Properties of Energy-Related Fluids. Annual Report, December 1, 1993--November 30, 1994.

17 Ǎug $93,24 \mathrm{p}, \mathrm{DOE} / \mathrm{ER} / 13823-\mathrm{T} 3$

Contract AI05-88ER13823

Sponsored by Department of Energy, Washington, DC

Keywords: "Refrigerants, "Measuring Instruments, Calorimeters, Densimeters, Dielectric Properties, Phase Studies, Progress Report, Solutions, Thermal Conductivity, Viscosimeters, "Thermophysical properties, EDB/440800.

Objectives are to develop state-of-the-art experimental apparatus for measuring the thermophysical properties of a wide range of fluids and fluid mixtures important to the energy chemical, and energy-related industries, and carry out benchmark measurements on key systems. Measurement capabilities to be developed cover transport properties, thermodynamic properties, phase equilibria properties, and dielectric properties. The new apparatus will make it possible to study a wide range of complex fluid systems under conditions that have been previously inaccessible. Speclfic measurement capabilities to be developed are: Thermal Conductivity Apparatus, Vibrating Wire Viscometer, Dual-Sinker Densimeter, High-Temperature Vibrating Tube Densimeter, Dynamic Phase Equilibria Apparatus, Apparatus for Dilute Solutions, Total-Enthalpy Flow Calorimeter, Dielectric Constant Apparatus. The research also includes benchmark experimental measurements on pure and mixed alternative refrigerants, aqueous solutions, and carefully selected systems consisting of species of diverse size (methane + neopentane) and polarity (methane + ammonia) important for development of predictive models for energy-related fluids. (ERA citation 19:030826)

\section{3,247}

\section{DE95002261 PC A02/MF A01}

National Inst. of Standards and Technology, Boulder,

Thermophysical properties of HCFC alternatives. Quarterly report, 1 July 1994--30 September 1994. Progress rept.

W. M. Haynes. Oct 94, 6p, DOE/CE/23810-48A Contract FG02-91CE23810

Sponsored by Department of Energy, Washington, DC Keywords: "Refrigerants, Performance, Progress Report, "Thermophysical properties, EDB/420400, EDB/ 320106

Numerous fluids and fluid mixtures have been identified as promising alternatives to the HCFC refrigerants, but, for many of them, reliable thermodynamic data do not exist. In particular, reliable thermodynamic properties data and models are needed to predict the performance of the new refrigerants in heating and cooling equipment and to design and optimize equipment to be reliable and energy efficient. The objective of this project is to measure, with high accuracy, selected thermodynamic properties data for two pure refrigerants and nine refrigerant blends; these data will be used to fit equations of state and other property models which can be used in equipment design. The new data will fill in gaps in the existing data and resolve problems and differences that exist in and between existing data sets. Most of the studied fluids and blends are potential replacements for HCFC-22 and/or R502; in addition, one pure fluid and one blend are potential replacements for CFC-13 in low temperature refrigeration ap plications.

\section{3,248}

DE 95011238 PC 3 MF AO

National Inst. of Standards and Technology (BFRL) Gaithersburg, MD.
Lean flammability limit as a fundamental refrigerant property. Phase 1, Interim technical report 1 October 1994--31 March 1995.

C. Womeldorf, M. King, and W. Grosshandler. 31 Mar 95, 30p, DOE/CE/23810-58.

Contract FG02-91CE23810

Sponsored by Department of Energy, Washington, DC.

Keywords: *Refrigerants, Chlorofluorocarbons, Flammability, Testing, EDB/320106.

Due to the ozone-depleting effects of commonly used chlorofluorocarbon refrigerants, safe environmentally friendly replacements must be found. HFC-32 (CH(sub 2) $F($ sub 2)) and other hydrochlorofluorocarbons are potential candidates; however, in contrast with the CFCs, many of these compounds are flammable. Test ing the flammability limits of these hydrochlorofluorocarbons using traditional ASTM E 681 methods has produced a range of limits depending upon the vessel and ignition source used. This project demonstrates the feasibility of defining a fundamenta flammability limit of HFC-32, that occurs at the limit of a zero strain rate and is independent of ignition source. Using a counterflow twin-flame burner to define extinction points for different strain rates, an extrapolation to zero strain rate is performed. Using this technique, pre32 and the critical flammability ratio of HFC-125 (C (sub 2) HF(sub 5)) in ETC-32 are reported.

\section{9}

DE96010433 PC A04/MF A01

National Inst. of Standards and Technology (CSTL), Boulder, CO. Thermophysics Div.

Thermophysical properties of HCFC alternatives. Quarterly report, October 1--December 31, 1995.

PROGRESS REPT

W. M. Haynes. Jan 96, 32p, DOE/CE/23810-66A. Contract FG02-91CE23810

Sponsored by Department of Energy, Washington, DC

Keywords: "Refrigerants, Air Conditioners, Chemica Composition, Chlorofluorocarbons, Density, Experimental Data, Fluorinated Aliphatic Hydrocarbons, Liquids, Material Substitution, Measuring Instruments, Progress Report, Refrigerating Machinery, Thermodynamic Model, Thermodynamic Properties, Vapo Pressure, Vapors, Tables(data), EDB/400201, EDB 320106, EDB/320302

Numerous fluids and fluid mixtures have been identified as promising alternatives to the HCFC refrigerants, but, for many of them, reliable thermodynamic data do not exist. In particular, reliable thermodynamic prop erties data and models are needed to predict the performance of the new refrigerants in heating and cooling equipment and to design and optimize equipment to be reliable and energy efficient. The objective of this project is to measure, with high accuracy, selected thermodynamic properties data for two pure refrig erants and nine refrigerant blends; these data will be used to fit equations of state and other property models which can be used in equipment design. The new data will fill in gaps in the existing data and resolve problems and differences that exist in and between existing data sets. Most of the studied fluids and blends are potential replacements for HCFC-22 and/or R502; in addition, one pure fluid and one biend are potential replacements for CFC-13 in low temperature refrigeration applications.

\section{3,250}

DE96010579 PC A05/MF A01

National Inst. of Standards and Technology (CSTL), Boulder, CO. Thermophysics Div.

Thermophysical properties of HCFC alternatives. Quarterly report, April 1--June 30, 1995

PROGRESS REPT

W. M. Haynes. Jul 95, 68p, DOE/CE/23810-61A Contract FG02-91CE23810

Sponsored by Department of Energy, Washington, DC

Keywords: "Refrigerants, Air Conditioners, Chlorofluorocarbons, Compiled Data, Density, Equilibrium, Experimental Data, Liquids Material Substitution, Mixtures, Progress Report, Refrigerating Machinery, Sound Waves, Specific Heat, Thermodynamic Model, Thermodynamic Properties, Vapor Pressure Vapors, Velocity, Tables(data), EDB/400201, EDB/ 320106, EDB/320302.

Numerous fluids and fluid mixtures have been identified as promising alternatives to the HCFC refrigerants, but, for many of them, reliable thermodynamic data do not exist. In particular, reliable thermodynamic prop- 


\title{
MATERIALS SCIENCES
}

\author{
Miscellaneous Materials
}

erties data and models are needed to predict the performance of the new refrigerants in heating and cooling equipment and to design and optimize equipment to be reliable and energy efficient. The objective of this project is to measure, with high accuracy, selected thermodynamic properties data for two pure refrigerants and nine refrigerant blends; these data will be used to fit equations of state and other property models which can be used in equipment design. The new data will fill in gaps in the existing data and resolve problems and differences that exist in and between existing data sets. Most of the studied fluids and blends a re potential replacements for HCFC-22 and/or R502; in addition, one pure fluid and one blend are potential replacements for $\mathrm{CFC}-13$ in low temperature refrigeration applications.

\section{3,251}

PB94-172426 Not available NTIS

National inst. of Standards and Technology (BFRL), Gaithersburg, MD. Building Environment Div.

Simultaneous Visual and Calorimetric Measurements of R11, R123, and R123/Alkylbenzene Nucleate Flow Boiling.

Final rept.

M. A. Kedzierski. 1993, 7p.

Pub. in Proceedings of NHTC Session on Alternative Refrigerants in Heat Transfer Equipment, Atlanta, GA. August 8-13, 1993, 7p. See also PB94-120756. Sponsored by Department of Energy, Washington, DC.

Keywords: "Refrigerants, "Nucleate boiling, "Environmental chemical substitutes, "Air pollution abatement, Mixtures, Performance standards, Lubricants, Freons, Chlorohydrocarbons, Fluorohydrocarbons, Benzenes, Heat measurement, Visual inspection, Reprints, Methane/trichlorofluoro, Ethane/dichloro-trifluoro, R11, R123.

Bubble formation during horizontal flow boiling of trichloromethane (R11), 1,1-dichloro-2,2,2trifluoroethane (R123) and fwo R123/alkylbenzene lubricant mixtures was investigated both visually and calorimetrically. Locally measured heat transfer coefficients were taken simultaneously with high speed motion picture images of the boiling process. The addition of a small amount $(0.55 \%)$ of alkylbenzene to R123 increases the number of active nucleation sites by approximately 5 sites/sq $\mathrm{cm}$ which corresponds to a $12 \%$ to $50 \%$ increase in the site density. The increase in the site density contributed to the enhancement of the heat transfer coefficient of the R $123 / 0.55 \%$ alkylbenzene mixture over that of the pure 123. Further increase in the amount of alkylbenzene to the R123 reduces the number of active sites to below that of pure R123 to approximately the value for that of R11. Consequently, the $0.55 \%$ lubricant mass fraction mixture than that of the $2 \%$ lubricant mass fraction mixture.

\section{3,252}

PB94-199775 Not available NTIS

National Inst. of Standards and Technology (NEL), Gaithersburg, MD. Building Environment Div.

Role of Refrigerant Mixtures as Alternatives to CFCs.

Final rept.

D. A. Didion, and D. B. Bivens. 1990, 13p.

Pub. in International Jnl. of Refrigeration 13, n3 p163. 175 May 90. See also PB92-170836.

Keywords: "Refrigerants, "Working fluids, *Azeotropes, Heat transfer fluids, Cooling systems, Refrigeration, Solutions(Mixtures), Air pollution abatement, Mixtures, Reprints, "Near-azeotropes, "Zeotropes.

A review of the state-of-the-art of refrigerant mixtures is presented. The categories of azeotropes, nearazeotropes and zeotropes are discussed separately as to their advantages as a refrigerant system working fluid and as to their requirements for adaptation. It may be possible to find azeotropes and near-zeotropes which could be candidates for alternatives for existing systems as well as new systems with little or no change in system performance. Zeotropes are not applicable for existing systems but offer the potential for significant performance improvements if the new systems are redesigned so as to incorporate the zeotropic attributes of two phase flow temperature glide and variable composition. Whereas, the azeotropes and nearazeotropes development are likely to have impact on the immediate ozone depletion crisis, the development will take longer but will be necessary for the greenhouse warming crisis.
03,253

PB94-199783 Not available NTIS

National Inst. of Standards and Technology (NEL), Gaithersburg, MD. Building Environment Div.

Role of R22 in Refrigerating and Air Conditioning Equipment.

Final rept.

D. A. Didion, R. Cohen, and D. R. Tree. 1990, 27p.

Pub. in Proceedings of the International Colloquium o Brussels 'Refrigeration and CFCs', p128-154 1990.

Keywords: "Refrigerants, "Refrigerating machinery, "Air conditioning equipment "Chlorofluorocarbons, Reprints, Heat transfer fluids, Economic analysis, Working fluids, Ozone depletion, Air pollution abatement, "R 22, Hydrofluorocarbons.

Refrigerant 22 is shown to be, like many other HCFC refrigerants, one of depletion potential, as a class, HCFC refrigerants, especially R22, are part of the interim solutions for the substitution of CFC refrigerants. Until refrigerants with no ozone depletion potential are developed and equipment designed and produced in quantity to use these refrigerants, the HCFC refrigerants will remain an important and vital refrigerant class. Except in a few applications, other factors such as global warming potential and efficiency, toxicity, safety, materials compatibility, and commercial availability of the refrigerant and of the refrigerating and air conditioning equipment make immediate replacement of R22 and all other HCFC refrigerants impossible. For some applications commitment of major capital investments and log lead times for design and production of new machinery systems using existing atmospherically benign alternatives are needed. Other applications require research for new refrigerants without ozone depletion potential, a process without a guarantee of success. The immediate replacement of R22 systems is not a viable choice.

\section{3,254}

PB94-199791 Not available NTIS

National Inst. of Standards and Technology (CSTL), Boulder, CO. Thermophysics Div.

Measurements of the Viscosities of Saturated and Compressed Fluid 1-chloro-1,2,2,2 tetrafluoroethane (R124) and Pentafluoroethane (R125) at Temperatures between 120 and $420 \mathrm{~K}$.

Final rept.

D. E. Diller, and S. M. Peterson. 1993, 12p.

Contract DE-A101-91CE23808

Pub. in International Jnl. of Thermophysics 14, n1 p55 66 Jan 93. Sponsored by Department of Energy, Washington, DC.

Keywords: "Viscosity, "Refrigerants, "Fluids, "Compressible fluids, "Saturation(Chemistry), Temperature dependence, Fluorohydrocarbons, Pressure, pentafluoro, Molar volume, R125, R124.

The shear viscosities of saturated and compressed fluid 1-chloro-1,2,2,-tetrafluoroethane (R124) and pentafluoroethane (R125) have been measured with two torsional crystal viscometers at temperatures be tween 120 and $420 \mathrm{~K}$ and at pressures up to $50 \mathrm{MPa}$. At small molar volumes, the fluidity (reciprocal viscos ity) increases linearly with molar volume at fixed temperature and weakly with temperature at fixed volume We have described this behavior with simple empirica equations and have compared the data of Shankland and of Ripple with them. The data of Ripple are in good agreement with our data for both fluids.

\section{3,255}

PB94-199890 Not available NTIS

National Inst. of Standards and Technology (NEL), Gaithersburg, MD. Building Environment Div.

Simplified Cycle Simulation Model for the Performance Rating of Refrigerants and Refrigerant Mixtures.

Final rept.

P. A. Domanski, and M. O. McLinden. 1993, 8p. Pub. in International Jnl. of Refrigeration 15, n2 p81 88 1992. See also PB93-130383. Sponsored by Envi ronmental Protection Agency, Washington, DC. and Electric Power Research Inst., Palo Alto, CA.

Keywords: "Refrigerants, "Thermodynamic properties, *Models "Computerized simulation Equations of state, Mixtures, Computer programs, Transport prop erties, Heat pumps, Refrigerators, Vapor compression refrigeration cycle, Reprints.

A simulation program, CYCLE11, which is useful for the preliminary evaluation of the performance of refrig- erants and refrigerant mixtures in the vapor compression cycle is described. The program simulates a theoretical vapor-compression cycle and departures from the theoretical cycle as occur in a heat pump and in a refrigerator. The cycles are prescribed in terms of the temperatures of the external heat transfer fluids with the heat exchangers generalized by their average effective temperature differences. The isenthalpic expansion process is assumed. The program includes a rudimentary model of a compressor and a representation of the suction line and liquid line heat exchange. Refrigerant thermodynamic properties are calculated using the Carnahan-Starling-DeSantes equation-ofstate. Refrigerant transport properties are not included in the simulations. The program can generate merit ratings of refrigerants for which limited measurement data are available. An example of simulation results stresses the need for careful application of simplified models and consideration for the involved assumptions.

\section{3,256}

PB94-212081 Not available NTIS

National Inst. of Standards and Technology (CSTL), Boulder, CO. Thermophysics Div.

Equation of State Formulation of the Thermodynamic Properties of R134a $(1,1,1,2$ Tetrafluoroethane).

Final rept.

M. L. Huber, and J. F. Ely. 1992, 8p.

Pub. in International Jnl. of Refrigeration 15, n6 p3934001992

Keywords: "Thermodynamic properties, "Refrigerants, Specific heat, Volume, Mathematical models, Equations of state, Vapor pressure, Density, Temperature, Reprints, "R134a, "Tetrafluoroethane.

New correlations for the thermodynamic properties of R134a are presented. A classical equation for the molar Helmholtz energy is used with temperature and density as the independent variables. The equation is accurate for both the liquid and vapor phases at pressures up to $70 \mathrm{MPa}$, and for a temperature range from the triple point to $450 \mathrm{~K}$. Temperatures a re given on the new International Temperature Scale of 1990 (ITS $90)$. The equation was developed by using experimental data for pressure-volume-temperature propenties, isochoric heat capacity, second virial coefficients, speed of sound and coexistence properties. Comparisons with experimental data and with two other equations of state are given. Ancillary equations representing the saturated liquid and vapor densities and the vapor pressure are also presented.

\section{3,257}

PB94-212099 Not available NTIS

National Inst. of Standards and Technology (CSTL), Boulder, CO. Thermophysics Div.

Prediction of Viscosity of Refrigerants and Refrigerant Mixtures.

Final rept.

M. L. Huber, and J. F. Ely. 1992, 10p.

Sponsored by Department of Energy, Washington, DC. Pub. in Fluid Phase Equilibria 80, p239-248 1992.

Keywords: "Viscosity, "Refrigerants, Mixtures, Equations of state, Thermodynamic properties, Mathematical models, Mass, Boundary conditions, Reprints, R134a, Tetrafluoroethane.

We have developed a predictive corresponding states model for the viscosity of pure refrigerants and refrigerant mixtures. The model uses extended corresponding states with R134a (1,1,1,2-tetrafluoroethane) as the reference fluid. The model uses equilibrium shape factors and an equivalent substance reducing ratio (ESRR) for the viscosity which involves the molecular mass. The 'mass' ESRR is found using viscosity data along the saturation boundary. We have fit these mass ESRR's to a general form involving reduced temperature and a structural factor. In addition, we have determined a set of universal coefficients for the mass ESRR that can be used to predict the viscosity of any halocarbon refrigerant given the critical parameters and the structural parameter. We give sample results comparing the model predictions with experimental data for pure fluids and a refrigerant mixture.

\section{3,258}

PB94-212107 Not available NTIS

National inst. of Standards and Technology (CSTL), Boulder, CO. Thermophysics Div. 
Prediction of the Thermal Conductivity of Refrigerants and Refrigerant Mixtures.

Final rept.

M. L. Huber, D. G. Friend, and J. F. Ely. 1992, 13p. Sponsored by Department of Energy, Washington, DC. Pub. in Fluid Phase Equilibria 80, p249-261 1992.

Keywords: "Refrigerants, "Mixtures, "Thermal conductivity, Thermodynamic properties, Working fluids, Mathematical models, Uncertainty, Reprints, R134a, Tetrafluoroethane.

We use an extended corresponding states model to predict the thermal conductivity of pure halocarbon refrigerants and refrigerant mixtures. The model uses R134a (1,1,1,2-tetrafluoroethane) as the reference fluid, and we present a correlation, including critical enhancement, for the thermal conductivity of R134a. We give sample results comparing the model predictions with experimental data for pure halocarbon refrigerants and refrigerant mixtures; typically, the uncertainty of the predictions is 5-10 percent.

\section{3,259}

PB94-212701 Not available NTIS

National Inst. of Standards and Technology (BFRL) Gaithersburg, MD. Building Environment Div.

Causes of the Apparent Heat Transfer Degradation for Refrigerant Mixtures.

Final rept.

M. A. Kedzierski, J. H. Kim, and D. A. Didion. 1992 10p.

Sponsored by Electric Power Research Inst, Palo Alto, $\mathrm{CA}$. and Department of Energy, Washington, DC.

Pub. in Proceedings of National Heat Transfer Conference and Exhibition (28th), San Diego, CA., August 9-12, 1992, p149-158.

Keywords: "Thermal degradation, "Refrigerants, "Heat transfer coefficients, Mixtures, Evaporation, Hea transfer, Working fluids, Mass transfer, Nucleate boiling, Reprints.

This paper presents an investigation into the causes of the apparent heat transfer degradation associated with honzontal-annular flow evaporation of refrigerant mixtures. The apparent heat transfer degradation is the difference between the measured heat transfer coefficient and the heat transfer coefficient that would be obtained from a linear interpolation of the single component values. The degradation is apparent since the linearly interpolated values have no physical basis. For horizontal-annular flow evaporation, most of the hea transfer degradation is a consequence of the use of the locally uniform equilibrium temperature in the measurement and calculation of the heat transfer coefficient. In reality, both circumferential and radial composition gradients can exist within the liquid film which cause temperature distributions that deviate significantly from a uniform saturation temperature. If the actual liquid-vapor interface temperatures (local vapor temperatures) were used in the calculation of the measured heat transfer coefficient for the impose hea flux condition, most of the apparent degradation would not exist. The remainder of the heat transfer degradation is due to nonlinear mixture property effects. Previously published measured heat transfer coefficients for three mixtures were investigated.

\section{3,260}

PB94-219375 (Order as PB94-219326, PC A05/ MF A02

National Inst. of Standards and Technology, Boulder, CO.

Refractive Indices of Fluids Related to Alternative Refrigerants.

T. J. Bruno, M. A. Wood, and B. N. Hansen. 1994, $4 \mathrm{p}$

Included in Jnl. of Research of the National Institute of Standards and Technology, v99 n3 p263-266 May/ Jun 94 .

Keywords: "Refrigerants, "Refractivity, Ethane, Ethylene, Ethers, Propane, Halohydrocarbons, Propyne.

As part of a comprehensive program to develop suitable methods of chemical analysis for alternative refrigerants and their products, the authors have compiled a database of spectral, chromatographic, and physical property data that can aid in compound identification. As a small part of this effort, they have measured the refractive indices of a number of such fluids for which data were unavailable. The measurements were performed on a commercially available, digital Abbe refractometer that was modified for the relatively low temperature measurements ( 0 deg $\mathrm{C}$ to $20 \mathrm{deg} \mathrm{C}$ ) that are sometimes required with the se samples.

\section{3,261}

PB95-107298 Not available NTIS

National Inst. of Standards and Technology (CSTL), Gaithersburg, MD. Process Measurements Div. Resistance Thermometers with Fast Response for Use in Rapidly Oscillating Gas Flows.

Final rept.

W. Rawlins, K. D. Timmerhaus, and R. Radebaugh 1992, 4p.

Pub. in Temperature - Its Measurement and Contro in Science and Industry, v6 p471-474 1992.

Keywords: "Thermometers, "Oscillating flow "Cryogenic cooling, "Refrigerators, "Working fluids, Temperature measurement, Effectiveness, Coolers, Gas flow, Refrigerating machinery, Reprints.

The paper describes a resistance thermometer that permits instantaneous measurement of gas temperatures with the use of a 4 micrometer diameter fungsten wire mounted on heavier wire supports. This configuration provides sufficient strength to survive oscillating flows of helium gas up $1030 \mathrm{~Hz}$ at pressures up to 2.2 $\mathrm{MPa}$. The probes are small enough to fit perpendicular to the flow in $3 \mathrm{~mm}$ diameter stainless steel tubes. Cus tom fittings designed to support the resistance thermometers introduce very little void volume to the system. Response time of the probes is approximately 260 microseconds, which permits resolution of temperature oscillations up to $30 \mathrm{~Hz}$ with negligible phase shifts. A very nearly linear response for these resistance thermometers is obtained from $80 \mathrm{~K}$ to $400 \mathrm{~K}$. Sensitivity of these thermometers decreases rapidly below about $60 \mathrm{~K}$. Results of dynamic temperature measurements obtained at the cold end of a pulse tube refrigerator will be shown.

\section{3,262}

\section{PB95-143301 PC A05/MF A02}

Gational Inst. of Standards and Technology

Gaishersburg, MD. Bulding EnvionmentDiv. cleate Flow Boiling.

M. A. Kedzierski, J. M. Crowder, A. M. Jacobi, and L. W. Zhang. Oct $94,99 p$, NISTIR-5519.

See also PB93-120756.

Keywords: "Refrigerants, "Nucleate boiling, "Flow visualization, "Image analysis, Heat flux, Computer programs, Flow rate, Heat transfer, Bubbles, Vaporization, Visual measurement.

This report provides a record of a visual measurement technique for the analysis of the bubbles formed by nucleate flow boiling. The purpose of measuring these bubbles is 10 extend the understanding of the boiling process. By studying the behavior of these vapor bubbles, a better understanding of the physics controlling boiling heat transfer will be gained. The boiling of refrigerants is simultaneously filmed with a high speed $16 \mathrm{~mm}$ camera while heat transfer measurements are taken. These tests are performed over a range of hea fluxes and flow velocities in order to study the effect of these conditions on the bubble dynamics. The analysis of the $16 \mathrm{~mm}$ films evolved from a manual to a computer assisted system, and finally to a digital image analysis system. The following is an overview of the filming apparatus and the bubble measurement schemes.

\section{3,263}

PB95-168688 Not available NTIS

National Inst. of Standards and Technology (CSTL) Boulder, CO. Thermophysics Div.

Reference Data for the Thermophysical Properties of Cryogenic Fluids.

Final rept.

W. M. Haynes, and D. G. Friend. 1994, 10p.

Pub. in Advances in Cryogenic Engineering, v39 p1865-1874 1994

Keywords: "Cryogenic fluids, "Refrigerants, "Thermophysical properties, "Reference materials, Thermodynamic properties, Pressure Temperature Volume, Specific heat, Thermal conductivity, Equations of state, Transport properties, Technology trans fer, Reprints, "Standard reference materials, US NIST, Phase equilibria, Sound speed profile.

The Thermophysics Division of the National Institute of Standards and Technology has long been involved in providing standard reference data for the thermophysical properties of cryogenic fluids. Comprehensive experimental facilities have been used to provide accurate data for Pressure-Vapor-Tempera- ture (PVT) relations, heat capacities, sound speeds, phase equilibria, and transport properties for a large variety of well characterized cryogenic fluid systems. This critically evaluated information on the thermophysical properties of fluids and fluid mixtures of cryogenic interest is used for custody transfer applications and for efficient design and operation of cryogenic processes in the chemical, natural gas, aerospace, environmental, refrigeration, and other energy related industries.

03,264

PB95-168878 Not available NTIS

National Inst. of Standards and Technology (CSTL) Boulder, CO. Thermophysics Div.

Measurements of Molar Heat Capacity at Constant Volume (Cv) for 1,1,1,2-Tetrafluoroethane (R134a). Final rept.

J. W. Magee. 1992, 9p.

Pub. in International Jnl. of Refrigeration 15, n6 p372 3801992

Keywords: "Specific heat, "Fluorohydrocarbons, "Refrigerants, Calorimetry Temperature dependence, Pressure dependence, Triple point, Phase transformations, Thermodynamic properties, Liquid phases, Vapor phases, Reprints, "R134a, "1,1,1,2 Tetrafluoroethane

The molar heat capacity at constant volume was measured by applying an adiabatic method. Heat capacities at 310 state conditions were measured. Measurements were made on liquid in equilibrium with its vapor and on compressed liquid samples of 1,1,1,2tetrafluoroethane (R134a), at temperatures from 172 to $343 \mathrm{~K}$ with pressures as high as $35 \mathrm{MPa}$. In the course of these measurements, a determination of the triple point temperature $(169.85+$ or $-0.01 \mathrm{~K})$ was made. For the high purity $(0.9999+)$ sample, results were obtained for two-phase, saturated liquid and single phase molar heat capacities as a function of measured temperature, pressure and density. The maximum probable uncertainty of the heat capacity values is estimated not to exceed + or $-0.5 \%$

03,265

PB95-168886 Not available NTIS

National Inst. of Standards and Technology (CSTL), Boulder, CO. Thermophysics Div.

Vapour Pressure Measurements on 1,1,1,2Tetrafluoroethane (R134a) from 180 to $350 \mathrm{~K}$.

Final rept.

J. W. Magee, and J. B. Howley. 1992, 3p

Pub. in International Jnl. of Refrigeration 15, n6 p362 3641992.

Keywords: "Vapor pressure, "Fluorohydrocarbons, "Refrigerants, Thermodynamic properties, Temperature dependence,

Measurements of vapor pressures were made for 11,1 2-tetrafluoroethane (R134a) from 180 to $350 \mathrm{~K}$ by using a static cell. Temperatures were measured with a platinum resistance thermometer to within an accuracy of + or $-0.03 \mathrm{~K}$. Pressures were measured with calibrated oscillating quartz-crystal pressure transducers with uncertainties estimated to range from 0.02 to $1.8 \mathrm{kPa}$.

\section{3,266}

PB95-168936 Not available NTIS

National Inst. of Standards and Technology (CSTL), Boulder, CO. Thermophysics Div.

Annex 18: An International Study of Refrigerant Properties.

Final rept.

M. O. McLinden. 1994, 4p.

Pub. in IEA Heat Pump Centre Newsletter 12, n1 p2831 Mar 94.

Keywords: "Fluorohydrocarbons, "Refrigerants, "Environmental chemical substitutes, "Thermophysical properties, Thermodynamic properties, Transport properties, Research programs, International cooperation, Alternatives, Reprints, "Hydrochlorofluorocarbons, Halons, Hydrofluorocarbons.

The article highlights the activities of Annex 18 'Thermophysical Properties of the Environmentally Accpetalbe Refrigerants', which was established in 1990. The objectives of the Annex are to foster coordination and cooperation among researchers in this field and to determine thermophysical properties leading to internationally accepted formulations for the leading al- 
ternative refrigerants. Tasks in support of these goals have included surveys of research, compilations of data, evaluations of equations of state, and the preparation of properties bulletins for HFC-134a and HCFC. 123.

\section{3,267}

PB95-168951 Not available NTIS

National Inst. of Standards and Technology (CSTL),

Boulder, CO. Thermophysics Div.

Development of a Dual-Sinker Densimeter for HighAccuracy Fluid P-V-T Measurements.

Final rept

M. O. McLinden, and N. V. Frederick. 1993, 16p.

Sponsored by Department of Energy, Washington, DC. neering Sciences (11th), Argonne, IL., May 3-5, 1993 p63-69 1994

Keywords: "Refrigerants, "Thermophysical properties *Fluorohydrocarbons, *Environmental chemical substitutes, Thermodynamic properties, Transport properties, Boiling points, Specific heat, Thermal conductiv ity, Equations of state, Vapor pressure, Triple point, prints, "Hydrofluorocarbons, Phase equilibria, Sound speed profile.

The status and available data for several of the leading 'new' or alternative refrigerants are summarized. The data considered include triple point, normal boiling point, and critical point parameters; the temperature dependence of the vapor pressure, ideal gas heat capacity, and saturated liquid density. Also considered are single phase Pressure-Vapor-Temperature ( $p-V$ $T)$, heat capacity, sound speed, viscosity, and thermal conductivity data. The fluids of interest are the HFCs $23,32,125,134 a, 134,143 a$, and 152a

\subsection{8}

PB95-168969 Not available NTIS

National Inst. of Standards and Technology (CSTL),

Boulder, CO. Thermophysics Div.

Need for, and Availability of, Working Fluid Property Data: Results from Annexes XIII and XVIII.

Final rept.

M. McLinden, and L. Vamling. 1993, 12p.

Pub. in Heat Pumps for Energy Efficiency and Environmental Progress, p115-126 1993.

Keywords: "Working fluids, "Thermodynamic properties, *Transport properties, Heat pumps, Equations of state, Refrigerants, Sensitivity analysis, Accuracy, Reprints, R134a, R123.

In these days of transition to new, more environmentally friendly working fluids, there is a definite need for good data and models of basic thermodynamic and transport properties of both pure compounds and mixtures to be used for cycle calculations and equipmen redesign. Two annexes of the IEA Program on Advanced Heat Pumps have dealt with such data, namely Annex XIII--State and Transport Properties of High Temperature Working Fluids and Nonazeotropic Mix tures and Annex XVIII--Thermophysical Properties of the Environmentally Acceptabie Refrigerants. In this paper, highlights of these two annexes are presented. A sensitivity analysis (from Annex XIII) gives an estimate of how accurately these basic data need to be known to reach a certain accuracy in heat pump coefficient of performance (COP) and heating or cooling ca pacity calculations. This analysis points out the importance of measured property data. Other projects to be highlighted are a comparison of equations of state with experimental density, vapor pressure, and heat capacity data for mixtures (XIII), a survey of current research on the properties of the 'new' refrigerants (XVIII), and an evaluation of equations of state for R134a and R123 which will lead to an international bulletin for the properties of these two alternative refrigerants (XVIII).

\subsection{9}

PB95-169058 Not available NTIS

National inst. of Standards and Technology (CSTL), Boulder, CO. Thermophysics Div.

Measurement of the Thermal Properties of Elec. trically Conducting Fluids Using Coated Transient Hot Wires.

Final rept.

R. A. Perkins. $1994,8 p$

Sponsored by Department of Energy, Washington, DC. Pub. in Proceedings of the Symposium on Energy Engineering Scien.

Keywords: *Thermal

"Fluorohydrocarbons, Thermal
Supercritical fluids, Refrigerants, Temperature dependence, Thermophysical properties, Reprints, "R134a, "Electrically conducting fluids, Transient hot wire technique.

Measurements of fluid thermal properties using the transient hot-wire technique are described. When bare hot wires are used in electrically conducting fluids, there are additional measurement uncertainties due to the formation of electric double layers on the surfaces of the wires and the cell wall. If the electrical conductivity of the fluid is large enough, there is also significant power generation in the fluid. These measurement uncertainties can be eliminated by electrically insulating the hot wires with a thin film. The use of tantalum hot wires with an anodized layer of tantalum pentoxide is demonstrated with measurements on nonpolar argon and polar 1,1,1,2-tetrafluoroethane (R134a). Although coated tantalum hot wires have been used previously in a transient mode to measure the thermal conductivity of liquids, this work is the first demonstration of the use of coated wires to measure thermal conductivity in the liquid, vapor, and supercritical gas phases.

\section{3,270}

PB95-169272 Not available NTIS

National Inst. of Standards and Technology (CSTL), Gaithersburg, MD. Thermophysics Div.

Measurements of the Vapor Pressures of Difluoromethane, Tetrafluoroethane, and Pentafluoroethane.

Final rept

L. A. Weber, and A. M. Silva. 1994, 5p

Pub. in Jnl. of Chemical and Engineering Data 39, n4 p808-812 Oct 94

Keywords: "Refrigerants, "Fluorohydrocarbons, *Thermophysical properties, Vapor pressure, Boiling points, Temperature measurement, Ebulliometry, Measuring methods, Reprints, "Difluoromethane, "Tetraftuoroethane, "Pentafluoroethane, R32, R124, R125.

The report presents new measurements of the vapor pressures of dibluoromethane (R32) from 235 to 265 $\mathrm{K}$, of 1-chloro-1,2,2,2-tetrafluoroethane (R124) from 220 to $286 \mathrm{~K}$, and of pentafluoroethane (R125) from 218 to $286 \mathrm{~K}$. Overall, pressures ranged from 13 to about $950 \mathrm{kPa}$. The report also presents vapor pressures of $\mathrm{R} 125$, calculated via thermodynamic relationships, for temperatures down to $170 \mathrm{~K}(2.3 \mathrm{kPa})$. The report also discusses the azeotropic mixture of R125 with chloropentafluoroethane (R115), and the authors correct their data for a small R115 impurity.

\section{3,271}

PB95-175154 Not available NTIS

National Inst. of Standards and Technology (CSTL), Gaithersburg, MD. Thermophysics Div

Vapor Pressures and Gas-Phase PVT Data for 1. Chloro-1,2,2,2-Tetrafluoroethane (R124).

Final rept.

S. J. Boyes, and L. A. Weber. 1994, 18p.

Contract DF-FG02-91CE23810

Sponsored by Department of Energy, Washington, DC. Pub. in International Jnl. of Thermophysics 15, n3 p443-460 May 94.

Keywords: "Fluorohydrocarbons, "Refrigerants, *Thermodynamic properties, Vapor pressure, Volume, Temperature, Gases, Vapor phases, Phase studies, Thermophysical properties, Reprints, Ethane/chlorotetraftuoro, Hydrofluorocarbons, R124, Virial coefficients.

The authors present new data for the vapor pressure and Pressure-Vapor-Temperature (PVT) surface of 1 chloro-1 2,22-tetrafluoroethane (designated R124 by the refrigeration industry) in the temperature range $278-423 \mathrm{~K}$. The PVT data are for the gas phase at densities up to 1.5 times the critical density. Correlating equations are given for the vapor pressures from 220 $\mathrm{K}$ to the critical temperature, $395.43 \mathrm{~K}$, and for the PVT surface at densities up to approximately 0.5 times the critical density. Second and third virial coefficients have been derived from the PVT measuremenis.

\section{3,272}

PB95-175196 Not available NTIS

National Inst. of Standards and Technology (CSTL), Boulder, CO. Thermophysics Div. $\begin{array}{lcc}\text { Retention of Halocarbons } & \text { on a } \\ \text { Hexafluoropropylene } & \text { Epoxide } & \text { Modified } \\ \text { Graphitized Carbon Black. Part 1. Methane-Based }\end{array}$ Graphitized Car

Compounds
Final rept.

T. J. Bruno, and M. Caciari. 1994, 10p.

Pub. in Jnl. of Chromatography A 672, p149-158 1994.

Keywords: "Refrigerants, "Chlorofluorocarbons, "Fluorocarbons, *Thermodynamic properties, Methane compounds, Halohydrocarbons, Chemical analysis, Gas chromatography, Temperature, Thermophysical properties, Carbon black, Environmental chemical substitutes, Reprints, *Halocarbons, Hexafluoropropylene, Tetrafluoromethane.

The retention characteristics of eight methane-based chlorofluorocarbon and fluorocarbon fluids have been studied as a function of temperature on a stationary phase consisting of a $5 \%(w / w)$ coating of a low-molecular-mass polymer of hexafluoropropylene epoxide on a graphitized carbon black. The fluids that were studied include chlorotrifluoromethane (R-13) tetrafluoromethane (R-14), dischlorofluoromethane ( $R$ 21), chlorodifluoromethane (R-22), trifluoromethane (R-23), difluoromethane (R-32), chloromethane (R-40), and fluoromethane (R-41). Qualitative features of the data are examined, and trends are identified. In addition, the data were fit to linear models for the purpose of predicting retention behavior of these compounds to facilitate chromatographic analysis

\section{3,273}

PB95-175386 Not available NTIS

National Inst. of Standards and Technology (CSTL), Boulder, CO. Thermophysics Div.

Measurements of the Viscosities of Saturated and Compressed Liquid 1,1,1,2-Tetrafluoroethane (R134a), 2,2-Dichloro-1,1,1-Trifluoroethane (R123) and 1,1-Dichloro-1-Fluoroethane (R141b).

Final rept.

D. E. Diller, A. S. Aragon, and A. Laesecke. 1993, $12 \mathrm{p}$.

Pub. in Fluid Phase Equilibria 88, p251-262 1993

Keywords: "Compressible fluids, "Refrigerants, "Viscosity, "Saturation(Chemistry), Fluorohydrocarbons, Temperature dependence, Shear flow, Volume, Reprints, “Ethane/tetrafluoro, "Ethane/ dichloro-difluoro, ${ }^{\star}$ Ethane/dichloro-fluoro, Ri34a, $\mathrm{R} 123, \mathrm{R} 141 \mathrm{~b}$, Molar volume.

The shear viscosity coefficients of saturated and compressed liquid 1,1,1,2-tetrafluoroethane (R134a), 2,2 dichloro-1,1,1-trifluoroethane (R123) and 1,1-dichloro1 -fluoroethane (R141b) have been measured with a torsional crystal viscometer at temperatures between 170 and $320 \mathrm{~K}$ and at pressures up to $30 \mathrm{MPa}$. The fluidities (reciprocal viscosity) increase linearly with molar volume at fixed temperature and increase weak ly with temperature at fixed volume. We have correlated our data using empirical fluidity-volume-temperature equations and have compared the data of others with the equations.

03,274

PB95-175691 Not available NTIS

National Inst. of Standards and Technology (CSTL) Boulder, CO. Thermophysics Div.

Coexisting Densities, Vapor Pressures and Critical Densities of Refrigerants R-32 and R-152a, at 300 $-385 \mathrm{~K}$.

Final rept.

C. D. Holcomb, V. G. Niesen, L. J. Van Poolen, and L Outcalt. $1993,13 p$

Contract DE-FG02-91CE23810

Sponsored by Department of Energy, Washington, DC Pub. in Fluid Phase Equilibria 91, p145-157 1993.

Keywords: *Refrigerants, "Critical point, "Vapor pres sure, "Thermodynamic $\quad$ properties, Equations of state, Phase studies, Temperature de pendence, Pressure dependence, Density(Mass $N$ olume), Reprints, "Difluoromethane, "Difluoroethane, R32, R152a, Hydrofluorocarbons, Coexisting density, Critical density.

Experimental measurement for the vapor pressures and coexisting densities are presented for the refrigerants R-32 (difluoromethane) and R-152a (1,1difluoroethane) from $300 \mathrm{~K}$ to near their respective critical points. In addition, the coexisting density measurements have been analyzed to determine an internally consistent critical density using the critical liquid volume fraction method. Experimental results have been 
correlated and are in good agreement with existing literature values for each compound.

\section{3,275}

PB95-175717 Not available NTIS

National Inst. of Standards and Technology (CSTL),

Boulder, CO. Thermophysics Div.

Predictive Extended Corresponding States Model for Pure and Mixed Refrigerants Including an Equa. tion of State for R134a.

Final rept.

M. L. Huber, and J. F. Ely. 1994, 14p.

Sponsored by Department of Energy, Washington, DC

Pub. in Rev. Int. Froid 17, n1 p18-311994.

Keywords: "Refrigerants, "Thermophysical properties, "Equations of state, "Fluorohydrocarbons, Thermodynamic properties, Saturation, Boundary conditions, Mixtures, Phase equilibria, Phase studies, Liquid phases, Vapor phases, Density(Mass/Nolume), Reprints, "R134a, "Tetrafluoroethane, Hydrofluorocarbons

The authors have developed a predictive corresponding states model for the thermophysical properties of pure refrigerants and refrigerant mixtures. The bulk phase properties such as the density, enthalpy and entropy are predicted using the principle of extended corresponding states, incorporating a recent 32-term modified Benedict-Webb-Rubin correlation for the $\mathrm{R} 134 \mathrm{a}(1,1,1,2$-tetrafluoroethane) reference fluid. In the work the authors present the coefficients for density-independent shape-factor correlations for 21 refrigerants, and a set of universal coefficients that can be used with any refrigerant given only the critical parameters and the acentric factor. The authors show comparisons with experimental density data for each of the 21 refrigerants. They also demonstrate the use of generalized coefficients for shape factors where only the critical parameters and acentric factors are known. In addition, they present comparisons of the volume prediction for two binary refrigerant mixtures and one ternary refrigerant mixture

\section{3,276}

PB95-176236 Not available NTIS

National Inst. of Standards and Technology (CSTL) Gaithersburg, MD. Thermophysics Div.

Estimating the Virial Coefficients of Small Polar Molecules.

Final rept

L. A. Weber. $1994,22 p$

Pub. in International Jnl. of Thermophysics $15, \mathrm{n} 3$ p461-482 May 94.

Keywords: "Refrigerants, "Thermophysical properties, Density, Thermodynamic properties, Estimating, Mathematical models, Evanescence, Halogenated hydrocarbons, Reprints, "Virial coefficients, "Fugacity coefficients, "Small polar molecules.

We adapt existing models for estimating the second and third virial coefficients of small molecules to the halogenated methanes and ethanes. We compare the results with the abundant new, high-quality PVT data resulting from the search for alternative refrigerants. The present model provides an accurate method for calculating densities, and therefore it should provide reliable thermodynamic properties and fugacity coefficients. We give equations and parameters useful for estimating the properties of pure refrigerants and their mixtures when no PVT data are available.

\section{3,277}

\section{PB96-117775 PC A99/MF E08}

National Inst. of Standards and Technology (BFRL), Gaithersburg, MD. Fire Science Div.

Fire Suppression System Performance of Alternative Agents in Aircraft Engine and Dry Bay Laboratory Simulations. SP890: Volume 1.

Special pub.

R. G. Gann. Nov 95, 792p, NIST/SP-890-V1.

Also available from Supt. of Docs. as SN003-00303371-5. See also PB96-117783. Sponsored by Wright Lab., Wright-Patterson AFB, OH., Naval Air Systems Command, Washington, DC, and Federal Aviation Administration Technical Center, Atlantic City, NJ.

Keywords: "Aircraft engines, "Fire suppression, "Environmental chemical substitutes, "Materials substitution, Halons, Alternatives, Aircraft fires, Engine nacelles, Inflight, Fire extinguishing agents, Fire fighting Performance evaluation, Chemical properties, Thermodynamic properties, Physical properties, Combus- tion kinetics, Fire tests, Fire research, Aviation safety, Dry bays.

This report documents the development of knowledge 1) to differentiate among chemicals, leading to selections of the optimal currently available options for in flight fire suppression, and (2) to assist in the development of engineering design criteria and suppression system certification.

\section{3,278}

PB96-117783 PC A99/MF AO6

National Inst. of Standards and Technology (BFRL), Gaithersburg, MD. Fire Science Div.

Fire Suppression System Performance of Alternative Agents in Aircraft Engine and Dry Bay Laboratory Simulations. SP 890: Volume 2.

Special pub.

R. G. Gann. Nov 95, 639p, NIST/SP-890-V2

Also available from Supt of Docs, as SNO03-003 03372-3. See also PB96-117775. Sponsored by Wright Lab., Wright-Patterson AFB, OH., Naval Air Systems Command, Washington, DC. and Federa Aviation Administration Technical Center, Atlantic City, NJ.

Keywords: "Aircraft engines, "Fire suppression, "Environmental chemical substitutes, "Materials substitution, Halons Alternatives, Aircraft fires Engine na celles, Inflight, Fire extinguishing agents, Fire fighting, Performance evaluation, Chemical properties, Thermodynamic properties, Physical properties, Combus tion kinetics, Fire tests, Fire research, Aviation safety, Dry bays.

This report documents the development of knowledge (1) to differentiate among chemicals, leading to selections of the optimal currently available options for inflight fire suppression, and (2) to assist in the develop ment of engineering design criteria and suppression system certification

\section{3,279}

P896-119573 Not available NTIS

National inst. of Standards and Technology (CSTL) Boulder, CO. Thermophysics Div.

Physical Properties of Alternatives to the Fully $\mathrm{Hal}$ ogenated Chlorofluorocarbons.

Final rept

M. O. McLinden $1989,24 \mathrm{p}$.

See also N92-15436/8

Pub. in Scientific Assessment of Stratospheric Ozone, Chapter 2, v2 p7-30 1989

Keywords: "Chlorofluorocarbons, "Physical properties *Thermophysical properties, Thermodynamic properties, Triple point, Boiling points, Temperature, Solubility, Vapor pressure, Critical point, Density(Mass/Nol. ume), Liquid phases, Hydrolysis, Reprints.

The properties of nine halogenated hydrocarbons are collected from a variety of sources, including unpublished data, evaluated and correlated. Considered are the triple point, normal boiling point, and critical point parameters and the temperature dependence of the vapor pressure, saturated liquid density, solubility in water and hydrolysis rates. The fluids, which are potential alternatives to the fully-halogenated chlorofluorocarbons, are R125, R22, R134a, R152a, $R 124, R 142 b, R 123$ and $R 141 b$; also included is the solvent menthyl choroform.

03,280

PB96-122114 (Order as PB96-117767, PC A08/

National Inst. of Standards and Technology, National Inst. of

Microform Calibration Uncertainties of Rockwell Diamond Indenters.

J. F. Song. F. F. Rudder, T. V. Vorburger, and J. H. Smith. 1995, 19p.

Included in Jnl. of Research of the National Institute of Standards and Technology, v100 n5 p543-561 Sep/ Oct 95.

Keywords: "Rockwell hardness, "Calibration stand ards, Reprints, Metals, Data covariances, Diamond pyramid hardness tests, Geometry, Mechanical tests, NIST(National Institute of Standards and Technology)

National and international comparisons in Rockwell hardness tests show significant differences. Uncertainties in the geometry of the Rockwell diamond indenters are largely responsible for these differences. By using a stylus instrument, with a series of calibration and check standards, and calibration and uncertainty cal- culation procedures, we have calibrated the microform geometric parameters of Rockwell diamond indenters These calibrations are traceable to fundamental standards. Under ISO and NIST guidelines for expressing measurement uncertainties, the calibration and uncertainty calculation procedures, error sources, and uncertainty components are described, and the expanded uncertainties are calculated.

03,281

PB96-146626 Not available NTIS

National Inst. of Standards and Technology (CSTL) Gaithersburg, MD. Thermophysics Div.

Interaction Coefficients for 15 Mixtures of Flammable and Non-Flammable Components.

Final rept

D. R. Defibaugh, and G. Morrison. 1995, 6p.

Pub. in International Jnl. of Refrigeration, v18 n8 p518 5231995

Keywords: "Refrigerants, "Thermodynamic properties, Environmental chemical substitutes, Alternatives, Equations of state, Interfacial tension, Pressure dependence, Temperature dependence, Ethanes, Propanes, Butanes, Liquid phases, Vapor phases, Flammability, Reprints, "Interaction coefficients.

Bubble pressures were measured for 15 binary mixtures, each composed mainly of one flammable and one non-flammable component. The mixtures were trichlorofluoromethane sopentane, pentafluoroethane $+1,1,1$-trifluoroethane, 1,1 dichloro-2,2,2-trifluoroethane + (1,1-dichloro-1fluoroethane or isopentane), 1,1,1,2-tetrafluoroethane $+(1,1$-difluoroethane or propane or cyclopropane or isobutane), difluoromethane + (pentafluoroethane or $1,1,1,2$-tetrafluoroethane or 1,1-difluoroethane). Also studied were mixtures of 1,1-difluoroethane + (cyclopropane or propane or butane or isobutane), which comprise two flammable components. The bubble pressures were used to determine interaction coefficients that characterize the non-ideal behavior of these fluid mixtures. The interaction coefficients are used in equation-of-state models for the thermodynamic properties of refrigerant mixtures.

03,282

PB96-159710 Not available NTIS

National Inst. of Standards and Technology (BFRL), Gaithersburg, MD. Fire Science Div.

Agent Screening for Halon 1301 Aviation Replace-

ment.

Final rept

W. Grosshandler, R. Gann, A. Hamins, J. Yang, M. Zachariah, M. Nyden, and W. Pitts. 1993, $9 p$. Pub. in International CFC and Halon Alternatives Conference, October 20-22, 1993, Washington, DC., 9p.

Keywords: "Environmental chemical substitutes, "Fire extinguishing agents, "Aircraft fires, Halons, Bicarbonates Halohydrocarbons Combustion kinetics, Two phase flow. Thermodynamic properties, Combustion chemistry, Fire suppression, Reprints, Halon 1301

A comprehensive experimental program is described in which eleven gaseous agents and sodium bicarbonate powder have been screened, so that the best three candidates for subsequent full scale air craft fire extinguishment evaluation can be identified. Because the effectiveness of a fire supression agent is known to be related to its thermodynamic properties, its behavior during two-phase flow, its interaction with flame chemistry, the timing of its release and the nature of the fire, a series of carefully designed experiments was conducted to examine each of these factors.

\section{3,283}

PB96-159728 Not available NTIS

National Inst. of Standards and Technology (BFRL) Gaithersburg, MD. Fire Science Div.

Validation of a Turbulent Spray Flame Facility for the Assessment of Halon Alternatives.

Final rept.

W. Grosshandler, C. Presser, and D. Lowe. 1993,

$8 p$.

Sponsored by Department of the Air Force, Wright-Patterson AFB, OH.

Pub. in Proceedings of the 'Halon Alternatives Technical Working Conference', Albuquerque, NM., May $12,1993,8 p$

Keywords: *Environmental chemical substitutes, "Fire extinguishing agents, "Aircraft fires, Material alternatives, Fire fighting, Fuel sprays, Turbulent flow, Fluorocarbons, Halohydrocarbons, Fire suppression, 


\section{MATERIALS SCIENCES}

\section{Miscellaneous Materials}

Flame propagation, Halons, Atomization, Reprints, Hydrochlorofluorocarbons.

The work discussed in this paper is part of a large effort at NIST focused on finding an alternative to halon 1301 for application to aircraft engine nacelle and dry bay in-flight fire protection. Alternative chemical compounds are sought which will perform similarly to halon 1301, and which do not create unacceptable safety, environmental, or systems compatibility problems. A cup burner, an opposed flow diffusion flame, a turbulent spray flame, and a deflagration/detonation tube are used to rank the relative combustion suppression effectiveness

\section{3,284}

PB96-164033 Not available NTIS

National Inst. of Standards and Technology (CSTL), Boulder, CO. Thermophysics Div.

Retention of Halocarbons on a Graphitized Carbon Black. 4. Propane-Based Compounds.

Final rept.

T. J. Bruno, K. H. Wertz, and M. Caciari. 1995, 10p.

See also Part 1, PB95-175196.

Pub. in Jnl. of Chromatography A, v708 p293-302 1995.

Keywords: "Refrigerants, "Chlorofluorocarbons, "Fluorocarbons, "Thermodynamic properties, Methane compounds, Halohydrocarbons, Chemical analysis, Gas chromatography, Temperature, Thermophysical properties, Reprints, "Foreign technology.

The retention Characteristics of 25 propane-based bromofluorocarbon, chlorocarbon, chlorofluorocarbon, and fluorocarbon fluids have been studied as a function of temperature on a stationary phase consisting of a $5 \%(\mathrm{~m} / \mathrm{m})$ coating of a low-molecular-mass polymer of hexafluoropropylene epoxide on a graphitized carbon black adsorbent. Measurements were performed at 0,20,40 and 60 degrees $C$ for R-245ca and R-245cb. Measurements were performed at 40,60,80, and 100 degress C for R-227ca, R-227ea, R-236ea, $R-236 f a, R-245 f a$, and R-263fb. Measurements were performed at $40,60,80$ and 100 degrees $C$ for $R$ $217 \mathrm{ba}, \mathrm{R}-254 \mathrm{cb}$ and $\mathrm{R}-1243 \mathrm{~b}$, and at $60,80,100$ and 120 degrees $\mathrm{C}$ for R-280da and R-217caB1. Measurements were performed at $80,100,120$, and 140 degrees C for R-215aa, R-216ba, R253fb, R-262da, and R-270aa. Measurements were performed at 100, 120 , 140 and 160 degrees C for R-215ba, R-225ca, R$225 \mathrm{cb}, \mathrm{R}-243 \mathrm{db}, \mathrm{R}-270 \mathrm{da}, \mathrm{R}-270 \mathrm{fa}$, and R-270fa, and $\mathrm{R}-270 \mathrm{fb}$. Relative retentions as a function of temperature were calculated with respect to the retentions of tetrafluoromethane (R-14) and hexafluoroethane (R116). Qualitative features of the data are examined, and trends are identified. In addition, the relative retention data were fitted to linear models for the purpose of predicting retention behavior of these compounds to facilitate chromatographic analysis.

\section{3,285}

PB96-164165 Not available NTIS

National Inst. of Standards and Technology (BFRL) Gaithersburg, MD. Fire Science Div.

In Search of Alternative Fire Suppressants.

Final rept.

W. L. IGrosshandler. 1995, 8p

Pub. in Thermal Science and Engineering Symposium in Honor of Chancello Chang-Lin Tien, Berkeley, CA., November 1995, p275-282

Keywords: "Fire extinguishing agents, "Alternatives "Aircraft fires, Environmental chemical substitutes, Material substitution, Fire suppresion, Fire fighting, Performance evaluation, Effectiveness, Halons, Reprints, Halon 1301

Halons had been the agents of choice for numerous fire protection applications because of their inherent ability to inhibit flames at low concentrations with no residue while exhibiting a number of additional strongly positive aftributes. To avoid costly mistakes in choosing replacements for aircraft applications, research has been conducted to determine the performance of different agents in extinguishing aircraft-lype fires. This paper describes the major elements of the overall program and the rationale of how an alternative to halon 1301 was chosen for aircraft applications.

\section{3,286}

PB96-167309 Not available NTIS

National Inst. of Standards and Technology (CSTL), Boulder, CO. Thermophysics Div.
Retention of Halocarbons on a
Hexafluoropropylene Graphitized Carbon Black. 3. Ethene-Based Compounds.

T. J. Bruno, and M. Caciari. 1994, 7p

See also PB95-175196 and PB96-164033

Pub. in Jnl. of Chromatography A, v686 p245-251 1994.

Keywords: "Refrigerants, "Chlorofluorocarbons, "Fluorocarbons, "Thermodynamic properties, Chemical analysis, Reprints, Gas chromatography, Temperature, Thermophysical properties, "Foreign technology, "Halocarbons, Relative retention.

The retention characteristics of 11 ethene-based chlorofluorocarbon, bromochlorofluorocarbon and fluorocarbon fluids have been studied as a function of temperature on a stationary phase consisting of a $5 \%(\mathrm{~m}$ m) coating of a low-molecular-mass polymer of hexafluoropropylene epoxide on a graphitized carbon black absorbent. Measurements were performed at $-20,0,20$ and 40 deg $C$ for trifluoroethene (R-1123) 1.1-difluoroethene(R1132a), and fluoroethene (R1112a), chlorotrifluoroethene (R-113), 2-chloro-1,1 difluoroethene (R-1122), 1-chloro-1-fluoroethene (R1121a), 2-bromo-1,1-difluoroethene (R-1122B1) and bromoethene (Vinly bromide, R-1140B1). Measurements were performed at $60,80,100$, and $120 \mathrm{deg}$ C fortrans-1,2-dichloro- 1,2-difluoroethene (R1112t) and cis-1,2-dichloro-1,2-difluoroethene (R-1112c). Ne retention volumes, corrected to a column temperature of 0 deg $\mathrm{C}$, were calculated from retention time measurements, the logarithms of which were fitted againstreciprocal thermodynamic temperature.

\section{3,287}

PB96-175724 Not available NTIS

National Inst. of Standards and Technology (BFRL), Gaithersburg, MD. Fire Science Div.

Suppression of High-Speed $\mathrm{C} 2 \mathrm{H} 4 / \mathrm{Air}$ Flames with C1-Halocarbons.

Final rept.

G. Gmurczyk, and W. Grosshandler. 1994, 7p. Sponsored by Department of the Air Force, Wright-Pat terson AFB, OH

Pub. in International Symposium on Combustion (25th), Irvine, CA., July 31-August 5, 1994, p14971503.

Keywords: "Fire suppression, "Halogenated hydrocarbons, "Combustion kinetics, Fire extinguishing agents, Halons, Performance evaluation, Turbulen flow, Flame propagation, Detonations, Flames, Ther modynamic properties, Heat flow, Reprints, Turbulent flames.

A primary objective of the work has been to determine the relative suppression efficiencies of different agents under highly dynamic situations, without the undue in fluence of either the ignition event or the mixing of the agent into the flame front. This was accomplished by generating a highly turbulent flame/quasi detonation in the driver section, which contain no suppressant, followed by measurements of the velocity and pressure ratio as the wave front entered the test section of the tube, which contained suppressant premixed with the same fuel/air combination. The experimental facility was successfully employed to clearly discriminate among the dynamic characteristics of the five compounds, revealing behavior distinct from what was observed in companion studies using atmospheric nonpremixed flames. The suppression process is strongly influenced by the concentration of an agent the structure and composition of a agent molecule, and the composition of the combustible mixture itself.

\section{3,288}

PB96-175732 Not available NTIS

National Inst. of Standards and Technology (BFRL), Gaithersburg, MD. Fire Science Div.

Effectiveness of Halon Alternatives in Suppressing Dynamic Combustion Process.

Final rept.

W. L. Grosshandler, C. Presser, and G. Gmurczyk. 1995, 21p.

Pub. in American Chemical Society Halon Replacements: Technology and Science Symposium, Washington, DC. August 21-25, 1994, p204-224 1995.

Keywords: "Aircraft fires, "Fire extinguishing agents, "Environmental chemical agents, "Combustion kinetics, Fire suppression, Alternatives, Halons, Fluorocarbons, Fire fighting, Fuel sprays, Turbulent flow, Thermodynamic properties, Detonations, Reprints, Explosiveness.
C3F8 is shown to require the least storage volume among twelve fluorocarbons for suppressing a quasidetonation. CF3I performs the best of the gaseous suppressants evaluated in a spray burner. Two experimental facilities are describes as part of an effort to identify suitable replacements for $\mathrm{CF} 3 \mathrm{Br}$ is aircraft applications. A turbulent spray burner simulates the hazards associated with a ruptured fuel line in an engine nacelle or dry bay. A deflagration/detonation tube evaluates the ability of a gaseous agent to attenuate the pressure build-up and Mach number of a quasi-detonation.

\section{3,289}

PB96-175740 Not available NTIS

National Inst. of Standards and Technology (CSTL), Gaithersburg, MD. Process Measurements Div.

Halon Thermochemistry: 'A Initio' Calculations of the Enthalpies of Formation of Fluoromethanes. Final rept.

R. J. Berry, D. R. F. Burgess, M. R. Nyden, M. R. Zachariah, and M. Schwartz. 1995, 6p.

Pub. in Jnl. of Physical Chemistry, v99 n47 p17145171501995

Keywords: "Fluorocarbons, "Thermochemistry, "Calculation methods, Thermodynamic properties, Thermophysical properties, Quantum chemistry, Molecule molecule interactions, Chemical bonds, Molecular orbitals, Reprints, "Enthalpy of formation.

Atomic equivalent (AEQ), BAC-MP4, G2(MP2), G2, CBS-4, CBS-Q, and CBS-QCI/APNO molecular orbital calculations were used to calculate enthalpies of formation in the series of fluoromethanes, $\mathrm{CH}$ (sub $x) F(\operatorname{sub}(4-x)), x=0-4$. Enthalpies of formation calculated with the $\mathrm{G} 2(\mathrm{MP} 2$ ) and $\mathrm{G} 2$ procedures exhibited systematic deviations from experiment which were linearly dependent upon the number of C-F bonds in the molecule. This technique had no significant effect on the quality of results from the AEQ, BAC-MP4, and CBS-4 methods.

\section{3,290}

PB96-176417 Not available NTIS

National Inst. of Standards and Technology (BFRL), Gaithersburg, MD. Fire Science Div.

Interaction of HFC-125, FC-218 and CF3I with High Speed Combustion Waves.

Final rept.

W. L. Grosshandler, and G. Gmurczyk. 1995, 9p. Sponsored by Naval Air Systems Command, Washington, DC

Pub. in International CFC and Halon Alternatives and Exhibition: Stratospheric Ozone Protection for the 90's, Washington, DC., October 23-25, 1995, p635-643.

Keywords: "Aircraft fires, "Fire extinguishing agents, "Combustion kinetics, Halogenated hydrocarbons, Fire suppression, Environmental chemical substitutes, Alteratives, Flame propagation, Fuel sprays, Propane, Thermodynamic properties, Halons, Overpressure, Reprints, "Turbulent flames, Explosiveness, Ethenes.

Live-fire full-scale testing has been conducted at Wright-Patterson Air Force Base to identify an agent to replace $\mathrm{CF}_{3} \mathrm{Br}$ (halon 1301) for suppressing fires in military aircraft dry bays. High pressures motivated the current study to determine the initial conditions which would lead to dangerous situations, and to explore a less extreme regime more representative of a realistic threat. Based upon over a hundred experiments with modified facility, it was possible to reconfirm the conclusion that FC-218 provides the most consistent performance over the widest range of fuel/air mixtures and tube geometries. The CF3l has the greatest positive impact at low partial pressure fractions, but exhibits non-monotonic behavior of flame speed and shock pressure ratio at increasing concentrations. The dangerously high over-pressures previously exhibited by HFC-125 were not obsenved during suppression under more moderate (and realistic) combustion conditions.

03,291

PB96-176425 Not available NTIS

National Inst. of Standards and Technology (BFRL) Gaithersburg, MD. Fire Science Div.

Suppression of Ignition Over a Heated Metal Surface.

Final rept.

A. Hamins, P. Borthwick, and C. Presser. 1995, 5p. Pub. in International Conference on Fire Research and Engineering. Orlando, FL., September 10-15, 1995 p77-81. 
Keywords: "Fire suppression, "Halogenated hydrocarbons, "Metals, "Surfaces, Fire extinguishing agents, Halons, Performance evaluation, Flames, Temperature, Combustion kinetics, Heat flow, Heat transmission, Thermal radiation, Thermodynamic properties, Fire fighting, Radiant heat flow, Reprints.

The objective of this work is to investigate the effectiveness of various agents in suppressing flame ignition. Experiments were conducted to determine the amount of agent needed to suppress the ignition of a gaseous propane flow over a heated metal disk. The metal surface was heated by a regulated power supply which provided up to $200 \mathrm{~W}$. With the fuel and oxidizer flowing, flame ignition occurred in a repeatable fashion by increasing the power through the metal disk. Various amounts of agent were added to the air flow and the temperature of the heated metal disk was measured at flame ignition using an optical pyrometer. The effectiveness of N2, HFC-125, HFC-227 and CF3l were compared in suppressing the ignition event.

03,292

PB96-176680 Not available NTIS

National Inst. of Standards and Technology (CSTL), Boulder, CO. Thermophysics Div.

Viscosity of 1,1,1,2,3,3-Hexafluoropropane and $1,1,1,3,3,3$-Hexafluoropropane at Saturated-Liquid Conditions from $262 \mathrm{~K}$ to $353 \mathrm{~K}$.

Final rept.

A. Laesecke, and D. R. Defibaugh. 1996, $4 p$

Pub. in Jnl. of Chemical and Engineering Data, v41 n1 p59-62 1996.

Keywords: "Refrigerants, "Viscosity, "Propanes, Alternatives, Material substitution, Environmental chemical substitutes, Volume, Vapor pressure, Free energy, Reprints, 1-1-1-2-3-3-hexafluoropropane, 1-1-1-3-3-3hexafluoropropane, R236ea, R236fa, Corresponding states, Saturated liquids

Viscosities are reported for two fluorinated propane isomers in the saturated-liquid state at temperatures from $262 \mathrm{~K}$ to $353 \mathrm{~K}$. The compounds are 1,1,1,2,3,3hexafluoropropane (R236ea) and 1,1,1,3,3,3hexafluoropropane (R236fa). The measurements were performed in a capillary viscometer designed for fluids with vapor pressures above ambient. A free-volume model was used to correlate the experimental results, and they were also compared with predictions from an extended corresponding states model.

03,293

PB96-176755 Not available NTIS

National Inst. of Standards and Technology (CSTL), Boulder, CO. Thermophysics Div.

Report of the Refrigeration, Air Conditioning and Heat Pumps Technical Options Committee.

Final rept.

M. O. McLinden, G. Angelino, D. Arnaud, J. M. Calm, E. Preisegger, R. Tillner-Roth, K. Watanabe, $P$.

Weiss, and S. Bhaduri. 1995, 24p.

Pub. in Assessment of the United Nations Environment Programme Montreal Protocol on Substances That Deplete the Ozone Layer, p31-54 1995.

Keywords: "Refrigerants, "Enviromental chemical substitutes,
Chlorofluorocarbons, Alternatives, Material substifution, Thermodynamic properties, Flammability, Toxicity, Reprints, Material compatibility.

The properties of refrigerants that may serve as replacements to the chlorofluorocarbons (CFCs) are considered. The characteristics required of a refrigerant are reviewed, and the data needed to evaluate possible alternative refrigerants are summarized. These data include thermophysical properties, toxicity and flammability data, materials compatibility information, and parameters characterizing environmental consequences. The available data for 45 potential replacement refrigerants are summarized.

\section{3,294}

PB96-176805 Not available NTIS

National Inst. of Standards and Technology (CSTL), Boulder, CO. Thermophysics Div.

International Standard Equation of State for the Thermodynamic Properties of Refrigerant 123 (2,2Dichloro-1,1,1-Trifluoroethane).

Final rept.

B. A. Younglove, and M. O. McLinden. 1994, 49p. Pub. in Jnl. of Physical and Chemical Reference Data, v23 n5 p731-779 1994.

Keywords: "Refrigerants, "Equations of state, "Thermodynamic properties, Alternatives, Material substitution, Enviroinmental chemical substitutes, Ethanes, Vapor pressure, Critical temperature, Specific heat, Density(Mass/Nolume), Standardization, Re prints, 2-2-dichloro-1-1-1-trifluoroethane, R123, Correlation density, Heat capacity, Speed of sound.

A modified Benedict-Webb-Rubin (MBWR) equation of state has been developed for Refrigerant $123(2,2$ dichloro- 1,1,1-trifluoroethane) basedon recently measured thermodynamic property data and data available fromthe literature. Single-phase pressurevolume-temperature (PVT), heat capacity, and sound speed data, as well as second virial, vapor pressure, and saturated liquid and saturated vapor density data were used with multiproperty linear least squares fitting techniques to fit the 32 adjustable coefficients of the MBWR equation. Coefficients for the equation of state and for ancillary equations representing the vaporpressure, saturated liquid and saturated vapor densities, and ideal gas heat capacity are given.

\section{3,295}

PB96-190095 Not available NTIS

National Inst. of Standards and Technology (MSEL), Boulder, $\mathrm{CO}$. Materials Reliability Div.

Mapping the Droplet Transfer Modes for an ER100S-1 GMAW Electrode.

Final rept.

P. R. Heald, R. B. Madigan, T. A. Siewert, and S. Liu. 1994, $7 p$.

See also PB91-174250.

Pub. in Welding Jnl., p38s-44s Feb 94.

Keywords: "Gas metal arc welding, "Electrodes, Spray transfer, Electric potential, Mapping, Transferring, Reprints, CTWD, GMAW(Gas metal arc welding), Metal transfer modes, Wire feed rate.

Welds were made with a 1.2-mm-diameter AWS ER100S-1 electrode using Ar-2\% O2 shielding gas to map the effects of contact-tube-to-work distance (13, 19 and $25 \mathrm{~mm}$ ), current, voltage, and wire feed rate on metal transfer. The droplet transfer modes were identified for each map by both the sound of the arc and images from a laser back-lit high-speed video system. The modes were correlated to digital records of the voltage and current fluctuations. The maps contain detailed information on the spray transfer mode, including the boundaries of drop spray modes. The metal ransfer mode boundaries shifted with an increase in contact-tube-to-work distance.

\section{3,296}

PB96-190145 Not available NTIS

National Inst. of Standards and Technology (MSEL), Boulder, CO. Materials Reliability Div.

Power Characteristics in GMAW: Experimental and Numerical Investigation.

Final rept.

P. G. Jonsson, J. Szekely, R. B. Madigan, and T. P. Quinn. 1995, 10p.

Pub. in Welding Jnl., p93s-102s Mar 95.

Keywords: "Power, "Gas metal arc welding, Numerical analysis, Electric power distribution, Input, Models, Electric potential, Reprints, GMAW(Gas metal arc welding), Arc column, Arc length.

The voltage and power distributions in gas metal arc welding (GMAW) were studied both experimentally and numerically. The principal voltage drop takes place in the arc, which also constitutes the dominant powe contribution. Within the arc, the dominating voltage contributions are from the arc column and the cathode fall, while the anode fall and the electrode regions are ess significant. The power input to the arc column increases with both increasing current and increasing arc length. These results indicate that it is critical to control the arc length in order to control the power input to the system.

\section{3,297}

PB96-190160 Not available NTIS

National Inst. of Standards and Technology (MSEL), Boulder, CO. Materials Reliability Div.

Sensing Droplet Detachment and Electrode Extension for Control of Gas Metal Arc Welding.

Final rept.

R. B. Madigan, T. P. Quinn, and T. A. Siewert. 1993,

Pub. in International Trends in Welding Science and Technology, Gatlinburg, TN., June 1-5, 1992, p99910021993

Keywords: "Gas metal arc welding, "Electrodes, Currents, Electric potential, Detectors, Luminous intensity,
Transferring, Drops(Liquid), Frequencies, Reprints, GMAW(Gas metal arc welding), Arc length, Arc current.

An arc light intensity sensor, in conjunction with voltage and current sensors, was evaluated to detect droplet detachment and electrode extension under spray transfer welding conditions. Arc length, electrode extension and droplet transfer frequency were obtained from high-speed video images of the process and correlated to arc current and arc light intensity measurements. The arc light intensity sensor was able to detect droplet frequency from variations in arc light intensity caused by changes in arc length due to droplet detachment. Arc length could be estimated using this regression within $1 \mathrm{~mm}$ with current ranging from 180 to 300 $A$ and arc length ranging from 4 to $12 \mathrm{~mm}$.

\section{3,298}

PB96-201033 Not available NTIS

National Inst. of Standards and Technology (CSTL), Gaithersburg, MD. Chemical Kinetics and Thermodynamics Div.

Temperature Dependence of the Gas and Liquid Phase Ultraviolet Absorption Cross Sections of
HCFC-123 (CF3CHCl2) and HCFC-142b (CH3CF2Cl).

Final rept.

A. K. Nayak, T. J. Buckley, M. J. Kurylo, and A. Fahr 1996, 8p.

Pub. in Jnl. of Geophysical Research, v101 nC4 p9055-9062 Apr 96.

Keywords: "Thermal properties, "Temperature dependence, "Ultraviolet absorption, "Absorption cross sections, Chlorofluorocarbons, Halogenated hydrocarbons, Vapor phases, Liquid phases

The absorption cross sections for HCFC-123 (CF3CHCl2) and HCFC-142b (CH3CF2Cl) have been measured in the gas and liquid phases over the temperature range of about $220-330 \mathrm{~K}$. The liquid phase results were converted into effective gas phase cross sections using a wavelength shift procedure, thereby extending the gas phase cross sections to longe wavelengths. The results are compared with other available data and lend increased confidence in atmos pheric lifetime calculations for these important indus trial alternatives to the fully halogenated chlorofluorocarbons.

03,299

PB97-118384 Not available NTIS

National Inst. of Standards and Technology (CSTL) Gaithersburg, MD. Thermophysics Div. Thermodynamic Properties
$1,1,1,2,3,3$-Hexafluoropropane.

Final rept

D. R. Defibaugh, K. A. Gillis, M. R. Moldover, J. W.

Schmidt, and L. A. Weber. 1996, 25p.

Pub. in Fluid Phase Equilibria, v122 p131-155 1996.

Keywords: "Thermodynamic properties, Vapor pressure Equations of state, Density(Mass Nolume), Refractive index, Critical temperature, Acoustic velocity, Surface tension, Specific heat, Critical pressure, Gas phases, Liquid phases, Phase studies, Ideal gas, Refrigerants, Refrigerating machinery, Environmental chemical substitutes, Reprints, "Hexafluoropropane, "HFC-236ea, Critical density.

The authors report the thermodynamic properties of $1,1,1,3,3$-Hexafluoropropane (known in the refrigeration industry as HFC-236ea) in the temperature and pressure region commonly encountered in thermal ma chinery. The properties are based on measurements of the vapor pressure, the density of the compressed liquid (PVT), the refractive index of the saturated liquid and vapor the critical temperature the speed of sound in the vapor phase, and the capillary rise. From these data, the authors deduce the ideal-gas heat-capacity, the saturated liquid and vapor densities, the equation of state of the vapor phase, the surface tension, and estimates of the critical pressure and density. The data determine the coefficients for a Carnahan-StarlingsDeSantis (CSD) equation of state.

03,300

PB97-118392 Not available NTIS

National Inst. of Standards and Technology (CSTL), Gaithersburg, MD. Thermophysics Div. 
Thermodynamic Properties of CHF2-CF2-CHF, 1,1,2,2,3-Pentafluoropropane.

Final rept.

D. R. Defibaugh, K. A. Gillis, M. R. Moldover, J. W. Schmidt, and L. A. Weber. 1996, 10p. Pub. in International Jnl. of Refrigeration, v19 n4 p284. 2941996.

Keywords: "Thermodynamic properties, Vapor pressure, Equations of state, Density(Mass/Nolume), Refractive index, Critical temperature, Acoustic velocity, Surface tension, Specific heat, Critical pressure, Gas phases, Liquid phases, Phase studies, Ideal gas, Refrigerants, Refrigerating machinery, Environmental chemical substitutes, Reprints, "Pentafluoropropane, "HFC-245ca.

The authors report the thermodynamic properties of $1,1,2,3,3$-pentafluoropropane (known in the refrigeration industry as HFC-245ea) in the temperature and pressure region commonly encountered in thermal machinery. The properties are based on measurements of the vapor pressure, the density of the compressed liquid, the refractive index of the saturated liquid and vapor, the critical temperature, the speed of sound in the vapor phase, and the capillary rise. From these data, the authors deduce the ideal-gas heat-capacity, the saturated liquid and vapor densities, the equation of state of the vapor phase, the surface tension, and estimates of the critical pressure and density. The data determine the coefficients for a Carnahan-StarlingsDeSantis (CSD) equation of state.

\section{3,301}

PB97-122261 Not available NTIS

National Inst. of Standards and Technology (CSTL) Gaithersburg, MD. Physical and Chemical Properties Div.

Environmental Aspects of Halon Replacements: Considerations for Advanced Agents and the Ozone Depletion Potential of CF31.

Final rept.

P. S. Connell, D. E. Kinnison, D. J. Bergmann, R. G. Daniel, C. K. Williamson, A. W. Miziolek, R. E. Huie, K. O. Patten, and D. J. Wuebbles. 1996, 15p.

Pub. in Halon Options Technical Working Conference, Albuquerque, NM., May 7-9, 1996, p585-609.

Keywords: "Environmental chemical substitutes, "Physical properties, "Chemical properties, "Environmental impacts, Halons, Materials replacement, Atmospheric reactivity, Chemical reactivity, Biodegradation, Iodine fluorides, Ozone depletion, Environmental persistance, Bioaccumulation, Biosphere, Reprints, " Trifluoromethyl iodide.

The introduction of a new chemical species into the environment raises the question of the ultimate fate of that compound. In most cases, any compound which is introduced into the environment can undergo various transformations, physical and chemical. Of considerable importance is accumulation in the biosphere, which can take place when the chemical is lipid soluble. In this case, the concentration of the substance through the food chain becomes important. In concert with these physical transformations are chemical transformations such as oxidation, photolysis, hydrolysis, and complexation. It is important to understand both the rates and the mechanisms of these transformations in order to evaluate the environmental impact of a chemical species which may be introduced into the environment.

\section{Nonferrous Metals \& Alloys}

\section{3,302}

AD-A279 180/4 PC A04/MF A01

National Bureau of Standards, Gaithersburg, MD Thermal Conductivity of Metals and Alloys at Low Temperatures. A Review of the Literature.

R. L. Powell, and W. A. Blanpied. 1 Sep 54, 72p.

Keywords: "Metals, "Alloys, "Thermal conductivity, "Low temperature, Construction materials, Dielectrics, Crystals, Chemical elements, Solids, Tables(Data), *Reviews.

No abstract available.
National Inst. of Standards and Technology, Boulder, CO. Cryogenic Engineering Lab.

Cryogenic Materials Data Handbook.

W. L. Burriss, and J. L. Mason. 14 Nov 61, 60p. Contract AF-04(647)-59-3

Keywords: "Aluminum, "Thermal properties, "Cryogenics, "Mechanical properties, Strength(Mechanics), Handbooks.

No abstract available.

\section{3,304}

\section{AD-A294 088/0 PC A04/MF A01}

National Inst. of Standards and Technology, Gaithersburg, MD. Synthesis of Metal and Intermetallic Composites.

D. S. Lashmore, C. R. Beauchamp, C. E. Johnson,

T. Moffat, and J. L. Mullen. 24 Feb 94, 72p.

Keywords: "Aluminum alloys, "Electrochemistry, * Manganese alloys, Metals, Reprints, Synthesis, Structural properties, Composite materials, Patents, Intermetallic compounds, Electrodeposition, Molten salts, Compilations, Pat cl 205/176.

Partial contents: Formation of the Al-Mn icosahedral phase by electrodeposition; The electrodeposition of an Aluminum-Manganese metallic glass from molten salts; Phase formation in electrodeposited and thermally annealed Al-Mn alloys: Structural study of electrodeposited Aluminum-Manganese alloys; Patents: Metal-coated fiber compositions containing alloys barrier layer; Cooperative research and development agreement. ig (abstract is modified and taken from document titles.).

\section{3,305}

AD-A306 625/5 PC A04/MF A01

National Inst. of Standards and Technology, Gaithersburg, MD.

Fracture Testing of Large-Scale Thin-Sheet Aluminum Alloy.

Final rept.

R. DeWit, R. J. Fields, S. R. Low, D. E. Harne, and T. Foecke. Feb 96, 32p, DOT/FAAAR-95/11 Contract DTGFA03-92-Z-00018

Keywords: "Aluminum alloys, "Fracture(Mechanics), "Sheets, "Thinness, Test and evaluation, Optical equipment, Models, Panels, Cracks, Sites, Test methods, Strength(Mechanics), Tension, Strain gages, Plastic properties, Linkages, Diagrams, Magnetic tape, Video tapes, Test fixtures, Motion picture film, R-curve, Antibuckling, Msd(Multiple site damage), Multiple site damage.

A series of fracture tests on large-scale, precracked, aluminum alloy panels were carried out to examine and characterize the process by which cracks propagate and link up in this material. Extended grips and test fixtures were specially designed to tension load the panel specimens in a $1780-\mathrm{kN}$ capacity universal testing machine. Single sheets of bare 2024-T3 aluminum alloy, approximately $4 \mathrm{~m}$ high, $2.3 \mathrm{~m}$ wide, and $1 \mathrm{~mm}$ thick were fabricated with simulated through cracks oriented horizontally at midheight. Using existing information, a test matrix was set up to explore regions of failure controlled by fracture mechanics, with additional tests near the boundary between plastic collapse and fracture. In addition, a variety of multiple site damage (MSD) configurations were included to distinguish between various proposed linkage mechanisms. All tests but one used antibuckling guides. Three specimens were fabricated with a single central crack, six others had multiple cracks on each side of the central crack, and one had a single crack but no antibuckling guides. The results of each fracture event were recorded on various media: film, video, computer, magnetic tape, and occasionally optical microscope. The video showed the crack tip with a load meter in the field of view, using motion picture film for one tip and super VHS video tape for the other. The computer recorded the output of the testing machine load cell, the stroke, and the twelve strain gages at 1.5 -second intervals. A wideband FM magnetic tape recorder was used to record data from the same sources. The data were analyzed by two different procedures: (1) the plastic zone model based on the residual strength diagram and (2) the R-curve.

\section{3,306}

DE94017331 PC A03/MF A01

National Inst. of Standards and Technology (MSEL), Gaithersburg, MD.
Evaluation of the Environmentally Induced Fracture Resistance of Ductile Nickel Aluminide. Technical Report Number 1, Final report. October-December 1990.

Progress rept

R. E. Ricker, U. Bertocci, J. L. Fink, and M. R

Stoudt. Jul 91, 21p, DOE/OR/21941-T1.

Contract Al05-90OR21941

Sponsored by Department of Energy, Washington, DC.

Keywords: "Aluminium Alloys, "Nickel Base Alloys, "Fracture Properties, Aluminium Chlorides, Boron Additions, Corrosion, Experimental Data, Hafnium Additions, Hydrogen Embrittlement, Iron Additions, Nickel Chlorides, PH Value, Progress Report, Sodium Chlorides, Sodium Sulfates, Tables(data), EDB/ 360103, EDB/360105.

Slow-strain-rate tensile tests and electrochemical experiments were performed in different aqueous solutions on ductile nickel aluminide, Ni(sub 3)Al+B, in order to evaluate the possibility of environmentally induced fracture of this material in neutral $\mathrm{pH}$ solutions as a result of hydrogen absorption. Two different processes were postulated that could lead to hydrogen absorption and embrittlement: (1) local acidification due to hydrolysis of the corrosion reaction products and (2) hydrogen reduction during the potential transient that accompanies film rupture and repair. Experiments were designed to evaluate each of these possibilities. First, slow strain rate tests were conducted in solutions with varying concentrations of metal ions and $\mathrm{pH}$ to determine the critical metal ion concentration and $\mathrm{pH}$ that result in hydrogen absorption and embrittlement of this material. Second the potential transient that follows the mechanical rupture of the protective surface film in different solutions was measured and the minimum potential during the transient was compared to the potential that results in a hydrogen fugacity large enough to cause cracking. The results indicate that hydrogen reduction, absorption, and embrittlement are not to be expected in neutral solutions as a result of local acidification during crevice corrosion or film rupture during crack propagation or cyclic loading.

\section{3,307}

DE94017351 PC A02/MF A01

National Inst. of Standards and Technology (MSEL), Gaithersburg, MD. Metallurgy Div.

Evaluation of the Electrochemical Behavior of Ductile Nickel Aluminide and Nickel in a pH 7.9 Solution. Technical Report Number 3, April-June 1991. D. M. Gram, U. Bertocci, and R. E. Ricker. Aug 91 , 10p, DOE/OR/21941-T3.

Contract Al05-90OR21941

Sponsored by Department of Energy, Washington, DC.

Keywords: "Aluminium Alloys, "Nickel Base Alloys, "Electrochemistry, Nickel, Doped Materials, Intermetallic Compounds, EDB/360104, EDB/400400.

The electrochemical behavior of ductile nickel aluminide has been examined in neutral solutions. Features observed in a certain potential range were characterized, and the potentiodynamic sweep parameters affecting them identified. Nickel aluminide behaves essentially as pure nickel; however, small differences were observed.

\section{3,308}

DE94018566 PC A01/MF A01

National Inst. of Standards and Technology, Gaithersburg, MD

Study of Laser Resonance lonization Mass Spectrometry Using a Glow Discharge Source. $X$. Xiong, J. M. R. Hutchinson, J. D. Fassett, W. A. Bowman, K. R. Hess. T. B. Lucatorto, and F. J.

Schima. 1994, 5p, DOE/ER/60447-T6.

Contract Al05-86ER60447

Sponsored by Department of Energy, Washington, DC.

Keywords: "Aluminium Base Alloys, "Copper Alloys, *Iron Alloys, Atomization, Data Acquisition Systems, Dye Lasers, Mass Spectrometers, Resonance Ionization Mass Spectroscopy, EDB/400102.

The mass spectra of a metal alloy sample consisting of $\mathrm{Al}, \mathrm{Cu}$ and $\mathrm{Fe}$ were studied using both glow discharge mass spectrometry (GDMS) and resonance ionization mass spectrometry (RIMS). Particular emphasis was placed on the reduction of isobaric interferences and discrimination between those ions formed by the discharge and those formed by the laser radiation. 
03,309

N94-25124/6

(Order as N94-25120/4, PC A11/

MF A03)

National Inst. of Standards and Technology, Gaithersburg, MD.

Dynamic Measurements of Thermophysical Properties of Metals and Alloys at High Temperatures by Subsecond Pulse Heating Techniques.

A. Cezairliyan. Nov $93,15 p$

In NASA. Lewis Research Center, Workshop on the Thermophysical Properties of Molten Materials $p$ 71. 85.

Keywords: "High temperature environments, "Pulse heating, "Thermophysical properties, "Liquid alloys, "Liquid metals, Dynamic characteristics, Electric potential, Electrical resistivity, Enthalpy, Phase transformations, Specific heat, Temporal resolution, Thermal expansion, Heat of fusion, Interfacial tension, Liquidus, Microgravity, Solidus, Spectral emission.

Rapid (subsecond) heating techniques developed at the National Institute of Standards and Technology for the measurements of selected thermophysical and related properties of metals and alloys at high temperatures (above $1000 \mathrm{C}$ ) are described. The techniques are based on rapid resistive self-heating of the specimen from room temperature to the desired high temperature in short times and measuring the relevant experimental quantities, such as electrical current through the specimen, voltage across the specimen, specimen temperature, length, etc., with appropriate time resolution. The first technique, referred to as the millisecond-resolution technique, is for measurements on solid metals and alloys in the temperature range $1000 \mathrm{C}$ to the melting temperature of the specimen. It utilizes a heavy battery bank for the energy source and the total heating time of the specimen is typically in the range of 100-1000 ms. Data are recorded digitally every $0.5 \mathrm{~ms}$ with a full-scale resolution of about one part in 8000 . The properties that can be measured with this system are as follows: specific heat, enthalpy, thermal expansion, electrical resistivity. normal spectral emissivity, hemispherical total emissivity, temperature and energy of solid-solid phase transformations, and melting temperature (solidus). The second technique, referred to as the microsecondresolution technique, is for measurements on liquid metals and alloys in the temperature range 1200 to $6000 \mathrm{C}$. It utilizes a capacitor bank for the energy source, and the total heating time of the specimen is typically in the range 50-500 micro-s. Data are recorded digitally every 0.5 micro-s with a full-scale resolution of about one part in 4000 . The properties that can be measured with this system are: melting temperature (solidus and liquidus), heat of fusion, specific heat, enthalpy, and electrical resistivity. The third technique is for measurements of the surface tension of liquid metals and alloys at their melting temperature. It utilizes a modified millisecond-resolution heating system designed for use in a microgravity environment.

03,310

N95-14548/8 (Order as N95-14522/3, PC A20/

MF A04)

National Inst. of Standards and Technology, Gaithersburg, MD. rectional Solidification.

S. R. Coriell, A. A. Chernov, B. T. Murray, and G. B. Mcfadden. Aug 94, 6p.

In NASA. Lewis Research Center, Second Microgravity Fluid Physics Conference p 175-180.

Keywords: *Anisotropy, "Binary alloys, "Convection, "Directional solidification (Crystals), "Interface stability, "Phase stability (Materials), Crystallography, Destabilization, Germanium alloys, Melts (Crystal growth) Morphology, Snear flow, Silicon alloys, Traveling waves.

For growth of a vicinal face at constant velocity, the effect of anisotropic interface kinetics on morphological stability is calculated for a binary alloy. The dependence of the interface kinetic coefficient on crystallographic orientation is based on the motion and density of steps. Anisotropic kinetics give rise to traveling waves along the crystal-melt interface, and can lead to a significant enhancement of morphological stability. The stability enhancement increases as the orientation approaches a singular orientation and as the solidification velocity increases. Shear flows interact with the traveling waves and, depending on the direction of the flow, may either stabilize or destabilize the interface. Specific calculations are carried out for germanium-silicon alloys.
03,311

N95-19494/0 (Order as N95-19468/4, PC A24/

MF A04) National Inst. of Standards and Technology,
Gaithersburg, MD.

Fracture Behavior of Large-Scale Thin-Sheet Aluminum Alloy.

R. Dewit, R. J. Fields, L. Mordfin, S. R. Low, and D.

Harne. Sep 94, 21p.

Contract DTFA03-92-Z-00018

In NASA. Langley Research Center, FAANNASA International Symposium on Advanced Structural Integrity Methods for Airframe Durability and Damage Tolerance, Part 2 p 963-983.

Keywords: "Aluminum alloys, "Cracks, "Failure "Fracture mechanics, "Fracturing, "Metal sheets, "Residual strength, Crack tips, Field of view, Loads (Forces), Magnetic recording, Microscopy, Strain gages.

A series of fracture tests on large-scale, pre-cracked, aluminum alloy panels is being carried out to examine and to characterize the process by which cracks propagate and link up in this material. Extended grips and test fixtures were specially designed to enable the panel specimens to be loaded in tension, in a 1780$\mathrm{kN}$-capacity universal testing machine. Twelve pane specimens, each consisting of a single sheet of bare 2024-T3 aluminum alloy, $3988 \mathrm{~mm}$ high, $2286 \mathrm{~mm}$ wide, and $1.016 \mathrm{~mm}$ thick are being fabricated with simulated through-cracks oniented horizontally at midheight. Using existing information, a test matrix has been set up that explores regions of failure that are controlled by fracture mechanics, with additional tests near the boundary between plastic collapse and fracture. In addition, a variety of multiple site damage (MSD) configurations have been included to distinguish between various proposed linkage mechanisms. All tests but one use anti-buckling guides. At this writ ing seven specimens have been tested. Three were fabricated with a single central crack, three others had multiple cracks on each side of the central crack, and one had a single crack but no anti-buckling guides. Each fracture event was recorded on film, video, computer, magnetic tape, and occasionally optical microscopy. The visual showed the crack tip with a load meter in the field of view, using motion picture film for one tip and SVHS video tape for the other. The computer recorded the output of the testing machine load cell, the stroke, and twelve strain gages at 1.5 second intervals. A wideband FM magnetic tape recorder was use to record data from the same sources. The data were analyzed by two different procedures: (1) the plastic zone model based on the residual strength diagram and (2) the R-curve. The first three tests were used to determine the basic material properties, and these results were then used in the analysis of the two subsequent tests with MSD cracks. There is good agreement between measured values and results obtained from the model.

\section{3,312}

PC A03/MF A01

National Inst. of Standards and Technology (CAML), Gaithersburg, MD. Applied and Computational Mathematics Div.

Boundary Conforming Grid Generation System for Interface Tracking

B. V. Saunders. Feb 94, 22p, NISTIR-5352.

Keywords: "Liquid-solid interfaces, Mapping(Transformations), Binary alloys, Tensor prod ucts, Spline functions, Coordinates, Directional solidification, Grid generation(Mathematics).

The development of an algebraic grid generation sys em that tracks a solid-liquid interface during directiona solidification of a binary alloy is discussed. A single mapping, constructed with tensor product $\mathrm{B}$-splines, is proposed for calculations of both shallow and deep solidification cells. The initial spline coefficients for the coordinate mapping are modified to minimize a discrete functional that regulates the smoothness and orthogonality of the mesh. The use of transfinite blending function interpolation to obtain an initial grid is examined.

03,313

PB94-172707 Not available NTIS

National Inst. of Standards and Technology (MSEL) Gaithersburg, MD. Metallurgy Div.
Effects of Elastic Stress on Phase Equilibrium in the Ni-V System.

Final rept.

M. J. Pfeifer, P. W. Voorhees, and F. S. Biancaniello. 1994, 6p.

Pub. in Scripta Metallurgica et Materialia 30, No. 6, p743-748 1994.

Keywords: "Elastic properties, "Stress analysis, "Phase diagrams, Nickel base alloys, Vanadium alloys, Phase transformations, Precipitation(Chemistry), points, Reprints, "Nickel vanadium, Congruent points.

Using our predictions of the effects of elastic stresses on the phase diagram of an alloy displaying a congruent point as a guide we studied experimentally the effects of elastic stress on the Ni-V phase diagram in the neighborhood of the Ni- $\mathrm{V}-->\mathrm{Ni} \mathrm{V}$ congruent point. The $\mathrm{Ni}-\mathrm{V}$--> Ni3V transformation has been well studied and displays a congruent point at 25 at $\% \mathrm{~V}, 1,045 \mathrm{C}$. These experiments have shown that the effects of elastic stress on phase equilibrium is observable in the $\mathrm{Ni}-\mathrm{V}$ system.

\section{3,314}

PB94-172764 Not available NTIS

National Inst. of Standards and Technology (IMSE), Gaithersburg, MD. Fracture and Deformation Div. Reaction Sensitivities of Al-Li Alloys and Alloy 2219 in Mechanical-Impact Tests.

Final rept.

N. J. Simon, J. D. McColskey, R. P. Reed, and C. Gracia-Salcedo. 1991, 23p

Pub. in Flammability Sensitivity of Materials in OxygenEnriched Atmospheres, ASTM STP 111, p193-215 1991. See also AD-A242 956.

Keywords: "Impact tests, "Flammability, Aluminum alloys, Lithium alloys, Chemical properties, Chemical reactions, Cracking(Fracturing), Mechanical tests, Deformation, Reprints, "Aluminum-Lithium alloys, "Oxygen compatibility.

Open-cup and pressurized mechanical impact tests were conducted at White Sands Test Facility (WSTF) on Al-Li alloys $(2090,8090$, and WL049) and Al Alloy 2219 in various tempers. Pressure, drop height (potential energy), and environment (gaseous (GOX) or liquid (LOX)) oxygen were variables in the test program. Reactions were detected in all alloys. The reaction sensitivity was found to vary with specimen absorbed energy. Large, macroreactions occurred on specimen shear lips, a region that has very high deformation and is exposed to striker pin-specimen friction. Small, microreactions were found to occur over many areas of the specimen. The effects of the test variables (pressure, drop height, LOX, GOX) on the frequency of macro- and microreactions is discussed. It is shown that the energy for fracture is always less than, or equal to, the energy for (oxygen) reaction.

03,315

PB94-172913 Not available NTIS

National Inst. of Standards and Technology (MSEL), Gaithersburg, MD. Metallurgy Div.

Intelligent Processing of Hot Isostatic Pressing.

Final rept.

R. J. Schaefer. 1990, 11p.

Pub. in Proceedings of Thermal Structures Conference (1st), Charlottesville, VA., November 13-15, 1990, p401-411.

Keywords: "Hot pressing, "Process control, "Metals "Intermetallic compounds, "Sensors, Microstructure, Metalworking, Artificial intelligence, Powder metallurgy, Mechanical working. Hot working, Reprints, *Intelligent processing, "Hot isostatic pressing.

Successful hot isostatic pressing (HIP) of metal or intermetallic powders requires that the product meet many goals with respect to shape, density, microstructure and cost. The development of sensors which can detect sample characteristics during HIP consolidation and process models which can predict now the sample characteristics will respond to changes in its environment raises the possibility of active control based on these characteristics. The report describes intelligent processing of hot isostatic pressing.

03,316

PB94-172962 Not available NTIS

National Inst. of Standards and Technology (MSEL),

Boulder, CO. Fracture and Deformation Div. 


\section{Nonferrous Metals \& Alloys} Temperature Increases in Aluminum Alloys during
Mechanical-Impact Tests for Oxygen Compatibility.

Final rept.

N. J. Simon, and R. P. Reed. 1991, 14p.

Pub. in Flammability Sensitivity of Materials in OxygenEnriched Atmospheres, ASTM STP 111, p367-380 1991.

Keywords: "Impact tests, "Aluminum alloys, "Fracture(Mechanics), "Temperature gradients, Thermal analysis, Cracking(Fracturing), Mechanical tests, Strain rate, Deformation, Reprints.

In mechanical-impact testing of $\mathrm{Al}-\mathrm{Li}$ alloys and $\mathrm{Al}$ alloy 2219 for oxygen compatibility, specimens absorb a significant amount of energy in a short time. The absorbed energy is sufficient to crack and, in many cases, produce large fractures (splits) in the specimens. The high strain rate from impact results in the formation of adiabatic shear bands under adiabatic conditions in the deformed areas. Large local temperature increases in the specimens occur during deformation. Localized melting is more prevalent in alloy 2219 , compared to $\mathrm{Al}-\mathrm{Li}$ alloys. Microreactions also occur more frequently in 2219, but larger, macroreactions occur more readily in the Al-Li alloys. Estimates of temperature increases assuming macroscopic homogeneous deformation during impact are presented. Characteristic times to propagate a thermal transient and to complete the mechanical deformation process are estimated. The difference between the alloy systems in this characteristic time to propagate a thermal disturbance is used to discuss the sizes and frequencies of macro- and microreactions.

03,317

PB94-198694 Not available NTIS

National Inst. of Standards and Technology (MSEL),

Gaithersburg, MD. Metallurgy Div.

Coherent Precipitates in the BCC/Orthorhombic Two Phase Field of the Ti-Al-Nb System.

Final rept.

L. A. Bendersky, W. J. Boettinger, and A. Roytburd.

1991, 11p.

Pub. in Acta Metallurgica et Materialia 39, n8 p1959

19691991

Keywords: "Aluminum intermetallics, "Niobium intermetallics, "Titanium intermetallics, Transmission electron microscopy, Binary systems(Materials) Orthorhombic lattices, Bcc lattices, Precipitates, Reprints.

Alloys of composition Ti2AINb and Ti4AINb3, cooled from $1400 \mathrm{C}$ and equilibrated at $700 \mathrm{C}$ for 26 days, were both found to consist of two phases: the Ti- and $\mathrm{Nb}$-rich bcc phase and the orthorhombic phase based on Ti2AINb. Depending on the alloy composition, each phase was observed as a precipitate with a plate morphology in the matrix of the other phase. In both cases, the phases share a common direction, (left bracket)011(right bracket)(bcc) (left bracket)001 (right bracket)(ort), and interface (habit plane, $(2(-1) 1)$ (bcc) $(1(-$ 1)0)(ort). Geometrical patterns of plates of the differen orientational variants were also observed. Analysis of the orientation relation, habit plane and plate patterns are consistent with the concept of a strain-type transition even though long range diffusion is required.

\section{3,318}

PB94-198702 Not available NTIS

National Inst. of Standards and Technology (MSEL), Gaithersburg, MD. Metallurgy Div.

Crystallographic Characterization of Some Intermetallic Compounds in the Al-Cr System. Final rept.

L. A. Bendersky, R. S. Roth, J. T. Ramon, and D.

Shechtman. 1991, $6 p$

Pub. in Metallurgical Transactions A 22, n1 p5-10 1991.

Keywords: "Aluminum intermetallics, "Crystal structure, Transmission electron microscopy, X-ray diffraction, Hexagonal lattices, Monoclinic lattices, Single crystals, Lattice parameters, Reprints, "Chromium intermeta!lics.

Two Al-Cr compounds known in literature as the nu and epsilon phases were studied in the present work by precession camera single crystal x-ray diffraction and transmission electron microscopy (TEM). The results are different from those reported in the literature. The epsilon phase was found to be hexagonal P6(3) $\mathrm{mmc}$, with lattice parameters $a=2.01 \mathrm{~nm}$ and $\mathrm{c}=2.48$ $\mathrm{nm}$. The phase is believed to be isomorphous to the
Al4Mn nu phase. The nu phase has monoclinic (pseudo-orthorhombic) $C$ centered Bravais lattice with a(3) approx. $=1.76 \mathrm{~nm}, \mathrm{~b}(3)$ approx $=3.05 \mathrm{~nm}, \mathrm{c}(3)$ approx. $=1.76 \mathrm{~nm}$ and beta approx. $=90 \mathrm{deg}$. Single crystal of the nu phase are usually twinned.

\section{3,319}

\section{PB94-198850 Not available NTIS}

National Inst. of Standards and Technology (MSEL)

Gaithersburg, MD. Reactor Radiation Div.

Structures of Sodium Metal.

Final rept.

R. Berliner, H. G. Smith, J. R. D. Copley, and J.

Trivisonno. 1992, 12p.

Pub. in Physical Review B 46, n22 p14 436-14 447 , 1 Dec 92 . Sponsored by Department of Energy, Washington, DC

Keywords: "Martensitic transformation, "Sodium, Neutron diffraction, Neutron scattering, Inelastic scattering, Stacking faults, Temperature dependence, Tempera ture range 0013-0065 K, Cryogenic temperature, Reprints.

The martensitic transformation in sodium metal has been studied using neutron-diffraction and neutron-inelastic-scattering techniques. In two separate experiments the transformation was observed on cooling near $32 \mathrm{~K}$. Measurements of the diffuse scattering, diffraction linewidths, quasielastic scattering, and the temperature dependence of the Sigma(4)(left bracket)hho(right bracket) phonon energies above the transformation show no evidence of transformation precursors. The sodium martensite appears as 24 rhombohedral variants, four about each bcc $(110)$, with reflections from their basal planes at $(1.018,0.92,+$ or $0.06),(0.92,1.018,+$ or -0.06$)$, and equivalent points. The layer stacking order of the variants is fixed by their relationship to the host bcc material. The crystallography of the low-temperature phase is shown to be a complex mixture of stacking-fault-affected rhombohedral polytypes of a particular 'almost-hexgonal' structure. These form a ladder of structures connected, one to another, by stacking faults. As the martensite is warmed and before the complete reversion to a bcc structure, the relative fraction of the different martensite phases changes. Near $55 \mathrm{~K}$, the longest-period polytypes are the most stable.

\section{3,320}

PB94-198900 Not available NTIS

National Inst. of Standards and Technology (MEL) Gaithersburg, MD. Automated Production Technology Div.

Dynamic Shear Modulus Measurements with Four Independent Techniques in Nickel-Based Alloys.

Final rept.

G. V. Blessing, A. Wolfenden, V. K. Kinra, M. R Harmouche, $P$. Terranova, Y. T. Chen, and V. Dayal. 1992, 3p.

Pub. in Jnl. of Testing and Evaluation 20, n4 p321-323 Jul 92.

Keywords: "Nickel alloys, "Shear properties, "Modulus of elasticity, "Vibration tests, *Nondestructive tests, Vibrational stress, Frequencies, Resonance testing, Mechanical properties, Resonant frequency, Reprints.

Experimental results are presented, on a round-robin basis, to study the effect of vibrational frequency on the dynamic shear moduli of two metals, Inconel 600 and Incoloy 907. Four separate laboratories, each using a different experimental technique, obtained results over the nominal frequency range of $7 \mathrm{kHz}$ to 10 $\mathrm{MHz}$. A brief discussion and comparison of the four techniques, both resonant and pulse, are included. No significant differences were observed for each alloy's modulus over this frequency range.

03,321

PB94-198934 Not available NTIS

National Inst. of Standards and Technology (MSEL),

Gaithersburg, MD. Metallurgy Div.

Thermodynamic Constraints on Non-Equilibrium Solidification of Ordered Intermetallic Compounds. Final rept.

W. J. Boettinger. 1991, 5p.

Keywords: "Intermetallic compounds, "Order-disorder transformations, "Solidification, Thermodynamic properties, Temperature, Trapping(Charged particles), Intertaces, Equilibrium, Freezing, Reprints.

Thermodynamic constraints on permissible values of temperature, liquid composition, solid composition and long range order parameter at the freezing interface during non-equilibrium solidification of intermetallic phases are defined in terms of the liquid and solid free energy functions.

03.322

PB94-198942 Not available NTIS

National Inst. of Standards and Technology (MSEL) Gaithersburg, MD. Metallurgy Div.

Disorder Trapping in Ni2TiAl.

Final rept.

W. J. Boettinger, L. A. Bendersky, J. Cline, J.A.

West, and M. J. Aziz. 1991, 4p.

Pub. in Materials Science and Engineering A 133 p592-595 Mar 91.

Keywords: "Nickel intermetallics, "Solidification, "Trapping(Charged particles), Interfaces, Melting Intermetallic compounds, Equilibrium, Order-disorder transformations, Reprints.

Pulsed laser melting and rapid resolidification can in duce disorder trapping in Ni2AITi at the solidification interface. The alloy formed from the melt with the nonequilibrium L2 structure and subsequently transformed to the equilibrium L2(sub 1) Heusler structure during cooling to room temperature.

\section{3,323}

PB94-199353 Not available NTIS

National Inst. of Standards and Technology (MSEL), Gaithersburg, MD. Metallurgy Div.

LMTO/CVM and LAPW/CVM Calculations of the Nickel Aluminide/Nickel Titanium Pseudobinary Phase Diagram.

Final rept

B. P. Burton, J. E. Osburn, and A. Pasturel. 1991

Pub. in Materials Research Society Symposia Proceedings, v213 p107-112 1991.

Keywords: Order disorder transformations, Aluminum intermetallics Nickel intermetallics, Titanium intermetallics, Phase diagrams, Reprints, "Nickel aluminides, "Nickel titanium, "Nickel aluminum titanium, Augmented plane wave method, Cluster variation method.

Linear Muffin Tin Orbital and Linearly Augmented Plane Wave calculations of equations of state were performed for observed and hypothetical ordered structures in the NiAl-NiTi System. Total energies were parameterized in both the Connolly and Williams and epsilon-G approximations, and the resulting parameters were used to calculate theoretical phase diagrams by the cluster variation method. Third nearest neighbor Al-Ti pairwise interactions are predicted to be strongly repulsive, and to be the major cause of observed immiscibility between $B 2$ ano L2(1) phases.

03.324

PB94-199403 Not available NTIS

National Inst. of Standards and Technology (IMSE) Gaithersburg, MD.

Modeling the Evolution of Structure in Unstable Solid Solution Phases by Diffusional Mechanisms. Final rept.

J. W. Cahn. 1991, 9p

Pub. in Scandinavian Jnl. of Metallurgy $20, n 1$ p9-17 1991.

Keywords: "Crystal-phase transformation, "Solid soluions, "Diffusion theory, Reprints, Nonlinear differentia equations, Order disorder transformations, Spinodal decomposition, Difference equations, Mathematical models, Alloys.

Certain phase changes evolve entirely by atom rearrangements among neighboring sites in a crysta structure and can be understood through the formulation and solution of a realistic diffusion equation. A portion of the 1956 thesis by Mats Hillert, published in 1961 under the title 'A Solid-solution Model for Inhomogeneous Systems', is the seminal theoretical paper in this subject. It deals with solid solutions that have atoms confined to lattice points with a one-dimensional concentration variation, that are unstable to ordering or phase separation, and that evolve entirely by atom exchanges. The results of the theory were applied to his experimental observations on a spinoda alloy. Subsequent theoretical studies have extended the theory to include many factors that make the theory more realistic. Among them is another of Mats Hillert's themes; the introduction of the effects of stresses that result from the diffusional processes. 
National Inst. of Standards and Technology (IMSE), Gaithersburg, MD.

Stability, Microstructural Evolution, Grain Growth, and Coarsening in a Two-Dimensional Two-Phase Microstructure.

Final rept.

J. W. Cahn. 1991, 11p

Pub. in Acta Metallurgica et Materialia 39, n10 p218921991991.

Keywords: *Grain growth, Binary systems(Materials), Grain boundaries, Two dimensional, Surface energy, Microstructure, Polycrystalline, Coarseness, Interfaces, Stability, Reprints.

The stability of microstructural features and how this affects grain growth and coarsening in a two-dimensional polycrystalline two-phase material with isotropic energies for the two grain boundaries and the interphase interface is examined using classical concepts of classical capilarity and surface energy driven processes. A catalog of stable microstructural features depends on surface-energy ratios; it is shown to include a four-grain junction that is stable for a range of surface-energy ratios, and, for a given set of ratios, is stable over a range of angles. It is the grain boundary analog of the line junctions among four phases, discussed by Gibbls. Three-grain junctions and grain boundaries had been known to have a limited range of stability. Inferences about grain growth, coarsening, and evolving morphology are obtained from the integrated curvature of the bounding surface of a grain which depends on the number and phase of neighboring grains, as well as the order of their arrangement. Under some conditions, a dispersion of grains of a boundary phase will tend to a uniform size; this phase resists coarsening.

\section{3,326}

PB94-199866 Not available NTIS

National Inst. of Standards and Technology (NEL)

Gaithersburg, MD. Precision Engineering Div.

Measuring the Stability of Three Copper Alloys.

Final rept.

T. Doiron, J. Stoup, P. Snoots, and G. Chaconas

1990, 8p.

Pub. in Proceedings of Society of Photo-Optical Instrumentation Engineers, San Diego, CA., July 12-13, 1990, v1335 p45-52.

Keywords: "Dimensional stability, "Copper alloys, *Experimental design, Dimensional measurement, Measuring instruments, Length, Probability theory Error analysis, Brass, Beryllium copper, Reprints, Tellurium copper alloys.

In the paper the authors report measurements of the dimensional stability of samples of brass, beryllium copper, and tellurium copper taken over an 18 month time span. Of the materials, brass was the most stable decreasing slightly in length at the rate of 1 part per million per year $(\mathrm{ppm} / \mathrm{y})$ with an uncertainty (3) of abou $1 \mathrm{ppm} / \mathrm{y}$. Tellurium copper shrank at an average rate of $2.4 \mathrm{ppm} / \mathrm{y}$ and beryllium copper at the rate of 5.8 $\mathrm{ppm} / \mathrm{y}$. In order to measure the instrumental uncertainty there were 4 samples of each material were measured, and the measurement scheme was designed to detect and correct for thermal dritt during measurements. The experiment design and the problems associated with these measurements and their associated uncertainties are discussed.

PB,327 $200326 \quad$ Not available NTIS

National Inst. of Standards and Technology (MSEL), Gaithersburg, MD. Metallurgy Div.

Micromechanics of Densification and Distortion.

Final rept.

R. B. Clough, and R. J. Schaefer. 1990, 17p

Pub. in Proceedings of International Conference on Isostatic Pressing (4th), Stratford-upon-Avon, UK, November 5-7, 1993, p38.1-38.17. Sponsored by Defense Advanced Research Projects Agency, Arlington, VA.

Keywords: "Metal powder, "Hot isostatic pressing "Densification, "Distortion, Compacting, Shear stress, Plastic properties, Finite element method, Porous materials, Powder metallurgy, Reprints.

While compaction under purely isostatic pressure is distortion-free, the introduction of shear stresses, e.g. by the canister, can cause a change in shape of a powder compact. The paper describes a plasticity theory for both shape and volume change of compacts. It is first formulated at a level sufficiently general to apply to both porous and particulate media. It is then combined with the Ashby micromechanical plasticity model for powder compaction, modified to allow for workhardening and applied to particulate compacts. It tests favorably against experimental data for a variety of powder compacts in uniaxial and isostatic stress states. The theory of Gurson for porous materials does not fit this data from particulate materials very well. The effect of shear stresses, generated by the containment cansiter, on shape change is then examined using data from copper powder compacts in copper canisters. The theory is available for finite element modeling.

\section{3,328}

PB94-200441 Not available NTIS

National Inst. of Standards and Technology (MSEL), Gaithersburg. MD. Metallurgy Div.

Effects of Crystalline Anisotropy and BuoyancyDriven Convection on Morphological Stability.

Final rept.

S. R. Coriell, G. B. McFadden, and R. F. Sekerka

1990, 8p.

Pub. in Proceedings of F. Weinberg International Symposium on Solidification Processing, Hamilton, Ontario, August 27-29, 1990, p44-51.

Keywords: *Tin base alloys, "Binary alloys, Indium alloys, Thermal conductivity, Fluid flow, Anisotropy, Convection, Stability, Reprints, "Directional solidification.

The effect of anisotropy of thermal conductivity and buoyancy-driven convection on morphological stability during directional solidification of a binary alloy is considered. Results are presented for dilute alloys of indium in tin. The onset of instability depends on crystallographic orientation, and can be oscillatory in time under certain conditions. Buoyancy-driven flow can result in a long wavelength instability that enhances morphological instability.

\section{3,329}

PB94-200474 Not available NTIS

National Inst. of Standards and Technology (IMSE),

Gaithersburg, MD. Metallurgy Div.

Assessment of the Al-Sb System.

Final rept.

C. A. Coughanowr, U. R. Kattner, and T. J.

Anderson. 1990, 10p.

Contract DE-FG05-86ER45276

Pub. in CALPHAD 14, n2 p193-202 1990. Sponsored by Department of Energy, Washington, DC.

Keywords: "Thermodynamic properties, "Phase diagrams, "Thermochemistry, Assessments, Antimony containing alloys, Aluminum alloys, Binary alloys, Reprints, "Aluminum antimony alloys.

An assessment of the thermochemistry and phase diagram data for the Al-Sb system using the Lukas optimization program is presented. A comparison between the Redlich-Kister and associate models is made.

\section{3,330}

PB94-203593 PC A03

National Inst. of Standards and Technology, Boulder, Co.

\section{Cryogenic Properties of Silver.}

D. R. Smith, and F. R. Fickett. Jan 94, 14p, NIST/

$\mathrm{TN}-1363$

Also available from Supt. of Docs as SNO03-00303265-4.

Keywords: "Cryogenics, "Silver, "Charts, Precious metals, Mechanical properties, Thermodynamic properties, Tensile properties, Resistivity, Low temperature, Cryochemistry, Stress-strain relationships.

The chart presents several cryogenic properties of silver.

\section{3,331}

PB94-211091 Not available NTIS

National Inst. of Standards and Technology (MSEL),

Gaithersburg, MD. Metallurgy Div.

Ordered omega-Derivatives in a Ti-37.5Al-2ONb at\% Alloy.

Final rept.

L. A. Bendersky, B. P. Burton, W. J. Boettinger, and

F. S. Biancaniello. 1990, $6 \mathrm{p}$.

Pub. in Scripta Metallurgica et Materialia 24, n8 p154115461990

Keywords: "Titanium containing alloys, "Titanium intermetallics, Molecular structure, Ternary compounds, Aluminum intermetallics, Niobium intermetallics, Transmission electron microscopy, Reprints, "Titanium aluminum niobium, "Omega phase.

In the present work we report finding a new Ti-Al-Nb phase which is apparently a more ordered derivative of the B82 phase. The phase was found as fine precipitates in the presumably $\mathrm{B} 8(2)$ matrix in $\mathrm{Ti}$ $37.5 \mathrm{Al}-20 \mathrm{Nb}$ at \% alloy. A possible structure of the new phase will be discussed based on the results of electron diffraction and high-resolution microscopy.

\section{3,332}

PB94-211638 Not available NTIS

National Inst. of Standards and Technology (MSEL) Gaithersburg, MD. Metallurgy Div.

Energy and Migration of Grain Boundaries in Polycrystals.

Final rept.

C. Handwerker. 1993, 11p.

Pub. in Interfaces: Structures and Properties, Chapter 20, p339-349 1993.

Keywords: "Grain boundanies, "Microstructure "Polycrystals, "Physical properties, "Diffusion, Scanning electron microscopy, Electron diffraction Backscattering, Surfaces, Reprints.

A challenge in the field of microstructure evolution is to understand the physical processes that govern microstructure evolution and the relationship between microstructure and the resulting physical properties of the polycrystal. In this paper two new scanning electron microscope (SEM) techniques for measuring two fundamental characteristics of grain boundaries are described. A metal reference line technique (MRL) has been developed for measuring surface-grain boundary dihedral angles from thermal grooves at a sample surface using an SEM. The second technique is the electron backscattered diffraction pattern (EBDP) for measuring grain texture and grain-grain misorientations.

\section{3,333}

PB94-211646 Not available NTIS

National Inst. of Standards and Technology (MSEL), Gaithersburg, MD. Metallurgy Div.

Fabrication of Platinum-Gold Alloys in Pre-His panic South America: Issues of Temperature and Microstructure Control.

Final rept.

C. Handwerker, H. Lechtman, R. Marinenko, D. Bright, and D. Newbury. 1991, 16p.

Pub. in Materials Research Society Symposia Pro. ceedings, v185 p649-6641991.

Keywords: "Metallurgy, "Platinum alloys, "Gold alloys, *Temperature *Microstructure, Sintering, Electron microscopy, Phase diagrams, Chemical composition, Fabrication, Historical aspects, Reprints.

The microstructures and compositions of sintered Pt Au objects from La Tolita, Ecuador, and of experimentally simulated Pt-Au alloy samples were analyzed using new electron microprobe microanalysis (EPMA) techniques and data from the Pt-Au phase diagram in an effort to determine the fabrication temperatures used by Pre-Hispanic South American smiths. A comparison of EPMA results from the simulated materials with the corresponding results from the La Tolita Pt Au objects suggests that the Pt-Au objects were never heated as high as $1100 \mathrm{C}$ and probably never contained a liquid phase. As illustrated by this comparison, the qualitative and quantitative information provided by these new digital acquisition and display techniques ta exceeds what conventional line scan and $x$-ray do maps could provide.

\section{3,334}

PB94-212040 Not a vailable NTIS

National Inst. of Standards and Technology (NML), Gaithersburg, MD. Chemical Process Metrology Div. In-situ Fume Particle Size and Number Density Measurements from a Synthetic Smelt.

Final rept.

J. C. L. Hsu, C. Presser, and D. T. Clay. 1990, 9p. Pub. in Proceedings of American Institute of Aero nautics and Astronautics/American Society of Mechan ical Engineers Thermophysics and Heat Transfer Conference, Seattle, WA., June 18-20, 1990, p67-75.

Keywords: "Particle size distribution, "Smelting, Polarization, Density, Fumes, Sodium, Potassium, Light scattering, Reprints.

In Kraft recovery furnaces, significant fuming takes place of sodium and potassium. It is essential to quan- 


\section{Nonferrous Metals \& Alloys}

tify the fume generation rate because fume particles coat heat transter tubes and piug flue gas passages. To address this problem an ensemble light scattering technique, based on measurement of the polarization ratio, was used to measure fume particle size and number density from a synthetic Kraft smelt. This insitu nonintrusive technique is used because of its application to high density particle-laden flows. Measurements were also carried out at different scattering angles in order to determine the imaginary part of the particle complex refractive index. In addition, fume particle size distributions obtained from scanning electron microscope images were correlated with the log-norma distribution function. Results indicated that an increase in nitrogen flow rate generated smaller fume particles, while a higher air flow rate produced larger fume particles. In addition, increased nitrogen flow rate increased fume mass density, but the air flow rate had a negligible effect. The fume particle geometric mean diameter $(\mathrm{Dg})$ was found to vary with gas flow rate between values of 0.2 and $0.3 \mathrm{~m}$, and number density varied between $3 \times 10(\exp 7)$ and $2 \times 10(\exp 8)$ particles/cc.

\section{3,335}

\section{PB94-212586 Not available NTIS}

National Inst. of Standards and Technology (MSEL), Gaithersburg, MD. Metallurgy Div.

Development in the Theory and Analysis of Eddy Current Sensing of Velocity in Liquid Metals.

Final rept.

A. H. Kahn, and L. C. Phillips. 1992, 12p.

Pub. in Res. Nondestr. Eval. 4, p237-2481992.

Keywords: "Velocity measurement, "Liquid metals *Eddy currents, Magnetic properties, Nondestructive tests, Magnetic fields, Velocity distribution, Magnetic coils, Flow measurement, Reprints, "Electromagnetic flowmeters.

We have performed a theoretical study of the magnetic fields produced by a single turn ac coil encircling a moving conductive rod or tube of flowing liquid metal. A solution exact to first order in the velocity has been obtained as a closed-form integral. Radial variation of the velocity profile is included. The solution allows calculation of the voltages induced in two secondary coils wired in opposition for application as a velocimeter. With secondary coils wired in series, the calculation provides the classical impedance vs. frequency curves which allow determination of sample conductivity and diameter. The solution has been used to opti mize design for a sensor to be used for monitoring speed and tube bore diameter in a liquid metal atomizer system. An additional solution has been obtained for arbitrary magnitude of velocity, but for constant velocity profile.

\section{3,336}

PB94-212636 Not available NTIS

National Inst. of Standards and Technology (MSEL) Gaithersburg, MD. Metallurgy Div.

Thermodynamic Calculation of the Ternary Ti-AlNb System.

Final rept.

U. R. Kattner, and W. J. Boettinger. 1992, 9p.

Pub. in Materials Science and Engineering A152, p9171992

Keywords: "Ternary alloy systems, "Titanium alloys, "Aluminum alloys, "Niobium alloys, Phase diagrams, Thermodynamics, Computation, Reprints, Phase equilibrium.

Phase equilibria of the ternary Ti-Al-Nb system are dominated by the large range of homogeneity of (beta$\mathrm{Ti}, \mathrm{Nb}$ ), the binary intermetallic compounds of the $\mathrm{Nb}$ $\mathrm{Al}$ and Ti-Al systems and the formation of two ternary compounds. The available ternary experimental data, together with a thermodynamic extrapolation of the ternary system from the binary systems, have been used to calculate the ternary phase diagram. The model descriptions of the Gibbs energies of most of these compounds are given by the existing calculations of the binary systems. In order to model a phase which is present in only one binary system, but has a ternary homogeneity range, a hypothetical phase with the same structure was analytically described for each binary system. Such a phase would, of course, be metastable in the other binary systems. Constraints on the Gibbs energies of formation were derived from the crystal structures of the corresponding ordered compounds. These same constraints were employed for the corresponding phases in the ternary system. In a final optimization step, ternary parameters were introduced and adjusted to the available experimental data.
The as-derived description of the ternary Ti-Al-Nb system can be used to estimate single or multiphase fields and thermodynamic quantities where no experimental data are yet available. It is also useful as an indicator of problem areas for which additional experimental data are required.

\section{3,337}

PB94-212644 Not available NTIS

National Inst. of Standards and Technology (MSEL), Gaithersburg, MD. Metallurgy Div.

Thermodynamic Assessment and Calculation of the Ti-Al System.

Final rept.

U. R. Kattner, J. C. Lin, and Y. A. Chang. 1992, 10p. Pub. in Metallurgical Transactions A 23A, p2081-2090 Aug 92

Keywords: "Binary alloy systems, "Titanium alloys, "Aluminum alloys, Least squares method, Phase diagrams, Thermodynamics, Intermetallics, Computation, Reprints.

A thermodynamic description of the Ti-Al system has been developed. Three different analytical descriptions were used to describe the three different types of phases occurring in the Ti-Al system: the stoichiometric compounds, the disordered solution phases, and the ordered intermetallic compounds which have homogeneity ranges. A least-squares technique was used to optimize the thermodynamic quantities of the analytical description using experimental data available in the literature. The calculated phase diagram, as well as the thermodynamic functions, agree well with the critically evaluated experimental data from the literature.

\section{3,338}

PB94-216694 Not available NTIS

National Inst. of Standards and Technology (IMSE), Boulder, CO. Fracture and Deformation Div.

Recommended Changes in ASTM Test Methods D2512-82 and G86-84 for Oxygen-Compatibility Mechanical Impact Tests on Metals.

Final rept.

G. W. McColskey, R. P. Reed, N. J. Simon, and J. W. Bransford. 1991, 28p.

Pub. in Flammability and Sensitivity of Materials in Oxygen-Enriched Atmospheres, American Society for Testing and Materials Special Technical Publication 1111, v5 p126-153 1991

Keywords: "Test methods, "Impact tests, "Aluminum alloys, "Lithium alloys, "Flammability, Energy absorption, Oxygen, Sensitivity, Ignition, sons, Reprints, ASTM Test Method D2512-82, ASTM Test Method G86-84, Oxygen compatibility mechanical impact tests.

A cooperative test program to assess the oxygen compatibility of aluminum-lithium alloys with White Sands Test Facility (WSTF), Marshall Space Flight Center (MSFC), and the National Institute of Standards and Technology (NIST) led to assessment of the mechanical impact test by NIST. There were substantially different variations in test parameters between the test laboratories; some of these led to significant variability in the actual energy absorbed by the specimens. Therefore, test results varied widely. To reduce the large disparities in interlaboratory test results, a number of changes are recommended in the current ASTM test standards.

\section{3,339}

PB95-107322 Not available NTIS

National Inst. of Standards and Technology (MSEL), Gaithersburg, MD. Ceramics Div.

Electric Field Effects on Crack Growth in a Lead Magnesium Niobate.

Final rept.

A. S. Raynes, G. S. White, and S. W. Freiman. 1990, $14 \mathrm{p}$

Sponsored by Office of Naval Research, Arlington, VA Pub. in Ceram. Trans. 15, p129-142 1990.

Keywords: "Crack propagation, "Electric fields, "Lead compounds, "Niobates, "Magnesium compounds, Strain(Mechanics), Field strength, Electrodes, Fracturing, Reprints, Electrostrictive strain.

The influence of an applied electric field on crack extension in a lead magnesium niobate ceramic was investigated in order to evaluate possible effects of electrostrictive strain on crack propagation. Cracks were generated by indenting polished specimen sur- faces equidistant between imbedded parallel electrodes. The field was applied before indentation and maintained for the duration of the measurement. Results showed that cracks emanating from indentations perpendicular to the applied field were consistently shorter than those from indentations made with no field present. No effect of field was observed on cracks parallel to the field. Crack length also appeared to depend on the indentation location relative to the electrodes. A qualitative model explaining these results is proposed

\section{3,340}

PB95-107348 Not available NTIS

National Inst. of Standards and Technology (IMSE) Boulder, CO. Fracture and Deformation Div.

Macro- and Microreactions in Mechanical-Impact Tests of Aluminum Alloys.

Final rept.

R. P. Reed, C. N. McCowan, J. D. McColskey, and N. J. Simon. 1991, 20p.

Pub. in Proceedings of the Symposium on Flammability and Sensitivity of Materials in Oxygen-Enriched Atmospheres, Cocoa Beach, FL., May 14-16, 1991. ASTM STP 1111, p240-259.

Keywords: "Aluminum alloys, "Lithium alloys, "Impact tests, "Accelerated life tests, Morphology, Metallography, Liquid oxygen, Oxygen, Pressure, Reprints, Macroreactions, Microreactions.

Open-cup and pressurized mechanical-impact tests have been conducted at White Sands Test Facility on selected tempers of Al-Li alloys (2090, 8090, WLO49) and $\mathrm{Al}$ alloy 2219. Both liquid (LOX) and gaseous (GOX) oxygen environments were used and pressure and drop height (potential energy) were varied in each environment. Reactions were observed in all alloys and tempers on both sides of the specimens. Reaction sizes, morphologies, and locations were characterized using a magnifying glass ( $2 X$ power), a light microscope (5 to 100X), and a scanning electron microscope $(100$ to $3000 X)$. Photomicrographs of various types of macro- and microreactions are presented and discussed. The variety of sizes of reactions poses a serious problem for the definition of a reaction in standards documents for LOX and GOX mechanical-impact qualification testing. This dilemma is discussed.

\section{3,341}

PB95-107355 Not available NTIS

National Inst. of Standards and Technology (IMSE), Boulder, CO. Fracture and Deformation Div.

Influence of Specimen Absorbed Energy in LOX Mechanical-impact Tests.

Final rept.

R. P. Reed, N. J. Simon, J. R. Berger, and J. D

McColskey. 1991, 18p.

Pub. in Proceedings of Symposium on Flammability and Sensitivity of Materials in Oxygen-Enriched Atmospheres, Cocoa Beach, FL., May 14-16, 1991, ASTM STP 1111, p381-398.

Keywords: "Impact tests, "Aluminum alloys, "Lithium alloys, *Energy absorption, Fracture mechanics, Liquid oxygen, Potential energy, Deformation, Strain(Mechanics), Reprints.

In mechanical impact tests, the potential energy of the plummet is divided between energy absorbed by the specimen, the plummet kinetic energy of rebound, and various losses in the test machine. Thus, all of the potential (or drop height) energy is not transferred to the specimen. The fracture and deformation characteristics and the reactability of metal specimens are dependent on the amount of energy that the specimen absorbs. The absorbed energy may be calculated either from knowledge of the potential energy and rebound height (assuming losses are negligible), or from the depth of striker-pin penetration into the specimen. The depth measurement gives an estimate of specimen strain; a reasonable estimate of force exerted on the specimen during deformation allows calculation of the work of deformation. The effects of the absorbed energy on fracture and deformation characteristics and on reactions in Al-Li alloys and alloy 2219 are discussed.

\section{3,342}

PB95-108577 Not available NTIS

National Inst. of Standards and Technology (NML), Gaithersburg, MD. Chemical Process Metrology Div. 
Parametric Investigation of Metal Powder Atomization Using Laser Diffraction.

Final rept.

C. Presser, S. Huzarewicz, F. Biancaniello, S. D.

Ridder, and H. G. Semerjian. 1990, 5p.

Pub. in Proceedings of Annual Conference on Extended Abstracts ILASS-Americas (4th), Hartford, CT., May 21-23, 1990, p187-191.

Keywords: "Atomization, "Powder metals, "Particle size distribution, *'Size determination, "Diffraction analysis, Reprints, Powder metallurgy, Solidification, Flow velocity, Microstructure, "Laser diffraction.

Special properties of rapidly solidified metal powders that are of particular interest to materials engineers include improved chemical homogeneity, novel microstructures, increased solubility and spherically smooth surfaces. These properties have a strong dependence on the solidification rate, and therefore on the particle size. Inert gas pressure, nozzle design and gas/melt flow rates can be used to control powder size. once the particular characteristics of a system are understood. A size, once the particular characteristics of a system are understood. A state-of-the-art sensor system is therefore required to provide high-speed, nonintrusive measurements of particle characteristics for process feedback and control. To address this challenge, a commercially-available particle size analyzer, based on laser diffraction is used to monitor the powder stream in the exhaust section of the Supersonic Inert Gas Metal Atomizer (SiGMA) at NIST. A line-ofsight measurement technique is preferred over one with a discrete point measurement, since this technique provides particle size information which is more representative of the entire flow stream passing through the atomizer exhaust section.

03,343
PB95-126355 Not available NTIS

National Inst. of Standards and Technology (MSEL), Gaithersburg, MD. Metallurgy Div.

Effect of Mn Content on the Microstructure of AIMn Alloys Electrodeposited at $150 \mathrm{C}$.

Final rept.

B. Grushko, and G. R. Stafford. 1988, 5p

Pub. in Israel Jnl. of Technology 24, n3-4 p523-527 1988.

Keywords: *Aluminum manganese alloys, *Electrodeposition, "Microstructure, "Manganese additions, Fused salts, $X$-ray diffraction, Substrates, Electron microscopy, Reprints.

Al-Mn alloys with compositions ranging between 0-50 $w t \% \mathrm{Mn}$ were electrodeposited at $150 \mathrm{C}$ onto copper substrates from a chloroaluminate molten salt electrolyte with controlled additions of $\mathrm{MnCl} 2$. The results of examination by scanning electron microscopy (SEM), transmission electron microscopy (TEM), energy dispersive $x$-ray analysis (EDAX) and $x$-ray diffraction are presented.

\section{3,344}

PB95-141057 Not available NTIS

National inst. of Standards and Technology (NEL), Gaithersburg. MD. Factory Automation Systems Div. Intelligent Control of an Inert Gas Atomization Process.

Final rept

S. A. Osella, S. D. Ridder, F. S. Biancaniello, and P. I. Espina. 1991, 4p

Pub. in Jnl. of the Minerals Metals and Materials Society 43, n1 p18-21 1991.

Keywords: "Metal powder, "Gas atomization, "Controllers, "Artificial intelligence, Process control, Computer aided manufacturing, Atomizers, Control systems, Rare gases, Feedback control, Cybernetics, Heuristic methods, Reprints, US NIST.

Intelligent control is an attempt to specify the function of a controller in ways which mimic the decision-making capabilities of humans. The National Institute of Standards and Technology's (NIST) program on Intelligent Processing of Metal Powders is a multi-disciplinary research initiative investigating the application of intelligent control technologies to improve the state-ofthe-art of metal powder manufacturing. This paper reviews the design of NIST's supersonic inert gas metal atomizer control system.

\section{3,345}

\section{PB95-147914 PC A04/MF A01}

National Inst. of Standards and Technology (CAML), Gaithersburg, MD. Applied and Computational Mathematics Div.
Phase-Field Model for Solidification of a Eutectic Alloy.

A. A. Wheeler, G. B. McFadden, and W. J.

Boettinger. Sep 94, 52p, NISTIR-5523.

Keywords: "Eutectic alloys, "Solidification, "Phase transformations, Liquid-soil interfaces, Crystallization, Concentration(Composition), Mathematical models, *Phase field models.

In this paper we discuss two phase-field models for solidification of a eutectic alloy, a situation in which a liquid may transform into two distinct solid phases. The first is based on a regular solution model for the solid with a chemical miscibility gap. This model suffers from the deficiency that, in the sharp interface limit, it approximates a free-boundary problem in which the surface energy of the solid/solid interface is zero and consequently mechanical equilibrium at a trijunction requires that the solid/solid interface has zero dihedral angle (locally planar). We propose a second model which uses two order parameters to distinguish the liquid phase and the two solid phases. We provide a thermodynamically consistent derivation of this phasefield model which ensures that the local entropy production is positive. We conduct a sharp interface asymptotic analysis of the liquid/solid phase transition and show it is governed by a free-boundary problem in which both surface energy and interface kinetics are present. Finally, we consider a sharp interface analysis of a stationary trijunction between the two solid phases and the liquid phase, from which we recover the condition that the interfacial surface tensions are in mechanical equilibrium (Young's equation). This sharp interface analysis compares favorably with numerical solutions of the phase-field model appropriate to a trijunction.

\section{3,346}

PB95-151346 Not available NTIS

National Inst. of Standards and Technology (MSEL), Gaithersburg, MD. Polymers Div.

New Alloys Show Extraordinary Resistance to Fracture and Wear.

Final rept.

R. M. Waterstrat. $1992,4 \mathrm{p}$.

Sponsored by American Dental Association Health Foundation, Chicago, IL.

Pub. in Jnl. of the American Dental Association 123, p33-36 Dec 92

Keywords: "Zirconium alloys, "Palladium alloys, "Ruthenium alloys, "Wear resistance, "Fracture strength, Implantation, Dental equipment, Medical equipment, Performance evaluation, Crack propagation, Reprints.

A newly invented alloy of zirconium, palladium and ruthenium holds great promise for use in high-strength orthodontic wires, dental and medical implants, and in other applications because of its extraordinary fracture-toughness and wear resistance. Cracks produced experimentally in alloy samples are apparently prevented from propagating by internal stress-induced transformations that occur at the crack tips. This type of behavior has been observed previously in certain industrial ceramics and alloy steels but is a relatively rare phenomenon. ADA and government scientists at the National Institute of Standards and Technology are particularly excited about the alloy because of the insights it might offer on the wear process mechanism and because of its practical implications.

\section{3,347}

PB95-151684 Not available NTIS

National Inst. of Standards and Technology (MSEL), Gaithersburg, MD. Metallurgy Div.

Investigation into a Practical Grain Growth Model for Hot Isostatic Pressing.

Final rept.

J. J. Wlassich, and R. B. Clough. 1989, 6p

Sponsored by Defense Advanced Research Projects Agency, Arlington, VA. and Air Force Office of Scientific Research, Bolling AFB, DC.

Pub. in Proceedings of International Conference on Hot Isostatic Pressing (2nd): Theory and Applications, Gaithersburg, MD., June 7-9, 1989, p123-128.

Keywords: *Grain growth, *Hot isostatic pressing, "Metals, Grain structure(Metallurgy), Sintering, Copper, Microstructure, Diffusion, Pressing(Forming), Reprints.

This paper describes an investigation into a practical model of grain growth for coupling with a microstructural model of densification during $\mathrm{Hot}$
Isostatic Pressing (HIP). Grain growth affects densification through plastic yielding and diffusive mechanisms, which are grain size dependent. Depending on the HIP conditions, grain growth can increase or inhibit densification. The grain growth formulation stated here contains relatively well-established parameters for many materiais. Coupled with the densification model it executes rapidly on modest computer resources, making the models amenable for further study into the effects of microstructural coarsening on densification. The grain growth model was compared to experiments using OFHC copper compacts that were sintering. Results show that the grain growth model can yield good results but is necessarily sensitive to the value for the activation energy of volume self diffusion.

03,348

PB95-152229 Not available NTIS

National Inst. of Standards and Technology (MSEL) Gaithersburg, MD. Ceramics Div.

Hybrid Method for Determining Material Properties from Instrumented Micro-Indentation Experiments. Final rept.

Y. M. Chen, A. W. Ruff, and J. W. Dally. 1994, 8 p Sponsored by National Science Foundation, Arlington, VA. and Office of Naval Research, Arlington, VA. Pub. in Jnl. Materials Research 9, n5 p1314-1321 May 94.

Keywords: "Indentation, "Copper, "Micromechanics, "Impact tests, Deformation, Finite element method, Penetration, Computational grids, Plastic properties, Elastic properties, Reprints, "EPIC computer program, "Hybrid methods.

The impact code EPIC was employed to study the relationship between the applied force and the penetration depth in a micrometer-scale indentation experiment with oxygen free high conductivity (OFHC) copper. EPIC is an elastic-plastic finite element code that uses a Lagrangian formulation and triangular mesh, which can accommodate large deformation without the need to remesh during the computation process. By fitting the force-penetration curves for a triangular indente with second degree polynomials, it was demonstrated that the fit changed with two material constants in the constitutive equation. A systematic procedure for determining the material constants is described that is based on matching either the slope or the curvature of the force penetration depth curves from numerical simulation and experiments. It is concluded that material constants can be determined from indentation data obtained using pyramidal or spherical indenters as well as a flat-ended indenter.

03,349

PB95-161782 Not available NTIS

National Inst. of Standards and Technology (MSEL) Gaithersburg, MD. Metallurgy Div.

Ultrasonic Method for Reconstructing the Two-Dimensional Liquid-Solid Interface in Solidifying Bodies.

Final rept

F. A. Mauer, S. J. Norton, Y. Grinberg, D. Pitchure,

and H. N. G. Wadley. 1991, 7p.

Pub. in Metallurgical Transactions B 22, n4 p467-473 1991.

Keywords: "Solidification, "Ultrasonic tests *Aluminum, Tomography, Time of flight spectrometers, Strands, Fluid boundaries, Least squares method, Reprints, "Liquid-solid interfaces.

A method is demonstrated for reconstructing the twodimensional boundary of the liquid core in a solidifying strand of aluminum using ultrasonic time-of-flight data. Approximating the boundary by an ellipse leads to a model that can be used to calculate the time-of-fligh along any path in the plane of the ellipse. The shape and location of the ellipse are specified by four free parameters. A least-squares algorithm is then used to find the set of four parameters that provides the best fit to the measured time-of-flight data on ten intersecting paths through the strand. For a strand with a 6 inch square cross section, the agreement between the actual interface and the computed interface was within about + or $-2 \%$. Reference data for the temperature dependence of longitudinal velocity for solid 1110 alu minum are included.

03,350

PB95-163028 Not available NTIS

National Inst. of Standards and Technology (NEL) Gaithersburg, MD. Applied and Computational Mathematics Div. 
Effects of Elastic Stress on the Stability of a SolidLiquid Interface.

Final rept.

B. J. Spencer, S. H. Davis, G. B. McFadden, and P.

W. Voorhees. $1990,2 \mathrm{p}$.

Sponsored by National Science Foundation, Washing ton, DC. and National Aeronautics and Space Administration, Washington, DC

Pub. in Applied Mechanics Reviews 43, n5 pS54-S55 May 90.

Keywords: "Liquid-solid interface, Binary alloys, Stability, Reprints, "Crystal-melt interface, Directional solidification, Elastic stresses.

The effects of elastic stress on the stability of solidliquid interfaces under a variety of conditions are discussed. In the cases discussed, the nonuniform composition field in the solid, which accompanies either the melting process or the development of a perturbation on the solid-liquid interface during solidification, generates nonhydrostatic stresses in the solid. Such compositionally generated elastic stresses have been shown experimentally to induce a solidifying solid-liquid interface to become unstable. We are in the process of analyzing the effects of these stresses on the conditions for morphological stability of a directionally solidified binary alloy.

\section{3,351}

PB95-164620 Not available NTIS

National Inst. of Standards and Technology (MEL), Gaithersburg, MD. Precision Engineering Div.

Surface Texture.

Final rept.

J. F. Song, and T. V. Vorburger. 1992, 12p.

Pub. in Metals Handbook, v18 p334-345 1992

Keywords: "Surface roughness, Metal working, Automotive components, Power spectra, Autocorrelation, Measurement, Calibration, Skewness, Tribology, Profiles, Pens, Uses, Reviews, Reprints, "Surface texture, Surface topography.

The field of surface texture measurement is reviewed with emphases on stylus techniques and applications to tribology and related problems. We define a number of useful surface statistical parameters including roughness average, rms roughness, skewness, and peak spacing as well as the power spectral density and autocorrelation functions. The strengths and limitations of stylus instruments are discussed in detail, along with procedures for calibrating them. Practical applications of these measurements include the study of lip seals, the study of wear by standard techniques, and many other problems in the automotive and metal working industries.

03,352

PB95-168415 Not available NTIS

National Inst. of Standards and Technology (EEEL), Boulder, CO. Electromagnetic Technology Div.

Effect of Microstructure on Phase Formation in the Reaction of Nb/Al Multilayer Thin Films.

Final rept.

K. Barmak, K. R. Coffey, D. A. Rudman, and S.

Foner. 1992, 6p.

Sponsored by Department of Energy, Washington, DC. Pub. in Materials Research Society Symposia Proceedings, v230 p61-66 1992.

Keywords: "Aluminum, "Niobium, Metal films, Thin films, Microstructure, Reprints, *Interfacial reactions, Phase formation, Multilayers.

We investigated the phase formation sequence in the reaction of multilayer thin films of $\mathrm{Nb} / \mathrm{Al}$ with overall compositions of 25 and 33 at.\% Al. We report novel phenomena which distinguish thin-film reactions unequivocally from those in bulk systems. For sufficiently thin layers, composition and stability of product phases are found to deviate significantly from that predicted from the equilibrium phase diagram. We demonstrate that in the $\mathrm{Nb} / \mathrm{Al}$ system, the length scales below which such deviations occur is about $150 \mathrm{~nm}$. We believe that these phenomena occur due to the importance of grain boundary diffusion and hence microstructure in these thin films.

\section{3,353}

PB95-168456 Not available NTIS

National Inst. of Standards and Technology (EEEL), Boulder, CO. Electromagnetic Technology Div.
First Nb/Al.

Final rept.

K. R. Coffey, K. Barmak, D. A. Rudman, and S.

Foner. 1992, 6p

Sponsored by Department of Energy, Washington, DC Pub. in Materials Research Society Symposia Proceedings, v230 p55-60 1992.

Keywords: "Niobium, Aluminum, Transmission electron microscopy, $X$ ray diffraction, Metal films, Thin films, Calorimetry, Annealing, Aluminum intermetallics, Niobium intermetallics, Reprints, *Interfacial reactions, Phase formation, Niobium aluminides, Multilayers.

Phase formation kinetics in the reaction of $\mathrm{Nb} / \mathrm{Al}$ multilayered thin films were investigated using scanning calorimetry, $x$-ray diffraction, and transmission electron microscopy. The first phase to form upon annealing the $\mathrm{Nb} / \mathrm{Al}$ layered structure is the $\mathrm{NbAI3}$ intermetallic. Its formation is clearly identified by the calorimetry to be a two-stage process, which has been modeled as the nucleation and three-dimensional growth to coalescence of the product phase in the plane of the initial interface, followed by the thickening of the product layer by one-dimensional growth perpendicular to the interface plane. For the initial reaction stage the reaction front velocity is higher than can be supported by diffusional transport within the lattice adjacent to the moving interface. Thus diffusion along nonequilionium interfaces must be the growth mechanism. The large volume fraction consumed during the initial reaction stage indicates a lower nucleation site density than expected at a $\mathrm{Nb} / \mathrm{Al}$ interface at local equilibrium, suggesting that the interface

03,354
PB95-176103 Not available NTIS

National Inst. of Standards and Technology (MSEL), Gaithersburg, MD. Reactor Radiation Div.

Small Angle Neutrons Scattering from ing.

Final rept.

P. G. Sanders, J. R. Weertman, J. G. Barker, and R. W. Siegel. 1993, 6p.

Pub. in Scripta Metallurgica et Materialia 29, p91-96 1993.

Keywords: "Neutron scattering, "Palladium, "Crystal defects, "Grain boundaries, Small angle scattering, Particle size, Mechanical properties, Crystal structure, Reprints, "Nanocrystalline materials.

Nanocrystalline materials, with grain sizes on the order of nanometers, have many interesting properties as a result of having confining grain sizes and a significant fraction of their atoms in grain boundary regions. Nanocrystalline materials may be synthesized by inert gas condensation and compaction, as developed by Gleiter and co-workers. While this processing method is very effective, it does produce specimens containing defects of various sizes. Evidence for the presence of these defects in nanocrystalline metals includes substantial deviations from theoretical density, positron lifetime measurements, and small angle neutron scattering (SANS) measurements. Previous SANS results have been interpreted primarily in terms of scattering from low density grain boundaries, although the contribution from pores has also been recognized. The presence of such defects is believed to have a strong influence on the apparent strength of nanocrystalline metals; therefore, any comprehensive study of mechanical behavior should include detailed information on the defect structure of the material. As part of a study of mechanical properties and their correlation with changes in internal structure, SANS measurements were made on a series of nanocrystalline palladium ( $n-P d$ ) samples. Preliminary results of the study are described.

\section{3,355}

PB95-181111 Not available NTIS

National Inst. of Standards and Technology (CAML), Gaithersburg, MD. Applied and Computational Mathematics Div.

Morphological Development of Second-Phase Particles in Elastically-Stressed Solids.

Final rept

P. W. Voorhees, G. B. McFadden, and W. C.

Johnson. $1992,14 \mathrm{p}$

Grant NSF-DMR89-57219

Sponsored by National Science Foundation, Washington, DC., National Aeronautics and Space Administration, Washington, DC., and Defense Advanced Research Projects Agency, Arlington, VA
Pub. in Acta Metall. Mater. 40, n11 p2979-2992 1992.

Keywords: "Particle shape, Boundary integral method, Particle motion, Thermodynamics, Coarseness, Elasticity, Morphology, Anisotropy, Dynamics, Reprints, Two phase systems, Ostwald ripening, Particle coarsening.

We examine the equilibrium shape and the dynamics of the morphological evolution of a coherent misfitting particle in an elastically anisotropic medium. No a prior assumptions are made on the possible particle morphologies; the particle evolves in a manner consistent with both the diffusion and elastic fields surrounding the particle and the thermodynamics of interfaces in stressed solids. Through these calculations we find the thermodynamically stable equilibrium shape of a misfitting particle. By thermodynamically stable we mean that all the equilibrium conditions, elastic, chemical and interfacial are satisfied simultaneously in contrast to previous treatments which determined the equilibrium shape by minimizing the sum of the elastic and interfacial energies for certain classes of particle shapes. We find that the equilibrium particle morphologies are not simple geometric shapes, have fourfold symmetry and a continuously varying interfacial curvature with position along the interface. Furthermore, the results show that the times required for a particle to evolve to its equilibrium morphology are likely to be within those which are accessible experimentally. In addition, we develop a general approach for determining the equilibrium shape of a particle in an elastically stressed solid.

\section{3,356}

PB96-102207 Not available NTIS

National Inst. of Standards and Technology (MSEL) Gaithersburg, MD. Metallurgy Div.

Radiance Temperatures (in the Wavelength Range $523-907 \mathrm{~nm}$ ) of Group IV B Transition Metals Titanium, Zirconium, and Hafnium at Their Melting Points by a Pulse-Heating Technique.

Final rept.

A. Cezairliyan, J. L. McClure, and A. P. Miller. 1994, $18 p$.

Sponsored by National Aeronautics and Space Administration, Washington, DC

Pub. in International Jnl. of Thermophysics, v15 n5 p993-1009 1994.

Keywords: "Emissivity, "Transition metals, "Melting points, "Pulse heating, Temperature measurement, Radiant flux density, Hafnium, Titanium, Zirconium, High temperature, Reprints.

The melting-point radiance temperatures (at six wavelengths in the range 523-907 $\mathrm{nm}$ ) of the Group IVB transition metals titanium, zirconium, and hafnium were measured by a pulse-heating technique. The method is based on rapid resistive self-heating of the specimen from room temperature to its melting point in less than $1 \mathrm{~s}$ and on simultaneously measuring the specimen radiance temperatures every $0.5 \mathrm{~ms}$ with a high-speed six-wavelength pyrometer. Melting was manifested by a plateau in the radiance temperatureversus-time function for each wavelength. The meltingpoint radiance temperatures for a given specimen were determined by averaging the measured temperatures along the plateu at each wavelength.

03,357

PB96-103007 Not available NTIS

National Inst. of Standards and Technology (CAML), Gaithersburg, MD. Applied and Computational Mathematics Div

Boundary Conforming Grid Generation System for Interface Tracking.

Final rept.

B. V. Saunders. $1995,17 p$.

See also PB94-158268.

Pub. in Computers and Mathematics with Applications $29, \mathrm{n} 10 \mathrm{p} 1-171995$.

Keywords: "Liquid solid interfaces, Reprints, Mapping(Transformations), Binary alloys, Tensor products, Spline functions, Coordinates, Directional solidification, Grid generation(Mathematics).

The development of an algebraic grid generation system to track a solid-liquid interface during directional solidification of a binary alloy is discussed. A single mapping, constructed with tensor product B-splines, is proposed for calculations of both shallow and deep solidification cells. The initial spline coefficients for the coordinate mapping are modified to minimize a discrete functional that regulates the smoothness and 
orthogonality of the mesh. The use of transfinite blending function interpolation to obtain an initial grid is examined.

\section{3,358}

PB96-109558 PC A06/MF A02

National Inst. of Standards and Technology, Gaithersburg, MD.

Factors Significant to Precracking of Fracture Specimens.

C. G. Interrante, and J. J. Filliben. Nov 89, 102p,

NISTIR-89/4214.

Keywords: "Fracture tests, "Impact tests, "Titanium, "Aluminum, "Steels, Standards, Cracking(Fracturing) Toughness, Fatigue(Materials), Stress analysis, Bend tests.

The significance of four controlled variables in the technique used to precrack Charpy specimens is determined by analyses of seven response variables computed from results of slow-bend tests of metallic mate rials. The results can be summarized in three majo conclusions: (1) All seven computed responses a re linearly related to crack size and the sensitivity to crack size depends on the choice of response parameter and on the material. (2) Precracking at either very high or very low levels of stress-intensity factor KF, are to be avoided. (3) For the three methods of notch prepara tion, the effects of notch preparation were largely not significant. This work is the result of a study conducted by ASTM Task Group E24.03.03 and members of eight participating laboratories.

\section{3,359}

\section{PB96-115050 PC A04/MF AO}

National Inst. of Standards and Technology (MSEL) Gaithersburg, MD. Office of Intelligent Processing of Materials.

Intelligent Processing of Materials, Technical Activities 1994 (NAS-NRC Assessment Panel, April 6 7, 1995).

Annual rept

D. E. Hall, and G. Birnbaum. 7 Apr 95, 70p, NISTIR5578 .

See also report for 1993, PB94-164183.

Keywords: "Materials, *Gas atomization, *Controllers, *Artificial intelligence, Process control, Computeraided manufacturing. Atomizers, Control systems, Rare gases, Ceramics, Metal powders, Polymers, Sensors, Grinding(Material removal), Research programs, Ultrasonic tests, Nondestructive tests.

This report discusses the technical activities during 1994 of the projects supported by the Office of Intelligent Processing of Materials in the Materials Science and Engineering Laboratories. The activities include large consortia and other large programs in metals and alloys, ceramics, polymer and polymer blends sensor research involving thermal wave and ultrasonic evaluation of ceramic grinding damage, eddy current temperature measurement during hot rolling of aluminum sheet, and metal sensors in the microstructural engineering of steel.

\section{3,360}

\section{PB96-123203 Not available NTIS}

National Inst. of Standards and Technology (PL), Gaithersburg, MD. Ionizing Radiation Div.

Study of Laser Resonance lonization Mass Spectrometry Using a Glow Discharge Source.

Final rept.

X. Xiong, J. M. R. Hutchinson, J. D. Fassett, W. A

Bowman, K. R. Hess, T. B. Lucatorto, and F.J.

Schima. $1995,4 p$

See also DE94018566.

Pub. in AIP Conference Proceedings, Bernkastel-kues, Germany, July 1994, v329 p316-319 1995.

Keywords: "Aluminum base alloys, "Copper alloys, "Iron alloys, Atomization, Data acquisition systems, Dye lasers, Mass spectrometers, Reprints, "Foreign technology.

The mass spectra of a metal alloy sample consisting of $\mathrm{Al}, \mathrm{Cu}$ and $\mathrm{Fe}$ were studies using both glow discharge mass spectrometry (GDMS) and resonance ionization mass spectrometry (RIMS). Particular emphasis was placed on the reduction of isobaric interferences and discrimination between those ions formed by the discharge and those formed by the laser radiation.
National Institute of Standards and Technology, Boulder, CO

\section{Low-Temperature Properties of Silver.}

D. R Smith, and F R Fickett. 1995, 53p.

Included in Jnl. of Research of the National Institute of Standards and Technology, v100 n2 p119-171 Mar/ Apr 95.

Keywords: "Mechanical properties, "Silver, Temperature, Physical properties, Conductivity, Cryogenics, Superconductors, Thermodynamics.

Pure silver is used extensively in the preparation of high-temperature superconductor wires, tapes, films, and other configurations in which the silver not only shields the superconducting material from the surrounding materials, but also provides a degree of flexibility and strain relief, as well as stabilization and lowresistance electrical contact. Silver is relatively expensive, but at this stage of superconductor development, its unique combination of properties seems to offer the only reasonable means of achieving usable lengths of conductor. In this role, the low-temperature physical (electrical, thermal magnetic, optical) and mechanical properties of the silver all become important. Here the authors present a collection of properties data extracted from the cryogenic literature and, to the extent possible, selected for reliability.

\section{3,362}

\section{PB96-134954 PC A05/MF A01}

National Inst. of Standards and Technology, Gaithersburg, MD.

Journal of Research of the National Institute of Standards and Technology, September-October 1993. Volume 98 , Number 5 .

B. N. Taylor, and D. R. Harris. 1993, 96p

See also PB94-108529.

No abstract available.

\section{3,363}

PB96-135231 Not available NTIS

National Inst. of Standards and Technology (MSEL) Boulder, CO. Materials Reliability Div.

Effects of Copper, Nickel and Boron on Mechanical Properties of Low-Alloy Steel Weld Metals Deposited at High Heat Input.

Final rept.

A. O. Kluken, T. A. Siewert, and R. Smith. 1994, 7p. Pub, in Welding Research Supplement, p193s-199s Aug 94.

Keywords: "Weld metal, "Heat, "Fracture resistance, "Submerged arc welding, Reprints, Mechanical prop erties, Tensile strength, High heat input.

The authors studied the effects of copper, nickel and boron on the mechanical properties of low-alloy steel weld metals deposited at high heat input $(4.8 \mathrm{~kJ} / \mathrm{mm})$ by the submerged arc process. The copper and nickel contents of the welds were systematically varied within the ranges of 0.03 to 0.89 wt- $\% \mathrm{Cu}$ and 0.01 to 1.54 wt.$\% \mathrm{Ni}$. In addition, several of these copper and nickel combinations were duplicated with welds containing 36 to $44 \mathrm{ppm} \mathrm{B}$. Tensile testing revealed yieled strengths in the range from 462 to $546 \mathrm{Mpa}$, and ultimate tensile strengths from 638 to $869 \mathrm{Mpa}$. The weld metal Charpy $\mathrm{V}$-notch (CVN) data showed a $35 \mathrm{~J}$ transition temperature, ITT, ranging from -28 to $55 \mathrm{C}$. Upon adding boron patches of intergranular fracture were present on the CVN fracture surfaces. This was particularly true when boron was added at the higher copper and nickel levels. Qualitative metallographic examinations revealed the presence of extensive amounts of particles at the prior austenite grain boundaries in boron containing welds. Furthermore, adding boron at the higher copper and nickel levels promoted the formation of the MAC (martensite-austenite-carbide) microconstituent. Also nickel seemed to promote formation of MAC in reheated weld metal. At this high heat input $(4.8 \mathrm{~kJ} / \mathrm{mm})$ additions of copper, nickel and boron did not improve the mechanical properties.

\section{3,364}

PB96-141015 Not available NTIS

National Inst. of Standards and Technology (MSEL), Boulder, CO. Materials Reliability Div.

Elastic Constants and Internal Friction of Polycrystalline Copper.

Final rept.

H. Ledbetter, C. Fortunko, and P. Heyliger. 1995, 2p. See also PB87-111662.

Pub. in Jnl. of Materials Research, v10 n6 p1352-1353 Jun 95 .
Keywords: "Elastic properties, "Copper, Internal friction, Reprints, Ultrasonics, Vibrations, Spectroscopy Koehler-Granato-Lucke model.

Using ultrasonic-resonance spectroscopy (URS), we measured the elastic constant $\mathrm{C}$ and companion internal friction Q-1 of isotropic polycrystalline copper. The annealed material was 0.9999 pure with equiaxed heavily twinned grains averaging about 75 micrometers diameter. The URS method offers the principal advantage of point contact or loose coupling, thus there was no contribution from a transducer-specimen bond and only small contributions from transducers and fixture. A second advantage is one measurement for all elastic constants and all associated internal frictions. The C's agree with established values. The Q1 's are much lower than pulse-echo-method values. Comparison of measured Q-1 with the KoehlerGranato-Lucke model permits estimating an effective dislocation-loop length. Q-1 (shear) exceeds Q-1 (Longitudinal) by a factor of about 1.5 .

\section{3,365}

PB96-141122 Not available NTIS

National Inst. of Standards and Technology (MSEL).

Boulder, CO. Materials Reliability Div.

Effect of Charpy $V$-Notch Striker Radii on the Absorbed Energy.

Final rept.

T. A. Siewert, and D. P. Vigliotti. 1995, 13p.

Pub, in Pendulum Impact Machines, p140-152 May 95.

Keywords: *Impact tests, "Energy absorption, Fracture mechanics, Strain(Mechanics), Reprints, Charpy Vnotch, Striker radius.

The two most common Charpy $V$-notch striker designs (8-mm and 2-mm radii on the striking edge) were compared using verification (reference-grade) impact specimens. Other variables in the test matrix included two different brands of $U$-type pendulum machines and four different specimen energy ranges (near 18, 45 , 100 , and $200 \mathrm{~J}$ ). In this comparative study, we found a very small difference betwenn the two striker designs and an even smaller difference between the two brands of machines. At $200 \mathrm{~J}$, the difference between the two striker designs was about $10 \mathrm{~J}$. This difference might not be important in most production testing, but must be considered in verification testing where the acceptable range may be 5 percent. The standard deviations of absorbed energy for the two strikes were similar, except at $200 \mathrm{~J}$ were the $2-\mathrm{mm}$ striker produced standard deviations about 3 times higher than the 8 $\mathrm{mm}$ striker.

\section{3,366}

PB96-146857 Not available NT!S

National Inst. of Standards and Technology (MSEL), Gaithersburg, MD. Reactor Radiation Div. Inelastic Neutron Scattering Study of Hydrogen in Nanocrystalline Pd.

Final rept.

U. Stuhr, H. Wipf, T. J. Udovic, J. Weissmuller, and

H. Gleiter $1995,4 p$

Pub. in NanoStructured Materials, v6 p555-558 1995

Keywords: "Neutron scattering, "Palladium, *Grain boundaries, Crystal structure, Reprints, "Foreign technology, "Nanocrystalline materials

The vibrational excitations and the position of hydrogen in nanocrystalline Pd was investigated by neutron spectroscopy. This study was restricted to low hydrogen concentrations where no hydrogen precipitation was observed at room temperature. The experiments show that most of the hydrogen is incorporated in the grain boundaries and at the inner surfaces of the grains. Surface modes of the hydrogen can be identified. Compared to coarse-grained $\mathrm{Pd}$, no change in the hydrogen solubility was found for the lattice regions of the nanocrystalline $\mathrm{Pd}$

\section{3,367}

PB96-161807 Not available NTIS

National Inst. of Standards and Technology (MSEL) Gaithersburg, MD

Nonlocal Effects of Existing Dislocations on CrackTip Emission and Cleavage.

Final rept.

V. Shastry, P. M. Anderson, and R. Thomson. 1994

$16 p$

Pub. in Jnl. of Materials Research, v9 n3 p812-827 Mar 94

Keywords: "Ductile brittle transition, "Crack propagation, Cleavage, Emission, Reprints Fractures(Materials), " Dislocations(Materials) 
This paper investigates the criterion for a ductile-tobrittle transition in materials, due to nonlocal shielding effects at the crack tip when the dislocation free zone (DFZ) size is small. It is found that both cleavage and emission criteria are altered by nonlocal shielding, but that the emission shift is dominant, and is always in the direction to increase the local critical stress intensity for emission, klle. The nonlocal shift varies with the sum sigma(gamma(sub us)dj) to the negative 3/2, over each dislocation (j), where gamma(sub us) is the unstable stacking fault energy, and dj is the distance from each dislocation to the crack tip. When there is a pileup of many shielding dislocations against a barrier near the crack tip, the total shift for the pileup varies as (gamma(sub us)d) to the minus i power. The most likely candidates for a brittle transition induced by the nonlocal shift are materials where barriers to dislocation motion exist within 10-100 nanometers of the crack tip, such as in thin films, multilayers, or ultrafine grain materials.

03,368

PB96-169057 PC A05/MF A01

National Inst. of Standards and Technology, Gaithersburg, MD.

Journal of Research of the National Institute of Standards and Technology, September/October 1993. Volume 98 , Number 5 .

1993, 96p.

See also PB96-169065 through PB96-169081 and PB94-108529. Also available from Supt. of Docs. as SN703-027-00054-7

Keywords: "Titanium alloys, "Aluminum alloys, "Niobium alloys, "Crystal-phase transformations, *Silicon carbides, "Silicon nitrides, "Corrosion, BCC lattices, HCP lattices, Orthorhombic lattices, Transmission electron microscopy, Heat exchangers, Combustion, Coal, Slags, Ashes, Integrated Services Digital Network.

Contents:

Articles--Transformation of BCC and B2 High

Temperature Phases to HCP and

Orthorhombic Structures in the Ti-Al-Nb

System. Part I--Microstructural Predictions

Based on a Subgroup Relation Between Phases;

Transformation of $\mathrm{BCC}$ and $\mathrm{B} 2$ High Temperature Phases to HCP and Orthorhombic Structures in the Ti-Al-Nb System. Part II--Experimental TEM Study of Microstructures;

Corrosion Characteristics of Silicon Carbide and Silicon Nitride. Conference Reports--North American Integrated Services Digital Network (ISDN) Users' Forum (NIUF):

News Briefs.

03,369

PB96-169057 PC A06/MF A01

National Inst. of Standards and Technology, Gaithersburg, MD.

Journal of Research of the National Institute of Standards and Technology, September/October 1993. Volume 98 , Number 5 .

1993, $96 p$

See also PB96-169065 through PB96-169081 and PB96-108529. Supersedes PB96-134954. Also available from Supt. of Docs. as SN-703-027-00054-7.

Keywords: "Titanium alloys, "Aluminum alloys, "Niobium alloys, "Crystal-phase transformations, "Silicon carbides, "Silicon nitrides, "Corrosion, BCC lattices, HCP lattices, Orthorhombic lattices, Transmission electron microscopy, Heat exchangers, Combustion, Coal, Slags, Ashes, "Integrated Services Digital Network.

Contents:

Calibration of Electret-Based Integral Radon Monitors Using NIST PolyethyleneEncapsulated 226Ra/222Rn Emanation (PERE) Standards;

Microstructural Characterization of CobaltTungsten Coated Graphite Fibers;

On Using Collocation in Three Dimensions and Solving a Model Semiconductor Problem

Precision Tests of a Ouantum Hall Effect Device DC Equivalent Circuit Using Double-Series and Triple-Series Connections:

Analysis of the $(5 d 2+5 d 6 s)-5 d 6 p$ Transition Arrays of Os VII and Ir VIII, and the 652 . $6 \mathrm{p} 2 \mathrm{P}$ Transitions of Ir IX.

National Inst. of Standards and Technology, Gaithersburg, MD.

Transformation of $\mathrm{BCC}$ and $\mathrm{B} 2$ High Temperature Phases to HCP and Orthorhombic Structures in the Ti-Al-Nb System. Part 1. Microstructural Predictions Based on a Subgroup Relation between Phases.

L. A. Bendersky, A. Roytburd, and W. J. Boettinger. 1993, 23p.

ncluded in Jnl. of Research of the National Institute of Standards and Technology, v98 n5 p561-583 Sep/ Oct 93

Keywords: "BCC lattices, "Orthorhombic lattices, Phases, Reprints, Structural relations, "Domain structures, Elastic accommodation, Space group relations.

Possible paths for the constant composition coheren transformation of $\mathrm{BCC}$ or $\mathrm{B} 2$ high temperature phases to low temperature HCP or Orthorhombic phases in the $\mathrm{Ti}-\mathrm{Al}-\mathrm{Nb}$ system are analyzed using a sequence of crystallographic structural relationships developed from subgroup symmetry relations. Symmetry elements lost in each step of the sequence determine the possibilities for variants of the low symmetry phase and domains that can be present in the microstructure. The orientation of interdomain interfaces is determined by requiring the existence of a strain-free interface beween the domains.

\section{3,371}

PB96-169073

MF A01)

National Inst. of Standards and Technology, Gaithersburg, MD.

Transformation of BCC and B2 High Temperature Phases to HCP and Orthorhombic Structures in the Ti-Al-Nb System. Part 2. Experimental TEM Study of Microstructures.

L. A. Bendersky, and W. J. Boettinger. 1993, 22p Included in Jnl. of Research of the National Institute of Standards and Technology, v98 n5 p585-606 Sep/ Oct 93

Keywords: "BCC lattices, "Orthorhombic lattices, Phases, Reprints, Structural relations, "Domain struc tures, Elastic accomodation, Space group relations.

Possible transformation paths that involve no long range diffusion and their corresponding microstructura details were predicted by Bendersky, Roytburd, and Boettinger for Ti-Al-Nb alloys cooled from the high temperature $\mathrm{BCC} / \mathrm{B} 2$ phase field into close-packed orthorhombic or hexagonal phase fields. These predictions were based on structural and symmetry rela tions between the known phases. In the present pape experimental TEM results show that two of the predicted transformation paths are indeed followed for different alloy compositions.

03,372

PB96-169081 (Order as PB96-169057, PC A05/

MF A01)

National Inst. of Standards and Technology, Gaithersburg, MD.

Corrosion Characteristics of Silicon Carbide and Silicon Nitride.

R. G. Munro, and S. J. Dapkunas. 1993, 25p.

Included in Jnl. of Research of the National Institute of Standards and Technology, v98 n5 p607-631 Sep/ Oct 93

Keywords: "Corrosion, "Silicon carbides, "Silicon nitrides, Ashes, Reprints, Ceramics, Coal

The present work is a review of the substantial effor that has been made to measure and understand the effects of corrosion with respect to the properties, performance, and durability of various forms of silicon carbide and silicon nitride. The review encompasses cor rosion in diverse environments, usually at temperatures of 1000 degrees $C$ or higher. The environments include dry and moist oxygen, mixtures of hot gaseous vapors, molten salts, molten metals, and complex environments pertaining to coal ashes and slags.

\section{3,373}

PB96-186069 Not available NTIS

National Inst. of Standards and Technology (MSEL) Boulder, CO. Materials Reliability Div.
Sensor System for Intelligent Processing of HotRolled Steel.

Final rept.

A. V. Clark, M. G. Lozev, B. J. Filla, and L. J. Bond. $1994,8 p$

Pub. in International Symposium on Nondestructive Characterization of Materials VI (6th), Honolulu, HI., June 7-11, 1993, p29-36 1994.

Keywords: "Acoustics, "Intelligent processing, "Steel, Ultrasonics, Reprints.

Microstructural engineering offers a means of reducing cycle time and improving product quality with reduced energy cost. Process modelling of hot-rolling of steel has advanced to the point where key metallurgical parameters (e.g. austenite grain size) can be predicted from computer models if other process parameters such as temperature are known. However, a need still remains to develop on-line sensors because sensors can rapidly acquire data for model validation, and could be combined with a process control strategy in feedforward or feedback loops. The authors report a study to develop an on-line sensor for autenite grain size measurement at temperatures up to 1000 degrees C. They are developing a system for measurement of attenuation in ultrasonic waves propagating through hot steel samples. Existing theories allow the authors to infer grain size for attenuation measurement. The authors' system consists of a high temperature trans. ducer acoustically coupled to a high strength, low loss buffer rod. The rod is to be pressed against a hot steel specimen sufficiently good contact to transmit ultrasound into the specimen. To avoid plastic deformation of the specimen, they implement computer control of the load applied to the buffer rod. Attenuation measurements were performed at room temperature on specimens of known grain size. Good agreement with optically measured grain size was obtained. The system is now being assembled for measurement at elevated temperatures.

\section{3,374}

PB96-190236 Not available NTIS

National Inst. of Standards and Technology (MSEL), Boulder, CO. Materials Reliability Div.

Aluminum-Lithium Alloys: Evaluation of Fracture Toughness by Two Test Standards, ASTM Method E 813 and E 1304 .

Final rept.

P. T. Purtscher, J. D. McColskey, and E. S. Drexler.

1992, 21p.

Pub. in Standard Technical Publication 1172, p1101301992.

Keywords: "Aluminum lithium alloys, "Fracture toughness, Standards, Cryogenic properties, Declaminations, Reprints, Alloy 2219.

Fracture toughness of three aluminum-lithium alloys and Alloy 2219 (a total of nine different plates) was measured with two different types of specimens and methods: (1) compact specimens using ASTM E 813 and (2) chevron-notched short-bar specimens using ASTM E 1304. The properties were measured in two orientations ( $T-L$ and $L-T$ ) and at three temperatures $(295,76$, and $4 \mathrm{~K})$. In general, the short-bar specimens exhibited a higher fracture toughness than the compact specimens. The difference between the two procedures was relatively constant, independent of test orientation and strength. However, when the specimens exhibited extensive declamations on the fracture surface, the compact specimens had a higher fracture loughness than the short bar specimens. The difference in fracture toughness measured by the two procedures is explained in terms of the alloys' crack growth behavior.

\section{3,375}

PB96-190244 Not available NTIS

National Inst. of Standards and Technology (MSEL). Boulder, CO Materials Reliability Div.

Light-Weight Alloys for Aerospace Applications II. Final rept.

P. T. Purtscher, and E. S. Drexler. 1991, 11p.

Pub. in Light-Weight Alloys for Aerospace Applications II, New Orleans, LA., February 17-21, 1991, p65،75.

Keywords: "Aluminum alloys, "Fracture toughness, Aerospace, Aluminum lithium, Chevron-notch, Cryogenic properties, Mechanical properties, Reprints.

The mechanical properties of $\mathrm{Al}-\mathrm{Li}$ alloys and alloy 2219 were measured in the through-thickness direction. The toughness was evaluated in the $S-L$ and $S$ T orientations according to ASTM Method E 1304 with 
chevron-notched specimens, $13 \mathrm{~mm}$ thick and $19 \mathrm{~mm}$ wide. The test temperatures were 295, 76, and $4 \mathrm{~K}$. Each alloy was tested after different thermomechanicai processing to vary the strength at a given temperature. The ultimate strength for all the materials increased with decreasing test temperature. The toughness varied significantly with chemical compositionn and aging treatment, but were inconsistent with respsect to test temperature.

\section{3,376}

PB96-204193 Not available NTIS

National Inst. of Standards and Technology (MSEL), Gaithersburg, MD. Ceramics Div.

Cavity Evolution during Tensil Creep of Si3N4. Final rept.

W. Luecke, S. M. Wiederhorn, B. J. Hockey, and G. G. Long. 1993, 6p.

Pub. in Materials Research Society Symposium Proceedings, Boston, MA., November 30-December 3 , 1992, v287 p467-472 1993

Keywords: "Silicon nitride, "Creep, "Cavities, Reprints, Lifetime, Rupture, Monkman-Grant.

The authors have characterized the evolution of cavities during tensile creep of a $\mathrm{Y}_{2} \mathrm{O} 3$-hot isostatically pressed Si3N4, using precision density measurements, small angle $x$-ray scattering (SAXS) and transmission electron microscopy (TEM). The cavities ae bimodally distributed in size. Lenticular, $200 \mathrm{~nm}$-size cavities are common, and lie primarily on two-grain boundaries. Irregularly shaped 500-1000 nm size cavities are rare and lie at multi-grain junctions, but comprise approximately half of the total volume fraction of cavities. Although the material shows a continuous decrease in strain rate with strain the cavity volume fraction evolves linearly with strain. Cavities account for approximately $85 \%$ of the total strain at any point during creep.

03,377

PB96-204466 Not available NTIS

National Inst. of Standards and Technology (MSEL), Gaithersburg, MD. Ceramics Div.

Experimental Assessment of Crack-Tip Dislocation Emission Models for an Al67Cr8Ti25 Intermetallic Alloy.

Final rept

W. G. Meng, M. D. Vaudin, M. F. Bartholomeusz,

and J. A. Wert. 1995, 13p.

Pub. in Metallurgical and Materials Transactions A, v26A p329-341 Feb 95

Keywords: "Dislocation emission, Reprints, Cleavage, "Intermetallic alloys, Electron backscatter pattern Grain orientations.

A potential explanation for the cleavage fracture of intermetallic alloys with low or moderate critical resolved shear stress (CRSS) is the existence of an energy barrier for crack-tip dislocation emission, as described by models that analyze the energetics of dislocation emission from crack tips. In the present study, an intermetallic alloy with the L1(2) crystal structure, Al67Cr8Ti25, has been used to experimentally assess th predictions of the Rice-Thomson dislocation-emission model. The assessment is performed in two ways. First model predictions of a fracture mode transition at elevated temperature are compared with experimental results. Bend tests performed at temperatures in the range of 293 to $1061 \mathrm{~K}$ reveal that the fracture mode of Al67Cr8Ti25 changes from predominately cleavage fracture at room temperature to a mixed mode of cleavage and intergranular fracture at intermediate temperatures and then to predominantly intergranular fracture at high temperatures. Second model predictions of the effect of grain orientation on the fracture mode are compared with experimental results.

\section{3,378}

PB97-119366 Not available NTIS

National Inst. of Standards and Technology (MSEL), Gaithersburg, MD. Metallurgy Div.

Electronic Structure and Phase Equilibria in Ternary Substitutional Alloys.

Final rept

A. J. S. Traiber, P. E. A. Turchi, R. M. Waterstrat, and S. M. Allen. 1996, 23p.

See also DE93013848.

Pub. in Jnl. of Physics: Condensed Matter, n8 p635763791996.

Keywords: "Iron alloys, "Titanium alloys, "Vanadium alloys, Binary alloy systems, Electronic structure, Mixing.
Perturbation theory, Phase stability, Reprints, "Foreign technology, "Ternary alloy systems.

A reliable and consistent scheme for studying phase equilibria in ternary substitutional alloys based on the tight-binding approximation is presented. With the electronic parameters obtained from linear muffin-tin orbital calculations, the authors show that the computed densities of states and band structures compare well with those obtained from more accurate ab initio calculations. Disordered alloys are studied within the tight-binding coherent-potential approximation formalism extended 10 multi-component alloys. The energetics of ordered systems is obtained through efective-pair interactions computed with the general perturbation method. Finally, partially ordered alloys are studied with a novel simplification of the molecular $\mathrm{CO}^{-}$ herent-potential approximation combined with the general perturbation method.

\section{Plastics}

03,379

PB94-211166 Not available NTIS

National Inst. of Standards and Technology (MSEL), Gaithersburg, MD. Polymers Div.

Torsional Dilatometer for Volume Change Measurements on Deformed Glasses: Instrument Description and Measurements on Equilibrated Glasses.

P. S. Duran and G. B. McKenna 1990, 27p.

Pub. in Jnl. of Rheology 34, n6 p813-839 1990.

Keywords: "Epoxy resins, "Volume, "Extensometers, "Torsion, Glass, Design criteria, Deformation, Rheology, Glass transition temperature, Aging

An automated mercury dilatometer has been designed and built for the purpose of making volume change measurements on cylindrical samples subjected to torsional deformations. In its current configuration the instrument takes readings of torque, normal force and volume change upon application of a twist at one end of the sample. Here we describe the instrument and preliminary measurements on epoxy glasses which have been equilibrated by annealing near to the glass transition. Our results show that the volume of the sample tested at temperafures ranging from $T$ (sub g)-10 to $T($ sub $g$ ) increases upon application of a torsiona deformation. This result is contrary to results reported in the literature for samples tested well below T(sub g).

\section{3,380}

PB94-213501 Not available NTIS

National Inst. of Standards and Technology (BFRL) Gaithersburg, MD. Fire Science Div.

Investigation of the Thermal Stability and CharForming Tendency of Cross-linked Poly(methyl methacrylate).

Final repi.

S. M. Lomakin, J. E. Brown, R. S. Breese, and M. R. Nyden. 1993, 15p.

Pub. in Polymer Degradation and Stability 41, p229. 2431993.

Keywords: "Polymethyl methacrylate, "Thermal stability, "Charring, Combustion products, Crosslinking. Thermal degradation. Pyrolysis Thermographs, Thermogravimetric analysis, Reprints.

The thermal degradation of two network copolymers of methyl methacrylate was studied as a function of the chemical nature of the cross-linking agent and the frequency of cross-links. Unlike the linear homopolymer. both the trimethylolpropane triacrlylate and trimethylolpropane trimethacrylate networks were lound to char when burned. The corresponding derivative thermograms indicate that there are dramatic differences in the thermal degradation of these polymers even in the absence of oxygen. These differences are interpreted in terms of a simple model for the kinetics of depolymerization.

03,381

PB95-126363 Not available NTIS

National Inst. of Standards and Technology (MSEL), Gaithersburg, MD. Polymers Div.
Influence of Physical Aging on the Yield Response of Model DGEBA + Poly(propylene oxide) Epoxy Glasses.

Final rept

C. G'Sell, and G. B. McKenna. 1992, $11 p$

Pub. in Polymer 33, n10 p2103-2113 1992

Keywords: "Aging tests(Materials), "Epoxy resins, "Polyoxypropylene, "Laminated glass, Kinetics, Plastic flow, Temperature, Crosslinking, Plasticity tests, Plastic analysis, Stress analysis, Reprints, DGEBA(Diglcidyl ether of Bisphenol).

Cylindrical specimens were prepared from two DGEBA amine-terminated PPO networks of differen crosslink density and subjected to quenching and isothermal aging treatments at different temperatures and aging times in the range from 0.1 to 1000 hours, The changes in the structure of the glasses during the aging towards equilibrium was subsequently assessed by the measurement of the yield stress in uniaxial compression at the same temperature as the aging. From the influence of temperature and strain rate on the above phenomena, the kinetics of physical aging were examined quantitatively in terms of the time-aging time-temperature equivalence principle. The results suggest that the increase in yield stress on aging is correlated to volume recovery and to the induced increase of the relaxation times as the polymeric glass evolves towards its equilibrium state.

03,382

PB95-140018 Not available NTIS

National Inst. of Standards and Technology (MSEL) Gaithersburg, MD Polymers Div.

Volume Recovery in Epoxy Glasses Subjected to Torsional Deformations: The Question of Rejuvenation.

Final repl

M. Santore, R. S. Duran, and G. B. McKenna.

$1991,5 p$

Pub. in Polymer 32, n13 p2377-2381 1991

Keywords: "Laminated glass, "Epoxy resins, "Aging tests(Materials), "Deformation, Dilatometry, Torsion, Mechanical properties, Volume, Viscoelasticity, Kinetics, Stress relaxation, Reprints, "Rejuvenation.

Torsional dilatometry was used to examine the me chanical properties of an epoxy glass during physical aging, i.e, after a quench from above the glass transition temperature to slightly below it. Volume changes in the sample were measured simultaneously with the viscoelastic responses in stress relaxation experiments as functions of the deformation magnitude. In this epoxy, the torsional deformations induce volume expansions which relax on a time scale similar to those of the torque relaxation. Mechanical stimuli only momentarily disrupt the volume evolution following a quench: the underlying volume recovery kinetics, which are much slower than the mechanical torque or volume relaxation, remain unaltered. Both the independence of $t^{*}$ of the magnitude of the strain and the fact that the volume recovery after a quench remains unaltered in spite of the imposition of mechanical deformations support the argument that mechanical stimuli neither alter the underlying (nonequilibrium) thermodynamic state of the glass nor erase physical aging

\section{3,383}

PB95-140026 Not available NTIS

National Inst. of Standards and Technology (MSEL) Gaithersburg, MD. Polymers Div.

Torsional Relaxation and Volume Response during Physical Aging in Epoxy Glasses Subjected to Large Torsional Deformations.

Final rept.

M. M. Santore, and G. B. McKenna. 1991, 6p.

Pub. in Materials Research Society Symposia Proceedings 215, p81-86 1991 .

Keywords: "Laminated glass, "Epoxy resins, *Aging tests(Materials), "Deformation, Dilatometry Torsion, Mechanical properties, Volume, Viscoelasticity, Stress relaxation, Reprints, "Rejuvenation.

We present torsional dilatometry experiments to simultaneously measure torque relaxation and volume re covery in an epoxy glass quenched from above its glass transition temperature to below it. Two strain histories are employed: one with strains of equal sizes and a second where small strains follow a large one. The baseline for the thermally induced volume recov ery is insensitive to intermittent torsional strains whose magnitude can be well into the non-linear regime. The to rque relaxations from equal intermittent mechanical 


\section{Plastics}

stimuli can be superposed by a time-aging time shift in a way that indicates fast changes cease to occur at approximately 10,000 seconds after the quench, a 'mechanical equilibration' time. Stimuli of different sizes can confound superposition via nonlinear effects, but do not affect the ultimate volume recovery of the glass or its mechanical equilibration time. Our results show the signature of rejuvenation; however, this may result from the nonlinear response of the material in a nonisochoric strain history. Our data firmly show that mechanical stimuli do not erase aging or rejuvenate this epoxy.

\section{3,384}

PB95-140901 Not available NTIS

National Inst. of Standards and Technology (MSEL) Gaithersburg, MD. Polymers Div.

Using Torsional Dilatometry to Measure the Effects of Deformations on Physical Aging.

Final rept.

M. Santore, R. Duran, and G. McKenna. 1990, 1p.

Pub. in Abstracts of Papers of the American Chemical Society 200, 344p Aug 90

Keywords: "Aging tests(Materials), "Epoxy resins, *Deformation, "Laminated glass, Dilatometry, Torsion, Mechanical properties, Volume, Reprints, "Rejuvenation.

An epoxy glass was studied via torsional dilatometry to test the 'rejuvenation' hypothesis, i.e. that large deformations retard physical aging. For example, rejuvenation predicts that samples subjected to deformations after a quench to below Tg will contract more slowly than those quenched without subsequent deformations. The model further implies that the mechanical properties should evolve more slowly toward equilibrium when deformations are imposed during aging. Our torsional dilatometry tests both premises: Simultaneous measurements of volume recovery and torque relaxation suggest that the application of large deformations does not directly influence the physical aging process. However, we do observe an interesting effect: the equilibration time based on the time required for the mechanical properties to cease evolving is at least one order of magnitude shorter than that evidenced by the volume recovery measurements.

03,385

\section{PB95-202990 Not available NTIS}

National Inst. of Standards and Technology (MSEL), Gaithersburg, MD. Reactor Radiation Div.

Hydration in Semicrystalline Polymers: SmallAngle Neutron Scattering Studies of the Effect of Drawing in Nylon-6 Fibers.

Final rept.

N. S. Murthy, and W. J. Orts. 1994, 9p.

Grant NSF-DMR-9122444

Sponsored by National Science Foundation, Arlington, VA.

Pub. in Jnl. of Polymer Science: Part B, Polymer Physics 32, p2695-2703 1994 .

Keywords: "Hydration, "Small angle scattering, "Nylon fibers, "Nylon 6, "Drawing. Neutron scattering, Thermoplastic resins, Amorphous materials, Crystallinity, Water, Polymer physics, Reprints.

Water absorbed by nylons appears to be partitioned into interlamellar and interfibrillar spaces. The amount of water in the interfibrillar region remains essentially unchanged with increasing draw ratio, whereas that in the interlamellar regions decreases with draw ratio; the latter accounts for the decrease in the water uptake in the drawn fibers. These results suggest that the amount of the amorphous material in the interfibrillar regions remains unchanged during drawing, and the increase in the crystallinity during drawing results from the incorporation of the amorphous chain segments in the interlamellar regions into the crystalline lamellae. Further, the interfibrillar water is more tightly bound than the interlamellar water. The length of the longitudinal channels into which water diffuses is about the same as that of the fibrils, and increases from ca. 1500 to $2000 \mathrm{~A}$ upon drawing. The longitudinal channels are highly oriented even in undrawn fibers, and their misorientation increases from 5 degrees to 15 degrees upon drawing. These channels can be described as surface fractals of dimension 3.4-3.6.

03,386

PB95-226866 PC A03/MF A01

National Inst. of Standards and Technology (PL),

Gaithersburg, MD. Radiometric Physics Div.
Standard Reference Materials: Polystyrene Films for Calibrating the Wavelength Scale of Infrared Spectrophotometers - SRM 1921.

Special pub.

D. Gupta, L. Wang, L. M. Hanssen, J. J. Hsia, and R. U. Datla. Apr 95, 49p, NIST/SP-260-122.

Also available from Supt. of Docs.

Keywords: "Polystyrene, "Fourier transformation, "Infrared spectrophotometers, Chemical analysis, Calibration standards, Optical measuring instruments, Standardization, Wavelengths

Standard Reference Material (SRM) 1921 is a matte finish polystyrene film and is intended for use in calibrating the wavelength scale of spectrophotometers in the infrared (IR) spectral region from $545.49 \mathrm{~cm}$ exp(1) to $3082.19 \mathrm{~cm} \exp (-1)(18.332$ micrometers to 3.2445 micrometers). Thirteen absorption peak positions obtained using a center-of-gravity method are certified. The expanded uncertainty value associated with these peak values are between $0.06 \mathrm{~cm} \exp (-1)$ and $0.66 \mathrm{~cm} \exp (-1)$, except for 1.84 at $2850.13 \mathrm{~cm}$ $\exp (-1)$ and 12.29 at $545.49 \mathrm{~cm} \exp (-1)$. This publication describes the IR spectrophotometer, instrument calibration, SRM material, measurement procedure, calibration of polystyrene films, and uncertainty determination.

03,387

PB96-123369 Not available NTIS

National Inst. of Standards and Technology (MSEL), Gaithersburg, MD. Polymers Div.

Flow-Induced Structure in Polymer. Chapter 1. An Introduction to Flow-Induced Structures in Polymers.

Final rept.

A. I. Nakantani. $1995,19 p$

Pub. in National Meeting of the American Chemical Society (208th), Washington, DC., August 21-25, 1994,
p1-19.

Keywords: "Polymers, Morphology, Blends, Solutions, Melts, Multicomponent systems, Reprints, "Flow induced structures.

An overview of flow-induced structures in polymers is presented and recent references to research in the areas of polymer solutions, homopolymer melts, polymer blends, block copolymers and multi-component systems, and liquid crystalline polymers a re given. The research area of flow-induced structures in polymers has expanded with the advent of in-situ measuring techniques. Studies of this type are useful for probing fundamental issues such as chain deformation, shifts in phase behavior, and the formation of novel shear induced structures. The applications addressed include the kinetics of morphology evolution and the rela. tionship between ultimate properties and morphology.

\section{3,388}

PB96-123377 Not available NTIS

National Inst. of Standards and Technology (MSEL), Gaithersburg, MD. Polymers Div.

Flow-Induced Structure in Polymers. Chapter 17 Phase-Separation Kinetics of a Polymer Blend SoIution Studied by a Two-Step Shear Quench.

Final rept.

A. I. Nakatani, D. S. Johnsonbaugh, and C. C. Han $1995,17 p$

Pub. in Symposium of the National Meeting of the American Chemical Society (208th), Washington, DC. August 21-25, 1994, p246-262 1995.

Keywords: "Polymers, Shear quench, Light scattering, Reprints, "Flow induced structures, Phase separation kinetics.

A polystyrene (PS) and polybutadiene (PB) blend (50:50 by weight) dissolved in dioctyl phthalate (DOP) ( $8 \%$ total polymer by weight) was subjected to a two step shear quench with a delay time between the two shear rates examine the phase separation kinetics by light scattering. The time dependence of the peak maximum position, qm, and the intensity of the peak maximum, I(qm), were measured. The growth rate after cessation from the second shear rate was unaffected by the delay time. The mechanism for the coarsening parallel to flow was coupled to the extent of coarsening which occurred normal to the flow direction during the second shear rate.

\section{3,389}

PB96-159777 Not available NTIS

National Inst. of Standards and Technology (BFRL)

Gaithersburg, MD. Fire Safety Engineering Div.
Effects of Sample Mounting on Flammability Properties of Intumescent Polymers.

Final rept.

T. Kashiwagi, and T. G. Cleary. 1993, 23p.

Pub. in Fire Safety Jnl., v20 n3 p203-225 1993.

Keywords: "Fire resistant materials, "Polymers, *Flammability testing, "Heat release rate, Sample preparation, Flame propagation, Burning rate, Radiative heat transfer, Heat flux, Combustion products, Fireproofing, Soot, Calorimetry, Heat of combustion, Reprints.

Various flammability properties of polycarbonate samples were measured with the cone calorimater and lateral ignition and flame spread (LIFT) devices at various external fluxes. Four different sample mountings were used with the cone calorimeter to investigate the ef fects of sample mounting on the flammability properties of these samples. The mounting configurations allowed the intumesced char to rise free. The results show that peak heat release rate curves were significantly affected by the sample mounting configuration but total heat released, effective heat of combustion, and soot yield were not significantly affected by the sample mounting configuration but total heat released, effective heat of combustion, and soot yield were not significantly affected. Tow sample mounting configurations were used, the standard method and the standard method with the addition of a wire grid to retain intumesced char. Significant differences in flame spread rates were observed between the two sample mounting configurations with and without the grid.

\section{3,390}

PB96-180229 Not available NTIS

National Inst. of Standards and Technology (MSEL), Gaithersburg, MD. Polymers Div

Advances in the Measurement of Polymer CTE: Micrometer- to Atomic-Scale Measurements.

Final rept.

M. Schen, F. I. Mopsik, W. Wu, G. T. Davis, W. Guthrie, W. E. Wallace, and N. C. Beck Tan. 1996, $3 p$.

Pub. in Polymer Preprints, v37 n1 p180-182 Mar 96 Keywords: "Polymer films, "Thermomechanical analyzer, Capacitance, Coefficient, Metrology, Reprints, -Coefficient or thermal expansion, $X$-ray reflectivity, Electronic packaging.

Today's microelectronics products rely heavily on the use of polymeric materials; whether to package and protect the integrated circuit or to manufacture printed wiring boards and assemblies. In this work, NIST is pursuing a three-pronged strategy to improve the quality of measurement techniques and resulting data for characterizing the out-of-plane, or z-axis, coefficient of thermal expansion (CTE) of polymer films. The strategy consists of effecting evolutionary improvements in commonly used measurement techniques and standards for measuring CTE using the thermomechanica analyzer (TMA), introducing revolutionary improvements in measuring CTE by developing and applying a new NIST capacitance technique that has vastly improved sensitivity and accuracy over the TMA or existing capacitance-based approaches, and utilizing $X$-ray reflectivity measurements to understand the behavior of ultra-thin polymer films (50 to $1000 \mathrm{~A})$.

03,391

PB97-112262 Not available NTIS

National Inst. of Standards and Technology (MSEL), Gaithersburg, MD. Polymers Div.

Lattice Model of a Hydrogen-Bonded Polymer Blend.

Final rept.

E. K. Hobbie, and C. C. Han. 1996, 11p.

Pub. in Jnl. of Chemical Physics, v105 n2 p738-748 Jul 96.

Keywords: "Polymer blends, "Hydrogen bonding, Lattice models, Phas

A mean-field lattice model is used to investigate some possible aspects of the low-temperature behavior of a lower critical solution temperature (LCST) polymer blend made miscible by a dilute distribution of a hydrogen-bonding comonomer. The nature of the binding interaction is examined for both a random comonomer distribution and for the case of identically end-modified chains. For the latter case, competition between the interaction responsible for the immiscibility of the homopolymer components and the attraction due to hydrogen bonding leads to a stable intermediate 
phase. For random distributions, polydispersity in the binding interaction restricts this intermediate phase to an extremely narrow window in reduced temperature, consistent with a 'smeared' transition. The free-energy landscape contains local minima separated by energy barriers.

\section{3,392}

PB97-112304 Not available NTIS

National Inst. of Standards and Technology (MSEL), Gaithersburg, MD. Polymers Div.

Vitrification and Crystallization of Organic Liquids Confined to Nanoscale Pores.

Final rept.

C. L. Jackson, and G. B. McKenna. 1996, 10p

Pub. in Chemistry of Materials, v8 n8 p2128-2137 1996.

Keywords: *Organic liquids, "Nanoscale pores, 0 terphenyl, Vitrification, Crystallization, Benzyl alcohol, Calorimetry, Glass transition temperature, Reprints.

The effect of finite size on the solidification of ofonfined in mode trolled pore glass (CPG) materials is described. These two organic liquids form either amorphous glasses or crystalline solids in the bulk upon cooling, depending on the rate of cooling and other factors. The solidification behavior of the liquid in the pores was studied as a function of pore diameter $(4-73 \mathrm{~nm})$, chemical surface treatment of the CPG and the degree of pore filling, by differential scanning calorimetry (DSC). The authors observe that the glass transition $\mathrm{Tg}$. shifts to a lower temperature as pore size decreases. This shift is independent of the degree of pore filling for both oterphenyl and benzyl alcohol, suggesting that a reduction in bulk density or a negative pressure effect is not the cause of the observed shift. The crystallization behavior of o-terphenyl and benzyl alcohol is also altered by confinement and strongly depends on the pore size and degree of pore filling.

03,393

PB97-112486 Not available NTIS

National inst. of Standards and Technology (MSEL), Gaithersburg, MD. Polymers Div.

Evidence of Crosslinking in Methyl Pendent PBZT Fiber.

Final rept.

V. R. Mehta, S. Kumar, M. B. Polk, T. D. Dang, D. L. Vanderhart, and F. E. Arnold. 1996, 11p.

Pub. in Jnl. of Polymer Science, Part B, Polymer Physics, v34 p1881-1891 1996.

Keywords: ${ }^{*}$ Crosslinking, "Methyl pendant PBZT, *Fibers, Reprints, Poly(p-phenylene benzobis thiazole).

In order to influence the compressive strength of the rigid rod polymeric fibers, methyl pendent poly ( $p$ phenylene benzodisthiazole) fibers have been heat treated in the 400 to 550 degrees $C$ temperature range in air and in nitrogen for varying times to achieve intermolecular crosslinking. These fibers have been examined using Fourier transform infrared (FTIR) spectroscopy, (13)C solid-state nuclear magnetic resonance (NMR) swelling behavior, and scanning electron microscopy. (13)C NMR has also been carried out on solutions of as-spun fibers. Fibers heat-treated at 400 degrees $\mathrm{C}$, both in nitrogen and in air, up to heat-treatment times of $60 \mathrm{~min}$ are insoluble in $99 \%$ chlorosulfonic acid, however, no direct evidence of crosslinking has been obtained for these fibers using spectroscopic techniques, suggesting that in these fibers the degree of crosslinking must be very low. Evidence that methyl groups are precursors to certain crosslinks was first seen via a weak methylene resonance in (13)C solid state NMR, corresponding to about $2 \%$ of the the original methyl intensity, in a sample heat-treated at 450 degrees $C$ in air.

03,394

PB97-112494 Not available NTIS

National Inst. of Standards and Technology (MSEL),

Gaithersburg, MD. Polymers Div.

Relaxation After a Temperature Jump Within the One Phase Region of a Polymer Mixture.

Final rept.

G. Merkle, B. Bauer, and C. Han. 1996, 4p.

Pub. in Jnl. of Chemical Physics, v104 n23 p96479650 Jun 96.

Keywords: *Polymer blends, *Hydrogen bonding, "Spinodal decomposition, Small angle neutron scattering, Concentration fluctuation growth, Reprints.
Small-angle neutron scattering was used to observe the relaxation of the structure factor from a smaller to a larger fluctuation state after a temperature jump within the one-phase region of a miscible polymer blend. The blend studied is a $60 / 40$ by weight mixture of a hydrogen bonded polymer blend which consists of hydroxy modified dueterated polystyrene and poly(butylmethacrylate). A peak clearly evolved during the transition from the initial state at Ti to the final state at Tf as predicted by linear as well as nonlinear theories. This relaxation process, which took more than 10 $h$, could be represented reasonably well by a simple linear approximation of the Chn-Hilliard-Cook $(\mathrm{CHC}$ ) theory without involving nonlinear calculations for the evolution of the dynamic structure factor. The meas urement offers an alternative method for estimating the order parameter relaxation rate in the one-phase region. Also, this demonstrates experimentally the correct relaxation (to a higher fluctuation state) mechanism predicted by the linear theory of $\mathrm{CHC}$ type.

\section{3,395}

PB97-113088 Not available NTIS

National Inst. of Standards and Technology (MSEL), Gaithersburg, MD. Polymers Div.

Dimensional Crossover in the Phase Separation Kinetics of Thin Polymer Blend Films.

Final rept.

L. Sung, A. Karim, J. F. Douglas, and C. C. Han. 1996, 4p.

Pub. in Physical Review Letters, v76 n23 p4368-4371 1996.

Keywords: "Thin films, "Phase separation, *Spinoda decomposition, Blend, Crossovers, Reprints.

The kinetics of phase separation in thin polymer blend films of polystyrene and polybutadiene on a silicon substrate is examined by optical microscopy of the free film boundary. The observations on 1000 and 200 Angstroms films are consistent with a crossover from three- to two-dimensional spinodal decomposition $\mathrm{k}$ netics in the (off-critical) viscous hydrodynamic regime. In this stage of phase separation the exponent $n$, characterizing the scale $R(t)$ approximately tn of the coarsening pattern, is predicted to change from 1 to a value near 0.46 upon lowering the dimensionality.

03,396

Not available NTIS

National Inst. of Standards and Technology (MSEL) Gaithersburg, MD. Polymers Div.

Swelling and Growth of Polymers, Membranes and Sponges.

Final rept.

J. F. Douglas. 1996, 13p.

Pub. in Physical Review E, v54 n3 p2677-2689 Sep

Keywords: "Polymers, "Membranes, "Sponges, Network swelling, Wiener sheet, Reprints, Branched polymers, Blocked copolymers.

Polymers can be formed into a wide range of structures depending on the monomer chemistry and the kinetic conditions of growth. A general model of polymers have higher-order connectivity is introduced that reduces to flexible linear polymers, membranes, and sponges as special cases. This 'Wiener sheet' model, which extends the conventional Wiener path model of linear polymers, is argued to describe various classes of branched polymers, as well as different types of interacting random surfaces. For example, lattice animals and percolation clusters are considered to be perforated sheets whose large-scale dimensions are described by the Wiener sheet model with excluded volume interactions. To within the approximations of the model calculations, the properties of the Wiener sheet 'membrane' are consistent with this correspondence. The influence of the excluded volume and the kinetics of growth of membrane and sponge structures are treated at a Flory-level approximation, although the Wiener sheet model should admit to a renormalizationgroup treatment as in the case of linear polymers. Predictions of the self-interacting Wiener sheet model are contrasted with an alternative and complementary random surface model introduced by Nelson and co-workers and are compared with recent simulations and ex periment.

\section{Refractory Metals \& Alloys}

03,397

PB94-172590 Not available NTIS

National Inst. of Standards and Technology (CSTL) Gaithersburg, MD. Thermophysics Div.

Radiance Temperature (in the Wavelength Range $519-906 \mathrm{~nm}$ ) of Tungsten at Its Melting Point by a Pulse-Heating Technique.

Final rept.

A. P. Miiller, and A. Cezairliyan. 1993, 14p

Pub. in International Jnl. of Thermophysics 14, n3 p511-524 1993. Sponsored by National Aeronautics and Space Administration, Washington, DC.

Keywords: "Tungsten, "Melting point, "Radiation pyrometers, Wavelengths, Radiance, Heating, Temperature measurement, Surface temperature Thermophysical properties, Uncertainty, Reprints.

Radiance temperatures (at six wavelengths in the range 519-906 nm) of tungsten at its melting point were measured by a pulse-heating technique. The method is based on rapid resistive self-heating of the specimen from room temperature to its melting point in less than $1 \mathrm{~s}$; and on simultaneously measuring the specimen radiance temperatures every $0.5 \mathrm{~ms}$ with a high-speed six-wavelength pyrometer. Melting was manifested by plateau in the radiance temperature versus time function for each wavelength. The melting-point radiance temperatures for a given specimen were determined by averaging the measured temperatures along the plateau at each wavelength. The melting-point radiance temperatures for tungsten were determined by averaging the results at each wavelength for 10 specimens (standard deviation in the range $0.5-1.1 \mathrm{~K}$ de pending on the wavelength) as follows: $3319 \mathrm{~K}$ at 519 $\mathrm{nm}, 3236 \mathrm{~K}$ at $615 \mathrm{~nm}, 3207 \mathrm{~K}$ at $652 \mathrm{~nm}, 3157 \mathrm{~K}$ at $707 \mathrm{~nm}, 3078 \mathrm{~K}$ at $808 \mathrm{~nm}$, and $2995 \mathrm{~K}$ at $906 \mathrm{~nm}$. Based on estimates of the random and systematic errors arising from pyrometry and specimen conditions, the total uncertainty in the reported values is about + or $-7 \mathrm{~K}$ at $653 \mathrm{~nm}$ and + or $-8 \mathrm{~K}$ at the other wavelengths.

\section{3,398}

PB94-200011 Not available NTIS

National Inst of Standards and Technology (CSTL), Gaithersburg, MD. Thermophysics Div.

Wavelength Dependence of Normal Spectral Emissivity of High-Temperature Metals at Their Melting Point.

Final rept

A. Cezairliyan, A. P. Miiller, F. Righini, and A. Rosso. 1991, 8p.

Pub. in High Temperatures - High Pressures 23, p325 332 1991. Sponsored by National Aeronautics and Space Administration, Washington, DC.

Keywords: "Refractory metals, "Spectral emittance, "Melting point, "Emissivity, "Wavelengths, Thermophysical properties, Literature surveys, Spectral emission, Spectral lines, Line spectra, Molybdenum, Niobium, Tantalum, Reprints

The literature data on normal spectral emissivity of seected high-temperature metals at their melting points are reviewed. This study is motivated by recent results published by a major laboratory which show a common wavelength-independent behavior for the emissivity (in the wavelength range $500-1,000 \mathrm{~nm}$ ) of several hightemperature metals (including $\mathrm{V}, \mathrm{Nb}, \mathrm{Mo}, \mathrm{Ta}$, and $\mathrm{W}$ ) at their melting points. Based on the present study of accurate data for $\mathrm{Ni}, \mathrm{Ti}, \mathrm{V}, \mathrm{Nb}, \mathrm{Mo}, \mathrm{Ta}$, and $\mathrm{W}$ reported in the literature it is concluded that the normal spectral emissivity of these high-temperature metals at their melting points is not constant but decreases with in creasing wavelength in the range $400-1,000 \mathrm{~nm}$.

\section{3,399}

PB94-200169 Not available NTIS

National Inst. of Standards and Technology (MSEL), Gaithersburg MD Reactor Radiation Div.

Textures of Tantalum Metal Sheets by Neutron Diffraction.

C. S. Choi H. J. Prask, and J. Orosz 1993, 8 p.

Pub. in Jnl. of Materials Science 28, p3283-3290 1993.

Keywords: *Tantalum, Neutron diffraction, Metal sheets, Orientation, Texture, Reprints.

The orientation distributions of six tantalum samples, TaPA, TaG1, TaG2, TaQ2-S1, TaQ2-S2 and TaQ2 S4, were studied by neutron diffraction and ODF analy- 
sis. The TaPA specimen is a commercial tantalum sheet with an unknown fabrication history. The TaG1 and TaG2 were fabricated from a powder metallurgical ingot by uniaxial compression, and the TaQ2 type samples were fabricated from commercial stock by simila uniaxial forging. TaQ2-S1 is the section closest to the center of the forged disc, S2 is the intermediate section, and S4 is the section adjacent to the periphery.

\section{3,400}

\section{PB94-216686 Not available NTIS}

National Inst. of Standards and Technology (CSTL), Gaithersburg, MD. Thermophysics Div.

Measurement of the Heat of Fusion of Tungsten by a Microsecond-Resolution Transient Technique. Final rept.

J. L. McClure, and A. Cezairliyan. 1993, 7p

Sponsored by National Aeronautics and Space Administration. Washington. DC.

Pub. in International Jni. of Thermophysics 14, n3 p449-455 1993.

Keywords: "Heat of fusion, 'Refractory metals, "Pulse heating, 'Tungsten, High temperature, Melting, Transients, Reprints.

A microsecond-resolution pulse-heating technique was used for the measurement of the heat of fusion of tung sten. The method is based on rapid (100 to 125 microseconds) resistive self-heating of a specimen by a high-current pulse from a capacitor discharge system and measuring current through the specimen and voltage across the specimen as functions of time. Melting of a specimen is manifested by changes in the slope of the electrical resistance versus time function. The time integral of the power absorbed by a specimen during melting yields the heat of fusion. Measurements gave a value of $48.7 \mathrm{~kJ} / \mathrm{mol}$ for the heat of fusion of tungsten with an estimated maximum uncertainty of + or - $6 \%$. The electrical resistivity of solid and liquid ured.

\section{PB95-202388 Not available NTIS}

National Inst. of Standards and Technology (MSEL) Gaithersburg, MD. Reactor Radiation Div.

Application of ODF to the Rietveld Profile Refinement of Polycrystalline Solid.

Final rept.

C. S. Choi, E. F. Baker, and J. Orosz. 1994, 9p.

Pub. in Advances in X-ray Analysis, v37 p49-57 1994

Keywords: "Nodular iron, "Tungsten, Diffraction patterns, Polycrystalline, Texture, Reprints, "Rietveld method, Orientation distribution functions.

The Rietveld profile refinement method is probably the most popular technique used for the crystallographic characterization of materials including crystal structures and phase analysis, but it has been used mostly with ideal powder sample, not with textured polycrystals, because effects of strong and complex textures. Most technological materials are fabricated by using thermo-mechanical forming processes, which inevitably produce strong and complex preferential orientations of the crystallites. Consequently, the diffraction patterns of a given technological material are no unique but vary considerably with the measuring direction. with intensity variations as large as factors of hundreds, depending on the degree of texture. Part I of this study describes the determination of the orientation distribution function (ODF) and its application to the Rietveld profile refinement of the highly textured tungsten sample. Part II demonstrates the application of the Rietveld method to the quantitative analysis of the austempered ductile irons.

\section{Wood \& Paper Products}

\section{3,402}

PB94-162500 PC A03/MF A01

National Inst. of Standards and Technology (TS)

Gaithersburg, MD. Office of Standards Services.

Voluntary Product Standard PS 20-94. American Softwood Lumber Standard.

Mar 94, 45p, NIST/PS-20/94

Supersedes PB87-158564. Also available from Supt of Docs as SN003-003-03257-3. Sponsored by American Lumber Standards Committee, Germantown, MD

Keywords: "Softwoods, "Lumber, "Standards, "Measurement, Requirements, Sizes, Quality,
Classifying, Marking, Inspection, Surface roughness, Industries, US NIST, Certification, Design criteria, Maintenance, Voluntary standards.

The Standard pertains to softwood lumber. It establishes standard sizes and requirements for development and coordination of the lumber grades of the various species, the assignment of design values when called for, and the preparation of grading rules applicable to each species. It provides for implementation of the Standard through an accreditation and certification program to assure uniform industry-wide grade marking and inspection. It establishes principal trade classifications and lumber sizes for yard, structural, factory and shop use and provides for the classification, measurement, grading and grade marking of rough and dressed sizes of lumber items. Terms and procedures are defined to provide a basis for the use of uniform methods in the grading, inspection, measurement, and description of softwood lumber. It also includes the organization and functions of the American Lumber Standard Committee, the Board of Review, and the National Grading Rule Committee. Commercial names of the principal softwood species, definitions of terms used in describing standard grades of lumber and commonly used industry abbreviations are also provided.

\section{3,403}

\section{PB94-212404 Not available NTIS}

National Inst. of Standards and Technology (NEL), Gaithersburg, MD. Fire Science and Engineering Div. Rate of Heat Release of Wood Products.

Final rept.

M. Janssens. 1991, 22p.

Pub. in Fire Safety Jnl. 17, n3 p217-238 1991.

Keywords: "Wood, "Fires, *Heat transfer, Burning rate, Calorimeters, Ignition, Combustion, Mathematical models, Pyrolysis, Reprints.

The importance of energy release rate in a fire is illus trated. Two ways are identified to calculate rate of heat release in compartment fires from wood products used as interior finish: (1) use of detailed mathematical models of wood pyrolysis and combustion; (2) use of experimental bench-scale rate of heat release data. Available wood pyrolysis models are briefly reviewed. The second option is looked at in more detail. Rate of heat release data is presented and analyzed for 22 different wood products which were tested on the NIST Cone Calorimeter over the past 6 years. The experimental data cover two orientations, a range of irradiance levels, conditioned and oven dried samples and wood species covering a wide range of densities. Oven dry density is used as the key independent variable to analyze the data. Some important conclusions are drawn concerning the influence of density, conditioning, irradiance and specimen orientation on the rate of heat release. Further analysis and more data are required to confirm the results and to provide an answer to the questions which could not be resolved.

\section{3,404}

PB95-125712 Not available NTIS

National Inst. of Standards and Technology (IMSE), Boulder, CO. Fracture and Deformation Div

Examination of Objects Made of Wood Using AirCoupled Ultrasound.

Final rept.

C. M. Fortunko, M. C. Renken, and A. Murray. 1990, $5 \mathrm{p}$.

Pub. in Proceedings of Institute of Electrical and Electronics Engineers Ultrasonics Symposium, Honolulu, HI., December 4-7, 1990, p1099-1103.

Keywords: "Wood, "Ultrasonic flow detection, *Grain boundaries, Nondestructive tests, Ultrasonic radiation, Ultrasonic tests, Acoustic measurement, Acoustic velocity, Acoustic properties, Wave propagation, Reprints

The speed of ultrasound is approximately $330 \mathrm{~m} / \mathrm{sec}$ in air and $1500 \mathrm{~m} / \mathrm{sec}$ in water. Many materials exhibit surface-acoustic-wave velocities in the $300-1500 \mathrm{~m} /$ sec range. For this reason, air appears to be the ideal coupling fluid for exciting and detecting flexural waves, which are generally slower than surface acoustic waves, and shear waves. Air coupling may also be ideal for launching and detecting the quasi-Iongitudinal plate wave in materials which can exhibit very low longitudinal phase velocities, such as balsa wood $(800 \mathrm{~m}$ sec). In this paper, we describe an experimental, aircoupled, ultrasonic measurement system that can be used to study the propagation of $\mathrm{MHz}$ signals in wooden plates ranging in thickness from $3 \mathrm{~mm}$ to $25 \mathrm{~mm}$. Also, we report experimental measurements of the am- plitude of through-transmitted, narrow-band, $0.5 \mathrm{MHz}$ signals as a function of the angle of incidence for different grain orientations.

03,405

PB 95-170429 PC A04/MF A01

National Inst. of Standards and Technology (TS), Gaithersburg, MD. National Voluntary Lab. Accredita tion Program

National Voluntary Laboratory Accreditation Program (NVLAP): Wood Based Products.

Handbook

L. I. Knab, L. S. Galowin, W. J. Rossiter, and W. A Hall. Nov 94, 74p, NIST/HB-150-9.

Also available from Supt. of Docs. as SN003-00303307-3. See also PB94-178225.

Keywords: "Laboratories, "Certification, "Wood products, "US NBS, Requirements, Procedures, Manuals, Quality assurance, Standardization, Licensure, Methodology, Calibration standards, Government policies, NVLAP(National Voluntary Laboratory Accreditation Program), National Institute of Standards and Technology, NIST

National Institute of Standards (NIST) Handbook 150 9 presents the technical requirements of the National Voluntary Laboratory Accreditation Program (NVLAP) for Wood Based Products. It is intended for information and use by staff of accredited laboratories, those laboratories seeking accreditation, other laboratory accreditation systems, users of laboratory services, and others needing information on the requirements for accreditation under the Wood Based Products program. The publication supplements NIST Handbook 150 NVLAP Procedures and General Requirements, which contains Part 285 of Title 15 of the U.S. Code of Federal Regulations (CFR) plus all general NVLAP procedures, criteria, and policies.

\section{3,406}

PB96-178900 PC A04/MF A01

National Inst. of Standards and Technology (TS) Gaithersburg, MD. Office of Standards Services.

Voluntary Product Standard PS 1-95 Construction and Industrial Plywood.

B. M. Meigs Mar $96,48 p$.

Supersedes PB84-216449, Product Standard PS 1-83. Also available from Supt. of Docs. as SNO03-00303404-5. Sponsored by Engineered Wood Association, Tacoma, WA

Keywords: "Plywood, "Standards, Test methods Specifications, Building codes, Definitions, Veneers.

The Standard pertains to construction and industrial plywood. It provides requirements for the principal types and grades, and it covers wood species, veneer grading, glue bonds, panel construction and workmanship dimensions and tolerances, grade marking, moisture content, and packing of plywood intended for construction and industrial uses. It includes test methods to determine product compliance and a glossary of trade terms and definitions. A quality certification program and information regarding reinspecting practices are also provided. The Standard, a revision of Voluntary Product Standard PS 1-83, Construction and Industrial Plywood, was developed by the Standing Committee for Voluntary Product Standard PS 1-83 in accordance with procedures of the U.S. Department of Commerce.

\section{General}

03,407

DE95017761 PC A03/MF A01

National Inst. of Standards and Technology,

MATHEMATICAL SCIENCES 
Report of the Federal Internetworking Requirements Panel.

31 May 94, 45p, DOE/ER/25188-T1.

Contract Al02-93ER25188

Sponsored by Department of Energy, Washington, DC.

Keywords: "Computer Networks, Computers, Performance Testing, Procurement, Recommendations, Security, Specifications, Standards, EDB/990200.

The Federal Internetworking Requirements Panel (FIRP) was established by the National Institute of Standards and Technology (NIST) to reassess Federal requirements for open systems networks and to recommend policy on the Government's use of networking standards. The Panel was chartered to recommend actions which the Federal Government can take to address the short and long-term issues of interworking and convergence of networking protocols--particularly the Internet Protocol Suite (IPS) and Open Systems Interconnection (OSI) protocol suite and, when appropriate, proprietary protocols. The Panel was created at the request of the Office of Management and Budget in collaboration with the Federal Networking Council and the Federal Information Resources Management Policy Council. The Panel's membership and charter are contained in an appendix to this report.

\section{Algebra, Analysis, Geometry, \& Mathematical Logic}

\section{3,408}

\section{PB94-152725 PC A03/MF A01}

National Inst. of Standards and Technology (CAML), Gaithersburg, MD.

Growth Surface for the Slopes at the Boundary of a Polygon.

J. Bernal. Feb 94, 22p, NISTIR-5344.

Keywords: "Computational geometry, Polygons, Slopes, Algorithms, Fortran, Theorems, Voronoi diagrams, Growth surfaces.

In this paper, we describe the growth surface for the slopes at the boundary of a polygon, and present a brute force algorithm for computing it.

\section{3,409}

PB94-172053 Not available NTIS

National Inst. of Standards and Technology (CAML), Gaithersburg, MD. Applied and Computational Mathematics Div.

Monte Carlo Approach to the Approximation of Invariant Measures.

Final rept.

F. Y. Hunt. 1994, 23p.

Pub. in Random and Computational Dynamics 2, n1 p111-133 1994. See also PB93-159069.

Keywords: Monte Carlo method, Numerical solution, Approximation, Optimization, Convergence, Reprints, "Invariant measures, Frobenius-Perron operator, Ulam method

Approximation of absolutely continuous measures of maps of the interval and the closely related tasks of computing Lyapunov exponents and metric entropy are accomplished in principle by iterating the map to produce a sufficiently long trajectory. There is an alternative approach based on approximating the fixed point of the Frobenius-Perron operator. The authors present a Monte-Carlo implementation of the original piecewise constant method proposed by Ulam. This method has the advantage of not requiring explicit evaluation of the elements of the approximate Frobenius-Perron operator. Convergence rates of Ulam's method and some recently proposed higher order variants are discussed. Finally Ulam's scheme is used to estimate the leading Lyapunov exponent of a one dimensional map with an absolutely continuous measure.

\section{3,410}

PB94-198660 Not available NTIS

National Inst. of Standards and Technology (CAML), Gaithersburg, MD. Applied and Computational Mathematics Div.

Computing S(alpha) Using Symbolic Monte Carlo. Final rept.

I. M. Beichl, and F. Sullivan. 1992, 7p.

Pub. in Physica D 60, p358-364 1992.
Keywords: "Computation, Monte Carlo method, Mapping(Transformations), Numerical solution, Reprints, Entropy functions, Symbolic dynamics.

We describe a method for carrying out a computation of the entropy function of the invariant set of a particular class of maps. We illustrate the method for the case of the quadratic and the Feigenbaum map. Elementary consequences of the symbolic dynamics associated with a map f lead to an algorithm that is a novel application of the Monte Carlo method. To sample, we use the fact that the salient features of the thermodynamic properties of the map are captured by its symbolic dynamics. In the symbolic domain, samples are chosen by manipulating character strings, rather than by performing floating-point calculations. Therefore, exact symbolic answers can be obtained by purely combinatorial methods. These symbolic answers then provide a recipe for performing high-precision numerical computations.

\section{3,411}

\section{PB94-216744 Not available NTIS}

National Inst. of Standards and Technology (NEL), Gaithersburg, MD. Mathematical Analysis Div

Boundary Integral Method for the Simulation of Two-Dimensional Particle Coarsening.

Final rept.

G. B. McFadden, P. W. Voorhees, R. F. Boisvert,

and D. I. Meiron. 1986, 28p.

Pub. in Jnl. of Scientific Computing 1, n2 p117-144 1986.

Keywords: *Boundary integral method, Time dependence, Two dimensional, Free boundaries, Integral equations, Iterative methods. Binary alloys, Coarseness, Simulation, Reprints, Ostwald ripening, Particle coarsening.

A boundary integral method for the solution of a timedependent free-boundary problem in a two-dimensional, multiply-connected, exterior domain is described. The method is based on an iterative solution of the resulting integral equations at each time step, previous time steps. The method is related to a technique discussed by Baker for the study of water waves. The discretization is chosen so that the solvability conditions required for the exterior Dirichlet problem do not degrade the convergence rate of the iterative solution procedure. Consideration is given to the question of vectorizing the computation. The method is applied to the problem of the coarsening of two-dimensional particles by volume diffusion.

\section{3,412}

\section{PB95-126165 Not available NTIS}

National Inst. of Standards and Technology (MEL) Gaithersburg, MD. Automated Production Technology Div.

Constructing Invariant Tori for Two Weakly Coupled van der Pol Uscillators.

Final rept.

D. E. Gilsinn. 1993, 20p.

Pub. in Nonlinear Dynamics 4, p289-308 1993.

Keywords: "Van der Pol differential equation, Partial differential equations, Fast Fourier transforms, Perturbation theory, Periodic functions, Nonlinear systems, Approximation, Toruses, Algorithms, Reprints, "Van der Pol oscillators, Invariant tori.

An algorithm is developed for the construction of an invariant torus of a weakly coupled autonomous oscillator. The system is put into angular standard form. The determining equations are found by averaging and are solved for the approximate amplitudes of the torus. A perturbation series is then constructed about the approximate amplitudes with unknown coefficients as periodic functions of the angular variables. A sequence of solvable partial differential equations is developed for determining the coefficients. The algorithm is applied to a system of nonlinearly coupled van der Pol equations and the first order coefficients are generated in a straightforward manner. The approximation shows both good numerical accuracy and reproducibility of the periodicities of the van der Pol system. A comparative analysis of integrating the van der Pol system with integrating the phase equations from the angular standard form on the approximate torus shows numerical errors of the order of the perturbation parameter epsilon $=0.05$ for integrations of up to 10,000 steps Applying fast Fourier transforms (FFT) to the numerica periodicities generated by integrating the van der Pol system near the torus reveals the same predominan frequencies found in the perturbation coefficients. Fi- nally an expected rotation number is found by integrating the phase equations on the approximate torus.

03,413

PB95-126249 Not available NTIS

National Inst. of Standards and Technology (EEEL) Boulder, CO. Electromagnetic Technology Div.

Accuracy of Eigenvalues: A Comparison of Two Methods.

Final rept

I. C. Goyal, R. L. Gallawa, and A. K. Ghatak. 1993,

$7 \mathrm{p}$.

Pub. in Jnl. of Mathematical Physics 34, n3 p11691175 Mar 93.

Keywords: "Differential equations, "Eigenvalues, Boundary value problems, Variational methods, Numerical solution, Perturbation, Comparative evaluations, Matrices, Éstimates, Accuracy, Reprints, Langer method, WKB method.

The problem of estimating the eigenvalues lambda of the equation (second derivative of psi with respect to $x)+($ lambda $f(x) p s i(x)=0$, where $f(x)$ is smooth and positive in the closed interval $(0,1)$ and the imposed boundary conditions are psi $(0)=p s i(I)=0$ is revisited. Two methods, the Wentzel-Kramers-Brillouin (WKB) method and Langer's method, which until very recently has been virtually ignored, are compared. The variational principle further improves the accuracy of the eigenvalues. The results obtained when the WKB and Langer's results are used as trial functions with the variational principle are also compared. Results are given in tabular form.

03,414

PB95-180923 Not available NTIS

National Inst. of Standards and Technology (BFRL) Gaithersburg, MD. Structures Div.

Melnikov Function and Homoclinic Chaos Induced by Weak Perturbations.

Final rept.

E. Simiu, and M. Frey. 1993, 3p.

Pub. in Physical Review E 48, n4 p3190-3192 Oct 93. Keywords: "Nonlinear differential equations, "Duffing differential equation, Dynamical systems, Perturbation, Chaos, Reprints, Melnikov functions, Homoclinic orbits.

The effect of noise on the possible occurrence of chaos in systems with a homoclinic orbit (e.g., the Duffing equation) was recently considered by Bulsara, Schieve, and Jacobs (Phys. Rev. A 41, 668 (1990)), and Schieve and Bulsara (Phys Rev. A 41, 1172 (1990)), who adopted an approach based on a redefinition of the Melnikov function. We show that this redefinition is unsatisfactory and leads to incorrect results.

\section{3,415}

PB95-198933 PC A03/MF A0

National Inst. of Standards and Technology (CAML), Gaithersburg, MD. Applied and Computational Mathematics Div.

Inserting Line Segments into Triangulations and Tetrahedralizations.

J. Bernal. Mar 95, 27p, NISTIR-5596.

Keywords: "Computational geometry, "Triangulation, *Tetrahedrons, Algorithms, Three dimensional models, Two dimensional models, "Segment insertion, Edge swapping, Delaunay triangulations, Voronoi diagrams. In this paper, we further develop an algorithm by Bernal, De Floriani, and Puppo, for inserting a line segment into a Constrained Delaunay triangulation. The new version of the algorithm inserts the line segment in exactly the same manner in which the old version does but has the additional capability that it does not delete the triangles intersected by the line segment but transforms them through edge-swapping. A 3-dimensional version of the algorithm without the optimization step for the Delaunay property is also presented for attempting to insert a line segment into a tetrahedralization. It is shown that for certain cases the failure of the 3-dimensional algorithm to insert a line segment is an indication that it can not be done. $\mathrm{Fi}$ nally, 3-dimensional problems that can be approached algorithmically as 2-dimensional problems are identified

\section{3,416}

PB95-202354 Not available NTIS

National Inst. of Standards and Technology (CAML), Gaithersburg, MD. Applied and Computational Mathematics Div. 
Overcoming Hoelder Continuity in III-Posed Continuation Problems.

Final rept.

A. S. Carasso. 1994, 23p

Pub. in Society for Industrial and Applied Mathematics Jnl. on Numerical Analysis 31, n6 p1535-1557 Dec 94.

Keywords: "Partial differential equations, Fast Fourier transforms, Image processing, Banach space, Numerical stability, Numerical solution, Computation, Reprints, "Image deblurring, III posed problems, Holder continuity, SECB constant, Tikhonov-Miller regularization.

Many ill-posed continuation problems in partial differential equations obey a logarithmic convexity inequality and can be stabilized in an appropriate Banach space by imposing an a priori bound on the solutions. The present paper analyzes the effects of prescribing a physically mutivated supplementary constraint, the so-called slow evolution from the continu ation boundary (SECB) constraint. When the SECB constraint is applicable, there results an improved stability estimate. This theoretical result is valid for a large class of ill-posed continuation problems. The computational significance of this result is demonstrated in the latter half of the paper. An important class of image deblurring problems is reformulated as a backwards in-time continuation problem for a generalized diffusion equation. A quadratic functional on $L(\sup 2)(R(\sup 2)$ ) is constructed for which the SECB deblurred image is the unique minimizer. An explicit formula is then obtained for SECB restoration in the Fourier transform domain, leading to a fast, practical, numerical restoration procedure involving fast Fourier transform (FFT) algorithms. For a $512 \times 512$ image, SECB restoration requires about 20 seconds of cpu time on current desktop workstations.

\subsection{7}

PB96-119557 Not available NTIS

National Inst. of Standards and Technology (CAML), Gaithersburg, MD. Applied and Computational Mathematics Div

Numerical Evaluation of Special Functions.

Final rept.

D. W. Lozier, and F. W. J. Olver. 1994, 47p

Pub in Proceedings of Symposia in Applied Mathematics, Vancouver, British Columbia, August $9-13$ 1993, v48 p 79-125

Keywords: * Numerical analysis, "Functions(Mathematics), "Computer software, Approximation, Algorithms, Transcendental functions Applications of mathematics, Computer applications, Reprints, "Special functions, Mathematical software.

Higher transcendental functions continue to play varied and important roles in investigations by engineers mathematicians, scientists and statisticians. The purpose of this paper is to assist in locating useful approximations and software for the numerical generation of these functions, and to offer some suggestions for future developments in this field.

\section{3,418}

\section{PB96-122858 Not available NTIS}

National Inst. of Standards and Technology (CAML), Gaithersburg, MD. Applied and Computational Mathematics Div.

Siow Evolution from the Boundary: A New Stabilizing Constraint in III-Posed Continuation Problems. Final rept.

A. S. Carasso. 1994, 5p.

Pub. in Proceedings of Symposia in Applied Mathematics, American Mathematical Society, Vancouver, Canada, August 8-14, 1993, v48 p269-273 1994.

Keywords: "Noise reduction, "Boundary value problems, Image resolution, Blurring, Constraints, Differential equations, Applications of Mathematics, Reprints, *Ill-posed continuation Holder continuity, SECB constraint, Tikhonov-Miller regularization, Image deblurring

A new a-priori constraint sharply reduces noise contamination in ill-posed continuation problems. One application concerns a significant class of image deblurring problems that can be formulated as ill-posed time-reversed parabolic initial value problems.

\subsection{9}

PB96-122874 Not available NTIS

National Inst. of Standards and Technology (EEEL), Gaithersburg, MD. Electricity Div.
Bounds on Frequency Response Estimates Derived from Uncertain Step Response Data. Final rept.

J. P. Deyst, and T. M. Souders. 1995, 6p.

Pub. in Institute of Electrical and Electronics Engineers Instrumentation and Measurement Technology Conference Proceedings, Waltham, MA., April 24-26, 1995, p252-257

Keywords: "Frequency response, "Probability theory, Dynamic response, Estimates, Boundaries, Step functions, Time, Reprints, Electrical measurment, Simulation, "Uncertainty bounds, Time domain.

A system's frequency response can be estimated from measurements of its step response; however, many error sources affect the accuracy of such estimates. This paper investigates the effects of incertainty in the knowledge of the step response. Methods for establishing uncertainty bounds for the frequency response estimates are developed, based on the corresponding time-domain uncertainties associated with the step-like waveform. Two methods are described. One is a provable upper bound that is often very conservative. The other is more realistic, but it is based on an unproved conjecture. End effects that influence the bounds are also considered. A simulated example of the bounds is presented.

\section{3,420}

PB96-155577 Not available NTIS

National Inst. of Standards and Technology (EEEL) Gaithersburg, MD. Electricity Div.

Effects of Nonmedel Errors on Model-Based Testing.

Final rept

G. N. Stenbakken. $1995,5 p$

Pub. in Institute of Electrical and Electronics Engineers Instrumentation/Measurement Conference, IMTC ' 95 , Watham, MA., April 24-26, 1995, p38-42.

Keywords: "Confidence intervals, Efficient testing Simulation, Reprints, Empirical modeling, Nonmodel error, Prediction intervals.

In previous work, methods have been developed for efficient testing of components and instruments that are based on models of these units. These methods allow for the full behavior of these units to be predicted from a small but efficient set of test measurements Such methods can significantly reduce the testing cost of such units by reducing the amount of testing required. But these methods are valid only as long as the model accurately represents the behavior of the units. Previous papers on this subject described many methods for developing accurate models and using them to develop efficient test methods. However, they gave little consideration to the problem of testing units which change their behavior after the model has been developed, for example, as a result of changes in the manufacturing process. Such changed behavior is referred to as nonmodel behavior or nonmodel error When units with this new behavior are tested with these more efficient methods, their predicted behavior can show significant deviations from their true behavior This paper describes how to analyze the data taken at the reduced set of measurements to estimate the uncertainty in the model predictions, even when the device has significant nonmodel error. Results of simulation are used to verify the accuracy of the estimates and to show the expected variation in the results for many modeling variables.

03,421

PB96-165931 PC A03/MF A01

National Inst. of Standards and Technology (CAML), Gaithersburg, MD. Applied and Computational Mathematics Div

Basic Linear Algebra Operations in SLI Arithmetic. M. A. Anuta, D. W. Lozier, N. Schabanel, and P. R. Turner. Mar 96, 20p, NISTIR-5811

Keywords: "Linear algebra, Algebra, "SLI arithmetric, Computer arithmetric, Level-index arithmetic.

Symmetric level-index arithmetic was introduced to overcome recognized limitations of floating-point systems, most notably overflow and underflow. The original recursive algorithms for arithmetic operations could be parallelized to some extent, particularly when applied to extended sums of products, and a SIMD software implementation of some of these algorithms is described. The main purpose of this paper is to present parallel SLI algorithms for arithmetic and basic linea algebra operations.
03,422

PB96-193750 PC A04/MF A01

National Inst. of Standards and Technology (CAML) Boulder, CO. Applied and Computational Mathematics Div.

Hybrid Gauss-Trapezoidal Quadrature Rules.

B. K. Alpert. Jul 96, 36p, NISTIR-5048.

Sponsored by Defense Advanced Research Projects Agency, Arlington, VA

Keywords: "Gaussian quadrature, "Convergence, Functions(Mathematics), Numerical methods, Numerical analysis, Algorithms.

A new class of quadrature rules for the integration of both regular and singular functions is constructed and analyzed. For each rule the quadrature weights are positive and the class includes rules of arbitrarily high order convergence. The quadratures result from alterations to the trapezoidal rule in which a small number of nodes and weights as the ends of the integration interval are replaced. The new nodes and weights are determined so that the asymptotic expansion of the resulting rule, provided by a generalization of the EulerMaclaurin summation formula, has a prescribed number of vanishing terms. The superior performance of the rules is demonstrated with numerical examples and application to several problems is discussed.

03,423

PB97-110167 Not available NTIS

National Inst. of Standards and Technology (PL), Gaithersburg, MD Ionizing Radiation Div.

Exact Series Solution to the Epstein-Hubbell Generalized Elliptic Type Integral Using Complex Variable Residue Theory.

Final rept.

J. D. Evans, 1993, 17p.

Pub. in Applied Mathematics and Computation, v53 p173-1891993.

Keywords: "Complex variable, "Disk source, "Elliptictype integral, Radiation field, Residue theory, Series solution, Reprints, Epstein-Hubbell integral.

In the paper, complex variable residue theory is used to arrive at an exact infinite series solution to the Epstein-Hubbell integral. This method of solution is differnet from those used to derive previous solutions to the Epstein-Hubbell integral, but produces exactly equivalent results. Several sets of numerical data are included that verify the results. Additional information on the improvement of convergence will be forthcoming at a later date.

\section{3,424}

PB97-111488 Not available NTIS

National Inst. of Standards and Technology (CAML), Gaithersburg, MD. Applied and Computational Mathematics Div

Heap of Data.

Final rept.

I. Beichl, and F. Sullivan. 1996, 4p.

Pub. in Institute of Electrical and Electronics Engineers Computational Science and Engineering, v3 n2 p11. 14, Summer 1996

Keywords: "Data structures, "Extremum values, Data processing, Maxima, Minima, Mathematical computations, Algorithms, Reprints.

The authors discuss the heap, a data structure that allows one to keep track of the maximum or minimum dynamically.

\section{Operations Research}

03,425

PB94-152709 PC A03/MF A01

National Inst. of Standards and Technology (CAML), Gaithersburg, MD

Submissions to a Planned Encyclopedia of Operations Research on Computational Geometry and the Voronoi/Delaunay Construct.

I. Beichl, J Bernal, C. Witzgall, and F. Sullivan. Jan $94,17 p$, NISTIR-5349

Sponsored by Institute for Defense Analysis, Bowie, MD. Supercomputing Research Center.

Keywords: "Computational geometry, Operations research, Computer applications, Encyclopedias, Set 
theory, Polygons, "Voronoi diagrams, "Delaunay triangulation.

This report contains two submissions to a prospective Encyclopedia of Operations Research. The first submission covers an entry on 'Computational Geometry'. The second submission addresses both the Voronoi Diagram and its dual, the Delaunay triangulation, and is therefore termed the 'Voronoi/Delaunay Construct'. The latter plays such an important role in Computafional Geometry and has so many potential applications in Operations Research, that a separate entry appears justified. Both submissions refer to other entries which may be included in the planned Encyclopedia.

03,426

PB94-198959 Not available NTIS

National Inst. of Standards and Technology (NEL), Gaithersburg, MD. Scientific Computing Div.

Algorithmic Enhancements to the Method of Centers for Linear Programming Problems.

Final rept.

P. T. Boggs, P. D. Domich, J. R. Donaldson, and C. Witzgall. 1989, 13p.

Pub. in ORSA Jnl. on Computing 1, n3 p159-171 1989.

Keywords: "Linear programming, Algorithms, Optimization, Convergence, Trajectory analysis, Ordinary differential equations, Two dimensional models, Reprints, "Method of centers, Interior point method.

Interior point algorithms to solving linear programming problems are considered. The techniques are derived from a continuous version of Huard's method of centers that yields a family of trajectories in the feasible region that all converge to an optimal solution. The tangential direction of these trajectories is the dual affine direction. Deficiencies in some of these trajectories are discussed, and the need to recenter is argued. Several new algorithms that use the dual affine direction and a recentering direction in a multi-direction approach are then derived. The most promising of these algorithms is based on minimizing the cost function on a sequence of two-dimensional cross sections of the feasible region. Numerical results are presented.

\section{3,427}

PB94-212347 Not available NTIS

National Inst. of Standards and Technology (NEL), ing.

Guidelines for Reporting Results of Computational Experiments. Report of the Ad hoc Committee. Final rept.

R. H. F. Jackson, P. T. Boggs, S. G. Nash, and S.

Powell. 1991, 13p.

Pub. in Mathematical Programming 49, p413-425 1991.

Keywords: "Mathematical programming, "Experimentation, "Reporting, "Guidelines, Algorithms, Computation, Technology transfer, Parallel processing, Reprints.

Controversy often surrounds the reporting of results from scientific experimentation Guidelines for the reporting of the results of experimentation have helped to reduce some common errors and to clarify some issues for all concerned. As experience grows in new areas, such as the reporting of the results of computational experiments, it is natural to attempt to update and revise the guidelines to stimulate further discussion and to improve the reporting of results. In this spirit, the Council of the Mathematical Programming Sociefy asked the Committee on Algorithms (COAL) to reconsider the existing guidelines and to offer extensions and modifications as deemed necessary. The proliferation of powerful desktop workstations, microcomputers, mainframes, supercomputers, and novel machines with some form of parallel architecture, will continue to place increasing pressure on developers of algorithms to support their ideas with the results of computational experiments. Our hope is that we can supply a document that authors, editors, and referees can agree upon, and one that can provide a solid basis for the presentation of results.

03,428

PB94-215688 PC A04/MF A01

Clemson Univ., SC. Dept. of Mathematical Sciences.
Time Dependent Vector Dynamic Programming Algorithm for the Path Planning Problem.

Master's thesis.

M. R. Wilson. Dec 93, 70p, NIST/GCR-93/636.

Grant NANBOD 1023

Portions of this document are not fully legible. Sponsored by National Inst. of Standards and Technology (BFRL), Gaithersburg, MD.

Keywords: "Dynamic programming, "Paths, "Operations research, Planning, Mathematical models, Algorithms, Vector processing, Fires, Buildings, Escape systems, Computer programs.

Dynamic programming is a modeling technique used for the decision making process. This method can be used to find the set of nondominated paths in a network with time dependent vector costs. In this report a dynamic programming algorithm and its implementation are discussed. An application to a fire egress problem is also included.

03,429

PB95-180089 Not available NTIS

National Inst. of Standards and Technology (CAML), Gaithersburg, MD. Applied and Computational Mathematics Div.

Interior-Point Method for Linear and Quadratic Programming Problems. (NIST Reprint).

Final rept.

P. T. Boggs, P. D. Domich, J. E. Rogers, and C.

Witzgall. 1991, $9 \mathrm{p}$

See also PB91-187815.

Pub. in Committee on Algorithms Newsletter 19, p3240, 1 Apr 91

Keywords: "Quadratic programming, Linear programming, Iteration, Algorithms, Mathematical programming, Reprints, "Interior point methods, Method of centers.

We have been working on a particular class of interior point methods for solving linear programming problems for several years. Our methods combine several search directions that are readily computed at each it. eration. The final step is then calculated by computing the step that solves the original problem restricted to the subspace spanned by these several directions. In this paper we propose an extension of these ideas to the case of convex quadratic programming.

03,430

PB95-180097 Not available NTIS

National Inst. of Standards and Technology (CAML), Gaithersburg. MD. Applied and Computational Mathematics Div.

Convergence Properties of a Class of Rank-Two Updates. (NIST Reprint).

Final rept.

P. T. Boggs, and J. W. Tolle. 1994, 26p

Contract AFOSR-ISSA-90-0004

See also PB91-187799. Sponsored by Air Force Office of Scientific Research, Bolling AFB, DC

Pub. in Society for Industrial and Applied Mathematics Jnl. of Optimization-4, n2 p262-287 May 94.

Keywords: "Optimization, "Convergence, "Numerical analysis, Algorithms, Approximation, Iteration, Matrices(Mathematics), Newton methods, Sequences(Mathematics), Reprints.

Many optimization algorithms generate, at each iteration, a pair (I(sub K), $H$ (sub K)) consisting of an approximation to the solution I(sub K) and a Hessian matrix approximation $\mathrm{H}$ (sub $\mathrm{K}$ ) that contains local secondorder information about the problem. Much is known about the convergence of $I$ (sub $K$ ) to the solution of the problem, but relatively little is known about the behavior of the sequence of matrix approximations. The sequence $H($ sub $K)$, generated by the extended Broyden class of updating schemes independently of the optimization setting in which they are used, is analyzed. Various conditions under which convergence is assured are derived, and the structure of the limits is delineated. Rates of convergence are also obtained. These results extend and clanify those already in the literature.

\section{3,431}

PB96-179528 Not available NTIS

National Inst. of Standards and Technology (CAML), Gaithersburg, MD. Statistical Engineering Div.

Move-to-Root Rule for Self-Organizing Trees with Markov Dependent Requests.

Final rept.

R. P. Dobrow. 1996, 15p

Pub. in Stochastic Analysis and Applications, v14 n1 p73-87 1996.
Keywords: "Search profiles, "Self organizing systems, "Heuristic methods, "Data structures, Markov chains, Eigenvalues, Search theory, Probability distribution functions, Trees(Mathematics), Costs, Reprints, Binary search trees, Move-to-root rule.

The move-to-root (MTR) heuristic is a self-organizing rule which attempts to keep a binary search tree in near-optimal form. It is a tree analogue of the well-studied move-to-front (MTF) scheme. The authors study a Markov move-to-root (MMTR) model, where the sequence of record requests is a Markov chain, and analyze several characteristics of the tree chain, including the stationary distribution, eigenvalues, and stationary expected search cost.

\section{Statistical Analysis}

03,432

PB95-104956 PC A11/MF A03

National Inst. of Standards and Technology (CAML), Gaithersburg, MD. Statistical Engineering Div.

Extreme Value Theory and Applications: Proceedings of the Conference on Extreme Value Theory and Applications, Volume 3. Held in Gaithersburg, Maryland in May 1993.

Special pub.

J. Galambos, J. Lechner, E. Simiu, and C. Hagwood. Aug $94,244 p$, NIST/SP-866

Also available from Supt. of Docs. as SN003-00303280-8.

Keywords: "Statistical analysis, "Meetings, Weibull density functions, Statistical distributions, Order statistics, Markov chains, Wind velocity, Reliability, Corrosion, Quantiles, "Extreme value theory.

These 27 manuscripts make up Volume III of the Proceedings of the National Institute of Standards and Technology (NIST) Conference on Extreme Value Theory. They have been selected for their important contribution to a number of specialized topics. Partia Contents: On the Record Values from Univariate Distributions; Composite Sampling and Extreme Values: Extremal Sojourn Times for Markov Chains; Extreme Analysis of Wave Pressure and Corrosion for Structural Life Prediction; The Point-Process Approach to the Directional Analysis of Extreme Wind Speeds; Approximate Extreme Value Analysis for a Rigid Block Under Seismic Excitation; Large Deviations for Order Statistics; Extreme Values in Business Interruption Insurance.

03,433

PB95-125761 Not available NTIS

National Inst. of Standards and Technology (CAML), Gaithersburg, MD. Statistical Engineering Div. Noise-Induced Chaos and Phase Space Flux.

Final rept.

M. Frey, and E. Simiu. 1993, 20p.

Pub. in Physica D 63, p321-340 1993

Keywords: "Dynamical systems, Stochastic processes, Random noise, Phase space, Excitation, Chaos, Reprints.

We study the effect of additive noise on near-integrable second-order dynamical systems whose unperturbed flows have homoclinic or heteroclinic orbits. The noise is represented by a type of Shinozuka stochastic process capable of arbitrarily closely approximating Gaussian noise with any specified spectrum. We derive a formula for the flux factor applicable for any as ymptotic mean stationary excitation. This derivation shows that, to first order, the effect of the external excitation on the system is mediated by a linear filter associated with the system homoclinic or heteroclinic orbit. It also shows that the stationary mean distribution of the filtered excitation determines the average phase space flux. This is true for both random and nonrandom excitations and indicates that, for the dynamical systems considered here, these two classes of excitation play substantially equivalent roles in the promotion of chaos.

03,434

PB95-150405 Not available NTIS

National Inst. of Standards and Technology (CAML), Gaithersburg, MD. Statistical Engineering Div. 


\section{Statistical Analysis}

Tolerance Intervals for the Distribution of True Valwes in the Presence of Measurement Errors.

Final rept.

C. M. Wang, and H. K. Iyer. 1994, 9p.

Pub. in Technometrics 36, n2 p162-170 May 94.

Keywords: "Statistical distributions, Confidence limits, Approximation, Variance(Statistics), Mathematical models, Growth, Reprints, "Tolerance intervals.

A tolerance-interval procedure for a distribution is derived based on mutually independent statistics. Application to the construction of tolerance intervals for the distribution of true values when observations are contaminated with measurement errors is explained. This includes the problem of constructing tolerance intervals in some growth-curve models. Numerical examples are given to illustrate the procedures.

\section{3,435}

PB95-151296 Not available NTIS

National Inst. of Standards and Technology (NEL), Gaithersburg, MD. Statistical Engineering Div.

Approximate Confidence Intervals on Linear Combinations of Expected Mean Squares.

Final rept.

C. M. Wang. 1992, 13p.

Pub in Jnl. of Statistical Computation and Simulation 43, p229-241 1992

Keywords: *Confidence limits, "Variance(Statistics), Mean square values, Approximation, Probability the ory, Mathematical models, Simulation, Reprints.

In this article, we develop new procedures for obtaining approximate confidence intervals on linear combinations of the expected mean squares. The proposed ap proximations are indexed by a 'tuning' constant. By choosing the appropriate constant, which can be easily determined from data, we show that the proposed methods produce confidence intervals that maintain the stated confidence level. Numerical examples compare the performance to other approximations.

\section{3,436}

PB95-151304 Not available NTIS

National Inst. of Standards and Technology (NEL) Gaithersburg, MD. Statistical Engineering Div.

Approximate Confidence Intervals on Positive Linear Combinations of Expected Mean Squares.

Final rept.

C. M. Wang. 1991, 16p.

Pub. in Communications in Statistics - Simulation 20 , n1 p81-961991.

Keywords: "Confidence limits, "Variance(Statistics) Mean square values, Approximation, Probability theory, Reprints.

In this article, we develop new procedures for obtaining approximate confidence intervals on positive linear combinations of the expected mean squares. The proposed approximations, in many cases, are the weighted average of liberal and conservative confidence lim its. By choosing the appropriate weight, which can be easily determined from data, we show that the proposed methods produce confidence intervals with confidence coefficients very close to the stated level. Numerical examples compare the performance to other approximations.

\section{3,437}

PB95-151312 Not available NTIS

National Inst. of Standards and Technology (CAML) Gaithersburg, MD. Statistical Engineering Div. Ranges of Confidence Coefficients for Final rept.

C. M. Wang. $1990,14 \mathrm{p}$.

Pub. in Communications in Statistics - Simulation 19 n4 p 1165-1178 1990

Keywords: "Confidence limits, Variance(Statistics), Mathematical models, Approximation, Reprints.

The among variance component is the balanced onefactor nested components-of-variance model is of interest in many fields of application. Expect for an artificial method that uses a set of random numbers which is of no use in practical situations, an exact-size confidence interval on the among variance has not yet been derived. This paper provides a detailed comparison of three approximate confidence intervals which possess certain desired properties and have been shown to be the better methods among many available approximate procedures. Specifically, the minimum and the maximum of the confidence coefficients for the one- and two-sided intervals of each method are obtained. The expected lengths of the intervals are also compared.

\section{3,438}

PB95-152286 Not available NTIS

National Inst. of Standards and Technology (CAML) Gaithersburg, MD. Statistical Engineering Div.

Representing a Large Collection of Curves: A Case for Principal Points.

Final rept.

B. D. Flury, and T. Tarpey. 1993, 3p

Pub. in American Statistician 47, n4 p304-306 1993.

Keywords: "Cluster analysis, "Principal components analysis, Curves, Reprints, Principal points.

A principal component-based method of selecting a few representative curves from a large collection of curves was previously proposed. We suggest a solution based on estimation of principal points.

\section{3,439}

PB95-153524 Not available NTIS

National Inst. of Standards and Technology (CAML), Gaithersburg, MD. Statistical Engineering Div.

Constant-Width Calibration Intervals for Linear Re. gression.

K. R. Eberhardt, and R. W. Mee. 1994, 9p.

Pub. in Jnl. of Quality Technology 26, n1 p21-29 Jan 94.

Keywords: "Linear regression, "Calibrating, Regression analysis, Mathematical models, Confidence limits, Independent variables, Dependent variables, Probability theory, Reprints, Tolerance intervals.

Calibration, in the sense of inverse regression, is wide ly used in measurement science and other applications. For univariate regression models, simultaneous calibration intervals enable one to construct confidence intervals for the unobserved values of the independent variable corresponding to an unlimited sequence of future observations of the dependent variable. The intervals considered have the interpretation that if the initial training sample belongs to a specified set $\mathrm{G}$ of 'good' outcomes, the conditional coverage probability for each future confidence interval will be at least the nominal value. The set $\mathrm{G}$ is constructed to occur with high probability. All methods for constructing calibration intervals currently in the literature are conservative in that they are obtained from simultaneous tolerance intervals for which the actual confidence level exceeds the nominal level. This work develops constant-width simultaneous tolerance intervals for which the bound on the nominal coverage probabilities is exact under normality. The resulting confidence intervals represent an attractive balance between efficiency and simplicity for linear calibration problems.

\section{3,440}

PB95-160594 PC A13/MF A03

National Inst. of Standards and Technology, Gaithersburg. MD

Journal of Research of the National Institute of Standards and Technology, July/August 1994. Volume 99, Number 4. Special Issue: Extreme Value Theory and Applications. Proceedings of the Conference on Extreme Value Theory and Applications, Volume 2. Held at Gaithersburg, Maryland, in May 1993.

May 93, 296p.

Also available from supt. of Docs. See also PB94219326 and PB95-104956.

Keywords: "Extreme-value problems, "Failure analysis, "Corrosion, "Meetings, Extremum values, Ocean waves, Radiation damage, Weibull density functions, Spacecraft electronic equipment, Microelectronics, Sequences(Mathematics), Multivariate analysis, Ground motion, Loads(Forces), Fatigue limit, Risk analysis, Wind velocity, Aerosols, Floods, Ozone, Uses, "Extreme value theory, Bayesian analysis, Seismic risk.

It appears that the authors live in an age of disasters: the Mississippi and the Missouri rivers flood millions of acres, earthquakes hit Tokyo and California, airplanes crash due to mechanical failure, and powerful windstorms cause increasingly costly damage. While these may seem to be unexpected phenomena to the man in the street, they are actually happening according to well defined rules of science known as extreme value theory. The Proceedings are published in three
Volumes, Volume I, published by Kluwer Academic Publishers, contains papers of general interest in ex treme value theory and practice. Volume II, this Specia Issue of the NIST Joumal of Research, contains pa pers deemed by the Committee to be most directly relevant to NIST's mission. Volume III, NIST Special Publication 866, contains papers selected for their important contribution to a number of specialized topics. Applications considered in Volume II include damage because of ocean waves, high winds, seismic waves, ionizing radiation, floods, and corrosion.

03,441

PB95-180352 Not available NTIS

National Inst. of Standards and Technology (CAML) Gaithersburg, MD. Statistical Engineering Div.

Existence and Nonexistence Theorems of Finite Di ameter Sequential Confidence Regions for Errorsin-Variables Models.

Final rept.

J. T. Hwang, and H. Liu. 1992, 11p.

Pub. in Statistics and Probability Letters 13, p45-55

Keywords: Stopping rules(Mathematics), Principa components analysis, Sequential analysis, Confidence limits, Existence therorems, Reprints, "Errors in variables models.

In errors-in-variables models, confidence sets (or intervals) for the key parameters with finite diameter and with asymptotic coverage probabilities greater than some nominal level 1-alpha have been constructed. Do these sets have good coverage probabilities for any fixed sample size. The answer may be no. In fact, in these models, any finite diameter confidence sets have zero minimum coverage probability, no matter how large the sample size is as long as it is fixed. The results apply to various models, including most linear and nonlinear errors-in-variables and inverse regression problems. Sequential approach appears to lie in between fixed-sample and asymptotic approaches. It seems to be interesting to investigate whether it is possible to construct good sequential confidence sets. In the paper, we focus on the errors-in-variables models and show that the answer is negative for K-stage, for any finite positive interger $K$, sequential sampling However, the answer is positive for fully sequential sampling.

\section{3,442}

PB95-181103 Not available NTIS

National Inst. of Standards and Technology (CAML), Gaithersburg, MD. Statistical Engineening Div.

Moments of the Quartic Assignment Statistic with an Application to Multiple Regression.

Final rept.

D. F. Vecchia, and H. K. Iyer. 1991, 17p.

Pub. in Communications in Statistics - Theory and Methods 20, n10 p3253-3269 1991.

Keywords: "Distribution moments, Nonparametric statistics, Statistical tests, Regression analysis, Permuta tions, Computation, Reprints, "Assignment models Pearson distributions.

We present the first three exact moments of the symmetric quartic assignment statistic. Efficient computational formulas have been derived to overcome severe difficulties in third moment calculations. Two examples illustrate applications of the quartic assignment statistic: evaluation of significant 'clustering' or 'mixing'; and distribution-free tests for equality of several planar regression models. This article extends previous results on the cubic assignment statistic in lyer and Vecchia (1989).

\section{3,443}

PB95-203360 Not available NTIS

National Inst. of Standards and Technology (CAML), Gaithersburg, MD. Statistical Engineering Div

Two Principal Points of Symmetric, Strongly Unimodal Distributions.

Final rept.

T. Tarpey. 1994, 5p.

Pub. in Statistics and Probability Letters 20, p253-257 1994.

Keywords: "Statistical distributions, Cluster analysis Mixtures, Variance(Statistics). Optimization, Reprints, *Principal points, Self-consistent points.

Examples illustrate that splitting a symmetric, unimoda distribution into two groups so as to minimize the sum of the within-group variances does not always occur at the mean. Strong unimodality is shown to be a suffi- 
cient condition for the optimal cutpoint to occur at the mean.

\section{3,444}

PB95-203436 Not available NTIS

National Inst. of Standards and Technology (CAML),

Gaithersburg, MD. Statistical Engineering Div.

Outlier-Resistant Methods for Estimation and Model Fitting.

Final rept.

D. F. Vecchia, and J. D. Splett. 1994, 10p.

Pub. in ISA Transactions 33, p411-420 1994.

Keywords: "Mathematical models, "Curve fitting, "Estimating, "Outliers(Statistics), Least squares method, Estimators, Robustness(Mathematics), Regression analysis, Computer software, Reprints, LMS(Least Median of Squares).

Least squares is perhaps the most widely used technique for model fitting. In this article, we illustrate the poor performance of least squares when there are spunous values, or outliers, in a sequence of measurements. A brief overview of three well-known classes of robust altematives to the least-squares mean is presented. For robust regression, a recent proposal called least median of squares (LMS) is described. LMS regression is compared to least-squares regression in an example involving the estimation of optical fiber geometry. References are provided for software that is available for robust estimation techniques surveyed in this article.

\section{3,445}

\section{PB96-111802 Not available NTIS}

National Inst. of Standards and Technology (CAML), Gaithersburg, MD. Statistical Engineering Div.

Taguchi's Parameter Design: A Panel Discussion. Final rept.

B. Abraham, J. Mackay, J. A. Nelder, R. N. Kacker J. Sacks, W. J. Welch, T. J. Lorenzen, A. C

Shoemaker, K. L. Tsui, R. H. Myers, G. G. Vining, S. Taguchi, J M Lucas, C. F. Wu, G. Box, and M S Phadke. 1992, 35p

Pub. in Technometrics, v34 n2 p127-161 May 92.

Keywords: "Parameters, "Statistical analysis, "Experimental design, Analysis of variance, Factora design, Reliability, Variations, Quality assurance Quality control, Process control, Signal-to-noise ratio, Performance evaluation, Reprints.

The statistical techniques used by Taguchi to implement parameter design have been the subject of much debate, however, and there has been considerable research aimed at integrating the parameter-design principles with well-established statistical techniques. On the other hand, Taguchi and his colleagues feel tha these research efforts by statisticians are misguided and reflect a lack of understanding of the engineering principles underlying Taguchi's methodology. This panel discussion provides a forum for a technical discussion of these diverse views. A group of practitioners cussion of these diverse views. A group of practitioners and Taguchi's methodology for implementing it. The topics covered include the importance of variation reduction, the use of noise factors, the role of interactions, selection of quality characteristics, signal-tonoise ratios, experimental strategy, dynamic systems, and applications. The discussion also provides an up. to-date overview of recent research on alternative methods of design and analysis.

\section{3,446}

\section{PB96-135090 Not available NTIS}

National Inst. of Standards and Technology (CAML), Gaithersburg, MD. Statistical Engineering Div.

Principal Points and Self-Consistent Points of Symmetric Multivariate Distributions.

Final rept

T. Tarpey. 1995, 13p.

Pub. in Jni. of Multivariate Analysis, v53 n1 p39-51 Apr 95.

Keywords: "Elliptical distributions, "Multivariate distributions, Reprints, "Principal points, Self consistent points, Unimodal distributions.

Principal points and self-consistent points are cluster means of a distribution and represent a generalization of the population mean from one to several points. Principal points and self-consistent points are studied for a class of strongly symmetric multivariate distributions. Elliptical distributions belong to the class of strongly symmetric distributions. Several results are given for principal points and self-consistent points of strongly symmetric multivariate distributions. One result relates self-consistent points to principal component subspaces. Another result provides a sufficient condition for any set of self-consistent points lying on a line to be symmetric to the mean of the distribution.

\section{3,447}

PB96-137740 Not available NTIS

National Inst. of Standards and Technology (CAML), Gaithersburg, MD.

Churchill Eisenhart, 1913-1994.

Final rept.

J. M. Cameron, and J. R. Rosenblatt. 1995, 2p.

Pub. in American Statistician, v49 n3 p243-244 Aug 94.

Keywords: Statistics, Biographies, Reprints, *Obituaries, *Churchill Eisenhart.

This document is an obituary for Churchill Eisenhart, 1913-1994.

\section{3,448}

PB96-141163 Not available NTIS

National Inst. of Standards and Technology (CAML),

Gaithersburg, MD. Statistical Engineering Div.

Anova Estimates of Variance Components for a Class of Mixed Models.

Final rept.

M. G. Vangel. 1995, 6p

Pub. in Proceedings of the Section on Physical and Engineering Sciences, Toronto, Canada, August 13-18, 1994, p91-96 1995.

Keywords: "Analysis of variance, Reprints, "Variance estimates, "Henderson method, Quadratic form, Singular value decomposition.

This article examines a simple and intuitively appealing way to estimate variance components in mixed models: perform as many ordinary least squares regres. sions as there are variance components, using dummy variables for all but one random effect in each regression. By equating these residual sums of squares to their expectations, unbiased estimates of all of the variance components can be obtained. This approach is a special case of Method 3 of Henderson (1953). For a class of 'nearly balanced' nested mixed models, having no interactions involving both fixed and random effects, the exact distributions (under normality) of residual sums of squares are derived.

\section{3,449}

PB96-141171 Not available NTIS

National Inst. of Standards and Technology (CAML), Gaithersburg, MD. Statistical Engineering Div.

One-Sided beta-Content Tolerance Intervals for Mixed Models.

Final rept.

M. G. Vangel. 1995, 7p

Pub. in Proceedings of the Section on Physical and Engineering Sciences, Toronto, Canada, Chapel Hill, NC., June 13-15, 1994, Birmingham, AL., P200-206 1995

Keywords: *Mathematical models, "Confidence limits, Variance(Statistics), Reprints, Approximation, *Material strength.

Lower confidence limits on lower tail quantiles of a population of material strength measurements are routinely used to characterize the strength of a material, particularly in air craft design. Composite materials typically exhibit conside rable between-batch variability so that the approach discussed in this article could have important applications. For example, if three batches of five specimens each are tested at each of four temperatures, and it is desired to determine as a function of temperature a lower confidence limit on the tenth percentile of the population corresponding to the strength of a specimen chosen at random from a randomly selected batch, then the proposed methodology could be applied.

03,450

PB97-118707 Not available NTIS

National Inst. of Standards and Technology (CAML),

Gaithersburg, MD. Statistical Engineering Div.

Developing Measurement for Experimentation.

Final rept.

W. Liggett. 1996, 26p

Pub. in Statistics in Quality, Chapter 10, p179-204 1996.

Keywords: *Experimental design, "Measurement, "Sensitivity, "Selectivity, Multivariate analysis, Analysis of variance, Accuracy, Estimating, Statistical analysis, Statistical inference, Reprints, "Homoscedasticity.

Measurement consists of producing, for each unit measured a response related to the unit property in question. The response may be multivariate. Development of a measurement system entails experiments involving responses obtained from sets of test units, with each set consisting of nearly identical units. These ex periments require a variety of statistical approaches, which this chapter brings together.

\section{MEDICINE \& BIOLOGY}

\section{Biochemistry}

\section{3,451}

DE96014476 PC A05/MF A01

National Inst. of Standards and Technology Gaithersburg, MD

Small genomes: New initiatives in mapping and sequencing. Workshop summary report. K. McKenney, and F. Robb. 1993, 57p, CONF. 9307232-SUMM

\section{Contract Al02-93ER61622}

Small genomes: new initiatives in mapping and sequencing, Rockville, MD (United States), 5-7 Jul 1993 Sponsored by Department of Energy, Washington, DC

Keywords: "DNA Sequencing, *Genetic Mapping, Biotechnology, Man, Meetings, EDB/550400, EDB 550200 .

The workshop was held 5--7 July 1993 at the Cente for Advanced Research in Biotechnology (CARB) and hosted by the University of Maryland Biotechnology Instifute (UMBI) and the National Institute of Standards and Technology (NIST). The objective of this workshop was to bring together individuals interested in DNA technologies and to determine the impact of these cur rent and potential improvements of the speed and costeffectiveness of mapping and sequencing on the planning of future small genome projects. A major goal of the workshop was to spur the collaboration of more diverse groups of scientists working on this topic, and to minimize competitiveness as an inhibitory factor to progress.

\section{3,452}

PB94-172145 Not available NTIS

National Inst of Standards and Technology (IMSE) Gaithersburg, MD. Polymers Div.

Calcium Phosphate Precipitation in Liposomal Suspensions.

Final rept.

E. D. Eanes. $1990,10 p$

Pub. in Materials Research Society Symposia Proceedings, v174 p15-24 1990. Sponsored by National Inst. of Dental Research, Bethesda, MD.

Keywords: "Liposomes, "Calcium phosphates, *Mineralization, Artificial membranes, Bones, In vitro analysis, Phosphatidycholines, Cholesterol, pH, Reprints, Diacetylphosphates.

Artificial lipid vesicles (liposomes) provide an in vitro approach for microencapsulating calcium phosphate precipitation reactions in a manner similar to that which occurs in matrix vesicles, the initial loci for extracellula mineralization in many skeletal tissues. Apatitic precipitates readily form within the aqueous interiors of liposomes prepared from phosphatidycholine dicetylphosphate, and cholesterol when the liposoma membranes enclosing pH 7.4 buffered $\mathrm{PO} 4$ solutions are made permeable to external $\mathrm{Ca} 2+$ with ionophores. If the external $\mathrm{Ca}$ solution is rendered metastable with PO4, the apatitic precipitates rapidly ex pand to outside the liposomes as well. The present paper describes the basic features of liposomal mineralization and presents some specific examples on how compositional alterations in the liposomal membrane and external $\mathrm{Ca}$ solution can affect the progress of this mineralization.

03,453

PB94-172509 Not available NTIS 


\section{Biochemistry}

National Inst. of Standards and Technology (MSEL), Gaithersburg, MD. Reactor Radiation Div.

Characterization of the Binding of Gallium, Platinum, and Uranium to Pseudomonas Fluorescens by Small-Angle $X$-ray Scattering and Transmission Electron Microscopy

Final rept.

S. Krueger, G. J. Olson, D. Johnsonbaugh, and T. J. Beveridge. 1993, 9p.

Pub. in Applied and Environmental Microbiology 59 n12 p4056-4064 Dec 93.

Keywords: "Gallium, "Platinum, "Uranium, "Pseudomonas fluorescens, "Radiation scattering *Electron microscopy, "Binding sites, $X$ rays, Cell membrane, Particle size, Peptidoglycans, Reprints.

Small-angle $X$-ray scattering (SAXS) was used to determine the binding of $\mathrm{Ga}, \mathrm{U}$, and $\mathrm{Pt}$ to Pseudomonas fluorescens in aqueous buffer. Atomic absorption spectrophotometry was used to quantify the heavy metals during bulk analysis, whereas transmission electron microscopy of whole mounts and thin sections was used to determine the locations of the cell-bound metal precipitates, as well as their sizes and physical structures. Energy-dispersive $X$-ray spectroscopy confirmed the compositions and identities of the precipitates and helped show that they were associated primarily with the envelope layers of the bacteria. Unlike $\mathrm{Ga}$ and $\mathrm{Pt}$, which were located only at the cell surface, $U$ was also found intracellularly in approximately killed and lysed the cells. Surface-bound Ga and $U$ were spread over the entire cell envelope (outer membrane-peptidoglycan-plasma membrane complex), whereas $P t$ was associated only with the lipopolysaccharide-rich, external face of the outer membrane. SAXS confirmed these data and showed that the bacteria were metal-enshrouded particles that were 1.0 to 1.5 micrometers in diameter. SAXS also provided a statistically significant representation of the bound metal precipitates, which ranged in size from 10 $\mathrm{nm}$ to 1 micrometers. The correlation between the microscopic data and the scattering data was extremely good. Since SAXS is performed in an aqueous milieu it yields a more representative picture of the physical state of the metal bound to cell surfaces.

\section{3,454}

PB94-198249 Not available NTIS

National Inst. of Standards and Technology (CSTL), Gaithersburg, MD. Biotechnology Div.

Calorimetric Determination of the Standard Transformed Enthalpy of a Biochemical Reaction at Specified $\mathrm{PH}$ and $\mathrm{pMg}$.

Final rept.

R. A. Alberty, and R. N. Goldberg. 1993,11p.

Pub. in Biophysical Chemistry 47, p213-223 1993.

Keywords: "Chemical equilibrium, " $\mathrm{pH}$, "Metals, "Ions, "Heat of reaction, Reprints, Calorimetry, Hydrogen ions, Enthalpy, Biochemistry, "Metallic ions.

In a biochemical reaction there is generally a change in the binding of hydrogen ions and metal ions. Therefore, calorimetric measurements of enthalpies of reaction have to be adjusted for the enthalpies of reaction of the hydrogen ions and metal ions produced or consumed with the buffer. It can be shown that this yields the standard transformed enthalpy of reaction that determines the change in the apparent equilibrium constant (written in terms of sums of concentrations of species of a reactant) with temperature at the chosen $\mathrm{pH}$ and concentration of free metal ion. The derivations are based on the assumption that the changes in $\mathrm{pH}$ and free metal ion concentrations in the calorimetric experiment are small. This assumption is experimentally realized if a solution is well buffered for hydrogen and metal ions. The derived equations are discussed in terms of the implications they have for the performance and interpretation of calorimetric measurements.

\section{3,455}

PB94-199593 Not available NTIS

National Inst. of Standards and Technology (CSTL) Gaithersburg, MD. Biotechnology Div

Thermodynamic Analysis of Heparin Binding to Human Antithrombin.

Final rept.

S. DeLauder, F. P. Schwarz, J. Williams, and D. H

Atha. 1992, $9 p$

Pub. in Biochimica et Biophysica Acta 1159, p141-149 1992

Keywords: "Heparin, *Antithrombin III,
*Thermodynamics, Binding sites, Differential scanning calorimetry, Protein denaturation, Protein folding, Reprints.

The binding of heparin to human antithrombin III (ATIII) was investigated by titration calorimetry (TC) and differential scanning calorimetry (DSC). TC measurements of homogeneous high-affinity pentasaccharide and octasaccharide fragments of heparin in $0.02 \mathrm{M}$ phosphate buffer and $0.15 \mathrm{M}$ sodium chloride $(\mathrm{pH} 7.3)$ yielded binding constants of $(7.1+$ or -1.3$)$ times 10 to the fifth power/M and $(6.7+$ or -1.2$)$ times 10 to the sixth power/M, respectively, and corresponding binding enthalpies of $-48.3+$ or -0.7 and $-54.4+$ or $5.4 \mathrm{~kJ} / \mathrm{mol}$. The binding enthalpy of heparin in phosphate buffer $(0.02 \mathrm{M}, 0.15 \mathrm{M} \mathrm{NaCl}, \mathrm{pH} 7.3)$ was estimated from TC measurements to be $-55+$ or $-10 \mathrm{~kJ}$ mol, while the enthalpy in Tris buffer $(0.02 \mathrm{M}, 0.15 \mathrm{M}$ $\mathrm{NaCl}, \mathrm{pH} 7.3)$ was $-18+$ or $-2 \mathrm{~kJ} / \mathrm{mol}$. The heparinbinding affinity was shown by fluorescence measurements not to change under these conditions. The 3 fold lower binding enthalpy in Tris can be attributed to the transfer of a proton from the buffer to the heparinATIII complex. DSC measurements of the ATIII unfolding transition exhibited a sharp denaturation peak a $329+$ or $-1 \mathrm{~K}$ with a van 't Hoff enthalpy of $951+$ or $89 \mathrm{~kJ} / \mathrm{mol}$, based on a two-state transition model and a much broader transition from 333 to $366 \mathrm{~K}$. The transition peak at $329 \mathrm{~K}$ accounted for $9-18 \%$ of the total ATIII. At sub-saturate heparin concentrations, the lower temperature peak became bimodal with the appearance of a second transition peak at $336 \mathrm{~K}$. At saturate heparin concentration only the $336 \mathrm{~K}$ peak was observed. This supports a two domain model of ATIII folding in which the lower stability domain (329 K) binds and is stabilized by heparin.

\section{3,456}

PB94-211042 Not available NTIS

National Inst. of Standards and Technology (NML), Gaithersburg, MD

Molar Absorptivities of Bilirubin (NIST SRM 916A) and Its Neutral and Alkaline Azopigments.

Final rept.

B. T. Doumas, B. W. Perry, R. B. McComb, K. L. J.

Vink, J. C. Koedam, R. C. Paule, A. Kessner, and H. L. Vader. $1990,4 p$

Pub. in Clinical Chemistry 36, n9 p1698-1701 1990

Keywords: "Pigments, "Azo compounds, "Bilirubin, "Absorptivity, Interlaboratory comparisons, Reprints, * Standard reference materials.

Three laboratories in the U.S. and two in the Netherlands determined molar absorptivities (epsilon) of bilirubin from the National Institute of Standards and Technology (NIST, the former National Bureau of Standards), Standard Reference Material SRM 916a. In caffeine reagent average epsilon values were 50,060 and $48,980 \mathrm{~L}$ times mol(sup -1) times $\mathrm{cm}$ (sup -1) at 432 and $457 \mathrm{~nm}$, respectively. The epsilon value of the blue azopigment, obtained with the reference method for total serum bilirubin was 76,490 L times mol(sup -1) times cm(sup -1) at $598 \mathrm{~nm}$. When the ad dition of alkaline tartrate was omitted, the molar absorptivity of the red azopigment was $56,600 \mathrm{~L}$ times mol(sup -1) times cm(sup -1) at $530 \mathrm{~nm}$.

\section{3,457}

\section{PB94-211190 Not available NTIS}

National Inst. of Standards and Technology (MSEL) Gaithersburg, MD. Polymers Div.

Mixed Phospholipid Liposome Calcification.

Final rept.

E. D. Eanes. 1992, 4p

Sponsored by National Inst. of Dental Research, Bethesda, MD

Pub. in Bone and Mineral 17, p269-272 1992.

Keywords: "Liposomes, "Phospholipids, "Calcification, Precipitation, Phosphatidylserines, Sphingomyelins, Reprints.

Synthetic lipid vesicle (liposome) suspensions have been used to experimentally model many of the cal cium phosphate precipitation steps observed in matrix vesicle (MV) calcification. In particular, precipitate development in liposomes can be made to preferentially follow the progression seen in MV, i.e. to occur initially in intraliposomal spaces and then to expand into the surrounding suspending medium. This paper reviews results from studies by us which show that certain phospholipid constituents of the liposomal membran can modulate this progression. Of greatest relevance to MV calcification is the observation that phosphatidylserine and sphingomyelin, two lipids selectively enriched in MV, slow the expansion of the precipitation from inside to outside the liposome.
03,458

PB94-212602 Not available NTIS

National Inst. of Standards and Technology (NML). Gaithersburg, MD. Ionizing Radiation Div.

Modification of Deoxyribose-Phosphate Residues by Extracts of Ataxia Telangiectasia Cells. Final rept.

L. R. Karam, P. Calsou, W. A. Franklin, T. Lindahl,

B. Painter and M. Olsson, $1990,8 p$.

Pub. in Mutation Research 236, n1 p19-26 1990

Keywords: "Ataxia telangiectasia, "Deoxyribose, "Phosphates, Molecular weight, Cell line, pH, Chromatography, Xeroderma pigmentosum, Bloom syndrome, Friedreich's ataxia, Werner's syndrome, Reprints.

The release of DNA 5'-terminal deoxyribose-phosphate residues from enzymatically incised apurinic/ apyrimidinic sites by human cell extracts has been under investigation. During the course of these studies, we observed that ataxia telangiectasia cell extracts modify deoxyribose-phosphate (dRp) residues by converting them to an adduct form, $d R p \cdot X$, which shows altered chromatographic properties on high pressure liquid chromatography (HPLC) analysis. The chemical nature of the adduct is as yet unknown, but $\mathrm{dRp}-\mathrm{X}$ is stable to both heat and acid. The modification requires an enzymatic activity and a low-molecular weight cofactor. Extracts of normal cells contain a dialyzable inhibitor that suppresses the reaction occurring with ataxia telangiectasia cell extracts. Formation of $\mathrm{dRp}$ $X$ has been observed in 7 out of 7 ataxia telangiectasia lymphoblastoid lines which represent at least 3 genetic complementation groups. Similar modification of $\mathrm{dRp}$ did not occur with extracts of cells of normal origin, nor those representing Fanconi's anemia, xeroderma pigmentosum, Bloom's syndrome, Werner's syndrome or Friedreich's ataxia.

\subsection{9}

PB94-212800 Not available NTIS

National Inst. of Standards and Technology (CSTL), Gaithersburg, MD. Biotechnology Div.

Apparent Molar Heat Capacities and Apparent Molar Volumes of Aqueous Glucose at Temperatures from $298.15 \mathrm{~K}$ to $327.01 \mathrm{~K}$.

Final rept.

N. Kishore, R. N. Goldberg, and Y. B. Tewari. 1993 , $13 p$. 8591993

Keywords: "Glucose, "Aqueous solutions, "Specific heat, "Density(MassNolume), "Volume, Temperature dependence, Thermodynamic properties, Error analysis, Reprints, "Molar volume, Thermodynamic excess properties.

Specific heat capacities, apparent molar heat capacities, densities, and apparent molar volumes were determined for aqueous glucose over the temperature range 298.15 to $327.0 \mathrm{~K}$. The molal concentrations of the solutions ranged from 0.20 to $1.54 \mathrm{~mol} / \mathrm{kg}$. The results were used to calculate c(sub $p, 2, \mathrm{~m})$ (sup o) and $V(s u b 2, m$ )(sup o) for glucose at $298.15 \mathrm{~K}$ and the first and second derivatives of these quantities with respect to temperature. The first virial coefficient in the expression for the excess molar volume was determined. The results were compared with published data. A discussion of systematic errors attributable to instrumental errors, sample impurities, and incomplete anomeric and relaxation in the solutions is given.

03,460

PB94-213113 Not available NTIS

National Inst. of Standards and Technology (CSTL), Gaithersburg, MD. Biotechnology Div.

Thermochemistry of the Reactions between Adenosine, Adenosine 5'-monophosphate, Inosine, and Inosine 5'-monophosphate; the Conversion of Lhistidine to (Urocanic Acid+Ammonia).

Final rept.

J. W. Larson, Y. B. Tewari, and R. N. Goldberg. 1993, 18p

Pub. in Jnt. of Chemical Thermodynamics 25, p73-90 1993.

Keywords: "Adenosine monophosphates, "Thermochemistry, "Chemical equilibrium, "Inosine monophosphate, "Thermodynamic properties, Gibbs free energy, Entropy, Enthalpy, Calorimetry, Histidine, Catalysis, Enzymes, Biochemistry, Reprints, Deamination, Dephosphorylation.

The results of equilibrium and calorimetric measurements are reported for a series of enzyme-catalyzed 
reactions involving deamination of adenosine- 5 ' monophosphate (AMP), adenosine, and L-histidine and the dephosphorylation of AMP and inosine-5'. monophosphate. The data have been analyzed with a model that described the complex equilibria in solution. Values for the standard molar enthalpies, Gibbs free energies, and entropy changes of the reactions were calculated and discussed in relation to thermodynamic cycle calculations involving the substances and other reactions of a chemically similar nature.

\section{3,461}

\section{PB94-216363 Not available NTIS}

National Inst. of Standards and Technology (NML), Gaithersburg, MD. Chemical Process Metrology Div. Gradient and an Alternating Electric Field.

Final rept.

V. S. Markin, T. Y. Tsong, R. D. Astumian, and B.

Robertson. 1990, $5 p$.

Pub. in Jnl. of Chemical Physics 93, n7 p5062-5066 1990.

Keywords: "Signal transduction, "Electric fields, "Proteins, Conformational changes, Gradients, Concentration(Composition), Membranes, Reprints, "Electroconformational coupling, "Alternating electric fields, Membrane transduction.

A four-state transport protein that has electric charges that move concomitantly with conformational changes can transduce energy in either direction between an alternating membrane potential and a concentration gradient. An alternating electric potential of very large amplitude is considered, and the rapid-equilibrium approximation is used for the conformational changes. This permits deriving simple expressions for the transport flux, the power supplied by the alternating potential, and the power applied to the concentration gradient.

\section{3,462}

\section{PB95-140497 Not available NTIS}

National Inst. of Standards and Technology (CSTL), Gaithersburg, MD. Biotechnology Div.

Determining Mobility from Homodyne ac Electrophoretic Light Scattering.

Final rept.

B. Robertson, and J. Hubbard. 1992, 4p.

Pub. in Jnl. of Colloid and Interface Science 153, n2 p457-460, 15 Oct 92

Keywords: Light scattering, Correlation functions, Alternating current, Electric fields, Suspensions, Reprints, "Electrophoretic mobility, Homodyne measurement.

The homodyne correlation function for light scattered from a suspension of particles in an oscillating field is derived, assuming there are two species of particles of the same size but with different mobilities. If the mobility of one species is known, the mobility of the other can be determined from the modulation of the correlation function. This method of determining mobility is insensitive to thermal convection effects, electroosmotic flow, and mechanical vibrations.

03,463
PB95-150801 Not available NTIS

National Inst. of Standards and Technology (CSTL), Boulder, CO. Biotechnology Div.

Thermochemistry of the Hydrolysis of L-arginine to (L-citrulline + Ammonia) and of the Hydrolysis of L-arginine to (L-ornithine + Urea).

Final rept.

Y. B. Tewari, N. Kishore, S. A. Margolis, R. N

Goldberg, and T. Shibatani. 1993, 13p

Pub. in Jnl. of Chemical Thermodynamics 25, p293. 3051993.

Keywords: "Arginine, "Heat measurement, "Hydrolysis, "Thermochemistry, Thermodynamics, Urea, Metabolic processes, Models, Chemical reactions, Enzymes, Ammonia, Catalysis, Biotechnology, Reprints, "Molar enthalpy, Microcalorimetry, L-arginine.

Molar enthalpies of reaction for the hydrolysis of $\mathrm{L}$ arginine (aq) to (L-ornithine(aq) + urea(aq)) and for the hydrolysis of $\mathrm{L}$-arginine $(\mathrm{aq})$ to (L-citrulline(aq) + ammonia(aq)) have been measured by microcalorimetry. These reactions are catalyzed, respectively, by arginase and by arginine deiminase. The effects of variations in $\mathrm{pH}$, temperature, and ionic strength of the molar enthalpies of reaction were studied. The results have been analyzed with a mode which accounts for the complex equilibria in solution. The results are discussed in terms of thermodynamiccycle calculations and in terms of the metabolic urea cycle

\section{3,464}

\section{PB95-162707 Not available NTIS}

National Inst of Standards and Technology (NML) Gaithersburg, MD. Chemical Thermodynamics Div.

Biological Thermodynamic Data for the Calibration of Differential Scanning Calorimeters: Dynamic Temperature Data on the Gel to Liquid Crystal Phase Transition of Dialkylphosphatidylcholine in Water Suspensions.

Final rept.

F. P. Schwarz. 1991, 19p.

Pub. in Thermochimica Acta 177, p285-303 Apr 91

Keywords: "Lipids, "Thermodynamics, "Heat measurement, Transition temperature, Specific heat, Transition points, Enthalpy, Liquid crystals, Chemical energy, Reprints, Diakylphosphatidylcholine, Differential scanning calorimetry, Gel to liquid crystal transition.

Heat capacity measurements of the gel to liquid crystalline transition of phosphocholine lipid suspensions in buffer solutions were performed by a differential scanning calorimeter over a range of experimental conditions. Since the temperatures at the transition peak maxima, i.e. transition temperatures, were observed to increase linearly with scan rate due to the response time of the DSC, they were corrected to zero scan rate and found to agree closely with the literature values.

\section{3,465}

PB95-162715 Not available NTIS

National Inst. of Standards and Technology (NML), Gaithersburg, MD. Chemical Thermodynamics Div. Thermodynamics of the Binding of Galactopyranoside Derivatives to the Basic Lectin Tetrogonolobus).

Final rept.

F. P. Schwarz, K. D. Puri, and A. Surolia. 1991, 7p Pub. in Jnl. of Biological Chemistry 266, n36 p24344243501991.

Keywords: "Thermodynamics, "Beans, "Heat measurement, Carbohydrates, Chemical energy, Chemical bonds, Bonding, Transition points, Transition temperature, Reprints, "Galactopyranoside, Winged bean Psophocarpus tetrogonolobus, Lectin, Differential scanning calorimetry, Binding energy.

The thermodynamics of the binding of galactose derivatives to the basic agglutinin from winged bean WBAI) in PBS (0.02 M sodium phosphate and 0.15 $M$ sodium chloride) have been investigated from 298.15 to $333.15 \mathrm{~K}$ by titration calorimetry and at the denaturation temperature by differential scanning calorimetry (DSC). WBAl is a dimer with one binding site per each identical sub-unit. The thermal transition consisted of two overlapping peaks over the $\mathrm{pH}$ range 5.6 to 7.4

03,466

PB95-163192 Not available NTIS

National Inst. of Standards and Technology (NML)

Gaithersburg, MD. Chemical Thermodynamics Div.

Glucose Permease of Bacillus Subtilis Is a Single Polypeptide Chain That Functions to Energize the Sucrose Permease.

Final rept.

S. L. Sutrina, P. Reddy, M. H. Saier, and J. Reizer. $1990,9 p$

Sponsored by California Univ., San Diego, La Jolla. Dept. of Biology.

Pub. in Jnl. of Biological Chemistry 265, n30 p858185891990.

Keywords: "Bacillus subtilis, "Peptides, Sucrose, Glucose Biological transport, Phosphorylation, Cell membrane, Phosphoenolpyruvate, Protein conformation, Reprints, "Glucose permease, "Sucrose permease.

Biochemical, immunological and sequence analyses demonstrated that the glucose permease of Bacillus subtilis, the glucose-specific Enzyme II of the phosphoenolpyruvate-dependent phosphotransferase system (PTS), is a single polypeptide chain with a Cterminal Enzyme III-like domain. A flexible hydrophilic linker, similar in length and amino acid composition to linkers previously identified in other regulatory or sensory transducing proteins, functions to tether the Enzyme III (glc)-like domain of the protein to the mem- brane-embedded Enzyme $\|$ (glc). Evidence is presented demonstrating that the Enzyme III(glc)-domain of the glucose permease plays a dual role and functions in the transport and phosphorylation of both glucose and sucrose. The sucrose permease appears to lack a sucrose-specific Enzyme III-like domain or a separate, soluble III(scr) protein. Enzyme II(scr) was capable of utilizing the III $(\mathrm{glc})$-like domain of the glucose permease regardless of whether the III(glc) polypeptide was provided as a purified, soluble protein as a membrane-bound protein within the same membrane as Enzyme ll(scr) or as a membrane-bound protein within membrane fragments different from those bearing Enzyme II(scr). These observations suggest that the III (glc)-like domain is an autonomous structural unit that assumes a conformation independent of the hydrophobic, $\mathrm{N}$-terminal intramembranal domain of Enzyme Il(glc).

\section{3,467}

PB95-163820 Not available NTIS

National Inst. of Standards and Technology (CSTL), Gaithersburg, MD. Thermophysics Div.

Affinity Chromatography on Inorganic Support Materials.

Final rept.

H. H. Weetall. 1993, 22p

Pub, in Molecular Interactions in Bioseparations, Chapter 3, p27-48 1993

Keywords: "Biochemistry, "Supports, "Inorganic compounds, "Ligands, Chromatographic analysis, Chemical bonds, Separations, Purification, Antigens, Antibodies, Enzymes, Nucleic acids, Reviews, Reprints, "Affinity chromatography.

Inorganic support materials have been used for chromatography for over 75 years. However, it is only been within the last 25 years that these materials have been applied to affinity separations in which the affinity ligand is covalently bonded to the support. This review will cover the application of inorganic silicas and metal oxides for coupling to ligands of various types for the isolation and purification of antigens, antibodies, enzymes, nucleic acids and related species. Specific protocols for the activation and coupling of ligands are presented. The review will not cover ion chromatography or use of hydrocarbon bonded phases for protein isolations, nor will it cover the application of ion exchange as a separation and purification method on inorganic supports. The review contains 77 references.

\section{3,468}

PB95-164547 Not available NTIS

National Inst. of Standards and Technology (MSEL), Gaithersburg, MD. Polymers Div.

Membrane-Mediated Precipitation of Calcium Phosphate in Model Liposomes with Matrix Vesicle-Like Lipid Composition.

Final rept

D. Skrtic, and E. D. Eanes. 1992, 11p.

Sponsored by National Inst. of Dental Research, Bethesda, MD.

Pub. in Bone and Mineral 16, p109-119 1992.

Keywords: "Calcium phosphates, "Membrane lipids, "Liposomes, "Precipitation(Chemistry), pH, Divalent cations, Phospholipids, In vivo analysis, Minerals, Biochemistry, Reprints, "Matrix vesicles, Apatite/hydroxy Serine/phosphatidyl, Sphingomyelin.

The present study examined calcium phosphate precipitation in aqueous suspensions of artificial liposomes which closely resembled matrix vesicles (MV) in membrane lipid composition. At $22 \mathrm{C}$, the liposomes per se did not initiate precipitation in the suspending medium for up to $120 \mathrm{~h}$ when the latter was made supersaturated with respect to hydroxyapatite (2.25 mM Ca(2+), $1.5 \mathrm{mM} \mathrm{PO4,} 240$ mosmol, $\mathrm{pH} 7.4$ ). Likewise the suspending medium remained stable for up to $72 \mathrm{~h}$ when precipitation was induced within the aqueous interiors of the liposomes by encapsulating $\mathrm{pH}$ 7.4-buffered $50 \mathrm{mM}$ PO4 solutions in the interior spaces and making the enclosing membranes permeable to external solution $\mathrm{Ca}(2+)$ ions with the ionophore X-537A. However, extraliposomal precipitation readily occurred under these latter conditions when phosphiatidylserine (PS) and sphingomyelin (Sph) were deleted from the MV-like lipid formulation used to prepare the liposomes. These results suggest that lipidic membrane constituents such as PS and Sph may have a controlling influence on MV-mediated calcification in vivo by affecting the release of intravesticularly formed mineral crystals into the extracellular matrix space where they can subsequently grow and proliferate. 


\section{Biochemistry}

03,469

PB95-169223 Not available NTIS

National Inst. of Standards and Technology (MSEL), Gaithersburg, MD. Polymers Div.

Effect of 1-Hydroxyethylidene-1,1-Bisphosphonate on Membrane-Mediated Calcium Phosphate Formation in Model Liposomal Suspensions.

Final rept.

D. Skrtic, and E. D. Eanes. 1994, 11p.

Sponsored by National Inst. of Dental Research, Bethesda, MD

Pub. in Bone and Mineral 26, p219-229 1994.

Keywords: "Calcium phosphates, "Membrane lipids, "Liposomes, Dental materials, Mineralization, Precipitation(Chemistry), Biochemistry, Physicochemical properties, In vivo analysis, Phosphatidyiserines, Matrix bands, Reprints, "Ethylidene bisphosphonate/hydroxy.

The bisphosphonate, 1-hydroxyethylidene-1,1bisphosphonate (HEBP), was examined for its effect on calcium phosphate precipitation in $\mathrm{pH} 7.4,22 \mathrm{C}$ suspensions of 7:2:1 phosphatidylcholine (PC):dicetylphosphate (DCP):cholesterol (Chol) and 7:1:1 PC:phosphatidylserine (PS):Chol liposomes. HEBP (0.5-50 micromol/l) in the suspending medium had little, if any, effect on precipitation that formed inside phosphate-rich $(50 \mathrm{mmol} / \mathrm{l})$ aqueous interiors of liposomes as a result of ionophore (X-537A) driven $2.25 \mathrm{mmol} / \mathrm{l} \mathrm{Ca}(2+)$ influxes from the medium. These results provide a possible physicochemical explanation for the suppression of matrix vesicle initiated mineralization in ectopically-induced osteoid tissue of HEBP treated mice. In particular, the liposome results suggest that membrane phosphatidylserine interactions with mineral may enhance HEBP's effectiveness in vivo.

03,470

PB95-180782 Not available NTIS

National Inst. of Standards and Technology (CSTL), Gaithersburg, MD. Biotechnology Div.

Supported Phospholipid/Alkanthiol Biomimetic Membranes: Insulating Properties.

Final rept.

A. L. Plant, M. Gueguetchkeri, and W. Yap. 1994,

Pub. in Biophysical Jnl. 67, p1126-1133 Sep 94.

Keywords: "Phospholipids, "Lipid bilayers, "Artificial membranes, Spectroscopy, Electron transfer, Gold, Melittin, Dielectrics, Insulation resistance, Conductiv ity, Resistance, Reprints, "Alkanethiol monolayers.

A novel model lipid bilayer membrane is prepared by the addition of phospholipid vesicles to alkanethiol monolayers on gold. This supported hybrid bilayer membrane is rugged, easily and reproducibly prepared in the absence of organic solvent, and is stable for very long periods of time. We have characterized the insulating characteristics of this membrane by examining the rate of electron transfer and by impedance spectroscopy. Supported hybrid bilayers formed from phospholipids and alkanethiols are pinhole-free and demonstrate measured values of conductivity and resistivity which are within an order of magnitude of that reported for black lipid membranes. Capacitance values suggest a dielectric constant of 2.7 for phospholipid membranes in the absence of organic solvent. The protein toxin, melittin, destroys the insulating capability of the phospholipid layer without significantly altering the bilayer structure. This model membrane will allow the assessment of the effect of lipid membrane perturbants on the insulating properties of natural lipid membranes.

\section{3,471}

PB96-102728 Not available NTIS

National Inst. of Standards and Technology (CSTL), Gaithersburg, MD. Biotechnology Div.

Bacteriorhodopsin Retains Its Light-Induced Proton-Pumping Function After Being Heated to $140 \mathrm{C}$. Final rept.

E. P. Lukashev, and B. Robertson. 1995, 4p

Pub. in Bioelectrochemistry and Bioenergetics 37, p157-160 1995

Keywords: "Photochemical reactions, "Protons, Reprints, Pumping, High temperature, Reactions, Photosynthetic bacteria, Membrane transport, "Bacteriorhodopsin, "Halobacterium.

Films of purple membrane of Halobacterium salinarium have been reported to keep their two-dimensional hex- agonal lattice structure and their optical absorbance spectrum even after being heated to 140 degrees $C$ for two days. Tests of the light-induced proton-pumping function of the bacteriorhodopsin (bR) molecule in the purple membrane were not reported. The present paper describes measurements of the light-induced proton-pumping by films of wild-type and $\mathrm{D} 96 \mathrm{H}$-mutant bR at room temperature after the films were heated to high temperatures. The measurements show that cycling bR through 140 deg $C$ does not reduce its protonpumping ability.

03,472

PB96-102900 Not available NTIS

National Inst. of Standards and Technology (CSTL), Gaithersburg, MD. Biotechnology Div.

Phos pholipid/Alkanethiol Bilayers for Cell-Surface Receptor Studies by Surface Plasmon Resonance. Final rept.

A. L. Plant, M. Brigham-Burke, E. C. Petrella, and D. J. O'Shannessy. 1995, 7p

Pub. in Analytical Biochemistry 226, p342-348 1995.

Keywords: "Phospholipids, "Lipid bilayers, "Artificial membranes, Reprints, Electron transfer, Conductivity, Resistance, Dielectrics, "Alkanethiol monolayers.

Supported hybrid bilayer membranes (HBM) composed of a monolayer of phospholipid and a monolayer of alkanetiol associated with a thin gold film on glass are useful as model lipid bilayer membranes for study ing membrane receptor-ligand and cell-cell binding events by surface plasmon resonance (SPR). Measurements of specific binding of proteins and lipid vescles to well-defined HBMs have been performed under conditions or continuous flow using a commercial SPR instrument (BIAcore). HBMs are shown to be stable in flow and to block nonspecific adsorption of proteins to the alkanethiol/gold surface. The use of such supported lipid bilayers in flow provides a means of conducting equilibrium and kinetic studies of models of ligand-cell and cell-cell interactions with receptors or ligands in a membrane environment. Compared to the extended dextran polymer layer that is currently used for surface modification of BIAcore 'sensor chips, 'the described HBMs provide a well-defined surface that will permit less ambiguous modeling of these important biological interactions.

\section{3,473}

PB96-111976 Not available NTIS

National Inst. of Standards and Technołogy (CSTL), Gaithersburg, MD. Biotechnology Div.

Detection of Aromatic Compounds Based on DNA Intercalation Using an Evanescent Wave Biosensor.

Fina! rept

P. C. Pandey, and H. H. Weetall. 1995, $6 \mathrm{p}$.

Sponsored by Environmental Protection Agency, Washington, DC

Pub. in Analytical Chemistry, v67 n5 p787-792, 1 Mar 95

Keywords: "Deoxyribonucleic acids, "Biosensors, "Aromatic compounds, Reprints, Flow injection analysis, "Intercalation, Wave sensors, Fluorophore, Fluorescence radiation.

A flow injection analysis system coupled with an evanescent wave biosensor employing total internationa reflection of fluorescence radiation for the detection of the compounds that intercalate within DNA is reported. A highly fluorescent intercalator, ethidium bromide, has been used as the reference compounds for the detection. The evanescent wave biosensor was developed using immobilized double-strand DNA (daDNA) over the surface of a cylindrical wave guide. The response of the DNA-modified fiber is significantly higher than the responce obtained with an unmodified fiber. The response of the biosensor at a constant concentration of ethidium bromide increases on increasing the concentration of immobilized dSDNA. At the steady-state response of the biosensor, obtained at a constant concentration of ethidium bromide, there is a decrease in the response to the injection of another DNA intercalator that competes for the intercalation sites on the ISDNA, displacing the ethidium bromide. This is immediately followed by recovery of the steady-state response. The decrease in the sensor response is linear function of the concentrations of injected intercalator. Responce curves for 9 , 10-anthraquinone2, 6-disulfonic acid, remazol brillant blue, decacyclene, and 4, 6-diamidino-2-phenylindole dihydrochloride are reported.
03,474

PB96-112040 Not available NTIS

National Inst. of Standards and Technology (CSTL), Gaithersburg, MD. Biotechnology Div.

Physicochemical Characterization of Low Molecular Weight Heparin.

Final rept.

D. Atha, B. Coxon, V. Reipa, and A. Gaigalas. 1995

列 364 Mar 95.

Keywords: "Heparin, "Light scattering, Reprints, Anticoagulants, Physicochemistry, HPLC, Raman spectroscopy.

Nuclear magnetic resonance spectroscopy (NMR), Raman spectroscopy, dynamic light scattering (DLS) and high performance exclusion chromatography (HPLC) were used to characterize two different commercial preparations of low molecular weight heparin produced by peroxide cleavage using nitrous acid. Proton NMR showed that the contamination by dermatam sulfate varied from $2 \%$ in the material produced by deaminative cleavage using nitrous acid, to $4 \%$ for the material produced by peroxide cleavage. The Raman spectra of the nitrous acid produced material showed an equivalent amount of $\mathrm{O}$ - sulfation to that in the material produced by peroxide, but about a $10 \%$ reduction in the content of $\mathrm{N}$-sulfated glucosamine, as expected from the deamination reaction. DLS and HPLC indicated the presence of less than $0.2 \%$ of very high molecular weight/aggregate material for the peroxide preparation as compared to $1 \%$ for the nitrous acid prepared material. The weight average molecular weight (Mw) determined from HPLC was 5900 Da for the nitrous acid prepared material and $6850 \mathrm{Da}$ for the peroxide produced material. The number average molecular weight $(\mathrm{Mn})$ calculated from this data was $5200 \mathrm{Da}$ for the nitrous acid preparation and $5300 \mathrm{Da}$ for the peroxide produced material. In addition, the nitrous acid prepared material exhibited a much narrower size distribution of oligomeric species, as evidenced by the polydispersity (MwMn) of 1.1 for the nitrous acid prepared material, as compared with a value of 1.3 for the peroxide prepared material.

03,475

PB96-112305 Not available NTIS

National Inst. of Standards and Technology (MSEL),

Gaithersburg, MD. Reactor Radiation Div.

Small Angle Neutron Scattering Studies of Structural Characteristics of Argarose Gels.

Final rept.

S. Krueger, A. P. Andrews, and R. Nossal. 1994

$10 p$.

Pub. in Biophysical Chemistry, v53 p85-94 1994

Keywords: "Neutron scattering, "Gels, Reprints, Structural analysis, "Agarose.

The $30 \mathrm{~m}$ small angle neutron scattering facility at the National Institutes of Standards and Technology has been used to examine neutron scattering from agarose gels formed in $\mathrm{D}(2) \mathrm{O}$. Differential scattering cross sections have been acquired over a continuous range of 0 between 0.005 and 0.3 Angstroms to the minus 1.power. Subtle changes in gel structure are observed when pre-gelation agarose concentration is varied. Similarly, except when the gelling solution is rapidly cooled to a low temperature, the rate at which the gels are formed does not seem to have much effect. Clearer evidence of structural rearrangement is observed when the solvent quality is changed by the addition of dimethyl sulfoxide, or when the temperature of the gel is elevated above 70 degrees $C$. These data are consistent with a description of a randomly structured polymer network containing discrete self-similar, hydrogenbonded, junctions normally of minimal thickness $=35$ 40 Angstroms.

\section{3,476}

PB96-122569 Not available NTIS

National Inst. of Standards and Technology (MSEL), Gaithersburg, MD. Reactor Radiation Div.

Extending the Angular Range of Neutron Reflectivity Measurements from Planar Lipid Bilayers: Applications to a Model Biological Membrane.

Final rept.

S. Krueger, J. F. Ankner, S. K. Staija, M. Colombini, C. F. Majkrzak, and D. Gurley. $1995,5 \mathrm{p}$ Pub. in Langmuir, v11 n8 p3218-3222 1995.

Keywords: "Lipids, "Neutron reflectivity, "Membranes, Measurement, Mitochondria, Reprints. 
A novel experimental setup has been used to measure the specularly reflected neutron intensity from a mode biological membrane containing components from the outer membrane of Neurospora crassa mitochondria. The specular reflectivity from a single bilayer membrane, formed by the fusion of vesicles onto a phospholipid monolayer supported on a flat substrate was measured in both D2O and $\mathrm{H} 20$ solvents. In D2O solvent, reflected neutron intensities down to 10 to the minus 6 power were measured for wavevector transfers out to $0.25 \mathrm{~A}-1$. A symmetric model for the neutron scattering length density profile perpendicular to the bilayer surface was constructed based on fits to the D2O data. The overall bilayer thickness was found to be 43 plus or minus $2 \mathrm{~A}$. The individual lipid head group and hydrocarbon tail layer thickness were 7.5 plus or minus 1.4 and 28.0 plus or minus $2.8 \mathrm{~A}$, respectively. The fitted results are consistent with the $\mathrm{H} 2 \mathrm{O}$ data. The integrity of the model membrane bilayer was confirmed by comparing its measured reflectivity to that obtained from a single lipid bilayer consisting of soybean phospholipids (asolectin) which was deposited on a flat substrate by the Langmuir-Blodgett technique.

03,477

PB96-123344 Not available NTIS

National inst. of Standards and Technology (CSTL), Gaithersburg, MD. Biotechnology Div.

Optical Control of Enzymatic Conversion of Sucrose to Giucose by Bacteriorhodopsin Incorporated into Self-Assembled Phosphatidylcholine Vesicles.

Final rept.

M. F. McCurley, and S. A. Glazier. 1995, 5p

Pub. in Analytica Chimica Acta, v311 p211-215 1995.

Keywords: "Enzymatic methods, *Vesicles, Bacteriorhodopsin, invertase, Reprints.

This report describes an alternative method to modulate enzyme activity optically through $\mathrm{pH}$ modulation. Bacteriorhodopsin (BR) is used to regulate the activity of an enzyme which it does not naturally regulate. In this study BR controlled the $\mathrm{pH}$-dependent conversion of sucrose to glucose by the enzyme, invertase. BR is oriented in the wall of phosphatidylcholine vesicles so that at $\mathrm{pH}$ values greater than 4-5 it pumps protons into the vesicles and at $\mathrm{pH}$ values less than 4 it pumps protons out of the vesicle when illuminated with light of the proper wavelength. The bulk-solution $\mathrm{pH}$ correspondingly changes as a result. At an intial $\mathrm{pH}$ of 5.57 the change in $\mathrm{pH}$ of the solution due to pumping by the bacteriorhodopsin-vesicle complex was $0.17 \mathrm{pH}$ units. To study this effect on the enzyme, samples containing BR vesicles and invertase were assayed for enzyme activity under dark and illuminated conditions. It was found that changes in enzyme activity, upon illumination, were generally larger than predicted for the corresponding changes in the bulk solution $\mathrm{pH}$ generated by the BR vesicles.

03,478

PB96-155460 Not available NTIS

National inst. of Standards and Technology (CSTL),

Gaithersburg, MD. Biotechnology Div.

Mapping Domains in Proteins: Dissection and Expression of 'Escherichia coli' Adenylyl Cyclase. Final rept.

P. Reddy, J. Hoskins, and K. McKenney. 1995, 5p.

Pub. in Analytical Biochemistry, n231 p282-286 1995.

Keywords: Oligonucleotides, Reprints, "Protein expression, "Domains, PRE expression vector.

The authors have used the pRE expression vector containing the Escherichia coli a denylyl cyclase gene (cya) with the unique Ndel restriction site CATATG at the initiation codon in conjunction with a family of self-complimentary oligonucleotides to create amino- and carboxy-terminal domains in adenylyl cyclase. The three sets of oligonucleotides contain a TAA translation stop condon in all reading frames flanked by the Ndel restriction endonuclease sequence and one or two nucleotides

$\left(5^{\prime}\right.$

NNCATATGTTAATTAATTAACATATGNN 3'). Ligation of one of these annealed oliognucleotides into a restriction site or creation of 5' TAA/CATATG 3' translation stop/Ndel restriction site along a gene in the $\mathrm{pRE}$ expression vector facilitates the premature termination of protein synthesis thus yielding amino-terminal domains. Removal of a fragment of the gene corresponding to the amino-terminal portion by $\mathrm{Ndel}$ restriction and ligation brings the 3 ' end of the gene in frame with the initiator ATG. With this strategy, expression of the carboxyl-terminal domain of a protein is possible which is otherwise not as simple as the expression of the amino-terminal domain. The feasibility of expression of any domain of protein is demonstrated using the cya gene to create several amino- and carboxyl-termina domains of adenylyl cyclase.

\section{3,479}

PB96-164132 Not available NTIS

National inst. of Standards and Technology (CSTL), Gaithersburg, MD. Biotechnology Div.

Novel Activities of Human Uracil DNA Nglycosylase for Cytosine-Derived Products of Oxidative DNA Damage.

Final rept.

M. Dizdaroglu, A. Karakaya, P. Jaruga, G.

Slupphaug, and H. E. Krokan. 1996, 5p.

See also PB96-110747.

Pub. in Nucleic Acids Research, v24 n3 p418-422 1996.

Keywords: "DNA damage, "Deoxyribonucleic acids, "Enzyme kinetics, Reprints, Isodialuric acid, Hydroxyl radical.

Uracil DNA N-glycosylase is a repair enzyme that releases uracil from the DNA. A major function of this enzyme is presumably to protect the genome from premutagenic uracil resulting from deamination of cytosine in DNA. Here, we report that human uracil DNA $\mathrm{N}$-glycosylase also recognizes three uracil derivatives that are generated as major products of cytosine in DNA by hydroxyl radical attack or other oxidative processes. DNA substrates were prepared by y-irradiation of DNA in aerated aqueous solution and incubated with human uracil DNA N-glycosylase, heat-inactivated enzyme or buffer. Ethanol-precipitated DNA and super natant fractions were then separated.

03,480

PB96-167226 Not available NTIS

National Inst. of Standards and Technology (CSTL), Gaithersburg, MD Biotechnology Div.

Structural Analysis of Heparin by Raman Spectroscopy.

Final rept.

D. H. Atha, A. K. Gaigalas, and V. Reipa. 1996, 5p Pub. in Jnl. of Pharmaceutical Sciences, v85 n1 p52 56 Jan 96

Keywords: "Heparin, "Raman spectroscopy Anticoagulants, Reprints, Physicochemistry.

The Raman spectra of commercially available heparin disaccharide standards exhibit bands associated with the $\mathrm{N}$-sulfate and the 6-O-sulfate groups of the glucosamine and the 2-O-sulfate of the iduronic acid. The $\mathrm{N}$-sulfate has a strong band at $1039 \mathrm{~cm}$ to the minus one. The 6-O-sulfate and the 2-O-sulfate exhibit bands at 1055 and $1065 \mathrm{~cm}$ to thh minus one, respectively. The pattern of these modes, which are assigned to the symmetric $\mathrm{SO}_{3}$ vibrations, was supported by semiempirical quantum mechanical calculations. The above bands were identified in the Raman spectrum of a commercial preparation of porcine mucosal heparin and were used to determine the relative proportion of the $\mathrm{N}$-sulfate, 6-O-sulfate, and 2-O-sulfate groups in the heparin molecule. The information, which is complementary to that obtained by NMR spectroscopy, is of particular importance in relation to biological activity.

03,481

PB96-167382 Not available NTIS

National inst of Standards and Technology (CSTL) Gaithersburg, MD. Biotechnology Div.

New Method for the Detection and Measurement of Polyaromatic Carcinogens and Related Compounds by DNA Intercalation.

Final rept.

J. J. Horvath, M Gueguetchkeri, A Gupta, D.

Penumatchu, and H. H. Weetall. 1995, 17p.

Sponsored by Environmental Protection Agency, Research Triangle Park, NC. Atmospheric Research and Exposure Assessment Lab.

Pub in National Meeting of the American Chemical Society (209th), Anaheim, CA. April 2-6, 1995, Biosenso and Chemical Sensor Technology, Process Monitoring and Control, ACS Symposium Series, Chapter 5, v613 p44-60.

Keywords: “Deoxyribonucieic acids, "Fluorescence, Measurement, Polarization, Reprints, "Polyaromatic carcinogens, DNA intercalation.

A simple and sensitive method for detection and quantification of carcinogens and related polyaromatic compounds by DNA intercalation has been devised. The experimental technique is based on the phenomenon of DNA intercalation using fluorescence polarization for quantitative measurements. The assay architecture is analogous to a protocol presently used for competitive immunoassays, whereby an intercalating dye of fluorochrome would compete with or be displaced by the analyte (test compound). Our competitive binding assay utilizes DNA binding sites and a fluorescent intercalating dye. The carcinogenic analytes investigated were benzo(a)pyrene, dibenz(a,h)anthracene, dibenz(a j)anthracene, benz(a)anthracene, benzo(j)fluoranthene, benzidine, aniline, parathion, pentachlorophenol and nitrobenzene. The non-carcinogenic hydrocarbons studies were naphthalene, an-

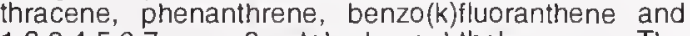
$1,2,3,4,5,6,7, \quad 8$-octahydronaphthalene. The intercalating dyes examined were acridine orange, ethidium bromide, proflavin and 4,6-diamidino phenylindale chloride (DAPI). Of the compounds studied, only the single ring compounds gave a negative response. All the other compounds investigated displaced the intercalated dye molecule. it has been determined that molecules require at least two adiacent benzene rings for intercalation to occur. The detection limits of this analytical method were between 10 to the minus 5th power $\mathrm{mol} / \mathrm{L}$ and 10 to the minus 8 power $\mathrm{mol} / \mathrm{L}$ for all materials tested.

\section{3,482}

PB96-190020 Not available NTIS

National Inst. of Standards and Technology (CSTL) Gaithersburg, MD. Analytical Chemistry Div.

Investigations of Sulfur Interferences in the Extraction of Methylmercury from Marine Tissues.

Final rept.

M. K. Behlke, P. C. Uden, and M. M. Schantz, 1996 ,

$2 p$.

Pub. in Analytical Communications, v33 p91-92 Mar 96.

Keywords: "Gas chromatography, "Calibration stand ards, "Sulfur, Methyl compounds, Mercury(Metal), Atomic spectra, Emission, Marine biology, Tissues(Biology), Reprints.

Gas chromatography with element-specific atomic emission detection was used to investigate sulfur inter ferences in the quantification of methylmercury in marine tissues. The elimination of sulfur-containing spe cies by treatment with copper powder was confirmed by sulfur-specific atomic emission detection.

\section{3,483}

PB96-200886 Not available NTIS

National Inst. of Standards and Technology (CSTL) Gaithersburg, MD. Biotechnology Div.

Quantitative Determination of Oxidative Base Damage in DNA by Stable Isotope-Dilution Mass Spectrometry.

Final rep

M. Dizdaroglu. 1993, $6 p$.

Pub. in Federation of European Biochemical Societies Letter, v315 n1 p1-61993.

Keywords: "Radiation damage, "DNA, "Spectrometry, Free radical, Hydroxyl radical, Modified base, Reprints, "Foreign technology, Isotope-labeled analogue, Selected-ion monitoring.

For understanding of the role of oxidative DNA damage in biological processes such as mutagenesis and carcinogenesis, it is essential to identify and quantify the type of DNA damage in cells. This can be achieved by gas chromatography/mass spectrometry. The present study describes the quantification of modified bases in DNA by isotope-dilution mass spectrometry with the use of stable isotope-labeled analogues as internal standards. A number of isotopically labeled DNA bases were synthesized. The mass spectra of their trimethylsilyl derivatives were recorded. Calibration plots were obtained for known quantities of modified bases and their isotope-labeled analogues. Quantification of various modified DNA bases by isotope-dilution mass spectrometry was demonstrated in isolated chromatin exposed to ionizing radiation. The results indicate that gas chromatography/stable isotope-dilution mass spectrometry is an ideally suited technique for selective and sensitive quantification of modifed bases in DNA

03,484

PB96-200894 Not available NTIS

National Inst. of Standards and Technology (CSTL), Gaithersburg, MD. Biotechnology Div. 


\section{Biochemistry}

Commentary: The Measurement of Oxidative Damage to DNA by HPLC and GC/MS Techniques.

Final rept.

B. Halliwell, and M. Disdaroglu. 1992, $13 p$.

Pub. in Free Rad. Res. Comms., v16 n2 p75-87 1992.

Keywords: "DNA, "Hydroxyl radical, Singlet oxygen, Mass spectrometry, Reprints, "Foreign technology, 8hydroxyguanine, Electro-chemical detection.

Oxidative damage to DNA has been measured by quantitating 8-hydroxy-2'-deoxyguanosine (8OHdGuo) after enzymic digestion of DNA, followed by HPLC separation and electrochemical detection. Alternatively, 8-hydroxyguanine (and a wide range of other base-derived products of free radical attack) may be measured after acidic hydrolysis of DNA or chromatin, followed by derivatization and gas-chromatography/ mass spectrometry. Both techniques have comparable sensitivity, but GC/MS enables determination of a wide variety of chemical changes to all four DNA bases and it can be applied to DNA-protein complexes. However, the two techniques do not always give similar results Potential reasons for this are discussed. Greater attention of methodological questions is required before using measurement of 8-OHdGuo as a 'routine' marker of oxidative DNA damage in vivo.

\section{3,485}

PB96-200928 Not available NTIS

National Inst. of Standards and Technology (CSTL)

Gaithersburg, MD. Biotechnology Div.

Intercomparison of DNA Sizing Ladders in Electrophoretic Separation Matrices and Their Potential for Accurate Typing of the D1S80 Locus. Final rept.

M. C. Kline, J. W. Redman, D. J. Reeder, and D. L. Duewer. 1996, 9p.

Pub. in Applied and Theoretical Electrophoresis, v6 p33-41 1996.

Keywords: "DNA, "Separation matrices, Acrylamide, Agarose, Basepair, Genetic markers, Quantitative analysis, Reprints, Electrophoretic mobility, Polymerase chain reaction.

The 100-1,000 basepair size typical of PCR-amplified DNA fragments demands high resolution electrophoretic gels for the adequate characterization of small differences among samples. We have studied the behavior of a number of commercial sizing ladders in three ladders in three classes of separation systems: polyacrylamides with discontinuous buffer, proprietary acrylamides with continuous buffer, and agarose-like materials with continuous buffer. None of the ladders examined perform adequately in any of these systems using vendor-supplied nominal ladder component basepair sizes. All ladders successfully types D1S80 alleles after calibration with the allelic ladder (replacing the nominal size values with the least squares estimate of allele/matrix-specific apparent sizes). Some ladders and matrices are qualitatively better than others. No one ladder proved consistently better than others; polyacrylamide gell with ribose modifier provided the most precise results in the study. Appropriate allelic ladders or a well defined subset of known alleles can serve as the calibration system.

\section{3,486}

PB97-119374 Not available NTIS

National Inst. of Standards and Technology (CSTL), Gaithersburg, MD. Biotechnology Div.

Positive and Negative Cooperativities at Subsequent Steps of Oxygenation Regulate the Allosteric Behavior of Multistate Sebacylhemoglobin.

Final rept.

R. Unger, A. Razynska, H. Kwansa, J. Collins, C

Fronticelli, M. Braxenthaler, J. Moult, X. Ji, G.

Gilliland, Z. Gryczynski, and E. Bucci. 1996, 8p.

Pub. in Biochemistry, v35 p3418-3425 1996.

Keywords: "Hemoglobin, Blood proteins, Oxygenation, Regulations, Reprints, "Allosteric effectors, Oxygen affinity, Sebacylhemoglobin.

Cross-linked human hemoglobin ( $\mathrm{Hba}$ ) is obtained by reaction with bis(3,5-dibromosalicyl) sebacate. Peptide maps and crystallographic analyses confirm the presence of the 10 carbon atom long sebacyl residue cross-linking the two beta82 lysines of the beta-cleft (DecHb). The Adair's constants obtained from the oxy. gen binding isotherms, show that at the first step of oxygenation normal hemoglobin and $\mathrm{DecHb}$ have a very similar oxygen affinity. In $\mathrm{DecHb}$ negative binding cooperativity is present at the second step of oxygen- ation, which has an affinity 27 times that of the second step. Positive cooperativity is present at the third binding step, whose affinity is 380 times that of the second step. The fourth binding step shows a weak negative cooperativity with an affinity one-half that of the third step. Crystals of deoxy-DecHb diffracted to 1.9 Angstroms resolution. The electron density map of deoxy-DecHb indicates the presence of the 10 carbon bridge between the beta82 lysines.

\section{Botany}

\section{3,487}

PB95-126280 Not available NTIS

National Inst. of Standards and Technology (NML), Gaithersburg, MD. Inorganic Analytical Research Div. Dissolution Problems with Botanical Reference Materials.

R. R. Greenberg, H. M. Kingston, R. L. Watters, and K. W. Pratt. $1990,5 p$.

Pub. in Fresenius Jnl. of Analytical Chemistry 338, n4 p394-398 1990.

Keywords: "Botany, "Dissolving, "Analytical techniques, "Chemical analysis, Trace elements, Absorption, Adsorption, Particulates, Leaves(Botany), Plants(Botany), Reprints, "Standard reference materials.

As part of the analytical research leading to the certification of the new Apple and Peach Leaves Standard Reference Materials (SRMS), a study was undertaken to evaluate different sample dissolution techniques for losses of analyte species. Possible loss mechanisms include absorption or adsorption of analyte elements at the walls of the sample decomposition vessels, and the formation or persistence of insoluble particulate material during sample dissolution. Results of this study indicated that significant fractions of some elements were present on particles after acid dissolution, despite visual indications that dissolution was complete. In addition, large amounts of some elements remained in the platinum crucibles used to fuse samples with lithium metaborate.

\section{3,488}

PB96-147061 Not available NTIS

National Inst. of Standards and Technology (CSTL), Gaithersburg, MD. Biotechnology Div.

Rapid Method for the Isolation of Genomic DNA from 'Aspergillus fumigatus'.

Final rept.

N. Bir, A. Paliwal, K. Muralidhar, P. Reddy, and P.

Usha Sarma. 1995, 11p.

Pub. in Preparative Biochemistry, n25(4) p171-181 1995.

Keywords: "Deoxyribonucleic acids, Genomes, Diagnosis, Reprints, "Aspergillus fumigatus, Polymerase chain reaction.

A majority of Aspergillus induced diseases are reported to be caused by Aspergillus fumigatus. In immunocompromized and post transplant cases it can lead to invasive aspergillosis. Due to this the molecular fingerprinting of aspergillus isolates by RFLP analysis and development of DNA diagnostic probes are gaining importance. Different methodologies are being adopted for extraction of the genomic DNA from fungus. The existing procedures for isolation of DNA are time consuming and range from several hours to few days. The most difficult step in the isolation of DNA from aspergillus species is to disrupt the tough chitin rich cell wall without causing damage to genomic DNA. The authors report here a rapid method for extraction of genomic DNA based on the cleavage of chitin with chirinase. The subsequent modification steps included are lysis and microwave treatment.

\section{3,489}

PB96-167267 Not available NTIS

National Inst. of Standards and Technology (CSTL), Gaithersburg, MD. Inorganic Analytical Research Div. Recent Developments in NIST Botanical SRMs.

Final rept.

D. A. Becker, and T. E. Gills. 1995, 3p.

See also PB93-153153.

Pub. in Fresenius J. Analytical Chemistry, v352 p1631651995.

Keywords: "Drying techniques, "Homogeneity, Reprints, "Foreign technology, "Standard reference mate- rials, "Botanical reference materials, Jet-milling techniques, Reference material preparation, Trace element analysis.

The National Institute of Standards and Technology (NIST) (formerly the Natioanl Bureau if Standards (NBS)) issued the first botanical reference material certified for elemental content in January 1971, as Standard Reference Material (SRM) 1571, Orchard Leaves. In the following years a total of nine additional botanical certified reference materials have been issued by NIST. Each of these materials was certified for major. minor and trace elements except for SRM 2695, certified for fluorine only Botanical SRMs issued since 1991 are significantly improved over previous materials in a number of ways. Probably the most significant change is the use of a jet-milling process to grind them to extremely fine particles. This has resulted in botanical SRMs wh significantly improved homogeneity. These NIST reference materials are described with information on homogeneity, drying techniques and grit content.

\section{3,490}

PB97-119036 Not available NTIS

National Inst. of Standards and Technology (CSTL). Gaithersburg, MD. Nuclear Methods Group. Results of the ASTM Nuclear Methods Intercomparison on NIST Apple and Peach Leaves Standard Reference Materials.

Final rept.

D. A. Becker. $1993,15 p$.

Pub. in Jnl. of Radioanalytical and Nuclear Chemistry, Articles, v168 n1 p169-183 1993.

Keywords: "Botany, "Analytical techniques, "Chemical analysis, Plants(Botany), Leaves(Botany), Trace elements, Comparisons, Absorption, Adsorption, Reprints, "Foreign technology, Standard reference materials.

The ASTM Task Group on Nuclear Methods of Chemical Analysis (E10.052 12) has conducted a trace element intercomparison among some of its members over the past two years. Eight non-NIST laboratories submitted data using nuclear techniques, with a total of 111 values for the apple leaves and 116 values for the peach leaves, on 46 to 50 elements, respectively. This intercomparison provided a unique opportunity for the analyical laboratories, because the analytical values submitted could be later compared to the NIST certified values. For the seven elements which were certified by NIST and had three or more intercomparison values, the results showed that: (1) $61 \%$ of all 56 intercomparison values submitted had results whose stated uncertainty overlapped the uncertainty limits of the NIST certified values, and (2) less than $6 \%$ of the intercomparison values had means which fell outside plus or minus $20 \%$ of the NIST values. In general, the intercomparison values submitted showed excellent agreement with the NIST values. However, many reported uncertainties accompanying intercomparison values appeared overly optimistic.

\section{Clinical Chemistry}

03,491

PB94-198579 Not available NTIS

National Inst. of Standards and Technology (CSTL), Gaithersburg, MD. Inorganic Analytical Research Div. Regulation of Lithium and Boron Levels in Normal Human Blood: Environmental and Genetic Considerations.

Final rept.

R. M. Barr, W. B. Clarke, R. M. Clarke, R. G. Downing, J. Venturelli, and G. R. Norman. 1993, $6 p$ Pub. in Jnl. of Laboratory and Clinical Medicine 121 n4 p614-619 Apr 93.

Keywords: "Lithium, "Boron, "Blood chemical analysis, "Genetics, "Environmental health, Mass spectroscopy, Carrier proteins, Water supply, Twins, Reprints.

Blood lithium levels may be both genetically and environmentally regulated. The genetic component is evidenced mainly from studies in twins who were either normal or had a manic-depressive disorder. An environmental contribution is adduced from the relationship between the blood lithium level and the amount of the element ingested. No such information is available for boron, another element present in ultra trace amounts in human blood. Unusually high levels of lithium and 
boron in the waters of northern Chile offer an opportunity to study the genetic and environmental regulation of these elements in the blood of healthy subjects. Samples of blood $(n=40)$ and water $(n=47)$ were collected at seven locations in the province of Tarapaca. Most of the healthy subjects were Aymara who had been resident in the respective communities for at least 3 years. Because some of the individual subjects $(n=15$ ) were first-degree relatives, a genetic component to the regulation of blood levels was explored. The variance in blood levels of lithium and boron was significantly greater between than within families $(p<0.0001)$. There are environmental and apparent genetic contributions to the regulation of blood levels of lithium and boron in healthy human subjects.

\section{3,492}

PB94-199379 Not available NTIS

National Inst. of Standards and Technology (NML), Gaithersburg, MD. Organic Analytical Research Div. Determination of 3-Quinuclidinyl Benzilate (Qnb) and Its Major Metabolites in Urine by Isotope Dilution Gas Chromatography Mass Spectrometry.

Final rept.

G. D. Byrd, R. C. Paule, L. C. Sander, H. T. Bausum, L. T. Sniegoski, and E. White. 1992, 6p.

Pub. in Jnl. of Analytical Toxicology 16, n3 p182-187 1992. Sponsored by Army Medical Bioengineering Research and Development Lab., Fort Detrick, MD.

Keywords: "Urinalysis, "Quinuclidinyl benzilate, "Metabolites, *Hallucinogens, Mass fragmentography, isotopes, Chemical analysis, Reprints, *3. Quinuclidinol, *Benzilic acid.

In response to the scheduled destruction of U. S. military stockpiles of the hallucinogenic agent 3quinuclidinyl benzilate (BZ), a specific confirmatory test for human exposure to $B Z$ was developed. The amount of the parent compound in the unine as well as the two major metabolites, 3-quinuclidinol (Q) and benzilic acid (BA), was determined since the exact relationship between $B Z$ dose and levels of $B Z$ and its metabolites in urine is not well known. BZ was determined in urine samples spiked at a target level of $0.5 \mathrm{ng} / \mathrm{mL}$ and the metabolites $B a$ and $Q$ were determined at a target level of $5 \mathrm{ng} / \mathrm{mL}$. The variabilities in the method are more or less evenly distributed between three imprecision categories (GC/MS measurement, sample preparation, and sample). The imprecision of a single measurement is about $15 \%$ for each analyte. The method developed uses solid phase extraction to isolate each analyte from the urine and isotope dilution GC/MS for quantitation. Each analyte is converted to its trimethylsilyl derivative for analysis. The analytical method was tested on 8 different urines spiked with known amounts of analytes near the target levels, at ten times the target levels, and blank (unspiked) urine samples.

\section{3,493}

PB94-200201 Not available NTIS

National Inst. of Standards and Technology (CSTL),

Gaithersburg, MD. Organic Analytical Research Div.

Determination of Oltipraz in Serum by HighPerformanace Liquid Chromatography with Optical Absorbance and Mass Spectrometric Detection.

Final rept.

R. G. Christensen, and W. Malone. 1992, 6p

Pub. in Jnl. of Chromatography 584, p207-212 1992. Sponsored by National Cancer Inst., Bethesda, MD.

Keywords: * Pesticides, "Blood chemical analysis, High pressure liquid chromatography, Mass spectroscopy, Isotopes, Reference standards, Reprints, "Oltipraz.

Three methods have been developed for the analysis of Oltipraz in serum. A method suitable for routine use employs spiking with a homologous internal standard, off-line solid-phase extraction, high-performance liquid chromatographic separation, and optical absorbance detection at $450 \mathrm{~nm}$. Method detection limit is about $1 \mathrm{ng} / \mathrm{ml}$. A second method, less susceptible to bias from co-eluting interferences, uses a stable isotope-labeled internal standard, similar extraction and separation, and detection by thermospray mass spectrometry. Method detection limit is about $0.2 \mathrm{ng} / \mathrm{ml}$. A third meth od was developed which can be used without specially synthesized internal standards. it uses on-line solidphase extraction, with quantification by comparison with external standards. Method detection limit is about $3 \mathrm{ng} / \mathrm{ml}$. Good agreement was observed between these methods and with similar and different methods run in other laboratories. Calibration curves were linear over the entire range which was investigated, i.e., up to $500 \mathrm{ng} / \mathrm{ml}$. Coefficients of variation were similar for all three methods, being about $5 \%$.

\section{3,494}

PB94-213493 Not available NTIS

National Inst. of Standards and Technology (CSTL), Gaithersburg, MD. Organic Analytical Research Div.
Liposome-Based Flow-Injection Immunoassay for Liposome-Based Flow-Injection Imm

Final rept.

L. Locascio-Brown, A. Plant, R. Chesler, R. Durst, M. Kroll, and M. Rudell. 1993, 6p.

Pub. in Clinical Chemistry 39, p386-391 1993.

Keywords: "Liposomes, "Flow injection analysis, *Theophylline, "Blood chemical analysis, Iarization immunoassay, Antibodies, Reprints.

We developed a method for quantitatively determining theophylline in serum using a heterogeneous immunoassay called flow-injection immunoanalysis. The reaction involves competition between serum theophylline and theophylline-labeled liposomes. Separation occurs on a solid-phase reactor column containing immobilized antibody to theophylline incorporated in a flow-injection system. Subsequent iysis of the bound liposomes provides sensitive detection of the analyte. Effective regeneration of the immobilized antibody activity allows the reactor to be reused for hundreds of sequential samples. Comparison of the results of the flow-injection immunoassay method with results obtained with a commercially available fluorescence polarization method showed an excellent correlation.

\section{3,495}

PB94-216330 Not available NTIS

National Inst. of Standards and Technology (NML) Gaithersburg, MD. Organic Analytical Research Div.

Ascorbic and Dehydroascorbic Acids Measured in Plasma Preserved with Dithiothreitol or Metaphorphoric Acid.

Final rept.

S. A. Margolis, R. C. Paule, and R. G. Ziegler. 1990,

Pub. in Clinical Chemistry 36, n10 p1750-1755 Oct 90.

Keywords: *Ascorbic acid, "Dehydroascorbic acid, "Blood chemical analysis, "Dithiothreitol, "Blood preservation, Reference standards, $\mathrm{pH}$, Temperature, Lyophilization, Accuracy, Precision, Stability, Reprints, Metaphosphoric acid.

The accurate and precise measurement of ascorbic and dehydroascorbic acids in human plasma requires reference materials of demonstrable stability and homogeneity, and analytical methodology of demonstrable accuracy and precision. We have developed rapid (8 min.), accurate (gravimetric and assessed values agree) and precise $(\mathrm{CV}<2 \%)$ method for ascorbic acid analysis. The stability and homogeneity of lyophilized plasma samples supplemented with ascorbic acid and dithiothreitol is sufficient for the materials to be used as control or reference materials. Plasma samples supplemented with ascorbic acid and dehydroascorbic acid, and preserved with 5\% metaphosphoric acid were used to develop an assay for dehydroascorbic acid and to study the stability of both ascorbic and dehydroascorbic acid under these conditions. The assay consists of measuring the native ascorbic acid, then reducing the dehydroascorbic acid at neutral $\mathrm{pH}$ with dithiothreitol and measuring the total ascorbic acid. The metaphosphoric acid treated samples were shown to be stable at $-70 \mathrm{C}$ and the stability decreased with temperature over the range of 4 to 50 C.

\section{3,496}

PB95-108767 Not available NTIS

National inst. of Standards and Technology (NML) Gaithersburg, MD. Gas and Particulate Science Div. Secondary Target X-Ray Excitation for In vivo Measurement of Lead in Bone.

Final rept.

P. A. Pella, and C. G. Soares. 1991, 6p.

Sponsored by Agency for Toxic Substances and Disease Registry, Atlanta, GA.

Pub. in Advances in X-ray Analysis 34, p293-298 1991.

Keywords: "Lead(Metal), "In vivo analysis, "Toxic sub stances, "X-ray analysis, "Bones, Biomedical measurement, Radiation dosage, Pollution sources, Excitation, Reprints.

Secondary target $x$-ray excitation (L-shell) was explored for possible application in $x$-ray systems designed for in vivo measurement of lead in bone (e.g. tibia bone) for screening purposes. Since $x$-ray output from secondary targets is due mostly to characteristic $x$-rays and is many times lower than from direct excitation from high power tubes, it was of interest to meas ure the $x$-ray dose. This consisted of the experimenta measurement of the $x$-ray fluorescence followed by ap propriate conversion to an effective equivalent dose. Minimum detection limits were calculated using simulated bone samples (i.e. bone ash) using different instrumental configurations. An overlay of lucite was used to simulate the absorption of the skin layer.

\section{3,497}

PB95-151486 Not available NTIS

National Inst. of Standards and Technology (NML) Gaithersburg, MD. Organic Analytical Research Div.

How to Verify Reference Materials.

Final rept.

M. Welch. 1989, 4p

Pub. in Forensic Urine Drug Testing, pp1, 5-7, Jun 89

Keywords: "Reference standards, "Verification "Urinalysis, "Quantitative chemical analysis, Laboratory tests, Test methods, Accuracy, Calibration, Quality control, Quality assurance, Laboratories, Reliability, Reprints.

The article describes the type of reference materials available for laboratories conducting urine drug testing. It describes how laboratories can check their standards to assure accuracy in their methods. Some description is provided on how NIST determines the purity of standard reference materials. The laboratories doing the drug testing do not have the time, facilities, or expertise to test their standards as thoroughly as is done at NIST. Some recommendations are provided concerning testing that these laboratories can and should perform on their standards.

03,498

PB95-161980 Not available NTIS

National Inst. of Standards and Technology (CSTL) Gaithersburg, MD. Biotechnology Div.

Amperometric Flow-Injection Analysis Biosensor for Glucose Based on Graphite Paste Modified with Tetracyanoquinodimethane.

Final rept.

P. C. Pandey, S. A. Glazier, and H. H. Weetall.

$1993,5 p$

Pub. in Analytical Biochemistry 214, p233-237 1993.

Keywords: "Flow injection analysis, "Glucose *Biosensors, Immobilized enzymes, Reproducibility of results, Oxidation, Reduction(Chemistry), Graphite, Blood, Humans,
*Tetracyanoquinodimethane.

A biosensor system using flow injection analysis (FIA has been developed for the analysis of glucose in human serum. The system consists of the enzyme glucose oxidase incorporated into graphite paste modified with the electroactive material tetracyanoquinodimethane (TCNQ). Data are presented to show the effect of sample injection volume and flow rate on the response of the FIA sensor. The biosensor exhibited excellent reproducibility for 800 in jections. The loss of response after 800 injections was due to leaching of TCNQ from the graphite paste. Each assay takes 3 min giving a sample throughput of 20 per hour at a flow rate of $30 \mathrm{ml} / \mathrm{h}$. The sensor was applied to the determination of glucose in human serum. The glucose measurements are in good agreement with those of a commercially available spectrophotometric method. Data showing the effect of interfering substances, ascorbic acid and acetaminophen, on the response of the sensor are also reported.

\section{3,499}

PB95-176160 Not available NTIS

National Inst. of Standards and Technology (CSTL) Gaithersburg, MD. Organic Analytical Research Div. Certification of Morphine and Codeine in a Human Urine Standard Reference Material.

Final rept.

S. S. C. Tai, R. G. Christensen, R. C. Paule, L. C. Sander, and M. J. Welch. 1994, 6p.

Pub. in Jnl. of Analytical Toxicology 18, p7-12 Jan/Fob 94.

Keywords: "Urinalysis, "Reference standards, "Morphine, "Codeine, "Chemical analysls, Humans Urine, Gas chromatography, Mass spectroscopy, Test methods, Analytical technlques, Certificatlon, Reprints, "Standard reference materlals, SRM 2381. 


\section{MEDICINE \& BIOLOGY}

\section{Clinical Chemistry}

The National Institute of Standards and Technology has developed and certified a Standard Reference Material, SRM 2381, for use in testing for bias in determinations of morphine and codeine in human urine. Three different analytical methods, employing GC/MS, LC/MS, and MS/MS, were used to certify the concentrations of each analyte. Results from the three methods were in good agreement and, therefore, were statistically combined to yield certified values of 138,293 , and $578 \mathrm{ng} / \mathrm{mL}$ for morphine and 134,283 , and 591 for codeine.

\section{3,500}

PB95-176251 Not available NTIS

National Inst. of Standards and Technology (CSTL), Gaithersburg, MD. Qrganic Analytical Research Div. Interlaboratory Comparison Studies on the Analysis of Hair for Drugs of Abuse.

Final rept.

M. J. Welch, L. T. Sniegoski, and C. C. Allgood

1993, $9 p$

Contracts IAA-89-IJ-CX-A031, RA-ND-92-36

Sponsored by National Inst. of Justice, Washington, DC. and National Inst. on Drug Abuse, Rockville, MD. Pub. in Forensic Science International 63, p295-303 1993

Keywords: "Hair, "Drug abuse, *Test methods, *Quantitative chemical analysis, *I terlaboratory comparisons, Laboratories, Detection, Cocaine, Morphine, Gas chromatography, Mass spectroscopy, Enzyme digestion, Acid extraction, Analytical techniques, Drug users, Reprints, Benzoylecgonine.

Eleven laboratories interested in the analysis of human hair for drugs of abuse participated in a study to determine how well drugs could be detected and quantified in hair. Samples sent to the participating laboratories included hair from drug users, drug-free hair, and hair into which drugs had been soaked. For the first exercise, the hair samples were sent as powders; for the second, they were in the form of short segments. Results from these studies have shown that the laboratories, with a few exceptions, have performed very well qualitatively. Various approaches were used to liberate drugs from the hair, with the most commonly used, acid extractions and enzyme digestions, producing similar results.

\section{3,501}

PB95-176269 Not available NTIS

National Inst. of Standards and Technology (CSTL), Gaithersburg, MD. Qrganic Analytical Research Div.

Hair Analysis for Drugs of Abuse: Evaluation of Analytical Methods, Environmental Issues, and Development of Reference Materials.

Final rep

M. J. Welch, L. Sniegoski, C. Allgood, and M. Habram. 1993, 10p.

Sponsored by National Inst. of Justice, Washington, DC. and National Inst. on Drug Abuse, Rockville, MD. Pub. in Jnl. of Analytical Toxicology 17, p389-398 Novi Dec 93

Keywords: "Hair, "Drug users, "Quantitative chemical analysis, "Detection, "Biomarkers, "Reference standards, Cocaine, Morphine, Codeine, Drug abuse, Certification, Test methods, Analytical techniques, Gas chromatography, Mass spectroscopy, Acid extraction, Enzyme digestion, Reprints, "Standard reference materials.

Methods for extraction of cocaine, some of its metabolites, morphine, and codeine from hair and methods for analyzing the extracts have been investigated. Results of these studies have shown that extractions with $0.1 \mathrm{~N} \mathrm{HCl}$ are efficient at removing the target compounds from hair and appear to be as effective as enzymatic digestions that dissolve the hair. To assist laboratories in evaluating the accuracy of their methods, two human hair reference materials with recommended concentrations of cocaine, benzoylecgonine, morphine, and codeine determined by GC/MS have been developed.

\section{3,502}

\section{PB95-200648 PC A07/MF A02}

National Inst of Standards and Technology (CSTL) Gaithersburg, MD. Qrganic Analytical Research Div. Methods for Analysis of Cancer Chemopreventive Agents in Human Serum.

Special pub.

J. B. Thomas, and K. E. Sharpless. Feb 95, 136p, NIST/SP-874.

Also available from Supt. of Docs. as SN003-00303321-9. Sponsored by National Cancer Inst., Bethesda, MD. Div. of Cancer Prevention and Control.
Keywords: "Trace elements, "Quantitative analysis "Serums, Analytical chemistry, Neoplasms, , Vitamins, Blood, Plasma, Glycyrrhetinic acid, Ascorbic acid, Dehydroascorbic acid, Vitamin K, Liquid chromatography, Measurement, Quality assurance, "Chemopreventive agents, Qltipraz, 4Hydroxyphenylretinamide.

The purpose of this manual is to summarize several years of methods development and refinement for the measurement of selected fat- and water-soluble vitamins, carotenoids, oltipraz, and glycyrrhetinic acid in serum and plasma as part of the National Institute of Standards and Technology (NIST)/National Cancer Institute (NCl) Micronutrients Measurement Quality Assurance Program. The manual is divided into six secuns, each containing a series of publications pertaining to the measurement of the specified analyte(s). A summary of factors that are important to the method performance for each procedure is provided. This summary is included to help the analyst focus on the steps needed to implement each procedure.

\section{3,503}

\section{PB96-119706 Not available NTIS}

National Inst of Standards and Technology (CSTL), Gaithersburg, MD. Qrganic Analytical Research Div.

History of NIST's Contributions to Development of Standard Reference Materials and Reference and Definitive Methods for Clinical Chemistry.

Final rept.

R. Schaffer, G. N. Bowers, and R. S. Melville. 1995 $7 p$.

Pub. in Clinical Chemistry, v41 n9 p1306-1312 1995

Keywords: *Calibration, ${ }^{*}$ Clinical chemistry, Chloride, Reprints, Cholesterol, Creatinine, Glucose, Lithium, Calcium, Standard Reference Materials, National Institute of Standards and Technology.

The issuance of cholesterol as a Standard Reference Material (SRM) in 1967 started the National Institute of Standards and Technology (NIST; then named the National Bureau of Standards) on a major effort to help clinical laboratories establish and improve the quality of measurements they make. NIST now issues three kinds of SRMs for that purpose: analyte samples of certified purity as primary standards, serum samples having certified analyte concentrations as accuracy controls, and materials certified for calibrating instruments, In working with clinical laboratory scientists to establish Reference Methods (RMs) for measuring the analytes NIST developed Definitive Methods (DMS) to use for evaluating RM accuracy and then used the DMs for assigning analyte values to its SRMS. The development of SRMs and DMs is discussed.

\section{3,504}

PB96-120555 PC A18/MF A04

National Inst. on Drug Abuse, Rockville, MD

Hair Testing for Drugs of Abuse: International Research on Standards and Technology.

E. J. Cone, M. J. Welch, and M. B. G. Babecki.

1995, 412p, NIH/PUB-95-3727.

Prepared in cooperation with National Inst. of Stand ards and Technology, Gaithersburg, MD

Keywords: "Hair, "Drug users, "Quantitative chemical analysis, Detection, Certification, Test methods, Analytical techniques, Gas chromatography, Mass spectroscopy, Acid extraction, Enzyme digestion, Standard reference materials.

\section{Partial Contents:}

introduction and Qverview:

National Institute on Drug Abuse Specia Publication on Hair Analysis for Drugs of Abuse

Mechanisms of Incorporation of Drugs Into Hair and the Interpretation of Hair Analysis Data;

How Environmental Drug Exposure Can Affect Hair Testing for Drugs of Abuse

Testing Human Hair for Qpiates and Cocaine by Gas Chromatography/Mass Spectrometry After Acid or Enzyme Hydrolysis;

Capillary Electrophoresis:

A Novel Tool for Toxicological Investigation:

Its Potential in the Analysis of Body Fluids and Hair;

Comparison of Different Extraction Procedures for Drugs in Hair of Drug Addicts

Man Scalp Hair as Biopsy Material Suitable for Quantitative Analysis in Therapeutic Drug Monitoring;

Neonatal Hair Analysis:

A Tool for the Assessment of In Utero Exposure to Drugs.
03,505

PB96-123807 Not available NTIS

National Inst. of Standards and Technology (CSTL),

Gaithersburg, MD. Organic Analytical Research Div.

NIST Reference Materials to Support Accuracy in Drug Testing.

Final rept.

M. J. Welch, P. Ellerbe, S. S. C. Tai, L. C. Sander,

C. S. Phinney, R. G. Christensen, and L. T.

Sniegoski. 1995, 5p.

Pub. in Fresenius Jnl. of Analytical Chemistry, v352 p61-65 1995.

Keywords: "Drug abuse, "Reference standards, "Quantitative chemical analysis, "Detection, Hair, Urinalysis, Drug users, Interlaboratory comparisons, ACcuracy, Quality assurance, Test methods, Analytical techniques, Cocaine, Morphine, Marijuana, Codeine, Mass spectroscopy, Gas chromatography, Reprints, Standard reference materials.

The National Institute of Standards and Technology (NIST) supports accuracy in drugs of abuse testing by proving Standard Reference Materials (SRMs) with certified concentrations of drugs of abuse in urine- and hair-based reference materials. NIST, working in collaboration with the College of American Pathologist (CAP), has developed urine-based SRMs for marijuana metobolite, cocaine metabolite, morphine and codeine and morphine glucuronide and CAP Reference Materials for amphetamines and phencyclidine. Certification measurements performed at NIST involve two independent methods for each analyte, one of which always uses GC/MS with the other usually being an LC method with either MS or UV detection. Work has recently been completed on a seven component drug in urine SRM. In addition NIST conducts research in the analysis of hair for drugs of abuse. To assist laboratories testing hair for that purpose, NIST has developed two drugs in hair reference materials.

\section{3,506}

\section{PB96-138425 Not available NTIS}

National Inst. of Standards and Technology (CSTL), Gaithersburg, MD. Qrganic Analytical Research Div. Determination of Vitamin $\mathrm{K} 1$ in Serum Using Catalytic-Reduction Liquid Chromatography with Fluorescence Detection.

Final rept.

W. A. MacCrehan, and E. Schonberger. 1995, 9p. Pub. in Jnl. of Chromatography B, Biomedical Applications, v670 p209-217 1995 .

Keywords: "Vitamin K, "Fluorescence detection, "Chromatography, Phylloquinone, Reverse phase separation, Solid phase fractionation, Reprints.

A new method for the liquid chromatographicfluorescene determination of serum vitamin $K$ is described using reduction of the $\mathrm{K}$-quinone to the fluorescent $\mathrm{K}$-hydroquinone. The reduction reaction occurs 'on-line' in the LC system using a catalytic reducer column and an alcohol mobile phase as reductant. A procedure for serum determination utilizes a liquid-liquid serum lipid extraction followed by normal-phase fractionation on a solid-phase extraction cartridge. The final measurement uses a reversed phase (C18) seperation with a ethanol-methanol mobile phase and provides a detection limit of approximately $20 \mathrm{pg} / \mathrm{ml}$.

\subsection{7}

PB96-159785 Not available NTIS

National Inst. of Standards and Technology (CSTL), Gaithersburg, MD. Inorganic Analytical Research Div. Development of a Standard Reference Material for ISE Measurements of Sodium and Potassium.

Final rept.

W. F. Koch, and R. C. Paule. 1989, 10p.

Pub. in IFCC Workshop, Stresa 1988, v10 p99-108 1989.

Keywords: "Blood, "Ion selective electrodes, Potassium, Reprints, Potentiometry, Serium, Sodium, Ultrafiltration, "Interiaboratory test, Standard Reference Material.

A research project, sponsored by the National Committee for Clinical Laboratory Standards and funded by eight manufacturers of clinical potentiometric instruments, is being conducted in the Electroanalytical Research Group at the National Institute of Standards and Technology (NIST, formerly NBS). The goal of this research is to bring conformity to the direct potentiometric measurements of sodium and potassium in human serum through the development of ref- 
erence materials. Currently, different manufacturers of clinical analyzers each recommend their own calibration standards. These materials range from simple aqueous solutions to complex mixtures containing bovine serum albumin. Hence, different electrolyte values for the same sample of human serum analyzed by dif ferent instruments are not uncommon. A series of fou interlaboratory tests has been carried out to quantify the problem and to identify the most suitable reference materials. Ideally, an accurate calibration standard should simulate the actual sample to minimize the effects of liquid junction, activity, protein volume, and other factors that affect the electrode potential. It also should also be easy to prepare in large quantities, be stable for long periods, and be convenient to use in hospital laboratory environment.

\section{3,508}

PB96-160692 Not available NTIS

National Inst. of Standards and Technology (CSTL) Gaithersburg, MD. Organic Analytical Research Div.

Certification of Phencyclidine in Lyophilized Human Urine Reference Materials.

Final rept.

S. S. C. Tai, R. G. Christensen, K. Coakley, M. J

Welch, P. Ellerbe, and T. Long. 1996, 7p.

Pub. in Jnl. of Analytical Toxicology, v20 p43-49 Jan/ Feb 96.

Keywords: "Drug abuse, "Calibration standards, "Quantitative chemical analysis, "Urine, Concentration(Composition), Test methods, Spectroscopic analysis, Chromatographic analysis, Certification, Interlaboratory compansions, Reprints, PCP(Phencyclidine), SEM 1511, CAP PCP RM.

The Natioanl Institute of Standards and Technology (formerly the National Bureau of Standards), in cooperation with the College of American Pathologists (CAP), has certified the concentrations of phencyclidine (PCP) in two new reference materials (RMs). One of these materials is Standard possibility of undetected bias, two independent analytical methods, employing gas chromatography-mass spectrometry and liquid chromatography-mass spectrometry and liquid chromatography-mass spectrometry, were used to certify PCP in these materials. Results from the two methods were in good agreement and were statistically combined to yield certified values.

\section{3,509}

\section{PB97-110449 Not available NTIS}

National Inst. of Standards and Technology (CSTL) Gaithersburg, MD. Analytical Chemistry Div.

Interlaboratory Studies on the Analysis of Hair for Drugs of Abuse: Results from the Fifth Exercise. Final rept.

M. J. Welch, and L. T. Sniegoski. 1996, 19p.

See also PB95-176251.

Pub. in Forensic Science International, p1-19 1996.

Keywords: "Hair, "Drug abuse, "Test methods, "Quantitative chemical analysis, "Interlaboratory comparisons, Laboratories, Detection, Cocaine, Morphine Gas chromatography, Mass spectroscopy, Reprints, "Foreign technology.

Fourteen laboratories interested in the analysis of human hair for drugs of abuse participated in a fifth interlaboratory study to determine how well drugs could be detected and quantified in hair. The drugs of above included cocaine, benzoylecgonine cocaethylene, codeine, morphine, and 6monoacetylmorphine. The size hair samples analyzed included three in the form of short segments and three in a powder form and included hair from drug users, drug-fortified hair (drug-free hair into which drugs had been soaked), and drug-free hair. Results from the study show that the laboratories performed well qualitatively, particularly for drug levels above $0.5 \mathrm{ng} / \mathrm{mg}$. While there was significant scatter in quantitative results, results were less scattered overall than had been seen previously. Various methods were used to extract the drugs from the hair, and the most commonly used approaches (i.e., acid extraction, methanol extractions, and enzyme digestions) have comparable results. For analysis of the extracts, gas chromatography/mass spectrometry (GC/MS) was used by all but one laboratory, which used a radioimmunoassay (RIA). Of the aboratories using GC/MS, some produced consistently good results, while others produced poorer quality results.
National Inst. of Standards and Technology (CSTL), Gaithersburg, MD. Analytical Chemistry Div.

Interlaboratory Studies on the Analysis of Hair for Drugs of Abuse: Results from the Fourth Exercise. Final rept.

L. T. Sniegoski, and M. J. Welch. 1996, 6p.

Pub. in Jnl. of Analytical Toxicology, v20 p242-247 Jul/ Aug 96

Keywords: "Drug abuse, "Reference materials, "Quantitative chemical analysis, "Detection, "Hair, Drug users, Interlaboratory comparisons, Accuracy, Quality assurance, Test methods, Analytical techniques, Cocaine, Morphine, Codeine, Mass spectroscopy, Gas chromatography, Reprints, Monoacetylmorphine.

Fourteen laboratories interested in the analysis of human hair for drugs of abuse participated in a fourth interlaboratory study to determine how well drugs could be detected and quantitated in hair. The drugs of abuse included cocaine, benzoylecgonine, monoacetylmorphine. The hair samples analyzed were monoacetylmorphine. The hair samples analyzed were drug users, soaked hair (drug-free hair into which drugs had been soaked), and drug-free hair. Results from the study show that the laboratories performed well qualitatively, but that there was a large amount of scatter in quantitative results.

\section{3,511}

PB97-112445 Not available NTIS

National Inst. of Standards and Technology (CSTL), Gaithersburg, MD. Analytical Chemistry Div.

Measurement of Ascorbic Acid in Human Plasma and Serum: Stability, Intralaboratory Repeatability, and Interlaboratory Reproducibility.

Final rept.

S. A. Margolis, and D. L. Duewer. 1996, 6p.

See also PB92-154442.

Pub. in Clinical Chemistry, v42 n8 p1257-1262 1996.

Keywords: "Ascorbic acid, "Blood chemical analysis, "Metaphosphoric acid, Blood plasma, Bias, Dithiothreitol, Liquid chromatography, Repeatability, Reproducibility, Reprints.

The authors demonstrate that total ascorbic acid (TAA, the sum of ascorbic acid and dehydroascorbic acid) in property prepared human plasma is stable at -70 degrees $C$ for at least 6 years when preserved with dithiothreitol. TAA in human plasma or serum preserved with metaphosphoric acid degrades slowly, at the rate of no more than $1 \%$ per year. As assessed from the stability data and from data obtained from 23 laboratories over a period of greater than 2 years, the intralaboratory repeatability of TAA measurements is approximately 2 micromol $/ L$, irrespective of TAA concentration. Nonchromatographic analytical methods involving dinitrophenylhydrazine and o-phenylenediamine yield biased results relative to chromatographic methods.

\section{3,512}

PB97-119333 Not available NTIS

National Inst. of Standards and Technology (CSTL), Gaithersburg, MD. Analytical Chemistry Div.

Liquid Chromatographic Determination of Carotenoids in Human Serum Using an Engineered C30 and a C18 Stationary Phase.

Final rept

K. E. Sharpless, T. J. Brown, L. C. Sander, and S. A Wise. 1996, 9p.

Pub. in Jnl. of Chromatography B: Biomedical Applications, v678 p187-195 Oct 96.

Keywords: "Trace elements, "Serums, "Quantitative analysis, Carotenoids, Liquid chromatography, Blood, Plamsa, Carbon, Reprints, "Chemopreventive agents

A C30 stationary phase was specifically engineered for carotenoid separations, and carotenoid measurements using this column are compared with those obtained using a somewhat more conventional $\mathrm{C} 18$ column Both methods were used to contribute measurements for the certification of carotenoids in Standard Reference Material 968b, Fat-Soluble Vitamins and Cholesterol in Human Serum. Analytes were extracted from the serum into hexane. Measurements on the C18 column were made using a gradient of acetonitrile methanol, and ethyl acetate, which is described in detail elsewhere. Measurements on the $\mathrm{C} 30$ column were made using a graident of water, methanol, and methyl tert.-butyl ether.

\section{Clinical Medicine}

\subsection{3}

AD-A279 120/0 PC A04/MF A01

National Bureau of Standards, Gaithersburg, MD. Report of the International Commission on Radiological Units and Measurements (ICRU), 1956. 10 Apr 57, 53p.

Keywords: "Radiology, "Measurement, "Handbooks, "Radiation dosage, "Radiation measuring instruments, Dose rate, Dosimetry, Radiation protection, Materials, Health physics instrumentation, ICRU(International Commissionon Radiological Units)

No abstract available.

\section{3,514}

PB94-185642 Not available NTIS

National Inst. of Standards and Technology (PL), Gaithersburg, MD. Ionizing Radiation Div.

Use of a Radiochromic Detector for the Determination of Stereotactic Radiosurgery Dose Characteristics.

Final rept.

W. L. McLaughlin, C. G. Soares, J. A. Sayeg, A. Wu, A. H. Maitz, E. C. McCullough, and R. W. Kline.

1994, 10p.
Pub. in Med. Phys. 21, n3 p379-388 Mar 94.

Keywords: "Radiosurgery "Radiotherapy dosage, *Detectors, Gamma rays, Densitometry, Lasers, Quality control, Precision, Resolution, Dosimetry, Reprints, Radiochromic films

The measurement of absorbed dose as well as dose distributions (profiles and isodose curves) for small radiation fields (as encountered in stereotactic surgery) has been difficult due to the usual large detector size or densitometer aperture $(>1 \mathrm{~mm}$ ) relative to the radiation field (as small as $4 \mathrm{~mm}$ ). The radiochromic directimaging film, when read with a scanning laser microdensitometer (laser beam diameter $0.1 \mathrm{~mm}$ ), overcomes this difficulty and has advantages over conventional film in providing improved precision, better tissue equivalence, greater dynamic range, higher spatial resolution, and room light handling. As a demonstration of suitability, the calibrated radiochromic film has been used to measure the dose characteristics for the 18-, 14-, 8-, and 4-mm fields from the gamma-ray stereotactic surgery units at the Mayo Clinic and the University of Pittsburgh. Intercomparisons of radiochromic film with conventional methods of dosimetry and vendor-supplied computational dose planning system values indicate agreement to within + or - $2 \%$ The dose, dose profiles, and isodose curves obtained with radiochromic film can provide high-spatial-resolution information of value for acceptance testing and quality control of dose measurement and/or calculation.

\section{3,515}

PB94-198470 Not available NTIS

National Inst. of Standards and Technology (NML)

Gaithersburg, MD. Organic Analytical Research Div.

Nutritional Status and Growth in Juvenile Rheumatoid Arthritis.

Final rept.

M. C. Bacon, P. H. White, D. J. Raiten, O. A

Levander, M. L. Taylor, R. N. Lipnick, S. Sami, N.

Craft, and S. Margolis. 1990, 10p.

Pub. in Seminars in Arthritis and Rheumatism 20, n2 p97-106 1990.

Keywords: "Nutrition, "Growth abnormalities, Reprints Anthropometry, Biochemistry, Vitamin and mineral preparations, Blood proteins, Nutritional requirements, "Juvenile rheumatoid arthritis.

Juvenile rheumatoid arthristis (JRA) has been associated with short stature. However, the specific cause is unknown. One hypothesis is that altered growth is due to inadequate nutrition. The authors looked at 34 children with JRA and 9 healthy controls using 3-day diet records, anthropometric measures and biochemical analysis of plasma protein, vitamin and mineral levels. The children with JRA were compared between groups according to type of JRA: systemic, polyarticular and pauciarticular. Significant differences in growth and biochemical status were found. Those with polyarticular or systemic types of JRA had lower levels of zinc, vitamins $A$ and $C$, and retinol binding protein. No direct relationship between intake and plasma levels of nutrients was found. However, alterations in nutritional needs according to disease type and severity could not be ruled out. 


\section{Clinical Medicine}

03,516

PB94-198827 Not available NTIS

National Inst. of Standards and Technology (NML), Gaithersburg, MD. Ionizing Radiation Div

Standard Reference Materials (SRM's) for Measuring Genetic Damage.

Final rept.

D. S. Bergtold, E. Holwitt, and M. G. Simic. 1990, 3p. Pub. in Fresenius Jnl. of Analytical Chemistry 338, n4 p383-385 1990.

Keywords: "DNA damage, "Radiotherapy, Biological markers, Reprints, "Standard reference materials, Hydroxyl radical, Thymine glycol, Thymidine glycol.

Standard reference materials (SRM's) were designed for the measurement of radiolytic products resulting from $\mathrm{OH}$ radical reaction with DNA in patients treated by radiation therapy. Deuterated thymine glycol and thymidine glycol are proposed as such SRM's; the synthesis of the former is described in detail. They might be of importance for optimizing the therapy.

03,517

PB94-219227 (Order as PB94-219219, PC A06/

MF A02)

National Inst. of Standards and Technology, Gaithersburg, MD.

Sealed Water Calorimeter for Measuring Absorbed Dose.

S. R. Domen. 1994, 21p.

Included in Jnl. of Research of the National Institute of Standards and Technology, v99 n2 p121-141 Mar/ Apr 94.

Keywords: "Calorimeters, "Radiation doses, "Water, Dissolved gases, Calorimetric dosemeters, Measuring instruments, Temperature effects, Thermistors, Exothermic reactions, Endothermic reactions, Heat defect, Convective barrier, National Institute of Standards and Technology.

The sealed water calorimeter is intended for direct measurement of absorbed dose to water. This calorimeter was used for a series of approximately 3700 measurements to investigate the so-called heat defect, that is, anomalous endothermic or exothermic effects caused by dissolved gases. The three systems investigated were high-purity water saturated with N2, H2, and mixtures of $\mathrm{H} 2 / \mathrm{O} 2$. The repeatability of measurements of absorbed dose rates for the $(60)$ Co teletherapy beam was studied with different water fillings and accumulated absorbed dose. Measurements with the $\mathrm{H} 2 / \mathrm{O} 2$ system varied with accumulated absorbed dose. Based on the measurements and theoretical considerations, it appears that the $\mathrm{H} 2$-saturated system is the best choice for eliminating the heat defect. Measurements with both the N2- and $\mathrm{H} 2$-saturated systems are in good agreement with those determined with a graphite and graphite-water calorimeter (for which there is no heat defect)

\section{3,518}

PB95-164604 Not available NTIS

National Inst. of Standards and Technology (PL), Gaithersburg, MD, lonizing Radiation Div.

Measurement of Radial Dose Distributions Around Small Beta Particle Emitters Using High Resolution Radiochromic Foil Dosimetry.

Final rept.

C. G. Soares, and W. L. McLaughlin. 1993, 6p.

Pub. in Radiation Protection Dosimetry 47, n1/4 p367. 3721993.

Keywords: "Radiation doses, "Beta particles, "Ophthalmology, Calibrating, Densitometry, Dosimetry, Strontium 90, Yttrium 90, Cobalt 60, Thulium 170, Reprints, "Radiochromic foils.

A method is described for the direct measurement of the radial dose distributions around small (from several $\mathrm{mm}$ down to tens of micrometers in diameter) beta particle emitters. The method employs the high resolution readout of radiochromic dye foils. These foils form a blue image upon irradiation and require no processing to stabilize the image. The color change (in units of absorbance) is nearly linear with absorbed dose over a range of 10 Gy to 1000 Gy and the response to electrons is nearly identical to (60)Co gamma radiation which makes the system easy to calibrate. Readout is performed with a laser scanning densitometer with a 100 micrometer spot size which can be stepped in increments of 40 micrometers in two dimensions. Examples of the results of measurements of $(90) \mathrm{Sr} /(90) \mathrm{Y}$ eye applicators as well as particle sources of $(90) \mathrm{Sr}$ $(90) \mathrm{Y},(60) \mathrm{Co}$, and $(170) \mathrm{Tm}$ are given.
03,519

PB96-102280 Not available NTIS

National Inst. of Standards and Technology (CSTL), Gaithersburg, MD. Analytical Chemistry Div

Isotope Dilution Mass Spectrometry as a Candidate Definitive Method for Determining Total Glycerides and Triglycerides in Serum.

Final rept.

P. Ellerbe, L. T. Sniegoski, and M. J. Welch. 1995

Pub. in Clinical Chemistry, v41 n3 p397-404 1995.

Keywords: "Isotope dilution, "Mass spectrometers, "Glycerol, "Cholesterol, Triglycerides, Isotope ratio, Test methods, Tracer techniques, Isotope applications, Gas chromatography, Blood serum, Humans, Reprints.

A new isotope dilution mass spectrometric method for total glycerides and triglycerides in human serum is described. Total glycerides are defined as the sum of tridi-, and monoglycerides plus free glycerol; triglycerides are defined as the pure triglyceride species. In both determinations, serum samples are supplemented by additon of (13C3) tripalmitin, processed, derivatized, and the abundance ratios of selected ions are determined. The $\mathrm{CV}$ for a single measurement ranged from $0.35 \%$ to $0.72 \%$, and the relative SEM ranged from $0.10 \%$ to $0.34 \%$; there was no significant bias in the measurements. The combination of high precision and absence of significant bias in the results qualify this method for consideration as a Definitive Method as defined by the National Committee for Clinical Laboratory Standards.

\section{3,520}

PB96-179486 Not available NTIS

National Inst. of Standards and Technology (EEEL), Gaithersburg, MD. Electronics and Electrical Engineering Lab. Office.

Preliminary Investigation of Oleoresin Capsicum Final rept

R. G. Christensen, and D. E. Frank. 1995, 20p.

Sponsored by National Inst. of Justice, Washington, DC.

Pub. in Preliminary Investigation of Oleoresin Capsicum, NIJ Report 100-95, 20p, 15 Apr 95.

Keywords: "Medicinal plants, *Peppers, "Oleoresins, Chemical composition, Toxicity, Reprints.

This report documents a preliminary investigation into the anlaytical characterization of Oleoresin Capsicum (OC), an oily extract of hot peppers that is increasingly used in law enforcement applications. Being a natural product, it is subject to variations in composition. The characteristic hot sensation or pungency is quantified in the industry in terms of 'Scoville Heat Units,' (SU). $\mathrm{SU}$ is defined as the dilution at which the pungency can barely be detected by a trained taster. Pure cap. saicin, the most pungent constituent, has an SU value of 16 times 10 to the sixth power $\mathrm{mL} / \mathrm{g}$ (milliliters of diluent per gram of base material). Recently, OC has come into use as a pain-producing agent in aerosol sprays used in an attempt to subdue violent individuals. This use raises the question of how to assign an objective index of potency to these sprays. Published information on the pharmacology of capsaicinoid compounds has been studied to examine correlations between pungency and pain production. Experiments in the analytical chemistry of OC have been carried out to check the feasibility of determining the concentrations of the pungent constituents.

\section{3,521}

PB97-110092 Not available NTIS

National Inst. of Standards and Technology (PL), Gaithersburg, MD. Ionizing Radiation Div.

Needs for Brachytherapy Source Calibrations in the United States.

Final rept.

B. M. Coursey, L. J. Goodman, D. D. Hoppes, C. G.

Soares, J. T. Weaver, R. Loevinger, and W. L.

McLaughlin. 1992, 5p.

Pub. in Nuclear Instruments and Methods in Physics Research, vA312 p246-250 1992.

Keywords: "Brachytherapy, "Dosimetry, “Gamma rays, Radiation, Radionuclides, Standards, Therapy, Reprints, "Foreign technology.

Brachytherapy sources of beta and gamma radiation ('brachy' is from the Greek, meaning 'near') have a long history of use in interstitial, intracavitary, intraluminal, and ocular radiation therapy. In the past the US national standards for these sources were often specified in activity of milligram radium equivalent. With the introduction of new radionuclide sources to replace radium. Source strength calibrations are now expressed as air kerma rate at a meter. In the paper. the authors review the NIST standards for brachytherapy sources, list some of the common radionuclides and source encapsulations in use in the US radiology community, and describe the latest NIST work, in collaroation with several US medical institutions, on a method of two-and three-dimensional dose mapping of brachytherapy sources using radiochromic films.

\section{3,522}

PB97-110100 Not available NTIS

National Inst. of Standards and Technology (PL) Gaithersburg, MD. Ionizing Radiation Div.

Radioassays of Yttrium-90 Used in Nuclear Medicine.

Final rept

B. M. Coursey, J. M. Calhoun, and J. T. Cessna

1993, 7p.

Pub. in International Jnl. of Nuclear Medicine and Biology, v20 p693-699 1993.

Keywords: "Nuclear medicine, "Radioassays, Standards, Liquid scintillation, Gamma counters, Dose calibrators, Reprints, "Foreign technology, "Yttrium-90, Cerenkov counting.

Yttrium-90 radioassays are required in nuclear medicine at the gigabecquerel activity level (Gbq) for measuring injected activity, and at the becquerel level for measuring individual tissue samples in biodistribution studies. A method of standardizing $90 \mathrm{Y}$ for activity using high-efficiency liquid-scintillation counting is described. Solution standards were used to establish the calibration factors for commercial radionuclide-calibrators. Detection efficiencies are also presented for liquid-scintillation counting. Nal(Ti) bremsstrahlung counting and Cerenkov counting.

\section{3,523}

PB97-110159 Not available NTIS

National Inst. of Standards and Technology (PL) Gaithersburg, MD. Ionizing Radiation Div.

Problem of Convection in the Water Absorbed Dose Calorimeter.

Final rept.

S. R. Domen, A. Krauss, and M. Roos. 1991, 9p. Pub. in Thermochimica Acta, v187 p225-233 1991.

Keywords: "Absorbed dose, "Calorimeters, "Convection, Convective barrier, Convective cooling, Thermistor water, Reprints, "Foreign technology.

The water absorbed dose calorimeter allows the water absorbed dose, the measure and in radiotherapy, to be measured in accordance with its definition. Its application, however, requires the suppression convection. In the present paper the authors investigate how far the convection problem may be solved by mechanical means, for the case of $60 \mathrm{C}$-gamma radiation.

\section{Cytology, Genetics, \& Molecular Biology}

03,524

PB94-172319 Not available NTIS

National Inst. of Standards and Technology (CSTL), Gaithersburg, MD. Biotechnology Div.

beta-D-Glucosyl-Hydroxymethyluracil: A Novel Modified Base Present in the DNA of the Parastic Protozoan T. brucei.

Final rept.

J. Gommers-Ampt, F. Van Leeuwen, A. de Beer, J.

Kowalak, P. Crain, P. Borst, J. Vliegenthart, and M.

Dizdaroglu. 1993, 8p.

Pub. in Cell 75, p1129-1136, 17 Dec 93.

Keywords: "Protozoan DNA, "Trypanosoma brucei, *Nucleotides, Surface antigens, Gene expression, Telomere, Reprints, *Uracil/5-((beta-D-glycopyranosyl oxy)-methyl)

We have previously shown that the DNA of the unicellular eukaryote $T$. brucei contains about $0.1 \%$ of a novel modified base, called $J$ The presence of $J$ correlates with a DNA modification associated with the silencing of telomeric expression sites for the variant sur- 
face antigens of trypanosomes. Here we show that $J$ is 5-((beta-D-glucopyranosyloxy)-methyl)-uracil (shortened to beta-D-glucosyl-hydroxymethyluracil), a base not previously found in DNA. We discuss putative pathways for the introduction of this base modification at specific positions in the DNA and the possible contribution of this modification to repression of surface antigen gene expression.

03,525

PB94-199825 Not available NTIS

National Inst. of Standards and Technology (CSTL), Gaithersburg, MD. Biotechnology Div.

Oxidative Damage to DNA in Mammalian Chromatin.

Final rept.

M. Dizdaroglu. 1992, 12p.

Pub. in Mutation Research 275, p331-342 1992. Sponsored by Department of Energy, Washington, DC.

Keywords: *DNA damage, "Chromatin, Mammals, Ionizing radiation, Hydrogen peroxide, Toxicology, Mass fragmentography, Cross-linking reagents, Cultured cells, In vivo analysis, In vitro analysis, Reprints.

Efforts have been made to characterize and measure DNA modifications produced in mammalian chromatin in vitro and in vivo by a vaniety of free radical-producing systems. Methodologies incorporating the technique of gas chromatography/mass spectrometry have been used for this purpose. A number of products from all four DNA bases and several DNA-protein cross-links in isolated chromatin have been identified and quantitated. Product formation has been shown to depend on the free radical-producing system and the presence or absence of oxygen. A similar pattern of DNA modifications has also been observed in chromatin of cultured mammalian cells treated with ionizing radiation or $\mathrm{H} 2 \mathrm{O} 2$ and in chromatin of organs of animals treated with carcinogenic metal salts.

\section{3,526}

PB94-199833 Not available NTIS

National Inst. of Standards and Technology (CSTL). Gaithersburg, MD. Biotechnology Div.

Modification of DNA Bases in Chromatin of Intact Target Human Cells by Activated Human Polymorphonuclear Leukocytes.

Final rept.

M. Dizdaroglu, R. Olinski, J. Doroshow, and S.

Akman. 1993, $4 \mathrm{p}$.

Pub. in Cancer Research 53, p1269-1272, 15 Mar 93.

Keywords: "DNA damage, "Tetradecanoylphorbol acetate, "Pharmacology, "Chromatin, "Leukocytes, Hydroxy compounds, Deoxyribonucleic acids, Mass fragmentography, Guanine, Adenine, Free radicals, In vivo analysis, Reprints.

The authors investigated whether phorbol-12-acetate13-myristate (PMA)-activated human polymorphonuclear leukocytes (PMNS) induce base modifications in target cell DNA in vivo. Human PMNs produced $9.4+$ or -0.8 (SD) nmol of $\mathrm{H}_{2} \mathrm{O} 2 / 10$ to the sixth power cells during $50 \mathrm{~min}$ of exposue to 2 micrograms/ml PMA and 13.7 + or $-2.8 \mathrm{nmol} / 10$ to the sixth power cells during exposure to PMA plus $5 \mathrm{mM}$ NaN3. Neither nonstimulated PMNs, nor PMA alone, nor NaN3 alone induced base modifications in chromatin-associated DNA of human Ad293 cells above control levels, when assayed by gas chromatography/ mass spectrometry with selected-ion monitoring, However, a 60-min exposure to $1.7+$ or -0.4 times 10 to the sixth power PMNs/ml in the presence of 2 micrograms/mI PMA induced a 2-3-fold increase in the level of all modified bases detected by gas chromatography/mass spectrometry with selected-ion monitoring. The guanine-denved products 8-
hydroxyguanine and 2,6-diamino-4-hydroxy-5hydroxyguanine and 2,6-diamino-4-hydroxy-54,6-diamino-5-formamidopyrimidine were induced to the highest levels among those bases detected. These data demonstrate that exposure to activated PMNs causes DNA base modifications in target cells in vivo typical of those induced by hydroxyl radical attack. The induction of potentially promutagenic modified bases may contribute to the mutagenicity of activated PMNs.

03,527

PB94-200375 Not available NTIS

National Inst. of Standards and Technology (CSTL),

Boulder, CO. Biotechnology Div.
Salt-PEG Two-Phase Aqueous Systems to Purify Proteins and Nucleic Acid Mixtures.

Final rept.

K. D. Cole. $1992,12 p$.

Pub. in Proceedings of Frontiers in Biochemistry II, Boulder, CO., June 17-21, 1990, p340-351 1992.

Keywords: "Proteins, "Nucleic acids, "Purification, Deoxyribonucleic acids, pH. Polyethylene glycols, Salts, Molecular weight, Protein denaturation, Reprints.

This paper examines the effect of variables on protein and nucleic acid partitioning in aqueous two-phase ex traction systems composed of salt and polyethylene glycol (PEG). The variables that can be changed in these systems include: $\mathrm{pH}, \mathrm{PEG}$ concentration, PEG molecular mass, salt type, salt concentration and addition of protein denaturants such as chaotropic salts and detergents. Methods for the preparation of high molecular mass DNA from crude mixtures are de scribed. These methods offer the advantages of simplicity, speed and use of nontoxic materials.

\section{3,528}

PB94-216322 Not available NTIS

National Inst. of Standards and Technology (CSTL), Gaithersburg, MD. Organic Analytical Research Div. Hydrolysis of Proteins by Mic rowave Energy.

Final rept.

S. A. Margolis, L. Jassie, and H. M. Kingston. 1991 3p.

Pub. in Jnl. of Aulomatic Chemistry 13, n3 p93-95 May/ Jun 91.

Keywords: "Bovine serum albumin, "Hydrolysis, "Microwaves, Valine, Isoleucine, Automation, Reprints Microwave energy, at manually-adjusted, partial power settings has been used to hydrolyze bovine serum albumin at $125 \mathrm{C}$. Hydrolysis was complete within $2 \mathrm{~h}$, except for valine and isoleucine which were completely liberated within $4 \mathrm{~h}$. The amino acid destruction was less than that observed at similar hydrolysis conditions with other methods and complete hydrolysis was achieved more rapidly. These results provide a basis for automating the process of amino acid hydrolysis.

\section{3,529}

PB95-150793 Not available NTIS

National Inst. of Standards and Technology (CSTL), Gaithersburg, MD. Biotechnology Div.

Application of Thermodynamics to Biotechnology. Final rept.

Y. B. Tewari, and R. N. Goldberg. 1991, 10p.

Pub. in Proceedings of International Enzyme Biotechnology Symposium, Veracruz, Mexico, October 1991, p1-10.

Keywords: "Thermodynamics, "Biotechnology, Temperature, Osmolar concentration, Heat, Enthalpy, Gibbs free energy, Chemical reactions, Reprints.

High product yield is important for the successful commercialization of any chemical reaction. The factors influencing the product yields are temperature, ionic strength, $\mathrm{pH}$, and the concentrations of the substrates and cofactors. Thermodynamics plays an importan role in understanding the interrelationships between these various factors. Thus, a knowledge of the Gibbs energy, enthalpy, and heat capacity change can be used to calculate the optimal product yield. This, in tum, is important in assessing the economic viability of the reaction. Thermodynamic data also provides the amount of heat evolved or absorbed in a reaction and defines the energy costs for that reaction. Thus, a reaction can be carried out under optimal condition to ensure maximum life and activity of the enzyme. In consideration of these points, a thermodynamic assessment will be made for several enzyme-catalyzed reactions of industrial importance.

\section{3,530}

PB95-151403 Not available NTIS

National Inst. of Standards and Technology (CSTL), Boulder, CO. Biotechnology Div.

Preparation of Immobilized Proteins Covalently Coupled Through Silane Coupling Agents to Inorganic Supports.

Final rept.

H. H. Weetall. 1993, 32p

Pub. in Applied Biochemistry and Biotechnology 41 p157-1881993.

Keywords: "Proteins, "Silane, Enzymes, Antibodies, Antigens, Molecular weight, Reprints, *Inorganic supports, Immobilization(Chemistry), Sol-gel.
Enzymes were first immobilized on inorganic supports through silane coupling agents over 25 yr ago. Since that initial report, literally hundreds of laboratories have utilized this methodology for the immobilization of enzymes, antigens, antibodies, receptors, and other high and low mol wt compounds. Today silane coupling is one of the commonly used techniques in the arsenal of the biochemist for the binding of material of all sorts to inorganic surfaces. Inorganic materials come in a vaniety of shapes, sizes, and characteristics. Sol-gel entrapped enzymes are also produced by the application of silane technology by the polymerization of the silane to form glass-like materials with entrapped protein. This review will discuss the general preparation and characterization of silane coupled proteins with special emphasis on enzymes and describe in detail the actual methods for the silanization and specific chemical coupling of proteins to the silanized carrier.

03,531

PB95-151411 Not available NTIS

National Inst. of Standards and Technology (CSTL), Gaithersburg, MD. Biotechnology Div.

Method for the Assay of Hydrolytic Enzymes Using Dynamic Light Scattering.

Final rept.

H. H. Weetall, and A. K. Gaigalas. 1993, 6p.

Pub. in Applied Biochemistry and Biotechnology 41 p139-1441993.

Keywords: "Enzymes, "Light scattering, Hydrolysis, Colloids, Substrate specificity, Reprints.

Dynamic light-scattering techniques have been successfully used for the assay of several hydrolytic enzymes. The enzymes were assayed using substratecoated colloidal particles. Hydrolysis of the substrate coat causes destabilization of the particles followed by particle aggregation. The rate of particle aggregation can be related to the initial concentration of added enzyme.

\section{3,532}

PB95-151429 Not available NTIS

National Inst. of Standards and Technology (CSTL). Gaithersburg, MD. Biotechnology Div.

Bacteriorhodopsin Immobilized in Sol-Gel Glass.

Final rept

H. Weetall, B. Robertson, D. Cullin, J. Brown, and M.

Walch. 1993, 3p.

Pub. in Biochimica et Biophysica Acta 1142, p211-213 1993

Keywords: "Bacteriorhodopsin, Light, pH, Spectros copy, Reprints, "Sol-gel glass.

The $96 \mathrm{~N}$ mutant of bacteriorhodopsin (BR) has been successfully trapped in a sol-gel glass. The protein retains its light-sensitive properties when immobilized at $\mathrm{pH}$ 9.0. Spectroscopic studies on the immobilized BR shows properties similar to those observed when in suspension.

\section{3,533}

\section{PB95-151544 Not available NTIS}

National Inst. of Standards and Technology (NML), Gaithersburg, MD. Chemical Thermodynamics Div.

Function of DnaJ and DnaK as Chaperones in Origin-Specific DNA Binding by RepA.

Final rept

S. Wickner, J. Hoskins, and K. McKenney. 1991, 3p Pub. in Nature 350, n6314 p165-167, 14 Mar 91.

Keywords: "Heat-shock proteins 60, "Heat-shock proteins 70, "Origin of replication, "Deoxyribonucleic acids, "DNA-binding proteins, Escherichia coli, Cell membrane, Biological transport, Plasmids, Phage lambda, Protein folding, Reprints

Heat-shock proteins are normal constituents of cells whose synthesis is increased on exposure to various forms of stress. They are interesting because of their ubiquity and high conservation during evolution. Two families of heat-shock proteins, hsp60s and hsp70s, have been implicated in accelerating protein folding and oligomerization and also in maintaining proteins in an unfolded state, thus facilitating membrane transport. The Escherichia coli hsp70 analogue, DnaK, and two other heat-shock proteins, DnaJ and GrpE, are required for cell viability at high temperatures and are involved in DNA replication of phage lambda and plasmids $P 1$ and $F$. These three proteins are involved in replication in vitro of P1 DNA along with many host replication proteins and the P1 RepA initiator protein. RepA exists in a stable protein complex with DnaJ containing a dimer each of RepA and DnaJ. We report 
here that DnaK and DnaJ mediate an alteration in the $\mathrm{P} 1$ initiator protein, rendering it much more active for oriP1 DNA binding.

\section{3,534}

PB95-151833 Not available NTIS

Nationa! Inst. of Standards and Technology (NML),

Gaithersburg, MD. Organic Analytical Research Div.

Potentiometric Enzyme-Amplified Flow Injection Analysis Detection System: Behavior of Free and Liposome-Released Peroxidase.

Final rept.

T. G. Wu, J. M. Bellama, and R. A. Durst. 1989, 18p.

Pub. in Analytical Letters 22, n5 p1107-1124 1989.

Keywords: "Flow injection analysis, "Potentiometric analysis, "Liposomes, "Peroxidases, "Enzymes, Electrodes, Immunochemistry, Reprints, Triton X-100.

A flow injection analysis (FIA) system was designed for the determination of free and liposome-released peroxidase activity. The inclusion of an extra washing channel in the FIA system provided a solution to problems of electrode fouling and slow recovery of response. The addition of Triton X-100 to acetate buffer resulted in an effective and reliable wash solution, and improved the electrode recovery rate by a factor of three. The sensitivity also increases by about $20 \%$ for the enzyme with $3 \mathrm{mU}$ activity by using the wash solution containing Triton $X-100$. Based on 16 replicate measures of the solution containing $3 \mathrm{mU}$ enzyme, the relative standard deviation of the enzyme determination was $1.5 \%$. Based on the sensitivity obtained with this prototype FIA system, it should be possible to adapt this approach to an immunochemical-based flow injection analysis system capable of measuring an analyte at the femtomole level.

\section{3,535}

PB95-153425 Not available NTIS

National Inst. of Standards and Technology (CSTL), Gaithersburg, MD. Biotechnology Div.

Substrate Specificity of the Escherichia coli Endonuclease III: Exicision of Thymine- and Cytosine-Derived Lesions in DNA Produced by Radiation-Generated Free Radicals.

Final rept.

M. Dizdaroglu, J. Laval, and S. Boiteux. 1993, 7p

Keywords: "Substrate specificity, "Escherichia coli, "Deoxyribonucleic acids, "Thymine, "Cytosine,
*Ionizing radiation, Free radicals, Mass fragmentography, DNA damage, DNA repair, Reprints, Endonuclease III.

The excision of modified bases from DNA by Escherichia coli endonuclease III was investigated. Modified bases were produced in DNA by exposure of dilute buffered solutions of DNA to ionizing radiation under oxic or anoxic conditions. The technique of gas chromatography/mass spectrometry (GC/MS) was used to identify and quantify 16 pyrimidine- and purinederived DNA lesions. DNA substrates were incubated either with the native enzyme or with the heat-inactivated enzyme. Subsequently, DNA was precipitated Pellets were analyzed by GC/MS after hydrolysis and derivatization. Supernatant fractions were analyzed after derivatization without hydrolysis. The results pro vided unequivocal evidence for the excision by $E$. col endonuclease III of a number of thymine- and cytosinederived lesions from DNA. None of the purine-derived lesions was excised by endonuclease III. The present work extends the substrate specificity of $E$ coli endonuclease III to another thymine-derived and fou cytosine-derived lesions. It is the first investigation of the substrate specificity of this repair enzyme in the context of a large number of pyrimidine- and purinederived lesions in DNA

\section{3,536}

PB95-163036 Not available NTIS

National Inst. of Standards and Technology (CSTL), Gaithersburg, MD. Biotechnology Div.

Electrophoretic Separations of Polymerase Chain Reaction: Amplified DNA Fragments in DNA Typing Using a Capillary Electrophoresis-Lased Induced Fluorescence System.

Final rept.

K. Srinivasan. 1993, 9p.

Sponsored by National Inst. of Justice, Washington,

Pub. in Jnl. of Chromatography A 652, p83-91 1993.

Keywords: "Polymerase chain reaction,

"Deoxyribonucleic acids, "Fluorescence spectroscopy,
Lasers, Ultraviolet spectroscopy, Apolipoproteins B Alleles, Viral DNA, Bacteriophages, Reprints, ${ }^{\star}$ Capillary electrophoresis.

Analysis of polymerase chain reaction (PCR)-amplified DNA fragments for human identification requires highresolution separation and efficient detection of amplified alleles. Capillary electrophoresis (CE) with its speed, automation, high resolution and efficiency shows promise for analyzing the amplified DNA fragments CE with UV detection, however, suffers from lack of detector sensitivity owing to the limited detection path length of the capillary. By the use o intercalating dyes (TOTO and YOYO) a laser-induced fluorescence (LIF) detection system can provide much greater sensitivity for detecting DNA fragments. Femtogram quantities of dsDNA (Phi X174 Haelll restriction digest mixture) per nanoliter of injected volume have been detected. Application of CE-LIF to analysis of PCR-amplified DNA fragments from three different genetic loci (apolipoprotein B, VNTR locus D1S80, mitochondrial DNA) is shown here. Further, the resolving power of a polymer-network capillary separation system is compared to that of a capillarygel separation system.

03,537

PB95-163291 Not available NTIS

National Inst. of Standards and Technology (NML) Gaithersburg, MD. Chemical Process Metrology Div.

Electropermeabilization of Cell Membranes: Effect of the Resting Membrane Potential.

Final rept.

E. Tekle, R. D. Astumian, and P. B. Chock. 1990, 6p Pub. in Biochemical and Biophysical Research Com munications 172, n1 p282-287 1990

Keywords: "Cell membrane, "Membrane potential, Electric fields, 3T3 cells, Fluorescent dyes, Plants(Botany), Electroporation, Erythrocyte membrane, Reprints.

Electric field induced permeabilization of cell membranes is a potentially important technique for biomedical research. Its application includes gene transfection and cell hybridization. To study the mechanism of this process, Mehrle et al. monitored the uptake of fluorescent indicator by plant protoplasts (Avena Mesophyll) and showed the permeabilization occurs predominantly on the hemisphere facing the positive electrode. However, Dimitrov and Sowers using erythrocyte ghosts loaded with fluorescent-labeled dextran demonstrated that the probes exit through the hemisphere facing the negative electrode. They proposed that electopores were symmetrically formed and electroosmosis is the dominant mechanism for molecular exchange in electroporation. To resolve these 'apparent' controversial observations, we conducted a systematic study of electroporation of NIH3T3 cells with varying electric field strength, waveform and frequency. Results indicate the electropermeabilization of cell membranes is affected by its resting potential and electroosmosis is not the dominant mechanism for the cellular uptake of foreign molecules in electroporation.

\section{3,538}

PB95-163382 Not available NTIS

National Inst. of Standards and Technology (NML) Boulder, CO Chemical Engineering Science Div.

Gravity Dependent Processes and Intracellular Motion.

Final rept.

P. Todd. 1991, 5p.

Contract NASA-W-16985

Sponsored by National Aeronautics and Space Administration, Washington, DC

Pub. in ASGSB Bulletin 4, n2 p35-39 Jul 91

Keywords: "Gravity, "Cells(Biology), "Cell movement, Cytoskeleton, Acceleration, Energy metabolism, Reprints.

The application of simple (and complicated) physical principles to the action of gravitational acceleration inside the cell depends heavily on our understanding of the physical properties of the cell interior. While substantial progress has been made in the last decade on understanding cytoskeletal structure and bioenergetics, the dynamics of intracellular and intercellular forces requires considerable further study.

03,539

PB95-163911 Not available NTIS

National Inst. of Standards and Technology (NML), Gaithersburg, MD. Center for Chemical Technology.
Deletion Analysis of the Mini-P1 Plasmid Origin of Replication and the Role of E.coli DnaA Protein. Final rept.

S. Wickner, J. Hoskins, D. Chattoraj, and K

McKenney. 1990, 6p

Pub. in Jnl. of Biological Chemistry 265, n20 p162216271990

Keywords: "Plasmids, "Origin of replication, "Sequence deletion, "Escherichia coli, Binding sites, In vivo analysis, Adenine nucleatides, Deoxyribonucleic acids, Bacteriophages, Reprints, "DnaA proteins.

The mini-PI plasmid origin of replication is contained on a 246 base pair (bp) piece of DNA. At one end there are five $19 \mathrm{bp}$ binding sites for the $\mathrm{Pl}$ initiator protein RepA and near the other end there are two 9 bp DnaA protein binding sites. To further define the limits of the origin we cloned the origin region in $\mathrm{M} 13$ and constructed deletions of either end. We sequenced the DNA and tested the RF 1 DNA of the deletion phages for their ability to support RepA-dependent DNA replication in an in vitro system. The origin that is functional in vitro could be reduced to $202 \mathrm{bp}$. It includes three intact and one incomplete RepA binding sites at one end and the two DnaA binding sites at the other end. When the two naturally occurring DnaA binding sites were replaced with one or two synthetic sites, only the construction containing two sites was active in vitro. We found that the minimal origin that is functional in vivo contains all of the five RepA and the two DnaA binding sites. Mini-PI plasmid replication both in vivo and in vitro requires two initiator proteins, the Escherichia coli DnaA protein and the PI RepA protein We have found that the ADP form of DnaA is as active as the ATP form of the protein in the in vitro replication of mini-PI. In contrast, only the ATP form is active for in vitro replication of plasmids carrying the $E$. coli origin.

03,540

PB95-175394 Not available NTIS

National Inst. of Standards and Technology (CSTL) Gaithersburg. MD. Biotechnology Div.

Chemical Determination of Oxidative DNA Damage by Gas Chromatography-Mass Spectrometry.

Final rept.

M. M. Dizdaroglu. 1994, 15p.

Sponsored by Department of Energy, Washington, DC Pub. in Methods in Enzymology 234, p3-17 1994.

Keywords: "DNA damage, "Mass fragmentography, Nucleosides, Oxidation, Nuclear proteins, Crosslinking(Chemistry), Free radicals, Isotopes, Reprints.

Measurement of oxidative DNA damage by gas chromatography-mass spectrometry (GC/MS) is described. The use of GC/MS with selected-ion monitoring facilities unequivocal identification and quantification of a large number of modified bases, nucleosides and DNA-protein cross-links that are produced in DNA or nucleoprotein by free radicals or by other DNA-damaging agents. Recently available stable isotope-labeled compounds permit precise quantification at low analyte concentrations. Methodologies involved are presented and discussed in detail.

03,541

PB95-175899 Not available NTIS

National Inst. of Standards and Technology (CSTL), Gaithersburg, MD. Biotechnology Div.

Optical Biosensor Using a Fluorescent, Swelling Sensing Element.

Final rept.

M. F. McCurley. 1994, 7p

Pub. in Biosensors and Bioelectronics 9, p527-533 1994.

Keywords: "Fluorescence, "Gels, "Polymers, *Fluorometry, Biotechnology, Fiber optics, Swelling, Enzymes, Crosslinking, Amines, Glucose oxidase, Reprints, "Optical biosensors.

An optical sensor based on coupling the swelling of a polymer gel to a change in fluorescence intensity is discussed. A fluorophore, an amine functional group and the enzyme glucose oxidase were each incorporated into a crosslinked polymer gel, which was formed on the end of a fiber optic rod While the amount of fluorophore remains constant, the gel volume changes in response to a change in the ionization state of the amine moiety. This change is related to glucose concentration. These effects were examined along with subsequent changes in the fluorescence of the gel. 
National Inst. of Standards and Technology (CSTL), Gaithersburg, MD. Surface and Microanalysis Science Div.

Interlaboratory Comparison of Autoradiographic DNA Profiling Measurements. 1. Data and Summary Statistics.

Final rept.

J. L. Mudd, F. S. Baechtel, D. L. Duewer, S. D.

Leigh, H. K. Liu, L. A. Currie, and D. J. Reeder.

1994, 15p.

Pub. in Analytical Chemistry 66, n20 p3303-3317, 15 Oct 94.

Keywords: *Deoxyribonucleic acids, *Autoradiography, *Interlaboratory comparisons, Reference standards, Restriction fragment length polymorphism, Base composition, Reproducibilints.

The data obtained during an interlaboratory precision study sponsored by the Technical Working Group of DNA Analysis Methods are summarized. These data are representative of DNA restriction fragment length polymorphism (RFLP) autoradiographic measurements using laboratory protocols based upon procedures validated by the Federal Bureau of Investigation. Measurement reproducibility for a given RFLP fragment is a nonlinear function of the fragment's size, with the logarithm of the sample standard deviation (SD) approximately proportional to fragment size. The leastsquares estimate of this relationship for fragments composed of 600 to 11,000 DNA base pairs (bp) is SD $=10.4 \times 10(\mathrm{sup}(\mathrm{bp} / 8300))$. Among-analyst and among-laboratory differences appear to contribute Autoradiographic imaging is a relatively small component of the overall variance for fragments $<$ or $=10,000$ bp. Currently available data are sufficient to establish reliable tolerances for the expected interlaboratory variation for any given RFLP band size in this size range. assuming that relevant laboratories continue to use RFLP analysis protocols that are within the current 'statistical population' (i.e., protocols similar to those used by the laboratories that participated in the interlaboratory study). This consistency may best be maintained through appropriate use of Standard Reference Materials, internal quality assurance programs, and external proficiency demonstrations.

03,543

PB96-110747 Not available NTIS

National Inst. of Standards and Technology (CSTL), Gaithersburg, MD. Biotechnology Div.

Novel Activity of E. coli uracil DNA N-glycosylase Excision of Isodialuric Acid (5,6-dihydroxyuracil), a Major Product of Oxidative DNA Damage, from DNA.

Final rept.

T. Zastawny, P. Doetsch, and M. Dizdaroglu. 1995,

Sponsored by Department of Energy, Washington, DC Pub. in FEBS Letters 364, p255-258 1995.

Keywords: "Carcinogenesis, "E coli, "DNA, Enzymes, DNA repair, Hydroxyl radicals, Isodialuric acid, Oxidation, Damage, Uracil glycosylase, Reprints, E. coli uracil, Isodialuric acid, N-glycosylase.

The authors describe a novel activity of $E$. coli uracil DNA N-glycosylase (UNG) that excises isodialuric acid from DNA. Isodialuric acid is formed in DNA as a major oxidative product of cytosine. DNA substrates, which were prepared by gamma-irradiation, were incubated with UNG. Following precipitation of DNA analyses of pellets and supernatant fractions by gas chromatography/mass spectrometry showed an efficient excision of isodialuric acid from DNA by UNG. None of the other 15 identified DNA base lesions was excised. The excision of isodialuric acid indicates that the nonaromaticity of a substrate may not be a limiting factor for UNG.

03,544

PB96-112081 Not available NTIS

National Inst. of Standards and Technology (CSTL),

Gaithersburg, MD. Biotechnology Div.

Rapid pH Change Due to Bacteriorhodopsin Measured with a Tin-Oxide Electrode.

Final rept.

B. Robertson, and E. P. Lukashev. 1995, 11p.

Pub. in Biophysical Jnl., v68 p1507-1517 Apr 95.

Keywords: *Bacteriorhodopsin, "Electrodes, Measurements, pH, Photosynthetic bacteria, Sensitivity, Membranes, Reprints, Tin oxide electrodes.

The photocurrent transient generated by bacteriorhodopsin $(\mathrm{bR})$ on a tin-oxide electrode is due to $\mathrm{pH}$ change and not to charge displacement as previously assumed. Films of either rendomly oriented of highly oriented purple membranes were deposited on transparent electrodes made of tin-oxide-coated glass. The membranes contained either wild-type or D96N mutant bR. When excited with yellow light through the glass, the bR pumps protons across the menbrane. The results is a rapid local $\mathrm{pH}$ change as well as a charge displacement. Experiments with these films show that it is the $\mathrm{pH}$ change rather than the displacement that produces the current transient. The calibration for the transient $\mathrm{pH}$ measurement is given. The sensitivity of a tin-oxide electrode to a transient pH change is very much larger than its sensitivity to a steady-state $\mathrm{pH}$ change.

\section{3,545}

PB96-112123 Not available NTIS

National Inst. of Standards and Technology (CSTL), Gaithersburg, MD. Analytical Chemistry Div.

Interlaboratory Comparison of Autoradiographic DNA Profiling Measurements. 2. Measurement Uncertainty and Its Propagation.

Final rept.

D. L. Duewer, L. A. Currie, D. J. Reeder, J. L. Mudd, S. D. Leigh, and H. K. Liu. 1995, $12 p$.

See also Part 1, PB95-175923.

Pub. in Analytical Chemistry, v67 n7 p1220-1231 1995.

Keywords: *Deoxyribonucleic acids, "Autoradiography, Interlaboratory comparisons, Reprints, Reference standards, Base composition, Measurement, "Band size.

Identifying the intrinsic sources of measurement uncertainty greatly facilitates control and further optimization of a measurement system. We have developed a model which quantitatively describes the observed interlaboratory variability autoradiographic DNA band sizing. The model focuses on optical imaging measurements of band position and the calibration techniques used to convert measured band position to reported band size. The imaging components of measurement variability is described as a $0.05-02 . \%$ standard deviation in determining the relative location of sample and calibration bands on a given film image. While developed solely with optical imaging information, the mode is consistent with interlaboratory band sizing measurement variability observed with pristine samples. This interlaboratory variability can be modeled as a 0.2 $0.4 \%$ standard deviation in the relative positions of sample and calibration bands across differen electrophoretic gels. Further band sizing protocol standardization among laboratories would thus be expected to achieve at best a 2 -fold reduction in interlaboratory.

\section{3,546}

PB96-123500 Not available NTIS

National Inst of Standards and Technology (CSTL) Gaithersburg, MD. Biotechnology Div.

Optical Properties of Triton X-100-Treated Purple Membranes Embedded in Gelatin Films.

Final rept.

D. W. Cullin, N. N. Vsevolodov, T. V. Dyukova, and

H. H. Weetall. 1995, $8 \mathrm{p}$.

See also PB96-119284.

Pub. in Supramolecular Science, v2 p25-32 1995.

Keywords: "Purple membranes, "Holography, *Geletin films, Optical properties, Reprints, "Bacteriorhodopsin. The purple membrane (PM) of the microorganism Halobacterium salinarium contains a hexagonally packed monolayer of the light-sensitive protein bacteriorhodopsin (BR). The optical characteristics of gelatin-immobilized PMs depend strongly on the chemcal environment of the PMs in the matrix. Here we present photoinduced absorptive and holographic characteristics of gelatin-embedded PMs solubilized with the non-ionic detergent, Triton X-100. The BR/detergent interaction was shown to slow the M-to-initial state transition of the photocycle and to increase the photosensitivity of the BR films. The lifetime of the holographic grating in Triton X-100-treated BR films was 2-3 times greater, when compared to the unmodified sample. Holographic grating growth times in BR films were shown to change depending on the extent of solubilization. The measured holographic sensitivity appeared to maximize in the range of Triton X-100/BR molar ratios from $15: 1$ to $25: 1$. The possible advanages of solubilized PM films as they are applied to optoelectronic devices are discussed.

03,547
PB96-135041 Not available NTIS
National Inst. of Standards and Technology (CSTL), Gaithersburg, MD. Biotechnology Div.

In situ Fluorescence Cell Mass Measurements of 'Saccharomyces cerevisiae' Using Cellular Tryptophan.

Jept

Harvath, S. A. Glazier, and C. J. Spangler.

1993, 5p.

Pub. in Biotechnology Progress, Nov/Dec Issue, v9 p666-670 1993

Keywords: "Spectroscopy, "Fluorescence, "Bioreactors, In situ, Sensors, Fermentation, Reprints, Cell mass.

This work describes a new spectroscopic optical fiber/ rod technique for in situ real time measurement of cel mass and product concentrations in bioreactors using intrinsic fluorescence. The variable excitation/emission wavelength capability of this sensor allows for the spe cies-selective measurement during fermentations. Cell mass (tryptophan) and product concentrations (pyridoxine) have been measured during fermentations of Saccharomyces cerevisiae. The effects of varying substrate concentration and oxygen concentration on the observed cell mass signals are eliminated by direct measurement of cell mass, as opposed to indirect measurement schemes such as those using NADH fluorescence. The sensor is robust and able to undergo many cycles of in situ steam sterilization without deg radation, and its fluorescence signal is linear with con centration for all species studied in this work. Trypto phan fluorescence from yeast is shown to be a better measure of cell mass than NADH fluorescence.

\section{3,548}

PB96-137799 Not available NTIS

National Inst. of Standards and Technology (MSEL) Gaithersburg, MD. Reactor Radiation Div.

Low-Frequency Excitations of Oriented DNA

Final rept

H. Grimm, P. M. Gehring, S. M. Shapiro, R. Kahn,

and A. Rupprecht. 1995,3p.

See also DE95000629.

Pub. in Physica B Condensed Matter, v213-214 p780 7821995

Keywords: "DNA, "Excitations, Thermal neutrons, Thermodynamic properties, Neutron scattering, Reprints, Proton self-correlations, Time-of-flight.

The self-correlation spectrum of DNA hydrogens has been measured by thermal neutron scattering for two orientations of the helical axis in the temperature range $200 \mathrm{~K}$ equal to or less than T equal to or less than 300 K. Typical spectra for glass formers are observed which show that DNA hydrogens become increasingly delocalized above T equals $200 \mathrm{~K}$. This self-correlation s essentially isotropic. Anisotropic contributions to the scattering are identified as being due too compressional waves along the helix and the low-lying optical band observed in Raman scattering. Both modes show strong coupling to relaxational modes at the zone center (10th layer line). It is argued that the observability of the optical band at this position is due to an antiparallel displacement of the base-pair centers along the helix direction.

03,549

PB96-160478 Not available NTIS

National Inst. of Standards and Technology (CSTL) Gaithersburg. MD. Biotechnology Div

Novel DNA N-Glycosylase Activity of E. coli T Endonuclease $V$ That Excises 4,6-Diamino-5 Formamidopyrimidine from DNA, a UV-Radiationand Hydroxyl Radical-Induced Product of Adenine. Final rept

M. Dizdaroglu, T. H. Zastawny, J. R. Carmical, and R. S. Lloyd. $1996,8 \mathrm{p}$

Pub. in Mutation Research DNA Repair, v362 p1-8 1996

Keywords: "Deoxyribonucleic acids, "Mutagenesis, Reprints, *Foreign technology, UV-radiation, Hydroxy radical.

We report on a novel activity of $\mathrm{T} 4$ endonuclease $\mathrm{V}$. This enzyme is well known to be specific for the excision of pyrimidine dimers from UV-irradiated DNA. In this work, we show that T4 endonuclease $V$ excise 4.6-diamino-5-for-mamidopyrimidine from DNA. 4,6Diamino-5-formamidopyrimidine is formed as a product of adenine in DNA upon action of hydroxyl radicals and upon UV-irradiation. DNA substrates were prepared by UV-or y-irradiation of DNA in aqueous solu tion. DNA substrates were incubated either with active 
T4 endonuclease $V$ or with heat-inactivated T4 endonuclease $V$ or without the enzyme. After incubation, DNA was precipitated and supernatant fractions were separated. Supernatant fractions after derivatization, and pellets after hydreolysis and derivatization were analyzed by gas chromatography. isotopedilution mass spectrometry. The results provide evidence for the excision of 4.6-diamino-5formamidopyrimidine by T4 endonuclease $V$ from both $y$-annd UV-irradiated DNA. Kinetics of excision were also determined. Fifteen other pyrimidine-and purinederived base lesions that were identified in DNA samples were not substrates for this enzyme. It was concluded that, in addition to its well known activity for py rimidine photodimers, T4 endonuclease $V$ possesses an $\mathrm{N}$-glycosylase activity for a major UV-radiation- and hydroxyl radical-induced monomeric product in DNA

\section{3,550}

PB96-161641 Not available NTIS

National Inst. of Standards and Technology (CSTL), Gaithersburg, MD. Biotechnology Div.

Genetically Engineered Pores for New Materials.

Final rept.

H. Bayley, S. Beznukhov, J. J. Kasianowicz, M.

Krishnasastry, and B. Walker. 1992, 1p.

See also PB96-161658

Pub. in Materials Research Society (Abstracts), Boston, MA., Nov/Dec 92, p568 Fall 92.

Keywords: "Exotoxins, "Hemolysis, "Ion channels, Reprints, Dynamics, Ion transport, Proteins, Protons, Noise analysis, Staphylococcus aureus.

Alpha-Hemolysin (alphaHL), which is secreted by Staphylococcus aureus as a single polypeptide chain of 293 amino acids, is capable of self-assembling into hexameric pores $-1.1 \mathrm{~nm}$ in internal diameter. Based on an understanding of the assembly mechanism and the functional properties of the pore, genetic manipulation and chemical modification will be used to construct new pores with potential applications in novel materials, such as monolayer coatings for selective electrodes. Details of the mechanism of self-assembly are being revealed by the isolation of mutant of recom binant alphaHL that become arrested at various stages in assembly, alphaHL first binds to membranes as a monomer, which undergoes a conformational change. It is then converted to a non-conducting hexamer and finally to the open pore.

\section{3,551}

PB96-161658 Not available NTIS

National Inst. of Standards and Technology (CSTL) Gaithersburg, MD. Biotechnology Div.

Genetically Engineered Pore as a Metal Ion Biosensor.

Final rept.

J. J. Kasianowicz, B. Walker, M. Krishnasastry, J.

Aghajanian, and H. Bayley. 1993, 1p.

See also PB96-161641.

Pub. in Materials Research Society (Abstracts), Boston, MA., Nov/Dec 1993, p480 Fall 93.

Keywords: "Biosensors, "Divalent cations, "Heavy metals, Reprints, Ion channels, Proteins, Toxins.

The authors are investigating the potential use of proteins that form pores in cell membranes as biosensors for heavy metals in the aqueous phase. Specifically the authors are producing genetically engineered versions of the alpha-hemolysin (alphaHL) from Staphlococcus aureus with properties that are sensitive to low concentrations of divalent cations. For example, in contrast to wild-type alphaHL, the assembly of one mutant (alphaHL-H5: residues 130-134 inclusive replaced with His) into pores was inhibited by $\mathrm{Zn}$ squared concentrations as low as 1.0 micro $M$ as judged by its ability to lyse rabbit RCBs and to increase the conductance of planar lipid bilayer membranes. Previously, the authors demonstrated two intermediates in the assembly of alphaHL a membrane-bound monomer and a hexameric prepore complex. Zn squared can act pn the alpha HL-H5 prepore complex to inhibit pore formation.

\section{3,552}

PB96-167093 Not available NTIS

National Inst. of Standards and Technology (CSTL), Boulder, CO. Thermophysics Div.

Determination of Total Protein Adsorbed on Solid (Membrane) Surface by a Hydrolysis Technique: Single Protein Adsorption.

Final rept.

M. K. Ko, K. D. Cole, and J. Pellegrino. 1994, 10 p.

Pub. in Jnl. of Membrane Science, v93 p21-30 1994
Keywords: "Adsorption, “Fouling organisms, "Proteins, Analytical methods, Reprints, Lactoglobulin, Hydrolysis, Polycarbonate, Ultrafiltration, "Biofouling, Bovine serum albumin

We developed a new analytical technique for single protein adsorption in the context of basic studies on membrane fouling. This method simply comoines two well-established techniques. $\mathrm{HCl}$ hydrolysis and reaction of the resultant amino acids with ninhydrin (10 to the minus 6 th power $\mathrm{M}$ sensitivity). The $\mathrm{HCl}$ hydrolysis step, using $6 \mathrm{~N} \mathrm{HCl}$ solution, breaks up the adsorbed protein into its constituent amino acids by hydrolyzing the peptide bonds, resulting in complete removal of the absorbed protein from the surface. After the $\mathrm{HCl}$ hydrolysis, the condition of the hydrolysate is adjusted for the ninhydrin derivatization by mixing with $6 \mathrm{~N} \mathrm{NaOH}$ and $6 \mathrm{~m}$ sodium acetate. The optimal $\mathrm{pH}$ for the ninhydrin derivatization reaction of the hydrolysate is approx. 6. This technique uses an unmodified protein and determines protein loading on the membrane's surface (external and/or pore wall), under static or filtration environments, in a consistent manner. This method provides an absolute measurement of the total protein adsorbed and holds good promise for high sensitivity, accuracy, and reproducibility at a relatively low cost Other amino acid analytical techniques (such as flourescence and HPLC) may be used to eliminate artifacts, improve sensitivity, and extend the aproach to multi-protein adsorption.

\section{3,553}

\section{PB96-167408 Not available NTIS}

National Inst. of Standards and Technology (CSTL), Gaithersburg, MD. Biotechnology Div.

Genetically Engineered Pores as Metal Ion Biosensors

Final rept

J. Kasianowicz, B. Walker, M. Krishnasastry, and H.

Bayley. 1994, 7p.

See also PB96-161641

Pub. in Materials Research Society Symposium Proceedings, v330 p217-223 1994 .

Keywords: "Hemolysin, "Dynamic range, Longevity, Response time, Selectivity, Sensitivity, Reprints, Staphylococcus aureus.

We are adapting proteins that form pores in lipid bilayers for use as components of biosensors. Specifically, we have produced genetically engineered variants of the alpha-hemolysin (alpha $\mathrm{HL}$ ) from Staphylococcus aureus with properties that are sensitive to low concentrations of divalent cations. For example, the pore-forming activity of one mutant (alpha HL-H5: residues $130-134$ inclusive replaced with histidine) is inhibited by $\mathrm{Zn2}+$ at concentrations as low as micromoles, as judged by the reduction in its ability to lyse rabbit red blood cells and to increase the conductance of planar lipid bilayer membranes. When alpha $\mathrm{HL}-\mathrm{H} 5$ is added to the aqueous phase bathing one side of a planar membrane, the subsequent addition of 100 micromoles $\mathrm{Zn} 2+$ to either side blocks the pores that form. This result suggests that at least part of the mutated region lines the channel lumen. $\mathrm{Ca} 2+$ and $\mathrm{Mg} 2+$ do not block the channel and therefore the $\mathrm{H} 5$ mutation confers a degree of analyte specificity to the alpha $\mathrm{HL}$ pore.

\section{3,554}

PB96-176557 Not available NTIS

National Inst. of Standards and Technology (CSTL), Gaithersburg, MD. Biotechnology Div.

Pore-Forming Protein with a Metal-Actuated Switch.

Final rept.

B. Walker, J. Kasianowicz, M. Krishnasastry, and $\mathrm{H}$. Bayley. 1994, 8p.

Pub. in Protein Engineering, v7 n5 p655-662 1992.

Keywords: "Histidine, "Mutagenesis, Pores, Sensors, Zinc, Reprints, Divalent metal ion.

Staphylococcal alpha-hemolysin, a pore-forming exotoxin, is a poplypeptide of 293 amino acids that is secreted by Staphylococcus aureus as a water-soluble monomer. It assembles to form hexameric pores in lipid bilayers. Previous studies of pore formation have established the involvement of a central glycine-rich loop. Here, we show that when five consecutive histidine residues replace amino acids 130-134 at the midpoint of the loop they provide a switch with which pore activity can be (1) turned off by micromolar concentrations of divalent zinc ions and (2) turned back on with the chelating agent EDTA.
03,555

PB96-190129 Not available NTIS

National Inst. of Standards and Technology (CSTL) Gaithersburg, MD. Biotechnology Div.

Repair of Products of Oxidative DNA Base Damage in Human Cells.

Final rept.

P. Jaruga, and M. Dizdaroglu. 1996, 6p.

Pub. in Nucleic Acids Research, v24 n8 p1389-1394 1996.

Keywords: "Deoxyribonucleic acid, "Damage, "Oxidation, "Cells(Biology), Humans, Hydroxyl radical, Mutagenesis, Kinetics, Hydrogen peroxide, Reprints, DNA repair, Repair kinetics, 8-Hydroxyguanine.

Oxidative DNA damage is the most frequent type of damage encountered by aerobic cells and may play an important role in biological processes such as mutagenesis, carcinogenesis and aging in humans. Oxidative damage generates a myriad of modifications in DNA. The authors investigated the cellular repair of DNA base damage products in DNA of cultured human ymphoblast cells, which were exposed to oxidative stress by by $\mathrm{H}_{2} \mathrm{O} 2$. This DNA-damaging agent is known to cause base modifications in genomic DNA of mammalian cells. The results showed a significan formation of these DNA base products upon $\mathrm{H} 2 \mathrm{O} 2$ treatment of cells.

03,556

PB97-111983 Not available NTIS

National Inst. of Standards and Technology (CSTL) Gaithersburg, MD. Biotechnology Div.

DNA Damage and DNA Sequence Retrieval from Ancient Tissues.

Final rept.

M. Hoss, P. Jaruga, T. H. Zastawny, M. Dizdaroglu, and S. Paabo. 1996, 4p.

Pub. in Nucleic Acids Research, v24 n7 p304-307 1996

Keywords: "DNA damage, Bones, Tissues, Archeological remains, Hydroxyl radical, Reprints, "Foreign technology, 8-hydroxyguanine.

Gas chromatography/mass spectrometry (GC/MS) was used to determine the amounts of eight oxidative base modifications in DNA extracted from 11 specimens of bones and soft tissues, ranging in age from 40 to 50,000 years. Among the compounds assayed hydantoin derivatives of pyrimidines were quantitatively dominant. From five of the specimens endogenous ancient DNA sequences could be amplified by PCR. The DNA from these specimens contained substantially lower amounts of hydantoins than the six specimens from which no DNA could be amplified. Other types of damage, e.g., oxidation products of purines, did not correlate with the inability to retrieve DNA sequences. Furthermore, all samples with low amounts of damage and from which DNA could be amplified stemmed from regions where low temperatures have prevailed throughout the burial period of the specimens.

\section{3,557}

PB97-119382 Not available NTIS

National Inst. of Standards and Technology (CSTL), Gaithersburg, MD. Biotechnology Div.

DnaJ, DnaK, and GrpE Heat Shock Proteins are Required in 'ori'P1 DNA Replication Solely at the RepA Monomerization Step.

Final rept.

S. Wickner, D. Skowyra, J. Hoskins, and K.

McKenney. 1992, 5p

Pub. in Proc. Natl. Acad. Sci., v89 p10345-10349 Nov 92.

Keywords: "Heat shock proteins, *Deoxyribonucleic acids, "Replication, "Monomerization, DNA Escherichia coli, Cell membrane, Biological transport, Reprints.

The authors have found that three Escherichia coli heat shock proteins, DnaK (the hsp 70 homolog) DnaJ, and GrpE, function in oriP1 DNA replication in vitro solely to activate DNA binding by the replication initiator protein RepA. Activation results from the conversion of $\mathrm{P} 1$ or P7 RepA dimers to monomers that bind with high affinity to the origin of replication of plasmid $P 1$. Thus, the essential role of these three hea shock proteins in this replication system is to change the quaternary structure of a single protein, RepA 


\section{Dentistry}

03,558

PB94-160843 PC A06/MF AO

National Inst. of Standards and Technology (MSEL), Gaithersburg, MD. Polymers Div.

Properties and interactions of Oral Structures and Restorative Materials. Annual Report for Period October 1, 1990 to September 30, 1991.

J. A. Tesk, J. M. Antonucci, J. W. Stansbury, Y Matsuya, H. Kikuchi, K. Asaoka, J. Tang, S. M Keeny, and M. Y. Chiang. May 92, 125p, NISTIR4841 .

Contract NIDR-Y01-DE30001

See also PB93-198836. Sponsored by National Inst. of Dental Research, Bethesda, MD.

Keywords: "Dental materials, "Permanent dental restoration. Biocompatible materials, Composite materials, Cements(Adhesives), Adhesives, Alloys, Fluorescent dyes, Sealants, Chemical analysis.

The research described herein is designed to achieve a number of objectives leading to improved dental restorative materials, techniques and applications of dental materials science for improved delivery of health care. The bulk of the research is related in one manner or another to dental composites, cements, adhesives, and sealants. Composite research focuses on improvements through the development of durable resin matrices, stronger, durable coupling between fillers and resins (defining the best overall combination of components) and optimal curing systems. A major emphasis of the programs is on the synthesis and applications of monomers which reduce polymerization shrinkage through the use of expanding monomers (or monomers which undergo much less shrinkage than conventional monomers). A second distinct area of effort focuses on dental alloy and ceramic systems. Weibull statistics and finite element stress analysis are employed for the determination of the strengths of $c e-$ ramic and ceramic-metal systems as affected by processing parameters and thermo-mechanical properties.

03,559

PB $95-150710 \quad$ Not available NTIS

National Inst. of Standards and Technology (MSEL), Gaithersburg, MD. Polymers Div.

Deposition of Loosely Bound and Firmly Bound Fluorides on Tooth Enamel by an Acidic Gel Containing Fluorosilicate and Monocalcium Phosphate Monohydrate.

Final rept.

S. Takagi, L. C. Chow, and B. A. Sieck. 1992, 7p

Sponsored by American Dental Association Health Foundation, Chicago, IL

Pub. in Caries Research 26, p321-327 1992.

Keywords: *Dental enamel, "Fluorides, "Mouthwashes, "Deposition, Enamel, Gels, Dental caries, Sodium fluorides, Tooth remineralization, In vivo analysis, Reprints, Phosphate fluoride, Calcium phosphate hydrate, Sodium hexafluorosilicate.

The amounts of loosely bound fluoride ( $F$ ) deposited on human enamel by 4-min and 2-hour treatments with either acidulated phosphate fluoride (APF) or a monocalcium phosphate monohydrate and sodium hexafluorosilicate (MCPM-SHFS)-containing gel were measured with the use of a constant-composition $F$ washing method. Enamel biopsies conducted before treatment and after washing were used to determine the firmly bound $\mathrm{F}$ uptake. The results showed that the MCPM-SHFS treatments produced significantly more loosely bound $F$ than did the APF treatments. The 4min treatment with either APF or MCPM-SHFS did not produce significant firmly bound $F$ deposition, but the 2-hour treatments did, with that produced by MCPMSHFS being significantly greater. The MCPM-SHFS gel, which had the same F content as APF and which may be applied to the proximal tooth surfaces in vivo without the use of a tray, has the potential to be more efficacious than APF because it deposits greater amounts of both loosely bound and firmly bound $F$.

\subsection{0}

PB95-150967 Not available NTIS

National Inst. of Standards and Technology (MSEL), Gaithersburg, MD. Polymers Div.

Influence of Natural and Synthetic Inhibitors on the Crystallization of Calcium Oxalate Hydrates.

Final rept.

B. B. Tomazic, M. E. Sheehan, and G. H. Nancollas 1992, 10p.

Sponsored by American Dental Association Health Foundation, Chicago, IL.
Pub. in World Jnl. Urol. 10, p216-225 1992.

Keywords: "Crystallization, "Reaction kinetics, *Dentistry, Polyacrylates, Molecular weight, Urine, Crystal growth, Crystal lattices, Ions, Heparins, Reprints, "Calcium oxalate hydrate, Polyaspartic acid, Polyglutamic acid

The kinetics of crystallization of calcium oxalate monohydrate was studied in the presence of polyacrylate, and the growth of both this salt and the trihydrate was investigated in the presence of polyaspartic acid, polyglutamic acid, and heparin. We monitored the crystallization reactions either by following the change in lattice-ion concentrations as a function of time or by maintaining constant supersaturation via the replacement of lattice ions during the experiments. In addition, the crystallization reactions of calcium oxalate monohydrate, dihydrate, and trihydrate were studied in the presence of urine fractions $(5 \%$ $\mathrm{v} / \mathrm{V}$ ) separated by molecular weight in the range of 1000-50000 Da from whole urine.

03,561

PB95-151205 Not available NTIS

National Inst. of Standards and Technology (MSEL), Gaithersburg, MD. Polymers Div.

Distribution of Fluoride in Saliva and Plaque Fluid After a $0.048 \mathrm{~mol} / \mathrm{L} \mathrm{NaF}$ Rinse.

Final rept.

G. L. Vogel, C. M. Carey, and J. Ekstrand. 1992, 5p. Sponsored by American Dental Association Health Foundation, Chicago, IL.

92.

Keywords: "Dental plaque, "Fluorides, "Saliva, "Microanalysis, Tooth, Sodium fluorides, Mouthwashes, Dental caries, Dental care, Reprints.

An ultramicro method has recently been described for measurement of plaque-fluid fluoride concentration. This method was used: (1) for exploration of the variation in fluoride concentration of plaque fluid collected from the same buccal tooth sites following a $0.048 \mathrm{~mol}$ $\mathrm{L} \mathrm{NaF}(0.2 \%)$ rinse, and (2) for examination of the distribution of fluoride in plaque fluid and saliva within on hour after this rinse. Results indicated an average coefficient of variation (CV) of $31 \%$ for plaque-fluid fluoride in triplicate samples recovered simultaneously from the buccal-proximal region of two teeth after the rinse. The variations suggest that an examination of plaque-fluid fluoride from specific tooth regions may be essential for understanding the effects of fluoride on the site-specificity of caries.

\section{3,562}

PB95-153052 Not available NTIS

National Inst. of Standards and Technology (MSEL) Gaithersburg, MD. Polymers Div.

Publication and Presentation Abstracts, 1993 (Published by Paffenbarger Research Center and Center of Excelience for Materials Science Research).

Final rept.

M. W. Chalkley, and W. A. Marjenhoff. 1993, 68p.

Sponsored by American Dental Association Health Foundation, Chicago, IL.

Pub. in Dental Materials Research Highlights, 68p Oct 93.

Keywords: "Dental materials, "Research projects, "Technology innovation, "Medical research, *Abstracts, Dental cements, Dental implantation, Biocompatible materials, Adhesives, Composite materials, Sealants, Temporary dental restoration, Permanent dental restoration, Dentistry, Reprints, Paffenbarger Research Center

The report is a compilation of abstracts published by Paffenbarger Research Center and Center of Exce lence for Materials Science Research staff in 1992

\section{3,563}

PB95-153169 Not available NTIS

National Inst. of Standards and Technology (MSEL), Gaithersburg, MD. Polymers Div.

Effects on Whole Saliva of Chewing Gums Containing Calcium Phosphates.

Final rept.

L. C. Chow, S. Takagi, R. J. Shern, B. A. Sieck, T.

H. Chow, and K. K. Takagi. 1994, 7p.

Sponsored by American Dental Association Health Foundation, Chicago, IL.

Pub. in Jnl. of Dental Research 73, n1 p26-32 Jan 94 Keywords: "Chewing gum, "Calcium phosphates, "Salivation, "Tooth remineralization, Additives, Weight,
$\mathrm{pH}$, Calcium, Phosphates, Reprints, Dicalcium phosphate anhydrous, Calcium phosphate hydrate Tetracalcium phosphate.

To evaluate chewing gums as a vehicle to increase salivary mineral saturation levels and enhance salivation, monocalcium phosphate monohydrate (MCPM) and an equimolar mixture of tetracalcium phosphate (TTCP) with dicalcium phosphate anhydrous (DCPA) were chosen as experimental chewing gum additives. The for weight, $\mathrm{pH}$, and total calcium ( $\mathrm{Ca}$ ) and phosphate for weight, $\mathrm{pH}$, and total calcium $(\mathrm{Ca})$ and phosphate
$(\mathrm{P})$ concentrations. The degree of saturation of tooth mineral was significantly increased by both experimental gums, with the greater increase being produced by the TTCP-DCPA gum. The MCPM gum produced a significantly greater saliva flow and a lower salivary $\mathrm{pH}$ than did the control and TTCP-DCPA gums. The results suggest that the experimental gums may be useful for promoting remineralization in general and for inducing salivation in xerostomic patients.

\section{3,564}

PB95-164448 Not available NTIS

National Inst. of Standards and Technology (MSEL), Gaithersburg, MD. Polymers Div.

Procedure for the Study of Acidic Calcium Phosphate Precursor Phases in Enamel Mineral Formation.

Final rept.

C. Siew, S. E. Gruninger, L. C. Chow, and W. E Brown. 1992, 5p

Sponsored by American Dental Association Health Foundation, Chicago, IL.

Pub. in Calcified Tissue International 50, p144-148 1992.

Keywords: "Dental enamel, "Acidification, "Minerals, Magnesium, Calcium phosphates, Fluorides, Biochemistry, Hydrolysis, In vitro analysis, In vivo analy sis, Reprints, "Octacalcium phosphate, Apatite/ hydroxy.

Considerable evidence suggests that an acidic calcium phosphate, such as octacalcium phosphate (OCP) or brushite, is involved as a precursor in enamel and other hard tissue formation. Additionally, there is in vitro evidence suggesting that fluoride accelerates and magnesium inhibits the hydrolysis of OCP to hydroxyapatite (OHAp). As the amount of OCP or brushite in enamel cannot be measured directly in the presence of an excess of hydroxyapatite, a procedure was developed that allows for their indirect in vivo quantification as pyrophosphate. This permits study of the effects of fluoride and magnesium ions on enamel mineral synthesis.

\section{3,565}

PB95-164505 Not available NTIS

National Inst. of Standards and Technology (MSEL), Gaithersburg, MD. Polymers Div.

Effects of Aluminum Oxalate/Glycine Pretreatment Solutions on Dentin Permeability.

Final rept

M. D. Simpson, J. A. Horner, P. D. Brewer, F.

Eichmiller, and D. H. Pashley. 1992, 5p.

Sponsored by American Dental Association Health Foundation, Chicago, IL.

Pub. in American Jnl. of Dentistry 5, n6 p324-328 Dec

Keywords: "Dentin permeability, "Glycine, "Solutions, In vitro analysis, $\mathrm{pH}$, Buffers, Reprints, "Aluminum oxalate, *Pretreatments, Smear layers.

Aluminum oxalate buffered with glycine to $\mathrm{pH} 0.5-2.5$ has been proposed as a dentin pretreatment for the Gluma bonding system. In this experiment, the effects of 1 -minute treatments of smear layers with these aluminum oxalates on the permeability of human dentin were determined in vitro. The aluminum oxalate solutions at $\mathrm{pH} 0.5 \cdot 1.5$ removed most of the original smear layer but occluded the tubules with crystalline deposits which decreased dentin permeability. Those solutions used at pH 2.0 and 2.5 increased dentin permeability. All dentin pretreatments increased dentin permeability when measured after a 24-hour storage period, especially the solutions at $\mathrm{pH} 2.0$ and 2.5 . The SEM correlates of these permeability changes indicated that these solutions remove the smear layer but reocclude the tubules with precipitates which are probably different forms of calcium oxalate, aluminum phosphate and calcium phosphates. 
National Inst. of Standards and Technology (MSEL), Gaithersburg, MD. Polymers Div.

Interaction of Chlorhexidine Digluconate with and Adsorption of Chlorhexidine on Hydroxyapatite.

Final rept.

D. N. Misra. 1994, 7p

Sponsored by American Dental Association Health Foundation, Chicago, IL.

Pub. in Jnl. of Biomedical Materials Research 28 p1375-1381 1994

Keywords: "Adsorption, "Hydroxyapatites, "Reaction kinetics, "Chlorhexidine, "Mouthwashes, "Dentistry, Dental cements, Aqueous solutions, Oral hygiene, Precipitation(Chemistry), Solubility, Reprints, "Chlorhexidine digluconate.

It is well known that chlorhexidine digluconate provides an effective microbicidal activity during oral rinsing, and therefore, it was considered worthwhile to inves tigate its interaction with hydroxyapatite on a fundamental level. The kinetics of uptake (or reaction) of the compound from aqueous solutions by synthetic hydroxyapatite was studied at $23 \mathrm{C}$ for four time periods by monitoring its concentration. All of the experimental facts can be qualitatively explained on the basis of the solubility considerations of hydroxyapatite and of chlorhexidine phosphate, the reaction product that slowly precipitates out of the solution. To explore the nature of interaction, the uptake of chlorhexidine base was studied from p-dioxane and it is irreversible. The uptake is total below a threshold equilibrium concentration and constant above it.

\section{3,567}

PB96-102140 Not available NTIS

National Inst. of Standards and Technology (MSEL), Gaithersburg, MD. Polymers Div.

Remineralization of Root Lesions with Concentrated Calcium and Phosphate Solutions.

Final rept.

L. C. Chow, and S. Takagi. 1995, 6p.

Sponsored by American Dental Association Health Foundation, Chicago, IL

Pub. in Dental Materials Jnl., v14 n1 p31-36 Jun 95

Keywords: "Teeth, "Enamels, "Calcium phosphates, Solutions, Diffusion, Caries, Teeth roots, Holes(Openings), Reprints, "Remineralization, Lesions, Root caries.

Remineralization of enamel lesions in vitro by use of sequential treatments with an alkaline $(\mathrm{pH} 9)$ phos phate $(1 \mathrm{~mol} / \mathrm{L})$ solution and slightly acidic $(\mathrm{pH} 6) \mathrm{cal}$ cium ( $1 \mathrm{~mol} / \mathrm{L})$ solution was reported to be relatively ineffective. An analysis of the diffusion processes that may occur during the remineralization treatments suggested that the driving force for diffusion of $\mathrm{Ca}$ into the lesion can be increased by making the calcium solution more alkaline than the phosphate solution. In the present study this modified treatment procedure was evaluated for remineralizing root lesion in vitro.

\section{3,568}

PB96-119250 Not available NTIS

National Inst. of Standards and Technology (MSEL) Gaithersburg, MD. Polymers Div.

Publications and Presentation Abstracts, 1995. (Published by Paffenbarger Research Center and Center of Excellence for Materials Science Research).

Final rept.

M. W. Chalkley, and W. A. Marjenhoff. 1995, 61p See also report for 1993, PB95-153052. Sponsored by American Dental Association Health Foundation, Gaithersburg, MD. Paffenbarger Research Center. Pub. in Dental Materials Research Highlights, 1995 Publications and Presentation Abstracts, p1-61 Oct 95

Keywords: "Dental materials, "Research projects, *Technology innovation, "Medical research, -Abstracts Dental cements, Dental implantation, Biocompatible materials, Adhesives, Composite materials, Temporary dental restoration, Permanent dental restoration, Reprints, Paffenbarger Research Center

This report is the annual compilation of abstracts pub lished or accepted for publication by Paffenbarger Research Center and Center of Excellence for Materials Science Research staff thus far in 1995.

\section{3,569}

PB96-119722 Not available NTIS

National Inst. of Standards and Technology (MSEL), Gaithersburg, MD. Polymers Div
In vitro Fracture Behavior of Ceramic and MetalCeramic Restorations.

Fina! rept.

T. B. Smith, J. R. Kelly, and J. A. Tesk. 1994, 7p.

Pub. in Jnl. of Prosthodontics, v3 n3 p138-144 Sep 94.

Keywords: "Dental ceramics, Reprints, In vitro analysis, Failure analysis, Metal ceramics.

Failed crowns and failure load data were studied to gain insights into the fracture behavior of prosthesis under incaisal-directed, load-to-failure testing. Incisor crowns ( $n-68$ ) were fabricated: two all-ceramic groups (feldspathic veneer on high-strength core), differing in core design, and two metal-ceramic groups, differing in metal oxidation time (30 seconds $v 3$ minutes). Crowns were loaded to failure on their indisal edge. Gross visual microscopic, and elemental microprobe analyses of failed crowns were coupled with Weibul analysis of the failure load data.

\section{3,570}

\section{PB96-123666 Not available NTIS}

National Inst. of Standards and Technology (MSEL), Gaithersburg, MD. Polymers Div.

Intensive Swimming: Can It Affect Your Patients' Smiles.

Final rept.

K. J. Rose, and C. M. Carey. 1995, 5p.

Pub. in Jnl. of the American Dental Association, v126 p1402-1406 Oct 95

Keywords: "Teeth, "Discoloration, *Swimming, Dental calculus, Calculi, Dental enamel, Pellicle, Chemical reactions, Oral intake, Chlorination, Exposure, Reprints Pool water

Athletes who swim intensively, such as those who swim laps more than six hours a week, may develop unusual yellowish brown or dark brown stains on their teeth. The authors hypothesize that long-term contact of the teeth with swimming pool water, as well as the mixture of oral fluids with swimming pool water, leads to the formation of these deposits. The authors report two cases of development of such stains.

\subsection{1}

PB96-135215 Not available NTIS

National Inst. of Standards and Technology (MSEL), Gaithersburg, MD. Polymers Div.

Effect of Supersaturation on Apatite Crystal Formation in Aqueous Solutions at Physiologic pH and Temperature.

Final rept.

K. Ishikawa, E. D. Eanes, and M. S. Tung. 1994, 8p. Pub. in Jnl. of Dental Research, v73 n8 p1462-1469 Aug 94

Keywords: "Apatites, "Calcification, "Crystal size, Reprints, Supersaturation, $X$ ray diffraction.

The importance of supersaturation in the dynamics of apatite precipitation from aqueous solutions is well-es-
tablished. To determine whether this parameter has a tablished. To determine whether this parameter has a the textural properties of this phase, such as crysta size and shape, the authors investigated mineral accretion in synthetic solutions seeded with $0.67 \mathrm{~g} / \mathrm{L}$ apatite over a range of supersaturations at $\mathrm{pH} 7.4$ and 37 degrees C. A dual specific-ion electrode-controlled titration method was used to maintain the seeded reactions under the following solution conditions: 1.0 to 1.8 $\mathrm{mmol} / \mathrm{L} \mathrm{Ca} 2+, 0.67$ to $1.2 \mathrm{mmol} / \mathrm{L}$ total phosphate (PO4), Ca/PO4 (initial) $=1.5,143 \mathrm{mmol} / \mathrm{L} \mathrm{KNO3,} \mathrm{and}$ $10 \mathrm{mmol} / \mathrm{L}$ HEPES. Samples were collected for chemica! and textural analyses when the seed apatite was reduced by new accretions to $1 / 2,1,4.1 / 8,1 / 16$, and $1 / 32$ of the total solids in suspension. All new accretions were found to be apatitic. At the lowest supersaturation, accretion occurred primarily by growth of the seed crystals. However, at the highes supersaturation examined, the crystals at the end of the experiments were actually smaller, on average, than the original seeds, even though the total mass in creased 32 -fold. The results suggest that proliferation of new crystals supplanted growth of the seed crystals as supersaturation was increased. The results also suggest that differences in tissue fluid supersaturation may contribute to the large disparity in dimensions between dentin and enamel apatite crystals

\section{2}

PB96-147012 Not available NTIS

National inst. of Standards and Technology (MSEL), Gaithersburg, MD. Polymers Div.
Bioactive Polymeric Dental Materials Based on Amorphous Calcium Phosphate.

Final rept.

J. M. Antonucci, D. Skrtic, and E. D. Eanes, 1994, $2 p$.

Pub. in Polymer Preprints, v35 n2 p460-461 1994.

Keywords: "Calcium phosphates, "Dental cements, Tooth remineralization, Physiologic calcification, Phototchemical reactions, Monomers, Amorphous compounds, Hydroxyapatites, Composite materials, Physicochemical properties, Biochemistry, Dental materials, Reprints, "Bioactive materials, Coupling agents, HAP(Hydroxyapatite).

Amorphous calcium phosphate (ACP) is an important intermediate in HAP formation, has received far less attention as a biomaterial. The two principal reasons for this are ACP's high solubility in aqueous media and its rapid conversion to HAP. These very properties, however, suggest its use as a bioactive filler in polymeric materials, e.g., composites, sealants, adhesives, that can preserve teeth against demineralization and possibly promote remineralization of defective tooth structure. In this study, several visible light curable composites based on several acrylic monomer systems and a stablized ACP were prepared, polymerized and evaluated for their ability to release calcium and phosphate ions in the presence of an aqueous environment in a sustained fashion and at levels adequate to favor HAP formation.

\subsection{3}

PB96-147020 Not available NTIS

National Inst. of Standards and Technology (MSEL), Gaithersburg, MD. Polymers Div.

Remineralizing Dental Composites Based on Amorphous Calcium Phosphate.

Final rept.

J. M. Antonucci, D. Skrtic, and E. D. Eanes. 1995,

$2 \mathrm{p}$.

Pub. in Polymer Preprints, Anaheim, California 1995 Meeting, v36 n1 p779-780 1995.

Keywords: "Calcium phosphates, "Mineralization, *Dental cements, Precipitation(Chemistry), Tooth remineralization. Physiologic calcification, Dental caries, Polymerization, Amorphous compounds, Composite materials, Hydroxyapatites, Biochemistry, Dental materials, Reprints, Acrylic monomers, HAP(Hydroxyapatite)

Amorphous calcium phosphate (ACP) has some unique properties that make it ideal for use as a remineralizing agent in polymeric composites. In this study, visible light curable composites based on an acrylic monomer system and either a stabilized ACP or a HAP particulate filler were evaluated for sustained release of $\mathrm{Ca}(2+)$ and $\mathrm{PO} 4$ ions into aqueous media at levels adequate for HAP formation, and for their abilities to remineralize caries-like lesions in extracted bovine incisors.

\section{3,574}

PB96-147095 Not available NTIS

National Inst. of Standards and Technology (MSEL), Gaithersburg, MD. Polymers Div.

Dynamics of Calcium Phosphate Precipitation.

Final rept.

E. D. Eanes. 1992, 17p

Pub. in Calcification in Biological Systems, Chapter 1, p1-17 1992

Keywords: *Calcium phosphates, "Precipitation(Chemistry), "Dental cements, Physicochemical properties, Biochemistry, Mineralization, Physiologic calcification, In vivo analysis, Synthetic materials, Dental materials, Reprints.

Many of the physicochemical principles of crystal nucleation and growth relevant to understanding biomineralization dynamics have been elucidated by solution studies on synthetic calcium phosphate analogs. These studies have also helped to delimit the range of action of specific biological substances. e.g., matrix macromolecules, that may be involved in initiating and regulating mineral formation in vivo. Together with our present knowledge of the ultrastructural features of skeleta! mineral and mineral-matrix associations, these synthetic studies have provided valuable insight into biomineral formation and the physiological constraints placed on it. This chapter reviews current knowledge in these areas.

O3,575
PB96-155544 Not available NTIS 
National Inst. of Standards and Technology (MSEL), Gaithersburg, MD. Polymers Div.

Polymeric Calcium Phosphate Composites with Remineralization Potential.

Final rept.

D. Skrtic, E. D. Eanes, and J. M. Antonucci. 1995, $16 p$.

Pub. in Industrial Biotechnological Polymers, Chapter 25, p393-408 1995

Keywords: "Teeth, "Enamels, "Calcium phosphate, Composites, Reprints, *Remineralization.

Calcium phosphates constitute an increasingly important class of inorganic dental materials. Their excellent compatibility with both hard and soft tissues as well as with other dental materials, especially polymeric resins and acids, has led to a number of possible prophylactic, prosthetic and restorative uses. Crystalline calcium phosphates such as hydroxyapatite (HAP) and various di-, tri- and tetracalcium phosphates have received the greatest consideration for such purposes Less attention in this regard has been given to the possible use of amorphous calcium phosphate (ACP), an important intermediate in the formation of apatite, as a dental material. One reason for this neglect is that the relatively high solubility of ACP and its conversion to apatite in aqueous environments poses limitations in applications where structural, mechanical and chemical stabilities are desired. These same properties however, may make ACP suitable as a potential mineralizing agent. When compounded with appropriate polymeric resins, ACP's bioactivity may be particularly useful in enhancing the performance of sealants and adhesives in preventing tooth demineralization or promoting its remineralization when defective.

\section{3,576}

PB96-164082 Not available NTIS

National Inst. of Standards and Technology (MSEL) Gaithersburg, MD. Polymers Div.

Publication and Presentation Abstracts, 1995.

Final rept.

M. W. Chalkley, and W. A. Marjenhoff. 1995, 61p See also PB95-153052. Sponsored by American Dental Association Health Foundation. Chicago, IL.

Pub. in Dental Materials Research Highlights, p1-61 Oct 95

Keywords: "Dental materials, "Research projects, "Technology innovation, "Medical research, "Abstracts, Dental cements, Dental implantation, Biocompatible materials, Adhesives, Composite materials, Sealants, Temporary dental restoration, Permanent dental restoration, Reprints, Paffenbarger Research Center.

This fourth compilation of Publication and Presentation Abstracts provides a summary of research conducted at the American Dental Association Health Foundation's (ADAHF) Paffenbarger Research Center (PRC) and the National Institute of Dental Research (NIDR)

- funded Center of Excellence for Materials Science Research (CEMSR) during the past year.

\section{3,577}

PB96-176623 Not available NTIS

National Inst. of Standards and Technology (MSEL) Gaithersburg, MD. Polymers Div.

Publication and Presentation Abstracts, 1994

Final rept.

M. W. Chalkley, and W. A. Marjenhoff. 1994, 74p.

See also report for 1993, PB95-153052 and report for 1995, PB96-164082.

Pub. in Dental Materials Research Highlights, p1-74 1994

Keywords: "Dental materials, "Research projects, *Technology innovation, "Medical research *Abstracts, Dental cements, Dental implantation, Biocompatible materials, Adhesives, Composite materials, Sealants, Temporary dental restoration, Permanent dental restoration, Dental equipment, Biomedical radiography, Mirrors, Resins, Reprints, Paffenbarger Research Center.

The American Dental Association (ADA) Health Foundation presents this third annual compilation of Publication and Presentation Abstracts of the Paffenbarge Research Center (PRC) and Center of Excellence for Materials Science Research. Among the innovations emanating from the ADA-NIST alliance are the panoramic radiograph; the turbine contra-angle handpiece; rhodium front-surface dental mirrors; dental composites; the first radiopaque mineral fillers (glasses) for composite restorative materials; and pit and fissure sealant resins and orthodontic bracket bonding resins.
03,578

PB96-180237 Not available NTIS

National Inst. of Standards and Technology (MSEL), Gaithersburg, MD. Polymers Div.

Fluoride Analytical Methods.

Final rept.

P. Venkateswarlu, and G. Vogel. 1996, 13p

Sponsored by American Dental Association Health Foundation, Chicago, IL.

Pub. in Fluoride in Dentistry, Chapter 2, p27-39 1996.

Keywords: "Dental, "Fluoride, Soil, Tissue, Analysis, Fluorine, Foods, Research, Reprints, "Foreign technology.

The emergence of fluoridation of public water supplies as a safe and economic mass caried-control measure was based on epidemiologic studies and on the concurrent elucidation of the tranpsort and metabolism of fluoride, the mechanism of action of fluoride compounds, their biological effects, and the environmental aspects of fluorides. The success of these studies depended, among other things, on the development of sound fluoride analytical methods. This involved fluorine analysis of water, soil, minerals, vegetation, foods and a host of other biological materials such as bone. teeth (enamel, dentin cementum) body fluids (urine, blood, saliva) and soft tissues (kidney, liver, muscle)

\section{3,579}

\section{PB96-204052 Not available NTIS}

National Inst. of Standards and Technology (MSEL), Gaithersburg, MD. Polymers Div.

New Surface-Active Comonomer for Adhesive Bonding.

Final rept

R. L. Bowen, P. S. Bennett, R. J. Groh, M. Farahani and F. C. Eichmiller 1996, 5p.

Pub. in Jnl. of Dental Research, v75 n1 p606-610 Jan

Keywords: "Adhesive bonding, "Amines, "Dentin, Reprints, Monomers, Chemical properties, Physical properties, Hydrogen atoms, Methyl groups.

Previous studies have indicated that chemical and physical characteristics of aromatic amines can be influenced by the nature of their substituents. The experimental question examined in the present study relate to the effects of replacing specific hydrogen atoms with methyl groups in a surface-active comonomr utilized in adhesive bonding protocols. N-2-propionic acid- $\mathrm{N}$ 3-(2-hydroxy- 1-methacryloxy)propyl=3 dimethylaniline sodium salt (N35A) was synthesized by an addition reaction of glycidyl methacrylate with the sodium salt of $\mathrm{N}$-(3,5-dimethylphenyl)alanine, which was formed by alkaline hydrolysis of ethyl- $\mathrm{N}-(3,5$ dimethylphenyl)alanate that was prepared by condensation ofethyl-2-bromopropionate with 3,5dimethylaniline.

03,580

PB97-111595 Not available NTIS

National Inst. of Standards and Technology (MSEL). Gaithersburg, MD. Polymers Div.

Calcium Phosphate Cements.

Final rept

L. C. Chow, and S. Takagi. 1996, 13p.

See also PB92-144443. Sponsored by American Dental Association Health Foundation, Chicago, IL.

Pub. in Cements Research Progress 1994, the American Ceramic Society, v8 p189-2011996.

Keywords: "Cements, "Calcium phosphate cements Biomaterials, Hydroxyapatite, Mechanical strength Setting time, Reprints.

Since their development in 1987 (1), self-hardening calcium phosphate cements (CPC) have been a subject of considerable interest in biomaterials research. The first CPC's consisted of tetracalcium phosphate (Ca4(PO4)2O or TTCP) and anhydrous dicalcium phosphate (CaHPO4 or DCPA), or TTCP and dicalcium phosphate dihydrate (ChH-PO4.2H2O or DCPD). These cements set in about 30 minutes afte mixing the powder with water or dilute acid (e.g., 25 $\mathrm{mmol} / \mathrm{L} \mathrm{H} 3 \mathrm{PO} 4$ ) and develop sufficient mechanica strength for many clinical applications.

\section{3,581}

PB97-112510 Not available NTIS

National Inst. of Standards and Technology (MSEL) Gaithersburg, MD. Polymers Div.
Adsorption of Polyacrylic Acids and Their Sodium Salts on Hydroxyapatite: Effect of Relative Molar Mass.

Final rept.

D. N. Misra. 1996, 8p

Sponsored by American Dental Association Health Foundation, Chicago, IL

Pub. in Jnl. of Colloid and Interface Science, Article No. 0380, v181 p289-296 1996

Keywords: "Polyacrylics, "Hydroxyapatites, "Molar mass, Molecular conformation, Acids Salts, Adsorption, Langmuir plot, Molecular association, Reprints.

The effect of molar mass on the adsorptive properties of polyacrylic acids and their sodium salts (polymers) has an important bearing on the adhesive mechanisms of restorative resins based on these or similar polymers. The adsorption isotherms of the aqueous solution of polymers were determined on synthetic hydroxyapatite at room temperature to elucidate the role of physico-chemical factors involved in the process. The adsorption of lower molar mass $(<90,000)$ polyacrylic acids shows that the isotherms first rise, reach a maximum, then decrease In contrast, a highe molar mass $(250,000)$ polyacrylic acid shows no maximum and follows a regular isotherm. The adsorption isotherms of sodium polyacrylates are regular, do not exhibit any maxima, and merge into each other for higher molar mass salts $(>60,000)$. The adsorption is irreversible for the acids as well as for the salts. The concentrations of calcium and phosphate ions were also determined in the filtrates in order to establish any possible correlation with the adsorbed amounts of polymers. The self-association of the polyacrylic molecules by intermotecular hydrogen bonding that may be responsible for the decrease in adsorption at higher concentrations may not play a significant role for higher molar mass acids where individual molecules can fold on themselves as intramolecular hydrogen bonding becomes more dominant.

03,582

PB97-118624 Not available NTIS

National Inst of Standards and Technology (MSEL) Gaithersburg, MD. Polymers Div

Posterior Restorative Materials Research.

Final rept.

F. C. Eichmiller, and W. A. Marjenhoff. 1996, 4p.

Keywords: "Dental materials, "Biocompatible materials, "Teeth, Calcium phosphates, Resins, Silver alloys, Glass ceramics, Protective coatings, Polymeriza tion, Shrinkage, Permanent dental restoration, Temporary dental restoration, Dental implantation, Reprints.

Novel materials and cutting-edge technologies developed at the American Dental Association Dental Health Foundation Center of Excellence for Materials Science Research are discussed. The search for better posterior restorative materials continues.

03,583

PB97-119010 Not available NTIS

National Inst. of Standards and Technology (MSEL) Gaithersburg, MD. Polymers Div.

Surface Roughness of Glass-Ceramic Insert. Composite Restorations: Assessing Several Polishing Techniques.

Final rept.

M. J. Ashe, G. A. Tripp, F. C. Eichmiller, L. A.

George, and J. C. Meiers. 1996, 6p.

Pub. in Jnl. of the American Dental Association, v127 n10 p1495-1500 1996

Keywords: ${ }^{\star}$ Ceramics, ${ }^{\star}$ Composites, Polishing, Profilometry, Surface roughness, Reprints.

The authors compared the effectiveness of seven polishing methods on glass-ceramic insert-composite restorations placed in plastic resin squares. The polishing methods used carbide dental finishing burs and diamond polishing paste, diamond abrasive finishing burs and diamond polishing paste, diamond abrasive finishing burs and composite resin finishing disks, diamond abrasive finishing burs and composite resin polishing points, diamond abrasive finishing burs only, diamond abrasive finishing burs followed by resin impregnated disks and an aluminum oxide polishing abrasive past, and diamond abrasive finishing burs followed by diamond polishing paste. All systems achieved comparable smoothness except the carbide finishing burs, which damaged the insert-composite margin 


\section{Dentistry}

\section{3,584 \\ PB97-119309 Not available NTIS}

National Inst. of Standards and Technology (MSEL), Gaithersburg, MD. Polymers Div.

Interaction of Citric Acid with Hydroxyapatite: Surface Exchange of lons and Precipitation of Calcium Citrate.

Final rept.

D. N. Misra. $1996,8 p$

Sponsored by American Dental Association Health Foundation, Chicago, IL

Pub. in Jnl. of Dental Research, v75 n6 p1418-1425 1996.

Keywords: "Calcium citrate, "Hydroxyapatite, "Citric acid, "Surface exchange, Formation, Interactions, Peridontal regeneration, Restorative bonding, Reprints.

The use of citric acid is efficacious and distinctive in the demineralization of dentinal root surfaces for periodontal regeneration and in the etching and conditioning of enamel or dentin for bonding restorative resins. To decipher the role of citric acid in these applications, it is important to have a basic understanding of its interaction with synthetic hydroxyapatite. The uptake or removal of citrate ions from aqueous solutions of citric adic ( 4 to $100 \mathrm{mmol} / \mathrm{L}, 10 \mathrm{~mL}$ ) by hydroxyapatite $(1 \mathrm{~g})$ was studied at 22 degrees $\mathrm{C}$ after a given reaction period (from $3 \mathrm{hr}$ to 11 days) by immediate spectrophotometric monitoring of the concentration of the fittrates $(214 \mathrm{~nm})$. The concentrations of calcium, phosphate and hydrogen ions were also determined in the same solutions. The interaction: (1) is a time-independent ionic exchange process with the substrate when the initial acid-concentrations are dilute ( 4 to $12.5 \mathrm{mmol} / \mathrm{L}$ ), and (2) is a reactive process that is time-dependent for higher acid concentrations. The exchange process shows an adsorption of about one citrate ion per (100) face of the unit cell of hydroxypatite for a maximally exchanged surface.

\section{3,585}

PB97-122238 Not available NTIS

National Inst. of Standards and Technology (MSEL),

Publication and Presentation Abstracts, 1996.

Final rept.

M. W. Chalkley, and W. A. Marjenhoff. 1996, 61p

See also PB96-164082. Sponsored by American Dental Association Health Foundation, Chicago, IL

Pub. in 1996 Publication and Presentation Abstracts, $61 \mathrm{p} 1996$.

Keywords: "Dental materials, "Research projects "Technology innovation, "Medical research, Biocompatible, Adhesives, Polymers, Composite materials, Sealants, Crystallography, Temporary dental restoration, Permanent dental restoration, Clinical medicine, Reprints.

The abstracts presented in the volume provide a summary of much of the research conducted during the past year at the American Dental Association Health Foundation's (ADAHF) Paffenbarger Research Center (PRC) and the National Institute of Dental Research (NIDR)-funded Center of Excellence for Materials Science Research (CEMSR). PRC research is primarily conducted in four areas: dental chemistry, polymer chemistry, clinical research, and dental crystallography.

\section{Immunology}

\section{3,586}

\section{PB94-200185 Not available NTIS}

National Inst. of Standards and Technology (NML), Gaithersburg, MD. Organic Analytical Research Div.

Planar Waveguide Optical Sensors.

Final rept.

S. Choquette, L. Locascio-Brown, and R. A. Durst.

1990, 9p

Pub. in Proceedings of Sensors Expo, Chicago, IL., September 11-13, 1990, p206A-1-206A-9.

Keywords: "Theophylline, *Optical waveguides, Planar structures, Fluorescence, Liposomes, Sensors, Reprints, "Ammonium ions, Immunosensors, Flow injection analysis.

The report addresses on-going research involving the development of optical waveguides as analytical sen- sors. Optical waveyuides were fabricated in our laboratory using a metal ion diffusion method, and were employed in independent assays for ammonium and theophylline. The assay for ammonium ion was performed in a flow injection analysis (FIA) system in which the waveguide was used as the detector for an absorbance-based measurement. Detection limits of this assay were $300 \mathrm{M}$, and the throughput for the system was 60 samples per hour. As a fluorescence sensor, anti-theophylline was immobilized to the surface of the waveguide to create an immunospecific senso for theophylline. Immunospecific binding of theophylline derivatized liposomes has been demonstrated, and work is progressing on developing a competitive assay for theophylline using planar waveguides.

\section{Microbiology}

\section{3,587}

PB94-212024 Not available NTIS

National Inst. of Standards and Technology (NML). Gaithersburg, MD. Chemical Process Metrology Div. In situ On-Line Optical Fiber Sensor for Fluorescence Monitoring in Bioreactors.

Final rept.

J. J. Horvath, and C. J. Spangler. 1992, 17p

Pub. in Front. Bioprocess. 2, Proc., p99-115 1992

Keywords: "Bioreactors, "Fluorescence spectroscopy, "Optical fibers, On-line systems, Pyridoxine, Tryptophan, NAD, Riboflavin, Saccharomyces cerevisiae, Reprints.

This work examines the fluorescence properties of nat ural cell components such as tryptophan pyridoxine $\mathrm{NADH}$ and riboflavin in batch fermentations of $S$. cerevisiae. Measurements were made using both an external flow-thru cuvette loop and an in-situ optical fiber/rod sensor. Pyridoxine gave the largest fluorescence signal; the spectrum of pyridoxine overlaps that of $\mathrm{NADH}$, complicating its interpretation. A good correlation was found between the dry weight of yeast and on-line fluorescence measurements of pyridoxine and tryptophan. The pyridoxine signal was linear with the dry mass of yeast and the tryptophan signal showed absorption effects starting at a dry mass of $0.5 \mathrm{~g} / \mathrm{l}$ in the cuvette. No deviations were observed with the in situ optical fiber/rod.

\section{3,588}

PB96-161732 Not available NTIS

National Inst. of Standards and Technology (CSTL), Gaithersburg, MD. Biotechnology Div.

Current Fluctuations Reveal Protonation Dynamics and Number of Ionizable Residues in the alpha Toxin Channel.

Final rept.

J. J. Kasianowicz, and S. M. Bezrukov. 1993, 1p.

Pub. in Biophysical Jnl., v64 pA344 Feb 93.

Keywords: "Ion channels, "Ion transport, "Noise analysis, Reprints, Proteins, Protons, Dynamics.

The I-V relationship of channels formed by Staphlococcus aureus alpha-toxin is $\mathrm{pH}$ dependent. To understand the nature of this effect, the authors measured the fluctuations in current through the open channel as a function of $\mathrm{pH}$. The low frequency noise levels in $\mathrm{NaCl}$ solutions exhibits a single, well-defined peak over the range 4.5 less than $\mathrm{pH}$ less than 7.5 . The noise can be described theoretically assuming the channel has several ionizable sites that bind protons with first-order kinetics, In the model, the association of a proton with each of the binding sites in the channe is assumed to increase the conductance in a stepwise manner. Data analysis shows that the residues responsible for the $\mathrm{pH}$ dependent noise have a $\mathrm{PKa}=5.5$ Moreover the rate constants for the association and dissociation of protons are $\mathrm{kg}=810$ to the 9 th power $M$ to the minus 1 power $S$ to the minus 1 power, and $\mathrm{KD}=10$ to the 5 th power, respectively.

\section{3,589}

PB96-161757 Not available NTIS

National Inst. of Standards and Technology (CSTL), Gaithersburg, MD. Biotechnology Div.

Protonation Dynamics in an Ion Channel Pore. Final rept.

J. J. Kasianowicz, and S. M. Bezrukov. 1993, 1p. Pub. in International Biophysics Conference (11th), Budapest, Hungary, July 25-30, 1993, p136.
Keywords: "Alpha toxin, "Ion channels, "Noise analysis, Reprints, Protons, Rate constant, Spectral analysis, "Foreign technology, Diffusion limited reactions, Ionizable group. Protein structure.

Staphylococcus aureus alpha-hemolysin (alpha-HL) forms channels that have a $\mathrm{pH}$ dependent I-V relationship. To evaluate the rate constants for these protonation reactions, the authors measured the fluctuations in current through fully open alpha-HL channels as a function of $\mathrm{pH}$ in the presence of $1 \mathrm{M} \mathrm{NaCl}$. Spectral analysis shows that the data are well described by a model in which protons bind independently to $n$ ionizable residues with first-order kinetics and that $\mathrm{pK}=5.5$, kon $=810$ to the 9 th power $M$ to the minus 1 power $S$ to the minus 1 power, koff $=2.5$ 10 to the 4th power $S$ to the minus 1 power and $n=$ 4 (Phys. Rev. Lett. 70). The value the authors obtain for the association rate constant, kon, is similar to the values measured by Eigen and co-workers for reactions between $\mathrm{H}_{+}$and free carboxyl or imidazole groups, which suggests that the protonation reactions inside these pores are diffusion limited.

03.590

PB96-163639 Not available NTIS

National Inst. of Standards and Technology (CSTL), Gaithersburg, MD. Biotechnology Div.

Autofluorescence Detection of 'Escherichia coli' on Silver Membrane Filters.

Final rept.

S. A. Glazier, and H. H. Weetall. 1994, 5p

Pub. in Jnl. of Microbiological Methods, v20 p23-27 1994

Keywords: "Bacteria, Reprints, Escherichia coli, "Foreign technology, "Epifluorescence.

This report describes the measurement of the limit of detection of the bacterium, Escherichia coli, on the surface of membrane filters employing autofluorescence. Investigation of detection of E. coli by this method was prompted by NASA's need for a method which could detect, principally, fecal coliforms in initially sterile storage water on-board the space station. This method was examined, with $E$ coli as a model, because it could be conducted rapidly with little or no need for disposables, large amounts of instrumentation, or constant human involvement. The autofluorescence of $E$. coli collected on the surface of silver membrane filters was quantitated by epifluorescence microscopy and related to a viable count of the bacteria. The fluorescence was excitated using a broad region of ultraviolet radiation from a mercury arc lamp (approx. 250-400 $\mathrm{nm}$ ) examined in the wavelength region of $495 \mathrm{~nm}$ and higher through a long pass optical filter. From these measurements, the limit of detection of viable $E$. coli collected on the membrane filters was fewer than 550 pius or minus 32 viable bacteria or 170,000 plus or minus $10,000 / \mathrm{ml}$ in terms of solution concentration.

\section{3,591}

PB96-163647 Not available NTIS

National Inst. of Standards and Techn

Gaithersburg, MD. Biotechnology Div.

Bacterial Enumeration in Storage Water.

Final rept.

S. A. Glazier, and H. H. Weetall. 1993, 33p

Pub. in Bacterial Enumeration in Storage Water, 33p, 17 Feb 93.

Keywords: "Bacteria, "Water analysis, "Fluorescence, Reprints, Enumeration, Microscopy.

This report describes the progress of a NASA funded project. The goal of this project is the enumeration of bacteria which are present in storage water. This work is part of NASA's efforts to prepare environmenta monitoring systems for the space station currently under construction. A prototype system for enumeration of bacteria in storage water is proposed based on the fiuorescence detection of single bacterial cells collected on a membrane filter. This method of bacterial detection was chosen because it conveniently concentrates the bacteria from the water and enumerates them without the need for disposable reagents and supplies. The report describes the model enumeration system proposed, explains the origins of bacterial cells by laser confocal microscopic imaging, explores the possibility of distinguishing between live and dead bacteria based on their native fluorescence, and details progress on the construction of a simple, compact bacterial enumeration system.

03,592

PB96-201017 Not available NTIS 
National Inst. of Standards and Technology (CSTL), Gaithersburg, MD. Biotechnology Div.

Escherichis coli Cyclic AMP Receptor Protein Mutants Provide Evidence for Ligand Contacts Important in Activation.

Final rept.

J. Moore, M. Kantorow, D. Vanderzwaag, and K. McKenney. 1992, 6p.

Pub. in Jnl. of Bacteriology, v174 n24 p8030-8035 Dec 92.

Keywords: "Protein mutants, "Ligand contacts, "Receptor proteins, Activation, Escherichis coli, Reprints, Cyclic AMP.

The three-dimensional model of the Escherichia col cyclic AMP (cAMP) receptor protein (CRP) shows that several amino acids are involved as chemical contacts for binding CAMP. We have constructed and characterized mutants at four of these positions, E72, R82, S83, and R123. The mutations were made in wild-type $\mathrm{crp}$ as well as a cAMP-independent crp, crp(star). The activities of the mutant proteins were characterized in vivo for their ability to activate the lac operon. These results provide genetic evidence to support that E72 and $R 82$ are essential and S83 and R123 are importnat in the activation of CRP by CAMP.

\section{Nutrition}

03,593

PB95-151692 Not available NTIS

National Inst. of Standards and Technology (TS) Gaithersburg, MD. Office of Measurement Services. Mixed Diet Reference Materials for Nutrient Analysis of Foods: Preparation of SRM-1548 Total Diet. Final rept.

W. R. Wolf, G. V. Iyengar, and J. T. Tanner. 1990, 3p.

Sponsored by Department of Agriculture, Beltsville, $\mathrm{MD}$. and Food and Drug Administration, Washington, DC.

Pub. in Fresenius Jnl. of Analytical Chemistry 338, n4 p473-475 1990

Keywords: "Food analysis, "Chemical analysis, "Diets, "Trace elements, "Nutritive value, Nutrients, Human nutrition, Fats, Proteins, Cholesterol, Caloric requirements, Reprints, "Standard reference materials, *SRM 1548.

Several mixed component diet materials have been available for use as Reference Materials for determination of elemental contents in foods. Wide acceptance of these materials has resulted in supplies being no longer available. A new mixed food material, SRM 1548 Total Diet has been prepared and characterized for a number of elemental and organic constituents o nutritional concern. SRM 1548 was prepared from foods obtained from collections of the USFDA's Total Diet Study, which are representative of foods consumed by the US population. SRM 1548 has been composited from these foods, proportional to a representative daily intake. The composited foods were blended, freeze-dried, reblended and bottled in six gram sample size. Homogeneity studies were carried out along with certification analyses for elements, cholesterol, caloric, and proximate content. This material is available from NIST and will be useful for multipurpose determinations of constituents in the naturally occurring range in foods and food related materials.

\section{Occupational Therapy, Physical Therapy, \& Rehabilitation}

03,594

PB96-147814 PC A05/MF A01

National Inst. of Standards and Technology (CSL) Gaithersburg, MD. Information Access and User Interface Div.
Virtual Environments for Health Care. A White Paper for the Advanced Technology Program (ATP), the National Institute of Standards and Technology.

Special pub.

J. Moline. Nov 95, 60p, NISTIR-5740.

Keywords: "Virtual reality, "Health, Surgery, Therapy, Education, Databases, Rehabilitation, "Augmented reality, "Telemedicine.

This report surveys the state of the art in applications of virtual environments (VE) and related technologies for healthcare. After a brief introduction, terms are defined and a review of the healthcare applications is presented. The report also discusses the value-added of these application, their limitations, and areas of current research and development concerning virtual environments for healthcare. Applications of virtual environments and related technologies are being developed for healthcare in the following areas: surgical procedures (remote surgery or telepresence, augmented or enhanced surgery, and planning and simulating procedures before surgery), medical therapy, medical diagnosis and monitoring (telemedicine), preventive medicine and patient education, medical education and training, visualization of massive medical databases, skills enhancement and rehabilitation, and architectural design for healthcare facilities. Virtual environments are benefiting healthcare. To date, the developments have improved the quality of healthcare and in the future will result in substantial cost savings. Tools are being refined or developed which respond to needs of present VE systems. However, additional large scale research is necessary in the following areas users' studies, use of robots for teleoperated procedures, enhancing system reality, and improving system functionality.

\section{Pathology}

\section{3,595}

PB96-201140 Not available NTIS

National Inst. of Standards and Technology (MSEL), Gaithersburg, MD. Polymers Div.

In vitro Inhibition of Membrane-Mediated Calcification by Novel Phosphonates.

Final rept

D. Skrtic, N. Eidelman, G. Golomb, E. Breuer, and E. D. Eanes. 1996, 8p.

Pub. in Calcified Tissue International, v58 p347-354 1996

Keywords: "Bone disorders, "Calcium metabolism, "Hydroxyapatite, Reprints, Geminal bisphosphonates.

The effects of a series of novel phosphonates on the kinetics of mineral development in an ionophoreprimed 7:2:1 phosphatidylcholine (PC): dicetylphosphate (DCP): cholesterol (Chol) liposomal model system are reported. When present at 2.5 micro $\mathrm{mol} / \mathrm{L}$ or $25 \mathrm{micro} \mathrm{mol} / \mathrm{L}$ concentrations in the solution surrounding the liposomes, the investigated phosphonates did not significantly delay the initial formation of hydroxyapatite-like calcium phosphate salts (HAP) within the liposomes or the penetration of HAP crystals through the enclosing membranes. However, the phosphonates variably retarded the subsequent growth and proliferation of the HAP crystals once they became directly exposed to the phosphonate-containing solution. The effectiveness of phosphonates in inhibiting extraliposomal precipitation strongly depended on their structure. The inhibitory action on active surface growth sites of released intraliposomal crystals was found to be the most effective if the phosphonate molecule contained two phosphonic groups linked to the same $\mathrm{C}$ atom. At a phosphonate concentration of 25 micro $\mathrm{mol} / \mathrm{L}$ the following general order of effectiveness was established: geminal bisphosphonate equal to or greater than geminal tetrakisphosphonate greater than bisacylphosphonates greater than monoacylphosphonate greater than bisalkylphosphonate.

\section{Pharmacology \& Pharmacological Chemistry}

03,596

PB94-172467 Not available NTIS

National Inst. of Standards and Technology (CSTL) Gaithersburg, MD. Biotechnology Div

Thermodynamics of the Hydrolysis of Penicillin G and Ampicillin.

Final rept.

N. Kishore, Y. B. Tewari, W. T. Yap, and R. N

Goldberg. 1994, 12p.

Pub. in Biophysical Chemistry 49, p163-174 1994

Keywords: "Penicillin G, "Ampicillin *Thermodynamics, "Hydrolysis, Calorimetry, High performance liquid chromatography, Enthalpy, betaLactamase, Reprints.

Apparent equilibrium constants and calorimetric enthalpies of reaction have been measured for the beta-lactamase catalyzed hydrolysis of penicillin $\mathrm{G}(\mathrm{aq})$ and ampicillin(aq) to penicillinoic $\operatorname{acid}(\mathrm{aq})$ and to ampicillinoic acid(aq), respectively. High-pressure liquid-chromatography and microcalorimetery were used to perform these measuremens. Calorimetric enthalpies of reaction for the beta-lactamase catalyzed hydrolysis of cephalosporin $\mathrm{C}$ have also been measured but the reaction products have not been identified and the measured enthalpies cannot be assigned to a specific reaction. Acidity constants for ampicillin, penicillin $\mathrm{G}$, ampicillinoic acid, and penicillinoic acid are also reported. A strain of energy of $116 \mathrm{~kJ}$ mol for the beta-lactam ring is obtained from thermochemical data.

03,597

PB95-175444 Not available NTIS

National Inst. of Standards and Technology (CSTL)

Gaithersburg, MD. Organic Analytical Research Div.

Determination of Amphetamine and Methamphetamine in a Lyophilized Human Urine Reference Material.

Final rept.

P. Ellerbe, T. Long, and M. J. Welch. 1993, 6p. Pub. in Jnl. of Analytical Toxicology 17, p165-170 May/ Jun 93

Keywords: "Amphetamine, "Methamphetamine, *Urine, "Reference standards, Mass fragmentography, Clinical chemistry, Humans, Freeze drying, Isotope dilution, Reprints, Heptafluorobutyryl derivative, $N$ trifluoroacetyl-1-prolyl chloride.

The concentrations of amphetamine and methamphetamine in a new lyophilized human urine reference material (RM) were determined at the National Institute of Standards and Technology in cooperation with the College of American Pathologists. In order to minimize the possibility of undetected bias, two different methods were developed. Both methods were based on isotope-dilution gas chromatography/mass spectrometry (GC/MS) with a deuterium-labeled standard. The first method used a solid phase extractor (SPE) to isolate the analytes, followed by the forming the heptafluorobutyryl (HFB) derivatives for measurement by GC/MS. The second method used another kind of SPE to isolate the analytes, followed by forming the $\mathrm{N}$-trifluoroacetyl-1-prolyl chloride (TPC) derivatives for measurement by GC/MS. The RM consists of three levels of amphetamine, three levels of methamphetamine, and a blank. For amphetamine, the concentrations are 291,558, and $1081 \mathrm{ng} / \mathrm{mL}$ of human urine. For methamphetamine, the concentrations are 311 573 , and $1137 \mathrm{ng} / \mathrm{mL}$ of human urine.

03,598

PB96-119383 Not available NTIS

National Inst. of Standards and Technology (CSTL) Gaithersburg, MD. Biotechnology Div.

Physical Characterization of Herparin by Light Scattering.

Final rept.

A. K. Gaigalas, J. B. Hubbard, R. LeSage, and D. H. Atha. $1995,5 p$

Pub. in Jnl. of Pharmaceutical Science, v84 n3 p355359 Mar 95.

Keywords: "Heparins, "Light scattering, "Transpor properties, Physical properties, Anticoagulants, Size determination, Diffusion coefficient, Charge density Electric charge, Reprints.

Dynamic and electrophoretic light scattering were used to measure the size and charge heterogeneity of a 
commercial preparation of heparin. For this preparation of porcine mucosal heparin $(M($ sub $r)=10-20$ $\mathrm{kDa}$ ), the diffusion coefficient was 1.2 plus or minus $0.5 \times 10(\exp -8)$ square $\mathrm{cm} / \mathrm{s}$ and the mobility was 4.4 plus or minus $0.9 \times 10(\exp -4)$ square $\mathrm{cm} N \mathrm{~s}$ for an unfiltered solution at $22 \mathrm{deg}$. $C$ in distilled water. This diffusion constant is 2 orders of magnitude smaller than expected for a molecule the size of heparin. A fast diffusion component of 5.8 plus or minus $1.0 \times 10(\exp$ -7) square $\mathrm{cm} / \mathrm{s}$, corresponding to the individual molecule, was observed in the presence of $2 \mathrm{M} \mathrm{NaCl}$ where single molecule motion is better observed. This indicates that a portion of the heparin population is in an aggregated state, which produces a higher scattering intensity than individual heparin molecules. The electrophoretic light-scattering measurements also indicate that the aggregate scattering species have very similar surface charge densities resulting from the aggregates being formed from heparin molecules. These results can provide a framework to interpret future light-scattering data for various preparations of heparin.

\section{Psychiatry}

\subsection{9}

AD-A280 082/9 PC A06/MF A02

National Bureau of Standards, Gaithersburg, MD

Screw-Thread Standards for Federal Services, 1957. Handbook H28 (1957), Part 2. Revised.

Apr 62, 114p.

Keywords: "Screw threads, "Standards, Gages, Handbooks, Screws, Fasteners, Couplers, Joints, Threaded products, Federal services.

No abstract available.

\section{Public Health \& Industrial Medicine}

\section{3,600}

PB94-154382 PC A04/MF A01

National Inst. of Standards and Technology (BFRL), Gaithersburg, MD. Building Materials Div.

Source of Phenol Emissions Affecting the Indoor Air of an Office Building.

Technical note.

J. W. Martin, F. R. Guenther, T. Nguyen, L. Oakley, W. S. Liggett, and E. Byrd. Feb $94,53 p$, NISTIR-

5353.

Sponsored by General Services Administration, Washington, DC

Keywords: "Phenols, "Indoor air pollution, "Office buildings, Occupational exposure, Building materials, Epoxy compounds, Floors, Occupational safety and health, Chemical analysis, Industrial hygiene, Environmental surveys, "Silver Spring(Maryland), Leveling materials.

For several years, National Oceanic and Atmospheric Administration (NOAA) employees occupying Floors 3 through 5 of the Silver Spring Metro Center Building One (SSMC-1) in Silver Spring. MD have complained about ailments which they have associated with poor indoor air quality. NOAA and the General Services Administration (GSA) commissioned at least six indoor air quality surveys to seek the causes of these complaints. In one of the later surveys (5), it was concluded that phenol emissions from an epoxy floor-leveling material used in leveling the floor slabs were causing the indoor air quality complaints from Floors 3 through 5 . To obtain an independent analysis and assessment, NOAA and GSA asked the National Institute of Standards and Technology (NIST) to ascertain whether phenol (or any other volatile organic compound) was being emitted from the epoxy floor-leveling material and, if so, to recommend remedial actions for mitigating or eliminating the emissions.

\section{3,601}

PB94-172038 Not available NTIS

National Inst. of Standards and Technology (BFRL), Gaithersburg, MD. Building Materials Div.
Lead Abatement in Buildings and Related Structures.

Final rept

K. Ashley, and M. E. McKnight. 1993, 8p.

Pub. in American Society for Testing and Materials Standardization News, p32-39 Dec 93.

Keywords: "Lead(Metal), "Health hazards, "Buildings, "Lead poisoning, Children, Legislation, Guidelines, Government agencies, Reprints.

The subject of lead-containing painted surfaces has received a great deal of attention in recent years. Much of this interest has been fueled by the potential hazard that this toxic metal poses to the developmental capabilities and health of young children. New federal and state guidelines and legislation concerning lead have spurred many activities in government agencies, academia, and the private sector. In 1988, the Agency for Toxic Substances and Disease Registry (ATSDR) provided in a report to Congress, a comprehensive review of the health risks that lead in anthropogenic sources pose to children. In 1991, the Centers for Disease Control (CDC) reduced the 'level of concern' of childhood blood lead concentrations from 25 to 10 micrograms per deciliter. According to the CDC report, millions of children in the United States are believed to be at risk of lead poisoning.

03,602

PB94-195047 PC A22/MF A04

National Inst. for Occupational Safety and Health, Cincinnati, OH. Div. of Standards Development and Technology Transfer.

Documentation for Immediately Dangerous to Life or Health Concentrations (IDLHs).

H. R. Ludwig, S. G. Cairelli, and J. J. Whalen. May $94,523 p$

See also PB95-100368

Keywords: "Occupational safety and health, *Acute exposure, "Toxicity, Regulations, Air pollution effects(Humans), Respirators, Toxic substances, Hazardous materials, Concentration(Composition), Classifications, Air pollution effects(Animals), Lethal doses, Respiration, "IDLH(Immediately Dangerous to Life or Health), "Immediately Dangerous to Life or Health, LC(Lethal concentration)

The 'immediately dangerous to life or health air concentration values (IDLHs)' used by the National Institute for Occupational Safety and Health (NIOSH) as respirator selection criteria were first developed in the mid-1970's. This report is a compilation of the rationale and sources of information used by NIOSH during the original determination of 387 IDLHs and their subsequent review and revision in 1994.

03,603

PB95-100368 PC\$14.00/MF A04

National Inst. for Occupational Safety and Health, Cincinnati, $\mathrm{OH}$. Div. of Standards Development and Technology Transfer

NIOSH Pocket Guide to Chemical Hazards.

Jun $94,457 p$

Also pub. as National Inst. for Occupational Safety and Health, Cincinnati, OH. Div. of Standards Development and Technology Transfer rept. no. DHHS/PUB/NIOSH94-116. Also available from Supt. of Docs.

Keywords: *Occupational safety and health, "Chemical compounds, "Health hazards, "Handbooks, Industrial hygiene, Exposure, Chemical properties, Physical properties, Concentration(Composition), Classifications, Toxic substances, Signs and symptoms, First aid, Nomenclature, Identifying, Tables(Data), Exposure limits, Listings

This guide is intended as a source of general industrial hygiene information for workers, employers, and occupational health professionals. It presents key information and data in abbreviated tabular form for 677 chemicals or substance groupings (e.g. manganese compounds, tellurium compounds, inorganic tin compounds, etc.) that are found in the work environment. The industrial hygiene information found in the Guide should help users recognize and control occupational chemical hazards. The chemicals or substances contained in this revision include all substances for which the National Institute for Occupational Safety and Health (NIOSH) has recommended exposure limits (RELs) and those with permissible exposure limits (PELs) as found in the Occupational Safety and Health Administration (OSHA) General Industry Air Contaminants Standard (29 CFR 1910.1000). This revision includes updated sampling and analytical methods, up- dated Department of Transportation (DOT) identification and guide numbers, current exposure limits, revised respirator selections, and revised IDLH (immediately dangerous to life or health concentration) values.

\section{3,604}

PB 95-267779 PC A02/MF A01

National Inst. for Occupational Safety and Health, Cincinnati, $\mathrm{OH}$. Div. of Standards Development and Technology Transfer.

NIOSH Comments to DOL on Risk Estimates from the Cadmium Cohort Study by L. Stayner, February 7, 1992.

7 Feb 92, 8p.

Keywords: "Occupational safety and health, "Testimony, "Risk assessment, "Cadmium, Epidemiology, Carcinogenesis, Mortality, Occupational exposure, Pulmonary neoplasms, Models, CAS 7440439.

This testimony includes a table summarizing the risk estimates from fitting the multistage model to a specific cadmium (7440439) cohort study. The risk estimates from fitting the multistage model to the cadmium study were intermediate between estimates derived from fitting the Cox and Poisson regression models. NIOSH further notes that the risk estimates from the Cost and Poisson models are about $50 \%$ higher than the estimates in the risk assessment report submitted earlie by NIOSH. The lifetime risk was calculated over the entire life span rather than the median life span. Lung cancer rates were used rather than respiratory cance rates. NIOSH assumed a five stage model for the analysis, as this is the number of stages generally observed in other multistage analyses of lung cancer. The model was fit by varing the stage affected by cadmium exposure and the model fit best when NIOSH assumed that cadmium affected the third stage.

\section{Radiobiology}

\section{3,605}

AD-A279 132/5 PC A03/MF A01

National Bureau of Standards, Gaithersburg, MD. $\mathrm{X}$-ray Protection.

1955, 50p

Keywords: “X rays, "Handbooks, "Radiation protection, Radiation shielding, Electromagnetic radiation, Ionizing radiation, Occupational exposure, MPD(Maximum Permissible Dose), Maximum permissible dose.

No abstract available.

\subsection{6}

AD-A279 181/2 PC A03/MF A01

National Bureau of Standards, Gaithersburg, MD $\mathrm{X}$-ray Protection Design.

H. O. Wyckoff, and L. S. Taylor. 9 May 52, 41 p.

Keywords: "X rays, "Radiation protection, Installation, Protective equipment.

No abstract available.

03,607

\section{AD-A279 261/2 PC A04/MF A01}

National Bureau of Standards, Gaithersburg, MD. Protection Against Radiations from Radium, Cobalt-60, and Cesium- 137 .

1 Sep 54, 67p, NBS-HB-54

Keywords: *Radium, "Radiation protection, “Cobalt 60 , *Cesium 137

No abstract available.

03,608

AD-A279 281/0 PC A05/MF A01

National Bureau of Standards, Gaithersburg, MD. Permissible Dose from External Sources of Ionizing Radiation.

24 Sep 54, 89p.

Keywords: "Ionizing radiation, Dose rate, $X$ rays, Radium.

No abstract available.

03,609

AD-A280 281/7 PC A04/MF A01 
National Bureau of Standards, Gaithersburg, MD Maximum Permissible Amounts of Radioisotopes in the Human Body and Maximum Permissible Concentrations in Air and Water.

20 Mar 53, 51p, HANDBOOK-52.

Keywords: "Human body, Concentration(Chemistry) Radiation protection, Air, Water, "Radioactive air pollutants, "Radioactive water pollutants, "Radioisotopes. No abstract available.

\section{3,610}

AD-A280 282/5 PC A06/MF A02

National Bureau of Standards, Gaithersburg, MD

Maximum Permissible Body Burdens and Maximum Permissible Concentrations of Radionuclides in Air and in Water for Occupational Exposure. 5 Jun 59, 104p, HANDBOOK-69.

Keywords: "Radiation protection "Exposure(Physiology), "Human body, "Radioactive air pollutants, "Radioactive water pollutants, Radioisotopes.

No abstract available.

\section{3,611}

AD-A286 681/2 PC A05/MF A01

National Bureau of Standards, Gaithersburg, MD.

Protection Against Neutron Radiation Up to $30 \mathrm{Mil}$ lion Electron Volts.

Handbook.

22 Nov 57, 95p.

Keywords: "Radiation, "Neutron radiography, "Radiation protection, Voltage, Electron irradiation.

No abstract available.

03,612
DE94014709 PC A01/MF A01

National Inst. of Standards and Technology (NEL), Gaithersburg, MD.

lonizing radiation-induced DNA damage and its repair in human cells. Progress report, (April 1,1993-February 28, 1994).

1994, 3p, DOE/ER/60826-T1.

Contract Al05-89ER60826

Sponsored by Department of Energy, Washington, DC Keywords: Endonucleases, Biochemical Reaction Kinetics, Carbon 13, Cytosine, Deuterium Compounds, Gas Chromatography, Guanine, Mass Spectroscopy, Molecular Biology Nitrogen 15 , Progress Report Ra. diolysis, Standards, Thymine, Uracils, "DNA damage "DNA repair, "lonizing radiation, EDB/560120, EDB/ 550200, Cells(Biology).

The excision of radiation-induced lesions in DNA by a DNA repair enzyme complex, namely the UvrABC nuclease complex, has been investigated. Irradiated DNA was treated with the enzyme complex. DNA fractions were analyzed by gas chromatography/isotopedilution mass spectrometry. The results showed that a number pyrimidine- and purine-derived lesions in DNA were excised by the UvrABC nuclease complex and that the enzyme complex does not act on radiation-induced DNA lesions as a glycosylase. This means that it does not excise individual base products, but it excises oligomers containing these lesions. A number of pyrimidine-derived lesions that were no substrates for other DNA repair enzymes investigated in our laboratory were substrates for the UvPABC nuclease complex.

\section{3,613}

DE95007065 PC A01/MF A01

National Inst. of Standards and Technology (IMSE), Gaithersburg, MD

Electron transport calculations with biomedical and environmental applications. Final report, December 23, 1992--January 31, 1994.

PROGRESS REPT.

S. M. Seltzer. 1995, 5p, DOE/ER/61529-T1

Contract AlO2-93ER61529

Sponsored by Department of Energy, Washington, DC

Keywords: "Man, "Radiation Hazards, "Radiation Transport, Biological Radiation Effects, Dosimetry. Mathematical Models, Progress Report, EDB/560100.

The general objective of this project has been to carry out studies of radiation interactions with matter, and of radiation transport in bulk media, in order to generate basic radiological physics information needed as input for: Biomedical radiation dosimetry, Assessment of radiation hazards in nuclear technology, Modeling of biological radiation action. This work has included the development of transport-theoretic methods, the compilation and critical evaluation of the underlying singlescattering cross sections, and the application of the transport methods to radiological physics problems. The project carried out for DOE has been closely related to other projects of the Radiation Interactions and Dosimetry Group of the Ionizing Radiation Division supported in-house by NIST (radiation standards for medical and industrial applications). It has shared with these projects mathematical methods, computer programs, and cross section data bases. The support from DOE has covered approximately $20 \%$ of the activities in the radiation transport area by the Radiation Theory Task. The research for DOE was carried out by S. M. Seltzer and M. J. Berger.

\subsection{4}

DE96007979 PC A02/MF AO1

National Inst. of Standards and Technology (PL), Gaithersburg, MD. Ionizing Radiation Div.

Experimental assessment of absorbed dose to mineralized bone tissue from internal emitters: An electron paramagnetic resonance study.

M. F . Desrosiers 1994, 70, DOE/EH/89334-T1.

Contract Al01-93EH89334

Sponsored by Department of Energy, Washington, DC

Keywords: "Electron spin resonance, "Bone Tissues, Humans, Radiation Doses, EDB/560161, EDB/ 560151, EDB/560101.

EPR resonances attributable to radiation-induced centers in hydroxyapatite were not detectable in bone samples supplied by the USTUR. These centers are the basis for imaging and dose assessment. Presumable, the short range of the alpha particles emitted precluded the formation of appreciable amounts of hydroxyapatite centers. However, one bone sample did offer a suggestion of hydroxyapatite centers and newly-developed methods to extract this information will be pursued.

\subsection{5}

\section{PB94-185139 Not available NTIS}

National Inst. of Standards and Technology (PL), Gaithersburg, MD. Ionizing Radiation Div.

Systematics of Alpha-Particle Energy Spectra and Lineal Energy $(Y)$ Spectra for Radon Daughters.

Final rept.

R. S. Caswell, L. R. Karam, and J. J. Coyne. 1994,

$4 p$.

Pub. in Radiation Protection Dosimetry 52, n1-4 p377. 380 1994. Sponsored by Department of Energy, Washington, DC

Keywords: "Bronchi, "Radon, "Alpha particles, "Biological radiation effects, "Daughter products, Carcinogenesis, Celis(Biology), Cell nucleus, Polonium 218, Polonium 214, Reprints, "Y spectra.

This study is concerned with the irradiation of target cells by alpha particles from radon daughters deposited in the bronchial airways, which may lead to cellular damage and carcinogenesis. Alpha particle energy spectra and y spectra at cell nuclei have been calculated for cell depths between 10 and 70 micrometers for the radon daughter nuclei, (218)Po, (214)Po, and for mixtures of the two. Calculations were performed for ainways of diameters $1.130,0.651,0.435$, and $0.198 \mathrm{~cm}$ (generations 2, 4, 6, and 10 of the $Y e h$ and Schum morphometry). The $y$ spectra assume a site size of 5 micrometers: the changes in the spectra are studied as functions of particular parameters. This information is useful as input to biophysical models of radon induced carcinogenesis. The significance of this work for the radon biological effectiveness problem is discussed.

\section{3,616}

PB94-199643 Not available NTIS

National Inst. of Standards and Technology (PL), Gaithersburg, MD Ionizing Radiation Div.

EPR Bone Dosimetry: A New Approach to Spectral Deconvolution Problems.

Final rept.

M. F. Desrosiers. 1993, 4p.

Pub. in Applied Radiation and Isotopes 44, n1/2 p81841993.

Keywords: "Bones, "Dosimetry, Electron paramagnetic resonance, Radiation accidents, Reprints.

Electron paramagnetic resonance (EPR) dosimetry was used to assess the dose to bone samples from radiation accident victims. Complications in the measurement process arose from overlapping resonances of multiple paramagnetic centers. A computer fitting routine was developed to enhance measurement of the EPR signal of interest, thereby improving the precision of the dose assessment.

\section{3,617}

Pot available NTIS

National Inst. of Standards and Technology (PL) Gaithersburg, MD. Ionizing Radiation Div.

Experimental Validation of Radiopharmaceutical Absorbed Dose to Mineralized Bone Tissue.

Final rept.

M. F. Desrosiers, M. J. Avila, D. A. Schauer, B. M.

Coursey, and N. J. Parks. 1993, 6p.

Pub. in Applied Radiation and Isotopes 44, n1/2 p4594641993

Keywords: "Radiopharmaceuticals, "Bones, "Dosimetry, *Physiologic calcification, Clinical trials, Electron paramagnetic resonance, Reprints.

Therapeutic and palliative uses of bone-seeking radiopharmaceuticals are undergoing clinical trials to human subjects. Radiation dosimetry for these applications is based on the Medical Internal Radiation Dosimetry (MIRD) schema. An experimental method for dosimetry of bone tissue based on electron paramagnetic resonance (EPR) spectrometry is described. Preliminary results for beagle bone exposed to radiopharmaceuticals under clinical conditions have in dicated that the EPR dose measurements give approximately the calculated dose, but suggest that the dose distribution may be non-uniform.

\section{3,618}

PB94-199676 Not available NTIS

National Inst. of Standards and Technology (NML), Gaithersburg, MD. Ionizing Radiation Div.

Radiation Doses.

Final rept.

M. F. Desrosiers, B. M. Coursey, M. J. Avila, and N. J. Parks. 1991, $2 p$

Pub. in Nature 349, n6307 p287-288 1991. Sponsored by California Univ., Davis. Lab. for Energy-Related Health Research

Keywords: "Radiation dosage, Electron paramagnetic resonance, Holmium 166, Reprints.

A radiopharmaceutical containing holmium-166m was administered to a beagle. A bone sample was removed and examined by electron paramagnetic resonance (EPR) spectroscopy. Radiation-induced paramagnetic centers were detected in the bone tissue. The goal o these and future studies will be to quantify these centers in terms of dose and correlate this estimate with theoretical estimates.

03,619

PB94-199700 Not available NTIS

National Inst. of Standards and Technology (PL),

Gaithersburg, MD. lonizing Radiation Div.

New EPR Dosimeter Based on Polyvinylalcohol.

Final rept.

M. F. Desrosiers, J. M. Puhl, and W. L. McLaughlin 1993, 2p.

Pub. in Applied Radiation and Isotopes 44, n1/2 p3253261993

Keywords: "Dosimetry, "Polyvinyl alcohol, Electron paramagnetic resonance, Dose-response relationships, Reprints.

A new dosimetry system based on the electron paramagnetic resonance (EPR) response of polyviny alcohol is presented. The dose response was meas ured from 10-100,000 Gy and the persistence of the signal was monitored over a period of 14 days.

\section{0}

PB94-211273 Not available NTIS

National Inst. of Standards and Technology (NML), Gaithersburg, MD. Ionizing Radiation Div.

National Quality Assurance Program for Personne Radiation Dosimetry: A Case History.

Final rept.

E. H. Eisenhower. 1988, 11p.

Pub. in Proceedings of the National Conference of Standards Laboratories Workshop and Symposium on Competitiveness in a World Market, Washington, DC. August 14-18, 1988, p18-1 - 18-11. 


\section{Radiobiology}

Keywords: "Quality assurance, "Personnel dosimetry, "Radiation dosage, "Dosimetry "Performance standards, Ionizing radiation, Occupational safety and health, Standards, Radiation protection, Health physics, Exposure, Case studies, Reprints.

The estimated 1.3 million workers who are potentially exposed to ionizing radiation in the United States must be monitored for exposure by wearing personnel dosimeters. Accurate measurement of radiation doses received by workers is needed because of the health, economic, and legal consequences of exposure to radiation. Because of a general concern about the reliability of reported doses, government and industry groups made several attempts during 25 years to establish dosimetry performance standards. In 1973 the Conference of Radiation Control Program Directors stated that the necessary degree of reliability was not being achieved, in spite of attempts to provide reliability testing of personnel dosimetry services. This paper describes the subsequent development of a national program which now accredits personnel dosimetry processors that satisfy a prescribed level of performance. Reasons are given why the present program is successful, in contrast to earlier attempts.

\section{3,621}

\section{PB94-211430 Not available NTIS}

National Inst. of Standards and Technology (MSEL), Gaithersburg, MD. Polymers Div.

New Method for Shielding Electron Beams Used for Head and Neck Cancer Treatment.

Final rept.

M. Farahani, F. C. Eichmiller, and W. L. McLaughlin. 1993, 5p.

Pub. in Medical Physics 20, n4 p1237-1241 Jul/Aug 93.

Keywords: "Head and neck neoplasms, "Electron beams, "Radiation shielding, "Radiotherapy, Copper, Silver, Resin matrix composites, Catalysts, Reprints.

Shields and stents of metals with high atomic number, which are custom cast in molds from the melt, are the materials most widely used to protect surrounding tissues during treatment of skin or oral lesions with therapeutic electron beams. An improved fabrication method is to mix a polysiloxane-metal composite, which is readily cast at room temperature by combining a metalpowder/polysiloxane resin mixture with a hardening catalyst. The purpose of the present study is to compare the shielding effectiveness of two different metalpolysiloxane composites with that of conventional cast Lipowitz metal $(50.1 \% \mathrm{Bi}, 26.6 \% \mathrm{~Pb}, 13.3 \% \mathrm{Sn}, 10 \%$ Cd). Also, a 2(sup 3) factorial experiment was run to investigate the effects and interactions of metal particle size (20-micrometers vs 100-micrometers diameter), the atomic weight of the metal (304 stainless steel vs $70 \% \mathrm{Ag}, 30 \% \mathrm{Cu}$ alloy), and the presence or absence of a layer of unfilled polymer added to the forward-scatter side of the shield. The composites of different thicknesses were made by blending $90 \%(w / w)$ metal powder separately with $10 \%$ polysiloxane base and catalyst. A thin GafChromic (TM) dosimeter film was placed between the shielding material and a polystyrene base to measure the radiation shielding effect of composite disc samples irradiated with a $6-\mathrm{MeV}$ electron beam normal to the flat surface of the disc. The results show that composite shields with the metal of higher atomic weight and density ( $\mathrm{Ag}-\mathrm{Cu}$ ) combined with an additional unfilled layer are more effective than the stainless-steel composite with a similar additional unfilled layer, in terms of diminishing the dose at the surface of the polystyrene backing material.

\section{3,622}

PB94-212933 Not available NTIS

National Inst. of Standards and Technology (NML),

Gaithersburg, MD. Ionizing Radiation Physics Div.

Summary of the Proceedings of the Workshop on Standard Phantoms for In-vivo Radioactivity Measurement.

G. H. Kramer, and K G W Inn 1991, 2p.

G. H. Kramer, and K. G. W. Inn. 1991, 2p.
Pub. in Health Physics 61, n6 p893-894 1991.

Keywords: "Meetings, "Anatomic models, "Standards, "Radioactivity, In vivo analysis, Quality assurance, Chest, Thyroid gland, Bone and bones, Tissue distribution, Reprints.

A three-day workshop on Standard Phantoms for Invivo Radioactivity Measurements was co-sponsored by the Bureau of Radiation and Medical Devices, the National Institute of Standards and Technology (NIST). U.S. Nuclear Regulatory Commission, and the U.S.
Department of Energy and held at NIST. It was established at the workshop that: (1) Standards Writing Groups must be organized to specify requirements for Torso, BOMAB, Thyroid, and Bone phantoms; (2) quality assurance of all manufacturing and radioactivity loading stages must include and stress documentation and be traceable to NIST; (3) radionuclides identified by the ANSI N13.30 draft standard are satisfactory; (4) the present Torso, BOMAB, and Thyroid reference phantoms are satisfactory as starting points for standard phantom research, development, and specification, and a set of $5,10,15$ year old and 95 percent adult male is sufficient to fulfill the needs of the community; and (5) radionuclides should be homogeneously distributed in the phantoms.

\section{3,623}

PB95-128658

\section{PC A06/MF A02}

National Inst. of Standards and Technology, Gaithersburg, MD. National Voluntary Lab. Accreditation Program.

National Voluntary Laboratory Accreditation Program: lonizing Radiation Dosimetry.

Handbook

P. R. Martin. Aug 94, 116p, NIST/HB-150-4.

Also available from Supt. of Docs. as SNO03-00303286-7. See also PB94-178225.

Keywords: "Personnel dosimetry, "Dosimetry, "Laboratories, "Handbooks, Dosimeters, Beta dosimetry, Gamma dosimetry, X-ray dosimetry, Standardization, Performance evaluation, Assessments, Calibration, Test methods, Government policies, *Nationa Voluntary Laboratory Accreditation Program, "NVLAP program, *lonizing radiation dosimetry, Traceability,

NIST Handbook 150-4 presents the technical requirements of the National Voluntary Laboratory Accredita tion Program (NVLAP) for Ionizing Radiation Dosim etry (formerly called the Personnel Radiation Dosimetry program). It is intended for information and use by staff of accredited laboratories, those laboratories seeking accreditation, other laboratory accreditation systems, users of laboratory services, and others needing information on the requirements for accreditaton under the Ionizing Radiation Dosimetr program. This publication supplements NIST Handbook 150, NVLAP Procedures and General Requirements, (PB94-178225) which contains Part 285 of Title 15 of the U.S. Code of Federal Regulations (CFR) plus all general NVLAP procedures, criteria, and policies. Handbook 150-4 contains information that is specific to the Dosimetry program and does not duplicate information contained in the Procedures and General Requirements. It is organized to cross-reference with Handbook 150; for example, Section 285.3 of Handbook 150 presents the description and goal of NVLAP. whereas Section 285.3 of Handbook 150-4 presents the description of the Dosimetry program. Where there is no material specific to the field of accreditation, the section number is omitted.

\section{3,624}

PB95-152260 Not available NTIS

National Inst. of Standards and Technology (PL) Gaithersburg, MD. Ionizing Radiation Div.

Neutron Energy Deposition on the Nanometer Scale.

Final rept.

J. J. Coyne, and R. S. Caswell. 1992, 4p.

Contract DÉ-A105-86ER60389

Sponsored by Department of Energy, Washington, DC. Office of Health and Environmental Research.

Pub. in Radiation Protection Dosimetry 44, n1/4 p49521992.

Keywords: Monte Carlo method, Tissues(Biology), Energy transfer, Neutrons, Radiation transport, Reprints, *Neutron dosimetry.

We have incorporated the synthesis of Monte Carlo results for proton tracks of Wilson, Metting, and Paretzke into our analytic method neutron microdosimetry code. Both 'crossers' and 'passers' are included. Results are calculated for neutrons in the energy range $1.05 \mathrm{MeV}$ to $14.5 \mathrm{MeV}$ for site diameters $2 \mathrm{~nm}$ to 1 micrometer.

\section{3,625}

PB95-152344 Not available NTIS

National Inst. of Standards and Technology (PL), Gaithersburg, MD. Ionizing Radiation Div.
Microdosimetry and Cellular Radiation Effects of Radon Progency in Human Bronchial Airways. Final rept.

W. Hofmann, M. Noesterer, M. G. Menache, J. J. Coyne, D. J. Crawtord-Brown, and R. S. Caswell. 1994, 5p.

Sponsored by Department of Energy, Washington, DC. Office of Energy Research. and Health Effects Research Lab., Research Triangle Park, NC

Pub. in Radiation Protection Dosimetry 52, n1-4 p3813851994.

Keywords: "Radon, "Biological radiation effects, "Microdosimetry, "Dose response relationships, "Bronchi, Alpha particles, Daughter products, Radionuclide migration, Respiration, Radiation induced neoplasms, Lung neoplasms, Carcinogenesis, Cells(Biology), Risk assessment, Humans, Reprints, "Cell damage.

The microdosimetric interaction model simulates individual interactions of alpha particles with nuclei of sensitive target cells in human bronchial airway generations 2, 4, 6 and 10. For a normalized source density of Po 218 and Po 214 alpha particles, number of hits, microdosimetric spectra and related parameters for cell nuclei located at various depths in bronchial epithelium are calculated. The lineal energy spectrum is a spherical nuclear target is then converted into probabilities for cell killing, mutation and transformation by multiplying the single event chord length distribution with event specific effect probabilities per unit track length as a function of LET. These effect probabilities are finally weighted by the depth-density distributions of basal and secretory cells. The predicted transformation probability is compared with other physical indicators of lung cancer risk, such as dose, dose equivalent, biologically weighted dose, mean lineal energy, and number of alpha particle hits.

\section{3,626}

PB95-153375 Not available NTIS

National Inst. of Standards and Technology (PL), Gaithersburg, MD. Ionizing Radiation Div.

Extrapolation Chamber Measurements on (90)Sr + (90)Y Beta-Particle Ophthalmic Applicator Dose Rates.

Final rept.

J. O. Deasy, and C. G. Soares. 1994, 9p.

Pub. in Medical Physics 21, n1 p91-99 Jan 94.

Keywords: "Ophthalmology, "Radiation doses, "Beta dosimetry "Extrapolation chambers, Strontium 90, Yttrium 90, Monte Carlo method, Electrodes, Accumulators, Ionizing radiation, Reprints, "Ophthalmic applicators, Collector electrodes.

Aspects of extrapolation chamber dose-rate measurements of (90) Sr + (90)Y beta-particle ophthalmic applicators are examined in this report, including the proper choice of collector electrode size, the gap width over which the measurement should be done the effect of the entrance window materials, and the stoppingpower ratio. Experiments, a simple analytic model for the effect of chamber geometry and nonzero gap width, and more detailed Monte Carlo simulations were used. The variation of the planar flux density as a function of angle for a thick (90) $\mathrm{Sr}+(90) \mathrm{Y}$ source was measured and used as input for the model. From Monte Carlo simulation, the dose rate for tissue irradiation falls off by $8 \%$ between the surface and a depth of $7 \mathrm{mg} / \mathrm{sq} \mathrm{cm}$. The derivative of chamber ionization as a function of gap width, needed for the dose-rate calibration, increases rapidly as the gap width decreases, typically by a factor of about 2 between gap widths of 1.5 and $0.15 \mathrm{~mm}$. The backscattering effect increases the derived surface dose by a factor of 1.46 . A satisfactory dose-rate extrapolation is obtained from gap widths of $0.1-0.25 \mathrm{~mm}$, where the total ionization current is observed to be nearly linear in gap width.

03,627

PB95-161741 Not available NTIS

National Inst. of Standards and Technology (CSTL), Gaithersburg, MD. Biotechnology Div.

DNA Base Damage Generated In vivo in Hepatic Chromatin of Mice upon Whole Body y-Irradiation. Final rept.

T. Mori, Y. Hori, and M. Dizdaroglu. 1993, 6p.

Sponsored by Department of Energy, Washington, DC. Pub. in International Jnl. of Radiation Biology 64, n6 p645-650 1993.

Keywords: "DNA damage, "Liver, "Chromatin, "Gamma rays, Dose-response relationships, Mutagenesis, Carcinogenesis, Mass fragmentography, Reprints, Hydroxyl radicals. 
DNA base lesions in hepatic chromatin formed upon whole-body irradiation of mice were studied. After gamma-irradiating (20-470 Gy) and killing animals, chromatin was isolated from their livers and analyzed by gas chromatography-mass spectroscopy (GC-MS) Five pyrimidine- and five purine-derived DNA lesions were identified and quantified. There were 5-hydroxy5-methylhydantoin, (hydroxymethyl)uracil, formamidopyrimidine hydroxy-adenine 5 -hydroxycytosine 4 - 6 -diamino-52,6-diamino-4-hydroxy-5mine ter two the amounts of these compounds were increased significantly over control levels in the dose range of 100-470 Gy. Above 200 Gy, a deviation from linearity was observed, although the yields were increased in most cases up to 470 Gy. The modified bases that were identified are typically produced by hydroxyl radical attack on the DNA bases. This may indicate a role for hydroxyl radicals in their formation in vivo. These lesions may play a role in the biologica consequences of ionizing radiation such as mutagenesis and carcinogenesis.

\section{3,628}

PB95-162632 Not available NTIS

National Inst. of Standards and Technology (PL), Gaithersburg, MD. Ionizing Radiation Div.

Radiation Process Data: Collection, Analysis, and Interpretation.

Final rept.

M. C. Saylor, S. W. Baryschpolec, L. M. Hurwitz, and W. L. McLaughlin. 1993, 21p

Sponsored by Johnson and Johnson, New Brunswick, NJ.

Pub. in Proceedings of International Kilmer Memorial Conference on Sterilization of Medical Products, Brussels, Belgium, June 13-15, 1993, p240-260.

Keywords: "Dosimetry, "Sterilization, "Data processing, Digital systems, Surveys, Radiation measuring instruments, Digital computers, Ultraviolet radiation, $\mathrm{Re}$ prints, Industrial sterilization.

Prior to 1984 , the capital expense tended to limit radiation-based sterilization technology's access to affordable, state-of-the-art digital technology in the form of programmable logic controllers (PLCs), computers and manufacturing-oriented programming languages. These components were utilized, however, in selected device manufacturing and pharmaceuticals sectors. The paper reviews key aspects of the role of radiation process data collection, analysis and interpretation at present and in the near future as radiation sterilization technologies continue to evolve.

\section{3,629}

\section{PB95-164554 Not available NTIS}

National Inst. of Standards and Technology Gaithersburg, MD. Occupational Health and Safety Div.

Characterization of a Health Physics Instrument Calibration Range.

Final rept.

L. A. Slaback, and C. G. Jones. 1988, 9p.

Pub. in Proceedings of Midyear Topical Meeting on Instrumentation (22nd), San Antonio, TX., December 4 8, 1988, p281-288.

Keywords: "Health physics, "Measuring instruments, "Calibrating, "Test facilities, "Occupational safety and health, Cesium 137, Radiation dosage, Radiation protection, Radiation shielding. Alternatives, Attenuators, Filtration, Reprints

The radiation field of an instrument calibration facility consisting of a $4 \mathrm{~m}$ range and an $80 \mathrm{Ci} \mathrm{Cs}-137$ source with various attenuators was characterized in terms of secondary field components such as backscatter and buildup; intensity of the radiation beam versus distance; actual effective energy of the filtered beams; useful field size and useful calibration distance as a function of detector size. The relative effectiveness of the attenuators with respect to an unfiltered beam is consistent at distances greater than one meter. At distances less than this, the relative effectiveness of the filters is not constant with up to an $8 \%$ change under certain conditions. Aluminum filtration was added to lead attenuators to correct for low energy emissions. This paper examines the scattered environment for a relatively small calibration room $(3 \mathrm{~m} \times 7 \mathrm{~m})$ and presents it as a viable alternative to a large facility. A PC computer program is available which fits the calibration data that are described here.
03,630

PB95-168472 Not available NTIS

National Inst. of Standards and Technology (PL). Gaithersburg, MD. Ionizing Radiation Div.

Angular Variation of the Personal Dose Equivalent $\mathrm{Hp}(0.07)$, for Beta Radiation and Nearly Monoer

sults.

T. A. da Silva, and C. G. Soares. 1994, 12p.

Pub. in Proceedings of Conference on Radiation Protection and Dosimetry (4th), Orlando, FL., October 23 27, 1994, p175-186.

Keywords: "Beta particles, "Electron beams, Reprints, Personal dose equivalent, Angular dependence, Monoenergetic.

Using the NIST standard beta-particle radiation sources, as well as the NIST nearly-monoenergetic electron beams, the angular variation of the personal dose equivalent, $\mathrm{H}$ (sub $\mathrm{p})(0.07)$, was experimentally determined. Measurements were performed with the NIST standard extrapolation chambers, and with thermoluminescent detectors covered with $7-\mathrm{mg} / \mathrm{sq} \mathrm{cm}$ thickness of tissue equivalent material on a slab phantom. Extrapolation chamber correction factors, which are functions of energy and angle of incidence, were determined.

\section{3,631}

\section{PB95-175428 Not available NTIS}

National Inst. of Standards and Technology (PL) Gaithersburg, MD. Ionizing Radiation Div.

Interpreting the Readings of Multi-Element Personnel Dosimeters in Terms of the Personal Dose Equivalent.

Final rept.

M. Ehrlich, and T. Soodprasert. 1994, 13p.

Pub. in Radiation Protection Management 11, n3 p3951 May/Jun 94.

Keywords: "Multi-element analysis, "Personnel dosimetry, "Dose equivalents, "Occupational exposure, Dosemeters, Radiation dose units, Radiation monitoring, Calibration, Performance evaluation, Algorithms Reprints, Angle dependence, Energy dependence, Photon dosimeters.

In 1989, one of the authors (M.E.) suggested an algo rithm for extending the well-known method of evaluating the readings of multi-filter and/or multi-element photon personnel dosimeters to take into account the dependence of their response both on energy and on angle, as is required for an interpretation in terms of the operational quantity "personal dose equivalent. This algorithm was tested on a widely used commercial photon dosimeter. At irradiation levels at which the dosimeter's less sensitive elements of low atomic numbers can be employed, the algorithm was found to be unnecessary for the interpretation of the readings in terms of the personal dose equivalent at a depth of 10 $\mathrm{mm}$ in tissue, over the entire photon-energy range and angles of photon incidence up to $60 \mathrm{deg}$.

\section{3,632}

PB95-175915 Not available NTIS

National Inst. of Standards and Technology (CSTL) Gaithersburg MD Biotechnology Div.

lonizing Radiation Causes Greater DNA Base Damage in Radiation-Sensitive Mutant M10 Cells That in Parent Mouse Lymphoma L5178Y Cells.

Final rept.

T. Mori, and M. Dizdaroglu. 1994, 6p.

Sponsored by Department of Energy, Washington, DC. Pub. in Radiation Research 140, p85-90 1994.

Keywords: "DNA damage, "Mutation, "Ionizing radiation, "Radiation tolerance, Dose-response relationships, Lymphoma, Cultured tumor cells, Chromatin, Mass fragmentography, Pyrimidines, Reprints.

The DNA base damage in radiation-sensitive mutant M10 cells and parent mouse lymphoma L $5178 \mathrm{Y}$ cells was studied. Cells were exposed to ionizing radiation in the dose range of 48 to 400 Gy. Chromatin was isolated from cells and analyzed by gas chromatographymass spectrometry. Ten DNA base products were identified and quantified. A dose-dependent formation of the products was observed. The yields of product in M10 cells were up to threefold greater than in L5178Y cells. Of the products measured, formamidopyrimidines had the highest difference in their yields between the two cell lines. The greater ini tial DNA base damage in M10 cells may play a role in their hypersensitivity to ionizing radiation.
03,633

Not available NTIS

National Inst. of Standards and Technology (PL) Gaithersburg, MD. Ionizing Radiation Div.

Intercomparison Study of (237) Np Determination in Artificial Urine Samples.

Final rept.

S. C. Lee, J. M. Robin Hutchinson, K. G. W. Inn, and M. Thein. 1995, 9p.

Pub. in Health Physics, v68 n3 p350-358 1995.

Keywords: "Neptunium 237, "Radioassay, "Bioassay "Unine, Urinalysis, Occupational exposure, Biological accumulation, Excretion, Radiochemical analysis, Qualitative chemical analysis, Alpha detection, Alpha particles, Coprecipatation, Anion exchanging, Re prints, Inductively coupled plasma mass spectroscopy. An intercomparison study of low-level Np-237 determination in artificial urine samples has been carried out. The purpose of this study was to find the 'optimal' method presently available for use in a routine in-vitro radiobioassay program for occupationally exposed workers. The solutions were submitted to 10 alpha-particle and 10 inductively coupled plasma-mass spec trometry (ICP-MS) laboratories of which six and fou laboratories, respectively, returned results. The best results obtained by ICP-MS were comparable with but not better than the most accurate results obtained by alpha-particle spectrometry. Alpha-particle spectrometry measurements overall gave consistently better agreement with known values.

\section{3,634}

PB96-123708 Not available NTIS

National Inst. of Standards and Technology (PL), Gaithersburg. MD. Ionizing Ra diation Div.

Comparison of NIST and Manufacturer Calibrations of $(90) \mathrm{Sr}+(90) \mathrm{Y}$ Ophthalmic Applicators.

Final rept.

C. G. Soares. 1995, 7p

Pub. in Medical Physics, v22 n9 p1487-14931995.

Keywords: "Calibration, "Radiation doses, Strontium 90, Yttrium 90, lonizing radiation, Reprints, ards and Technology.

Since the resumption of the NIST calibration service for $90 \mathrm{Sr}+90 \mathrm{Y}$ beta-particle ophthaimic applicators, 65 sources have been calibrated using the revised tech nique (C. G. Soares, Med. Phys. 18, 787-793(1991)) For 59 of these sources, the manufacturer's calibration results were available for comparison to the NIST calibration results were available for comparison to the NIST calibration results. The 59 sources represent eight different manufactures, only one of which is still selling new sources. Manufacturer calibration dates range from the present back to 1954. The resuls of the comparisons are presented, broken down by both manufacturer and calibration date: the re are interesting and significant trends in both, with average differences of $20 \%$ not uncommon. The obsolete unit, "roentgenequivalent-beta' (reb), in which some of the manufacturer calibrations are expressed, is discussed and a factor $(0.00982$ Gy reb sub -1$)$ for its conversion to $a b-$ sorbed dose is suggested.

\section{3,635}

PB96-141288 Not available NTIS

National Inst. of Standards and Technology (PL), Gaithersburg, MD. lonizing Radiation Div.

Research and Development Activities in Electron Paramagnetic Resonance Dosimetry. Final rept.

M. F. Desrosiers, G. Burlinska, P. Kuppusamy, F. P. Auteri, M. R. McClelland, C. E. Dick, W. L.

McLaughlin, J. Zweier, and D. M. Yaczko. 1995, 4p.

Pub. in International Meeting on Radiation Processing (9th), Istanbul, Turkey, September 11-16, 1994, Radiation Physics and Chemistry, v46 n4-6 p1181-1184 Oct 95

Keywords: "Irradiation, Electron paramagnetic reso nance, Reprints, Gamma rays, Electron beams, Quartz, "Foreign technology, "Dosimetry, "Alanine, Spin trapping.

This work describes ongoing activities in Electron Paramagnetic Resonance (EPR) dosimetry at the National Institute of Standards and Technology. Progress in the commercialization of the alanine-EPR system will be discussed along wih basic research in highdose dosimetry, including EPR imaging. 


\section{Radiobiology}

National Inst. of Standards and Technology (PL), Gaithersburg, MD. Ionizing Radiation Div.

Investigation of Applicability of Alanine and Radiochromic Detectors to Dosimetry of Proton Clinical Beams.

Final rept.

D. Nichiporov, V. Kostijuchenko, J. M. Puhl, C. E. Dick, W. L. McLaughlin, T. Kojima, B. M. Coursey, S. Zink, D. L. Bensen, and M. F. Desrosiers. 1995, 8p. Pub. in Applied Radiation and Isotopes, v46 $\mathrm{n} 12$ p1355-1362 Dec 95.

Keywords: *Alanine, *Dosimetry, *Proton accelerators, Detectors, Films, Proton beams, Radiotherapy, Radiation dosage, Interlaboratory comparisons, Clinical medicine, Reprints, Radiochromic films, LET.

Cancer therapy studies using proton accelerators are underway in several major medical centers in the U.S. Russia, Japan and elsewhere. To facilitate dosimetry intercomparisons between these laboratories, alaninebased detectors produced at the National Institue of Standards and Technology and commercially available radiochromic films were studied for their possible use as passive transfer dosimeters for clinical proton beams. Evaluation of characteristics of these instruments, including the LET dependence of their response of proton energy, was carried out at the Institute of Theoretical and Experimental Physics.

\section{3,637}

PB96-160254 Not available NTIS

National Inst. of Standards and Technology (PL), Gaithersburg, MD. Ionizing Radiation Div.

External Gamma-ray Counting of Selected Tissues from a Thorotrast Patient.

Final rept.

C. W. Mays, R. L. Aamodt, K. G. W. Inn, V. G. lyengar, F. J. Schima, L. S. Slaback, J. W. Tracy, T P. Lynch, K. L. Mossman, D. R. Brown, and R. R. Greenberg. 1992, 8p.

Pub. in Workshop Proceedings, Health Physics, v63 n1 p33-40 1992

Keywords: *Health physics, Patients, Dose, Tissue sampling, Reprints, "Thorotrast.

Results of gamma-ray measurements of selected tissues from a patient who was injected with Thorotrast almost 36 y ago are reported. The purposes of this study were: (1) to determine the relative tissue distribution and activities of specific radionuclides in the Th decay chain, specifically $\mathrm{Ra}$ (as measured by $\mathrm{Ac}$ ), $\mathrm{Pb}$, and $\mathrm{Ra}$ (measured directly and as measured by $\mathrm{Pb}$ ), and (2) to evaluate the level of radioactive disequilibrium among the daughter products. The spleen and liver had the highest concentrations of radioactivity. Bone also appears to be a long-term sink for Th daughter products based on estimates from a small portion of one rib. Larynx and esophagus contained measurable activity, which may have been due to their proximity to the "Thorotrastoma."

\section{3,638}

PB97-110035 Not available NTIS

National Inst. of Standards and Technology (PL), Gaithersburg, MD. Ionizing Radiation Div.

Radon in the Lung.

Final rept.

R. S. Caswell, and L. R. Karam. 1993, 7p.

See also PB95-152344

Pub. in Radon Research Notes, p6-12 Nov 93.

Keywords: *Radon, "Lungs, "Dose response relationships, Bronchi, Respiration, Computer codes, Reprints, *Cell damage, RADONA

A computer code, RADONA (radon-analytic), has been developed to investigate the physics of the interaction of radon in the lung. This code (1) calculates the slowing-down spectra of alpha particles at the locations of the cells in the bronchial epithelium and (2) calculates y (lineal energy) spectra at specified cell depths and for site sizes in the range of 0.5 to 10 micrometers. The authors are primarily concerned with cylindrical airways.

03,639

PB97-110373 Not available NTIS

National Inst. of Standards and Technology $(\mathrm{PL})$, Gaithersburg, MD. Ionizing Radiation Div.
EPR Dosimetry of Cortical Bone and Tooth Enamel Irradiated with $X$ and Gamma Rays: Study of Energy Dependence.

Final rept.

D. A. Schauer, M. F. Desrosiers, F. G. Le, S. M

Seltzer, and J. M. Links. 1994, 8p.

Sponsored by Navy Health Sciences Education and Training Command, Bethesda, MD. and Department of Energy, Washington, DC. Office of Health and Environmental Research.

Pub. in Radiation Research, v138 p1-8 1994.

Keywords: "X rays, "Tooth enamel, "Cortical bone, "Dosimetry, Energy dependent, Gamma rays, Reprints.

Previous investigators have reported that the radiationinduced EPR signal intensity in compact or cortical bone increases up to a factor of two with decreasing photon energy for a given absorbed dose. If the EPR signal intensity was dependent on energy, it could limit the application of EPR spectrometry and the additive reirradiation method to obtain dose estimates. The au thors have recently shown that errors in the assumptions governing conversion of measured exposure to absorbed dose can lead to similar 'apparent' energydependence results. They hypothesized that these previous results were due to errors in the estimated dose in bone, rather than the effects of energy dependence per se. To test this hypothesis the authors studied human adult cortical bone from male and female donors ranging in age from 23 to 95 years, and bovine tooth enamel, using 34 and $138 \mathrm{keV}$ average energy $X$-ray beams and (137) Cs (662 keV) and (60) Co (1250 keV) gamma rays.

03,640

PB97-110381 Not available NTIS

National Inst. of Standards and Technology (PL),

Gaithersburg, MD. Ionizing Radiation Div.

Exposure-to-Absorbed-Dose Conversion for Human Adult Cortical Bone.

Final rept.

D. A. Schauer, S. M. Seltzer, and J. M. Links. 1993

$5 p$

Sponsored by Navy Health Sciences Education and Training Command, Bethesda, MD. and Department of Energy, Washington, DC. Office of Health and Environmental Research.

Pub. in Applied Radiation and Isotopes, v44 n3 p4854891993.

Keywords: "Bones, "Photons, Humans, Dose-absorbed, Exposure, Reprints, F-factors, Mass energyabsorption coefficients.

The conversion of measured exposure to absorbed dose at a point in bone, under conditions of electron equilibrium, involves a factor (the f-factor) which is proportional to the ratio of the spectrum-averaged photon energy-absorption coefficient for bone to that for air. The paper gives mass energy-absorption coefficients and f-factors for three compositions of human adult compact or cortical bone recommended in publications by the ICRU and ICRP, for photon energies from $1 \mathrm{keV}$ to $1.5 \mathrm{MeV}$. Spectrum-averaged f-factor results reflect: (1) the rather large differences due to the differing calcium contents among the recommended compositions for bone; and (2) the generally poor predictions obtained when replacing a broad energy spectrum by an equivalent photon energy.

03,641

PB97-119259 Not available NTIS

National Inst. of Standards and Technology (PL) Gaithersburg, MD. Ionizing Radiation Div.

Novel Radiochromic Films for Clinical Dosimetry Final rept.

W. L. McLaughlin, J. M. Puhl, M. Al-Sheikhly, A Kovacs, L. Wojnarovits, D. F. Lewis, C. A. Christou, and A. Miller. 1996, 6p.

Pub. in Radiation Protection Dosimetry, v66 n1-4 p263-268 1996.

Keywords: "Dosimetry, Radiotherapy, Radiation dos age, Clinical medicine, Absorption, Gamma rays, $\mathrm{Re}$ prints, "Foreign technology, "Radiochromic films.

New transparent radiochromic films, GafChromic MD55 and NMD-55, which turn from colorless to deep blue upon irradiation, have been designed particulariy for measuring radiation therapy absorbed doses (1 Gy to $100 \mathrm{~Gy}$ ). They are also useful for high resolution mapping of dose distributions, radiographic imaging, treatment planning dosimetry, beam penumbra measurements, and interface dosimetry with ionizing photons, electrons and protons. The gamma ray responses are linear with dose in terms of increase of optical absorbance at 670.633 , and $600 \mathrm{~nm}$ and are independent of absorbed dose rate and relative humidity. The radiochromic images show a slight gradual post-irradiation increase in absorbance especially during the first $24 \mathrm{~h}$. In addition, there is a small but predictable variation of sensitivity with temperature, both during iraddiation and during spectrophotometry. The films also have a slight sensitivity to ultraviolet radiation (250 to $350 \mathrm{~nm}$ ) in direct sunlight. Experiments with $X$ ray beams show no appreciable energy dependence relative to dose in water at photon energies greater than $100 \mathrm{keV}$, but they have a sensitivity that gives readings of about $60 \%$ of the dose in water for photons at 20 to $40 \mathrm{keV}$.

\section{3,642}

PB97-122246 Not available NTIS

National Inst. of Standards and Technology (PL), Gaithersburg, MD.

Flat and Curved Crystal Spectrography for Mammographic $X$-ray Sources.

Final rept.

C. T. Chantler, R. D. Deslattes, A. Henins, and L. T.

Hudson. 1996, 14p.

Pub. in British Jnl. of Radiology, v69 p636-649 Jul 96

Keywords: "X ray, "Mammography, "Spectrography, Crystals, Voltage calibration, Analytical equations, Reprints.

The demand for improved spectral understanding of mammographic $X$-ray sources and non-invasive voltage calibration of such sources has led to research into applications using curved crystal spectroscopy. Recent developments and the promise of improved precision and control are described. Analytical equations are presented to indicate effects of errors and alignment problems in the flat and curved crystal systems. These are appropriate for all detection systems. Application to and testing of spectrographic detection (using standard X-ray film) is presented. Suitable arrangements exist which can be used to measure X-ray tube voltages well below $1 \mathrm{kV}$ precision in the operating range of $20-35 \mathrm{kV}$.

\section{3,643}

PB97-122444 Not available NTIS

National Inst. of Standards and Technology (CSTL), Gaithersburg, MD. Biotechnology Div.

DNA Base Damage in Lymphocytes of Cancer Patients Undergoing Radiation Therapy.

Final rept.

R. Olinski, T H Zastawny, M. Foksinski, M

Dizdaroglu, W. Windorbska, and P. Jaruga. 1996, 9p.

Pub. in Cancer Letters, v106 p207-215 1996.

Keywords: *Radiation therapy, "DNA damage, "Lymphocytes, Patients, Cancer, Free radicals, Hydroxyl radical, Reprints, *Foreign technology.

The authors investigated DNA base damage in genomic DNA of lymphocytes of cancer patients undergoing radiation therapy. Lymphocyte chromatin samples were analyzed by gas chromatography/isotope-dilution mass spectrometry for DNA base damage. The results provided evidence for formation of typical hydroxyl radical-induced base modifications in genomic DNA of lymphocytes. Different levels of DNA products in individuals were observed and, in the case of some patients, there was no significant product formation, possibly resulting from differences between individuals and between the types of radlation exposures. Decreases in product levels after an initial increase by radiation exposure were observed. This may indicate the removal of modified bases from lymphocyte DNA by cellular repair.

\section{Toxicology}

03,644

PB94-182003 Not available NTIS

National Inst. of Standards and Technology (CSTL), Gaithersburg, MD. Biotechnology Div. 
Tert-Butyl Hydroperoxide-Mediated DNA Base Damage in Cultured Mammalian Cells.

Final rept.

S. Altman, T. Zastawny, L. Randers, J. Remacle, M. Dizdaroglu, G. Rao, Z. Lin, and J. Lumpkin. 1994,

$10 \mathrm{p}$.

Contract NIH-RR-06562

Pub. in Mutation Research 306, p35-44 1994. Sponsored by National Institutes of Health, Bethesda, MD

Keywords: "DNA damage, "Mutagens, Cultured cells, Mass spectrometry, Escherichia coli, Dose-response relationships, Hybridomas, Free radical scavengers, Chromatin, Reprints, "Tert-butyl hydroperoxide.

Tert-Butyl hydroperoxide has been utilized to study the effect of oxidative stress on living cells; however its effect on DNA bases in cells has not been characterized. In the present work, we have investigated DNA base damage in mammalian cells exposed to this organic hydroperoxide. SP2/0 derived murine hybridoma cells were treated with 4 concentrations of tert.-butyl hydroperoxide for varying periods of time. Chromatin was isolated from treated and control cells and subsequently analyzed by gas chromatography-mass spectrometry with selected-ion monitoring for DNA-base damage. Quantification of damaged DNA bases was achieved by isotope-dilution mass spectrometry. The amounts of 8 products were significantly higher than control levels in cells treated with tert-butyl hydroperoxide at a concentration range of 0.01-0.1 $\mathrm{mM}$. At concentrations from 1.0 to $10 \mathrm{mM}$, product formation was inhibited and the amounts of products were similar to those in control cells. The bimodal nature of the dose-response may be qualitatively analogous to previous reports of bimodal killing of $E$. coli bacteria by hydrogen peroxide. The nature of the identified DNA base lesions suggests the involvement of the hydroxyl radical in their formation. The inhibition of product formation at high concentrations of tert.-butyl hydroperoxide may be explained by the scavenging of tert.-butoxyl radical by tert.-butyl hydroperoxide resulting in inhibition of oxidative stress. The plausibility of the scavenging mechanism was evaluated with a mathematical simulation of the dose-response for DNA damage in solutions containing hydrogen peroxide.

\section{3,645}

PB94-198231 Not available NTIS

National Inst. of Standards and Technology (CSTL), Gaithersburg, MD. Biotechnology Div.

Copper Ion-Mediated Modification of Bases in DNA in Vitro by Benzoyl Peroxide.

Final rept.

S. A. Akman, T. W. Kensler, J. H. Doroshow, and M. Dizdaroglu. 1993, 4p.

Pub. in Carcinogenesis 14, n9 p1971-1974 1993.

Keywords: "DNA damage, "Copper, "Mutagens, "Benzoyl peroxide, In vitro analysis, Ions, Mass fragmentography, Free radical scavengers, Dimethyl sulfnxide, Reprints.

The mouse skin tumor promoter benzoyl peroxide (BzPO), in conjunction with $\mathrm{Cu}(\mathrm{l})$, causes promutagenic damage in DNA. Because free radical intermediates are produced by the reaction of BzPO with $\mathrm{Cu}(\mathrm{I})$, the authors sought to determine whether BzPO plus Cu(I) caused DNA base damage typical of that caused by the hydroxyl radical. A broad range of modified DNA bases were measured by GC-MS with selected-ion monitoring after exposure of purified plasmid pCMV beta gal DNA to BzPO + or - Cu(I). Exposure to BzPO/Cu(I) caused up to 20-fold increases in the levels of adenine-derived modified bases, up to 4 -fold increases in guanine- and cytosine-derived modified bases, and only a <2-fold increase in thymine-derived modified bases. The guanine-derived modified base 8-hydroxyguanine was elevated to the highest net amount. Exposure to BzPO alone or $\mathrm{Cu}(\mathrm{l})$ alone induced only minor ( $<<2$-fold) DNA base modification. Also benzoic acid, the major non-radical metabolite of $\mathrm{BzPO}$, or BzPO plus $\mathrm{Fe}(\mathrm{II})$ were ineffective at inducing DNA base modification. The hydroxyl radical scavenger dimethyl sulfoxide inhibited BzPO/ $\mathrm{Cu}(\mathrm{l})$-induced base modification by $10-50 \%$. These data suggest that the reaction of BzPO with $\mathrm{Cu}(\mathrm{I})$ generates hydroxyl radical or a similarly reactive intermediate which causes DNA base damage. This damage may be responsible for $\mathrm{BzPO} / \mathrm{Cu}(\mathrm{I})$-mediated mutagenesis

\section{3,646}

PB94-212628 Not available NTIS

National Inst. of Standards and Technology (CSTL), Gaithersburg, MD. Biotechnology Div.
Nickel(II)-Mediated Oxidative DNA Base Damage in Renal and Hepatic Chromatin of Pregnant Rats and Their Fetuses. Possible Relevance to Carcinogenesis.

Final rept.

K. Kasprzak, B. Diwan, J. Rice, M. Dizdaroglu, M Misra, and R. Olinski. 1992, 7p

Pub. in Toxicology 5, n6 p809-815 Nov/Dec 92.

Keywords: "DNA damage, "Nickel, "Liver, "Kidney, Teratogens, "Chromatin, Carcinogens, Fetus, Oxidation, Reprints.

DNA base damage was studied in renal and hepatic chromatin of nickel(II)-injected pregnant female F344/ $\mathrm{NCr}$ rats and their fetuses under conditions leading to nitiation of sodium barbital-promotable renal tumors. but not liver tumors, in the male offspring. Pregnant rats were given a total of 90 or 180 micromol of nickel(II) acetate/kg body wt in a single ip dose on day 17 or in 2 or 4 ip doses between days 12 and 18 of gestation. Control rats received 180 micromol of sodium acetate/kg body wt. The animals were killed 24 or $48 \mathrm{~h}$ after the last injection. Chromatin was isolated from livers and kidneys from both adults and fetuses and analyzed by gas chromatography/mass spectrometry with selected ion monitoring. Eleven products derived from the purine and pyrimidine bases in DNA bases were identified and quantified. Nickel(II) exposure increased the content of these products, especially those derived from purines, in both renal and hepatic chromatin of pregnant rats. The major difference between these two organs was the content of $8-\mathrm{OH}$ Gua, which increased greatly in the kidney but remained unchanged in the liver. In the corresponding etal organs, the relative increases in 8-OH-Gua were comparable to the findings in adults. Fetal kidney DNA was relatively higher in pyrimidine-derived products (especially thymine glycol and 5-hydroxyhydantoin and lower in purine-derived products (except for $8-\mathrm{OH}$ Gua) than fetal hepatic DNA. No consistent dose effect of nickel(II) on the amounts of the DNA base products recovered from either organ was observed in either the dams or their fetuses. The present results indicate possible involvement of oxidative DNA base damage in the mechanism of nickel(II) carcinogenesis in the rat kidney.

\section{3,647}

PB95-150025 Not available NTIS

National Inst. of Standards and Technology (CSTL), Gaithersburg, MD. Biotechnology Div.

Oxidative DNA Base Damage in Renal, Hepatic, and Pulmonary Chromatin of Rats After Intraperitoneal Injection of Cobalt (II) Acetate.

Final rept.

K. Kasprzak, T. Zastawny, S. North, J. Rice, M.

Dizdaroglu, C. Riggs, and B. Diwan. 1994, $7 p$.

Pub. in Chemical Research in Toxicology $7, n 3$ p329 335 May/Jun 94

Keywords: "DNA damage, "Chromatin, "Liver, "Kidney, "Lung, "Mutagens, Rats, Mass fragmentography, Sex factors, Oxidation, Carcinogens, Reprints, "Cobalt acetate.

DNA base damage was studied in renal, hepatic, and pulmonary chromatin of male and female $\mathrm{F} 344 / \mathrm{NCr}$ rats that had been given either 50 or 100 micromol of Co(II) acetate/kg body wt in a single ip dose and killed 2 or 10 days later Control rats received 200 micromo of sodium acetate $/ \mathrm{kg}$ body wt. Chromatin was isolated from organs and analyzed by gas chromatography! mass spectrometry with selected ion monitoring. Eleven products derived from purine and pyrimidine bases in DNA were quantified. The response was organ-specific. Eight of the DNA base products in renal chromatin of CO(II)-treated rats, five in hepatic chromatin, and two in pulmonary chromatin were increased by $30 \%$ to more than $200 \%$ over control levels with increasing CO(II) dose. The renal and hepatic, but not pulmonary, DNA base damage tended to increase with time No significant differences in response were found between male and female rats. The bases determined were typical products of hydroxyl radical attack on DNA, suggesting a role for this radical in the mechanism(s) of DNA damage caused by Co(II) in vivo. Some of these bases have been shown previously to be promutagenic. The present results imply involvement of oxidative DNA damage in $\mathrm{Co}(\mathrm{II})$-induced genotoxic and carcinogenic effects.

03,648

PB95-150363 Not available NTIS

National Inst. of Standards and Technology (CSTL) Gaithersburg. MD. Biotechnology Div.
DNA Base Modifications in Renal Chromatin of Wistar Rats Treated with a Renal Carcinogen, Ferric Nitrilotriacetate.

Final rept

S. Toyokuni, T. Mori, and M. Dizdaroglu. 1994, 6p.

Sponsored by Department of Energy, Washington, DC

Pub. in International Jnl. of Cancer 57, p 123-128 1994

Keywords: "DNA damage, "Carcinogens, "Chromatin "Kidney, Wistar rats, Mass fragmentography, Proximal kidney tubules, Necrosis, Mutagens, Carcinogenesis, Reprints, "Ferric nitrilotriacetate.

Ferric nitrilotriacetate ( $\mathrm{Fe}$-NTA) causes renal proximal tubular necrosis, a consequence of iron ion-mediated free-radical-associated damage, that finally leads to a high incidence of renal adenocarcinoma in male rats and mice. We have investigated the levels of typical hydroxyl radical-induced DNA base modifications in renal chromatin of male Wistar rats treated with a single or repeated administrations of Fe-NTA. Five pyrimidine-derived and 5 purine-derived modified DNA bases were identified and quantified by gas chromatography/ mass spectrometry with selected-ion monitoring. The amounts of most of these compounds were significantly increased over control levels in renal chromatin of Fe-NTA-treated rats as measured 3 and 24 hrs after treatment. Elevated levels of modified bases were accompanied by proximal tubular necrosis. Presence of modified DNA bases concomitant with necrosis and regeneration of the renal proximal tubules may be a critical step in Fe-NTA-induced carcinogenesis.

03,649

PB96-112115 Not available NTIS

National Inst. of Standards and Technology (CSTL), Gaithersburg, MD. Biotechnology Div.

Treatment of Wistar Rats with a Renal Carcinogen, Ferric Nitrilotriacetate, Causes DNA-Protein CrossLinking between Thymine and Tyrosine in Their Renal Chromatin.

Final rept.

S. Toyokuni, T. Mori, H. Hiai, and M. Dizdaroglu.

1995, 5p.

Pub. in International Jnl. of Cancer, v62 p309-313, 14 Sep 95

Keywords: "DNA damage, "Carcinogens, "Chromatin, "Kidney, Reprints, Wistar rats, Carcinogenesis, Necrosis, Proximal kidney tubules, "Ferric nitrilotriacetate.

Ferric nitrilotriacetate (Fe-NTA) induces renal proximal tubular damage associated with lipid peroxidation and oxidative DNA base modifications that finally leads to a high incidence of renal adenocarcinoma in rodents. In the present study, we report on the in vivo formation of DNA-protection cross-links (DPCs) involving thymine and tyrosine in the renal chromatin of Wistar rats treated with single or repeated i.p. administration of $\mathrm{Fe}$ NTA. Analyses of chromatin samples by gas chromatography/mass spectrometry revealed a significant increase in the amount of 3-((1,3-dihydro- 2,4 dioxopyrimidin- 5-yl)-methyl)-L-tyrosine (Thy-Tyr cross-link) 23 and $48 \mathrm{hr}$ after the administration of $\mathrm{Fe}$ NTA. At19th day of FE-NTA treatment, the amount of Thy-Tyr cross-link decreasedto the control level, indicating the presence of cellular repair activity. Thy-Ty cross-link may play a role in the genetic alteration of this renal carcinogenesis model, since mitoses for reregeneration of renal proximal tubules were closely associated with the increase in DPCs.

\section{3,650}

PB96-123260 Not available NTIS

National Inst. of Standards and Technology (CSTL), Gaithersburg, MD. Biotechnology Div.

Further Development of the $\mathrm{N}$-Gas Mathematica Model: An Approach for Predicting the Toxic Potency of Complex Combustion Mixtures.

Final rept.

B. C. Levin, E. Braun, M. Navarro, and M. Paabo. 1995, 19p.

Pub. in Fire and Polymers II, Chapter 20, p293-311 Oct

Keywords: "Combustion, Mathematical models, Carbon dioxide, Carbon monoxide, Complex mixtures, $\mathrm{Hy}$ drogen chloride, Hydrogen cyanide, Toxicity, Reprints, "N-gas model.

A methodology has been developed for predicting smoke toxicity based on the toxicological interactions of complex fire gas mixtures. This methodology consists of burning materials using a bench-scale method that simulates realistic fire conditions, measuring the concentrations of the following primary fire gases - $\mathrm{CO}$, 


\section{Toxicology}

$\mathrm{CO} 2, \mathrm{O} 2, \mathrm{HCN}, \mathrm{HCl}, \mathrm{Hbr}$, and $\mathrm{NO} 2$ - and predicting the toxicity of the smoke using an empirical mathematical model called the N-Gas Model. The model currently in use is based on toxicological studies of the first six of the above listed primary gases both as individual gases and complex mixtures. The predicted toxic po tency (based on this N-Gas Model) is checked with a small number of animal (Fischer 344 male rats) tests to assure than an unanticipated toxic gas was not generated. The results indicate whether the smoke from a material or product is extremely toxic (based on mass consumed at the predicted toxic level) or unusually toxic (based on mass consumed at the predicted toxic level) or unusually toxic (based on the gases deemed responsible). The predictions based on bench-scale laboratory tests have been verified with full-scale room burns of a limited number of materials of widely differing characteristics chosen to challenge the system.

\section{3,65}

PB96-137724 Not available NTIS

National Inst. of Standards and Technology (CSTL) Gaithersburg, MD. Biotechnology Div.

Formation of DNA-Protein Cross-Links in Cultured Mammalian Cells Upon Treatment with Iron Ions. Final rept.

S. A. Altman, T. H. Zastawny, L. Randers-Eichhorn, M. Dizdaroglu, G. Rao, M. A. Cacciuttolo, and S. A. Akman $1995,6 p$.

Pub. in Free Radical Biology and Medicine, v19 n6 p897-902 1995 .

Keywords: "DNA-binding proteins, "Crosslinking, "Chromatin, "Iron, Toxicity, Reprints, Hydroxyl radical Oxidative stress, Free radicals, Thymine-tyrosine crosslinks.

Formation of DNA-protein crosslinks (DPCs) in marnmalian cells upon treatment with iron or copper ions was investigated. Cultured murine hybridoma cells were tested with $\mathrm{Fe}(\mathrm{II})$ or $\mathrm{Cu}(\mathrm{II})$ ions by addition to the culture medium at various concentrations. Subsequently, chromatin samples were isolated from treated and control cells. Analyses of chromatin samples by gas chromatography/mass spectrometry after hydrolysis and derivatization revealed a significant increase over the background amount of 3-((1,3-dihydro- 2,4 dioxopyrimidin- 5-yl)-methyl)-L-tyrosine (Thy-Tyr crosslink) in cells treated with Fe(II) ions in the concentration range of 0.01 to $1 \mathrm{mM}$. In contrast $\mathrm{Cu}$ (II) ions at the same concentrations did not produce this DPC in cells. No DNA base damage was observed in cells treated with $\mathrm{Cu}(\mathrm{II})$ ions, either. Preincubation of cells with ascorbic acid or coincubation with dimethyl sulfoxide did not significantly alleviate the $\mathrm{Fe}$ (II) ion-mediated formation of DPCs. In addition, a modified fluorometric analysis of DNA unwinding assay was used to detectDPCs formed in cells. Fe(II) ions caused significant formation of DPCs, but CU(II) ions did not The nature of the Fe(II)-mediated DPCs suggeststhe involvement of the hydroxyl radical in their formation The Thy-Tyr crosslink may contribute to pathological processes associated with free radical reactions.

\section{3,652}

PB96-161740 Not available NTIS

National Inst. of Standards and Technology (CSTL) Gaithersburg, MD. Biotechnology Div.

Protonation Dynamics of the alpha-Toxin Ion Channel from Spectral Analysis of $\mathrm{pH}$-Dependent Current Fluctuations.

Final rept

J. J. Kasianowicz, and S. M. Bezrukov. 1995, $12 p$.

Pub. in Biophysical Jnl., v69 p94-105 Jul 95.

Keywords: "Conductance fluctuations, "Amino acids, "Noise analysis, Reprints, "Deuterium isotope effects, Ionization dynamics, Protein ion channels.

To probe protonation dynamics inside the fully open alpha-toxin ion channel, the authors measured the $\mathrm{pH}$ dependent fluctuations in its current In the presence of $1 \mathrm{M} \mathrm{NaCl}$ dissolved in $\mathrm{H} 2 \mathrm{O}$ and positive applied potentials (from the side of protein addition), the low frequency noise exhibited a single well defined peak between $\mathrm{pH} 4.5$ and 7.5. A simple model in which the current is assumed to change by equal amounts upon the reversible protonation of each of $N$ identical ionizable residues inside the channel describes the data well. These results, and the frequency depend ence of the spectral density at higher frequencies, allow the authors to evaluate the effective $\mathrm{pK}=5.5$ as well as the rate constants for the reversible protonation reactions: kon $=8 \times 10$ to the 9 th power $M$ to the minus 1 power $S$ to the minus 1 power and Koff $=2.5 \times 10$ to the 4 th power $S$ to the minus 1 power
The estimate of kon is only slightly less than the diffusion-limited values measured by others for protonation reactions for free carboxyl or imidazole residues. Substitution of $\mathrm{H} 2 \mathrm{O}$ by $\mathrm{D} 2 \mathrm{O}$ caused a 3.8 -fold decrease in the dissociation rate constant and shifted the $\mathrm{pK}$ to 6.0 . The decrease in the ionization rate constants caused by $\mathrm{H} 2 \mathrm{O} / \mathrm{D} 30$ substitution permitted the reliable measurement of the characteristic relaxation time over a wide range of $\mathrm{D}+$ concentrations and voltages. The dependence of the relaxation time on $\mathrm{D}+$ concentration strongly supports the first order reaction model.

\section{General}

\section{3,653}

PB95-189510 PC A03/MF A01

National Inst. of Standards and Technology (CSL), Gaithersburg, MD. Computer Security Div.

Assessment of the DOD Goal Security Architecture (DGSA) for Non-Military Use.

A. E. Oldehoeft. Nov $94,34 p$, NISTIR-5570.

See also PB94-170362 and PB95-109203. Sponsored by Defense Information Systems Agency, Arlington,

Keywords: "US DOD, "Computer security, *Commercial sector, Systems engineering, Data processing security, Standards, Computer networks, Information systems, Telecommunication, Computer systems hardware, Computer software, "DGSA(DoD Goal Security Architecture), "DoD Goal Security Architecture, ISO(International Standards Organization), ISA(Internet Security Architecture), DCE(Distributed Computing Environment).

The purpose of this study is to assess the potential of the DOD Goal Security Architecture (DGSA) as a model and framework for the development of non-military computer information security architectures. To achieve this goal, this study presents an overview of the DGSA and provides a cursory comparison of some of the features of the DGSA with several prominent, proposed non-military security architectures including: the International Standards Organization (ISO): SES$A M E$ : the Internet Security Architecture (ISA); and Open Systems Foundation (OSF) Distributed Computing Environment (DCE). The study concludes the DGSA has the potential for being applicable in both the civilian and commercial sectors. However, there are some attending restrictions that may prevent its acceptance. These restrictions are detailed in the Summary Observations.

\section{3,654}

PB95-203790 PC A03/MF A01

National Inst. of Standards and Technology (MEL) Gaithersburg, MD. Intelligent Systems Div.

Robotics Application to Highway Transportation. Volume 1. Final Report.

Rept. for Sep 92-Nov 93

E.W. Kent Jan 95, 34p, FHWA/RD-94/053.

Contract DTFH61-92-Y-00160

See also PB95-170551, PB95-171633 and PB95193173. Sponsored by Federal Highway Administration, McLean, VA. Office of Advanced Research.

Keywords: "Air Force training, "United States Air Force Acadre "Career development, Military training Air Force planning, Air Force personnel, Education, Schools, Careers, Curriculum development, Military personnel, Military planning.

The Office of the Registrar, Academic Affairs Division (DFRC) publishes the Curriculum Handbook at the beginning of each calendar year. Together with the USAFA Catalog published by the Office of Administrations (RR), it documents the curriculum of the United States Air Force Academy as approved by the Academy Board. The Curriculum Handbook contains general information concerning graduation requirements, academic registration procedures, course offerings, academic majors, and academic probation.
03,655

Not available NTIS National Inst. of Standards and Technology (EEEL) Boulder, CO. Electromagnetic Fields Div. NIST and the Navy: Past, Present and Future. Final rept.

G. R. Reeve, and D. S. Friday. 1995, 9p. Sponsored by American Society of Naval Engineers Alexandria, VA. and Society of Logistics Engineers, New Carrollton, MD.

Pub. in Proceedings of the Test and Calibration Symposium, Washington, DC., November 30-December 1 1994, p229-237.

Keywords: "Navy, "Interagency cooperation, US DOD Technology innovation, Naval research, Research support, Research programs, Support services, Program management, History, Reprints, "US NIST.

The National Institute of Standards and Technology (NIST) has had an historic and fruitful relationship with the Department of Defense, its predecessors, and the three military services, particularly the Navy. In this paper, we will outline some of the historic support, current collaborations, and what areas of technology may require our support for the Navy of the future.

\section{Logistics, Military Facilities, \& Supplies}

\section{3,656}

AD-A280 223/9 PC A06/MF A02

National Bureau of Standards, Gaithersburg, MD.

1950 Supplement to Screw-Thread Standards for Federal Services. 1944.

20 Mar 51, 119p.

Keywords: "Federal law, "Standards, "Screw threads, Handbooks, Department of Defense.

No abstract available.

\section{3,657}

PB94-962000 Contact NTIS for subscription

National Inst. of Standards and Technology, Gaithersburg, MD.

CALS-Automated Interchange of Technical Information.

Irregular repts.

1994, open series.

Supersedes PB93-962000

Paper copy available on Standing Order, deposit account required (minimum deposit \$100 U.S., Canada and Mexico; all others $\$ 200$ ). Single copies also available in paper copy or microfiche.

Keywords: "Standards, "Specifications, Digital data, Manuals, Computer graphics, Computer aided design, Computer aided manufacturing, File management systems, Automation, Information processing, "CALS(Computer-aided Acquisition and Logistics Support)

The publication is the parent document for a family of military specifications through which the Computeraided Acquisition and Logistics Support (CALS) standards will be published. It provides the 'enveloping' rules for organizing files of digital data into a complete document--for example, a technical manual composed of MIL-M 28001 text, MIL-D-28003 Vector Graphics, and MIL-R-28001 Raster Graphics.

\section{3,658}

PB94-962100 Contact NTIS for subscription information and price.

National Inst. of Standards and Technology, Gaithersburg, MD

CALS-Digital Representation for Communication of Product Data: IGES Application Subsets.

Irregular repts.

1994, open series.

Supersedes PB93-962100.

Paper copy available on Standing Order, deposit account required (minimum deposit $\$ 100$ U.S., Canada, and Mexico; all others $\$ 200$ ). Single copies also available in paper copy or microfiche.

Keywords: "Computer aided design, Computer aided manufacturing, Graphic arts, Numerical control, Engineering drawings, Data transmission, 
"CALS(Computer-aided Acquisition and Logistics Support), "IGES(Initial Graphics Exchange Specification).

The publication defines subsets of the Initial Graphics Exchange Specification (IGES) for technical illustration, engineering drawings, electrical/electronic applications, and numerical control manufacturing, IGES is a neutral format for digital interchange of Product Definition Data between dissimilar computer aided design systems.

\section{3,659}

PB94-962200 Contact NTIS for subscription

information and price. National Inst. of Standards and Technology, CALS-Markup Requirements and Generic Style Specifications for Electronic Printed Output and Exchange of Text.

Irregular repts.
1994 , open series.

1994, open series.
Supersedes PB93-962200

Paper copy available on Standing Order, deposit account required (minimum deposit \$100 U.S., Canada and Mexico; all others \$200). Single copies also available in paper copy and microfiche.

Keywords: "Publishing, "Automation, Text processing, Computer aided design, Computer aided manufacturing, "CALS(Computer-aided Acquisition and Logistics Support), Department of Defense.

The publication defines standard Department of Defense (DoD) requirements for all steps involved in automated publishing of page-oriented (i.e., printed) technical publications. For exchange of source data (prior to document composition it defines a common implementation of the Standard Generalized Markup Language (SGML). For composition processing functions, it defines an Output Specification of typographic tags and format rules, and for display of the composed document, it provides options for use of commercial Page Description ranguages.

\section{3,660}

P894-962300 Contact NTIS for subscription information and price.

National Inst. of Standards and Technology, Gaithersburg, MD

CALS-Raster Graphics Representation Binary Format Requirements.

Irregular repts.

1994 , open series

Supersedes PB93-962300

Paper copy available on Standing Order, deposit account required (minimum deposit $\$ 100$ U.S., Canada and Mexico; all other $\$ 200$ ). Single copies also available in paper copy or microfiche.

Keywords: "Computer aided design, "Computer graphics, File management systems, Documents, Facsimile communication, Image processing, Standards, Computer aided manufacturing, ${ }^{*} \mathrm{CALS}$ (Computer-aided Acquisition and Logistics Support), Department of Defense.

The publication defines Department of Defense (DoD) technical requirements for raster (bit-map) graphics that have been compressed to reduce file size and transmission time. Computer-aided Acquisition and Logistics Support (CALS) must apply raster graphics to both office documents and facsimiles (for which the international standard that MIL-R-28002 implements was prepared), and for engineering drawings and other oversize documents. An option is included to use tiling in which the raster image is divided into a series of tiles that can be individually processed to reduce throughout and terminal storage requirements.

\section{3,661}

PB94-962400 Contact NTIS for subscription information and price.

National Inst. of Standards and Technology, Gaithersburg, MD.

CALS-Digital Representation for Communication of Illustration Date: CGM Application Profile. Irregular repts.

1994 , open series

Supersedes PB93-962400.

Paper copy available on Standing Order, deposit account required (minimum deposit $\$ 100$ U.S., Canada, and Mexico; all others $\$ 200$ ). Single copies also available in paper copy or microfiche.

Keywords: "Computer aided design, "Computer graphics, Manuals, Graphic arts, Work stations, Digital data,
Two dimensional models, Computer aided manufacturing, "CALS(Computer-aided Acquisition and Logistics Support), Computer graphics metafile.

The publication defines use of Computer Graphics Metafile (CGM) for two dimensional vector (line segment) picture descriptions or illustrations in technical manuals. Whereas Initial Graphics Exchange Specification (IGES) has its principal use within computeraided design, CGM is becoming widely available for authoring and graphic art work stations.

\section{3,662}

P894-962500 Contact NTIS for subscription

information and price. National Inst. of Standards and Technology,
Gaithersburg, MD.

CALS-Department of Defense Computer Aided ACquisition Logistic Support (CALS).

Irregular repts.

1994, open series.

Supersedes PB93-962500

Paper copy available on Standing order, deposit account required (minimum deposit $\$ 100$ U.S., Mexico, and Canada; all others $\$ 200$ ). Single copies also available in paper copy or microfiche.

Keywords: *Weapons system, "Logistics management, Acquisition, Information services, Contract administration, Computer aided design, Computer aided manufacturing, "CALS(Computer-aided Acquisition and Logistics Support), Department of Defense.

The guide assists weapon system acquisition managers to understand when, where, and how to apply Computer-Aided Acquisition and Logistics Support (CALS) capabilities efficiently to support their information interchange and access requirements, and how to define their functional requirements for integration of the contractor process (such as Reliability and Maintainability analysis) that create and use the information.

\section{3,663}

PB94-962600 Contact NTIS for subscription

information and price.

National Inst. of Standards and Technology, Gaithersburg, MD

CALS-Contractor Integrated Technical Information Service (CITIS), Functional Requirements.

Irregular repts.

1994, open series

Supersedes PB93-962600.

Paper copy available on Standing Order, deposit account required (minimum deposit \$100 U.S., Canada, and Mexico; all others $\$ 200$ ). Single copies also available in paper copy or microfiche.

Keywords: "Information services, "Requirements, On line systems, Contractors, US DOD, "CALS(Computeraided Acquisition and Logistics Support), "CITIS(Contractor Integrated Technical Information Service).

The publication describes functional requirements for the Department of Defense's (DoD's) new ComputerAided Acquisition and Logistics Support (CALS) acquisition policy which gives preference to contractor information services and online access instead of data deliverables. Contractor Integrated Technical Information Service (CITIS) addresses how DoD will buy information services instead of what form the deliverable will be in whether paper or digital based. it defines things a contractor must do, such as planning, analysis, and submitting proposals and things the service must do, such as managing data, and providing access to the data tailored to meet a government Concept of Operations.

\section{3,664}

PB95-105029 PC AO4/MF A01

National Inst. of Standards and Technology (CAML), Gaithersburg, MD. Office of Applied Economics.

Present Worth Factors for Life-Cycle Cost Studies in the Department of Defense (1995).

Final rept.

S. R. Petersen. Oct 94, 63p, NISTIR-4942-2.

See also PB94-109238. Sponsored by Assistant Secretary of Defense (Production and Logistics), Washington, DC.

Keywords: *Life cycle costs, *Present worth, *Energy supplies, "Military facilities, Benefit cost analysis, Economic analysis, Energy conservation, Buildings, Construction, Return on investment, Cost engineering, Fuels, Regional analysis, Department of Defense, Tables(Data), Military Construction Program.
This document provides 47 tables of present worth factors to be used in computing the present worth of future costs (or cost reductions) in economic analyses of design decisions for projects in the Department of Defense (DoD) Military Construction Program. These facors are especially useful for the life-cycle cost analysis f investments in buildings or building systems which are intended to reduce future operating, maintenance, repair, replacement, and energy costs over the life of the facility. The tables include present worth factors for both one-time costs and annually recurring costs based on the Federal Energy Management Program (FEMP) discount rate of 3.0\% (FY 1995) for energyrelated studies and on the Office of Management and Budget (OMB) discount rate of $2.5 \%$ and $2.8 \%$ for short-term and long-term non-energy studies, respectively. Forecasts of future energy prices used in the calculation of present worth factors for energy costs were provided by the Energy Information Administration.

\section{3,665}

PB95-162723 Not available NTIS

National Inst. of Standards and Technology (NEL), Gaithersburg, MD. Robot Systems Div.

Vehicle-Command Center Communications in a Robotic Vehicle System.

Final rept.

H. A. Scott. 1992, 18p.

Pub. in Automation in Construction 1, n3 p267-284 Dec 92

Keywords: "Ground vehicles, "Automatic control, Robotics, Remote control, Communications central Army, Real time operations, Data links, Telecommunication, Reprints, Remote vehicles, Robotic vehicles Real time control, Remote data links.

The U.S. Army Laboratory Command is developing a testbed for cooperative, real-time control of multiple land vehicles from a remotely located command center. The paper analyzes the characteristics of the communication link between the vehicle and the remote command center. Areas addressed include the com munication technology, the information content and required rates, the control system communication requirements, communications support of the development effort and examination of available standards.

\section{3,666}

PB95-962000 Contact NTIS for subscription information and price.

National Inst. of Standards and Technology, Gaithersburg, MD

CALS-Automated Interchange of Technical Information.

Irregular repts.

1995, open series.

Supersedes PB94-962000

Paper copy available on Standing Order, deposit ac count required (minimum deposit $\$ 100$ U S Canada and Mexico; all others $\$ 200$ ). Single copies also available in paper copy or microfiche.

Keywords: "Standards, "Specifications, Digital data Manuals, Computer graphics, Computer aided design, Computer aided manufacturing, File management systems, Automation, Information processing, ${ }^{+} \mathrm{CALS}$ (Continuous Acquisition and Life-Cycle Support)

The publication is the parent document for a family of military specifications through which the Continuous Acquisition and Life-Cycle Support (CALS) standards will be published. It provides the 'enveloping' rules for organizing files of digital data into a complete document--for example, a technical manual composed o MIL-M 28001 text, MIL-D-28003 Vector Graphics, and MIL-R-28001 Raster Graphics.

\section{3,667}

PB95-962100 Contact NTIS for subscription information and price.

National Inst. of Standards and Technology,

CALS-Digital Representation for Communication of Product Data: IGES Application Subsets.

rregular repts.

1995 , open series.

Supersedes PB94-962100.

Paper copy available on Standing Order deposit account required (minimum deposit $\$ 100$ U.S. Canada and Mexico; all others $\$ 200$ ). Single copies also available in paper copy or microfiche.

Keywords: "Computer aided design, Computer aided manufacturing, Graphic arts, Numerical control, Engi- 
neering drawings, Data transmission, ${ }^{*}$ CALS (Continuous Acquisition and Life-Cycle Support), "IGES(Initial Graphics Exchange Specification).

The publication defines subsets of the Initial Graphics Exchange Specification (IGES) for technical illustration, engineering drawings, electrical/electronic applications, and numberical control manufacturing, IGES is a neutral format for digital interchange of Product Definition Data between dissimilar computer aided design systems.

\section{3,668}

PB95-962200 Contact NTIS for subscription

information and price.

National Inst. of Standards and Technology, Gaithersburg, MD.

CALS-Markup Requirements and Generic Style Specifications for Electronic Printed Output and Exchange of Text.

Irregular repts.

1995, open series.

Supersedes PB94-962200.

Paper copy available on Standing Order, deposit account required (minimum deposit $\$ 100$ U.S., Canada, and Mexico; all others \$200). Single copies also available in paper copy and microtiche.

Keywords: "Publishing, "Automation, Text processing, Computer aided design, Computer aided manufacturing, "CALS(Continuous Acquisition and Life-Cycle Support), Department of Defense.

The publication defines standard Department of Defense (DoD) requirements for all steps involved in automated publishing of page-oriented (i.e., printed) technical publications. For exchange of source data (prior to document composition it defines a common imple. mentation of the Standard Generalized markup Language (SGML). For composition processing functions, it defines an Output Specification of typographic tags and format rules, and for display fo the composed document, it provides options for use of commercial Page Description languages.

\section{3,669 \\ PB95-962300 Contact NTIS for subscription}

information and price.

National Inst of Standards and Technology, Gaithersburg, MD.

CALS-Raster Graphics Representation Binary Format Requirements.

Irregular repts.

1995, open series

Supersedes PB94-962300.

Paper copy available on Standing Order, deposit account required (minimum deposit $\$ 100$ U.S., Canada, and Mexico; all other \$200). Single copies also available in paper copy or microfiche.

Keywords: "Computer aided design, ${ }^{*}$ Computer graphics, File management systems, Documents, Facsimile communication, Image processing, Standards, Computer aided manufacturing, "CALS (Conînuous Acquisition and Life-Cycle Support), Department of Defense.

The publication defines Department of Defense (DoD) technical requirements for raster (bit-map) graphics that have been compressed to reduce file size and transmission time Continuous Acquisition and LifeCycle Support (CALS) must apply raster graphics to both office documents and facsimiles (for which the international standard that MIL-R-28002 implements was prepared), anf for engineering drawings and other oversize documents. An option is included to use tiling, in which the raster image is divided into a series of tiles that can be individually processed to reduce through. out and terminal storage requirements.

\section{3,670}

PB95-962400 Contact NTIS for subscription information and price

National Inst. of Standards and Technology, Gaithersburg, MD.

CALS-Digital Representation for Communication of Illus tration Date: CGM Application Profile.

Irregular repts

1995 , open series.

Supersedes PB94-962400.

Paper copy available on Standing Order, deposit account required (minimum deposit $\$ 100$ U.S., Canada and Mexico; all others \$200). Single copies also available in paper copy or microfiche.

Keywords: "Computer aided design, "Computer graphics, Manuals, Graphic arts, Work stations, Digital data,
Two dimensional models, Computer aided manufacturing, ${ }^{*}$ CALS(Continuous Acquisition and Life-Cycle Support), Computer graphics metafile.

The publication defines use of Computer Graphics Metafile (CGM) for two dimensional vector (line segment) picture descriptions or illustrations in technica manuals. Whereas Initial Graphics Excahnge Specification (IGES) has its principal use within computeraided design, CGM is becoming widely available for authoring and graphic art work stations.

03,671

PB95-962500 Contact NTIS for subscription information and price.

National Inst. of Standards and Technology, Gaithersburg, MD.

CALS-Department of Defense Computer Aided Acquisition Logistic Support (CALS).

Irregular repts.

1995, open series.

Supersedes PB94-962500.

Paper copy available on Standing order, deposit account required (minimum deposit \$100 U.S., Mexico, and Canada; all others $\$ 200$ ). Single copies also available in paper copy or microfiche.

Keywords: "Weapons system, "Logistics management, Acquisition, Information services, Contract administration, Computer aided design, Computer aided manu facturing, "CALS(Continuous Acquisition and LifeCycle Support), Department of Defense.

The guide assists weapon system acquisition managers to understand when, where, and how to apply Computer-Aided Acquisition and Logistics Suppor (CALS) capabilities efficiently to support their information interchange and access requirements, and how to define their functional requirements for integration of the contractor process (such as Reliability and Maintainability analysis) that create and use the information.

\section{3,672}

PB95-962600 Contact NTIS for subscription

information and price.

National Inst. of Standards and Technology, Gaithersburg, MD.

CALS-Contractor Integrated Technical Information Service (CITIS), Functional Requirements.

Irregular repts.

1995, open series.

Supersedes PB94-962600.

Paper copy available on Standing Order, deposit account required (minimum deposit \$100 U.S., Canada, and Mexico; all others \$200). Single copies also available in paper copy or microfiche.

Keywords: "Information services, "Requirements, On line systems, Contractors, US DOD, port) *CITIS (Contractor Integrated Technical Information Service).

The publication describes functional requirements for the Department of Defense's (DoD's) new Continuous Acquisition and Life-Cycle Support (CALS) acquisition policy which gives preference to contractor information services and online access instead of data deliverables. Contractor Integrated Technical Information Service (CITIS) addresses how DoD will buy information services instead of what form the deliverable will be in, whether paper or digital based. it defines things a contractor must do, such as planning, analysis, and submitting proposals and things the service must do, such as managing data, and providing access to the data tailored to meet a government Concept of Operations.

\section{3,673}

PB96-106869 PC A04/MF A01

National Inst. of Standards and Technology (CAML) Gaithersburg, MD. Office of Applied Economics.

Present Worth Factors for Life-Cycle Cost Studies in the Department of Defense (1996).

\section{S. R. Petersen. Oct 95, 62p, NISTIR-4942-3.}

See also report for 1995, PB95-105029. Sponsored by Federal Energy Management Program, Washington, DC.

Keywords: "US DOD, "Life cycle costs, "Energy supplies, Present worth, Cost analysis, Economic analysis, Cost engineering, Return on investment, Cost reduction, Cost estimates, Fuels, Natural gas, Electric power, Disillates, Residual oils, Liquefied petroleum gas, Steam, Coal, Tables(Data), Military Construction Program.
This document provides 47 tables of present worth factors to be used in computing the present worth of future costs (or cost reductions) in economic analyses of design decisions for projects in the DOD Military Construction Program. These factors are especially useful for the life-cycle cost analysis of investments in buildings or building systems which are intended to reduce future operating, maintenance, repair, replacement, and energy costs over the life of the facility. The tables include present worth factors for both one-time costs and annually recurring costs, based on the FEMP discount rate of $4.1 \%$ (FY 1996) for energy-related studies and on the OMB discount rate of $4.6 \%$ and $4.9 \%$ for short-term and long-term non-energy studies, respectively. Forecasts of future energy prices used in the calculation of present worth factors for energy costs were provided by the Energy Information Administration.

03,674

PB96-112651 PC A04/MF A01

National Inst. of Standards and Technology (NCSL), Gaithersburg, MD. Systems and Network Architecture Div.

Federal Implementation Guideline for Electronic Data Interchange: ASC X12 003040 Transaction Set 838 Trading Partner Profile (Vendor Registration), Implementation Convention.

Special pub.

J. P. Favreau. Aug 95, 61p, NIST/SP-881/1.

Also available from Supt. of Docs. as SN003-00300363-4. See also PB96-111190, AD-A263 350/1 and AD-A263 445/9.

Keywords: "Computer programs, "Information exchange *Acquisitions, Standards, Databases, Logistics management, Industries, Corporations, Procurement, "EDI(Electronic Data Interchange), "Elect! nic Data Interchange.

The ASC X12 003040 Transaction Set 838, Trading Partner (Vendor Registration), Implementation Convention defines the Federal Government interpretation of the use of ASC $\times 12003040$ standards. This implementation convention provides the information necessary for the user to be able to develop an interface program between the computer application and the ASC $\times 12$ translator.

\section{3,675}

PB96-155023 PC A05/MF A01

National Inst. of Standards and Technology (EEEL), Boulder, CO. Electromagnetic Fields Div.

TEM/Reverberating Chamber Electromagnetic Radiation Test Facility at Rome Laboratory.

M. L. Crawford, B. F. Riddle, and D. G. Camell. Jan 96, 75p, NISTIR-5002

Keywords: $\quad$ *Reverberation chambers, "Electromagnetic testing, Electromagnetic susceptibility, Vulnerability, Test facilities, Performance evaluation, Radio frequencies, Electromagnetic pulses, Rome Air Development Center, Griffis Air Force Base, Weapons systems, Magnetic permeability, Electric equipment.

This report summarizes the measurement and evaluation of a TEM/reverberating chamber. This chamber was developed as a single, integrated facility for testing radiated electromagnetic compatibility/vulnerability (EMCN) of large systems over the frequency range of $10 \mathrm{kHz}$ to $18 \mathrm{GHz}$ or higher. The facility consists of a large shielded enclosure configured as a transverse electromagnetic (TEM) transmission line-driven reverberating chamber. TEM mode test fields are generated at frequencies below multimode cutoff, and modestirred test fields are generated at frequencies above multimode cutoff. The report discusses the basis for such a development including the theoretical concepts, the advantages and limitations, the experiments approach for evaluating the operational parameters, and the procedures for using the chamber to perform EMC/ $\checkmark$ measurements. Both the chamber's $\mathrm{cw}$ and pulsed if characteristics are measured and analyzed. 


\section{NATURAL RESOURCES \& EARTH SCIENCES}

\section{Cartography}

\section{3,676 \\ PB94-172079 Not available NTIS}

National Inst. of Standards and Technology (TS), Gaithersburg, MD. Metric Program.

Metrication.

Final rept.

G. P. Carver. 1993, 6p.

Pub. in Point of Beginning magazine, p74-78, 81, Oct Nov 93. See also PB93-188969.

Keywords: "Surveying(Geographic), "Mapping "Metrication, Government policies, Metric system Standardization, Conversion, USA, Reprints.

The metric system is one key to success in the global marketplace, where non-metric products are becoming increasingly unacceptable. Procurement is the primary federal tool for encouraging and helping U.S. industry to convert voluntarily to the metric system. Common sense, based upon the knowledge, experience, and needs of an industry, should be the determinant in choosing a practical conversion approach. In the United States, besides the perceived unwillingness of the customer, and certain legal definitions in some states, there are no major impediments to conversion of surveying and mapping to metric units. Instead, there are good reasons for changing, including an opportunity to rethink many industry standards and to take advantage of size standardization. Also, when surveying and mapping services adopt the metric system, they will come into conformance with federal agencies engaged in similar activities.

\section{3,677}

PB95-150942 Not available NTIS

National Inst. of Standards and Technology (NCSL) Gaithersburg, MD. Information Systems Engineering Div.

Standards: A Cardinal Direction for Geographic Information Systems.

Final rept.

H. Tom. $1988,11 p$

Pub. in Proceedings of Annual Conference of the Regional Information Systems Association (26th), Los Angeles, CA., August 7-11, 1988, v2 p142-152.

Keywords: "Geographic information systerns, "Computer applications, "Standards, Computer aided mapping, Automatic mapping, Digital data, Systems integration, Interoperability, Reprints, Digital spatial ap plications.

Geographic Information Systems reflect the changing role of computer technology within earth science disciplines. Successful use of computer technology includes the ability to interchange or interface computer data, software, and hardware. This ability, in large part, is predicated on standards. Intensifying the need for standards is the changing use of computer technology to provide information. Standardization of fundamental parameters and the implementation, integration, and support of standards facilitate system integration and interoperability. A clear understanding of standards is decisive in determining the impact of geographic information systems. The focus is primarily on the applicability, strategy, and commitment regarding standards pertaining to geographic information systems.

\section{3,678}

PB95-163390 Not available NTIS

National Inst. of Standards and Technology (NCSL), Gaithersburg, MD. Information Systems Engineering Div.

Geographic Information Systems Standards: A Federal Perspective.

Final rept

H. Tom. 1990, 3p.

Pub. in GIS World 3, n2 p50-52 1990

Keywords: *Geographic information systems, "Computer aided mapping, "Standards, Automatic mapping. Digital data, Computer applications, Systems integration, Interoperability, Information technology, Reprints, Digital spatial data.

Spatial data standards are associated with defining, describing, and processing spatial data. Information technology (IT) standards are generic computer standards. IT standards specifically implemented or generically configured for GIS usage can also be considered GIS standards. The adaptation of IT standards provides GIS with an existing set of common computer standards for enabling integration within a GIS. These standards aiso facilitate interoperability between different GIS or between a GIS and its overall corporate computer environment.

\section{3,679}

PB96-135108 Not available NTIS

National Inst. of Standards and Technology (CSL), Gaithersburg, MD. Information Systems Engineering Div.

Spatial Information and Technology Standards Evolving

Final rep

H. Tom. 1991, 3p.

Pub. in 1991-92 International GIS Sourcebook, Chapter 3, p422-424 Aug 91

Keywords: "Digital data, "Geographic information sys tems, "Standards, Computer aided mapping, Automatic mapping Systems integration, Interoperability, Computer applications, Reprints, Digital spatial data.

Standards typically evoke different responses in observers. Such a range of reactions also is experienced in the world of GIS standards. Support at both ends of this spectrum is evident, yet a balanced perspective of GID standards is lacking.

\section{3,680}

PB96-159629 Not available NTIS

National Inst. of Standards and Technology (TS), Gaithersburg, MD. Metric Program

METRICATION: An Economic Wake-Up Call for Surveyors and Mappers.

Final rept.

G. P. Carver. $1993,8 p$.

See also PB93-188969.

Pub. in Point of Beginning (P.O.B.), v19 n1 p74-81 Oct 93.

Keywords: "Mapping, "Surveying, "Metric system, Metrication, Measurement, Conversion, International system of units, Cartography, Reprints.

The 'modernized' metric system is the international System of Units or SI (from the French Le Systeme Internationale d'Unites). It includes the product stand. ards and preferred sizes that are accepted by industries and governments throughout the world.

\section{Geology \& Geophysics}

\section{3,681}

PB94-198686 Not available NTIS

National Inst. of Standards and Technology (PL), Boulder, CO. Quantum Physics Div.

Integrated Laser Doppler Method for Measuring Planetary Gravity Fields.

Final rept.

P. L. Bender. 1992, 10p.

Pub in Proceedings of International Association of Geodesy Symposium on Gravity Field Determination from Space and Airborne Measurements, Vienna, Austria, August 20, 1991, p63-72 1992

Keywords: "Gravitational fields, "Earth gravitation, "Lunar gravitation, "Planetary gravitation, Polar orbits, Satelite tracking, High resolution, Geodynamics, Reprints, Laser doppler techniques.

Four main approaches have been applied to, or studied extensively for use in high-resolution determination of the global gravity fields of the Earth, other planets, or the Moon. These approaches are the following: laser or microwave tracking of spacecraft orbiting the Earth; microwave tracking from the Earth of spacecraft orbiting other planets or the Moon; satellite-to-satellite microwave tracking; and gravity gradiometry from an orbiting spacecraft. The paper will discuss another possibility which has been studied much less extensively. It is the use of integrated laser Doppler meas- urements between two spacecraft in essentially the same nearly polar orbit. in this case, the shot noise in the measurement system can be extremely low, even for $5 \mathrm{~cm}$ diameter transmit and receive apertures and 1 milliwatt of laser power. For the highest accuracy, it is assumed that a Disturbance Reduction System would be used to strongly reduce the disturbing forces on a freely floating and carefully shielded test mass inside each spacecraft. As one example, the authors take $50 \mathrm{~km}$ spacecraft separation and a spurious acceleration level of $5 \times 10($ sup -10) $\mathrm{m} / \mathrm{s}(\sup 2) / \mathrm{Hz}$ (sup $0.5)$ from 0.005 to $0.1 \mathrm{~Hz}$. The achievable accuracy with laser Doppler measurements is then expected to be 10(sup -5) Eotvos $/ \mathrm{Hz}(\sup 0.5)$ in this frequency range for the difference of the line-of-sight test mass accelerations divided by the spacecraft separation.

03,682

PB95-175030 Not available NTIS

National Inst. of Standards and Technology (MSEL), Boulder, CO. Materials Reliability Div.

Crystal Structure and Compressibility of 3:2 Mullite.

Final rept.

D. Balzar, and $H$. Ledbetter. 1993, 5p.

Pub. in American Mineralogist 78, p1192-1196 1993.

Keywords: "Mullite, "Crystal structure, "Compressibility, $X$ ray diffraction, Ionic crystals, Crystal models, Bulk modulus, Ultrasonic radiation, Aluminum oxide, Silicon dioxide, Reprints.

The crystal structure of 3:2 mullite (3Al2O3 (center dot) $2 \mathrm{SiO} 2$ ) has been refined by Rietveld refinement of $X$ ray powder diffraction data. The average structure is described successfully by previously published models for 2:1 mullite. Bond lengths of tetrahedral cations are slightly shorter, because of the smaller ionic radii of $\mathrm{Si}(4+)$ relative to $\mathrm{Al}(3+)$. Using ultrasonic methods, we determined the bulk modulus (reciprocal compress ibility). The result, $174 \mathrm{GPa}$, is slightly lower than that of a prediction based on a rigid-ion ionic crystal model: $190 \mathrm{GPa}$

03,683

PB95-202495 Not available NTIS

National Inst. of Standards and Technology (PL), Boulder, CO. Quantum Physics Div.

Lunar Laser Ranging: A Continuing Legacy of the Apollo Program.

Final rept.

J. O. Dickey, P. L. Bender, J. E. Faller, J. G. Ries, P. J. Shelus, C. Veillet, A. L. Whipple, J. R. Wiant, J. G. Williams, C. F. Yoder, Newhall, and R. L. Ricklefs.

1994, 9p.

See also PB91-203026

Pub. in Science 265, p482-490, 22 Jul 94.

Keywords: "Lunar rangefinding, "Geodynamics, Apollo $\ddagger 1$ flight, Laser range finders, Equivalence principle, Lunar rotation, Gravitation, Moon, Uses, Reprints.

On 21 July 1969, during the first manned lunar mission, Apollo 11, the first retroreflector array was placed on the moon, enabling highly accurate measurements of the Earthmoon separation by means of laser ranging. Lunar laser ranging (LLR) turns the Earthmoon system into a laboratory for a broad range of investigations, including astronomy, lunar science, gravitational physics, geodesy, and geodynamics. Contributions from LLR include the three-orders-of-magnitude improvement in accuracy in the lunar ephemeris, a several-orders-of-magnitude improvement in the measurement of the variations in the moon's rotation, and the verification of the principle of equivalence for massive bodies with unprecedented accuracy Lunar laser ranging analysis has provided measurements of the Earth's precession, the moon's tidal acceleration, and lunar rotational dissipation. These scientific results, current technological developments, and prospects for the future are discussed.

\section{3,684}

PB95-202651 Not available NTIS

National Inst. of Standards and Technology (PL), Boulder, CO. Quantum Physics Div.

Calibration of a Superconducting Gravimeter Using Absolute Gravity Measurements.

Final rept.

J. Hinderer, N. Florsch, J. Makinen, H. Legros, and

J. E. Faller. 1991, 7p.

Pub. in Geophys. Jnt. Int. 106, p491-497 1991.

Keywords:

"Gravimeters,

"Calibration,

Superconducting devices, Sensitivity analysis, Uncer- 


\section{Geology \& Geophysics}

tainty, Precision, Tests, Reprints, "Superconducting gravimeters, Absolute gravity.

A $24 \mathrm{hr}$ continuous parallel registration between an absolute free-fall gravimeter and a rela tive cryogenic gravimeter is analyzed. Different adjustment procedures $(L(1), L(2)$ norms) are applied to the sets of absolute and relative readings in order to estimate the value of the calibration factor of the superconducting meter, as well as its uncertainty. In addition, a sensitivity test is performed to investigate the influence of some parameters (like the laser frequency and its short-term drift) upon this factor. The precision in the calibration facto is found to be better than 1 per cent, but systematic effects related to the short time interval may add an other one and half per cent uncertainty. From preliminary results, it appears that this calibration experimen leads to a close agreement between the values of the gravimetric factor for the reference tidal wave $O(1)$ observed with the superconducting meter and the theoretical value (Dehant-Wahr body tide + ocean loading).

\section{3,685}

PB95-203048 Not available NTIS

National Inst. of Standards and Technology (PL), Boulder, CO. Quantum Physics Div.

Continuous Gravity Observations Using Joint Institute for Laboratory Astrophysics Absolute Gravimeters.

Final rept.

T. M. Niebauer, and J. E. Faller. 1992, $9 p$

Pub. in Jnl. of Geophysical Research 97, nB9 p12,427 12,435, 10 Aug 92

Keywords: *Gravimeters, *Gravity, Calibration, Correction, Reprints, Absolute gravity.

We discuss two different 1-month gravity records taken with two different Joint Institute for Laboratory Astrophysics (JILA) gravimeters. These records were corrected for the effects of Earth tides, ocean loading. local and global barometric effects, and polar motion. These data allowed precise determinations of the local air pressure admittance. We also obtained a value for the second-order gravimetric factor which agreed with the theoretical value to about $0.3 \%$ where the predominant uncertainty is the ocean load modeling. We note that absolute gravimeters have a sensitivity to time varying gravity that is comparable to that obtained with superconducting relative gravimeters. The sensitivity of these data emphasizes the need for better modeling of ocean loads, air pressure effects, and even manmade environmental noise.

\section{3,686}

PB96-102611 Not available NTIS

National Inst. of Standards and Technology (PL), Boulder, CO. Time and Frequency Div.

Measurement and Interpretation of Tidal Tilts in a Small Array.

Final rept

M. L. Kohl, and J. Levine. 1995, 13p.

Pub. in Jnt. of Geophysical Research, v100 nB3 p3929-3941, 10 Mar 95.

Keywords: "Tiltmeters, "Strain measurement, Reprints, Geodynamics, Boreholes, Sensitivity, "Earth tides, Tilt tides

Strain-induced tilts caused by variations in materials properties can produce large perturbations in the til field near a material discontinuity. This is evidenced in data from a closely spaced array of borehole tiltmeters that has been operating at Pinon Flat Geophysical Observatory in southern California since 1987. The array is composed of three borehole tiltmeters located at depths of 24,36 , and $120 \mathrm{~m}$. The tiltmeters have a sensitivity of a few nanoradians. Tidal measurements show differences between the boreholes of up to $50 \%$. The largest percent difference occurs in the semidiurnal band where the tilt field measured in the $36-\mathrm{m}$ borehole differs by approximately $10 \mathrm{nrad}$ at the M2 frequency. This difference has been modeled as a strain-induced tilt caused by the weathering layer.

\section{3,687}

\section{PB96-102660 Not available NTIS}

National Inst. of Standards and Technology (PL), Boulder, CO. Time and Frequency Div.

Measurement of Very Low Frequency Vibrations. Final rept.

J. Levine. 1994, 10p

Pub. in Proceedings of Society of Photo-Optical Instrumentation Engineers: Vibration Monitoring and Control, San Diego, CA., July 28-29, 1994, v2264 p160169 .
Keywords: "Low frequency vibrations, "Tiltmeters, *Strain measurement, Reprints, Boreholes, Geodynamics, Sensitivity, Laser strainmeter.

We have developed a number of instruments that are capable of measuring vibrations at very low frequencies (below $1 \mathrm{~Hz}$ ). Two of these instruments are a laser strainmeter, which can respond to strains with frequencies ranging from 0 to about $100 \mathrm{~Hz}$ and a borehole tiltmeter which can respond to deflections of the vertical or horizontal accelerations in the frequency range from 0 to about $1 \mathrm{~Hz}$. We have also modified commercial gravity meters (vertical accelerometers) and have used them to make measurements at comparable frequencies. Most of our work has been motivated by geophysical considerations including the dynamics of the earth in seismically active regions, but the instrumentation might also be useful in vibration monitoring for other purposes.

\section{3,688}

PB96-102991 Not available NTIS

National Inst. of Standards and Technology (PL), Boulder, CO. Quantum Physics Div.

Intracomparison Tests of the FG5 Absolute Gravity Meters.

Final rep

G. S. Sasagawa, F. Klopping, T. M. Niebauer, J. E. Faller, and R. L. Hilt. 1995, 4p.

Pub. in Geophysical Research Letters 22, n4 p461. 464, 15 Feb 95

Keywords: "Gravimeters, "Calibration, Reprints, *Absolute gravity

The FG5 series absolute gravimeters have an estimated instrumental accuracy of 1-2 microGal. A number of instrument comparisons were conducted with six FG5 instruments over a period of 9 months; the predecessor series JILA4 instrument was also used. The standard deviation of mean g values (averaged over 24-48h), as observed by different instruments, is 1.8 microGal. Observations taken within $48 \mathrm{~h}$ typically agree within 2 microGal, and the maximum observed disagreement is 6 microGal for two observations taken 37 days apart.

\section{3,689}

PB96-137732 Not available NTIS

National Inst. of Standards and Technology (CSTL),

Gaithersburg, MD. Thermophysics Div.

Temperature and Frequency Dependence of Anelasticity in a Nickel Oscillator.

Final rept.

R. F. Berg. 1995, 6p.

Pub. in Review of

Keywords: *Creep, *Rock mechanics, Flexural properties, Oscillators, Transfer function, Reprints, *Anelasticity.

The frequency dependance of the real and imaginary parts of a nickel oscillator's transfer function is described over 3 decades in frequency by the use of simple expressions. These expressions incorporate only the resonance frequency wO the quality factor $Q$, and a characteristic exponent Beta determined by a single measurement of creep. They are based on the ansatz phi $(w)=Q(-1)(w / w 0)$-Beta, where psi is the imaginary part of the spring constant. Over a $100 \mathrm{~K}$ range of temperature $T$ the exponent Beta $=0.18$ was constant even though $Q(T)$ changed by a factor of 8 . These expres. sions are potentially useful for accurately describing a mechanical oscillator whose transfer function must be modeled at frequencies tar below wo. Examples include accelerometers based on a flexure element and suspensions for interferometric gravitational wave detectors.

03,690

PB96-165972 PC A05/MF A01

Colorado School of Mines, Golden. Div. of Engineering.

Preliminary Processing of the Lotung LSST Data.

S. D. Glaser, and A. L. Leeds. Mar 96, 59p. NIST/

GCR-96/690

Contract NIST-60NANB4D1677

See also PB93-178606. Sponsored by National Inst of Standards and Technology (BFRL), Gaithersburg, MD.

Keywords: "Soil mechanics, "Nuclear power plants, *Earthquake engineering *Soil structure interactions, Soil dynamics, Soil profiles, Seismic effects, Earthquakes, Ground motion, Seismic waves, Strain meas- urement, Site characterization, Earthquake magnitude Piezometers, Data collection, Data processing, Lotung(Taiwan)

Possibly the best set of data for earthquake excitation of soils exists for the test site operated by the Electric Power Research Institute (EPRI) and the Taiwan Power Company at Lotung Taiwan. At this site, two locations are instrumented with three-component accelerometers at depths of $47,17,11,6$ meters, and at the surface. One array is in the free-field while the other is adjacent to a one-quarter scale nuclear containment vessel. The site is also well instrumented with piezometers at various depths and locations. The report summarizes the data and signal processing that was done to the EPRI Lotung data at the Colorado School of Mines. The over 2000 files were organized into MATLAB experiments by event. The provided acceleration data were carefully double integrated to yield velocity and displacement time history records.

\section{3,69}

PB96-214747 PC A13/MF A03

Bechtel Corp., San Francisco, CA.

Energy-Based Method for Liquefaction Potential Evaluation. Phase 1. Feasibility Study.

F. Ostadan, N. Deng, and I. Arango. Aug 96, 264p NIST/GCR-96/701.

Figures in this document may not be legible in microfiche. Sponsored by National Inst. of Standards and Technology (BFRL), Gaithersburg, MD

Keywords: "Liquefaction, "Ground motion, "Earthquakes, Pore pressure, Strain measurement, Earthquake magnetic, Seismic waves, Strain energy release rate, Strain rate, Shear strain, Vibration dampling, Stress-strain relations, Acceleration, Sands, Seismic effects, Soil sampling, Laboratory tests, Soil mechanics.

The purpose of this study is to evaluate the feasibility of the development and application of the strain energy method for general use. The study is expected to continue with two additional phases that will develop generic strain energy, liquefaction curves as a function of the most relevant soil properties and generic strain energy demand as a function of seismicity data and a wide range of site soil data and profiles. The limited scope of the feasibility study did not permit laboratory testing for the purpose of the strain energy computation. Available laboratory data were used for this purpose.

\section{Hydrology \& Limnology}

\section{3,692}

PB96-202452 PC A04/MF AO

National Oceanic and Atmospheric Administration, Ann Arbor, MI. Great Lakes Environmental Research Lab.

Lake Erie Water Temperature Data, Put-in-Bay, Ohio, 1918-1992.

Technical memo.

M. J. McCormick. Aug 96, 41p, NOAA-TM-ERL

GLERL-97.

See also PB96-202445.

Keywords: "Lake Erie, "Water temperature, "Data collections, Great Lakes, Ohio, Trends, Climate change, Graphs(Charts), Put-In-Bay(Ohio).

Water temperature data from several sites around the Great Lakes were obtained to determine if any regional climatic changes or trends could be detected based upon these data sets. This technical memorandum contains data for Put-In-Bay, Ohio on Lake Erie. Water temperature data were measured at the Put-In-Bay fish hatchery on South Bass Island on Western Lake Erie. The hatchery data were collected by the Ohio Department of Natural Resources, Lake Erie Fisheries Unit, Sandusky Bay, from 1918 through 1992.

\section{3,693}

PB96-204425 Not available NTIS

National Inst. of Standards and Technology (MSEL), Gaithersburg, MD. Ceramics Div

Adhesion, Contact Electrification, and Acid-Base Properties of Surfaces.

Final rept.

A. Grabbe, D. T. Smith, and R. G. Hom. 1995, 8p.

See also PB93-125235. 
Pub. in AMP Jnl. of Technology, v4 p95-102 Jun 95. Keywords: "Electrification, "Electrical insulation, "Adhesion, "Static electricity, Reprints, Silicon dioxide, Mica, Charge transport, Electric discharges.

When two different materials contact and separate, an electrical charge transfer from one surface to the other can take place because of the different electronic structures of the two materials. In the paper, it is shown that the same can occur between two surfaces of the same material, if one of the two surfaces carries a monolayer of a chemi-sorbed species. A surface force apparatus is used for studying contact electrification and resulting adhesion between two surfaces. It is shown that the interaction between a silica surface presenting an amine, i.e., basic, termination to a silanol terminated, i.e., acidic surface, is vastly greater than between two ordinary silica surfaces. The increased adhesion demonstrates the connection between acidbase interactions and contact electrification.

\section{Mineral Industries}

03,694

PB94-185386 Not available NTIS

National Inst. of Standards and Technology (NEL), Gaithersburg, MD. Robot Systems Div.

Task Decomposition Methodology for the Design of a Coal Mining Automation Hierarchical RealTime Control System.

Final rept.

H. M. Huang, and R. Quintero. 1990, 9p.

Pub. in Proceedings of the International Institute of Electrical and Electronics Engineers Symposium on Intelligent Control, p884-892 1990. Sponsored by Bureau of Mines, Pittsburgh. PA.

Keywords: "Coal mining, "Control systems, Reprints, Hierarchies, Automation, Real time systems, Planning, Artificial intelligence, Sumps, Coal mines, "Task decomposition.

The paper describes a systematic approach to hierarchical task decomposition and planning. Such a methodology can be used to design a complex system which receives goals from the external world, performs intelligent planning, and commands the actuators to achieve the goals. The application of this task decomposition methodology is illustrated in the paper through the design of a coal mining automation hierarchical real-time control system.

03,695

PB94-193976 PC A04/MF A01

Purdue Univ., Lafayette, IN. Thermal Sciences and Propulsion Center.

Investigation of Oil and Gas Well Fires and Flares. Final rept.

P. Dutta, Y. R. Sivathanu, and J. P. Gore. Jun 94,

68p, NIST/GCR-94/653.

Grant NIST-60NANB1D1172

Sponsored by National Inst. of Standards and Technology (BFRL), Gaithersburg, MD.

Keywords: "Natural gas wells, "Diffusion flames, "Oil wells, "Radiative heat transfer Atomizers, Soot, Heat flux, Burners, Sprayers, Mathematical models, Jet flow, "Well fires, "Well flares, Effervescent atomization. A theoretical and experimental study of jet flames with applications to large fires resulting from oil well and gas well accidents is reported. The results have been used in the interpretation of the single point radiation heat flux data collected around well fires in Kuwait. Based on the high liquid loading involved in actual well fires, a new device called effervescent atomizer/burner was successfully designed, constructed and tested during the grant period. Measurements of flame heights, radiative heat loss fractions, emission temperatures, and path integrated transmittances were completed for nine crude oil and methane/air flames in the 10-20 KW range. The significant accomplishments during the grant include: (1) Development of a technique to find total radiative heat loss from turbulent jet flames based on measurements of heat flux at a single location; (2) Design and successful operation of an effervescent atomizer/burner. The burner also allows laboratory measurements of such flames for the first time; and (3) Study of global properties of the high liquid loading jet flames have shown that their lengths are affected by two-phase flow effects and that their soot loading and radiant output is lower than equivalent pool flames.
03,696

PB94-203429 PC A02/MF A01

National Inst. of Standards and Technology (MEL), Gaithersburg, MD. Robot Systems Div.

Integration of Servo Control into a Large-Scale Control System Design: An Example from Coal Mining.

J. A. Horst. Jun 94, 10p, NISTIR-5446

Keywords: "Continuous miners, "Systems integration, "Servocontrol, "Motion simulators, "Robotics, "Coal mining, Control systems design, Systems engineering, Cutting machines, Controllers, Mining engineering, Computerized simulation, Autonomous navigation, Mathematical models, "Real-time Control Systems, Coal interface detection, Proportional-plus-Integra!plus Derivative controllers

Closed loop control of cutting drum height of a continuous mining machine (CM) using a coal interface detection (CID) sensor is described. The control is accom plished using a discrete-time Proportional-plus-Integral-plus-Derivative (PID) controller. The mining machine, sensor, and mine environment are simulated. The dynamic model of the cutting drum arm (boom) and motors is described and a discrete time version of the solution to the plant equation of boom angle and angular velocity is derived. Cutting drum height control is placed in the context of an existing large-scale Realtime Control System (RCS) for CM control, simulation, and animation. RCS is a paradigm for defining, describing, and designing intelligent control systems developed at the Robot Systems Division of the National Institute of Standards and Technology (NIST).

\section{3,697}

PB94-203536 PC A03/MF A01

National Inst. of Standards and Technology (MEL), Gaithersburg, MD. Robot Systems Div.

Environment Simulation for a Continuous Mining Machine.

J. A. Horst. Jun 94, 11p, NISTIR-5449.

Keywords: "Continuous miners, "Mining equipment, "Coal mining, "Motion simulators, "Robotics, Computerized simulation, Mining engineering, Systems engineering, Cutting machines, Mathematical models, Controllers, Autonomous navigation, Collisions.

A simulator is described for the motion of a continuous mining machine (CM) in a coal mine. Of special interest is the motion of the $\mathrm{CM}$ following collision with an immovable obstacle in the mine. This type of constrained motion effected our choice of presentation of the $\mathrm{CM}$ and its environment. The CM is represented as a two dimensional polygonal hull and the mine obstacles are given two separate representations, certainty grids and obstacle boundary curves, each of which efficiently perform specific tasks. The object boundary curve representation is most useful for determining motion following a collision and the certainty grid is most usefu for determining whether and where a collision has occurred.

\section{3,698}

PB94-212065 Not available NTIS

National Inst. of Standards and Technology (NEL), Gaithersburg, MD. Robot Systems Div.

Hierarchical Real-Time Control System for Use with Coal Mining Automation.

Final rept.

H. M. Huang. 1990, 12p

Sponsored by Bureau of Mines, Pittsburgh, PA

Pub. in Proceedings of Conference on the Use of Computers in the Coal Industry (4th), Morgantown, WV. June 20-22, 1990, p3-14

Keywords: "Coal mining *Automation *Real time operation, "Hierarchies, "Control systems, Signal processing, Systems engineering, Artificial intelligence, Man machine systems, Reprints.

This paper describes a systematic approach to the design of a hierarchical control system for mining automation. In particular, the methodology can be used to design a complex system which receives goals from the external world, evaluates the current world situation through an interactive sensory data assimilation process, performs intelligent planning, and commands the actuators to achieve its goals. The operator interaction capability is also emphasized.

\section{3,699}

PB96-210760 PC A07/MF A02

National Inst. of Standards and Technology (BFRL) Gaithersburg, MD. Structures Div.
Report of a Workshop on Requalification of Tubular Steel Joints in Offshore Structures. Held in Houston, Texas on September 5-6, 1995.

Final rept.

A. W. Taylor. Jun 96, 105p, NISTIR-5877.

Sponsored by Minerals Management Service, Herndon, VA. Technology Assessment and Research Branch.

Keywords: "Offshore structures, "Joints(Junctions), "Meetings, Pipes(Tubes), Inspection, Loads(Forces) Stress analysis, Houston(Texas)

This report is a summary of a workshop title Requalification of Tubular Steel Joints in Offshore Structures, held September 5 and 6, 1995 in Houston, Texas. The workshop was sponsored by the U.S. Minerals Management Service, and the National Institute of Standards and Technology. This report contains the papers presented at the workshop, summary of the workshop discussions, and the conclusions reached by the workshop participants. The major issues discussed at the workshop included tubular joint characterization computational methods, tubular joint failure definition condition, condition assessment, and code requirements/technology transfer.

\section{Natural Resource Surveys}

\section{3,700}

PB94-203528 PC A02/MF A01

National Inst. of Standards and Technology (MEL), Gaithersburg, MD. Robot Systems Div.

Continuous Mining Machine Control Using the Real-Time Control System.

J. A. Horst, and A. J. Barbera. Jun 94, 10p, NISTIR5448 .

Prepared in cooperation with Advanced Technology and Research Corp., Laurel, MD.

Keywords: "Continuous miners, *Real time operation, "Control systems design, ${ }^{*}$ Coal mining, "Motion simulators, "Robotics, Mining equipment, Underground mining, Computerized simulation, Systems engineering, Mining engineering, Controllers, Autonomous navigation, "Real-time Control Systems.

Application of the Real-time Control System (RCS) reference model to a simulated underground coal mining machine is described RCS is characterized by explicit software modules that perform tasks decomposition sensory processing, and world modeling functions at different hierarchical levels. We use a detailed and sharply defined approach to RCS design characterized by task-oriented problem analysis, generic software objects, rule-based control (using finite state machines), cyclic execution of manually scheduled processes, and generic communications interfaces.

\section{Navigation Systems}

03,701

PB94-211885 Not available NTIS

National Inst. of Standards and Technology (NEL) Gaithersburg, MD. Robot Systems Div.

Real-Time Vision for Autonomous and Teleoperated Control of Unmanned Vehicles. Final rept.

M. Herman, J. S. Albus, and T. H. Hong. 1991, 36p. Sponsored by Army Research Lab., Adelphi, MD and Defense Advanced Research Projects Agency, Arlington, $V A$.

Pub. in Active Perception and Robot Vision, 36p 1991

Keywords: *Autonomous navigation, "Computer vision, ${ }^{*}$ Real time operation, "Telerobotics, Remotely piloted 


\section{Navigation Systems}

vehicles, Architecture(Computers), Robotics, Control systems design, Hierarchies, Teleoperators, Pattern recognition, Video compression, Reprints, "Unmanned vehicles.

This paper focuses on two related topics: a control system architecture that unifies autonomous and teleoperated control of unmanned vehicles, and examples of how real-time vision processing fits into this architecture. The National Institute of Standards and Technology (NIST) hierarchical real-time control sys. tem architecture and its application to unmanned vehicles is presented. The paper then discusses recent work at NIST in real-time vision for both teleoperated and autonomous vehicles. For teleoperated vehicles, we describe a system for video compression for low data rate remote vehicle driving. For autonomous vehicles, we describe passive range extraction from optical flow for applications such as target extraction and identification, vehicle driving, and terrain mapping. We also describe how each of these vision systems fits into the control system architecture.

\section{3,702}

PB94-211893 Not available NTIS

National Inst. of Standards and Technology (NEL), Gaithersburg, MD. Robot Systems Div.

Real-Time Vision for Unmanned Vehicles.

Final rept

M. Herman, J. S. Albus, and T. H. Hong. 1990, 12p.

Sponsored by Army Research Lab., Adelphi, MD and Defense Advanced Research Projects Agency, Arlington, VA

Pub. in Proceedings of Sensors Expo, Chicago, IL. September 1990, p206c-1-206c-12.

Keywords: "Autonomous navigation, "Computer vision, "Real time operation, "Telerobotics, Architecture(Computers), Robotics, Remotely piloted vehicles, Control systems design, Teleoperators, Pattern recognition, Video compression, Reprints, *Unmanned vehicles.

This paper provides examples of how real-time vision may be used for controlling both teleoperated and autonomous vehicles. For teleoperated vehicles, we describe a system for video compression for low data rate remote vehicle driving. For autonomous vehicles, we describe passive range extraction from optical flow for applications such as target extraction and identification, vehicle driving, and terrain mapping.

03,703

PB95-220414 PC A03/MF A01

National Inst. of Standards and Technology (MEL), Gaithersburg, MD. Intelligent Systems Div.

Outline of a Multiple Dimensional Reference Model Architecture and a Knowledge Engineering Methodology for Intelligent Systems Control.

H. M. Huang. Apr 95, 18p, NISTIR-5643.

Keywords: "Underwater navigation, "Submarines, "Real time operations, "Automatic control, Computer systems programs, Systems engineering, Controllers, Simulations, Computer models, "Autonomous navigation.

The authors outline a multiple dimensional reference model architecture and a methodology for representing and developing intelligent systems. The reference model architecture features multiple dimensions enabling modeling the multiple aspects of complex systems. The canonical form within this framework facilitates open and scalable system architecture. The welldefined structures facilitate efficient knowledge engineering processes. We describe a submarine automation model performing real-time control to illustrate the application of this reference model architecture.

\section{NUCLEAR SCIENCE \& TECHNOLOGY}

\section{General}

\section{4}

PB96-157847 Not available NTIS

National Inst. of Standards and Technology (PL), Gaithersburg, MD. Electron and Optical Physics Div. Laser-Synchrotron Hybrid Experiments: A Photon to Tickle, A Photon to Poke.

Final rept

D. L. Ederer, J. E. Rubensson, D. R. Mueller, J. Jai, Q. Y. Dong, T. A. Callcott, G. L. Carr, G. P. Williams C. J. Hirschmugl, S. Etemad, A. Inam, D. B. Tanner, R. Shuker, and W. L. O'Brien. 1992, 7p.

Pub. in Nuclear Instruments and Methods in Physics Research, Section A, vA319 p250-256 1992.

Keywords: "Laser synchrotron coincidence, *Infrared absorption, "Pulse synchronization, Reprints, AL2O3 exciton, Nanosecond scale dynamical process, Phase shifting pulses.

In this paper we present the preliminary results from a new experimental technique to synchronize the pulses from a mode-locked Nd-YAG laser to the light pulses in the VUV storage ring at the National Synchrotron Light Source (NSLS). We describe a method to electronically change the delay time between the lase pulses and the synchrotron pulses. We also illustrate a method to overlap the synchrontron pulses with the laser pulses in space and time. Preliminary results will be presented for two experiments.

\section{Fusion Devices (Thermonuclear)}

03,705

PB95-147351 PC A18/MF A04

National Inst. of Standards and Technology (MSEL) Gaithersburg, MD. Materials Reliability Div.

Irradiation Damage in Inorganic Insulation Materials for ITER Magnets: A Review.

N. J. Simon. Sep 94, 424p, NISTIR-5025.

See also PB93-206928. Sponsored by Department of Energy, Washington, DC. Office of Fusion Energy.

Keywords: "Thermonuclear reactor materials, "Iter tokamak, "Superconducting magnets, "Electrical insulation, "Radiation damage, "Literature surveys, Aluminum oxide, Aluminum nitrides, Magnesium oxides, Silicon dioxide, Zirconium oxides, Porcelain, Mica, Spinel, Quartz, Toroidal configuration, Neutron irradiation Crystal defects, Amorphous materials, Ceramics, Mag nesium aluminates, Amorphization.

A literature search on the irradiation resistance of inorganic insulators for the ITER toroidal field superconducting magnets is reported. The materials included $\mathrm{Al} 2 \mathrm{O} 3$, AIN, MgO, porcelain, $\mathrm{SiO} 2, \mathrm{MgAl} 2 \mathrm{O} 4$ $\mathrm{ZrO} 2$, and mica. No in situ measurements of the 4-K compressive, shear, and electrical breakdown strengths after $4-\mathrm{K}$ neutron irradiation were retrieved. Limited optical and thermal property measurements made on these insulators after cryogenic irradiations indicate that recovery occurs in ceramic oxides at ambient temperatures. Host atom amorphization has been demonstrated in $\mathrm{Al} 2 \mathrm{O} 3$ at $77 \mathrm{~K}$, but was not successful at $300 \mathrm{~K}$. Apparently host ion amorphization does not occur in most ceramic oxides at $300 \mathrm{~K}$. Amorphization has evidently not been attempted in AIN, MgO, or MgAl2O 4 at $77 \mathrm{~K}$. Silicate-bonded structures, such as mica, porcelain, and silica, do amorphize at ambient temperature and are, at present, considered unacceptable unless proven otherwise by in situ electrical and mechanical property testing after 4-K irradiation. Zirconia, even if stabilized, will probably transform under neutron irradiation and is, therefore considered unsuitable. Mica layers delaminated under low energy electron bombardment. Natural mica layers are porous, owing to spontaneous and induced tracks from $U$ impurities. Tracks caused a decrease in elec- trical breakdown strength. Simulation of neutron irradiation by other radiation species is also reviewed in this report.

03,706

PB95-198768 PC A1O/MF A03

National Inst. of Standards and Technology (MSEL), Boulder, CO. Materials Reliability Div.

Cryogenic Properties of Inorganic Insulation Materials for ITER Magnets: A Review.

N. J. Simon. Dec 94, 218p, NISTIR-5030

Sponsored by Department of Energy, Washington, DC Office of Fusion Energy.

Keywords: "ITER tokamak, "Superconducting magnets, "Electrical insulation, Literature surveys, Electrical properties, Mechanical properties, Thermal properties, Aluminum nitrides, Aluminum oxides, Magnesium oxides, Silicon dioxide, Zirconium oxides, Porcelain, Cryogenics, Spinel, Mica, Reviews, Temperature range 0000-0013 $\mathrm{K}$, Temperature range 0013$0065 \mathrm{~K}$, Temperature range 0065-0273 K, Temperature range 0273-0400 K, Graphs(Charts), Magnesium aluminates.

Results of a literature search are reported on the cryogenic properties of candidate inorganic insulators for the toroidal field magnets of the International Thermonuclear Experimental Reactor. The materials investigated include: $\mathrm{Al} 2 \mathrm{O} 3$ AIN $\mathrm{MgO}$ porcelain, $\mathrm{SiO} 2$, $\mathrm{MgAl2O} 4, \mathrm{ZrO} 2$, and mica. A graphical presentation is given of mechanical, elastic, electrical, and thermal properties between 4 and $300 \mathrm{~K}$. A companion report reviewed the low temperature irradiation resistance of these materials.

\section{Isotopes}

03,707

PB96-159637 Not available NTIS

National Inst. of Standards and Technology (PL), Gaithersburg, MD. lonizing Radiation Div.

System for intercomparing Standard Solutions of Beta-Particle Emitting Radionuclides.

Final rept.

J. M. Calhoun, J. T. Cessna, and B. M. Coursey. $1992,7 \mathrm{p}$

Pub. in Nuclear Instruments and Methods in Physics Research, Section A (ICRM Symposium Issue), Chapter A312, p114-120 1992

Keywords: "Radioisotopes, "Beta particles, "Aqueous solutions, Technetium 99, Tritium, Carbon 14, Hexadecane, Liquid scintillation, Quantum efficiency, Comparisons, Radiochemistry, Reference standards, Reprints, "Standard reference materials.

A system for intercomparing standard solutions of pure beta-particle emitting radionuclides is described. The CIEMAT/NIS technique of beta-particle efficiency tracing is based on establishing a parameter in a simple calculational model, using a ( $\mathrm{H}-3)$ standard with comparable quenching. Measurements were made under similar geometrical and quenching conditions for each radionuclide with a commercial scintillator and conventional liquid-scintillation counter with two phototubes operating in coincidence. The technique was then tested at different sites in the area using a set of flamesealed vials and state-of-the-art liquid-scintillation counters.

\section{3,708}

PB96-159751 Not available NTIS

National Inst. of Standards and Technology (PL), Gaithersburg MD Ionizing Radiation Div.

\section{International Radon-in-Air Measurement} Intercomparison Using a New Transfer Standard. Final rept.

J. M. R. Hutchinson, J. Cessna, R. Colle, and P. A. Hodge. 1992, 15p

Pub. in Applied Radiation and Isotopes, Chapter 12. v43 n12 p175-189 1992

Keywords: "Radon 222, "Interlaboratory comparisons, "Air sampling, Radiation monitoring, Photon emission scanning, Ionization chambers, Calibration standards, Standardization, Quality control, Reprints.

A new secondary measuring system for Rn22-in-air that can be used as a very efficacious transfer calibration standard for measurement intercomparisons be- 
tween laboratories has been developed and extensively tested. The system is based on measurement of the photon emission rate of radon samples con tained in spherical $35-\mathrm{mL}$ glass ampoules with a $\mathrm{Nal}(\mathrm{TI})$ well counter. The system is calibrated agains the NIST pulse-ionization-chamber (PIC) national radon measuring system, and has an overall uncer tainty of approximately 2 percent. An internationa intercomparison of 11 laboratories, including NIST, using this system was performed.

\section{3,709}

PB97-110084 Not available NTIS

National Inst. of Standards and Technology (PL), Gaithersburg, MD. Ionizing Radiation Div.

Liquid-Scintillation Counting Techniques for the Standardization of Radionuclides Used in Therapy. Final rept.

B. M. Coursey, J. M. Calhoun, J. Cessna, M. P. Unterweger, D. B. Golas, and F. J. Schima. 1994, $5 \mathrm{p}$. Pub. in Nuclear Instruments and Methods in Physics Research A, v339 p26-30 1994.

Keywords: "Scintillation counting, "Nuclear medicine, *Radionuclides, Radiopharmaceuticals, Standards, Therapy, Reprints, "Foreign technology.

Radionuclides are increasingly used in therapeutic nuclear medicine. The CIEMAT/NIST method of stand ardizing high-energy beta-particle emitters is being applied to a list of candidate radionuclides developed by the US nuclear medicine community Standards and standard methods are needed by the pharmaceutical manufacturers in North America before these nuclides can be widely distributed. Solutions standardized-by liquid-scintillation counting are used to establish counting efficiencies for Cerenkov counting and $\mathrm{Nal}(\mathrm{TI})$ well crystals, and potentiometer settings for commercial radionuclide calibrators. Results are presented for a number of beta-particle-emitting radionuclides.

\section{Nuclear Instrumentation}

\section{3,710}

\section{AD-A286 647/3 PC A05/MF A0}

National Bureau of Standards, Gaithersburg, MD Measurement of Absorbed Dose of Neutrons, and of Mixtures of Neutrons and gamma rays.

G. S. Hurst, R. S. Caswell, F. C. Maienschein, H. H. Rossi, and J. A. Sayeg. 3 Feb 61, 94p, NBS-HB-75, NCRP-25

Keywords: "Scintillation counters, Dosimetry, Gamma rays, Neutrons Proportional counters, lonization chambers, Radiation shielding, Inelastic scattering, Fast neutrons, Dosimeters, Handbooks, Photoelectric effect(Gamma rays), Radiation effects, Neutron scat tering, *Neutron dosimetry, "Gamma dosimetry, Radiation doses

No abstract available.

\section{3,711}

DE96010065 PC A03/MF A01

National Inst. of Standards and Technology, Gaithersburg, MD.

Improving measurement quality assurance for photon irradiations at Department of Energy facilities. Final technical report.

PROGRESS REPT.

1996, 24p, DOE/EH/89321-T1.

Contract Al01-93EH89321

Sponsored by Department of Energy, Washington, DC.

Keywords: *Calibration Standards, "Dosemeters, Personnel Dosimetry, Photon Beams, Progress Report Quality Assurance, Radiation Protection, US DOE, X Radiation, EDB/440102.

For radiation-instrument calibration to be generally acceptable throughout the US, direct or indirect traceability to a primary standard is required. In mos instances, one of the primary standards established at NIST is employed for this purpose. The Department of Energy Laboratory Accreditation Program (DOELAP) is an example of a program employing dosimetn based on the NIST primary photon-, beta particle- and neutron-dosimetry standards. The NIST primary dosimetry standards for bremsstrahlung were first established in the 1950s. They have been updated since then on several occasions. In the 1970s, Technical Committee 85 of the International Standards Organization (ISO) started its work on establishing sets of internationally acceptable, well-characterized photon beams for the calibration of radiation-protection instruments. It is the intent of this paper to make a detailed comparison between the current NIST and the most up-to-date ISO techniques. At present, 41 bremsstrahlung techniques are specified in ISO 4037 while NIST supports a total of 32 techniques. Given the existing equivalences, it makes sense to try to extend the NIST techniques to cover more of the ISO Narrow Spectrum and High Air-Kerma Rate Series. These extensions will also allow the possibility for use of ISO beam techniques in future revisions of the DOELAP standard which has been suggested by DOE. To this end, NIST was funded by DOE to procure material and make adaptations to the existing NIST $x$-ray calibration ranges to allow NIST to have the capability of producing all the ISO bremsstrahlung techniques. The following sections describe the steps that were taken to achieve this.

\section{3,712}

PB95-126272 Not available NTIS

National Inst. of Standards and Technology (NML), Gaithersburg, MD. Ionizing Radiation Div.

Review of the USCEANIST Measurement Assurance Program for the Nuclear Power Industry.

Final rept.

D. H. Gray, D. B. Golas, and J. M. Calhoun. 1991

Pub. in Radioact. Radiochem. 2, n1 pp30, 34-41 1991

Keywords: "Nuclear power, "Radiation measurement, *Quality assurance, Electric power industry, Electric utilities, Energy sources, Laboratories. Proving, Tracing, Radioactivity, Reprints, "USCEA(US Council for Energy Awareness), "US Council for Energy Awareness, "US NIST.

Beginning in 1987 the U.S. Council for Energy Awareness (USCEA) in cooperation with the National Institute of Standards and Technology (NIST) established a measurement assurance program for the nuclear power industry. It was designed to serve the needs of the utility companies, source suppliers, and service laboratories which were not addressed by other programs in existence at that time. The program distributes various samples prepared at NIST to all participants, with the NIST value undisclosed until the measurement result from the participant is reported. In another part of the program, source suppliers also submit samples of their standards to NIST for verification and traceability. The mechanics of the program are described, and the results from the first three years of the program are summarized.

\section{3,713}

PB95-151106 Not available NTIS National Inst. of Standards and Technology (PL), Gaithersburg, MD. Ionizing Radiation Div.

Electron and Proton Dosimetry with Custom-Developed Radiochromic Dye Films.

Final rept.

R. M. Uribe, C. Vargas-Aburto, W. L. McLaughlin, M. L. Walker, and C. E. Dick. 1993, 4p

$\mathrm{Pub}$, in Radiation Protection Dosimetry 47, n1/4 p6936961993

Keywords: "Electron dosimetry, "Proton dosimetry, *Film dosimetry, MeV range 1-10, Electron irradiation, Proton irradiation, Fabrication, Sensitivity, Reprints, "Radiochromic dyes, "Radiochromic dosimeters.

The systematic study and identification of the mechanisms responsible for changes occurring in radiochromic dye films (RDFs) as a result of exposure to ionizing radiation, requires the possibility of varying a number of parameters during their fabrication. A series of RDFs were developed with thicknesses in the range of 15 to 60 micrometers. The amount of dye precursor and stabilizers was varied in a systematic way. A series of unexposed films was irradiated with $1 \mathrm{MeV}$ electrons and $10 \mathrm{MeV}$ protons. Optical characterization of the films was performed. Irradiations were carried out in the dose range from 0 to $440 \mathrm{kGy}$. The fabrication techniques, the irradiation procedures, and the optical characterization of the films are described Conclusions are presented regarding the useful dose range for the RDFs, their sensitivity to different types of radiation, and a comparison is made with other similar film dosemeters used in the materials processing industry.

\section{3,714}

PB95-162954 Not available NTIS

National Inst. of Standards and Technology (NML) Gaithersburg, MD. lonizing Radiation Div.

Dose Mapping of Radioactive Hot Particles Using Radiochromic Film.

Final rept.

C. G. Soares, B. M. Coursey, F. F. McWilliams, and M. J. Scannell. $1990,2 p$.

Pub. in Radioact. Radiochem. 1, n2 p14, 16-17 1990.

Keywords: "Dosimetry, "Radioactivity, "Cobalt 60 Dosage, Films, Radioactive isotopes, Beta particles, Radiation protection, Reprints, Hot particles, Radiochromic films, Dose profile, Scanning densitometer.

An attempt to standardize hot particle beta dosimetry is addressed through the generation and characterization of (60)CO micro-spheres, An electrical arc, rapid solidification process of a cobalt alloy was used to produce uniform spherical particles of various diameters ranging from 50 to 250 microm. Neutron activation of these particles yielded essentially pure $(60) \mathrm{CO}$ in the microcurie range. The particles were characterized by physical size and activity; dose rates were determined using extrapolation chamber and radiochromic dye film measurements.

03,715

PB96-135173 Not available NTIS

National Inst. of Standards and Technology (PL) Gaithersburg, MD. Ionizing Radiation Div.

Anionic Triphenylmethane Dye Solutions for LowDose Food Irradiation Dosimetry.

Final rept.

N. B. El-Assy, Y. D. Chen, M. L. Walker, M. A

Sheikhly, and W. L. McLaughlin. 1995, 9p.

Pub. in Radiation Physics and Chemistry, v45 n4-6 p1189-1197 Oct 95

Keywords: *Dosimetry, "Food irradiation, Reprints, Gamma radiation, Radiation processing, "Foreign technology, "Dye bleaching, Fast Green FCF, Light Green SF, Radiolytic bleaching.

The radiolytic bleaching of aryl sulfonic-substituted para-diethyl-amino triphenylmethane dye solutions can be used for dosimetry in the absorbed dose range 10 to $400 \mathrm{~Gy}$. The sulfonic anions provide solubility of these acid dyes in water. Two of these dyes are supplied as stable greenish-blue biological stains when dissolved in weakly-acidic aqueous solution. Light Green SF Yellowish and East Green FCF. They have respectively, linear molar absorption coefficents of $7.14 \times 10$ to the 3rd power (at $\mathrm{pH} 5.4$ ) and $10.0 \times 10$ to the 3rd power (at $\mathrm{pH} \mathrm{4.2)} \mathrm{sq.} \mathrm{m.} \mathrm{mol} \mathrm{to} \mathrm{the} \mathrm{minus}$ one power, when measured at the peaks of the primary absorption bands, $630 \mathrm{~nm}$ and $622 \mathrm{~nm}$, respectively. The bleaching due to irradiation with gamma rays shows a linear function with a positive slope between the negative algorithm of the absorbance and the absorbed dose. The effect of $\mathrm{pH}$ on the response is studied, as well as the effects of light and temperature on pre- and post-irradiation stability. A mechanism, based mainly on radiolytic oxidation of the protonated phenolic or sulfonated phenyl group by $\mathrm{OH}$, with the abstraction of $\mathrm{H}$-atom to water, is postulated for neutral to slightly acidic aerated aqueous solutions.

\section{3,716}

PB96-135249 Not available NTIS

National Inst. of Standards and Technology (PL) Gaithersburg, MD. Ionizing Radiation Div.

Alcohol Solutions of Triphenyl-Tetrazolium Chlo ride as High-Dose Radiochromic Dosimeters. Final rept.

A. Kovacs, L. Woinarovits, N. B. El-Assy, M. L. Walker, W. L. McLaughlin, H. Y. Afeefy, and M. AtSheikhly. $1995,9 p$.

Pub. in International Meeting on Radiation Processing (9th), Istanbul, Turkey, September 11-16, 1994, Radiation Physics and Chemistry, v46 n4-6 p1217-1225 Oct 95

Keywords: * Radiochromic dosimeters, "Dosimetry, Reprints, Dyes, Formazan, Gamma rays, "Foreign technology, "Triphenyl tetrazolium chloride.

The radiolytic reduction of colorless tetrazolium salts in aqueous solution to the highly colored formazan dye is well-known acid-forming radiation chemical reaction Radiochromic thin films and three-dimensional hydrocolloid gels have been used for imaging and mapping absorbed dose distributions. The high solubility of 


\section{Nuclear Instrumentation}

2.3.5-triphenyl-tetrazolium chloride (TTC) in alcohols provides a useful liquid dosimeter ( $45 \mathrm{mM}$ TTC in aerated ethanol) and shows a linear response of absorbance increase (lambda(sub max) $=480 \mathrm{~nm}$ ) with dose over the range 1-16 kGy. The linear molar absorption coefficient lambda for the formazan at the absorption peak is $1.5 \times 10$ to the third power sq. $\mathrm{m}$. mol to the minus one power, and the radiation chemical yield for the above solution is $\mathrm{G}$ (formazan) $=0.014$ micromoles $\mathrm{J}$ to the minus 1 power. The irradiation temperature coefficient is about 0.8 percent per degree Celsius rise in temperature over the temperature range $0-30$ degrees $C$ but is much larger between 30 degrees and 60 degrees $C$.

\section{3,717}

PB96-135280 Not available NTIS

National Inst. of Standards and Technology (PL), Gaithersburg, MD. Ionizing Radiation Div.

Dosimetry Systems for Radiation Processing.

Final rept.

W. L. McLaughlin, and M. F. Desrosiers. 1995, 12p.

See also PB92-117118.

Pub. in International Meeting on Radiation Processing (9th), Istanbul, Turkey, September 11-16, 1994, Radiation Physics and Chemistry, v46 n4-6 p1163-1174 Oct 95.

Keywords: "Dosemeters, "Radiation monitors, "Calibration, "Quality assurance, Gamma dosimetry, Electron dosimetry, Calorimetric dosemeters, Radiation doses, Dose rates, Dose-response relationships, Thin films, Solids state devices, Reprints, Radiation processing.

Dosimetry serves important functions in radiation processing, where large absorbed doses and dose rates from photon and electron sources have to be meas ured with reasonable accuracy. Proven dosimetry systems are widely used to perform radiation measurements in development of new processes, validation, qualification, and verification (quality control) of established processes and archival documentation of dayto-day and plant-to-plant processing uniformity. Proper calibration and traceability of routine dosimetry systems to standards are crucial to the success of many large-volume radiation processes. Recent innovations and advances in performance of systems that enhance radiation measurement assurance and process diagnostics include dose-mapping media (new radiochomic film and solutions), optical waveguide systems for food irradiation, solid-state devices for realtime and passive dosimetry over wide dose-rate and dose ranges, and improved analytical instruments and data acquisitions.

\section{3,718}

PB96-135298 Not available NTIS

National Inst. of Standards and Technology (PL), Gaithersburg, MD. lonizing Radiation Div.

Temperature and Relative Humidity Dependence of Radiochromic Film Dosimeter Response to Gamma and Electron Radiation.

Final rept.

W. L. McLaughlin, J. M. Puhl, and A. Miller. 1995

$7 p$.

Pub. in International Conference on Radiation Processing (9th), Istanbul, Turkey, September 11-16, 1994, Radiation Physics and Chemistry, v46 n4-6 p1227. 1233 Oct 95

Keywords: "Colonimetric dosemeters, "Environmental effects, "Temperature effects, "Relative humidity, Gamma dosimetry, Electron dosimetry, Radiation monitors, Electron beams, Radiation doses, Gamma radiation, Thin films, Reprints, Radiation processing.

Nylon-base radiochromic films are commercially-available, thin dosimeters that are widely used in radiation processing. Based on some earlier studies, their re sponse functions have been reported to be dependent on the temperature and relative humidity during irradiation. The present study investigates differences in response over practical ranges of temperature, relative humidity, dose, and for different recent batches of films of both types. It is observed that for each new batch of film to be used for radiation processing, the effects of such parameters on response to both gamma rays and electrons should be investigated.

\section{3,719}

PB96-135306 Not available NTIS

National Inst. of Standards and Technology (PL)

Gaithersburg, MD. Ionizing Radiation Div.
Real Time Monitoring of Electron Processors. Final rept.

S. V. Nablo, D. R. Kneeland, and W. L. McLaughlin. $1995,7 p$

Pub. in International Meeting on Radiation Processing (9th), Istanbul, Turkey, September 11-16, 1994, Radiation Physics and Chemistry, v46 n4-6 p1377-1383 Oct 95.

Keywords: "Dosimeters, "Electron processors, Radiation dosage, Real time operation, Reprints, Diagnosis, Performance, "Foreign technology.

A real time radiation monitor (RTRM) has been developed for monitoring the dose rate (current density) of electron beam processors. The system provides continuous monitoring of processor output, electron beam uniformity, and an independent measure of operating voltage or electron energy. In view of the device's ability to replace labor-intensive dosimetry in verification of machine performance on a real-time basis, its application to providing archival performance data for in-line processing is discussed.

03,720

PB96-146725 Not available NTIS

National Inst. of Standards and Technology (PL), Gaithersburg, MD. Ionizing Radiation Div.

Orientation Effects on ESR Analysis of AlaninePolymer Dosimeters.

Final rept.

T. Kojima, S. Kashiwazaki, H. Tachibana, W. L.

McLaughfin, R. Tanaka, and M. F. Desrosiers. 1995

Pub. in Applied Radiation and Isotopes, v46 n12 p1407-1411 Dec 95.

Keywords: *Dosimeters, "Elcetron paramagnetic resonance, "Alanine, "Polymers, Thin films, Orientation, Pressing(Forming), Extrusion, Shape, Reprints, ESR(Electron spin resonance), Film dosimeter, Rod dosimeter.

Orientation effects during electron spin resonance (ESR) measurement are studied on alanine-polymer dosimeters prepared by different molding procedures (press-molding or extrusion), and also according to different shapes (rods with different lengths and thinfilms). The variation in ESR signal amplitude was measured by rotating a dosimeter around the vertical mid-axis and at a right angle to the magnetic field in the ESR cavity, at a temperature of $22 \mathrm{deg} C$. Orientation effects for rod dosimeters with both molding procedures (press and extrusion), and with different lengths, were negligible. Orientation effects on the film dosimeters can be eliminated, however, when they are positioned vertically and parallel to the mid-axis of the ESR cavity.

03,721

PB97-110514 Not available NTIS

National Inst. of Standards and Technology (PL) Gaithersburg, MD. Ionizing Radiation Div.

USCEANNIST Measurement Assurance Programs for the Radiopharmaceutical and Nuclear Power Industries.

Final rept.

D. B. Golas. $1993,16 p$.

See also PB95-126272.

Pub. in Proceedings of the Workshop on Measurement Quality Assurance for lonizing Radiation, Gaithersburg, MD., March 16-18, 1993, p191-206.

Keywords: "Nuclear power plants, *Radiopharmaceuticals, "Radiation measurement, Radiation monitoring, Laboratories, Public utilities, Nuclear medicine, Tracer techniques, Dosimetry, Radiochemistry, Quality assurance, Reprints.

In cooperation with the U.S. Council for Energy Awareness (USCEA), the National Institute of Standards and Technology (NIST) supervises and administers two measurement assurance programs for radioactivity measurement traceability. One in existence since the mid 1970s, provides traceability to suppliers of radiochemicals and radiopharmaceuticals, dose calibrators, and nuclear pharmacy services. The second program, begun in 1987, provides traceability to the nuclear power industry for utilities, source suppliers, and service laboratories. Each program is described. and the results of measurements of samples of known, but undisclosed activity, prepared at NIST and measured by the participants are presented.
National Inst. of Standards and Technology (CSTL), Gaithersburg, MD.

Conference Report: Calorimetry Conference (50th). Final rept.

E. S. Domalski, R. N. Goldberg, and P. A. G. O'Hare. 1996, 6p

Pub. in Calorimetry Conference (50th), Gaithersburg, MD., July 23-28, 1995, Jnl. of Research of the National Institute of Standards and Technology, v101 n1 p63681996.

Keywords: "Calorimetry, "Thermodynamics *Meetings, Temperatures, Chemistry, Chemical engineering, Biochemistry, Reprints.

Calorimetry and thermodynamics are used in a wide variety of basic and applied sciences. This was re. flected in the seemingly disparate topics dealt with at the conterence: low- and high-temperature physics, inorganic and organic chemistry, solution chemistry, chemical engineering, biochemistry, and biology. Accordingly, the conference was organized into 11 symposia covering a variety of topics.

\section{Radiation Shielding, Protection, \& Safety}

03,723

PB95-126132 Not available NTIS

National Inst. of Standards and Technology (NML) Gaithersburg, MD. Ionizing Radiation Div.

Neutron Leakage Benchmark for Criticality Safety Research.

Final rept.

D. M. Gilliam, V. Spiegel, C. M. Eisenhauer, J. Tang, E. Quang, and J. Briesmeister. 1990, 3p.

Pub. in Transactions of the American Nuclear Society 62, p340-342 1990.

Keywords: "Neutron leakage, "Criticality, Interlaboratory comparisons, Monte Carlo method, Fis sion neutrons, Neutron transport, Neutron sources, Californium 252, Subcriticality, Measurements, Benchmarks, Arrays, Water, Computation, Reprints

An interlaboratory program has been undertaken to investigate the consistency of direct measurements and rigorous calculations of neutron leakage from a moderated fission neutron source for the purpose of improving the calculations of criticality for arrays of individually sub-critical components. The present work is intended to check the possibility that one problem with these calculations may be associated with the estimation of neutron leakage from individual components, which could lead to misrepresented source terms for other components of the array. Results of calculations and measurements are presented, and discrepancies are noted.

\section{3,724}

PB95-161121 Not available NTIS

National Inst. of Standards and Technology (MSEL), Gaithersburg, MD. Metallurgy Div.

Information Retrieval Using Key Words and a Structured Review.

Final rept.

C. G. Interrante, C. A. Messina, and A. C. Fraker.

1993, $10 p$

Sponsored by Nuclear Regulatory Commission, Washington, DC. Div. of High-Level Waste Management.

Pub. in Standardizing Terminology for Better Communication: Practice, Applied Theory, and Results, ASTM STP 1166, p242-251 1993

Keywords: *High level radioactive wastes, "Radioactive waste storage, "Information retrieval, "Databases, Information systems, Database management, Standardized terminology, Reviewing, Reliability, Literature surveys, Radioactive waste disposal, containment systems, Failure mode analysis, Reprints, Controlled vocabulary.

A database has been developed to assist the Nuclear Regulatory Commission in assessing the efficacy of proposed plans for radionuclide containment for longterm storage of high-level nuclear waste (HLW). Important elements of this database are reviews and evalua tions of available literature on a waste package for HLW. The review process and database have been de. veloped and tailored to quickly provide information to answer questions about materials or components. about environments, and about interactions among the 
components of a waste package. Descriptions are given of the structure of the database and of the document review process and methods of searching.

\section{Radioactive Wastes \& Radioactivity}

03,725

PB95-220448 PC A04/MF AO1

National Inst. of Standards and Technology (BFRL), Gaithersburg, MD. Structures Div.

Prediction of Cracking in Reinforced Concrete Structures.

N. J. Carino, and J. R. Clifton. Apr 95, 58p, NISTIR5634.

Sponsored by Nuclear Regulatory Commission, Washington, DC.

Keywords: "Cracking(Fracturing), "Concrete structures, "Predictions, "Low-level radioactive wastes, Creep properties, Shrinkage, Construction materials, Radioactive waste disposal, Permeability, Tensile properties, Reinforced concrete.

The useful life of a buried concrete, containment structure for low level nuclear waste may be controlled by the loss of its load-bearing capacity or an increase in permeability. The latter factor is controlled by the general degradation of the concrete and by the presence of discrete cracks resulting from externally applied loads or from restraint to normal volume changes. To be able to predict the effects of cracks on permeability. it is necessary to understand the causes and mechanisms of discrete crack formation in reinforced concrete structures. The objective of this report is to provide an overview of the design and behavior of reinforced concrete members and to discuss the factors affecting the formation of cracks in hardened concrete.

\section{3,726}

\section{PB95-231593 PC A05/MF A01}

National Inst. of Standards and Technology (BFRL), Gaithersburg, MD. Building Materials Div.

4SIGHT Manual: A Computer Program for Modelling Degradation of Underground Low Level Waste Concrete Vaults.

K. A. Snyder, and J. R. Clifton. Jun 95, 80p, NISTIR5612 .

Sponsored by Nuclear Regulatory Commission, Washington, DC. Div. of Engineering.

Keywords: "Low-level radioactive wastes, "Radioactive waste disposal, "Underground disposal, "Concretes, *Performance evaluation, Service life, Leaching, Precipitation(Chemistry), Dissolution, Sulfates, Ion transport, Computer models, One-dimensional models, 4SIGHT computer program.

A computer program has been written to facilitate performance assessment of concrete vaults used in Low Level Waste (LLW) disposal facilities. The computer program is a numerical computer model of degradation in concrete. A one-dimensional finite different equation is used to propagate ions by precipitation/dissolution of available salts. The precipitation/dissolution of salts, in turn, changes the transport properties, which changes the rate of ion transport. The result is a model which incorporates the synergism of multiple degradation mechanisms.

\section{3,727}

PB95-260816 PC A03/MF A01

National Inst. of Standards and Technology (BFRL), Gaithersburg, MD. Building Materials Div.

Long-Term Performance of Engineered Concrete Barriers.

J. R. Clifton, J. M. Pommersheim, and K. Snyder. Jul $95,22 p$, NISTIR-5690.

Prepared in cooperation with Bucknell Univ., Lewisburg, PA.

Keywords: "Low-level radioactive wastes, "Radioactive waste disposal, "Concretes, Service life, Sulfates, Leaching, Cracking(Fracturing), Accelerated tests.

This paper describes research being carried out at NIST on the long-term pertormance of concrete for constructing low-level nuclear waste (LLW) disposal facilities. Three major research needs have been identified which are: validation of service life models; development of performance criteria for materials and systems to repair concrete before closure of concrete vaults; and development of an expert system to dissiminate knowledge on concrete durability for constructing concrete vaults

\section{Reactor Engineering \& Nuclear Power Plants}

03,728

NÚREG/CP-0136 PC A16/MF A03

National Inst. of Standards and Technology.

Gaithersburg, MD. Digital Systems Reliability and

Nuclear Safety Workshop. Held in Rockville, Maryland on September 13-14, 1993.

D. R. Wallace, B. B. Cuthill, L. M. Ippolito, and L. Beltracchi. Mar 94, 356p, NIST-SP-500-216.

Also available from Supt. of Docs. Prepared in co. operation with Nuclear Regulatory Commission, Washington, DC. Office of Nuclear Regulatory Research.

Keywords: "Meetings, "Digital systems, "Reactor safety, "Nuclear power plants, Information exchange, Software engineering, Computer software, Computer software management, Program verification(Computers).

The United States Nuclear Regulatory Commission (NRC) in cooperation with the National Institute of Standards and Technology conducted the Digital Systems Reliability and Nuclear Safety Workshop on September 13-14, 1993, in Rockville, Maryland. The workshop provided a forum for the exchange of information among experts within the nuclear industry, experts from other industries, regulators and academia. The information presented at this workshop provided indepth exposure of the NRC staff and the nuclear industry to digital systems design safety issues and also provided feedback to the NRC from outside experts re garding identified safety issues, proposed regulatory positions, and intended research associated with the use of digital systems in nuclear power plants. Technical presentations provided insights on areas where current software engineering practices may be inadequate for safety-critical systems, on potential solutions for development issues, and on methods for reducing risk in safety-critical systems. The report contains an analysis of results of the workshop, the papers presented, panel presentations, and summaries of discussions at this workshop.

\section{3,729}

PB95-151619 Not available NTIS

National Inst. of Standards and Technology (MSEL), Gaithersburg, MD. Reactor Radiation Div.

Liquid-Hydrogen Cold Neutron Source for the NBSR.

Final rept.

R. E. Williams, J. M. Rowe, and P. Kopetka. 1992

$3 p$

66. $169-1711992$

Keywords: "NBSR reactor, "Neutron sources, "Liquid hydrogen, "Cold neutrons, Research reactors, Neutron scattering, Thermosyphons, Moderators, Safety, Reprints.

The NBSR is a 20-MW research reactor operated by the National Institute of Standard and Technology. It was designed with a $55 \mathrm{~cm}$ diameter beam port for the purpose of installing a D20-ice cold neutron source completed in 1987. A liquid hydrogen cold neutron source is being developed to replace the D20 ice in order to increase the cold-neutron yield. A simple, passively-safe system has been designed, with multiple barriers preventing air from mixing with hydrogen. A thermosiphon will be used to maintain the liquid hydrogen inventory in the moderator chamber. The hydrogen condenser is cooled by a $3.5-\mathrm{kW}$ helium refrigerator. A ballast tank is connected to the condenser so the entire hydrogen inventory can expand freely into the tank, providing completely passive protection against refrigerator failures.

03.730

PB95-152955 Not available NTIS

National Inst. of Standards and Technology (MSEL), Gaithersburg, MD. Reactor Radiation Div.

Nuclear Heat Load Calculations for the NBSR Cold Neutron Source Using MCNP

M. Blau, J. M. Rowe, and R. E. Williams. 1993, 2p.

Pub. in Transactions of the American Nuclear Society 69, p404-405 1993.
Keywords: "NBSR reactor, "Neutron sources, "Liquid hydrogen, "Moderators, Spherical configuration, Annular shape, Reactor cores, Heavy water, Monte Carlo method, Cold neutrons, Heating load, Computation, Reprints.

The existing D20-ice cold neutron source in the 20MW NBSR will be replaced in 1994 with a liquid-hydrogen (LH2) source to increase the yield of cold neutrons (lambda greater than $0.4 \mathrm{~nm}$ ). Two series of Monte Carlo calculations using MCNP were performed to determine the optimum cold moderator geometry, and to verify its pertormance. Only the region near the cryostat was modeled for the first series of calculations, leading to the choice of a spherical annulus for the LH2 source. A complete MCNP model of the core was subsequently developed.

03,731

PB95-163978 Not available NTIS

National Inst. of Standards and Technology (MSEL), Gaithersburg, MD. Reactor Radiation Div.

Cold Neutron Gain Calculations for the NBSR Using MCNP.

Final rept.

R. E. Williams, M. Blau, and J. M. Rowe. 1993, 3p.

Pub. in Transactions of the American Nuclear Society 69, p401, 403-404 1993

Keywords: "NBSR reactor, "Neutron sources, "Cold neutrons, Monte Carlo method, Mathernatical models, Spherical configuration, Annular space, Liquid hydrogen, Reactor cores, Moderators, Gain, Reprints, MCNP model.

The existing D2O-ice cold neutron source in the 20MW National Bureau of Standards reactor (NBSR) will be replaced in 1994 with a liquid-hydrogen (LH2) source to increase the yield of cold neutrons (lambda $>0.4 \mathrm{~nm}$ ). Two series of Monte Carlo calculations using MCNP were performed to determine the optimum cold moderator geometry and to verify its performance. Only the region near the cryostat was modeled for the first series of calculations, leading to the choice of a spherical annulus for the $\mathrm{LH} 2$ source. A complete MCNP model of the core was subsequently developed.

\section{3,732}

PB95-209888 PC A08/MF A02

National Inst. of Standards and Technology (MSEL), Gaithersburg, MD. Reactor Radiation Div.

Reactor Radiation Technical Activities, 1994. NAS NRC Assessment Panel, April 6-7, 1995.

J. M. Rowe, and T. M. Raby. 1994, 154p, NISTIR5583

See also PB92-133024

Keywords: "Nuclear reactors, "Research projects, *Nondestructive tests, "Neutron activation analysis, Cold neutrons, Irradiation, Standards, Radioisotopes, Neutron radiography, Neutron diffraction, Crystal struc ture, Measurement, Calibrating, US NBS Microanalysis, NBSR reactor, US NIST, Molecular dynamics.

This report summarizes all the programs which use the NIST reactor It covers the period for October 1993 through September 1994. The programs range from the use of neutron beams to study the structure and dynamics of materials through nuclear physics and neutron standards to sample irradiations for activation analysis, isotope production, neutron radiography, and nondestructive evaluation.

03,733

PB96-138599 Not available NTIS

National Inst. of Standards and Technology (MSEL) Gaithersburg, MD. Reactor Radiation Div.

MCNP Model of the National Bureau of Standards Reactor (NBSR) Core.

Final rept.

R. E. Williams. 1995, 2p

Pub. in Transactions of the American Nuclear Society San Francisco, CA., October 29-November 2, 1995 v73 n0003-18x p397-398 1995

Keywords: "NBSR reactor, "Reactors, "Cold neutrons, Monte Carolo method Reactor cores, Reprints, "Criticality calculations, MCNP model, National Institute of Standards and Technology(NIST).

The National Bureau of Standards reactor (NBSR) is a $20-\mathrm{MW}$ research reactor operated for the U.S. Department of Commerce at the National Institute of Standards and Technology in Gaithersburg, Maryland 


\section{Reactor Engineering \& Nuclear Power Plants}

Earlier this year, a liquid hydrogen cold neutron source was installed in the NBSR to increase the yield of long wavelength (lambda equal to or greater than $0.4 \mathrm{~nm}$ ) neutrons. As part of the design effort for the new cold source, Monte Carlo simulations of the core and the cryogenic beam port were performed using the MCNP code. The model has been steadily improved and, where possible, compared with measurements.

\section{3,734}

PB96-160890 Not available NTIS

National Inst. of Standards and Technology (CSL) Gaithersburg, MD. Systems and Software Technology Div.

Assessing Functional Diversity by Program Slic. ing.

Final rept

K. B. Gallagher, J. R. Lyle, D. Wallace, and L

Ippolito. 1993, 1p.

Pub. in Transactions of the Water Reactor Safety Information Meeting (21st), Bethesda, MD., October 25-27, 1993, p9-1.

Keywords: "Nuclear power plants, "Electronic security, 'Software engineering, Safety programs, Validation, Debugging(Computers), Computer program verification, Software reliability, Computer program integrity, Computer programming, Mathematical calculations, Reprints, "Program slicing, Functional diversity.

One of the most commonly invoked safeguards against design basis events in nuclear power plants is the use of functional diversity. When auditors for the United States Nuclear Regulatory Commission (NRC) examine safety systems for nuclear power plants, they also inspect the software embedded within the safety systems to ensure functional diversity. For computer software, this means that there is no common code shared among diverse computations. The steps the auditor must take include identifying the types of system haz ards that could occur, using techniques, like fault tree analysis, to locate software that should be examined further, and then inspecting the code for diversity. This paper describes an approach using a concept called program slicing to support auditors.

\section{3,735}

PB96-161351 Not available NTIS

National Inst. of Standards and Technology (CSL), Gaithersburg, MD. Systems and Software Technology Div.

Analysis of Standards for the Assurance of High Integrity Software.

Final rept.
D. R. Wallace, D. R. Kuhn, L. M. Ippolito, and L.

Beltracchi. 1992, $21 \mathrm{p}$

See also PB95-173084 and PB95-251674

Pub. in International Symposium on Nuclear Power Plant Instrumentation and Control, Tokyo, Japan, May 18-22, 1992, p1-21

Keywords: "Computer program integrity, "Computer program verification, "Validation, "Nuclear power plants, Software reliability, Software engineering, Configuration management, Safety programs, Standards, Specifications, Computer information security, Electronic security, Ouality assurance, Reprints, High integrity

This study examines standards, draft standards, and guidelines (all of which will hearafter be referred to as documents) that provide requirements for the assurance of software in safety systems in nuclear power plants. The study focuses on identifying the attributes necessary in a standard for providing reasonable assurance for software in nuclear systems. The documents vary widely in their requirements and the precision with the requirements are expressed. Recommendations are provided for guidance for the assurance of high integrity software.

\section{3,736}

PB96-161369 Not available NTIS

National Inst. of Standards and Technology (CSL), Gaithersburg, MD. Systems and Software Technology Div.

Control and Instrumentation: Standards for HighIntegrity Software.

Final rept.

D. R. Wallace, D. R. Kuhn, L. M. Ippolito, and L.

Beltracchi. 1994, 12p.

Pub. in Nuclear Safety, v35 n1 p86-97 Jan-Jun 94.

Keywords: "Nuclear power plants, "Electronic security, *Safety programs, "Software reliability, Software engi- neering, Computer program integrity, Configuration management, Computer information security Standards, Requirements, Software development, Functional analysis, Ouality assurance, Reprints, High integrity.

This article describes a study that examines standards draft standrads, and guidelines (all of which will hereafterbe referred to as documents) that provide requirements for the assurance of software in safety systems in nuclear power plants. The study focuses on identifying, for developers of standards, the elements to be addressed in a standard for providing reasonable assurance of software in safety systems in nuclea power plants. The documents vary widely in their requirements and the precision with which the requirements are eexpressed. Recommendations are outlined for guidance for the assurance of high-integrity software.

\section{3,737}

PB96-161872 Not available NTIS

National Inst. of Standards and Technology (MSEL)

Gaithersburg, MD. Reactor Radiation Div.

Upgrade and Modernization Projects at the NBSR. Final rept.

R. E. Williams. 1995, 2p

Pub. in Transactions of the American Nuclear Society, v72 n0003-018X p312-313 1995.

Keywords: "Heat exchangers, "Nuclear reactors, 'Refueling system, Reprints, NBSR, Cold Neutron Research Facility(CNRF)

The National Bureau of Standards (now the National Institute of Standards and Technology (NIST)) reactor (NBSR), a 20-MW research reactor operated by NIST has become the leading U.S. laboratory in research using nuclear methods. About 1000 scientists from 200 industries, government and foreign laboratories, and universities conducted experiments at the NBSR last year Since 1990, when the first instruments in the Cold Neutron Research Facility (CNRF) became available the number of research participants has doubled. A major program of modernization and facility upgrade was initiated to meet this growing demand and to ensure safe and reliable reactor operations for 30 additional yr.

\section{3,738}

PB96-167259 Not available NTIS

National Inst. of Standards and Technology (CSTL) Gaithersburg, MD. Analytical Chemistry Div. New NIST Rapid Pneumatic Tube System.

Final rept.

D. A. Becker, R. M. Lindstrom, J. K. Langland, and

R. R. Greenberg. 1996, 7p.

Pub. in Jnl. of Trace and Microprobe Techniques, v14 n1 p1-7 1996.

Keywords: "Neutron activation analysis, Neutron irradiation, Reprints, Epithermal irradiation, Nuclear reac tors, "Foreign technology, "Pneumatic tube terminal, Rapid activation analysis.

The Nuclear Methods Group of NIST is developing a new rapid pneumatic tube irradiation system to be inserted into our $20 \mathrm{MW}$ nuclear reactor. The terminal will be inserted into an existing horizontal pneumatic tube location, the only one of five pneumatic tubes which has not been operational since the reactor up grade from 10 to $20 \mathrm{MW}$ ten years ago. This new terminal will contain two irradiation locations, one at the tip in a void area between several fuel elements for epithermal fluence optimization, the second in a D2O tank midway in the terminal to optimize the therma fluence. Estimated neutron fluence rates (in n-cm-2s 1) are $9 E 13$ thermal/3E13 epithermal for the tip irradiation location inside the D2O tank.

\section{9}

PB96-176615 Not available NTIS

National Inst. of Standards and Technology (MSEL), Gaithersburg, MD. Reactor Radiation Div.

Magnetic Structure Determination for Annealed Ni80Fe20/Ag Multilayers Using Polarized-Neutron Reflectivity.

Final rept.

J. A. Borchers, P. M. Gehring, C. F. Majkrzak, K. R. Coffey, M. A. Parker, J. K. Howard, J. F. Ankner, and T. L. Hylton. 1995, 6p.

Pub. in Materials Research Symposium Proceedings, Boston, MA., November 1994, v386 p577-582 1995

Keywords: "Magnetoresistance, "Neutron reflectivity, Reprints, "Antiferromagnetic coupling, Metallic multilayers.
Sputtered Ni80Fe20/Ag multilayers, annealed postgrowth, show giant magnetoresistive (GMR) effects at unusually low magnetic fields (approx. $5 \mathrm{Oe}$ ). Structural characterization by cross-sectional TEM and $x$ ray diffraction indicates that the $\mathrm{Ag}$ preferentially diffuses into the Ni80Fe20 layers at the interfaces. Using polarized-neutron specular reflectivity the authors have obtained magnetization depth profiles for a series of annealed (Ni80Fe20(20A)/Ag(40A)) 4 multilayers. Though GMR in related materials is associated with coherent antiferromagnetic alignment of the ferromagnetic layers, specular neutron data for the Ni80Fe20/ Ag multilayers show no trace of half-order spin-flip intensity characteristic of this simple structure. In small applied fields, transverse scans at the half-order position show a broad feature which disappears upon saturation. These data suggest that while the Ni80Fe20 moments are antiferromagnetically correlated along the growth axis, the in-plane magnetic domains are only of micron-order size and are thus not apparent in a specular measurement.

03,740

PB96-176631 Not available NTIS

National Inst. of Standards and Technology (MSEL), Gaithersburg, MD. Reactor Radiation Div.

Energy Distributions of Neutrons Scattered from Solid $\mathrm{C} 60$ by the Beryllium Detector Method.

Final rept.

J. R. D. Copley, D. A. Neumann, and W. A.

Kamitakahara. $1995,9 p$

Pub. in Canadian Jnl. of Physics, v73 p763-771 1995.

Keywords: "Fullereness, *Spectroscopy, Reprints, *Foreign technology, Internal mode dynamics, Neutron scattering, Vibrational spectrometer, C60.

The authors measured the energy distribution scattered from polycrystalline $\mathrm{C} 60$, using a high-resolution filter-analyzer spectrometer. In the energy range 30 $90 \mathrm{meV}$ (242-726 cm to the minus one power) the authors observed a rich spectrum that the authors fitted to a sum of 15 Gaussian functions, each of which is assigned to one or a set of several degenerate normal vibrational modes of the C60 molecule. The authors also observed two broad features in the energy range from $90-130 \mathrm{meV}(726-1049 \mathrm{~cm}$ to the minus one power). The authors' results are generally in excellent agreement with published spectroscopic data. Detailed comparisons with the results of several first-principles calculations suggest that present-day theories can predict the internal vibrational frequencies of $\mathrm{C} 60$ rather well, at least in the 30-90 meV range of energies.

03,741

PB96-193644 PC A07/MF A02

National Inst. of Standards and Technology (MSEL), Gaithersburg, MD.

Reactor Radiation Technical Activities, 1995.

Rept. for Oct 94-Sep 95.

L. K. Clutter. 1996, 108p, NISTIR-5751.

See also PB95-209888

Keywords: "Nuclear reactors, "Research projects "Nondestructive tests, "Neutron activation analysis, Cold neutrons, Irradiation, Standards, Radioisotopes, Neutron radiography, Neutron diffraction, Crystal structure, Measurement, Calibrating, US NIST, Microanalysis.

The report summarizes all the programs that use the NIST reactor. It covers the period for October 1994 through September 1995. The programs range from the use of neutron beams to study the structure and dynamics of materials through nuclear physics and neutron standards to sample irradiations for activation analysis, isotope production, neutron radiography, and nondestructive evaluation.

03,742

PB96-193784 PC A07/MF A02

National Inst. of Standards and Technology (BFRL), Gaithersburg, MD. Fire Science Div.

Methodology for Developing and Implementing Alternative Temperature-Time Curves for Testing the Fire Resistance of Barriers for Nuclear Power Plant Applications.

L. Y. Cooper, and K. D. Steckler. May 96, 117p, NISTIR-5842

Sponsored by Nuclear Regulatory Commission, Washington, DC. Office of Nuclear Reactor Regulation.

Keywords: "Nuclear power plants, "Barriers, "Fire protection, "Performance testing, Fire tests, Flammability, Ratings, Scenarios, Threats, Reactor safety, Fire safe- 
ty, Exposure, Fire resistance, Test methods, Temperature-time curves, ASTM E 119 Standard.

The aim of the current study is to propose a methodology for developing and implementing NPP-specific descriptions of fire environments and associated ASTMtype furnace test methods. Here the terminology ASTM E 119-type test is used to refer to a test method that basically follows the ASTM E 119 test procedures, but where the ASTM E 119 standard temperature-time curve is replaced with a relevant, NPP-specific, alternative curve. The approach taken in the current study consists of three steps or tasks: (1) review the history of the ASTM E 119 temperature-time curve to assess its current applicability and limitations in simulating real fires; (2) review the history of efforts to develop alternative curves and the methodologies used; and (3) use the findings from (1) and (2), knowledge of NPP construction, fuel types and loads, and state-of-the-art fire science to propose a methodology for developing and implementing NPP-specific descriptions of fire environments and associated ASTM-type temperature-time curves and test methods.

\section{Reactor Materials}

03,743

PB96-148127 Not available NTIS

National Inst. of Standards and Technology (MSEL), Gaithersburg, MD. Ceramics Div.

High-Temperature Furnace for In situ Small-Angle Neutron Scattering during Ceramic Processing. Final rept.

H. M. Kerch, H. E. Burdette, and G. G. Long. 1995

$7 \mathrm{p}$.

Pub. in Jnl. of Applied Crystallography, v28 p604-610 1995.

Keywords: "Furnances, "Research and development, "Ceramics, Neutron scattering, Experimental data, Nuclear reactors, Sintering, Glasses, Silicon dioxide, Reprints, "Small angle neutron scattering, SANS, SANS furnace, MSANS.

The design and operation of a new research furnace, optimized for small-angle neutron scattering experiments at a steady-state nuclear reactor with a cold source, are presented. The apparatus enables the study of thermal processing of materials, such as the sintering behavior in ceramics, through the measurement of their microstructural parameters (e.g. pore sizes, pore volumes and pore surface areas) during thermal treatment. Small-angle neutron scattering measurements can be performed in situ in an oxidizing, reducing or neutral environment at temperatures up to $2000 \mathrm{~K}$.

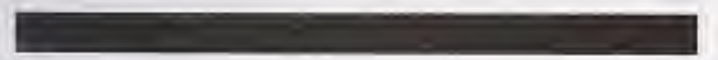

\section{OCEAN SCIENCES \& TECHNOLOGY}

\section{Biological Oceanography}

\section{3,744}

PB95-162640 Not available NTIS

National Inst. of Standards and Technology (CSTL), Gaithersburg, MD. Organic Analytical Research Div. Determination of PCBs and Chlorinated Hydrocarbons in Marine Mammal Tissues.

Final rept.

M. M. Schantz, B. J. Koster, S. A. Wise, and P. R Becker. 1993, 23p.

Pub. in Science of the Total Environment 139/140, p323-345 1993.

Keywords: "Chlorinated aromatic hydrocarbons, "Marine animals, "Pesticides, Chromatographic analysis, Tissue culture, Liver, Kidney, Muscles, Mammals, Reprints, PCBs(Polychlorinated biphenyls), Blubber, Northern fur seal, Ringed seal, Belukha whale.

Selected tissues (blubber, liver, kidney and muscle) from marine mammals, which were collected as part of the Alaska Marine Mammal Tissue Archival Project (AMMTAP), were analyzed for polychlorinated biphenyl (PCB) congeners and chlorinated pesticides. Concentrations of these compounds in the different tissues were compared and blubber was selected as the primary tissue for organic contaminant analyses for the AMMTAP based on higher levels (1-2 orders of magnitude) in this tissue compared to liver, kidney and muscle.

\section{3,745}

PB96-103023 Not available NTIS

National Inst. of Standards and Technology (CSTL), Gaithersburg, MD. Organic Analytical Research Div. Certification of Polychlorinated Biphenyl Congeners and Chlorinated Pesticides in a Whale Blubber Standard Reference Material.

Final rept.

M. M. Schantz, B. J. Koster, L. M. Oakley, S. B. Schiller, and S. A. Wise. 1995, 10p.

Pub. in Analytical Chemistry 67, n5 p901-910, 1 Mar 95.

Keywords: "Polychlorinated biphenyls, "Pesticides, "Whales, "Animal tissues, "Bioaccumulation, Marine mammals, Water pollution effects (Animals), Reference standards, Gas chromatography, Mass spectroscopy, Electron capture, Chemical analysis, Quallty assurance, Reprints, "Blubber, Standard reference materials.

A Standard Reference Material (SRM) made from whale blubber has been developed for the validation of methods used for the determination of polychlorinated biphenyl (PCB) congeners and chlorinated pesticides. This material, which is a frozen blubber tissue homogenate, was analyzed using three different analytical techniques. These techniques were based on gas chromatography with electron capture detection on two stationary phases with different selectivity for the seperation of PCB congeners and gas chromatography with mass spectrometric detection. The results from these three techniques were in good agreement and were combined to provide certified concentrations for 27 PCB congeners and 15 chlorinated pesticides.

\section{Marine Engineering}

03,746

PB94-174505 PC A04/MF A01

National Inst. of Standards and Technology (MSEL), Gaithersburg, MD. Metallurgy Div.

Characterization of the Hydrogen Induced Cold Cracking Susceptibility at Simulated Weld Zones in HSLA-100 Steel.

M. R. Stoudt, and R. E. Ricker. 1994, 71p, NISTIR5408.

See also AD-A224 341. Sponsored by Office of Naval Research, Arlington, VA.

Keywords: "Alloys, "Carbon steels, "Crack resistance, "Welding, "Naval vessels, Fracture(Mechanics), Fabrication, Impact strength, Hydrogen embrittlement Cold forming. Mechanical properties, Structural steel Strength(General), Corrosion, Sea water, Performance evaluation, Comparison, Strain rate, Tests, Electrochemistry.

The relative susceptibilities to hydrogen induced cold cracking were evaluated for $\mathrm{HY}-100$, a steel presently in service in naval applications and for HSL A-100. The martensitic microstructure of the HY-100 undergoes
wide variations in the heat affected zone during welding which strongly influence the resistance of that alloy to cold cracking. The HSLA-100, a low carbon, precipitation hardened steel with similar strength and toughness to that of the $H Y-100$, possesses a significantly lower degree of hardenability which results in minima microstructural variations in the heat affected zone under the same simulated welding conditions. The mechanical properties of the base metal and the heat affected zone created during a simulated, single welding pass were characterized by the slow strain rate technique for both steels in an inert environment and in artificial seawater under free corrosion and controlled hydrogen fugacities. The electrochemical behaviors of both steels were also evaluated in artificial seawater.
National Inst. of Standards and Technology (NEL), Gaithersburg, MD. Robot Systems Div.

Intelligent Control for Multiple Autonomous Undersea Vehicles.

Final rept.

M. Herman, J. S. Albus, and T. H. Hong. 1990, 48p Pub. in Neural Networks for Control, Chapter 18, p427. 4741990.

Keywords: "Autonomous navigation, "Underwater vehicles, "Artificial intelligence, Neural nets, Architecture(Computers), Control systems design, Remotely piloted vehicles, Real time operation Hierarchies, Robotics, Models, Reprints, "Multiple Autonomous Undersea Vehicles project, "MAUV project.

Intelligent control for autonomous vehicles in a natural, potentially hostile environment requires a system that integrates artificial intelligence with modern control theory, arid that is implemented on parallel, possibly special-purpose hardware. Issues dealing with the requirements of such a system are discussed in the context of the Multiple Autonomous Undersea Vehicles (MAUV) project. The MAUV control system and its implementation are also presented. The goal of the MAUV project was to have multiple undersea vehicles exhibiting intelligent, autonomous, cooperative behavior. The MAUV control system is hierarchically structured and incorporates sensing, world modeling, planning and execution. The levels in the hierarchy include: mission, group, vehicle task, elemental action, primitive action, and servo. Issues of real-time planning and dynamic replanning in unstructured environments are discussed. A multi-level world model that supports real-time planning is also described. Finally, timing is sues, implementation, and initial experimental results are presented

\section{3,748}

PB95-251633 PC A03/MF A01

National Inst. of Standards and Technology (MEL), Gaithersburg, MD. Intelligent Systems Div.

Submarine Automation: Demonstration No. 5.

Rept. for Oct 92-Nov 93.

H. M. Huang, R. Quintero, and K. Young. 1995, 21p, NISTIR-5676, ARPA-7829-2

See also PB93-184257. Prepared in cooperation with PdMA Corp., Rockville, MD. Sponsored by Defense Advanced Research Projects Agency, Arlington, VA.

Keywords: *Submarines, "Automation, "Demonstration projects, Computer applications, ARPA(Advanced Research Projects Agency)

The major objectives of this project are to: Demonstrate the application of the NIST RCS to submarine automation; and Refine and document the RCS methodology. This cycle of the submarine automation project emphasizes: Continuing investigating and developing the human computer interface $(\mathrm{HCl})$; Expanding the submarine control system, the simulator, and the animator to include engineering supporting systems; Demonstrating reusing the existent automated maneuvering system software; and Refining the RCS methodology

03,749

PB96-123112 Not available NTIS

National Inst. of Standards and Technology (BFRL), Gaithersburg, MD. Structures Div.

Stranding Experiments on Double Hull Tanker Structures.

J L. Rodd, M. P. Phillips, and E. D. Anderson 1994 $17 \mathrm{p}$.

Pub. in Advanced (Unidirectional) Double-Hull Technical Symposium, Gaithersburg, MD., October 25-26, 1994, p1-17.

Keywords: "Oil tankers, "Double hulls, Ship structural components, Tanker ships, Ship hulls, Losses, Structural failures, Design analysis, Failure analysis, Crashworthiness, Ship accidents, Reprints, "Strandings, "Groundings.

Three different hull designs were evaluated in the 30,000-40,000 lon range: a baseline conventional double hull bottom with transverse and longitudinal framing, an advanced double hull (ADH) with unidirectional longitudinal web, and a variant of the $\mathrm{AH}$ design incorporating curved plates. A large fabricated 'rock' was used to indent each structure vertically, causing first rupture of the outer shell, and then rupture of the inner shell. Inner shell rupture occurred at model loads of up to $750,000 \mathrm{lbs}$, and the rock intrusion into the ship was nearly twice the double hull spacing. The results 
are proving to be vital in validating numerical modeling tools needed to develop reliable design and analysis methods. In addition, efforts are already underway to develop an ADH crash worthiness system that can minimize or eliminate rupture of the inner shell during the high energy dissipation that occurs during a grounding.

\section{3,750}

\section{PB96-155759 Not available NTIS}

National Inst. of Standards and Technology (BFRL), Gaithersburg, MD. Structures Div.

Transitions to Chaos Induced by Additive and Multiplicative Noise.

Final rept.

E. Simiu, and M. Frey. 1994, 4p

Pub. in Proceedings of the Toyota Conference (7th)

'Towards the Harnassing of Chaos', Amsterdam, p4054081994.

Keywords: "Noise, "Building technology, "Chaos, Reprints, Transitions, Stochastic processes, "Foreign technology.

For a class of multistable systems, deterministic and stochastic chaos are closely related mathematically; necessary condition for the occurrence of noise-induced chaos with sensitive dependence on initial conditions can be derived from the generalized Melnikov function. Proof that this condition is applicable requires the approximate representation of the noise in the form of a modified Shinozuka process or other uniformly continuous and uniformly bounded processes. Additive and/or multiplicative Gaussian noise with any spectra density can be accommodated, as can other types of noises, including shot noise and non-Gaussian noise. We review recent results, including a successful verfication of our Melnikov-based approach against results based on a solution of the Fokker-Planck equation. We conclude by briefly describing ongoing research.

\section{3,75}

PB96-195516 PC A03/MF A01

National Inst. of Standards and Technology (MEL), Gaithersburg, MD. Intelligent Systems Div.

Operator Experience with a Hierarchical Real-Time Control System (RCS)

H. M. Huang. Jun 96, 19p, NISTIR-5862.

Keywords: ".Submarines, "Real time control, Automatic control, Computer architecture, Human computer interface, Artificial intelligence, Knowledge bases, Computer programming, Underwater vehicles, Autonomous navigation

The issue of operator interface in the RCS hierarchica control systems is the focus of this report. In a submarine automation project, the authors have studied the logical structure of the operator interface function and the way it should be integrated to existing systems. A design issue is to allow operators to be involved in system operations at in different degrees. In some situations, the operators are requested to perform certain manual operations and report the status back to the control systems. In some other situations, the operators are required to make some decisions for the controllers. At different control levels, operators require different information. A system developer may require total different types of information.

\section{Physical \& Chemical Oceanography}

\section{3,752}

PB94-199122 Not available NTIS

National Inst. of Standards and Technology (MSEL), Gaithersburg, MD. Polymers Div.

Atmospheric and Marine Trace Chemistry: Interfacial Biomediation and Monitoring.

Final rept.

F. E. Brinckman, and G. J. Olson. 1990, 14p

Pub. in Marine Chemistry 30, n1-3 p147-160 1990 Sponsored by International Union of Pure and Applied Chemistry, Stockholm (Sweden).

Keywords: "Air-water interfaces, "Metalloids, "Metals, "Marine chemistry, "Environmental monitoring, *Environmental impact assessments, Trace elements Geochemistry, Biotechnology, State of the art, Natural emissions, Pollutants, Metal containing organic compounds, Reprints.
Valid monitoring and assessment of metals and metalloids active in our planetary environment is now established with analytical methodology employing ultratrace (microgram/L) molecular speciation of illustrative organometallic substances. During the past 20 years, such measurements have placed into realistic context the vital interplay betwen abiotic geochemical sources and man's technological effluents, in terms of the frequent rate-deterring and extensive biogeorganometallic chemistry dictating heavy element transport and speciation, especially at bioactive surface microlayers featuring heightened bioprocessing of both depositional and emissive fluxes of metals and metalloids. The report places its overview of this current state of art with air-sea measurement capability for monitoring such biogenic organometal(loid)s, within the context of independently established predictive mimetic aqueous chemistry of metals and metalloids, with implications for marine biotechnology.

\section{Ammunition, Explosives, \& Pyrotechnics}

\subsection{3}

AD-A295 896/5 PC A03/MF A01

National Inst. of Standards and Technology (NML), Gaithersburg, MD. Molecular Physics Div.

Thermal Decomposition Pathways in Nitramine Propellants.

Final rept. 1 Jan 92-31 Dec 94.

F. J. Lovas, and R. D. Suenram. 4 Apr 95, 45p, ARO-29596.2-CH Contract ARO-MIPR-126-94

Keywords: "Thermal properties, "Propellants, "Decomposition, "Nitramines, Spectroscopy, Vapors, Test methods, Microwaves, Chemical composition, Amines, Organic compounds, Rdx, Reactants(Chemistry), Pyrolysis, Nitro radicals, Microwave spectroscopy, Pathways.

We have investigated intermediates and products in the thermal decomposition of RDX vapor, using a variety of experimental microwave techniques previously employed in our laboratory in studies of pyrolysis decomposition of organic amines. We used microwave spectroscopy to determine the chemical composition of the decomposition of nitramines by pyrolysis methods and identify the products in the thermal decomposition processes. The objective was to determine the validity of proposed decomposition mechanisms, and to identify new reaction products or pathways. jg p.1.

\section{3,754}

PB94-200177 Not available NTIS

National Inst. of Standards and Technology (MSEL), Gaithersburg, MD. Reactor Radiation Div.

Texture Study of Two Molybdenum Shaped Charge Liners by Neutron Diffraction.

Final rept.

C. S. Choi, H. J. Prask, J. Orosz, and E. L. Baker.

1993, 7p.

Pub. in Jnl. of Materials Science 28. p3557-3563 1993.

Keywords: "Shaped charges, "Cavity liners, "Molybdenum, Neutron diffraction, Conical bodies, Texture, Reprints.

The textures of two different conical shaped liners, fabricated by the same forging processes from arc-cast and powder-sintered ingots, were investigated by using neutron-diffraction measurements and three-dimensional orientation-distribution-function (ODF) analysis. The major textures of both liners could be described by the $(111)<u v w>$ and $(100)<u v w>$ type. The two liners had essentially identical texture at the $8 \mathrm{~cm}$ position (measured from the base of the cone) with strong sheet-type texture components, i.e. $(111)<(-$ 1) $01>,(111)<(-1) 10>$ and $(100)<011>$. However, the dominant textures at the $3 \mathrm{~cm}$ positions were <111> and $\langle 100\rangle$ fiber textures with the fiber axes oriented parallel to the normal direction in both liners. A strong cube texture was observed at the $3 \mathrm{~cm}$ position of the arc-cast liner but it was not observed for the powdersintered liner. The arch-cast liner had a generally higher degree of texture than the powder-sintered liner.

03,755

PB95-108437 Not available NTIS

National Inst. of Standards and Technology (MSEL) Gaithersburg, MD. Ceramics Div.

1,4-Dinitrocubane and Cubane under High Pressure.

Final rept.

G. J. Piermarini, S. Block, R. Damavarapu, and S.

lyer. $1991,6 p$.

Pub. in Propeliants, Explosives, Pyrotechnics 16 p188-1931991.

Keywords: "High pressure tests, "Cubane, Chemical reactions, Phase transformations, Fourier transform spectrometers, Pyrolysis, Optical microscopy, Pyrotechnic devices, Reprints, "Cubane/dinitro, Energetic materials.

The effects of pressure on the chemical reactivity, phase behavior and thermal decomposition of energetic materials can be studied by a combination of measurement techniques in conjunction with a high pressure diamond anvil cell and the ruby fluorescence method of pressure measurement. These techniques include: (1) optical polarizing microscopy and (2) Fourier transform infrared (FTIR) spectroscopy. Recent results on the study of 1,4-dinitrocubane and cubane will be presented.

\section{3,756}

PB95-202396 Not available NTIS

National Inst. of Standards and Technology (MSEL), Gaithersburg, MD. Reactor Radiation Div.

Microstructure Study of Molybdenum Liners by Neutron Diffraction.

Final rept.

C. S. Choi, E. L. Baker, and J. Orosz. 1994, 8p.

Pub. in Nondestructive Characterization of Materials VI, p637-6441994.

Keywords: "Shaped charges, *Cavity liners, Neutron diffraction, Microstructure, Molybdenum, Reprints, Orientation distribution functions, Plastic strain ratio, $R$ values.

The crystallites orientation distributions of three different conical molybdenum shaped-charge liners, labeled $\mathrm{COO}, \mathrm{C} 2 \mathrm{O}$ and $\mathrm{C} 40$, fabricated with different thermo-mechanical treatment processes, were investigated by using neutron diffraction measurements and three-dimensional ODF (orientation distribution function) analysis. Two specimens were obtained from each liner, one from $25 \mathrm{~mm}$ below the apex and the other from $25 \mathrm{~mm}$ above the liner base. Results are discussed.

\section{Armor}

03,757

PB94-158573 PC A03/MF A01

National Inst. of Standards and Technology (EEEL), Gaithersburg, MD

Study to Determine the Most Important Parameters for Evaluating the Resistance of Soft Body Armor to Penetration by Edged Weapons.

N. J. Calvano. Jul 93, 47p, NISTIR-4895.

See also PB87-105524. Sponsored by National Inst of Justice, Washington, DC.

Keywords: "Body armor, "Puncture resistance, Blades, Knives, Police, Law enforcement officers, Protective clothing, Impact tests, Lightweight armor, Polyaramid fabrics.

The paper describes tests that were conducted to determine the most important parameters for measuring the resistance of soft body armor to penetration by edged weapons. Samples consisting of multiple layers of polyaramid fabric were tested against the following variables: blade, geometry, backing material, conditioning, impact angle, and velocity. Stab and slice tests were conducted. Some commercial armors were also tested. The commercial armors were constructed of ultra-high molecular weight polyethylene, polyaramid fabric, and combinations of ultra-high molecular weight polyethylene, polyaramid fabric, and titanium. Results 
indicate that penetration is a strong function of blade geometry. Backing material, conditioning, and impact angle appeared to have only a minimal affect upon the results.

\section{Combat Vehicles}

03,758
N94-34037/9 (Order as N94-34019/7, PC A21/

MF A04) National Inst. of Standards and Technology,
Gaithersburg, MD.

Ground Vehicle Control at NIST: From Teleoperation to Autonomy.

K. N. Murphy, M. Juberts, S. A. Legowik, H. A. Scott, S. Szabo, M. Nashman, and $H$. Schneiderman. Jan 94, $6 \mathrm{p}$

In NASA. Johnson Space Center, the Seventh Annual Workshop on Space Operations, Applications, and Research (Soar 1993), Volume 1 p 137-142.

Keywords: "Communication equipment, "Ground based control, "Inertial navigation, "Real time operation, "Teleoperators, Military operations, Radio communication, Communication networks, Highways, Measuring instruments, Steering, "Military vehicles, "Autonomous navigation.

NIST is applying their Real-time Control System (RCS) methodology for control of ground vehicles for both the U.S. Army Research Lab, as part of the DOD's Unmanned Ground Vehicles program, and for the Department of Transportation's Intelligent Vehicle/Highway Systems (IVHS) program. The actuated vehicle, a military HMMWV, has motors for steering, brake, throttle, etc. and sensors for the dashboard gauges. For military operations, the vehicle has two modes of operation: a teleoperation mode--where an operator remotely controls the vehicle over an RF communications network; and a semi-autonomous mode called retro-traverse--where the control system uses an inertial navigation system to steer the vehicle along a prerecorded path. For the IVHS work, intelligent vision processing elements replace the human teleoperator to achieve autonomous, visually guided road following.

03,759

PB95-163200 Not available NTIS

National Inst. of Standards and Technology (NEL), Gaithersburg, MD. Robot Systems Div.

Control System Architecture for a Remotely Operated Unmanned Land Vehicle.

Final rept.

S. Szabo, H. A. Scott, K. Murphy, and S. A. Legowik. $1990,8 \mathrm{p}$

Put. in Proceedings of Institute of Electrical and Electronics Engineers International Symposium on Intelligent Control (5th), Philadelphia, PA., September 5-7, 1990, p876-883.

Keywords: "Control systems, "Systems architecture, Military vehicles, Remote control, Teleoperators, Real time operation, Unmanned, Ground vehicles, Robot dynamics, Systems engineering, Reprints, "Mobile robots.

The U.S. Army Laboratory Command, as part of its Robotics Initiative, is developing a testbed for cooperative, real-time control of unmanned land vehicles. The National Institute of Standards and Technology is supporting this program by developing the vehicle control system using an architecture based on the Real-time Control System (RCS). RCS is a hierarchical, sensorybased control system, initially developed for the control of industrial robots and automated manufacturing systems. In this application, RCS controls all vehicle mobility functions, coordinates the operations of the other subsystems on the vehicle, and communicates between the vehicle and the remote operator control sta. tion. The paper reviews the overall control system architecture and the design of the mobility and communication functions.

\section{Underwater Ordnance}

03,760

PB95-182390 PC A05/MF A01
National Inst. of Standards and Technology (EEEL), Boulder, CO. Electromagnetic Fields Div. Assessment of Data by a Second-Order Transfer Function.

Technical note.

D. G. Camell, and M. T. Ma. Oct 94, 78p, NIST/TN1372.

Also available from Supt. of Docs. as SN003-00303303-1. See also PB95-161485.

Keywords: *Electromagnetic interference, "Torpedoes, Electromagnetic pulses, Convolution integrals, Continuous radiation, Resonant frequency, Transfer functions, Laplace transformation, Linear systems, Ap proximation, Magnitude, Phase, Graphs(Charts), Impulse response.

A newly developed theory for predicting the response of a linear system to an electromagnetic pulse, based only on the measured continuous-wave magnitude, is applied to the problem of possible electromagnetic interferences at a sensitive part of a torpedo. The measured magnitude representing the system's transfer function is deduced first from the measured response at this sensitive point to a known cw source, supplied by the Naval Surface Warfare Center. We de rive an analytic expression for the magnitude square of the transfer function to approximate the measured data and obtain a system transfer function in terms of the complex frequency, from which we predict the system's cw phase characteristics and its multiple solutions due to a given impulse source.

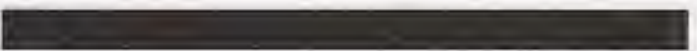 \\ PHOTOGRAPHY \& RECORDING DEVICES}

\section{Holography}

03,761

PB96-119284 Not available NTIS

National Inst. of Standards and Technology (CSTL), Gaithersburg, MD. Biotechnology Div.

Holographic Properties of Triton X-100-Treated Bacteriorhodopsin Embedded in Gelatin Films.

Final rept.

D. W. Cullin, N. N. Vsevolodov, and T. V. Dyukova. 1995, 4p.

Pub. in BioSystems, v34 n2,3 p 141-144 Jul 95.

Keywords: "Bacteria, "Thin films, "Gelatins, "Holographic spectroscopy, Surfactants, Biotechnology, Diffraction, Phases, Amplitudes, Kinetics, Concentration(Composition), Fabricatións, Reprints, "Triton X-100, "Bacteriorhodopsin.

Bacteriorhodopsin (bR) thin films have been fabricated with varying amounts of the detergent Triton $X-100$ to measure the effects of this additive on the holographic performance of these thin films. Holographic spectroscopy is used to measure the effect of these detergents on the overall diffraction efficiency as well as on the phase and amplitude components of the overall signal. The diffracted rise and decay kinetics of these materials will also be presented as a function of varying detergent concentration. This research also studied the effect of this additive on the absorptive properties of bR-based thin films. Comparison of the two com plimentary sets of data are drawn.

\section{3,762}

PB96-167366 Not available NTIS

National Inst. of Standards and Technology (EEEL), Boulder, CO. Electromagnetic Fields Div.

Planar Near-Field Measurements and Microwave Holography for Measuring A perture Distribution on a $60 \mathrm{GHz}$ Active Array Antenna.

Final rept.

J. Guerrieri, N. Canales, K. MacReynolds, and D.

Tamura. 1995, 6p.

Pub. in Annual Meeting and Symposium (17th), Antenna Measurement Techniques 1995 Association, Williamsburg. VA., November 13-17, 1995, p295-300. Keywords: *Amperture amplitude distribution, Reprints, "Planar near-field method, Aperture phase distribution, Black transform, Phase-shifter settings.
This paper discusses results of a recent attempt to measure aperture distribution of a small active steerable array antenna at $60 \mathrm{GHz}$ using planar near-field measurements and the back transform. Using a procedure which exercises every phase shifter without steering the antenna beam, it is possible to isolate problems with individual bits in the phase shifters. From calculation of the aperture fields for each case we hope to infer the individual phase shifter bit loss. We will also discuss problems which arose in the measurement because of the short wavelength signal-to-noise ratio and small number of elements.

\section{Recording Devices}

\section{3,763}

PB95-180907 Not available NTIS

National Inst. of Standards and Technology (EEEL), Boulder, CO. Electromagnetic Technology Div.

Comparison of Magnetic Fields of Thin-Film Heads and Their Corresponding Bit Patterns Using Magnetic Force Microscopy.

Final rept.

P. Rice, B. Hallett, and J. Moreland. 1994, 3p. Pub. in Institute of Electrical and Electronics Engineers Transactions on Magnetics 30, n6 p4248-4250 Nov 94.

Keywords: *Recording heads, "Magnetic fields, Imaging techniques. Thin films, Magnetization, Comparison, Reprints, Magnetic force microscopy, Magnetic imaging, Hard disks, Bit patterns.

We have used de-mode magnetic force microscopy to image the magnetic fringing fields of several thin-film heads and the bit patterns written with these heads. The images were taken with Si3N4 tips coated with 10$\mathrm{nm} \mathrm{Fe}$ and 5-nm Au. The heads and disks are typical industry standards. The heads had a variety of pole piece configurations. A large track separation was used so that the erase bands could be thoroughly studied. We were surprised to discover magnetic fields that correspond to layers in the alumina overcoat near the pole pieces. The magnetic force microscope images of the bit pattern show a definite twist at the track edge that points toward the trailing pole piece. We also observed disk magnetization patterns that remained after an ac erase procedure.

\section{PHYSICS}

\section{General}

\section{3,764}

\section{AD-A278 130/0 PC A05/MF A01}

National Bureau of Standards, Gaithersburg, MD. Atomic Energy Levels As Derived from the Analyses of Optical Spectra. Volume 1. Section 1. The Spectra of Hydrogen, Deuterium, Helium, Lithium Beryllium, Boron, Carbon, Nitrogen, Oxygen, and Fluorine.

C. E. Moore. 15 Apr 48, 79p, NBS-CIRCULAR-467.

Keywords: "Atomic energy levels, "Atomic spectra, Atomic structure, Energy levels, Spectra, Optical analysis, Hydrogen, Deuterium, Helium, Lithium, Beryllium, Boron, Carbon, Nitrogen, Oxygen, Fluorine.

No abstract available.

\section{3,765}

\section{AD-A278 139/1 PC A04/MF A01}

National Bureau of Standards, Boulder, $C O$

$X$-ray Attenuation Coefficients from $10 \mathrm{Kev}$ to 100 Mev.

G. W. Grodstein. 30 Apr 57, 58p, NBS-CIRCULAR

583.

Keywords: "X rays, "Radiation attenuation, "Gamma rays, Narrow beams(Radiation), Photoelectric effoct, Photons, Interactions, Attenuation coefficients.

A tabulation of attenuation coefficients of $X$-rays and gamma rays from 0.01 to $100 \mathrm{Mev}$ for 29 materlals is 


\section{PHYSICS}

\section{General}

presented. A summary of information on the probability of the basic interaction processes of photons with matter and a detailed analysis of experimental and theo retical evidence are included. Present information on the basic processes is adequate for many applications; however, improved theory and additional experimental data are needed in certain areas. A comparison of calculated and experimental coefficients points up this need.

\section{3,766}

AD-A279 282/8 PC A15/MF A03

National Bureau of Standards, Gaithersburg, MD

Technologic Papers of the Bureau of Standards: Number 170. Pyrometric Practice.

P. D. Foote, C. O. Fairchild, and T. R. Harrison. $1948,326 p$

Keywords: "Optical pyrometers, "Measuring instruments, "Standards, Radiation pyrometers, High temperature, Thermometers, Melting point, Thermoelectricity, Resistance thermometers, Recording systems, "Temperature measurement, Techno logical papers

No abstract available.

\section{3,767}

\section{AD-A279 289/3 PC A03/MF A01}

National Bureau of Standards, Gaithersburg, MD

$X$-Ray Attenuation Coefficients from $10 \mathrm{kev}$ to 100 Mev.

R. T. McGinnies. 30 Oct 59, 15p, NBS-CIRC-583-
SUPP.

Keywords: "X-rays, X-ray scattering, Photons, Incoherent scattering, Attenuation, Coefficients, Absorption, Tables(Data), X-ray absorption.

No abstract available.

\section{3,768}

AD-A280 290/8 PC A04/MF A01

National Bureau of Standards, Gaithersburg, MD $X$-Ray Attenuation Coefficients from $10 \mathrm{KEV}$ to 100 MEV.

G. W. Grodstein. 30 Apr 57, 58p.

Keywords: "X-ray absorption analysis, X-ray scattering, C;ross sections, Radiation attenuation, Gamma ray absorption coefficients, Tables(Data), Water, Hydrogen, Potassium, Air, X-ray attenuation coefficients.

A tabulation of attenuation coefficients of $X$-rays and gamma rays from 0.01 to $100 \mathrm{Mev}$ for 29 materials is presented. A summary of information on the probability of the basic interaction processes of photons with matter and a detailed analysis of experimental and theoretical evidence are included. Present information on the basic processes is adequate for many applications however, improved theory and additional experimental data are needed in certain areas. A comparison of calculated and experimental coefficients points up this need. (Author).

\section{3,769}

\section{AD-A281 167/7 PC A07/MF A02}

National Bureau of Standards, Gaithersburg, MD.

Bibliography of the Physical Equilibria and Related Properties of Some Cryogenic Systems.

T. M. Flynn. May 60, 130p, NBS-TN-56.

Keywords: ${ }^{*}$ Cryogenics, Bibliographies, Carbon diox de, Carbon monoxide, Ethanes, Helium, Hydrogen Methane, Mixtures, Nitrogen, Propane, Liquid phases, Vapor phases, Physical properties, Pressure, Volume, Temperature, "Physical equilibrium.

A bibliography of approximately 700 references is presented on the physical equilibria and related properties of several important cryogenic systems. The systems considered are the pure components and mixtures of Hydrogen, Helium, Nitrogen, Carbon Dioxide, Carbon Monoxide, Methane, Ethane, and Propane.

\section{3,770}

AD-A286 701/8 PC A03/MF A01

National Bureau of Standards, Boulder, $\mathrm{CO}$

Precise Measurement of Heat of Combustion with a Bomb Calorimeter.

R. S. Jessup. 2 Sep $60,28 p$.

Keywords: "Heat of combustion, "Calorimeters, Meas urement, Reprints, Standards, Liquids, Hydrocarbons,
Fuels, Computations, Organic compounds, Chemical compounds, Solid fuels, Precision, Water, Combustion, Oxygen, Pressure, "Bomb calorimeter, Glass bulbs.

No abstract available.

\section{3,771}

\section{AD-A292 502/2 PC A01/MF A01}

National Bureau of Standards, Gaithersburg, MD.

Penetration and Diffusion of Hard X-rays through Thick Barriers. III. Studies of Spectral Distributions.

P. R. Karr, and J. C. Lamkin. 15 Dec 49, 3p

Pub. in The Physical Review, v76 n12 p1843-1845, 15 Dec 49 .

Keywords: "Gamma rays, "Spectral energy distribution, "Compton scattering, Mathematical models, Reprints, Thickness, Penetration Barriers, Photons, Diffusion, Integral equations, Monochromatic light, Laplace transformation, Photon beams, Photoelectric effect(Gamma rays), Gamma ray spectra.

Two kinds of spectral distributions of gamma-rays are discussed. These are spectra found in an infinite medium with an uniformly distributed monochromatic source and equilibrium spectra obtained in an artificial penetration problem. Curves are shown for various media and for various energies of the source photons. The relations of these spectra to the general problem of gamma-ray penetration is discussed. (AN).

\section{3,772}

\section{AD-A295 314/9 PC A01/MF A01}

National Bureau of Standards, Gaithersburg, MD

Further Calculations of $X$-ray Diffusion in an Infinite Medium.

L. V. Spencer, and F. Stinson. 15 Feb 52, 3p. Pub. in Tax Physical Review, v85 n4 p662-664, 15 Feb

Keywords: * $X$ ray scattering, "Diffusion, Sources, Computations, Spectra, Polynomials, Expansion, Homogeneity, Reprints.

The problem of $x$-ray diffusion in an infinite homogeneous medium can be solved numerically by a method of expansion into suitable polynomial systems. This method has already been applied to determine the spectrum of scattered radiation as a function of distance from the radiation source for several cases of interest. This note presents further numerical applications of the polynomial method.

\section{3,773}

AD-A295 578/9 PC A03/MF A01

National Inst. of Standards and Technology (NML), Gaithersburg, MD. Molecular Physics Div.

Infrared and Near-Infrared Spectra of HCC and DCC Trapped in Solid Neon.

D. Forney, M. E. Jacox, and W. E. Thompson. 1995, 38p, ARO-30094.4-CH.

Contract ARO-MIPR-107-94

Pub. in Jnl. of Molecular Spectroscopy, v170 p178 214, 1995

Keywords: "Trapping(Charged particles), "Solids, *Near infrared radiation, *Neon, *Infrared spectra, Reprints, Vibration, Vapor phases, Spectra, Shifting, Isotopes, Ground state, Absorption, Energy levels, Low frequencies, Chemical shifts.

Spectra have been recorded from 700 to $12 \mathrm{000} / \mathrm{cm}$ for HCC and DCC trapped in solid neon. In this region, which includes not only the ground-state absorptions but also all of the absorptions of appreciable intensity in the highly perturbed A (2)pi state, all of the matrix shifts are to lower frequencies, and none of them exceeds $30 / \mathrm{cm}$. The X(OnO) and X(On1) progressions of HCC, recently reported in the photoelectron spectrum of HCC-, also appear in the infrared spectrum The spectra of the singly and doubly carbon-13 substituted species of $\mathrm{HCC}$ and of doubly carbon- 13 substituted DCC are presented. Except in a few regions of very strong mixing of energy levels of the $X$ and $A$ states, correlations of the HCC and DCC bands with their carbon-13 substitution counterparts are possible and the resulting isotopic shift data are useful in assigning the vibronic spectra. Assignments are proposed for a considerable number of HCC and DCC bands. These assignments are aided by the carbon13 shift data, by the results of recent ab initio calculations, and, where they are available, by the upper-state symmetries of bands observed in the gas phase. Absorptions of $\mathrm{C} 2, \mathrm{C} 2-, \mathrm{C} 4, \mathrm{HC} 4, \mathrm{HCCO}$, and $\mathrm{C}_{2} \mathrm{H}_{3}$ isolated in a neon matrix are also identified. jg.
03.774

AD-A296 061/5 PC A03/MF A01

National Inst. of Standards and Technology (NML), Gaithersburg, MD. Molecular Physics Div.

Spectroscopic Study of Reaction Intermediates and Mechanisms in Nitramine Decomposition and Combustion.

Final rept 20 Mar 92-19 Mar 95

M. B. Jacox. 20 May $95,40 p, A R O-30094.5-\mathrm{CH}$.

Contract ARO-MIPR-107-94

Keywords: "Spectroscopy, "Combustion "Decomposition, "Nitramines, Water, Solids, Short range(Time), Spectra, Responses, Diagnostic techniques, Free radicals, Neon, Infrared spectra, Nitromethane, Photodecomposition, Molecular ions.

The infrared spectra of reaction intermediates trapped in solid neon were studied in order to support the development of diagnostics for short-lived species which are reaction carriers in nitramine decomposition and combustion and to derive information about reactions which a re important in the condensed-phase decomposition of nitramines. Nitromethane and monomethylnitramine were used as model compounds in these studies. Evidence was obtained for the formation of water complexes with both of these species. The results support the water-catalyzed decomposition mechanism for nitramines that was proposed by Melius. Studies of the photodecomposition of isotopically substituted monomethylnitramine demonstrate that four different groups of products are formed. Tentative spectral assignments are made for the aci-isomer of monomethylnitramine and for CH3NHONO. The final photodecomposition products are $\mathrm{CH} 4, \mathrm{NO}, \mathrm{CH} 3 \mathrm{OH}$, and $\mathrm{N} 20$. Other studies have provided evidence for the formation of a weakly bonded complex of $\mathrm{H} 2$ with $\mathrm{H} 20$, as well as spectral data for the $\mathrm{HCC}$ free radical and for the $\mathrm{H} 2 \mathrm{O}+, \mathrm{NO} 2+, \mathrm{NO}(2-$ ), and $\mathrm{NO}(3-)$, molecular ions.

\section{3,775}

\section{DE94005988 PC A02/MF A01}

National Inst. of Standards and Technology (EEEL), Boulder CO Electromagnetic Technology Div.

Magnetic Characteristics and Measurements of Filamentary $\mathrm{Nb}-\mathrm{Ti}$ Wire for the Superconducting Super Collider.

R. B. Goldfarb, and R. L. Spomer. 1989, 9p, CONF890701-32

\section{5-85ER40240}

International cryogenic materials conference, Los Angeles, CA (United States), 24-28 Jul 1989. Sponsored by Department of Energy, Washington, DC

Keywords: "Niobium Alloys, "Superconducting Super Collider, "Superconducting Wires, "Titanium Alloys, Evaluation, Magnetic Flux, Magnetic Properties, Magnetization, Superconducting Magnets, EDB/430300.

In synchrotron accelerator applications, such as the Superconducting Super Collider (SSC) superconducting magnets are cycled in magnetic field. Desirable properties of the magnets include field uniformity, field stability with time, small residual field, and fairly small energy losses upon cycling. This paper discusses potential sources of problems in achieving these goals, describes important magnetic characteristics to be considered, and reviews measurement techniques for magnetic evaluation of candidate SSC wires. Instrumentation that might be practical for use in a wire-fabrication environment is described. The authors report on magnetic measurements of prototype SSC wires and cables and speculate on causes for instability in multipole fields of dipole magnets constructed with such cables.

\section{6}

\section{DE95004446 PC A03/MF A01}

National Inst. of Standards and Technology (PL), Gaithersburg, MD. Electron and Optical Physics Div. Electron-atom collision studies using optically state selected beams. Progress report, May 15, 1987--May 14, 1988.

R. J. Celotta, and M. H. Kelley. 15 Nov 88, 36p DOE/ER/13520-T1.

Contract Al01-86ER 13520

Sponsored by Department of Energy, Washington, DC. Keywords: "Electron-Atom Collisions, "Sodium, EV Range 10-100, Elastic Scattering, Electron Reactions, Optical Pumping, Polarized Beams, Progress Report, EDB/664300, Electron spin polanization, Electron scattering.

This report discusses progress made during the current contract period on the authors research program 
to study collisions between spin-polanized electrons and optically prepared atoms. The objective of this work is to stimulate a deeper theoretical understanding of the electron-atom interaction by providing more complete experimental measurements on colliding systems. By preparing the internal states of the collision partners before scattering, they are able to extract substantially more information about the scattering process than is available from more conventional measurements of differential cross sections. The authors are principally interested in observing the role played by spin in low energy electron-atom collisions. The additional information provided by these spin-dependent measurements can greatly enhance understanding of both exchange and the spin-orbit interaction in the scaltering process. They have made substantial progress in the past three years in their measurements both of elastic and superelastic scattering of spin-polarized electrons from optically pumped sodium.

\section{3,777}

\section{DE95004447 PC A03/MF A01}

National Inst. of Standards and Technology (PL), Gaithersburg, MD. Electron and Optical Physics Div. Electron-atom collision studies using optically state selected beams. Progress report, May 15 , 1988--May 14, 1991.

R. J. Celotta, and M. H. Kelley. 15 Nov 90, 29p, DOE/ER/13520-T2

\section{Contract Al01-86ER13520}

Sponsored by Department of Energy, Washington, DC.

Keywords: "Sodium, Electron reactions, EV Range 10100, Elastic Scattering, Polarized Beams, Progress Report, "Electron-atom collisions, EDB/664300, Electron spin polarization, Electron scattering.

This report discusses progress made during the current contract period on the authors research program to study collisions between spin-polarized electrons and spin-polarized atoms. The objective of this work is to stimulate a deeper theoretical understanding of the electron-atom interaction by providing the most complete possible experimental characterization of the colliding system. Through the use of optical state preparation techniques to prepare both the incident electrons and atom in well-defined initial states, the authors are able to extract substantially more information about the scattering process than is available from more conventional measurements of differential scattering cross sections. Their primary interest is to study in detail the role played by spin in electron-atom collisions at low to intermediate energies. The additional information provided by these spin-dependent measurements greatly enhances understanding of both exchange and the spin-orbit interaction in the scattering process. During this three-year period they have made substantial progress in measurements of both elastic and superelastic scattering of spin-polarized electrons from optically pumped sodium. The elastic scattering measurements in particular were made possible by several important improvements to the experimental apparatus.

\section{3,778}

\section{DE95011352 PC A03/MF A01}

National Inst. of Standards and Technology, Boulder, Co.

Experimental plan to determine the performance of the Oak Ridge National Laboratory Cold Neutron Moderator. Final report, September 1, 1993--November 30, 1993.

PROGRESS REPT.

P. J. Giarratano, and J. D. Siegwarth. 10 Nov 93 , 21p, DOE/OR/22121-T1.

Contract Al05-93OR22121

Sponsored by Department of Energy, Washington, DC.

Keywords: *Deuterium, "Moderators, Cold Neutrons, Hydraulics, Mathematical Models, Neutron Beams, Neutron Source Facilities, ORNL, Performance, Properties, Waste Heat, EDB/430100, EDB/663610.

This paper outlines an experimental plan to test the thermohydraulic concept of the proposed Oak Ridge National Laboratory Cold Neutron Moderator. The goals, approach, description of the experimental apparatus, and proposed budget and duration are pre-
sented.

\section{3,779}

A04)
National Inst. of Standards and Technology, Boulder, CO. Implementation of a Standard Format for GPS Common View Data.

M. A. Weiss, and C. Thomas. May 95, 14p.

In NASA. Goddard Space Flight Center, the 26TH Annual Precise Time and Time Interval (Ptti) Applications and Planning Meeting $p$ 75-88.

Keywords: "Data processing, *Global positioning system, "Standardization, "Time signals, Receivers, Time measurement.

A new format for standardizing common view time transfer data, recommended by the Consultative Committee for the Definition of the Second is being implemented in receivers commonly used for contributing data for the generation of International Atomic Time. We discuss three aspects of this new format that potentially improve GPS common-view time transfer: (1) the standard specifies the method for treating short term data, (2) it presents data in consistent formats including needed terms not previously available, and (3) the standard includes a header of parameters important for the GPS common-view process. In coordination with the release of firmware conforming to this new format the Bureau International des Poids et Mesures will release future international track schedules consistent with the new standard.

03,780

PB94-140605 (Order as PB94-140555, PC A06/ MF A02)

National Inst. of Standards and Technology, Gaithersburg, MD.

Wavelengths and Energy Levels of Neutral $\operatorname{Kr}(84)$ and Level Shifts in All Kr Even Isotopes.

V. Kaufman. 1993, 8p.

Included in Jnl. of Research of the National Institute of Standards and Technology, v98 n6 p717-724 Nov/ Dec 93.

Keywords: "Atomic energy levels, "Krypton 84, *Isotope effect, Spectral shift, Krypton 86, Krypton 82 Krypton 80, Krypton 78, Interferometry, Wavelengths. Interferometrically-measured wavelengths of 109 lines of neutral $\mathrm{Kr}(84)$ are compared with those of $\mathrm{Kr}(86)$. Sixty energy levels of neutral $\mathrm{Kr}(84)$ derived from those wavelengths and $25 \operatorname{Kr}(86)-\operatorname{Kr}(84)$ isotope shifts previously measured are given along with their shifts from the energy levels of $\operatorname{Kr}(86)$. Twenty levels of each of $\mathrm{Kr}(82), \mathrm{Kr}(80)$, and $\mathrm{Kr}(78)$ are also evaluated using isotope-shift information in the literature. The differences between the experimentally observed shifts and the normal mass shift leave large negative residuals which are accounted for by ionization energy differences and by the specific mass shift. It appears that the volume effect causes only a very small, if any, energy level shift.

\section{3,781}

PB94-142486 PC A03/MF A01

National Inst. of Standards and Technology (MEL), Gaithersburg, MD. Automated Production Technology

Mass Unit Disseminated to Surrogated Laboratories Using the NIST Portable Mass Calibration Package.

R. M. Schoonover, J. E. Taylor, and J. Rothleder.

Feb 94, 18p, NISTIR-5290.

See also PB89-132336.

Keywords: "Calibration, Interlaboratory comparisons, Primary standards, Portable equipment, Computer applications, Uncertainty, Balances, Buoyancy, "Mass standards, California State Laboratory, US NIST.

Over a ten-year period, three mass calibration packages were circulated to selected laboratories by the Mass Group at NIST. Initially the objective was to determine if the NIST mass calibration service could be disseminated to qualified users via a 'do-it-yourself' package. However, it became obvious that the technique could be very useful in the training of personnel and the examination of laboratories. The first package of 1-kilogram artifacts was circulated to see what problems might arise in their calibration. The second package was designed to overcome the difficulties encountered and to ensure the quality of the next package. The third and final package incorporates improve ments and demonstrates the method with the calibration of the California State primary mass standards from $1 \mathrm{~kg}$ to $1 \mathrm{mg}$. This report looks briefly at the entire project experience with comments about the pitfalls and benefits of calibrations by surrogate laboratories and other likely uses of the method.

\section{3,782}

PB94-152691 PC A03/MF A01

National Inst. of Standards and Technology (CSTL), Gaithersburg, MD. Process Measurements Div.

Assessment of Uncertainties of Thermocouple Calibrations at NIST.

Internal rept.

D. Ripple, G. W. Burns, and M. G. Scroger. Jan 94 37p, NISTIR-5340.

Keywords: "Thermocouples, "Calibration, Tempera ture measurement, Uncertainty, Estimates, International Temperature Scale of 1990, ITS-90, US NIST

The uncertainties of thermocouple calibrations performed at the National Institute of Standards and Technology are described in this report, and they are expressed in a manner consistent with the newly-adopted NIST standard for the expression of uncertainties. The adoption of the International Temperature Scale of 1990 (ITS-90) has resulted in alterations in data analysis techniques that affect the uncertainties, and these are described also.

03,783

PB94-156957 PC A08/MF A02

Maryland Univ., College Park. Dept. of Mechanical Engineering.

Transient Cooling of a Hot Surface by Droplets Evaporation.

Final rept.

P. Tartarini, Y. Liao, C. Kidder, and M. di Marzo. Apr 93, 170p, NIST/GCR-93/623

Contract NIST-NANB8H0840

Also pub. as Maryland Univ., College Park. Dept. of Mechanical Engineering rept. no. REPT-91-1. See also PB93-189421. Sponsored by National Inst. of Standards and Technology (BFRL), Gaithersburg, MD.

Keywords: "Fire suppression, "Evaporative cooling, "Spray cooling, "Cooling, "Surfaces, Computerized simulation, Thermal conductivity, Temperature distribution, Computer programs, Solid fuels, Transients, Aluminum, Conduction, Water, "Droplet-solid interactions, Macor.

The long term objective of the study of droplet-solid interaction is to obtain information applicable to the ex tinguishment of fire through a droplet array (e.g. spray) The solids of concern include low thermal conductivity materials, typical of fire applications. The research described hereafter constitutes portion of an extensive research program aimed at developing accurate droplet cooling models of burning solid fuel surfaces. This research is only concerned with the evaporation of single droplets on a hot surface. Limited experiments are also performed in the nucleate boiling range. This report consists of three different portions; each portion focuses on an independent issue of the research program. In Part A the major progresses in the numerical simulation of a single droplet evaporation are described. In Part B the experimental program conducted with radiant heat input for single droplets evaporating on Macor is described. In Part C a novel model is presented, which extends the single droplet results to a multi-droplet system.

\section{3,784}

PB94-163037 PC A03/MF A01

National Inst. of Standards and Technology Gaithersburg, MD.

Examination of Parameters That Can Cause Error in Mass Determinations.

R. M. Schoonover, and F. E. Jones. Feb 94, 14p, NISTIR-5376.

Keywords: Weight measurement, Error analysis, Stainless steels, Platinum, Iridium, Silicon, Buoyancy, Uncertainty, Metrology, Comparison, Calibration, "Mass standards, Kilogram.

The parameters that can cause error in mass determinations are examined and estimates of the magnitude of consequent errors are given. We examine the possible errors in the embodiment of the kilogram in the platinum-iridium (Pt-Ir) artifact. We trace the propagation of errors from the Pt-Ir artifact to the system of stainless steel artifacts through mass comparison. We examine in detail such parameters as buoyancy corrections, including uncertainties in measurement of en vironmental variables.

\section{3,785}

PB94-163052 PC A03/MF A01

National Inst. of Standards and Technology, Gaithersburg, MD. 


\section{General}

Electronic Balance and Some Gravimetric Applications. (The Density of Solids and Liquids, Pycnometry and Mass)

R. M. Schoonover, M. S. Hwang, J. Taylor, and C.

Smith. c1993, 26p, NISTIR-5375.

See also PB91-112854. Prepared in cooperation with Industrial Technology Research Inst., Hsinchu (Taiwan). Center for Measurement Standards. and Alu minum Co. of America, Alcoa Center, PA. Alcoa Tech nical Center.

Keywords: *Density measurement, Pycnometers, Calibration, Gravimetry, Liquids, Solids, Water, Air, Silicon, Algorithms, "Electronic balances, Hydrostatic weighing, Pycnometry.

In recent years the electronic force balance has been perfected to a degree that it can replace the mechanical balance in both precision and capacity. Hence, the mechanical balance is rapidly disappearing from the scene. The work reported here describes the use of the electronic balance in some high precision gravimetric applications. The balance has been examined from the user's viewpoint and its use is illustrated in measuring solid and liquid densities and mass. The density assigned to a silicon crystal is in good agreement with its accepted value to within $2.4 \mathrm{ppm}$. Likewise, the water density measurements substantiate Kell's equation for the density of water near 23 degrees Celsius. (Copyright (c) ISA, 1993.)

\section{3,786}

PB94-163060 PC A03/MF A01

National Inst. of Standards and Technology, Gaithersburg, MD

Piggyback Balance Experiment: An Illustration of Archimedes' Principles and Newton's Third Law.

R. M. Schoonover. Feb 94, 13p, NISTIR-5377.

Keywords: "Buoyancy, Weight measurement, Gravimetry, Force, Mass, Metrology, Newton third law, Archimedes principles, Electronic balances, Piggyback balance experiment, Mass measurement.

The lifting force of a fluid upon an immersed body was analyzed and reported by Archimedes in the third cen tury B.C. In these modern times many people employed in metrology find it difficult to grasp the concept of buoyancy as it applies to mass measurement. Antiquated techniques in use today propagate ignorance of the concept. The modern electronic balance makes possible a simple, educational iwo-balance experiment that provides the observer with a clear picture of the buoyant force and Newton's third law. The experiment, when performed carefully, yields an excellent volume determination of the demonstration mass and brings together the SI units of mass, length and temperature and the derived units of force, pressure and density.

03,787

PB94-163078 PC A03/MF A01

National Inst. of Standards and Technology, Gaithersburg, MD

Determination of Density of Mass Standards: Requirement and Method.

Conference proceedings.

R. M. Schoonover, M. S. Hwang, and R. Nater. Feb

$94,22 \mathrm{p}$, NISTIR-5378.

See also PB91-112854. Prepared in cooperation with Industria! Technology Research Inst., Hsinchu (Tai wan). and Mettler-Toledo A.G., Greifensee (Switzerland).

Keywords: "Density measurement, Weight indicators, Error analysis, Calibration, Silicon, Hydrostatic weighing, Electronic balances.

The usual method of a mass assignment to an artifact is by comparing the forces exerted on a balance pan. The observed difference between the standard mass and the unknown object is then expressed as the solu tion of the two force equations and includes terms fo their respective displacement volumes, i.e, densities. With the advent of commercially available balances with precision near the 1 part per billion level (ppb). the user must pay particular attention to the artifact density and its associated error if the available precision is to be meaningful. Described here is the need and a method to determine the artifact density with an error analysis that leads to the selection of the appropriate equipment. Measurements of a $200 \mathrm{~g}$ silicon crystal of known density were performed to demonstrate the accuracy of the method.
National Inst. of Standards and Technology (CAML), Gaithersburg, MD.

Complete Reduction of the Euler-Poinsot Problem. Final rept.

A. Deprit, and A. Elipe. 1993, 26p.

Pub. in Jnl. of the Astronautical Sciences 41, n4 p603628 Oct-Dec 93.

Keywords: Rigid structures, Hamiltonians, Dynamics, Reprints, "Euler-Poinsot problem, Serret-Andoyer var'ables.

The authors propose a new way of defining the SerretAndoyer variables that does not call on spherical trigonometry. The authors use those variables to presen a complete solution of the Euler-Poinsot problem in the phase space determined by the components of the angular momentum along the principal axes of inertia. The authors use the solution to convert directly the Serret-Andoyer variables into action- and angle-variables, thereby making the Hamiltonian dependent on only two momenta.

\section{3,789}

PB94-172376 Not available NTIS

National Inst. of Standards and Technology (NML) Gaithersburg, MD. Atomic and Plasma Radiation Div. Electron Screening Correction to the Self Energy in High-Z Atoms.

Final rept.

P. Indelicato, and P. J. Mohr. 1991, 3p.

Pub. in Proceedings of International Conference on Atomic Physics (12th), Ann Arbor, MI., July 31-August 3, 1990, p501-5031991.

Keywords: Quantum electrodynamics, Coulomb field Self-energy, Correction, Reprints, "Electron screening.

The paper describes a calculation of the electron screening effect on the self energy in an external Coulomb field, and gives preliminary results. The calculation is done to first order in the perturbing potential and to all orders in the strong Coulomb field

\section{3,790}

\section{PB94-172830 Not available NTIS}

National Inst. of Standards and Technology (NML) Gaithersburg, MD. Atomic and Plasma Radiation Div. New Critical Review of Experimental Stark Widths and Shifts.

Final rept.

W. L. Wiese, and N. Konjevic 1991, 2p.

Pub. in Proceedings of International Conference on Spectral Line Shapes (10th), Austin, TX., 1990, v6 p63-64 1991.

Keywords: "Stark effect, Experimental data, Line broadening, Spectral lines, Positive ions, Neutral atoms, Reviews, Reprints.

A critical review and tabulation of the available experimental data on Stark widths and shifts for spectral lines of non-hydrogenic neutral atoms and positive ions has been carried out. The review covers the period from 1983 through the end of 1988 and represents a continuation of earlier critical reviews through the year 1982

03,791

\section{PB94-172855 Not available NTIS}

National Inst. of Standards and Technology (PL) Gaithersburg, MD. Ionizing Radiation Div.

Measurement and Calibration of Large-Area AlphaParticle Sources at NIST

Final rept.

M. P. Unterweger, J. M. R. Hutchinson, and P. A

Hodge. 1994, $7 p$

Pub. in Nuclear instruments and Methods in Physics Research A 339, p109-1151994.

Keywords: "Alpha sources, "Calibration, Plutonium 238, Plutonium 239, Monitoring, Alpha particles, Reprints, $\mathrm{X}$-ray emission.

Work has been performed at NIST to develop and calibrate large-area (238)Pu alpha-particle sources and related measuring equipment. A large-area-source $X$ ray counting system and a 2 pi(alpha) internal gas-proportional counting system were used for the primary calibration of these sources. Studies have been performed to relate these calibrations to the measurement of thin, large area (239)Pu sources, which are of primary interest but are generally unavailable in the necessary high specific activities required. Two systems have been characterized for the monitoring and measurement of large-area alpha sources: (1) a source-de- tector mounting unit developed at NIST to calibrate monitoring systems by mounting a standard source of plutonium at an accurately calibrated distance from the user's alpha-monitoring detector; (2) a diffused-junction silicon semiconductor detector which is used as a transfer standard from low to high activity of largearea alpha-particle sources. A determination of the $L$ $X$-ray emission probability for (239)Pu has been made.

\section{3,792}

PB94-172889 Not available NTIS

National Inst. of Standards and Technology (NML), Gaithersburg, MD. Center for Atomic, Molecular and Optical Physics.

Report on the Meeting of the CCU (10th) (of the International Committee of Weights and Measures). Held on July 10-11, 1990

Final rept.

B. N. Taylor. $1990,1 \mathrm{p}$

Pub. in Metric Today 25, n4, 3, Jul/Aug 90.

Keywords: "Metric system, International system of units, Symbols, Meetings, Reprints, ${ }^{*}$ Consultative Committee on Units, International Committee of Weights and Measures, Liter.

This is a brief summary of the 10 th meeting of the Consultative Committee on Units (CCU) of the International Committee of Weights and Measures (CIPM) held 1011 July 1990 at the International Bureau of Weights and Measures (BIPM). The topics covered at the meeting included revisions to the 5 th edition of the brochure 'International System of Units (SI)' published by the BIPM, the symbol for the liter, and new SI prefixes for 10 (sup -21), 10(sup 21), 10(sup -24), and 10(sup -24)

\section{3,793}

PB94-172897 Not available NTIS

National Inst. of Standards and Technology (NML) Gaithersburg, MD. Electron and Optical Physics Div. National Institute of Standards and Technology Resonance lonization Spectroscopy/Resonance Ionization Mass Spectroscopy Data Service.

Final rept.

E. B. Saloman. 1990,4p

Contract DE-AI05-86ER-60447

Pub. in Proceedings of International Symposium on Resonance Ionization Spectroscopy and Its Applica tions (5th), Varese, Italy, September 16-21, 1990, p4548. Sponsored by Department of Energy, Washington, DC.

Keywords: "Resonance ionization mass spectroscopy, "Mass spectroscopy, "Data bases, Chemical analysis Reprints, "Resonance ionization spectroscopy, US NIST.

A resonance ionization spectroscopy data service has been established at the National Institute of Standards and Technology. It publishes formatted data sheets which provide the atomic and laser physics information to apply RIS/RIMS to a given element. A first group of ten data sheets has been published. It covers As, $\mathrm{B}, \mathrm{Cd}, \mathrm{C}, \mathrm{Ge}, \mathrm{Au}, \mathrm{Fe}, \mathrm{Pb}, \mathrm{Si}$, and $\mathrm{Zn}$. A second group has been submitted for publication. It covers $\mathrm{Al}$, $\mathrm{Ca}$ $\mathrm{Cs}, \mathrm{Cr}, \mathrm{Co}, \mathrm{Cu}, \mathrm{Kr}, \mathrm{Mg}, \mathrm{Hg}$, and $\mathrm{Ni}$. More will be produced in the future.

\section{3,794}

PB94-172905 Not available NTIS

National Inst. of Standards and Technology (PL), Gaithersburg, MD. Radiometric Physics Div.

Realization of New NIST Radiation Temperature Scales for the $1000 \mathrm{~K}$ to $3000 \mathrm{~K}$ Region, Using Absolute Radiometric Techniques.

Final rept.

R. D. Saunders, B. C. Johnson, V. Sapritsky, and K D. Mielenz. $1992,5 p$

Pub. in ITS Measurement and Control in Science and Industry, Temperature, v6 p221-225 1992.

Keywords: "Temperature scales, "Temperature measurements, Temperature range $1000-4000 \mathrm{~K}$. High temperature, Melting points, Uncertainty, Gold, Reprints, Radiance temperatures.

The National Institute of Standards and Technology (NIST) has developed a radiance temperature measurement technique using absolute optical detectors fo measuring the freezing temperature of gold with a 3 (sigma) uncertainty of + or -0.34 K (K. D. Mielenz, R D Saunders, and J B Shumaker, J Res Natl. Inst Stand. Technol. 95, 49 (1990)). The significance of this approach is discussed for the measurement of the radiance temperature of sources in the range of $1000 \mathrm{~K}$ to $3000 \mathrm{~K}$, and a comparison of the current method 
complete with uncertainty estimates, is presented. The proposed experimental apparatus is described, and progress to date is reported.

03,795

PB94-172996 Not available NTIS

National Inst. of Standards and Technology (NML) Gaithersburg, MD. Center for Atomic, Molecular and Optical Physics.

Fine-Structure Constant

Final rept.

B. N. Taylor. 1991, 7p.

Pub. in Numerical Data and Functional Relationships in Science and Technology - Units and Fundamental Constants in Physics and Chemistry, Chapter 3.2.9, p3-125 - 3-131 1991

Keywords: "Sommerfeld constant, "Fundamental con stants, Quantum electrodynamics, Josephson effect, Reprints, *Fine structure constant, Quantum Hall effect.

The paper briefly reviews the history of the fine-structure constant alpha and discusses its relationship to other fundamental constants and quantities of physics, including the Josephson and von Klitzing constants, the gyromagnetic ratio of the proton, and the electron magnetic moment anomaly. It summarizes and compares the values of alpha presently available having an uncertainty of 2 parts in 10(sup 7) or less, and briefly discusses the experiments from which they are obtained and their major sources of uncertainty. A list of useful references is given.

03,796

PB94-176088 PC A10/MF A03

National Inst of Standards and Technology (PL),

Gaithersburg, MD

Physics Laboratory Technical Activities, 1993.

K. B. Gebbie. Mar 94, 224p, NISTIR-5363.

Presented to the Board on Assessment of NIST Programs, National Research Council, May 2-3, 1994 See also report for 1992, PB93-178648. Sponsored by National Research Council, Washington, DC. Board on Assessment of NIST Programs.

Keywords: "Physics, Atomic physics, Molecular physcs, Fundamental constants, Frequency standards, Time standards, Quantum theory, Ionizing radiation, Calibration, Metrology, Radiometry, Optics, Electrons, Measurement, Progress report.

This report summarizes research projects, measurement method development, calibration and testing, and data evaluation activities that were carried out during calendar year 1993 in the NIST Physics Laboratory. These activities fall in the areas of electron and optical physics, atomic physics, molecular physics, radiometric physics, quantum metrology, ionizing radiation, ime and frequency, quantum physics, and fundamental constants.

03,797

PB94-185295 Nòt available NTIS

National Inst. of Standards and Technology (PL), Gaithersburg, MD. Atomic Physics Div.

Investigation of LS Coupling in Boronlike Ions.

Final rept.

S. Glenzer, H. J. Kunze, J. Musielok, Y. K. Kim, and W. L. Wiese. 1994, 7p.

Pub. in Physical Review A 49, n1 p221-227 Jan 94.

Keywords: "L-S coupling, Reprints, Light elements, Transition probabilities, "Boron-like ions.

In order to test the validity of the LS-coupling approximation for light elements, the line-intensity ratios of the $3 s-3 p$ and $3 p-3 d$ multiplets in $C$ II through $F V$ and $C$ II through Ne VI, respectively, have been investigated experimentally with emission sources. Also, calculations with multiconfiguration Dirac-Fock wave functions have been performed. Within the errors of the experimental procedure, no significant violations of the LS. coupling approximation were found for the lines of the $3 s-3 p$ and $3 p-3 d$ multiplets in $C \|$ through $F$ V. How ever, in the case of $\mathrm{Ne} \mathrm{VI,} \mathrm{small} \mathrm{deviations} \mathrm{of} \mathrm{meas}$ ured intensities from those obtained by applying the LS-coupling approximation were observed.

03,798

PB94-185311 Not available NTIS

National Inst. of Standards and Technology (EEEL)

Boulder, CO. Electromagnetic Technology Div.
Anharmonic Oscillator Analysis Using Modified Airy Functions.

Final rept.

I. C. Goyal, S. Roy, A. K. Ghatak, and R. L. Gallawa. 1992, 4p

Pub. in Canadian Jnl. of Physics 70, p1218-1221 1992

Keywords: "Anharmonic oscillators, Reprints, Schrodinger equation, WKB approximation, Wave equations Airy function, Numerical solution, WKB-J approximation.

The authors have applied the modified Airy function (MAF) method to the analysis of an anharmonic oscilla tor, characterized by the potential $\mathrm{V}(\mathrm{x})=\mathrm{k}$ ( $\mathrm{x}$ squared) 2 + alpha ( $x$ (sup 4)) $k>0$ alpha $>0$. The MAF method gives an accurate closed-form expression for the wave function as well as very accurate eigenvalues with much less numerical effort than the five-term (eighthorder) WKBJ approximation. The application of the first-order perturbation correction to the MAF eigenvalues makes them even more accurate than those obtained by the five-term WKBJ approximation.

\section{3,799}

PB94-185345 Not available NTIS

National Inst. of Standards and Technology (PL), Boulder, CO. Quantum Physics Div.

Backscattering in Electron-Impact Excitation of Multiply Charged lons.

Final rept.

X. Q. Guo, E. W. Bell, J. S. Thompson, R. A

Phaneuf, A. C. H. Smith, G. H. Dunn, and M. E.

Bannister, 1993, 10p.

Contract DE-A 105-86ER53237

Pub. in Proceedings of International Conference on the Physics of Highly Charged Ions (6th), Manhattan, KS. September 28-October 2, 1992, p463-472 1993. Sponsored by Department of Energy, Washington, DC.

Keywords: "Electron-ion collisions, "Argon ions, Reprints, Multicharged ions, Electron scattering, Electron impact, Inelastic scattering, Backscattering, Excited states, Cross sections, Excitation.

For the first time, evidence for strong back-scattering of electrons during near threshold excitation of highly charged ions has been observed. By measuring the apparent cross section for the electron-impact excitation of $\mathrm{Ar}(7+)(3 \mathrm{~s}->3 \mathrm{p})$ as a function of center-of-mass velocity, over $90 \%$ of the inelastically scattered electrons were found to be deflected by more than $90 \mathrm{de}$ grees in the center-of-mass frame. This unusual angu lar scattering has not been previously observed for atoms or ions, but may be typical for multiply charged ions. Our observation of backscattering has been confirmed recently by close-coupling calculations and distorted-wave calculations. Absolute total excitation cross sections measured in an energy range extending to $2.2 \mathrm{eV}$ above threshold are also reported and are in agreement with close-coupling calculations.

\section{3,800}

PB94-185576 Not available NTIS

National Inst. of Standards and Technology (BFRL) Gaithersburg, MD. Fire Safety Engineering Div.

Intercomparison of Internal Proportional Gas Counting of (85) $\mathrm{Kr}$ and (3) $\mathrm{H}$.

Final rept.

J. L. Makepeace, F. E. Clark, J. L. Picolo, M. P. Unterweger, N. Coursol, and E. Gunther. 1994, 6p Pub. in Nuclear Instruments and Methods in Physics Research A 339, p343-348 1994

Keywords: "Krypton 85, "Tritium, Interlaboratory comparisons, Proportional counters, Uncertainty, Reprints, Intercomparison.

An international intercomparison of internal proportional gas counting of (85) Kr and (3) H has been carried out under the auspices of EUROMET, a European Collaboration in Measurement Standards. A sample of (85) $\mathrm{Kr}$ mixed with inactive krypton gas was measured at three laboratories and a sample of (3) $\mathrm{H}$ mixed with nitrogen gas was measured at four laboratories. In each case, agreement was achieved within the stated uncertainties. The experimental techniques employed at each of the participating laboratories are described and a statement of the results and conclusions is provided.

\section{3,801}

PB94-185709 Not available NTIS

National Inst. of Standards and Technology (PL)

Gaithersburg, MD. Electron and Optical Physics Div.
Verification of the Ponderomotive Approximation for the ac Stark Shift in Xe Rydberg Levels. Final rept.

T. R. O'Brian, J. B. Kim, G. Lan, T. J. Mclirath, and T. B. Lucatorto. 1994, 4p.

Pub. in Physical Review A 49, n2 pR649-R652 Feb 94 Sponsored by Department of Energy, Washington, DC

Keywords: "Rydberg states, "Xenon, Alternating current, Laser radiation, Stark effect, Perturbation theory Reprints.

We report direct measurements of the ac Stark shifts of Xe Rydberg levels in high-intensity laser fields (up to $6 \mathrm{GW} / \mathrm{sq} \mathrm{cm}$ ), demonstrating the expected near ponderomotive behavior. These measurements provide extensive unambiguous evidence for the validity of perturbative calculations of the nonresonant ac Stark shift at these intensities.

03,802

PB94-185741 Not available NTIS

National Inst. of Standards and Technology (PL) Gaithersburg, MD. Ionizing Radiation Div.

Intermediate Structure in the Neutron-Induced Fis. sion Cross Section of $236 \mathrm{U}$.

Final rept.

W. E. Parker, J. E. Lynn, G. L. Morgan, N. W. Hill, P

W. Lisowski, and A. D. Carlson. 1994,6p.

Pub. in Physical Review C 49, n2 p672-677 Feb 94

Keywords: "Uranium 236 target, "Fission cross sections, "Neutron reactions, Intermediate structure, Intermediate resonance, Reprints.

Neutron-induced fission of (236) $U$ has been measured at the Los Alamos Neutron Scattering Center with a white neutron source using a fast parallel plate ionization chamber at a flight path of about $56 \mathrm{~m}$. In the reso nance and the intermediate resonance region, very litthe of the previously reported structure was detected. Only five resonance structures were observed. Additionally, the width of the $5.45 \mathrm{eV}$ resonance is approximately 100 times smaller than previously reported. An explanation for the discrepancies between old data and data reported here is discussed. New fission widths for resonances from $5.45 \mathrm{eV}$ to $10.4 \mathrm{keV}$ are reported. The new data are in agreement with theoretical estimates

03,803

PB94-187713 PC A03/MF A01

National Inst. of Standards and Technology, Gaithersburg, MD.

Use of the Electronic Balance for Highly Accurate Direct Mass Measurements Without the Use of External Mass Standards.

R. M. Schoonover, and F. E. Jones. May 94, 20p, NISTIR-5423.

Keywords: Weight measurement, Calibration, Uncertainty, "Mass measurement, Electronic balances, Traceability.

The object of the paper is to show how to make highly accurate mass measurements using an appropriate electronic balance without the use of external mass standards. The method eliminates the need for a set of external mass standards and, therefore, the need to have these mass standards calibrated periodically at a standards laboratory. Traceability to NIST can be achieved by periodic calibration of the built-in mass standard of the balance by a standards laboratory and by self-verification of balance linearity and function. The method benefits the user with lower cost and a higher measurement rate. Presented here is a detailed analysis of the method a determination of measurement uncertainty, and the traceability path. Lastly, the method is illustrated by specific examples including the calculation of uncertainty.

03,804

PB94-193760 PC A06/MF A02

National Inst of Standards and Technology (PL), Gaithersburg, MD. Ionizing Radiation Div.

Bibliography of Photon Total Cross Section (Attenuation Coefficient) Measurements $10 \mathrm{eV}$ to 13.5 GeV, 1907-1993.

J. H. Hubbell. May 94, 102p, NISTIR-5437.

See also PB87-116141.

Keywords: "Photon cross sections, *Far ultraviolet radiation, "X rays, "Gamma rays, "Bibliographies, Vacu um ultraviolet radiation, Extreme ultraviolet radiation, Absorption coefficients, Total cross sections, Brems strahlung, Attenuation coefficients. 


\section{General}

A bibliography is presented of papers reporting absolute measurements of photon (XUV, X-ray, gamma-ray, bremsstrahlung) total interaction cross sections or attenuation coefficients for the elements and some compounds. The energy range covered is from $10 \mathrm{eV}$ to above $10 \mathrm{GeV}$. These papers are part of the reference collection of the National Institute of Standards and Technology Photon and Charged Particle Data Center. They cover the period from 1907 through 1993 and into 1994. Thus this report is an update of the 1986 earlier report NBSIR 86-3461 (PB87-116141), and also includes additional papers dating back as far as 1966 which have since been found or brought to the attention of the author Included with each reference are annotations specifying the energy range covered and the substances studied. This updated bibliography now includes 573 non-duplicative references to available measured data, plus 42 references to critical evaluations and review articles.

\section{3,805}

PB94-194073 PC A03/MF A01

National Inst. of Standards and Technology (BFRL), Gaithersburg, MD. Building Environment Div.

Single-Phase Heat Transfer and Pressure Drop Characteristics of an Integral-Spine-Fin Within an Annulus.

M. A. Kedzierski, and M. S. Kim. Jun 94, 37p, NISTIR-5454.

Sponsored by Department of Energy, Washington, DC Building Equipment Div.

Keywords: "Fins, "Heat transfer, "Pressure gradients, "Annular flow, Nusselt number, Friction factor, Hea transfer coefficients, Mathematical models, Test facilities, Skin friction.

The laminar, single phase heat transfer and friction characteristics of an integral-spine-fin pipe within an annulus are presented. The heat-transfer coefficient was determined using a modified version of the Wilson Plot method. The test fluid was pumped through the annulus of a straight, $3 \mathrm{~m}$ test section. Three fluids were investigated: (1) tap water, (2) $34 \%$ ethylene glycol/ water mixture, and (3) $40 \%$ ethylene glycol/water mix ture. These fluids produced a significant variation in the Prandtl number so that its exponential dependence could be determined. The annulus-Reynolds numbers were varied from 100 to $\$ 400$ to obtain the Reynolds number exponent. An empirical correlation for the Nusselt number was developed which accounts for the development of the thermal boundary layer. An empirical correlation for the friction factor is also presented. It is demonstrated that the spines enhance the heat transfer through additional surface area and fluid mix ing

\section{3,806}

PB94-198587 Not available NTIS

National Inst. of Standards and Technology (PL), Boulder, CO. Ouantum Physics Div.

Characteristics of Light Emission After Low-Energy Electron Impact Excitation of Caesium Atoms. Final rept.

K. Bartschat, U. Thumm, and D. W. Norcross. 1992, $6 p$

Contract DE-A105-86ER53237

Pub. in Jnl. of Physics B: Atomic and Molecular Opt Physics 25, pL641-L646 1992. Sponsored by Department of Energy, Washington, DC. Office of Energy Research.

Keywords: *Electron-atom collisions, "Polarized light, "Cesium, EV range 1-10, Electron scattering, Electron impact, Atomic excitations, Excited states, R matrix, Reprints.

Results of a recent calculation for e-Cs scattering, carried out in a fully relativistic (Dirac R-matrix) framework, are used to calculate the polarization of the light emitted by excited cesium atoms in an energy range from threshold to $2.7 \mathrm{eV}$. They are compared with previous semirelativistic Breit-Pauli calculations and the available experimental data. The results indicate that the Percival-Seaton theory approximating relativistic scattering amplitudes by recoupling amplitudes from a non-relativistic LS calculation is not valid for low-energy e-Cs scattering.

\section{3,807}

Pot available NTIS

National Inst. of Standards and Technology (MSEL) Gaithersburg, MD. Reactor Radiation Div.
Scattering Properties of the Leveled-Wave Model of Random Morphologies.

Final rept.

N. F. Berk. 1991, 11p.

Pub. in Physical Review A 44, n8 p5069-5079, 15 Oct

Keywords: *Scattering, Light scattering, Neutron scattering, X-ray scattering, Random processes, Standing waves, Porous materials, Morphology, Reprints, Leveled wave model.

In the leveled-wave model, random porous morphologies are simulated by discrete maps of continuous stochastic standing waves generated by adding sinusoids over suitable distributions of wave vectors and phase constants. The mathematical structures obtained this way appear to be useful models for certain kinds of random geometries, such as the bicontinuous morphologies that occur in microemulsions and porous glasses. Properties of the leveled-wave method are developed and the scattering behavior is derived. Scattering from an arbitrary three-phase system can be analyzed by adding different instances of scattering from a generalized film morphology in which the scattering phase is confined to the interior of the interspatial region bounded by two leveled-wave interfaces that are separated everywhere. General results for this interspace scattering are derived. Fractal surfaces, dimension $2=$ or $<D=$ or $<3$, are incorporated into the leveled-wave scheme by using a wave-number distribution having an appropriate long-tailed asymptotic behavior. Scenarios are discussed for the scattering as $D \rightarrow 2$ from above and for $\mathrm{D} \rightarrow 3$ from below. For $\mathrm{D}=3$ the asymptotic scattering falls faster than algebraically as the scattering wave vector $\mathrm{O} \rightarrow$ infinity.

\section{3,808}

\section{PB94-198918 Not available NTIS}

National Inst. of Standards and Technology (PL), Gaithersburg, MD. Atomic Physics Div.

Evaluation of Two-Photon Exchange Graphs for Highly-Charged Heliumlike Ions.

Final rept.

S. A. Blundell, P. J. Mohr, W. R. Johnson, and J.

Sapirstein. 1993, 12p.

Pub. in Physical Review A 48, n4 p2615-2626 Oct 93

Keywords: "Multicharged ions, Ouantum electrodynamics, Perturbation theory, Ground state, Graphs, Reprints, "Helium-like ions, Two photon exchange.

Contributions of one-loop ladder and crossed-ladder graphs to the ground-state energy of heliumlike ions are calculated in Furry representation OED. With the aid of a contour rotation, the graphs are evaluated to all orders in $Z$ (aipha) in the range $Z=10-110$. Particular attention is given to the relation of this work to recent many-body perturbation theory and configurationinteraction calculations.

\section{3,809}

PB94-198991 Not available NTIS

National Inst. of Standards and Technology (NML),

Boulder, CO. Time and Frequency Div.

Liquid and Solid Atomic Ion Plasmas.

Final rept.

J. J. Bollinger, S. L. Gilbert, D. J. Heinzen, W. M

Itano, and D. J. Wineland. 1990, 11p.

Pub. in Proceedings of American Physical Society Topical Conference on Atomic Processes in Plasmas (7th), Gaithersburg, MD., October 2-5, 1989, p152-162 1990

Keywords: Strongly coupled plasmas, Atomic spectroscopy, Laser spectroscopy, Ion storage, Atomic ions, Beryllium ions, Beryllium 9, Reprints, *Ion plasmas, Penning traps, Laser cooling.

Atomic ions which are stored in electromagnetic traps can be viewed as one component plasmas. When the ions are laser cooled they become strongly coupled (gamma > 100) and exhibit liquid-like and solid-like behavior. Experimental evidence for this behavior is discussed for nonneutral (9)Be(1+) ion plasmas stored in a Penning ion trap.

03,810

PB94-199080 Not available NTIS

National Inst. of Standards and Technology (NML), Gaithersburg, MD. Temperature and Pressure Div.
Precision Nuclear Orientation Measurements for Determining Mixed Magnetic Dipole/Electric Quadrupole Hyperfine Interactions.

Final rept.

W. D. Brewer, P. Roman, and H. Marshak. 1989, 7p. Pub. in Hyperfine Interactions 51, n1-4 p1131-1137 Jun 89.

Keywords: "Terbium 160, Nuclear alignment, Oriented nuclei, Gamma radiation, Hyperfine structure, Single crystals, Temperature dependence, Magnetic dipole moments, Ouadrupole moments, Temperature range 0000-0013 K, Least squares method, Reprints.

The authors report the temperature dependence of the gamma-ray anisotropies from oriented (160)Tb nucle produced by neutron activation of the central portion of a high-purity single crystal Tb slab. The magnetically saturated sample was studied over a wide temperature range from $18 \mathrm{mK}$ to $150 \mathrm{mK}$. The temperatures were determined using precision resistance thermometry with in situ calibration by a magnetically shielded sixelement superconducting fixed point device. Temperature stability during data acquisition was better than $0.1 \%$, and least-squares fitting of the resulting temperature dependences of $0 \mathrm{deg}$ and $90 \mathrm{deg}$ anisotropies allowed both the magnetic dipole and the electric quadrupole hyperfine interaction frequencies to be determined with good accuracy. The weighted averages for 18 gamma rays are $\mathrm{nu}(\mathrm{M})=1393.8$ (8.1) $\mathrm{MHz}$ and $n u(P)=178.0(2.1) \mathrm{MHz}$, in excellent agreement with NMR results on ion-implemented samples.

\section{3,811}

PB94-199445 Not available NTIS

National Inst. of Standards and Technology (IMSE) Gaithersburg, MD. Office of Nondestructive Evaluation.

Collision-Induced Emission in the Fundamental Vibration-Rotation Band of $\mathrm{H} 2$.

Final rept.

G. E. Caledonia, R. H. Krech, T. Wilkerson, R. L.

Taylor, and G. Birnbaum. 1991, 8p.

Pub. in Physical Review A 43, n11 p6010-6017 1991.

Keywords: *Atom-molecule collisions, "Hydrogen, Infrared spectra, Emission spectra, Band spectra, Shock waves, Temperature range 0273-0400, Temperature range 0400-1000, Dipole moments, Neon, Argon, Xenon, Reprints.

Measurements of collision-induced emission in the fundamental vibration-rotation band of hydrogen are presented for argon, xenon, and neon collision partners. These absolute, spectrally-resolved infrared measurements were performed at high densities behind reflected shock waves over the temperature range of 900 to $3,400 \mathrm{~K}$. The emission was found to be dominated by $Q$ branch transitions at high temperature due primarily to the dipole moment induced by the overlap between the electron clouds of the collision pair. The strength of this interaction was evaluated from the data and compared with similar evaluations determined from low temperature absorption studies.

03,812

PB94-199486 Not available NTIS

National Inst. of Standards and Technology (CAML) Gaithersburg. MD. Applied and Computational Mathematics Div.

Slowly Divergent Space Marching Schemes in the Inverse Heat Conduction Problem.

Final rept.

A. S. Carasso. $1993,16 p$

Pub. in Numerical Heat Transfer 23, ptB p111-126 1993.

Keywords: *Conduction, Reprints, One dimensional calculations. Finite difference theory, Rocket nozzles. Divergence, Lax-Richtmyer theory, Tikhonov regularization, III posed problems, Inverse problems

Recently developed 'slowly divergent' space marching difference schemes, coupled with Tikhonov regularization, can solve the one-dimensional inverse heat conduction problem at values of the nondimensional time step (Delta) $t(+)$ as low as (Delta) $t(+)=0.0003$. A Lax Richtmyer analysis is used to demonstrate dramatic differences in error amplification behavior among various space marching algorithms, for the same problem, on the same mesh; maximum error amplification factors may differ by more than 10 orders of magnitude at parameter values that are of interest in rocket nozzle applications. Slowly divergent schemes are characterized by their damping behavior at high frequencies. $A$ widely used benchmark problem, where the surface 
temperature gradient is a step function, provides a basis for evaluating Tikhonov-regularized marching computations.

\section{3,813}

PB94-199510 Not available NTIS

National Inst. of Standards and Technology (NML),

Gaithersburg, MD. Ionizing Radiation Div.

Neutron Standard Cross Sections in Reactor Physics: Need and Status.

Final rept.

A. D. Carlson. $1990,2 p$

Pub. in Transactions of the American Nuclear Society $62, p 525-5261990$

Keywords: "Neutron cross sections, "Neutron reactions, "Reactor physics, "Standards, Uranium 235 target, Lithium 6 target, Helium 3 target, Boron 10 target, Gold, Carbon, Hydrogen, Reprints.

Topics covered include: highlights of the evaluation process for the ENDF/B-VI standards evaluation; relation of the standards to reactor physics; highlights of work done on standards since the completion of the standards evaluation.

\section{3,814}

PB94-199585 Not available NTIS

National Inst. of Standards and Technology (PL)

Gaithersburg, MD. Atomic Physics Div.

Isotope Shifts and Hyperfine Splittings of the 398.8-nm Yb I Line.

Final rept.

K. Deilamian, J. D. Gillaspy, and D. E. Kelleher

$1993,5 p$

Pub. in Jnl. of the Optical Society of America B 10, n5 p789-793 May 93

Keywords: "Ytterbium, "Spectral lines, "Isotope effect, Reprints, Hyperfine structure, Electron transitions, Line width, Ytterbium 168, Ytterbium 170, Ytterbium 171, Ytterbium 174, Photon burst spectroscopy.

We report the first observation to our knowledge of the 6s(2) Singlet $S(0)$ - 6s6p singlet $P$ (sub 1, sup 0) trans tion $(398.8 \mathrm{~nm})$ of $(168) \mathrm{Yb}$. The relative position of this spectral line was directly determined by using a laser sideband technique to simultaneously probe pairs of lines. The hyperfine structure of (171) Yb and the isotope shifts of (170) Yb, and (171) Yb, and (174) Yb were determined to an accuracy of better than $6 \mathrm{MHz}$ and found to differ by as much as $23 \mathrm{MHz}$ from previous results. By using photon-burst spectroscopy to carry out these measurements, we obtained subnatura linewidths and fully resolved the (170) $\mathrm{Yb}$ and (171) $\mathrm{Yb}$ lines, which otherwise overlap in fluorescence.

\section{3,815}

PB94-199627 Not available NTIS

National Inst. of Standards and Technology (PL) Gaithersburg, MD. Quantum Metrology Div.

Experimental Aspects and Z-Dependent System atics in One- and Two-Electron lons and Single Vacancy Systems.

Final rept.

R. D. Deslattes. $1993,7 p$.

Pub. in Physica Scripta T46, p9-15 1993.

Keywords: "X-ray spectra, "Multicharged ions, Reprints, Heavy ions, $K$ lines, "Hydrogen-like ions, "Helium-like ions, Radiative corrections.

Some predominantly experimental considerations concerning both few electron highly charged ion spectra and inner-shell vacancy spectra are discussed in relation to the currently available theoretical approaches. For the cases of $\mathrm{H}$-like and $\mathrm{He}$-like spectra, theoretica machinery appears to be well in hand particularly in comparison to the relatively sparse experimental database which is also of limited accuracy in mos cases. Owing particularly to some impressive recent theoretical progress on the problem of nearly neutra ions containing inner-shell vacancies, the traditional $X$ ray data suitably filtered and strengthened by modern measurements, appear to offer some quite useful in sights in spite of their formidable complexity. With the advent of new storage ring facilities and advanced technology ion sources as well as a new effort on transuranic $\mathrm{X}$-ray spectra, one can reasonably foresee an upcoming period of fruitful activity.

\section{3,816}

PB94-199734 Not available NTIS

National Inst. of Standards and Technology (PL), Boulder, CO. Quantum Physics Div.
Polarization of Light Emitted After Positron Impact Excitation of Alkali Atoms.

Final rept.

K. M. DeVries, K. Bartschat, R. P. McEachran, and

A. D. Stauffer. 1992, 4p.

Grant NSF-PHY-9014103

Pub. in Jnl. of Physics B: At. Mol. Opt. Phys, 25, pL653-L656 1992. Sponsored by National Science Foundation, Arlington, VA.

Keywords: "Positron-atom collisions, "Alkali metals, "Polarized light, Reprints, Linear polarization, EV range 10-100, EV range 1-10, Excitation, Lithium, Sodium, Potassium, Rubidium, Percival-Seaton hypothesis, Close coupling approximation.

Recent two-state and five-state close-coupling calculations for positron-alkali scattering are used to predict the linear polarization of the resonance line (np) double $\mathrm{P}(\mathrm{O})$-> (ns) double $\mathrm{S}$ emitted by the alkali atoms $\mathrm{Li}$ $\mathrm{Na}, \mathrm{K}$ and $\mathrm{Rb}$ after positron impact excitation. The collision energies considered range from threshold (about $2 \mathrm{eV}$ ) to $40 \mathrm{eV}$. The results are interpreted in terms of elementary collision theory, including depolarization of the line radiation through fine- and hyperfine-structure effects. Given the size of the polarizations predicted and the recent developments in positron physics, an experimental test of our results should be pos. sible. This should provide an independent test of the theoretical model, in addition to the cross sections considered so far.

\section{3,817}

PB94-199742 Not available NTIS

National Inst of Standards and Technology (NML)

Gaithersburg, MD. Quantum Metrology Div.

Current Results and Future Prospects for a Neutron Lifetime Determination Using Trapped Protons.

Final rept.

M. S. Dewey. 1991, 13p.

Pub. in Proceedings of International Symposium on Capture Gamma-ray Spectroscopy and Related Topics (7th), Pacific Grove, CA., October 14-19, 1990 , p774-786 1991.

Keywords: "Neutron lifetime, "Neutron decay, Reprints, Trapping(Charged particles), Neutron beams, Particle decay, Measurement, Protons, Penning traps.

The availability of an accurate value for tau(n) has important implications for tests of the standard V - A theory of semi-leptonic weak processes, cosmology, and astrophysics. The lack of agreement between values of $g(A) / g(V)$ derived from recent lifetime measurements and values obtained from recent beta-decay asymmetry experiments hints at a problem with the current theoretical framework. In this neutron beam
measurement, the lifetime is determined by counting decay protons stored in a Penning trap whose magnetic axis coincides with the neutron beam axis. The recent result, $\operatorname{tau}(r)=893.5+$ or $-5.3 \mathrm{~s}$, is based upon data accumulated during one reactor cycle at the Institut Laue-Langevin in Grenoble, France. In the next two years a final accuracy approaching is will be sought

\section{3,818}

PB94-199841 Not available NTIS

National Inst. of Standards and Technology (PL), Boulder, CO. Quantum Physics Div.

Absolute Cross-Section Measurements for Electron-Impact Ionization of $\mathrm{C} 1(+1)$

Final rept.

N. Djuric, E. W. Bell, E. Daniel, and G. H. Dunn.

1992, 5p

Contract DE-A 105-86ER53237

Pub. in Physical Review A 46, n1 p270-274, 1 Jul 92. Sponsored by Department of Energy, Washington, DC. Office of Energy Research.

Keywords: *Electron-ion collisions, "Chlorine ions, $\mathrm{Re}$ prints, EV range 100-1000, EV range 10-100, Colliding beams, Cross sections, Electron impact, Ionization.

Absolute cross sections for electron-impact ionization of $\mathrm{Cl}(1+)$ have been measured from threshold to 200 $\mathrm{eV}$ with the use of the crossed-beams technique. The cross section shows a peak value of $12.8 \times 10$ (sup -17) $\mathrm{sq} \mathrm{cm}$ at about $70 \mathrm{eV}$. Results are compared to the semiempirical prediction formula of Lotz, with scaled cross sections for ions in the same isoelectronic sequence, and with other recent measurements. Expansion coefficients and formulas for generating ionization rate coefficients in the electron temperature range 10 thousand $=$ or $<\mathrm{T}=$ or $<10$ million $\mathrm{K}$ are presented
03,819

PB94-199916 Not available NTIS

National Inst. of Standards and Technology (PL) Gaithersburg, MD. Ionizing Radiation Div.

Neutron Measurement Intercomparisons Sponsored by CCEMRI, Section 3 (Neutron Measurements).

Final rept

R. S. Caswell, and V. E. Lewis. 1992, 6p.

Pub. in Radiation Protection Dosimetry 44, n1/4 p1051101992

Keywords: Reprints, Interlaboratory comparisons, Neutron sources, Neutron fluence, Radiation sources, *Neutron measurement, Systematic errors Intercomparison.

A large number of neutron measurement comparisons has been out under the sponsorship of Section III (Neutron Measurements) of the Consultative Committee for Measurement Standards of lonizing Radiations (CCEMRI) since Section III became active in 1961. These have included a series of comparisons of radionuclide neutron source emission rate, neutron fluence, and neutron dose measurements with the participation of laboratories worldwide. The paper discusses these comparisons and what has been learned from them. Future plans of Section III and neutron measurement services available from BIPM to national laboratories are also given.

03,820

PB94-200078 Not available NTIS

National Inst. of Standards and Technology (PL) Gaithersburg, MD. Quantum Metrology Div.

Improvements in Computation of Form Factors.

Final rept.

C. T. Chantler. 1993, $8 \mathrm{p}$.

. in Radiation Keywords: "Form factors, EV range 10-100, EV range sion relations, Cross sections, $X$ rays, Computation Reprints, Anomalous dispersion.

Tables for form factors and anomalous dispersion are of wide general use in the UV X-ray and gamme-ray communities, and have existed for a considerable period of time. The generality of these works has entailed numerous simplifications compared to detailed relativistic S-matrix calculations. However, the latter calculations do not appear to give convenient tabular ap plication for the range of $Z$ and energy of general interest, whereas the former tables have large regions of limited validity throughout the range of $Z$ and energies, and in particular have limitations with regard to extrapolation to energies outside tabulated ranges. Intermediate theoretical and procedural assumptions limit the precision and applicability of available tabulation and procedures. The paper identifies regions of $Z$ and energy where these assumptions fail and the improvement which can be achieved by their avoidance, both in tabulated ranges and by extrapolation. Particular concern involves the application of the dispersion relation to derive Re(f) from photoelectric absorption crosssections. Revised formulae can lead to significant qualitative and quantitative improvement, particularly above 30-60 keV energies, near absorption edges, o at $30-3000 \mathrm{eV}$ energies.

03,82

PB94-200086 Not available NTIS

National Inst. of Standards and Technology (PL) Gaithersburg, MD. Quantum Metrology Div.

Photographic Response to X-Ray Irradiation. 1. Es timation of the Photographic Error Statistic and Development of Analytic Density-Intensity Equations.

Final rept.

C. T. Chantler. 1993, $27 \mathrm{p}$

Pub. in Applied Optics 32, n13 p2371-2397, 1 May 93 See also Part 2, PB94-200094 and Part 3, PB94 200102

Keywords: *X rays, "Vacuum ultraviolet radiation, Photographic analysis, Reprints, KeV range 01-10, $\mathrm{KeV}$ range $10-100$, EV range $1-10$, EV range 10-100, EV range 100-1000, Optical density, Photographic emulsions, Linearization, Estimates, Intensity, Beam foil spectra, DEF-392 emulsion.

Formulations for specular optical density as a function of incident $x$-ray intensity are shown to be inadequate, theoretically and compared with available data. Ap- 


\section{PHYSICS}

\section{General}

proximations assuming low intensities, grain densities, or energies yield significant error in typical emulsions. Unjustifiable simplifications limit analysis and consequent results. The avoidance of assumptions leads to models for rough and smooth emulsion surfaces which correspond to Kodak 101-01 and DEF-392 emulsion types. The self-consistent use of spherical grains yields scaling that is dependent on emulsion roughness. The authors obtained improvement over standard formulations, avoiding the empirical character of earlier models and associated parameterization. The correlation of grain locations and occluded emulsion area is approximated within monolayer depths but neglected between layers. Effects of the incident angle from a broad source, scattering and photoelectrons are considered. The models presented herein apply to the vacuum UV and $x$-ray energies from $9 \mathrm{eV}$ to $20 \mathrm{keV}$ and may be preferred over alternative models at lower energies, densities greater than unity, emulsions with high grain fractions, or where interpolation over energy ranges is desired.

\section{3,822}

PB94-200094 Not available NTIS

National Inst. of Standards and Technology (PL), Gaithersburg, MD. Quantum Metrology Div.

Photographic Response to X-Ray Irradiation. 2. Correlated Models.

Final rept.

C. T. Chantler. 1993, 13p.

Pub. in Applied Optics 32, n13 p2398-2410, 1 May 93. See also Part 1, PB94-200086 and Part 3, PB94200102.

Keywords: "X rays, "Vacuum ultraviolet radiation, *Photographic analysis, Reprints, $\mathrm{KeV}$ range $01-10$ $\mathrm{KeV}$ range 10-100, EV range 1-10, EV range 10-100, EV range 100-1000, Optical density, Photographic emulsions, Photographic grain, Statistics, Models, DEF-392 emulsion.

In the paper models from the first paper are generalized so that they include the correlation of attenuation coefficients and coverages with emulsion depth. They avoid further assumptions and can provide physically meaningful parameters (as opposed to earlier studies); thus closer agreement with experimental measurements is obtained. The difficulty in estimating correlated overlap functions is discussed. Error estimates resulting from grain statistics are generalized and computed in a self-consistent manner. Contributions to granularity from densitometer and grain statistics have been shown to be significant or dominant in most emulsion types. The formulation derives reliable error estimates. Correlated models are important for thick emulsions such as DEF-392, whereas integral formalisms may be as useful for thin emulsions. In agreement with the first paper, reciprocity failure appears to be negligible for $U V$ or $x$-ray energies above $9 \mathrm{eV}$.

\section{3,823}

PB94-200102 Not available NTIS

National Inst. of Standards and Technology (PL), Gaithersburg, MD. Quantum Metrology Div.

Photographic Response to X-Ray Irradiation. 3 Photographic Linearization of Beam-Foil Spectra. Final rept.

C. T. Chantler, J. D. Silver, and D. D. Dietrich. 1993 , $11 \mathrm{p}$.

Pub. in Applied Optics 32, n13 p2411-2421, 1 May 93 See also Part 1, PB94-200086 and Part 2, PB94200094.

Keywords: "X rays, "Photographic analysis, Reprints, Optical density, Linearization, Statistics, Models, Beam foil spectra.

In the paper models for the relation of specular density to incident (x-ray) intensity with uncertainties are applied to experimental data, indicating methods for the correction of additional effects. Linearization and error calculations are simplified by double linear interpolation, and the effect of this is quantified. Relative firstorder intensities are determined directly. Secondary linearization or calculation for higher-order lines gives correction factors that yield absolute and relative higher-order intensity ratios. The effects of energy and angle on linearization are included. Densitometry uncertainty is estimated and quantified.

03,824

PB94-200334 Not available NTIS

National Inst. of Standards and Technology (CAML). Gaithersburg, MD. Statistical Engineering Div.
Analysis of Scattering Asymmetry Statistics When Background Corrected Counts Are Negative.

Final rept.

K. J. Coakley. 1993, 4p.

Pub. in Review of Scientific Instruments $64, n 7$ p18951898 Jul 93

Keywords: *Signal processing, Reprints, Background noise, Confidence limits, Polarized beams, Scattering, Uncertainty, Asymmetry, Correction, Estimates, Poisson processes, Bootstrap technique.

An asymmetry statistic of physical interest is an estimate of the ratio of the difference and the sum of the Poisson rate parameters for two scattering processes Typically, an additive background signal contributes to measurements of each signal. Background is meas ured in a third experiment. Data are corrected by subtracting measured background. When the measured background is larger than one of the other measurements, background-correlated counts are negative For this case, it would seem that no useful information can be extracted from the experiment. Here, asymmetry and an associated uncertainty interval are estimated for such cases using a bootstrap approach.

\section{3,825}

PB94-200342 Not available NTIS

National Inst. of Standards and Technology (CAML), Gaithersburg, MD. Statistical Engineering Div.

Uncertainty Intervals for Polarized Beam Scattering Asymmetry Statistics.

Final rept.

K. J. Coakley, J. J. McCelland, M. H. Kelley, and R. L. Celotta. 1993, 7p.

Pub. in Review of Scientific Instruments $64, n 7$ p1888 1894 Jul 93.

Keywords: *Polarized beams, "Signal processing, Reprints, Monte Carlo method, Confidence limits, Asymmetry, Uncertainty, Statistics, Background noise, Correction, Bootstrap technique, Poisson processes.

In many scattering experiments, the quantity of most direct physical interest is a measure of the difference between two closely related scattering signals, each generated by a Poisson scattering process. The difference is often expressed in terms of an asymmetry statistic, that is, the difference normalized to the sum of the two signals, corrected for an additive back ground contribution. Typically, a propagation of errors approach is used to compute confidence intervals for asymmetry. However, these confidence intervals are not reliable in general. In this work, generally accurate confidence intervals for asymmetry are obtained using a parametric bootstrap approach. Based on the observed data, data are simulated using a Monte Carlo resampling scheme. The resampled data sets satisfy a constraint that ensures that background-corrected count rates are not negative.

03,826

PB94-200417 Not available NTIS

National Inst. of Standards and Technology (MSEL)

Gaithersburg MD Reactor Radiation Div.

Acceptance Diagram Analysis of the Performance of Multidisk Neutron Velocity Selectors.

Final rept.

J. R. D. Copley. 1993, 10p.

Pub. in Nuclear Instruments and Methods in Physics Research A 332, p511-5201993.

Keywords: Reprints, Neutron scattering, Neutron beams, Performance, Design, Algorithms, *Neutron velocity selectors, Acceptance diagrams, Multidisk rotors, Neutron monochromators.

Transmission and absorption properties of helica multidisk neutron velocity selectors can be calculated using the method of acceptance diagrams. In this approach, neutron trajectories are characterized by transverse spatial coordinate and by a coordinate that is linearly related to wavelength and angle relative to the selector axis. It is then possible to classify neutron trajectories according to the number of absorbing sectors that they cross within the sector. In this way the authors can determine the extent to which a given se lector is successful in eliminating neutrons that get through the selector other than via one of the intended helical channels. The authors describe the design algorithm for selectors such as those in use at the $\mathrm{Na}$ tional Institute of Standards and Technology, and the authors discuss the properties of a selector built according to this algorithm. The authors also consider what happens when the direction of the neutron beam is reversed. The problem of optimizing the design of a multidisk velocity selector is briefly discussed.
03,827

PB94-200425 Not available NTIS National Inst. of Standards and Technology (MSEL) Gaithersburg, MD. Reactor Radiation Div. Acceptance Diagram Analysis of the Performance of Vertically Curved Neutron Monochromators.

Final rept.

J. R. D. Copley. 1991, 11p.

Pub. in Nuclear Instruments and Methods in Physics Research A301, p191-2011991.

Keywords: Reprints, Neutron scattering, Neutron guides, Beam forming, Bragg reflection, Thermal neutrons, *Neutron monochromators, Acceptance diagrams.

Vertically curved monochromator crystals are commonly used to increase the intensity in thermal neutron scattering applications. Such devices are found on spectrometers installed both at nonreflecting beam tubes and at totally reflecting guide tubes. The authors present an analysis of the performance of vertically curved monochromators located at instruments of both types. The acceptance diagram approach gives considerable insight into the behavior of vertically curved monochromators. The analysis can be extended to more complicated systems, which closely represent what is acutally found at a neutron scattering installation.

\section{3,828}

PB94-200433 Not available NTIS

National Inst. of Standards and Technology (MSEL), Gaithersburg, MD. Reactor Radiation Div.

Joy of Acceptance Diagrams.

Final rept.

J. R. D. Copley. 1993, 16p.

Pub. in Jnl. of Neutron Research 1, n2 p21-36 1993.

Keywords: Reprints, Neutron scattering, Neutron guides, Ray tracing, Phase space, Collimators, "Acceptance diagrams, Neutron velocity selectors, Neutron monochromators.

An acceptance diagram is a convenient and instructive way to represent spatial/angular neutron distributions within a neutron scattering instrument. In complicated systems, ray-tracing is used to calculate acceptance diagrams whereas in simple systems an analytical approach is appropriate. In the latter case, the action of a device such as a collimator or reflecting guide corresponds to one or more rather simple operations on the entrance acceptance diagram. Acceptance diagrams can also be used to investigate the behavior of devices such as focusing monochromators and velocity selectors.

\section{3,829}

PB94-200482 Not available NTIS

National Inst. of Standards and Technology (NML), Gaithersburg, MD. Ionizing Radiation Div.

Assay of the Eluent from the Alumina-Based Tungsten-188-Rhenium-188 Generator.

Final rept.

B. M. Coursey, J. M. Calhoun, J. Cessna, M. P. Unterweger, D. B. Golas, A. P. Callahan, S Mirzadeh, F. F. Knapp, D. D. Hoppes, and F. J. Schima. 1990, 9p.

Pub. in Radioact. Radiochem. 1, n4, 38, p42-49 1990.

Keywords: "Rhenium 188, Radiopharmaceuticals, Liquid scintillators, Decay schemes, Half life, Standardization, Tungsten 188, Radioisotopes, Reprints.

The alumina-based (188)W-(188)Re generator from ORNL can be eluted with saline to provide the (188)Re daughter as a short-lived high-energy beta-particle emitter which shows great promise for radiolabelling therapeutic radiopharmaceuticals. In this work, key parameters in the decay scheme of the (188)Re have been measured, and standard methods for quantitating the eluent have been developed. Rhenium-188 has been standardized for activity by the CIEMAT/NIST method of 4 (pi) (beta) liquid-scintillation efficiency tracing with tritium, with an uncertainty (equivalent to one standard deviation) of $0.3 \%$. Half-life measurements with a pressurized ionization chamber gave a $T(1 / 2)$ $=17.01+$ or -0.01 hours. Photon emission rates were measured with semi-conductor detectors, and the probability of emission of the principal gamma ray at $155 \mathrm{keV}$ was found to be $\mathrm{P}($ gamma $)=0.1588+$ or 0.0011

03,830

PB94-200490 Not available NTIS 
National Inst. of Standards and Technology $(P L)$, Gaithersburg, MD. Ionizing Radiation Div.

Standardization and Decay Scheme of Rhenium186.

Final rept.

B. M. Coursey, J. Cessna, E. Garcia-Torano, D. H. Gray, D. D. Hoppes, J. M. Los Arcos, M. T. MartinCasallo, F. J. Schima, M. P. Unterweger, D. B. Golas, and A. Grau Malonda. 1991, 5p. Pub. in Applied Radiation and Isotopes 42, n9 p8658691991.

Keywords: "Rhenium 186, Liquid scintillators, Gamma radiation, Half life, Decay schemes, Standardization, Radioactivity, Reprints.

Rhenium- 186 has been standardized for activity by the CIEMAT/NIST method of 4(pi)(beta) liquid-scintillation efficiency tracing with tritium, with an uncertainty (equivalent to $1 \mathrm{SD}$ ) of $1.61 \%$. Half-life measurements with pressurized ionization chamber give $\operatorname{a} \operatorname{Tau}(1 / 2)=$ $89.25+$ or $-0.07 \mathrm{~h}$. Photon emission rates were measured with semiconductor detectors, and the probability of emission of the principal gamme-ray at $137 \mathrm{keV}$ was found to be $\mathrm{P}(\mathrm{gamma})=0.0945+$ or -0.0016

03,831

PB94-200615 Not available NTIS

National Inst. of Standards and Technology (NML), Gaithersburg, MD. Center for Radiation Research. Accuracy-Weighted Variational Principle for Degenerate Continuum States.

Final rept.

M. Danos. 1990, 4p.

Pub. in Jnl. of Mathematical Physics 31, n11 p258825911990

Keywords: Variational principles, Numerical solution, Ritz method, Accuracy, Reprints, "Degenerate states, Continuum states, Degeneracy.

A variational principle for continuum states is given which permits numerical solution by the Ritz method It allows to maximize the accuracy of the solution in pre-selected regions of space, and also allows the selection of that solution from the perhaps infinitely degenerate solution set which is needed in the particular application.

03,832

PB94-200623 Not available NTIS

National Inst. of Standards and Technology (NML), Gaithersburg, MD. Center for Radiation Research.

Comprehensive Theory of Nuclear Effects on the Intrinsic Sticking Probability. 1.

Final rept.

M. Danos, L. C. Biedenharn, and A. Stahlhofen.

$1989,12 p$

Pub. in AlP Conf. Proc. Muon-Catal. Fusion, v181 p308-319 1989. See also Part 2, PB94-20063t.

Keywords: "Muon-catalyzed fusion, Nuclear fusion, Nuclear forces, Reprints, Intrinsic sticking fraction.

A comprehensive, inherently many-body, reaction theory for an accurate calculation of the intrinsic sticking fraction for $\left(\mathrm{dt}\left(\mathrm{m}^{\circ}\right)\right)$ fusion is outlined. The nonpertubative treatment of the long-range Coulomb force and its interference with the short range nuclear force is emphasized.

\section{3,833}

\section{PB94-200631 Not available NTIS}

National Inst. of Standards and Technology (NML). Gaithersburg, MD. Center for Radiation Research. Comprehensive Theory of Nuclear Effects on the Intrinsic Sticking Probability. 2.

Final rept.

M. Danos, L. C. Biedenharn, and A. Stahlhofen

1989, 10p

Pub. in AIP Conf. Proc. Muon-Catal. Fusion, v181 p320-329 1989. See also Part 1, PB94-200623.

Keywords: "Muon-catalyzed fusion, Nuclear fusion, Reprints, Intrinsic sticking fraction.

An accurate calculation of the intrinsic sticking fraction for (dt(mu)) fusion requires the development of a comprehensive, non-perturbative, inherently many-body, reaction theory. The authors identify and discuss the key problems underlying their construction of this theory.

\section{3,834}

PB94-211109

Not available NTIS

National Inst. of Standards and Technology (PL), Boulder, CO. Quantum Physics Div.
Three-Vector Correlation Theory for Orientation/ Alignment Studies in Atomic and Molecular Collisions.

Final rept.

J. P. J. Driessen, and L. Eno. 1992, 10p.

Contract NSF-PHY90-12244

Sponsored by National Science Foundation, Washington, DC

Pub. in Jnl. of Chemical Physics 97, n8 p5532-5541, 15 Oct 92

Keywords: "Molecular collisions, "Atomic collisions, Colliding beams, Cross sections, Orientation, Align ment, Calcium, Helium, Reprints.

The laboratory integral cross section under crossedbeam conditions is examined for collisions between structured and an unstructured species, where the orientation (or alignment) of the initial and final states is determined with respect to arbitrarily configured laboratory axes. Three parameters are necessary to characterize the mutual orientation of the relevant axes in this case and these parameters then also characterize the cross section. The laboratory cross section is expanded in terms of a more fundamental (angle-independent) set of cross sections as determined in the socalled collision frame, where projections of the structured species are taken with respect to the direction of the initial relative velocity vector. Drawing upon a number of symmetry relations, we count the numbers of fundamental cross sections for arbitrary initial and final angular momenta. Then we consider the explicit dependence on angular parameters which can be anticipated for the laboratory cross section. Finally, we raise a number of experimental considerations in attempting to determine the fundamental cross sections. These considerations are 'fleshed out' by applying ou cross-section analysis to a recent three-vector correlation experiment involving collisions between $\mathrm{Ca}$ and $\mathrm{He}$.

\section{3,835}

PB94-211117 Not available NTIS

National Inst. of Standards and Technology (NML) Boulder, $\mathrm{CO}$. Time and Frequency Div.

NIST Optically Pumped Cesium Frequency Standard.

Final rept

R. E. Drullinger, D. J. Glaze, J. L. Lowe, and J. H. Shirley. 1991 3p.

Pub. in Institute of Electrical and Electronics Engineers Transactions on Instrumentation and Measurement 40, n2 p162-164 1991

Keywords: "Cesium frequency standards, "Frequency standards, Optical pumping, Performance, Design, Tests, Reprints, US NIST.

The National Institute of Standards and Technology is developing an optically-pumped, cesium-beam primary frequency standard. The design follows from an analy sis of systematic errors found in cesium beam standards. The details of the design, performance of major sub-systems, and preliminary tests of the standard are presented.

03,836

PB94-211158 Not available NTIS

National Inst. of Standards and Technology (PL), Boulder, CO. Quantum Physics Div.

Early Dielectronic Recombination Measurements: Singly Charged lons.

Final rept.

G. H. Dunn. 1992, 17p

Contract DE-A105-86ER53237

Sponsored by Department of Energy, Washington, DC. Office of Energy Research.

Pub. in Recombination of Atomic lons, p115-131 1992.

Keywords: "Dielectronic recombination, "Atomic ions, Electric fields, Cross sections, Magnesium ions, $\mathrm{Ca}$ cium ions, Carbon ions, Autoionization, Measurement Reviews, Reprints.

Dielectronic recombination (DR) cross sections measurements on singly charged ions are reviewed, including a perspective on events leading to the exciting breakthroughs that occurred in 1982-83 for the study of this process. The DR mechanism is discussed along with the effect of electric fields on the process. The measurements on $\mathrm{Mg}$ ions in variable fields and the measurements of the effects of fields on the inverse process of autoionization are cited as the definitive ex perimental work on the field effects on DR. The quite spectacular disagreements between theory and experiment for DR cross section in $\mathrm{C}$ and $\mathrm{Ca}$ ions remain iment for DR cross sect
a perplexing mystery.
03,837

PB94-211182 Not available NTIS National Inst. of Standards and Technology (PL), Boulder, CO. Quantum Physics Div.

Fine Structure of Negative lons of Alkaline-EarthMetal Atoms.

Final rept.

V. A. Dzuba, V. V. Flambaum, G. F. Gribakin, and D. P. Sushkov. $1991,5 p$

Pub. in Physical Review A 44, n5 p2823-2827, 1 Sep 91.

Keywords: "Calcium ions, "Strontium ions, "Barium ions, "Radium ions, "Fine structure, Electron scattering, Spin orbit interactions, Negative ions, Perturbation theory, Reprints.

Fine-structure intervals $n p($ sub 1/2)-np(sub 3/2) (np(sub 1/2) is the ground state) are calculated for negative ions using relativistic many-body perturbation theory: $\mathrm{Ca}(1-)$ 4p, 56/cm; $\mathrm{Sr}(1-)$ 5p, 178/cm; $\mathrm{Ba}(1-) 6 \mathrm{p}$, about $460 / \mathrm{cm}$; and $\mathrm{Ra}(1-) 7 \mathrm{p}, 1,341 / \mathrm{cm}$. Comparison of the fine-structure interval with affinity: $\mathrm{Ca}(1-) \mathrm{H} \%$, $\mathrm{Sr}(1-) 22 \%, \mathrm{Ba}(1-) \quad 30 \%$; for $\mathrm{Ra}(1-)$ the fine-structure interval is larger than the affinity. Thus the $\mathrm{Ra}(1-)$ (sup 7)p(sub $3 / 2$ ) state is a resonance in the continuous spectrum with energy $0.018 \mathrm{eV}$. This means that the relativistic spin-orbit interaction plays a determining role in slow electron-radium scattering.

\section{3,838}

PB94-211265 Not available NTIS

National Inst. of Standards and Technology (NML), Gaithersburg, MD. lonizing Radiation Div.

Slab Transmission and Reflection for Point Source and Point Detector.

Final rept.

C. M. Eisenhauer. 1990, $2 p$.

Pub. in Transactions of the American Nuclear Society 62, p449-450 1990

Keywords: "Neutron transport, "Photon transport, Monte Carlo method, Gamma rays, Point sources, Polyethylene, Reflection, Slabs, Reprints.

Using Monte Carlo calculations, we show that the transmission of scattered neutrons or gamma rays from a point source, through a plane slab of infinite extent, to a point detector depends on the orientation of the slab but varies very little with the slab position. This statement is also true for reflected radiation if the source is replaced by its image source and the results are interpreted in terms of a transmission problem. We also show that the transition from a slab of small extent (narrow beam conditions) to a slab of infinite extent (broad beam conditions) can be characterized by a simple function of the single scatter angle. This function, too, can be applied to reflected radiation by invoking the image source. Typical results are presented for polyethylene.

\section{3,839}

PB94-211406 Not available NTIS

National Inst. of Standards and Technology (NML), Gaithersburg, MD. Ionizing Radiation Div.

Anomalous Odd- to Even-Mass Isotope Ratios in Resonance Ionization with Broad-Band Lasers.

Final rept.

W. M. Fairbank, M. T. Spaar, J. E. Parks, and J. M.

R. Hutchinson. 1989, 4p

Contract DE-Al-05-86-ER-60447

Sponsored by Department of Energy, Washington, DC. Pub. in Physical Review A 40, n4 p2195-2198, 15 Aug 89.

Keywords: "Molybdenum, "Tin, Laser radiation, Isotope ratio, Fractionation, Anomalies, Reprints, Resonance ionization

The response of even- and odd-mass isotopes is found to be very different in several schemes for resonance lonization of Mo and Sn, even when the laser linewidth is greater than the span of the hyperfine structure and isotope shifts.

\subsection{0}

PB94-211414 Not available NTIS

National Inst. of Standards and Technology (PL), Boulder, CO. Quantum Physics Div.

\section{Earth-Based Gravitational Experiments.}

Final rept.

J. E. Faller. 1990, 4p.

Pub. in Proceedings of International Conference on General Relativity and Gravitation (12th), Boulder, CO., July 2-8, 1989, p345-348 1990. 


\section{PHYSICS}

\section{General}

Keywords: "Gravitation, General relativity, Ground based, Experimentation, Reprints.

Though historically, the solar system has been the principal area for testing theories of gravitation, we seem to be at the end of the golden age of solar-system tests (Reasenberg (1987)). The classical effects have now been measured within the limits of today's technology and further significant improvements cannot be expected in the near term. Future space-based experiments such as GPB, Gravity Probe B; LAGOS, Laser Gravitational-wave Observatory in Space; and POINTS, the (proposed) Astrometric Optical Interferometer, await further technological as well as engineering developments and logistic (launch) support to deliver them to the laboratory of space. Recent years have seen, however, ground-based gravitational experimentation undergo a resurgence, driven by new experimental capabilities and by new theoretical work.

03,841

PB94-211505 Not available NTIS

National Inst. of Standards and Technology (NML) Gaithersburg, MD. Radiometric Physics Div.

Photoionization of Small Molecules Using Synchrotron Radiation.

Final rept.

T. A. Ferrett, and A. C. Parr. 1988, 4p.

Pub. in Proceedings of Society of Photo-Optical Instru mentation Engineers - X-ray and Vacuum Ultraviole Interaction Data Bases, Calculations, and Measurements, Los Angeles, CA., January 14-15, 1988, v911 p19-22.

Keywords: "Carbon monoxide, "Carbon dioxide, "Photoionization, EV range 10-100, Vibrational states, Synchrotron radiation, Angular distribution, High reso. lution, Molecular dynamics, Molecular ions, Autoionization, Reprints.

We report here new high-resolution vibrationally re solved studies of photoionization in the molecules CO and $\mathrm{CO} 2$ using synchrotron radiation in the photon-energy range 10-30 eV. The detailed decay dynamics of autoionizing resonances in $\mathrm{CO}$ and the origin of symmetry-forbidden vibrational transitions in the ( $C$ bar) state of $\mathrm{CO} 2(1+)$ are discussed. These examples illus trate the need for experimental work with both high optical resolution and electron resolution sufficient to resolve the complex resonances in molecules and the vibrational dependence of molecular dynamics.

03,842

Pot available NTIS

National Inst. of Standards and Technology (NML) Gaithersburg, MD. Atomic and Plasma Radiation Div. Atomic Transition Probability Ratios between Some Ar I 4s-4p and 4s-5p Transitions.

Final rept.

T. D. Hahn, and W. L. Wiese. 1990,3p

Pub. in Physical Review A 42, n9 p5747-5749 1990 Keywords: "Argon, Atomic energy levels, Electron transitions, Transition probabilities, Visible spectra, Line spectra, Neutral atoms, Ratios, Reprints.

All recent determinations of the atomic transition probabilities for the prominent $\mathrm{Ar} I 4 S-4 p$ lines in the red part of the visible spectrum have been carried out separately from other determinations of the equally prominent $4 \mathrm{~s}-5 \mathrm{p}$ lines in the blue part of the spectrum. A cross-connection of these data is thus desirable to establish their mutual consistency. We have measured several transition probability ratios for $4 s-4 p$ to $4 s-5 p$ transitions and find very close consistency.

\section{3,843}

PB94-211562 Not available NTIS

National Inst. of Standards and Technology (NML) Gaithersburg, MD. Atomic and Plasma Radiation Div. Ion Broadening Parameters for Several Argon and Carbon Lines.

Final rept.

T. D. Hahn, and L. A. Woltz. 1990, 4p.

Pub. in Physical Review A 42, n3 p1450-1453 1990.

Keywords: "Line broadening, "Carbon, "Argon, Spectral lines, Neutral atoms, Asymmetry, Reprints, Ion broadening.

The ion broadening parameters of several argon and carbon spectral lines are determined by fitting asymmetric theoretical profiles to spectral line profiles obtained in a wall-stabilized arc.
National Inst. of Standards and Technology (MSEL), Gaithersburg, MD. Metallurgy Div.

High Temperature.

Final rept.

J. W. Hastie, D. W. Bonnell, and J. B. Berkowitz.

$1991,6 \mathrm{p}$

Pub. in Encyclopedia of Physics, p497-502 1991.

Keywords: "High temperature, Temperature measurement, Materials, Flames, Fusion, Plasma, Reprints.

This article defines the condition of high temperature its measurement, and its significance to physics and interdisciplinary areas of materials and chemical sciences

\section{3,845}

\section{PB94-211752 Not available NTIS}

National Inst. of Standards and Technology (NML) Gaithersburg, MD. Radiation Source and Instrumentation Div.

Drill-Hearn-Gerasimov Sum Rule.

Final rept.

E. Hayward. 1992, 6p.

Pub. in Proceedings of Course (7th) and the Winter Course (2nd) of the International School of Intermediate Energy Nuclear Physics, L'Aquila and Folgaria, Italy, July 16-26, 1990 and January 27-February 3, 1991, p164-1691992.

Keywords: "Sum rules, Dispersion relations, Optical theorem, Low-energy theorem, Reprints.

The Drell-Hearn-Gerasimov sum rule is derived and discussed. The much earlier work of Karliner is pointed out.

\section{3,846}

PB94-211760 Not available NTIS

National Inst. of Standards and Technology (NML), Gaithersburg, MD. Radiation Source and Instrumentation Div.

Polarizability of the Nucleon.

Final rept.

E. Hayward. 1992, 22p

Pub. in Proceedings of Seventh Course and Second Winter Course of the International School of Intermediate Energy Nuclear Physics, L'Aquila and Folgaria, Italy, July 16-26, 1990 and January 27-February 3 1991, p126-1471992.

Keywords: "Neutrons, "Protons, Polarization characteristics, Absorption cross sections, Scattering cross sections, Low-energy theorem, Dispersion relations, Optical theorem, Nucleons, Reprints, Photon absorption.

The conflict between the polarizabilities of the nucleon derived from the total photon absorption cross section and the photon scattering cross section is discussed. The electric and magnetic dipole cross sections for the proton and neutron are illustrated. Expressions for the photon scattering cross sections for the proton and neutron are given including the scattering cross section for the point nucleon and for plane polarized incident photons. Possible explanations for this discrepancy are discussed.

\section{3,847}

PB94-211786 Not available NTIS

National Inst. of Standards and Technology (NML), Gaithersburg, MD. Molecular Physics Div.

Asymptotic Wave Function Splitting Procedure for Propagating Spatially Extended Wave Functions: Application to Intense Field Photodissociation of H2(+).

Final rept.

R. W. Heather 1991, 14p

Pub. in Computer Physics Communications $63, \mathrm{n} 1-3$ p446-459 1991

Keywords: "Hydrogen ions 2 plus, "Photodissociation, Wave functions, Reprints.

We describe an efficient procedure for propagating a spatially extended wave function evaluated on a small grid The procedure involves the repeated splitting of the wave function into a part residing mainly in the interaction region (small fragment separation) and parts residing solely in the asymptotic region (large fragment separation), and propagating each part separately. The total wave function can be reassembled at a later time by adding the individual asymptotic pieces, propagated to a common final time with a free particle propagator, to the part of the wave function remaining in the interaction region. This procedure is well suited to propagation methods that evaluate the wave function on a spatial grid, because the amount of asympotic region included in the grid, and therefore the computational effort, can be minimized (the smaller the asymptotic region, the more frequent the splitting).

\section{3,848}

PB94-211851 Not available NTIS

National Inst. of Standards and Technology (NML) Gaithersburg, MD. Electron and Optical Physics Div. Design and Characterization of X-Ray Multilayer Analyzers for the $50-1000$ eV Region.

Final rept.

B. L. Henke, E. M. Gullikson, J. Kerner, A. L. Oren and R. L. Blake. 1990, 64p

Pub. in Jnl. of X-ray Science and Technology 2, n1 p17-80 Mar 90.

Keywords: "X-ray optics, "Analyzers, EV range 1001000 , EV range 10-100, X-ray analysis, Spectroscopy, Reflectance, Design, Reprints.

Detailed absolute reflectivity characterizations are presented for selected examples of multilayers, which have been semi-empirically determined for Mica, KAP and the fabricated Langmuir-Blodgett and sputtered multilayer analyzers with d-spacings in the 10-200 A range. Design requirements for absolute spectrometry are established. Efficient analytical multilayer reflectivity models are derived and parameterized (based upon a modification of the Darwin-Prins model for the lowenergy $x$-ray region) -- including, for the sputtered multilayers, parameters for defining interface structure. The dependence of the reflectivity characteristics, high-order Bragg diffraction suppression, and over-al efficiency upon the model parameters is analyzed. A special spectrograph and procedure for the absolute measurement of the relevant reflectivity cha racteristics are described. Detailed measurements and semi-empirical characterizations are presented.

03,849

PB94-212248 Not available NTIS

National Inst. of Standards and Technology (PL), Gaithersburg, MD. Quantum Metrology Div.

$K$ alpha Transitions in Few-Electron lons and in Atoms.

Final rept.

P. Indelicato. 1990, 12p.

Pub. in Proceedings of International Conference on $X$ ray and Inner-Shell Processes (15th), Knoxville, TN. July 1990, p591-602.

Keywords: "K lines, Atomic energy levels, Electron transitions, Heavy elements, Reprints, Hydrogen-like ions, Helium-like ions

The study of the 1s level in heavy atoms or ions provides very important tests of QED and relativistic corrections in strong electromagnetic fields. Such studies have been conducted mainly by measuring $2 p-1$ s transitions in one and two-electron ions and $\mathrm{K}$ transitions in atoms. In this paper I will discuss the status and the merit of the different approaches, and show detailed comparisons between experiment and theory in all three cases

03,850

PB94-212271 Not available NTIS

National Inst of Standards and Technology (PL), Boulder, CO. Time and Frequency Div.

Quantum Projection Noise: Population Fluctuations in Two-Level Systems.

Final rept.

W. M. Itano, J. C. Bergquist, J. J. Bollinger, F. L. Moore, M. G. Raizen, D. J. Wineland, J. M. Gilligan, and D. J. Heinzen. 1993, 17p.

Pub. in Physical Review A 47, n5 p3554-3570 May 93. Keywords: "Quantum mechanics, "Ion storage, Laser spectroscopy, Frequency standards, Internal energy, Beryllium ions, Beryllium 9, Mercury ions, Mercury 199 Reprints, Penning traps, Ion traps, Quantum noise.

Measurements of internal energy states of atomic ions confined in traps can be used to illustrate fundamental properties of quantum systems, because long relaxation times and observation times a re available. In the experiments described here, a single ion or a few identical ions were prepared in well-defined superpositions of two internal energy eigenstates. The populations of the energy levels were then measured. For an individual ion, the outcome of the measurement is uncertain unless the amplitude for one of the two eigenstates is zero, and is completely uncertain when the magnitudes of the two amplitudes are equal. In one experiment, 
a single $(199) \mathrm{Hg}(1+)$ ion, confined in a linear if trap was prepared in various superpositions of two hyperfine states. In another experiment, groups of (9) $\mathrm{Be}(1+)$ ions, ranging in size from about 5 to about 400 ions, were confined in a Penning trap and prepared in various superposition states. The measured population fluctuations were greater when the state amplitudes were equal than when one of the amplitudes was nearly zero, in agreement with the predictions of quantum mechanics. These fluctuations, which we call quantum projection noise, are the fundamental source of noise for population measurements, with a fixed number of atoms. These fluctuations are of practical importance, since they contribute to the errors of atomic frequency standards.

\section{3,851}

PB94-212560 Not available NTIS

National Inst. of Standards and Technology (NML), Gaithersburg, MD. Molecular Physics Div.

Theory of Atomic Collisions at UItracold Temperatures.

Final rept.

P. S. Julienne, R. Heather, and J. Vigue. 1991, 21p. Pub. in Proceedings of International Conference on Atomic Physics (12th), Ann Arbor, MI., July 31-August 3, 1990, p116-136 1991 . Keywords: “Atomic collisions, Temperature range ium, Cesium, Reprints, Ultracold atoms, Atom traps.

Recent developments in laser cooling and trapping of neutral atoms have opened the new field of atomic collisions at ultracold temperatures, $T<1 \mathrm{mK}$. There are many new challenges and opportunities for both theory and experiment. We will outline some of the key problems in this field, and present calculations on several collisional processes which have been observed in atom traps.

03,852

PB94-212651 Not available NTIS

National Inst. of Standards and Technology (EEEL),

Boulder, CO. Electromagnetic Technology Div.

Chaos in a Computer-Animated Pendulum.

Final rept.

R. L. Kautz. 1993, 9p.

Pub. in American Jnl. of Physics 61, n5 p407-415 May 93.

Keywords: *Pendulums, "Chaos, Computer animation Strange attractors, Symmetry breaking, Frequency multipliers, Josephson effect, Demonstrations, Trajectories, Reprints, Chaotic motion, State space.

A classroom demonstration based on computer animation illustrates chaotic motion in a driven pendulum Generated by a 76 line BASIC program that runs on $\mathrm{PC}$-compatible computers, the animation shows four simultaneous displays, including the pendulum and its trajectory in state space. The program can be used to illustrate periodic attractors, symmetry breaking, period doubling, and chaos.

\section{3,853}

PB94-212727 Not available NTIS

National Inst. of Standards and Technology (NML) Gaithersburg, MD. Atomic and Plasma Radiation Div. Autoionizing Resonances in Electric Fields.

Final rept.

D. E. Kelleher, E. B. Saloman, and J. W. Cooper. $1991,4 \mathrm{p}$.

Pub. in Proceedings of International Conference on Coherent Radiation Processes in Strong Fields (1st) Washington, DC., June 18-22, 1990, p665-678 1991

Keywords: *Autoionization, Electric fields, Direct current, Resonance, Reprints.

We present a brief overview of observed effects of d.c. electric fields on autoionizing resonances in the region above the first ionization threshold. The types of phenomena fall into three broad categories: (I) field broadening (and narrowing), (II) field-induced interferences and transitions, and (III) field-induced Stark manifolds. Theoretical methods for dealing with these phenomena have been developed, and comparisons with their predictions will be discussed.

\section{3,854}

PB94-212784 Not available NTIS

National Inst. of Standards and Technology (NML) Gaithersburg, MD. Atomic and Plasma Radiation Div.
Relativistic and Quantum Electrodynamic Effects in Highly-Charged lons.

Final rept.

Y. K. Kim. $1990,21 p$

Pub. in AIP Conference Proceedings on At. Processes Plasmas, v206 p19-39 1990

Keywords: "Multicharged ions, "Atomic structure, "Atomic spectra, Quantum electrodynamics, Relativistic effects, Transition probabilities, Lamb shift, Corrections, Reprints.

Recent spectroscopic data clearly indicate that both relativistic and quantum electrodynamic (OED) effects are indispensable in understanding the spectra of highly-charged ions. OED effects are discernible even in spectra involving $\mathrm{M}$ - and $\mathrm{N}$-shell electrons. Current understanding and theoretical capability in predicting relativistic and OED corrections in atomic structure are reviewed.

\section{3,855}

PB94-212842 Not available NTIS

National Inst. of Standards and Technology (PL), Gaithersburg, MD. Atomic Physics Div.

Atomic Branching Ratio Data for Carbon-Like lons. Final rept.

J. Z. Klose, T. M. Deters, J. R. Fuhr, and W. L.

Wiese. 1993, 6p.

Pub. in Jnl. of Ouantitative Spectroscopy and Radiative Transfer (JOSRT) 50, n1 p1-6 1993.

Keywords: *Branching ratio, "Multicharged ions, Vacuum ultraviolet radiation, Transition probabilities, High temperature, Spectral lines, Calibration, Radiometry, Plasma, Reprints, ${ }^{*}$ Carbon-like ions.

The branching-ratio technique for radiometric calibrations in the vacuum ultraviolet spectral region is briefly reviewed. A list of transitions suitable for use of the technique is given for carbon-like ions, along with pertinent data for their application.

\section{3,856}

PB94-213055 Not available NTIS

National Inst. of Standards and Technology (MSEL),

Gaithersburg, MD. Ceramics Div

High Resolution Hard X-Ray Microscope.

Final rept.

M. Kuriyama, R. Dobbyn, and R. Spal. 1990, 11p.

Pub. in Rigaku Jnl. 7, n2 p5-15 1990.

Keywords: Hard $\mathrm{x}$ radiation, $\mathrm{X}$-ray optics, $\mathrm{X}$-ray diffraction, $X$-ray detection, Modulation transfer function, Spatial resolution, High resolution, Microradiography Tomography, Uses, Reprints, "X-ray microscopes, Microtomography, Multilayers

A high resolution hard $x$-ray microscope is described. This system is capable of detecting line features as small as 0.5 micrometer in width. The spatial resolution determined by the modulation transfer function is 1.0 micrometer. Three types of two-dimensional image detectors are discussed and compared for use with hard $x$-rays in high resolution. Principles of $x$-ray image magnification are also discussed, based on $x$-ray optics and diffraction physics. The effect of the x-ray source size is discussed as an important factor limiting spatial resolution. Some examples of applications are demonstrated in microradiography and diffraction imaging (topography). High resolution tomography has now become a reality.

03,857

PB94-213063 Not available NTIS

National Inst. of Standards and Technology (CSTL), Boulder, CO. Thermophysics Div.

Thermal Conductivity of R134a.

Final rept.

A. Laesecke, R. A. Perkins, and C. A. Nieto de

Castro. 1992, 12p.

Sponsored by Environmental Protection Agency, Washington, DC

Pub. in Fluid Phase Equilibria 80, p263-274 1992.

Keywords: "Fluorohydrocarbons, "Thermal conductivity, Supercritical fluids, Refrigerants, Substitutes, Temperature dependence, Reprints, "R134a, Ethane/ tetrafluoro.

Thermal conductivity measurements are reported for the alternative refrigerant 1,1,1,2-tetrafluoroethane (R134a). These measurements were made over the temperature range from 200 to $390 \mathrm{~K}$ at pressures to $70 \mathrm{MPa}$. Data are reported for the liquid, vapor, and supercritical fluid phases. A significant critical en- hancement is observed for R134a A thermal conductivity surface, which fits our data to + or $-3.8 \%$ a $95 \%$ confidence, is provided for R134a. Correlations are also provided for the saturated vapor and saturated liquid thermal conductivity of R134a. Comparisons are made with other available thermal conductivity data fo the dilute gas, saturated vapor, and saturated liquid.

03,858

PB94-213154 Not available NTIS

National Inst. of Standards and Technology (MSEL) Gaithersburg, MD. Reactor Radiation Div.

Elastic Deformation of a Monolithic Perfect Crystal Interferometer: Implications for Gravitational Phase Shift Experiments.

Final rept.

H. P. Layer, and G. L. Greene. 1991, 5p.

Pub. in Physics Letters A 155, n8,9 p450-454, 27 May 91.

Keywords: *Gravitation, Finite element method, Elastic deformation, Phase shift, Distortion, Neutrons, Re prints, Silicon interferometers.

A calculation of the gravitationally induced distortion of a monolithic silicon interferometer using the finite element method has been used to analyze the data from a gravitational phase shift experiment using neutrons. The observed dependence of the $X$-ray phase shift on interferometer rotational angle has been reproduced. An additional interferometer distortion has been identified which can explain the discrepancy between the observed neutron phase shift and the theoretical value.

\section{3,859}

PB94-213238 Not available NTIS

National Inst. of Standards and Technology (NML), Gaithersburg, MD. Electron and Optical Physics Div. Ultracold Collisions: Associative Ionization in a Laser Trap.

Final rept.

P. Lett, P. Jessen, C. Westbrook, P. Jullienne, P.

Gould, S. Rolston, and W. Phillips. 1991, 2p.

Sponsored by Office of Naval Research, Arlington, VA Pub. in Light Induced Kinetic Effects on Atoms, Ions and Molecules, p181-182 1991.

Keywords: Reprints, "Associative ionization, Lase cooling, Laser trapping, Ultracold atoms, Optical molasses.

Experimental investigations of associative ionization in Elaser trap are reported. The recent prediction by Julienne of a laser intensity modified reaction rate has been confirmed. In addition, a new channel for the reaction involving long-range molecular states has been discovered.

\section{3,860}

PB94-213279 Not available NTIS

National Inst. of Standards and Technology (PL) Gaithersburg, MD. Quantum Metrology Div. High-Energy Behavior of the
Photoionization of Helium from 2 to $12 \mathrm{keV}$. Final rept.

J. C. Levin, I. A. Sellin, B. M. Johnson, N. Berrah, Y. Azuma, H. G. Berry, D. H. Lee, D. W. Lindle, and R. D. Miller. 1993, 4p. Sponsored by Department of Energy, Washington, DC. Pub. in Physical Review A 47, n1 pR16-R19 Jan 93. Keywords: "Photoionization, "Helium, KeV range 1-10, $\mathrm{KeV}$ range 10-100, Synchrotron radiation, Correlation, Reprints.

We report that the ratio of double-to-single photoionization of $\mathrm{He}$ at several photon energies from 2 to $12 \mathrm{keV}$. By time-of-flight methods, we find a ratio consistent with an asymptote at $1.5 \%+$ or $-0.2 \%$, es sentially reached by $\mathrm{h}(\mathrm{nu})$ approx $=4 \mathrm{keV}$. Fair agreement is obtained with older shake calculations of Byron and Joachain (Phys. Rev. 164, 1 (1967)), of Aberg (Phys. Rev. A 2, 1726(1970)), and with recent manybody perturbation theory (MBPT) of Ishihara, Hino, and McGuire (Phys. Rev. A 44, 6980 (1991)). The result lies below earlier MBPT calculations by Amusia et al. (J. Phys. B 8, $1248(1975)$ ) (2.3\%), and well above semiempirical predictions of Samson (Phys. Rev. Lett. $65,2861(1990))$, who expects no asymptote and predicts sigma $(\mathrm{He}(2+)) / \mathrm{sigma}(\mathrm{He}(1+))=0.3 \%$ at $12 \mathrm{keV}$.

03,861

PB94-213337 Not available NTIS

National Inst. of Standards and Technology (NML) Gaithersburg, MD. Molecular Spectroscopy Div. 


\section{PHYSICS}

\section{General}

\section{Molecular Spectroscopy.}

Final rept.

D. R. Lide, and A. Weber. 1991, 17p.

Pub. in Encyclopedia of Physics, p737-753 1991.

Keywords: "Molecular spectroscopy, Rotational states, High resolution, Electronic spectra, Microwave spectra, Infrared spectra, Raman spectra, Laser spectroscopy, Perturbation, Reprints, Molecular constants.

This article is a revised version of an earlier one on molecular spectroscopy. Experimental techniques are discussed and the advantages of using narrow band lasers for high resolution investigations are illustrated.

\section{3,862}

PB94-213360 Not available NTIS

National Inst. of Standards and Technology (NML).

Gaithersburg, MD. Quantum Metrology Div.

Polarized X-Ray Emission Spectroscopy.

Final rept.

D. W. Lindle, P. L. Cowan, T. Jach, R. E. LaVilla,

and R. D. Deslattes. 1989, 5p

Pub. in Nuclear Instruments and Methods in Physics Research B40-41, p257-261 Apr 89.

Keywords: "X-ray fluorescence, *X-ray spectroscopy, Polarization(Waves), Synchrotron radiation, Excited states, Methyl chloride, Reprints, Inner-shell spectroscopy, X-ray emission.

Strongly polarized $x$-ray fluorescence from gas-phase molecules has been observed by selectively exciting near core level ionization thresholds using monochromatized synchrotron radiation. Both the degree and the direction of the polarization are very sensitive to the incident excitation energy, and the symmetry of the occupied and unoccupied molecular orbitals involved in the excitation/fluorescence process. Illustration of the phenomenon will be made using $\mathrm{Cl} \mathrm{K}$ edge excitation on the molecule methyl chloride, $\mathrm{CH} 3 \mathrm{Cl}$. The possibility of extracting orientational, geometrical, and orbital-symmetry information directly from experiment will be discussed, and the importance of the rapid time scale of the $x$-ray fluorescence decay (about $10 \mathrm{fs}$ ), which effectively precludes diso rientation effects, will be stressed. Expectations are that x-ray polarized fluorescence spectroscopy will enjoy wide applicability to, for example, solids, surfaces and interfaces, oriented molecules such as surface adsorbates, and active sites in macromolecules.

\section{3,863}

PB94-213378 Not available NTIS

National Inst. of Standards and Technology (NML), Gaithersburg. MD. Quantum Metrology Div.

Atomic, Molecular, and Optical Physics with $X$ rays.

Final rept.

D. W. Lindle, and B. Crasemann. 1991, 5p.

Pub. in Nuclear Instruments and Methods in Physics Research B 56-7, p441-445 May 91 .

Keywords: "Molecular spectroscopy, "Atomic spectroscopy, "Synchrotron radiation, Synchrotron radiation sources, Advanced Light Source, X-ray sources, Uses, Reprints.

With the advent of third-generation synchrotron-radiation sources now being built, research in the area of atomic, molecular, and optical physics using $x$-rays rom these insertion-device-based facilities is expected to experience a renaissance. Many of the most soughtafter experimental goals in this area of research will become possible or even routine. To highlight some of the exciting possibilities, some specific examples are discussed, such as the $x$-ray and Auger resonantRaman effect and polarized molecular $\mathrm{x}$-ray emission. Plans for implementation of an $x$-ray synchrotron-radiation beamline dedicated to atomic, molecular, and optical physics at the Advanced Light Source are presented, with emphasis on the enhanced capabilities that will be available at this state-of-the-art facility.

\section{3,864}

PB94-216041 Not available NTIS

National Inst. of Standards and Technology (CSTL), Gaithersburg, MD. Inorganic Analytical Research Div. Measurement of $\mathrm{CO}$ Pressures in the Ultrahigh Vacuum Regime Using Resonance-Enhanced Multiphoton-Ionization Time-of-Flight Mass Spectroscopy.

J. P. Looney, J. E. Harrington, K. C. Smyth, T. R. O'Brian, and T. B. Lucatorto. 1993, 10p.

Pub. in Jnl. of Vacuum Science and Technology A 11 , n6 p3111-3120 Nov/Dec 93
Keywords: "Carbon monoxide, "Pressure measure ment, Time-of-flight method, Two photon absorption Ultrahigh vacuum, Laser radiation, Photoionization, Reprints.

An evaluation is made of measurements of $\mathrm{CO}$ pressures in the UHV regime using resonance-enhanced multiphoton ionization coupled with time-of-flight mass spectroscopy (REMPI-TOFMS). It has been found that once the REMPI-TOFMS system has been calibrated quantitative measurement of $\mathrm{CO}$ pressures as low as 10 (sup -10) $\mathrm{Pa}$ is possible, even in overwhelming N2 backgrounds. With compensation for laser pulse en ergy variations, we find measurements with uncertainties of $10 \%-15 \%$ are possible for pressures down to 10(sup -7) $\mathrm{Pa}$, and an ultimate detection limit for $\mathrm{CO}$ pressures of 10(sup -10) Pa for our measurement system. In this study, the REMPI-TOFMS system was calibrated using a pressure division technique along with a spinning rotor gage. The ionization of $\mathrm{CO}$ is achieved using $230 \mathrm{~nm}$ radiation to excite the $B$ singlet Sigma $(1+)$ state of CO at $10.8 \mathrm{eV}$ via a two-photon absorption and then ionizing some of the excited state molecules by the absorption of an additional photon from the laser beam.

\section{3,865}

PB94-216124 Not available NTIS

National Inst of Standards and Technology (CSTL) Gaithersburg. MD. Thermophysics Div.

Analytical Method of Determining the Heat Capacity at High Temperatures from the Surface Tem perature of a Cooling Sphere.

Final rept

R. A. MacDonald. 1992, 7p

Pub. in High Temperature-High Pressures 24, p127 1331992.

Keywords: "Specific heat, *Spheres, "Radiant cooling "Surface temperature, "Molybdenum, Thermophysica properties, High temperature, Temperature dependence, Emissivity, Reprints, Time dependent temperature profiles.

The feasibility of determining thermophysical properties at high temperatures from measurements of the surface temperature of a radiating sphere is investigated. For a sphere initially maintained in a steady state but with no heat supplied after some time, $t=0$ the subsequent temperature profile in the sphere has been calculated as a function of time. The material properties of the sphere: thermal conductivity, heat ca pacity at constant pressure, thermal expansion, and emissivity, are assumed to have a quadratic depend ence on temperature over a limited temperature range (several hundred degrees). The sensitivity of the temperature profile to the material parameters has been examined for values representative of molybdenum. The surface temperature shows some sensitivity to the heat capacity and to the emissivity but it is quite insensitive to the thermal conductivity and thermal expansion. In spite of the rather small effect, a method for determining the heat capacity, given the surface tem perature of the sphere as a function of time, has been developed on the basis of these calculations for the full range of temperature, $1500-3000 \mathrm{~K}$

\section{3,866}

PB94-216215 Not available NTIS

National Inst. of Standards and Technology (MSEL) Gaithersburg, MD. Reactor Radiation Div.

Supermirror Transmission Polarizers for Neutrons. Final rept.

C. F. Maikrzak, V. Nunez, J. R. D. Copley, J. F.

Ankner, and G. C. Greene. 1992, 17p.

Pub. in Proceedings of Society of Photo-Optical Instrumentation Engineers - Neutron Optical Devices an Applications, San Diego, CA., July 22-24, 1992, v1738 p90-106.

Keywords: "Polarizers, Polarization(Spin alignment) Cold neutrons, Thermal neutrons, Silicon coatings, Iron, Monochromators, Reprints, "Neutron polarizers, *Supermirrors, Accordion configuration.

We describe several geometrical configurations of supermirror arrays for polarizing cold and sub-therma neutrons in transmission with high efficiency, which is particularly important in applications where several polarizers occur in series. The measured polarizing efficiency and reflectivity of Fe-Si supermirror coating which can be used in these devices are also reported.

\section{3,867}

PB94-216439 Not available NTIS
National Inst. of Standards and Technology (PL), Boulder, CO. Quantum Physics Div.

Atomic Beam Splitters and Mirrors by Adiabatic Passage in Multilevel Systems.

Final rept.

P. Marte, P. Zoller, and J. Hall. 1992, 10p

Sponsored by National Science Foundation, Washington, DC. and Office of Naval Research, Arlington, VA. Pub in Proceedings Foundations of Quantum Mechanics Workshop, Santa Fe, NM., June 1991, p2983071992.

Keywords: "Atomic beams, "Beam splitters, Beam optics, Laser spectroscopy, Raman effect, Deflection, Reprints, "Beam mirrors, Atomic interferometry.

We study atomic beam deflection by adiabatic passage between Zeeman ground-levels via Raman transitions induced by counterpropagating Sigma + - polarized lasers. We show that complete population transfer between the ground states can be achieved which corresponds to the scattering of the atomic wave packet into a single final momentum state by absorption and induced emission of laser photons. Although the lasers can be resonant, the excited state(s) are never populated during the adiabatic transfer which suppresses the effects of spontaneous emission and preserves the coherence of the atomic wave function. This scheme has attractive features as a beam splitter and mirror for atomic interferometry.

03,868

PB94-216611 Not available NTIS

National Inst. of Standards and Technology (NML) Gaithersburg, MD. Center for Radiation Research. Theoretical Aspects of Tagged Photon Facilities. Final rept.

C. Maximon. 1990,17p.

Pub. in Proceedings of Seminar on Electromagnetic Interactions of Nuclei at Low and Medium Energies (7th), Moscow, USSR, December 12-14, 1988, p2172331990.

Keywords: "Tagged photon method, Polarization, Marking, Reprints.

The idea behind photon tagging is conceptually simple; a signal from a detector looking at one of the particles involved in the photon production process provides information on both the energy and the time of creation of each photon, thereby 'tagging' the energy of each of the photons in a continuous spectrum. $A$ fast time coincidence between the signal from the tagging detector and the signal from the nuclear reaction product detector indicates that the reaction was initiated by the associated photon and serves to identify the energy of the photon that initiated the reaction. Three tagging techniques have been implemented at a number of accelerator laboratories.

03,869

PB94-216652 Not available NTIS

National Inst. of Standards and Technology (PL), Gaithersburg, MD. Electron and Optical Physics Div. Determination of Complex Scattering Amplitudes in Low-Energy Elastic Electron-Sodium Scattering. Final rept.

J. J. McClelland, S. R. Lorentz, R. E. Scholten, M. H. Kelley, and R. J. Celotta. 1992, 4p.

Sponsored by Department of Energy, Washington, DC. Pub. in Physical Review A 46, n9 p6079-6082, 1 Nov 92.

Keywords: "Electron-atom collisions, "Sodium, Scattering cross sections, Scattering amplitudes, Electron scattering. Elastic scattering, EV range 1-10, EV range 10-100, Reprints.

Measurements of spin-resolved elastic electron-sodium scattering have been carried out at incident energies of 4.1 and $12.1 \mathrm{eV}$, and the ratio of triplet to singlet scattering cross sections has been obtained at each energy. The ratio is used to provide a determination of not only the magnitudes of the triplet and singlet amplitudes, but also the cosine of the relative phase ditference between them. These determinations of magnitude and relative phase represent the most detailed characterization to date of electron-atom scattering from a 'one-electron' target

03,870

PB94-216660 Not available NTIS

National inst. of Standards and Technology (NML), Gaithersburg. MD. Electron and Optical Physics Div. 
Laser Focusing of Atoms: A Particle Optics Approach.

Final rept.

J. J. McClelland, and M. R. Scheinfein. 1990, $1 p$.

Pub. in Proceedings of International Conference on Atomic Physics (12th), Ann Arbor, MI., July 29-August 3, 1990, $1 \mathrm{p}$.

Keywords: "Atomic beams, "Focusing, Photon-atom interactions, Laser radiation, Reprints, Laser focusing, Atomic diffraction.

The use of a TEM(sub 01, sup ") ('donut') mode laser beam has been proposed as a means of focusing an atomic beam to extremely small spot sizes. In the initial analysis, Balykin and Letokhov showed that when a donut mode laser beam is focused to a small spot of order 1 micrometer, and an atom beam is passed concentrically through the focus, the dipole force on the atoms has the correct radial dependence to produce first order focusing. They then estimated the effects of atomic diffraction (due to the deBroglie wavelength of the atom), spherical and chromatic aberration, and diffusion arising from spontaneous emission. It was found that spot sizes of order a few Angstroms could be obtained with reasonable values for atomic beam collimation and monochromaticity, and laser power and detuning.

03,871

PB95-107025 Not available NTIS

National Inst. of Standards and Technology (PL), Boulder, CO. Quantum Physics Div.

Intermolecular HF Motion in Ar(sub n)HF Micromatrices $(n=1,2,3,4$, ): Classical and Quantum Calculations on a Pairwise Additive Potential Surface.

Final rept.

A. Mcllroy, and D. J. Nesbitt. 1992, 13p.

Grant NSF-CHE90-00641

Sponsored by National Science Foundation, Washington, DC.

Pub. in Jnl. of Chemical Physics 97, n9 p6044-6056, 1 Nov 92.

Keywords: "Hydrogen fluoride, "Argon, "High resolution, "Librational motion, Matrix isolation, Van der Waals Forces, Quantum mechanics, Three body problem, Isomers, Supersonic aircraft, Infrared radiation, Microwaves, Molecular interactions, Reprints, Argon clusters.

The availability of pairwise additive 'two-body' potentials for van der Waals systems from near-IR, far-IR and microwave data permits detailed prediction of librational behavior for isolated $\mathrm{HF}$ chromophores solvated by successive numbers of rare gas Ar atoms. The paper describes theoretical calculations of $\operatorname{Ar}(n) H F$ equilibrium structures and intermolecular HF vibrational frequencies based on an $\operatorname{Ar}(n) \mathrm{HF}$ 'twobody' potential energy surface developed from previously determined $\mathrm{Ar}$-Ar and Ar-HF potentials. Isomeric structures are predicted from local minima on these multidimensional surfaces, and are found to be in excellent qualitative agreement with near-IR observations of $\operatorname{Ar}(n) \mathrm{HF}$ clusters with $\mathrm{n}=1,2,2$ and $4 \mathrm{Ar}$ atoms. Quantum mechanical calculations are performed for the HF librational and van der Waals stretching modes against a rigid $\operatorname{Ar}(n)$ frame. In the limit of sufficient Ar atoms to fill the first coordination sphere around the HF, the calculations indicate a nearly perfect concellation of angular anisotropy for $\mathrm{HF}$ librational motion, consistent with the nearly free internal rotation of the HF observed in cryogenic Ar matrices.

03,872

PB95-107363 Not available NTIS

National Inst. of Standards and Technology (PL), Gaithersburg, MD. Ionizing Radiation Div.

Thin Dyed-Plastic Dosimeter for Large Radiation Doses.

Final rept.

F. Abdel Rehim, S. Ebraheem, W. Z. Ba, and W. L.

McLaughlin. 1992, 8p.

Pub. in Applied Radiation and Isotopes 43, n12 p150315101992.

Keywords: "Gamma dosimetry, "Film dosimetry, "Dosimeters, Polyvinyl chloride, Phthalocyanines, Polymeric films, Gamma radiation, Spectrophotometry, Performance, Stability, Dyes, Reprints, Radiation processing, Absorbed dose.

A commercially available, thin, dyed-plastic film (mean thickness 30 micrometers) having a transparent green color, changes color progressively to brown shades and then to a deep red shade upon irradiation at successively higher values of absorbed dose (gamma radiation) in the range 8-60 kGy. The analysis for dosimetry is made with spectrophotometric measurements (at visible light wavelengths) of pre-irradiation absorbance, $A(0)$, and radiation-induced absorbance, $A(1)$. Either the wavelength of the maximum of the $a b$ sorption band that bleaches upon irradiation (wavelength $=425 \mathrm{~nm}$ ) or the wavelengths of the maxima of the two overlapping radiation-induced absorption bands ( wavelength $=525$ or $556 \mathrm{~nm}$ ) are used to make these measurements. The most reproducible calibration consists of the ratio, $A(1) / A(0)$, at a given waveength, as a function of the absorbed dose. The new film dosimeter is flexible, rugged, and relatively stable at temperatures between $-80 \mathrm{C}$ and $+50 \mathrm{C}$, and in daylight. The radiation-induced color, in terms of the absorbance, $A(1)$, at selected wavelengths, is unstable for about two days subsequent to irradiation, after which the values of $A(1) / A(0)$ remain stable for at least several months, even under illumination with white fluorescent or indirect daylight.

\section{3,873}

PB95-108411 Not available NTIS

National Inst. of Standards and Technology (NML) Gaithersburg, MD. Center for Atomic, Molecular and Optical Physics.

Heterodyne Measurement of the Fluorescence Spectrum of Optical Molasses.

Final rept

W. D. Phillips, C. I. Westbrook, R. N. Watts, P. D Lett, P. L. Gould, S. L. Rolston, and C. E. Tanner. 1989, 4p.

Sponsored by Office of Naval Research, Arlington, VA Pub. in Proceedings of International Conference on Laser Spectroscopy (9th), Bretton Woods, NH., June 18-23, 1989, p8-11.

Keywords: "Resonance fluorescence, Experiment design, Line narrowing, Spectral analysis, Heterodyning, prints, "Optical molasses, Laser cooling, Laser trapping, Atom traps.

The authors describe a heterodyne experiment to measure the spectrum of fluorescence of the atoms confined in optical molasses.

\section{3,874}

PB95-108692 Not available NTIS

National Inst. of Standards and Technology (NML), Gaithersburg, MD. Ionizing Radiation Div.

Preparation and Characterization of (6) LiF and (10)B Reference Deposits for the Measurement of the Neutron Life time.

Final rept.

J. Pauwels, R. Eykens, A. Lamberty, R. Scott, J. Byrne, P. Dawber, D. Gilliam, J. Van Gestel, and H Tagziria. 1991, 8p.

Pub. in Nuclear instruments and Methods in Physics Research A 303, n1 p133-140 1991

Keywords: "Neutron lifetime, "Lithium fluorides, "Lithium 6, "Boron 10 Vacuum deposition, Preparation, Characteristics, Measurement, Deposits, Silicon, Wafers, Reprints.

In beam experiments, the achievement of an accurate neutron lifetime measurement depends heavily on the use of well characterized boron and/or lithium reference deposits to assess the neutron density. Sets of $(6) \mathrm{LiF}$ and (10)B reference deposits on silicon wafers were prepared by vacuum deposition using planetary rotating substrate holders. The uniformity of deposition was investigated using visible light spectrophotometry and accurate dimensional characterization was carried out on the basis of travelling $\mathrm{mi}-$ croscope and talistep measurements. The results obtained agreed well with theoretical calculations based on the geometry of the evaporation system. The deposits prepared in successive evaporation runs were then characterized by neutron induced charged particle measurements calibrated by isotope dilution mass spectrometry. (6) Li and (10)B surface densities are certified with accuracies of $0.35-0.50 \%$ and 0.30 $0.41 \%$, respectively. An intercomparison of the (6)Li and (10)B calibrations confirms the consistency of the measurements.

\section{3,875}

PB95-108874 Not available NTIS

National Inst. of Standards and Technology (NML) Gaithersburg, MD. Center for Atomic, Molecular and Optical Physics.

\section{Atoms in Optical Molasses.}

Final rept

W. D. Phillips, P. D. Lett, S. L. Rolston, C.

Westbrook, C. Salomon, J. Dalibard, A. Clairon, S. Guellati, C. E. Tanner, and R. Watts. 1991, 10p.

Pub. in Proceedings of the Conference on Light In duced Kinetic Effects on Atoms, Ions and Molecules, Elba, Italy, May 2-5, 1990, p25-34.

Keywords: Temperature range 0000-0013 K, Temperature measurement, Neutral atoms, Reprints, "Optical molasses, Polarization gradient cooling, Laser cooling, Atom cooling, Sodium atoms, Cesium atoms, Atomic fountains, Laser trapping.

Temperature measurements on atoms released from optical molasses are found to agree very well with the predictions of the theoretical models of polarization gradient cooling. This agreement extends over a wide range of laser intensity and detuning and even covers values of those parameters where it was originally believed the cooling would not be effective. The lowest temperatures achieved in a 3-D optical molasses are 25 microK and 2.5 microK for sodium and cesium respectively. In each case, this is equivalent to an rms velocity of about three times the recoil velocity.

\section{3,876}

PB95-108882 Not available NTIS

National Inst. of Standards and Technology (NML) Gaithersburg, MD. Center for Atomic, Molecular and Optical Physics

Atoms in Optical Molasses: Applications to Frequency Standards.

Final rept.

W. D. Phillips, P. D. Lett, S. L. Rolston, C. I.

Westbrook, C. Salomon, J. Dalibard, A. Clairon, S

Guellati, C. E. Tanner, and R. N. Watts. 1990, 4p.

Pub. in Proceedings of the European Frequency and Time Forum (4th), Neuchatel, Switzerland, March 1315, 1990, p273-276.

Keywords: "Frequency standards, "Time standards, Laser spectroscopy, Trapped particles, Quantum optics, Neutral atoms, Temperature range 0000-0013 K, Reprints, "Optical molasses, Laser trapping, Laser cooling, lon traps, Atomic fountains.

Recent progress in laser cooling and trapping of neutral atoms is discussed with a view toward future applications to time and frequency standards. Advantages and discadvantages of neutral atom standards with respect to ion standards are discussed. Prospects for a neutral atom 'atomicfountain' clock are examined and appear to be promising.

03,877

PB95-108890 Not available NTIS

National Inst. of Standards and Technology (NML), Gaithersburg, MD. Center for Atomic, Molecular and Optical Physics

Optical Molasses: Cold Atoms for Precision Measurements.

Final rept

W. D. Phillips, P. D. Lett, S. L. Rolston, C. I. Westbrook, C. Salomon, J. Dalibard, A. Clairon, S Sponsored by Office of Naval Research, Arlington, VA. Pub, in Institute of Electrical and Electronics Engineers Transactions on Instrumentation and Measurement 40 , n2 p78-80 Apr 91

Keywords: Atomic spectroscopy, Frequency standards, Neutral atoms, Temperature range 0000-0013 K, Reprints, "Optical molasses, Laser cooling, Ultracold atoms, Cesium atoms, Precision measurements

Reduction of atomic kinetic motion by laser cooling has important applications to atomic frequency standards and other precision measurements. The optical molasses technique, used for cooling neutral atoms, has produced temperatures below 3 microK for cesium atoms, corresponding to an rms velocity of about $13 \mathrm{~mm} / \mathrm{s}$. This is considerably lower than the lower limit originally thought to apply to laser cooling and is apparently the lowest kinetic temperature ever measured for three-dimensional cooling. New laser cooling mechanisms have been identified to explain the low temperature.

National Inst. of Standards and Technology (NML), Gaithersburg, MD. Electron and Optical Physics Div. 
Optical Molasses: The Coldest Atoms Ever. Final rept.

W. Phillips, P. Lett, S. Rolston, C. Westbrook, C Salomon, J. Dalibard, A. Clairon, S. Guellati, C. Tanner, and R. Watts. 1991, 3p.

Pub. in Physica Scripta T34, p20-22 1991

Keywords: Temperature range 0000-0013 K, Atomic clocks, Doppler effect, Neutral atoms, Reprints *Optical molasses, Laser cooling, Atom cooling, Sodium atoms, Cesium atoms, Ultracold atoms, Atomic fountains.

Optical molasses is a three-dimensional (3-D) configuration of laser beams used to laser-cool and to confine neutral atoms. Atoms laser cooled in optical molasses reach temperatures much lower than the limit given by the original theories of laser cooling based on the Doppler effect. This cooling below the Doppler-Cooling limit is now seen as being due to new laser cooling mechanisms not present in the original theories. The dependence of the a tomic temperature on parameters such as laser intensity and detuning shows good agreement between calculations performed in 1-D and experiments performed in 3-D. For cooling of $\mathrm{Na}$ and Cs atoms, the lowest observed temperatures correspond to rms velocities between three and four times the single photon recoil velocity. For Cs the tempera ture is $2.5+$ or -0.6 microK and is the lowest temperature ever measured for $3-\mathrm{D}$ cooling.

\section{3,879}

\section{PB95-125639 Not available NTIS}

National Inst. of Standards and Technology (NML), Gaithersburg, MD. Chemical Process Metrology Div. Development of a Temperature Scale below $0.5 \mathrm{~K}$. Final rept.

W. E. Fogle, J. H. Colwell, and R. J. Soulen. 1990,

2p.

Keywords: *Temperature scales, "Cryogenic temperature, Temperature range 0000-0013 K, Temperature measurement, Noise thermometers, Melting points Reprints, Fixed points.

We summarize our most recent results on the develop ment of an absolute temperature scale below $0.5 \mathrm{~K}$ It is the most recent of several experiments at our laboratory and represents both our best effort and the one in which all available thermometers were simultaneously and fully operational. Perhaps the most inter esting conclusion reached by us so far is that the temperature scale below 15 mK may be quite different than has been developed by other laboratories, particularly those engaged in low-temperature physics projects such as (3)He research.

\section{3,880}

PB95-125886 Not available NTIS

National Inst. of Standards and Technology (PL), Boulder, CO. Quantum Physics Div.

Associative lonization in Collisions of Slowed and Trapped Sodium.

Final rept

A. Gallagher. 1991, $11 p$

Pub. in Physical Review A 44, n7 p4249-4259, 1 Oct 91

Keywords: "Atom-atom collisions, "Atomic collisions, Energy transfer, Ionization, Photoabsorption, Reprints, *Sodium atoms, Atom traps

Collisions of trapped atoms occur over time intervals that are often longer than radiative lifetimes. This leads to radiative interruption of excited-state collision dy namics and constrains collisional interactions to atom pairs that are in close proximity at the time of excitation. Using classical dynamics, I show that this causes $\mathrm{Na}$ associative ionization to be dominated by a four-step process in which the longer-range $\mathrm{Na}$ (star)$\mathrm{Na}$ interaction initiates inward motion that is completed as $\mathrm{Na}$ (star) + $\mathrm{Na}$ (star) $->\mathrm{Na} 2(1+)+\mathrm{e}$. Thus low-temperature associative ionization does not result from $\mathrm{Na}($ star) + Na(star) collisions. I predict strong single and two-wavelength dependencies, which have a mo lecular character due to the close proximity at the time of photoabsorption. These are amenable to experimental observation. I also calculate saturation that results from the depletion of interacting pairs. This produces very-low-power saturation and explains the as sociative ionization measurements of Gould et al. (Phys. Rev. Lett. 60, 788 (1988)).

National Inst. of Standards and Technology $(P L)$, Gaithersburg, MD. Atomic Physics Div. Temperature of Optical Molasses for Two Different Atomic Angular Momenta.

Final rept.

C. Gerz, T. Hodapp, P. Jessen, C. Westbrook, K Molmer, K. Jones, and W. Phillips. 1993, 6p. Sponsored by Office of Naval Research, Washington, DC.

Pub. in Europhysics Letters 21, n6 p661-666 1993

Keywords: Fokker-Planck equation, Isotope effects, Temperature dependence, Quantum optics, Neutral atoms Rubidium 85, Rubidium 87, Reprints, "Rubidium atoms, Laser cooling, Optical molasses.

We have measured the temperature of laser-cooled $\mathrm{Rb}$ atoms in optical molasses as a function of laser intensity and detuning. For both (85) Rb and $(87) \mathrm{Rb}$, cooled on the $F=3->F^{\prime}=4$ and $F=2->F^{\prime}=3$ transitions, respectively, the temperatures are proportional to the ratio of laser power and detuning for a wide range of these parameters. We observe a small but significant difference between the two isotopes. We also show the results of three-dimensional semi-classical numerical calculations. Our results favor a model which includes atomic localization in optical standing waves.

\section{3,882}

PB95-126298 Not available NTIS

National Inst. of Standards and Technology (NML), Gaithersburg, MD. Quantum Metrology Div. Some Aspects of Fundamental Neutron Physics. Final rept.

G. L. Greene. 1990, 9p.

Pub. in Proceedings of Rencontre Moriond New Exotic Phenom., v25 p163-171 1990.

Keywords: "Neutron physics, Sommerfeld constant, Neutron lifetime Cold neutrons, Fundamental constants, Reprints, Gravitational phase shift, Fine structure constants, Neutron interferometry.

Low energy neutrons provide a tool of great versatility in the investigation of fundamental interactions. Recent experimental work with 'cold' neutrons have addressed such issues as the study of weak interactions, nuclear physics and astrophysics. They have also been employed in the study of invariance principles and for the determination of fundamental constants. This contribution focuses on three particular areas of investigation which reflect this broad nature of neutron physics. The examples discussed include gravitational phase shift experiments with neutron interferometers, the measurement of the neutron lifetime, and the determination of the fine structure constant by a new method

03,883

PB95-126405 Not available NTIS

National Inst. of Standards and Technology (PL), Boulder, CO. Quantum Physics Div.

Evidence for Significant Backscattering in NearThreshold Electron-Impact Excitation of $\operatorname{Ar}(7+)(3 s$ yields $3 p$ ).

Final rept.

X. Q. Guo, E. W. Bell, J. S. Thompson, R. A Phaneuf, A. C. H. Smith, G. H. Dunn, and M. E.

Bannister 1993, 4p.

Contract DE-Al05-86ER53237

Sponsored by Department of Energy, Washington, DC. Office of Energy Research.

Pub. in Physical Review A 47, n1 pR9-R12 Jan 93.

Keywords: *Electron-ion collisions, "Electron scattering, *Backscattering, *Argon ions, Total cross sections, Multicharged ions, Electron impact, Excited states, Inelastic scattering, Excitation, R matrix, Reprints, Sodium-like ions.

Measurements of absolute total cross sections for electron-impact excitation of $\operatorname{Ar}(7+)(3 s-->3 p)$ using a merged-beams electron-energy-loss technique show that near threshold, the inelastically scattered electrons are ejected primarily in the backward direction. This unusual angular scattering has not been previously observed for atoms or ions, but may be typical for multiply charged ions. The total cross sections, measured over an energy range to $2.2 \mathrm{eV}$ above threshold, agree with seven-state R-matrix close-coupling calculations. Both close-coupling and distortedwave calculations also confirm the backscattering observed in these measurements.

\section{3,884}

PB95-135570 PC A03/MF A01

National Inst. of Standards and Technology (CSTL), Boulder, CO. Process Measurements Div.
Thermal Hydraulic Tests of a Liquid Hydrogen Cold Neutron Source.

J. D. Siegwarth, D. A. Olson, M. A. Lewis, P. Kopetka, J. M. Rowe, and R. E. Williams. Jul 94 , 28p, NISTIR-5026.

Keywords: "NBSR reactor, "Neutron sources, "Cold neutrons, "Moderators, Liquid hydrogen, Hydraulic tests, Thermal testing, Scale models, Thermosyphons.

We have built and tested a simplified full scale model of the planned liquid hydrogen moderator for the cold neutron source of the NIST reactor. The heat load to the liquid hydrogen will be removed to an external refrigerator by means of a thermosyphon. Our experiments showed that this system could be filled, would operate stably, and the gas volume in the moderator chamber would not exceed 20 percent. Results of the tests suggested some improvements to the original designs. The circulation of liquid hydrogen proved to be self-regulating even when the available liquid inflow rate was sufficient to flood the vapor line.

03,885

PB95-140059 Not available NTIS

National Inst. of Standards and Technology (PL), Gaithersburg, MD. Radiometric Physics Div.

Precision High Temperature Blackbodies. Final rept. V. I. Sapritsky, B. C. Johnson, R. D. Saunders, B. B. Klevnoy, V. I. Shapoval, I. A. Dmitriev, L. M.

Buchnev, A. V. Prochorov, L. V. Vlasov, and K. A.

Sudarev. 1993, 9p.

Pub. in Proceedings of Society of Photo-Optical Instrumentation Engineers: Ultraviolet Technology IV, San Diego, CA., July 20-21, 1992, v1764 p323-331 1993 Keywords: "Blackbody radiation, *Radiation sources, Temperature range 1000-4000 K, High temperature, Ultraviolet radiation, Precision, Design, Reprints.

This paper reviews the research and design of high temperature blackbody sources for the temperature interval from $2000 \mathrm{~K}$ to $3000 \mathrm{~K}$. Sources with large apertures are addressed specifically, as these are well suited to the important problem of spectral irradiance scale realizations.

\section{3,886}

PB95-140422 Not available NTIS

National Bureau of Standards (NML), Gaithersburg, MD. Ionizing Radiation Div.

Measurement of the $(235) \mathrm{U}(\mathbf{n}, \mathbf{f})$ Reaction from Thermal to $1 \mathrm{keV}$.

Final rept.

R. A. Schrack. 1988, 4p.

Pub in Proceedings of International Conference on Nuclear Data for Science and Technology, Mito, Japan, May 30, 1988, 4p.

Keywords: *Fission cross sections, "Neutron reactions *Uranium 235, Milli eV range, EV range 01-10, EV range 10-100, EV range 100-1000, Accelerator facilities, Nuclear fission, Measurement, Boron 10, Reprints.

The neutron induced fission cross section for (235) U has been measured from 02 to 1000 electron volts at the NBS electron LINAC facility. This cross section, especially the integrals from 7.8 to $11 \mathrm{eV}$ and from 100 to $1000 \mathrm{eV}$ is widely used as a standard to normalize shape experiments. A special ion chamber incorporating both the (235) $U$ foils and the (10)B foils used to monitor the neutron beam was built. The geometry of the chamber and the data taking system was designed to give the effect of a common environment and flight path so as to reduce or eliminate background and energy assignment problems. The shape measurements were normalized at thermal. Results are compared to other recent measurements, and ENDF/B-V.

\section{3,887}

PB95-140869 Not available NTIS

National Inst. of Standards and Technology (PL), Gaithersburg, MD. Atomic Physics Div.

Wavelengths and Isotope Shifts for Lines of Astrophysical Interest in the Spectrum of Doubly lonized Mercury (Hg III).

Final rept.

C. J. Sansonetti, and J. Reader. 1993, 5p.

Sponsored by National Aeronautics and Space Administration, Washington, DC.

Pub. in Physical Review A 47, n4 p3080-3084 Apr 93.

Keywords: *Mercury ions, *Ultraviolet spectra, *Isotope effect, `Spectral shift, "Peculiar stars, Stellar spectra, 
Mercury isotopes, Line spectra, Wavelengths, Radio frequency discharge, Multicharged ions, Reprints, Isotope abundance.

Wavelengths and isotope shifts have been measured for the 1738.4- 1738.5-, 1740.2-, and 2354.2-A lines of $\mathrm{Hg}$ III with an uncertainty of $0.002 \mathrm{~A}$. The lines were excited in a pulsed radio-frequency discharged and observed with a $10.7-\mathrm{m}$ normal-incidence vacuum spectrograph. Observations were made with lamps containing natural $\mathrm{Hg},(198) \mathrm{Hg}$, and (204) Hg. By using wellestablished relative isotope shifts, wavelengths of these four lines for $(196) \mathrm{Hg},(200) \mathrm{Hg},(202) \mathrm{Hg}$, and the centers of gravity for (199) $\mathrm{Hg}$ and (201) $\mathrm{Hg}$ were also determined. The results provide important data for determining elemental and isotopic abundances of $\mathrm{Hg}$ from spectra of $\mathrm{Hg}$-rich chemically peculiar stars

03,888

PB95-140877 Not available NTIS

National Inst. of Standards and Technology (PL), Gaithersburg, MD. Atomic Physics Div.

Spectrum and Energy Levels of Triply Ionized Barium (Ba IV).

Final rept.

C. J. Sansonetti, J. Reader, A. Tauheed, and Y. N

Joshi. 1993, 6p.

Pub. in Jnl. of the Optical Society of America B 10, n1 p7-12 Jan 93

Keywords: "Barium ions, "Atomic energy levels, *UItraviolet spectra, Vacuum ultraviolet radiation, Ionization potentials, Electron transitions, Line spectra, Multicharged ions, Reprints.

The spectrum of $\mathrm{Ba}$ IV was observed with sliding sparks and low-inductance vacuum sparks on 10.7 . and $3-m$ normal-incidence vacuum spectrographs. Thirty-nine lines between 463 and $923 \mathrm{~A}$ are classified as transitions between levels of the $5 s(2) 5 p(5)$ doublet $P$ ground term and levels of the $5 s 5 p(6), 5 s(2) 5 p(4) 5 d$, and $5 s(2) 5 p(4) 6 s$ configurations. These configurations are theoretically interpreted, and the energy parameters determined from least-squares fits to the observed levels are compared with Hartree-Fock calculations. The ionization energy is revised to $379,300+$ or $-2700 / \mathrm{cm}(47.03+$ or $-0.33 \mathrm{eV})$

03,889

PB95-141206 Not available NTIS

National Inst. of Standards and Technology (CSTL), Gaithersburg, MD. Inorganic Analytical Research Div. Neutron Focusing Lens Using Polycapillary Fibers. Final rept

H. Chen, D. F. R. Mildner, and Q. F. Xiao. 1994, 3p. Contracts DE-FG02-91ER81220, DE-FG02

91ER81411

Sponsored by Department of Energy, Washington, DC. Pub. in Applied Physics Letters 64, n16 p2068-2070, 18 Apr 94.

Keywords: "Neutron beams, "Cold neutrons, "Focusing, Borosilicate glass, Grazing incidence, Thermal neutrons, Neutron transport. Mirrors, Lenses, Reprints, *Neutron focusing, Multiple reflection.

Multiple mirror reflection at small grazing angles from the smooth surfaces of the narrow channels of polycapillary fibers can be used to transport, bend, and focus thermal neutron beams. We report the results of the focusing of a polychromatic cold neutron beam using a compact lens of borosilicate fibers and with a focal distance of $57 \mathrm{~mm}$. The intensity profile of the beam at the focus is approximately conical in shape with a full width at half-maximum of $0.49 \mathrm{~mm}$, and with an average gain in intensity of about 20 . These experimental results agree well with those obtained by computer simulation.

\section{3,890}

PB95-143194 PC A09/MF A02

Maryland Univ., College Park. Dept. of Mechanical Engineering.

Transient Cooling of a Hot Surface by Droplets Evaporation.

Final rept.

G. White, S. Tinker, and M. di Marzo. Dec 93, 178p, NIST/GCR-94/662

Grant NIST-70NANB1H1173

See also PB90-227968. Sponsored by National Inst. of Standards and Technology (BFRL), Gaithersburg, $M D$.

Keywords: "Droplets, "Surface temperature, "Evaporative cooling, "Hot surfaces, Heat transfer, computer programs, Heat flux, Green's function,
Water, Vaporizing, Thermal conductivity, Numerical in tegration.

A computer code is developed and tested which simu lates the transient evaporation of a single liquid drople from the surface of a semi-infinite solid subject to radiant heat input from above. For relatively low temperature incident radiation, it is shown that the direct $a b$ sorption of radiant energy by the droplet can be treated as purely boundary conditions, while a model for highe as purature incident radiation would require the addition of constant heat source terms. The heat equation is numerically coupled between the liquid and solid domains by using a predictor-corrector scheme. Three one-dimensional solution schemes are used within the droplet: a start-up semi-infinite medium solution, a ridiagonal Crank-Nicholson transient solution, and steady-state solution. The solid surface temperatures at each time step are calculated through careful nu merical integration of an axisymmetric Green's func tions solution with the forcing function given by the past lower droplet surface and solid-vapor boundary hea fluxes. The time step is increased after a sensitive initial period to allow for reasonable run times. Two ge ometry models are included which give the drople height as a function of current droplet volume and initia wetted radius; the second allows inclusion of the ef fects of initial contact angle and receding angle. Using water as the liquid and Macor, a low-thermal conductivty material, as the solid, the program output was com pared to the experimental results in this line of research. They correlate well to the experiments in which the critical geometric shape factor and evaporation time were most easily measured.

\section{3,891}

PB95-150298 Not available NTIS

National Inst. of Standards and Technology (PL) Gaithersburg, MD. Quantum Metrology Div.

Resonance and Threshold Effects in Polarized $X$ Ray Emission from Atoms and Molecules.

Final rept.

S. H. Southworth. 1994, 6p.

Sponsored by Department of Energy, Washington, DC Pub. in Nuclear Instruments and Methods in Physics Research B 87, p247-252 1994.

Keywords: “X-ray spectra, *Polarized electromagnetic radiation, Inner-shell excitation, Molecular spectra, Atomic spectra, X-ray absorption, Molecular orbitals, Angular distribution. Threshold effects, $\mathrm{K}$ shell, Anisotropy, Resonance, Gases, Reprints, "X-ray emission.

Strongly anisotropic and polarized $\mathrm{K}-\mathrm{V}$ (valence to $\mathrm{K}$ vacancy) $X$-ray emission has been observed from gas phase molecules following excitation of a K-shell electron to valence-like subthreshold resonances using a narrow bandwidth, linearly polarized $X$-ray beam. Distinctively different polarizations and angular distributions are observed for emission involving molecular orbitals of different symmetries. A classical model of the X-ray absorption/emission process accurately describes the observed radiation patterns. Relatively weak polarizations and anisotropies are observed following excitation of atomic subthreshold resonances, due to averaging over unresolved final states. However, resonance and threshold studies of atomic X-ray spectra illustrate the inelastic resonance scattering process and the onset of characteristic fluorescence.

\section{3,892}

NB95-150470 Not available NTIS

National Inst. of Standards and Technology (MSEL), Gaithersburg, MD. Reactor Radiation Div.

Grazing Incidence Prompt Gamma Emissions and Resonance-Enhanced Neutron Standing Waves in a Thin-Film

Final rept.

H. Zhang, P. D. Gallagher, S. K. Satija, T. P. Russell, P. Lambooy, E. J. Kramer, F. S. Bates, R. M.

Lindstrom, and R. L. Paul. 1994, 4p.

Pub. in Physical Review Letters 72, n19 p3044-3047. 9 May 94.

Keywords: "Neutron capture gamma rays, "Prompt gamma radiation, "Polymeric films, Grazing incidence, Thin films, Measurement, Gadolinium, Resonance, Reprints, "Neutron standing waves, Neutron depth profiling, Neutron reflectivity.

We report simultaneous measurements of neutroncapture prompt gamma emissions as well as neutron reflectivity on a polymer film with an embedded $\mathrm{Gd}$ layer. Enhanced gamma-ray signals and reduced neuron reflectivity were observed when the neutron stand ing waves were resonantly amplified in the film. Fitting of both the gamma-ray and reflectivity data is of significant aid in uniquely characterizing the depth profile of the polymer film as well as locating the Gd layer.

03,893

PB95-150546 Not available NTIS

National Inst. of Standards and Technology (PL), Boulder, CO. Quantum Physics Div

Excitation of Balmer Lines in Low-Current Discharges of Hydrogen and Deuterium.

Final rept.

Z. Stokic, M. M. F. R. Fraga, J. Bozin, B. M.

Jelenkovic, V. Stojanovic, and Z. L. Petrovic. 1992

Pub. in Physical Review A 45, n10 p7463-7468, 15 May 92

Keywords: "Gas discharges, "Hydrogen plasma, Deuterium plasma *Balmer lines, Electron-ion collisions, Electron impact, Gas ionization, Ionization coef ficients, $\mathrm{H}$ alpha line, Excitation, Reprints

Measurements have been made of electron-impact onization and excitation of Balmer lines in low-current steady-state discharges of hydrogen and deuterium Results were obtained from spatial scans of $\mathrm{H}$ (alpha) lines for $\mathrm{E} / \mathrm{N}$ ranging from $250 \mathrm{Td}$ to $10 \mathrm{kTd}$. Here $\mathrm{E}$ is the electric field $\mathrm{N}$ is the gas density and 1 $T d=10(\exp -21) V$ meters squared. Ionization and excitation coefficients versus $\mathrm{E} / \mathrm{N}$ are presented for $\mathrm{E} / \mathrm{N}$ be tween 250 and 1,800 $\mathrm{Td}$, and Nd (where d denotes the gap length) between $2.3 \times 10(\exp 22)$ and $1.7 \times$ 10(exp 21) per square meter. Excitation coefficients obtained for $\mathrm{H}$ (alpha) and D(alpha) are placed on an absolute scale using a standard tungsten lamp calibrated against the blackbody radiation standard. The ionization coefficients are compared with previous ex perimental and theoretical data, while the excitation coefficients are compared with the calculated values.

\section{4}

PB95-150553 Not available NTIS

National Inst. of Standards and Technology (PL), Boul der, CO. Quantum Physics Div.

Fast Computer Evaluation of Radiative Properties of Hydrogenic Systems.

Final rept.

P. J. Storey, and D. G. Hummer. 1991, 13p.

Contract NASA-NAGW-766, Grant NSF-AST88

02937

Sponsored by National Aeronautics and Space Administration, Washington, DC. and National Science Foundation, Washington, DC

Pub. in Computer Physics Communications 66, p129 1411991

Keywords: Hypergeometric functions, Einstein coeffi cients, Oscillator strengths, Computer calculations, Cross sections, Photoionization, Reprints, *Hydrogenlike ions, Gaunt factor.

Three subroutines are described for the very fast caculation of bound-bound, bound-free, and free-free cross-sections for nonrelativistic hydrogenic systems of arbitrary nuclear charge and reduced mass. The first wo are essentially exact, being based on recursion relations which are known to be stable. The third evalu ates the thermally-averaged free-free Gaunt factor by means of a two-dimensional Chebyshev expansion calculated by numerical evaluation of the cross-sections expressed as hypergeometric functions, augmented by other analytical approximations.

03,895

PB95-150629 Not available NTIS

National Inst. of Standards and Technology (PL) Gaithersburg MD Atomic Physics Div.

Improved Wavelengths for Prominent Lines of $\mathrm{C}$ XVI to $\mathrm{Cr}$ XXII.

Final rept

J. Sugar, V. Kaufman, and W. L. Rowan. 1993, 3p. Sponsored by Department of Energy, Washington, DC. Pub. in Jnl. of the Optical Society of America B 10, n1 p13-15 Jan 93.

Keywords: "Chromium ions, "Ultraviolet spectra, * $X$ ray spectra, Extreme ultraviolet radiation, Soft $x$-rays Multicharged ions, Spectral lines, Wavelengths, Atom ic energy levels, TEXT devices, Reprints

New measurements of 34 spectral lines of highly ionized $\mathrm{Cr}$ ions in the range of 100-280 $\mathrm{A}$ have been made with a wavelength uncertainty of + or $-5 \mathrm{~mA}$. The light source was the TEXT tokamak at the University of Texas at Austin. Lines of Li-like to F-like $\mathrm{Cr}$ are included with visually estimated relative intensities, the 


\section{General}

best previous measurements, and their energy-level classifications. The uncertainties of the best earlier measurements were reported as + or - 20-30 mA, but much larger errors were found in some cases.

\section{3,896}

PB95-150637 Not available NTIS

National Inst. of Standards and Technology (PL), Gaithersburg, MD. Atomic Physics Div. Observation of Pd-Like Resonance Lines Through $\mathrm{Pt}(32+)$ and $\mathrm{Zn}$-Like Resonance Lines of $\mathrm{Er}(38+)$ and $\mathrm{Hf}(42+)$.

Final rept.

J. Sugar, V. Kaufman, and W. L. Rowan. 1993, 3p. Sponsored by Department of Energy, Washington, DC Pub. in Jnl. of the Optical Society of America B 10, n5 p799-801 May 93

Keywords: "Erbium ions, "Ytterbium ions, "Hafnium ions, *Tungsten ions, "Platinum ions, *Resonance lines, Multicharged ions, Isoelectronic sequence, Plasma spectra, TEXT devices, Reprints, "Palladium-like ions, Zinc-like ions.

Spectra of highly ionized $\mathrm{Er}, \mathrm{Yb}, \mathrm{Hf}, \mathrm{W}$, and $\mathrm{Pt}$ were observed by injecting these elements into the plasma of the TEXT tokamak. Resonance lines of the $4 \mathrm{~d}(10)$ $4 d(9) 4 f$ transition array in the $\mathrm{Pd} \mathrm{I}$ isoelectronic sequence were identified by comparison with plots of observed-minus-calculated transition energies. These plots were based on data previously known through $\mathrm{Ho}(21+)$. They were fitted to low-order polynomials that permitted accurate predictions of wavelengths to $\mathrm{Bi}(37+)$. In addition, resonance lines of $\mathrm{Zn}$-like $\mathrm{Er}(38+)$ and $\mathrm{Hf}(42+)$ were observed

\section{3,897}

PB95-150645 Not available NTIS

National Inst. of Standards and Technology (PL) Gaithersburg, MD. Atomic Physics Div.

Rb-Like Spectra: Pd X to Nd XXIV.

Final rept.

J. Sugar, V. Kaufman, and W. L. Rowan. 1992, 3p Sponsored by Department of Energy, Washington, DC. Pub. in Jnl. of the Optical Society of America B 9, n11 p1959-1961 Nov 92

Keywords: "Ultraviolet spectra, "Palladium ions, *Neodymium ions, Extreme ultraviolet radiation, Isoelectronic sequence, TEXT devices, Plasma spectra, Multicharged ions, Wavelengths, Reprints, "Rubidium-like ions

Spectra of selected elements from Pd to Nd were observed with the TEXT tokamak at the University of Texas in Austin. Lines of the Rb $\mathrm{l}$ isoelectronic sequence were identified and wavelengths compared with calculated values obtained with the Cowan relativistic Hartree-Fock code. We made plots of observed minus calculated (O - C) transition energies in order to obtain smoothed wavelengths and interpolate values for elements not observed.

\section{3,898}

PB95-150652 Not available NTIS

National Inst. of Standards and Technology (PL), Gaithersburg, MD. Atomic Physics Div

Rh I Isoelectronic Sequence Observed from $\operatorname{Er}(23+)$ to $\operatorname{Pt}(33+)$.

Final rept.

J. Sugar, V. Kaufman, and W. L. Rowan. 1993, 3p.

Sponsored by Department of Energy, Washington, DC. Pub. in Jnl. of the Optical Society of America B 10, n11 p1977-1979 Nov 93.

Keywords: "Erbium ions, *Ytterbium ions, "Hafnium ions, "Tungsten ions, "Platinum ions, " $X$ ray spectra, Soft $x$-rays, Multicharged ions, Isoelectronic sequence TEXT devices, Plasma spectra, Metal films, Laser ablation, Reprints, "Rhodium-like ions.

The TEXT tokamak was used to obtain spectra of highly ionized $\mathrm{Er}, \mathrm{Yb}, \mathrm{Hf}, \mathrm{W}$, and $\mathrm{Pt}$. Injection of these elements into the plasma was achieved by laser ablation of thin films of metal deposited on glass slides and mounted at the inner wall of the vessel. The spectra were photographed with a $2.2-\mathrm{m}$ grazing-incidence spectrograph set at a grazing angle of $4 \mathrm{deg}$. These spectra occur in a very small wavelength interval, for example about $2 \mathrm{~A}$ at $50 \mathrm{~A}$ in $\mathrm{W}$. In this spectral range we previously classified lines of the $\mathrm{Ag}$ and $\mathrm{Pd}$ isoelectronic sequences. We have now classified lines of the Rh isoelectronic sequence $4 d(9)-4 d(8) 4 f$, which accounts for the remaining strong lines in this dense but well-resolved group. No lines of this array have previously been identified. They were classified by com- parison of observed and calculated transition energies along the isoelectronic sequence.

\section{3,899}

PB95-150660 Not available NTIS

National Inst. of Standards and Technology (PL),

Gaithersburg, MD. Atomic Physics Div.

Spectra of Ag I Isoelectronic Sequence Observed from $\operatorname{Er}(21+)$ to $\mathrm{Au}(32+)$.

Final rept.

J. Sugar, V. Kaufman, and W. L. Rowan. 1993, 5p.

Pub. in Jnl. of the Optical Society of America B 10, n8 p1321-1325 Aug 93

Keywords: “Erbium ions, “Gold ions, "Hafnium ions, *Platinum ions, "Tungsten ions, "Ytterbium ions, " $X$-ray spectra, "Ultraviolet spectra, Extreme ultraviolet radiation, Soft $x$ rays, Multicharged ions, Isoelectronic sequence, TEXT devices, Plasma spectra, Metal films, Laser ablation, Reprints, "Silver-like ions.

Spectra of highly ionized $\mathrm{Er}, \mathrm{Yb}, \mathrm{Hf}, \mathrm{W}, \mathrm{Pt}$, and $\mathrm{Au}$ were observed with the TEXT tokamak at the University of Texas at Austin. These metals were injected into the tokamak plasma by laser ablation of thin-film targets. Spectra were photographed with a 2.2-m grazingincidence spectrograph in the range 40-300 A. Three strong doublets of the $\mathrm{Ag} \mathrm{I}$ isoelectronic sequence were identified: $4 \mathrm{~d}(10) 4 \mathrm{f}-4 \mathrm{~d}(9) 4 \mathrm{f}$ (singlet P) $4 \mathrm{f}$ doublet $D$, doublet $F$ and doublet $G$. Interpolated and extrapolated wavelengths in this sequence were derived from plots of the difference between observed and calculated values for the transition energies. The correspondence of the $W$ wavelengths with previously reported tokamak spectra is shown.

\section{3,900}

\section{PB95-150769 Not available NTIS}

National Inst. of Standards and Technology (PL) Gaithersburg, MD. Atomic Physics Div. Analysis of the $5 s(2) 5 p(2)-$
$(5 s 5 p(3)+5 s(2) 5 p 5 d+5 s(2) 5 p 6 s)$ Transitions of Four-Times lonized Xenon (Xe V).

Final rept.

A. Tauheed, Y. N. Joshi, V. Kaufman, J. Sugar, and E. H. Pinnington. 1993, 5p

Pub. in Jnl. of the Optical Society of America B 10, n4 p561-565 Apr 93.

Keywords: *Ultraviolet spectra, "Xenon ions, Far ultraviolet radiation, Electron transitions, Multicharged ions, Line spectra, Reprints.

The spectrum of xenon was photographed in the range 350-2000 A with 3-m normal-incidence and 10.7-m normal-incidence spectrographs. The source used in both cases was a gas-triggered spark. The Xe V spectrum was distinguished by observation of spectral changes with variation of spark current. The $5 s(2) 5 p(2)$ $(5 s 5 P(3)+5 s(2) 5 p 5 d+5 s(2) 5 p 6 s)$ transition array of $\mathrm{Xe} V$ was identified and analyzed. Calculations were carried out to assist in the analysis and to help us to interpret the results. An earlier analysis was revised and extended, and 73 lines are now classified. A value for the ionization energy of $436,700+$ or $-400 / \mathrm{cm}$ was determined.

\subsection{1}

PB95-150868 Not available NTIS

National Inst. of Standards and Technology (PL), Boulder, CO. Quantum Physics Div.

Relativistic Effects in Spin-Polarization Parameters for Low-Energy Electron-Cs Scattering.

Final rept.

U. Thumm, K. Bartschat, and D. W. Norcross. 1993, $12 p$.

Contract DE-Al05-86ER53237

Sponsored by Department of Energy, Washington, DC Office of Energy Research.

Pub. in Jnl. of Physics B: Atomic and Molecular Optical Physics 26, p1587-1598 1993.

Keywords: "Electron-atom collisions, "Electron scattering, "Cesium, Relativistic effects, Inelastic scattering Elastic scattering, EV range 1-10, Polarized targets, $R$ matrix, Excitation, Reprints, “Electron spin polarization.

Based on a recent Dirac R-matrix (close-coupling) calculation, we present results for various spin-polarization parameters for elastic and inelastic scattering of slow (E(kin) approx $=$ or $<2 \mathrm{eV}$ ) polarized electrons from unpolarized and polarized neutral Cs atoms in their ground state. Our results allow for a quantitative estimate of the importance of relativistic effects, and our calculated parameters clearly deviated from predictions obtained by just jj-recoupling the results of a nonrelativistic calculation.
03,902

PB95-151122 Not available NTIS

National Inst. of Standards and Technology (EEEL), Gaithersburg, MD. Electricity Div.

Kinetic-Energy Distributions of $K(+)$ in Argon and Neon in Uniform Electric Fields.

Final rept.

R. J. Van Brunt, and J. K. Olthoff. 1993, 3p.

Pub. in Proceedings of International Seminar on Electron and Ion Swarms (8th), Trondheim, Norway, July 15-17, 1993, p76-78.

Keywords: *Potassium ions, Mass spectroscopy, Kinetic energy, Electric fields, Drift tubes, Argon, Neon, Reprints, lon energy analysis.

Kinetic-energy distributions have been measured for the $\mathrm{K}(1+)$ ions traveling in a drift tube in argon or neon buffer gases. Measurements were made at different electric field-to-gas density ratios (E/N). The distributions were observed to deviate from a Maxwellian dependence at high $\mathrm{E} / \mathrm{N}$ for neon buffer gas.

03,903

PB95-151221 Not available NTIS

National Inst. of Standards and Technology (PL), Gaithersburg, MD. Atomic Physics Div.

Hyperfine Effects and Associative Ionization of Uitracold Sodium.

Final rept

M. Wagshul, K. Helmerson, P. Lett, R. Heather, P.

Jullienne, S. Rolston, and W. Phillips. 1993, 4p.

Sponsored by Office of Naval Research, Arlington, VA Pub. in Physical Review Letters 70, n14 p2074-2077. 5 Apr 93.

Keywords: "Atomic collisions, "Sodium, Hyperfine structure, Laser spectroscopy, Reprints, Associative ionization, Ultracold atoms, Laser cooling, Millikelvin temperature.

We observe a new resonance structure in the associative ionization spectrum of laser-cooled sodium due to the collision of two atoms in different ground hyperfine states. This associative ionization is due to doubly resonant excitation at moderate internuclear separation through intermediate molecular states. Substructure within this resonance is evidence for the role of molecular bound states in this process. We present calculations modeling the effect of ground-state hyperfine structure, the importance of which was unanticipated in earlier theoretical studies.

03,904

PB95-151239 Not available NTIS

National Inst. of Standards and Technology (PL), Boulder, CO. Quantum Physics Div.

Electron-Impact Excitation of $\mathrm{Si}(3+)(3 \mathrm{~S}$ yields 3P) Using a Merged-Beam Electron-Energy-Loss Technique.

Final rept.

E. K. Wahlin, J. S. Thompson, G. H. Dunn, A. C. H.

Smith, R. A. Phaneuf, and D. C. Gregory. 1991, 4p.

Contract DE-Al01-76PR06010

Sponsored by Department of Energy, Washington, DC. Pub. in Physical Review Letters 66, n2 p157-160, 14 Jan 91.

Keywords: "Electron-ion collisions, "Silicon ions, Multicharged ions, Electron impact, Cross sections, Excitation, Reprints, Electron energy loss spectroscopy.

For the first time, absolute cross sections for electronimpact excitation of a multiple charged ion have been measured using an electron-energy-loss technique. Cross sections for $e+\operatorname{Si}(3+)$ 3s doublet $S(1 / 2) \quad \cdots$ e+Si $(3+) 3 p$ doublet $P(1 / 2,3 / 2) \cdot 8.88 \mathrm{eV}$ have been measured with an accuracy of + or $-20 \%$ (at $90 \%$-confidence level) over a narrow energy range (t or -0.6 $\mathrm{eV}$ ) about the threshold energy with an energy resolution of $0.2 \mathrm{eV}$. Results are in good agreement with close-coupling calculations.

03,905
PB95-151254 Not available NTIS

National Inst. of Standards and Technology (PL), Gaithersburg, MD. Atomic Physics Div.

Magneto-Optical Trapping of Metastable Xenon: Isotope-Shift Measurements.

Final rept.

M. Walhout, H. J. L. Megens, A. Witte, and S. L.

Rolston. 1993, 4p.

Sponsored by Office of Naval Research, Arlington, VA. Pub. in Physical Review A 48, n2 pR879-R882 Aug 93. 
Keywords: "Trapped particles, "Xenon isotopes, "Isotope effect, "Magnetooptics, Metastable state Zeeman effect, Spectral shift, Hyperfine structure, Reprints, Atom traps, Laser cooling.

We have magneto-optically trapped the nine stable isotopes of xenon. Using the Zeeman slowing method to decelerate a beam of xenon atoms in the metastable 6s 3/2 (3/2)(sub 2) state (notation representing $\mathrm{nl}$ $\mathrm{J}($ core $)(\mathrm{K}=\mathrm{J}($ core $)+\mathrm{I})($ sub $\mathrm{J})$ ), we load our trap to a collisionally limited density of more than $10(\exp 10)$ atoms/cc. The two odd isotopes are trapped withou a repumping frequency, even though they have hyperfine structure. The sensitivity of the trapping process to the laser frequency is exploited to make accurate measurements of the isotope shifts for the $6 \mathrm{~s} 3 /$ 2 (3/2)(sub 2) -.> 6p 3/2 (5/2)(sub 3) laser-cooling transition and of the hyperfine constants for (131)Xe.

03,906

PB95-151353 Not available NTIS

National Inst. of Standards and Technology (CSTL),

Gaithersburg, MD. Thermophysics Div.

Measurements of the Virial Coefficients and Equation of State of the Carbon Dioxide + Ethane System in the Supercritical Region.

Final rept.

L. A. Weber. 1992, 22p.

Sponsored by Gas Research Inst., Chicago, IL.

Pub. in International Jnl. of Thermophysics 13, n6 p1011-1032 Nov 92

Keywords: *Virial equation, "Binary mixtures, "Carbon dioxide, "Ethane, "Equations of state, Density(Mass volume), Critical pressure, Critical temperature, Thermophysical properties, Temperature dependence, Reprints, Phase boundaries.

Gas-phase densities of the system carbon dioxide + ethane were measured with a Burnett apparatus at 320 $\mathrm{K}$ and at pressures up to approximately $10 \mathrm{MPa}$. Measurements were made on systems having carbon diox ide mole fractions of $0,0.25166,0.49245,0.73978$, and 1 . Second and third virial coefficients were determined for each composition, and the cross virial coefficients were calculated. Comparisons were made with other recent high-quality measurements on this sys tem. For each mixture composition PdT measurements were made on five isochores having densities within + or $-30 \%$ of the critical density. Temperatures varied from 288 to $320 \mathrm{~K}$. The two-phase boundary was determined and estimates are given for $\mathrm{T}_{\mathrm{C}}$ and $\mathrm{Pc}$ for each composition.

\section{3,907}

PB95-151494 Not available NTIS

National Inst. of Standards and Technology (NML) Gaithersburg, MD. Radiometric Physics Div.

High Resolution Angle Resolved Photoelectron Spectroscopy Study of N2.

Final rept.

J. B. West, M. A. Hayes, A. C. Parr, T. A. Ferrett, J.

L. Dehmer, X. M. Hu, G. V. Marr, J. E. Hardis, and

S. H. Southworth. 1990, 3p.

Pub. in Physica Scripta 41, n4 p487-489 1990.

Keywords: *Photoelectron spectroscopy *Photoionization, "Nitrogen, Near infrared radiation, High resolution, Rydberg series, Nitrogen ions, Autoionization, Reprints.

This is a preliminary report on a series of measurements, using high optical resolution, on the photoionization of the nitrogen molecule in the wavelength region $740-780 \mathrm{~A}$ where there are at least five Ryberg series converging onto the Ist excited state of $\mathrm{N} 2(1+)$ A doublet $\mathrm{Pi}(\mathrm{u})$. An advanced electron spectrometer system is used which provides measurements of the angular distribution parameter beta for three members of the ground state of the ion, on a wavelength mesh sufficient to show variations in beta over the width of the autoionizing lines. This study was undertaken to assist in the identification of these series, and to encourage further theoretical work on interaction between electronic and vibrational motion in the region of autoionizing resonances.

\section{3,908}

PB95-151502 Not available NTIS

National Inst. of Standards and Technology (NML), Gaithersburg, MD. Center for Atomic, Molecular and Optical Physics.

Laser Cooling.

Final rept.

C. I. Westbrook, and W. D. Phillips. 1991, 3p.

Pub. in McGraw-Hill Yearbook of Science and Technology, p208-210 1991.
Keywords: Temperature range 0000-0013 K, Range(Extremes), Atomic mobilities, Atomic clocks, Doppler effect, Limits, Reprints, "Laser cooling, Miliikelvin temperature, Photon recoil.

Laser cooling can reduce the thermal motion of atoms in a gas. Such cooling has achieved temperatures less than one millikelvin. The traditional view of laser cooling involves the Doppler-effect induced velocity dependence of light absorption and predicts a limit on the lowest achievable temperature. The energy imparted to an atom from a single photon recoil aiso implies a separate limit. Recent experiments have shown that both of these limits can be broken with the help of new laser cooling mechanisms.

\section{3,909}

\section{PB95-151551 Not available NTIS}

National Inst. of Standards and Technology (NML), Gaithersburg, MD. Atomic and Plasma Radiation Div. Comprehensive Spectroscopic Data Tabulations and Progress in the Compilation of Atomic Transition Probabilities.

Final rept.

W. L. Wiese. 1991, 10p

Pub. in Jnl. de Physique IV 1, nC1 p287-296 Mar 91.

Keywords: "Atomic spectroscopy, "Transition probabilities, Atomic energy levels, Wavelengths, Reprints, *Spectroscopic data, Critical data tables, US NIST.

The critical data compilation work as well as the bibliographical efforts of two data centers on atomic spectroscopy at the National Institute of Standards and Technology (formerly the National Bureau of Standards) are briefly reviewed. A complete listing of all current compilations on wavelengths, energy levels and transition probabilities is given. A recently completed large tabulation of atomic transition probabilities for the Fe-group elements comprising about 18000 lines is discussed in some detail, and several data comparisons are presented in order to provide an impression of the current status of these atomic data.

\section{3,910}

\section{PB95-151585 Not available NTIS}

National Inst. of Standards and Technology (NML), Gaithersburg, MD. Atomic and Plasma Radiation Div. Spectroscopic Data Tables for Highly-Ionized Atoms.

Final rept

W. L. Wiese, J. R. Fuhr, W. C. Martin, A. Musgrove, and J. Sugar. $1991,2 p$.

Pub. in Zeitschrift fur Physik D 21, pS147-S148 1991.

Keywords: "Atomic spectroscopy, "Multicharged ions, Atomic energy levels, Transition probabilities, Wavelengths, Tables(Data), Reprints, "Spectroscopic data, Critical data tables, US NIST.

Two data centers at the National Institute of Standards and Technology (NIST) are engaged in the critical compilation of atomic spectroscopic data including those for highly ionized atoms: the Atomic Energy Levels Data Center and the Data Center for Ato mic Transition Probabilities. Several major compilations have been recently completed, centered on the iron-group elements.

\section{3,911}

\section{PB95-151627 Not available NTIS}

National Inst. of Standards and Technology (PL), Boulder, CO. Time and Frequency Div.

Trapped Atoms and Laser Cooling.

Final rept.

D. J. Wineland. $1992,8 p$.

Pub. in Elementary Modern Physics, Chapter 4, p1561631992

Keywords: "Trapped particles, Laser spectroscopy, Atomic spectroscopy, Atomic clocks, lon storage, Textbooks, Reprints, ${ }^{\star}$ Laser cooling, Ion traps, Millikelvin temperature.

This short article is an 'essay' in the elementary physics text Physics by P A. Tipler It is intended as supplementary material to the regular text, but refers to the text material when appropriate. Topics discussed include atomic clocks, atomic spectroscopy, ion trapping, laser cooling, and laser spectroscopy.

\section{3,912}

PB95-151635 Not available NTIS

National Inst. of Standards and Technology (PL), Boulder, CO. Time and Frequency Div.
Spin Squeezing and Reduced Quantum Noise in Spectroscopy.

Final rept.

D. J. Wineland, J. J. Bollinger, W. M. Itano, F. L.

Moore, and D. J. Heinzen. 1992, 4p.

Pub. in Physical Review A 46, n11 pR6797-6800, 1 Dec 92

Keywords: *Atomic spectroscopy, Atomic clocks, Frequency standards, Reprints, Squeezed states(Quantum theory), Quantum noise, Correlated states.

We investigate the quantum-mechanical noise in spectroscopic experiments on ensembles of $\mathrm{N}$ twolevel (or spin-1/2) systems where transitions are detected by measuring changes in state population. By preparing correlated states, here called squeezed spin states, we can increase the signal-to-nose ratio spectroscopy (by approximately $N(\exp 1 / 2)$ in certain cases) over that found in experiments using uncorrelated states. Possible experimental demonstrations of this enhancement are discussed.

03,913

PB95-151999 Not available NTIS

National Inst. of Standards and Technology (NML) Gaithersburg, MD. Chemical Process Metrology Div.

Multiphoton Ionization Spectroscopy Measurements of Silicon Atoms during Vapor Phase Synthesis of Ceramic Particles.

Final rept.

M. R. Zachariah, and R. G. Joklik. 1990, 7p

Pub. in Jnl, of Applied Physics 68, n1 p311-317 1990

Keywords: "Vapor phases, "Silicon, *Particle production, "Ceramics, "Ionization, "Chemical vapor deposition, Chemical reactors, Silanes, Spectrum analysis, Concentrating, Clumps, Particle size distribution, Reprints, "Silicon atoms, Multiphoton ionization spectroscopy.

Resonance Enhanced Multi-Photon Ionization spectroscopy has been applied to the problem of detection of gas phase species in a dense particle forming flow. Relative silicon atom profiles of an atmospheric pres sure flame reactor producing silica particles have been made using a ' $2+1$ ' ionization scheme. The results have shown that silicon atoms are confined to a very narrow time window in the reactor (less than $15 \mathrm{msec}$ ). In addition, the non-resonant background Indicated the presence of ionizable clusters and could be a valuable tool for obsening the presence and location of small clusters. The effect of varying silane loading changed both the location and magnitude of the silicon concentration observed. The results suggest that chain branching chemistry is important in the production of silicon and that enhancement of pyrolytic relative to oxidative processes occurs as the silane loading is increased

03,914

PB95-152161 Not available NTIS

National Inst. of Standards and Technology (PL), Boulder, CO. Quantum Physics Div.

High-Precision Calculations of Cross Sections for Low-Energy Electron Scattering by Ground and Excited State of Sodium.

Final rept.

H. L. Zhou, D. W. Norcross, and B. L. Whitten. 1993 $10 \mathrm{p}$

Grant NSF-PHY86-04504

Sponsored by National Science Foundation, Washington, DC.

Pub. in Proceedings of International Symposium on Correlations and Polarization in Electronic and Atomic Collisions and (e,2e) Reactions (6th), Adelaide, Australia, July 18-21, 1992, p39-48.

Keywords: *Electron-atom collisions, "Electron scattering, "Sodium, Scattering cross sections, Excited states, Ground states, Precision, Orientation, Polarization(Spin alignment), Reprints.

We report progress in a detailed series of calculations intended to provide a comprehensive set of results against which a wide variety of completed, in progress and proposed measurements can be compared. A high emphasis has been put on numerical accuracy, technical convergence in various parameters (e.g. state ex pansions and partial wave summations), and inclusion of physical effects (e.g. exchange and core polarization).

03,915
PB95-152203 Not available NTIS 


\section{PHYSICS}

\section{General}

National Inst. of Standards and Technology (NML), Gaithersburg, MD. Time and Frequency Div

Atomic Oxygen Fine Structure Splittings with Tunable Far Infrared Spectroscopy.

Final rept.

L. R. Zink, K. M. Evenson, F. Matsushima, T. Nelis, and R. Robinson. 1991, 2p.

Pub. in Astrophysical Jnl. 371, n2 pL85-L86 1991.

Keywords: "Oxygen atoms, "Fine structure, Far infrared radiation, Infrared spectroscopy, Interstellar matter, Atmospheric composition, Ground states, Reprints.

We have measured the atomic oxygen (16)O ground state fine structure splittings using a tunable far infrared spectrometer. The triplet $P(0)$ - triplet $P(1)$ splitting is 2,060,069.09 (10) $\mathrm{MHz}$ and the triplet $P(1)$ - triple $\mathrm{P}(2)$ splitting is $4,744,777.49$ (16) $\mathrm{MHz}$. These frequencies are important for the study of the earth's atmosphere and the interstellar medium.

\section{3,916}

PB95-152245 Not available NTIS

National Inst. of Standards and Technology (CAML),

Gaithersburg, MD. Statistical Engineering Div.

Effect of Splitting on Estimation of Emission Rate Profiles from Neutron Depth Profiling Spectra.

Final rept

K. J. Coakley. 1994, 5p

Pub. in Proceedings of 1993 Section on Physical and Engineering Sciences of the American Statistical Association, San Francisco, CA., August 8-12, 1993, p77821994.

Keywords: Stopping rules(Mathematics), Iterative methods, Estimating, Reprints, "Neutron depth profiling, Cross validation, Data splitting, EM algorithm.

Based on Neutron Depth Profiling spectra, emission rate profiles are estimated using a data splitting and cross-validation approach, In the procedure, data are split into a variable number of parts. From each part, emission rate profiles are estimated using the iterative EM algorithm. The algorithm is halted according to a cross-validation criterion. The estimates from all parts are averaged. As the split becomes finer, the stopping point is reached sooner and the estimate gets smoother. Mean and variance curves are computed for simulated data. An experimental data set is analyzed.

\section{3,917}

PB95-152252 Not available NTIS

National Inst. of Standards and Technology (MSEL) Gaithersburg, MD. Reactor Radiation Div.

Analysis of the Effectiveness of Oscillating Radial Collimators in Neutron Scattering Applications.

Fina! rept.

J. R. D. Copley, and J. C. Cook. 1994, 11p.

Pub. in Nuclear Instruments and Methods in Physics Research A 345, p313-3231994.

Keywords: "Neutron scattering, "Collimators, Time-offlight method, Neutron diffraction, Transmissivity, Effectiveness, Reprints, Visibility functions, Radial collimators.

We describe a formalism which allows the transmission properties of oscillating radial collimators to be discussed using the concept of 'visibility' functions. We develop a small angle approximation to the theory which proves to be valuable in describing typical instrumental situations. Independent Monte Carlo (ray-tracing) simulations give very good agreement with analytical results and provide a means for investigating situa tions where an analytical treatment would be extremely complicated.

03,918

PB95-152906 Not available NTIS

National Inst. of Standards and Technology (PL), Boulder, CO. Quantum Physics Div.

Electron-Impact Ionization of $\operatorname{In}(+)$ and $X e(+)$.

Final rept.

E. W. Bell, N. Djuric, and G. H. Dunn. 1993, 6p.

Contract DE-A105-86ER53237

Sponsored by Department of Energy, Washington, DC Pub. in Physical Review A 48, n6 p4286-4291 Dec 93.

Keywords: "Electron-ion collisions, "Indium ions, * Xenon ions, lonization cross sections, EV range 10 100 , EV range 100-200, Electron impact, Excited states, Colliding beams, Autoionization, Reprints.

Absolute ionization cross sections for $\ln (1+)$ and $\mathrm{Xe}(1+)$ by electron impact have been measured from below threshold to $200 \mathrm{eV}$ using the crossed-beams technique. The cross sections for $\operatorname{In}(1+)$ were possibly enhanced by indirect ionization processes. The excitation of the ion from the $4 d(10) 5 S(2)$ ground state to the $4 d(9) 5 S(2) 5 P$ state followed by autoionization has been postulated. The $\operatorname{In}(1+)$ cross sections show a peak value at about $80 \mathrm{eV}$. The cross sections for $\mathrm{Xe}(1+)$ peak at a value at about $35 \mathrm{eV}$. Also presented are ionization-rate coefficients and fitting parameters for both ions for temperatures in the range 10(exp 4) $\mathrm{K}=$ or $<\mathrm{T}=$ or $<10(\exp 7) \mathrm{K}$

\section{3,919}

Not available NTIS

National Inst. of Standards and Technology (CSTL), Gaithersburg, MD. Thermophysics Div.

Thermal Equilibration Near the Critical Point: Effects Due to Three Dimensions and Gravity.

Final rept.

R. F. Berg. 1993, 7p

Contract NASA-C-32009-M

Sponsored by National Aeronautics and Space Administration, Washington, DC.

Pub. in Physical Review E 48, n3 p1799-1805 Sep 93.

Keywords: “Critical point, "Liquid-vapor interfaces, Density distribution, Thermodynamic properties, Heat transfer, Relaxation(Mechanics), Temperature gradients, Reprints, "Thermal equilibrium.

Two calculations are presented that clarify how the density profile equilibrates near the liquid-vapor critical point. Both use the equation of heat transfer recently improved to account for the large compressibility near the critical point. Previous work by others indicated that in one dimension the slowest mode of this equation relaxes at a rate four times faster than that predicted by the older, usual equation of heat transfer. However, this is not always true in higher dimensions. The first calculation demonstrates this for the cases of isobaric modes excited by temperature gradients in a rectangle and in a thin disk. For thin experimental cells with isothermal walls the slowest mode is accurately estimated by the usual heat-transfer equation. The second calculation indicates that gravity-induced stratification plays an insignificant role in determining the final relaxation rate. This is done by estimating the size of the $V$ (center dot) Del $P$ term in the improved heat-transfer equation.

03,920

PB95-152963 Not available NTIS

National Inst. of Standards and Technology (PL), Boulder, CO. Time and Frequency Div.

Electrostatic Modes of Ion-Trap Plasmas.

Final rept.

J. Bollinger, D. Heinzen, F. Moore, D. Dubin, W

Itano, and D. Wineland. 1993, 21p.

Sponsored by Office of Naval Research, Arlington, VA. and National Science Foundation, Washington, DC

Pub. in Physical Review A 48, n1 p525-545 Jul 93.

Keywords: "Trapped particles, "Ion storage, Beryllium ions, Electrostatics, Reprints, Nonneutral plasmas, Ion plasmas. Ion traps, Laser cooling, Penning traps, Paul traps.

The electrostatic modes of a non-neutral plasma confined in a Penning or Paul (r) trap are discussed in the limit that the Debye length is small compared to the plasma dimensions, and the plasma dimensions are small compared to the trap dimensions. In this limit, the plasma shape is spheroidal and analytic solutions exist for all of the modes. The solutions for the modes of a Paul-trap plasma are a special case of the modes of a Penning-trap plasma. A simple derivation of some of the low-order quadrupole modes is given. Experimental measurements of these mode frequencies on plasmas of laser-cooled $\mathrm{Be}(+)$ ions in a Penning trap agree well with the calculations. A general discussion of the higher-order modes is given. The modes provide a nondestructive method for obtaining information on the plasma density and shape. In addition, they may provide a practical limit to the density and number charged particles that can be stored in a Penning trap.

03,921

PB95-153045 Not available NTIS

National Inst. of Standards and Technology (CSTL), Gaithersburg, MD. Thermophysics Div.

Dynamic Technique for Measuring Normal Spectral Emissivity of Electrically Conducting Solids at High Temperatures with a High-Speed Spatial Scanning Pyrometer.

Final rept.

A. Cezairliyan, and A. P. Miiller. 1993, 6p.

Sponsored by National Aeronautics and Space Administration, Washington, DC.
Pub. in International Jnl. of Thermophysics 14, n5 p1109-1114 1993

Keywords: "Temperature measurement, "Spectral emission, "Molybdenum, Temperature range 1000 . $4000 \mathrm{~K}$, High temperature, Optical measurement, Blackbody radiation, Electric conductors, Pyrometers, Emissivity, Reprints.

A dynamic (subsecond) technique is described for measuring normal spectral emissivity of electrically conducting solids at high temperatures, primarily in the range $1800 \mathrm{~K}$ up to near their melting point. The basic method involves resistively heating a tubular specimen from ambient temperature through the temperature range of interest in less than $1 \mathrm{~s}$ by passing an electrical current pulse through it, while using a high-speed spatial scanning pyrometer to measure spectral radiance temperatures along a $25-\mathrm{mm}$ length on the specimen. This portion of the specimen includes a small rectangular hole that approximates a blackbody cavity Measurements of spectral radiance temperature of the specimen surface as well as specimen true temperature enable the determination of the normal spectral emissivity of the surface via Planck's law. The applicability of the technique is demonstrated by measurements performed on molybdenum in the range 1900 . $2850 \mathrm{~K}$.

03,922

PB95-153078 Not available NTIS

National Inst. of Standards and Technology (CSTL),

Gaithersburg, MD. Inorganic Analytical Research Div. Neutron Focusing Lens Using Polycapillary Fibers. Final rept.

H. Chen, D. F. R. Mildner, and Q. F. Xiao. 1994, 3p Contracts DE-FG02-91ER81220, DE-FG02-

91ER81411

Sponsored by Department of Energy, Washington, DC Pub. in Applied Physics Letters 64, n16 p2068-2070, 18 Apr 94.

Keywords: "Neutron beams, "Neutron transport, "Cold neutrons, Neutron guides, Grazing incidence Borosilicate glass, Reprints, *Neutron focusing lenses, Neutron reflection, Polycapillary fibers.

Multiple mirror reflection at small grazing angles from the smooth surfaces of the narrow channels of polycapillary fibers can be used to transport, bend, and focus thermal neutron beams. We report the results of the focusing of a polychromatic cold neutron beam using a compact lens of borosilicate fibers and with a focal distance of $57 \mathrm{~mm}$. The intensity profile of the beam at the focus is approximately conical in shape with a full width at half-maximum of $0.49 \mathrm{~mm}$, and with an average gain in intensity of about 20 . These experimental results agree well with those obtained by computer simulation.

03,923

PB95-153268 Not available NTIS

National Inst. of Standards and Technology (NML), Gaithersburg, MD. Center for Atomic, Molecular and Optical Physics.

New Mechanisms for Laser Cooling.

Final rept.

C. N. Cohen-Tannoudji, and W. D. Phillips. 1990, 8p. Pub. in Physics Today 43, n10 p33-40 1990.

Keywords: Laser spectroscopy, Optical pumping, Trapped particles, Reprints, "Laser cooling, Millikelvin temperature, Photon recoil.

Well known physical effects, such as optical pumping and light-shifts, have unexpectedly conspired to improve, by orders of magnitude, the performances of laser cooling and to produce the lowest kinetic temperatures ever measured.

03,924

PB95-153433 Not available NTIS

National Inst. of Standards and Technology (PL),

Gaithersburg, MD. Atomic Physics Div.

Hydrogen Balmer Alpha Line Shapes for Hydrogen-Argon Mixtures in a Low-Pressure of Discharge.

Final rept.

S. Djurovic, and J. R. Roberts. 1993, 8p.

Pub. in Jnl. of Applied Physics 74, n11 p6558-6565, 1 Dec 93.

Keywords: "Hydrogen plasma, "Balmer lines, "Atomatom collisions, "Ion-atom collisions, Radio frequency discharge, Line broadening, Doppler effect, Plasma spectra, Argon plasma, Line shape, Gas mixtures, Reprints, Reference cells. 
The spectral and spatial profiles of atomic hydrogen emission (the Balmer lines $\mathrm{H}$ (alpha) and $\mathrm{H}$ (beta)) from a low-pressure of (13.56 MHz) discharge in $\mathrm{H} 2+\mathrm{Ar}$ mixtures have been studied. The plasma emission was observed in a direction normal to the applied electric field. The $H$ (alpha) profiles exhibit central narrow components and wide components which are due to Doppler broadening. Comparisons of the $\mathrm{H}$ (alpha) profiles in a pure hydrogen plasma with those in $\mathrm{H} 2+\mathrm{Ar}$ mixtures show that collisions of molecular hydrogen ions and hydrogen atoms with argon atoms play a significant role in the production of the $H$ (alpha) profile shapes.

\section{3,925}

PB95-153532 Not available NTIS

National Inst. of Standards and Technology (CSTL), Gaithersburg, MD. Surface and Microanalysis Science Div.

Semiclassical Explanation of the Generalized Ramsauer-Townsend Minima in Electron-Atom Scattering.

Final rept.

W. F. Egelhoff. 1993, 4p.

Pub. in Physical Review Letters 71, n18 p2883-2886, 1 Nov 93.

Keywords: "Electron-atom collisions, "Ramsaver effect, Electron scattering, Reprints, Semiclassical methods.

The generalized Ramsauer-Townsend minima which occur, at certain scattering angles, in the intensity of electrons elastically scattered by atoms have been a subject of interest in atomic physics for over sixty years. While quantum mechanical calculations predict these minima with great accuracy, no clear, simple, intuitively appealing description of the underlying scattering processes has been given. It is shown here for the first time that simple semiclassical calculations provide such a description.

\section{3,926}

PB95-153615 Not available NTIS

National Inst. of Standards and Technology (PL), Gaithersburg, MD. Ionizing Radiation Div.

Polarization Effects on Multiple Scattering Gamma Transport.

Final rept.

J. E. Fernandez, J. H. Hubbell, A. L. Hanson, and L. V. Spencer. 1993, 52p.

Pub. in Radiation Physics and Chemistry $41, n 4 / 5$ p579-630 1993.

Keywords: "Polarization(Waves), "Gamma ray scattering, "X-ray scattering, "Photon-electron collisions, Polarized electromagnetic radiation, Photoelectron emission, Rayleigh scattering, Compton effect, Multiple scattering, Polarized beams, Backscattering, Reprints.

The scattering of $X$-rays and gamma-rays are events that have strong dependencies on the polarization of the incident and scattered photons. Because of this, scattering problems that can be solved without explicit reference to the state of polarization of the incident and scattered radiation are exceptional. This article reviews available information on polarization effects arising when photons in the $\mathrm{X}$-ray and gamma-ray regime undergo photoelectric effect, coherent (Rayleigh) scattering and incoherent (Compton) scattering by atomic electrons. In addition to descriptions and discussion of these effects, we study the backscattering of gammarays from an infinite thickness target excited with a plane slant monodirectional and monochromatic source, using the Boltzmann transport theory and the mathematical representation of polarization introduced by Stokes. Results from this model, for both unpolarized and polarized gamma-ray sources, are compared with computations performed neglecting or averaging polarization effects, showing the limitations of such approximations.

\section{3,927}

PB95-161105 Not available NTIS

National Inst. of Standards and Technology (MSEL),

Gaithersburg, MD. Reactor Radiation Div.

Crystal Structure of Annealed and As-Prepared $\mathrm{HgBa} 2 \mathrm{CaCu} 2 \mathrm{O} 6+$ delta Superconductors.

Final rept.

Q. Huang, J. W. Lynn, R. L. Meng, and C. W. Chu

1993, 9p.

Pub. in Physica C 218, p356-364 1993.

Keywords: "Electron beams, "Calibration,
"Calorimeters, "Radiation doses, MeV range 1-10,

Keywords: "Electron beams, "Calibration,
"Calorimeters, "Radiation doses, MeV range 1-10,

"Calibration,
MeV range 10-100, Temperature measurement, Industrial production, Thermistors, Reprints, "Graphite calorimeters, Radiation processing.

A multi-body graphite calorimeter has been designed for the absolute calibration of high-intensity electron beams in the energy regime of 2 to $12 \mathrm{MeV}$. This novel calorimeter consists of eight thermally and electrically insulated disks of high-purity graphite that serve as the active calorimetric bodies, arranged in a stacked array and oriented so that the flat surfaces are perpendicular to the electron-beam axis. Calibrated thermistors imbedded in the disks act as temperature sensors. The emperature of each disk is measured in real-time during irradiation by a scanning multichannel digital meter interfaced with a computer-based data acquisition system. The resultant data provide a depth-dose profile from which the electron energy can be calculated. Calorimeters of this type would be useful in standardiz ing the absorbed dose to passive routine dosimeters in the range of $100 \mathrm{~Gy}$ to $50 \mathrm{kGy}$, typical of that delivered by industrial processing electron beams.

\section{3,928}

PB95-161147 Not available NTIS

National Inst. of Standards and Technology (PL), Boulder, CO. Time and Frequency Div.

Quantum Measurements of Trapped lons.

Final rept.

W. M. Itano, J. C. Bergquist, J. J. Bollinger, F. L. Moore, M. G. Raizen, D. J. Wineland, J. M. Gilligan, and D. J. Heinzen. 1993, 15p.

Sponsored by Office of Naval Research, Arlington, VA Pub. in Vistas in Astronomy 37, p169-183 1993.

Keywords: "Ion storage, "Quantum mechanics Trapped particles, Beryllium ions, Mercury ions, Reprints, "Quantum measurements, Quantum jumps.

When a quantum state is subjected to a measurement and the state is not an eigenstate of the dynamical variable being measured, the outcome is unpredictable. Only the probabilities of the various possible outcomes are predicted by theory. This phenomenon is someimes discussed in terms of 'wavefunction collapse. Trapped ions can be used for real, as opposed to 'gedanken' demonstrations of this basic process. In the experiments described here, a single ion, or a few identical ions, were prepared in well defined superpositions of two internal energy eigenstates. The populations of the energy levels were then measured. When the state amplitudes were equal, the population fluctuations were greater than when one of the amplitudes was nearly zero, in agreement with the predictions of quantum mechanics. In other experiments, such as those with atomic beams, the number of atoms under observation fluctuates, and this obscures the luctuations from other sources. However, if the number of atoms is small and constant, the fundamental quantum mechanical fluctuations can be observed.

\section{3,929}

PB95-161220 Not available NTIS

National Inst. of Standards and Technology (NML), Gaithersburg, MD. Molecular Physics Div.

Laser Assisted Collisions at Ultracold Temperatures.

Final rept.

P. S. Julienne, R. Heather, and J. Vigue. 1990, 15p. Sponsored by Air Force Office of Scientific Research Bolling AFB, DC

Pub. in Spectral Line Shapes, v6 p191-205 1990.

Keywords: "Photon-atom collisions, "Atomic collisions, Molecular spectroscopy, Alkali metals, Bound state, Neutral atoms. Temperature range 0000-0013 K Dimers, Reprints, Laser cooling, Laser trapping, Atom traps, Ultracold atoms, Millikelvin temperature, Associative ionization, Photoassociation spectroscopy.

Recent developments in laser cooling and trapping of neutral atoms have opened the new field of atomic collisions at ultracold temperatures, $T<1 \mathrm{mk}$. This field offers many new challenges and opportunities for both theory and experiment in the general area of spectral line shapes. We will outline here some of the key problems in this field, and present calculations on several collisional processes which have been observed in atom traps. First the very unusual energy, time and distance scales encountered in ultracold collisions will be described. Second, the molecular physics of excited plus ground alkali dimers will be examined, and the Tdependent rate coefficients will be calculated for processes which lead to loss of alkali atoms from an atom trap. Finally, a new kind of free-bound molecular spectroscopy, photoassociation spectroscopy, will be de- scribed which should permit the observation of long range molecular bound states extremely close to the molecular dissociation limit.

\section{3,930}

\section{PB95-161527 Not available NTIS}

National Inst. of Standards and Technology (NML), Gaithersburg, MD. Chemical Process Metrology Div Practical Applications of the ITS-90: Inherent Uncertainties.

Final rept.

B. W. Mangum, E. R. Pfeiffer, and G. F. Strouse. 1991, 12p.

Pub. in Proceedings of Measurement Science Conference, Anaheim, CA., January 31-February 1, 1991 $12 \mathrm{p}$.

Keywords: "Temperature measurement, Temperature range 0013-0065 $\mathrm{K}$, Temperature range 0065-0273 K Temperature range $0273-0400 \mathrm{~K}$, Temperature range 0400-1000 K, Resistance thermometers, Platinum, Error analysis, Calibration, Uncertainty, Reprints, International Temperature Scale of 1990, "ITS-90, Fixed points.

We report results of a study of the non-uniqueness of the ITS-90 at temperatures in the range $13.8033 \mathrm{~K}$ to $933.473 \mathrm{~K}(660.323 \mathrm{C}$ ) of a variety of capsule-type and long-stem type standard platinum resistance thermometers (SPRTs). The capsule SPRTs were calibrated by comparison techniques and at some fixed points of the ITS-90; the long-stem SPRTs were calibrated at the relevant defining fixed points In addition, we presen results on subrange inconsistencies for several partly overlapping ranges of the ITS-90. Error propagation curves and errors introduced by extrapolations will be discussed also.

\section{3,931}

PB95-161634 Not available NTIS

National Inst. of Standards and Technology (PL) Gaithersburg, MD. Ionizing Radiation Div.

ESR-Based Analysis in Radiation Processing.

Final rep

W. L. McLaughlin, M. F. Desrosiers, and M. C. Saylor. 1993, 27p

Sponsored by Johnson and Johnson, New Brunswick

Pub. in Proceedings of International Kilmer Memoria Conference on the Sterilization of Medical Products, Brussels, Belgium, June 13-15, 1993, p213-239.

Keywords: *Electron spin resonance, "Dosimetry, Radiation doses, Radiation effects, Nuclear medicine, Sterilization, Food irradiation, Polymers, Alanines, Uses, Reprints, "Radiation processing, "ESR dosimetry.

Electron spin resonance (ESR) spectrometry has attracted attention in radiation processing. Not only is ESR analysis of alanine achieving success as a ref erence dosimetric system, but ESR also provides a means of evaluating short- and long-term effects of radiation on polymers, composites and ceramics, and in the post-irradiation analysis and imaging of absorbed dose in teeth and bone. The broad range of doses (10(exp -1) - 10(exp 8) Gy) and dose rates (up to 10 (exp 8) Gy/s) measurable with relatively high precision and accuracy, and the small size, ruggedness, and inexpensiveness of sensor materials, such as Lalpha-alanine pellets and films and other sensor materials (e.g., hydroxyapatite, sugar, quartz, cellulose, etc.), make ESR dosimetry attractive for radiation sterilization dosimetry, radiation treatment planning, nuclear medicine dosimetry, food irradiation and the study of radiation effects on materials. Within the next few years, there may well be impact of this ESR-based analysis on international radiation standards practices and $2 \mathrm{D}$ and $3 \mathrm{D}$ imaging of radiation dose distributions, especially as the trend toward lowering the cost of the analytical equipment and simplifying its operation continues. ESR dosimetry may, in fact, be the future method of choice for interlaboratory transfer metrology process validation, and measurement quality assurance.

\section{3,932}

PB95-161667 Not available NTIS

National Inst. of Standards and Technology (CSTL) Gaithersburg, MD. Thermophysics Div.

Measurement of Surface Tension of Tantalum by a Dynarnic Technique in a Microgravity Environment.

A.P. Miiller and A. Cezairliyan. 1993, 13p.

Pub. in International Jnl. of Thermophysics 14, n5 p1063-1075 1993. 


\section{General}

Keywords: "Interfacial tension, "Tantalum "Weightlessness simulation, "High temperature, Melting points, Research aircraft, Dynamic tests,

A dynamic technique has been used in a microgravity environment to measure the surface tension of tantalum at its melting point. The basic method involves resistively heating a tubular specimen from ambient temperature to temperatures above its melting point in about $1 \mathrm{~s}$ by passing an electrical current pulse through it, while simultaneously measuring the pertinent experimental quantities with millisecond resolution. A balance between the magnetic and the surface tension forces acting on the specimen is achieved by splitting the current after it passes through the specimen tube and returning a fraction of the current along the tube axis and the remaining fraction concentrically outside of the specimen. Values for surface tension are determined from measurements of the equilibrium dimensions of the molten specimen tube and the magnitudes of the currents. Rapid melting experiments were performed during microgravity simulations with NASA's KC-135 aircraft and the results were analyzed, yielding a value of $2.07+$ or $-0.06 \mathrm{~N} / \mathrm{m}$ for the surface tension of tantalum at its melting point

\section{3,933}

PB95-161774 Not available NTIS

National Inst. of Standards and Technology (PL), Gaithersburg, MD. Electron and Optical Physics Div. Spin-Resolved Elastic Scattering of Electrons from Sodium.

Final rept.

S. R. Lorentz, R. E. Scholten, J. J. McClelland, M. H. Kelley, and R. J. Celotta. 1993, 7p

Sponsored by Department of Energy, Washington, DC. Pub. in Physical Review A 47, n4 p3000-3006 Apr 93. Keywords: "Electron-atom collisions, "Sodium, Polanzation(Spin alignment), Optical pumping, EV range 1-10, EV range 10-100, Electron scattering, Elastic scattering, Asymmetry, Reprints.

Angle-resolved ratios of the separate triplet and singlet spin channel cross sections have been measured for elastic scattering of spin-polarized electrons from optically pumped spin-polarized sodium atoms. The tripletto-singlet ratios are reported at incident energies of $4.1,10.0$ and $20.0 \mathrm{eV}$ for scattering angles from 20 deg to $140 \mathrm{deg}$. The data demonstrate that spin-exchange scattering plays an important role in the description of electron scattering from sodium at these several close-coupling theoretical calculations.

\section{3,934}

PB95-161840 Not available NTIS
National Inst. of Standards and Techr

National Inst. of Standards and Technology (PL), Gaithersburg, MD. Atomic Physics Div.

sigma+-sigma- Optical Molasses in a Longitudinal Magnetic Field.

Final rept.

M. Walhout, J. Dalibard, S. L. Rolston, and W. D.

Phillips. 1992, 11p.

Sponsored by Office of Naval Research, Arlington, VA Pub. in Jnl. of the Optical Society of America B 9, n11 p1997-2007.

Keywords: Fokker-Planck equation, Diffusion coefficient, Magnetic fields, Numerical solution, Magnetooptics, Reprints, "Optical molasses, "Atom cooling, Pola rized gradient cooling, Atom traps.

We investigate the effect of a longitudinal magnetic field on the orientational cooling mechanism that operates both in sigma $(+)$ - sigma $(-)$ optical molasses and in a magneto-optical trap. For an atom with different Lande $\mathrm{g}$ factors in its ground and excited states, singlephoton scattering and stimulated Raman scattering are resonant at different field-dependent velocities. The sharp polarization-gradient feature in the force-versusvelocity curve may therefore be translated to a velocity at which the Doppler force is dominant. In addition to this, we find that the momentum diffusion coefficient can grow as the field is increased. By numerically solving a semiclassical Fokker-Planck equation, we show that these effects lead to a field-induced inhibition of efficient sub-Doppler cooling for atoms with unequal $g$ factors. Our treatment also enables us to discuss other phenomena that are accessible only if both Doppler and polarization-gradient effects are included in the calculation.
National Inst. of Standards and Technology (EEEL), Gaithersburg, MD. Electricity Div.

Kinetic-Energy Distributions of lons Sampled from Argon Plasmas in a Parallel-Plate, Radio-Frequency Reference Call.

Final rept.

J. K. Olthoff, R. J. Van Brunt, S. B. Radovanov, J. A Rees, and R. Surowiec. 1994, $11 \mathrm{p}$.

Pub. in Jnl. of Applied Physics 75, n1 p115-125 Jan 94.

Keywords: "Radio frequency discharge, "Gas discharges, "Argon ions, Argon plasma, Kinetic energy, Reprints, Reference cells.

Kinetic-energy distributions are presented for ions sampled from 13.56- $\mathrm{MHz}$ discharges in argon in a capacitively-coupled, parallel-plate, Gaseous Electronics Conference (GEC) radio-frequency reference cell. The cell was modified to allow sampling of ions through an orifice in the grounded electrode. Kinetic-energy distributions are presented for $\operatorname{Ar}(1+), \operatorname{Ar}(2+), \operatorname{Ar} 2(1+)$, and $\mathrm{ArH}(1+)$, and several trace ions for plasma pressures ranging from $1.3 \mathrm{~Pa}$, where ion-atom collisions in the plasma sheath are not important, to $33.3 \mathrm{~Pa}$, where collisions are important. Applied peak-to-peak radio-frequency (ff) voltages of 50,100 , and $200 \mathrm{~V}$ were used, and the current and voltage waveforms at the powered electrode were measured. Dependences of the ion fluxes, mean energies, and kinetic-energy distributions on gas pressure and applied rf voltage are interpreted in terms of possible ion-collision processes.

\section{3,936}

PB95-162053 Not available NTIS

National Inst. of Standards and Technology (PL), Gaithersburg, MD. Quantum Metrology Div.

Production and Characterization of lon Beam Sputtered Multilayers.

Final rept.

J. Pedulla, and R. D. Deslattes. 1994, 11p.

Pub. in Society of Photo-Optical Instrumentation Engineers Proceedings, v2011 p299-309 Jan 94.

Keywords: "X-ray optics, Extreme ultraviolet radiation, Sputtering, Polishing, Reprints, Ion beam deposition, Multilayers.

We report on the application of low pressure ion beam sputtering combined with simultaneous (neutralized) ion beam polishing to the production of multilayer structures for $x$-ray optics. Initial examination of these structures by high resolution diffractometry at $0.154 \mathrm{~nm}$ indicates that the structures exhibit a high degree of structural perfection.

03,937

PB95-162574 Not available NTIS

National Inst. of Standards and Technology (NML), Gaithersburg, MD. Electron and Optical Physics Div. Labeling Conventions in Isoelectronic Sequences -Reply.

E. B Saloman, and Y K Kim $1990,2 p$

Pub. in Physical Review A 42, n3 p1824-1825 1990.

Keywords: "Isoelectronic sequence, Atomic energy levels, Relativistic effects, Multicharged ions, Electron correlation, Labelling, Reprints, "Sulfur-like ions.

We agree with the Comment by Maniak and Curtis (Phys. Rev. Al (1990)) that qualitative isoelectronic behavior of low-lying energy levels of sulfur-like ions with high nuclear charges, Z, can easily be understood using single ij configuration labels. However, such labels are, in general, useful only for high-Z ions. We do not recommend mixing numerical results from multiconfiguration calculations with those from single configuration calculations because such a mixed usage may introduce artificial discontinuities in Z dependence, defeating the main advantage of studies along an isoelectronic sequence.

03,938

PB95-162657 Not available NTIS

National Inst. of Standards and Technology (NML),

Gaithersburg, MD. Electron and Optical Physics Div.

Surface Magnetic Microstructural Analysis Using Scanning Electron Microscopy with Polarization Analysis (SEMPA)

Final rept.

M. R. Scheinfein, J. Unguris, D. T. Pierce, and R. J. Celotta. 1990, 2p.

Pub. in Proceedings of International Congress for Elec tron Microscopy (12th), Seattle, WA., August 12-18, 1990, p216-217.
Keywords: "Electron microscopes, "Magnetic materials, "Polarization, Ferromagnetism, Microstructure, Images, Domain walls, Secondary emission, Electrons, Reprints, SEMPA(Scanning electron microscope with polarization analysis), Scanning electron microscope, Secondary electrons.

High resolution imaging of magnetic microstructure has important ramifications for both fundamental stud ies of magnetism and the technology surrounding the magnetic recording industry. A focused beam of electrons excites secondary electrons on a ferromagnet's surface. The secondaries leave the solid with an electron spin polarization which is characteristic of the net spin density in the ferromagnet. This is related directly to the sample magnetization. By scanning the beam and analyzing the secondary electron spin polarization at each point, a magnetization map of the ferromagnet's surface is generated.

\section{3,939}

PB95-162970 Not available NTIS

National Inst. of Standards and Technology (NML), Gaithersburg, MD. Chemical Process Metrology Div. Systematic Studies of the Effect of a Bandpass Filter on a Josephson-Junction Noise Thermometer. Final rept.

R. J. Soulen, W. E. Fogle, and J. H. Colwell. 1991, $4 \mathrm{p}$.

Pub. in Institute of Electrical and Electronics Engineers Transactions on Magnetics 27, n2 p2920-2923 1991.

Keywords: "Bandpass filters, "Noise temperature, * Superconductivity, Temperature measurement, Temperature scales, Fixed points(Mathematics), Variance(Statistics), Josephson junctions, Reprints, "Noise thermometers, SQUID noise thermometer.

The authors present the results of an extensive study of the effect of a filter upon the performance of a resistive SQUID noise thermometer used to define an absolute temperature scale below $1 \mathrm{~K}$. Agreement between the model for this effect and the experimental results indicates that the temperature scale defined by this noise thermometer is accurate to $0.1 \%$.

03,940

PB95-162988 Not available NTIS

National Inst. of Standards and Technology (CSTL) Gaithersburg, MD. Process Measurements Div.

Systematic Studies of the Effect of a Post-Detection Filter on a Josephson-Junction Noise Thermometer.

R. J. Soulen, W. E. Fogle, and J. H. Colwell. 1990,

$2 p$.

Pub. in Physica B 165, p111-112 Aug 90.

Keywords: "Filters, "Noise temperature, "Thermometers, Temperature measurement, Temthermometers, SQUID noise thermometer.

The authors present the results of an extensive study of the effect of a filter upon the performance of a resistive SQUID noise thermometer used to define an absolute temperature scale below $1 \mathrm{~K}$. Agreement between the theory and the experiment indicates that the scale defined by this noise thermometer is accurate to $0.1 \%$.

\section{3,941}

\section{PB95-163226 Not available NTIS}

National Inst. of Standards and Technology (NML). Gaithersburg, MD. Ionizing Radiation Div.

Problems Related to the Determination of Mass Densities of Evaporated Reference Deposits.

Fina! rept.

H. Tagziria, J. Pauwels, J. Verdonk, D. M. Gilliam, R. D. Scott, J. Byrne, P. Dawber, J. Van Gestel, and R. Eykens. 1991, 10p.

Pub. in Nuclear Instruments and Methods in Physics Research A 303, n1 p123-132 1991

Keywords: Experiment design, Lithium fluorides, Lithium 6. Boron 10, Reprints, "Reference deposits, Surface density, Mass density.

The accurate characterization of the surface density of thin deposits is of highest importance in certain experiments. However, if accuracies better than + or $-0.5 \%$ are to be quoted, careful consideration of secondary effects is necessary, as these effects may generate serious errors. The details of the radial surface density distribution, the formation of edge effects, and the evaluation of effective deposit diameters are discussed for the case of measurements and observations made 
during the preparation and characterization of (6)LiF and (10)B reference deposits, which were employed in a recent determination of the free neutron lifetime. Theoretical considerations are in excellent agreement with the experimental data obtained.

\section{3,942}

PB95-163283 Not available NTIS

National Inst. of Standards and Technology (NML), Gaithersburg, MD: Center for Atomic, Molecular and Optical Physics.

How Accurate Are the Josephson and Quantum Hall Effects and QED.

Final rept.

B. N. Taylor, and E. R. Cohen. 1991, 5p.

Pub. in Physics Letters A 153, n6-7 p308-312 1991.

Keywords: “Quantum electrodynamics, "Josephson effect, Fundamental constants, Sommerfeld constant, Accuracy, Reprints, "Quantum Hall effect, Von Klitzing constant, Fine structure constant.

We assess the accuracy of the theories of the Josephson and quantum Hall effects by testing precisely the exactness of the theoretically predicted relations $K(J)$ $=2 \mathrm{e} / \mathrm{h}$ and $\mathrm{R}(\mathrm{K})=\mathrm{h} / \mathrm{e}$ (squared), where $\mathrm{K}(\mathrm{J})$ and $\mathrm{R}(\mathrm{K})$ are the phenomenological Josephson and von Klitzing constants, respectively. Using the reported results of the most accurate determinations of various fundamental constants and related quantities currently available, we find $K(J) /(2 \mathrm{e} / \mathrm{h})=1-(219+$ or -103$) X$ $10(\exp -9)$ and $R(K) /(h / e($ squared $))=1+(60+$ or -22$)$ $X 10(\exp -9)$. If, as theory predicts, the relations are assumed to be exact, quantum electrodynamics (QED) may be tested precisely by comparing the QED value of the inverse fine-structure constant obtained from the electron magnetic moment anomaly, with the essentially QED-independent value derived from all of the other data.

\section{3,943}

PB95-163879 Not available NTIS

National Inst. of Standards and Technology (NML), Gaithersburg, MD. Center for Atomic, Molecular and Optical Physics.

Measurements of Fluorescence from Cold Atoms: Localization in Three-Dimensional Standing Waves.

Final rept.

C. I. Westbrook, P. S. Jessen, C. E. Tanner, R. N. Watts, W. D. Phillips, P. D. Lett, and S. L. Rolston

1991, 16p.

Pub. in AlP Conf. Proc. At. Phys. 12, v233 p89-104 1991.

Keywords: "Resonance fluorescence, Optical heterodyning, Three dimensional, Standing waves, Power spectra, Reprints, "Ultracold atoms, Doppler velocity spectroscopy, Optical molasses, Laser cooling, Laser trapping.

We have developed a heterodyne detector to measure the power spectrum of the resonance fluorescence from laser cooled atoms. With this detector we have demonstrated that cooled atoms can be localized in a three-dimensional optical standing wave. We have also used it to measure the temperature of the atoms in optical molasses and our results are consistent with those measured by other methods.

\section{3,944}

\section{PB95-163887 Not available NTIS}

National Inst. of Standards and Technology (NML), Gaithersburg, MD. Center for Atomic, Molecular and Optical Physics.

Localization of Atoms in a Three-Dimensional Standing Wave.

Final rept.

C. I. Westbrook, R. N. Watts, C. E. Tanner, P. D.

Lett, P. L. Gould, S. L. Rolston, and W. D. Phillips

1990, 4p

Pub. in Physical Review Letters 65, n1 p33-36 1990.

Keywords: Optical heterodyning, Resonance fluorescence, Standing waves, Three dimensional, Reprints, "Sodium atoms, "Atom traps, Optical molasses, Dicke narrowing, Laser cooling, Laser trapping.

We have a heterodyne technique to observe the spectrum of resonance fluorescence from sodium atoms in optical molasses. The spectra show a very narrow feature which we attribute to Dicke narrowing of the fluorescence of atoms localized to less than a wavelength in three dimensional potential wells.

\section{3,945}

PB95-163986 Not available NTIS
National Inst. of Standards and Technology (NML) Gaithersburg, MD Inorganic Analytical Research Div. Measurement of the $(93) \mathrm{Nb}(\mathrm{n}, 2 \mathrm{n})(92 \mathrm{~m}) \mathrm{Nb}$ Cross Section in a (235) U Fission Spectrum.

Final rept.

T. G. Williamson, and G. P. Lamaze. 1990, 5p.

Sponsored by Virginia Univ., Charlottesville. Dept. of Nuclear Engineering and Engineering Physics.

Pub. in Proceedings of ASTM-EURATOM Symposium (7th) on Reactor Dosimetry, Strasbourg, France, August 27-31, 1990, p371-375.

Keywords: "Niobium 93 target, "Neutron reactions, "Neutron cross sections, Neutron monitors, Fast neutrons, Fission spectra, Metastable state, Radioactivation, Dosimetry, Úranium 235, Niobium 92, Reprints.

When niobium is used as a fast neutron monitor, the isotopes $(93 \mathrm{~m}) \mathrm{Nb}$ and $(92 \mathrm{~m}) \mathrm{Nb}$ are produced. Much interest has been placed on the (93) $\mathrm{Nb}\left(n, n^{\prime}\right)(93 \mathrm{~m}) \mathrm{Nb}$ reaction because of the long half life of the product isotope and the low threshold energy of the reaction. On the other hand, the $(93) \mathrm{Nb}(n, 2 n)(92 \mathrm{~m}) \mathrm{Nb}$ reaction product has a half life of 10.1 days and the reaction has a threshold energy of about $9 \mathrm{MeV}$. It is useful for relatively short irradiations and for cases in which information is desired about high energy neutrons, such as in fusion applications. The spectrum-averaged cross section for the reaction $(93) \mathrm{Nb}(n, 2 n)(92 \mathrm{~m}) \mathrm{Nb}$ in a (235) $\mathrm{U}$ fission spectrum has been measured. The measured value is $0.433+$ or $-0.017 \mathrm{mb}$. Comparisons are made with calculated values.

\section{3,946}

\section{PB95-164497 Not available NTIS}

National Inst. of Standards and Technology (NEL) Gaithersburg, MD. Center for Manufacturing Engineering.

\section{Metrology}

J. A Simpson. 1991, 3p

Pub. in Encyclopedia of Physics, p723-725 1991

Keywords: "Metrology, Units of measurement, Nomenclature, Interferometry Quality control, Symbols, Mass, Time, Reprints, Weights and measures.

Metrology is the science of measurement, and if broady construed would encompass the bulk of experimental physics. The term is usually used in a more restricted sense to refer to that portion of measurement science used to provide, maintain, and disseminate consistent set of units; to provide support for the enforcement of equity in trade by weights and measures laws: or as an adjunct to quality control in manufacturing.

\section{3,947}

PB95-164646 Not available NTIS

National Inst. of Standards and Technology (PL), Boulder, CO. Quantum Physics Div.

Validation of the Inverse Square Law of Gravitation Using the Tower at Erie, Colorado, USA.

Final rept.

C. Speake, J. Faller, J. Y. Cruz, and J. C. Harrison.

1990, 3p.

Sponsored by Defense Mapping Agency, Fairfax, VA Pub. in Gravity, Gradiometry, and Gravimetry, Symposium No. 103, Edinburgh, Scotland, August 8-10, 1989, p17-191990

Keywords: "Gravitation, "Gravity, Basic interactions, Validation, Measurement, Towers, Tests, Reprints, Inverse square laws, Fifth force.

Eckhardt et al (1988) point out that gravity measurements on towers provide a straightforward way of testing Newton's inverse square law of gravitation, whose validity in the distance range of $10-1000 \mathrm{~m}$ has been repeatedly questioned during the last decade. Eckhardt et al (loc cit) and Romaides et al (1989) have interpreted their measurements on the $600 \mathrm{~m}$ WTVD tower in North Carolina as evidence for nonNewtonian gravitation. This paper describes a simila experiment using a $300 \mathrm{~m}$ meteorological tower at Erie on the plains of Colorado some $20 \mathrm{~km}$ east of Boulder. Gravity was measured at eight levels and in contrast with the results from North Carolina these measurements are in excellent agreement with values obtained by upward continuation of surface values using Newton's inverse square law.

\section{3,948}

PB95-168514 Not available NTIS

National Inst. of Standards and Technology (PL), Boulder, CO. Time and Frequency Div.
Interference in the Resonance Fluorescence of Two Trapped Atoms.

Final rept.

U. Eichmann, J. C. Bergquist, J. J. Bollinger, M. G.

Raizen, D. J. Wineland, J. M. Gilligan, and W. M.

Itano. 1994, 6p

Pub. in Proceedings of International Conference on Laser Spectroscopy ( $\$ 1$ th), Hot Springs, VA., June 13$18,1993, \mathrm{p} 43-481994$

Keywords: *Optical interference *Resonance fluorescence, Trapped particles, Polarization(Waves), Lase radiation, Mercury ions, Ion storage, Reprints, Laser cooling, Atom traps, Complementarity.

We report the observation of interference in the resonance fluorescence of two localized atoms irradiated by laser light. The fringe visibility can be explained in terms of $X$-ray scattering by a harmonic crystal and by simple 'which-path' arguments for the scattered photons. Polarization-sensitive detection of the resonance fluorescence demonstrates complementarity without invoking the position-momentum uncertainty relation.

03,949

PB95-16869s Not available NTIS

National Inst. of Standards and Technology (MEL)

Gaithersburg, MD. Precision Engineering Div.

Force Calibrations in the Nanonewton Regime.

Final rept.

L. P. Howard, and E. C. Teague. 1994, 5p.

Pub. in Proceedings of American Society for Precision Engineering Annual Meeting, Cincinnati, $\mathrm{OH}$., October 2-7, 1994, p13-17.

Keywords: "Atomic force microscopy, "Calibration Electrical measurement, Prototypes, Tribology, Pens, Reprints, "Force measurement, "Nanohardness NanoNewton forces, Nanotechnology, Force balances.

The paper describes an instrument and experiments for calibrating forces encountered with atomic force microscopes (AFMs) and nanohardness instruments. Calibration needs of such instruments are examined and a prototype calibration instrument is briefly intro duced. Our force balance performs nulling measurements using a capacitance gage for displacement detection, a magnet and coil actuator for application of nulling forces and a Proportional Integral Derivative (PID) control system. Experiments using electrica power measurements to calibrate the force balance magnet and coil actuator are described. Our experiments were modeled after Absolute Ampere and kilogram comparisons to $\mathrm{S} 1$ electrical units. Actual applied forces of tens of microNewtons were compared against the electrical calibrations to an agreement of approximately $3 \%$. For forces of tens of microNewtons, type A uncertainties of approximately $14 \mathrm{nN}$ were observed with estimated type B uncertainties of about $1.7 \%$ as a coefficient of variation $(\mathrm{CV})$

03,950

PB95-168746 Not available NTIS

National Inst. of Standards and Technology (PL), Boul der, CO. Time and Frequency Div.

Laser Cooling of Trapped Ions.

Final rept.

W. M. Itano, J. C. Bergquist, J. J. Bollinger, and D. J Wineland. 1992, 19p.

Sponsored by Office of Naval Research, Arlington, VA and Air Force Office of Scientific Research, Bolling AFB, DC

Pub. in Proceedings of International School of Physics: Laser Manipulation of Atoms and Ions, Varenna, Italy July 9-19, 1991, p519-537 1992.

Keywords: "Ion storage, Trapped particles, Atomic spectroscopy, Hyperfine structure, Laser spectros copy, Reprints, "Laser cooling, Nonneutral plasmas Penning traps, Paul traps.

Laser cooling techniques which have been applied to trapped ions are reviewed. We describe cooling in terms of mode coupling by a parametric drive. Experiments and theory which illustrate Doppler cooling, resolved-sideband cooling and Sisyphus cooling are described. Differences of laser cooling in Penning and Paul traps are discussed. Sympathetic cooling of one ion species by another is also discussed.

03,951

PB95-168910 Not available NTIS

National Inst. of Standards and Technology (EEEL) Boulder, CO. Electromagnetic Technology Div 


\section{PHYSICS}

\section{General}

Metrological Accuracy of the Electron Pump.

Final rept.

J. M. Martinis, M. Nahum, and H. D. Jensen. 1994,

$4 \mathrm{p}$.

Sponsored by Office of Naval Research, Arlington, VA Pub. in Physical Review Letters 72, n6 p904-907, 7 Feb 94.

Keywords: Electron pumping, Electron tunneling, Error analysis, Metrology, Accuracy, Reprints, "Electron pumps.

We have operated a five-junction electron pump with an error for transferring electrons of approximately 0.5 part per 10(exp 6). The error predicted from existing theory is several orders of magnitude smaller, thus implying that our present understanding of the Coulomb blockade is incomplete. We conjecture that the errors arise from photon-assisted tunneling, where the photon energy is supplied by noise from the environment.

\section{3,952}

PB95-169322 Not available NTIS

National Inst. of Standards and Technology (PL), Boulder, CO. Time and Frequency Div.

Recent Experiments on Trapped lons at the $\mathrm{Na}$ tional Institute of Standards and Technology.

Final rept.

D. J. Wineland, J. C. Bergquist, J. J. Bollinger, J. M. Gilligan, M. G. Raizen, D. J. Heinzen, C. S. Weime C. H. Manney, W. M. Itano, and F. L. Moore. 1992, $15 \mathrm{p}$.

Sponsored by Office of Naval Research, Arlington, VA and Air Force Office of Scientific Research, Bolling AFB, DC.

Pub. in Proceedings of International School of Physics: Laser Manipulation of Atoms and Ions, Varenna, Italy, July 9-19, 1991, p553-567 1992

Keywords: "Ion storage, Trapped particles, Hyperfine structure, Atomic spectroscopy, Laser spectroscopy, High resolution, Radiation pressure, Optical spectra, Beryllium ions, Beryllium 9, Mercury ions, Mercury 199 Reprints, Laser cooling, Penning traps, Paul traps.

These lecture notes summarize the work performed by the lon Storage group of NIST in the year preceeding July, 1991. Specific topics discussed are (1) the measurement of a hyperfine pressure shift in (9) $\mathrm{Be}(1+)$; (2) construction and testing of a Paul trap with linear geometry; (3) density limitations in a Penning trap; (4) a search for anomalous spin-dependent forces using spectroscopy of (9) $\mathrm{Be}(1+)$ ions; (5) the theory of Sisyphus cooling for a bound atom; (6) the observation of 'atomic projection' noise; (7) a precision measurement of the $\mathrm{g}(\mathrm{j})$ factor of $\mathrm{Mg}(1+)$; (8) subharmonic excitation of a single electron; (9) observation of time varying radiation pressure forces; and (10) high resolution optical spectroscopy of $(199) \mathrm{Hg}(1+)$ ions.

\subsection{3}

PB95-169330 Not available NTIS

National Inst. of Standards and Technology (PL), Boulder, CO. Time and Frequency Div.

High-Resolution Atomic Spectroscopy of LaserCooled lons.

Final rept.

D. J. Wineland, J. C. Bergquist, J. J. Bollinger, J. M. Gilligan, M. G. Raizen, C. S. Weimer, C. H. Manney, W. M. Itano, and F. L. Moore. 1992, $14 p$

Sponsored by Office of $\mathrm{Naval}$ Research, Arlington, VA. and Air Force Office of Scientific Research, Bolling AFB, DC

Pub. in Proceedings of International School of Physics: Laser Manipulation of Atoms and Ions, Varenna, Italy, July 9-19, 1991, p539-552 1992.

Keywords: "Ion storage, *Atomic spectra, Atomic spectroscopy, High resolution, Hyperfine structure, Laser spectroscopy, Beryllium ions, Beryllium 9, Mercury ions, Mercury 199, Reprints, Laser cooling, Penning traps, Paul traps, Nonneutral plasmas.

Techniques for obtaining high-resolution atomic spectra of laser-cooled ions are illustrated by on-going ex periments at NIST. In one experiment, an if oscillator is locked to a nuclear spin-flip hyperfine transition (frequency approx $=3.03 \times 10(\exp 8) \mathrm{Hz})$ in $(9) \mathrm{Be}(1+)$ ions which are stored in a Penning trap and sympathetically laser-cooled. Stability is better than $3 x$ 10 (exp -12) tau (sup -1/2) and uncertainty in Doppler shifts is estimated to be less than $5 \times 10(\exp -15)$. In a second experiment, a stable laser is used to probe an electric quadrupole transition (frequency approx $1.07 \times 10(\exp 15) \mathrm{Hz})$ in a single laser-cooled $(199) \mathrm{Hg}(1+)$ ion stored in a Paul trap. The measured
$Q$ value of this transition is approximately 10(exp 13) Future possible experiments are also discussed.

\section{3,954}

PB95-169348 Not available NTIS

National Inst. of Standards and Technology (PL), Boulder, CO. Time and Frequency Div. Laser-Cooled Positron Source.

Final rept.

D. J. Wineland, C. S. Weimer, and J. J. Bollinger.

1993, 11p.

Pub. in Hyperfine Interactions 76, p115-125 1993.

Keywords: "Positron sources, Ion storage, Beryllium ions, Beryllium 9, Feasibility, Reprints, Laser cooling Penning traps, Millikelvin temperature, Nonneutral plasmas.

We examine, theoretically, the feasibility of producing a sample of cold $(=$ or $<4 \mathrm{~K}$ ), high-density (approx $10(\exp 10) / \mathrm{cc})$ positrons in a Penning trap. We assume (9) $\mathrm{Be}(1+)$ ions are first loaded into the trap and lasercooled to approximately $10 \mathrm{mK}$ where they form a uniform density column centered on the trap axis Positrons from a moderator are then injected into the trap along the direction of the magnetic field through an aperture in one endcap of the trap so that they intersect the (9)Be(1+) column. Positron/(9)Be $(1+)$ Coulomb collisions extract axial energy from the positrons and prevent them from escaping back out the entrance aperture. Cooling provided by cyclotron radiation and sympathetic cooling with the laser-cooled (9)Be(1+) ons causes the positrons to eventually coalesce into a cold column along the trap axis. We present estimates of the efficiency for capture of the positrons and estimates of densities and temperatures of the resulting positron column. Positrons trapped in this way may be interesting as a source for antihydrogen production, as an example of a quantum plasma and as a possible means to produce a bright beam of positrons by leaking them out along the axis of the trap.

\section{3,955}

PB95-175022 Not available NTIS

National Inst. of Standards and Technology (PL), Gaithersburg, MD. Ionizing Radiation Div

Multi-Stage, Position Stabilized Vibration Isolation System for Neutron Interferometry.

Final rept.

M. Arif, D. E. Brown, G. L. Greene, R. Clothier, and K. Littrell. 1994, 7p.

Pub. in Proceedings of Society of Photo-Optical Instrumentation Engineers: Vibration Monitoring and Control, San Diego, CA., July 28-29, 1994, v2264 p20-26

Keywords: "Vibration isolators, Computerized control systems, Pneumatic servomechanisms, Degrees of freedom, Proximlty devices, Interferometers, Inclinometers, Metrology, Design, Reprints, "Neutron interferometry, CNRF facility, US NIST.

A two stage, position stabilized vibration isolation system has been constructed and is now in operation a the Cold Neutron Research Facility of the National Institute of Standards and Technology, Gaithersburg Md. The system employs pneumatic isolators with a multiple input/multiple output pneumatic servo system based upon pulse width modulation control loops. The first stage consists of a $40,000 \mathrm{~kg}$ reinforced concrete table supported by pneumatic isolators. A large environmentally isolated laboratory enclosure rests on the concrete table. The second stage consists of a 3000 $\mathrm{kg}$ granite optical table located within the enclosure and supported by another set of pneumatic isolators. The position of the two stages is monitored by proximity sensors and inclinometers with 12 degrees of free dom. The system controls 12 independent pneumatic airsprings. The signals from these sensors are fed into a personal computer based control system. The contro system has maintained the position of the two stages to better than 1 micrometer in translation and 5 (micro)rad in orientation for a period of a few months. $A$ description of the system and its characteristics is given.

\section{3,956}

PB95-175105 Not available NTIS

National Inst. of Standards and Technology (PL), Boulder, CO. Time and Frequency Div.

Experimental Results on Normal Modes in Cold, Pure lon Plasmas.

Final rept.

J. Bollinger, D. Heinzen, F. Moore, D. Wineland, C.

Weimer, and W. M. Itano. 1993, 6p.

Sponsored by Office of Naval Research, Arlington, VA
Pub. in Proceedings of Conference on Strongly Coupled Plasma Physics, Rochester, NY, August 17-21, 1992, p393-398 1993

Keywords: "Ion storage, "Beryllium ions, Strongly coupled plasmas, Cold plasmas, Beryllium 9 Trapping(Charged particles), Reprints, Electrostatic modes, Normal modes, Nonneutral plasmas, Penning traps, Ion plasmas, Ion traps.

Experimental measurements of some of the low order electrostatic modes of a nonneutral plasma of (9) $\mathrm{Be}(1+)$ ions stored in a Penning trap are discussed. The ions are laser-cooled and typically are strongly coupled with couplings as large as 200 to 300 . The measured mode frequencies agree well with a cold fluid calculation. The modes provide a nondestructive method for obtaining information on the plasma density and shape. In addition, the mode excitation by field asymmetries may provide a practical limit to the density and number of charged particles that can be stored in a Penning trap. Future measurements of the damping of the modes should provide information on the plasma viscosity in a strongly correlated plasma.

\section{3,957}

PB95-175113 Not available NTIS

National Inst. of Standards and Technology (PL), Boulder, CO. Time and Frequency Div.

Non-Neutral Ion Plasmas and Crystals, Laser Cooling, and Atomic Clocks.

Final rept.

J. J. Bollinger, D. J. Wineland, and D. H. E. Dubin

1994, 12p.

Sponsored by Office of Naval Research, Arlington, VA Pub. in Phys. Plasmas 1, n5 p1403-1414 May 94.

Keywords: "Ion storage, *Atomic clocks, Strongly coupled plasmas, Brillouin flow, Trapping(Charged particles), Reprints, Penning traps, Paul traps, Ion plasmas, lon traps, Millikelvin temperature, Laser coc ng, Nonneutral plasmas.

Experimental work which uses Penning and Paul traps to confine non-neutral ion plasmas is discussed. Penning traps use a static uniform magnetic field and a static electric field to confine ions. The Paul trap uses the ponderomotive force from inhomogeneous radiofrequency fields to confine ions to a region of minimum field strength. In many atomic physics experiments, these traps are designed to produce a harmonic restoring force for small numbers of stored ions $(<10,000)$. Under these conditions and at low temperatures, both traps produce plasmas with simple shapes whose mode properties can be calculated exactly. Laser cooling has been used to reduce the temperature of trapped ions to less than $10 \mathrm{mK}$ with ion spacings less than 20 micrometers. At such temperatures and interion spacings, the Coulomb potential energy between nearest neighbor ions is greater than the ion thermal energy and the ions exhibit spatial correlations characteristic of a liquid or crystal. Laser beams also apply a torque which, by changing the plasma angular momentum, changes the plasma density. Atomic clocks are an important application of ion trap plasmas. Better control of the plasma dynamics will reduce fluctuations in the relativistic time dilation, yielding better clocks.

\section{3,958}

PB95-176137 Not available NTIS

National Inst. of Standards and Technology (PL), Gaithersburg, MD. Quantum Metrology Div.

Electron-Ion-X-ray Spectrometer System

Final rept.

S. H. Southworth, M. A. MacDonald, T. LeBrun, and R. D. Deslattes. 1994, 5p.

Contract DE-W-31-109-ENG-38

Sponsored by Department of Energy, Washington, DC. Pub. in Nuclear Instruments and Methods in Physics Research A 347, p499-5031994.

Keywords: *Electron spectrometers, "Mass spectrometers, "X ray spectrometers, "Spectrometers, Innershell excitation, Electron spectroscopy, Ion spectroscopy, $X$ ray spectroscopy, Coincidence spectrometry, Fluorescence spectroscopy, Synchrotron radiation, Electron spectra, Design, Reprints.

We describe a spectrometer system developed for electron, ion, and X-ray spectroscopy of gas-phase atoms and molecules following inner-shell excitation by tunable synchrotron radiation. The instrumentation and experimental methods are discussed, and examples are given of electron spectra and coincidence spectra between electrons and fluorescent X-rays. 
03,959

PB95-176244 Not available NTIS

National Inst. of Standards and Technology (PL), Boulder CO. Time and Frequency Div.

Electrostatic Modes as a Diagnostic in PenningTrap Experiments.

Final rept.

C. S. Weimer, J. J. Bollinger, F. L. Moore, and D. J. Wineland. 1994, 12p.

Sponsored by Office of Naval Research, Arlington, VA. Pub. in Physical Review A 49, n5 p3842-3853 May 94.

Keywords: *Electron clouds, Diagnostic techniques, Reprints, "Electrostatic modes, Nonneutral plasmas, Penning traps.

A subset of the electrostatic modes of a cold cloud of electrons, a non-neutral electron plasma, trapped in a Penning trap has been observed and identified using a recent theoretical model. The detection of these modes is accomplished using electronic techniques which could apply to any ion species. The modes are observed in the low-density, low-rotation limit of the cloud where the cloud approaches a two-dimensional charged disk. We observe both axially symmetric and asymmetric drumhead modes. The shape, rotation frequency, and density of the cloud are found in a realtime nondestructive manner by measuring the frequency of these modes. In addition, it is found that radio-frequency sideband cooling compresses the cloud, increasing its density. The ability to measure and control the density of a trapped ion cloud might be useful for experiments on low-temperature ion-neutral-atom collisions, recombination rates, and studies of the confinement properties of non-neutral plasmas.

03,960

PB95-176293 Not available NTIS

National Inst. of Standards and Technology (PL), Boulder, CO. Time and Frequency Div.

Squeezed Atomic States and Projection Noise in Spectroscopy.

Final rept.

D. J. Wineland, J. J. Bollinger, W. M. Itano, and D. J. Heinzen, 1994, 22p.

Sponsored by Office of Naval Research, Arlington, VA. Pub. in Physical Review A 50, n1 p67-88 Jul 94.

Keywords: "Atomic spectroscopy, Atomic clocks, Trapped particles, Reprints, 'Squeezed states(Quantum theory), Quantum noise, Atom traps.

We investigate the properties of angular-momentum states which yield high sensitivity to rotation. We discuss the application of these 'squeezed-spin' or correlated-particle states to spectroscopy. Transitions in an ensemble of $\mathrm{N}$ two-level (or, equivalently, spin-1/ 2) particles are assumed to be detected by observing changes in the state populations of the particles (population spectroscopy). When the particles' states are detected with $100 \%$ efficiency, the fundamental limiting noise is projection noise, the noise associated with the quantum fluctuations in the measured populations. If the particles are first prepared in particular quantummechanically correlated states, we find that the signalto-noise ratio can be improved over the case of initially uncorrelated particles. We have investigated spectroscopy for a particular case of Ramsey's separated oscillatory method where the radiation pulse lengths are short compared to the time between pulses.

03,961

PB95-180055 Not available NTIS

National Inst. of Standards and Technology (PL), Gaithersburg, MD. Radiometric Physics Div.

Inner-Valence States $\mathrm{CO}(+)$ between $22 \mathrm{eV}$ and 46

eV Studied by High Resolution Photoelectron Spectroscopy and ab Initio Cl Calculations.

Final rept.

P. Baltzer, M. Lundqvist, B. Wannberg, M. A. Hayes, J. B. West, M. R. F. Siggel, A. C. Parr, J. L. Dehmer, L. Karlsson, and M. Larsson. 1994, 18p.

Contract DE-W-31-109-ENG-38

Sponsored by Department of Energy, Washington, DC. Pub. in Jnl. of Physics B: Atomic and Molecular Optical Physics 27, p4915-4932 1994

Keywords: "Carbon monoxide, Photoelectron spectroscopy, Vibrational spectra, Vibrational states, Angular distribution, High resolution, Branching ratio, EV range 10-100, Molecular ions, Synchrotron radiation, Rydberg states, Helium ions, Photoionization, Reprints.

Photoionization of the $\mathrm{CO}$ molecule and inner-valence states of $\mathrm{CO}(1+)$ between 22 and $45 \mathrm{eV}$ have been studied by means of photoelectron spectroscopy using both synchrotron radiation and He II radiation. Vibrational structure has been resolved in many bands up to $45 \mathrm{eV}$. CASSCF (complete active space self-consistent field) and $\mathrm{MRCl}$ (multireference configuration interaction) calculations of potential curves in the 22 $30 \mathrm{eV}$ range have been pertormed and these have been used to predict vibrational levels and FranckCondon factors. In this energy range three valence states, have been identified, and spectroscopic constants have been determined for the first two of these. Above $30 \mathrm{eV}$, all valence states have been found to be repulsive. In addition to the broad bands expected for these states, several progressions of narrow lines are observed, most probably reflecting transitions to Rydberg states.

\section{3,962}

PB95-180295 Not available NTIS

National Inst. of Standards and Technology (CAML), Gaithersburg, MD. Applied and Computational Mathematics Div.

Laplace's Equation and the Dirichlet-Neumann Map in Multiply Connected Domains. (NIST Reprint)

Final rept.

A. Greenbaum, L. Greengard, and G. B. McFadden. 1993, 12p

See also DE91011069. Sponsored by National Aeronautics and Space Administration, Washington, DC Microgravity Science and Applications Div. and Defense Advanced Research Projects Agency, Arlington, VA.

Pub. in Jnl. of Computational Physics 105, n2 p267278 Apr 93.

Keywords: "Laplace equation, Boundary integral method, Dirichlet problem, Integral equations, Iterative methods, Computational fluid dynamics, Computation, Matrices, Reprints, Ostwald ripening.

A variety of problems in materials science and fluid dynamics require the solution of Laplace's equation in multiply connected domains. Integral equation methods are natural candidates for such problems as they discretize the boundary alone, require no special effor for free boundaries, and achieve superalgebraic convergence rates on sufficiently smooth domains in two space dimensions, regardless of shape Current integral equation methods for the Dirichlet problem, however, require the solution of $M$ independent problems of dimension $\mathrm{N}$, where $\mathrm{M}$ is the number of boundary components and $\mathrm{N}$ is the total number of points in the discretization. In this paper, we present a new boundary integral equation approach, valid for both interior and exterior problems, which requires the solution of a single linear system of dimension $N+M$. We solve this system by making use of an iterative method (GMRES) combined with the fast multipole method fo the rapid calculation of the necessary matrix vector products. For a two-dimensional system with $200 \mathrm{com}$ ponents and 100 points on each boundary we gain a speedup of a factor of 100 from the new analytic formulation and a factor of 50 from the fast multipole method.

\section{3,963}

PB95-180436 Not available NTIS

National Inst. of Standards and Technology (PL), Gaithersburg, MD. Atomic Physics Div.

Atomic Branching Ratio Data for Oxygen-Like Species.

Final rept

J. Z. Klose, T. M. Deters, J. R. Fuhr, and W. L.

Wiese. 1994, 19p.

See also PB94-212842

Pub. in Jnl. of Quantitative Spectroscopy and Radiative Transfer 52, n5 p601-619 1994

Keywords: *Branching ratio, Vacuum ultraviolet radiation, Transition probabilities, High temperature, Spectral lines, Neon ions, Oxygen atoms, Radiometry, Plasma, Reprints, *Oxygen-like ions.

The branching ratio technique for radiometric calibrations in the vacuum ultraviolet spectral region is briefly reviewed. Lists of transitions suitable for use of the technique are given for oxygen-like species $(\mathrm{O} I$ and Ne III) along with pertinent data for their application.

\section{3,964}

PB95-180600 Not available NTIS

National Inst. of Standards and Technology (CSTL) Gaithersburg, MD. Thermophysics Div.
Laser Photoionization Measurements of Pressure in Vacuum.

Final rep

J.P. Looney. 1994, 8 p.

Pub. in Jnl. of the Vacuum Society of Japan $37, n^{9}$ p703-710 1994

Keywords: *Pressure measurement, *Ultrahigh vacuum, Laser applications, Reprints, "Laser photoionization.

For some time, laser photoionization has been used to detect low concentrations of atoms, molecules, and radical intermediates in a variety of applications, in cluding analytical chemistry, combustion, plasma and CVD diagnostics. Several of these studies have shown that laser photoionization has the fundamental sensitivity and selectivity necessary for species-specific partial pressure measurement in the ultrahigh vacuum regime. This body of work has, in part, spurred interest in the application of laser photoionization to measurements of pressures in the UHV regime. In this paper we discuss the results of the recent research con ducted at NIST on the use of laser photoionization for quantitative pressure measurements and the factors which limit the pressure measurements based upon this technique.

\section{3,965}

PB95-180790 Not available NTIS

National Inst. of Standards and Technology (PL), Boulder, CO. Time and Frequency Div.

Progress on a Cryogenic Linear Trap for (199) $\mathrm{Hg}(+)$ lons.

Final rept.

M. E. Poitzsch, J. C. Bergquist, W. M. Itano, and D.

J. Wineland. 1994, 3p.

Sponsored by Office of Naval Research, Arlington, VA Pub in Proceedings of Institute of Electrical and Electronic Engineers International Frequency Control Sym posium, Boston, MA., June 1-3, 1994, p744-746.

Keywords: "Ion storage, "Mercury ions, Mercury 199 Temperature range 0000-0013 K, Extremely high frequency, Frequency standards, Prototypes, Reprints, "Ion traps, Laser cooling.

We have observed linear 'crystals' of up to tens of laser-cooled $(199) \mathrm{Hg}(1+)$ ions in a new cryogenic linear if ion trap that operates at liquid helium temperature and is designed for use as a prototype $40.5 \mathrm{GHz}$ frequency standard with high accuracy and stability.

03,966

PB95-180915 Not available NTIS

National Inst. of Standards and Technology (EEEL) Boulder, CO. Electromagnetic Technology Div.

Flexible-Diaphragm Force Microscope.

Final rept

P. Rice, and J. Moreland. 1994, $2 p$

Pub. in Jnl. of Vacuum Science and Technology B 12 n4 p2465-2466 Jul/Aug 94

Keywords: "Atomic force microscopy, *Diaphragms(Mechanics) *Microscopes, Scanning tunneling microscopy, Disc recording systems, Polyamide resins, Low temperature, Flexibility, Reprints Surface topography, Hard disks.

A flexible polyimide diaphragm was used in place of the usual cantilever for atomic force microscopy. Images of hard disk surface features are presented, demonstrating the practicality of the method.

03,967

PB95-180956 Not available NTIS

National Inst. of Standards and Technology (PL) Gaithersburg, MD. Ionizing Radiation Div.

Comparison of NIST and ISO Filtered Bremsstrahlung Calibration Beams.

Final rept.

C. G. Soares, and M. Ehrlich. 1994, 16p.

Contract DE-Al01-93EH89321

Sponsored by Department of Energy, Washington, DC Pub. in Proceedings of Conference on Radiation Protection and Dosimetry (4th), Orlando, FL., October 23 27, 1994, p457-472

Keywords: "Bremsstrahlung, $X$ ray spectra Interlaboratory comparisons, Calibration, Standards, Revisions, ISO, Reprints, US NIST.

A detailed comparison is made between the 32 currently available National Institute of Standards and

Technology (NIST) bremsstrahlung techniques and the 41 International Standards Organization (ISO) brems- 


\section{General}

strahlung techniques recommended for use in the lat est revision of ISO Standard 4037 . The comparison is made in terms of measured and theoretical spectral distributions, and measured half-value layers. Some historical background on the choice of the respective techniques is also given. With the goal of greater international uniformity, a program is underway at NIST to implement the ISO techniques. The scope of the work and the current status of this effort is presented.

\section{3,968}

PB95-180964 Not available NTIS

National Inst. of Standards and Technology (PL),

Gaithersburg, MD. Ionizing Radiation Div.

Calibration of Dosimeters for the Cryogenic Irradiation of Composite Materials Using an Electron Beam.

Final rept.

A. Spindel, M. L. Tupper, W. L. McLaughlin, H. L.

Whittaker, and T. Overett. 1994, 4p.

Contract DE-AC35-89ER40486

Sponsored by Department of Energy, Washington, DC Pub. in Nuclear Instruments and Methods in Physics Research B 94, p150-1531994.

Keywords: "Electron dosimetry, "Dosimeters, "Calibration, Superconducting composites, Superconducting magnets, Temperature range 0000$0013 \mathrm{~K}$, MeV range 10-100, Radiation effects, Electron irradiation, Electron beams, Accelerators, Reprints, Radiochromic films.

In order to evaluate materials such as superconducting magnet components for use in the high-dose radiation environment of the large recirculating charged-particle accelerators such as CERN's Large Hadron Collider (Switzerland/France), CEBAF (Virginia) HERA (Hamburg, Germany), Fermilab's Tevatron (Illinois), and the recently cancelled Superconducting Super Collider (Texas), a study of the radiation resistance of these materials was carried out. These materials must with stand absorbed doses as large as $10(\exp 7)$ Gy at a temperature of $4 \mathrm{~K}$ and were tested under these conditions using an electron beam from a $20 \mathrm{MeV}$ linear accelerator. This paper describes the dosimetry at such very large doses and how the dose was delivered to the samples.

\section{3,969}

\section{PB95-181244 Not available NTIS}

National Inst. of Standards and Technology (PL), Boulder, CO. Time and Frequency Div.

High-Resolution Diode-Laser Spectroscopy of Cal-

cium.

Final rept.

A. S. Zibrov, R. W. Fox, R. Ellingsen, G. M. Tino, L. Hollberg, C. S. Weimer, and V. L. Velichansky. 1994, $5 \mathrm{p}$.

Sponsored by Air Force Office of Scientific Research Bolling AFB, DC

Pub. in Applied Physics B 59, p327-331 1994.

Keywords: "Laser spectroscopy, "Calcium, Semiconductor lasers, High resolution, Line width, Visible radiation, Reprints, *Frequency references.

Saturated-absorption signals on the calcium $657 \mathrm{~nm}$ transition are observed by direct absorption using diode lasers and a high flux atomic-beam cell. Linewidths as narrow as $65 \mathrm{kHz}$ are observed with a high signal-to-noise ratio. Prospects for using this system as a compact wavelength/frequency reference are considered.

\section{3,970}

PB95-202214 Not available NTIS

National Inst. of Standards and Technology (PL), Boulder, CO. Quantum Physics Div.

Core Potentials for Quasi-One-Electron Systems.

Final rept.

B. J. Albright, K. Bartschat, and P. R. Flicek. 1993,

$8 p$.

Grant NSF-PHY-9014103

Sponsored by National Science Foundation, Washington, DC.

Pub. in Jnl. of Physics B: Atomic and Molecular Optical Physics 26, p337-344 1993.

Keywords: "Atomic structure, Ionization potentials, Electron orbitals, Barium ions, Cesium, Indium, Sodium, Thallium, Reprints, Core potentials.

We have developed a method to calculate core potentials for quasi-one-electron systems and the corresponding single electron orbitals. It is shown that the approximate inclusion of exchange effects between the valence electrons and the core removes the unphysical structure in the potential function that is characteristic for potentials calculated by only including the effect of core polarization due to the valence electrons. Excellent agreement with experimental ionization potentials is achieved, and example results for various systems such as $\mathrm{Na}, \mathrm{Cs}, \mathrm{Ba}(1+)$, In and $\mathrm{Tl}$ are presented.

\section{3,971}

PB95-202248 Not available NTIS

National Inst. of Standards and Technology (PL), Boulder, CO. Quantum Physics Div.

Reduction of Light-Assisted Collisional Loss Rate from a Low-Pressure Vapor-Cell Trap.

Final rept.

M. H. Anderson, W. Petrich, J. R. Ensher, and E. A.

Cornell. 1994, 4p.

Grant NSF-PHY90-12244

Sponsored by National Science Foundation, Arlington,

VA. and Office of Naval Research, Arlington, VA.

Pub. in Physical Review A 50, n5 pR3597-R3600 Nov 94

Keywords: *Rubidium 87, Trapped particles, Laser radiation, Magnetooptics, Lifetime, Reduction, Losses, Reprints, "Atom traps, Collisional energy transfer.

We have demonstrated an order of magnitude increase in the number and in the lifetime of magneto optically trapped (87)Rb atoms collected from a lowpressure vapor. The improvement results from the reduction of light-assisted collision losses and is achieved by shelving the atomic population into the ower hyperfine ground state. Large fractional popu lations of the lower hyperfine ground state have been obtained by blocking the hyperfine repumping light in the region of the trapped atoms and by application of laser light that optically pumps the atoms into that state. We have observed trap lifetimes in excess of 700 $s$ at our highest vacuum levels. At the rubidium pressure that maximizes the product of the number and lifetime of trapped atoms we observe a lifetime of $240 \mathrm{~s}$ with $5 \times 10$ (exp 7) trapped atoms, under conditions for which an ordinary magneto-optical trap has a lifetime of only $14 \mathrm{~s}$ with $3 \times 10(\exp 6)$ atoms.

\section{3,972}

PB95-202271 Not available NTIS

National Inst. of Standards and Technology (PL), Boulder, CO. Quantum Physics Div.

Resonance Structure and Absolute Cross Sections in Near-Threshold Electron-Impact Excitation of the $4 s(2)$ (1)S yields $4 s 4 p(3) P$ Intercombination Transition in $\mathrm{Kr}(6+)$

Final rept.

M. E. Bannister, X. Q. Guo, T. M. Kojima, and G. H.

Dunn. 1994, 3p.

Contract DE-A105-86ER53237

Sponsored by Department of Energy, Washington, DC Pub. in Physical Review Letters 72, n21 p3336-3338, 23 May 94.

Keywords: "Electron-ion collisions, "Krypton ions, Tota cross sections, Multicharged ions, Electron transitions, Electron impact, Excitation, Reprints, Zinc-like ions.

First measurements of absolute total cross sections for electron-impact excitation of an intercombination transition to a nonradiating state of an ion are reported. The cross sections for near-threshold excitation of the $4 s(2)$ singlet $S \rightarrow-4 s 4 p$ triplet $P$ transition of $\mathrm{Kr}(6+)$ are dominated by dielectronic resonances. The measurements can serve as a benchmark for theoretical predictions by dielectronic resonance structure in the excitation of multicharged ions.

03,973

PB95-202289 Not available NTIS

National Inst. of Standards and Technology (PL), Boulder, CO. Quantum Physics Div

Short-Range Correlation and Relaxation Effects on the $(6 \mathrm{p}(2))(1)$ SO Autoionizing State of Atomic Barium.

Final rept.

K. Bartschat and C. H. Greene, 1993, 8p.

Sponsored by National Science Foundation, Washing ton, DC

Pub. in Jnl. of Physics B: Atomic and Molecular Optical Physics 26, pL109-L116 1993.

Keywords: "Photoionization, "Autoionization, "Barium, Excited states, R matrix, Correlation, Relaxation, Reprints, Basis sets.

The discrepancy between previous R-matrix calcula tions using fixed boundary conditions and the eigenchannel method for the photoionization spectrum of barium is resolved In particular, it is shown that the position of the $(6 \mathrm{p}(2))$ singlet $S(0)$ resonance that re sults in a maximum of the experimental photoionization cross section near $44,800 / \mathrm{cm}$ relative to the $(s(2))$ singlet $S(0)$ ground state of barium cannot be obtained by a standard close-coupling expansion involving only physical bound states; instead, the correct representation of short-range correlation effects and the relaxation of the $6 p$ orbital requires the inclusion of doubly excited configurations of the continuum-continuum type.

03,974

PB95-202297 Not available NTIS

National Inst. of Standards and Technology (PL), Boulder, CO. Quantum Physics Div

Connection between Superelastic and Inelastic Electron-Atom Collisions Involving Polarized Collision Partners.

Final rept.

K. Bartschat, and D. H. Madison. 1993, 2p

Sponsored by National Science Foundation, Washing ton, DC

Pub. in Physical Review Letters 48, n1 p836-837 Jul 93.

Keywords: "Electron-atom collisions, "Polarized beams, "Sodium, Electron transitions, Electron scattering, Elastic scattering, Inelastic scattering, Polarization(Spin alignment), Low energy, Reprints.

It is shown how the results of a recent experiment by Jiang, Zuo, Vuskovic, and Bederson (Phys. Rev. Lett. 68,915 (1992)), who investigated low-energy electron scattering from laser-excited polarized sodium atoms in the initial $(3 p)$ doublet $P($ sub $3 / 2$, sup 0$)(F=3, M(F)$ $=3$ ) state can be related to the inelastic $3 S \rightarrow 3 P$ transition involving initially unpolarized electron and atom beams. Hence, this method can provide an independent check of the traditional electron-scattering experiment with unpolarized beams.

03,975

PB95-202305 Not available NTIS

National Inst. of Standards and Technology (PL), Boulder, CO. Quantum Physics Div.

Femtosecond Time-Resolved Molecular Multiphoton lonization: The Na2 System.

Final rept.

T. Baumert, M. Grosser, R. Thalweiser, and G

Gerber. 1991, 4p.

Pub. in Physical Review Letters 67, n27 p3753-3756, 30 Dec 91

Keywords: "Sodium, Multi-photon processes, Molecular ions, Molecular beams, Excited states, Diatomic molecules, Autoionization, Reprints, "Multiphoton ionization, Femtosecond pulses, Time-of-flight spectroscopy

We report here the first experimental study of femtosecond time-resolved molecular multiphoton ionization. Femtosecond pump-probe techniques are combined with time-of-flight spectroscopy to measure transient ionization spectra of $\mathrm{Na} 2$ in a molecularbeam experiment. The wave-packet motions in different molecular potentials show that incoherent contributions from direct photoionization of a singly excited state and from excitation and autoionization of a bound doubly excited molecular state determine the observed transient ionization signal.

\section{3,976}

PB95-202362 Not available NTIS

National Inst. of Standards and Technology (MSEL), Gaithersburg, MD. Reactor Radiation Div.

Roles of Local Classical Acceleration and Spatial Separation in the Neutral Particle Analogs of the Aharonov-Bohm Phases.

Final rept.

R. C. Casella. 1994, 5p

Pub. in Physical Review Letters 73, n22 p2941-2945, 28 Nov 94.

Keywords: "Aharonov-Bohm effect, Neutral particles, Quantum theory, Acceleration, Spin, Reprints, Neutron interferometry.

The author shows that neither local nonuniform classical acceleration nor spatial separation of the quantized spin components plays an essential role in a number of neutron interferometer experiments designed to detect phases phi $(+-)(B, E)$ akin to the Aharonov-Bohm phases. These neutral particle phases, which simply manifest quantum interference in spin space, can also 
be detected by single beams, as exemplified by a recent breakthrough experiment by Sangster et al.

03,977

PB95-202438 Not available NTIS

National Inst. of Standards and Technology (PL), Boulder, CO. Quantum Physics Div.

Analyses of Recent Experimental and Theoretical Determinations of e-H2 Vibrational Excitation Cross Sections: Assessing a Long-Standing Controversy.

Final rept.

R. W. Crompton, and M. A. Morrison. 1993, 27p

Grant NSF-PHY-9108890

Sponsored by National Science Foundation, Washington, DC.

Pub. in Australian Jnl. of Physics 46, p203-229 1993.

Keywords: "Electron-molecule collisions, "Hydrogen, Vibrational states, Colliding beams, Cross sections, Reprints, Vibrational excitation.

During the last four years, we have undertaken a major effort to resolve a serious, long-standing discrepancy between various experimental and theoretical determinations of the cross section for the upsilon $(0)=0$ has involved crossed electron-beam molecular-beam measurements of relative angular distributions, measurements of transport coefficients in mixtures of $\mathrm{H} 2$ and various rare gases, and ab initio theoretical calculations using a vibrational close-coupling formalism with an exact treatment of non-local exchange effects. The discrepancy remains unresolved--a fact with potentially wide-ranging consequences for beam experiments, theory, and the unfolding of inelastic electronmolecule cross sections from swarm data. New analyses of the transport data and the application of a new method of extrapolating angular distributions beyond the range of measurement sheds light on this disagreement and its implications.

03,978

PB95-202446 Not available NTIS

National Inst. of Standards and Technology (PL), Boulder, CO. Quantum Physics Div.

Test of Newton's Inverse Square Law of Gravitation Using the 300-m Tower at Erie, Colorado.

Final rept.

J. Y. Cruz, J. C. Harrison, C. C. Speake, P. T. Keyser, J. E. Faller, J. Makinen, R. B. Beruff, T. M

Niebauer, and M. P. McHugh. 1991, 20p.

Pub. in Jnl. of Geophysical Research 96, nB12 p10,073-20,092, 10 Nov 91.

Keywords: "Gravitation, "Gravity, Basic interactions, Measurement, Validation, Towers, Tests, Reprints, Inverse square laws, Fifth force.

Gravity was measured at eight different heights on a 300-m meteorological tower, using electrostatically nulled LaCoste and Romberg gravimeters. The observed values were corrected for tides, temperature, and gravimeter screw errors, and tested for systematic effects due to (wind-induced) tower motion (no such effects were found). These corrected resuits were compared with values predicted by means of Newton's inverse square law from surface gravity values. The differences exhibit no systematic trends, and their rms value is only 10 microGal, well within the errors of the experiment, as the estimated measurement errors increase from 9 microGal at the lowest platform to 14 microGal at the top and those of the predictions from 10 microGal to 23 microGal. These results set new constraints on the magnitude of any non-Newtonian gravitational force; if such a force is derived from a Yukawa potential, the absolute value of alpha must be less than 0.00 l for lambda $=1,000 \mathrm{~m}$.

03,979

PB95-202453 Not available NTIS

National Inst. of Standards and Technology (PL), Boulder, CO. Quantum Physics Div.

Three-Vector Correlation Study of Orientation and Coherence Effects in Na(3p, (2)P1/2 inversely maps (2) P3/2)+He: Semiclassical and Quantum Calculations.

Final rept.

R. de Vivie-Riedle, J.P. J. Driessen, and S. R

Leone. 1993, 16p.

Grant NSF-PHY90-12244

Sponsored by National Science Foundation, Washington, DC

Pub. in Jnl. of Chemical Physics 98, n3 p2038-2053, 1 Feb 93.
Keywords: "Atom-atom collisions, "Atomic collisions, Sodium, Atomic energy levels, Electron tro $\mathrm{MeV}$ range 10-100, MeV range 100-1000, Colliding beams, Fine structure, Laser radiation, Cross sections, Quantum mechanics, Polarization, Orientation, Coherence, Alignment, Correlation, Helium, Reprints.

Multistructure cross sections, of both conventional and coherence types, are calculated for the fine structure transition $\mathrm{Na}$ (doublet $\mathrm{P}(1 / 2)<-$-doublet $\mathrm{P}(3 / 2)$ ) $+\mathrm{He}$ in an energy range of $10-200 \mathrm{meV}$. The cross sections are related to conditions of a crossed beams experiment in which the three controlled vectors are the initial relative velocity and the two polarization directions of excitation and probe laser beams. Both semiclassica and quantum mechanical calculations are performed The semiclassical method is employed to interpret and visualize the collision mechanism leading to the multistructure cross sections. In addition, the validity of the approximations used in the semiclassical mode is investigated.

\section{3,980}

PB95-202503 Not available NTIS

National Inst. of Standards and Technology (PL), Boulder, CO. Quantum Physics Div.

Absolute Cross-Section Measurements for Electron-Impact Single lonization of $\mathrm{Se}(+)$ and $\mathrm{Te}(+)$

Final rept.

N. Djuric, E. W. Bell, and G. H. Dunn. 1994, 5p. Contract DE-A 105-86ER53527

Sponsored by Department of Energy, Washington, DC. Office of Fusion Energy.

Pub. in International Jnl. of Mass Spectroscopy and Ion Processes 135, p207-211 1994

Keywords: "Electron-ion collisions, "Selenium ions, Tellurium ions, Electron impact, Colliding beams, Cross sections, EV range 10-100, EV range 100-1000, Ionization, Reprints, Arsenic-like ions, Antimony-like ions.

The crossed beams technique has been used to meas ure absolute cross-sections for the single ionization of $\mathrm{Se}(1+)$ and $\mathrm{Te}(1+)$ at electron energies from threshold to $200 \mathrm{eV}$. The peak cross-sections obtained are 16.6 $x$ 10(exp-17) sq $\mathrm{cm}$ at about $53 \mathrm{eV}$ for $\mathrm{Se}(1+)$ and 24.3 $x$ 10(exp-17) sq $\mathrm{cm}$ at about $43 \mathrm{eV}$ for $\mathrm{Te}(1+)$. The measured $\mathrm{Se}(1+)$ cross-sections are bracketed from above by directionization cross-sections from configuration-averaged distorted-wave calculations of Pindzola (unpublished work) and from below by results using the single parameter semi-empirical formula of Lotz (Z. Phys., 216 (1968) 241), with both calculations giving results in moderate agreement with the meas urements. In contrast, comparisons of measured $\mathrm{Te}(1+)$ cross-sections with similar calculations are generally poor. Ionization-rate coefficients for a Maxwellian electron-temperature distribution calculated from the experimental data presented

\section{3,981}

\section{PB95-202511 Not available NTIS}

National Inst. of Standards and

Crossed-Beams Measurements of Absolute Cross Sections for Electron Impact lonization of S(+) Final rept.

N. Djuric, E. W. Bell, and G. H. Dunn. 1993, 5p.

Contract DE-A105-86ER53237

Sponsored by Department of Energy, Washington, DC

Office of Energy Research.

Pub. in International Jns. of Mass Spectrometry and Ion Processes 123, p187-191 1993

Keywords: "Electron-ion collisions, "Sulfur ions, EV range 10-100, EV range 100-1000, Electron impact Cross sections, Colliding beams, Ionization coefficients, Reprints, Phosphorus-like ions.

Absolute cross sections for electron impact ionization of $S(1+)$ have been measured in the electron energy range from $30-250 \mathrm{eV}$ using the crossed beams technique. Results are compared with scaled cross sections from other P-like ions, with a semiempirical formula and with other recently reported measurements. Expansion coefficients and formulas for generating ionization rate coefficients in the electron temperature range $10,000 \mathrm{~K}=$ or $<\mathrm{T}=$ or $<1$ million $\mathrm{K}$ are presented.

\section{3,982}

Not available NTIS

National Inst. of Standards and Technology (PL), Boulder, CO. Quantum Physics Div.
Absolute Cross Sections for Electron-Impact Single lonization of $\mathrm{Si}(+)$ and $\mathrm{Si}(2+)$.

Final rept.

N. Djuric, E. W. Bell, X. Q. Guo, M. E. Bannister, M.

S. Pindzola, D. C. Griffin, G. H. Dunn, and R. A.

Phaneuf. 1993, 8p.

Contract DE-A 105-86ER53237

Sponsored by Department of Energy, Washington, DC Office of Fusion Energy.

Pub. in Physical Review A 47, n6 p4786-4793 Jun 93.

Keywords: "Electron-ion collisions, "Silicon ions, Electron impact, Cross sections, Colliding beams, Ionization, Reprints, Aluminum-like ions, Magnesium-like ions.

Absolute cross sections for electron-impact single ionization of $\mathrm{Si}(1+)$ and $\mathrm{Si}(2+)$ have been measured using crossed beams of ions and electrons and calculated using a configuration-average distorted-wave method. Corrections have been made for metastable components and small tractions of nitrogen impurities in the incident ion beams. Excitation-autoionization measurably enhances the cross sections of both $\mathrm{Si}(1+)$ and $\mathrm{Si}(2+)$. Ionization rate coefficients and fitting parameters are presented for the experimental data.

\section{3,983}

\section{PB95-202545 Not available NTIS}

National Inst. of Standards and Technology (PL), Boulder, CO. Quantum Physics Div.

$\mathrm{n}$-Vector Correlations in Collision Dynamics with Atomic Orbital Alignment: The Importance of Co herence Denoting Azimuthal Structure for $n$ (greater than or equal to) 3.

Final rept.

J. P. J. Driessen, and S. R. Leone. 1992, 11p.

Pub. in Jnl. of Physical Chemistry 96, n15 p6136-6146 1992

Keywords: "Atomic collisions, "Calcium, Vectors(Mathematics), Atomic orbitals, Polarized light, Laser radiation, Energy transfer, Orientation, Alignment, Coherence, Reprints.

An introduction is presented of vector correlations in collision experiments involving atomic orbital alignment or orientation. At present, aligned or oriented species can be prepared (or probed) with 'relative' ease using polarized laser beams. First, an extensive expose o the necessary mathematical formalisms for two- and three-vector correlations is given, and then experimental examples for atomic $\mathrm{Ca}$ are discussed to elucidate the theory. It is demonstrated both theoretically and experimentally that azimuthal structure about the initial relative velocity vector (called coherence) be comes important when three or more vector quantities are controlled in the collision process.

\section{3,984}

PB95-202552 Not available NTIS

National Inst. of Standards and Technology (PL), Boulder, CO. Quantum Physics Div.

Resonant Two-Color Detachment of $\mathrm{H}(-)$ with Excitation of $H(n=2)$.

Final rept.

N. Y. Du, A. F. Starace, and N. A. Cherepkov. 1993, $13 p$.

Contract DE-FG02-88ER13955

Sponsored by Department of Energy, Washington, DC. Pub. in Physical Review A 48, n3 p2413-2425 Sep 93

Keywords: "Hydrogen ions, "Electron detachment, *Photodetachment, Polarized light Cross sections, Negative ions, Excitation, Reprints, Two photon processes

The cross sections for resonant two-color, two-photon detachment of $\mathrm{H}(1-)$ with excitation of the degenerate $H(2 s)$ and $H(2 p)$ levels are calculated within a semiempirical adiabatic hyperspherical representation The first photon with energy omega(1) $=0.4017$ au is resonant with the well-known Feshbach (sup 1) $P$ (sup o) resonance below the $H(n=2)$ threshold. The second photon, with energy omega(2) = or > $0.12605 \mathrm{au}$, scans the energy region above the $\mathrm{H}(\mathrm{n}$ = 2) threshold over which long-range dipole-field-induced cross-section oscillations are predicted to occur. Such Gailitis-Damburg oscillations have not yet been observed experimentally. Results for various pairs of light polarization for the two photons are presented.

03,985

PB95-202578 Not available NTIS

National Inst of Standards and Technology (PL) Gaithersburg, MD. Electron and Optical Physics Div. 


\section{PHYSICS}

\section{General}

Numerical Solution of the Nonlinear Schroedinger Equation for Small Samples of Trapped Neutral Atoms.

Final rept

M. A. Edwards, and K. Burnett. 1995, 5p.

Grant NSF-PHY-9206769

Sponsored by National Science Foundation, Arlington, VA

Pub. in Physical Review A 51, n2 p1382-1386 Feb 95

Keywords: "Schrodinger equation, Nonlinear differential equations, Bose-Einstein condensation, Trapped particles, Neutral atoms, Numerical solution, Reprints, "Atom traps, Laser traps, Laser cooling, Gross Pitaevskii equation.

We present a numerical technique to solve the time independent nonlinear Schrodinger equation with an external potential. We apply it to the case of a dilute Bose-condensed assembly of trapped neutral atoms where the potential varies on the same scale as the condensate. This situation should soon be accessible to experimental observation.

\section{3,986}

PB95-202594 Not available NTIS

National Inst. of Standards and Technology (PL), Boulder, CO. Quantum Physics Div.

Long-Range Parity-Nonconserving Interaction.

Final rept.

V. V. Flambaum. 1992, 6p.

Grant NSF-PHY89-04035

Sponsored by National Science Foundation, Washington, DC Pub. in Physical Review A 45, n9 p6174-6179, 1 May

Keywords: *P invariance, "Parity, Weak interactions, Polarization characteristics, Charged particles, Vectors(Mathematics), Molecules, Atoms, Solids, Interactions, Reprints.

There is a long-range parity-nonconserving (PNC) interaction between a charged particle and a composite system (atom, nucleus) induced by the usual contact weak interaction inside this system: $\mathrm{U}=(\mathrm{H} X$ E) $($ dot) (beta(sub nu)) approx. $=1 / R($ sup 4$)$ or $1 / R($ sup $5)$, where $\mathrm{R}$ is distance, $\mathrm{E}$ and $\mathrm{H}$ are electric and magnetic fields of the particle, and $\mathrm{J}$ and $\mathrm{B}$ (beta(sub nu) are the angular momentum and PNC vector polarizability of the system. The mechanisms of en hancement of PNC vector polarizability are considered. PNC effects in atoms, molecules, and solids are discussed (P-odd and T-even electric dipole moment, $P$-odd electric field, etc)

\section{3,987}

PB95-202628 Not available NTIS

National Inst. of Standards and Technology (PL), Boulder, CO. Ouantum Physics Div.

Quantum Mechanics of a Solid-State Bar Gravitational Antenna.

Final rept.

L. P. Grishchuk. 1992, 8p.

Pub. in Physical Review D 45, n8 p2601-2608, 15 Apr

92

Keywords: "Gravitational wave antennas, "Quantum mechanics, Absorption cross sections, Solid state devices, Bars, Reprints.

A quantum-mechanical treatment of a bar gravitational antenna is presented. The theory takes into account the crystalline structure of the bar and collective behavior of its mass elements. The low-frequency and highfrequency (Debye) modes of oscillations are considered. It is shown that the quantum-mechanical derivation of the absorption cross section of the gravitational antenna agrees totally with the classical result. The recent claims of a significant (six orders of magnitude) quantum-mechanical enhancement of the cross section are shown to be incorrect.

\section{3,988}

PB95-202701 Not available NTIS

National Inst. of Standards and Technology (PL), Boulder, CO. Quantum Physics Div.

Modified Effective Range Theory as an Alternative to Low-Energy Close-Coupling Calculations.

Final rept

W. A. Isaacs, and M. A. Morrison. 1992, 23p.

Grant NSF-PHY-8505438

Sponsored by National Science Foundation, Washington, DC

Pub. in Jnl. of Physics B: Atomic and Molecular Optical

Physics 25, p703-725 1992.
Keywords: "Electron-molecule collisions, "Effective range theory, "Hydrogen, "Nitrogen, Electron scattering, Cross sections, Low energy, Feasibility, Reprints. We investigate the feasibility of using modified effective range theory to 'project' results of theoretical scattering calculations to energies so low that such calculations are numerically intractable. In particular, this the ory, as developed by Fabrikant in the body-frame fixednuclei formulation of electron scattering from non-pola molecules, provides expansions of the reactance matrix that enable determination of the scattering length (and other parameters as needed) and total cross sections with greater accuracy and over a larger range of energies than do conventional implementations of such expansions for the total cross section. We have explored the viability, accuracy and potential problems of this stratagem for the e-H2 and e-N2 systems; in both cases, careful use of these expansions enables calculation of highly accurate cross sections at very low energies without resource to numerically problematic, CPU-intensive close-coupling calculations.

\section{3,989}

PB95-202719 Not available NTIS

National Inst. of Standards and Technology (PL), Boulder, CO. Quantum Physics Div

Slow-Electron Collisions with $\mathrm{CO}$ Molecules in an Exact-Exchange Plus Parameter-Free Polarization Model.

Final rept.

A. Jain, and D. W. Norcross. 1992, 13p.

Contract DE-FC05-85ER-250000

Sponsored by Department of Energy, Washington, DC Office of Energy Research.

Also pub. as Physical Review A 45, n3 p1644-1656, 1 Feb 92

Keywords: "Electron-molecule collisions, "Carbon monoxide, Electron scattering, Electron collisions, Milli EV range, EV range 1-10, Momentum transfer, Cross sections, Reprints.

We report low-energy $(0.001$ to $10-\mathrm{eV})$ electron-CO scattering cross sections obtained using an exact-exchange (via a separable-exchange formulation) plus a parameter-free correlation-polarization model in the fixed-nuclei approximation (FNA). The differential, total, and momentum-transfer cross sections are reported for rotationally elastic, inelastic, and summed processes. To remove the limitations of the FNA with respect to the convergence of total and differentia cross sections, the multipole-extracted-adiabaticnuclei approximation is used.

03,990

PB95-202727 Not available NTIS

National Inst. of Standards and Technology (PL), Boulder, CO. Quantum Physics Div.

Superconducting Resonator and a Cryogenic GaAs Field-Effect Transistor Amplifier as a SingleIon Detection System.

Final rept.

S. R. Jefferts, T. Heavner, P. Hayes, and G. H

Dunn. 1993, 4p.

Grant NSF-PHY90-12244

Sponsored by National Science Foundation, Washington, DC

Pub. in Review of Scientific Instruments 64 , n3 p737. 740 Mar 93

Keywords: "Ion detection, Field effect transistors, Transisior amplifiers, Gallium arsenides, Superconducting devices, Resonators, Inductors, Reprints, "Single ion detectors.

A current measuring system capable of detecting single ions is described. The detector operates at a frequency of nominally $900 \mathrm{kHz}$ and uses a superconducting inductor with high $\mathrm{Q}(\mathrm{Q}$ approx = 8,500 ) and a cryogenic GaAs field-effect transistor (FET) amplifier with noise temperature less than 0.3 $K$. Noise measurements of the FET are presented, along with details of the construction of the superconducting inductor.

03,991

PB95-202818 Not available NTIS

National Inst. of Standards and Technology (PL), Boulder, CO. Quantum Physics Div.

Probing Bose-Einstein Condensed Atoms with Short Laser Pulses.

Final rept.

M. Lewenstein, and L. You. 1993, 4p.

Pub. in Physical Review Letters 71, n9 p1339-1342, 30 Aug 93.
Keywords: Bose-Einstein condensation, Light scattering, Light pulses, Laser radiation, Reprints, "Atom traps, Optical probes.

We propose a method of probing a system of cooled atoms in a trap using short laser pulses. Above the critical temperature for Bose-Einstein condensation, such a system scatters very weakly. Coherent scattering occurs primarily in the forward direction. Below the critical temperature, the number of scattered photons increases dramatically and the scattered light is emitted in the solid angle determined by the size of the condensate.

\section{3,992}

PB95-202867 Not available NTIS

National Inst. of Standards and Technology (PL), Boulder, CO. Quantum Physics Div.

Variationally Stable Treatment of Two- and ThreePhoton Detachment of $\mathbf{H}(-)$ Including Electron-Correlation Effects.

Final rept

C. Liu, B. Gao, and A. F. Starace. 1992, 14p.

Pub. in Physical Review A 46, n9 p5985-5998, 1 Nov 92.

Keywords: "Hydrogen ions, Electron affinity, Electron correlation, Electron detachment, Negative ions, Hyperspheres, Reprints, Keldysh approximation, Hyperspherical coordinates.

A variationally stable, adiabatic hyperspherical treatment of two- and three-photon detachment of $\mathrm{H}(1-)$ is presented. Results are compared with analytic predictions of a zero-range potential model of $\mathrm{H}(1-)$. De tailed comparisons are made also with other theoretical results which include the effects of electron correlations. We predict analytically (and demonstrate numerically) an extreme sensitivity of the theoretical predictions to any errors in the value of the electron affinity employed. In an Appendix we show that the low-intensity limit of the Keldysh treatment of detachment of an electron bound in a zero-range potential agrees with the results of a perturbative treatment.

03,993

PB95-202883 Not available NTIS

National Inst. of Standards and Technology (PL), Boul der, CO. Quantum Physics Div.

Alignment in Two-Step Pulsed Laser Excitation of Rydberg Levels in Light Atoms: The Example of Sodium.

Final rept

K. B. MacAdam, and M. A. Morrison. 1993, 14p.

Grants NSF-PHY-9122377, NSF-PHY-9108890

Sponsored by National Science Foundation, Washington DC

Pub. in Physical Review A 48, n2 p1345-1358 Aug 93

Keywords: "Rydberg states, "Sodium, Atomic energy levels, Laser radiation, Pulsed lasers, Density matrix, Excitation, Alignment, Atomic orbitals, Reprints.

Aligned atomic Rydberg states of sodium can be prepared using two-step excitation from the ground state by linearly polarized pulsed lasers. Information that is normally inaccessible, e.g., sublevel partial cross sections in charge-transfer experiments, can be obtained when aligned targets are used. The calculations of or bital alignment must carefully allow for fine and hyperfine structure, laser linewidths, pulse widths and delays, sublevel coherences, and other factors. In this paper we derive the orbital alignments and time-averaged d-state sublevel populations for 3 doublet $S(1 / 2)$ $\rightarrow 3$ doublet $P(J$ sub 1$) \rightarrow n$ doublet $D$ excitations in Na using angular-momentum and density-matrix methods. We consider both quadrupole alignment $A(\sup (2))$ and hexadecapole alignment $A(\sup (4))$, with excitation through either $J(1)=1 / 2$ or $3 / 2$ intermediate states considered on the same footing. We show sublevel populations for absolute values of $M(L)=0,1$, and 2 analytically and graphically.

\section{3,994}

PB95-202917 Not available NTIS

National Inst. of Standards and Technology (PL), Boulder, CO. Quantum Physics Div.

Threshold Electron Excitation of $\mathrm{Na}$.

Final rept.

B. Marinkovic, P. Wang, and A. Gallagher. 1992, 5p. Contract DE-FG02-87ER13720

Sponsored by Department of Energy, Washington, DC. Office of Energy Research.

Pub. in Physical Review A 46, n5 p2553-2557, 1 Sep 92. 
Keywords: "Electron-atom collisions, "Sodium, Electron collisions, Electronic states, Threshold effects, Ex. cited states, Excitation, Reprints, Collisional energy transfer.

Electron collisional excitation of the 4D, 5D, 4P, and $6 S$ states of $\mathrm{Na}$ has been measured with about 30meV energy resolution. Very rapid, unresolved threshold onsets are seen for all but the 4P state, and a nearthreshold resonance is suggested by the $5 \mathrm{D}$ data. However, only weak undulations in the cross sections are observed above threshold.

\section{3,995}

PB95-202958 Not available NTIS

National Inst. of Standards and Technology (PL), Boulder, CO. Ouantum Physics Div.

Collisional Energy Transfer between Excited-State Strontium and Noble-Gas Atoms.

Final rept.

D. A. Miller, L. You, J. Cooper, and A. Gallagher

1992, $7 \mathrm{p}$

Grant NSF-PHY90-12244

See also PB91-204164. Sponsored by National Science Foundation, Washington, DC.

Pub. in Physical Review A 46, n3 p1303-1309, 1 Aug 92.

Keywords: "Atomic collisions, "Strontium, Atomic energy levels, Oscillator strengths, Excited states, Pulsed lasers, Radiative lifetime, Transition probabilities, Rare gases, Reprints, Collisional energy transfer.

Inert-gas induced collisional transfer between the first four excited states of $\mathrm{Sr}$ has been measured by determining excited-state densities following pulsed laser excitation of the 5 singlet $P$ state. We observe 5 singlet $P$ collisional transfer exclusively to the 4 triplet $D$ state, with (about $800 \mathrm{~K}$ ) rate coefficients ranging from 0.24 to $2.0 \times 10(\exp -11) \mathrm{cc} / \mathrm{s}$ for the various gases. We also measure 4 singlet $D(2)$ transfer primarily to the 4 triplet $\mathrm{D}$ state, with rate coefficients of 0.65 to $6.8 \times 10(\exp$ $-11) \mathrm{cc} / \mathrm{s}$. The 4 triplet $D$ state radiative lifetime is observed to be $2.9+$ or -0.2 microsec

\section{3,996}

PB95-202974 Not available NTIS

National Inst. of Standards and Technology (PL), Boulder, CO. Ouantum Physics Div.

Importance of Bound-Free Correlation Effects for Vibrational Excitation of Molecules by Electron Impact: A Sensitivity Analysis.

Final rept.

M. A. Morrison, and W. K. Trail. 1993, 13p.

Grant NSF-PHY90-12244

Sponsored by National Science Foundation, Washington, DC.

Pub. in Physical Review A 48, n4 p2874-2886 Oct 93.

Keywords: "Electron-molecule collisions, "Hydrogen, Electron scattering, Electron impact, Colliding beams, Cross sections, Sensitivity analysis, Correlation, $\mathrm{Re}$ prints, Vibrational excitation.

One of the most interesting, important, and problematic components of interaction potentials for electron-atom and -molecule scattering arises from many-body ef fects in the near-target region. Such 'core-polarization' effects are of particular concern for vibrational-excitation calculations, where these short-range bound free correlation and nonadiabatic velocity-dependent effects have remained resistant to rigorous treatmen being represented instead by approximations or model potentials. In order to provide guidance for assessing such potentials and insight into the nature of these many-electron effects, we have investigated the sensitivity to core polarization of total, momentum-transfer rotational-excitation, and vibrational-excitation e-H2 cross sections. The sensitivity analysis for the latter cross section also comments on a long-standing, severe discrepancy between cross sections determined in various crossed-beam experiments and by transport analysis of swarm data for this simplest electron-neutral-molecule system.

\section{3,997}

PB95-203030 Not available NTIS

National Inst. of Standards and Technology (PL), Boul. der, CO. Ouantum Physics Div.

Energy-Pooling Collisions in Barium.

Final rept.

J. A. Neuman, A. Gallagher, and J. Cooper. 1994

9p.

Grant NSF-PHY90-12244

Sponsored by National Science Foundation, Arlington, VA.
Pub. in Physical Review A 50, n2 p1292-1300 Aug 94.

Keywords: "Barium, "Atomic collisions, Atomic energy levels, Excited states, Energy transfer, Reprints, Rate coefficients.

Rate coefficients for energy-pooling collisions between low-lying excited states of Ba have been measured The 6s6p triplet $\mathrm{P}(1)$ level in $\mathrm{Ba}$ is pumped by a $\mathrm{cW}$ diode laser, and radiative decay and collisions with buffer-gas atoms also populate the 5 d6s (singlet D) and 5 d6s (triplet D) metastable levels. The densities of these low-lying excited states are measured by the absorption of lines from a Ba hollow-cathode lamp, and the states populated by energy-pooling collisions are studied by comparing their fluorescence intensity to that of the laser-excited level. The rate coefficients are on the order of gas-kinetic rates, and they are not strongly dependent on energy defect, spin changes, or angular-momentum changes.

03,998

PB95-203097 Not available NTIS

National Inst. of Standards and Technology (PL), Boulder, CO. Ouantum Physics Div.

Relative Photoionization and Photodetachment Cross Sections for Particular Fine-Structure Transitions with Application to $\mathrm{Cl} 3 \mathrm{~s}$-subshell Photoionization.

Final rept.

C. Pan, and A. F. Starace. 1993, 10p.

Grant NSF-PHY-9108002

Sponsored by National Science Foundation, Washington, DC.

Pub. in Physical Review A 47, n1 p295-304 Jan 93.

Keywords: "Photoionization, "Photodetachment, "Chlorine, Branching ratio, Cross sections, Electron transitions, Fine structure, Negative ions, Positive ions, Reprints.

The relative photoionization cross section for starting from a particular fine-structure level of the initial state of an atomic system and leading to a particular finestructure level of the residual ion is presented for the case of atomic systems having one open subshell using a simple theoretical model in which emphasis is placed on the analysis of geometrical effects. This model parametrizes photoionization cross sections in terms of LS-dependent, single-configuration dynamical amplitudes, unlike previous treatments which assume LS-independent transition amplitudes from the start. In special cases our general formulas are shown to re duce to those of previous workers. We highlight photoionization of inner $s$ subshells in open-shell atoms and show that when the total angular momenta for the initial atomic and final ionic fine-structure levels differ by more than $1 / 2$ then such transitions are quasiforbidden. This prediction is tested in detail for the case of $\mathrm{Cl} 3 \mathrm{~s}$-subshell photoionization by comparison of our single-configuration results with the multiconfiguration, eigenchannel R-matrix results of Robicheaux and Greene. All of our results apply also to photoionization of open-shell positive ions or photodetachment of open-shell negative ions.

\section{3,999}

PB95-203105 Not available NTIS

National Inst. of Standards and Technology (PL), Boulder, CO. Ouantum Physics Div.

Behavior of Atoms in a Compressed Magneto-Optical Trap.

W. Petrich, M. H. Anderson, J. R. Ensher, and E. A. Cornell. 1994, 4p.

Grant NSF-PHY90-12244

Sponsored by National Science Foundation, Arlington, VA. and Office of Naval Research. Arlington, VA

Pub. in Jnl. of the Optical Society of America B 11, n8 p1332-1335 Aug 94.

Keywords: Trapped particles, Laser radiation, Magnetic fields, Magnetooptics, Reprints, "Atom traps, Laser cooling.

We investigate the behavior of a cloud of atoms in a magneto-optical trap, which--after collection--is compressed when the field gradients of the trap magnetic field are increased. We measure sizes and shapes of the atom cloud as a function of laser detuning, magnetic field gradient, and number of trapped atoms. A transient density increase of more than an order of magnitude has been achieved. Moreover, reproducible Gaussian density distributions are observed at large detunings and intermediate magnetic-field gradients, permitting an accurate determination of density.

\section{4,000}

PB95-203113 Not available NTIS

National Inst. of Standards and Technology (PL), Boulder, CO. Ouantum Physics Div.

Dielectronic Capture Processes in the Electron-Impact lonization of $\mathrm{Sc}(2+)$.

Final rept

M. S. Pindzola, T. W. Gorczyca, N. R. Badnell, G.

Hofmann, B. Weissbecker, K. Tinschert, E. Salzborn, A. Multer, G. H. Dunn, D. C. Griffin, and M. Stenke. 1994, 6p.

Pub. in Physical Review A 49, n2 p933-938 Feb 94.

Keywords: "Electron-ion collisions, "Electron capture 'Scandium ions, Ionization cross sections, Electron impact, Colliding beams, Excited states, Reprints.

Theoretical and experimental results are presented for the electron-impact ionization of $\mathrm{Sc}(2+)$ in the nearthreshold energy region where strong resonance features due to dielectronic capture processes are found The indirect ionization contributions are calculated in the close-coupling approximation. Both absolute and scan measurements for the ionization cross section are obtained in a crossed-beams experimental geometry. The overall agreement between theory and experiment is good, once a sufficient number of singly excited states are included in the close-coupling expansion. The additional states allow a proper theoretical determination of the decay pathways available to the resonances formed following dielectronic capture of the incident electron.

\section{4,001}

PB95-203147 Not available NTIS

National Inst. of Standards and Technology (PL), Boulder, CO. Ouantum Physics Div.

Charge Cloud Distribution of Heavy Atoms After Excitation by Polarized Electrons.

Final rept.

A. Raeker, K. Blum, and K. Bartschat. 1993, 18p. Grant NSF-PHY-901414103

Sponsored by National Science Foundation, Washington, DC

Pub. in Jnl. of Physics B: Atomic, Molecular and Optical Physics 26, p1491-1508 1993

Keywords: *Electron-atom collisions, Polarized beams, Electron impact, Heavy elements, Charge distribution, Multipoles, Mercury, Thallium, Reprints, Electron spin polarization.

We consider the charge cloud distribution of heavy atoms after impact excitation by polarized electrons. A general formula in terms of state multipoles is derived and interpreted with regard to ratios of principal axes and three independent angles of rotation. Finally, the theory is illustrated by numerical results for excitation of mercury and thallium.

04,002

PB95-203162 Not available NTIS

National Inst. of Standards and Technology $(\mathrm{PL})$, Boulder, CO. Ouantum Physics Div

Pulsed Laser Photolysis Time-Resolved FT-IR Emission Studies of Molecular Dynamics.

Final rept.

S. A. Rogers, and S. R. Leone. 1993, 8p.

Contract DE-FG02-883413860

Sponsored by Department of Energy, Washington, DC Pub. in Applied Spectroscopy 47, n9 p1430-1437 1993.

Keywords: "Molecular dynamics, Pulsed lasers, Excimer lasers, Energy transfer, Photolysis, Molecula collisions, Chemical radicals, Reprints, Fourier transform infrared spectroscopy, Argon fluoride lasers, Photofragmentation.

Time-resolved Fourier transform infrared (FT-IR) emis sion experiments are used to study photofragmentation processes, single collision reac tions, energy transfer events, and laser-initiated radical-radical reactions. In the experimental apparatus, $200-\mathrm{Hz}$ ArF excimer laser is coupled to a commercial $0.01 / \mathrm{cm}$ resolution Fourier transform infrared spectrometer. Fringes from the He:Ne reference laser are used for time synchronization of the laser pulses to the FT-IR mirror retardation. We report here on the comprehensive details of our experimental apparatus and discuss several of the processes studied with the use of this apparatus.

O4,003
PB95-203261 Not available NTIS 
National Inst. of Standards and Technology (PL), Boulder, CQ. Quantum Physics Div.

Laser Preparation and Probing of Initial and Final Orbital Alignment in Collision-Induced Energy Transfer Ca(4s5p,(1)P1) + He yields Ca(4s5p,(3)P2) + He.

C. J. Smith, J. P. J. Driessen, L. Eno, and S. R.

Leone. 1992, 13p.

Grant NSF-PHY90-12244

Sponsored by National Science Foundation, Washing ton, DC.

Pub. in Jnl. of Chemical Physics 96, n11 p8212-8224 1 Jun 92.

Keywords: "Atom-atom collisions, "Atomic collisions, *Calcium, Atomic energy levels, Atomic orbitals, Angular momentum, Colliding beams, Cross sections, Laser radiation, Pulsed laser, Polarized light, Alignment, Correlation, Helium, Reprints.

In a crossed beam atomic energy transfer experiment relative cross sections are measured between initial and final magnetic substates of atomic orbitals in a three vector correlation experiment. A pulsed laser beam prepares $\mathrm{Ca}(4 \mathrm{~s} 5 \mathrm{p}$ singlet $\mathrm{P}(1))$ in a single magnetic sublevel (vertical stroke)i(1) $\mathrm{m}(\mathrm{i})>$ with respect to the laser polarization vector. Subsequent collision with $\mathrm{He}$ at a well-defined relative velocity yields $\mathrm{Ca}(4 \mathrm{~s} 5 \mathrm{p}$ triplet $P(2)$ ). The near-resonant $\mathrm{Ca}(4 \mathrm{~s} 5 \mathrm{P}$ triplet $\mathrm{P}(2))$ is probed by a second polarized pulsed laser, revealing its magnetic sublevel (vertical stroke) $\mathrm{j}(2) \mathrm{m}(\mathrm{f})>$ distribution with respect to the probe laser polarization vector. The experiment is analyzed in the collision frame where the direction of the initial relative velocity vector serves as the quantization axis.

\section{4,004}

PB95-203279 Not available NTIS

National Inst. of Standards and Technology (PL), Boulder, CQ. Quantum Physics Div.

Initial and Final Orbital Alignment Probing of the Fine-Structure-Changing Collisions among the $\mathrm{Ca}$ $(4 s)(1)(4 p)(1),(3) P J$ States with He: Determination of Coherence and Conventional Cross-Sections.

Final rept.

C. J. Smith, E. M. Spain, M. J. Dalberth, S. R.

Leone, and J.P. J. Driessen. 1993, 11p.

Grant NSF-PHY90-12244

Sponsored by National Science Foundation, Washington, DC. Div. of Physics.

Pub. in Jnl. of the Chemical Society, Faraday Transactions 89, n10 p1401-14111993.

Keywords: "Atom-atom collisions, "Atomic collisions, ${ }^{\star}$ Calcium, Atomic energy levels, Atomic orbitals, Lase radiation, Polarized light, Fine structure Qrientation(Direction), Cross sections, Alignment, $\mathrm{He}$ lium, Colliding beams, Reprints, Collisional energy transfer.

A laser pump-probe experiment is used to study the orbital alignment effects, orientation effects and vector correlations of collisional transfer of the $\mathrm{Ca}(4 \mathrm{~s})(1)$ $(4 p)(1)$, triplet $P(1)$ state to the $\mathrm{Ca}(4 s)(1)(4 p)(1)$, triple $P(2,0)$ levels. The experiment is configured in a single collision crossed-beam arrangement between $\mathrm{Ca}$ and $\mathrm{He}$, and multi-structure cross-sections are determined using appropriate combinations of linear and circular laser light for the pump/probe steps. Real and imaginary parts of coherence cross-sections are obtained along with the conventional population cross-sections for the $m(1)-->m(2)$ magnetic sublevel transitions into the triplet $P(2)$ level.

\section{4,005}

PB95-203402 Not available NTIS

National Inst. of Standards and Technology (PL), Boulder, CQ Quantum Physics Div.

Angle-Differential and Momentum-Transfer Cross Sections for Low-Energy Electron-Cs Scattering.

Final rept.

U. Thumm, and D. W. Norcross. 1993, 12p.

Contract DE-A 105-86ER53237

Sponsored by Department of Energy, Washington, DC. Qffice of Energy Research.

Pub. in Physical Review A 47, n1 p305-316 Jan 93.

Keywords: "Electron-atom collisions, *Cesium, Electron scattering, Elastic scattering, Inelastic scattering Momentum transfer, EV range 170, Electron collisions, Cross sections, Relativistic effects, Excitation, Reprints.

Based on a previous Dirac R-matrix calculation, we have derived elastic and inelastic angle-differential and elastic momentum-transfer cross sections for slow electrons ( $E($ kin $)=$ or $<2.8 \mathrm{eV}$ ) colliding with neutra Cs atoms. Qur results for the angle-differential cross sections are in good agreement with scaled experi mental data and, depending on the incident electron energy, in qualitative or fair quantitative agreemen with previously published theoretical work. The inelas tic angle-differential cross sections for sextet $p(1 / 2)$ and sextet $p(3 / 2)$ excitation differ by more than a statis tical branching ratio due to relativistic effects. For the momentum-transfer cross sections, we hope to resolve existing discrepancies in the literature and to provide more reliable input for transport calculations.

\section{4,006}

PB95-203410 Not available NTIS

National Inst. of Standards and Technology (PL), Boulder, CQ. Quantum Physics Div.

Relativistic R-Matrix Calculations for Electron - Alkali-Metal-Atom Scattering: Cs as a Test Case.

Final rept.

U. Thumm, and D. W. Norcross. 1992, 22p.

Contract DE-A105-86ER532337

Sponsored by Department of Energy, Washington, DC Qffice of Energy Research.

Pub. in Physical Review A 45, n9 p6349-6370, 1 May 92.

Keywords: "Electron-atom collisions, "Alkali metals, ${ }^{*}$ Cesium, Milli EV range, EV range 1-10, Electron scattering, Electron collisions, Relativistic effects, Cross sections, R matrix, Low energy, Multiplets, Reprints.

We have reformulated the Dirac R-matrix method for low-energy electron scattering by (effectively) oneelectron, alkali-metal-like systems and developed an independent computer program for this special purpose. A highly accurate and relativistic representation of the target was used to perform a multichannel closecoupling calculation of cross sections for electron scattering. Qur results include the negative-ion affinity and elastic, inelastic, and total cross sections for inciden electrons with 0 to $2.8 \mathrm{eV}$ kinetic energy. We find that core-polarization and relativistic effects lead to multiplets of very narrow triplet $P(j), J=0,1,2$ shape resonances: the effect of the induced core polarization on the electron-electron correlation leads to $6 \mathrm{~s} 6 \mathrm{p}$ triplet $\mathrm{P}$ (sub i, sup 0) resonances in contrast to previously predicted bound states of $\mathrm{Cs}(1-)$, and relativistic interactions are responsible for the autoionizing decay of $6 \mathrm{p}(2)$ triplet $P(J)$ states below the first excitation threshold

04,007

PB95-203477 Not available NTIS

National Inst. of Standards and Technology (PL), Boulder, CQ. Quantum Physics Div.

Short-Pulse Detachment of $\mathrm{H}(-)$ in the Presence of a Static Electric Field.

Final rept.

Q. Wang, and A. F. Starace. 1993, 4p.

Grant NSF-PHY-9108002

Sponsored by National Science Foundation, Washington, DC

Pub. in Physical Review A 48,m n3 pR1741-R1744 Sep 93.

Keywords: "Hydrogen ions, "Electron detachment, ${ }^{*}$ Photodetachment, Electric fields, Negative ions, Cross sections, Laser radiation, Reprints, Laser photoionization.

Quantum interference effects occurring in photodetachment of $\mathrm{H}(1-)$ in the presence of a uniform static electric field are shown theoretically to be controllable through use of short laser pulses having characteristic times comparable to photodetached electron reflection times. In particular, calculated cross sections for single-photon detachment by two laser pulses that are delayed and phase shifted relative to one another are shown to oscillate as a function of the relative phases of the laser pulses at fixed photodetached electron energy.

\section{4,008}

PB95-203493 Not available NTIS

National Inst. of Standards and Technology (PL), Boulder, CQ. Quantum Physics Div.

Oscillator Strengths and Radiative Branching Ratios in Atomic Sr.

Final rept.

H. G. C. Werij, C. H. Greene, C. E. Theodosiou, and A. Gallagher. 1992, 13p.

\section{Grant NSF-PHY90-12244}

Sponsored by National Science Foundation, Washington, $\mathrm{DC}$.
Pub.

Keywords: "Qscillator strengths, *Strontium, Transition probabilities, Branching ratio, $R$ matrix, Reprints, Radiative transitions.

Tables of radiative transition rates (A) are provided for allowed singlet and triplet transitions between Sr states below $n$ approx. $=11$. These are obtained from a combination of R-matrix, multichannel-quantum-defect-theory (MQDT), and modified Coulomb-approximation calculations, plus branching-ratio measurements. Measurements of some spin-changing branchings and of the 4 singlet $D(2)-5$ singlet $S(0)$ quadrupole transition rate are also reported. Lifetime and A-value measurements from the literature are included in the tables and compared to the calculations. These and the present measurements provide the most comprehensive test to date of calculated transition probabilities for divalent atoms. The MQDT calculation achieves $0-20 \%$ agreement with experiment in the great majority of cases tested.

04,009

PB95-203600 Not available NTIS

National Inst. of Standards and Technology (PL), Boulder, CO. Quantum Physics Div.

Suppression of lonization in One- and Two-Dimensional Model Calculations.

Final rept.

L. You, J. Mastowski, and J. Cooper. 1992, 7p.

Grant NSF-PHY90-12244

Sponsored by National Science Foundation, Washington, DC.

Pub. in Physical Review A 45, n5 p3203-3209, 1 Mar 92.

Keywords: Qne-dimensional calculations, Two-dimensional calculations, Two dimensional models, Mathematical models, Multi-photon processes, Reprints, *Ionization suppression, Multiphoton ionization

We study numerically ionization from a model atom induced by a strong high-frequency field. Both one- and two-dimensional models are considered. We discuss the physics of ionization suppression in strong fields and point out the differences between one- and higherdimensional models. We also discuss the applicability of classical modeling.

04,010

PB95-203816 Not available NTIS

National Inst. of Standards and Technology (PL). Boulder, CQ. Quantum Physics Div.

Precision Lifetime Measurements of Cs 6p (2)P1/ 2 and $6 p(2) P 3 / 2$ Levels by Single-Photon Counting.

Final rept.

L. Young, W. T. Hill, S. J. Sibener, C. E. Wieman, S

R. Leone, S. D. Price, and C. E. Tanner. 1994, 8p.

Contract DE-W-31-109-ENG-38

Sponsored by National Science Foundation, Arlington VA and Department of Energy, Washington, DC. Pub. in Physical Review A 50, n3 p2174-2181 Sep 94. Keywords: "Atomic energy levels, ${ }^{\star}$ Cesium, Mode locked lasers, Excited states, Atomic beams, P invariance, Lifetime, Parity, Reprints, Titanium sapphire lasers, Femtosecond pulses, Photon counting.

Time-correlated single-photon counting is used to measure the lifetimes of the $6 p$ doublet $P(1 / 2)$ and $6 p$ doublet $P(3 / 2)$ levels in atomic $C s$ with accuracies of approx. $0.2-0.3 \%$. A high-repetition-rate, femtosecond self-mode-locked Ti:sapphire laser is used to excite Cs produced in a well-collimated atomic beam. The time interval between the excitation pulse and the arrival of a fluorescence photon is measured repetitively until the desired statistics are obtained. The lifetime results are $34.75(7)$ and $30.41(10)$ ns for the $6 p$ doublet $P(1 / 2)$ and $6 p$ doublet $P(3 / 2)$ levels, respectively. These lifeimes fall between those extracted from ab initio manybody perturbation-theory calculations by Blundell Johnson, and Sapirstein (1991) and V. A. Dzuba et al (1989) and are in all cases within $0.9 \%$ of the calculated values. The measurement errors are dominated by systematic effects, and methods to alleviate these and to approach an accuracy of $0.1 \%$ are discussed. The technique is a viable alternative to the fast-beam laser approach for measuring lifetimes with extreme accuracy.

04,011

PB95-203824 Not available NTIS

National Inst. of Standards and Technology (PL), Boulder, CO. Quantum Physics Div. 
Hyperfine-Structure Studies of Zr II: Experimental and Relativistic Configuration-Interaction Results. Final rept.

L. Young, C. A. Kurtz, D. R. Beck, and D. Datta. 1993, 9p.

Contract DE-W-31-109-ENG-38

Contract DE-W-31-109-ENG-38
Sponsored by Department of Energy, Washington, DC. Office of Basic Energy Sciences.

Pub. in Physical Review A 48, n1 p173-181 Jul 93.

Keywords: "Zirconium ions, "Hypertine structure, Atomic energy levels, Atomic spectroscopy, Atomic structure, Metastable state, Electric moments, Quadrupole moments, Experimental data, Zirconium 91 , Reprints, Ab initio calculations.

We report an experimental and theoretical study of the hyperfine structure (hfs) in various metastable levels in (91) Zr II. Hyperfine structures in 11 levels arising from the $4 d(3)$ and $4 d(2) 5$ s configurations were measured using the laser-rf double-resonance method in a collinear laser-ion-beam geometry. The his $A$ and $B$ constants were measured to a precision of 4 and 11 $\mathrm{kHz}$, respectively. Less precise values for his constants for nine upper levels in the $4 d(2) 5 p$ configuration were derived from optical spectra. Theoretically, the A and $B$ constants for the metastable levels having $J=0.5$ and 1.5 were calculated using a relativistic configuration-interaction $(\mathrm{RCl})$ approach. The calculations confirm a previous report that the level at $17,614.00 / \mathrm{cm}$ reported in Moore's Atomic Energy Levels, Vol. II (US Government Printing Office, Washington, DC, 1971) is spurious.

04,012

P895-203840 Not available NTIS

National Inst. of Standards and Technology (PL), Boulder, CO. Quantum Physics Div.

Improved Hyperfine Measurements of the Na NP Excited State Through Frequency-Controlled Dopplerless Spectroscopy in a Zeeman MagnetoOptic Laser Trap.

Final rept.

M. Zhu, C. W. Oates, and J. L. Hall. 1993, 3p.

Contract No0014-89-J-1227, Grant NSF-PHY9O-

12244

Sponsored by National Science Foundation, Washington, DC. and Office of Naval Research, Arlington, VA Pub. in Optics Letters 18, n14 p1186-1188, 15 Jul 93

Keywords: "Sodium, Atomic spectroscopy, Excited states, Hyperfine structure, High resolution, Frequency standards, Magnetooptics, Reprints, "Atom traps, Laser traps, Dopplerless spectroscopy.

We report what is to our knowledge the first Dopplerless high-resolution optical spectroscopy based on unperturbed atoms left nearly at rest when a vapor cell magneto-optic trap is switched off. A lamb$\mathrm{da}=285 \mathrm{~nm}$ beam excited the $3 \mathrm{~s}-->5 \mathrm{p}$ transition in our sample of laser trapped/cooled $\mathrm{Na}$ atoms from which we collected fluorescence. Excited-state 5 doublet $P(3 / 2)$ and 5 doublet $P(1 / 2)$ hyperfine interaction constants are derived with 4- and 47-fold increases, respectively, in accuracy. Future prospects for vaporcell based optical spectroscopy and frequency standards are considered.

04,013

PB95-220539 PC A06/MF A02

National Inst. of Standards and Technology (PL), Gaithersburg, MD. Ionizing Radiation Div.

Tables of X-ray Mass Attenuation Coefficients and Mass Energy-Absorption Coefficients $1 \mathrm{keV}$ to 20 $\mathrm{MeV}$ for Elements $Z=1$ to 92 and 48 Additional

Substances of Dosimetric Interest.
J.H. Hubbell, and S. M. Seltzer. May 95, 116p, NISTIR-5632.

Keywords: " $X$ rays, "Gamma rays, $\mathrm{KeV}$ range $1-10$, $\mathrm{KeV}$ range $10-100, \mathrm{KeV}$ range $100-1000$, MeV range 1-10, MeV range 10-100, Gamma dosimetry, X-ray dosimetry, Radiation shielding, Chemical elements, Bremsstrahlung, Tables(Data), Graphs(Charts), "Mass attenuation coefficients, "Mass energy-absorption coefficients.

Tables and graphs of the photon mass attenuation coefficient mu/rho and the mass energy-absorption coefficient $\mathrm{my}(\mathrm{en}) / \mathrm{rho}$ are presented for all of the elements $\mathrm{Z}=1$ to 92 , and for 48 compounds and mixtures of radiological interest. The tables cover energies of the photon ( $x$ ray, gamma ray, bremsstrahlung) from $1 \mathrm{keV}$ to $20 \mathrm{MeV}$. The mu/rho values are taken from the curren photon interaction database at the National Institute of Standards and Technology, and the $\mathrm{mu}(\mathrm{en}) /$ /ho values are based on the new calculations by Seltzer described in Radiation Research 136, 147 (1993). These tables of $\mathrm{my} / \mathrm{rho}$ and $\mathrm{mu}(\mathrm{en}) / \mathrm{rho}$ replace and extend the tables given by Hubbell in the International Journal of Applied Radiation and Isotopes 33, 1269 (1982).

\subsection{4}

PB95-242384 PC A04/MF A01

National Inst. of Standards and Technology, Gaithersburg, MD.

DETAN 95: Computer Code for Calculating Spectrum-Averaged Cross Sections and Detector Responses in Neutron Spectra.

C. M. Eisenhauer. Mar 95, 70p, NISTIR-5622.

Keywords: "Neutron cross sections, "Neutron spectra, "Programming manuals, Neutron detectors, Spectrum analysis, Personnel dosimetry, Fission spectra, Response functions, Computer programs, Tables(Data), Computation, "DETAN 95 computer code.

DETAN 95 is a computer code for converting an input neutron spectrum to a spectrum or detector response function expressed in arbitrary energy-group structure. The input spectrum may be expressed either in analytic form or in some energy-group structure. The code also calculates spectrum-averaged neutron cross sections or responses for neutron personnel detectors. The code includes 625-group neutron cross sections for a number of reactions common to materials dosimetry. Other energy-dependent cross sections or response functions supplied by the user may be used to calculated spectrum-averaged values.

\section{4,015}

PB95-255923

PC A03/MF A01

National Inst. of Standards and Technology (CSTL), Gaithersburg, MD. Process Measurements Div.

Reproducibility of the Temperature of the Ice Point in Routine Measurements.

Technical note.

B. W. Mangum. Jun 95, 27p, NIST/TN-1411.

Also available from Supt. of Docs. as SNO03-00303347-2.

Keywords: "Temperature measurement, "Water, Resistance thermometers, Triple point, Reproducibility, "Ice point, Fixed points

This note presents results on the reproducibility and temperature of the ice point of water. The data were obtained over the years 1963 to 1970 , in 1992 and in 1995. The data from 1963 to 1970 are from resistance measurements of a standard platinum resistance thermometer in ice points, made for the purpose of obtaining the reference value for the thermometer to be used for determining temperatures of calibration of liquid-inglass thermometers. In 1992 and in 1995, measurements of the temperature of the ice point were made, using water (and ice) from different sources. The value of the temperature determined for the ice point prepared from the authors' highest-purity water is in excellent agreement with that determined previously. Also, the reproducibility of routine measurements at the ice point are consistent with what has been generally accepted, but heretofore undocumented.

\section{4,016}

P895-261905 (Order as PB95-261897, PC A07/ MF A02)

Bureau International des Poids et Mesures, Sevres (France).

Determining the Magnetic Properties of $1 \mathrm{~kg}$ Mass Standards.

\section{R. S. Davis. 1995, 17p.}

Included in Jnl. of Research of the National Institute of Standards and Technology, v100 n3 p209-225 May/ Jun 95.

Keywords: "Magenetic properties, "Mass, "Metrology, Calibration standards, Magnetic permeability, US NBS, National Institute of Standards and Technology, Susceptometer.

Magnetic interactions may lead to errors in precision mass metrology. An analytical description of such magnetic errors is presented in which the roles of both the volume magnetic susceptibility and permanent magnetization are discussed. The same formalism is then used to describe in detail the calibration and operation of a susceptometer developed at the Bureau International des Poids et Mesures (BIPM).
National Inst. of Standards and Technology (PL), Boulder, CO. Quantum Physics Div.

Merged-Beams Energy-Loss Technique for Electron-lon Excitation: Absolute Total Cross Sections for $\mathrm{O}(5+)(2 s$ yields $2 p)$.

Final rept.

E. W. Bell, X. Q. Guo, J. L. Forand, J. S. Thompson, G. H. Dunn, M. E. Bannister, D. C. Gregory, R. A. Phaneuf, A. C. H. Smith, A. Muller, C. A. Timmer, E. K. Wahlin, B. D. DePaola, D. S. Belic, K. Rinn, and D. R. Swenson. 1994, 12p.

Contract DE-A105-86ER53237

Sponsored by Department of Energy, Washington, DC Pub. in Physical Review A 49, n6 p4585-4596 Jun 94 Keywords: "Beams(Radiation), "Energy dissipation, "Electron-ion coupling, Cross sections, Oxygen, Ionization, Backscattering, Spectroscopy, Reprints, Merged beams, $\mathrm{O}(5+)$.

A merged-beams electron-energy-loss technique is described, by which absolute cross sections can be measured for near-threshold electron-impact excitation of multiple charged ions. The experimental data are in good agreement with a seven-state close-coupling throughout the energy range of the experiment. Results agree with calculations showing that more than $90 \%$ of the electrons causing excitation are ejected in the backward direction in the center-of-mass frame. This backscattering is shown in both quantum-mechanical and semiclassical calculations. Evidence is observed for high-lying metastable autoionizing states with a life-time of approximately 0.9 microseconds which are made to ionize by electron impact.

04,018

PB96-102074 Not available NTIS

National Inst. of Standards and Technology (CAML) Gaithersburg, MD. Applied and Computational Mathematics Div.

Faster Monte Carlo Simulations.

Final rept.

J. L. Blue, I. Beichl, and F. Sullivan. 1995, 2p See also PB95-136370.

Pub. in Physical Review E 51, n2 pR867-R868 Feb 95. Keywords: "Monte Carlo method, "Simulation, Crystal growth, Kinetics, Computerized simulation, Equilibrium equations, Reprints, Computer time.

For Monte Carlo simulations of systems of size M, either kinetic simulations or equilibrium simulations that use the method of Bortz, Kalos, and Liebowitz the best computer time per event has been O(M(sup 1/2)). The authors present two methods whose computer time pe event is $O(M(\sup 1 / k))$ or $O(\log M)$. In practice, for typical simulation sizes, $\mathrm{K}=4$ or $\mathrm{K}=5$ is fastest, requiring even less computer time than the $\mathrm{O}(\log M)$ method. For typical simulation sizes, the authors are able to achieve speedup factor of 5 to 7 over the O(M(sup 1/2)) technique.

\section{4,019}

P896-102090 Not available NTIS

National Inst. of Standards and Technology (CSTL), Gaithersburg, MD. Thermophysics Div.

Vapour Pressures and Gas-Phase ( $\mathrm{p}$, rho $n, T)$ Values for CF3CHF2'R125).

Final rept.

S. J. Boyes, and L. A. Weber. $1995,12 \mathrm{p}$

Pub. in Jnl. of Chemical Thermodynamics 27, p163 1741995.

Keywords: "Vapor pressure "Gases, "Fluorinated celiphatic hydrocarbons, Refrigerants, Pressure, Temperature, Density(Mass/volume), Virial equation, Ther modynamic properties, Reprints, "Pentafluoroethane $\mathrm{R} 125$ refrigerant.

The authors present new vapor pressures and the ( $p$, $p($ sub $n), T$ ) surface of CF3CHF2 (pentafluoroethane, designated $\mathrm{R} 125$ by the refrigeration industry) in the temperature range $T=273 \mathrm{~K}$ to $363 \mathrm{~K}$. The $\mathrm{p}$, $\mathrm{p}$ (sub n), T) measurements are for the gas phase at densities up to approximately the critical density. The static vapor pressures reported here have been combined with previous ebulliometric measurements made in this laboratory at lower temperatures. Correlating equations are given for the vapour pressures from $T=220$ $\mathrm{K}$ to the critical temperature: $\mathrm{T}$ (sub $\mathrm{c}$ ) $-339.30 \mathrm{~K}$. A virial equation has been used to correlate the $(p, p$ (sub n), T) surface at amount-of-substance densities up to $1600 \mathrm{~mol} / \mathrm{cubic} \mathrm{m}$. Second and third virial coefficients have been derived from the ( $p, p(s u b n), T)$ measurements. 


\section{PHYSICS}

\section{General}

National Inst. of Standards and Technology (PL), Gaithersburg, MD. Quantum Metrology Div. Energy Dependences of Absorption in Beryllium Windows and Argon Gas.

Final rept

C. T. Chantler, and J. L. Staudenmann. $1995,4 \mathrm{p}$

Pub. in Review of Scientific Instruments 66, n2 p16511654 Feb 95.

Keywords: "Energy dependence, "Beryllium, "Argon, *Absorption, Gas detectors, Coefficients, Synchrotron radiation, $\mathrm{X}$ rays, Form factors, Particle properties, $\mathrm{Re}$ prints.

In part of an ongoing work on $x$-ray form factors, new absorption coefficients are being evaluated for all elements, across the energy range from below $100 \mathrm{eV}$ to above $100 \mathrm{keV}$. These new coefficients are applied herein to typical problems in synchrotron radiation stations, namely the use of beryllium windows and argon gas detectors. Results are compared with those of other authors. The electron-ion pair production process in ionization chambers is discussed, and the effects of $3 d$-element impurities are indicated.

\section{4,021}

PB96-102199 Not available NTIS

National Inst. of Standards and Technology (MSEL), Gaithersburg, MD. Reactor Radiation Div.

Transmission Properties of Short Curved Neutron Guides. Part 1. Acceptance Diagram Analysis and Calculations.

Final rept.

J. R. D. Copley. 1995, 9p.

Pub. in Nuclear Instruments and Methods in Physics Research A, v355 p469-477 1995.

Keywords: "Transmittance, "Neutron guides, Neutron reflectors, Beam acceptance, Optical properties Gamma rays, Performance evaluation, Reprints, "Curved guide, "Acceptance diagrams, Ray-tracing

A curved neutron guide is considered 'short' if there are direct lines of sight through the device Such guides have the disadvantage that they transmit high energy neutrons and gamma rays, which are generally unwanted, as well as the desired low energy neutrons. Nevertheless short curved guides have several uses at thermal neutron research facilities because they can be combined with sections of straight guide to produce composite guides through which there is no line of sight. The authors analyze the transmission properties of short curved neutron guides using the method of acceptance diagrams, and they present the results of several calculations of their performance.

\section{4,022}

PB96-102330 Not available NTIS

National Inst. of Standards and Technology (BFRL), Gaithersburg, MD. Structures Div.

Fluctuations in Probability Distribution on Chaotic Attractors.

Final rept.

M. Franaszek. 1995, 7p.

Pub. in Physics Letters A 203, p115-121 1995

Keywords: "Probability distribution functions, "Variations, "Chaos, "Strange attractors, Perturbation, Periodic variations, Noise, Amplitudes, Time series analysis, Stochastic processes, Reprints

Fluctuations in probability distribution are used to study the influence of a weak periodic perturbation on chaotic system. A detailed analysis of one very long time senes reveals a great sensitivity to external perturbation. The case of large amplitude stochastic perturbation is also discussed. It is shown that the fractal geometry of an experimental attractor may be visualized up to length scales smaller than noise amplitude.

\section{4,023}

PB96-102348 Not available NTIS

National Inst. of Standards and Technology (PL), Boulder, CO. Quantum Physics Div.

Kinetics and Dynamics of Vibrationally State Resolved Ion-Molecule Reactions: $(14) \mathrm{N} 2(+)(v=1$ and $2)$ and $(15) \mathrm{N} 2(+)(v=0,1$ and 2$)$ with $(14) \mathrm{N} 2$.

Final rept

M. J. Frost, S. Kato, V. M. Bierbaum, and S. R

Leone. 1994, 9p.

Contract AFOSR-FA9620-92J-0072, Grant NSF-

PHY90-12244

Sponsored by Air Force Office of Scientific Research,

Bolling AFB, DC. and National Science Foundation,

Washington, $\mathrm{DC}$.

Pub. in Jnl. of Chemical Physics 100, n9 p6359-6367,

1 May 94.
Keywords: "Charge transfer, "Ions, "Reaction kinetics, Molecular interactions, Nitrogen isotopes, Lasers, Ionic reactions, Vibration, Reprints.

Vibrationally state-selected measurements of the kinetics and dynamics of $14 \mathrm{~N} 2(1+)(\mathrm{nu}=1$ and 2$)$ and $15 \mathrm{~N} 2(1+)(\mathrm{nu}=0,1$, and 2$)$ in collisions with (14)N2 are made using a selected ion flow tube (SIFT), laser induced fluorescence (LIF) technique at thermal energies. Kinetics are measured by monitoring the LIF signal amplitudes of $\mathrm{N} 2(1+)(\mathrm{nu})$ as a function of (14) N2 concentration, added after ion injection. By comparison with the known N2(1+)(nu = 1) + Ar rate, the $(15) \mathrm{N} 2(1+)(\mathrm{nu}=0)+14 \mathrm{~N} 2$ rate constant is found to be one-half of the Langevin collision rate or $4.2+$ or $-0.2 \times 10(\exp -10)$ cubic $\mathrm{cm} / \mathrm{molecule} / \mathrm{s}$. This suggests that the reaction proceeds via an $\mathrm{N} 4(1+)$ energized adduct in which charge is shared on a time scale shorter than the adduct lifetime. The removal rates of (14) N2 $(1+)(\mathrm{nu}=1$ and 2 ) reactions by (14)N2 are also found to proceed at one-half of the Langevin collision rate. Thus product channels that remove vibrational energy from the ion upon dissociation of the adduct account for $50 \%$ of the collision probability.

\section{4,024}

PB96-102744 Not available NTIS

National Inst. of Standards and Technology (CSTL), Gaithersburg, MD. Thermophysics Div.

Analytical Method for Determining Thermal Conductivity from Dynamic Experiments.

Final rept.

R. A. MacDonald. 1995, 8p

Sponsored by National Aeronautics and Space Administration, Washington, DC

Pub. in International Jnl. of Heat and Mass Transfer, v38 n14 p2549-2556 1995.

Keywords: "Thermal conductivity, "Rods, "Tungsten Temperature dependence, Specific heat, Surface temperature, Profiles, Cylindrical bodies, High temperaperature, Profiles, Cylindrical bodies, High temperaRadiative heat transfer, Thermodynamics, Reprints

The temperature profile along a cylindrical rod or tube as it cools has been obtained by numerical solution of the second-order time-dependent partial differentia equation for heat conduction. The surface temperature is calculated for given temperature dependent values of the heat capacity, hemispherical total emissivity thermal expansion and thermal conductivity and, by adjusting the thermal conductivity, is fitted to experimental temperature profiles from preliminary measurements on a cooling cylindrical tungsten rod. The surface temperature of the rod is quite sensitive to the thermal conductivity but the accuracy of the predicted thermal conductivity cannot be assessed without further experimentation.

\section{4,025}

\section{PB96-102751 Not available NTIS}

National Inst. of Standards and Technology (PL) Gaithersburg, MD. Quantum Metrology Div.

Evolution of X-ray Resonance Raman Scattering into $X$-ray Fluorescence from the Excitation of Xenon Near the L3 Edge.

Final rept.

M. A. MacDonald, S. H. Southworth, J. C. Levin, T. Lebrun, Y. Azuma, P. L. Cowan, B. A. Karlin, A Henins, and R. D. Deslattes. 1995, 6p.

Pub. in Physical Review A, v51 n5 p3598-3603 May 95.

Keywords: "Xenon, "Raman spectra, "X ray fluorescence, Synchrotion radiation, Absorption spectra Edges, Experimental data, Reprints, Absorption edge, L3 edge.

Near an absorption edge, $x$-ray emission cannot be treated separately from the absorption process itself a scattering formalism must be used. Experimental data have been recorded showing $x$-ray emission from xenon following excitation by tunable synchrotron radiation below and above the L3 absorption edge. Complete data sets are presented for $\mathrm{Xe} \mathrm{La1,2}$ and Lbeta2,15 emission from $10 \mathrm{eV}$ below to $40 \mathrm{eV}$ above the L3 edge. In accord with the resonant-inelastic-scattering model, the observed $x$-ray emission evolves from resonant Raman scattering into characteristic fluorescence as the excitation energy is scanned from below to above the absorption edge.

\section{4,026}

PB96-102850 Not available NTIS

National Inst. of Standards and Technology (EEEL) Gaithersburg, MD. Electricity Div.
Effect of Electrode Material on Measured Ion Energy Distributions in Radio-Frequency Discharges. Final rept.

J. K. Olthoft, R. J. Van Brunt, and S. B. Radovanov. 1995, 3p.

Pub. in Applied Physics Letters, v67 n4 p473-475, 24 Jul 95.

Keywords: "Electrodes, "Ions, "Radio frequency discharge, Reprints, Ion energy analysis, Distribution, Discharges, Surface charging.

Evidence is presented for a significant influence of electrode surface material and condition on the measurement of the kinetic energies of ions sampled from discharges through a orifice in the electrode. Significant differences in ion energy shifts and/or discrimination of low-energy ions are found using aluminum and stainless-steel electrodes in a radio-frequency $(\mathrm{rf})$ discharge cell. It is argued that the observed differences in energy shifts may be attributable in part to differences in charging oxide layers on the electrode surface around the sampling orifice.

\section{4,027}

PB96-102918 Not available NTIS

National Inst. of Standards and Technology (CSTL), Gaithersburg, MD. Surface and Microanalysis Science Div.

Energy Calibration of X-ray Photoelectron Spectrometers: Results of an Interlaboratory Comparison to Evaluate a Proposed Calibration Procedure. Final rept.

C. J. Powell. 1995, 12p.

Pub. in Surface and Interface Analysis, v23 p121-132 1995.

Keywords: "Spectrometers, "Calibration, Reprints, Copper, Gold, Silver, $\mathrm{X}$ rays, Photoelectrons, "Foreign technology, "Binding energy.

Results are reported of an interlaboratory comparison conducted to evaluate a proposed procedure for calibration of the binding energy (BE) scales on $x$-ray photoelectron spectrometers. The calibration was performed at two points on the BE scale (the Au $4 f 7 / 2$ and $\mathrm{Cu} 2 \mathrm{p} 3 / 2$ lines) and checks were made of the assumption of BE-scale linearity from measurements on other lines. It is shown here that small offsets can arise if peaks are located with assumed backgrounds on nonzero slope or if multipeak fits are made to Cu L3VV and Ag M4VV Auger spectra.

\section{4,028}

PB96-102942 Not available NTIS

National Inst. of Standards and Technology (PL) Gaithersburg, MD. Atomic Physics Div.

Time-Resolved Balmer-Alpha Emission from Fast Hydrogen Atoms in Low Pressure, Radio-Frequency Discharges in Hydrogen.

Final rept.

S. B. Radovanov, K. Dzierzega, J. R. Roberts, and J. K. Olthoff. 1995, 3p.

Pub. in Applied Physics Letters, v66 n20 p2637-2639, 15 May 95.

Keywords: "Hydrogen, "Radiofrequency discharges, Reprints, Atoms, Balmer lines, Low pressure, "Doppler broadened emission.

Doppler-broadened $\mathrm{H}$ (alpha) emission $(656.28 \mathrm{~nm}$ ) detected from a $13.56 \mathrm{MHz}$, parallel-plate, radio-frequency discharge in hydrogen indicates the presence of fast excited $\mathrm{H}$ atoms throughout the discharge volume. Time and spatially resolved measurements of the Doppler-broadened emission indicate that the fast $\mathrm{H}$ atoms are formed primarily at the surface of the powered electrode with kinetic energies exceeding $120 \mathrm{eV}$

\section{4,029}

PB96-102959 Not available NTIS

National Inst. of Standards and Technology (EEEL) Gaithersburg, MD. Electricity Div.

Ion Kinetic-Energy Distributions and Balmer-alpha (Halpha) Excitation in Ar-H2 Radio-Frequency Discharges.

S. B. Radovanov, J. K. Olthoff, R. J. Van Brunt, and S. Djurovic. $1995,12 p$

Pub. in Jnl. of Applied Physics, v78 n2 p746-757, 15 Jul 95.

Keywords: "Radiofrequency charges, "Gas discharges, "Argon ions, Reprints, Hydrogen, Balmer lines, Neutrals, Electrodes, Ion kinetic energy.

Excited neutrals and fast ions produced in a $13.56 \mathrm{MHz}$ radio-frequency discharge in a $90 \%$ argon $-10 \%$ hydro- 
gen gas mixture was investigated, respectively, by spatially and temporally resolved optical emission spectroscopy, and by mass-resolved measurements of ion kinetic energy distributions at the grounded electrode. The electrical characteristics of the discharge were also measured and comparisons are made with results obtained for discharges in pure $\mathrm{H} 2$ under comparable conditions. Measurements of Balmer-alpha emission show Doppler-broadened emission that is due to the excitation of fast atomic hydrogen neutrals formed from ion neutralization processes in the discharge.

\section{4,030}

PB96-103106 Not available NTIS

National Inst. of Standards and Technology (PL), Boulder, CO. Quantum Physics Div.

Low-Energy-Electron Collisions with Sodium Elastic and Inelastic Scattering from the Ground State.

Final rept.

W. K. Trail, M. A. Morrison, H. L. Zhou, K. B. MacAdam, T. L. Goforth, D. W. Norcross, B. L. Whitten, and K. Bartschat. 1994, 26p.

Sponsored by National Science Foundation, Arlington, $\checkmark A$.

Pub. in Physical Review A 49, n5 p3620-3645 May 94.

Keywords: *Cross sections, *Electron-atom collisions, "Elastic scattering, "Inelastic scattering, Sodium, R matrix, Angular momentum, Electron diffraction, Experimental data, Data bases, Reprints.

The electron-Na system is the prototype for nonrelativistic scattering of a charged particle from a quasi-one-electron system. At scattering energies below several eV, e-Na cross sections are particularly sensitive to the exchange interaction and manifest a rich variety of near-threshold structures in various spin channels. By applying the nonperturbative coupledchannel R-matrix method in carefully converged calculations the authors have generated a comprehensive data base of accurate scattering quantitities for studying these phenomena and for comparison to presen and future experimental data. In addition to conventional integrated and differential cross sections, the authors consider partial cross sections for changes in the projection of the spin and orbital angular momentum of the $\mathrm{Na}$ valence electron, comparing results for all these quantities to experimental data where available.

\section{4,031}

PB96-103122 Not available NTIS

National Inst. of Standards and Technology (PL), Boulder, CO. Time and Frequency Div.

Aging, Warm-Up Time and Retrace; Important Characteristics of Standard Frequency Generators.

Final rept.

J. Vanier, J. J. Gagnepain, W. J. Riley, F. L. Walls, and M. Granveaud. 1992, $9 p$.

Pub. in Proceedings of Institute of Electrical and Electronics Engineers Frequency Control Symposium, Hershey, PA., May 22-29, 1992, p807-815 1992.

Keywords: "Frequency, "Standards, *Pulse generators, Frequency measurement, Environmental effects, Sensitivity, Aging(Materials), Reporting, Reprints, Retrace, Warm-up.

The report is an effort to produce an IEEE standard providing guidelines for standardized methods of defining, measuring and reporting environmental sensitivities of precision frequency generators. The present report covers the subject of aging, warm-up time and retrace.

\section{4,032}

PB96-110739 Not available NTIS

National Inst. of Standards and Technology (PL), Boulder, CO. Quantum Physics Div.

Intensity-Dependent Scattering Rings in High Order Above-Threshold Ionization.

Final rept.

B. Yang, K. J. Schafer, B. Walker, L. F. DiMauro, K C. Kulander, and P. Agostini. 1993, $4 p$.

Sponsored by Department of Energy, Washington, DC. Pub. in Physical Review Letters 71, n23 p3770-3773, 6 Dec 93.

Keywords: "Photoionization, "Electron scattering, *Xenon, "Krypton, Photoelectrons, Angular distribution, Ponderomotive forces, Electrodynamics, Rings, Wave functions, Reprints, Multiphoton processes, Photoelectron angular distributions.
Angular distributions of high energy photoelectrons from $\mathrm{Xe}$ and $\mathrm{Kr}$, excited by a $50 \mathrm{ps}, 1.05$ micro $\mathrm{m}$ laser are presented. In $\mathrm{Xe}$, strong, narrow, rings about 45 deg. off the polarization axis appear in a limited energy range centered around $\mathrm{U}(\mathrm{sub} p)$, where $\mathrm{U}(\mathrm{sub} p)$ is the ponderomotive energy. This effect is much weaker in $\mathrm{Kr}$. Single active electron calculations agree well with these observations. The authors conclude that the rings result from single-electron ionization dynamics. most likely involving rescattering from the ion core of the tunneling component of the continuum wave function.

\section{4,033}

PB96-111612 Not available NTIS

National Inst. of Standards and Technology (PL), Gaithersburg, MD. Ionizing Radiation Div.

Monte Carlo and Analytic Methods in the Transport of Electrons, Neutrons, and Alpha Particles.

Final rept.

R. S. Caswell, and S. M. Seltzer. 1994, 17p.

Pub. in Computational Approaches in Molecular Radiation Biology, p115-131 1994

Keywords: “Alpha particles, "Monte Carlo method Photons, Electrons, Neutrons, Biological effects, Computer programs, Radiation transport, Energy, Deposition, Reprints.

In this paper the authors discuss Monte Carlo calculational methods and analytic methods, and methods combining features of both, developed over the past decades at the National Institute of Standards and Technology. These include the Monte Carlo program ETRAN for electrons and photons developed by Berger and Seltzer: the neutron analytic method developed by Caswell and Coyne, and the incorporation into that program of the synthesis of Monte Carlo results for proton tracks of Wilson and Paretzke. Some comparisons with experimental results and with other calculations are given. Also, some applications of the calculational results to the prediction of biologial effects using biophysical models are given.

\section{4,034}

PB96-111661 Not available NTIS

National Inst. of Standards and Technology (CAML), Gaithersburg, MD. Applied and Computational Mathematics Div.

Nonequilibrium Thermodynamic Theory of Viscoplastic Materials.

Final rept.

B. Bernstein, and J. T. Fong. 1993, 9p.

Pub. in Jnl. of Applied Physics, v74 n4 p2220-2228, 15 Aug 93

Keywords: "Thermodynamic equilibrium, "Elasticity, Mechanical properties, Thermodynamic properties Entropy, Free energy, Creep, Fatigue, Models, Reprints

A nonequilibrium thermodynamic theory for predicting the mechanical behavior of materials beyond the elastic range is formulated. The theory incorporates the idea of a concealed parameter alpha, originally due to Bridgman where the constitutive equation are governed by (1) a thermodynamic potential such as a generalized Gibbs function, $G$ or Helmholtz free-energy function, $F$, each with an explicit dependence on alpha and (2) a prescription for alpha, the time rate of change of alpha, such that alpha is directly proportional to the negative of G(sub alpha) or F(sub alpha), the partial derivative of $G$ or $F$ with respect to alpha, respectively.

\section{4,035}

PB96-111828 Not available NTIS

National Inst. of Standards and Technology (MSEL), Gaithersburg, MD. Reactor Radiation Div.

Vibrational Excitations and the Position of Hydrogen in Nanocrystalline Palladium.

Final rept.

U. Stuhr, H. Wipf, T. J. Udovic, J. Weissmuller, and H. Gleiter. $1995,12 p$

Pub. in Jnl. of Physics: Condensed Matter, v7 p219 2301995

Keywords: "Palladium, "Hydrogen atoms, "Vibrational spectroscopy, Position(Location), Grain boundaries, Solubility, Crystal lattices, Neutron scattering, Absorption, Reprints, "Nanocrystals, "Hydrogen absorption.

The vibrational excitations and the position of hydrogen in nanocrystalline palladium were investigated by means of inelastic neutron-scattering and $\mathrm{H}$ solubility measurements. The study focuses on the $\mathrm{H}$ concentration regime (less than or equal to 4.8 at. $\% \mathrm{H}$ ) where, at room temperature, no precipitation of the $\mathrm{H}$ in a hydride phase (Beta-phase) was observed. In this concentration regime, the solubility measurements show an enhanced $\mathrm{H}$ solubility relative to coarse-grained $\mathrm{Pd}$. The neutron-scattering experiments show that this additional $\mathrm{H}$ is incorporated in the grain boundaries and at the surface of the grains. Surface modes of $\mathrm{H}$ at approximately 90 and $120 \mathrm{meV}$ were identified. Compared to coarse-grained $\mathrm{Pd}$, no change in the $\mathrm{H}$ solubility was found for the crystal lattice of the nanosized grains.

\section{4,036}

\section{PB96-111836 Not available NTIS}

National Inst of Standards and Technology (MSEL)

Gaithersburg, MD. Reactor Radiation Div.

Magnetic Structure and Spin Dynamics of the Pr and $\mathrm{Cu}$ in $\mathrm{Pr} 2 \mathrm{CuO} 4$.

Final rept.

I. W. Sumarlin, J. W. Lynn, T. Chattopadhyay, J. L.

Peng, S. N. Barilo, and D. I. Zhigunov. 1995, 15p.

Pub. in Physical Review B, v51 n9 p5824-5838, 1 Mar 95.

Keywords: "Magnetic properties, "Spin dynamics, "Praseodymium, "Copper, Crystal structure, Magnons, Atomic interactions, Velocity, Phase diagrams, Temperature dependence, Zero point energy, Field theory, Neutron scattering, Reprints, "Zero-field ordered moment.

Neutron-scattering techniques have been used to study the magnetic structure and spin dynamics of the $\mathrm{Pr}$ and $\mathrm{Cu}$ spins in Pr2CuO4. In the ordered state the $\mathrm{Cu}$ spin-wave velocity $\mathrm{c}$ has been determined to be $0.85+$ or $-0.08 \mathrm{eV}$ Angstroms, which corresponds to an in-plane nearest-neighbor exchange constant $\mathrm{J}=$ $130+$ or -13 meV. A spin-wave gap of approximately $5 \mathrm{meV}$ has been observed, corresponding to a reduced anisotropy constant alpha(sub parrallel sign) $=(\mathrm{J}$ $J(\sup x y)) / J$ of about $2 \times 10(\exp -4)$. In the paramagnetic regime the evolution of the Cu spin-correlation length with temperature is adequately described by the renormalized classical theory for the quantum nonlinear sigma model.

04,037

PB96-111885 Not available NTIS

National Inst. of Standards and Technology (PL), Boulder, CO. Quantum Physics Div.

Electron-Ion Collisions in the Plasma Edge.

Final rept.

G. H. Dunn. 1992, 15p

Pub. in Nuclear Fusion A and M Suppl., v2 p25-39 1992

Keywords: "Electron ion collisions, "Data bases, Reprints, Plasma diagnostics, Modelling, Fusion plasmas, *Plasma edge.

The electron-ion collisional database required for modelling and diagnostics of the fusion plasma edge is briefly examined from the points of view of (1) experimental and theoretical means for obtaining the data, (2) relevant physical processes, (3) limitations on the data, and (4) availability and quality of the data for relevant species. Both molecular and atomic ions are considered.

\section{4,038}

PB96-111992 Not available NTIS

National Inst. of Standards and Technology (EEEL), Boulder, CO. Electromagnetic Technology Div.

Comparison of Photodiode Frequency Response Measurements to $40 \mathrm{GHz}$ between NPL and NIST. Final rept.

A. D. Gifford, D. A. Humphreys, and P. D. Hale. 1994, 2p.

Pub. in Electronics Letters, v31 n5 p397-398, 2 Mar 95.

Keywords: "Photodiodes, Measurements, Reprints, Comparison, *Frequency responses.

The authore report the first comparison, at the national standards level, of photodiode frequency response measurements at wavelengths of $1,285,1319$ and 1.531 micrometers. A photodiode was measured up to $40 \mathrm{GHz}$ and the results were normalized to $1.319 \mathrm{mi}-$ crometers using a model of the device. The average scatter in the results was plus or minus $0.12 \mathrm{~dB}$ (2omega) below $20 \mathrm{GHz}$ and plus or minus $0.21 \mathrm{~dB}$ from 20 to $33 \mathrm{GHz}$.

\section{4,039}

PB96-112073 Not available NTIS 


\section{PHYSICS}

\section{General}

National Inst. of Standards and Technology (PL), Boulder, CO. Time and Frequency Div.

Quantum-Limited Cooling and Detection of RadioFrequency Oscillations by Laser-Cooled lons.

Final rept.

D. J. Heinzen, and D. J. Wineland. 1990, 18p.

Pub. in Physical Review A, v42 n5 p2977-2994, 1 Sep 90

Keywords: *Radio frequency detection, Reprints, Quantum measurements, Mass spectroscopy, "Ion traps, *Laser cooling, Squeezed states.

A single trapped ion, laser cooled into its quantum ground state of motion, may be used as a very-lowtemperature detector of radio-frequency signals applied to the trap end caps. If the signal source is a resonant oscillator of sufficiently high $Q$, the source may also be placed in its quantum ground state by coupling to the ion. Parametric couplings may be used to cool and detect source modes other than the mode directly coupled to the ion. A theoretical analysis of these cooling and detection processes is presented, and as an example, their application to single trapped electron and proton spectroscopy is examined. Squeezing and low noise detection of one quadrature component of the source oscillation are also discussed. The tech niques discussed here may lead to radio-frequency measurements of improved accuracy and sensitivity. Cooling and detection of vibrations of macroscopic oscillators also appear possible.

\section{4,040}

PB96-112164 Not available NTIS

National Inst. of Standards and Technology (PL), Boulder, CO. Time and Frequency Div.

Precise Spectroscopy for Fundamental Physics.

Final rept.

W. M. Itano J C. Bergquist J J Bollinger F L Moore, M. G. Raizen, D. J. Wineland, J. M. Gilligan, and D.J Heinzen. 1993, 10p.

Pub. in Hyperfine Interactions, v78 p211-220 1993.

Keywords: "Beryllium ions, "Ion storage, Reprints, Lorentz invariance, Spin, Interactions, Quantum mechan-

We have applied experimental techniques that were developed for use in atomic frequency standards and clocks to investigations of local Lorentz invariance, the linearity of quantum mechanics, and anomalous longrange-spin-dependent forces. These experiments used a hyperfine transition in 9Be+ ions in a Penning trap. Recently, we have studied hyperfine transitions in $199 \mathrm{Hg}+$ ions in a linear it trap. $\mathrm{Hg}+$ ions might be used for similar investigations in the future.

\subsection{1}

PB96-112347 Not available NTIS

National Inst. of Standards and Technology (CSTL), Gaithersburg, MD. Inorganic Analytical Research Div. Analytical Applications of Guided Neutron Beams. Final rept.

R. M. Lindstrom, E. A. Mackey, and R. L. Paul. 1994. $7 p$.

Pub. in Biological Trace Element Research, p47-53 1994.

Keywords: "Cold neutrons, "Neutron guides, Reprints, Neutron beams, Neutron scattering, Prompt gamma radiation, "Foreign technology.

Guided beams of thermal and cold neutrons have become available to analysts at several reactors during the past decade. The very pure beams from these guides have led to lower backgrounds and higher sensitivities for prompt-gamma activation analysis (PGAA), and thus to new applications for this technique. For analytical accuracy, the details of neutron scattering within the sample need to be taken into account; this consideration is especially important for most materials of biological origin

04,042

PB96-119631 Not available NTIS

National Inst. of Standards and Technology (PL), Gaithersburg, MD. Quantum Metrology Div.

Strangeness Flow Difference in Nuclear Collisions at $15 \mathrm{~A}$ and $200 \mathrm{~A} \mathrm{GeV}$.

Finai rept.

J. Rafelski, and M. Danos, 1994, 4p.

Pub. in Physical Review C, v50 n3 p1684-1687 Sep

Keywords: "Nuclear collisions, Reprints, Particle production, "Strangeness flow.
The authors show existence of an important difference between strange-particle production in Si-Au collisions at $15 \mathrm{~A} \mathrm{GeV}$ (AGS) and in S-A collisions at $200 \mathrm{~A} \mathrm{GeV}$ (CERN), with $A=32$ and $A=0(200)$.

04,043

PB96-119763 Not available NTIS

National Inst. of Standards and Technology (EEEL), Boulder, CO. Electromagnetic Technology Div. VAMAS Intercomparison of Critical Current Meas urements on NB3Sn Superconductors: A Summary Report.

Final rept.

H. Wada, C. R. Walters, L. F. Goodrich, and

Tachikawa. 1994, 10p

See ałso PB89-202147.

Pub. in Cryogenics, v34 n11 p899-908 1994.

Keywords: Reprints, Electrical measurement "Superconducting wires, "Niobium stannides, "Critical current, Niobium tin, Interlaboratory comparisons.

The paper is a summary of an international collaboration endorsed by VAMAS to study problems associated with critical current measurements in Nb3Sn superconductors and provide guidelines for a standard measurement. Two series of critical current measurements were implemented. In the fifth series, three different sample conductors were used and participants made measurements using their own techniques. As a result, coefficients of variation for these samples at $12 \mathrm{~T}$ turned out the be $8-29.9 \%$. A major source of these variations was attributed to strain sensitivity of the Nb3Sn conductors. Thus, the second series of measurements were done on one sample conducto and under specified measurement conditions, particu larly in terms of specimen strain. The coefficient of variation decreased to $2.2 \%$, which is regarded as a rea sonable base for future establishment of an international standard measurement method.

\section{4,044}

PB96-119789 Not available NTIS

National Inst. of Standards and Technology (PL), Boulder, CO. Time and Frequency Div.

High-Order Multipole Excitation of a Bound Electron.

Final rept.

C. S. Weimer, F. L. Moore, and D. J. Wineland.

1993, 4p.

Pub. in Physical Review Letters, v70 n17 p2553-2556, 26 Apr 93

Keywords: *Multipole excitation, Reprints, "Parametric processes, Penning trap, Single electron.

The nonlinear resonant response of a bound electron to a time-varying spatially inhomogeneous electric field was studied experimentally. By use of the artifical atom 'geonium' (an electron bound in a Penning trap), the authors observed up to ninth-order multiple (pentacosiododecapole) coherent excitation of the electron's magnetron motion, and up to third-order ( $\mathrm{Oc}-$ tuple) excitation of the cyclotron motion. Also, by applying two fields simultaneously, the authors have observed coherent stimulated Raman Excitation of the electron's motion.

04,045

PB96-122106 (Order as PB96-117767, PC A08/ MF A02)

National Inst. of Standards and Technology, Gaithersburg. MD

Potential and Current Distributions Calculated Across a Quantum Hall Effect Sample at Low and High Currents.

M. E. Cage, and C. F. Lavine. 1995, 13p.

Included in Jnl. of Research of the National Institute of Standards and Technology, v100 n5 p529-541 Sep/ Oct 95

Keywords: "Hall effect, "Quantum theory, Potential, Charge distribution, Current distribution, Electron gas Reprints. NIST(Nationai Institute of Standards and Technology)

The potential and current distributions are calculated across the width of a quantum Hall effect sample for applied currents between 0 microA and 225 microA. For the first time, both a confining potential and a current-induced charge-redistribution potential are used The confining potential has a parabolic shape, and the charge-redistribution potential is logarithmic. The solution for the sum of the two types of potentials is unique at each current, with no free parameters. The calculated potential distributions are in excellent agree- ment with contactless electro-optic effect laser beam measurements of Fontein et al.

04,046

PB96-122148 (Order as PB96-117767, PC A08/

MF A02)

National Inst. of Standards and Technology, Gaithersburg, MD.

Third Generation Water Bath Based Blackbody Source.

J. B. Fowler. 1995, 9p.

Included in Jnl. of Research of the National Institute of Standards and Technology, v100 n5 p591-599 Sep/ Oct 95.

Keywords: "Blackbody radiation, "Apertures, Reprints, Cavities, Emissivity, Temperature, Reflectance, Radiometry, Measuring instruments, NIST(National Institute of Standards and Technology).

A third generation water bath based blackbody source has been designed and constructed in the Radiometric Physics Division at the National Institute of Standards and Technology Gaithersburg, MD. The goal of this work was to design a large aperture blackbody source with improved temporal stability and reproducibility compared with earlier designs, as well as improved ease of use. These blackbody sources operate in the $278 \mathrm{~K}$ to $353 \mathrm{~K}$ range with water temperature combined standard uncertainties of $3.5 \mathrm{mK}$ to $7.8 \mathrm{mK}$. The calculated emissivity of these sources is 0.9997 with a relative standard uncertainty of 0.0003 . With $50 \mathrm{~mm}$ limiting aperture at the cavity; entrance, the emissivity increases 0.99997

\section{4,047}

PB96-122742 Not available NTIS

National Inst. of Standards and Technology (PL), Boulder, CO. Quantum Physics Div.

Orbital Alignment and Vector Correlations in Inelastic Atomic Collisions.

Final rept.

E. M. Spain, C. J. Smith, M. J. Dalberth, and S. R

Leone. 1993, 9p.

Pub. in International Conference on the Physics of Electronic and Atomic Collisions (18th), Invited Papers, Aarhus, Denmark, v295 p675-683 Jul 93.

Keywords: *Calcium, "Inelastic scattering, "Atomic collisions, Atomic orbitals, Alignment, Coherence, Vector analysis, Lasers, Reprints.

We present a brief commentary on two-, three-, and four-vector correlations in the inelastic scattering of orbitally aligned calcium atoms.

\section{4,048}

PB96-122916 Not available NTIS

National Inst. of Standards and Technology (PL), Boul-

der, $\mathrm{CO}$. Quantum Physics Div.

Atom Cooling and Trapping, and Collisions of Trapped Atoms.

Final rept.

A. Gallagher. 1992, 14p.

Pub. in International Conference on the Physics of Electronic and Atomic Collisions (17th), Invited Papers, Brisbane, Australia, July 10-16, 1991, Section 3, p139152

Keywords: "Atomic collisions, "Energy transfer, Sodium, Densities, Temperatures, Reprints, "Foreign technology, Atom cooling, Trapped atoms.

Methods currently used to optically cool and trap atoms, and the resulting trap densities and temperatures are discussed. Next, the types of cold-atom collision processes that occur in these traps are discussed, with emphasis on their differences compared to more familiar atomic collisions at thermal and higher energies. A semiclassical model and calculations for traploss and associative ionization, due to excited state collisions of these very cold atoms, is then described.

\section{4,049}

PB96-123476 Not available NTIS

National Inst. of Standards and Technology (EEEL), Gaithersburg, MD. Electricity Div.

Noise Characteristics Below $1 \mathrm{~Hz}$ of Zener DiodeBased Voltage Reference.

Final rept.

A. F. Clark, and R. L. Steiner. 1995, 90p.

Pub in Bulletin of the American Physical Society Meeting, San Jose, CA., March 13-17, 1995, v40 n1 90p. Keywords: "Calibration, "Low frequency noise, "Voltage reference, Zener diode, Reprints. 
The short- and mid-term noise of voltage references based on Zener diodes are the limiting factors in the accuracy of their application as voltage standards. Although these references are relatively rugged, survive the rigors of shipping, and have predictable long-term behavior, they do have noise in the voltage output values with a time dependence about the same as the desired measurement intervals. We have characterized several of these references in the frequency range of 10 to the minus 1 power to 10 to the minus 6 power $\mathrm{Hz}$ (time intervals of seconds to months) by direct comparison to a Josephson array voltage standard at the 10 and 1 volt level with nanvolt precision. Several distincty different regions of noise characteristics are observed. Comparisons are made with $1 / f$ and other expected behaviors and resulting accuracy limitations discussed

\section{4,050}

PB96-123518 Not available NTIS

National Inst. of Standards and Technology (PL), Gaithersburg, MD. Atomic Physics Div

Search for Small Violations of the Symmetrization Postulate in an Excited State of Helium.

Final rept.

K. Deilamian, J. D. Gillaspy, and D. E. Kelleher.

1995, 4p.

Pub. in Physical Review Letters, v74 n24 p4787-4790 Jun 95.

Keywords: "Excited states, "Helium, "Laser spectroscopy, Reprints, Pauli Exclusion Principles, Symmetrization postulate.

We have searched for the existence of the permutation symmetric 1s2siS state of helium in an atomic beam. Such a state directly violates the symmetrization postulate (SP) of quantum mechanics and implies a breakdown of the Pauli exclusion principle. Our data constrain recent SP-violating models at the $5 \mathrm{ppm}$ level. This is the first experiment to look systematically for an SP-violating state with no multiple occupancy of a quantum state.

04,051

PB96-123658 Not available NTIS

National Inst. of Standards and Technology (EEEL), Gaithersburg, MD. Electricity Div

Ion Kinetics and Symmetric Charge-Transfer Collisions in Low-Current, Diffuse (Townsend) Discharges in Argon and Nitrogen.

Final rept.
S. B. Radovanov, R. J. Van Brunt, and J. K. Olthoff. 1995, 11 p.

Pub. in Physical Review E, v51 n6 p6036-6046 Jun 95.

Keywords: "Discharge, "Argon, "Nitrogen, Gas mixture, Hydrogen, Ion energy, Optical emission, Radio frequency, Reprints, Balmer alpha, Doppler broadened emission.

Translational kinetic-energy distributions of mass-selected ions have been measured in diffuse, low-current Townsend-type discharges at high electric field-to-gas density ratios $(E / N)$ in the range of $1 \times 10$ to the minus $18-2 \times 10$ to the minus $17 \mathrm{Vm} 2(1-20 \mathrm{kTd})$. The discharges were generated in Ar and N2 under uniformfield conditions and ion energies were measured using a cylindrical-mirror energy analyzer coupled to a quadrupole mass spectrometer. The mean ion energies determined from measured energy distributions of $\mathrm{Ar}+$ in $\mathrm{Ar}$ and $\mathrm{N} 2+$ in $\mathrm{N} 2$ are compared with the mean energies predicted from solutions of the Boltzmann transport equation based on the assumption that symmetric resonant charge transfer is the predominant ionneutral interaction. The results for $\mathrm{Ar}+$ and $\mathrm{N} 2+$ are consistent with predictions made using a constant (energy independent) cross section for which an effective ion temperature can be defined. However, for both ions, the measured mean energies tend to fall increasingly below the predicted values as $\mathrm{E} / \mathrm{N}$ increases.

04,052

PB96-123799 Not available NTIS

National Inst. of Standards and Technology (PL), Gaithersburg, MD. Atomic Physics Div.

Relativistic Modifications of Charge Expansion Theory.

Final rept.

A. W. Weiss, and Y. K. Kim. 1995, 7p.

Pub. in Physical Review A, v51 n6 p4487-4493 Jun 95.

Keywords: "Atomic structure, Relativistic effects, Reprints, "Electron correlation, "Charge expansion theory.
We examine the effects of relativity on the high- $Z$ (where $Z$ denotes nuclear charge) behavior of isoelectronic sequences and the modifications require of the traditional charge expansion theory. We propose that the idea of a complex be refined as the set of all configurations with the same occupation of angular momentum quantum numbers $j$ as well as the same principal quantum numbers $n$. This leads to asymptotic, high-Z grouping of states that might cut across the conventional LS-coupled states, grouping together levels from different configurations, as long as the $n$ and $j$ occupation numbers remain the same. This re grouping substantially reduces the asymptotic configuration interaction from the predicted by a nonrelativistic theory. Frequently correlation configurations that are significant in low- $Z$ ions disappear entirely in high- $Z$ jons.

04,053

PB96-128129 PC A04/MF AO

National Inst. of Standards and Technology (BFRL), Gaithersburg, MD. Building Environment Div.

Calorimetric and Visual Measurements of R123 Pool Boiling on Four Enhanced Surfaces.

M. A. Kedzierski. Nov 95, 62p, NISTIR-5732.

Sponsored by Department of Energy, Washington, DC.

Keywords: "Pool building, "Heat transfer, Surface treatment, "Electric resistance heating, R-123 refrigerant, TURBO-B tubes.

Pool boiling of R-123 on four commercial enhanced surfaces was investigated both calorimetrically and vis ually. The four surfaces were: (1) Turbo-BIITM-LP, (2) The surfaces were either machined (4) GEWA-TTM a flat thick OFHC copper plate. This permitted 20 sheathed thermocouples to be embedded in the copper for accurate heat transfer measurements. The diference between electric resistance and fluid heating was investigated. The fluid heating condition results in heat fluxes that are as much as $32 \%$ greater than those obtained by electric resistance heating. Hysterisis effects near the results in heat fluxes that are as much as $32 \%$ greater than those obtained by electric resistance heating. Hysterisis effects near the onset of nucleate boiling were also investigated. The boiling was visually recorded with $16 \mathrm{~mm}$ high speed film. Mechanistic descriptions of the boiling activity are given for each surface.

\section{4,054}

PB96-135256 Not available NTIS

National Inst. of Standards and Technology (PL),

Gaithersburg, MD. Ionizing Radiation Div.

Oscillometric and Conductometric Analysis of Aqueous and Organic Dosimeter Solutions.

Final rept.

A. Kovacs, I. Slezsak, W. L. McLaughlin, and A

Miller. 1995, 5p.

Pub. in International Meeting on Radiation Processing (9th), Istanbul, Turkey, September 11-16, 1994, v46 4-6 p1211-1215 Oct 95.

Keywords: *Chlorobenzene, "Dosimetry "Conductivity, Reprints, "Foreign technology, "Ethano monochlorobenzene, Electron beam dosimetry, Gamma ray dosimetry, Oscillometry.

Conductometric and oscillometric evaluation methods have earlier been developed to determine absorbed dose in the ethanol-monochlorobenzene dosimeter soution. Recent investigations on the same solution as well as on alanine solutions included the study of the possible use of different type 'conductometric' electrodes and the study of the effect of frequency on the sensitivity of the method. On the basis of these invesigations an oscillometric reader has been designed and tested. The same evaluation methods have been tested on the irradiated aqueous alanine solutions, aiming also at the study of the applicable concentration of the alanine solute and the dose (1 to $50 \mathrm{kGy}$ ) and dose-rate range using both electron and gamma radiation.

\section{4,055}

PB96-135272 Not available NTIS

National Inst. of Standards and Technology (PL), Gaithersburg, MD. Ionizing Radiation Div. Calorimeters for Calibration of High-Dose Dosimeters in High-Energy Electron Beams. Final rept

W. L. McLaughlin, M. L. Walker, and J. C. Humphreys. 1995, 8p.

Pub. in International Meeting on Radiation Processing (9th), Istanbul, Turkey, September 11-16, 1994, Radi- ation Physics and Chemistry, v46 n4-6 p1235-1242 Oct 95.

Keywords: "Calorimeters, "Electron beams, Reprints Dosimeters, Calibration, Radiation processing, "Foreign technology, "Beam calibration.

Graphite calorimeters in both single-slab and modular multiple-slab disk geometries have been designed and used for measuring absorbed doses in graphite in intense electron beams of 2 to $12 \mathrm{MeV}$ energy. The mod ular system has also been used with two to eight graphite elements to measure electron-beam depthdose curves and to calibrate the response functions of radiochromic-film and alanine-pellet dosimeters. An advantage of this new design is that such calibrations can be accomplished without requiring electron-beam current or charge monitoring, since the calorimeter modules adjacent to the dummy graphic element used to hold the dosimeters during calibration provide simultaneous calorimetric dose monitoring. Temperature dependence corrections for dosimeter response are also simplified in this way.

04,056

PB96-138441 Not available NTIS

National Inst. of Standards and Technology (PL) Gaithersburg, MD. Atomic Physics Div.

Observation and Visible and uv Magnetic Dipole Transitions in Highly Charged Xenon and Barium. Final rept

C. A. Morgan, F. G. Serpa, E. Takacs, J. Sugar, J. R. Roberts, C. M. Brown, U. Feldman, E. S. Meyer, and J. D. Gillaspy. 1995, 4p.

Pub. in Physical Review Letters, v74 n10 p1716-1719 Mar 95

Keywords: "lons, "Charged ions, Reprints, "Atomic spectroscopy, "Electron Beam Ion Trap(EBIT).

The authors have observed an unusual transition which is predicted to result in visible and near-uv emission from very highly charged titaniumlike ions spanning the entire upper half of the periodic table Measurements of the wavelengths of the 3d45D2-5D3 transitions in $\mathrm{Ba}+34$ and $\mathrm{Xe}+32$ are in surprisingly poor agreement with ab initio calculations. This work was carried out in an electron beam ion trap and demonstrates that such a device can be an important too for visible spectroscopy of highly charged ions.

04.057

PB96-138466 Not available NTIS

National Inst. of Standards and Technology (PL) Gaithersburg, MD. Atomic Physics Div.

Atomic Transition Probabilities and Tests of the Spectroscopic Coupling Scheme for N I.

Final rept.

J. Musielok, W. L. Wiese, and G. Veres. 1995, 10p Pub. in Physical Review A, v51 n5 p3588-3597 May 95.

Keywords: Emission, Spectrum, Reprints, "Atomic transition probabilities, "Spectroscopic coupling, LS coupling, Neutral nitrogen.

With a wall-stabilized arc source, the authors have measured the relative transition probabilities of 100 lines of neutral nitrogen in the visible and near-infrared spectrum and have normalized their data to an abso ute scale utilizing four recent lifetime results. The au thors estimate that the expanded uncertainties of their data are in the range of plus or minus $11-15 \%$. For a number of $3 s-3 p$ and $3 p-3 d$ multiplets. They have measured complete sets of lines and observe that most $3 p-3 d$ multiplets show considerable departure from LS coupling, while the 3s-3p multiplets adhere to it within plus or minus $20 \%$. Agreement between intermediate coupling calculations and their experimental data for the 3p-3d multiplets is noticeably better, but significant differences are still encountered for two multiplets originating from the 3d4P level.

04,058

PB96-146675 Not available NTIS

National Inst. of Standards and Technology (EEEL) Boulder, CO Electromagnetic Fields Div.

Electric Dipole Excitation of a Long Conductor in a Lossy Medium.

Final rept

D. A. Hill. $1995,19 p$.

Pub. in Electromagnetics, v15 p301-319 1995.

Keywords: "Electric charge, "Dipoles, "Conductors, Electormagnetic wave transmission, Lossy medla, Fre. quencies, Numerical analysls, Reprints. 


\section{General}

Excitation of currents on an infinitely long conductor is analyzed for horizontal electric dipole or line sources and for plane-wave, far-field source. Any of these sources can excite strong currents which produce strong scattered fields for detection. Numerical results for these sources indicate that long conductors produce a strong anomaly over a broad frequency range. The conductor can be either insulated or bare, to model either ungrounded or grounded conductors.

\section{4,059}

\section{PB96-146683 Not available NTIS}

National Inst. of Standards and Technology (EEEL), Gaithersburg, MD. Electricity Div.

Evidence for inelastic Processes for $\mathrm{N}(+) 3$ and $\mathrm{N}(+) 4$ from ion Energy Distributions in He/N2 Radio Frequency Glow Discharges.

Final rept.

H. H. Hwang, J. K. Olthoff, R. J. Van Brunt, S. B.

Radovanov, and M. J. Kushner. 1996, 6p

Pub. in Jnl. of Applied Physics, v79 n1 p93-98 Jan 96.

Keywords: "Ion distribution, "Radio frequency discharge, "Glow discharges, Microelectronics, Gases, Mixtures, Helium, Nitrogen, inelastic collisions, interactions, Fabrication, Reprints, IEDs(Ion energy distributions), ion molecule reactions.

The ion energy distributions (IEDs) striking surfaces in if glow discharges are importatnt in the context of plasma etching during the fabrication of microelectronics devices. In discharge sustained in molecular gases of multicomponent gas mixtures, the shape of the IED and the relative magnitudes of the ion fluxes are sensitive to ion-molecule collisions which occur in the presheath and sheath. Ions which collisionlessly traverse the sheaths or suffer only elastic collisions arrive at the substrate with a measurably different IED than do ions which undergo inelastic collisions. In this article we present measurements and results from parametric calculations of IEDs incident on the grounded electrode of a $i f$ glow discharge sustained in a $\mathrm{He} / \mathrm{N} 2$ gas mixture while using a Gaseous Electronics Conference Reference Cell (33.3 $\mathrm{Pa}, 13.56 \mathrm{MHz})$.

\section{4,060 Not available NTiS}

National Inst. of Standards and Technology (EEEL), Boulder, CO. Electromagnetic Fields Div.

Accuracy in Time Domain Transmission Line Measurements.

Final rept

L. A. Hayden, and R. B. Marks. 1994, 3p.

Pub. in Institute of Electrical and Electronics Engineers Topical Meeting on Electrical Performance of Electronic Packaging (3rd), Monterey, CA., November 24, 1994, p176-178.

Keywords: "Transmission lines, *Signals, "Time, Accuracy, Joining, Signal generators, Network analysis, domain network analysis).

This paper examines time domain methods for characterizing signal propagation in uniform transmission lines. The impact of the limitations associated with time domain instrumentation and methodologies are examined and guidelines for minimizing errors are presented.

\section{4,061}

\section{PB96-148176 Not available NTIS}

National inst. of Standards and Technology (EEEL),

Boulder, CO. Electromagnetic Fields Div.

High-Speed Interconnection Characterization Using Time Domain Network Analysis.

Final rept

R. B. Marks, D. C. DeGroot, and J. A. Jargon. 1995,

Pub. in Advancing Microelectronics, v22 n6 p35-39 Nov/Dec 95.

Keywords: "Time, "Calibration standards, "Packaging, "Microelectronics, Network analysis, Errors, Joining, Measurement, Reprints, "Time domain, TDNA(Time Domain Network Analysis).

Time domain network analysis (TDNA) has become a realistic competitor to conventional automatic network analyzers. Off-line processing of data from fast digital sampling oscilloscopes can provide measurements of network parameters with an accuracy that is acceptable for many packaging and interconnection problems at frequencies from dc to over $10 \mathrm{GHz}$. Since many packaging laboratories have ready access to the required instruments, TDNA brings many advanced measurement capabilities into the hands of engineers to whom a conventional network analyzer is unavailable.

\section{4,062}

PB96-155502 Not available NTIS

National Inst. of Standards and Technology (EEEL) Boulder, CO. Optoelectronics Div.

Simuitaneous Laser-Diode Emission and Detection for Fiber-Optic Sensor Applications.

Final rept.

K. B. Rochford, and A. H. Rose. 1995, 3p

Pub. in Optics Letters, v20 n20 p1205-1207 Oct 95.

Keywords: "Detectors, Polarization, Responsitivity Emission, Detection, Reprints, "Fiber optic sensors, Laser diodes.

The simultaneous emission and detection of radiation with a semiconductor laser is investigated. Measured signal-to-noise ratios of up to $56 \mathrm{~dB}$ demonstrate tha self-detecting devices are adequate for sensor applications with discrete measurands. The authors observed a strong polarization dependence, which can cause response fluctuations, and suggest methods to minimize these fluctuations. This technique could be used for lower-cost sensors without splitters and detectors.

\section{4,063}

PB96-155775 Not available NTIS

National Inst. of Standards and Technology (BFRL) Gaithersburg, MD. Structures Div.

Necessary Condition for Homoclinic Chaos in duces by Additive Noise.

Final rept.

E. Simiu, M. Frey, and M. Grigoriu. $1992,8 p$.

Pub. in Proceedings of the International Conference on Computational Stochastic Mechanics (1st), Corfu, Greece, September 17-19, 1991, p889-896 1992

Keywords: *Chaos, "Noise, Reprints, "Foreign technology, Duffing equation, Dynamical systems, Homoclinic orbits, Nonlinear differential equations.

The effect of noise on the possible occurrence of chaos in systems with a homoclinic orbit was recently inves tigated in the literature on the bais of a redefinition of the Meinikov function. The purpose of this note is to show that, even in the case of deterministic equations, this redefinition is not consistent with the geometry of the perturbed orbits and would therefore lead to incor rect solutions. The possibility is then explored in devel oping a necessary condition for the occurrence of homoclinic chaos in forced systems perturbed additively by a commonly used approximate representation of white noise.

\section{4,064}

\section{PB96-156047 Not available NTIS}

National Inst. of Standards and Techno

Gaicl for Calculating Virial Coefficients of Natu Gas Hydrocarbons with impurities.

Final rept.

L. A. Weber. 1995, 12p

Pub. in Fluid Phase Equilibria, v111 p15-26 1995.

Keywords: "Activity coefficient, Vapor pressure, Reprints, "Foreign technology, "Ebulliometry, "Refrigerant mixtures, Vapor liquid equilibrium

A model for calculating second and third virial coefficients, originally developed to describe the behavior of polar halocarbon refrigerants and their mixtures, has been applied to systems of natural gas hydrocarbons and their common impurities, namely $\mathrm{H} 2 \mathrm{~S}, \mathrm{H} 2 \mathrm{O}, \mathrm{CO} 2$ $\mathrm{CO}$, and N2. It can be used to correlate data, point out probable errors and provide predictions where data are nonexistent or unreliable. it also provides a means for calculating accurate gas-phase densities, thermodynamic properties, fugacities, and 'calculated data' which can be incorporated into global equations of state for phase equilibrium calculations. Examples are shown for some of the pure components and for the binary systems $\mathrm{C} 2 \mathrm{H} 6+\mathrm{H}_{2} \mathrm{~S}$ and $\mathrm{CH}_{4}+\mathrm{H}_{2} \mathrm{O}$. in the case $\mathrm{H} 2 \mathrm{O}$, terms are included that account for association. Comparisons are made with available data, and predicted values are shown where no data exist.

\section{4,065}

PB96-156203 Not available NTIS

National Inst. of Standards and Technology (PL) Gaithersburg, MD. Electron and Optical Physics Div.
Simple Variable Line Space Grating
Monochromator for Synchrotron Light Source Monochromat
Beamlines.

Final rept.

T. A. Callcott, W. L. O'Brien, J. J. Jia, R. N. Watts, D. R. Mueller, Q Y Dong, and D. L. Ederer 1992 7p.

Pub. in Nuclear instruments and Methods in Physics Research, vA319 p128-134 1992.

Keywords: "Monochromators, "Spectrometers, Synchrotrons, Vacuum ultraviolet, Reprints, Soft $x$ ray, Varied line spaced grating.

In this paper, the authors present and analyze an improved spectrometer design that has evolved from studies of the focal properties of transmission gratings in converging light beams. This design uses a variable line space grating at a fixed angle of incidence to focus the diffracted light in one dimension, producing resolutions in excess of 1000 over a large wavelength region. Attractive features of this concept include high throughput, simple wavelength scanning, simple optical elements (plane gratings and plane and toroidal mirrors), and relatively inexpensive realizations. After presenting a mathematical analysis of the primary design features, the authors discuss implementations of this design both as a spectrometer and as two versions of beamline monochromators. Design parameters for the two monochromators will be given.

04,066

PB96-157813 Not available NTIS

National Inst. of Standards and Technology (CAML), Gaithersburg, MD. Statistical Engineering Div.

Modeling Detector Response for Neutron Depth Profiling.

Final rept

K. J. Coakley, R. G. Downing, G. P. Lamaze, C.

Ronning, H. C. Hofsass, and J. Biegel. 1995, 8p.

Pub. in Nuclear Instruments and Methods in Physics Research, Section A, vA366 p137-1441995.

Keywords: "Detector response, Semiconductors Straggling, Reprints, "Foreign technology, "Neutron depth profiling, Diamond-like carbon, Statistical modeling.

In Neutron Depth Profiling (NDP), inferences about the concentration profile of an element in a material are based on the energy spectrum of charged particles emitted due to specific nuclear reactions. The detector response function relates the depth of emission to the expected energy spectrum of the emitted particles. Here, the detector response function is modeled for arbitrary source and detector geometries based on a model for the stopping power of the material, energy straggling, multiple scattering and random detector measurement error. At the the NIST Cold Neutron Research Facility, a NDP spectrum was collected for a diamond-like carbon (DLC) sample doped with boron. A vertical slit was placed in front of the detector for collimation. Based on the computed detector response function, a model for the depth profile of boron is fit to the observed NDP spectrum. The contribution of straggling to overall variability was increased by multiplying the Bohr Model prediction by a ramp factor. The adjustable parameter in the ramp was selected to give the best agreement between the fitted profile and the expected shape of the profile. The expected shape is determined from experimental process control measurements.

\section{4,067}

PB96-157839 Not available NTIS

National inst. of Standards and Technology (PL), Gaithersburg, MD. Electron and Optical Physics Div. Al L2,3 Core Excitons in AlxGa1-x as Studied by Soft-X-ray Refiection and Emission.

Final rept.

Q. Y. Dong, W. L. O'Brien, J. J. Jia, T. A. Callcott, and D. L. Ederer. 1992, 7p.

Pub. in Physical Review B, v46 n23 p15 116-15 122 Dec 92

Keywords: "X ray emission, Reprints, "Aluminum gallium arsenide, Absorption spectra, Core exciton.

The Al L2.3 soft-x-ray reflection and emission spectra of AlxGa1-xAs have been measured for $x$ between 0.17 and 1.0 . The valence-band maximum was monitored via the emission spectra using a fit based on band-structure parameters. The dominant features in the reflection spectra are produced by the $L 2.3$ core excitons. Since both the reflection spectra and emission spectra were obtained using the same soft-x-ray spectrometer, the energies of the excitons above the 
valence-band maximum are acurately obtained. The known values of the optical band gap, as a function of $x$, are then used to determine the core exciton binding energy. The core excitons are found to follow and lie above the conduction-band minimum by $0.3 \mathrm{eV}$ as the Al composition changes. Our results are compared to various core exciton theories and to previous experiments.

\section{4,068}

PB96-157870 Not available NTIS

National Inst. of Standards and Technology (CAML), Gaithersburg, MD. Statistical Engineering Div.

Stochastic Modeling of a New Spectrometer.

Final rept.

C. Hagwood, K. Coakley, A. Negiz, and K. Ehara.

1995, 17p.

Pub. in Aerosol Science and Technology, n23 p6116271995

Keywords: "Aerosol spectrometers, Equations, Reprints, "Stochastic differential, "Transfer function, Exit probability.

A new spectrometer for classifying aerosol particles according to specific masses is being considered (Ehara et at. 1995). The spectrometer consists of concentric cylinders which rotate. The instrument is designed so that an electric field is established between the cylinders. Thus, aerosol particles injected into the spectrometer are subjected to a centrifugal force and an electric force. Depending on the balance between these two forces, as well as Brownian motion, charged particles either pass through the space between the cylinders or stick to either cylinder wall. Particles which pass through are detected. Given the rotation rate, voltage drop and physical dimensions of the device, we calculate the probability of detection in terms of particle density, diameter and charge.

04,069

PB96-157912 Not available NTIS

National Inst. of Standards and Technology (PL), Gaithersburg, MD. Electron and Optical Physics Div. Soft-X-ray-Émission Investigation of Cobalt Implanted Silicon Crystals.

Final rept.

J. J. Jia, T. A. Callcott, W. L. O'Brien, J. E

Rubensson, D. L. Ederer, Z. Tan, F. Namavar, J. I.

Budnick, Q. Y. Dong, and D. R. Mueller. 1991, 5p.

Pub. in Jnl. of Applied Physics, v69 n11 p7800-7804 Jun 91.

Keywords: "X ray emissions, "Semiconductors, Core excitation, Silicide, Reprints, Band structure, Ion implantation.

The Si L2,3 emission spectra of silicon crystals implanted with $C o$ at doses of $(1-8) \times 10$ to the 17 th power $\mathrm{Co} / \mathrm{cm} 2$ have been examined using soft-x-ray-emission (SXE) spectroscopy. At the lowest dose, the spectra are little modified from that of crystalline Si, indicating that only a small fraction of $S i$ is in the form of silicides within the probe depth of SXE spectroscopy. For higher doses and implant profiles with Co extending to the surface, there is clear evidence ordered CoSi2 combined with richer Co phases, but little evidence for pure Si or for ordered regions of COSi.

\section{4,070}

PB96-157920 Not available NTIS

National Inst. of Standards and Technology (PL), Gaithersburg, MD. Electron and Optical Physics Div. Soft-X-ray-Emission Spectra of Solid Kr and Xe.

Final rept

J. J. Jia, W. L. O'Brien, T. A. Callcott, D. R. Mueller, D. L. Ederer, Q. Y. Dong, and J. E. Rubensson.

1991, 4p.

Pub. in Physical Review Letters, v67 n6 p731-734 Aug 91.

Keywords: "Electron structure, "X ray emissions, Reprints, "Rare gas solids

We present the first soft-x-ray-emission spectra for solid $\mathrm{Kr}(4 \mathrm{p}-3 \mathrm{~d})$ and $\mathrm{Xe} \mathrm{(5p-4d)}$ that are free of complicating satellite spectra. Monochromatic synchrotronradiation excitation is used to suppress the satellites. The data are analyzed to determine the P3/2 bandwidth for both elements; these results a re compared with both photoemission data and available theoretical calculations. Bandwidths are found to be larger than those predicted by most electronic structure calculations.
National Inst. of Standards and Technology (PL), Gaithersburg, MD Electron and Optical Physics Div. Soft-X-ray-Emission Studies of Bulk Fe3Si, FeSi, and FeSI2, and Implanted Iron Silicides.

Final rept.

J. J. Jia, T. A. Callcott, W. L. O'Brien, D. L. Ederer, Z. Tan, J. I. Budnick, Q. Y. Dong, and D. R. Mueller. 1992, 6p

Pub. in Physical Review B, v46 n15 p9446-9451 Oct

Keywords: "Electronic structure, "Iron silicides, " $X$ ray emissions, Reprints, Iron implanted silicon, Partial density.

Bulk iron silicides and implanted iron silicides have been studied by soft-x-ray emission (SXE) spectroscopy. The Si L2,3 emission spectra of these materials are measured For bulk silicides, these spectra provide a measure of s-and d-type partial density of states (PDOS) localized on the Si sites. We compare them with available band-structure calculations and also with photoemission measurements. For implanted systems, the Si L2,3 emission spectra provide useful information about the silicide formation process with the variation of implant doses.

\section{4,072}

\section{PB96-157946 Not available NTIS}

National Inst. of Standards and Technology (PL)

Gaithersburg, MD. Electron and Optical Physics Div.

Influence of Coadsorbed Potassium on the Elec tron-Stimulated Desorption of $F(+), F(-)$, and $F\left({ }^{*}\right)$ from PF3 on Ru(0001).

Final rept.

S. A. Joyce, C. Clark, V. Chakarian, T. E. Madey, P.

Nordlander, B. Maschhoff, H. S. Tao, D. K. Shuh,

and J. A. Yarmoff. 1992, 9p.

Pub. in Physical Review B, v45 n24 p14 264-14 272 Jun 92

Keywords: "Charge transfer, "Fluorine, "Photoemission, Ruthenium, Reprints, PF3, Stimulated desomtion.

The electron-stimulated-desorption ion angular distributions (ESDIAD) of positive ions, negative ions, and metastable species from $P F$ and $K$ coadsorbed on a $\mathrm{Ru}(0001)$ surface are measured. In the absence of $K$ only positive- and negative-ion ESDIAD is observed showing highly anisotropic, off-normal fluorine-ion emission ( $F+, F-)$ from PF3, which demonstrates that $P F$ bonds are inclined away from the surface normal In the presence of $K$, ESDIAD patterns of $F_{+}, F_{-}$, and metastable $F($ star) display only normal emission, suggesting a reorientation or chemical reaction which results in fluorine bonding perpendicular to the surface. Thermal desorption spectrometry, Auger electron spectroscopy, and soft-x-ray photoemission (SXPS) are used to determine the surface chemistry as an aid in intepreting the ESDIAD results. The SXPS data show that in the presence of potassium, PF3 dissoci ates, possibly forming KF-like species, which suggests that an alkali-metal-induced chemical reaction is responsible for the changes observed in ESDIAD. The yields of the various desorbed fluorine species vary strongly with potassium coverage; the results for $F$ and $F(s t a r)$ are interpreted in terms of a charge-trans fer model.

\section{4,073}

PB96-157953 Not available NTIS

National Inst. of Standards and Technology (PL), Gaithersburg, MD. Electron and Optical Physics Div.
RIS Measurement of AC Stark Shifts and RIS Measurement of AC Stark Shift
Photoionization Cross Sections in Calcium.

Final rept.

J. B. Kim, X. Xiong, T. R. O'Brian, T. J. Mcllrath, and T. B. Lucatorto. 1992, 4p

Pub. in Inst. Phys. Conf. Ser. No. 128, RIS 92, Santa $\mathrm{Fe}, \mathrm{NM}$., May 24-29, 1992, p59-62

Keywords: "Calcium, Reprints, "AC Stark shifts, Intense laser fields, Multiphoton ionization, Photo-ionization, Resonance ionization spectroscopy, Rydberg levels.

The ac Stark shifts of four high-lying $\mathrm{Ca}$ levels in in tense laser fields ( 10 to the 8th power to 10 to the 10 th power W/sq. $\mathrm{cm}$ ) are studied using two-photon ionization spectroscopy. The two-photon Rabi rates and photoionization cross sections for excited states are also measured, and compared to values obtained from detailed modeling of the RIS profiles. Although three Rydberg levels studied display the expected pondermotive energy shifts, a fourth doubly-excited level deviates significantly from the ponderomotive behavior.

04,074

PB96-157979 Not available NTIS

National Inst. of Standards and Technology (PL) Gaithersburg, MD. Electron and Optical Physics Div. Comment On: Two-Photon Absorption Series of Calcium.

J. R. Krumrine, A. Musgrove, and T. B. Lucatorto.

1995, 2p.

Pub. in Canadian Jnl. of Physics, v73 p403-404 1995. Keywords: "Calcium, "Energy levels, "Laser spectroscopy, Series, Spectra, Quantum defects, Reprints, "Foreign technology.

Using two-photon absomtion laser spectroscopy of calcium. Makdisi and Bhatia presented a well-resolved series that they tentatively assigned as 4S2 1SO - 4sn 1F3 ( $n=33-59)$. We find this assignment to be erro neous. Calculations of quantum defects and a comparison of their observed energy levels with previously determined values reveal that the spectra actually represent the 4s2 1 SO - 4snp $1 P 1$ series $(n=34-60)$.

04,075

PB96-157987 Not available NTIS

National Inst. of Standards and Technology (PL) Gaithersburg, MD. Electron and Optical Physics Div. Laser Modification of Ultracold Collisions: Experiment.

Final rept.

P. D. Lett, P. S. Jessen, W. D. Phillips, P. L. Gould S. L. Rolston, and C. I. Westbrook. 1991, 4p. Pub. in Physical Review Letters, v67 n16 p2139-2142 Oct 91.

Keywords: "Atomic collisions, "Laser cooling, Optica molasses, Trapped atoms, Reprints, "Ultracold atoms, Associative ionization, Laser trapping, Low energy collisions.

Julienne recently predicted a dramatic laser-intensitydependent modification of the associative ionization (AI) rate in ultracold collisions. We observe such a modification, but with a behavior inconsistent with the originally proposed mechanism. Furthermore, we find resonant structure in the spectrum of $\mathrm{Al}$ rate versus laser frequency, showing the importance of molecula bound states in the Al process. These observations are explained in a new theoretical treatment by Julienne and Heather (preceding Letter)

\subsection{6}

PB96-158019 Not available NTIS

National Inst. of Standards and Technology (PL), Gaithersburg, MD. Electron and Optical Physics Div. Barium Contributions to the Valence Electronic Structure of YBa2Cu307-delta, PrBa2Cu307-delta, and Other Barium-Containing Compounds.

Final rept.

D. R. Mueller, J. S. Wallace, J. J. Jia, T. A. Callcott K. E. Miyano, T. A. Ederer, W. L. O'Brien, and Q. Y Dong. 1995, 7p.

Pub. in Physical Review B, v52 n13 p9702-9708 Oct 95.

Keywords: "Barium partial density, "Photon beam excitation, "Soft x ray emission, Reprints, BaF2, BaTiO3, $\mathrm{PrBa2Cu} 307, \mathrm{YBA} 2 \mathrm{Cu} 307$

Monochromatic photon beams were used to excite barum NIV,V soft $x$-ray emission spectra from YBa2Cu307-delta, prBa2Cu307-delta, BaF2, and $\mathrm{BaTiO} 3$ Near threshold excitation was used to demonstrate that small contributions to the barium NV and NIV emission spectra in the energy region above the $5 p-->4 d$ core-core transitions do not arise as satellite emission from transitions in multiply excited atoms but rather occur as a result of transitions from the valence states. The emission spectrum of YBa2Cu307-delta and $\mathrm{PrBa} 2 \mathrm{Cu} 3 \mathrm{O}$-delta reveals a contribution to the electronic density of states at the barium site in the region near the Fermi level. The YBa2Cu307-delta compound is a superconductor and $\mathrm{PrBa} 2 \mathrm{Cu} 3 \mathrm{O} 7$ delta is an insulator. It has been proposed that the difference between them is due to mixing of praseodymium and barium among the sites occupied by yttrium and barium, with an accompanying change in electronic structure. However, the authors' measure ments indicate that the barium partial density of states for the two compounds are essentially identical.

O4.077
PB96-158027 Not available NTIS 
National Inst. of Standards and Technology (PL), Gaithersburg. MD. Electron and Optical Physics Div. Cooper M(sub II,III) X-ray-Emission Spectra of Copper Oxides and the Bismuth Cuprate Superconductor.

Final rept

D. R. Mueller, J. Wallace, D. L. Ederer, Q. Y. Dong,

T. A. Callcott, J. J. Jia, and W. L. O'Brien. 1992, 6p. Pub. in Physical Review B, v46 n17 p11 069-11 074 Nov 92.

Keywords: "Cupric oxide, "Electron density, Cuprous oxide, Reprints, "X ray fluorescence, Superconductivity bismuth cuprate.

Photon-excited soft x-ray emission spectra for $\mathrm{CuO}$, $\mathrm{Cu} 2 \mathrm{O}$, and bismuth cuprate in the region of the $\mathrm{Cu} 3 \mathrm{~d}-$ $\rightarrow 3 p$ transitions were measured to obtain bulk-sensitive electronic partial density-of-states information. For $\mathrm{CuO}$ and $\mathrm{Cu} 2$ transitions from the valence levels into the $\mathrm{Mn}$ and $\mathrm{Mm}$ levels were clearly resolved. The emission features were separated by the $2.3-\mathrm{eV}$ spinorbit interaction of the copper $3 p 1 / 2$ and $3 p 3 / 2$ states and appeared in the statistical intensify ratio. The copper Mm emission spectrum of the superconductor is broader than the spectra $\mathrm{CuO}$ and $\mathrm{Cu} 2 \mathrm{O}$. For all three compounds the shape of the $\mathrm{Mm}$ emission spectrum extracted from the data is qualitively similar to calculations of the copper single-particle local density of slates. No evidence of multiplet structure was observed in the emission spectrum of CuO in spite of the open $\mathrm{d}$ shell in the ground state. The measurements are compared with published copper $\mathrm{L}$ emission data and photoemission results.

\section{4,078}

PB96-158035 Not available NTIS

National Inst. of Standards and Technology (PL). Gaithersburg, MD. Electron and Optical Physics Div. RIS Measurements of the AC Stark Shift.

Final rept

T. O'Brian. 1993, 2p.

Pub. in Resonances (Newsletter of the Instifute for Resonance Ionization Spectroscopy), v1 n3 p2-3 Spring 1993.

Keywords: "Spectroscopy, Reprints, "AC Stark shift, *Rydberg atoms, Resonance ionization spectroscopy. The ac Stark shift in xenon Rydberg levels is directly measured using resonance ionization spectroscopy. The authors report excellent agreement between the measured shifts and the near-ponderomotive shifts predicted by detailed theory of the resonant ionization process.

\section{4,079}

PB96-158043 Not available NTIS

National Inst. of Standards and Technology (PL), Gaithersburg, MD. Electron and Optical Physics Div. Intermediate Coupling in L2-L3 Core Excitons of $\mathrm{MgO}, \mathrm{Al} 2 \mathrm{O} 3$, and $\mathrm{SiO} 2$.

Final rept.

W. L. O'Brien, J. J. Jia, Q. Y. Dong, D. L. Mueller, D. L. Ederer, T. A. Callcott, and J. E. Rubensson. 1991, $6 p$.

Pub. in Physical Review B, v44 n3 p1013-1018 Jul 91.

Keywords: "Absorption spectra, "Reflection spectra,

The $\mathrm{L} 23$ reflection spectra for single-crystal $\mathrm{MgO}$, alpha-Al2O3, and alpha-SiO2 are measured. These reflection spectra are used to generate absorption spectra that agree well with electron-total-yield and electron-energy-loss measurements. Exciton features are shown to exist in the band gap by comparing the valence-band maximum plus optical band gap to the observed transition energies. The energy splitting on the L2-L3 excitons, as seen in reflection, is clearly resolved for $\mathrm{Al} 2 \mathrm{O} 3$ and $\mathrm{SiO} 2$, while in electron-total-yield measurements the doublets are not resolved for $\mathrm{Al} 2 \mathrm{O} 3$. This resolution allows the calculation of the exchange energy using intermediate-coupling theory.

\section{4,080}

\section{PB96-158092 Not available NTIS}

National Inst. of Standards and Technology (PL), Gaithersburg, MD. Electron and Optical Physics Div. New NIST/ARPA National Soft X-ray Reflectometry Facility.

Final rept.

C. Tarrio, R. N. Watts, T. B. Lucatorto, J. Jia, M.

Haass, and T. A. Calcott. 1994, $6 p$.

Pub. in Jnl. of X-ray Science and Technology, v4 p961011994.
Keywords: "Lithography, "Metrology, Monochromator, Reflectometry, Synchrotron, Reprints, "Soft x ray.

The authors have recently begun a series of upgrades to the NIST/ARPA National Reflectometry Facility at the Synchrotron Ultraviolet Radiation Facility. The facility currently consists of a new monochromator and the original sample manipulator which allows the authors to measure optical components less than $10 \mathrm{~cm}$ in diameter. The monochromator offers high throughput and modest resolution over the wavelength range 3.5 $40 \mathrm{~nm}$. In the next year the authors will be installing a sample manipulator that will be able to accommodate the much larger optics that will be used in future $x$-ray projection lithography and astronomy instruments. The authors offer preliminary measurements of the throughput and resolution of the new monochromator.

\section{4,081}

\section{PB96-159678 Not available NTIS}

National Inst. of Standards and Technology (EEEL), Gaithersburg, MD. Semiconductor Electronics Div.

Boron-Implanted 6H-SiC Diodes.

Final rept.

M. Ghezzo, D. M. Brown, E. Downey, J. Kretchmer and J. J. Kopanski. 1993, 3p

Pub, in Applied Physics Letters, v63 n9 p1206-1208 Aug 93.

Keywords: "Ion implantation, Reprints, "High temperature electronics, SiC, Silicon carbide.

Ion implanted planar $p-n$ junctions are important for silicon carbide discrete devices and integrated circuits. Conversion to $\mathrm{p}$-type of $\mathrm{n}$-type $6 \mathrm{H}$-SiC was observed for the first time using boron implantation. Diodes were fabricated with boron implants at 25 and 1000 degrees $\mathrm{C}$, followed by 1300 degrees $\mathrm{C}$ post-implant annealing in a furnace. The best diodes measured at 21 degrees $C$ exhibited an ideality factor of 1.77 , reverse bias leakage of 10 to the minus 10 th power $\mathrm{A} / \mathrm{sq} . \mathrm{cm}$. at.-10 $V$, and a record high (for a SiC-implanted diode) breakdown voltage of $--650 \mathrm{~V}$

\subsection{2}

PB96-159801 Not available NTIS

National Inst. of Standards and Technology (CSTL), Gaithersburg, MD. Surtace and Microanalysis Science Div.

Dynamics of Hydrogen Interactions with Si(100) and $\mathrm{Si}(111)$ Surfaces.

Final rept.

K. W. Kolasinski. 1995, 57p

Pub. in International Jnl. of Modern Physics B, v9 n21 p2753-2809 1995.

Keywords: "Internal state distribution, "Molecular beam scattering, Reaction dynamics, Reprints, Silicon, Ad sorption, Desorption, Hydrogen, "Foreign technology, Disilane.

Experimental and theoretical work probing the dynamics of dissociative adsorption and recombinative desorption of hydrogen at Si(100) and Si(111) surfaces is reviewed. Whereas molecular beam experiments demonstrate that molecular excitations do aid in overcoming a substantial activation barrier toward adsorption, desorbed molecules are found to 'iave a total energy content only slightly above the equilibrium expectation at the surface temperature. A consistent interpretation of the ad/desorption dynamics is arrived at which requires neither a violation of microscopic reversibility nor defect-mediated processes. An essential element of this model is that surface atom relaxations play an essential role in the dynamics such that ditferent portions of the potential energy hypersurface govern the results of adsorption and desorption experiments. The 'lost' energy, i.e. that portion of the activation energy not evident in the total energy of the desorbed molecules. is deposited in the surface coordinates where it is inaccessible to experiments that probe the desorbates final state.

\section{4,083}

PB96-160221 Not available NTIS

National Inst. of Standards and Technology (EEEL) Gaithersburg, MD. Semiconductor Electronics Div.

Stacking Fault Pyramid Formation and Energetics in Silicon-on-Insulator Material Formed by Multiple Cycles of Oxygen Implantation and Annealing.

Final rept.

J. D. Lee, J. C. Park, D. Venables, S. J. Krause, and

P. Roitman. 1993, 3p.

Pub. in Applied Physics Letters, v63 n24 p3330-3332, 13 Dec 93.
Keywords: "Silicon defects, Reprints, "Silicon on insulator, Oxygen implanted silicon, Stacking faults, Transmission electron microscopy.

The defect microstructure of silicon-on-insulator wafers produced by multiple cycles of oxygen implantation and annealing was studied with transmission electron microscopy. The dominant defects are stacking fault pyramids (SFPs), $30-100 \mathrm{~nm}$ wide, located at the upper buried oxide interface at a density of approx. 10 to the 6th power $\mathrm{cm}$ to the minus 2 power. The defects are produced by the expansion and interaction of narow stacking fault (NSF) ribbons pinned to residual precipitates in the top silicon layer. Consideration of the energetics of the transformaion from a collection of four NSF ribbons to a single SFP indicates that the reaction is energetically favorable below a critical NSF length. Thus small defects are stable as SFPs while large defects are stable as NSF ribbons.

\section{4,084}

PB96-160247 Not available NTIS

National Inst. of Standards and Technology (EEEL), Gaithersburg, MD. Semiconductor Electronics Div. RII Spectroscopy of Trap Levels in Bulk and LPE $\mathrm{Hg} 1-\mathrm{xCdxTe}$.

Final rept.

C. L. Littler, X. N. Song, Z. Yu, D. G. Seiler, J. L.

Elkind, and J. R. Lowney. 1993, 5p.

Pub. in Semiconductor Science and Technology, v8 pS317-S321 1993.

Keywords: "Magnetoabsorption, "Liquid phase epitaxy One photon, Trap levels, Impurities, Defects, Reprints *Foreign technology, HgCdTe, Mercury interstitials.

Resonant impact ionization (RII) spectroscopy, a new technique for studying low concentrations of trap levels in narrow-gap $\mathrm{Hg}(1-x) \mathrm{Cd}(x) \mathrm{Te}$, has been used to investigate impurity and defect levels in both bulk and liquid phase epitaxy (LPE) crystals of this ternary material. Mercury interstitials, deliberately introduced into bulk samples with $x$ equals 0.22 and $x$ equals 0.24 , provide direct evidence for the formation of trap levels near 45 and $61 \mathrm{meV}$ above the valence band edge for these $x$-value samples. The $61 \mathrm{meV}$ level in the $x$ equals 0.24 ( $\mathrm{Eg}=121 \mathrm{meV}$ at $5 \mathrm{~K}$ ) sample is seen to enhance the transition strength of resonant two-photon magneto-absorption (TPMA) observed at high fields by acting as a near-resonant intermediate state for the two-photon process. In the LPE material, samples which have received just a post-anneal in an $\mathrm{Hg}$-saturated atmosphere show strong (RII) structure due to defect levels at equals $60 \mathrm{meV}$ above the valence band, whereas samples which are both doped with indium and post-annealed show only intrinsic one-photon magneto-absorption structure.

\section{4,085}

PB96-160296 Not available NTIS

National Inst. of Standards and Technology (PL) Gaithersburg, MD. Electron and Optical Physics Div. Phonon Relaxation in Soft-X-ray Emission of Insulators.

W. O'Brien, J Jia O Y Dong D L Ederer, D. R Mueller, C. C. Kao, T. A. Callcott, and K. E. Miyano. 1993, 4p.

Pub. in Physical Review B, v47 p140-143 1993.

Keywords: "Insolators, "Phonons, Photon excitation, Reprints, Soft $x$ ray emission, Stoke shift, Synchrotron radiation

Phonon-relaxation effects on the $\mathrm{L} 2.3$ soft-x-ray emis sion of $\mathrm{MgO}, \mathrm{Al}_{2} \mathrm{O}_{3}$, and $\mathrm{SiO} 2$ are measured by $\mathrm{com}$ paring soft-x-ray emission spectra to photoelectron spectra. The observed shifts of the soft-x-ray emission spectra relative to the photoelectron spectra are identified as Stokes shifts and are described in terms of partial phonon relaxation. The observed Stokes shifts are $0.5,0.9$ and $1.2 \mathrm{eV}$ for $\mathrm{MgO}, \mathrm{Al} 2 \mathrm{O} 3$, and $\mathrm{SiO} 2$, respectively. Similar measurements on $\mathrm{Mg}, \mathrm{Al}$, and $\mathrm{Si}$ show no detectable Stokes shift within the experimental uncertainty of $0.2 \mathrm{eV}$. The larger Stokes shifts observed in the oxides are due to the longer core-hole lifetime the faster phonon relaxation, and larger phonon relaxation energy in the oxides.

\section{4,086}

PB96-160312 Not available NTIS

National Inst. of Standards and Technology (PL), Gaithersburg, MD. Atomic Physics Div. 
Laser-Cooled Neutra! Atom Frequency Standards. Final rept

S. L. Rolston, and W. D. Phillips. 1991, 9p.

Pub. in Proceedings of the Institute of Electrical and Electronics Engineers, v79 n7 p943-951 Jul 91.

Keywords: *Atomic fountain, *Frequency standards, "Laser cooling, Microwave standards, Optical clock, Reprints, Two-photon spectroscopy.

Recent advances in laser cooling, including temperatures as low a few microkelvin, and the demonstration of the ability to launch atoms while maintaining these low temperatures, now allows the realization of atomic fountain frequency standards. An analysis of a Cs microwave fountain predicts potential stability anmd accuracy improvements of a factor of 100 over present laboratory standards, with an ultimate accuracy near 10 to the 16 th power. An optical frequency standard based on a two-photon transition in xenon is analyzed and shown to have potential stability 5 orders of magnitude better than current Cs standards, and a possible accuracy of 10 to the 18th power. Neutral atom frequency standards will have much better signal-to-noise (S/N) ratios than ion standards, but suffer from large systematic effects, making the two technologies complementary in many ways.

04,087

PB96-160338 Not available NTIS

National Inst. of Standards and Technology (PL), Gaithersburg, MD. Electron and Optical Physics Div. Improved Reflectometry Facility at the National Institute of Standards and Technology.

Final rept.

C. Tarrio, R. N. Watts, T. B. Lucatorto, J. Jia, M.

Haass, and T. A. Calcott. 1993,6p.

Pub. in Society of Photo-Optical Instrumentation Engineers Proceedings of the International Society for Optical Engineering; Multilayer and Grazing Incidence $X$ ray EUV Optics II, San Diego, CA., July 14-16, 1993 , v2011 p534-539.

Keywords: "Extreme ultraviolet, "Lithography, "Reflectometers, Optics, Reprints, Monochromator, Soft $x$ ray, Synchrotron.

We have recently completed construction of a high throughput, modest resolution soft $x$-ray monochromator installed at the Synchrotron Ultraviolet Radiation Facility of the National Institute of Standards and Technology. Although this monochromator will be used primarily to characterize the optical properties of multilayer-coated $x$-ray optics, the versatility of the instrument will enable us to measure such properties as reflectivity, transmission, diffraction, and scatter of a variety of component. We present measurements of monochromator throughput and resolution.

04,088

PB96-160379 Not available NTIS

National Inst. of Standards and Technology (PL), Gaithersburg, MD. Electron and Optical Physics Div. NIST Metrology for Soft X-ray Multilayer Optics.

Final rept.

R. N. Watts, D. L. Ederer, T. B. Lucatorto, and M.

Isaacson. 1991, 3p.

Pub. in OSA Proceedings on Soft-X-ray Projection Lithography, Monterey, CA., April 10-12, 1991, v12 p142-144.

Keywords: "X ray optics, "Nanostructure, "Lithography, Reflectivity, Multilayers, Metrology, Figure, Reprints.

We describe the capabilities of the exciting NIST soft $X$-ray reflectometry program and outline our proposed new characterization facility

04,089

PB96-160445 Not available NTIS

National Inst. of Standards and Technology (PL),

Gaithersburg, MD. Electron and Optical Physics Div.

Appearance Intensities for Multiply Charged lons in a Strong Laser Field.

Final rept.

M. Brewczyk, K. Rzazewski, and C. W. Clark. 1995 ,

$6 \mathrm{p}$

Pub. in Physical Review A, v52 n2 p1468-1473 Aug

Keywords: "Nonlinear optics, Ionization, Reprints, Atoms, Lasers, "Thomas-Fermi model.

We study multiple ionization of atoms by a strong laser field using a time-dependent approach based on the Thomas-Fermi model. The evolution of the electron density under the influence of transient, linearly polarized radiation field is determined by numerical solution of hydrodynamic equations on a two-dimensional grid. We find a threshold 'appearance intensity' for a given charge state, i.e., the laser intensity at which such charge state first appears, which is in reasonable agreement with experimental results for high charge states.

\section{4,090}

PB96-160700 Not available NTIS

National Inst. of Standards and Technology (MEL) Gaithersburg, MD. Precision Engineering Div.

Generating and Measuring Displacements Up to $0.1 \mathrm{~m}$ to an Accuracy of $0.1 \mathrm{~nm}$ : Is It Possible.

Final rept.

E. C. Teague. 1993, 42p

Pub. in Society of Photo-Optical Instrumentation Engineers Handbook 'The Technology of Proximal Probe Lithography', Part 3, p322-363 Jun 93.

Keywords: "Metrology, *Displacement, Workplace layout, Interferometry, Accuracy, Motion studies, Lithography, Reproducibility, Reprints, Positioning uncertainty, Abbe offset, Proximal probe lithography.

Four major tasks of accurate positioning are examined: generating highly repeatable motion in a workspace constructing a metrology frame from highly-stable, accurate lines and planes, realizing a metric in the workspace, and linking the workpiece to the coordinate system with a probe. Optical scales, $x$-ray interferometry, optical heterodyne interferometry, Fabry-Perot etalons, and impedance-based transducers are evaluated as possible means to realize a metric. The major error sources, practical limitations, and guidelines for establishing a coordinate reference frame are described. The repeatability of sliding bearings and flexure bearings is examined and this combined with cosine and Abbe offset errors is used to estimate uncertainties in generating and measuring linear motion. Finally, the effects of probe-substrate forces and energies of interactions on positioning accuracy are discussed.

\section{4,091}

PB96-161617 Not available NTIS

National Inst. of Standards and Technology (MEL), Gaithersburg, MD. Precision Engineering Div.

Residual Error Compensation of a Vision-Based Coord inate Measuring Machine.

Final rept.

M. V. Ananda, J. Raja, T. Doiron, and J.

Zimmerman. 1993, 5p

Pub. in Proceedings of the American Society for Precision Engineering Fall Meeting, p66-70 1993.

Keywords: "Calibration, *Metrology, Reprints, "Dimensional metrology, Error compensation, Grid plate.

The commercial equipment for measuring lithography patterns has reached an accuracy level which makes it difficult to provide two dimensional artifact of higher accuracy for use as calibration standards. Because of the simpler nature of one-dimensional measurements, it is still possible to measure one line of a grid plate to a higher accuracy than is possible using the two dimensional measurement machines. The main idea of this paper is to introduce the idea of using a partially calibrated grid plate (one of two lines calibrated) and a series of measurements of the whole plate on the two dimensional machine to produce a calibration of both the grid plate and the machine. The authors' procedure involves measurement of a grid plate in three specified orientation. The measurement data is used to build an error compensation model. The following sections give a brief description. Software compensation of a coordinate measuring machine has become an accepted practice to enhance the accuracy of a CMM. In addition to parametric error compensation schemes, research work in self calibration schemes have also been carried out in recent years. Hocken and Borchardt used the measurements of an artifact in two specified onientations to model squareness and scale error. Belforte, et al. Developed a model that included the twenty one error components. They used legendre polynomials to model the errors. The self calibration methods eliminate the need for calibrated artifacts, but require the dimensional stability of the artifacts during the measurement. Raugh has also developed a method that falls under this category. The authors' method is an extension of the Hocken-Borchardt method.

04,092
PB96-161674 Not available NTIS
National Inst. of Standards and Technology (CSTL), Gaithersburg. MD. Biotechnology Div.

Current Noise Reveals Protonation Kinetics and Number of Ionizable Sites in an Open Protein lon Channel.

Final rept.

S. M. Bezrukov, and J. J. Kasianowicz. 1993, 4p. Pub. in Physical Review Letters, v70 n15 p2352-2355 Apr 93.

Keywords: *Ionization, *Kinetics, "Noise analysis, $R e$ prints, Protons, * Foreign technology, Protein structure.

In analogy to current fluctuations found in solid state electronic microstructure devices, excess noise generated by the reversible ionization of sites in a transmembrane ionic channel was observed. By analyzing the $\mathrm{pH}$-dependent fluctuations in the current through fully open single channels formed by the alpha-toxin protein, the authors were able to evaluate the protonation rate constants, the number of sites participating in the protonation process, and the effect of recharging a single site on the channel conductance.

\section{4,093}

PB96-161682 Not available NTIS

National Inst. of Standards and Technology (CSTL), Gaithersburg, MD. Biotechnology Div.

Noise Analysis of lonization Kinetics in a Protein lon Channel.

Final rept

S. M. Bezrukov, and J. J. Kasianowicz. 1993, 4p. Pub. in Proceedings of the International Conference on Noise Analysis in Physical Systems and 1/f Fluctua tions (12th), p677-680 1993.

Keywords: "Chemical reactions, "Ion channel, "Noise analysis, Current, Reprints, Fluctuations, Kinetics, Ionization, Protein structure, Spectral density.

The authors observed excess current noise generated by the reversible ionization of sites in a transmembrane protein ion channel, which is analogous to current fluctuations found recently in solid state microstructure electronic devices. Specifically the current through fully open single channel formed by Staphylococcus aureus alpha-toxin show $\mathrm{pH}$ dependent fluctuations. The authors show that noise analysis of the open channel current can be used to evaluate the ionization rate constants, the number of sites participating in the ionization process, and the effect of recharging a single site on the channel conductance.

\section{4,094}

PB96-161708 Not available NTIS

National Inst. of Standards and Technology (PL), Gaithersburg, MD. Ionizing Radiation Div.

Measurement of the Neutron Lifetime.

Final rept

M. S. Dewey, D. M. Gilliam, W. M. Snow, and G. L. Greene 1992 . $8 p$

See also PB91-118026. Sponsored by Department of Energy, Washington, DC

Pub. in Proceedings of the Weak and Electromagnetic 1992

Keywords: *Neutrons, Measurement, Counting, Reprints, Penning traps, Lifetime.

Neutron beta-decay is a proto-typical semi-leptonic weak interaction whose rate is of considerable theoretical importance. Accurate measurements of the neutron decay rate along with Big Bang nucleosynthesis cal culations and measurements of light element abundances constrain the number of light neutrino species, Nv. This constraint currently agrees with the number of neutrino species inferred from the recent precision measurements of the decay width of the $Z$ boson. A resolution of the current disagreement among experimental values for the neutron lifetime Tn, the neutron beta asymmetry $\mathrm{An}$, and fr-values of pure Fermi $\mathrm{O}_{+}$ goes to $\mathrm{O}+$ superallowed beta-transitions might require extensions to the Standard Model. In the coming year the authors hope to improve by a factor of four the accuracy of their previous measurement of the neutron lifetime (5.3 goes to approximately $1.5 \mathrm{~s}$ ). The current status and future prospects for the measurement are discussed.

\section{4,095}

PB96-161716 Not available NTIS

National Inst. of Standards and Technology (MEL), Gaithersburg, MD. Precision Engineering Div. 


\section{PHYSICS}

\section{General}

SPC Artifact for Automated Solder Joint Inspection.

Final rept.

T. Doiron, D. A. Pinsky, S. Chen, and M. Plott. 1993 , $8 p$.

Pub. in Measurement, v12 p1-8 1993.

Keywords: "Solder joints, *Inspections, Reprints, *Foriegn technology, Dimensional metrology, Interim artifact, Statistical process control.

Automated inspection of printed wiring assemblies, particularly those using surface mount technology, has become practical with the advent of high-speed vision processing for dimensional measurements on each feature coupled with artificial intelligence tools to make decisions based on the measurements. In this paper the authors present accuracy tests of such a system based on the measurement of known artifacts which simulate the geometry of typical solder joints.

\section{4,096}

PB96-161724 Not available NTIS

National Inst. of Standards and Technology (MEL),

Gaithersburg, MD. Precision Engineering Div.

Length Metrology of Complimentary Small Plastic Rulers.

Final rept.

D. T. Doiron, and T. D. Doiron. 1994, 7p.

Pub. in Proceedings of the Measurement Science Conference, Pasadena, CA., January 1994, $7 p$.

Keywords: "Measurement systems, "Rulers, ${ }^{\star}$ Metrology, Reprints, "Scale error Standards.

The national measurement system concerns the relationships between individual measurements and the specific units of measure. In some cases, gage blocks for example, there are standards, a large body of scientific research, and considerable effort taken to trace the accuracy of blocks used in manufacturing back to the unit of length. The international trend to laboratory accreditation (NVLAP, NAMAS, etc.) is a formalization of the idea of assuring measurement accuracy by assessment of laboratory practice and evaluation of a measurement system's relation to the unit of length.

\section{4,097}

PB96-161781 Not available NTIS

National inst. of Standards and Technology (CSTL), Gaithersburg, MD. Biotechnology Div.

Nonequilibrium Statistical Mechanics.

Final rept.

B. Robertson. 1993, 2p

Pub. in Physics and Probability, p251-260 1993

Keywords: "Statistical mechanics, "Temperature, *Thermodynamics, Reprints, "Foreign technology, *Nonequilibrium entropy, "Correlation function, Projection operators.

The derivation of the equations of nonequilibrium statistical mechanics is reviewed. These exact closed equations are valid for nonlinear systems arbitrarily far from equilibrium. In the short-correlation-time and local limit they reduce to the equations of nonequilibrium thermodynamics with molecular expression for the transport coefficients.

\section{4,098}

PB96-161799 Not available NTIS

National Inst. of Standards and Technology (PL),

Gaithersburg, MD. Ionizing Radiation Div.

Measurement of the $(10) B(n$, alpha1gamma $)(7) L i$

Cross Section in the 0.3 to $4 \mathrm{MeV}$ Neutron Energy Interval.

R. A. Schrack, O. A. Wasson, D. C. Larson, J. K

Dickens, and J. H. Todd. 1992, 3p.

Pub. in Nuclear Data for Science and Technology, Forschungszentrum Julich, Federal Republic of Germany, May 13-17, 1991, p507-509 1992

Keywords: "Germanium detector, "Neutron standards, Cross sections, Reprints, *Foreign technology, "Black detectors, Monte Carlo.

A relative cross section measurement of the $10 \mathrm{~B}(\mathrm{n}$, alpha, gamma)7Li cross section was made using the ORELA neutron source. The reaction was measured by observing the $478 \mathrm{keV}$ photon using a $30 \%$ efficiency germanium detector. The neutron flux was monitored with a high efficiency plastic scintillator. Data analysis required the use of extensive Monte Carlo calculations for corrections to the data. The measured cross section differs as much as $40 \%$ from the ENDF/
$B-V I$ evaluation for neutron energies greater than 1.5 MeV.

\section{4,099}

PB96-161864 Not available NTIS

National Inst. of Standards and Technology (MSEL) Gaithersburg, MD. Polymers Div.

Gas Absorption during Ion-Irradiation of a Polymer Target.

Final rept.

W. E. Wallace, T. T. Chiou, J. B. Rothman, and R. J. Composto. $1995,5 p$

Pub. in Nuclear Instruments and Methods in Physics Research B, v103 p435-439 May 95.

Keywords: "Blistering, "Polyimide, "Gas absorption Reprints, Water absorption, "Foreign technology, "Ion beam modification.

The presence of a high partial pressure of deuterated water in the vacuum of the scattering chamber of an $\mathrm{MeV}$ ion accelerator system was shown to promote the incorporation of deuterium into a thin polyimide target subjected to $1.3 \mathrm{MeV} 4 \mathrm{He}+$ irradiation. When the back ground pressure in the chamber was low, $2.7 \times 10$ to the minus 5 th power $\mathrm{Pa}(2 \times 10$ to the minus 7 th power Torr), the polyimide was shown by forward recoil spectrometry to evolve hydrogen when ion bombarded. This was an expected, and commonly observed phe nomenon. However, when the pressure in the scattering chamber was raised to $1.3 \times 10$ to the minus power $\mathrm{Pa}(1 \times 10$ to the minus 4 power Torr) by the addition of $\mathrm{D} 2 \mathrm{O}$ vapor through a leak valve, deuterium was readily incorporated into the target in the ion-irradiated area. The degree to which the polyimide film ab sorbed gas from the vacuum of the scattering chambe was unexpected. The deuterium/hydrogen atomic concentration ratio reached 0.235 after a dose of $3.5 \times 10$ to the 15 th power ions/sq. $\mathrm{cm}$. The absorption of deuterium was accompanied by blistering in the polyimide at high ion fluences.

\section{4,100}

PB96-161989 Not available NTIS

National Inst. of Standards and Technology (MSEL) Gaithersburg, MD.

Interfacial Crack in a Two-Dimensional Hexagonal Lattice.

Final rept

R. Thomson, and S. J. Zhou. 1994, 11p.

Pub. in Physical Review B, v49 n1 p44-54 Jan 94

Keywords: "Crack propagation, Fracture, Interfaces Dislocation, Reprints, "Interfacial crack, "Lattices.

In this paper we compare a set of atomic calculations of interfacial crack structure and properties with the predictions of an augmented elastic theory. Our intent is to critique the elastic predictions, especially the mode conversion and displacement closure oscillatio features of the elastic theory. A simple physical picture is developed based on a crack stability diagram, using two sets of stress intensity axes. The first set is the normal applied stress intensity $\mathrm{K}$ and the second is a local stress intensity factor $k$, defined to describe the physics of the core region. The Griffith condition is different from that for dislocation emission. In each case the effective core size is much smaller than the physical core size, which means that the mode shift at the crack tip is considerably larger than would be expected on the basis of linear elasticity. However, with appropriately defined condition is also surprisingly well satis fied in the mode-ll emission configuration. The crack is found never to exhibit displacement oscillations, in part, because of the necessary condition that the Griffith condition be satisfied at the crack tip, and in part because the amount of shear in the core is limited by dislocation emission.

\section{4,101}

PB96-162003 Not available NTIS

National Inst. of Standards and Technology (MSEL) Gaithersburg, MD

Dislocation Core-Core Interaction and Peierls Stress in a Model Hexagonal Lattice.

Final rept.

S. J. Zhou, A. E. Carlsson, and R. Thomson. 1994

Pub. in Physical Review B, Condensed Matter, Third Series, v49 n10 p6451-6456 Mar 94.

Keywords: "Dislocation core, Fracture, Reprints, *Lattices, "Atomistic calculations, Peierls Stress.

A series of atomistic calculations is performed in orde to explore dislocation core-core interactions and the
Peierls stress in a model hexagonal lattice. The method of calculation is the lattice Green's function method, using several pair potentials with variable parameters. We confirm that dislocation cores broaden as a pair of dislocations with opposite sign move closer to each other. Continuum theories are surprisingly accurate in describing the dislocations-dislocation interaction force even in the range of strong core-core overlap. However, our atomistic calculations show that while the relation between the Peierls stress and dislocation width is exponential as the Peierls-Nabarro model predicts, that model underestimates the Peierls stress by nearly a factor of 10 to the 4th power.

04,102

PB96-163746 Not available NTIS

National Inst. of Standards and Technology (PL) Gaithersburg, MD. Electron and Optical Physics Div. Charge-Transfer-Induced Multiplet Structure in the $\mathrm{N} 4,5 \mathrm{O} 2,3$ Soft-X-ray Emission Spectrum of Lanthanum.

Final rept.

D. R. Mueller, C. W. Clark, D. L. Ederer, Q. Y. Dong T. A. Callcott, J. J. Jia, and W. L. O'Brien. 1995, 5p.
Pub. in Physical Review A, v52 n6 p4457-4461 Dec 95.

Keywords: "Lanthanum, "Multiplet structures, *Emission spectrum, Reprints, "Soft $x$-ray emission spectra.

Soft-x-ray emission spectra for the La N4.5O2.3 transitions in lanthanum aluminate $\mathrm{LaAlO} 3$ include contributions from several relatively intense multiplet lines near $80 \mathrm{eV}$. The appearance of this spectrum contrasts sharply with the three diagram lines observes for the $\mathrm{N} 4.5 \mathrm{O} 2.3$ emission spectrum of $\mathrm{Ba}$ in $\mathrm{BaF} 2$. An atomic model of the La N4.5O2.3 transition, assuming single occupancy of the $4 f$ shell by a spectator electron, provides a good approximation of the measured spectrum. Emission features due to the low-energy 3PO and 3D0 terms of the $4 \mathrm{~d} 94 \mathrm{f}$ configuration are also apparent in the laAlO3 emission spectra, but not in emission spectra from BaF2. Reflection measurements indicate that the $4 f$ level is not occupied in the ground state in LaAlO3. The data suggest that charge transfer from ligand orbitals following creation of a $4 \mathrm{~d}$ vacancy prior to the emission process is the predominant mechanism giving rise to the required $4 f$ occupancy.

\section{4,103}

PB96-163761 Not available NTIS

National Inst. of Standards and Technology (CSTL), Boulder, CO. Thermophysics Div.

Non-Newtonian Flow between Concentric Cylinders Calculated from Thermophysical Properties Obtained from Simulations.

Final rept.

A. P. Narayan, J. C. Rainwater, and J. M. Hanley.

1995, 7p

Pub. in International Jnl. of Thermophysics, v16 $\mathrm{n} 2$ p347-353 Mar 95

Keywords: "Colloidal suspension, " Rheology, Reprints, ${ }^{*}$ Foreign technology, ${ }^{*}$ Couette flow, Shear thickening, Soft sphere fluid, Weissenberg effect.

A study of the Weissenburg effect (rod climbing in a stirred system) based on nonequilibrium molecular dynamics (NEMD) is reported. Simulation results from a soft-sphere fluid are used to obtain a self-consistent free-surface profile of the fluid of finite compressibility undergoing Couette flow between concentric cylinders. A numerical procedure is then applied to calculate the height profile for a hypothetical fluid with thermophysical properties of the soft-sphere liquid and of a dense colloidal suspension. The height profile calculated is identified with shear thickening and the forms of the visometric functions. The maximum climb occurs between the cylinders rather than at the inner cylinder.

04,104

PB96-163779 Not available NTIS

National Inst. of Standards and Technology (EEEL), Gaithersburg, MD. Semiconductor Electronics Div. 2D-Scanning Capacitance Microscopy Measurements of Cross-Sectioned VLSI Teststructures. Final rept.

G. Neubauer, and A. Erickson. 1996, 4p

Pub in Proceedings of the International Workshop on Semiconductor Characterization: Present Status and Future Needs, Gaithersburg, MD., January 30-February 2, 1995, p318-321 1996. 
Keywords: "Microscopy, Measurements, Reprints, Electrical data, Topographic data, ${ }^{\star}$ Scanning capacitance microscopy, 2-D dopant profiling.

The authors have developed a setup which uses scanning capacitance microscopy (SCM) to obtain electrical data of cross-sectioned samples while simultaneously acquiring conventional topographical AFM data. The results presented here include 2D SCM maps of crosssections of blanket implanted, annealed Si wafers as well as test structures on Si. The authors found the technique to be sensitive over several orders of magnitude of carrier density concentrations less than E15 to $\mathrm{E} 20$ atoms/sq. $\mathrm{cm}$., with a lateral resolution of 20 $150 \mathrm{~nm}$, depending on probe tip and dopant level. The authors find excellent agreement of total implant depth obtained from SCM signals of cross-sectioned samples with conventional Secondary lon Mass Spectrometry (SIMS) profiles of the same sample.

\section{4,105}

PB96-167119 Not available NTIS

National Inst. of Standards and Technology (CSTL),

Gaithersburg, MD. Analytical Chemistry Div.

Loss-Free Counting at IRI and NIST.

Final rept.

R. M. Lindstrom, M. J. J. Ammerlaan, and S. S.

Then. 1996, 9p.

Pub. in Jnl. of Trace and Microprobe Techniques, v14 n1 p67-75 1996.

Keywords: *Gamma spectrometry, Accuracy, Reprints, "Foreign technology, "Loss free counting, Neutron activation analysis.

Loss-free counting has been successfully implemented for neutron activation analysis with short-lived nuclides at the Interfaculty Reactor Institute (IRI) and at the Natioanl Institute of Standards and Technology (NIST) In the system built at IRI, the LFC is integrated through a buffer module to a Unix workstation; the NIST system uses standard Canberra equipment and VMS software. Procedures are described for the calibration and validation of LFC in these two environments, with emphasis on accuracy and on control of the quality of the measurements.

04,106

PB96-167317 Not available NTIS

National Inst. of Standards and Technology (CSTL),

Boulder, CO. Thermophysics Div.

Dynamic Scaling in an Aggregating $2 D$ LennardJones System.

Final rept

B. D. Butler, H. J. M. Hanley, D. Hansen, and D. J.

Evans. $1995,4 p$

Pub. in Physical Review Letters, v74 n22 p4468-4471

May 95.

Keywords: "Fractal aggregation, "Gelaton, Molecular dynamics, Reprints, Scaling, Spinodal decomposition.

The evolution of a 2D Lennard-Jones system, quenched from the fluid to below the triple point, is simulated by molecular dynamics. We show that the structure factor obeys the scaling relation $\mathrm{S}(\mathrm{q} / \mathrm{qm}(\mathrm{t}))$ approx. $q m(t)$-dfS $(q / g m)$. Here qm is the location of the low angle peak in $S(q)$, df equals 1.85 plus or minus 0.05 is a fractal dimension, and $S(\mathrm{q} / \mathrm{gm})$ is a time-independent characteristic function which peaks at qm. The quenching process is thermodynamically similar to the formation of a gel from a sol. Hence the relation suggests that a characteristic fractal dimension of even a dense gel can be derived from measurements of the time evolution of $S(q)$.

\section{4,107}

PB96-167341 Not available NTIS

National Inst. of Standards and Technology (MSEL), Gaithersburg, MD. Ceramics Div.

Preparation and Crystal Structure of Sr5TiNb4017. Final rept.

A. R. Drews, W. Wong-Ng, R. S. Roth, and T. A.

Vanderah. 1996, 10p.

Pub. in Materials Research Bulletin, v31 n2 p153-162 1996.

Keywords: "Ceramics, "Crystal growth, "X ray diffraction, Reprints, Crystal structure.

The compound Sr5TiNb4O17 was prepared and its crystal structure determined by single-crystal $X$-ray diffraction This compound crystallizes with an orthorhombic unit cell (space group Pnnm (No. 58); a equals 5.6614(4), b equals $32.515(7)$, c equals $3.9525(3)$ Angstroms; $Z$ equals 2$)$. The structure con- sists of alternating perovskite-like slabs oftset with respect to each other by $\mathrm{a} / 2$ and $\mathrm{c} / 2$ The $\mathrm{Sr} 2+$ ions $00-$ cupy two 12 -fold coordinated sites within the slabs and a distorted $(7+1)$-fold coordinated site in the gap separating the slabs. Nb5+ and Ti4+ are distributed among the octahedral sites of the sperovskite slabs with preferential odering of Ti4+ on octahedral sites on the center of the slabs adjacent to the gap. This compound is the $n$ equals 5 member of a structural series $\mathrm{AnBnO}(3 n+29)$ ( $\mathrm{A}$ equal $\mathrm{Sr}$; B equal $\mathrm{Ti}, \mathrm{Nb}$ ) where $\mathrm{n}$ is the number of perovskite layers within each slab.

\section{4,108}

PB96-172358 PC A11/MF A03

National Inst. of Standards and Technology $(P L)$, Boulder, CO. Time and Frequency Div.

Trapped Ions and Laser Cooling 4: Selected Publications of the lon Storage Group of the Time and Frequency Division, NIST, Boulder, Colorado.

Technical note.

J. C. Bergquist, J. J. Bollinger, W. M. Itano, C. R Monroe, and D. J. Wineland. Jan 96, 205p, NIST/TN 1380.

Also available from Supt. of Docs. as SN003-003 03395-2. See also PB92-189547. Sponsored by Office of Naval Research, Arlington, VA. and Army Research Office, Research Triangle Park, NC.

Keywords: "Ion storage, "Trapped particles, Atoms, Lasers, Cooling, Atomic clocks, Spectroscopy, Frequency standards, Lights scattering, spectroscopy, NIST(National Institute of Standards and Technology).

This collection of papers represents the work of the lon Storage Group, Time and Frequency Division National Institute of Standards and Technology, from May 1992 to January 1996. It follows the collections of papers contained in NBS Technical Note 1086, Trapped Ions and Laser Cooling (June 1985), NIST Technical Note 1324, Trapped Ions and Laser Cooling II (September 1988), and NIST Technical Note 1353, Trapped Ions and Laser Cooling III (April 1992)

\section{4,109}

PB96-176490 Not available NTIS

National Inst. of Standards and Technology (CSTL) Gaithersburg, MD. Process Measurements Div.

Electrical Characteristics of Argon Radio Frequency Glow Discharges in an Asymmetric Cell. Final rept

M. A. Sobolewski. 1995, 17p

Pub. in Institutue of Electrical and Electronics Engineers Transactions on Plasma Science, v23 n6 p10061022 Dec 95

Keywords: "Current, "Discharges, Diagnostics, Electrical, Impedance, Plasma, Radio frequency, Voltage Reprints, "Gaseous Electronics Conference.

Measurements of the current and voltage at both electrodes of a parallel-plate, capacitively coupled RF discharge cell (the Gaseous Electronics Conference Reference Cell) were combines with measurements of the voltage on a wire inserted into the glow region between the electrodes, for argon discharges at pressures of 1.3-133 $\mathrm{Pa}$ and peak-to-peak applied voltages equal to or less than $400 \mathrm{~V}$. Together, these measurements determined the RF voltage current, impedance, and power of each sheath of the plasma. Simple powe laws were found to describe changes in sheath impedances observed as voltage and pressure were varied. An equivalent circuit model for the electrical behavior of the discharge was obtained. The equivalent circuit model can be used to relate the electrical data to plasma properties such as electron densities, ion currents, and sheath widths. The results differ from models previously proposed for asymmetric RF discharges, and the implications of this disagreement are discussed.

\section{4,110}

PB96-176540 Not available NTIS

National Inst. of Standards and Technology (EEEL), Boulder, CO. Optoelectronics Div.

Automated Measurement of Nonlinearity of Optical Fiber Power Meters.

Final rept.

I. Vayshenker, S. Yang, X. Li, and T. R. Scott. 1995, $8 p$.

Pub. in Society of Photo-Optical Instrumentation Engi-

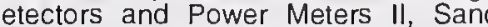
Diego, CA., July 11-12, 1995, v2550 p12-19.

Keywords: "Optical fibers, "Fiber optics, Nonlinearity, Power calibration, Power meter, Reprints.
We have developed a system for measuring the nonlinearity of optical power meters or detectors over a dynamic range of more than $60 \mathrm{~dB}$ at telecommunications wavelengths. This system uses optical fiber components and is designed to accommodate common optical power meters and optical detectors. It is based on the triplet supernosition method The system also measures the range discontinuity between neighboring power ranges or scale settings of the optica power meter. We have developed an algorithm to trea both the nonlinearity and the range discontinuity in logically consistent manner. Measurements with this system yield correction factors for powers in all ranges. The measurement system is capable of producing results which have standard deviations as low as $0.02 \%$. With slight modification the system can operate ove a $90 \mathrm{~dB}$ dynamic range at telecommunications wavelengths. This measurement system provides accurate determination of optical power meter or detector nonlinearity; the characterized detectors then can be used for such applications as absolute power and at tenuation measurements.

04,111

PB96-176698 Not available NTIS

National Inst of Standards and Technology (CSTL).

Boulder, CO. Thermophysics Div.

Small-Angle Neutron Scattering (SANS) Study of Worm-Like Micelles Under Shear.

Final rept

M. Y. Lin, H. J. M. Hanley, G. C. Straty, S. K. Sinha, D. G. Peiffer, and M. W. Kim. 1994, 10 p.

Pub. in International Jnl. of Thermophysics, v15 n6 p1169-1178 Nov 94.

Keywords: " Neutron scattering, "Shear alignment, Anisotropy, Couette flow, Polymers, Rods, Reprints, Polydispersity, Worm-like micelles

The structure of a cationic worm-like cylindrical micelle was investigated by SANS (small-angle neutron scattering). Intensities from $0.1 \%$ by weight solutions in $\mathrm{D} 2 \mathrm{O}$, at rest and under shear, were measured on the NIST Cold Neutron Research Facility 30-m spectrometer in the wave vector range 0.03 equal to or less than Q $(\mathrm{nm}$ to the minus one power) equal to or less than 2.0. Scattered intensity patterns from the solutions subjected to shears equal to or greater than $40 \mathrm{~s}$ to the minus one power showed pronounced anisotropy, but such anisotropy could not be detected below this apparent threshold shear. The threshold was characterized by a relaxation time since anisotropy was detected only after several minutes of shearing. In contrast, the anisotropy was apparent immediately the shear was applied at the higher shears. The data were analyzed based on the assumption that the micelles behave as rigid rods. Estimates of the radii and length under shear are given. Polydispersity in rod length is discussed, and the authors argue that it contributes significantly to the scattering patterns.

\section{4,112}

PB96-180021 Not available NTIS

National Inst. of Standards and Technology (MSEL), Gaithersburg, MD. Reactor Radiation Div.

Dynamics of $\mathrm{Mu}(+)$ in $\mathrm{Sc}$ and $\mathrm{ScH}$.

Final rept

F. N. Gygax, A. Amato, R. Feyerherm, T. J. Udovic, G. Solt, A. Schenck, and I. S. Anderson. 1996, 4p. Pub. in Jnl. of Alloys and Compounds, v231 p248-251 1995.

Keywords: "Muon spin rotations, "Crystals, Relaxation rate, Reprints, Dynamics, Locations, Magnetic fields.

Muon spin rotation (microSR) experiments on singlecrystal samples of $\mathrm{ScHx}$, with $\mathrm{x}=0,0.05$, and 0.10 and 0.25 , were performed in order to investigate the muon location and possible systematic variations in muen dynamics in these systems. The angular and magnetic field dependence of the microSR relaxation rates shows that at temperatures below $50 \mathrm{~K}$ the muon is localized at the interstitial tetrahedral $(T)$ sites. The results for pure Sc, covering a wide range of high and ow fields, are coherent with the picture that the muon performs fast tunnelling between two adjacent $T$ sites, separated by $2 z=0.18 \mathrm{c}$, above and below the $a-b$ plane. This value for the muon position along the $c$ axis is remarkably near the $S z=0.194 \mathrm{c}$ found for D in Sc by neutron diffraction. The anisotropy of the relaxation rate at low magnetic fields varies sensitively with the $\mathrm{H}$ concentration, indicating a change in the ratio of the radial, muon-induced and the partly inherent, uniaxial electric field gradients at the position of the Sc nucle 


\section{General}

04,113

PB96-180062 Not available NTIS

National Inst. of Standards and Technology (MSEL) Gaithersburg, MD. Polymers Div.

Electrostatic Rigidity of Polyelectrolytes from Reparametrization Invariance.

Final rept

A. L. Kholodenko, J. F. Douglas, and T. A. Vilgis.

1996, 7p.

Pub, in Macromolecules Theory Simul., v5 p121-127 1996.

Keywords: "Persistence length, "Polyelectrolytes, Electrostatics, Rigidity, Reprints, "Reparametrization invariance.

The persistence length of a polyelectrolyte chain can be represented as $I p=10+$ le where lo is the bare persistence and le is the electrostatic contribution coming from the effects of electrostatic chain self-interactions. Using a reparametrization-invariant path integral model of semiflexible polymers the authors find that le depends on the ionic strength I as le approximately to the minus $1 / 2$. This result accords with experimental observations and recent Monte Carlo simulations. Reparametrization-invariance is apparently an essential constraint in selecting acceptable models of semiflexible polymers.

\section{4,114}

\section{PB96-180096 Not available NTIS}

National Inst. of Standards and Technology (EEEL) Gaithersburg, MD. Electricity Div.

Quantum Hall Effect-Based Resistance Standard: Capabilities and Implementation.

Final rept.

K. C. Lee. $1996,6 p$

See also PB95-171419.

Pub. in NASA Metrology and Calibration 1995, Proceedings of the Annual Workshop (18th), Greenbelt, MD., April 18-20, 1995, p1-6.

Keywords: “Digital multimeter, Reprints, "Quantum Hall effect, "Resistance standard.

In order to support modern 8 and one-half digit digital multimeters and high accuracy calibrators capable of delivering uncertainties of the order of parts per million (ppm), primary standards laboratories must be able to provide calibration uncertainties less than $0.1 \mathrm{ppm}$. Quantum Hall effect-based resistance standards can provide these low uncertainties, but at present require very sophisticated and costly equipment. This talk describes the quantum Hall effect (QHE), the equipment necessary to use it as a resistance standard, and some of the challenges in making a QHE-based resistance standard commercially viable.

\section{4,115}

PB96-180112 Not available NTIS

National Inst. of Standards and Technology (EEEL), Gaithersburg, MD. Semiconductor Electronics Div

Assessing MOS Gate Oxide Reliability on Wafer Level with Ramped/Constant Voltage and Current Stress.

Final rept.

A. Martin, J Suehle, P. Chaparala, C. Messick, P O'Sullivan, and A Mathewson. 1996, 11p.

Pub. in Proceedings of the Institute of Electrical and Electronics Engineers International Integrated Reliability Workshop Final Report, Lake Tahoe, CA., October 22-25, 1996, p81-91.

Keywords: "Accelerated testing, "Oxide breakdown Reliability, Reprints, "MOS gate oxides.

In this study time to breakdown distributions are compared for MOS gate oxides which were stressed with a constant voltage (or current) stress or a pre-stressing voltage (or current) ramp followed by a constant voltage (or current) stress. Results show clearly that a pres-stress can increase time to breakdown. This increase is discussed and it is shown that it is dependent on oxide thickness, pre-stressing ramp rate and the processing conditions. The current-time (or voltagetime) characteristics of the constant stress are inves tigated and it is observed that charge trapping in the oxide is the reason for the time to breakdown increase. The pre-stressed oxide clearly shows a different initial charge trapping characteristic than the non prestressed oxide. The measurement results are discussed and it will be demonstrated that the common understanding of oxide breakdown cannot explain the observed results. Therefore, a new parameter will be proposed which is related to oxide degradation and breakdown and which has to be considered in combined ramped/constant stress measurements.

04,116

PB96-180120 Not available NTIS

National Inst. of Standards and Technology (PL), Gaithersburg, MD. Atomic Physics Div.

Designations of $d s(2) p$ Energy Levels in Neutral Zirconium, Hafnium, and Rutherfordium ( $Z=104)$. Final rept.

W. C. Martin, and J. Sugar. 1996, 4p.

Pub. in Physical Review A, v53 n3 p1911-1914 Mar 96.

Keywords: "Atomic energy levels, "Electron configuration, "Energy-level calculations, Atomic spectroscopy, Hafnium, Level designation, Rutherfordium, Reprints.

The authors have examined available data for the oddpanty energy-level structures in $\mathrm{Zr}$ and $\mathrm{Hf}$, stimulated by the designations of four predicted 6d7s27p levels in the homologous atom rutherfordium (Rf, $Z=104$ ) by Eliav et al. (Phys. Rev. Lett. 74, 1079 (1995)). The authors point out some errors and deficiencies in the $\mathrm{Zr}$ data and give the results of Hartree-Fock calculations for $\mathrm{Hf} 5 \mathrm{~d} 6 \mathrm{~s} 26 \mathrm{p}-6 \mathrm{p}$ and Rf $6 \mathrm{~d} 7 \mathrm{~s} 27 \mathrm{p}$ levels. Configuration interactions within the $(d+s) 3 p$ complexes were included. The resulting eigenvectors allow meaningful LS-coupling designations for most of the levels belonging mainly to $\mathrm{Hf} 5 \mathrm{~d} 6 \mathrm{~s} 26 \mathrm{p}$ and for most of the predicted $\mathrm{Rf}$ levels belonging mainly to $6 \mathrm{~d} 7 \mathrm{~s} 27 \mathrm{p}$. Some changes in the designations assigned to these levels in the literature are suggested: in particular, the lowest level of both $\mathrm{Hf} 5 \mathrm{~d} 626 \mathrm{p}$ and $\mathrm{Rf} 6 \mathrm{~d} 7 \mathrm{~s} 27 \mathrm{p}$ is most appropriately designated 3F02. The authors point out the need for systematic whole-row studies of the low oddparity configurations in $4 \mathrm{~d}$ - and $5 \mathrm{~d}$-shell spectra.

04,117

PB96-180203 Not available NTIS

National Inst. of Standards and Technology (PL), Gaithersburg, MD. Atomic Physics Div.

Hyperfine Structure Investigations and Identification of New Energy Levels in the lonic Spectrum of (147) Pm

Final rept.

R. Otto, H. Huhnermann, J. Reader, and J. F. Wyart. 1996, 13p.

Pub. in Jnl. of Physics B, Atomic and Molecular Physics, v28 p3615-3627 1995

Keywords: "Energy levels, "Hyperfine structures, "Promethium, Spectrum, Reprints.

Hyperfine structure splittings have been measured for 75 lines of singly ionized promethium $147 \mathrm{Pmll}$ with a hollow cathode discharge and a Fabry-Perot interferometer. Magnetic dipole and electric quadrupole splitting factors $A$ and $B$ have been determined for 10 levels of the $4 \mathrm{f} 56 \mathrm{~s}$ configuration, 2 levels of $4 \mathrm{f} 5 \mathrm{~d}$, and 57 upper undesignated levels. The A-factors are in general agreement with calculated values. With the aid of Hartree-Fock calculations and Zeeman-effect data, four new levels of the $4 \mathrm{f} 5(6 \mathrm{H}) 5 \mathrm{~d} 7 \mathrm{~K}$ term were located. The strong lines terminating on these $7 \mathrm{~K}$ levels originate from levels of $4 f 5(6 \mathrm{H}) 6 \mathrm{p} 7 \mathrm{l}$, which were already known, but had not been designated. An unidentified line at 556.17 nm previously observed with collinear laser ion-beam spectroscopy is now classified as $4 \mathrm{f} 5(6 \mathrm{H}) 5 \mathrm{~d} 7 \mathrm{~K} 6-4 \mathrm{f} 5(6 \mathrm{H}) 6 \mathrm{p} 7 \mathrm{I5}$.

\section{4,118}

PB96-180211 Not available NTIS

National Inst. of Standards and Technology (PL), Gaithersburg, MD. Atomic Physics Div

Measurements of the Resonance Lines of $(6) \mathrm{Li}$ and (7) Li by Doppler-Free Frequency-Modulation Spectroscopy.

Final rept.

C. J. Sansonetti, B. Richou, R. Engleman, and L. J. Radziemski. 1995, 7p.

Pub. in Physical Review A, v52 n4 p2686-2688 Oct 95.

Keywords: "Atomic spectra, "Isotope shift, "Laser spectroscopy, Lithium, Wavelengths, Reprints.

Using Doppler-free frequency-modulation spectroscopy, the authors have measured most of the hyperfine components and the centers of gravity of the D1 line of $7 \mathrm{Li}$ and the D2 line of $6 \mathrm{Li}$. By using previously measured fine- and hyperfine-structure splittings, the authors have also determined centers of gravity for the $\mathrm{D} 2$ line of $7 \mathrm{i}$ and the $\mathrm{D} 1$ line of $6 \mathrm{Li}$. The present results are more accurate than previously reported values by a factor of more than 100 . The wave numbers are $7 \mathrm{Li}$ D2: $14,903.983,468$ (14) $\mathrm{cm}$ to the minus 1 power; $7 \mathrm{Li}$
D1: $14,903.648,130(14) \mathrm{cm}$ to the minus 1 power; $6 \mathrm{Li}$ D2: $14.903 .632,116(18) \mathrm{cm}$ to the minus 1 power; $6 \mathrm{Li}$ D1: $14,903.296,792(23) \mathrm{cm}$ to the minus 1 power. Values for the isotope shifts of the resonance lines of $0.351,338(21) \mathrm{cm}$ to the minus 1 power for the D1 line and $0.351,352(15) \mathrm{cm}$ to the minus 1 power for the D2 line have been derived. All uncertainties represent a $95 \%$ level of confidence.

04,119

PB96-186085 Not available NTIS

National Inst. of Standards and Technology (PL), Gaithersburg, MD. Ionizing Radiation Div

Alpha-Particle and Electron Capture Decay of (209)Po.

Final rept

F. J. Schima, and R. Colle. 1996, 5p.

Pub. in Nuclear Instruments and Methods in Physics Research A, v369 p498-502 Feb 96.

Keywords: "Electron capture decay, Alpha decay, Reprints, Photon spectrometry, Radioactivity, "Foreign technology, "Internal conversion coefficients, Bismuth209, Lead-205, Polonium-209.

Gamma-ray and $\mathrm{K}$ alpha $\mathrm{X}$-ray emissions have been measured from a very pure 209 Po source containing less than $0.13 \% 208$ Po activity and no detectable 210 Po (equal to or less than ( $2 \times 10$ to the minus 4 power) \%). The alpha-particle emission rate for this source has previously been determined. Data are presented that confirm alpha decay to the $205 \mathrm{~Pb}$ excited level at $262.8 \mathrm{keV}$, with an alpha-particle emission probability ( + or - standard uncertainty) of $0.00559+$ or -0.00008 . The ratio of $\mathrm{K}$-shell electron capture to total electron capture for the second forbidden unique electron capture decay to the $896.6 \mathrm{keV}$ level in 209 $\mathrm{Bi}$ was determined to be $0.594+$ or -0.018 . The electron capture decay fraction was found to be 0.00454 + or -0.00007 , while the probabilities per decay for the $896.6,262.8$, and $260.5 \mathrm{keV}$ gamma rays and the $\mathrm{BI}$ $\mathrm{K}$ alpha and $\mathrm{PB} \mathrm{K}$ alpha $\mathrm{X}$-rays were measured as $0.00445+$ or $-0.00007 \cdot 0.00085+$ or -0.00002 , $0.00254+$ or $-0.00003,0.00202+$ or -0.00005 , and $0.00136+$ or -0.00005 , respectively.

04,120

PB96-186101 Not available NTIS

National Inst. of Standards and Technology (PL), Gaithersburg, MD. lonizing Radiation Div.

Scattered Fractions of Dose from 18 and 25 MV Xray Radiotherapy Linear Accelerators.

Final rept.

J. Shobe, J. E. Rodgers, P. L. Taylor, J. Jackson, and G. Popescu. 1996, 3p.

Keywords: "Radiotherapy, "Linear accelerator scatter, Neutrons, Shielding, Reprints, "Foreign technology, "Scattered fractions of dose.

Over the years, measurements have been made at few energies to estimate the scattered fraction of dose from the patient in medical radiotherapy operations. This information has been a useful aid in the determination of shielding requirement for these facilities. With these measurements, known characteristics of photons, and various other known parameters, Monte Carlo codes are being used to calculate the scattered fractions and hence the shielding requirements for the photons of other energies commonly used in radiotherapeutic applications. The National Institute of Standards and Technology (NIST) acquired a Sagittaire medical linear accelerator (linac) which was previously located at the Yale-New Haven Hospital. This linac provides an $x$-ray beam of $25 \mathrm{MV}$ photons and electron beams with energies up to $32 \mathrm{MeV}$. The housing on the gantry was permanently removed from the accelerator during installation. A varian Clinac 1800 linear accelerator was used to produce the $18 \mathrm{MV}$ photons at the Frederick Memorial Hospital Regional Cancer Therapy Center in Frederick, MD. This paper presents a study of the photon dose scattered from a patient in typical radiation treatment situations as it relates to the dose delivered at the isocenter in water. The results of these measurements will be compared to Monte Carlo calculations. Photon spectral measurements were not made at this time. Neutron spectral measurements were made on this Saggitaire machine in its previous location and that work was not repeated here, made at this time. Neutron spectral measurements were made on this Sagittaire machine in its previous location and that work was not repeated here, although a brief study of the neutron component of the 18 and $25 \mathrm{MV}$ linacs was performed utilizing thermoluminescent dosimetry (TLD) to determine the isotropy of the neutron dose. 
04,121

PB96-186127 Not available NTIS

National Inst. of Standards and Technology (PL), Gaithersburg, MD. Electron and Optical Physics Div. Coarsening of Unstable Surface Features during Fe(001) Homoepitaxy.

Final rept.

J. A. Stroscio, D. T. Pierce, M. D. Stiles, and A.

Zangwill. 1995, 4p.

Pub. in Physical Review Letters, v75 n23 p4246-4249 Dec 95

Keywords: "Surface morphology, "Epitaxial growth, Reprints, STM, RHEED, Fe homoepitaxy, Non selfaffine surface, Nonequilibrium growth.

The evolution of the surface potential during homoepitaxial growth of $\mathrm{Fe}(001)$ is studied by scanning tunneling microscopy and reflection high energy electron diffraction. The observed morphology exhibits a non-self-affine collection of moundlike features that maintain their shape but coarsen as growth proceeds. The characteristic feature separation $L$ is set in the submonolayer regime and increases with thickness t as $L(t)$ approx. $t 0.16$ plus or minus 0.04 . During the coarsening phase, the mounds are characterized by a magic slope and a lack of reflection symmetry. These observations are shown to be described by a continuum growth equation without capillarity.

\section{4,122}

PB96-190152 Not available NTIS

National Inst. of Standards and Technology (PL), Gaithersburg, MD. Atomic Physics Div.

Atomic Branching Ratio Data for Nitrogen-Like Species.

J. Z. Klose, J. R. Fuhr, and W. L. Wiese. 1996, 18p

Pub. in Jnl. of Quantitative Spectroscopy and Radiative Transfer (JQSRT), v55 n4 p413-430 1996

Keywords: *Atoms, *Branching ratio, High temperature, Nitrogen ions, Plasmas(Physics), Spectral lines, Vacuum ultraviolet radiation, Transition probabilities, Radiometry, Reprints, “Nitrogen-like ions.

The branching ratio technique for radiometric calibrations in the vacuum ultra-violet spectral region is briefly reviewed. Lists of transitions suitable for use with the technique are given for nitrogen-like species (NI, O II, and NeIV) along with pertinent data for their application.

\section{4,123}

PB96-200290 Not available NTIS

National Inst. of Standards and Technology (PL), Boulder, CO. Quantum Physics Div.

Comment on $<<A$ Dynamic Electric Trap for Ground-State Atoms $>>$.

Final rept.

E. A. Cornell. 1995, 2p

Grant NSF-PHY90-12244

Sponsored by National Science Foundation, Arlington, VA.

Pub. in Europhysics Letters, v30 n7 p439-440 1995. Keywords: "Trapping(Charged particles), Electric fields, Dynamics, Gravitational fields, Ground state, Equilibrium(General), Reprints, "Atom traps, "Foreign technology.

Riis and Barnett have examined the motion of a polarizable atom in the electric field in the vicinity of a sphere with time-arying charge. The configuration they examined may be experimentally useful but their paper propagates a common misconception about the mechanism of dynamic stabilization. Dynamic stabilization is an important techique in atom and ion trapping, and the purpose of the comment is to clear up the misconception.

\section{4,124}

PB96-200316 Not available NTIS

National Inst. of Standards and Technology (PL), Boulder, CO. Quantum Physics Div.

Alignment Probing of Rydberg States by Stimulated Emission.

Final rept.

E. M. Spain, M. J. Dalberth, P. D. Kleiber, J. P. J.

Driessen, S. R. Leone, and S. S. Op de Beek. 1995

$10 p$.

Grant NSF-PHY90-12244

Sponsored by National Science Foundation, Arlington.

Pub. in Jnl. of Chemical Physics, v102 n24 p9522. 9531, 22 Jun 95
Keywords: "Calcium, "Collision, Lasers, Alignment, Reprints, "Rydberg states.

The possibility of probing the collisions of aligned Rydberg atoms by stimulated emission is assessed with studies of a polarized state and a new measurement of a collisional alignment effect in atomic $\mathrm{Ca}$. The stimulated emission method uses a laser to dump the desired state to a lower level which subsequently fluoresces. The technique can be used to obtain populations and polarization dependent information. First the method is tested by applying it to an aligned $\mathrm{Ca}(4 \mathrm{~s} 17 \mathrm{d1D}$ ) state. Alignment curves are measured when the initial state is prepared with both parallel and perpendicular relative polarizations. The experimentally observed alignment compares well with that derived from theoretical considerations of a saturated stimulated transition. Second, a two-vector collisional alignment experiment (initial state and relative velocity vector) is performed to study the energy transfer process and alignment effects are measured by both stimulated emission and conventional direct fluorescence detection.

\section{4,125}

PB96-200357 Not available NTIS

National Inst. of Standards and Technology (PL), Boulder, CO. Quantum Physics Div.

Pairwise and Nonpairwise Additive Forces in Weakly Bound Complexes: High Resolution Infrared Spectroscopy of ArnDF $(n=1,2,3)$.

Final rept.

J. T. Farrell, S. Davis, and D. J. Nesbitt. 1995, 16p Pub. in Jnl. of Chemical Physics, v103 n7 p2395-2411 1995.

Keywords: "Spectroscopy, "Intermolecular forces, Infrared, Reprints, "Nonpainwise additive forces, Potential energy surface, Vander Waals, Argon hydrogen fluoride.

High resolution infrared spectra of the $V D F=1<-0$ stretch in ArnDF $(n=1-3)$ have been recorded using a slit-jet infrared spectrometer. Analysis of the rotationally resolved spectra provides vibrationally averaged geometries and vibrational origins for a DF chromophore sequentially 'solvated' by Ar atoms. Calculations using pairwise additive Ar-Ar and Ar-DF potentials predict lowest energy equilibrium structures consistent with the vibrationally averaged geometries inferred spectroscopically. Variational calculations by Ernesti and Hutson using pairwise additive potentials predict rotational constants which are in qualitative agreement with, but consistently larger than, the experimental values. The inclusion of nonpairwise additive (three-body) terms improves the agreement, though still not to within the uncertainty of the pair potentials.

\section{4,126}

PB96-200720 Not available NTIS

National Inst. of Standards and Technology (PL), Boulder, CO. Quantum Physics Div

Stable, Tightly Confining Magnetic Trap for Evaporative Cooling of Neutral Atoms.

Final rept.

W. Petrich, M. H. Anderson, J. R. Ensher, and E. A. Cornell. 1995, 4p.

Pub. in Physical Review Letters, v74 n17 p3352-3355 Apr 95.

Keywords: "Magnetic traps, "Evaporative cooling, Atoms, Reprints, "Bose condensation, Orbiting potential.

We describe a new type of magnetic trap whose timeaveraged, orbiting potential (TOP) supplies tight and harmonic confinement of atoms. The TOP trap allows for long storage times even for cold atom samples by suppressing the loss due to nonadiabatic spin flips which limits the storage time in an ordinary magnetic quadrupole trap. In preliminary experiments on evaporative cooling of $87 \mathrm{Rb}$ atoms in the TOP trap, we obtain a phase-space density enhancement of up to 3 orders of magnitude and temperatures as low as $200 \mathrm{nK}$.

\section{4,127}

PB96-200944 Not available NTIS

National Inst. of Standards and Technology (EEEL), Gaithersburg, MD. Electricity Div.

Quantum Hall Effect-Based Resistance Standard (Quantum Hall Res).

Final rept.

K. C. Lee. $1996,18 p$

See also PB96-180096.

Pub. in Proceedings of the Annual National Aeronautics and Space Administration Metrology and Cali- bration Workshop (19th), NASA Kennedy Space Center, FL., February 26-March 1, 1996, p577-594.

Keywords: *Digital multimeter, Temperatures, Magnetic fields, Reprints, "Quantum Hall effect, *Resistance standard.

The present technology for observing the quantum Hall effect requires that a quantum Hall resistor $(\mathrm{OHR}$ ) be maintained at temperatures below $1.4 \mathrm{~K}$, and in magnetic fields between $5 \mathrm{~T}$ and $15 \mathrm{~T}$. Furthermore, in contrast with wire-wound standard resistors that can pass milli-amperes of current, present quantum Hall resistors are limited to currents between 25 micro Angstroms and 60 micro Angstroms. This means that expensive cryogenic systems and super conducting solenoids are required to produce the conditions for the $\mathrm{QHE}$ to be observed. The very limited current-carrying capacity of present QHRs requires highly specialized and complex measurement systems that can compare the low voltages developed across them to the voltages developed across the resistors being calibrated. The primary objective of the project is to develop QHRs that can operate at lower magnetic fields, higher temperatures, and higher currents, in order to reduce the costs and complexity of the cryogenic system and the measurement system. A secondary objective is to develop and improve simple measurement techniques that can calibrate wire-wound resistors using QHRs with combined relative uncertainties less than $0.1 \mathrm{ppm}$, even with the present limitations on the current that can be passed through the devices.

04,128

PB96-200985 Not available NTIS

National Inst. of Standards and Technology (EEEL) Gaithersburg, MD. Semiconductor Electronics Div. New Oxide Degradation Mechanism for Stresses in the Fowler-Nordheim Tunneling Regime.

Final rept.

A. Martin, J. S. Suehle, P. Chaparala, P. O'Sullivan, and A. Mathewson. 1996, 10p.

Pub. in Proceedings of the International Reliability Physics Conference, Dallas, TX., April 30-May 2, 1996, p67-76.

Keywords: "Oxides, *Tunneling, Reliability, Degradation, Stresses, Reprints, "Fowler-Nordheim.

In the study, voltage and current stress measurements in the Fowler-Nordein regime, performed on gate oxides $(9 \mathrm{~nm}-28 \mathrm{~nm})$, indicated that a ramped pre-stress prior to a constant stress can increase the time to breakdown in some cases. In the literature oxide breakdown is said to be related to a fixed amount of trapped oxide charge or to a fixed amount of generated traps in the oxide. However, these models cannot explain our experimental observations. Current-time, current-charge voltage-time characteristics and results of high frequency pre-stresses have been extensively studied in order to gain information about the charge trapping properties of the virgin and pre-stressed oxides. It is concludes from experimental results that the rate of initial positive charge build up in the oxide during the constant stress is a key factor for oxide degradation and breakdown.

\section{4,129}

PB96-201066 Not available NTIS

National Inst. of Standards and Technology (MEL),

Gaithersburg, MD. Precision Engineering Div.

Modification of a Commercial SEM with a Computer Controlled Cathode Stabilized Power Supply. Final rept

M. T. Postek, W. J. Keery, and A. E. Vladar. 1993

Pub. in Scanning, v15 p208-211 1993.

Keywords: "Accelerating voltage, "Scanning electron microscopes, Anode, Cathode, Computer control, Reprints, Wehnelt.

Modification of the high-voltage circuitry of the thermionic electron gun of a commercial scanning electron microscope (SEM) to a fully computer-controlled, cathode-stabilized system is described. The modification permits automatic filament saturation and monitoring. Using this system, variations and drifts in the emission current over time can be adjusted and compensated automatically with a feedback loop in the computercontrolled system. This change also enables the accurate determination and setting of the primary electron beam energy. Therefore, the accelerating voltage is known for comparison to Monte Carlo computer modeling of the electron beam specimen interaction. 
04,130

PB96-201173 Not available NTIS

National Inst. of Standards and Technology (PL), Gaithersburg, MD. Electron and Optical Physics Div. Spin-Dependent Interface Transmission and $\mathbf{R e}$ flection in Magnetic Multilayers (Invited) Final rept.

M. D. Stiles. 1996, 6p.

Pub. in Jnl. of Applied Physics, v79 n8 p5805-5810 Apr 96.

Keywords: "Interface transmission, "Magnetic multilayers, Reflection, Exchange coupling, Reprints, Magnetoresistance, Magnetotransport.

First-principles calculations of transmission and reflec tion from $\mathrm{Ag} / \mathrm{Fe}, \mathrm{Au} / \mathrm{Fe}$ Cu/Co, and $\mathrm{Cu} / \mathrm{Ni}$ interfaces show very strong spin dependence that differs significantly from expectations based on free electron ap proximations. The results can be used to understand both the giant magnetoresistance and the oscillatory exchange coupling observed in magnetic multilayers of these materials. The spin dependent of the reflection probabilities is strong enough to give a large gian magnetoresistance even if there is no spin-dependent defect scattering. The calculated reflection amplitudes determine the strength of the oscillatory exchange coupling.

\section{4,131}

PB96-201207 Not available NTIS

National Inst. of Standards and Technology (PL) Gaithersburg, MD. Radiometric Physics Div.

Vibrationally Resolved Photoelectron Angular Distributions and Branching Ratios for the Carbon Dioxide Molecule in the Wavelength Region 685-795 Angstrom.

Final rept

J. B. West, M. A. Hayes, M. R. F. Siggel, A. C. Parr, J. E. Hardis, J. L. Dehmer, and P. M. Dehmer. 1996, $12 p$

Pub. in Jnl. of Chemical Physics, v104 n11 p39233934 Mar 96

Keywords: "Branching ratios, "Asymmetry parameters, "Spectroscopy, Cross sections, Ionization, Reprints, Pholoelectron spectroscopy.

Measurements of vibrational branching ratios and photoelectron angular distributions have been made in the regions for the Tanaka-Ogawa, Linkholm, and Henning series for the $\mathrm{CO} 2$ molecule. The behavior of these parameters was found to be sensitive to which particular resonance is excited, with considerable in ensity going into vibrational modes other than the symmetric stretch. An initial analysis of some of the data taken is presented.

\section{4,132}

Not available NTIS

National Inst. of Standards and Technology (PL), Gaithersburg, MD. Electron and Optical Physics Div. Nanostructure Fabrication via Laser-Focused Atomic Deposition (Invited)

Final rept.

R. J. Celotta, R. Gupta, R. E. Scholten, and J. J.

McClelland. 1996, 5p.

Pub. in Jnl. of Applied Physics, v79 n8 p6079-6083 Ap 96.

Keywords: "Nanostructure, "Atomic deposition, Reprints, Fabrication, Atoms, "Laser focusing

Nanostructured materials and devises will play an important role in a variety of future technologies, including magnetics. The authors describe a method for nanostructure fabrication based on the use of lase light to focus neutral atoms. The method uses neither a mask nor a resist, but relies on the direct deposition of atoms to form permanent structures. Since the atomic de Broglie wavelength is of picometer order, the size of structurs produced is not significantly limited by diffraction, as in optical lithography Lines as narrow as $38 \mathrm{~nm}$ full width at half maximum spaced by 213 $\mathrm{nm}$ have been produced and the authors have demonstrated the production of a two-dimensional array of dots. The highly parallel process of nanostructure formation and the intrinsic accuracy of the optical wavelength that determines structure spacing suggest a number of interesting applications, including calibration standards for various types of microscopy, lithography and micromeasurement systems. Possible magnetic applications include the production of arrays of mag netic elements, laterally structures giant magnetoresistive devices, and the patterning of magnetic media.
04,133

PB96-204144 Not available NTIS

National Inst. of Standards and Technology (PL), Gaithersburg, MD. Electron and Optical Physics Div. Properties of a Bose-Einstein Condensate in an Anisotropic Harmonic Potential.

Final rept.

M. Edwards, R. J. Dodd, C. W. Clark, P. A

Ruprecht, and K. Burnett. 1996, 4p.

Pub. in Physical Review A, v53 n4 pR1950-R1953 Apr 96.

Keywords: "Laser cooling, Reprints, Lifetime, Rubidium, Vortex, "Bose-Einstein condensation, Non-linear Schrodinger equation.

The authors present results on the size, density, chemical potential, and lifetime of a Bose-Einstein condénsate as a function of a number of condensate atomes for parameters appropriate to the time-averaged orbiting potential trap in which a Bose condensate of 87Rb atoms was recently formed. These results were obtained by solving the time-independent nonlinear Schrodinger equation with anisotropic harmonic trapping potential. The authors also find vortex solutions for the trap and the critical rotation frequency required for their formation.

04,134

PB96-204201 Not available NTIS

National Inst. of Standards and Technology (PL), Gaithersburg, MD. Radiometric Physics Div.

Mode-Locked Lasers for High-Accuracy Radiometry

Final rept

T. R. Gentile, and C. L. Cromer. 1996. 3p. Pub. in Metrologia, v32 p585-587 1995/96.

Keywords: *Radiometry, "Lasers, Reprints, Cryogenics, Linearity, Mode-locked laser, NIST.

The use of a mode-locked laser as a source for highaccuracy calibration of transfer standards to a cryogenic radiometer is discussed. The critical issue is the relative response of the transfer standard to the modelocked laser light as compared with continuous-wave laser light As a first test, the author have measured the linearity of the response of silicon photodiode lighttrapping detectors using mode-lock laser light at a wavelength of $532 \mathrm{~nm}$.

\section{4,135}

PB97-110175 Not available NTIS

National Inst. of Standards and Technology (PL), Gaithersburg, MD. Electron and Optical Physics Div. Stable Silicon Photodiodes for Absolute Intensity Measurements in the VUV and Soft X-ray Regions. Final rep

E. M. Gullikson, R. Korde, L. R. Canfield, and R. E. Vest. 1996, 4p

Pub. in Jnl. of Electron Spectroscopy and Related Phenomena, v80 p313-316 1996

Keywords: "X-rays, "Photodiodes, Silicon, Vacuum ultraviolet, Absolute detectors, Reprints, "Foreign technology.

Stable silicone photodiodes with $100 \%$ internal quanlum efficiency have been developed for the vacuum ultraviolet and soft $x$-ray regions. It is demonstrated that the response of these detectors can be reasonably well represented by a simple model for photon energies above $40 \mathrm{eV}$. The measured efficiency is consistent with a constant electron-hole pair creation energy for Si above $40 \mathrm{eV}$. Radiation damage is demonstrated to result in loss of carriers to recombination at the front surface. The uniformity of the diodes is shown to be better than $0.1 \%$ RMS at $110 \mathrm{eV}$.

\section{4,136}

Not available NTIS

National Inst. of Standards and Technology (CSTL), Gaithersburg, MD. Biotechnology Div.

Hydrodynamic Friction of Arbitrarily Shaped Brownian Particles.

Final rept.

J. B. Hubbard, and J. F. Douglas. 1993, 4p 93.

Keywords: "Brownian motion, "Hydrodynamic friction Capacitance, Wiener sausage, Reprints.

The authors present a simple and accurate method of estimating the translational hydrodynamic friction on rigid Brownian particles of arbitrary shape. The Brownian friction coefficient if take the form $\mathrm{f}=6(\mathrm{pi}) \mathrm{nC}$ (omega), where $\mathrm{C}$ (omega) is mathematically equivalent to the electrostatic capacitance of the particle omega in units where the caacity of a sphere equals its radius. This formula is particularly useful for particles consisting of a few globular subunits, for which slender body approximations are not very accurate.

04,137

PB97-110399 Not available NTIS

National Inst. of Standards and Technology (PL), Gaithersburg, MD. Ionizing Radiation Div.

Calculation of Photon Mass Energy-Transfer and Mass Energy-Absorption Coefficients.

Final rept.

S. M. Seltzer. 1993, 24p

See also PB92-181106.

Pub. in Radiation Research, v136 p147-170 1993.

Keywords: "Photons, "Energy absorption, "Energy ransfer, Bremsstrahlung, Cross sections, Calculations, Reprints, Positron annihilation.

Calculations of mass energy-transfer and mass energy-absorption coefficients for photon energies from $1 \mathrm{keV}$ to $100 \mathrm{MeV}$ have been developed, based on a re-examination of the processes involved after the initial photon interaction. The probabilities for the initial interaction are from the current photon interaction cross-section database at the National Institute of Standards and Technology. The calculation then take into account (1) electron binding effects on the Compton-scattered photon distribution; (2) the complete cascade of fluorescence emission after ionization events in any atomic subshell, including those associated with incoherent scattering and triplet production; and (3) the radiative energy losses of the secondary electrons and positrons slowing down in the medium, including the emission of bremsstrahlung, characteristic $x$-rays from mpact ionization, and positron in-flight as well as atrest annihilation quanta. Consideration of the processes in (3) goes beyond the continuous-slowing-down approximation and includes the effects of energy-loss straggling. Results for the mass energy-absorption coefficient are compared with those from recent tabulations.

04,138

PB97-110407 Not available NTIS

National Inst of Standards and Technology (PL). Gaithersburg, MD. Ionizing Radiation Div.

Electron-Photon Monte Carlo Calculations: The ETRAN Code.

Final rept.

S. M. Seltzer. 1991, 24p

Pub. in Applied Radiation and Isotopes, v42 n10 p9179411991.

Keywords: *Photons, *Electrons, Positron, Bremsstrahlung, Reprints, "Foreign technology, "ETRAN code, Monte Carlo transport code.

The paper briefly describes the Monte Carlo code ETRAN, developed for the solution of coupled electron-photon transport problems. The authors focus on the multiple-scattering distributions and underlying cross sections that form the basis of current versions of ETRAN, without details on sampling algorithms or numerical procedures.

\section{4,139}

PB97-110563 Not available NTIS

National Inst. of Standards and Technology (MEL) Gaithersburg. MD. Precision Engineering Div.

Point Charges, Radiation Reaction, and Quantum Mechanics.

Final rept.

E. Marx. $1993,5 p$

Pub. in Institute of Electrical and Electronic Engineers EMC Symposium, Anaheim, CA. August 17-2i, 1992 p508-5121993.

Keywords: *Point charges, *Radiation reactions, "Quantum mechanics, Electrodynamics, Reprints, Aharonov-Bohm effect, Cerenkov radiation.

Moving point charges generate nonsinusoidal electromagnetic fields, and the motion of the charges is in turn determined by the fields. The full dynamical problem includes radiation reaction, which is discussed here. Nonsinusoidal fields are also associated with relativis. tic quantum mechanics, where the notion of causality is extended. Cerenkov radiation and the AharonovBohm effect are two other topics. 
04,140

PB97-110571 Not available NTIS

National Inst. of Standards and Technology (MEL), Gaithersburg, MD. Precision Engineering Div.

Positronium in Relativistic Quantum Mechanics. Final rept.

E. Marx. 1992, 11p.

Pub. in Jnl. of the Franklin Institute, v329 n5 p869-879 1992.

Keywords: *Quantum mechanics, "Positronium, Bound states, Pair annihilation, Electromagnetic interactions, Reprints, Klein-Gordan equation.

The problem of bound states of a charged particle and its antiparticle is formulated in the framework of the relativistic quantum mechanics of a single scalar particle interacting with the electromagnetic field. The Coulomb interaction between the particle and the antiparticle gives rise to the bound states, and the coupling of the wave function to the radiation field is responsible for the decay of the pair into electromagnetic energy. The contribution of a pion-pion interaction is discussed briefly. The theory is extended to the spin-1/2 electron.

04,141

PB97-110589 Not available NTIS

National Inst. of Standards and Technology (MEL), Gaithersburg, MD. Precision Engineering Div.

Relativistic Quantum Mechanics of Interacting Particles.

Final rept.

E. Marx. 1992, 17p

Pub. in Jnl. of the Franklin Institute, v329 n4 p637-653 1992.

Keywords: "Quantum mechanics, "Particles, "Electromagnetic interactions, Probability amplitudes, Field theory, Identical particles, Reprints, Klein-Gordon equation.

The relativistic quantum mechanics of several charged scalar particles interacting with a dynamical electromagnetic field is presented in the framework of the many-amplitudes formalism. Each particle and its antiparticle are represented by a seaparate wave function that satisties the Klein-Gordon equation and which contributes to the charge-current density that is a source of the electromagnetic field. The probability amplitude for several particles or antiparticles is the symmetrized product of the single-particle probability amplitudes.

\section{4,142}

PB97-110605 Not available NTIS

National Inst. of Standards and Technology (MEL) Gaithersburg, MD. Precision Engineering Div.

Spinor Equations in Relativistic Quantum Mechanics.

Final rept.

E. Marx. 1992, 13p

Pub. in Jnl. of Mathematical Physics, v33 n6 p22902302 Jun 92.

Keywords: "Quantum mechanics, "Spinor equations, Wave functions, Formulas, Weyl equations, Reprints.

A set of two first-order equations for classical spinor fields are used to formulate the relativistic quantum mechanics (RQM) of a single massive spin-one half particle. These equations are generalized Weyl equations and they are not equivalent to the Dirac equation. They lead to a conserved charge that is not positive definite, thus allowing for a probabilistic interpretation fo the wave function in RQM. The charge is also conserved when the fields interact with a scalar field through the charge-current densities.

\section{4,143}

PB97-111256 Not available NTIS

National Inst. of Standards and Technology (PL), Gaithersburg, MD. Atomic Physics Div.

Beam Line for Highly Charged lons.

Final rept.

A. I. Pikin, C. A. Morgan, E. W. Bell, J. D. Gillaspy,

L. P. Ratliff, and D. A. Church. 1996, 6p

Pub. in Review of Scientific Instruments, v67 $n 7$ p2528-2533 Jul 96

Keywords: "Beam lines, "Ion optics, "Highly charged ions, Electron beams, Reprints, Electron beam ion trap.

The design and operation of a beam line for transporting and charge-to mass selecting highly charged ions extracted from the National institute of Standards and Technology electron beam ion trap (EBIT) are described. This beam line greatly extends the range of experiments possible at this facility. Using the transport system, pure beams of low-energy, highly charged ions up to $\mathrm{Xe}(44+)$ have been produced with substantially higher fluxes than previously reported from an EBIT source. Design choices and computer modeling for the various components of the beam line are explained in detail.

\section{4,144}

PB97-111264 Not available NTIS

National Inst. of Standards and Technology (EEEL), Gaithersburg, MD. Semiconductor Electronics Div.

Quantum Conductance Fluctuations in the LargerSize-Scale Regime.

Final rept.

C. A. Richter, D. G. Seiler, and J. G. Pellegrino.

1996, 5p.

Pub. in Physical Review B, v53 n19 p13 086-13 090 May 96.

Keywords: *Conductance,

*Fluctuations,

Heterostructure, Magnetoresistance, Mesoscopic, Reprints, "GaAs/AlGaAs, UCF.

The authors report the results of experimental studies of 'universal' conductance fluctuations in a variety of millimeter-sized GaAs/AlxGal-xAs heterostructures. The ability to observe these mesoscopic fluctuations in traditionally macroscopic semiconductor devices is due to the enhanced sensitivity of the magnetic field modulation measurement technique, which allows a coherent interference effect to be observed and studied in the large-size-scale regime where both the sample length and width are much greater than the quantum scattering lengths.

\section{4,145}

PB97-111306 Not available NTIS

National Inst. of Standards and Technology (EEEL),

Boulder, CO. Electromagnetic Technology Div.

Size Effects and Giant Magnetoresistance in Unannealed NiFe/Ag Multilayer Stripes.

Final rept.

S. C. Sanders, R. W. Cross, S. E. Russek, A.

Roshko, and J. O. Oti. 1996, 3p.

Pub. in Jnl. of Applied Physics, v79 n8 p6240-6242 Apr 96.

Keywords: "Magnetoresistance, "Magnetostatics, Annealing, Barkhausen noise, Multilayers, Size effects, Thin films, Reprints, NiFe/Ag

The authors have observed giant magnetoresistance (GMR) in unannealed NiFe/Ag multilayer thin-film stripes. Rectangular stripes having constant thickness and a constant 11:1 length-to-width aspect ratio, but varying widths down to 0.5 micrometers were measured. Two types of multilayer configurations were tested, a system of five NiFe/Ag bilayers with $5.5-\mathrm{nm}$-thick $\mathrm{Ag}$ spacer layers, and a system of nine bilayers with 4.4-nm-thick Ag layers. In contrast to the characterstics of annealed NiFe/Ag multilayer stripes, the unannealed stripes produced increasing GMR ratios for decreasing stripe sizes, with the 0.5 -micrometer-wide stripe of the five-bilayer system exhibiting a deltaR/R of $2.5 \%$. Barkhausen noise and response broadening also increased with decreasing stripe size, however. The results are discussed in terms of magnetostatic coupling of the NiFe layers within the stripes.

\section{4,146}

PB97-111413 Not available NTIS

National Inst. of Standards and Technology (CSTL), Gaithersburg, MD. Thermophysics Div.

Commercial Helium Permeation Leak Standards: Their Properties and Reliability.

Final rept.

P. J. Abbott, and S. A. Tison. 1996, $5 p$.

Pub. in Jnl. of Vacuum Science and Technology A, v14 n3 p1242-1246 May/Jun 96.

Keywords: *Helium, *Permeation leaks, Decay rates, Stability, Properties, Reliability, Reprints.

Standard leaks or leak artifacts are used extensively in industrial and research environments, typically for the calibration of helium leak detectors. The most commonly used leak is the helium permeation type, which uses a glass, quartz, or polymer barrier to restrict the flow of helium via diffusion. Physical leaks, on the other hand, use a physically restrictive element to limit the flow. The article will discuss the properties and reliability of helium permeation standard leaks, based on eight years of accumulated calibration data. Physical helium leaks will not be discussed since relatively few have been calibrated at the National Institute of Standards and Technology.

04,147

PB97-111421 Not available NTIS

National Inst. of Standards and Technology (MSEL), Gaithersburg, MD

Extrapolation of the Heat Capacity in Liquid and Amorphous Phases.

Final rept.

J. Agren, B. Cheynet, M. T. Clavaguera-Mora, U. Kattner, F. Sommer, K. Hack, and J. Hertz. 1996, 32p.

Pub. in Calphad, v19 n4 p449-480 1995.

Keywords: "Amorphouse phases, "Heat capacity, Extrapolation, Liquid phase, Phase stability, Two-states model, Reprints.

Various methods of extrapolating the thermodynamic properties of the liquid will be discussed. A phenomenological method based on polynomials as well as a more physical method based on an extended two-state Schottky formalism will be presented and their application to some substances will be demonstrated.

\section{4,148}

\section{PB97-111496 Not available NTIS}

National Inst. of Standards and Technology (EEEL), Boulder, CO. Electromagnetic Technology Div. Pulse-Driven Programmable Josephson Voltage Standard.

Final rept.

S. P. Benz, and C. A. Hamilton. 1996, 3p.

Pub. in Applied Physics Letters, v68 n22 p3171-3173 May 96.

Keywords: *Josephson junctions, *Voltage standards, Digital-to-analog converters, Programmable pulses, Pulses, Reprints.

A voltage standard based on a series array of pulsebiased, nonhysteretic Josephson junctions is proposed. The output voltage can be rapdily and continuously programmed over a wide range by changing the pulse repetition frequency. Simulations relate the circuit margins to pulse height, width, and frequency. Ex perimental results on a prototype circuit confirm the expected behavior.

\section{4,149}

PB97-111868 Not available NTIS

National Inst. of Standards and Technology (MSEL), Gaithersburg, MD. Reactor Radiation Div

X-ray Reflectivity Determination of Interface Roughness Correlated with Transport Properties of (AIGa)As/GaAs High Electron Mobility Transistor Devices.

Final rept.

J. A. Dura, J. G. Pellegrino, and C. A. Richter. 1996 ,

Pub. in Applied Physics Letters, v69 n8 p1134-1136 Aug 96.

Keywords: “ $X$ ray reflectivity *Aluminum gallium arsenide, "Gallium arsenide, Interface roughness, Mobility, Molecular beam epitaxy, Transport properties, Reprints.

To explore the role of interface scattering in high electron mobility transistor (HEMT) device pe:formance, a series of samples consisting of both a superlattice and a HEMT structure were grown by molecular beam epitaxy (MBE) at temperatures ranging from 500 to 630 degrees $\mathrm{C}$. Hall mea surements indicate a trend toward higher mobilities in samples growth at higher temperatures. Subsequent $x$-ray reflectivity measurements were made, and the data were fitted by least-squares refinement of a calculated reflectivity curve determined from a model of the sample structure to obtain the composition profile along the growth direction. These results indicate smoother interfaces for the samples with higher mobilities.

04,150

PB97-111934 Not available NTIS

National inst. of Standards and Technology (MSEL) Gaithersburg, MD. Polymers Div.

Intrinsic Conductivity of Objects Having Arbitrary Shape and Conductivity.

Final rept.

E. J. Garboczi, and J. F. Douglas. 1996, 12p.

Pub. in Physical Review E, v53 n6 p6169-6180 1996 


\section{PHYSICS}

\section{General}

Keywords: "Intrinsic conductivity, "Conductivity, Arbitrary, Shapes, Reprints.

The authors study the electrical conductivity omega of a dispersion of randomly oriented and positioned particle inclusions having common shape and conductivity omega(p), suspended in an isotropic homogeneous matrix of conductivity omega(0). For this problem, the mixture conductivity is a scalar and the authors concentrate on the leading order concentration virial coefficient, the 'intrinsic conductivity' (omega). Results for (omega) are summarized for limiting cases where there is a large mismatch between the conductivities of the inclusions and the suspending matrix. For a general particle shape, the authors then treat the more difficult case of arbitrary relative conductivity delta equal or minus omega(p)/omega(0) through the introduction of a Pade approximant that incorporates (exact or numerical) information for (omega(delta)) in the delta $\rightarrow$ infinity, delta $\rightarrow 0+$, and delta approximately 1 limits Comparison of this approximation for (omega(delta)) to exact and finite element calculations for a variety of particle shapes in two and three dimensions shows excellent agreement over the entire range of delta. This relation should be useful for inferring particle shape and property information from conductivity measurements on dilute particle dispersions. The leading order concentration virial coefficient for other mixture properties (thermal conductivity, dielectric constant, refrac tive index, shear modulus, bulk modulus, viscosity, etc.) are equally well described by a similar Pade approximant.

\section{4,151}

PB97-111942 Not available NTIS

National Inst. of Standards and Technology (MSEL) Gaithersburg, MD. Reactor Radiation Div.

Observation of Two Length Scales Above ( $T$ sub $\mathrm{N})$ in a Holmium Thin Film.

Final rept.

P. M. Gehring, A. Vigliante, D. F. McMorrow, G Helgesen, R. A. Cowley, R. C. C. Ward, M. R. Wells, D. Gibbs, and C. F. Majkrzak. 1996, 7p

Pub. in Physica B, v221 p398-404 1996.

Keywords: "Thin films, "Holmium, Two length scales, Neutron scattering, Reprints, "Foreign technology.

Two-axis neutron-scattering measurements on a $1 \mathrm{mi}-$ crometer thick holmium film reveal the presence of two magnetic correlation lengths above TN that are qualitatively similar to those recently observed in X-ray and neutron scattering studies of bulk hoimium and terbium. The scattering profile is well described by a broad Lorentzian plus a narrow Lorentzian-squared line-shape with widths that, for temperatures sufficiently close to TN, differ by a factor of ten or more.

\section{4,152}

\section{PB97-112239 Not available NTIS}

National Inst. of Standards and Technology (PL) Gaithersburg, MD. Quantum Metrology Div

Systematic Correction in Bragg $X$-ray Diffraction of Flat and Curved Crystals.

Final rept

C. T. Chantler, and R. D. Deslattes. 1995, 25p.

Pub. in Review of Scientific Instruments, v66 n11 p5123-5147 Nov 95.

Keywords: "Systematic corrections, "Bragg x-ray diffraction, "Crystals, Curved crystals, Flat crystals, Refractive index shifts, Spectral wavelengths, Foreign technology, Reprints.

Measurements of spectral wavelengths in Bragg diffraction from crystals often require refractive index corrections to allow a detailed comparison of experiment with theory. These corrections are typically 100-300 ppm in the $x$-ray regime, and simple estimates may sometimes be accurate to $5 \%$ or better. The inadequacies of these estimates are discussed. Even with a possibly improved index of refraction estimate, this cor rection is insufficient since additional systematics in the diffraction process occur at or above this level. For example, asymmetries of diffraction profiles with pi-polarized radiation or due to three-beam diffraction can approach the magnitude of refractive index corrections for flat or curved crystals. The depth of penetration of the $x$-ray field inside curved crystals, the shift of the mean angle to the diffracting planes, and lateral shifts around the crystal surface are rarely considered but can dominate over refractive index corrections, particularly for high-order diffraction or medium-energy $x$ rays. Shifts and nonlinearities arise when diffracting surfaces lie off the Rowland circle, and exhibit strong and rapidly varying angular dependencies.
04,153

PB97-112346 Not available NTIS

National Inst. of Standards and Technology (EEEL), Boulder, CO. Electromagnetic Technology Div

Bias Current Dependent Resistance Peaks in NiFe/ Ag Giant Magnetoresistance Multilayers.

Final rept.

L. S. Kirschenbaum, C. T. Rogers, P. D. Beale, S. E Russek, and S. C. Sanders. 1996, 3p

Pub. in Applied Physics Letters, v68 n22 p3099-3101 May 96.

Keywords: "Thin films, "Magnetoresistance, "Currents, Multilayers, Peaks, Magnetic fields, Reprints, NiFe-Ag.

The authors show that thin-film Ni82Fe18/Ag multilayer structures display multiple peaks in their magnetoresistance curves when biased at current densities above $10(6) \mathrm{A} / \mathrm{cm}(2)$. These peaks appear for annealed and unannealed structures, and their number is correlated with the number of NiFe layers. At high bias currents, the peak positions shift linearly with the internal magnetic field created by the bias current. The peak positions extrapolate to nonzero fields at zero bias currents, providing an upper bound on the magnetic layer-layer coupling strength of $J(0)$ approximately equal to $10(-20) \mathrm{J}(\mathrm{k}(\mathrm{B}) \times 700 \mathrm{~K})$. The peak positions do not shift with temperature over the range 200-375 K; their widths narrow with increasing temperature. The single-domain magnetic moment micro is estimated as $1017 \mathrm{~J} / \mathrm{T}$ from the peak widths of approximately $0.8 \mathrm{kA} / \mathrm{m}$.

\section{4,154}

PB97-112387 Not available NTIS

National Inst. of Standards and Technology (MSEL), Gaithersburg, MD. Reactor Radiation Div.

Isolated Spin Pairs and Two-Dimensional Magnetism in $\mathrm{SrCr}$ (sub 9p) Ga(sub 12-9p)019.

Final rept.

S. H. Lee, C. Broholm, G. Aeppli, A. Taylor, T. G.

Perring, and B. Hessen. 1996, $4 \mathrm{p}$

Pub. in Physical Review Letters, v76 n23 p4424-4427 Jun 96.

Keywords: "Neutron scattering, "Ligand environment, *Magnetism, Sub-lattices, Dispersionless, Inelasticity, Reprints.

The authors show by inelastic neutron scattering that the frustrated magnet SrCr9pGa12-9p019 ( $p=$ $0.92(5))$ has dispersionless magnetic excitations at energies $18.6(1)$ and $37.2(5) \mathrm{meV}$. The wave vector and temperature dependence of the excitations as well as the ratio $1: 2$ of excitation energies is perfectly accounted for by isolated antiferromagnetically exchange coupled pairs of spins $s=3 / 2$. Consideration of the layered structure of magnetic chromium ions and the ligand environment indicates that these pairs are adjacent spins in neighboring triangular lattice planes which separate two-dimensional sublattices of interacting kagome-triangle-kagome layers.

\section{4,155}

PB97-112403 Not available NTIS

National Inst. of Standards and Technology (PL),

Gaithersburg, MD. Molecular Physics Div.

Matrix Isolation Study of the Interaction of Excited Neon Atoms with 03: Infrared Spectrum of $\mathrm{O}($ (sub $3)(-))$ and Evidence for the Stabilization of O2... O ((sub 4)(+)).

Final rept.

C. L. Lugez, W. E. Thompson, and M. E. Jacox.

1996, 8p.

Pub. in Jnl. of Chemical Physics, v105 n6 p2153-2160 Aug 96

Keywords: "Neon atoms, "Matrix isolation, Infrared spectrum, Photodissociation, Photionization, Reprints.

When a Ne:O3 sample is codeposited at approximately $5 \mathrm{~K}$ with neon atoms that have been excited in a microwave discharge, the most prominent infrared absorptions of the resulting solid are contributed by trans-and cyc-O4(=) and by $\mathrm{O} 3(-)$. The failure to detect infrared absorptions of $\mathrm{O} 3(+)$ is consistent with the initial formation of that species in one or more dissociative excited states. The v3 absorption of O3(-) appears at $796.3 \mathrm{~cm}(-1)$, close to its position in earlier argonmatrix experiments in which photoionization of an alkali metal atom provided the electron source and in which diffusion of the atomic cation would result in the stabilization of appreciable $\mathrm{M}+\mathrm{O} 3(-)$. The identification of O3(-) isolated in solid neon is supported by observations of $\mathrm{O} 3(-)$ generated from isotopically substituted
Ne:O2: 220 samples, also codeposited with excited neon atoms. An upper bound of $810 \mathrm{~cm}$ to the minus 1 power is estimated for the gas-phase band center of $v 3$ of $\mathrm{O} 3(-)$. Infrared absorption which grow on mild warmup of the sample are tentatively assigned to an O2...O4(+) complex.

04,156

PB97-112429 Not available NTIS

National Inst. of Standards and Technology (MSEL), Gaithersburg, MD. Reactor Radiation Div.

Unconventional Ferromagnetic Transition in $\mathrm{La}\left(\right.$ sub 1-x) $\mathrm{Ca}$ (sub x) $\mathrm{MnO}_{3}$

Final rept.

J. W. Lynn, R. W. Erwin, J. A. Borchers, Q. Huang

and A. Santoro. 1996, 4p.

Pub. in Physical Review Letters, v76 n21 p4046-4069 May 96.

Keywords: *Quasielastic component, "Polycrystalline sotropic, Ferromagnetic, Despersion relation, $\mathrm{Re}$ prints

Neutron scattering has been used to study the magnetic correlations and long wavelength spin dynamics of $\mathrm{La}(1-x) \mathrm{Ca}(x) \mathrm{MnO}_{3}$ in the ferromagnetic regime (0 equal to or less than $1 / 2)$. For $x=1 / 3(T(C)=250 \mathrm{~K})$ where the magnetoresistance effects are largest the system behaves as an ideal isotropic ferromagnet at low $\mathrm{T}$, with a gapless (less than $0.04 \mathrm{meV}$ ) dispersion relation $E=D q 2$ and $D(T-0)$ approximately equal to 170 meV Angstroms. However, an a nomalous strongly field-dependent diffusive component develops above approximately $200 \mathrm{~K}$ and dominates the fluctuation spectrum at $T \rightarrow T(C)$. This component is not present at lower $\mathrm{x}$.

\section{4,157}

PB97-112585 Not available NTIS

National Inst. of Standards and Technology (EEEL), Boulder, CO. Electromagnetic Technology Div.

Modeling Effects of Temperature Annealing on Giant Magnetoresistive Response in Discontinuous Multilayer NiFe/Ag Films.

Final rept.

J. O. Oti, and Y. K. Kim. 1996, 3p

Pub. in Jnl. of Applied Physics, v79 n8 p5596-5598 Apr 96

Keywords: "Magnetoresistance, "Grain clusters, "Multilayer films, Anisotropy, Exchange, Magnetostatic, Reprints.

The giant magnetoresistive (GMR) behaviors of discontinuous double-layer giant magnetoresistive films with different microstructure arising from different annealing conditions, are calculated using a numerical micromagnetic model. The effect of magnetic grain growths in the perpendicular and lateral directions in the magnetic layers, and the formation and growth of grain clusters were studied. The GMR responses of the films are analyzed in terms of magnetostatic interactions between the magnetic layers and the microstructural geometric effects on the transport properties of the samples.

04,158

PB97-112593 Not available NTIS

National Inst. of Standards and Technology (EEEL), Boulder, CO. Electromagnetic Technology Div Simulating Device Size Effects on Magnetization Pinning Mechanisms in Spin Valves.

Final rept.

J. O. Oti, R. W. Cross, S. E. Russek, and Y. K. Kim. 1996, 3p.

Pub. in Jnl. of Applied Physics, v79 n8 p6386-6388 Apr 96.

Keywords: "Spin valves, "Magnetization, Anisotropy, Exchange, Magnetoresistance, Magnetostatic, Reprints

The effects of magnetostatic interactions on the gian magnetoresistive (GMR) response of $\mathrm{NiFe} / \mathrm{Cu} / \mathrm{NiFe}$ spin valves are studied using an analytical model. The model is applicable to devices small enough for the magnetic layers to exhibit single-domain behavior. Devices having lengths in the track-width direction of 10 micrometers and interlayer separations of $4.5 \mathrm{~nm}$ are studied Stripe heights are varied from 0.5 to 2 micrometers. The magnetization of one magnetic layer is pinned by a transverse pinning field that is varied from 0 to $24 \mathrm{kA} / \mathrm{m}$ (300 Oe). GMR curves for transverse fields are calculated. At zero external field and magnetization of the layers shows a tendency to align themselves antiparallel in the transverse direction. This 
results in an offset from the ideal biasing of the device. Broadening of the curves due to shape anisotropy occurs with decreasing stripe height and increasing magnetic layer thickness, and the magnetization in the pinned layer becomes less stable.

04,159

PB97-113021 Not available NTIS

National Inst. of Standards and Technology (PL), Gaithersburg, MD. Radiometric Physics Div.

Results of a NIST/NNIIOFI Comparison of SpectralRadiance Measurements.

Final rept.

R. D. Saunders, C. E. Gibson, K. D. Mielenz, B. B.

Khlevnoy, S. N. Mekhontsev, C. D. Harchenko, V. I

Sapritsky, and K. A. Sudarev. 1996, 5p.

Pub. in Metrologia, v32 p449-453 1995/96.

Keywords: "Spectral radiance, "Radiometry, Temperature scales, Intercomparison, Ribbon filament lamps, Reprints, NISTNNIIOFI

A comparison of the spectral-radiance scales of the National Institute of Standards and Technology (NIST) and the All-Russian Research Institute for Optophysical measurements (NVIIOFI) was carried ou in 1989 and 1990 . The spectral range of the comparison was between $240 \mathrm{~nm}$ and $2400 \mathrm{~nm}$. The transfer standards were argon-filled tungsten ribbon filament lamps. The NIST N NIIOFI agreement was $0.5 \%$ to $1 \%$ in the $240 \mathrm{~nm}$ to $800 \mathrm{~nm}$ range, and better than $0.5 \%$ between $900 \mathrm{~nm}$ and $2400 \mathrm{~nm}$. On average, the spectral radiances measured at the NVIIOFI were slightly higher than the NIST values.

04,160

PB97-113070 Not available NTIS

National Inst. of Standards and Technology (MSEL), Gaithersburg, MD. Reactor Radiation Div.

Colossal Magnetoresistance without $\mathrm{Mn}(3+) / \mathrm{Mn}(4$

Double Exchange in the Stoichiometric Pyrochlore TI2Mn2O7.

Final rept.

M. A. Subramanian, B. H. Toby, A. P. Ramirez, G. H.

Kwei, W. J. Marshall, and A. W. Sleight. 1996, 4p.

Pub. in Science, v273 p81-84 Jul 96

Keywords: "Magnetic structure, "Magnetoresistance, "Neutron diffraction, Pyrochlore, Single crystal diffraction, Reprints.

Structural analysis with powder neutron and singlecrystal $x$-ray diffraction data for a sample of the $\mathrm{Tl} 2 \mathrm{Mn} 2 \mathrm{O} 7$ pyrochlore, exhibiting colossal magnetoresistance (CMR), shows no deviations from ideal stoichiometry. Furthermore, the analysis gives an $\mathrm{Mn-O}$ distance of 1.90 Angstroms, significantly shorter than the $\mathrm{Mn}-\mathrm{O}$ distances in the range 1.94 to 2.00 Angstroms observed in phases based on LaMnO3 perovskites which show CMR. Both the stoichiometry and shorter $\mathrm{Mn}-\mathrm{O}$ dsitance in TI2Mn2O7 indicate oxi dation states very close to $\mathrm{TI} 2 \mathrm{Mn} 2 \mathrm{O} 7$. Thus $\mathrm{TI} 2 \mathrm{Mn} 2 \mathrm{O} 7$ has neither mixed valency for a double exchange magnetic interaction or a Jahn-Teller cation such as $\mathrm{Mn}(3+)$. Both were thought to play an essential role in CMR materials. The authors propose an alternate mechanism for CMR in T12Mn2O7 based on magnetic ordering driven by superexchange and strong spin fluctuation scattering above Tc. Thus the pyrochlores represent a distinct new class of CMR materials.

04,161

PB97-113104 Not available NTIS

National Inst. of Standards and Technology (PL), Gaithersburg, MD. Atomic Physics Div.

Polarization Measurements on a Magnetic Quadrupole Line in Ne-Like Barium. Final rept.

E. Takacs, E. S. Meyer, J. D. Gillaspy, L. T. Hudson, R. D. Deslattes, C. M. Brown, J. M. Laming, J. R. Roberts, and C. T. Chantler. 1996, 9p.

Pub. in Physical Review A, v54 n2 p1342-1350 Aug 96

Keywords: "Polarization, "X ray spectroscopy, Measurement, Charged ions, Reprints, "Electron beam ion trap(EBIT)

The authors have measured the absolute polarization of the $(2 p(-1) 3 / 23 s 1 / 2) \mathrm{J}=2$ to (2p6) $\mathrm{J}=0$ Ne-like barium $\mathrm{M} 2$ line, excited in an electron beam ion trap (EBIT) at a variety of energies. The authors find strong evidence for the existence of resonant excitation processes which are not explained by collisional-radiative calculations even when the polarization arising from impact excitation is included. At energies well away from where the resonances occur, the agreement between experiment and theory is good

04,162

PB97-113161 Not available NTIS

National Inst. of Standards and Technology (PL) Gaithersburg, MD. Radiometric Physics Div.

Comparison of Filter Radiometer Spectra Responsivity with the NIST Spectral-Irradiance and Illuminance Scales.

Final rept.

B. K. Tsai, B. C. Johnson, R. D. Saunders, and C. L. Cromer. 1996, 5p.

Pub. in Metrologia, v32 p473-477 1995/96.

Keywords: "Scales, "Radiometers, "Spectral compara tor, "Photometers, Detectors, Filters, Illuminance, Irradiance, Quartz halogen lamp, Reprints, "Foreign tech nology.

Narrowband filter radiometers are being used in the development of a new method of irradiance scale realization at the National Institute of Standards and Technology (NIST). To improve the accuracy of the curren spectral-irradiance scale, the authors propose to eliminate several steps from the calibration process and to use the filter radiometers and a high-temperature black body in realizing the scale. Ultimately, the filter radiometers will be calibrated against a trap detecto measured at the NIST High Accuracy Cryogenic Radiometer, which maintains an expanded uncertainty at $633 \mathrm{~nm}$ of $0.0266 \%$ for a coverage factor of $k=2$. In the work, the filter and detector were calibrated as a unit using the NIST Detector Comparator Facility, and the aperture areas were measured by flux comparison with a uniform source. Six filter radiometers covering the spectral range $350 \mathrm{~nm}$ to $1100 \mathrm{~nm}$ were used to measure a known irradiance source; the measurements agreed with predictions to within $1 \%$

\section{4,163}

PB97-113229 Not available NTIS

National Inst. of Standards and Technology (CSTL) Gaithersburg, MD. Thermophysics Div.

Design of a High-Pressure Ebulliometer, with Vapor-Liquid Equilibrium Results for the Systems $\mathrm{CHF} 2 \mathrm{Cl}+\mathrm{CF}_{3}-\mathrm{CH}_{3}$ and $\mathrm{CF}_{3}-\mathrm{CH}_{2} \mathrm{~F}+\mathrm{CH}_{2} \mathrm{~F}_{2}$

Final rept.

L. A. Weber, and A. M. Silva. 1996, 16p.

Pub. in International Jnl. of Thermophysics, v17 n4 p873-888 Jul 96.

Keywords: "Ebulliometers, "Phase equilibrium, *Refrigerant mixtures, Activity coefficient, Reprints, Chlorodifluoromethane, Difluoromethane, 1-1-1 trifluoroethane, 1-1-1-2-tetrafluoroethane.

The authors give a description of the design and operation of a new high-pressure metal ebulliometer which is capable of operating at pressures to at least $3 \mathrm{MPa}$ in the range 220-400 K. Infinite dilution activity coefficients are presented for the system $\mathrm{CHF} 2 \mathrm{Cl}=\mathrm{CF} 3$ $\mathrm{CH} 3$ at $275 \mathrm{~K}$ and for the system $\mathrm{CF} 3 \cdot \mathrm{CH} 2 \mathrm{~F}=\mathrm{CH} 2 \mathrm{~F} 2$ at 260,280 , and $300 \mathrm{~K}$. The Wilson activity coefficient model and a virial coefficient model are applied to these systems, and the phase equilibrium condition are calculated. The results are shown to agree we with predicted and with published measured values. The excess enthalpy is calculated and compared with results using a Peng-Robinson equation of state. Vapor densities of the dew curves are given.

04,164

PB97-113278 Not available NTIS

National Inst. of Standards and Technology (MSEL), Gaithersburg, MD. Reactor Radiation Div.

Diffraction of Neutron Standing Waves in Thin Films with Resonance Enhancement.

Final rept.

H. Zhang, S. K. Satija, P. D. Gallagher, C. P. Flynn, J. F. Ankner, J. A. Dura, and K. Ritley. 1996, 5p. Pub. in Physica B, v221 p450-454 1996.

Keywords: "Thin films, "Standing waves, "Neutrons, Diffraction, Resonance, Reprints

Diffraction on neutron standing waves in thin films has been demonstrated for the first time with experiments on an epitaxial $\mathrm{Y} / \mathrm{Gd} / \mathrm{Y} / \mathrm{Nb} / \mathrm{Al} 2 \mathrm{O} 3$ sample. Resonance enhancement in the diffraction intensity has been observed. The new diffraction scheme for neutrons and $x$-rays provides an oscillatory spatial concentration in thin films, in contrast to the evanescent diffraction which provides an exponential concentration. It also discriminates against diffraction background from sub strate, and may be especially useful when diffraction in transmission geometry is not available due to high absorption of substrate.

04,165

PB97-114474 PC A04/MF A01

National Inst. of Standards and Technology (PL), Gaithersburg, MD. Ionizing Radiation Div.

Experimentally Measured Total X-ray Attenuation Coefficients Extracted from Previously Unprocessed Documents Held by the NIST Photon and Charged Particle Data Center.

J. H. Hubbell. Sep 96, 37p, NISTIR-5893.

Keywords: "Bibliographies, "Photon cross sections, " $X$ rays, Tables(Data), Nuclear data collections, Data bases.

This report lists and annotates 47 previously unprocessed original-source documents held by the NIST Photon and Charged Particle Data Center (PCPDC), containing experimentally measured absolute values of the mass attenuation coefficient, or total photon interaction cross section for one or more elemental materials. The data from these documents had not been previously extracted for purposes of entering into the PCPDC $x$-ray attenuation measurement data base. Tables of the extracted data, with all photon energies converted to multiples of eV (electron volts), and all photon cross sections converted to units of barns/atom, are given. In some cases, photon wavelengths in angstroms or $\mathrm{nm}$ (nanometers) are given in the source documents instead of photon energies, in which case the reciprocal conversion to photon energies (in eV) has been made. A total of 3735 data points were extracted and are presented.

04,166

PB97-118657 Not available NTIS

National Inst. of Standards and Technology (PL), Gaithersburg, MD. Molecular Physics Div

Bimolecular Interactions in (Et)3SiOH:Base: $\mathrm{CCl} 4$ Hydrogen-Bonded Solutions Studied by Deactivation of the Free OH-Stretch Vibration.

Final rept.

W. T. Grubbs, T. P. Dougherty, and E. J. Heilweil. 1995, 4p.

Pub in Jnl, of the American Chemical Society, v117 n48 p11989-11992 1995.

Keywords: "Bimolecular reaction, "Hydrogen bonding, Complexes, Diffusing control, Infrared, Picoseconds, Reprints, Time-resolved spectroscopy, Vibrational relaxation.

Picosecond infrared (PI) pump-probe measurements of the $\mathrm{OH}$-stretch $(\mathrm{v}=1)$ population lifetime were performed for uncomplexes ( $\mathrm{Et}) 3 \mathrm{SiOH}$ present in dilute, room temperature, tertiary ( $\mathrm{Et}) 3 \mathrm{SiOH}$ :base: $\mathrm{CCl} 4$ hy drogen-bonded solutions (base = acetonitrile, tetrahydrofuran, and pyridine). When base is present in solution, the intrinsic $\mathrm{OH}$-stretch T1 vibrational population lifetime (183 plus or minus $6 \mathrm{ps}$ for $(\mathrm{Et}) 3 \mathrm{SiOH}$ in $\mathrm{CCl} 4$ ) is reduced by bimolecular ( $\mathrm{Et}) 3 \mathrm{SiOH}$ :base hydrogenbonding encounters. The base concentration dependence of the 'free' $\mathrm{OH}$-stretch vibrational deactivation rate is analyzed by a Stern-Volmer kinetic model and a least-squares fit to all the data yielded a single rate constant $k(B M)=1.2$ plus or minus $0.2 \times 10$ to the 10 th power $\mathrm{dm}$ to the 3rd power mole $(-1) \mathrm{s}(-1)$ for (Et)3SiOH:base bimolecular encounters. This vlaue is in agreement with estimate sfor the bimolecular diffusion limit, $k(B M)$ was found to be the same for all (Et)3SiOH:base interactions studied, suggesting that the bimolecular $\mathrm{OH}$-stretch deactivation mechanism is relatively insensitive to the proton-accepting strength of the base.

04,167

PB97-118699 Not available NTIS

National Inst. of Standards and Technology (MSEL), Gaithersburg, MD. Metallurgy Div.

Radiance Temperatures at $1500 \mathrm{~nm}$ of Niobium and Molybdenum at Their Melting Points by a PulseHeating Technique.

Final rept.

E. Kaschnitz, and A. Cezairliyan. 1996, 10p.

See also PB92-154095.

Pub. in International Workshop on Subsecond Thermophysics (4th), Koln, Germany, June 27-29, 1995. International Jnl. of Thermophysics, v17 n5 p1069-1078 Sep 96

Keywords: "Niobium, "Temperature measurement, Pulse heating, High temperature, Near infrared radiation, Visible radiation, Melting, Reprints, *Foreign technology, "Radiance temperatures. 


\section{PHYSICS}

\section{General}

Radiance temperatures at $1500 \mathrm{~nm}$ of niobium and molybdenum at their melting points were measured by a pulse-heating technique. The method is based on rapid resistive self-heating of the strip-shaped specimen from room temperature to its melting point in less than $1 \mathrm{~s}$ and measuring the specimen radiance temperature every $0.5 \mathrm{~nm}$ with a high-speed infrared pryometer. Melting of the specimen was manifested by a plateau in the radiance temperature-versus-time function. The melting-point radiance temperature for a given specimen was determined by averaging the measured values along the plateau. A total of 12 to 13 experiments was performed for each metal under investigation. The melting-point radiance temperatures for each metal were determined by averaging the results of the individual specimens.

\section{4,168}

PB97-118723 Not available NTIS

National Inst. of Standards and Technology (PL), Gaithersburg. MD. Radiometric Physics Div.

Microwave Spectrum and Structure of $\mathrm{CH} 2 \mathrm{O}-\mathrm{H} 2 \mathrm{O}$. Final rept.

F. J. Lovas, and C. L. Lugez. 1996, 4p

Pub. in Jnl. of Molecular Spectroscopy, Article No. 0210, v179 p320-323, 1996

Keywords: "Microwave spectrum, "Dipole moment, Dimer, Formaldehyde, Structure, Water, Reprints, *Formaldehyde-water complex, Rotational spectrum.

The microwave spectrum of the formaldehyde-water complex $(\mathrm{CH} 2 \mathrm{O}-\mathrm{H} 2 \mathrm{O})$ has been studied with a pulsedbeam Fourier transform Fabry-Perot cavity spectrometer. Both a-type and b-type transitions were observed for each of the isotopic species studied. To provide additional structural information, the spectra of $\mathrm{H} 2 \mathrm{O}$. $\mathrm{HDO}$, and D2O substituents combined with $\mathrm{H} 2 \mathrm{CO}$ and $\mathrm{D} 2 \mathrm{CO}$ were assigned. Measurement of the dipole moment components yielded the values micro $a=3.379(13) \times t 0$ to the minus 30 power C.m(micro $a=1.043(4) D)$ and micro $b=2.54(20) \times 10$ to the minus 30 power C.m (micro $b=0.76(6) \mathrm{D}$ ) and indicated that the dipole moment vectors are anti-aligned. The molecular structure derived from the moments of inertia has a center of mass separation of 2.00(3)Angstroms with the C2v symmetry axes of the monomers oriented at 19.3 degrees from parallel with the dipole moments opposed. The complex is quite strongly bound with a harmonic pseudodiatomic stretching force constant, $\mathrm{k} 2=8.93 \mathrm{~N} / \mathrm{m}$, and hydrogen bond lengths of approximately 2.68 Angstroms between the water oxygen atom and a $\mathrm{CH}_{2}$ hydrogen atom, and 2.03 Angstroms between a water hydrogen atom and the oxygen atom of $\mathrm{H} 2 \mathrm{CO}$. Expanded uncertainties (coverage factor $k$ $=2$ i.e., two standard deviations) are shown in parentheses for each experimental value reported above.

\section{4,169}

PB97-118756 Not available NTIS

National Inst. of Standards and Technology (PL), Gaithersburg, MD. Ionizing Radiation Div.

Colour Centres in LiF for Measurement of Absorbed Doses Up to 100 MGy.

Final rept.

W. L. McLaughlin. 1996, 4p

Pub. in Radiation Protection Dosimetry, v66 n1-4 p197-200 1996

Keywords: "Lithium fluoride, "Dosimeters, "Radiation damage, Measurements, Absorbed doses, Reprints.

Radiation damage studies on electronic components, polymers, composites, ceramics, metals, and insulating materials exposed to very large doses of ionizing photons and particles (i.e. electrons, protons, and neutrons) require reproducible and accurate passive dosimetry. Absorbed doses in the range 10 to the 2nd power $-2 \times 10$ to the 6 th power Gy have previously been measured with optical quality, undoped LiF by spectrophotometry of radiation-induced absorption bands of stable color centers. These include the relatively stable $F$ center (247 nm), $M$ center $(443 \mathrm{~nm})$, R1 center $(315 \mathrm{~nm})$, R2 center $(374 \mathrm{~nm})$, and $\mathrm{N} 2$ center (547 nm). It is now possible to extend this range to 10 to the 8th power Gy by spectrophotometry of the $N 1$ center $(517 \mathrm{~nm})$ and an ' $X$ ' Center $(785 \mathrm{~nm})$. These dosemeters can be used from cryogenic $(4 \mathrm{~K})$ to relatively high radiation temperatures (up to $500 \mathrm{~K}$ ). the radiation-induced coloration can be bleached for re. use of the dosemeters by a two hour thermal annealing treatment at $830 \mathrm{~K}$
National Inst. of Standards and Technology (PL), Gaithersburg, MD. Molecular Physics Div. Rotational Spectra of $\mathrm{CH} 3 \mathrm{CCH}-\mathrm{NH} 3, \mathrm{NCCCH}-\mathrm{NH} 3$ and $\mathrm{NCCCH}-\mathrm{OH} 2$

Final rept

R. M. Omron, A. R. Hight Walker, G. Hilpert, G. T. Fraser, and R. D. Suenram. 1996, 9p.

Pub. in Jnt. of Molecular Spectroscopy, v179 p85-93 1996.

Keywords: "Cyanoacetylene, "Hydrogen bonding "Methlacetylene, Microwave spectroscopy, Complexes, Reprints.

Microwave spectra of $\mathrm{NCCCH}-\mathrm{NH} 3, \mathrm{CH} 3 \mathrm{CCH}-\mathrm{NH} 3$ and $\mathrm{NCCCH}-\mathrm{OH} 2$ have been recorded using a pulsednozzle Fourier-transform microwave spectrometer The complexes NCCCH-NH3 are found to have symmetric-top structures with the acetylenic proton hydrogen-bonded to the nitrogen of the $\mathrm{NH} 3$. The data fo $\mathrm{CH} 3 \mathrm{CCH}-\mathrm{NH} 3$ are further consistent with free or nearly ree internal rotation of the methyl top against the ammonia top. For $\mathrm{NCCCH}-\mathrm{OH} 2$, and acetylenic proton is hydrogen-bonded to the oxygen of the water. The complex has a dynamical C2 structure, as evidenced by the presence of two nuclear-spin modifications of the complex. The hydrogen bond lengths and hydrogenbond stretching force constants are 2.212 Angstroms and $10.8 \mathrm{~N} / \mathrm{m}, 2.322$ Angstro $\mathrm{ms}$ and $6.0 \mathrm{~N} / \mathrm{m}$, and 2.125 Angstroms and $9.6 \mathrm{~N} / \mathrm{m}$, for $\mathrm{NCCCH}-\mathrm{NH} 3, \mathrm{CH} 3 \mathrm{CCH}$ $\mathrm{NH} 3$, and $\mathrm{NCCCH}-\mathrm{OH} 2$, respectively. For the cyanoacetylene complexes, these bond lengths and force constants lie between the values for the related hydrogen cyanide and acetylene complexes of $\mathrm{NH}$ and $\mathrm{H} 2 \mathrm{O}$. The $\mathrm{NH} 3$ bending and weak-bond stretching force constants of $\mathrm{CH} 3 \mathrm{CCH}-\mathrm{NH}_{3}$ are less than those found in $\mathrm{NCCCH}-\mathrm{NH} 3, \mathrm{NCH}-\mathrm{NH}_{3}$, and $\mathrm{HCCH}-\mathrm{NH} 3$, suggesting that the hydrogen bonding interaction is particularly weak in $\mathrm{CH} 3 \mathrm{CCH}-\mathrm{NH} 3$. The weakness of this hydrogen bond is partially a consequence of orientation of monomer electric dipole moments in the complex In $\mathrm{CH} 3 \mathrm{CCH}-\mathrm{NH} 3$ the antialigned monomer dipole moments leads to a repulsive dipole-dipole interaction energy, while in $\mathrm{NCH}-\mathrm{NH} 3$ and $\mathrm{NCCCH}$ $\mathrm{NH} 3$ the aligned dipoles give an attraction interaction

\section{4,171}

PB97-119028 Not available NTIS

National Inst. of Standards and Technology (PL) Gaithersburg, MD. Ionizing Radiation Div.

Effect of Formulation Changes on the Response to lonizing Radiation of Radiochromic Dye Films.

Final rept.

M. Barcelo, R. M. Uribe, W. L. McLaughlin, and G.

Belmont. 1996, 6p.

Pub. in Radiatio

Keywords: "Film dosimetry, "Gamma radiation, *Radiochromic films, Ultraviolet radiation, Radiation processing, Reprints, "Foreign technology Pararosaniline cyanide.

Radiochromic dye films have been studied with the purpose of optimizing the formulation in order to increase their sensitivity to ultraviolet and ionizing radiation. The films consist of certain substituted triphenylmethane leucodyes incorporated in an acid solution with polyacetal resins, which can be cast into thin colorless, tansparent films. Upon irradiation they become deeply colored due to dye formation (withou development); they are analyzed for dosimetry by absorption spectrophotometry at the visible wavelength of maximum absorption, in terms of increase of sponse functions were obtained for different films, in which the concentrations of each of the constituents the dye precursors, as well as the types of acids and solvents were varied. A new formulation is suggested for radiochromic dye films useful in the dose range from 5 kGy to $35 \mathrm{kGy}$

04,172
PB97-119051 Not available NTIS

National Inst. of Standards and Technology (PL) Gaithersburg, MD. Ionizing Radiation Div

Measurements of the $(235) \cup(n, f)$ Cross Section in the 3 to $30 \mathrm{MeV}$ Neutron Energy Region.

Final rept

A. D. Carlson, O. A. Wasson, P. W. Lisowski, J. L Ullmann, and N. W. Hill. 1991, 3p.

Pub. in Proceedings of the International Conference on Nuclear Data for Science and Technology, Julick FRG, May 13-17, 1991, p518-520.

Keywords: "Standard cross section, "Neutron energy, "Fission chamber, Telescopes, Measurements Fluence, Reprints, "Foreign technology
To improve the accuracy of the $235 U(n, f)$ cross section, measurements have been made of this standard cross section at the target 4 facility at Los Alamos $\mathrm{Na}$ tional Laboratory (LANL). The data were obtained at the 20 -meter flight path of that facility. The fission reaction rate was determined with a fast parallel plate ionization chamber and the neutron fluence was measured with an annular proton recoil telescope. The measurements provide the shape of the $235 \mathrm{U}(n, f)$ cross section relative to the hydrogen scattering cross section for neutron energies from about 3 to $30 \mathrm{MeV}$ neutron energy. The data have been normalized to the very accurately known value near $14 \mathrm{MeV}$. The results are in good agreement with the ENDF/B-VI evaluation up to about $15 \mathrm{MeV}$ neutron energy. Above this energy differences as large as $5 \%$ are observed.

\section{4,173}

PB97-119069 Not available NTIS

National Inst. of Standards and Technology (PL), Gaithersburg, MD. Ionizing Radiation Div.

Measurements of the $(237) \mathrm{Np}(\mathrm{n}, \mathrm{f})$ Cross Section. Final rept.

A. D. Carlson, W. E. Parker, P. W. Lisowski, N. W Hill, K. Meggers, G. L. Morgan, and S. J. Seestrom. 1994, 7p.

Sponsored by Department of Energy, Washington, DC. Pub. in American Society for Testing and MaterialsEuration Symposium on Reactor Dosimetry (8th), Vail, CO., August 29-September 3, 1993, p704-710 Dec 94.

Keywords: "Fission cross section, "Neutron scattering, "Dosimetry, Measurements, Resonance, Reprints.

Measurements have been made of the neutron induced fission cross section of $237 \mathrm{~Np}(\mathrm{n}, \mathrm{f})$ at the LoS Alamos Neutron Scattering Center utilizing a white neutron source and a fast parallel multiplate ionization chamber. The $10 \mathrm{~B}(\mathrm{n}, \mathrm{a})$ standard cross section was used to establish the energy dependence of the neutron fluence and a very accurately known fission resonance integral for $235 \mathrm{U}$ was used for normalization. The measurements in the resonance region, from $5 \mathrm{eV}$ to $5 \mathrm{keV}$, indicate that the ENDF.B-VI values are too low by about a factor of three.

\section{4,174}

PB97-119077 Not available NTIS

National Inst. of Standards and Technology (CSTL), Gaithersburg, MD. Thermophysics Div. Influence of Envelopes Geometry on the Sensitivity of 'Nude' Ionization Gauges.

Final rept.

A. R. Fillippelli. 1996, 5p.

Pub. in Jnl. of Vacuum Science and Technology A, v14 n5 p2953-2957 Sep/Oct 1996.

Keywords: "Envelopes, "Ionization gauges, "Extractors, Nude, Sensitivity, Reprints, Bayard-Alpert.

This article presents the results of some measurements of the influence of envelope size and shape on the N2 sensitivity of two nominally identical 'nude' extractor ionization gauges, and three common 'nude' versions of the Bayard-Alpert ionization gauge. Measurement were made over the pressure range 10 to the minus 7 power - 10 to the minus 1 power $\mathrm{Pa}$, using typical gauge operating parameters and with vacuum chamber and gauge envelope at ground potnetial. Sensitivity values corresponding to three different envelopes used in this work differed by as much as a factor of 2 (comparing maximum value to minimum value for each gauge) for the Bayard-Alpert gauges. For the extractor gauges, the diferences were as large as $7 \%$.

\section{4,175}

PB97-119085 Not available NTIS

National Inst. of Standards and Technology (PL), Gaithersburg, MD. Ionizing Radiation Div.

Mass Assay and Uniformity Test of Boron Targets by Neutron Beam Methods.

Final rept.

D. M. Gilliam, G. P. Lamaze, M. S. Dewey, and G. L. Greene. 1993, 5p

Pub. in Nuclear Instruments and Methods in Physics Research A, v334 p149-1531993.

Keywords: "Ionization chambers, "Neutron beams, "Boron targets, Deposits, University, Vacuum evaporation, Reprints.

The areal densities $(\mathrm{g} / \mathrm{cm} 2)$ of enriched boron and lithium fluoride targets were compared to those of reference deposits from the Central Bureau of Nuclear Measurements (CBNM, Geel, Belgium) by chargedparticle counting at the NIST Research Reactor. The 
uniformity of the target thicknesses was also investigated by scans with a small-diameter, intense neutron beam. The neutron uniformity measurements agree satisfactorily with previously reported interferometric results and no pattern of uniformity variation except the expected, small radial dependence was found. In a separate series of measurements, the boron areal density ratios of a number of the deposits were determined using a new double ionization chamber. The new double ionization chamber permits a comparison of two targets directly without a beam monitor and withou sensitivity to the exact placement of the samples. The new device needs only a very simple data acquisition system and requires less neutron beam intensity than the previously employed systems with arrays of surface barrier detectors. The ionization chamber counting losses from the combined effects of self-absorption and sub-threshold pulse-height formation were determined by comparison with ratio measurements made by the conventional surface barrier detector technique.

04,1776

PB97-119275 Not available NTIS

National Inst. of Standards and Technology (MSEL) Gaithersburg, MD. Reactor Radiation Div.

Orientational Fluctuations, Diffuse Scattering, and Orientational Order in Solid C60.

Final rept.

K. H. Michel, and J. R. D. Copley. 1996, 4p.

Pub. in Proceedings of the International Winterschoo on Electronic Properties of Novel Materials Fullerenes and Fullerene Nanostructures, p1-4 1996, World Scientific

Keywords: "Diffuse scattering. "Mode coupling, *Fluctuations, "Orientational disorder, Scattering cross section, Carbon, Reprints, *Foreign technology.

The authors present a theory of the orientational dynamics of solid $\mathrm{C} 60$ which takes into account the cou pling of orientational modes belonging to various symmetries and manifolds. More coupling strongly enhances the transition temperature and the discontinuity of the order parameter at the first order phase transition to the Pa3 structure. The diffuse scattering intensity in the disordered phase is calculated and com pared with experiments

04,177

PB97-122345 Not available NTIS

National Inst. of Standards and Technology (CSTL), Gaithersburg MD Process Measurements Div.

Generalized Optical Theorem for On-Axis Gaussian Beams.

Final rept.

G. Gouesbet, C. Letellier, G. Grehan, and J. T.

Hodges. 1996, 21p.

Pub. in Optics Communications, v125 p137-157 1996.

Keywords: "Optical theorems, "Beams, "Gaussian beams, Plane waves, Finite scatters, Reprints, "Foreign technology

The optical theorem for an incident plane was interacting with a finite scatterer is generalized to the case of on-axis Gaussian beams, taken as examples of shaped beams. The results are restricted in the pape o homogeneous spheres. The beams are defined by using (1) the standard description (2) the localized approximation and (3) the modified localized approximation. Rigorous expressions generalizing the classical plane wave case expression are given and numerically discussed. A recently proposed approximation is investigated in order to point out assumptions underlying its validity.

04,178
PB97-122360 Not available NTIS

National Inst. of Standards and Technology (PL), Gaithersburg, MD. Atomic Physics Div.

Measurement of the Atomic $\mathrm{Na}(3 \mathrm{P})$ Lifetime and of Retardation in the Interaction between Two Atoms Bound in a Molecule.

Final rept.

K. M. Jones, P. S. Julienne, P. D. Lett, C. J.

Williams, W. D. Phillips, and E. Tiesinga. 1996, 5p.

Pub. in Europhysics Letters, v35 n2 p85-90 1996.

Keywords: "Atomic interactions, "Lifetimes, "Retardation, Atoms, Molecules, Measurements, Reprints, "Foreign technology.

From molecular spectroscopy of the $\mathrm{Na} 2$ purely longrange $\mathrm{O}-\mathrm{g}$ state the authors determine the $\mathrm{Na}(3 \mathrm{P})$ lifetime and measure the predicted but previously unobserved effect of retardation in the interaction be- tween two atoms. The lifetime tau(P3/2) $=16.230(16)$ ns helps to remove a longstanding discrepancy between experiment and theory. Electron cloud overlap is unimportant in the $\mathrm{O}-\mathrm{g}$ state ( $\mathrm{R}$ greater than 55ao) and the spectrum is calculated, ab initio, from atomic properties. By measuring the binding energies the 120 $\mathrm{MHz}$ correction due to retardation of the resonantdipole R-3 interaction is confirmed.

\section{4,179}

PB97-122386 Not available NTIS

National Inst. of Standards and Technology (BFRL), Gaithersburg, MD. Building Environment Div.

Single-Phase Heat Transfer and Pressure Drop Characteristics of an Integral-Spine Fin Within an Annulus.

Final rept.

M. A. Kedzierski, and M. S. Kim. 1996, 10p.

See also PB94-194073.

Pub. in Jnl. of Enhanced Heat Transfer, v3 n3 p201 2101996.

Keywords: *Fins, "Heat transfer, "Pressure gradients, "Annular flow, Nusselt number, Friction factor, Hea transfer coefficients, Mathematical models, Test facilities, Reprints.

The laminar, single-phase heat transfer and friction characteristics of a porcupine-like surface (integralspine fin) within an annulus are presented. The heat transfer coefficient was determined using a modified version of the Wilson Plot method on a $3 \mathrm{~m}$ test sec tion. Three fluids were investigated: (1) water, (2) $34 \%$ ethylene glycol/water mixture, and (3) $40 \%$ ethylene glycol/water mixture. These fluids produced a sufficien variation in the Prandtl number, so that its exponential dependence could be determined. The annulus Reynolds numbers were varied from 100 to 1400 to obtain the Reynolds number exponent. An empirical correlation for the Nusselt number that accounts for the deveopment of the thermal boundary layer is presented. An empirical correlation for the Fanning friction factor is also provided. It is shown that the spines enhance the heat transfer through additional surface area and fluid mixing.

04,180

\section{PB97-122436 Not available NTIS}

National Inst. of Standards and Technology (PL) Gaithersburg MD lonizing Radiation Div.

Complex Time Dependence of the EPR Signal of Irradiated L-alpha-alanine.

Final rept.

V. Y. Nagy, and M. F. Desrosiers. 1996, 5p.

Pub. in Applied Radiation Isotopes, v47 n8 p789-793 1996

Keywords: "Alanine, *EPR signal, Irradiation, Amplitude, Measurements, Reprints, "Foreign technology, ${ }^{\star}$ Complex time dependence.

Measurements of the EPR signal amplitude of gammairradiated L-alpha-alanine with use of an adjacent reference sample have revealed variations in the signal intensity within hours and days after irradiation. The character of the time dependence of the amplitude varies with dose and the amplitude changes reach 1 $1.5 \%$. This observation favors the hypothesis that irradiated alanine contains several paramagnetic centers. Usefulness of adjacent reference samples in alanine dosimetry is also demonstrated.

\section{Acoustics}

04,181

PB94-172137 Not available NTIS

National Inst. of Standards and Technology (MEL) Gaithersburg, MD. Automated Production Technology Div.

Ultrasonic Measurements of Surface Roughness.

Final rept

G. V. Blessing, J. A. Slotwinski, D. G. Eitzen, and H. M. Ryan. 1 Jul $93,5 p$

Pub. in Applied Optics 32, n19 p3433-3437, 1 Jul 93.

Keywords: "Surface roughness, "Ultrasonic tests, *Profilometers, Wave propagation, Sound transmission, Test methods, Surface properties, Real time, Process control, Acoustic properties, Scattering, Reflection, Reprints.

Pulsed ultrasound propagating in water was used a megahertz carrier frequencies (nominally 10-50 MHz) to reflect and scatter from rough surfaces in the same way as light. We have considered noncontact ultrasonic techniques as complementary to optical techniques in several ways: (a) for specific applications such as wet surfaces, (b) for rougher surfaces with average roughness, $\mathrm{Ra}>$ or $=0.1$ micrometer, and (c) for (simultaneous) profilometry by time-of-flight measurements. Stylus and ultrasonic data are compared. An example of application to the manufacturing environment is for on-line, real-time sensor feedback and process control in the cutting or grinding of metals and ceramics.

04,182

PB94-199007 Not available NTIS

National Inst. of Standards and Technology (MSEL), Boulder, CO. Materials Reliability Div.

Absorption of Ultrasonic Waves in Air at High Frequencies (10-20 MHz).

Final rept.

L. J. Bond, C. H. Chiang, and C. M. Fortunko 1992 $10 p$.

Pub. in Jnl. Acoust. Soc. Am. 92, n4 p2006-2015 Oct 92

Keywords: *Ultrasonic radiation, "Acoustic absorption, *Air, $\mathrm{MHz}$ range 01-100, Acoustic measurement, Acoustic velocity, Acoustic waves, High frequency, Room temperature, Reprints.

A high power and sensitive gated radio-frequency (if) measurement system has been used to determine the absorption of compressional waves in air, in the frequency range from 10-20 $\mathrm{MHz}$. The measured absorption, between 80 and $90 \mathrm{kPa}$ and at room temperature ( $15 \mathrm{deg} C$ to $25 \mathrm{deg} C$ ), has been found to be in good agreement with that predicted, by extrapolation, from low-frequency (below $1 \mathrm{MHz}$ ) data for the combination of the classical and rotational loss effects. The velocity of ultrasound in air in the frequency range from 10-18 $\mathrm{MHz}$ has also been measured and is in good agreement with that predicted from theory. Normalized velocity and attenuation data plotted against Knudsen numbers and a rarefaction parameter are in good agreement with those obtained at reduced pressure and lower frequencies.

04,183

PB94-199015 Not available NTIS

National Inst. of Standards and Technology (MSEL),

Boulder, CO. Materials Reliability Div.

Absorption of Sound in Gases between 10 and 25 MHz: Argon.

Final rep

L. J. Bond, C. H. Chiang, C. M. Fortunko, and J. D. McColskey. 1991, 5p.

Pub. in Proceedings of Ultrasonics Symposium, Orlando, FL., December 5-8, 1991, p1069-1073.

Keywords: "Ultrasonic radiation, "Acoustic absorption, *Argon $\mathrm{MHz}$ range 01-100, Acoustic measurement, Acoustic velocity, Temperature dependence, Pressure dependence, High frequency, Reprints

A measurement system has been developed to investigate the technology required for high-power gas-coupled ultrasonics and the phenomenology of finite-amplitude of ultrasonic waves in gases at high frequencies (> $10 \mathrm{MHz}$ ) and pressures up to $10 \mathrm{MPa}$ (100 bar) In this paper, results of measurements of compression wave absorption and velocity in argon between 10 and $25 \mathrm{MHz}$, with various combinations of pressure, temperature and power level are presented. For small-amplitude waves, the resulting data are in good agreement with that calculated using ideal gas equations and fundamental thermophysical gas constants. At higher power levels, non-linear phenomena are observed.

04,184

PB94-199023 Not available NTIS

National Inst. of Standards and Technology (MSEL), Boulder, CO. Materials Reliabillty Div.

interaction of Rayleigh Waves with a Rib Attached to a Plate.

Final rept.

L. J. Bond, and J. Taylor. 1991, 8p.

Pub. in Ultrasonics 29, p451-458 Nov 91

Keywords: "Rayleigh waves, "Wave propagation, *Acoustic emissions, Plates, Ribs, Surface acoustic waves, Models, Finite difference method, Interactions, Reprints.

An analysis of the interaction of a pulse of Rayleigh waves on a plate with a rectangular flange or rib at- 
tached in the far field of its acoustic emission (AE) source is presented. A finite difference (FD) model is used to give numerical visualizations of the development of the complete wave-field propagating through the structure and also to give displacement data for selected points. An 'energy partition model', for Rayleigh wave propagation across the rib has been developed and is described. Ray theory wave arrival time calculations are given for the propagation of various modeconverted components transmitted across the flange. A series of experimental measurements of $A E$ wave propagation across a T-butt welded rib on a plate were made and these are compared with the model predictions. The practical implications of these findings for acoustic emission monitoring of large structures of complex shape are discussed.

\section{4,185}

PB94-212537 Not available NTIS

National Inst. of Standards and Technology (MSEL), Gaithersburg, MD. Metallurgy Div.

Ultrasonic Spectroscopy of Metallic Spheres Using Electromagnetic-Acoustic Transduction.

Final rept.

W. L. Johnson, S. J. Norton, F. Bendec, and R.

Pless. 1992, 6p.

Pub. in J. Acoust. Soc. Am. 91, n5 p2637-2642 May 92

Keywords: "Ultrasonic spectroscopy, Vibrational spectra, Resonant frequency, Magnetic fields, Elastic properties, Aluminum, Spheres, Reprints.

An ultrasonic technique for studying vibrational resonant modes of metallic spheres is presented. The technique employs electromagnetic-acoustic transduction with a configuration consisting of a sample surrounded by a coil in a static magnetic field. Resonance spectra from 0.5 to $4.5 \mathrm{MHz}$ with the coil axis parallel and perpendicular to the magnetic field are measured for a $3.145-\mathrm{mm}$-diam sphere of polycrystalline 2024 aluminum. Elastic constants calculated from the resonant peak frequencies are consistent with results obtained using an ultrasonic pulse-echo system. This new technique has advantages over pulse-echo and conventional resonance techniques for experiments where high absolute accuracy is necessary or where samples are heated far above room temperature.

\section{4,186}

PB95-151957 Not available NTIS

National Inst. of Standards and Technology (CSTL), Boulder, CO. Thermophysics Div.

Speed-of-Sound Measurements in Liquid and Gaseous Air.

Final rept.

B. A. Younglove, and N. V. Frederick. 1993,9p.

Pub. in International Jnl. of Thermophysics 13, n6 p1033-1041 Nov 92

Keywords: "Acoustic velocity, "Acoustic resonators "Air, "Acoustic measurements, "Time of flight spectrometers, Sound waves, Sound transmission, Isotherms, Vapor phases, Reprints, *Liquified air, AIRPROPS model.

The speed of sound in air has been measured along isotherms for a 'standard air' mixture $(0.7811$ N2 + $0.2097 \mathrm{O} 2+0.0092 \mathrm{Ar}$ ) in the gas and liquid phases at pressure to $14 \mathrm{MPa}$. A cylindrical resonator was used in the vapor and supercritical gas phases, and a time-of-flight system was used for measurements of the liquid phase. Data were obtained for the liquid phase at $90,100,110,120$, and $130 \mathrm{~K}$. Data were taken at $110,120,130,135,140,150,200$, and 300 $K$ in the vapor and supercritical gas phases. These experimental results were compared to a predictive computer model, namely, AIRPROPS.

\section{4,187}

PB95-162905 Not available NTIS

National Inst. of Standards and Technology (MSEL), Gaithersburg, MD. Metallurgy Div.

Leady Axisymmetric Modes in Infinite Clad Rods. Part 1.

Final rept.

J. A. Simmons, E. Drescher-Krasicka, and H. N. G.

Wadley. 1992, 30p

Pub. in Jnl. of the Acoustical Society of America 92, n2 p1061-1090 1992.

Keywords: "Waves, "Clad metals, "Rods, *Computation, Composite materials, Reinforcing materials, Wave propagation, Phase velocity, Nondestructive tests, Aluminum, Silicon carbides, Re- prints, Leaky waves, Clad rods, Radial-axial modes, Ultrasonic waves, Interface waves.

A detailed computational study is presented for the radial-axial modes-both leaky and non-leaky-in an infinitely clad isotropic rod. The complex phase velocities of leaky modes are located using an application of the argument principle. Particle orbits are determined, and leaky modes are shown to have an asymptotic leakage angle away from the interface. By using the homotopic methods of varying densities and elastic constants clad-rod modes are compared with those in a bare rod. The topology of the clad-rod mode dispersion diagram differs qualitatively from that of a bare rod, even when the cladding has negligible density, with no velocity cutoffs and with wave mode knitting. Comparison is also given with modes occuring in a cladding without a rod present (a tunnel) and for a planar interface. Most leaky modes can be correlated with rod modes; only a limited number of tunnel modes exist. Energy flow contours within modes are also calculated. The local energy velocity, which generalizes group velocity, can vary considerably in the radial direction for bare rod modes. For leaky modes the contours are quite complex due to the cylindrical geometry, giving rise to apparent shifts in wave position across the interface.

\section{4,188}

\section{PB95-182234 PC A05/MF A01}

National Inst. of Standards and Technology (TS), Gaithersburg, MD. National Voluntary Lab. Accreditation Program.

National Voluntary Laboratory Accreditation Program Acoustical Testing Services.

Handbook.

P. R. Martin, and R. J. Peppin. Dec 94, 88p, NIST/ HB-150-8.

Also available from Supt. of Docs. as SN003-003 03314-6. See also PB91-107524. Sponsored by Nuclear Regulatory Commission, Rockville, MD.

Keywords: "Acoustic testing, "Acoustic measurement, "Laboratories, Acoustic absorption, Acoustic attenuation, Sound transmission, Noise reduction, Performance evaluation, Test methods, Ear protection, Hearing. Calibration, National Voluntary Laboratory Accreditation Program, NVLAP program.

NIST Handbook 150-8 presents the technical requirements of the National Voluntary Laboratory Accreditation Program (NVLAP) for Acoustical Testing Services It is intended for information and use by staff of accredited laboratories, those laboratories seeking accreditation, other laboratory accreditation systems, users of laboratory services, and others needing information on the requirements for accreditation under this program. This publication supplements NIST Handbook 150 NVLAP Procedures and General Requirements, which contains Part 285 of Title 15 of the U.S. Code of Federal Regulations (CFR) plus all general NVLAP procedures, criteria, and policies. Handbook $150-8$ contains information that is specific to the Acoustical Testing Services program and does not duplicate information contained in the Procedures and General Requirements. It is organized to cross-reference with Handbook 150; for example, Section 285.3 of Handbook 150 presents the description and goal of NVLAP, whereas Section 285.3 of Handbook $150-8$ presents the description of the Acoustical Testing Services program. Where there is no material specific to the field of accreditation, the section number is omitted.

\section{4,189}

\section{PB96-102413 Not available NTIS}

National Inst. of Standards and Technology (CSTL), Gaithersburg, MD. Thermophysics Div.

Thermodynamic Properties of Two Gaseous Halo. genated Ethers from Speed-of-Sound Measurements: Difluoromethoxy-Difluoromethane and 2 . Difluoromethoxy-1,1,1-Trifluoroethane.

Final rept.

K. A. Gillis. 1994, 27p

Contracts N0002490MP33493, DW13935108-01-0

Sponsored by Department of the Navy, Washington DC. and Environmental Protection Agency, Washington, DC

Pub. in International Jnl. of Thermophysics 15, n5 p821-847 Sep 94.

Keywords: $\quad$ Thermodynamic properties, "Halohydrocarbons, *Ethers, *Gases, "Acoustic velocity, Specific heat, Ideal gas, Virial coefficients, Intermolecular forces, Reprints, Difluoromethoxy-
difluoromethane, trifluoroethane.

The authors present measurements of the speed of sound in gaseous difluoromethoxy-difluoromethane
(CHF2-O-CHF2) and 2-difluoromethoxy- 1,1,1trifluoroethane (CF3-CH2-O-CHF2). These measurements were performed in an all-metal apparatus between 255 and $384 \mathrm{~K}$. The authors have obtained ideal-gas heat capacities and second acoustic virial coefficients from analysis of these measurements. Two methods of correlating the second acoustic virial coefficients, a squarewell model of the intermolecular interaction and a function due to Pitzer and Curl, are presented.

\section{4,190}

\section{PB96-204508 Not available NTIS}

National Inst. of Standards and Technology (MEL), Gaithersburg, MD. Automated Production Technology Div.

Determination of Acoustic Center Correction for Type LS2aP Condensor Microphones.

Final rept.

R. P. Wagner, and V. Nedzelnitsky. 1995, 1p.

Pub. in Jnl. of the Acoustical Society of America, v98 n5 pt2 p2917 Nov 95.

Keywords: "Acoustical instruments, "Microphones, Reprints, Calibration, Acoustic center.

Acoustic center corrections for microphones are necessary for accurate free-field calibration by the reciprocity technique. Such correction for several pairs of the relatively new type LS2aP microphone (IEC Std. 1094-1: 1992) were obtained by utilizing the theoretical inverse relationship between the sound pressure amplitude at the receiving microphone and the distance between the acoustic centers of the source and receiving microphones. For a nominally $10-\mathrm{V}$ sinusoidal rms excitation of the source microphone, source-to-receiver voltage ratios were measured with a dynamic signal analyzer at $500 \mathrm{~Hz}$ intervals in the extended frequency range ( 2 to 50$) \mathrm{kHz}$. This procedure allowed all the data for a microphone pair to be gathered within several hours as a function of microphone diaphragm separation at $10-\mathrm{mm}$ intervals from 101 to $311 \mathrm{~mm}$. At each frequency, these ratios were corrected for atmospheric effects, including attenuation of sound, and then fit to a straight line (ratio versus diaphragm separation) Acoustic center corrections were calculated from the derived values of the fit parameters. These corrections agree with appropriately scaled values (IEC Publication 486-1974) for 'I-in.' microphones with recessed diaphragms. Physical phenomena that cause small deviations from the linear fits will be discussed along with uncertainty estimate, and effects of spatially truncating data.

04,191

PB'96-204516 Not available NTIS

National Inst. of Standards and Technology (MSEL), Gaithersburg, MD. Ceramics Div.

Thermal Wave NDE of Advanced Materials Using Mirage Effect Detection.

Final rept.

L. Wei, and G. S. White. 1993, 8p

Pub. in Proceedings of the International Conference on Photoacoustics, Maine, 1993, p1-8

Keywords: "Meetings, *Mirage detection, "Therma waves, Reprints, Advanced materials, Nondestructive evaluation, Thermal property.

The dramatic improvement in modern materials has driven the search for improved measurement techniques, for both the detection of flaws which will pre vent or limit applications and for the evaluation of basic physical parameters of the materials themselves. The mirage thermal wave technique has been used successfully to meet both requirements. Because thermal waves are sensitive to changes in the heat flow in a material, the thermal wave technique is complementary to techniques such as ultrasonics and light scattering measurements, which are sensitive to changes in elastic and optical properties, respectively.

\section{Fluid Mechanics}

\section{4,192}

AD-A278 249/8 PC A03/MF A01

National Bureau of Standards, Gaithersburg, MD Characteristics of Turbulence in a Boundary Layer with Zero Pressure Gradient.

P. S. Klebanoff. 1955,22 p, NASA-L-1247.

Keywords: "Turbulent boundary layer, "Pressure gra dients, Turbulent flow, Zero pressure gradients. 
No abstract available

\section{4,193}

AD-A286 680/4 PC A04/MF A01

National Bureau of Standards, Gaithersburg, MD. Survey of the Literature on Heat Transfer from Solid Surfaces to Cryogenic Fluids.

Technical note

R. J. Richards, W. G. Steward, and R. B. Jacobs Oct $61,51 \mathrm{p}$.

Keywords: "Cryogenics, "Fluids, "Heat transfer, "Solids, "Surfaces, " Low temperature, "Bibliographies, Computations, Formulations, Literature surveys, Liquid helium, Liquid hydrogen, Liquid oxygen, Convection, Nucleate boiling.

A bibliography of 156 references on heat transfer from solid surfaces to fluids and related phenomena is presented. Heat transfer data obtained from experimental work on cryogenic fluids are presented in graphical form. The theoretical and empirical formulations appearing in the references are presented. In those cases where sufficient information is available to make numerical computations, the formulations are presented graphically to permit comparison with the results of the experimental work.

\section{4,194}

\section{AD-A297 391/5 PC A03/MF A01}

National Bureau of Standards, Gaithersburg. MD.

Air Flow in the Boundary Layer of an Elliptic Cylinder

G. B. Schubauer. 1939, 25p, NBS-652.

Document partially illegible.

Keywords: "Air flow, "Boundary layer, Velocity, Position(Location) Intensity Turbulence, Turbulent flow, Turbulent boundary layer, Variations, Cylindrical bodies, Scale, Airspeed, Transitions, Hot wire, Ellipses.

The boundary layer of an elliptic cylinder of major and minor axes 11.78 and 3.98 inches, respectively, was investigated in an air stream in which the turbulence could be varied. Conditions were arranged so that the flow was two-dimensional with the major axis of the ellipse parallel to the undisturbed stream. Speed distributions across the boundary layer were determined with a hot-wire anemometer at a number of positions about the surface for the lowest and highest intensities of turbulence, with the air speed in both cases sufficiently high to produce a turbulent boundary layer over the downstream part of the surface. The magnitude and the frequency of the speed fluctuations in the boundary layer were also measured by the use of the conventional type of hot-wire turbulence apparatus. Stream turbulence was found to affect both the nature of transition from to turbulent flow in the layer and the position on the surface at which transition occurred. Transition was then investigated in detail with stream turbulence of several different scales and intensities. It was found that the position of transition could be ex pressed as a function of the intensity divided by the fifth root of the scale. (KAR) P.5.

\section{4,195}

\section{DE94017817 PC A02/MF A01}

National Inst. of Standards and Technology (NEL), Gaithersburg, MD. Thermophysics Div.

Ebulliometers for Measuring the Thermodynamic Properties of Fluids and Fluid Mixtures.

L. A. Weber, and A. M. Silva. 1994, 8p, CONF-

$9404137-5$

Contract Al05-88ER13823

Symposium on Energy Engineering Sciences (12th), Argonne, IL (United States), 27-29 Apr 1994. Sponsored by Department of Energy, Washington, DC.

Keywords: "Boiling Points, *Fluids, "Temperature Measurement, "Thermodynamic properties, Design, Measuring Instruments, Measuring Methods, Mixtures, Vapor Pressure. EDB/440500.

The design and operation of two ebulliometers is described. One is constructed of glass and is used for measuring vapor pressures of fluids at low reduced temperatures and pressures. The other is constructed of metal. It can be used for vapor pressure measurements, and also for the study of fluid mixture thermodynamics through the determination of the activity coefficients at infinite difution. The advantages and potential problems associated with ebulliometers are described, and typical results are given for the properties of alternative refrigerants.
04,196

PB94-160736 PC A04/MF A01

National Inst. of Standards and Technology (CSTL), Gaithersburg, MD. Process Measurements Div. Summary Report of NIST's Industry-Government Consortium Research Program on Flowmeter Installation Effects with Emphasis on the Research Period, January-September 1991: The Reducer. G. E. Mattingly, and T. T. Yeh. Feb 92, 58p, NISTIR4779

See also PB92-149848 and PB94-149855.

Keywords: "Flow meters, "Pipe flow, Flow measurement, Turbulence, Reynolds number, Velocity distribution, Orifice meters, Orifices, Pipes(Tubes), Research programs.

This report presents recent results obtained in a consortium-sponsored research program on flowmeter in stallation effects being conducted at NISTGaithersburg, MD. The objective of this research program is to produce improved flowmeter performance when meters are installed in non-ideal conditions. Ideal meter installation conditions are those where long straight lengths of constant diameter piping precede the meter locations. Actual installations seldom conform to these conditions. This research effort has also included experimental studies of the flow into and out of several tube bundle flow conditioners. These results have produced, for the first time, detailed descriptions of the effects these devices have on swirling pipe flows. Specifically, this report contains measurements of the pipeflows produced downstream of the conventional reducer. Results are given for the performance characteristics of a range of orifice meter geometries and a specific turbine meter in these pipe flows. Also included are the velocity profile measurements downstream of the tee used as an elbow and several arrangements of tube bundles installed downstream of the single elbow and the tee used as an elbow.

\section{4,197}

PB94-198405 Not available NTIS

National Inst. of Standards and Technology (CSTL) Boulder, CO. Thermophysics Div

Transient Methods for Thermal Conductivity.

Final rept.

M. J. Assael, C. A. Nieto de Castro, H. M. Roder, and W. A. Wakeham. 1991, 32p.

Pub. in Measurement of the Transport Properties of Fluids, Chapter 7, p163-194 1991.

Keywords: "Thermal conductivity, "Transport properties, "Hot wire "Fluids, "Measuring instruments, ACcuracy, Reliability, Thermophysical properties, Temperature measurement, Reprints.

The chapter summarizes the transient hot-wire technique for the measurement of thermal conductivity of fluids. A description is given for specific examples of instruments of proven reliability for both electrically conducting and electrically non-conducting fluids. The theory and working equations of the method are given, as is the accuracy of the technique.

\section{4,198}

\section{PB94-211380 Not available NTIS}

National Inst. of Standards and Technology (CSTL),

Boulder, CO. Thermophysics Div.

Conditions for Existence of a Reentrant Solid Phase in a Sheared Atomic Fluid.

Final rept.

D. J. Evans, S. T. Cui, H. J. M. Hanley, and G. C

Straty. $1992,4 p$.

Sponsored by Department of Energy, Washington, DC.

Pub. in Physical Review A 46, n10 p6731-6734, 15 Nov 92

Keywords: Small angle scattering, Neutron scattering, Molecular dynamics, Suspensions, Simulation, Shear rate, Reprints, *Atomic fluids.

A nonequilibrium molecular-dynamics method is proposed to simulate the behavior of simple fluids under high shear rates. A thermostat that makes no assumptions regarding the form of the streaming velocity of the fluid is introduced. Simulations are carried out for a Weeks-Chandler-Andersen dense liquid. With the form of the streaming velocity partially or wholly constrained, we find evidence for a reentrant solid or string phase, but the phase disappears if constraints are removed. Small-angle neutron-scattering data from a sheared colloidal suspension are presented. There is no evidence of a steady-state shear-induced structure in the suspension.
04,199

PB94-212420 Not available NTIS

National Inst. of Standards and Technology (CSTL) Gaithersburg, MD. Thermophysics Div.

Global Thermodynamic Behavior of Fluid Mixtures in the Critical Region.

Final rept.

G. X. Jin, S. Tang, and J. V. Sengers. 1993, 15p

Contract DE-FG05-88ER13902

Sponsored by Department of Energy, Washington, DC. Pub. in Physical Review E 47, n1 p388-402 Jan 93.

Keywords: "Fluids, "Thermodynamic properties, ${ }^{*}$ Mixtures, ${ }^{*}$ Critical point, Equations of state, Free energy, Ethane, Carbon dioxide, Thermophysical properties, Reprints.

In a previous publication (Z. Y. Chen, A. Abbaci, S. Tang and J V Sengers Phys Rev. A 42, 4470 (1990)) a renormalized Landau expansion was constructed for the thermodynamic free energy of onecomponent fluids that incorporates the crossover from singular thermodynamic behavior at the critical point to regular behavior far away from the critical point. In the present paper the approach is extended to obtain a crossover free energy for binary fluid mixtures in the region around the vapor-liquid critical line. The thermodynamic equations thus obtained are compared with experimental equation-of-state and specific-heat data for mixtures of carbon dioxide and ethane.

\section{4,200}

PB94-212818 Not available NTIS

National Inst. of Standards and Technology (NML) Gaithersburg, MD. Chemical Process Metrology Div. Evolution of a Turbulent Boundary Layer Induced by a Three-Dimensional Roughness Element. Final rept.

P. S. Klebanoff, W. G. Cleveland, and K. D.

Tidstrom. 1992, 87p

Sponsored by Calspan Field Services, Inc., Arnold AFS, TN. AEDC Div.

Pub. in Jnl. of Fluid Mechanics 237, p101-187 Apr 92.

Keywords: "Turbulent boundary layer, "Boundary layer transition, "Vortex generators, "Roughness, Reynolds number, Vortices, Unsteady flow. Steady flow. Three dimension flow, Vortex shedding, Reprints

An experimental investigation of the transition from a laminar to a fully-developed turbulent boundary layer induced by a three dimensional roughness element in a zero pressure gradient boundary layer, incorporating an extensive bibliography, is described. The critica roughness Reynolds number at which turbulence is regarded as originating at the roughness was determined for the roughness elements herein considered and evaluated in the context of data existing in the literature. The effect of a steady and oscillatory free stream velocity on eddy shedding was also investigated and whether the eddy shedding is governed by an inflectional instability is examined. Distributions of mean velocity and turbulence intensity demonstrating the evolution toward a fully developed turbulent bound ary layer were measured at various Reynolds numbers. A two region model is postulated for the evolutionary change toward a fully developed turbulent boundary layer: an inner region where the turbulence is generated by the complex interaction near the surface of hairpin eddies with pre-existing stationary vortces, and an outer region where the hairpin eddies deform and generate turbulent vortex rings. The structure of the resulting fully developed turbulent boundary layer is discussed in the light of the proposed model for the evolutionary process.

\section{4,201}

PB95-105540 PC A05/MF A0

National Inst. of Standards and Technology (CSTL), Gaithersburg, MD. Process Measurements Div.

Flow Conditioner Tests for Three Orifice Flow. meter Sizes.

Technical note.

J. L. Scott, and M. A. Lewis. May 94, 87p, NIST/TN1367.

Also available from Supt. of Docs. as $\mathrm{SNOO}^{-003}$ 03287-5. Sponsored by Gas Research Inst., Chicago,

Keywords: "Orifice flow, "Flowmeters, Flow measurement, Discharge coefficient, Flow resistance, Orifices, Pipe flow, Turbulent flow, Reynolds number, "Flow conditioners, Beta ratio.

Tests sponsored by the Gas Research Institute were performed with orifice flowmeters of three nominal 


\section{Fluid Mechanics}

sizes: 52,104 and $154 \mathrm{~mm}$ (2, 4, and 6 in). Tests with the $52 \mathrm{~mm}$ orifice meter included establishing a baseline curve for 6 beta ratios and then conducting flow conditioner performance tests with an elbow located 17 pipe diameters (17D) upstream of the orifice plate. Results from this laboratory indicate that beta ratios of 0.54 or less do not require flow conditioning in this configuration and the 19 tube bundle flow conditioner at 12D produces the best results for larger beta ratios For the $104 \mathrm{~mm}$ orifice meter in the same piping configuration, flow conditioning is necessary for beta ratios of 0.55 and greater. Meter performance is less sensitive to the location of the 7 tube bundle than the 19 tube bundle flow conditioner, but the influence of the flange tape location is greater. Testing of two beta ratios in the $154 \mathrm{~mm}$ orifice meter in a baseline configuration and with a tee located $18 \mathrm{D}$ from the orifice plate indicates that placing a 19 tube bundle flow conditioner at $12 \mathrm{D}$ produces discharge coefficients equivalent to those measured in baseline conditions. Flow rates for all tests were in the turbulent regime.

\section{4,202}

PB95-107256 Not available NTIS

National Inst. of Standards and Technology (MSEL), Gaithersburg, MD. Metallurgy Div.

Reconstructing Stratified Fluid Flow from Reciprocal Scattering Measurements.

Final rept.

S. J. Norton. 1991, 6p.

Pub. in Jnl. of the Acoustical Society of America 89, n6 p2567-2572 1991

Keywords: "Fluid flow, "Acoustic scattering, "Stratification, Inhomogeneity, Wave propagation, Flow measurement, Acoustic properties, Defects, Acoustic signals, Reprints.

The authors show how an inversion formula can be derived for reconstructing the magnitude and direction of stratified fluid flow from two scattering measurements. The measurements are recorded by transmitting an acoustic pulse from a source below the region of stratified flow and then recording the scattered wave at another point horizontally displaced from the source. A second measurement is performed with the source and receive interchanged and the result is subtracted from the first scattering measurement. This difference is, within the Born approximation, independent of scattering that arises from stationary inhomogeneities and thus solely sensitive to scattering due to flow. As a byproduct, the sum of the two scattering measurements with source and receiver interchanged yields a quantity sensitive only to scattering from stationary inhomogeneities. This allows the stationary inhomogeneities to be recovered independently of flow.

\section{4,203}

PB95-125787 Not available NTIS

National Inst. of Standards and Technology (CSTL), Boulder, CO. Thermophysics Div.

Thermophysical Property Computer Packages from NIST.

Final rept.

D. G. Friend, M. L. Huber, and J. S. Gallagher. 1992, $6 p$.

Pub. in Proceedings of American Society of Mechanical Engineers Winter Annual Meeting Computerized Thermophysical Property Packages, Anaheim, CA., November 8-13, 1992, p13-18.

Keywords: "Thermophysical properties, *Data bases, *Fluids, *Mixtures, Working fluids, Cryogenic fluids, Refrigerants, Water, Transport properties, Reprints.

The Fluid Mixtures Data Center and the Thermophysics Division are currently supporting, and continuing development on several computerized packages to calculate the thermophysical properties of commercially important fluid systems. The NIST Thermophysical Properties of Hydrocarbon Mixtures Database (SUPERTRAPP) and the NIST Mixture Property Database (DDMIX) are based on extended corresponding states models. The NIST Thermophysical Properties of Fluids Database
(MIPROPS) computes properties of 17 pure fluids, generally of cryogenic importance, based on validated wide ranging thermophysical properties correlations. The NIST Thermodynamic Properties of Refrigerants and Refrigerant Mixtures Database (REFPROP) computes thermodynamic and transport properties of existing as well as proposed environmentally acceptable alternative refrigeration fluids and mixtures. Finally, the NIST Thermophysical Properties of Water Database interactively calculates properties using the most re- cent formulation approved by the International Association for the Properties of Water and Steam.

\section{4,204}

PB95-143061 PC A06/MF A02

National inst. of Standards and Technology (CSTL),

Gaithersburg, MD. Process Measurements Div.

Summary Report of NIST's Industry-Government Consortium Research Program on Flowmeter Installation Effects: The 45 Degree Elbow.

Technical note.

T. T. Yeh, and G. E. Mattingly. Sep 94, 108p, NIST/ TN-1408

Also available from Supt. of Docs. as SN003-003 03296-4. See also PB94-160736.

Keywords: "Flow meters, "Pipe bends, "Pipe flow, * Meetings, Flow measurement, Turbulence, Velocity distribution, Orifice meters, Órifices, Pipes(Tubes), Governmentindustry relations, Research programs, Concentric tube bundle flow conditioners.

The report presents recent results obtained in a consortium-sponsored research program on flowmeter installation effects being conducted at National Institute of Standards and Technology (NIST)-Gaithersburg, MD, presented at the meeting of consortium participants in November 1992. This project is supported by an industry-government consortium and has been underway for several years. This piping element tested and reported here is the conventional 45 degree elbow; the conventional 19- and 7-tube concentric tube bundle flow conditioners were also tested. The LDV velocity measurements are reported for the pipeflows produced downstream of the 45 degree elbow with and without the 19-tube tube bundles. The performance characteristics of a range of orifice meters with various pressure tap geometries and a specific turbine meter are obtained downstream of the 45 degree elbow with and without the 19- and 7-tube bundle flow conditioners

\section{4,205}

PB95-150280 Not available NTIS

National Inst. of Standards and Technology (BFRL), Gaithersburg, MD. Building Materials Div.

Cross-Property Relations and Permeability Estimation in Model Porous Media.

Final rept.

L. M. Schwartz, N. Martys, D. P. Bentz, E. J.

Garboczi, and S. Torquato. 1993, 8p.

Sponsored by Department of Energy, Washington, DC. Pub. in Physical Review E 48, n6 p4584-4591 Dec 93 Keywords: "Porous materials, "Permeability, "Stokes law(Fluid mechanics), Solubility, Stokes flow, Diffusion, Nuclear magnetic resonance, Electrical resistivity, Reprints.

Results from a numerical study examining cross-property relations linking fluid permeability to diffusive and electrical properties are presented. Numerical solutions of the Stokes equations in three-dimensional consolidated granular packings are employed to provide a basis of comparison between different permeability estimates. Estimates based on the lambda parameter (a length derived from electrical conduction) and on $\mathrm{d}(\mathrm{sub} \mathrm{c})$ (a length derived from immiscible displacement) are found to be considerably more reliable than estimates based on rigorous permeability bounds related to pore space diffusion. We propose two hybrid relations based on diffusion which provide more accurate estimates than either of the rigorous permeability bounds.

\section{4,206}

PB95-150892 Not available NTIS

National Inst. of Standards and Technology (CSTL). Gaithersburg, MD. Thermophysics Div.

Experimental Data and Theoretical Modeling of Gas Flows Through Metal Capillary Leaks.

Final rept.

S. A. Tison. 1993, 5p

Pub. in Vacuum 44, n11/12 p1171-1175 1993.

Keywords: "Flow rate, "Capillary tubes, "Leak detectors, "Gas flow, Flow measurement, Leakage, Viscous flow, Vacuum gages, Vacuum apparatus, Slip flow, Calibration, Pressure measurement, Transition flow, Reprints.

Metal capillary tubes are commonly used as leak elements to admit known flows of gases into vacuum systems for calibration of vacuum gaging equipment. In many instances it is desired to generate flow rates over a range of three or more decades, preferably with a single leak element. The generation of flow rates over wide ranges is possible with metal capillary leaks, but in most cases the conductance of the leak element will need to be measured as a function of the relevant pressures due to the changing of the flow regimes. Many fits to experimental data and theoretical models exist for predicting the flow rate through tubes, but their validity is not well established in this study, measured conductances of stainless steel tubes for flow rate of $10(\exp -8)$ to 10(exp -14) $\mathrm{mol} / \mathrm{s}$ with several inert gases are compared with various experimental and theoretical models of gas flow in the molecular, viscous and transition flow regimes. Characteristics of crimped metal capillaries are also examined over this range of flows.

04,207

PB95-162301 Not available NTIS

National Inst. of Standards and Technology (BFRL), Gaithersburg, MD. Fire Science Div.

Global Density Effects on the Self-Preservation Behavior of Turbulent Free Jets.

Final rept.

C. Richards, and W. Pitts. 1993, 19p.

Pub. in Jnl. of Fluid Mech. 254, p417-435 1993.

Keywords: "Turbulent jets, "Free jets, "Jet mixing flow, "Density(Mass/volume), Jet flow, Turbulent flow, Concentration(Composition), Boundary conditions, Nozzle flow, Light scattering, Axisymmetric flow, Density measurement, Velocity distribution, Reprints, "Global density ratio, "Self-preservation behavior.

An experimental investigation was designed to test the hypothesis that all axisymmetric turbulent free jets become asymptotically independent of the source conditions and may be described by classical similarity analysis. Effects of initial conditions were studied by varying jet exit boundary conditions and the global density ratio. The exit velocity profile and turbulence level was changed by using both pipe and nozzle flow hardware. Initial density differences were imposed by using three gases: helium, methane, and propane. The scalar field (concentration) in the momentum-dominated regime of the far field (10 to 60 jet exit diameters downstream) of turbulent free jets was characterized using Rayleigh light scattering as the diagnostic. The results show that regardless of the initial conditions axisymmetric turbulent free jets decay at the same rate, spread at the same angle, and both the mean and r.m.s. values collapse in a form consistent with full self-preservation. The means and fluctuations follow a law of full selfpreservation in which two virtual origins must be specified.

\section{4,208}

\section{PB95-181145 Not available NTIS}

National Inst. of Standards and Technology (CAML), Gaithersburg, MD. Applied and Computational Mathematics Div.

Convective Stability in the Rayleigh-Benard and Directional Solidification Problems: High-Frequency Gravity Modulation.

Final rept. R. Coriell. 1991, 12p.

Sponsored by National Aeronautics and Space Administration, Washington, DC. and Defense Advanced Research Projects Agency, Arlington, VA

Pub. in Physics of Fluids A 3, n12 p2847-2858 Dec 91.

Keywords: Rayleigh-Benard convection, Gravitational effects, Asymptotic series, Numerical solution, Flow stability, Solutes, Rayleigh number, Schmidt number, Reprints, "Directional solidification, Floquet theory.

The effect of vertical, sinusoidal, time-dependent gravitational acceleration on the onset of solutal convection during directional solidification is analyzed in the limit of large modulation frequency Omega. When the unmodulated state is unstable, the modulation amplitude required to stabilize the system is determined by the method of averaging, and is O(Omega). Comparison of the results from the averaged equations with numerical solutions of the full linear stability equations (based on Floquet theory) show that the difference is $O(O m e g a(S u p 1 / 2)$ ). When the unmodulated state is stable, resonant modes of instability occur at large modulation amplitude. These are analyzed using matched asymptotic expansions to elucidate the boundary-layer structure for both the Rayleigh-Benard and directional solidification configurations. 
National Inst. of Standards and Technology (MSEL), Gaithersburg, MD. Polymers Div.

Probabilistic Computation of Poiseuille Flow Velocity Fields.

Final rept.

F. Y. Hunt, J. F. Douglas, and J. Bernal. 1995, 16p. 2401 May 95.

Keywords: "Open channel flow, "Laminar flow, Probability theory, Fractals, Pipe flow, Cross sections, Torsion, Rigidity, Reprints.

Velocity fields for Poiseuille flow through tubes having general cross sections are calculated using a path integral method involving the first-passage times of random walks in the interior of the cross sectional domain of the pipe. This method is applied to a number of ex amples where exact results are available and to more complicated geometries of practical interest. These examples include a tube with 'fractal' cross section and open channel flows. The calculations demonstrate the feasibility of the probabilistic method for pipe flow an other applications having an equivalent mathematical description (e.g., torsional rigidity of rods, membrane deflection).

04,210

PB96-102579 Not available NTIS

National Inst. of Standards and Technology (MSEL), Gaithersburg, MD. Metallurgy Div.

Measurements of Thermophysical Properties of Nickel Near Its Melting Temperature by a Microsecond-Resolution Transient Technique. Final rept.

E. Kaschnitz, J. L. McClure, and A. Cezairliyan.

1994, 10p.

Pub. in International Jnl. of Thermophysics $15, \mathrm{n} 4$ p757-766 Jul 94.

Keywords: "Thermophysical properties, "Nickel, "Melting points, Electrical resistivity, Enthalpy. Heat of fusion, High temperature, Specific heat, Liquid phases, Pulse heating, Reprints, Transient techniques.

A microsecond-resolution capacitor discharge system was used to heat nickel specimens rapidly to temperatures several hundred degrees above the melting point. From time-resolved measurements of current, voltage, and radiance temperature, selected thermophysical properties of nickel at its melting temperature and in the liquid state were determined. The properties measured include enthaipy and electrical resistivity at the beginning and end of melting, heat of fusion, and enthalpy, specific heat capacity, and electrical resistivity in the liquid phase up to $2000 \mathrm{~K}$.

04,211

PB96-113543 (Order as PB96-113535, PC A05/ MF A01

National Physical Lab., New Delhi (India)

Intercomparison between NPL (India) and NIST (USA) Pressure Standards in the Hydraulic Pressure Region Up to $26 \mathrm{MPa}$.

J. K. N. Sharma, K. K. Jain, C. D. Ehrlich, J. C

Houck, and D. B. Ward. 1994, 5p

Prepared in cooperation with National Inst. of Standards and Technology, Gaithersburg, MD

Included in Jnl. of Research of the National Institute of Standards and Technology, v99 n6 p725-729 Nov? Dec 94.

Keywords: "Hydraulic fluids, "Pressure, "Standards, Interlaboratory comparison. Pistons, Pressure measurement, India, United States, Pneumatics, Manometers, Pressure gages, NIST(National Institute of Standards and Technology).

Results are presented of an intercomparison of pressure measurements between the National Physica Laboratory (NPL), India, and the National Institute of Standards and Technology (NIST), USA, using piston guage pressure standards over the range $6 \mathrm{MPa}$ to 26 $\mathrm{MPa}$. The intercomparison, using the NPL piston gauge pressure standard, with a normal effective area of $8.4 \times 10(\exp -5)$ square $\mathrm{m}$., and the NIST piston gauge pressure standard, with a nominal effective area of $2.0 \times 10(\exp -5)$ square $m$., was carried out at the NPL. The intercomparison data obtained show a relative difference of $1 \times 10(\exp -6)$ in the zero-pressure effective area A(sub 0 ) of the NPL standard as obtained by the NIST standard.

04,212

PB96-119565 Not available NTIS

National Inst. of Standards and Technology (CSTL)

Gaithersburg, MD. Thermophysics Div.
Long-Lived Structures in Fragile Glass-Forming Liquids.

Final rept.

A. I. Mel'cuk, R. A. Ramos, H. Gould, W. Klein, and

R. D. Mountain. $1995,4 p$.

Pub. in Physical Review Letters, v75 n13 p2522-2525, 25 Sep 95.

Keywords: "Molecular structure, "Supercooling, "Liquids, "Glass, "Lennard-Jones potential, Computerized simulation, Clumps, Monte Carlo method, Thermodynamic properties, Thermal stability, Kinetics, Two dimensional, Reprints, "Molecular dynamics, "Fragile glass, Voronoi analysis

We present molecular dynamics results for the existence of long-lived clusters near the glass transition in a two component, two-dimensional Lennard-Jones supercooled liquid. Several properties of this system are similar to a mean-field glass-forming liquid near the spinodal. This similarity suggests that the glass 'transition' in the supercooled liquid is associated with an incipient thermodynamic instability. Our results also suggest that single particle properties are not relevant for characterizing the instability, but are relevant to the kinetic transition that occurs at a lower temperature than the glass transition.

04,213

PB96-122726 Not available NTIS

National Inst. of Standards and Technology (BFRL), Gaithersburg, MD. Structures Div.

Non-Gaussian Noise Effects on Reliability of Multistable Systems.

Final rept.

E. Simiu, and M. Grigoriu. 1993, 7p.

Pub. in Proceedings of the International Conference on Offshore Mechanics and Arctic Eng., OMAE (12th), Glargow, Scotland, June 20-24, 1993, v2 p65-71 1993.

Keywords: "Chaos, "Noise, "Dynamical systems, "Ocean engineering, Stability, Elastic properties, Nonlinear systems, Stochastic processes, Branching(Mathematics), Reliability, Reprints, "Nongaussian effects, Fluidelasticity.

For certain types of compliant structures the designer must consider limit states associated with the onset of fluidelastic instability. For a wide class of dynamical systems, a fundamental connection between deterministic and stochastic chaos allows the application of this condition to obtain probabilities that chaotic motions with jumps cannot occur in multistable systems excited by processes with tail-limited marginal distributions.

04,214

PB96-123427 Not available NTIS

National Inst. of Standards and Technology (CAML), Gaithersburg, MD. Applied and Computational Mathematics Div.

Lubrication Theory for Reactive Spreading of a Thin Drop.
Final rept.

R. J. Braun, B. T. Murray, W. J. Boettinger, and G. B. McFadden. 1995, 14p.

See also PB95-189460.

Pub. in Phys. Fluids, v7 n8 p1797-1810 Aug 95.

Keywords: "Contact line, "Lubrication theory, Reactive spreading, Solder, Reprints, ${ }^{\star}$ Foreign technology.

Solder drops spreading on metallic substrates are a reactive form of the wetting problem. A metallic component may diffuse in the liquid toward a metal substrate where it is consumed by a reaction that forms a solid intermetallic phase. The resulting spatial variation in the composition of the drop may cause composition gradients along the free surface of the drop. Together with any thermal gradients along the free surface. Marangoni effects may, in turn, modify the bulk transport in the spreading drop. Motivated by this situation, we extend lubrication theory for the spreading of thin drops in the presence of gravity and thermocapillarity to include mass transport and equations for the free surface shape and concentration field. Numerical solu. tions for the nonreactive (single component) drop agree well with previous theory. In the reactive case, we are only able to compute results for parameters outside of the range for solder materials. Including reactive effects in the model impacts the flow patterns and spreading rates at relatively early times; but by the end of the spreading, solutal effects have died out in the model.

\section{4,215}

PB96-123534 Not available NTIS
National Inst. of Standards and Technology (MSEL), Gaithersburg, MD. Polymers Div.

Book Review: Aspects and Applications of the Random Walk.

Final rept.

J. Douglas. 1995, 4p

Pub. in Jnl. of Statistical Physics, v79 n1-2 p497-500 1995.

Keywords: "Random walk, "Hydrodynamics, "Computational fluid dynamics, Random processes, Phase transitions, Dynamical systems, Equilibrium, Stochastic processes, Numerical analysis, Mathematical models, Reviews, Reprints.

Scientists have often pondered the unreasonable effectiveness' of mathematics in describing the complex cooperative phenomena which occur at many scales in our physical environment - processes ranging from subatomic to cosmic dimensions. The success of random walk models, and the limit theorems associated with these walks in describing the origin of various kinds of cooperative phenomena (phase transitions, fluid flow,..) is similarly remarkable and we can expec the perspective and tools of random walk theory to offer clues into the microscopic origin of large-scale regularities in the natural world and into the success of hydrodynamic mathematical models introduced to describe dynamic and equilibrium cooperative phenomena.

\section{4,216}

PB96-156013 Not available NTIS

National Inst. of Standards and Technology (CSTL)

Gaithersburg, MD. Thermophysics Div.

mproved Gas Flow Measurements for Next-Generation Processes.

Final rept.

S. A. Tison. 1996, $5 p$

Pub. in Proceedings of the International Workshop on Semiconductor Characterization: Present Status and Future Needs, Gaithersburg, MD., January 30-February 2, 1995, p497-501 1996.

Keywords: "Gas flow, Semiconductors, Meters, Reprints, Mass flow controller.

Many semiconductor processes require stable and known flows of gas be delivered to the processing chamber. Gas types and flow rates are process de. pendent, but it is clear that next-generation processes will require flow measurements that are two to three decades lower than current requirements. Specifically, the Semi-Sematech-sponsored Mass Flow Controlle Working Group recently identified the need for flow measurements with an accuracy of $1 \%$ or better to be extended to cover the range $7 \times 10$ to the minus 8 th power to $7 \times 10$ to the minue 5 th power mol/s $(0.1$ to $100 \mathrm{sccm}$, standard cubic centimeters per minute). At least two problems must be overcome if this goal is to be attained. Achieving $1 \%$ accuracy in the process chamber will require reference standards with an accuracy of $0.2 \%$ or better, but adequate reference stand ards do not exist over this range. Further, the thermal mass flow controllers (TMFCs) used to measure and control the process gases are generally calibrated with nitrogen and 'corrected' for other gases, but the correction factors are not well understood and of questionable reliability. The National Institute of Standards and Technology (NIST) is addressing both of these problems by developing new flow standards and by investigating the performance of TMFCs. This paper will present data on the performance of five TMFCs, from different manufacturers, with full scale rangers of 1.5 $\times 10$ to the minus 6 th power $\mathrm{mol} / \mathrm{s}$ to $3.7 \times 10$ to the minus 6 th power $\mathrm{mol} / \mathrm{s}$ (2 to $5 \mathrm{sccm}$ ).

\section{4,217}

PB96-161880 Not available NTIS

National Inst. of Standards and Technology (CSTL), Gaithersburg, MD. Thermophysics Div.

Electric Field Effects on a Near-Critical Fluid in Microgravity.

Final rept.

G. Zimmerli, R. A. Wilkinson, R. A. Ferrell, H. Hao,

and M. R. Moldover. 1995, $6 p$.

See also N95-25878.

Pub. in Proceedings of the National Heat Transfer Conference (30th), Portland, OR., August 6-8, 1995, v3 p 121-126.

Keywords: "Critical flow, "Electric fields, "Electrostriction, "Microgravity, Reprints, Sulfur hexafluoride, Meetings, Critical point, Density distribution, Interferometry.

The authors have studied the effects of an electric field on a sample of SF6 fluid in the vicinity of the liquid 


\section{Fluid Mechanics}

vapor critical point The authors measured the isothermal increase of the density of a near-critical sample as a function of the applied electric field. In agreement with theory, this electrostriction effect diverges near the critical point as the isothermal compressibility diverges. Also as expected, turning on the electric field in the presence of density gradients can induce flow within the fluid, in a way analogous to turning on grav ity. These effects were observed in a microgravity environment by using the Critical Point Facility which flew onboard the Space Shuttle Columbia in July 1994 as part of the Second International Microgravity Laboratory Mission. Both visual and interferometric images of wo separate sample cells were obtained by means of video downlink.

\section{4,218}

PB96-176789 Not available NTIS

National Inst. of Standards and Technology (CSTL), Gaithersburg, MD. Process Measurements Div. Thermal Anemonetry for Mass Flow Measurement in Oscillating Cryogenic Gas Flows.

Final rept.

W. Rawlins, R. Radebaugh, and K. D. Timmerhaus. 1993, 7p.

Pub. in Review of Scientific Instruments, v64 n11 p3229-3235 Nov 93

Keywords: "Oscillating flow, *Anemometers, Reprints, Pulse tube refrigerator, Cryocoolers, Constant temperature anemometer, Resistance temperature detectors.

Constant temperature anemometers and resistance emperature detectors have been adapted to measure the instantaneous mass flow rates and tempeatures in oscillating gasflows at frequencies up to $30 \mathrm{~Hz}$ and emperatures down to $70 \mathrm{~K}$. These devices were used to study the behavior of the oscillating working fluid (helium in a regenerative refrigerator called an orifice pulse tube refrigerator with little instrusion in the sys tem. The probes have proved to be very robust with no probe breakage even under the severe operating conditions imposed by the oscillating mass flow. Calibration procedures are presented for correcting the anemometer output associated with the oscillating temperatures and pressures in such systems. Under these conditions the sensors appear to have an uncertainty of less than $1.6 \%$. Typical results obtained with these sensors are presented in this paper. These sensors could be used in many other applications for studies of oscillating systems where instantaneous mass flow rates and temperatures are required.

\section{4,219}

PB96-180195 Not available NTIS

National Inst. of Standards and Technology (BFRL), Gaithersburg, MD. Fire Science Div.

Tomographic Reconstruction of the Moments of Local Probability Density Functions in Turbulent Flow Fields.

Final rept

M. R. Nyden, P. Vallikul, and Y. R. Sivathanu. 1996 ,

\section{0.}

Pub. in Jnl. of Quantative Spectroscopy Radiation Transfer, v55 n3 p345-356 1996.

Keywords: "Turbulent flow, "Flow fields, "Probability density functions, Turbulent heat transfer, Turbulen jets, Flame propagation, Jet mixing flow, Jet flow, Fourier transformation, Reprints, Ethenes.

An algorithm for the tomographic reconstruction of the individual moments of the probability density functions describing the local transmittance of radiation through a turbulent flow field is advanced. The new method, which is based on Fourier inversion, is applicable to asymmetric (as well as, to axiymmetric) flows. The validity of the method is examined by comparing reconstructed moments of the local probability functions in a buoyant propene/air flame and an ethene/air je flame to the corresponding values obtained from optical probe measurements.

\section{4,220}

PB96-204417 Not available NTIS

National Inst. of Standards and Technology (CSTL), Gaithersburg, MD. Thermophysics Div.

Greenspan Acoustic Viscometer for Gases.

Final rept.

K. A. Gillis, J. B. Mehl, and M. R. Moldover. 1996

Pub. in Review of Scientific Instruments, v67 n5 p1850-1857 May 96.
Keywords: "Viscometers, "Transport properties, Reprints, Viscosity, Speed-of-sound, Thermophysical properties, "Helmholtz resonators.

Double Helmholtz acoustic resonators, first proposed by Greenspan for measuring the viscosity of gases, were tested with helium, argon, and propane. Two different resonators were tested extensively with all three gases. For each of these instruments, the results for the viscosities of the three gases were consistent within plus or minus $5 \%$ at pressures spanning the range $25-1000 \mathrm{kPa}$. Without calibration, the viscosities deduced from one viscometer were systematically $1 \%$ larger than data from the literature; the viscositites from the second viscometer were systematically $3 \%$ larger than data from the literature. If the systematic differences were removed for each viscometer by calibration with a single gas at a single temperature and pressure, then nearly all the results for both instruments would have fallen within plus or minus $0.5 \%$ of the data from the literature. In these viscometers, the gases are in contact with robust metal parts only; thus, these instruments are applicable to a very wide variety of gases over a very wide range of temperatures.

\section{4,221}

PB97-111504 Not available NTIS

National Inst. of Standards and Technology (CSTL), Gaithersburg, MD. Thermophysics Div. Internal Waves in Xenon Near the Critical Point.

Final rept.

R. F. Berg, M. J. Lyell, G. B. McFadden, and R. G. Rehm. 1996, 12p.

Pub. in Physics of Fluids, v8 n4 p1464-1475 Jun 96. Keywords: "Critical points, "Internal waves, "Density profiles, Mode symmetry, Stratified fluid, Reprints, Brunt-Vaisala frequency.

Just above the liquid-vapor critical point, a fluid's large compressibility causes a stable stratification in which the density varied by as much as $10 \%$ in $1 \mathrm{~cm}$. This stratification supports internal gravity waves which the authors observe with an oscillator immersed in a nearcritical xenon sample. The authors found the number and frequencies of the observable modes depended on the sample cell's orientation, with only two modes seen in the horizontal cell. The frequencies of the two modes had different temperature dependences: with decreasing temperature, the higher frequency increased monotonically from 0.7 to $2.8 \mathrm{~Hz}$, but the lower frequency varied nonmonotonically, with a maximum of $1.0 \mathrm{~Hz}$ at $20 \mathrm{mK}$ above the critical temperature. These temperature dependences continued to $20 \mathrm{mK}$ below the critical temperature, where the xenon was separated into liquid and vapor phases. The authors calculated these two frequencies by solving the eigenvalue problem of internal waves in a box containing a stratified fluid. The fluid's density profile was obtained from xenon's equation of state. The calculated and measured frequencies agree to within $15 \%$. Analytical calculations based on simple approximations of the density profile provide insight into the observed temperature dependences.

\section{Optics \& Lasers}

\section{4,222}

N94-23605/6

MF A04)

National Inst. of Standards and Technology,

Gaithersburg, MD.

Activities of NIST (National Inst. Of Standards and Technology).

1992, 14p.

In NASA. Goddard Space Flight Center, the Fifth Calibration/Data Product Validation Panel Meeting $14 \mathrm{p}$.

Keywords: "Black body radiation, "Calibrating, "Earth observing system (Eos), "Infrared radiometers, "Remote sensors, "Standardization, Instrument compensation, Radiance, Radiant flux density, Temperature measurement, US NIST.

The Radiometric Physics Division of the NIST is responsible for the national standards in radiation thermometry, spectroradiometry, photometry, and spectrophotometry; dissemination of these standards by providing measurement services to customers requiring calibrations of the highest accuracy; and conducting fundamental and applied research to develop ducting fundamental and applied research to develop
the scientific basis for future measurement services. Its relevance to EOS/TIR calibration includes calibrating unknown blackbody for radiance using a well-characterized NIST blackbody source by matching the radian fluxes with an IR radiometer. The TIR Round Robin is used to verify the calibration of the sources that are used for the absolute radiometric calibration of the individual EOS sensors.

04,223

PB94-140555 PC A06/MF AO2

National Inst. of Standards and Technology,

Gaithersburg, MD. Standards and Technology, November-December Standards and Technology,
1993. Volume 98 , Number 6 .

$1993,125 p$

See also PB94-140563 through PB94-140605 and PB94-108529. Also available from Supt. of Docs. as SN703-027-00055-5

Keywords: "Research, Atomic energy levels, Mass spectroscopy, Chlorine 36, Tritium, Thermodynamic properties, Infrared filters, Light scattering, Isotope of fect, Krypton 84, Smoke, Standards, Accelerator mass spectroscopy.

Contents:

(36) $\mathrm{Cl} / \mathrm{Cl}$ Accelerator-Mass Spectrometry Standards--Verification of Their Serial-DilutionSolution Preparations by Radioactivity Measurements:

Pressure-Volume-Temperature Relations in Liquid and Solid Tritium;

Filter Transmittance Measurements in the Infrared;

On Two Numerical Techniques for Light Scattering by Dielectric Agglomerated Structures;

Wavelengths and Energy Levels of Neutral $\mathrm{Kr}(84)$ and Level Shifts in All Kr Even Isotopes.

04,224

PB94-140589 (Order as PB94-140555, PC A06)

MF A02)

National Inst. of Standards and Technology, Gaithersburg, MD

Filter Transmittance Measurements in the Infrared. A. L. Migdall, A. Frenkel, and D. E. Kelleher. 1993, $7 p$

Included in Jnl. of Research of the National Institute of Standards and Technology, v98 n6 p691-697 Nov/ Dec 93.

Keywords: "Infrared filters, Intermediate infrared radiation, Carbon dioxide lasers, Optical heterodyning, Laser radiation, Light modulation, Transmittance, Attenuators.

The authors have set up a novel direct detection system to measure filter transmittances over an attenuation range of at least 5 decades, with relative combined standard uncertainties as low as $0.5 \%$ ( 1 sigma) per decade, in the 9 micrometer to 11 micromete spectral region. This system, using an apparatus originally designed for a heterodyne measurement of transmittance, achieves higher accuracy at the expense of a reduced dynamic range. The high modulation frequency and narrow bandwidth of the system allow thermal background radiation to be suppressed and high accuracy to be achieved. The authors correct for the non-ideal natures of the detector and attenuators. In particular, the detector position is scanned to reduce the effect of its spatial nonuniformity and the deflection of the transmitted beam caused by the nonparallel surfaces of the filter. The authors discuss the sources of systematic errors and the methodology to reduce their contribution.

\section{4,225}

PB94-140597

MF A02) State Univ. . University Park. Dept. of Enwerience and Mechanics.

wo Numerical Techniques for Light Scattering by Dielectric Agglomerated Structures.

A. Lakhtakia, and G. W. Mulholland. 1993, $18 p$.

Prepared in cooperation with National Inst. of Stand ards and Technology, Gaithersburg, MD.

Included in JnI. of Research of the National Institute of Standards and Technology, v98 n6 p699-716 Nov/ Dec 93.

Keywords: "Light scattering, "Smoke, Method of moments, Maxwells equations, Agglomerates, Combus tion, Fires, Soot, Coupled dipole method, Dielectric spheres. 
Smoke agglomerates are made of many soot spheres, and their light scattering response is of interest in fire research. The numerical techniques chiefly used for theoretical scattering studies are the method of moments and the coupled dipole moment. The two methods have been obtained in this tutorial paper directly from the monochromatic Maxwell curl equations and shown to be equivalent. The effects of the finite size of the primary spheres have been numerically delineated.

04,226

PB94-185246 Not available NTIS

National Inst. of Standards and Technology (PL), Gaithersburg, MD. Atomic Physics Div.

Spectrum and Energy Levels of Five-Times-lonized Niobium (Nb VI).

Final rept.

J. O. Ekberg, and J. Reader. 1994, 13p.

Pub. in Jnl. of the Optical Society of America B 11, n3 p415-427 Mar 94. Sponsored by Department of Energy, Washington, DC

Keywords: "Atomic energy levels, "Ultraviolet spectra, *Niobium ions, Vacuum ultraviolet radiation, Atomic spectra, Line spectra, Hyperfine structure, Ionization, Reprints, Krypton-line ions.

The spectrum of the kryptonlike ion $\mathrm{Nb} \mathrm{VI}$ was observed from 325 to $2,700 \mathrm{~A}$ with sliding-spark discharges on 10.7-m normal-incidence and grazing-incidence spectrographs. Experimental energies were determined for all levels of the $4 s(2) 4 p(6), 4 s(2) 4 p(5) 4 d$, $4 f, 5 s, 5 p, 5 d, 5 g, 6 s$, and $4 s 4 p(6) 4 d$ configurations as well as for some levels of the $4 \mathrm{~s}(2) 4 \mathrm{p}(5) 6 \mathrm{~g}$ and $6 \mathrm{~h}$ configurations. A total of 303 lines are now classified as transitions between 99 observed levels. Large hyperfine splittings were found for several levels of the $4 p(5) 5 s$ and $5 p$ configurations. The observed configurations were theoretically interpreted by means of Hartree-Fock calculations and least-squares fits of the energy parameters to the observed levels. An improved value of the ionization energy was determined from the $4 p(5) 5 \mathrm{~g}, 6 \mathrm{~g}$, and $6 \mathrm{~h}$ configurations.

\section{4,227}

\section{PB94-188240 PC A03/MF AO}

National Inst. of Standards and Technology (PL), Gaithersburg, MD. Radiometric Physics Div.

NIST Response to the Fifth CORM Report on the Pressing Problems and Projected Needs in Optical Radiation Measurements.

A. C. Parr, and J. J. Hsla. May 94, 18p, NISTIR-

5420

Keywords: "Optical measurement, "Radiometry, "Metrology, Optical properties, Visible radiation, Infrared radiation, Infrared detectors, Irradiance, Reflectance, Photometry, *CORM(Council for Optical Radiation Measurements), Council for Optical Radiation Measurements, Standard reference materials, Spectroradiometry.

The Council for Optical Radiation Measurements (CORM) issued its Fifth Report in September 1989. Some of the concerns addressed in the CORM report required a response from NIST measurements services. Herein is documented NIST's endeavors to assist the optical radiation measurement community by acting on some of the CORM requests.

\section{4,228}

PB94-191707 PC A03/MF A0

National Inst. of Standards and Technology (PL),

Gaithersburg. MD. Radiometric Physics Div.

Optical Metrology and More. Programs and Services of the Radiometric Physics Division, Physics Laboratory.

S. Bruce. May 94, 24p, NISTIR-5429.

Color illustrations reproduced in black and white.

Keywords: "Optical measurement, "Radiometry, Temperature range 1000-4000 K. Temperature measurement, Ultraviolet radiation, Visible radiation, Infrared radiation, Infrared detectors, Light scattering, Light sources, Calibration, Photometry, Metrology, "Radiometric Physics Division, US NIST

The Radiometric Physics Division is the primary unit within NIST for carrying out the basic mission of promoting accurate and useful optical radiation measurements in the ultraviolet, visible, and infrared regions. This brochure describes the programs and projects that comprise the work represented by this division.

04,229

PB94-199098 Not available NTIS
National Inst. of Standards and Technology (PL), Gaithersburg, MD. Atomic Physics Div.

Development and Calibration of UV/VUV Radiometrlc Sources.

Final rept.

J. M. Bridges. $1993,9 p$

Pub. in Proceedings of Society of Photo-Optical Instrumentation Engineers - Ultraviolet Technology IV, San Diego, CA., July 20-21, 1992, v1764 p262-270 1993 Sponsored by Office of Space Science and Applications, Washington, DC

Keywords: "Vacuum ultraviolet radiation, *Ultraviolet radiation, "Radiometry, *Calibration, SURF II storage ring, Electric arcs, Irradiance, Radiance, Reprints, Hydrogen arcs, Argon arcs, US NIST.

A program exists at NIST to calibrate radiometric sources for the spectral range from $118-350 \mathrm{~nm}$. These include deuterium lamps, hollow-cathode lamps, RFexcited dimer lamps, and wall-stabilized argon arcs. Sources have been calibrated for and used by researchers in solar physics, astrophysics, atmospheric physics (ozone measurements), magnetically controlled fusion, and photobiology. The argon arcs were developed in our laboratory, and provide intense sources of both radiance and irradiance. Calibrations are performed relative to two primary sources, a wallstabilized hydrogen arc and a 12,000 K black-body line arc, both developed in our laboratory. Also we recently have begun periodic calibrations on the NIST storage ring, SURF II, to ensure consistency between our respective radiometric bases Various sources have been calibrated for space applications, including several which are flyable. Also, some development and testing of radiometers for semiconductor lithography were recently carried out with an intense argon arc source.

\section{4,230}

PB94-199163 Not available NTIS

National Inst. of Standards and Technology (PL) Gaithersburg, MD. Radiometric Physics Div.

Overview of Radiometric Program of the NIST Thermal Imaging Laboratory.

Final rept.

R. J. Bruening. 1991, 11p.

Pub. in Proceedings of MICOM Logistics Research and Development Workshop (2nd), Huntsville, AL., August 27-28, 1991, p503-513. Sponsored by Naval Wartare Assessment Center/Metrology Engineering Center, Corona, CA.

Keywords: *Radiometry, Infrared radiometers, Infrared detectors, Infrared cameras, Temperature range 0273 $0400 \mathrm{~K}$, Blackbody radiation, Heat pipes, Calibration Reprints, Thermal Imaging Laboratory, US NIST.

Radiometric calibrations performed in the NIST Ther mal Imaging Laboratory now support infrared detecting devices that operate in the temperature range of 0 to $100 \mathrm{C}$, including devices that produce pictures of ambient temperature scenes. The laboratory provides characterization of the temperature and temperature-differences of infrared blackbody sources, and characterization of radiometers. Research into the quantitative performance of infrared cameras, and use of higher temperature calibrated heat pipe sources (to $1000 \mathrm{C}$ ) is planned.

04,231

PB94-207636 PC A10/MF A03

National Inst. of Standards and Technology (EEEL),

Boulder, CO. Electromagnetic Technology Div.

Technical Digest: Symposium on Optical Fiber Measurements (8th), 1994. Held in Boulder, Colorado on September 13-15, 1994.

Special pub. (Final)

G. W. Day, D. L. Franzen, and R. K. Hickernell. Sep 94, 233p, NIST/SP-864

Also available from Supt. of Docs. as SN003-003 03277-8. See also PB93-102259. Prepared in co operation with Lasers and Electro-Optics Society (IEEE), Piscataway, NJ. and Optical Society of America, Washington, DC.

Keywords: "Fiber optics, "Optical fibers, "Optical measurement, "Meetings, Optical polarization, Electrooptics, Transmission lines, Reviews, US NBS Integrated optics, National Institute of Standards and Technology.

The Digest contains the manuscripts of 50 papers, 10 invited and 40 contributed, presented at the eighth Symposium on Optical Fiber Measurements, September 13-15, 1994 in Boulder, Colorado. The most signifi- cant theme of the symposium is the present importance of polarization measurements. More than $20 \%$ of the papers are concerned either with polarization measurements, especially polarization mode dispersion and polarization dependent loss, or the characterization of fibers and components with special polarization properties. Optical time-domain reflectometry (OTDR) measurements are another important theme, as they have been since the first Symposium in 1980 and the characterization of optical fiber amplifiers continues to be important. Nonlinear processes in fiber seem to be a growing interest Approximately two thirds of the papers come from outside of the United States, up from about half at the seventh Symposium. Ten countries are represented in the program.

04,232

PB94-211224 Not available NTIS

National Inst of Standards and Technology (NML) Gaithersburg, MD. Radiometric Physics Div.

Update on the Low Background IR Calibration Facility at the National institute of Standards and Technology.

Final rept.

S. C. Ebner, A. C. Parr, and C. C. Hoyt. 1989, 12p. Pub. in Proceedings of Society of Photo-Optical Instrumentation Engineers - Imaging Infrared: Scene Simulation, Modeling, and Real Image Tracking, Orlando, FL. March 30-31, 1989, v1110 p49-60.

Keywords: *Infrared sources, ${ }^{*}$ Calibration, Temperature range 0013-0065 K Blackbody radiation Research facilities, Radiometry, Irradiance, Reprints, US NIST.

Details will be given about the recently completed facilty for the calibration of infrared sources in a low back ground environment. The basic components of the facility are a large ( $60 \mathrm{~cm}$ diameter by $152 \mathrm{~cm}$ long) stainless steel vacuum chamber housed in a soft-wall cleanroom. A low background environment inside the chamber is achieved by cooling internal cryoshields to temperatures less than $20 \mathrm{~K}$ using a closed cycle helium refrigerator. Sources of up to $30 \mathrm{~cm}$ square can be inserted into the chamber for calibration. Total radiant power from the blackbodies is measured with an Absolute Cryogenic Radiometer. Plans will be discussed for future enhancement of the system allowing for measurement of spectral and angular distribution of the emitted radiation and possible experiments which could utilize the full capabilities of this system.

04,233

PB94-211232 Not available NTIS

National Inst. of Standards and Technology (PL), Gaithersburg, MD. Radiometric Physics Div.

Comparison of Regular Transmittance Scales of Four National Standardizing Laboratories. Final rept.

K. E. Eckerle, J. Bastie, J. Zwinkels, V. Sapritsky and A. Ulyanov. 1993, 6p.

Pub. in COLOR Research and Application 18, n1 p35 40 Feb 93.

Keywords: $\quad$ "Transmittance, International, Interlaboratory comparisons, Spectrophotometers, Standardization, Optical filters, Uncertainty, Reprints.

A comparison of the regular spectral transmittance scales of the Nationa! Institute of Standards and Technology (USA), Institut National de Metrologie (France) National Research Council (Canada), and All-Union Research Institute for Optical and Physical Measurements (CIS) was accomplished using neutral glass filters with transmittances ranging from approximately 0.92 to 0.001 . Storing the filters for almost four years produced no conclusive evidence of improvement over a previous interchange between NIST and three different national standardizing laboratories when the filters were stored for only 30 days. The agreement ranges from $0.01 \%$ to $0.3 \%$ depending on the laboratory and the filter used. This interchange is part of an ongoing efiort to obtain international standardization.

04,234

PB94-211349 Not available NTIS

National Inst. of Standards and Technology (PL), Gaithersburg, MD. Radiometric Physics Div.

Longterm Changes of Silicon Photodiodes and Their Use for Photometric Standardization.

Final rept.

G. Eppeldauer. 1990, 6p.

90. 


\section{Optics \& Lasers}

Keywords: "Photometry, "Photodiodes, Long term effects, Optical measurement, Silicon diodes, Standardization, Calibration, Accuracy, Reprints

A secondary standard silicon photodiode matched with a V-lambda filter was calibrated against primary standard self-calibrated inversion layer silicon photodiodes, to achieve a high accuracy photometer, according to the new definition of the candela (the photometric base unit). The measured several percent/year specula spectral reflectance change of the windowless primary standard photodiodes was eliminated by their repeated self-calibration. This self-calibration also eliminated the measured several tenth of a percent/year spectral response change of the secondary standard silicon photodiode. The secondary standard detector could be a nonunity quantum efficiency light detector. The spectral response calibration of the V-lambda matched detector of medium spectral mismatch against the absolute spectral responses of three self-calibrated photodiodes resulted in a standard deviation of $0.17 \%$ in luminous flux (lumen) calibration. Also illuminance (lux) and light intensity (candela) calibrations were derived from the above primary photometric calibration. It is shown that the $V$-lambda matched photometer with the above spectral calibration can be used for accurate photometric measurements for all kinds of light sources of known spectral power distribution.

\section{4,235}

PB94-211570 Not available NTIS

National Inst. of Standards and Technology (PL), Boulder, CO. Quantum Physics Div.

Dreams About the Next Generation of Super-Stable Lasers.

Final rept.

J. L. Hall, M. Zhu, and D. Hils. 1992, 8p

Contracts AFOSR91-0283, No0014-89-J-1227

Sponsored by Office of Naval Research, Arlington, VA., National Science Foundation, Arlington, VA., and Air Force Office of Scientific Research, Bolling AFB, DC.

Pub. in Proceedings of International Conference on Laser Spectroscopy (10th), Font-Romeu, France, June 1991, p83-90 1992.

Keywords: "Laser stability, "Laser spectroscopy, High resolution, Beam cooling, Tunable lasers, Calcium, Silver, Reprints, Laser cooling.

Even if we propose using general, widely-tunable lasers for our ultra-resolution dream spectroscopy, we show that the $S / N$ available from a cavity locking scheme is adequate to define sub-milliHertz laser linewidths. We prove that present External Stabilizer servo technology is adequate to track an error signal precisely enough that $97 \%$ of the emitted power lies within the narrow spectral feature defined by phaseor cavity-locking. Cavity stability of + or $-5 \mathrm{kHz}$ for several weeks is documented, and efforts to eliminate the influence of laboratory vibrations are described. The paper concludes with a dream: application of these lasers to two delicious laser-cooled species, $\mathrm{Ag}$ and $\mathrm{Ca}$.

\section{4,236}

PB94-211935 Not available NTIS

National Inst. of Standards and Technology (EEEL) Boulder, CO. Electromagnetic Technology Div. Pump-Induced Dispersion of Erbium-Doped Fiber Measured by Fourier-Transform Spectroscopy.

Final rept.

R. K. Hickernell, K. Takada, M. Yamada, M. Shimizu, and M. Horiguchi. 1993, 3p.

Sponsored by Nippon Telegraph and Telephone Corp., Ibaraki. Opto-Electronics Labs.

Pub. in Optics Letters 18, n1 p19-21, 1 Jan 93.

Keywords: Near infrared radiation, Light amplifiers, Optical measurement, Doped materials, Erbium ions, Interferometry, Dispersion, Reprints, *Fiber amplifiers, Fourier transform spectroscopy, Group index.

We report the measurement of group index and dispersion in an erbium-doped fiber amplifier by Fourier transformation of low-coherence interferograms. In germania-codoped fiber whose background dispersion was -14 ps/(km nm), we measured resonant gain-induced changes as high as 9 and $-12 \mathrm{ps} /(\mathrm{km} \mathrm{nm})$ near 1.536 micrometers. The interferometric measurements agree with calculations based on a Kramers-Kronig transformation of absorption and emission spectra.

\section{4,237}

PB94-212511 Not available NTIS

National Inst. of Standards and Technology (NML), Gaithersburg, MD. Radiation Source and Instrumentation Div.
NIST-NRL Free-Electron Laser Facility.

Final rept.

R. G. Johnson, R. L. Ayres, J. B. Broberg, B. C. Johnson, E. R. Lindstrom, D. L. Mohr, J. E. Rose, J. K. Whittaker, N. D. Wilkin, M. A. Wilson, S. Penner, C. M. Tang, P. Sprangle, R. I. Cutler, and P. H. Debenham. 1990, 12p.

Pub. in Society of Photo-Optical Instrumentation Engineers - Free-Electron Lasers and Applications, Los Angeles, CA., January 18-19, 1990, v1227 p14-25.

Keywords: "Free electron lasers, "Research facilities, Naval Research Laboratory, Racetrack microtrons, Tunable lasers, Infrared lasers, Electron accelerators, Ultraviolet lasers, Wiggler magnets, Uses, Reprints, US NIST.

A free-electron laser (FEL) facility is being constructed at the National Institute of Standards and Technology (NIST) in collaboration with the Naval Research Laboratory (NRL). The FEL will be driven by the electron beam from the NIST racetrack microtron (RTM). The anticipated performance of the FEL is given. Initial operation of the FEL is scheduled for 1991. The NISTNRL FEL will provide a powerful, tunable light source for research in biomedicine, materials science, physics, and chemistry.

\section{4,238}

PB94-212529 Not available NTIS

National Inst. of Standards and Technology (NML), Gaithersburg, MD. Radiation Source and Instrumentation Div.

Hybrid Undulator for the NIST-NRL Free-Electron Laser.

Final rept.

R. G. Johnson, D. L. Mohr, M. A. Wilson, B. Ng, K.

Pub. in Procenner, and F. C. Younger. 1990, $5 p$. Laser Conference (11th), Naples, FL., August $28-$ September 1, 1989, p592-596 1990.

Keywords: "Free electron lasers, "Wiggler magnets, Permanent magnets, Hybrid structures, Tunable lasers, Magnetic fields, Cobalt alloys, Samarium alloys, Reprints, US NIST

The National Institute of Standards and Technology (NIST) and the Naval Research Laboratory (NRL) free electron laser (FEL) will use a 3.64-m hybrid undulator that is being constructed at Brobeck Division of Maxwell Laboratories. The undulator has a period of 2.8 $\mathrm{cm}$, a variable gap with a $1.0-\mathrm{cm}$ minimum, a peak magnetic field of $0.54 \mathrm{~T}$, and a total number of periods of 130 . The magnetic design uses SmCo permanent magnets and vanadium permendur pole pieces. Provision has been made to operate the undulator at half its length to enhance lasing at longer wavelengths. Remote control of gap and taper will permit on-line tuning of the wavelength. Vacuum chambers for both full and half length operation are available. The oval aperture of the vacuum chambers is $0.86 \mathrm{~cm}$ by $1.6 \mathrm{~cm}$. A system to measure the magnetic field along the undulator is included in the structure. A full-scale, one-period model of the magnetic design has been tested and the results exceed specifications. The mechanical structure of the undulator is nearly complete as is the control system. Construction of the magnet assemblies is underway.

\subsection{9}

PB94-212545 Not available NTIS

National Inst. of Standards and Technology (EEEL), Boulder, CO. Electromagnetic Technology Div.

\section{Beam Analysis Round Robin.}

Final rept.

R. D. Jones, and T. R. Scott. 1992, 12p

Pub. in Proceedings of Society of Photo-Optical Instrumentation Engineers - Laser Energy Distribution Profiles: Measurement and Applications, Boston, MA., November 16-18, 1992, v1834 p60-71.

Keywords: "Laser beams, Statistical analysis, Reprints, Beam analysis, Round robins.

We present the results of a round robin in which six U.S. manufacturers of beam analysis instruments participated. Following a procedure recommended by the International Organization for Standardization (ISO), participants used a camera, pinhole, slit, or knife-edge to determine several parameters characterizing the beam of a common laser source. Their results and ours are displayed graphically and analyzed statistically. Agreement on beam width measurements is in a range around five percent relative standard deviation while significantly less agreement exists for other quantities calculated within the ISO procedure.
04,240

PB94-212552 Not available NTIS

National Inst. of Standards and Technology (EEEL) Boulder, CO. Electromagnetic Technology Div. Laser-Beam Analysis Pinpoints Critical Parameters.

Final rept.

R. D. Jones, and T. R. Scott. 1993, 8p.

Pub. in Laser Focus World, p123-130 Jan 93.

Keywords: "Laser beams, Beam profiles, Optical measurements, Reprints, Beam analysis.

We demonstrate the need for measuring laser beam parameters such as diameter, divergence and propagation constant. These terms are defined and methods for their measurement are described. We make the point that assumption of ideal beam properties can result in degraded optical performance.

\section{4,241}

PB94-213097 Not available NTIS

National Inst. of Standards and Technology (MEL), Gaithersburg, MD. Precision Engineering Div.

Normal-Incidence Complex-Index Refractometry.

Final rept.

R. D. Larrabee. $1992,8 p$.

Pub. in Proceedings of Society of Photo-Optical Instrumentation Engineers - Laser-Induced Damage in Optical Materials, v1848 p86-93 1992.

Keywords: "Refractive index, "Refractivity, "Optical measurement, Electromagnetic absorption, Optical materials, Optical prisms, Complex numbers, Reprints.

Traditional refractometry of bulk transmissive optical materials is usually restricted to the lossless case where the material can be completely characterized by a real index of refraction. This paper is concerned with the measurement of both the real and the imagir ary parts of the index of refraction (i.e., including any l. ss) using a single prism-shaped specimen of homogeneous material. The normal-incidence configuration is analyzed and discussed in this paper because of its mathematical simplicity compared to the minimum-deviation configuration. The measurement of the imaginary part of the index of refraction (i.e. loss) is practical when the longest and shortest optical paths through the prism differ by an absorption length or more. The measurement of the real part of the index can ignore loss and still be accurate to better than one part per million if the power absorption length in the prism material is longer than 60 wavelengths in that material. An equation to determine the real part of the index of refraction is given when the loss must be taken into consideration.

\section{4,242}

PB94-213246 Not available NTIS

National Inst. of Standards and Technology (NEL), Gaithersburg, MD. Precision Engineering Div.

Light Scattering from Glossy Coatings on Paper. Final rept.

T. R. Lettieri, E. Marx, J. F. Song, and T. V.

Vorburger. 1991, 9p.

Pub. in Applied Optics 30, n30 p4439-4447 1991.

Keywords: "Light scattering, "Surface roughness, "Coatings, Optical measurement, Laser radiation, Angular distribution, Autocorrelation, Texture, Pens, Reprints, Angle resolved scattering, Glossy paper.

The application of angle-resolved light scattering (ARLS) to the measurement of the surface roughness of coatings on glossy paper was investigated. To this end, ARLS patterns were measured for laser light scattered from several glossy paper samples, and these experimental patterns were compared to those calculated using a theoretical model based on plane-wave scattering from an isotropic, two-dimensional, rough surface. The calculated patterns were computed using as input, mechanical stylus data for the rms roughnesses and the autocorrelation lengths of the coatings. For all of the paper samples measured, as well as for all of the incidence angles used, there was very good agreement between the experimental and the calculated patterns, indicating that ARLS may be used to determine the coating roughness parameters. As a check on these results, measurements were also made with a commercial optical surface probe; these data compared favorably with both the ARLS and the stylus results. 
National Inst. of Standards and Technology (NEL), Gaithersburg, MD. Precision Engineering Div.

Determination of Surface Roughness from Scattered Light.

Final rep

E. Marx, L. X. Cao, and T. Vorburger. 1989, 4p. Pub. in Proceedings of International Symposium Digest - Antennas and Propagation, San Jose, CA., June 26-30, 1989, v1 p192-195.

Keywords: *Surface roughness, "Light scattering, Optical measurement, Data processing, Autocorrelation, Pens, Reprints.

The surface roughness and correlation length are determined from measurements of scattered light by fitting the data of the spectrum calculated for a random rough surface. The results are compared to values determined from stylus measurements.

04,244

PB94-216538 Not available NTIS

National Inst. of Standards and Technology (MEL), Gaithersburg, MD. Precision Engineering Div.

Autocorrelation Functions from Optical Scattering for One-Dimensionally Rough Surfaces.

Final rept.

E. Marx, B. Leridon, T. R. Lettieri, J. F. Song, and T. V. Vorburger. $1993,10 p$

Pub. in Applied Optics 32, n1 p67-76, 1 Jan 93.

Keywords: * Surface roughness, "Light scattering, One dimensional, Fourier transformation, Autocorrelation, Pens, Reprints, Angle resolved scattering, Fraunhofer approximation.

The relationship between the height autocorrelation function of a one-dimensionally rough surface and the Fourier transform of the intensity distribution of the light scattered by that surface is tested experimentally. The theory is derived by using the Fraunhofer approximation, without recourse to the inconsistent Kirchhoff boundary conditions. In spite of the limitations imposed by the approximations used, the results obtained from optical data agree well with those obtained from stylus data, even for an autocorrelation length as small as the optical wavelength. However, this method should be limited to surfaces with rms roughness smaller than approximately 0.14 times the wavelength of light.

04,245

PB94-216546 Not available NTIS

National Inst. of Standards and Technology (NEL), Gaithersburg, MD. Precision Engineering Div.

Light Scattered by Coated Paper.

Final rept.

E. Marx, J. F. Song, T. V. Vorburger, and T. R.

Lettieri. 1990, $9 \mathrm{p}$

Pub. in Proceedings of Society of Photo-Optical Instrumentation Engineers - Optical Testing and Metrology III: Recent Advances in Industrial Optical Inspection, San Diego, CA., July 8-13, 1990, v1332 p826-834.

Keywords: "Light scattering, "Surface roughness, "Coatings, Optical measurement, Laser radiation, Angular distribution, Autocorrelation, Profiles, Pens, Reprints, Angle resolved scattering, Glossy paper.

Angle-resolved light scattering (ARLS) was used to investigate the roughness of coatings on glossy paper. The angular spectra were measured for laser light scattered from several glossy paper samples, and these spectra were compared to those calculated using the Beckman model of a surface that is isotropic, random, and rough in two dimensions. A surface was characterized by its rms roughness and autocorrelation function, which were determined from surface profiles measured with a stylus instrument. There was very good agreement between the measured and the computed spectra. The surfaces are too rough to produce a specular beam and we cannot get an accurate value of the rms roughness from the angular spectrum, but ARLS provides information about the coating roughness when the measured spectra are compared to computed ones.

04,246

PB94-219391 (Order as PB94-219326, PC A05/

MF A02) National Inst. of Standards and Technology,
Gaithersburg, $M D$.

Comments on the Paper 'Wolf Shifts and Their Physical Interpretation under Laboratory Conditions'

K. D. Mielenz. 1994, 2p.

Included in Jnl. of Research of the National Institute of Standards and Technology, v99 n3 p281-282 May' Jun 94
Keywords: "Spectral shift, Light transmission, Conservation laws, Spectroscopy, Radiometry, Diffraction, Coherence, Invariance, "Wolf shifts.

In a recent paper in the Journal of Research of the National Institute of Standards and Technology, K. D. Mielenz has criticized the generally accepted interpretation of a phenomenon discovered a few years ago, regarding frequency shifts of spectral lines due to coherence properties of sources. In these comments the authors show that much of the criticism is invalid.

\section{4,247}

PB94-219409 (Order as PB94-219326, PC A05/ MF A02)

National Inst. of Standards and Technology, Gaithersburg, MD

Reply to Professor Wolf's Comments on My Paper on Wolf Shifts.

K. D. Mielenz. 1994, 1p.

Included in Jnl, of Research of the National Institute of Standards and Technology, v99 n3 p283 May/Jun 94.

Keywords: "Spectral shift, Light transmission, Conservation laws, Spectroscopy, Radiometry, Diffraction, Coherence, Invariance, "Wolf shifts.

The authors addressed the following comments: (1) Does the spectrum of partially coherent light change on propagation in free space. (2) Do the theory of partial coherence and the classical Huyghens-FresnelKirchhoff diffraction theory give different results in sityations that involve incoherent physical sources. Which of them should be applied for solutions of practical problems. (3) Are traditional radiometric practices af licted by previously unknown errors due to the partial coherence of light.

\section{4,248}

Not available NTIS

National Inst. of Standards and Technology (PL), Boulder, CO. Quantum Physics Div.

Efficient $\mathrm{Br}\left(^{*}\right)$ Laser Pumped by Frequency-Doubled Nd: YAG and Electronic-to-Vibrational Transfer-Pumped $\mathrm{CO} 2$ and $\mathrm{HCN}$ Lasers.

Final rept.

R. L. Pastel, G. D. Hager, H. C. Miller, and S. R.

Leone. 1991, 5p.

Sponsored by Phillips Lab., Kirtland AFB, NM. and National Science Foundation, Washington, $D C$

Pub. in Chemical Physics Letters 183, n6 p565-569, $13 \operatorname{Sep} 91$.

Keywords: *Carbon dioxide lasers, Intermediate infrared radiation, Near infrared radiation, Infrared lasers, Gas lasers, Optical pumping, lodine bromides, YAG lasers, Photodissociation, Reprints, "Bromine lasers, "Hydrogen cyanide lasers.

A photolytic, repetitively pulsed $\operatorname{Br}(4$ doublet $P(1 / 2)$. 4 doublet $P(3 / 2)$ ) laser at 2.714 micrometers is demonstrated by photodissociation of $\mathrm{IBr}$ at $532 \mathrm{~nm}$ using frequency-doubled Nd:YAG pump laser. Pulse energies of $3 \mathrm{~mJ}$ at $10 \mathrm{~Hz}$ are achieved, resulting in a maximum pump energy conversion efficiency of $1.5 \%$. The authors also demonstrate lasing on ro-vibrational transitions of $\mathrm{CO} 2$ at 4.3 micrometers and $\mathrm{HCN}$ at $3.9 \mathrm{mi}$ crometers by electronic-to-vibrational transfer from photolytically generated $\mathrm{Br}$ (star)

\section{4,249}

PB95-125910 Not available NTIS

National Inst. of Standards and Technology (EEEL)

Boulder, CO. Electromagnetic Technology Div.

Complex Propagation Constants for Nonuniform Optical Waveguides: Calculations.

inal rept.

R. L. Gallawa. 1993, 4p.

Pub. in Microwave and Optical Technology Letters 6 , n8 p490-493, 20 Jun 93.

Keywords: "Optical waveguides, *Optical fibers, *Fiber optics, Refractive index, Light transmission, Plana structures, Galerkin method, Attenuation, Reprints, Hermite-Gauss functions, Basis functions.

A method of calculating the complex propagation constant of a planar optical waveguide under very genera but weakly guiding conditions is derived. The method, based on Galerkin's formalism using HermiteGaussian basis functions, allows a nonuniform and complex refractive index profile. The real and imaginary parts of the index are allowed to vary independently and arbitrarily as a function of position. The planar waveguide is used so the results can be com- pared with exact solutions. The method is more general than this, however, and can be used with any geometry. The results are inherently stationary.

04,250

PB95-125936 Not available NTIS

National Inst. of Standards and Technology (EEEL) Boulder, CO. Electromagnetic Technology Div.

Fiber Spot Size: A Simple Method of Calculation. Final rept

R. L. Gallawa, I. C. Goyal, and A. K. Ghatak. 1993 $6 p$.

Pub. in Jnl. of Lightwave Technology 11, n2 p192-197 Feb 93.

Keywords: "Optical fibers, "Fiber optics, Sizes(Dimensions), Symbolic programming, Radiation patterns, Galerkin method, Far field, Algorithms, Reprints, Laguerre-Gauss functions, Spot size, Mode field diameter.

The ability to integrate the Laguerre-Gauss functions in closed form is exploited to allow a simple but accurate evaluation of single-mode fiber spot size using Galerkin's method. The method avoids the need for numerical integration in a broad class of refractive-index profiles. Its simplicity depends on the use of a patternmatching algorithm to avoid the numerical integration normally called for. The algorithm is very fast and gives exact results. The development of symbolic computer languages makes this approach especially easy. We used a symbolic program to predict the spot size and the far-field radiation pattern and compared the results with the exact values, getting excellent results.

04,251

PB95-125944 Not available NTIS

National Inst. of Standards and Technology (EEEL)

Boulder, CO. Electromagnetic Technology Div

Modal Properties of Circular and Noncircular Optical Waveguides.

Final rept.

R. L. Gallawa, I C. Goyal, and A. K. Ghatak 1992

$26 p$.

Pub. in Fiber and Integrated Optics 11, p25-50 1992

Keywords: "Optical waveguides, Circular configura tion, Rectangular configuration, Trigometric functions, Variational methods, Orthogonal functions, Galerkin method, Wave equations, Reprints, Laguerre-Gauss functions, Hermite-Gauss functions, Basis functions, Modal analysis.

We give a review and a comparison of recent methods of analyzing circular and noncircular optical waveguides. Comparison among competing methodologies is made as follows: Galerkin's method is used with Laguerre-Gauss basis functions in circular geometry to examine the modal solution in a step index fiber, and comparison with the exact solution is made. A W-fiber, which has no exact solution, is then examined. Rectangular geometry is considered, and discus sion centers on the use of Galerkin's method using trigonometric basis functions and Hermite-Gauss basis functions. The difficulty arising from the use of basis functions that do not decay exponentially for large argument (trigonometric functions) is illustrated. Finally, a square step index waveguide is used to illustrate a comparison between a variational method that uses the Gaussian approximation as the starting point, and Galerkin's method using Hermite-Gauss basis functions. We conclude that the variational method does well in predicting the propagation constant beta but does not do well in predicting the modal field.

04,252

PB95-126082 Not available NTIS

National Inst. of Standards and Technology (EEEL) Boulder, CO. Electromagnetic Technology Div.

Frequency Stabilization of a Fiber Laser to Rubid jum: A High-Accuracy $1.53 \mathrm{mu} m$ Wavelength Standard.

Final rept.

S. L. Gilbert. 1993, 8p.

Pub. in Proceedings of Society of Photo-Optical Instru mentation Engineers Frequency-Stabilized Lasers and Their Applications, Boston, MA., November 16-18, 1992, v1837 p146-153 1993

Keywords: "Erbium glass lasers, "Frequency stability, *Frequency standards, Near infrared radiation, Infrared spectroscopy, Electron transitions, Rubidium 87, Sta bilization, Optical communication, Laser spectroscopy, Reprints, "Wavelength standards, Fiber lasers, Lase cooling, Atom traps 


\section{Optics \& Lasers}

Spectroscopy of the rubidium $5 \mathrm{P}(3 / 2) \rightarrow->\mathrm{D}(5 / 2)$ transition near 1.529 micrometers has been performed using a single-longitudinal-mode erbium-doped fiber laser. Rubidium atoms were laser-cooled and confined in a vapor-cell Zeeman optical trap. This produced a dense sample of cold atoms and reduced the Doppler broadening of the transition to less than the natural linewidth. Transition linewidths of $10 \mathrm{MHz}$ were observed on the $5 P(3 / 2) \rightarrow 4 D(5 / 2)$ transition and the fiber laser was actively stabilized to the $5 P(3 / 2), F=$ $3 \rightarrow 4 D(5 / 2), F^{\prime}=3$ line of $(87) R b$.

04,253

PB95-126090 Not available NTIS

National Inst. of Standards and Technology (EEEL), Boulder, CO. Electromagnetic Technology Div.

Laser Cooling and Trapping for the Masses.

Final rept.

S. L. Gilbert, and C. E. Wieman. 1993, 7p.

Pub. in Optics and Photonics News, p8-14 Jul 93

Keywords: Temperature range 0000-0013 K, Laser spectroscopy, Semiconductor lasers, Frequency standards, Trapped particles, Beam cooling, Uses, Reprints, "Laser cooling, "Laser trapping, Atoms traps, Wavelength standards, Fiber lasers.

In this article, we describe laser trapping techniques and discuss current applications of this technology, ranging from frequency and wavelength standards to basic physics research.

04,254

\section{PB95-126256 Not available NTIS}

National Inst. of Standards and Technology (EEEL), Boulder, CO. Electromagnetic Technology Div.

Approximate Solution to the Scalar Wave Equation for Optical Waveguides.

Final rept.

I. C. Goyal, R. L. Gallawa, and A. K. Ghatak. 1991,

Pub. in Applied Optics 30, n21 p2985-2989, 20 Jul 91.

Keywords: "Optical waveguides, "Wave equations, WKB approximation, Circular configuration, Planar structures, Integrated optics, Scalars, Reprints.

We consider an approximate solution to the wave equation appropriate to the optical waveguides encountered in practice. The refractive-index profile may be arbitrary, and the geometry may be two or three dimensional. A circular or a planar waveguide could thus be treated by this method. The technique is more accurate and more useful than the WKB method, which is often used in problems of this type, because the technique is valid even at the turning points, where the WKB solution fails. The fields and the propagation constraints of the lowest-order modes for two profiles are calculated, and they compare well with the exact solutions. The solutions that we proposed are, in fact, not new. However, insofar as we know, they are unknown and unused by the optics community.

\section{4,255}

\section{PB95-128633 PC A03/MF A01}

National Inst. of Standards and Technology (PL), Boulder, CO. Time and Frequency Div.

Sub-Doppler Frequency Measurements on OCS at 87 Thz (3.4 micrometers) with the co Overtone Laser: Considerations and Details.

Technical note.

A. Dax, J. S. Wells, L. Hollberg, A. G. Maki, and W.

Urban. Jul 94, 34p, NIST/TN-1365.

Also available from Supt. of Docs. as SN003-00303285-9. Prepared in cooperation with Bonn Univ. (Germany, F.R.). Inst. fuer Angewandte Physik.

Keywords: "Carbon oxysulfide, "Frequency measurement, Intermediate infrared radiation, Carbon monoxide lasers, Optical heterodyning, Electron transitions, Calibration standards, Frequency synthesizers, "Carbonyl sulfide, Polarization spectroscopy, Saturated absorption spectroscopy.

The authors have investigated two techniques for mak ing sub-Doppler frequency measurements with the $\mathrm{CO}$ overtone laser. They studied three OCS transitions whose frequencies overlap either directly with CO (Delta) $v=2$ overtone transition frequencies or with the overtone lines after they have been shifted by an acousto-optic modulator. The authors have inves tigated both conventional saturated-absorption and an optical heterodyne polarization. While they eventually used the latter technique for their measurements, it is too cumbersome to use as a conventional laser stabilization tool. Saturation absorption is considerably simpler and has potential for a more accurate measurement, if some technical problems are overcome This becomes more important in the light of a potential use of the CO (Delta) v=2P(sub 26)(9) transition in a new frequency chain. This transition was used for the OCS P(27) 10(sup 0) 1-00(sup 0)0 measurement. Polarization spectroscopic techniques with optical heterodyne detection were used to observe the features and then to provide the discriminant for locking the overtone laser to the OCS transitions. A CO2 laser synthesizer was used for the frequency measurement basis.

\section{4,256}

PB95-140158 Not available NTIS

National Inst. of Standards and Technology (EEEL), Boulder, CO. Electromagnetic Technology Div.

Metrology Applications of Mode-Locked Erbium Fiber Lasers.

Final rept.

J. B. Schlager, P. D. Hale, and D. L. Franzen. 1992, $1 \mathrm{p}$

Pub. in Proceedings of Conference on Precision Electromagnetic Measurements, Paris, France, June 9-12, 1992, $1 \mathrm{p}$

Keywords: "Mode locked lasers, "Erbium glass lasers, Near infrared radiation, Pulse compression, Light sources, infrared lasers, Metrology, Solitons, Reprints, Fiber lasers, Femtosecond pulses, Optical sampling.

Mode-locked erbium fiber lasers (MLEFLs) are a compact source of short optical pulses around $1540 \mathrm{~nm}$. NIST has developed an 'all' fiber design consisting of commercially available pigtailed components. Soliton pulse shaping helps produce sub-picosecond pulses. Characterization of high-speed detectors and fast optical waveforms has been realized using MLEFLs as a sampling pulse source.

\section{4,257}

PB95-140836 Not available NTIS

National Inst. of Standards and Technology (EEEL), Boulder, CO. Electromagnetic Technology Div.

Rare-Earth-Doped Waveguide Devices: The Poten. tial for Compact Blue-Green Lasers.

Final rept.

N. A. Sanford, J. A. Aust, D. R. Larson, and K. J.

Malone. 1992, 4p

Pub. in Technical Digest Compact Blue-Green Lasers, Santa Fe, NM., February 20-21, 1992, p1-4.

Keywords: "Waveguide lasers, Lithium niobates, Doped materials, Electron transitions, Optical pumping, Visible radiation, Erbium, Reprints, "Blue green lasers, Titanium sapphire lasers.

Er-doped LiNbO3 channel waveguides produce efficient upconversion luminescence from two bands centered at $520 \mathrm{~nm}$ and $550 \mathrm{~nm}$ due to radiative decay from the doublet $H(11 / 2)$ and quartet $S(3 / 2)$ levels, respectively. The doping density of the rare earth was approximately $10(\exp 19) / \mathrm{cu} \mathrm{cm}$. Pumping experiments were performed near $800 \mathrm{~nm}$ and again near $970 \mathrm{~nm}$ with a cw Ti:sapphire laser. The excitation paths are given. The integrated luminescence efficiency, defined as the total visible output (guided and unguided) divided by the absorbed pump power in the waveguide, was approximately $1 \%$. Laser action in the visible due to a single-wavelength pump appears to be prevented by parasitic nonradiative decay from the intermediate levels.

\section{4,258}

PB95-140844 Not available NTIS

National Inst. of Standards and Technology (EEEL), Boulder, CO. Electromagnetic Technology Div.

Linewidth Narrowing in an Imbalanced Y-Branch Waveguide Laser

Final rept.

N. A. Sanford, J. A. Aust, K. J. Malone, and D. R. Larson. 1993, 3p.

Pub. in Optics Letters 18, n4 p281-283, 15 Feb 93

Keywords: "Neodymium lasers, "Waveguide lasers, "Line narrowing, "Line width, Near infrared lasers, Optical pumping, Glass lasers, Reprints, Titanium sapphire lasers.

A $Y$-branch channel waveguide laser whose branch segments were mismatched in length by $2.4 \%$ was fabricated by electric-field-assisted ion exchange in $\mathrm{Nd}$ doped, mixed alkali-silicate glass. The laser output wavelength was centered at $1057.3 \mathrm{~nm}$, and the linewidth was $0.4 \mathrm{~nm}$ FWHM. Our similarly fabricated single-channel Fabry-Perot lasers and balanced $Y$ - branch lasers display linewidths of 3-4 nm. Pumping was performed with a $\mathrm{CW}$ Ti:sapphire laser operating at $785 \mathrm{~nm}$. The imbalanced $Y$-branch laser reached threshold with an absorbed pump power of $48 \mathrm{~mW}$ when a $2 \%$ transmitting output coupler was used. The slope efficiency was $2 \%$. An extended cavity was used to imbalance the arms in a second laser by a ratio of 2.8:1. This device displayed a linewidth of approximately $3.7 \mathrm{GHz}$ FWHM. The linewidth narrowing of these coupled-cavity lasers is analogous to that seen in a Michelson laser.

04,259

PB95-140851 Not available NTIS

National Inst. of Standards and Technology (EEEL) Boulder, CO. Electromagnetic Technology Div.

Nd:LiTaO3 Waveguide Laser.

Final rept.

N. A. Sanford, J. A. Aust, K. J. Malone, D. R. Larson, and A. Roshko. 1992, 3p.

Pub. in Optics Letters 17, n22 p1578-1580, 15 Nov 92

Keywords: "Neodymium lasers, "Waveguide lasers, Near infrared radiation, Lithium tantalates, Infrared lasers, Wafers, Reprints.

Waveguide lasers operating near 1092 and $1076 \mathrm{~nm}$ were fabricated in Z-cut Nd-Ti codiffused LiTaO3. The $\mathrm{Nd}$ diffusion was at $1400 \mathrm{C}$ for $120 \mathrm{~h}$. Samples from two wafers were examined. The Nd film starting thickness was $7 \mathrm{~nm}$ in wafer 1 and $15 \mathrm{~nm}$ in wafer 2 . T stripes, 8-15 micrometers wide, were diffused at 1500 $\mathrm{C}$ for $4 \mathrm{~h}$ for wafer 1 (130-nm stripe thickness) and 2 $\mathrm{h}$ for wafer 2 (100-nm stripe thickness). Pumping was at $750 \mathrm{~nm}$. Threshold occurred at $330 \mathrm{~mW}$ of absorbed pump power for the best waveguides from wafer 1 and $100 \mathrm{~mW}$ for the best waveguides from wafer 2 . The slope efficiency of the latter was $0.07 \%$.

04,260

PB95-141123 Not available NTIS

National Inst. of Standards and Technology (NML) Gaithersburg, MD. Electron and Optical Physics Div. Perturbative Calculation of the AC Stark Effect by the Complex Rotation Method.

Final rept.

L. Pan, K. T. Taylor, and C. W. Clark. 1991, 12p.

Pub. in Physical Review A 43, n11 p6272-6283 1991

Keywords: "Stark effect, Frequency dependence, Multi-photon processes, Alternating current, Perturbation theory, Ground state, Hamiltonians, Greens function, Computation, Reprints, *Hydrogen atoms, Complex rotation method, Multiphoton ionization.

We have calculated the high-order AC Stark effect for the ground state of atomic hydrogen by applying Rayleigh-Schrodinger perturbation theory to a complex-rotated Hamiltonian. We present results for the level shift and multiphoton ionization rate, which include effects of above-threshold ionization.

\section{4,261}

PB95-150173 Not available NTIS

National Inst. of Standards and Technology (PL) Gaithersburg, MD. Radiometric Physics Div.

Integrating Sphere Simulation: Application to Total Flux Scale Realization.

Final rept.

Y. Ohno. 1994, 11p.

Pub. in Applied Optics 33, n13 p2637-2647, 1 May 94.

Keywords: "Optical measurement, Computerized simulation, Ray tracing, Light sources, Calibration, Photometry, Lumens, Reprints, "Luminous flux, "Integrating spheres.

A method is proposed for realizing the total flux scale of light sources by use of an integrating sphere with an opening to introduce a known amount of flux from a luminous intensity standard or a spectral irradiance standard lamp placed outside the sphere. Compute simulations were made on several models of an integrating sphere, designed to compare the total flux of a test lamp inside the sphere with the flux introduced from an external source. The author describes the theory and algorithm of the simulation, presents the results of the simulation for varying conditions of sphere geometry such as size and location of the baffles, internal source, and wall reflectance, and predicts that one of the models has sufficient accuracy to calibrate lamps for total flux.

04,262

PB95-150496 Not available NTIS 
National Inst of Standards and Technology (EEEL) Boulder, CO. Optical Electronic Metrology Group.

Multiwavelength Birefringent-Cavity Mode-Locked Fibre Laser.

Final rept.

H. Takara, S. Kawanishi, M. Saruwatari, and J. B

Schlager. 1992, 2p.

Sponsored by Yokosuka Electrical Communication Lab. (Japan). Transmission Systems Labs.

Pub. in IEE Electronics Letters 28, n25 p2274-2275 Dec 92 and Proceedings of Conference on Lasers and Electro-Optics, Anaheim, CA., May 10-15, 1992, p250.

Keywords: "Erbium glass lasers, "Mode locked lasers *Ring lasers, Laser cavities, Light pulses, Picosecond pulses, Doped materials, Birefringence, Synchronism, Reprints, Multiwavelengths

Generation of simultaneous four wavelength optica pulses is successfully demonstrated using a nove multiwavelength mode-locked $\operatorname{Er}(3+)$-doped fiber ring laser with two $45 \mathrm{deg}$ concatenated, birefringent fibers in the cavity. Wavelength separations of 0.9-2.7 nm and pulse durations of 28-55 ps are obtained.

\section{4,263}

PB95-150702 Not available NTIS

National Inst. of Standards and Technology (EEEL),

Boulder, CO. Electromagnetic Technology Div.

Direct Dispersion Measurement of Highly-ErbiumDoped Optical Amplifiers Using a Low Coherence Reflectometer Coupled with Dispersive Fourier Spectroscopy.

Final rept

K. Takada, T. Kitagawa, K. Hattori, R. Hickernell; M. Yamada, and M. Horiguchi. 1992, 3p.

Sponsored by Nippon Telegraph and Telephone Corp. Ibaraki. Opto-Electronics Labs.

Pub. in Electronics Letters 28, n20 p1889-1891, 24 Sep 92.

Keywords: "Light amplifiers, "Optical measurement, Optical fibers, Planar structures, Refractive index, Erbium additions, Doped materials, Wave dispersion, spectroscopy, Group delay.

The group delay and dispersion, including the erbium ion contributions, of the highly erbium-doped silica planar waveguide amplifier and multicomponent glass fiber amplifiers are directly measured at different pump powers using a low coherence reflectometer and dispersive Fourier spectroscopy. This method derives the refractive index spectra of these amplifiers directly from the produced reflectograms without any physical or mathematical assumptions. The dispersion of the planar waveguide amplifier at $500 \mathrm{~mW}$ pumping changes between +300 and $-200 \mathrm{ps} / \mathrm{km} / \mathrm{nm}$ with a 0.4 wt $\%$ erbium concentration.

\section{4,264}

PB95-150736 Not available NTIS

National Inst. of Standards and Technology (CSTL), Gaithersburg, MD. Surface and Microanalysis Science Div.

Use of Sum Rules on the Energy-Loss Function for the Evaluation of Experimental Optical Data.

Final rept.

S. Tanuma, C. J. Powell, and D. R. Penn. 1993, 15p. Pub. in Jnl. of Electron Spectroscopy and Related Phenomena 62, p95-109 1993.

Keywords: "Optical data, "Sum rules, "Aluminum, "Iridium, "Molybdenum, "Silicon, "Titanium, "Tungsten, EV range 10-100, EV range 100-1000, Electron spectroscopy, Energy losses, Reprints, Energy loss functions.

We present an evaluation of optical data for $\mathrm{Al}, \mathrm{Si}, \mathrm{Ti}$ Mo, W, and Ir based on two sum rules for the energyloss function, the familiar f-sum rule and another sum rule based on a limiting form of the Kramers-Kronig integral. These sum rules were used to evaluate sets of energy-loss function data constructed first from tabulated optical data which have been supplemented by interpolations in the 40-100 eV range for Ti, Mo, W and $\operatorname{Ir}$. A second set of energy-loss function data was constructed for each material by substituting energy loss function values calculated from the optical data of Windt et al. (Appl. Opt., 27(1988) 246, 279) in the 10$525 \mathrm{eV}$ range. The deviations in the results of the sumrule tests with the second set of optical data were about twice those found for the first set. We conclude that the first set of optical data is preferred over the second set.
National Inst. of Standards and Technology (MEL). Gaithersburg, MD. Precision Engineering Div.

Regimes of Surface Roughness Measurable with Light Scattering.

Final rept.

T. V. Vorburger, E. Marx, and T. R. Lettieri. 1993,

Pub. in Applied Optics 32, n19 p3401-3408, 1 Jul 93.

Keywords: "Surface roughness, "Light scattering, *Optical measurement, Directional measurement. Angular distribution, Fourier transformation, Autocorrelation, Reflectance, Reprints, Angle resolved scattering.

In this paper we summarize a number of previous experiments on the measurement of the roughness of metallic surfaces by light scattering. We identify several regimes that permit measurement of different surface parameters and functions, and we establish approximate limits for each regime. Using a straightforward criterion, we calculate that the smooth-surface regime in which the angular distribution of scattered light is closely related to the power spectral density of the roughness, ranges over $0<$ sigma/lambda approx $=$ or $<0.05$, where sigma is the rms roughness and lambda is the optical wavelength. Above that, the surface autocorrelation function may be calculated from a Fourier transform of the angular distribution over 0 $<$ sigma/lambda approx $=$ or $<0.14$. Then comes the specular regime where the specular beam can still be identified and measured over $0<$ sigma/lambda approx $=$ or $<0.3$. For all of these regimes and for rougher surfaces too, the rms width of the scatter distribution is proportional to the rms slope of the surface.

\section{4,266}

PB95-151940 Not available NTIS

National Inst. of Standards and Technology (EEEL), Boulder, CO. Electromagnetic Technology Div.

Optical Fiber Geometry: Accurate Measurement of Cladding Diameter.

Final rept.

M. Young, S. E. Mechels, and P. D. Hale. 1993, 2p. Pub. in Proceedings of Conference on Precision Electromagnetic Measurements, Paris, France, June 9-12, 1992, p202-203.

Keywords: *Optical fibers, "Optical microscopes, "Optical measurement, "Dimensional measurement, "Micrometers Optical interferometers, Fiber optics, White light, Diameters, Artifacts, Reprints, "Claddings, Scanning confocal microscopes.

This paper reports progress toward developing an artifact standard for video microscopes devoted to measuring optical fiber geometry. Specifically, we have developed three devices, a contact micrometer a scanning confocal microscope, and a white-light interference microscope, that are capable of absolute measurements with accuracy between 50 and $100 \mathrm{~nm}$.

\section{4,267}

PB95-153334 Not available NTIS

National Inst of Standards and Technology (MSEL). Gaithersburg MD Ceramics Div.

Moisture and Water-Induced Crack Growth in Optical Materials.

Final rept.

D. C. Cranmer, S. W. Freiman, G. S. White, and A. S. Raynes. 1990, 12p.

Pub. in Proceedings of Society of Photo-Optical Instrumentation Engineers: Optical Surfaces Resistant to Severe Environments, San Diego, CA., July 11-12, 1990, v1330 p152-163.

Keywords: "Optical materials, "Crack propagation, "Water erosion, "Moisture, Environment effects, Fracture properties, Fatigue(Materials), Sensitivity, Zinc sulfides, Silicon dioxide, Titanium dioxide, Glass, Reprints

A number of optically important materials such as $\mathrm{ZnS}$, $\mathrm{SiO} 2, \mathrm{SiO} 2-\mathrm{TiO} 2, \mathrm{GaAs}$, and heavy metal fluoride (e.g., ZBLAN) glasses are subject to moisture-and/or liquid water-induced crack growth. A notable exception to this behavior appears to be Si. Such environmentally enhanced crack growth can lead to ultimate failure in service at stresses well below those expected from normal strength tests. The sensitivity of a material to water can be obtained by determining a crack growth parameter. N . This parameter can be combined with other easily obtainable fracture information which include measures of the strength and strength distribution to create a lifetime design diagram using fracture mechanics concepts. Methods for determining these fracture parameters including direct crack growth measurements and dynamic fatigue are reviewed, and the influence of environmental water on the materials is discussed. Crack growth mechanisms including physical (dielectric) and chemical reaction mechanisms are discussed, and lifetime design diagrams which can be used to determine stress levels in service are presented.

\section{4,268}

PB95-153714 Not available NTIS

National Inst. of Standards and Technology (EEEL) Boulder, CO. Electromagnetic Technology Div.

High-Resolution Spectroscopy of Laser-Cooled Rubidium in a Vapor-Cell Trap.

Final rept.

S. L. Gilbert. 1993, $1 p$.

Pub. in Proceedings of Quantum Electronics and Lase Science Conference, Baltimore, MD., May 2-7, 1993 , p212.

Keywords: "Laser spectroscopy, "Rubidium, Erbium glass lasers, Near infrared rediation, Optical communication, High resolution, Electron transitions, Frequency stability, Trapped particles, Reprints, Fiber lasers, Atom traps, Laser cooling.

High resolution spectroscopy was performed using a 1.529 micrometer fiber laser to probe the $5 \mathrm{P}(3 / 2)$--> $4 \mathrm{D}(5 / 2)$ transition in rubidium atoms confined in a vapor-cell trap. Line shapes and fiber laser stabilization are discussed.

04,269

PB95-153771 Not available NTIS

National Inst. of Standards and Technology (PL), Boulder, CO. Quantum Physics Div.

Frequency Stabilized Lasers: A Parochial Review. Final rept.

J. L. Hall. $1993,14 p$

Contracts N00014-89-J-1227, AFOSR91-0283

Sponsored by Office of Naval Research, Arlington, VA., Air Force Office of Scientific Research, Bolling AFB, DC., and National Science Foundation, WashingAFB, DC.

Pub. in Proceedings of Society of Photo-Optical Instrumentation Engineers: Frequency-Stabilized Lasers and Their Applications, Boston, MA., November 16-18, 1992, v1837 p2-15 1993.

Keywords: *Laser stability, "Frequency stability, Atomic clocks, Frequency standards, Laser spectroscopy Laser cavities, High resolution, Uses, Reprints, Atomic interferometry, Atom traps.

This article is meant to serve as a useful introduction to the art of stabilized lasers, especially for those who are entering this activity from other fields. With the current explosion of interest in atom trapping techniques we can look forward to major progress in the narrowine laser/super-sharp absorber high resolution spectroscopy business. Applications range from atomic clocks to cold atom collision physics to tests of special relativity. The combination of ultra-stable lasers with cold atom interferometry will be especially powerful in offering new tests of atomic charge neutrality and of time reversal invariance via new limits on atomic elec tric dipole moments. Remarkably, a practical instrument for oil and gas prospecting might be based on a laser-diode/atom-interferometric measurement of local ' $g$ '.

\section{4,270}

PB95-161204 Not available NTIS

National Inst. of Standards and Technology (PL), Gaithersburg, MD. Radiometric Physics Div.

Method of Realizing Spectral Irradiance Based on an Absolute Cryogenic Radiometer.

Final rept.

B. C. Johnson C. Cromer, R Saunders, Sapritsky, G. Dezsi, G. Eppeldauer, and J. Fowler. 1993, 7p

Pub. in Metrologia 30, p309-315 1993

Keywords: Blackbody radiation, Cryogenic temperature, Radiometers, Reprints, "Irradiance measure ment.

A technique is presented for realizing spectral irradiance using a large-area, high temperature, uniform, black-body source and filter-radiometers that are calibrated using a High Accuracy Cryogenic Radiometer. The method will be studied by calibrating irradiance lamps with this new technique and comparing the results with those obtained by the method currently em ployed at the National Institute of Standards and Tech- 
nology (NIST). Progress to date and preliminary results are presented. The ultimate goal of the program is to reduce the measurement uncertainties in the spectral irradiance scales that are made available to industry by calibrating deuterium and tungsten-halogen irradiance lamps.

\section{4,271}

PB95-161329 Not available NTIS

National Inst. of Standards and Technology (EEEL)

Boulder, CO. Electromagnetic Technology Div.

Bending-Induced Phase Shifts in Dual-Mode Planar Optical Waveguides.

Final rept.

A. Kumar, and R. L. Gallawa. $1993,3 p$

Pub. in Optics Letters 18, n17 p1415-1417, 1 Sep 93.

Keywords: "Optical waveguides, "Bending, Refractive index, Phase shift, Planar structures, Dual mode, Reprints, Waveguide sensors.

We examine the manner in which the effective index for each of the two modes of a bent dual-mode planar waveguide changes with curvature. We find that the bending-induced changes in the effective indices depend strongly on core-cladding index contrast and the value of $V$. For waveguides with large contrast, the changes in effective indices are such that the change in the phase difference between the modes is positive (or negative) at large (or small) values of $\mathrm{V}$. The change becomes zero at a $V$ value that depends on the waveguide parameters and curvature. If the index contrast is small, the bending-induced phase difference may change sign with increase in curvature. This might help in ultimately explaining the bipolar phase shift seen in a recent experiment. The results of our study can be used to increase or decrease the bending sensitivity of dual-mode optical-waveguide sensors and devices.

04,272

PB95-161519 Not available NTIS

National Inst. of Standards and Technology (EEEL), Boulder, CO. Electromagnetic Technology Div

Optical Fiber Geometry by Gray-Scale Analysis with Robust Regression.

Final rept.

L. Mamileti, C. M. J. Wang, M. Young, and D. F

Vecchia. 1992, 4p.

Pub. in Applied Optics 31, n21 p4182-4185, 20 Jul 92.

Keywords: "Optical fibers, "End effects, "Image analysis, Elliptical configuration, Regression analysis, Fiber optics, Geometry, Reprints, Gray scale.

We have used least-median-of-squares (LMS) regres. sion to analyze gray-scale images of optical fiber ends. This regression is a form of robust regression that ignores outlying data points. We fitted ellipses to the images of each of two fiber ends by using LMS and leastsum-of-squares regression. The two methods yielded nearly identical results on a pristine fiber end, but the LMS method was far superior on a damaged fiber end, even though we made no effort to filter the outlying data points.

\section{4,273}

PB95-161949 Not available NTIS

National Inst. of Standards and Technology (PL), Gaithersburg, MD. Radiometric Physics Div.

Detector-Based Candela Scale and Related Photometric Calibration Procedures at NIST.

Final rept.

Y. Ohno, C. L. Cromer, J. E. Hardis, and G.

Eppeldauer. 1994, 10p

Pub. in Jnl. of the Illuminating Engineering Society, p89-98 1994.

Keywords: "Calibration, "Accuracy, "Luminosity, "Photometers, Light sources, Lumens, Detectors, Uncertainty, Standards, Luminous intensity, Spectrum analysis, Luminance, Illuminance, Flux density, Reprints, "Candela scale.

The candela scale, one of the SI base units, has been realized by using absolutely calibrated detectors rather than sources. A group of eight photometers were constructed using silicon photodiodes, precision apertures, and glass filters for V(lambda) match. Their absolute spectral responsivities were calibrated against the NIST absolute spectral responsivity scale. The measurement chain has been significantly shortened compared with the old scale based on a black body. This resulted in improving the calibration uncertainty to be $0.46 \%$ ( 2 sigma), a factor-of- 2 improvement. This revision has made various photometric calibrations at
NIST more versatile and flexible. Luminous intensity of light sources, ranging from 10(exp -3) to 10(exp 4) candelas, is directly calibrated with the standard photometers which have a linear response over that range. Illuminance meters are calibrated directly against the standard photometers. The luminance scale has also been realized on the detector base using an integrating sphere source. The total flux ranging from 10(exp -2) to 10(exp 5) lumens can be meas ured in a $2 \mathrm{~m}$ integrating sphere using a photometer of the same design. These revisions accompanied significant improvement of the calibration accuracy.

04,274

PB95-162020 Not available NTIS

National Inst. of Standards and Technology (EEEL), Boulder, CO. Electromagnetic Technology Div.

Bragg Gratings in Optical Fibers Produced by a Continuous-Wave UItraviolet Source.

Final rept.

H. Patrick, and S. L. Gilbert. 1993, 1p.

Pub. in Proceedings of Conference on Lasers and Electro-Optics, Baltimore, MD., May 2-7, 1993, p508.

Keywords: *Gratings(Spectra), *Optical fibers, Continuous radiation, Near ultraviolet radiation, Laser rad ation. Frequency multipliers, Argon lasers, Germanium additions, Doped materials, Reprints, *Bragg gratings.

Bragg gratings with a reflectance of $50 \%$ were written in Ge-doped fiber by exposure to continuous-wave 244 $\mathrm{nm}$ light. The ultraviolet light was produced by frequency doubling argon-ion laser light in a buildup cavity.

\subsection{5}

PB95-162038 Not available NTIS

National Inst. of Standards and Technology (EEEL), Boulder, CO. Electromagnetic Technology Div.

Growth of Bragg Gratings Produced by Continuous-Wave Ultraviolet Light in Optical Fiber.

Final rept.

H. Patrick, and S. L. Gilbert. 1993, 3p.

Pub. in Optics Letters 18, n18 p1484-1486, 15 Sep 93.

Keywords: "Gratings(Spectra), *Optical fibers, Continuous radiation, Near ultraviolet radiation, Germanium additions, Doped materials, Refractive index, Reprints, "Bragg gratings.

We have written Bragg gratings of as much as $94 \%$ reflectance in germanium-doped optical fiber by twobeam interference of $244-\mathrm{nm}$ continuous-wave UV light. We measured grating reflectance as a function of exposure time for UV light intensities ranging from 1.5 to $47 \mathrm{~W} / \mathrm{sq} \mathrm{cm}$. The observed dependence of index modulation on time and intensity does not agree with the predictions of a model based on depletion of a de fect population by one-photon absorption.

\section{4,276}

PB95-162350 Not available NTIS

National Inst. of Standards and Technology (PL), Boulder, CO. Quantum Physics Div.

Stabilization and Precise Calibration of a Continuous-Wave Difference Frequency Spectrometer by Use of a Simple Transfer Cavity.

Final rept.

E. Riedle, S. H. Ashworth, J. T. Farrell, and D. J.

Nesbitt. 1994, 7 p

Grants NSF-CHE90-00641, NSF-PHY90-12244

Sponsored by National Science Foundation, Washington, DC

Pub. in Review of Scientific Instruments $65, n 1 p 42$ 48 Jan 94

Keywords: "Spectrometers, "Calibration, Continuous radiation, Difference frequency, Molecular spectra Near infrared radiation, Intermediate infrared radiation, Helium neon lasers, Argon lasers, Ring lasers, Dye la sers, Frequency stability, Reprints, Transfer cavities.

A novel, simple, and inexpensive calibration scheme for a continuous-wave difference frequency spectrometer is presented, based on the stabilization of an open transfer cavity by locking onto the output of a polanization stabilized HeNe laser. High frequency, acoustic fluctuations of the transfer cavity length are com pensated with a piezoelectric transducer mounted mirror, while long term drift in cavity length is controlled by thermal feedback A single mode $\mathrm{Ar}(1+)$ laser, used with a single mode ring dye laser in the difference frequency generation of 2-4 micrometers light, is then ocked onto a suitable fringe of this stable cavity achieving a very small long term drift and furthermore reducing the free running $\operatorname{Ar}(1+)$ linewidth to about 1
$\mathrm{MHz}$. The dye laser scan provides tunability in the difference frequency mixing process, and is calibrated by marker fringes with the same stable cavity. Due to the absolute stability of the marker cavity, precise frequency determination of near infrared molecular transitions is achieved via interpolation between these marker fringes. It is shown theoretically that the residual error of this scheme due to the dispersion of air in the transfer cavity is quite small, and experimentally that a frequency precision on the order of $1 \mathrm{MHz}$ per hour is routinely obtained with respect to molecular transitions.

04,277

PB95-163606 Not available NTIS

National Inst. of Standards and Technology (PL), Boulder, CO. Time and Frequency Div.

Laser Spectroscopy of Carbon Monoxide: A Frequency Reference for the Far Infrared.

Final rept.

T. D. Varberg. 1992, 2p.

Pub. in Proceedings of Conference on Precision Electromagnetic Measurements, Paris, France, June 9-12, 1992, p145-146.

Keywords: "Carbon monoxide, "Laser spectroscopy, "Far infrared radiation, Rotational spectra, Frequency standards, Reprints, Reference materials.

The purely rotational spectrum of carbon monoxide in the far infrared has been accurately measured. The set of calculated transitions derived from a fit to these data forms the world's most accurate far infrared frequency reference.

\section{4,278}

PB95-164000 Not available NTIS

National Inst. of Standards and Technology (NML) Gaithersburg, MD. Center for Atomic, Molecular and Optical Physics.

XUV Characterization Comparison of Mo/Si Multilayer Coatings.

Final rept.

D. L. Windt, W. K. Waskiewicz, G. D. Kubiak, T. W.

Barbee, and R. N. Watts. 1991, 9p.

Sponsored by A.T. and T. Bell Labs., Murray Hill, NJ. Pub. in Proceedings of Society of Photo-Optical Instrumentation Engineers: X-ray/EUV Optics for Astronomy, Microscopy, Polarimetry, and Projection Lithography, San Diego, CA., July 9-13, 1990, v1343 p2742821991.

Keywords: "Optical coatings, "Reflectance, Extreme ultraviolet radiation, Comparative evaluations, Optical measurement, Soft x-rays, Reflectometers, Molybdenum, Silicon, Reprints, Multilayers, X-ray mirrors, Systematic errors.

The reflectance of seven Mo/Si multilayer coatings made at AT\&T Bell Labs and at Lawrence Livermore National Laboratories (LLNL) have been measured using three different reflectometers, in order to determine whether reflectance measurements made using different reflectometers yield consistent results. By comparing the deduced adjustable parameters used to fit the measured reflectances with those calculated from a model based on recursive application of the modified Fresnel equations, we conclude that the measurements made with the three reflectometers are inconsistent. We attribute the discrepancies to systernatic measurement errors, including those associated with the spectral purity of the incident radiation.

\section{4,279}

PB95-164182 Not available NTIS

National Inst. of Standards and Technology (EEEL), Boulder, CO. Electromagnetic Technology Div.

Vector Theory of Diffraction by a Single-Mode Fiber: Application to Mode-Field Diameter Meas. urements.

Final rept.

M. Young, and R. C. Wittman. 1993, 3p.

Pub. in Optics Letters 18, n20 p1715-1717, 15 Oct 93.

Keywords: "Optical fibers, "Wave diffraction, "Diffraction, Vector fields, Far field, Obliqueness, Integrals, Fiber optics, Reprints, Single mode fibers, Modefield diameter, Obliquity factors.

We derive a far-field diffraction integral by (vector) electromagnetic theory the result differs from the (scalar) Rayleigh-Sommerfeld or Kirchhoff result in that it depends on polarization. For unpolarized light, we derive a factor that replaces the usual obliquity factor. Omitting this factor can cause an error of the same order as the measurement uncertainty in determining 
the mode-field diameter of a single-mode fiber from farfield data.

04,280

PB95-168464 Not available NTIS

National Inst. of Standards and Technology (EEEL), Boulder, CO. Electromagnetic Technology Div. Interlaboratory Comparison of Polarization-Holding Parameter Measurements on HighBirefringence Optical Fiber.

Final rept.

Pub. in Proceedings of Optical Fibre Measurement Conference, Torino, Italy, September 21-22, 1993 p177-180.

Keywords: *Optical fibers, Interlaboratory comparisons, Optical measurement, Polarization(Waves) Fiber optics, Birefringence, Reprints, $H$ parameter.

We report the results of a preliminary interlaboratory companison of polarization-holding parameter ( $h$-parameter) measurements, in which seven participants measured three coils of fiber using the participants normal procedures. The variations in results (one standard deviation) among the three coils ranged from $13 \%$ to $50 \%$

04,281

PB95-168548 Not available NTIS

National Inst. of Standards and Technology (PL), Boulder, $\mathrm{CO}$. Time and Frequency Div.

New cw CO2 Laser Lines: The 9-mu m Hot Band. Final rept.

K. M. Evenson, C. C. Chou, B. W. Bach, and K. G. Bach. 1994, 2p.

Pub. in Institute of Electrical and Electronics Engineers Jnl. of Quantum Electronics 30, n5 p1187-1188 May 94.

Keywords: *Carbon dioxide lasers, "Infrared spectra, Intermediate infrared radiation, Frequency measurement, Continuous radiation, Gratings(Spectra), Line spectra, Reprints.

We have made what we think is the first observation of the oscillation of the 9-micrometer hot-band lines 01(sup 1) $\rightarrow-$ (left bracket)11(sup 1)0, 03(sup 1) 0(right bracket)(sub II) of $\mathrm{CO} 2$. We have observed 40 lines with a maximum power of $8 \mathrm{~W}$. They will provide a new source of laser radiation for spectroscopy. The set of lines has been positively identified by directly measuring the frequencies of two of the lines with a heterodyne technique using a $\mathrm{CO} 2$ laser standard.

04,282

PB95-168571 Not available NTIS

National Inst. of Standards and Technology (EEEL), Boulder, CO. Electromagnetic Technology Div.

Mode Coupling and Loss on Tapered Optical Waveguides.

Final rept.

R. L. Gallawa, A. Kumar, and A. Weisshaar. 1994,

$3 p$

Pub. in Proceedings of Integrated Photonics Research Topical Meeting, San Francisco, CA., February 17-19, 1994, p57-59.

Keywords: *Optical waveguides, "Dielectric waveguides, Coupled modes, Galerkin method, Approximation, Tapering, Reprints.

Tapered dielectric waveguides have been analyzed using a variety of methods including coupled mode theory, a step tapered configuration, a method that uses a ray-optics model, and the beam propagation method We also use a step taper approach, approximating the smooth taper with a series of discrete steps. Our method accounts for the interaction between modes and is capable of tracking the propagation through the taper by using an expansion of the field on each side of the step; we use basis functions that are known to approximate the field very accurately. Integration is avoided in evaluating the coupling efficiency across the step.

\section{4,283}

PB95-168589 Not available NTIS

National Inst. of Standards and Technology (EEEL),

Boulder, CO. Electromagnetic Technology Div.

Symbolic Programming with Series Expansions: Applications to Optical Waveguides.

Final rept.

R. L. Gallawa, A. Kumar, and A. Weisshaar. 1994

Pub. in Proceedings of Annual Review of Progress in Applied Computational Electromagnetics (10th), Monterey, CA., March 21-26, 1994, p475-481.
Keywords: *Optical waveguides, "Optical fibers, "Symbolic programming, Series expansion, Approximation, Eigenvalues, Eigenvectors, Reprints, Single mode fibers.

We discuss the utility of symbolic computer languages in the context of optical fiber analysis. The symbolic Mapping command, for example, is useful whenever a series expansion approach is used in eigenvalue problems if the basis functions are integrable in closed form. We show how this command allows a simple but accurate evaluation of single-mode fiber parameters in most cases of practical interest. The Replacement command is also demonstrated in tracking the variation of fiber operational parameters as a function of the V-parameter. The saving in CPU time is substantial.

\section{4,284}

PB95-168597 Not available NTIS

National Inst. of Standards and Technology (EEEL),

Boulder, CO. Electromagnetic Technology Div.

Comparison of UV-Induced Fluorescence and Bragg Grating Growth in Optical Fiber.

Final rept.

S. L. Gillbert, and H. Patrick. 1994, 1p.

Pub. in Proceedings of Conference on Lasers and Electro-Optics, Anaheim, CA., May 8-13, 1994, v8 p244.

Keywords: *Optical fibers, *Fluorescence, Near ultraviolet radiation, Continuous radiation, Optical measurement. Time dependence, Refractive index Gratings(Spectra), Doped materials, Germanium additions, Darkening, Reprints, "Bragg gratings.

We have measured the time dependence of the 400 $\mathrm{nm}$ fluorescence of Ge-doped optical fiber illuminated with continuous-wave $244 \mathrm{~nm}$ light. Our results differ from previous measurements that used pulsed $242 \mathrm{~nm}$ light.

\section{4,285}

PB95-168639 Not available NTIS

National Inst. of Standards and Technology (EEEL), Boulder, CO. Electromagnetic Technology Div.

Improved Variational Analysis of Inhomogeneous Optical Waveguides Using Airy Functions.

Final rept

I. C. Goyal, R. L. Gallawa, and A. K. Ghatak. 1993

$4 p$. 1578 in $\mathrm{Jnl}$.

Keywords: *Optical waveguides, Variational methods, Airy function Planar structures, Light transmission, Approximation, Inhomogeneity, Eigenvalues, Reprints.

Variational trial fields that are based on modified Airy functions are proposed to obtain the propagation characteristics of inhomogeneous planar optical waveguides. We compare with other recently proposed trial fields to demonstrate the improved accuracy obtained through the use of these Airy function trial fields. The probable reason that the proposed fields are better suited than others is that, unlike the others, they depend on the profile shape. The argument of the Airy function trial field is also sensitive to the rate of chang of the profile. The fields are thus better matched to the exact field, improving the variational results.

\section{4,286}

PB95-168753 Not available NTIS

National Inst. of Standards and Technology (PL), Boulder, CO. Time and Frequency Div.

Light Scattered from Two Atoms.

Final rept.

W. M. Itano, U. Eichmann, J. C. Bergquist, M. G. Raizen, D. J. Wineland, J. J. Bollinger, and J. M. Gilligan. 1994, 8p.

Sponsored by Office of Naval Research, Arlington, VA Pub. in Proceedings of the International Conference on Lasers '93, Lake Tahoe, NV., December 6-9, 1993 p412.4191994

Keywords: *Optical interference, "Light scattering Trapped particles, Laser radiation, Mercury ions, Ion storage, Photon-atom collisions, Ouantum mechanics, Ouantum optics, Reprints, Polarization dependence, Laser cooling, Atom traps, Complementarity.

We have observed interference fringes, like those in Young's classic experiment, in the laser light scattered by two trapped atoms. The interference fringes are present only in one polarization of the scattered light. The polarization dependence is related to the complementarity principle, which forbids the simultaneous observation of wave-like and particle-like aspects of light. The interference fringes are due to processes in which a single photon scatters from two atoms. We describe methods which might be used to observe other interference effects due to two photons scattering from two atoms.

04,287

PB95-168779 Not available NTIS

National Inst. of Standards and Technology (EEEL)

Boulder, CO. Electromagnetic Technology Div.

Widths and Propagation of a Truncated Gaussian Beam.

Final rept.

R. D. Jones, and T. R. Scott. 1994, 12p.

Pub. in Proceedings of Workshop on Laser Beam Characterization (2nd), Berlin, Germany, May 30-June 1, 1994, p161-172.

Keywords: "Laser beams, Light transmission, Beam profiles, Near field, Far field, Slits, Irradiance, Truncation, Reprints, Gaussian beams, Beam divergence.

We calculated irradiance profiles resulting from the truncation of a gaussian beam by hard-edged slits transmitting 95 or 99 percent of the incident power. Three definitions--second moment, slit, and knife edge--were used to obtain widths of the near and far field profiles. From these widths, we calculated the propagation factors of the beam, with and without a lens second moment widths are indeterminate due to their dependence on the extent of integration. Scanning slit and knife-edge widths are measurable, but do not, in general, fit the hyperbolic propagation equation Least-squares fits of the measurements can result in propagation factors better than the ideal limit. Beam divergence values calculated from single beam width measurements, or from least-squares fits of many data, differ by several percent.

\section{4,288}

PB95-168795 Not available NTIS

National Inst. of Standards and Technology (EEEL)

Boulder, CO. Electromagnetic Technology Div.

Bending-Induced Loss in Dual-Mode Rectangular Waveguides.

Final rept.

A. Kumar, and R. L. Gallawa. 1994, 3p.

Pub. in Optics Letters 19, n10 p707-709, 15 May 94. Keywords: "Optical waveguides, Rectangular waveguides, Elliptical configuration, Dual mode, Reprints, Bending losses.

We examine how the bending-induced mode losses in a dual-mode rectangular-core waveguide vary with bend orientation. Bending about the minor axis (case (i)) and bending about the major axis (case (ii)) are considered. The second (LP(11)) mode is more lossy in case (i) than in case (ii), while the reverse is true for the first (LP(01)) mode. Further, in case (i) the LP(11)-mode loss is larger than than LP(01)-mode loss, but in case (ii) the LP(01)-mode loss is, surprisingly, larger than the LP(11)-made loss. LP(11)-mode loss is consistent with the recent experimental results. This study should be useful in designing efficient elliptical-core fiber components such as LP(11)-mode strippers based on differential mode loss of the first two modes.

04,289

PB95-168803 Not available NTIS

National Inst. of Standards and Technology (EEEL),

Boulder, CO. Electromagnetic Technology Div.

Bent Rectangular Core Waveguides: An Accurate Perturbation Approach.

Final rept.

A. Kumar, and R. L. Gallawa. 1994, 5p.

Pub. in Microwave and Optical Technology Letters 7. n6 p281-285, 20 Apr 94

Keywords: "Dielectric waveguides, "Optical waveguides, "Optical fibers, Rectangular waveguides, Perturbation theory, Waveguide bends, Reprints.

We discuss a method of evaluating the effective indices of quasi-modes of a bent rectangular core waveguide taking into account the correct dielectric constant of the corner regions, which is ignored in earlier methods. At small bend curvatures and low $\mathrm{V}$ values, the corner regions contribute much more to the effective index change than the bending itself. (Copyright(c) 1994 John Wiley \& Sons, Inc)

04,290

PB95-169256 Not available NTIS 


\section{PHYSICS}

\section{Optics \& Lasers}

National Inst. of Standards and Technology (EEEL), Boulder, CO. Electromagnetic Technology Div. Optical Power Meter Calibration Using Tunable Laser Diodes.

Final rept.

I. Vayshenker, X. Li, and T. R. Scott. 1994, 10p

Pub. in Proceedings of National Computer Systems

Laboratory Workshop and Symposium 'The Role of

Metrology in a Changing World', Chicago, IL., July 31-

August 4, 1994, p363-372.

Keywords: "Optical measurement, "Power meters, "Calibration, Near infrared radiation, Semiconductor lasers, Tunable lasers, Metrology, Standards, Reprints, "Optical power.

We describe a measurement system developed by NIST to calibrate optical power meters using either collimated-beam or connectorized-fiber configurations. This calibration system uses tunable laser diodes which operate in the three fiber optics windows of 850 1,310 , and $1,550 \mathrm{~nm}$. This paper describes the stand ards, techniques, and systems involved in these calibrations.

\section{4,291}

PB95-169355 Not available NTIS

National Inst. of Standards and Technology (EEEL), Boulder, CO. Electromagnetic Technology Div.

Optical Detector Nonlinearity: A Comparison of Five Methods.

Final rept.

S. Yang, I. Vayshenker, X. Li, and T. R. Scott. 1994,

$2 p$

Pub. in Proceedings of Conference on Precision Elec tromagnetic Measurements Digest, Boulder, CO., June 27-July 1, 1994, p455-456.

Keywords: "Optical detectors, "Infrared detectors, *Power meters, Optical measurement, Mathematica models, Computerized simulation, Nonlinearity, Reprints, Optical power.

We derived a set of unified equations for five methods to evaluate nonlinearity of power meters and detectors. We performed computer simulations of these methods The simulations assist in design of a measurement system to meet a target accuracy. Measurements verified the simulations.

\section{4,292}

PB95-169363 Not available NTIS

National Inst. of Standards and Technology (PL), Boulder, CO. Time and Frequency Div.

Far Infrared Laser Frequencies of (13) $\mathrm{CD} 3 \mathrm{OH}$

Final rept.

L. R. Zink, G. P. Galvao, K. M. Evenson, and E. C.

C. Vasconcellos. 1994, 2p.

Pub. in Jnl. of Ouantum Electronics 30, n6 p1361-1362 Jun 94

Keywords: "Laser radiation, "Methyl alcohol, * Methanol, Far infrared radiation, Infrared lasers, Optical pumping, Deuterium compounds, Carbon 13 Heterodyning, Reprints.

We have measured 30 far-infrared laser frequencies of optically pumped (13) $\mathrm{CD} 3 \mathrm{OH}, 13$ of which are new lines. The frequencies range from 0.5 to $4.9 \mathrm{THz}$ with the majority between 0.75 and $1.5 \mathrm{THz}$. Two frequency stabilized $\mathrm{CO} 2$ lasers were used as standards for the heterodyne measurements.

04,293

PB95-169371 PC A07/MF A02

National Inst. of Standards and Technology,

Gaithersburg, MD

Journal of Research of the National Institute of Standards and Technology, September/October 1994. Volume 99, Number 5 .

$1994,148 \mathrm{p}$

See also PB95-169389 through PB95-169405 and PB95-160594. Also available from Supt. of Docs. as SN703-027-00060-1.

Keywords: "Meetings, "US NIST, "Research and development, "Periodicals, Optics, Electron microscopes, Microelectronics, Systems engineering, Information systems, Standards, Computer security.

Contents include articles on Tilt Effects in Optical Angle Measurements; Optical Characterization in Microelectronics Manufacturing; and Critical Issues in Scanning Electron Microscope Metrology. Also, there are Conference Reports on the 16th National Computer Security Conference and Systems Integration
Needs of U.S. Manufacturers. Various News Briefs are included as well.

04,294

PB95-169389

MF A02)

National Inst of Standards and Technology,

Gaithersburg, MD.

Tilt Effects in Optical Angle Measurements.

Y. H. Oueen. 1994, 12p

Included in Jnl. of Research of the National Institute of Standards and Technology, v99 n5 p593-604 Sep/ Oct 94.

Keywords: "Calibrating, "Angles(Geometry), "Collimators, Tilt, Errors, Vector analysis, Tests.

Vector analysis is used to determine the quantitative error in angle calibration using autocollimators. This error is caused by tilt in the mount upon which the artifact is placed. For tilt angles that are less than 1 degree, the error can be simplified to be the product of a coefficient and three terms. The three terms are: (1) the square of the tilt, (2) the sine of the artifact's nominal angle, and (3) the cosine of the artifact's nominal angle plus two times the artifact's position angle. It is shown that the error can be eliminated by placing the artifact at designated periodic positions.

04,295

PB95-173068 PC A04/MF A01

National Inst. of Standards and Technology (EEEL),

Boulder, CO. Electromagnetic Technology Div.

Video Microscopy Applied to Optical Fiber Geometry Measurements.

Technical note.

N. A. Brilliant, B. K. Alpert, and M. Young. Nov 94

56p, NIST/TN-1369.

Also available from Supt. of Docs. as SN003-033103.

Keywords: "Fibers, "Optical equipment, "Microscopes, Fiber optics, Telecommunication, Micrometers, Measuring instruments, Gray scales, Coherence, Geometry, Images, "Video microscope, National Institute of Standards and Technology.

The report describes a video microscope used to measure the mean diameter and noncircularity of the cladding of optical fibers. Discribed is an unsuccessful attempt to perform absolute measurements. When the microscope was calibrated with a fiber of known diameter, however, the cladding diameter could be measured within 0.1 micro $\mathrm{m}$

\section{4,296}

PB95-175469 Not available NTIS

National Inst. of Standards and Technology (PL), Boulder, CO. Time and Frequency Div.

Optical Probing of Cold Trapped Atoms.

Final rept.

R. W. Fox, S. L. Gilbert, L. Hollberg, J. H. Marquardt, and H. G. Robinson. 1993, 3p

Sponsored by Air Force Office of Scientific Research Bolling AFB, DC . and Naval Command, Control and Ocean Surveillance Center, San Diego, CA.

Pub. in Optics Letters 18, n17 p1456-1458, 1 Sep 93

Keywords: "Rubidium, "Cesium, Electron transitions Excited states, Beam cooling, Neutral atoms, Semiconductor lasers, Glass lasers, Reprints, Laser trapping, Laser cooling, Atom traps.

Transitions between excited states of laser-cooled and laser-trapped rubidium and cesium atoms are probed by use of fiber and diode lasers. High-resolution Dopper-free spectra are detected by observation of the absorption and fluorescence of light from the intermediate level of two-step cascade systems. The optical doubleresonance spectra show Autler-Townes splitting in the weak probe limit and more complicated spectra for a strongly coupled three-level system.

\section{4,297}

PB95-175477 Not available NTIS

National Inst. of Standards and Technology (PL), Boulder, CO. Time and Frequency Div.

High-Sensitivity Spectroscopy with Diode Lasers. Final rept.

R. W. Fox, H. G. Robinson, A. S. Zibrov, J. Magyar, L. W. Hollberg, N. Mackie, and J. Marquardt. 1992,

$6 p$.

Sponsored by National Aeronautics and Space Administration, Washington, DC. and Air Force Office of Scientific Research, Bolling AFB, DC.
Pub. in Proceedings of Society of Photo-Optical Instrumentation Engineers: Frequency-Stabilized Lasers and Their Applications, Boston, MA., November 16-18, 1992, v1837 p360-365.

Keywords: "Laser spectroscopy "Semiconductor lasers, Laser stability, Laser cavities, Line spectra, Line width, Visible spectra, High sensitivity, High resolution, Red(Color), Calcium, Cesium, Reprints.

Linewidth reduction of an extended cavity diode laser at $657 \mathrm{~nm}$ was accomplished by negative feedback to an intra-cavity ADP crystal. High resolution $(170 \mathrm{kHz}$ wide) saturated absorption signals were recorded of the calcium intercombination line which is of interest for a frequency standard. The spectrum of the red 6 doublet $P(3 / 2)-9$ doublet $S(1 / 2)$ cesium line in a mag. neto-optical cell trap was also investigated.

\section{4,298}

PB95-175501 Not available NTIS

National Inst. of Standards and Technology (EEEL)

Boulder, CO. Electromagnetic Technology Div.

Calculated Fiber Attenuation: A General Method Yielding Stationary Values.

Final rept.

R. L. Gallawa, I. C. Goyal, and A. K. Ghatak. 1993, $5 p$

Pub. in Jnl. of Lightwave Technology 11, n12 p19001904 Dec 93.

Keywords: "Optical fibers, "Transmission loss, "Refractive index, Galerkin method, Complex numbers, Fiber optics, Attenuation, Computation, Reprints. A method of calculating the attenuation constant of an optical fiber under very general but weakly guiding conditions is derived. The method, based on Galerkin's formalism, allows a nonuniform and complex refractive-index profile. The real and imaginary parts of the refractive index are allowed to vary independently and arbitrarily as a function of radius. The result is the predicted complex propagation constant. The results are inherently stationary.

\section{4,299}

PB95-175519 Not available NTIS

National Inst. of Standards and Technology (EEEL), Boulder, CO. Electromagnetic Technology Div.

Fibre Splice Loss: A Simple Method of Calculation. Final rept.

R. L. Gallawa, A. Kumar, and A. Weisshaar. 1994,

$8 p$

Pub. in Optical and Ouantum Electronics 26, pS165S172 1994

Keywords: "Optical fibers, "Transmission loss, *Splices, Galerkin method, Fiber optics, Computation, Splicing, Eigenvectors, Reprints, Single mode fibers. We evaluate the loss encountered when splicing between two circular single-mode fibers with unmatched parameters. Our method represents a significant improvement in simplicity over other methods, with only an insignificant degradation of accuracy. We use Galerkin's method, but expand the field of both fibers in terms of the same set of basis functions, leading to considerable simplicity: the overlap integral is simply the inner (dot) product of the eigenvectors. Integration is thus avoided. We assume that weakly guiding conditions prevail.

04,300

PB95-175881 Not available NTIS

National Inst. of Standards and Technology (PL), Boulder, CO. Time and Frequency Div.

Absolute Frequency Measurements of Methanol from 1.5 to $6.5 \mathrm{THz}$.

Final rept.

F. Matsushima, K. Evenson, and L. Zink. 1994, 14p Pub. in Jnl. of Molecular Spectroscopy 164, p517-530 1994

Keywords: "Methanol, "Infrared spectra, "Frequency measurement, Far infrared radiation, Forbidden transitions, Carbon dioxide lasers, Rotational spectra, Reprints, Frequency references.

Frequencies of $445 \mathrm{CH} 3 \mathrm{OH}$ rotational transitions between 1.5 and $6.5 \mathrm{THz}$ have been measured with an accuracy of one part in 10(exp 9). The far-infrared radiation used for the measurements was generated from the radiation of two $\mathrm{CO} 2$ lasers using a MIM diode as a nonlinear mixer. The high resolution and sensitivity of the spectrometer also enabled us to observe a series of forbidden O-branch transitions ((Delta) $n=1$ and (Delta) $K=0$ ) for $J=12$ to 26 . 
04,301

PB 95-175949 Not available NTIS

National Inst. of Standards and Technology (MEL),

Gaithersburg, MD. Precision Engineering Div.

Rapid Post-Polishing of Diamond-Turned Optics.

Final rept.

R. E. Parks, and C. J. Evans. 1994, 5p.

Pub. in Precision Engineering 16, n3 p223-227 Jul 94

Keywords: “Polishing, Asphericity, Finishes, Reprints "Optical surfaces, Diamond turning, Surface finish, Electroless nickel.

A simple technique for post-polishing single-point, diamond-turned optics is described. Synthetic fabricfaced laps are used. Surface finish converges to a limiting value set by process parameters. Lap construction and diamond grit size affects both convergence rate and ultimate finish.

\section{4,302}

PB95-176111 Not available NTIS

National Inst. of Standards and Technology (EEEL),

Boulder, CO. Electromagnetic Technology Div.

High-Sensitivity Optical Sampling Using an Erbium-Doped Fiber Laser Strobe.

Final rept.

J. B. Schlager, P. D. Hale, and D. L. Franzen. 1993,

Pub. in Microwave and Optical Technology Letters 6 , n15 p835-837 Dec 93.

Keywords: Near infrared radiation, Distributed feedback lasers, Erbium glass lasers, Mode locked lasers, Infrared lasers, High sensitivity, Picosecond pulses, Laser amplifiers, Real time, Light pulses, Stroboscopes, Reprints, "Optical sampling, Indium gallium arsenide phosphides.

Optical pulses at a repetition frequency of $1 \mathrm{GHz}$ from a gain-switched InGaAsP distributed-feedback laser diode at 1.3 micrometers are optically sampled with compressed pulses from a mode-locked erbium-doped fiber laser amplifier. High signal-to-noise ratios down to 21 -(micro) $W$ average laser-diode power with temporal resolutions close to the sampling pulse duration of 4 ps are achieved. The sampled waveform is displayed at a repetition rate of $3 \mathrm{~Hz}$ or higher to give a real-time measurement.

\section{4,303}

PB95-176152 Not available NTIS

National Inst. of Standards and Technology (PL), Boulder, CO. Time and Frequency Div.

Improved Rubidium Frequency Standards Using Diode Lasers with AM and FM Noise Control.

Final rept.

C. Szekely, and R. E. Drullinger. 1992, 7p.

Pub. in Proceedings of Society of Photo-Optical Instrumentation Engineers: Frequency-Stabilized Lasers and Their Applications, Boston, MA., November 16-18, 1992, v1837 p299-305.

Keywords: ${ }^{\star}$ Rubidium frequency standards, "Frequency standards, Semiconductor lasers, Optical pumping, Noise reduction, Reprints.

Diode-laser-pumped, rubidium cell frequency standards have potential short-term stability that is vastly better than their lamp-pumped counterparts. However, $A M$ and FM noise in monolithic laser diodes limit their performance. With extended-cavity, grating-feedback diode lasers, the AM and FM noise can be controlled. In the preliminary work reported here, we have used such a laser to make measurements in two different rubidium cell systems. Measured line $Q$ and signal-tonoise ratios corresponding to sigma(sub y) (tau) $=4 X$ $10(\exp -13)$ tau(sup -1/2) in a commercial, buffer gas type standard and sigma(sub y) (tau) $=2 \times 10(\exp -13)$ tau(sup -1/2) in an evacuated, wall-coated cell are demonstrated. We believe both of these numbers can be improved significantly in a fully engineered and optimized standard.

04,304
PB95-176186 Not available NTIS

National Inst. of Standards and Technology (PL), Boulder, CO. Time and Frequency Div.

Frequency-Stabilized LNA Laser at 1.083 mum: Application to the Manipulation of Helium 4 Atoms. Final rept.

N. Vansteenkiste, C. Gerz, R. Kaiser, A. Aspect, L.

Hollberg, and C. Salomon. 1991, 22p.

Pub. in Jnl. Phys. II France 1, p1407-1428 1991.

Keywords: *Ring lasers, Infrared lasers, Near infrared radiation, Gas discharges, Atomic beams, Metastable
State, Frequency stability, Helium 4, Reprints, "Helium spectroscopy, Laser cooling, Optical molasses.

We describe a single mode LNA ring laser, which emits $60 \mathrm{~mW}$ at 1.083 micrometers. Thanks to a high frequency F.M. saturated absorption technique, this lase is directly locked to an atomic line in a (4) He discharge cell. The residual frequency jitter is $130 \mathrm{kHz}$. We then present two examples of laser manipulation of helium 4 atoms in the 2 triplet S(1) metastable state, using this laser : a density increase of the atomic beam by a factor of 8 , with one-dimensional optical molasses, and a selective deflection of the metastable helium beam using a laser beam with curved wavefronts.

\section{4,305}

PB95-176228 Not available NTIS

National Inst. of Standards and Technology (PL), Boulder, CO. Time and Frequency Div.

Precise Optical Frequency References and Difference Frequency Measurements with Diode La sers.

Final rept.

S. Waltman, A. Romanovsky, J. Wells, M. P. Sassi,

H. G. Robinson, R. W. Fox, and L. Hollberg. 1992 ,

$6 \mathrm{p}$

Sponsored by National Aeronautics and Space Administration, Washington, DC. and Air Force Office of Scientific Research, Bolling AFB, DC.

Pub. in Proceedings of Society of Photo-Optical Instrumentation Engineers: Frequency-Stabilized Lasers and Their Applications, Boston, MA., November 16-18, 1992, v1837 p386-391.

Keywords: "Frequency measurement, "Difference fre quency, Near infrared radiation, Semiconductor lasers, Electron transitions, Schottky diodes, Wavelengths, Heterodyning, Reprints, Frequency references.

Heterodyne methods have been used in conjunction with molecular calculations to accurately determine the wavelengths of more than 35,000 infrared transitions. We have used high speed whisker contact Schottky diodes to extend this technology to the 0.8 micromete spectral region. Using microwave harmonic mixing, we demonstrate that it is possible to detect beat notes be tween diode lasers to frequencies as high as $400 \mathrm{GHz}$.

\section{4,306}

PB95-176319 Not available NTIS

National Inst. of Standards and Technology (EEEL) Gaithersburg, MD. Office of Law Enforcement Stand ards.

Spectrally Smooth Reflectances That Match.

Final rept.

J. A. Worthey. 1994, 2p.

Pub. in Color Research and Application 19, n5 p395 396 Oct 94

Keywords: "Spectral reflectance, Color vision, Illuminating, Reprints, Metamerism.

This letter to the editor is a comment on William A Thornton, 'Intersections of spectral power distributions of lights that match, Color Res. App. 18(6): 399-410. The present author expresses support for criticisms explained at length by two non-NIST authors in a separate letter. Thornton claims generality for a result that in fact depends on arbitrary assumptions.

\section{4,307}

PB95-180030 Not available NTIS

National Inst. of Standards and Technology (PL) Gaithersburg, MD. Radiometric Physics Div.

Rayleigh Scattering Limits for Low-Leve Bidirectional Reflectance Distribution Function Measurements.

Final rept.

C. Asmail, J. Hsia, A. Parr, and J. Hoeft. 1994, 8p. Pub. in Applied Optics 33, n25 p6084-6091, 1 Sep 94.

Keywords: "Light scattering, *Rayleigh scattering, Optical measurement, Optical paths, Angular distribution, Surfaces, Nitrogen, Helium, Air, Reprints, BSDF(Bidirectional Scattering Distribution Function).

The objective is to estimate the Rayleigh limit in bidirectional reflectance distribution function (BRDF) measurements caused by air in the laboratory, the wavelength, and the path length of light in the receive field of view. Moreover, we intend to show the trend or the reduction of this limit by introducing a medium with small refractive index and by using a longer waveength. Although the BRDF typically describes the an gular distribution of scattered light from surfaces, the expression describing the equivalent BRDF caused by the optical scattering from gas molecules in the optica path is derived through the use of the Rayleigh scattering theory. The instrumentation is described, and the experimental results of the equivalent BRDF caused by gas scattering from molecules in clear air, nitrogen and helium gases are reported. These results confirm the trends of the prediction.

04,308

PB95-180048 Not available NTIS

National Inst. of Standards and Technology (EEEL) Boulder, CO. Electromagnetic Technology Div. Passively Q-Switched Nd-Doped Waveguide Laser Final rept.

J. A. Aust, K. J. Malone, D. L. Veasey, N. A

Sanford, and A. Roshko. 1994,3p.

Pub. in Optics Letters 19, n22 p1849-1851, 15 Nov 94

Keywords: "Waveguide lasers, "Neodymium lasers, Solid state lasers, Q switched lasers, Infrared lasers, Glass lasers, Near infrared radiation, Intergrated optics, Reprints.

A passively Q-switched waveguide laser operating at 1.054 micrometers has been demonstrated in a Nddoped phosphate glass. The channel waveguide was fabricated by K-ion exchange from a nitrate melt. Pas sively Q-switched pulses were achieved by placement of an acetate sheet containing an organic saturableabsorbing dye within the laser cavity. The resulting pulse train consisted of puises with a FWHM of about $25 \mathrm{~ns}$ and peak powers of $3.04 \mathrm{~W}$. With an $80 \%$ trans mitting output coupler, cw operation of the laser pro vided a $5.2 \mathrm{~mW}$ of output power at 1.054 micrometers for $229 \mathrm{~mW}$ of absorbed $794-\mathrm{nm}$ pump power.

04,309

PB95-180261 Not available NTIS

National Inst. of Standards and Technology (PL) Gaithersburg, MD. Radiometric Physics Div.

Broadband High-Optical-Density Filters in the In frared.

Final rept

A. Frenkel, and Z. M. Zhang. 1994, 3p.

Pub. in Optics Letters 19, n18 p1495-1497, 15 Sep 94

Keywords: "Infrared filters, Intermediate infrared radiation, Near infrared radiation, Cadmium tellurides, Zinc selenides, Metal films, Thin films, Substrates, Broadband, Reprints, Lexan(Trademark), Multilayers.

A new concept for the design of broadband (2-25 micrometers and beyond) neutral-density filters in the infrared region without etaloning effects is proposed and demonstrated. One important aspect of the technique is to use metallic-thin-film (10-200-nm-thick) multilaye combinations deposited onto different substrates (ZnSe, CdTe, Lexan). Neutral-density filters with optical densities as high as 4 are designed and built in the broadband region (2-25 micrometers). Another key in novation is the use of ultrathin substrates (about 100 $\mathrm{nm}$ thick) for elimination of etaloning effects normally present in thick $(>0.5-\mathrm{mm}$ ) substrates. Neutral-density filters with such ultrathin substrates are also designed and investigated.

04,310

PB95-180394 Not available NTIS

National Inst. of Standards and Technology (EEEL) Boulder, CO. Electromagnetic Technology Div.

Error Propagation in Laser Beam Spatial Parameters.

Final rept

R. D. Jones, and T. R. Scott. 1994, 10p.

Sponsored by Army Test Measurements and Diagnostic Equipment Activity, Redstone Arsenal, AL.

Pub. in Optical and Quantum Electronics 26, p25-34 1994.

Keywords: "Laser beams, Light transmission, Beam profiles, Spatial distribution, Semiconductor lasers, Error analysis, Uncertainty, Reprints, Beam analysis.

We have performed a propagation-of-errors analysis on two methods used to determine the spatial parameters of a laser beam. We measured diameters of a diode laser beam focused by a $993 \mathrm{~mm}$ focal length lens. Measurement uncertainties of less than $1 \%$ can result in uncertainties greater than $200 \%$ in locating the beam waist of the laser. We compare the inherent uncertainties in the spatial parameters as obtained by the two methods. Longer focal length lenses and lens position can reduce this magnification of uncertainty, but would require large propagation distances. 
04,311

PB95-180485 Not available NTIS

National Inst. of Standards and Technology (EEEL), Boulder, CO. Electromagnetic Technology Div.

Modal Characteristics of Bent Dual Mode Planar Optical Waveguide.

Final rept.

A. Kumar, R. L. Gallawa, and I. C. Goyal. 1994, 4p. Pub. in Jnl. of Lightwave Technology 12, n4 p621-624 Apr 94.

Keywords: *Optical waveguides, "Waveguide bends Planar structures, Modal response, Dual mode, Bending, Reprints.

Modal characteristics of bent dual-mode planar optical waveguides are obtained. The bending-induced changes in the modal power distribution is found to be quite different for the two modes. Surprisingly, unlike the fundamental mode, bending causes the fractional modal power for the second mode to increase in the inner core-half and to decrease in the outer core-half of the waveguide. Interestingly, this leads to a decrease in effective index of the second mode due to bending at sufficiently high $V$-values.

\section{4,312}

PB95-180634 Not available NTIS

National Inst. of Standards and Technology (PL), Boulder, CO. Time and Frequency Div.

Improved Molecular Constants and Frequencies for the $\mathrm{CO} 2$ Laser from New High-J Regular and Hot-Band Frequency Measurements.

Final rept.

A. G. Maki, C. C. Chou, K. M. Evenson, L. R. Zink, and J. T. Shy 1994, $14 \mathrm{p}$.

Pub. in Jnl. of Molecular Spectroscopy 167, p211-224 1994.

Keywords: "Carbon dioxide lasers, "Frequencies, Intermediate infrared radiation, Frequency measurement, Electron transitions, Carbon 12, Carbon 13, Oxygen 16, Oxygen 18, Reprints, Molecular constants.

New frequencies are given for the $(12) \mathrm{C}(16) \mathrm{O} 2$, (13) $\mathrm{C}(16) \mathrm{O} 2$, (12) $\mathrm{C}(18) \mathrm{O} 2$, and (13) $\mathrm{C}(18) \mathrm{O} 2$ regular band laser transitions and for the hot-band transitions of $(12) \mathrm{C}(16) \mathrm{O} 2$. These frequencies are based on a new least-squares analysis of all of the frequency measurements of these four molecular species including new high-J measurements reported here and recent absolute frequency measurements. Fourteen new high-J transitions of the regular (12) $\mathrm{C}(16) \mathrm{O} 2$ laser bands have been observed, the lasers have been stabilized with sub-Doppler saturated 4.3-micrometer fluorescence, and their frequencies have been measured. Nine of these transitions fill the gap between the 9.4and 10.4 -micrometer bands. New trequency measurements are reported for 84 hot-band lines, which were also included in the reanalysis of the $\mathrm{CO} 2$ laser trans:
tions.

\section{4,313}

PB95-180709 Not available NTIS

National Inst. of Standards and Technology (PL), Boulder, CO. Time and Frequency Div.

Stabilization of 3.3 and $5.1 \mathrm{~m}$ Lead-Salt Diode Lasers by Optical Feedback.

Final rept.

M. Murtz, M. Schaefer, M. Schneider, U. Schiessl, M. Tacke, J. S. Wells, and W. Urban. 1992, 6p.

Sponsored by National Aeronautics and Space Administration, Washington, DC. Upper Atmospheric Research Program.

Pub. in Optics Communications 94, n6 p551-556 1994.

Keywords: "Semiconductor lasers, "Laser stability, Intermediate infrared radiation, Lead inorganic salts, Frequency stability, Line narrowing, Molecular spectroscopy, Infrared lasers, Tunable lasers, Feedback, Reprints.

Optical stabilization of tunable lead-salt diode lasers (TDL) in the mid-infrared is presented tor the first time By introducing an external feedback mirror both the linewidth is substantially narrowed and the frequency of the TDL is stabilized and controlled via this mirror in a frequency-offset locked scheme. We achieved narrowing of the linewidth by 1-2 orders of magnitude or better. The TDL is offset-locked to a CO gas laser by a heterodyne technique: the beatnote between the two lasers is used to control the length of the external resonator. By this scheme we gain the capability of absolute frequency measurements with sub-Doppler accuracy. The improved spectral properties of the diode laser provide a new tool for high-resolution molecular spectroscopy in the mid-infrared.

04,314

PB95-180857 Not available NTIS

National Inst. of Standards and Technology (PL) Gaithersburg, MD. Atomic Physics Div.

Laser-Produced and Tokamak Spectra of Lithiumlike Iron, $\mathrm{Fe}(23+)$

Final rept.

J. Reader, J. Sugar, N. Acquista, and R. Bahr. 1994, $5 p$

Sponsored by Department of Energy, Washington, DC. Pub. in Jnl. of the Optical Society of America B 11, n10 p1930-1934 Oct 94

Keywords: *Iron ions, "Ulitraviolet spectra, Laser-produced plasma, Multicharged ions, Tokamak devices, Electron transitions, Extreme ultraviolet radiation, Line spectra, Energy levels, Reprints, "Lithium-like ions.

Spectra of highly ionized iron were generated in a laser-produced plasma and a tokamak. In the spectra from the laser-produced plasma, new lines of $\mathrm{Fe}(23+)$ were observed that were identified as $3 s-4 p, 3 p-4 d$ and $3 d-4 f$ transitions. The measured wavelengths of these lines were combined with measurements of the $2 s-2 p$ resonance lines measured in the tokamak plasma and with earlier measurements of $n=2$ to $n=3$ transitions to yield an improved system of energy levels for $\mathrm{Fe}(23+)$. The measured wavelengths are compared with ab initio theoretical values calculated with a Dirac-Fock code. With the $4 f$ energy levels, which are nearly hydrogenic, an improved ionization energy of $16,503,000+$ or $-1400 / \mathrm{cm}(2046.11+$ or $-0.17 \mathrm{eV})$ was determined.

04,315

PB95-180899 Not available NTIS

National Inst. of Standards and Technology (EEEL) Boulder, CO. Electromagnetic Technology Div.

Antenna-Coupled High-Tc Air-Bridge Microbolometer on Silicon.

Final rept.

J. P. Rice, E. N. Grossman, and D. A. Rudman

1994, 3p.

Pub. in Applied Physics Letters 65, n6 p773-775, 8 Aug 94.

Keywords: *Bolometers, Temperature range 0065 $0273 \mathrm{~K}$ High temperature superconductors, Superconducting devices, Infrared detectors, Reprints, "Microbolometers, Silicon substrates.

An antenna-coupled high-T(c) superconducting microbolometer on a silicon substrate, operating at infrared wavelengths, is described. This detector incorporates a silicon-micromachined yttria-stabilized zirconia air bridge at the feed of a planar lithographic antenna to simultaneously minimize the thermal conductance and the heat capacity of the bolometer. A an operating temperature of $87.4 \mathrm{~K}$, the optica responsivity measured using a $300-\mathrm{K}$ blackbody source over a $0.2-2.9 \mathrm{THz}$ bandwidth is $2900 \mathrm{~V} / \mathrm{W}$, the optical noise-equivalent power (NEP) is $9 \times 10$ (exp 12) $\mathrm{W} / \mathrm{Hz}(\exp 1 / 2)$ and the time constant is $<10$ microsec. This NEP is nearly a factor of 2 lower than the previous record for a liquid-nitrogen-cooled therma detector, and the time constant is several orders of magnitude shorter.

\section{4,316}

PB95-181236 Not available NTIS

National Inst. of Standards and Technology (PL), Gaithersburg, MD. Radiometric Physics Div.

Thermal Modeling of Absolute Cryogenic Radiometers.

Final rept.

Z. M. Zhang, R. U. Datla, S. R. Lorentz, and H. C. Tang. 1994, 6p.

Pub. in Jnl. of Heat Transfer 116, p993-998 Nov 94. Keywords: *Infrared radiometers, *Radiometers, Finite element method, Resistance thermometers, Temperature sensors, Superconducting devices, Cryogenic temperature, Recommendations, Germanium, Reprints.

This work consists of a detailed thermal modeling of two different radiometers operated at cryogenic temperatures. Both employ a temperature sensor and an electrical-substitution technique to determine the absolute radiant power entering the aperture of a receiver Their sensing elements are different: One is a germanium resistance thermometer, and the other is a superconducting kinetic-inductance thermometer. The finite element method is used to predict the transient and steady-state temperature distribution in the receiver. Recommendations are given based on the modeling for future improvement of the dynamic response of both radiometers.

04,317

PB95-202255 Not available NTIS

National Inst. of Standards and Technology (PL), Boulder, CO. Ouantum Physics Div.

High-Efficiency, High-Power Difference-Frequency Generation of 0.9-1.5 mu $\mathrm{m}$ Light in BBO.

Final rept.

S. Ashworth, C. Iaconis, O. Votava, and E. Riedle. 1993, 6p.

Grant NSF-PHY90-12244

Sponsored by National Science Foundation, Washing ton, DC

Pub. in Optics Communications 97, p109-114 1993.

Keywords: "Near infrared radiation, Difference frequency, High power, Nonlinear optics, Light pulses Laser radiation, Neodymium lasers, YAG lasers, Dye lasers, Reprints, *Barium borates, Barium beta borates, Nanosecond pulses.

An efficient method for generation of high energy pulsed infrared light between 0.9 and 15 micrometers is described. The technique uses difference frequency mixing of pulsed, visible dye and Nd:YAG laser light in a $10 \mathrm{~mm}$ long BBO crystal. Ouantum efficiencies of up to $23 \%$ and infrared pulse energies up to $4.5 \mathrm{~mJ}$ are demonstrated. The low shot-to-shot flucluations of difference frequency generation in BBO make this technique an attractive alternative to the conventional optical parametric oscillator or Raman shifting methods that are currently used to access this spectral region.

04,318

PB95-202636 Not available NTIS

National Inst. of Standards and Technology (PL), Boulder, CO. Ouantum Physics Div.

Failures of the Four-Wave Mixing Model for Cone Emission.

Final rept

R. C. Hart, L. You, A. Gallagher, and J. Cooper. 1994, 7p

Pub. in Optics Communications 111, p331-337 1994. Keywords: Four wave mixing, Optical pumping Blue(Color), Pulsed lasers, Dye lasers, Strontium, Vapors, Reprints, *Cone emission.

Strontium vapor is pumped by an unfocused, singlemode, pulsed dye laser tuned near the $461 \mathrm{~nm}$ resonance transition. With blue laser detuning the previously reported red-shifted conical emission is present. We simultaneously resolve in angle and in frequency the emissions from this intense, near-resonant pumping of a 'two-level' atom. This quantitative spectral and angular discrimination provides uniquely detailed measurements and demonstrates a serious lack of understanding of this highly nonlinear phenomenon.

\section{4,319}

PB95-202891 Not available NTIS

National Inst. of Standards and Technology (PL), Boulder, CO. Ouantum Physics Div.

Narrow-Band Tunable Diode Laser System with Grating Feedback, and a Saturated Absorption Spectrometer for Cs and Rb.

Final rept

K. B. MacAdam, A. Steinbach, and C. Wieman 1992, 14p.

Contract N00014-91-J-1006, Grant NSF-PHY9O12244

Sponsored by Office of Naval Research, Arlington, VA. Pub. in American Jnl. of Physics 60, n12 p1098-1111 Dec 92.

Keywords: *Semiconductor lasers, *Tunable lasers, *Spectrometers, Near infrared radiation, Red(Color), Absorption spectra, Atomic spectroscopy, Instruction manuals, Construction, Operation, Rubidium, Cesium, Reprints, Optical feedback.

Detailed instructions for the construction and operation of a diode laser system with optical feedback are presented. This system uses feedback from a diffraction grating to provide a narrow-band continuously tuneable source of light at red or near-IR wavelengths. These instructions include machine drawings for the parts to be constructed, electronic circuit diagrams, and prices and vendors of the items to be purchased. It is also explained how to align the system and how to use it to observe saturated absorption spectra of atomic cesium or rubidium. 
04,320

PB95-203071 Not available NTIS

National Inst. of Standards and Technology (EEEL), Boulder, CO. Electromagnetic Technology Div. Decrease of Fluorescence in Optical Fiber during Exposure to Pulsed or Continuous-Wave Ultraviolet Light.

Final rept.

H. Patrick, S. L. Gilbert, and A. Lidgard. 1994, $8 p$.

Pub. in Optical Materials 3, p209-216 1994

Keywords: "Optical fibers, "Radiation effects, "Fluorescence, Near ultraviolet radiation, Refractive index, Germanium additions, Boron additions, Doped matenals, Blue(Color), Silica, Reprints, Bragg gratings.

We exposed optical fibers to UV light and simultaneously measured the intensity of the blue fluorescence from the fiber core. Two silica glass fibers with different core dopants were investigated: a germanium-doped fiber and a germanium-boron-codoped fiber. The fibers were exposed transversely to pulsed or continuous-wave $244 \mathrm{~nm}$ light for times ranging from a few minutes to over an hour. For all UV intensities and exposure times used, the fluorescence decreased during UV exposure. For a given fiber, the fractional decrease in fluorescence seen from the side of the fiber was dependent only on the total UV fluence.

04,321

PB95-203352 Not available NTIS

National Inst. of Standards and Technology (PL), Boulder, CO. Quantum Physics Div.

Frequency Shifting of Pulsed Narrow-Band Laser Light in a Multipass Raman Cell.

Final rept.

R. Sussmann, T. Weber, E. Riedle, and H. J.

Neusser. 1992, 7p.

Pub. in Optics Communications 88, p408-414 1992

Keywords: "Laser radiation, ${ }^{*}$ Frequency shift, *Raman effect, Molecular spectroscopy, High resolution, Light sources, Light pulses, Optical pumping, Carbon dioxide, Hydrogen, Benzene, Narrowband, Reprints.

A multipass cell is described which allows efficient stimulated Raman frequency shifting for low pump laser intensities and low gas pressures. The latter is important for Raman shifting of narrow-band Fouriertransform limited light pulses (Delta $\mathrm{nu}=75 \mathrm{MHz}$ ). It is shown that frequency broadening of the Raman shifted light can be largely avoided in the Dicke narrowing regime at low pressures. For $75 \mathrm{MHz}$ pump pluses and an $\mathrm{H} 2$ density of 2.5 amagat, we found a negligible broadening to $90 \mathrm{MHz}$ of the stimulated Stokes light This is far below the value of $250 \mathrm{MHz}$ expected from spontaneous emission. The narrow-band Stokes pulses achieved in $\mathrm{CO} 2$ enabled us to measure the pressure shift coefficient $(-0.71 \times 10$ (sup -2$) / \mathrm{cm} /$ amagat) of this gas. It is demonstrated, for the example of benzene, that our technique provides a very practical light source for high resolution molecular spectroscopy.

\section{4,322}

PB95-203469 Not available NTIS

National Inst. of Standards and Technology (PL), Boulder, CO. Quantum Physics Div.

Resonance Fluorescence with Squeezed-Light Excitation.

Final rept.

R. Vyas, and S. Singh. 1992, 15p.

Sponsored by National Science Foundation, Washington, DC.

Pub. in Physical Review A 45, n11 p8095-8109, 1 Jun 92.

Keywords: "Resonance fluorescence, Parametric oscillators, Excitation, Reprints, Squeezed light.

Resonance fluorescence from a single two-level atom driven by a beam of squeezed light is studied in the weak-field limit. We consider the situation where the atom is coupled to the ordinary vacuum and only a few field modes corresponding to the driving field are squeezed. The field produced by the degenerate optical parametric oscillator is used as the driving field. Heisenberg equations of motion are solved in the steady-state and analytic expressions for the fluores cent-light intensity and the spectrum of fluorescent light are derived. We also consider photon statistics of fluorescent light. In particular, squeezing, antibunching and sub-Poissonian statistics of fluorescent photons are discussed, and analytic expressions for the quadrature variance and the two-time intensity correlation function are presented. Contrary to the case of coherent excitation, the second-order intensity correlation function does not factorize. This and other differences are discussed, and curves are presented to illustrate the behavior of various quantities. We also present results for thermal excitation of the atom.

\section{4,323}

PB95-203543 Not available NTIS

National Inst. of Standards and Technology (PL), Boulder, CO. Quantum Physics Div.

High-Resolution Optical Multiplex Spectroscopy.

Final rept.

M. P. Winters, C. W. Oates, J. L. Hall, and K. P.

Dinse. 1992, 9p.

Contract N00014-89-J-1227, Grant NSF-PHY90-

12244

Sponsored by National Science Foundation, Washing ton, DC. and Office of Naval Research, Arlington, VA Pub. in Jnl. of the Optical Society of America B 9, n4 p498-506 Apr 92.

Keywords: "Laser spectroscopy, Laser radiation, Frequency stability, High resolution, Multiplexing, lodine, Reprints, Stochastic excitation.

We report on recent refinements in the development of high-resolution optical multiplex spectroscopy using stochastic excitation. We discuss the attainment of higher excitation bandwidths through the use of faster digital noise sources and digital filtering techniques, the active suppression of residual amplitude modulation noise, laser-frequency stabilization using an external stabilizer, and the performance of various dataanalysis methods.

\section{4,324}

PB95-203550 Not available NTIS

National Inst. of Standards and Technology (EEEL)

Boulder, CO. Electromagnetic Fields Div.

Integral Occurring in Coherence Theory.

Final rept.

R. C. Wittmann, and B. K. Alpert. 1994, 1p.

Pub. in Society for Industrial and Applied Mathematics Review 36, n4 p655-62 Dec 94

Keywords: *Coherent light, "Integrals, Bessel functions, Reprints.

We evaluate a multi-dimensional integral involving cylindrical Bessel functions. This integral arose during study of optical coherence.

\section{4,325}

PB95-203584 Not available NTIS

National Inst. of Standards and Technology (PL), Boulder, CO. Quantum Physics Div.

Cone Emission from Laser-Pumped Two-Level Atoms. 1. Quantum Theory of Resonant Light Propagation.

Final rept.

L. You, J. Mostowski, and J. Cooper. 1992, 22p.

See also Part 2, PB95-203592. Sponsored by National Science Foundation, Washington, DC

Pub. in Physical Review A 46, n5 p2903-2924, 1 Sep 92.

Keywords: Four wave mixing, Resonance fluorescence, Laser pumping, Quantum theory, Light transmission, Reprints, "Cone emission.

This is the first of a series of papers on the theory of cone emission from a laser-pumped two-level atomic medium. Starting from microscopic quantum theory for both the active medium and the field, equations for the slowly varying macroscopic quantities are derived. In the steady-state limit, which experimentally corresponds to cw excitation, we find that the sources for the paraxial fields of interest have a long coherence length despite the random nature of spontaneous emission. These radiation sources enter into the equations for the fields in the same form as the source generated by four-wave mixing and therefore may be considered as a 'spontaneous fou r-wave mixing,' since the photons emitted in the Rabi sidebands are correlated. This has far-reaching consequences on the physics of cone emission and also sheds some new light on the fluorescence from strongly driven atomic systems.

\section{4,326}

PB95-203592 Not available NTIS

National Inst. of Standards and Technology (PL), Boulder, CO. Quantum Physics Div.
Cone Emission from Laser-Pumped Two-Level Atoms. 2. Analytical Model Studies.

Final rept.

L. You, J. Mostowski, and J. Cooper. 1992, 14p.

See also Part 1, PB95-203584. Sponsored by National Science Foundation, Washington, DC

Keywords: Four wave mixing. Spontaneous emission, Cherenkov radiation, Resonance fluorescence, Laser

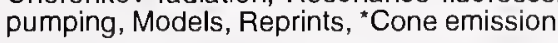

We establish a mechanism, which supports Cherenkov-type radiation, as a source of cone emission. This can be considered as an additional mechanism to the previously discussed four-wave mixing (4WM) and initial encoding and follow-up refraction effects. The radiation source is provided by spontaneous emission of the driven atoms at the frequencies of interest. Due to the medium dispersion, the pump field propagates faster than the phase velocity of the field that is generated at the Rabi sideband at lower frequency. Cherenkov radiation is then possible if the source has a long correlation length, which is indeed the case despite the random nature of spontaneous emission. Coherent superposition of the source for the lower-frequency field is in an off-axis direction given by the Cherenkov radiation condition. Differences between $4 \mathrm{WM}$ and Cherenkov-type models are consequently resolved.

04,327

PB95-203832 Not available NTIS

National Inst. of Standards and Technology (PL), Boulder, CO. Quantum Physics Div.

Stabilization of Optical Phase/Frequency of a Laser System: Application to a Commercial Dye Laser with an External Stabilizer.

Final rept.

M. Zhu, and J. L. Hall. 1993, 15p.

Contract N00014-89-J-1227, Grant NSF-PHY90-

12244

Sponsored by Office of Naval Research, Arlington, VA. and National Science Foundation, Washington, DC Pub. in Jnl. of the Optical Society of America B 10, n5 p802-816 May 93

Keywords: "Continuous wave lasers, "Dye lasers, * Laser stability, Phase locked systems, Frequency stability, Power spectra, Stabilization, Reviews, Reprints.

We present a comprehensive and quasi-tutorial review of the theory for analyzing the optical power spectrum of an optical field that has noise modulations of both the amplitude and the phase. We also present experimental results of the frequency stabilization of a commercial dye laser to a high-finesse Fabry-Perot cavity (0.49- $\mathrm{Hz}$ resulting full linewidth) and of the optical phase locking of the dye laser to a second reference laser (putting $97 \%$ of the optical power into the carrier) using an external stabilizer scheme. This external optical phase/frequency stabilization technique can be applied to virtually any cw laser system.

\section{4,328}

PB95-260758 PC A06/MF A02

National Inst. of Standards and Technology (PL), Gaithersburg, MD. Radiometric Physics Div.

45 deg/0 deg Reflectance Factors of Pressed Polytetrafluoroethylene (PTFE) Powder.

Technical note.

P. Y. Barnes, and J. J. Hsia. Jul 95, 110p, NIST/TN 1413

Also available from Supt. of Docs. as SN003-00303352-9. $\begin{array}{ll}\text { Keywords: } & \text { *Reflectance, } \\ \text { "Powder(Particles), } & \text { "Polytetrafluoroethylene, }\end{array}$ Reflectometers, Synthetic resins, Difluoro compounds, Quality control, Optical measuring instruments, *Reflectance factors, PTFE resins.

Pressed polytetrafluoroethylene (PTFE) powder is used for $45 \mathrm{deg}$. $/ 0 \mathrm{deg}$. reflectance factor standards. The radiometric and spectrophotometric measurements community such as the Council for Optical Radiation Measurements (CORM) has demonstrated the need for such a standard and its application to quality control and quantity assessment. This publication briefly describes the instrumentation used for the 45 deg./ 0 deg. reflectance factor measurements of pressed PTFE powder from $380 \mathrm{~nm}$ to $770 \mathrm{~nm}$. Also, the variations of $45 \mathrm{deg} . / 0 \mathrm{deg}$. reflectance factor with sample preparation and materials are discussed. The expanded uncertainty at a coverage factor of two for 
the $45 \mathrm{deg} . / 0 \mathrm{deg}$. reflectance factors of pressed PTFE powder range from 0.009 to 0.017

04,329

PB95-261913 (Order as PB95-261897, PC A07)

MF A02)

National Inst. of Standards and Technology, Gaithersburg, MD.

Intercomparison of Photometric Units Maintained at NIST (USA) and PTB (Germany), 1993.

Y. Ohno, and G. Sauter. 1995, $13 p$.

Prepared in cooperation with Physikalisch-Technische Bundesanstalt, Brunswick (Germany, F.R.). Abt. Optik und Photometrie.

Included in Jnl. of Research of the National Institute of Standards and Technology, v100 n3 p227-239 May/ Jun 95.

Keywords: "Interlaboratory comparisons, "Calibration standards, "Photometers, "Luminous intensity, Lumens, Flux density, Canada, United States, Germany(Unified). Photometric responsivity, National Institute of Standards and Technology, PTB(Germany).

A bilateral intercomparison of photometric units between NIST, USA and PTB, Germany has been conducted to update the Knowledge of the relationship between the Photometric units diseminated in each country. The luminous intensity unit (cd) and the luminous flux unit ( $\mathrm{Im}$ ) maintained at both laboratories are compared by circulating transfer standard lamps. Also, the photometric responsivity $s(s u b v$ ) is compared by circulating a lambda-corrected detector with a built-in current-to-voltage converter. The results show that the difference of luminous intensity unit between NIST and PTB, (PTB-NIST)/NIST, is $0.2 \%$ with a relative expanded uncertainty (coverage factor $k=2$ ) of $0.24 \%$. The difference is reduced significantly from that at the 1985 CCPR intercomparison $(0.9 \%)$

\section{4,330}

PB96-102215 Not available NTIS

National Inst. of Standards and Technology (PL), Boulder, CO. Time and Frequency Div.

Sub-Doppler Frequency Measurements on OCS at $87 \mathrm{THz}(3.4 \mathrm{mu} \mathrm{m})$ with the CO Overtone Laser.

Final rept.

A. Dax, J. S. Wells, L. Hollberg, A. G. Maki, and W.

Urban. 1994. 13p.

See also PB95-128633. Sponsored by National Aeronautics and Space Administration, Washington, DC.

Pub. in Jnl. of Molecular Spectroscopy 168 , p416-428 1994.

Keywords: *Carbonyl compounds, “Sulfides, "Lasers, *Frequency measurement, Calibrating, Standards, Polarization, Molecular spectroscopy, Wavelengths, Tables(Data), Reprints, "Sub-Doppler frequencies.

Sub-Doppler frequency measurements have been made on three transitions of OCS in the $87-\mathrm{THz}$ region (near $29001 / \mathrm{cm}$ ). The CO overtone laser was used as the saturating laser. Polarization spectroscopic techniques utilizing optical heterodyne detection were used to observe the features and subsequently provide the discriminant for locking the overtone laser to the OCS transitions. A CO2 laser synthesizer was used to measure the frequency of the $\mathrm{CO}$ overtone laser and thereby measure the frequencies of the OCS lines. The resulting frequencies of the three new measurements are given. These new numbers have been fitted along with more than 5700 other data points in our OCS data bank and improved constants have been obtained These latest constants are used to calculate updated calibration tables containing values with much smaller uncertainties; three such tables are included.

\section{4,331}

\section{PB96-102553 Not available NTIS}

National Inst. of Standards and Technology (EEEL)

Boulder, CO. Optoelectronics Div.

Characterization of a Clipped Gaussian Beam.

Final rept.

$1995,15 p$

Pub. in Proceedings of Society of Photo-Optical Instrumentation Engineers: Beam Control, Diagnostics, Standards, and Propagation, San Jose, CA., February 6-7, 1995, v2375 p360-374.

Keywords: *Laser beams, Light transmission, Beam profiles, Divergence, Irradiance, Wave propagation, Width, Slits, Diffraction, Reprints, Times diffraction limit.

The authors calculated irradiance distributions resulting from clipping a Gaussian beam with hard-edged slits transmitting 99 or 95 percent of the incident power The authors determined the widths -- second momen (with 8- and 12-bit resolution), scanning slit, and knifeedge -- of these profiles at several distances from the source, both with and without a focusing lens. When using a lens, they had the option of determining the characterization parameters from two beam width measurements and one axial distance, or from a least squares fit of several beam width measurements and axial distances. Finite resolution of irradiance, inheren in any measurement device, truncates integrals necessary for calculation of the second moment of irradiance distributions.

04,332

PB96-102736 Not available NTIS

National Inst. of Standards and Technology (PL), Boulder CO Quantum Physics Div.

Delivering the Same Optical Frequency at Two Places: Accurate Cancellation of Phase Noise Introduced by an Optical Fiber or Other Time-Varying Path.

Final rept

L. S. Ma, P. Jungner, J. Ye, and J. L. Hall. 1994, 3p. Sponsored by Office of Naval Research, Arlington, VA and National Science Foundation, Arlington, VA.

Pub. in Optics Letters 19, n2 p1777-1779, 1 Nov 94 Keywords: "Phase noise, ${ }^{\star}$ Doppler radar, Reprints Cancellation, Noise, "Optical radar, Optical fiber.

Although a single-mode optical fiber is a convenient and efficient interface/connecting medium, it intro duces phase-noise modulation, which corrupts highprecision frequency-based applications by broadening the spectrum toward the kilohertz domain We describe a simple double-pass fiber noise measurement and control system, which is demonstrated to provide millihertz accuracy of noise cancellation.

\section{4,333}

PB96-102843 Not available NTIS

National Inst. of Standards and Technology (PL), Gaithersburg, MD. Radiometric Physics Div.

New Method for Realizing a Luminous Flux Scale Using an Integrating Sphere with an External Source.

Final rept

Y. Ohno. 1995, 10p.

Pub. in Jnl. of the Illuminating Engineering Society 24 n1 p106-115 1995.

Keywords: "Luminous flux, *Optical measurement, $R e$ prints, Lumens, Light sources, Computerized simulation, Integrating spheres, Calibration.

A method is proposed to realize a total luminous flux scale (lumens) using an integrating sphere with a known amount of flux introduced from an externa source. The integrating sphere features an opening for a detector, a test lamp in its center, a baffle between the test lamp and the detector. A second opening is used to introduce flux from an external source, and is appropriately baffled from the test lamp. Flux from the external source is introduced into the sphere through limiting aperture of known area. The flux entering the sphere is determined from knowledge of the illuminance on the aperture plane and the area of the aperture. The total flux from the test lamp is deduced by comparison to the flux from the external source. Theoretical analysis was first made by computer simulation based on a ray tracing technique. Several integrating sphere designs were analyzed under various conditions, and based on the results of the simulation, an integrating sphere $50 \mathrm{~cm}$ in diameter was built to conduct experimental analysis. The characteristics of the sphere were measured and compared with the simulation results. The total luminous flux values of severa miniature lamps measured with this method were found to agree within $0.5 \%$ with measurements using goniophotometric technique. This method can be ap plied also to realize a total spectral radiant flux scale.

04,334

PB96-111638 Not available NTIS

National Inst. of Standards and Technology (PL) Gaithersburg, MD. Atomic Physics Div.

Improved Wavelengths for Prominent Lines of $\mathrm{Fe}$ $\mathrm{XX}$ to $\mathrm{Fe} X \mathrm{X}$ III.

Final rept.

J. Sugar, and W. L. Rowan. 1995, 3p.

Pub. in Jnl. of the Optical Society of America B, v12 n8 p1403-1405, 13 Aug 95.

Keywords: *Iron, "Line spectra "Wavelengths, Ioniza tion, Laser plasmas, Intensity, Energy levels, Tokamak devices, Electron transitions, Charged particles, Reprints.

New measurements of 26 spectral lines of highly ionized $\mathrm{Fe}$ in the range of 91.263 Angstroms have been carried out with a wavelength uncertainty of + or - 5 mAngstroms. The light source was the TEXT tokamak at the University of Texas in Austin. Lines of Be-like, $\mathrm{C}$-like, and $\mathrm{N}$-like $\mathrm{Fe}$ are included, with visually estimated intensities, the best previous measurements, and energy-level classifications. Level values are derived from these wavelengths and from known magnetic dipole (M1) lines.

04,335

PB96-111950 Not available NTIS

National Inst. of Standards and Technology (EEEL), Boulder, CO. Electromagnetic Technology Div.

Glasses for Waveguide Lasers.

Final rept.

K. J. Malone, D. L. Veasey, N. A. Sanford, and J. S. Hayden. 1994, 13p.

Pub. in Proceedings of Society of Photo-Optical Instrumentation Engineers: Properties and Characteristics of Glass III, San Diego, CA., July 28-29, 1994, v2887 p75-87.

Keywords: *Glass, "Optical systems, Reprints, Ion exchange, Neodymium, Erbium, Waveguide lasers.

Waveguide lasers formed by ion exchange in rateearth-doped glasses have emerged as an attractive new technology on the threshold of commercial insertion. These devices can be used as both laser oscillators and optical amplifiers. In this article, we review ion exchange and glass composition. We then discuss the performance of ion-exchanged waveguide lasers made in silicate and phosphate glasses.

\section{4,336}

PB96-113535 PC A05/MF A01

Nationa! Inst. of Standards and Technology, Gaithersburg, MD.

Journal of Research of the National Institute of Standards and Technology, November/December 1994. Volume 99 , Number 6.

1994, 92p.

See also PB96-113543 through PB96-113584 and PB95-169371. Also available from Supt. of Docs.

Keywords: "Research and development, "Metrology, "Standards, Interlaboratory comparisons, Quality assurance, Pressure, Temperature, Optical equipment, Radiators, Magnetohydrodynamics, NIST(National Institute of Standard and Technology).

No abstract available.

\section{4,337}

PB96-113576 (Order as PB96-113535, PC A05/

MF A01)

National Inst. of Standards and Technology,

Gaithersburg, MD.

Beamcon III, a Linearity Measurement Instrument for Optical Detectors.

A. Thompson, and H. M. Chen. 1994, 5p.

Prepared in cooperation with Alabama Univ. in Hunts ville. Dept. of Electrical and Computer Engineering.

Included in Jnl. of Research of the National Institute of Standards and Technology, v99 n6 p751-755 Nov/ Dec 94

Keywords: "Optical detection, "Beams(Radiation), "Linearity, Detectors, Radiation measuring instruments, Silicon, Photodiodes, "Beam addition method, NIST(National Institute of Standards and Technology).

The design and operation of Beamcon III, the latest linearity measurement instrument using the beam addition method in the detector metrology program at the National Institute of Standards and Technology, is described. The primary improvements in this instrument are the reduction of stray radiation to extremely low levels by using three well-baffled chambers, a larger dynamic range, and an additional source entrance port. A polynomial response function is determined from the data obtained by this instrument using a leastsqueares method. The linearity of a silicon photodiodeamplifer detector system was determined to be within $0.054 \%$ (2 sigma estimate) over nine decades of signal

04,338

PB96-119292 Not available NTIS

National Inst. of Standards and Technology (EEEL) Boulder, CO. Optoelectronics Div. 
Magnetooptic Effects.

Final rept.

M. N. Deeter, G. W. Day, and A. H. Rose. 1995, 36p. Pub. in CRC Handbook of Laser Science and Technology, Supplement 2: Optical Materials, Section 9.1, p367-402 1995 .

Keywords: "Magnetooptics, "Faraday effect, "Kerr magnetooptical effect, Liquid crystals, Birefringence, Magnetohydrodynamics, Ferromagnetic materials, Paramagnetic materials, Diamagnetism, Ferrimagnetic materials, Antiferromagnetism, Reprints.

We review the orgins and applications of various magneto-optic effects, including the Faraday and Kerr effects, and magnetic linear birefringence. Mathematically, we show how all of these effects can be derived from a single dielectric tensor. We then consider the characteristics of magneto-optic effects in specific material classes, including diamagnetic, paramagnetic, ferromagnetic, ferrimagnetic, and antiferromagnetic materials. Relevant data for each class are summanzed in tabular format. Finally, both scientific and commerical applications of magneto-optic materials are described.

\section{4,339}

PB96-119581 Not available NTIS

National Inst. of Standards and Technology (PL), Boulder, CO. Quantum Physics Div

Doppler-Free Spectroscopy of Large Polyatomic Molecules and van der Waals Complexes.

Final rept.

H. J. Neusser, E. Riedle, T. Weber, and E. W.

Schlag. 1992, 7p.

Pub. in Topics in Applied Physics, v70 p205-212 1992.

Keywords: "Tunable lasers, "Laser spectroscopy "Benzene, "Polyatomic molecules, Spectral line width, Molecular structure, Energy distribution, Electronic spectra, Ultraviolet spectra, Van der Waals forces, Chemical bonds, Coupling, Reprints, *Doppler-free spectroscopy, Molecular clusters, Coriolis coupling

It is shown that high-resolution spectroscopy with tunable dye lasers leads to rotationally resolved electronic spectra of large molecular systems. Two-photon absorption of narrow-band cw light in an external cavity eliminates Doppler broadening in the S(sub 1) inversely maps to S(sub 0) transition of the prototype organic molecule benzene. Several thousands of rotational lines in the room temperature spectrum are analyzed, providing spectroscopic constants with a hitherto inaccessible precision. Investigations of the homogeneous linewidth of individual rotational transitions reveals that Coriolis coupling plays an important role in the intramolecular energy redistribution process in this moloecule. Rotationally resolved UV spectra of benzene-noble-gas van der Waals clusters were measured. These measurements yield precise information on the van der Waals bond lengths and structures of these complexes.

\section{4,340}

PB96-119599 Not available NTIS

National Inst. of Standards and Technology (EEEL) Boulder, CO. Electromagnetic Technology Div

Using Secondary lon Mass Spectrometry (SIMS) to Characterize Optical Waveguide Materials.

Final rept.

S. W. Novak, J. M. Zavada, and K. Malone. 1994,

2p.

Pub. in Proceedings of the Annual MAS Meeting (28th), Microbeam Analysis Society, New Orleans, LA July 31-August 5, 1994, p167-168.

Keywords: "Optical waveguides, "Mass spectroscopy, Measuring instruments, Lithium niobates, Oxides, Glass, Diffusion, Ion implantation, Refractivity, Tita nium, Silver, Reprints, SIMS(Secondary Ion Mass Spectrometry).

Secondary ion mass spectrometry (SIMS) has proven to be a versatile technique to obtain depth resolved in formation on optical waveguides in materials such as $\mathrm{LiNbO3}, \mathrm{LiTaO} 3$ and glasses. Optical waveguides are typically formed by diffusing an element into the material to cause a localized change in its refractive index. In LiNbO3 this is done using Ti indiffusion from a deposited metal film or proton exchange in an acid bath. Optical waveguides in glasses have been made using $\mathrm{Ag}$ indiffusion from $\mathrm{AgNO} 3$ solutions. SIMS instruments have been used to obtain depth profiles of planar waveguides using either a normal-incidence electron gun in a magnetic sector SIMS instrumenta or a quadrupole-based SIMS instrument.
04,341

PB96-119623 Not available NTIS

National Inst. of Standards and Technology (PL), Boulder, $\mathrm{CO}$. Time and Frequency Div

Far Infrared Laser Frequencies of $\mathrm{CH} 3 \mathrm{OD}$ and $\mathrm{N} 2 \mathrm{H} 4$.

Final rept.

H. E. Radford, K. M. Evenson, F. Matushima, T. J.

Sears, L. R. Zink, and G. P. Galvao. 1991, 6p

Pub. in International Jnl. of Infrared and Millimeter Waves, v12 n10 p1161-1166 1991.

Keywords: "Lasers, "Methyl alcohol, "Hydrazine, Optical pumping, Reprints, Frequency measurements, Molecular laser lines.

The frequencies of 26 laser lines with wavelengths be tween 57 and 534 um have been measured in the optically pumped laser gases $\mathrm{CH} 3 \mathrm{OD}$ and $\mathrm{N} 2 \mathrm{H} 4$. A pair of stabilized cw $12 \mathrm{CO} 2$ lasers was used as a frequency standard for the heterodyne frequency measurements. Seven of the 26 lines are new.

\section{4,342}

PB96-119672 Not available NTIS

National Inst. of Standards and Technology (EEEL),

Boulder, CO. Electromagnetic Technology Div.

Standard Polarization Components: Progress Toward an Optical Retardance Standard.

Final rept.

K. B. Rochford, P. A. Williams, A. H. Rose, G. W.

Day, I. G. Clarke, and P. D. Hale. 1994, 7 .

Pub. in Society of Photo-Optical Instrumentation Engineers, Polarization Analysis and Measurement II, San Diego, CA., July 25-27, 1994, v2265 p2-8.

Keywords: "Birefringence, Reprints, Polarization, Metrology, Waveplate, "Optical retardance, Quartwave retarder.

NIST is developing a quarterwave linear retarder designed to have a retardance stable within 0.1 degree over a vaniety of operational and environmental conditions. In this paper the authors review several design strategies and early results of this effort. These have led to a promising prototype design consisting of a double rhomb TIR retarder constructed from a low stressoptic glass. The authors also review several measurement methods that are used in their evaluations.

\section{4,343}

PB96-119680 Not available NTIS

National Inst. of Standards and Technology (EEEL), Boulder, CO. Electromagnetic Technology Div. Improved Annealing Technique for Optical Fiber. Final rept.

A. H. Rose, Z. B. Ren, and G. W. Day. 1994, 4p

Pub. in International Conference on Optical Fibre Sensors (10th), Glasgow, Scotland, UK, October 11-13, 1994, v2360 p306-309.

Keywords: "Birefringence, Reprints, *Foreign technology, ${ }^{*}$ Current sensing, "Fiber annealing

The authors demonstrate that twisting a fiber a few turns per meter before it is annealed largely eliminates the residual linear bierefringence. This dramatically improves the yield of annealed coils for current sensing and makes it possible to use fibers that previously could not be successfully annealed. It is believed that twisting is effective because the residual birefringence is associated with core ellipticity and this contribution is averaged to near zero by twisting. The authors also show the temperature stability of sensors made with this new technique.

\section{4,344}

PB96-119805 Not available NTIS

National Inst. of Standards and Technology (EEEL), Boulder, CO. Electromagnetic Technology Div.

Standard Reference Materials for Optical Fibers and Connectors.

Final rept.

M. Young. 1995, 2p.

Pub. in Proceedings: Optical Fiber Communication Conference, San Diego, CA., February 26-March 3 , 1995, v8 p239-240.

Keywords: "Microscopes, *Optical fibers, Reprints, Conforal, Gray scale, Interference, Micrometers, Video, Communications.

This paper outlines the development of a standard of fiber cladding diameter and the three instruments - a micrometer, a scanning confocal microscope, and a white-light interference microscope - that were crucial to that development. An international round robin yielded agreement generally with 0.1 micometers. The authors are planning other artifact standards.

\section{4,345}

PB96-122643 Not available NTIS

National Inst. of Standards and Technology (EEEL), Boulder, CO. Electromagnetic Technology Div.

Decay of Bragg Gratings in Hydrogen-Loaded Optical Fibers.

Final rept.

H. Patrick, and S. L. Gilbert. 1995, 2p

Pub. in OFC ' 95 Optical Fiber Communication, San Diego, CA., February 26-March 3, 1995, v8 p179-180 1995.

Keywords: "Optical fibers, "Hydrogen "Gratings(Spectra), Annealing, Doping, Photosensitivity, Ultraviolet radiation, Reflectance, Reprints, Bragg gratings, Doped fibers, Hydrogen-loading.

Bragg gratings written in hydrogen-loaded optical fibers showed a larger decrease in reflectivity than gratings in non-hydrogen-loaded fibers after 10 hours at 100 degrees $C$ and after 18 days at room temperature.

04,346

PB96-122957 Not available NTIS

National Inst. of Standards and Technology (EEEL)

Boulder, CO. Electromagnetic Technology Div.

Growth Characteristics of Fiber Gratings.

Final rept.

S. L. Gilbert, and H. Patrick. 1994, $2 p$

Pub. in Proceedings of the LEOS ' 94 Symposium on Photoinduced Devices in Optical Fibers and Wavequides (IEEE Lasers and Electro-Optics Society 1994 Annual Meeting) Boston, MA. October 31-November 3, 1994, p131-132.

Keywords: "Gratings, *Fiber optics, Bragg angle, Optical fibers, Ultraviolent radiation, Reprints, "Fiber gratings.

We have measured the time and UV intensity dependence of the growth of Bragg gratings in germaniumdoped and germanium-boron-codoped optical fiber We compare this with the change in blue fluorescence and theoretical predictions. We find that the index change during UV exposure is correlated with a decrease in fluorescence and that a simple one-photon model for defect absorption does not explain the time and intensity dependence of the grating growth.

04,347

PB96-122973 Not available NTIS

National Inst. of Standards and Technology (PL), Boulder, CO. Quantum Physics Div.

Introduction to Phase-Stable Optical Sources.

Final rept.

J. L. Hall, and M. Zhu. 1992, 32p.

Contract AFOSR91-0283, Grant NSF-PHY90-12244 Sponsored by National Science Foundation, Washing ton, DC.. Air Force Office of Scientific Research, Bolling AFB, DC. and Office of Naval Research, Arlington, VA.

Pub. in Proceedings of the International School of Physics 'Enrico Fermi', Course CXVIII, Laser Manipulation of Atoms and Ions, Varenna, Italy, July 9-19, 1991, p671-702

Keywords: "Lasers, Reprints, "Foreign technology, * Superstable lasers, High resolution laser spectroscopy, Laser cooled atoms.

This lecture deals with one of the impressive and exciting topics of our time--the development and application of superstable lasers. Their relevance to this schoo arises in large measure from the incipient marriage of such stable laser sources with the remarkable samples of free-floating quantum absorbers which can be assembled. Manipulated and cooled using light forces on atoms--the later forming the main course of our study here. In addition, such gas samples and stable lase sources represent now in many cases the tools of choice in ultra-precise experiments designed to test our cherished beliefs about the fundamental physical laws. Indeed, a large number of important and Impres sive measurements have already been carrled out using stable-laser techniques. Still, many of us in the field believe that the best is just anead, becoming freshly enabled in a significant number of examples by progress in the 'atom manipulatlon business'. 
04,348

PB96-123609 Nol available NTIS

National Inst. of Standards and Technology (EEEL)

Boulder, CO. Electromagnetic Technology Div.

Optical Sampling Using Nondegenerate Four-Wave Mixing in a Semiconductor Laser Amplifier.

Final rept.

M. Jinno, J. B. Schlager, and D. L. Franzen. 1994,

$2 p$.

See also PB96-122502

Pub. in Electronics Letters, v30 n18 1489-1490 Sep 94.

Keywords: *Laser amplifiers, Semiconductors, Reprints, "Multiwave mixing, "Optical sampling, High speed optical techniques.

Picosecond optical sampling using nondegenerate lour-wave mixing in a semiconductor laser amplifier (SLA) is demonstrated for the first time. High-peakpower pulses and electrical gating of the SLA produce an optical sampling signal-to-noise ratio.

04,349

PB96-126180 PC A05/MF A01

National Inst. of Standards and Technology,

Journal of Research of the National institute of Standards and Technology, March/April 1995. Volume 100, Number 2.

1995, 96p.

See also PB96-126198, PB96-126206, and PB96113535. Also available from Supt. of Docs.

Keywords: "Meetings, "Research and development, *Periodicals, "National Institute of Standards and Technology(NIST).

The Journal of Research of the National Institute of Standards and Technology features advances in measurement methodology and analyses consistent with the NIST responsibility as the nation's measurement science laboratory. It includes reports on instrumentation for making accurate and precise measurements in fields of physical science and engineering, as well as the mathematical models of phenomena which enable the predictive determination of information in regions where measurements may be absent. Papers on critical data, calibration techniques, quality assurance programs, and well-characterized reference materials reflect NIST programs in these areas. Special issues of the Journal are devoted to invited papers in a particular field of measurement science. Occasional survey articles and conference reports appear on topics related to the Institute's technical and scientific programs.

\section{4,350}

PB96-135199 Not available NTIS

National Inst. of Standards and Technology (PL), Boulder, CO. Quantum Physics Div.

Frequency-Stabilized Lasers: A Driving Force for New Spectroscopies.

Final rept.

J. L. Hall. 1994, 23p

See also PB95-153771.

Pub. in 1992 International School of Physics 'Enrico Fermi', Italy, p217-239 1994

Keywords: "Laser stability, "Laser spectroscopy, * Standards, Frequency stability, High resolution, Optical measurement, Laser cavities, Reprints, Saturated absorption spectroscopy.

It is particularly appropriate to consider the evolution of stabilized lasers within the context of a Fermi School concerned with the Frontiers of Laser Spectroscopy, as the stabilization of lasers has clearly provided one of the stimulating influences for the development of new spectroscopic methods.

\subsection{1}

PB96-137757 Not available NTIS

National Inst. of Standards and Technology (MEL), Gaithersburg, MD. Precision Engineering Div.

Visualization of Surface Figure by the Use of Zernike Polynomials.

Final rept.

C. J. Evans, R. E. Parks, P. J. Sullivan, and J. S

Taylor. $1995,5 p$

Pub. in Applied Optics, v34 n34 p7815-7819 Dec 95.

Keywords: "Interferometers, "Optical testing, Reprints, Zernike polynomials, Power spectral density

Commercial software in modern interferometers used in optical testing frequently fit the wave-front or sur- face-figure error to Zernike polynomials; typically 37 coefficients are provided. We provide visual represen tation of these data in a form that may help optical fabricators decide how to improve their process or how to optimize system assembly.

\section{4,352}

PB96-138482 Not available NTIS

National Inst. of Standards and Technology (MEL), Gaithersburg, MD. Precision Engineering Div.

Test of a Slow Off-Axis Parabola at its Center of Curvature.

Final rept.

R. E. Parks, C. J. Evans, and L. Shao. 1995, 5p.

Pub. in Applied Optics, v34 n31 Nov 95.

Keywords: *Optical testing, Interferometry, Reprints, "Zernike polynomials, "Off-axis aspherics.

The authors describe the interferometric testing of a slow ( $F / 16$ at the center of curvature) off-axis parabola, intended for use in an $x$-ray spectrometer, that uses a spherical wave front matched to the mean radius of the asphere. The authors find the figure error in the off-axis mirror by removing the theoretical difference between the off-axis segment and the spherical reference from the measured wave-front error. This center of curvature test is easy to perform because the spherical reference wave front has no axis and thus alignment is trivial. The authors confirm that the test results are the same as the double-pass null test for a parabola that uses a plane autocollimating mirror. They also determine that the off-axis section apparently warped as the result of being cut from the symmetric parent part

\section{4,353}

PB96-140371 Not available NTIS

National Inst. of Standards and Technology (EEEL) Boulder, CO. Electromagnetic Technology Div. Inexpensive Laser Cooling and Trapping Experiment for Undergraduate Laboratories.

Final rept.

C. Wieman, G. Flowers, and S. Gilbert. 1995, 14p. Pub. in American Jnl. of Physics, v63 n4 p317-330 Apr 95

Keywords: "Laboratories, Spectroscopy, Rubidium Reprints, "Laser cooling, "Laser trapping, Vapor cell trap.

We present detailed instructions for the construction and operation of an inexpensive apparatus for laser cooling and trapping of ribidium atoms. This apparatus allows one to use the light from low power diode lasers to produce a magneto-optical trap in a low pressure vapor cell. We present a design which has reduced the cost to less than $\$ 3000$ and does not require any matching or glassblowing skills in the construction. It has the additional virtues that the alignment of the trapping laser beams is very easy, and the rubidium pressure is conveniently and rapidly controlled. These features make the trap simple and reliable to operate, and the trapped atoms can be easily seen and studied. With a few milliwatts of laser power we are able to trap $4 \times 10$ to the 7th power atoms for $3.5 \mathrm{~s}$ in this apparatus. A step-by-step procedure is given for construction of the cell, setup of the optical system, and operation of the trap. A list of parts with prices and vendors is given in the Appendix.

\section{4,354}

\section{PB96-140405 Not available NTIS}

National Inst. of Standards and Technology (EEEL), Boulder, CO. Optoelectronics Div.

Thermal Modeling and Analysis of Laser Calorimeters.

Final rept.

Z. M. Zhang, D. J. Livigni, R. D. Jones, and T. R.

Scott. 1995, 11p.

Pub. in ASME/AIAA National Heat Transfer Conference, Portland, OR., August 6-9, 1995, AIAA 95. $3520, p^{1-11}$

Keywords: "Calorimeters, "Laser radiation, Measurements, Thermal analysis, Reprints.

We performed detailed thermal analysis and modeling of the C-series laser calorimeters at the National Institute of Standards and Technology for calibrating laser power or energy meters. A finite-element method was employed to simulate the space and time dependance of temperature at the calorimeter receiver. The inequivalence in the temperature response caused by different spatial distributions of the heating power was determined. The inequivalence between electrical power applied to front and rear portions of the receiver is approximately 1.7 percent, and the inequivalence between the electrical and laser heating is estimated to be less than 0.05 percent. The computational results are in good agreement with experiments at the 1 percent level. The effects of the deposited energy, power duration, and relaxation time on the calibration factor and cooling constant were investigated. This paper provides information for the future design improvement on the laser calorimeters.

04,355

PB96-141031 Not available NTIS

National Inst. of Standards and Technology (EEEL), Boulder, CO. Optoelectronics Div.

Deep-UV Excimer Laser Measurements at NIST.

Final rept.

R. W. Leonhardt, and T. R. Scott. 1995, 12p

Pub. in Society of Photo-Optical Instrumentation Engineers: The International Society for Optical Engineer ing, Santa Clara, CA., February 20-22, 1995, v2439 p448-459.

Keywords: "Eximer lasers, "Beamsplitters, ${ }^{*}$ Calorimeters, Dosage, Reprints, Volume absorbers.

The National Institute of Standards and Technology has designed and built two electrically calibrated laser calorimeters as primary standards for absolute energy measurements at the wavelength of $248 \mathrm{~nm}$. Under the sponsorship of SEMATECH, NIST developed the calorimeters to improve measurements of dose energy in excimer laser based microlithography. The calorimeter system can be used to calibrate transfer standards which in furn can be used to calibrate detectors employed for energy measurements of semiconductor wafer exposure. The excimer calorimeter uses a glass filter which functions as a volume absorber that allows collection of nanosecond pulses of laser radiation without suffering damage. The measurement range of the calorimeters is 0.3-25 J, but can be extended to $1 \mathrm{~mJ}$ with beamsplitters. Electrical calibration of the calorimeters shows a standard deviation in the calibration factor of less than 0.5 percent for entire energy range. The total uncertainty of typical power and energy meter calibrations is approximately 2 percent.

04,356

\section{PB96-141072 Not available NTIS}

National Inst. of Standards and Technology (PL), Gaithersburg, MD. Electron and Optical Physics Div. Atom-Optical Properties of a Standing-Wave Light Field.

Final rept.

J. J. McClelland. $1995,8 p$

Pub. in Jnl. of the Optical Society of America B, v12 n10 p1761-1768 Oct 95.

Keywords: "Atomic optics, "Lithography, Lasers, Standing waves, Reprints, Dipole force, Paraxial approximation.

The focusing of atoms to nanometer-scale dimensions by a near-resonant standing-wave light field is examined from a particle optics perspective. The classical equation of motion for atoms traveling through the lens tormed by a node of the standing wave is derived and converted to a spatial trajectory equation. A paraxial solution is obtained, which results in simple expressions for the focal properties of the lens, useful for estimating its behavior. Aberrations are also discussed and an exact numerical solution of the trajectory equation is presented. The effects on focal linewidth of angular collimation and velocity spread in the atomic beam are investigated, and it is shown that angular collimation has much more significant effect than velocity spread, even when the velocity spread is thermal.

04.357

PB96-141197 Not available NTIS

National Inst. of Standards and Technology (EEEL) Boulder, CO. Electromagnetic Technology Div. Vector and Quasi-Vector Solutions for Optical Waveguide Modes Using Efficient Galerkin's Method with Hermite-Gauss Basis Functions.

Final rept.

A. Weisshaar, R. L. Gallawa, and I. C. Goyal. 1995,

$6 p$.

Pub. in Jnl. of Lightwave Technology, v13 n8 p17951800 Aug 95.

Keywords: "Optical waveguides, Galerkin method, Reprints, *Scalar approximation, "Polarization, Vector solution, Basis functions.

An efficient vector formulation and corresponding quasi-vector formulation for the analysis of optica 
waveguides are presented. The proposed method is applicable to a large class of optical waveguides with general refractive index profile in a finite region of arbitrary shape and surrounded by a homogenous cladding. The vector formulation is based on Galerkin's procedure using Hermite-Gauss basis functions. It is shown that use of Hermite-Gauss basis functions leads to a significant increase in computational efficiency over trigonometric basis functions. The quasi-vector solution is obtained from the standard scalar formulation by including a polarization correction. The accuracy of the scalar, vector, and quasi-vestor solutions is demonstrated by companison with the exact solution for the fundamental mode in a circular fiber. Comparison of the model solutions obtained with the various methods for optical wave-guides with square, rectangular, circular, and elliptical core demonstrate the accuracy and advantage of the quasi-vector soiution.

\section{4,358}

\section{PB96-147103 Not available NTIS}

National inst. of Standards and Technology (PL), Gaithersburg, MD. Radiometric Physics Div.

Absolute Response Calibration of a Transfer Standard Cryogenic Bolometer.

Final rept.

G. Eppeldauer, A. L. Migdall, T. R. Gentile, and C. L. Cromer. 1995, 11p.

Pub. in Proceedings Reprint, Society of Photo-Optical Instrumentation Engineers: The International Society for Optical Engineering, San Diego, CA., July 11-12, 1995, v2550 p36-46.

Keywords: *Boiometers, "Calibration, Standards, Reprints, Cryogenic radiometer, infrared detector, Spectral response.

A cryogenic bolometer has been developed for use as a transfer standard in an ambient temperature infrared detector response comparator facility at the National institute of Standards and Technology (NIST). issues affecting calibration of the bolometer response were studied and a calibration procedure was developed. These issues included frequency dependence, stability, repeatability, and spectral flatness. The relative spectral response as determined from the reflectance of the bolometer absorber and the transmittance of the bolometer window. The bolometer calibration has been tied to the NIST primary standard High Accuracy Cryogenic Radiometer (HACR) using a silicon trap detector and a pyroelectric detector.

\section{4,359}

\section{PB96-148028 Not availabie NTIS}

National inst. of Standards and Technology (EEEL),

Boulder, CO. Electromagnetic Technoiogy Div.

Partially Coherent Transmittance of Dielectric Lamellae.

Final rept.

E. N. Grossman, and D. G. McDonaid. 1995, 7p

Pub. in Optical Éngineering, v34 n5 p1289-1295 May 95.

Keywords: "Dielectrics, "Lamella, "Transmittance, Interferometers, Fourier transformations, Coherence, Surface roughness, Microstructure, Reprints, Nonparalleiism, Fabry-Perot fringes.

The authors derive an analytic formula for the transmittance of a dielectric lamella when the interference between successive internal reflections is only partially spatially coherent. This allows effects such as surface roughness and nonparalielism, which produce cumulative distortions in the phase front with each reflection, and which result in a loss of fringe contrast at high frequencies, to be accounted for quantitatively. The transmittance of a Si lamella agrees with the authors' formula to within the accuracy of the data, which is dominated by systematic instrumental effects.

\section{4,360}

PB96-154422 PC A05/MF A01

National Inst. of Standards and Technology (EEEL), Boulder, CO. Optoelectronics Div.

Optical Fiber, Fiber Coating, and Connector Ferrule Geometry: Results of Interlaboratory Measurement Comparisons.

Technical note.

T. J. Drapela, D. L. Franzen, and M. Young. Nov 95, 66p, NiSTTN-1378

Aiso available from Supt. of Docs. as SN003-00303384-7.

Keywords: "Interlaboratory comparisons, "Fibers, "Geometry, Calibration, Coatings, Optical fibers, Con- nectors, Glass fibers, Parameterization, Diameter, Standards, Fiber connectors ferrule, Geometric parameters, Pin gage, NiST(National Institute of Standards and Technology)

Interlaboratory measurement comparisons, dealing with geometrical parameters of optical fibers, fibe coatings, and fiber connector ferrules (including stee pin gages used to determine ferrule inside diameter) have been coordinated by National institute of Standards and Technology (NIST). The international fibe (giass) geometry comparison showed better agreement among participants, for all measured parameters than in previous comparisons. Many participants' tes set were calibrated for fiber cladding diameter meas urements by means of calibration artifacts from NiST or other national standards laboratories; there was significantly better agreement among those participants than among participants whose test sets were not calibrated. NIST is developing ferrule, pin gage, and coat ing calibration artifacts.

\section{4,361}

PB96-155437 Not availabie NTIS

National Inst. of Standards and Technology (EEEL), Bouider, CO. Optoelectronics Div.

Annealing of Bragg Gratings in Hydrogen-Loaded Optical Fiber.

Finai rept.

H. Patrick, S. L. Gilbert, A. Lidgard, and M. D.

Gallagher. 1995, 6p.

Pub. in Jnl. of Applied Physics, v78 n5 p2940-2945 Sep 95.

Keywords: "Annealing, "Optical fibers, Change, Re prints, "Bragg gratings, Photo induced index, Hydrogen loaded fiber.

The authors have conducted a detailed study of the thermal stability of Bragg gratings written in hydrogenloaded and unloaded germanium-doped optical fiber. interference of either continuous-wave or pulsed ultraviolet light was used to induce the index modulation gratings. Some gratings were kept at room temperature and others were annealed at fixed temperatures for 10-20 h. For temperatures between room temperature and 350 degrees $C$, gratings in the hydrogen-loaded fiber showed significantly greater decay than those in the unloaded counterpart. The ultraviolet-induced index modulation in hydrogen-loaded fiber was reduced by $40 \%$ after $10 \mathrm{~h}$ at 176 degrees $\mathrm{C}$, whereas it was reduced by only $5 \%$ in unloaded fiber under the same conditions. The annealing behavior of gratings written using the pulsed source was identical to that of gratings written with the continuous-wave source, and the thermal stability of gratings in hydrogen-loaded fiber did not depend on the magnitude of the index modulation.

\section{4,362}

PB96-155445 Not availabie NTIS

National Inst. of Standards and Technology (EEEL)

Boulder, CO. Optoelectronics Div.

Comparison of UV Photosensitivity and Fluorescence during Fiber Grating Formation.

Finai rept.

H. Patrick, and S. Gilbert. 1995, 4p.

See also PB95-168597

Pub. in Proceedings of the Photosensitivity and Quad ratic Nonlinearity in Glass Waveguides, Fundamentals and Applications Topical Meeting, Portland, OR., September 9-11, 1995, p148-151.

Keywords: "Optical fibers, "Fluorescence, Near ultraviolet radiation, Reprints, Continuous radiation, Optica measurement, Fiber gratings, "Bragg gratings.

The authors have conducted a comparison of the UV photosensitivity of optical fiber with the blue fluores cence emitted during the exposure. In a survey of ten non-hydrogen-loaded germanium-doped fibers, the authors measured the UV photosensitivity and monitored the blue fluorescence during the growth of fibe gratings. The authors' goal was to determine whether the initiai fluorescence, or the change in the fluorescence during exposure, is correlated with the index change. This provides insight into the underlying physical mechanism of UV-induced index change and also determines whether the blue fluorescence can be used as an indicator of the photosensitivity of a fiber.

\section{4,363}

PB96-157995 Not available NTIS

National Inst. of Standards and Technology (PL) Gaithersburg, MD. Electron and Optical Physics Div.
Configuration-Dependent AC Stark Shifts in Caicium.

Final rept.

Q. Li, T. R. O'Brian, T. B. Lucatorto, X. Xiong, T. J

Mcllrath, and J. B. King. 1992, 3p.

Pub. in Optics Letters, v17 n19 p1373-1375 Oct 92

Keywords: "Calcium, "Laser spectroscopy, Light shifts Reprints, "AC stark effect, Configuration intervention Rydberg levels.

The energy shifts caused by intense laser radiation (10 to the 8th power to 10 to the 10 th power W/sq. $\mathrm{cm}$ ) are investigated for several high-lying levels of calcium in order to determine the ac Stark shift of a loosely bound electron in a strong electromagnetic field. Energy shifts are measured for the closely spaced levels $4 s 9 s$ 1So. 4s8d 1D2. 4s10s 1 So. and $3 d 5 s$ dd2 by using resonance ionization spectroscopy. The levels associated with Rydberg configurations show the expected pondermotive energy shifts, but the 3d5s 1D2 shift is approximately half as large as the ponderomotive shifts.

04,364

PB96-159611 Not available NTIS

National Inst. of Standards and Technology $(\mathrm{PL})$, Gaithersburg, MD. Electron and Optical Physics Div. Soft-X-ray Damage to p-terphenyl Coatings for Detectors.

Final rept.

E. L. Benitez, M. L Dark, D. E. Husk, S. E

Schnatterly, and C. Tarrio. 1994, 3p.

Pub. in Applied Optics, v33 n10 p1854-1856 Apr 94.

Keywords: "Detectors, "Soft $x$ ray, Efficiency, Phosphor, Rerints, Reprints, P-terphenyl, Photoluminescence, Photon damage.

The organic phosphor p-terphenyl is used as a wavelength-converter coating in some soft-x-ray detectors. The authors have measured the absolute photoluminescent efficiency of p-terphenyl as a functon of incident photon energy from 36 to $191 \mathrm{eV}$. The authors have also measured changes in the efficiency caused by soft-x-ray fluence (total photons absorbed per unit area) at several photon energies in this range. The authors find that efficiency drops rapidly as a function of fluence, with the rate of decrease increasing with higher soft-x-ray energies.

\section{4,365}

PB96-159736 Not availabie NTIS

National Inst. of Standards and Technology (PL), Gaithersburg, MD. Electron and Optical Physics Div. Influence of Electricai Isolation on the Structure and Reflectivity of Multilayer Coatings Deposited on Dielectric Substrates.

Final rept.

G. Gutman, J. Keem, J. Wood, C. Tarrio, and R.

Watts. $1993,4 p$

Pub. in Applied Optics, v32 n34 p6981-6984 Dec 93.

Keywords: "Soft x ray, Cha racterization, Reprints, Substrates, Multilayers, Performance, "Electrically isolated.

Multilayers prepared with electrically isolated or grounded surfaces during deposition are shown to have dramatically different hard-x-ray, soft-x-ray, and neutron reflectivity characteristics. The effect has been observed for (100) silicon wafers, fused silica, and borate glass substrates of different sizes and with different surface roughness and flatness for multilayer structures prepared by if and dc magnetron sputtering.

04,366

PB96-159744 Not available NTIS

National inst. of Standards and Technology (PL) Gaithersburg, MD. Electron and Optical Physics Div. New Method for Achieving Accurate Thickness Control for Uniform and Graded Multilayer Coatings on Large Flat Substrates.

Final rept.

G. Gutman, J. Keem, K. Parker, C. Tarrio, J. L.

Wood, and R. Watts. 1993, 11p.

Pub. in Conference on Multilayer and Grazing Incidence X-ray/EUV Optics for Astronomy and Projection Lithography, No. 1742, San Diego, CA., July 19-22, 1992, p373-381.

Keywords: "Deposition, "Soft x ray, Multilayer, Sputtering, Uniformity, Reprints.

High performance in normal incidence Soft X-Ray optical systems requires accurate control of the d-spacing 


\section{Optics \& Lasers}

across the surface of each mirror in the system. As first step towards being able to fabricate any desired $\mathrm{d}$-spacing variation, the authors demonstrate the ability to produce large $(25 \mathrm{~mm} \times 150 \mathrm{~mm})$ flat Mo/Si multilayer coated mirrors with a d-spacing uniformity of +l $-0.4 \%$. Instead of applying the approach most often taken to minimize the $d$-spacing variation physical shielding of the deposition source, the authors use a mask with a corrected profile positioned just in fron of the rotating substrate to compensate for the non uniform deposition flux. Results obtained from hard (lambda $=0.154 \mathrm{~nm}$ ) and soft (wavelength of interest) X-Ray mapping of the surface are presented along with a discussion of the technique used to control the $d$ spacing distribution.

\section{4,367}

PB96-160387 Not available NTIS

National Inst. of Standards and Technology (PL) Gaithersburg, MD. Electron and Optical Physics Div. Upgraded Facility for Multilayer Mirror Characterization at NIST.

Final rept.

R. N. Watts, D. L. Ederer, R. D. Deslattes, C. J Evans, T. V. Vorburger, T. B. Lucatorto, and W. T. Estler. 1991, 7p.

Pub. in Society of Photo-Optical Instrumentation Engineers 1547, Multilayer Optics for Advanced X-ray Ap plication, San Diego, CA., July 21-26, 1991, p159-165.

Keywords: "Surface figure, *Reflectometry, Character izations, Reprints, Diffractometry, Surface finish, XUV multilayer optics.

In response to the needs of the emerging field of normal incidence, soft $X$-ray optics, a field with applica tions ranging from extreme ultraviolet (XUV) solar im aging to $X$-ray lithography, the National Institute of Standards and Technology (NIST) has initiated an XUV multilayer and optical substrate characterization program. In this paper, we give an overview of the present capabilities of the NIST facility and discuss some of the proposed improvements, concentrating on the new soft $X$-ray reflectometry facility being built at the SURF-II electron storage ring

\section{4,368}

PB96-160395 Not available NTIS

National Inst. of Standards and Technology (PL) Gaithersburg, MD. Electron and Optical Physics Div. Soft X-ray Reflectometry Program at the National Institute of Standards and Technology.

Final rept.

R. Watts, T. Lucatorto, and C. Tarrio. 1993, 9p.

See also PB96-158092

Pub. in Conference on Multilayer and Grazing Incidence X-ray/EUV Optics for Astronomy and Projection Lithography, No. 1742, San Diego, CA., July 19-22, 1992, p345-353 1993.

Keywords: *Monochromator, "Optics, "Reflectometers Multilayer, Reprints, Reflectivity, Soft $x$ ray.

In response to the metrology needs of the soft $X$-ray community, the National Institute of Standards and Technology (NIST) has initiated a program devoted to the characterization of multilayer coated optics in the $4.40 \mathrm{~nm}$ region. In this paper, we describe the synchrotron based XUV reflectometers in use and unde construction at NIST. We review the characteristics of the Synchrotron Ultraviolet Radiation Facility storage ring (SURF II), dicuss the capabilities of the existing reflectometry facility, and present the final design parameters, expected performance, and construction sta tus of a new reflectometry beam line.

\section{4,369}

PB96-161963 Not available NTIS

National Inst. of Standards and Technology (CSTL) Gaithersburg, MD. Process Measurements Div.

Electrical Measurements for Monitoring and Control of if Plasma Processing.

Final rept

M. A. Sobolewski, and J. R. Whetstone. 1993, 12p. Pub. in Proceedings of the Society of Photo-Optical In strumentation Engineers, San Jose, CA., September 21-23, 1992, Advanced Techniques for Integrated Circuit Processing II, v1803 p309-320 1993.

Keywords: "Gaseous electronics, "Electrical measurements, "Plasma, Argon, Current, Discharge, Reprints, Impedance, Real-time, Transient, Voltage.

We have investigated the possibility of using current and voltage measurements for real-time monitoring and control of radio-frequency discharges. Specifically, we have equipped a Gaseous Electronics Conference (GEC) if Reference Cell with a computer-controlled measurement system that samples the voltage and current waveforms at the cell power input and Fourier analyzes these waveforms to obtain the amplitude and phase of their fundamental and harmonic components. The system accounts for errors introduced by the stray impedance of the cell, yielding corrected values that more accurately reflect the values of voltage and current at electrode surfaces in contact with the plasma. These corrected values are monitored to reveal changes in fundamental plasma parameters such as shealth voltages, shealth fields, and shealth (dark space) thickness. Futhermore, the corrected values serve as better control parameters than the raw values of voltage, current or power, measured externally. The time required for the acquisition and analysis of a pair of current and voltage waveforms is approximately one second, making these measurements suitable for realtime sensing and control applications.

\section{4,370}

PB96-163688 Not available NTIS

National Inst. of Standards and Technology (CSTL), Gaithersburg, MD. Biotechnology Div.

Elastic Scattering from Spheres under Non PlaneWave Illumination.

Final rept.

J. T. Hodges, G. Grehan, C. Presser, and H. G. Semerilan. 1993, 15p

Pub. in Laser Applications in Combustion and Combustion Diagnostics, Los Angeles, CA., January 16-23, 1993, SPIE Proceedings, v1862 n33 p -94-308.

Keywords: "Light scattering, "Elastic scattering, Reprints, Measurements, Gaussian laser beams, LorenzMie theory, Laser beam illumination.

Measurements of the scattered light intensity from individual droplets illuminated by focused Gausssian laser beams are presented. The results are compared with both the generalized Lorenz-Mie theory and classical Lorenz-Mie theory of elastic scattering. The data are shown to be consistent with the generalized theory. Besides experimental results, computations indicate that differences between the two theoretical approaches exist. It is shown that for sufficiently small beam diameter, characteristic of focused laser between the two theoretical approaches exist. It is shown that for sufficiently small beam diameter, characteristic of focused laser beam illumination, the departure of elastic scattering from that predicted by the classical Lorenz-Mie Theory can be significant.

\section{4,371}

PB96-164140 Not available NTIS

National Inst. of Standards and Technology $(\mathrm{PL})$, Population Trapping in Short-Pulse Multiphoton Ionization.

Final rept.

M. Edwards, and C. W. Clark. 1996, 12p

Pub. in Optical Society of America B, v13 n1 p101-112 Jan 96.

Keywords: "Multiphoton ionization, Reprints, *Complex resonance energies, "Excitodion spectra, Floquet analysis, Photoelectron spectra, Population trapping.

We have studied population trapping in a one-dimensional model interacting with a short-pulse high-intensity laser, using Floquet analysis and direct numerical integration of the time-dependent Schrodinger equation for a range of peak pulse intensities. We find that photoelectrons are efficiently produced only by pulses whose peak intensities lie in a narrow range around the resonant intensity for a given intermediate state. Excited-state populations, however, are generated throughout a larger volume than are photoelectrons. Interpretations of recent experiments are examined in light of this result.

\section{4,372}

PB96-167325 Not available NTIS

National Inst. of Standards and Technology (EEEL), Gaithersburg, MD. Semiconductor Electronics Div. Double-Modulation and Selective Excitation Photoreflectance for Wafer-Level Characterization of Quantum-Well Laser Structures.

Final rept.

D. Chandler-Horowitz, D. W. Berning, J. G.

Pellegrino, J. H. Burnett, and P. M. Amirtharaj. 1996

$5 p$.

Pub. in Proceedings of the International Workshop on Semiconductor Characterization: Present Status and
Future Needs, Gaithersburg, MD., January 30-February 2, 1995, p639-643 1996

Keywords: "Laser structures, "Photoreflectance, Luminescence, Reprints, Pump beams, Bandgap, Double modulation, Quantum-well.

A double-modulation photoreflectance (PR) procedure is presented, where both the probe and pump beams are modulated, and the photoreflectance signal can be isolated from the luminescence and the scattered pump beam signals. The PR signal is separated from the other two signals through detection at the sum frequency. A careful choice of frequencies and specially design filters and tuned amplifiers were needed to achieve optimum operation. A couple system, along with the necessary circuits, is presented and applied to the characterization of a highly luminescent quantum-well laser structure. The freedom allowed by such a system to easily accommodate any pump wavelength is an important feature. We have exploited this added versatility, and the ordering of the bandgap of the multiple layers required in complex laser structures, to extract the bandgap and alloy composition of each of the constituent regions as well as the built-in strain in the pseudomorphic quantum-well.

\section{4,373}

PB96-175690

(Order as PB96-175666, PC A07)

MF A02

National Institute of Standards and Technology, Boulder, $\mathrm{CO}$

Theory of Electron Beam Moire.

D. T. Read, and J. W. Dally. 1996, 29p.

Prepared in cooperation with Maryland Univ., College Park. Dept. of Mechanical Engineering.

Included in Jnl. of Research of the National Institute of Standards and Technology, v101 n1 p47-76 Jan/ Feb 96.

Keywords: "Experimental mechanics, "Electronic beams, Contract, Division, Fringe, Multiplication, Pitch, Rotation, Spatial frequency, Stress analysis, Reprints.

When a specimen surface carrying a high-frequency line grating is examined under a scanning electron microscope (SEM) moire fringes are observed at several different magnifications. The fringes are characterized by their spatial frequency, orientation, and contrast. These features of the moire pattern depend on the spatial frequency mismatch between the specimen grating and the raster scan lines, the diameter of the electron beam, and the detailed topography of the lines on the specimen.

04,374

PB96-176433 Not available NTIS

National Inst. of Standards and Technology (PL) Gaithersburg, MD. Radiometric Physics Div.

Realization of NIST 1995 Luminous Flux Scale Using the Integrating Sphere Method.

Final rept.

Y. Ohno. 1996, 10p

See also PB96-102843.

Pub. in IESNA Annual Conference, New York, NY., August 1-4, 1995, Jnl. of the Illuminating Engineering Society, v25 n1 p13-22 1996

Keywords: "Optical measurement, Lumens, Light sources, Calibration, Photometry, Radiant flux density, Scale, Units of measurement, Standards, Photometers, Reprints, "Luminous flux, Integrating spheres.

The NIST IUminous flux scale was last realized in 1985 using a goniophotometer. A new theoretical method for luminous flux scale realization using an integrating sphere has been studied at NIST. An experimental realization predicted sufficient accuracy of this method for standards use. This method (called integrating sphere method) uses an integrating sphere with an opening to introduce a known amount of flux from an external source, which is compared to the liminous flux of an internal source to be calibrated.

04,375

PB96-176466 Not available NTIS

National Inst. of Standards and Technology (PL), Gaithersburg, MD. Atomic Physics Div.

Irradiances of Spectral Lines in Mercury Pencil Lamps.

Final rept.

J. Reader, C. J. Sansonetti, and J. M. Bridges. 1996,

$6 p$.

See also PB96-176474.

Pub. in Applied Optics, v35 n1 p78-83, 1 Jan 96. 
Keywords: "Irradiances, Reprints, "Mercury pencil lamps, "Spectral lines.

The irradiances of 37 spectral lines emitted by mercury pencil-type lamps were measured by companison with calibrated continuum sources. The lines span the region $230-590 \mathrm{~nm}$. For the 14 most prominent lines the absolute irradiances should be useful for radiometric calibrations at an uncertainty level of approx. $15 \%(95 \%$ confidence). The ratios of the irradiances for this same group of lines are significantly more reproducible; they should be useful at an uncertainty level of approx. $10 \%$.

04,376

PB96-176474 Not available NTIS

National Inst. of Standards and Technology (PL) Gaithersburg, MD. Atomic Physics Div.

Wavelengths of Spectral Lines in Mercury Pencil Lamps.

Final rept.

C. J. Sansonetti, M. L. Salit, and J. Reader. 1996 $4 p$

Keywords: "Wavelengths, Spectral lines, Reprints "Fourier-transform spectroscopy, "Mercury pencil lamp.

The wavelengths of 19 spectral lines in the region 253$579 \mathrm{~nm}$ emitted by $\mathrm{Ho}$ pencil-type lamps were measured by Fourier-transform spectroscopy. Precise calibration of the spectra was obtained with wavelengths of $198 \mathrm{Hg}$ as external standards. Our recommended values should be useful as wavelength-calibration standards for moderate resolution spectrometers at an uncertainty level of $0.0001 \mathrm{~nm}$.

\section{4,377}

PB96-179536 Not available NTIS

National Inst. of Standards and Technology (MEL), Gaithersburg, MD. Precision Engineering Div.

Test Optics Error Removal.

Final rept.

C. J. Evans, and R. N. Kestner. 1996, 7p.

Pub. in Applied Optics, v35 n7 p1015-1021, 1 Mar 96.

Keywords: "Optical interferometers, "Instrument errors, *Geometrical aberrations, Optical tests, Optical measurement, Optical correction procedure, Symmetry, Surfaces, Wave fronts, Configuration management, Reprints, Zernike polynomials.

Wave-front or surface errors may be divided into rotational symmetric and nonrotationally symmetric terms. It is shown that if either the test part or the reference surface in an interferometric test is rotated to $N$ equally spaced positions about the optical axis and the resulting wave fronts are averaged, then errors in the rotated member with angular orders that are not interger multiples of the number of positions will be removed.

04,378

PB96-179585 Not available NTIS

National Inst. of Standards and Technology (PL) Gaithersburg, MD. Radiometric Physics Div.

National Institute of Standards and Technology High-Accuracy Cryogenic Radiometer.

Final rept.

T. R. Gentile, J. M. Houston, J. E. Hardis, C. L.

Cromer, and A. C. Parr. 1996, 13p.

Pub. in Applied Optics, v35 n7 p1056-1068 Mar 96.

Keywords: "Cryogenics, "Radiometry, Standards, Reprints. "Electrical substitution, National Institute of Standards and Technology, High-accuracy.

A high-accuracy cryogenic radiometer has been developed at the National Institute of Standards and Technology to serve as a primary standard for optical power measurements. This instrument is an electrical-substitution radiometer that can be operated at cryogenic temperatures to achieve a relatively standard uncertainty of $0.021 \%$ at an optical power level of $0.8 \mathrm{~mW}$. The construction and operation of the high-accuracy cryogenic radiometer and the uncertainties in optical power measurements are detailed.

04,379

PB96-200704 Not available NTIS

National Inst. of Standards and Technology (PL), Boulder, CO. Quantum Physics Div.
Gravitational Sisyphus Cooling of (87)Rb in a Magnetic Trap.

Final rept.

N. R. Newbury, C. J. Myatt, E. A. Cornell, and C. E. Wieman. 1995, 4p.

Pub. in Physical Review Letters, v74 n12 p2196-2199 May 95.

Keywords: "Magnetic traps, "Laser cooling, "Nuetron atoms, Reprints.

We describe a method for cooling magnetically trapped $87 \mathrm{Rb}$ atoms by irreversibly cycling the atoms between two trapped states. The cooling force is proportional to gravity. The atoms are cooled to $1.5 \mathrm{micro} \mathrm{K}$ in the vertical dimension. We have extended this cooling method to two dimensions through anharmonic mixing, achieving a factor of 25 increase in the phase space density over an uncooled sample. The cooling method should be an important intermediate step toward achieving a Bose-Einstein condensate of Rb atoms.

\section{4,380}

PB96-200738 Not available NTIS

National Inst. of Standards and Technology (EEEL), Boulder, CO. Optoelectronics Div.

Self-Calibrated Intelligent Optical Sensors and Systems.

Final rept.

A. H. Rose, and J. C. Wyss. 1996, 7p.

Pub. in Proceedings of the Self-Calibrated Intelligent Optical Sensors and Systems Conference, Philadelphia, PA., October 25-26, 1996, v2594 p142-148 1996.

Keywords: "Temperature sensors, "Calibration, Self repairing devices, Computerized control systems, Reprints, "Optical thermometers, "Self calibration, Optical fiber sensors, Fiber optic sensors.

A computer controlled optical thermometer has been built to demonstrate a self-calibrating optical sensor. The self-calibrating thermometers records the temperature with a fiber-optic polarimetric temperature sensor. The wavelength sensitivity of the polarimetric sensor is used to facilitate the recalibration. The system contains an optical source which can be tuned over approximately a $9 \mathrm{~nm}$ wavelength range, and a monochromator to measure any shifts in the wavelength of the laser. The monochromator is calibrated with the spectrum of a neon discharge lamp.

\section{4,381}

PB96-201165 Not available NTIS

National Inst. of Standards and Technology (EEEL), Boulder, CO. Electromagnetic Technology Div.

Electronically Tunable Fiber Laser for Optical Pumping of (3) He and (4) He.

Final rept.

E. F. Stephens, H. Patrick, and S. L. Gilbert. 1996

$2 p$

Pub. in Review of Scientific Instruments, v67 n3 p843844 Mar 96.

Keywords: "Optical pumping, "Fiber lasers, "Helium, "Laser spectroscopy, Fiber grating, Neodymium, Reprints, Bragg grating, Nd-doped optical fiber.

We present in the paper a low threshold, highly stable, integrated fiber laser cavity that uses an electronically tunable internal Bragg grating. The fiber laser produced over $5 \mathrm{~mW}$ with a spectral width of about $5 \mathrm{GHz}$ at $1083 \mathrm{~nm}$. The laser was used to achieve $30 \%$ polarization of the $23 \mathrm{~S} 1$ metastable states of $4 \mathrm{He}$ in a weak rf discharge cell. (Copyright (c) 1996 American Institute of Physics.)

\section{4,382}

PB97-108559 PC A04/MF A01

National Inst. of Standards and Technology (PL), Gaithersburg, MD. Optical Technology Div.

National Measurement System for Radiometry, Photometry, and Pyrometry Base Upon Absolute Detectors.

Technical note.

A. C. Parr. Sep 96, 37p, NIST/TN-1421.

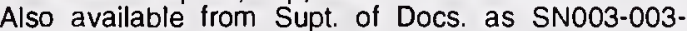
03429-1.

Keywords: "Radiometers, "Photometry, "Pyrometry, Procedures, Calibrations, Cryogenic radiometers, National Institute of Standards and Technology.

Advancements in the performance and ease of use of absolute photodetectors based upon electrical substitution principals offers the possibility of a simplified and more accurate way to transfer the fundamental radio- metric photometric and pyrometric units from NIST to the U.S. technical establishment. The history of electrical substitution radiometers at NIST is briefly re viewed and the implementation of the latest generation absolute cryogenic radiometer is discussed. Procedures to maintain fundamental units based upon a detector approach are reviewed in a general way with references to the technical literature for detailed discussion. It is proposed that NIST customers consider adopting the suggestions made in this technical note to simplify and improve their optical radiation based calibrations.

\section{4,383}

PB97-108583 PC A12/MF A03

National Inst. of Standards and Technology (EEEL), Boulder, CO. Optoelectronics Div.

Technical Digest: Symposium on Optical Fiber Measurements (9th), 1996. Held in Boulder, Colorado on October 1-3, 1996.

Special pub.

G. W. Day, D. L. Franzen, and P. A. Williams. Oc 96, 226p, NIST/SP-905.

Details in illustrations may not be fully legible in microfiche. Also available from Supt of Docs. as $\mathrm{SNOO}_{3}$ 003-03425-8. See also PB94-207636. Prepared in cooperation with Lasers and Electro-Optics Society (IEEE), Piscataway, NJ. and Optical Society of America, Washington, DC

Keywords: "Fiber optics, "Optical fibers, "Optical measurement, "Meetings, Optical polarization Electrooptics, Transmission lines, Reviews, Nonlinea optics, Integrated optics, National Instifute of Standards and Technology.

Measurements of polarization mode dispersion (PMD) and nonlinear processes in optical fibers are two of the major topics in this digest of papers presented at the ninth Symposium on Optical Fiber Measurements, Held October 1-3, 1996, at the laboratories of the $\mathrm{Na}$ tional Institute of Standards and Technology in Boulder, Co. Summaries of all the papers presented at the Symposium-10 invited and 39 contributed-are in cluded. The statistical nature of PMD complicates its measurement and makes verification of accuracy difficult. This is reflected in the content of the papers, many of which focus on comparisons of measurement lechniques. In wavelength division multiplexed systems employing optical amplification, high powers can be lead to problems from nonlinear processes such as Brillouin scattering and four-wave mixing. The importance of these issues is seen by the inclusion of several papers regarding the measurement of nonlinea coefficients and effective area. Strong internationa participation has been maintained, as about two thirds of the paper in this digest originated outside of the United States.

04,384

PB97-110209 Not available NTIS

National Inst of Standards and Technology (MEL) Gaithersburg, MD. Precision Engineering Div.

Model of an Optical Roughness-Measuring Instrument.

Final rept.

A. B. Kiely, T. R. Lettieri, and T. V. Vorburger. 1991,

Pub. in Proceedings of the International Conference on the Metrology and Properties of Engineering Surfaces (5th), Leicester, England, April 1991, 4p

Keywords: "Light scattering, "Optical metrology, Roughness, Surface finish, Texture, Models, Reprints.

The authors have developed a nonlinear model of commercial optical instrument for measuring the rootmean-square slopes of rough surfaces. The model im proves upon previous ones in that it accounts for certain instrumental factors which affect the optical roughness measurements.

04,385

PB97-110415 Not available NTIS

National Inst. of Standards and Technology (MEL) Gaithersburg, MD. Precision Engineering Div. Optical Scattering from Moderately Rough Surfaces.

Final rept.

T. V. Vorburger, E. Marx, and T. R. Lettieri. 1991, 3p.

Pub. in ICO Topical Meeting on Atmospheric, Volume and Surface Scattering Propagation, Florence, Italy, August 27-30, 1991, p205-207. 


\section{PHYSICS}

\section{Optics \& Lasers}

Keywords: "Optical scattering, "Surface roughness, Light scattering, Optical metrology, Reprints, Fraunhofer diffraction.

Light-scattering techniques have become importan tools for the study of surface finish, and several such techniques have been developed. The choice depends, among other factors, upon the degree of roughness: for very smooth surfaces a technique such as total integrated scatter can be used, while for rougher surfaces angle-resolved light scattering should be applicable. In the paper, the authors discuss some of the work being conducted at the National Institute of Standards and Technology which applies laser ligh scattering to the measurement and understanding of the topographic properties of moderately rough surfaces.

\section{4,386}

PB97-110506 Not available NTIS

National Inst. of Standards and Technology (MEL), Gaithersburg, MD. Precision Engineering Div.

Compensation for Errors Introduced by Nonzero Fringe Densities in Phase-Measuring Interferometers.

Final rept.

C. J. Evans $1993,4 \mathrm{p}$

Pub. in Annals of the CIRP, v42 n1 p577-580 1993. Keywords: *Optical interferometers, *Instrument errors, ${ }^{*}$ Geometric abberations, Optical tests, Optica measurement, Surfaces, Optical correction procedures, Reprints.

Interferometers offer rapid full aperture measurement of optics. Flats, spheres and conics can be measured to high accuracy with computer controlled systems using high density detectors. In principle, aspheres that depart from the base sphere or conic by several micrometers could also be measured. The paper demonstrates that substantial errors may be incurred and describes simple tests to evaluate the scale of those errors. The paper then discusses the error sources and presents a method for compensating for those errors

\section{4,387
PB97-110548 Not available NTIS}

National Inst. of Standards and Technology (MEL), Gaithersburg, MD. Precision Engineering Div. Light Scattering by Sinusoidal Surfaces: Illumination Windows and Harmonics in Standards.

Final rept.

E. Marx, T. R. Lettieri, and T. V. Vorburger. 1995,

\section{9p.}

Pub. in Applied Optics, v34 n7 p1260-1277 Mar 95.

Keywords: "Light scattering, "Sinusoidal surfaces, Windowing effects, Harmonics, Bidirectional reflectance, Distribution function, Reprints, Angle-resolved scattering.

Sinusoidal surfaces can be used as material standards to help calibrate instruments that measure the angula distribution of the intensity of light scattered by arbitrary surfaces, because the power in the diffraction peaks varies over several orders of magnitude. The calculated power in the higher-order diffraction peaks from sinusoidal surfaces expressed in terms of Besse functions is much smaller than the values determined from angular distributions that are measured or computed from measured profiles, both of which are determined mainly by the harmonic contents of the profile. The finite size of the illuminated area, represented by an illumination window, gives rise to a background that is much larger than the calculated power in the higherorder peaks. For a rectangular window of a size equal to an even number of periods of the sinusoid, a computation of the power distribution produces minima at or near the location of the diffraction angles for higherorder diffraction angles.

\section{4,388}

PB97-110597 Not available NTIS

National Inst. of Standards and Technology (MEL), Gaithersburg, MD. Precision Engineering Div.

Sinusoidal Surfaces as Standards for BRDF Instruments.

Final rept.

E. Marx, T. R. Lettieri, T. V. Vorburger, and M.

McIntosh. 1991 7p.

Pub. in Proceedings Reprint, Optical Scatter: Applications, Measurement, and Theory, San Diego, CA., July 24-26, 1991, v1530 p15-21.

Keywords: *Sinusoidal surfaces, "Lasers, "Electromagnetic scattering, Diffraction peaks, Reprints, BRDF standards, Kirchhoff approximation.
The study of light scattered by sinusoidal surfaces shows that such a configuration can be used as a material standard to help calibrate instruments that measure the BRDF of arbitrary surfaces. Measured and computed values of the power scattered into the diffraction peaks show good agreement, and such calculations can be further improved and used to verify the standards.

\section{4,389}

\section{PB97-111215 Not available NTIS}

National Inst. of Standards and Technology (MEL), Gaithersburg, MD. Precision Engineering Div.

Windowing Effects on Light Scattering by Sinusoidal Surfaces.

Final rept

E. Marx, and T. V. Vorburger. 1993, 14p.

Pub. in Proceedings Reprint, Optical Scattering: Applications, Measurement, and Theory II, San Diego, CA. v1995 p1-14 1993.

Keywords: "Sinusoidal surfaces, "Window functions, *Light scattering, Diffraction peaks, Rough surfaces, Reprints, BRDF.

A window function is used to represent the variation in the intensity of the radiation in a light beam. Windowing effects are factored into computations of the angular distribution of light scattered by a sinusoidal surface. These effects, as well as those of harmonics and noise, are shown for the angular distributions computed from simulated profiles. The integrated power in each diffraction peak computed for perfect sinusoids, measured profiles, and simulated profiles with different window functions is compared to that of a measured angular distribution.

\section{4,390}

PB97-111272 Not available NTIS

National Inst. of Standards and Technology (EEEL), Boulder, CO. Optoelectronics Div

Magneto-Optic Rotation Sensor Using a Laser Diode as Both Source and Detector.

Final rept.

K. B. Rochford, and A. H. Rose. 1996, 4p.

Pub. in International Conference on Optical Fiber Sensors (11th), Sapporo, Japan, May 21-24, 1996, p686689.

Keywords: *Detectors, "Fiber optic sensors, "Laser diodes, Rotation sensors, Self-detection, Reprints, *Foreign technology

The authors show that a laser diode can serve as both the emitter and detector in a diffraction-based magneto-optic rotation sensor Self-detection devices have sufficient linearity and stability for applications with discrete measurands. Signal-to-noise ratios greater than $50 \mathrm{~dB}(1 \mathrm{~Hz}$ bandwidth) are possible for binary measurands, and a fiber optic rotation sensor with a signal-to-noise ratio of $18 \mathrm{~dB}$ in a $1.25 \mathrm{kHz}$ bandwidth is demonstrated.

\section{4,391}

PB97-111280 Not available NTIS

National Inst. of Standards and Technology (MEL), Gaithersburg, MD. Precision Engineering Div.

Laser Cooling and the Recoil Limit.

Final rept.

S. L. Rolston, P. D. Lett, and W. D. Phillips. 1989,

$6 p$.

Pub. in Conference on Quantum Electronics and Laser Science, Baltimore, MD., April 24-28, 1989, Technical Digest Series, $v 126 p$.

Keywords: *Laser cooling, "Recoil limits, Cooling limits, Doppler cooling, Optical molasses, Radiation pressure, Reprints.

The effect of a finite recoil on the laser cooling process is studied with a Monte Carlo simulation. The authors find non-Maxwell-Boltzmann velocity distributions, and mean energies no lower than the recoil energy.

\section{4,392}

PB97-111330 Not available NTIS

National Inst. of Standards and Technology (MEL), Gaithersburg, MD. Precision Engineering Div.

Numerical Reference Models for Optical Metrology Simulation.

Final rept.

G. L. Wojcik, J. Mould, E. Marx, and M. P. Davidson. 1992, 13p.

Pub. in Society of Photo-Optical Instrumentation Engineers Microlithography '92: IC Metrology, Inspection, and Process Control VI, v1673-06 p 1-131992.
Keywords: *Electromagnetic scattering, "Optical measurement, Integral equations, Finite element analysis Maxwell equations, Computerized simulation, $\mathrm{Re}$ prints, Numerical reference models.

Optical modeling on the computer can aid R\&D efforts to enhance metrology methods, and similarly for lithography, alignment, and particulate monitoring. However, full exploitation of optical modeling is hindered by the lack of appropriate benchmarks for verifying algorithms and evaluating approximations. To help remedy this situation the authors describe a preliminary set of sca lar, 2D numerical reference models (NRMs). These in clude isolated thin and thick lines, periodic lines, and an isolated trench. Scattered fields are compared fo three different solution methods, based on time-domain finite elements, boundary integrals, and a waveguide model. Correlation is good in general, although important differences are seen in both code accuracy and performance. NRM generalizations are suggested that accommodate 3D effects, imaging, and experiment verification.

04,393

PB97-111470 Not available NTIS

National Inst. of Standards and Technology (PL) Gaithersburg, MD. Electron and Optical Physics Div. Closed Loop Controller for Electron-Beam Evaporators.

Final rept.

A. Band, and J. A. Stroscio. 1996, 4p.

Pub. in Review of Scientific Instruments, v67 n6 p2366-2369 Jun 96.

Keywords: "Evaporators, ${ }^{\star}$ Closed loop controllers *Electron-beam heating, Instruments, Process control lers, Reprints

A simple instrument for automatically controlling the deposition rate of an electron-beam evaporator is described. The design incorporates a commercially available, microprocessor based, proportional-integral-differential process controller that provides loop contro and automatic determination of optimal poportional, in tegral, and differential loop constants. A logarithmic amplifier is used to linearize the overall loop response. The controller is used in conjunction with a compact electron-beam heated evaporator

04,394

PB97-112254 Not available NTIS

National Inst. of Standards and Technology (CSTL) Gaithersburg, MD. Process Measurements Div.

Laser Bandwidth Effects in Quantitative Cavity Ring-Down Spectroscopy.

Final rept.

J. T. Hodges, J. P. Looney, and R. D. van Zee.

1996, 5p.

Pub. in Applied Optics, v35 n21 p4112-4116 Jul 96

Keywords: *Lasers, "Spectroscopy, "Cavity ringdown Absorption, Bandwidths, Oxygen, Reprints.

The authors have investigated the effects of laser bandwidth on quantitative cavity ring-down spectroscopy using the $(r) R$ transitions of the $b(v=0)<--X(v=0)$ band of molecular oxygen. It is found that failure to account properly for the laser bandwidth leads to systematic errors in the number of densities determined from measured ring-down signals. When the frequency-integrated expression for the ring-down signal is fitted and measured laser line shapes are used, excellent agreement between measured and predicted number den sities is found

\section{4,395}

PB97-112288 Not available NTIS National Inst. of Standards and Technology (CSTL), Gaithersburg, MD. Process Measurements Div.

Forward Scattering of a Gaussian Beam by a Nonabsorbing Sphere.

Final rept.

J. T. Hodges, G. Grehan, G. Gouesbet, and C. Presser. 1995, 13p.

Pub. in Applied Optics, v34 n12 p2120-2132 Apr 95

Keywords: "Gaussian beams, "Light scattering, *Spheres, Diffractions, Reprints, Lorenz-Mie theory.

The forward scattering of a Gaussian laser beam by a spherical particle located along the beam axis is analyzed with the generalized Lorenz-Mie theory (GLMT) and with diffraction theory. Forward-scattering and near-foward-scattering profiles from electrodynamically levitated droplets, 51.6 micrometers in diameter, are also presented and compared 
with GLMT-based predictions. The total intensity in the forward direction, formed by the superposition of the incident and the scattered fields, is found to correlate with the particle-extinction cross section, the particle dimater, and the beam width. Based on comparison with the GLMT, the diffraction solution is accurate when beam widths that are approximately greater than or equal to the particle diameter are considered and when large particles that have an extinction efficiency near the asymptotic value of 2 are considered. However, diffraction fails to describe the forward intensity for more tightly focused beams. The experimental observations, which are in good agreement with GLMTbased predictions, reveal that the total intensity profile about the forward direction is quite sensitive to particle axial position within a Gaussian beam.

04,396

PB97-116057 PC A06/MF A01

National Inst. of Standards and Technology (EEEL) Boulder, CO. Electromagnetic Technology Div.

Metrology for Electromagnetic Technology: A Bibliography of NIST Publications.

A. G. Bradford. Sep 96, 79p, NISTIR-5051.

See also PB96-128269.

Keywords: "Superconductivity, "Electromagnetic fields, "Bibliographies, Cryogenics, Publishing, Employees, Scientists, Lasers, Optical communication, Metrology, US NIST.

This bibliography lists the publications of the personnel of the Electromagnetic Technology Division of NIST during the period from January 1970 through publication of this report. A few earlier references that are directly related to the present work of the Division are also included. The bibiliography includes publications in cryoelectronic metrology and superconductor and magnetic measurements.

\section{4,397}

PB97-118640 Not available NTIS

National Inst. of Standards and Technology (PL), Gaithersburg, MD. Radiometric Physics Div.

Realization of a Scale of Absolute Spectral Response Using the NIST High Accuracy Cryogenic Radiometer.

Final rept.

T. R. Gentile, J. M. Houston, and C. L. Cromer.

1996, 12p.

See also PB96-179585.

Pub. in Applied Optics, v35 n22 p4392-4403 Aug 96.

Keywords: "Cryogenics, "Radiometry, Standards, Reprints, "Electrical substitution, National Institute of Standards and Technology, High-accuracy.

Using the National Institute of Standards and Technology high-accuracy cryogenic radiometer (HACR), the authors have realized a scale of absolute spectral response between 406 and $920 \mathrm{~nm}$. The HACR, an electrical-substitution radiometer operating at cryogenic temperatures, achieves a combined relative standard uncertainty of $0.021 \%$. Silicon photodiode light-trapping detectors were calibrated against the HACR with a typical relative standard uncertainty of $0.03 \%$ at nine laser wavelengths between 406 and 920 $\mathrm{nm}$. Modeling of the quantum efficiency of these detectors yields their responsivity throughout this range with comparable accuracy.

\section{4,398}

PB97-119390 Not available NTIS

National Inst. of Standards and Technology (EEEL), Boulder, CO. Optoelectronics Div.

Anomalous Relation between Time and Frequency Domain PMD Measurements.

Final rept.

P. A. Williams, and P. R. Hernday. 1995, 4p.

Pub, in Optical Fibre Measurement Conference (3rd), Liege, Belgium, September 25-26, 1995, 4p.

Keywords: "Polarization mode dispersion, *Fiber optics, *Optical fibers, Electrooptics, Fourier transform, Reprints, "Foreign technology, Differential group delay, Jones matrix eigenanalysis.

The authors report nearly simultaneous measurements of polarization mode dispersion (PMD) in various samples of highly mode-coupled single-mode fiber using the measurement methods of Jones matrix eigenanalysis (JME) and Fourier-transformed wavelength scanning. The ratio of the PMD values resulting from these two methods differs by approximately $10 \%$ from current theoretical predictions. The measurements are verified by demonstrating the theoretical agreement between the JME and wavelength scanning extremum counting results.

\section{4,399}

PB97-122212 Not available NTIS

National Inst. of Standards and Technology (EEEL), Gaithersburg, MD. Electricity Div.

Interface-Filter Characterization

Spectraradiometers and Colorimeters.

Final rept.

P. A. Boynton, Y. Ohno, and E. F. Kelley. 1996, 4p. Pub. in Society for Information Display (SID) Symposium, Digest of Technical Papers, San Diego, CA., May 12-17, 1996, p207-210.

Keywords: "Spectroradiometers, "Colorimeters, *Filters, "Interface filters, Displays, Measurements, Reprints.

Spectroradiometers and colorimeters are used in display measurements to measure color in one of several color space coordinate systems. How accurately these instruments can measure the color coordinates can be simply tested by using interference filtes. Error sources within the measuring system are identified which could explain several observed anomalies.

\section{4,400}

PB97-122378 Not available NTIS

National Inst. of Standards and Technology (PL), Gaithersburg, MD. Electron and Optical Physics Div. Large Local-Field Corrections in Optical Rotatory Power of Quartz and Selenium.

Final rept.

L. Jonsson, Z. H. Levine, and J. W. Wilkins. 1996

$4 \mathrm{p}$.

Pub. in Physical Review Letters, v76 n8 p1372-1375 Feb 96.

Keywords: "Rotary powers, "Corrections, "Ouartz, "Selenium, Local fields, Nonconductors, Calculations, Reprints.

The authors show that local fields can increase the rotatory power rho of nonconductors by a factor of 10-in contrast to the typical $10 \%$ effect in other properties. The authors present calculations for quartz and $\mathrm{Se}$, and a general method to estimate the size of local-field corrections. Notably, only scalar local fields are needed despite the vector character of light. A self-energycorrected local-density band structure yields corrections to rho of a factor of +7 in quartz and -4 in Se. These values are $30 \%$ above experiment for quartz, and, for one sign choice, within the $50 \%$ error bars for Se.

\subsection{1}

PB97-122469 Not available NTIS

National Inst. of Standards and Technology (PL), Gaithersburg, MD. Radiometric Physics Div.

Photonic Band-Structure Effects for Low-IndexContrast Two-Dimensional Lattices in the Near Infrared.

Final rept.

A. Rosenberg, R. J. Tonucci, H. B. Lin, and E. L.

Shirley. 1996, 4p.

Pub. in Physical Review B, v54 n8 pR5195-R5198 Aug 96.

Keywords: "Lattices, "Low index contrast, Attenuation, Two dimensional, Reprints, "Photonic band structure.

The authors demonstrate that two-dimensional periodic dielectric structures with low index contrast give rise to remarkable, easily measurable photonic bandstructure effects. The structures consist of triangular arrays of glass cylinders embedded in a glass matrix, having centr-to-center nearest-neighbor separations from 1.08 micrometers to 0.54 micrometers. The cylinders and the matrix are composed of different glasses, but the difference between the indices of refraction is as low as 0.02 throughout the relevant spectral region. The attenuation features corresponding to the boundaries of the first Brillouin zone appear in the near-infrared, at photon energies between $0.4 \mathrm{eV}$ and $0.9 \mathrm{eV}$.

\section{Plasma Physics}

\section{4,402}

DE $94004400 \quad P C$ A02/MF A01
National Inst. of Standards and Technology, Gaithersburg, MD

Determination of Atomic Data Pertinent to the Fusion Energy Program. Progress Report for FY 92. 1992, 6p, DOE/ER/53237-T1.

Contract Al05-86ER53237

Sponsored by Department of Energy, Washington, DC

Keywords: "Argon lons, "Atoms, "Electron-Atom Collisions, "Electron-Ion Collisions, "Ions, "Oxygen Ions, *Thermonuclear Reactors, Calculation Methods, Cross Sections, Data Compilation, Energy Levels, Excitation, ITER Tokamak, Ionization, J Codes, Plasma Diagnostics, Plasma Impurities, Polarization, Progress Report, Research Programs, Scattering, Spectra TEXT Devices, TFTR Tokamak, Wavelengths, EDB/ 700380 .

In the pursuit of the general goal to provide essential atomic radiation and collision data for the fusion energy program, the following progress was achieved at NIST during FY 92: (1) identification of spectral lines of impurity in ions (from $\mathrm{Er}$ to $\mathrm{Bi}$ ); (2) spectroscopic data compilations of critically evaluated wavelengths and energy levels of atoms and ions; (3) electron-ion collision experiments; (4) electron impact excitation theory; and (5) electron-impact ionization theory.

04,403

DE95011593 PC AO2/MF AO1

National Inst. of Standards and Technology, Gaithersburg, MD

Measurements of quantum electrodynamic sensitive transitions in $\mathrm{Na}$-like and $\mathrm{Cu}$-like ions: Fina report, 1 October 1993--29 September 1994.

PROGRESS REPT.

J. Reader. 1994, 7p, DOE/SF/20143-T2

Contract FG03-93SF20143

Sponsored by Department of Energy, Washington, DC

Keywords: "Iron lons, "Laser-Produced Plasma, Cations, Emission Spectra, Energy Levels, Energy Level Transitions, Experimental Data, ICF Devices, Modifications, Plasma Diagnostics, Progress Report, Research Programs, Theoretical Data, Tokamak Devices, X-Ray Lasers, Tables(data), EDB/700320, EDB $700380, \mathrm{EDB} / 426002$

The object of this research was to use the GDL lase at the Laboratory for Laser Energetics at the University of Rochester to measure the energies of spectral transitions that would be of importance for testing the accuracy of calculations used to predict properties of plas mas found in inertial fusion experiments as well as in tokamaks and $x$-ray lasers. The general method to be used for this experiment was to focus the beam from the GDL laser to a small point so as to create a laserproduced plasma of the material of interest. Light from the plasma was to be photographed with a $2.2-\mathrm{m}$ graz ing incidence spectrograph transported to Rocheste from NIST. The region of observation was 10-300 angstroms. In the initial phase of the work, a series of spectrograms were made of highly ionized iron. For his, a special target chamber was fabricated at NIST and interfaced to the light beam from the GDL laser. The results for iron provided valuable data for Li-like iron, $\mathrm{Fe}(\mathrm{sup} 23+$ ). Fig. 2 shows an example of the spectrum obtained in the region of 30-40 angstroms. The lower spectrum shows intense lines of $\mathrm{Fe}$ (sup $23+$ ), obtained by focusing the GDL beam tightly onto the target surface. For the upper spectrum the GDL beam was focused slightly in front of the target, producing lines of $\mathrm{Fe}$ (sup 23+) that are much reduced in intensity. The lines of $\mathrm{Fe}($ sup 23+) in the 30-40 angstrom region were observed for the first time in this ex periment. Measurement of their wavelengths provided the following comparison with ab initio values calculated with the Dirac-Fock quantum mechanics computer code of Desclaux. After completing the observations for Li-like iron, the GDL laser was shut down for the planned upgrade to much higher energy. Extensive work was undertaken to interface the NIST targe chamber to the 30-cm-diameter beam of the upgraded GDL laser. The NIST system is now ready for use with the upgraded GDL laser.

04,404

PB94-172327 Not available NTIS

National Inst. of Standards and Technology (PL) Gaithersburg, MD. Atomic Physics Div.

Gaseous Electronics Conference Radio-Frequency Reference Cell: A Defined Parallel-Plate Radio-Frequency System for Experimental and Theoretical Studies of Plasma-Processing Discharges. Final rept.

$1994,15 p$

Pub. in Rev. Sci. Instrum. 65, n1 p140-154 Jan 94 
Keywords: "Radio frequency discharge, "Glow discharges, Electrical measurement, Interlaboratory comparisons, Argon plasma, Reprints, Reference celis.

A 'reference cell' for generating radio-frequency ( $\mathrm{f}$ ) glow dicharges in gases at a frequency of $13.56 \mathrm{MHz}$ is described. The reference cell provides an experimental platform for comparing plasma measurements carried out in a common reactor geometry by differen experimental groups, thereby enhancing the transfer of knowledge and insight gained in if discharge studies. The results of performing ostensibly identical measurements on six of these cells in five different laboratories are analyzed and discussed. Measurements were made of plasma voltage and current cha racteristics fo discharges in pure argon at specific values of applied voltages, gas pressures, and gas flow rates. Data are presented on relevant electrical quantities derived from Fourier analysis of the voltage and current wave forms. Amplitudes, phase shifts, self-bias voltages, and powe dissipation were measured.

\section{4,405}

\section{PB94-185048 Not available NTIS}

National Inst. of Standards and Technology (PL), Boulder, CO. Quantum Physics Div.

Physics and Prospects of Inertial Confinement Fusion.

Final rept.

M. M. Basko. 1993, 10p.

Pub. in Plasma Phys. Control. Fusion 35, pB81-B90 1993.

Keywords: "Inertial confinement fusion, Plasma confinement, Trends, Reprints, Prospects.

Some key physical aspects of the inertial confinement fusion (ICF) are discussed. The minimum scale of ICF microexplosions is determined by the ability to implode spherical shells with high radial convergence ratios $C(R)$ and high initial aspect ratios $A(R O)$. The attainable values of $C(R)$ are limited by large-scale drive asymmetries, while the values of $A(R O)$ are constrained by the Rayleigh-Taylor instability. Under the indirect drive approach to ICF, it is easier to achieve the required uniformity of the drive pressure, but the penalty is a factor 4-5 reduction of the target energy gain as compared to the direct drive option. Spark ignition is a crucial issue for indirect drive targets (at least for those to be used in power reactors), while the tar gets driven directly by heavy ion beams could, in principle, use a less demanding volume ignition scheme.

\section{4,406}

PB94-185717 Not available NTIS

National Inst. of Standards and Technology (EEEL) Gaithersburg, MD. Electricity Div.

Use of an Ion Energy Analyzer: Mass Spectrometer to Measure Ion Kinetic-Energy Distributions from RF Discharges in Argon-Helium Gas Mixtures.

Final rept.

J. K. Olthoff, R. J. Van Brunt, S. B. Radovanov, and

J. A. Rees. $1994,6 p$.

Pub. in IEE Proc.-Sci. Meas. Technol. 141, n2 p105110 Mar 94.

Keywords: "Helium ions, "Argon ions, Radio frequency discharge, Mass spectroscopy, Metastable state, Kinetic energy, Helium plasma, Argon plasma, Gas mixtures, Semiconductors, Reprints, Ion energy analysis, Plasma processing.

A mass spectrometer equipped with an electrostatic ion energy analyzer has been used to measure the kinetic-energy distributions of ions sampled through an orifice in the grounded electrode of a parallel-plate radio-frequency (RF) discharge cell. Kinetic-energy distributions are presented for $\operatorname{Ar}(1+), \operatorname{Ar} 2(1+), \operatorname{Ar}(2+)$, $\mathrm{He}(1+)$, and $\mathrm{ArHe}(1+)$ sampled from argon-helium plasmas with helium concentrations ranging from 0-95 mole percent, applied peak-to-peak RF voltages of 200 $\mathrm{V}$, and gas pressures of $13.3 \mathrm{~Pa}$. Variations in the ion kinetic-energy distributions and ion fluxes observed fo different gas mixtures demonstrate the ability of this diagnostic technique to monitor plasma conditions and to investigate the ion kinetic processes occurring in the system.

\section{4,407}

PB94-211497 Not available NTIS

National Inst. of Standards and Technology (NML) Gaithersburg, MD. Quantum Metrology Div.
Magnetic Dipole Line from U LXXI Ground-Term Levels Predicted at 3200 Angstroms.

Final rept.

U. Feldman, P. Indelicato, and J. Sugar. 1991, 3p. Pub. in Jnl. of the Optical Society of America B 8, n1 p3-5 Jan 91. Sponsored by Department of Energy, Washington, DC.

Keywords: "M1-transitions, "Uranium ions, Near ultraviolet radiation, Plasma diagnostics, Line spectra, Wavelengths, Reprints, Titanium-like ions.

Magnetic dipole (M1) lines above 2,500 A are convenient diagnostic tools for high-energy, low-density plasmas such as those of tokamaks. We found a sequence of such lines arising from transitions between two levels of the $3 d(4)$ quintet $D$ ground term of titanium-like ions. From $N d X X X I X$ to $U L X X I$, the calculated wavelengths range from 3,557 to $3,200 \mathrm{~A}$ with ionization energies of 2.7 to $8.2 \mathrm{keV}$. These lines have transition rates of 480 to $262 / \mathrm{sec}$. The calculations were made with the multiconfiguration Dirac-Fock code of Desclaux (J. Phys. B 20,651 (1987)).

\section{4,408}

PB94-212719 Not available NTIS

National Inst. of Standards and Technology (NML), Gaithersburg, MD. Atomic and Plasma Radiation Div. Attempts at Extending the Unified Theory to InAttempts at Extending the
clude Many-Body Effects.

Final rept.

D. E. Kelleher, and J. Cooper. 1990, 2p.

Pub. in Proceedings of internationai Conference on Spectral Line Shapes (10th), Austin, TX., June 25-29, 1990, p78-79.

Keywords: "Hydrogen plasma, Many-body problem, Ion collisions, Line broadening, Stark effect, Reprints, Hydrogen-like ions, lon dynamics.

As a rule, hydrogen and hydrogen-like systems are the most sensitive to collisional Stark broadening in plasmas. The I-degeneracy in hydrogen gives rise to a firstorder Stark effect. This same fact accounts for the long-range of the interaction between the hydrogenic radiator and the charged perturbers in the plasma, and thus for the fact that strong ion collisions which overlap in time are likely to occur, except at very low densities.

\section{4,409}

PB95-141008 Not available NTIS

National Inst. of Standards and Technology (EEEL), Gaithersburg. MD. Electricity Div.

Ion Kinetic-Energy Distributions in Argon if Glow Discharges.

Final rept.

J. K. Olthoff, R. J. Van Brunt, and S. Radovanov 1992, 9p

Sponsored by SEMATECH, Austin, TX.

Pub. in Jnl. of Applied Physics 72, n10 p4566-4574, 15 Nov 92.

Keywords: "Argon ions, *Argon hydrides, *Glow discharges, Radio frequency discharge, Plasma sheaths, Charge exchange, Kinetic energy, Reprints.

Kinetic-energy distributions have been measured for different mass-selected ions sampled from 13.56 $\mathrm{MHz}$ if glow discharges in argon inside a 'GEC if reference cell.' The electrode geometry of this cell produces an asymmetric discharge and the cell is operated in a pressure regime where ion-molecule collisions in the sheath region of the discharge a re significant. Ions are sampled from the side of the plasma perpendicular to the interelectrode axis using an electrostatic energy analyzer coupled to a quadrupole mass spectrometer. Kinetic-energy distributions for $\operatorname{Ar}(+), \operatorname{Ar} 2(+), \operatorname{Ar}(++)$, and $\mathrm{ArH}(+)$ are presented as functions of applied if voltage, gas pressure, and distance of the mass spectrometer entrance aperture from the edge of the electrodes.

\section{4,410}

\section{PB95-151569 Not available NTIS}

National Inst. of Standards and Technology (NML), Gaithersburg, MD. Atomic and Plasma Radiation Div. Spectroscopic Data for Fusion Edge Plasmas. Final rept.

W. L. Wiese. 1992, 7p.

Pub. in Nuclear Fusion 2, p7-13 1992

Keywords: *Plasma diagnostics, "Spectroscopic analysis, Atomic energy levels, Atomic ions, Transition probabilities, Neutral atoms, Iron ions, Wavelengths, Tables(Data), Reprints, Fusion edge plasmas.

The status of spectroscopic data for neutral atoms and moderately charged atomic ions of interest to fusion edge plasmas is reviewed. A table is presented which lists references to all current critical compilations on energy levels, wavelengths and transition probabilities. The critical assessment of spectroscopic data is dis. cussed mainly with respect to atomic transition probabilities, as the typical uncertainties for these are stil quite large. For the specific case of neutral iron and its low ions ( $\mathrm{Fe} \mathrm{I}-\mathrm{Fe} \mathrm{V}$ ), the scope and quality of evaluated spectroscopic data is discussed in detail.

04,411

PB95-151577 Not available NTIS

National inst. of Standards and Technology (NML) Gaithersburg, MD. Atomic and Plasma Radiation Div Spectroscopic Diagnostics of Low Temperature Plasmas: Techniques and Required Data.

Final rept.

W. L. Wiese. 1991, 11p.

Pub. in Spectrochimica Acta B 46, n6-7 p831-841 1991.

Keywords: "Cold plasmas, "Plasma diagnostics, *Spectroscopic analysis, Atomic spectroscopy, Transition probabilities, Electron density, Stark effect, Argon, Iron, Reprints.

An overview of plasma diagnostic techniques based on atomic spectroscopy, interferometry, and laser scattering is given. Two widely used spectroscopic techniques--the Boltzmann-plot or slope method for excitation temperature measurements, and Stark width determinations for electron density measurements--are discussed in detail. As these techniques depend critically on the availability of accurate atomic data, specifically transition probabilities and Stark broadening parameters, the numerical data are reviewed with respect to availability and quality. For the important spectra of Ar $I$ and $\mathrm{Fel}$, tables of the most accurate available transition probabilities--typically with uncertainties of + or $-10 \%$ or less--are given, which are based on very recent critical compilations. Some specific data needs are pointed out which would significantly contribute to improve the accuracy of the diagnostic techniques.

\section{4,412}

PB95-162962 Not available NTIS

National Inst. of Standards and Technology (CSTL), Gaithersburg, MD. Process Measurements Div.

Electrical Sensors for Monitoring if Plasma Sheaths.

M. A. Sobolewski, and J. K. Olthoff. 1994, 11p. Pub. in Proceedings of Society of Photo-Optical Instrumentation Engineers: Microelectronic Processes, Sensors, and Controls, Monterey, CA., September 27-29, 1992, v2091 p290-300 1994.

Keywords: "Plasma sheath, "Argon, *Radiofrequencies, Sensors, Glow discharges, Electric current, Electrical impedance, Electrical properties, Electric potential, Kinetics, Distribution functions, Reprints.

The authors have investigated the use of radio-frequency $(\mathrm{r})$ current and voltage measurements to monitor the electrical characteristics of if plasmas and to predict changes in ion kinetic energy distributions. These studies were performed at 2.7 and $13.3 \mathrm{~Pa}(20$ and $100 \mathrm{mTorr}$ ) in a Gaseous Electronics Conference (GEC) RF Reference Cell in mixtures of argon with oxygen, nitrogen and water. Simultaneous with the electrical measurements, the kinetic energy distributions of ions at the grounded electrode were obtained using a mass spectrometer equipped with an ion energy analyzer. It is expected that the measurement techniques described here could be extended to monitor the sheath above a wafer during plasma etching to obtain information about changes in the condition of the wafer surface and energies of ions bombarding it.

\section{4,413}

PB96-111927 Not available NTIS

National inst. of Standards and Technology (EEEL) Gaithersburg, MD. Electricity Div.

Kinetic Energy Distribution of Ions Produced from Townsend Discharges in Neon and Argon.

Final rept.

M. V. V. S. Rao, S. B. Radovanov, R. J. Van Brunt, and J. K. Olthoff. 1995, $1 \mathrm{p}$.

Pub. in International Conference on the Physics of Electronic and Atomic Collisions (19th), Whistler, British Columbia, Canada, July 26-August 1, 1994, p392 1995.

Keywords: *Gas discharges, "Kinetic energy, "Charge transfer, Argon, Neon, Reprints, Mass spectrometry, Townsend discharge, Electric fields, Ions. 
Results are reported from measurements of the kinetic energy distributions of mass selected ions from diffuse, Townsend discharges in neon and argon at high electric field-to-gas density $(E / N)$ ratios from $3 \times 10$ to the minus 18 th power $V \mathrm{~m} 2$ to $6 \times 10$ to the minus 18 th power $V \mathrm{~m} 2$. The ion energies were measured using a cylindrical mirror analyzer coupled to a quadruple mass spectrometer. The measured ion energy distributions are compared with predictions from solving the Boltzmann transport equation assuming that symmetric resonant charge transfer is the dominant ion-neutra interacton. Results for $\mathrm{Ne}+$ in $\mathrm{Ne}$ show evidence of deviations from equilibrium.

\section{4,414}

PB96-119391 Not available NTIS

National Inst. of Standards and Technology (PL), Gaithersburg, MD. Atomic Physics Div.

Visible and UV Light from Highly Charged Ions: Exotic Matter Advancing Technology

Final rept.

J. D. Gillaspy. 1995, 2p.

Pub. in Institute of Electrical and Electronics Engineers Leos Newsletter, v9 n4 p19-20 Aug 95.

Keywords: "Charged particles, "Ions, "Plasma diagnostics, Atomic properties, Spectroscopy, Nuclear fusion, Electron beams, Ion traps(Instrumentation), Reprints, HCls(Highly charged ions), EBIT(Electron beam ion trap).

This news article discusses one of the applications of our research on highly charged ions: an optical diagnostic for extremely hot plasmas, such as those found in fusion devices. Our results were published in Phys. Rev. Letters earlier this year and are reviewed and updated, and put into perspective for a more general au dience.

\section{4,415}

PB96-123351 Not available NTIS

National Inst. of Standards and Technology (EEEL), Gaithersburg, MD. Electricity Div.

Kinetic Energy Distributions of $H(+), H 2(+)$, and $\mathrm{H} 3(+)$ from a Diffuse Townsend Discharge in $\mathrm{H} 2$ at High $E / N$.

M. V. V. S. Rao, R. J. Van Brunt, and J. K. Olthoff. 1995, 2p.

Pub. in International Symposium on Electron- and Photon-Molecule Collisions and Swarms, Berkley, CA. July 22-25, 1995, $\mathrm{pH}-9$.

Keywords: "Hydrogen ions, "Gas discharges, Kinetic energy, Distribution functions, Townsend discharge. Reprints.

The translational kinetic-energy distributions of the predominant ions, $\mathrm{H}+, \mathrm{H} 2+$, and $\mathrm{H} 3+$, formed in a diffuse Townsend discharge in $\mathrm{H} 2$ were measured for electric field-to-gas density ratios $(E / N)$ from $7 \times 10$ to the minus 19 th power $V m 2$ to $2 \times 10$ to the minus 18 th power Vm2. The discharge was generated in a parallel-plate electrode gap for conditions corresponding to the 'left-hand' side of the Paschen minimum where the electric field in the gap is expected to be constan and uniform, i.e., the discharge is characterized by a well defined $\mathrm{E} / \mathrm{N}$. The discharge cell configuration and ion measurement system are similar to those de scribed previously. The ions were sample through a $0.1 \mathrm{~mm}$ diameter orifice in the center of the stainlesssteel cathode. Simultaneous analyis of ion energy and mass was performed using a cylindrical mirror analyze coupled to a quadrupole mass spectrometer.

\section{4,416}

PB96-138532 Not available NTIS

National Inst. of Standards and Technology (EEEL), Gaithersburg, MD. Semiconductor Electronics Div. Nonequilibrium Total-Dielectric-Function Ap proach to the Electron Boltzmann Equation for Inelastic Scattering in Doped Polar Semiconductors. Final rept.

B. A. Sanborn. 1995, 9p

Pub. in Physical Review B, v51 n20 p14 247-14 255 May 95.

Keywords: "Dielectric functions, "Semiconductors, Inelastic scattering, Reprints, "Doped polar semiconductors, Boltzmann equation.

The paper describes a simple and general method for deriving the inelastic collision term in the electron Boltzmann equation for scattering from a coupled electron-phonon system and applies the method to the case of doped polar semiconductors. In the Born ap. proximation, the inelastic differential scattering rate W(inel) can be expressed in terms of the nonequilibrium total dynamic dielectric function, which includes both electronic and lattice contributions. Within the random-phase approximation W(inel) separates into two componenents: an electron-electron interaction containing the nonequilibrium distribution function for excitations of the electron gas and a Frohlich interaction including the phonon distribution function and self-energy due to polarization of the electrons. Each of these two interactions is screened by only the electronic part of the total dielectric function, which contains the high-frequency dielectric constant, unlike commonly used expressions that contain the static dielectric constant.

04.417

PB96-141130 Not available NTIS

National Inst. of Standards and Technology (EEEL),

Boulder, CO. Electromagnetic Technology Div

Apparatus for Resistance Measurement of Short, Small-Diameter Conductors.

Final rept.

C. A. Thompson. 1994, 3p.

Pub. in Institute of Electrical and Electronics Engineers Transactions on Instrumentation and Measurements, v43 n4 p675-677 Aug 94.

Keywords: "Electrical resistivity, "Conductors, Copper, Reprints, Fibers, Measurement, Resistance.

A system for determining the dc resistance of individual conductors 2 micrometers in diameter and 0.5-1 mm in length is described. The system uses a four-wire measurement, computerized data acquisition, and unique sample handling and contacting methods. To demonstrate system operation, data from measurements made on small-diameter copper wires are presented. These wires were first measured in long lengths on another system and then cut into short lengths and remeasured on this system. The results from these two measurement systems show that this system is an effective tool for determining the resistance per unit length of short, small-diameter conductors.

\section{4,418}

PB96-204458 Not available NTIS

National Inst. of Standards and Technology (MSEL), Gaithersburg, MD. Polymers Div.

Shear Suppression of Critical Fluctuations in a $\mathrm{Di}$ luted Polymer Blend.

Final rept.

E. K. Hobbie, A. I. Nakatani, H. Yajima, J. F

Douglas, and C. C. Han. 1996, 3p.

Pub. in Physical Review E, v53 n5 p4322-4324 May 96.

Keywords: "Phase separation, "Dituted blends, Reprints, Neutron scattering, Light scattering, "Fisher renormalization.

Small-angle neutron scattering has been combined with equilibrium dynamic light scattering to study shear-induced mixing in a diluted high-molecularweight polymer blend. The data show an enhancement of critical fluctuations upon dilution and are found to collapse onto a universal scaling curve containing no free parameters. The scaling curve is motivated by the theoretical predictions of Onuki and Kawasaki for undiluted binary mixtures. The data also appear to suggest that 'Fisher renormalization' is relevant in this diluted pseudobinary polymer mixture, consistent with a previous dynamic-light-scattering study.

04,419

PB97-111231 Not available NTIS

National Inst. of Standards and Technology (MSEL), Gaithersburg, MD. Ceramics Div.

Characterization and Processing of Spray-Dried Zirconia Powders for Plasma Spray Application. Final rept.

P. Pei, S. G. Malghan, S. J. Dapkunas, and P. H. Zajchowski. 1996, $9 p$.

Pub. in Jnl. of Thermal Spray Technology, v5 n3 p343351 Sep 96

Keywords: "Plasma spray, "Zirconia powder, "Spray dried powder, Powder characterization, Thermal barrier coatings, Thermal rupture test, Reprints.

The correlation between the performance of plasma spray coatings and feedstock powder properties is not fully understood. To demonstrate this correlation, eight spray-dried zirconia powders containing a mass fraction of $20 \%$ Y2O3(yttria) were characterized with re- spect to their physical, bulk chemical, and surface chemical properties. The same powders were plasma spray deposited as coatings, and their relative performance was evaluated using a thermal rupture test developed by Pratt and Whitney. The specific powder prop erties studied were chemical composition, binder con tent, particle size distribution, powder morphology, interface chemistry, thermogravimetry, phase composition, and specific surface area. Among the characterization data, the binder-related properties of the powder correlated most strongly with the thermal rup ture test data. Specifically, higher binder contents were associated with poor thermal rupture test performance.

\section{Radiofrequency Waves}

\section{4,420}

AD-A292 471/0 PC A02/MF AO1

National Bureau of Standards, Boulder, CO. Central Radio Propagation Lab.

Higher-Order Approximations in Ionospheric Wave-Propagation.

J. Feinstein. Jun 50, 10p

Pub. in Jnl. of Geophysical Research, v55 n2 p161 170 , Jun 50

Keywords: "Approximation, *Ionospheric propagation Velocity, Mathematical models, Electromagnetic fields, Ionosphere, Reprints, Vibration, Equations of motion Time dependence, Electromagnetic wave transmission, Drift, Resonance, Perturbations, Transverse waves, Maxwells equations, Plane waves, Difference frequency.

It is found that the ionosphere, even when considered homogeneous, is a linear medium for the propagation of electromagnetic waves only as a first approximation Second-order terms give rise to harmonics, and to sum and difference frequencies when low independent waves traverse the same physical region. These new frequencies are, in general, of the nature of forced vibrations, except in the case where their propagation characteristics are those of a natural mode capable of existing in the region. A resonance effect then occurs the new wave increasing its energy at the expense of the interacting waves, and assuming an independent existence. While these effects couple energy from the primary wave, they do not affect its propagation characteristics. As a result of the degeneracy of the determinantal equation for the propagation constant the introduction of any disturbing physical effects, such as a layer drift velocity, raises the degree of the equation, resulting not merely in changes in the values of the propagation constants of the usual modes, but, in addition, introducing new ones. For the usual disturbing effects encountered in the ionosphere, the energy content of these new modes is negligibly small. (AN).

\subsection{1}

PB94-211950 Not available NTIS

National Inst. of Standards and Technology (EEEL) Boulder, CO. Electromagnetic Fields Div.

Electromagnetic Scattering by a Periodic Surface with a Wedge Profile.

Final rept.

D. A. Hill. 1992, 18p.

Pub. in Electromagnetics 12, p247-264 1992

Keywords: "Electromagnetic scattering Polarization(Waves), Two dimensional, Numerical solution, Perturbation theory, Absorbers(Materials), Matrices, Electromagnetic absorption, Reprints, Dielectric wedges, Periodic surfaces, Extended boundary conditions, Ill-conditioned problems(Mathematics)

Two-dimensional scattering from a periodic surface with a wedge profile is treated for both TE and TM polarization. The interface can be either perfectly conducting or penetrable, and the extended boundary condition (EBC) is used in both cases. The method results in simple matrix elements and is easy to apply. Comparison with a perturbation solution shows that the EBC method has greater accuracy and a greater range of validity. Even so, EBC becomes numerically ill conditioned for surface heights that are a significant fraction of the period or the wavelength. Numerical results are presented for conservation of power, and some comparisons are made with previous results for sinusoidal surfaces. Some applications to if absorber are also presented. 
04,422

PB94-216512 Not available NTIS

National Inst. of Standards and Technology (NEL) Gaithersburg, MD. Precision Engineering Div.

Alternative Single Integral Equation for Scattering by a Dielectric.

Final rept.

E. Marx. 1991, 4p.

Pub. in Proceedings of Antennas and Propagation Society Symposium 1991 Digest, London, Ontario, Canada, June 24-28, 1991, p1635-1638

Keywords: "Electromagnetic scattering, "Integral equations, Boundary conditions, Cylindrical bodies, Helmholtz equations, Delta function, Dielectrics, Reprints.

A single integral equation for electromagnetic scattering by a dielectric cylindrical body is derived. The unknown is the jump in the auxiliary field across the boundary. The delta-function behavior of the derivative of the solution of the Helmholtz equation is studied in detail.

04,423

PB95-161675 Not available NTIS

National Inst. of Standards and Technology (EEEL) Gaithersburg, MD. Electricity Div

ELF Electric and Magnetic Field Measurement Methods.

Final rept.

M. Misakian. 1993, 6p.

Pub. in Proceedings of International Symposium on Electromagnetic Compatibility, Dallas, TX., August 9 13, 1993, 6p.

Keywords: "Extremely low frequencies, "Magnetic fields, "Electric fields, ${ }^{*}$ Frequency measurement, Harmonic generation, Harmonics, Measuring methods, Calibration, Instrumentation, Standards, Reprints, Power frequency, Harmonic fields.

The paper surveys the instrumentation, calibration procedures, measurement techniques, and standards which can be used to characterize extremely low frequency (ELF) electric and magnetic fields. While the focus of the paper is on power frequency and power frequency harmonic fields, the measurement methods discussed are appropriate in principle for other ELF frequencies.

\section{4,424}

PB95-261939 (Order as PB95-261897, PC A07/

MF A02)

National Institute of Standards and Technology, Boulder, CO.

Low-Frequency Model for Radio-Frequency Absorbers.

J. Randa. 1995, 11p.

Included in Jnl. of Research of the National Institute of Standards and Technology, v100 n3 p257-267 May/ Jun 95.

Keywords: "Law frequencies, "Radio frequencies, ${ }^{\star}$ Absorbers(Equipment), "Models, Electromagnetic absorption, Anechoic chambers, National Institute of Standards and Technology.

A simple model is developed to characterize the behavior of radio-frequency absorbers at low frequency. The absorber is represented by a flat, homogeneous, isotropic slab of lossy material, with effective constitutive parameters are determined by a fit to measured data. Excellent fits are obtained in the two applications considered. The model is intended for use in the characterization of absorber-lined chambers at low frequency

04,425

\section{PB96-112222 Not available NTIS}

National Inst. of Standards and Technology (PL). Gaithersburg, MD. Ionizing Radiation Div.

Study of Multiple Scattering Background in Compton Scatter Imaging.

Final rept.

G. Barnea, C. E. Dick, A. Ginzburg, E. Navon, and S. M. Seltzer. 1995, 8p.

Pub. in NDT and $E$ International, v28 n3 p155-162 1995

Keywords: "Electromagnetic scattering, "Compton effect, Reprints, Monte Carlo method, Computerized simulation, "Foreign technology, "Multiple scattering.

The multiple scattering background in Compton scatter imaging at $662 \mathrm{keV}$ is studied, both experimentally and by Monte Carlo radiation transport calculations as a function of the scattering angle, scattering material (aluminum, brass and tin) and object thickness. A dou ble-peak structure was observed in the pulse-heigh distribution for the thicker brass and tin objects and at the larger scattering angles (90 degrees and 120 de grees). In addition to the Compton peak, a second peak appeared at a higher energy. Monte Carlo transport simulations have revealed the origin of the second peak: photons that have scattered exactly twice before reaching the detector. The ratio of the multiple-scattered radiation to the total radiation detected was calculated as a function of the energy-window width around the Compton peak and scattering angle. The results of this study may help in the design of future Compton scatter imaging apparatus.

04,426

PB96-138474 Not available NTIS

National Inst. of Standards and Technology (EEEL) Boulder, CO. Electromagnetic Fields Div.

Accurate Computations of Radar Cross Sections of Simple Objects.

Final rept.

L. A. Muth, and J. Gary. 1994, 5p.

Pub. in International Microwave Conference (10th)

MIKON - 94, Ksiaz Castle, Poland, May 30-June 2, 1994, v1 p298-302.

Keywords: "Radar cross sections, "Measurement, Two dimensional models, Electromagnetic scattering Boundary conditions, Impedance, Computationa grids, Helmholtz equations, Error analysis, Reprints.

A comparison of two methods for the computation of the radar cross section is given. The first method is by Mei and the second by Keller and Givoli. Both direct methods solve a discrete version of the Helmholtz equation in a small domain containing the scatterer using absorbing outer boundary conditions. The solutions for a plane wave scattered from a two-dimensional infinite cylinder are better than $0.10 \mathrm{~dB}$ when compared to the analytic solutions.

\section{4,427}

PB96-148077 Not available NTIS

National Inst. of Standards and Technology (EEEL), Boulder, CO. Electromagnetic Fields Div.

Spatial Correlation Function for Fields in a Rever beration Chamber.

Final rept.

D. A. Hill. 1995, 1p

Pub. in Institute of Electrical and Electronics Engineers Transactions on Electromagnetic Compatability, v37 n1 p1 Feb 95

Keywords: "Plane waves, "Reverberation, "Chambers, Spatial filtering, Acoustic properties, Wave scattering, Correlation, Reprints, Reverberation chamber, Plane wave spectrum, Spatial correlation.

The plane-wave spectrum representation has been found useful for providing a mathematical description for the response of a receiving antenna or other test object in a reverberation chamber and for calculating the quality factor $(\mathrm{O})$ for reverberation chambers of arbitrary geometries. The purpose of this correspondence is to show that the plane-wave spectrum representation can also be used to provide a simple derivation for the spatial correlation function of the fields. The identical correlation function has been derived via a cavity mode representation and has been tested ex perimentally.

\section{4,428}

PB97-110274 Not available NTIS

National Inst. of Standards and Technology (MEL), Gaithersburg, MD. Precision Engineering Div.

Hypersingular Single Integral Equation and the Dielectric Wedge.

Final rept.

E. Marx. 1992, 4p.

Pub. in Institute of Electrical and Electronics Engineers Antennas and Propagation Society International Symposium, Chicago, IL., July 18-25, 1992, v4 p1865 1868, 1992 Digest.

Keywords: "Dielectric wedge, "Electromagnetic scattering, Far fields, Hypersingular integrals, Integra equations, Sharp edges, Reprints.

The theory of the behavior of electromagnetic fields near the edge of a dielectric wedge is poorly understood and disagrees with numerical results. Here the authors compute the fields scattered by a finite dielectric wedge and compared the results obtained by using two different integral equations. The unknown boundary function is either the jump $n$ in the normal derivative of the auxiliary field across the boundary or the jump 0 in the field itself. In the latter case, one of the integrals is hypersingular.

04,429

PB97-110522 Not available NTIS

National Inst. of Standards and Technology (MEL),

Gaithersburg, MD. Precision Engineering Div.

Causality and Maxwell's Equations.

Final rept.

E. Marx. 1993, 5p

Pub. in Institute of Electrical and Electronics Engineers International EMC Symposium, Anaheim, CA., August $17-21,1992$.

Keywords: "Electromagnetic scattering, "Integral equations, Maxwell's equations, Algorithms, Reprints, ${ }^{\star}$ Causality

Causality is addressed in the context of the principles of electromagnetism for nonsinusoidal fields. Topics include Maxwell's equations, integral equations for scattering, stepping-in-time algorithms, dispersive media, and Green's functions.

04,430

PB97-110530 Not available NTIS

National Inst. of Standards and Technology (MEL), Gaithersburg, MD. Precision Engineering Div.

Electromagnetic Scattering from a Dielectric Wedge and the Single Hypersingular Integral Equation.

Final rept.

E. Marx. $1993,8 p$

See also PB97-110274

Pub. in Institute of Electrical and Electronics Engineers Transactions on Antennas and Propagation, v41 n8 p1001-1008 Aug 93.

Keywords: "Electromagnetic scattering, "Dielectrics, Integral equations, Reprints, Dielectric wedges.

Electromagnetic fields scattered by a finite dielectric wedge are computed using a hypersingular integral equation (HIE). The results are compared with those obtained previously using a singular integral equation (SIE) and with the theory that predicts that the fields near the edge of the wedge behave like static fields. The HIE produces more consistent results than the SIE, probably because the unknown boundary function tends to a constant near the edge instead of diverging. The two numerical methods agree reasonably well, and these results agree only in part with the static field behavior

\section{Solid State Physics}

\section{4,431}

AD-A280 150/4 PC A05/MF A01

National Bureau of Standards, Gaithersburg, MD. Atomic Energy Levels in Crystals.

Monograph rept.

J. L. Prather. 24 Feb 61, 90p.

Keywords: "Atomic energy levels, Crystals, Perturbations, Ions, Electrostatics, Charged particles, Symmetry, Angular momentum, Selection rules(Physics), Dipole moments, Magnetic dipoles, Ouadrupole moment, Transitions, Absorption spectra, Optical properties, Nuclear electric moments, Spectroscopy, $\mathrm{N}$ 99057.

No abstract available.

04,432

DE95015476 PC A04/MF AO

National Inst. of Standards and Technology (EEEL), Boulder, CO. Electromagnetic Technology Div.

Electromechanical properties of superconductors for DOE fusion applications.

J. W. Ekin, S. L. Bray, C. L. Lutgen, and W. L. Bahn Jan 94, 53p, NISTIR-5013.

Contract Al01-84ER52113

Sponsored by Department of Energy, Washington, DC.

Keywords: "Superconducting Magnets,

"Superconductors, Current Density, Electromechanics, Leading Abstract, Magnetic Fields, Niobium Base Alloys, Stresses, Thermonuclear Reactors, Tin Alloys EDB/700430, EDB/665412. 
The electrical performance of many superconducting materials is strongly dependent on mechanical load. This report presents electromechanical data on a broad range of high-magnetic-field superconductors. The conductors that were studied fall into three general categories: Candidate conductors, experimental conductors, and reference conductors. Research on candidate conductors for fusion applications provides screening data for superconductor selection as well as engineering data for magnet design and performance analysis. The effect of axial tensile strain on criticalcurrent density was measured for several $\mathrm{Nb}$ (sub 3) Sn candidate conductors including the US-DPC (United States Demonstration Poloidal Coil) cable strand and an ITER (International Thermonuclear Experimental Reactor) candidate conductor. Also, data are presented on promising experimental superconductors that have strong potential for fusion applications. Axial strain measurements were made on a V(sub 3)Ga tape conductor that has good performance at magnetic fields up to $20 \mathrm{~T}$. Axial strain data are also presented for three experimental $\mathrm{Nb}$ (sub 3) Sn conductors that contain dispersion hardened copper reinforcement for increased tensile strength. Finally, electromechanical characteristics were measured for three different $\mathrm{Nb}$ (sub 3) Sn reference conductors from the first and second VAMAS (Versailles Project on Advanced Materials and Standards) international $\mathrm{Nb}$ (sub 3) Sn criticalcurrent round robins. Published papers containing key results, including the first measurement of the transverse stress effect in $\mathrm{Nb}$ (sub 3 ) $\mathrm{Sn}$, the effect of stress concentration at cable-strand crossovers, and electromechanical characteristics of $\mathrm{Nb}$ (sub 3)Al, are included throughout the report.

\subsection{3}

DE95016656 PC A01/MF A01

National Inst. of Standards and Technology, Boulder, CO.

VAMAS interlaboratory comparisons of critical current vs. strain in $\mathrm{Nb}$ (sub 3) Sn.

J. W. Ekin. 1989, 5p CONF-8902131-3.

Contract Al01-84ER52113

Japan-US workshop on high field superconductors materials and standard procedures for high-field superconducting materials testing (6th), Boulder, CO (United States), 22-24 Feb 1989. Sponsored by Department of Energy, Washington, DC.

Keywords: "Niobium Base Alloys, "Tin Alloys, "Meetings, Comparative Evaluations, Critical Current, Current Density, Intermetallic Compounds, Magnetic Fields, Strains, Superconducting Magnets, Tensile Properties, Wire, EDB/360107, EDB/360103, EDB/ 665412.

A comparison is made of measurements of the effect of axial tensile strain on the critical current of multifilamentary $\mathrm{Nb}$ (sub 3) Sn superconductors by three different laboratories. Two of the laboratories used short sample testing apparatus wherein a straight section of conductor was cooled in a force-free state. One of the laboratories used a spring apparatus wherein a long sample was reacted in a coil shape and attached to a spring sample holder. The agreement beween the results for the two laboratories that used the straight sample apparatus was quite good, within $15 \%$ for all three conductors at $15 \mathrm{~T}$, except at very high strain for one conductor which had an upper critical field close to the measurement field. To make a comparison with the data obtained using the spring method, it was necessary to fit the data to the compressive prestrain determined using the straight-sample technique. With such a fit the agreement was variable, between 15 and $25 \%$ depending on the conductor. Values of the prestrain and irreversible strain obtained from the straight sample data agreed within $0.06 \%$ and $0.05 \%$ respectively. Values of the maxi (strain-free) upper critical fields agreed within several tenths of a esla.

\section{4,434}

DE95016659 PC A01/MF A01

National Inst. of Standards and Technology, Boulder co.

ransverse stress effect on the critical current of internal tin and bronze process $\mathrm{Nb}$ (sub 3 ) $\mathrm{Sn}$ superconductors.

J. W. Ekin, S. L. Bray, P. Danielson, D. Smathers and R. L. Sabatini. 1989, 4p, CONF-8902131-2. Contract Al01-84ER52113

Japan-US workshop on high field superconductors materials and standard procedures for high-field superconducting materials testing (6th), Boulder, CO (United States), 22-24 Feb 1989. Sponsored by Department of Energy, Washington, DC.
Keywords: "Niobium Alloys, "Tin Alloys, Comparative Evaluations, Critical Current Fabrication, Intermetallics, Stresses, Superconducting Magnets, Tensile Properties, Wire, EDB/360107, EDB/360103, $E D B / 665412$

The effect of transverse stress on the critical current density, $J$ (sub c), has been shown to be significant in bronze process $\mathrm{Nb}$ (sub 3) $\mathrm{Sn}$, with the onset of significant degradation at about $50 \mathrm{Mpa}$. In an applied field of $10 \mathrm{~T}$, the magnitude of the effect is about seven times larger for transverse stress than for axial tensile stress. In a subsequent study, similar results were observed in another bronze process $\mathrm{Nb}$ (sub 3 ) Sn conductor made by a different manufacturer. The mechanism accounting for the transverse stress effect and its large magnitude compared with the axial tensile effect is still the subject of speculation. In an attempt to better understand the nature of the effect, The authors have undertaken a series of experiments to determin whether the transverse stress effect depends on the grain morphology of the $\mathrm{Nb}$ (sub 3) $\mathrm{Sn}$ reaction layer in the superconductor. To do this, the authors have measured the effect in an internal tin conductor with excess tin, which yields a more equiaxed $\mathrm{Nb}$ (sub 3 ) $\mathrm{Sn}$ grain morphology than for bronze process $\mathrm{Nb}$ (sub 3)Sn, where the grains are more columnar. The results for the effect of transverse compression on the $\mathrm{J}$ (sub c) of a round bronze process $\mathrm{Nb}$ (sub 3) $\mathrm{Sn}$ wire are given. The data are probably applicable to a wide variety of $\mathrm{Nb}$ (sub 3) $\mathrm{Sn}$ conductors for magnet engineering.

\section{4,435}

PB94-145984 PC A01/MF A01

Nation

Alternating-Field Susceptometry and Magnetic Susceptibility of Supercondoctors. Presented at Office of Naval Research Workshop on Magnetic Susceptibility of Superconductors and Other Spin Systems. Held in Berkeley Springs, West Virginia on 20 May 1991.

R. B. Goldfarb, M. Lelental, and C. A. Thompson Oct $91,36 \mathrm{p}$, NISTIR-3977

Sponsored by Eastman Kodak Co., Rochester, NY

Keywords: *Magnetic susceptibility, *Superconductors, High temperature superconductors, Superconducting Meissner effect, Alternating current, Calibration, Diamagnetism, Demagnetization, Granular materials, Diamagnetism, Demagnetization, Granular materials,
Sensitivity, Design, Reviews, Bibliographies, "Susceptometers.

The review critically analyzes current practice in the design, calibration, sensitivity determination, and operation of alternating-field susceptometers, and examines applications in magnetic susceptibility measurements of superconductors. Critical parameters of the intrinsic and coupling components of granula superconductors may be deduced from magnetic sus ceptibility measurements. The onset of intrinsic diamagnetism corresponds to the initial decrease in electrical resistivity upon cooling, but the onset of intergranular coupling coincides with the temperature for zero resistivity. The lower critical field may be determined by the field at which the imaginary part of susceptibility increases from zero. Unusual features in the susceptibility of superconductor films, such as a magnetic moment that is independent of film thickness and the variation of susceptibility with angle, are related to demagnetization. Common misunderstandings of the Meissner effect, magnetic units, and formula conversions are discussed. There is a comprehensive summary of critical-state formulas for slabs and cylinders, including new equations for complex susceptibility in large alternating fields Limitations on the use of the critical-state model for deducing critical current density are listed and the meaning of the Imaginary part of sus ceptibility is considered.

\section{4,436}

PB94-160744 PC A03/MF A01

National Inst. of Standards and Technology (CAML), Gaithersburg, MD. Applied and Computational Mathematics Div.

Computation of Dendrites Using a Phase Field Model.

A. A. Wheeler, B. T. Murray, and R. J. Schaefer. Jul 92, 42p, NISTIR-4894.

Keywords: "Dendritic crystals, ${ }^{*}$ Crystal growth "Solidification, Finite difference theory, Mathematica models, Two-dimensional calculations, Numerical solution, Interfaces, Nickel, Spheres, Free boundary problems, Phase field models.
A phase field model is used to numerically simulate the solidification of a pure material. We employ it to compute growth into an undercooled liquid for a one-dimensional spherically symmetric geometry and a planar two-dimensional rectangular region. The phase field model equations are solved using finite difference techniques on a uniform mesh. For the growth of a sphere, the solutions from the phase field equations for sufficiently small interface widths are in good agreement with a numerical solution to the classical sharp interface model obtained using a Green's function approach. In two dimensions, we simulate dendritic growth of nickel with four-fold anisotropy and investigate the effect of anisotropy level on the growth of a dendrite. The quantitative behavior of the phase field model is evaluated for varying interface thickness and spatial and temporal resolution. Results are discussed.

\section{4,437}

PB94-161502 PC A08/MF A02

National Inst. of Standards and Technology (MSEL), Gaithersburg. MD. Reactor Radiation Div.

NIST Reactor: Summary of Activities October 1992 through September 1993.

Internal rpt.

C. L. O'Connor. Feb 94, 155p, NISTIR-5362.

See also PB93-162873.

Keywords: "NBSR reactor, Research reactors, Activation analysis, Cold neutrons, Crystal structure, Neutron diffraction, Neutron radiography, Nondestructive tests, Analytical chemistry, YBCO superconductors, Fullerenes, Hydrogen, Magnetism, Polymers, Macromolecules, Microstructure, Thin films, Neutron dosimetry, Multilayers.

This report summarizes all of the programs which use the NIST reactor. It covers the period for October 1992 through September 1993. The programs range from the use of neutron beams to study the structure and dynamics of materials through nuclear physics and neutron standards to sample irradiations for activation analysis, isotope production, neutron radiography, and nondestructive evaluation.

\section{4,438}

PB94-169745 Not available NTIS

National Inst. of Standards and Technology, Gaithersburg, MD.

Precision Comparison of the Lattice Parameters of Silicon Monocrystals.

E. G. Kessler, A. Henins, R. D. Deslattes, L. Nielsen, and M. Arif. 1994, 18p.

Included in Jnl of Research of the National Institute of Standards and Technology, v99 n1 p1-18 Jan/Feb 94.

Keywords: "Lattice parameters, "Silicon, Interlaboratory comparisons, $X$-ray spectrometers, $X$ ray diffraction, Michelson interferometers, Bragg angle, Single crystals, Comparators, Monocrystals, Precision, Uncertainty, Reprints, US NIST.

The lattice spacing comparator established at the $\mathrm{Na}$ tional Institute of Standards and Technology to measure the lattice spacing differences between nearly perfect crystals is described in detail. Lattice spacing differences are inferred from the measured differences in Bragg angles for different crystals. The difference in lattice spacing between silicon samples used at Physikalisch-Technische Bundesanstalt (PTB) and NIST has been measured with a relative uncertainty of $1 \times 10(-8)$. The measurement is consistent with absolute lattice spacing measurements made at PTB and NIST. Components of uncertainty associated with systematic effects due to misalignments are derived and estimated.

\section{4,439}

PB94-172129 Not available NTIS

National Inst. of Standards and Technology (MSEL), Gaithersburg, MD.

RDFs and Fe-Fe Pair Correlations in an AICuFe Icosahedral Alloy by Double Isotopic Substitution. Final rept.

R. Bellissent, B. Mozer, Y. Calvayrac, D. Gratias, and J. W. Cahn. 1993, 4p.

Pub. in Jnl. of Non-Crystalline Solids 153/154, p1-4 1993.

Keywords: Aluminum copper alloys, Iron alloys, Neutron diffraction, Crystal structure, Reprints, *Ouasicrystals, Icosahedral phase, Radial distribution functions.

Short range order in an AICuFe quasicrystal was investigated using the double isotopic substitution of $\mathrm{Fe}$ in 
order to determine partial radial distribution (pair correlation) functions of the system. The experiment was carried out on the 7c2 spectrometer at Orphee designed for studies of atomic local order in liquid and amorphous systems. Very accurate total radial distribution functions were calculated. All of the observed pair distances, up to about $10 \mathrm{~A}$, are consistent with the atomic positions of the model proposed by CornierQuiquandon et al. (Phys. Rev. B44 (1991) 2071). Partial Fe-Fe pair correlation functions have been extracted and show that Fe atoms occupy only a subset of sites

04,440

PB94-172210 Not available NTIS

National Inst. of Standards and Technology (NML), Gaithersburg, MD. Gas and Particulate Science Div. Raman and Fluorescence Spectra Observed in Laser Microprobe Measurements of Several Compositions in the Ln-Ba-Cu-O System.

F. S. Etz, T. D. Schroeder, and W. Wong-Ng. 1990,

Pub. in Proceedings of Annual Conference of the Microbeam Analysis Society (25th), Seattle, WA., August 12-18, 1990, p243-247.

Keywords: "Superconductors, Rare earth compounds, Vibrational spectra, Raman spectra, Microanalysis, Microprobes, Fluorescence, Impurities, Reprints, Erbium barium cuprates, Europium barium cuprates.

The superconducting and related phases in the Ln-Ba$\mathrm{Cu}-\mathrm{O}$ system are investigated by Raman microprobe measurements to obtain their vibrational Raman spectra and any structure-specific fluorescence spectra from active lanthanide ( $L n)$ or rare earth ions. These studies are aimed at relating the observed optical spectra to the compositional and structural aspects of these materials. Specific examples are discussed with special reference to the erbium and the europium analogs of the yttrium $1: 2: 3$ superconductor. Highlighted are the results from certain phases that are known to constitute potential impurity phases in the superconducting compositions. The microanalytical implications of these results are discussed.

04,441

PB94-172475 Not available NTIS National Inst. of Standards and Technology (MSEL),

Neutron-Spectroscopy Study of the Hydrogen Vibrations in Hydrogen-Doped YBa2Cu3OX.

Final rept

U. Knell, H. Wipf, H. J. Lauter, T. J. Udovic, and J. J. Rush. 1993, 7p.

Pub. in Jnl. of Phys.: Condens. Matter 5, p7607-7613 1993.

Keywords: "YBCO superconductors, Electron phonon interactions, Neutron spectroscopy, Orthorhombic lattices, Tetragonal lattices, Doped materials, Cryogenic temperature, Reprints, *Hydrogen vibrations.

The authors studied $\mathrm{H}$ vibrations in $\mathrm{H}$-doped $\mathrm{YBa} 2 \mathrm{Cu} 3 \mathrm{O}(\mathrm{x}) \mathrm{H}(\mathrm{y})(\mathrm{y}=0.6)$ by neutron spectroscopy. Neutron spectra were taken at 10 and $14 \mathrm{~K}$ from both orthorhombic ( $x$ approx equals 6.9) and tetragonal ( $x$ approx equals 6.3) samples. The spectra exhibit large $\mathrm{H}$-induced intensities in the energy range between 40 and $130 \mathrm{meV}$. In comparison to orthorhombic samples, the $H$ vibrations in tetragonal samples are shifted to lower energies by about $11 \%$. This shift is opposite to the spectral shift of the lattice vibrations between $\mathrm{H}$. free orthorhombic and tetragonal $\mathrm{YBa} 2 \mathrm{Cu} 3 \mathrm{O}(\mathrm{x})$, frequently attributed to differences in the electron-phonon coupling. The results show that any such differences in the electron-phonon coupling are not apparent in the $\mathrm{H}$ vibrations. The results also support previous suggestions that the $\mathrm{H}$ occupies sites in the close neighborhood of the $\mathrm{Cu}(1)$ atomic planes.

\section{PB94-172558 Not available NTIS}

National Inst. of Standards and Technology (MSEL),

Gaithersburg, MD. Reactor Radiation Div.

Structural and Magnetic Ordering in Iron Oxide/

Nickel Oxide Multilayers by X-ray and Neutron Diffraction (Invited).

Final rept.

D. M. Lind, S. P. Tay, S. D. Berry, J. A. Borchers,

and $R$. W. Ewrin. 1993, 6p.

Contract N00014-92-J-1356, Grant NSF-DMR-

9206870

Pub. in Jnl. of Applied Physics 73, n10, p6886-6891,

15 May 93. Sponsored by Office of Naval Research,
Arlington, VA. and National Science Foundation, Arlington, VA.

Keywords: "Iron oxides, "Nickel oxides, "Superlattices, Antiferromagnetic materials, $X$ ray diffraction, Neutron diffraction, Magnetization, Thin films, Reprints, Magnetic ordering, Exchange interactions, Multilayers.

Presented are studies of the magnetic and structural ordering in superlattices composed of $\mathrm{Fe} 3 \mathrm{O} 4$ and $\mathrm{NiO}$ and their study by a variety of techniques including $x$ ray and neutron diffraction, and SQUID magnetometry, $X$-ray diffraction indicates that structures with individual layer thicknesses down to $8.5 \mathrm{~A}$ grow as single crystals in registry with the substrate lattice with a layer-thickness-dependent tetragonal lattice distortion due to epitaxial and interfacial lattice mismatch. The lattice coherence of the $\mathrm{Fe} 3 \mathrm{O} 4$ layers, however, is degraded by stacking faults between adjacent spinel layers. Neutron diffraction indicates that the $\mathrm{NiO}$ orders antiferromagnetically along the (in brackets: 111) direction with a magnetic coherence that extends over several superlattice bilayers, and the presence of an enhancement in the NiO Neel temperature in thin layered superlattices. These results are compared with SQUID magnetometry, which shows large anisotropy energies, but a lack of favored magnetization direction, indicating that the magnetic ordering in these systems is dominated by the exchange coupling across the interfaces.

\section{4,443}

PB94-172665 Not available NTIS

National Inst. of Standards and Technology (IMSE) Gaithersburg, MD. Reactor Radiation Div.

Enhanced Curie Temperatures and Magnetoelastic Domains in Dy/Lu Super Lattices and Films.

Final rept.

R. S. Beach, J. A. Borehers, A. Matheny, B. Everitt K. Pettit, J. J. Rhyne, C. P. Flynn, R. W. Erwin, and M. B. Salamon. 1993, $4 p$

Pub. in Physical Review Letters 70, No. 22, pp3502 3505 May 31, 1993. Sponsored by National Science Foundation, Arlington, VA.

Keywords: *Dysprosium, "Lutetium, "Superlattices, Curie temperature, Magnetostriction, Thin films, Ferromagnetic materials, Alignment, Reprints.

We have grown high quality superlattices of Dy with nonmagnetic Lu and find that the $2.4 \%$ epitaxial compression nearly doubles the ferromagnetic $T(c)$ of Dy with little change in the Neel temperature. A helimagnetic phase exists over a narrow temperature range. Below $\mathrm{T}(\mathrm{c})$ in superlattices, $300 \mathrm{~A}$ orthorhombic domains form despite epitaxial constraints, each with a magnetostrictive distortion comparable to that of bulk Dy. For the thinnest intervening Lu layers, individual ferromagnetic Dy blocks have parallel alignment; the remaining samples show antiparallel alignment, coherent over many bilayer periods.

\section{4,444}

PB94-173044 Not available NTIS

National Inst. of Standards and Technology (MSEL) Gaithersburg, MD. Reactor Radiation Div.

Dispersions of Magnetic Excitations of the Pr Ions in Pr2CuO4.

Final rept.

W. Sumarlin, J. W. Lynn, and T. Chattpadhyay.

1994, 5p.
Pub. in Physica C 219, p195-199 1994.

Keywords: "Superconductors, Praseodymium ions, Crystal field, Dispersion, Excitons, Reprints, "Praseodymium cuprates, Exchange interactions.

The authors have used inelastic neutron scattering techniques to measure the dispersion of the singletdoublet magnetic excitons of the $\operatorname{Pr}(3+)$ ions in the singlet ground state system Pr2CuO4. The excitons exhibit significant dispersion both within the basa plane as well as along the c-axis direction, directly demonstrating the Pr-Pr exchange interactions. These exchange interactions must be mediated through the
$\mathrm{CuO} 2$ layers involved in the formation of the superconducting state.

\section{4,445}

PB94-173069 Not available NTIS

National Inst. of Standards and Technology (PL) Gaithersburg, MD. Electron and Optical Physics Div.
Homoepitaxial Growth of Iron and a Real Space View of Reflection-High-Energy-Electron Diffraction.

Final rept

J. A. Stroscio, D. T. Pierce, and R. A. Dragoset.

1993, 4p

Pub. in Physical Review Letters 70, n23 p3615-3618,

7 Jun 93. Sponsored by Office of Naval Research, Arlington, VA.

Keywords: "Iron, "Epitaxial growth, Scanning tunneling microscopy, Whiskers(Single crystals), Electron diffraction, Surface diffusion, Temperature range 0273$0400 \mathrm{~K}$, Temperature range $0400-1000 \mathrm{~K}$, Metal films, Reprints.

The authors report real space views of the homoepitaxial growth of $\mathrm{Fe}$ on $\mathrm{Fe}(001)$ whiskers observed by scanning tunneling microscopy. A measure of the surface diffusion of the $\mathrm{Fe}$ atoms is obtained over the temperature range of 20-250 deg C. The effect of the diffusion kinetics is observed in the surface morphology as a decrease in the intertace width with temperature. Measurements of reflection-high-energyelectron diffraction during growth allow a comparison of real and reciprocal space techniques.

\section{4,446}

PB94-185162 Not available NTIS

National Inst. of Standards and Technology (EEEL) Boulder, CO. Electromagnetic Technology Div.

Effects of Critical Current Density, Equilibrium Magnetization and Surface Barrier on Magnetization of High Temperature Superconductors.

Final rept.

D. X. Chen, R. W. Cross, and A. Sanchez. 1993, 9p Pub. in Cryogenics 33, n7 p695-703 1993.

Keywords: "High temperature superconductors "Magnetization, YBCO superconductors, Critical current, Surface barrier, Mathematical models, Tempera ture range 0065-0273 K, Current density, Reprints, Yttrium barium cuprates.

An extended critical state model which includes the effects of bulk critical current density, equilibrium magnetization and surface barrier is developed for the magnetization of superconductors. The equilibrium magnetization and surface barrier are modeled by an applied field dependent surface supercurrent density i(sub $\mathrm{s})(\mathrm{H})$, whose presence changes the boundary field of the bulk. The volume supercurrents flow with a density equal to the internal field dependent critical current density $\mathrm{J}($ sub $\mathrm{c})(\mathrm{H}(\mathrm{sub} \mathrm{i}))$. The magnetization $M$ is produced by both supercurrents. For the $M(H)$ curve computation, exponential-type $\mathrm{J}$ (sub $\mathrm{c}(\mathrm{H}$ (sub i)) and $($ sub $s)(H)$ values are used for the general case of an infinite sample of rectangular cross-section. A comparison between the experimental magnetization curves of a sintered $\mathrm{YBa} 2 \mathrm{Cu} 3 \mathrm{O} 7$ superconductor at 76 $K$ and the model fit shows that $j(s u b s)(H)$ is null for the coupling matrix, whereas a non-zero j(sub $s)(H)$ is needed for the grains. The model fit for the irreversible magnetization of the grains is improved by including a surface barrier to the entry and exit of flux.

04,447

PB94-185329 Not available NTIS

National Inst. of Standards and Technology (EEEL) Gaithersburg, MD. Semiconductor Electronics Div. Optical Conductivity of Single Crystals of Ba1 $x M x B i O 3(M=K, R b, x=0.04,0.37)$.

Final rept

S. Guha, D. Peebles, V. Browning, M. Norton, T. Wieting, and D. Chandler-Horowitz, 1993, 11p. Pub. in Jnl. of Superconductivity 6, n5 p339-349 1993

Keywords: *Infrared reflectance, Reprints, Single crystals, Room temperature, Superconductors, Ellipsometry, Polarons, Phonons, "Potassium barium bismuthates, "Rubidium barium bismuthates, Optical conductivity

Reflectance data (0.001-4.0 eV) from several large (a typical surface area $3 \times 3 \mathrm{sq} \mathrm{mm}$ ) single crystals of $\mathrm{Ba}(\mathrm{I}-\mathrm{x}) \mathrm{K}(\mathrm{x}) \mathrm{BiO} 3(\mathrm{x}=0.04,0.37)$ (BKBO) and $\mathrm{Ba}(\mathrm{I}$ x) $\mathrm{Ab}(\mathrm{x}) \mathrm{BiO} 3(\mathrm{x}=0.37)$ (BABO) were obtained by Fourier Transform infrared (FTIR) and ellipsometric methods. Normal-state optical conductivities of these samples were obtained from infrared and ellipsometric measurements using a Kramers-Kronig transform. A broad mid-IR band was observed that peaked at 0.3 $\mathrm{eV}$ for BKBO and at $0.16 \mathrm{eV}$ for BRBO at room tem perature. Each band was fitted with two Lorentz oscillators. The optical mass of the charge carriers was obtained from a Drude fit, and was found to be large. 
These overdamped charge carriers can be viewed as polarons with a large effective mass. An optic phonon mode at $325 / \mathrm{cm}$ was also observed in the metallic phase. Interpretations of low-temperature measurements on BKBO and BRBO were complicated due to the change of color of the sample from bluish-green to bronze-red. Upon warming, samples revert to their original bluish-green color.

\section{4,448}

PB94-185428 Not available NTIS

National Inst. of Standards and Technology (MSEL), Gaithersburg, MD. Metallurgy Div.

Interfacial Free Energies from Substrate Curvature Measurements of the Creep of Multilayer Thin Films.

Final rept.

D. Josell. $1994,8 p$

Pub. in Acta Metali. Mater. 42, n3 p1031-1038 1994

Keywords: "Free energy, Creep properties, Thin films, Substrates, Interfaces, Reprints, Biaxial curvature, Multilayers.

The equilibrium biaxial curvature of a multilayer thin film adhering to a free standing substrate is determined when plastic flow is possible in the multilayer. The relationship found between the equilibrium biaxial curvature and the interfaces in the multilayer is modified to account for grain boundaries in the layers. Two possible experiments that use the resulting relationship to determine interfacial free energies are described.

04,449

PB94-185477 Not available NTIS

National Inst. of Standards and Technology (PL), Boulder, CO. Quantum Physics Div.

Temperature Dependence and Anharmonicity of Phonons on $\mathrm{Ni}(110)$ and $\mathrm{Cu}(110)$ Using Molecular Dynamics Simulations.

Final rept.

D. D. Koleske, and S. J. Sibener. 1993, 10p.

Pub. in Surface Science 298, p215-224 1993. Sponsored by National Science Foundation, Washington DC. and Air Force Office of Scientific Research, Washington, DC.

Keywords: "Nickel, "Copper, "Phonons, Temperature dependence. Molecular dynamics, Surface potential, Simulation, Reprints, Anharmonicity.

Molecular dynamics simulations were performed for $\mathrm{Ni}(110)$ and $\mathrm{Cu}(110)$ using Finnis-Sinclair model potential. During the simulations the temperature dependencies of the mean-square displacements (MSD), the layer-by-layer stress tensors, and the surface phonon spectral densities were measured. The simulation results imply that the $\mathrm{Ni}(110)$ and $\mathrm{Cu}(110)$ surfaces do not extensively roughen before the onset of adatom-defect formation, and, in confirmation of experimental findings, that the rapid decrease of specular intensity for helium or electron scattering at elevated temperatures is due to the influence of anharmonicity in the surface potential.

04,450

PB94-185659 Not available NTIS

National Inst. of Standards and Technology (MSEL), Gaithersburg, MD. Metallurgy Div.

Enhanced Magnetocaloric Effect in Gd3Ga5xFex012.

Final rept.

R. D. McMichael, J. J. Ritter, and R. D. Shull. 15

May 93, 3p

Pub. in Jnl. of Applied Physics 73, nt0 p6946-6948, 15 May 93.

Keywords: Temperature range 0013-0065 K, Gadolinium-gallium garnet, Magnetic refrigerators, Solid clusters, Entropy, Reprints "Gadolinium gallium ferrates, "Magnetocaloric effect, Cryogenic refrigerators, Exchange interactions, Nanostructures.

The working refrigerant material in the majority of magnetic refrigerators has been Gd3Ga5012 (GGG) which has an upper temperature limit near $15 \mathrm{~K}$. In this paper we report on the field-induced adiabatic magnetic entropy change, Delta $S($ sub $m)(H, T)$, of a series of ironsubstituted gadolinium garnets (GGIG) Gd3Ga(5$x) \mathrm{Fe}(x) \mathrm{O} 12$ which have the potential to increase the working temperature range or to reduce the field requirements of cryogenic magnetic refrigeration. Depending on Fe concentration, $x$, the entropy change of these materials at applied fields of 0.9 and $5.0 \mathrm{~T}$ is much greater than that of GGG, especially at tempera tures above $15 \mathrm{~K}$. At low Fe concentrations, the results are consistent with formation of magnetically ordered clusters of spins at low temperatures. Room temperature electron paramagnetic resonance measurements show that $\mathrm{Fe}(3+)$ ions mediate exchange interactions which are responsible for clustering at low temperatures.

\section{4,45}

PB94-185667 Not available NTIS

National Inst. of Standards and Technology (MSEL) Gaithersburg, MD. Metallurgy Div.

Magnetocaloric Effect in Rapidly Solidified Nd-FeAl-B Materials.

Final rept.

R. D. McMichael, R. D. Shull, L. H. Bennett, C. D.

Fuerst, and J. F. Herbst. 1993, 7p.

Pub. in NanoStructured Materials 2, p277-283 1993.

Keywords: Magneto-thermal effects, Magnetic refrigerators, Magnetic fields, Neodynium containing alloys,

Boron containing alloys, Aluminum alloys, Iron alloys, Temperature range 0273-0400 K, Temperature range 0400-1000 K, Temperature dependence, Magnetization, Entropy, Reprints, “Magnetocaloric effect, Rapid solidification.

We have measured the temperature and field dependence of the magnetization of several rapidly solidified $\mathrm{Nd}(0.14)(\mathrm{Fe}(1-\mathrm{x}) \mathrm{Al}(\mathrm{x}))(0.80) \mathrm{B}(0.06)$ alloys in the 290$500 \mathrm{~K}$ temperature range at fields from 0 to $0.9 \mathrm{~T}$. At room temperature the materials are ferromagnetic. Calculations of the magnetocaloric effect from the magnetization data clearly show a peak in the field-induced entropy change, Delta $S$, at a critical temperature $T(p)$, which varies with aluminum content and is identified as the Curie temperature, $T(c)$, by differential scanning calorimetry measurements. The magnitude, shape and width of the Delta $S$ vs. T curves differ greatly from that predicted for elemental ferromagnets, but are consistent with curves predicted for magnetic nanocomposites. This similarity suggests that the magnetic moments are (1) grouped in clusters with moments on the order of $20-50 \mathrm{mu}(\mathrm{sub} \mathrm{B}$ ) and (2) that these materials may be potential candidates for high temperature magnetic refrigerants.

\section{4,452}

PB94-185840 Not available NTIS

National Inst. of Standards and Technology (EEEL), Gaithersburg, MD. Semiconductor Electronics Div.

Correction to the Decay Rate of Nonequilibrium Carrier Distributions Due to Scattering-in Processes.

Final rept.

B. A. Sanborn, B. Y. K. Hu, and S. Das Sarma

1994, 3p

Pub. in Physical Review B 49, n11 p7767-7769, 15 Mar 94.

Keywords: "Charge carriers, Electron scattering, Doped materials, Semiconductors, Correction, Reprints.

We show that, for doped semiconductor structures at nonzero temperatures, processes which scatter electrons into a state (vertical stroke) $\mathrm{K}>$ can contribute strongly to the decay of a nonequilibrium electron occupation of (vertical stroke) $\mathrm{K}>$. For electrons, the decay rate gamma $(K)$ is given by the sum of the total scattering-out and scattering-in rates of state (vertical stroke) $\mathrm{K}>$. The scattering-in term is safely neglected in the nondegenerate and highly degenerate limits, but it increases gamma (K) of low-energy electrons injected into semidegenerate systems (between these limits) by the factor $(1-f(0)(K))$ (sup -1). We show that the degree of degeneracy of a system of fermions depends strongly on the system dimension, so that doped systems of reduced dimension can be semidegenerate, with the two scattering rates comparable, even at high temperature.

\section{4,453}

PB94-185857 Not available NTIS

National Inst. of Standards and Technology (MSEL), Gaithersburg, MD. Metallurgy Dlv.

Magnetocaloric Effect of Ferromagnetic Particles. Final rept.

R. D. Shull. 1993, 2p.

Pub. in Institute of Electrical and Electronics Engineers Transactions on Magnetics 29, n6 p2614-2615 Nov 93

Keywords: Magneto-thermal effects, Gadoliniumgallium garnet, Magnetic refrigerators, Superparamagnetism, Solid clusters, Entropy, Reprints, "Gadolinium gallium ferrates, "Magnetocaloric effect, Nanocomposites.
The entropy change accompanying the removal of an applied magnetic field (i.e., the magnetocaloric effect) has been calculated for a system of magnetic spins independent of each other and also clustered together into independently-acting magnetic particles. Meanfield theory calculations have also been made for interacting, single magnetic spins and also for interacting magnetic particles. In both cases, there are found temperature and field regimes where the entropy changes are larger when the spins are coupled together into nanometer-sized magnetic particles. Entropy change data is shown for a new Gd3Ga(5-x)Fe(x)O(12) ( $X=$ or $<2.5$ ) magnetic nanocomposite, confirming the calculations and possessing magnetocaloric effects larger than the presently preferred low temperature paramagnetic refrige rant, gadolinium gallium garnet.

04,454

PB94-185865 Not available NTIS

National Inst. of Standards and Technology (MSEL)

Gaithersburg, MD. Metallurgy Div.

Viewpoint: Nanocrystalline and Nanophase Materials.

Final rept.

R. D. Shull. 1993, 4p

Pub. in Jnl. of NanoStructured Materials 2, p213-216 1993.

Keywords: Nucleation, Magnetism, Metals, Ceramics, Reprints, "Nanomaterials, Nanocomposites, Nanophases.

Nanocrystalline materials represent one of the most active laboratories in recent times for the atomic tailor ing of materials with specific properties and property combinations. However, it is still in its infancy; its emergence as a major materials science thrust has just begun. At this stage in its development, there have been glimpses of exciting new properties (e.g. superplasticity, giant magnetoresistance, transparency in opaque ceramics, enhanced homogeneity, unusually soft ferromagnetism, and giant magnetocaloric ef fects) possessed by materials containing some critica length which has been reduced to nanometer dimensions. But, for many of these cases, it still remains to be determined whether the property changes are due to new physics of the mechanisms at small dimensions or to an extension of larger-scale systematics to small sizes.

\section{4,455}

PB94-185923 Not available NTIS

National Inst. of Standards and Technology (PL) Gaithersburg, MD. Electron and Optical Physics Div, Scaling of Diffusion-Mediated Island Growth in Iron-on-Iron Homoepitaxy.

Final rept.

J. A. Stroscio, and D. T. Pierce. 1994, 4P.

Pub. in Physical Review B 49, n12 p8522-8525, 15 Mar 94. Sponsored by Office of Naval Research, Arlington, VA.

Keywords: "Epitaxial growth, "Iron, Whiskers(Single crystals), Scanning tunneling microscopy, Critical size, Nucleation, Metal films, Scaling, Reprints Homoepitaxy.

An analysis of the island size and separation distribu tions of $\mathrm{Fe}$ islands, observed in the initial stages of growth in the homoepitaxy of Fe on Fe(001) whiskers, shows scaling properties recently predicted in nucleation and growth theories. A critical size of one atom, where islands greater than the critical size undergo irreversible nucleation, is supported by the measured scaling functions for the $\mathrm{Fe}$ on $\mathrm{Fe}$ system in the temperature range of $20-250 \mathrm{C}$.

04,456

PB94-187663 PC A04/MF A01

National Inst. of Standards and Technology (MSEL) Gaithersburg, MD. Ceramics Div

Workshop on Characterizing Diamond Films (3rd) Held in Gaithersburg, Maryland on February 23-24, 1994.

A. Feldman, S. Holly, C. A. Klein, and G. Lu. Apr 94, $56 \mathrm{p}$, NISTIR-5418.

See also PB92-205426 and PB93-207157. Prepared in cooperation with Rockwell International, Canoga Park, CA., Raytheon Co., Lexington, MA. Research Div., and Norton Diamond Film, Northboro, MA

Keywords: "Diamond films, "Meetings, Chemical vapor deposition, Thermal conductivity, Thermal diffusivity, Raman spectroscopy, Fracture properties, Thin films, Polycrystalline, Polishing, Brazing, Stresses, Strains, Standards. 


\section{Solid State Physics}

The third in a series of workshops was held at NIST on February 23rd and 24th, 1994 to discuss the characterization of diamond films and the need for standards in diamond technology. The audience targeted for this workshop were producers and potential users of chemical vapor deposition (CVD) diamond technology in the United States. Three technical topics that have relevance to applications of CVD diamond films were discussed: characterizing brazing and polishing, standardization of thermal conductivity measurement, and characterizing stress, strain, and fracture.

\section{4,457}

PB94-198355 Not available NTIS

National Inst. of Standards and Technology (MSEL), Gaithersburg, MD. Reactor Radiation Div.

Combined Low- and High-Angle X-Ray Structural Refinement of a Co/Pt(111) Multilayer Exhibiting Perpendicular Magnetic Anisotropy.

Final rept.

J. F. Ankner, J. A. Borchers, R. F. C. Farrow, and R. F. Marks. 1993, 3p

Pub. in Jnl. of Applied Physics 73, n10 p6427-6429, 15 May 93.

Keywords: "Cobalt, "Platinum, Molecular beam epitaxy, Magnetic anisotropy, X-ray diffraction, X-ray reflection, Metal films, Superlattices, Substrates, Interfaces, Sapphire, Reprints, Multilayers.

We have used Cu K(alpha) radiation to measure both specular reflectivity (000) and longitudinal Bragg diffraction (222) from a Co/Pt multilayer grown by molecular-beam epitaxy on a sapphire (0001) substrate. By refining both low- and high-angle profiles, we are able to separate the effects of surface morphology from microstructure. Our results indicate mixing at the interfaces consistent with the existence of alloy or compound formation.

\section{4,458}

PB94-198363 Not available NTIS

National Inst. of Standards and Technology (MSEL), Gaithersburg, MD. Reactor Radiation Div.

Magnetic Dead Layer in Fe/Si Multilayer: Profile Refinement of Polarized Neutron Reflectivity Data. Final rept.

J. F. Ankner, C. F. Majkrzak, and H. Homma. 1993,

$2 p$.

Pub. in Jnl. of Applied Physics 73, n10 p6436-6437, 15 May 93.

Keywords: "Silicon, "Iron, Polarized beams, Metal films, Antiferromagnetism, Interfaces, Thin films, Reprints, Neutron reflectivity, Polarized neutrons, Multilayers

We have used polarized neutron reflectometry to study the magnetic structure of an Fe/Si multilayer film. By simultaneous refinement of both plus and minus reflectivities, we have extracted separate nuclear and magnetic scattering density profiles that include a 6A-thick magnetically dead layer in $\mathrm{Fe}$ at the interface. This result supports the contention that the antiferromagnetic coupling reported in this system is mediated by the presence of $\mathrm{Fe}$ in the $\mathrm{Si}$ interlayers.

\section{4,459}

PB94-198421 Not available NTIS

National Inst. of Standards and Technology (MSEL),

Gaithersburg, MD. Ceramics Div.

Weak-Link-Free Behavior of High Angle YBa2Cu307-x Grain Boundaries in High Magnetic Fielas

Final rept.

S. E. Babcock, X. Y. Cai, D. L. Kaiser, and D. C.

Larbalestier. 1990, 3p

Pub. in Nature 347, n6289 p167-169 1990.

Keywords: "YBCO superconductors, "Grain boundaries, High temperature superconductors, Transport properties, Critical current, Current density, Magnetic fields, Bicrystals, Reprints, "Yttrium barium cuprates, Weak links

The transport properties across grain boundaries in several bi-crystals of YBa2Cu3O(7-delta) have been measured. In three cases, including two high angle grain boundaries, no weak link behavior was observed. These results remove an important restriction suggested by earlier thin film bi-crystal results, showing that at least some high angle grain boundaries can transmit supercurrents in high magnetic fields at $77 \mathrm{~K}$. Such results are conceptually important in searching for ways to improve the J(ct) characteristics of bulk, polycrystalline high temperature superconductors for high field applications.
04,460

PB94-198512 Not available NTIS

National Inst. of Standards and Technology (MSEL), Boulder, CO. Materials Reliability Div.

Profile Fitting of X-Ray Diffraction Lines and Fourier Analysis of Broadening.

Final rept.

D. Balzar. 1992, 12p

Pub. in Jnl. of Applied Crystallography 25, p559-570 1992.

Keywords: "X-ray diffraction, "Line broadening, Reprints, High temperature superconductors, Distribution functions, Fourier analysis, Lanthanum strontium cuprates, Warren-Averbach method, Voigt functions, Microstains.

The extraction of pure-specimen $X$-ray diffraction-line broadening is described using a convolution of the instrumental profile and an exact Voigt function. Real Fourier coefficients were computed from the Cauchy and Gauss integral breadths and were input for Warren-Averbach analysis. Smooth surface-weighted and volume-weighted column-length distribution functions were obtained and errors in root-mean-square strains as well as effective domain sizes were evaluated. The method was applied to two cubic structures with aver age volume-weighted domain sizes up to $3600 \mathrm{~A}$ as well as patterns of tetragonal and orthorhombic ( $\mathrm{La}$ $\mathrm{Sr}$ 2CuO4, which exhibit weak line broadenings and highly overlapping reflections. Comparison with the integral-breadth methods is given. Reliability of the method is discussed in the case of a cluster of overlap. ping peaks

\section{4,46}

PB94-198520 Not available NTIS

National Inst. of Standards and Technology (MSEL), Boulder, CO. Materials Reliability Div.

Microstrains and Domain Sizes in Bi-Cu-O Superconductors: An X-Ray Diffraction PeakBroadening Study.

Final rept.

D. Balzar, and H. Ledbetter. 1992, 2p

Pub. in Jnl. of Materials Science Letters 11, p141914201992

Keywords: "BSCCO superconductors, High temperature superconductors, X-ray diffraction, Size determination, Domains, Reprints, *Bismuth strontium calcium cuprates, Warren-Averbach method, Microstrains.

We present an $x$-ray diffraction peak-broadening analysis of three Bi-Cu-O high-T(c) superconductors. The diffraction line profiles were fitted with a convolution of instrumental and specimen functions. The specimen's particle size and strain contributions to the peak-broadening were analyzed with the Warren-Averbach method. We found that for both the 2212 and $2223 \mathrm{Bi}-\mathrm{Cu}$ O superconductors, microstrain along the c-axis is larger than in the basal plane, because of larger stacking disorder. In the specimen containing both phases strain is relieved by the macroscopic intergrowth of two phases within the same grain, and effective domain sizes are smaller than in one-phase specimens.

\section{4,462}

PB94-198538 Not available NTIS

National Inst. of Standards and Technology (MSEL), Boulder, CO. Materials Reliability Div.

Voigt-Function Modeling in Fourier Analysis of Size- and Strain-Broadened X-Ray Diffraction Peaks.

Final rept.

D. Balzar, and H. Ledbetter. 1993, 7p.

Pub. in Jnl. of Applied Crystallography 26, p97-103 1993.

Keywords: *X-ray diffraction, Reprints, Fourier analysis, Strains, "Voight functions, Warren-Averbach method

With the assumption that both size- and strain-broadened profiles of the pure-specimen function are described with a Voigt function, it is shown that the analysis of Fourier coefficients leads to the WarrenAverbach method of separation of size and strain contributions. The analysis of size coefficients shows that the 'hook' effect occurs when the Cauchy content of the size-broadened profile is underestimated. The ratio of volume-weighted and surface-weighted domain sizes can range from about 1.31 , for the minimum allowed Cauchy content, to 2 , when the size-broadened profile is given solely by a Cauchy function. If the dis- tortion coefficient is approximated by a harmonic term, mean-square strains decrease linearly with increasing the average distance. The local strain is finite only in the case of purely Gaussian strain broadening, because strains are then independent of averaging dis tance.

\section{4,463}

PB94-198553 Not available NTIS

National Inst. of Standards and Technology (NML) Gaithersburg, MD. Electron and Optical Physics Div. Experimental Constraints on Some Mechanisms for High-Temperature Superconductivity.

Final rept.

T. W. Barbee, M. L. Cohen, and D. R. Penn. 1991 $7 p$.

Pub. in Physical Review B 44, n9 p4473-4479 1991. Keywords: "High temperature superconductors, Elec tron phonon interactions, Isotope effect, Spectral shift, Transition temperature, Specific heat, Cuprates, Reprints.

Three years after its discovery many mechanisms have been suggested to explain the phenomenon of high-temperature superconductivity (HTS) in oxides. We propose that experimental data, along with theoretical models, can place constraints on or exclude many of these mechanisms. The isotope shift a, the transition temperature $T(0)$, the ratio of the electronic specific heat jump to the normal state specific heat (Delta) C/(gamma)T(o) and the superconducting gap ratio 2 (Delta) $/ k(B) T(c)$ are considered. We find limits on the applicability of solely phonon-based and combined phonon-electronic mechanisms.

\section{4,464}

PB94-198744 Not available NTIS

National Inst. of Standards and Technology (MSEL) Gaithersburg, MD. Metallurgy Div.

Meissner, Shielding, and Flux Loss Behavior in Single-Crystal YBa2Cu3O6+X.

Final rept.

L. H. Bennett, L. J. Swartzendruber, and D. L

Kaiser. 1991, 2p

Pub. in Jnl. of Applied Physics 69, n8 p4889-4890 1991.

Keywords: "Meissner effect, "Ytterbium compounds, Twinning, Flux(Rate), Crystal properties, Magnetic properties, Temperature, Reprints.

Meissner effect measurements were performed on a nearly cubic single crystal of YBCO, which exhibited a predominant (110) twin habit, by cooling the crystal from above $T(c)$ to $10 \mathrm{k}$ in an applied field, $H$, of 10 Oe. A significant difference was observed for $\mathrm{H}$ applied parallel and perpendicular to the $c$ axis, but little difference could be seen for $\mathrm{H}$ perpendicular and parallel to the twin boundaries. After cooling from above T(c) in $10 \mathrm{Oe}$ to $10 \mathrm{~K}$ the field was reduced to zero. The magnetization changed sign, increasing by approx. 15 $G$, which is the value expected for complete flux expulsion by a crystal of this geometry. Magnetization measurements were then made as the temperature was raised back to $100 \mathrm{~K}$. Again, there is a significant difference for the results with $H$ parallel and perpendicular to the c axis, especially above $60 \mathrm{~K}$, with only a small (but possibly meaningful) difference for perpendicular and parallel to the twin boundaries.

\section{4,465}

PB94-198751 Not available NTIS

National Inst. of Standards and Technology (NML), Gaithersburg, MD. Surface Science Div. Magnetic Properties of Pd/Co Multilayers.

Final rept

W. R. Bennett, C. D. England, D. C. Person, and C. M. Falco. 1991, 7p

Pub. in Jnl. of Applied Physics 69, n8 p4384-4390 1991

Keywords: *Palladium, *Cobalt, Reprints, Magnetic an isotropy, Temperature dependence, Domain walls, Metal films, Thin films, Interfaces, Magnetization, Multilayers.

Measurements of the magnetic anisotropy of a series of $\mathrm{Pd} / \mathrm{Co}$ multilayers grown by magnetically enhanced dc triode sputter deposition revealed an interface contribution of $0.53 \mathrm{ergs} / \mathrm{sq} \mathrm{cm}$ and a net volume anisotropy of $-13.2 \times 10$ (sup 6 ) ergs/cc. A characteristic reduction of perpendicular anisotropy for ultrathin Co layers $(<4 A)$ is explained by using a pair interaction model to evaluate a thickness dependence of the interface anisotropy. Temperature dependence of saturation 
magnetization of a $\mathrm{Pd}(11 \mathrm{~A}) / \mathrm{Co}(7.8 \mathrm{~A})$ multilayer demonstrates complete mixing of $\mathrm{Pd}$ and $\mathrm{Co}$ by $380 \mathrm{C}$.

04,466

PB94-198769 Not available NTIS

National Inst. of Standards and Technology (NML), Gaithersburg, MD. Surface Science Div.

Concurrent Enhancement of Kerr Rotation and Antiferromagnetic Coupling in Epitaxial $\mathrm{Fe} / \mathrm{Cu} / \mathrm{Fe}$ Structures.

Final rept.

W. R. Bennett, W. Schwarzacher, and W. F

Egelhoff. 1990, 4p.

Pub. in Physical Review Letters 65, n25 p3169-3172 1990. Keywords: $\quad$ Kerr $\quad$ magnetooptical effect,
"Antiferromagnetism, "Copper, "Iron, Metal films, Epitaxy, Reprints, Multilayers.

The authors report the observation of major enhancements in the magneto-optical polar Kerr rotation in the $\mathrm{Fe} / \mathrm{Cu} / \mathrm{Fe}$ system. These enhancements are as much as a factor of two greater than those anticipated due to recently reported plasma resonance effects. A close connection between these enhancements and antiferromagnetic coupling between the two Fe films is established by the observation of concurrent oscilla tions in the magnitude of these two effects as a function of Cu thickness.

04,467

\section{PB94-198868 Not available NTIS}

National Inst. of Standards and Technology (CAML) Gaithersburg, MD. Applied and Computational Mathematics Div.

Non-Equilibrium Thermodynamic Theory of Viscoplastic Materials.

Final rept.

B. Bernstein, and J. T. Fong. 1993, 9p

Pub. in Jnl. of Applied Physics 74, n4 p2220-2228, 15 Aug 93

Keywords: "Viscoplasticity, Constitutive equations, Continuum mechanics, Creep properties, Mechanical properties, Fatique(Materials), Thermodynamic, Irreversible processes, Reprints.

A nonequilibrium thermodynamic theory for predicting the mechanical behavior of materials beyond the elastic range is formulated. The theory incorporates the idea of a 'concealed' parameter alpha, originally due to Bridgman (Rev. Mod. Phys. 22, 56 (1950)), where the constitutive equations are governed by (1) a thermodynamic potential such as a generalized Gibbs function, $G$, or Helmholtz free-energy function, $F$, each with an explicit dependence on alpha, and (2) a prescription for (alpha dot), the time rate of change of alpha, such that (alpha dot) is directly proportional to the negative of $G$ (alpha) or F(alpha), the partial derivative of $G$ or $F$ with respect to alpha, respectively. The theory is found to be consistent with (1) the second law of thermodynamics regarding entropy production 2) the concept of Lyapunov stability at equilibrium; (3) the rule of invariance with respect to a transformation of parameters; and (4) the powerful law of invariance with respect to the Legendre transformation. Significance of the new formulation is discussed by solving a class of one-dimensional creep and fatigue modeling problems and by comparing the new theory with several similar approaches in the literature.

04,468

PB94-199031 Not available NTIS

National Inst. of Standards and Technology (EEEL), Boulder, CO. Electromagnetic Technology Div.

Intrinsic Stress in DC Sputtered Niobium.

Final rept.

P. A. A. Booi, C. A. Livingston, and S. P. Benz.

1993, 3p

Pub. in Institute of Electrical and Electronics Engineers Transactions on Applied Superconductivity, n2 p30293031 Jun 93.

Keywords: "Niobium, Metal films, Direct current, Transition temperature, Stresses, Reprints, Target erosion, Sputtered films.

The intrinsic mechanical stress of dc magnetron-sputered $\mathrm{Nb}$ films is characterized as a function of sputtering parameters and target erosion. The zero-stress point shifts to lower cathode voltages as the target erodes. The zero-stress point was always characterized by the same cathode current-Ar pressure relationship.
04,469

PB94-199072 Not available NTIS

National Inst. of Standards and Technology (CAML) Gaithersburg, MD. Applied and Computational Mathematics Div.

Asymptotic Behavior of Modulated Taylor-Couette Flows with a Crystalline Inner Cylinder.

Final rept.

R. J. Braun, G. B. McFadden, B. T. Murray, M. E Selleck, S. R. Coriell, and M. E. Glicksman. 1993 $13 p$

Pub. in Phys. Fluids A 5, n8 p1891-1903 Aug 93. See also PB93-139061.

Keywords: "Crystal growth, "Solidification, Coaxia configuration. Prandtl number, Asymptotic series, Hydrodynamics, Cylinders, Instability, Reprints, Crystal-melt interface, Taylor-Couette flow, Floquet theory.

The linear stability of a modulated Taylor-Couette sys tem when the inner cylindrical boundary consists of a crystalline solid-fiquid interface is considered. Both ex perimentally and in numerical calculations, it is found that the two-phase system is significantly less stable than the analogous rigid-walled system for materials with moderately large Prandtl numbers. A numerical treatment based on Floquet theory is described, which gives results that are in good agreement with preliminary experimental findings. In addition, this instability is further examined by carrying out a formal asymptotic expansion of the solution in the limit of large Prandt number. It is surprising that the determination of the linear stability of the two-phase system is considerably simpler than that of the rigid-walled system, despite the complications introduced by the presence of the crystal-melt interface.

04,470

PB94-199148 Not available NTIS

National Inst. of Standards and Technology (MSEL) Gaithersburg, MD. Ceramics Div.

Microstructure and Ferroelectric Properties of Lead Zirconate-Titanate Films Produced by Laser Evaporation.

Final rept.

P. S. Brody, J. M. Benedetto, B. J. Rod, P. K

Schenck, C. K. Chiang, W. Wong-Ng, K. W. Bennett and L. P. Cook. 1991, 4p.

Pub in Proceedings of Institute of Electrical and Electronics Engineers Symposium on Applications of Ferroelectrics (7th), Urbana-Champaign, IL., June 68, 1990, p181-1841991.

Keywords: "Lead zirconate titanates, "PZT, Reprints, Ferroelectric materials, Dielectric properties, Laser ablation, Thin films, Microstructure, Neodymium lasers, Polarization, X-ray diffraction, Silicon substrates.

Lead titanate-zirconate films were produced by laserinduced vaporization from a PZT target with the use of a focused q-switched Nd:YAG laser. Deposition was onto room-temperature platinum-covered silicon substrates. Although the films were initially amorphous as indicated by $x$-rays, they crystallized when annealed. EDX results showed that the film and targets were similar in composition. SEM and optical dark field micrographs showed a structure which might be interpreted as a sintered assemblage of particulate. A pattern of platinum electrodes was sputter deposited onto each sample to form parallel plate capacitors for investigating dielectric and ferroelectric properties. For a 2.5-micrometer-thick film annealed at $700 \mathrm{C}$, the relative dielectric constant was approximately that of the bulk target. Hysteresis loops were obtained The remanent polarization was $5.1 \mathrm{microC} / \mathrm{sq} \mathrm{cm}$ measured at a $160 \mathrm{kV} / \mathrm{cm}$ peak sinusoidal field.

\section{4,471}

PB94-199239 Not available NTIS

National inst. of Standards and Technology (CAML) Gaithersburg, MD. Applied and Computational Mathe matics Div.

Laser Melting of Thin Silicon Films.

Final rept.

L. N. Brush, G. B. McFadden, and S. R. Coriell

1991, 21p.

Pub. in Jnl. of Crystal Growth 114, p446-466 1991

Keywords: *Silicon films, Reprints, Laser-radiation heating, Liquid-solid interfaces, Boundary integral method, Boundary conditions, Numerical solution, Melting, Stability.

Thin silicon films on a cooled substrate are often found to develop two-phase lamellar structures upon radi- ative heating. Jackson and Kurtze developed a two dimensional model for the process in which the heated film consists of alternating parallel bands of liquid and solid phases separated by planar solid-liquid interfaces. To understand the cellular or dendritic structures that sometimes are observed in these interfaces, they also performed a linearized morphological stability analysis and obtained the conditions for the growth or decay of infinitesimal perturbations to the interface. In this work the authors extend that analysis to finite amplitudes by developing a boundary integral representation of the thermal field, and obtain steady-state numerical solutions for nonplanar solid-liquid interfaces. Eigenvalue calculations to determine the linear stability of the nonplanar solutions are also performed. The effects of the lateral boundary conditions on the stability of the solutions are investigated as well.

\section{4,472}

PB94-199395 Not available NTIS

National Inst. of Standards and Technology (EEEL) Gaithersburg, MD. Electricity Div

Quantized Dissipation of the Quantum Hall Effect at High Currents.

Final rept.

M. E. Cage. 1993, 3p

Pub in Institute of Electrical and Electronics Engineers Transactions on Instrumentation and Measurement 42 n2 p176-178 Apr 93. See also PB93-150712

Keywords: "Hall effect, Two dimensional, Electric current, Electron gas, Quantization, Dissipation, Reprints, *Quantum Hall effect.

Quantized dissipative voltage states are observed when large currents are passed through a high-quality quantized Hall resistance device. These dissipative states are interpreted as occurring when electrons are excited to higher Landau levels and then return to the original Landau level. We show that the quantization is more complicated than previously thought. For example, the quantization can be a function of magnetic field. Therefore, the dissipative voltage quantization can, in general, be difficult to verify and determine.

04,473

PB94-199411 Not available NTIS

National Inst. of Standards and Technology (MSEL), Gaithersburg, MD

Singularities in Minimum Surface Energy Problems and Their Influence in Surface Motion.

Final rept

J. W. Cahn. 1992, 3p

Pub. in Proceedings of Computational Crystal Growers Workshop, Minneapolis, MN., February 22-28, 1992 p28-30.

Keywords: "Minimal surfaces, "Surface energy, Reprints. Three dimensional, Singularity, Froth, Soap bubbles.

Singularities can arise in area minimizing problems in three dimensions when three or more contiguous volumes separated by surfaces are considered. In a soap froth, the catalog of singularities has two members: a trijunction curve, where three bubbles meet with the three surface separating them meeting with 120 degree dihedral angles along a curve, and a quadrijunction point, where four bubbles and four trijunctions curves meet along tetrahedral angles and where there are six surfaces, each spanning a pair of trijunction curves (Taylor). For the soap froth, all surfaces have the same energy per unit area, and thus surface energy minimization is the same as area minimization. Furthermore, no energy is assigned to the point or line singularities. Recent research has focussed on the singularities that arise when the problem is altered to add certain complications, some realistic and some hypothetical.

\section{4,474}

PB94-199635 Not available NTIS

National Inst. of Standards and Technology (NML),

Gaithersburg, MD. Quantum Metrology Div.

Status of a Silicon Lattice Measurement and Dissemination Exercise.

Final rept

R. D. Deslattes, and E. G. Kessler. 1991, 6p.

Pub. in Institute of Electrical and Electronics Engineers Pranstrumentation and Measurement 40 n2 p92-97 1991.

Keywords: ${ }^{\star} C r y s t a l$ lattices, ${ }^{\star}$ Silicon, Single crystals, Optical interferometers, Comparators, Reprints, X-ray interferometers. 
Although no longer explicitly directed toward measu rement of the Avogadro constant, we continue significant effort on lattice period measurement in monocrystalline silicon. There are currently operating an up-graded $x$ ray/optical interferometer and a recently established lattice comparator. We report the status of measurements with these two instruments.

\section{4,475}

PB94-199767 Not available NTIS

National Inst. of Standards and Technology (MSEL), Gaithersburg, MD. Polymers Div.

Hysteresis Measurements of Remanent Polarization and Coercive Field in Polymers.

Final rept.

B. Dickens, E. Balizer, A. S. DeReggi, and S. C.

Roth. 1992, 7p.

Pub. in Jnl. of Applied Physics 72, n9 p4258-4264, 1 Nov 92 . Sponsored by Naval Surtace Wartare Center, Silver Spring, MD.

Keywords: "Polymers, "Ferroelectric materials, "Hysteresis, "Polarization(Charge separation) Reprints, Electric potential, Electric conductivity, "Remanent polarization, Coercive fields

An experimental method is described which allows es timation of remanent polarization and coercive fields without assuming functional forms for the capacitive and electrical resistance terms. The method can be used to measure polarization in specimens with voltage-dependent conductivity (often arising from the presence of ions in the specimens), voltage-dependent capacitance, and significant amounts of space charge. It consists of: (1) performing bipolar current/voltage hysteresis loops to allow a steady state of remanent polarization and space charge to build up in the specimen, and (2) following a bipolar loop with two or more unipolar loops in the first unipolar loop. Both sinusoida and linear time-dependent applied voltages may be used. Automatic data processing of hysteresis loops is described for cases in which specimen behavior may be considered to be ideal.

\section{4,476}

PB94-199924 Not available NTIS

National Inst. of Standards and Technology (PL), Gaithersburg, MD. Quantum Metrology Div.

Diffraction of X-rays at the Far Tails of the Bragg Peaks.

Final rept.

A. Caticha. 1993, 8p

Contract AFOSR 88-0018

Pub in Physical Review B 47, n1 p76-83, 1 Jan 93. Sponsored by Air Force Office of Scientific Research, Bolling AFB, DC. and Defense Advanced Research Projects Agency, Arlington, VA.

Keywords: *X-ray diffraction, Bragg reflection, Bragg angle, Reprints, Truncation rod scattering.

It is known that the kinematical and dynamical theories of diffraction by perfect crystals predict the same values for the intensities scattered into the vicinity of the Bragg peaks whenever those intensities are low. Here we address the question of whether this is also true throughout the region between two Bragg peaks. We show that the two theories give equivalent results for the weak intensities in the far tails of the Bragg peaks provided better approximations for both the shape of the dispersion surface and the boundary conditions are used: One needs to take into account the asymptotic sphericity of the dispersion surface and the difference between electric and displacement fields. This results in a nontrivial transmission coefficient for the diffracted electric field as it leaves the crystal. By explicitly showing the equivalence of kinematical and dynamical re sults, this work provides additional theoretical support for the kinematical approach usually adopted to describe the so-called truncation-rod-scattering.

\subsection{7}

PB94-200045 Not available NTIS

National Inst. of Standards and Technology (NML), Gaithersburg, MD. Radiation Physics Div.

\section{Electronic Correlations and Satellites in} Superconducting Oxides.

Final rept.

K. J. Chang. M. L. Cohen, and D. R. Penn. 1988, 6p. Pub. in Physical Review B38, n13 p8691-8696 1988.

Keywords: "YBCO superconductors, High temperature superconductors, Electron correlation, Band theory, $\mathrm{S}$ matrix, Satellites, Reprints, "Lanthanum strontium cuprates, "Yttrium barium cuprates.
The satellite observed at a binding energy of 12 to 13 $\mathrm{eV}$ below the Fermi level in the high $T(\mathrm{c})$ oxide superconductors, $\mathrm{La}(2-\mathrm{x}) \mathrm{Sr}(\mathrm{x}) \mathrm{CuO} 4$ and YBa2Cu3O(7-delta), is viewed as originating from a state with two holes bound at the same Cu site. As in the case of Ni metal, the satellite is caused by an intra $\mathrm{d}$-shell shake-up process into a $\mathrm{Cu} 3 \mathrm{~d}(8)$ final state and its intensity is enhanced at resonance because of a super Koster-Kronig transition. Based on the t-matrix approach for the hole self-energy, the authors study the effect of electronic correlations on the one-electron band structure. They examine the position of the satellite and find a large Coulomb interaction energy of $5 \mathrm{eV}$ at the $\mathrm{Cu}$ site when the experimental satellite position is duplicated by the theory. Since this energy is comparable with the $3 d$ bandwidth, the twohole bound state is a high energy excitation. This indicates that in the ground state a creation of two holes at the $\mathrm{Cu}$ site is unlikely and thus holes are formed at the $\mathrm{O}$ sites when $\mathrm{Sr}$ is substituted for La in La2CuO4 and when the oxidation is increased in YBa2CuO(7delta).

\section{4,478}

\section{PB94-200128 Not available NTIS}

National Inst. of Standards and Technology (EEEL), Boulder, CO. Electromagnetic Technology Div.

Surface Barrier and Lower Critical Field in YBa2Cu307-delta Superconductors.

Final rept.

D. X. Chen, R. B. Goldfarb, R. W. Cross, and A

Sanchez. 1993, 5p.

Pub. in Physical Review B 48, n9 p6426-6430, 1 Sep 93.

Keywords: "YBCO superconductors, High temperature superconductors, Ginzburg-Landau theory, Temperature dependence, Surface barrier, Critical field, Magnetization, Reprints, "Yttrium barium cuprates.

The fields for first vortex entry and last vortex exit, $H(1)$ and $H(2)$, and the lower critical field $H(C 1)$ for a grainaligned, sintered YBa2Cu3O(7-delta) superconductor have been determined from saturated magnetichysteresis loops using an extended critical-state model. For fields oriented along the grain $c$ axis, $H(1)$ increases with decreasing temperature, showing an upturn below $50 \mathrm{~K}$, whereas $H(2)$ remains small and positive, in general agreement with the theory of Bean= Livingston surface barriers. $H(C 1)$ has a Bardeen-Cooper-Schrieffer temperature dependence above $50 \mathrm{~K}$, but it rises at low temperatures. For fields oriented in the ab plane, $H(C 1)$ has a similar temperature depend. ence, but surface barriers are not evident in the magnetization.

\section{4,479}

\section{PB94-200144 Not available NTIS}

National Inst. of Standards and Technology (CSTL), Gaithersburg, MD. Surface and Microanalysis Science Div.

$X-R a y$ Photoelectron and Auger Electron Spectroscopy Study of Ultraviolet/Ozone Oxidized P2S5/ (NH4)2S Treated GaAs(100) Surfaces.

\section{Final rept.}

M. Chester, T. Jach, and J. A. Dagata. 1993, 7p. Pub. in Vacuum Science and Technology $A 11, n 3$ p474-480 May/Jun 93.

Keywords: "Gallium arsenides, "Surface analysis, "Oxidation, X-ray photoelectron spectroscopy, Auger electron spectroscopy, Temperature dependence, Temperature range 0273-0400 $\mathrm{K}$, Temperature range 0400-1000 K. Thermal stability, Passivation, Annealing, Reprints.

In the paper the authors discuss the results of a study undertaken to investigate the composition and thermal stability of ultraviolet/ozone oxidized, P2S5/(NH4)2S treated GaAs(100) surfaces. In particular, the authors have used $x$-ray photoelectron spectroscopy and Auger electron spectroscopy to probe the oxide and interface at room temperature and as a function of annealing temperature. The room temperature data indicate that $S$ is buried between the oxide overlayer and the GaAs substrate. This oxide contains a vaniety of $\mathrm{As}$ and $\mathrm{Ga}$ bonding configurations which, after moderate annealing, are transformed into thermally more stable phases, such as As2O3 and Ga2O3. Complete desorption of the oxide occurs after annealing at 600 $\operatorname{deg} \mathrm{C}$. Annealing the as-oxidized surface to high temperatures also has a profound effect on the $S$.

\section{4,480}

PB94-200151 Not available NTIS
National Inst. of Standards and Technology (CSTL), Gaithersburg, MD

Grazing-Incidence X-Ray Photoemission Spectros copy Investigation of Oxidized GaAs(100): A Novel Approach to Nondestructive Depth Profifing

Final rept.

M. J. Chester, T. Jach, and S. Thurgate. 1993, 5p Pub. in Jnl. of Vacuum Science and Technology B 11 n4 p1609-1613 Jul/Aug 93.

Keywords: "Gallium arsenides, "Surface analysis, $X$ ray photoelectron spectroscopy, Grazing incidence, Oxidation, Reprints, Depth profiles.

When a beam of $x$ rays (about $1 \mathrm{keV}$ ) impinges on a flat surface at grazing angles (approx $=$ or $<3 \mathrm{deg}$ ) the $x$ rays undergo total external reflection. Under these conditions, the penetration depth of the $x$ rays can become comparable to photoelectron escape depths and the photoelectron yields from the surface are enhanced. As the incidence angle of the $x$ rays is increased, and $x$-ray penetration depth increases and the photoelectron yields contain a larger contribution from deeper layers within the sample. By exploiting this depth-dependence of photoelectron yields as a function of incident $x$-ray beam angle, it is possible to obtain information about the depth distribution of the photoelectron emitting atoms. The authors have used this novel application of grazing-incidence $x$-ray photoelectron spectroscopy (GIXPS) to study the ultraviolet oxidized $\mathrm{GaAs}(100)$ surface. Much insight into the composition and depth-dependence of these oxide phases can be obtained by directly comparing the spectra collected at different $x$-ray incidence angles.

04,481

PB94-200276 Not available NTIS

National Inst. of Standards and Technology (MSEL) Gaithersburg, MD. Reactor Radiation Div.

Incorporation of Gofd into YBa2Cu307: Structure and TC Enhancement.

Final rept.

M. Z. Cieplak, G. Xiao, C. L. Chien, W. Bryden, J. K. Stalick. J. J. Rhyne, A. Bakhshai, and D. Artymowicz. 1990, 9p.

Grant NSF-DMR88-22559

Pub. in Physical Review B 42 n10 p6200-6208, 1 oct 90. Sponsored by National Science Foundation, Arling. ton, VA.

Keywords: "YBCO superconductors, "Doped matenals, "Gold, Orthorhombic lattices, X-ray diffraction, Neutron diffraction, Transition temperature, Crysta structure, High temperature superconductors, Reprints, "Yttrium banium cuprates.

The structural and superconducting properties of golddoped YBa2Cu3O7 compounds have been studied with $x$-ray and neutron diffraction, optical microscopy, weight-loss analysis, magnetization, and resistivity measurements. The solubility of $\mathrm{Au}$ in $\mathrm{YBa} 2 \mathrm{Cu} 3 \mathrm{O} 7$ is closed to 10 at. \%, below which all samples are thermally stable up to the processing temperature (950 C). Metallic Au grains precipitate out when the Au conten exceeds 10 at. \%. Analysis of the $x$-ray and neutronscattering intensities shows that Au substitutes for the Cu(1) chain site exclusively and has a probable valence state of $A u(3+)$. The main effect of Au doping is a substantial uniaxial lattice expansion, while the orthorhombic structure is preserved. The authors present a detailed set of structural data including the $\mathrm{Cu}-\mathrm{O}$ bond lengths and interplanar distances in a $\mathrm{Au}-$ doped sample. Unlike all other known dopants on $\mathrm{Cu}$ sites, the Au dopant is not detrimental to the superconducting properties. On the contrary, $T(c)$ is slightly enhanced over that of YBa2Cu307. Such an enhancement, observed for the first time for a Cu site dopant, is intrinsic and reproducible. The authors discuss the possible causes of the $T(c)$ increase and the features of $\mathrm{Au}$ dopant useful in the investigation of high-T(c) superconductivity.

04,482

PB94-200284 Not available NTIS

National Inst. of Standards and Technology (MSEL) Gaithersburg, MD. Reactor Radiation Div.

Unexpected Effects of Gold on the Structure Superconductivity, and Normal State of YBa2Cu307.

M. Z. Cieplak, G. Xiao, C. L. Chien, J. K. Stalick, and M. Z. Cieplak, G. Xiao,
J. J. Rhyne. 1990, 3p.

J. J. Rhyne. 1990, 3p.

Pub. in Applied Physics Letters 57, n9 p934-936 Aug 90. Sponsored by National Science Foundation, Arlington, VA. 
Keywords: "YBCO superconductors, "Doped materials, "Gold, High temperature superconductors, X-ray diffraction, Neutron diffraction, Transition temperature, Crystal structure, Reprints, "Yttrium barium cuprates, Rietveld method.

Using high-resolution $x$-ray and neutron diffraction measurements, the authors have shown that gold can be doped into $\mathrm{YBa} 2 \mathrm{Cu} 3 \mathrm{O} 7(1: 2: 3)$ up to $10 \%$ per formula unit, exclusively at the Cu(1) sites. Au doping causes a larger uniaxial expansion along the $c$ axis accompanying an increase in the $\mathrm{Cu}(1)-\mathrm{O}(1)$ bond length and a decrease in the $\mathrm{Cu}(2)-\mathrm{O}(1)$ bond length. The superconducting transition temperature $(T(c))$ is enhanced upon Au doping and the normal-state transport properties are altered even for small levels of $A u$ doping. The widely held belief in the inert nature of $A u$ with regard to high T(c) superconductors is therefore invalid. These findings also raise questions concerning results using single crystals grown from gold crucibles.

\section{4,483}

PB94-200359 Not available NTIS

National Inst. of Standards and Technology (EEEL), Boulder, CO. Electromagnetic Technology Div.

High-Frequency Linear Response of Anisotropic Type-II Superconductors in the Mixed State.

Final rept.

M. W. Coffey. 1993, 9p.

Pub. in Physical Review B 47, n18 p12 284-12 287, 1 May 93.

Keywords: "Type 2 superconductors, "Superconductors, Penetration depth, High frequency Effective mass, Response functions, Anisotropy, Com plexity, Linearity, Tensors, Vortices, Reprints, Surface impedance.

Effective-mass anisotropy is incorporated into a selfconsistent phenomenological theory of the high frequency electrodynamic response of type-II superconductors in the mixed state. The theory accounts for two-fluid effects, including a possible anisotropic normal-fluid contribution, in addition to nonlocal vortex interaction. The approach, applicable to the modeling of a wide range of complex response functions, is illustrated in the calculation of the complex penetration depths and surface impedance for uniaxially anisotropic type-II superconductors.

\section{4,484}

PB94-200367 Not available NTIS

National Inst. of Standards and Technology (EEEL),

Boulder, CO. Electromagnetic Technology Div.

Nonlinear Response of Type-ll Superconductors in the Mixed State in Slab Geometry.

Final rept.

M. W. Coffey. 1993, 4p.

Pub. in Physical Review B 47, n22 p15 298-15 301 1 Jun 93. Sponsored by Department of Energy, Washington, DC

Keywords: $\quad$ "Type 2 superconductors, *Superconductors, Penetration depth, Magnetic permeability, Boundary conditions, Electrical conductivity, Electrodynamics, Complexity, Nonlinearity, Vortices Reprints.

The nonlinear response of a type-II superconductor of finite thickness arising from vortex motion is investigated. The results of the phenomenological theory extend the complex of magnetic permeability and conductivity to a specific regime of nonlinear response. Explicit expressions for the complex penetration depths, amplitudes, fields, and densities for the second-harmonic response with various boundary conditions are presented.

\section{4,485}

PB94-200458 Not available NTIS

National Inst. of Standards and Technology (MSEL), Gaithersburg, MD

Neutron Scattering Structural Study of AlCuFe Quasicrystals Using Double Isotopic Substitution. Final rept.

M. Cornier-Quiquandon, R. Bellissent, Y. Calvayrac, B. Mozer, J. W. Cahn, and D. Gratias. 1993, 5p. Pub. in Jnl. of Non-Crystalline Solids 153 and 154 p10-14 1993.

Keywords: "Crystal structure, Aluminum alloys, Copper alloys, Iron alloys, Correlation functions, Isotope exchange, Neutron diffraction, Reprints, *Quasicrystals lcosahedral phase.

Neutron diffraction data with double isotopic substitution using two isotopes each of $\mathrm{Fe}$ and $\mathrm{Cu}$ has been obtained on six samples of quasicrystalline AlCuFe with a 6D face-centered icosahedral symmetry. Calculations of heteroatomic and homoatomic pair correlation functions for this structure have been made. The authors find that there is a localized position of copper on a $6 \mathrm{D}$ body center site which gives a clear but still qualitative picture of the structure with iron, copper, and aluminum chemically ordered and occupying successive concentric 'shells' in the large atom surfaces centered at the two major atomic positions, $n(1)$ $=$ left bracket $0,0,0,0,0,0$ right bracket and $n(2)=$ left bracket $1,0,0,0,0,0$ right bracket, (indexed with respect to a primitive unit cell) in this $6 \mathrm{D} \mathrm{NaCl}$ type structure. There is an indication that some of the aluminum is mixed with the iron (or is encapsulated in a small volume inside an iron surface)

\section{4,486}

PB94-200557 Not available NTIS

National Inst. of Standards and Technology (NML), Gaithersburg, MD. Electron and Optical Physlcs Div. Anharmonic Phonons and the Isotope Effect in Superconductivity.

Final rept.

V. H. Crespi, M. L. Cohen, and D. R. Penn. 1991

$4 \mathrm{p}$

Pub. in Physical Review B 43, n16 p2921-2924 1991.

Keywords: "High temperature superconductors, "Phonons, High-Tc superconductors, Isotope effects, Electron phonon interactions, Schrodinger equation, Cuprates, Reprints, Anharmonicity.

Anharmonic ionic potentials are examined to study the unusual isotope effect exponents for the high-T(c) oxides. Numerical solutions of Schrodinger's equation for a double well potential produce electron-phonon coupling constants lambda in the range 1.5 to 4 for a material with a vanishing isotope effect parameter alpha. However, low phonon frequencies limit $T(c)$. Within our approximations, double well potential cannot produce alpha, $>1 / 2$. However, a negative quartic perturbation to a harmonic well can increase alpha above $1 / 2$. In the extreme strong coupling limit, alapha is $1 / 2$ regardless of anharmonicity.

\section{4,487}

PB94-211059 Not available NTIS

National Inst. of Standards and Technology (NML), Gaithersburg, MD. Inorganic Analytical Research Div. Measurement of Boron at Silicon Wafer Surfaces by Neutron Depth Profiling.

Final rept.

R. G. Downing, J. P. Levine, T. Z. Hossain, J. B.

Russell, and G. P. Zenner. 1990, 3p.

Pub. in Jnl. of Applied Physics 67, n8 p3652-3654, 15 Apr 90.

Keywords: "Silicon, "Boron, Secondary ion mass spectroscopy, Concentration(Composition), Boron 10 target, Neutron reactions, Measurement, Aerosols, Surfaces, Wafers, Reprints, Neutron depth profiling.

The thermal neutron reaction (10) $B(n$, alpha) $(7) L i$ is used to measure the boron concentration on the surface of silicon wafers. The technique, referred to as neutron depth profiling (NDP), requires no special sample preparation. Boron is determined on the as-received wafers at a level of 10(sup 12) to 10(sup 13) atoms $/ \mathrm{sq} \mathrm{cm}$. A boron level of about $2 \times 10$ (sup 12) atoms $/ \mathrm{sq} \mathrm{cm}$ is found at the wafer surface after oxidation, epitaxial, or polycrystalline silicon deposition. Additional measurements are given from SIMS measurements of multilayer structures on silicon also showing the presence of boron. Ambient air appears to be a significant source of the boron.

\section{4,488}

\section{PB94-211174 Not available NTIS}

National Inst. of Standards and Technology (NML), Gaithersburg, MD. Surface Science Div.

Brillouin Light Scattering Intensities for Thin Magnetic Films with Large Perpendicular Anisotropies. Final rept.

J. R. Dutcher, W. F. Egelhoff, J. F. Cochran, and I. Jacob. $1989,3 p$

Pub. in Physical Review B 39, n14 p430-432 1989

Keywords: "Magnetic films, "Light scattering, "Brillouin effect, Metal films, Thin films, Magnetization, Anisotropy, Copper, Iron, Reprints.

The intensity of light scattered from thermal magnetic waves in thin magnetic films with large perpendicular uniaxial anisotropies was calculated. The anisotropies were large enough to pull the magnetization out of the sample plane. The intensity of light scattered from the surface magnetic mode in a 3.0 monolayer thick fcc $\mathrm{Fe}$ film grown on $\mathrm{Cu}(001)$ was measured as a function of the applied in-plane d.c. magnetic field. The measured intensities are in excellent agreement with those calculated using the magnetic parameters determined rom the measured values of the surface mode frequency. Both the frequency and intensity data indicate that the magnetization of the Fe film was perpendicular to the sample plane in zero applied field.

\section{4,489}

PB94-211216 Not available NTIS

National Inst. of Standards and Technology (EEEL), Gaithersburg, MD. Electricity Div.

Half-Integral Constant Voltage Steps in High-TC Grain Boundary Junctions.

Final rept.

E. A. Early, A. F. Clark, and K. Char. 1993, 3p. Pub. in Applied Physics Letters 62, n25 p3357-3359, 21 Jun 93

Keywords: "Superconducting junctions, High temperature superconductors, YBCO superconductors, Grain boundaries, Superhigh frequency, Josephson effect, Magnetic fields, Reprints, Ytrium barium cuprates.

A novel effect from microwave radiation near $9.3 \mathrm{GHz}$ applied to high-T(c) YBa2Cu3O(7-delta) single grain boundary junctions was observed. In addition to the usual Shapiro steps resulting from the ac Josephson effect, constant voltage steps with voltages halfway between the voltages of the Shapiro steps were present. The widths of these 'half-integral' steps were measured as a function of microwave power, and the influence of a magnetic field was investigated. From previous results on high-T(c) grain boundary junctions and a comparison of the results presented here with singleand multiple-junction effects in low-T(c) materials, we conclude that the half-integral steps are likely to be a result of grain boundaries being composed of multiple junctions in parallel.

\section{4,490}

PB94-211281 Not available NTIS

National Inst. of Standards and Technology (EEEL), Boulder, CO. Electromagnetic Technology Div.

Critical Magnetic-Field Angle for High-Field Current Transport in $\mathrm{YBa} 2 \mathrm{Cu} 307$ at $76 \mathrm{~K}$.

Final rept.

J. W. Ekin. 1992, 4p

Pub. in Cryogenics 32, n11 p1089-1092 1992.

Keywords: "YBCO superconductors, "Critical current High temperature superconductors, Temperature range 0065-0273 K, Flux pinning, Reprints, “ Yttrium barium cuprates, High magnetic fields.

High magnetic field $(0.5 \mathrm{~T}$ to $9 \mathrm{~T})$ measurements of the in-plane transport critical current density $\mathrm{J}(\mathrm{c})$ of $\mathrm{YBa} 2 \mathrm{Cu} 3 \mathrm{O} 7$ at liquid nitrogen temperature are reported as a function of the orientation of magnetic field $B$ with respect to the a,b-plane. In contrast to earlie results at lower fields $(<3 \mathrm{~T})$, the measurements re ported here at high fields reveal a $J(c)$ versus angle curve with a head-and-shoulders shape with two angular regions having distinctly different behavior. For magnetic field oriented close to the a,b-plane, a sharp, narrow intrinsic-pinning peak in $\mathrm{J}(\mathrm{c})$ about the $\mathrm{B}$ perpendicular c-axis is observed; on either side of the peak, $J(c)$ is still relatively high and nearly independent of magnetic field magnitude and angle. However beyond a critical angle theta(c), the $\mathrm{J}(\mathrm{c})$ versus theta dependence crosses over to a markedly different behavior, characterized by a decrease in the sharpness of the voltage-current characteristic and a rapid decrease in $\mathrm{J}(\mathrm{c})$. To take advantage of the high, nearly field-independent $J(c)$ on either side of the intrinsic pinning peak, magnet design will need to allow an adequate angular margin to avoid the pinning transition at theta(c)

\section{4,491}

Not available NTIS

National Inst. of Standards and Technology (NEL), Boulder, CO. Electromagnetic Technology Div.

Superconducting Materials: Specification.

Final rept

J. W. Ekin. 1992, 6p.

Pub. in Concise Encyclopedia of Magnetic and Superconducting Materials, p578-583 1992.

Keywords: "Superconductors, Mechanical properties Critical current, Specifications, Stabilizers, Standards, Reprints.

The specification of superconductors is needed both in the purchase of practical superconductors, as well 
as in the characterization of superconductor materials for research. In the case of research, the emphasis is usually in specifying parameters that are intrinsic to the material such as the critical temperature $T(c)$ and upper critical field $H(c 2)$. In the case of procuring superconductors for practical applications, the primary concern is, rather, with the extrinsic parameters such as the physical shape of the conductor, critical current, matrix resistivity, and mechanical properties. The emphasis of this article is on practical superconductors and, consequently, specification of the extrinsic parameters. The extrinsic parameters are described under three general headings: physical, electrical, and mechanical specification.

\subsection{2}

PB94-211307 Not available NTIS

National Inst. of Standards and Technology (NEL), Boulder, CO. Electromagnetic Technology Div.

Transport Critical Current of Aligned Polycrystalline Yttrium Barium Copper Oxide (YBa2Cu3O7-delta).

Final rept.

J. W. Ekin. 1990, 4p.

Pub. in Prog. High Temp. Supercond. 25, p23-26 1990. Keywords: "YBCO superconductors, "Critical current, High temperature superconductors, Magnetic fields, Direct current, Polycrystalline, Reprints, Yttrium barium cuprates, Grain boundaries, Weak links.

A study of grain alignment and its effect on the $d c$ transport critical current in fine-grained bulk Y1Ba2Cu3O(7-delta) is reported in magnetic fields from 10(sup -4) $\mathrm{T}$ to $26 \mathrm{~T}$. This paper presents a summary of the main results. The data are interpreted as evidence for two parallel components for intergranular current conduction, one consisting of weak-linked material, the other behaving like intrinsic intragranular material that is not weak-linked. A comparison with unaligned $\mathrm{YBa} 2 \mathrm{Cu} 3 \mathrm{O} 7$ indicates that the volume fraction of such nonweak-linked material is significantly enhanced by grain alignment, but still only 0.01 to $0.1 \%$ of the grain boundary area.

04,493

PB94-211315 Not available NTIS

National Inst. of Standards and Technology (EEEL),

Boulder, CO. Electromagnetic Technology Div.

Effect of Axial Strain on the Critical Current of AgSheathed Bi-Based Superconductors in Magnetic Fields Up to $25 \mathrm{~T}$.

Final rept.

J. W. Ekin, D. K. Finnemore, Q. Li, J. Tenbrink, and W. Carter. 1992, 3p.

Pub. in Applied Physics Letters 61, n7 p858-860, 17 Aug 92.

Keywords: "BSCCO superconductors, " Critical current, *Axial strain, High temperature superconductors, Superconducting wires, Magnetic fields, Silver, Reprints, "Bismuth strontium calcium cuprates.

The irreversible strain limit epsilon(irrev) for the onse of permanent axial strain damage to $\mathrm{Ag}$-sheathed $\mathrm{Bi} 2 \mathrm{~S} 2 \mathrm{Ca} 1 \mathrm{Cu} 2 \mathrm{O}(8+\mathrm{x})$ and $\mathrm{Bi} 2 \mathrm{Sr} 2 \mathrm{Ca} 2 \mathrm{Cu} 3 \mathrm{O}(10+\mathrm{x})$ superconductors has been measured to be in the range of $0.2 \%-0.35 \%$. This strain damage onset is about an order of magnitude higher than for bulk sintered $\mathrm{Y}$-, Bi-, or TI-based superconductors and is approaching practical values for magnet design. The measurements show that the value of epsilon(irrev) is not dependent on magnetic field, nor does the critical current depend on strain below epsilon(irrev) at least up to $25 \mathrm{~T}$ at $4.2 \mathrm{~K}$. Both of these factors indicate that the observed strain effect in Ag-sheathed Bi-based superconductors is not intrinsic to the superconductor material. Rather, the effect is extrinsic and arises from superconductor fracture. Thus, the damage onset is amenable to further enhancement. Indeed, the data suggest that subdividing the superconductor into fine filaments or adding $\mathrm{Ag}$ to the superconductor powder prior to processing significantly enhances the damage threshold epsilon(irrev) to above $0.6 \%$.

\section{4,494}

PB94-211323 Not available NTIS

National Inst. of Standards and Technology (EEEL) Boulder, CO. Electromagnetic Technology Div. In situ Noble Metal YBa2Cu307 Thin-Film Contacts.

Final rept.

J. W. Ekin, S. E. Russek, C. C. Clickner, and B.

Jeanneret. 1993, 3p

Pub. in Applied Physics Letters 62, n4 p369-371, 25 Jan 93.
Keywords: "YBCO superconductors, *Electric contacts, High temperature superconductors, Thin films, Metal films, Silver, Gold, Reprints, "Yttrium barium cuprates, Ohmic contacts.

Thin-film contacts to $\mathrm{YBa2Cu} 3 \mathrm{O} 7$ have been fabricated by an in situ noble-metal process and patterned down to $2 \times 2$ sq micrometers; at this small size, the contacts carry transport current over 10(sup 6) A/sq cm while maintaining a specific contact resistivity rho(sub c) in the 10(sup -8) to 10(sup -9) ohm sq cm range. No oxygen annealing was used in the processing, thus avoiding the problem of silver or gold agglomeration, as well as preserving a sharp interface for Josephsondevice applications. Rho(sub c) was measured to increase only about $25 \%$ as temperature was increased from 4 to $90 \mathrm{~K}$. The measurements were carried out on a series of film morphologies using both superconductor-normal metal and superconductornormal metal-superconductor test structures; a carefully designed test pattern was used to correct for spreading conduction in the noble-metal contact layer. The contacts were ohmic with voltage-current characteristics that were linear over more than four orders of magnitude.

\section{4,495}

\section{PB94-211356 Not available NTIS}

National Inst. of Standards and Technology (IMSE), Gaithersburg, MD. Reactor Radiation Div.

Magnetoelasticity in Rare-Earth Multilayers and Films.

Final rept.

R. W. Erwin, J. J. Rhyne, J. Borchers, C. P. Flynn, M. B. Salamon, and R. Du. 1990, 6p.

Pub. in Proceedings of Neutron Scattering for Materials Science Symposium, Boston, MA., November 27 30, 1989, p133-1381990.

Keywords: "Magnetostriction, *Dysprosium, "Yttrium, *Erbium, Phase transformations, Neutron scattering, Critical field, Metal films, Epitaxy, Reprints, Multilayers

The magnetic structure has been measured with neutron scattering and SQUID magnetometry for a number of epitaxial multilayers and films of the magnetic rareearths dysprosium and erbium grown with non-magnetic yttrium. In almost all cases the first order ferromagnetic phase transitions are suppressed, although the Neel temperatures are within $5 \%$ of the bulk values. The observed critical fields are explained by including epitaxial constraints with the bulk theory of magnetoelasticity.

\section{4,496}

PB94-211869 Not available NTIS

National Inst. of Standards and Technology (MSEL), Gaithersburg, MD. Reactor Radiation Div.

Fast-Ion Conduction and Disorder in Cation and Anion Arrays in Y2(ZryTi(1-y))2O7 Pyrochlores Induced by Zr Substitution: A Neutron Rietveld Analysis.

Final rept.

C. Heremans, B. J. Wuensch, J. K. Stalick, and E.

Prince. 1993, 6p.

Pub. in Materials Research Society Symposium Proceedings, v293 p349-354 1993.

Keywords: *Order-disorder transformations, *Ionic conductivity, "Crystal structure, Zirconium additions, Reprints, Yttrium zirconate titanates, Rietveld method, Pyrochlore structure.

Structural refinements have been carried out on the Y2(Zr(y)Ti(1-y))2O7 system for increasing amounts of $\mathrm{Zr}$ substitution. The structure changes from an ordered pyrochlore structure (superstructure of the fluorite structure) to an anion-deficient fluorite structure. Separate processes govern the disordering of the cation and anion arrays. In particular, the anions begin to disorder at lower values of $y$ than the cations. For intermediate compositions, a minor amount of fluorite phase coexists with the primary partially-disordered pyrochlore phase.

\section{4,497}

PB94-211919 Not available NTIS

National Inst. of Standards and Technology (MSEL), Boulder, CO. Materials Reliability Div.

Elastic Constants of Isotropic Cylinders Using Resonant Ultrasound.

Final rept.

P. R. Heyliger, A. Jilani, H. Ledbetter, R. G. Leisure and C. L. Wang. 1993, $6 \mathrm{p}$

Pub. in Jnl. of Acoust. Soc. Am. 94, n3 p1482-1487 1993.
Keywords: "Resonant frequency, "Elastic properties, ${ }^{*}$ Cylindrical bodies, *Isotropic media, Equations of motion, Ultrasonic frequencies, Free vibration, Solid mechanics, Reprints.

A method of ultrasonic resonance is used to determine the elastic constants of isotropic cylinders. As applied in this study, this method implies simultaneous application of approximate techniques to solve for the equations of motion of the cylinder and an experimental measurement of the natural frequencies of free vibration. Optimal estimates for the elastic constants are obtained by minimizing the differences between these values. Two separate examples are considered in this study, with promising results.

\section{4,498}

PB94-211992 Not available NTIS

National Inst. of Standards and Technology (MSEL), Gaithersburg, MD.

Microstructural Evolution in Two-Dimensional Two-Phase Polycrystals.

Final rept.

E. A. Holm, D. J. Srolovitz, and J. W. Cahn. 1993,

$18 p$

Pub. in Acta Metallurgica Materials 41, n4 p1119-1136 1993.

Keywords: "Microstructure, "Crystal growth, *Polycrystals, Crystal structure, Phase transformations, Crystallization, Thermodynamic properties, Crystal lattices, Crystal field theory, Reprints.

In two-dimensional polycrystals composed of alphaphase and beta-phase grains the stability of threegrain junctions and four-grain junctions depends on the interfacial energies. A computer simulation which generates thermodynamically consistent microstructures for arbitrary interfacial energies has been used to investigate microstructural evolution in such polycrystals when phase volume is not conserved. Since grain shapes, phase volume, and phase arrangements are dictated by interfacial energies, clustered-, altematingisolated-, and single-phase microstructures occur in different interfacial energy regimes. Despite great differences in microstructure, polycrystals which contain only three-grain junctions evolve with normal grain growth kinetics. In contrast, structures containing flexible four-grain junctions eventually stop evolving. We conclude that two dimensional polycrystals continually evolve when grain junction angles are thermodynamically fixed, while grain growth ultimately ceases when grain junction angles may vary. Predictions concerning three-dimensional and phase-volume conserved systems are made.

04,499

PB94-212073 Not available NTIS

National Inst. of Standards and Technology (MSEL), Gaithersburg, MD. Reactor Radiation Div.

Neutron Powder Diffraction Study of the Crystal Structure of YSr2AICu2O7.

Final rept.

Q. Huang, S. A. Sunshine, R. J. Cava, and A

Santoro, 1993, 8p.

Pub. in Jnl. of Solid State Chemistry 102, p534-541 1993.

Keywords: "Crystal structure, Orthorhombic lattices, Neutron diffraction, Reprints, "Yttrium strontium aluminum cuprates.

The structure of $\mathrm{YSr} 2 \mathrm{AlCu} 2 \mathrm{O} 7$ has been analyzed by neutron powder diffraction techniques. This compound crystallizes with space group $P 4 / \mathrm{mmm}$ symmetry and with lattice parameters $a=3.8646(1), c=11.1139$ (3)A The general structural features of $Y \mathrm{Sr} 2 \mathrm{AlCu} 207$ are similar to those found for the 123 superconductor $\mathrm{YBa} 2 \mathrm{Cu} 3 \mathrm{O}(6+\mathrm{X})$, and in particular the sequence of layers is the same in the two materials, with SrO substitut ing for $\mathrm{BaO}$. The $\mathrm{Al}$ ions substitute for the copper ions of the chain sites of the 123 parent structure and have tetrahedral coordination. The tetrahedra form chains propagating along the $\mathrm{a}$ and $\mathrm{b}$ axes on the basal plane of the unit cell. Thus the structure is made of short range domains within which the local symmetry is orthorhombic. All of the oxygen atoms in this compound have a disordered configuration around the specialized positions of the 123 structure. This arrangement results in tilting of the CuO5 pyramids and in significant distortions of the coordination polyhedra of the other cations. 
National Inst. of Standards and Technology ( $\mathrm{PL}$ ) Gaithersburg, MD. Quantum Metrology Div. Photoelectron Spectroscopic Study of the Valence and Core-Level Electronic Structure of $\mathrm{BaTiO}_{3}$ Final rept

L. T. Hudson, R. L. Kurtz, S. W. Robey, D. Temple, and R. L. Stockbauer. 1993, 7p.

Pub. in Physical Review B 47, n3 p1174-1180, 15 Jan 93.

Keywords: "Barium titanates, "Electronic structure Photoelectron spectroscopy, Single crystals, Band theory, Photoemission, Valence. Surfaces, Reprints.

We present valence and core-level photoemission measurements from vacuum-fractured, single-crystal barium titanate. These results resolve contradicton measurements in the literature which have employed other methods of sample surface preparation. The valence-shell electronic structure is compared with previously published results of band structure and cluste calculations. Resonant photoemission is used to probe the covalent coupling between titanium and oxygen in the cubic and tetragonal phases of this ionic compound. Photoelectron spectra of the Ti $2 p$ and $\mathrm{O}$ Is core levels reveal the valence of these two ions to be TiO2-like. Valence, core, satellite, and Auger transitions are also assigned and tabulated.

\section{4,501}

\section{PB94-212156 Not available NTIS}

National Inst. of Standards and Technology (PL) Gaithersburg, MD. Quantum Metrology Div.

Surface Core-Level Shifts of Barium Observed in Photoemission of Vacuum-Fractured BaTiO3 (100). Final rept.

L. T. Hudson, R. L. Kurtz, S. W. Robey, D. Temple, and R. L. Stockbauer. 1993, 7p.

Pub. in Physical Review B 47, n16 p10 832-10 838, 15 Apr 93

Keywords: *Barium titanates, X-ray photoelectron spectroscopy, Ultraviolet spectroscopy, Electronic structure, Band theory, Photoemission, Oxides, Re prints, Core level shift.

When clean, vacuum-fractured surfaces of $\mathrm{BaTiO} 3$ are analyzed using ultraviolet and $\mathrm{x}$-ray photoelectron spectroscopy (UPS and XPS), more than one set of barium core levels is observed. Sputtering the surface with ions removes lower-binding-energy intensity within the barium line shapes in UPS spectra while little change is noted in XPS. Sputtering of the surface also causes band bending and the creation of band-gap surface states of $\mathrm{Ti} 3 \mathrm{~d}$ character. From a comparison of UPS and XPS Ba $4 d$ line shapes from a vacuumfractured surface, the lower-binding-energy component is assigned to barium with 12-fold oxygen coordination representative of bulk stoichiometry; the higherbinding-energy intensity originates from undercoordinated barium in the region of the sample surface. Reasonable values for the energy-dependent attenuation length of electrons in $\mathrm{BaTiO} 3$ are derived from the relative intensities of bulk and surface components using a simple model of electron attenuation.

\section{4,502}

PB94-212230 Not available NTIS

National Inst. of Standards and Technology (EEEL) Boulder, CO. Electromagnetic Technology Div.

Flux Expulsion at Intermediate Fields in Type-II Superconductors.

Final rept.

O. B. Hyun. 1993, $7 p$.

Pub. in Physica C 206, p169-175 1993.

Keywords: "BSCCO superconductors, "YBCO superconductors, "Niobium stannides, "Superconductors, High temperature superconductors, Type 2 superconductors, Temperature dependence, Critical current, Current density, Magnetic fields, Magnetic flux, Magnetization, Reprints, *Bismuth strontium calcium cuprates, "Y ttrium barium cuprates.

The flux expulsion behavior at an intermediate field $\mathrm{H}$ was studied for type-II superconductors Nb3Sn, $\mathrm{YBa} 2 \mathrm{Cu} 3 \mathrm{O} 7$, and $\mathrm{Bi2S} 2 \mathrm{CaCu} 2 \mathrm{O} 8$. Based on a temperature-dependent critical-state model, magnetization as a function of temperature, $M(T)$ for the zero-fieldcooled (ZFC) measurement was used to predict flux expulsion in the field-cooled (FC) measurement and to construct the internal flux profiles for both ZFC and FC at an applied field of $1 \mathrm{~T}$. The model includes empirical relations of the temperature dependence of the reversible magnetization, and the critical current density. Various $M(T)$ patterns are demonstrated by the exponent $\mathrm{n}$, varying from 2 for $\mathrm{Nb} 3 \mathrm{Sn}$ to over 20 for Bi2Sr2CaCu2O8.

04,503

PB94-212263 Not available NTIS

National Inst. of Standards and Technology (EEEL),

Boulder, CO. Electromagnetic Technology Div.

Offset Susceptibility of Superconductors.

Final rept.

T. Ishida, R. B. Goldfarb, A. B. Kos, and R. W.

Cross. 1992, 4p.

Pub. in Physical Review B 46, n18 p12 080-12 083, 1 Nov 92

Keywords: *High temperature superconductors, "YBCO superconductors, "Superconductors, Temperature dependence, Magnetic fields, Fourier analysis, Magnetometers, Reprints, "Yttrium barium cuprates, ${ }^{\star}$ Offset susceptibility.

The novel concept of offset susceptibility chi(O) is presented for superconductors in applied fields $H(d c)$ $\mathrm{H}(\mathrm{ac}) \mathrm{sin}$ (omega)t. Chi(O) represents the dc component of the time-dependent magnetization $M(t)$. For sintered $\mathrm{YBa}$ CU307, chi(0) was measured as a function of $\mathrm{H}(\mathrm{ac}), \mathrm{H}(\mathrm{dc})$, and temperature using a Hall probe magnetometer and Fourier analysis. For intragranular diamagnetism, chi( 0 ) behaves as the real part of complex fundamental susceptibility chi'(1), confirming a London-like field penetration. However, chi $(0)$ arising from intergranular coupling is positive at temperatures just below the onset of the coupling transition, in good agreement with theoretical predictions of a simplified Kim critical-state model of magnetization.

\section{4}

PB94-212412 Not available NTIS

National Inst. of Standards and Technology (NML), Gaithersburg, MD. Electron and Optical Physics Div. Local Partial Densities of States in $\mathrm{Ni}$ and $\mathrm{Co}$ Silicides Studied by Soft X-Ray Emission Spectroscopy.

Final rept.

J. J. Jia, T. A. Callcott, W. L. O'Brien, D. R. Mueller

D. L. Ederer, J. E. Rowe, Q. Y. Dong, and J. E.

Rubensson. 1991, 8p.

Pub. in Physical Review B 43, n6 p4863-4870 1991.

Keywords: *Cobalt silicides, "Nickel silicides, X-ray spectroscopy, X-ray fluorescence, Soft x-rays, Emission spectra, Band theory, Reprints, Density of states.

The $\mathrm{Ni}$ and $\mathrm{Co}$ mono- and di-silicides are studied by soft $x$-ray emission spectroscopy The Si L $(2,3)$ spectra, which provide a measure of the partial densities of states of $s$ and d angular momentum symmetry $((s+d)$-PDOS) localized on the Si site, are compared with available theoretical calculations, and discussed in terms of the contribution of s electrons to the bonding. The $\mathrm{Ni}$ and $\mathrm{Co} \mathrm{M}(2,3)$ emission spectra are also obtained, and analyzed to provide information about the d-PDOS localized at the metal site. These spectra are discussed in terms of $\mathrm{p}$ - $\mathrm{d}$ bonding in the silicides.

04,505

PB94-212578 Not available NTIS

National Inst. of Standards and Technology (NEL),

Gaithersburg, MD. Statistical Engineering Div.

Efficient Experiment to Study Superconducting Ceramics.

Final rept.

R. N. Kacker, E. S. Sagergren, M. D. Hill, E. R.

Fuller, W. Wong-Ng, and C. K. Chiang. 1991, 16p.

Pub. in Communications in Statistics, Theory and Methods 20, n2 p441-456 1991.

Keywords: "High temperature superconductors, Experimental design, Formulations, Collinearity, Processing, Approximation, Polynomials, Ceramics, Mixtures, Reprints. Scheffe method.

Materials formulation and processing research are important industrial processes and most materials knowhow comes from physical experiments Our impression, based on discussions with materials scientists, is that statistically planned experiments are infrequently used in materials research. This scientific and engineering area provides an excellent opportunity for both using the available techniques of statistically planned experiments, including mixture experiments, and identifying opportunities for collaborative research leading to further advances in statistical methods for scientists and engineers. This paper describes an application of Scheffe's (1958) simplex approach for mixture experiments to formulation of high-temperature superconducting compounds. This example has given us better appreciation of the needs of materials scientists and has provided us opportunities for furthe collaborative research.

\section{4,506}

Not available NTIS

National Inst. of Standards and Technology (EEEL) Boulder, CO. Electromagnetic Technology Div Effect of Thermal Noise on Shapiro Steps in HighTc Josephson Weak Links.

Final rept.

R. L. Kautz, R. H. Ono, and C. D. Reintsema. 1992 $3 p$

Sponsored by Defense Advanced Research Projects Agency, Arlington, VA

Pub. in Applied Physics Letters 61, n3 p342-344, 20 Jul 92.

Keywords: "Josephson junctions, Temperature range 0013-0065 K, Temperature range 0065-0273 K, High emperature superconductors, Monte Carlo method, YBCO superconductors, Thermal noise, Silver alloys, Gold alloys, Simulation, Reprints, *Shapiro steps, Weak links, Yttrium barium cuprates.

The amplitudes of Shapiro steps are measured at temperatures up to $77 \mathrm{~K}$ for a superconductor-norma metal-superconductor Josephson weak link fabricated from YBa2Cu3O(7-delta) with a Ag-Au alloy bridge. The step amplitudes are found to be in agreement with calculations based on the resistively shunted-junction model when the Johnson noise of the junction resist ance is included.

04,507

PB94-212685 Not available NTIS

National Inst. of Standards and Technology (EEEL) Boulder, CO. Electromagnetic Technology Div Self-Heating in the Coulomb-Blockade Elec. trometer.

R. L Kautz, G. Zimmerli, and J. M. Martinis. 1993

$11 \mathrm{p}$

Contract N00014-92-F-0003

Sponsored by Office of Naval Research, Arlington, VA Pub. in Jnl. of Applied Physics 73, n5 p2386-2396, 1 Mar 93.

Keywords: "Electron temperature, Electron-phonon coupling, Electron tunneling, Hot electrons, Elec trometers, Aluminum, Reprints.

A detailed comparison between theory and experiment is used to demonstrate the presence of self-heating in the Coulomb-blockade electrometer. When three different heating models are considered, the best fit with experimental electrometer characteristics is obtained for a model in which the electron temperature of the sland electrode is delermined by heat transfer to the lattice via electron-phonon coupling. In the successfu model, the temperature of the island electrons is related to the power dissipated in the island and the temperature of the phonons. The best fit between theory and experiment yields a value of electron-phonon coupling parameter for the electron-phonon coupling in aluminum. Our calculations show that the electron temperature of the island commonly exceeds $100 \mathrm{mK}$ even when the lattice remains at $35 \mathrm{mK}$.

\section{4,508}

PB94-212743 Not available NTIS

National Inst of Standards and Technology (MSEL) Gaithersburg, MD. Ceramics Div.

Compositional Homogeneity in Processing Precursor Powders to the Ba2YCu307-x High TC Superconductor.

Final rept.

J. F. Kelly, and J. J. Ritter. 1992, 5p.

Pub. in Jnl. of Materials Research $7, n 3$ p580-584 1992.

Keywords: "YBCO superconductors, *Composition(Property), High temperature superconductors, Scanning electron spectroscopy, $X$ ray spectroscopy, Powder(Parics, Reprints, "Yttrium barium cuprates

We have used scanning electron microscopy (SEM) and energy dispersive $x$-ray spectroscopy (EDS) to study compositional homogeneity at several stages in the processing of powders to the Ba2YCu3Ox high $T$ (c) superconductor. We investigated the effects of solution $\mathrm{pH}$ and jonic concentration in the precipitation preparation of mixed hydroxycarbonates of $\mathrm{Ba}, \mathrm{Y}$, and $\mathrm{Cu}$ as precursor to the Ba2YCu3O(7-x) high $\mathrm{T}(\mathrm{c})$ 


\section{PHYSICS}

\section{Solid State Physics}

superconductor. Our results show that both powder homogeneity and particle morphology are strongly dependent on these parameters. Additionally, studies of the compacted powders by EDS mapping at successive stages of processing show the development of phase inhomogeneities, even in homogeneous starting powders.

\section{4,509}

PB94-212768 Not available NTIS

National Inst. of Standards and Technology (NML), Gaithersburg. MD. Electron and Optical Physics Div. Correlations of Modulation Noise with Magnetic Microstructure and intergranular interactions for CoCrTa and CoNi Thin Film Media.

Final rept.

M. R. Khan, S. Y. Lee, S. L. Duan, D. E. Speliotis M. R. Scheinfein, J. L. Pressesky, and N. Heiman. 1991, 3p

Pub. in Jnl. of Applied Physics 69, n8 p4745-4747 1991

Keywords: "Cobalt alloys, Chromium containing alloys, Nickel containing alloys, Tantalum containing alloys, Magnetic alloys, Magnetic anisotropy, Magnetic films, Remanent magnetism, Coercive force. Thin films, Demagnetization, Microstructure, Reprints, Modulation noise.

This paper reports on two thin film media alloys $\mathrm{Co}(86) \mathrm{Cr}(12) \mathrm{Ta}(2)$ and $\mathrm{Co}(75) \mathrm{Ni}(25)$ which have very different noise characteristics. The magnetic microstructure of these films were observed with SEMPA. The variance of the magnetization across the transition region was calculated. The origin of anisotropy was determined by measuring the temperature coefficients of $H(c)$ and $M(s)$. The interaction strengths between grains, $M(H)$, were obtained from the measurements of the reverse demagnetization remanence $\mathrm{Md}(\mathrm{H})$, and the forward magnetization remanence $\mathrm{Mr}(\mathrm{H})$. We found that the CoNi film showed a greater degree of interparticle interaction which may explain the observed cross-bit linkages in SEMPA image, larger RMS transition variation, and higher modulation noise.

\section{4,510}

PB94-213048 Not available NTIS

National Inst. of Standards and Technology (MSEL), Gaithersburg, MD. Ceramics Div.

Materiais Science with SR Using X-Ray Imaging: Spatial-Resolution/Source Size.

Final rept.

M. Kuriyama. 1991, 12p.

Pub. in Nuclear Instruments and Methods in Physics Research 303, n3 p503-514 1991.

Keywords: "Materials science, "Synchrotron radiation, "X-ray imagery, Synchrotron radiation sources, X-ray diffraction, Spatial resolution, Single crystals, Microradiography, Polycrystalline, Reprints, Multilayers.

Some examples of applications of synchrotron radiation to materials science demonstrate the importance of microstructure information within structural as well as functional materials in order to control their properties and quality as designed for industrial purposes. To collect such information, $x$-ray imaging in quasi real time ( 50 - 100 sec time intervals) is required in either the microradiographic mode or the diffraction (in transmission) mode. New measurement technologies based on imaging are applied to polycrystalline materials, single crystal materials and multilayered device materials to illustrate what kind of synchrotron radiation facility is most desirable for materials science and engineering.

\section{4,511}

\section{PB94-213089 Not available NTIS}

National Inst. of Standards and Technology (CSTL), Gaithersburg, MD. Inorganic Analytical Research Div. Analysis of Boron in CVD Diamond Surfaces Using Neutron Depth Profiling.

Final rept.

G. P. Lamaze, R. G. Downing, L. Pilione, A. Badzian and T. Badzian. 1993, 6p.

Sponsored by Office of Naval Research, Arlington, VA Pub. in Applied Surface Science 65/66, p587-592 1993.

Keywords: "Diamond films, "Surface analysis, "Boron, Chemical vapor deposition, Concentration(Composition). Semiconducting films, Cold neutrons, Doped materials, Single crystals, Thin films, Reprints, Neutron depth profiling, Depth profiles, CNRF facility

Neutron depth profiling (NDP) is a method of near surface analysis for isotopes that undergo neutron-induced positive $\mathrm{Q}$-value charged particle reactions, e.g., (n,alpha), (n,p). Because of its large cross section and large $Q$-value, the $(10) B(n$,alpha)(7) Li reaction has been widely employed for NDP studies. We have used the NDP technique to study the concentration and distribution of boron in CVD diamond layers. The meas urements were made using the new Cold Neutron Depth Profiling instrument at the NIST Cold Neutron Research Facility. The diamond samples were prepared at the Materials Research Laboratory at the Pennsylvania State University. The doped diamond single crystal films were grown by microwave plasma chemical vapor deposition from a mixture of methane hydrogen and diborane. Natural type lla crystals cut along the (001) plane were used as substrates. Results of the measurements are presented.

\section{4,512}

PB94-213121 Not available NTIS

National Inst. of Standards and Technology (IMSE) Gaithersburg, MD. Metallurgy Div.

Magnetic and Structurai Properties of Electrodeposited Copper-Nickel Microlayered Alloys.

Final rept

D. S. Lashmore, R. R. Oberle, L. H. Bennett, M. P.

Dariel, L. Romankiw, L. Swartzendruber, and $U$.

Atzmony. 1990, 11p.

Pub. in Proceedings of Symposium on Magnetic Materials, Processes, and Devices, Hollywood, FL., Octo ber 15-19, 1989, p347-357 1990.

Keywords: "Electrodeposited coatings, "Superlattices, $X$-ray diffraction, Magnetic properties, Magnetic films, Metal films, Thin films, Electrodeposition, Copper, Nickel, Reprints, Multilayers.

Magnetic measurements of $\mathrm{Ni}-\mathrm{Cu}$ compositionally modulated alloys prepared by electrochemical deposi tion suggest a high degree of perfection is achieved. The magnetic behavior closely resembles that of bulk nickel and suggests little interdiffusion between the copper and the nickel. The high value of the saturation magnetization implies that no magnetic dead layer ex ists at the nickel/copper interface for wavelengths greater than $2.5 \mathrm{~nm}$. A magnetic after effect has been found which increases with decreasing temperature and is a maximum at the coercive field. The electrochemical technique has progressed to the point where third order satellites are now commonly observed, suggesting a very square composition profile. Continuous layers of copper as thin as $5 \mathrm{~A}$ (about 2 monolayers) have been produced

\section{4,513}

PB94-213196 Not available NTIS

National Inst. of Standards and Technology (MSEL), Boulder, CO. Materials Reliability Div.

Elastic Constants of Polycrystaliine $\mathrm{Y} 1 \mathrm{Ba} 2 \mathrm{Cu} 3 \mathrm{OX}$. Final rept.

H. Ledbetter, 1992, 3p.

Pub. in Jnl. of Materials Research 17, n11 p2905-2907 Nov 92.

Keywords: "YBCO superconductors, "Elastic properties. High temperature superconductors, Ultrasonic radiation, Acoustic velocity, Debye temperature, Bulk modulus, Ionic crystals, Polycrystalline, Reprints, "Yttrium barium cuprates, Oxygen content.

We measured the elastic constants of polycrystalline Y1Ba2Cu30x with eleven oxygen contents varying from 6.2 to 6.9. Reported properties include sound velocities; bulk, shear, Young's moduli; Poisson ratio; Debye temperature. After correction to the void-free state, the measurements fail to show a systematic dependence on oxygen content. Focusing on the bulk modulus, all measurements fall below an ionic-crystal model prediction, by $5-50 \%$. We attribute this macroscopic softness to either microcracks or weak grainboundary mechanical linkages. For the case $x=6.9$, we suggest a set of intrinsic elastic constants.

04,514

PB94-213220 Not available NTIS

National Inst. of Standards and Technology (MSEL), Boulder, CO. Materials Reliability Div.
Correlation between Tc and Elastic Constants of (La-M)2CuO4.

Final rept.

H. Ledbetter, S. Kim, and A. Roshko. 1992, 3p.

Pub. in Zeitschrift fuer Physikalische B - Condensed Matter 89, p275-277 1992.

Keywords: "Superconductors, High temperature superconductors, Electron phonon interactions, Ultrasonic radiation, Acoustic velocity, Debye temperature, Elastic properties, Transition temperature, Correlation, Reprints, "Lanthanum calcium cuprates, "Lanthanum barium cuprates, "Lanthanum strontium cuprates.

By an ultrasonic method, we measured the elastic constants of polycrystalline $\mathrm{La}(1.85) \mathrm{M}(0.15) \mathrm{CuO} 4$ with $\mathrm{M}=\mathrm{Ca}, \mathrm{Ba}, \mathrm{Sr}$. These materials show zero-resistance normal-superconductive transition temperatures of 19 28 , and $36 \mathrm{~K}$. Versus $\mathrm{T}(\mathrm{c})$, all the elastic stiffnesses - bulk modulus, Young modulus, shear modulus - increase. Derived from the elastic constants and average atomic volume, the Debye temperature increases linearly with $\mathrm{T}(\mathrm{c})$. In conventional BCS superconductors, T(c) decreases with increasing 1 Theta(D).

\section{4,515}

\section{PB94-213352 Not available NTIS}

National Inst. of Standards and Technology (MSEL), Boulder, CO. Materials Reliability Div.

Elastic Constants and Debye Temperature of Y1Ba2Cu3Ox: Effect of Oxygen Content.

Final rept

S. Lin, M. Lei, and H. Ledbetter. 1993, 4p.

Pub. in Materials Letters 16, p165-168 1993.

Keywords: "YBCO superconductors, "Elastic properties, High temperature superconductors, Ultrasonic radiation, Debye temperature. Bulk modulus, Temperature range 0000-0013 $\mathrm{K}$, Temperature range 0013-0065 K, Temperature range 0065-0273 K, Temperature range 0273-0400 K, Polycrystalline, Reprints, Yttrium barium cuprates, Oxygen content.

Using ultrasonic methods, we measured the elastic constants of polycrystalline $\mathrm{Y} 1 \mathrm{Ba} 2 \mathrm{Cu} 3 \mathrm{O} x$ between 295 and $4 \mathrm{~K}$. For a single specimen, using vacuum annealing and oxygen annealing, we varied the oxygen content in four steps from $x=6.2$ to $x=6.9$. Elastic stiffnesses that depend mainly on shear modes increase monotonically with increasing oxygen content. Stiffnesses that depend on dilatation modes increase up to about $x=6.7$ and then decrease. The elastic Debye temperature, which depends mainly on shear modes, increases monotonically, in agreement with the specific-heat Debye temperature. An ionic-model calculation for the bulk modulus and Debye temperature predicts a monotonic increase for both. We conjecture that the irregular oxygen-content dependence of the dilatation-mode-related elastic constants arises from changes in copper-oxygen valences.

04,516

PB94-216116 Not available NTIS

National Inst. of Standards and Technology (MSEL), Gaithersburg. MD. Reactor Radiation Div.

Polarization Analysis of the Magnetic Excitations in invar and Non-Invar Amorphous Alloys.

Final rept.

J. W. Lynn, N. Rosov, and G. Fish. 1993, 3p.

15 May 93.

Keywords: "Invar, Phosphorus containing alloys Boron containing alloys, Nickel alloys, Iron alloys, Polarized beams, Neutron scattering, Inelastic scattering Amorphous materials, Spin waves, Ferromagnetic materials, Reprints, *Amorphous ferromagnets, Magnetic excitations, METGLAS 2826

Conventional spin wave theory works remarkably well in describing the spin dynamics of both Invar and nonInvar isotropic ferromagnets, with the important exception that for invar systems the magnetization de creases much more rapidly with temperature than can be explained based on the measured spin wave dispersion relations. We have been carrying out triple-axis polarized inelastic neutron scattering experiments on the amorphous ferromagnets Fe86B14 (Invar system) and Fe40Ni40P14B6 (METGLAS (R(circled)) 2826) in order to separate the longitudinal magnetic fluctuations from the transverse (spin wave) excitations, and thereby determine if the presence of Iongitudinal excitations might resolve this discrepancy. The present measurements exhibit longitudinal excitations below $t(\mathrm{c})$, but in both materials. Possible interpretations of the results are discussed. 
04,517

PB94-216207 Not available NTIS

National Inst. of Standards and Technology (IMSE), Gaithersburg, MD. Reactor Radiation Div. Neutron Scattering Studies of Surfaces and Interfaces.

Final rept.

C. F. Majkrzak, and G. P. Felcher. 1990, 7p

Pub. in MRS Bulletin 15, n11 p65-71 Nov 90.

Keywords: "Surfaces, "Interfaces, Neutron diffraction, Grazing incidence, Magnetic materials, Superconductors, Polymers, Reprints, Neutron reflectometry, Multilayers.

During the past decade, scientific and technological in terest in the properties of surfaces and interfaces has grown at an astounding rate. Electron, atomic beam, and $x$-ray diffraction techniques have been used to study surface or near surface problems. Neutrons can also be used in many cases and because neutrons can distinguish between different isotopes of the same element, most notably hydrogen and deuterium, as well as couple to atomic magnetic moments via a dipolar interaction, they can be an indispensable and complementary probe. The purpose of this paper is to give a concise description of the methodology of neutron reflectometry, multilayer diffraction, and grazing angle diffraction, including specific examples of the characterization of magnetic, superconducting, and polymeric surfaces or interfaces.

04,518

PB94-216314 Not available NTIS

National Inst. of Standards and Technology (MSEL) Gaithersburg, MD. Reactor Radiation Div.

Crystal Structure of $\mathrm{Pb} 2 \mathrm{Sr} 2 \mathrm{YCu} 3 \mathrm{O} 8+$ delta with delta=1.32, 1.46, 1.61, 1.71, by Powder Neutron Diffraction.

Final rept

M. Marezio, A. Santoro, J. J. Capponi, Q. Huang, R J. Cava, and O. Chmaissem. 1992, 10p.

Pub. in Physica C 199, p365-374 1992.

Keywords: "Crystal structure, Neutron diffraction, Reprints, "Lead strontium yttrium cuprates, Oxygen content

The structures of $\mathrm{Pb} 2 \mathrm{Sr} 2 \mathrm{YCu} 3 \mathrm{O}(8$ + delta) with delta $=1.46,1.61,1.71$, have been determined by powder neutron diffraction data. As previously shown for $\mathrm{Pb} 2 \mathrm{Sr} 2 \mathrm{YCu} 3 \mathrm{O}(9.32)$, the extra oxygen atoms are incorporated on the Cu layer sandwiched between two $\mathrm{PbO}$ layers. The structural refinements indicated that the most stable structure is that of Pb2Sr2YCu3O(9.5) in which the two possible oxygen sites on the Cu laye are filled up to $75 \%$. These extra oxygen atoms give rise to superstructures and change the coordination of the cations belonging to the $(\mathrm{PbO})(\mathrm{Cu})(\mathrm{PbO})$ blocks. In Pb2Sr2YCu3O(9.5) the Cu cations inside these blocks are surrounded by an oxygen pyramid, while the $\mathrm{Pb}$ cations are surrounded by seven, eight, or nine oxygen atoms, depending upon the specific site in the superstructure. At the same time, the valences of these cations increase from $1+$ to $2+$ for $\mathrm{Cu}$ and from $2+$ to $4+$ for half of the $\mathrm{Pb}$. The oxidation of the $\mathrm{Pb}$ cations hinders the charge transfer from the $\mathrm{CuO}(1.5)$ layers to the superconducting $(\mathrm{CuO} 2)(\mathrm{Y})(\mathrm{CuO} 2)$ blocks and the oxidation of $\mathrm{Pb} 2 \mathrm{Sr} 2 \mathrm{YCu} 3 \mathrm{O} 8$ does not lead to superconducting state. Every sample we analyzed should be considered either an oxygen-deficient compound or one containing an excess oxygen with respect to the $\mathrm{Pb} 2 \mathrm{Sr} 2 \mathrm{YCu} 3 \mathrm{O}(9.5)$ structure

\section{4,519}

\section{PB94-216553 Not available NTIS}

National Inst. of Standards and Technology (IMSE), Gaithersburg, MD. Fracture and Deformation Div. Atomic Theory of Fracture of Brittle Materials: Ap. plication to Covalent Semiconductors.

Final rept.

K. Masuda-Jindo, V. K. Tewary, and R. Thomson.

1991, 14p

Pub. in Jnl. of Materials Research 6, n7 p1553-1566 1991

Keywords: "Brittle fracturing, "Atomic theory, "Silicon, Perturbation theory, Greens function, Chemical bonds, Semiconductors, Cracks, Reprints, LCAO theory.

Using the lattice Green's function approach and LCAO (linear combination of atomic orbitals) electron theory, we investigate the atomistic configuration and lattice trapping of cracks in Si. The LCAO electron theory coupled to second order perturbation (SOP) theory has been used to derive explicit expressions for the bond breaking non-linear forces between Si atoms. We calculate the cracked lattice Green's functions for a crack on the (111) plane and lying in the (110) direction. With the non-linear forces acting in a cohesive region nea the crack tips, the crack structure is then calculated. The calculated structure possesses a crack opening at the Griffith load which should allow penetration of typical external molecules to the crack tip at the Griffith loading. Other consequences for chemical reactions a the crack tip are discussed in the light of these results. The lattice trapping is low, only a few percent of the Griffith load

\section{4,520}

PB94-216678 Not available NTIS

National Inst. of Standards and Technology (PL) Gaithersburg, MD. Electron and Optical Physics Div. Simple, Compact, High-Purity Cr Evaporator for UItrahigh Vacuum.

Final rept.

J. J. McClelland, J. Unguris, R. E. Scholten, and D. T. Pierce. 1993, 2p

Pub. in Jnl. of Vacuum Science and Technology A 11 n5 p2863-2864 Sep/Oct 93.

Keywords: "Evaporators, "Chromium, Ultrahigh vacuum, Metal films, Thin films, Filaments, Reprints.

A simple, compact $\mathrm{Cr}$ evaporator is constructed by electroplating $\mathrm{Cr}$ metal onto the tip of a $\mathrm{W}$ hairpin filament. At $5 \mathrm{~cm}$ from the evaporator, deposition rates up to $10 \mathrm{~nm} / \mathrm{min}$ (flux approx $=10$ (sup 19) atoms $/ \mathrm{sq}$ $\mathrm{m} / \mathrm{s}$ ) have been obtained, with total deposition thick ness in excess of $400 \mathrm{~nm}$. Auger analyses of thin films deposited in ultrahigh vacuum show impurities below detectability.

04,521

PB94-216736 Not available NTIS

National Inst. of Standards and Technology (NEL) Gaithersburg, MD. Applied and Computational Mathematics Div.

Effect of Modulated Taylor-Couette Flows on Crystal-Melt Interfaces: Theory and Initial Experiments. Final rept.

G. B. McFadden, B. T. Murray, S. R. Coriell, M. E.

Glicksman, and M. E. Selleck. 1992, 20p.

Pub. in Proceedings of IMA Workshop on the Evolution of Phase Boundaries, September 17-21, 1990, p81 1001992

Keywords: "Crystal growth, "Solidification, Cylindrica configuration, Free boundaries, Reprints, "Crystal-me interface, "Taylor-Couette flow, Floquet theory, Two phase systems.

An important problem in the process of crystal growth from the melt phase is to understand the interaction of the crystal-melt interface with fluid flow in the melt. This area combines the complexities of the NavierStokes equations for fluid flow with the nonlinear behavior of the free boundary representing the crystalmelt interface. Some progress has been made by studying explicit flows that allow a base state corresponding to a one-dimensional crystal-melt interface with solute and/or temperature fields that depend only on the distance from the interface. This allows the strength of the interaction between the flow and the interface to be assessed by a linear stability analysis of the simple base state. The case of a time-periodic Taylor-Couette flow interacting with a cylindrical crystalline interface is currently being investigated both experimentally and theoretically; some preliminary results are given here. The results indicate that the effect of the crystal-melt interface in the two-phase system is to destabilize the system by an order of magnitude relative to the single-phase system with rigid walls.

\section{4,522}

PB95-107207 Not available NTIS

National Inst. of Standards and Technology (NML) Gaithersburg, MD. Gas and Particulate Science Div.

Monte Carlo Electron Trajectory Simulation of $\mathrm{X}$ Ray Emission from Films Supported on Substrates.

Final rept.

D. E. Newbury, R. L. Myklebust, and E. B. Steel. 1990, 4p

Pub. in Microbeam Anal. 25, p127-130, 1990

Keywords: "Monte Carlo method, "X-ray analysis, Reprints, Thin films, Microanalysis, Electron probes, Sili con, Calibration standards, Trajectories, Simulation, Multicomponent targets, Scanning electron micros copy, National Institute of Standards and Technology, SRM 2063

The development of a Monte Carlo electron trajectory simulation for application to scanning electron microscopy and $x$-ray microanalysis involves several possible choices of elastic scattering and energy loss models, as well as for inner shell ionization. The problem of establishing proper weighting for the simulation of multicomponent targets must also be considered. While there is an extensive base of pure element experimental data for comparison to calculations, relatively little data is available on multi-component targets.

04,523

PB95-107306 Not available NTIS

National Inst. of Standards and Technology (MSEL), Gaithersburg, MD. Ceramics Div.

Improved Crystallographic Data for Aluminum Niobate (AINbO4).

Final rept.

C. J. Rawn, R. S. Roth, and H. F. McMurdie. 1991

$2 \mathrm{p}$.

Pub. in Powder Diffr. 6, n1 p48-49 1991.

Keywords: "Lattice parameters, Least squares method, X-ray diffraction, Orthorhombic lattices, Single crystals, Reprints, "Aluminum niobates.

The compound AINbO4 has been studied by single crystal $x$-ray precession photographs and $x$-ray powder diffraction. Unit cell dimensions were calculated using a least squares analysis that refined to a Delta 2 theta deg of no more than $0.03 \mathrm{deg}$. An orthorhombic cell was found with space group $\mathrm{C} 2 / \mathrm{m}, a=121558(5) \mathrm{A}$, $\mathrm{B}=3.7345$ (2) A, $\mathrm{c}=6.4886(3) \mathrm{A}$, and beta $=107.613(4)$ deg.

04,524

PB95-107371 Not available NTIS

National Inst. of Standards and Technology (MSEL), Gaithersburg, MD. Reactor Radiation Div

Rietveld Analysis of $\mathrm{NaxWO}+x / 2 . y \mathrm{H} 2 \mathrm{O}$, Which Has the Hexagonal Tungsten Bronze Structure. Final rept.

K. P. Reis, E. Prince, and M. S. Whittingham. 1992

$6 p$.

Pub. in Chem. Mater. 4, n2 p307-312 1992.

Keywords: "Sodium tungstates, "Crystal structure, Hexagonal lattices, Neutron diffraction, Hydrates, Deuterium compounds, Reprints, Rietveld method.

Powder neutron diffraction and Rietveld analysis were used to investigate the crystal structures of hydrated and deuterated samples of $\mathrm{Na}(x) \mathrm{WO}(3+\mathrm{x} / 2)$ (dot)yH2O ( $x$ approx $=0.17, y$ approx $=0.23$ ). The compound, which crystallizes with the symmetry of space group $\mathrm{P} 6 / \mathrm{mmm}$, is related to the hexagonal tungsten bronze structure but differs from it due to the presence of sodium and oxygen along the hexagonal tunnel. Some of the oxygens appear in the structure as water molecules. The oxygen is disordered along the $z$ axis in the hexagonal cavity with sodium in the hexagonal window.

\section{4,525}

PB95-108429 Not available NTIS

National Inst. of Standards and Technology (PL), Gaithersburg, MD. Electron and Optical Physics Div. Magnetic Moments in Cr Thin Films on Fe(100). Final rept.

D. T. Pierce, R. J. Celotta, and J. Unguris. 1993, 3p. Sponsored by Office of Naval Research, Arlington, VA. Pub. in Jnl. of Applied Physics 73, n10 p6201-6203, 15 May 93.

Keywords: *Magnetic moments, "Chromium, Whiskers(Single crystals), Polarization(Spin alignment), Antiferromagnetic materials, Scanning electron microscopy, Spin waves, Metal films, Thin films, Epitaxy, Iron, Reprints, *Surface magnetism, Electron spin polarization, Secondary electrons.

The magnetism at the surface of a $\mathrm{Cr}$ film grown epitaxially on a $\mathrm{Fe}(100)$ whisker is observed as a function of $\mathrm{Cr}$ thickness by scanning electron microscopy with polarization analysis. Use of a wedge-shaped film of linearly increasing thickness allows the magnetism to be followed continuously for $75 \mathrm{Cr}$ layers. Over the temperature range measured from just below the Neel temperature of bulk $\mathrm{Cr}, \mathrm{T}(\mathrm{N})$, to $1.8 \mathrm{~T}(\mathrm{~N})$, the surface magnetic moment is seen to persist and change direction with each additional $\mathrm{Cr}$ layer, but there are phase slips in this antiferromagnetic ordering. These are con- 


\section{Solid State Physics}

sistent with an incommensurate spin density wave (SDW) in the Cr film having a wavelength of 40 layers at $T(N)$. An irregularity in the antiferromagnetic stacking order in the first four layers is discussed and the behavior of the moments at the subsequent phase slip is examined. The limitations which prevent the determination of the surface magnetic moment from the spin polarization of secondary electrons are discussed.

04,526

PB95-108601 Not available NTIS

National Inst. of Standards and Technology (MSEL),

Gaithersburg. MD. Reactor Radiation Div.

Mathematical Aspects of Rietveld Refinement.

Final rept.

E. Prince. 1993, 12p.

Pub. in Rietveld Method, Chapter 3, p43-54 1993.

Keywords: Least squares method, X-ray diffraction, Neutron diffraction, Crystal structure, Data processing, Reprints, "Rietveld method, Parameter estimation.

The method of least squares is a powerful technique for estimating the parameters of a model given a set of observations. In the Rietveld method, the observations are the intensities of neutrons or x-rays in a powder diffraction pattern, and the model predicts these intensities as a function of background, peakshape and crystallographic parameters. The loss of information due to overlapping peaks can be compensated for by incorporating additional information, such as chemical composition, noncrystallographic symmetry and rigid body motion, in the model by means of constraints and restraints. Careful attention to the efficiency and stability of refinement procedures is important. Statistical analysis can be used to assess the uncertainties in the parameters of a refined model and to compare two or more models.

04,527

PB95-125647 Not available NTIS

National Inst. of Standards and Technology (MSEL),

Boulder, CO. Materials Reliability Div

Asymmetry between Flux Penetration and Flux Expulsion in TI-2212 Superconductors.

Final rept

M. Foldeaki, H. Ledbetter, M. Paranthaman, H. M

Duan, and A. M. Hermann. 1993, 5p.

Pub. in Jnl. of Superconductivity 6, n3 p185-189 1993.

Keywords: *High temperature superconductors, Temperature range 0013-0065 K, Irreversible processes, Time dependence, Magnetic hysteresis, Flux pinning, Direct current, Single crystals, Polycrystals, Asymmetry, Reprints, "Thallium barium calcium cuprates.

dc magnetic hysteresis as well as flux penetration and flux expulsion were investigated in $\mathrm{TI}(2-$ y) $\mathrm{Ba} 2 \mathrm{CaCu} 2 \mathrm{O}(8-\mathrm{x})$ polycrystals and monocrystals. All measurements were performed at $35 \mathrm{~K}$ and in the 0$5 \mathrm{~T}$ field range. Hysteresis measurements revealed an irreversibility field of about $2 \mathrm{~T}$. Existing models predict identical field-cooled (fc) and zero-field-cooled ( $\mathrm{zfc}$ ) magnetizations and vanishing time dependence above this field. Although the identical fc and zfc magnetizations are in fact observed, the time dependence vanishes only for flux penetration after zero-field cooling: a remanence is preserved after field cooling and decays with a finite relaxation rate. Activation energies calculated on the basis of the thermal activation model display a pronounced field dependence, and are lower for flux penetration than for flux expulsion in high fields $(\mathrm{H}=$ or $>3 \mathrm{~T})$ for all orientations. This behavior of extreme layered superconductors contradicts classical theoretical models and questions the original definition of the irreversibility line as well. All of our results are consistent with the recent theory of lock-in transition, and can be well interpreted by using those principles.

\section{4,528}

PB95-126033 Not available NTIS

National Inst. of Standards and Technology (MSEL), Gaithersburg, MD. Metallurgy Div.

Nature of (001) Tilt Grain Boundaries in YBa2Cu3O6+X.

Final rept.

F. W. Gayle, and D. L. Kaiser. 1991, 8p

Pub. in Jnl. of Materials Research 6, n5 p908-915 1991.

Keywords: "YBCO superconductors, "Grain boundaries, High temperature superconductors, Crystalphase transformations, Orthorhombic lattices, Tetragonal lattices, Twinning, Reprints, "Yttrium barium cuprates, Tilt boundaries.
Fifty-two facetted grain boundary segments ((left bracket) 001 (right bracket) tilt boundaries) in clusters of bulk-scale YBa2Cu3O(7-x) crystals having coincident $c$-axes were characterized by optical microscopy techniques. Grain boundary orientations were widely distributed, with a slight favoring of (110), (310), and (510) boundary planes. All grain boundaries, excep those far from the symmetric condition and those with (110) facets, exhibited well-developed matching of the twin domains across the boundary. It is suggested that this newly-reported phenomenon of twin pattern matching occurs due to a local coordination of the tetragonal $\rightarrow>$ orthorhombic transformation strain across grain boundaries and may be beneficial with regard to electrical transport in highly-textured polycrystalline material.

04,529

PB95-126074 Not available NTIS

National Inst. of Standards and Technology (EEEL) Gaithersburg, MD. Electricity Div.

Cryogenic Precision Capacitance Bridge Using a Single Electron Tunneling Electrometer.

Final rept

R. N. Ghosh, E. R. Williams, A. F. Clark, and R. J

Soulen. 1993, $2 p$

Pub. in Proceedings of the International Conference on Low Temperature Physics (20th), Eugene, OR., August 4-11, 1993.

Keywords: "Capacitance bridges, "Electrometers, Cryogenic equipment, Precision, Design, Reprints, "Electron charge, Single electron tunneling.

The electronic charge can be determined by placing a known number of electrons on a calibrated capacito and measuring the resulting voltage. Single electron tunneling (SET) electrometers with sufficient sensitivity for this application have been fabricated. We report on the design and preliminary results of a capacitance bridge experiment using an SET electrometer to measure two capacitors in a dilution refrigerator. This includes discussion of issues such as the leakage rate of a capacitor at cryogenic temperatures and the optimum coupling of the electrometer to the bridge circuit.

\section{4,530}

PB95-126116 Not available NTIS

National Inst. of Standards and Technology (NML), Gaithersburg, MD. Gas and Particulate Science Div Image Depth Profiling SIMS: An Evaluation for the x Single Crystal Superconductors.

Final rept.

G. Gillen, D. L. Kaiser, and J. S. Wallace. 1991, 8p. Pub. in Surface and Interface Analysis 17, $\mathrm{n} 1 \mathrm{p} 7-14$ 1991.

Keywords: "YBCO superconductors, Secondary ion mass spectroscopy, High temperature superconductors, Single crystals, Oxygen 18, Diffusion, Reprints, ${ }^{\star} Y$ ttrium barium cuprates, Depth profiles.

An analytical strategy for quantifying the diffusion of (18)O in small, melt-grown single crystal YBa2Cu3O(7 x) superconductors by image depth profiling SIMS (Secondary Ion Mass Spectroscopy) is presented. This technique was used to obtain data for both in-depth (along the $\mathrm{c}$ axis) and lateral (in the ab plane) diffusion from one superconducting crystal. Cross sectional images, generated from the image depth profiling data, revealed features of the diffusion processes which would not have been apparent in a standard depth profile. Retrospective selected area depth profiling was used to remove the influence of background species, edge effects, and imperfections in the crystals, result ing in processed depth profiles with greater dynamic range. Finally, the influence of detector artifacts on the analytical results were evaluated.

04,531

PB95-126199 Not available NTIS

National Inst. of Standards and Technology (EEEL), Boulder, CO. Electromagnetic Technology Div.

Magnetic Measurement of Transport Critical Current Density of Granular Superconductors.

Final rept.

R. B. Goldfarb, R. W. Cross, L. F. Goodrich, and N. F. Bergren. 1993, 5p

Pub. in Cryogenics 33, n1 p3-7 1993.

Keywords: "High temperature superconductors *Critical current, Granular materials, Magnetic measurement, Current density, Magnetization, Reprints.
We describe two magnetic techniques that may be used to determine the transport critical current density $\mathrm{J}(\mathrm{ct})$ of granular superconductors by measuring the intergranular magnetization of a sample. In the first method, magnetization critical current density $\mathrm{J}(\mathrm{cm})$ is used to estimate $\mathrm{J}(\mathrm{ct})$ by isolating the intergranular magnetization and applying the critical state model. In the second method, magnetic detection is used to measure $\mathrm{J}(\mathrm{ct})$ directly: intergranular magnetization hysteresis loops are obtained while increasing a transport current through a sample. The critical current density $\mathrm{J}(\mathrm{ct})$ is that value of transport current density which causes the intergranular magnetization to collapse at a given magnetic field and temperature. Both methods give values of $\mathrm{J}(\mathrm{ct})$ in fair agreement with values obtained from conventional transport measurements of $J(c t)$. Magnetization was measured with both extraction and Hall probe magnetometers.

\section{4,532}

PB95-126215 Not available NTIS

National Inst. of Standards and Technology (MSEL) Gaithersburg, MD. Metallurgy Div.

Inelastic Neutron Scattering Measurements of Phonons in Icosahedral Al- $\mathrm{Li}-\mathrm{Cu}$.

Final rept.

A. I. Goldman, C. Stassis, R. Bellissent, F. W. Gayle H. Moudden, and N. Pyka. 1991, 4p.

Pub. in Physical Review B 43, n10 p8763-8766 1991

Keywords: Aluminum copper alloys, Lithium alloys Neutron scattering, Inelastic scattering, Icosahedrons, Reprints, "Quasicrystals, Phonon dispersion.

We describe the results of inelastic neutron scattering measurements on a single grain of the icosahedral alloy Al-Li-Cu. Within experimental error the acoustic phonon dispersion at small, but finite, $q$ is independent of direction, confirming the isotropic elasticity expected for icosahedral crystals. By following the longitur" nal phonon dispersion curve along a five-fold axis, we van identify the location of a "quasi-Brillouin zone boundary.' Furthermore, the measurements suggest the presence of an optical or localized mode along the twofold axis at approximately $10 \mathrm{meV}$.

\section{4,533}

PB95-126223 Not available NTIS

National Inst. of Standards and Technology (EEEL), Boulder, CO. Electromagnetic Technology Div. $\mathrm{n}$-Value and Second Derivative of the Final rept.

L. F. Goodrich, A. N. Srivastava, M. Yuyama, and H. Wada. 1993, 4p.

Pub. in Institute of Electrical and Electronics Engineers Transactions on Applied Superconductivity $3, n 1$ p1265-1268 Mar 93.

Keywords: "Superconductors, High temperature superconductors, BSCCO superconductors, Critical current, Electric current, Transition points, Voltage, Copper, Simulators, Data acquisition, Reprints, Niobium titanium.

We sludied the $n$-value ( $V$ varies as I(sup $n$ )) and second derivative of $V$ with respect to I of the voltagecurrent curve of high and low temperature superconductors and superconductor simulators. We used these parameters for diagnosing problems with sample heating and data acquisition, and as indicators of the superconducting-to-normal state transition. The superconductor simulator may be useful in testing the measurement system integrity and reducing measure ment variability as its characteristics are highly repeatable.

\section{4,534 \\ PB95-126389 Not available NTIS}

National Inst. of Standards and Technology (NML), Gaithersburg, MD. Gas and Particulate Science Div. Transition Metal Implants in In0.53GA0.47As.

Final rept

S. M. Gulwadi, M. V. Rao, A. K. Berry, H. B. Dietrich, D. S. Simons, and P. H. Chi. 1991, 6p.

Pub. in Jnl. of Applied Physics 69, n8 p4222-4227 1991

Keywords: *Indium gallium arsenides, *Ion implanta tion, Secondary ion mass spectroscopy, Semiconductor doping, Chromium ions, Iron ions, Phosphorus ions, Vanadium ions, Annealing, Reprints, Photoreflectance.

Single-and multiple-energy $\mathrm{Fe}, \mathrm{Cr}$, and $\mathrm{V}$ implants are performed into InGaAs. Annealing of the implanted InGaAs samples results in redistribution of the im- 
planted atoms, as determined by secondary ion mass spectrometry. Coimplantation of $\mathrm{Fe}$ with $\mathrm{P}$ does not prevent this redistribution. A transport equation calculation of $\mathrm{Fe}$-implantation-induced stoichiometric disturbances in InGaAs is done. The lattice quality of implanted InGaAs is investigated by photoreflectance measurements. Fe-implanted InGaAs has a resistivity close to the intrinsic limit, whereas $\mathrm{Cr}$ - and $\mathrm{V}$-implanted InGaAs have a lower resistivity than the unimplanted material.

\section{4,535}

PB95-126397 Not available NTIS

National Inst. of Standards and Technology (NML), Gaithersburg, MD. Gas and Particulate Science Div. Range Statistics and Rutherford Backscattering Studies on Fe-Implanted In0.53Ga0.47As.

Final rept.

S. M. Gulwadi, M. V. Rao, D. S. Simons, C. Caneau, H. B. Dietrich, O. W. Holland, and W. P. Hong. 1991, $6 p$.

Pub. in Jnl. of Applied Physics 69, n1 p162-167 1991. Keywords: *Indium gallium arsenides, Secondary ion mass spectroscopy, $\mathrm{KeV}$ range $10-100, \mathrm{KeV}$ range 100-1000, MeV range 1-10, Semiconductor doping, Doped materials, Radiation damage, Rutherford scattering, Ion implantation, Iron ions, Backscattering, Reprints, Amorphization.

Single-energy Fe-implantation at energies in the range $50 \mathrm{keV}-2 \mathrm{MeV}$ to achieve a peak Fe-concentration of $1-2 \times 10$ (exp 18)/cu $\mathrm{cm}$ is performed into undoped (n-type) In-GaAs layers grown on InP:Fe. The first four statistical moments of the Fe-profiles measured by secondary ion mass spectrometry are determined. The Pearson IV distribution calculated from these moments matches the implant-profile closely. Samples implanted with $\mathrm{Fe}$ to doses in the range $5 \times 10(\exp 12)$ $-2 \times 10(\exp 15) / \mathrm{sq} \mathrm{cm}$ at $380 \mathrm{keV}$ are analyzed by Rutherford backscattering measurements to study ioninduced damage. For $380 \mathrm{KeV}$ implants, amorphization takes place at a dose of $=6 \times 10(\exp$ $13) / \mathrm{sq} \mathrm{cm}$.

04,536

PB95-136628 PC A03/MF A01

National Inst. of Standards and Technology (CAML) Gaithersburg, MD. Applied and Computational Mathematics Div.

xi-Vector Formulation of Anisotropic Phase-Field Models: 3-D Asymptotics.

A. A. Wheeler, and G. B. McFadden. 17 Oct 94, 25p, NISTIR-5505.

Sponsored by Bristol Univ. (England). School of Mathematics.

Keywords: "Solidification, Liquid-solid interfaces, Surface energy, Differential geometry, Asymptotic series, Vector analysis, Three dimensional, Anisotropy, "Phase field models, Allen-Cahn equation, GibbsThomson equation, $X i$ vector.

In the paper the authors present a new formulation of a large class of phase-field models, which describe solidification of a pure material and allow for both surface energy and interface kinetic anisotropy, in terms of the Hoffman-Cahn xi-vector. The Hoffman-Cahn vi-vector has previously been used in the context of sharp interface models, where it provides an elegant tool for the representation and analysis of interfaces with anisotropic surface energy. The authors show that the usual gradient-energy formulations of ansotropic phase-field models are expressed in a natural way in terms of the vi-vector when approximately interpreted. The authors use this new formulation of the phase-field equations to provide a concise derivation of the Gibbs-ThomsonHerring equation in the sharp-interface limit in three dimensions.

\section{4,537}

PB95-140034 Not available NTIS

National Inst. of Standards and Technology (MSEL). Gaithersburg, MD. Reactor Radiation Div.

Defective Structures of Barium Yttrium Copper Oxide (Ba2YCu3OX) and Ba2YCu3-yMyOz ( $\mathrm{M}=\mathrm{Fe}$, Co, Al, Ga, ..).

Final rept.

A. Santoro. $1992,44 p$.

Pub. in Chem. Supercond. Mater., p146-189 1992.

Keywords: "YBCO superconductors, "Crystal defects, High temperature superconductors, Vacancies(Crystal defects), Twinning, Substitutes, Reprints, "Yttrium barium cuprates, Oxygen transport.
The structural determination of $\mathrm{Ba} 2 \mathrm{YCu} 3 \mathrm{O}(\mathrm{x})$ for 6.8 or $<x=$ or $<7.0$ was completed in several labora tories by Rietveld analysis of powder neutron diffraction data. The neutron diffraction experiments confirmed the space group Pmmm and the main structura features found by $x$-rays by Siegrest et al., but revealed that some of the oxygen assignments made in the $x$ ray studies were not entirely correct. The refined structural parameters obtained in four of these neutron diffraction analyses are given and the structure of Ba2YCu307.0 is schematically illustrated where it is compared with the parent structure of perovskite. Data collected at low temperatures revealed that no phase transitions take place in going from room temperature down to $5 \mathrm{~K}$

04,538

PB95-140042 Not available NTIS

National Inst. of Standards and Technology (IMSE),

Gaithersburg, MD. Reactor Radiation Div.

Neutron Powder Diffraction Study of the Structures of La1.9Ca1.1Cu2O6 and La1.9Sr1.1Cu2O6+Delta. Final rept.

A. Santoro, F. Beech, and R. J. Cava. 1990, 6p Pub. in Materials Research Society Symposia Proceedings 166, p187-1921990.

Keywords: “Crystal structure, Tetragonal lattices, Lattice parameters, Neutron diffraction, Superconductors, Reprints, "Lanthanum calcium c.uprates, "Lanthanum strontium cuprates, Reitveld method, Powder diffraction.

The title compounds are structurally isomorphous and crystallize with the symmetry of space group $14 / \mathrm{mmm}$. The lattice parameters are $\mathrm{a}=3.8248(1), \mathrm{c}=$ 19.4286(5)A for $\mathrm{La}(1.9) \mathrm{Ca}(1.1) \mathrm{Cu} 2 \mathrm{O} 6$ and $\mathrm{a}$ $3.8601(1), \mathrm{C}=19.9994(5) \mathrm{A}$ for $\mathrm{La}(1.9) \mathrm{Sr}(1.1) \mathrm{Cu} 2 \mathrm{O}(6$ + delta). The structure can be easily derived from that of $\mathrm{La} \mathrm{CuO} 4$ by substituting the layers of composition CuO2 present in La2CuO4 with a block of three layers having composition and sequence (CuO2) - (R) (CuO2), where $\mathrm{R}$ is $\mathrm{Ca}, \mathrm{Sr}$, and/or La. Although the general structural configuration is the same for the $\mathrm{Ca}$ and the $\mathrm{Sr}$ compounds, the distribution of the metal atoms is different in the two cases.

\section{4,539}

PB95-140067 Not available NTIS

National Inst. of Standards and Technology (MSEL), Gaithersburg, MD. Reactor Radiation Div.

Normal Modes and Structure Factor for a Canted Spin System: The Generalized Villain Model.

Final rept.

W. M. Saslow, and R. W. Erwin. 1992, 10p

Pub. in Physical Review B 45, n9 p4759-4768, 1 Mar 92

Keywords: Structure factors, Heisenberg model, Antiferromagnetism, Ferromagnetism, Spin waves, Hamiltonians, Reprints, “Villain model, Normal modes.

The generalized Villain model has a rich variety of pos sible structures, ranging from a pure ferromagnet to a pure antiferromagnet. In between these extremes, the system is canted. Using a Heisenberg exchange Hamiltonian and linearized spin-wave theory, we have studied the normal modes and structure factors for this model. The exchange ratio $\mathrm{J} / \mathrm{J}$ and the external field have both been varied. The effect of canted spin structures on the modes and structure factors is readily seen. We believe that it is now a practical matter to study the normal modes and structure factors for more complicated systems described by this type of model.

04,540

PB95-140471 Not available NTIS

National Inst. of Standards and Technology (MSEL) Gaithersburg, MD. Ceramics Div.

Study of the Hydroxycarbonate Precursor Route to the YBa2Cu3O7-x High Tc Superconductor.

Final rept.

J. J. Ritter, and J. F. Kelly. 1989, 8p.

Pub. in Proceedings of Symposium on High Tempera

ture Superconducting Compounds: Processing and Related Properties, Las Vegas, NV., February 27-28, 1989, p303-310.

Keywords: "YBCO superconductors, "Precipitation(Chemistry), "Precursors, High temperature superconductors, Chemical composition, Synthesis(Chemistry), Particle size, Polycrystalline, Reprints, *Yttrium barium cuprates, Hydroxycarbonates.

The precipitation of the mixed hydroxycarbonates of $\mathrm{Ba}, \mathrm{Y}$ and $\mathrm{Cu}$ as precursors to the $\mathrm{YBa} 2 \mathrm{Cu} 3 \mathrm{O}(7-\mathrm{x})$ high $T(c)$ superconductor is an attractive route to this material. However, studies in our laboratory indicate that powder homogeneity is strongly dependent upon ionic concentrations and system $\mathrm{pH}$. Moreover, we have assessed the composition of the precipitated moieties. These data have been incorporated into controlling the precipitation process in order to optimize particle size and powder homogeneity. Further processing of these powders has yielded polycrystalline solids with sharp transitions near $95 \mathrm{~K}$ and high transport current densities.

04,541

PB95-140505 Not available NTIS

National Inst. of Standards and Technology (CSTL), Gaithersburg, MD. Surface and Microanalysis Science Div.

Substitution-Induced Midgap States in the Mixed Oxides RxBa1-ChiTiO3-Delta, with $R=Y$, La, and Nd.

Final rept.

S. W. Robey, L. T. Hudson, C. Eylem, and B

Eichorn. 1993, 7p

Pub. in Physical Review B 48, n1 p562-568, 1 Jul 93.

Keywords: "Electronic structure, "Band theory, Photoelectron spectroscopy, Ultraviolet spectroscopy, Barium titanates, Binding energy, Substitutes, Reprints, "Yttrium barium titanates, *Lanthanum barium titanates, "Neodymium barium titanates, Metal insulator transition, Mixed oxides.

Changes induced in the electronic structure of $\mathrm{BaTjO} 3$ by substitutions of $R=Y$, $L a$, or $N d$ for $B a$ to form the mixed oxides, $\mathrm{R}(\mathrm{x}) \mathrm{Ba}(1-x) \mathrm{TiO}(3-$ delta) have been investigated using ultraviolet photoelectron spectroscopy. Substitution of formally $\mathrm{R}(3+)$ ions for $\mathrm{Ba}(2+)$ leads to the introduction of filled states in the band gap that are shown by resonant-photoemission measurements to have significant $\mathrm{Ti} 3 \mathrm{~d}$ character, consistent with a Mott-Hubbard-insulator description for these oxides. Various contributions to the binding energy and width of these states are considered. It is suggested that the dominant factor is electron-electron correlation and this leads to the estimates $U$ approx equals Delta approx equals $3 \mathrm{eV}$ for this system, where $U$ is the correlation energy for the $3 \mathrm{~d}$ electrons and Delta is the charge-transfer energy.

04,542

PB95-140513 Not available NTIS

National Inst. of Standards and Technology (MSEL), Gaithersburg, MD. Reactor Radiation Div. Incommensurate Magnetic Order in UPtGe. Final rept.

R. A. Robinson, A. C. Lawson, J. W. Lynn, and K. H J. Buschow. 1993, 4p.

Sponsored by Department of Energy, Washington, DC. Pub. in Physical Review B 47, n10 p6138-6141, 1 Mar 93.

Keywords: Orthorhombic lattices, Platinum intermetallics, Ternary compounds, Neel temperature, Neutron diffraction, Magnetic moments, Reprints, *Uranium platinum germanides, *Magnetic ordering, Uranium intermetallics, Germanium intermetallics, Powder diffraction.

The orthorhombic uranium (1:1:1) ternary compound UPtGe has been studied by means of neutron powder diffraction, using both the time-of-flight method at a spallation source and the constant-wavelength reactor method. The material exhibits incommensurate magnetic order with $q=(0,0.5543,0)$, with a uranium moment at the lowest temperature of 1.11 mu(sub B) and a Neel temperature of $51 \mathrm{~K}$. The moments are confined to the bc plane, which includes the propagation vector for the incommensurate modulation, and they rotate within that plane as one moves parallel to the $b$ axis. The magnitude of the ordered uranium moment is the only order parameter in the problem.

04,543

PB95-140521 Not available NTIS

National Inst. of Standards and Technology (MSEL), Gaithersburg, MD. Reactor Radiation Div.

Crystallographic and Magnetic Properties of UAUSn.

Final rept.

R. A. Robinson, J. W. Lynn, V. Nunez, A. C. Lawson, K. H. J. Buschow, and H. Nakotte. 1993, 5p.

Pub. in Physical Review B 47, n9 p5090-5094, 1 Mar 93.

Keywords: *Crystal structure, Antiferromagnetic materials, Gold intermetallics, Tin intermetallics, Neutron 


\section{PHYSICS}

\section{Solid State Physics}

diffraction, Magnetic properties, Reprints, *Uranium gold stannides, Uranium intermetallics, Powder diffraction, Uranium palladium stannides, Uranium copper stannides.

The uranium intermetallic compound UAuSn has been studied by means of neutron powder diffraction. It crystallizes in the Caln2 structure type, with space group $\mathrm{P} 6(3) / \mathrm{mmc}$, in agreement with previous $\mathrm{x}$-ray work Below $37 \mathrm{~K}$, it exhibits long-range antiferromagnetic order, in a double-sized orthohexagonal magnetic unit cell. At the lowest temperature measured, the uranium moment is $1.10 \mathrm{mu}(\mathrm{sub} B$ ). The magnetic structure is collinear with moments parallel to the orthorhombic $b$ axis and the magnetic space group is $C(p) m^{\prime} \mathrm{cm}^{\prime}$. We discuss the $\mathrm{Au} / \mathrm{Sn}$ disorder in terms of metallic radil and discuss the consequences of this symmetry on the collinearity of the magnetic structure. These results are compared with work on the isostructura! compounds UPdSn and UCuSn.

\section{4,544}

PB95-140539 Not available NTIS

National Inst. of Standards and Technology (EEEL), Boulder, CO. Electromagnetic Technology Div.

Influence of Deposition Parameters on Properties of Laser Ablated YBa2Cu307-Delta Films.

Final rept.

A. Roshko, D. A. Rudman, L. R. Vale, H. Beck, L. F.

Goodrich, and J. Moreland. 1993, 4p.

Pub. in Institute of Electrical and Electronics Engineers Transactions on Applied Superconductivity $3, n 1$ p1590-1593 Mar 93

Keywords: "YBCO superconductors, *Superconducting films, "Laser ablation, High temperature superconductors, Scanning tunneling microscopy, Temperature dependence, Transition tempera*Yttrium barium cuprates, Lanthanum aluminates.

YBa2Cu3O(7-delta) films have been laser ablated under a variety of conditions, onto four different substrate materials. Using scanning tunneling microscopy it was observed that the films grow by an island nucleation and growth mechanism. The properties of the films were siudied as functions of the deposition conditions. Films on LaAlO3 had the best and most reproducible properties. The superconducting transition temperatures of films deposited on LaAlO3 proved to be fairly insensitive to the substrate temperature during deposition; $T$ (c) $>90 \mathrm{~K}$ were obtained for films deposited over a temperature range from 760 to $850 \mathrm{C}$. The oxygen partial pressure during the deposition had a large effect on the transition temperature; the highest $\mathrm{T}$ (c)s were obtained for films deposited in $26.7 \mathrm{~Pa}$ (200 mTorr) ox ygen.

\section{4,545}

PB95-140547 Not available NTIS

National Inst. of Standards and Technology (MSEL) Gaithersburg, MD. Reactor Radiation Div. PrBa2Cu306+x.

Final rept.

N. Rosov, J. W. Lynn, G. Cao, J. E. Crow, J. W.

O'Reilly, and P. Pernambuco-Wise. 1992, 8p.

Pub. in Physica C 204, p171-178 1992.

Keywords: Neel temperature, Neutron diffraction, Sin gle crystals, Antiferromagnetic materials, Copper, Reprints, "Praseodymium barium cuprates, *Magnetic ordering.

Neutron diffraction has been employed to investigate the magnetic ordering of the Cu spins in a single crystal of PrBa2Cu3O $(6+x)$. At small $x$ we have observed a Neel temperature $T(N)$ of $370 \mathrm{~K}$, where both the chain and plane $\mathrm{Cu}$ spins order simultaneously. For $\mathrm{x}$ approx 0.6 , the Cu planes still order at $\mathrm{T}(\mathrm{N})$ about $370 \mathrm{~K}$; how ever, the ordering temperature of the chain spins has decreased to about $160 \mathrm{~K}$. The observed intensities of the magnetic reflections cannot be explained by keep ing the chain layer $\mathrm{Cu}$ spin direction in the ab plane rather the chain moments must have a component along the c direction. We discuss these results in light of recent optical reflectivity measurements on PrBa2Cu3O $(6+x)$ by Takenaka et al. (Phys. Rev. B 46 (1992) 5833)

\section{4,546}

\section{PB95-140554 Not available NTIS}

National Inst. of Standards and Technology (MSEL), Gaithersburg, MD. Reactor Radiation Div
Crystal Structure and Magnetic Ordering of the Rare-Earth and $\mathrm{Cu}$
$\mathrm{RBa} 2 \mathrm{Cu} 2 \mathrm{NbO}$ ( $\mathrm{R}=\mathrm{Nd}, \mathrm{Pr})$.

Final rept.

N. Rosov, J. W. Lynn, H. B. Radousky, P. Klavins, R. N. Shelton, M. Bennahmias, and T. J. Goodwin.

1993, 9p.

Pub. in Physical Review B 47, n22 p15 256-15 265, 1 Jun 93.

Keywords: "Crystal structure, Neutron diffraction, Magnetic moments, Reprints, "Neodymium barium copper niobates, "Praseodymium barium copper niobates, "Magnetic ordering

Using neutron diffraction, we have studied the crystal structure and magnetic ordering of the rare-earth and $\mathrm{Cu}$ moments in the layered perovskite systems NdBa2Cu2NbO8 (Nd 2:2:1:8) and PrBa2Cu2NbO8 (Pr $2: 2: 1: 8)$. These are systems similar to $R$ $\mathrm{Ba} 2 \mathrm{Cu} 3 \mathrm{O}(6+\mathrm{X})(\mathrm{R}$ 1:2:3:(6+X)) for which the chain $\mathrm{Cu}$ $\mathrm{O}$ layers have been fully replaced by layers of $\mathrm{Nb}-\mathrm{O}$ octahedra. Powder profile refinements below $20 \mathrm{~K}$ show that both $N d 2: 2: 1: 8$ and $\operatorname{Pr} 2: 2: 1: 8$ are in space group $14 / \mathrm{mcm}$, because the near-neighbor $\mathrm{Nb}-\mathrm{O}$ octahedra are rotated about the $c$ axis in opposite senses. The $\mathrm{Cu}$ moments are ordered below 375(10) $\mathrm{K}$ for Nd 2:2:1:8 and $340(15) \mathrm{K}$ for $\operatorname{Pr} 2: 2: 1: 8$, with saturation moments in both cases of $0.5(1)$ mu(sub B). The near-neighbor $\mathrm{Cu}$ spins are aligned antiparallel, just as for the Cu 'plane' ordering in $R$ 1:2:3: $(6+X)$.

\section{4,547}

PB95-140562 Not available NTIS

National Inst. of Standards and Technology (MSEL), Gaithersburg, MD. Reactor Radiation Div.

Quasielastic and Inelastic Neutron-Scattering Studies of ((CD3)3ND)FeCI3.2D2O: A One-Dimensional Ising Ferromagnet.

Final rept.

N. Rosov, J. W. Lynn, J. J. M. Williams, and C. P

Landee. 1993, 3p.

Pub. in Jnl. of Applied Physics 73, n10 p6081-6083, 15 May 93.

Keywords: Orthorhombic lattices, Cryogenic temperature, Hydrogen bonds, Deuterium compounds, Iron compounds, Neutron scattering, Inelastic scattering, one dimensional, Hydrates, Reprints, "Ferrous trimethylammonium chloride, Ising ferromagnets.

Ferrous trimethylammonium chloride (FeTAC) is a member of a family of compounds that consist of chains of bihalide-bridged metal ions aligned along the $b$ axis of the orthorhombic unit cell. FeTAC orders three dimensionally below $T(N)=3.16 \mathrm{~K}$, with the moments, which are canted from the $\mathrm{b}$ axis by approx 20 deg, coupled ferromagneticality along the $b$ axis and staggered along the (left bracket) 101 (right bracket) direction. The order parameter follows the exact Onsager solution for a rectangular 2D Ising lattice below $T / T(N)$ approx 0.95 . However, quasielastic scattering measurements in the vicinity of $T(N)$ show that the correlations do not evolve as do those of the $2 \mathrm{D}$ Ising antiferromagnets ErBa2Cu307 and $\mathrm{K} 2 \mathrm{CoF} 4$. Above $T(N)$, magnetic excitations along the $b$ axis that follow the dispersion relation of a $1 \mathrm{D}$ ferromagnet with a gap of 3.7 meV have been observed.

\section{4,548}

PB95-140810 Not available NTIS

National Inst. of Standards and Technology (EEEL),

Boulder, CO. Electromagnetic Technology Div.

Thermally Activated Hopping of a Single Abrikosov Vortex.

Final rept.

S. C. Sanders, J. Sok, D. K. Finnemore, and Q. Li.

1993, 5p.

Pub. in Physical Review B 47, n14 p8996-9000, 1 Apr

Keywords: Joseph junctions, Lead(Metal), Metal films, Thin films, Flux pinning, Superconductors, Reprints, "Abrikosov vortices, Thermal activation.

Thermally activated hopping of a single Abrikosov vortex has been investigated for a thin $\mathrm{Pb}$ film that was decorated with an artificial pinning structure. To determine the location of the vortex, the $\mathrm{Pb}$ film is fabricated to be one electrode of a cross-strip superconductor/ normal-metal/insulator/superconductor (SNIS') Josephson junction. Distortions in the Fraunhofer pattern specify the vortex location. As the temperature is raised toward $T(c)$, the vortex depins from the artificial pinning site and reproducibly moves through the same sequence of other pinning sites before it leaves the function area of the $\mathrm{Pb}$ film. The first thermal depinning occurs when the order parameter of the bulk
superconductor is about $20 \%$ of the $T=O$ value. The trajectory is not random.

04,549

PB95-141016 Not available NTIS

National Inst. of Standards and Technology (NEL), Boulder, CO. Electromagnetic Technology Div.

Critical Current Behavior of Ag-Coated YBa2Cu307-x Thin Films.

Final rept.

R. H. Ono, J. A. Beall, T. E. Harvey, M. W. Cromar L. F. Goodrich, J. Moreland, A. Roshko, T. C. Stauffer, C. D. Reintsema, and M. Johansson. 1991, $4 \mathrm{p}$.

Pub. in Institute of Electrical and Electronics Engineers Transactions on Magnetics 27, n2 p1471-1474 Mar 91. Keywords: $\quad$ "YBCO superconductors,

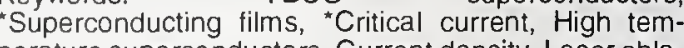
perature superconductors, Current density, Laser ablation, Metal films, Thin films, Interfaces, Silver, Reprints, *Yttrium barium cuprates, Bilayers.

We have studied the behavior of high-quality YBa2Cu3O(7-delta) (YBCO) thin films with $\mathrm{Ag}$ overlayers. In some cases, the $\mathrm{Ag}$ was diffused into the high-T(c) film post-annealing. We chose to study $\mathrm{Ag}$ in detail because of its widespread use as contact metallization and our earlier studies of proximity effects in YBCO. The details of transport critical current measurements are presented. The $\mathrm{Ag}$ coatings can reduce normal state resistance while not degrading $J(c)$.

04,550

PB95-141024 Not available NTIS

National Inst. of Standards and Technology (NEL) Boulder, CO. Electromagnetic Technology Div.

Magnetic Field Dependence of the Critical Current Anisotropy in Normal Metal-YBa2Cu307-delta Thin Film Bilayers.

Final rept.

R. H. Ono, L. F. Goodrich, J. A. Beall, M. E

Johansson, and C. R. Reintsema 1991, 3p.

Pub. in Applied Physics Letters 58, n11 p1205-1207 1991.

Keywords: "YBCO superconductors, "Critical current, *Superconducting films, High temperature superconductors, Magnetic fields, Current density, Metal films, Thin films, Anisotropy, Laser ablation, Interfaces, Silver, Reprints, “Yttrium barium cuprates, Bilayers.

We have measured the transport critical current density $(\mathrm{J}(\mathrm{c})$ ) in epitaxial-quality films of $\mathrm{YBa} 2 \mathrm{Cu} 3 \mathrm{O}(7$ delta) which were covered by thin $(10 \mathrm{~nm}) \mathrm{Ag}$ films The films, both with and without $\mathrm{Ag}$, had $\mathrm{J}(\mathrm{C})$ values greater than 10 (exp 6) $\mathrm{A} / \mathrm{sq} \mathrm{cm}$ in liquid nitrogen. The effect of the $\mathrm{Ag}$ was to greatly reduce the dependence of $\mathrm{J}(\mathrm{c})$ on external magnetic fields, but only in the case where the field was oriented in the plane of the film perpendicular to the $\mathrm{c}$ axis. It is unlikely that the effect is simply due to altered surface pinning, although qualitative agreement with critical state models is observed.

\section{4,551}

PB95-141065 Not available NTIS

National Inst. of Standards and Technology (EEEL),

Boulder, CO. Electromagnetic Technology Div.

Micromagnetic Model of Dual-Layer Magnetic-Recording Thin Films.

Final rept.

J. O. Oti. 1993, $11 p$

Pub. in Institute of Electrical and Electronics Engineers Transactions on Magnetics 29, n2 p1265-1275 Mar 93.

Keywords: *Magnetic films, Magnetic recording, Magnetic anisotropy, Thin films, Magnetostatics, Cobalt alloys, Reprints, Micromagnetic models, Exchange interactions.

A micromagnetic model of dual-layer magnetic-recording thin films is described. The model, which is capable of simulating magnetic layers having different magnetic and geometric properties, is applied to the study of the magnetic properties of dual-layer media characterized by a three-dimensional isotropic distribution of anisotropy axes in both layers, using parameters typical of cobalt-alloy films. In the absence of exchange interactions between the layers, a correlation is found between squareness ratios, average magnetostatic energy densities, and structural dimensions of the media. An in-phase magnetization reversal of the layers is found to occur with increasing interlayer exchange 
coupling. A complex relationship is found between coercivity and media parameters.

04,552

PB95-141073 Not available NTIS

National Inst. of Standards and Technology (EEEL) Boulder, CO. Electromagnetic Technology Div. Micromagnetic Simulations of Tunneling Stabilized Magnetic Force Microscopy.

Final rept

J. O. Oti, and P. Rice, 1993, 3p.

Pub. in Jnl. of Applied Physics 73, n10 p5802-5804, 15 May 93

Keywords: Electron tunneling, Magnetic films, Simulation, Images, Reprints, "Magnetic force microscopy, "Garnet films, Micromagnetics.

A micromagnetic model simulating the images produced by tunneling stabilized magnetic force microscopy is described. The images are related to the force interactions between the fringing fields from the imaged surface and stray fields from the sensing probe. The model, which allows for variations in the magnetization states of the probe, is, used to examine the dependence of the interaction forces on varying probefilm separations for a magnetic garnet film. The results show that the images are sensitive to separation and that the changing probe magnetization plays an important role in determining the final image.

04,553

PB95-141081 Not available NTIS

National Inst. of Standards and Technology (EEEL)

Boulder, CO. Electromagnetic Technology Div.

Experimental Verification of a Micromagnetic Model of Dual-Layer Magnetic Films.

Final rept.

J. O. Oti, and S. E. Russek. 1993, 3p.

Pub. in Jnl. of Applied Physics 73, n10 p5845-5847 15 May 93

Keywords: "Magnetic films, Thin films, Cobalt alloys, Nickel alloys, Magnetostatics, Chromium, Reprints, *Micromagnetic models, Exchange interactions, Multilayers.

A micromagnetic model has been used to characterize the magnetic properties of dual-layer magnetic films. The model calculations, with experimentally deter mined input parameters, have been compared with measurements on fabricated Co alloy dual-layer films Calculations are done with a more general version of a previous micromagnetic model, modified to allow the variation of certain media parameters which were previously held constant. Each of the experimental media consists of a bilayer of 30-nm-thick Co(0.75) $\mathrm{Ni}(0.25)$ magnetic films separated by a $\mathrm{Cr}$ decoupling layer. The calculations predict a split in the coercivities of the layers for small $\mathrm{Cr}$ thicknesses which is observed experimentally. The model correlates an observed increase in media squareness ratio and coercivity, as the $\mathrm{Cr}$ thickness is increased, with diminishing exchange and magnetostatic interactions between the magnetic layers.

\section{4,554}

PB95-141149 Not available NTIS

National Inst. of Standards and Technology (NEL) Boulder, CO. Electromagnetic Technology Div. Trapped Vortices in a Superconducting Microbridge.

Final rept

G. S. Park, C. E. Cunningham, B. Cabrera, and M.

E. Huber. 1991, 4p

Sponsored by Stanford Univ., CA. Dept. of Physics.

$P u b$. in Institute of Electrical and Electronics Engineers Transactions on Magnetics 27, n2 p3021-3024 1991. Keywords: Frequency dependence, Optical switching, Noise reduction, SQUID devices, Laser radiation, Ligh pulses, Magnetometers, Niobium, Reprints, "Flux vortices, "Flux trapping, Microbridges.

We are experimenting with optical switches for use in a noise reduction device for SQUID magnetometers. Laser light pulsed onto a Nb microbridge drives it momentarily normal and changes the quantum flux state of a superconducting circuit. For certain laser pulse parameters, a vortex is sometimes trapped in the microbridge and is detected by a SQUID coupled to the circuit. The trapping frequency and vortex position were studied using various waveforms.
National Inst. of Standards and Technology (NML) Gaithersburg, MD. Surface Science Div.

Variation in Magnetic Properties of Cu/Fcc (001) Sandwich Structures.

Final rept.

W. Schwarzacher, J. Penfold, I. Jacob, R. F. Willis W. Allison, R. C. Ward, and W. F. Egelhoff. 1989,

$4 p$.

Pub. in Solid State Communications 71, n7 p563-566 1989

Keywords: Polarization(Spin alignment), Magnetic moments, Fcc lattices, Sandwich structures, Magnetization, Copper, Magnetic films, Metal films, Thin films, Epitaxy, Reprints, *Ion films, Ultrathin films, Neutron reflection.

Spin-polarized neutron reflection is used to determine the magnetization of ultrathin epitaxial fcc Fe films sandwiches by $\mathrm{Cu}(001)$. A $3.0 \mathrm{ML}$ film had a measured average magnetic dipole moment per atom of 1.9 + or - 0.6 Bohr magnetons/atom which is the highest value yet observed for an fcc Fe film and is consistent with the high spin-moment phase predicted for bulk fcc iron. This state appears to be unstable since increasing the film thickness or using a higher growth temperature leads to a considerable decrease in the observed moment

\section{4,556}

PB95-150017 Not available NTIS

National Inst. of Standards and Technology (PL), Gaithersburg, MD. Electron and Optical Physics Div. Effects of Interfacial Roughness on the
Magnetoresistance of Magnetic Metallic Magnetoresist

Final rep

R. Q. Hood, L. M. Falicov, and D. R. Penn. 1994, $10 \mathrm{p}$

Contract DE-AC03-76SF00098

Sponsored by Department of Energy, Washington, DC Pub. in Physical Review B 49, n1 p368-377, 1 Jan 94

Keywords: "Magnetoresistance, "Superlattices, Boltzmann equation, Chromium, Copper, Iron, Re prints, "Interface roughness, Magnetic multilayers.

The Boltzmann equation is solved for a system consisting of a ferromagnetic-normal-metallic multilayer. The in-plane magnetoresistance of $\mathrm{Fe} / \mathrm{Cr}$ and $\mathrm{Fe} / \mathrm{Cu}$ superlattices is calculated for (1) varying interfacial geometric random roughness with no lateral coherence, (2) correlated (quasiperiodic) roughness, and (3) varying chemical composition of the interfaces. The interplay between these three aspects of the interfaces may enhance or suppress the magnetoresistance, depending on whether it increases or decreases the asymmetry in the spin-dependent scattering of the conduction electrons. Properties of the interfaces relevant to the giant negative magnetoresistance are discussed.

\section{4,557}

\section{PB95-150074 Not available NTIS}

National Inst. of Standards and Technology (MSEL), Gaithersburg, MD. Reactor Radiation Div.

Magnetic Neutron Scattering (Invited).

Final rept.

J. W. Lynn. 1994, 5p.

Pub. in Jnl. of Applied Physics 75, n10 p6806-6810, 15 May 94

Keywords: "Neutron scattering, "Magnetism, Neutron spectrometers, Inelastic scattering, Order parameters, Polarized beams, Critical point, Form factors, Uses Reprints, Spin dynamics.

The application of neutron scattering techniques to magnetic problems is reviewed. We will first discuss diffraction techniques used to solve magnetic structures, as well as to measure magnetic form factors, order parameters, critical phenomena, and the scattering from low-dimensional systems. We will also discuss inelastic scattering techniques, including polarized beam methods, used to determine the spin dynamics of various materials. Information will be provided about the types of spectrometers available at the user-oriented national facilities located at Argonne National Laboratory, Brookhaven National Laboratory, Los Alamos National Laboratory, The National Institute of Standards and Technology, and Oak Ridge National Laboratory, as well as the spectrometers at the Missouri University Research Reactor.

04,558

PB95-150082 Not available NTIS
National Inst. of Standards and Technology (MSEL), Gaithersburg, MD. Reactor Radiation Div.

Polarization Analysis of the Magnetic Excitations in Fe65Ni35 Invar.

Final rept.

J. W. Lynn, N. Rosov, M. Acet, and H. Bach. 1994,

$3 p$.

Pub. in Jnl. of Applied Physics 75, n10 p6069-6071, 15 May 94.

Keywords: "Invar, Iron alloys, Nickel containing alloys, Polarization characteristics, Polarized beams, Neutron beams, Spin waves, Temperature dependence, Single crystals, Amorphous materials, Comparative evaluations, Reprints, "Magnetic excitations.

Triple-axis inelastic polarized neutron measurements have been performed as a function of temperature on a single crystal of the Invar alloy $\mathrm{Fe}(65) \mathrm{Ni}(35)$ to distinguish longitudinal from transverse magnetic excitations in the magnetically ordered phase. Well below the Curie temperature of $501 \mathrm{~K}$ the magnetic excitation spectrum is dominated by conventional transverse spin-wave excitations, which in fact follow the predictions of spin-wave theory very well. In particular, we find no evidence for propagating longitudinal excitations in this system, ir sharp contrast to the behavior observed in the amorphous Invar $\mathrm{Fe}(86) \mathrm{B}(14)$ material as well as the non-Invar amorphous system $\mathrm{Fe}(40) \mathrm{Ni}(40) \mathrm{P}(14) \mathrm{B}(6)$.

04,559

PB95-150116 Not available NTIS

National Inst. of Standards and Technology (EEEL),

Gaithersburg, MD. Electricity Div.

Superconducting Energy Gap of Bulk UBe13.

Final rept.

J. Moreland, A. F. Clark, R. J. Soulen, and J. L.

Smith. 1994, 2p

Pub. in Physica B 194-196, p1727-1728 1994

Keywords: "Energy gap, "Superconductors, Superconducting junctions, Temperature dependence, BCS theory, Reprints, "Uranium beryllium, Point contacts, Uranium intermetallics, Beryllium intermetallics.

The superconducting energy gap, Delta, of bulk UBe13 was measured as a function of temperature. Junctions were made by breaking a narrow region of a specimen in a vacuum and then repositioning the broken ends to form a mechanically adjustable break junction point contact. We concluded that the 2 Delta(O)/ $/(B) T(C)=$ 4.2 by fitting the data to the BCS form, assuming a T(C) of $0.80 \mathrm{~K}$

04,560

PB95-150157 Not available NTIS

National Inst. of Standards and Technology (EEEL) Gaithersburg, MD. Semiconductor Electronics Div Spectroscopic Ellipsometry Determination of the Properties of the Thin Underlying Strained Si Layer and the Roughness at SiO2/Si Interface.

Final rept.

N. V. Nguyen, D. Chandier-Horowitz, P. M.

Amirtharaj, and J. G. Pellegrino. 1994, 3p.

Pub. in Applied Physics Letters 64, n20 p2688-2690, 16 May 94

Keywords: "Silicon dioxide, "Oxide films, Dielectric films, Ellipsometry, Substrates, Strains, Silicon, Reprints, "Interface roughness.

The existence of both the strain and microroughness at the interface of thermally grown SiO2 films on Si was ascertained unambiguously for the first time by high accuracy spectroscopic ellipsometry. The dielectric function of the interface was determined by a comprehensive data analysis procedure. By carefully examining the dielectric function obtained by our model, the strain was seen to cause a red shift of $0.042 \mathrm{eV}$ of the interband critical point $E(1)$ compared with the bulk silicon value. The thickness of the interface region was found to be $2.2 \mathrm{~nm}$ of which a significant part is due to the strain.

\section{4,56}

\section{PB95-150165 Not available NTIS}

National Inst. of Standards and Technology (CSTL), Gaithersburg, MD. Surface and Microanalysis Science Div.

Spot-Profile-Analyzing LEED Study of the Epitaxial Growth of $\mathrm{Fe}, \mathrm{Co}$, and $\mathrm{Cu}$ on $\mathrm{Cu}(100)$.

Final rept.

G. L. Nyberg, M. T. Kief, and W. F. Egelhoff. 1993,

$11 \mathrm{p}$

Pub. in Physical Review B 48, n19 p14 509-14 519

15 Nov 93 


\section{Solid State Physics}

Keywords: "Epitaxial growth, "Cobalt, "Copper, "Iron, Temperature range 0065-0273 $\mathrm{K}$, Temperature range 0273-0400 K, Temperature dependence, Thermal diffusion, Metal films, Thin films, Microstructure, Substrates, Reprints, Low energy electron diffraction, Henzler rings, Ultrathin films.

The structure of epitaxial films of $\mathrm{Fe}, \mathrm{Co}$, and $\mathrm{Cu}$ grown at $80-300 \mathrm{~K}$ on $\mathrm{Cu}(100)$ has been investigated using a spot-profile-analyzing low-energy electron-diffraction (LEED) instrument. In all three systems rings appear around the substrate LEED spots, although the rings differ in intensity and in diameter depending upon the variables of film thickness and deposition temperature. Rings of this type have been studied extensively by Henzler et al and correlated with the mean separation between islands. Much can be inferred about the growth mechanism through a study of these Henzler rings. The rings contract radially with increasing deposition temperature or with increasing annealing temperature as thermally activated diffusion permits the formation of larger islands with greater distances between them. The value of the mean separation suggests that upon consideration these atoms do not come to rest at the immediate site of impact but instead experience some very transient type of mobility associated with the impact and accommodation process.

\section{4,562}

PB95-150181 Not available NTIS

National Inst. of Standards and Technology (PL), Gaithersburg, MD. Electron and Optical Physics Div. Influence of $\mathrm{Cr}$ Growth on Exchange Coupling in $\mathrm{Fe} / \mathrm{Cr} / \mathrm{Fe}(100)$

Final rept.

D. T. Pierce, J. A. Stroscio, J. Unguris, and R. J

Celotta. 1994, 90

Sponsored by Office of Naval Research, Arlington, VA.

Pub. in Physical Review B 49, n20 p14 564-14 572 . 15 May 94

Keywords: "Epitaxial growth, "Chromium, "Iron, Scanning electron microscopy, Scanning tunneling microscopy, Polarization characteristics, Temperature range 0273-0400 K, Exchange interactions, Metal films, Magnetization, Substrates, Reprints, Magnetic multilayers, Exchange coupling.

Scanning electron microscopy with polarization-analysis (SEMPA) measurements of the dependence of the oscillations of the exchange coupling in $\mathrm{Fe} / \mathrm{Cr} / \mathrm{Fe}(100)$ structures on the $\mathrm{Cr}$ growth temperature are correlated with the thickness fluctuations in $\mathrm{Cr}$ films measured by scanning tunneling microscopy (STM) at similar growth temperatures. Layer-by-layer growth was observed by STM for $\mathrm{Cr}$ deposition on very flat $\mathrm{Fe}(100)$ whiskers at deposition temperatures $=$ or $>300 \mathrm{C}$. The SEMPA measurements of the magnetization of the Fe overlayer as a function of $\mathrm{Cr}$ spacer-layer thickness at this temperature could be simulated well by oscillatory coupling with periods $2.105+$ or $-0.005 \mathrm{~d}$ and $12+$ or $1 d$, where $d$ is the layer spacing. Rougher $\mathrm{Cr}$ growth limited by diffusion kinetics, occurs at lower growth temperatures giving a distribution of thicknesses in the growth front as determined by STM. We modeled the Fe magnetization for lower-temperature $\mathrm{Cr}$ growth by assuming that the exchange coupling at each discrete $\mathrm{Cr}$ thickness is the same as found for layer-by-layer growth. Important characteristic length scales and the role of biquadratic coupling in the SEMPA measurements are addressed.

\section{4,563}

PB95-150223 Not available NTIS

National Inst. of Standards and Technology (MSEL), Gaithersburg, MD. Reactor Radiation Div.

Temperature Dependence of the Magnetic Excitations in Ordered and Disordered Fe72Pt28. Final rept.

N. Rosov, J. W. Lynn, J. Kastner, H. Bach, E. F. Wassermann, and T. Chattopadhyay. 1994, 3p. Pub. in Jnl. of Applied Physics 75, n10 p6072-6074,
15 May 94 .

Keywords: "Platinum containing alloys, "Iron alloys, Temperature dependence, Neutron scattering, Inelas tic scattering, Single crystals, Spin waves, Reprints, "Magnetic excitations.

We have performed inelastic neutron scattering meas urements of the long-wavelength spin wave excitations of both ordered and disordered $\mathrm{Fe}(72) \mathrm{Pt}(28)$ single crystals below their critical temperatures, $\mathrm{T}(\mathrm{C})=510$ and $375 \mathrm{~K}$, respectively. The spin waves followed the expected $E=D$ (q squared) dependence, and the temperature-dependent spin stiffness D decreased as $T$ /
$T(C)(\exp 5 / 2)$, as expected for an isotropic ferromagnet. The extrapolated zero-temperature spin stiffness was $D=98(4) \mathrm{meV}$ (A squared) and 107(1) me V (A squared) for the disordered and ordered alloy, respectively. These values are significantly higher than the zero-temperature stiffness as determined by magnetization measurements.

\section{4,564}

PB95-150272 Not available NTIS

National Inst. of Standards and Technology (PL) Gaithersburg, MD. Electron and Optical Physics Div. Nanostructure Fabrication via Direct Writing with Atoms Focused in Laser Fields.

Final rept.

R. E. Scholten, J. J. McClelland, E. C. Palm, A. Gavrin, and R. J. Celotta. 1994, 4p.

Grant NSF-PHY93-12572

Sponsored by National Science Foundation, Arlington, $\checkmark A$.

Pub. in Jnl. of Vacuum Science and Technology B 12 n3 p1847-1850 May/Jun 94.

Keywords: Scanning electron microscopy, Atomic force microscopy, Laser radiation, Fabrication, Lithogulation, "Nanostructures, Atom optics, Laser cooling

The techniques of atom optics can be applied during the deposition of atoms onto a surface to produce nanostructures. A laser is used to form a standing wave intensity pattern in front of the substrate. An atom beam, which has been collimated by optical means, is focused onto the substrate by dipole forces from the standing wave pattern so as to deposit a series of lines spaced by half the laser wavelength. We describe the fabrication of a $\mathrm{Cr}$ nanograting formed using this new technique. The experimental arrangement for deposition and the optical collimation of the atom beam are described. Scanning electron microscopy (SEM) and atomic force microscopy (AFM) images of the resulting nanostructures are presented.

\section{4,565}

PB95-150306 Not available NTIS

National Inst. of Standards and Technology (MSEL), Gaithersburg, MD. Ceramics Div.

Effect of a Crystal Monochromator on the Local Angular Divergence of an X-Ray Beam.

Final rept.

R. D. Spal. 1994, $7 p$

Pub. in Acta Crystallographica A50, p295-301 1994.

Keywords: "X-ray monochromators, "X-ray optics, $X$ ray diffraction, Synchrotron radiation, Incident radi-

The performance of an X-ray optical system often de pends critically on the local angular divergence of the $\mathrm{X}$-ray beam. For example, in systems for radiography, tomography and diffraction topography the angular divergence of the incident beam at a point in the sample determines the limiting spatial resolution. In this paper formulas are derived for the local divergence in the difracted beam of the non-dispersive asymmetric reflection double-flat-crystal monochromator, illuminated by synchrotron or characteristic radiation. The formulas are analyzed to determine the general behavior of the local divergence as a function of the asymmetry factors of the crystal refiections. For synchrotron radiation, one surprising conclusion is that the local divergence of the magnifying monochromator is always greater than that of the symmetric monochromator, significantly so for even moderate magnification factors

\section{4,566}

\section{PB95-150314 Not available NTIS}

National Inst. of Standards and Technology (PL). Gaithersburg, MD. Electron and Optical Physics Div. Exchange Coupling in Magnetic Heterostructures. Final rept.

M. D. Stiles. 1993, 21p

Pub. in Physical Review B 48, n10 p7238-7258, 1 Sep 93.

Keywords: Exchange interactions, Fermi surfaces, Oscillations, Reprints, “Magnetic heterostructures, Magnetic multilayers, Exchange coupling

Many structures consisting of magnetic layers separated by a nonmagnetic spacer layer show an oscillatory exchange coupling. This behavior is explained in terms of a simple model that shows that the Fermi surface of the spacer-layer material is responsible for the oscillatory coupling. The periods of the oscillatory coupling are set by extremal spanning vectors of the Fermi surface of the spacer-layer material. The strength of the coupling depends both on the geometry of the Fermi surface and on the reflection amplitudes for electrons scattering from the interfaces between the spacer layers and the magnetic layers. To test this and related models, the extremal spanning vectors and the associated Fermi-surface geometrical factors have been calculated for a large set of spacer-layer materials and interface orientations. These models are at least consistent with the experimental data. All measured oscillation periods are consistent with the calculated penods, but particularly for transition metals there are many more periods calculated than are seen experimentally.

\section{4,567}

PB95-150322 Not available NTIS

National Inst. of Standards and Technology (PL). Gaithersburg, MD. Electron and Optical Physics Div. Growth of Iron on Iron Whiskers.

Final rept.

J. A. Stroscio, and D. T. Pierce. $1994,6 \mathrm{p}$

Pub. in Jnl. of Vacuum Science and Technology B 12, n3 p1783-1788 May/Jun 94.

Keywords: "Epitaxial growth, *Iron, Whiskers(Single crystals), Scanning tunneling microscopy, Electron diffraction, Surface roughness, Surface diffusion, Temperature range $273-400 \mathrm{~K}$, Temperature range 400$1000 \mathrm{~K}$, Temperature dependence, Metal films, Thin films, Islands, Scaling, Reprints.

Real space views of the homoepitaxial growth of $\mathrm{Fe}$ on $\mathrm{Fe}(001)$ whiskers is reported, observed by scanning tunneling microscopy (STM) during the initial stages of growth. Scaling of the $\mathrm{Fe}$ island sizes and separation distribution are observed as a function of the diffusion rate to the deposition rate. A measure of the surface diffusion of the $\mathrm{Fe}$ atoms is obtained over the temperature range of 20-250 C from the temperature dependence of the island density. The effect of the diffusion kinetics is also observed in the surface morphology as a decrease in surface roughness with increasing temperature in thin Fe films. A comparison of real and reciprocal space techniques is obtained from a comparison of STM images and reflection-high-energy-electron-diffraction measurements during growth.

\section{4,568}

PB95-150330 Not available NTIS

National Inst. of Standards and Teshnology (PL), Gaithersburg, MD. Electron and Optical Physics Div. Scanning Tunneling Microscopy Study of the Growth of $\mathrm{Cr} / \mathrm{Fe}(001)$ : Correlation with Exchange Coupling of Magnetic Layers.

Final rept.

J. A. Stroscio, D. T. Pierce, J. Unguris, and R. J.

Celotta. 1994, 4p.

Sponsored by Office of Naval Research, Arlington, VA. Pub. in Jnl. of Vacuum Science and Technology B 12, n3 p1789-1792 May/Jun 94.

Keywords: "Epitaxial growth, *Chromium, Scanning tunneling microscopy, Whiskers(Single crystals), Temperature range 400-1000 K. Temperature dependence, Electron diffraction, Exchange interactions, Film thickness, Metal films, Substrates, Reprints, Magnetic multilayers, Exchange coupling.

Scanning tunneling microscopy (STM) and reflection high-energy electron diffraction (RHEED) were used to study the epitaxial growth of $\mathrm{Cr}$ on $\mathrm{Fe}(001)$ whiskers as a function of the $\mathrm{Fe}$ whisker temperature during growth. The STM images give real space views of the morphology of $\mathrm{Cr}$ growth, which can be correlated with the nature of the RHEED intensity oscillations. Layer by layer growth is found for $\mathrm{Cr}$ deposition on an $\mathrm{Fe}(001)$ surface at $300 \mathrm{C}$, and very rough growth, limited by diffusion kinetics, is observed at lower temperatures. The variation in the interlayer exchange coupling in $\mathrm{Fe} / \mathrm{Cr} / \mathrm{Fe}$ sandwiches as a function of the thickness of the $\mathrm{Cr}$ interlayer, which has been found to depend strongly on the growth temperature of the $\mathrm{Cr}$ interlayer, can be explained by the thickness fluctuations determined from the STM measurements of Cr films grown at different temperatures.

\section{4,569}

PB95-150371 Not available NTIS

National Inst. of Standards and Technology (PL) Gaithersburg, MD. Electron and Optical Physics Div. Oscillatory Exchange Coupling in $\mathrm{Fe} / \mathrm{Au} / \mathrm{Fe}(100)$ Final rept.

J. Unguris, R. J. Celotta, and D. T. Pierce. 1994, 3p. Sponsored by Office of Naval Research, Arlington, VA 
Pub. in Jnl. of Applied Physics 75, n10 p6437-6439, 15 May 94.

Keywords: *Epitaxial growth, "Gold, "Iron, Scanning electron microscopy, Polarization characteristics, Electron diffraction, Exchange interactions, Fermi surfaces, Metal films, Thin films, Substrates, Reprints, Magnetic multilayers, Exchange coupling.

Scanning electron microscopy with polarization analysis was used to investigate the interlayer exchange coupling in $\mathrm{Fe} / \mathrm{Au} / \mathrm{Fe}(100)$ sandwich structures. The films were epitaxially grown on single-crystal $\mathrm{Fe}(100)$ substrates. Electron diffraction measurements revealed that the Au spacer film grew with a surface reconstruction consistent with that observed for bulk $\mathrm{Au}$ crystals. The exchange coupling oscillates between primarily ferromagnetic and antiferromagnetic coupling for Au spacer layers up to 65 layers $(13 \mathrm{~nm})$ thick, but a significant biquadratic coupling component was also observed. The oscillatory coupling exhibited two components with periods of $2.48+$ or -0.05 layers $(0.506$ + or $-0.010 \mathrm{~nm})$ and $8.6+$ or -0.3 layers $(1.75+$ or $-0.06 \mathrm{~nm}$ ). The measured periods are in excellent agreement with those calculated from spanning vectors of the Au Fermi surface.

\section{4,570}

\section{PB95-150413 Not available NTIS}

National Inst. of Standards and Technology (EEEL), Gaithersburg, MD. Semiconductor Electronics Div. Band-to-Band Photoluminescence and Luminescence Excitation in Extremely Heavily CarbonDoped Epitaxial GaAs.

Final rept

L. Wang, N. M. Haegel, and J. R. Lowney. 1994,

10p.

Sponsored by National Science Foundation, Arlington, $\checkmark A$.

Pub. in Physical Review B 49, n16 p10 976-10 985, 15 Apr 94

Keywords: "Gallium arsenides, "Photoluminescence, "Band structure of solids, Carbon additions, Doped crystals, Energy gap, Fermi level, Emission spectra, rowing, Density of states

Heavily carbon-doped GaAs samples with doping levels as high as $4.1 \times 10(\exp 20) / c c$ were studied by photoluminescence and luminescence excitation spectroscopies. Luminescence and absorption band gaps were determined, from which the positions of the affect the observed emission spectra including diffraction and refraction of the luminescence, substrate effects, and lattice contraction in the carbon-doped fects, and lattice contraction in the carbon-doped
epilayer, were examined. A first-principles calculation was performed to calculate the density of states, the band gap, the Fermi energy, and the emission spectrum. Comparison of the calculation results with the experiments shows that the theoretical model we use is a good approximation for describing band structure for doping levels higher than $10(\exp 20) / \mathrm{cc}$. However, the discrepancy between the measured and the calculated emission spectra indicates that the usual assumptions such as constant momentum matrix elements and quasiequilibrium distribution of photoexcited electrons in the conduction band may not be appropriate in treating the transition processes in highly degenerate semiconductors.

\section{4,571}

\section{PB95-150512 Not available NTIS}

National Inst. of Standards and Technology (CAML), Gaithersburg, MD. Applied and Computational Mathematics Div.

Self-Avoiding Surfaces, Topology, and Lattice Animals.

Final rept.

A. L. Stella, E. Orlandini, I. Beichl, T. L. Einstein, F. Sullivan, and M. C. Tesi. 1992, 4p. Pub. in Physical Review Letters 69, n25 p3650-3653,
21 Dec 92.

Keywords: "Surfaces, Monte Carlo method, Cubic lattices, Topology, Simulation, Reprints, "Self-avoiding surfaces, Oct trees.

With Monte Carlo simulation, we study closed selfavoiding surfaces (SAS) of arbitrary genus on a cubic lattice. The gyration radius and entropic exponents are $v=0.506+$ or -0.005 and theta $=1.50+$ or -0.06 respectively. Thus, SAS behave like lattice animals (LA) or branched polymers at criticality. This result, contradicting previous conjectures, is due to a mechanism of geometrical redundancy, which is tested by exact renormalization on a hierarchical vesicle model. By mapping SAS into restricted interacting site $L A$, we conjecture $v($ sub theta $)=1 / 2$ phi(sub theta $)=1$, and theta(sub theta) $=3 / 2$ at the LA theta point.

\section{4,572}

PB95-150587 Not available NTIS

National Inst. of Standards and Technology (PL) Gaithersburg, MD. Electron and Optical Physics Div. Atomic Manipulation of Polarizable Atoms by Electric Field Directional Diffusion.

Final rept.

J. A. Stroscio, L. J. Whitman, R. A. Dragoset, and R. J. Celotta. 1992, 4p.

Pub. in Nanotechnology 3, p133-136 1992.

Keywords: "Alkali metals, Scanning tunneling microscopy, Electric fields, Gallium arsenides, Polarization, Semiconductors, Adsorbates, Diffusion, Cesium, Surfaces, Reprints, "Atomic manipulation, Nanostructures.

We present a brief summary of the properties of alkali metals on III-V(110) surfaces, as studied with the scanning tunneling microscope (STM). These include the non-metallic properties found in the one- and two-dimensional phases, and the directional diffusion of the alkali atoms due to the electric field gradient in the STM junction.

\subsection{3}

PB95-150595 Not available NTIS

National Inst. of Standards and Technology (PL), Boulder, CO. Ouantum Physics Div.

Nanoscale Study of the As-Grown Hydrogenated Amorphous Silicon Surface.

Final rept.

G. C. Stutzin, R. M. Ostrom, A. Gallagher, and D. M

Tanenbaum. 1993, 10p

Contract SERI-DD-1-11001-1(XG1-1216)

Sponsored by Solar Energy Research Inst., Golden, CO.

Pub. in Jnl. of Applied Physics 74, n1 p91-100, 1 Jul 93.

Keywords: "Amorphous silicon, *Surface analysis, "Silicon, Radio frequency discharge, Scanning tunneling microscopy, Gallium arsenides, Topography, Substrates, Single crystals, Reprints, Nanostructures.

A scanning tunneling microscope has been used to study the topography of the as-grown surface of device-quality, intrinsic, hydrogenated amorphous silicon deposited by if discharge from silane. The substrates were atomically flat, oxide-free, single-crystal silicon or gallium arsenide. No evidence for island formation or nanoscale irregularities was seen in studies of 100-Athick films on either silicon or gallium arsenide. The topography of 1000- and 4000-A-thick films has much variation many regions can be characterized as 'rolling hills, "but atomically flat areas have also been observed nearby. Generally, it appears that surface diffusion plays a role in smoothing the film topography. In most regions, the observed slopes were $10 \%$ or less from horizontal, but some steep-sided valleys, indicating incipient voids, were observed. The effect of the finite size of the scanning tunneling microscope probe tip is considered; this has an effect on the observed images in some cases.

\section{4,574}

\section{PB95-150686 Not available NTIS}

National Inst. of Standards and Technology (MSEL),

Gaithersburg, MD. Reactor Radiation Div.

Phonon Density of States in $\mathrm{R}_{2} \mathrm{CuO} 4$ and Superconducting R1.85Ce0.15CuO4 $(R=\mathrm{Nd}, \mathrm{Pr})$. Final rept.

I. W. Sumarlin, J. W. Lynn, D. A. Neumann, J. L.

Peng, Z. Y. Lin, J. J. Rush, and C. K. Loong. 1993,

$10 p$.

Pub. in Physical Review B 48, n1 p473-482, 1 Jul 93.

Keywords: 'Superconductors, "Phonons, Time-of-flight spectrometers, Neutron scattering, Inelastic scattering, Electron tunneling, Crysta! field, Neodymium ions, Praseodymium ions, Excitation, Reprints, "Neodymium cerium cuprates, "Praseodymium cerium cuprates, "Neodymium cuprates, "Praseodymium cuprates, Density of states.

Inelastic-neutron-scattering measurements of the generalized phonon density of states (GDOS) for the electron-doped superconductors $\mathrm{Nd}(1.85) \mathrm{Ce}(0.15) \mathrm{CuO} 4$ and $\operatorname{Pr}(1.85) \mathrm{Ce}(0.15) \mathrm{CuO} 4$ have been carried out with the use of both a filter-detector method and time-of- flight spectroscopy. A measurement of the GDOS for the insulating $\mathrm{Pr} 2 \mathrm{CuO} 4$ was also done for comparison with that of $\operatorname{Pr}(1.85) \mathrm{Ce}(0.15) \mathrm{CuO} 4$, while a more limited set of data was obtained for $\mathrm{Nd}_{2} \mathrm{CuO} 4$. We have also determined the ground-state crystal-field excitations associated with the rare-earth ions $(\mathrm{Nd}(3+)$ $\operatorname{Pr}(3+))$.

04,575

PB95-150843 Not available NTIS

National Inst. of Standards and Technology (EEEL) Boulder, CO. Electromagnetic Technology Div Insulating Nanoparticles on YBa2Cu3O7-delta Thin Films Revealed by Comparison of Atomic Force and Scanning Tunneling Microscopy

Final rept

R. E. Thomson, J. Moreland, N. Missert, B. F. Cole D. A. Rudman, and S. Sanders. 1993, 3p.

Pub. in Applied Physics Letters 63, n5 p614-616, 2 Aug 93.

Keywords: "High temperature superconductors, *Superconducting films, "YBCO superconductors, Atomic force microscopy, Scanning tunneling microscopy, Surface analysis, Thin films, Topography, Reprints, "Yttrium barium cuprates, Nanostructures.

The surface topography of $\mathrm{YBa} 2 \mathrm{Cu} 3 \mathrm{O}$ (7-delta) thin films has been studied with both atomic force micros. copy (AFM) and scanning tunneling microscopy (STM). The AFM images reveal a high density of smal distinct nanoparticles, $10-50 \mathrm{~nm}$ across and 5-20 nm high, which do not appear in STM images of the same samples. In addition, we have shown that scanning the STM tip across the surface breaks off these particles and moves them to the edge of the scanned area, where they can later be imaged with the AFM.

04,576

PB95-150850 Not available NTIS

National Inst. of Standards and Technology (MSEL) Gaithersburg, MD

Lattice Imperfections Studied by Use of Lattice Green's Functions.

Final rept.

R. Thomson, S. Zhou, A. Carlsson, and V. Tewary. 1992, 10p

Pub. in Physical Review B 46, n17 p10 613-10 622, 1 Nov 92.

Keywords: "Crystal defects, "Crack propagation, "Greens function, Crystal dislocations, Hexagonal lattices, Fractures(Materials), Numerical solution, Interfaces, Reprints.

This paper explores the use of lattice Green's functions for calculating the static structure of defects in lattices, in that the atoms of the lattice interact with their neighbors with an arbitrary nonlinear (short-range) potential.

The method is hierarchical, in which Green's functions are calculated for the perfect lattice, for increasingly complicated defect lattices, and finally the nonlinea structure problem is iterated until a converged solution is found. As an illustration of the method, we report numerical calculations for an interfacial crack emitting dislocations from an interface between two joined twodimensional hexagonal lattices. The supercell size was $4 \times 10(\exp 6)$, and the crack length was 101 lattice spacings. After the Green's functions were obtained for the defective lattice, the dislocation and crack strucures were obtained in a minute or less, making pos sible detailed studies of the defects with various external loads, force laws, defect relative positions, etc. with negligible computer time.

04,577

PB95-151320 Not available NTIS

National Inst. of Standards and Technology (NEL) Gaithersburg, MD. Statistica! Engineering Div.

Technique to Evaluate Benchmarks: A Case Study Using the Livermore Loops.

Final rept.

J. C. M. Wang, J. M. Gary, and H. K. Iyer. 1990,

$16 \mathrm{p}$.

Sponsored by Department of Energy, Washington, DC Pub. in International JnI. of Superconductor Application 4, n4 p40-55 1990

Keywords: Principle components analysis Least squares method, Data reduction, Data analysis, Cluster analysis, Kernel functions, Superconductors, Reprints, "Livermore loops.

This paper is devoted to an analysis of the data from the Livermore kernels benchmark. We will show that in the sense of least squares prediction the dimension 


\section{Solid State Physics}

of these data is rather small; a reduction of the data to dimension four has about the same predictive power as the original data. Two techniques are used that reduce the 72 kernel timings for each machine to a few scores by which the machine is characterized. The first is based on a principal component analysis, the second on a cluster analysis of the kernels. The validity of the reduction to lower dimension is checked by various means. The possible use of the Livermore data to predict the running time of larger codes is demonstrated.

\section{4,578}

PB95-151536 Not available NTIS

National Inst. of Standards and Technology (NML), Gaithersburg, MD. Electron and Optical Physics Div. Manipulation of Adsorbed Atoms and Creation of New Structures on Room-Temperature Surfaces with a Scanning Tunneling Microscope.

Final rept

L. J. Whitman, J. A. Stroscio, R. A. Dragoset, and R J. Celotta. 1991, 5p.

Pub. in Science 251, n4998 p1206-1210, 8 Mar 91.

Keywords: Scanning tunneling microscopy, Room temperature Indium antimonides, Gallium arsenides, Adsorbates, Semiconductors, Diffusion, Cesium, Surfaces, Alkali metals, Reprints, “Atomic manipulation, Nanostructures, Nanoclusters.

A general method of manipulating adsorbed atoms and molecules on room-temperature surfaces with the use of a scanning tunneling microscope is described. By applying an appropriate voltage pulse between the sample and probe tip, adsorbed atoms can be induced to diffuse into the region beneath the tip. The field-induced diffusion occurs preferentially toward the tip during the voltage pulse because of the local potential energy gradient arising from the interaction of the adsorbate dipole moment with the electric field gradient at the surface. Depending upon the surface and pulse parameters, cesium (Cs) structures from one nanometer to a few tens of nanometers across have been created in this way on the (110) surfaces of gallium arsenide (GaAs) and indium antimonide (InSb), including structures that do not naturally occur.

\section{4,579}

PB95-151700 Not available NTIS

National Inst. of Standards and Technology (MSEL), Gaithersburg, MD Ceramics Div.

$X$-Ray Characterization of the Crystallization Process of High-Tc Superconducting Oxides in the SrBi-Pb-Ca-Cu-O System.

Final rept.

W. Wong- $\mathrm{Ng}, \mathrm{C}$. K. Chiang, S. W. Freiman, M. D.

Hill, L. P. Cook, and N. M. Hwang. 1990, 6p.

Pub. in Materials Research Society Symposia Proceedings, v169 p123-128 1990.

Keywords: "High temperature superconductors, *BSCCO superconductors, "Crystallization, Scanning electron microscopy, X-ray diffraction, Heat treatment, Lead additions, Microstructure, Glass, Reprints, "Bismuth lead strontium calcium cup rates.

Phase assemblages in two $\mathrm{Sr}-\mathrm{Bi}-\mathrm{Pb}-\mathrm{Ca}-\mathrm{Cu}-\mathrm{O}$ glasses and their crystallization into glass-ceramics through a sequence of heat treatments are described Samplers were heat treated at various temperature based on differential thermal analysis (DTA) of the as-quenched glasses. X-ray powder diffraction was used to identify the compounds in each sample; and the different phases formed in these two samples were compared. Results of microstructural characterization of selected samples using scanning electron microscopy are discussed.

\section{4,580}

PB95-151718 Not available NTIS

National Inst. of Standards and Technology (MSEL), Gaithersburg, MD. Ceramics Div.

Crystal Chemistry and Phase Equilibrium Studies of the $\mathrm{BaO}(\mathrm{BaCO}$ )-R2O3-CuO Systems. 5. Melting Relations in $\mathrm{Ba2}(\mathrm{Y}, \mathrm{Nd}, \mathrm{Eu}) \mathrm{Cu} 3 \mathrm{O} 6+\mathrm{X}$.

Final rept.

W. Wong-Ng, L. P. Cook, M. Hill, B. Paretzkin, and E. R. Fulier. 1990, 6 .

See also PB95-151742, Part 2, PB95-151734 and Part 4 , PB95-151759.

Pub. in Materials Research Society Symposia Proceedings, v169 p81-86 1990.

Keywords: "YBCO superconductors, "Crystal chemistry, "High temperature superconductors, Scanning electron microscopy, $X$ ray diffraction Recrystallization, Europium additions, Neodymium additions, Melting, Grain growth, Microstructure, Reprints, Yttrium barium cuprates, Europium barium cuprates, Neodymium barium cuprates, Phase equilibrium, lonic size.

The influence of the ionic size of the lanthanides $R$ on melting relations of $\mathrm{Ba} 2 \mathrm{RC} u 3 \mathrm{O}(6+\mathrm{x})$, where $\mathrm{R}=\mathrm{Y}$, $\mathrm{E}_{\mathrm{U}}$ and $\mathrm{Nd}$, was studied and compared with that of a high $T$ (c) superconductor mixed-lanthanide phase $\mathrm{Ba} 2(Y(0.75) \mathrm{Eu}(0.125) \mathrm{Nd}(0.125)) \mathrm{Cu} 3 \mathrm{O}(6+\mathrm{x})$. These materials have been characterized by a variety of methods including scanning electron microscopy (SEM). Single phase samples of $\mathrm{Ba} 2(Y(0.75) \mathrm{Eu}(0.125) \mathrm{Nd}(0.125)) \mathrm{Cu} 3 \mathrm{O}(6+\mathrm{x})$ were an nealed at 1004,1040,1052,1060,1078, 1107 and $1160 \mathrm{C}$ and quenched into a helium gas containe cooled by liquid nitrogen. The SEM micrographs of these samples showed the progressive changes in features of the microstructures from sintering and grain growth through melting and then recrystallization from the melt. The addition of the SEM technique in conjunction with X-ray diffraction has been helpful in the study of phase equilibria in this system.

\section{1}

PB95-151726 Not available NTIS

National Inst. of Standards and Technology (MSEL) Gaithersburg, MD. Ceramics Div.

X-Ray-Diffraction Study of a Thermomechanically Detwinned Single Crystal of $\mathrm{YBa} 2 \mathrm{Cu} 3 \mathrm{O} 6+\mathrm{x}$.

Final rept.

W. Wong-Ng, F. W. Gayle, D. L. Kaiser, S. F.

Watkins, and F. R. Fronczek. 1990, 4p.

Pub. in Physical Review B 41, n7 p4220-4223, 1 Ma 90.

Keywords: "High temperature superconductors, *YBCO superconductors, * ${ }^{*}$ nystal structure, Temperafure range 0065-0273 K. Room temperature, X-ray diffraction, Single crystals, Orthorhombic lattices, Gold additions, Thermomechanics, Reprints, "Yttrium barium cuprates, Detwinning.

A twin-free single crystal of $\mathrm{YBa} 2 \mathrm{Cu} 3 \mathrm{O}(6+\mathrm{x})$ has been studied at room temperature and $115 \mathrm{~K}$ by $\mathrm{x}$-ray diffraction analysis. The crystal was grown from an $Y$-BaCu-O melt in a gold crucible, leading to a $2 \%$ substitution of $\mathrm{Au}$ for $\mathrm{Cu}$. Twins were removed from the asgrown crystal by a novel thermomechanical process. resulting in an orthorhombic ( $\mathrm{Pmmm})$, single-domain crystal. Oxygen positions and occupancies in the $\mathrm{Cu}$ 0 basal plane have been refined, showing that the $\mathrm{O}$ (5) site is completely vacant, and the $\mathrm{O}(4)$ atoms are offset from the crystallographic mirror plane positions by $0.15 \mathrm{~A}$ in a zig-zag fashion. Gold, which is a common impurity in crystals grown by the present technique, was found to occupy $\mathrm{Cu}(1)$ sites only. Weak superlattice reflections suggest a possible three-dimensional ordering of $\mathrm{O}$ and/or Au. To our knowledge. this paper reports the first high-resolution $x$-ray study of an intentionally detwinned, superconducting $\mathrm{Ba} 2 \mathrm{Cu} 3 \mathrm{O}(6+\mathrm{x})$ single crystal.

\section{4,582}

PB95-151734 Not available NTIS

National inst. of Standards and Technology (MSEL) Gaithersburg, MD. Ceramics Div.

Crystal Chemistry and Phase Equilibrium Studies of the BaO-R2O3-CuO Systems. 2. X-Ray Charac terization and Standard Patterns of BaR2CuO4, $\mathrm{R}=$ Lanthanides.

Final rept.

W. Wong-Ng, and B. Paretzkin. 1991, 3p.

See also PB95-151742, Part 4, PB95-151759 and Part 5, PB95-151718. Sponsored by Electric Power Research Inst., Palo Alto CA.

Pub. in Powder Diffraction 6, n4 p187-189 Dec 91.

Keywords: *Rare earth compounds, "Crystal structure Cnystal chemistry Orthorhombic lattices, X-ray diffraction, Barium oxides, Reprints, Phase equilibrium.

The compounds BaR2O4, where R = La, Nd, Sm, Gd, Eu, Dy, Ho and Er have been prepared from a stoichiometric mixture of $\mathrm{BaCO} 3$ and lanthanide oxides, and characterized by $X$-ray powder diffraction. Standard $X$ ray patterns of these phases were prepared. In general, BaR2O4 crystallizes in the pervoskite-related $\mathrm{CaFe} 2 \mathrm{O} 4$ structure which is orthorhombic with a space group Pnam. The cell parameters of these compounds from $R=E r$ to $L a$ were found. When $R=T m$, Lu and $\mathrm{Yb}$, the $\mathrm{BaO} \mathrm{R} 2 \mathrm{O} 3$ composition produced the mixture Ba3R4O9 and the unreacted lanthanide oxide Under the present experimental conditions, the compound
BaRO3 was the predominant component when $\mathrm{R}=\mathrm{Ce}$, $\mathrm{Pr}$, and $\mathrm{Tb}$.

\section{4,583}

PB95-151767 Not available NTIS

National Inst. of Standards and Technology (MSEL), Gaithersburg, MD. Ceramics Div.

X-Ray Powder Diffraction Data for $\mathrm{BaCu}(\mathrm{C} 2 \mathrm{O} 4) 2.6 \mathrm{H} 2 \mathrm{O}$.

Final rept

W. Wong-Ng, W. E. Rhine, and R. B. Hallock. 1991,

Pub. in Powder Diffraction 6, n1 p50 Mar 91.

Keywords: *Crystal structure, "X-ray diffraction, Hydrates, Reprints, "Barium copper oxalates, Powder patterns.

An indexed powder diffraction pattern and related crystallographic data are reported for a new phase, $\mathrm{BaCu}(\mathrm{C} 2 \mathrm{O} 4) 2$ (dot) $6 \mathrm{H} 2 \mathrm{O}$, which was a major product in the coprecipitation of barium and copper oxalates.

\section{4,584}

PB95-152104 Not available NTIS

National Inst. of Standards and Technology (MSEL), Gaithersburg, MD. Reactor Radiation Div.

Coupled-Bilayer Two-Dimensional Magnetic Order of the Dy lons in Dy2Ba4Cu7015.

Final rept.

H. Zhang, J. W. Lynn, and D. E. Morris. 1992, 10p. Pub. in Physical Review B 45, n17 p10 022-10 031, 1 May 92.

Keywords: "High temperature superconductors, "Dysprosium ions, Neutron scattering. Two dimensional, Antiferromagnetism, Reprints, "Dysprosium barium cuprates, "Magnetic ordering, Bilayers.

Neutron scattering has been used to investigate the magnetic ordering of the Dy ions in the superconducting $(\mathrm{T}(\mathrm{c})$ approx $=60 \mathrm{~K})$ Dy2Ba4Cu70 15 $(2: 4: 7)$ material. A modulated saw-tooth scattering profile is observed, indicative of a coupled-bilayer two-dimensional (2D) system in which the nearest-neighbor Dy spins within the $a-b$ plane are coupled antiferromagnetically, with the moment direction along the $c$ axis. The a-b-plane spin configuration is identical to the one which has been observed for the related DyBa2Cu3O7 (1:2:3) and Dy2Ba4Cu8016 (2:4:8) materials. We anticipate that a similar coupled-bilayer $2 \mathrm{D}$ behavior should occur in other R2Ba4Cu7015 systems ( $\mathrm{R}=$ rare-earth element except $\mathrm{Er}$ ) which exhibit the same $a-b$-plane spin configuration as the Dy.

04,585

PB95-152302 Not available NTIS

National Inst. of Standards and Technology (MSEL). Gaithersburg, MD. Reactor Radiation Div.

Anomalous Dispersion and Thermal Expansion in Lightly-Doped KTa1-xNbxO3.

Final rept.

P. M. Gehring, H. Chou, S. M. Shapiro, J. Toulouse, D. Rytz, L. A. Boatner, J. A. Hriljac, and D. H. Chen. 1993,12p.

Sponsored by Department of Energy, Washington, DC Pub. in Ferroelectrics 150, p47-58 1993.

Keywords: "Potassium tantalates, "Thermal expansion, Temperature dependence, Cryogenic temperature, X-ray diffraction, Neutron scattering, Inelastic scattering, Niobium additions, Lattice parameters, Cubic lattices, Single crystals, Anomalies, Reprints, "Potassium tantalate niobates, Phonon dispersion.

The temperature dependences of the lattice constant and TA phonon dispersion in dilute single crystals of $\mathrm{KTa}(1-x) \mathrm{Nb}(\mathrm{x}) \mathrm{O} 3$ with 0.012 less than or equal to $\mathrm{x}$ less than or equal to 0.09 have been studied using $x$ ray diffraction and neutron scattering techniques, respectively. For $x$ less than or equal to 0.06 an anomalous low-temperature expansion of the cubic lattice parameter is observed at a temperature $\mathrm{T}$ (sub $\mathrm{min}$ ) that increases monotonically with $\mathrm{Nb}$ concentration, with no measurable change in symmetry down to $10 \mathrm{~K}$. Concurrent with $\mathrm{T}$ (sub $\mathrm{min}$ ) is a monogeneous broadening of the $x$-ray Bragg peaks which persists down to 10 $K$, as well as a strong relief of extinction seen by neutron diffraction. Inelastic neutron scattering measurements reveal that the coupling between TA and TO modes, first observed in pure KTaO3, is both wavevector and concentration dependent. 
National Inst. of Standards and Technology (MSEL), Gaithersburg, MD. Reactor Radiation Div. Neutron-Scattering Studies of the Two Magnetic Correlation Lengths in Terbium.

Final rept.

K. Hirota, G. Shirane, P. M. Gehring, and C. F.

Majkrzak. 1994, 12p.

Pub. in Physical Review B 49, n17 p11 967-11 978, 1 May 94.

Keywords: "Terbium, Transition temperature, Neutron scattering, Reprints, "Magnetic correlation lengths.

Extensive neutron-scattering experiments have been performed in order to characterize the nature of the two correlation lengths observed in $\mathrm{Tb}$, a phenomenon common to $\mathrm{Ho}$ as well as $\mathrm{SrTiO}_{3}$ In the vicinity of the transition temperature, each of those crystals exhibits an anomalous two-component q profile in the critical scattering; the usual broad peak and an unexpected additional narrow peak. In order to clarify the spatia origin of the narrow component, the $(0,0$,delta) magnetic satellite peak of $\mathrm{Tb}$ has been closely examined using a very narrow neutron beam realized by a high spatial resolution reflectometer at the National Institute of Standards and Technology, which can produce welldefined beam widths of $0.3 \mathrm{~mm}$ and less. The small scattering angle (theta approx $=1.35 \mathrm{deg}$ ) and the narrow beam width result in an extraordinarily fine $q$ and $E$ resolution. As recently reported, we have confirmed that the intensity of the narrow component is enhanced near the edge of the crystal; it demonstrates that the major part is located within the near surface volume or 'skin' of the crystal.

04,587

PB95-152971 Not available NTIS

National Inst. of Standards and Technology (MSEL), Gaithersburg, MD. Reactor Radiation Div.

'Phase Transitions in Antiferromagnetic Superlattices'.

Final rept.

J. A. Borchers, and R. W. Erwin. 1993, 1p.

Pub. in Physical Review B 48, n9 p6711, 1 Sep 93

Keywords: "Phase transformations, "Antiferromagnetic materials, "Superlattices, Mean-field theory, Neutron diffraction, Cobalt oxides, Iron oxides, Nickel oxides, Reprints, Magnetic insulators.

Using a mean-field approach, Carrico and Camley (Phys. Rev. B 45, 13117 (1992)) have determined the magnetic behavior expected for superlattices consisting of uniaxial antiferromagnets. They state that the resulting phase transitions in a real system cannot be observed by standard, bulk measurements of the magnetization. In fact, the predictions of their model can be explicitly tested using neutron-diffraction techniques, which have already been applied toward the niques, which have already been applied toward the
study of antiferromagnetic $\mathrm{NiO} / \mathrm{CoO}$ and $\mathrm{Fe} 3 \mathrm{O} 4 / \mathrm{NiO}$ superlattices.

04,588

PB95-153060 Not available NTIS

National Inst. of Standards and Technology (EEEL),

Boulder, CO. Electromagnetic Technology Div.

Effects of Critical Current Density, Equilibrium Magnetization and Surface Barrier on Magnetization of High Temperature Superconductors.

Final rept.

D. X. Chen, R. W. Cross, and A. Sanchez. 1993, 9p. Pub. in Cryogenics 33, n7 p695-703 1993.

Keywords: "High temperature superconductors, "HighTC superconductors, "Magnetization, Critical current, Current density, surface barrier, YBCO superconductors, Reprints, Yttrium barium cuprates.

An extended critical state model which includes the efects of bulk critical current density, equilibrium magnetization and surtace barrier is developed for the magnetization of superconductors. The equilibrium magnetization of superconductors. The equilibrium applied field dependent surface supercurrent density (s) $(H)$ whose presence changes the boundary field of the bulk. The volume supercurrents flow with a density equal to the internal field dependent critical current density $\mathrm{J}(\mathrm{c})(\mathrm{Hi})$. The magnetization $\mathrm{M}$ is produced by both supercurrents. For the $M(H)$ curve computation exponential-type $\mathrm{J}(\mathrm{C})(\mathrm{Hi})$ and $\mathrm{j}(\mathrm{s})(\mathrm{H})$ values are used or the general case of an infinite sample of rectangular cross-section. A comparison between the experimental magnetization curves of a sintered $\mathrm{YBa} 2 \mathrm{Cu} 3 \mathrm{O} 7$ superconductor at $76 \mathrm{~K}$ and the model fit shows that $(\mathrm{s})(\mathrm{H})$ is null for the coupling matrix, whereas a nonzero $j(s)(H)$ is needed for the grains. The model fit for the irreversible magnetization of the grains is improved by including a surface barrier to the entry and exit of flux.

04,589

PB95-153128 Not available NTIS

National Inst. of Standards and Technology (CSTL), Gaithersburg, MD. Surface and Microanalysis Science Div.

Grazing-Incidence X-Ray Photoelectron Spectroscopy: A Novel Approach to Thin Film Characterizaion.

Final rept

M. J Chester and T Jach. $1993,9 p$

Pub. in Physical Review B 48, n23 p17-262 - 17-270, 15 Dec 93.

Keywords: "X-ray photoelectron spectroscopy, "Chemical composition, "Thin films, Concentration(Composition), Grazing incidence, Gallium arsenides, Gallium oxides, Intert

At the energies of interest in $x$-ray photoelectron spectroscopy (XPS), total external reflection of the $x$-ray trom a smooth surface at small incidence angles. The penetration of the $x$ rays into the material is strongly attenuated at these angles and surface sensitivity is enhanced in the XPS yields. As the incidence angle is increased, the $x$ rays penetrate more deeply into the material and the XPS signal contains a large contribution from the bulk. By exploiting this angle-dependent $x$-ray penetration depth, it is possible to obtain depth-dependent XPS spectra from which the concentration profiles of the photoelectron-emitting atom can be inferred. In this paper we develop a general formalism for calculating grazing-incidence XPS (GIXPS) yields from multilayer media. A quantitative analysis of GIXPS spectra acquired from an oxidized GaAs(100) surface that was annealed to remove oxidized As will be discussed. The results show that this annealed oxide is composed of $\mathrm{Ga} 2 \mathrm{O} 3$ and that the oxide-GaAs substrate interface is rough.

\subsection{0}

PB95-153250 Not available NTIS

National Inst. of Standards and Technology (EEEL), Boulder, CO. Electromagnetic Technology Div.

Transverse Thermomagnetic Effects in the Mixed State and Lower Critical Field of High-Tc Superconductors.

Final rept.

M. W. Coffey. 1993, 5p.

Pub. in Physical Review B 48, n13 p9767-9771, 1 Oct 93.

Keywords: "High temperature superconductors *Thermomagnetic effects, Ettingshausen effect, Nerns effect, Type 2 superconductors Critical field, Penetration depth, Temperature dependence, Thermodynamics, Vortices, Reprints.

Transverse thermomagnetic effects (Ettingshausen Nernst effects) are discussed for a variety of phenome nological models of high-T(c) and other layered superconductors. The use of the temperature-dependent vortex-line energy in determining the transport entropy is stressed, leading to predictions and possibilities for additional experiments. The dynamics of both Abrikosov and Josephson vortices is considered.

\section{4,591}

PB95-153318 Not available NTIS

National Inst. of Standards and Technology (MSEL) Gaithersburg, MD. Metallurgy Div.

Morphological Stability.

Final rept

S. R. Coriell, and G. B. McFadden. 1993, 73p.

Sponsored by National Aeronautics and Space Administration, Washington, DC. and Defense Advanced Research Projects Agency, Arlington, VA

Pub. in Handbook of Crystal Growth, Chapter 12, v1 p785-857 1993

Keywords: "Crystal growth, "Solidification, Liquid-solid interfaces, Binary alloys, Morphology, Stability, Reviews, Reprints, Crystal-melt interface.

During crystal growth, a smooth crystal-fluid interface is subject to instability and may become cellular or dendritic. Linear morphological stability theory described the conditions under which the interface becomes unstable. The theory is applicable to first order phas transformation in general, but in this article we will trea the growth of a crystal from a liquid phase. There ar many reviews of morphological stability. In this article we will review the basic theory and discuss extension of the theory with emphasis on recent developments. A number of important topics will not be treated in this article, but will be covered in other articles in this volume. Effects of fluid flow are treated in the Chapter by Davis. Dendritic growth is treated in the Chapter by Billia and Trivedi. We will also assume that effects of crystalline anisotropy are small enough that facets or missing interface orientations do not occur.

04,592

PB95-153490 Not available NTIS

National Inst. of Standards and Technology (MSEL), Gaithersburg, MD. Reactor Radiation Div.

Epitaxial Integration of Single Crystal C60.

Final rept.

J. A. Dura, P. M. Pippenger, N. J. Halas, S. C. Moss, X. Z. Xiong, and P. C. Chow. 1993, 3p.

Sponsored by National Science Foundation, Washington, DC. and Office of Naval Research. Arlington, VA Pub. in Jnl. of Applied Physics Letters 63, n25 p34433445, 20 Dec 93

Keywords: "Buckminsterfullerene, "Fullerenes, "Epitaxial growth, ${ }^{\text {"Single }}$ crystals, antimonides, Thin films, Substrates, Antimony, Reprints, Low energy electron diffraction, Carbon films.

Single crystal thin films of (111) oriented $C(60)$ are grown on epitaxial layers of single crystal antimony. The $C(60) / S b$ epitaxy is confirmed by low-energy electron diffraction which indicates that the (left bracket) (110) (right bracket) in-plane directions are parallel in the two layers. X-ray diffraction shows that the $C(60)$ film is entirely (111) oriented and of high quality with sharp Bragg peaks and narrow mosaic spread. In this study, the $\mathrm{Sb}$ films were grown on GaSb, to which they are lattice matched; however, as Sb can be epitaxially grown on surfaces with a large lattice mismatch, this technique may be applied to integrate $C(60)$ single crystals onto many substrates or devices with a surface having sixfold symmetry.

04,593

PB95-153540 Not available NTIS

National Inst. of Standards and Technology (EEEL).

Boulder, CO. Electromagnetic Technology Div.

Even-Odd Asymmetry of a Superconductor Revealed by the Coulomb Blockade of Andreev Reflection.

Final rept.

M. T. Eiles, J. M. Martinis, and M. H. Devoret. 1993 ,

Contract NOOO14-92-F-0003

Sponsored by Office of Naval Research, Arlington, VA Pub. in Physical Review Letters $70, n 12$ p1862-1865, 22 Mar 93.

Keywords: *Superconductors, Superconducting junctions, Symmetry breaking, Asymmetry, Reprints, Single electron tunneling, Andreev reflection.

We have measured at low temperatures the current through a submicrometer superconducting island connected to two normal metal leads by ultrasmall tunnel junctions. As the bias voltage is lowered well below twice the superconducting energy gap, the current changes from being e periodic with gate charge to $2 e$ periodic. This behavior is clear evidence that there is a difference in the total energy between the ground states of an even and odd number of electrons on the island. The 2e-periodic current peaks are the first observation of the Coulomb blockade of Andreev reflection.

04,594

PB95-153565 Not available NTIS

National Inst. of Standards and Technology (EEEL), Boulder, CO. Electromagnetic Technology Div.

Improved Uniaxial Strain Tolerance of the Critical Current Measured in Ag-Sheathed

Final rept.

J. W. Ekin, S. L. Bray, T. A. Miller, D. K. Finnemore, and J. Tenbrink. 1992, 3p.

Sponsored by Office of Naval Research, Arlington, VA Pub. in Advances in Cryogenic Engineering Materials, v38 ptB p 1041-1043 1992

Keywords: "High temperature superconductors, *BSCCO superconductors, Superconducting magnets, Critical current, Axial strain, Electromechanics, Tolerances, Silver, Reprints, "Bismuth strontium calcium cuprates. 


\section{Solid State Physics}

Practical electromechanical properties of a high $T(c)$ superconductor have been measured for $\mathrm{Ag}$-sheathed Bi2Sr2Ca1Cu2O $(8+x)$ superconductors at high magnetic fields up to $25 \mathrm{~T}$. A melt-processed powder-inlube $\mathrm{Bi2S} 2 \mathrm{Ca} 1 \mathrm{Cu} 2 \mathrm{O}(8+\mathrm{x})$ conductor had an irreversible strain of $0.2 \%$ for the onset of permanent damage and a $50 \%$ critical-current-degradation strain of $0.36 \%$. Measurements of a discontinuous filament melt-processed Ag-sheathed Bi2Sr2Ca1Cu2O $(8+x)$ superconductor show an irreversible strain of $0.6 \%$ and a $50 \%$ degradation strain of about $1 \%$. These strain damage thresholds are about an order of magnitude higher than for high-temperature superconductors made by bulk sintering and are reaching practical values for magnet design.

\section{4,595}

PB95-153649 Not available NTIS

National Inst. of Standards and Technology (EEEL), Boulder, CO. Electromagnetic Technology Div.

Surface Topography and Ordering-Variant Segregation in GalnP2.

Final rept.

D. J. Fnedman, J. G. Zhu, A. E. Kibbler, J. M. Olson,

and J. Moreland. 1993, 3p.

Sponsored by Department of Energy, Washington, DC. Pub. in Applied Physics Letters 63, n13 p1774-1776, 27 Sep 93

Keywords: Atomic force microscopy, Vapor phase epitaxy, Electron diffraction, Gallium arsenides, Substrates, Reprints, "Gallium indium phosphides, "Surface topography, Nomarski microscopy, Epilayers. Using transmission electron diffraction dark-field imaging, atomic force microscopy (AFM), and Nomarski microscopy, we demonstrate a direct connection between surface topography and cation site ordering in GalnP2. We study epilayers grown by organometallic vapor-phase epitaxy on GaAs substrates oriented 2 deg off (100) towards (110). Nomarski microscopy shows that, we growth proceeds, the surface of ordered material forms faceted structures aligned roughly along left bracket 011 right bracket. A comparison with the dark-field demonstrates that the left bracket 1-11 right bracket and left bracket 11-1 right bracke ordering variants are segregated into complementary regions corresponding to opposite-facing facets of the surface structures. This observation cannot be rationalized with the obvious but naive model of the surface topography as being due to faceting into low-index planes. However, AFM reveals that the facets are in fact not low-index planes, but rather are tilted 4 deg from (100) towards (111)(sub B). This observation explains the segregation of the variants: the surface facets act as local (111)(sub B)-misoriented growth surfaces which select only one of the two variants.

\section{4,596}

PB95-153698 Not available NTIS

National Inst. of Standards and Technology (MSEL),

Gaithersburg, MD. Reactor Radiation Div.

Origin of the Second Length Scale Above the Magnetic Spiral Phase of Tb.

Final rept.

P. M. Gehring, K. Hirota, C. F. Majkrzak, and G.

Shirane. 1993, 4p.

Pub. in Physical Review Letters 71, n7 p1087-1090, 16 Aug 93.

Keywords: "Terbium, Neutron scattering, Line shape, Holmium, Reprints, Critical scattering.

High q-resolution neutron-scattering measurements of the critical scattering in Tb above the magnetic-spiral phase transition temperature T(s) exhibit a two-component line shape, as recently documented in $\mathrm{Ho}$. This implies the existence of a second length scale. By using a narrow beam only 300 micrometers wide, and then translating the crystal through the beam, we have established that the origin of the second length scale lies within the near-surface volume or 'skin' of the Tb crystal. This is manifested by a large enhancement of the scattering intensity at the c-axis face of the cubeshaped crystal.

\section{4,597}

PB95-153722 Not available NTIS

National Inst. of Standards and Technology (EEEL), Boulder, CO. Electromagnetic Technology Div.

Volume Magnetic Hysteresis Loss of Nb3Sn Superconductors as a Function of Wire Length. Final rept.

R. B. Goldfarb. 1993, 1p.

Sponsored by Department of Energy, Washington, DC.
Pub. in Proceedings of U.S.-Japan Workshop (8th) on High-Field Superconducting Materials, Wires and Conductors and Standard Procedures for High-Field Superconducting Wires Testing, Madison, Wl., March 17-19, 1993, p72.

Keywords: "Superconducting wires, "Magnetic hysteresis, "Niobium stannides, Temperature range 0000-0013 K, SQUID devices, AC losses, Reprints, "Niobium ten, Multifilaments.

The magnetic hysteresis losses of samples of reacted multifilamentary Nb3Sn superconductor wire in coiled and straight geometries were measured at $4.1 \mathrm{~K}$ with a superconducting-quantum-interference-device (SQUID) magnetometer and an extraction dc magnetometer (DCM) for fields perpendicular to the wire axis. There were significant differences in the volume energy dissipation for the various samples. These differences are a function only of the length of the wire samples and are independent of whether the samples are straight segments or coils. As sample length increases from $2.6 \mathrm{~mm}$, losses per total sample volume, for complete field cycles of + or $-3 T$, increase nonlinearly from 0.19 to an asymptotic value of 0.43 $\mathrm{MJ} / \mathrm{cu} \mathrm{m}$. A coiled sample with wire length $3 \mathrm{~cm}$ has $97 \%$ of the losses of a coiled sample with wire length $15 \mathrm{~cm}$ or greater. Losses are very length-dependent below $1 \mathrm{~cm}$.

\section{4,598}

PB95-161113 Not available NTIS

National Inst. of Standards and Technology (PL), Gaithersburg, MD. Ionizing Ra diation Div.

Calibration of High-Energy Electron Beams by Use of Graphite Calorimeters.

Final rept.

J. C. Humphreys, M. L. Walker, J. M. Puhl, C. E.

Dick, and W. L. McLaughlin. 1993, 7p.

Pub. in Nuclear Instruments and Methods B 83, p5635691993

Keywords: "High temperature superconductors, "Crystal structure, Tetragonal lattices, Neutron diffraction, Oxygen additions, Annealing, Temperature dependence, Reprints, "Mercury barium calcium cuprates.

Neutron profile refinements have been carried out on powders of as-prepared and oxygen annealed samples of the layered $\mathrm{HgBa} 2 \mathrm{CaCu} 2 \mathrm{O}(6+$ delta $)(1212)$ cuprate superconductors. The crystal structure is tetragonal $\mathrm{P} 4 / \mathrm{mmm}$ over the range of temperatures 10 $\mathrm{K}$ to $298 \mathrm{~K}$ investigated, with no structural transitions of any kind being detected. The extra oxygen that electrically dopes these materials is found to reside in the centered position of the $\mathrm{Hg}$ plane, and no other excess oxygen is present in these samples. In addition, we found no conclusive evidence for mixing of the $\mathrm{Hg}$ and Cu cations. In the as-prepared sample we found delta $=0.22$, while the oxygen content increased substantially to delta $=0.35$ with annealing. This extra oxygen had only a small effect on the superconductivity, broadening the transition somewhat and shifting $T$ (c) from $112 \mathrm{~K}$ to $120 \mathrm{~K}$. The incorporation of extra oxygen also had the effect of constricting the lattice parameters by about $0.1 \%$. The overall behavior is similar to the single-layer $\mathrm{Hg}$ - 1201 system.

\section{4,599}

PB95-161139 Not available NTIS

National Inst. of Standards and Technology (EEEL), Boulder, CO. Electromagnetic Technology Div.

Harmonic and Static Susceptibilities of YBa2Cu3O7.

Final rept

T. Ishida, R. B. Goldtarb, S. Okayasu, T. Arndt, W Schauer, Y. Kazumata, and J. Franz. 1993, 29p. Pub. in Materials Science Forum 137-139, p103-131 1993.

Keywords: "High-temperature superconductors, "Magnetic susceptibility, "YBCO superconductors, Alternating current, Direct current, Sintered materials, Superconducting films, Single crystals, Flux pinning, Temperature dependence, Thin films, Comparison, Reprints, "Yttrium barium cuprates, Gadolinium barium cuprates, Magnetic field dependence.

Intergranular properties of the sintered superconductor YBa2Cu3O7 have been studied in terms of complex harmonic magnetic susceptibility chi(sub $n)=$ (chi prime)(sub $n$ ) - i(chi double prime)(sub $n$ ) ( $n$ integer) as well as DC susceptibility chi(dc). As functions of temperature T, (chi prime)(sub 1) and (chi, double prime)(sub 1) depend on both the AC magnetic-field amplitude $\mathrm{H}(\mathrm{ac})$ and the magnitude of a superimposed DC field $H(d c)$. Only odd-harmonic susceptibilities are observed below the critical temperature, $T(\mathrm{c})$, for zero $H(d c)$ while both odd and even harmonics are observed for nonzero $H(d c)$. With $T$ constant, odd-harmonic susceptibilities are even functions of $\mathrm{H}(\mathrm{dc})$ whereas even-harmonic susceptibilities are odd functions of $H(d c)$. In contrast, even-harmonic susceptibilities measured for a $\mathrm{GdBa}$ 2Cu3O7 thin film and an YBa2Cu3O7 single crystal are not prominen due to missing weak links, whereas odd-harmonic susceptibilities are remarkable. A survey of several models for the harmonic response of superconductors is presented.

\section{4,600}

PB95-161154 Not available NTIS

National Inst. of Standards and Technology (CSTL), Gaithersburg, MD. Surface and Microanalysis Science Div.

Grazing Angle X-Ray Photoemission System for Depth-Dependent Analysis.

Final rept.

T. Jach, M. J. Chester, and S. M. Thurgate. 1994, Pub. in Review of Scientific Instruments 65, n2 p339342 Feb 94.

Keywords: "X-ray photoelectron spectroscopy, "X-ray spectrometers, "Gallium arsenides, "Surface analysis, Grazing incidence, Photoemission, Oxidation, Oxides, Reprints, Depth dependence, Depth profiles.

We have developed an $\mathbf{x}$-ray photoelectron spectrometer system which combines an adjustable grazing incidence angle source with reflected beam detection When operated about the critical angle, this combination permits a variation of the $\mathrm{x}$-ray penetration depth which can be monitored by means of the reflectivity. At angles of incidence less than the critical angle, the sampling depth of the photoemission is diminished, but the photoemission from the surface is enhanced due to the constructive interference of the incident and reflected $\mathrm{x}$-ray beams. When used with $\mathrm{Mg} \mathrm{K}$ (alpha) radiation $(E$ (sub gamma $)=1253.6 \mathrm{eV}$ ), the spectrometry system obtalns useful distributions of chemical species in surface layers of 10-40 A thickness. We present data showing the depth dependence obtained with the spectrometer of different oxides in a sulfide-treated oxidized GaAs (100) surface.

\section{4,601}

PB95-161279 . Not available NTIS

National Inst. of Standards and Technology (MSEL), Gaithersburg, MD. Reactor Radiation Div. Hydrogen in YBa2Cu3Ox: A Neutron Spectroscopy and a Nuclear Magnetic Resonance Study.

Final rept.

U. Knell, C. Heid, H. Wipf, H. J. Lauter, T. J. Udovic, and J. J. Rush. 1993, 5p.

Pub. in Zeitschrift fur Physikalische Chemie 179, p3974011993.

Keywords: "High temperature superconductors, "YBCO superconductors, "Hydrogen additions, Spin lattice relaxation, Nuclear magnetic resonance, Neutron spectroscopy, Neutron scattering, Inelastic scattering, Relaxation time, Vibrational spectra, Doped materials, Reprints, "Yttrium barium cuprates.

The high temperature superconductor $\mathrm{YBa} 2 \mathrm{Cu} 3 \mathrm{O}(\mathrm{x})$ absorbs considerable amounts of hydrogen if it is kep at elevated temperatures in a hydrogen gas atmosphere In parallel neutron spectroscopic and nuclear magnetic resonance experiments, we studied the vibrational spectra and the spin lattice relaxation time of the protons in $\mathrm{YBa} 2 \mathrm{Cu} 3 \mathrm{O}(\mathrm{x})$ samples that were doped with hydrogen due to the absorption of hydrogen gas. Both the vibrational spectra and the spin lattice relaxation time are a sensitive probe for the local environment of the protons. We carried out analogous measurements on samples with a similar proton concentration due to the uptake of water vapor. These experiments demonstrated a totally different local environment of the protons. This fact is a strong indication that the protons absorbed from the hydrogen gas atmosphere did not react with the oxygen of the sample under formation of molecular water.

04,602

PB95-161451 Not available NTIS

National Inst. of Standards and Technology (NEL), Boulder, CO. Electromagnetic Technology Div. 
Grain Alignment and Transport Properties of Bi2Sr2CaCu2O8 Grown by Laser Heated Float Zone Method.

Final rept.

J. Luo, X. P. Jiang, H. M. Chow, T. P. Orlando, D. A Rudman, M. J. Cima, and J. M. Graybeal. 1991, 4p. Pub. in Institute of Electrical and Electronics Engineers Transactions on Magnetics 27, n2 p1499-1502 Mar 91.

Keywords: "High temperature superconductors, "BSCCO superconductors, "Grain structure, ${ }^{*} \mathrm{Critical}$ current, Grain boundaries, Orientation(Direction), Xray analysis, Temperature dependence. Transport properties, Zone melting, Float zones, Laser heating, Alignment, Anisotropy, Reprints, "Bismuth strontium calcium cuprates, Magnetic field dependence.

Single phase $\mathrm{Bi} 2 \mathrm{Sr} 2 \mathrm{CaCu} 2 \mathrm{O} 8$ bulk superconductor ( $\mathrm{T}(\mathrm{c})$ approx $=82 \mathrm{~K}$ ) has been grown by laser heated floating zone method. The samples are highly textured with the grains, typically 100 micrometers in diameter with the grains, typically 100 micrometers in diameter
and $0.5 \mathrm{~cm}$ long, elongated along the crystal a-axis which is the preferred growth direction. Our X-ray analysis and transport measurements indicate that the $b$, $c$ axes are also orientated from grain to grain in the plane perpendicular to the growth direction. The upper limit of the misalignment between the grains is estimated to be less than 2 degrees from the measured upper critical field anisotropic ratio. The critical current density for such highly textured polycrystalline is anisotropic and limited mainly by the weak links at the grain boundaries. The grain boundary effects will be discussed on the basis of the measured temperature and magnetic field dependence of the critical current.

04,603

PB95-161600 Not available NTIS

National Inst. of Standards and Technology (NEL), Boulder, CO. Electromagnetic Technology Div.

Effects of Anneal Time and Cooling Rate on the Formation and Texture of $\mathrm{Bi} 2 \mathrm{Sr} 2 \mathrm{CaCu} 2 \mathrm{O} 8$ Films. Final rept.

M. M. Matthiesen, J. M. Graybeal, T. P. Orlando, J. B. Vander Sande, and D. A. Rudman. 1991, 4p.

Pub. in Institute of Electrical and Electronics Engineers Transactions on Magnetics 27, n2 p1223-1226 Mar 91

Keywords: "High temperature superconductors, "Superconducting films, "BSCCO superconductors, Time dependence, Cooling rate, Microstructure, Annealing, Reprints, "Bismuth strontium calcium cuprates, Sputtered coatings, Surface texture.

The effects of anneal time and cooling rate on the formation and texturing of superconducting Bi2Sr2CaCu2O8 (BSCCO) films were investigated. Samples were prepared by sputter depositing amorphous BSCCO films, annealing them at $870 \mathrm{C}$ in flowing $20 \%$ O2-80\% Ar for 30,60 , or 180 minutes and then cooling them. Two cooling rates were investigated: a fast cool of $80 \mathrm{C} / \mathrm{min}$ and a slow cool of 9 $\mathrm{C} / \mathrm{min}$. It was observed that effective coupling between superconducting 2,212 grains occurs when films exhibit a minimum amount of (001) texturing. In addition, the nucleation and growth kinetics are significantly different for the isothermal and cooling regimes of thermal processing. Consequently, anneal time and cooling rate play distinct roles in the achievement of the appropriate texture and microstructure in the films: longer anneal times increase the volume fraction of the 2,212 phase while slower cooling rates enhance grain growth and texturing.

04,604

PB95-161618 Not available NTIS

National Inst. of Standards and Technology (PL) Gaithersburg, MD. Electron and Optical Physics Div. Laser-Focused Atomic Deposition.

Final rept.

J. J. MCClelland, R. E. Scholten, E. C. Palm, and R. J. Celotta. 1993, 4p

Pub. in Science 262, p877-880, 5 Nov 93.

Keywords: Photon-atom collisions, Atomic force microscopy, Atomic beams, Standing waves, Laser radiation, Line width, Lithography, Substrates, Silicon, Reprints, "Atom optics, "Nanostructures, "Laser deposition, Laser focusing, Chromium atoms.

The ability to fabricate nanometer-sized structures that are stable in air has the potential to contribute significantly to the advancement of new nanotechnologies and our understanding of nanoscale systems. Laser light can be used to control the motion of atoms on a nanoscopic scale. Chromium atoms were focused by a standing-wave laser field as they deposited onto a silicon substrate. The resulting nanostructure consisted of a series of narrow lines covering 0.4 millimeter by 1 millimeter. Atomic force microscopy measurements showed a line width of $65+$ or -6 nanometers, a spacing of 212.78 nanometers, and a height of 34 + or - 10 nanometers. The observed line widths and shapes are compared with the predictions of a semiclassical atom optical model.

\section{4,605}

PB95-161659 Not available NTIS

National Inst. of Standards and Technology (MEL) Gaithersburg, MD. Precision Engineering Div.

Comparison of Elastic and Plastic Contact Models for the Prediction of Thermal Contact Conductance.

T. H. MCWaid, and E Marschall. 1993, 8p.

Pub. in Warme- und Stoffubertragung 28, p441-448 1993.

Keywords: *Contact resistance, *Thermal conductors, Plastic properties, Elastic properties, Plastic deforma"Contact models, Asperity.

Experimentally determined thermal contact resistance conductance) data are compared with predictions based on two different theories. One of the theories assumes elastic contact, while the other theory is based on the assumption of plastic contact. Even though the high plastic index calculated for the contacting surtace suggested that contacting asperities would deform plastically, the experimental data generally agree better with the predictions obtained using the elastic contact model than with the predictions obtained using the plastic contact model.

\section{4,606}

PB95-161709 Not available NTIS

National Inst. of Standards and Technology (EEEL) Boulder, CO. Electromagnetic Technology Div.

Tunneling Spectroscopy of Thallium-Based HighTemperature Superconductors.

Final rept.

J. Moreland. 1994, 9p

in Thallium-Based

High-Temperature

Puperconductors, p569-577 1994

Keywords: "High temperature superconductors, "Thallium compounds, Bound state, Interfaces, Reprints, "Thallium barium calcium cuprates, "Tunneling spectroscopy, "Electron tunneling spectroscopy.

This chapter focuses on electron tunneling spectroscopy (ETS) of the thallium based high-temperature superconductor (HTS) materials. A summary of published results is included with comments regarding interpretations of each measurement. Other phenomena that may have subsequent bearing on the interpretation of tunneling spectra are addressed briefly including a linear background conductance, Coulomb block ade effects, and bound states at superconductor/normal metal (SN) interfaces. This chapter does not address many of the Josephson like devices based on weak-links, point contacts, microbridges, or step-edge junctions

\section{4,607}

\section{PB95-161717 Not available NTIS}

Noulonal Inst. of Standards and Technology

Stabilized Magnetic-Force Micr Final rept.

J. Moreland. 1993, 2p.

Pub. in Proceedings of the Annual Meeting of the Microscopy Society of America (51st), Cincinnati, $\mathrm{OH}$., August 2-6, 1993, p1034-1035.

Keywords: Scanning tunneling microscopy. High temperature superconductors, Superconducting films, Magnetic flux, Domain walls, Magnetic recording, Reprints, "Magnetic force microscopy, Bloch walls, Garnet films.

Magnetic force microscopy (MFM) can be done by making a simple change in conventional scanning tunneling microscopy (STM) where the usual rigid STM tip is replaced with a flexible magnetic tip. STM images acquired this way show both the topography and the magnetic forces acting on the flexible tip. The $z$-motion of the STM piezo tube scanner flexes the tip to balance the magnetic force so that the end of the tip remains a fixed tunneling distance from the sample surface. We present a review of some 'tunneling-stabilized' MFM (TSMFM) images showing magnetic bit tracks on a hard disk, Bloch wall domains in garnet films, and flux patterns in high-T(c) superconductor films. The image resolution of TSMFM is routinely 0.1 micrometer using Au coated magnetic tips cut from $\mathrm{Ni}$ or Fe films. Recen results show that a TSMFM resolution of less than 40 $\mathrm{nm}$ is possible with micromachined cantilevers coated with a very thin Au-Fe bilayer.

\section{4,608}

PB95-161733 Not available NTIS

National Inst. of Standards and Technology (EEEL),

Boulder, CO. Electromagnetic Technology Div.
Magnetic Force Microscopy of Flux in Magnetic Force

Final rept.

J. Moreland, P. Rice, and A. Wadas. 1993, 4p.

Pub. in Proceedings of the International Workshop on Superconductivity, Hakodate, Japan, June 28-July 1 , 1993, p77-80

Keywords: "Magnetic flux, "Superconductors, Scanning tunneling microscopy, Imaging techniques, Image analysis, Penetration, Reprints, *Magnetic force microscopy.

We are developing a novel form of magnetic force $\mathrm{mi}$ croscopy (MFM) which is well suited for low-temperature imaging of magnetic flux lines penetrating superconductors. This method is based on standar scanning tunneling microscopy (STM) where the usual rigid tunneling tip is replaced with a flexible magnetic tip. We present a discussion of the interpretations of these images which includes theoretical aspects of MFM of flux lines in superconductors and experimental details regarding MFM which we believe are critical for flux imaging.

04.609

PB95-161832 Not available NTIS

National Inst. of Standards and Technology (MSEL).

Gaithersburg, MD. Reactor Radiation Div.

New Exact Solution of the One-Dimensional Schrodinger Equation and Its Application to Polarized Neutron Reflectometry.

Final rept.

H. Zhang, and J. W. Lynn. $1993,4 p$.

93.

Keywords: "Schrodinger equation, "Superconductors, Penetration depth, One dimensional, Magnetization, Neutron scattering, Reprints, "Polarized neutron reflectometry.

An analytic expression for the polarized neutron reflectivity R(sup +,-) (theta, la mbda) from a superconductor with penetration depth lambda is derived as an exact solution of the 1D Schroedinger equation in the continuum limit. The down-spin solution R(sup -) (theta lambda) reveals a surprising oscillatory dependence on lambda within a narrow angular range immediately above the total reflection angle, and in fact vanishe when lambda and $\mathrm{H}$ satisfy certain conditions. This exact solution is applicable to other scattering problems with a general exponential dependence in the potential.

04,610

PB95-162111 Not available NTIS

National Inst. of Standards and Technology (CAML), Gaithersburg, MD. Applied and Computational Mathematics Div.

Micromagnetic Structure of Domains in Co/Pt Multilayers. 1. Investigations of Wall Structure. Final rept

R. Ploessi, J. N. Chapman, M. R. Scheinfein, $H$ Hoffmann, J. L. Blue, and M. Mansuripur 1993, $7 p$. Pub. in Jnl. of Applied Physics 74, n12 p7431-7437, 15 Dec 93

Keywords: "Magnetic domains, "Domain walls, Electron microscopy, Metal films, Cobalt, Platinum, Reprints, Micromagnetics, Multilayers.

An analysis of the micromagnetic structure of domains and domain walls in Co/Pt multilayer films is reported Magneto-optically written domains have been imaged motransmission electron microscope by using the modified differential phase contrast mode of Lorentz electron microscopy. These have been compared with computer-simulated images based on a two-dimensional model of a circular, perpendicular magnetized domain with a Bloch-like wall structure. Agreement is found for the domain and stray field contrast but the absence of wall contrast in the experimental images indicates a more complex wall structure 


\section{PHYSICS}

\section{Solid State Physics}

in the multilayer than was assumed by the model. In a further series of calculations the magnetic microstructure of a Co/Pt multilayer was modeled by solving the Landau-Lifshitz-Gilbert equations. These suggest that the wall structure varies throughout the thickness of the multilayer, allowing significant saving of magnetostatic energy through the establishment of flux closure paths close to the walls, and are consistent with experimental observations.

\section{4,611}

PB95-162293 Not available NTIS

National Inst. of Standards and Technology (MSEL), Gaithersburg, MD. Reactor Radiation Div.
Magnetic Rare Earth Artificial Metallic Superlattices.

Final rept.

J. J. Rhyne, R. W. Erwin, J. A. Borchers, C. P. Flynn, M. B. Salamon, and R. Du. 1991, 20p.

Pub. in Proceedings of NATO Advanced Study Institute on the Science and Technology of Nanostructured Magnetic Materials, Crete, Greece, June 24-July 6, 1990, p117-136 1991.

Keywords: "Rare earth elements, "Superlattices, Magnetic anisotropy, Magnetic films, Magnetic materials, Magnetostriction, Exchange interactions, Neutron diffraction, Metal films, Magnetism, Reviews, Reprints, *Artificial superlattices, Multilayers

Intense interest has been generated over the past sev eral years in the growth and properties of layered mag netic materials, both from a fundamental point of view and for applications. For the purposes of this review the term artificial metallic superlattice will be reserved for true three dimensionally coherent layered structures, while the term multilayer will be used for layered structures in which coherence is present in less than three dimensions. The recent refinements in computer controlled molecular beam epitaxy (MBE) techniques for the growth of single crystal artificial superlattices of two or more distinct compositions have opened up vast possibilities for the production of tailor-made artificia superlattices with controlled film thicknesses down to atomic dimensions and with highly reproducible stack ing sequences. This has provided previously unavailable opportunities to examine problems of interaction ranges, tunneling distances, and other coherent phenomena which are dependent on the superlattice periodicity. In particular, the development starting in 1984 of MBE growth procedures for single crystal rare earth metal superlattices has permitted prototypical tests for verifying many of the theoretical concepts of magnetic exchange, anisotropy, and magnetostriction effects in the rare earths which could not previously be examined in as controlled a way using conventional bulk materials.

\section{4,612}

\section{PB95-162384 Not available NTIS}

National Inst. of Standards and Technology (CSTL) Gaithersburg, MD. Surface and Microanalysis Science Div.

Resonant-Photoemission Investigation of the Heusler Alloys Ni2MnSb and NiMnSb.

Final rept.

S. W. Robey, L. T. Hudson, and R. L. Kurtz. 1992

Pub. in Physical Review B 46, n18 p11 697-11 704 1 Nov 92.

Keywords: "Electronic structure, "Photoemission, Heusler alloys, Magnetic alloys, Valence bands, Band theory, Binding energy, Reprints, *Nickel manganese antimonides, Density of states.

Photoemission was used to investigate the electronic density of states (DOS) in Ni2MnSb and NiMnSb. Comparisons with calculated band structures reveal reasonable agreement, but there are indications that the calculations overemphasize $\mathrm{Ni} 3 \mathrm{~d}$ contributions at some binding energies. Resonant effects at the $\mathrm{Mn} 3 \mathrm{p}$ core threshold were employed to obtain information on the $\mathrm{Mn} 3 \mathrm{~d}$ partial densities of states and these indicate that $\mathrm{Mn} 3 \mathrm{~d}$ character is spread throughout the valence bands, in agreement with theory. These effects are strongest in the bottom of the valence band around a binding energy of $3.1 \mathrm{eV}$ and produce structure which agrees well with the theoretical Mn 3d DOS, but are weak for other portions of the calculated Mn DOS. These results are discussed in the context of models for the formation of localized moments in these mate rials.
National Inst. of Standards and Technology (MSEL), Gaithersburg, MD. Metallurgy Div.

Temperature Dependence of Vortex Twin Boundary Interaction in Yttrium Barium Copper Oxide (YBa2Cu3O6+x).

Final rept.

A. Roitburd, L. J. Swartzendruber, D. L. Kaiser, F. W. Gayle, and L. H. Bennett. 1990, 1p.

Pub. in Physical Review Letters 64, n24 p2962 1990.

Keywords: "High temperature superconductors, "YBCO superconductors, "Flux pinning, Temperature dependence, Temperature range 0065-0273 K, Magnetic fields, Twinning, Reprints, "Yttrium barium cuprates.

It is shown that the interaction energy between flux vortices and twin boundaries changes sign from negative to positive as the temperature is lowered. For small applied fields $(\mathrm{H}<2 \mathrm{Hc} 1)$ the crossover temperature is approximately $70 \mathrm{~K}$.

04,614

PB95-162541 Not available NTIS

National Inst. of Standards and Technology (EEEL),

Boulder, CO. Electromagnetic Technology Div.

Growth of Laser Ablated YBa2Cu307-delta Films as Examined by Rheed and Scanning Tunneling Microscopy

Final rept

S. E. Russek, A. Roshko, S. C. Sanders, J.

Moreland, D. A. Rudman, and J. W. Ekin. 1993, 6p.

Contract DE-Al05-89ER14044

Sponsored by Department of Energy, Washington, DC. Pub. in Materials Research Society Symposia Proceedings, v285 p305-310 1993.

Keywords: "High temperature superconductor, "Superconducting films, "YBCO superconductors, Scanning tunneling microscopy, Electron diffraction, Crystal growth, Laser ablation, Thin films, Morphology, Reprints, "Yttrium barium cuprates, Surface structure.

Using scanning tunneling microscopy (STM) and reflection high energy electron diffraction (RHEED) we have examined the growth morphology, surface structure, and surface degradation of laser ablated YBa2Cu30(7-delta) thin films. Films from $5 \mathrm{~nm}$ to $1 \mathrm{mi}$ crometer thick were studied. The films were deposited on $\mathrm{MgO}$ and LaAIO3 substrates using two different excimer laser ablation systems. Both island nucleated and spiral growth morphologies were observed depending on the substrate material and deposition rate used. The initial growth mechanism observed for a 5 $10 \mathrm{~nm}$ thick film is replicated through different growth layers up to thicknesses of $200 \mathrm{~nm}$. Beyond $200 \mathrm{~nm}$, the films show some a-axis grains and other outgrowths. The thinnest films $(5-10 \mathrm{~nm})$ show considerable surface roughness on the order of $3-4 \mathrm{~nm}$. For both growth mechanisms the ledge width remains approximately constant (about $30 \mathrm{~nm}$ ) and the surface roughness increases as the film thickness increases.

04,615

PB95-162624 Not available NTIS

National Inst. of Standards and Technology (MSEL),

Gaithersburg, MD. Reactor Radiation Div.

Description of Layered Structures: Applications to High Tc Superconductors.

Final rept.

A. Santoro. $1993,6 p$

Pub. in Jnl. of Alloys and Compounds 197, p153-158 1993.

Keywords: "Superconductors, "Oxides, "Classifying, Structural analysis, Chemical composition, Gradients, Crystal structure, Crystallography, Perovskites, Rock salt, Reprints.

The layered structures of the known superconducting copper oxides can be described in terms of alternating slices having the rock salt and perovskite structure. This layer by layer representation of the crystal structures of oxide superconductors offers a conventient method for classifying and comparing to one another these important materials, and for predicting new compounds which may exhibit interesting electronic properties.

\section{4,616}

PB95-162749 Not available NTIS

National Inst. of Standards and Technology (EEEL), Boulder, CO. Electromagnetic Technology Div.
Critical Current Density, Irreversibility Line, and Flux Creep Activation Energy in Silver-Sheathed Bi2Sr2Ca2Cu2Ox Superconducting Tapes.

Final rept.

D. Shi, Z. Wang, S. Sengupta, S. X. Dou, H. K. Liu, Y. C. Guo, M. Smith, and L. F. Goodrich. 1993, 3p. Contract DE-W-31-109-ENG-38

Sponsored by Department of Energy, Washington, DC.

Pub. in Institute of Electrical and Electronics Engineers Transactions on Applied Superconductivity $3, n 1$ p1194-1196 Mar 93

Keywords: "Superconductors, "Films, "Magnetism Microstructure, Creep rate, Flux(Rate), Current density, Transport properties, Data processing, Magnetic hysteresis, Reprints, Flux creep.

Transport data, magnetic hysteresis and flux creep activation energy experimental results are presented fo silver-sheathed high-Tc Bi2Sr2Ca2Cu3Ox superconducting tapes The $110 \mathrm{~K}$ superconducting phase was formed by lead doping in a Bi-Sr-Ca-Cuphase was formed by lead doping in a Bi-Sr-Ca-Cumeasured at $4.0 \mathrm{~K}$ to be $0.7 \times 10(5) \mathrm{A} / \mathrm{sq} . \mathrm{cm}$ (the corresponding critical current is $74 \mathrm{~A}$ ) at zero field and 1.6 $x$ 10(4) $\mathrm{A} / \mathrm{sq} \mathrm{cm}$ at $12 \mathrm{~T}$ for $\mathrm{H}$ (double vertical bars)ab. Excellent grain alignment in the $a-b$ plane was achieved by a short-melting method, which considerably improved the critical current density and irreversibility line.

04,617

PB95-162780 Not available NTIS

National Inst. of Standards and Technology (MSEL) Gaithersburg, MD. Metallurgy Div.

Nanocomposite Magnetic Materials.

Final rept.

R D Shull and L. H Bennett 1992,6p.

Pub. in Nanostructured Materials 1, p83-88 1992.

Keywords: "Composite materials, "Magnetic materials, *Electrodeposition, Anisotropy, Glass, Magnetic properties, Magnetism, Copper, Nickel, Iron oxides, Silica gel, Sputtering, Reprints, Magnetic spin glass.

Composites of dissimilar materials wherein the sizes of the constituents are on the order of a few nanometers (Nanocomposites) are found to possess properties similar to, but different from, the properties of the individual constituents. When at least one of the constituents possesses a permanent magnetic moment, the magnetization statics and dynamics of the nanocomposite can be very different from that of the magnetic species in bulk form. In addition, the sensitivity of these magnetic properties to composition and morphology allows the class of magnetic nanocomposites to serve as a laboratory for the atomic engineering of materials with specific magnetic characteristics.

\section{4,618}

PB95-162798 Not available NTIS

National Inst. of Standards and Technology (MSEL), Gaithersburg, MD. Metallurgy Div.

Magnetocaloric Effect in Nanocomposites.

Final rept

R. D. Shull, L. J. Swartzendruber, and L. H. Bennett. 1991, 15p.

Pub. in Proceedings of International Cryocoolers Conference (6th), Plymouth, MA., October 25-26, 1990 p231-2451991.

Keywords: "Superconductors, "Magnetic properties Microstructure, Magnetic field, Barium, Yttrium, Cop per oxides, Currents, Vortices, Reprints, Twins BaYCuO, Vortex pinning, Critical currents, Magnetocaloric effect.

The magnetic properties of nanocomposites containing extremely small magnetic particulates dispersed in a nonmagnetic matrix can be very different than those of the bulk magnetic material. Here we consider the consequences of such a microstructure on the magnetocaloric effect. Upon the application of an external magnetic field, the magnetic spins in a materia tend to align with the field, thereby reducing the magnetic entropy of the spin system. Creation of a nanocomposite with many very small ferromagnetic regions provides an effective enhanced magnetic moment which can result in certain temperature and field ranges in an increased dT over that provided by eithe paramagnetic or ferromagnetic materials, with potential application to magnetic refrigeration.

04,619

PB95-162806 Not available NTIS 
National Inst. of Standards and Technology (MSEL), Gaithersburg, MD. Polymers Div.

Preparation of 2-Dimensional Ultra Thin Polystyrene Film by Water Casting Method.

Final rept.

K. Shuto, Y. Oishi, T. Kajiyama, and C. C. Han.

1993, 10p

Pub. in Polymer Jnl. 25, n3 p291-300 1993.

Keywords: "Thin films, "Polystyrene, "Neutron reactions, Electron microscopy, Neutron scattering, Polymers, Gyration, $\mathrm{X}$ rays, Reprints, TEM(Transmission electron microscopy), Water casting method, Taggedpolymer method, Radius of gyration.

The preparation condition of polystyrene (PS) ultra thin film by a water casting method was investigated on the basis of transmission electron microscopic (TEM) observations. Homogeneous ultra thin films were prepared from a $0.2-2.0$ wt\% cyclohexanone solution of PS owing to moderate spreadability of the solution on the water surface. The spreading state of the solution on the water surface depending on the dissipation speed of solvent and entanglements among PS chains were in strong relation to formation of the homogeneous ultra thin film. X-Ray interference measurements revealed that the thickness of the ultra thin film was smaller than the dimension of an unperturbed PS chain.

04,620

PB95-162822 Not available NTIS

National Inst. of Standards and Technology (MSEL) Gaithersburg, MD. Ceramics Div.

Ca1-xCuO2, a NaCuO2-Type Related Structure.

Final rept.

T. Siegrist, R. S. Roth, C. J. Rawn, and J. J. Ritter.

1990, 3p.

Pub. in Chemistry of Materials 2, n2 p192-194 1990

Keywords: "Crystal structure, "Superconductors, $X$ ray diffraction, Crystallography, Calcium, Copper oxides Sodium, Reprints.

The crystal structure of $\mathrm{Ca}(1-\mathrm{x}) \mathrm{CuO}(2)$, a low temperature phase in the Ca-Cu-O system, has been studied by X-ray diffraction. The structure contains edge shared chains of $\mathrm{CuO} 4$ squares, forming linea (CuO2)-chains and is related to the $\mathrm{NaCuO2-type.} \mathrm{In}$ contrast to the NaCuO2-type, the calcium atoms are not well localized and various superstructures caused by different $\mathrm{Ca}$ ordering are observed.

04,621

PB95-163002 Not available NTIS

National Inst. of Standards and Technology (NML), Gaithersburg, MD. Quantum Metrology Div.

Anisotropy of Polarized $X$-ray Emission from Atoms and Molecules.

Final rept.

S. H. Southworth, D. W. Lindle, R. Mayer, and P. L.

Cowan. 1991, 5p.

Pub. in Nuclear Instruments and Methods in Physics Research B 56-7, p304-308 May 91

Keywords: "Anisotropy, "Polarization, "X rays, Atoms, Molecules, $X$ ray analysis, Reprints, $X$ ray emission Strongly anisotropic, polarized $\mathrm{Cl}$ Kbeta $\mathrm{x}$-ray emission has been observed from gas-phase $\mathrm{CF} 3 \mathrm{Cl}$ following selective resonant excitation with a linearly polarized $x$-ray beam. Distinctively different polarizations and angular distributions result for emission involving molecular orbitals of different symmetries. A classical mode of the $x$-ray absorption/emission process well describes the observed radiation patterns. A small anisotropy also was measured for Ar Kalpha emission following resonant excitation. The observation of only a smal anisotropy in this case is attributed to the averaging out of the individual anisotropies from unresolved final states. The authors review the experimental methods and results and discuss theoretical models used to describe polarization and anisotropy effects in $x$-ray absorption and emission from atoms and molecules.

04,622

PB95-163358 Not available NTIS

National inst. of Standards and Technology (EEEL)

Boulder, CO. Electromagnetic Technology Div.

Observation of Insulating Nanoparticles on YBCO Thin-Films by Atomic Force Microscopy.

Final rept.

R. E. Thomson, J. Moreland, N. Missert, D. A.

Rudman, and S. C. Sanders. 1993, 2p.

Pub. in Proceedings of International Workshop on Superconductivity, Hokkaido, Japan, June 28-July 1 $1993, \mathrm{p} 242-243$
Keywords: "High temperature superconductors, "YBCO superconductors, "Superconducting films, "Surface analysis, Scanning tunneling microscopy, Atomic force microscopy, Image analysis, Thin films, Reprints, "Yttrium barium cuprates, Surface topography, Nanostructures.

The surface topography of $\mathrm{YBa} 2 \mathrm{CU} 3 \mathrm{O}$ (7-delta) thin films was studied with both atomic force microscopy (AFM) and scanning tunneling microscopy (STM). The AFM images revealed a high density of small distinct nanoparticles, $10-50 \mathrm{~nm}$ across and 5-20 nm high, which did not appear in STM images of the same samples. Scanning the STM tip across the surface broke off particles and moved them to the edge of the scanned area, where they were later imaged with the AFM

\section{4,623}

PB95-163366 Not available NTIS

National Inst. of Standards and Technology (NEL) Gaithersburg, MD. Building Materials Div.

Elastic Properties of Central-Force Networks with Bond-Length Mismatch.

Final rept.

M. F. Thorpe, and E. J. Garboczi. 1990, 13p.

Pub. in Physical Review B 42, n13 p8405-8417 1990

Keywords: "Elastic properties, "Crystal lattices, "Crystal defects, Crystal structures, Bonding. Crystallography, Strain rate, Stress analysis, Reprints, "Bond-length mismatch.

We study a triangular network containing two kinds of Hooke springs with different natural lengths. If the two spring constants are the same, we can solve the mode exactly and show that Vegard's law is obeyed, irrespective of whether the bonds are arranged randomly or in a correlated way. A more complete description of these networks is obtained through the mean lengths and length fluctuations associated with the two kinds of bonds, and the strain energy. The complete distribution of bond lengths is obtained numerically and shows an interesting and unexpected symmetry for the random case. Finally, we show that numerical results for a similar system, but with different force constants as well as different natural lengths, can be well accounted for by using an effective medium theory which reduces to the exact results when the two spring constants are made equal. We show that these lattices can be described very accurately up to about $50 \%$ length mismatches, when pleating occurs and the lattices develop local instabilities.

04,624

PB95-163499 Not available NTIS

National Inst. of Standards and Technology (MSEL), Gaithersburg, MD. Metallurgy Div.

Determination of Thermoactivation Parameters of Vortex Mobility in YBa2Cu3O7 Using Only Magnetic Measurements.

Final rept.

M. J. Turchinskaya, A. L. Roytburd, L. H. Bennett, L. J. Swartzendruber, and K. Sawano. 1991, 10p.

Pub. in Physica C 182, n4-6 p297-306 1991.

Keywords: "High temperature superconductors, *YBCO superconductors, Type 2 superconductors, Magnetic measurement, Magnetic induction, Activation energy, Time dependence, Single crystals, Reprints, "Yttrium barium cuprates, Vortex mobility.

A method for determination of the basic parameters of vortex mobility from the magnetic induction vs. time curve obtained from a zero-field cooled superconductor is presented. Three stages of magnetizing are considered. Measurement of the log-creep rate allows determination of the activation moment alpha, while the study of the kinetics during the approach to equilibrium provides the relaxation time $r$, and the equilibrium inductions $\mathrm{B}(\mathrm{o})$. Then the activation energy $U$ can be calculated. These two stages were obtained both for three different samples of YBa2Cu3O7: a single crystal, a sintered powder, and a sample prepared by the quench-melt-growth (QMG) process. The QMG sample was perfect enough to give reliable quantitative results.

04,625

PB95-163556 Not available NTIS

National Inst. of Standards and Technology $(\mathrm{PL})$, Gaithersburg, MD. Electron and Optical Physics Div. SEMPA Studies of Oscillatory Exchange Coupling. Final rept.

J. Unguris, D. T. Pierce, R. J. Celotta, and J. A.

Stroscio. 1993, 12p.

Sponsored by Office of Naval Research, Arlington, VA.
Pub. in Magnetism and Structure in Systems of Reduced Dimension, p101-112 1993.

Keywords: *Exchange interactions, "Magnetism, Scanning electron microscopy, Whiskers(Single crystals) Fermi surfaces, Metal films, Polarization, Epitaxy, Iron, Chromium, Silver, Spacers, Reprints, Oscillatory coupling, Multilayers.

We have used scanning electron microscopy with polarization analysis (SEMPA) to investigate the magnetic exchange coupling between an Fe film and an Fe whisker separated by either a Cr or Ag spacer layer. The thickness dependence of the oscillatory coupling in these atomically well ordered, epitaxial films was precisely measured. The periodicity of exchange coupling is consistent with predictions based on Fermi surface spanning vectors of the interlayer material.

04,626

PB95-163564 Not available NTIS

National inst. of Standards and Technology (MSEL), Gaithersburg, MD. Metallurgy Div.

Experimental Verification of a Vector Preisach Model.

Final rept.

F. Vajda, J. Oti, M. Pardavi-Horvath, L. H. Bennett,

E. Della Torre, and L. J. Swartzendruber. 1991, 4p. Pub. in Jnl. of Applied Physics 69, n8 p4501-4504 1991

Keywords: "Magnetic recording, Remanent magnetism, Coercive force, Experimentation, Verification, Particulates, Reprints, "Preisach model, Angular dependence, Micromagnetics.

A vector moving Preisach model has been developed that describes partially oriented particulate media by pseudo-particles, consisting of a distribution of several basic particles with various orientations of easy axes. The model uses switching modes calculated by a micro-magnetic simulation of physical particles. In order to test this model, the angular dependence of the magnetic properties was measured by a VSM for several samples of commercial recording media that had different degrees of particle orientation. The experimental results are in good agreement with the orientation dependence of the squareness, remanence, and coercivity, as predicted by the model

04,627

PB95-163721 Not available NTIS

National Inst. of Standards and Technology (NEL), Boulder, CO. Electromagnetic Technology Div.

High Critical Temperature Superconductor Tunneling Spectroscopy Using Squeezable Electron Tunneling Junctions.

Final rept.

T. Walsh, J. Moreland, R. H. Ono, T. Harvey, C.

Reintsema, T. S. Kalkur, J. A. Beall, and M. Cromar.

1991, 4p

Pub. in Institute of Electrical and Electronics Transactions on Magnetics 27, n2 p840-843 Mar 91

Keywords: "High temperature superconductors, "Tunnel junctions, Electron tunneling, Temperature dependence, BSCCO superconductors, YBCO superconductors, Energy gap, Electrodes, Niobium, Cuprates, Reprints, Tunneling spectroscopy.

Tunneling spectroscopy measurements were performed on squeezable electron tunneling (SET) junctions using $\mathrm{Bi}-\mathrm{S} r-\mathrm{Ca}-\mathrm{Cu}-\mathrm{O}, \mathrm{Y}-\mathrm{Ba}-\mathrm{Cu}-\mathrm{O}$, and $\mathrm{Nb}$ electrodes in a variety of combinations. A zero-bias conductance peak has been seen repeatedly in the current-voltage $(1(V))$ and the conductance-voltage $(G(V))$ characteristics. A model is presented to explain this conductance peak in terms of quasi-particle tunneling phase diffusion, and a supercurrent. Two additional structures have been seen repeatedly in $I(V)$ and $G(B)$. One of these structures has the characteristics of an energy gap feature. The other structure, which can mimic the gap feature, is explained in terms of the switching to the voltage state of a grain boundary junc tion that is in series with the SET junction. The dependence of these features upon temperature and the force applied to the junction are examined.

\section{4,628}

PB95-163739 Not available NTIS

National Inst. of Standards and Technology (NEL)

Boulder, CO. Electromagnetle Technology Div. 


\section{Solid State Physics}

Tunneling Measurement of the Zero-Bias Conductance Peak and the Bi-Sr-Ca-Cu-O Thin-Film Energy Gap.

Final rept.

T. Walsh, J. Moreland, R. H. Ono, and T. S. Kalkur

1991, $4 p$.
Pub. in Physical Review Letters 66, n4 p516-519 1991.

Keywords: "High temperature superconductors, "Superconducting junctions, "Superconducting films, "BSCCO superconductors, "Energy gap, Temperature ing. Thin films, Reprints, "Bismuth strontium calcium cuprates.

We have used squeezable electron tunneling junctions, at $4 \mathrm{~K}$, to examine the zero-bias conductance peak that has been found in high temperature superconductor tunnel junction spectra by a number of researchers. In addition, peaks in the differential conductance-voltage characteristic have been found repeatedly between 46 and $64 \mathrm{mV}$. We interpret the voltages at which these peaks occur to be the gap voltage 2 delta $(4 \mathrm{~K})$. The zero-bias conductance peak can be explained in terms of a supercurrent and thermal excitations, both quasi-particles and phase diffusion.

\section{4,629}

PB95-164224 Not available NTIS

National Inst. of Standards and Technology (MSEL), Gaithersburg, MD. Reactor Radiation Div.

Analytic Calculation of Polarized Neutron Reflectivity from Superconductors.

Final rept.

H. Zhang, and J. W. Lynn. 1993, 12p.

Pub. in Physical Review B 48, n21 p15 893-15 904, 1 Dec 93.

Keywords: "Superconducting films, "Superconductors, Polarized beams, Penetration depth, Angle of incidence, Schrodinger equation, Magnetic fields, Thick films, Meissner effect, Ferromagnetic materials. Magnetization, Reprints, "Neutron reflection, "Polarized neutron reflectometry.

We have obtained an analytic expression for the reflectivity $R($ sup +- )(theta, lambda) of polarized neutrons from a superconductor in the Meissner state, where lambda is the magnetic-field penetration depth and theta is the incident angle of the neutron beam. The result is derived as an exact solution of the 1D Schroedinger equation in the continuum limit, with an interaction potential. The solution for $R($ sup +- ) (theta lambda) reveals surprising features in its lambda dependence that have not been discovered in previous numerical studies. In particular, R(sup -)(theta, lambda) displays an oscillatory dependence on lambda within a narrow angular range immediately above the total reflection angle, instead of a monotonic dependence as inferred from earlier numerical calculations. The solution also reveals that complete transmission for the down-spin state $\left(R\left(\right.\right.$ sup $\left._{-}\right)($theta, lambda $\left.)=0\right)$ may occur when lambda and $\mathrm{H}$ (the applied magnetic field) satisfy certain conditions.

\section{4,630}

PB95-164240 Not available NTIS

National Inst. of Standards and Technology (IMSE), Gaithersburg, MD

Dislocation Emission at Ledges on Cracks.

Final rept.

S. J. Zhou, and R. Thomson. 1991, 15p.

Pub. in Jnl. of Materials Research 6, n3 p639-653 1991.

Keywords: "Dislocations, "Emission, " "Crack tips, "Ledges, Brittleness, Fracture(Mechanics) Cracking(Fracturing), Crack initiation, Solid mechanics, Reprints.

In this paper, we propose that, depending on their height, ledges on cracks can be efficient sources of dislocations at loadings well below the critical loading for homogeneous emission. Detailed three dimensional elastic calculations are presented supporting this proposition. There are two configurations for emission; one which blunts the crack, and one we call a jogging configuration. By our calculations, the blunting configuration should be the more efficient source; however, jogging dislocations are more frequently observed, experimentally. We find that the ledge is only a finite source of dislocations, in the sense that the ledge height decreases as the dislocations are emitted. The atomic configuration at the ledge is composed of finite lengths of real dislocations, which is the reason why such dislocations can be emitted easier than ho- mogeneously produced dislocations. The stresses at the ledge tip produce a cloud of bound dislocations near the ledge, pinned at the ledge ends, so that the ledge crack configuration becomes delocalized. Delocalization of the pile-up dislocations in the jogging direction may explain why the jogging dislocations are more frequently seen. Implications for DFZ's and ductile transitions are discussed.

\section{4,631}

PB95-164257 Not available NTIS

National Inst. of Standards and Technology (IMSE), Gaithersburg, MD.

Shielding of Cracks in a Plastically Polarizable Material.

Final rept.

S. J. Zhou, and R. Thomson. 1991, 10p.

Pub. in Jni. of Materials Research 6, n8 p1763-1772 1991.

Keywords: "Shielding, " Crack tips, "Fracture strength, *Dislocations, Plastic properties, Mechanical properties, Polarization(Waves), Dipoles, Emission, Brittleness, Reprints, "Plastically polarizable materials.

In this paper, we address some fundamental questions regarding the response of a crack to externally generated dislocations. We note that since dislocations formed at external sources in the material must be in the form of loops or dipoles, that the theory must be couched in terms of crack shielding in a plastically polarizable medium. There are strong analogies to dielectric theory. We prove two general theorems: (1) Dipoles formed in the emission geometry relative to a crack tip always antishield the crack, and (2) when dipoles are induced during uniform motion through a uniformly plastically polarizable material, then the shielding is always positive. We illustrate these general theorems with a number of special cases for fixed and polarizable sources. Finally, we simulate the self consistent time dependent response of a crack to a polarizable source as the crack moves past it. The results show the crack is initially antishielded, but that positive shielding always dominates during later stages of configuration evolution. The crack may be arrested by the source, or it may break away from it, depending upon the various parameters--source strength and geometry, dislocation mobility, Griffith condition for the crack, etc. The results indicate that the time dependence of crack shielding in the presence of a nonuniform cases of brittle transitions in materials.

\section{4,632}

PB95-164406 Not available NTIS

National Inst. of Standards and Technology (PL), Gaithersburg, MD. Quantum Metrology Div.

Lattice Position of Si in GaAs Determined by X-Ray Standing Wave Measurements.

Final rept.

A. Shih, P. Cowan, S. Southworth, B. Karlin, F.

Moore, E. Dobisz, H. Dietrich, L. Fotiadas, and C.

Hor. 1993, 8p.

Pub. in Jnl. of Applied Physics 73, n12 p8161-8168, 15 Jun 93.

Keywords: "Gallium arsenides, "Silicon additions, Molecular beam epitaxy, Synchrotron radiation, Ion implantation, Thermal diffusion, Standing waves, Position finding, Crystal lattices, X-ray analysis, Doped crystals, Impurities, NSLS, Reprints, "Lattice sites.

The $\mathrm{x}$-ray standing wave (XSW) technique was applied to determine the lattice location of Si impurity atoms in GaAs(100) crystals. The synchrotron radiation of X24A at the national synchrotron light source was used to set up backreflection XSW, an experimental geometry which drastically relaxes the otherwise stringent requirement on the lattice perfection. Specifically, the lattice sites were determined with respect to the (left bracket) 311 (right bracket) reflection planes which differentiate a Ga site from an As site. With the aid of an appropriate choice of the $x$-ray fluorescence filter. we were able to study GaAs(100) samples with very low levels of Si impurities. On a sample doped with 4 $x 10(\exp 18) / c c$ Si during the molecular-beam epitaxy growth, we found that the Si atoms predominantly occupied the Ga sites. On both an ion-implanted sample after annealing and a sample with $\mathrm{Si}$ impurities introduced by thermal diffusion, about $30 \%$ of the Si atoms occupied the Ga sites, and the rest occupied random sites. The As site occupation was less than $6 \%$. Suggestions are made for further experiments with improved sensitivity
04.633

PB95-164521 Not available NTIS

National Inst. of Standards and Technology (MSEL), Gaithersburg, MD. Reactor Radiation Div.

Field Dependence of the Magnetic Ordering of $\mathrm{Cu}$ in $\mathrm{R} 2 \mathrm{CuO} 4(\mathrm{R}=\mathrm{Nd}, \mathrm{Sm})$.

Final rept.

S. Skanthakumar, J. W. Lynn, J. L. Peng, and Z. Y. Li. $1993,3 p$.

Pub. in Jnl. of Applied Physics 73, n10 p6326-6328, 15 May 93.

Keywords: Neutron diffraction, Electron spin, Spin orientation, Copper, Reprints, "Neodymium cuprates, "Samarium cuprates, "Magnetic ordering, Magnetic field dependence.

We have used neutron diffraction techniques to study the field dependence of the magnetic ordering of $\mathrm{Cu}$ spins in R2CuO4 ( $\mathrm{R}=\mathrm{Nd}, \mathrm{Sm}$ ) in order to distinguish between the proposed collinear and noncollinear spin structures. In the proposed collinear spin structure, there are two separate domains with the spins either along the (110) or along the (1-10) directions, while in the noncollinear model there is a single domain with the alternate-layer spins along the (100) and (010) directions, respectively. If a magnetic field is applied along the (1-10), strong hysteresis effects are anticipated for the collinear spin structure due to domain repopulation, while such effects are not expected for the noncollinear spin structure. Our field dependen data do not show any hysteresis effects associated with the pure $\mathrm{Cu}$ ordering, which strongly suggest that the noncollinear spin structure is correct for the magnetic spin configuration of the Cu spins of both compounds. Hysteresis effects in a field are observed in $\mathrm{Sm} 2 \mathrm{CuO} 4$ near and below the $\mathrm{Sm}$ ordering temperature, and these are most likely caused by the interaction between $\mathrm{Sm}$ and Cu sublattices.

04,634

PB95-164539 Not available NTIS

National Inst. of Standards and Technology (MSEL), Gaithersburg, MD. Reactor Radiation Div.

Observation of Noncollinear Magnetic Structure for the Cu Spins in Nd2CuO4-Type Systems.

Final rept.

S. Skanthakumar, J. W. Lynn, J. L. Peng, and Z. Y. Li. 1993, 4p

Pub. in Physical Review B 47, n10 p6173-6176, 1 Mar 93.

Keywords: Spin orientation, Neutron diffraction, Electron spin, Copper, Reprints, "Neodymium cuprates, "Samarium cuprates, Magnetic field dependence, Magnetic ordering.

Field-dependent neutron-diffraction measurements have been taken on $\mathrm{Nd}_{2} \mathrm{CuO} 4$ and $\mathrm{Sm}_{2} \mathrm{CuO} 4$ in order to distinguish between the proposed collinear and noncollinear spin structures for the $\mathrm{Cu}$ ions. For magnetic fields applied along the (110) direction, both systems exhibit Bragg intensities that are continuous and reversible with $\mathrm{H}$. For $\mathrm{Nd} 2 \mathrm{CuO} 4$ we have also taken data for fields applied within an angle alpha of the (100) direction, and we have found that the intensities of the magnetic reflections increase or decrease depending on the sign of alpha. Both of these observations are only consistent with the noncollinear spin model.

\section{4,635}

PB95-164562 Not available NTIS

National Inst. of Standards and Technology (CSTL) Gaithersburg, MD. Surface and Microanalysis Science

Reactive Coevaporation of DyBaCuO Superconducting Films: The Segregation of Bulk Impurities on Annealed $\mathrm{MgO}(100)$ Substrates.

Final rept.

R. V. Smilgys, S. W. Robey, C. K. Chiang, and T. J. Hsien. 1993, $6 \mathrm{p}$.

Pub. in Jnl. of Vacuum Science and Technology A 11, n4 p1361-1366 Jul/Aug 93

Keywords: "Superconducting films, "Magnesium oxides, "Surface analysis, High temperature superconductors, Substrates, Thin films, Microstructure, Annealing, Impurities, Calcium, Reprints. "Dysprosium barium cuprates.

This article reports results from a study on the effect bulk impurities in $\mathrm{MgO}$ substrates may have when substrates are annealed to improve film microstructure and transport properties. DyBaCuO thin films are prepared by molecular beam reactive coevaporation on 
both annealed and unannealed $\mathrm{MgO}(100)$. While predeposition annealing has led to improved film quality for some groups, under our conditions just the opposite occurs. We point out the existence of $\mathrm{Ca}$ segregation to the MgO surface as a factor that needs investigation because $\mathrm{Ca}$ is a common contaminant in commercially supplied MgO. Atomic force microscope im ages show decoration of annealed substrates with submicron size particles that may be composed of the segregated $\mathrm{Ca}$. These particles seem to underlie the distinct differences seen in the surface microstructure of films on annealed and unannealed substrates.

\section{4,636}

PB95-168431 Not available NTIS

National Inst. of Standards and Technology (EEEL), Boulder, CO. Electromagnetic Technology Div.

In situ Observation of Surface Morphology of InP Grown on Singular and Vicinal (001) Substrates.

Final rept.

K. A. Bertness, C. Kramer, J. M. Olson, and J.

Moreland. 1994, 6p.

Pub. in Jnl. of Electronic Materials 23, n2 p195-200 1994.

Keywords: "Indium phosphides, "Vapor phase epitaxy, Atomic force microscopy, Optical microscopy, Surface roughness, Orientation(Direction), Light scattering, Laser radiation, Substrates, Reprints, In situ monitoring, Surface topography.

Surface morphology of InP layers is monitored during organometallic vapor phase epitaxy using an in situ diffuse laser light scattering technique. Changes in the diffuse scatter signal are noted for several substrate orientations near the (001) plane and at various growth temperatures. The diffuse scatter signal is shown to be a semi-quantitative indicator of surface roughness through post-growth examination of the samples with phase contrast optical microscopy and atomic force microscopy. Singular substrates consistently have almos featureless surfaces and very little diffuse scattering during growth. Vicinal substrates display a more complicated morphological evolution which cannot be deduced from the diffuse scattering alone but which does produce characteristic changes in diffuse scattering.

04,637

PB95-168522 Not available NTIS

National Inst. of Standards and Technology (EEEL)

Boulder, CO. Electromagnetic Technology Div.

Combined Josephson and Charging Behavior of the Supercurrent in the Superconducting SingleElectron Transistor.

Final rept.

T. M. Eiles, and J. M. Martinis. 1994, 280p.

Contract ONR-00014-92-F-0003

Sponsored by Office of Naval Research, Arlington, VA Pub. in Physical Review B 50, n1 p627-630, 1 Jul 94.

Keywords: "Josephson junctions, Josephson effect Cooper pairs, Superconductivity, Reprints, "Single electron transistors, Coulomb blockage, Supercurrent, Nanoelectronics, Shapiro steps.

Measurements of two ultrasmall Josephson junctions connected in series show a supercurrent with magnitude much greater than previously reported for similar devices. The dependence of the supercurrent on gate charge shows agreement with predictions made by Averin and Likharev that are based on a conjugate relationship between the junction phase and charg variables. The transport of Cooper pairs through the two junctions makes the devices behave effectively as a single junction whose critical current is modulated by the gate charge.

\section{4,638}

PB95-168613 Not available NTIS

National Inst. of Standards and Technology (EEEL) Boulder, CO. Electromagnetic Technology Div.

Alternating-Field Susceptometry and Magnetic Susceptibility of Superconductors.

Final rept.

R. B. Goldfarb, and M. Lelental. 1991, 32p.

See also PB94-145984

Pub. in Magnetic Susceptibility of Superconductors and Other Spin Systems, p49-80 1991.

Keywords: "Magnetic susceptibility, *Superconductors, Superconducting films, Alternating current, Granula materials, Critical field, Critical current, Meissner ef fect, Demagnetization, Thin films, Sensitivity, Operation, Calibration, Design, Reviews, Reprints, Susceptometers.
This review critically analyzes current practice in the design, calibration, sensitivity determination, and operation of alternating-field susceptometers, and examines applications in magnetic susceptibility measurements of superconductors. Critical parameters of the intrinsic and coupling components of granular superconductors may be deduced from magnetic susceptibility measurements. The onset of intrinsic diamagnetism corresponds to the initial decrease in electrical resistivity upon cooling, but the onset of intergranular coupling coincides with the temperature for zero resistivity. The lower critical field may be determined by the field at which the imaginary part of susceptibility increases from zero. Unusual features in the susceptibility of superconductor films, such as a magnetic moment that is independent of film thickness and the variation of susceptibility with angle, are related to demagnetization.

\section{4,639}

PB95-168621 Not available NTIS

National Inst. of Standards and Technology (EEEL), Boulder, CO. Electromagnetic Technology Div.

High Current Pressure Contacts to Ag Pads on Thin Film Superconductors.

Final rept

L. F. Goodrich, A. N. Srivastava, T. C. Stauffer, A. Roshko, and L. R. Vale. 1994, 4p.

Pub. in Institute of Electrical and Electronics Engineers Transactions on Applied Superconductivity 4, n2 p6164 Jun 94.

Keywords: "High temperature superconductors, "Superconducting films, "YBCO superconductors, "Electric contacts, "Contact resistance, Soldered joints, Critical current, Electrical resistance, Thin films, Indium, Silver, Reprints, "Yttrium barium cuprates, *Pressure contacts.

High current, low resistance, nonmagnetic, and nondestructive pressure contacts to $\mathrm{Ag}$ pads on YBa2Cu3O(7-delta) (YBCO) thin film superconductors were developed in this study. The contact resistance reported here includes the resistance of the current lead/Ag pad interface, the $\mathrm{Ag}$ pad/YBCO interface, and the bulk resistance of the contact material. This total contact resistance is the relevant parameter which determines power dissipation during critical-current measurements. It was found that regardless of the optimization of the $\mathrm{Ag}$ pad/YBCO interface through annealing, a pressure contact can yield a lower total resistance than a soldered contact. The lowest resistance obtained with pressure contacts was 3 (micro)ohms (for a $2 \times 4$ sq mm contact). These contacts may be useful for many different high temperature superconductor (HTS) studies where high-current contacts with low heating are needed.

\section{4,640}

PB95-168670 Not available NTIS

National Inst. of Standards and Technology (EEEL), Boulder, CO. Electromagnetic Technology Div

Suitability of Metalorganic Chemical Vapor Deposition-Derived PrGaO3 Films as Buffer Layers for YBa2Cu307-x Pulsed Laser Deposition.

Final rep

B. Han, D. A. Neumayer, T. J. Marks, V. P. Dravid D. A. Rudman, and H. Zhang. 1993, 3p.

Pub. in Applied Physics Letters 63, n26 p3639-3641, 27 Dec 93.

Keywords: *YBCO superconductors, High temperature superconductors, Metalorganic chemical vapor deposition, Temperature range $0400-1000$ K, films, Substrates, Reprints, "Praseodymium gallates, films, Substrates, Reprints, "Praseodymium gallates, Buffer layers.

Phase-pure thin films of the $\mathrm{YBa} 2 \mathrm{Cu} 3 \mathrm{O}(7-\mathrm{x})$ (YBCO) attice matched and low loss tangent perovskite insulator PrGaO3 have been grown in situ on single-crystal (110) LaAlO3 substrates by metalorganic chemical vapor deposition (MOCVD). Films were grown at temperatures of 750-800 C using beta-diketonate precursors $\mathrm{M}(\mathrm{dpm}) 3(\mathrm{M}=\mathrm{Pr}, \mathrm{Ga}$; $\mathrm{dpm}=$ dipivaloylmethanate). YBCO films were then grown on the MOCVD-derived $\mathrm{PrGaO} 3$ by pulsed laser deposition (PLD). Scanning electron microscopy reveals that the PrGaO3 films have smooth, featureless surfaces. As assessed by $x$-ray diffraction, the $\mathrm{PrGaO} 3$ films grow epitaxially on LaAlO3 with a high degree of (001) and/ or (110) plane orientation parallel to the substrate surface, and the subsequent $Y B C O$ films grow with a (OOI) orientation. Rocking curve and phi-scan analyses reveal that the PrGaO3 and YBCO films grow epitaxially.
YBCO films grown by PLD on the MOCVD-derived PrGaO3 exhibit $\mathrm{T}(\mathrm{c})=91 \mathrm{~K}$ and $\mathrm{J}(\mathrm{c})=6 \times 10(\exp 6)$ $\mathrm{A} / \mathrm{sq} \mathrm{cm}$ at $77 \mathrm{~K}$ in zero field.

\section{4,641}

PB95-168829 Not available NTIS

National Inst. of Standards and Technology (MSEL), Boulder, CO. Materials Reliability Div.

Relationship between Bulk-Modulus Temperature Dependence and Thermal Expansivity.

Final rept.

H. Ledbetter 1994,5p

Pub. in Physica Status Solidi (b) 181, p81-85 1994.

Keywords: *Bulk modulus, "Thermal expansion, Zero point energy, Temperature dependence, Debye temperature, Gruneisen constant, Elastic properties, Metals, Reprints.

The relationship between two anharmonic physical properties is considered: the bulk-modulus temperature dependence and the thermal expansivity. For the first Varshni's relationship is used, which derives from an Einstein-oscillator model of crystals. For the second, a relationship from Slater is used with a simila basis. The ration of the two properties is found to be gamma +1 , where gamma denotes the usua Gruneisen parameter. Varshni's adjustable parameter $\mathrm{s}$ is interpreted in terms of the Gruneisen parameter, the Einstein temperature, and the atomic volume Applied to metals, our relationship works well for those with s-p-electron bonding, but poorly for those where d-electrons contribute to bonding.

\section{4,642}

PB95-168837 Not available NTIS

National Inst. of Standards and Technology (MSEL) Boulder, CO. Materials Reliability Div.

Low-Temperature Elastic Constants of Y1Ba2Cu3O7.

Final rept.

H. Ledbetter, M. Lei, A. Hermann, and Z. Sheng.

1994, $7 p$.
Pub. in Physica C 225, p397-403 1994.

Keywords: "YBCO superconductors, "Elastic properties, High temperature superconductors, Temperaure range 0000-0013 $\mathrm{K}$, Temperature range 0013 $0065 \mathrm{~K}$, Temperature range 0065-0273 K, Temperarure range 0273-0400 K, Temperature dependence, Polycrystalline, Ultrasonic tests, Bulk modulus, Shear modulus, Young modulus, Poisson ratio, Reprints, "Yttrium barium cuprates.

Using ultrasonic methods, we studied the $4-295 \mathrm{~K}$ elastic constants of a 'good' Y1Ba2Cu3O 7 polycrystal. We report the bulk, shear, Young moduli and the Poisson ratio. Except for the Poisson ratio, all elastic constants show smooth temperature behavior. Near T(c) during cooling, the Poisson ratio decreases irregularly. Focusing on the bulk modulus $B$, we use simple thermodynamics to show that the harmonic-observed decrement at $\mathrm{T}=\mathrm{O}$ corresponds to expectation, but the tem perature effect $\mathrm{dB} / \mathrm{dT}$ is too large by about a factor of two. We attribute the irregular slope to oxygen-atom reordering. Against many reports, our bulk-modulus value is approximately that found in monocrystals or that predicted by an ionic-crystal model calculation.

04,643

PB95-168977 Not available NTIS

National Inst. of Standards and Technology (EEEL), Boulder, CO. Electromagnetic Technology Div.

Microwave Properties of YBa2Cu307-x Films at 35 $\mathrm{GHz}$ from Magnetotransmission and Magnetoreflection Measurements.

E. K. Moser, W. J. Tomasch, M. J. McClorey, C. L. Petiette-Hall, S. M. Schwarzbek, J. K. Furdyna, and M. W. Coffey. 1994, 10p.

Sponsored by Department of Energy, Washington, DC Pub. in Physical Review B 49, n6 p4199-4208, 1 Feb 94. superconductors, Extremely high frequency, Microwave transmission, Temperature dependence, Type 2 superconductors, Penetration depth, Thin films, Substrates, Reprints, Magnetic field dependence, Surface impedance, Vortex dynamics, Lanthanum aluminates, Magnetoreflectance.

Microwave transmission and reflection measurements are performed simultaneously on thin films (approx $1,800 \mathrm{~A}$ ) of $\mathrm{YBa} 2 \mathrm{Cu} 3 \mathrm{O}(7-\mathrm{x})$ having their $\mathrm{c}$ axis per- 
pendicular to the LaAlO3 substrate. We determine power transmission and reflection fractions, Tau(T,H and $R(T, H)$ as functions of temperature and static magnetic field $(\mathrm{O}<\mathrm{H}<5.4 \mathrm{~T})$, the latter being applied perpendicular to the film. A guided-wave model, which we develop for our experimental situation, is the basis of a computational algorithm that extracts quantitative values of the complex penetration depth (lambda bar) or alternatively the complex surface impedance $Z$ (s), from Tau and $\mathrm{R}$ data. This inversion algorithm is successful when applied to test data generated from phenomenological theory describing the electrodynamic response of a type-II superconductor. The procedure is applied to $\operatorname{Tau}(T, H)$ and $R(T, H)$ measure ments to infer the temperature-and field-dependen complex penetration depth (lambda bar)(T,H) that completely characterizes the superconductive film's electromagnetic response.

\section{4,644}

PB95-168993 Not available NTIS

National Inst. of Standards and Technology (EEEL),

Boulder, CO. Electromagnetic Technology Div

Hot-Electron Microcalorimeters for X-ray and Phonon Detection.

Final rept.

M. Nahum, J. M. Martinis, and S. Castles, 1993, 6p. Pub. in Jnl. of Low Temperature Physics 93, n3/4 p733-738 1993

Keywords: "X ray detectors, "Superconducting junctions, "Calorimeters, Electron temperature, Electron funneling, Hot electrons, Metal films, Dark matter, Tunnel junctions, Microcalorimetry, Prototypes, Reprints, "Phonon detectors, "Microcalorimeters.

We propose a novel hot-electron microcalorimeter for measurements of $x$-rays or phonons produced by the interaction of a high energy particle with the underlying substrate. This type of detector uses a normal metal film to absorb the incoming excitation which subsequently heats the electrons above the lattice temperature. The temperature of the electrons is measured from the current - voltage characteristics of a superconductor-insulator-normal metal tunnel junction where part of the absorber forms the normal electrode. We present simple calculations of the energy sensitivity of the junction and of the uitimate performance of $x$-ray and phonon detectors. We also present preliminary measurements of prototype devices which were used to test the basic detector physics.

04,645

PB95-169017 Not available NTIS

National Inst. of Standards and Technology (EEEL)

Boulder, CO. Electromagnetic Technology Div.

Proposed Antiferromagnetically Coupled DualLayer Magnetic Force Microscope Tips.

Final rept.

J. O. Oti, P. Rice, and S. Russek. 1994, 3p

Pub. in Jnl. of Applied Physics 75, n10 p6881-6883, 15 May 94.

Keywords: Magnetic films, Exchange interactions, Antiferromagnetism, Magnetostatics, Resolution, Reprints, "Magnetic force microscopy, "Microscope tips, Dual layers, Micromagnetics.

A magnetic force microscope tip designed from duallayer magnetic films of antiferromagnetically coupled magnetic layers is proposed. A theoretical analysis of the possible advantages of such a tip over conventional single-layer tips is given, using an extension to dual layers of a previously described micromagnetic model of single-layer tips. In contrast to single-layer tips, the magnetic domains of dual-layer tips are less sensitive to the fringing fields of the specimen, and the tips' stray fields are greatly reduced, thus minimizing the likelihood of erasure of the sample magnetization. These properties of dual-layer tips should lead to improved resolution of magnetic force microscopy images.

\section{4,646
PB95-1}

\section{PB95-169025 Not available NTIS}

National Inst. of Standards and Technology (EEEL) Boulder, CO. Electromagnetic Technology Div.

Magnetic and Magnetoresistive Properties of Inhomogeneous Magnetic Dual-Layer Films.

Final rept

J. O. Oti, S. E. Russek, and S. C. Sanders. 1994, 3p. Pub. in Jnl. of Applied Physics 75, n10 p6519-6521 15 May 94.

Keywords: "Magnetoresistance, "Magnetic films, Magnetic anisotropy, Coercive force, Exchange inter- actions, Cobalt alloys, Nickel alloys, Metal films, Magnetostatics, Chromium, Simulation, Reprints, Dual layers, Micromagnetics, Multilayers.

Magnetic and magnetoresistive properties of sputtered Co alloy dual-layer films are compared with micromagnetic simulations. The simulations elucidate the details of the switching behavior of the dual-layer films as a function of the interlayer exchange and magnetostatic interactions. The simulations have led to a conceptual understanding of the coercive field splitting caused by the interlayer interactions. A calculation of the anisotropic magnetoresistance (AMR) has been included in the simulations. The AMR provides a second independent macroscopic quantity (in addition to the average magnetization) which can be measured and compared with the micromagnetic simulations. The AMR is more sensitive to the micromagnetic structure perpendicular to the applied field and is a better test of the accuracy of the micromagnetic model. The simulations qualitatively describe the measured AMR data on CoNi-Cr-CoNi dual layers.

\subsection{7}

PB95-169033 Not available NTIS

National Inst. of Standards and Technology (MSEL), Boulder, CO. Materials Reliability Div.

Enhanced Flux Pinning via Chemical Substitution in Bulk Superconducting T1-2212.

Final rept.

M. Paranthaman, M. Foldeaki, D. Balzar, A. M. Hermann, H. Ledbetter, and A. J. Nelson. 1994, 7p. Pub. in Supercond. Sci. Technol. 7, p227-233 1994.

Keywords: "Flux pinning, High temperature superconductors, Temperature range 0000-0013 K Penetration depth, Magnetic hysteresis, Magnesium additions, Direct current, Doped materials, Critical current, Crystal defects, Reprints, "Thallium barium calcium cuprates.

The flux-pinning characteristics in both pure and doped superconducting TI2Ba2CaCu2O8 (referred to as TI2212) systems were studied via DC magnetic hysteresis as well as flux-penetration and flux-expulsion measurements. Our results demonstrate an increase in both intrinsic pinning and lock-in transition of flux lines as a result of increased density of atomicsize structural defects via Mg doping in TI-2212.

04,648

PB95-169157 Not available NTIS

National Inst. of Standards and Technology (EEEL) Boulder, CO. Electromagnetic Technology Div.

Insulating Boundary Layer and Magnetic Scattering in YBa2Cu307-delta/Ag Interfaces Over a Contact Resistivity Range of 10(-8) - 10(-3) Omega $\mathrm{cm}(2)$.

Final rept.

S. C. Sanders, S. E. Russek, C. C. Clickner, and J. W. Ekin. 1994, 3p.

Contract ARPA-7975-01

Sponsored by Advanced Research Projects Agency, Arlington, VA.

Pub. in Applied Physics Letters 65, n17 p2232-2234, 24 Oct 94.

Keywords: "YBCO superconductors, "Electron tunneling, High temperature superconductors, layer, Thin films, Interfaces, Silver, Reprints, "Yttrium barium cuprates, Contact resistivity.

We have measured interface transport in thin-film YBa2Cu3O(7-delta)/Ag interfaces having resistivities ranging from 10 (exp -8) to 10 (exp -3) ohm sq cm. Anal$y$ sis of the interface I-V data indicates that tunneling is the predominant transport mechanism even for the in situ interfaces having contact resistivities of $1-7 X$ 10(exp -8) ohm sq cm. Zero-bias conductance peaks are also observed for the entire range of interface resistivity. The similarity of the zero-bias conductance peaks among these widely varying interfaces suggests that the low-temperature interface transport is governed by the same mechanism in each case. These conductance peaks are analyzed in the framework of the Appelbaum-Anderson model for tunneling assisted by magnetic scattering from isolated magnetic spins in the interface.

\section{4,649}

PB95-171963 PC A05/MF A01

National Inst. of Standards and Technology (EEEL), Gaithersburg, MD. Electricity Div.
From Superconductivity to Supernovae: The Ginzburg Symposium. Report on the Symposium Held in Honor of Vitaly L. Ginzburg. Held in Gaithersburg, Maryland on May 22, 1992.

A. F. Clark V. L Ginzburg, L. P. Gor'kov, V. Z. Kresin, J. D. Kurfess, R. Ramaty, W. A. Little, and K. Kellermann. Nov $94,79 p$, NISTIR-5556.

Keywords: "High temperature superconductors, "Gamma ray astronomy, "Meetings, High-Tc superconductors, Electron tunneling, Radio galaxies, Radio sources(Astronomy), Gamma ray observatory, Cosmic rays, Quasars, Cuprates, "Ginzburg symposium.

A one day symposium honoring Professor Vitaly $\mathrm{L}$. Ginzburg and his many contributions to the world of physics was held at the National Institute of Standards and Technology. An additional purpose was to explore future directions for research in the context of his extensive involvement in the rapid developments in physics in the last fifty years. This transcription of the proceedings includes invited talks on some of those recent developments in such fields as superconductivity and astrophysics. Prof. Ginzburg's own address 'What problems of physics and astrophysics seem now to be especially important and interesting' was the focus for discussion on the future directions of physics research. Titles of the other papers given at the symposium are as follows: New Phenomena in Proximity Effect Tunneling in High TC Superconductors: Radio Galaxies, Quasars, and Superluminal Radio Sources; Exotic Properties of High TC Materials and the Ginzburg Parameter; Gamma Ray Astrophysics and Early Results of the Compton Observatory; Some Puzzles of the Normal State of High TC Oxides; Galactic Annihilation Radiation.

\section{4,650}

PB95-175089 Not available NTIS

National Inst. of Standards and Technology (EEEL), Boulder, CO. Electromagnetic Technology Div.

Thermal Noise in High-Temperature Superconducting-Normal-Superconducting StepEdge Josephson Junctions.

Final rept.

S. J. Berkowitz, W. J. Skocpol, P. M. Mankiewich, P. A. Rosenthal, L. R. Vale, R. H. Ono, and N. A.

Missert. 1994, 3p.

Pub. in Jnl. of Applied Physics 76, n2 p1337-1339, 15 Jul 94

Keywords: "Josephson junctions, "Thermal noise, High temperature superconductors superconductors, Reprints, Yttrium barium cuprates, RSJ model.

We have fabricated $\mathrm{YBa} 2 \mathrm{Cu} 3 \mathrm{O}$ (7-delta)-normal metalYBa2Cu3O(7-delta) step-edge Josephson junctions that fit the resistively shunted junction model with Johnson-Nyquist thermal noise. The I-V curves are well fit over a large temperature range for junctions of varying critical current values. There is good agreement between the fitted thermal noise temperature and the measured ambient temperature. This is strong evidence that these junctions are not dominated by superconducting shorts longer than the superconducting coherence length.

04,651

PB95-175295 Not available NTIS

National Inst. of Standards and Technology (EEEL), Boulder CO. Electromagnetic Technology Div.

Thin Film Reaction Kinetics of Niobium/Aluminum Multilayers.

Final rept.

K. R. Coffey, K. Barmak, D. A. Rudman, and S.

Foner. 1992, 9p.

Pub. in Jnl. of Applied Physics 72, n4 p1341-1349, 15 Aug 92.

Keywords: "Aluminum alloys, "Niobium alloys, "Phase studies, Transmission electron microscopy, $X$ ray diffraction, Reaction kinetics, Metal films, Thin films, Nucleation, Calorimetry, Superconductors, Reprints, "Niobium aluminides, Multilayers.

Phase formation kinetics in $\mathrm{Nb} / \mathrm{Al}$ multilayered thin films having overall compositions of $25,33,50$, and 75 at. \% Al have been investigated using scanning calorimetry, $x$-ray diffraction, and cross-sectional transmission electron microscopy. The first phase to form upon annealing the $\mathrm{Nb} / \mathrm{Al}$ layered structure of all samples is the NbAl3 intermetallic. Calorimetry clearly identifies the NbAl3 formation to be a two-stage process. The first stage is the formation of a planar layer 
by nucleation and growth to coalescence while the second stage is the thickening of the planar layer. The nex phase observed in samples of 25 and 33 at. \% Al is the A15 superconducting phase, Nb3Al. The Nb3A growth completes a first reaction stage similar to the $\mathrm{NbAl3}$, but the subsequent thickening reaction stage is not observed without simultaneous Nb2Al growth. The high interface velocities denived from the calonimetry for formation of both $\mathrm{NbAl} 3$ and the $\mathrm{A} 15$ $\mathrm{Nb3Al}$ indicate that atomic transport must be by grain boundary diffusion.

\section{4,652}

PB95-175303 Not available NTIS

National Inst. of Standards and Technology (EEEL) Boulder, CO. Electromagnetic Technology Div.

Aspects of a Deformable Superconductor Model for the Vortex Mass.

Final rept.

M. W. Coffey. 1994, 9p.

Pub. in Jnl. of Low Temperature Physics 96, n1/2 p81891994.

$\begin{array}{lll}\text { Keywords: } & \text { "Superconductivity, } & \text { Typer } 2 \\ \text { superconductors, Mathematical models, Temperature }\end{array}$ superconductors, Mathematical models, Temperature Mass, Reprints, Vortex dynamics.

A deformable superconductor model for the vortex mass per unit length mu(sub d) In a type-ll superconductor is discussed. A new identity for the inertial vortex mass in this model is presented which holds for an arbitrary quasiparticle fraction when the ionic displacement is irrotational. This result is used to show unphysical behavior in the temperature dependence of the ionic-strain-field vortex mass and is key in resolving this difficulty. A possibility for the expenmental observation of the strain field mechanism is discussed.

\section{4,653}

PB95-175311 Not available NTIS

National Inst. of Standards and Technology (EEEL), Boulder, CO. Electromagnetic Technology Div.

Deformable Superconductor Model for the Fluxon Mass.

Final rept.

M. W. Coffey. 1994, 4p.

Pub. in Physical Review B 49, p9774-9777, 1 Apr 94. Keywords: "Superconductivity, Type 2 superconductors, Mathematical models, Quasi-particles, Elasticity, Mass, Reprints, "Fluxons, Vortex dynamics.

Outstanding difficulties in a deformable type-II superconductor model for the fluxon inertial mass per unit length mu(sub d) are resolved. An identity for the inertial mass, valid for an arbitrary quasiparticle fraction when the ionic displacement field is irrotational plays a critical role in the analysis. This approach avoids previously employed approximations, leading to qualitatively different results, including a fluxon mass which properly vanishes at the transition temperature and which has a greatly reduced magnitude. A fram $\theta$ work for the solution of the elasticity equation for an isotropic superconductor is presented and the close re lation between mu(sub d) and the ionic strain field is shown.

04,654

PB95-175352 Not available NTIS

National Inst. of Standards and Technology (EEEL) Boulder, CO. Electromagnetic Technology Div.

Increased PinnIng Energies and Critical Current Densities in Heavy-Ion-Irradlated $\mathrm{BI} 2 \mathrm{Sr} 2 \mathrm{CaCu} 2 \mathrm{O}$ Single Crystals.

Final rept.

J. A. Cutro, D. A. Rudman, T. P. Orlando, A. E White, E. M. Gyorgy, J. V. Waszczak, R. J. Felder, R. B. van Dover, and L. F. Schneemeyer. 1993, 3p. Pub. in Applied Physics Letters 62, n7 p759-761, 14 Feb 93.

Keywords: "BSCCO superconductors, "Radiation effects, "Ion irradiation, "Flux pinning, "Critical current Hydrogen ions 1 plus, Helium ions, Argon lons, Single crystals, Crystal defects, High temperature superconductors, Light ions, Heavy ions, Reprints, "Bismuth strontium calcium cuprates, Flux vortices.

We report a significant increase in the pinning energy of vortices in single-crystal $\mathrm{Bi} 2 \mathrm{Sr} 2 \mathrm{CaCu} 2 \mathrm{O} 8$ when irradiated with heavy ions such as $\operatorname{Ar}(1+)$. This is in contrast with the results of light ion $(\mathrm{H}(1+), \mathrm{He}(1+))$ irradiations which give pinning energies comparable with those of unirradiated crystals. The stronger pinning is attributed to defects larger than point defects, e.g., clusters or amorphized regions. As a result of higher plnning energies, critlcal currents persist at markedly hlgher temperatures and fields.

\section{4,655}

PB95-175360 Not available NTIS

Natlonal inst. of Standards and Technology (EEEL). Gaithersburg, MD. Semiconductor Electronics Div. Characterization of the $\mathrm{ZnSe/GaAs}$ Interface Layer by TEM and Spectroscopic Ellipsometry.

Final rept.

R. Dahmani, L. Salamanca-Riba, D. P.

Beesabathina, B. T. Jonker, N. V. Nguyen, and D.

Chandler-Horowitz. 1993, 4p.

Grant NSF-DMR90-20304

Sponsored by National Science Foundation, Washington, DC.

Pub. in Materials Research Society Symposia Proceedings, v280 p271-274 1993.

Keywords: "Zinc selenides, "Gallium arsenides, "Interfaces, Transmission electron microscopy, Molecular beam epitaxy, Transition layers, Thin films, Ellipsometry, Substrates, Reprints.

The interface between $\mathrm{ZnSe}$ thin films and GaAs substrates is characterized by High Resolution Transmission Electron Microscopy and room temperature Spectroscopic Ellipsometry. The films were grown on (001) GaAs by Molecular Beam Epitaxy. A three-phase model is used in the reduction of the ellipsometric data, from which the presence of a transition layer of Ga2Se3, with a thickness of less than $1 \mathrm{~nm}$, is confirmed. These results corroborate the high resolution transmisslon electron microscopy images obtained from the same samples.

04,656

PB95-175378 Not available NTIS

National Inst. of Standards and Technology (EEEL). Gaithersburg, MD. Semiconductor Electronics Div.

Determination of the Optical Constants of ZnSe Films by Spectroscopic EIllpsometry.

Final rept

R. Dahmani, L. Salamanca-Riba, N. V. Nguyen, D

Chandler-Horowltz, and B. T. Jonker. 1994, 4p.

Grant NSF-DMR90-20304

Sponsored by National Science Foundation, Artington, VA. and Offlce of Naval Research, Artington, VA. Pub. in JnI. of Applied Physics 76, $n 1$ p514-517, 1 Jul 94.

Keywords: "Zinc selenides, Molecular beam epitaxy, Semiconducting films, Dielectric properties, Complex numbers, Refractive index, Energy gap, Thin films, Gallium arsenides, Ellipsometry, Substrates, Reprints.

Spectroscopic ellipsometry was used to determine the real and imaginary parts of the dielectric function of $\mathrm{ZnSe}$ thin films grown on (001) GaAs substrates by molecular-beam epitaxy, for energies between 1.5 and $5.0 \mathrm{eV}$. A sum of harmonic oscillators is used to fit the dielectric function in order to determine the values of the threshold energies at the critical polnts. The fundamental energy gap was determined to be at $2.68 \mathrm{eV}$. The $E(0)+D e l t a(0)$ and $E(1)$ points were found to be equal to 3.126 and $4.75 \mathrm{eV}$, respectively. Below the fundamental absorption edge, a Sellmeir-type function was used to represent the refractive index. At the critical points, $E(0)$ and $E(0)+$ Delta(0), the fitting was improved by using an explicit function combining the contributions of these two points to the dielectric function.

\section{4,657}

PB95-175410 Not available NTIS

National Inst. of Standards and Technology (EEEL), Gaithersburg, MD. Electricity Div.

Evidence for Parallel Junctions Within High-Tc Grain-Boundary Junctions.

Final rept.

E. A. Early, R. L. Steiner, A. F. Clark, and K. Char. 1994, 10p.

Pub. in Physical Review B 50, n13 p9409-9418, 1 Oct 94.

Keywords: "Josephson junctions, High temperature superconductors, Altemating current, YBCO superconductors, Grain boundaries, Josephson effect, Parallel orientation, Microwaves, Reprints, Grain boundary junctions, Ytrium barium cuprates.

Half-integral constant voltage steps were observed in many high-T(c) grain-boundary Josephson junctions of $\mathrm{YBa} 2 \mathrm{Cu} 3 \mathrm{O}$ (7-delta) when a microwave field was ap- plied. Five distinct observed behaviors of the widths of both integral and half-integral steps as a function of microwave amplitude, Delta I(dc)(I(ac)), are reproduced by simulations of two or three junctions in parallel. This provides quantitative evidence that a single high-T(c) grain-boundary junction is composed of several junctions in parallel. These junctions are formed by the overlap of superconducting filaments on either side of the grain boundary, and the spacing between ones with relatively large critical currents is approximately 20 micrometers.

\section{4,658}

\section{PB95-175436 Nol available NTIS}

National Inst. of Standards and Technology (EEEL) Boulder, CO. Electromagnetic Technology Div.

Thermal Enhancement of Cotunneling in UItraSmall Tunnel Junctions.

Final rept.

T. M. Eiles, G. Zimmerli, H. D. Jensen, and J. M. Martinis. 1992, 4p.

Contract ONR-NO0014-92-F-0003

Sponsored by Olfice of Naval Research, Arlington, VA Pub. in Physical Review Letters 69, n1 p148-151, 6 Jul 92.

Keywords: "Electron tunneling, Tunnel junctions, Reprints, Single electron tunneling, Coulomb blockade, Thermal enhancement.

We have measured the cotunneling current below the Coulomb blockade threshold in a circuit of two ultrasmall metallic tunnel junctions. The thermal enhancement of cotunneling, as well as the island-charge dependence, is in excellent agreement with theory. Circuit parameters were measured enabling theoretical and experimental values for the cotunneling current to be compared without adjustable parameters. This comparison yielded agreement within the $15 \%$ uncertainty to the experiment. Data showing the cotunneling current near the threshold voltage are also presented.

\subsection{9}

P895-175543 Not available NTIS

National Inst. of Standards and Technology (EEEL), Boulder, CO. Electromagnetic Technology Div.

Standard Reference Devices for High Temperature

Superconductor Critical Current Measurements.

Final rept.

L. F. Goodrich, A. N. Srivastava, and T. C. Stauffer. $1993,7 p$

Pub. in Cryogenics 33, n12 p1142-1148 1993

Keywords: "High temperature superconductors "Electrical measurement, "Critical current, BSCCO superconductors, High-Tc superconductors, Performance evaluation, Design, Reprints, "Standard reference devices.

Obtaining repeatable critical current measurements for a high temperature superconductor (HTS) is a challenging task, as HTSs are highly susceptible to degradation due to mechanical stress, moisture, thermal cycling and aging. This paper discusses the development of a high temperature superconducting standard reference device (SRD) to address these measurement concerns, and gives preliminary data on its characteristics. An SRD is an HTS specimen that has had its critical current /(C) non-destructively evaluated. Because HTSs are sensitive to mechanical alterations, minor changes in sample preparation or mounting procedure could yield large changes in the measured critical current. Preliminary data on SRDs made using $\mathrm{Bi}$ based oxide tapes (2212) with an Ag substrate are presented. Differences between two consecutive measurements of /(c) can typically change by $40 \%$; these deviations have been reduced to about $4 \%$.

\section{4,660}

PB95-175725 Not available NTIS

National Inst. of Standards and Technology (EEEL),

Boulder, CO. Electromagnetic Technology Div.

Experimental Aspects of Flux Expulsion in TypeII Superconductors.

Final rept.

O. B. Hyun. 1993, 5p

Pub. in Physical Review B 48, n2 p1244-1248, 1 Ju 93.

Keywords: "YBCO superconductors, "Niobium stannides, High temperature superconductors, Type 2 superconductors, Temperature dependence, Experimental data, Meissner effect, Magnetic flux, Flux pinning, Magnetization, Diamagnetism, Reprints, "Yttrium banum cuprates. 


\section{Solid State Physics}

Experimental aspects of flux expulsion in Nb3Sn and YBa2Cu3O7 type-II superconductors are presented. There is a clear distinction in magnetization between field-cooled measured-upon-cooling (FCC) and fieldcooled measured-upon-warming (FCW) results. This thermal hysteresis, predicted in the temperature-dependent critical-state model at low fields by Clem and Hao, was observed for measuring fields up to about $0.5 \mathrm{~T}$. The model explains the observation of increases in diamagnetism after field cooling and thermal cycling. The thermal hysteresis, together with weak links, accounts for the occurrence of a negative peak in FCW magnetization. The FCC.FCW bifurcation observed for a $0.1 \mathrm{mT}$ field down to $5 \mathrm{~K}$ might imply that flux lines are not completely frozen below the temperature at which the lower critical field is equal to the measuring field, but are expelled from the sample even in the Meissner state.

\section{4,661}

\section{PB95-175840 Not available NTIS}

National Inst. of Standards and Technology (EEEL),

Gaithersburg, MD. Semiconductor Electronics Div.

Magneto-Transport Properties of $\mathrm{HgCdTe}$.

Final rept.

J. R. Lowney, J. S. Kim, and D. G. Seiler. 1994, 7p.

Pub. in EMIS Data Reviews, p264-270 1994.

Keywords: "Mercury cadmium tellurides, Shubnikov-de Haas effect, Hall effect, Magnetoresistivity, Reprints.

Magnetotransport techniques are widely used to characterize the electrical properties of HgCdTe. The Halleffect method is the most common method of determining the carrier density and mobility of semiconductor materials. Multicarrier characterization techniques are needed for determination of carrier densities and mobilities in a multicarrier system present in layeredstructures or in a variety of complex semiconductor materials. Because of the importance of these methods for proper electrical characterization of complex $\mathrm{HgCdTe}$ single crystals or layered-structures, we discuss this topic pedagogically with the primary objective of promulgation of this emerging tool to the $\mathrm{HgCdTe}$ characterization community. We also discuss magnetoresistance and magnetoresistance-based techniques such as the Shubnikov-de Haas effect and magnetophonon effect, which have been used to characterize $\mathrm{HgCdTe}$ materials. These techniques are ca pable of providing very accurate data if applied with care and proper analysis.

\section{4,662}

\section{PB95-175865 Not available NTIS}

National Inst. of Standards and Technology (EEEL),

Boulder, CO. Electromagnetic Technology Div.

Effect in Environmental Noise on the Accuracy of Coulomb-Blockade Devices.

Final rept.

1993, 4p

Contract ONR-N00014-92-F-0003

Sponsored by Office of Naval Research, Arlington, VA Pub. in Physical Review B 48, n24 p18 316-18 319 , 15 Dec 93.

Keywords: Electron tunneling, Thermal noise, Performance, Accuracy, Reprints, "Coulomb blockade, Electron pumps.

We calculate how noise generated by a finite environmental impedance limits the ultimate performance of Coulomb-blockade devices and possible current or charge standards. We have expressed the environmental theory of the Coulomb blockade in terms of the spectral density of the voltage noise arising from the environment, and have calculated the resulting singlejunction tunneling, and two-junction cotunneling rates. These rates are used to predict the tunneling rate of electrons through a five-junction pump and may explain the anomalously large rates that are observed experimentally.

\section{4,663}

PB95-175873 Not available NTIS

National Inst. of Standards and Technology (EEEL), Boulder, CO. Electromagnetic Technology Div.

Testing for Metrological Accuracy of the Electron Pump.

Final rept.

J. M. Martinis, M. Nahum, and H. D. Jensen. 1994,

$2 p$

Contract ONR-N00014-92-F-0003

Sponsored by Office of Naval Research, Arlington, VA.

Pub. in Physica B 194-196, p1045-1046 1994
Keywords: Metrology, Accuracy, Tests, Reprints "Electron pumps, Single electron tunneling, Coulomb blockage.

We have measured the electron leakage rate through a 5 junction electron pump. Our observed rate of about $0.3 \mathrm{e} / \mathrm{s}$ is several orders of magnitude larger than predicted by thermal activation or cotunneling rates. Our results may be explained by the environmental theory of the Coulomb blockade where noise from the environment causes photon assisted tunneling.

\section{4,664}

PB95-175931 Not available NTIS

National Inst. of Standards and Technology (EEEL), Boulder, CO. Electromagnetic Technology Div.

Numerical Micromagnetic Techniques and Their Applications to Magnetic Force Microscopy Calculations.

O. Oti. 1993,6p.

Pub. in Institute of Electrical and Electronics Engineers Transactions on Magnetics 29, n6 p2359-2364 Nov 93

Keywords: Exchange interactions, Magnetic anisotropy, Magnetic films, Numerical solution, *Micromagnetics, Magnetic force microscopy.

Numerical micromagnetics is a flexible and powerful means of designing and characterizing magnetic devices. This paper presents an overview of numerical methods of solution of micromagnetics problems. The modeling of exchange, anisotropy and magnetostatic interaction fields in magnetic films, and micromagnetic modeling of magnetic force microscopy are discussed.

\section{4,665}

PB95-176061 Not available NTIS

National Inst. of Standards and Technology (EEEL), Boulder, CO. Electromagnetic Technology Div.

dc Magnetic Force Microscopy Imaging of ThinFilm Recording Head.

Final rept.

P. Rice, J. Moreland, and A. Wadas. 1994, 3p.

Pub. in Jnl. of Applied Physics 75, n10 p6878-6800 15 May 94.

Keywords: "Recording heads, "Imaging techniques Atomic force microscopy, Magnetic fields, Magnetic films, Thin films, Direct current, Tips, Reprints, Magnetic force microscopy, Surface topography.

Using a new form of magnetic force microscope (dc MFM) magnetic force images of a thin-film recording head have been made. Using dc MFM, atomic force microscope images are presented of the surface topography and magnetic forces taken simultaneously allowing direct correlation of magnetic fields to the pole pieces. Magnetic force images of the head at typical head-to-disk spacings are presented. The tips used for these images had two different magnetic coatings.

04,666

PB95-176079 Not available NTIS

National Inst. of Standards and Technology (EEEL)

Boulder, CO. Electromagnetic Technology Div,

High Temperature Superconductor-Normal MetalSuperconductor Josephson Junctions with High Characteristic Voltages.

Final rept.

P. A. Rosenthal, E. N. Grossman, R. H. Ono, and L. R. Vale, 1993, 3p.

Pub. in Applied Physics Letters 63, n14 p1984-1986, 4 Oct 93.

Keywords: "Josephson junctions, High temperature superconductors, Superconducting films, Submillimeter waves, Far infrared radiation, YBCO superconductors, Epitaxy, Reprints, Yttrium barium cuprates.

We have fabricated step edge superconductor-normal metal-superconductor microbridges using YBa2Cu3O(7-x) (YBCO) and noble metals with critical current-normal resistance $(\mathrm{l}(\mathrm{C}) \mathrm{R}(\mathrm{N}))$ products as high as $10 \mathrm{mV}$ and normal resistances up to $38 \mathrm{ohms}$. Our fabrication process achieves high values of the I(c) $R(N)$ product by exploiting the anisotropy in the properties of epitaxial YBCO films, allowing contact only between normal metal and superconductor through the crystalline axes which support the largest Josephson coupling. This results in a dramatic in crease in the normal resistance of a junction withou decreasing its critical current. We discuss the role of the superconductor-normal metal boundary resistance on the junction electrical properties. We have coupled submillimeter wave if currents quasioptically into junctions integrated at the feeds of noble metal planar log periodic antennas and have induced up to 7 Shapiro steps in the current-voltage characteristics with a 760 $\mathrm{GHz}$ beam from a far infrared laser.

\section{4,667}

PB95-176095 Not available NTIS

National Inst. of Standards and Technology (EEEL), Boulder, CO. Electromagnetic Technology Div.

Surface Degradation of Superconducting YBa2Cu307-delta Thin Films.

Final rept.

S. E. Russek, S. C. Sanders, A. Roshko, and J. W.

Ekin. 1994, 3p.

Contract ARPA-7975-01

Sponsored by Advanced Research Projects Agency, Arlington, VA.

Pub. in Applied Physics Letters 64, n26 p3649-3651, 27 Jun 94.

Keywords:

*YBCO

superconductors, *Superconducting films, "Surface reactions, *Contact resistance, High temperature superconductors, Scanning tunneling microscopy, Electron diffraction, Time dependence, Amorphous state, Surface layers, Thin films, Carbon dioxide, Vacuum, Nitrogen, Oxygen, Air, Reprints, "Yttrium barium cuprates, Surface degradation.

The surface degradation of c-axis oriented YBa2Cu3O(7-delta) thin films due to air, $\mathrm{CO} 2, \mathrm{~N} 2, \mathrm{O} 2$, and vacuum exposure has been studied with reflection high-energy electron diffraction (RHEED), scanning tunneling microscopy, and contact resistivity measurements. The formation of an amorphous surface reaction layer upon exposure to air and $\mathrm{CO} 2$ is monitored with RHEED and correlated with an increase in contact resistivity. The contact resistivity of samples exposed to air increases with time. Surfaces exposed to $\mathrm{CO} 2$ show a similar degradation while surfaces exposed to N2 showed a slightly different degradation mechanism. Vacuum exposed surfaces show little increase in contact resistivity, indicating no long-term surface oxygen loss.

04,668

PB95-176129 Not available NTIS

National Inst. of Standards and Technology (BFRL), Gaithersburg, MD. Building Materials Div.

Transport and Diffusion in Three-Dimensional Composite Media.

Final rept.

L. M. Schwartz, F. Auzerais, J. Dunsmuir, S.

Torquato, N. Martys, and D. P. Bentz. 1994, 9p.

Pub. in Physica A 207, p28-36 1994.

Keywords: "Diffusion, "Transport properties, "Porous materials, Permeability. Three dimensional models, Grain boundaries Nuclear magnetic resonance, Porosity, Microstructure, Fluid flow, Reprints, " $X$ ray microtomography.

We will review recent progress in our understanding of classical transport in porous media. Theoretical concepts will be illustrated with two distinct kinds of calculations. The first involve the grain consolidation model and are based on a particular multisize packing of spherical grains. The computational methods developed here are sufficiently accurate that we propose to combine them with direct measurements of the pore and grain geometry based on X-ray microtomography. Our preliminary results indicate that this approach may well play an important part in future studies of transport in porous media.

\section{4,669}

PB95-176210 Not available NTIS

National Inst. of Standards and Technology (EEEL), Boulder, CO. Electromagnetic Technology Div.

Magnetic Force Microscopy Images of Magnetic Garnet with Thin-Film Magnetic Tip.

Final rept.

A. Wadas, J. Moreland, P. Rice, and R. Katti. 1994, 3p.

Pub. in Applied Physics Letters 64, n9 p1156-1158, 28 Feb 94.

Keywords: "Ferrite garnets, "Magnetic domains, *Imaging techniques, Atomic force microscopy, Magnetic films, High resolution, Silicon nitrides, Substrates, Thin films, Tips, Reprints, Magnetic force microscopy, Surface topography.

We present magnetic force microscopy images of YGdTmGa/YSmTmGa magnetic garnet, using a thin 
magnetic film deposited on Si3N5 atomic force microscopy tips. We have found correlations between the topography and the magnetic domain structure. We show that by using either magnetized $\mathrm{Fe}$-Ni bilayer tips versus unmagnetized single layer $\mathrm{Fe}$ tips that the image contrast shows domains versus domain walls, respectively.

\section{4,670}

PB95-176327 Not available NTIS

National Inst. of Standards and Technology (EEEL), Boulder, CO. Electromagnetic Technology Div. Noise in the Coulomb Blockade Electrometer. Final rept.

G. Zimmerli, T. M. Eiles, R. L. Kautz, and J. M.

Martinis. 1992, 3p.

Contract ONR-N00014-92-F-0003

Sponsored by Office of Naval Research, Arlington, VA Pub. in Applied Physics Letters 61, n2 p237-239, 13 Jul 92.

Keywords: "Electrometers, Tunnel junctions, Shot noise, Reprints, ${ }^{*}$ Coulomb blockade electrometers, Single electron tunneling, Quantum noise.

We have measured the noise of a Coulomb blockade electrometer. Below $100 \mathrm{~Hz}$, the noise referred to the input charge has a $1 / f$ power spectrum with a charge noise of $3 \times 10(\exp -4)$ e/ $\mathrm{Hz}($ sup 1/2) and an energy sensitivity $E(N)$ of $3 \times 10$ (exp 4)(Planck's constant) at $10 \mathrm{~Hz}$. The $1 / \mathrm{f}$ noise probably results from the stochastic occupation of charge traps which could in principle be eliminated. The theoretical noise floor is set by shot noise, and indirect measurements show that this contribution to $E(N)$ can be as small as 1.5 (Planck's constant), suggesting that the electrometer will be a quantum limited amplifier if the $1 / f$ noise can be eliminated.

04,671

PB95-176335 Not available NTIS

National Inst. of Standards and Technology (EEEL)

Boulder, CO. Electromagnetic Technology Div.

Voltage Gain in the Single-Electron Transistor.

Final rept.

G. Zimmerli, R. L. Kautz, and J. M. Martinis. 1992

$3 p$.

Contract ONR-N00014-92-F-0003

Sponsored by Office of Naval Research, Arlington, VA Pub. in Applied Physics Letters 61, n2 p2616-2618, 23 Nov 92.

Keywords: Tunnel junctions, Voltage gain, Electrometers, Reprints, *Single electron transistors, Single electron tunneling, Coulomb blockade.

We report the first observation of voltage gain in the capacitively coupled single-electron transistor (SET). Using parallel-plate and interdigital geometries for the gate capacitor $(\mathrm{C}(\mathrm{g})=1.2$ and $0.4 \mathrm{fF})$ and ultrasmal tunnel junctions with capacitances near $0.2 \mathrm{fF}$, we find maximum voltage gains of 2.8 and 1.5 , respectively. The leakage resistance of the gate is of the orde 10 (exp 12) ohms for the parallel-plate capacitor and greater than $10(\exp 18)$ ohms for the interdigital capacitor

\section{4,672}

PB95-180071 Not available NTIS

National Inst. of Standards and Technology (EEEL) Boulder, CO. Electromagnetic Technology Div. Increased Transition Temperature in In situ Coevaporated YBa2Cu307-delta Thin Films by Low Temperature Post-Annealing.

Final rept.

S. J. Berkowitz, E. De Obaldia, K. F. Ludwig, R. H.

Ono, J. A. Beali, L. R. Vale, D. A. Rudman, M. L.

O'Malley, L. M. Drabeck, P. A. Polakos, W. J.

Skocpol, and P. M. Mankiewich. 1994, 3p.

Pub. in Applied Physics Letters 65, n12 p1587-1589, 19 Sep 94.

Keywords:

"YBCO

superconductors

*Superconducting films, High-Tc superconductors, Transition temperature, Thin films, Annealing, Strains Reprints, "Yttrium barium cuprates.

In situ coevaporated YBa2Cu3O(7-delta) thin films have a slightly depressed transition temperature $T(c)$, though they have excellent radio-frequency surface resistance characteristics. These films consistently have less orthorhombic strain than laser ablated or post-annealed films. Low temperature $(320-420$ C) post-annealing of in situ coevaporated films in $100 \mathrm{kPa}$ of $\mathrm{O} 2$ raised $\mathrm{T}$ (c) to values as high as $91.5 \mathrm{~K}$ with some increase in the orthorhombic strain. All measured thin films show less variation of $T(c)$ with orthorhombic strain than does bulk material.

\section{4,673}

PB95-180170 Not available NTIS

National Inst. of Standards and Technology (EEEL), Boulder, CO. Electromagnetic Technology Div.

Crossover in the Pinning Mechanism of Anisotropic Fluxon Cores.

Final rept.

L. D. Cooley, A. Gurevich, and D. C. Larbalestier.

1994, 4p.

Sponsored by Department of Energy, Washington, DC. Pub. in Proceedings of International Workshop on Critical Currents in Superconductors (7th), Alpbach, Austria, January 24-27, 1994, p573-576

Keywords: *Flux pinning, *Superconductors, Josephson effect, Crystal defects, Anisotropy, Reprints, Fluxons.

We propose that thin planar defects have a strong magnetic flux-pinning force. Magnetic pinning results from the anisotropic nature of a fluxon when the core is situated in the plane of the defect, and is a consequence of nonlocal Josephson electrodynamics. Since the magnetic pinning force approaches the maximum core-pinning force, a crossover from core pinning to magnetic pinning is predicted when the proximity coupling becomes strong. This model is supported by flux-pinning measurements of an artificial pinning center superconducto

\section{4,674}

PB95-180188 Not available NTIS

National Inst of Standards and Technology (EEEL),

Boulder, CO. Electromagnetic Technology Div. Size and Self-Field Effects in Giant Magnetoresistive Thin-Film Devices.

Final rept.

R. W. Cross, S. E. Russek, S. C. Sanders, S. A Hossian, M. R. Parker, and J. A. Barnard. 1994, 3p. Pub. in Institute of Electrical and Electronics Engineers Transactions on Magnetics 30, n6 p3825-3827 Nov 94. Keywords: "Magnetıc devices, "Magnetoresistance, Sizes(Dimensions), Magnetic films, Metal films, Thin films, Magnetization, Cobalt alloys, Iron alloys, Nickel alloys, Copper, Silver, Reprints, Magnetic multilayers.

Giant magnetoresistance (GMR) was measured as a function of device size for patterned $\mathrm{NiCoFe} / \mathrm{Cu}$ and $\mathrm{NiFe} / \mathrm{Ag}$ films. For the quasi-granular NiCoFe/Cu films, the normalized maximum change in resistivity (Delta)rho/rho was $8 \%$ for most of the samples. For the NiFe/Ag films, antiparallel alignment was achieved through magnetostatic coupling, not exchange fields, with a (Delta) rho/rho of $4.5 \%$. The films were patterned into stripes with Au current leads for size-effect measurements. The height of the stripes varied from 0.5 to 16 micrometers and the track width varied from 1 to 16 micrometers. Discrete switching events and anomalous low-field dips in the response were observed for both materials for small device sizes.

\section{4,675}

PB95-180204 Not available NTIS

National Inst. of Standards and Technology (EEEL), Boulder, CO. Electromagnetic Technology Div.

Novel Bulk Iron Garnets for Magneto-Optic Magnetic Field Sensing.

Final rept.

M. N. Deeter, S. Milian Bon, G. W. Day, G. Diercks,

and S. Samuelson. 1994, 3p.

Pub. in Institute of Electrical and Electronics Engineers Transactions on Magnetics 30, n6 p4464-4466 Nov 94. Keywords: *Ferrite garnets, Yttrium iron garnets, Bismuth additions, Gallium additions, Faraday effect, Frequency response, Response functions, Magneto-optics, Substitutes, Sensitivity, Reprints, "Magnetic field sensors.

We report measurements of the magneto-optic response function and frequency response for three bulk iron garnet crystals grown by a flux technique. The samples were the product of an intensive effort to develop iron garnet compositions with properties specifically optimized for magnetic field sensing. Sensitivity enhancement was achieved through both bismuth substitution (for increasing the saturation Faraday rotation) and gallium substitution (for reducing the saturation magnetization). Frequency response measurements indicate that bismuth substitution actually improves performance (compared to unsubstituted yttrium iron garnet) in contrast with gallium, which causes substantial degradation.

\subsection{6}

PB95-180220 Not available NTIS

National Inst. of Standards and Technology (CSTL), Gaithersburg, MD. Surface and Microanalysis Science Div.

$X$-ray Photoelectron and Auger Electron Forward Scattering: A Structural Diagnostic for Epitaxial Thin Films.

Final rept

W. F. Egelhoff. 1994, 83p

See also PB91-112136 and PB91-112136.

Pub. in Ultrathin Magnetic Structures I: An Introduction

to Electronic, Magnetic, and Structural Properties, Chapter 5.2, p220-302 1994

Keywords: *X ray photoelectron spectroscopy, "Auge electron spectroscopy, *Magnetic films, *Forward scattering, *Epitaxial growth, Electron-atom collisions, EV range 100-1000, Electron scattering, Stacking faults, Magnetism, Quantum mechanics, Thin films, Reviews, Reprints

The technique of X-ray photoelectron and Auger electron forward scattering has developed in the past few years into one of the most valuable structura diagnostics for investigating the early stages of epitaxial thin-film growth. A review is given here of this technique with special emphasis on its application to epitaxial films of interest to the thin-film magnetism community. The aim of this review is to provide an eas ily readable, self-contained account directed primarily at experimentalists, with the hope of making forward scattering accessible to new or occasional users. Some familiarity with XPS and Auger spectroscopy is assumed, as is an elementary knowledge of quantum mechanics.

04,677

PB95-180253 Not available NTIS

National Inst. of Standards and Technology (MSEL), Gaithersburg, MD. Materials Reliability Div.

Magnetic Susceptibility of Pr2-xCexCuO4 Monocrystals and Polycrystals.

Final rept.

M. Foldeaki, H. Ledbetter, and Y. Hidaka. 1994, 8p See also PB92-175264.

Pub. in Jnl. of Magnetism and Magnetic Materials 138 p139-146 1994

Keywords: "Magnetic susceptibility, "Superconductors, Temperature dependence, Crystal field, Single crys tals, Polycrystals, Magnetic moments, Reprints, "Praseodymium cerium cuprates.

We measured the temperature dependence of the magnetic susceptibility of $\mathrm{Pr}(2-x) \mathrm{Ce}(x) \mathrm{CuO} 4$ monocrystals and polycrystals in the composition range $x=0-0.18$. For all compositions, the susceptibility curves reveal strong crystal-field effects and are evaluated in terms of Van Vleck's equation for mag netic susceptibility. At temperatures above $100 \mathrm{~K}$, the behavior could be described by a Curie-Weiss-like equation, the parameters of which allow us to characterize the crystal-field interactions. The concentration dependence of these parameters is much stronger in the monocrystals, with the apparent magnetic momen going through a sharp maximum near $x=0.1$, the composition of the onset of superconductivity after a reducing anneal. The concentration dependence of the mag netic moment can be qualitatively interpreted as a con ribution of charge-carrying spins in localized states, mainly through initiating $\mathrm{Cu}(2+)-\operatorname{Pr}(3+)$ interactions. With increasing $\mathrm{Ce}$, these additional spins can not remain localized, thus the magnetic moment decreases, and a reducing anneal results in superconductivity.

\section{4,678}

PB95-180287 Not available NTIS

National Inst. of Standards and Technology (MSEL), Gaithersburg, MD. Reactor Radiation Div.

Crystal Structure and Magnetic Properties of CuGeO3.

Final rept.

M. Green, M. Kurmoo, J. Stalick, and P. Day. 1994

Pub. in Jnl. of the Chemical Society, Chemical Communications, p1995-19961994.

Keywords: *Crystal structure, Temperature range 0000-0013 K, Temperature range 0013-0065 K, Room temperature, Magnetic susceptibility, Magnetic prop erties, Neutron diffraction, Reprints, "Copper germanates, Rietveld method.

The crystal structure of $\mathrm{CuGeO}$, which has been re ported to undergo a spin-Peierls transition at $14 \mathrm{~K}$, is 


\section{Solid State Physics}

determined by neutron powder diffraction at 300, 20 and $4.2 \mathrm{~K}$ and the structure refined in the space group Pmma at all three temperatures, le without displacement of the $\mathrm{Cu}$ atoms from a uniform one-dimensional chain; anisotropic broadening of (hol) reflections, observed in all three data sets, is successfully modeled by an orthorhombic micro-strain along a and c, i.e. perpendicular to $\mathrm{Cu}-\mathrm{Cu}$ chains. Below $10 \mathrm{~K}$ the magnetic susceptibility fits very well to the Bulaevskii model, indicating a ratio of 0.69 between the $\mathrm{Cu}-\mathrm{Cu}$ exchange constants in the dimerised chain and a mean exchange constant of $88 \mathrm{~K}$

\section{4,679}

PB95-180303 Not available NTIS

National Inst. of Standards and Technology (MSEL), Gaithersburg, MD. Reactor Radiation Div.

Observation of Oscillatory Magnetic Order in the Antiferromagnetic Superconductor HoNi2B2C.

Final rept.

T. E. Grigereit, J. W. Lynn, Q. Huang, J. J.

Krajewski, W. F. Peck, A. Santoro, and R. J. Cava

1994, 4p.

Grant NSF-DMR93-02380

Sponsored by National Science Foundation, Arlington, VA.

Pub. in Physical Review Letters 73, n20 p2756-2759, 14 Nov 94.

Keywords: "Superconductors, Temperature range 0000-0013 K, Phase transformations, Antiferromagnetic materials, Neutron scattering, Oscillations, Reprints, "Holmium nickel boron carbides, "Magnetic ordering.

The nature of the holmium order in the reentrant superconductor $(T(c)=7.5 \mathrm{~K})$ HoNi2B2C is revealed by neutron scattering. Upon cooling, a transversely polarized oscillatory magnetic state is formed ( $T(M)$ approx $=8 \mathrm{~K}$ ), characterized by a wave vector $(0,0, Q(c))$ where $Q(c)$ approx $=0.05 / \mathrm{A}$ is only weakly dependent on temperature and field. At the reentrant superconducting transition (about $5 \mathrm{~K}$ ), the amplitude of the oscillatory state abruptly decreases in favor of a commensurate antiferromagnet, whereby superconductivity is restored and coexists with antiferromagnetism at low temperatures.

04,680

PB95-180345 Not available NTIS

National Inst. of Standards and Technology (CAML), Gaithersburg, MD. Applied and Computational Mathematics Div.

Analytical Expressions for Barkhausen Jump Size Distributions.

Final rept.

F. Y. Hunt, and R. D. McMichael. 1994, 3p

Pub. in Institute of Electrical and Electronics Engineers Transactions on Magnetics 30, n6 p4356-4358 Nov 94.

Keywords: "Barkhausen effect, Langevin equation, Markov chains, Random walk, Bessel functions, Reprints.

In previous calculations of Barkhausen jump size distributions, the Langevin equation was used to describe the pinning field $h(c)$. In this paper, $h(c)$ is modeled by discretized random walks which are used to obtain analytical expressions for the Barkhausen jump size distribution, $P($ tau $)$. For a bounded random walk which reduces to the Langevin function in the continuum limit. $P($ tau $)$ is a sum of exponentials which is compared to functions of the form $P(t a u)=$ tau(sup -alpha) exp(-tau/ tau(0)). The scaling exponent changes from alpha approx $=1.5$ for small jumps to alpha approx $=1.0$ for jumps larger than the correlation length. For an unbounded random walk with exponentially distributed distances between steps in $\mathrm{h}(\mathrm{c}), \mathrm{P}(\mathrm{tau})$ is shown to be proportional to a modified Bessel function which, for long jumps, is asymptotically a pure power law, tau(exp $-3 / 2)$. This suggests that the scaling exponent shift and the exponential cutoff are caused by correlations in $h(c)$.

\section{4,681}

PB95-180402 Not available NTIS

National Inst. of Standards and Technology (EEEL), Boulder, CO. Electromagnetic Technology Div.

Quasipotential and the Stability of Phase Lock in Nonhysteretic Josephson Junctions.

Final rept.

R. L. Kautz. 1994, 7p

Sponsored by Army Test Measurements and Diagnostic Equipment Activity, Redstone Arsenal, AL

Pub. in Jnl. of Applied Physics 76, n9 p5538-5544, 1 Nov 94
Keywords: "Josephson junctions, Josephson effect, Activation energy, Phase locked systems, Therma noise, Stability, Reprints, Voltage standards, Johnson noise, State space, Quasipotentials.

The principle of minimum available noise energy is used to calculate the quasipotential over the state space of a nonhysteretic Josephson junction driven by a if bias. This potential surface provides an intuitive picture of the dynamics of phase lock and defines a stability parameter, the activation energy for thermally induced phase slippage, which determines the optimum operating conditions for a proposed programmable voltage standard.

04,682

PB95-180410 Not available NTIS

National Inst. of Standards and Technology (EEEL), Boulder, CO. Electromagnetic Technology Div. Large-Amplitude Shapiro Steps and Self-Field Effects in High-Tc Josephson Weak Links.

Final rept.

R. L. Kautz, S. P. Benz, and C. D. Reintsema. 1994 $3 p$.

Sponsored by Army Test Measurements and Diagnostic Equipment Activity, Redstone Arsenal, AL.

Pub. in Applied Physics Letters 65, n11 p1445-1447 Sep 94.

Keywords: "Josephson junctions, High-Tc superconductors, Temperature range 0013-0065 K YBCO superconductors, Reprints, *Shapiro steps, Yttrium barium cuprates, Voltage standards, Weak links. We determine contiguous Shapiro steps of orders 0 and 1 having amplitudes of $1 \mathrm{~mA}$ in a $\mathrm{YBa2Cu} 3 \mathrm{O}(7$ delta) step-edge junction operated at $38 \mathrm{~K}$. A wide unction model that includes self-field effects explains why the observed step amplitudes are smaller than expected from the resistively shunted point-junction model. In spite of their reduced amplitudes, the observed steps are suitable for use in a proposed rapidly programmable Josephson voltage standard.

\section{4,683}

PB95-180493 Not available NTIS

National Inst. of Standards and Technology (MSEL) Gaithersburg, MD. Materials Reliability Div.

Dependence of $T c$ on Debye Temperature Theta(sub D) for Various Cuprates.

Final rept

H. Ledbetter. 1994, 2p.

Pub. in Physica C 235-240, p1325-1326 1994

Keywords: "High-Tc superconductors, "Critical temperature, "Debye temperature, Temperature dependence, Mercury inorganic compounds, YBCO superconductors, BSCCO superconductors, Electron phonon interactions, Reprints, Thallium barium calcium cuprates, Lanthanum cuprates.

The author extends the $T(c)$-Theta(D) relationship established by Ledbetter and coworkers for $L a-O$ and $Y$ $\mathrm{O}$, and by Dominec for $\mathrm{Bi}-\mathrm{O}$, to include $\mathrm{TI}-\mathrm{O}$ and $\mathrm{Hg}$ O compounds. In BCS-McMillan materials, Theta(D) occurs both in the pre-exponential factor in the expression T(C) approx = Theta(D) exp (-1/lambda) and in Mc Millan's expression for the electron-phonon paramete lambda $=\mathrm{C} / \mathrm{M}<$ omega squared $>$, where < omega $>$ approx Theta(D). In BCS materials, T(c) increases with decreasing Theta(D), that is with lattice softening. In all five cuprate superconductors listed above, the opposite occurs: T(c) increases with lattice stiffening. From these results, the author draws two principal conclusions. First, T(c) depends on phonons because it depends on Theta(D), the quintessential phonon parameter. Second, the $T(c)-T h e t a(D)$ relationship for high-T(c) oxide superconductors differs dramatically from that for conventional BCS materials.

\section{4,684}

\section{PB95-180527 Not available NTIS}

National Inst. of Standards and Technolog

MD. Materials Reliability Div.

Elastic Constants of a Material with Orthorhombic Symmetry: An Alternative Measurement Approach. Final rept.

M. Lei, H. Ledbetter, and Y. Xie. 1994, 4p.

Pub. in Jnl. of Applied Physics 76, n5 p2738-2741, 1 Sep 94.

Keywords: "Elastic properties, Fiber reinforced composites, Orthorhombic lattices, Ultrasonic radiation, Measurement, Anisotropy, Reprints.

An alternative approach to measuring the complete anisotropic elastic constants $\mathrm{C}(\mathrm{ij})(\mathrm{S}(\mathrm{ij}))$ of an orthorhombic-symmetry material is proposed. This approach requires measurements along only principal directions: 100 . In this approach, a pulse-echo (megahertz-frequency) method was used to measure $C(11)$, $C(22), C(33), C(44), C(55)$, and $C(66)$. A resonance (kilohertz-frequency) method was used to measure three elastic compliances: $S(11), S(22)$, and $S(33)$. Combining the $C$ (ii) with the $S$ (ii), the complete sets of $C(i i)$ and $S(i j)$ were obtained. This approach avoids the troublesome, error-prone determination of off-diagonal $\mathrm{C}(\mathrm{ij})(\mathrm{S}(\mathrm{ij}))$ by the usual measurements along nonprincipal directions. The approach was applied to a boron-aluminum composite where the off-diagonal terms remain uncertain. In principle, the approach applies to any orthorhombic-symmetry material.

\section{4,685}

PB95-180659 Not available NTIS

National Inst. of Standards and Technology (PL), Gaithersburg, MD. Electron and Optical Physics Div. Laser Focused Atomic Deposition.

Final rept.

J. J. McClelland, R. E. Scholten, R. Gupta, and R. J Celotta. 1994, 4p.

Pub. in Proceedings of Society of Photo-Optical Instrumentation Engineers: Laser Techniques for Surface Science, Los Angeles, CA., January 27-29, 1994, v2125 p324-327.

Keywords: "Photon-atom interactions, Vapor deposition, Standing waves, Surface layers, Laser beams, Lithography, Arrays, Reprints, "Laser focusing, "Nanostructures, Chromium atoms, Silicon substrates, Atom optics, Microfabrication.

We have demonstrated the use of a standing-wave laser beam to focus chromium atoms as they deposit onto a silicon surface. A permanent array of $\mathrm{Cr}$ lines has been fabricated, with line width $65 \mathrm{~nm}$, spacing $213 \mathrm{~nm}$, and height $34 \mathrm{~nm}$. The array covers an area of $0.4 \mathrm{~mm} \times 1 \mathrm{~mm}$, and was deposited in approximately 10 minutes. The lines made in this way constitute a proof-of-principle of an entirely new approach to nanostructure fabrication, with potential for extremely small feature size coupled with massive parallelism.

\section{4,686}

PB95-180717 Not available NTIS

National Inst. of Standards and Technology (MSEL), Gaithersburg, MD. Reactor Radiation Div. Magnetic Properties of Single-Crystalline UCu3AI2. Final rept

H. Nakotte, E. Bruck, J. H. V. J. Brabers, V.

Sechovsky, K. H. J. Buschow, A. V. Andreev, R. A

Robinson, A. Purwanto, J. W. Lynn, K. Prokes, and

F. R. de Boer. 1994, 3p

See also DE93016607.

Pub. in Institute of Electrical and Electronics Engineers Transactions on Magnetics 30, n2 p1217-1219 Mar 94. Keywords: Temperature range 0000-0013 K, Aluminum intermetallics, Hexagonal lattices, Magnetic susceptibility, Neutron diffraction, Temperature dependence, Single crystals, Magnetic fields, Reprints, "Uranium copper aluminides, Copper intermetallics, Uranium intermetallics, Powder patterns.

UCu3Al2 crystallizes in an ordered variant of the hexagonal CaCu5 structure. By neutron powder-diffraction, the $U$ atoms were found to occupy the la sites, while the $2 \mathrm{c}$ sites are occupied by $\mathrm{Cu}$ atoms only and a random occupation of the $3 \mathrm{~g}$ sites by the remaining $\mathrm{Cu}$ and $\mathrm{Al}$ is found. The magnetic susceptibility, measured on a single crystal grown by the Czochralski triarc technique is found to be maximal within the hexagonal basal plane with a maximum at about $10 \mathrm{~K}$. For fields applied within the basal plane, the magnetization at $4.2 \mathrm{~K}$ exhibits a slight $\mathrm{S}$-shape starting slightly below $15 \mathrm{~T}$. No such anomalies are found for fields applied along the $\mathrm{c}$-axis where the magnetic response is found to be much lower. No additional magnetic peaks, which could be related with long-range antiferromagnetic ordering, were detected in the neutron powder-patterns at low temperatures.

04,687

PB95-180725 Not available NTIS

National Inst. of Standards and Technology (EEEL)

Boulder, CO. Electromagnetic Technology Div.

Epitaxial Growth and Characterization of the Ordered Vacancy Compound Culn3Se5 on GaAs (100) Fabricated by Molecular Beam Epitaxy.

Final rept.

A. J. Nelson, M. Bode, G. Horner, K. Sinha, and J.

Moreland. 1994, 5p.

Pub. in Materials Research Society Symposia Proceedings, v340 p599-603 1994. 
Keywords: "Epitaxial growth, Molecular beam epitaxy, Transmission electron microscopy, Atomic force $\mathrm{mi}$ croscopy, X ray diffraction, Raman spectra, Gallium arsenides, Single crystals, Stacking faults, Energy gap, Photoluminescence, Substrates, Films, Reprints, ${ }^{*}$ Copper indium selenides.

Epitaxial growth of the ordered vacancy compound OVC) Culn3Se5 has been achieved on GaAs (100) by molecular beam expitaxy (MBE) from Cu2Se and In $2 \mathrm{Se} 3$ sources. Electron probe microanalysis and $X$ ray diffraction have confirmed the composition for the 1-3-5 OVC phase and that the film is single crystal Culn3Se5 (100). Transmission electron microscopy TEM) characterization of the material also showed it to be single crystalline. Structural defects in the layer consisted mainly of stacking faults. Photoluminescence (PL) measurements performed at $7.5 \mathrm{~K}$ indicate that the bandgap is $1.28 \mathrm{eV}$. Raman spectra reveal a strong polarized peak at $152 / \mathrm{cm}$ which is believed to arise from the totally symmetric vibration of the Se atoms in the lattice. Atomic force microscopy reveals faceting in a preferred (100) orientation.

04,688

PB95-180881 Not available NTIS

National Inst. of Standards and Technology (MSEL), Gaithersburg, MD. Reactor Radiation Div.

High Resolution Inelastic Neutron Scattering Study of Phonon Self-Energy Effects in YBCO.

Final rept

D. Reznik, B. Keimer, F. Dogan, and I. A. Aksay.

1994, 2p.

Keywords: "YBCO superconductors, "Phonons, Temperature range 0013-0065 $\mathrm{K}$. Temperature range 0065-0273 K, High resolution, Neutron scattering, Inelastic scattering, High-Tc superconductors, Self-energy, Single crystals, Reprints, "Yttrium barium cuprates.

We report preliminary results of the high resolution inelastic neutron scattering measurements of the 42.5 meV optical phonon branch in YBCO whose behavio at momentum transfer $q=0$ has been extensively studat momentum transter $q=0$ has been extensively studa large $(75 \mathrm{~g})$ single crystal of $\mathrm{YBa}$ 2Cu3O(7-x) (T(c) approx $=90 \mathrm{~K}$ ) with the resolution of $2 \mathrm{meV}$ (full width at half maximum). In cooling from $100 \mathrm{~K}$ to $50 \mathrm{~K}$, we observe a small softening of the phonon energy at $q=0.25,0.25,0$, but no significant linewidth change

04,689

PB95-180980 Not available NTIS

National Inst. of Standards and Technology (EEEL) Boulder, CO. Electromagnetic Technology Div

Scanning Tunneling Microscopy of the ChargeDensity-Wave Structure in 1 T-TaS2.

Final rept.

R. E. Thomson, B. Burk, A. Zettl, and J. Clarke

994, 18p

Contract DE-AC03-76F00098, Grant NSF-DMR9017254

Sponsored by Department of Energy, Washington DC., Office of Naval Research, Arlington, VA., and Naional Science Foundation, Arlington, $V A$

Pub. in Physical Review B 49, n24 p16 899-16 916 15 Jun 94

Keywords: *Tantalum sulfides, Temperature range 0065-0273 K, Temperature range 0273-0400 K, Scanning tunneling microscopy, Fourier transformation, Domain structure, Reprints.

Using scanning tunneling microscopy (STM), we studied 1T-TaS2 in all four of its charge-density-wave CDW) supporting phases over the temperature range 360-143 K. Special attention was given to the search for discommensurate structures and the distinguishing of true CDW discommensurations and domains from apparent discommensurations and domains formed by interference between the CDW and the atomic lattice. In the lowest-temperature commensurate (C) phase, we find that the CDW is in the commensurate configuration as expected. In the nearly commensurate (NC) phase, the CDW is in a true domain structure as evidenced by satellite spots in the Fourier transforms of the STM images. In the triclinic (T) phase, the STM data indicate that the CDW is in a striped domain phase, which is significantly different from the domain model previously proposed. Finally, in the high-temperature incommensurate (I) phase, we find unexpected satellite spots in the STM Fourier transforms, suggesting that a CDW modulation is also present in this phase.
04,690

PB95-181186 Not available NTIS

National Inst. of Standards and Technology (EEEL)

Boulder, CO. Electromagnetic Technology Div.

Novel YBa2Cu307-x and YBa2Cu307-x/Y4Ba3O9 Multilayer Films by Bias-Masked 'On-Axis' Magnetron Sputtering.

Final rept

J. H. Xu, G. G. Zheng, A. M. Grishin, J. Moreland, B

M. Moon, and K. V. Rao. 1994, 3p.

Pub. in Applied Physics Letters 64, n14 p1874-1876, 4 Apr 94.

Keywords: "YBCO superconductors

*Superconducting films, "Dielectric films, High temperature superconductors, Scanning tunneling microscopy, Strontium titanates, Magnetrons, Sputtering, Substrates, Reprints, *Yttrium barium cuprates, Lanthanum aluminates, Multilayers

In situ YBa2Cu3O(7-x) (YBCO) films have been fabricated on $\mathrm{SrTiO} 3(001)$ and $\mathrm{LaAlO} 3$ (001) substrate by on-axis biased-radio-frequency magnetron sputtering in $\mathrm{Ar}-10 \% \mathrm{O} 2$ at total pressures as low as $3 \mathrm{~Pa}$ $0.03 \mathrm{mbar}$ ) and a deposition rate $210 \mathrm{~nm} / \mathrm{h}$. Negative oxygen ion-resputtering has been considerably reduced by introducing a biased copper mask between the substrate and target. The surface morphology and physical properties of the films are greatly improved on applying a positive dc substrate bias with respect to grounded deposition chamber. We have obtained superconducting YBCO films with transport critical current as high as $10(\exp 6) \mathrm{A} / \mathrm{sq} \mathrm{cm}$ at $77 \mathrm{~K}$ and low normal-state resistivity by this approach. However, films deposited by negative dc bias under identica sputtering conditions are insulating. We identify the in sulating films 10 be c-axis oriented $\mathrm{Y} 4 \mathrm{Ba} 3 \mathrm{O} 9$ (YBO) films. Furthermore, YBCO films could be grown on the YBO layers without any degradation of T(sub C) and $\mathrm{c}$-axis orientation. This novel bias sputtering feature gives us a unique opportunity to produce superconductor/insulator, $\mathrm{YBCO} / \mathrm{YBO}$, multilayers from a single YBCO target.

04,691

PB95-202370 Not available NTIS

National Inst. of Standards and Technology (PL), Boulder, CO. Quantum Physics Div.

Laser Vacuum Ultraviolet Single Photon Ionization Probing of III-V Semiconductor Growth.

Final rept.

S. M. Casey, A. L. Alstrin, A. K. Kunz, and S. R.

Leone. 1994, 10p.

Contract ACS-PRF-27135-AC6, Grant NSF-PHY9012244

Sponsored by National Science Foundation, Arlington VA. and Petroleum Research Fund, Washington, DC Pub. in Proceedings of Society of Photo-Optical Instru mentation Engineers - Ultraviolet Technology V, San Diego, CA., July 26-27, 1994, v2282 p39-48.

Keywords: *Molecular beam epitaxy, "Epitaxial growth, "Gallium arsenides, Time-of-flight mass spectrometers, Vacuum ultraviolet radiation Neodymium lasers YAG lasers, Laser radiation, Photoionization, Reprints.

Epitaxial growth of III-V semiconductor materials is probed in a molecular beam epitaxy reactor by single photon ionization of the gaseous fluxes using vacuum ultraviolet (VUV) laser radiation. The ninth harmonic of the Nd:YAG laser is produced by frequency tripling the output to $355 \mathrm{~nm}$ and then to $118 \mathrm{~nm}$ in a Xe/Ar mixture. Together with a time-of-flight mass spectrometer this radiation is used to selectively probe the gaseou fluxes of Ga, As, As2, and As4 during molecular bear epitaxy of III-V materials. The essential aspects of the method and details of calibration procedures to obtain relative fluxes are described. Recent work to correlate the flux determinations with Reflection High Energy Electron Diffraction (RHEED) oscillations during GaAs epitaxial growth will be discussed.

\section{4,692}

PB95-202487 Not available NTIS

National Inst. of Standards and Technology (PL) Gaithersburg, MD. Quantum Metrology Div. Early History and Future Outlook for the X-ray Crystal Density Method.

Final rept.

attes $1994,7 p$

Pub. in Metrologia 31, p173-179 1994.

Keywords: *Crystal structure, $X$ ray diffraction, Historical aspects, Reprints, $X$ ray interferometry, Avogadro constant.
It is useful to recall that the (late nineteenth-century) Baltimore Lectures of Lord Kelvin indicated that the likely sizes of atomic particles spanned almost two decades. Yet in the early years of the present century, Sir William Bragg's ansatz, together with the oil-drop e, gave the first reliable estimate of the scale of crystal interplanar spacings. The converse process of using an XRCD approach to obtain a value for the Avogadro constant was prior to the advent of $x$-ray interferometry, limited by the need to connect optical and $x$-ray wavelengths before using the latter to estimate unit cell dimensions in crystals. Other limitations of these early measurements included the use of water as a density standard and the assignment of molar masses to individual specimens based on geochemical abundance averages. All these difficulties were overcome, in principle, with the application of x-ray/optical interferometry to the determination of lattice periods, the use of solid object density standards, and the determination of densities and isotopic abundances on individual monocrystalline specimens. While the present-day situation is addressed in other contributions to this workshop, the present essay attempts to place some of the early work in context and to look also to the future.

\section{4,693}

\section{PB95-202560 Not available NTIS}

National Inst. of Standards and Technology (MSEL),

Gaithersburg, MD. Reactor Radiation Div.

Epitaxial Growth of Sb/GaSb Structures: An Example of V/III-V Heteroepitaxy.

Final rept.

J. A. Dura, A. Vigliante, T. D. Golding, and S. C.

Moss. $1995,7 p$

Sponsored by

Pub. in Jnl. of Applied Physics 77, n1 p21-27, 1 Jan

Keywords: "Gallium antimonides, "Gallium arsenides "Antimony, "Molecular beam epitaxy, "Epitaxial growth, Semiconducting films, $X$ ray diffraction, Crystal growth, Reprints, Heteroepitaxial films,

The requirements for heteroepitaxial growth of $\mathrm{Sb}$ on both GaSb and GaAs, and the subsequent growth of GaSb on Sb, using molecular-beam epitaxy are described. These systems serve as examples of the heteroepitaxy of group- $V$ elements with III-V compounds, i.e., between materials using different bonding and possessing different electronic properties. The quality of the films was determined using high-resolution four-circle $x$-ray diffraction, and comparisons were made between different structures. GaSb was found to grow (III) oriented on $\mathrm{Sb}$ (III) with an inverted stacking sequence. A simple epitaxial model is proposed to explain this.

\section{4,694}

PB95-202602 Not available NTIS

National Inst. of Standards and Technology (PL), Boul-

der, CO. Quantum Physics Div. Enhancement of
Hole Dispersion and Antiferromagnetic Interaction of Localized Spins in High-Tc Superconductors.

Final rept.

V. V. Flambaum and O. P. Sushkov. 1992, 7p.

Pub. in Physica C 190, p453-459 1992

Keywords: *High-Tc superconductors, "Hole mobility, Spin spin interactions, Holes(Electron deficiencies), Etfective mass, Hubbard model, Band theory, Antiferromagnetism, Cuprates, Reprints.

In the framework of the extended Hubbard model (copper-oxygen and oxygen-oxygen hopping), the energy and effective mass of a mobile hole is expressed in terms of hopping parameters and the correlators between spins of the background $(S(n) S(n+1))$. Due to interaction of the mobile hole with localized holes, its effective mass increases about four times.

\subsection{5}

PB95-202768 Not available NTIS

National Inst. of Standards and Technology (MSEL), Boulder, CO. Materials Reliability Di

Off-Diagonal Orthorhombic-Symmetry Elastic Constants.

Final rept

S. Kim. $1994,2 p$.

Pub. in Applied Physics Letters 65, n23 p2949-2950, 5 Dec 94

Keywords: *Elastic properties, Orthorhombic lattices, Cubic lattices, Tetragonal lattices, Hexagonal lattices, 
Ultrasonic radiation, Young modulus, Fiber composites, Gallium, Indium, Lithium, Zinc, Stiffness, Reprints.

We derive analytical expressions for off-diagonal elastic stiffnesses $C(12), C(13)$, and $C(23)$ in terms only of easily and precisely measurable diagonal elastic stiffnesses and Young moduli. We verify the expressions for several symmetry classes: cubic (Li), hexagonal ( $\mathrm{Zn}$ ), tetragonal (In), orthorhombic (Ga), and a weakly orthorhombic fiber composite: B/AI.

\section{4,696}

PB95-203063 Not available NTIS

National Inst. of Standards and Technology (PL), Boulder, CO. Quantum Physics Div.

Construction of Silicon Nanocolumns with the Scanning Tunneling Microscope.

Final rept.

R. M. Ostrom, D. M. Tanenbaum, and A. Gallagher. 1992, 3p.

Pub. in Applied Physics Letters 61, n8 p925-927, 24 Aug 92.

Keywords: Scanning tunneling microscopy, Construction, Reprints, *Silicon nanocolumns, "Nanostructures, Nanolithography, Nanotechnology.

Voltage pulses to a scanning tunneling microscope (STM) are used to construct silicon columns of 30-100 A diameter and up to $200 \mathrm{~A}$ height on a silicon surface and on the end of a tungsten probe. These nanocolumns have excellent conductivity and longevity, and they provide an exceptional new ability to measure the shapes of nanostructures with a STM. This construction methodology and these slender yet robust columns provide a basis for nanoscale physics, lithography, and technology.

\section{4,697}

\section{PB95-203345 Not available NTIS}

National Inst. of Standards and Technology (PL), Boulder, CO. Quantum Physics Div.

Deposition Rates in Direct Current Diode Sputtering.

Final rept.

G. C. Stutzin, K. Rozsa, and A. Gallagher. 1993

10p.

Contract SERI-DD-1-11001-1

Sponsored by Solar Energy Research Inst., Golden, CO.

Pub. in Jnl. of Vacuum Science and Technology A 11 n3 p647-656 May/Jun 93.

Keywords: *Sputtering, Electric discharges, Direct current, Thin films, Silicon, Reprints, Deposition rates, Sputtered films.

A physical model for anode deposition rates in parallelplate, dc-diode sputtering is presented and tested. The deposition rate has been measured as a function of the discharge voltage, the anode-cathode gap and the pressure, using silicon or molybdenum cathodes with argon. Deposition rates as high as $23 \mathrm{~A} / \mathrm{s}$ were obtained using $3 \mathrm{kV}$, and it is shown that deposition efficiency is similar to that obtained with magnetrons. The deposition rate varies inversely with anode-cathode gap at constant pressure and voltage and almost linearly with power density for discharge voltages above a threshold which depends on the cathode material. These observations are explained by the model, which can be used to estimate the deposition rates for any cathode material and gas for which the ion sputtering yields are known. Potential advantages and complications of $\mathrm{dc}$ diode sputtering for thin film deposition are discussed, and optimum conditions for high, uniform deposition rates are described.

\section{4,698}

PB95-203378 Not available NTIS

National Inst. of Standards and Technology (MSEL), Gaithersburg, MD

Linking Anisotropic Sharp and Diffuse Surface Motion Laws via Gradient Flows.

Final rept.

J. E. Taylor, and J. W. Cahn. 1994, 15p.

Sponsored by National Science Foundation, Arlington, VA. and Advanced Research Projects Agency, Arlington, VA.

Pub. in Jnl. of Statistical Physics 77, n1/2 p183-197 1994.

Keywords: *Surface diffusion, Partial differential equations, Laplacian, Curvature, Anisotropy, Reprints.

We compare four surface motion laws for sharo surfaces with their diffuse interface counterparts by means of gradient flows on corresponding energy functionals. The energy functionals can be defined to give the same dependence on normal direction for the energy of sharp plane surfaces as for their diffuse counterparts. The anisotropy of the kinetics can be in corporated into the inner product without affecting the energy functional.

\section{4,699}

PB95-203394 Not available NTIS

National Inst. of Standards and Technology (EEEL), Boulder, CO. Electromagnetic Technology Div.

Surface Modification of YBa2Cu307-delta Thin Films Using the Scanning Tunneling Microscope: Five Methods.

Final rept.

R. E. Thomson, J. Moreland, and A. Roshko. 1994,

$13 p$

Pub. in Nanotechnology 5, p57-69 1994.

Keywords: "YBCO$$
994 .
$$
superconductors, Scanning tunneling microscopy Thin films, Reprints, "Yttrium barium cuprates, *Surface modification, Nanolithography.

We have investigated using the scanning tunneling microscope (STM) as a tool for surface modification of YBa2Cu3O(7-delta) (YBCO) thin films and have identified five distinct methods whereby the STM tip can modify the superconductor surface. (i) By lowering the tunneling resistance we make the tip scratch or 'mill' the sample surface mechanically. (ii) By increasing the bias voltage above about $4 \mathrm{~V}$ we can modify the surface by an apparent electron beam damaging process. (iii) By increasing the bias voltage above $10 \mathrm{~V}$ and rais ing the tunneling current, we can cause a more dramatic effect which is probably due to a thermal process. (iv) By operating the STM in a damp carbon dioxide atmosphere we can cause the STM tip to etch the surface electrochemically. (v) Finally, we have some preliminary data suggesting that the high field under an extremely sharp tip displaces the oxygen atoms in the YBCO lattice. Examples of each of these techniques are shown and discussed.

\section{4,700}

PB95-220430 PC A09/MF A02

National Inst. of Standards and Technology (MSEL) Gaithersburg, MD. Reactor Radiation Div.

NIST Reactor: Summary of Activities, October 1993 through September 1994.

C. L. O'Connor. Apr 95, 184p, NISTIR-5594.

Keywords: "NBSR reactor, Research reactors, Activation analysis, Cold neutrons, Crystal structure, Neutron diffraction, Neutron radiography, Nondestructive tests, Boron nitrides, Surface chemistry, Polymers.

The report summarizes all the programs which use the NIST reactor. It covers the period for October 1993 through September 1994. The programs range from the use of neutron beams to study the structure and dynamics of matierls through nuclear physics and neutron standards to sample irradiations for activation analysis, isotope production, neutron radiography, and nondestructive evaluation.

\section{4,701}

\section{PB95-255204 PC A99/MF E11}

National Inst. of Standards and Technology Gaithersburg, MD.

Proceedings of the Applied Diamond Conference 1995: Applications of Diamond Films and Related Materials International Conference (3rd). Held in Gaithersburg, Maryland, on August 21-24, 1995.

Special pub.

A. Feldman, Y. Tzeng, W. A. Yarbrough, M

Yoshikawa, and M. Murakawa. Aug 95, 975p, NIST/ SP-885, ISBN-1-886843-01-5.

Also available from Supt. of Docs. See also PB95256053. Prepared in cooperation with Pennsylvania State Univ., University Park., Nippon Inst. of Tech. Saitama., Auburn Univ., AL. and Tokyo Inst. of Technology (Japan).

Keywords: "Diamonds, "Thin films, "Meetings, Vapor deposition, Electron emission, Cutting tools, Crys tallization, Nucleation, Optics, Brazing, Wear, Composite materials, Modeis.

Interest in commercializing diamond technology and related materials technologies continues to grow. This can be attested to by the large number of abstracts received for this meeting, the Third International Conference on the Applications of Diamond Films and Re- lated Materials. More than 300 abstracts were received. Cutting tools coated with CVD diamond are of immediate commercial interest. Other applications, such as diamond windows and diamond heat spreaders, are developing but at a slower pace. Interest in cubic boron nitride is receiving more attention because of its resistance to oxidation and because it can be used on tools to machine ferrous materials.

04,702

PB96-102066 Not available NTIS

National Inst. of Standards and Technology (EEEL), Boulder, CO. Electromagnetic Technology Div

Step-Edge and Stacked-Heterostructure High-Tc Josephson Junctions for Voltage-Standard Arrays. Final rept.

S. P. Benz, C. D. Reintsema, R. H. Ono, G. F

Virshup, J. N. Eckstein, and I. Bozovic. 1995, 4p

Sponsored by Department of the Army, Washington DC., Naval Research Lab., Washington, DC. and Office of Naval Research, Arlington, VA.

Pub. in Institute of Electrical and Electronics Engineers Transactions on Applied Superconductivity, v5 n2 p2915-2918 Jun 95.

Keywords: "Josephson junctions, "Electric potential, "Arrays, "Transition temperature, Comparative evaluations, High temperature, Bias, YBCO superconductors, Epitaxy, Heterojunction devices, Electric current, Resistance, Reprints, SNS junctions, Shapiro steps, Voltage-standa rd arrays.

The authors have explored two high-transition temperature Josephson junction technologies for application in voltage standard arrays: step-edge junctions made with TBa2Cu3O(7-delta) and Au normal-metal bridges, and stacked series arrays of Josephson junctions in selectively doped, epitaxially grown $\mathrm{Bi} 2 \mathrm{Sr} 2 \mathrm{CaCu} 2 \mathrm{O} 8$ heterostructures. For both kinds of junctions, Shapiro steps induced by a microwave bias were characterized as a function of power. The authors compare the two technologies with respect to critical current and normal resistance uniformity, maximum achievable critical current, critical-current normal resistance product, and operating temperature.

\section{4,703}

PB96-102157 Not available NTIS

National Inst. of Standards and Technology (EEEL) Gaithersburg, MD. Electricity Div.

Application of Single Electron Tunneling: Precision Capacitance Ratio Measurements.

Final rept.

A. F. Clark, N. M. Zimmerman, E. R. Williams, F. C. Wellstood, C. J. Lobb, R. J. Soulen, A. Amar, and D. Song. 1995, 3p.

Pub. in Applied Physics Letters, v66 n19 p2588-2590, 8 May 95.

Keywords: "Metrology, "Electron tunneling, *Capacitance, Capacitors, Electric bridges, Cryogenic equipment, Precision, Ratios, Electron spectroscopy, Superconductivity, Electrometers, Reprints, SET(Single electron tunneling).

A metrological application is reported of the single electron tunneling (SET) phenomena: a precise measurement of the ratio of two cryogenic capacitors. The measurement used a superconducting SET electrometer as the null detector for a capacitance bridge. A 3-ppm level of imprecision has been achieved in the measurement of the capacitance ratio from 100 to $1000 \mathrm{~Hz}$. Further improvements can be made in the attempt to obtain an imprecision of 10(exp-8) at lower frequencies, sufficient for the metrological measurement of capacitance or the fine-structure constant using a SET pump.

04,704

PB96-102264 Not available NTIS

National Inst. of Standards and Technology (EEEL), Gaithersburg, MD. Electricity Div.

Physical Basis for Half-Integral Shapiro Steps in a DC SQUID.

Final rept.

E. A. Early, A. F. Clark, and C. J. Lobb. 1995, 13p. Pub. in Physica C, v245 p308-320 1995.

Keywords: *SQUID(Detectors), *Direct current, "Josephson junctions, Alternating current, Dynamic characteristics, Equations of motion, Projectory analysis, Energy storage, Potential, Reprints, DC SQUID, AC Josephson effect, Shapiro steps

The dynamics of a DC SQUID is analogous to the classical dynamics of a particle subject to conservative, 
damping, and driving forces in two dimensions. The equations of motion define a trajectory on a potentialenergy surface derived from the conservative forces, the components of which correspond to different forms of stored energy in the SQUID. In the presence of a periodic driving force, half-integral Shapiro steps are possible when the trajectory follows a zig-zag path between minima of the potential surface. This description of the dynamics in terms of a potential surface provides an intuitive, physical basis for previous simulation results on half-integral Shapiro step in a DC SQUID.

\section{4,705}

PB96-102272 Not available NTIS

National Inst. of Standards and Technology (EEEL),

Boulder, CO. Electromagnetic Technology Div.
Stacked Series Arrays of High-Tc Trilayer Josephson Junctions.

Final rept.

J. N. Eckstein, I. Bozovic, G. F. Virshup, R. H. Ono,

and S. P. Benz. 1995, 4p.

Pub. in Institute of Electrical and Electronics Engineers Transactions on Applied Superconductivity, v5 n2 p3284-3287 Jun 95

Keywords: "Josephson junctions, "Molecular beam epitaxy, Phase locked systeins, High temperature, Current density, Superconductors, Alternating current, Wafers, Layers, Reprints.

The authors report on the properties of stacked series arrays of trilayer Josephson junctions grown by atomic layer-by-layer molecular beam epitaxy. Trilayer Josephson junctions oriented so that the current travels in the $\mathrm{c}$-axis direction have been described previously. Series arrays are made by placing more than one barrier layer in the Ba2Sr2CaCu2O8-based, (2212) epitaxial structure. Single molecular layers of 2212 doped with Dy to reduce the local carrier concentration are used as barriers, and are placed very close to each other, e.g., separated by only a few molecular layers of the superconducting phase. Phase locking of a.c. Josephson currents has been observed. The critica current density of such junctions has been observed to be very uniform on wafers that are free of second phase defects, and operation up to $60 \mathrm{~K}$ has been obtained.

\subsection{6}

PB96-102421 Not available NTIS

National Inst. of Standards and Technology (MSEL), Gaithersburg, MD. Reactor Radiation Div.

Lattice Dynamics of $\mathrm{Ba} 1-x \mathrm{~K} \times \mathrm{BiO} 3$.

Final repl.

M. A. Green, K. Prassides, P. Day, and D. A.

Neumann. 1995, 2p

Pub. in Synthetic Metals, v71 p1619-1620 1995.

Keywords: "Crystal lattices, "Superconductivity, "Density measurement, Temperature, Doped materials, Energy gaps(Solid state), Energy levels, Transitions, Reprints.

Neutron weighted density-of-states measurements of $\mathrm{Ba}(1-\mathrm{x}) \mathrm{K}(\mathrm{x}) \mathrm{BiO} 3$ are presented which show pronounced changes as a function of both temperature and $\mathrm{Bi}$ oxidation states. Information is gained on both the structural and metal-insulator transitions occurring with increased potassium doping. Phonon anomalies associated with the appearance of a superconducting energy gap gives strong evidence of electron-phonon coupling and superconductivity via a conventional BCS mechanism.

\section{4,707}

\section{PB96-102439 Not available NTIS}

National Inst. of Standards and Technology (MSEL), Gaithersburg, MD. Reactor Radiation Div.

Structure and Conductivity of Layered Oxides $(\mathrm{Ba}, \mathrm{Sr}) \mathrm{n}+1(\mathrm{Sn}, \mathrm{Sb}) \mathrm{nO} \mathrm{n}+1$

Final rept.

M. A. Green, K. Prassides, P. Day, and J. K. Stalick. 1995, 2p.

Pub. in Synthetic Metals, v71 p1617-1618 1995.

Keywords: "Crystal structure, "Superconductivity, "Neuron diffraction, Oxides, Metal powder, Energy gaps(Solid state), Hueckel theory, Barium oxides, Strontium compounds, Tin compounds, Antimony compounds, Reprints.

Structures of various members of the RuddlesdonPopper homologous series, (BaSr) $n+1(\mathrm{Sn}, \mathrm{Sb}) \mathrm{nO} 3 n+1$ solved by Rietveld refinement of powder neutron diffraction data are summarized. Predictions of band gaps are made on the basis of extended Huckel calculations.
04,708

PB96-102447 Not available NTIS

National Inst. of Standards and Technology (MSEL)

Gaithersburg, MD. Reactor Radiation Div.

Lattice Dynamics of Semiconducting, Metallic, and Superconducting $\mathrm{Ba} 1-\mathrm{xK} \times \mathrm{BiO} 3$ Studied by Inelastic Neutron Scattering.

Final rept.

M. A. Green, K. Prassides, D. A. Neumann, and P.

Day. $1995,6 p$

Pub. in Chemistry of Materials, v7 p888-893 1995

Keywords: "Crystal lattices, "Superconductors, "Semiconductors(Materials), "Neutron scattering, Chemical composition, Phase diagrams, Temperature, Energy levels, Phase transformations, Barium oxides, Potassium, Bismuth oxides, BCS theory, Reprints.

Inelastic neutron scattering has been used to study the lattice dynamics in $\mathrm{Ba}(1-\mathrm{x}) \mathrm{K} \times \mathrm{BiO} 3$ over a wide range of compositions, covering regions of the (composition (x), temperature (T)) phase diagram displaying semiconducting, metallic and superconducting behavior. Modes are assigned using interatomic potentials calculations, which show good agreement with experiment. The oxygen phonon modes are very sensitive to changes in composition and oxidation state of the Bi ions. Phonon anomalies observed in both the oxygen TO bending (about $33 \mathrm{meV}$ ) and stretching (about $62 \mathrm{meV}$ ) modes as a function of temperature are discussed within the context of the BCS theory. Novel behavior is shown by the LO oxygen bending mode at about $42 \mathrm{meV}$ that shows a strong dependence on composition, proposed to arise from the occurrence of structural phase transitions in this system.

\section{4,709}

PB96-102462 Not available NTIS

National Inst. of Standards and Technology (EEEL), Boulder, CO. Electromagnetic Technology Div.

$30 \mathrm{THz}$ Mixing Experiments on High Temperature Superconducting Josephson Junctions.

Final rept.

E. N. Grossman, L. R. Vale, D. A. Rudman, K. M.

Evensen, and L. R. Zink. 1995, 4p.

Pub. in Institute of Electrical and Electronics Engineers Transactions on Applied Superconductivity, v5 n2 p3061-3064 Jun 95.

Keywords: "Josephson junctions, "Superconductors *Heterodyning, "Hot electrons, High temperature, Infrared radiation, Signal mixing, Bias, Microwaves, Reprints, SNS Josephson junctions, High TC Josephson junctions.

The authors have investigated YBz2Cu3O7-delta superconductor- normal-superconductor Josephson junctions as mixers of $30 \mathrm{THz}$ radiation. The authors have directly observed (2nd order) differencefrequencies from $10 \mathrm{MHz}$ to $12.8 \mathrm{GHz}$ be. tween two $\mathrm{CO} 2$ laser lines. Applying a third microwave signal to the junction, the authors have observed $\mathrm{CO} 2$ laser difference frequencies up to $27 \mathrm{GHz}$. The d.c. bias dependence of the difference frequency signal, as well as other evidence, suggests two distinct mixing mechanisms: hot-electron mixing in the junction banks at high d.c. biases, and bolometric Josephson mixing at low d.c. biases. The latter is the first observation of Josephson mixing at $\mathrm{CO} 2$ laser frequencies in high$\mathrm{T}$ (sub $\mathrm{C}$ ) junctions. The Josephson mixing has generated observable mixing products up to 6 th order.

\section{4,710}

PB96-102470 Not available NTIS

National Inst. of Standards and Technology (CSTL),

Gaithersburg, MD. Inorganic Analytical Research Div. Effect of Stoichiometry on the Phases Present in Boron Nitride Thin Films.

Final rept.

L. B. Hackenberger, L. J. Pilione, R. Messier, and G

P. Lamaze. 1994, 7p

Pub. in Jnl. of Vacuum Science and Technology A 12, n4 p1569-1575 Jul/Aug 94.

Keywords: "Boron nitrides, "Thin films, "Stoichiometry, Nondestructive tests, Neutron absorption, Chemical composition, Solids, Phase, Chemical analysis, Reprints, NDP(Neutron depth profiling)

Boron nitride thin films were deposited by ion-assisted evaporation and characterized by neutron depth profiliny (NDP), a nondestructive method for the composition:al analysis of solids. The phases present in the films we:a determined by infrared spectroscopy. Examination of $t r i$ data presented here and compari- son with the work of other authors revealed that stoichiometric or nearly stoichiometric films contained the greatest amount of the cubic phase. This led to the proposition that film stoichiometry is one of the factors that stabilize cubic boron nitride in boron nitride thin films. A shift in the position of the cubic boron nitride infrared absorption was also observed by the present authors which was related to film stoichiometry.

04,711

PB96-102512 Not available NTIS

National Inst. of Standards and Technology (MSEL) Gaithersburg, MD. Reactor Radiation Div.

Oxygen Dependance of the Crystal Structure of $\mathrm{HgBa} 2 \mathrm{CuO} 4+$ Superconductivity.

Final rept.

Q. Huang, J. W. Lynn, Q. Xiong, and C. W. Chu

1995, 9p.

Pub. in Physical Review B, v52 n1 p462-470 Jul 95

Keywords: "Mercury oxides, "Crystal structure "Superconductivity, Phase transformations, Mercury compounds, Oxygen, Barium, Copper, Lattice parameters, Doped crystals, Temperature, Reprints.

Powder neutron-diffraction profile refinement techniques have been used to investigate the oxygen dependence of the crystal structure and its effect on the superconducting phase transition in high-quality samples of the superconductor $\mathrm{HgBa} 2 \mathrm{CuO}(4+$ delta) $(0.04$ less than or equal to delta less than or equal to 0.23 ) The sustem remains tetragonal (space group $\mathrm{P} 4$ $\mathrm{mmm}$ ) over the full range of temperature $(10-300 \mathrm{~K}$ ) and oxygen concentration explored. The a-axis lattice parameter decreases smoothly with increasing delta while the c-axis lattice parameter exhibits a maximum. The extra oxygen in the material is found to randomly occupy the centered $\mathrm{O}(3)$ site $(1 / 2,1 / 2,0)$ in the $\mathrm{Hg}$ layer, and no other additional site for the extra oxygen was found in the structure. There is also no mixing of the cations on the $\mathrm{Cu}$ and $\mathrm{Hg}$ sites.

04,712

PB96-102538 Not available NTIS

National Inst. of Siandards and Technology (EEEL) Boulder, CO. Electromagnetic Technology Div. Self-Biasing Cryogenic Particle Detector Utilizing Electrothermal Feedback and a SQUID Readout. Final rept.

K. D. Irwin, S. W. Nam, B. Cabrera, R. P. Welty, M. Martinis, B. Chugg, and S. G. Park. 1995, 4p. Pub. in Institute of Electrical and Electronics Engineers Transactions on Applied Superconductivity, v5 n2 p2690-2693 Jun 95

Keywords: "SQIDS(Detectors), "Radiation counters "Phonons, Transitions, Edges, Tungsten, Thin films, Silicon, Substrates, Feedback Temperature, Superconductors, Reprints.

The authors are developing and testing a new type of superconducting transition edge sensor for phonon mediated particle detection. This sensor consists of a superconducting tungsten thin film deposition on a silicon substrate. The temperature of the film is held constant within the superconducting transitions ( $T$ (sub $\mathrm{C}$ ) $=70 \mathrm{mK}$ ) by a feedback process, with the substrate temperature well below the film temperature. Phonon energy deposited in the film is removed by a reduction in feedback Joule heating, which is measured using a series array of DC SQUIDs. The resulting signals show improvements in linearity and signal-to-noise ratio over the authors' previous transition edge sensors.

04,713

PB96-102546 Not available NTIS

National Inst. of Standards and Technology (CSTL), Gaithersburg, MD. Thermophysics Div.

Simulation of $\mathrm{C} 60$ Through the Plastic Transition Temperatures.

Final rept.

S. D. Johnson, R. D. Mountain, and P. H. E. Meijer 1995, 3p.

Pub. in Jnl. of Physical Chemistry, v103 n3 p1106 1108 Jul 95

Keywords: "Carbon 60, "Transition temperature "Plastic properties, Molecular interactions, Solid phase, Phase transformations, Solid phase, Phase transformations, Models, Simulation, Charge distribu tion, Crystal structure, Reprints, Fullerene, Molecular dynamics.

An interaction model is presented that accounts for the phase transitions in a crystalline $\mathrm{C} 60$ at 90 and 250 


\section{PHYSICS}

\section{Solid State Physics}

$K$. This was obtained by a molecular dynamics simula tion of the $\mathrm{C} 60$ crystal. These transitions are used as indirect evidence of the appropriateness of the charge distribution. Additional support of the proposed charge distribution comes from the agreement of multiple energies with theoretical considerations.

\section{4,714 \\ PB96-102587 Not available NTIS}

National Inst. of Standards and Technology (EEEL), Boulder, CO. Electromagnetic Technology Div.

Phase Locking in Two-Dimensional Arrays of Josephson Junctions: Effect of Critical-Current Nonuniformity.

Final rept.

R. L. Kautz. 1995, 5p.

Pub. in Institute of Electrical and Electronics Engineers Transactions on Applied Superconductivity, v5 n2 p2702-2706 Jun 95.

Keywords: "Josephson junctions, "Microwave oscillators, "Phase locked systems, Arrays, Nonuniformity, Electric current, Reprints.

Numerical simulations are used to study mutual phase locking in two-dimensional arrays of Josephson junctions for parameters typical of successful millimeterwave oscillators. Such arrays are shown to be very tolerant of random critical-current nonuniformities. How ever, comparison with an equivalent series array reveals that the locking between rows in a twodimensonal array is principally due to feedback through the external load and not to internal coupling between rows.

\section{4,715}

\section{PB96-102595 Not available NTIS}

National Inst. of Standards and Technology (MSEL), Boulder, CO. Materials Reliability Div.

Tensile Deformation-Induced Microstructures in Free-Standing Copper Thin Films.

Final rept.

R. R. Keller, J. M. Phelps, and D. T. Read. 1994, 6p. Pub. in Materials Research Society Symposia Proceedings, v338 p227-232 1994

Keywords: "Thin films, "Copper, "Microstructure, *Tensile deformation, Dislocations(Materials), Fractures(Materials), Reliability, Electron microscopy, Loads(Forces), Plastic deformation, Reprints, TEM(Transmission electron microscopy).

Existing work in the mechanical behavior of thin films focuses mainly on measurement of macroscopic properties without string correlation to microstructural features. The authors used transmission electron microscopy (TEM) to characterize the microstructures of freestanding copper thin films both before and after monotonic tensile deformation in an ex-situ thin film tensile testing system, as well as during in-situ loading in the TEM. The defect structures contributing to plastic deformation were investigated with an emphasis on comparison to mechanisms known to operate in bulk copper. The thin film exhibited much lower ductility (approximately $1 \%$ ) than that normally observed in bulk form (greater than $40 \%$ ). The predominant plastic deformation mechanisms did not include the typical dislocation activity that occurs in bulk copper, but rather greatly inhibited dislocation interactions typical of stages I and II hardening only.

\section{4,716}

\section{PB96-102603 Not available NTIS}

National Inst. of Standards and Technology (EEEL),

Boulder, CO. Electromagnetic Technology Div.

Magnetostriction and Giant Magnetoresistance in Annealed NiFe/Ag Multilayers.

Final rept.

Y. K. Kim, and S. C. Sanders. 1995, 3p.

Pub. in Applied Physics Letters 66, n8 p1009-1011, 20

Feb 95.

Keywords: "Magnetostriction, "Magnetoresistivity, *Annealing. "Thin films, Temperature dependence, Nickel compounds, Iron compounds, Gold, Stress relieving, Sensors, Magnetic tape, Recording heads, Reprints, GMR, Multilayers.

Magnetostriction data are reported for NiFe/Ag multilayer thin films displaying giant magnetoresistance. Magnetostriction and magnetoresistance were meas ured as functions of annealing temperature for $\mathrm{NiFe} /$ $\mathrm{Ag}$ samples having different numbers of $\mathrm{NiFe} / \mathrm{Ag}$ bilayers and Ag spacer thicknesses. They varied systematically with annealing temperature in a manner consistent with residual stress reduction and microstructural changes such as grain-boundary diffusion and grain growth. Zero magnetostriction concurrent with high magnetoresistance ratio $(5 \%)$ and field sensitivity $(7.5 \%(\mathrm{kA} / \mathrm{m})(0.6 \% / \mathrm{Oe}))$ was observed for an optimal multilayer configuration and annealing temperature. This combination of zero magnetostriction and high magnetoresistive response makes the NiFe/ Ag multilayer system an attractive candidate for highperformance magnetic recording read-head sensors.

\section{4,717}

PB96-102819 Not available NTIS

National Inst. of Standards and Technology (PL), Boulder, CO. Time and Frequency Div.

Optically Stabilized Tunable Diode-Laser System for Saturation Spectroscopy.

Final rept.

M. Murtz, M. Schaefer, T. George, J. S. Wells, and

W. Urban. $1995,7 p$

Pub. in Applied Physics B, v60 p31-37 1995.

Keywords: "Saturation spectroscopy, "Lead salt diode lasers, "Laser applications, Tunable lasers, Diodes, Lasers, Reprints, "Foreign technology, Optical Feedback.

We present an optically stabilized lead-salt diode-laser system which is the nucleus of a very-high resolution instrument for sub-Doppler molecular spectroscopy in the mid-infrared. By application of external optical reedback, we have narrowed the diode-laser linewidth by two orders of magnitude, yielding a spectral width of less than $200 \mathrm{kHz}$. The diode-laser frequency is stabilized and controlled via the external reflector by variable-frequency offset-locking the diode-laser to a CO laser frequency. This substantial improvement in the spectral properties enabled us to perform a Lamb-dip experiment on a carbonyl sulfide (OCS) absorption line near $1985 \mathrm{~cm} \cdot 1$.

\section{4,718}

\section{PB96-102827 Not available NTIS}

National Inst. of Standards and Technology (EEEL),

Boulder, CO. Electromagnetic Technology Div.

Electronic Microrefrigerator Based on a Normal-Insulator-Superc onductor Tunnel Junction.

Final rept.

M. Nahum, T. M. Eiles, and J. M. Martinis. 1994, 3p. Pub. in Applied Physics Letters, v65 n24 p2123-2125 Dec 94

Keywords: "Tunneling(Electronics), "Superconductors, "Refrigerators, Reprints, Junctions, Electro phonon coupling.

We present measurements on a novel electronic microrefrigerator that can cool conduction electrons significantly below the lattice temperature. A normalinsulator-superconductor tunnel junction is used to extract electrons from the normal metal electrode whose energy is higher than the Fermi energy. Electrons with an average energy equal to the Fermi energy are returned to the metal by a superconducting contact. Consequently, the high-energy thermal excitations are removed from the normal metal, thus cooling the electrons. For lattice temperatures higher than $100 \mathrm{mK}$ the data can be explained by a simple theory incorporating the BCS density of states in the superconducting electrode and the coupling between electrons and phonons. At lower temperatures our measurement suggests that the electron energies in the normal electrode depart strongly from an equilibrium distribution.

04,719

PB96-103015 Not available NTIS

National Inst. of Standards and Technology (EEEL), Boulder, CO. Electromagnetic Technology Div. Superconducting Integrated Circuit Fabrication with Low Temperature ECR-Based PECVD SiO2 Dielectric Films.

Final rept.

J. E. Sauvageau, C. J. Burroughs, P. A. A. Booi, J. A. Koch, M. W. Cromar, and S. P. Benz. 1995, 7p Pub. in Institute of Electrical and Electronics Engineers Transactions on Applied Superconductivity 5, n2 p2303-2309 Jun 95.

Keywords: "Josephson junctions, "Integrated circuits, *Superconductors, "Fabrication, Low temperature, Very large scale integration, Thin films, Vapor deposition, Cyclotron resonance, Silicon dioxide, Experimental design, Statistical quality control, Reprints.

A superconducting integrated circuit fabrication process has been developed to encompass a wide range of applications such as Josephson voltage standards, VLSI scale array oscillators, SQUIDS, and kinetic-inductance-based devices. An optimal Josephson junction low temperature processing for all deposition and etching steps. This low temperature process involves an electron cyclotron resonance-based plasma-enhanced chemical vapor deposition of $\mathrm{SiO} 2$ films for interlayer dielectrics. Experimental design and statistical process control techniques have been used to ensure high quality oxide films. Oxide and niobiom etches include endpoint detection and controlled overetch of all films. An overview of the fabrication process is presented.

04,720

PB96-103056 Not available NTIS

National Inst. of Standards and Technology (PL), Boulder, CO. Quantum Physics Div.

Nanoscale Study of the Hydrogenated Amorphous Silicon Surface.

Final rept.

D. M. Tanenbaum, A. Laracuente, and A. C.

Gallagher. 1994, $6 \mathrm{p}$.

Contract NREL-XG-1-1216

See also PB95-150595. Sponsored by National Renewable Energy Lab., Golden, CO.

Pub. in Materials Research Society Symposia Proceedings, v336 p49-54 1994

Keywords: * Surface roughness, *Films, " Silicon, Amorphous materials, Vapor deposition, Topology, Microscopy, Crystal growth, Voids, Substrates, Reprints, Hydrogenated amorphous silicon, Scanning tunneling $\mathrm{mi}$ croscopy.

A scanning tunneling microscope has been used to study the topology of the surface of device-quality, hydrogenated amorphous silicon deposited by if discharge from silane or hot wire CVD. The substrates were oxide-free single-crystal silicon or GaAs. Films studied were either grown in the authors' laboratory and observed with no air exposure, or grown at other aboratories producing device-quality photovoltaic cells and viewed after air exposures of less than 30 minutes. Thin films $(10 \mathrm{~nm})$ representing early growth stages appear significantly smoother than the thicker films. The topology of thick films (greater than $50 \mathrm{~nm}$ ) has large variations over individual samples. Overall surface roughness measured on sub-micron areas of the authors' films is very inhomogeneous.

\section{4,721}

PB96-103130 Not available NTIS

National Inst. of Standards and Technology (EEEL), Boulder, CO. Electromagnetic Technology Div. Recent Results in Magnetic Force Microscopy. Final rept.

A. Wadas, P. Rice, and J. Moreland. 1994, 5p.

Pub. in Jnl. of Applied Physics A, v59 p63-67 1994

Keywords: *Microscopy, *Magnetic properties, Domain structure, Magnetic disks, Permalloy, Iron alloys, Single crystals, Topography, Reprints, "Atomic force microscopy, MFM(Magnetic force microscopy), Magnetic garnet.

The authors present domain wall images obtained by using Magnetic Force Microscope (MFM) on magnetic samples like: double layer of permalloy alloy, magnetic hard disk. BaFe12019 single crystal and TGdTmGa/ TSmTMGa magnetic garnet. The authors have imaged topography and magnetic forces of the same area. The Fe double- and single-layer thin films tips have been prepared to achieve high sensitivity $(10(\exp -12) N)$ and high resolution of MFM.

04,722

PB96-111679 Not available NTIS

National Inst. of Standards and Technology (EEEL) Boulder, CO. Electromagnetic Technology Div.

Stable Phase Locking in a Two-Cell Ladder Array of Josephson Junctions.

Final rept.

B. H. Larsen, and S. P. Benz. 1995, 3p.

Pub. in Applied Physics Letters, v66 n23 p3209-3211 Jun 95.

Keywords: "Josephson junctions, "Arrays, Magnetic fields, Stability, Numerical analysis, Oscillators, Reprints, " Phase locking

The stability of the periodic solution of the two-cell ladder array has been numerically investigated in order to explore intrinsic phase-locking mechanisms relevant to arrays and stacked junction oscillators. In zero magnetic field the periodic in-phase solution of the system 
is neutrally stable. However, this solution is stable over a finite voltage range when an applied control current exceeds a critical value. The dependence upon system parameters of the boundaries of the stable range and the critical control current is investigated. Finally, the influence of the control current on the microwave power in a typical range of stability is calculated.

04,723

PB96-111745 Not available NTIS

National Inst. of Standards and Technology (EEEL), Boulder, CO. Electromagnetic Technology Div. Temperature Dependence and Magnetic Field Mod. ulation of Critical Currents in Step-Edge SNS YBCO/Au Junctions.

Final rept.

N. Missert, L. R. Vale, R. H. Ono, R. E. Thomson, S J. Berkowitz, C. D. Reintsema, and D. A. Rudman.

1995, 4p.

Pub. in Institute of Electrical and Electronics Engineers Transactions on Applied Superconductivity, v5 n2 p2969-2972 Jun 95

Keywords: "Josephson junctions, "Critical current, "Temperature dependence, "Magnetic fields, Gold, Modulation, Electrical properties, Transport properties, Superconducting devices, YBCO superconductors, Reprints, Step-edge SNS YBCO/Au junctions.

The authors compare the electrical transport properties of superconductor-normal metal-superconductor SNS step-edge YBCO/Au junctions where the Au is deposited at $100 \mathrm{deg} C$ and $600 \mathrm{deg} C$. For both types of junctions the authors observe Resistively Shunted Junctions (RSJ) current-voltage characteristics. The critical currents Ic in all cases are similar for a given YBCO thickness to step height ratio, while the normal resistance $R($ sub $n$ ) for the Au deposited at $600 \mathrm{C}$ is consistently $25 \%$ lower. The normalized temperature dependence of the I(sub c)R(sub $n$ ) product is identical for all junctions with Au deposited at high temperatures and varies among junctions on a single chop for $\mathrm{Au}$ deposited at $100 \mathrm{deg}$ C. Low magnetic field modulation of the critical current can show either the expected Fraunhoffer-like pattern or a double-junction modulation for both types of devices. The modulation period is consistently a factor of three lower for the high-tem perature deposited $\mathrm{Au}$.

04,724

PB96-111752 Not available NTIS

National Inst. of Standards and Technology (EEEL) Boulder, CO. Electromagnetic Technology Div,

Critical Current and Normal Resistance of High-Tc Step-Edge SNS Junctions.

Final rept.

C. D. Reintsema, R. H. Ono, G. Barnes, G. Kunkel,

D. A Rudman, L. R Vale, N Missert, P. A.

Rosenthal, L. Borcherdt, and T. E. Harvey. 1995, 5p. Pub. in Institute of Electrical and Electronics Engineers Transactions on Applied Superconductivity, v5 n2 p3405-3409 Jun 95.

Keywords: "Josephson junctions, "High temperature, "Critical current, "Electric conductivity, Superconducting devices, Geometry, Technology utilization, Reprints, High-Tc, Step-edge SNS junctions, I(sub C)R(sub n) product.

The authors have fabricated high-Tc superconductornormal- superconductorJosephson junctions with a variety of controlled geometries and measuredthe resulting dependences of critical current and normal resistance. These studies show that the authors can adjust their junction parametersover orders of magnitude, thus allowing the authors to tailor the junctions for a variety of applications.

\section{4,725}

PB96-111794 Not available NTIS

National Inst. of Standards and Technology (MSEL) Gaithersburg, MD. Ceramics Div.

Crystal Structure of a New Monoclinic Form of Potassium Dihydrogen Phosphate Containing Orthophosphacidium lon, (H4PO4)(sup +1).

Final rept.

M. Mathew, and W. Wong-Ng. 1995, 5p.

Pub, in Jnl. of Solid State Chemistry, v114 p219-223 1995.

Keywords: *Crystal structure, *Potassium inorganic compounds, *Phosphate inorganic compounds, " $X$ ray diffraction, Hydrogen bonds, lons, Lattice parameters, Monoclinic lattice, Three dimensional, Crystallography, Reprints, "Potassium dihydrogen phosphate, "Orthophosphacidium ion.

The crystal structure of a new monoclinic form of pothassium dihydrogen phosphate, $\mathrm{KH} 2 \mathrm{PO} 4$, has been determined by single crystal $x$-ray diffraction. Crystals are monoclinic, space group P2/c with $a=7.4399(7)$, $\mathrm{b}=7.2634(9), \mathrm{c}=9.3629(13)$ Angstroms, Beta $=$ 127.696(8) degrees, $V=400.35$ (9) cubic Angstroms, $17.9 \mathrm{~cm}(\mathrm{xp}-1) \mathrm{F}(000)$ Mg/cubic $\mathrm{m}$ for $Z=4$, $\mathrm{mu}=$ ture was refined to $R=0.022$ and $R w=0.038$ for 740 ture was refined to $R=0.022$ and $R w=0.038$ for
reflections with greater I than or equal to 3 sigma(I).

\section{4,726}

\section{PB96-112024 Not available NTIS}

National Inst. of Standards and Technology (EEEL),

Boulder, CO. Electromagnetic Technology Div.

Temperature and Field Dependence of Flux Pinning in $\mathrm{NbTi}$ with Aritificial Pinning Centers.

Final rept.

S. L. Wipf, and L. D. Cooley. 1994, 4p.

Pub. in Proceedings of the International Workshop on Critical Currents in Superconductors, Alpback, Austria January 24-27, 1994, p613-616.

Keywords: *Flux pinning, *Superconducters, Reprints, Niobium titanium, Efficiency, Force, "Foreign technology.

An experimental study of artificial pinning centers in $\mathrm{NbTi}$ covered a large range of many parameters: size of pints from $65 \mathrm{~nm}$ down to $7.7 \mathrm{~nm}$ (Pins spaced at twice their size in hexagonal array), critical current densities at tempertures between $2 \mathrm{~K}$ and $\mathrm{Tc}$ at fields up to Bc2. The objective of this paper is to understand mainly the field dependence of flux pinning in this material with a view to achieve optimal performance in a practical conductor. We conclude that in these samples the optimal pinning at 5T is between 14 and 28 $\mathrm{nm}$ pin size and that the pinning is within a factor of 4 plus or minus 1 of the ultimate possible.

\section{4,727}

\section{PB96-112032 Not available NTIS}

National Inst. of Standards and Technol

Distribution of Dielectric Relaxation Times and the Moment Problem.

Final rept.

J. Baker-Jarvis, and M. D. Janezic. 1994, 2p.

Pub. in Proceedings of the Conference on Precision Electromagnetic Measurements (CPEM), Boulder CO., June 27-July 1, 1994, p356-357.

Keywords: "Permittivity, Reprints, Maximum entry method, "Dielectric relaxation, "Moment problem.

A procedure for deconvolving time-dependent distribution functions from permittivity data is developed. The problem is reduced to solving an associated moment problem. This moment problem is then solved using a maximum-entropy procedure. A new algorithm for approximating the associated Lagrange multipliers in the distribution function is also outlined. The method is applied to permittivity modeling both for in-band and out-of-band regions. The numerical results indicate that the technique has potential for deconvolving the distribution function from the permittivity data.

\section{4,728}

PB96-112065 Not available NTIS

National Inst. of Standards and Technology (EEEL)

Boulder, CO. Electromagnetic Technology Div

SUSAN: SUperconducting Systems ANalysis by Low Temperature Scanning Electron Microscopy (LTSEM).

T. Doderer, D. Hoffman, R. P. Huebener, S.

Lachenmann, D. Quenter, J. Schmidt, S. Stehle, J.

Niemeyer, R. Popel, S. P. Benz, P. A. A. Booi, N.

Kirchmann, and C. A. Krulle. 1993, 4p.

Pub. in Institute of Electrical and Electronics Engineers Transactions on Applied Superconductivity, v3 n1 p2724-2727 Mar 93

Keywords: "Elecron microscopy, "Superconducting magnets, Reprints, Low temperature, Integrated circuits, "2D arrays, "Fluxon oscillations, "Josephson junctions.

We used the technique of Low Temperature Scanning Electron Microscopy for spatially resolved investigations of both Josephson junctions and superconducting integrated circuits during their operation with a spatial resolution of about 1 micrometer. Two examples of our studies will be presented. With single Josephson tunnel junctions of various geometries we studied different dynamics states such as fluxon oscillations or unidirectional flux flow. With an integrated circuit consisting of a two-dimensional array of tunnel junctions and an if detection circuit we investigated the rf-properties of the coupling circuit and confirmed the existence of an impedance mismatch and a geometrical standing wave in the blocking capacitor.

04,729

PB96-112321 Not available NTIS

National Inst. of Standards and Technology (MSEL) Gaithersburg, MD. Polymers Div.

Measured Stopping Powers of Hydrogen and Helium in Polystyrene Near Their Maximum Values. Final rept.

L. Leblanc, G. G. Ross, and W. E. Wallace 1995

Pub. in Nuclear Instruments and Methods in Physics Research B, v95 p457-462 1995.

Keywords: "Energy losses, "Backscattering, "Stopping power, Reprints, Ion beams, Polymers, Hydrogen, $\mathrm{He}$ -

Rutherford backscattering at two different angles on multilayer targets is used to measure the stopping powers of hydrogen and helium ions in polystyrene in the energy range near their maximum values. In the energy range from 40 to $300 \mathrm{keV} / \mathrm{amu}$, the hydrogen stopping power reaches a maximum value of $9.8 \mathrm{eV}$ 10 to the 15th power atoms $/ \mathrm{cm}$. sq. at $81 \mathrm{keV} / \mathrm{amu}$. As the energy increases from 25 to $90 \mathrm{keV} / \mathrm{amu}$, the helium stopping power increases from 15.7 to $26.0 \mathrm{eV} /$ (10 to the 15th power atoms/cm.sq.). Identical results (in keV/amu energy units) were obtained for $2 \mathrm{H}$ and $1 \mathrm{H}$, as well as for $4 \mathrm{He}$ and $3 \mathrm{He}$. The hydrogen and helium measured stopping power values are compared to the results calculated from two empirical models (Bragg's rule and the cores-and-bonds model). A function has been fit to the experimental data, including those previosly published, which permits rapid calculation of the stopping powers.

04,730

(Order as PB96-113535, PC A05/

MF A01)

National Inst. of Standards and Technology, Gaithersburg, MD

Spectroscopic Study of Quantized Breakdown Voltage States of the Quantum Hall Effect.

C. F. Lavine, M. E. Cage, and R. E. Elmquist. 1994,

$8 p$.

Included in Jnl. of Research of the National Institute of Standards and Technology, v99 n6 p757-764 Nov/ Dec 94.

Keywords: "Spectroscopic analysis, "Electric potential "Electrical faults, "Hall effect, Quantum theory, Semiconductors(Materials), Electron gas, Spectra, Solid-state plasma, Energy losses, Dissipation factor Histograms, Two dimensional, NIST(National Institute of Standards and Technology).

Quantized breakdown voltage states are observed in a second, wide, high-quality GaAs/AIGaAs sample made from another wafer, demonstrating that quantization of the longitudinal voltage drop along the sample is a general feature of the quantum Hall effect in the breakdown regime. The voltage states are interpreted in a simple energy conservation model as occuring when electrons are excited to higher Landau levels and then return to the original level. $A$ spectroscopic study of these dissipative voltage states reveals how well they are quantized. The statistical variations of the quantized voltages increase linearly with quantum number.

04,731

Not available NTIS

National Inst. of Standards and Technology (EEEL), Boulder, CO. Electromagnetic Technology Div.

Measurement of the Weak-Localization Complex Conductivity at $1 \mathrm{Ghz}$ in Disordered $\mathrm{Ag}$ Wires. Final rept.

J. B. Pieper, J. C. Price, and J. M. Martinis. 1992

Pub. in Physical Review B, v45 n7 p3857-3860 Feb 92

Keywords: "Magnetoconductance, Reprints, "Electron diffusion constant, *Weak localization

The authors have measured the complex magnetoconductivity of disordered quasi-dimensiona $\mathrm{Ag}$ wires at $1 \mathrm{GHz}$ and at $200 \mathrm{~Hz}$. Because $1 \mathrm{GHz}$ is of the same order as the dephasing rate, the authors have been able to observe the frequency dependence 


\section{Solid State Physics}

of weak localization. Both the real and imaginary parts of the conductivity at $1 \mathrm{GHz}$ are in good agreement with theory. The data yield an accurate value for the electron diffusion constant, which is not well determined by dc measurements, and support the theory of weak localization at finite frequency.

\section{4,732}

PB96-119417 Not available NTIS

National Inst. of Standards and Technology (PL) Gaithersburg, MD. Electron and Optical Physics Div Nanofabrication of a Two-Dimensional Array Using Laser-Focused Atomic Deposition.

Final rept.

R. Gupta, J. J. McClelland, Z. J. Jabbour, and R. J. Celotta. 1995, 3p.

Pub. in Applied Physics Letters, Chapter 67, v10 p1378-1380, 4 Sep 95.

Keywords: "Chromium, *Deposition, *Laser radiation, "Lithography, "Atomic beams, Fabrication, Silicon, Substrates, Two dimensional, Arrays, Microstructure, Lithography, Reprints, "Atom lithography Nanofabrication, Nanostructures.

Fabricaion of a two-dimensional array of nanometerscale chromium features on a silicon substrate by laser-focused atomic deposition is described. Features 13 plus or minus $1 \mathrm{~nm}$ high and having a full-width at half maximum of 80 plus or minus $10 \mathrm{~nm}$ are fabricated in a square array with lattice constant $212.78 \mathrm{~nm}$, determined by the laser wavelength. The array covers an area of approximately 100 micro $\mathrm{m} \times 200$ micro $\mathrm{m}$. Issues associated with laser-focusing of atoms in a twodimensional standing wave are discussed, and potential applications and improvements of the process are mentioned.

\section{4,733}

PB96-119441 Not available NTIS

National Inst. of Standards and Technology (MSEL), Gaithersburg, MD. Ceramics Div.

Effect of Sm2BaCuO5 on the Properties of Sintered (Bulk) YBa2Cu306+X

Final rept.

M. D. Hill, J. E. Blendell, C. K. Chiang, L. Chacon, J. F. Kelly, L. H. Bennett, and J. J. Ritter. $1995,5 p$. Pub. in Physica C, n251 p361-365, 7 Jul 95.

Keywords: *Critical current, *Superconductors, *Sintering, Transport properties, Current density, Superconductivity, Solid solutions, Microstructure, Samarium, Barium oxides, Copper oxides, Yttrium oxides, Reprints, "YBCO superconductors, High temperature superconductors, Weale-links.

The addition of Sm2BaCuO5 to $\mathrm{YBa} 2 \mathrm{Cu} 3 \mathrm{O}(6+\mathrm{x})$ has been shown to increase the transport critical current density. Because Sm2BaCuO5 and $\mathrm{CuO}$ do not coexist at equilibrium, additions of $\mathrm{Sm} 2 \mathrm{BaCuO} 5$ to $\mathrm{YBa} 2 \mathrm{Cu} 3 \mathrm{O}(6+\mathrm{x})$ cause a reaction with the $\mathrm{Cu}$ rich liquid phase usually present during sintering. This forms crystalline phases that do not completely cover the grain boundaries. Sm was found to be homogeneously distributed in the YBa2Cu3O $(6+x)$, matrix. Additions of $5 \mathrm{~mole} \% \mathrm{Sm} 2 \mathrm{BaCuO} 5$ and sintering temperatures of 950 deg. C are effective in increasing the transport critical current density.

\section{4,734}

PB96-119615 Not available NTIS

National Inst. of Standards and Technology (CSTL),

Boulder, CO. Process Measurements Div.

Building a Better Crycooler.

Final rept.

R. Radebaugh. 1992, 4p.

Pub. in Superconductor Industry, p22-25 Fall 92

Keywords: "Cryogenics, Reprints, Electronics, Pulse tube, Refrigeration, Superconductors, "Cryocoolers, Brayton, Gifford-McMahon, Joule-Thomson, Stirling.

The lack of satisfactory cryocoolers designed specifically for superconducting electronics has hindered the marketability of superconducting devices. This article reviews the current status of the five most common cryocoolers: Joule-Thomson, Brayton, Stirling, GiffordMcMahon, and pulse tubes. Advantages and disadvantages of each type of commercially available cryocoolers are given. Most of these cryocoolers have been developed for the cooling of infrared sensors and can sometimes be adapted for use with superconducting electronics. Current areas of research are discussed and some projections for the future are given.
04,735

PB96-119656 Not available NTIS

National Inst. of Standards and Technology (EEEL), Gaithersburg, MD. Semiconductor Electronics Div. Mesoscopic Conductance Fluctuations in Large Devices.

Final rept.

C. A. Richter, D. G. Seiler, and J. C. Pellegrino.

$1995,4 p$

Pub. in Workbook of the International Conference on the Physics of Semiconductors (22nd), Vancouver British Columbia, Canada, August 15-19, 1994, v3 p1967-19701995

Keywords: "Conductance, "Fluctuations, Reprints, *Mesoscopic.

An ac magnetic field modulation and lock-in amplifier technique was used to study 'universal' conductance fluctuations in GaAs/AIGaAs devices. The enhanced measurement sensitivity of this technique permits universal conductance fluctuations to be observed and studied in a new large-size-scale regime where devices have been considered macroscopic. It generally has been assumed that a finite temperature, coherent quantum interference effects could not be observed in devices of this large size.

\section{4,736}

PB96-119664 Not available NTIS

National Inst. of Standards and Technology (EEEL), Boulder, CO. Electromagnetic Technology Div.

Faraday Effect Sensors for Magnet Field and Electric Current.

Final rept.

K. B. Rochford, G. W. Day, M. N. Deeter, and A. H

Rose. 1994, 4p

Pub. in Proceedings of DoD Fiber Optics '94, 'Optical Networks in the Concept of a Global Grid', McLean, VA., March 22-24, 1994, p21-24.

Keywords: "Faraday effect, Reprints, Iron garnets, Magnetic field sensors, Magneto-optics, Optical fiber sensors, "Electric current sensors.

Recent research at NIST has resulted in significant fundamental and practical improvements in magnetooptics sensors used to measure magnetic field and electric current. This paper reviews these developments and considers prospects for further gains.

\subsection{7}

PB96-122783 Not available NTIS

National Inst. of Standards and Technology (MSEL), Gaithersburg, MD. Polymers Div.

Novel Method for Determining Thin Film Density by Energy-Dispersive X-ray Reflectivity.

Final rept.

W. E. Wallace, and W. L. Wu. 1995, 3p.

Pub. in Applied Physics Letters, v67 n9 p1203-1205 Aug 95.

Keywords: "Thin films, " $X$ ray density measurement Reflectance, $X$ rays, Wavelengths, Alignment, Silicon dioxide, Single crystals, Reprints, Spin-on-glass, Polysilsesquioxane.

A technique utilizing the reflection of $x$-rays to determine material density at flat surfaces is described. The effects of sample misalignment limit the accuracy of $x$ ray reflectivity as typically practiced. These effects may be properly accounted for by measuring the critical angle for reflection at many different $x$-ray wavelengths simultaneously from which an extrapolation of the position of the critical angle at infinite wavelength may be made. This extrapolation has the effect of correcting for sample misalignment. Use of the technique is demonstrated for single-crystal silicon surfaces and for silica spin-on-glass thin films.

\section{4,738}

PB96-123617 Not available NTIS

National Inst. of Standards and Technology (EEEL), Gaithersburg, MD. Semiconductor Electronics Div.

Characterization of Liquid-Phase Epitaxially Grown HgCdTe Films by Magnetoresistance Measurements.

Final rept.

J. S. Kim, D. G. Seiler, L. Colombo, and M. C. Chen. 1995, 6p.

Pub. in Jnl. of Electronic Materials, v24 n9 p1305-1310 1995.

Keywords: *Magnetoresistance, *Films, Epitaxy, Magnetic properties, Reprints, $\mathrm{HgCdTe}$
In this paper, we demonstrate that measurements of the magnetoresistance can be used as a valuable alternative to conventional characterization tools to study transport properties of advanced semiconducting materials, structures, or devices. We have measured magnetoresistance on two different systems, namely, three liquid-phase epitaxially grown HgCdTe Films and two GaAs-based high-electron-mobility-transistor (HEMT) structures. The results are analyzed by using a two-carrier model as a reference in the context of the reduced-conductivity-tensor scheme. The HEMT data are in quantitative agreement with the two-carrier model, but the $\mathrm{HgCdTe}$ data exhibit appreciable deviations from the model. The observed deviations strongly indicate a mobility spread and material complexity in the $\mathrm{HgCdTe}$ samples which are probably associated with inhornogeneities and the resulting anomalous electrical behavior.

04,739

PB96-123641 Not available NTIS

National Inst. of Standards and Technology (EEEL) Boulder, CO. Electromagnetic Technology Div.

Hot-Electron Microcalorimeters as High-Resolution X-ray Detectors.

Final rept.

M. Nahum, and J. M. Martinis. 1995, 3p.

See also PB95-168993.

Pub. in Applied Physics Letters, v66 n23 p3203-3205 Jun 95.

Keywords: "X-ray detectors, "Superconducting junctions, "Calorimeters, Electron temperature, Hot electrons, Metal films, Reprints, "Phonon detectors, *Microcalorimeters.

Measurements are presented on a novel microcalorimeter for the detectionof $x$-rays. This detector uses a normal metal film deposited on a thin membrane to absorb x-ray photons. The subsequent temperature rise of theelectrons is measured from the current-voltage characteristics of a normal-insulatorsuperconductor tunnel junction, where part of the absorber forms the normal electrode. A superconductingquantum- interference-device is used as a low-noise high-bandwidth readout for the junction. We have measured an energy resolution of $22 \mathrm{eV}$ full width at half-maximum and a time constant of 15 micro $s$ for a detector operating at $80 \mathrm{mK}$ and having a $0.5 \mathrm{microm}$ eter thick Au adsorber with an area of $100 \times 100 \mathrm{mi}-$ crometer square.

04,740

PB96-123773 Not available NTIS

National Inst. of Standards and Technology (EEEL) Boulder, CO. Optoelectronics Div.

Distributed Feedback Lasers in Rare-Earth-Doped Phosphate Glass.

Final rept.

D. L. Veasey, K. J. Malone, J. A. Aust, N. A.

Sanford, and A. Roshko. 1995, 3p.

Pub. in Proceedings of the European Conference on Integrated Optics, Delft, The Netherlands, April 3-6, 1995, p579-581.

Keywords: "Grating, "Lasers, *Glass, Phosphate glass, Waveguide, Reprints, *Foreign technology

We have successfully demonstrated waveguide lasers operating at 1056.1,1058.2, and $1062.6 \mathrm{~nm}$ in $\mathrm{Nd}$ doped phosphate glass. The waveguides were fabricated by potassium ion exchange from a nitrate melt. Distributed feedback grating patterns were holographically written at $458 \mathrm{~nm}$ in photoresist spun on the sample surfaces. The gratings were developed, coated with chromium at a 60-degree evaporation angle, and were then transferred into the glass by argon ion sputtering. Typical laser threshold was 32 $\mathrm{mW}$ of absorbed pump power at $805 \mathrm{~nm}$ with a corresponding slope efficiency of $2 \%$.

\section{4,741}

PB96-131594 PC A04/MF A01

National Inst. of Standards and Technology (CAML), Gaithersburg, MD. Applied and Computational Mathematics Div.

Anisotropy of interfaces in an Ordered Alloy: A Multiple-Order-Parameter Model.

R. J. Braun, J. W. Cahn, G. B. McFadden, and A. A Wheeler. Apr 95, 70p, NISTIR-5641.

Prepared in cooperation with Delaware Univ., Newark Dept. of Mathematical Sciences. Sponsored by Southampton Univ. (England). Faculty of Mathematical Studies. 
Keywords: "Face centered cubic lattices, "Alloys, Anisotropy, Lattice parameters, Crystal symmetry, Phase transformations, Binary systems(Materials).

The authors develop a diffuse-interface theory of ordering of a binary alloy on a face-centered-cubic (FCC) crystal lattice. A continuum, multiple-order-parameter theory is used that incorporates the underlying symmetries of the FCC crystal in both the bulk and gradientenergy terms of the free energy. With the theory the authors are able to compute interphase and antiphase boundaries for general orientations with respect to the fixed crystal axes. The authors are able to compute the structure of these interfaces, which compare very well with previous calculations by Kikuchi and Cahn using the cluster variation method. For the parameters the authors have studied, they find that the anisotropy of the surface free energy is not sufficiently strong to cause edges or corners in equilibrium shapes.

04,742

PB96-135033 Not available NTIS

National Inst. of Standards and Technology (EEEL)

Boulder, CO. Electromagnetic Technology Div.

Coexistence of Grains with Differing Orthorhombicity in High Quality YBa2Cu307-delta Thin Films.

Final rept.

E. I. de Obaldia, K. F. Ludwig, S. J. Berkowitz, P. M.

Mankiewich, D. A. Rudman, A. Roshko, R. Moerman,

L. Vale, R. H. Ono, A. M. Clark, and W. J. Skocpol.

1994, 3p

Pub. in Applied Physics Letters, v65 n26 p3395-3397 Dec 94.

Keywords: "Thin films, " $X$ ray diffraction, "Lasers, Reprints, "Pulsed laser deposition, YBCO.

High quality films of YBa2Cu3O7-sigma on LaAIO3 have been grown by pulsed-laser deposition at oxygen pressures of 2.4-54 Pa (25-400 mTorr). X-ray diffraction reveals the coexistence of grains that align with the substrate axes (axial grains). The axial grains are tetragonal while the diagonal grains achieve lattice parameters close to bulk $\mathrm{YBa} 2 \mathrm{Cu} 307$. The relative proportion of axial grains accounts for the measured variations of normal-state conductance and superconducting critical current density from film to film, based on a simple two-dimensional model of randomly positioned, insulating axial grains.

\section{4,743}

PB96-135223 Not available NTIS

National Inst. of Standards and Technology (EEEL),

Boulder, CO. Electromagnetic Technology Div.

Accuracy of the Electron Pump.

Final rept.

H. D. Jensen, and J. M. Martinis. 1992, 21p.

See also PB95-168910 and PB95-175873.

Pub. in Physical Review B, v46 n20 p407-427 Nov 92.

Keywords: Metrology, Reprints, Tests, Accuracy, "Electron pumps, Single electron tunneling, Coulomb blockage, Cotunneling.

The authors calculate the accuracy of the single electron pump numerically and analytically. With a biasing of the device that the authors describe as optimal, the accuracy is computed with finite temperature cotunneling rates that systematically include all possible cotunneling processes. A simple graphical representation of the operation of the pump illustrates when cotunneling processes become active. The au thors show that the accuracy is limited by cotunneling, thermal activation, and operating the device at too high a frequency; simple approximation formulas are given for these errors. Metrological accuracy is attainable for devices with five or more junctions and with param eters that are experimentally attainable.

\section{4,744}

PB96-135348 Not available NTIS

National Inst. of Standards and Technology (EEEL), Boulder, CO. Electromagnetic Technology Div

Mutual Phase Locking in Systems of High-Tc Superconductor-Normal Metal-Superconductor Junctions.

Final rept.

C. D. Reintsema, R. H. Ono, T. E. Harvey, N.

Missert, and L. R. Vale. 1994, 11p.

Pub. in International Society for Optical Engineering Los Angeles, CA., January 25-27, 1994, v2160 p208218 Jul 94.

Keywords: "High temperature superconductors, *Superconductors, Reprints, *Phase locking, *SNS junctions.
The authors have investigated the interaction between high critical temperature (high-Tc) superconductor-normal metal-superconductor step-edge junctions coupled through a non-superconducting feedback loop. The authors have characterized the strength of the interaction as a function of frequency and temperature for both a circuit without a groundplane and an all highTc multilayer circuit incorporating a superconducting ground plane. The authors observed relative locking strengths (the ratio of the measured locking current IL to the junctions average critical current Ic) as large as $\mathrm{IL} / \mathrm{IC}=9 \%$ and peak locking frequencies as high as 1.06 $\mathrm{THz}$. The maximum temperature at which locking occurred was $35 \mathrm{~K}$. An analysis of the temperature de pendence of the locking current accounting for therma fluctuations in the context of Johnson noise from resis tive elements in the circuit agrees well with the authors' experimental observations.

04,745

Not available NTIS

National Inst. of Standards and Technology (PL) Gaithersburg, MD. Electron and Optical Physics Div. Influence of Thickness Fluctuations on Exchange Coupling in $\mathrm{Fe} / \mathrm{Cr} / \mathrm{Fe}$ Structures.

Final rept.

J. A. Stroscio, D. T. Pierce, J. Unguris, and R. J.

Celotta. 1995, 8p

Pub. in Ultimate Limits of Fabrication and Measurement, p181-188 1995

Keywords: "Chromium, "Exchange coupling Magnetism, Reprints, Iron, Electron microscopy, Polarization, Microscopy, "Foreign technology.

Realizing the ultimate limits in fabrication requires the controlled placement of material at the single atomic layer limit. This precision is required for both semiconductor and magnetic devices, the two largest indus tries racing towards the Ultimate Limits in fabrication. In this report the authors summarize results on the effects of thickness fluctuations on the exchange coupling in $\mathrm{Fe} / \mathrm{Cr} / \mathrm{Fe}$ trilayer structures. This work demonstrates the importance of controlling thin film thick ness at the single atomic layer level in magnetic sys tems.

04,746

PB96-137773 Not available NTIS

National Inst. of Standards and Technology (EEEL)

Boulder, CO. Electromagnetic Technology Div.

Panel Discussion on Units in Magnetism.

Final rept

R. B. Goldfarb. 1995, 12p

Pub. in Magnetic and Electrical Separation, v6 p105 1161995

Keywords: "Magnetism, Units of measurement, Symbols, Reprints, Conversion factors.

An evening panel discussion on magnetic units, attended by 150 participants, was held at the 1994 Join MMM-Intermag Conference in Albuquerque, New Mex ico, USA. The season was organized by C.D. Graham Jr., and moderated by R.B. Goldfarb. The panel members were asked to describe the use of magnetic unit in the countries, and to make appropriate comments and recommendations In addition to units, severa panelists talked about distinction between magnetic induction $B$ and magnetic field strength $H$, and the conversion of equations. After the panelists' opening statements, the floor was opened for questions and discussion from the audience. Below are the panelists' summaries of their remarks. By agreement with authors, this article is not subject to copyright.

\section{4,747}

PB96-138417 Not available NTIS

National Inst. of Standards and Technology (EEEL).

Boulder, CO. Electromagnetic Technology Div.

Low Noise YBa2Cu307-x-SrTiO3-YBa2Cu3O7-x Mulitlayers for Improved Superconducting Magnetometers.

Final rept.

F. Ludwig, D. Koelle, E. Dantsker, J. Clarke, R. E Thomson, D. T. Nemeth, and A. H. Miklich. 1995, 3p. Pub. in Applied Physics Letters, v66 n3 p373-375 Jan 95.

Keywords: "Magnetometers, Multilayers, Noise, Trilayers, Reprints, "Flux transformers, YBCO.

We have fabricated YB2Cu307-x-SrTiO3 YBa2Cu3O7-x (YBCO-STO-YBCO) trilayers, in which each layer is patterned photolithographically, capping the first $Y B C O$ film with an in situ STO film. Atomic force microscopy demonstrates that the capping process dramatically improves the quality of the surface of the second layer, allowing the growth of an uppe YBCO film with a substantially reduced level of lowfrequency flux noise. A magnetometer with a multiturn flux transformer coupled to a dc superconducting quantum interference device achieved a magnetic field noise of $74 \mathrm{fT} \mathrm{Hz-1/2}$ at $1 \mathrm{~Hz}$, improving to $31 \mathrm{fT} \mathrm{Hz}$ $1 / 2$ at $1 \mathrm{kHz}$

04,748

PB96-138458 Not available NTIS

National Inst. of Standards and Technology (MSEL) Gaithersburg, MD. Reactor Radiation Div. High-Energy

Final rept.

A. H. Moudden, P. M. Gehring, G. Shirane, B.

Hennion, Y. Endoh, I. Tanaka, H. Kojima, M.

Matsuda, and L. Vasiliu-Doloc. 1995, 3p.

Pub. in Physica B Condensed Matter, v213-214 p72 741995.

Keywords: "Superconducting, Transverse, Longitudinal, Reprints, "Phonon dispersion.

The authors have measured two phonon-dispersion curves above $50 \mathrm{meV}$ in a superconducting ( $\mathrm{TC}=33 \mathrm{~K}$ ) single crystal of La1.8Sr0.15CuO4. In the normal phase $(T=45 \mathrm{~K}$ ), the authors show that the $A 1$ mode compatible with the Raman $A 1 \mathrm{~g}(425 \mathrm{~cm}-1)$ mode is weakly dispersive, falling from $53.7 \mathrm{meV}$ at the zone center to about $51 \mathrm{meV}$ at the zone boundary along the tetragonal (0.0) direction. For the A1 mode compatible with the infra-red A2u (480 cm to the minus one) mode, they observe a significant dispersion of the photonon energy which drops monotonically from 60 to $56 \mathrm{meV}$. The global features of these dispersion curves were similar to those previously reported in studies of both pure and $10 \% \mathrm{Sr}$ doped La2CuO4 However significant differences in the shape of the dispersion of the highest A1 branch is shown, as well as a significant softening with increasing carrier concentration.

04,749

PB96-138524 Not available NTIS

National Inst. of Standards and Technology (EEEL),

Gaithersburg, MD. Semiconductor Electronics Div. Phonon Modes, and Mobility in n-Type GaAs.

Final rept.

B. A. Sanborn. 1995, 9p.

Pub. in Physical Review B, v51 n20 p14 256-14 264 May 95.

Keywords: "Electron scattering, Mobility, Reprints, "Electron-electron interactions, GaAs, Coupled modes, Boltzmann equation, Dynamic screening.

The paper investigates the mobility of electrons scattering from the coupled system of electrons and longitudinal-optical (LO) phonons in n-type GaAs. The Boltzmann equation is solved exactly for low electric fields by an iterative method, including electron-electron and electron-LO-phonon self-energy is treated in the plasmon-pole approximation. Scattering from ionized impurities screened in static RPA is calculated with phase-shift cross sections and scattering from RPA screened deformation potential and piezoelectric acoustic phonons is included in the elastic approximation. The results show that dynamic screening and plasmon-phonon coupling significantly modify inelastic scattering at low temperatures and densities. The effect on mobility is obscured by ionized impurity scattering in conventionally doped material, but should be important in modulation doped structures. For uncompensated bulk n-type GaAs, the RPA phase-shift model for electron-impurity scattering gives lower drift mobilities than the standard Thomas-Fermi or Born calculations, which are high compared to experiment.

04,750

PB96-138557 Not available NTIS

National Inst. of Standards and Technology (EEEL), Boulder, CO. Electromagnetic Technology Div

Dependence of Contrast on Probe/Sample Spacing with the Magneto-Optic Kerr-Effect Scanning NearField Optical Microscope (MOKE-SNOM).

Final rept.

T. J. Silva, and A. B. Kos. 1995, 7p.

Pub. in Proceedings - The Society of Photo-Optical Instrumentation Engineering Near-Field Optics, San Diego, CA., July 9-10, 1995, v2535 p2-8 1995.

Keywords: "Light scattering, Near-field, Reprints, *Magneto-optic, Surface plasmons. 
A magneto-optic Kerr-effect scanning near-field optical microscope is used to image a stripe domain wall in a Co/Pt multilayer sample. The microscope is an improved version of a type previously reported, which uses light scattering from surface plasmons in 20-40 $\mathrm{nm}$ Ag particles as a near-field probe. Data are presented for both the probe intensity and polarization contrast as a function of probe/sample spacing. Oscillatory behavior in both sets of data is reasonbly explained with a simplified model of optical interference.

04,751

PB96-138573 Not available NTIS

National Inst. of Standards and Technology (EEEL),

Boulder, CO. Electromagnetic Technology Div.

Development of Highly Conductive Cantilevers for Atomic Force Microscopy Point Contact Measurements.

Final rept.

R. E. Thomson, and J. Moreland. 1995, 3p.

Pub. in Jnl. of Vacuum Science and Technology B, v13 n3 p1123-1125 May/Jun 95.

Keywords: "Cantilevers, Contact resistance, Point contact, Reprints, *Atomic force microscopy.

Several techniques for improving the electrical conductivity of micromachined silicon cantilevers for atomic force microscopy point contact measurements were investigated. The techniques studied included sputtering or evaporating thin layers of gold, platinum or silver onto the lower surface of the cantilever to create a conducting metal layer, and doping the cantilevers with phosphorus. It was found that the lowest resistance contacts to a gold surface can be made by the meta coated tips, which can make stable point contacts with resistances as low as 30 omega at a tip sample force of $15 \mathrm{mu} \mathrm{N}$.

04,752

PB96-141098 Not available NTIS

National Inst. of Standards and Technology (EEEL),

Boulder, CO. Electromagnetic Technology Div.

Evidence for Tunneling and Magnetic Scattering at 'In situ' YBCO/Noble-Metal Interfaces.

Final rept.

S. C. Sanders, S. E. Russek, C. C. Clickner, and J.

W. Ekin. 1995, 6p

Pub. in Institute of Electrical and Electronics Engineers Transactions on Applied Superconductivity, v5 n2 p2404-2407 Jun 95

Keywords: "Conductance, "Electrical transport, Contacts, Reprints, Tunneling, Interfaces, Noble metals `YBCO superconductors.

We report low-temperature conductance data for in situ YBa2Cu3O7-sigma (YBCO)/Au, and YBCO/Pt planar $\mathrm{c}$-axis interfaces. Analysis of the conductance data for these interfaces, which have resistivities as low as 1 $x 10$ to the minus 8 th power omega-cm2, indicates that tunneling is the predominant transport mechanism. Zero-bias conductance peaks are present for all of the in situ interfaces. These peaks are analyzed in the framework of the Appelbaum model and are attributed to the presence of isolated magnetic spins at the interface. The presence and similarity of the peaks for each noble-metal overlayer supports the hypothesis that the magnetic spins are inherent to the YBCO surface.

\section{4,753}

PB96-141155 Not available NTIS

National Inst. of Standards and Technology (MSEL), Gaithersburg, MD. Reactor Radiation Div.

Neutron-Powder-Diffraction Study of the LongRange Order in the Octahedral Sublattice of LaD2.25.

Final rept.

T. J. Udovic, Q. Huang, J. J. Rush, J. Schefer, and I. S. Anderson. 1995, $11 \mathrm{p}$

Pub. in Physical Review B, v51 n18 p12 116-12 126 1995.

Keywords: *Crystal structure, "Lanthanum deuteride, Neutron diffraction, Reprints, Rare earths, Long range order.

Neutron-powder-diffraction patterns of the superstoichiometric rare-earth dideuteride LaD2.25 were measured between 15 and $400 \mathrm{~K}$. Profile refinements indicated that, above approximately $345 \mathrm{~K}$, the LaD2.25 structure is cubic ( $\mathrm{Fm} 3 \mathrm{~m}$ ) with deuterium fully occupying the tetrahedral $(t)$ interstices of the fcc La lattice and the excess deuterium occupying a portion of the octahedral $(0)$ interstices with full statistical disorder. As the temperature is decreased below approxi- mately $345 \mathrm{~K}$, the LaD2.25 structure undergoes a transformation to tetragonal symmetry concomitant with the onset of deuterium long-range order $(14 / \mathrm{mmm})$ in the o sublattice. Fully developed long-range order is established near $230 \mathrm{~K}$ and ideally corresponds to the occare-earth-deuterium systems possessing similar D/Metal stoichiometric ratios. This ordering is ac companied by an outward expansion of the cubic ensemble of eight t-site deuterium atoms surrounding each o-site deuterium (Do) atom. Moreover, the c-directed La-Do bond distances are decreased by a displacement of the La atoms toward the Do atoms.

04,754

PB96-141205 Not available NTIS

National Inst. of Standards and Technology (MSEL), Gaithersburg, MD. Reactor Radiation Div. Photoluminescence Spectra of Epitaxial Single Crystal C60.

Final rept.

R. D. Averitt, P. M. Pippenger, V. O. Papanyan, N. J. Halas, J. A. Dura, and P. Nordlander. 1995, $6 \mathrm{p}$. Pub. in Chemical Physics Letters, n242 p592-597 Sep 95.

Keywords: "Epitaxial growth, "Single crystals, Thin films, Optical properties, Carbon, Temperature, Reprints, "Photoluminescence.

We report the photoluminescence spectrum of well characterized, epitaxially grown single crystal C60 thin fims. The highly regular and reproducible spectral features observed can be explained by a simple molecular model which takes into account enhanced coupling of an excited $\mathrm{C} 60$ molecule with its nearest neighbor. This model provides an identification of all the observed spectral features at low temperature including a qualitative understanding of the relative peak height ratios, as well as the observed temperature dependene of the PL spectrum.

\section{4,755}

PB96-141213 Not available NTIS

National Inst. of Standards and Technology (EEEL), Boulder, CO. Electromagnetic Technology Div.

Quench Energy and Fatigue Degradation Properties of $\mathrm{Cu}$ - and $\mathrm{Al} / \mathrm{Cu}-\mathrm{Stab}$ lized Nb-Ti Epoxy-Impregnated Superconductor Coils.

Final rept.

S. L. Bray, J. W. Ekin, D. J. Waltman, and M. J.

Supercynski. 1995, 4p.

Pub. in Institute of Electrical and Electronics Engineers Transactions on Applied Superconductivity, v5 ptl n2 p222-225 Jun 95

Keywords: “Superconductors, „Coils, Superconducting devices, Reprints, Fatigue degradation, NbTi, Residual resistivity, Stability strain, Quench energy.

In comparative measurements of small-scale epoxyimpregnated Cu-stabilized and Al/Cu-stabilized Nb-Ti test coils at $4 \mathrm{~K}$ and $5 \mathrm{~T}$, the heat energy required to quench the $\mathrm{Al} / \mathrm{Cu}$-stabilized coil was 4 to 12 times greater than for the Cu-stabilized coil, depending on the relative operating current. Also, the coils' stabilizer resistivity (p) was measured as a function of mechanical fatigue to test for strain-induced degradation. The $\mathrm{p}$ of the $\mathrm{Cu}$-stabilized coil is relatively unaffected by fatigue, while that of the Al/Cu-stabilized coil increases with fatigue. However, in these coils, having a typical stabilizer:superconductor ratio of $4: 1$, the degradation of the Al/Cu-stablized coil beings to saturate after several hundred fatigue cycles: after 2000 fatigue cycles to $0.2 \%$ strain, the $p$ of the Al/Cu-stabilized coil is still 2.6 times lower than the p of the $\mathrm{Cu}$-stabilized coil.

\section{4,756}

PB96-141221 Not available NTIS

National Inst. of Standards and Technology (PL), Boulder, CO. Time and Frequency Div.

Atomic Iron in Its (5)D Ground State: A Direct Measurement of the $J=0$ inverted arrow 1 and $J$ $=1$ inverted arrow 2 Fine-Structure Intervals (1.2). Final rept.

J. M. Brown, and K. M. Evenson. 1995, 4p.

Pub. in Astrophysical Jnl., v441 pL97-L100 Mar 95.

Keywords: "Iron, "Magnetic resonance, Fine structure, Reprints, Atoms.

The $J=0<-1$ and $J=1<-2$ fine-structure transitions in atomic $\mathrm{Fe}$ in its grond $5 \mathrm{D}$ state have been detected in the laboratory by far-infared laser magnetic resonance. The fine-structure intervals have been measured accurately as 2696.3942(22) and 5519.9397(18) $\mathrm{GHz}$, respectively; these values correspond to wave- lengths of 111.18273 and 54.31082 micrometers, with an uncertainty of about $2 \times$ time 10 to the minus 5th power micrometers.

\section{4,757}

PB96-141247 Not available NTIS

National Inst. of Standards and Technology (PL), Gaithersburg, MD. Electron and Optical Physics Div. Using Atom Optics to Fabricate Nanostructures. Final rept.

R. J. Celotta, J. J. McClelland, R. E. Scholten, and R. Gupta. 1995, 4p.

Pub. in Ultimate Limits of Fabrication and Measurement, p75-78 1995

Keywords: Photon-atom collisions, Atomic force microscopy, Reprints, Microstructures, "Nanostructures, "Atom optics, Chromium atoms.

The drive for further miniaturization of electronic and magnetic devices continues to place increasing demands on the authors' abilities to fabricate very small structures and to understand and exploit the physical laws applicable in such devices. Optical lithographic techniques continue to be refined and limitations imposed by diffraction are driving the wavelength of the photons utilized toward the $x$-ray region. Still shorter wavelength particles, high energy electrons, are used for the highest resolution mask making. A logical extension of the progression would involve using atoms, because of their short de Broglie wavelength, as the pattem generating particle, either to expose a resist, or to deposit structures directly. The authors' are exploring how the techniques of atom optics, a field paralleling electron optics but utilizing neutral atoms instead of electrons, can be used to fabricate structures on the nanometer scale. This article is a brief summary of the authors' research on this topic. A more detailed treatment can be found elsewhere.

\section{4,758}

PB96-141312 Not available NTIS

National Inst. of Standards and Technology (EEEL), Boulder, CO. Electromagnetic Technology Div.
Oxygen Annealing of Ex-situ YBCO/Ag Thin-Film

Interfaces.

Final rept.

J. W. Ekin, C. C. Clickner, S. E. Russek, and S. C

Sanders, 1995, 4 p.

Pub. in Institute of Electrical and Electronics Engineers Transactions on Applied Superconductivity, v5 pt3 n2 p2400-2403 Jun 95.

Keywords: "YBCO superconductors, "Electric contacts, Thin films, Silver, Magnetic scattering, Reprints, *Anneling.

The resistivity of $\mathrm{YBCO} / \mathrm{Ag}$ interfaces has been measured for different oxygen annealing temperatures for a series of ex-situ fabricated thin-film contacts having sizes ranging from 16 micrometers $\times 16$ micrometers down to 4 micrometers $x 4$ micrometers. The interface resistivity began to decrease after annealing at 300 deg $C$ for 10 mlnutes in one atmosphere oxygen. After annealing at $400 \operatorname{deg} C$, the contact resistivity decreased by several orders of magnitude to the 10 to the minus 7 power Omega- $(\mathrm{cm})$ squared range. The 500-nm thick Ag layer showed massive surface diffusion and agglomeration for annealing temperatures above $-400 \mathrm{deg} \mathrm{C}$; this temperature thus represents a practical limit for oxygen annealing the TBCO/Ag interface system for more than 10 minutes. Rapid cooling of the chip after annealing led to a severe loss of critical current density in the YBCO layer, which could be restored by reannealing and cooling at a slower rate of $50 \mathrm{deg} \mathrm{C} / \mathrm{min}$.

04,759

PB96-141338 Not available NTIS

National Inst. of Standards and Technology (MSEL), Boulder, CO. Materials Reliability Div.

Ultrasonic-Resonance Spectroscopy of Bulk and Layered Solids.

C. Fortunko $\mathrm{H}$ Ledbetter, and $\mathrm{P}$. Heyliger $1995,6 p$. Pub in Intemational Symposium on Surace Waves in Solid and Layered Structures and National Conference on Acoustoelectronics, Moscow-St. Petersburg, May 17-23, 1994, p430-435 1995.

Keywords: "Resonat frequency, "Composites, Copper, Elastic constants, Layered solids, Thin films, Superconductors, Reprints, "Foreign technology, "Ultrasonic-resonance spectroscopy.

The authors used ultrasonic-resonance spectroscopy (URS) to determine the complete elastic-stiffness. The 
authors show spectra for five materials: polycrystal and monocrystal copper, laminated composite B1/Al, a seven-layer structure aramid-epoxy/Al, a $\mathrm{YBa} 2 \mathrm{Cu} 3 \mathrm{O} 7$ superconductive film on a $\mathrm{SrTiO}_{3}$ substrate. Frequencies involved are $100 \mathrm{kHz}-5 \mathrm{MHz}$. Specimen sizes are approximately $1 \mathrm{~cm}$. The URS approach of fers the principal advantage of one measurement for a multicomponent tensor property: for orthotropic symmetry, nine independence components.

\section{4,760}

PB96-141346 Not available NTIS

National Inst. of Standards and Technology (PL), Gaithersburg, MD. Electron and Optical Physics Div. Domain Structures in Magnetoresistive Granular Metals.

Final Gept. M. H. Kelley, J. Q. Xiao, and C. L. Chien. A. Gavrin,

Pub. in Applied Physics Letters, v66 n13 p1683-1685 Mar 95.

Keywords: "Electron microscopy, * Granular materials, Polarizaton analysis, Annealing.

The author imagined the magnetic domain structure of several heterogeneous CovAg1-x alloys by using scanning electron microscopy with polarization analysis. These images show that extended domain structures exist in both the as-deposited samples and in samples annealed at moderate temperatures. This suggests that a significant fraction of the cobalt in these materials does not contribute to the giant magnetoresistance Only those samples annealed at 600 deg $\mathrm{C}$ and containing less than $40 \%$ cobalt by volume show no domain structure.

\section{4,761}

PB96-141387 Not available NTIS

National Inst. of Standards and Technology (EEEL)

Boulder, CO. Electromagnetic Technology Div,

Theory of the Magneto-Optic Kerr Effect in the Near Field.

Final rept.

V. A. Kosobukin. 1995, 7p

Pub. in Proceedings of the International Society for Optical Engineering Near-Field Optics, San Diego, CA. July 9-10, 1995, v2535 p9-15.

Keywords: "Magneto-optical effects, Kerr effect, Near field, Reprints, Light scattering, Surace plasmons

A theory is developed for the magneto-optic Kerr effect (MOKE) excited via an optical near field. The model under consideration includes a semi-infinite ferromagnet and a small non-magnetic metal particle which is located nearby and possesses a long-living surface plasmon. A multiple-scattering formulation of the problem is given. Considering the Rayleigh scattering of light and the magneto-optical light polarization conversion as elementary events, all the essential optical processes are classified, and those which contribute to the near-field excitation, is treated self-consistently to all orders in light-matter interaction, and the magneto-optical interaction is described in the first order approximation of perturbation theory. The scattered light field is discussed with special emphasis on the change of optical polarization due to MOKE.

\section{4,762}

PB96-146691 Not available NTIS

National Inst. of Standards and Technology (EEEL), Boulder, CO. Electromagnetic Technology Div.

Low Magnetostriction in Annealed NiFe/Ag Giant Magnetoresistive Multilayers.

Final rept.

Y. K. Kim, S. C. Sanders, and S. E. Russek. 1995

3p.

Tra. In Institute of Electrical and Electronics Engineers 95.

Keywords: "Magnetoresistivity, *Annealing, *Thin films, Temperature dependence, Nickel compounds, Iron compounds, Silver, Sensors, Magnetic tape, Record. ing heads, Reprints, "Magnetorestriction, Multilayers. Systematic changes were observed in magnetostriction and magnetoresistance ratio for $\mathrm{NiFe} / \mathrm{Ag}$ multilayers as a function of annealing temperature. Optimal multilayer configurations (number of bilayers and Ag layer thickness) can be engineered to achieve zero magnetostriction concurrent with high magnetoresistance sensitivity. This feature makes the $\mathrm{NiFe/Ag} \mathrm{multilayers} \mathrm{potentially} \mathrm{useful} \mathrm{for} \mathrm{high-perform-}$ ance magnetic recording read-sensors.
04,763

PB96-146717 Not available NTIS

National Inst. of Standards and Technology (EEEL)

Boulder, CO. Electromagnetic Technology Div.

Telegraph Noise in Silver-Permalloy Giant Magnetoresistance Test Structures.

Final rept.

L. S. Kirschenbaum, C. T. Rogers, S. E. Russek, and S. C. Sanders. 1995, 3p.

Pub. in Institute of Electrical and Electronics Engineers Transactions on Magnetics, v31 n6 p3943-3945 Nov 95.

Keywords: "Magnetoresistivity, "Noise, "Permalloys(Trademark), Magnetic domains, Ferromagnetic materials, Telegraph systems, Electrical properties, Reprints, GMR, RTF(Random telegraph fluctuator), Multilayers

We report noise data for discontinuous Ni82Fe18/Ag multilayer test structures. Examination of the nois data for this material indicates that random telegraph fluctuator (RTF) noise of the resistance is the predominant noise source. Analysis of the RTF noise in these structures presents an opportunity to estimate mag. netic domain or magnetic cluster strengths and the domain-domain interactions.

\section{4,764}

PB96-146824 Not available NTIS

National Inst. of Standards and Technology (MSEL) Gaithersburg, MD. Reactor Radiation Div.

Statistical Descriptors in Crystallography. 2. Report of a Working Group on Expression of Uncer tainty in Measurement.

Final rept.

D. Schwarzenbach, S. C. Abrahams, H. D. Flack, E.

Prince, and A. J. C. Wilson. 1995, 5p.

See also PB89-201826.

Pub. in Acta Crystallographica, vA51 p565-569 1995

Keywords: "Crystallography, "Uncertainty analysis Terminology, Statistical mechanics, Experimenta data, Computations, Standard deviation, Error analy sis, Statistics, Nomenclature, Reprints.

The present publication updates an earlier report of the IUCr Subcommittee on Statistical Descriptors (Schwarzenbach, Abrahams, Flack, Gonschorek Hahn, Huml, Marsh, Prince, Robertson, Rollett and Wilson (1989): Acta Cryst. A45, 63-75). This new report presents the concepts of standard uncertainty, of combined stanard uncertainty, and of Type A and Type $B$ evaluations of standard uncertainties. It expands the earlier dictionary of statistical terms, recommends replacement of the term estimated standard deviation e.s.d.) by standard uncertainty (s.u.) or by combined standard uncertainty (c.s.u.) in statements of the statistical uncertainties of data and results, and requests complete description of the experimental and computa tional procedures used to obtain all results submitted to IUCr publications.

\section{4,765}

PB96-146832 Not available NTIS

National Inst. of Standards and Technology (CAML) Gaithersburg, MD. Computer Systems and Communications Div.

Stagnant Film Model of the Effect of Natural Convection on the Dendrite Operating State.

Final rept.

R. F. Sekerka, S. R. Coriell, and G. B. McFadden. 1995, 7p.

Pub. in Jnl. of Crystal Growth, v154 p370-376 Apr 95

Keywords: "Dendritic crystals, "Crystal growth, "Films Mathematical models, Convection, Heat transfer, Nusselt number, Fluid dynamics, Reprints.

We develop a simple model of the influence of natura convection on the selection of the operating state (dendrite tip velocity, $V$, and tip radius, rho) for dendritic growth of a pure material. We hypothesize that the im portant aspects if natural convection can be accounte for by considering the global convection that would occur in the vicinity of a sphere of radius $R$ that characterizes the size of a dendritic array that is growing from a point source. We estimate the thickness, delta, of a stagnant boundary layer surrounding this sphere by matching the value of the Nusselt number obtained from the heat transfer literature. We solve the steadystate problem of a paraboloidal dendrite at temperature $\mathrm{T}$ (sub $\mathrm{M}$ ) growing toward a confocal paraboloid at temperature $T$ (sub infinity) located at a distance delta from perature
04,766

Not available NTIS

National Inst. of Standards and Technology (EEEL)

Boulder, CO. Electromagnetic Technology Div.

Magnetoresistance of Thin-Film NiFe Devices Ex hibiting Single-Domain Behavior.

Final rept.

R. W. Cross, J.

K. Kim. 1995, 3p.

Pub. in Institute of Electrical and Electronics Engineers Transactions on Magnetics, v31 n6 p3358-3360 Nov

Keywords: "Magnetoresistivity, "Thin films, Nicke compounds, Iron compounds, Magnetization, Hysteresis, Devices, Single domain, Reprints, Barkhausen.

Rectangular NiFe stripes as small as $1 \times 5$ microm were fabricated and characterized as a function of film thickness. Gold current leads were sputtered and patterned onto the stripes so that magnetoresistance measurements could be performed. A uniform in-plane magnetic field was applied transverse to the stripe length and at various angles from the perpendicular $\mathrm{d}$ rection. For film thicknesses greater than $10 \mathrm{~nm}$, the magnetoresistance for all of the devices had large jumps and hysteresis due to domain formation. As the thickness of the film decreased below $10 \mathrm{~nm}$, the domain structure disappeared for stripe heights 2 microm or less. Theoretical calculations of the magnetization reversals were obtained using a numerical implementation of the Stoner-Wohlfarth model for the switching of a single-domain ellipsoidal particle.

\section{4,767}

PB96-147160 Not available NTIS

National Inst. of Standards and Technology (EEEL) Boulder, CO. Electromagnetic Technology Div.

II-3: Critical Current Measurement Methods: Quan titative Evaluation.

Final rept

L. F. Goodrich, and A. N. Srivastava. 1995, 5p. See also PB93-153369.

Pub. in Cryogenics, v35 VAMAS Supplement, pS19 S23 1995

Keywords: "Superconductors, "Critical current, Electrical measurement, Comparative evaluations, $R$ prints, "Foreign technology, Thermal contraction.

The critical current Ic of a superconductor is a quantitative evaluation of its current carrying capacity, and is defined as the current at which a specified electric field criterion Ec, or resistivity criterion Pc is achieved in the specimen. Typical electric field and resistivity criteria are 10 to the minus one microm and 10 to the minus 14 th power omega $m$, respectively. The voltagecurrent $(\mathrm{V}-1)$ characteristic of the superconductor can be modelled by the empirical equation $\mathrm{V}=\mathrm{VO}(\mathrm{III})$ sup $n$ where 10 is the observed current at voltage Vo. The value of $n$ can be thought of as a 'figure of merit' for the conductor, and reflects the abruptness of the transition from the superconducting to the normal state, with typical values ranging from 10 to 100 .

04,768

PB96-147178 Not available NTIS

National Inst. of Standards and Technology (EEEL) Boulder CO. Electromagnetic Technology Div.

First VAMAS USA Interlaboratory Comparison of High Temperature Superconductor Critical Current Measurements.

Final rept.

L. F. Goodrich, J. A. Wiejaczka, A. N. Srivastava, T. C. Stauffer, and L. T. Medina. 1995, 4p.

Pub. in Institute of Electrical and Electronics Engineers Transactions on Applied Superconductivity, v5 pt1 n2 p552-555 Jun 95.

Keywords: "High-Tc superconductors, "Critical current, Comparative evaluations, Damage, Homogeneity, Routing, Standards, Reprints.

The authors conducted an interlaboratory comparison of critical current (Ic) measurements on measurements from six participating US laboratories, with NIST as the central, organizing laboratory. A number of specimens were prepared with different degrees of instrumentation to isolate sources of variability. Mos of the specimens were pre-measured by NIST to reduce uncertainties due to sample variability. Different specimen routing patterns among the laboratories were implemented to isolate sources of variability due to the specimen's measurement history. 
04,769

PB96-147186 Not available NTIS

National Inst. of Standards and Technology (EEEL),

Boulder, CO. Electromagnetic Technology Div.

II-5: Thermal Contraction of Materials Used in Nb3Sn Critical Current Measurements.

Final rept.

L. F. Goodrich, and A. N. Srivastava. 1995, 4p.

Pub. in Cryogenics, v35 VAMAS Supplement, p\$29-

S32 1995.

Keywords: *Superconductors, Comparative evaluations, Contraction, Electrical measurement, Reprints, "Foreign technology, "Niobium stannoides, "Critical current.

It is typical for Nb3Sn-Cu superconductor specimens to be wound into coils on tubular measurement mandrels for critical current measurements. If the thermal contraction of the mandrel is different from that of the specimen, either tensile or compressive axial strain could develop upon cooling from room temperature to liquid helium temperature, thus affecting the measured critical current Ic1.2. The amount of strain depends on the magnitude of the differential contraction, the relative strength of the specimen and mandrel, and the mechanical coupling between the specimen and its mandrel.

\section{4,770}

PB96-147194 Not available NTIS

National Inst. of Standards and Technology (EEEL), Boulder, CO. Electromagnetic Technology Div.

USA Interlaboratory Comparison of Superconductor Simulator Critical Current Measurements.

Final rept.

L. F. Goodrich, J. A. Wiejaczka, A. N. Srivastava, T.

C. Stauffer, and L. T. Medina. 1995, 4p.

Pub. in Institute of Electrical and Electronics Engineers Transactions on Applied Superconductivity, v5 n2 pt1 p548-551 Jun 95.

Keywords: "Superconductors, Comparative evaluations, Variability, Simulators, Standards, Reprints, ${ }^{*}$ Critical current.

An interlaboratory comparison of critical current (IC) measurements was conducted on the superconductor simulator, which is an electronic circuit that emulates the extremely nonlinear voltage-current characteristic of a superconductor. These simulators are high precision instruments, and are useful for establishing the integrity of part of a superconductor measurement system. The study includes measurements from participating US laboratories, with NIST as the central, organizing laboratory. This effort was designed to determine the sources of uncertainty in Ic measurements due to uncertainties in the measurement apparatus, technique, or the analysis system.

\section{4,771}

PB96-148010 Not available NTIS National Inst. of Standards and Technology (EEEL),
Boulder, CO. Electromagnetic Technology Div.

Microwave Noise in High-Tc Josephson Junctions. Final rept.

E. N. Grossman, L. R. Vale, and D. A. Rudman

1995, 3p. Pub. in Applied Physics Letters, v66 n13 p1680-1682
Mar 95 .

Keywords: "Josephson junctions, "YBCO superconductors, Noise, Mixing circuits, Heterodyning, Reprints, SNS junctions, Terahertz, Mixer.

The authors have measured the noise of $\mathrm{YBa} 2 \mathrm{Cu} 307-$ delta superconductor- normal-superconductor (SNS) junctions whose high normal-state resistances and characteristic frequencies make them suitable for $\mathrm{THz}$ frequency mixers. By directly measuring the $1 \mathrm{GHz}$ powerspectral density delivered to a low-noise 50 Ohms radiometer system, thenoise could be measured over a wide range of $\mathrm{dc}$ voltage and temperature without complications due to $1 / f$ noise, and without invoking any specific model. The effective noise temperature of the normal resistanceis approximately equal to the physical temperature at high temperatures,but approaches a limiting value at low temperatures, implying an excess current noise of unknown origin.

\section{4,772}

PB96-148143 Not available NTIS

National Inst. of Standards and Technology (EEEL), Boulder, CO. Electromagnetic Technology Div.
V-6: Effects of Temperature Variation.

Final rept

H. Kirchmayr, and L. F. Goodrich. 1995, 2p.

Pub. in Cryogenics, VAMAS Supplement, v35 pS99. S100 1995

Keywords: *Superconductors, "Low temperature tests, "Interlaboratory comparisons, Temperature dependence, Liquid helium, Pressure, Cryostats, Reproducibility, Niobium stannides, Reprints, Critical current, Mandrel.

The critical current (IC) of a NbTi or Nb3Sn superconductor specimen depends on its temperature. The pressure, and thus the liquid helium temperature, may vary between laboratories due to different altitudes and different cryostats used for the Ic measurement. Thus, it is necessary to study the temperature dependence of the specimen's critical current. This paper presents measurements of Ic as a function of temperature for tests carried out at the Technical University of Vienna (TUW) and the National Institute of Standards and Technology (NIST).

\section{4,773}

PB96-148150 Not available NTIS

National Inst. of Standards and Technology (EEEL), Gaithersburg, MD. Semiconductor Electronics Div. Scanning Capacitance Microscopy Measurements and Modeling: Progress Towards Dopant Profiling of Silicon.

Final rept.

J. J. Kopanski, J. F. Marchiando, and J. R. Lowney. 1995, 8p.

Pub. in Proceedings of the International Workshop on the Measurement and Characterization of Ultra-Shallow Doping Profiles in Semiconductors (3rd), Research Triangle Park, NC., March 20-22, 1995, p9.1-9.8.

Keywords: "Microscopy, "Scanning, *Semiconductor devices, "Silicon, Dopes, Profiles, Experimental data, Models, Reprints, AFM(Atomic force microscope), SCM(Scanning capacitance microscope).

A scattering capacitance microscope (SCM) has been implemented by interfacing a commercial contactmode atomic force microscope (AFM) with a high-sensitivity capacitance sensor. SCM has promise as a next-generation dopant-profiling technique because the measurement is inherently two dimensional, has a potential spatial resolution limited by tip diameter of at least $20 \mathrm{~nm}$, and requires no current carrying metalsemiconductor contact. Different capacitance images have been made with the SCM of a variety of bulkdoped samples and in the vicinity of pn junctions and homojunctions. Also, a computer code has been written that can numerically solve Poisson's equation for a model SCM geometry by using the method of collocation of Gaussian points.

04,774

PB96-148184 Not available NTIS

National Inst. of Standards and Technology (EEEL), Boulder, CO. Electromagnetic Technology Div.

Effects of Substrate Surface Steps on the Microstructure of Epitaxial Ba2YCu3O7-x Thin Films on (001) LaAlO3.

Final rept.

P. C. McIntyre, M. J. Cima, and A. Roshko. 1995, $10 p$.

Pub. in Jnl. of Crystal Growth, v\$49 p64-73 1995.

Keywords: "Epitaxy, "YBCO superconductors, "Thin films, Critical temperature, High temperature, Substrates, Crystal growth, Morphology, Reprints.

The effect of steps in the (001) surface of LaAlO3 substrates on the microstructural evolution of heteroepitaxial Ba2YCu3O7(7-x) (BYC) thin films were characterized by high resolution transmission electron microscopy (TEM) and atomic force microscopy (AFM). Native substrate surface steps acted as nucleation sites for both the c-axis normal and c-axis in-plane (a-axis normal) orientations in BYC films prepared by post-deposition annealing of a precursor film. The density of C-axis in-plane BYC grains varied dramatically across the surface of the substrates. In several samples. The spatial pattern of $\mathrm{c}$-axis in-plane regions present in the BYC films was similar to the twin structure present in the LaAlO3 substrates.

\section{4,775}

PB96-155585 Not available NTIS

National Inst. of Standards and Technology (PL), Gaithersburg, MD. Electron and Optical Physics Div.
Tunneling Spectroscopy of bcc(001) Surface States.

Final rept.

J. A. Stroscio, D. T. Pierce, A. Davies, and R. J.

Celotta. 1995, 4p.

Pub. in Physical Review Letters, v75 n16 p2960-2963 Oct 95 .

Keywords: "Tunneling spectroscopy, "Surface states, Band structure, Reprints, Bcc (001) surfaces, $\mathrm{Cr}, \mathrm{Fe}$.

The authors have discovered an intense, sharp feature in tunneling spectra of the $\mathrm{Fe}(001)$ and $\mathrm{Cr}(001)$ surfaces that derives from a bcc surface state near the Fermi level. Band-structure calculations show that this state is a general feature of $\mathrm{bcc}(001)$ surfaces, and that it originates from a nearly unperturbed d orbital extending out into the vacuum region. This general feature should permit chemical identification with atomic spatial resolution on bcc(001) surfaces. The surface state is in the minority spin band for $\mathrm{Fe}$ and $\mathrm{Cr}$, so it should be useful for future spin-polanized tunneling experiments.

04,776

PB96-156104 Not available NTIS

National Inst. of Standards and Technology (MSEL), Gaithersburg, MD. Reactor Radiation Div.

Fast-Ion Conducting Y2(ZryTi1-y)207 Pyrochlores: Neutron Rietveld Analysis of Disorder Induced by Zr Substitution.

Final rept.

C. Heremans, B. J. Wuensch, J. K. Stalick, and E.

Prince. 1995, 14p.

Pub in Jnl. of Solid State Chemistry, v117 p108-121 1995.

Keywords: "Order-disorder transformations, "Ionic conductivity, "Crystal structure, Zirconium additions, Reprints, Yttrium zirconate titanates, Rietveld method, Pyrochlore structure.

The structure of Y2(ZrvTi1-y)2Ov solid solutions progressively changes, with increasing $y$, from an ordered pyrochlore structure space group Fd3m, to a deflectfluorite structure space group $\mathrm{Fm} 3 \mathrm{~m}$, at $\mathrm{y}=0.90$. The anion array consists of three independent sites $O(1)$ $\mathrm{O}(2)$, and $\mathrm{O}(3)$, occupying positions $48 \mathrm{8}, 8 \mathrm{a}$ and $8 \mathrm{~b}$ respectively, of which $8 \mathrm{~b}$ is unoccupied in a fully ordered pyrochlore. Rietveld powder-profile analysis of data collected with 1.5453-A thermal neutrons was used to determine the structure state of four samples with increasing $\mathrm{Zr}$ content $(\mathrm{y}=0.30,0.45,0.60$, and $0.90)$. Refinements that employed only the pyrochlore superstructure intensity data provided weighted profile residuals that ranged 8.06 to $8.67 \%$ compared with expected values of 7.13 to $7.87 \%$ derived from counting statistics.

04,777

PB96-161278 Not available NTIS

National Inst. of Standards and Technology (MSEL) Gaithersburg, MD. Ceramics Div.

Powder X-ray Diffraction Data for Ca2Bi2O5 and C4Bi6013.

Final rept.

C. J. Rawn, R. S. Roth, and H. F. McMurdie. 1992,

3p.

Pub. in Powder Diffraction, v7 n2 p109-111 1992.

Keywords: *Crystal growth, Reprints, "X ray diffraction, "Space group symmetry, Unit cell dimensions, $X$ ray powder diffraction, CA2Bi205, CA4Bi6013.

Single crystals and powder samples of $\mathrm{Ca} 2 \mathrm{Bi5O} 5$ and Ca4Bi6013 have been synthesized and studied using single crystal $\mathrm{X}$-ray diffraction as well as $\mathrm{X}$-ray and neutron powder diffraction. Unit cell dimensions were calculated using a least squares analysis that refined to a delta 2 theta of no more than 0.03 degrees. A triclinic cell was found with space group $\mathrm{P1} a=$ $10.1222(7), b=10.1466(6), c=10.4833$ (7) Angstroms. Alpha $=116.912(5)$, Beta $=107.135(6)$ and gamma $=$ $92.939(6), Z=6$ for the Ca2Bi2O5 compound. An orthorhombic cell was found with space group C2mm, $a=17.3795(5), b=5.9419(2)$ and $c=7.2306(2)$ Angstroms, $Z=2$ for the Ca4Bi6O13 compound.

04,778

PB96-161948 Not available NTIS

National Inst. of Standards and Technology (EEEL), Gaithersburg, MD. Semiconductor Electronics Div. 
Business and Manufacturing Motivations for the Developing of Analytical Technology and Metrology for Semiconductors.

Final rept.

T. J. Shaffner, A. C. Diebold, R. C. McDonald, D. G. Seiler, and W. M. Bullis. 1996, 10p.

Pub. in Proceedings of the International Workshop on Semiconductor Characterization: Present Status and Future Needs, Gaithersburg, MD., January 30-February 2, 1995, p1-10 1996.

Keywords: "Semiconductors, "Metrology, "Circuit technology, Analytical technology, Characterization, Microelectronics, Process development, Reprints.

Semiconductor characterization is an indispensable enabler of all modern microelectronics and optoelectronic circuit, and is in the critical path for maintaining the steady decline in cost-per-function of silicon integrated circuit technology. It is also driving new developments in compound semiconductor materials and devices (III-V and II-VI). In this overview, we present a perspective on measurement technology relative to business and economic challenges of the semiconductor industry, and illustrate the key role metrology plays in modern process development and manufacturing. We also describe how new characterization techniques evolve for semiconductor applications.

\section{4,779}

PB96-164058 Not available NTIS

National Inst. of Standards and Technology (EEEL), Gaithersburg, MD. Semiconductor Electronics Div. Use of Pressure for Quantum-Well Band-Structure Characterization.

Final rept.

J. H. Burnett, P. M. Amirtharaj, H. M. Cheong. B.

Elman, W. Paul, and E. S. Koteles. 1996, 5p.

Pub. in Proceedings of the International Workshop on Semiconductor Characterization: Present Status and Future Needs, Gaithersburg, MD., January 30-February 2, 1995, p634-638 1996.

Keywords: "Spectroscopy, Reprints, GaAs, Heterostructure, Pressure, Stress, "Quantum-well, HgCdTe, Photoluminescence, Uniaxial pressure.

We discuss the use of externally applied pressure for determining the origin of the peaks in absorption spectra (and related spectra) of semiconductor heterostructures. This technique depends on the different effective mass dependences of the various types of heterostructure energy levels involved in $a b$ sorption transitions, such as electron, heavy-hole, light-hole, exciton and defect levels, and the dissimilar pressure dependences of the various masses. Measurements of the hydrostatic or uniaxial pressure dependences of the spectral peaks thus distinguish peaks associated with these different types of energy levels. The approach is demonstrated for GaAs/ AlGaAs quantum wells and $\mathrm{HgTe} / \mathrm{HgCdTe}$ superlattices using hydrostatic pressure. We also briefly discuss the use of externally applied uniaxial pressure for distinguishing heavy- and light-hole-related peaks, and using the heavy-hole/light-hole splitting for determining quantitatively the amount of built-in uniaxial strain in the heterostructure layers.

\section{4,780}

PB96-164074 Not available NTIS

National Inst. of Standards and Technology (PL) Gaithersburg, MD. Electron and Optical Physics Div. SEMPA Studies of Exchange Coupling in Magnetic Multilayers.

Final rept.

R. J. Celotta, D. T. Pierce, and J. Unguris. 1995, 4p. Pub. in MRS Bulletin, v20 n10 p30-33 Oct 95.

Keywords: *Exchange coupling, "Magnetic multilayers, Reprints, "Scanning electron microscopy, Reflection high energy diffraction, Scanning tunneling microscopy.

In the late 1980 's, a number of exciting yet puzzling observations resulted from experiments investigating the couplng between two ferromagnetic layers separated by a nonferromagnetic spacer layer. A pioneering experiment by Grunberg et al. showed that $\mathrm{Fe}$ layers separated by a thin $\mathrm{Cr}$ spacer aligned with antiparalle magnetization, but with Au as the spacer layer, a parallel alignment occured. The long-range magnetic dipole from each layer would tend to explain antiparallel alignment; small pinholes in the space layer would produce parallel alignment. Alternatively, the layers might be coupled through the spacer-layer conduction electrons by the Ruderman-Kittel-KasuyaYosida (RKKY) effects. This was expected to produce an oscillation in coupling as the spacer thickness in creased that is an oscillation between parallel and antiparallel alignment. Oscillatory coupling was first observed by Parkin et al. Researchers had also found that, at spacer thickness where antiparallel alignment occured, the $\mathrm{Fe} / \mathrm{Cr} / \mathrm{Fe}$ system can exhibit a giant magnetoresistance (GMR) effect, that is, an anomalously large change in resistance when a magnetic field is applied. The potential technological importance of the GMR effect to magnetic sensing and magnetic information storage added further impetus to the already rapidly growing area of research in magnetic multilayers.

04,781

PB96-164207 Not available NTIS

National Inst of Standards and Technology (EEEL) Gaithersburg, MD. Semiconductor Electronics Div.

Scanning Capacitance Microscopy Measurements and Modeling for Dopant Profiling of Silicon.

Final rept.

J. J. Kopanski, J. F. Marchiando, and J. R. Lowney. 1996, 5p.

See also PB96-148150

Pub. in Proceedings of the International Workshop on Semiconductor Characterization: Present Status and Future Needs, Gaithersburg, MD., January 30-February 2, 1995, p308-312 1996

Keywords: "Microscopy, "Scanning "Seniconductor devices, "Silicon, Dopes, Profiles, Experimental data, Models, Reprints, AFM(Atomic force microscope), SCM(Scanning capacitance microscope)

A scanning capacitance microscope (SCM) for the twodimensional profiling of dopants in silicon has been implemented by interfacing a commercial atomic force microscope (AFM) with a high densitivity capacitance sensor. The AFM is used to control the position of a conducting tip and measure surface topography, while other electonics are used to control and record a simultaneous measurement of the capacitance-voltage (CV) response between the tip and a semiconductor. The operation of the SCM has been quantified by acquiring differential capacitance images of a variety of latera pn-junctions and uniformly doped silicon samples. The shape of the $C-V$ response between the AFM tip and oxidized silicon has been measured. Also, a compute code has been written to solve poisson's equation for the SCM geometry in three dimensions by using the method of collocation of Gaussian points. The purpose of the SCM modeling is to extract the dopant profile from the SCM measurement.

\section{4,782}

PB96-176763 Not available NTIS

National Inst. of Standards and Technology (MSEL) Gaithersburg, MD. Ceramics Div.

Reference Relations for the Evaluation of the Materials Properties of Orthorhombic YBa2Cu3OX Superconductors.

Final rept.

R. G. Munro, and H. Chen. 1996, 6p.

Pub. in Jnl. of the American Ceramic Society, v79 n3 p603-608 1996

Keywords: "Ceramics, *Superonductors, Reprints, Critical temperature, Lattice parameters.

Several expressions, suitable as reference relations, pertaining to the composition and temperature dependencies of the properties of orthorhombic YBA2Cu3O superconductors have been derived from statistical assessments of collections of data from independent experimental studies. Relations are given for (1) the critical temperature vs oxygen contents, (2) the lattice parameters at room temperature vs oxygen content, (3) the lattice parameters at fixed oxygen content vs temperature, (4) the anisotropic thermal expansion vs temperature, (5) the isotropic polycrystalline thermal expansion vs temperature, and (6) the mass density vs temperature.

\section{4,783}

PB96-176797 Not available NTIS

National Inst. of Standards and Technology (MSEL) Gaithersburg, MD. Reactor Rádiation Div.

Characterization of the Structure of LaD2.50 by Neutron Powder Diffraction.

Final rept.

T. J. Udovic, Q. Huang, and J. J. Rush. 1996, 9p.

Pub. in Jnl. of Solid State Chemistry, Article No. 0096 , v122 p151-159 1996
Keywords: "Neutron diffraction, Reprints, "Lanthanum deuteride, Long range order, Metal hydride, Phase transition.

Neutron powder diffraction (NPD) measurements of the rare-earth deuteride LaD2 50 were undertaken be tween 10 and $430 \mathrm{~K}$. Rietveld refinements indicated that, above approximately $385 \mathrm{~K}$, the LaD2.50 structure is cubic ( $\mathrm{Fm} 3 \mathrm{~m})$ with deuterium fully occupying the terrahedral $(t)$ interstices of the fcc La lattice and the excess deuterium occupying a portion of the octahedral (o) interstices with full statistical disorder. As the temperature is decreased below approximately $385 \mathrm{~K}$ the LaD2.50 structure undergoes a tetragonal distortion concomitant with the onset of deuterium longrange order (141amd) in the o sublattice, similar to previous structural results for the light-rare-earth deuterides RD2 $+x$ for 0.3 less than $x$ less'than 0.5 . Fully developed long-range order is established in the vicinity of 200 to $230 \mathrm{~K}$

04,784

PB96-179494 Not available NTIS

National Inst. of Standards and Technology (MSEL), Gaithersburg, MD. Ceramics Div.

Texture Measurement of Sintered Alumina Using the March-Dollase Function.

Final rept.

J. P. Cline, M. D. Vaudin, J. E. Blendell, K. J.

Bowman, N. Medendorp, C. A. Handwerker, and R.

Jiggets. 1994, $6 p$

Pub. in Advances in X-ray Analysis, $\sqrt{ } 37$ p473-478 1994

Keywords: "Alumina, Texture, Reprints, "MarchDollase, "Pole figures, Rietveld refinement, $X$ ray diffraction.

The Rietveld method entails the calculation of a powder diffraction pattern from crystallographic microstructural and equipment characteristics. These characteristics are related to the form of the pattern through a series of model functions. The difference between an observed and calculated pattern is then min mized by sequentially refining the physical parameters contained within the model functions to obtain an accurate and precise desceiption of the specimen. A pow der diffraction pattern from a specimen exhibiting crystallographic texture, or preferred orientation, will display intensity values which differ systematically from those calculated for a specimen of random orientation. This systematic discrepancy can be addressed by in corporating into the Rietveld refinement a model function for sample texture. A successful model for texture will accurately assess the phase abundance and degree of texture from both oriented and randomized specimens. In this study the authors use the MarchDollase model function to characterize texture development in sintered alumina with respect to processing variables and sintering time.

\section{4,785}

PB96-179551 Not available NTIS

National Inst. of Standards and Technology (MSEL) Gaithersburg, MD. Polymers Div.

Dielectric Behavior of a Polycarbonate/Polyester Mixture Upon Transesterification.

Final rept.

B. J. Factor, F. I. Mopsik, and C. C. Han. 1996, 3p.

Pub. in Macromolecules, v29 n6 p2318-2320 1996.

Keywords: "Dielectric spectroscopy, Polycarbonates Polyesters, Reprints, "Transesterfication.

Dielectric relaxation spectroscopy provides a sensitive measure of the local environment surrounding molecu lar dipoles. In the examination of polymers, it is cus tomary to record the complex dielectric constant $e(s t a r)(v)=e^{\prime}(v)+i e^{\prime}(v)$ as a function of frequency $v$. The form of the spectra can often be related to aspects of specimen structure. For example the distribution of local environments that arise due to concentration fluctuations in polymer blends and diblock copolymers has been characterized using this technique. Further information concerning the dipolar magnitude as well as local geometric constraints can also be obtained through accurate measurement of the dielectric dispersion magnitude.

\section{4,786}

PB96-183090 PC A04/MF A01

National Inst. of Standards and Technology (MSEL),

Gaithersburg, MD. Ceramics Div. 
Workshop on Characterizing Diamond Films (4th). Held in Gaithers burg, Maryland on March 4-5, 1996. A. Feldman, A. P. Malshe, and J. E. Graebner. Apr 96, 33p, NISTIR-5837

See also report on 3rd Workshop, PB94-187663. Prepared in cooperation with Arkansas Univ., Little Rock. Keywords: "Diamonds, "Films, "Meetings, Cutting tools, Adhesion, Chemical vapor deposition, Interlaboratory comparisons, Thermal conductivity, Thermal diffusivity, Tungsten carbides, Round robin tests.

The fourth in a series of workshops was held at NIST on March 4th and 5th, 1996 to discuss the characterization of diamond films and the need for standards in diamond technology. The audience targeted for this workshop were the producers and potential users of chemical vapor deposited (CVD) diamond technology in the United States. Two technical topics that have relevance to applications of CVD diamond were discussed; adhesion of CVD diamond to cutting tool materials and standardization of thermal conductivity measurement for heat spreading applications. A new roundrobin of thermal conductivity measurements was planned which will utilize specimens having significantly better homogeneity than those used in a previous round robin.

\section{4,787}

PB96-190186 Not available NTIS

National Inst. of Standards and Technology (EEEL), Boulder, CO. Electromagnetic Technology Div. Epitaxial Nucleation and Growth of Chemically Derived $\mathrm{Ba} 2 \mathrm{YCu} 3 \mathrm{O} 7-\mathrm{x}$ Thin Films on (001) SrTiO3.

Final rept.

P. C. Mclntyre, M. J. Cima, and A. Roshko. 1995,

$11 \mathrm{p}$.

Pub. in Jnl. of Applied Physics, v77 n10 p5263-5272 May 95.

Keywords: *Epitaxy, "Nucleation, "Thin films, "YBCO superconductors, Growth, Organometallic compounds, Microstructure, Barium oxides, Copper oxides, Yttrium Microstructure,
oxides, Reprints.

The nucleation and growth kinetics of epitaxial $\mathrm{Ba} 2 \mathrm{YCu}^{\mathrm{O}}(7-\mathrm{x})$ thin films prepared on (001) $\mathrm{SrTiO}$ by postdeposition annealing of a chemically derived intermediate layer were investigated in specimens quenched from the growth anneal. The films were produced by spin-on deposition of a metal-organic precursor solution at room temperature and ambient pressure, and subsequent postdeposition annealing to form $\mathrm{Ba} 2 Y$ cu3O(7-x) (BYC). Integrated $x$-ray intensities of reflections from the majority, c-axis-normal epitaxial BYC in the films were analyzed as a function of time at annealing temperature. The linear transformation $\mathrm{ki}-$ netics suggest the growth process is controlled by molecular processes at the BY/intermediate interface, and is not rate limited by diffusion.

\section{4,788}

\section{PB96-193776 PC A03/MF A01}

National Inst. of Standards and Technology (CAML), Gaithersburg, MD. Applied and Computational Mathematics Div

Notion of a xi-Vector and a Stress Tensor for a General Class of Anisotropic Diffuse Interface Models.

A. A. Wheeler, and G. B. McFadden. Apr 96, 29p NISTIR-5848.

Prepared in cooperation with Southampton Univ. (England). Faculty of Mathematical Studies.

Keywords: "Surface diffusion, "Anistropy, *Interfaces, *Stress tensors, Binary systems(Materials), Phase transitions, Vector analysis, Surface energy, Three dimensional, Phase field models, Gibbs-Thomson equation, $X i$ vectors.

The authors consider a general class of multiple-orderparameter models which represent the diffuse interface of a first-order phase transition with anisotropic surface energy and kinetics. They show that in the sharp interface limit, it is possible to construct a xi-vector and they derive the three-dimensional form of the Gibbs-Thomson equation. Moreover they develop the notion of a stress tensor for diffuse interface theories and show that it may be used to derive the equilibrium conditions for edges in interfaces as well as multiple functions. The authors also show that when edges form in the special case of a phase-field model, a weak shock forms in which the spatial derivatives of the phase field are not continuous, and they derive the jump conditions.
04,789

PB96-195268 PC A03/MF A01

National Inst. of Standards and Technology (EEEL), Gaithersburg, MD. Office of Microelectronics.

National Semiconductor Metrology Program, Project Portfolio FY 1996.

D. S. Yaney, and A. D. Settle-Raskin. Jun 96, 28p

NISTIR-5851.

See also PB96-175856.

Keywords: "Semiconductor devices, "Metrology, Integrated circuits, Dielectrics, Chemical analysis, Research projects, *Semiconductor materials, Calibration.

The National Semiconductor Metrology Program (NSMP) is a NIST-wide effort designed to meet the highest priority measurement needs of the semiconductor industry as expressed by the National Technology Roadmap for Semiconductors and other authoritative industry sources. The NSMP was established in 1994 with a strong focus on mainstream siticon CMOS technology and an ultimate funding goal of $\$ 25$ million annually. Current annual funding of approximately $\$ 11$ million supports the 23 internal projects which are summarized in this Project Portfolio booklet.

04,790

PB96-200183 Not available NTIS

National Inst. of Standards and Technology (EEEL),

Boulder, CO. Electromagnetic Technology Div.

High-Tc Multilayer Step-Edge Josephson Junctions and SQUIDs.

Final rept.

N. Missert, T. E. Harvey, R. H. Ono, and C. D.

Reintsema. 1993, 3p.

Pub. in Applied Physics Letters, v63 n12 p1690-1692 Sep 93.

Keywords: "Josephson junctions, *Yttrium barium copper oxides, "High temperature superconductors, Fabrication, Thin films, Strontium titanates, Neodymium oxides, Gadolinium oxides, Silver, Reprints, Superconducting Quantum Interference Devices, SQUIDS(Superconducting Quantum Interference Devices), Yttrium barium cuprates, SNS junctions.

The authors have developed a reliable process to fabricate high-quality YBa2Cu3O(7-x) (YBCO) superconductor normal metal-superconductor (SNS) step-edge junctions and SQUIDs over YBCO ground planes. These multilevel circuits employ thin films of $\mathrm{SrTiO}_{3}$ and $\mathrm{NdGaO} 3$ as the insulating layer between the active device and the ground plane and use $\mathrm{Ag}$ as the normal metal in the Josephson junction. The reproducibility and uniformity of the junctions are better than our single-level devices grown directly on step edges cut into single-crystal substrates. Here the critical current variation among junctions on a single wafer is less than a factor of 2 . Junctions grown on thin-film step edges of $\mathrm{SrTiO}_{3}$ have critical currents near $2 \mathrm{MA}$ at $4 \mathrm{~K}$, while those grown on NdGaO3 step edges have critical currents near $0.5 \mathrm{~mA}$ at $4 \mathrm{~K}$

\section{4,791}

PB96-200340 Not available NTIS

National Inst. of Standards and Technology (PL), Boulder, CO. Quantum Physics Div.

Collisions of Electrons with Highly-Charged lons. Final rept.

G. H. Dunn, N. Djuric, Y. S. Chung, M. Bannister,

and A. C. H. Smith. 1995, 7p.

See also DE95007393.

Pub. in Nuclear Instruments and Methods in Physics Research B, v98 p107-113 1995.

Keywords: *Gold, "Multicharged ions, "Electron ion collisions, "Ion atom collisions, Electron cyclotron resonance, Excitation, Ion collisions, Ion sources, Ionization, Surfaces, Reprints, *Foreign technology.

The progress report, in addition to describing recent work in the authors' laboratory, highlights the general character and importance of electron collisions with highly-charged ions $(\mathrm{HCl})$ and describes major advances in the field since the previous conference on the subject Thus, while the authors' principle efforts are currently directed toward investigation of excitation of $\mathrm{HCl}$, there have been very notable advances by others in the areas of ionization and recombination and an attempt is made to put these into context of the field in general. Also, for the first time, significant progress is now being made by others in experimental investigation of elastic scattering electrons from $\mathrm{HCl}$.
04,792

PB96-200399 Not available NTIS

National Inst. of Standards and Technology (EEEL), Boulder, CO. Electromagnetic Technology Div.

Hot-Electron Microcalorimeter for X-ray Detection

Using a Superconducting Transition Edge Sensor with Electrothermal Feedback.

Final rept.

K. D. Irwin, G. C. Hilton, J. M. Martinis, and B.

Cabrera. 1996, 3p.

Pub. in Nuclear Instruments and Methods in Physics Research A, v370 p177-179 Apr 96.

Keywords: "X-ray detectors, *Superconductivity junctions, ${ }^{\star}$ Calorimeters, Electron temperature, Metal films, Hot electrons, Reprints, "Phonon detectors, "Micro calorimeters.

The authors investigate a hot-electron microcalorimeter for X-ray detection. The X-ray $a b$ sorber consists of a normal metal film in thermal and electrical contact with a superconducting transitionedge sensor. The sensor is formed by a proximity-effect bilayer of aluminum and silver, with a sharp superconducting transition near $100 \mathrm{mK}$. Energy from $X$-rays absorbed in the normal film is removed by a reduction of the Joule heating in the proximity bilayer due to electrothermal feedback and measured using a SQUID. The feedback mode of operation allows the measurement of incident energy with no free parameters and should lead to improvement in detector resolution over existing hot-electron microcalorimeters.

\section{4,793}

PB96-200670 Not available NTIS

National Inst. of Standards and Technology (EEEL), Boulder, CO. Electromagnetic Technology Div. Hot-Electron-Microcalorimeters with $0.25 \mathrm{~mm}(2)$ Area.

Final rept.

J. M. Martinis. 1996, 2p.

Pub. in Nuclear Instruments and Methods in Physics Research A, v370 p171-172 Apr 96

Keywords: “ $X$-ray detectors, "Superconducting junctions, "Calorimeters, Reprints, "Microcalorimeters, Phonon detectors.

The author presents measurements on hot-electron microcalonimeter with a normal-insulator superconductor tunnel-junction thermometer that is used for the detection of X-rays. With an absorber area of $0.5 \mathrm{~mm}$ by $0.5 \mathrm{~mm}$, pulses of 20 - micrometers in duration were observed that gave a $30 \mathrm{eV}$ FWHM resolution for $6 \mathrm{keV} X$-rays and an $18 \mathrm{eV}$ resolution for heat pulses. This detector has sufficient resolution, detector area, and speed to warrant application in materials analysis.

\section{4,794}

PB96-200712 Not available NTIS

National Inst. of Standards and Technology (EEEL), Boulder, CO. Electromagnetic Technology Div.

Controlling the Critical Current Density of HighTemperature SNS Josephson Junctions.

Final rept.

R. H. Ono, L. R. Vale, C. D. Reintsema, G. Kunkel,

and S. J. Berkowitz. 1995, 3p.

Pub. in International Superconductive Electronics Conference (5th) (ISEC '95), Nagoya, Japan, September 18-21, 1995, p114-116.

Keywords: *Josephson junctions, "Current density, High temperature, Superconductors, Reprints, SNS junctions.

High-Tc Josephson junctions have been made using a step-edge super-conductor-normal-conductor (SNS) process where the critical current density has been controlled by the geometric length of the $\mathrm{N}$ region. The authors will discuss techniques for controlling the critjcal current while simultaneously adjusting the normal resistances.

\section{4,795}

PB96-200746 Not available NTIS

National Inst. of Standards and Technology (EEEL),

Boulder, CO. Electromagnetic Technology Div.

Magnetic Flux Pinning in Epitaxial YBa2Cu3O7delta Thin Films.

Final rept.

A. Roshko, L. F. Goodrich, D. A. Rudman, R.

Moerman, and L. R. Vale. 1995, 4p.

Pub. in Jnl. of Electronic Materials, v24 n12 p191919221995. 
Keywords: "Flux pinning, "Thin films, Morphology, Dislocations, Microstructures, Reprints, Scanning tunneling microscopy, Nucleation mechanism.

The influence of microstructure on the critical current density of laser ablated $\mathrm{YBa} 2 \mathrm{Cu} 3 \mathrm{O} 7$ thin films has been examined. Scanning tunneling microscopy was used to examine the morphologies of $\mathrm{YBa} \mathrm{BCu} 307$ films and the morphology data were then correlated with measurements of the critical current density. The films were found to grow by an island nucleation and growth mechanism. The critical current densities of the films are similar to those of films with screw dislocation growth, indicating that screw dislocation growth is not necessary for good pinning. The data suggest that the critical current density in applied magnetic field may be higher in films with higher densities of growth features.

\section{4,796}

PB96-201058 Not available NTIS

National Inst. of Standards and Technology (EEEL), Gaithersburg, MD. Semiconductor Electronics Div. Time-Resolved Measurements of the Polarization State of Four-Wave Mixing Signals from GaAs Multiple Quantum Wells.

Final rept.

A. E. Paul, J. A. Bolger, A. L. Smirl, and J. G.

Pellegrino. 1996, 10p

Pub. in Jnl. of the Optical Society of America B, v13 n5 p1016-1025 May 96.

Keywords: "Gallium arsenides, "Aluminum gallium arsenides, "Quantum wells, "Polarization, Optical properties, Excitons, Reprints, Four wave mixing.

The complete polarization state of the degenerate fourwave mixing signal from a GaAs/Al2Ga 1-xAs multiple quantum well is determined by time resolution of al four of its Stokes parameters as a function of the relative angle between the two linear input polarizations. The degree of ellipticity and the orientation of the polarization ellipse are both observed to vary dramatically in time, and the temporal evolution is found to be consistent with previous measurements of the time-integrated Stokes parameters and to provide new constraints for physical models. The results are shown to be qualitatively consistent with a phenomenological model requiring the inclusion of both many-body interactions and biexcitonic effects.

04,797

PB96-204474 Not available NTIS

National Inst. of Standards and Technology (MSEL), Gaithersburg, MD. Ceramics Div.

Alvin Van Valkenburg and the Diamond Anvil Cell. Final rept.

G. J. Piermarini. 1993, 6p.

Pub. in High Pressure Research, v11 p279-284 1993.

Keywords: "High pressure, "Diamond anvil cells, Reprints, Infrared spectroscopy, Invention, X-ray diffraction, Ruby fluorescence.

A history of the development of the diamond anvil high pressure cell at the National Institute of Standards and Technology (formerly NBS) in the late 1950's is given along with a description of the contributions made by the four co-inventors of the device.

\section{4,798}

PB97-109011 PC A11/MF A03

National Inst. of Standards and Technology, Gaithersburg, MD.

Journal of Research of the National Institute of Standards and Technology, May/June 1996. Volume 101, Number 3 . Special Issue: NIST Workshop on Crystallographic Databases.

1996, 224p.

See also PB97-109029 through PB97-109227 and PB96-177381. Also available from Supt. of Docs. as SN703-027-00070-9.

Keywords: "Crystallography, *Information systems, Crystal structure, Data base management.

\section{Contents:}

CRYSTMET-The NRCC Metals Crystallographic Data File;

Inorganic Crystal Structure Database (ICSD) and Standardized Data and Crystal Chemica Characterization of Inorganic Structure Types (TYPIX)--Two Tools for Inorganic Chemists and Crystallographers;

Evaluation of Crystallographic Data with the Program DIAMOND:

The Cambridge Structural Database (CSD)
Current Activities and Future Plans; The Protein Data Bank:

Current Status and Future Challenges:

The Nucleic Acid Database:

Present and Future;

The Powder Diffraction File:

Past, Present, and Future;

NIST Crystallographic Databases for Research and Analysis;

Conventional and Eccentric Uses of Crystallographic Databases in Practica Materials Identification Problems:

Using NIST Crystal Data Within Siemens Software for Four-Circle and SMART CCD Diffractometers;

Phase Identification in a Scanning Electron Microscope Using Backscattered Electron Kikuchi Patterns

The Biological Macromolecule Crystallization Database and NASA Protein Crystal Growth Archive;

Investigations of the Systematics of Crystal Packing Using the Cambridge Structural Database:

Troublesome Crystal Structures:

Prevention, Detection, and Resolution;

CIF (Crystallographic Information File)

A Standard for Crystallographic Data Interchange

The Role of Journals in Maintaining Data Integrity:

Checking of Crystal Structure Data in Acta Crystallographica;

Electronic Publishing and the Journals of the American Chemical Society

How the Cambridge Crystallographic Data Center Obtains its Information

Data Import and Validation in the Inorganic Crystal Structure Database:

World Wide Web for Crystallography

Workshop Highlights.

\section{4,799}

(Order as PB97-109011, PC A11/ MF A03)

National Research Council of Canada, Ottawa (On tario). Scientific Numeric Database Service.

CRYSTMET: The NRCC Metals Crystallographic Data File.

G. H. Wood, J. R. Rodgers, S. R. Gough, and P. Villars. $1996,11 p$

Prepared in cooperation with Materials Phases Data Bank, Vitznau (Switzerland).

Included in Jnl of Research of the National Institute of Standards and Technology, v101 n3 p205-215 May/ Jun 96.

Keywords: "Crystallography, "Metals, "Minerals, "Information systems, Alloys, Intermatallic compounds, Numeric data, Crystal structure, Online systems, Searching, Software tools, Database management.

CRYSTMET is a computer-readable database of critically evaluated crystallographic data for metals (including alloys, intermerallics and minerals) accompanied by pertinent chemical, physical and bibliographic information. It currently contains about 60.000 entries and covers the literature exhaustively from 1913. Scientific editing of the abstracted entries, consisting of numerous automated entries, consisting of numerous automated and manual checks, is done to ensure consistency with related, previously published studies, to as sign structure types where necessary and to help guarantee the accuracy of the data and related information. Analyses of the entries and their distribution across key journals as a function of time show interesting trend in the complexity of the compounds studied as well as in the elements they contain. Two applications of CRYSTMET are the identification of unknowns and the prediction of properties of materials. CRYSTMET is available either online or via license of a private copy from the Canadian Scientific Numeric Database Serv ice (CAN/SND). The indexed online search and analysis system is easy and economical to use yet fast and powerful. Development of a new system is under way combining the capabilities of ORACLE with the flexibility of a modern interface based on the Netscape browsing tool.

04,800

PB97-109086 (Order as PB97-109011, PC A11/ MF AOS)

JCPDS-International Centre for Diffraction Data, Newtown Square, PA
Powder Diffraction File: Past, Present, and Future. D. K. Smith, and R. Jenkins. 1996, 13p.

Included in Jnl. of Research of the National Institute of Standards and Technology, v101 n3 p259-271 May/ Jun 96.

Keywords: *Crystallography, "X-ray powder diffraction, "Information systems, Crystal structure, Databas management.

The Powder Diffraction file has been thte primary reference for Powder Diffraction Data for more than half a century. The file is a collection of about 65,000 reduced powder patterns stored as sets of $d / l$ data along with the appropriate crystallographic, physical and experimental information. This paper reviews the development and growth of the PDF and discusses the role of the ICDD in the maintenance and dissemination of the file.

04,801

PB97-109094 (Order as PB97-109011, PC A11) MF A03)

National Inst. of Standards and Technology, Gaithersburg, MD

NIST Crystallographic Databases for Research and Analysis

A. D. Mighell, and V. L. Karen. 1996, 8p.

Included in $\mathrm{Jnl}$. of Research of the National Institute of Standards and Technology, v101 n3 p273-280 May/ Jun 96.

Keywords: "Crystallography, "Information systems, *Information centers, Crystal structure, Chemical composition, Physical properties, Bibliographies, Software tools, US National Institute of Standards and Technology.

The NIST Crystal and Electron Diffraction Data Center builds a comprehensive database with evaluated chemical, physical, and crystallographic information on all types of well-characterized substances. The data are evaluated and standardized by specially designed computer programs as well as by experts in the field. From its master database, the Data Center produces NIST Crystal Data and an Electron Diffraction Database with over 220,000 and 81,000 entries, respectively. These distribution databases are made available to the scientific community via CD-ROM, scientific community via CD-ROM, scientific instruments and online systems. In addition, the Data Center has developed theory and software that can be used for establishing all types of lattice relationships, for the determination of symmetry, for the identification of unknowns using lattice matching techniques, and for data evaluation.

04,802

PB97-109102 (Order as PB97-109011, PC A11/ MF A03)

Amoco Corp., Naperville, IL. Naperville Analytical Technical Lab. Services.

Conventional and Eccentric Uses of Crystallographic Databases in Practical Materials Identification Problems.

J. A. Kaduk. 1996, 14p.

Included in Jnl. of Research of the National Institute of Standards and Technology, v101 n3 p281-294 May/ Jun 96

Keywords: "Crystallography, "Materials, "Information systems, Crystal structure, Cobalt compounds, Copper aluminum barates, Magnesium chloride tetrahydrate, Palladium chlorides, Terephthalic acid, Vanadium phosphates, Uses, Database management.

The crystallographic database are powerfull and costeffective tools for solving materials identification problems, both individually and in combination. Examples of the conventional and unconventional use of the databases in solving practical problems involving organic, coordination, and inorganic compounds are provided. The creation and use of fully-relational versions of the Powder Diffraction File and NIST Crystal Data are described.

04,803

PB97-109110 (Order as PB97-109011, PC A11 MF A03)

Siemens Energy and Automation, Madison, WI.

Using NIST Crystal Data within Siemens Software for Four-Circle and SMART CCD Diffractometers. S. K. Byram, C. F. Campana, J. Fait, and R. A Sparks. $1996,6 p$.

Included in Jnl. of Research of the National Institute of Standards and Technology, v101 n3 p295-300 May/ Jun 96 
Keywords: "Crystallography, "X-ray diffractometers, "Information systems, Information retrieval, Single crystals, Crystal structure.

NIST Crystal Data developed at The National Institute for Standards and Technology has been incorporated with Siemens single crystal software for data collection on four-circle and two-dimensional CCD diffractometers. Why this database is useful in the process of single crystal structure determination, and how the database is searched, are described. Ideas for future access to this and other databases are presented.

\section{4,804}

PB97-109128 (Order as PB97-109011, PC A11/ MF A03)

Sandia National Labs., Albuquerque, NM. Materials and Process Sciences Center.

Phase Identification in a Scanning Electron Microscope Using Backscattered Electron Kikuchi Patterns.

R. P. Goehner, and J. R. Michael. 1996, 8p.

Included in Jni. of Research of the National Institute of Standards and Technology, v101 n3 p301-308 May/ Jun 96.

Keywords: "Crystallography, "Scanning election microscopy, "Microstructure, Crystal structure, Change coupled devices, Detectors, Metals, Welds, BKD(Backscatter Kikuchi diffraction), Backscatter Kikuchi diffraction

Backscattered electron Kikuchi patterns (BEKP) suitable for crystallographic phase analysis can be collected in the scanning electron microscope (SEM) with a newly developed charge coupled device (CCD) based detector. Crystallographic phase identification using BEKP in the SEM is unique in that it permits high magnification images and BEKP's to be collected from a bulk specimen. The combination of scanning electron microscope (SEM) imaging, BEKP, and energy dispersive $x$-ray spectrometry holds the promise of a powerful new tool for materials science.

\section{4,805}

PB97-109169

(Order as PB97-109011, PC A11/

MF A03)

McMaster Univ., Hamilton (Ontario). Brockhouse Inst. for Materials Research.

CIF Crystallographic Information File: A Standard for Crystallographic Data Interchange.

I. D. Brown. 1996, 6p.

Included in Jnl. of Research of the National Institute of Standards and Technology, v101 n3 p341-346 May/ Jun 96.

Keywords: "Crystallography, "Information systems, "Standards, Dictionaries, Crystal structure, Databases.

The Crystallographic Information File (CIF) uses the self-defining STAR file structure. This requires the creation of a dictionary of data names and definitions. A basic dictionary of terms needed to describe the crystal structures of small molecules was approved in 1991 and is currently used for the submission of papers to Acta Crystallographica C. A number of extensions to this dictionary are in preparation. By storing the dictionary itself as a STAR file, the definitions and relationships in the CIF dictionary become computer interpretable. This offers many possibilities for the automatic handling of crystallographic information.

04,806

PB97-109177 (Order as PB97-109011, PC A11/ MF A03)

International Union of Crystallography, Chester (England)

Role of Journals in Maintaining Data Integrity: Checking of Crystal Structure Data in 'Acta Crystallographica'.

B. McMahon. 1996, 9p.

Included in Jnl. of Research of the National Institute of Standards and Technology, v101 n3 p347-355 May' Jun 96.

Keywords: "Crystallography, "Journals, Quality control, Publications, Crystal structure, Error analysis.

Ouality control of the papers in its journals is a major concern of the International Union of Crystallography. Recent technology development, not least the emergence of a standard data interchange file format, have facilitated the checking of numerical data in a paper, and its error-free transference to the printed page. Consequently, database holdings derived from IUCr journals will be of greater accuracy. Other publishers of crystallographic data may benefit from these innovations.

04,807
PB97-109185 (Order as PB97-109011, PC A11/ MF A03)

American Chemical Society, Washington, DC

Electronic Publishing and the Journals of the American Chemical Society.

J. D. Spring, and L. R. Garson. 1996, 4p.

Included in Jnl. of Research of the National Institute of Standards and Technology, v101 n3 p357-360 Mayl Jun 96.

Keywords: "Crystallography, “Journals, Crystal structure, Publications, Color graphics, Services, CDROMs, Electronic journals, American Chemical Society.

The American Chemical Society is developing a number of initiatives that implement emerging electronic technologies in order to provide a broad range of products and services to members and subscribers. Examples of products currently available, or under development, for access via the World Wide Web include supporting information for journals, Electronic ads, color graphics and entire journals. Other activities employ email, CD-ROMs, and softcopy text

\section{4,808}

PB97-109193 (Order as PB97-109011, PC A11/

MF A03)

Cambridge Crystallographic Data Centre (England).

How the Cambridge Crystallographic Data Centre Obtains Its Information.

D. G. Watson. 1996, 3p.

Included in $\mathrm{Jnl}$. of Research of the National Institue of Standards and Technology, v101 n3 p361-363 May/ Jun 96.

Keywords: "Crystallography, "Journals, "Information systems, "Data validation, Publications, Crystal structure, Records(Files), Databases.

This paper is concerned with the acquisition of supplementary information, both hardcopy and electronic. Special arrangements with major journals are discussed and plans announced for the deposition of private communications using an electronic deposition form.

04,809

PB97-109201 (Order as PB97-109011, PC A11/ MF A03)

Fachinformationszentrum Karlsruhe, Gesellschaft fuer Wissenschaftlich-Technische Information m.b.H., Eggenstein-Leopoldshafen (Germany, F.R.)

Data Import and Validation in the Inorganic Crystal Structure Database.

H. Behrens. 1996, 9p.

Included in Jnl. of Research of the National Institute of Standards and Technology, v101 n3 p365-373 May/ Jun 96 .

Keywords: "Crystallography, "Information systems, "Data validation, "Inorganic compounds, Crystal structure, Publications, Bibliometrics.

In the following paper the input procedures for the Inorganic Crystal Structure Database (ICSD) will be outlined. The input flow of the data is explained. Since the data have been excerpted from journal articles a bibliometric analysis of the relevant literature is presented. The types of data and the form in which they are recorded are discussed. Finally, illustrations are given of the importance of data checking and the data checking procedures a re described in detail.

\section{4,810}

PB97-10921

MF A03)

Geneva Univ. (Switzerland). Lab. de Cristallographie. World Wide Web for Crystallography.

H. D. Flack. 1996, 6p.

Included in Jnl. of Research of the National Institute of Standards and Technology, v101 n3 p375-380 May/ Jun 96.

Keywords: "Crystallography, "Information systems, Crystal structure, "Internet, World Wide Web, Elec tronic publications.

Some characteristics of the World Wide Web (WWW) and its Virtual Library (W3VL) are described. Aspects of the setting up, maintenance, future development and objectives of the World Wide Web Virtual Library: Crystallography are detailed. An overview of the successful use of WWW in the organization of two crystallographic conferences and one entirely electronic conference is given. A revolution in scientific publication is under way with the introduction of WWW and CD-ROM technologies and a few of the points important to crystallography are touched upon. An application to distance teaching in crystallography is described. There is no mention of WWW applications to crystallographic databases in this paper as others at the Workshop have adequately described their work.

04,811

PB97-109227 (Order as PB97-109011, PC A11/

National Inst. of Standards and Technology, National Inst. of

Workshop Highlights.

V. L. Karen, and A. Mighell. 1996, 1p.

Included in Jnl. of Research of the National Institute of Standards and Technology, v101 n3 p381-418 May/ Jun 96.

Keywords: "Crystallography, "Research projects, "Information systems, "Databases, Crystal structure, Workshops, Abstracts.

The ongoing computer revolution is causing changes in all crystallographic data activities and is blurring distinctions between what have traditional been independent efforts. As computer technology removes barriers in data storage, retrieval, and networking, unprecedented opportunities arise to achive the dream of all crystallographers: easy access to all crystallographic data. One topic stressed was the importance of quality throughout all stages of the data flow including collec. tion, publication, and incorportaion into a database. A second major focus was software tools. Many L' ers emphasized that use and accepatance of crystallographic databases by the general scientific community is directly related to the availability and quality of the search tools. A third theme was related to access and availability of all the information. Among the questions raised were: how data will be made available to the user community, and 'Who will be the power brokers in the future technologies. Issues such as these demonstrate the need among the representatives of the various data activities for future meetings to foster a continuing dialog. In addition, many attendees emphasized the need for much greater cooperation among the data centers, among the journals and the date centers, and among users and the data centers.

04,812

PB97-112411 Not available NTIS

National Inst. of Standards and Technology (MSEL) Gaithersburg, MD. Reactor Radiation Div.

Neutron Scattering Study of Antiferromagnetic Order in the Magnetic Superconductors RNi2B2C. Final rept.

J. W. Lynn, O. Huang, S. K. Sinha, R. Nagarajan, C. Godart, Z. Hossain, and L. C. Gupta. 1996, 3p.

Pub. in Physica B, v223 and 224 p66-68 1996.

Keywords: "Superconductors, "Neutron scattering, * Magnetic order, Reprints.

Neutron diffraction has been used to investigate the magnetic order and crystallography of RNi2B2C. For $R=D y$ the magnetic order that develops at $10 \mathrm{~K}$ consists of ferromagnetic sheets of spins in the tetragonal $a-b$ plane which are stacked antiferromagnetically along the c-axis. An identical magnetic order is observed for $\mathrm{R}=\mathrm{Ho}$ at low $\mathrm{T}$, while from approximately $5 \mathrm{~K}$ to $\mathrm{T}(\mathrm{N})$ approximately equal to $8 \mathrm{~K}$ each layer along the $\mathrm{c}$-axis is rotated by 163 degrees to form a spiral state, which strongly couples the magnetic order to the superconductivity. Small a-axis peaks are also observed in a narrow temperature range, but the c-axis peaks are dominant. In ErNi2B2C(Tc $=11 \mathrm{~K})$ an antiferromagnetic a-axis spin density wave stage develops below $T(N)=6.8 \mathrm{~K}$, which readily coexists with the superconductivity indicating that weak coupling occurs between this type of magnetic state and the superconducting state. There is no evidence of magnetic order for either $R=C$ e or $R=Y$.

04,813

PB97-113286 Not available NTIS

National Inst. of Standards and Technology (EEEL), Gaithersburg, MD. Electricity Div. 

Results of Capacitance Ratio Measurements for
the Single Electron Pump-Capacitor Charging Experiment.

Final rept.

N. M. Zimmerman, A. F. Clark, and E. R. Williams.

1996, 2p.

See also PB96-102157.

Pub. in Conference on Precision Electromagnetic Measurements, Braunschweig, Germany, June 17-20, 1996, p505-506.

Keywords: "Metrology, "Electron tunneling, "Capacitance, Electric bridges, Precision, Ratios, Capacitors, Reprints, "Foreign technology, SET(Single electron tunneling).

The authors report on a metrological application of the single electron tunneling (SET) phenomena: a precise measurement of the ratio of two cryogenic capacitors. The measurement used a superconducting SET electrometer as the null detector for a capacitance bridge. They have achieved a 3-ppm level of imprecision in the measurement of capacitance ratio from 100 to $1000 \mathrm{~Hz}$. They used custom-made cryogenic vacuumgap capacitors, which have a leakage resistance of no less than 10(19) omega. Further improvements can be made in the attempt to obtain an imprecision of 10 8) at lower frequencies, sufficient for the metrologica measurement of capacitance or the fine-structure constant using an SET pump.

04.814

PB97-118335 Not available NTIS

National Inst. of Standards and Technology (EEEL), Gaithersburg, MD. Semiconductor Electronics Div.

Majority and Minority Electron and Hole Mobilities in Heavily Doped Gallium Aluminum Arsenide. Final rept.

H. S. Bennett. 1996, 10p.

See also PB91-236968

Pub. in Jnl. of Applied Physics, v80 n7 p3844-3853 Oct 96.

Keywords: "Gallium arsenides, "Electron mobility, *Hole mobility, Minority carriers, Semiconductor doping. Doped materials, Reprints.

The majority electron and minority hole mobilities were calculated in Gal-yAlyAs for donor densities between 10 to the 16 th power and 10 to the 19 th power to the minus 3 power. Similarly, the majority hole and minority electron mobilities were calculated for acceptor densities between 10 to the 16 th power and 10 to the 20 th power to the minus 3 power. The mole fraction of AIAS $y$, varies between 0.0 and 0.3 in these calculations. Al the important scattering mechanisms have been included. The ionized impurity and carrier-carrier scattering processes were treated with a quantum-mechani$\mathrm{cal}$, phase-shift analysis. These calculations are the first to use a phase-shift analysis for minority carriers scattering from majority carriers in ternary compounds such as Gal-yAlyAs. The results are in good agreement with experiment for majority mobilities and predict that at high dopant densities minority mobilities should increase with increasing dopant density for a shor range of densities. This effect occurs because of the reduction of plasmon scattering and the removal of carriers from carrier-carrier scattering due to the Pauli exclusion principle.

\section{4,815}

PB97-119408 Not available NTIS

National Inst. of Standards and Technology (EEEL), Boulder, CO. Electromagnetic Technology Div.

Vortex Images in Thin Films of YBa2Cu3O(sub 7. $x$ ) and Bi2Sr2Ca1Cu2O(sub $8+x$ ) Obtained by LowTemperature Magnetic Force Microscopy. Final rept.

C. W. Yuan, Z. Zheng, A. L. deLozanne, J. N Eckstein, M. Tortonese, and D. A. Rudman. 1996 $4 \mathrm{p}$.

Pub. in Jnl. of Vacuum Science and Technology, V14 n2 p1210-1213 Mar 96.

Keywords: "Microscopy, "Thin films, "Magnetic force microscopy, "Superconductors, High temperature, Vortices, Reprints.

The authors have imaged vortices in superconducting thin films with a low-temperature magnetic force microscope that utilizes microfabricated piezoresistive cantilevers with built-in tips. The films of $\mathrm{YBa} 2 \mathrm{Cu} 3 \mathrm{O} 7-\mathrm{x}$ and $\mathrm{Bi} 2 \mathrm{Sr} 2 \mathrm{Ca} 1 \mathrm{Cu} 2 \mathrm{O} 8+x$, are made by laser ablation and molecular beam epitaxy, respectively. The vortices usually appear as round features in the noncontac image with a diameter of about 1 micrometer or slightly less. In some cases the position of the vortices is cor related to defects observed in the topographic image of the same area. The vortices move sometimes, especially after taking a topographic (contact mode) scan.

04,816

PB97-122550 Not available NTIS

National Inst. of Standards and Technology (CAML), Gaithersburg, MD. Applied and Computational Mathematics Div.

Xi-Vector Formulation of Anisotropic Phase-Field Models: 3-D Asymptotics.

Final rept

A. A. Wheeler, and G. B. McFadden. 1996, 15p. Pub. in European Jnl. of Applied Mathematics, v7 p367-381 1996.

Keywords: "Solidification, Liquids-solid interfaces, Surface energy, Differential geometry, Asymptotic series, Vector analysis, Reprints, "Foreign technology, "Phase field models, Allen-Cahn equation, Gibbs-Thomson equation.

In the paper the authors present a new formulation of a large class of phase-field models, which describe solidification of a pure material and allow for both surface energy an interface kinetic anisotropy, in terms of the Hoffman-Cahn epsilon-vector. The Hoffman-Cahn epsilon-vector has previously been used in the context of sharp interface models, where it provides an elegant tool for the representation and analysis of interfaces with anisotropic surface energy. The authors show that the usual gradient-energy formulations of anisotropic phase-field models are expressed in a natural way in terms of the epsilon-vector when apprpriately interpreted. They use this new formulation of the phasefield equations to provide a concise derivation of the Gibbs-Thomson-Herring equation in the sharp-interace limit in three dimensions.

\section{Structural Mechanics}

\section{4,817}

P894-198298 PC A01

National Inst. of Standards and Technology (NEL), Gaithersburg, MD. Structures Div.

Foias-Temam Approximations of Attractors for Galloping Oscillators.

Final rept.

B. Alibe, G. R. Cook, and E. Simiu. 1991,5p.

Pub. in Proceedings of American Society of Civil Engineers Engineering Mechanics Specialty Conference, Mechanics Computing in 1990's and Beyond, Columbus, $\mathrm{OH}$., May 20-22 1991, p781-785.

Keywords: "Oscillations, "Structural analysis, "Nonlinear equations, Nonlinear systems, Approximation, Self excitation, Structural engineering, Analysis(Mathematics), Reprints, "Autonomous galloping oscillators, "Foias-Temam approximation.

A method for the algebraic approximation of attractors recently developed by Foias and Temam is adapted for application to autonomous galloping oscillators. We compare results obtained by algebraic approximation on the one hand and numerical integration on the other. We conclude with an assessment of the limitations of the method as applied to our systems.

\section{4,818}

PB94-200250 Not available NTIS

National Inst. of Standards and Technology (MSEL), Gaithersburg, MD. Ceramics Div.

Application of a Simple Technique for Estimating Errors of Finite-Element Solutions Using a General-Purpose Code.

Final rept.

T. J. Chuang, J. T. Tang, and J. T. Fong. 1990, $11 \mathrm{p}$. Pub. in Proceedings of Pressure Vessels and Piping Conference, Nashville, TN., June 17-21, 1990, p105115.

Keywords: "Fracture mechanics, "Error analysis, "Finite element method, "Stress analysis, Glass, Computational grids, Plates, Computerized simulation, Mathematical models, Estimates, Reprints

A simple error-estimate technique using commercially available general purpose finite element codes to solve a class of engineering problems is presented. The technique is based on the assumption that the class of engineering problems to be solved can be simplified to a new class having known analytical solutions. $A$ two-phase procedure is developed where a series of successively-refined mesh designs with acceptable error estimates for the simplified class of problems is used to guide the finite element modeling of the actual engineering problem. To illustrate the procedure, an application in the preliminary design of a circular glass plate with a center hole, a 3-sector configuration, and reinforcing beams at all edges of the sectors, is reported. Error estimates and rates of convergence for parameters of practical interest are derived from numerical results using a finite element code named ANSYS (versions $4.3 \mathrm{~A}$ and 4.4 ) as implemented on a Sun3-110 workstation.

\section{4,819}

P895-107330 Not available NTIS

National Inst. of Standards and Technology (MSEL) Boulder, CO. Materials Reliability Div.

Potential Drop in the Center-Cracked Panel with Asymmetric Crack Extension.

Final rept.

D. T. Read, and M. Pfuff. 1991, 11p

Sponsored by GKSS - Forschungszentrum Geesthacht G.m.b.H., Hamburg (Germany, F.R.)

Pub. in International Jnl. of Fracture 48, p219-229 1991

Keywords: "Crack propagation, "Fracture strength *Electric potential, "Nondestructive tests, Tensile tests, Fracturing, Asymmetry, Panels, Calibration, Reprints, Johnson formula.

The well-known Johnson formula relates the electrical potential drop across a symmetric center crack in a current-carrying tensile panel to the crack length. To allow individual measurement of the crack growth at both ends of such a crack, the potential measured be tween contact points located on opposite sides and equidistant from the panel center line has been studied. Because this potential must ideally be identically zero for symmetric crack growth, it is termed the asymmetric potential. Its sign and magnitude are related to the direction and extent of asymmetric crack growth It was found that contact points directly above the ends of the initial symmetric crack provide a signal which is well-suited to the determination of the asymmetry of the crack extension. This information is complementary to the total crack length information obtained using the Johnson formula. Experimental measurements and theoretical results were used to produce calibration formulae relating the asymmetric potential to the asymmetry of the crack dimensions in center-cracked panels

\section{4,820}

P895-162897 Not available NTIS

National Inst. of Standards and Technology (NEL) Gaithersburg MD Structures Div.

Algebraic Approximation of Attractors for Galloping Oscillators

Final rept.

E. Simiu, G. R. Cook, and B. Alibe. 1991, 6p.

Pub. in Jnl. of Sound and Vibration 146, n1 p170-175 1991.

Keywords: "Oscillators, "Structural engineering, *Nonlinear algebraic equations, Oscillations, Numerical analysis, Nonlinear systems, Fluidelasticity, Dynamic structural analysis, Approximations, Analysis(Mathematics), Reprints, "Foias-Teman approximation, "Autonomous galloping oscillators, Attractors, Bilinear operator

A constructive method for approximating attractors was recently developed by Foias and Temam, who applied it to systems in which the nonlinearity is due to a bilinear operator. The purpose of the note is to tes the practical applicability of the method to autonomous galloping oscillators, in which the nonlinearity, is due to seventh degree polynomials. The authors give revised versions of two of the expressions given in the original method. They then compare results obtained by algebraic approximation on the one hand and numerical integration on the other. The authors conclude with an assessment of the limitations of the method as applied to the systems

04,821

P895-202347 Not available NTIS

National Inst. of Standards and Technology (MSEL), Gaithersburg, MD 


\section{PHYSICS}

\section{Structural Mechanics}

Exponentially Rapid Coarsening and Buckling in Coherently Self-Stressed Thin Plates.

Final rept.

J. W. Cahn, and R. Kobayashi. 1995, 14p.

Sponsored by Defense Advanced Research Projects Agency, Arlington, VA

Pub. in Acta Metall. Mater. 43, n3 p931-944 1995.

Keywords: "Buckling, "Coarseness, "Nonlinear systems, "Thin plates, Diffusion, Elastic properties, Wetting. Stress analysis, Decomposition, Reprints.

The nonlinear equations that couple diffusion and stress are solved by computation for one-dimensional spinodal decomposition and coarsening in a thin plate. The vicinity of two critical values of the stress parameter are explored. At small values of elastic selfstresses, coarsening changes to exponentially fast from the exponentially slow dynamics expected for one-dimensions in the absence of stress. Coarsening is by rapid thickening of a single layer of a different phase from each of the two plate surfaces, leading to bending of the plate. Even though the diffusion equation is the same for bulk and thin plate and changes type at the coherent spinodal transition, in a thin plate the changes near this transition are gradual; the difference in behavior is due to the boundary conditions. Furthermore elasto-chemical equilibria through this transition are completely continuous. If the elastic term is large compared to the wetting term, surface wetting layers of phase of lower energy are found to disappear late in the coarsening.

\section{4,822}

PB96-111943 Not available NTIS

National Inst. of Standards and Technology (BFRL), Gaithersburg, MD. Structures Div

Noise Modeling and Reliability of Behavior Prediction for Multi-Stable Hydroelastic Systems.

Final rept.

E. Simiu, and M. Frey. 1992, 6p.

Pub. in Proceedings of the International Conference on Offshore Mechanics and Arctic Eng. (OMAE) (11th) Alberta, Canada, June 7-12, 1992, Safety and Reliability, v2 p39-44.

Keywords: "Oscillators, "Hydroelasticity, Modelling, Noise, Hydrodynamics, Prediction, Behavior, Reprints, *Hydroelastic systems.

This paper reviews results of experiments conducted on a simple multi-stable hydroelastic (galloping) oscillator. These results show that a noise may cause a multi-stable hydroelastic system to exhibit chaotic behavior, and that in some instances such behavior cannot be predicted reliably unless noise effects are carefully accounted for. We then present results of a theoretical investigation of a simple, paradigmatic multi-stable system, the Duffing-Holmes oscillator. The results of this investigation show that for the system being considered, noise promotes the occurance of chaotic behavior associated with Smale horseshoes. This theoretical investigation is the first phase of an effort to develop analytical tools for predicting reliably the potential for chaotic behavior of actual hydro elastic sys. tems such as deep-water compliant platforms.

\section{4,823}

PB96-122718 Not available NTIS

National Inst. of Standards and Technology (BFRL), Gaithersburg. MD. Structures Div.

Chaotic Motions of Coupled Galloping Oscillators and Their Modeling as Diffusion Progresses. Final rept.

E. Simiu, G. R. Cook, and B. Alibe. 1991, 8p.

See also PB93-153245.

Pub. in International Conference on Applications of Statistics and Probability (6th), Mexico City, Mexico, June 17-21, 1991, v2 p993-1000.

Keywords: "Chaos, "Diffusion, "Oscillators, Models, Elastic properties, Stability, Stochastic processes, Differential equations, Structural engineering, Hydroelasticity, Reliability, Nonlinear systems, Reprints, "Galloping oscillators, "Fluidelasticity.

According to numerical investigations recently reported in the literature certain types of compliant offshore structures may experience undesirable hydroelastic responses, including chaotic responses. The question arises whether such responses can be predicted reliably by using current fluidelastic models. We addressed this question in the specific case of a paradigmatic, strongly nonlinear hydroelastic oscillator exhibits strongly chaotic behavior. It is then shown that modeling the hydroelastic system as a nonlinear diffusion process helps to improve substantially the reliability of the predictions.

04,824

PB96-160650 Not available NTIS

National Inst. of Standards and Technology (CAML), Gaithersburg, MD. Statistical Engineering Div.

Exits in Multistable Systems Excited by Coin-Toss Square-Wave Dichotomous Noise: A Chaotic Dynamics Approach.

Final rept

Y. R. Sivathanu, C. Hagwood, and E. Simiu. 1995, $7 \mathrm{p}$.

Pub. in Physical Review E, v52 n5 p4669-4675 Nov 95.

Keywords: "Stochastic processes, "Structural engineering, "Noise, Chaos, Construction, Functions(Mathematics), Functional analysis, Reprints, Melnikov function.

We consider a wide class of multistable systems perturbed by adissipative term and coin-toss square-wave dichotomous noise. These systems behave like their harmonically or quasiperiodically driven counterparts: depending upon the system parameters, the steadystate motion is confined to one well for all time or experiences exits from the wells. This similarity suggests the application to the stochastic systems of a Melnikov approach originally developed for the deterministic case. The noise induces a Melnikov process that may be used to obtain a simple condition guaranteeing the nonoccurrence of exits from a well.

\section{Police, Fire, \& Emergency Services}

04,825

PB94-207461 PC A09/MF A02

National Inst. of Standards and Technology (BFRL), Gaithersburg, MD. Structures Div.

Northridge Earthquake 1994: Performance of Structures, Lifelines, and Fire Protection Systems. Special pub.

D. Todd, N. Carino, R. M. Chung, W. D. Walton, J. D. Cooper, R. Nimis, H. S. Lew, and A. W. Taylor. May $94,185 p$, NIST/SP-862

Also available from Supt. of Docs. as SN003-003 03264-6. See also PB94-157666 and PB94-161114 Prepared in cooperation with Federal Highway Administration, Washington, DC.

Keywords: "Earthquake engineering, "Damage assessment, Highway bridges, California, Seismic design, Overpasses, "Northridge(California).

A magnitude 6.8 (Ms) earthquake centered under the community of Northridge in the San Fernando Valley shook the entire Los Angeles metropolitan area at $4: 31$ a.m. local time on Monday, January 17, 1994. Moderate damage to the built environment was widespread; severe damage included collapsed buildings and highway overpasses. A multi-agency team, organized under the auspices of the Interagency Committee on Seismic Safety in Construction and headed by the National Institute of Standards and Technology, arrived at the earthquake site within days of the event to document the effects of the earthquake. The team focused on the effects to the built environment with the goal of capturing perishable data and quickly identifying situations deserving in-depth study. The report includes a summary of the team's observations.

\section{SPACE TECHNOLOGY}

\section{General}

04,826

PB96-161955 Not available NTIS

National Inst. of Standards and Technology (CSTL), Gaithersburg, MD. Process Measurements Div. SSME LOX Duct Flowmeter Design and Test Results.

Final rept.

J. D. Siegwarth. $1994,8 p$.

Pub. in Advanced Earth-to-Orbit Propulsion Technology 1994 NASA Conference, Huntsville, AL., May 17-19, 1994, Publication 3282, v1 p 105-112.

Keywords: "Flowmeters, Liquid oxygen, Reprints, Water, "Space Shuttle, "Vortex, Vortex shedding.

A vortex shedding flowmeter designed to measure Liquid oxygen flow in the RS007031 duct of the Space Shuttle Main Engine has been built and tested using water and liquid nitrogen (LN2). The best meter performance was obtained with an elbow located a few diameters upstream and the vane axis perpendicular to the plane of the bend rather than with a long straight section upstream of the meter. At either the same flow velocity or the same Reynolds number, the meter performance was better for LN2 than for water. A flowmeter vane of circular cross section has been developed for the $28 \mathrm{~mm}$ branch of the RS007032 duct. The performance of these meters approaches that of commercial flowmeters.

\section{Astronautics}

04,827

N95-14084/4

(Order as N95-14062/0, PC A20/

MF A04)

National Inst. of Standards and Technology, Gaithersburg, MD.

Partial Pressure Analysis in Space Testing.

C. R. Tilford. Nov $94,13 p$.

In NASA. Goddard Space Flight Center, Eighteenth Space Simulation Conference: Space Mission Success Through Testing p 257-269. Sponsored by Jhu. Keywords: "Contamination, "Gas pressure, "Instrument errors, "Measuring instruments, "Partial pressure, "Space environment simulation, Calibrating, Vacuum systems, Exposure, Handbooks, Pressure vessels, Quadrupoles, Residual gas, Sensitivity.

For vacuum-system or test-article analysis it is often desirable to know the species and partial pressures of the vacuum gases. Residual gas or Partial Pressure Analyzers (PPA's) are commonly used for this purpose. These are mass spectrometer-type instruments, most commonly employing quadrupole filters. These instruments can be extremely useful, but they should be used with caution. Depending on the instrument design, calibration procedures, and conditions of use, measurements made with these instruments can be accurate to within a few percent, or in error by two or more orders of magnitude. Significant sources of error can include relative gas sensitivities that differ from handbook values by an order of magnitude, changes in sensitivity with pressure by as much as two orders of magnitude, changes in sensitivity with time after exposure to chemically active gases, and the dependence of the sensitivity for one gas on the pressures of other gases. However, for most instruments, these errors can be greatly reduced with proper operating procedures and conditions of use. In this paper, data are presented illustrating performance characteristics for different instruments and gases, operating parameters are recommended to minimize some errors, and calibrations procedures are described that can detect and/or correct other errors.

04,828

N96-15584/1 (Order as N96-15552, PC A20/MF A04) 
National Inst. of Standards and Technology, Gaithersburg, MD.

Ignition and Subsequent Transition to Flame Spread in a Microgravity Environment.

T. Kashiwagi, K. Mcgrattan, and H. Baum. Aug 95,

$6 \mathrm{p}$

In NASA. Lewis Research Center, the 3RD International Microgravity Combustion Workshop p 207212.

Keywords: "Computerized simulation, "Flame propagation, "Flames, "Flow distribution, "Gravitational ef fects, "Heat flux, "Ignition, "Microgravity, Extinguishing, Fire prevention, Fires, Three dimensional flow, Time dependence, Two dimensional flow.

The fire safety strategy in a spacecraft is (1) to detect any fire as early as possible, (2) to keep any fire as small as possible, and (3) to extinguish any fire as quickly as possible. This suggests that a material which undergoes a momentary, localized ignition might be tolerable but a material which permits a transition to flame spread would significantly increase the fire hazard. Therefore, it is important to understand how the transition from localized ignition to flame spread occurs and what parameters significantly affect the transition. The fundamental processes involved in ignition and flame spread have been extensively studied, but they have been studied separately. Some of the steady state flame models start from ignition to reach a steady state, but since the objective of such a calculation is to obtain the steady state flame spread rate, the calculation through the transition process is made without high accuracy to save computational time. We have studied the transition from a small localized ignition at the center of a thermally thin paper in a microgravity environment. The configuration for that study was axisymmetric, but more general versions of the numerical scheme have been developed by including the effects of a slow, external flow in both two and three dimensions. By exploiting the non-buoyant nature of the flow, it is possible to achieve resolution of fractions of millimeters for 3D flow domains on the order of 10 centimeters. Because the calculations are time dependent, we can study the evolution of multiple flame fronts originating from a localized ignition source. The interaction of these fronts determines whether or not they will eventually achieve steady state spread. Most flame spread studies in microgravity consider two-dimensional flame spread initiated by ignition at one end of a sample strip with or against a slow external flow. In this configuration there is only one flame front. A more realistic scenario involves separate, oppositely directed fronts in two dimensions, or a continuous, radially directed front in three dimensions. We present here some results of both the two and three dimensional codes.

\section{4,829}

PB96-103072 Not available NTIS

National Inst. of Standards and Technology (CSTL), Gaithersburg, MD. Thermophysics Div.

Partial Pressure Analysis in Space Testing.

Final rept.

C. R. Tilford. 1995, $6 p$.

See also N95-14084.

Pub. in Jnl. of the IES, p30-35 May/Jun 95.

Keywords: "Partial pressure, "Space, "Tests, Measuring instruments, Vacuum systems, Gases, Monitoring, Accuracy, Error, Calibrating, Quadrupoles, Filters, Reprints, "Space testings.

For vacuum-system or test-article analysis it is often desirable to know the species and partial pressures of the vacuum gases. Residual Gas or Partial Pressure Analyzers (PPAs) are commonly used for this purpose. These are mass spectrometer-type instruments, most commonly employing quadrupole filters. Depending on the instrument design, calibration procedures, and conditions of use, measurements made with these instruments can be accurate to within a few percent, or in error by two or more orders of magnitude. In this paper, data are presented illustrating performance characteristics for different instruments and gases, operating parameters are recommended to minimize some errors, and calibrations procedures are described that can detect and/or correct other errors.

\section{Extraterrestial Exploration}

04,830

PB95-163234

Not available NTIS
National Inst. of Standards and Technology (MSEL), Gaithersburg, MD. Materials Reliability Div. In-Space We

D. Tamir, T Siewert, K Matsubuchi, T. Eagar, L.

Flanigan, and R. Su. 1993, 8p.

Pub. in Proceedings of Space Congress 'Yesterday's Vision is Tomorrow's Reality' (13th), Cocoa Beach, FL., April 27-30, 1993, p1-9-1-16.

Keywords: "Space industrialization, "Aerospace environments, "Welding, "Lunar exploration, Space shuttle missions, Space manufacturing, Space laboratories, Space stations, Space processing, Plasma arc welding, Laser welding, Gas tungsten arc welding, Electron beam welding, Space exploration, Reprints, Space Exploration Initiative.

This paper establishes the value of having an in-space welding capability and identifies its applications, both near-term for Shuttle-Spacelab missions and Space Station Freedom, and longer-term for the First Luna Outpost and Manned Mission to Mars. The leading candidate technologies, consisting of Electron Beam Gas Tungsten Arc, Plasma Arc, and Laser Beam are examined against the criteria for an in-space welding system. Research and development work to date, striv ing to achieve an in-space welding capability, is reviewed. Finally, a series of strategic NASA flight experiments is discussed as the remaining development pability, which can fully serve the Space Exploration Initiative. This paper summarizes the visions and realities associated with in-space welding.

\section{Manned Spacecraft}

04,831

PC A03/MF A01

National Inst. of Standards and Technology (MEL) Gaithersburg, MD. Robot Systems Div.

Overview of NASREM: The NASAINBS Standard Reference Model for Telerobot Control System Architecture.

J. S. Albus, R. Quintero, and R. Lumia. Apr 94, 14p, NISTIR-5412

See also PB88-123773 and PB89-193940.

Keywords: "Architecture(Computers), "Robot control, "Space station freedom, "Telerobotics, Control systems design, Robots, Systems engineering. Memory(Computers), Computer systems design "Computerized control systems, NASANBS standard reference model.

The NASA/NBS Standard Reference Model for Telerobot Control System Architecture (NASREM) was developed by the National Institute of Standards and Technology (NIST) for the National Aeronautics and Space Administration (NASA) to provide a software control system architecture guideline for use by development contractors charged with building the Flight Telerobot Servicer (FTS) control system as part of the Freedom Space Station project. The original NASREM document describes a conceptual or domain-independent architecture, and suggests the outline of a functional or domain-specific architecture for FTS. This paper presents an overview of the NASREM conceptual architecture and reviews subsequent work at NIST in defining a functional architecture for the servo and primitive levels. This work suggests outlines for software and hardware architecture specifications, and software development environments to complement the NASREM conceptual and functional architectures.

\section{4,832}

PB94-216082 Not available NTIS

National Inst. of Standards and Technology (NEL), Gaithersburg, MD. Robot Systems Div.

Evolution of the Flight Telerobotic Servicer.

Final rept.

R. Lumia. $1990,4 p$

Pub. in Proceedings of International Symposium on $\mathrm{Ar}$ tificial Intelligence, Robotics and Automation in Space, Kobe, Japan, November 18-20, 1990, p69-72.

Keywords: "Space Station Freedom, "Manipulators, Automation, Robotics, Robot control, Telerobotics, Reprints, ${ }^{*}$ FTS(Flight Telerobotic Servicer), "Flight Telerobotic Servicer, NASREM(NASANNIST Standard Reference Model)

The Flight Telerobotic Servicer (FTS) is a two armed manipulator which will be used to build and maintain
Space Station Freedom. One of the goals of the project is to be able to upgrade the capabilities of the FTS by incorporating new technology. To achieve this goal the FTS is using the NASANIIST Standard Reference Model for Telerobot Control System Architecture (NASREM) for its functional architecture. While using NASREM helps integrate new technology into the system, the decisions concerning the precise technology needing development must be addressed. In this paper, an approach to the technological evolution of the FTS will be explored. The approach begins with detailed scripts of representative FTS activities. These scripts are analyzed to determine the generic or common actions performed by the FTS. Then, technological alternatives are described in terms of a decision tree format.

\section{4,833}

PB95-151510 Not available NTIS

National Inst. of Standards and Technology (NEL), Gaithersburg, MD. Robot Systems Div.

Mapping Processes to Processors for SpaceBased Robot Systems.

Final rept.

T. E. Wheatley. 1989, 4p.

Pub. in Institute of Electrical and Electronics Engineers International Conference on Systems Engineering, Fairborn, OH., August 24-26, 1989, p65-68.

Keywords: "Robots, "Space stations, "Computers, Resource allocation, Robot control, Telerobotics, Manipulators, Computer architecture, Algorithms, Servocontrol, Reprints

The NASA/NBS Standard Reference Model for Telerobot Control System Architecture (NASREM) has been adapted by NASA for use in the Flight Telerobotic Servicer, a two armed telerobotic manipulator which will build and maintain the Space Station. NASREM provides the paradigm that allows standard interfaces to be defined so that functionally equivalent software and hardware modules can be interchanged. This paper examines the mapping of these logical modules onto a functional computer architecture. Interfaces must be first defined which are capable of supporting the algorithms in the literature. Atter interface definition, the specific computer architecture for the implementation can be determined. An example is shown mapping the SERVO level of NASREM onto a set of computers utilizing the knowledge of the dominant response time required to aid in the selection process.

\section{4,834}

PB96-111810 Not available NTIS

National Inst. of Standards and Technology (MSEL), Gaithersburg, MD. Materials Reliability Div.

Wear Mechanism Maps of $440 \mathrm{C}$ Martensitic Stainless Steel.

Final rept.

A. J. Slifka, T. J. Morgan, R. Compos, and D. K.

Chaudhuri. 1993, 5p.

Pub. in Wear, v162-164 p614-168 1993.

Keywords: "Martensitic stainless steels, "Wear, "Tribology, "Space shuttle main engine, "Turbine pumps, "Liquid oxygen, Wear tests, Temperature, Loads(Forces), Velocity, Sliding friction, Oxidation, Reprints, SSME(Space Shuttle Main Engine), HPOTP(High Pressure Oxygen Turbopump), Wear maps, Wear mechanisms.

AISI $440 C$ martensitic stainless steel is the material of choice for the high pressure oxygen turbopump (HPOTP) of the space shuttle main engine (SSME) Tests have been done over a range of sliding speeds from 0.5 to $2.0 \mathrm{~m} / \mathrm{s}$, initial hertzian stresses from 0.915 to $3.66 \mathrm{GPa}$, and bulk temperatures from $-184 \mathrm{deg}$. C (liquid oxygen temperature) to 760 deg. C. which cover the variations thought to exist in the HPOTP. Data are presented in the form of contour wear maps to allow a more direct view of the interdependencies of the major tribological variables that can be obtained with wear data presented vs. a single variable. Wear maps are used as a tool to aid in the determination of regions dominated by a given wear mode.

\section{4,835}

PB96-155809 Not available NTIS

National Inst. of Standards and Technology (BFRL), Gaithersburg, MD. Fire Safety Engineering Div. 


\section{Manned Spacecraft}

Transition from Localized Ignition to Flame Spread Over a Thin Cellulosic Material in Microgravity. Final rept.

K. B. McGrattan, K. Nakabe, H. R. Baum, and T

Kashiwagi. 1993, 4p.

Pub. in Eastern States Section of the Combustion Institute Technical Meeting, Princeton, NJ., October 1993, Book of Abstracts, p409-412.

Keywords: "Cellulose, "Fire prevention, "Ignition, Combustion, Flame spread, Reprints, Microgravity.

Ignition and flame spread processes are complicated by strong coupling between chemical reactions and transport processes, not only in the gas phase but also in the condensed phase. In most previous studies, ignition and flame spread were studied separately with the result that there has been little understanding of the transition from ignition to flame spread. In fire safety applications this transition is crucial to determine whether a fire will be limited to a localized, temporary burn or whether it will grow to become a large fire. In order to understand the transition to flame spread, the transient mechanisms of ignition and subsequent spread must be studied. However, there have been no definitive experimental or modeling studies because of the complexity of the buoyancy-induced flow near the heated sample surface. One must solve the full NavierStokes equations over an extended region to represent accurately the highly unstable buoyant plume and entrainment of surrounding gas. To avoid the complicated nature of the plume problem under normal gravity, previous detailed radiative ignition models were assumed to be one-dimensional or were applied at a stagnation point. Unfortunately, these models could not be extended to include the transition to flame spread.

\section{4,836}

PB96-160270 Not available NTIS

National Inst. of Standards and Technology (BFRL), Gaithersburg, MD. Fire Science Div.

Ignition and Subsequent Flame Spread Over a Thin Cellulosic Material.

Final rept.

K. Nakabe, H. R. Baum, and T. Kashiwagi. 1992

$13 p$.

Also available as N93-20205 (Order as N93-20178). Pub. in International Microgravity Combustion Workshop (2nd), Cleveland, OH., September 15-17, 1992, p167-179.

Keywords: "Cellulose, "Fire prevention, "Fires, *Flame propagation, *Ignition, *Reduced gravity, Gravitation, Mathematical models, Buoyancy, Entrainment, Reprints, "Spacecraft construction materials.

Both ignition and flame spread on solid fuels are processes that not only are of considerable scientific interest but that also have important fire safety applications. Both types of processes, ignition and flame spread, are complicated by strong coupling between chemical reactions and transport processes, not only in the gas phase but also in the condensed phase. In most prephase but also in the condensed phase. In most pre-
vious studies, ignition and flame spread were to flame spread studied separately with the result that there has been little understanding of the transition from ignition limited to flame spread. In fire safety applications this transistion is crucial to determine whether a fire will be limited to a localized, temporary burn or will transition into a growth mode with a potential to become a large fire. In order to understand this transition, the transient mechanisms of ignition and subsequent flame spread must be studied. However, there have been no definitive experimental or modeling studies, because of the complexity of the flow motion generated by buoyancy near the heated sample surface. One must solve the full Navier-Stokes equations over an extended region to represent accurately the highly unstable buoyant plume and entrainment of surrounding gas from far away.

\subsection{7}

PB96-160288 Not available NTIS

National Inst. of Standards and Technology (BFRL), Gaithersburg, MD. Fire Science Div.

Ignition and Transition to Flame Spread Over a Thermally Thin Cellulosic Sheet in a Microgravity Environment.

Final rept.

K. Nakabe, K. B. McGrattan, T. Kashiwagi, G.

Kushida, H. R. Baum, and H. Yamashita. 1994, 14p.

See also PB96-155809

Pub. in Combustion and Flame, v98 n4 p361-374 1994

Keywords: "Cellulose, "Fire prevention, "Ignition, Combustion, Flame spread, Reprints, Microgravity.
An axisymmetric, time-dependent model is developed describing auto-ignition and subsequent transition to flame spread over a thermally-thin cellulosic shee heated by external radiation in a quiescent microgravity environment. Due to the unique combination of a microgravity environment and low Reynolds number associated with the slow, thermally induced flow, the resulting velocity is taken as a potential flow. A onestep global gas phase oxidaton reaction and three global degradation reactions for the condensed phase are used in the model. A maximum external radiant flux of $5 \mathrm{~W} / \mathrm{sq}$. cm (Gaussian distribution) with $21 \%, 30 \%$, and $50 \%$ oxygen concentrations is used in the calculations. The results indicate that autoignition is observed for $30 \%$ oxygen concentrations but the transition to the flame spread does not occur. For $50 \%$ oxygen the transition is achieved. A delailed discussion of the transition from ignition to flame spread is given as an aid to understanding this process. Also, a comparison is made between the axisymmetric configuration and a two-dimensional (line source) configuration.

\section{Space Safety}

\section{4,838}

PB95-171039 PC A04/MF A01

National Inst. of Standards and Technology (PL) Gaithersburg, MD. Ionizing Radiation Div.

Updated Calculations for Routine Space-Shielding Radiation Dose Estimates: SHIELDOSE-2.

S. M. Seltzer. Dec 94, 59p, NISTIR-5477.

Contract NASA-T9311R

See also PB80-176860. Sponsored by National Aeronautics and Space Administration, Houston, TX. Lyndon B. Johnson Space Center.

Keywords: "Spacecraft shielding, "Radiation dosage Extraterrestrial radiation, Electron irradiation, Proton ir radiation, Bremsstrahlung, Cosmic rays, Aluminum, Graphite, Silicon, Water, Gallium arsenides, Air, Calcium fluorides, Silicon dioxide, Computer programs Fortran, Graphs(Charts), "SHIELDOSE-2 computer program.

New, more-extensive, depth-dose distributions for electrons, electron-bremsstrahlung, and protons have been calculated, based on improvements in cross-section information since the development of the original SHIELDOSE code. The new database covers inciden electron energies from $5 \mathrm{keV}$ to $50 \mathrm{MeV}$, with the bremsstrahlung tail calculated for depths out to $50 \mathrm{~g} /$ $\mathrm{sq} \mathrm{cm}$, and incident proton energies from $10 \mathrm{keV}$ to $10 \mathrm{GeV}$. Effects of nuclear interactions on proton depth-dose distributions in aluminum shields have been estimated, and options are provided to include these approximations in test calculations. In addition to the absorbed dose in aluminum, the dose in smal volumes of graphite, $\mathrm{Si}$, air, bone, CaF2, GaAs, LiF $\mathrm{SiO} 2$, tissue or water can be evaluated. The functionality of new code is much the same as the old code; however, the resultant dose estimates as a func tion of depth in aluminum spacecraft can be somewhat different. Through the use of a companion code based on approximate transformations, results can be extended beyond the dose as a function of depth in plane slabs and at centers of solid spheres to include the dose at off-center points in a solid sphere and at the inner surface of spherical shells.

\section{Spacecraft Trajectories \& Flight Mechanics}

\section{4,839}

\section{PB96-102165 Not available NTIS}

National Inst. of Standards and Technology (CAML), Gaithersburg, MD. Applied and Computational Mathematics Div

Frozen Orbits for Satellites Close to an Earth-Like Planet.

Final rept.

S. Coffey, A. Deprit, and E. Deprit. 1994, 36p

Pub. in Celestial Mechanics and Dynamical Astronomy 59, p37-72 1994

Keywords: "Artificial satellites, "Satellite orbits, Hamiltonian functions, Inclination, Eccentricity, Mission planning, Space missions, Surveys, Reprints, "Satellite theory.
The authors say that a planet is Earth-like if the coefficient of the second order zonal harmonic dominates all other coefficients in the gravity field. This paper concerns the zonal problem for satellites around the Earthlike planet, all other perturbations excluded. By numerical continuation the authors have discovered three families of frozen orbits in the full zonal problem under consideration; (1) a family of stable equilibria starting from the equatorial plane and tending to the critical inclination; (2) an unstable family arising from the bifurcation at the critical inclination; (3) a stable family also arising from that bifurcation and terminating with a polar orbit. Except in the neighborhood of the critical inclination, orbits in the stable families have very small eccentricities, and are thus well suited for survey missions.

\section{Unmanned Spacecraft}

04,840

PB95-140984 Not available NTIS

National Inst. of Standards and Technology (EEEL), Gaithersburg, MD. Electricity Div.

Metrology Requirements of Future Space Power Systems.

Final rept.

Sponsored by Defense Nuclear Agency, Washington, DC.

Pub. in Space Nuclear Power Systems, Chapter 11 , p95-101 1992.

Keywords: "Spacecraft power supplies, "Metrology, Strategic defense initiative, Antimissile defense, Reliability, Calibration, Sensors, Reprints.

Anticipated metrology requirements of future space power systems have been researched in a program initiated at the National Institute of Standards and Technology. These requirements have been compared with existing state-of-the-art measurement capabilities, and inadequacies in present measurement techniques are discussed. Particular attention is paid to the difficulties of determining measurement reliabilities for long-term, unattended sensor operation.

\section{4,841}

PB96-179437 Not available NTIS

National Inst. of Standards and Technology (PL) Gaithersburg, MD. Radiometric Physics Div.

Organization and Implementation of Calibration in the EOS Project. Part 1.

Final rept.

J. J. Butler, and B. C. Johnson. 1996, 6p.

Pub. in The Earth Obsenver, v8 n1 p22-27 Jan/Feb 96.

Keywords: "Landsat satellites, "Radiometers, *Calibration, Remote sensors, Multispectral band scanners, Radiometric resolution, Spectral resolution, Satellite observation, Earth observations(From space), Data quality, Standardization, Testing, Comparison, Reprints.

The Earth Observing System (EOS) is an international multi-satellite program in global remote sensing of the Earth. The simultaneous goals of acquiring accurate data over many years and correctly identifying systematic effects depend crucially on: (1) calibrating all instruments against a set of recognized physical standards, (2) carefully characterizing the instruments' performances at the system level, (3) adhering to good measurement practices and established protocols, (4) intercomparing instrument measurements where possibie, and (5) establishing traceability for all instruments to the common scale of physical quantities maintained at the national standards laboratories.

04,842

PB97-112213 Not available NTIS

National Inst. of Standards and Technology (PL) Gaithersburg, MD. Optical Technology Div.

Calibration in the Earth Observing System (EOS) Project. Part 2. Implementation.

Final rept.

J. J. Butler, and B. C. Johnson. 1996, 6p.

See also PB96-179437.

Pub. in The Earth Obsener, p26-31 1996.

Keywords: "Earth observing system(EOS), "Radiometers, "Calibration, Satellite observation, Remote sensors, Radiometry, Requirements, Testing, Implementation, Remote sensing, Data quality, Reprints. 
The article constitutes part 2 of a two-part article describing the overall organization and implementation of a calibration program in the EOS project based on requirements initially established in 1989. The article completes the description of the implementation of the program by outlining the EOS approaches to pre-flight and on orbit calibration.

04,843

PB97-113013 Not available NTIS

National Inst. of Standards and Technology (PL). Gaithersburg, MD. Optical Technology Div.

NIST Thermal Infrared Transfer Standard Radiometer for the Earth Observing System (EOS) Program.

Final rept.

J. P. Rice, and B. C. Johnson. 1996, 5p

Pub. in The Earth Observer, p31-35 1996

Keywords: "Earth observing system(EOS), "Infrared radiometers, "Calibration standards, Blackbody radiation, Infrared radiation, Thermometers, Temperature control, Heat transfer, Reprints.

A program to establish radiometric traceability between Earth Observing System (EOS) instrument calibration facilities and the National Institute of Standards and Technology (NIST) radiance scale is underway. To ensure the high accuracy required for instruments used in the National Aeronautics and Space Administration's (NASA's) EOS program, the output of the working standards will be compared to the radiance scale maintained at NIST. Plans are in place for NIST to provide similar radiometers for the EOS program: the Visible Transfer Radiometer (VXR), the Short-wave Infrared Transfer Radiometer (SWIXR), and the Thermal-infrared Transfer Radiometer (TXR). This article describes the TXR.

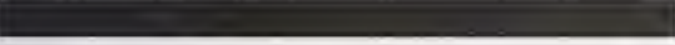 \\ TRANSPORTATION}

\section{Air Transportation}

04,844

PB94-213857 PC A06/MF A02

National Inst. of Standards and Technology (BFRL), Gaithersburg, MD.

Feasibility of Fire Evacuation by Elevators at FAA Control Towers.

J. H. Klote, B. M. Levin, and N. E. Groner. Aug 94, $112 p$, NISTIR-5445.

Prepared in cooperation with George Mason Univ., Fairfax, VA. Sponsored by Federal Aviation Administration, Washington, DC. Engineering and Environmental Safety Div.

Keywords: "Airport towers, "Air traffic control, "Fire safety, *Evacuating(Transportation), Elevators(Lifts), US FAA, Feasibility, Emergencies.

Throughout most of the world, warning signs next to elevators indicated they should not be used in fire situ ations. Because these elevators have not been designed for fire evacuation, they should not be used for fire evacuation. However, the idea of using elevators for fire evacuation has gained considerable attention. The Federal Aviation Administration (FAA) has sponsored a project to study the feasibility of elevator emergency evacuation at air traffic control towers. This paper describes this project including (1) a general discussion of elevator evacuation, (2) presentation of conceptual criteria for such elevator evacuation systems and (3) application of that criteria to several ATCTs.

\section{Global Navigation Systems}

\section{4,845}

PB94-215712 PC A04/MF A01

National Inst. of Standards and Technology (PL), Boulder, CO. Time and Frequency Div.
Analytical Estimation of Carrier Multipath Bias on GPS Position Measurements.

Technical note.

C. M. Volk, and J. Levine. Apr 94, 67p, NIST/TN-

1366

Also available from Supt. of Docs. as SN003-00303266-2. Prepared in cooperation with Joint Inst. for Lab. Astrophysics, Boulder, CO.

Keywords: *Global positioning system, "Multipath transmission, "Position finding. Phase measurement, Mathematical models, Error analysis, Degradation, Accuracy, Bias.

Multipath is one of the factors degrading the accuracy of position measurements obtained with the Global Positioning System (GPS). We investigate the effects of multipath on the carrier phase measurements and the resulting bias on relative GPS positions for observation times longer than several hours. We first model the phase error due to multipath from a single plane reflector in terms of satellite reflector geometry. The model is then employed to estimate upper limits of the multipath bias for general receiver environments. We also consider bias due to multipath from the flat ground, from nearby objects and from tilted ground, obtaining formulae for each situation that depend only on reflector characteristics and geometry. We find that the multipath phase error gives rise to vertical biases smaller than $2 \mathrm{~mm}$ and horizontal biases smaller than $1 \mathrm{~mm}$ if carrier-phase observations are averaged for at least several hours and if some basic precautions are taken regarding the placement of the receiving antenna.

\section{Pipeline Transportation}

04,846

PB94-161999 PC A05/MF A01

National Inst. of Standards and Technology (BFRL), Gaithersburg, MD.

Earthquake Resistant Construction of Gas and Liquid Fuel Pipeline Systems Serving, or Regulated By, the Federal Government.

Earthquake hazard reduction series no. 67

F. Y. Yokel, and R. G. Mathey. Jul 92, 79p. NISTIR4795, FEMA-233

See also PB94-161817. Sponsored by Federal Emergency Management Agency, Washington, DC.

Keywords: "Pipelines, "Storage tanks, "Earthquake re sistant structures, Seismic design, Design standards, Building codes, Piping systems, Pipe joints, Welded joints, Dynamic structural analysis, Natural gas dis tribution systems, Liquefied natural gas, Oil lines, Gas mains, Soil-Structure interactions, Earthquake engineering, Lifeline systems.

The vulnerability of gas and liquid fuel pipeline systems to damage in past earthquakes, as well as available standards and technologies that can protect these facilities against earthquake damage are reviewed. An overview is presented of measures taken by various Federal Agencies to protect pipeline systems under their jurisdiction against earthquake hazards. It is concluded that the overall performance of pipeline systems in past earthquakes was relatively good, however, older pipelines and above-ground storage tanks were damaged in many earthquakes. Standards and regulations for liquid fuel pipelines contain only general references to seismic loads. Standards and regulations for above-ground fuel storage tanks and for liquefied natural gas facilities contain explicit seismic design provisions. It is recommended that a guideline for earthquake resistant design of gas and liquid fuel pipeline systems be prepared for Federal Agencies to ensure a uniform approach to the protection of these systems.

\section{4,847}

PB96-147129 Not available NTIS

National Inst. of Standards and Technology (MSEL), Boulder, CO. Materials Reliability Div.

Gas-Coupled, Pulse-Echo Ultrasonic Crack Detection and Thickness Gaging.

Final rept.

C. M. Fortunko, R. E. Schramm, C. M. Teller, W. P Dube, M. C. Renken, G. M. Light, and J. D.

McColskey. 1995, 8p.

Pub. in Review of Progress in Quantitative Nondestructive Evaluation, Snowmass Village, CO., July 31-August 5, 1994, v14A p951-958 1995.
Keywords: "Nondestructive tests, "Crack detection, Reprints, "Thickness gages, Ultrasonic thickness gages, Gas-coupled, Pulse-echo.

Ultrasonic inspection is a standard method to assess the integrity of large-diameter oil pipelines. However, similar methods applied to natural-gas pipelines present a considerably greater challenge; gas is apoor coupling agent for the probing ultrasonic signals be iween the transducer and the pipe wall. Natural gas exhibits a very low specific acoustic impedance (300 Rayls for methane at atmospheric pressure) compared to oil (1.5 Mrayls and higher). Consequently, large ultrasonic-signal transmission losses occur at the trans ducer/gas and pipe-wall/gas interfaces. To circumven this obstacle, past exploratory developments included the use of a liquid-filled wheel, electromagnetic- acoustic-transducer (EMAT), and liquid-slug technology While prototypes of high-speed, in-line inspection sys tems employing such principles do exist, all exhibit serious operational shortcomings that prevent wide spread commercial exploitation.

\section{Railroad Transportation}

\section{4,848}

PB94-152006 PC A11/MF AO3

National Inst. of Standards and Technology (BFRL), Gaithersburg, MD. Fire Safety Engineering Div.

Fire Safety of Passenger Trains: A Review of Current Approaches and of New Concepts.

Technical note (Final)

R. D. Peacock, R. W. Bukowski, W. W. Jones, J. E Brown, P. A. Reneke, and V. Babrauskas. Jan 94 239p, NIST/TN-1406

Also available from Supt. of Docs. as SN003-003 03246-8. Sponsored by Department of Transportation, Washington, DC

Key'vords: "Fire safety, *Trains, "Passengers, Smoke Fire tests, Evacuating(Transportation), Rapid transit systems

New alternative technologies have been developed which can be used to increase intercity passenger train operating speeds. These technologies include steelwheel-on-rail and magnetic levitation (maglev) systems. Fire safety is an area of particular interest for these technologies, as well as for conventional intercity and sommuter trains. While the historical fire record has been very good and few serious passenger train fires have occurred, minor incidents could develop into potential life-threatening events. The report presents a detailed comparison of the fire safety approaches used in the United States, France, and Germany. The strengths and weaknesses of current methods for measuring the fire performance of rail transportation systems are evaluated. An optimum systems approach to fire safety which addresses typical passenger train fire scenarios is analyzed and recommendations are presented to address the current state-of-the-art in materials lesting.

04,849

PB95-140430 Not available NTIS

National Inst. of Standards and Technology (MSEL) Boulder, CO. Materials Reliability Div.

Ultrasonic Measurement of Residual Stress in Railroad Wheel Rims.

Final rept.

R. E. Schramm, A. V. Clark, and T. J. McGuire.

$1992,5 p$

Sponsored by Federa! Railroad Administration, Washington, DC

Pub. in Proceedings of International Wheelset Congress (10th), Sydney, Australia, September 27-October 1, 1992, p151-155.

Keywords: "Nondestructive tests, "Ultrasonic flow detection, "Railroad trucks, "Stress measurement "Wheels, Acoustic measurement, Accuracy, Signal processing, Ultrasonics, Transducers, Residual stress, Data analysis, Birefringence, Reprints.

This work discusses three possible acoustic measure ment techniques to detect and quantify the stress railroad wheel rims. The goal is to develop instrumentation useful for nondestructive testing during field inspection for potentially dangerous conditions. In each case, the application of novel noncontact transducers (EMATs) takes advantage of minimal surface preparation and the elimination of fluid couplants. Each tech- 


\section{Railroad Transportation}

nique depends on the very precise (one part in 10(exp 4)) timing of signal arrival. Elastic changes caused by stress generate very small, but detectable velocity changes. However, other factors, notably changes in metallurgical texture, will similarly alter the velocity. Initial tests indicate the possibility of determining the size of this texture effect and reducing the uncertainty in measured stress to about $55 \mathrm{MPa}$ or less. This accuracy would allow judgment on the safety of continued operation of a wheel. For one method, a birefringence approach, we have constructed a prototype that is now at a railroad research center. Ultrasonic data collected with this gear correlate well with those from destructive sawcutting for the four wheels we have examined so far. Work on the design and construction of trans ducers and data analysis continues.

\section{4,850}

PB95-162046 Not available NTIS

Not available NTIS
National Inst. of Standards and Technology (BFRL) Gaithersburg, MD. Fire Safety Engineering Div.

New Concepts for Fire Protection of Passenger Rail Trans portation Vehicles.

Final rept.
R. D. Peacock, R. W. Bukowski, W. W. Jones, and

P. A. Reneke. 1993, 10p.

Sponsored by John A. Volpe National Transportation Systems Center, Cambridge, MA.

Pub. in Proceedings of Fire and Materials, Internationa Conference and Exhibition (2nd), Arlington, VA. September 23-24 1993p171-180.

Keywords: "Rail transportation, "Fire safety, "Fire protection, Railroad passenger senvice, Railroad cars, Passenger compartments, Trains, Fire prevention, Fire hazards, Fire tests, Test methods, Smoke, Reprints. Recent advances in guided ground transportation, fire test methods, and hazard analysis necessitate re-examination of requirements for fire safety. A comparison of the approaches used in the United States, Germany, and France is presented. With the strengths and weaknesses of current methods for measuring the fire performance of materials used in rail transit systems re viewed, a direction is suggested in which most fire science-oriented organizations in the world are clearly headed - fire hazard and fire risk assessment methods supported by measurement methods based on heat release rate.

04,851
PB95-162673 Not available NTIS

National Inst. of Standards and Technology (MSEL) Gaithersburg, MD. Materials Reliability Div

Noncontact Ultrasonic Inspection of Train Rails for Stress.

Final rept.

R. E. Schramm, A. Van Clark, T. J. McGuire, P. T

Purtscher, B. J. Filla, and D. V. Mitrakovic. 1993,

$10 p$

Contract DTF53-91-X-0068

Sponsored by Federal Railroad Administration, Washington, DC.

Pub. in Proceedings of International Conference on Rail Quality and Maintenance for Modern Railway Op eration, Delft, The Netherlands, June 1992, p99-108 1993.

Keywords: "Railroad tracks, "Acoustic measurement "Nondestructive tests, Ultrasonic frequencies, Ultrasonic tests, Texture, Stresses, Stress analysis, Sound transducers, Reprints, EMAT.

The paper discusses acoustic techniques to quantify stress, the construction of transducers, and initial measurements on a short test section of railroad track. Our goal is to develop instrumentation useful for nondestructive testing during field inspection for potentially dangerous conditions, such as those generated by thermal stress. The application of unconventional noncontact transducers (EMATs) takes advantage of minimal surface preparation and the elimination of fluid couplants.

\section{4,852}

PB95-169199 Not available NTIS

National Inst. of Standards and Technology (MSEL)

Boulder, CO. Materials Reliability Div.

Ultrasonic Measurement of Residual Stress in Cast Steel Railroad Wheels.

Final rept.

R. E. Schramm, A. V. Clark, and J. Szelazek. 1994, $6 \mathrm{p}$.

Contract FRA-DTF53-91-X-0068

Sponsored by Federal Railroad Administration, Washington, DC.
Pub. in Proceedings of Pressure Vessels and Piping Conference, Minneapolis, MN., June 19-23, 1994, p157-162.

Keywords: "Residual stress, "Ultrasonic tests, "Railroad cars, "Wheels, Nondestructive tests, Transducers, Inspection, Steels, Transportation safety, Detection, Cracks, Defects, Reprints, "Cast steel railroad wheels.

Residual hoop stresses in the rims of railroad wheels may change from compressive to tensile during their lifetime, a potentially hazardous condition that could lead to wheel failure by cracking. The current U.S. regulation calls for the measurement of discoloration in the wheel plate indicating a history of heating that may lead to unsafe stress. Quantitative measurement by ultrasonic methods is an attractive alternative. Stress influences elastic parameters and causes small changes in sound velocity. We did an extensive series of measurements around the circumference of ten cast-steel wheels; two were as-made, while the others received induction heating at three different energy levels to generate stresses similar to those of in-service wheels. Two different ultrasonic instruments gave comparable results. Destructive evaluation of stress in three wheels by sawcutting showed a good correlation between flange-tip opening displacement and the ultrasonic measurements. This suggests a reliable method and a field-usable instrument for quantitative nondestructive inspection. This could contribute to the safety and dependability of freight rail systems, and reduce costs due to failures and the unnecessary replacement of good wheels.

\section{4,853}

PB96-102868 Not available NTIS

National Inst. of Standards and Technology (BFRL), Gaithersburg, MD. Fire Safety Engineering Div.

Concepts for Fire Protection of Passenger Rail Transportation Vehicles: Past, Present, and Future.

Final rept.

1995, 17p.

See also PB95-162046 and PB95-180774.

Pub. in Fire and Materials, v19 p71-87 1995.

Keywords: "Railroad passenger service, "Fire protection, "Risk assessment, "Safety engineering, Rail transportation, Passenger transportation, Fire safety, Transportation safety, Railroad cars, Trains, Fire prevention, Fire hazards, Reprints, Heat release rate.

Recent advances in passenger rail transportation, fire test methods, and hazard analysis necissitate re-examination of requirements for fire safety. The strengths and weaknesses of current methods for measuring the fire performance of rail transportation systems are evaluated. A systems approach to fire safety which addresses typical passenger train fire scenarios is analyzed. A rationale is presented for the direction in which most fire science-oriented organizations in the world are clearly headed - the use of fire hazard and fire risk assessment methods supported by measurement methods based on heat release rate.

\section{4,854}

PB96-106992 PC A04/MF A01

National Inst. of Standards and Technology (MSEL), Boulder, CO. Materials Reliability Div.

Residual Stress in Induction-Heated Railroad Wheels: Ultrasonic and Saw Cut Measurements. Report No. 28

Rept. for 30 May $91-30$ Sep 94

R. E. Schramm, J. Szelazek, and A. V. Clark. May

95, 60p, NISTIR-5038.

Sponsored by Federal Railroad Administration, Washington, DC.

Keywords: "Railroad trains, "Wheels, "Nondestructive tests, Ultrasonic tests, Residual stress, Stress measurement, Mathematical models, Cross sections, Service life.

This is Report Number 28 in a series covering research performed by the National Institute of Standards and Technology for the Federal Railroad Administration. The report covers a project by the Materials Reliability Division to develop and test an ultrasonic system to measure residual stress in the rims of railroad wheels.

04,855

PB96-141114 Not available NTIS

National Inst. of Standards and Technology (MSEL), Boulder, CO. Materials Reliability Div.
Safety Assessment of Railroad Wheels by Residual Stress Measurements.

Final rept.

R. E. Schramm, A. V. Clark, and J. Szelazek. 1995

$12 \mathrm{p}$.

Pub. in Proceedings of Society of Photo-Optical Instrumentation Engineers Conference on Nondestructive Evaluation of Aging Infrastructure, Oakland, CA., June 6-8, 1995, v2458 p97-108 1995.

Keywords: "Railroad trucks, "Wheels, "Stress analysis, Detection, Defects, Inspection, Ultrasonic tests, Nondestructive tests, Residual stresses, Acoustic properties, Elastic properties, Railroad cars, Reprints, Electromagnetic acoustic transducers.

Residual stresses in railroad wheels may change from compressive to tensile; this change could lead to wheel failure. The current U.S. regulation calls for the measurement of discoloration indicating heating that may lead to unsafe stress. Quantitative measurement by ultrasonic methods is an attractive alternative, since stress causes small changes in sound velocity. We did an extensive series of measurements on ten cast-steel wheels. Two different ultrasonic instruments gave comparable results. Destructive evaluation of stress in three wheels showed a good correlation with the ultrasonic measurements.

\section{4,856}

PB96-141254 Not available NTIS

National Inst. of Standards and Technology (MSEL), Boulder, CO. Materials Reliability Div.

Safety Assessment of Railroad Wheels Through Roll-by Detection of Tread Cracks.

Final rept.

A. V. Clark, R. E. Schramm, S. R. Schaps, and B. J. Filla. $1995,11 \mathrm{p}$

Pub. in Proceedings of Society of Photo-Optical Instrumentation Engineers Conference on Nondestructive Evaluation of Aging Railroads, Oakland, CA., June 68, 1995, p109-119.

Keywords: "Railroad trucks, "Wheels, "Cracks, "Detection, Defects, Inspection, Rolling stock, Ultrasonic tests, Nondestructive tests, Railroad cars, Reprints, Electromagnetic acoustic transducers.

A prototype system has been developed for roll-by inspection of cracks in the trends of freight car wheels. The system uses noncontacting electromagneticacoustic transducers (EMATs) which generate and receive surface waves propagating along the trend surface. If a defect is present it reflects an echo back to the EMAT. The EMAT itself consists of a coil and permanent magnet which are embedded in a rocking shoe'. The rocking shoe is suspended by a spring sys tem in a recess machined into the outer surface of a rail plug. When the wheel is centerend over the shoe, it activates a trigger circuit which energizes the EMAT and causes propagation of the surface wave. The detected echoes are analyzed by a microprocessor which characterizes the wheel as being safe or unsafe. The crack detector system has been subjected to field testing using actual locomotive and rolling stock. It has been able to detect both artificial and real defects at roll-by speeds of $25 \mathrm{~km} / \mathrm{h}$ (15 mph).

04,857

PB96-183199 PC A04/MF A01

National Inst. of Standards and Technology (MSEL) Boulder, CO. Materials Reliability Div.

Dynamometer-Induced Residual Stress in Railroad Wheels: Ultrasonic and Saw Cut Measurements. Report No. 30.

R. E. Schramm, J. Szelazek, and A. V. Clark. Ma 95, 44p, NISTIR-5043.

See also PB91-222653. Sponsored by Federal Railroad Administration, Washington, DC. Office of Research and Development.

Keywords: "Railroad trains, "Nondestructive tests, "Wheels, Rolling stock, Ultrasonic tests, Residual stress, Piezoelectric gages, Thermal stresses.

The effect of stress on elastic parameters causes a small change in sound wave velocity. This acoustoelastic effect is the basis for a method of nondestructive evaluation (NDE). The authors used two types of ultrasonic transducers, piezoelectric and electromagnetic, to measure both thickness-averaged and near-surface stresses in the rims of twenty unused cast-steel raitroad wheels. The manufacturer mounted these wheels on a unique dynamometer and induced thermal damage by dragging tread breaks to simulate in-service conditions that might generate tensile hoop 
stress. After the authors' ultrasonic nondestructive tests, they cut eighteen wheels with a saw along a radius to measure flange tip opening and verify the stress state.

\section{Road Transportation}

\section{4,858}

PB94-177060 PC A10/MF A03

National Inst. of Standards and Technology (EEEL), Gaithersburg, MD. Electricity Div.

Advanced Components for Electric and Hybrid Electric Vehicles. Workshop Proceedings. Held in Gaithersburg, Maryland on October 27-28, 1993.

Special pub.

K. L. Stricklett, A. H. Cookson, R. W. Bartholomew, and T. Leedy. Mar 94, 217p, NIST/SP-860.

Also available from Supt. of Docs. as SN003-00303253-1.

Keywords: "Hybrid electric-powered vehicles, "Electric-powered vehicles, "Meetings, Electric batteries, Standards, Electric propulsion, Energy storage, Energy conversion, Electrochemistry, Engine components, Automotive engineering.

This is a key period in the development of electric and hybrid electric vehicles. The landmark 1990 legislation in California requires that 2 percent of new automobiles be zero emission vehicles in 1998 , rising to 10 percent in the year 2005. This can only be met by electric vehicles. The purpose of the workshop was to concentrate on the technologies to improve the design, performance, manufacturability, and economics of the critical components for the next generation of electric and hybrid electric vehicles for the year 2000 and beyond. The workshop began with invited speakers to cover the general topics of impact of the California legislation, Federal agency programs, development of standards, infrastructure needs, advanced battery development, and the imperatives for commercial success of electric and hybrid electric vehicles. Working sessions were five parallel meetings on Energy Conversion Systerns, Energy Storage Systems, Electric Propulsion Systems, Controls and Instrumentation, and Ancillary Systems.

04,859

PB94-195914 PC A05/MF A01

National Inst. of Standards and Technology (MEL),

Gaithersburg, MD. Robot Systems Div.

Recommendations on Selection of Vehicle-toRoadside Communications Standards for Commercial Vehicle Operations.

$H$. Scott, K. Stouffer, and P. Rowe. Jun 94, 80p,

NISTIR-5444.

Keywords: "Highway communication, "Standards, "Data transmission, "Commercial vehicles, Radio links, Communication systems, Transponders, Communication equipment, Communications networks, Automatic highways, Intelligent Vehicle Highway Systems.

The National Institute of Standards and Technology (NIST) Robot Systems Division is participating in a Federal Highway Administration (FHWA) program that is to lead to the recommendation of standards in Vehicle-to-Roadside Communications (VRC) equipment used in commercial vehicle operations (CVO). There is substantial motivation to develop these standards, both on the part of the government and the CVO community. A standardized VRC system will allow states to check credentials, weight, and safety parameters while commercial vehicles travel at highway speeds of up to $160 \mathrm{~km} / \mathrm{h}$. This will improve the current situation in which substantial time and productivity is lost while commercial vehicles stop and wait for inspections. More sophisticated systems may employ weigh-in-motion equipment, on-vehicle sensors to determine brake temperature and other parameters in real-time, and other subsystems (navigation, etc.) capable of generating relevant data. This communications link will serve an important role in passing data between various onboard intelligent control system computers and the roadside systems.

\section{4,860}

PB95-188827 PC A05/MF A01

National Inst. of Standards and Technology (MEL), Gaithersburg, MD. Intelligent Systems Div.
Vehicle-to-Roadside Communications for Com. mercial Vehicle Operations: Requirements and Approaches.

H. Scott, K. Stouffer, and P. Rowe. Feb 95, 88p, NISTIR-5593.

See also PB94-195914

Keywords: "Highway communication, "Commercial ve hicles, "Data transmission, Transponders, Standards, Communications networks, Communication systems, Automatic highways, Communication equipment, Radio finks, Intelligent Vehicle Highway Systems.

The National Institute of Standards and Technology (NIST) Robot Systems Division is participating in a Federal Highway Administration (FHWA) program that is to lead to the recommendation of standards in Vehicle-to-Roadside Communications (VRC) equipment. A standardized VRC system will allow states to check credentials, weight, and safety parameters while commercial vehicles travel at highway speeds of up to 160 $\mathrm{km} / \mathrm{h}$. This will improve the current situation in which substantial time and productivity is lost while commercial vehicles stop and wait for inspections. More sophisticated systems may employ weight-in-motion equipment, on-vehicle sensors to determine brake temperature and other parameters in real-time, and other subsystems (navigation, etc.) capable of generating relevant data. This communications link will serve an important role in passing data between various onboard intelligent control system computers and the roadside systems. Numerous incompatible VRC designs and protocols exist, or are being developed. The deployment of incompatible systems will not result in transparent borders, where a truck can pass, for example, from one state to another without stopping. A standard is to be developed or chosen that will meet current and projected requirements, such as those of the Intelligent Vehicle Highway System (IVHS). NIST interacted with various users, VRC equipment manufacturers, IVHS planners, and other government agencies to determine the current and projected VRC requirements. Fifteen systems and six protocols were examined in this study.

\section{4,861}

PB95-197455 PC A03/MF A01

National Highway Traffic Safety Administration, Washington, DC. Traffic Safety Programs.

Model Minimum Performance Specifications for Lidar Speed Measurement Devices.

Technical rept Apr 92-Dec 94 (Final).

J. Worthy, G. Lieberman, and B. Moran. Jan 95, 50p, DOT-HS-808 214

Prepared in cooperation with National Inst. of Standards and Technology (EEEL), Gaithersburg, MD. Office of Law Enforcement Standards.

Keywords: *Specifications, "Optical radar, *Speed indicators, Speed limit effectiveness, Speed limits, Electronic monitoring systems, Traffic law enforcement, Laser applications, Traffic law violations, Highway safety, Velocity measurement, "LIDAR(Light Detection and Ranging).

This technical report provides an overview of the circumstances leading to a request from the International Association of Chiefs of Police to the National Highway Traffic Safety Administration (NHTSA) for the development of performance specifications for LIDAR (LIght Detection And Ranging) aka Laser, devices used in speed limit enforcement. Chapter I provides an overview and description of the use of lidar speed measurement devices in speed limit enforcement. This chapter also contains NHTSA's recommendations concerning the use of lidar in speed limit enforcement and for the training of law enforcement officers using these devices. Chapter II contains the model minimum performance specifications for the lidar along with the protoco for use in the laboratory and field testing of these de vices to determine their compliance with the model performance specifications.

\section{Transportation Safety}

04,862

PB94-194065 PC A03/MF A01

National Inst. of Standards and Technology (BFRL), Gaithersburg, MD.
Risk Analysis for the Fire Safety of Airline Passengers.

R. L. Smith. Jun $94,40 p$, NISTIR-5441.

Contract DTFA03-92-Z-00018

Sponsored by Federal Aviation Administration Technical Center, Atlantic City, $\mathrm{NJ}$.

Keywords: "Fire safety, "Airlines, Passengers, Aircraf fires, Software, Risk analysis, Decision theory, Water

The purpose of this report is to describe the National Institute of Standards and Technology's work to date relating to the general methodology being developed for the project Risk Analysis for the Fire Safety of Airline Passengers and the software being used to facilitate this methodology. The approach selected involved the use of influence diagrams. Therefore, a brief dis cussion of influence diagrams is given. The status of their application to the water mist system for passenger planes is given and the overall approach to carrying out the project is described. An example is included that shows how the process works, but the case is fictional, not intended to be realistic.

\section{4,863}

PB95-180774 Not available NTIS

National Inst. of Standards and Technology (BFRL), Gaithersburg, MD. Fire Safety Engineering Div.

New Concepts for Fire Protection of Passenger Rail Transportation Vehicles. (NIST Reprint).

Final rept.

R. D. Peacock, R. W. Bukowski, W. W. Jones, and

P. A. Reneke. 1994, 10p.

See also PB95-162046.

Pub. in Proceedings of International Symposium on Fire Safety Science (4th), Ottawa, Ontario, Canada June 13-17, 1994, p1007-1016.

Keywords: "Fire safety, "Rail transportation, "Risk as sessment, "Transportation safety, Fires, Railroad cars, Test methods, Standards, Burning rate, Smoke, Combustion, Reprints, "Heat release rate.

Recent advances in guided group transportation, fire test methods, and hazard analysis necessitate re-examination of requirements for fire safety. Several stud ies have indicated nearly random ability of current tests to predict actual fire behavior. A comparison of the approaches used in the United States, Germany, and France is presented. With the strengths and weak nesses of current methods for measuring the fire performance of materials used in rail transit systems re viewed, a direction is suggested in which most fire science-oriented organizations in the world are clearly headed--fire hazard and fire risk assessment methods supported by measurement methods based on heat release rate.

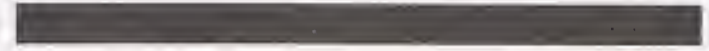 \\ URBAN \& REGIONAL TECHNOLOGY \& DEVELOPMENT}

\section{General}

04,864

AD-A297 420/2 PC A06/MF A02

National Inst. of Standards and Technology,

Properties of Working Fluids for Thermoacoustic Refrigerators.

Annual rept. 1 Oct $94-30$ Sep 95

M. R. Moldover, and K. A. Gillis. 1 Aug 95, 108p Contract N00014-93-F-0101

Keywords: *Acoustic data, "Refrigerants, "Refrigeration systems, *Thermophysical properties, Computer programs, Density, Temperature, Vapor phases, Gas dynamics, Thermal conductivity, Pressure, Fluids, Helium, Viscosity, Acoustic velocity, Enthalpy, Xenon, Entropy, Prandtl number, Viscometers "Thermoacoustics, Nobleg computer program.

The objective of this project is to provide thermophysical property data for candidate working 


\section{General}

fluids in a form suitable for optimizing the design of thermoacoustic refrigerators. The data will be provided in a computer program that calculates the properties of the most promising gas mixtures. This package will consist of currently available data from the literature (provided that it is of sufficient quality) and our own measurements (when insufficient literature data exist). The properties provided are viscosity, thermal conductivity, Prandtl number, density, speed-of-sound, specific heat, enthalpy, and entropy for temperatures between -20 and $30 \mathrm{C}$ and pressures up to 20 bars. We will also explore methods of varying the composition of the working fluid to maintain a constant speed of sound over the operating range of the refrigerator. (MM).

\section{4,865}

FIPS PUB 55-3 PC E04

National Inst. of Standards and Technology (CSL), Gaithersburg, MD.

Guideline: Codes for Named Populated Places, Primary County Divisions, and Other Locational Entities of the United States, Puerto Rico, and the Outlying Areas. Category: Data Standards and Guidelines. Subcategory: Representations and Codes.

Federal information processing standards.

28 Dec 94, 30p.

Supersedes FIPS PUB 55-2.

Three ring vinyl binder also available, North American Continent price $\$ 7.00$; all others write for quote.

Keywords: "Geocoding, "Census tracts, "Classification, "Geography, Counties, Cities, Urban areas, Municipalities, Communities, Airports, National parks, Military facilities, Data files, Data processing, United States, Federal Information Processing Standards, Data standards and guidelines, Representations and codes, Populated places, Data elements.

The publication, which supersedes FIPS PUB 55-2 and 55DC-4, provides a 2-character FIPS State code and a 5-character FIPS numeric code to uniquely identify each entity contained in a list of names of incorporated places, other communities and settlements, primary county divisions (such as townships, New England towns, and census county divisions), American Indian and Alaska Native areas, airports, military bases, national parks, post offices, and other locational entities (except natural or physical features). A 2-character class code distinguishes the different types of geographic entities. The purpose of the codes is to promote the interchange of formatted, machine-sensible data

\section{4,866}

FIPS PUB 55-DC3 PC E99

National Inst. of Standards and Technology,

Gaithersburg, MD.

Guideline: Codes for Named Populatd Places, Primary County Divisions, and Other Locational Entities of the United States, Puerto Rico, and the Outlying Areas. Category: Data Standards and Guidelines; Subcategory: Representation and Codes.

Federal information processing standards.

28 Dec 94, 3976p.

Supersedes FIPS PUB 55-2.

Three ring vinyl binder also available; North American Continent price $\$ 7.00$; all others write for quote.

Keywords: "Geocoding, "Census tracts, *Classification, *Geography, Counties, Cities, Urban areas, Municipalities, Communities, Airports, National parks, Military facilities, Data files, Data processing, United States, Federal Information Processing Standards, Data standards and guidelines, Representations and codes, Populated places, Data elements.

The publication, which supersedes FIPS PUB 55-2 and 55DC-4, provides a 2-character FIPS state code and a 5-character FIPS numeric code to uniquely identify each entity contained in a list of names of incorporated places, other communities and settlements, primary county divisions (such as townships, New England towns, and census county divisions), American Indian and Alaska Native areas, airports, military bases, national parks, post offices, and other locational entities (except natural or physical features)

\section{Fire Services, Law Enforcement, \& Criminal Justice}

04,867

PB95-151379 Not available NTIS

National Inst. of Standards and Technology (CAML), Gaithersburg, MD

Evaluating Investments in Law Enforcement Equipment: An Annotated Bibliography.

Final rept.

S. F. Weber, B. C. Lippiatt, and K. S. Johnson. 1989,

$27 \mathrm{p}$.

Pub. in National Institute of Justice Report 900-89, 27p 1989.

Keywords: "Bibliographies, "Protective equipment, "Police vehicles, "Law enforcement, "Investments, Ammunition, Weapons, Police patrol cars, Operations research, Information systems, Cost benefit analysis, Economic analysis, Reprints, Police equipment.

The report is a guide to the literature and annotated bibliography on evaluating investments in law enforce ment equipment. Each entry includes the complete citation, an abstract with key words, and in most cases, information on availability. The search strategy is documented and a subject index and lists of references by author and by subject area are included. The main cat egories are: (1) evaluation methods; (2) police vehicles; (3) police armament; (4) Automatic Vehicle Monitoring (AVM) systems; and (5) police information systems. All 43 included publications both address eco nomic issues such as costs or benefits and are relevant to current decisions on procuring police equip ment. The search focused on three primary sources: (1) the NCJRS (National Criminal Justice Reference Service) database; (2) the Criminal Justice Periodica Index (CJPI); and (3) the NTIS (National Technical Information Service) database.

\section{4,868}

PB95-162913 Not available NTIS

National Inst. of Standards and Technology (NEL), Gaithersburg, MD. Fire Science and Engineering Div. Exposure: An Expert System Fire Code.

Final rept.

R. L. Smith. 1991, 15p

Pub. in Fire Technology 27, n2 p145-159 May 91

Keywords: "Fire codes, "Artificial intelligence, "Building codes, Fire spread, Computer programs, Exposure, Cost effectiveness, Walls, Fire safety, Reprints, *Building fires, *Expert systems, Combustibles.

The report addresses the issue of developing an exper or knowledge-based system that deals with the problem of preventing the spread of fire between buildings. A knowledge-based program, EXPOSURE, has been developed that facilitates using more appropriate technology expanding the problem domain, and providing cost-effective solutions. EXPOSURE can solve the problem of the prevention of the spread of fire between buildings for the case when the exposed building has combustible walls. The use of the expert system EX POSURE and NFPA $80 \mathrm{~A}$ produce significantly different recommended minimum separation between buildings. In one case the separation required by NFPA $80 A$ was more than five times greater than what EXPOSURE recommended. The program demonstrates that significant cost savings in achieving the desired level of fire safety and in assuring the levels of safety can be obtained by use of expert system fire codes.

\section{4,869}

PB95-162939 Not available NTIS

National Inst. of Standards and Technology (BFRL) Gaithersburg, MD. Building and Fire Research Lab. Office.

Forum for International Cooperation on Fire Research.

Final rept.

J. E. Snell. 1993, 9p.

Pub. in Proceedings of Nordic Fire Safety Engineering Symposium, Development and Verification of Tools for Performance Codes, Espoo, Finland, August 30-September 1, 1993, 9p.

Keywords: *Fire research, "International cooperation, "Meetings, Fire safety engineering, Norway, Sweden Finland, Reprints, "Nordic countries, FORUM research group.

The paper describes the Forum for International Cooperation on Fire Research (FORUM) and its rule in the Nordic Fire Safety Engineering Symposium. The FORUM is an informal group of individuals who direct fire research programs and laboratories. This group shares a common commitment to the advance of fire safety engineering through cooperation in research and its application. The paper suggests actions needed to accelerate the advance of fire safety engineering.

\section{4,870}

PB95-162947 Not available NTIS

National Inst. of Standards and Technology (NEL), Gaithersburg, MD. Center for Fire Research.

Fresh Look at Strategies for Fire Safety.

Final rept.

J. E. Snell. 1990, 10p

Pub. in Proceedings of Interflam "90 Conference, Can terbury, England, September 3-6, 1990, p305-314.

Keywords: "Fire research, "Technology innovation, *Fire safety, Cost effectiveness, Fire losses, Fire codes, International cooperation, Reprints, Fire research strategies.

Recently, in the U.S.A., fire deaths and injuries have plateaued (and may even be increasing) and the costs of fire safety to society have been increasing and in most cases well ahead of inflation. The paper presents a preliminary analysis of this situation from the perspective of the potential contribution of fire research. It points to the need for major technical innovations in five areas to provide the basis for legitimizing fire prevention technology, or what we have termed the technologies for assured fire safety. These technologies may complement and strengthen the traditional fire safety strategies, further reducing the losses to fire and the economic burdens of fire on society.

\section{4,871}

PB96-154570 PC A03/MF A01

National Inst. of Standards and Technology (BFRL), Gaithersburg, MD. Office of Applied Economics.

AutoBid 2.0: The Microcomputer System for Police Patrol Vehicle Selection.

S. F Weber. Feb 96, 12p, NISTIR-5787.

Sponsored by National Inst. of Justice, Washington, DC

Keywords: "Police patrol cars, "Fleet management, *Acquisition, *Decision support systems, Selection, Ranking, Ratings, Factor analysis, Cost benefit analysis, Performance evaluation, Procurement, Decision making Optimization, Police vehicles, User manuals(Computer programs), AutoBid computer program, Police equipment, Multiattribute analysis.

This report is the user manual for a microcomputer system designed to help police fleet administrators select the patrol vehicle that is best suited to their needs. The system is called AutoBid and uses vehicle performance test data for police patrol package models published annually by the Michigan State Police. The system offers two vehicle selection methods: performance-based and value-based. Performance selection is based on both vehicle test scores alone. It ranks vehicles by their overall test performance independent of cost Value selection is based on both vehicle cost and test scores. It identifies which vehicle is the 'Best Buy' in terms of the lowest cost for equivalent test performance and ranks the vehicles by the bid price adjusted for performance. Either or both of these methods may be used for a given fleet acqusition decision.

\section{4,872}

PB96-178918 PC A05/MF A01

National Inst. of Standards and Technology (EEEL), Gaithersburg, MD. Office of Law Enforcement Standards.

Directory of Law Enforcement and Criminal Justice Associations and Research Centers.

Special pub

S. Lyles, M. Leach, and R. Joel. Mar 96, 65p, NIST/ SP-480/20-ED-1996.

Supersedes PB86-213089. Also available from Supt. of Docs. as SN003-003-03403-7.

Keywords: "Directories, "Law enforcement, *Organizations, United States, Criminal justice.

This is a directory of organizations that are active in one or more areas of the criminal justice system, including law enforcement, courts, corrections and rehabilitation. The directory lists national organizations primarily, but also includes regional organizations and local organizations of special interest as well as international organizations which have a significant number of American members, a U.S. chapter or subcommit- 
tee, or are doing work applicable to law enforcement in this country. The types of national law enforcement and criminal justice organizations listed in this directory are limited by the criterion that they be nonprofit. Included in this category are professional and volunteer social action associations, research centers (usually connected with a university), and government agencies. Strictly social or fraternal organizations are not listed.

\section{Urban Administration \& Planning}

04,873

FIPS PUB 8-6 PC E09

National Inst. of Standards and Technology (CSL), Gaithersburg, MD.

Metropolitan Areas (Including MSAs, CMSAs, PMASs, and NECMAs). Category: Data Standards and Guidelines; Subcategory: Representations and Codes.

Mar 95, 133p

Supersedes FIPS PUB 8-5. Also available from Supt. of Docs.

Keywords: "Metropolitan areas, "Geocoding, "Classification, "Geography, Metropolitan statistical areas, Standard Metropolitan Statistical Areas, New
England, Cities, Counties, Urban areas, States(United States), Federal Information Processing Standards, Data standards and guidelines, Representation and codes, Consolidated Metropolitan Statistical Areas, Primary Metropolitan Statistical Areas, Data elements.

The standard specifies titles, components, and identification codes for the Metropolitan Areas (MAs) of the United States and Puerto Rico, including units called Metropolitan Statistical Areas (MSAS), Consolidated Metropolitan Statistical Areas (CMSAS), and Primary Metropolitan Statistical Areas (PMSAs), and related units called New England County Metropolitan Areas (NECMAS). The general concept underlying Metropoli$\tan$ Areas is that of a core are containing a large population nucleus together with adjacent communities having a high degree of economic and social integration with that core. The revision incorporates minor editorial changes and technical changes that have been issued in change notices and supersedes FIPS PUB 8-5 in its entirety.

04,874
PB95-503280 Diskette $\$ 59.00$

National Inst. of Standards and Technology, Gaithersburg, $M D$.

FIPS PUB 8-6, Metropolitan Areas (for Microcomputers).

Data file.

May 95,1 diskette

This product contains text only. Customers must provide their own search and retrieval software.
The datafile is on one $31 / 2$ inch DOS diskette, 1.44M high density. File format: WordPerfect 5.1. Also available in paper copy, order number FIPS PUB 8-6.

Keywords: “Data file, "Metropolitan areas, "Geocoding "Classification, "Geography, Metropolitan statistical areas, Statistical Metropolitan Statistical Areas, New England, Cities, Countries, Urban areas, States(United States), Diskettes, Federal Information Processing Standards, Data standards and guidelines, Representation and codes, Consolidated Metropolitan Statistical Areas, Primary Metropolitan Statistical Areas, Data elements.

This standard specifies titles, components, and identification codes for the Metropolitan Areas (MAs) of the United States and Puerto Rico, including units called Metropolitan Statistical Areas (MSAs), Consolidated Metropolitan Statistical Areas (CMSAs), and Primary Metropolitan Statistical Areas (PMSAs), and related units called New England County Metropolitan Areas (NECMAs). The general concept underlying MAs is that of a core area containing a large population nucleus together with adjacent communities having a high degree of economic and social integration with that core. This revision incorporates technical changes which reflect revised definitions by the Office of Management and Budget. 



\section{SAMPLE ENTRY}

SMITH, R. L.

Performance Parameters of Fire Detection Systems

PB94-194339
Author name(s)

Title

00,123
NTIS order number

Abstract number
AAMODT, R. L.

External Gamma-ray Counting of Selected Tissues from a Thorotrast Patient.

03,637

ABBOTT, P. J.

Comments on the Stability of Bayard-Alpert Ionization Gages.

02,673

Commercial Helium Permeation Leak Standards: Their Properties and Reliability.

PB97-111413

04,146

Influence of the Filament Potential Wave Form on the Sensitivity of Glass-Envelope Bayard-Alpert Gages.

PB95-175014

02,657

Long-Term Stability of Bayard-Alpert Gauge Performance: Results Obtained from Repeated Calibrations against the National Institute of Standards and Technology Primary Vaum Standard.

02,676

ABDEL REHIM, $F$.

Thin Dyed-Plastic Dosimeter for Large Radiation Doses. PB95-107363 03,872

ABOLA, E. E.

Protein Data Bank: Current Status and Future Challenges. PB97-109060

00,517

ABRAHAM, 8.

Taguchi's Parameter Design: A Panel Discussion.

PB96-111802

03,445

ABRAHAMS, S. C.

Statistical Descriptors in Crystallography. 2. Report of a Working Group on Expression of Uncertainty in Measurement.

04,764

ABRAMOWITZ, S.

Conformational Alterations of Bovine Insulin Adsorbed on a Silver Electrode.

PB96-161773

00,510

Thermodynamic Properties of Gas Phase Species of Importance to Ozone Depletion.

00.126
ABRAMS, $M$.

Proceedings of the Workshop on the Federal Criteria for Information Technology Security. Held in Ellicol City, Maryland on June 2-3, 1993.

PB94-162583

01,575

ABRAMS, M. D

Head Start on Assurance: Proceedings of an Invitational Workshop on Information Technology (IT) Assurance and Trustworthiness. Held in Williamsburg, Virginia on March 21-23, 1994.

ACET, $M$.

01,586

Potarization Analysis of the Magnetic Excitations in

Fe65Ni35 Invar.
PB95-150082

ACQUISTA, N.

Laser-Produced and Tokamak Spectra of Lithiumlike Iron,

$\mathrm{Fe}(23+)$.
$\mathrm{PB} 95-180857$

04,314

ADAIR, $D$.

Electro-Optical Sensor for Surface Displacement Measurements of Compliant Coatings.

PB94-198223

02,123

ADAMS, J.

Electric-Field Strengths Measured Near Personal Transceivers.

PB94-172020

01,564

ADAMS, J. W.

Aperture Excitation of Electrically Large, Lossy Cavities. PB94-145711 00,029 Characterization of Unknown Linear Systems Based on Measured CW Amplitude.

PB95-161485

01,897

ADAMS, V. H.

Natural Convection from an Array of Electronic Packages Mounted on a Horizontal Board in a Narrow Aspect Ratio Enclosure.

PB96-164017

02,087

ADAMUTI-TRACHE, $M$.

Segmental Concentration Profiles of End-Tethered Polymers with Excluded-Volume and Surface Interactions

PB97-119002

00,654
ADVANI, S. G.

Interaction between Micro and Macroscopic Flow in RTM Preforms.

PB95-162012

03,159

AEPPLI, G.

Isolated Spin Pairs and Two-Dimensional Magnetism in SrCr(sub 9p)Ga(sub 12-9p)019.

PB97-112387

04,154

AFEEFY, H. Y.

Alcohol Solutions of Triphenyl-Tetrazolium Chloride as High-Dose Radiochromic Dosimeters.

PB96-135249

03,716

AGHAJANIAN, $J$

Genetically Engineered Pore as a Metal Ion Biosensor. PB96-161658

03,551 AGOSTINI, P.

Intensity-Dependent Scattering Rings in High Order AboveThreshold lonization.

PB96-110739

04,032

AGREN, J.

Extrapolation of the Heat Capacity in Liquid and Amorphous

PBases. 11142

04,147

AHMED, G. N.

Calculating Flame Spread on Horizontal and Vertical Sur-

faces.

00,335

AIZED, $D$.

Comparing the Accuracy of Critical-Current Measurements Using the Voltage-Current Simulator.

PB96-119219

02,227

AKE, T. B.

First Results from the Goddard High-Resolution Spectro. graph: The Chromosphere of Tauri.

PB94-199528

00,054

AKMAN, $\mathrm{S}$.

Modification of DNA Bases in Chromatin of Intact Targat Human Cells by Activated Human Polymorphonuclear Leukocytes

PB94-199833

03,526 
AKMAN, S. A.

Copper Ion-Mediated Modification of Bases in DNA in Vitro by Benzoyl Peroxide.

03,645

Formation of DNA.Protein Cross-Links in Cultured Mammalian Cells Upon Treatment with Iron Ions.

03,651

AKSAY, I. A.

High Resolution Inelastic Neutron Scattering Study of Phonon Self-Energy Effects in YBCO

04,688

q Dependence of Self-Energy Effects of the Plane Oxygen Vibration in $\mathrm{YBa} 2 \mathrm{Cu} 3 \mathrm{O} 7$

PB96-138516

01,096

AL-NAAFA, M. A.

Brownian Diffusion of Hard Spheres at Finte Concentra-

PB95-164307

00.975

AL-SHEIKHLY, $M$

Alcohol Solutions of Triphenyl-Tetrazolium Chloride as High-Dose Radiochromic Dosimeters.

03,716 Anıonic Triphenylmethane Dye Solutions for Low-Dose

Food Irradiation Dosimetry

03,715

PB97-119259

03,641

Polymerization Initiation by $\mathrm{N}$-p-Tolylglycine: Free-Radical

Reactions Studied by Pulse and Steady-State Radiolysis.

01,269

Radiochromic Solid-State Polymerization Reaction.

PB95-180683

01,271

Radiochromic Solid-State Polymerization Reaction. PB96-180146

01,290

ALBERS, J.

Exact Recursion Relation Solution for the Steady-State Surface Temperature of a General Multilayer Structure.

02,376

Exact Solution of the Steady-State Surface Temperature for a General Multilayer Structure.

PB95-152773

02,337

JEDEC 'TCR' Interlaboratory Experiment: Lessons Learned.

PB95-203188

02,371

Semiconductor Measurement Technology: HOTPAC. Pro-

grams for Thermal Analysis Including Version 3.0 of the

TXYZ Program, TXYZ30, and the Thermal MultiLayer Pro-

gram, TML.

02,374

ALBERS, $\mathrm{J}$. C

Santa Ana Fire Department Experiment at 1315 South Bristol, July 14,1994

00,389

Santa Ana Fire Department Experiment at 1315 South Bristol, July 14, 1994. (Reprint)

PB'96-102934

00,207

Santa Ana Fire Department Experiments at South Bristol Street

PB96-154810

00,305

ALBERTY, R. A.

Calorimetric Determination of the Standard Transformed Enthalpy of a Biochemical Reaction at Specified $\mathrm{PH}$ and PMg.

03,454

ALBRIGHT, B. J.

Core Potentials for Ouasi-One-Electron Systems. PB95-202214

03,970

ALBUS, C

Proceedings of the Annual Manufacturing Technology Conference (2nd): Toward a Common Agenda. Held in Gaithersburg, Maryland on April 18-20, 1995.

Publications of the Manufacturing Engineering Laboratory Covering the Period January 1989-September 1992.
PB94-165966

ALBUS, C. F.

Automated Manufacturing Research Facility 1994 Annual Repo

PB95-209854

00,015

Proceedings of the Manufacturing Technology Needs and Issues: Establishing National Priorities and Strategies Conference. Held in Gaithersburg, Maryland on April 26-28, 1994

PB95-206181

02,930

ALBUS, J. S.

Hierarchical Interaction between Sensory Processing and World Modeling in Intelligent Systems.

PB94-198256

01,580

Intelligent Control for Multiple Autonomous Undersea Vehi-

PB94-211877

03,747

NIST Support to the Next Generation Controller Program: 1991 Final Technical Report

PB94-163490

02,808

Overview of NASREM: The NASANBS Standard Reference Model for Telerobot Control System Architecture.

04,831
Real-Time Vision for Autonomous and Teleoperated Control of Unmanned Vehicles.

03,701

4-211885

03,702

Peference Model Architecture for Intelligent Systems De-

PBg5-143137

01,789

Role of World Modeling and Value Judgment in Perception.

PB94-198264

Theory of Intelligent Systems.

01.58

PB94. 198272

01,582

Toward a Reference Model Architecture for Real-Time Intelligent Control Systems (ARTICS)

PB94-172046

02,932

ALCOCK, C. B.

Thermodynamic Properties of the Group IIA Elements.

PB94. 160983

ALDERMAN, D. F.

Airborne Asbestos Analysis: National Voluntary Laboratory

$\begin{array}{ll}\text { Accreditation Program. } & 02,566\end{array}$

National Voluntary Laboratory Accreditation Program: Bulk

$\begin{array}{ll}\text { Asbestos Analysis. } & 02,541 \\ \text { PB95-138129 }\end{array}$

National Voluntary Laboratory Accreditation Program NVLAP): Fasteners and Metals.

02881

PB97-114185

Observations of Partial Discharges in Hexane Under High Magnification.

ALFASSI, Z. B.

01,900

Oxidation of Ferrous and Ferrocyanide Ions by Peroxyl

Radicals.
PB97-122402

01,191

Temperature Dependence of the Rate Constants for Reac tion of Dihalide and Azide Radicals with Inorganic Reduc-

PB95-162756

00,964

Temperature Dependence of the Rate Constants for Reaction of Inorganic Radicals with Organic Reductants. PB94-198280

ALGEO, M. E. A.

Process for Selecting Standard Reference Algorithms for Evaluating Coord

02,629

State-of-the-Art Survey of Methodologies for Representing Manufacturing Process Capabilities.

PB94-187655

02,812

ALIBE, B.

Algebraic Approximation of Attractors for Galloping Oscillators.

B95-162897

04,820

Chaotic Motions of Coupled Galloping Oscillators and Thei Modeling as Diffusion Progresses.

PB96-122718

04,823

Foias-Temam Approximations of Attractors for Galloping Oscillators.

ALKAN DONMEZ, $M$.

04,817

Real Time Compensation for Tool Form Errors in Turning Using Computer Vision.

PB95-107231

02,945

ALLAN, D. W.

Confidence on the Second Difference Estimation of Frequency Drift.

PB95-151460 01,532

Precision Oscillators: Dependence of Frequency on Temperature, Humidity and Pressure.

PB94-198306

02,031

Smart Clock: A New Time

01,530

ALLEN, A. J.

Analysis of Small-Angle Scattering Data Dominated by Multiple Scattering for Systems Containing Eccentrically tiple Scattering for Syster

PB96-160411

03,075

Anisotropy of the Surfaces of Pores in Plasma Sprayed Alumina Deposits

ALLEN, L. P.

03,126

Effect of Anneal Temperature on Si/Buried Oxide Interface Roughness on SIMOX

PB96-112206

02,382

ALLEN, O. E.

Time-Domain Antenna Characterizations. PB95-152781

02,003

ALLEN, $\mathrm{R}$. Resolution of DNA in the Presence of Mobility Modifying
Polar and Nonpolar Compounds by Discontinuous Electrophoresis on Rehydratable Polyacrylamide Gels. PB95-152799

00,590

ALLEN, R. A.

Application of the Modified Voltage-Dividing Potentiometer to Overlay Metrology in a CMOS/Bulk Process.
PB94-181997

Comparisons of Measured Linewidths of Sub-Micrometer Lines Using Optical, Electrical, and SEM Metrologies.

PB95-152807 02,338

Electrical Test Structure for Improved Measurement of Feature Placement and Overlay in Integrated Circuit Fabrication Processes.

Electrical Test Structure for Overlay Metrology Referenced to Absolute Length Standards.

PB95-152278

02,336

Electrical Test Structures Replicated in Silicon-on-Insulator Materia

$\begin{array}{ll}\text { PB97-111827 } & 02,454\end{array}$

Enhanced Voltage-Dividing Potentiometer for High-Precision Feature Placement Metrology.

PB96-164025

02,428

Hybrid Optical-Electrical Overlay Test Structure.

02,450

PB96-204136

connect

Measurement of

PB96-148168

02,420

Microelectronic Test Structures for Feature Placement and Electrical Linewidth Metrology

PB95-180568

Microelectronic Test Structures for Overlay Metrology.

PB96-164249

New Test Structure for Nanometer-Level Overlay and Feature-Placement Metrology.

PB95-175345

02,363

Test Structures for the In-Plane Lacations of Projected Feafures with Nanometer-Level Accuracy Traceable to a Coordinate Measurement System.

PB94-200565

02,313

ALLEN, S. M.

Electronic Siructure and Phase Equilibria in Ternary Substitutional Alloys.

03,378

ALLGOOD, C.

Hair Analysis for Drugs of Abuse: Evaluation of Analytical Methods, Environmental issues, and Development of Reference Materials.

PB95-176269

03,501

ALLGOOD, C. C.

Interlaboratory Comparison Studies on the Analysis of Hair for Drugs of Abuse.

PB95-17625

03,500

ALLISON, W.

Variation in Magnetic Properties of Cu/Fcc (001) Sandwich Structures.

ALPERT, B. K

Hybrid Gauss-Trapezoidal Quadrature Rules.

PB96-193750

Integral Occurring in Coherence Theory

04,324

Video Microscopy Applied to Optical Fiber Geometry Measurements.

PB95-173068

04,295

PERT, R. L.

Prediction of Fire Dynamics. 
Vibrational Distributions of As2 in the Cracking of As4 on Si(100) and Si(111)

PB94-198314

00,784

ALTAFIM, R. A. C.

Electrohydrodynamic Instability and Electrical Discharge Initiation in Hexane.
PB96-186119

02,244

ALTMAN, 5 .

Tert-Butyl Hydroperoxide-Mediated DNA Base Damage in Cultured Mammalian Cells

PB94-182003

03,644

ALTMAN, S. A.

Formation of DNA-Protein Cross-Links in Cultured Mammalian Cells Upon Treatment with Iron Ions.

03,651

ALVAREZ, $R$.

Recently Developed NIST Food Related Standard Reference Materials.

PB94-198322 ronmental Pollutants.

PB94-198330

02,518

ALVORD, D. M.

CFAST Output Comparison Method and lts Use in Comparing Different CFAST Versions.
PB96-109541

00,401

Feasibility and Design Considerations of Emergency Evacuation by Elevators.

PB94-163441

00,287

AMAR, A.

Application of Single Electron Tunneling: Precision Capacitance Ratio Measurements.

PB96-102157

04,703

AMATO, A.

Dynamics of $\mathrm{Mu}(+)$ in $\mathrm{Sc}$ and $\mathrm{ScHx}$.

PB96-180021

04,112

AMBUEL, L. M

Proceedings of the Workshop on the Federal Criteria for Information Technology Security. Held in Ellicot City. Maryand on June 2-3, 1993.

PB94-162583

01,575

\section{AMIRTHARAJ, P. M.}

Buffer Layer Modulation-Doped Field-Effect-Transistor Interactions in the Al0.33Ga0.67As/GaAs Superlattice System.

PB96-102876 02,380

Comparison of Techniques for Nondestructive Composition Measurements in CdZnTe Substrates.

PB96-103098

02,703

Double Modulation and Selective Excitation Photoreflectance for Characterizing Highly Luminescen Semiconductor Structures and Samples with Poor Surface Morphology.
PB97-111439 Double-Modulation and Selective Excitation Photoreflectance for Wafer-Level Characterization of Quanium-Well Laser Structures.

PB96-167325

04,372

High-Accuracy Principal-Angle Scanning Spectroscopic Ellipsometry of Semiconductor Interfaces.

Interface Roughness of Short-Period AIAs/GaAs Superlattices Studied by Spectroscopic Ellipsometry.
PB95-107215 137 Interface Sharpness during the Initial Stages of Growth of Thin, Short-Period III-V Superlattices.

02,139

Interface Sharpness in Low-Order III-V Superlattices

PB95-108775

02,138

Spectroscopic Ellipsometry Determination of the Properties of the Thin Underlying Strained Si Layer and the Roughness at $\mathrm{SiO} 2 / \mathrm{Si}$ interface.

04,560

Use of Pressure for Quantum-Well Band-Structure Characterization.

PB96-164058

AMMERLAAN, M. J. J.

04,779

Loss-Free Counting at IRI and NIST.

PB96-167119

04,105

AMMON, H. L.

Crystal Structure of Calcium Adipate Monohydrate.
PB94-216579

Crystal Structure of Decacalcium Tetrapotassium Hexakis (Pyrophosphate) Nonahydrate.

PB96-141064

01,099

ANANDA, M. V.

Residual Error Compensation of a Vision-Based Coordinate Measuring Machine.

PB96-161617

04,091

ANASTASIADIS, S. H

Morphology of Symmetric Diblock Copolymers as Revealed by Neutron Reflectivity.

PB95-140075

01,234

Temperature Dependence of the Morphology of Thin Diblock Copolymer Films as Revealed by Neutron ReflectivPB94-172756

01,199
ANDERSEN, $\mathbf{N}$.

Can Quantum Mechanical Description of Electron-Sodium Collisions Be Considered Complete. Present Status and Fulure Prospects for $3 s<->3 p$ Transitions.

PB94-185014

00,768

ANDERSON, A. C.

Use of Ion Scattering Spectroscopy to Monitor the Nb Target Nitridation during Reactive Sputtering.

PB94-172525

00,761

ANDERSON, D. B.

Evaluation of Corrosion Data: A Review.

PB94-198348

03,187

NDERSON, D. L.

Effects of Target Shape and Neutron Scattering on Element Sensitivities for Neutron-Capture Prompt Gamma-ray Activation Analysis.

00,558

Neutron Capture Prompt Gamma-Ray Activation Analysis at the NIST Cold Neutron Research Facility.

PB94-213394

00,556

Use of Neutron Beams for Chemical Analysis at NIST.

PB97-112437

ANDERSON, D. $M$.

Diffuse-Interface Description of Fluid Systems.

00,652

ANDERSON, E.

Performance of HUD-Affiliated Properties during the January 17,1994 Northridge Earthquake.

PB95-174488

00,443

Thermodynamics of Enzyme-Catalyzed Reactions: Part 1 Oxidoreductase

ANDERSON, E. D.

Stranding Experiments on Double Hull Tanker Structures. PB96-123112

00,737

ANDERSON, E. H.

Room-Temperature Thermal Conductivity of Expanded Pol-

ystyrene Board for a Standard Reference Material.
PB96-193693

ANDERSON, $\mathrm{H}$. M.

Dusty Plasma Studies in the Gaseous Electronics Conference Reference Cell.

PB96-113410

02,396

Gaseous Electronics Conference Radio-Frequency Reference Cell: A Defined Parallel-Plate Radio-Frequency System for Experimental and Theoretical Studies of PlasmaProcessing Discharges.

PB94-172327

04,404

ANDERSON, I. S

Characterization of the Structure of TbD2.25 at $70 \mathrm{~K}$ by Neutron Powder Diffraction.

01,130

Characterization of the Vibrational Dynamics in the OctaheCharacterization of the Vibrational Dynamics in the Octahe-
dral Sublattices of LaD2.25 and LaH2.25.
PB96-123724

01,091

Dynamics of $\mathrm{Mu}(+)$ in $\mathrm{Sc}$ and $\mathrm{ScH}$

04,112

Local-Mode Dynamics in YH2 and YD2 by Isotope-Dilution Neutron Spectroscopy

PB95-181012

01,017

Neutron-Powder-Diffraction Study of the Long-Range Order in the Octahedral

04,753

Neutron Spectroscopic Comparison of beta-Phase Rare Earth Hydrides.

01,134

Neutron Spectroscopic Comparison of Rare-Earth/Hydrogen alpha-Phase Systems.

00,970

Neutron Spectroscopic Evidence of Concentration-Dependent Hydrogen Ordering in the Octahedral Sublattice of beta

PB95-181020

01,018

Vibrations of Hydrogen and Deuterium in Solid Solution with

Lutetium.

01,019

ANDERSON, $\mathbf{M} . \mathbf{H}$.

Behavior of Atoms in a Compressed Magneto-Optical Trap.
PB95-203105

Reduction of Light-Assisted Collisional Loss Rate from a

Low-Pressure Vapor-Cell Trap.
PB95-202248

03,971

Stable, Tightly Confining Magnetic Trap for Evaporative Cooling of Neutral Atoms.

PB96-200720

04,126

ANDERSON, $P$.

Fracture in Multilayers.

PB96-163613

ANDERSON, P. M.

Nonlocal Effects of Existing Dislocations on Crack-Tip Emission and Cleavage.

ANDERSON, R. C.

Product Realization Process Modeling: A Study of Requirements, Methods

02,836

ANDERSON, T. J.

Assessment of the Al-Sb System.

03,329

ANDREEV, A. V.

Magnetic Properties of Single-Crystalline UCu3Al2.

ANDREWS, A. P.

Small Angle Neutron Scattering Studies of Structural Characteristics of Argarose Gels.

ANDREWS, $M$.

03,475

Histopathology, Blood Chemistry, and Physiological Status of Normal and Moribund Striped Bass ("Morone saxatilis') Involved in Summer Mortality ('Die-Otf') in the SacramentoSan Joaquin Delta of California.

PB94-198157

00,034

ANDULIS, $\mathrm{C}$

Deuterium in the Local Interstellar Medium: Its

Cosmological Signiticance.
PB95-202842

00,081

Redshitts in Ste

00,104

Relationship between Radiative and Magnetic Fluxes for Three Active Solar-Type Dwarts

PB96-119540

00,097

Transition Regions of Capella.

00.105

Transition Regions of Capella (1995)

PB96-176714

00,108

Ground Improvement Techniques for Liquefaction Remedi-

ation Near Existing Litelines.

PB96-128111

01,350

ANGELINI, $P$.

Matrix Grain Bridging Contribution to the Toughness of Whisker Reinforced Ceramics.

PB94-198645

03,134

ANGELINO, G

Report of the Refrigeration. Air Conditioning and Heat Pumps Technical Options Committee.

03,293

Evaluated Bimolecular Ion-Molecule Gas Phase Kinetics of Positive lons for Use in Modeling Planetary Atmospheres, Cometary Comae, and Interstellar Clouds.

PB94-168598

ANKNER, J. F.

00,753

Antiferromagnetic Interlayer Correlations in Annealed

Ni80Fe20/Ag Multilayers
PB97-122220

03,109

Combined Low- and High-Angle X-Ray Structural Refinement of a $\mathrm{Co} / \mathrm{Pt}(111)$ Multilayer Exhibiting Perpendicular Magnetic Anisotropy.

PB94-198355

04,457

Compatibilization of Polymer Blends by Complexation. 2. Kj-

netics of Interfacial Mixing
PB97- 111900

01,295

Diffraction of Neutron Standing Waves in Thin Films with Resonance Enhancement.

04,164

Extending the Angular Range of Neutron Reflectivity MeasExtending the Angular Range of Neutron Reflectivity Meas-
urements from Planar Lipid Bilayers: Applications to a Model Biological Membrane.

PB96-122569 


\section{PERSONAL AUTHOR INDEX}

ANTONUCCI, J.

Ring-Opening Dental Resin Systems Based on Cyclic

Acetals.
PB95-162251

00,162

ANTONUCCI, J. M.

Bioactive Polymeric Dental Materials Based on Amorphous Calcium Phosphate.

PB96-147012

Dental Materials.

03,572

(172871

00,142

Effects of Surface-Active Resins on Dentin/Composite Bonds.

00,156

Evaluation of Methylene Lactone Monomers in Dental Res-

00,164

Facile Synthesis of Novel Fluorinated Multifunctional

Acrylates.
PB94-198389 01,207

Formation of Hydroxyapatite in a Polymeric Calcium Phosphate Cement

00,173

Polymeric Calcium Phosphate Cements Derived from Poly(methyl vinyl ether-maleic acid)

00,180

Polymeric Calcium Phosphate Composites with Remineralization Potential.
PB96-155544

Properties and Interactions of Oral Structures and Restorative Materials. Annual Report for Period October 1, 1990 to September 30, 199

PB94-160843

03,558

Remineralizing Dental Composites Based on Amorphous Calcium Phosphate.

PB96-147020

03,573

ANUTA, M. A

Basic Linear Algebra Operations in SLI Arithmetic.

PB96-165931

03,421

MasPar MP. 1 as a Computer Arithmetic Laboratory.

MasPar MP. 1 as a Computer Arithmetic Laboratory.

MasPar MP.1 as a Computer Arithmetic Laboratory.

APPEL, N.

01,627

Group 1 for the Process Engineering Data STEP Application Protocol.

PB97-116073

ARAGON, A. S.

Measurements of the Viscosities of Saturated and Com pressed Liquid 1,1,1,2-Tetrafluoroethane (R134a), 2,2Dichloro-1,1,1-Trifluoroethane (R123) and 1,1-Dichloro-1Fluoroethane (R141b)

PB95-175386

03,273

AAGON, B. P.

Inductively Coupled Plasma Source for the Gaseous Elecronics Conference RF Reference Cell.

PB96-11339

ARANGO, I

02,394

Energy-Based Method for Liquefaction Potential Evaluation Phase 1. Feasibility Study.

PB96-214747

03,691

ARBON, R. E.

Calibration and Performance of GafChromic DM-100 Radiochromic Dosemeters.

PB97-119291

ARCHER, D. G.

00,703

Enthalpy Increment Measurements from $4.5 \mathrm{~K}$ to $350 \mathrm{~K}$ and the Thermodynamic Properties of the Titanium Silicide Ti5si3(cr)

PB96-204037

00,679

Enthalpy Increment Measurements from 4.5 to $318 \mathrm{~K}$ fo Bismuth (cr). Thermodynamic Properties from $0 \mathrm{~K}$ to the Melting Point.

PB96-204011

01,166

Enthalpy Increment Measurements from 4.5 to $350 \mathrm{~K}$ and the Thermodynamic Properties of Titanium Disilicide(cr) to $1700 \mathrm{~K}$

PB96-204029

00,678

Isopiestic Investigation of the Osmotic and Activity Coefficients of Aqueous $\mathrm{NaBr}$ and the Solubility of $\mathrm{NaBr} 2 \mathrm{H} 2 \mathrm{O}$ (cr) at $298.15 \mathrm{~K}$ : Thermodynamic Properties of the $\mathrm{NaBr}+\mathrm{H} 2 \mathrm{O}$ System over Wide Ranges of Temperature and Pressure. PB97.110365

01.175

Thermodynamic Properties of Synthetic Otavite, $\mathrm{CdCO} 3(\mathrm{cr})$

Enthalpy Increment Measurements from $4.5 \mathrm{~K}$ to $350 \mathrm{~K}$.
PB97-111447

Thermodynamic Properties of Synthetic Sapphire (alpha Al2O3), Standard Reference Material 720 and the Effect of Temperature-Scale Differences on Thermodynamic Properties.

PB94-168564

00,750

ARD, C. K.

Comparison of Techniques for Nondestructive Composition Measurements in CdZnTe Substrates.

PB96-103098

02,703

International Marine-Atmospheric (222)Rn Measurement Intercomparison in Bermuda. Part 2. Results for the Participating Laboratories.

00,115
ARIF, $M$.

Multi-Stage, Position Stabilized Vibration Isolation System or Neutron Interferometry.

03,955

Precision Comparison of the Lattice Parameters of Silicon Monocrystals.
PB94-169745

04,438

ARKIN, E. M.

Point Probe Decision Trees for Geometric Concept Classes.

PB96-160817

01,612

ARNAUD, D.

Report of the Refrigeration, Air Conditioning and Heat Pumps Technical Options Committee.

03,293

ARNDT, $T$.

Harmonic and Static Susceptibilities of $\mathrm{YBa} 2 \mathrm{Cu} 3 \mathrm{O} 7$

04.599

ARNOLD, F. E.

Evidence of Crosslinking in Methyl Pendent PBZT Fiber

PB97-112486

ARONOFF, $R$.

Analysis of ANSI ASC $\times 12$ and UN/EDIFACT Electronic Data Interchange (EDI) Standards.

PB95-220554

01.729

Measuring Nondipolar Asymmetries of Photoelectron Angular Distribution

ARTYMOWICZ, D.

01,193

Incorporation of Gold into YBa2Cu3O7: Structure and Tc

Enhancement.

04,481

ARVAY, E.

dc Method for the Absolute Determination of Conductivities of the Primary Standard KCl Solutions frem OC to $50 \mathrm{C}$

PB94-219342

02,644

ASAOKA, K.

Behavior of a Calcium Phosphate Cement in Simulated Blood Plasma In vitro.

00,165

Effect of Transformation of Alloy on Transient and Residual Stresses in a Porcelain-Metal Strip.

00,143

Evaluation of Fracture Toughness and Residual Stress in Dental Porcelain by Indentation-Microfracture Method.
PB95-125613 00,154

tress in

Evaluation of Fracture Toughness and Residual Str

PB95-152831

00,159

Influence of Tempering Method on Residual Stress in Dental Porcelain.

00,138

Properties and Interactions of Oral Structures and Restorative Materials. Annual Report for Period October 1, 1990 to September 30, 1991

PB94-160843

03,558

ASCARRUNZ, $F$

Frequency Synthesis and Metrology at $10(-17)$ and Beyond.
PB97.113187

ASCARRUNZ, F. G.

Effect of Harmonic Distortion on Phase Errors in Frequency Distribution and Synthesis.

ASCARUNZ, F. G.

Investigations of $A M$ and $P M$ Noise in X-Band Devices

PB95-180022

01,563

SHE, M. J.

Surface Roughness of Glass-Ceramic Insert. Composite Restorations: Assessing Several Polishing Techniques.

PB97-119010

03,583

ASHFOLD, M. N. R.

Photodissociation of Ammonia at 193.3nm: Rovibrational State Distribution of the NH2(A(2)A1) Fragment. PB95-151775

00,937

Resonance Enhanced Multiphoton Ionization Spectroscopy of the PF Radical.

PB97-119119

00,702

EY, K.

Lead Abatement in Buildings and Related Structures.

PB94-172038

High-Efficiency, High-Power Difference-Frequency Generation of 0.9-1.5 mu $\mathrm{m}$ Light in BBO.

PB95-202255

04,317

High-Resolution Infrared Spectroscopy of DF Trimer: A Cyclic Ground State Structure and DF Stretch Induced Intramolecular Vibrational Coupling.

PB95-150678

00,920

ASHWORTH, S. H.

Stabilization and Precise Calibration of a Continuous-Wave Difference Frequency Spectrometer by Use of a Simple Transfer Cavity

ASMAIL, C.

04,276

Rayleigh Scattering Lımıs for Low-Level Bidirectional Reflectance Distribution Function Measurements.

04,307

ASPECT, A

Frequency-Stabilized LNA Laser at 1.083 mum: Application to the Manipulation of Helium 4 Atoms.

PB95-176186

04,304

ASSAEL, M. J.

Standard Reference Data for the Thermal Conductivity of

PB96-145875

Status of the Round Robin on the Transport Properties of

PB96-167218

01,152

Transient Methods for Thermal Conductivity.

04,197

PB94-198405

ASTUMIAN, R. D.

Electropermeabilization of Cell Membranes: Effect of the Resting Membrane Potential.

PB95.163291

Energy Transduction between a Concentration Gradient and an Alternating Electric Field.

PB94-216363

03,461

Imposed Oscillations of Kinetic Barriers Can Cause an Enzyme to Drive a Chemical Reaction Away from Equilibrium.
01,137 Michaelis-Menten Equation for an Enzyme in an Oscillating Electric Field

00,906

Ouadratic Response of a Chemical Reaction to External Oscillations.

ATALLA, R. H.

01,138

Solid State (13)C NMR and Raman Studies of Cellulose Triacetate: Oligomers, Polymorphism, and Inferences about Chain Polarity

ATHA, D.

Physicochemical Characterization of Low Molecular Weight

PB96-112040

03,474

ATHA, D. H.

Physical Characterization of Herparin by Light Scattering.
PB96-119383 SB96-119383

P3,480 Thermodynamic Analysis of Heparin Binding to Human Antithrombin.
PB94-199593

ATZMONY, $U$.

Magnetic and Structural Properties of Electrodeposited Copper-Nickel Microlayered Alloys.

PB94-21312

04,512

Growth of Epitaxial KNbO3 Thin Films

PB96-135181
AUSLOOS, P. J.

02,409

Ionization Energy of Sulfur Pentafluoride and the Sulfur Pentafluoride-Fluorine Atom Bond Dissociation Energy.
PB95-162814

AUST, J. A

Distributed Feedback Lasers in Rare-Earth-Doped Phosphate Glass.

04,740

Linewidth Narrowing in an Imbalanced $Y$-Branch Waveguide Laser

PB95-140844 
AVEDISIAN, C. T.

Analysis of Droplet Arrival Statistics in a Pressure-Atomized Spray Flame.

01,352

Combustion of Methanol and MethanolDodecanol Spray Flames.

PB95-108544

02,478

Effect of Dodecanol Content on the Combustion of Methanol Spray Flames.

01,389

Role of Combustion on Droplet Transport in Pressure-Atomized Spray Flames.

01,434

Study of Droplet Transport in Alcohol-Based Spray Flames Using Phase/Doppler Interferometry.

PB95-108551

02,479

AVERITT, R. D.

Photoluminescence Spectra of Epitaxial Single Crystal C60.

PB96-141205

AVGOLOUPIS, $S$.

Rotational Modulation and Flares on RS Canum Venaticorum and BY Draconis Stars. XVI. IUE Spectros. copy and VLA Observations of $\mathrm{C} 1182(=\mathrm{V} 1005$ Orionis) in
October 1983. PB94-185626

00,050

AVILA, M. J.

Experimental Validation of Radiopharmaceutical Absorbed Dose to Mineralized Bone Tissue.

PB94-199668

03,617

Radiation Doses.

PB94-199676

03,618

Surface Transverse Wave Oscillators with Extremely Low Thermal Noise Floors.

01,967

AVRAMOV, $S$.

Automatic Calibration of Inductive Voltage Dividers for the NASA Zeno Experiment.

PB95-152849

02,041

Automatic Inductive Voltage Divider Bridge Operates from $10 \mathrm{~Hz}$ to $100 \mathrm{kHz}$

02.032

Inductive Voltage Divider Calibration for the NASA Flight

Experiment.

PB95-152856

02,042

AVRAMOV-ZAMUROVIC, $S$.

Binary versus Decade Inductive Voltage Divider Comparison and Error Decomposition.

02,071

Exploring the Low-Frequency Performance of Thermal Converters Using Circuit Models and a Digitally Synthesized

PB97-112551

02,848

Low Voltage S

02,100

Proposed Tests to Evaluate the Frequency-Dependent Capacitor Ratio for Single Electron Tunneling Experiment.
PB97-111454 PB97-111454

$24 \mathrm{GHz}$ Josephson Array Voltage Standard.

PB94-211588

02,033

AXELBAUM, R. L.

In-situ Studies of a Novel Sodium Flame Process for Synthesis of Fine Particles.

00,681

Optical and Modeling Studies of Sodium/Halide Reactions tor the Formation of Titanium and Boron Nanoparticles. 00,682
PB97-113054

AXLEY, J. W.

New Mass Transport Elements and Components for the NIST IAQ Model.

PB95-255899

NIST-NRL Free-Electron Laser Facility.

PB94-21251

02,558

AYRES, T.

04,237

Sleuthing the Dynamo: HST/FOS Observations of UV Emis.

sions of Solar-Type Stars in Young Clusters.

PB96-122817

00.098

AYRES, T. R.

Deuterium and the Local Interstellar Medium: Properties for the Procyon and Capella Lines of Sight.

00,111

Distant Future of Solar Activity: A Case Study of Beta Hydri. 3. Transition Region, Corona, and Stellar Wind. PB94-185220

00,049

Distant Future of Solar Activity: A Case Study of beta Hydri. 3. Transition Region, Corona, and Stellar Wind.

00,074

B95-153441

00.086

PB96-102033

Goddard High-Resolution Spectrograph Observations of the Local Interstellar Medium and the Deuterium/Hydrogen

Ratio along the Line of Sight Toward Capella.

00,066
Hydrogen Lyman-alpha Emission of Capella.

Relationship between Radiative and Magnetic Fluxes for Three Active Solar-Type Dwarts.

00,097

Riass Coronathon: Joint $X$-ray and Ultraviolet Observations of Normal F-K Stars.

PB96-200217

Transition Regions of Capella.

PB96-123336

Transition Regions of Capella (1995).

PB96-176714

00,109

AZIZ, M. J.

Disorder Trapping in Ni2TiAl.

AZIZ, R. A.

Ab initio Calculations for Helium: A Standard for Transport Property Measurements.

PB96-102041

00,105

AZUMA, $Y$.

Evolution of $X$-ray Resonance Raman Scattering into $X$-ray Fluorescence from the Excitation of Xenon Near the L3

PBg6-102751 04,025

High-Energy Behavior of the Double Photoionization of He-

lium from 2 to $12 \mathrm{keV}$.

03,860

BA, w. $z$.

Thin Dyed-Plastic Dosimeter for Large Radiation Doses.

BABA, T.

High-Temperature Laser-Pulse Thermal Diffusivity ApparaPB94.185147

02,631

Thermal Diffusivity of POCO AXM-5Q1 Graphite in the Range 1500 to $2500 \mathrm{~K}$ Measured by a Laser-Pulse Technique.

BABCOCK, S. E.

Electromagnetic Coupling Character of (001) Twist Boundaries in Sintered Bi2Sr2CaCu2O8+x Bicrystals. Weak-Link-Free Behavior of High Angle YBa2Cu3O7-x Grain Boundaries in High Magnetic Fields.
PB94-198421

BABECKI, M. B. G.

Hair Testing for Drugs of Abuse: International Research on Standards and Technology.

PB96-120555

BABRAUSKAS, $v$.

Developing Rational Pertormance-Based Fire Safely Requirements in Model Building Codes. PB95-175220

00,200 Effective Measurement Techniques for Heat. Smoke, and
Toxic Fire Gases.
PB94-198439
01,369

Effects of Specimen Edge Conditions on Heat Release PBate. 152864

00,375

Fire Satety of Passenger Trains: A Review of Current Approaches and of New Concepts.

PB94-152006

04,848

Modern Test Methods for Flammability

PB94-198447

01,370

Standardization of Formats and Presentation of Fire Data The FDMS.

PB94-198462

01,371

Toxicity, Fire Hazard and Upholstered Fumiture. PB94-198454

00,289

BACH, B. W.

New cw CO2 Laser Lines: The 9-mu $m$ Hot Band. PB95-168548

04,281

BACH, $\mathrm{H}$.

Polarization Analysis of the Magnetic Excitations in Fe65Ni35 Invar.
PB95.150082

Temperature Dependence of the Magnetic Excitations in Ordered and Disordered Fe72Pt28.

PB95-150223

BACH, K. G.

New cw CO2 Laser Lines: The 9-mu $m$ Hot Band. PB95-168548

04,563

BACHEM, E.

Extension of Heterodyne Frequency Measurements on OCS to $87 \mathrm{THz}(2900 \mathrm{~cm}(-1))$

PB94-200680

BACKES, P. G.

Unified Telerobotic Architecture Project (UTAP) Standard Intertace Envir

BACON, D.

02,938

Crystal Packing Interactions of Two Different Crystal Forms of Bovine Ribonuclease A.

PB95-152823

00,943

BACON, M. C.

Nutritional Status and Growth in Juvenile Rheumatoid Arhritis.

PB94-198470

BADNELL, N. R.

Dielectronic Capture Processes in the Electron-Impact Ionization of $\mathrm{Sc}(2+)$.

BADZIAN, A.

Analysis of Boron in CVD Diamond Surfaces Using Neutron Depth Profiling.

PB94-213089

BADZIAN, T.

Analysis of Boron in CVD Diamond Surfaces Using Neutron PB94-213089.

BAECHTEL, F. S

Interlaboratory Comparison of Autoradiographic DNA Profiling Measurements. 1. Data and Summary Statistics. PB95-175923

03,542

BAGCHI, $\mathrm{S}$.

Mechanism of Defect Formation in Low-Dose Oxygen Implanted Silicon-on-Insulator Material.

PB97-111462

02,453

BAGG, T. C.

Dilemma-Preservation versus Access.

PB94-198488

02,711

Security in Open Systems

BAGWILL, R. H.

Electronic Implementors' Workshop.

PB95-210936

Sharing Information via the Internet: An Infoserver Case Study.

PB96-13151

01,493

BAHEL, A.

Energy Dependence of Collision Characteristics in Molecule-Surtace Collisions.

BAHN, $W$. L

Electromechanical properties of superconductors for DOE fusion applications.

DE95015476

04,432

Electromechanical Properties of Superconductors for DOE Fusion Applications.

PB94-13967

02,250

BAHR, R.

Laser-Produced and Tokamak Spectra of Lithiumlike Iron, $\mathrm{Fe}(23+)$

BAKER, E. F.

Application of ODF to the Rietveld Profile Refinement of Polycrystalline Solid

PB95-202388

03,401

BAKER, E. L.

Microstructure Study of Molybdenum Liners by Neutron Dit fraction.

PB95-202396

03,756

Texture Study of Two Molybdenum Shaped Charge Liners by Neutron Diffraction.

BAKER-JARVIS, J.

Analysis of an Open-Ended Coaxial Probe with Lift-Off for Nondestructive Testing.

PB96-135116

01,940

Applicability of Effective Medium Theory to Ferroelectric Ferrimagnetic Composites with Composition and Frequency-Dependent Complex Permittivities and Permeabilities.

Dielectric and Magnetic Measurements from $-50 \mathrm{C}$ to $200 \mathrm{C}$ and in the Frequency Band $50 \mathrm{MHz}$ to $2 \mathrm{GHz}$. PB96-191382

Distribution of Dielectric Relaxation Times and the Moment Problem.

PB96-112032

04,727

Effective Medium

01,893

Preparation, Crystal Structure, Dielectric Properties, and Magnetic Behavior of Ba2Fe2Ti4O13. 
BALASUBRAMANIAN, R.

Comparison of Techniques for Nondestructive Composition Measurements in CdZnTe Substrates.

PB96-103098

BALASUBRAMANIAN, $V$.

Energy Dependence of Collision Characteristics in Molecule-Surface Collisions.

PB94-198504

00,786

BALDONI, J. G.

Tensile Creep of Whisker Reinforced Silicon Nitride.

PB94-211984

03,142

BALDWIN, J. R.

Standard Reference Materials: Glass Filters as a Standard Reference Material for Spectrophotometry - Selection. Preparation, Certification, and Use of SRM 930 and SRM 1930

PB94-188844

00,536

BALIZER, E.

Mysteresis Measurements of Remanent Polarization and Coercive Field in Polymers.

PB94-199767

BALLINGER, R. G.

Charpy Impact Test as an Evaluation of $4 \mathrm{~K}$ Fracture Toughness.

BALLSCHMITER, $\mathrm{K}$.

03,219

Comparison of Methods for Gas Chromatographic Determination of PCBs and Chlorinated Pesticides in Marine Reference Materials.

PB95-140091

02,584

BALSARA, N. P.

Thermodynamic Interactions and Correlations in Mixtures of Two Homopolymers and a Block Copolymers by Small Angle Neutron Scattenng.

PB95.152872

01,247

Thermodynamic Interactions in Model Polyolefin Blends Obtained by Small-Angle Neutron Scattering.

01,208

BALTATU, M. E.

Viscosity of Defined and Undefined Hydrocarbon Liquids Calculated Using an Extended Corresponding-States Model

BALTZER, $P$.

Inner-Valence States $\mathrm{CO}(+)$ between $22 \mathrm{eV}$ and $46 \mathrm{eV}$ Studied by High Resolution Photoelectron Spectroscopy and $a b$ initio $\mathrm{Cl}$ Calculations.

PB95-180055

03,961

BALZAR, D

Accurate Modeling of Size and Strain Broadening in the Rietveld Refinement: The 'Double-Voigt' Approach.

PB96-200225

00.664

Crystal Structure and Compressibility of 3:2 Mullite.

PB95-175030

03,682

Enhanced Flux Pinnıng via Chemical Substitution in Bulk Superconducting T1-2212.

Microstrains and Domain Sizes in Bi-Cu-O Superconductors: An X-Ray Diffraction Peak-Broadening Stud

PB94-198520 04,461

Profile Fitting of X-Ray Diffraction Lines and Fourier Analysis of Broadening.

04,460

Residual Stresses in Aluminum-Mullite (alpha-Alumına) Composites.

PB95-152880

03.155

Voigt-Function Modeling in Fourier Analysis of Size- and Strain-Broadened X-Ray Diffraction Peaks.

PBin-Broade

04,462

\section{BAN, Y. B.}

Neutron Scattering Study of Shear Induced Turbidity in Pol. ystyrene/Dioctyl Phthalate Solutions at High Shear Rates. PB94-172624
01,197 Neutron Scattering Study of Shear Induced Turbidity in Polystyrene Dissolved in Dioctyl Phthalate.

PB95-161865

01,256

\section{BAND, A} Closed Loop Controller for Electron-Beam Evaporators.
PB97-111470 04,393

BAND, Y. B.

Density Matrix Calculation of Population Transfer between Vibrational Levels of $\mathrm{Na} 2$ by Stimulated Raman Scattering with Temporally Shifted Laser Beams.

00,787

Excitons in Complex Quantum Nanostructures.

PB97.118343

01,184

BANDY, $H$. T.

Compensation of Errors Detected by Process-Intermittent

PB97-110472

Data Management for Error Compensation and Process Control.

02,847

PIECS: A Software Program for Machine Tool ProcessIntermittent Error Compensation.

02,842
BANG, C. A.

Thermal Isolation of High-Temperature Superconductıng Thin Films Using Siticon Wafer Bonding and Micromachining

PB96-135017

02,408

NNISTER, $M$.

Collisions of Electrons with Highly-Charged lons.

PAN96-200340

04,791

Absolute Cross Sections for Electron-Impact Single Ionization of $\mathrm{Si}(+)$ and $\mathrm{Si}(2+)$

PB95-202529

03,982

Backscattering in Electron-Impact Excitation of Multiply Charged lons
PB94-185345

03,799

Evidence for Significant Backscattering in Near-Threshold Electron-Impact Excitation of $\operatorname{Ar}(7+)(3$ s yields $3 p)$.
PB95-126405

03.883
Ion Ex.

Merged-Beams Energy-Loss Technique for Electron-Ion Excitation: Absolute Total Cross Sections for $\mathrm{O}(5+)$ (2s yields
2p).
PB96-102058

Resonance Structure and Absolute Cross Sections in NearThreshold Electron-Impact Excitation of the $4 s(2)$ (1) $S$ yields $4 \mathrm{~s} 4 \mathrm{p}$ (3)P Intercombination Transition in $\operatorname{Kr}(6+)$.
PB95-202271

BARBEE, T. W.

Experimental Constraints on Some Mechanisms for HighTemperature Superconductivity.

04,463

XUV Characterization Comparison of Mo/Si Multilayer Coatings.

PBPB-16

04,278

ERA, A. J.

Continuous Mining Machine Control Using the Real-Time Control System.

PB94-203528

03,700

Effect of Formulation Changes on the Response to lonizing Radiation of Radiochromic Dye Films.

PB97-119028

04,171

BARCO, M. T.

Wear of Human Enamel against a Commercial Castable Ceramic Restorative Material.

PB95.161972

00,161

BARD, A

Microlithography by Using Neutral Metastable Atoms and Self-Assembled Monolayers.

PB96-190038

02.441

BARILO, S. N.

Magnetic Structure and Spin Dynamics of the $\mathrm{Pr}$ and $\mathrm{Cu}$ in

PB96-111836

04.036

ARKER, E.

EDI and EFT Security Standards.

PB96-122833

01,605

BARKER, J. G.

Instrumental Smearing Effects in Radially Symmetric SmallAngle Neutron Scattering by Numerical and Analytical Methods.

96- 160429

02,984

Small Angle Neutrons Scattering from Nanocrystalline Palladium as a Function of Annealing.

PB95-176103

03,354

BARKLEY, J.

Distributed Communication Methods and Role-Based ACcess Control for Use in Health Care Applications.

PB96-183165

Security in Open Systems.

PB95-105383

01,508

BARKLEY, J. F.

01,473

Comparing Remote Procedure Calls: Open Network Computing, Distributed Computing Environment and International Organization for Standardization.

PB95-194205

01,724

BARKMEYER, E.

Control Entity Interiace Specification.

PB94-191715

02,815

BARKMEYER, E. J.

Requisite Elements. Rationale, and Technology Overview or the Systems Intergration for Manufacturing Applications (SIMA) Program. Background Study

PB96- 112685

02,831

Effect of Microstructure on Phase Formation in the Reaction of $\mathrm{Nb} / \mathrm{Al}$ Multilayer Thin Films.

PB95-168415 03,352

First Phase Formation Kinetics in the Reaction of Nb/Al.

$\begin{array}{ll}\text { PB95-168456 03.353 } & \end{array}$

Thin Film Reaction Kinetics of Niobium/Aluminum Multilayers.

BARNARD, J. A.

Size and Self-Field Effects in Giant Magnetoresistive ThinFilm Devices.

04.674

BARNEA, G.

Study of Multiple Scattering Background in Compton Scatter Imaging.

PB96-112222

BARNES, C. $S$.

04,425

Binary Decision Clustering for Neural Network Based Optical Character Recognition.

01,848

Binary Decision Clustering for Neural-Network-Based Optical Character Recognition.

PB96-186184

01,857

Face Recognition Technology for Law Enforcement Applications.

BARNES, F.

01,837

Dielectric Properties of Thin Film SrTiO3 Grown on LaAlO3 with YBa2Cu3O7-x Electodes.

PB95-181160
BARNES, G.

Critical Current and Normal Resistance of High-Tc StepEdge SNS Junctions.

PB96-111752

04,724

ARNES, $J$

Precision Oscillators: Dependence of Frequency on Temperature, Humidity and Pressure.

PB94-198306

Synthesis of Hybrid Organic-Inorganic Materials from

Interpenetrating Polymer Network Chemistry.
PB96-180054

02,031

BARNES, K. A

02,994

Flow-Induced Structure in Polymers: Chapter 16. Shear-Induced Changes in the Order-Disorder Transition Temperature and the Morphology of a Triblock Copolyme

PB96-123237

03,127

Shear-Excited Morphological States in a Triblock Copoly-

PB94-172392

01,196

Shear-Induced Martensitic-Like Transformation in a Block Copolymer Melt.

PB96-119508

BARNES, P. Y.

01,277

$45 \mathrm{deg} / 0$ deg Reflectance Factors of Pressed Polyletrafluoroethylene (PTFE) Powder.

PB95-260758

BARNETT, J. P.

04.328

Using Emulator/Testers for Commissioning EMCS Software, Operator Training, Algorithm Development, and Tuning Local Control Loops.

BAROGHEL-BOUNY, $v$.

00,245

Modelling Drying Shrinkage of Cement Paste and Mortar. Part 1. Structural Models from Angstroms to Millimeters. PB96-135132

BAROUCH, E.

Numerical Simulation of Submicron Photolithographic Processing.

BARR, R. M.

02,310

Regulation of Lithium and Boron Levels in Normal Human Blood: Environmental and Genetic Considerations.

PB94-198579 


\section{PERSONAL AUTHOR INDEX}

BECKER, D. A

Polarization of Light Emitted After Positron Impact Excitation of Alkali Atoms

03,816

Relativistic Effects in Spin-Polarization Parameters for LowEnergy Electron-Cs Scattering

03,901 PB95-150868

Short-Range Correlation and Relaxation Effects on the (6p (2))(1) SO Autoionizing State of Atomic Barium.

BARYSCHPOLEC, S. W.

Radiation Process Data: Collection, Analysis, and Interpretation

PB95-162632

03,628

BASCH, $\mathrm{H}$.

Strong Hydrogen Bond in the Formic Acid-Formate Anion PBg4-198595

00,788

Structure of Glycine-Water H-Bonded Complexes.

PB94-198603

00,789

ASKO, M. M.

Physics and Prospects of Inertial Confinement Fusion.
PB94-185048 BASRI, G.

Sleuthing the Dynamo: HST/FOS Observations of UV Emissions of Solar-Type Stars in Young Clusters.

PB96-122817

00,098

BASRI, G. S.

Far-Ultraviolet Flare on a Pleiades G Dwart

PB96-102033

BASSHAM, L. E.

Precise Identification of Computer Viruses.

PB96-160825

00,086

STEP

PB94-139649

tions of

02,766

BASTIAN, M. J.

Laser-Induced Fluorescence Measurements of Rotationally Resolved Velocity Distributions for $\mathrm{CO}(+)$ Drifted in $\mathrm{He}$ PB94-213139

00,848

BASTIAN, T. S.

Class of Radio-Emitting Magnetic B Stars and a Wind-Fed Magnetosphere Model.

00,067

Radio Emıssion from Chemically Peculiar Stars PB94-213469

00,068

BASTIE, J.

Comparison of Regular Transmittance Scales of Four National Standardizing Laboratories.

BATES, F. S.

Grazing Incidence Prompt Gamma Emissions and Resonance-Enhanced Neutron Standing Waves in a Thin-Film.

PB95-150470 03,892

BATRA, P.

Optical Density Measurements of Laser Eye Protection Materials

PB96-190301

00,190

BAUER, B.

Relaxation After a Temperature Jump Within the One Phase Region of a Polymer Mixture.

PB97-112494

03.394

Cylinder Wipe Air-Drying Intaglio Ink Vehicles for U.S. Currency Inks.

Electron Beam Crosslinking of Poly(vinylmethyl ether).

PB94-185550

01.205

Grafted Interp

01,200

Slow Dynamics of Segregation in Hydrogen-Bonded Polymer Blends.

01.281

Synthesis of Hybrid Organic-Inorganic Materials from Interpenetrating Polymer Network Chemistry.

02.994

Dispersion and Deposition of Smoke Plumes Generated in Massive Fires

PB95-126066

02,540

Ignition and Subsequent Transition to Flame Spread in a Microgravity Environment.

04,828

Smoke Emission from Burning Crude Oil

PB96-122890

01,407

BAUM, H. R.

Application of Boundary Element Methods to a Transient Axis-Symmetric Heat Conduction Problem.

PB94-212693

01,375

Gravity-Current
PB96-147046

01,415

Heat Transfer in an Intumescent Material Using a Three-Dimensional Lagrangian Model.

00,408

Ignition and Subsequent Flame Spread Over a Thin Cellulosic Material.

04,836
Ignition and Transition to Flame Spread Over a Thermally Thin Cellulosic Sheet in a Microgravity Environment. PB96-160288

04,837

In situ Burning of Oil Spills: Mesoscale Experiments and Analysis.

02,587

Large Eddy Simulations of Smoke Movement in Three Di-

PB96-190012

01,426

Mathematica! Modeling and Computer Simulation of Fire

Phenomena.
PB95-180063 00,384

Numerical Simulation of Rapid Combustion in an Underground Enclosure.

PB96-183132

01,424

Transition from Localized Ignition to Flame Spread Over a Thin Cellulosic Material in Microgravity.

PB96-155809

04.835

Transport by Gravity Currents in Building Fires.

BAUM, M. S

01,441

Federal Certification Authority Liability and Policy: Law and Policy of Certificate-Based Public Key and Digital Signa-

PB94-191202

01,578

BAUMERT, $T$.

Femiosecond Time-Resolved Molecular Multiphoton Ioniza. tion: The Na2 System.

PB95-202305 03,975

Femtosecond Time-Resolved Wave Packet Motion in Molecular Multiphoton Ionization and Fragmentation.

PB94-198611

00,790

\section{BAUMONT, $F$.}

Preliminary Comparison of Time Transfers via LASSO, GPS and Two-Way Satellite

and Two-Way

01,529

BAUR, W. H.

Neutron Powder Diffraction Study of a Na, Cs-Rho Zeolite.

PB94-198629

00,791

BAUSUM, H. T.

Determination of 3-Ouinuclidinyl Benzilate (Onb) and Its Major Metabolites in Urine by Isotope Dilution Gas Chroma lography Mass Spectrometry.

PB94-199379

03,492

\section{BAYLEY, $\mathrm{H}$.}

Genetically Engineered Pore as a Metal Ion Biosensor.

PB96-161658 03,55

Genetically Engineered Pores as Metal Ion Biosensors.

PB96-167408

03,553

Genetically Engineered Pores for New Materials.

PB96-161641

Pore-Forming Protein with a Metal-Actuated Switch

B96-176557

03,550

EACH, R. $\mathbf{S}$

Enhanced Curie Temperatures and Magnetoelastic Domains in Dy/Lu Super Lattices and Films.

PB94-172665

04,443

BEAHN, T. J.

Magneto-Optic Magnetic Field Sensor with $1.4 \mathrm{pT} / \mathrm{square}$ root of $1(\mathrm{~Hz})$ Minimum Detectable Field at $1 \mathrm{kHz}$.

PB94-19955

02,125

BEALE, P. D.

Bias Current Dependent Resistance Peaks in NiFe/Ag Giant Magnetoresistance Multilayers.

04,153

BEALL, J.

Dielectric Properties of Thin Film $\mathrm{SrTiO}_{3}$ Grown on $\mathrm{LaAlO} 3$ with YBa2Cu3O7-x Electodes.

PB95-181160

02,267

BEALL, J. A.

Characterization of a Tunable Thin Film Microwave YBa2Cu3O7-x/SrTiO3 Coplanar Capacito

02,264

Critical Current Behavior of Ag-Coated YBa2Cu3O7-x Thin Films.

PB95-141016 04.549

Ferroelectric Thin Film Characterization Using Superconducting Microstrip Resonators.

02,270

High Critical Temperature Superconductor Tunneling Spectroscopy Using Squeezable Electron Tunneling Junctions. PB95-163721

Increased Transition Temperature in In situ Coevaporated YBa2Cu307-delta Thin Films by Low Temperature Post-An nealing.

B95-180071

04,672

Magnetic Field Dependence of the Critical Current Anisotropy in Normal Metal-YBa2Cu3O7-delta Thin Film Bilayers. Microwave Properties of Voltage-Tunable $\mathrm{YBa} 2 \mathrm{Cu} 307$. delta/SrTiO3 Coplanar Waveguide Transmission Lines. PB96-141262 02,235

Tunable High Temperature Superconductor Microstrip Resonators

02,048

BEAN, J.W.

Optical Performance of Commercial Windows.

PB95-208757

00,392

BEAN, V. E.

Development of the NIST Transient Pressure and Temperature Calibration Facility

00,626

Executive Summary: Proceedings of the Workshop on the Executive Summary: Proceedings of the Workshop on the Measurement of Transient Pressure and Temperature.
PB96-160841 NIST Measurement Services: NIST Pressure Calibration Service

PB94-164043

02,892

Pressure Measurements with the Mercury Melting Line Reterred to ITS-90.

PB96-161005

01,136

BEARDEN, D. W

Influence of Surface Interaction and Chain Stiffness on Polymer-Induced Entropic Forces and the Dimensions of Confined Polymers.

PB94-185469

01.203

ARY, E. S.

Comparative Strategies for Correction of Interferences in Isotope Dilution Mass Spectrometric Determination of Vana-

PB94-18526

00,531

BEATON, S. P.

Determination of the Molecular Parameters of $\mathrm{NiH}$ in its (2)Delta Ground State by Laser Magnetic Resonance

PB95-107116 00,869

High-Order Harmonic Mixing with GaAs Schottky Diodes.

PB95-108585

01,528

Rotational Spectroscopy of the $\mathrm{CoH}$ Radical in Its Ground (3) Phi State by Far-Infrared Laser Magnetic Resonance:

Determination of Molecular Parameters.

PB95-175048

00,992

Rotational Spectrum of Copper Hydride Using Tunable Fa Infrared Radiation.

PB94-198637

BEAUCHAMP, C. R.

00,792

Electrochemical Synthesis of Metal and Intermetallic Composites

AD-A294 088/0

03,304

EAVER, E. A

Observations of $3 \mathrm{C} 273$ with the Goddard High Resolution Spectrograph on the Hubble Space Telescope.

PB95-20232

00,076

AZLEY, W. G.

Framework for Information Technology Integration in Process Plant and Related Industries.

PB94-219086

02,772

BECHER, P. F

Matrix Grain Bridging Contribution to the Toughness of Whisker Reinforced Ceramics.

PB94-198645

03,134

BECHIS, D. J. 
Determination of 21 Elements by INAA for Certification of SRM 1570a, Spinach

PB96-167242

00,698

New NIST Rapid Pneumatic Tube System.

PB96-167259

03,738

Recent Developments in NIST Botanical SRMs

PB96-167267

03,489

Resolution of Discrepant Analytical Data in the Certification of Platinum in Two Automobile Catalyst SRMs.

00,638

Results of the ASTM Nuclear Methods Intercomparison on NIST Apple and Peach Leaves Standard Reference Mate-

03,490

Role of Certified Reference Materials in Trace Analysis $\begin{array}{ll}\text { Ouality Assurance. } & \\ \text { PB97-110019 } & 00,650\end{array}$

00,650
Unique Ouality Assurance Aspects of INAA for Reference Unique Ouality Assurance Aspects of INAA for Reference
Material Homogeneity and Certification.
PB96-200811 00,699

BECKER, P. R.

Alaska Marine Mammal Tissue Archival Project: Specimen Inventory.

02,589

Concentrations of Chlorinated Hydrocarbons, Heavy Metals and Other Elements in Tissues Banked by the Alaska Ma-
rine Mammal Tissue Archival Project.

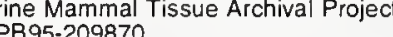

02.590

Determination of Inorganic Constituents in Marine Mamma

PB95-152047

00,589

Determination of PCBs and Chlorinated Hydrocarbons in Marine Mammal Tissues.

PB95-162640

03,744

Relationship of Silver with Selenium and Mercury in the Liver of Two Species of Toothed Whales (Odontocetes). PB96-167275

Trace Element Concentrations in Cetacean Liver Tissues Archived in the National Marine Mammal Tissue Bank.
PB96-167127

02,595

Discovery of an X-Ray Selected, Radio-Loud Ouasar at $z=3.9$

PB94-198652

00,052

New High-Redshitt Damped Lyman-alpha Absorption Systems and the Redshift Evolution of Damped Absorbers. PB95-203501

00,083

BECKERLEY, J. G

Preliminary List of References Containing Compilations of Data on Properties of Materials.

AD-A302 670/5

03,243

Preliminary Subject and Authors Index to Compilations of

Data on Properties of Materials.

03,242

$\mathrm{BEECH}, \mathrm{F}$.

Neutron Powder Diffraction Study of the Structures of La 1.9Ca1.1Cu2O6 and La1.9Si1.1Cu2O6+Delta.

PB95-140042

04,538

BEERS, J.

Verification of Revised Water Vapour Correction to the Index of Refraction of Air.

PB96-161666

02,680

BEERS, J. S.

Evolution of Automatic Line Scale Measurement at the $\mathrm{Na}$ ional Institute of Standards and Technology.

PB95-108809

02,897

Gage Block Handbook

PB95-251716

02,667

Measuring Long Gage Blocks with the NIST Line Scale InPB95-242400

02,665

BEESABATHINA, D. P.

Characterization of the $\mathrm{ZnSe} / \mathrm{GaAs}$ Interface Layer by TEM and Spectroscopic Ellipsometry.

PB95- 175360

04,655

BEGLEY, E. F.

Database Development and Management (Project A.2.2) The Annual Report for 1992-1993.

PB97-110290

Guide to Locating and Accessing Computerized Numeric Materials Databases.

PB96-204045

03.007

Multimedia Tutorial on Phase Equilibria Diagrams.

PB96-200829

03,088

Structural Ceramics Database. Topical Report, June 1989 May 1991.

03.060

BEHLKE, M. K.

Investigations of Sulfur Interferences in the Extraction of Methylmercury from Marine Tissues.

03,482

BEHRENS, $\mathrm{H}$.

Data Import and Validation in the Inorganic Crystal StrucPB 97.109201

04,809

\section{BEICHL, I.}

Data-Parallel Algorithm for Three-Dimensional Delaunay Triangulation and Its Implementation.

PB95-163309

01,714

Faster BKL Monte Carlo Simulations.

PB95-136370

01,706

Faster Monte Carlo Simulations.

B96-102074

Heap of Data.

PB97.111488

Making Connections.

Self-Avoiding Surfaces, Topology, and Lattice Animals

PB95-150512

04,571

Submissions to a Planned Encyclopedia of Operations Research on Computational Geometry and the Voronoi/ Delaunay Construct.

PB94-152709

03,425

Tree-Lookup for Partial Sums Or: How Can I Find This Stuff

PBickly.

01,770

BEICHL, I. M.

Computing S(alpha) Using Symbolic Monte Carlo

PB94-198660

Parallel Monte Carlo Simulation of MBE Growth

PB96-122841

03,410

BELIC, D. S

Merged-Beams Energy-Loss Technique for Electron-Ion Excitation: Absolute Total Cross Sections for $O(5+)$ (2s yields

2p).

04,017

\section{BELL, B.}

Exploring the Low-Frequency Performance of Thermal Converters Using Circuit Models and a Digitally Synthesized Source.

PB97-112551

02,848

BELL, $D$.

Thermodynamics of Enzyme-Catalyzed Reactions: Part 1. Oxidoreductases.
PB94-162252

BELL, E. W.

00,737

Absolute Cross-Section Measurements for Electron-Impact lonization of $\mathrm{C} 1(+1)$

PB94-199841

03,818

Absolute Cross-Section Measurements for Electron-Impact Single lonization of $\mathrm{Se}(t)$ and $\mathrm{Te}(t)$.

PB95-202503

03,980

Absolute Cross Sections for Electron-Impact Single Ionization of $\mathrm{Si}(t)$ and $\mathrm{Si}(2+)$

PB95-202529

03,982

Backscattering in Electron-Impact Excitation of Multiply Charged lons

Beam Line for Highly Charged lons.

03,799

PB97-111256

04,143

Crossed-Beams Measurements of Absolute Cross Sections

for Electron Impact Ionization of $\mathrm{S}(+)$

PB95-202511

03,981

Electron-Impact Ionization of $\ln (+)$ and $\mathrm{Xe}(+)$.

00,770

Electron-Impact Ionization of $\ln (+)$ and $X e(+)$.

03,918

PB95-152906

Evidence for Significant Backscattering in Near-Threshold Electron-Impact Excitation of $\operatorname{Ar}(7+)(3 s$ yields $3 p)$.

PB95-126405

03,883

Merged-Beams Energy-Loss Technique for Electron-Ion Excitation: Absolute Total Cross Sections for $\mathrm{O}(5+)$ (2s yields 2p).

PB96-102058

04,017

BELLAMA, J. $M$.

L-threo-beta-Hydroxyhistidine, an Unprecedented Iron(III) lon-Binding Amino Acid in a Pyoverdine-type Siderophore irom Pseudomonas fluorescens 244.

PB94.211620

00,553

Potentiometric Enzyme-Amplified Flow Injection Analysis Detection System: Behavior of Free and Liposome-Released Peroxidase.

PB95-151833

03,534

Total Surface Areas of Group IVA Organometallic Compounds: Predictors of Toxicity to Algae and Bacteria. PB94-211331

00,814

BELLISSENT, $R$.

Inelastic Neutron Scattering Measurements of Phonons in cosahedral Al-Li-Cu.

Neutron Scattering Structural Siudy of AlCuFe Ouasicrystals Using Double Isotopic Substitution.

RDFs and $\mathrm{Fe} \cdot \mathrm{Fe}$ Pair Correlations in an $\mathrm{AlCuFe}$ Icosahedral Alloy by Double Isotopic Substitution. B94-172129

04,439

BELMONT, G.

Effect of Formulation Changes on the Response to lonizing Radiation of Radiochromic Dye Films.

04,171

BELOV, S. P

Tunneling-Rotation Spectrum of the Hydrogen Fluoride

PB94-198678

00,793

BELTRACCHI, L.

Analysis of Standards for the Assurance of High Integrity

$\begin{array}{ll}\text { PB96-161351 03,735 } & \end{array}$

Control and Instrumentation: Standards for High-Integrity

03,736

Proceedings of the Digital Systems Reliability and Nuclear

Safety Workshop. Held in Rockville, Maryland on Septem-

ber 13-14, 1993.

NUREG/CP.0136

03,728

Standards for High Integrity Sottware.

PB96-161385

01,764

BENCK, E. C.

Optical and Mass Spectrometric Investigations of lons and Neutral Species in SF6 Radio-Frequency Discharges.

PB97-111918

01,985

BENDEC, $F$.

Ultrasonic Spectroscopy of Metallic Spheres Using Electro.

PB94-212537

\section{PER, $P$.}

Low-Frequency, Active Vibration Isolation System

PB95-203303

04,185

Path Aimospheric Refraction Calibrator: An Instrumen Microwave Propagation Delays Induced by Atmospheric Water Vapor.

PB 95-151270

01,476

Integrated Laser Doppler Method for Measuring Planetary Gravity Fields.

PB94-198686

03,681

Lunar Laser Rangıng: A Continuing Legacy of the Apollo Program.

BENDERSKY, L. A.

03,683

Coherent Precipitates in the BCC/Orthorhombic Two Phase Field of the Ti-Al-Nb System.

PB94-198694

03,317

Crystallographic Characterization of Some Intermetallic Compounds in the Al-Cr System.

PB94-198702

03,318

Disorder Trapping in Ni2TiAl.

Ordered omega-Derivatives in a Ti-37.5Al-20 Nb at\% Alloy. 
Standard Reference Materials for the Determination of Polycyclic Aromatic Hydrocarbons in Environmental Samles - Current Activities.

PB95-151668

BENNETT, G. E.

Time and Frequency: Bibliography of NIST Publications,

PB95-220463

BENNETT, H. S.

Can Displays Deliver a Full Measure: Manufacturing. 02,185

Effects of Heavy Doping on Numerical Simulations of Gallium Arsenide Bipolar Transistors.

PB95-150975

02,334

Majority and Minority Electron and Hole Mobilities in Heavily Doped Gallium Aluminum Arsenide.

04,814

Majority and Minority Mobilities in Heavily Doped Silicon for Device Simulations

PB94-198728

02,31

Making Displays Deliver a Full Measure.

01,490

Physics for Device Simulations and Its Verification by Meas-

PBrements.

02,327

Meas-

Physics tor

PB95-152914

02,339

BENNETT, J.

Integration of Scanning Tunneling Microscope Nanolithography and Electronics Device Processing.
PB95-153359 347

Relative Sensitivity Factors and Useful Yields for a Microfocused Gallium Ion Beam and Time-of-Flight Secondary Ion Mass Spectrometer.

PB94-198736

BENNETT, K. W.

00.541

Microstructure and Ferroelectric Properties of Lead Zirconate-Titanate Films Produced by Laser Evaporation.

PB94-199148

04,470

BENNETT, L. H.

Determination of Thermoactivation Parameters of Vortex Mobility in YBa2Cu3O7 Using Only Magnetic Measure-

Effect of Sm2BaCuO5 on the Properties of Sintered (Bulk)

PB96-119441

Experımental Verification of a Vector Preisach Model.

04,626

Langevin Approach to Hysteresis and Barkhausen Modeling

PB94-185675

03,206

Magnetic and Structural Properties of Electrodeposited Copper-Nickel Microlayered Alloys.

04,512

Magnetocaloric Effect in Nanocomposites.

04,618

Magnetocaloric Effect in Rapidly Solidified Nd-Fe-Al-B Ma-

terials.
PB94-185667 04,457

Meissner, Shielding, and Flux Loss Behavior in Single-Crystal YBa2Cं $u 306+x$

PB94-198744

04,464

Monte Carlo and Mean-Field Calculations of the Magnetocaloric Effect of Ferromagnetically Interacting Clusters.

03,201

Nanocomposite Magnetic Materials.

PB95-162780

04.617

Temperature Dependence of Vortex Twin Boundary Interaction in Yttrium Barium Copper Oxide (YBa2Cu3O6+x).

PB95-162442

04,613

BENNETT, P. $S$.

New Surface-Active Comonomer for Adhesive Bonding.
PB96-204052

BENNETT, W. $\mathbf{R}$.

Concurrent Enhancement of Kerr Rotation and Antiferromagnetic Coupling in Epitaxial $\mathrm{Fe} / \mathrm{Cu} / \mathrm{Fe}$ Structures. PB94-198769 04,466

Magnetic Properties of $\mathrm{Pd} / \mathrm{Co}$ Multilayers.

PB94-198751

04,465

Fabrication of Flaw-Tolerant Aluminum-Titanate-Reinforced

Alumina.
PB95-162533 03,161

Flaw-Insensitive Ceramics.

03,095

Flaw-Tolerance and Crack-Resistance Properties of Alumina-Aluminum Titanate Composites with Tailored Microstructures.

03,101

Loading Device for Fracture Testing of Compact Tension Specimens in the Scanning Electron Microscope.

PB95-162434

02,652

Model for Toughness Curves in Two-Phase Ceramics. 1.

Basic Fracture Mechanics.

03,085
Model for Toughness Curves in Two-Phase Ceramics. 2 Microstructural Variables.

03,078

Objective Evaluation of Short-Crack Toughness Curves Using Indentation Flaws: Case Study on Alumina-Based Ceramics.

PB96-179429

03,079

BENOIT, $H$

Neutron Scattering by Multiblock Copolymers of Structure A-B)N-A.

PB94-211547

01,219

ENSEN, D.

Commentary on 'Optimization of Experimental Parameters or the EPR Detection of the Cellulosic Radical in Irradiated Foodstuffs'.

BENSEN, D. L

00,043

Investigation of Applicability of Alanine and Radiochromic Detectors to Dosimetry of Proton Clinical Beams. PB96-146782

03,636

\section{BENT, B. E.}

Silicon Surface Chemistry by IR Spectroscopy in the Mid- to Far-IR Region: $\mathrm{H} 2 \mathrm{O}$ and Ethanol on $\mathrm{Si}(100)$

PB96-138565

01,097

BENTZ, D.

Development of a Method for Measuring Water-Stripping Resistance of Asphalt/Siliceous Aggregate Mixtures.

PB96-197249

01,348

Development of a Method for Measuring Water-Stripping Resistance of Asphalt/Siliceous Aggregate Mixtures.

PB96-202296

01,329

Digitized Simulation of Mercury Intrusion Porosimetry.

01,304

Percolation and Pore Structure in Mortars and Concrete. PB95-150439

00,370

NTZ, D. P.

Application of Digital-Image-Based Models to Microstructure, Transport Properties, and Degradation of Cement-Based Materials.

00,406

Calculation of the Thermal Conductivity and Gas Permeability in a Uniaxial Bundle of Fibers.

PB95-180931

03,058

Cellular Automaton Simulations of Cement Hydration and Microstructure Development.

PB95-175055

01,320

Computer Simulation of the Diffusivity of Cement-Based Materials

PB95-125985

00,362

Computer Simulations of Binder Removal from 2-D and 3-D Model Particulate Bodies.

PB97-121339

00,418

Cross-Property Relations and Permeability Estimation in Model Porous Media.

04,205

Diffusion Studies in a Digital-Image-Based Cement Paste Microstructural Model

PB94-198801

01,312

Digital Simulation of the Aggregate-Cement Paste Interfacial Zone in Concrete.

PB95-125993

00,363

Digitized Direct Simulation Model of the Microstructural Development of Cement Paste.

PB94-198777

01,309

Digitized Simulation Model for Microstructural Development. PB94-198785

01310

Evolution of Porosity and Calcium Hydroxide in Laboratory Concretes Containing Silica Fume.

01,321

Fundamental Computer Simulation Models for CementBased Materials.

PB95.126009

00,364

Hydraulic Radius and Transport in Reconstructed Mode Three-Dimensional Porous Media.

PB96-123419

00.403

Interfacial Transport in Porous Media: Application to dc

Electrical Conductivity of Mortars.

Modelling Drying Shrinkage of Cement Paste and Mortar

Part 1. Structural Models from Angstroms to Millimeters.

PB96-135132

03.074

Modelling the Leaching of Calcium Hydroxide from Cement Paste: Effects on Pore Space Percolation and Diffusivity.

Multi-Scale Picture of Concrete and Its Transport Properties: Introduction for Non-Cement Researchers.

PB97-115802

03,107

Survey of Recent Cementitious Materials Research in Western Europe

00,353

Transport and Diffusion in Three-Dimensional Composite Media.

PB95-176129

04,668

Unreacted Cement Content in Macro-Defect-Free Composites: Impact on Processing-Structure-Property Relations.
PB96-141270

Water Permeability and Chloride Ion Diffusion in Portland Cement Mortars: Relationship to Sand Content and Critical Pore Diameter

PB96-148036

03,193

BENZ, S. P.

Characterization of the Emission from 2D Array Josephson

Oscillators.

02,054

Design of High-Frequency, High-Power Oscillators Using Josephson-Junction Arrays.

PB96-200258

02,094

Direct Observation of Vortex Dynamics in Two-Dimensional

Josephson-Junction Arrays.

PB96-102223

02,067

Emission Linewidth Measurements of Two-Dimensional Array Josephson Oscillators.

PB95-175139

02,055

Experimental Results on Single Flux Quantum Logic.

PB95-175071 02,053

Frequency Dependence of the Emission from 2D Array Josephson Oscillators.

PB95-175147

02,056

High-Frequency Oscillators Using Phase-Locked Arrays of Josephson Junctions.

High Power Generation with Distributed Josephson-Junction Arrays.

High-Power, High-Frequency Oscillators Using Distributed Josephson-Junction Arrays.

6-200266

02,095

Intrinsic Stress in DC Sputtered Niobium

PB94-199031

04,468

Large-Amplitude Shapiro Steps and Self-Field Effects in High-Tc Josephson Weak Links.

95-180410

04,682

Metallic-Barrier Junctions for Programmable Josephson Voltage Standards.

PB96-200134

02,089

Novel Vortex Dynamics in Two-Dimensional Josephson Arrays.

02,091

Observation of Vortex Dynamics in Two-Dimensional Josephson-Junction Arrays.

PB95-168811

02,050

Phase-Locked Oscillator Optimization for Arrays of Josephson Junctions.

PB95-169314 02,052

Pulse-Driven Programmable Josephson Voltage Standard.

04,148

Resonances in Two-Dimensional Array Oscillator Circuits.

PB96-102082 02,066

Stable Phase Locking in a Two-Cell Ladder Array of Josephson Junctions.

PB96-111679 04.722 
BERGER, P. W.

General Types of Information Services. PB96-147053

BERGERHOFF, G.

Evaluation of Crystallographic Data with the Program DIAMOND.

PB97-109045

00,649

BERGGREN, K. K

Microlithography by Using Neutral Metastable Atoms and Self-Assembled Monolayers.

PB96-190038

BERGMANN, D.J.

Environmental Aspects of Halon Replacements: Ccnsiderations for Advanced Agents and the Ozone Depletion Potential of CF31.

BERGQUIST, J. C.

03,301

High-Resolution Atomic Spectroscopy of Laser-Cooled lons.

PB95-169330 03,953

Interference in the Resonance Fluorescence of Two Trapped Atoms.

PB95-168514

03,948

Laser Cooling of Trapped lons.

Light Scattered from Two Atoms.

PB95-168753

Precise Spectroscopy for Fundamental Physics.

03,950

PB96-112164

04,286

PB95-180790

03,965

Quantum Measurements of Trapped Ions.

PB95-161147

03,928

Ouantum Projection Noise: Population Fluctuations in Two.

Level Systems.

03,850

Recent Experiments on Trapped lons at the National Institute of Standards and Technology.

03,952

Trapped lons and Laser Cooling 4: Selected Publications of the Ion Storage Group of the Time and Frequency Division, NIST, Boulder, Colorado.

PB96-172358

04,108

BERGREN, N. F.

Magnetic Measurement of Transport Critical Current Density of Granular Superconductors.

PB95-126199

04,531

BERGTOLD, D. S

Standard Reference Materials (SRM's) for Measuring Genetic Damage.
PB94-198827

03.516

BERK, N. F.

Analysis of SANS from Controlled Pore Glasses.

PB94-198843

03,035

Analysis of Small-Angle Scattering Data Dominated by Multiple Scattering for Systems Containing Eccentrically Shaped Particles or Pores.

PB96-160411

03,075

Characterization of Chemically Modified Pore Surfaces by Small Angle Neutron Scattering.

00,898

Neutron Spectroscopic Comparison of Rare-Earth/Hydrogen alpha-Phase Systems.

PB95-163523

00,970

Scattering Properties of the Leveled-Wave Model of Random Morphologies.

$\begin{array}{ll}03,807 & \end{array}$

Structure of a Triglyceride Microemulsion: A Small Angle Neutron Scattering Study.

PB96-112255

01,077

VITZ, J. B.

High Temperature

BERKOWITZ, S. J.

03,844

Coexistence of Grains with Differing Orthorhombicity in High Ouality YBa2Cu3O7-delta Thin Films.

PB96-135033

04,742

Controlling the Critical Current Density of High-Temperature SNS Josephson Junctions.

PB96-200712

04,794

Increased Transition Temperature in In situ Coevaporated YBa2Cu3O7-delta Thin Films by Low Temperature Post-Annealing.

nealing.

04,672

Temperature Dependence and Magnetic Field Modulation of Critical Currents in Step-Edge SNS YBCO/Au Junctions.
PB96-111745

Thermal Noise in High-Temperature Superconducting-Normal-Superconducting Step-Edge Josephson Junctions. PB95-175089

04,650

BERLINER, $R$

Structures of Sodium Metal.
PB94-198850

PB94-198850

03,319

Effect of Liftoff on Accuracu of Phase Velocity Measurements Made with Electromagnetic-Acoustic Transducers.
PB96-186044
02,280
Methods to Improve the Accuracy of On-Line Ultrasonic Measurement of Steel Sheet Formability.

PB96-186051

02,281

BERMAN, H. M.

Nucleic Acid Database: Present and Future.

.

00,518

BERN, H. A

Histopathology, Blood Chemistry, and Physiological Status of Normal and Moribund Striped Bass ('Morone saxatilis') Involved in Summer Mortality ('Die-Off') in the SacramentoSan Joaquin Delta of California.

PB94-198157

00,034

BERNAL,

Growth Surtace for the Slopes at the Boundary of a PolygBon. 152725

03,408

Inserting Line Segments into Triangulations and Tetrahedralizations. Probabilistic Computation of Poiseuille Flow Velocity Fields. PB96-102520 04,209 Submissions to a Planned Encyclopedia of Operations Research on Computational Geometry and the Voronoi/ Delaunay Construct.

PB94-152709

03,425

BERNDT, C. C.

Anisotropy of the Surfaces of Pores in Plasma Sprayed Alumina Deposits.

PB96-123211

03,126

BERNDT, $M$.

Evaluation of Crystallographic Data with the Program DIA-

MOND.

BERNING, D. $W$.

Double-Modulation and Selective Excitation Photoreflectance for Wafer-Level Characterization of Ouantum-Well Laser Structures.

PB96-167325

04,372

\section{BERNSTEIN, B.}

Non-Equilibrium Thermodynamic Theory of Viscoplastic Ma-

PB94-198868

04,467

Nonequilibrium Thermodynamic Theory of Viscoplastic Materials.
PB96-111661

04,034

PC-Based Prototype Expert System for Data Managemen and Analysis of Creep and Fatigue of Selected Materials a Elevated Temperatures.

PB94-172251

03,202

BERRAH, N.

High-Energy Behavior of the Double Photoionization of $\mathrm{He}$ lium from 2 to $12 \mathrm{keV}$

PERRY, A. K.

03,860

Transition Metal

PB95-126389

04,534

BERRY, H. G.

High-Energy Behavior of the Double Photoionization of $\mathrm{He}$ lium from 2 to $12 \mathrm{keV}$

PB94-213279

03,860

BERRY, R. J.

Halon Thermochemistry: 'Ab Initio' Calculations of the Enthalpies of Formation of Fluoromethanes.

03,289

BERRY, S. D

Structural and Magnetic Ordering in Iron Oxide/Nickel Oxide Multilayers by $X$-ray and Neutron Diffraction (Invited)

PB94-172558

\section{BERTNESS, K. A.}

In situ Observation of Surface Morphology of InP Grown on Singular and Vicinal (001) Substrates.

PB95-168431

04,636

BERTOCCI, U.

Evaluation of the Electrochemical Behavior of Ductile Nickel Aluminide and Nickel in a pH 7.9 Solution. Technical Report Number 3, April-June 1991.

DE94017351

03,307

Evaluation of the Environmentally Induced Fracture Resistance of Ductile Nickel Aluminide. Technical Report Number 1. Final report. October-December 1990.

03,306

Modeling Polarization Curves and Impedance Spectra for Simple Electrode Systems.

PB94-198876

03,188

BERUBE, J.

Compatibility Analysis of the ANSI and ISO IRDS Services Interfaces.
PB94-163474

01,805

BERUFF, R. B.

Test of Newton's Inverse Square Law of Gravitation Using the 300-m Tower at Erie, Colorado.

PB95-202446

03,978

\section{BESSON, R}

New Model of 1/F Noise in Baw Ouartz Resonators.

PB96-112248

02,383

BEVERIDGE, T. J.

Characterization of the Binding of Gallium, Platinum, and Uranium to Pseudomonas Fluorescens by Small-Angle $X$ ray Scattering and Transmission Electron Microscopy.
PB94-172509
03,453

BEZNUKHOV, $\mathbf{S}$.

Genetically Engineered Pores for New Materials.

PB96-161641

BEZRUKOV, S. M.

03,550

Current Fluctuations Reveal Protonation Dynamics and Number of lonizable Residues in the alpha-Toxin Channel. PB96-161732

Current Noise Reveals Protonation Kinetics and Number of Ionizable Sites in an Open Protein lon Channel.

PB96-161674

04,092

Noise Analysis of Ionization Kinetics in a Protein Ion Chan-

PB96-161682

04,093

Protonation Dynamics in an Ion Channel Pore.

PB96-161757

03,589

Protonation Dynamics of the alpha-Toxin Ion Channel from Spectral Analysis of pH-Dependent Current Fluctuations.

BHADURI, S.

Report of the Refrigeration, Air Conditioning and Heat Pumps Technical Options Committee.

PB96-176755

03,293

BIANCANIELLO, $F$

Parametric Investigation of Metal Powder Atomization Using Laser Diffraction

BIANCANIELLO, F. S.

03.342

Beneficial Etfects of Nitrogen Atomization on an Austenitic Stainless Steel.

PB94-212396

03,209

Development of Adaptive Control Strategies for Inert Gas Atomization.

02,823

Effect of Backfill and Atomizing Gas on the Powder Porosity and Mechanical Properties of $304 \mathrm{~L}$ Stainless Steel.

PB94-185394

03,205

Effects of Elastic Stress on Phase Equilibrium in the Ni-V System.
PB94-172707

03,313

Intelligent Control of an Inert Gas Atomization Process.

PB95-141057

Optimization of Inert Gas Atomization.

PB95-107405

01,377

PBdered omega-

Process Modeling and Control of Inert Gas Atomization

PB95-162343

BICKHAM, D.

Biological Macromolecular Crystallization Database: A Tool for Developing Crystallization Strategies.

PB95-126157

00,897

BIEDENHARN, L C

Comprehensive Theory of Nuclear Effects on the Intrinsic Sticking Probability. 1 .

PB94-200623

03.832

Comprehensive Theory of Nuclear Effects on the Intrinsic 
BINKLEY, D.

Slicing in the Presence of Parameter Aliasing PB96-160858

BINKLEY, D. W.

Application of the Pointer State Subgraph 10 Static Program Slicing

PB96-167838

01,768

$\mathrm{C}++$ in Safety Critical Systems.

01,750

Unravel: A CASE Tool to Assist Evaluation of High Integrity Software. Volume 1. Requirements and Design.

01,736

Unravel: A CASE Tool to Assist Evaluation of High Integrity Software. Volume 2. User Manual.

PB95-267894

01,737

BIR, N.

Rapid Method for the Isolation of Genomic DNA from 'Aspergillus fumigatus

PB96-14706

03,488 Effect of Cross-Links on the Miscibility of a Deuterated Polybutadiene and Protonated Polybutadiene Blend.
PB94-212438

Design and Construction of a Liquid Hydrogen Temperature Refrigeration System.

02,619

Helium Refrigeration and Liquefaction Using a Liquid Hydrogen Refrigerator for Precooling.

02,749

AD-A286 683/8

Location of a (1)A(sub g) State in Bithiophene

PB95-202313

BIRNBAUM, G.

01,025

Collision-Induced Emission in the Fundamental VibrationRotation Band of $\mathrm{H} 2$.

03,811

Intelligent Processing of Materials, Technical Activities 1994 (NAS-NRC Assessment Panel, April 6-7, 1995).

PB96-115050

03,359

Memory Function Approach to the Shape of Pressure Broadened Molecular Bands.

PB95-152930

00,944

BISS, B.

Response Comparison of Electret Ion Chambers, LiF TLD, and HPIC

PB96-190103

02,578

BISWAS, P.

In situ Characterization of Vapor Phase Growth of Iron Oxide-Silica Nanocomposites: Part 1. 2-D Planar Laser-Induced Fluorescence and Mie Imaging

PB97-112478

03,185

Role of Refrigerant Mixtures as Alternatives to CFCs

Role of Refr

03,252

BLAAUW, $M$.

Local Area Networks in NAA: Advantages and Pitfalls.

PB94-172095

00,527

BLACK, D. R.

Diffraction Imaging of Polycrystalline Materials

PB94-198884

02,971

Evolution of the Pore Size Distribution in Final-Stage Sintering of Alumina Measured by Small-Angle X-ray Scattering. (Reannouncement with New Availability Information) AD-A249 178/5 03,023 Structures of Vapor-Deposited Yttria and Zirconia Thin Films.

PB94-216025

03,041

BLACKBURN, D. L.

Natural Convection trom an Array of Electronic Packages Mounted on a Horizontal Board in a Narrow Aspect Ratio Enclosure.

$\begin{array}{ll}\text { PB96-164017 } & 02,087\end{array}$

Simulating the Dynamic Electro Thermal Behavior of Power Electronic Circuits and Systems.

PB95-161014

02,345

Status and Trends in Power Semiconductor Devices.
PB95-175097

Thermal Component Models for Electro-Thermal Network Simulation.

PB95-161022

02,346

LAIR, J. J.

Uncertainties of Frequency Response Estimates Derived from Responses to Uncertain Step-Like Inputs.

01,984

BLAIR, W. P.

Rapid Decline in the Optical Emission from SN 1957D in

PB94-216033

00,070

BLAIR, W. R.

Certification of the Standard Reference Material 1473a, a Low Density Polyethylene Resin.

01,282

Cylinder Wipe Air-Drying Intaglio Ink Vehicles for U.S. Currency Inks.

03,115
Preparation and Monitoring of Lead Acetate Containing Prinking Water Solutions for Toxicity Studies.

00,538

\section{BLAKE, R. L.}

Design and Chatacterization of X-Ray Multilayer Analyzers or the $50 \cdot 1000 \mathrm{eV}$ Region

PB94-211851

03,848

BLAKE, T. A.

Infrared and Microwave Spectroscopy of the Argon Propyne Dimer.

PB94-198892

00,794

OLACK, T. $V$

Observations of Partial Discharges in Hexane Under High Magnification.

BLANCA ROS, $M$ X-ray Observation of Electroclinic Layer Constriction and
Rearrangement in a Chiral Smectic-A Liquid Crystal. Rearrangement

01,100

BLANPIED, W. A.

Thermal Conductivity of Metals and Alloys at Low Temperaures. A Review of the Literature.

AD-A279 180/4

03,302

BLASCHKO, $O$.

Neutron Spectroscopic Comparison of Rare-Earth/Hydrogen alpha-Phase Systems.

00,970

Vibrations of Hydrogen and Deuterium in Solid Solution with

$\begin{array}{ll}\text { Lutetium. } & \\ & 01,019\end{array}$

BLAU, $M$.

Cold Neutron Gain Calculations for the NBSR Using MCNP.

PB95-163978

Nuclear Heat Load Calculations for the NBSR Cold Neutron Source Using MCNP.

PB95-152955

BLENDELL, J. E.

Effect of Sm2BaCuO5 on the Properties of Sintered (Bulk) YBa2Cu3O6+X

04,733

Stability and Surface Energies of Wetted Grain Boundaries in Aluminum Oxide.

PB95-202750

03,059

Texture Measurement of Sintered Alumina Using the March-Dollase Function

04,784

BLESSING, G. V.

Design, Construction and Application of a Large Aperture Lens-Less Line-Focus PVDF Transducer.

02,765

Dynamic Shear Modulus Measurements with Four Independent Techniques in Nickel-Based Alloys.

03,320

Material Characterization By a Time-Resolved and Polarization-Sensitive Ultrasonic Technique.

PB97-122576

02,764

NIST Calibration of ASTM E127-Type Ultrasonic Reference Blocks

PB94-191640

02,702

Transient Analysis of a Line-Focus Transducer Probing a Liquid/Solid Interface

PB97-118681

02,763

Ultrasonic Measurement of Sheet Anisotropy and Formability.

PB95-153235

03,213

Ultrasonic Measurements of Surface Roughness.

PB94-172137

04,181

Uitrasonic NDE of Sprayed Ceramic Coatings

02,761

BLETZINGER, P.

Gaseous Electronics Conference Radio-Frequency Reference Cell: A Defined Parallel-Plate Radio-Frequency System for Experimental and Theoretical Studies of PlasmaProcessing Discharges.

04,404

BLOCK, S.

1.4-Dinitrocubane and Cubane under High Pressure.

B $95-108437$

BLOMQUIST, B. W.

International Marine-Aimospheric (222)Rn Measurement Intercomparison in Bermuda. Part 2. Results for the Participating Laboratories.

PB96-175682

BLOMQUIST, D. S.

Quality in Automated Manufacturing

BLOOM, H. M.

Formulation of Position on U.S. Standards Role in Enterprise Integration.

PB95-105052

02,773

Technical Program Description Systems Integration for Manufacturing (SIMA)

02,819

Technical Program of the Factory Automation Systems Division 1993

PB94-160819

02,805

BLUE, J. L.

Effect of Training Dynamics on Neural Network Perform ance.

PB95-267845

01,852

Faster BKL Monte Carlo Simulations.

PB95-136370

01,706

Faster Monte Carlo Simulations.

PB96-102074

04,018

Improving Neural Network Performance for Character and Fingerprint Classification by Altering Network Dynamics.

Improving Neural Network Performance for Character and Fingerprint Classification by Altering Network Dynamics. PB96-123195

01,856

Micromagnetic Structure of Domains in Co/Pt Multilayers. 1

Investigations of Wall Structure.

NIST Form-Based Handprint Recognition System.

PB94-217106

04,610

Parallel Monte Carlo Simulation of MBE Growth. PB96-122841

01,838 Fluorescence in a Cathodic Sputtering Cell.

00,598

BLUM, $\mathrm{K}$.

Charge Cloud Distribution of Heavy Atoms After Excitation by Polarized Electrons.

PB95-203147

04,001

Catastrophic Failures Propagate Field of Fracture Mechanics.

BLUNDELL, S. A.

Evaluation of Two-Photon Exchange Graphs for HighlyCharged Heliumlike Ions.

POATNER, A.

03,808

Anomalous Dispersion and Thermal Expansion in Lightly Doped KTa1-xNbxO3

PB95-152302

BOCHMANN, G. V.

Open Issues in OSI Protocol Development and Conformance Testing

BODARKY, S.

Guide to Configuration Management and the Revision Con-

trol System for Testbed Users.
PB94-150919

01,678

BODE, $M$.

Epitaxial Growth and Characterization of the Ordered Va cancy Compound Culn3Se5 on GaAs (100) Fabricated by Molecular Beam Epitaxy.

PB95-180725

04,687

BOETTINGER, $W . \mathrm{J}$.

Coherent Precipitates in the BCC/Orthorhombic Two Phase

Field of the Ti-Al-Nb System.

PB94-198694

03,317

Disorder Trapping in Ni2TiAl.

PB94-198942

03,322

Lubrication Theory for Reactive Spreading of a Thin Drop.

PB95-189460 02,865

Lubrication Theory for Reactive Spreading of a Thin Drop

PB96-123427 04,214

Ordered omega-Derivatives in a Ti-37.5Al-2ONb at\% Alloy.

PB94-211091 03,33

Phase-Field Model for Solidification of a Eutectic Alloy 


\section{PERSONAL AUTHOR INDEX}

Interior-Point Method for Linear and Quadratic Programming Problems. (NIST Reprint)

PB95.180089

03,429

BOGGS, S. A.

Transient Errors in a Precision Resistive Divider.

PB97-111512

BOISVERT, R. F.

Boundary Integral Method for the Simulation of Two-Dimensional Particle Coarsening.

PB94-216744

03,411

Portable Vectorized Software for Bessel Function Evaluation.

01,690

PB94-198975

01.689

PB94-198967

Virtual Software Repository System.

PB94-198983

01,691

\section{BOITEUX, $\mathrm{S}$.}

Substrate Specificity of the Escherichia coli Endonuclease III: Exicision of Thymine- and Cytosine-Derived Lesions in DNA Produced by Radiation-Generated Free Radicals.

PB95-153425

03,535

BOLAND, T

Asynchronous Transfer Mode Procurement and Usage

Guide

Data Communications Strategy.

02,738

ISDN LAN Bridging

PB95-154696

01,477

BOLGER, J. A.

Time-Resolved Measurements of the Polarization State of

Four-Wave Mixing Signals from GaAs Multiple Quantum

PB96-201058

04.796

OLLINGER, J.

Electrostatic Modes of Ion-Trap Plasmas

PB95-152963

03,920

Experimental Results on Normal Modes in Cold, Pure Ion Plasmas.

BOLLINGER, J. J.

03,956

Electrostatic Modes as a Diagnostic in Penning-Trap Experiments

PB95-176244

03,959

High-Resolution Atomic Spectroscopy of Laser-Cooled lons.

PB95-169330 03,953

Interference in the Resonance Fluorescence of Two

Trapped Atoms.

PB95-168514

03,948

Laser-Cooled Positron Source.

PB95.169348

03,954

Laser Cooling of Trapped Ions.

PB95-168746

03,950

Light Scattered from Two Atoms.

95-168753

04,286

Liquid and Solid
PB94-198991

03,809

Non-Neutral Ion Plasmas and Crystals, Laser Cooling, and Atomic Clocks.

Precise Spectroscopy for Fundamental Physics.

PB96-112164

03,957

Ouantum Measurements of Trapped lons.

04,040

PB95-161147

03,928

Quantum Projection Noise: Population Fluctuations in Two-

Level Systems.

03,850

Recent Experiments on Trapped Ions at the National Insttute of Standards and Technology.

03,952

Spin Squeezing and Reduced Quantum Noise in Spectros.

copy

03,912

Squeezed Atomic States and Projection Noise in Spectros. PB95-176293

03,960

Trapped lons and Laser Cooling 4: Selected Publications of the Ion Storage Group of the Time and Frequency Division, NIST, Boulder, Colorado.

PB96-172358

04,108

BOLLONG, A. B.

Comparison of Techniques for Nondestructive Composition Measurements in CdZnTe Substrates.

PB96-103098

02.703

BOND, L J.

Absorption of Sound in Gases between 10 and $25 \mathrm{MHz}$

04,183

PB94-199015

Absorption of Ultrasonic Waves in Air at High Frequencies (10-20 MHz).

04,182

Interaction of Rayleigh Waves with a Rib Attached to a Plate

04,184
Sensor System for Intelligent Processing of Hot-Rolled

PBg6-186069

03,373

Ultrasonic Technique for Sizing Voids Using Area Functions.

PB95-151916

BONNELL, D. W.

High Temperature.

02,904

PB94-211737

03,844

Recent Experimental and Modeling Developments in High Temperature Thermochemistry.

PB94-172343

00,759

Survey of Steel Moment-Resisting Frame Buildings Affected by the 1994 Northridge Earthquake. PB95.211918

00,451

BOOI, A. A. P.

High Power Generation with Distributed Josephson-Junction

PB97-111520

02,099

BOOI, P. A. A.

Characterization of the Emission from 2D Array Josephson

$\begin{array}{ll}\text { Oscillators. } & 02,054\end{array}$

Design of High-Frequency, High-Power Oscillators Using Josephson-Junction Arrays.

02,094

Direct Observation of Vortex Dynamics in Two-Dimensional Josephson-Junction Arrays.

PB96-102223

02,067

Emission Linewidth Measurements of Two-Dimensional Array Josephson Oscillators.

PB95-175139 02,055

Frequency Dependence of the Emission from 2D Array Josephson Oscillators

02,056

High-Frequency Oscillators Using Phase-Locked Arrays of Josephson Junctions.

02,080

High-Power, High-Frequency Oscillators Using Distributed Josephson-Junction Arrays.

PB96-200266

02,095

Intrinsic Stress in DC Sputtered Niobium.

PB94-199031

04,468

Novel Vortex Dynamics in Two-Dimensional Josephson Ar-

rays. 200167

02,091

Observation of Vortex Dynamics in Two-Dimensional Josephson-Junction Arrays.

02,050

Phase-Locked Oscillator Optumization for Arrays of Josephson Junctions.

02,052

P95-169314

Resonances in Two-Dimensional Array Oscillator Circuits.
PB96-102082

PB96-102082

th Low

Superconducting Integrated Circuit Fabrication with

PB96-103015

04,719

SUSAN: SUperconducting Systems ANalysis by Low Temperature Scanning Electron Microscopy (LTSEM).

PB96-112065

04,728

BOOKBINDER, J.

Sleuthing the Dynamo: HST/FOS Observations of UV Emis-

sions of Solar-Type Stars in Young Clusters.

00,098

BOOKBINDER, J. A.

Far-Ultraviolet Flare on a Pleiades G Dwart

PB96-102033

00,086

Four Years of Monitoring alpha Orionis with the VLA:

Where Have All the Flares Gone.

00,048

Search for Radio Emission from the 'Non-Magnetic' Chemically Peculiar Stars

PB96-102249

00,087

Displacement Method for Machine Geometry Calibration.

PB95.152088 02,946

Estimation of Measurement Uncertainty of Small Circular Features Measured by CMMs.

PB95-267928

02,918

Interim Testing Artifact (ITA): A Performance Evaluation System for Coordinate Measuring Machines (CMMS). User

PB95-210589

02,914

NIST SRM 9983 High-Rigidity Ball-Bar Stand. User Manual.

02,669

Some Considerations for Interim Testing of Coordinate Measuring Machine Performance Using a Specitic Artifact. PB95-108858

02,898

BORCHARDT, B. R.

User's Guide to NIST SRM 2084: CMM Probe Performance Standard.

PBg 206109

02,709

Critical Current and Normal Resistance of High-Tc StepEdge SNS Junctions.

04,724

BORCHERDT, L. J.

High-Tc Superconducting Antenna-Coupled Microbolometer on Silicon.

BORCHERS, J.

Magnetoelasticity in Rare-Earth Multilayers and Films.

PB94-211356

02,166

ORCHERS, J. A

Combined Low- and High-Angle X-Ray Structural Refine-

ment of a Co/Pt(111) Multilayer Exhibiting Perpendicular

Magnetic Anisotropy.

PB94-198355

04,457

Comment on 'Phase Transitions in Antiferromagnetic Superlattices'.

Magnetic Rare Earth Artificial Metallic Superlattices.

04,587

PB95-162293

04,611

Magnetic Structure Determination for Annealed Ni80Fe20/

Ag Multilayers Using Polarized-Neutron Reflectivity.

PB96-176615

03,739

Structural and Magnetic Ordering in Iron Oxide/Nickel Oxide Multilayers by $\mathrm{X}$-ray and Neutron Diffraction (Invited).

04,442

Unconventional Ferromagnetic Transition in La(sub 1 . x) $\mathrm{Ca}(\mathrm{sub} x) \mathrm{MnO} 3$.

PB97-112429

04,156

REHERS, J. A.

Enhanced Curie Temperatures and Magnetoelastic Domains in Dy/Lu Super Lattices and Films.

BORST, $P$.

beta-D-Glucosyl-Hydroxymethyluracil: A Novel Modified Base Present in the DNA of the Parastic Protozoan T. brucei.

PB94-172319

03,524

BORSUK, G.

Beyond the Technology Roadmaps: An Assessment of Electronic Materials Research and Development.

PB96-165998

BORTFELD, D. P.

Display-Measurement Round-Robin

PB96-119227

BORTHWICK, P.

Suppression of Ignition Over a Heated Metal Surface. PB96-176425

BORYSOW, J.

N2(a'(sup 1) Sigma(sub g)(sup + ) ) Metastable Collisiona Destruction and

BOSE, J.

Error Protecting Characteristics of CDMA and Impacts on

PB96-122452 
BOWERS, G.

Frozen Human Serum Reference Material for Standardization of Sodium and Potassium Measurements in Serum or Plasma by Ion-Selective Electrode Analyzers.

00,532

BOWERS, G. N

History of NIST's Contributions to Development of Standard Reference Materials and Reference and Definitive Methods for Clinical Chemistry.

PB96-119706

03,503

BOWERS, W. J.

Development of the NIST Transient Pressure and Temperature Calibration Facility.

00,626

Simultaneous Forward-Backward Raman Scattering Studies Simultaneous Forward-Backward Raman Scattering Studies
of D2 Broadened by D2, He, and Ar.
PB95-162459
00,961

BOWMAN, K. J.

Texture Measurement of Sintered Alumina Using the March-Dollase

04,784

BOWMAN, W. A

Study of Laser Resonance Ionization Mass Spectrometry Using a Glow Discharge Source.

DE94018566

03,308

Study of Laser Resonance Ionization Mass Spectrometry Using a Glow Discharge Source.

03,360

BOX, G.

Taguchi's Parameter Design: A Panel Discussion.

03,445

BOYD, C. F.

Fire Protection Foam Behavior in a Radiative Environment.

PBER $C$.

00,237

Minimum Mass Flux Requirements to Suppress Burning Surfaces with Water Sprays.

01,425

BOYES, S. J.

Interatomic Potential of Argon

PB95-141180

00,912

Interatomic Potential of Argon

PB95-152989

00.945

Vapor Pressures and Gas-Phase PVT Data for 1-Chloro1,2,2,2-Tetrafluoroethane (R124).

PB95-175154

03,271

Vapour Pressures and Gas-Phase $(p$, rho $n, T)$ Values for CF3CHF2(R125)

BOYKO, K. C.

Experimental Investigation of the Validity of TDDB Voltage

Acceleration Models.
PB94-185949

02,304

Field and Temperature Acceleration of Time-Dependent Di-

electric Breakdown in Intrinsic Thin SiO2.

02,305

BOY $94-185956$

Perception of Clamp Noise in Television Receivers.

PB96-119433

01,489

OYNTON, P. A

Display-Measurement Round-Robin.
PB96-119227

02,186

Interface-Filter Characterization of Spectraradiometers and PB97-122212

04,399

Survey of the Components of Display-Measurement StandPB96-122528

02,188

Survey of the Components of Display-Measurement Standards.

02,209

BOZIN, J.

Excitation of Balmer Lines in Low-Current Discharges of Hydrogen and Deuterium.

PB95-150546

03,893

BOZOVIC, I.

Stacked Series Arrays of High-Tc Trilayer Josephson Junctions.

PB96-102272 04,705

Step-Edge and Stacked-Heterostructure High-Tc Josephson Junctions for Voltage-Standard Arrays.

PB96-102066

04,702

BRABERS, J. H. V. J.

Magnetic Properties of Single-Crystalline UCu3Al2.
PB95-180717

BRACHA, E.

Channel Coding for Code Excited Linear Prediction (CELP) Encoded Speech in Mobile Radio Applications. $\quad 01,475$

BRADFORD, A. G.

Metrology for Electromagnetic Technology: A Bibliography of NIST Publications.

04,396

Metrology for Electromagnetic Technology: A Bibliography of NIST Publications, September 1995

01,938
BRADLEY, P.

Energy Flows in an Orifice Pulse Tube Refrigerator

B95-169082

Graded and Nongraded Regenerator Performance. PB95-169090

02,752

BRADY, $K$.

Distributed Communication Methods and Role-Based ACcess Control for Use in Health Care Applications.

PB96-183165

01,508

BRADY, K. G.

Conformance Testing and Specification Management PB97-113781

User's Guide for the PHIGS Validation Tests (Version 2.1). PB94-165206

BRAKE, M. L.

Gaseous Electronics Conference Radio-Frequency Reference Cell: A Defined Parallel-Plate Radio-Frequency System for Experimental and Theoretical Studies of PlasmaProcessing Discharges.

04,404

Reactive Ion Etching in the Gaseous Electronics Conerence RF Reference Cell.

PB96-113402

02,395

BRAND, P. C.

Determination of the Residual Stresses Near the Ends of Skip Welds Using Neutron Diffraction and X-ray Diffraction Procedures.

02,868

BRANDENBURG, $K$.

Evaluation of Crystallographic Data with the Program DIA-

PB97-109045

00,649

RANDT, J. C.

Observations of $3 \mathrm{C} 273$ with the Goddard High Resolution Spectrograph on the Hubble Space Telescope.

00,076

Observing Stellar Coronae with the Goddard High Resolution Spectrograph. I. The dMe Star AU Microscopil.

PB96-102777

00,092

BRANECKY, R. J.

Evaluation of Wear Resistant Ceramic Valve Seats in GasFueled Power Generation Engines. Topical Report, December 1991-April 1994.

PB95-200218

02,466

BRANSFORD, J. W.

Recommended Changes in ASTM Test Methods D2512-82 and G86-84 for Oxygen-Compatibility Mechanical Impact Tests on Metals.

PB94-216694

03,338

BRANSTAD, D

Introduction to Secure Telephone Terminals.

PB97-110498

01,512

BRANSTAD, D. K

Report of the NIST Workshop on Digital Signature Certificate Management. Held on December 10-11, 1992

PB94-135001

01,574

Report of the NIST Workshop on Key Escrow Encryption. Held in Gaithersburg, Maryland on June 8-10, 1994.

PB94-209459 01,584

Response to Comments on the NIST Proposed Digital Signature Standard.

PB96-161815

BRAUN, E.

Early Detection of Room Fires Through Acoustic Emission. (NIST Reprint)

00,298

Fire Performance of an Interstitial Space Construction SysPB95-188918

00,390

Further Development of the N-Gas Mathematical Model: An Approach for Predicting the Toxic Potency of Complex Combustion Mixiures.

PB96-123260

03,650

New Approach for Reducing the Toxicity of the Combustion Products from Flexible Polyurethane Foam.

01,411

Ouantifying the Ignition Propensity of Cigarettes.

PB96-155411

00,306

Relating Bench
PB95-125977

00,361

BRAUN, L. M.

Model for Toughness Curves in Two-Phase Ceramics. 1. Basic Fracture Mechanics.

03,085

Model for Toughness Curves in Two-Phase Ceramics. 2. Microstructural Variables.

PB96-163795

03,078

Need for Advanced Characterization Techniques in Product Manufacturing: A Case Study on Ceramic Matrix Compos-

ites.

03,089

Objective Evaluation of Short-Crack Toughness Curves Using Indentation Flaws: Case Study on Alumina-Based Ceramics.

PB96-179429

03,079

BRAUN, R. J.

Anisotropy of Interfaces in an Ordered Alloy: A MultipleOrder-Parameter Model.

PB96-131594

04,741

Asymptotic Behavior of Modulated Taylor-Couette Flows with a Crystalline Inner Cylinder.

PB94-199072 04,469

Lubrication Theory for Reactive Spreading of a Thin Drop.

PB95-189460 02,865

Lubrication Theory for Reactive Spreading of a Thin Drop.

PB96-123427

04,214

BRAUN, $W$.

Reaction Rate Determinations of Vinyl Radical Reactions with Vinyl, Methyl, and Hydrogen Atoms.

BRAXENTHALER, M.

00,815

Positive and Negative Cooperativities at Subsequent Steps of Oxygenation Regulate the Allosteric Behavior of Multistate Sebacylhemoglobin.

PB97-119374

03,486

BRAY, S. L

Critical-Current Degradation in Nb3 Al Wires Due to Axial

PB95-202784 02,226

Effect of Magnetic Field Orientation on the Critical Current of HTS Conductor and Coils

PB96-141189

02,956

Electromechanical properties of superconductors for DOE usion applications.

DE95015476 04,432

Electromechanical Properties of Superconductors for DOE Fusion Applications.

PB94-139672

02,250

mproved Uniaxial Strain Tolerance of the Critical Current Measured in Ag-Sheathed Bi2Sr2Ca1Cu2O8+x Superconductors.

Ouench Energy and Fatigue Degradation Properties of $\mathrm{Cu}$ and $\mathrm{Al} / \mathrm{Cu}-\mathrm{Stablized} \mathrm{Nb}-\mathrm{Ti}$ Epoxy-Impregnated Superconductor Coils

PB96-141213

04,755

Transverse stress effect on the critical current of internal tin and bronze process $\mathrm{Nb}$ (sub 3 ) Sn superconductors.

16659

BREEN, J. J.

Application of a Novel Slurry Furnace AAS Protocol for PB96-112354

BREESE, R. S

Investigation of the Thermal Stability and Char-Forming Tendency of Cross-linked Poly(methyl methacrylate). 03,380 PB94-213501

BREHOB, E.

Turbulent Upward Flame Spread on a Vertical Wall under External Radiation.

BREITENBERG, $M$

Electrical Product Requirements (Especially Ouality Requirements) in the United States

PB96-119235

01,929

Ouestions and Answers on Ouality, the ISO 9000 Standard Series, Ouality System Registration, and Related Issues. More Ouestions and Answers on the ISO 9000 Standard Series and Related Issues.

00,495

Survey on the Implementation of ISO/IEC Guide 25 by National Laboratory Accreditation Programs.

PB94-210150

00,479

BREUER, E. 


\section{PERSONAL AUTHOR INDEX}

Report of the National Conference on Weight and Measures (78th). Held in Kansas City, MO. on July 18-22, 1993. PB94-138989 02,623 Report of the National Conference on Weights and Measures (79th). Held in San Diego, California on July 17-21,

PB95-169819

02,656

BRIDGES, J. M

Development and Calibration of UVNUV Radiometric Sources.

PB94-199098

04,229

Irradiances of Spectral Lines in Mercury Pencil Lamps.

PB96-176466

04,375

BRIESMEISTER, J.

Neutron Leakage Benchmark for Criticality Safety Research.

03,723

BRIGGS, M. E.

Susceptibility Critical Exponent for a Nonaqueous Ionic Binary Mixture Near a Consolute Point

PB95.152112

00,938

BRIGHAM-BURKE, M

Phospholipid/Alkanethiol Bilayers for Cell-Surface Receptor Studies by Surface Plasmon Resonance.

PB96-102900

03,472

Fabrication of Platinum-Gold Alloys in Pre-Hispanic South America: Issues of Temperature and Microstructure Control. PB94-21164

03,333

BRIGHT, D. S.

Compositional Mapping of the Microstructure of Materials.

PB95-107199 00,565

Concentration Histogram Imagıng: A Scatter Diagram Technique for Viewing Two or Three Related Images.

PB94-199114 00,542 Object Finder for Digital Images Based on Multiple Thresholds, Connectivity, and Internal Structures.

PB94-199106

01,833

Study of Diffusion Zones with Electron Microprobe Compositional Mapping.

PB94-216348

00,559

BRILL, W. J.

Learning to Change: Opportunities to Improve the Performance of Smalier Manufacturers.

PB94-166212

00,010

BRILLIANT, N. A.

Video Microscopy Applied to Optical Fiber Geometry MeasUrements.

04,295

BRINCKMAN, F. E.

Atmospheric and Marine Trace Chemistry: Interfacial Biomediation and Monitoring.

PB94-199122

03,752

Bıleaching of Cobalt from Smelter Wastes by 'Thiobacillus ferrooxidans'

02,582

Total Surface Areas of Group IVA Organometallic Compounds: Predictors of Toxicity to Algae and Bacteria.

pounds: Predicto

00,814

BROADHURST, M. G.

Spatial Dependence of Electrical Fields Due to Space Charges in Films of Organic Dielectrics Used for Insulation of Power Cables.

PB94-199130

02,214

BROBERG, J, B.

NIST-NRL Free-Electron Laser Facility.

PB94-21251

04,237

BROCHERS, J. A.

Antiferromagnetic Interlayer Correlations in Annealed Ni80Fe20/Ag Multilayers.

$\mathrm{PB}: 97-12222$

03,109

BROCK, C. P.

Investigations of the Systematics of Crystal Packing Using the Cambridge Structural Database.

PB97-10914

BROCKER, C. W.

Design of a High-Flux Backscattering Spectrometer for Ultra-High Resolution Inelastic Neutron Measurements.

PB96-173577

02,992

BRODY, P. S.

Microstructure and Ferroelectric Properties of Lead Zirconate-Titanate Films Produced by Laser Evaporation.

PB:34-199148

04,470

BROHOLM, C.

Isolated Spin Pairs and Two-Dimensional Magnetism in SrCr(sub 9p) Ga(sub 12-9p)O19.

PB97-112387

04,154

BROMAGE, G.

First Results from a Coordinated ROSAT, IUE, and VLA Study of RS CVn Systems.

BROMAGE, G. E

00,069

Rotational Modulation and Flares on RS Canum Venaticorum and BY Draconis Stars. XVIII. Coordinated VLA, ROSAT, and IUE Observations of RS CVn Binaries.

PB96-102322

00,089
Volume-Limited ROSAT Survey of Extreme Ultraviole Emission from all Nondegenerate Stars within 10 Parsecs. PB96-103189

BROMBACHER, W. G.

Methods of Measuring Humidity and Testing Hygrometers. AD.A278 851/1

BROMBACKER, W. G.

Guide to Instrumentation Literature.

BROOKS, D. B

Physical Properties of Some Purified Aliphatic HydroAD-A297 265/1

BROSIUS, J. W.

Observing Stellar Coronae with the Goddard High Resolution Spectrograph. I. The dMe Star AU Microscopii.

PB96-102777

00,092

BROWN, A.

Deuterium and the Local Interstellar Medium: Properties fo the Procyon and Capella Lines of Sight.

00,11

Deuterium in the Local Interstellar Medium: Its Cosmological Significance.

00,08 nary II Pegasi in August 1989. 1. Observational Data.
PBg4-211067

Far-Ultraviolet Flare on a Pleiades G Dwart.

PB96-102033 00,086

First Results from a Coordinated ROSAT, IUE, and VLA Study of RS CVn Systems.

PB94-213477 00,069

First Results from the Goddard High-Resolution Spectrograph: The Chromosphere of Tauri.

00,054

GHAS Observations of Cool, Low-Gravity Stars. 1. The Far-

Ultraviolet Spectrum of alpha Orions (M2 lab).

Goddard High-Resolution Spectrograph Observations of the Local Interstellar Medium and the Deuterium/Hydrogen Ratio along the Line of Sight Toward Capella.

00,066

High Sensitivity Survey of Radio Continuum Emission in Herbig Ae/Be Stars.

PB94-185915

00,051

Hydrogen Lyman-alpha Emission of Capella.

00,075

Next Generation Computer Resources: Reference Model for

Project Support Environments (Version 2.0).
PB94-143401

Observing Stellar Coronae with the Goddard High Resolu-

tIon Spectrograph. I. The dMe Star AU Microscopii.
PB96-102777

00,092

Riass Coronathon: Joint $\mathrm{X}$-ray and Ultraviolet Observations of Normal F-K Stars.

00,109

Rotational Modulation and Flares on RS Canum Venaticorum and BY Draconis Stars. XVIII. Coordinated VLA, ROSAT, and IUE Observations of RS CV n Binaries.
PB96-102322

Sleuthing the Dynamo: HST/FOS Observations of UV Emissions of Solar-Type Stars in Young Clusters.

00,098

Transition Regions of Capella

PB96-123336

00,105

Transition Regions of Capella (1995).

00,108

Volume-Limited ROSAT Survey of Extreme Ultraviole Emission from all Nondegenerate Stars within 10 Parsecs. PB96-103189

00,093

BROWN, C. L.

Histopathology, Blood Chemistry, and Physiological Status of Normal and Moribund Striped Bass ('Morone saxatilis') Involved in Summer Mortality ("Die-Off') in the SacramentoSan Joaquin. Delta of California.

PB94-198157

BROWN, C. M.

00,034

Observation and Visible and uv Magnetic Dipole Transitions

in Highly Charged Xenon and Barium.

PB96-138441

04,056

Polarization Measurements on a Magnetic Ouadrupole Line in Ne-Like Barium.
PB97.113104

\section{BROWN, D. E.}

04,161

Multi-Stage, Position Stabilized Vibration isolation System for Neutron Interferometry.

03,955

BROWN, D. M.

Boron-Implanted 6H-SiC Diodes.

PB96-159678

04,081

BROWN, D. R.

External Gamma-ray Counting of Selected Tissues from a Thorotrast Patient

PB96-160254

03,637

Germanium Detector Optimization of MDA for Efficiency vs

Low Intrinsic Background.

00,543

BROWN, G. A.

Electron and Hole Trapping in Irradiated SIMOX, ZMR and BESOI Buried Oxides.

PB96-160320

BROWN, H. J.

Aging Effects on XRF Measurements of Solder Coatings.
PB95-140927

BROWN, I. D.

CIF Crystallographic Information File: A Standard for Crystallographic Data Interchange.

PB97-109169

04,805

BROWN, J.

Bacteriorhodopsin Immobilized in Sol-Gel Glass.

PB95-151429

03.532

BROWN, J. E.

Computer-Aided Molecular Design of Fire Resistant Aircraft Materials.

PB96-160601 00,025

Fire Safety of Passenger Trains: A Review of Current Approaches and of New Concepts.

PB94-152006

04,848

Gas Phase Oxygen Effect on Chain Scission and Monomer Content in Bulk Poly(methyl methacrylate) Degraded by External Thermal Radiation.

PB96-204078

01,293

Investigation into the Flammability Properties of Honeycomb Composites.

03,152

Investigation of the Thermal Stability and Char-Forming Tendency of Cross-linked Poly(methyl methacrylate).
PB94-213501 380

New Generation of Fire Resistant Polymers. Part 1. Com. puter-Aided Molecular Design.

PB96-160593

01,419

BROWN, J. M.

Atomic Iron in Its (5)D Ground State: A Direct Measurement of the $\mathrm{J}=0$ inverted arrow 1 and $\mathrm{J}=1$ inverted arrow 2 Fine-Structure Intervals (1.2)

PB96-141221

04,756

Atomic Sulfur: Frequency Measurement of the $\mathrm{J}=0$ inversely maps 1 Fine-Structure Transition at 56.3 Microns by Laser Magnetic Resonance.

PB95-180105

01,007

Detection of $\mathrm{OH}_{+}$in Its a(1)Delta State by Far Infrared Laser Magnetic Resonance.

PB95-181087

01,021

Determination of the Molecular Parameters of $\mathrm{NiH}$ in Its (2)Delta Ground

Fine-Structure Intervals of (14)N(+) By Far-Infrared Laser Magnetic Resonance.

PB95-175162

00,993

Laser Magnetic-Resonance Measurement of the (3)P1 (3) P2 Fine-Structure Splittings in (17)O and (18)O

00,994

Measurement of the $\mathrm{J}=2$ less than 1 Fine-Structure Interval or (28)Si and (29)Si in the Ground (3)P State.

00,771

Rotational Spectroscopy of the $\mathrm{CoH}$ Radical in Its Ground Rotational Spectroscopy of the $\mathrm{CoH}$ Radical in Its Ground
(3) Phi State by Far-Intrared Laser Magnetic Resonance: (3) Phi State by Far-Infrared Laser Magn

Determination
PB95-175048

00,992

BROWN, L. 
Octacalcium Phosphate Carboxylates. 1. Preparation and Identification.
PB95-161535

00,660

Octacalcium Phosphate Carboxylates. 2. Characterization and Structural Consideration

00,955

PB95-161543
Octacalcium Phosphate Carboxylates. 5. Incorporation of Excess Succinate and Ammonium lons in the Octacalcium

Phosphate Succinate Structure.
PB95-168894

00,166

Octacalcium Phosphate. 3. Intrared and Raman Vibrational Spectra.

00,756

Periapical Tissue Reactions to a Calcium Phosphate Cement in the Teeth of Monkeys.

00,149

Physicochemical Properties of Calcific Deposits Isolated from Porcine Bioprosthetic Heart Valves Removed from Patients Following 2-13 Years Function.

00,184

Procedure for the Study of Acidic Calcium Phosphate Precursor Phases in Enamel Mineral Formation.
PB95-164448 PB95- 164448
03,564 Selective Inhibition of Crystal Growth on Octacalcium Phos-
phate and Nonstoichiometric Hydroxyapatite by

Pyate and
PBg4-211257 at Physiological Concentration.

BROWNING, D. F.

PC-Based Spinning Rotor Gage Controller.

BROWNING, $M$.

Status of Electrocomposites.
PB94-212453

BROWNING, $V$.

Optical Conductivity of Single Crystals of Ba1$\times M \times B i O 3(M=K, R b$
$P B 94-185329$ BRUCE, $\mathbf{S}$.

Optical Metrology and More. Programs and Services of the Radiometric Physics Division, Physics Laboratory.

BRUCE S.

Assessing the Credibility of the Calorific Content of Muncipal Solid Waste.

00,147

02,609

Developing Ouality System Documentation Based on ANSI NCSL 2540-1-1994: The Optical Technology Division's Effort.

01,869

NIST High Accuracy Scale for Absolute Spectral Response from $406 \mathrm{~nm}$ to $920 \mathrm{~nm}$.

01,865

BRUCK, E.

Magnetic Properties of Single-Crystalline UCu3AI2.

BRUENING, R. J.

04,686

Overview of Radiometric Program of the NIST Thermal $1 \mathrm{~m}$ aging Laboratory.
PB94-199163

BRUNO, T. J.

04,230

Applications of the Vortex Tube in Chemical Analysis Applications of the Vortex Tube in Chemical Analysis. Part 2. Applications
PB96-112107

00,615

Chromatographic Cryofocusing and Cryotrapping with the Vortex Tube.
PB95-180113

00,604

Constituents and Physical Properties of the $\mathrm{C} 6+$ Fraction of Natural Gas. Topical Report, April-June 1994.
PB95-136644 02,483 Fugacity Coefficients of Hydrogen in (Hydrogen + Butane).
PB95-175212 High-Pressure Equilibrium Cell for Solubility Measurements in Supercritical Fluids.

00,998

Measurement of Diffusion in Fluid Systems: Applications to the Supercritical Fluid Region

PB95-175188

02,490

Measurement of Diffusion in Supercritical Fluid Systems: A Review.
PB94-199189

00,795

Permeation Tube Approach to Long-Term Use of Automatic Sampler Retention Index Standards.

00,639

Process Gas Chromatography Detector for Hydrocarbons Based on Catalytic Cracking.
PB95.141099

02,485

Refractive Indices of Fluids Related to Alternative Refrigerants.

03,260

Retention of Halocarbons on a Hexafluoropropylene Epoxide Modified Graphitized Carbon Black. Part 1. MethaneBased Compound

Retention of Halocarbons on a Hexafluoropropylene Epox-

03,272 ide-Modified Graphitized Carbon Black. 3. Ethene-Based Compounds.
PB96-167309

03,286
Retention of Halocarbons on a Hexafluoropropylene Epoxide-Modified Graphitized Carbon Black. 4. Propane-Based Compounds.

PB96-164033

03,284

Simple and Efficient Low-Temperature Sample Cell for Infrared Spectrophotometry.

Simple and Efficient Methane-Marker Devices for Chromatographic Samples.

PB96-164041

00,635

Simple, Inexpensive Apparatus for Sample Concentration

PB94-199205 00,546

Solubilities of Copper(II) and Chromium(III) betaDiketonates in Supercritical Carbon Dioxide.

01,147

Solubility Measurement by Direct Injection of SupercriticalFluid Solutions into a HPLC System.

00,997

Summary of the Patent Literature of Supercritical Fluid Technology.

Supercritical Fluid Extraction of Biological Products.
PB95-175204

Thermophysical Properties of Fluids for the Gas Industry.

Thermophysical Properties of Fluids for the Gas Industry. Final Report, February 1, 1988-August 31, 1993

PB94-146677

02,472

Thermophysical Property Data for Supercritical Fluid Extraction Design.

BRUSCHKE, M. V.

00,668

Report on the Workshop on Manufacturing Polymer Com posites by Liquid Molding. Held in Gaithersburg, Maryland on September 20.22, 1993.

PB94-160066

03,131

BRUSH, L. N.

Laser Melting of Thin Silicon Films.

04,47

BRUSIL, P.

Integrated Network Management

PB94-199247

01,583

BRUST, $J$

Excitation Transfer in Barium by Collisions with Noble Gases.

PB96-200274

01,163

BRYANT, G. W.

Excitons in Complex Ouantum Nanostructures.

PB97.118343

Ouantum Dots in Ouantum Well Structures.

01,184

PB97-118350

01,185

Theory for Ouantum-Dot Ouantum Wells: Pair Correlation and Internal Ouantum Confinement in Nanoheterostruch

PB96-179445

02,437

BRYDEN, $W$.

Incorporation of Gold into YBa2Cu3O7: Structure and Tc Enhancement.

BRYNER, N. P.

Carbon Monoxide Production in Compartment Fires: Reduced-Scale Enclosure Test Facility.

PB95-231700

01,394

Ground-Based Smoke Sampling Techniques Training Course and Collaborative Local Smoke Sampling in Saud

PB94-143542

02,532

Radiometric Model of the Transmission Cell-Reciprocal Nephelometer.

00,124

Scaling Compartment Fires: Reduced- and Full-Scale Enclosure Burns
PB96-175708

BUCCI, E.

Positive and Negatıve Cooperativities at Subsequent Steps of Oxygenation Regulate the Allosteric Behavior of Multistate Sebacylhemoglobin.

PB97-119374

03,486

'ICHNEV, L. M

Precision High Temperature Blackbodies

PB95-140059

All-Metal Collection System for Preparative-Scale Gas
Chromatography: Purification of Low-Boiling-Point ComChromatography: Purification of Low-Boiling-Point Com-
pounds. pounds.

00,619

Assessing the Credibin: Muncipal Solid Waste.

PB94-199882

02,581

Calculation of Higher Heating Values of Biomass Materials and Waste Components from Elemental Analyses.
PB94-199254 PB94-199254

Ionization Energies, Appearance Energies and Thermochemistry of CF2O and FCO.

PB97-111538

Sultur Dioxide Capture in the Combustion of Mixtures of Lime, Refuse-Derived Fuel, and Coal.

02,534

Temperature Dependence of the Gas and Liquid Phase UI-

traviolet Absorption Cross Sections of HCFC-123 (rF3 CHCl2) and $\mathrm{HCFC}-142 \mathrm{~b}(\mathrm{CH} 3 \mathrm{CF} 2 \mathrm{Cl})$.

PB96-201033

BUDNICK, J. 1.

Soft-X-ray-Emission Investigation of Cobalt Implanted Silicon Crystals.

04,069

Solt-X-ray-Emission Studies of Bulk Fe3Si, FeSi, and FeSi2, and Implanted Iron Silicides.

PB96-157938

BUDOWLE, B.

Resolution of DNA in the Presence of Mobility Modifying Polar and Nonpolar Compounds by Discontinuous Electrophoresis on Rehydratable Polyacrylamide Gels.

BUFFA, G.

Self Broadening in the nu1 Band of $\mathrm{NH} 3$.

PB94-216371

00,590

BUHLER, $B$.

Ferntosecond Time-Resolved Wave Packet Motion in Molecular Multiphoton Ionization and Fragmentation.

PB94-198611

00,790

BUIE, E. A.

Usability Engineering: Industry-Government Collaboration for System Effectiveness and Efficiency.

BUIE, M. J.

Reactive Ion Etching in the Gaseous Electronics Conterence RF Reference Cell.

PB96-113402

BUKOWSKI, R. W.

Analysis of the Happyland Social Club Fire with HAZARD I.
PB94-199270 Developing Rational Performance-Based Fire Safety Requirements in Model Building Codes.

PB95-175220

00,200

Earthquake and Fire in Japan: When the Threat Became a Reality.

PB95-175238

00,201

Field Modeling: Simulating the Effect of Sloped Beamed

Ceilings on Detector and Sprinkler Response.
PB96-122866

01,406

Fire Codes for Global Practice.

PB96-102108

00,205

Fire Protection Engineering Tools. Simple Tools: The EquaPB96-156179

00,221

Fire Safety Engineering in the Pursuit of PerformanceBased Codes: Collected Papers.

PB97-114482

00,235

Fire Safety of Passenger Trains: A Review of Current Approaches and of New Concepts.

PB94-152006

04,848

How to Evaluate Alternative Designs Based on Fire Model-

PB96-102116

00,206

New Concepts for Fire Protection of Passenger Rail Trans. portation Vehicles.

PB95-162046

04,850

New Concepts for Fire Protection of Passenger Rail Transportation Vehicles. (NIST Reprint).

04,863

Predictıng the Fire Performance of Buildings: Establishing Appropriate Calculation Methods for Regulatory Applica-

PB96-141239

00,316

Protecting Your Family from Fire.

PB96-156187

Review of Internatioanl Fire Risk Predictions Methods.

Studies Assess Performance of Residential Detectors. PB94-199262

00,222

Verification of a Model of Fire and Smoke Transport

PB95-108718

00,290

BULJAN, S. T.

Tensile Creep of Whisker Reinforced Silicon Nitride 
BUNCH, W. R.

AMRF Composite Fabrication Workstation

PB94-17268

02,810

BUNTIN, S. A.

Laser-Induced Desorption of NO from Si(111): Effects of Coverage on NO Vibrational Populations.

00,959

Photodecomposition Dynamics of $\mathrm{Mo}(\mathrm{CO}) 6 / \mathrm{Si}(111) 7 \times 7$ : CO Internal State and Transiational Energy Distributions--Trans-

lation.

PB94-199288

00,687

Photodecomposition of Mo(CO)6/Si(111) 7×7: Co State-Resolved Evidence for Excited State Relaxation and Quench-

ing. $\mathrm{PB}$ - 180154

01,009

Photodesorption Dynamics of $\mathrm{CO}$ trom Si(111): The Role of Surface Defects.
PB96-111646

03,066

BUR, A. J.

Fluorescence Anisotropy Measurements on a Polymer Melt as a Function of Applied Shear Stress.

01,209

In-Line Optical Monitoring of Injection Molding.

PB94-185105

01,201

Observations of Shear Induced Molecular Orientation in a Polymer Melt Using Fluorescence Anisotropy MeasurePB94-199304

01,210

Observations of Shear Stress and Molecular Orientation Using Fluorescence Anisotropy Measurements.

01,211 (1993

BURCH, D.

Parametric Study of Wall Moisture Contents Using a Revised Variable Indoor Relative Humidity Version of the 'Moist' Transient Heat and Moisture Transter Model.

PB97-122535

00.419

BURCH, D. M.

Analysis of Moisture Accumulation in a Wood-Frame Wall Subjected to Winter Climate.

00,338

Controlling Moisture in the Walls of Manufactured Housing.
PB95-105136

Empirical Validation of a Transient Computer Model for Combined Heat and Moisture Transfer.

00,416

Experimental Verification of a Moisture and Heat Transfer

Model in the Hygroscopic Regime.

PB97-111546 $\quad 00,309$

Heat and Moisture Transter in Wood-Based Wall Construction: Measured versus Predicted.

PB95-200655

00,391

Manufactured Housing Walls That Provide Satistactory

Moisture Performance in All Climates.

PB95-178885

00,383

Mathematical Analysis of Practices to Control Moisture in the Roof Cavities of Manufactured Houses.

00,278

Water-Vapor Measurements of Low-Slope Roofing Materials.

PB95-251617

00,399

BURDETTE, H. E.

Diffraction Imaging of Polycrystalline Materials.

02,971
High-Temperature Furnace for In situ Small-Angle Neutron Scattering during Ceramic Processing.

PB96-148127

03,743

BURGES, C. J. C.

Second Census Optical Character Recognition Systems Conterence.

BURGESS, D.

01,832

Fluorinated Hydrocarbon Flame Suppression Chemistry.
PB94-185113

Gas Phase Reactions Relevant to Chemical Vapor Deposition: Numerical Modeling

PB94-199346

03,117

Incinerability of Perchloroethylene and Chlorobenzene

PB95-163457

01,388

Thin Film Thermocouples for Measurement of Wall Temperatures in Internal Combustion Engines.

peratures in In

01,449

BURGESS, D. B.

Gas Phase Reactions Relevant to Chemical Vapor Deposition: Optical Diagnostics.

PB94-199338

03,116

BURGESS, D. R. F.

Halon Thermochemistry: 'Ab Initio' Calculations of the Enthalpies of Formation of Fluoromethanes.

PB96-175740

03,289

Thermochemical and Chemical Kinetic Data for Fluorinated Hydrocarbons.

PB95-260618 01,056

UV-Photopatterning of Alkylthiolate Monolayers Selt-Assembled on Gold and Silver.

PB95-150751

00,924
BURK, $B$.

Scanning Tunneling Microscopy of the Charge-DensityWave Structure in 1 T.TaS2.

PB95-180980

BURKE, R. W.

Standard Reference Materials: Glass Fillers as a Standard Reterence Material for Spectrophotometry - Selection, Preparation, Certification, and Use of SRM 930 and SRM 1930.
PB94-188844
00,536

BURKETT, W.

Group 1 for the Plant Spatial Configuration STEP Application Protocol.

02,789

BURKHOLDER, J. B.

Infrared Spectrum of OCIO in the 2000/cm(-1) Region: The 2 (nu sub 1) and (nu sub $1+$ nu sub 3 ) Bands.

00,908

Intensities and Dipole Moment Derivatives of the Fun damental Bands of (35) $\mathrm{ClO} 2$ and an Intensity Analysis of the nu1 Band.

00,909

BURLINSKA, G

Research and Development Activities in Electron Paramagnetic Resonance Dosimetry.

PB96-141288

03,635

BURNETT, J. H.

Double-Modulation and Selective Excitation Photoreflectance for Wafer-Level Characterization of Quantum-Well Laser Structures.

PB96-167325

04,372

Use of Pressure for Quantum-Well Band-Structure Characterization.

BURNETT, $K$.

Numerical Solution of the Nonlinear Schroedinger Equation for Small Samples of Trapped Neutral Atoms.

03,985

PB95-202578

Properties of a Bose-Einstein Condensate in an Anisotropic
Harmonic Potential.
PB96-204144 PB96-204144

BURNS, G. W.

Assessment of Uncertainties of Thermocouple Calibrations at NIS

PB94-152691

03,782

BUROW, M.

Determination of Inorganic Constituents in Marine Mammal Tissues.

PB95-152047 00,589

Development of Frozen Whale Blubber and Liver Reference Materials for the Measurement of Organic and Inorganic Contaminants.

00,587

BURR, C. R.

Structural and Magnetic Properties of $\mathrm{CuCl} 2$ Graphite Intercalation Compounds.

PB96-119748

03,020

BURR, W. E.

Comparison of FDDI Asynchronous Mode and DQDB Queue Arbitrated Mode Data Transmission for Metropolitan Area Network Applications.

PB96-160452

01,498

NIST Workshop on the Computer Interface to Flat Pane Displays. Held in San Jose, Calitornia on January 13-14, PB95-136388

01,625

Planning for the Fiber Distributed Data Interface (FDDI)

PB94-135761

01,621

Public Key Infrastructure Invitational Workshop. Held in McLean, Virginia on September 28, 1995.

01,616

PB96-166004 Evaluation of Wear Resistant Ceramic Valve Seats in Gas-
Fueled Power Generation Engines. Topical Report, DecemFueled Power Generat

PB95-200218

02,466

BURRIS, S. B.

Proficiency Tests for the NIST Airborne Asbestos Program -

PB94-193828

00,537

Proficiency Tests for the NIST Airborne Asbestos Program -

PB94-194362

00,539

BURAISS, $W$.

Cryogenic Materials Data Handbook.

AD-A286 675/4

03,303

BURPOUGHS, C. J. Automated Josephson Integrated Circuit Test System.
PB95-175246 057

Experimental Results on Single Flux Quantum Logic. BQ5-175071

Josephson D/A Converter with Fundamental Accuracy. PB96-148044

Pertormance and Reliability of NIST $10-\mathrm{V}$ Josephson Ar-

rays.

02,419 Superconducting Integrated Circuit Fabrication with Low
Temperature ECR-Based PECVD SiO2 Dielectric Films. PB96-103015

BURROUGHTS, c. J.

04.719

Optimization of ECR-Based PECVD Oxide Films for Superconducting Integrated Circuit Fabrication.

PB95-169165

02,051

BURAOWS, J. H.

Information Technology Standards in a Changing World: The Rose of the Users.

PB96-160866

URTON, B. P

Ca4Bi6013: A Compound Containing an Unusually Low Bismuth Coordination Number and Short Bi Bi Contacts.
PB95-141131
00,911

00,911

LMTO/CVM and LAPW/CVM Calculations of the Nicke PB94-199353 Ordered omega-Derivatives in a Ti-37.5Al-2ONb at\% Alloy. PB94-211091 03,331 Phase Equilibria in the Systems $\mathrm{CaO}-\mathrm{CuO}$ and $\mathrm{CaO}-\mathrm{Bi} 2 \mathrm{O} 3$. PB95-140570

BURTON, $R$

Real Time Compensation for Tool Form Errors in Tuming Using Computer Vision.

BUSATTO G.

Experimental Study of Reverse-Bias Failure Mechanisms in Bipolar Mode JFET (BMFET).

PB95-152997

BUSCHE, R. $M$.

Opportunities for Innovation: Biotechnology.

BusCHOW, K. H. J.

Crystallographic and Magnetic Properties of UAuSn PB95-140521

Incommensurate Magnetic Order in UPtGe

PB95-140513

Magnetic Properties of Single-Crystalline UCu3AI2.

PB95-180717

02,340

BUSH, M. T.

Effect of CF3H and CF3Br on Laminar Diffusion Flames in Normal and Microgravity.

PB96-161831

Effect of CF3H and CF3Br on Laminar Diffusion Flames in Normal and Microgravity.

PB96-161849

01,421

BUSHBY, S. T.

Testing Conformance and Interoperability of BACnet (Trade Name) Building Automation Products.

PB97-111553

BUSSIBA, A.

Charpy Specimen Tests at $4 \mathrm{~K}$.

PB96-190335

03,002

BUTCHER, $K$.

Checking the Net Contents of Packaged Goods as Adopted by the 79 th National Conference on Weights and Measures, 1994, Third Edition, Supplement 4.

PB95-182226

00,484

BUTCHER, K. S.

Uniform Laws and Regulations in the Areas of Legal Metrology and Motor Fuel Quality as Adopted by the 80th National Conference on Weights and Measures 1995. 1996

PB96-172309

02,927

Uniform Laws and Regulations in the Areas of Legal Metrology and Motor Fuel Quality, 1994 as Adopted by the 79th National Conference on Weights and Measures 1994.

PB95-174470

02,909 
Interface Properties for Ceramic Composites from a Single Fiber Pull-Out Test.

03,135

Micro-Mechanical Aspects of Asperity-Controlled Friction in Fiber-Toughened Ceramic Composites.

03,136

PB94-199536

BUTLER, J. J.

Calibration in the Earth Observing System (EOS) Project. Part 2. Implementation

04,842

Organization and Implementation of Calibration in the EOS Project. Part 1

PB96-179437

BUTLER, K. M.

04.841

Heat Transfer in an Intumescent Material Using a Three-Dimensional Lagrangian Model.

T. A.

00,408

BUTLER, T. A.

Preparation and Certification of a Rhodium Standard Reference Material Solution.

PB94-185071

00,529

BUTTERBAUGH, J. W.

Gaseous Electronics Conference Radio-Frequency Reference Cell: A Defined Parallel-Plate Radio-Frequency System for Experimental and Theoretical Studies of PlasmaProcessing Discharges.

PB94-172327

04,404

BUXTON, G. V.

Critical Review of Rate Constants for Reactions of Tran. sients from Metal lons and Metal Complexes in Aqueous Solution.

PB96-1

01,109

BYRAM, S. K.

Using NIST Crystal Data within Siemens Software for FourCircle and SMART CCD Diffractometers.

PB97-109110

04,803

BYRD, E.

Degradation of Powder Epoxy Coated Panels immersed in a Saturated Calcium Hydroxide Solution Containing Sodium Chloride.

PB96-101050

01,344

Development of a Method for Measuring Water-Stripping Resistance of Asphalt/Siliceous Aggregate Mixtures.
PB96-197249

Development of a Method for Measuring Water-Stripping Resistance of Asphalt/Siliceous Aggregate Mixtures. 01,329 Development of a Test Method for Leaching of Lead from Lead-Based Paints Through Encapsulants.

PB96-154984

03,128

Materials-Science Based Approach to Phenol Emissions from a Flooring Material in an Office Building.
PB97-118749 Source of Phenol Emissions Affecting the Indoor Air of an Office Building.

PB94-154382

03,600

BYRD, G. D.

Determination of 3-Ouinuclidinyl Benzilate (Onb) and Its Major Metabolites in Urine by Isotope Dilution Gas Chromatography Mass Spectrometry.

03,492

BYRNE, J.

Preparation and Characterization of (6) LiF and (10)B Reference Deposits for the Measurement of the Neutron Lifetime. Problems Related to the Determination of Mass Densities of

Evaporated Reference Deposits.
PB95-163226

03.941

BYRNE, P. B.

Dynamic Phenomena on the RS Canum Venaticorum Binary II Pegasi in August 1989. 1. Observational Data.
PB94-211067

Observing Stellar Coronae with the Goddard High Resolution Spectrograph. I. The dMe Star AU Microscopii.
PB96-102777 Rotational Modulation and Flares on RS Canum Venaticorum and BY Draconis Stars. XVI. IUE Spectroscopy and VLA Observations of C1182(=V 1005 Orionis) in October 1983

CABEZAS, $H$

00,050

Statistical Thermodynamics of Phase Separation and Ion Partitioning in Aqueous Two-Phase Systems.

01,212

CABLE, J. S.

One Gigarad Passivating Nitrided Oxides for $100 \%$ Internal Ouantum Efficiency Silicon Photodiodes.

Ouantum Efficien
PB94-185485

CABRERA, $B$.

02,119

Hot-Electron Microcalorimeter for X-ray Detection Using a Superconducting Transition Edge Sensor with Electrothermal Feedback.

PB96-200399

04,792

Noise Reduction in Low-Frequency SOUID Measurements with Laser-Driven Switching.

02,081
Self-Biasing Cryogenic Particle Detector Utilizing Electrothermal Feedback and a SOUID Readout.
PB96-102538 Trapped Vortices in a Superconducting Microbridge.
PB95-141149 04,554 CACCIUTTOLO, M. A.

Formation of DNA-Protein Cross-Links in Cultured Mammalian Cells Upon Treatment with Iron Ions.

PB96-137724

03,651

\section{CACIARI, $M$.}

Retention of Halocarbons on a Hexafluoropropylene Epoxide Modified Graphitized Carbon Black. Part 1. MethaneBased Compounds.

PB95-175196

03,272

Retention of Halocarbons on a Hexafluoropropylene Epox ide-Modified Graphitized Carbon Black. 3. Ethene-Based Compounds.

03,286

Retention of Halocarbons on a Hexafluoropropylene Epox ide-Modified Graphitized Carbon Black. 4. Propane-Based Compounds.

CAGAN, $J$.

HVAC CAD Layout Tools: A Case Study of University/lndustry Collaboration

CAGE, M. E.

Anomalous Behavior of a Ouantized Hall Plateau in a HighMobility Si Metal-Oxide-Semiconductor Field-Effect TransisPB95-164174

02,354

Design Challenges in a Commercial Ouantum Hall EffectBased Resistance Standard.

02,263

Evidence That Voltage Rather Than Resistance is Ouantized in Breakdown of the Ouantum Hall Effect.

01,868

179163

Potential and Current Distributions Calculated Across Ouantum Hall Effect Sample at Low and High Currents.
PB96.122106 Precision Tests of a Ouantum Hall Effect Device DC Equivalent Circuit Using Double-Series and Triple-Series Connec. PB96.159256 01,953

Ouantized Dissipation of the Ouantum Hall Effect at High Currents.

PB94-199395 04,472

Sources of Uncertainty in a DVM-Based Measurement System for a Ouantized Hall Resistance Standard.

PB94-219334

01,884

Spectroscopic Study of Ouantized Breakdown Voltage States of the Ouantum Hall Effect.

04,730

Using Ouantized Breakdown Voltage Signals to Determine the Maximum Electric Fields in a Ouantum Hall Effect Sample.

02,375

CAHN, J. W.

Anisotropy of Interfaces in an Ordered Alloy: A Multiple-

Order-Parameter Model
PB96-131594

04,741

Evolution Equations for Phase Separation and Ordering in Binary Alloys.

02,979

Exponentially Rapid Coarsening and Buckling in Coherently Self-Stressed Thin Plates.

PB95-202347

04,821

Linking Anisotropic Sharp and Diffuse Surface Motion Laws via Gradient Flows.

Microstructural Evolution in Two-Dimensional Two-Phase Polycrystals.

Modeling the Evolution of Structure in Unstable Solid Solvtion Phases by Diffusional Mechanisms.

PB94-199403

03,324

Neutron Scattering Structural Study of AICuFe Ouasicrystals Using Double isotopic Substitution.
PB94-200458 04,485
PB94-200458

PBs4-198561

02,310

RDFs and Fe-Fe Pair Correlations in an AICuFe icosahedral Alloy by Double Isotopic Substitution.
PB94-172129 04,439
Singularities in Minimum Surface Energy Problems and Their Influence in Surface Motion

PB94-199411

04,473

Stability, Microstructural Evolution, Grain Growth, and Coarsening in a Two-Dimensional Two-Phase Microstructure

PB94-19942

03,325

CAI, $\mathrm{H}$.

Deformation and Fracture of Mica-Containing Glass-Ceramics in Hertzian Contacts.

PB96-179452

Flaw Tolerance and Toughness Curves in Two-Phase Particulate Composites: SiC/Glass System.

03.081

Indentation Fatigue: A Simple Cyclic Hertzian Test for Measuring Damage Accumulation in Polycrystalline Ceram ics.

03,084

Model for Microcrack Initiation and Propagation beneath Hertzian Contacts in Polycrystalline Ceramics.

CAI, X. Y.

Electromagnetic Coupling Character of (001) Twist Bound

aries in Sintered Bi2Sr2CaCu2O8+x Bicrystals.
PB96-176573

01,963

Weak-Link-Free Behavior of High Angle $\mathrm{YBa} 2 \mathrm{Cu} 3 \mathrm{O} 7-\mathrm{x}$ Grain Boundaries in High Magnetic Fields.

PB94-198421

04,459

CAILLAULT, J.P

IUE Observations of Solar-Type Stars in the Pleiades and the Hyades.

CAIRELLI, S. G.

Documentation for Immediately Dangerous to Life or Health Concentrations (1DLHs)

PB94-195047

03,602

CAKIROGLU, $F$

Comparisons of Some NIST Fixed-Point Cells with Similar Cells of Other Standards Laboratories.

PB97-119242

00,655

CALCOTT, T. A.

Improved Reflectometry Facility at the National Institute of Standards and Technology.

PB96-160338

04,087

New NIST/ARPA National Soft X-ray Reflectometry Facility.

PB96-158092

CALEDONIA, G. E.

Collision-Induced Emission in the Fundamental VibrationRotation Band of $\mathrm{H} 2$

PB94-199445

03,811

CALHOUN, J. M.

Assay of the Eluent from the Alumina-Based Tungsten-188Rhenium-188 Generato

PB94-200482

03,829

Liquid-Scintillation Counting Techniques for the Standardization of Radionuclides Used in Therapy

PB97-110084

03,709

Radioassays

Review of the USCEANIST Measurement Assurance Pro.

gram for the Nuclear Power Industry.

03,712

System for Intercomparing Standard Solutions of Beta-Particle Emitting Radionuclides.

PB96-159637

03,707

\section{CALLAHAN, A. P.}

Assay of the Eluent from the Alumina-Based Tungsten-188Rhenium-188 Generato

PB94-200482

03,829

CALLCOTT, T. A.

Al L2,3 Core Excitons in AlxGa1-x as Studied by Soft-X-ray Reflection and Emission.

PB96-157839

04,067

Barium Contributions to the Valence Electronic Structure of YBa2Cu307-delta, PrBa2Cu307-delta, and Other Barium Containing Compounds.

B96-158019

04,076

Charge-Transfer-Induced Multiplet Structure in the PB96-163746

04,102

Cooper M(sub II,III) X-ray-Emission Spectra of Copper Oxides and the Bismuth Cuprate Superconductor. 
CALSOU, $P$

Modification of Deoxyribose-Phosphate Residues by Extracts of Ataxia Telangiectasia Cells.

03,458

CALVANO, N. J.

Study to Determine the Most Important Parameters for Evaluating the Resistance of Soft Body Armor to Penetration by Edged Weapons.

PB94-158573

CALVAYRAC, $Y$.

Neutron Scattering Structural Study of AlCuFe Ouasicrystals Using Double Isotopic Substifution.

PB94-200458

RDFs and Fe-Fe Pair Correlations in an AlCuFe Icosahedral Alloy by Double Isotopic Substitution. PB94-172129

04,439

\section{CAMELL, D.}

Standard Source Method for Reducing Antenna Factor Errors in Shielded Room Measurements.

CAMELL, D. G.

Assessment of Data by a Second-Order Transfer Function. PB95-182390

03,760

Data Evaluation of a Linear System by a Second-Order

Transfer Function.
PB96-200282

Measurements of Shielding Effectiveness and Cavity Characteristics of Airplanes.

00,030

Radiated Emissions and Immunity of Microstrip Transmission Lines: Theory and Measurements.

02,238

TEM/Reverberating Chamber Electromagnetic Radiation

Tesi Facility at Rome Laboratory.
PB96-155023

CAMERON, J. M.

Churchıll Eisenhart, 1913-1994.

PB96-137740

CAMPANA, C. F.

03,447

Usıng NIST Crystal Data withın Siemens Software for FourCircle and SMART CCD Diffractometers.

PB97-109110

CAMPBELL, $D$.

Effect of Three Sterilization Techniques on Finger Pluggers. PB94-216090

CAMPBELL, E. J.

Experimental Sidies of Line Shapes from a Balle-Flygare Spectrometer.

CAMPISI, G. J.

00,796

Electron and Hole Trapping in Irradiated SIMOX, ZMR and BESOI Buried Oxides.

PB96-160320

01,956

CAMUS, $P$. $P$

Performance of a Reftectron Energy Compensating Mirror PB94-199460

00,547

CAMUS, T. A.

Calculating Time-to.Contact Using Real-Time Ouantized Optical Flow.

CANALES, N.

01,604

Planar Near-Field Measurements and Microwave Holography for Measuring Aperture Distribution on a $60 \mathrm{GHz} A \mathrm{C}$. tive Array Antenna.

PB96-167366

03,762

CANDELA, G. T.

Bringing Natural Language Information Retrieval Out of the Closet.

PB94-172335

Comparison of FFT Fingerprint Filtering Methods for Neural Network Classification.

01,840

NIST Form-Based Handprint Recognition System.

PB94-217106

01,838

PCASYS: A Pattern-Level Classification Automation System for Fingerprints.

CANEAU, $\mathrm{C}$.

01,853

Range Statistics and Rutherford Backscattering Studies on Fe. Implanted In0.53Ga0.47As.

PB95-126397

04,535

CANFIELD, L. R

One Gigarad Passivating Nitrided Oxides for $100 \%$ Internal Ouantum Efficiency Silicon Photodiodes.

PB94-185485

02,119

Silicon Photodiodes Optimized for EUV and Soft X-Ray Regions.

02.124

Stable Silicon Photodiodes for Absolute Intensity Measurements in the VUV and Solt $X$-ray Regions.

PB97-110175

04,135

CANTILLO, A.

National Status and Trends Program Specimen Bank: Sampling Protocols, Analytical Methods, Results, and Archive PB97-119226

02,598
CAO, G.

Magnetic Ordering of the $\mathrm{Cu}$ Spins in $\mathrm{PrBa} 2 \mathrm{Cu} 3 \mathrm{O} 6+x$. PB95-140547

04,545

CAO, L. $X$.

Determination of Surface Roughness from Scattered Light. PB94-216520

Light.

CAPPARELLI, $\mathbf{R}$.

Displacement Method for Machine Geometry Calibration. PB95-152088

02,946

\section{CAPPELLETTI, R. L.}

Phase Transitions in Solid C70: Supercooling, Metastable Phases, and Impurity Effect.

PB95-150090 00,914

Rotational Dynamics of Solid C70: A Neutron-Scattering Study.

00,755

Rotational Dynamics of Solid C70: A Neutron-Scattering

$\begin{array}{ll}\text { Study. } & 00,949\end{array}$

CAPPONI, J. J.

Crystal Structure of Pb2Sr2YCu3O8+delta with delta=1.32, $1.46,1.61,1.71$, by Powder Neutron Diffraction.

PB94-216314

04,518

CARASSO, A. S.

Image Restoration and Diffusion Processes.

PB95-153003

01,843

Infinite Divisibility and the Identification of Singular Waveorms

02,701

Overcoming Hoelder Continuity in III-Posed Continuation Problems.

PB95-202354

03,416

Slow Evolution from the Boundary: A New Stabilizing Constraint in III-Posed Continuation Problems.

03,418

Slowly Divergent Space Marching Schemes in the Inverse

$\begin{array}{ll}\text { Heat Conduction Problem. } & 03,812 \\ \text { PB94-199486 }\end{array}$

CAREY, C. M.

Cyclic Polyamine Ionophore for Use in a Dibasic Phosphate-Selective Electrode.

01,008

Distribution of Fluoride in Saliva and Plaque Fluid After a $0.048 \mathrm{~mol} / \mathrm{L} \mathrm{NaF}$ Rinse.

PB95-151205

03,561

Intensive Swimmıng: Can It Affect Your Patients' Smiles.

PB96-123666

03,570

CARINO, N.

Northridge Earthquake, 1994. Performance of Structures,

Lifelines and Fire Protection Systems. 00,421

Northridge Earthquake 1994: Performance of Structures,

Lifelines, and Fire Protection Systems.
PB94-207461

04,825

Performance of HUD-Affiliated Properties during the January 17, 1994 Northridge Earthquake.

PB95-174488

00,443

CARINO, N. J.

Detection of Voids in Grouted Ducts Using the Impact-Echo Method.

PB94-18512

01,306

Effects of Testing Variables on the Measured Compressive Strength of High-Strength ( $90 \mathrm{MPa}$ ) Concrete.

00,445

Effects of Testing Variables on the Strength of HighStrength (90 Mpa) Concrete Cylinders.

00,456

High-Performance Concrete: Research Needs to Enhance Its Use.

PB95-180147

01,322

Maturity Functions for Concrete Made with Various Cements and Admixtures.

PB94-199502

Maturity Method.

PB94-199494

01,314

Nondestructive Testing of Concrete: History and Challenges.

00,385

Prediction of

PB95-220448

PB96-112180 01,324

Recent Development in Nondestructive Testing of Concrete.

PB96-122445
01,325

Shear Design of High-Strength Concrete Beams: A Review of the State-of-the-Art.

01,330

CARLSON, A. D.

Intermediate Structure in the Neutron-Induced Fission Cross Section of 236

03,802

Measurements of the $(235) \mathrm{U}(\mathrm{n}, \mathrm{f})$ Cross Section in the 3 to $30 \mathrm{MeV}$ Neutron Energy Region.

04,172

Measurements of the $(237) N p(n, f)$ Cross Section.

Neutron Standard Cross Sections in Reactor Physics: Need and Status.

PB94-199510

CARLSON, N. M.

03,813

Ultrasonic Sensing of GMAW: Laser/EMAT Defect Detection System.

CARLSSON, A.

Lattice Imperfections Studied by Use of Lattice Green's Functions.

CARLSSON, A. E.

Dislocation Core-Core Interaction and Peierls Stress in a Model Hexagonal Lattice.

PB96-162003

Novel DNA N-Glycosylase Activity of E. coli T4 Endonuclease $V$ That Excises 4,6-Diamino-5 Formamidopyrimidine from DNA, a UV-Radiation- and Hydroxyl Radical-Induced Product of Adenine.

PB96-160478

03,549

CARNAHAN, L.

Security in Open Systems.

(105383

01,473

ARNAHAN, L. J.

Guideline for the Analysis of Local Area Network Security. Category: Computer Security, Subcategory: Risk Analysis and Contingency Planning.

FIPS PUB 191

01,799

Keeping Your Site Comfortably Secure: An Introduction to Internet Firewalls.

CARNEY, D.

02,730

Next Generation Computer Resources: Reference Model for Project Support Environments (Version 2.0).

PB94-143401

01,677

CARO, T.

Evaluation of Survey Procedures for Determining Occupant Load Factors in Contemporary Office Buildings.

PB97-11622

00,238

CARO, T. C.

Survey of Fuel Loads in Contemporary Office Buildings. PB97-114235

CAROSELLA, C. A.

Effect of Beam Voltage on the Properties of Aluminum Nitride Prepared by Ion Beam Assisted Deposition. PB97-118616

01,995

CARPENTER, J. A.

Dielectric Properties Measurements and Data.

PB94-172186

01,876

Measurements of Properties of Materials in Electronic Pack-

PBing.

01,973

NIST/NCMS Program on Electronic Packaging: First Up-

PB96-204086

03,008

Overview of U.S. Government Advanced Packaging Programs.

PB96-200845

02,443

Photonic Materials: A Report on the Results of a Workshop. Held in Gaithersburg, Maryland on August 26-27, 1992, Volume 1.

CARPENTER, K. G.

First Results from the Goddard High-Resolution Spectrograph: The Chromosphere of Tauri. 


\section{PERSONAL AUTHOR INDEX}

CERNY, E. H.

Micro-Mechanical Aspects of Asperity-Controlled Friction in Fiber-Toughened Ceramic Composites.

PB94-199536

Transient Subcritical Crack-Growth Behavior in Trans-
formation-Toughened Ceramics.
PB94-200656
PART,038 PB94-200656

03,038

Interdigitated Stacked P-I-N Multiple Quantum Well ModulaPB97-112296

02,455

Scaling of the Nonlinear Optical Cross Sections of GaAsAIGaAs Multiple Ouantum-Well Hetero n-i-p-i's.

PB96-102793

CARVER, G. P.

Federal Metric Progress in 1993

Metric for Success

PB94.187630

02,600

Metric Path to Global Markets and New Jobs: A Questionand-Answer and Thematic Discussion.

Metrication.

PB94-172079

02,601

METRICATION: An Economic Wake-Up Call for Surveyors and Mappers.
PB96-159629

03,680

Program of the Manufacturing Engineering Laboratory. 1995. Infrastructural Technology, Measurements, and Standards for the U.S. Manufacturing Industries.

PB95-188835

02,754

CASASSA, M. P.

Fragment Energy and Vector Correlations in the OvertonePumped Dissociation of $\mathrm{HN} 3 \times(1) \mathrm{A}^{\prime}$

00,802

Fragment State Correlations in the Dissociation of NO. HF $(v=1)$.
PB95.164430

00,982

Product Kinetic Energles, Correlations, and Scattering Anisotropy in the Bimolecular Reactor $\mathrm{O}((1) \mathrm{D})+\mathrm{H} 2 \mathrm{O}$ yields PB94-212792

00.838

Product State Correlations in the Reaction of $O((1) D)$ and $\mathrm{H} 2 \mathrm{O}$ in Bimolecular Collisions and in $03 . \mathrm{H} 2 \mathrm{O}$ Clusters-Translation.

PB95-153011

00,946

Vibrational Predissociation Dynamics of Overtone-Excited HN3.

00,691

CASELLA, R. C

Roles of Local Classical Acceleration and Spatial Separation in the Neutral Particle Analogs of the Aharonov-Bohm Phases.

PB95-202362

03,976

CASEY, S. M.

Laser Vacuum Ultraviolet Single Photon Ionization Probing of III-V Semiconductor Growth.

PB95-202370

04,691

Single Photon Ionization, Laser Optical Probe Technique for Semiconductor Growth.

PB95-202776

01,032

CASHMAN, J. R.

Histopathology, Blood Chemistry, and Physiological Status of Normal and Moribund Striped Bass ('Morone saxatilis') Involved in Summer Mortality ('Die-Off') in the SacramentoSan Joaquin Delta of California

PB94-198157

00,034

Interim Testing Artifact (ITA): A Performance Evaluation

Interim Testing Artifact (ITA): A Performance Evaluation
System for Coordinate Measuring Machines (CMMs). User

PB95-210589 02,914

NIST SRM 9983 High-Rigidty Ball-Bar Stand. User Manual.

PB95-255840 02,669

Two New Probes for a Coordinate Measuring Machine.

PBS5-16

02,653

User's Guide to NIST SRM 2084: CMM Probe Performance Standard.

PB94-206109

02,709

ASS, G. R.

Sources of Urban Contemporary Carbon Aerosol.

PB95-175659

CASSEL, K. W.

Gravity-Current Transport in Building Fires.

Transport by Gravity Currents in Building Fires.

PB97-119325

CASSIDY, M. M. Field Evaluation of the System for Calibration of the Mar-
shall Compaction Hammer. shall Compaction
PB95-190674

01,323

System for Calibration of the Marshall Compaction Hammer.

PB94-145661

01,303

Hot-Electron Microcalorimeters for $\mathrm{X}$-ray and Phonon De-

tection

04,644
CASWELL, R. S.

Measurement of Absorbed Dose of Neutrons, and of Mixtures of Neutrons and gamma rays.

03,710

Microdosimetry and Cellular Radiation Effects of Radon Progency in Human Bronchial Airways.

03,625

Monte Carlo and Analytic Methods in the Transport of Electrons, Neutrons, and Alpha Particles.

04,033

Neutron Energy Deposition on the Nanometer Scale. PB95-152260

03,624

Neutron Measurement Intercomparisons Sponsored by CCEMRI, Section 3 (Neutron Measurements).

03,819

Radon in the Lung.

PB97.110035

03,638

Systematics of Alpha-Particle Energy Spectra and Lineal Energy $(Y)$ Spectra for Radon Daughters.

PB94-185139

03,615

Diffraction of X-rays at the Far Tails of the Bragg Peaks
PB94-199924

CATRON, B. A.

Generic Manufacturing Controllers.

02,818

Implementing a Transition Manager in the AMRF Cell Con

troller.

02,817

Crystal Structure of Pb2Sr2YCu3O8+delta with delta $=1.32$ Cry

PB94-216314
Neutron Powder Diffraction Study of the Crystal Structure of Neutron Powder Diffraction Study of the Crystal Structure of
YSr2AICu2O7.
PB94-212073
04,499

Neutron Powder Diffraction Study of the Structures of La 1.9Ca1.1Cu2O6 and La1.9Sr1.1Cu2O6+Delta.

PB95-140042

04,538

Observation of Oscillatory Magnetic Order in the Antiferromagnetic Superconductor HoNi2B2C.
PB95-180303

CAVALLI, A. R.

Formal Methods in Conformance Testing: Result and PerFormal Methods in Conformance Testing: Result and Per-
Spectives.
PB95-153029 01,710

CAVANAGH, R. R.

01,710

Characterization of the Interaction of Hydrogen with Iridium Clusters in Zeolites by Inelastic Neutron Scattering SpecPB95-180741

01,013

Dynamics of Nonthermal Reactions: Femtosecond Surface Chemistry.

00,688

Hot Carrier Excitation of Adlayers: Time-Resolved Measurement of Adsorbate-Lattice Coupling.

00,758

Laser-Induced Desorption of NO from Si(111): Effects of Coverage on NO Vibrational Populations.

00,959

Neutron and Raman Spectroscopies of 134 and $134 \mathrm{a}$ Hydrofluorocarbons Encaged in Na-X Zeolite.

03,00

Photodecomposition Dynamics of Mo(CO)6/Si(111) $7 \times 7$ : CO internal State and Translational Energy Distributions--TransInternal State and Translational Energy Distributions--TransPB94-199288

00,687

Photodecomposition of $\mathrm{Mo}(\mathrm{CO}) 6 / \mathrm{Si}(111) 7 \times 7$ : CO State-Resolved Evidence for Excited State Relaxation and OuenchPB95-180154

01,009

Photodesorption Dynamics of CO from Si(111): The Role of Surface Defects

Picosecond Measurement of Substrate-to-Adsorbate Energy Transfer: The Frustrated Translation of $\mathrm{CO} / \mathrm{Pt}(111)$--Trans-

PB95-126041

00,895

Time-Resolved Measurements of Energy Transfer at Surfaces.

00,913

Time-Resolved Measurements of Energy Transter at Sur-

PB95-153037

00,947

Time-Resolved Probes of Surface Dynamics.

PB94-199957 00,803

Ultrafast Time-Resolved Infrared Probing of Energy Transfer at Surfaces.

00,620

Vibrational Relaxation Measurements of Carbon Monoxide on Metal Clusters.

PB94-211810

00,820

CAVCEY, K. H.

Crosstalk between Microstrip Transmission Lines.

Pr-135639

Crosstalk between Microstrıp Transmission Lines. (NIST Reprint).

02,225

Radiated Emissions and Immunity of Microstrip Transmission Lines: Theory and Measurements.

PB96-162649

02,238

CAVICCHI, R.

Reactivity of Pd and Sn Adsorbates on Plasma and Thermally Oxidized $\mathrm{SnO} 2(1$ to

PB94-199973 Tin Oxide Gas Sensor Fabricated Using CMOS Micro-
Hotplates and In-situ Processing. PB95-150603

\section{CELEBI, $M$.} Comparison of Responses of a Select Number of Buildings
to the $10 / 17 / 1989$ Loma Prieta (California) Earthquake and Low-Level Amplitude Test Results.

PB96-159645

00,467

Dynamic Characteristics of Five Tall Buildings during Strong and Low-Amplitude Motions.

94.199981

00,427

Response of Buildings to Ambient Vibration and the Loma Prieta Earthquake: A Comparison.

CELOTTA, R, J.

Atomic Manipulation of Polarizable Atoms by Electric Field Directional Diffusion.

PB95-150587 04,572

Determination of Complex Scattering Amplitudes in Low-Energy Elastic Electron-Sodium Scattering

03,869

Electron-atom collision studies using optically state selected

beams. Progress report, May 15, 1987--May 14, 1988.
DE95004446

03,776

Electron-atom collision studies using optically state selected beams. Progress report, May 15, 1988--May 14, 1991. Influence of $\mathrm{Cr}$ Growth on Exchange Coupling in $\mathrm{Fe} / \mathrm{Cr}$ /

Fe(100).

04,562

Influence of Thickness Fluctuations on Exchange Coupling in $\mathrm{Fe} / \mathrm{Cr} / \mathrm{Fe}$ Structures.

PB96-135371

04,745

Laser-Focused Atomic Deposition.

PB95-161618

04,604

Laser Focused Atomic Deposition

PB95-180659

04,685

Magnetic Moments in $\mathrm{Cr}$ Thin Films on Fe(100)

PB95-108429

04,525

Manipulation of Adsorbed Atoms and Creation of New Structures on Room-Temperature Surfaces with a Scanning B 95-151536

Nanofabrication of a Two-Dimensional Array Using LaserFocused Atomic Deposition

PB96-119417

04,732

Nanostrucfure Fabrication via Direct Writing with Atoms Fo. cused in Laser Fields.

Nanostructure Fabrication via Laser-Focused Atomic Deposition (invited)

PB96-204094

04,132

Oscillatory Exchange Coupling in $\mathrm{Fe} / \mathrm{Au} / \mathrm{Fe}(100)$

Scanning Tunneling Microscopy Study of the Growth of $\mathrm{Cr} /$ Fe(001): Correlation with Exchange Coupling of Magnetic

PB95-150330

04,568

SEMPA Studies of Exchange Coupling in Magnetic Multilayers.
PB96-164074 
CERNYAR, E. W.

Importance of Unraveling Memory Propagation Effects in Interpreting Data on Partial Discharge Statistics.

PB95.163572

01,901

\section{CESSNA, J.}

Assay of the Eluent from the Alumina-Based Tungsten-188Rhenium- 188 Generato

PB94-200482

03,829

International Radon-in-Air Measurement Intercomparison Using a New Transfer Standard.

03,708

Liquid-Scintillation Counting Techniques for the Standard zation of Radionuclides Used in Therapy.

Standardization and Decay Scheme of Rhenium-186. PB94-200490

03,830

CESSNA, J. T.

Radioassays of Yttrium-90 Used in Nuclear Medicine.
PB97-110100 PB97-110100 03,522 ticle Emitting Radionuclides.

PB96-159637

03,707

CEZAIRLIYAN, A

Dynamic Measurements of Thermophysical Properties of Metals and Alloys at High Temperatures by Subsecond Pulse Heating Techniques.

N94-25124/6

03,309

Dynamic Technique for Measuring Normal Spectral Emissivity of Electrically Conducting Solids at High Tempera-
tures with a High-Speed Spatial Scanning Pyrometer. tures with a High

High-Speed Spatial Scanning Pyrometer.

03,921

PB94-200003

02,636

High-Temperature Laser-Pulse Thermal Diffusivity Appara-

PB94-185147

02.631

Issues in High-Speed Pyrometry.

02,693

Measurement of Surface Tension of Tantalum by a Dynamic Technique in a Microgravity Environment.
PB95-161667

Measurement of the Heat of Fusion of Tungsten by a Microsecond-Resolution Transient Technique.

03,400

Measurements of Thermophysical Properties of Nickel Nea Its Melting Temperature by a Microsecond-Resolution Transient Technique.

Sient Technique.
PB96-102579

04,210

Millisecond-Resolution Pulse Heating System for SpecificHeat Measurements at High Temperatures.

PB94-199999

02,635

Radiance Temperature (in the Wavelength Range 519-906 $\mathrm{nm}$ ) of Tungsten at its Melting Point by a Pulse-Heating Technique.

03,397

Radiance Temperatures at $1500 \mathrm{~nm}$ of Niobium and Molyb denum at Their Melting Points by a Pulse-Heating Technique.
PB97-118699

04,167

Radiance Temperatures (in the Wavelength Range 523-907 $\mathrm{nm}$ ) of Group IV B Transition Metals Titanium, Zirconium, and Hafnium at Their Melting Points by a Pulse-Heating Technique. PBechnique.

03,356

Radiance Temperatures (in the Wavelength Range 523-907 nm) of Group IVB Transition Metals Titanium, Zirconium, and Hafnium at Their Melting Points by a Pulse-Heating Technique.
PB96-135025

02,677

Simultaneous Measurement of Normal Spectral Emissivity by Spectral Radiometry and Laser Polarimetry at High Tem by Spectral Radiometry and Laser Polarimetry at High Temperatures in Pulse-Heating

02,694

Thermal Diffusivity of POCO AXM-501 Graphite in the Range 1500 to $2500 \mathrm{~K}$ Measured by a Laser-Pulse Tech-

PB94-185022

03,013

Wavelength Dependence of Normal Spectral Emissivity of High-Temperature Metals at Their Melting Point PB94-200011

03,398

CHABAL, Y.J.

Silicon Surface Chemistry by IR Spectroscopy in the Mid- to Far-IR Region: $\mathrm{H} 2 \mathrm{O}$ and Ethanol on $\mathrm{Si}(100)$.

PB96-138565

01,097

CHABAN, E. E.

Silicon Surface Chemistry by IR Spectroscopy in the Mid- to Far-IR Region: $\mathrm{H} 2 \mathrm{O}$ and Ethanol on $\mathrm{Si}(100)$.

PB96-138565

07,097

CHACON, L.

Effect of Sm2BaCuO5 on the Properties of Sintered (Bulk) YBa2Cu3O6+x

CHACONAS, $G$

Measurıng the Stability of Three Copper Alloys

CHACONAS, $K$.

Three Dimensional Position Determination from Motion

PB95-107108
ChaKARIAN, $v$.

Influence of Coadsorbed Potassium on the Electron-Stimulated Desorption of $F(+), F(\cdot)$, and $F(*)$ from $P F 3$ on $\mathrm{Ru}(0001)$.

PB96-157946

04,072

CHAKOUMAKOS, B. C.

Preparation, Crystal Structure, Dielectric Properties, and Magnetic Behavior of Ba2Fe2Ti4O13.

PB96- 186176

CHAKRABARTI, C.

01,162

VLSI Architectures for Template Matching and Block Match-

ing.

CHALKLEY, M. W.

01,834

Publication and Presentation Abstracts, 1993. (Published by Paffenbarger Research Center and Center of Excellence for Materials Science Research).

PB95-153052

03,562

Publication and Presentation Abstracts, 1994.

PB96-176623

Publication and Presentation Abstracts, 1995

03,577

6- 164082

Publication and Presentation Abstracts, 1996. PB97-122238

03,576

Publications and Presentation Abstracts, 1995. (Published by Patfenbarger Research Center and Center of Excellence for Materials Science Research)

PB96-119250

CHAMBERS, G. P.

03,568

Pure Element Sputtering Yield Data: Appendix 4.

PB94-20003

00,805

Flaw-Tolerance and Crack-Resistance Properties of Alumina-Aluminum Titanate Composites with Tailored Microstructures.

03,101

Interface Properties for Ceramic Composites from a Single Fiber Pull-Out Test

PB94-199361

03,135

CHAN, W. R.

Experimental and Numerical Studies on Two-Dimensional Gravity Currents in a Horizontal Channel.

PB94-165941

01,359

CHANDLER-HOROWITZ, D.

Characterization of the $\mathrm{ZnSe} / \mathrm{GaAs}$ Interface Layer by TEM and Spectroscopic Ellipsometry.

04,655

Comparison of Techniques for Nondestructive Composition Measurements in CdZnTe Substrates.

PB96-103098

02,703

Determination of the Optical Constants of $\mathrm{ZnSe}$ Films by Spectroscopic Ellipsometry

04,656

Double Modulation and Selective Excitation Photoreflectance for Characterizing Highly Luminescent Morphology.

02,452

Double-Modulation and Selective Excitation Photoreflectance for Wafer-Level Characterization of Ouantum-Well Laser Structures.

PB96-16732

04,372

High-Accuracy Principal-Angle Scanning Spectroscopic Ellipsometry of Semiconductor Intertaces. 02,427 Interface Roughness-Induced Changes in the Near-E(sub 0) Spectroscopic Behavior of Short-Period AIAs/GaAs Superlattices.

PB94-185154

02,118

Optical Conductivity of Single Crystals of Ba1$x M \times B i O 3(M=K, R b, x=0.04,0.37)$.

PB94-185329

04,447

Spectroscopic Ellipsometry Determination of the Properties of the Thin Underlying Strained Si Layer and the Roughness at SiO2/Si Intertace.

PB95-150157

04,560

CHANDRASEKHARAIAH, M. S.

Disilicides of Tungsten, Molybdenum, Tantalum, Titanium, Cobalt, and Nickel, and Platinum Monosilicide: A Survey of Their Thermodynamic Properties.

PB94-168580

00,752

CHANG, K. J.

Electronic Correlations and Satellites in Superconducting

PB94-200045

04,477

HANG, R. F.

High-Speed Spatial Scanning Pyrometer.

PB94-200003 eter

PB96-146618

01,123

CHANG, $S$.

Security in Open Systems

PB95-105383

01,473

Preliminary Functional Specifications of a Prototype Electronic Research Notebook for NIST.

00,012

CHANG, S. S.

Effect of Curing History on Ultimate Glass Transition Tem. Hork Structure of Crosslinking Polym PB94-200052

CHANG, $Y$.

Introduction to Traffic Management for Broadband ISDN. PB94-142494

CHANG, Y. A.

Thermodynamic Assessment and Calculation of the Ti-Al System.

CHANG, Y. M.

NIST Capacitance Measurement Assurance Program PB94-200060 02,254

NIST Measurement Assurance Program for Capacitance Standards at $1 \mathrm{kHz}$

CHANTLER, C. T.

02,276

Energy Dependences of Absorption in Beryllium Windows and Argon Gas.

04,020

Flat and Curved Crystal Spectrography for Mammographic

$X$-ray Sources.

PB97-122246

Improvements in Computation of Form Factors.

03,642

PB94-200078

03,820

Photographic Response to X-Ray Irradiation. 1. Estimation of the Photographic Error Statistic and Development of AnaIytic Density-Intensity Equations.

03,821

Photographic Response to X-Ray Irradiation. 2. Correlated

Models.

03,822

Photographic Response to X-Ray Irradiation. 3. Photographic Linearization of Beam-Foil Spectra.

03,823

Polarization Measurements on a Magnetic Ouadrupole Line in Ne-Like Barium.

PB97-113104

04,161

Systematic Correction in Bragg $\mathrm{X}$-ray Diffraction of Flat and Curved Crystals

04,152

Theoretical Form Factor, Attenuation and Scattering Tabulation for $Z=1-92$ from $E=1-10 \mathrm{eV}$ to $E=0.4-1.0 \mathrm{MeV}$

PB96-145594

01,104

PARALA, $P$.

Assessing MOS Gate Oxide Reliability on Wafer Level with Ramped/Constant Voltage and Current Stress.

B96-180112

04,115

in Intrinsic Thin $\mathrm{SiO}$.

PB97-122527

Electric Field Dependent Dielectric Breakdown of Intrinsic $\mathrm{SiO} 2$ Films Under Dynamic Stress.

B96-204102

02,449

Experimental Investigation of the Validity of TDDB Voltage Acceleration Models.

02,304

Field and Temperature Acceleration of Time-Dependent Di- 
CHAR, $K$.

Evidence for Parallel Junctions Within High-Tc GrainBoundary Junctions.

04,657

Half-Integral Constant Voltage Steps in High-Tc Grain Boundary Junctions.

PB94-211216

04,489

CHASE, M. W.

NIST Standard Reference Data Products Catalog, 1994.

PB94-151842

00,727

Numeric Data Distribution: The Vital Role of Data Exchange in Today's World

N95-15937/2 02,622

Thermodynamic Properties of Gas Phase Species of Importance to Ozone Depletion.

PB94-198215

00,126 Thermodynamic Properties of the Group IIA Elements.
PB94-160983

Representing Designs with Logic Formulations of Spatial Relations.

PB97-111561 02,792

Using Logic to Specify Shapes and Spatial Relations in De-

sign Grammars.

02,793

CHATTOPADHYAY, T.

Magnetic Structure and Spin Dynamics of the $\mathrm{Pr}$ and $\mathrm{Cu}$ in PB96-111836

04,036

Temperature Dependence of the Magnetic Excitations in Ordered and Disordered Fe72Pt28.

04,563

CHATTORAJ, D.

Deletion Analysis of the Mini-P1 Plasmid Origin of Replication and the Role of E.coli DnaA Protein.

PB95-163911

03,539

CHATTPADHYAY, T.

Dispersions of Magnetic Excitations of the Pr lons in Pr2CuO4.

CHAUDHURI, D. $K$

Tribological Behavior of 440/Diamond-Like-Carbon Film Couples.

PB96-119714

03,019

Tribometer for Measurements in Hostile Environments.

PB95-180949 02,967

Wear Mechanism Maps of $440 \mathrm{C}$ Martensitic Stainless Steel.

PB96-111810

CHAUDURI, D. $K$.

Friction and Oxidative Wear of $440 \mathrm{C}$ Ball Bearing Steels Under High Load and Extreme Bulk Temperatures.
PB95-175253

CHEETHAM, A.K.

Determination of Complex Structures from Powder Diffraction Data: The Crystal Structure of La3Ti5A115037.
PB95-202966

CHEIM, L. A. V.

Correlations between Electrical and Acoustic Detection of Partial Discharge in Liquids and Implications for Continuous Data Recording

PB96-204490

02,248

Face Recognition Technology for Law Enforcement Applications.

PB94-207768 01,837

General Motion Model and Spatio-Temporal Filters for Computing Optical Flow

Human and Machine Recognilion of Faces: A Su

PB96-111687 01,854

Image Gradient Evolution: A Visual Cue for Danger.

PB96-154562

Motion-Model-Based Boundary Extraction.

PB95-189502 01,849

Reliable Optical Flow Algorithm Using 3-D Hermite Polynomials.

PB94-145620

CHELTON, D. B.

Design and Construction of a Liquid Hydrogen Temperature

Refrigeration System
AD-A286 618/4

02,619

CHEN, C. F.

Cavitation Damage During Flexural Creep of SiAION-YAG Ceramics.

PB94-200110

03,036

CHEN, D. H.

Anomalous Dispersion and Thermal Expansion in LightlyDoped KTa1-xNbxO3.

PB95-152302

04,585

CHEN, D. $X$

Effects of Critical Current Density, Equilibrium Magnetization and Surface Barrier on Magnetization of High Tempera. ture Superconductors.

04,446
Effects of Critical Current Density, Equilibrium Magnetization and Surface Barrier on Magnetization of High Tempera. ture Superconductors.

PB95-153060

04,588

Surface Barrier and Lower Critical Field in $\mathrm{YBa} 2 \mathrm{Cu} 3 \mathrm{O} 7$. delta Superconductors

04,478

CHEN, $\mathrm{H}$.

Neutron Focusing Lens Using Polycapillary Fibers.
PB95-141206

03,889

Neutron Focusing Lens Using Polycapillary Fibers.

895-153078

Reference Relations for the Evaluation of the Materials Properties of Orthorhombic YBa2C $330 \times$ Superconductors.
PB96-176763 04,782

\section{CHEN, H. M.}

Beamcon III, a Linearity Measurement instrument for OptiCal Detectors.

CHEN, L. T.

04,337

Determination of Sulfur in Fossil Fuels by Isotope Dilution Thermal lonization Mass Spectrometry.

PB96-141379

CHEN, M. C.

Characterization of Liquid-Phase Epitaxially Grown $\mathrm{HgCdTe}$

Films by Magnetoresistance Measurements.
PB96-123617

04,738

Characterization of LPE HgCdTe Film by Magnetoresistance.
PB96-157961 02,197

Electrical Characterization of Liquid-Phase Epitaxially Grown Single-Crystal Films of Mercury Cadmium Telluride

by Variable-Magnetic-Field Hall Measurements.

02,177

CHEN-MAYER, $\mathrm{H}$.

Use of Neutron Beams for Chemical Analysis at NIST.
PB97-112437 00,652

CHEN, $\mathrm{S}$.

SPC Artifact for Automated Solder Joint Inspection.
PB96-161716

04,095

Densification of Nano-Size Powders. 1994 Report.

DE94013486

Equipment for Investıgation of Cryogenic Compaction of Nanosize Silicon Nitride Powders. 1993 Report DE94013593

03,028

CHEN, Y.

Localization Model of Rubber Elasticity: Comparison with Torsional Data for Natural Rubber Networks in the Dry State.

PB95-107033

03,195

CHEN, Y. D.

Anionic Triphenylmethane Dye Solutions for Low-Dose Food Irradiation Dosimetry.

PB96-135173

03,715

CHEN, Y. M.

Fracture Mechanics Analysis of Near-Surface Cracks.
PB94-172400

PB94-172400 03,230 strumented Micro-Indentation Experiments.

PB95-152229

03,348

CHEN, Y. T.

Dynamic Shear Modulus Measurements with Four Independent Techniques in Nickel-Based Alloys.

PB94-198900

03,320

CHENG, G. W.

Facile Synthesis of Novel Fluorinated Multifunctional Acrylates.

CHENG, H. S

01,207

Mechano-Chemical Model: Reaction Temperatures in a Concentrated Contact

CHENG, $Y$.

03,227

Prediction of Strengthening Due to $\mathrm{V}$ Additions in DirectCooled Ferrite-Pearlite Forging Steels.

PB96-190251

03.220

CHENG, Y. W.

Hot-Deformation Apparatus for Thermomechanical Process-

ing Simulation

03,207

Microstructure and Tensile Properties of Microalloyed Steel

Forgings.
PB94-172715 03,204

Prediction of the Strength Properties for Plain-Carbon and Vanadium Micro-Alloyed Ferrite-Pearlite Steel.

PB96-123393

03,216

CHEOK, G.

Performance of HUD-Affiliated Properties during the January 17,1994 Northridge Earthquake.

CHEOK, G. S.

00,443

Model Precast Concrete Beam-to-Column Connections Subject to Cyclic Loading

00,438

Partially Prestressed and Debonded Precast Concrete

PB95-153102

00,439

Performance of $1 / 3$-Scale Model Precast Concrete BeamColumn Connections Subjected to Cyclic Inelastic Loads Report No. 4.

PB95-179024

00.444

Seismic Performance Behavior of Precast Concrete Beam-

Column Joints.

00,440

Simplified Design Procedure for Hybrid Precast Concrete Connections.

PB96-154836

00,405

Strengthening Methodology for Lightly Reinforced Concrete Frames: Recommended Design Guidelines for Strengthening with infill Walls.

00,454

CHEONG, H. M.

Use of Pressure for Quantum-Well Band-Structure Characterization.

- 164058

04,779

CHERBAUCICH, C.

Approach to Setting Performance Requirements for Automated Evaluation of the Parameters of High-Voltage Im-

pulses.

CHEREPKOV, N. A

Resonant Two-Color Detachment of $\mathrm{H}(-)$ with Excitation of $\mathrm{H}(\mathrm{n}=2)$

PB95-202552

03,984

CHERNIAVSKY, J. C.

High Integrity Software Standards Activities at NIST.
PB96-112214

Report of a Workshop on the Assurance of High Integrity Software.

PB96-161377

01,763

CHERNOV, A. A.

Convection and Morphological Stability During Directional Solidification

N95- $14548 / 8$

CHERRY, S. M

03,310

Summaries of Center for Fire Research In-House Projects and Grants: 1990

CHESLER, R.

00,286

Liposome-Based Flow-Injection Immunoassay for Determin ing Theophylline in Serum

CHESLER, S.N

03,494

ELER, S. N.

Standard Reference Materials for the Determination of Polycyclic Aromatic Hydrocarbons in Environmental Samples - Current Activities.

PB95-151668

00,586

Supercritical Fluid Extraction-Immunoassay for the Rapid Screening of Cocaine in Hair.

00,637

CHESTER, $M$.

X-Ray Photoelectron and Auger Electron Spectroscopy Study of UltraviolevOzone Oxidized P2S5/(NH4)2S Treated GaAs $(100)$ Surfaces.

CHESTER,

04,479

TER, M. J.

Grazing Angle X-Ray Photoemission System for Depth-De. pendent Analysis
PB95-161154

04,600

Grazing-Incidence X-Ray Photoelectron Spectroscopy: A Novel Approach to Thin Film Characterization.

Grazing Incidence X-ray Photoemission and Its Implementation on Synchrotron Light Source X-ray Beamlines.

PB95-175766

01,001

Grazing-Incidence X-Ray Photoemission Spectroscopy Investigation of Oxidized GaAs(100): A Novel Approach to Nondestructive Depth Profiling.

200151

CHEUNG, S. $K$.

Interdigitated Stacked P-I-N Multiple Quantum Well Modulator. 
Efficient Experiment to Study Superconducting Ceramics. Efficient Experiment to Study Superconducting Ceramics.
PB94-212578 04,505 Microstructure and Ferroelectric Properties of Lead Zirconate-Titanate Films Produced by Laser Evaporation. PB94-199148

04,470

Reactive Coevaporation of DyBaCuO Superconducting Films: The Segregation of Bulk Impurities on Annealed $\mathrm{MgO}(100)$ Substrates.

04,635

X-Ray Characterization of the Crystallization Process of High-Tc Superconducting Oxides in the $\mathrm{Sr}-\mathrm{Bi}-\mathrm{Pb}-\mathrm{Ca}-\mathrm{Cu}-\mathrm{O}$

PB95-151700

04,579

CHIANG, M. Y.

Properties and Interactions of Oral Structures and Restorative Materials. Annual Report for Period October 1, 1990 to September 30, 1991

PB94-160843

03,558

CHIANG, M. Y. M.

Hygrothermal Effects on the Performance of Polymers and Polymeric Composites: A Workshop Report. Held in Gaithersburg, Maryland on September 21-22, 1995.
PB96-183207 CHIEH, $K$.

$24 \mathrm{GHz}$ Josephson Array Voltage Standard.

PB94-211588

02,033

CHIEN, C. L.

Domain Structures in Magnetoresistive Granular Metals.
PB96- 141346

PB96-141346 04,760

Incorporation of Gold into YBa2Cu3O7: Structure and $\mathrm{TC}_{\mathrm{C}}$
Enhancement.

Enhancement

04,481

Unexpected Effects of Gold on the Structure, Superconductivity, and Normal State of YBa2Cu3O7.
PB94-200284 CHILD, M. S.

Rotational-RKR Inversion of Intermolecular Stretching Potentials: Extension to Linear Hydrogen Bonded Complexes. PB95-203014

CHILDERS, C. B.

AC-DC Difference Characteristics of High-Voltage Thermal Converters.

02,083

Frequency Extension of the NIST AC-DC Difference Cali-

bration Service for Current.
PB95-161253

High-Current Thin Film Multijunction Thermal Converters and Multi-Converter Modules.

CHIN, J.W.

01,989

Materials Aspects of Fiber-Reinforced Polymer Composites In Infrastructure.

PB96-210695

CHIOU, T. T.

Gas Absorption during Ion-Irradiation of a Polymer Target:

PB96-161864

CHIRICO, R. D.

Thermodynamic Properties of Alkenes (Mono-Olefins Larger Than C4).

CHMAISSEM, 0 .

Crystal Structure of Pb2Sr2YCu3O8+delta with delta $=1.32$, $1.46,1.61,1.71$, by Powder Neutron Diffraction

PB94-216314

СНОСК, P. B.

04,518

Electropermeabilization of Cell Membranes: Effect of the Resting Membrane Potential.

CHOI, $\mathrm{B}$.

Distributed Architecture for Standards Processing PB96-164181

03,537

CHOI, C. S.

Application of ODF to the Rietveld Profile Refinement of Polycrystalline Solid

PB95-202388

03,401

Methyl Torsional Levels of Solid Acetonitrile ( $\mathrm{CH} 3 \mathrm{CN})$ : A Neutron Scattering Study.

00,926

Microstructure Study of Molybdenum Liners by Neutron Dif-

iraction.

03,756

Neutron Diffraction Texture Study of Deformed Uranium Plates.

03,010

PB97-111587

Texture Study of Two Molybdenum Shaped Charge Liners
by Neutron Diffraction.
PB94-200177 PB94-200177

Textures of Tantalum Metal Sheets by Neutron Diffraction.
PB94-200169 CHOI, $M$.

Simultaneous Optical Measurement of Soot Volume Fraction, Temperature, and $\mathrm{CO} 2$ in Heptane Pool Fire.

PB96-102132

01,397

CHONG, R. A.

Viscosity of Defined and Undefined Hydrocarbon Liquids Calculated Using an Extended Corresponding-States Model. PB96-167234 02,498

\section{CHOO, R. T. C.}

Mathematical Models of Transport Phenomena Associated with Arc-Welding Processes: A Survey.

02,870

CHOQUETTE, $S$.

Planar Waveguide Optıcal Sensors.

PB94-200185

03,586

CHOQUETTE, S. J.

Embossable Grating Couplers for Planar Waveguide Optical

PB96-190277 00,641

Instrument for Evaluating Phase Behavior of Mixtures for Supercritical Fluid Experiments.

00,606

CHOU, C. C.

Improved Molecular Constants and Frequencies for the $\mathrm{CO} 2$ Laser from New High-J Regular and Hot-Band Frequency Measurements.

PB95-180634

04,312

New cw CO2 Laser Lines: The 9-mu $m$ Hot Band.

PB95-168548

04,281

CHOU, $\mathbf{H}$.

Anomalous Dispersion and Thermal Expansion in LightlyDoped KTa1-xNbxO3.

PB95-152302

04,585

\section{CHOU, Y.}

Chip Morphology, Tool Wear and Cutting Mechanics in Finish Hard Turning

CHOUDHARY, L.

Copolymerization of N-Phenyl Maleimide and gammaMethacryloxypropyl Trimethoxysilane.

01,248

Thermal Behavior of 4-Maleimidophenyl Glycidyl Ether Resins.

PB95-153151 01,249

Thermal Behaviour of Methyl Methacrylate and N-Phenyl Maleimide Copolymers.

PB95-152237

01,246

CHOUDHARY, $V$

Copolymerization of N-Phenyl Maleimide and gammaMethacryloxypropyl Trimethoxysilane.

01,248

Thermal Behavior of 4-Maleimidophenyl Glycidyl Ether Res-

PB95-153151

01,249

CHOW, A. F.

Growth of Epitaxial KNbO3 Thin Films.

PB96-135181

02,409

\section{CHOW, H. M}

Grain Alignment and Transport Properties of Bi2Sr2CaCu2O 8 Grown by Laser Heated Float Zone Meth-

Od.

04,602

CHOW, L. C.

Behavior of a Calcium Phosphate Cement in Simulated Blood Plasma in vitro.

PB95-168712

00,165

Calcium Phosphate Cements.

PB97-111595

Dental Materials.

PB94-172871

03,580

Deposition of Loosely Bound and Firmly Bound Fluorides on Tooth Enamel by an Acidic Gel Containing Fluorosilicate

and Monocalcium Phosphate Monohydrate.
PB95-150710

03,559

Effects on Whole Saliva of Chewing Gums Containing Calclum Phosphates.

PB95-153169

03,563

Formation of Hydroxyapatite in a Polymeric Calcium Phosphate Cement

PB95-180642

00,173

Formation of

00,170

Periapical Tissue Reactions to a Calcium Phosphate $\mathrm{Ce}$ ment in the Teeth of Monkeys.

PB94-212008

00.149

Physical and Chemical Properties of Resin-Reinforced Calcium Phosphate Cements.

00,171

PB95-180212

Polymeric Calcium Phosphate Cements Derived from Poly(methyl vinyl ether-maleic acid).

00,180

Procedure for the Study of Acidic Calcium Phosphate Precursor Phases in Enamel Mineral Formation.

03,564

PB95-164448

Phos-

Properties and

phate Cements.
PB96-123229

00,178

Reinforcement of Cancellous Bone Screws with Calcium Phosphate Cement.

PB96-158001

00,179

Remineralization of Root Lesions with Concentrated Calcium and Phosphate Solutions.

03,567

CHOW, P. C.

Epitaxial Integration of Single Crystal C60

PB95-153490

04,592

CHOW, T. H.

Effect of Ethanol on the Solubility of Hydroxyapatite in the System $\mathrm{Ca}(\mathrm{OH}) 2 \cdot \mathrm{H} 3 \mathrm{PO} 4 \cdot \mathrm{H} 2 \mathrm{O}$ at $25 \mathrm{C}$ and $33 \mathrm{C}$.

Effects on Whole Saliva of Chewing Gums Containing Calcium Phosphates.

PB95-153169

03,563

CHRISTENSEN, D. $\mathrm{H}$.

Characterization of Vertical-Cavity Semiconductor Struc-

PB94-200193

02,126

Comparative Photoluminescence Measurement and Simulation of Vertical-Cavity Semiconductor Laser Structures. 5-169173

02,169

Correlation of Optical, X-ray, and Electron Microscopy

Measurements of Semiconductor Multilayer Structures.
PB95-175279

Cross-Sectional Photoluminescence and Its Application to Buried-Layer Semiconductor Structures.

PB96.141106

02,415

Determination of the Complex Refractive Index of Individual Ouantum Wells from Distributed Reflectance.

PB95-175642

02,176

Measurement and Simulation of Photoluminescence Spectra from Vertical-Cavity Quantum-Well Laser Structures.

PB95-16918

02,170

Vertical-Cavity Optoelectronic Structures: CAD, Growth, and Structural Characterization.

02,148

Vertical-Cavity Semiconductor Lasers: Structural Characterization, CAD, and DFB Structures.

PB95-153193

02,150

Vertical-Cavity Semiconductor Structures: Materials Charac-

terization.

CHRISTENSEN, R. G.

Certification of Morphine and Codeine in a Human I 'rine Standard Reference Material.

PB95-176160

03,499

Certification of Phencyclidine in Lyophilized Human Urine Reference Materials.

PB96-160692

03,508

Determination of Oltipraz in Serum by High-Performanace

Liquid Chromatography with Optical Absorbance and Mass

Spectrometric Detection.

PB94-200201

Flow Immunoassay Using Solid-Phase Entrapment.

PB96-200951

00,642

Instrument for Evaluating Phase Behavior of Mixtures for

Supercritical Fluid Experiments.

PB95- $180758 \quad 00,606$

NIST Reference Materials to Support Accuracy in Drug

Testing.
PB96-123807

03,505

Preliminary Investigation of Oleoresin Capsicum.

PB96-179486

03,520

CHRISTIDES, C.

Neutron-Scattering Study of C60(n-) $(n=3,6)$ Librations in Al- 
CHU, C. W.

Crystal Structure of Annealed and As-Prepared $\mathrm{HgBa} 2 \mathrm{CaCu} 2 \mathrm{O} 6$ +delta Superconductors. 03.927 Oxygen Dependance of the Crystal Structure of $\mathrm{HgBa2CuO4}+$ and lis Relation to Superconductivity.
$\mathrm{PB} 96.102512$

\section{4,711}

Decomposition of SF6 and Production of S2F10 in Power Arcs.

PB96-122619 01,084

Investigation of S2F10 Production and Mitigation in Compressed SF6-Insulated Power Systems.

PB94-212388

02,467

Investigation of $\mathrm{S}_{2} \mathrm{~F} 10$ Production and Mitigation in Compressed SF6- Insulated Power Systems

02,468

$\mathrm{CHU}, \mathrm{J} . \mathrm{L}$.

Asymmetric Tip Morphology of Creep Microcracks Growing Along Bimaterial Interiaces.

PB94-200243

03,138

Diffusive Crack Growth at a Bimaterial interface
PB96-204110

03,090

Photodecomposition of $\mathrm{Mo}(\mathrm{CO}) 6 / \mathrm{Si}(111)$ 7×7: CO State-RePhotodecomposition of $\mathrm{Mo}(\mathrm{CO}) 6 / \mathrm{Si}(111) 7 \times 7$ : CO State-Re-
solved Evidence for Excited State Relaxation and Ouenching.

01,009 Photodesorption Dynamics of $\mathrm{CO}$ from Si(111): The Role of Surface Defects.

CHUANG, T. J.

03,066

Analysis of Creep in a Si-SiC C-Ring by Finite Element Method

PB94-200268

03,037

Application of a Simple Technique for Estimating Errors of Finite-Element Solutions Using a General-Purpose Code.

Asymmetric TIp Morphology of Creep Microcracks Growing Along Bimaterial Interfaces.
PB94-200243

03,138

Cavitation Damage During Flexural Creep of SiAION-YAG Ceramics.

03,036

Crack Growth Resistance of Strain-Softening Materials

under Flexural Loading
PB94-200227

02,972

Creep and Creep Rupture of Structural Ceramics.

03,093

Diffusive Crack Growth at a Bimaterial Interface

PB96-204110

03,090

Generic Model for Creep Rupture Lifetime Estimation on $\mathrm{Fi}$ brous Ceramic Composites.

(200235

03,137

Life Prediction of a Continuous Fiber Reinforced Ceramic Composite Under Creep Conditions.

PB96-204128

03,091

HUGG, B.

Selt-Biasing Cryogenic Particle Detector Utilizing Electrothermal Feedback and a SQUID Readout.
PB96-102538

CHUN, S. W.

04,712

Vapor-Liquid Equilibria of Mixtures of Propane and Isomeric Hexanes.
PB95-175287

00,995

CHUNG, $\mathbf{R}$.

January 17, 1995 Hyogoken-Nanbu (Kobe) Earthquake. Performance of Structures, Lifelines and Fire Protection Systems. Executive Summary and Paper

PB97.104160

00,475

Performance of HUD-Affiliated Properties during the January 17, 1994 Northridge Earthquake

PB95-174488

00,443

HUNG, R. M.

Ground Improvement Techniques for Liquefaction Remediation Near Existing Lifelines

01,350

Method of Estimating the Parameters of Tuned Mass Dampers for Seismic Applications.

PB96-167820

00,473

Northridge Earthquake, 1994. Performance of Structures, Lifelines and Fire Protection Systems

00,421

Northridge Earthquake 1994: Performance of Structures, Lifelines, and Fire Protection Systems.

PB94-20746

04,825

Post-Earthquake Fire and Lifelines Workshop. Held in Long Beach, Callifornia on January 30-31, 1995. Proceedings.
PB96-117916

00,209

Proceedings of a Workshop on Developing and Adopting Seismic Design and Construction Standards for Lifelines. Held in Denver, Colorado on September 25-27, 1991

PB97-115794

01,302

CHUNG, Y.S.

Collisions of Electrons with Highly-Charged lons.
PB96-200340

04,791
CHURCH, D. A.

Beam Line for Highly Charged lons.

PB97-111256

04,143

CHURNEY, K. L.

Assessing the Credibility of the Calorific Content of Muncipal Solid Waste.

02,581

Sulfur Dioxide Capture in the Combustion of Mixtures of Lime, Refuse-Derived Fuel, and Coal.

PB94-155587

02,534

CICHY, M. A.

Neutron-Scattering Study of Librations and Intramolecular Phonons in Rb2.6K0.4C60.

PB95-162269

00,958

CIEPLAK, M. $Z$.

Incorporation of Gold into YBa2Cu3O7: Structure and Tc Enhancement.
PB94-200276

04,481

Unexpected Effects of Gold on the Structure,

Superconductivity, and Normal State of YBa2Cu3O7. 04,482

CIGLR, J.L

National Voluntary Laboratory Accreditation Program: Procedures and General Requirements.

PB94-178225

02,630

IMA, M. J.

Effects of Substrate Surface Steps on the Microstructure of

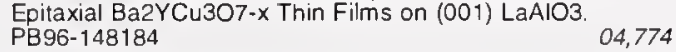

04,774
Derived

Epitaxial Nucleation and Growth of Chemically Derived
Ba2YCu307-x Thin Films on (001) SrTiO3. PB96-190186

Grain Alignment and Transport Properties of
Bi2Sr2CaCu2O8 Grown by Laser Heated Float Zone Meth$\mathrm{Bi2} \mathrm{Sr} 2 \mathrm{CaCu} 2 \mathrm{O} 8$ Grown by Laser Heated Float Zone MethPB95-161451

04,602

\section{CINCOTTA, A.}

Distributed Communication Methods and Role-Based Ac-

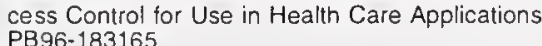

01,508

CIRAC, J. I.

Phase Shifts and Intensity Dependence in Frequency-Modulation Spectroscopy.

PB96-103205

01,071

CIVIS, S.

Perpendicular C-H Stretching Band nug/nu13 and the Torsional Potential of Dimethylacetylene.

PB97-122451

01,192

\section{CLAGUE, F. R.}

Calibration Service for Coaxial Reference Standards for Microwave Power

PB96-162722

01,958

Coaxial Reference Standard for Microwave Power

PB94-193786

01,880

Developing a NIST Coaxial Microwave Power Standard at

PB95-202412 01.914

Method to Determine the Calorimetric Equivalence Correction for a Coaxial Microwave Microcalorimeter.

01,913

New Coaxial Microwave Microcalorimeter Evaluation Tech-

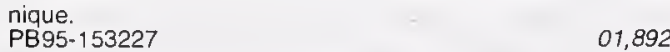

NIST Model PM2 Power Measurement System for $1 \mathrm{~mW}$ at

$1 \mathrm{GHz}$.
PB94-135803

CLAIRON, A.

Atoms in Optical Molasses

$\begin{array}{ll}\text { PB95-108874 } & 03,875\end{array}$

Atoms in Optical Molasses: Applications to Frequency Standards.

03,876

Optical Molasses: Cold Atoms for Precision Measurements.

PB95-108890 03,877

Optical Molasses: The Coldest Atoms Ever.

PB95-108908

03,878

CLARK, A. F.

Accuracy Comparisons of Josephson Array Systems.
PB95-164687 02,047

Application of Single Electron Tunneling: Precision Capacitance Ratio Measurements.

04,703

Cryogenic Precision Capacitance Bridge Using a Single Electron Tunneling Electrometer.

PB95-126074

04,529

Cryogenic Precision Capacitance Bridge Using a Single Electron Tunneling Electrometer.

PB95-152310

02,040

Cryogenic Precision Capacitance Bridge Using a Single Electron Tunneling Electrometer.

02,072

Evidence for Parallel Junctions Within High-Tc GrainBoundary Junctions

04,657

From Superconductivity to Supernovae: The Ginzburg Sym posium. Report on the Symposium Held in Honor of Vitaly

L. Ginzburg. Held in Gaithersburg, Maryland on May 22 ,

PB95-171963 04,649

Half-Integral Constant Voltage Steps in High-Tc Grain Boundary Junctions.

PB94-211216

04,489

Noise Characteristics Below $1 \mathrm{~Hz}$ of Zener Diode-Based Voltage Reference.

PB96-123476

04,049

Physical Basis for Half-Integral Shapiro Steps in a DC SOUID

PB96-102264 04,704

Proposed Tests to Evaluate the Frequency-Dependent Capacitor Ratio for Single Electron Tunneling Experiment

PB97-111454 01,982

Results of Capacitance Ratio Measurements for the Single Electron Pump-Capacitor Charging Experiment

PB97-113286

04,813

Superconductıng Energy Gap of Bulk UBe13.

04,559

CLARK, A. M.

Coexistence of Grains with Differing Orthorhombicity in High Ouality YBa2Cu3O7-delta Thin Films.

PB96-135033

04,742

CLARK, A. V

Application of Electromagnetic-Acoustic Transducers for Nondestructive Evaluation of Stresses in Steel Bridge Struc-

PB96-167978

01,301

Determination of Sheet Steel Formability Using Wide Band

Electromagnetic-Acoustic Transducers.
PB96-186036

02,279

Dynamometer-Induced Residual Stress in Railroad Wheels: Ultrasonic and Saw Cut Measurements. Report No. 30

PB96-183199

04,857

Effect of Liftoff on Accuracu of Phase Velocity Measurements Made with Electromagnetic-Acoustic Transducers.
PB96-186044

PB96-186044
Methods to Improve the Accuracy of On-Line Uitrasonic Measurement of Steel Sheet Formability.

PB96-186051

02,281

Residual Stress in Induction-Heated Railroad Wheels: Ultrasonic and Saw Cut Measurements. Report No. 28.

PB96-106992

04,854

Safety Assessme

PB96-141114

4,855

Safety Assessment of Railroad Wheels Through Roll-by Detection of Tread Cracks

PB96-141254

04,856

PB96-186069

03,373

Ultrasonic Measurement of Residual Stress in Cast Stee Railroad Wheels.

PB95-169199

04,852

Ultrasonic Measurement of Residual Stress in Railroad Wheel Rims.

Ultrasonic Measurement of Sheet Anisotropy and Formability.

PB95-153235

03,213 
CLARK, N. A

Dielectric Spectroscopic Determination of Temperature Behavior of Electroclinic Parameters in the Liquid Crystal havior

PB96-140397

01,098

Shear-Induced Melting of Two-Dimensional Solids.

PB96-112057

01,075

Studies of the Higher Order Smectic Phase of the Large Electroclinic Effect Material W317.

PB95-151601

00,935

X-ray Observation of Electroclinic Layer Constriction and Rearrangement in a Chiral Smectic-A Liquid Crystal.

PB96-141080

01,100

CLARK, $\mathbf{R}$.

HVAC CAD Layout Tools: A Case Study of University/lndustry Collaboration.

PB97-11222

00,281

\section{CLARK, R. A.}

Characterization of Cytochrome c/Alkanethiolate Structures Prepared by Self.Assembly on Gold.

PB95-164638

00,987

CLARK, S. N.

APDE Demonstration System Architecture. National PDES Testbed Report Series.

PB94-154325

02,767

Object-Oriented Tel/Tk Binding for Interpreted Control of the NIST EXPRESS Tookit in the NIST STEP Application Pro-

tocol Development Environment.
PB96-141049

02,785

CLARKE, I.

Effect of Semiconductor Laser Characteristics on Optical Fiber Sensor Performance.

PB95-169132

CLARKE, I. G.

Polarization Insensitive $3 \times 3$ Sagnac Current Sensor Using Polarizing Spun High-Biretringence Fiber

02,187

Standard Polarization Components: Progress Toward an Optical Retardance Standard.

PB96-119672

04,342

CLARKE, J.

Fabrication Issues in Optımizing YBa2Cu307-x Flux Transformers for Low $1 / \uparrow$ Noise.

PB95-175857

02,059

Low Noise YBa2Cu3O7-x-SrTiO3-YBa2Cu3O7-x Mulitlayers for Improved Superconducting Magnetometers.

04,747

PB96-138417

Density-

Scanning Tunneling Microsc
Wave Structure in $1 \mathrm{~T}-\mathrm{TaS} 2$

PB95-180980

04,689

CLARKE, R. M.

Regulation of Lithium and Boron Levels in Normal Human Blood: Environmental and Genetic Considerations.

PB94-198579

CLARKE, S. M.

Structure and Rheology of Hard-Sphere Systems.

PB96-167333

00,662

CLARKE, W. B.

Determination of Boron and Lithium in Diverse Biological Matrices Using Neutron Activation - Mass Spectrometry (NA-MS)

PB94-212289

00,554

Regulation of Lithium and Boron Levels in Normal Human Blood: Environmental and Genetic Considerations.

PB94-198579

03,491

Clavaguera-MORA, M. T.

Extrapolation of the Heat Capacity in Liquid and Amorphous Phases.

PB97-111421

04,147

CLAY, D. T.

In-situ Fume Particle Size and Number Density Measurements from a Synthetic Smelt.

PB94-212040

03,334

CLEARY, T. G

Effect of Fuel Tank Rupture Mode on the Ignitability of Expelled Fuel.

PB97-110043

01,444

Effects of Sample Mounting on Flammability Properties of Intumescent Polymers.

03,389

Flammability Characterization with the Lift Apparatus and the Cone Calorimeter.

PB97-110050

01,435

Flow of Alternative Agents in Piping

00,022

Generation and Characterization of Acetylene Smokes.

PB94-200292

01,372

Heat Flux from Flames to Vertical Surfaces.

01,438

Influence of Ignition Source on the Flaming Fire Hazard of Upholstered Furniture. (NIST Reprint).

PB95-180162

00,297

Letter Report on Flame Spread Testing of a Composite Material.

01,436
Ultrafine Combustion Aerosol Generator PB94-200300

01,373

CLEMENTS, $\mathrm{A}$.

Preliminary Comparison of Time Transfers via LASSO, GPS and Two-Way Satellite.

PB95-151098

01,529

CLEVELAND, W. G.

Evolution of a Turbulent Boundary Layer Induced by a Three-Dimensional Roughness Element.

04,200

CLICKNER, C. C

Evidence for Tunneling and Magnetic Scatttering at 'In situ' YBCO/Noble-Metal Interfaces.

PB96-141098

04,752

In situ Noble

04,494

Insulating Boundary Layer and Magnetic Scattering in YBa2Cu307-delta/Ag Interfaces Over a Contact Resistivity Range of $10(-8) \cdot 10(-3)$ Omega $\mathrm{cm}(2)$.

PB95-169157

04,648

Oxygen Annealing of Ex-situ YBCO/Ag Thin-Film Interfaces. PB96-141312

04,758

CLIFTON, C. L.

Electron Transfer Reaction Rates and Equilibria of the Carbonate and Sulfate Radical Anions.

PB94-212180

00,829

Kinetics of the Reaction of the Sulfate Radical with the Oxalate Anion.

PB97-119127

01,186

Kinetics of the Self-Reaction of Hydroxymethylperoxyl Radicals.

00,827

Temperature Dependence of the Rate Constants for Reac tions of the Sulfate Radical, SO4-, with Anions.

PB94-212172

00,828

CLIFTON, J.

Optimization of Highway Concrete Technology

PB94-182995

01,333

Warping of T

CLIFTON, J.R.

4SIGHT Manual: A Computer Program for Modelling Degradation of Underground Low Level Waste Concrete Vaults. PB95-231593 Corrosion Resistant Epoxy-Coated Reinforcing Steel.
PB94-185618
01,307

01,307

Diagnosis of Causes of Concrete Deterioration in the MLP. 7A Parking Garage.

01,318

Expansion of Cementitious Materials Exposed to Sulfate Solutions

PB94-185782

02,577

High-Performance Concrete: Research Needs to Enhance lis Use.

01,322

Highway Concrete (HWYCON) Expert System User Reference and Enhancement Guide.

PB94-215670

01,316

Long-Term Performance of Engineered Concrete Barriers. PB95-260816

03,727

Microstructural Features of Some Low Water/Solids, Silica

Fume Mortars Cured at Different Temperatures.
PB94-160777

00,330

Prediction of Cracking in Reinforced Concrete Structures.

PB95-220448 03,725

Sulfate Attack of Cementitious Materials: Volumetric Relations and Expansions.

PB94-187317

03,232

CLINE, J.

Disorder Trapping in Ni2TiAl.

PB94-198942

03,322

CLINE, J.P.

Ceramic Characterization

DE94013170

03,026

Characterization of Phase and Surface Composition of Silicon Carbide Platelets.

03,043

Epitaxial Growth of $\mathrm{BaTiO}$ Thin Films at $600 \mathrm{C}$ by Metalorganic Chemical Vapor Deposition.

03,071

Introduction of a NIST Instrument Sensitivity Standard Reference Material for X-Ray Powder Diffraction.
PB94-200318

00,807

Texture Measurement of Sintered Alumina Using the March-Dollase Function.

PB96-179494

04,784

CLOTHIER, $\mathbf{R}$.

Multi-Stage, Position Stabilized Vibration Isolation System for Neutron Interferometry.

PB95-175022

03,955

CLOUGH, R. B.

Investıgation into a Practical Grain Growth Model for Hot sostatic Pressing

03,347

Micromechanics of Densification and Distortion PB94-200326

CLOWNEY, L.

Nucleic Acid Database: Present and Future.

PB97-109078

00,518

UTTER, L. K.

Reactor Radiation Technical Activities, 1995.

PB96-193644

03,741

Certification of Phencyclidine in Lyophilized Human Urine Reference Materials

66-16069

03,508

Stochastic Modeling of a New Spectrometer.

PB96-157870

04,068

COAKLEY, K. J.

Analysis of Scattering Asymmetry Statistics When Back-

ground Corrected Counts Are Negative.

PB94-200334

03,824

Effect of Splitting on Estimation of Emission Rate Profiles from Neutron Depth Profiling Spectra.

Modeling Detector Response for Neutron Depth Profiling

PB96-157813

04,066

Uncertainty Intervals for Polarized Beam Scattering Asymmetry Statistics

COCHRAN, J. F.

03,825

Brillouin Light Scattering Intensities for Thin Magnetic Films with Large Perpendicular Anisotropies.

COFER, $\mathbf{S}$.

04,488

Hierarchical Ada Robot Programming System (HARPS): A Complete and Working Telerobot Control System Based on the NASREM Model.

PB94-213162

COFFEN, L.

02,934

Approach to Setting Performance Requirements for Automated Evaluation of the Parameters of High-Voltage Im-

pulses.

COFFEY, K. R.

Antiferromagnetic Interlayer Correlations in Annealed Ni80Fe20/Ag Multilayers.

03,109

Effect of Microstructure on Phase Formation in the Reaction of $\mathrm{Nb} / \mathrm{Al}$ Multilayer Thin Films.

PB95-168415

03,352

First Phase Form

AlAl.

Magnetic Structure Determination for Annealed Ni80Fe2O/ Ag Multilayers Using Polarized-Neutron Reflectivity.

PB96-176615

03,739 
Electronic Correlations and Satellites in Superconducting

Experimental Constraints on Some Mechanisms for HighTemperature Superconductivity.

04,463 PB94-198553

Phonon

Total-Dielectric-Fun
Response in Solids

Response in

01,067

COHEN, $R$.

Role of R22 in Refrigerating and Air Conditioning Equip.

PB94-199783

03,253

COHEN-TANNOUDJI, C. N.

New Mechanisms for Laser Cooling.

PB95-153268

03,923

COHEN, $Y$.

Effect of Hydrodynamic Interactions on a Terminally Anchored Bead-Rod Model Chain.

01,237

Methods to Improve the Accuracy of On-Line Uitrasonic Measurement of Steel Sheet Formability.

02,281

Response of a Terminally Anchored Polymer Chain to Simple Shear Flow.

PB95-108668

01,233

COHN, S. M

Economic, Energy, and Environmental Impacts of the Energy-Related Inventions Program.

DÉ94-017162

00,008

COHN, T. B.

International Green Building Conference and Exposition (2nd). Held in Big Sky, Montana on August 13-15, 1995. PB95-25360

02,525

COLBERT, J.

What Is a 'Standard Reference Material' - What Is Any Reference Material.

COLBERT, J. C.

Assessing the Credibility of the Calorific Content of Muncipal Solid Waste.

02,581

Standard Reference Materials: Glass Filters as a Standard Reference Material for Spectrophotometry - Selection, Preparation, Certification, and Use of SRM 930 and SRM 1930.
PB94-188844
00,536 COLE, B. F.

Insulating Nanoparticles on YBa2Cu3O7-delta Thin Films Revealed by Comparison of Atomic Force and Scanning Tunneling Microscopy.

PB95.150843

04.575

COLE, K. D.

Determination of Total Protein Adsorbed on Solid (Membrane) Surface by a Hydrolysis Technique: Single Protein Adsorption.

PB96-167093

03,552

Phase Composition, Viscosities, and Densities for Aqueous Two-Phase Systems Composed of Polyethylene Glycol and Various Salts at $25 \mathrm{C}$

00,986

Salt-PEG Two-Phase Aqueous Systems to Purify Proteins and Nucleic Acid Mixtures.

COLEMAN, T.

03,527

Checking the Net Contents of Packaged Goods as Adopted by the $79^{\text {th }}$ National Conference on Weights and Measures, 1994, Third Edition, Supplement 4

PB95-182226

00,484

COLLE, $R$.

$36 \mathrm{Cl} / \mathrm{Cl}$ Accelerator-Mass-Spectrometry Standards: Verification of Their Serial-Dilution-Solution Preparations by Radioactivity Measurements.

PB94-140563

00.524

63Ni Half-Life: A New Experimental Determination and Critical Review.

PB97-111603

00,700

Alpha-Particle and Electron Capture Decay of (209)Po. PB96-186085 04,119

Calibration of Electret-Based Integral Radon Monitors Using NIST Polyethylene-Encapsulated (226)Ra/(222)Rn Emanation (PERE) Standards.
PB96-159223

01,950

International Marine-Atmospheric (222)Rn Measurement Intercomparison in Bermuda. Part 1. NIST Calibration and Methodology for Standardized Sample Additions.

PB96- 175674

00,114

International Marine-Atmospheric (222)Rn Measurement Intercomparison in Bermuda. Part 2. Results for the Participating Laboratories

00,115

International Radon-in-Alr Measurement Intercomparison

Using a New Transfer Standard.
PB96-159751

03,708

Long-Term Stability of Carrier-Free Polonium Solution PB94-185170

00,685

Nickel-63 Standardization: 1968-1995.

00,701
COLLICA, L. A.

ISDN Conformance Testing.

01,478

ISDN Conformance Testing Guidelines: Guidelines for Implementors of ISDN Customer Premises Equipment to Conform to Both National ISDN-1 and North American ISDN Users' Forum Layer 3 Basic Rate Interface Basic Call Control Abstract Test Suites.

PB94-219094

01,471

\section{PLINS, B. L.}

Consensus Process in Standards Development.

B96-179502

00,136

Helping to Reduce Technical Barriers to Trade.

00,491

Papers Presentations Shine.

00,244

Performance of Compact Fluorescent Lamps at Different Ambient Temperatures.

PB95-175329

00,258

Post-Occupancy Evaluation of the Forrestal Building.
PB97-111298
00,280 PB97-111298 00,280 \begin{tabular}{l} 
Psychological Aspects of Lighting: A Review of the Work of \\
CIE TC 3.16 . \\
PB $94-172160$ \\
\hline
\end{tabular}

Psychological Aspects of Lighting: A Review of the Work of CIE TC 3.16
PB95- 153276

COLLINS, $J$.

00,254

Positive and Negative Cooperativities at Subsequent Steps of Oxygenation Regulate the Allosteric Behavior of Multistate Sebacylhemoglobin.

03,486

COLOMBINI, $M$

Extending the Angular Range of Neutron Reflectivity Measurements from Planar Lipid Bilayers: Applications to a Model Biological Membrane.

PB96-122569

03,476

COLOMBO, L

Characterization of Liquid-Phase Epitaxially Grown $\mathrm{HgCdTe}$ Films by Magnetoresistance Measurements.
PB96-123617 04,738

Characterization of LPE HgCdTe Film by Magnetoresistan

02,197

Electrical Characterization of Liquid-Phase Epitaxially Grown Single-Crystal Films of Mercury Cadmium Telluride by Variable-Magnetic-Field Hall Measurements. PB95-175782

02,177

COLTON, R. J.

Summary Report: Workshop on Industrial Applications of Scanned Probe Microscopy (2nd). A Workshop Co-Sponsored by NIST, SEMATECH, ASTM E42.14, and the American Vacuum Society. Held in Gaithersburg, Maryland on
May 2-3, 1995 .

00,509

Workshop Summary Report: Industrial Applications of Scanned Probe Microscopy. A Workshop Co-sponsored by NIST, SEMATECH, ASTM, E42.14, and the American Vacuum Society. Held in Gaithersburg, Maryland on March 24 25,1994

PB $95-170387$

00,506

COLWELL, J. H

Development of a Temperature Scale below 0.5 K.
PB95-125639

Systematic Studies of the Effect of a Bandpass Filter on a PB95-162970

03,939

Systematic Studies of the Effect of a Post-Detection Filter on a Josephson-Junction Noise Thermometer.

03,940

COMAS, $\mathrm{J}$.

Influence of Lattice Mismatch on Indium Phosphide Based High Electron Mobility Transistor (HEMT) Structures Observed in High Resolution Monochromatic Synchrotron XRadiation Diffraction Imaging.

PB95-164679

02,357

Interface Roughness, Composition, and Alloying of LowOrder AIAs/GaAs Superlattices Studies by X-ray Diffraction.
PB96-160262

Interface Sharpness during the Initial Stages of Growth of Thin, Short-Period III.V Superlattices.

PB95-108783

02,139

Interface Sharpness in Low-Order III-V Superlattices.
PB95-108775

02,138

Scaling of the Nonlinear Optical Cross Sections of GaAsAlGaAs Multiple Ouantum-Well Hetero $n$-i-p-i's.

PB96-102793

02,183

COMPOS, $\mathbf{R}$

Tribological Behavior of 440/Diamond-Like-Carbon Film Couples.

PB96-119714

03,019

Tribometer for Measurements in Hostile Environments.

PB95-180949 02,967

Wear Mechanism Maps of $440 \mathrm{C}$ Martensitic Stainless Steel. PB96-111810

COMPOSTO, R. J. Gas Absorption during lon-irradiation of a Polymer Target.
PB96-161864

CONE, E. J.

Hair Testing for Drugs of Abuse: International Research on Standards and Technology.

CONLEY, J. F.

Electron Traps, Structural Change, and Hydrogen Related SIMOX Defects.

02,312

Evidence for a Deep Electron Trap and Charge Compensation in Separation by Implanted Oxygen Oxides.

PB95-175337

CONNELL, P. S.

02,362

Environmental Aspects of Halon Replacements: Considerations for Advanced Agents and the Ozone Depletion Potential of CF3

COOK, G. R.

03,301

Algebraic Approximation of Attractors for Galloping Oscillators

Chaotic Motions of Coupled Galloping Oscillators and Their Modeling as Diffusion Progresses.

PB96-122718

04,823

Experimental and Numerical Chaos in Continuous Systems: Two Case Studies.

Foias-Temam Approximations of Attractors for Galloping Oscillators.

COOK, J. C.

Analysis of the Effectiveness of Oscillating Radial Collimators in Neutron Scattering Applications.

PB95-152252

03,917

COOK, L.

Single-Photon Ionization and Detection of $\mathrm{Ga}$, In, and As(sub n) Species in GaAs Growth

PB95-152815

00,591

COOK, L. P.

Crystal Chemistry and Phase Equilibrium Studies of the $\mathrm{BaO}(\mathrm{BaCO} 3)-\mathrm{R} 2 \mathrm{O} 3-\mathrm{CuO}$ Systems. 5. Melting Relations in $\mathrm{Ba} 2(Y, \mathrm{Nd}, \mathrm{Eu}) \mathrm{Cu} 3 \mathrm{O} 6+\mathrm{x}$

PB95-151718

04,580

Microstructure and Ferroelectric Properties of Lead Zirconate-Titanate Films Produced by Laser Evaporation.

PB94-199148

04,470

$X$-Ray Characterization of the Crystallization Process of System

95-151700

COOKSON, A. H.

Advanced Components for Electric and Hybrid Electric Vehicles. Workshop Proceedings. Held in Gaithersburg, Maryland on October 27.28, 1993.

PB94-177060

04,858

President's Column 'Editorial'.

PB96-164090

02,239

President's Column for Dielectrics and Electrical Insulation Society Newsletter.

(0.0409.

01,882

OKSY, A. L

Fine-Structure Intervals of (14)N(+) By Far-Infrared Laser Magnetic Resonance.

PB95-175162 
COOPER, J. D.

Northridge Earthquake 1994: Performance of Structures, Lifelines, and Fire Protection Systems

PB94-207461

04,825

COOPER, J.P.

Learning to Change: Opportunities to Improve the Performance of Smaller Manufacturers.

PB94-166212

00,010

COOPER, J.W

Autoionizing Resonances in Electric Fields.

PB94-212727

03,853

COOPER, L. Y.

Calculating Combined Buoyancy- and Pressure-Driven Flow Through a Shallow. Horizontal, Circular Vent; Application to Problem of Steady Burning in a Ceiling-Vented Enclosure.
PB96-164108 Combined Buoyancy- and Pressure-Driven Flow Through a Shallow, Horizontal, Circular Vent.
PB94-2io077 Combined Buoyancy and Pressure-Driven Flow Through a Shallow, Horizontal, Circular Vent.

00,410

PB96-164116

Fire-Plume-Generated Ceiling Jet Characteristics and ConTwo-Layer Fire Environment: Uniform Temperature Ceiling

00,382

PB95-164711

Generation Rate and Distribution of Products of Combustion

in Two-Layer Fire Environments: A Model and Applications.
PB96-102173

Interaction of an Isolated Sprinkler Spray and a Two-Laye

Compartment Fire Environment. Phenomena and Model Simulations.

01,437

Methodology for Developing and Implementıng Alternative Temperature-Time Curves for Testing the Fire Resistance of Barriers for Nuclear Power Plant Applications.

PB96-193784

03,742

Simulating Smoke Movement through Long Vertical Shafts in Zone-Type Compartment Fire Models.

00,368

Some Factors Affecting Design of a Furniture Calorimeter Hood and Exhaus

PB94-139193

00,285

Some Factors Affecting the Design of a Calorimeter Hood and Exhaust.

00,302

VENTCF2: An Algorithm and Associated FORTRAN 77 Subroutine for Calculating Flow through a Horizontal Ceiling/Floor Vent in a Zone-Type Compartment Fire Model.

PB94-210127

00,345

COPLEN, T.

New IUPAC Guidelines for the Reporting of Stable Hydrogen, Carbon, and Oxygen Isotope-Ratio Data. Letter to the

PB95-261962

COPLEY, J. R. D

01,058

Acceptance Diagram Analysis of the Pertormance of Multidisk Neutron Velocity Selectors.

03,826

Acceptance Diagram Analysis of the Performance of Vertically Curved Neutron Monochromators.

PB94-200425

03,827

Analysis of the Effectiveness of Oscillating Radial Collimators in Neutron Scattering Applications.

tors in Neutron
PB95-152252

03,917

Discontinuous Volume Change at the Orientational-Ordering Transition in Solid C60.

PB94-211828

00,821

Energy Distributions of Neutrons Scattered from Solid C60 by the Beryllium Detector Method

03,740

Inelastic Neutron Scattering Studies of Rotational Excitations and the Orientational Potential in $\mathrm{C} 60$ and $\mathrm{A} 3 \mathrm{C} 60$ Compounds.

PB94-172673

00,763

Joy of Acceptance Diagrams.

PB94-200433

03,828

Neutron and X-Ray Scattering Cross Sections of Orientationally Disordered Soild C60.

Neutron-Scattering Study of $\mathrm{C60}(n-)(n=3,6)$ Librations in A1. kali-Metal Fullerides.

PB94-200219

00,806

Neutron-Scattering Study of Librations and Intramolecular Phonons in Rb2.6K0.4C60.

PB95-162269

00,958

Orientational Fluctuations, Diffuse Scattering, and Orientational Order in Solid C60.

PB97-119275

04,176

Rotational Dynamics of $\mathrm{C} 60$ in Na2RbC60

00,948

Rotational Dynamics of Solid C70: A Neutron-Scattering

Study.
PB94-172178

Rotational Dynamics of Solid C70: A Neutron-Scattering Study.

00,949
Scattering and Absorption Effects in Neutron Beam Activation Analysis Experiments.

PB94-216140

00,557

Simulations of Neutron Focusing with Curved Mirrors.

PB96-176649

02,200

Structure and Dynamics of Buckyballs

00.950

Structures of Sodium Metal.

PB94-198850

03,319

Supermirror Transmission Polarizers for Neutrons. PB9 4-216215

03,866

Transmission Properties of Short Curved Neutron Guides. Part 1. Acceptance Diagram Analysis and Calculations. Part 1. Acceptance Diagram Analysis and Calculations.
PB96-102199 021 X-ray Powder Diffraction from Carbon Nanotubes and Nanoparticles.

PB96-102975

03,064

CORBIN, D. R.

Neutron and Raman Spectroscopies of 134 and 134a Hydrofluorocarbons Encaged in $\mathrm{Na}-\mathrm{X}$ Zeolite.

PB96-186168

03,001

RDARA, $F$

Precision Oscillators: Dependence of Frequency on Temperature, Humidity and Pressure.

PB94-198306

02,031

DTS, B. F.

Effect of Annealing Ambient on the Removal of Oxide Precipitates in High-Dose Oxygen Implanted Silicon.

PB95-164356

02,356

CORIELL, S. R

Asymptotic Behavior of Modulated Taylor-Couette Flows with a Crystalline Inner Cylinder.

04,469

Convection and Morphological Stability During Directional Solidification

N95-14548/8

03,310

Convective Stability in the Rayleigh-Benard and Directional Solidification Problems: High-Frequency Gravity Modulation. 04,208

Effect of Modulated Taylor-Couette Flows on Crystal-Melt Interfaces: Theory and Initial Experiments.

04,521

Effects of Crystalline Anisotropy and Buoyancy-Driven Convection on Morphological Stability.

PB94-200441

03,328

Laser Melting of Thin Silicon Films

PB94-199239

04,471

Morphological Stability.

Rayleigh Instability for a Cylindrical Crystal-Melt Interface.
PB95-180667

Stagnant Film Model of the Effect of Natural Convection on the Dendrite Operating State.

PB96-14683

04,765

CORMIER, A

Frozen Human Serum Reference Material for Standardization of Sodium and Potassium Measurements in Serum or Plasma by Ion-Selective Electrode Analyzers.

PB94-185337

00,532

CORNELL, E. A.

Behavior of Atoms in a Compressed Magneto-Optical Trap. 03,999

Comment on $\ll$ A Dynamic Electric Trap for Ground-State Atoms $>>$

PB96-200290

04,123

Gravitational Sisyphus Cooling of (87)Rb in a Magnetic Trap.

04,379

Reduction of Light-Assisted Collisional Loss Rate from a Low-Pressure Vapor-Cell Trap.

PB95-202248

03.971

Stable, Tightly Confining Magnetic Trap for Evaporative Cooling of Neutral Atoms.

\section{CORNIER-QUIQUANDON, M.}

Neutron Scattering Structural Study of AlCuFe Ouasicrystals Using Double Isotopic Substitution. PB94-200458

CORRUCCINI, R. J.

Reference Tables for Thermocouples.

AD-A279 948/4

04,126

COSANDEY, F.

Imaging of Fine Porosity in a Colloidal Silica: Potassium Silicate Gel by Defocus Contrast Microscopy.

PB94-212750

03,039

COTELL, C. M.

Interface Sharpness during the Initial Stages of Growth of Thin, Short-Period III-V Superlattices.

PBO5-108783

02,139

COTSORADIS, $C$

NIST Handbook 44, 1996: Specifications, Tolerances, and Other Technical Requirements for Weighing and Measuring Devices as Adopted by the 80th National Conference on Weights and Measures, 1995.

02,926

COTTS, E. J.

Enthalpy Increment Measurements from $4.5 \mathrm{~K}$ to $350 \mathrm{~K}$ and the Thermodynamic Properties of the Titanium Silicide Tissis

PB96-204037

00,679

Enthalpy Increment Measurements from 4.5 to $350 \mathrm{~K}$ and the Thermodynamic Properties of Titanium Disilicide(cr) to $1700 \mathrm{~K}$

00,678

COUDERT, L. $H$

2-Tunneling Path Internal-Axis-Method-Like Treatment of the Microwave Spectrum of Divinyl Ether.

PB94-200466

00,808

COUGHANOWR, C. A

Assessment of the Al-Sb System.

PB94-200474

03,329

COURMONT, $\mathrm{s}$.

Diffusion of Water along 'Closed' Mica Interfaces.

PB96-180039

02,993

Pressurized Internal Lenticular Cracks at Healed Mica Inter-

faces.

80252

COURSEY, B. $M$.

Assày of the Eluent from the Alumina-Based Tungsten-188-

Rhenlum-188 Generator.

Dose Mapping of Radioactive Hot Particles Using Radiochromic Film

03,714

Experimental Validation of Radiopharmaceutical Absorbed

Dose to Mineralized Bone Tissue.

03,617

PB94-199668

Investigation of Applicability of Alanine and Radiochromic
Detectors to Dosimetry of Proton Clinical Beams.

Detectors to Dosimetry of Proton Clinical Beams.
PB96-146782

Liquid-Scintillation Counting Techniques for the Standard-

ization of Radionuclides Used in Therapy.

03,709

PB97-110084

United

PB97-110092

03,521

Overview of a Radiation Accident at an Industrial Accelera-

tor Facility.

02,612

Radiation Accident at an industrial Accelerator Facility.

PB95-140117

02,575

Radiation Doses.

Radioassays of Yttrium-90 Used in Nuclear Medicine.

03,618

PB97-110100

Role of the Office of Radiation Measurement in Quality As.

surance.

Standardization and Decay Scheme of Rhenium-186.

00,689

PB94-200490

03,830 
CROSS, R. W.

CoX, D. M.

Discontinuous Volume Change at the Orientational-Ordering Transition in Solid $\mathrm{C} 60$

00,821

COX, S. D.

Inelastic Neutron Scattering Studies of Nonlinear Optical Materials: $p$-Nitroaniline Adsorbed in ALPO-5.

COXON, 8.

00,874

L-threo-beta-Hydroxyhistidine, an Unprecedented Iron(III) lon-Binding Amino Acid in a Pyoverdine-type Siderophore from Pseudomonas fluorescens 244

PB94-211620

00,553

Physicochemical Characterization of Low Molecular Weight Heparin.

03,474

Two-Dimensional POMMIE Carbon-Proton Chemical Shift Correlated (13)C NMR Spectrum Editing.
PB94-200508

PB94-200508

00,809

Microdosimetry and Cellular Radiation Effects of Radon

Progency in Human Bronchial Airways.

03,625

Neutron Energy Deposition on the Nanometer Scale.

PB95-152260

03,624

Systematics of Alpha-Particle Energy Spectra and Lineal Energy (Y) Spectra for Radon Daughiers.

CRAFT, N.

03,615

Development of Engineered Stationary Phases for the Separation of Carotenoid Isomers.

PB95-150249

00,578

Nutritional Status and Growth in Juvenile Rheumatoid Ar-

thritis

PB94-198470

03,515

CRAFT, N. E.

Carotenoid Reversed-Phase High-Performance Liquid Chro. matography M
PB94-200516

00.549

Device for Subambient Temperature Control in Liquid Chromatography.

High-Performance Liquid Chromatography of Phytoplankton Pigments Using a Polymeric Reversed-Phase C18 Column PB95-151130 00,583

Individual Carotenoid Content of SRM 1548 Total Diet and Influence of Storage Temperature, Lyophilization, and Irradiation on Dietary Carotenoids

00,033

Liquid Chromatographic Method for the Determination of Carotenoids, Retinoids, and Tocopherols in Human Serum and in Food.

CRAFTON, H. C.

Physical Properties of Some Purified Aliphatic HydroCarbons. $205 / 1$

00,657

CRAIG, D.

Guidelines for the Development of Mapping Tables.

PB96-154539

02,786

CRAIG, R. M.

Interlaboratory Comparison of Polarization-Holding Parameter Measurements on High-Birefringence Optical Fiber. PB95-168464

04,280

CRAIN, $\mathbf{P}$.

beta-D-Glucosyl-Hydroxymethyluracil: A Novel Modified Base Present in the DNA of the Parastic Protozoan T. brucei.

PB94-172319

03,524

CRANKSHAW, O. $S$.

Proficiency Tests for the NIST Airborne Asbestos Program, 1993.

PB96-106463

CRANMER, D. C.

Assessment of Testing Methodology for Ceramic Matrix Composites.

O3, 139

PB95-153334

04,267

Perspective on Fiber Coating Technology.

PB94-200540

03,118

Tensile Creep Testing of Structural Ceramics.

03,105

Transient Creep Behaviour of Hot Isostatically Pressed Silicon Nitride.

CRASEMANN, 8.

Atomic, Molecular, and Optical Physics with X-rays. PB94-213378

$03,08 \hat{0}$

CRAWFORD-BROWN, D. J.

Microdosimetry and Cellular Radiation Effects of Radon Progency in Human Bronchial Airways.
PB95-152344

CRAWFORD, M. K.

03,625

Neutron and Raman Spectroscopies of 134 and 134a

Hydrofluoroca
PB96-186168

03,001
CRAWFORD, M. L.

Alternative EMC Compliance Test Facilities. PB96-200324

Aperture Coupling to a Coaxial Air Line: Theory and Experiment.

PB94-211968

02,216

Aperture Excitation of Electrically Large, Lossy Cavities

PB94-145711 00,029

Aperture Excitation of Electrically Large, Lossy Cavities.

PB95-175675

00,031

Band-Limited. White Gaussian Noise Excitation for Reverberation Chambers and Applications to Radiated Suscepti-

bility Testing.
PB96-165410 01,960

Measurements of Shielding Effectiveness and Cavity Char-

acteristics of Airplanes.
PB94-210051

00,030

TEM/Reverberatıng Chamber Electromagnetic Radiation Test Facility at Rome Laboratory.

PB96-155023

CRAWFORD, T. M.

Observation of the Transverse Second Harmonic MagnetoOptic Kerr Effect from Ni81Fe19 Thin Film Structures. PB96-200332

01,971

CREECY, R.

Second Census Optical Character Recognition Systems Conference.

01,832

CREEGAN, K. M.

Discontinuous Volume Change at the Orientational-Ordering Transition in Solid $\mathrm{C} 60$

94-21182

00,821

CRESPI, V. H.

Anharmonic Phonons and the Isotope Effect in Superconductivity.

CRESSWELL, M. W

Application of the Modified Voltage-Dividing Potentiometer to Overlay Metrology in a CMOS/Bulk Process.

PB94-181997

02,302

Design Guide for CMOS-On-SIMOX. Test Chips NIST3 and NIST 4 .

PB94-163458

02,297

Electrical Test Structure for Overlay Metrology Referenced to Absolute Length Standards.

B95-152278

Electrical Test Structures Replicated in Silicon-on-Insulator

Material.
PB97-111827 02,454

Enhanced Voltage-Dividing Potentiometer for High-Precision Feature Placement Metrology.

PB96-164025

02,428

Hybrid Optical-Electrical Overlay Test Structure.

PB96-204136

02,450

Measurement of Patterned Film Linewidth for Interconnect Characterization

02,420

Metrology Standards for Advanced Semiconductor Lithography Referenced to Atomic Spacings and Geometry.
PB96-160718

Microelectronic Test Structures for Feature Placement and Electrical Linewidth Metrology.

PB95-180568

02,367

Microelectronic Test Structures for Overlay Metrology.
PB96-164249

New Test Structure for Nanometer-Level Overlay and Feature-Placement Metrology.

PB95-175345

02,363

Test Structures for the In-Plane Locations of Projected Features with Nanometer-Level Accuracy Traceable to a Coordinate Measurement System.

PB94-200565

02,313

CRISCO, J. J.

Reinforcement of Cancellous Bone Screws with Calcium Phosphate Cement.

PB96-158001

00,179

CRISSMAN, J. M.

Performance of Plastic Packaging for Hazardous Materials Transportation. Part 1. Mechanical Properties.
AD-A301 258/0

02,580 Cryogenic Blackbody Calibrations at the National Institute of
Standards and Technology Low Background Infrared CaliStandards and Technology Low Background Infrared Call-
bration Facility. PB94-169802

02,117

Measurement and Uncertainty of a Calibration Standard for the Scanning Electron Microscope.

00,560

CROCHIERE, S. M

Characterization of Vertical-Cavity Semiconductor Struc-

tures.

02,126

Vertical-Cavity Optoelectronic Structures: CAD, Growth, and

Structural Characterization.

02,148

Vertical-Cavity Semiconductor Lasers: Structural Characterization, $\mathrm{CAD}$, and DFB Structures.

153193

02,150

CROMAR, $M$

High Critical Temperature Superconductor Tunneling Spec troscopy Using Squeezable Electron Tunneling Junctions.

CROMAR, M. W

Critical Current Behavior of Ag-Coated YBa2Cu3O7-x Thin

PB95-141016 04,549

Optimization of ECR-Based PECVD Oxide Films for Superconducting Integrated Circuit Fabrication.

02,051

Superconducting Integrated Circuit Fabrication with Low Temperature ECR-Based PECVD SiO2 Dielectric Films. PB96-103015

CROMER, C.

Method of Realizing Spectral Irradiance Based on an Absolute Cryogenic Radiometer.

PB95-161204

04.270

CROMER, C. L.

Absolute Response Calibration of a Transfer Standard Cryogenic Bolometer

04,358

Comparison of Filter Radiometer Spectral Responsivity with the NIST Spectral-|rradiance and Illuminance Scales. the NIST Spectral-Irradiance and Iliminance Scales.
PB97-113161

Detector-Based Candela Scale and Related Photometric Calibration Procedures at NIST.

PB95-161949

04,273

Improved Dose Metrology in Optical Lithography.

02,439

PB96-179510

04,134

PB96-204201

Natronal Institute of Standards and Technology High-Accu-
racy Cryogenic Radiometer. racy Cryogenic Radiometer

NIST Detector-Based Luminous Intensity Scale.

01,864

NIST High Accuracy Scale for Absolute Spectral Response

from $406 \mathrm{~nm}$ to $920 \mathrm{~nm}$

01,865

Realization of a Scale of Absolute Spectral Response Using the NIST High Accuracy Cryogenic Radiometer.

CROMPTON, R. W.

04,397

Analyses of Recent Experimental and Theoretical Determinations of e-H2 Vibrational Excitation Cross Sections: As sessing a Long-Standing Controversy.

PB95-202438

03,977

Mes, $D$

Measurements of Permittivity and the Dielectric Loss Tan gent of Low Loss Dielectric Materials with a Dielectric Resonator Operating on the Higher Order Te(sub o gamma delta) Modes.

delta) Modes.

CROSBY, F.J.

Modulation of Fossil Fuel Production by Global Tempera ture Variations, 2

PB94-146636

02,533

\section{ROSS, R. W.}

Effects of Critical Current Density, Equilibrium Magnetization and Surface Barrier on Magnetization of High Temperaure Superconductors.

PB94-185162

04,446

Effects of Critical Current Density, Equilibrium Magnetization and Surface Barrier on Magnetization of High Temperature Superconductors

PB95-153060

04,588 


\section{CROUCH, A. E.}

Assessment of Technology for Detection of Stress Corrosion Cracking in Gas Pipelines. Final Report, July 1993March 1994.

PB94-206646

CROVETTO, R.

Standard States, Reference States and Finite-Concentration Effects in Near-Critical Mixtures with Applications to Aqueous Solutions.

00.979

Thermodynamic Behavior of the $\mathrm{CO}_{2}-\mathrm{H}_{2} \mathrm{O}$ System from 400 to $1000 \mathrm{~K}$ up to $100 \mathrm{MPa}$ and $30 \%$ Mole Fraction of $\mathrm{CO} 2$.

PB94-162245

00,736

CROW, J. E.

Magnetic Ordering of the Cu Spins in PrBa2Cu3O6+x.
PB95-140547

CROWDER, J. $M$.

Visual Measurement Technique for Analysis of Nucleate Flow Boiling.

CRUPE, W. E.

03,262

Application of the Electronic Balance in High Precision Pycnometry.

CRUZ, J. $Y$.

00.534

Test of Newton's Inverse Square Law of Gravitation Using the 300-m Tower at Erie, Colorado.

PB95-202446

03,978

Validation of the Inverse Square Law of Gravitation Using the Tower at Erie, Colorado, USA

PB95-164646

03,947

CUGINI, J.

Common Criteria: On the Road to International Harmoni-

zation.

PB96-123484 01,606

Functional Security Criteria for Distributed Systems.

PB96-123492

01,607

CUGINI, J. A.

Proceedings of the Workshop on the Federal Criteria for Information Technology Security. Held in Ellicot City. Mary. and on June 2-3, 1993.

PB94-16258

01,575

CUGINI, J. V.

Graphical Conceptual Navigation as a Presentation Technique for a Graphics Standard.

User's Guide for the PHIGS Validation Tests (Version 2.1).

PB94-165206 01,682

CUI, J. P.

Homogeneous Gas Phase Decyclization of Tetralin and Benzocyclobutene.

00,928

Rate Constants for Hydrogem Atom Attack on Some

Chlorinated Benzenes at High Temperature.

PB94-200581

00,810

CUI, S. T.

Conditions for Existence of a Reentrant Solid Phase in a Sheared Atomic Fluid.

PB94-211380

04,198

CULLIN, D.

Bacteriorhodopsin Immobilized in Sol-Gel Glass.

PB95-151429

CULLIN, D. W.

Holographic Properties of Triton X-100-Treated Bacteriorhodopsin Embedded in Gelatin Films.

Optical Properties of Triton X-100-Treated Purple Membranes Embedded in Gelatin Films.

PB96-123500

CUNNINGHAM, C. E.

03,546

Noise Reduction in Low-Frequency SQUID Measurements with Laser-Driven Switching.

Trapped Vortices in a Superconducting Microbridge.

PB95-141149

04,554

Abstract and Index Collection in the Research Information
Center of the National Institute of Standards and TechCenter of the National Institute of Standards and Tech PB94-152204

02,744

Abstract and Index Collection in the Research Information Center of the National Institute of Standards and TechPB95-232633

02,741

Databases Available in the Research Information Center of the National Institute of Standards and Technology.
PB95-128641

02,724

Databases Available in the Research Information Center of the National Institute of Standards and Technology (December 1995).

CUNNINGHAM, M.

02,734

Parametric Study of Wall Moisture Contents Using a Revised Variable Indoor Relative Humidity Version of the 'Moist' Transient Heat and Moisture Transfer Model.

PB97-122535

00,419
CURRIE, L. A.

Comparative Study of $\mathrm{Fe}-\mathrm{C}$ Bead and Graphite Target Performance with the National Ocean Sciences AMS (NOSAMS) Facility Recombinator Ion Source. PB95-175790

00,693

Distinguishing the Contributions of Residential Wood Combustion and Mobile Source Emissions Using Relative Concentrations of Dimethylphenanthrene Isomers.

02.563

PB96-13512

Importance of Chemometrics in Biomedical Measurements.
PB94-200599

Interlaboratory Comparison of Autoradiographic DNA Profiling Measurements. 1. Data and Summary Statistics. PB95-175923 03,542 Interlaboratory Comparison of Autoradiographic DNA Profiling Measurements. 2. Measurement Uncertainty and Its Propagation

03,545

Metrological Measurement Accuracy: Discussion of 'Measurement Error Models' by Leon Jay Gleser

00,551

Radiocarbon Measurements of Atmospheric Volatile Organic Compounds: Quantifying the Biogenic Contribution.
PB97-122352

Sources of Urban Contemporary Carbon Aerosol.

CURT

Thermodynamics of (Germanium + Selenium): A Review and Critical Assessment.

PB97-112536

01,182

CUTHILL, $B$

PB96-131545

02,733

Information Technology Engineering and Measurement Model: Adding Lane Markings to the Information SuperPBgh-143145

01.474

Making Sense of Software Engineering Environment Framework Standards.

PB95-105037

01,701

CUTHILL, B. B.

Guide 10 Software Engineering Environment Assessment and Evaluation.

01.676

Proceedings of the Digital Systems Reliability and Nuclear Safety Workshop. Held in Rockville, Maryland on September 13-14, 1993.

03,728

Reference Information for the Software Verification and Validation Process.

PB96-188164

01,773

Using a Multi-Layered Approach to Representing Tort Law Cases for Case-E. ased Reasoning.

CUTLER, R.

00.135

NIST-NRL Free-Electron Laser Facility.

PB94-21251

04,237

Increased Pinning Energies and Critical Current Densities in Heavy-Ion-Irradiated Bi2Sr2CaCu2O8 Single Crystals.

04,654
PB95-175352

cYPHER,

Error Protecting Characteristics of CDMA and Impacts on Speech.

PB96-122452

01,491

Framework for National Information infrastructure Services. PB95-103719

02,723

Provision of Isochronous Service on IEEE 802.6

PB96-160635

01,501

Standardization

01,499

D.ORAZIO, $\mathrm{P}$.

Frozen Human Serum Reference Material for Standardization of Sodium and Potassium Measurements in Serum or Plasma by Ion-Selective Electrode Analyzers.

00,532

DA SILVA, T. A.

Angular Variation of the Personal Dose Equivalent,

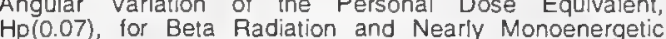
$\mathrm{Hp}(0.07)$, for Beta Radiation and
Electron Beams: Preliminary Results.

PB95-168472

03,630

DABROWSKI, C

Context Analysis of the Network Management Domain Conducted as Pan of the Domain Analysis Case Study.
PB94-142528

Domain Analysis of the Alarm Surveillance Domain. Version 1.0. Conducted as Part of the Domain Analysis Case Study PB95-136339

01.705

Glossary of Software Reuse Terms.

PB95-178992

01,720

Application of Exper System to Select Data Sources from Chemical Information Databases. PB95-12565

0,505

Neutron Scattering Study of the Orientation of a Liquid Crystalline Polymer by Shear Flow.

01.270

Phase Behavior of a Hydrogen Bonding Molecular ComposPB94-185188

01,202

DAGALAKIS, N

NIST Support to the Next Generation Controller Program: 991 Final Technical Report.

PB94-163490

02,808

DAGATA, J, A.

Integration of Scanning Tunneling Microscope Nanolithography and Electronics Device Processing. PB95-153359

Junction Locations by Scanning Tunneling Microscopy: InAir-Ambient Investigation of Passivated GaAs pn Junctions. PB94-185964

Scanning Tunneling Microscopy and Fabrication of Nanometer Scale Structures at the Liquid-Gold Interface.

00,904

Summary Report: Workshop on Industrial Applications of Scanned Probe Microscopy (2nd). A Workshop Co-Sponsored by NIST, SEMATECH, ASTM E42.14, and the American Vacuum Society. Held in Gaithersburg, Maryland on May 2-3, 1995

00,509

Workshop Summary Report: Industral Applications of Scanned Probe Microscopy. A Workshop Co-sponsored by Macuum Society. Held in Gaithersburg. Maryland on March 24

B $95-170387$

00,506

X-Ray Photoelectron and Auger Electron Spectroscopy Study of UltravioletOzone Oxidized P2S5/(NH4)2S Treated GaAs(100) Surfaces.

PB94-200144

04,479

\section{DAHMANI, $R$}

Characterization of the $\mathrm{ZnSe} / \mathrm{GaAs}$ Interface Layer by TEM Chectroscopic Ellipsometry.

Determination of the Optical Constants of ZnSe Films by Spectroscopic Ellipsometry

DAI, Z

Mixing and Radiation Properties of Buoyant Luminous

PB96-202254

01.432

Mixing and Radiation Properties of Buoyant Turbulent Diffusion Flames.

01,396

Radiation and Mixing Properties of Buoyant Turbulent Diffusion Flames.

DALBERTH, M. J.

01,360

Alignment Probing of Rydberg States by Stimulated EmisPB96-200316

04,124

Initial and Final Orbital Alignment Probing of the Fine-Structure-Changing Collisions among the $\mathrm{Ca}(4 \mathrm{~s})(1)(4 \mathrm{p})(1)$, (3)PJ States with He: Determination of Coherence and Conventional Cross-Sections.

PB95-203279

04,004

Orbital Alignment and Vector Correlations in Inelastic Atom. ic Collisions.

DALGARNO, A

04,047

Papers on the Symposium on Collision Phenomena in Astrophysics, Geophysics, and Masers.

AD-A280 291/6

00,047

DALIBARD, J

Atoms in Optical Molasses.

03,875

Atoms in Optical Molasses: Applications to Frequency 
DAMAZO, B

Open Architectures for Machine Control PB94-135621

DANG, T. D.

Evidence of Crosslinking in Methyl Pendent PBZT Fiber. PB97-112486

DANG, T. T.

International Marine-Atmospheric (222)Rn Measurement Intercomparison in Bermuda. Part 2. Results for the Participating Laboratories.

PB96-175682

00,115

Absolute Cross-Section Measurements for Electron-Impact Absolute Cross-Sectior
lonization of $\mathrm{C} 1(+1)$

PB94-199841

DANIEL, R. G.

Environmental Aspects of Halon Replacements: Considerations for Advanced Agents and the Ozone Depletion Po tential of CF3

PB97-122261

03,301

DANIELSON, B. L.

Low-Coherence Interierometric Measurement of Group

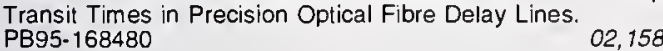

DANIELSON, P.

Transverse stress effect on the critical current of internal tin and bronze process $\mathrm{Nb}$ (sub 3 ) Sn superconductors. DE95016659

04,434

DANOS, $M$.

Accuracy-Weighted Variational Principle for Degenerate

Continuum States.
PB94-200615 03,831

Comprehensive Theory of Nuclear Effects on the Intrinsic Sticking Probability. 1.

03,832

PB94-200623

Comprehensive Theory of Nuclear Effects on the Intrinsic
Sticking Probability. 2.

Sticking Probability. 2

03,833

Strangeness Flow Difference in Nuclear Collisions at $15 \mathrm{~A}$ and $200 \mathrm{~A} \mathrm{GeV}$

PB96-119631

04,042

Fabrication Issues in Optimizing YBa2Cu307-x Flux Trans Fabrication Issues in Optimizing YBa2Cu3O7-x Flux Trans-
formers for Low I/ Nolse.
PB95-175857
L2,059

02,059

Low Noise YBa2Cu3O7-x-SrTiO3-YBa2Cu3O7-x Mulitlayers

for Improved Superconducting Magnetometers. $\quad 04,747$

DAOU, J. N.

Neutron Spectroscopic Comparison of Rare-Earth/Hydrogen alpha-Phase Systems

PB95-163523

Densification of Nano-Size Powders. 1994 Report.

Densification

DAPKUNAS, $\mathbf{S}$. J.

Ceramics Technical Activities, 1995

PB96-193677

00,970

Characterization and Processing of Spray-Dried Zirconia Powders for Plasma Spray Application

04,419

Corrosion Characteristics of Silicon Carbide and Silicon

Nitride.
PB96-169081 03,372

Equipment for Investigation of Cryogenic Compaction of Nanosize Silicon Nitride Powders. 1993 Report.
DE94013593 03,028

03,028

Materials Databases.
Maccessing Computerized Numeric PB96-204045

03,007

Measurement Methods and Standards for Processing and Application of Thermal Barrier Coatings.

01,447

NMR Characterization of Injection-Moulded Alumina Green Compacts. Part 2. T2-Weighted Proton Imaging.
PB96-201181 Review of Corrosion Behavior of Ceramic Heat Exchanger Materals: Corrosion Characteristics of Silicon Carbide and Silicon Nitride. Final Report, September 11, 1992--March 11,1993

DÉ93041307

03,228

Ambient Temperature Synthesis of Bulk Intermetallics.
PB95-169074 00,168

Magnetic and Structural Properties of Electrodeposited Cop-

per-Nickel Microlayered Alloys.

PB94-213121

04,512

DARK, M. L.

Soft-X-ray Damage to p-terphenyl Coatings for Detectors. PB96-15961

Optimization of Highway Concrete Technology

DAS, A.

01,333

Thermodynamic and Thermophysical Properties of Organic Nitrogen Compounds. Part 11. 1- and 2-Butanamine, 2-Meth-
yl-1-Propanamine, 2-Methyl-2-Propanamine, Pyrrole, 1-, 2and 3-Methylpyrrole. Pyridine, 2-, 3-, and 4-Methylpyridine Pyrrolidine, Piperidine, Indole, Quinoline, Isoquinoline, Acridine, Carbazole, Phenanthridine, 1- and 2Naphthalenamine, and 9-Methylcarbazole.
PB94-162294

00,741

DAS SARMA, $S$.

Correction to the Decay Rate of Nonequilibrium Carrier Disrributions Due to Scattering-in Processes.

PB94-185840

DASTIDAR, P.

HVAC CAD Layout Tools: A Case Study of University/Industry Collaboration

7-112221

00,281

\section{DATLA, R. U.}

Cryogenic Blackbody Calıbrations at the National Institute o Standards and Technology Low Background Infrared Calibration Facility.

PB94-169802

02,117

Development of Neutral-Density Infrared Filters Using Me-

$\begin{array}{ll}\text { tallic Thin Films. } & \\ \text { PB96-180286 } & 02,998\end{array}$

Metrology Issues in Terahertz Physics and Technology.
PB96-128277

Standard Reference Materials: Polystyrene Films for Cali-

brating the Wavelength Scale of Infrared $\begin{array}{ll}\text { Spectrophotometers - SRM } 1921 . & 03,386\end{array}$

Thermal Modeling of Absolute Cryogenic Radiometers.

PB95-181236 04,316

\section{DATTA, D.}

Hypertine-Structure Studies of Zr II: Experimental and Relativistic Configuration-Interaction Results.

PB95-203824

04.01

DATTA, $\mathrm{S}$.

Cast-Iron Elastic Constants: Effect of Graphite Aspect

Ratio. 213212

03,211

Elastic Properties of $\mathrm{Al} 2 \mathrm{O} 3 / \mathrm{Al}$ Composites: Measurements and Modeling.

03,157

Elastic Properties of Uniaxial-Fiber Reinforced Composites: General Features

PB94-200649

03,140

\section{SKARDT, R. H.}

Transient Subcritical Crack-Growth Behavior in Transormation-Toughened Ceramics.

PB94-200656

DAVID, W. I. F.

Structure and Dynamics of Buckyballs

ABID-153292

Vibrations of S1((1)B2u) p-Difluorobenzene - d4. S1-So Fluorescence Spectroscopy and ab Initio Calculations

PB95-202586

\section{DAVIDSON, M. P.}

Numerical Reference Models for Optical Metrology Simula tion.

04,392

DAVIES, A

Tunneling Spectroscopy of bcc(001) Surface States. PB96-155585

Use of Ionospheric Data in GPS Time Transfer

PB95-163853
AVIES, $M$.

01,540

Effects of Spindle Dynamic Characteristics on Hard Turning

PB96-12298

DAVIES, M. A.

Chip Morphology, Tool Wear and Cutting Mechanics in Finsh Hard Turning

DAVIS, D. D.

Calibration of GPS Equipment in Japan

PB95-151452

01,531

High Resolution Time Interval Counter

PB96-138607

01,531

Smart Clock: A New Time.

PB95-151445

01,495

DAVIS, G.

Standards Development in North America for Performance of Whole Buildings and Facilities.

PB94-185196

00,312

DAVIS, G. T.

Advances in the Measurement of Polymer CTE Micrometer- to Atomic-Scale Measurements.
PB96-180229 03,390

03,390

PB96-122692

02,405

Piezoelectric and Pyroelectric Polymers.

PB95-153367

01,250

Thermal Stability of Internal Electric Field and Polarization Polymethylmethacrylate.

PB95- 151072

01,240

DAVIS, M. W.

Room-Temperature Thermal Conductivity of Expanded Polystyrene Board for a Standard Reference Material.

PB96-193693

00,412

DAVIS, R. S.

Determining the Magnetic Properties of $1 \mathrm{~kg}$ Mass Stand-

PB95-261905

Mass and Density Determinations.

PB94-200672

00,504

Report of Density Intercomparisons Undertaken by the Working Group on Density of the CCM.

PB94-200664

DAVIS, $S$.

Pairwise and Nonpairwise Additive Forces in Weakly Bound Complexes: High Resolution Infrared Spectroscopy of ArnDF $(n=1,2,3)$
PB96-200357

04,125

DAVIS, S. H.

Effects of Elastic Stress on the Stability of a Solid-Liquid Interface.

PB95-163028

03,350

AVIS, $W$

Analysis of High Bay Hangar Facilities for Detector Sensitivity and Placement

PB96-190210

01,429

Field Modeling: Simulating the Effect of Sloped Beamed

Ceilings on Detector and Sprinkler Response.
PB96-122866

01,406

NASA Fire Detector Study

01,423

Use of Computer Models to Predict Temperature and Smoke Movement in High Bay Spaces. Smoke Movem
PB94-145976

00,191

DAWBER, $P$.

Preparation and Characterization of (6) LiF and (10)B Reference Deposits for the Measurement of the Neutron Life-

PB95-108692

03,874

Problems Related to the Determination of Mass Densities of Evaporated Reference Deposits.

PB95-163226

03,941

DAX, A.

Extension of Heterodyne Frequency Measurements on OCS to $87 \mathrm{THz}(2900 \mathrm{~cm}(-1))$

PB94-200680

00,811

Sub-Doppler Frequency Measurements on OCS at 87 Thz (3.4 micrometers) with the CO Overtone Laser: Considerations and Details.

PB95-128633

04,255

Sub-Doppler Frequency Measurements on OCS at $87 \mathrm{THz}$ (3.4 mu m) with the CO Overtone Laser.

PB96-102215

04,330

DAY, G. W.

Eftect of Semiconductor Laser Characteristics on Optical Fiber Sensor Períormance.

02,167

Faraday Effect Current Sensor with Improved SensitivityBandwidth Product

PB95-203154

02,180

Faraday Effect Current Sensors.

PB94-200698

02,127

Faraday Effect Sensors: A Review of Recent Progress

Faraday Effect Sensors for Magnet Field and Electric Current

PB96-119664 
Optoelectronics at NIST

PB96-200860

02,202

Polarization Dependence of Response Functions in $3 \times 3$ Sagnac Optical Fiber Current Sensors.

PB95.16242

02,154

Polarization Dependence of Response Functions in $3 \times 3$ Sagnac Optical Fiber Current Sensors.

02.189

Polarization Insensitive $3 \times 3$ Sagnac Current Sensor Using Polarizing Spun High-Birefringence Fiber.

02,187

PB96-119276

Self-Calibrating Fiber Optic Sensors: Potential Design Methods

02,172

Self Calibrating Fiber Optic Sensors: Potential Design Meth-

PBs. 169306

02,173

Standard Polarization Components: Progress Toward an Optıcal Retardance Standard

04,342

Studies of the Higher Order Smectic Phase of the Large

Studies of the Higher Order Smectic Phase of the Large

$\begin{array}{ll}\text { Electroclinic Effect Material W317. } & \\ \text { PB95-151601 } & 00,935\end{array}$

Submicroampere-Per-Root-Hertz Current Sensor Based on the Faraday Effect in Ga: YIG.

PB95-162467

02,155

Technical Digest: Symposium on Optical Fiber Measurements (8th), 1994. Held in Boulder, Colorado on September $13-15,1994$.

04,231

Technica! Digest: Symposium on Optical Fiber Measurements (9th), 1996. Held in Boulder, Colorado on October 1 . 3. 1996 .

PB97-108583

04,383

Wideband Current and Magnetic Field Sensors Based on Iron Garnets.
PB96-200878

01,975

DAY, J. M

Internal Droplet Circulation Induced by Surface-Driven Rotation.

02,500

DAY, P.

Crystal Structure and Magnetic Properties of $\mathrm{CuGeO}$.

PB95-180287

04,678

Lattice Dynamics of $\mathrm{Ba} 1-x \mathrm{~K} \times \mathrm{BiO} 3$.

04,706

Lattice Dynamics of Semiconducting. Metallic, and Superconducting $\mathrm{Ba} 1-\times \mathrm{Kx} \times \mathrm{BiO} 3$ Studied by Inelastic Neutron Scattering

PB96-102447

04,708

Structure and Conductivity of Layered Oxides $(\mathrm{Ba}, \mathrm{Sr}) \mathrm{n}+1(\mathrm{Sn}, \mathrm{Sb}) \mathrm{nO} 3 \mathrm{n}+1$

04.707

DAYAL, $V$.

Dynamic Shear Modulus Measurements with Four Inde-

pendent Techniques in Nickel-Based Alloys.

03,320

DAYHOFF, R. E.

Prototyping a Graphical User Interiace for DHCP.

PB96-160544

DAYWITT, W. C.

Single-Port Technique for Adaptor Efficiency Evaluation.

PB96-17644

02,088

DE BEER, A.

beta-D-Glucosyl-Hydroxymethyluracil: A Novel Modified Base Present in the DNA of the Parastic Protozoan T. brucel.

PB94-172319

03,524

DE BIEVRE, $P$

New Values for Silicon Reference Materials, Certified for Isotope Abundance Ratios. Letter to the Editor.

PB94-219268

00,863

DE BOER, F. R.

Magnetic Properties of Single-Crystalline UCu3Al2.

DE HAAN, G.

04,686

Perception of Clamp Noise in Television Receivers.

PB96-119433

DE LUCA, N. P.

Milliwatt Mixer for Small Fluid Samples.

DE LUCIA, F. C.

Millimeter- and Submillimeter-Wave Spectrum of trans-Ethyl Alcohol.

PB96-145578

01,102

DE MARCHI, A.

New 5 and $10 \mathrm{MHz}$ High Isolation Distribution Amplifie

PB96-190202

01,510

DE NATALE, $P$.

Rotational Far Infrared Spectrum of (13)CO.

DE OBALDIA, E.

Increased Transition Temperature in In situ Coevaporated YBa2Cu307-delta Thin Films by Low Temperature Post-An.

PB95-18007

04,672
DE OBALDIA, E. I.

Coexistence of Grains with Differing Orthorhombicity in High Quality YBa2Cu3O7-delta Thin Films.

PB96-135033

04,742

DE RIS, J.

Prediction of Fire Dynamics.

PB94-193620

00,336

E ROOS, J. L.

Principle of Congruence and Its Application to Compressible States.

PB96-102892

01,068

DE SWAAN ARONS, $J$.

Principle of Congruence and Its Application to Compressible States.
PB96-102892

01,068

DE VIVIE-RIEDLE, $R$.

Three-Vector Correlation Study of Orientation and Coherence Effects in $\mathrm{Na}(3 \mathrm{p},(2) \mathrm{P} 1 / 2$ inversely maps (2) $\mathrm{P} 3 / 2)+\mathrm{He}$

Semiclassical and Quantum Calculations.

PB95-202453

DE VREEDE, $J$.

Intercomparison of Thermal Converters at NIM, NIST, PTB, SIRI and VSL from 10 to $100 \mathrm{MHz}$.

PB94.172459

02,027

DE VREEDE, J.P. M.

Intercomparison of NIST, NPL, PTB, and VSL Thermal Voltage Converters from $100 \mathrm{kHz}$ to $1 \mathrm{MHz}$

PB94-172442

02,026

DEAL, $\mathrm{S}$.

Evaluating Small Board and Care Homes: Sprinklered vs. Nonsprinklered Fire Protection.

PB94-206356

00,195

Santa Ana Fire Department Experiment at 1315 South Bris tol, July 14, 1994.

Santa Ana Fire Department Experiment at 1315 South Bristol, July 14, 1994. (Reprint)

PB'96-102934

00,207

DEAL, S. P.

Numerical Simulation of Rapid Combustion in an Underground Enclosure.

PB96-183132

01,424

EAN, B. E.

Correlation of $\mathrm{HgCdTe}$ Epilayer Defects with Underlying Substrate Defects by Synchrotron X-Ray Topography.
PB94-200714 129

DEAN, J. W.

Design and Construction of a Liquid Hydrogen Temperature System

AD-A286 618/4

02,619

Helium Refrigeration and Liquefaction Using a Liquid Hydrogen Refrigerator for Precooling.

AD-A286 $683 / 8$

02,749

Tabulation of the Thermodynamic Properties of Normal Hy drogen from Low Temperatures to $300 \mathrm{~K}$ and from 1 to 100 Atmospheres.

AD-A279 $951 / 8$

00,713

\section{DEARDEN, D. V.}

New Rydberg States of Aluminum Monofluoride Observed by Resonance-Enhanced Multiphoton Ionization Spectroscopy

DEASY, J. 0.

00,797

Extrapolation Chamber Measurements on (90) $\mathrm{Sr}+(90) \mathrm{Y}$ Beta-Particle Ophthalmic Applicator Dose Rates.

PB95-153375

DEBENHAM, P. H.

NIST-NRL Free-Electron Laser Facility.

PB94-212511

DEBIEVRE, $P$.

Reference Materials by Isotope Dilution Mass Spectrometry. PB95-153383

Measuring Matching Wear Scars on Balls and Flats.
PB95-151528

DEETER, M. N.

Domain Effects in Faraday Effect Sensors Based on Iron Garnets.

Faraday Effect Current Sensor with Improved SensitivityBandwidth Product

PB95-203154

Faraday Effect Current Sensors

02,180

B94-200698

02,127

Faraday Effect Sensors: A Review of Recent Progress.

PB94-200706

02,128

Faraday Effect Sensors for Magnet Field and Electric Current.

Fiber-Optic Faraday-Effect Magnetic-Field Sensor Based on Flux Concentrators

PB96-200308

02,201

High Frequency Magnetic Field Sensors Based on the Faraday Effect in Garnet Thick Films.

02,282

High Speed, High Sensitivity Magnetic Field Sensors Based on the Faraday Effect in Iron Garnets.

02,151

Magneto-Optic Magnetic Field Sensor with 1.4pT/square root of $1(\mathrm{~Hz})$ Minimum Detectable Field at $1 \mathrm{kHz}$. 02,125 Magneto-Optic Magnetic Field Sensors Based on Uniaxial Iron Garnet Films in Optical Waveguide Geometry.
PB95-153409 Iron Garnet Films in Optic Field Sensors Based on

PB95-168498

02,159

Magnetooptic Effects

PB96-119292

04,338

Novel Bulk Iron Garnets for Magneto-Optic Magnetic Field

04,675

Submicroampere-Per-Root-Hertz Current Sensor Based on the Faraday Effect in Ga: YIG.

02,155

Wideband Current and Magnetic Field Sensors Based on Iron Garnets.

DEFIBAUGH, D. E.

Vapor Pressure of 1,1,1,2,2-Pentafluoropropane.

PB97-113237

01,975

DEFIBAUGH, D. R.

Compressed Liquid Densities, Saturated Liquid Densities, and Vapor Pressures of 1,1-Difluoroethane.

01,173

Interaction Coefficients for 15 Mixtures of Flammable and Non-Flammable Components.

PB96-146626

03,281

Thermodynamic Properties of CF3-CHF-CHF2, 1,1,1,2,3,3Hexafluoropropane.

03,299

Thermodynamic Properties of CHF2-CF2-CHF, 1,1,2,2,3Pentafluoropropane

PB97-118392

03,300

Thermodynamic Properties of CHF2-O-

CHF2,Bis(difluoromethyl) Ether.

PB94-199569

Thermodynamic Properties of Difluoromethane.

PB94-185204

00,798

Vapor Pressure of Pentafluorodimethyl Ether.

00,772

PB96-201199

00,677

Vapor Pressure of 1,1-dichloro-2,2,2-trifluoroethane (R123)

PB95-126231
00,899

Virial Coefficients of Five Binary Mixtures of Fluorinated Methanes and Ethanes.

PB96-156054

01,128

Viscosity of $1,1,1,2,3,3$-Hexafluoropropane and 1,1,1,3,3,3-

Hexafluoropropane at Saturated-Liquid Conditions from 262

$\mathrm{K} 10353 \mathrm{~K}$.

DEGROOT, D. C.

03,292

High-Speed Interconnection Characterization Using Time Domain Network Analysis.

04.061

Microwave Properties of Voltage-Tunable YBa2Cu3O7delta/SrTiO3 Coplanar Waveguide Transmission Lines.
PB96-141262

Optımizing Time-Domaın Network Analysis.

02,085

DEHMER, J. L

High Resolution Angle Resolved Photoelectron Spectros- 
DEILAMIAN, $K$.

Isotope Shifts and Hyperfine Splittings of the 398.8-nm Yb I

03,814

PB94-199585

04,050

Search for Small Violations of thin

in an Excited
PB $96-123518$

DEISSLER, A.

Liquid Chromatographic Determination of Polycyclic Aromatic Hydrocarbon Isomers of Molecular Weight 278 and 302 in Environmental Standard Reference Materials. PB95-164042

02,523

DEITERS, U. $K$.

Application of the Taylor Dispersion Method in Supercritical Fluids.

PB95-164323

00,977

DELAETER, J. $R$.

Reference Materiais by Isotope Dilution Mass Spectrometry.
PB95-153383

DELANEY, H. D.

Stacking the Cards in Europe: One Company's Story.
PB97-110126

DELAUDER, $S$.

Thermodynamic Analysis of Heparin Binding to Human Antithrombin

03,455

DELAUTER, L

Fire Performance of an Interstitial Space Construction System.

00,390

DELLA TORRE, E.

Experimental Verification of a Vector Preisach Mode PB95-163564

DELOZANNE, A. L.

Vortex Images in Thin Films of YBa2Cu3O(sub 7-x) and Bi2Sr2Ca1Cu2O(sub $8+x$ ) Obtained by Low-Temperature Magnetic Force Microscopy

EMATTEIS, $R$.

04,815

Comparisons of Some NIST Fixed-Point Cells with Similar Cells of Other Standards Laboratories.

PB97-119242

00,655

DEMIRALP, $R$

Certification of Standard Reference Material (SRM) 1941 a Organics in Marine Sediment.

02,593

Concentrations of Chlorinated Hydrocarbons, Heavy Metals and Other Elements in Tissues Banked by the Alaska Marine Mammal Tissue Archival Project.

PB95-209870

02,590

Determination of Inorganic Constituents in Marine Mammal

Tissues.
PB95- 152047

00,589

Development of Frozen Whale Blubber and Liver Reference Materials for the Measurement of Organic and Inorganic Contaminants.

00,587

Relationship of Silver with Selenium and Mercury in the Liver of Two Species of Toothed Whales (Odontocetes).

PB96-167275 02,596

Trace Element Concentrations in Cetacean Liver Tissues Archived in the National Marine Mammal Tissue Bank.
PB96-167127

DEMPSEY, $\mathbf{R}$.

First Results from a Coordinated ROSAT, IUE, and VLA Study of RS CVn Systems.

DEMPSEY, R. C.

00,069

Riass Coronathon: Joint $\mathrm{X}$-ray and Ultraviolet Observations of Normal F-K Stars.

PB96-200217

00,109

ROSAT All-Sky Survey of Active Binary Coronae. 1. Ouiescent Fluxes for the RS Canum Venaticorum Systems.
PB95-202479 00,077 ROSAT All-Sky Survey of Active Binary Coronae. 2. Coronal Temperatures of the RS Canum Venaticorum Systems.
PB94-199601 Rotational Modulation and Flares on RS Canum Venaticorum and BY Draconis Stars. XVIII. Coordinated VLA, ROSAT, and IUE Observations of RS CVn Binaries. PB96-10232

DENENBERG, $R$.

Z39.50 Implementation Experiences.

PB96-114939

00,089

DENG, $N$.

01,816

Energy-Based Method for Liquefaction Potential Evaluation. Phase 1. Feasibility Study.

PB96-214747

03,691

DENNIS, T. J. $S$.

Rotational Dynamics of Solid C70: A Neutron-Scattering Study

00,755

Rotational Dynamics of Solid C70: A Neutron-Scattering Study.

00,949
DENNO, $P$.

Dynamic Objects and Meta-Level Programming of an EXPRESS Language Environment.

01,774

DENNO, P. O.

Aspects of a Product Model Supporting Apparel Virtual Enerprises.

PB96-183231

02,790

DEPAOLA, B. D.

Merged-Beams Energy-Loss Technique for Electron-Ion Excitation: Absolute Total Cross Sections for $\mathrm{O}(5+)$ (2s yields 2p).

PB96-102058

04,017

DEPONDT, P.

Maximum Entropy as a Tool for the Determination of the CAxis Profile of Layered Compounds.

00,800

DEPRIT, A.

Complete Reduction of the Euler-Poinsot Problem.

PB94-172152

03,788

Frozen Orbits for Satellites Close to an Earth-Like Planet. PB96-102165

04,839

DEPRIT, E.

Frozen Orbits for Satellites Close to an Earth-Like Planet.

PB96-102165

04.839

DEREGGI, A.

Effect of DC Tests on Induced Space Charge

B94-172350

DEREGGI, A. $S$.

Hysteresis Measurements of Remanent Polarization and Coercive Field in Polymers.

PB94-199767

04,475

Spatial Dependence of Electrical Fields Due to Space Charges in Films of Organic Dielectrics Used for Insulation of Power Cables.

PB94-199130

02,214

Thermal Pulse Study of the Polarization Distributions Produced in Polyvinylidene Fluoride by Corona Poling at Constant Current.

01,195

Thermal Stability of Internal Electric Field and Polarization Distribution in Blend of Polyvinylidene Fluoride and Polymethylmethacrylate.

PB95-151072

01,240

DERKSON, G.

Effects of Calcium Phosphate Solutions on Dentin Permeability.

00,157

DERR, L. S.

Bibliography of the NIST Optoelectronics Division PB96-128210

02,193

DESAl, P. G.

Unreacted Cement Content in Macro-Defect-Free Composites: Impact on Processing-Structure-Property Relations.

ites: Impact on Processing-Structure-Property Relations.
PB96-141270 03,174

DESCHAMPS, M. F.

Approach to Setting Performance Requirements for Automated Evaluation of the Parameters of High-Voltage $1 \mathrm{~m}$ -

pulses.

01,878

DESJARLAIS, A. O.

Water-Vapor Measurements of Low-Slope Roofing Mate-

PBials. 251617

00,399

DESLATTES, R. D.

Crystal Diffraction Spectrometry for Accurate, Non-Invasive
$\mathrm{kV} V / S$ pectral Measurement for Improvement of MammokV/Spectral Measurement for Improvement of Mammo graphic Image Quality.

00,721

Early History and Future Outlook for the X-ray Crystal Density Method

04,692

Electron-Ion-X-ray Spectrometer System.

PB95-176137

03,958

Evolution of $X$-ray Resonance Raman Scattering into $X$-ray Fluorescence from the Excitation of Xenon Near the L Edge.

96-102751

04,025

Experimental Aspects and Z-Dependent Systematics in One- and Two-Electron lons and Single Vacancy Systems.

Flat and Curved Crystal Spectrography for Mammographic

$\begin{array}{ll}\text { X-ray Sources. } & 03,642 \\ \text { PB97-122246 } & \end{array}$

Noninvasive High-Voltage Measurement in Mammography by Crystal Diffraction Spectroscopy.

00,160

Polarization Measurements on a Magnetic Quadrupole Line in Ne-Like Barium.

PB97-113104

04,161

Polarized X-Ray

03,862

Precision Comparison of the Lattice Parameters of Silicon Monocrystals.

04,438

Production and Characterization of Ion Beam Sputtered Multilayers.

Status of a Silicon Lattice Measurement and Dissemination Exercise.

PB94-199635

04,474

Systematic Correction in Bragg $X$-ray Diffraction of Flat and Curved Crystals.

Upgraded Facility for Multilayer Mirror Characterization at PB96-160387

DESROSIERS, $M$

Commentary on 'Optimization of Experimental Parameters for the EPR Detection of the Cellulosic Radical in Irradiated Foodstuffs'

PB96-164124

00,043

DESROSIERS, M. F.

Complex Time Dependence of the EPR Signal of Irradiated L-alpha-alanine.

B 97-122436

04,180

Dosimetry Systems for Radiation Processing

EPR Bone Dosimetry: A New Approach to Spectral Deconvolution Problems.

PB94-199643

03,616

EPR ated with $\mathrm{X}$ and Gamma Rays: Study of Energy Dependence.

PB97-110373

03,639

ESR-Based Analysis in Radiation Processing. PB95-161634

03,931

Estimation of the Absorbed Dose in Radiation-Processed Food. 1. Test of the EPR Response Function by a Linear Regression Analysis

00,039

Estimation of the Absorbed Dose in Radiation-Processed Food. 2. Test of the EPR Response Function by an Exponential Fitting Analysis.

PB94-199650

00,036

Estimation of the Absorbed Dose in Radiation-Processed

Food. 3. The Effect of Time of Evaluation on the Accuracy of the Estimate.

PB94-199684 00,037

Estimation of the Absorbed Dose in Radiation-Processed Food. 4. EPR Measurements on Eggshell.

PB94-199692

00,038

Experimental assessment of absorbed dose to mineralized bone tissue from internal emitters: An electron paramagnetic resonance study.

DE96007979

03,614

Experimental Validation of Radiopharmaceutical Absorbed Dose to Mineralized Bone Tissue.

PB94-199668

03,617

Inter-Laboratory Trials of the EPR Method for the Detection

of Irradiated Meats Containing Bone.

00,042

Investigation of Applicability of Alanine and Radiochromic Detectors to Dosimetry of Proton Clinical Beams.

PB96-146782

New EPR Dosimeter Based on Polyvinylalcohol.

PB94-199700

03,636

Dosimeters.

PB96.146725

Polymer

Overview of a Radiation Accident at an Industrial Accelerator Facility.
PB97-122485

Radiation Accident at an Industrial Accelerator Facility

PB95-140117 
DEVOE, J. R.

Establishing Quality Measurements for Inorganic Analysis of Biomaterials.
PB94-199726

DEVORET, M. H.

Even-Odd Asymmetry of a Superconductor Revealed by the Coulomb Blockade of Andreev Reflection.

PB95-153540

Observation of Hot-Electron Shot Noise in a Metallic ResisPB.

DEVRIES, K. $M$.

Polarization of Light Emitted After Positron Impact Excitation of Alkali Atoms.

PB94-199734

DEWEESE, M. E.

Metrology for Electromagnetic Technology: A Bibliography of NIST Publications.

DEWEY, M. S.

02,116

Current Results and Future Prospects for a Neutron Lifetime Determination Using Trapped Protons.

03,817

Mass Assay and Uniformity Test of Boron Targets by Neutron Beam Methods

PB97-119085

04,175

Measurement of the Neutron Lifetime.

DEWIT, $R$.

Fracture Behavior of Large-Scale Thin-Sheet Aluminum Alloy.

Fracture Testing of Large-Scale Thin-Sheet Aluminum Alloy. Fracture Testing of Large-Scale Thin-Sheet Aluminum Alloy.
AD-A306 625/5 Fracture Testing of Large-Scale Thin-Sheet Aluminum Alloy.
PB95-242368 DEXTER, A. L.

Use of Building Emulators to Evaluate the Performance of Building Energy Management Systems.

PB96-11190

DEYST, J. P.

00,269

Bounds on Frequency Response Estimates Derived from Uncertain Step Response Data.

Bounds on Least-Squares Four-Parameter Sine-Fit Errors Due to Harmonic Distortion and Noise.

PB96-141304

01,609

Uncertainties of Frequency Response Estimates Derived from Responses to Uncertain Step-Like Inputs.

PB97-111843

01,984

Wideband Sampling Voltmeter.

01,990

DEZSI, G.

High Accuracy Measurement of Aperture Area Relative to a Standard Known Aperture.

PB95-261954

01,919

Method of Realizing Spectral irradiance Based on an Abso-

lute Cryogenic Radiometer.
PB95-16 1204

04,270

DHAR, S.

Procedure for Product Data Exchange Using STEP Devel. oped in the AutoSTEP Pilot.

PB96-183058

02,843

DI MARZIO, E. A.

Glass Temperature of Polymer Blends: Comparison of Both the Free Volume and the Entropy Predictions with Data.

PB95-140190

01,236

Mixing Plate-Like and Rod-Like Molecules with Solvent: A Test of Flory-Huggins Lattice Statistics.

PB96-126206

DI MARZO, M.

Application of Boundary Element Methods to a Transient Axis-Symmetric Heat Conduction Problem.

01,375

Fire Protection Foam Behavior in a Radiative Environment.
PB97-116131 00,237

Sparse Water Sprays in Fire Protection.

00,237

Transient Cooling of a Hot Surface by Droplets Evaporation

Transient Cooling of a Hot Surface by Droplets Evaporation.
PB94-156957 PB94-156957 03,783
Transient Cooling of a Hot Surface by Droplets Evaporation. PB95-143194 Water Droplet Evaporation from Radiantly Heated Solids. PB95-217147

00,394

DIAMOND, $\mathrm{S}$.

Interaction between Naphthalene Sulfonate and Silica Fume

in Portland Cement Pastes.
PB94-199759

01,315

DIAMONDSTONE, B. I.

CSTL Technical Activities 1991
PB94-160769

DIANOUX, A.J.

Inelastic-Neutron-Scattering Studies of Poly(p-phenylene vinylene).
PB95-180766

01,014
DIAZ, C.

Algorithm Testing and Evaluation Program for Coordinate Measuring Systems: Long Range Plan.

02,915

Algorithm Testing and Evaluation Program for Coordinate Measuring Systems: Testing Methods

02,666

Concept for an Algorithm Testing and Evaluation Program at NIST

PB94-163029

02,890

Process for Selecting Standard Reference Algorithms for Evaluating Coordinate Measurement Software.

02,629

PB94-173754

Calibration of High-Energy Electron Beams by Use of

ICK, C. E. Graphite Calorimeters.

04,598

Electron and Proton Dosimetry with Custom-Developed Radiochromic Dye Films. PB95-151106

03,713

Image Information Transfer Properties of $X$-Ray Intensifying Screens in the Energy Range from 17 to $320 \mathrm{keV}$.
PB95-126173

Investigation of Applicability of Alanine and Radiochromic Detectors to Dosimetry of Proton Clinical Beams.
PB96-146782
03,636

eleraOverview of a Radiation Accident at an Industrial Accelera-
tor Facility.
PB97-122485
02,612

Radiation Accident at an Industrial Accelerator Facility.
PB95-140117

Research and Development Activities in Electron

Paramagnetic Resonance Dosimetry.

Study of Multiple Scattering Background in Compton Scatter Imaging

PB96-112222

04,425

DICKENS, B.

Cylinder Wipe Air-Drying Intaglio Ink Vehicles for U.S. Currency Inks.

03,115

Effect of DC Tests on Induced Space Charge.

PB94-172350

02,212

Grafted Interpenetrating Polymer Networks.

01,200

Hysteresis Measurements of Remanent Polarization and Coercive Field in Polymers.

04,475

Modified Surface-Active Monomers for Adhesive Bonding to Dentin

PB95-151163
NIR-Spectroscopic Investigation of Water Sorption Characteristics of Dental Resins and Composites.

00, 189

PB95-151171

NIST/NCMS Program on Electronic Packaging: First Up-
date.
PB96-204086
03,008

PB96-204086
Physical 008

cium Phosphate Cements.

PB95-180212

00,171

Preparation and Characterization of Cyclopolymerizable

Resin Formulations
PB96-146840

01,285

Spatial Dependence of Electrical Fields Due to Space Charges in Films of Organic Dielectrics Used for Insulation of Power Cables.

DICKENS, J. K.

Measurement of the $(10) B(n$, alpha1gamma)(7)Li Cross Section in the $0.3104 \mathrm{MeV}$ Neutron Energy Interval.

PB96-161799

DICKENS, S. $V$.

Effect of Two Initiator/Stabilizer Concentrations in a Metal

Primer on Bond Strengths of a Composite to a Base Metal Alloy.

72723

00,141

DICKENS-VENZ, $S$.

Physical and Chemical Properties of Resin-Peinforced Calcium Phosphate Cements.

PB95-180212

00,171

DICKEY, J. 0.

Lunar Laser Ranging: A Continuing Legacy of the Apollo Program.

PB95-202495
DIDION, D. A.

03,683

Causes of the Apparent Heat Transfer Degradation for Refrigerant Mixtures.

PB94-212701

03,259

Role of R22 in Refrigerating and Air Conditioning Equip-

PB94-199783 03,253

Role of Refrige 03,252

Siudy of Heat Pump Pertormance Using Mixtures of R32/ R134a and R32/R125/R134a as 'Drop-In' Working Fluids for R22 with and Without a Liquid-Suction Heat Exchanger. PB94-218559 02,503

Study to Determine the Existence of an Azeotropic R-22 'Drop-In' Substitute.

PB96-16781?

02,568

IEBOLD, A. C

Business and Manufacturing Motivations for the Developing of Analytical Technology and Metrology for Semiconductors. PB96-161948

Characterization of Two-Dimensional Dopant Profiles: Status and Review.

PB96-119300 02,400

Characterization of Two-Dimensional Dopant Profiles: Status and Review.

02,451

Summary Report: Workshop on Industrial Applications of Scanned Probe Microscopy (2nd). A Workshop Co-Sponsored by NIST, SEMATECH, ASTM E42.14, and the American Vacuum Society. Held in Gaithersburg. Maryland on May 2-3, 1995

Workshop Summary Report: Industrial Applications of Scanned Probe Microscopy. A Workshop Co-sponsored by unm $25,1994$.

PB95-170387

00,506

DIERCKS, G.

Novel Bulk Iron Garnets for Magneto-Optic Magnetic Field PB95-180204

04,675

DIETENBERGER, M. A.

Calculating Flame Spread on Horizontal and Vertical Sur-

PB94-187283

DIETRICH, D. D.

Photographic Response to X-Ray Irradiation. 3. Photographic Linearization of Beam.Foil Spectra.

PB94-200102

03,823

DIETRICH, H.

Lattice Position of Si in GaAs Determined by X-Ray Stand-

ing Wave Measurements.

B 95-164406

DIETRICH, H. B.

Range Statistics and Rutherford Backscattering Studies on Fe-Implanted In0.53Ga0 47As.

PB95-126397

04,535

Transition Metal Implants in In0.53GA0.47As.

PB95-126389

DIKKERS, R. D.

Proceedings of a Workshop on Developing and Adopting

Seismic Design and Construction Standards for Lifelines.

Held in Denver,
PB97-115794

DILL, J.

Crystal Packing Interactions of Two Different Crystal Forms of Bovine Ribonuclease A.

PB95-152823

00,943

DILLER, D. E.

Measurements of the Viscosities of Saturated and Compressed Fluid 1-chloro-1,2,2,2-tetrafluoroethane (R124) and Pentafluoroethane (R125) at Temperatures between 120 and $420 \mathrm{~K}$.

PB94-199791 03,254

Measurements of the Viscosities of Saturated and Compressed Liquid 1,1,1,2-Tetrafluoroethane (R134a), 2,2-
Dichloro-1,1,1-Trifluoroethane (R123) and 1,1-Dichloro-1Dichloro-1,1,1-Trifluoroe
Fluoroethane (R141b).

PB95-175386

03,273

DIMARZIO, E. A.

Peeling a Polymer from a Surface or from a Line. PB94-199809

DIMAURO, L. F.

Intensity-Dependent Scattering Rings in High Order AbovePhreshold lonization

DIMITRIOU, $\mathbf{P}$ 
Goddard High-Resolution Spectrograph Observations of the Local Interstellar Medium and the Deuterium/Hydrogen Ratio along the Line of Sight Toward Capella.

00,066

ISDAROGLU,

Commentary: The Measurement of Oxidative Damage to DNA by HPLC and GC/MS Techniques.

DITMARS, D. A

03,484

Calibration Standards for Differential Scanning Calorimetry 1. Zinc Absolute Calorimetric Measurement of Enthalpy of Fusion and Temperature of Fusion HM

00,801

DITMANN, $\mathrm{s}$.

Measurement Comparability, Traceability, and Measurement Assurance Programs.

DIWAN, B.

02,692

Nickel(II)-Mediated Oxidative DNA Base Damage in Renal and Hepatic Chromatin of Pregnant Rats and Their Fetuses. Possible Relevance to Carcinogenesis.

03,646

Oxidative DNA Base Damage in Renal, Hepatic, and Pulmonary Chromatin of Rats After Intraperitoneal Injection of Cobalt (II) Acetate.

PB95-150025

03,647

DIXON, R. N.

Resonance Enhanced Multiphoton Ionization Spectroscopy of the SnF Radical.

PB97-111223

01,176

Increasing the Value of Atomic Force Microscopy Process

Increasing the Value of Atomic Force Microscopy Process
Metrology Using a High-Accuracy Scanner, Tip Character-

Metrology Using a High-Accuracy Scanner, Tip Character-
ization, and Morphological Image Analysis.
PB96-190293

02,758

Progress Toward Accurate Metrology Using Atomic Force Microscopy.

DIZDAROGLU, $M$

beta-D-Glucosyl-Hydroxymethyluracil: A Novel Modified Base Present in the DNA of the Parastic Protozoan T.

PB94-172319 03.524

Copper Ion-Mediated Modification of Bases in DNA in Vitro by Benzoyl Peroxide.

PB94-198231 03.645

DNA Base Damage Generated In vivo in Hepatic Chromatin of Mice upon Whole Body y-Irradiation.

03,627

DNA Base Damage in Lymphocytes of Cancer Patients Undergoing Radiation Therapy.

03,643

DNA Base Modifications in Renal Chromatin of Wistar Rats Preated with a Renal Carcinogen, Ferric Nitrilotriacetate.

DNA Damage and DNA Sequence Retrieval from Ancient Tissues.
PB97-111983

03,556

Formation of DNA-Protein Cross-Links in Cultured Mammalian Cells Upon Treatment with Iron lons.

03,651

onizing Radiation Causes Greater DNA Base Damage in

Radiation-Sensitive Mutant M10 Cells That in Parent Mouse Lymphoma L5178Y Cells.

Modification of DNA Bases in Chromatin of Intact Targe Human Cells by Activated Human Polymorphonuclear Leu-

PB94-199833

03,526

Nickel(II)-Mediated Oxidative DNA Base Damage in Renal and Hepatic Chromatin of Pregnant Rats and Their Fetuses. Possible Relevance to Carcinogenesis. Novel Activities of Human Uracil DNA N-glycosylase for Cytosine-Derived Products of Oxidative DNA Damage.
PB96-164132

Novel Activity of E. coli uracil DNA N-glycosylase Excision of Isodialuric Acid (5,6-dihydroxyuracil), a Major Product of Oxidative DNA Damage, from DNA.

PB96-110747 Damage, from DNA. 03.543

Novel DNA N-Glycosylase Activity of $E$. coli T4 Endonuclease $V$ That Excises 4,6-Diamino-5 Formamidopyrimidine from DNA, a UV-Radiation- and Hydroxyl Radical-Induced Product of Adenine.

03,549

Oxidative Damage to DNA in Mammalian Chromatin.

PB94-199825 03,525

Oxidative DNA Base Damage in Renal, Hepatic, and Pulmonary Chromatin of Rats After Intraperitoneal Injection of Cobalt (II) Acetate.

PB95-150025

03,647

Quantitative Determination of Oxidative Base Damage in DNA by Stable Isotope-Dilution Mass Spectrometry

PBepair of Products of Oxidative DNA Base Dama Human Cells.

amage in -190129

03,555

Substrate Specificity of the Escherichia coli Endonuclease 1II: Exicision of Thymine- and Cytosine-Derived Lesions in DNA Produced by Radiation-Generated Free Radicals.

PB95-153425

03,535
Tert-Butyl Hydroperoxide-Mediated DNA Base Damage in Cultured Mammalian Cells.

03,644

Treatment of Wistar Rats with a Renal Carcinogen, Ferric Nitrilotriacetate, Causes DNA-Protein Cross-Linking beNitrilotriacetate, Causes DNA-Protein Cross-Linking be-
tween Thymine and Tyrosine in Their Renal Chromatin. tween Thymine and Tyrosine in Their Renal Chromatin.
PB,649-112115

\section{DIZDAROGLU, M. $M$.}

Chemical Determination of Oxidative DNA Damage by Gas Chromatography-Mass Spectrometry.

PB95. 175394

03.540

DJURIC, $N$.

Absolute Cross-Section Measurements for Electron-Impact onization of $\mathrm{C} 1(+1)$

$\begin{array}{ll}\text { PB94-199841 } 03,818 & \end{array}$

Absolute Cross-Section Measurements for Electron-Impact Single lonization of $\mathrm{Se}(+)$ and $\mathrm{Te}(+)$.

03,980

Absolute Cross Sections for Electron-Impact Single Ionization of $\mathrm{Si}(+)$ and $\mathrm{Si}(2+)$

PB95-202529

03,982 Collisions of Electrons with Highly-Charged Ions.
PB96-200340

Sections

Crossed-Beams Measurements of Abso

PB95-202511

03,981

Electron-Impact Ionization of $\ln (+)$ and $\mathrm{Xe}(+)$.

PB94-185089

00,770

Electron-Impact lonization of $\ln (+)$ and $\mathrm{Xe}(+)$

PB95-152906

03,918

DJUROVIC, $\mathrm{s}$.

Hydrogen Balmer Alpha Line Shapes for Hydrogen-Argon Mixtures in a Low-Pressure if Discharge.

03,924

Ion Kinetic-Energy Distributions and Balmer-alpha (Halpha) Excitation in Ar-H2 Radio-Frequency Discharges. PB96-102959

04,029

DMITRIEV, I. A.

Precision High Temperature Blackbodies.

PB95-14005

03,885

DOBBYN, R.

High Resolution Hard X-Ray Microscope.

PB94-213055

03,856

DOBBYN, R. C.

Correlation of $\mathrm{HgCdTe}$ Epilayer Defects with Underlying Substrate Defects by Synchrotron X-Ray Topography. PB94-200714

(1)

DOBISZ, E.

Lattice Position of $\mathrm{Si}$ in GaAs Determined by X-Ray Standing Wave Measurements.

PB95-164406

04,632

DOBISZ, E. A.

Integration of Scanning Tunneling Microscope

Nanolithography and Electronics Device Processing.
PB95-153359

02,341

DOBLECKI, W

Effect of Three Sterilization Techniques on Finger Pluggers.
PB94-216090

DOBROW, R.P.

Move-to-Root Rule for Self-Organizing Trees with Markov Dependent Requests.

PB96-179528

03,431

DOBRY, R.

Functional Security Criteria for Distributed Systems.

DODD, R. J.

01,607

Properties of a Bose-Einstein Condensate in an Anisotropic Harmonic Potential.

PB96-204144

04,133

DODERER, T.

Direct Observation of Vortex Dynamics in Two-Dimensional Josephson-Junction Arrays.

02,067

Frequency Dependence of the Emission from 2D Array Josephson Oscillators.

02,056

Novel Vortex Dynamics in Two-Dimensional Josephson Ar-

rays.

02,091

Observation of Vortex Dynamics in Two-Dimensional Josephson-Junction Arrays.

02,050

SUSAN: SUperconducting Systems ANalysis by Low Temperature Scanning Electron Microscopy (LTSEM).

PB96-112065

DOETSCH, P.

Novel Activity of E. coli uracil DNA N-glycosylase Excision of Isodialuric Acid (5,6-dihydroxyuracil), a Major Product of Oxidative DNA Damage, from DNA.

PB96-110747

03,543

DOGAN, F.

High Resolution Inelastic Neutron Scattering Study of Phonon Self-Energy Effects in YBCO.

04,688

q Dependence of Self-Energy Effects of the Plane Oxygen Vibration in $\mathrm{YBa} 2 \mathrm{Cu} 3 \mathrm{O} 7$

PB96-138516

01,096

DOIRON, D. T.

Length Metrology of Complimentary Small Plastic Rulers. DOIRON, T.

Gage Block Handbook

PB95-251716

02,667

Gage Block Standards, Measurement Capabilities and LabOratory Accreditation

Measuring the Stability of Three Copper Alloys.

02,757

PB94-199866

03,326

Residual Error Compensation of a Vision-Based Coordinate Measuring Machine

SPC Artifact for Automated Solder Joint Inspection.

PB96-161716

04,091

Verification of Revised Water Vapour Correction to the

Index of Refraction of Air

DOIRON, T. D.

Computer Vision Based Tool Setting Station.

Length Metrology of Complimentary Small Plastic Rulers.

DOLEV, A.

NIST High-Accuracy Sampling Wattmeter.

DOLS, W. S.

Air Change Effectiveness Measurements in Two Modern

Office Buildings.
PB94-185766
Environmental Evaluation of a New Federal Otfice Bulding.

Environmental Evaluation of a New Federal Office Building.

Field Measurements of Ventilation and Ventilation Effectiveness in an Otfice/Library Building.

PB95-108833 00,247

Indoor Air Quality Commissioning of a New Office Building.
00,262

Indoor Air Ouality Commissioning of a New Office Building.

PB97-110142
Measurements of Outdoor Air Distribution in an Otfice Build-

ing. 05 -210944 00,264

Study of Ventilation and Carbon Dioxide in an Office Build-

PB.95-150140

02,542

Ventilation Effectiveness Measurements in Two Modern Of. fice Buildings.

DOMALSKI, E. S.

Assessing the Credibility of the Calorific Content of Muncipal Solid Waste.

PB94-199882

02,581

Conference R

03,722

Estimation of the Heat Capacities of Organic Liquids as a Function of Temperature Using Group Additivity. I. Hydro carbon Compounds

00,739

Estimation of the Heat Capacities of Organic Liquids as a Function of Temperature Using Group Additivity. II. Compounds of Carbon, Hydrogen, Halogens, Nitrogen, Oxygen,
and Sulfur.
PB94-162286
00,740

Estimation of the Thermodynamic Properties of C-H-N-O-SHalogen Compounds at $298.15 \mathrm{~K}$

DOMANSKI, P. A.

Intracycle Evaporative Cooling in a Vapor Compression

Cycle.
PB97-116107

02,762

Simplified Cycle Simulation Model for the Performance Rat-

ing of Refrigerants and Refrigerant Mixtures.
PB94-199890

03,255

Theoretical Evaluation of the Vapor Compression Cycle with a Liquid-Line/Suction-Line Heat Exchanger, Economizer, and Ejector.

DOMEN, S. R.

02,607

Problem of Convection in the Water Absorbed Dose Calo. 
DONAHUE, D. J.

Radiocarbon Measurements of Atmospheric Volatile Organic Compounds: Quantifying the Biogenic Contribution.
PB $92,574-122352$ DONALDSON, J. R.

Algorithmic Enhancements to the Method of Centers for Linear Programming Problems.

PB94-198959

03,426

DONG, Q. Y.

Al L2,3 Core Excitons in AlxGa 1-x as Studied by Soft-X-ray Reflection and Emission.

PB96-157839

04,067

Barium Contributions to the Valence Electronic Structure of YBa2Cu307-delta, PrBa2Cu307-delta, and Other Barium-

Containing Compounds.
PB96-158019 04,076

Charge-Transfer-Induced Multiplet Structure in the $\mathrm{N} 4,5 \mathrm{O} 2,3$ Soft-X-ray Emission Spectrum of Lanthanum.
$\mathrm{PB} 96-163746$

Cooper M(sub II,IiI) X-ray-Emission Spectra of Copper OXides and the Bismuth Cuprate Superconductor.

04,077

Intermediate Coupling in L2-L3 Core Excitons of $\mathrm{MgO}$, $\mathrm{Al} 2 \mathrm{O} 3$, and $\mathrm{SiO}_{2}$

04,079

Laser-Synchrotron Hybrid Experiments: A Photon to Tickle, A Photon to Poke.

03.704

PB96-157847

Local Partial Densities of States in $\mathrm{Ni}$ and $\mathrm{Co}$ Silicides
Studied by Soft X-Ray Emission Spectroscopy.
PB94-212412 PB94-212412

Phonon Relaxation in Soft-X-ray Emission of Insulators.

Simple Variable Line Space Grating Monochromator for Synchrotron Light Source Beamlines.

04,065

Soft-X-ray-Emission Investigation of Cobalt Implanted Siljcon Crystals.

04,069

Soft-X-ray-Emission Spectra of Solid $\mathrm{Kr}$ and $\mathrm{Xe}$.

04,070

Soft-X-ray-Emission Studies of Bulk Fe3Si, FeSi, and FeSi2, and Implanted Iron Silicides.

PB96-157938

04,071

DONG, $X$.

Mechanism of Mild to Severe Wear Transition in Alpha-Alumina.

03.233

Tribological Characteristics of Alpha-Alumina at Elevated Temperatures.

DONGARRA, J.

02.963

IML++ v. 1.2 Iterative Methods Library Reference Guide.
PB96-195219

DONMEZ, A.

Displacement Method for Machine Geometry Calibration.
PB95-152088

Effects of Spindle Dynamic Characteristics on Hard Turning.

PB96-122981 02,699.
0

DONMEZ, M. A.

Development of a New Ouality Control Strategy for Automated Manufacturing.

PB96-160486 02,840

Evaluation of a Tapered Roller Bearing Spindle for HighPrecision Hard Turning Applications.

02,700 Integrated Inspection System for Improved Machine Performance.

02,959

Prediction of Geometric-Thermal Machine Tool Errors by Artificial Neural Networks.

PB94-186673

02,943

DOOM, S. S

Proficiency Tests for the NIST Airborne Asbestos Program PB94. 193828

DOORN, S. S.

Proficiency Tests for the NIST Airborne Asbestos Program,

PB94-188836 00,535

Proficiency Tests for the NIST Airborne Asbestos Program . 1992.

PB94-194362

00,539

DORKO, W. D.

NIST Traceable Reference Material Program for Gas Standards: Standard Reference Materials.

PB96-210786

00,644

Standards for Atmospheric Measurements PB95-163622

DOROFEEVA, O. $v$.

02,547

Ideal Gas Thermodynamic Properties of Sulphur Heterocyclic Compounds.

DOROSHOW, J.

Modification of DNA Bases in Chromatin of Intact Target Human Cells by Activated Human Polymorphonuclear Leu-

kocytes.
PB94-199833

03,526
DOROSHOW, J. H.

Copper Ion-Mediated Modification of Bases in DNA in Vitro by Benzoyl Peroxide.

DOSCHEK, G.

03,645

Sleuthing the Dynamo: HST/FOS Observations of UV Emis-

sions of Solar-Type Stars in Young Clusters.

DOSCHEK, G. A

00,098

Far-Ultraviolet Flare on a Pleiades G Dwarf,

PB96-102033

00,086

Critical Current Density, Irreversibility Line, and Flux Creep

Activation Energy in Silver-Sheathed Bi2Sr2Ca2Cu2Ox

Activation Energy in
Superconducting Tapes.

04,616

DOUGHERTY, T. P.

Bimolecular Interactions in (Et) $3 \mathrm{SiOH}: \mathrm{Base}: \mathrm{CCl} 4$ Hydrogen

Bonded Solutions Studied by Deactivation of the Free $\mathrm{OH}$.

Stretch Vibration
PB97-118657

DOUGHTY, D. A.

04,166

Plasma Chemistry in Disilane Discharges.

02,514

Plasma Chemistry in Silane/Germane and Disilane/Germane Mixtures.
PB95-202537

DOUGLAS, J.

Book Review: Aspects and Applications of the Random PBak. 123534

Book Review: Statistical Physics of Macromolecules.

PB96-123526

04,215

Dynamic Light-Scattering Study of a Diluted Polymer Blend Near Its Critical Point.

Generalized Stokes-Einstein Equation for Spherical Particle Suspensions.
PB95-202743

01,031

How Far Is Far from Critical Point in Polymer Blends. Lattice Cluster Theory Computations for Structured Monomer Compressible Systems.

Non-Perturbative Relation between the Mutual Diffusion Coefficient, Suspension Viscosity, and Osmotic Compress ibility: Application to Concentrated Protein Solutions.

PB96-102355

01,062

Thermodynamic Properties of Dilute and Semidilute Solutions of Regular Star Polymers.

PB96-146808

01,284

DOUGLAS, J. F.

Competition between Hydrodynamic Screening ('Draining') and Excluded Volume Interactions in an Isolated Polymer

PB95-175402

01,265

Dimensional Crossover in the Phase Separation Kinetics of Thin Polymer Blend Films.

PBin Polymer

03,395

Etfect of Swelling on the Elasticity of Rubber: Localization

Model Description.
PB94-211034

Electrostatic Rigidity of Polyelectrolytes from Reparametrization Invariance.
PB96-180062

Examination of the $1 / d$ Expansion Method from Exact Enu-

meration for a Self-Interacting Self-Avoiding Walk.
PB95-175733

Geometrical Percolation Threshold of Overlapping

Ellipsoids.

lapping

Hydrodynamic Friction of Arbitrarily Shaped Brownian Particles.

PB97-110191

04,136

Hypercubic Lattice SAW Exponents nu and gamma : 3.99 Dimensions Revisited.

01,215

Influence of Shear on the Ordering Temperature of a Triblock Copolymer Melt

PB96-163753

01,288

Influence of Surface Interaction and Chain Stitfness on Polymer-Induced Entropic Forces and the Dimensions of Confined Polymers.

01,203

Intrinsic Conductivity of Objects Having Arbitrary Shape and Conductivity.
PB97-111934

04,150

Intrinsic Viscosity and the Polarizability of Particles Having a Wide Range of Shapes.

03,170

Localization Model of Rubber Elasticity: Comparison with Torsional Data for Natural Rubber Networks in the Dry

State.
PB95-107033

03,195

Modification of the Phase Stability of Polymer Blends by Diblock Copolymer Additivies.

03,172

Neutron Reflectivity Study of the Density Profile of a Model End-Gratted Polymer Brush: Influence of Solvent Quality.
PB95-202735

Neutron Scattering Study of Shear Induced Turbidity in Polystyrene/Dioctyl Phthalate Solutions at High Shear Rates. PB94-172624 01,197
0 Neutron Scattering Study of Shear Induced Turbidity in Polystyrene Dissolved in Dioctyl Phthalate.

01,256

Phase Separation in Thin Film Polymer Blends With and Without Block Copolymer Additives.

PB96-204482 01,294

Probabilistic Computation of Poiseuille Flow Velocity Fields. PB96-102520 04,209

Response to 'Draining in Dilute Polymer Solutions and Renormalization

01,283

Segmental Concentration Profiles of End-Tethered Polymers with Excluded-Volume and Surface Interactions. 00,654 Self-Avoiding-Walk Contacts and Random-Walk Self-Intersections in Variable Dımensionality

sections in Variable Dimensionality.
PB96-102231

01,276

Shear-Induced Changes in the Order-Disorder Transition Temperature and the Morphology of a Triblock Copolymer.

Shear Suppression of Critical Fluctuations in a Diluted Polymer Blend.

PB96-204458

04,418

Swelling and Growth of Polymers, Membranes and

Sponges.

03,396

Topological Influences on Polymer Adsorption and Desorption Dynamics.
PB94-212479

DOUMAS, B. T.

01,227

Molar Absorptivities of Bilirubin (NIST SRM 916A) and Its Neutral and Alkaline Azopigments.

PB94-211042

03,456

Boron-Implanted 6H-SiC Diodes.

PB96-159678

DOWNEY, L. L.

Design and Development of an Information Retrieval System for the EAMATE Data Volume 2 of 2. Appendices. PB94-168390 00,487

Usability Engineering: Industry-Government Collaboration for System Etfectiveness and Etticiency.

PB97-122287

01,514

DOWNING, R. G.

Analysis of Boron in CVD Diamond Surfaces Using Neutron Depth Profiling

PB94-213089

04,511

Determination of Boron and Lithium in Diverse Biological Matrices Using Neutron Activation - Mass Spectrometry (NA-MS)

(NA-MS).

00,554

Measurement of Boron at Silicon Wafer Surfaces by Neu-

tron Depth Profiling
PB94-211059

04,487

Modeling Detector Response for Neutron Depth Profiling

PB96-157813 04,066

Regulation of Lithium and Boron Levels in Normal Human Blood: Environmental and Genetic Considerations.

PB94-198579

03,491

Use of Neutron Beams for Chemical Analysis at NIST. PB97-112437

DOYLE, J. G.

Dynamic Phenomena on the RS Canum Venaticorum Binary II Pegasi

00,056

Rotational Modulation and Flares on AS Canum Venaticorum and BY Draconis Stars XVI. IUE Spectroscopy and VLA Observations of $C 1182(=V 1005$ Orionis) in October 1983

PB94- 185626
DOYLE, J. R.

00,050 
Manipulation of Adsorbed Atoms and Creation of New Structures on Room-Temperature Surfaces with a Scanning Tunneling Microscope

04,578

DRAKE, S. A

Class of Radio-Emitting Magnetic B Stars and a Wind-Fed Magnetosphere Model

PB94-21345

00,067

Efficient Way of Identifying New Active Stars: A VLA Survey of X-ray Selected Active Stellar Candidates.

00,099

Four Years of Monitoring alpha Orionis with the VLA: Where Have All the Flares Gone.

PB94-185212

00,048

Radio Continuum and X-Ray Properties of the Coronae of RS Canum Venaticorum and Related Active Binary Sys.

00,057

PB94-211083

Radio Emission from Chemically Peculiar Stars.
PB94-213469

Search for Radio Emission from the 'Non-Magnetic' Chemi-

Cally Peculiar Stars.

00,087

$X$-ray Emission from Chemically Peculiar Stars.

PB96-102256

00,088

DAPELA, T. J.

Optical Fiber, Fiber Coating, and Connector Ferrule Geometry: Results of Interlaboratory Measurement Comparisons.
PB96-154422 DRAVID, V. P.

Suitability of Metalorganic Chemical Vapor Deposition-De-
rived PrGaO3 Films as Buffer Layers for YBa2Cu3O7-x rived PrGaO3 Films as

PB95-168670

04,640

DRAVINS, $D$.

Distant Future of Solar Activity: A Case Study of Beta Hydri. 3. Transition Region, Corona, and Stellar Wind.

00,049

PB94-185220

Distant Future of Solar Activity: A Case Study of beta Hydri.
3. Transition Region, Corona, and Stellar Wind.
PB95-153441 PB95-153441

0074

Formal Specification and Verification of Control Software for Cryptographic Equipment

01,585

Guideline for the Use of Advanced Authentication Technology Alternatives. Category: Computer Security. Subcategory: Access Control.

01,798

DRAYER, D. E.

Compilation of the Physical Equilibria and Related Properties of the Hydrogen-Carbon Monoxide System.
AD-A286 603/6

DRESCHER-KRASICKA, E.

Leady Axisymmetric Modes in Infinite Clad Rods. Part 1.
PB95-162905 PB95-162905

REWS, A. R.

Preparation and Crystal Structure of Sr5TiNb4O17.

PBEXLER, E. $\mathbf{S}$.

Aluminum-Lithium Alloys: Evaluation of Fracture Toughness by Two Test Standards, ASTM Method E 813 and E 1304 .

03,375

Light-Weight Alloys for Aerospace Applications II.

PB96-190244

DRIESSEN, J.P. J.

Alignment Probing of Rydberg States by Stimulated Emission. 200316

04,124

Initial and Final Orbital Alignment Probing of the Fine-Structure-Changing Collisions among the $\mathrm{Ca}(4 \mathrm{~s})(1)(4 \mathrm{p})(1)$, (3)P States with $\mathrm{He}$ : Determination of Coherence and Conventional Cross-Sections.

PB95-203279

04,004

Laser Preparation and Probing of Initial and Final Orbital Alignment in Collision-Induced Energy Transfer $\mathrm{Ca}(4 s 5 p,(1) \mathrm{P} 1)+\mathrm{He}$ yields $\mathrm{Ca}(4 s 5 p,(3) \mathrm{P} 2)+\mathrm{He}$.
$\mathrm{PB95-203261}$

$\mathrm{n}$-Vector Correlations in Collision Dynamics with Atomic Orbital Alignment: The Importance of Coherence Denoting Azimathal Structure for $n$ (greater than or equal to) 3.

PB95-202545

03,983

Three-Vector Correlation Study of Orientation and Coherence Effects in $\mathrm{Na}(3 \mathrm{p},(2) \mathrm{P} 1 / 2$ inversely maps (2)P3/2) $+\mathrm{He}$ Semiclassical and Quantum Calculations.

03,979

Three-Vector Correlation Theory for Orientation/Alignment Studies in Atomic and Molecular Collisions.

PB94-211109

03,834

DROUIN, $N$.

Computing Effects and Error for Large Synthetic Perturba. tion Screenings
PB94-139623

DROUIN, N. V.

Using S-Check, Alpha Release 1.0.

01,767
DRULLINGER, R. E.

Diode-Laser Pumped, Rubidium Cell Frequency Standards.
PB95-163218

Error Analysis of the NIST Optically Pumped Primary Frequency Standard

01,535

Hybrid Digital/Analog Servo for the NIST-7 Frequency Standard.

01,544

Improved Rubidium Frequency Standards Using Diode Lasers with $A M$ and FM Noise Control.

04,303

Microwave Leakage as a Source of Frequency Error and Long-Term Instability in Cesium Atomic-Beam Frequency Standards.

01,541

NIST Optically Pumped Cesium Frequency Standard. 03,835

NIST-7, the New US Primary Frequency Standard.

PB95-153458

01,534

Reducing the Effect of Local Oscillator Phase Noise on the

Frequency Stability of Passive Frequency Standards.
PB95-180972

01,545

Ultra-High Stability Synthesizer for Diode Laser Pumped PB94-216066

01,527

Velocity Distribution of Atomic Beams by Gated Optical Pumping.

01,542

DU, N. Y

Resonant Two-Color Detachment of $\mathrm{H}(-)$ with Excitation of $H(n=2)$.

DU, $\mathrm{R}$.

03,984

Magnetic Rare Earth Artificial Metallic Superlattices.

PB95-162293

Magnetoelasticity in Rare-Earth Multilayers and Films.

PB94-211356

04,495

DUAN, H. M.

Asymmetry between Flux Penetration and Flux Expulsion in TI-2212 Superconductors.

PB95-125647

04,527 Correlations of Modulation Noise with Magnetic
Microstructure and Intergranular Interactions for CoCrTa and CoNi Thin Film Media. and CoNi Thin

04,509

DUBE, W. P.

Gas-Coupled, Pulse-Echo Ultrasonic Crack Detection and Thickness Gaging

PB96-147129

04,847

DUBEY, I. P.

Energy Dependence of Collision Characteristics in Molecule-Surface Collisions.

PB94-19850

00,786

Electrostatic Modes of Ion-Trap Plasmas.

PB95-152963

03,920

DUBIN, D. H. E.

Non-Neutral Ion Plasmas and Crystals, Laser Cooling, and Atomic Clocks
PB95-175113

03,957

DUBS, R. L.

Intersystem Crossing in Collisions of Aligned $\mathrm{Ca}(4 \mathrm{~s} 5 \mathrm{p}(1) \mathrm{P}$ + He: A Half Collision Analysis Using Multichannel Quantum Defect Theory.

Defect Theory.

00,813

Multichannel Quantum Defect Half Collision Analysis of K2 Photodissociation Through the B1Pi(sub u) State.

PB94-211125

00,812

DUDOWICZ, J.

How Far Is Far from Critical Point in Polymer Blends. Lat tice Cluster Theory Computations for Structured Monomer, tice Cluster Theory Compt
Compressible Systems.

Compressible
PB94-211141

01,217

Modification of the Phase Stability of Polymer Blends by Diblock Copolymer Additivies.

PB96-123542

03,172

DUEWER, D. L

Intercomparison of DNA Sizing Ladders in Electrophoretic Separation Matrices and Their Potential for Accurate Typing of the D1S80 Locus.

PB96-200928

03,485

Interlaboratory Comparison of Autoradiographic DNA Profiling Measurements. 1. Data and Summary Statistics. PB95-175923

03,542

Interlaboratory Comparison of Autoradiographic DNA Profiling Measurements. 2. Measurement Uncertainty and Its Propagation

03,545

Measurement of Ascorbic Acid in Human Plasma and Serum: Stability, Intralaboratory Repeatability, and Interlaboratory Reproducibility.

03,511

PB97-112445

Population Distributions and Intralaboratory Reproducibility
for Fat-Soluble Vitamin-Related Compounds in Human for Fat-Soluble Vitamin-Related Compounds in Human PB96-155536

00,624

DUFAUX, D. P.

In-situ Studies of a Novel Sodium Flame Process for Synthesis of Fine Particles.

00,681

Optical and Modeling Studies of Sodium/Halide Reactions for the Formation of Titanium and Boron Nanoparticles. for the Formatio

DUFFEY, M. R.

Product Realization Process Modeling: A Study of Requirements, Methods and Research Issues.

PB96-147962

02,836

DUNCAN, W. M.

Comparison of Techniques for Nondestructive Composition Measurements in CdZnTe Substrates.

PB96-103098

02,703

PB95-169397

DUNGAN, D. F.

Applicability of Effective Medium Theory to Ferroelectric/ Ferrimagnetic Composites with Composition and Frequency-Dependent Complex Permittivities and PB96-157854

DUNN, G. H.

Absolute Cross-Section Measurements for Electron-Impact lonization of $\mathrm{C} 1(+1)$

03,818

Absolute Cross-Section Measurements for Electron-Impact Single lonization of $\mathrm{Se}(+)$ and $\mathrm{Te}(+)$.

PB95-202503

03,980

Absolute Cross Sections for Electron-Impact Single Ionization of $\mathrm{Si}(+)$ and $\mathrm{Si}(2+)$

03,982

Backscattering in Electron-Impact Excitation of Multiply Charged lons
PB94-185345

03,799

Collisions of Electrons with Highly-Charged Ions.

PB96-200340

04,791

Crossed-Beams Measurements of Absolute Cross Sections for Electron Impact Ionization of $\mathrm{S}(+)$

PB95-202511

03,981

Dielectronic Capture Processes in the Electron-Impact Ion-

ization of $\mathrm{Sc}(2+)$.
PB95-203113 04,000

Early Dielectronic Recombination Measurements: Singly

Charged lons.

03,836

Electron-Impact Excitation of Si(3+)(3S yields $3 \mathrm{P})$ Using a Merged-Beam Electron-Energy-Loss Technique.

PB95-151239

03,904

Electron-Impact

00,770

Electron-Impact Ionization of $\ln (+)$ and $\mathrm{Xe}(+)$

PB95-152906

03,918

Plectron-Ion Coli

04,037

Evidence for Significant Backscattering in Near-Threshold Electron-Impact Excitation of $\mathrm{Ar}(7+)(3 \mathrm{~s}$ yields $3 \mathrm{p})$

03.883

Merged-Beams Energy-Loss Technique for Electron-Ion Excitation: Absolute Total Cross Sections for $\mathrm{O}(5+)$ (2s yields 2p).

04,017

Resonance Structure and Absolute Cross Sections in NearThreshold Electron-Impact Excitation of the $4 s(2)$ (1)S yields 4s4p (3)P Intercombination Transition in $\mathrm{Kr}(6+)$.
PB95-202271 972

Superconducting Resonator and a Cryogenic GaAs FieldEffect Transistor Amplifier as a Single-Ion Detection Sys- 


\section{PERSONAL AUTHOR INDEX} Photoluminescence Spectra of Epitaxial Single Crystal C60
PB96-141205 04,754 $X$-ray Reflectivity Determination of Interface Roughness Correlated with Transport Properties of (AIGa)As/GaAs High Electron Mobility Transistor Devices.

PB97-111868

04.149

DURAN, $R$.

Using Torsional Dilatometry to Measure the Effects of Deformations on Physical Aging.

PB95-14090

03,384

DURAN, R. S.

Aging in Glasses Subjected to Large Stresses and Defor-

PB95-107041

03,235

ists on Deformed Glasses: Instrument Description and Measurements on Equilibrated Glasses.

PB94-211166

03,379

Volume Recovery in Epoxy Glasses Subjected to Torsional Deformations: The Question of Rejuvenation.

03,382

DURST, $R$.

Liposome-Based Flow-Injection Immunoassay for Determining Theophylline in Serum

PB̈94-213493

03,494

URS, R. A.

Planar Waveguide Optical Sensors.

03,586

Potentiometric Enzyme-Amplified Flow Injection Analysis Detection System: Behavior of Free and Liposome-Released Peroxidase.

PB95-151833

03,534

DUTCHER, J. R.

Brillouin Light Scattering Intensities for Thin Magnetic Films with Large Perpendicular Anisotropies.

04,488

DUTHINH, D.

Shear Design of High-Strength Concrete Beams: A Review of the State-of-the-Art.

01,330

DUTTA, P.

Investigation of Oil and Gas Well Fires and Flares.

PB94-193976

03,695

DUVALL, K. C.

Role of the Office of Radiation Measurement in Quality As surance.

PB94-212255

DYUKOVA, T. V.

Holographic Properties of Triton X-100-Treated Bacteriorhodopsin Embedded in Gelatin Films.
PB96-119284 Optical Properties of Triton X-100-Treated Purple Membranes Embedded in Gelatin Films.

03,546

PB96-123500

Retinal-Protein Complexes as Optoelectronic Components.

DZIERZEGA, $\mathrm{K}$.

Time-Resolved Balmer-Alpha Emission from Fast Hydrogen Atoms in Low Pressure, Radio-Frequency Discharges in Hydrogen.

DZIUBA, R. F.

Automated Resistance Measurements at NIST

PB96-119326

04,028

parator.

Loading Effects in Resistance Scaling

PB97-111884 urement Systems.

02,288

NIST Comparison of the Quantized Hall Resistance and the Realization of the SI Ohm Through the Calculable Capaci-

PB97-119184

02,291

NIST Comparison of the Quantized Hall Resistance and the Realization of the SI Ohm Through the Calculable Capacitor. Conference Proceedings, June 17-20, 1996.

Pressure Dependencies of Standard Resistors.

PB95-153516

Resistors

PB97-111876

02,257

DZUBA, V. A.

02,284

Fine Structure of Negative Ions of Alkaline-Earth-Metal

Atoms.

EAGAR, T.

In-Space Welding: Visions and Realities

PB95-163234

03,837

EANES, E. D.

Behavior of a Calcium Phosphate Cement in Simulated Blood Plasma In vitro.

PB95.168712

00,165
Bioactive Polymeric Dental Materials Based on Amorphous Calcium Phosphate.

03,572

Calcium Phosphate Precipitation in Liposomal Suspensions.

PB94-172145 03,452

Critical Evaluation of the Purification of Biominerals by $\mathrm{Hy}$ pochlorite Treatment

B95-150959

00,186

Dynamics of Cal

03,574

Effect of Supersaturation on Apatite Crystal Formation in Aqueous Solutions at Physiologic $\mathrm{pH}$ and Temperature.

PB96-135215

03,571

Effect of 1-Hydroxyethylidene-1,1-Bisphosphonate on Membrane-Mediated Calcium Phosphate Formation in Model Liposomal Suspensions.

PB95-169223

03,469

In vitro Inhibition of Membrane-Mediated Calcification by Novel Phosphonates.

PB96-201140

03,595

Membrane-Mediated Precipitation of Calcium Phosphate in Model Liposomes with Matrix Vesicle-Like Lipid Composi-

tion.

PB95-164547

03,468

Mixed Phospholipid Liposome Calcification

03,457

Polymeric Calcium Phosphate Composites with Remineralization Potential.
PB96-155544

Proteoglycan Inhibition of Calcium Phosphate Precipitation in Liposomal Suspensions.

PB94-211208

00,658

Remineralizing Dental Composites Based on Amorphous Calcium Phosphate.

PB96-147020

EARLY, E. A.

03,573

Evidence for Parallel Junctions Within High-Tc GrainBoundary Junctions.

Half-Integral Constant Voltage Steps in High-Tc Grain Boundary Junctions.

PB94-211216 04,489

Irradiance of Horizontal Quartz-Halogen Standard Lamps.

PB96-179130 01,866

Physical Basis for Half-integral Shapiro Steps in a DC

SQUID

PB96-102264

04,704

Report on USDA Ultraviolet Spectroradiometers.

PB96-214648

00,125

Voltage Ratio Measurements of a Zener Reference Using a Digital Voltmeter

EARLY, G.

01,906

Relationship of Silver with Selenium and Mercury in the Liver of Two Species of Toothed Whales (Odontocetes).

PB96-167275

EASTERLING, R. G.

Agile Manufacturing from a Statistical Perspective.

ATON, P. E.

02,596

Neutron Scattering Study of the Lattice Modes of Solid Cubane.

PB96-147152

EBBERTS, D. F

Room Temperature Thermal Conductivity of Fumed-Silica Insulation for a Standard Reference Material.

PB95-152039

EBBESEN, T. $W$.

Rotational Dynamics of $\mathrm{C} 60$ in Na2RbC 60

PB95-153201

00,374

BBETS, D. C.

First Results from the Goddard High-Resolution Spectrograph: The Chromosphere of Taur.

PB94-199528

00,054

Observations of $3 \mathrm{C} 273$ with the Goddard High Resolution Spectrograph on the Hubble Space Telescope.

PB95-202321

EBERHARDT, K. R.

Combining Data from Independent Chemical Analysis Meth-

PB95-140141

00,572

Constant-Width Calibration Intervals for Linear Regression.

PB95-153524 03,439

Quantifying the Ignition Propensity of Cigarettes.

PB96-155411

00,306

Update on the Low Background IR Calibration Facility at the National Institute of Standards and Technology.

PB94-211224

EBRAHEEM, $S$.

Thin Dyed-Plastic Dosimeter for Large Radiation Doses.

EBRAHIM EID, S. E.

Radiation-Chemical Reaction of 2,3,5-Triphenyl-Tetrazolium Chloride in Liquid and Solid State.

01,124

EBY, R. K.

Application of Thermal Analysis Techniques to the Characterization of EPDM Roofing Membrane Materials.

Use of Thermal Mechanical Analysis to Characterize Ethylene-Propylene-Diene Terpolymer (EPDM) Roofing Membrane Materials.

ECKERLE, $K$.

Standards for Corrected Fluorescence Spectra.

PB95-150835

00,360

ECKERLE, K. E.

00,581

Comparison of Regular Transmittance Scales of Four $\mathrm{Na}$ tional Standardizing Laboratories.

PB94-211232

04,233

ECKSTEIN, J. N.

Stacked Series Arrays of High-Tc Trilayer Josephson Junc-

PB96-102272

04,705

Step-Edge and Stacked-Heterostructure High-Tc Josephson Junctions for Voltage-Standard Arrays.

04,702

Vortex Images in Thin Films of $\mathrm{YBa} 2 \mathrm{Cu} 3 \mathrm{O}($ sub $7-x$ ) and
Bi2Sr2Ca1Cu2O (sub $8+x$ ) Obtained by Low-Temperature Bi2Sr2Ca1Cu2O (sub 8+x) Obtained by Low-Temperalure
Magnetic Force Microscopy. PB97-119408

ECONOMOU, D. J.

Two-Dimensional Self-Consistent Radio Frequency Plasma Simulations Relevant to the Gaseous Electronics Conference RF Reference Cell.

PB96-113436

02,398

Effect of Beam Voltage on the Properties of Aluminum

Nitride Prepared by lon Beam Assisted Deposition.
PB97-118616

\section{EDERER, D. L}

01,995

Al L2,3 Core Excitons in AlxGa1-x as Studied by Soft-X-ray Retlection and Emission.

B 96-157839

Charge-Transfer-Induced Multiplet Structure in the $\mathrm{N} 4,5 \mathrm{O} 2,3$ Soft-X-ray Emission Spectrum of Lanthanum. PB96-163746

04,102

Cooper M(sub II,III) X-ray-Emission Spectra of Copper Oxides and the Bist

04,077

Intermediate Coupling in L2-L3 Core Excitons of $\mathrm{MgO}$, $\mathrm{Al} 2 \mathrm{O} 3$, and $\mathrm{SiO} 2$

PB96-158043

04,079

Laser-Synchrotron Hybrid Experiments: A Photon to Tickle, A Photon to Poke.

03,704

Local Partial Densities of States in $\mathrm{Ni}$ and Co Silicides Studied by Soft X-Ray Emission Spectroscopy.

PB94-212412

04,504

NIST Metrology for Soft X-ray Multilayer Optics.

PB96-160379 04,08

Phonon Relaxation in Soft-X-ray Emission of Insulators.

PB96-160296

04,085

Simple Variable Line Space Grating Monochromator for Synchrotron Light Source Beamlines.

04,065

Soft-X-ray-Emission Investigation of Cobalt Implanted Silicon Crystals.
PB96-157912

Soft-X-ray-Emission Spectra of Solid $\mathrm{Kr}$ and $\mathrm{Xe}$.

04,069 
Properties of a Bose-Einstein Condensate in an Anisotropic Harmonic Potential.

PB96-204144

04,133

EDWARDS, M. A.

Numerical Solution of the Nonlinear Schroedinger Equation

for Small Samples of Trapped Neutral Atoms.
PB95-202578

EDWARDS, W. E.

Physicochemical Characterization of Natural and Bioprosthetic Heart Valve Calcific Deposits: Implications for Prevention.

PB96-156039

EGELHOFF, W. F.

00,187

Brillouin Light Scattering Intensities for Thin Magnetic Films with Large Perpendicular Anisotropies.

PB94-211174

04,488

Concurrent Enhancement of Kerr Rotation and Antiferromagnetic Coupling in Epitaxial Fe/Cu/Fe Structures.

Semiclassical Explanation of the Generalized RamsauerTownsend Minima in Electron-Atom Scatleing.

PB95-153532

03,925

Spot-Profile-Analyzing LEED Study of the Epitaxial Growth of $\mathrm{Fe} . \mathrm{Co}$, and $\mathrm{Cu}$ on $\mathrm{Cu}(100)$.

04,561

Variation in Magnetic Properties of Cu/Fcc (001) Sandwich

Structures.
PB95-141164 04,555

$X$-ray Photoelectron and Auger Electron Forward Scattering: A Structural Diagnostic for Epitaxial Thin Films.

PB95-180220

04,676

EGGERS, D. F.

Infrared and Microwave Spectroscopy of the Argon Propyne Dimer
PB94-198892

00,794

EHARA, $K$.

Experimental Optimization of Peak Shape with Application Experimol Generation.

PB94-185535

00,501

Stochastic Modeling of a New Spectrometer.

04,068

EHLEN, M. A.

Economics of New-Technology Materials: A Case Study of FRP Bridge Decking

EHRLICH, C.

01,349

Working Conference on Global Growth of Technology: Is America Prepared. Held in Gaithersburg, Maryland on December 7. 1995.

PB96-210059

EHRLICH, C. D.

00,018

Intercomparison between NPL (India) and NIST (USA) Pressure Standards in the Hydraulic Pressure Region Up to PB96-113543

04,211

Intercomparison of the Effective Areas of a Pneumatic PisIon Gauge Determined by Different Techniques.

02,640

Look at Uncertainties over Twenty Decades of Pressure MBo5-168506

02,655

Operational Mode and Gas Species Effects on Rotational Drag in Pneumatic Dead Weight Pressure Gages.

PB95-140182

00,903

EHRLICH, $M$

Comparison of NIST and ISO Filtered Bremsstrahlung Catibration Beams
PB95-180956

03,967

Interpreting the Readings of Multi-Element Personnel Dosimeters in Terms of the Personal Dose Equivalent.

PB95-175428

03,631

EHRSTEIN, J. R

Development of a Standard Reference Material for Meas. urement of Interstitial Oxygen Concentration in Semiconductor Silicon by Infrared Absorption.

02,404

PB96-122668

Methodology for the Certification of Reference Specimens for Determination of Oxygen Concentration in Semiconductor Silicon by Infrared Spectrophotometry.

PB96-155478

02,421

EICHHORN, R. M.

Spatial Dependence of Electrical Fields Due to Space Charges in Films of Organic Dielectrics Used for Insulation of Power Cables.

PB94-199130

EICHMANN, U.

Interference in the Resonance Fluorescence of Two Trapped Atoms.

PB95-168514

03,948

PB95-168753

04,286

EICHMILLER, $F$

Diagnosis and Treatment of an Oral Base-Metal Contact Lesion Following Negative Dermatologic Patch Tests. PB95-180626 00,172

Effect of Two Initiator/Stabilizer Concentrations in a Metal Primer on Bond Strengths of a Composite to a Base Metal Alloy.

00,141
Effects of Aluminum Oxalate/Glycine Pretreatment Solutions

on Dentin Permeability.
PB95.164505

03,565

EICHMILLER, F. C.

Clinical Perspective on Dentin Adhesives.

PB94-211240

00,146

Dental Applications of Ceramics.

00,177

Dental Materials.

PB94-172871

00,142

Effect of Three Sterilization Techniques on Finger Pluggers.

Effects of Surface-Active Resins on Dentin/Composite

Bonds.
PB95-140448 00,156

New Method for Shielding Electron Beams Used for Head

and Neck Cancer Treatment.

PB94-211430

03,621

New Surface-Active Comonomer for Adhesive Bonding.
PB96-204052

Posterior Restorative Materials Research

03,582

PB97-118624

Reduction of Marginal Gaps in Composite Restorations by
Use of Glass-Ceramic Inserts.
PB96-102405 PB96-102405

Surface Roughness of Glass-Ceramic Insert. Composite

Restorations: Assessing Several Polishing Techniques.
PB97-119010 03,583

Tapered Cross-Pin Attachments for Fixed Bridges.

PB94-185238

00,185

Wear of Enamel against Glass-Ceramic, Porcelain, and Amalgam.
PB96-179593

EICHORN, B.

03,082

Substitution-Induced Midgap States in the Mixed Oxides

RxBa1-ChiTiO3-Delta, with $\mathrm{R}=\mathrm{Y}, \mathrm{La}$, and $\mathrm{Nd}$.

PB95-140505

04,541

\section{EIDELMAN, $N$.}

In vitro Inhibition of Membrane-Mediated Calcification by 1140

PB96-201140

03,595

Selective Inhibition of Crystal Growth on Octacalcium Phosphate and Nonstoichiometric Hydroxyapatite by Pyrophosphate at Physiological Concentration.
PB94-211257

00,147

EILES, M. T.

Even-Odd Asymmetry of a Superconductor Revealed by the Coulomb Blockade of Andreev Reflection

PBos-153540

04,593

EILES, T. M.

Combined Josephson and Charging. Behavior of the Supercurrent in the Superconducting Single-Electron TranSistor.

04,637

Electronic Microrefrigerator Based on a Normal-InsulatorSuperconductor Tunnel Junction.

PB96-102827

04,718

Noise in the Cou

04,670

Thermal Enhancement of Cotunneling in Ultra-Small Tunnel Junctions

PB95-175436

EINSTEIN, T. L.

Self-Avoiding Surfaces, Topology, and Lattice Animals.

PB95-150512

\section{EISENHAUER, C. $M$.}

DETAN 95: Computer Code for Calculating Spectrum-Averaged Cross Sections and Detector Responses in Neutron

04,014

Neutron Leakage Benchmark for Criticality Safety Re-

search

03,723

Slab Transmission and Reflection for Point Source and

Point Detector.

PB94-211265

EISENHOWER, E

Role of the Office of Radiation Measurement in Ouality As-

Surance.

00,689

EISENHOWER, E. $\mathrm{H}$.

National Ouality Assurance Program for Personnel Radiation Dosimetry: A Case History.

PB94-211273

EITZEN, D. G.

Ultrasonic Measurements of Surface Roughness.

PB94-172137

03,620

EKBERG, J. 0 .

Spectrum and Energy Levels of Five-Times-lonized Niobium (Nb VI).

EKIN, J. W.

04,226

Critical-Current Degradation in $\mathrm{Nb3}$ Al Wires Due to Axial and Transverse Stress.

02,226

Critical Magnetic-Field Angle for High-Field Current Transport in $\mathrm{YBa} \mathrm{Cu} 3 \mathrm{O} 7$ at $76 \mathrm{~K}$

04,490

Effect of Axial Strain on the Critical Current of Ag-Sheathed Bi-Based Superconductors in Magnetic Fields Up to $25 \mathrm{~T}$. Bi-Based Superconductors in Magnetic Fields Up to $25 \mathrm{~T}$.
PB94-211315

Effect of Magnetic Field Orientation on the Critical Current of HTS Conductor and Coils.

PB96-141189

02,956

Electromechanical properties of superconductors for DOE usion applications.

E95015476

04.432

Electromechanical Properties of Superconductors for DOE Fusion Applications

PB94-139672

02,250

Evidence for Tunneling and Magnetic Scatttering at 'In situ' BCO/Noble-Metal Interfaces.

PB96-141098

04,752

Growth of Laser Ablated YBa2Cu3O7-delta Films as Examined by Rheed and Scanning Tunneling Microscopy. PB95-162541 Measured in Ag-Sheathed Bi2Sr2Ca1Cu2O8+x Superconductors

PB95.153565

In situ Noble Metal YBa2Cu3O7 Thin-Film Contacts.

04,594

Insulating Boundary Layer and Magnetic Scattering in YBa2Cu307-delta/Ag Interfaces Over a Contact Resistivity Range of $10(-8)-10(-3)$ Omega $\mathrm{cm}(2)$.

PB95.169157

04,648

Oxygen Annealing of Ex-situ YBCO/Ag Thin-Film Interfaces.

04,758

Preparation of Low Resistivity Contacts for High-Tc Superconductors.

PB95-153557

02,258

Uuench Energy and Fatigue Degradation Properties of Cuand $\mathrm{Al} / \mathrm{Cu}$-Stablized $\mathrm{Nb}-\mathrm{Ti}$ Epoxy-Impregnated Superconductor Coils.

PB96-141213

Superconducting Materials: Specification.

PB94-211299

04,755

Surface Degradation of Superconducting YBa2Cu3O7-delta Thin Films.

Transport Critical Current of Aligned Polycrystalline Ytrium Barium Copper Oxide ( $Y B a 2 C u 307-$ delta)

PB94-211307

04,492

Transverse stress effect on the critical current of internal tin and bronze process $\mathrm{Nb}$ (sub 3 ) Sn superconductors.

DE95016659 strain in $\mathrm{Nb}$ (sub 3) Sn.

DE95016656

04,433

STRAND, J.

Distribution of Fluoride in Saliva and Plaque Fluid After a $0.048 \mathrm{~mol} / \mathrm{L} \mathrm{NaF}$ Rinse.

PB95-151205 


\section{PERSONAL AUTHOR INDEX}

Design Guide for CMOS-On-SIMOX. Test Chips NIST3 and NIST4.

PB94-163458

02,297

Electrical Test Structure for Overlay Metrology Referenced to Absolute Length Standards.

02,336

Enhanced Voltage-Dividing Potentiometer for High-Precision Feature Placement Metrology.

PB96-164025

02,428

New Test Structure for Nanometer-Level Overlay and Feature-Placement Metrology.

02,363

Test Structures for the In-Plane Locations of Projected Features with Nanometer-Level Accuracy Traceable to a Coordinate Measurement System.

PB94-200565

02,313

ELLERBE, $P$.

Certification of Phencyclidine in Lyophilized Human Urine Reference Materials.

PB96-160692

03,508

Determination of Amphetamine and Methamphetamine in a Lyophilized Human Urine Reference Material.

03,597

Isotope Dilution Mass Spectrometry as a Candidate Definitive Method for Determining Total Glycerides and Triglycerides in Serum.
PB96-102280

03,519

NIST Reference Materials to Support Accuracy in Drug Testing.

03,505

ELLINGSEN, $R$.

High-Resolution Diode-Laser Spectroscopy of Calcium.

PB95-181244

03,969

ELLIOT, D. S.

Phase Shitts and Intensity Dependence in Frequency-Modulation Spectroscopy

PB96-103205

01,071

ELLSWORTH, J.

Correlation of $\mathrm{HgCdTe}$ Epilayer Defects with Underlying Substrate Defects by Synchrotron X-Ray Topography.

PB94-200714

02,129

ELMAN, $B$

Use of Pressure for Ouantum. Well Band-Structure Characterization.

ELMOUIST, R. E

04,779

High-Temperature Superconductor Cryogenic Current Comparator.

02, 074

Leakage Current Detection in Cryogenic Current Compara. tor Bridges.

02,024

Loading Effects in Resistance Scaling.

02,285

NIST Comparison of the Ouantized Hall Resistance and the Realization of the SI Ohm Through the Calculable CapaciPB97-119184

02,291

NIST Comparison of the Quantized Hall Resistance and the Realization of the SI Ohm Through the Calculable Capacitor. Conference Proceedings, June 17-20, 1996.

PB97-119192

02,292

Precision Tests of a Ouantum Hall Effect Device DC Equivalent Circuit Using Double-Series and Triple-Series Connec-

PB96-159256

01,953

Progress on the Ouantized Hall Resistance Recommended Intrinsic/Derived Standards Practice.

PB96-122460

02,403

Spectroscopic Study of Ouantized Breakdown Voltage States of the Ouantum Hall Effect.

PB96-113584

04,730

ELSTON, H. J.

Vibrations of $\mathrm{S} 1((1) \mathrm{B} 2 \mathrm{u}) \mathrm{p}$-Difluorobenzene - d4. S1-SO Fluorescence Spectroscopy and ab Initio Calculations.

PB95-20258

01,028

ELTA, M. E.

Gaseous Electronics Conference Radio-Frequency Reference Cell: A Defined Parallel-Plate Radio-Frequency System for Experimental and Theoretical Studies of PlasmaProcessing Discharges.

PB94-172327

04,404

ELY, J. F.

Equation of State Formulation of the Thermodynamic Properties of R134a $(1,1,1,2$-Tetrafluoroethane).

PB94-212081

03,256

New Data and Correlations for the Custody Transfer of Natural Gas Liquids.

02,499

Prediction of the Thermal Conductivity of Refrigerants and Refrigerant Mixtures.

PB94-212107

03,258

Prediction of Viscosity of Refrigerants and Refrigerant Mix-

03,257

Predictive Extended Corresponding States Model for Pure and Mixed Refrigerants Including an Equation of State for

03,275

PA-38
Thermodynamic Properties of the Methane-Ethane System. PB95-125779

00891

\section{EMBREE, E.}

Degradation of Powder Epoxy Coated Panels Immersed in a Saturated Calcium Hydroxide Solution Containing Sodium

PB96-101050 01,344

Non-Osmotic, Defect-Controlled Cathodic Disbondment of a Coating from a Steel Substrate.

03,120

Performance of Tape-Bonded Seams of EPDM Membranes: Comparison of the Peel Creep-Rupture Response of Tape Bonded and Liquid-Adhesive-Bonded Seams.

PB96-183249

EMENHISER, $C$.

03,012

solation and Structural Elucidation of the Predominant Geometrical Isomers of alpha-Carotene.

PB96-190061

00,640

EMMERICH, S. J

Application of a Multizone Airflow and Contaminant Dispersal Model to Indoor Air Ouality Control in Residential Buildings.

02,555

Effectiveness of a Heat Recovery Ventilator, an Outdoor Air Intake Damper and an Electrostatic Particulate Filter at Controlling Indoor Air Ouality in Residential Buildings. 96-146642

02,564

Indoor Air Ouality Impacts of Residential HVAC Systems Phase II.B Report: IAO Control Retrofit Simulations and PB96-106877

02,559

Indoor Air Ouality Impacts of Residential HVAC Systems, Phase 1 Report: Computer Simulation Plan.
PB95-135596
00,249

stems

ndoor Air Ouality Impacts of Residential HVAC Systems. PB95-178893 Multizone Modeling of Three Residential Indoor Air Ouality Control Options.

02,565

Multizone Modeling of Three Residential Indoor Air Ouality Control Options.

02,567

Workplan to Analyze the Energy Impacts of Envelope Airtightness in Office Buildings.

PB96-154463

ENDOH, Y.

High-Energy Phonon Dispersion in La1.85Sro.15CuO4. B96-138458

00,273

ENG, G.

Microbial Degradation of Polysulfides and insights into Thei

$\begin{array}{ll}\text { Possible Occurrence in Coal. } & 02,488\end{array}$

Total Surface Areas of Group IVA Organometallic Compounds: Predictors of Toxicity to Algae and Bacteria.

NGEN,

00,814

Terminal Invari

Terminal Invariant Description of Amplifier Noise

PB95-153573

Magnetic Properties of $\mathrm{Pd} / \mathrm{Co}$ Multilayers.

ENGLEMAN, $\boldsymbol{R}$

Measurements of the Resonance Lines of (6) $\mathrm{Li}$ and (7) Li by Doppler-Free Frequency-Modulation Spectroscopy.

PB96-180211

ENGLERT, G.

Isolation and Structural Elucidation of the Predominant Geometrical Isomers of alpha-Carotene.

PB96-190061

00,640

ENO, L.

Laser Preparation and Probing of Initial and Final Orbita Alignment in Collision-Induced Energy Transfer $\mathrm{Ca}(4 s 5 p,(1) \mathrm{P} 1)+\mathrm{He}$ yields $\mathrm{Ca}(4 s 5 p,(3) \mathrm{P} 2)+\mathrm{He}$

PB95-203261

04,003

Three-Vector Correlation Theory for Orientation/Alignment Studies in Atomic and Molecular Collisions.

PB94-211109

ENSHER, J. $R$.

Behavior of Atoms in a Compressed Magneto-Optical Trap. PB95-203105

Reduction of Light-Assisted Collisional Loss Rate from Low-Pressure Vapor-Cell Trap.

Table Tightly Confining Magnetic Trap for Evaporative Cooling of Neutral Atoms.

04,126

Liquid Chromatographic Method for the Determination of Carotenoids, Retinoids, and Tocopherols in Human Serum and in Food.

PB95-153599

00,593

Liquid Chromatography: Laser-Enhanced Ionization Spectrometry for the Speciation of Organolead Compounds.

trometry for the

EPPELDAUER, G

Absolute Response Calibration of a Transfer Standard Cryogenic Bolometer.

04.358

Detector-Based Candela Scale and Related Photometric Calibration Procedures at NIST.

PB95-161949

04,273

Longterm Changes of Silicon Photodiodes and Their Use for Photometric Standardization.

04,234

Method of Realizing Spectral Irradiance Based on an Absolute Cryogenic Radiometer.

PB95-161204

NIST Detector-Based Luminous Intensity Scale.

04,270

PB96-179114

EPSTEIN, M. S.

01,864

Application of a Novel Slurry Furnace AAS Protocol for Rapid Assessment of Lead Environmental Contamination.

02,526
Spectral Interference in the Determination of Arsenic in High-Purity Lead and Lead-Base Alloys Using Electrothermal Atomic Absorption Spectrometry and Zeeman-Effect Background Correction

PB96-112099

00,614

ERICKSON, A.

2D-Scanning Capacitance Microscopy Measurements of Cross-Sectioned VLSI Teststructures.

PB96-163779

Antiferromagnetic Interlayer Correlations in Annealed Ni80Fe20/Ag Multilayers.

PB97-122220

03,109

Comment on 'Phase Transitions in Antiferromagnetic Superlattices'
PB95-152971

04,587

Enhanced Curie Temperatures and Magnetoelastic Do-

mains in Dy/Lu Super Lattices and Films.

PB94-172665

04,443

Magnetic Rare Earth Artiticial Metallic Superlattices.

PB95-162293

04,611

Magnetoelasticity in Rare-Earth Multilayers and Films.

Normal Modes and Structure Factor for a Canted Spin System: The Generalized Villain Model.

04,539

Unconventional Ferromagnetic Transition in La(sub 1 . x) $\mathrm{Ca}(\mathrm{sub}$ x) $\mathrm{MnO} 3$.

ESCALANTE, E.

Corrosion Resistance of Materials for Renovation of the United States Botanic Garden Conservatory.

04,156

PB94-154390

00,032

Evidence

RBodium.

ESCRIBANO, $\mathrm{R}$

03,191

Infrared Spectrum of OCIO in the $2000 / \mathrm{cm}(-1)$ Region: The 2(nu sub 1) and (nu sub $1+$ nu sub 3 ) Bands.

00,908

Intensities and Dipole Moment Derivatives of the Fundamental Bands of (35) $\mathrm{ClO} 2$ and an Intensity Analysis of the nu1 Band

PB95-141040

00,909

ESPINA, P. I.

Development of Adaptive Control Strategies for Inert Gas PB95-162335 
Chip Morphology, Tool Wear and Cutting Mechanics in Finish Hard Turning.
PB97-112247

03,106

Compensation for Errors Introduced by Nonzero Fringe Densities in Phase-Measuring Interferometers.

PB97-110506

04,386

Damage Processes in Ceramics Resulting from Diamond Tool Indentation and Scratching in Various Environments. PB96-102983

03,065

Fabrication of Optics by Diamond Turning

02,954

Japan Technology Program Assessment: Precision Engineering/Precision Optics in Japan.

PB95- 171112

02,884

Rapid Post-Polishing of Diamond-Turned Optics.

PB95-175949

04,301

Test of a Slow Off-Axis Parabola at Its Center of Curvature

PB96-138482

04,352

Test Optics Error Removal.

PB96-179536 04,377

Upgraded Facility for Multilayer Mirror Characterization at

PB96-160387

04,367

Visualization of Surface Figure by the Use of Zernike Polynomials.

PB96-137757

04,351

EVANS, D.

Smoke Emission from Burning Crude Oil.

PB96-122890

01,407

EVANS, D. D

Application of Boundary Element Methods to a Transient Axis-Symmetric Heat Conduction Problem.

PB94-212693

07,375

Development of Hazard Assessment and Suppression Technology for Oil and Gas Well Blowout and Diverter

01,408

PB96-122965

wait Oi

Flame Heigh

PB96.119342

01,404

In situ Burning of Oil Spills: Mesoscale Experiments and Analysis.

PB95-163747

02,587

Large Fire Exper

01,427

Smoke Plume Trajectory from in situ Burning of Crude Oi in Alaska: Field Experiments.

PB96-131560

Suppression Research: Strategies.

PB94-211372

02.594

EVANS, D. J.

00,346

Conditions for Existence of a Reentrant Solid Phase in a Sheared Atomic Fluid.

04,198

Dynamic Scaling in an Aggregating 20 Lennard-Jones Sys-

PB96-167317

04,106

Simulation and SANS Studies of Gelation Under Shear.

PB96-167176

01,150

Testing the Sensitivity of Accelerometers Using Mechanical Shock Pulses Under NIST Special Publication 250 Test No. 240405

PB96-179544

02,683

EVANS, $\mathrm{J}$.

Overview of U.S. Government Advanced Packaging Pro-

grams.

02,443

EVANS, J. D.

Exact Series Solution to the Epstein-Hubbell Generalized Elliptic Type Integral Using Complex Variable Residue The PBy $97-110167$

03,423

EVANS, R. D.

NIST/NCMS Program on Electronic Packaging: First Update

PB96-204086

EVENSEN, K. M.

03,008

$30 \mathrm{THz}$ Mixing Experiments on High Temperature Superconducting Josephson Junctions.

04,709

EVENSON, $\mathrm{K}$.

Absolute Frequency Measurements of Methanol from 1.5 to $6.5 \mathrm{THz}$.

PB95-175881

04,300

EVENSON, K. M.

Atomic Iron in Its (5)D Ground State: A Direct Measurement of the $\mathrm{J}=0$ inverted arrow 1 and $\mathrm{J}=1$ inverted arrow 2 Fine-Structure Intervals $(1.2)$

PB96-141221

04,756

Atomic Oxygen Fine Structure Splittings with Tunable Far Infrared Spectroscopy.

PB95-152203

03,915

Atomic Sulfur: Frequency Measurement of the $J=0$ inversely maps 1 Fine-Structure Transition at 56.3 Microns by Lase Magnetic Resonance.

PB95-180105

01,007
Detection of $\mathrm{OH}+$ in Its a(1)Delta State by Far Infrared Laser Magnetic Resonance.

01,021

Determination of the Molecular Parameters of $\mathrm{NiH}$ in Its (2)Delta Ground State by Laser Magnetic Resonance. PB95-107116 00,869 Far Infrared Laser Frequencies of $\mathrm{CH} 3 \mathrm{OD}$ and $\mathrm{N} 2 \mathrm{H} 4$. PB96-119623 04,341 Far Infrared Laser Frequencies of $(13) \mathrm{CD} 3 \mathrm{OH}$.

04,292 Improved Molecular Constants and Frequencies for the CO2 Laser from New High-J Regular and Hot-Band Frequency Measurements.

04,312

Laboratory Measurements for the Astrophysical Identification of $\mathrm{MgH}$.

00,073

Laser Magnetic-Resonance Measurement of the (3)P1 (3)P2 Fine-Structure Splittings in (17) O and (18)O PB95-175170

00,994

Measurement of the $\mathrm{J}=2$ less than 1 Fine-Structure Interval or (28) Si and (29) $\mathrm{Si}$ in the Ground (3)P State.

00,771

Pew cw CO2

04,281

Pure Rotational Spectra of CuH and CuD in Their Ground States Measured by Tunable Far-Infrared Spectroscopy.
PB95-176194

Rotational Far Infrared Spectrum of (13) CO

00,941

Rotational Spectroscopy of the CoH Radical in Its Ground Rotational Spectroscopy of the CoH Radical in Its Ground
(3)Phi State by Far-Infrared Laser Magnetic Resonance: Determination of Molecular Parameters.

PB95-175048

00,992

Rotational Spectrum of Copper Hydride Using Tunable Far Infrared Radiation.

PB94-198637

00,792

Rotational Spectrum of $\mathrm{OH}$ in the $\mathrm{v}=0-3$ Levels of Its Ground State
PB95-176202

EVERHART, $K$

01,006

Computer Security Training and Awareness Course Compendium.

EVERITT, 8.

01,589

Enhanced Curie Temperatures and Magnetoelastic Domains in Dy/Lu Super Lattices and Films.

PB94-172665

04,443

EWRIN, R. W.

Structural and Magnetic Ordering in Iron Oxide/Nickel Oxide Multilayers by X-ray and Neutron Diffraction (Invited).

PB94-172558

EYGENRAAM, $M$

Decomposition of SF6 and Production of S2F10 in Power Arcs.

PB96-122619

01,084

\section{EYKENS, $R$.}

Preparation and Characterization of (6) LiF and (10)B Refo erence Deposits for the Measurement of the Neutron Lifetime

PB95-108692

03,874

Problems Related to the Determination of Mass Densities of Evaporated Reference Deposits

PB95-163226

03,941

LEM, C.

Substitution-Induced Midgap States in the Mixed Oxides RxBa1-ChiTiO3-Delta, with R=Y, La, and No

PB95-140505

FACTOR, B. J.

Dielectric Behavior of a Polycarbonate/Polyester Mixture Upon Transesterification.

PB96-17955

FAETH, G. M.

Mixing and Radiation Properties of Buoyant Luminous Flame Environments

PB96-202254

01,432

Mixing and Radiation Properties of Buoyant Turbulent Diffusion Flames.

PB95-242327

01,396

Radiation and Mixing Properties of Buoyant Turbulent Diffusion Flames.
PB94-165974

01,360

FAHR, A.

Deuterium Isotope Effect in Vinyl Radical Combination/ Dispropor tionationReaction

B96-204151

01.167

Experimental Determination of the Rate Constant for the Reaction of $\mathrm{C} 2 \mathrm{H} 3$ with $\mathrm{H} 2$ and Implications for the Partitioning of Hydrocarbons in Atmospheres of the Outer Planets. PB97-122295

00,112

Reaction Rate Determinations of Vınyl Radical Reactions with Vinyl, Methyl, and Hydrogen Atom

00,815

Temperature Dependence of the Gas and Liquid Phase UItraviolet Absorption Cross Sections of HCFC-123 (CF3CHCl2) and $\mathrm{HCFC}-142 \mathrm{~b}(\mathrm{CH} 3 \mathrm{CF} 2 \mathrm{Cl})$.

PB96-201033

03,298

Temperature Dependence of the Ultraviolet Absorption Cross Section of CF31

01,168

Temperature Dependent Ultraviolet Absorption Cross SectIons of Propylene, Methylacetylene and Vinylacetylene. PB96-204177 01,169

UV Absorption Cross Sections of Methylchlorotorm: Temperature-Dependent Gas and Liquid Phase Measurements.
PB96-201041
00,643

FAHY, R. F.

Enhancement of EXIT89 and Analysis of World Trade Center Data.

PB96-202247

00,231

FAIRBANK, W. M.

Anomalous Odd- to Even-Mass Isotope Ratios in Reso-

nance Ionization with Broad-Band Lasers.
PB94-211406

FAIRCHILD, C. 0.

Technologic Papers of the Bureau of Standards: Number 170. Pyrometric Practice.

AD-A279 282/8

03,766

FAIT, J.

Using NIST Crystal Data within Siemens Software for Four-

Circle and SMART CCD Diffractometers

PBg7-1

04,803

FALCO, C. M.

Interfaces in Mo/Si Multilayers.

PB96-160668

02,423

Magnetic Properties of $\mathrm{Pd} / \mathrm{Co}$ Multilayers.

PB94-198751

04,465

FALICOV, L. M

Effects of Interfacial Roughness on the Magnetoresistance of Magnetic Metallic Multilayers.

PB95-150017

04,556

FALLER, J.

Low-Frequency, Active Vibration Isolation System.

PB95-203303

02,710

Validation of the Inverse Square Law of Gravitation Using the Tower at Erie, Colorado, USA

PB95-164646

03,947

FALLER, J. E.

Calibration of a Superconducting Gravimeter Using Absolute Gravity Measurements.

PB95-202651

03,684

Continuous Gravity Observations Using Joint Institute for Laboratory Astrophysics Absolute Gravimeters.

PB95-203048

03,685

Earth-Based Gravitational Experiments.

PB94-211414

03,840

Intracomparison Tests of the FG5 Absolute Gravity Meters.

PB96-102991 03,688

Lunar Laser Ranging: A Contınuing Legacy of the Apollo 
FARABAUGH, E. N. Lineshape Analysis of the Raman Spectrum of Diamond
Films Grown by Hot-Filament and Microwave-Plasma wn by Hot-Filam

$\begin{array}{ll}\text { Chemical-Vapor Deposition. } & 03,016\end{array}$

Structures of Vapor-Deposited Yttria and Zirconia Thin Films.

Studies of Defects in Diamond Films and Particles by Raman and Luminescence Spectroscopies.

03,017

Surface Roughness Evaluation of Diamond Films Grown on Substrates with a High Density of Nucleation Sites.

PB95-162418

03,018

FARAH, O. G.

Framework for National Information Infrastructure Services.
PB95-103719
02,723

FARAHANI, $M$.

New Method for Shielding Electron Beams Used for Head and Neck Cancer Treatment.

PB94-211430

New Surface-A

03,579

Polymerization Initiation by N-p-Tolylglycine: Free-Radical Reactions Studied by Pulse and Steady-State Radiolysis.
PB $95-180014$

PARELL J.T.

High-Resolution Infrared Overtone Spectroscopy of ArHF via Nd:YAG/Dye Laser Difference Frequency Generation.

PB94-211448

High-Resolution Intrared Overtone Spectroscopy of N2-HF Vibrational Red Shifts and Predissociation Rate as a Function of HF Stretching Ouanta.

PB96-102298
High-Resolution Infrared Spectroscopy of DF Trimer: A CyHigh-Resolution Infrared Spectroscopy of DF Trimer: A Cy-
clic Ground State Structure and DF Stretch Induced Intramolecular Vibrational Coupling.

PB95-150678

00,920

Pairwise and Nonpairwise Additive Forces in Weakly Bound Complexes: High Resolution Infrared Spectroscopy of ArnDF $(n=1,2,3)$

04,125

Slit Jet Intrared Spectroscopy of Hydrogen Bonded N2HF Isotopomers: Rotational Rydberg-Klein-Rees Analysis and H/D Dependent Vibrational Predissociation Rates.

PB95-161873

00,956

Stabilization and Precise Calibration of a Continuous-Wave Difference Frequency Spectrometer by Use of a Simple Transier Cavity.
PB95-162350

FARROW, R. F. C.

04,276

Combined Low- and High-Angle X-Ray Structural Refinement of a Co/Pt(111) Multilayer Exhibiting Perpendicular Magnetic Anisotropy

FARROW, R. L.

04,457

Measurement of the Self Broadening of the $\mathrm{H} 2 \mathrm{O}(0-5)$ Raman Transitions from 295 to $1000 \mathrm{~K}$.

00,884

FARVARDIN, $N$.

Channel Coding for Code Excited Linear Prediction (CELP) Encoded Speech in Mobile Radio Applications.

PB95-143178

01,475

FASSETT, J.

Working Conference on Global Growth of Technology: Is America Prepared. Held in Gaithersburg, Maryland on December 7, 1995.

PB96-210059

00,018

FASSET, J. D.

Comparative Strategies for Correction of Interferences in Isotope Dilution Mass Spectrometric Determination of Vana-

PB94-185261

00,531

Isotopic and Nuclear Analytical Techniques in Biological Systems: A Critical Study. 10. Elemental Isotopic Dilution Systems: A Critical Study. 10. Elemental Isotopic Dilution
Analysis with Radioactive and Stable Isotopes (Technical

PB96-164157

00,696

Study of Laser Resonance Ionization Mass Spectrometry Using a Glow Discharge Source.

DE94018566

03,308

Study of Laser Resonance lonization Mass Spectrometry Using a Glow Discharge Source.

PB96-123203

Traceability to the Mole: A New Initiative by CIPM.
PB95-180246

03,360

FAUST, $\mathbf{B}$.

Interim Testing Artifact (ITA): A Performance Evaluation System for Coordinate Measuring Machines (CMMs). User

PB95-210589

02,914

FAVREAU, J. P.

Federal Implementaion Guideline for Eletronic Data Interchange. ASC X12 003050 Transaction Set 865 Purchase Order Change Acknowledgement/Request - Seller Initiated. ( Convention.

01,825
Federal Implementation Guideline for Electronic Data Interchange: ASC X12 003040 Transaction Set 838 Trading Partner Profile (Confirmation of Vendor Registration). Implementation Convention

01,813

Federal Implementation Guideline for Electronic Data InterFederal Implementation Guideline for Electronic Data InterPartner Profile (Vendor Registration), Implementation Con-

vention.

03,674

Federal Implementation Guideline for Electronic Data Interchange: ASC $\times 12003050$ Transaction Set 836 Procure. ment Notices. Implementation Convention.

01,827

Federal Implementation Guideline for Electronic Data InterFederal Implementation Guideline for Electronic Data Inter-
change. ASC X 12003050 Transaction Set 840 Request for change. ASC $\times 12003050$ Transaction Set 840 Request for
Quotation. Implementation Convention.
PB96-172531
01,824

01,824

Federal Implementation Guideline for Electronic Data Interchange. ASC $X_{12} 003050$ Transaction Set 843 Response to Request for Ouotation. Implementation Convention

01,822

Federal Implementation Guideline for Electronic Data Interchange. ASC $\times 12003050$ Transaction Set 850 Award Instrument. Implementation Convention.

01,814

Federal Implementation Guideline for Electronic Data Interchange. ASC X12 003050 Transaction Set 855 Purchase change. ASC $\times 12003050$ Transaction Set 855 Purchase
Order Acknowledgment: Implementation Convention. Order Acknowledgment: Implementation Convention.
PB96-172374 823 Federal Implementation Guideline for Electronic Data Interchange. ASC X12 003050 Transaction Set 860 Modifications to Award Instrument. Implementation Convention. PB96-114921

01,815

Five O's (Cues) of the U.S. GOSIP Testing Program. 01,826 01,826
FB96-175716 spectives.

PB95-153029

01,710

Formal Multi-Layer Test Methodology and Its Application to OSI

PB94-172194

02,718

Industry/Government Open Systems Specitication Testing Framework. Version 1.0

01,809

ISO/IEC Workshop on Worldwide Recognition of OSI Test Results Regional Progress - North America.

02,719

Lessons from the Establishment of the U.S. GOSIP Testing

Program.
PB96-119359

01,817

National Voluntary Laboratory Accreditation Program. GOSIP: Government Open Systems Interconnection Protile.
PB95-267993

Open Issues in OSI Protocol Development and Conform. ance Testing.

PB96-122908

01,818

U.S. GOSIP Testing Program.

01,807

FAWZY, W. M. P-Type Doubling in the Infrared Spectrum of NO-HF.
PB94-211463
00,817

\section{Part 1.}

Thermodynamics of Enzyme-Catalyzed Reactions: Part 1. Oxidoreductases.

00,737

FEDOR, L. $S$.

Dual-Frequency Millimeter-Wave Radiometer Antenna for Airborne Remote Sensing of Atmosphere and Ocean. PB96-112289
Dual Frequency $\mathrm{mm}$-Wave Radiometer Antenna for Airborne Remote Sensing of Almosphere and Ocean.
PB95-180378

FEFNEY A. B

APDE Demonstration System Architecture. National PDES Testbed Report Series.

PB94-154325

02,767

Challenges to the National Information Infrastructure: The Barriers to Product Data Sharing National PDES Testbed

Report Series
PB95-136347

02,776

Guidelines for the Development of Mapping Tables.
PB96-154539 Issues and Recommendations for a STEP Application Protocol Framework. National PDES Testbed.

PB94-160868

FEINSTEIN, J.

Higher-Order Approximations in lonospheric Wave-PropagaAD-A292 471/0

04,420

FELCHER, G. P.

Neutron Scattering Studies of Surfaces and Interfaces. PB94-216207 04,517

FELDER, R. J.

Increased Pinning Energies and Critical Current Densities in Heavy-Ion-Irradiated Bi2Sr2CaCu2O8 Single Crystals.
PB95-175352

FELDMAN, A.

Analysis of Thermal Wave Propagation in Diamond Films.

PB94-211471

03,014

Applications of Diamond Films and Related Materials: International Conference (3rd). Held in Gaithersburg, Maryland on August 21-24, 1995. Supplement to NIST Special Publi-

PB95-256053 03,063

Lineshape Analysis of the Raman Spectrum of Diamond

Films Grown by Hot-Filament and Microwave-Plasma

Chemical-Vapor Deposition.

Proceedings of the Applied Diamond Conference 1995: Applications of Diamond Films and Related Materials International Conference (3rd). Held in Gaithersburg, Maryland, on August 21-24, 1995

04,701

Structures of Vapor-Deposited Yttria and Zirconia Thin Films.
PB94-216025

03,041

Studies of Defects in Diamond Films and Particles by Raman and Luminescence Spectroscopies.

03,017

Surface Roughness Evaluation of Diamond Films Grown on Substrates with a High Density of Nucleation Sites.
PB95-162418

Thermal Wave Propagation in Diamond Films.

PB94-211489

03,015

Workshop on Characterizing Diamond Films (3rd). Held in

Gaithersburg, Maryland on February 23-24, 1994.

04,456

Workshop on Characterizing Diamond Films (4th). Held in Gaithersburg, Maryland on March 4-5, 1996.

PB96-183090

FELDMAN, $U$.

Magnetic Dipole Line from U LXXI Ground-Term Levels Predicted at 3200 Angstroms.

4-211497

04,407

Observation and Visible and uv Magnetic Dipole Transitions in Highly Charged Xenon and Barium

04,056

FELL, N. F.

Embossable Grating Couplers for Planar Waveguide Optical Sensors.

PB96-19027
FELLER, I.

Learning to Change: Opportunities to Improve the Perform-

ance of Smaller Manufacturers.

PB94-166212

00,010

High Spectral Purity X-Band Source.

PB95-163713

02,045

FENG, D.

dc Method for the Absolute Determination of Conductivities

of the Primary Standard $\mathrm{KCl}$ Solutions from OC to $50 \mathrm{C}$.

FENG, L.

Addition of $M$ and L-Series Lines to NIST Algorithm for Calculation of X-Ray Tube Output Spectral Distributions. PB95-108742

FENG, S. C.

Dimensional Inspection Planning Based on Product Data Standards.

02,910

Machining Process Planning Activity Model for Systems Integration.

U.S. Navy Coordinate Measuring Machines: A Study of Needs

FENG, $Y$.

02,807

Compatibilization of Polymer Blends by Complexation. 2. Kinetics of Interfacial Mixing.

PB97-111900 
Observations of Partial Discharges in Hexane Under High Magnification.
PB95-163127

01,900

Report on the Workshop on Advanced Digital Video in the National Information Infrastructure. Held in Washington, D.C. on May 10-11, 1994 .

PB95-103677

01,472

Summary Report on the Workshop on Advanced Digital Video in the National Information Infrastructure.

PB96-141320

01,497

Three-Axis Coil Probe Dimensions and Uncertainties during Measurement of Magnetic Fields from Appliances.

PB94-219359

FERAUDY, D.

Preliminary Comparison of Time Transters via LASSO, GPS and Two-Way Satellite.

FEREK, R. J.

Smoke Plume Trajectory from In situ Burning of Crude Oil: Field Experiments

PB96-200993

02,597

FERNANDES, $G$.

Comparison of POSIX Open System Environment (OSE) and Open Distributed Processing (ODP) Reference Models. PB96-131495

Distributed Systems: Survey of Open Management Approaches.

FERNANDEZ, D. P.

01,746

Database for the Static Dielectric Constant of Water and

PB96-145586

01,103

Measurements of the Relative Permittivity of Liquid Water at Frequencies in the Range of 0.1 to $10 \mathrm{kHz}$ and at Temperatures between 273.1 and 373.2 K at Ambient Pressure.

PB96-119375

01,078

Static Dielect
PB96-123559

01,090

FERNANDEZ, J. E.

Polarization Effects on Multiple Scattering Gamma Trans-

Port.

03,926

FERNANDEZ-PELLO, A. C.

Fire Propagation in Concurrent Flows.

PB94-193844

01,365

FERRARIS, C. F.

Alkali-Silica Reaction and High Performance Concrete.
PB96-131537

Guide to a Format for Data on Chemical Admixtures in a Materials Property Database.

01,327

Guide to a Format for Data on Chemical Admixtures in a
Materials Property Database. (Reannouncement with new Materials Property Database. (Reannouncement with new
abstract). abstract).

01,328

Measurement of Rheological Properties of High Pertormance Concrete: State of the Art Report.

00,414

Testing of Selected Self-Leveling Compounds for Floors.
PB95-220455

Warping of Terrace Pavers at the U.S. Capitol Building

PB96-193651

FERRE, E. S.

Investigations of AM and PM Noise in X-Band Devices.

PB95-180022

02,062

FERRE-PIKAL, E. S

Design Criteria for BJT Amplifiers with Low $1 / 4$ AM and PM Noise.

02,442

PB96-200365

ansistor

Origin of $1 / /$

Amplitiers.
PB96-200787

02,096

Reducing the $1 / f$ AM and PM Noise in Electronics for Precision Frequency Metrology.

PB97-113195

02,102

FERRELL, R. A.

Electric Field Effects on a Near-Critical Fluid in Microgravity.
PB96-161880

PB96- 161880
FERRETT, T. A

High Resolution Angle Resolved Photoelectron Spectros-

copy Study of N2
PB95-151494

03,907

Photoionization of Small Molecules Using Synchrotron Radi-

ation. 211505

03,841

Vibronic Coupling and Other Many-Body Effects in the 4sigmag(-1) Photoionization Channel of $\mathrm{CO} 2$.

4sigmag(-1) Pho
PB95-162509

00,962

FETTERS, L. J.

Neutron Reflectivity of End-Grafted Polymers: Concentration and Solvent Ouality Dependence in Equilibrium Conditions. PB94-185758

01,206

Neutron Reflectivity Study of the Density Profile of a Model End-Grafted Polymer Brush: Influence of Solvent Quality.

PB95-202735
Thermodynamic Interactions in Model Polyolefin Blends Obtained by Small-Angle Neutron Scattering.

PB94-198496

Dynamics of $\mathrm{Mu}(+)$ in $\mathrm{Sc}$ and $\mathrm{ScHx}$

PB96-18002

01,208

PB96- 180021

04,112

Visual Pursuit Systems.

PB95-143285

FICK, S. E.

NIST Power Reference Source.

PB94-211513

Ultrasound Power Measurement Techniques at NIST

PB96-179569

FICKET, F. R.

Cryogenic Properties of Silver.

PB94-203593 acterization.

Low-Temperature Properties of Silver.

PB96-126198

Roles of Copper in Applied Superconductivity. PB94-211521

01,841

00,148

FIELD, $B$.

Report on the Workshop on Advanced Digital Video in the National Information Intrastructure. Held in Washington, D.C. on May 10-11, 1994.

PB95-103677

01,472

Summary Report on the Workshop on Advanced Digital

Video in the National Information Infrastructure.

PB96-141320

01,497

FIELD, B. F.

Can Displays Deliver a Full Measure: Manufacturing.

PB96-111935

02,185

Making Displays Deliver a Full Measure.

PB96-122411 01,490

Nonlinear Color Transformations in Real Time Using a Video Supercomputer.

PB96-123021

02,191

Perception of Clamp Noise in Television Receivers PB96-119433

01,489

Specification for Interoperability between Ballistic Imaging Systems. Part 1. Cartridge Cases.

PB96-195524

01,860

FIELD, J.

One-Electron Oxidation of Metalloporphycenes as Studied by Radiolytic Methods.

PB97-111967

FIELDS, A. B.

01,179

Fracture of Silicon Nitride and Silicon Carbide at Elevated Temperatures.

FIELDS, $R$

03,179

Void Shape in Sintered Titanium

PB96-141023

FIELDS, R. J.

Effect of Backfill and Atomizing Gas on the Powder Porosity and Mechanical Properties of $304 \mathrm{~L}$ Stainless Steel.

PB94-185394

Fracture Behavior of Large-Scale Thin-Sheet Aluminum

N95-19494/0

03,311

Fracture Testing of Large-Scale Thin-Sheet Aluminum Alloy. AD-A306 625/5 03,305 Fracture Testing of Large-Scale Thin-Sheet Aluminum Alloy.
PB95-242368
00,024

FILIPPELLI, A. R.

Comments on the Stability of Bayard-Alpert Ionization Gages.

PB96-103080

FILLA, B. J.

02,673

Noncontact U

04,85

Safety Assessment of Railroad Wheels Through Roll-by Detection of Tread Cracks.

PB96-141254

04,856

Sensor System for Intelligent Processing of Hot-Rolled Steel.

03,373

B96-186069

FILLIBEN, J. J.

Factors Significant to Precracking of Fracture Specimens. PB96-109558

FILLIPPELLI, A. R.

Influence of Envelopes Geometry on the Sensitivity of

'Nude' Ionization Gauges.
PB97-119077

04,174

Long-Term Stability of Bayard-Alpert Gauge Performance: Results Obtained from Repeated Calibrations against the National Institute of Standards and Technology Primary Vacuum Standard.

PB96-123567

02,676

FILLMORE, C. L.

High Temperature Reactions of Uranium Dioxide with Various Metal Oxides.

00,717

FILOR, D.

Enthalpy Increment Measurements from $4.5 \mathrm{~K}$ to $350 \mathrm{~K}$ and the Thermodynamic Properties of the Titanium Silicide Ti5Si3(cr).

FINE, J.

Pure Element Sputtering Yield Data: Appendix 4

PB94-200037

00,679

FINK, J. L.

Analysis of Failed Dry Pipe Fire Suppression System Couplings from the Filene Center at Wolf Trap Farm Park for the Performing Arts.

PB94-164407

00.331

Comparison of the Corrosion Rates of FeAl, Fe(sub 3)Al and Steel in Distilled Water and $0.5 \mathrm{M}$ Sodium Chloride. Technical Report Number 2, January--March 1991.

DE94017332

03.186

Evaluation of the Environmentally Induced Fracture Resistance of Ductile Nickel Aluminide. Technical Report Number 1, Final report. October-December 1990

DE94017331

03,306

Evidence of Film-Induced Cleavage by Electrodeposited Rhodium.

PB95-162327

03,191

FINK, S. E.

Transient Analysis of a Line-Focus Transducer Probing a Liquid/Solid Interface.

PB97-118681

02,763

FINKELSTEIN, $R$.

Robotics Application to Highway Transportation. Volume 2. Literature Search

PB95-170551

01,337

Effect of Axial Strain on the Critical Current of Ag-Sheathed Bi-Based Superconductors in Magnetic Fields Up to $25 \mathrm{~T}$.
PB94-211315 Improved Uniaxial Strain Tolerance of the Critıcal Current Measured in Ag-Sheathed Bi2Sr2Ca1Cu2O8+x Superconductors

PB95-153565

04,594

Thermally Activated Hopping of a Single Abrikosov Vortex

PB95-140810

04,548

FIOCK, E. F.

Bibliography of Books and Published Reports on Gas Turbines, Jet Propulsion, and Rocket Power Plants.

AD-A278 $138 / 3$

01,445

Bibliography of Books and Published Reports on Gas Turbines, Jet Propulsion, and Rocket Power Plants, January 1950 through December 1953

AD-A278 213/4

01,446

FIREBAUGH, S. L.

Measurement of $\mathrm{S}_{2} \mathrm{OF}_{10}$, and $\mathrm{S}_{2} \mathrm{O}_{2} \mathrm{~F}_{10}$ Production Rates from Spark and Negative Glow Corona Discharge in SF6/ O2 Gas Mixtures.

PB96-123740

01,093

Discontinuous Volume Change at the Orientational-Ordering

Transition in Solid $\mathrm{C} 60$ 
Composite Materials for Offshore Operations: Proceedings of the International Workshop (1st). Held in Houston, Texas on October 26-28, 1993

03,169

Hemispherical Test Fixture for Measuring the Wavefields Generated in an Anisotropic Solid.

03,181

High-Sensitivity Acoustic Emission Sensor/Proamplifier Sub.

systems.

02,900

Selection of Appropriate Ultrasonic System Components for NDE of Thick Polymer-Composites.

PB94-185279

FITZPATRICK, G. J.

Active High Voltage Divider with 20-PPM Uncertainty.
PB97-119317

Approach to Selting Performance Requirements for Automated Evaluation of the Parameters of High-Voltage Immated Evaluation of the Parameters of High-Voltage Impulses

01,878

Comparative Measurements of High-Voltage Impulses Using a Kerr Cell and a Resistor Divider.

02,028

Investıgation of the Effects of Aging on the Calibration of a Investigation of the Effects of Aging on the Calibration of a
Kerr-Cell Measuring System for High Voltage Impulses.
PB94-172384 02,025 PB94-172384

Optical Current Transducer for Calibration Studies. PB94-185907

Transient Errors in a Precision Resistive Divider. PB97-111512

02,121

FLACH, D. R.

Custom Integrated Circuit Comparator for High-Performance Sampling Applications.

PBg4-213147

02,320

FLACK, H. D.

Statistical Descriptors in Crystallography. 2. Report of a Working Group on Expression of Uncertainty in Measure-

PB96-146824

04,764

World Wide Web for Crystallography.

PB97-109219

04,810

FLAHAVIN, E.

Multi-Agency Certification and Accreditation (C and $A$ ) Process: A Worked Example.

PB95-171955

01,601

FLAHAVIN, E. C.

TMACH Experiment Phase 1. Preliminary Developmental Evaluation.

01,618

FLAHAVIN, E. E.

Concept Paper: An Overview of the Proposed Trust Technology Assessment Program.

PB96-160882

01,614

Guidance to Federal Agencies on the Use of Trusted Systems.

PB95-163440

01,597

FLAITZ, C. M.

Diagnosis and Treatment of an Oral Base-Metal Contact Lesion Following Negative Dermatologic Patch Tests.

PB95-180626

00,172

FLAMBAUM, V. V.

Fine Structure of Negative Ions of Alkaline-Earth-Metal PB94-211182

03,837

Hole Dispersion and Enhancement of Antiferromagnetic Interaction of Localized Spins in High-Tc Superconductors.

Long-Range Parity-Nonconserving Interaction. PB95-202594

03,986

FLANIGAN, L.

In-Space Welding: Visions and Realities.

PB95-163234

04,830

FLATER, D.

Coping with Different Retrieval Methods in Next Generation Networks.

PB95-168555

02,726

FLAUD, J. M.

Reanalysis of the (010), (020), (100), and (001) Rotational Levels of (32)S(16)O2.

PB95-125621

00,887

FLEISCHMANN, C. M.

Backdraft Phenomena.

PB94-193927

01,366

FLEMING, R. F.

Dead Time, Pileup, and Accurate Gamma-Ray Spectrometry. 167101

00,697

FLEMING, T.

First Results from a Coordinated ROSAT, IUE, and VLA Study of RS CVn Systems.

00,069

FLEMING, T. A.

Riass Coronathon: Joint $\mathrm{X}$-ray and Ultraviolet Observations of Normal F.K Stars.

PB96-200217

00,109
ROSAT All-Sky Survey of Active Binary Coronae. 1. Ouiescent Fluxes for the RS Canum Venaticorum Systems.
PB95-202479 00,077

ROSAT All-Sky Survey of Active Binary Coronae. 2. Coronal Temperatures of the RS Canum Venaticorum Systems.

FLETCHER, R. A.

Complementary Molecular Information on Phthalocyanine Compounds Derived from Laser Microprobe Mass Spectrometry and Micro-Raman Spectroscopy.

00,757

4.172269

Generation and Characterization of Acetylene Smokes.
PB94-200292

Measurement of the Uniformity of Particle Deposition of Fil-

ter Cassette Sampling in a Low Velocity Wind Tunnel.

PB95-163754

02,549

Ultrafine Combustion Aerosol Generator.

PB94-200300

01,373

Core Potentials for Ouasi-One-Electron Systems.

PB95-202214

03,970

FLIK, M. I.

Thermal Isolation of High-Temperature Superconducting Thin Films Using Silicon Wafer Bonding and Micromachining.

FLORKOWSKI, D. R.

02,408

Four Years of Monitoring alpha Orionis with the VLA Where Have All the Flares Gone.

PB94-185212

00,048

FLORSCH, N.

Calibration of a Superconducting Gravimeter Using Absolute Gravity Measurements.

PB95-202651

03,684

FLORUSSE, L. J.

Principle of Congruence and Its Application to Compressible States

PB96-102892

01,068

FLOWERS, G.

Inexpensive Laser Cooling and Trapping Experiment for Undergraduate Laboratories.

04,353

FLOYD, F. L

Methodologies for Predicting the Service Lives of Coating Systems.

FLUCK, E.

Inorganic Crystal Structure Database (ICSD) and Standardized Data and Crystal Chemical Characterization of Inorganic Structure Types (TYPIX): Two Tools for Inorganic Chemists and Crystallographers.

PB97-109037

00,648

FLURY, B. D.

Representing a Large Collection of Curves: A Case for Principal Points.

PB95-152286

FLYNN, C. P.

Diffraction of Neutron Standing Waves in Thin Films with

Resonance Enhancement
PB97-113278

04,164

Enhanced Curie Temperatures and Magnetoelastic Domains in Dy/Lu Super Lattices and Films.

PB94-172665

04,443

Magnetic Rare Earth Arificial Metallic Superlattices.

PB95- $\$ 62293$ 04,611

Magnetoelasticity in Rare-Earth Multilayers and Films. PB94-211356

FLYNN, G. W.

Silicon Surface Chemistry by IR Spectroscopy in the Mid- to Far-IR Region: $\mathrm{H} 2 \mathrm{O}$ and Ethanol on $\mathrm{Si}(100)$

PB96- $\$ 38565$

01,097

FLYNN, K. M.

Localization Model of Rubber Elasticity: Comparison with Torsional Data for Natural Rubber Networks in the Dry State.

PB95-107033

03,195

FLYNN, T. M.

Bibliography of the Physical Equilibria and Related Properties of Some Cryogenic Systems.

03,769

Compilation of the Physical Equilibria and Related Properties of the Hydrogen-Carbon Monoxide System. AD-A286 603/6

00,716

FOECKE, T.

Fracture Testing of Large-Scale Thin-Sheet Aluminum Alloy.
AD-A306 625/5

Fracture Testing of Large-Scale Thin-Sheet Aluminum Alloy. PB95-242368

FOEST, $R$.

Optical and Mass Spectrometric Investigations of lons and Neutral Species in SF6 Radio-Frequency Discharges. PB97-111918

FOGLE, W. E.

Development of a Temperature Scale below $0.5 \mathrm{~K}$.

PB95-125639

Systematic Studies of the Effect of a Bandpass Filter on a Josephson-Junction Noise Thermometer.

03,939

Systematic Studies of the Effect of a Post-Detection Filter on a Josephson-Junction Noise Thermometer.

PB95-162988

03,940

FOKSINSKI, $M$.

DNA Base Damage in Lymphocytes of Cancer Patients Un. dergoing Radiation Therapy.

FOLDEAKI, $M$.

Asymmetry between Flux Penetration and Flux Expulsion in TI-2212 Superconductors

PB95-125647

04,527

Enhanced Flux Pinning via Chemical Substitution in Bulk Superconducting T1-2212.

04,647

Magnetic Susceptibility of $\mathrm{Pr} 2 \cdot \times \mathrm{CexCuO} 4$ Monocrystals and Polycrystals.

FOLEY, G. M.

High-Speed Spatial Scanning Pyrometer

PB94-200003

02,636

FOLEY, J. T.

Theoretical Analysis of the Coherence-Induced Spectral Shift Experiments of Kandpal, Vaishya, and Joshi. PB94-219383

00,561

FOLEY, R. P.

Prediction of Strengthening Due to $\mathrm{V}$ Additions in DirectCooled Ferrite-Pearlite Forging Steels.

PB96-190251

03,220

Prediction of the Strength Properties for Plain-Carbon and Vanadium Micro-Alloyed Ferrite-Pearlite Steel.

PB96-123393

03,216

FOLMAR, L. C.

Histopathology, Blood Chemistry, and Physiological Status of Normal and Moribund Striped Bass ('Morone saxatilis') Involved in Summer Mortality ('Die-Off') in the SacramentoSan Joaquin Delta of California.

PB94-198157

00,034

FONER, $\mathrm{S}$.

Effect of Microstructure on Phase Formation in the Reaction of $\mathrm{Nb} / \mathrm{Al}$ Multilayer Thin Films.

PB95-168415

03,352

First Phase Formation Kinetics in the Reaction of $\mathrm{Nb} / \mathrm{Al}$

PB95-168456 03,353

Thin Film Reaction Kinetics of Niobium/Aluminum Multilayers.
PB95-17529

FONG, E.

Interoperability Experiments with CORBA and Persistent Object Base Systems.

PB96-183140

01,772

Preliminary Functional Specifications of a Prototype Electronic Research Notebook for NIST.

00,012

Report on Application Integration Architectures (AIA) Workshop. Held in Dallas, Texas on February 8-12, 1993. PB94-142536

FONG, E. N.

Application of Expert System to Select Data Sources from Chemical Information Databases. 
FORNEY, $D$.

Infrared and Near-Intrared Spectra of HCC and DCC Trapped in Solid Neon.

AD-A295 578/9

03,773

Mid- and Near-Infrared Spectra of Water and Water Dimer Isolated in Solid Neon.

PB95-125662

00,888

Vibrational Spectra of Molecular lons Isolated in Solid Neon: $\mathrm{HCCH}+$ and HCC-. (Reannouncement with New Availability Information).

AD-A253 551/6

00,707

Vibrational Spectra of Molecular lons Isolated in Solid Neon. X. $\mathrm{H} 2 \mathrm{O}(+), \mathrm{HDO}(+)$, and $\mathrm{D} 2 \mathrm{O}(+)$.

00,889

Vibrational Spectra of Molecular Ions isolated in Solid Neon. XI. NO2(+), NO2 (-), and NO3(-).

00,890

Vibrational Spectra of Molecular lons Isolated in Solid Neon. 11. NO2 (+), NO2(-), and NO3(-)

AD-A275 828/2

00,708

FORNEY, G. P.

Analyzing and Exploiting Numerical Characteristics of Zone PB96-102314

01,400

Computing Radiative Heat Transfer Occurring in a Zone Fire Model.

01,399

Computing the Effect of Sprinkler Sprays on Fire Induced Gas Flow.

00,404

Field Modeling: Simulating the Effect of Sloped Beamed Ceilings on Detector and Sprinkler Response. $\quad 01,406$
PB96-122866

Compart-

mentovement in $P$ sed

PB94-172418

00,332

Pressure Equations in Zone-Fire Modeling

PB96-102967

00,208

FORTUNKO, $\mathrm{C}$.

Artificial Crack in Steel: An Ultrasonic-Resonance-Spectroscopy and Modeling Study.

03,241

Compressibility of Polycrystal and Monocrystal Copper: Acoustic-Resonance Spectroscopy.

02,990

Elastic Constants and Internal Friction of Polycrystalline

Copper. 14015

03,364

Orthotropic Elastic Constants of a Boron-Aluminum FiberReinforced Composite: An Acoustic-Resonance-Spectroscopy Study.
PB96-200175

03,182

Ultrasonic-Resonance Spectroscopy of Bulk and Layered Solids.

PB96-141338

04,759

FORTUNKO, C. M.

Absorption of Sound in Gases between 10 and $25 \mathrm{MHz}$ Argon.

PB94-199015

04,183

Absorption of Ultrasonic Waves in Air at High Frequencies (10-20 MHz)

Appropriate Ultrasonic System Components for NDE of Thick Polymer Composites.

PB95-125696

03,148

Assessment of Technology for Detection of Stress Corrosion Cracking in Gas Pipelines. Final Report, July 1993 March 1994.

02,475

Determination of Sheet Steel Formability Using Wide Band Electromagnetic-Acoustic Transducers.

02,279

Examination of Objects Made of Wood Using Air-Coupled Ultrasound.

PB95-125712 03,404

Gas-Coupled, Pulse-Echo Ultrasonic Crack Detection and Thickness Gaging.

04,847

Hemispherical Test Fixture for Measuring the Wavefields Generated in an Anisotropic Solid.

PB96-190087

03,181

High-Sensitivity Acoustic Emission Sensor/Preamplifier Subyystems.

PB95-125704

02,900

Selection of Appropriate Ultrasonic System Components for NDE of Thick Polymer-Composites

PB94-185279

03,133

Well-Shielded EMAT for On-Line Ultrasonic Monitoring of GMA Welding.

02,879

FosSUM, B. $M$

Learning to Change: Opportunities to Improve the Performance of Smaller Manufacturers.

00,010

FOSTER, $N$.

Development of the National Marine Mammal Tissue Bank.

PB95-161402
FOTI, J.

Preliminary Functional Specifications of a Prototype Electronic Research Notebook for NIST.

PB94-20775

00,012

FOTIADAS, L.

Lattice Position of $\mathrm{Si}$ in GaAs Determined by X-Ray Standing Wave Measurements.

ing Wave Meas

FOUQUET, $Y$.

04,632

Hardware Measurement Techniques for High-Speed Net-

PB96-160551

01,500

FOWELL, A. J.

Fire Hazard Model Developments and Research Efforts at

00,407

PB96-159652

ent Ac-

International Organization for Standardization: Current Ac-
tivities in Fire Safety Engineering.

ivities in Fire Safety Engineering.

National Planning for Construction and Building $R$ and $D$.

PB96-137104

00,324

Program of the Subcommittee on Construction and Building.

PB94-193646 00319

Program of the Subcommittee on Construction and Building (July 1994).

PB95-122537

00,321

Rationale and Preliminary Plan for Federal Research for Construction and Building.

00,322

White Papers Prepared for the White House: Construction Industry Workshop on National Construction Goals. Held on December 14-16, 1994

PB95-216891

01,299

FOWLER, B. O.

Composition and Solubility Product of a Synthetic Calcium Hydroxyapatite.

PB96-180104 02,995

Crystal Structure of Calcium Succinate Monohydrate.

$\begin{array}{ll}\text { PB95-168928 } & 00,167\end{array}$

Octacalcium Phosphate Carboxylates IV. Kinetics of Formation and Solubility of Octacalcium Phosphate Succinate.
PB94-185600
00,776

Octacalcium Phosphate Carboxylates. 1. Preparation and Identification.

00,660

Octacalcium Phosphate Carboxylates. 2. Characterization

and Structural Consideration.
PB95-161543

00,955

Octacalcium Phosphate Carboxylates. 5. Incorporation of Excess Succinate and Ammonium lons in the Octacalcium Phosphate Succinate Structure.

PB95-168894

00,166

Octacalcium Phosphate. 3. Infrared and Raman Vibrational Spectra.

PB94-172244

00,756

FOWLER, J.

Method of Realizing Spectral Irradiance Based on an Absolute Cryogenic Radiometer.

161204

04,270

FOWLER, J. B.

High Accuracy Measurement of Aperture Area Relative to a Standard Known Aperture.

Third Generation Water Bath Based Blackbody Source.

PB96-122148

01,919

FOWLER, J. E.

Systems Integration for Manufacturing Applications Program S95 Annual Report.

Variant Design for Mechanical Artifacts-A State of the Art Survey.

02,768

FOX, D.

Dynamic Phenomena on the RS Canum Venaticorum Binary II Pegasi in August 1989. 1. Observational Data. 211067

00,056

First Results from a Coordinated ROSAT, IUE, and VLA Study of RS CVn Systems.

00,069

FOX, D. C.

Rotational Modulation and Flares on RS Canum Venaticorum and BY Draconis Stars. XVIII. Coordinated VLA, ROSAT, and IUE Observations of RS CVn Binaries. PB96-102322

00,089

R. W.

Diode Laser as a Spectroscopic Tool.

PB95-175485 00,600

High-Resolution Diode-Laser Spectroscopy of Calcium.

PB95-181244

03,969

High-Sensitivity Spectroscopy with Diode Lasers.

PB95-175477

04,297

Optical Probing of Cold Trapped Atoms.

Precise Optical Frequency References and Difference Frequency Measurements with Diode Lasers.

04,305

FOY, B. R.

Fragment Energy and Vector Correlations in the OvertonePumped Dissociation of HN3X(1)A'.

PB94-199908

00,802

Vibrational Predissociation Dynamics of Overtone-Excited $\mathrm{HN} 3$.

FRAGA,

00,691

GA. M.

Excitation of Balmer Lines in Low-Current Discharges of Hydrogen and Deuterium.

\section{FRAIME, L.}

03,893

Proceedings of the Workshop on the Federal Criteria for In formation Technology Security. Held in Ellicot City, Maryland on June 2-3, 1993

01,575

RAKER, A. C.

Dental Materials
PB94-172871

00,142

Information Retrieval Using Key Words and a Structured Review.

PB95-161121

03,724

FRALEY, K. L.

Advanced Mass Calibration and Measurement Assurance

PB95-253571

02,492

FRANASZEK, $M$.

Fluctuations in Probability Distribution on Chaotic Attractors.
PB96-102330
04,022

FRANCHUK, C. A.

Economic, Energy, and Environmental Impacts of the Energy-Related Inve

FRANCIS, M. H

Comparison of k-Correction and Taylor-Series Correction for Probe-Position Errors in Planar Near-Field Scanning.

PB96-147137

02,012

Comparison of Ultralow-Sidelobe-Antenna Far-Field Pat-

terns Using the Planar-Near-Field Method and the Far-Field

PB96-200373

02,015

Dual-Frequency Millimeter-Wave Radiometer Antenna for Airborne Remote Sensing of Atmosphere and Ocean.

PB96-112289 02,009

Dual Frequency mm-Wave Radiometer Antenna for Airborne Remote Sensing of Atmosphere and Ocean.

Planar Near-Field Measurements of Low-Sidelobe Anten

PB94-219235

02,001

Proposed Analysis of RCS Measurement Uncertainty

PB95-203568

FRANCONI, B.

Binder Characterization and Evaluation by Nuclear Magnetic Resonance Spectroscopy.

PB94-193471

01,334

FANK, D. E.

Preliminary Investigation of Oleoresin Capsicum.

PB96-179486

FRANK, $H$.

03,520

Report on the Workshop on Advanced Digital Video in the National Information Infrastructure. Held in Washington, D.C. On May 10-11, 1994

01,472 


\section{PERSONAL AUTHOR INDEX}

Precise Laser-Based Measurements of Zero-Dispersion Wavelength in Single-Mode Fibers.

01,511

Technical Digest: Symposium on Optical Fiber Measure Technical Digest: Symposium on Optical Fiber Measure-
ments (8th), 1994. Held in Boulder, Colorado on September $13-15,1994$.

04,231

Technical Digest: Symposium on Optical Fiber Measurements (9th), 1996. Held in Boulder, Colorado on October 1 3. 1996 .

PB97-108583

04,383

FRASER, G. T.

Infrared Spectra of van der Waals Complexes of Importance in Planetary Atmospheres.

PB95-125738

00,07

Microwave and Submillimeter Spectroscopy of Ar-NH3 States Correlating with $\mathrm{Ar}+\mathrm{NH} 3(\mathrm{j}=1, \mathrm{k}=1)$.

Microwave Spectrum and Structure of $\mathrm{CH}_{3} \mathrm{NO}_{2} \cdot \mathrm{H} 2 \mathrm{O}$.

AD-A296 $377 / 5$

00,719

Molecular-Beam Optothermal Spectrum of the $\mathrm{OH}$ Stretching Band of Methanol.

PB94-212826

00,839

P-Type Doubling in the Infrared Spectrum of NO-HF.

PB94-211463 00,817

Rotational Spectra of $\mathrm{CH} 3 \mathrm{CCH}-\mathrm{NH}_{3}, \mathrm{NCCCH}-\mathrm{NH} 3$, and $\mathrm{NCCCH}-\mathrm{OH} 2$
$\mathrm{~PB} 97-118798$

RATELLO, $\mathrm{V}$.

04,170

Magneto-Optic Magnetic Field Sensors Based on Uniaxial Iron Garnet Films in Optical Waveguide Geometry.

Iron Garnet Fil

02,152

FRATELLO, V. J.

High Frequency Magnetic Field Sensors Based on the Faraday Effect in Garnet Thick Films.

02,282

Magneto-Optic Magnetic Field Sensors Based on Uniaxia Iron Garnet Films in Optical Waveguide Geometry. PB95-168498

\section{FRECHETTE, M. F.}

02,159

Investigation of $\mathrm{S} 2 \mathrm{~F} 10$ Production and Mitigation in Compressed SF6- Insulated Power Systems.

PB96-155528

02,468

FRECHETTE, $\mathbf{S}$.

NIST SIMA Interactive Management Workshop. Held in Fort Belvoir, Virginia on November 14-16, 1994

PB96-154877

02,838

Open Architectures for Machine Control.

PB94-135621

02,942

FRECHETTE, S.P.

Interoperability Requirements for CAD Data Transfer in the AutoSTEP Project.

PB97-114268

02,796

FREDERICK, $\mathrm{N}$

Distributed measurements of tracer response on packed bed flows using a fiberoptic probe array. Final report

DE95013079

00,667

FREDERICK, N. V.

Development of a Dual-Sinker Densimeter for High-Accuracy Fluid P-V-T Measurements.

PB95-168951

03,267

Development of a Dual-Sinker Densimeter for High-Accuracy Fluid P-V-T Measurements. Appendix A.

02,620

Speed-of-Sound Measurements in Liquid and Gaseous Air.
PB95-151957

\section{FREDERIKSE, H. P. R.}

Analysis of Thermal Wave Propagation in Diamond Films.

PB94-21147

03,014

Thermal Wave Propagation in Diamond Films. PB94-211489

03,015

FREE, G. M.

Measurements of the Characteristic Impedance of Coaxial Air Line Standards.

PB95-16878

02,221

FREED, K.

How Far Is Far from Critical Point in Polymer Blends. Lattice Cluster Theory Computations for Structured Monomer. Compressible Systems.

PB94-211141

01,217

\section{FREED, K. F.}

Competition between Hydrodynamic Screening ('Draining') and Excluded Volume Interactions in an Isolated Polymer

PB95-175402

01,265

Examination of the V/d Expansion Method from Exact Enumeration for a Self-Interacting Self-Avoiding Walk.

PB95-175733

01,266

Hypercubic Lattice SAW Exponents nu and gamma : 3.99 Dimensions Revisited

01,215

Modification of the Phase Stability of Polymer Blends by Diblock Copolymer Additivies.

03,172

Response to "Draining in Dilute Polymer Solutions and Renormalization

01,283
FREEMAN, J. J.

Standard of Attenuation for Microwave Measurements. AD-A297 905/2

FREIMAN, S. W.

Ceramics Technical Activities, 1995

PB96-193677

03,087

Electric Field Effects on Crack Growth in a Lead MagnePBium Niobate.

03,339

Environmentally Enhanced Fracture of Ceramics.

PB95-125746

03,046

Moisture and Water-Induced Crack Growth in Optical Mate-

Molecular Orbital Calculations of Bond Rupture in Brittle

00,973

Molecular Orbital Study of Water Enhanced Crack Growth

Process.

PB95-164067

03,240

Photonic Materials: A Report on the Results of a Workshop. Held in Gaithersburg, Maryland on August 26-27, 1992, Vol-

02,114

$X$-Ray Characterization of the Crystallization Process of High-Tc Superconducting Oxides in the Sr-Bi-Pb-Ca-Cu-O Systern.

PB95-151700

04,579

RENCH, J. D.

Creep Rupture of MoSi2/SiCp Composites.

PB95-152294

03.154

Sources of Strain-Measurement Error in Flag-Based Extensometry.

03,108

FRENKEL, A.

Broadband High-Optical-Density Filters in the Infrared

PB95-180261

04,309

Filter Transmittance Measurements in the Infrared.

PB94-140589 04,224

Thermal and Nonequilibrium Responses of

Superconductors for Radiation Detectors.
PB95-164232

FRENKEL, $M$.

Thermodynamic and Thermophysical Properties of Organic Nitrogen Compounds. Part II. 1- and 2-Butanamine, 2-Meth yl-1-Propanamine, 2-Methyl-2-Propanamine, Pyrrole, 1-, 2and 3-Methylpyrrole, Pyridine, 2-, 3-, and 4-Methylpyridine dine, Carbazole, Phenanthridine, 1- and 2Naphthalenamine, and 9-Methylcarbazole. 1- and Naphthalenamine, and 9-Methylcarbazole.
PB94-162294 FREY, $M$.

Deterministic and Stochastic Chaos.

PB96-156138

Melnikov Function and Homoclinic Chaos Induced by Weak Perturbations.

PB95-180923

03,414

Necessary Condition for Homoclinic Chaos Induces by Ad-

ditive Noise.

Noise-Induced Chaos and Phase Space Flux

PB95-125761

04,063

Noise-Induced Transitions to Chaos.

03,433

Noise Modeling and Reliability of Behavior Prediction for Multi-Stable Hydroelastic Systems.

PB96-111943

04,822

Spectrum of the Stochastically Forced Duffing-Holmes Os-

00,216

Transitions to Chaos Induced by Additive and Multiplicative

PB96-155759

03,750

FREY, M. $\mathbf{R}$.

Capacity of the Lp Norm-Constrained Poisson Channel.

PB95-125753

FREYER, G. J.

01,515

Band-Limited, White Gaussian Noise Excitation for Reverberation Chambers and Applications to Radiated Susceptibility Testing.

FRIDAY, D. S.

NIST and the Navy: Past, Present and Future.

PB96-119649

01,960

RIEDMAN, C.

Reinforcement of Cancellous Bone Screws with Calcium Phosphate Cement

PB96-15800

00,179

FRIEDMAN, D. J.

Surface Topography and Ordering-Variant Segregation in GalnP2.

PB95-153649

04,595

FRIEND, D. G.

Calculation of Enthalpy and Entropy Differences of Near-

Critical Binary Mixtures with the Modified Leung-Griffiths

PB95-108635

00,885

Composition Dependence of a Field Variable Along the Binary Fluid Mixture Critical Locus.

01,003

Critical Lines for Type-II| Aqueous Mixtures by Generalized Corresponding-States Models.

01,063

Prediction of the Thermal Conductivity of Refrigerants and Refrigerant Mixtures.

03,258

Reference Data for the Thermophysical Properties of Cryogenic Fluids.

PB95-168688 03,263

Thermodynamic Properties of the Methane-Ethane System.

PB95-125779

Thermophysical Property Computer Packages from NIST.

PB95-125787 04,203

Thermophysical Property Standard Reference Data from NIST.

PB96-167358

01,153

\section{FRIEND, J. P.}

International Marine-Atmospheric (222)Rn Measurement intercomparison in Bermuda. Part 2. Results for the Participating Laboratories.

PB96-175682

00,115

FROHNSDORFF, $G$

Suggestions for a Logically-Consistent Structure for Service Life Prediction Standards.

PB95-125795

00,358

FROHNSDORFF, G. J. C.

Performance Approach to the Development of Criteria for Low-Sloped Roof Membranes.

PB94-160751

FRONCZEK, F. R.

$X$-Ray-Diffraction Study of a Thermomechanically Detwinned Single Crystal of YBa2Cu3O6+X.

PBg 15

Portsmouth Fastener Manufacturing Workstation. User's Manual.

PB95-147922

02,860

FRONTICELLI, C.

Positive and Negative Cooperativities at Subsequent Steps of Oxygenation Regulate the Allosteric Behavior of Multistate Sebacylhemoglobin.

PB97-11937

03,486

FROST, M. J.

Kinetics and Dynamics of Vibrationally State Resolved lonMolecule Reactions: (14) N2 $(+)(v=1$ and 2) and 15) $\mathrm{N} 2(+)(\mathrm{v}=0,1$ and 2) with (14) N2.

04,023

Selected Ion Flow Tube-Laser Induced Fluorescence Instrument for Vibrationally State-Specific Ion-Molecule Reac-

PB94-185444 
FULCRAND, $\mathrm{H}$.

Oxidation of Cafteic Acid and Related Hydroxycinnamic Acids.

00,651

FULLER, E. R

Ceramic Characterization.

DE94013170

03,026

Crystal Chemistry and Phase Equilibria Studies of the $\mathrm{BaO}(\mathrm{BaCO} 3)-\mathrm{R} 2 \mathrm{O} 3-\mathrm{CuO}$ Systems. 4. Crystal Chemistry and Subsolidus Rich Region of the Ternary Diagrams, $\mathrm{R}=$ Lanthanides.
PB95-151759
00,936 Crystal Chemistry and Phase Equilibrium Studies of the $\mathrm{BaO}(\mathrm{BaCO}$ ) - $\mathrm{R} 2 \mathrm{O} 3-\mathrm{CuO}$ Systems. 5. Melting Relations in $\mathrm{Ba} 2(\mathrm{Y}, \mathrm{Nd}, \mathrm{Eu}) \mathrm{Cu} 3 \mathrm{O} 6+\mathrm{x}$

PB95-151718

04,580

Determination of Fiber-Matrix Interfacial Properties of Importance to Ceramic Composite Toughening.

03,149

Efficient Experiment to Study Superconducting Ceramics.

PB94-212578

Interface Properties for Ceramic Composites from a Single Fiber Pull-Out Tes

PB94-199361

03,135

Matrix Grain Bridging Contribution to the Toughness of Whisker Reinforced Ceramics.

03,134

Micro-Mechanical Aspects of Asperity-Controlled Friction in Fiber-Toughened Ceramic Composites.

PB94-199536

03,136

FULLER, S. $K$.

Benefits and Costs of Research: Two Case Studies in Building Technology.

00,230

Life-Cycle Costing Manual for the Federal Energy Management Program. 1995 Edition.

PB96-172317 02,511

Life-Cycle Costing Workshop for Energy Conservation in Buildings: Student Manual.

PB95-175006

00,257

FURDYNA, J. K

Microwave Properties of YBa2Cu3O7-x Films at $35 \mathrm{GHz}$ from Magnetotransmission and Magnetoreflection Measurements.
PB95-168977

04,643

FURLANI, C. $M$.

National PDES Testbed: An Overview.

PB95-125829

02,775

FURUKAWA, G. T.

Preliminary Results of a Comparison of Water Triple-Point Cells Prepared by Different Methods.

PB96-161344

00,634

FURUTA, $R$

Important Papers in the History of Document Preparation Systems: Basic Sources.

G'SELL, C.

Influence of Physical Aging on the Yield Response of Model DGEBA + Poly(propylene oxide) Epoxy Glasses.

PB95-126363

03,381

GADALLA, N. A. M.

Thermodynamic and Thermophysical Properties of Organic Nitrogen Compounds. Part II. 1- and 2-Butanamine, 2-Methyl-1-Propanamine, 2-Methyl-2-Propanamine, Pyrrole, 1-, 2-, and 3-Methylpyrrole, Pyridine, 2-, 3-, and 4-Methylpyridine, Pyrrolidine, Piperidine, Indole, Ouinoline, Isoquinoline, Acridine, Carbazole, Phenanthridine, 1- and 2. Naphthalenam

00,741

GADDY, G. D.

Application of Thermal Analysis Techniques to the Charac-

terization of EPDM Roofing Membrane Materials.
PB95-125845

00,359

Use of Thermal Mechanical Analysis to Characterize Ethylene-Propylene-Diene Terpolymer (EPDM) Roofing Membrane Materials.

GADZUK, J. W.

00,360

Energy Dependence of Collision Characteristics in Molecule-Surface Collisions.

00,786

Single-Atom Point Source for Electrons: Field-Emission Resonance Tunneling in Scanning Tunneling Microscopy.
PB95-125860 GAGNE- 125860

Aging, Warm-Up Time and Retrace; Important Characteris-

tics of Standard Frequency Generators.

04,031

Environmental Sensitivities of Ouartz Oscillators.

PB96-103148

02,271

New Model of 1/F Noise in Baw Ouartz Resonators. PB96-112248

02,383

GAIGALAS, A.

Conformational Alterations of Bovine Insulin Adsorbed on a

Silver Electrod

00,510
Physicochemical Characterization of Low Molecular Weight Heparin.

PB96- 112040

GAIGALAS, A. $K$.

Aggregation Kinetics of Colloidal Particles Destabilized by Enzymes.

00,894

Method for the Assay of Hydrolytic Enzymes Using Dynamic Light Scattering.

03,53

Non-Perturbative Relation between the Mutual Diffusion Coefficient, Suspension Viscosity, and Osmotic Compressibility: Application to Concentrated Protein Solutions. ibility: Application to Concentrated Protein Solutions. 01,062
PB96-102355 Physical Characterization of Herparin by Light Scattering
PB96-119383

Structural Analysis of Heparin by Raman Spectroscopy. PB96-167226

GAIGNEBET, $J$

Preliminary Comparison of Time Transfers via LASSO, GPS and Two-Way Satellite.

GAITAN, $M$.

CMOS Circuit Design for Controlling Temperature in

Micromachined Devices
PB96-156088

02,196

Efficient Method to Compute the Maximum Transient Drain

Current Overshoot in Silicon on Insulator Devices.
PB94-172483 High-Level CAD Melds Micromachined Devices with Foundries.

02,321

Interaction of Stoichiometry, Mechanical Stress, and Intertace Trap Density in LPCVD Si-rich SiNx-Si Structures.

MEMS in Standard CMOS VLSI Technology. $\quad 02,377$ PB96-102363
Micromachined Coplanar Waveguides in CMOS Tech-

nology
PB97-119283

02,456

Micromachined Display Output for a Cellular Neural Network.

02,422

Multijunction Thermal Converters by Commercial CMOS Fabrication.

02,343

Performance of Commercial CMOS Foundry-Compatible Multijunction Thermal Converters.

PB95-153656

02,342

Realizing Suspended Structures on Chips Fabricated by PMOS Foundry Processes Through the MOSIS Service.

Test Structures for Determining Design Rules for Microelectromechanical-Based Sensors and Actuators.
PB95-150488 105 PB95-150488 02,105 Tin Oxide Gas Sensor Fabricated Using CMOS MicroHotplates and In-situ Processing.

PB95-150603

00,580

GALAMBOS, $J$

Extreme Value Theory and Applications: Proceedings of the Conference on Extreme Value Theory and Applications, Volume 3. Held in Gaithersburg, Maryland in May 1993. PB95-104956

GALLAGHER, A.

Associative Ionization in Collisions of Slowed and Trapped Sodium

03,880

Atom Cooling and Trapping, and Collisions of Trapped Atoms.

PB96-122916

04,048

Atomic-scale characterization of hydrogenated amorphoussilicon films and devices. Annual subcontract report, 14 February $1994-14$ April 1995.

February 1994
DE95009287

02,294

Collisional Energy Transfer between Excited-State Strontium and Noble-Gas Atoms.

PB95-202958

03,995

Construction of Silicon Nanocolumns with the Scanning Tunneling Microscope.

PB95-203063

04,696

PB95-203345

Energy-Pooling Collisions in Barium.

PB95-203030

04,697

PBailures of the

mission.

Nanoscale Study of the As-Grown Hydrogenated Amorphous Silicon Surface.

PB95-150595 04,573

Oscillator Strengths and Radiative Branching Ratios in

Atomic Sr.
PB95-203493

04,008

Plasma Chemistry in Disilane Discharges.

PB94-211075

02,514

Plasma Chemistry in Silane/Germane and Disilane/Germane Mixtures.

01,027

Threshold Electron Excitation of $\mathrm{Na}$

PB95-202917

03,994

GALLAGHER, A. C.

Excitation Transfer in Barium by Collisions with Noble

Gases.

01,163

Growth and Nucleation of Hydrogenated Amorphous Silicon on Silicon (100) Surfaces.

02,991

Nanoscale Study of the Hydrogenated Amorphous Silicon Surface.

PB96-103056

04,720

GALLAGHER, J. G.

REFPROP Refrigerant Properties Database: Capabilities, Limitations, and Future Directions.

PB96- 167150

01,149

Critical Lines for Type-III Aqueous Mixtures by Generalized Corresponding-States Models.

Formulation of the Refractive Index of Water and Steam.

PB95-140133

00,900

Standard States, Reference States and Finite-Concentration Effects in Near-Critical Mixtures with Applications to Aque ous Solutions.

Thermodynamic Behavior of the $\mathrm{CO} 2-\mathrm{H} 2 \mathrm{O}$ System from 400 to $1000 \mathrm{~K}$, up to $100 \mathrm{MPa}$ and $30 \%$ Mole Fraction of $\mathrm{CO} 2$.

PB94-162245

00,736

Thermophysical Property Computer Packages from NIST.

PB95-125787

04,203

GALLAGHER, J. W.

Journal of Physical and Chemical Reference Data, Volume

22. No. 1, January/February 1993.
PB94-160975

00,729

Journal of Physical and Chemical Reference Data, Volume 22. No. 2, March/April 1993.

PB94-162211

00,733

Journal of Physical and Chemical Reference Data, Volume 22, No. 3, May/June 1993

B94-162260

00,738

Journal of Physical and Chemical Reference Data, Volume 22, No. 4, July/August 1993

00,743

Journal of Physical and Chemical Reference Data, Volume 22, No. 5, September/October 1993.

PB94-162336

00,745

Journal of Physical and Chemical Reference Data, Volume 22. No. 6, November/December 1993.

PB94-168556

Journal of Physical and Chemical Reference Data, Volume

24, No. 1, January/February 1995.
PB96-145560

01,101

Journal of Physical and Chemical Reference Data. Volume 24, No. 2, March/April 1995

01,105

Journal of Physical and Chemical Reference Data, Volume 24, No. 3, May/June 1995

PB96-145842

01,108

Journal of Physical and Chemical Reference Data, Volume 24, No. 4, July/August 1995

01,112

Journal of Physical and Chemical Reference Data, Volume

24, No. 5, September/October 1995.
PB'96-145925 
Grazing Incidence Prompt Gamma Emissions and Resonance-Enhanced Neutron Standing Waves in a Thin-Film. PB95-150470 03,892

Observed Frustration in Confined Block Copolymers. PB95-150033

01,238

GALLAS, M. R.

Bulk Modulus and Young's Modulus of Nanocrystalline gamma-Alumina.

PB96-204185

03,092

Fabrication of Transparent gamma-Al2O3 from Nanosize Particles.

PB95-175493

03,054

\section{GALLAWA, R. L.}

Accuracy of Eigenvalues: A Comparison of Two Methods. $\begin{array}{ll}\text { PB95-126249 03,413 } & \end{array}$ Anharmonic Oscillator Analysis Using Modified Airy Functions.

03,798

Approximate Solution to the Scalar Wave Equation for Opti-

cal Waveguides.
PB95-126256

Bending-Induced Loss in Dual-Mode Rectangular Waveguides.

04,288

Bending-Induced Phase Shifts in Dual-Mode Planar Optical Waveguides.

Bent Rectangular Core Waveguides: An Accurate Perturbation Approach.

PB95-168803

04,289

Calculated Fiber Attenuation: A General Method Yielding Stationary Values.

04,298

Complex Propagation Constants for Nonuniform Optical Waveguides: Calculations.

Tav-125910

Fiber Spot Size: A Simple Method of Calculation.

PB95-125936

Fibre Splice Loss: A Simple Method of Calculation PB95-175519

04,250

Improved Variational Analysis of Inhomogeneous Optical Waveguides Using Airy Functions.

PB95-168639

04,285

International Intercomparison of Detector Responsivity at 1300 and $1550 \mathrm{~nm}$.

PB95-125928 02,140

International Intercomparison of Detector Responsivity at 1300 and $1550 \mathrm{~nm}$.

02,141

LP11-Mode Leakage Loss in Coated Depressed Clad Fi-

bers.

02,145

Modal Characteristics of Bent Dual Mode Planar Optical Waveguide.
PB95-180485

04,311

Modal Properties of Circular and Noncircular Optical Waveguides.

Mode Coupling and Loss on Tapered Optical Waveguides.
PB95-168571

Modified Airy Function Method for the Analysis of Tunneling Problems in Optical Waveguides and Quantum Well StrucPB94-185824

02,120

Symbolic Programming with Series Expansions: Applications to Optical Waveguides.

PB95-168589

04,283

Vector and Ouasi-Vector Solutions for Optical Waveguide Modes Using Efficient Galerkin's Method with HermiteGauss Basis Functions.

B 96-141197

04,357

\section{GALLER, M. A}

Computer Simulations of Binder Removal from 2-D and 3-D Model Particulate Bodies.

GALOWIN, L.

Laboratory Accreditation for Testing Energy Efficient Lighting.

Proceedings of the Open Forum on Laboratory Accreditation at the National Institute of Standards and Technology, October 13,1995

PB96-210141

02,686

GALOWIN, L. S.

Efficiency of Electric Motors. National Voluntary Lab. ACcreditation Program (NVLAP).

02,107

National Voluntary Laboratory Accreditation Program: Carpet and Carpet Cushion.

00,295

National Voluntary Laboratory Accreditation Program: Energy Efficient Lighting Products.

02,642

National Voluntary Laboratory Accreditation Program (NVLAP): Wood Based Products.

03,405
Water Efficient Plumbing Fixtures through Standards and Test Methods.

GALT, D.

Characterization of a Tunable Thin Film Microwave YBa2Cu3O7-x/SrTiO3 Coplanar Capacitor.

02,264

Dielectric Properties of Thin Film SrTiO3 Grown on LaAlO3 with YBa2Cu3O7-x Electodes.
PB95-181160

Ferroelectric Thin Film Characterization Using Superconducting Microstrip Resonators.

PB96-102389 02,270

Tunable High Temperature Superconductor Microstrip Resonators.

PB95-168423

02,048

GALVAO, G. P.

Far Infrared Laser Frequencies of $\mathrm{CH} 3 \mathrm{OD}$ and $\mathrm{N} 2 \mathrm{H} 4$. PB96-119623 PB95-169363

GAMMON, R. W

04,292

Binary versus Decade Inductive Voltage Divider Comparison and Error Decomposition.

02,071

inductive Voltage Divider Calibration for the NASA Flight Experiment.

02,042

Susceptibility Critical Exponent for a Nonaqueous Ionic Binary Mixture Near a Consolute Point.

GANGOPADHYAY, A.

00,938

Control of Friction and Wear of Alpha-Alumina with a Composite Solid-Lubricant Coating.

PB95-125969

03,225

\section{ANGULI, B.}

Kinetics and Mechanism of the Collision-Activated Dissociation of the Acetone Cation

PB94-21646

00,859

GANN, $R$.

Agent Screening for Halon 1301 Aviation Replacement.

PB96-159710

03,282

GANN, R. G.

Effect of Fuel Tank Rupture Mode on the Ignitability of Expelled Fuel.

PB97-110043

01,444

Evaluation of Alternative In-Flight Fire Suppressants for Full-Scale Testing in Simulated Aircraft Engine Nacelles and Dry Bays.

Fire Suppression System Performance of Alternative Agents in Aircraft Engine and Dry Bay Laboratory Simulations. SP890: Volume 1.

PB96-117775

03,277

Fire Suppression System Performance of Alternative Agents in Aircraft Engine and Dry Bay Laboratory Simulations. SP 890: Volume 2

03,278

Flame Retardants - Overview.

PB94-185287

01,363

Innovation in the Japanese Construction Industry: A 1995 Appraisal.

PB96-177373

00,225

PB97-122311

NIST Research on Less-Flammable Materials.

PB97-118632

Quantifying the Ignition Propensity of Cigarettes.

PB96-155411

Relating Bench-Scale and Full-Scale Toxicity Data. PB95-125977

01,442

Variationally Stable Treatment of Two- and Three-Photon Detachment of $\mathrm{H}(-)$ Including Electron-Correlation Effects. Detachment of $\mathrm{H}(-)$ Including Electron-Correlation Effects.
PB95-202867 GARBOCZI, E.

Digitized Simulation of Mercury Intrusion Porosimetry.
PB94-172236

Percolation and Pore Structure in Mortars and Concrete.

PB95-150439

GARBOCZI, E. J.

Application of Digital-Image-Based Models to Cement-Based Materials.

PB96-156161

00,406

Cellular Automaton Simulations of Cement Hydration and Microstructure Development.

01,320

Computer Simulation of the Diffusivity of Cement-Based

Materials.

00,362

Cross-Property Relations and Permeability Estimation in Model Porous Media.

PB95-150280

04,205

Diffusion Studies in a Digital-Image-Based Cement Paste Microstructura! Model.

PB94-198801

01,312

Digital Simulation of the Aggregate-Cement Paste Interfacial Zone in Concre

00,363

Digitized Direct Simulation Model of the Microstructural Development of Cement Paste.

PB94-198777

01,309

Digitized Simulation Model for Microstructural Development.

PB94-198785 01,310

Elastic Properties of Central-Force Networks with BondLength Mismatch.

PB95-163366 04,623

Fundamental Computer Simulation Models for Cement-

Based Materials.
PB95-126009

Geometrical Percolation Threshold of Overlapping Ellipsoids.

PB96-102397

03,167

Interfacial Transport in Porous Media: Application to dc Electrical Conductivity of Mortars.

PB96-146816

01,326

Intrinsic Conductivity of Objects Having Arbitrary Shape and Conductivity.

04,150

Intrinsic Viscosity and the Polarizability of Particles Having a Wide Range of Shapes.

PB96-119318

03,170

Modelling Drying Shrinkage of Cement Paste and Mortar. Part 1. Structural Models from Angstroms to Millimeters.
PB96-135132
03,074

Modelling the Leaching of Calcium Hydroxide from Cemen Paste: Effects on Pore Space Percolation and Diffusivity. Multi-Scale Picture of Concrete and Its Transport Prop. erties: Introduction for Non-Cement Researchers.

PB97-115802

03,107

Water Permeability and Chloride Ion Diffusion in Portland Cement Mortars: Relationship to Sand Content and Critical Pore Diameter.

GARCIA-TORANO, E.

Standardization and Decay Scheme of Rhenium-186.

PB94-200490

03,830

GARDENER, J. L.

International Intercomparison of Detector Responsivity at 1300 and $1550 \mathrm{~nm}$.

PB95-125928

02,140

International Intercomparison of Detector Responsivity at 1300 and $1550 \mathrm{~nm}$.

PB95-126017

02,141

UuILO J. J.

Guidelines for the Evaluation of Electronic Data Interchange Products.

PB96-172325

GARMER, D.

Active Site Ionicity and the Mechanism of Carbonic Anhydrase.

GARRETSON, S. P.

Learning to Change: Opportunities to Improve the Performance of Smaller Manufacturers.

PB94-166212

00,010

GARRETT, J. $\mathrm{H}$.

Development of Computer-Based Models of Standards and Attendant Knowledge-Base and Procedural Systems.

PB96-155783

00.464

Distributed Architecture for Standards Processing

PB96-164181

00,276

GARRIS, M. D.

Analysis of a Biologically Motivated Neural Network for Character Recognition. 
tem for Experimental and Theoretical Studies of Plasma. Processing Discharges.

PB94-172327

GARSON, L. R.

Electronic Publishing and the Journals of the American Chemical Society.

PB97-10918

\section{GARVEY, $M$.}

Precision Oscillators: Dependence of Frequency on Temperature, Humidity and Pressure.

02,031

GARY, D.

Rotational Modulation and Flares on RS Canum Venaticorum and BY Draconis Stars. XVI. IUE Spectros copy and VLA Observations of $\mathrm{C} 1182(=\mathrm{V} 1005$ Orionis) in October 1983.

GARY, J.

00,050

Accurate Computations of Radar Cross Sections of Simple

Objects.

04,426

Graded and Nongraded Regenerator Performance

PB95-169090

02,753

GARY, J. M

Technique to Evaluate Benchmarks: A Case Study Using the Livermore Loops.

PB95-151320

04,577

GASS, S. I.

Development and Validation of Multicriteria Ratings: A Case Study.

PB95-126025

00,004

GATES, B. C.

Characterization of the Interaction of Hydrogen with Iridium Clusters in Zeolites by Inelastic Neutron Scattering Spectroscopy.
PB95-180741

01.013

GATES, R. S.

Boundary Lubrication of Silicon Nitride.

PB95-213583

03,226

Silicon Nitride Boundary Lubrication: Effect of Oxygenates.

PB96-111711 03,068

Silicon Nitride Boundary Lubrication: Lubrication Mechanism of Alcohols.

PB96-111703

03,067

GAVIN, R. J.

Portsmouth Fastener Manufacturing Workstation. User's Manual.

PB95-147922

02,860

GAVRIN, A.

Domain Structures in Magnetoresistive Granular Metals.
PB96-141346

Nanostructure Fabrication via Direct Writing with Aloms Focused in Laser Fields.

cused in Lase
PB95-150272

04,564

GAYLE, F. W.

Inelastic Neutron Scattering Measurements of Phonons in Icosahedral Al-Li-Cu.

PB95-126215

04,532

Nature of (001) Till Grain Boundaries in YBa2Cu3O6+X.

PB95-126033

04,528

Temperature Dependence of Vortex Twin Boundary Inter-

action in Yitrium Barium Copper Oxide (YBa2Cu3O6+x).

PB95-162442 04,613

$X$-Ray-Diffraction Study of a Thermomechanically Detwinned Single Crystal of YBa2Cu3O6+X

PB95-151726

04,581

GAYLEY, $K$.

Goddard High-Resolution Spectrograph Observations of the Local Interstellar Medium and the Deuterium/Hydrogen Ratio along the Line of Sight Toward Capella.

00,066

GAYLEY, K. G.

Hydrogen Lyman-alpha Emission of Capella.
PB95-202263

GEBASE, L.

Analyzing Electronic Commerce.

PB94-219102

GEBBIE, K. B.

Physics Laboratory Technical Activities, 1993

PB94-176088

GEHRING, P. M.

Anomalous Dispersion and Thermal Expansion in Lightly-

PB95-152302

04,585

Antiferromagnetic Interlayer Correlations in Annealed Ni80Fe20/Ag Multilayers.

03,109

Design of a High-Flux Backscattering Spectrometer for Ultra-High Resolution Inelastic Neutron Measurements. High-Energy Phonon Dispersion in La 1.85Sr0.15CuO 4. PB96-138458
Magnetic Structure Determination for Annealed Ni80Fe20/ Ag Multilayers Using Polarized-Neutron Reflectivity. PB96-176615

03,739

Neutron-Scattering Studies of the Two Magnetic Correlation Lengths in Terbium.

04,586

Neutron Scattering Study of the Lattice Modes of Solid Cubane.

PB96-147152

01.126

Observation of Two Length Scales Above ( $T$ sub $N$ ) in a Holmium Thin Film.

PB97-111942 04,151

Origin of the Second Length Scale Above the Magnetic Spiral Phase of Tb.

GEIST, J.

NIST Form-Based Handprint Recognition System

PB94-217106

(1)

Second Census Optical Character Recognition Syster Conference.

\section{GELBIN, A.}

Nucleic Acid Database: Present and Future. PB97-109078

01.832

GENIS, A.

Effect of Anneal Temperature on Si/Buried Oxide Interface Roughness on SIMOX

PB96-112206

\section{GENTILE, T. R.}

Absolute Response Calibration of a Transfer Standard Cryogenic Bolometer.

PB96. 147103

04,358

Mode-Locked Lasers for High-Accuracy Radiometry.

PB96-204201

04,134

National Institute of Standards and Technology High-Accuracy Cryogenic Radiometer.

04,378

Realization of a Scale of Absolute Spectral Response Using the NIST High Accuracy Cryogenic Radiometer.

PB97-118640

04,397

GENTRY, J. W.

Generation and Characterization of Acetylene Smokes. PB94-200292

Measurement of the Uniformity of Particle Deposition of Filter Cassette Sampling in a Low Velocity Wind Tunnel.

PB95-163754

Ultrafine Combustion Aerosol Generator.

02,549

PB94-200300

01,373

ORG, E.

Report on the Workshop on Advanced Digital Video in the National Information Infrastructure. Held in Washington D.C. on May 10-11, 1994

B95-103677

01,472

Summary Report on the Workshop on Advanced Digital Video in the National Information Infrastructure.

01,497

Dental Applications of Ceramics.

PB96-122940

00,177

Dental Materials.

PB94-172871

00,142

Paffenbarger Research Center: The Cutting Edge of Denta Science.
PB94-216355

00,151

Reduction of Marginal Gaps in Composite Restorations by

Use of Glass-Ceramic Inserts.

00,174

Surface Roughness of Glass-Ceramic Insert. Composite Restorations: Assessing Several Polishing Techniques.

PB97.119010

03,583

Wear of Enamel against Glass-Ceramic, Porcelain, and Amalgam.

\section{GEORGE, T.}

03,082

Optically Stabilized Tunable Diode-Laser System for Saturation Spectroscopy

PB96-102819

04,717

GERARDO, J. B.

Gaseous Electronics Conference Radio-Frequency Reference Cell: A Defined Parallel-Plate Radio-Frequency System for Experimental and Theoretical Studies of PlasmaProcessing Discharges.

PB94-172327

\section{GERBER, G.}

Femtosecond Time-Resolved Molecular Multiphoton Ionization: The Na2 System.

PB95-202305

03,975

Femtosecond Time-Resolved Wave Packet Motion in Molecular Multiphoton Ionization and Fragmentation.

PB94-198611

00,790

Photoelectron Spectroscopy of Negatively Charged Bismuth Clusters: $\mathrm{Bi}(-) 2, \mathrm{Bi}(-) 3$, and $\mathrm{Bi}(-) 4$

00,880

Photoelectron Spectroscopy of Small Antimony Cluster Anions: $\mathrm{Sb}(-), \mathrm{Sb} 2(-), \mathrm{Sb} 3(-)$, and $\mathrm{Sb} 4(-)$

01,045

GERHARDT, $R$.

Imaging of Fine Porosity in a Colloidal Silica: Potassium Silicate Gel by Defocus Contrast Microscopy.

ERHARDT, R. A.

03,039

Small-Angle Neutron Scattering Characterization of Processing/Microstructure Relationships in the Sintering of Crystalline and Glassy Ceramics. (Reannouncement with New Availability Information)

AD-A249 510/9

03,025

\section{ER, T. A.}

Hot Carrier Excitation of Adlayers: Time-Resolved Measurement of Adsorbate-Lattice Coupling.

00,758

Picosecond Measurement of Substrate-to-Adsorbate Energy Transfer: The Frustrated Translation of CO/Pt(111)--Trans-

PB95-126041

00,895

Specular and Diffuse Reflection Measurements of Electronic Displays.

02,208

Ultrafast Time-Resolved Infrared Probing of Energy Transfer at Surfaces.
PB96-123443

GERZ, C.

Frequency-Stabilized LNA Laser at 1.083 mum: Application to the Manipulation of Helium 4 Atoms.

PB95-176186

04,304

Temperature of Optical Molasses for Two Different Atomic Angular Momenta.

PB95-126058

GETTINGS, R. J.

Tensile Creep of Silicide Composites.

GEYER, R. G.

03,881

Analysis of an Open-Ended Coaxial Probe with Lift-Off for Nondestructive Testing.

PB96-135116

01,940

Applicability of Effective Medium Theory to Ferroelectric/ Ferrimagnetic Composites with Composition and Frequency-Dependent Complex Permittivities and
Permeabilities. PB96-157854

01,945

Dielectric Properties of Materials at Cryogenic Temperatures and Microwave Frequencies.

PB95-202610

02,369

Dielectric Properties of Single Crystals of $\mathrm{Al} 2 \mathrm{O} 3, \mathrm{LaAlO} 3$, $\mathrm{SrTiO3,}$ and $\mathrm{MgO}$ at Cryogenic Temperatures.

Effective Medium Theory for Ferrite-Loaded Materials.

PB95-154662 01,893

Influence of Films' Thickness and Air Gaps in Surface $1 \mathrm{~m}$ pedance Measurements of High Temperature Superconductors Using the Dielectric Resonator Technique. Measurements of Permittivity and the Dielectric Loss Tangent of Low Loss Dielectric Materials with a Dielectric Resonator Operating on the Higher Order Te(sub 0 gamma

PB96-111869

02,273

Microwave Dielectric Properties of Anisotropic Materials at Cryogenic Temperatures.

02,412

NIST 60-Millimeter Diameter Cylindrical Cavity Resonator: 


\section{PERSONAL AUTHOR INDEX}

GHONIEM, A.

Smoke Emission from Burning Crude Oil.

PB96-122890

GHONIEM, A. F.

01,407

Computational Model for the Rise and Dispersion of WindBlown, Buoyancy-Driven Plumes. Part 2. Linearly Stratified Atmosphere.

00,119

Dispersion and Deposition of Smoke Plumes Generated in Massive Fires.

\section{GHOSH, R. N.}

Cryogenic Precision Capacitance Bridge Using a Single Electron Tunneling Electrometer

PB95-126074

04,529

Cryogenic Precision Capacitance Bridge Using a Single Electron Tunneling Electrometer.

PB95-152310

02,040

Cryogenic Precision Capacitance Bridge Using a Single Electron Tunneling Electrometer.

02,072

GHOSHTAGORE, R. N

Electrical Test Structures Replicated in Silicon-on-Insulator Material.

PB97-111827 02,454

Measurement of Patterned Film Linewidth for Interconnect Characterization.

PB96-148168

02,420

GIACOMETTI, J. A.

Thermal Pulse Study of the Polarization Distributions Produced in Polyvinylidene Fluoride by Corona Poling at ConStant Current.

GIAMPAPA, M. S

01,195

Relationship between Radiative and Magnetic Fluxes for Three Active Solar-Type Dwarts.

Three Active Sol

00,097

GIARRATANO, P. J. Experimental plan to determine the performance of the Oak report, September 1, 1993--November 30, 1993.

report, Septemb

03,778

GIBBS, D.

Observation of Two Length Scales Above ( $T$ sub $N$ ) in a Holmium Thin Film

PB97-111942

04,151

GIBSON, C.

Intercomparison of the ITS-90 Radiance Temperature Scales of the National Physical Laboratory (U.K.) and the National Institute of Standards and Technology. PB96-113550

02,674

GIBSON, C. E.

Results of a NIST/NNIIOFI Comparison of Spectral-Radiance Measurements.

7-113021

04,159

GIBSON, D. M

Rotational Modulation and Flares on RS Canum Venaticorum and BY Draconis Stars. XVI. IUE Spectroscopy and VLA Observations of C1182(=V 1005 Orionis) in October 1983.

GIBSON, K. A.

00,050

Bibliography of the NIST Electromagnetic Fields Division Publications.

PB94-165990

01,875

Bibliography of the NIST Electromagnetic Fields Division Publications.

PB95-13556

01,886

GIER, T. E.

Structural and Chemical Investigations of $\mathrm{Na} 3(\mathrm{ABO})$ ) 3.4H2O-Type Sodalite Phases.
$\mathrm{PB} 95-180733$

Tetrahedral-Framework Lithium Zinc Phosphate Phases: Tetrahedral-Framework Lithium Zinc Phosphate Phases:
Location of Light-Atom Positions in LiZnPO4 H2O by PowLocation of Light-Atom Positions in LiZnPO4 H2O by Powder Neutron Diffraction and
LiZnPO4 by ab Initio Methods.

LiZnPO4 by ab

GIFFORD, A.

01,129

Photodetector Frequency Response Measurements at NIST, US, and NPL, UK: Preliminary Results of a Stand-

ards Laboratory Comparison.

02,175

GIFFORD, A. D.

Comparison of Photodiode Frequency Response Measurements to $40 \mathrm{GHz}$ between NPL and NIST.

PB96-111992

04,038

GILBERT, D. M.

Study of Federal Agency Needs for Information Technology Security.

PB94-193653

GILBERT, $M$.

01,579

Group 1 for the Process Engineering Data STEP Application Protocol.

GILBERT, S.

02,797

Comparison of UV Photosensitivity and Fluorescence during Fiber Grating Formation.

04,362
Inexpensive Laser Cooling and Trapping Experiment for Undergraduate Laboratories.

PB96-140371

04,353

GILBERT, S. L.

Annealing of Bragg Gratings in Hydrogen-Loaded Optical

PBiber.

04,361

Bragg Gratings in Optical Fibers Produced by a Continuous-Wave Ultraviolet Source.

04,274

Comparison of UV-Induced Fluorescence and Bragg Grating Growth in Optical Fiber.

04,284

Decay of Bragg Gratings in Hydrogen-Loaded Optical Fi-

bers.

PB96-122643

04,345

Decrease of Fluorescence in Optical Fiber during Exposure to Pulsed or Continuous-Wave Ultraviolet Light.

04,320

Electronically Tunable Fiber Laser for Optical Pumping of (3) $\mathrm{He}$ and (4) $\mathrm{He}$

B 946-201165

Frequency Stabilization of a Fiber Laser to Rubidium: A High-Accuracy $1.53 \mathrm{mu} \mathrm{m}$ Wavelength Standard

PB95-126082

04,252

Growth Characteristics of Fiber Gratings.

PB96-122957

04,346

Growth of Bragg Gratings Produced by Continuous-Wave Ultraviolet Light in Optical Fiber.

04,275

5-162038

idium in

High-Resolution Spec

a Vapor-Cell Trap.
PB95-153714

Laser Cooling and Trapping for the Masses.

04,268

PB95-126090

Liquid and Solid Atomic Ion Plasmas.

PB94-198991

03,809

Optical Probing of Cold Trapped Atoms.

04,296

GILES, N. C.

Comparison of Techniques for Nondestructive Composition Measurements in CdZnTe Substrates.

GILLASPY, J. D.

Beam Line for Highly Charged lons.

PB97-111256

02,703

Line.

PB94-199585

$\mathrm{nm} \mathrm{Yb}$

Microlithography by Using Neutral Metastable Atoms and

Self-Assembled Monolayers.
PB96-190038

02,441

Observation and Visible and uv Magnetic Dipole Transitions

in Highly Charged Xenon and Barium.
PB96-138441

Polarization Measurements on a Magnetic Quadrupole Line in Ne-Like Barium

04,161

Search for Small Violations of the Symmetrization Postulate in an Excited State of Helium.

PB96-123518

04,050

Visible and UV Light from Highly Charged lons: Exotic Mat ter Advancing Technology.

GILLEN, G.

04,414

Image Depth Profiling SIMS: An Evaluation for the Analysis of Light Element Diffusion in YBa2Cu307-x Single Crystal Superconductors.

04,530

Molecular Ion Imaging and Dynamic Secondary Ion Mlass Spectrometry of Organic Compounds.

PB95-126124

00,571

Use of Kinetic Energy Distributions to Determine the Relative Contributions of Gas Phase and Surface Fragmentation in KeV lon Sputtering of a Quaternary Ammonium Salt. PB95-126108

GILLEN, J, G.

Epitaxial Growth of $\mathrm{BaTiO} 3$ Thin Films at $600 \mathrm{C}$ by Metalorganic Chemical Vapor Deposition.

PB96-122510

03,07

UV-Photopatterning of Alkylthiolate Monolayers Self-Assembled on Gold and Silver.

PB95-150751

GILLESPIE, A.

Measurement and Reduction of Alignment Errors of the NIST Watt Experiment

01,987

NIST Watt Balance: Progress Toward Monitoring the Kilo-

gram.

01,991

NIST Watt Experiment: Monitoring the Kilogram.

PB97-122329

01,997

GILLIAM, D.

Preparation and Characterization of (6)LiF and (10)B Reftime.

PB95-108692

03,874

GILLIAM, D. M.

Mass Assay and Uniformity Test of Boron Targets by Neu-

PB97-119085

04,175

Measurement of the Neutron Lifetime

04,094

Neutron Leakage Benchmark for Criticality Safety Research

03,723

Problems Related to the Determination of Mass Densities of Evaporated Reference Deposits.

PB95-163226

GILLIAND, G. L.

Crystal Packing Interactions of Two Different Crystal Forms Cf Bovine Ribonuclease A.

PB95-152823

00,943

GILLIES, C. $W$.

Rotational Spectrum and Structure of a Weakly Bound Complex of Ketene and Acetylene.

PB95-126140

00,896

GILLIES, J. Z.

Rotational Spectrum and Structure of a Weakly Bound Complex of Ketene and Acetylene.

PB95-126140

00,896

GILLIGAN, J. M.

High-Resolution Atomic Spectroscopy of Laser-Cooled lons. PB95-169330

03,953

nterference in the Resonance Fluorescence of Two

Trapped Atoms.

03,948

Light Scattered from Two Atoms.

PB95-168753

04,286

Precise Spectroscopy for Fundamental Physics.

PB96-112164

04,040

Quantum Measurements of Trapped Ions.

Quantum Projection Noise: Population Fluctuations in TwoLevel Systems.

Recent Experiments on Trapped lons at the National Institute of Standards and Technology.

PB95-169322

03,952

Using Archie to Find Files on the INTERNET.

PB95-168605

02,727

AND, D.

Catalogue of Electromagnetic Environment Measurements, $30-300 \mathrm{~Hz}$.
$\mathrm{PB} 96-155452$

01,943

Condensed Catalogue of Electromagnetic Environment Measurements, $30-300 \mathrm{~Hz}$

PB95-162210

01,899

GILLILAND, G.

Positive and Negative Cooperativities at Subsequent Steps of Oxygenation Regulate the Allosteric Behavior of 
Certitying the Chemical Composition of a Biological Material: A Case Study.

Recent Developments in NIST Botanical SRMS.

00,636

PB96-167267

03,489

Comparison of Finite Element and Analytic Calculations of Comparison of Finite Element and Analytic Calculations of
the Resonant Modes and Frequencies of a Thick Shell the Resonant Modes and Frequencies of a Thick Shell
Sphere.
PB94-160785 02,626

02,626 Compensation of Errors Detected by Process-Intermitten
Gauging.
PB97-110472 02,846

Constructing Invariant Tori for Two Weakly Coupled van der Pol Oscillators.
PB95-126165

03,412

Data Management for Error Compensation and Process Control.

PB97-110480

02,847

PIECS: A Soltware Program for Machine Tool ProcessIntermittent Error Compensation.

02,842

Prediction of Geometric-Thermal Machine Tool Errors by Artificial Neural Networks.

PB94-186673

02,943

GINGOLD, D

Diffusion Studies in a Digital-Image-Based Cement Paste Microstructural Model.

PB94-198801

GINLEY, R. A.

NIST $30 \mathrm{MHz}$ Linear Measurement System.

PB94-169752

GINZBURG, A.

Image Information Transfer Properties of $X$-Ray Intensifying Screens in the Energy Range from 17 to $320 \mathrm{keV}$.

PB95-126173

00,155

Study of Multiple Scattering Background in Compton Scatter Imaging.

04,425

GINZBURG, V. L

From Superconductivity to Supernovae: The Ginzburg Symposium. Report on the Symposium Held in Honor of Vitaly L. Ginzburg. Held in Garthersburg, Maryland on May 22, PB95-171963

04,649

\section{GIRARD, J. E.}

Comparison of Selectivities for PCBs in Gas Chromatography for a Series of Cyanobiphenyl Stationary Phases.
PB96-119458

Enhanced Detection of PCR Products Through Use of TOTO and YOYO Intercalating Dyes with Laser Induced Fluorescence - Capillary Electrophoresis.

00,599 PB95-164653

00,599

Ouantitative Analysis of Selected PCB Congeners in Marin Stationary Phase.

PB96-111737

02,591

GIVEN, J. A.

Critical Behavior of lonic Fluids

PB95-164331

00,978

Critical Lines for Type-III Aqueous Mixtures by Generalized Corresponding-States Models.

PB96-102371

01,063

GJERTSON, $W$.

Catalogue of Electromagnetic Environment Measurements, $30-300 \mathrm{~Hz}$.

01,943

Condensed Catalogue of Electromagnetic Environment Measurements, $30-300 \mathrm{~Hz}$

PB95-162210

GLADHILL, R. L.

01,899

Program Handbook: Requirements for Obtaining NIST APproval/Recognition of a Laboratory Accreditation Body Under P.L. 101-592. The Fastener Ouality Act.

PB94-210143

02,859

LLASER, M. A.

Shear-Induced Melting of Two-Dimensional Solids.
PB96-112057

GLASER, S. D.

Estimation of System Damping at the Lotung Site by Application of System Identification.

PB96-214697

Preliminary Processing of the Lotung LSST Data.

PB96-165972

01,351

GLAZE, D. J.

Error Analysis of the NIST Optically Pumped Primary Frequency Standard.

PB95-153482

01,535

NIST Optically Pumped Cesium Frequency Standard. NIST.7, the New US Primary Frequency Standard. PB95-153458

GLAZIER, S. A.

Amperometric Flow-Injection Analysis Biosensor for Glucose Based on Graphite Paste Modified with Tetracyanoqu
PB95-161980

03,498
Autofluorescence Detection of 'Escherichia coli' on Silver Membrane Filters.

Bacterial Enumeration in Storage Water.

03,590

PB96-163647

03,591

Feasibility of Fluorescence Detection of Tetracycline in Media Mixtures Employing a Fiber Optic Probe.

PB96-163654

00,511

Fluorescence Measurements of Tetracycline in High Cell

Mass for Fermentation Monitoring.
PB95-175709

00,601

In situ Fluorescence Cell Mass Measurements of 'Saccharomyces cerevisiae' Using Cellular Tryptophan.

Novel Amperometric Immunosensor for Procainamide Employing Light Activated Labels

PB96-163662

00,512

Optical Control of Enzymatic Conversion of Sucrose to Glucose by Bacteriorhodopsin Incorporated into Self-Assembled Phosphatidylcholine Vesicles. PB96-123344

03,477

GLEITER, $\mathrm{H}$.

Inelastic Neutron Scattering Study of Hydrogen in Nanocrystalline Pd

03,366

Vibrational Excitations and the Position of Hydrogen in Nanocrystalline Palladium.

PB96-111828

04,035

GLENN, K. R.

RDI-SIM ECMA Inter-Domain Routing Protocol Simulation Tool.

PB94-17230

01,683

GLENZER, $S$.

Investigation of LS Coupling in Boronlike Ions.

PB94-185295

03,797

GLICKSMAN, M. E

Asymptotic Behavior of Modulated Taylor-Couette Flows with a Crystalline Inner Cylinder.

04,469

Effect of Modulated Taylor-Couette Flows on Crystal-Melt Interfaces: Theory and Initial Experiments.

PB94-216736

04,521

GLIGOR, $V$.

Functional Security Criteria for Distributed Systerns. PB96-123492

01,607

Proceedings of the Workshop on the Federal Criteria for Information Technology Security. Held in Ellicot City, Maryd on June 2-3, 1993

PB94-162583

01,575

GLINKA, C. J.

Analysis of SANS from Controlled Pore Glasses.

03,035

Characterization of Chemically Modified Pore Surfaces by Small Angle Neutron Scattering.

PB95-126181

00,898

Small Angle Neutron Scattering Study of the Structure and Formation of MCM-41 Mesoporous Molecular Sieves.

$\begin{array}{ll}\text { PB97-122337 } & 03,110\end{array}$

Small Angle Neutron Scattering Study of the Structure and Formation of Ordered Mesopores in Silica.

PB96-111919

03,069

GLOTZER, S. C.

Mixing Plate-Like and Rod-Like Molecules with Solvent: A Test of Flory-Huggins Lattice Statistics.

PB96-126206

03,173

GLOVER, M.

Suppression of Elevated Temperature Hydraulic Fluid and JP-8 Spray Flames.

PB95-181095

00,021

Effectiveness of Halon Alternatives in Suppressing Dynamic Effectiveness of Halon Alternatives in Suppressing Dynamic
Combustion Process.
PB96-175732
03,288

03,288
Iron

Inhibition of Premixed Methane-Air Flames by Iron
Pentacarbonyl.
PB96-163712
00,513

Interaction of HFC-125, FC-218 and CF3I with High Speed Combustion Waves.

03,290

Parametric Study of Hydrogen Fluoride Formation in Suppressed Fires.

00,514

Suppression Effectiveness of Extinguishing Agents under Highly Dynamic Conditions.

00,020

Suppression of High-Speed $\mathrm{C} 2 \mathrm{H} 4 /$ Alr Flames with C1Halocarbons.

03,287

Suppression of High Speed Turbulent Flames in a Detonation/Deflagration Tube.

PB95-231817

01,395

GOCKENBACH, E

Digital Techniques in HV Tests - Summary of 1989 Panel Session.

02,035

GODART, C.

Neutron Scattering Study of Antiferromagnetic Order in the Magnetic Superconductors RNi2B2C

PB97-112411

GODEFROID, $M$.

Fundamental Torsion Band in Acetaldehyde.

04,812

OEHNER, R. P.

Phase Identification in a Scanning Electron Microscope Using Backscattered Electron Kikuchi Patterns.

04,804

GOFORTH, T. L.

Low-Energy-Electron Collisions with Sodium: Elastic and Inelastic Scattering from the Ground State.

PB96-103106

Anisotropy of the Surfaces of Pores in Plasma Sprayed Alumina Deposits.

GOLAS, D. B.

Assay of the Eluent from the Alumina-Based Tungsten-188Rhenium-188 Generator.

PB94-200482

03,829

Liquid-Scintillation Counting Techniques for the Standardization of Radionuclides Used in Therapy.

PB97-110084

03,709

Review of the USCEA/NIST Measurement Assurance Pro-

gram for the Nuclear Power Industry.

B95-126272

03,712

Standardization and Decay Scheme of Rhenium-186. PB94-200490

USCEANIST Measurement Assurance Programs for the Radiopharmaceutical and Nuclear Power Industries.

03,721

\section{GOLDBERG, R. N.}

Apparent Molar Heat Capacities and Apparent Molar Volumes of Aqueous Glucose at Temperatures from 298.15 K to $327.01 \mathrm{~K}$.

PB94-212800

03,459

Application of Thermodynamics to Biotechnology

PB95-150793

03,529

Calorimetric Determination of the Standard Transformed Enthalpy of a Biochemical Reaction at Specified $\mathrm{PH}$ and pMg.

198249

03,454

Conference Report: Calorimetry Conference (50th).

PB97-122279

03,722

Equilibrium and Calorimetric Investigation of the Hydrolysis

of L-Tryptophan to (Indole + Pyruvate + Ammonia).
PB95-163317

00,661

Thermochemistry of the Hydrolysis of L-arginine to (L. citrulline + Ammonia) and of the Hydrolysis of L-arginine to L-ornithine + Urea)

PB95-150801

03,463

Thermochernistry of the Reactions between Adenosine, Adenosine 5'-monophosphate, Inosine, and Inosine 5' monophosphate; the Conversion of L-histidine to (Urocanic

PB94-213113

Thermodynamic and NMR Study of the Interactions of Cyclodextrins with Cyclohexane Derivatives. 
Workshop on Magnetic Susceptibility of Superconductors and Other Spin Systems. Held in Berkeley Springs, West Virginia on 20 May 1991.

04,435

Alternating-Field Susceptometry and Magnetic Susceptibility of Superconductors.

PB95-168613

04,638

Harmonic and Static Susceptibilities of $\mathrm{YBa2Cu3O}$.
PB95-161139

PB95-161139
04,599 $\mathrm{Nb}-\mathrm{Ti}$ Wire for the Superconducting Super Collider. $\begin{array}{ll}\text { DE94005988 } & 03,775\end{array}$ Magnetic Measurement of Transport Critical Current Density of Granular Superconductors.

PB95-126199

Oftset Susceptibility of Superconductors.

04,531

B94-212263

04,503

Panel Discussion on Units in Magnetism.

04,746

Preparation, Crystal Structure, Dielectric Properties, and Magnetic Behavior of Ba2Fe2Ti4O13

01,162 PB96-186176

Reduction of Interfilament Contact Loss in Nb3Sn
Superconductor Wires.
PB95-175535 PB95-175535

Cu307.

Surface Barrier and Lower Critical Field in YBa2Cu3O7 delta Superconductors.

04,478

Volume Magnetic Hysteresis Loss of Nb3Sn

Superconductors as a Function of Wire Length.
PB95-153722

Ouantum Dynamics of Renner-Teller Vibronic Coupling: The Predissociation $\mathrm{HCO}$.

PB94-185303

GOLDFINE, A.

00,773

Compatibility Analysis of the ANSI and ISO IRDS Services Interfaces.

01,805

Information Resource Dictionary System (IRDS): A Status Report

PB95-126207

01,810

GOLDING, T.

Epitaxial Growth of Sb/GaSb Structures: An Example of V/ III-V Heteroepitaxy.

04,693

GOLDMAN, A. I.

Inelastic Neutron Scattering Measurements of Phonons in cosahedral Al-Li-Cu.

PB95-126215

GOLDSTEIN, R. E.

Nonlinear Dynamics of Stiff Polymers.

PB96. 122478

GOLMIE, $\mathbf{N}$.

NIST ATM Network Simulator: Operation and Programming, Version 1.0 .

PB96-106851

01,487

GOLOMB, G.

In vitro Inhibition of Membrane-Mediated Calcification by Novel Phosphonates.

PB96-201140

GOMAA, $\mathrm{H}$

03,595

Knowledge-Based Approach for Automating a Design Method for Concurrent and Real-Time Systems.

01,780

GOMMERS-AMPT, J.

beta-D-Glucosyl-Hydroxymethyluracil: A Novel Modified Base Present in the DNA of the Parastic Protozoan T.

brucel.

GONZALEZ, E. J.

03,524

Low temperature fabrication from nano-size ceramic pow ders

DE95013505

03,029

GONZALEZ, J. A

Center for Electronics and Electrical Engineering Technical Progress Bulletin Covering Center Programs, October 10 Progress Bulletin Covering Center Programs, October to December, with 1991 CEEE Events Calendar.
PB94-159787

Electronics and Electrical Engineering Laboratory Technical Progress Bulletin Covering Laboratory Programs, January to March 1991, with 1991 EEEL Events Calendar.

PB94-145968

02,113

Electronics and Electrical Engineering Laboratory Technical Progress Bulletin Covering Laboratory Programs, January to March 1992, with 1992/1993 EEEL Events Calendar. PB95-210480

01,917 GOODMAN, A. A.

Magnetic Fields in Star-Forming Regions: Observations. PB96. 123005

GOOMAN L J.

Needs for Brachytherapy Source Calibrations in the United States.

GOODRICH, L. F.

03,521

Anomalous Switching Phenomenon in Critical-Current Measurements When Using Conductive Mandrels.

PB96-13778
Comparing the Accuracy of Critical-Current Measurements Using the Voltage-Current Simulator.

02,227

Critical Current Behavior of Ag-Coated $\mathrm{YBa} 2 \mathrm{Cu} 3 \mathrm{O} 7-\mathrm{x}$ Thin Films.

PB95-141016

04,549

Critical Current Density, Irreversibility Line, and Flux Creep Activation Energy in Silver-Sheathed Bi2Sr2Ca2Cu2Ox Superconducting Tapes.

PB95-162749

04,616

First VAMAS USA Interlaboratory Comparison of High Temperature Superconductor Critical Current Measurements. $\begin{array}{ll}\text { PB96-147178 } & 04,768\end{array}$

High Current Pressure Contacts to Ag Pads on Thin Film Superconductors.

PB95-168621 04,639

II-3: Critical Current Measurement Methods: Ouantitative Evaluation.

04,767

11-5: Thermal Contraction of Materials Used in Nb3Sn Critical Current Measurements.

PB96-147186 04,769

Influence of Deposition Parameters on Properties of Laser Ablated YBa2Cu3O7-Delta Films.

PB95-140539

04,544

Magnetic Field Dependence of the Critical Current Anisotropy in Normal Metal-YBa2Cu307-delta Thin Film Bilayers. PB95-141024

Magnetic Flux Pinning in Eptaxial YBa2Cu3O7-delta Thin

PB96-200746 04,795

Magnetic Measurement of Transport Critical Current Density of Granular Superconductors.

PB95-126199

$n-V a l u e$ and Second Derivative of the Superconductor Volt-

age-Current Characteristic.
PB95-126223

04,533

Simple and Repeatable Technique for Measuring the Critical Current of Nb3Sn Wires.

PB96-119409 02,229

Standard Reference Devices for High Temperature Superconductor Critical Current Measurements.

PB95-175543

04,659

Superconductor Critical Current Standards for Fusion Applications. Final Progress Report, October 1993-July 1994.

PB95-169538 02,222

USA Interlaboratory Comparison of Superconductor Simula-

tor Critical Current Measurements

PB96-147194

04,770

V.6: Effects of Temperature Variation.

PB96-148143 04,772

VAMAS Intercomparison of Critical Current Measurements on NB3Sn Superconductors: A Summary Report. PB96-119763

04,043

GOODRICH, M. T. Point Probe Decision Trees for Geometric Concept Classes.
PB96-160817

01,612

OODWIN, A. R. H.

Database for the Static Dielectric Constant of Water and Steam.

01,103

PB96-145586

00,952

Dielectric Studies of Fluids with Reentrant Resonators.

Dielectric Studies of Fluids with Reentrant Resonators. Ap

Dielectric Studies of Fluids with Reentrant Resonators. Ap-
pendix B.
DE93019683
03,244

Ebulliometric Measurement of the Vapor Pressure of Difluoromethane.

00.931

Measurements of the Relative Permittivity of Liquid Water at Frequencies in the Range of 0.1 to $10 \mathrm{kHz}$ and at Temperatures between 273.1 and 373.2 $\mathrm{K}$ at Ambient Pressure.

PB96-119375

01,078

Static Dielectric Constant of Water and Steam.

01,090

PB96-123559
Vapor Pressure of 1,1-dichloro-2,2,2-trifluoroethane (R123).

PB95-126231

00,899

GOODWIN, T.

Crystal Structure and Magnetic Ordering of the Rare-Earth and $\mathrm{Cu}$ Moments in $\mathrm{RBa} 2 \mathrm{Cu} 2 \mathrm{NbO} 8(\mathrm{R}=\mathrm{Nd}, \mathrm{Pr})$.

PB95-140554

04,546

GOR'KOV, L. P.

From Superconductivity to Supernovae: The Ginzburg Symposium. Report on the Symposium Held in Honor of Vitaly L. Ginzburg. Held in Gaithersburg, Maryland on May 22,

PB95-171963

04,649

GORCZYCA, T. W.

Dielectronic Capture Processes in the Electron-Impact Ionization of $\mathrm{Sc}(2+)$
$\mathrm{PB} 95-203113$

GORDON, G. E.

04,000

Effects of Target Shape and Neutron Scattering on Element Sensitivities for Neutron-Capture Prompt Gamma-ray ActiSensitivities for $\mathrm{N}$ vation Analysis.

00,558

Trace Elements Associated with Proteins. Neutron Activa tion Analysis Combined with Biological Isolation Tech-

PB95-16310

00,597

GORE, J.

Estimate of Flame Radiance via a Single Location Measure.

PB94-211596

02,476

Measurement of Radiative Feedback to the Fuel Surface of a Pool Fire.

PB94-211604

02,477

ORE, J.P.

Development of Hazard Assessment and Suppression Technology for Oil and Gas Well Blowout and Diverter

PB96-122965

01,408

Investigation of Oil and Gas Well Fires and Flares. PB94-193976

Structure and Radiation Properties of Pool Fires.

PB94-193802

03,695

GOTT, J. E.

Analysis of High Bay Hangar Facilities for Detector Sensitivity and Placement.

PB96-190210
OTTSCHO, R. A

Gaseous Electronics Conference Radio-Frequency Reference Cell: A Defined Parallel-Plate Radio-Frequency System for Experimental and Theoretical Studies of PlasmaProcessing Discharges.

PB94-172327

04,404

GOUESBET, G.

Forward Scattering of a Gaussian Beam by a Nonabsorbing PB97-1122

04,395

Generalized Optical Theorem for On-Axis Gaussian Beams. PB97-122345

GOUGH, S. R.

CRYSTMET: The NRCC Metals Crystallographic Data File. PB97-109029 04.799

GOULD, $H$.

Long-Lived Structures in Fragile Glass-Forming Liquids. PB96-119565

GOULD, $P$.

Ultracold Collisions: Associative Ionization in a Laser Trap.
03,859 994-213238

Heterodyne Measurement of the Fluorescence Spectrum of Optical Molasses.

PB95-108411

03,873

Laser Modificatic

04,075

Localization of Atoms in a Three-Dimensional Standing Wave.

PB95. 163887

03,944

GOVINDAN, T. $R$.

One-Dimensional Modeling Studies of the Gaseous Elecronics Conference RF Reference Cell.

PB96-113428 
Contact Electrification Induced by Monolayer Modification of a Surface and Relation to Acid-Base Interactions. PB94-185378

GRACIA-SALCEDO, C

Reaction Sensitivities of Al-Li Alloys and Alloy 2219 in Mechanical-Impact Tests.

PB94-172764

03.314

GRADY, W. M.

Surging the Upside-Down House: Measurements and Modeling Results

PB96-180138

Workshop on Characterizing Diamond Films (4th). Held in Gaithersburg, Maryland on March 4-5, 1996. PB96-183090

04,786

GRAESSLEY, $w$. $w$.

Thermodynamic Interactions in Model Polyolefin Blends Obtained by Small-Angle Neutron Scattering.

01,208

GRAETTINGER, T. M.

Growth of Epitaxial KNbO3 Thin Films.

PB96-135181

GRAHAM, J. R.

Unravel: A CASE Tool to Assist Evaluation of High Integrity Software. Volume 1. Requirements and Design. 01,736 Unravel: A CASE Tool to Assist Evaluation of High Integrity Sottware. Volume 2. User Manual.

01,737

PB95-267894 Evaluation of the Electrochemical Behavior of Ductile Nickel
Aluminide and Nickel in a $\mathrm{pH} 7.9$ Solution. Technical Report Number 3, April-June 1991.

Number 3, A

03,307

GRANICK, S.

Topological Influences on Polymer Adsorption and Desorption Dynamics

PB94-212479

01,227

GRANTHAM, R. E.

Standard of Attenuation for Microwave Measurements.

AD-A297 905/2

01,517

GRANVEAUD, $M$.

Aging, Warm-Up Time and Retrace; Important Characteristics of Standard Frequency Generators.

PB96-103122

04,031

Preliminary Comparison of Time Transfers via LASSO, GPS and Two-Way Satellite.

PB95-151098

01,529

GRATIAS, D.

Neutron Scattering Structural Study of AlCuFe Quasicrystals Using Double Isotopic Substitution. RDFs and $\mathrm{Fe}-\mathrm{Fe}$ Pair Correlations in an AlCuFe Icosahedral Alloy by Double Isotopic Substitution.
PB94-172129

GRAU MALONDA, A. Standardization and Decay Scheme of Rhenium-186.
PB94-200490
03,830

03,830

GRAVES, D. B.

Gaseous Electronics Conference Radio-Frequency Reference Cell: A Defined Parallel-Plate Radio-Frequency System for Experimental and Theoretical Studies of PlasmaProcessing Discharges.
PB94-172327

GRAVES, R. S.

04,404

Comparison of Heat-Flow-Meter Tests from Four Labora-

PB95-126264

00,365

GRAY, B. J.

Electronic Implementors' Workshop.

PB95-210936

01,484

GRAY, D. H.

Review of the USCEANIST Measurement Assurance Program for the Nuclear Power Industry.

PB95-126272

03,712

Standardization and Decay Scheme of Rhenium-186.

PB94-200490

03,830

GRAY, M.

Fokker-Planck Description of Multivalent Interactions

PB95-108478

00,879

GRAY, M. M.

National Voluntary Laboratory Accreditation Program POSIX. Portable Operating System Interface.

Testers Open Dialogue at Inaugural NIST Workshop 02,661 PB95-175550

GRAY, S. K.

Quantum Dynamics of Renner-Teller Vibronic Coupling: The Predissociation $\mathrm{HCO}$

00.773

GRAYBEAL, J. M.

Effects of Anneal Time and Cooling Rate on the Formation and Texture of $\mathrm{Bi} 2 \mathrm{Sr} 2 \mathrm{CaCu} 2 \mathrm{O} 8$ Fitms.

04,603 Grain Alignment and Transport Properties of
Bi2Sr2CaCu2O8 Grown by Laser Heated Float Zone MethPB95-161451

04,602

GREEN, J. W.

Corrected Optical Pyrometer Readings

AD-A279 949/2

02,615

GREEN, M.

Crystal Structure and Magnetic Properties of $\mathrm{CuGeO}_{3}$

PB95-180287

04,678

GREEN, M. A.

Lattice Dynamics of $\mathrm{Ba} 1-\mathrm{xK} \times \mathrm{BiO}$

PB96-102421

04,706

Lattice Dynamics of Semiconducting. Metallic, and Superconducting Ba1-xKxBiO3 Studied by Inelastic Neutron Scattering.

B96-102447

Structure and Conductivity of Layered Oxides

Ba, Sr) $n+1(\mathrm{Sn}, \mathrm{Sb}) \mathrm{nO} 3 \mathrm{n}+1$

PB96-102439

GREEN, T.

Hierarchical Ada Robot Programming System (HARPS): A Complete and Working Telerobot Control System Based on

PB94-213162

GREENBAUM, A.

02,934

Laplace's Equation and the Dirichlet-Neumann Map in Multiply Connected

03,962

GREENBERG, K. E.

Gaseous Electronics Conference Radio-Frequency Reference Cell: A Defined Parallel-Plate Radio-Frequency System for Experimental and Theoretical Studies of PlasmaProcessing Discharges.

PB94-172327

04,404

Gaseous Electronics Conference RF Reference Cell: An Introduction.

02,387

Inductively Coupled Plasma Source for the Gaseous Electronics Conference RF Reference Cell.

PB96-113394

02,394

Optical Diagnostics in the Gaseous Electronics Conference Reference Cell.

GREENBERG, $\mathbf{N}$.

Frozen Human Serum Reference Material for Standardization of Sodium and Potassium Measurements in Serum or Plasma by Ion-Selective Electrode Analyzers.

PB94-185337

GREENBERG, $\mathbf{R}$.

00,532

Development of Frozen Whate Blubber and Liver Reterence Materials for the Measurement of Organic and Inorganic Contaminants

GREENBERG, R. R

00,587

Certification of Standard Reference Material (SRM) 1941a

Organics in Marine Sediment.

PB96- 123690

02,593

Concentrations of Chlorinated Hydrocarbons, Heavy Metals and Other Elements in Tissues Banked by the Alaska Marine Mammal Tissue Archival Project.

PB95-209870

02,590

Dissolution Problems with Botanical Reference Materials.

03.487

External Gamma-ray Counting of Selected Tissues from a Thorotrast Patient

PB96-160254

03,637

Measuring Hydrogen by Cold-Neutron Prompt-Gamma Activation Analysis.

00,612

Neutron Capture Prompt Gamma-Ray Activation Analysis a the NIST Cold Neutron Research Facility.

PB94-213394

00,556

New NIST Rapid Pneumatic Tube System.

PB96-167259

03,738

Trace Element Concentrations in Cetacean Liver Tissues Archived in the National Marine Mammal Tissue Bank.

PB96-167127

02,595

Use of Neutron Beams for Chemical Analysis at NIST.

PB97-112437

GREENE, C. H.

Oscillator Strengths and Radiative Branching Ratios in

Atomic Sr.

Short-Range Correlation and Relaxation Effects on the $(6 p(2))(1)$ SO Autoionizing State of Atomic Barium.

PB95-202289

03,973

, G. C.

Supermirror Transmission Polarizers for Neutrons.

PB94-216215

03,866

GREENE, G. L.

Elastic Deformation of a Monolithic Perfect Crystal Interferometer: Implications for Gravitational Phase Shift Experiments.

PB94-213154

03,858

Mass Assay and Uniformity Test of Boron Targets by Neu. tron Beam Methods

04,175

Measurement of the Neutron Lifetıme.

6-161708

Multi-Stage, Position Stabilized Vibration Isolation System for Neutron Interferometry.

PB95-175022

03,955

Some Aspects of Fundamental Neutron Physics.

95-126298

03,882

Laplace's Equation and the Dirichlet-Neumann Map in Multiply Connected Domains. (NIST Reprint).

PB95-180295

03,962

PENHALL, C. A.

Confidence on the Modified Allan Variance and the Time Pariance.

GREENSPAN, L.

Evaluation of a Tapered Roller Bearing Spindle for HighPrecision Hard Turning Applications.

PB96-160494

02,700

GREGORY, D. C.

Electron-Impact Excitation of Si(3+)(3S yields 3P) Using a Merged-Beam Electron-Energy-Loss Technique.

03,904

Merged-Beams Energy-Loss Technique for Electron-Ion Excitation: Absolute Total Cross Sections for $O(5+)(2$ s yields citation: Absolute Total Cross Sections for $\mathrm{O}(5+)$ (2s yields 2p).

GREHAN, G.

04,017

Elastic Scattering from Spheres under Non Plane-Wave lliumination.

Forward Scattering of a Gaussian Beam by a Nonabsorbing Sphere.

PB97-112288

04,395

Generalized Optical Theorem for On-Axis Gaussian Beams. B97-122345

GREY, C. P.

Neutron and Raman Spectroscopies of 134 and 134a Hydrofluorocar b

GRIBAKIN G. F.

Fine Structure of Negative Ions of Alkaline-Earth-Metal Atoms.

PB94-211182

03,837

GRIESMEYER, J. M.

Integrating Automated Systems with Modular Architecture. PB95-150231

GRIFFIN, D. C

Absolute Cross Sections for Electron-Impact Single Ioniza. fion of $\mathrm{Si}(+)$ and $\mathrm{Si}(2+)$

PB95-202529

03,982

Dielectronic Capture Processes in the Electron-Impact Ionization of $\mathrm{Sc}(2+)$

GRIFFIN, G. D.

04,000

Investigation of S2F10 Production and Mitigation in Compressed SF6-Insulated Power Systems.

Pres-212388

Investigation of S2F10 Production and Mitigation in Compressed SF6- Insulated Power Systems.

PB96-155528

GRIGEREIT, T. E.

Observation of Oscillatory Magnetic Order in the Antiferromagnetic Superconductor HoNi2B2C.

Vortex Dynamics and Melting in Niobium.
PB96- 112362 
Planar Near-Field Measurements of Low-Sidelobe AntenPBas.

02,001

GRINBERG, Y.

Uitrasonic Method for Reconstructing the Two-Dimensional Liquid-Solid Interface in Solidifying Bodies.

PB95-161782

03,349

GRISHCHUK, L. P.

Ouantum Mechanics of a Solid-State Bar Gravitational Antenna.

PB95-202628

03,987

GRISHIN, A. M.

Novel YBa2Cu3O7-x and YBa2Cu3O7-x/Y4Ba3O9 Multilayer Films by Bias-Masked 'On-Axis' Magnetron Sputtering.
PB95-181186

Pinch Effect in Commensurate Vortex-Pin Lattices.
PB96-147079
01,125

GRITZO, L.

Materials and Fire Threat.

PB97-122311

GRODKOWSKI, J.

One-Electron Oxidation of Metalloporphycenes as Studied by Radiolytic Methods.

PB97-111967

01,179

GRODSTEIN, G. W.

X-ray Attenuation Coefficients from $10 \mathrm{Kev}$ to $100 \mathrm{Mev}$.
AD-A278 $139 / 1$

$X$-Ray Attenuation Coefficients from $10 \mathrm{KEV}$ to $100 \mathrm{MEV}$.

AD-A280 290/8 03,768

GROH, R. J.

New Surface-Active Comonomer for Adhesive Bonding.
PB96-204052

PB96-204052

Feasibility and Design Considerations of Emergency Evacu-

ation by Elevators.

PB94-163441

00,287

Feasibility of Fire Evacuation by Elevators at FAA Control

Towers.

PB94-213857

04,844

Human Factors Considerations for the Potential Use of Elevators for Fire Evacuation of FAA Air Traffic Control Tow-

ers.

01,300

GROSS, J.

Performance of HUD-Affiliated Properties during the Janu-

ary 17, 1994 Northridge Earthquake.

GROSS, J. G.

Growing Significance of CIB.
PB95-126306

00,443

Standards Development in North America for Performance of Whole Buildings and Facilities.

PB94-185196

00,312

ROSS, J. L.

Ashland Tank Collapse Investigation.

02,481

Ashland Tank-Collapse Investigation: Closure by Authors.

PB95-126322 02,482

Extreme Wind Estimates by the Conditional Mean

Exceedance Procedure.
PB95-220471

00,120

Extreme Winds Estimation by 'Peaks Over Threshold' and

Epochal Methods.
PB96-159686

00,468

Modeling of Extreme Loading by 'Peaks Over Threshold'

PB96-159694 00,469

Survey of Steel Moment-Resisting Frame Buildings Affected by the 1994 Northridge Earthquake.

00,451

Workgroup Summary Report: Plastic Hinge-Based Techniques for Advanced Analysis.

PB96-159702

\section{GROSSER, M.}

Femtosecond Time-Resolved Molecular Multiphoton Ionization: The Na2 System

PB95-202305 03.975

Femtosecond Time-Resolved Wave Packet Motion in Molecular Multiphoton Ionization and Fragmentation.

PB94-198611

00,790

GROSSHANDLER, $w$

Acoustic Emission of Structural Materials Exposed to Open Flames.

PB95-164810

Agent Screening for Halon 1301 Aviation Replacement

PB96-159710 03,282

Assessing Halon Alternatives for Aircraft Engine Nacelle Fire Suppression.

01,401

Assessment of Technologies for Advanced Fire Detection.

PB95-126330 00,294

Early Detection of Foom Fires Through Acoustic Emission. (NIST Reprint).

00,298 Lean flammability limit as a fundamental refrigerant prop-
erty. Phase 1, Interim technical report, 1 October 1994--31 March 1995.

DE95011238

03,248

Protection of Data Processing Equipment with Fine Water Pprays.

02,610

Suppression Effectiveness of Extinguishing Agents under Highly Dynamic Conditions.

00,020

Suppression of Elevated Temperature Hydraulic Fluid and JP-8 Spray Flames.

PB95-181095

00,021

Suppression of High-Speed $\mathrm{C} 2 \mathrm{H} 4 /$ Air Flames with $\mathrm{C} 1$ Halocarbons.

03,287

Turbulent Spray Burner for Assessing Halon Alternative Fire Suppressants.

01,385

Validation of a Turbulent Spray Flame Facility for the Assessment of Halon Alternatives.

B 96 - 159728

03,283

GROSSHANDLER, W. L.

Effectiveness of Halon Alternatives in Suppressing Dynamic Combustion Process.

03,288

Evaluation of Alternative In-Flight Fire Suppressants for Evaluation of Alternative In-Flight Fire Suppressants for
Full-Scale Testing in Simulated Aircraft Engine Nacelles and Dry Bays.

B $94-203403$

00,023

Plow of Alternativ

00,022

Interaction of HFC-125, FC-218 and CF3l with High Speed Combustion Waves

PB96-176417

03,290

Minimum Mass Flux Requirements to Suppress Burning Surfaces with Water Sprays.

01,425

Proceedings of the 1995 Workshop on Fire Detector Research. Held on February 6-7, 1995.

PB95-270062

02,611

Review of Measurements and Candidate Signatures for Early Fire Detection

00,300

Solid Propellant Gas Generators: Proceedings of the 1995 Workshop. Held in Gaithersburg, Maryland on June 28-29,

PB96-131479

01,412

Suppression of High Speed Turbulent Flames in a Detonation/Deflagration Tube.

PB95-231817

01,395

GROSSMAN

High-Tc Superconducting Antenna-Coupled Microbolometer on Silicon.

GROSSMAN, E.

02,166

Metrology Issues in Terahertz Physics and Technology. GROSSMAN, E. N

$30 \mathrm{THz}$ Mixing Experiments on High Temperature Superconducting Josephson Junctions.

PB96-102462

04,709

Antenna-Coupled High-Tc Air-Bridge Microbolometer on Sil-

icon. 180899

04,315

Extended $\mathrm{CO}(7$ yields 6) Emission from Warm Gas in

Prion.

00,090

Far-Infrared Kinetic Inductance Detector.

PB95-126348

(0)

Heterodyne Mixing and Direct Detection in High Tempera. ture Josephson Junctions.

PB96-147202

01,565

High Temperature Superconductor-Normal MetalSuperconductor Josephson Junctions with High Characteristic Voltages.

PB95-176079

04,666

Microwave Noise in High-Tc Josephson Junctions.
PB96-148010

Niobium Microbolometers for Far-Infrared Detection.

PB96-111729

02,184

Optical Performance of Photoinductive Mixers at Terahertz Frequencies.

02,161

Partially Coherent Transmittance of Dielectric Lamellae.

PB96-148028

04,359

Superconducting Kinetic Inductance Radiometer.
PB95-140083

02,144
Terahertz Detectors Based on Superconducting Kinetic Inductance.

PB95-168647

02,160

Terahertz Shapiro Steps in High Temperature SNS Josephson Junctions
PB95-169140

GROSVENOR, J.

02,168

Applicability of Effective Medium Theory to Ferroelectric/

Ferrimagnetic Composites with Composition and Fre-

quency-Dependent Complex Permittivities and Permeabilities
PB96-157854

01,945

GROSVENOR, J. H.

Dielectric and Magnetic Measurements from $-50 \mathrm{C}$ to $200 \mathrm{C}$ and in the Frequency Band $50 \mathrm{MHz}$ to $2 \mathrm{GHz}$.

PB96-191382

02,245

Transmission/Reflection and Short-Circuit Line Methods for Measuring Permittivity and Permeability.

PB94-165537

02,211

GROTE, M. D.

Display-Measurement Round-Robin.

PB96-119227

02,186

Survey of the Components of Display-Measurement Stand-

PB96-122528

02,188

Survey of the Components of Display-Measurement Standards

GROTHER, P. J.

Binary Decision Clustering for Neural Network Based Optical Character Recognition.

cal Character

01,848

Binary Decision Clustering for Neural-Network-Based Opti-

cal Character Recognition.

PB96-186184

01,857

Comparison of FFT Fingerprint Filtering Methods for Neural

Network Classification.

PB95-136362 01,840

Generalized Form Registration Using Structure-Based Techniques.

PB96-191374

01,858

NIST Form-Based Handprint Recognition System.

PB94-217106

01,838

PCASYS: A Pattern-Level Classification Automation System

for Fingerprints.

01,853

Second Census Optical Character Recognition Systems Conference.

GRUBBS, W. T.

Bimolecular Interactions in (Et)3SiOH:Base: $\mathrm{CCl} 4$ Hydrogen-

Bonded Solutions Studied by Deactivation of the Free $\mathrm{OH}$ -

Stretch Vibration

PB97-118

04,166

Preliminary Comparison of Time Transfers via LASSO, GPS and Two-Way Satellite.

PB95-151098

01,529

GRUNINGER, S. E.

Procedure for the Study of Acidic Calcium Phosphate Precursor Phases in Enamel Mineral Formation.

PB95-164448

GRUSHKO, B.

Effect of Mn Content on the Microstructure of Al-Mn Alloys

Electrodeposited at 1500 .

PB95-126355

GRYCZYNSKI, $Z$.

Positive and Negative Cooperativities at Subsequent Steps of Oxygenation Regulate the Allosteric Behavior of Multistate Sebacylhemoglobin

PB97-119374

GUANG-QIU, T.

Wide Band Active Current Transformer and Shunt.

PB95-126371

GUEGUETCHKERI, $M$

New Method for the Detection and Measurement of Polyaromatic Carcinogens and Related Compounds by DNA Intercalation.

PB96-167382 
Source of Phenol Emissions Affecting the Indoor Air of an Office Building.
PB94.154382

03,600

GUERRIERI, J.

Planar Near-Field Measurements and Microwave Holog. raphy for Measuring Aperture Distribution on a $60 \mathrm{GHz} A \mathrm{C}$ tive Array Antenna.

PB96-167366

03,762

GUERRIERI, J. R.

New Extrapolation/Spherical/Cylindrical Measurement Facility at the National Institute of Standards and Technology PB95-153755

GUHA, S.

Optical Conductivity of Single Crystals of Ba1$x M \times B i O 3(M=K, R b, x=0.04,0.37)$.
PB94-185329 GUIBERTEAU,

Effect of Grain Size on Hertzian Contact Damage in Alu-

mina.

03,083

Indentation Fatigue: A Simple Cyclic Hertzian Test for
Measuring Damage Accumulation in Polycrystalline CeramMeasuring Damage Accumulation in Polycrystalline Ceram-
ics.

Model for Microcrack Initiation and Propagation beneath

Hertzian Contacts in Polycrystalline Ceramics.
PB96-163704

GUILLOT, $B$.

Dipole Moments in Rare Gas interactions

PB94-212982

GULDI, D. M.

00,844

One-Electron Oxidation of Metalloporphycenes as Studied by Radiolytic Methods.

01,179

\section{GULLIKSON, E. M.}

Design and Characterization of X-Ray Multılayer Analyzers for the $50-1000$ eV Region.

03,848

Stable Silicon Photodiodes for Absolute Intensity Measurements in the VUV and Soft X-ray Regions.

PB97-110175

04,135

GULWADI, S. M.

Range Statistics and Rutherford Backscattering Studies on Fe-Implanted In $0.53 \mathrm{GaO} .47 \mathrm{As}$

04,535

Transition Metal Implants in In0.53GAO 47 As.

PB95-126389

04,534

GUNARATNA, $P$.

Frozen Human Serum Reference Material for Standardization of Sodium and Potassium Measurements in Serum or Plasma by Ion-Selective Electrode Analyzers.

PB94-185337

00,532

GUNTHER, E.

Intercomparison of Internal Proportional Gas Counting of (85) $\mathrm{Kr}$ and (3) $\mathrm{H}$.

GUO, X. $\mathbf{Q}$.

03,800

Absolute Cross Sections for Electron-Impact Single Ionization of $\mathrm{Si}(+)$ and $\mathrm{Si}(2+)$

PB95-202529

03,982

Backscattering in Electron-Impact Excitation of Multiply Charged lons.

03,799

Evidence for Significant Backscatterıng in Near-Threshold Electron-Impact Excitation of $\mathrm{Ar}(7+)(3 \mathrm{~s}$ yields $3 p)$. PB95-126405 Merged-Beams Energy-Loss Technique for Electron-Ion Ex-
citation: Absolute Total Cross Sections for $O(5+)$ (2s yields citation: Absolute Total Cross Sections for $\mathrm{O}(5+)$ (2s yrelds 2p).

04,017

Resonance Structure and Absolute Cross Sections in NearThreshold Electron-Impact Excitation of the $4 \mathrm{~s}(2)$ (1)S yields $4 s 4 \mathrm{p}$ (3) P Intercombination Transition in $\mathrm{Kr}(6+)$

PB95-202271

03,972

GUO, Y. C. Critical Current Density, Irreversibility Line, and Flux Creep
Activation Energy in Silver-Sheathed Bi2Sr2Ca2Cu2Ox Superconducting Tapes.

PB95-162749

04,616

GUPTA, A.

New Method for the Detection and Measurement of Polyaromatic Carcinogens and Related Compounds by DNA Intercalation.

PB96-167382

03,481

GUPTA, A. K.

Analysis of Droplet Arrival Statistics in a Pressure-Atomized Spray Flame.

01,352

Combustion of Methanol and Methanol/Dodecanol Spray Flames.

PB95-108544

02,478

Effect of Dodecanol Content on the Combustion of Methanol Spray Flames

PB95-176020

01,389

Rote of Combustion on Droplet Transport in Pressure-AtomPB96-204433

01,434
Structure of a Swirl-Stabilized Kerosene Spray Flame.
PB95-108569 480 Study of Droplet Transport in Alcohol-Based Spray Flames Using Phase/Doppler Interferometry.

GUPTA, D.

02,479

Standard Reference Materials: Polystyrene Films for Calibrating the Wavelength Scale of Infrared PB95-226866

GUPTA, L. C.

Neutron Scattering Study of Antiferromagnetic Order in the Magnetic Superconductors RNi2B2C.

PB97-1124

GUPTA, R.

04,812

Laser Focused Atomic Deposition.

PB95-180659

04,685

Nanolabrication of a Two-Dimensional Array Using LaserFocused Atomic Deposition

PB96-119417

04,732

Nanostructure Fabrication via Laser-Focused Atomic Deposition (Invited)

04,132

Using Atom Optics to Fabricate Nanostructures.

GUREVICH A.

04,757

Crossover in the Pinning Mechanism of Anisotropic Fluxon Cores.
PB95-180170

\section{GURLEY, D.}

Extending the Angular Range of Neutron Reflectivity Measurements from Planar Lipid Bilayers: Applications to a Model Biological Membrane.

PB96-122569

03,476

GURNELL, A. W.

Hybrid Optical-Electrical Overlay Test Structure.
PB96-204136

GURVICH, L. V.

Ideal Gas Thermodynamic Properties of Sulphur Heterocyclic Compounds.

GUTHRIE, W.

Advances in the Measurement of Polymer CTE Micrometer- to Atomic-Scale Measurements.

PB96-180229

GUTHRIE, W. F.

Effects of Testing Variables on the Measured Compressive Strength of High-Strength (90 MPa) Concrete.
PB95-179040

Effects of Testing Variables on the Strength of HighStrength (90 Mpa) Concrete Cylinders.

PB96-112198

00,456 Hybrid Optical-Electrical Overlay Test Structure.
PB96-204136

02,450

Study on the Reuse of

PB96-122130

00,402

GUTMAN, D.

Kinetics of the Reaction of $\mathrm{CCl} 3-\mathrm{Br}-2$ and the Thermochemistry of CCl3 Radical and Cation.

PB94-212115

00,82

GUTMAN, G.

Influence of Electrical Isolation on the Structure and Reflectivity of Multilayer Coatings Deposited on Dielectric Sub-

New Method for Achieving Accurate Thickness Control for Uniform and Graded Multilayer Coatings on Large Flat Sub-

Strates.
PB96-159744

GUTTMAN, B.

Computer Security: An Introduction to Computer Security. The NIST Handbook

PB96-131610

01,608

Computer Security: Generally Accepted Principles and Practices for Securing Information Technology Systems.

PB97-110811

01,619

Security Program Management.
PB96-156112

GUTTMAN, C. M.

01,610

Certification of the Standard Reference Material 1473a, a Low Density Polyethylene Resin.

01,282

Determination of the Weight Average Molecular Weight of

Two Poly(Ethylene Oxides), SAM 1923 and SRM 1924.
PB94-217031

01,230

Peeling a Polymer from a Surface or from a Line.

PB94-199809

01,213

Performance of Plastic Packaging for Hazardous Materials Transportation. Part 1. Mechanical Properties.

02,580

Recertification of the Standard Reference Material 1475A, a Linear Polyethylene Resin.

02,628

GYGAX, F. N.

Dynamics of $\mathrm{Mu}(+)$ in $\mathrm{Sc}$ and $\mathrm{ScHx}$

04,112

GYORGY, E. M.

High Frequency Magnetic Field Sensors Based on the Far aday Effect in Garnet Thick Films.

02,282

Increased Pinning Energies and Critical Current Densities in Heavy-Ion-Irradiated Bi2Sr2CaCu2O8 Single Crystals. PB95-175352

\section{HAASS, $M$}

Improved Reflectometry Facility at the National Institute of Standards and Technology.

PB96-160338

04,087

New NIST/ARPA National Soft $X$-ray Reflectometry Facility. PB96-158092

HABRAM, $M$.

Hair Analysis for Drugs of Abuse: Evaluation of Analytica Methods, Environmental Issues, and Development of Reference Materials.
PB95-176269

HACK, K.

Extrapolation of the Heat Capacity in Liquid and Amorphous Phases.

PB97-11142

04,147

ABENBERER, L. B.

Effect of Stoichiometry on the Phases Present in Boron Nitride Thin Films

HACKLEY, V. A.

04,710

Effects of Soxhlet Extraction on the Surface Oxide Layer of Silicon Nitride Powders

PB95-175584

03,057

Electroacoustic Characterization of Particle Size and Zeta Potential in Moderately Concentrated Suspensions.

PB96-119425

01,079

Electrokinetic Sonic Analysis of Silicon Nitride Suspensions

PB96-123575 03,073

Polyelectrolytes as Dispersants in Colloidal Processing of Silicon Nitride Ceramics.

PB95-175568

03,055

Standard Reference Material for the Measurement of Par-

ticle Mobility by Electrophoretic Light Scattering.

PB96-102488

00,609

Surface Chemical Interactions of Si3N4 with Polyelectrolyte Deflocculants.
PB95-175576

03,056

Surface Chemistry of Silicon Nitride Powder in the Presence of Dissolved lons.

01,073

\section{HACKMAN, C.}

Confidence on

HADDAD, J. W.

Comparing the Accuracy of Critical-Current Measurements Using the Voltage-Current Simulator

HADDON R

Neutron-Scattering Study of $\mathrm{C} 60(n-)(n=3,6)$ Librations in $\mathrm{Al}$ kali-Metal Fullerides.

PB94-200219

00,806

HADJICHRISTIDIS, N.

Thermodynamic Interactions in Model Polyolefin Blends Obtained by Small-Angle Neutron Scattering.

01,208

HADZIIOANNOU, G.

Neutron Scattering by Multiblock Copolymers of Structure (A-B)N-A

B94-211547

01,219

HAEGEL, N. M.

Band-to-Band Photoluminescence and Luminescence Excitation in Extremely Heavily Carbon-Doped Epitaxial GaAs. PB95-150413

04,570 
Ion Broadening Parameters for Several Argon and Carbon

Lines.

03,843

HAILER, A. W.

Proteoglycan Inhibition of Calcium Phosphate Precipitation In Liposomal Suspensions.

PB94-211208

00,658

HAIR, D.

Dynamic Light-Scattering Study of a Diluted Polymer Blend Near Its Critical Point.

PB95-151890

01,245

HAIR, D. W.

Crossover to Strong Shear in a Low-Molecular-Weight Critical Polymer Blend

HAKIM, E. B.

01,222

Overview of U.S. Government Advanced Packaging Programs.

HALAMICKOVA, P.

02,443

Water Permeability and Chloride Ion Diffusion in Portland Cement Mortars: Relationship to Sand Content and Critical Pore Diameter

HALAS, N. J.

03,193

Epitaxial Integration of Single Crystal C60.

PB95-153490 04,592

Photoluminescence Spectra of Epitaxial Single Crystal C60.

PB96-141205 04,754

HALE, P. D.

Accurate Characterization of High Speed Photodectors.

Comparison of Photodiode Frequency Response Measurements to $40 \mathrm{GHz}$ between NPL and NIST.

PB96-111992 04,038

Fiber Laser Strobe.

PB95-176111 04,302

Metrology Applications of Mode-Locked Erbium Fiber La-

sers.

04,256

Optical Fiber Geometry: Accurate Measurement of Cladding Diameter.

PB95-151940

04.266

Photodetector Frequency Response Measurements at NIST, US, and NPL, UK: Preliminary Results of a Standards Laboratory Comparison.

02,175

Standard Polarization Components: Progress Toward an Optical Retardance Standard

PB96-119672

04,342

HALL, D. E.

Intelligent Processing of Materials, Technical Activities 1994 (NAS-NAC Assessment Panel, April 6-7, 1995)

PB96-115050

03,359

HALL, H. G.

Learning to Change: Opportunities to Improve the Performance of Smaller Manufacturers.

PB94-166212

00,010

HALL, J.

Atomic Beam Splitters and Mirrors by Adiabatic Passage in Multilevel Systems

HALL, J. L.

03,867

Delivering the Same Optical Frequency at Two Places: Accurate Cancellation of Phase Noise Introduced by an Optical Fiber or Other Time-Varying Path.

PB96-102736

04,332

Dreams About the Next Generation of Super-Stable Lasers.
PB94-211570

Frequency-Stabilized Lasers: A Driving Force for New

Spectroscopies.
PB96-135199

$0.4,350$

Frequency Stabilized Lasers: A Parochial Review.

PB95-153771

04,269

High-Resolution Optical Multiplex Spectroscopy.

04,323

Improved Hyperfine Measurements of the Na NP Excited State Through Frequency-Controlled Dopplerless Spectroscopy in a Zeeman Magneto-Optic Laser Trap.

PB95-203840

04,012

Introduction to Phase-Stable Optical Sources.

04,347

Low-Noise High-Speed Diode Laser Current Controller.

PB95-202826 02,178

Phase Shifts and Intensity Dependence in Frequency-Modulation Spectroscopy.

01,071

Stabilization of Optical Phase/Frequency of a Laser System: Application to a Commercial Dye Laser with an External Stabilizer.

PB95-203832

HALL, W.

04,327

Natıonal Voluntary Laboratory Accreditation Program: Energy Efficient Lighting Products.

02,642
HALL, W. A.

Efficiency of Electric Motors. National Voluntary Lab. Accreditation Program (NVLAP).

02,107

National Voluntary Laboratory Accreditation Program: Carpet and Carpet Cushion.

00,295

National Voluntary Laboratory Accreditation Program (NVLAP): Wood Based Products.

03,405

HALLER, $W$.

Analysis of SANS from Controlled Pore Glasses. PB94-198843

03,035

Real-Time Small-Angle X-Ray Scattering Study of the Early Stage of Phase Separation in the SiO2-BaO-K20 System.

HALLETT, $B$.

Comparison of Magnetic Fields of Thin-Film Heads and Their Corresponding Bit Patterns Using Magnetic Force Microscopy.

03,763

HALLIWELL, $B$.

Commentary: The Measurement of Oxidative Damage to DNA by HPLC and GC/MS Techniques.

03,484

HALLOCK, R. B.

$X$-Ray Powder Diffraction Data for $\mathrm{BaCu}(\mathrm{C} 2 \mathrm{O} 4) 2.6 \mathrm{H} 2 \mathrm{O}$.

PB95-151767 04,583

HALPERN, C.

Bibliography of Books and Published Reports on Gas Turbines, Jet Propulsion, and Rocket Power Plants. 01,445 Bibliography of Books and Published Reports on Gas Turbines, Jet Propulsion, and Rocket Power Plants, January 1950 through December 1953

AD-A278 213/4

01,446

\section{HAMANN, W. F.}

Self Calibrating Fiber Optic Sensors: Potential Design Methods

PB95-169306

02,173

HAMBRIGHT, P.

Metalloporphyrin Sensitized Photooxidation of Water to Oxygen on the Surface of Colloidal Iridium Oxides - Photochemical and Pulse Radiolytic Studies.

PB95-107082

00,868

One-Electron Oxidation of Nickel Porphyrins. Effect of Structure and Medium on Formation of Nickel(IIi) Porphyrin or Nickel(II) Porphyrın pi-Radical Cation.

PB95-107058

00,865

Site of One-Electron Reduction of Ni(II) Porphyrins. Forma. tion of Ni(I) Porphyrin of Ni(II) Porphyrin pi-Radical Anion. PB95-107066

00,866

HAMEL, J. F.

Supercritical Fluid Extraction of Biological Products.

PB95- 175204

00,040

HAMILTON, B. E

Learning to Change: Opportunities to Improve the Performance of Smaller Manufacturers.

PB94-166212

00,010

HAMILTON, C. A

$24 \mathrm{GHz}$ Josephson Array Voltage Standard.

PB94-211588

02,033

Automated Josephson Integrated Circuit Test System.

Experimental Results on Single Flux Ouantum Logic.

PB95-17507

02,057

Josephson D/A 02,053 PB96-148044

Josephson Voltage Standard Based on Single-Flux-Ouantum Voltage Multiplier

PB95-175600

02,058

Performance and Reliability of NIST $10-\mathrm{V}$ Josephson Arrays.

02,419

Pulse-Driven Programmable Josephson Voltage Standard. PB97-111496

04,148

Voltage-Standard Devices.

PB96-102496

01,920

HAMINS, A.

Agent Screening for Halon 1301 Aviation Replacement.

03,282 Combustion of a Polymer (PMMA) Sphere in Microgravity.
N96-15569/2 N96-15569/2 01,354 Estimate of Flame Radiance via a Single Location Measure PB94-211596

02,476

Estimate of the Effect of Scale on Radiative Heat LosS Fraction and Combustion Efficiency. PB95-150447

02,486

Measurement of Radiative Feedback to the Fuel Surface of a Pool Fire.

02,477

Simultaneous Optıcal Measurement of Soot Volume Fraction, Temperature, and $\mathrm{CO} 2$ in Heptane Pool Fire.

PB96-102132

01,397

Suppression of Ignition Over a Heated Metal Surface. PB96-176425

AMMER, L. H.

Display-Measurement Round-Robin

PB96-119227

02,186

HAMMOND, B.

Second Census Optical Character Recognition Systems Conference.

PB94-188711

01,832

AMSTAD, M. A.

High-Sensitivity Acoustic Emission Sensor/Preamplifier Subsystems.
PB95-125704

02,900

HAN, B.

Suitability of Metalorganic Chemical Vapor Deposition-De-
rived PrGaO3 Films as Buffer Layers for YBa2Cu3O7-x Pulsed Laser Deposition.

PB95-168670

04,640

HAN, C.

Dynamic Light-Scattering Study of a Diluted Polymer Blend Near Its Critical Point.

PB95-151890

01,245

Molecular Weight Dependence of the Lamellar Domain Spacing of ABC Triblock Copolymers and Their Chain Conformation in Lamellar Domains.

01,254

Relaxation After a Temperature Jump Within the One

Phase Region of a Polymer Mixture.

PB97-112494

03.394

HAN, C. C.

Anisotropic Phase Separation Kinetics in a Polymer Blend Solution Following Cessation of Shear Studied by Light Scattering.

PB95.151247

Compatibilization of Polymer Blends by Complexation. 2. Kinetics of Interfacial Mixing.

01,295

Crossover to Strong Shear in a Low-Molecular-Weight Critical Polymer Blend.

Dielectric Behavior of a Polycarbonate/Polyester Mi 'ure Upon Transesterification

04,785

Dimensional Crossover in the Phase Separation Kinetics of Thin Polymer Blend Films.

03,395

Effect of Cross-Links on the Miscibility of a Deuterated Polybutadiene and Protonated Polybutadiene Blend

PB94-212438

01,225

Flow-Induced Structure in Polymers: Chapter 16. Shear-In(he ture and the Morphology of a Triblock Copolymer.

ture and the

03,127

Flow-Induced Structure in Polymers. Chapter 17. PhaseSeparation Kinetics of a Polymer Blend Solution Studied by a Two-Step Shear Ouench.

PB96-123377

03,388

Influence of Shear on the Ordering Temperature of a

Triblock Copolymer Melt.

01,288

Inversion of the Phase Diagram from UCST to LCST in

Deuterated Polybutadiene and Protonated Polybutadiene

Blends.

PB94-212446

Lattice Model of a Hydrogen-Bonded Polymer Blend.

PB97-112262 
Preparation of 2-Dimensional Ultra Thin Polystyrene Film by Water Casting Method.

04,619

SANS Studies of Space-Time Organization of Structure in Polymer Blends.

01,251

Shear Dependence of Critical Fluctuations in Binary Poly-

Mer Mixtures by Small Angle Neutron Scattering.
PB94-211612

01,220

Shear-Excited Morphological States in a Triblock CopolyPB94-172392

01,196

Shear-Induced Changes in the Order-Disorder Transition Temperature and the Morphology of a Triblock Copolymer.
PB97-1 18772

Shear-Induced Martensitic-Like Transformation in a Block Copolymer Melt.

Copolymer Melt.

01,277

Shear-Induced Mixing in Polymer Blends

01,287

Shear Suppression of Critical Fluctuations in a Diluted Polymer Blend.

04,418

Slow Dynamics of Segregation in Hydrogen-Bonded Polymer Blends.
PB96-12359

01,281

Small Angle Neutron Scattering Study on Poly(N-Isopropyl Acrylamide) Gels Near Their Volume-Phase Transition Temperature.

01,263

Small-Angle Neutron Scattering Study on Weakly Charged Temperature Sensitive Polymer Gels.

01,264

Small-Angle $X$-Ray and Neutron Scattering Study of Block Copolymer/Homopolymer Mixtures

01,221

Structural Stabilization of Phase Separating PC/Polyester Blends through Interfacial Modification by Transesterification PBeaction.

01,239

Thermodynamic Interactions and Correlations in Mixtures of Two Homopolymers and a Block Copolymers by Small Angle Neutron Scattering
PB95-152872

01,247

Thermodynamic Interactions in Model Polyolefin Blends Obtained by Small-Angle Neutron Scattering.

01,208

Time Dependent Small Angle Neutron Scattering Behavior in Triblock Copolymers Under Steady Shear.

01,198

Time-Resolved Small-Angle Neutron Scattering Study of Spinodal Decomposition in Deuterated and Protonated Polybutadiene Blends. 1. Effect of Initial Thermal Fluctuations.

HANCOCK, D. K.

01,252

L-threo-beta-Hydroxyhistidine, an Unprecedented Iron(III) lon-Binding Amino Acid in a Pyoverdine-type Siderophore from Pseudomonas fluorescens 244

PB94-211620

00.553

ANDEL, P. H.

New Model of $1 / F$ Noise in Baw Ouartz Resonators.
PB96-112248 HANDWERKER, $\mathrm{C}$

Energy and Migration of Grain Boundaries in Polycrystals.
PB94-211638

Fabrication of Platinum-Gold Alloys in Pre-Hispanic South America: Issues of Temperature and Microstructure Control. America: Issues of Temperature and Microstructure Control.
PB94-211646
03,333 HANDWERKER, C. A.

Determination of the Prior-Austenitic Grain Size of Selected Steels Using a Molten Glass Etch

Electronics Packaging Materials Research at NIST.
PB96-122692 02,405
Stability and Surface Energies of Wetted Grain Boundaries in Aluminum Oxide.

PB95-202750

03,059

Study of Diffusion Zones with Electron Microprobe Compositional Mapping
PB94-216348

00,559

Texture Measurement of Sintered Alumina Using the March-Dollase Function.

PB96-179494

04,784

HANIQUE, E.

Approach to Setting Performance Requirements for Automated Evaluation of the Parameters of High-Voltage $1 \mathrm{~m}$ pulses.

HANLEY, H. J. M.

01,878

Conditions for Existence of a Reentrant Solid Phase in a Sheared Atomic Fluid.

04,198

Contrast Matched Studies of a Sheared Binary Colloidal Suspension,
PB95-150561

00,918

Density Dependence of Fluid Properties and Non-Newtonian Flows: The Weisenberg Effect.

01,140
Dynamic Scaling in an Aggregating 2D Lennard-Jones SysPB96-167317

04,106

Partial Scattered Intensities from a Binary Suspension of Polystyrene and Silica.

00,996

Shear-Induced Melting of Two-Dimensional Solids.

PB96-112057

01,075

Simulation and SANS Studies of Gelation Under Shear

PB96-167176 01,150

Small-Angle Neutron Scattering (SANS) Study of WormLike Micelles Under Shear.

PB96-176698 04,111

Small Angle Neutron Scattering Study of a Clay Suspension

Under Shear.
PB96-167374

Small-Angle Neutron-Scattering Study of Dense Sheared

PB96-167184

01,151

Structure and Rheology of Hard-Sphere Systems. PB96-167333

00,662

HANLEY, J. M.

Non-Newtonian Flow between Concentric Cylinders Calculated from Thermophysical Properties Obtained from Simulations.

PB96-163761

04,103

HANSEN, B. N.

High-Pressure Equilibrium Cell for Solubility Measurements in Supercritical Fluids.

Refractive Indices of Fluids Related to Alternative Refrigerants.

PB94-219375

03,260

Solubilities of Copper(II) and Chromium(III) betaDiketonates in Supercritical Carbon Dioxide.

Solubility Measurement by Direct Injection of SupercriticalFluid Solutions into a HPLC System.

PB95-175626

00,997

HANSEN, D.

Dynamic Scaling in an Aggregating 2D Lennard-Jones Sys-

tem.

04,106

Simulation and SANS Studies of Gelation Under Shear

PB96-167176

01,150

HANSEN, H. $J$.

Comparison of Finite Element and Analytic Calculations of the Resonant Modes and Frequencies of a Thick Shel Sphere

02,626

HANSFORD, $W$

Realizing Suspended Structures on Chips Fabricated by PB94-193984

01,881

HANSMA, P. K

Scanning Tunneling Microscopy and Fabrication of Nanometer Scale Structures at the Liquid-Gold Interface.
PB95-140414

HANSON, A. L.

Polarization Effects on Multiple Scattering Gamma Trans. port.

HANSON, D. W.

Satellite Two-Way Time Transfer: Fundamentals and Recent Progress
PB95-161089

HANSON, $W$.

01,536

Precision Oscillators: Dependence of Frequency on Temperature, Humidity and Pressure.

PB94-198306

02,031

Preliminary Comparison of Time Transfers via LASSO, GPS and Two-Way Satellite

HANSSEN, L. M.

Development of Neutral-Density Infrared Filters Using Metallic Thin Films

PB96-180286

Standard Reference Materials: Polystyrene Films for Calibrating the Wavelength Scale of Infrared Spectrophotometers - SRM 1921.

$\mathrm{HAO}, \mathrm{H}$.

Electric Field Effects on a Near-Critical Fluid in Microgravity.

HAPIOT, $P$.

Changes in the Redox State of Iridium Oxide Clusters and Their Relation to Catalytic Water Oxidation: Radiolytic and Electrochemical Studies.

PB95-107017

00,864

Oxidation of Caffeic Acid and Related Hydroxycinnamic Acids.

P97-111975

00,651

Oxidation of 10-Methylacridan, a Synthetic Analogue of $\mathrm{NADH}$ and Deprotonation of Its Cation Radical. Convergen Application of Laser Flash Photolysis and Direct and Redox

Catalyzed Electrochemistry to the Kinetics of Deprotonation of the Cation Radical.

PB94-198371

00,785

ARARY, $\mathrm{H}$

Integration of Scanning Tunneling Microscope Nanolithography and Electronics Device Processing.
PB95-153359 PB95-153359 02,34 served by Scanning Tunneling Microscopy.

PB95-107389

01,232

RARY, H. H.

Scanning Tunneling Microscopy and Fabrication of Nanometer Scale Structures at the Liquid-Gold Interface. PB95-14041

U.S. Navy Coordinate Measuring Machines: A Study of Needs.

994-162831

02,807

HARCHENKO, C. D.

Results of a NISTNNIIOFI Comparison of Spectral-Radiance Measurements.

PB97-113021

HARDING, L. B.

04,159

Ouantum Dynamics of Renner-Teller Vibronic Coupling: The Predissociation $\mathrm{HCO}$

HARDIS, J. E.

Detector-Based Candela Scale and Related Photometric Calibration Procedures at NIST.

PB95-161949

04,273

High Resolution Angle Resolved Photoelectron SpectrosCopy Study of N2.

03,907

Improving Color Measurements of Displays

02,203

National Institute of Standards and Technology High-Accuracy Cryogenic Radiometer.

PB96-179585

04,378

NIST Detector-Based Luminous Intensity Scale

PB96-179114

01,864

Vibrationally Resolved Photoelectron Angular Distributions and Branching Ratios for the Carbon Dioxide Molecule in

the Wavelength Region 685-795 Angstrom.
PB96-201207

04,131

Vibronic Coupling and Other Many-Body Effects in the 4 sigmag(-1) Photoionization Channel of $\mathrm{CO} 2$.

PB95-162509

00,962

HARDY, S. C.

Metallurgy Technical Activities 1994 (NAS-NRC Assessment Panel, April 6-7, 1995)

PB96-136981

Metallurgy. Technical Activities, 1995

HAREWOOD, P. M.

02,981

Estimation of the Absorbed Dose in Radiation-Processed Food. 4. EPR Measurements on Eggshell.

PB94-199692

HARGIS, P. J.

00,038

Gaseous Electronics Conference Radio-Frequency Reference Cell: A Defined Parallel-Plate Radio-Frequency Sys tem for Experimental and Theoretical Studies of PlasmaProcessing Discharges.

Processing Disch

HARLOW, R. L.

Troublesome Crystal Structures: Prevention, Detection, and Resolution.

HARMAN, D.

Lab Report Special Section: Natural Language Processing and Information Retrieval Group Information Access an User Interfaces Division, National Institute of Standards and Technology.

02,742

Panel: Building and Using Test Collections.

PB97-118673

02,743

HARMAN, D. K.

Bringing Natural Language Information Retrieval Out of the

Closet.
PB94-172335

02,720

Overview of the Text REtrieval Conference (3rd) (TREC-3)

Held in Gaithersburg, Maryland on November 2-4, 1994. 
Wire Bonding to Multichip Modules and Other Soft SubStrates.

96-135207

02,082

HARMOUCHE, M. R

Dynamic Shear Modulus Measurements with Four Independent Techniques in Nickel-Based Alloys.

PB94-198900

HARNE, D.

Fracture Behavior of Large-Scale Thin-Sheet Aluminum Alloy.

03,311

HARNE, D. E.

Fracture Testing of Large-Scale Thın-Sheet Aluminum Alloy.

Fracture Testing of Large-Scale Thin-Sheet Aluminum Alloy.
PB95-242368

PB5-242368

Effects of Spindle Dynamic Characteristics on Hard Turning. PB96-122981 02,699

Evaluation of a Tapered Roller Bearing Spindle for HighPrecision Hard Turning Applications.

PB96-160494

02,700

HARRIMAN, A.

Metalloporphyrin Sensitized Photooxidation of Water to Oxygen on the Surtace of Colloidal Iridium Oxides - Photochemical and Pulse Radiolytic Studies.

00,868

Radiation Chemistry of Cyanine Dyes: Oxidation and ReRuction of Merocyanine 540 .
PB94-211661 00.818

Solution

Reduction of Dinitrogen to Ammonia in Aqueous Solution
Mediated by Colloidal Metals. PB95-107074

Site of One-Electron Reduction of Ni(II) Porphyrins. Formation of Ni(I) Porphyrin of Ni(II) Porphyrin pi-Radical Anion.
PB95-107066 00,866 HARRIMAN, P. Changes in the Redox State of Iridium Oxide Clusters and
Their Relation to Catalytic Water OxIdation: Radiolytic and Electrochemical Studies.

00,864

HARRINGTON, J. E.

Greatly Enhanced Soot Scattering in Flickering Ch4/Air Diffusion Flames.
PB94.172988

Laser Imaging of Chemistry-Flowfield Interactions: Enhanced Soot Formation in Time-Varying Diffusion Flames.
PB94-185352

Laser-Induced Fluorescence Measurements of Formaldehyde in a Methane/Air Diffusion Flame.

PB94-211679

01,374

Measurement of CO Pressures in the Ultrahigh Vacuum Regime Using Resonance-Enhanced Multiphoton-Ionization Time-of-Flight Mass Spectroscopy

PB94-216041

03,864

Ouantitative Measurements of Enhanced Soot Production in a Flickering Methane/Air Diftusion Flame.

PB95-203246

01,393

ARRIS, D. R

Journal of Research of the National Institute of Standards and Technology. March/April 1994. Volume 99, Number 2.

Journal of Research of the National Institute of Standards and Technology. May/June 1994. Volume 99, Number 3.

PB94-219326

02,643

Journal of Research of the National Institute of Standards and Technology, September-October 1993. Volume 98 , Number 5.

PB96-134954

03,362

HARRIS, G. L.

Advanced Mass Calibration and Measurement Assurance Program for State Calibration Laboratories.

PB95-253571

02,492

Ensurıng Accuracy and Traceabulity of Weighing Instruments.

PB94-211687
Evaluation and Accreditation of State Calibration Labora-

PB97-110183

00,486

Specifications and Tolerances for Reference Standards and Field Standard Weights and Measures. 2. Specifications and Tolerances for Fleld Standard Measuring Flasks. 6 - 178926

02,682

State Weights and Measures Laboratories: Program HandPB96-214705

02,687

State Weights and Measures Laboratories: State Standards Program Description and Directory. 1994 Edition.

PB94-207727
HARRIS, J. R.

02,895

De Facto Microzonation through the Use of Soils Factors in Design Triggers.

HARRIS, R. H.

00,462

New Approach for Reducing the Toxicity of the Combustion Products from Flexible Polyurethane Foam

01,411
Ouantifying the Ignition Propensity of Cigarettes. PB96-155411

00,306

HARRISON, J. C

Test of Newton's Inverse Square Law of Gravitation Using the 300-m Tower at Erie, Colorado.

03,978

Validation of the Inverse Square Law of Gravitation Using the Tower at Erie, Colorado, USA

PB95-164646

HARRISON, T. R.

03,947

Technologic Papers of the Bureau of Standards: Number 170. Pyrometric Practice.

AD-A279 282/8

03,766

HARRISON, W. T. A.

Crystal Structure of a New Sodium Zinc Arsenate Phase Solved by 'Simulated Annealing'. PB95-107124

00,870

Structural and Chemical Investigations of Na3(ABO4)3.4H2O-Type Sodalite Phases. PB95-180733

01,012

Tetrahedral-Framework Lithium Zinc Phosphate Phases: Location of Light-Atom Positions in LiZnPO4 H2O by Powder Neutron Diffraction and Structure Determination of LiZnPO4 by ab Initio Methods.

PB96-160510

01,129

HART, R. C.

Failures of the Four-Wave Mixing Model for Cone Emission.

HARTMAN, A. W.

Geometric Characterization of Rockwell Diamond Indenters.
PB95-203287
02,950

Microform Calibrations in Surface Metrology.

02,951

Particle Size Standards and Their Certification at NIST

PB94-211695

HARTSON, H. R.

Usability Engineering: Industry-Government Collaboration for System Effectiveness and Efficiency.

PB97-122287

HARVATH, J. J.

In situ Fluorescence Cell Mass Measurements of

'Saccharomyces cerevisiae' Using Cellular Tryptophan.

03,547

HARVEY, A. H.

Standard States, Reference States and Finite-Concentration Effects in Near-Critical Mixtures with Applications to Aqueous Solutions.

PB95-164349.

00,979

Supercritical Solubility of Solids from Near-Critical DiluteMixture Theory.
PB94-211703

00,819

\section{HARVEY, T.}

High Critical Temperature Superconductor Tunneling Spectroscopy Using Squeezable Electron Tunneling Junctions.

HARVEY, T. E.

Critical Current and Normal Resistance of High-Tc StepEdge SNS Junctions.

04,724

Critical Current Behavior of Ag-Coated YBazCu3O7-x Thin

PBilms. 141016

04,549

Ferroelectric Thin Film Characterization Using Superconducting Microstrip Resonators.
PB96-102389 02,270
High-Tc Multilayer Step-Edge Josephson Junctions and SOUIDs.

PB96.200183

04,790

Mutual Phase Locking in Systems of High-Tc Superconductor-Normal Metal-Superconductor Junctions.
04,744

Phase Locking in Two-Junction Systems of High-Temperature Superconductor-Normal Metal-Superconductor Junctions.

PB95-176053 Superconductor/Semiconductor Integration.
PB94-211711

\section{HASCALL, V. C.}

Proteoglycan Inhibition of Calcium Phosphate Precipitation in Liposomal Suspensions.

PB94-211208

00,658

Eftect of Cross-Links on the Miscibility of a Deuterated Polybutadiene and Protonated Polybutadiene Blend. PB94-212438

01,225

Inversion of the Phase Diagram from UCST to LCST in Deuterated Polybutadiene and Protonated Polybutadiene Blends.

PB94-212446

01,226

SANS Studies of Space-Time Organization of Structure in Polymer Blends.

Small-Angle X-Ray and Neutron Scattering Study of Block Copolymer/Homopolymer Mixtures.

PB94-211729

01,221

Time-Resolved Small-Angle Neutron Scattering Study of Polybutadiene Bends 1. Ettect of Inirial Thermal Fluctua.

PB95-161196

01,252

HASHIMOTO, T

Effect of Cross-Links on the Miscibility of a Deuterated Polybutadiene and Protonated Polybutadiene Blend.

PB94-212438

01,225

Inversion of the Phase Diagram from UCST to LCST in Deuterated Polybutadiene and Protonated Polybutadiene

PB94-212446

01,226

SANS Studies of Space-Time Organization of Structure in Polymer Blends.

01,251

Small-Angle X-Ray and Neutron Scattering Study of Block Copolymer/Homopolymer Mixtures.

01,221

Time-Resolved Small-Angle Neutron Scattering Study of Spinodal Decomposition in Deuterated and Protonated Polybutadiene Blends. 1. Effect of Initial Thermal FluctuaPB95-161196

01,252

HASTIE, J. W.

High Temperature.

PB94-211737

03,844

Recent Experimental and Modeling Developments in High Temperature Thermochemistry.

00,759

Vapor Transport in Materials and Process Chemistry. PB94-211745

HATAT, J. L.

Preliminary Comparison of Time Transfers via LASSO, GPS and Two-Way Satellite.

PB95-151098

01,529

HATCH, C. R.

Network Brokers Handbook: An Entrepreneurial Guide to Cooperative Strategies for Manufacturing Competitiveness.

PB95-219325

HATFIELD, M. O.

Band-Limited, White Gaussian Noise Excitation for Reverberation Chambers and Applications to Radiated Susceptibility Testing.

HATTORI, K.

01,960

Direct Dispersion Measurement of Highly-Erbium-Doped

Optical Amplifiers Using a Low Coherence Reflectometer

Coupled with Dispersive Fourier Spectroscopy.

PB95-150702

04,263

HAUSNER, $\mathrm{H}$.

Analysis of Physical Properties of Ceramic Powders in an International Interlaboratory Comparison Program.

PB95-161501

03,050

HAVES, $P$.

Reproducibility of Tests on Energy Management and Control Systems Using Building Emulators.

00,260

Use of Building Emulators to Evaluate the Performance of Building Energy Management Systems.

PB96-111901

00,269

HAYDEN, J. S.

Glasses for Waveguide Lasers.

PB96-111950

04,335

Integrated Optic Laser Emitting at 905, 1057, $1356 \mathrm{~nm}$. PB94-216298 02,136 Integrated Opt
PB94-216280

02,135 
Vibrationally Resolved Photoelectron Angular Distributions and Branching Ratios for the Carbon Dioxide Molecule in the Wavelength Region 685-795 Angstrom.

PB96-201207

04,131

HAYES, $P$.

Superconducting Resonator and a Cryogenic GaAs Field Effect Transistor Amplifier as a Single-Ion Detection Sys-

PB95-202727

03,990

HAYNES, G. A.

Flame Heights and Heat Release Rates of 1991 Kuwalt Oi Field Fires.

PB96-119342

01,404

HAYNES, W. M.

Isochoric ( $p \cdot p \cdot T)$ Measurements on Liquid and Gaseous Air from 67 to $400 \mathrm{~K}$ at Pressures to $35 \mathrm{MPa}$

PB96-167390

01,154

New Data and Correlations for the Custody Transfer of Natural Gas Liquids.

02,499

Reference Data for the Thermophysical Properties of Cryo-

genic Fluids.
PB95-168688

Thermophysical Properties of Fluids for the Gas Industry.

Thermophysical Properties of Fluids for the Gas Industry. Final Report. February 1, 1988-August 31, 1993.

Thermophysical properties of HCFC alternatives. Ouarterly report, April 1--June 30, 1995

DE96010579

03,250

Thermophysical properties of HCFC alternatives. Ouarterly report, October 1--December 31, 1995

03,249

Thermophysical properties of HCFC alternatives. Ouarterly report, 1 July 1994--30 September 1994.

Thermophysical Properties of HFC-143a and HFC-152a Ouarterly Report, 1 July 1993--30 September 1993 DE94004236

$0.3,245$

HAYS, M. J.

Certification of Polycyclic Aromatic Hydrocarbons in a Marine Sediment Standard Reference Material.

02,592

Certification of Standard Reference Material (SRM) 1941a, Organics in Marine Sediment

PB96-123690

02,593

HAYWARD, E.

Drill-Hearn-Gerasimov Sum Rule.

PB94-211752

03,845

Polarizability of the Nucleon

PB94-211760

03,846

HAZEN, D. A.

Dual-Frequency Millimeter-Wave Radiometer Antenna for Airborne Remote Sensing of Atmosphere and Ocean.
PB96-112289

Dual Frequency mm-Wave Radiometer Antenna for Airborne Remote Sensing of Atmosphere and Ocean.
PB95-180378

02,006

Effect of Microstructure on the Wear Transition of Zirconia.

Toughened Alumina.
PB94-211778

$\begin{array}{rr}\text { PB94-211778 } & 03,141 \\ \text { Wear Transitions in Monolithic Alumina and Zirconia-Alu- }\end{array}$

Wear Transitions in Monolithic Alumina and Zirconia-Alu-
mina Composites.
PB96-103163
03,168

HE, Y. Z.

Rate Constants for Hydrogem Atom Attack on Some Chlorinated Benzenes at High Temperature.

PB94-200581

00,810

HEALD, A. L.

Learning to Change: Opportunities to Improve the Performance of Smaller Manufacturers

HEALD, P. R.

00,010

Droplet Transfer Modes for a MIL 100S-1 GMAW Electrode.

PB95-209300 02,867
Mapping

Mapping the Droplet Transfer Modes for an ER100S-1 GMAW Electrode.

PB96-190095

03,295 Goddard High-Resolution Spectrograph Observations of the
Local Interstellar Medium and the Deuterium/Hydrogen Ratio along the Line of Sight Toward Capella.

00,066

Observations of 3C 273 with the Goddard High Resolution Spectrograph on the Hubble Space Telescope.

PB95-202321

00.076

HEARING, E. D.

Estimation of the Thermodynamic Properties of C-H-N-O-SHalogen Compounds at $298.15 \mathrm{~K}$

PB94-162328

00,744

HEATHER, $R$

Hyperfine Effects and Associative Ionization of Uliracold Sodium.

03,903
Laser Assisted Collisions at Ultracold Temperatures. PB95-161220

03,929

Theory of Atomic Collisions at Ultracold Temperatures.

PB94-212560

HEATHER, R. W.

Asymptotic Wave Function Splitting Procedure for PropagatSpatially Extended Wave Functions: Application to In ing Spatially Extended Wave Function

PB94-211786

HEATON, H. T.

Role of the Office of Radiation Measurement in Ouality As-

PB94-212255

HEAVNER, T.

Superconducting Resonator and a Cryogenic GaAs FieldEffect Transistor Amplifier as a Single-Ion Detection SysPB95-202727

HEBNER, G. A.

Gaseous Electronics Conference Radio-Frequency Reference Cell: A Defined Parallel.Plate Radio-Frequency System for Experimental and Theoretical Studies of PlasmaProcessing Discharges.

PB94-172327

04,404

Inductively Coupled Plasma Source for the Gaseous Electronics Conterence RF Reference Cel

02,394

Optical Diagnostics in the Gaseous Electronics Conference Reference Cell.

PB96-113352

02,390

HEBNER, $R$.

Transfer of Technology from Defense to Civilian Sectors.
PB94.185360

00,01

HEBNER, R. E.

Directions in MEMS Research Application Development.

Metrology Requirements of Future Space Power Systems.

PB95-140984

HECKERT, N. A.

Assessment of 'Peaks Over Threshold' Methods for Estimating Extreme Value Distribution Tails.

PB95-161360

00,441

Estimates of Hurricane Wind Speeds by the 'Peaks Ove Threshold' Method.

PB96-162540

00,471

Extreme Wind Distribution Talls: A 'Peaks Over Threshold' Approach

Extreme Wind Estimates by the Conditional Mean Exceedance Procedure.

PB95-220471

00,120

Extreme Winds Estimation by 'Peaks Over Threshold' and Epochal Methods.

00,468

Graphical Analysis of the CCRL Portland Cement Proficiency Sample Database (Samples 1-72). (Part 1. Univariant Analysis of Portland Cement)

PB94-196557

01,308

Modeling of Extreme Loading by 'Peaks Over Threshold' Methods.

PB96-159694

HEFNER, A. R

Electro-Thermal Simulation of an IGBT PWM Inverter.

PB94-185592

Investigation of the Power Insulated Gate Bipolar Transistor (IGBT).

PB94-211794

Modeling Buffer Layer IGBT's for Circuit Simulation

PB96-164173

Modeling Butfer Layer IGBTs for Circuit Simulation. PB95-153805

Simulating the Dynamic Electro Thermal Behavior of Power Simstronic Circuits and Systems.

PB95-161014

Thermal Component Models for Electro-Thermal Network Simulation.

HEID, C.

02,346

Hydrogen in YBa2Cu3Ox: A Neutron Spectroscopy and a Nuclear Magnetic Resonance Study.

PB95-161279

04,601

HEIDENREICH, J.E.

Gaseous Electronics Conference Radio-Frequency Reference Cell: A Defined Parallel-Plate Radio-Frequency System for Experimental and Theoretical Studies of PlasmaProcessing Discharges.

PB94-172327

04,404

EILES, $\mathrm{C}$.

Interstellar Disk-Halo Connection in Galaxies: Review of Observational Aspects

00,058

Magnetic Fields in Star-Forming Regions: Observations

PB96-123005

00,100

HEILWEIL, E. J.

Bimolecular Interactions in (Et)3SiOH:Base: $\mathrm{CCl} 4$ Hydrogen Bonded Solutions Studied by Deactivation of the Free OHStretch Vibration.

04,166

Hot Carrier Excitation of Adlayers: Time-Resolved Measurement of Adsorbate-Lattice Coupling.

00,758

Picosecond Measurement of Substrate-to-Adsorbate Energy Transfer: The Frustrated Translation of CO/P:(111)--Trans-

PB95-126041 00,895

Time-Resolved Measurements of Energy Transfer at Surfaces. 141198 00.913

Time-Resolved Measurements of Energy Transfer at Surfaces. 153037 00,947

Time-Resolved Probes of Surface Dynamics. 00,803

Vibrational Relaxation Measurements of Carbon Monoxide on Metal Clusters.

PB94-211810

00,820

HEIMAN, $N$.

Correlations of Modulation Noise with Magnetic and $\mathrm{CoNi}$ Thin Film Media.

PB94-212768

04,509

HEINEY, P. A.

Discontinuous Volume Change at the Orientational-Ordering Transition in Solid C60.

PB94-211828

00,821

Phase Transitions in Solid C70: Supercooling, Metastable Phases, and Impurity Effect.

PB95-150090

00,914

HEINZ, T. F.

Dynamics of Nonthermal Reactions: Femtosecond Surface Chemistry.

PB94-199965

00,688

HEINZEN, D.

Electrostatic Modes of Ion-Trap Plasmas.

PB95-152963

03,920

Experimental Results on Normal Modes in Cold. Pure Ion Plasmas.

PB95-175105

HEINZEN, D. J.

Liquid and Solid Atomic Ion Plasmas.

PB94-198991

03,956

Precise Spectroscopy for Fundamental Physics.

PB96-112164

03,809

Duantum-Limited Cooling and Detection of Radio-Frequency Oscillations by Laser-Cooled lons.

B96-112073

Ouantum Measurements of Trapped Ions.

PB95-161147

Ouantum Projecti

Level Systems.

03,850

Recent Experiments on Trapped Ions at the National Insti-

tute of Standards and Technology.
PB95-169322

03,952

Spin Squeezing and Reduced Ouantum Noise in Spectros

PB95-151635

03,912

Squeezed Atomic States and Projection Noise in SpectrosPB95-176293

03,960

HELFAND, D. J.

Discovery of an X-Ray Selected, Radio-Loud Ouasar at $z=3.9$.
$P B 94-198652$ 
HELMERSON, $\mathrm{K}$.

Hyperfine Effects and Associative Ionization of Ultracold Sodium.

PB95-151221

03,903

HEMPELMANN, $\mathrm{R}$.

Low-Energy Vibrations and Octahedral Site Occupation in Nb95V5H(D)y

HENINS, A.

Evolution of X-ray Resonance Raman Scattering into X-ray Fluorescence from the Excitation of Xenon Near the L3

PB96-102751

Flat and Curved Crystal Spectrography for Mammographic X-ray Sources.
PB97-122246

03,642

Noninvasive High-Voltage Measurement in Mammography by Crystal Diffraction Spectroscopy

PB95.153417

00,160

Precision Comparison of the Lattice Parameters of Silicon Monocrystals.

PB94-169745

04,438

Design and Characterization of X-Ray Multilayer Analyzers for the $50 \cdot 1000 \mathrm{eV}$ Region.

03,848

HENNION, B.

High-Energy Phonon Dispersion in La1.85Sr0.15CuO4. PB96-138458

HENS, K. F.

NMR Characterization of Injection-Moulded Alumina Green Compacts. Part 2. T2-Weighted Proton Imaging

01,165

HERBST, E.

Millimeter- and Submillimeter-Wave Spectrum of trans-Ethy| Alcohol

PB96-145578

01,102

HERBST, J. F.

Magnetocaloric Effect in Rapidly Solidified Nd-Fe-Al-B Materials.

04,451

PB94-1

Fast-Ion Conducting Y2(ZryTi1-y)207 Pyrochlores: Neutron Rietveld Analysis of Disorder Induced by Zr Substitution.

PB96-156104 04,776

Fast-Ion Conduction and Disorder in Cation and Anion Arrays in Y2(ZryTi(1-y))207 Pyrochlores Induced by $\mathrm{Zr}$ Substitution: A Neutron Rietveld Analysis.

HERMAN, $\mathrm{H}$.

04,496

Anısotropy of the Surfaces of Pores in Plasma Sprayed Alumina Deposits

HERMAN, $M$.

03,126

Fundamental Torsion Band in Acetaldehyde.

PB94-212834

00,840

General Motion Model and Spatio-Temporal Filters for Computing Optical Flow.

Image Gradient Evolution: A Visual Cue for Danger.

PB96-154562

01,847

Vision Algorithms.

02,936

Integrated Vision Touch-Probe System for Dimensional In-

PB95-255832

02,917

Intelligent Control for Multiple Autonomous Undersea Vehicles.

PB94-211877

03,747

Motion-Model-Based Boundary Extraction.

PB95-189502

01,849

Real Time Differential Range Estimation Based on TimeSpace Imagery Using PIPE

01,844

Real-Time Implementation of a Differential Range Finder.

PB95-108650 01,839

Real-Time Obstacle Avoidance Using Central Flow Divergence and Peripheral Flow

02,937

Real-Time Vision for Autonomous and Teleoperated Control of Unmanned Vehicles.

PB94-211885

03,701

Real-Time Vision for Unmanned Vehicles.

PB94-211893

03,702

Reliable Optical Flow Algorithm Using 3-D Hermite Poly-

nomials.
PB94-145620 01,829

Unified Approach to Camera Fixation and Vision-Based Road Following.

PB95-162244

Visual Road Following without 3-D Reconstruction.

01,594

HERMAN, S.

Perception of Clamp Noise in Television Receivers.

PB96-119433

01,591

PA-58
HERMANN, A.

Low-Temperature Elastic Constants of $\mathrm{Y} 1 \mathrm{Ba} 2 \mathrm{Cu} 3 \mathrm{O} 7$.

PB95-168837

04,642

HERMANN, A. M.

Asymmetry between Flux Penetration and Flux Expulsion in Tl-2212 Superconductors.

04,527

Enhanced Flux Pinning via Chemical Substitution in Bulk Superconducting T1-2212

PB95-169033

04,647

HERNDAY, P. R.

Anomalous Relation between Time and Frequency Domain PMD Measurements.

PB97-119390

04,398

HERRON, J. T.

International Conference on Chemical Kinetics (2nd). Held in Gaithersburg, Maryland on July 24-27, 1989.

PB94-211901

00,822

Plasma Chemical Model for Decomposition of SF6 in a Negative Glow Corona Discharge.

01,020

95-181053

Progress in the Development of a Chemical Kinetic
Database for Combustion Chemistry.
PB95-151056
01,384

PB95-151056

(1)

CSTL Technical Activities 1991

PB94-160769

00,728

HERTZ, J.

Extrapolation of the Heat Capacity in Liquid and Amorphous Phases.

PB97-11142

04,147

HESS, K. R.

Study of Laser Resonance Ionization Mass Spectrometry Using a Glow Discharge Source.
DE94018566

rometry

Study of Laser Resonance Ionization Mass Spectrometry
Using a Glow Discharge Source. PB96-123203

03,360

HESS, $V$.

Beyond the Technology Roadmaps: An Assessment of Electronic Materials Research and Development.
PB96-165998

HESSEL, M. M.

Conceptual Design Plan for the National Advanced Manufacturing Testbed

PB95-231866

02,828

\section{(}

Isolated Spin Pairs and Two-Dimensional Magnetism in

$\mathrm{SrCr}($ sub $9 p) \mathrm{Ga}$ (sub 12-9p) O19.
PB97-112387

04,154

HEYES, D.

Structure and Rheology of Hard-Sphere Systems.

00,662

\section{HEYLIGER, P.}

Artificial Crack in Steel: An Ultrasonic-Resonance-Spectroscopy and Modeling Study.

PB96-141395 03,241

Compressibility of Polycrystal and Monocrystal Copper: Acoustic-Resonance Spectroscopy

PB96-164223

02,990

Elastic Constants and Internal Friction of Polycrystalline Copper.

03,364

Orthotropic Elastic Constants of a Boron-Aluminum FiberReinforced Composite: An Acoustic-Resonance-SpectrosCopy Study.
PB96-200175

03,182

Ultrasonic-Resonance Spectroscopy of Bulk and Layered Solids

04,759

\section{HEYLIGER, P. R.}

Elastic Constants of Isotropic Cylinders Using Resonant Ultrasound.

PB94-211919

04,497

HIAI, $\mathrm{H}$.

Treatment of Wistar Rats with a Renal Carcinogen, Ferric Nitrilotriacetate, Causes DNA.Protein Cross-Linking between Thymine and Tyrosine in Their Renal Chromatin

PB96-112115

03,649

\section{HICHO, G. E.}

Determination of the Prior-Austenitic Grain Size of Selected Steels Using a Molten Glass Etch.

03,208

Determination of the Residual Stresses Near the Ends of Skip Welds Using Neutron Diffraction and X-ray Diffraction

$\begin{array}{ll}\text { Procedures. } & 02,868\end{array}$

Effect of Backfill and Atomizing Gas on the Powder Porosity and Mechanical Properties of 304L Stainless Steel.

PB94-185394

HICKERNELL, $\mathbf{R}$

Direct Dispersion Measurement of Highly-Erbium-Doped Optical Amplifiers Using a Low Coherence Reflectometer Coupled with Dispersive Fourier Spectroscopy. 04,263

HICKERNELL, R. K.

Characterization of Vertical-Cavity Semiconductor Structures.

02,126

Comparative Photoluminescence Measurement and Simula-

tion of Vertical-Cavity Semiconductor Laser Structures.
PB95-169173 169

Correlation of Optical, $X$-ray, and Electron Microscopy Measurements of Semiconductor Multilayer Structures.
PB95-175279

Determination of the Complex Refractive Index of Individual Quantum Wells from Distributed Reflectance.

PB95-175642

02,176

Measurement and Simulation of Photoluminescence Spectra from Vertical-Cavity Quantum-Well Laser Structures.
PB95-169181

Optical Fiber Sensors: Accelerating Applications in Navy Ships.

02,632

Pump-Induced Dispersion of Erbium-Doped Fiber Measured by Fourier-Transform Spectroscopy.

PB94-211935

04,236

Technical Digest: Symposium on Optical Fiber Measurements (8th), 1994. Held in Boulder, Colorado on September $13-15,1994$.

04,231

Vertical-Cavity Optoelectronic Structures: CAD, Growth, and Structural Characterization.

02,148

Vertical-Cavity Semiconductor Lasers: Structural Character-

ization, $C A D$, and DFB Structures.

02,150

Vertical-Cavity Semiconductor Structures: Materials Characterization.

PB95-153185

02,149

HIDAKA, Y.

Magnetic Susceptibility of $\mathrm{Pr2}-x \mathrm{CexCuO} 4$ Monocrystals and Polycrystals.

HIDNERT, $P$.

Density of Solids and Liquids.
AD-A278 $517 / 8$

04,677

HIGGINS, J. B.

Small Angle Neutron Scattering Study of the Structure and Formation of MCM-41 Mesoporous Molecular Sieves.

PB97-122337

HIGGINS, K

Rechargeable Batteries for Personal/Portable.

PB96-164231

HIGHT WALKER, $A . P$

Rotational Spectra of $\mathrm{CH} 3 \mathrm{CCH}-\mathrm{NH} 3, \mathrm{NCCCH}-\mathrm{NH} 3$, and $\mathrm{NCCCH}-\mathrm{OH} 2$.
PB97-118798

HILDEMANN, L. M.

Sources of Urban Contemporary Carbon Aerosol.

HILL, D. A.

Aperture Coupling to a Coaxial Air Line: Theory and Experi-

ment. 211968 02,216

Aperture Excitation of Electrically Large, Lossy Cavities. PB94-145711 00.029 Aperture Excitation of Electrically Large, Lossy Cavities. PB95-175675 00,031 Bistatic Scattering of Absorbing Materials from 30 to 1000 PB95-150934

Crosstalk between Microstrip Transmission Lines. 
Time-Domain Antenna Characterizations.

PB95-152781

02,003

HILL, J. R.

Correlation of Optical, X-ray, and Electron Microscopy Measurements of Semiconductor Multilayer Structures.
PB95-175279 Vertical-Cavity Semiconductor Lasers: Structural Characterization, CAD, and DFB Structures.

02,150

PB95-153193

02,150

Vertical-Cavition.

PB95-153185

02,149

HILL, M.

Crystal Chemistry and Phase Equilibrium Studies of the $\mathrm{BaO}(\mathrm{BaCO} 3)-\mathrm{R} 2 \mathrm{O} 3-\mathrm{CuO}$ Systems. 5. Melting Relations in $\mathrm{Ba2}(\mathrm{Y}, \mathrm{Nd}, \mathrm{Eu}) \mathrm{Cu} 3 \mathrm{O} 6+\mathrm{x}$

PB95-151718

04,580

HILL, M. D.

Effect of Sm2BaCuO5 on the Properties of Sintered (Bulk) YBa2Cu3O6+X
PB96-119441

04,733

Efficient Experiment to Study Superconducting Ceramics.

$X$-Ray Characterization of the Crystallization Process of High-Tc Superconducting Oxides in the $\mathrm{Sr}-\mathrm{Bi} \cdot \mathrm{Pb}-\mathrm{Ca} \cdot \mathrm{Cu}-\mathrm{O}$ Pystem.

04.579

HILL, N. W.

Intermediate Structure in the Neutron-Induced Fission Cross Section of $236 \mathrm{U}$

03,802

Measurements of the $(235) \mathrm{U}(\mathrm{n}, \mathrm{f})$ Cross Section in the 3 to $30 \mathrm{MeV}$ Neutron Energy Region.

PB97-119051

04,172

Measurements of the (237) Np(n,f) Cross Section.
PB97-119069

PB97-11906

Test Procedures for Advanced Insulation Panels. PB97-111892

00,415

HILL, S. M.

Full-Scale Room Fire Experiments Conducted at the University of Maryland
PB97-116081

00,236

HILL, T. A.

Application of the Modified Voltage-Dividing Potentiometer

to Overlay Metrology in a CMOS/Bulk Process.
PB94-181997 PB94-181997

Precision Lifetime Measurements of Cs $6 p(2) \mathrm{P} 1 / 2$ and $6 p$

(2)P3/2 Levels by Single-Photon Counting.
PB95-203816 PB95-203816

HILLERY, B. $\mathrm{R}$

Comparison of Selectivities for PCBs in Gas Chroma. tography for a Series of Cyanobiphenyl Stationary Phases.

Quantitative Analysis of Selected PCB Congeners in Marine Matrix Reference Materials Using a Novel Cyanobiphenyl Stationary Phase.

PB96-111737

02,591

Interfaces in Mo/Si Multilayers.

PB96-160668

02,423

HILPERT, G.

Rotational Spectra of $\mathrm{CH} 3 \mathrm{CCH}-\mathrm{NH} 3, \mathrm{NCCCH}-\mathrm{NH} 3$, and PBg7-118798

HILS, D.

04,170 Dreams About the Next Generation of Super-Stable Lasers.
PB94-211570
04,235

04,235

HILT, R. L.

Intracomparison Tests of the FG5 Absolute Gravity Meters. PB96-102991

03,688

HILTON, G. C.

Hot-Electron Microcalorimeter for $X$-ray Detection Using a Superconducting Transition Edge Sensor with

$\begin{array}{ll}\text { Electrothermal Feedback. } & 04,792\end{array}$ PB96-200399

Calibration of a Superconducting Gravimeter Using Absolute Gravity Measurements.

lute Gravity
PB95-202651

03,684

HINKEN, J. H.

Dielectric Properties of Single Crystals of Al2O3, LaAlO3,

$\mathrm{SrTiO3,}$ and $\mathrm{MgO}$ at Cryogenic Temperatures.

HIROSAWA,

02,266

Rotational Dynamics of $\mathrm{C} 60$ in Na2RbC60

PB95-153201

00,948

HIROTA, K.

Neutron-Scattering Studies of the Two Magnetic Correlation Lengths in Terbium

PB95-152328

04,586

Origin of the Second Length Scale Above the Magnetic SpiPB95-153698

04,596
HIRSCHMUGL, C. J.

Laser-Synchrotron Hybrid Experiments: A Photon to Tickle, A Photon to Poke.

HISSONG, D. W.

03,704

Critical Properties and Vapor-Liquid Equilibria of the Binary System Propane + Neopentane

PB95-175683

00,999

HIXENBAUGH, G. W.

Science. Technology, and Competitiveness: Retrospective on a Symposium in Celebration of NIST's 90th Anniversary and the 25th Anniversary of the Gaithersburg Laboratories, November 14-15, 1991

02,696

HO, J.

Photoelectron Spectroscopy of Negatively Charged Bismuth Clusters: $\mathrm{Bi}(-) 2, \mathrm{Bi}(-) 3$, and $\mathrm{Bi}(-) 4$.

PB95-108494

00,880

Photoelectron Spectroscopy of Small Antimony Cluster Anions: $\mathrm{Sb}(-)$, Sb2(-), Sb3(-), and Sb4(-)

HOBBIE, E. K.

01,045

Crossover to Strong Shear in a Low-Molecular-Weight Critical Polymer Blend.

PB94-211976

01,222

Lattice Model of a Hydrogen-Bonded Polymer Blend.

PB97-112262

03,391

Shear-Induced $M$

01,287

Shear Suppression of Critical Fluctuations in a Diluted Polymer Blend.

PB96-204458

04,418

Slow Dynamics of Segregation in Hydrogen-Bonded Polymer Blends.

01,281

\section{HOBBS, T.}

Response Comparison of Electret Ion Chambers, LiF TLD, and HPIC.

PB96-190103

02,578

HOBISH, M. K.

Metrology Issues in Terahertz Physics and Technology.
PB96-128277

HOCHGREB, $\mathrm{S}$

Effect of CF3H and CF3Br on Laminar Diffusion Flames in

Normal and Microgravity.

01,420

Effect of CF3H and CF3Br on Lamınar Diffusion Flames in Normal and Microgravity.

01,421

HOCKEN, $R$.

Displacement Method for Machine Geometry Calibration. PB95-152088

02,946

HOCKEY, B.

Fabrication of Transparent gamma-Al2O3 from Nanosize

Particles.
PB95-175493 03,054

Low temperature fabrication from nano-size ceramic powders.

03,029

HOCKEY, B. J.

Cavitation Contributes Substantially to Tensile Creep in Silicon Nitride.

PB96-122577

03,171

Cavity Evolution during Tensil Creep of Si3N4

PB96-204193

03,376

Creep and Creep Rupture of Structural Ceramics

Fracture of Silicon Nitride and Silicon Carbide at Elevated Temperatures.
PB96-180260

03,179

High Temperature Degradation of Structural Composites

PB94-172848

03,132

Stability and Surface Energies of Wetted Grain Boundaries in Aluminum Oxide.

PB95-202750

03,059

Tensile Creep of Whisker Reinforced Silicon Nitride

PB94-211984

03,142

Transient Creep Behaviour of Hot Isostatically Pressed Silicon Nitride.

$\begin{array}{ll}\text { PB96-180278 03,086 } & \end{array}$

Wear Transitions in Monolithic Alumina and Zirconia-Alumina Composites.

PB96-103163

03,168

HODAPP, T.

Temperature of Optical Molasses for Two Different Atomic Angular Momenta.

PB95-126058

03,881

HODGE, P. A

International Marine-Atmospheric (222)Rn Measurement Intercomparison in Bermuda. Part 1. NIST Calibration and Methodology for Standardized Sample Additions.

PB96-175674

00,114

International Radon-in-Air Measurement Intercomparison

Using a New Transfer Standard.

PB96-159751

03,708

Measurement and Calibration of Large-Area Alpha-Particle Sources at NIST.

PB94-172855

03,791

HODGES, J. T.

Analysis of Droplet Arrival Statistics in a Pressure-Atomized Spray Flame.

PB97-112270

01,352

Effect of Finite Beam Width on Elastic Light Scattering from Droplets.

01,144

Elastic Scattering from Spheres under Non Plane-Wave Illumination.

PB96-163688

04,370

Forward Scattering of a Gaussian Beam by a Nonabsorbing Sphere.
PB97-112288

04,395

Generalized Optical Theorem for On-Axis Gaussian Beams.

PB97-122345 04,177

Internal Droplet Circulation Induced by Surface-Driven Rotation.

PB97-119267 02,500

Laser Bandwidth Effects in Ouantitatıve Cavity Ring-Down Spectroscopy

04,394

Role of Combustion on Droplet Transport in Pressure-Atomized Spray Flames.

PB96-204433

01,434

\section{ODGES, R.}

Report on Application Integration Architectures (AIA) Work shop. Held in Dallas, Texas on February 8-12, 1993.

PB94-142536

HODGKIN, S. T.

01,803

Volume-Limited ROSAT Survey of Extreme Ultraviolet Emission from all Nondegenerate Stars within 10 Parsecs. PB96-103189

00,093

HOEFT, J.

Rayleigh Scattering Limits for Low-Level Bidirectional Reflectance Distribution Function Measurements.

PB95.180030

04,307

\section{HOEFT, $S$.}

Complementary Molecular Information on Phthalocyanine Compounds Derived from Laser Microprobe Mass Spectrometry and Micro-Raman Spectroscopy.

PB94-172269

00,757

HOFFMAN, D

SUSAN: SUperconducting Systems ANalysis by Low Temperature Scanning Electron Microscopy (LTSEM).

PB96-112065

04,728

HOFFMAN, D. M.

ISDN Conformance Testing Guidelines: Guidelines fo Implementors of ISDN Customer Premises Equipment to Conform to Both National ISDN-1 and North American ISDN Users' Forum Layer 3 Basic Rate Interface Basic Call Control Abstract Test Suites.

PB94-219094

01,471

\section{FMAN, J.}

Comparison of Ultralow-Sidelobe-Antenna Far-Field Patterns Using the Planar-Near-Field Method and the Far-Field

PB96-200373 02,015

Planar Near-Field Measurements of Low-Sidelobe Anten- 


\section{PERSONAL AUTHOR INDEX}

HOLLAND, J. Z

Rare-Earth Isotopes as Tracers of Particulate Emissions: An Urban Scale Test

02,535

HOLLAND, L. A.

dc Method for the Absolute Determination of Conductivities of the Primary Standard KCl Solutions from OC to $50 \mathrm{C}$.

PB94-219342

HOLLAND, O. W.

02,644

Range Statistics and Rutherford Backscattering Studies on Fe-Implanted In0.53Ga0.47As.

PB95-126397

04,535

HOLLBERG, L.

Diode Laser as a Spectroscopic Too

PB95-175485

00,600

Frequency-Stabilized LNA Laser at 1.083 mum: Application to the Manipulation of Helium 4 Aloms.

PB95-176186

High-Resolution Diode-Laser Spectroscopy of Calcium

PB95-181244

04,304

Optical Probing of Cold Trapped Atoms

03,969

PB95-175469

04,296

Precise Optical Frequency References and Difference Frequency Measurements with Diode Lasers

PB95-176228

04,305

Sub-Doppler Frequency Measurements on OCS at 87 Thz (3.4 micrometers) with the CO Overtone Laser: Considerations and Details

04,255

Sub-Doppler Frequency Measurements on OCS at $87 \mathrm{THz}$

$(3.4 \mathrm{mu} \mathrm{m})$ with the CO Overtone Laser.

PB96-102215

HOLLBERG, L. W.

High-Sensitivity Spectroscopy with Diode Lasers.

PB95-175477

04,330

HOLLERBACH, $U$

04,297

Numerical Simulation of Submicron Photolithographic ProcPBsing.

02.310

HOLLY, S.

Workshop on Characterizing Diamond Films (3rd). Held in Gaithersburg, Maryland on February 23-24, 1994

PB94-187663

04,456

HOLM, E. A

Microstructural Evolution in Two-Dimensiona! Two-Phase Polycrystals.

HOLMES, W. T.

04,498

Evaluation and Strengthening Guidelines for Federal Build ings: Assessment of Current Federal Agency Evaluation Programs and Rehabilitation Criteria and Development of Typical Costs for Seismic Rehabilitation.

00,425

Evaluation and Strengthening Guidelines for Federal Buildings: Identification of Current Federal Agency Programs. ings: Identification of Current Federal Agency Programs.
PB94-176278
00,424 HOLWITT, E.

Standard Reference Materials (SRM's) for Measuring Genetic Damage

03,516

HOMMA, $\mathrm{H}$.

Magnetic Dead Layer in Fe/Si Multilayer: Profile Refinement of Polarized Neutron Reflectivity Data.

PB94-198363

04,458

HONG, C. Y.

Periapical Tissue Reactions to a Calcium Phosphate Cement in the Teeth of Monkeys

PB94-212008

00,149

HONG, T.

Real-Time Implementation of a Differential Range Finder.

PB95-108650 01,839

Real-Time Obstacle Avoidance Using Central Flow Divergence and Peripheral Flow.
PB95-198677

HONG, T. H.

02,937

General Motion Model and Spatio-Temporal Filters for Computing Optical Flow.

01,847

Image Gradient Evolution: A Visual Cue for Danger.

Integrated Vision Touch-Probe System for Dimensional Inspection Tasks

$\begin{array}{ll}\text { PB95-255832 02,917 } & \end{array}$

Intelligent Control for Multiple Autonomous Undersea Vehi-

PB94-211877

03,747

Motion-Model-Based Boundary Extraction.

PB95-189502

01,849

Real Time Differential Range Estimation Based on TimeSpace Imagery Using PIPE

PB95-161808

01,844

Real-Time Vision for Autonomous and Teleoperated Control of Unmanned Vehicles.

PB94-211885

03,701

PB94-211893

03,702
Reliable Optical Flow Algorithm Using 3-D Hermite Polynomials.

01,829

HONG, W. P.

Range Statistics and Rutherford Backscattering Siudies on Fe-Implanted In $0.53 \mathrm{Ga0} .47 \mathrm{As}$

PB95-126397

04,535

HONG, Y. C.

Periapical Tissue Reactions to a Calcium Phosphate Cement in the Teeth of Monkeys.

PB94-212008

00,149

HONG, $Z$.

Associated Object Model for Distributed Systems. PB94-212016

01,694

HOOD, R. Q

EHects of Interfacial Roughness on the Magnetoresistance Magnetic Metallic Multilayers.

HOOKS, B. M.

04,556

Flaw Tolerance and Toughness Curves in Two-Phase Particulate Composites: SiC/Glass System.

PB96-179460

03,081

HOPE, G. A.

Thermodynamic Properties of Silicides. 5. Standard Molar Enthalpy of Formation at the Temperature $298.15 \mathrm{~K}$ of Trimolybdenum Monosilicide Mo3Si Determined by Fluorine-Combustion Calorimetry.

PB97-119358

01,190

HOPKINS, D.

Predicting the Ignition Time and Burning Rate of Thermoplastics in the Cone Calorimeter.

PB96-154794

01,418

\section{HOPKINS, M. B.}

Langmuir Probe Measurements in the Gaseous Electronics Conference RF Reference Cell.

PB96-113386

02,393

HOPP, T. H.

Performance Measures for Geometric Fitting in the NIST AIgorithm Testing and Evaluation Program for Coordinate Measurement System

122122

01,745

Representation of Axes for Geometric Fitting.

01,782

Requisite Elements, Rationale, and Technology Overview Requisite Elements, Rationale, and Technology Overview
for the Systems Intergration for Manufacturing Applications
(SIMA) Program. Background Study.
PB96-112685 PB96-112685

02,83

Sensitivity of Three-Point Circle Fitting.

02,901

Statistical Ouality Control Technology in Japan.

PB94-199064

02,708

User's Guide to 'SuperFit' Modeling Software for CMM Probe Lobing.

02,921

HOPPES, D. D.

Assay of the Eluent from the Alumina-Based Tungsten-188Rhenium-188 Generator.

PB94-200482

03,829

Needs for Brachytherapy Source Calibrations in the United States.

PB97-110092

New and Revised Half-Life Measurement Results.

PBg6-160346

03,521

Standardization and Decay Scheme of Rhenium-186 PB94-200490

00,695

HOR, C.

Lattice Position of Si in GaAs Determined by X-Ray Standing Wave Measurements.

B95-164406

04.632

Surface Geometry of BaO on W(100): A Surface-Extended $X$-Ray-Absorption Fine-Structure Study.

PB95-164414

00,980

HORI, Y.

DNA Base Damage Generated In vivo in Hepatic Chromatin of Mice upon Whole Body y-Irradiation.

PB95-161741

03,627

HORIGUCHI, $M$.

Direct Dispersion Measurement of Highly-Erbium-Doped Optical Amplifiers Using a Low Coherence Reflectomete Coupled with Dispersive Fourier Spectroscopy.

PB95-150702

04,263

Pump-Induced Dispersion of Erbium-Doped Fiber Measured by Fourier-Transform Spectroscopy.

PB94-211935

04,236

HORLICK, J.

National Voluntary Laboratory Accreditation Program: Electromagnetic Compatibility and Telecommunications. FCC Methods.

PB95-242376

02,664

National Voluntary Laboratory Accreditation Program. GOSIP: Government Open Systems Interconnection Profile. PB95-267993

01,486

National Voluntary Laboratory Accreditation Program: POSIX. Portable Operating System Interface.

02,661

NVLAP Procedures U.S. Code of Federal Regulations. Title 15, Subtitle A, Chapter 2, Part 7. (Effective December 1984; Amended September 1990)

HORN, $R$.

Diffusion of Water along 'Closed' Mica Interlaces.

PB96-180039

02,627

HORN, R. G.

Adhesion, Contact Electrification, and Acid-Base Properties

of Surtaces.

PB96-204425

03,693

Contact Electrification Induced by Monolayer Modification of a Surface and Relation to Acid-Base Interactions.

PB94-185378

03,034

Pressurized Internal Lenticular Cracks at Healed Mica Inter-

$\begin{array}{ll}\text { PB96-180252 02,997 } & \end{array}$

Surface Forces and Adhesion between Dissimilar Materials Measured in Various Environments.

PB94-172970

03,033

HORNER, G.

Epitaxial Growth and Characterization of the Ordered Vacancy Compound Culn3Se5 on GaAs (100) Fabricated by Molecular Beam Epitaxy

PB95-180725

04,687

HORNER, J. A.

Effects of Aluminum Oxalate/Glycine Pretreatment Solutions on Dentin Permeability.

PB95-164505

03,565

HORST, J. A.

Certainty Grid to Object Boundary Algorithm.

PB94-203510

01,835

Continuous Mining Machine Control Using the Real-Time Control Syster

03,700

Environment Simulation for a Continuous Mining Machine.

PB94-203536

03.697

Integration of Servo Control into a Large-Scale Control System Design: An Example from Coal Mining.

PB94-203429

03,696

HORVAI, G

Flow Immunoass

96-200951

00,642

ORVATH, J. J.

Feasibility of Fluorescence Detection of Tetracycline in Media Mixtures Employing a Fiber Optic Probe.

PB96-163654

Fluorescence Measurements of Tetracycline in High Cell Mass for Fermentation Monitoring

00,601

In situ On-Line Optical Fiber Sensor for Fluorescence Monitoring in Bioreactors.

PB94-212024

New Method for the Detection and Measurement of 


\section{PERSONAL AUTHOR INDEX}

HUANG, Q.

HOUCK, J. C.

Intercomparison between NPL. (India) and NIST (USA) Pressure Standards in the Hydraulic Pressure Region Up to $26 \mathrm{MPa}$

PB96-113543 04,211

Intercomparison of the Effective Areas of a Pneumatic Piston Gauge Determined by Different Techniques.

PB94.212370

02,640

HOUGEN, J. T.

Molecular-Beam Optothermal Spectrum of the $\mathrm{OH}$ Stretching Band of Methanol.

00,839

12826

P-Type Doubling in the infrared Spectrum of NO-HF.
PB94-211463

Use of Extended Permutation-Inversion Groups in Constructing Hyperfine Hamiltonians for Symmetrical-Top Internal Rotor Molecules Like H3C-SiH3.

PB94-212032

00,823

HOUSE, J. M.

Optimal Control of Building and HVAC Systems.

PB96-141353

00,272

HOUSTON, J. M.

National Institute of Standards and Technology High-Accuracy Cryogenic Radiometer.
PB96-179585

04,378

Realization of a Scale of Absolute Spectral Response Using the NIST High Accuracy Cryogenic Radiometer.

PB97-118640

04,397

HOVEL, H. J.

Nano-Defects in Commercial Bonded SOI and SIMOX

PB96-123674

02,407

HOWARD, F. L.

Physical Properties of Some Purified Aliphatic Hydrocarbons.
AD-A297 265/1

00,657

HOWARD, J. K.

Antiferromagnetic Interlayer Correlations in Annealed Ni80Fe20/Ag Multilayers.

03,109

PB97-122220

Magnetic Structure Determination for Annealed Ni80Fe20/
Ag Multilayers Using Polarized-Neutron Reflectivity.
PB96-176615
03,739

PB96-176615

HOWARD, L. P.

Force Calibrations in the Nanonewton Regime.

HOWE, D.

03,949

Confidence on the Second Difference Estimation of Fre-

quency Drift.
PB95-151460

01,532

HOWE, D. A.

Satellite Two-Way Time Transfer: Fundamentals and Recent Progress
PB95. 161089

01,536

Wavelet Analysis for Synchronization and Timekeeping.
PB96-200381

01,558

Wavelet Variance, Allan Variance, and Leakage.

HOWE, J.

01,509

Resonance Enhanced Multiphoton Ionization Spectroscopy of the PF Radical.

00,702

HOWE, J. E.

Extended $\mathrm{CO}(7$ yields 6) Emission from Warm Gas in Orion

HOWELL, B.

00,090

Polyethylene Crystallized from an Entangled Solution Observed by Scanning Tunneling Microscopy.

HOWLEY, J. $B$.

Isochoric ( $\mathrm{p}-\mathrm{p}-\mathrm{T}$ ) Measurements on Liquid and Gaseous Air from 67 to $400 \mathrm{~K}$ at Pressures to $35 \mathrm{MPa}$.

PB96-167390 01,154

Vapour Pressure Measurements on 1,1,1,2Tetrafluoroethane (R134a) from 180 to $350 \mathrm{~K}$.
PB95.168886

HOYT, C. C.

Update on the Low Background IR Calibration Facility at the

National Institute of Standards and Technology.
PB94-211224

04,232

HOZUMI, $N$

Effect of DC Tests on Induced Space Charge. PB94-172350

02,212

HRILJAC, J. A.

Anomalous Dispersion and Thermal Expansion in LightlyDoped KTa1-XNbXO3.

PB95-152302

04,585

HSIA, J.

Rayleigh Scattering Limits for Low-Level Bidirectional Reflectance Distribution Function Measurements.
PB95-180030

HSIA, J. J.

$45 \mathrm{deg} / 0 \mathrm{deg}$ Reflectance Factors of Pressed Polytetrafluoroethylene
PB95-260758 Standard Reference Materials: Polystyrene Films for Cali-
brating the Wavelength Scale of Infrared Spectrophotometers - SRM 1921

PB95-226866

03,386

HSIAO-YU, C.

Variations in Size Measurements by Indicating Gaging Sys-

PB95-163614

02,864

HSIEH, S. H.

Nucleic Acid Database: Present and Future.

PB97-109078

00,518

Reactive Coevaporation of DyBaCuO Superconducting

Reactive Coevaporation of DyBaCuO Superconducting $\mathrm{MgO}(100)$ Substrates
Films: The Segregation

MgO(100) Subsir

04,635

HSIN, Y. E.

Acid Gas Production in Inhibited Diffusion Flames. PB95-180576

01,390

HSING, K.

AnalysIs of ANSI ASC $\times 12$ and UN/EDIFACT Electronic Data Interchange (EDI) Standards.

01,729

HSLA, J. J.

NIST Response to the Fitth CORM Report on the Pressing Problems and Projected Needs in Optical Radiation Measurements.

PB94-188240

04,227

HSU, J. C. L.

In-situ Fume Particle Size and Number Density Measurements from a Synthetic Smelt.

PB94-212040

03,334

HSU, N. N.

Design, Construction and Application of a Large Aperture Lens-Less Line-Focus PVDF Transducer.

PB97.122584

02,765

Material Characterization By a Time-Resolved and Polarization-Sensitive Ultrasonic Technique.

PB97-122576

02,764

Transient Analysis of a Line-Focus Transducer Probing a Liquid/Solid Interface.

02,763

HSU, S. M.

Analysis of Physical Properties of Ceramic Powders in an International Interlaboratory Comparison Program.

PB95-161501

03,050

Asperity-Asperity Contact Mechanisms Simulated by a TwoBall Collision Apparatus.

P95- 164158

02,966

Boundary Lubrication of Silicon Nitride.

03,226

Ceramic Powders Characterization: Results of an International Laboratory Study.

02,672

Chemical Effect in Ceramics Grinding

PB97-122592

03,113

Chemically Assisted Machining of Si3N4.

PB96-122999

03,072

Deposit Forming Tendencres of Diesel Engine Oils-Correlation between the Two-Peak Method and Engine Tests.

PB95-152138

01,452

Effect of Microstructure on the Wear Transition of Zirconia.

Toughened Alumina.
PB94-211778

03,141

Evaluation of Wear Resistant Ceramic Valve Seats in GasFueled Power Generation Engines. Topical Report, December 1991-April 1994.

PB95-200218

02,466

Glimpse of Materials Research in China: A Report from an Interagency Study Team on Materials Visiting China from June 19, 1995 to June 30, 1995

PB96-112677

02,978

Mechano-Chemical Model: Reaction Temperatures in a Concentrated Contact.

03,227

PB96-119466

New Method to Evaluate Deposit Forming Tendencies of Liquid Lubricants by Differential Scanning Calorimetry.
PB95-152120

Silicon Nitride Boundary Lubrication: Effect of Oxygenates.

PB96-111711 03,068

Silicon Nitride Boundary Lubrication: Lubrication Mechanism of Alcohols.

PB96-111703

03,067

Tribochemical Reaction of Stearic Acid on Copper Surface Studied by Surface Enhanced Raman Spectroscopy.

Tribological Characteristics of Alpha-Alumina at Elevated Temperatures
PB94-211018

02,963

Variances in the Measurement of Ceramic Powder Properties.

03,100

Wear Mechanism Maps of Ceramics.

PB94-172368

03,229

Wear Model for Alumina Sliding Wear.

03,239

Wear Modeling of Si-Based Ceramics.

03,112

Wear Transitions in Monolithic Alumina and Zırconia.Alumina Composites.

PB96-103163

03,168

Chemically Assisted Machining of Si3N4.

PB96-122999

03,072

HU, B. Y. K.

Correction to the Decay Rate of Nonequilibrium Carrier Distributions Due to Scattering-in Processes.

PB94-185840

04,452

HU, J. T.

Structural Heterogeneity in Epoxies.

PB95-151866

01,243

High Resolution Angle Resolved Photoelectron Spectroscopy Study of N2.
PB95-151494

03,907

HU, Z. S.

Tribochemical Reaction of Stearic Acid on Copper Surface Studied by Surface Enhanced Raman Spectroscopy

PB94-212057

02,964

HUANG, D.

Intercomparison of Thermal Converters at NIM, NIST, PTB, SIRI and VSL from 10 to $100 \mathrm{MHz}$

HUANG, D. $\mathrm{X}$.

02,027

AC-DC Difference Characteristics of High-Voltage Thermal

$\begin{array}{ll}\text { Converters. } & \\ \text { PB96-148093 } & 02,083\end{array}$

Development of Thin-Film Multijunction Thermal Converters at NIST

PB97-112338

02.286

Integrated Thin-Film Micropotentiometers

PB96-146709

02,109

Multijunction

PB95-153664

CMOS

Performance of Commercial CMOS Foundry-Compatible Multijunction Thermal Converters.

PB95-153656

02,342

Performance of Multilayer Thin-Film Multjunction Thermal Converters.

HUANG, $\mathrm{H}$. M.

Certainty Grid to Object Boundary Algorithm.

02,084

Hierarchical Real-Time Control System for Use with Coal Mining Automation

PB94-212065 03,698

Operator Experience with a Hierarchical Real-Time Control System (RCS)

03,751

Outline of a Multiple Dimensional Reference Model Architecture and a Knowledge Engineering Methodology for Intelligent Systems Control.

PB95-220414 


\section{PERSONAL AUTHOR INDEX}

Observation of Oscillatory Magnetic Order in the Antiferromagnetic Superconductor HoNi2B2C. $\quad 04,679$ Oxygen Dependance of the Crystal Structure of $\mathrm{HgBa} 2 \mathrm{CuO} 4+$ and Its Relation to Superconductivity. $\mathrm{HgBa} 2 \mathrm{CuO} 4+$ and Its Relation to Superconductivity.
PB96-102512 Preparation, Crystal Structure, Dielectric Properties, and Magnetic Behavior of Ba2Fe2Ti4O13.

PB96-186176

01,162

Unconventional Ferromagnetic Transition in La(sub 1 x) $\mathrm{Ca}(\mathrm{sub}$ x) $\mathrm{MnO}$

HUANG, $X$. $R$.

04,156

Interdigitated Stacked P-I-N Multiple Ouantum Well Modulator.

PB97-112296

02,455

\section{HUBBARD, J}

Determining Mobility from Homodyne ac Electrophoretic Light Scattering.

03,462

Non-Perturbative Relation between the Mutual Diffusion Coefficient. Suspension Viscosity, and Osmotic Compressibility: Application to Concentrated Protein Solutions.

PB96-102355

HUBBARD, J. B.

Electrolytes Constrained on Fractal Structures: DebyeHuckel Theory.

01,174

Fluctuation Dominated Recombination Kinetics with Traps.

PB95-107264

00,875

Fokker-Planck Description of Multivalent Interactions.
PB95-108478

Hydrodynamic Friction of Arbitrarily Shaped Brownian Par-

PB97-110191

04,136

Physical Characterization of Herparin by Light Scattering.

PB96-119383

HUBBELL, J. H.

Bibliography of Photon Total Cross Section (Attenuation Coefficient) Measurements $10 \mathrm{eV}$ to $13.5 \mathrm{GeV}, 1907-1993$

efficient) Meas

03,804

Experimentally Measured Total X-ray Attenuation Coefficients Extracted from Previously Unprocessed Documents Held by the NIST Photon and Charged Particle Data CenPB97-114474

04,165

Polarization Effects on Multiple Scattering Gamma Trans-

PBrt.

03,926

Tables of $\mathrm{X}$-ray Mass Attenuation Coefficients and Mass Energy-Absorption Coefficients $1 \mathrm{keV}$ to $20 \mathrm{MeV}$ for Elements $Z=1$ to 92 and 48 Additional Substances of Dosimetric Interest.

PB95-220539

04,013

HUBER, M. E.

Noise Reduction in Low-Frequency SOUID Measurements with Laser-Driven Switching.

PB96-135165

Trapped Vortices in a Superconducting Microbridge.

PB95-141149

02,08

HUBER, M. L.

Equation of State Formulation of the Thermodynamic Prop erties of R134a (1,1,1,2-Tetrafluoroethane)

03,256

Prediction of the Thermal Conductivity of Refrigerants and Refrigerant Mixtures. Refrigerant Mixtures.
PB94-212107

03,258

Prediction of Viscosity of Refrigerants and Refrigerant Mix

PB94-212099

03,257

Predictive Extended Corresponding States Model for Pure and Mixed Refrigerants Including an Equation of State for

PB95-175717

03,275

REFPROP Refrigerant Properties Database: Capabilities, Limitations, and Future Directions.

PB96-167150

01,149

Thermodynamic Properties of R134a(1,1,1,2 Tetrafluoroethane).
PB95-168704 00,988 Thermophysical Property Computer Packages from NIST

PB95-125787 04,203

Thermophysical Property Standard Reference Data from NIST.

PB96-167358

01,153

Viscosity of Defined and Undefined Hydrocarbon Liquids Calculated Using an Extended Corresponding-States Model. PB96-167234

02,498

HUCKER, T.

Standardised Computer Data File Format for Storage, Transport, and Off-Line Processing of Partial Discharge

PB96-122486

01,930

HUDGENS, J. W.

Electronic Spectra of $\mathrm{CF} 2 \mathrm{Cl}$ and $\mathrm{CFCl} 2$ Radicals Observed PB95-151023

00,927
Experimenta! and Abinitio Studies of Electronic Structures of the $\mathrm{CCl} 3$ Radical and Cation.

PB94-212131

00,826

Kinetics of the Reaction of $\mathrm{CCl} 3-\mathrm{Br}-2$ and the Thermochemistry of $\mathrm{CCl} 3$ Radical and Cation.

00,824

Multiphoton Ionization of SiH3 and SiD3 Radicals: Electronic Spectra, Vibrational Analyses of the Ground and Rydberg States, and Ionization Potential.

00,837

Multiphoton Ionization of $\mathrm{SiH} 3$ and SiD3 Radicals. 2. Three Multiphoton Ionization of $\mathrm{SiH} 3$ and SiD3 Radicals. 2. Three
Photon Resonance-Enhanced Spectra Observed between Photon Resonance-Enhanced Spectra Observed between
460 and $610 \mathrm{~nm}$.
PB94-212487
PB, 835

0,835

New Electronic States of NH and ND Observed from 258 to $288 \mathrm{~nm}$ by Resonance Enhanced Multiphoton Ionization Spectroscopy.
PB94-212495

00,836

New Rydberg States of Aluminum Monofluoride Observed by Resonance-Enhanced Multiphoton Ionization Spectroscopy.

PB94-199544

00,797

Resonance Enhanced Multiphoton Ionization Detection of GeF and $\mathrm{GeCl}$ Radicals.

PB94-212123 00.825

Resonance Enhanced Multiphoton Ionization Spectroscopy of the PF Radical.

PB97-119119

00,702

Resonance Enhanced Multiphoton lonization Spectroscopy of the SnF Radical.

01,176

Resonance Enhanced Multiphoton Ionization Spectroscopy of 2-Butene-1-yl (C4H7) between 455-485 $\mathrm{nm}$.

00.670

UUDSON, L.

Crystal Diffraction Spectrometry for Accurate, Non-Invasive kV/Spectral Measurement for Improvement of Mammographic lmage Ouality.

00,721

HUDSON, L. T.

Flat and Curved Crystal Spectrography for Mammographic X-ray Sources
PB97-122246

03,642

Photoelectron Spectroscopic Study of the Valence and Core-Level Electronic Structure of $\mathrm{BaTiO}$.

Polarization Measurements on a Magnetic Ouadrupole Line in Ne-Like Barium

PB97-113104

04.161

Resonant-Photoemission Investigation of the Heusler Alloys $\mathrm{Ni2MnSb}$ and NiMnSb.

PB95-162384

04,612

Substitution-Induced Midgap States in the Mixed Oxides RXBa1-ChiTiO3-Delta, with $R=Y, L a$, and $N d$.

04,541

Surface Core-Level Shifts of Barium Observed in Photoemission of Vacuum-Fractured $\mathrm{BaTiO}$ (100)

PBission of Vacu

04,501

\section{HUEBENER, R. P}

Direct Observation of Vortex Dynamics in Two-Dimensional Josephson-Junction Arrays.

02,067

Frequency Dependence of the Emission from 2D Array Josephson Oscillators.

02,056

Novel Vortex Dynamics in Two-Dimensional Josephson Ar-

rays.

02,091

Observation of Vortex Dynamics in Two-Dimensional Josephson-Junction Arrays.

PB95-168811

02,050

SUSAN: SUperconducting Systems ANalysis by Low Temperature Scanning Electron Microscopy (LTSEM) PB96-112065

04,728

\section{HUERTA-GARNICA, $M$.}

Comparison of Finite Element and Analytic Calculations of the Resonant Modes and Frequencies of a Thick Shell

PB94-160785

02,626

HUHNERMANN, $\mathrm{H}$.

Hyperfine Structure Investigations and Identification of New Energy Levels in the Ionic Spectrum of (147) Pm.

PB96.180203

04,117

\section{HUIE, R. E.}

Atmospheric Lifetimes of HFC-143a and HFC-245fa: Flash Photolysis Resonance Fluorescence Measurements of the OH Reaction Rate Constants.

PB97-112577

00,118

Atmospheric Reactivity of alpha-Methyl-Tetrahydrofuran. PB95-163705

02,548

Electron Transfer Reaction Rates and Equilibria of the Carbonate and Sulfate Radical Anions.

PB94-212180

00,829

Environmental Aspects of Halon Replacements: Considerations for Advanced Agents and the Ozone Depletion Potential of CF3l

03,301

Experimental Determination of the Ionization Energy of $\mathrm{O}(\mathrm{X}($ sup 2)II(sub 3/2)) and Estimations of Delta(sub f) $\mathrm{H}$ (sup deg)(sub 0$)(1 \mathrm{O}($ sup -$))$ and $\mathrm{PA}(1 \mathrm{O})$

PB96-146899

Ferric Ion Assisted Photooxidation of Halocetates.

B97-112460

00,694 PB96-148101

Gas Phase Reactivity Study of $\mathrm{OH}$ Radicals with 1,1Dichloroethene and cis-1,1-Dichloroethene and Trans-1,2Chloroethene over the Temperature Range $240-400 \mathrm{~K}$. B95-152146

Ionization Energies, Appearance Energies and Thermochemistry of CF2O and FCO. Kinetics of the Reaction of the Sulfate Radical with the Oxalate Anion.

PB97-119127

01,186

Kinetics of the Self-Reaction of Hydroxymethylperoxyl Radicals. Oxidation of Ferrous and Ferrocyanide lons by Peroxyl Radicals

PB97-12240?

01,191

Rate Constants for the Gas Phase Reactions of the $\mathrm{OH}$ with $\mathrm{CF} 3 \mathrm{CF} 2 \mathrm{CHCl} 2$ (HCFC-225ca) and CF2CICF2CHCIF (HCFC-225cb).

PB95-152153

00,940

Reaction of Nitric Oxide with Organic Peroxyl Radicals

PB95-141107

00,910

Reaction of NO with Superoxide.

00,830

Solvent Effects in the Reactions of Peroxyl Radicals with Organic Reductants. Evidence for Proton Transfer Mediated Electron Transter

PB95-107157

00,873

Temperature Dependence of the Rate Constants for ReacTen of Dinalide and Azide Radicals with Inorganic Redu tants

PB95-162756

00,964

Temperature Dependence of the Rate Constants for Reaction of Inorganic Radicals with Organic Reductants.

PB94-198280

00,783

Temperature Dependence of the Rate Constants for Reactions of the Carbonate Radical with Organic and Inorganic Reductants.

00,831

Temperature Dependence of the Rate Constants for Reactions of the Sulfate Radical, SO4-, with Anions

PB94-212172

00,828

Temperature Dependence of the Ultraviolet Absorption Cross Section of CF31.

PB96. 204169

01,168

HULL, J. J.

Second Census Optical Character Recognition Systems Conference. 
Distributed Systems: Survey of Open Management Ap-

proaches.
PB96-128202 01,746

Open System Environment Implementors Workshop (OIW); Standardization Role Defined.

PB96-180047

01,828

HUNSTON, D. L.

Micromechanics of Fracture in Rubber-Toughened Epoxies.

Review of Cure Monitoring Techniques for On-Line Process Control.

PB94-216728

03,145

Structural Heterogeneity in Epoxies.

PB95-151866

01,243

Water Adsorption at a Polyimide/Silicon Water Interface.

PB96-103197

01,070

HUNT, F. Y.

Analytical Expressions for Barkhausen Jump Size Distribu-

tions. 18034504,680

Monte Carlo Approach to the Approximation of Invariant Measures.

PB94-172053

03,409

Probabilistic Computation of Poiseuille Flow Velocity Fields.

PB96-102520

\section{HUNTER, C. $R$}

Estimation of the Absorbed Dose in Radiation-Processed Food. 1. Test of the EPR Response Function by a Linear Regression Analysis.

PB94-199718

00,039

HUO, a.

Small Angle Neutron Scattering Study of the Structure and Formation of MCM-41 Mesoporous Molecular Sieves.

PB97-122337 03,110

Small Angle Neutron Scattering Study of the Structure and

Formation of Ordered Mesopores in Silica.

PB96-111919

03,069

HURLY, J. J.

Absence of Quantum-Mechanical Effects on the Mobility of Argon lons in Helium Gas at $4.35 \mathrm{~K}$

PB97-122543

01,194

HURST, G. S.

Measurement of Absorbed Dose of Neutrons, and of Mixtures of Neutrons and gamma rays.

AD-A286 647/3

03,710

HURST, W. S.

Development of the NIST Transient Pressure and Temperature Calibration Facility

00,626

Q Branch Lineshape Functions for CARS Thermometry.

PB96-160643

Simultaneous Forward-Backward Raman Scattering Studies of $\mathrm{D} 2$ Broadened by $\mathrm{D} 2, \mathrm{He}$, and $\mathrm{Ar}$.

00,961

HURWITZ, L. M.

Radiation Process Data: Collection, Analysis, and Interpreation

03,628

HUSK, D. E.

Soft-X-ray Damage to p-terphenyl Coatings for Detectors.

PB96-159611

HUTCHINGS, J. B.

Observations of $3 \mathrm{C} 273$ with the Goddard High Resolution Spectrograph on the Hubble Space Telescope.

PB95-202321

HUTCHINSON, J. M. R.

Anomalous Odd- to Even-Mass Isotope Ratios in Reso-

nance lonization with Broad-Band Lasers.
PB94-211406

03,839

Atom-counting standards and Doppler-free resonance ionization mass spectroscopy. (Progress report)

00,723

Calibration of Electret-Based Integral Radon Monitors Using NIST Polyethylene-Encapsulated (226)Ra/(222)Rn Emanation (PERE) Standards.

PB96-159223

01,950

International Marine-Atmospheric (222)Rn Measurement Intercomparison in Bermuda. Part 1. NIST Calibration and Methodology for Standardized Sample Additions.

Methodology

00,114

International Marine-Atmospheric (222)Rn Measurement Intercomparison in Bermuda. Part 2. Results for the Partici-

pating Laboratories.
PB96-175682

00,115

International Radon-in-Air Measurement Intercomparison

Using a New Transfer Standard.

03,708

PB96-159751

03,708

Measurement and Calibration of Large-Area Alpha-Particle Sources at NIST.

PB94-172855

03,791

Study of Laser Resonance lonization Mass Spectrometry

Using a Glow Discharge Source.

03,308

Study of Laser Resonance Ionization Mass Spectrometry Using a Glow Discharge Source.

03,360
HUTSON, J. M.

Signatures of Large Amplitude Motion in a Weakly Bound Complex: High-Resolution IR Spectroscopy and Quantum Calculations for $\mathrm{HeCO} 2$.

PB95-203485

01,054

Spectroscopic Puzzle in ArHF Solved: The Test of a New

PB94-216058 00,850

Vibrational Dependence of the Anisotropic Intermolecular Potential of Ar-HCl.

PB95-202685

01,029

Vibrational Dependence of the Anisotropic Intermolecular Potential of Ar-HF.

HUTTER, A. R

01,030

International Marine-Atmospheric (222)Rn Measuremen Intercomparison in Bermuda. Part 2. Results for the Participating Laboratories.

HUTTER, E.

00,115

Use of Building Emulators to Evaluate the Performance of Building Energy Management Systems.

PB96-11 190

HUTTON, D. R.

Estimation of the Absorbed Dose in Radiation-Processed Food. 1. Test of the EPR Response Function by a Linear Regression Analysis.

PB94-199718

HUZAREWICZ, S.

Flame Synthesis of High Tc Superconductors.
PB95-151981

00,039

00,659

Parametric Investigation of Metal Powder Atomization Using Laser Diffraction.

HWANG, C. S.

03,342

Epitaxial Growth of $\mathrm{BaTiO}_{3}$ Thin Films at $600 \mathrm{C}$ by Metalorganic Chemical Vapor Deposition.

03,071

HWANG, $H$.

Evidence for Inelastic Processes for $\mathrm{N}(+) 3$ and $\mathrm{N}(+) 4$ from Ion Energy Distributions in He/N2 Radio Frequency Glow Discharges.

HWANG, I. $S$.

04,059

Charpy Impact Test as an Evaluation of $4 \mathrm{~K}$ Fracture Toughness.

PB96-190194

03,219

Fatigue Crack Thresholds of a Nickel-iron Alloy for Superconductor Sheaths at $4 \mathrm{~K}$.

03,223

HWANG, J. T.

Existence and Nonexistence Theorems of Finite Diameter Sequential Confidence Regions for Errors-in-Variables Mod els.

PB95-180352

03,441

Application of the Electronic Balance in High Precision Pycnometry.

00,534

Determination of Density of Mass Standards: Requirement and Method.

03,787

Electronic Balance and Some Gravimetric Applications.

(The Density of Solids and Liquids, Pycnometry and Mass).
PB94-163052
03,785 PB94-163052

HWANG, N. M.

Phase Equilibria in the Systems $\mathrm{CaO} \cdot \mathrm{CuO}$ and $\mathrm{CaO}-\mathrm{Bi} 2 \mathrm{O} 3$ 03,048 $X$-Ray Characterization of the Crystallization Process of High-Tc Superconducting Oxides in the Sr-Bi-Pb-Ca-Cu-O System.

PB95-151700

04,579

HYATT, J. A.

Solid State (13)C NMR and Raman Studies of Cellulose Triacetate: Oligomers, Polymorphism, and Inferences about Chain Polarity

HYER, C. W.

01,289

Directory of U.S. Private Sector Product Certification Pro-

PBrams. 15074

02,688

HYLTON, T. L.

Antiferromagnetic Interlayer Correlations in Annealed Ni80Fe20/Ag Multilayers.

PB97-122220

03,109

Magnetic Structure Determination for Annealed Ni80Fe20/ Ag Multilayers Using Polarized-Neutron Reflectivity.

PB96-176615

03,739

HYUN, O. B.

Experimental Aspects of Flux Expulsion in Type-II Superconductors.

04,660

Flux Expulsion at Intermediate Fields in Type-II

Superconductors

PB94-212230

04,502

High-Efficiency, High-Power Difference-Frequency Generation of $0.9-1.5 \mathrm{mu}$ Light in BBO.

04,317

IDAISS, I. $M$

Assessment of Site Response Analysis Procedures. PB95.210928

IGA, I.

Shape-Resonance-Enhanced Continuum-Continuum Coupling in Photoionization of $\mathrm{CO} 2$.

PB95-16447

00,983

ILAVSKY, J.

Anisotropy of the Surfaces of Pores in Plasma Sprayed Alu-

mina Deposits.

03,126

IMAE, $M$.

Use of Ionospheric Data in GPS Time Transfer.

PB95. 163853

01,540

MHOFF, J.

Radiation-Driven Winds of Hot Luminous Stars $X$. The Determination of Stellar Masses Radii and Distances from Terminal Velocities and Mass-Loss Rates.

INAM, A.

Laser-Synchrotron Hybrid Experiments: A Photon to Tickle, A Photon to Poke.

PB96-157847

03,704

INCE, A. T.

Comparisons of Some NIST Fixed-Point Cells with Similar Cells of Other Standards Laboratories.

PB97-119242

INDELICATO, P.

Electron Screening Correction to the Self Energy in High-Z

$\begin{array}{ll}\text { PB94-172376 03,789 } & 0\end{array}$

$K$ alpha Transitions in Few-Electron lons and in Atoms.

PB94-212248 03,849

Magnetic Dipole Line from U LXXI Ground-Term Levels Predicted at 3200 Angstroms.

PB94-2114

04,407

Process Gas Chromatography Detector for Hydrocarbons Based on Catalytic Cracking.

NGUSCIO, M.

Rotational Far Infrared Spectrum of (13) CO

PB95-152187

02,485

INN, K. G. W.

Atom-counting standards and Doppler-free resonance ionization mass

00,723

External Gamma-ray Counting of Selected Tissues from a Thorotrast Patient.

03,637

Intercomparison Study of (237)Np Determination in Artificial Urine Samples

PB96-102645

03,633

Pilot Studies for Improving Sampling Protocols.

PB97-118715

02,530

Role of the Office of Radiation Measurement in Ouality Assurance

00,689

Summary of the Proceedings of the Workshop on Standard Phantoms for In-vivo Radioactivity Measurement. PB94-212933

03,622

INTERRANTE, C. G.

Factors Significant to Precracking of Fracture Specimens. 
IRWIN, K. D.

Hot-Electron Microcalorimeter for X-ray Detection Using a Superconducting Transition Edge Sensor with

Self-Biasing Cryogenic Particle Detector Utilizing Electrothermal Feedback and a SOUID Readout. $\quad 04,712$

ISAACS, W.A.

Modified Effective Range Theory as an Alternative to LowEnergy Close-Coupling Calculations.

PB95-202701

03,988

SAACSON, $M$

NIST Metrology for Soft X-ray Multilayer Optics.

ISHIBASHI, T.

Etfects of Heavy Doping on Numerical Simulations of Gallium Arsenide Bipolar Transistors.

PB95-150975

02,334

ISHIDA, $\mathrm{H}$

Upward Flame Spread along the Vertical Corner Walls (Oc-

tober 1993).
PB94-206299 00,340

ISHIDA, T.

Harmonic and Static Susceptibilities of $\mathrm{YBa} 2 \mathrm{Cu} 3 \mathrm{O} 7$.

PB95-161139

Oftset Susceptibility of Superconductors.

PB94-212263

04,599

ISHIKAWA, K.

Behavior of a Calcium Phosphate Cement in Simulated Blood Plasma In vitro.

PB95-16871

00,165

Effect of Supersaturation on Apatite Crystal Formation in Aqueous Solutions at Physiologic pH and Temperature.

PB96-135215

03,571

Formation of Hydroxyapatite in Cement Systems PB95-175261

00,170

Properties and Mechanisms of Fast-Setting Calcium Phosphate Cements.

ISHIKAWA, Y.

00,178

Behavior of a Calcium Phosphate Cement in Simulated Blood Plasma In vitro.

PB95-168712

00,165

Properties and Mechanisms of Fast-Setting Calcium Phosphate Cements.

ISHINABE, T.

00,178

Examination of the V/d Expansion Method from Exact Enumeration for a Self-Interacting Self-Avoiding Walk. 01,266

Hypercubic Lattice SAW Exponents nu and gamma : 3.99 Dimensions Revisited

01,215

PB94-211026

elf-Inter-

sections in Variable Dimensionality.
sandom-Walk Self-Inter-

PB96-102231

01,276

ITANO. W

Electrostatıc Modes of Ion-Trap Plasmas.

PB95-152963

Obtaining and Installing a Public Domain TEX.

PB95-175741

03,920

ITANO, W. M.

Accurate Measurement of Time.

PB96-119482

Atomic Clock

Atomic Clock.

Experimental Results on Normal Modes in Cold, Pure Ion Plasmas.

PB95-175105

03,956

Getting Started on Mosaic.

01,721

High-Resolution Atomic Spectroscopy of Laser-Cooled Ions.

PB95-169330 03,953

Interference in the Resonance Fluorescence of Two Trapped Atoms.

PB95-168514

Laser Cooling of Trapped Ions.

03,948

Light Scattered from Two Atoms.

03,950

PB95-168753

04,286

Liquid and Solid Atomic Ion Plasmas.

03,809

Precise Spectroscopy for Fundamental Physics.

04,040

PB96-112164

Progress on a Cryogenic Linear Trap for $(199) \mathrm{Hg}(+)$ lons.
PB95-180790

Ouantum Measurements of Trapped Ions.

PB95-161147

03,928

Ouantum Projection Noise: Population Fluctuations in TwoLevel Systems

03,850

Recent Experiments on Trapped lons at the National Institute of Standards and Technology.

03,952
Retrieving Articles from the Internet (without a UNIX Workstation). Part 1. File Formats and Software Tools.
PB95-168720

Retrieving Articles from the Internet (without a UNIX Workstation). Part 2. An Example.

02,729

Spin Squeezing and Reduced Ouantum Noise in SpectrosCopy. 151635

Squeezed Atomic States and Projection Noise in Spectros. Copy.

03,960

Trapped lons and Laser Cooling 4: Selected Publications of the Ion Storage Group of the Time and Frequency Division. NIST, Boulder, Colorado.

PBO6-172358

04,108

ITKIN, V.

Thermodynamic Properties of the Group IIA Elements. PB94-160983

00,730

ITOH, K.

Reduction of Intertilament Contact Loss in Nb3Sn Superconductor Wires.

PB95-175535

02,223

IULIANO, M. J.

Overview of the Manufacturing Engineering Toolkit Prototype.
PB96-128228

02,833

\section{IVES, L. K.}

Abrasive Wear by Diesel Engine Coal-Fuel and Related Particles.

PB95-104915 01,450

Generation and Characterization of Acetylene Smokes.

(20)-200292

01,372

Ultrafine Combustion Aerosol Generator

PB94-200300

01,373

NGAR, G. V

Determination of Boron and Lithium in Diverse Biological Matrices Using Neutron Activation - Mass Spectrometry PB94-212289

00,554

Mixed Diet Reference Materials for Nutrient Analysis of Foods: Preparation of SRM-1548 Total Diet.

PB95-151692

03,593

IYENGAR, V. G.

External Gamma-ray Counting of Selected Tissues from a Thorotrast Patient.

PB96-160254

\section{IYER, H. K.}

03,637

Moments of the Ouartic Assignment Statistic with an Application to Multiple Regression.

PB95-181103

03,442

Technique to Evaluate Benchmarks: A Case Study Using the Livermore Loops.

04,577

Tolerance Intervals for the Distribution of True Values in the Presence of Measurement Errors.

PB95-150405

03,434

IYER, $S$.

1,4-Dinitrocubane and Cubane under High Pressure.

PB95-108437

03.755

IZWORSKI, N.

Methods to Improve the Accuracy of On-Line Ultrasonic Measurement of Steel Sheet Formability.

PB96-186051

JABBOUR, Z. J.

Nanofabrication of a Two-Dimensional Array Using LaserFocused Atomic Deposition.

PB96-119417

04,732

JABLONSKI, A

Elastic-Electron-Scattering Effects on Angular Distributions in X-ray Photoelectron Spectroscopy.

PB95-175758

01,000

Formalism and Parameters for Ouantitative Surface Analysis by Auger Electron Spectroscopy and X-Ray Photoelectron Spectroscopy

JACH, T.

00,832

Grazıng Angle X-Ray Photoemission System for Depth-De-

pendent Analysis.
PB95-161154 04,600

Grazing-Incidence X-Ray Photoelectron Spectroscopy: A Novel Approach to Thin Film Characterization.

04,589

Grazing Incidence X-ray Photoemission and Its Implementation on Synchrotron Light Source $X$-ray Beamlines. PB95-175766

01,001

Grazing-Incidence X-Ray Photoemission Spectroscopy Investigation of Oxidized GaAs(100): A Novel Approach to Nondestructive Depth Profiling.

PB94-200151

04,480

Polarized X-Ray Emission Spectroscopy.

03,862

$\mathrm{X}$-Ray Photoelectron and Auger Electron Spectroscopy Study of UltravioletOzone Oxidized P2S5/(NH4)2S Treated GaAs(100) Surfaces.

04,479

JACKSON, C. L.

Anomalous Freezing and Melting of Solvent Crystals in Swollen Gels of Natural Rubber.

01,223

Flow-Induced Structure in Polymers: Chapter 16. Shear-Induced Changes in the Order-Disorder Transition Temperature and the Morphology of a Triblock Copolymer.

PB96-123237

03,127

Glass Transition of Organic Liquids Confined to Small PB94-212305

00,833

Influence of Shear on the Ordering Temperature of a Triblock Copolymer Melt.

PB96-163753

01,288

Melting Behavior of Organic Materials Confined in Porous Solids

PB94-212313

00,834

Morphology and Phase Separation Kinetics of a Compatibilized Blend.

PB97-119135

01,297

Polymer Liquid Crystalline Materials.

PB94-212339

01,224

mer. 172392

01,196

Shear-Induced Changes in the Order-Disorder Transition Temperature and the Morphology of a Triblock Copolymer. PB97-118772

03,130

Cop-Induced Mar

PB96-119508

01,277

Synthesis of Hybrid Organic-Inorganic Materials from Interpenetrating Polymer Network Chemistry.

02,994

Vitrification and Crystallization of Organic Liquids Confined to Nanoscale Pores.

PB97-112304

03,392

JACKSON, J.

Scattered Fractions of Dose from 18 and 25 MV X-ray Radiotherapy Linear Accelerators.

PB96-186101

04,120

KSON, J. K.

Characterization of Modified FEL Ouartz-Halogen Lamps for Photometric Standards.

PB97-112544

JACKSON, $M$.

00,282

Acoustic

PBames. 16481

JACKSON, M. A per, Chlorinated Polyvinyl Chloride and Polybutylene, in

Residential and Light Hazard Installations.
PB95-182267

00,299

JACKSON, R. H. F.

Guidelines for Reporting Results of Computational Experiments. Report of the Ad hoc Committee.

PB94-212347

03,427

JACOB, I.

Brillouin Light Scattering Intensities for Thin Magnetic Films with Large Perpendicular Anisotropies.

PB94-211174 
JACOX, M. B.

Spectroscopic Study of Reaction Intermediates and Mechanisms in Nitramine Decomposition and Combustion.

nisms in Nitram
AD-A296 $061 / 5$

\section{JACOX, M. E.}

Infrared and Near-Infrared Spectra of HCC and DCC Trapped in Solid Neon

AD-A295 578/9

03,773

Matrix Isolation Study of the Interaction of Excited Neon Atoms with BCl3: Infrared Spectra of $\mathrm{BCl}(\mathrm{sub} 3$, sup + ) $\mathrm{BCl}$ (sub 2, sup +), and $\mathrm{BCl}$ (sub 3 , sup -)

PB97-119143

01,187

Matrix Isolation Study of the Interaction of Excited Neon Atoms with $\mathrm{O} 3$ : Infrared Spectrum of $\mathrm{O}(($ sub 3$)(-))$ and Evidence for the Stabilization of O2... $(($ sub 4$)(+))$.

04,155

Mid- and Near-Infrared Spectra of Water and Water Dimer Isolated in Solid Neon.

PB95-125662

00,888

Production and Spectroscopy of Small Polyatomic Molecular lons Isolated in Solid Neon. (Reannouncement with New Availability Information).

AD-A234 043/8

00,704

Vibrational Spectra of Molecular lons Isolated in Solid Neon: HCCH+ and HCC-. (Reannouncement with New Availability Information).

AD-A253 551/6

00,707

Vibrational Spectra of Molecular Ions Isolated in Solid Neon. X. $\mathrm{H} 2 \mathrm{O}(+), \mathrm{HDO}(+)$, and $\mathrm{D} 2 \mathrm{O}(+)$

00,889

Vibrational Spectra of Molecular Ions Isolated in Solid Neon. XI. NO2(+). NO2(-), and NO3(-)

00,890

Vibrational Spectra of Molecular Ions Isolated in Solid Vibrational Spectra of Molecular lons Isolated in Solid
Neon. 6 . $\operatorname{CO} 4(-)$. (Reannouncement with New Availability Information).

00,705

Vibrational Spectra of Molecular lons Isolated in Solld Neon. 7. $\mathrm{CO}(+), \mathrm{C} 2 \mathrm{O} 2(+)$, and $\mathrm{C} 2 \mathrm{O} 2(-)$. (Reannouncement with New Availability Information)

00,706

Vibrational Spectra of Molecular Ions Isolated in Solid Neon. 11. NO2(+), NO2(-), and NO3(-). AD-A275 828/2 00,708

Vibrational Spectra of Molecular lons Isolated in Solid Neon. 13. lons Derived from $\mathrm{HBr}$ and $\mathrm{HI}$.

PB97-119234

01,188

JAFARABAD, K. R.

Enzyme and Protein Mass Transfer Coefficient in Aqueous

Two Phase Systems. 1. Spray Extraction Columns.
PB95-161162

00,594

JAFFE, D. T.

Extended $\mathrm{CO}(7$ yields 6) Emission from Warm Gas in Orion

PB96-102504

00,090

JAHANMIR, S.

Control of Friction and Wear of Alpha-Alumina with a Composite Solid-Lubricant Coating.

03,225

Evaluation of Thermal Wave Imaging for Detection of Machining Damage in Ceramics.

Fracture Mechanics Analysis of Near-Surface Cracks. PB94-172400 03,230 Mechanism of Mild to Severe Wear Transition in Alpha-Alu-

mina. 212354

03,233

Tribological Characteristics of Alpha-Alumina at Elevated Temperatures.
PB94-211018

02,963

Tribology Education: Present Status and Future Challenges. PB94-212362

02,965

JAI, J.

Laser-Synchrotron Hybrid Experiments: A Photon to Tickle, A Photon to Poke.

PB96-157847

03,704

JAIN, A.

Slow-Electron Collisions with CO Molecules in an Exact-Exchange Plus Parameter-Free Polarization Model.

PB95-202719

03,989

JAIN, K. K.

Intercomparison between NPL (India) and NIST (USA) Pressure Standards in the Hydraulic Pressure Region Up to $26 \mathrm{MPa}$.

PB96-113543

04,211

Intercomparison of the Effective Areas of a Pneumatic Piston Gauge Determined by Different Techniques.

PB94-212370

02,640

JAIN, M. K.

Characterization of Phase and Surface Composition of Silicon Carbide Platelets.

PB94-216264

03,043

JAJA, J.

Using Synthetic-Perturbation Techniques for Tuning Shared Memory Programs (Extended Abstract).

01,685
Using Synthetic Perturbations and Statistical Screening to Assay Shared-Memory Programs.

01,740

VLSI Architectures for Template Matching and Block Matching.

\section{JAMES, D. R.}

Investigation of S2F10 Production and Mitigation in Compressed SF6-Insulated Power Systems.

PB94-212388

02,467

Investigation of S2F10 Production and Mitigation in Compressed SF6- Insulated Power Systems.

PB96-155528

02,468

ANET, $S$.

Second Census Optical Character Recognition Systems PB94-188711

01,832

JANET, $S$. A.

NIST Form-Based Handprint Recognition System.

PB94-217106

JANEZIC, M. D.

Analysis of an Open-Ended Coaxial Probe with Litt-Off for Nondestructive Testing.

01,940

Distribution of Dielectric Relaxation Times and the Moment Distribution of Dielectric Relaxation Times and the Moment
Problem. PB96-112032

04,727

NIST 60-Millimeter Diameter Cylindrical Cavity Resonator Performance Evaluation for Permittivity Measurements.

PB94-151776

02,251

Open-Ended Coaxial Probes for Nondestructive Testing of Substrates and Circuit Boards.

02,078

Transmission/Reflection and Short-Circuit Line Methods for Measuring Permittivity and Permeability

JANIK, D.

02,211

Intercomparison of Thermal Converters at NIM, NIST, PTB, SIRI and VSL from 10 to $100 \mathrm{MHz}$.

PB94-172459

02,027

NOWSKI, G. M.

Beneficial Effects of Nitrogen Atomization on an Austenitic Stainless Stee

03,209

Effect of Backtill and Atomizing Gas on the Powder Porosity and Mechanical Properties of $304 \mathrm{~L}$ Stainless Steel.

PB94-185394

03,205

JANSEN, $W$.

Framework for National Information Infrastructure Services.
PB95-103719
02,723

JANSEN, W. A.

Conformance Assessment of Transport Layer Security Implementations.

01,576

Conformance Testing of a Lower Layer Security Protocol.

PB94-185402

01,577

SDNS Security Management

PB95-161170

01,592

Taxonomy for Security Standards.

01,602

PBNSEN

Rate of Heat Release of Wood Products.

PB94-212404

03,403

Standardization of Formats and Presentation of Fire Data -

The FDMS.

PB94-198462

01,371

JANZEN, A. R

Ab initio Calculations for Helium: A Standard for Transport Property Measurements.

PB96-102041

01,060

JARGON, J. A

$30 \mathrm{MHz}$ Comparison Receiver

PB96-200407

01,972

Coaxial Line-Reflect-Match Calibratlon

PB96-200118

02,246

Electrical Measurements of Microwave Flip-Chip InterPBnections.

02,436

High-Speed Interconnection Characterization Using Time Domain Network Analysis.

04,061

Microwave Characterization of Flip-Chip MMIC Compo-

PB96-176722

02,434

Microwave Characterization of Flip-Chip MMIC Interconnections.

02,435

NIST $30 \mathrm{MHz}$ Linear Measurement System.

PB94-169752

02,020

Revised Uncertainty Analysis for the NIST 30-MHz Attenuation Calibration System.

PB95-16876

01,907

Time Domain Network Analysis Using the Multiline TRL

PB95-202925

02,065

Two-Tier Multiline TRL for Calibration of Low-Cost Network Analyzers.

PB96-157888

01,947

JARRETT, D. G.

Automated Guarded Bridge for Calibration of Multimegohm Standard Resistors.

PB97-119150

02,289

Automatic Inductive Voltage Divider Bridge Operates from $10 \mathrm{~Hz}$ to $100 \mathrm{kHz}$

02,032

Constant Temperature and Humidity Chamber for Standard Resistors.

PB96-122494

02,275

Low Thermal Guarded Scanner for High Resistance Meas-

urement Systems.

02,288

Resistance Measurements from $10 \mathrm{M}$ Omega to $1 \mathrm{~T}$ Omega at NIST

PB97-119168

02,290

ARUGA, $P$.

DNA Base Damage in Lymphocytes of Cancer Patients Undergoing Radiation Therapy

PB97-122444

03,643

DNA Damage and DNA Sequence Retrieval from Ancient

Tissues.

03,556

Novel Activities of Human Uracil DNA N-glycosylase for $\mathrm{Cy}$ -

tosine-Derived Products of Oxidative DNA Damage.
PB96-164132

03,479

Repair of Products of Oxidative DNA Base Damage in

Human Cells.

03,555

JASON, N. H.

BFRL Fire Publications, 1993

PB94-164191

00,192

Building and Fire Research Laboratory Publications, 1993.

PB95-143202 00,369

Building and Fire Research Laboratory Publications, 1994.

PB95-226684 00,398

Evolution of a United States Information System.

PB96-157896

02,713

FIREDOC Users Manual, 3rd Edition.

01,378

In Situ Burning Oil Spill Workshop Proceedings. Held in Orlando, Florida on January 26-28, 1994

PB95-104907

Information Resources for the Fire Community.

PB96-148119

Information Transfer in the 21 st Century.

PB96-157904

Locating Fire Engineering Information.

PB95-161188

02,583

00,211

Locating Fire Information

PB96-190137

02,714

Beach, California on January 30-31, 1995. Proceedings. 


\section{PERSONAL AUTHOR INDEX}

Superconducting Resonator and a Cryogenic GaAs Field. Effect Transistor Amplifier as a Single-Ion Detection Sys. tem.

PB95-202727

03,990

\section{JEFFERY, A.}

Conversion of a 2-Terminal-Pair Bridge to a 4-Terminal-Pair Bridge for Increased Range and Precision in Impedance Measurements.

PB97-119176

02,103

NIST Comparison of the Ouantized Hall Resistance and the Realization of the SI Ohm Through the Calculable CapaciPB97-119184

02,291

NIST Comparison of the Ouantized Hall Resistance and the Realization of the SI Ohm Through the Calculable Capacitor. Conference Proceedings, June 17-20, 1996. tor. Conference

02,292

Precision Tests of a Ouantum Hall Effect Device DC Equivalent Circuit Using Double-Series and Triple-Series Connec.

PB96-159256

01,953

Proposed Tests to Evaluate the Frequency-Dependent Ca. pacitor Ratio for Single Electron Tunneling Experiment. PB97-111454

01,982

JELENKOVIC, $B$.

Atomic-scale characterization of hydrogenated amorphous. silicon films and devices. Annual subcontract report, 14 February 1994--14 April 1995.

DE95009287

02,294

\section{JELENKOVIC, B. M.}

Excitation of Balmer Lines in Low-Current Discharges of Hydrogen and Deuterium.

PB95-150546

03,893

JEMIAN, P. $R$.

Evolution of the Pore Size Distribution in Final-Stage Sintering of Alumina Measured by Small-Angle X-ray Scattering (Reannouncement with New Availability Intormation) AD-A249 178/5

03,023

JENKINS, $R$.

Introduction of a NIST Instrument Sensitivity Standard Reference Material for X-Ray Powder Diffraction.

PB94-200318

Powder Diffraction File: Past, Present, and Future. PB97-109086

04,800

JENNINGS, D. A

Laboratory Measurements for the Astrophysical Identification of MgH.

00,073

JENNINGS, $\boldsymbol{H}$.

Calculation of the Thermal Conductivity and Gas Permeability in a Uniaxial Bundie of Fibers.

PB95-180931

03,058

\section{JENNINGS, H. M.}

Diffusion Studies in a Digital-Image-Based Cement Paste

Microstructural Mode
PB94-198801

01,312

Modelling Drying Shrinkage of Cement Paste and Mortar. Part 1. Structural Models from Angstroms to Millimeters.
PB96-135132 03,074 PB96-135132

Binder Characterization and Evaluation by Nuclear Mag-

netic Resonance Spectroscopy.
PB94-193471

01,334

JENSEN, H. D.

Accuracy of the Electron Pump.

Metrological Accuracy of the Electron Pump.

04,743

PB95-168910

03,951

Performance of the Electron Pump with Stray Capacitances

PB96-200902 01,976

Testing for Metrological Accuracy of the Electron Pump.

PB95-175873 04,663

Thermal Enhancement of Cotunneling in Ultra-Small Tunnel Junctions.

JESPERSEN, J.

04,658

Preliminary Comparison of Time Transters via LASSO, GPS

and Two-Way Satellite.

01,529

JESPERSEN, J. L.

Satellite Two-Way Time Transfer: Fundamentals and Recent Progress

01,536

JESSEN, $P$.

Temperature of Optical Molasses for Two Different Atomic Angular Momenta.

$\begin{array}{ll}\text { PB95-126058 } 03,881 & \end{array}$

Ultracold Collisions: Associative Ionization in a Laser Trap.

PB94-213238 03,859

JESSEN, P. S.

Laser Modification of Ultracold Collisions: Experiment.
PB96-157987

Measurements of Fluorescence from Cold Atoms: Localization in Three-Dimensional Standing Waves.

03,943
JESSUP, R. S.

Precise Measurement of Heat of Combustion with a Bomb Calorimeter.

AD-A286 701/8

03,770

AET, K. L.

Preparation and Monitoring of Lead Acetate Containing

Drinking Water Solutions for Toxicity Studies.
PB94-193885

JI, $x$.

Positive and Negative Cooperativities at Subsequent Steps of Oxygenation Regulate the Allosteric Behavior of Multistate Sebacylhemoglobin

PB97-119374

03,486

J|A, J.

Improved Reflectometry Facility at the National Institute of Standards and Technology.

PB96-160338

04,087

New NIST/ARPA National Soft X-ray Reflectometry Facility

PB96-158092 04,080

Phonon Relaxation in Soft-X-ray Emission of Insulators

PB96-160296

04,085

JIA, J. J.

Al L2,3 Core Excitons in AlxGa1-x as Studied by Sott-X-ray Reflection and Emission

PB96-157839

04,067

Barium Contributions to the Valence Electronic Structure of YBa2Cu307-delta, PrBa2Cu307-delta, and Other BariumContaining Compounds.

$\begin{array}{ll}\text { PB96-158019 } & 04,076\end{array}$

Charge-Transfer-Induced Multiplet Structure in the N4,5O2,3 Soft-X-ray Emission Spectrum of Lanthanum.
PB96-163746

Cooper M(sub II,III) X-ray-Emission Spectra of Copper OX ides and the Bismuth Cuprate Superconductor.
PB96-158027

Intermediate Coupling in L2-L3 Core Excitons of $\mathrm{MgO}$ $\mathrm{Al} 2 \mathrm{O} 3$, and $\mathrm{SiO} 2$

PB96-158043

04,079

Local Partial Densities of States in $\mathrm{Ni}$ and Co Silicides Left $X$-Ray Emission Spectroscopy.

04,504

Simple Variable Line Space Grating Monochromator for Synchrotron Light Source Beamlines.

04,065

Soft-X-ray-Emission Investigation of Cobalt Implanted Silicon Crystals

PB96-157912

04,069

PB96-157920

04,070

Soft-X-ray-Emission Studies of Bulk Fe3Si, FeSi, and FeSi2, and Implanted Iron Silicides.

PB96-157938

04,071

JIANG, X.P. Grain Alignment and Transport Properties of
Bi2Sr2CaCu2O8 Grown by Laser Heated Float Zone Meth-

Od.

04,602

JIGGETS, R.

Texture Measurement of Sintered Alumina Using the March-Dollase Function.

PB96-179494

04,784

JILANI, A.

Elastic Constants of Isotropic Cylinders Using Resonant Ultrasound.

PB94-211919

04,497

JIN, G. $X$.

Critical Scaling Laws and a Classical Equation of State

PB95-169249 00,990

Global Thermodynamic Behavior of Fluid Mixtures in the Critical Region.
PB94-212420

04,199

INNAI, $\mathrm{H}$

Effect of Cross-Links on the Miscibility of a Deuterated Polybutadiene and Protonated Polybutadiene Blend.
PB94-212438 Inversion of the Phase Diagram from UCST to LCST in Deuterated Polybutadiene and Protonated Polybutadiene Blends.

PB94-212446

01,226

SANS Studies of Space-Time Organization of Structure in Polymer Blends.

PB95-153789

01,251

Time-Resolved Small-Angle Neutron Scattering Study of Spinodal Decomposition in Deuterated and Protonated Polybutadiene Blends. 1. Effect of Inıtial Thermal Fluctuations.

PB95-161196

01,252

JINNO, $\mathrm{M}$.

Millimeter-Resolution Optical Time-Domain Reflectometry Using a Four-Wave Mixing Sampling Gate.

02,190

Optical Sampling Using Nondegenerate Four-Wave Mixing in a Semiconductor Laser Amplifier.

PB96-122502

02,076

Optical Sampling Using Nondegenerate Four-Wave Mixing in a Semiconductor Laser Amplifier.

04,348

JOARDER, K.

New Method to Calculate Looming for Autonomous Obstacle Avoidance.

PB95-171435

01,600

B, V. A

2nu9 Band of Propyne-d3

JOEL, $\mathbf{R}$.

00,985

Directory of Law Enforcement and Criminal Justice Associations and Research Centers.

PB96-178918

04,872

JOHANSSON, $M$.

Critical Current Behavior of Ag-Coated YBa2Cu307-x Thin Films.

(141016

04,549

JOHANSSON, M. E.

Effects of Etching on the Morphology and Surface Resistance of YBa2Cu3O7-delta Films.

PB96-135355

02,410

Magnetic Field Dependence of the Critical Current Anisotropy in Normal Metal-YBa2Cu3O7-delta Thin Film Bilayers. PB95-141024

JOHNK, $R$.

Aperture Excitation of Electrically Large, Lossy Cavities.

PB95-175675 00,031

Crosstalk between Microstrip Transmission Lines. (NIST

PB95-180337

02,225

OHNK, R. T.

Alternative Contour Technique for the Efficient Computation of the Effective Length of an Antenna.

PB96-141361

02,011

00,029

Crosstalk between Microstrip Transmission Lines.

PB94-135639

02,210

Measurements of Shielding Effectiveness and Cavity Char-

acteristics of Airplanes.

PB94-210051 00,030

Rapid Evaluation of Mode-Stirred Chambers Using Impulsive Waveforms.

PB96-210026

01,979

Time-Domain Measurements of the Electromagnetic Backscatter of Pyramidal Absorbers and Metallic Plates. PB94-185410

JOHNSON, A. L.

Structure of Molecules on Surfaces as Determined Using Electron-Stimulated Desorption.

PB94-216165

00,852

JOHNSON, B. C.

Calibration in the Earth Observing System (EOS) Project. Part 2. Implementation

PB97-112213

04,842

Comparison of Filter Radiometer Spectral Responsivity with the NIST Spectral-Irradiance and Illuminance Scales. PB97-113161

04,162

Intercomparison of the ITS-90 Radiance Temperature Scales of the National Physical Laboratory (U.K.) and the National Institute of Standards and Technology. 
JOHNSON, D. L.

Calculation of the Thermal Conductivity and Gas Permeability in a Uniaxial Bundle of Fibers.

PB95-180931

JOHNSON, D. R.

Aid for Smaller Businesses.

03,058

PB94-21246

00,492

Montgomery Education Connection and Resource Education Awareness Partnership Making Connections between Local Schools and NIST Volunteers.

PB96-159769

00,134 Topological Influences on Polymer Adsorption and
Desorption Dynamics. Desorption Dynamics.

01,227

JOHNSON, J. A.

Ultrasonic Sensing of GMAW: Laser/EMAT Defect Detection System.

JOHNSON, J. W.

02,878

Summary of the Apparent Standard Partial Molal Gibbs Free Energies of Formation of Aqueous Species, Minerals, and Gases at Pressures 1 to 5000 Bars and Temperatures 25 to $1000 \mathrm{C}$.

JOHNSON, K.S.

01,113

Evaluating Investments in Law Enforcement Equipment: An Annotated Bibliography.

04,867

Ada Compiler Validation Summary Report: Certificate Number 940902S1.11376. UNISYS Corporation IntegrAda fo Windows NT, Version 1.0. Intel Deskside Server for Intel Pentium $60 \mathrm{MHz} \Rightarrow$. Intel Deskside Server with Inte Pentium $60 \mathrm{MHz}$

AD-A288 572/1 $\quad 01,659$

Ada Compiler Validation Summary Report: Certificate Number $940902 S 1.11377$ UNISYS Corporation. IntegrAda for Windows NT, Version 1.0. Intel Deskside Server with Intel $80486 \mathrm{D} \times 266 \Rightarrow$ Intel Deskside Server with Intel $804860 \times 266$

$804860 \times 266$.

JOHNSON, R. D.

01,658

Electronic Spectra of $\mathrm{CF} 2 \mathrm{Cl}$ and $\mathrm{CFCl} 2$ Radicals Observed by Resonance Enhanced Multiphoton Ionization.

00,927

Experimental and Abinitio Studies of Electronic Structures Experimental and Abinitio Studies or Electronic Structures PB94-212131 00,826 Thermochemistry of CF2O and FCO. Kinetics of the Reaction of CCl3-Br-2 and the Thermochemistry of CCl3 Radical and Cation. PB94-212115

00,824

Multiphoton Ionization of $\mathrm{SiH} 3$ and $\mathrm{SiD} 3$ Radicals: Electronic Spectra, Vibrational Analyses of the Ground and Rydberg States, and Ionization Potential.

PB94-212503

00,837

Multiphoton Ionization of $\mathrm{SiH} 3$ and $\mathrm{SiD} 3$ Radicals. 2. Three Photon Resonance-Enhanced Spectra Observed between 460 and $610 \mathrm{~nm}$.

00,835

New Electronic States of NH and ND Observed from 258 to $288 \mathrm{~nm}$ by Resonance Enhanced Multiphoton Ionization PB94-212495

00,836

New Rydberg States of Aluminum Monofluoride Observed by Resonance-Enhanced Multiphoton Ionization Spectros Copy. 199544

00.797

Resonance Enhanced Multiphoton Ionization Detection of $\mathrm{GeF}$ and $\mathrm{GeCl}$ Radicals.

PB94-212123

00,825

Resonance Enhanced Multiphoton Ionization Spectroscopy of the SnF Radical.

01,176

Resonance Enhanced Multiphoton Ionization Spectroscopy of 2-Butene-1-yl $(\mathrm{C} 4 \mathrm{H} 7)$ between $455-485 \mathrm{~nm}$. PB95-151031

00,670

JOHNSON, R. G

Hybrid Undulator for the NIST-NRL Free-Electron Laser.

B94-212529

04,238

NIST-NRL Free-Electron Laser Facility.

PB94-212511

04,237

Simulation of C60 Through the Plastic Transition Tempera-

Simulation of C60 Through the Plastic Transition Tempera-
tures.
PB96-102546
04,713

JOHNSON, W. C.

Morphological Development of Second-Phase Particles in Elastically-Stressed Solids.

B 95-181111

03,355

JOHNSON, W. L.

Ultrasonic Spectroscopy of Metallic Spheres Using Electromagnetic-Acoustic Transduction.

04,185
JOHNSON, W. R.

Evaluation of Two-Photon Exchange Graphs for HighlyCharged Heliumlike lons.

03,808

PB94-198918

JOHNSONBAUGH, D. Characterization of the Binding of Gallium, Platinum, and
Uranium to Pseudomonas Fluorescens by Small-Angle Xray Scattering and Transmission Electron Microscopy

PB94-172509

03,453

JOHNSONBAUGH, D. S.

Flow-Induced Structure in Polymers. Chapter 17. PhaseSeparation Kinetics of a Polymer Blend Solution Studied by a Two-Step Shear Quench.

PB96-123377

03,388

JOHNSSON, E. L.

Carbon Monoxide Production in Compartment Fires: Reduced-Scale Enclosure Test Facility.

01,394

Greatly Enhanced Soot Scattering in Flickering Ch4/Air Diffusion Flames.

01,361

Scaling Compartment Fires: Reduced- and Full-Scale Enclosure Burns

PB96-175708

00,224

Study of Technology for Detecting Pre-Ignition Conditions of Cooking-Related Fires Associated with Electric and Gas Ranges and Cooktops, Phase 1 Report.

PB96-128095

JOHNSTON, A. D.

Physical and Chemical Properties of Resin-Reinforced Calcium Phosphate Cements.

PB95-180212

00,171

JOKLIK, R. G.

Multiphoton Ionization Spectroscopy Measurements of Silicon Atoms during Vapor Phase Synthesis of Ceramic Par-

PB95-151999

03,913

JONES, A.

Japan Technology Program Assessment. Simulation: Stateof the-Art in Japan.

PB95-217097

02,827

JONES, C. A.

Dielectric Measurements on Printed-Wiring and Circuit Boards, Thin Films, and Substrates: An Overview.
PB96-147038

02,384

PB96-112297

JONES, C. G.

Characterization of a Health Physics Instrument Calibration

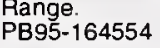

03,629

JONES, D.

Economic, Energy, and Environmental Impacts of the Energy-Related Inventions Program.

DE94-01716

00,008

JONES, F.E.

Examination of Parameters That Can Cause Error in Mass Determinations.

03,784

Use of the Electronic Balance for Highly Accurate Direct Mass Measurements Without the Use of External Mass Standards.

PB94-1877 13

03,803

JONES, G. A.

Comparative Study of $\mathrm{Fe}-\mathrm{C}$ Bead and Graphite Target Performance with the National Ocean Sciences AMS (NOSAMS) Facility Recombinator Ion Source. PB95-175790

00,693

JONES, G. R.

Display-Measurement Round-Robin.

02,186

Specular and Diffuse Reflection Measurements of Electronic Displays.

PB97-119200

02,208

Survey of the Components of Display-Measurement Standards.

PB96-122528

02,188

Survey of the Components of Display-Measurement Standards.

02,209

JONES, $K$.

Temperature of Optical Molasses for Two Different Atomic Angular Momenta.

PB95-126058

03,881

JONES, K. $M$.

Measurement of the Atomic $\mathrm{Na}(3 \mathrm{P})$ Lifetime and of Retardation in the Interaction between Two Atoms Bound in a Molecule.

PB97-122360

04,178

JONES, M. C.

Brownian Diffusion of Hard Spheres at Finite ConcentraPB95-164307

00,975

Distributed measurements of tracer response on packed bed flows using a fiberoptic probe array. Final report.

DE95013079

00,667

JONES, R. D.

Beam Analysis Round Robin.

PB94-212545

Error Propagation in Laser Beam Spatial Parameters.

04,310

aser-Beam Analysis Pinpoints Critical Parameters.

04,240

Thermal Modeling and Analysis of Laser Calorimeters.

PB96-140405 04,354

Widths and Propagation of a Truncated Gaussian Beam

$\begin{array}{ll} & 04,287\end{array}$

JONES, $S$.

Report on the NIST Low Accelerating Voltage SEM Magnification Standard Interiaboratory Study.

PB96-201074

02,445

JONES, S. N.

Electrical Method for Determining the Thickness of Metal Films and the Cross-Sectional Area of Metal Lines.
PB95-203170

Scanning Electron Microscope Magnification Calibration Interlaboratory Study.

PB96-20108

01,164

JONES, W. W.

Calculating Flame Spread on Horizontal and Vertical Sur-

faces.

00,335

Fire Safety of Passenger Trains: A Review of Current Approaches and of New Concepts

PB94-152006

04,848

Improvement in Predicting Smoke Movement in Compartmented Structures.

00,332

New Concepts for Fire Protection of Passenger Rail Trans-

portation Vehicles.
PB95-162046 04,850

New Concepts for Fire Protection of Passenger Rail Transportation Vehicles. (NIST Reprint).

PB95-180774 04,863

Verification of a Model of Fire and Smoke Transport.

PB95.108718

00,357

ONKER, B. T.

Characterization of the ZnSe/GaAs Interface Layer by TEM and Spectroscopic Ellipsometry

04,655

Determination of the Optical Constants of ZnSe Films by Spectroscopic Ellipsometry.

04.656

JONNALAGADDA, S. V

Thermodynamic Interactions and Correlations in Mixtures of Two Homopolymers and a Block Copolymers by Small Angle Neutron Scattering.

ONSSON, L.

Large Local-Field Corrections in Optical Rotatory Power of Quartz and Selenium.

PB97-122378

JONSSON, P. G.

Mathematical Models of Transport Phenomena Associated with Arc-Welding Processes: A Survey.

PB96-135058

02,870

Power Characteristics in GMAW: Experimental and Numerical Investigation

03,296

JOSELL, D.

Interfacial Free Energies from Substrate Curvature Measurements of the Creep of Multilayer Thin Films

PB94-185428

JOSEPHSON, E. S.

Estimation of the Absorbed Dose in Radiation-Processed 


\section{PERSONAL AUTHOR INDEX}

JOSHI, Y.

Natural Convection from an Array of Electronic Packages Mounted on a Horizontal Board in a Narrow Aspect Ratio Enclosure.

PB96-164017

02,087

\section{OSHI, Y. N.}

Analysis of the (5d(2)+5d6s)-5d6p Transition Arrays of Os

VII and Ir VIII, and the 6s (2) S-6p (2)P Transitions of Ir IX.
PB96-159264

01,954

Analysis of the $5 s(2) 5 p(2)-(5 s 5 p(3)+5 s(2) 5 p 5 d+5 s(2) 5 p 6 s)$ Transitions of Four-Times lonized Xenon (Xe V).

03,900

Spectrum and Energy Levels of Triply lonized Barium (Ba IV)

PB95-140877

03,888

JOUBRAN, $R$.

Structure of a Triglyceride Microemulsion: A Small Angle Neutron Scattering Study.

01,077

JOYCE, S. A.

Desorption Induced by Electronic Transitions.

PB94-216173

00.853

Influence of Coadsorbed Potassium on the Electron-Stimu lated Desorption of $F(+), F(-)$, and $F\left({ }^{*}\right)$ from $P F 3$ on $\mathrm{Ru}(0001)$

PB96-157946

04,072

Role of Adsorbed Alkalis in Desorption Induced by Electronic Transitions.

00,762

Structure of Molecules on Surfaces as Determined Using Electron-Stimulated Desorption.

PB94-216165

00,852

JUERTS, $M$.

Ground Vehicle Control at NIST: From Teleoperation to Autonomy.

N94-34037/9

03,758

JUDGE, $P$.

Transition Regions of Capella.

PB96-123336

00,105

Transition Regions of Capella (1995).

00,108

JUDISH,

Multi-State Two-Port: An Altemative Transfer Standard

PB95-168530

02,049

JUDY, D. C

Electrical Breakdown in Transformer Oil in Large Gaps. PB95-150579

01,889

JUHASZ, E.

dc Method for the Absolute Determination of Conductivities of the Primary Standard $\mathrm{KCl}$ Solutions from $0 \mathrm{C}$ to $50 \mathrm{C}$

PB94-219342

JULIENNE, $P$.

Ultracold Collisions: Associative Ionization in a Laser Trap.

PB94-213238

JULIENNE, P. $S$

Density Matnx Calculation of Population Transfer between Vibrational Levels of $\mathrm{Na} 2$ by Stimulated Raman Scattering with Temporally Shitted Laser Beams.

PB94-198546

00,787

Excitons in Complex Ouantum Nanostructures.

01,184

PB97-118343

Intersystem Crossing in Collisions of Aligned $\mathrm{Ca}(4 \mathrm{s5p}$ (1)P + He: A Half Collision Analysis Using Multichannel Ouantum Defect Theory.

00,813

Laser Assisted Collisions at Ultracold Temperatures. PB95-161220

03,929

Measurement of the Atomic $\mathrm{Na}(3 \mathrm{P})$ Lifetime and of Retardation in the Interaction between Two Atoms Bound in a Molecule.

PB97-122360

04,178

Multichannel Quantum Defect Half Collision Analysis of K2 Photodissociation Through the B1Pi(sub u) State.

PB94-211125

00,812

Theory of Atomic Collisions at Ultracold Temperatures. PB94-212560

03,851

JULL, A. J. T.

Radiocarbon Measurements of Atmospheric Volatile Organic Compounds: Ouantifying the Biogenic Contribution.

02,574

Hyperfine Effects and Associative Ionization of Ultracold So-

dium.

03,903

JUN, J. S.

Metrology Standards for Advanced Semiconductor Lithog raphy Referenced to Atomic Spacings and Geometry.
PB96-160718

JUNG, H. J.

Comparisons of Some NIST Fixed-Point Cells with Similar Cells of Other Standards Laboratories.

Direct Comparison of Three PTB Silver Fixed-Point Cells with the NIST Silver Fixed-Point Cell.

00,628
JUNGNER, $P$.

Delivering the Same Optical Frequency at Two Places: Accurate Cancellation of Phase Noise Introduced by an Optcal Fiber or Other Time-Varying Path.

PB96-102736

04,332

Observations of $3 \mathrm{C} 273$ with the Goddard High Resolution Spectrograph on the Hubble Space Telescope.

PB95-202321

00.076

JUROSHEK, J.

Multi-State Two-Port: An Alternative Transfer Standard PB95-168530

02,049

JUROSHEK, J. R.

Measurements of the Characteristic Impedance of Coaxial Air Line Starıdards.

PB95-168787

02,221

KABIRI-BADR, $M$.

Statistical Thermodynamics of Phase Separation and Ion Partitioning in Aqueous Two-Phase Systems.

PB94-199387

01,212

KACKER, $\mathbf{R}$

Computing Effects and Error for Large Synthetic Perturbation Screenings.

01,675

Scalability Test for Parallel Code.

PB96-146758

01,749

Simple Scalabilit

01,688

Study on the Reuse of Plastic Concrete Using Extended Set-Retarding Admixtures.

PB96-122130

00,402

PBnthetic-Perturb

Time-Perturbation Tuning of MIMD Programs
PB94-164399

01,687

Time-Perturbation Tuning of MIMD Programs.

01,681

Using Synthetic-Perturbation Techniques for Tuning Shared Memory Programs (Extended Abstract)

01,685

Using Synthetic Perturbations and Statistical Screening to Assay Shared-Memory Programs.

PB96-103031

KACKER, R. N.

Efficient Experiment to Study Superconducting Ceramics.

Statistical Analysis of Parameters Affecting the Measurement of Particle-Size Distribution of Silicon Nitride Powders ment of Particle-Size Distributio

by Sedigraph (T
PB94-216249

Statistical Ouality Control Technology in Japan.

PB94-199064

03,042

Taguchi's Parameter Design: A Panel Discussion. PB96- 111802

02,708

KADUK, J. A.

Conventional and Eccentric Uses of Crystallographic Databases in Practical Materials Identification Problems. PB97-109102

KAESER, R. S.

ITS-90 Calibration Facility.

PB96-160916

00,627

Optimization of Highway Concrete Technology.

PB94-182995
KAETZEL, L. J.

Highway Concrete (HWYCON) Expert System User Reference and Enhancement Guide.

PB94-215670

01,316

KAFAFI, S. A.

Carbon Acidities of Aromatic Compounds. 1. Effects of InRing Aza and External Electron-Withdrawing Groups PB94-216595

00,860

Experimental and Abinitio Studies of Electronic Structures of the $\mathrm{CCl} 3$ Radical and Cation.

PB94-212131

00,826

Thermal Decomposition of Hydroxy- and Methoxy-Substituted Anisoles.

00,767

KAHN A. H.

Development in the Theory and Analysis of Eddy Current Sensing of Velocity in Liquid Metals.

PB94-21258

03,335

Low-Frequency Excitations of Oriented DNA.

AISER, D.

03,548

Epitaxial Growth of $\mathrm{BaTiO} 3$ Thin Films at $600 \mathrm{C}$ by Metalorganic Chemical Vapor Deposition.

03,071

Image Depth Profiling SIMS: An Evaluation for the Analysis Image Depth Profiling SIMS: An Evaluation for the Analysis Superconductors
PB95-126116

04.530

Meissner, Shielding, and Flux Loss Behavior in Single-Crystal YBa2Cu3O6+x.

04,464

Nature of (001) Tilt Grain Boundaries in YBa2Cu3O6+x.

04,528 Temperature Dependence of Vortex Twin Boundary Interaction in Yttrium Barium Copper Oxide (YBa2Cu3O6+x).
PB95-162442

Weak-Link-Free Behavior of High Angle YBa2Cu3O7-x Grain Boundaries in High Magnetic Fields.

PB94-198421

04,459

X-Ray-Diffraction Study of a Thermomechanically Detwinned Single Crystal of YBa2Cu3O6+x.

PB95-151726

04,581

KAISER, $\mathbf{R}$.

Frequency-Stabilized LNA Laser at 1.083 mum: Application to the Manipulation of Helium 4 Atoms.

PB95-176186

KAJIYAMA, T.

Preparation of 2-Dimensional Ultra Thin Polystyrene Film by Water Casting Method.

PB95-162806

KALCEFF, M. A.

Deformation and Fracture of Mica-Containing Glass-Ceramics in Hertzian Contacts.

PB96-179452

03,080

KALKUR, T. S.

High Critical Temperature Superconductor Tunneling Spectroscopy Using Squeezable Electron Tunneling Junctions. PB95-163721 04,627

Tunneling Measurement of the Zero-Bias Conductance Peak and the Bi-Sr-Ca-Cu-O Thin-Film Energy Gap.

PB95-163739

04,628

Weatherability of Plastic Materials.

AD-A301 675/5

02,968

KAMITAKAHARA, W. A.

Discontinuous Volume Change at the Orientational-Ordering Transition in Solid $\mathrm{C} 60$

PB94-211828

00,821

Energy Distributions of Neutrons Scattered from Solid C60 by the Beryllium Detector Method

03,740

Inelastic Neutron Scattering Studies of Rotational Exciations and the Orientational Potential in $\mathrm{C} 60$ and A3C60 Compounds.

PB94-172673

00,763

Neutron-Scattering Study of Librations and Intramolecular Phonons in Rb2.6K0.4C60.

00,958

Neutron Scattering Study of the Lattice Modes of Solid

Pubane.

01,126

KANA, T. M.

High-Performance Liquid Chromatography of Phytoplankton Pigments Using a Polymeric Reversed-Phase $\mathrm{C}_{18} \mathrm{Column}$. PB95-151130

KANDA, $M$.

Alternative Contour Technique for the Etficient Computation of the Effective Length of an Antenna.

PB96-141361 


\section{PERSONAL AUTHOR INDEX}

KAUTZ, R. L.

KAO, J. Y.

Evaluation of GSA Maintenance Practices of Large Centrifugal Chillers and Review of GSA Refrigerant Management Practices.

PB94-143344. 02,502

Factors Affecting the Energy Consumption of Two Refrigerator-Freezers.
PB97- 112312

00,311

Using Emulators to Evaluate the Performance of Building Energy Management Systems.

Energy Managem
PB95-175774

00,259

KAPLAN, B.

SQA Standards and Total Quality Management.

PB96-111844

01,743

KAPLAN, C. $R$.

Computations of Enhanced Soot Production in Time-Varying $\mathrm{CH} 4 /$ Air Diffusion Flames.

01.440

KARAKAYA, A.

Novel Activities of Human Uracil DNA N-glycosylase for Cytosine-Derived Products of Oxidative DNA Damage.

PB96-164132

03,479

Modification of Deoxyribose-Phosphate Residues by Extracts of Ataxia Telangiectasia Cells.

PB94-212602

03,458

Radon in the Lung

PB97-110035

03,638

Systematics of Alpha-Particle Energy Spectra and Lineal

Energy (Y) Spectra for Radon Daughters.

KARASZ, F. A.

03.615

Inelastic-Neutron-Scattering Studies of Poly(p-phenylene vinylene).

PB95-180766

01.014

KAREN, P.

Neutron Powder Diffraction Study of the Nuclear and Magnetic Structures of the Oxygen-Deficient Perovskite YBaCuCoO5

KAREN, V. L.

00,954

Neutron Powder Diffraction Study of the Nuclear and Magnetic Structures of the Oxygen-Deficient Perovskite YBaCuCOO5.

PB95-161097 00.954

NIST Crystallographic Databases for Research and AnalyPBis. 109094

Workshop Highlights.

04,801

04,811

KARIM, A.

Compatibilization of Polymer Blends by Complexation. 2. Kinetics of Interfacial Mixing.

PB97-111900

01,295

Dimensional Crossover in the Phase Separation Kinetics of Thin Polymer Blend Films.

03,395

Neutron Reflectivity Study of the Density Profile of a Model End-Grafted Polymer Brush: Influence of Solvent Ouality.
PB95-202735

Phase Separation in Thin Film Polymer Blends With and Without Block Copolymer Additives.

KARKI, S.

01,294

Reproducibility of Tests on Energy Management and Control Systems Using Building Emulators.

PB95-175980

00,260

KARLIN, B.

Lattice Position of Si in GaAs Determined by X-Ray Standing Wave Measurements.

04,632

KARLIN, B. A.

Evolution of X-ray Resonance Raman Scattering into X-ray Fluorescence from the Excitation of Xenon Near the L3 Edge.

02751

04,025

Inner-Valence States $\mathrm{CO}(+)$ between $22 \mathrm{eV}$ and $46 \mathrm{eV}$ Studied by High Resolution Photoelectron Spectroscopy and $\mathrm{ab}$ Initio Cl Calculations.

PB95-180055

03,961

KARR, P. $R$.

Penetration and Diffusion of Hard X-rays through Thick Barriers. III. Studies of Spectral Distributions.

AD-A292 502/2

03,771

ARTHA, V. B.

2nu9 Band of Propyne-d3

PB95- 164513

00,985

Tunneling-Rotation Spectrum of the Hydrogen Fluoride

Dimer.

00,793

KASCHNITZ, E. Measurements of Thermophysical Properties of Nickel Near
Its Melting Temperature by a Microsecond-Resolution Transient Technique.
PB96-102579

04,210 Radiance Temperatures at $1500 \mathrm{~nm}$ of Niobium and Molyb-
denum at Their Melting Points by a Pulse-Heating Tech-

nique.
PB97-1 18699

04,167

KASHIWAGI, T.

Effects of Molecular Weight and Thermal Stability on Polymer Gasification.

PB94-212610

01,228

Effects of Sample Mounting on Flammability Properties of Intumescent Polymers.

03,389

Estimate of Flame Radiance via a Single Location Measure

ment in Liquid Pool Flames.
PB94-211596

02,476

Estimate of the Effect of Scale on Radiative Heat Loss Fraction and Combustion Efficiency.

02,486

PB95-150447

Gas Phase Oxygen Effect on Chain Scission and Monome
Content in Bulk Poly(methyl methacrylate) Degraded by Exernal Thermal Radiation.

PB96-204078

01,293

Heat Transier in an Intumescent Material Using a Three-Dimensional Lagrangian Model.

PB96-164066

00,408

Ignition and Subsequent Flame Spread Over a Thin Cellúlosic Material.

04,836

Ignition and Subsequent Transition to Flame Spread in a Microgravity Environment.

04,828

Ignition and Transition to Flame Spread Over a Thermally Thin Cellulosic Sheet in a Microgravity Environment.

PB96-160288

04,837

Measurement of Radiative Feedback to the Fuel Surface of a Pool Fire.

02,477

Polymer Combustion and Flammability: Role of the Condensed Phase.

01,279

Simultaneous Optical Measurement of Soot Volume Frac tion, Temperature, and $\mathrm{CO} 2$ in Heptane Pool Fire.

PB96-102132

01,397

Transition from Localized Ignition to Flame Spread Over a Thin Cellulosic Material in Microgravity

PB96-155809

04,835

KASHIWAZAKI, S.

Orientation Effects on ESR Analysis of Alanine-Polymer Dosimeters.

KASIANOWICZ, J.

Genetically Engineered Pores as Metal lon Biosensors. PB96-167408

Pore-Forming Protein with a Metal-Actuated Switch.

PB96-176557

KASIANOWICZ, J. J.

Current Fluctuations Reveal Protonation Dynamics and Number of Ionizable Residues in the alpha-Toxin Channel.

PB96-161732

Current Noise Reveals Protonation Kinetics and Number of lonizable Sites in an Open Protein Ion Channel.

PB96-161674

04,092

PB96-161658

Genetically Engıneered Pores for New Materials.
PB96-161641

03,551

Noise Analysis of Ionization Kinetics in a Protein Ion Chan-

PB96-161682

04,093

Protonation Dynamics in an Ion Channel Pore.

03,589

PB96-161757

Protonation Dynamics of the alpha-Toxin Ion Channel from Spectral Analysis of $\mathrm{pH}$-Dependent Current Fluctuations.

PB96-161740

KASPRZAK, $K$

Nickel(II)-Mediated Oxidative DNA Base Damage in Renal and Hepatic Chromatin of Pregnant Rats and Their Fetuses. Possible Relevance to Carcinogenesis.

PB94-212628

03,646

Oxidative DNA Base Damage in Renal, Hepatic, and Pulmonary Chromatin of Rats After Intraperitoneal Injection of Cobalt (II) Acetate.

PB95-150025

03,647

KASSOFF, J. M.

Appearance Potentials of Ions Produced by Electron-Impact Induced Dissociative Ionization of SF6, SF4, SF5Cl, S2F10. $\mathrm{SO} 2$, SO2F 2 , SOF2, and SOF 4 .

01,080

KASTNER, J.

Temperature Dependence of the Magnetic Excitations in Ordered and Disordered Fe72Pt28.

PB95.150223

04,563

KATO, $\mathrm{S}$.

Kinetics and Dynamics of Vibrationally State Resolved IonMolecule Reactions: $(14) \mathrm{N} 2(+)(\mathrm{v}=1$ and 2) and $(15) \mathrm{N} 2(+)(v=0,1$ and 2$)$ with $(14) \mathrm{N} 2$

04,023

Selected Ion Flow Tube-Laser Induced Fluorescence Instrument for Vibrationally State-Specific Ion-Molecule Reac-

PB94-185444

00,774

KATTI, R.

Magnetic Force Microscopy Images of Magnetic Garnet with Thin-Film Magnetic Tip.

PB95-176210

04,669

KATTNER, U.

Extrapolation of the Heat Capacity in Liquid and Amorphous Phases.

KATTNER, U. R.

04,147

Assessment of the Al-Sb System.

PB94-200474

03.329

Thermodynamic Assessment and Calculation of the Ti-Al

System.

$\begin{array}{ll}\text { PB94-212644 03,337 } & \end{array}$

Thermodynamic Calculation of the Ternary Ti-Al-Nb Sys-

PB94-212636

03,336

ATZ, S.

Glossary of Software Reuse Terms.

PB95-178992

KATZ, S. B.

Context Analysis of the Network Management Domain. Conducted as Part of the Domain Analysis Case Study.

PB94-142528

KAUFFMAN, D. A

Determination of the Prior-Austenitic Grain Size of Selected Steels Using a Molten Glass Etch.

PB94-211927

KAUFMAN, $v$.

03,208

Analysis of the $5 s(2) 5 p(2)-(5 s 5 p(3)+5 s(2) 5 p 5 d+5 s(2) 5 p 6 s)$ Transitions of Four-Times lonized Xenon $(X e V)$.

PB95-150769

03,900

Compilation of Energy Levels and Wavelengths for the Spectrum of Singly-lonized Oxygen (O II).

00,746

Improved Wavelengths for Prominent Lines of $\mathrm{Cr} X \mathrm{VI}$ to $\mathrm{Cr}$

PB95-150629 03,895

Observation of Pd-Like Resonance Lines Through $\mathrm{Pt}(32+)$ and $\mathrm{Zn}$-Like Resonance Lines of $\mathrm{Er}(38+)$ and $\mathrm{Hf}(42+)$

PB95-150637

Rb-Like Spectra: Pd X to Nd XXIV.

PB95-150645

03,896 $\mathrm{Pt}(33+)$

Spectra of $\mathrm{Ag}$ I Isoelectronic Sequence Observed from

Er(21+) to $\mathrm{Au}(32+)$
PB95-150660

03,899 tra of Sulfur (S I through $S \times V I$ ).

Wavelengths and Energy Levels of Neutral $\operatorname{Kr}(84)$ and Level Shifts in All Kr Even Isotopes.

PB94-14060
KAUTZ, R. L.

$24 \mathrm{GHz}$ Josephson Array Voltage Standard.

PB94-211588

03,780

Chaos in a Computer-Animated Pendulum. 


\section{PERSONAL AUTHOR INDEX}

\section{KAVOOSI, F}

Application of Boundary Element Methods to a Transien Axis-Symmetric Heat Conduction Problem.

01,375

Multiwavelength Birefringent-Cavity Mode-Locked Fibre

Laser.

04,262

KAWI, S.

Characterization of the Interaction of Hydrogen with Iridium Clusters in Zeolites by Inelastic Neutron Scattering Spectroscopy.

01,013

KAY, J. G.

International Marine-Atmospheric (222)Rn Measuremen Intercomparison in Bermuda. Part 2. Results for the Participating Laboratories.

PB96-17568

00,115

\section{KAY, W. B.}

Critical Properties and Vapor-Liquid Equilibria of the Binary System Propane + Neopentane.

00,999

Vapor-Liquid Equilibria of Mixtures of Propane and Isomeric

Hexanes.
PB95-175287

00,995

KAZUMATA, $Y$.

Harmonic and Static Susceptibilities of YBa2Cu3O7.

PB95-161139

KEARNEY, P. A.

Interfaces in Mo/Si Multilayers.

PB96-160668

KECK, J.

Proposed International Interactive Courseware Standard. PB96-123161

KEDER, N. L.

Structural and Chemical Investigations of $\mathrm{Na}$ 3(ABO4)3.4H2O-Type Sodalite Phases.

PB95-180733

01,012

KEDZIERSKI, M. A.

Calorimetric and Visual Measurements of R123 Pool Boiling on Four Enhanced Surfaces.

04,053

Causes of the Apparent Heat Transfer Degradation for Refrigerant Mixtures.

PB94-212701

Desıgn and Machıning of Copper Specimens with Micro Holes for Accurate Heat Transfer Measurements

PB95-180428

Effect of Inclination on the Performance of a Compac Brazed Plate Condenser and Evaporator.

PB96-136973

Enhancement of R123 Pool Boiling by the Addition of N.

Hexane.
PB96-165956

02,605

Simultaneous Visual and Calorimetric Measurements of $\mathrm{R} 11, \mathrm{R} 123$, and $\mathrm{R} 123 / \mathrm{Alkyl}$ benzene Nucleate Flow Boiling.

Single-Phase Heat Transfer and Pressure Drop Characteristics of an Integral-Spine-Fin Within an Annulus.

Single-Phase Heat Transfer and Pressure Drop Characterstics of an Integral-Spine Fin Within an Annulus.

Visual Measurement Technique for Analysis of Nucleate Flow Boiling.

03,262

KEEM, J.

Influence of Electrical Isolation on the Structure and Reflectivity of Multilayer Coakings Deposited on Dielectric Sub-

PB96-159736

04,365

New Method for Achieving Accurate Thickness Control for Uniform and Graded Multilayer Coatings on Large Flat Substrates.

PB96-159744

04,366

KEENY, S. M.

Properties and Interactions of Oral Structures and Restora tive Materials. Annual Report for Period October 1, 1990 to September 30,1991

PB94-160843

03,558

KEERY, W. J.

Modification of a Commercial SEM with a Computer Controlled Cathode Stabilized Power Supply.

Report on the NIST Low Accelerating Voltage SEM Magnification Standard Interlaboratory Study.

02,445

Scanning Electron Microscope Magnification Calibration Interlaboratory Study.

01,164

SEM Linewidth Metrology of X-ray Lithography Masks.

PB96-201108

01,164

P-ray Mask Metrology: The Development of Liner

Standards for X-ray Lithography.

PB95-162129

\section{KEIMER, B.}

High Resolution Inelastic Neutron Scattering Study of Self-Energy Effects in YBCO.

04,688

q Dependence of Self-Energy Effects of the Plane Oxygen Vibration in YBa2Cu3O7.

PB96-138516

\section{KELLEHER, D. E.}

01,096

Attempts at Extending the Unified Theory to Include ManyPB94-21271

04,408

Autoionizing Resonances in Electric Fields

03,853

PB94-212727

04,224

Filter Transmit

Isotope Shifts and Hypertine Splittings of the $398.8 \cdot \mathrm{nm} \mathrm{Yb}$ | Line. 199585

03,814

Search for Small Violations of the Symmetrization Postulate in an Excited State of Helium.

PB96-123518

04,050

KELLER, L.

Evaluation of a Tapered Roller Bearing Spindle for HighPrecision Hard Turning Applications

PB96-160494

02,700

KELLER, R. R.

Scanning Electron Microscopy Observations of Misfit Dislocations in Epitaxial In0.25Ga0.75As on GaAs(001). PB96-200159

03,004

Tensile Deformation-Induced Microstructures in Free-Standing Copper Thin Films.

KELLERMANN, $K$

04,715

From Superconductivity to Supernovae: The Ginzburg Symposium. Report on the Symposium Held in Honor of Vitaly L. Ginzburg. Held in Gaithersburg. Maryland on May 22, (1995.

04,649

\section{KELLETT, B. J.}

Dynamic Phenomena on the RS Canum Venaticorum Binary If Pegasi in August 1989. 1. Observational Data. Volume-Limited ROSAT Survey of Extreme Ultraviolet Emission from all Nondegenerate Stars within 10 Parsecs.
PB96-103189
00,093

KELLEY, E. F.

Can Displays Deliver a Full Measure: Manufacturing.
PB96-111935 Display-Measurement Round-Robin.

02,185 PB96-119227

02,186

Interface-Filter Characterization of Spectraradiometers and Colorimeters

04,399

Making Displays Deliver a Full Measure.

01,490

Nonlinear Color Transformations in Real Time Using a Video Supercomputer.

PB96-123021

02,191

Observations of Partial Discharges in Hexane Under High Magnification.
PB95-163127

01.900

Specification for Interoperability between Ballistic Imaging Systems. Part 1. Cartridge Cases.

01,860

Specular and Diffuse Reflection Measurements of Electronic DBisplays.

02,208

Survey of the Components of Display-Measurement Stand-

PB96-122528

02,188

Survey of the Components of Display-Measurement Standards.

02,209

KELLEY, M. H.

Determination of Complex Scattering Amplitudes in Low-Energy Elastic Electron-Sodium Scattering

PB94-216652

03,869

Domain Structur

Electron-atom collision studies using optically state selected beams. Progress report, May 15, 1987--May 14, 1988

DE95004446

03,776

Electron-atom collision studies using optically state selected beams. Progress report. May 15, 1988--May 14, 1991

DE95004447

03,777

Spin-Resolved Elastic Scattering of Electrons from Sodium. PB95-161774 03,933 Uncertainty Intervals for Polarized Beam Scattering Asymmetry Statistics.

03,825

KELLEY, R, J.

Electromagnetic Coupling Character of (001) Twist Boundaries in Sintered Bi2Sr2CaCu2O8+x Bicrystals.

PB96-176573

01,963

KELLOG, G. J.

Observed Frustration in Confined Block Copolymers.
PB95-150033

KELLY, G.

Use of Building Emulators to Evaluate the Performance of Building Energy Management Systems.

PB96-111901

00,269

KELL.Y, G. E.

Factors Affecting the Energy Consumption of Two Refrigerator-Freezers
PB 97.112312

00,311

Performance Testıng of a Family of Type I Combination Ap-

pliance.

02,505

Predicting the Energy Performance Ratings of a Family of Type I Combination Appliances.

PB95-105524

02,504

Using Emulator/Testers for Commissioning EMCS Software Operator Training. Algorithm Development, and Tuning Local Control Loops.

Using Emulators to Evaluate the Performance of Building Energy Management Systems.

KELLY, J.

00,259

Statistical Analysis of Parameters Affecting the Measurement of Particle-Size Distribution of Silicon Nitride Powders by Sedigraph (Trade Name).

PB94-216249

03,042

KELLY, J. F.

Compositional Homogeneity in Processing Precursor Powders to the Ba2YCu3O7-x High Tc Superconductor B94-212743

Effect of Sm2BaCuO5 on the Properties of Sintered (Bulk) PB96-119441

04,733

Interface Modification and Characterization of Silicon Carbide Platelets Coated with Alumina Particles.

PB95-108734

03,121

Loading Device for Fracture Testing of Compact Tension Specimens in the Scanning Electron Microscope.

02,652

Study of the Hydroxycarbonate Precursor Route to the YBa2Cu3O7-x High Tc Superconductor.

PB95-140471

KELLY, J. $R$.

Dental Materials.

BB94-172871

04,540 PBacture Toughness for Ceramics.
PB92434

03,032

Evaluation of Fracture Toughness and Residual Stress in Dental Porcelain by Indentation-Microfracture Method. PB95-125613

00,154

Evaluation of Fracture Toughness and Residual Stress in Dental Porcelain by Indentation-Microfracture Method. PB95-152831

Failure of All-Ceramic Fixed Partial Dentures 'In vitro' and 'In vivo': Analysis and Modeling. 
Study of Potential Applications of Automation and Robotics Technology in Construction. Maintenance and Operation of Highway Systems: A Final Report. Volume 1

01,341

Study of Potential Applications of Automation and Robotics Technology in Construction, Maintenance and Operation of Highway Systems: A Final Report. Volume 2

01,343

Study of Potential Applications of Automation and Robotics Technology in Construction. Maintenance and Operation of

Highway Systems: A Final Report. Volume 3.
PB95-251690

01,342

KENT, E. W

Robotics Application to Highway Transportation. Volume 1 Final Report

PB95-203790

03,654

Robotics Application to Highway Transportation. Volume 2. Literature Search

01,337

Robotics Application to Highway Transportation. Volume 3 Proposed Research Topics and CostBenefit Evaluations by PB95-171633

01,338

Robotics Application to Highway Transportation. Volume 4. Proposals for Potential Research

PB95-193173

01,339 Study of Potential Applications of Automation and Robotics
Technology in Construction, Maintenance and Operation of

Highway Systems: A Final Report. Volume 4
PB95-251641

01,340

Workshop on the Application of Virtual Reality to Manufacturing. Final Report. Held in Gaithersburg, Maryland on August 9, 1994.

KERCH, $\mathrm{H}$.

02,825

Imaging of Fine Porosity in a Colloidal Silica: Potassium Silicate Gel by Defocus Contrast Microscopy.

PB94-212750

03,039

KERCH, H. M.

High-Temperature Furnace for In situ Small-Angle Neutron Scattering during Ceramic Processing.

PB96-148127

03,743

KERNER, J.

Design and Characterization of X-Ray Multilayer Analyzers for the $50-1000 \mathrm{eV}$ Region.

03,848

Silicon Photodiodes Optimized for EUV and Soft X-Ray Regions.

02,124

KERSTEL, E

Sub-Doppler, Intrared Laser Spectroscopy of the Propyne 2nu1 Band: Evidence of Z-Axis Coriolis Dominated Intramolecular State Mixing in the Acetylenic $\mathrm{CH}$ Stretch Overtone.

PB95-202941

KESSLER, E. G.

Precision Comparison of the Lattice Parameters of Silicon

Monocrystals.

01,037

04,438

Status of a Silicon Lattice Measurement and Dissemination PB94.199635

KESSNER, A.

Molar Absorptivities of Bilirubin (NIST SRM 916A) and Its Neutral and Alkaline Azopigments.

PB94-211042

03,456

Test Optics Error Removal

KEY, T. S.

Surging the Upside-Down House: Looking into Upsetting Reference Voltages

PB96-112313

02,385

KEYSER, P. T.

Test of Newton's Inverse Square Law of Gravitation Using the 300-m Tower at Erie, Colorado.

PB95-202446

03,978

KHAIKIN, G. I.

Oxidation of Ferrous and Ferrocyanide lons by Peroxyl Radicals.

KHAN. M. R.

01,191

Correlations of Modulation Noise with Magnetic Microstructure and Intergranular Interactions for $\mathrm{CoCrTa}$ and CoNi Thin Film Media.

PB94-212768

KHLEVNOY, B. B.

04,509

Results of a NISTNNIIOFI Comparison of Spectral-Radiance Measurements.

PB97-113021

04,159

KHODAKOVSKII, I. L

Thermodynamic Properties of the Aqueous lons $(2+$ and $3+)$ of Iron and the Key Compounds of Iron.

PB96-145958

KHOLODENKO, A.

01,119

Generalized Stokes-Einstein Equation for Spherical Particle

Suspensions.

01,031

\section{KHOLODENKO, A. L}

Electrostatic Rigidity of Polyelectrolytes from Reparametrization Invariance.
PB96-180062

Influence of Surface Interaction and Chain Stiffness on Polymer-Induced Entropic Forces and the Dimensions of Confined Polymers.

KHORRAMSHAHGOL, $R$.

01,203

SQA Standards and Total Quality Management. PB96-111844

KIBBLER, A. E.

01.743

Surface Topography and Ordering-Variant Segregation in

KIDDER, C.

04,595

Transient Cooling of a Hot Surface by Droplets Evaporation.
PB94.156957

KIEF, M. T.

03,783

Spot-Profile-Analyzing LEED Study of the Epitaxial Growth of $\mathrm{Fe}, \mathrm{Co}$, and $\mathrm{Cu}$ on $\mathrm{Cu}(100)$

KIELY, A. B.

04,561

Model of an Optical Roughness-Measuring Instrument. PB97-110209 04,384

KIKUCHI, $\mathbf{H}$.

Properties and Interactions of Oral Structures and Restorative Materials. Annual Report for Period October 1, 1990 to September 30, 1991

PB94-160843

03,558

KILE, L. L.

Programmable Guarded Coaxial Connector Panel.

PB96-122544

02,108

CCOTE, $H$

Distributed Architecture for Standards Processing

(LMER,

00,276

AMRF Composite Fabrication Workstation.

PB94-172681

02,810

KIM, B. S.

Intracycle Evaporative Cooling in a Vapor Compression Cycle.
PB97-116107

02,762

KIM, D. Y.

Stability and Surface Energies of Wetted Grain Boundarles

in Aluminum Oxide.

03,059

KIM, G. J.

Combining interactive Exploration and Optimization for AsSB97-112320

KIM, J. B.

02,794

RIS Measurement of AC Stark Shifts and Photoionization

Cross Sections in Calcium
PB96-157953

04,073

Verification of the Ponderomotive Approximation for the ac

Stark Shift in Xe Rydberg Levels.
PB94-185709

03,801

KIM, J. H.

Causes of the Apparent Heat Transfer Degradation for Retrigerant Mixtures.

PB94-212701

03,259

KIM, J. S.

Characterization of Liquid-Phase Epitaxially Grown HgCdTe PB96-123617

04,738

Characterization of LPE HgCdTe Film by Magnetoresistance.
PB96-157961 Electrical Characterization of Liquid-Phase Epitaxially Grown Single-Crystal Films of Mercury Cadmium Telluride
by Variable-Magnetic-Field Hall Measurements. PB95-175782

02.177

Electrical Characterization of Narrow Gap n-Type Bulk HgCdTe Single Crystals by Variable-Magnetic-Field Hall Measurements and Reduced-Conductivity-Tensor Analyses.
PB96-164199

Magneto-Transport Properties of $\mathrm{HgCdTe}$

PB95-175840 04,661

Multicarrier Characterization Method for Extracting Mobilities and Carrier Densities of Semiconductors from Variable Magnetic Field Measurements.

PB94-212776

02,317

\section{KIM, M. S.}

Single-Phase Heat Transfer and Pressure Drop Characteristics of an Integral-Spine-Fin Within an Annulus. PB94-194073

03,805

Single-Phase Heat Transfer and Pressure Drop Characteristics of an Integral-Spine Fin Within an Annulus. PB97-122386

04,179

Study to Determine the Existence of an Azeotropic R-22 'Drop-In' Substitute.

KIM, M. W.

02,568

Small-Angle Neutron Scattering (SANS) Study of WormLike Micelles Under Shear.

PB96-176698
PB

04,111

KIM, S

Artificial Crack in Steel: An Ultrasonic-Resonance-Spectroscopy and Modeling Study.

03,241

Compressibility of Polycrystal and Monocrystal Copper:

Acoustic-Resonance Spectroscopy.

02,990

Correlation between Tc and Elastic Constants of (La-

PB94-213220 04,514

Off-Diagonal Orthorhombic-Symmetry Elastic Constants.

PB95-202768

04,695

Void Shape in Sintered Titanium.

PB96-14 1023

02,705

KIM, S. Y

Strategy to Support Multipoint Communication Service Over Native ATM Service.

KIM, Y. K.

Investigation of LS Coupling in Boronlike Ions

PB94-185295

01,507

Labeling Conventions in Isoelectronic Sequences - Reply.
PB95-162574

Low Magnetostriction in Annealed NiFe/Ag Giant

Magnetoresistive Multilayers.

Magnetoresistance of Thin-Film NiFe Devices Exhibiting

Single-Domain Behavior.
PB96-147087

Magnetostriction and Giant Magnetoresistance in Annealed NiFe/Ag Multilayers.

Modeling Effects of Temperature Annealing on Giant Magnetoresistive Response in Discontinuous Multilayer NiFe/Ag Films.

PB97-112585

04,157

Observation of the Transverse Second Harmonic Magneto-

Optic Kerr Effect from Ni81Fe19 Thin Film Structures.

PB96-200332

Highly-

Charivistic and

PB94-212784

03,854

Relativistic Modifications of Charge Expansion Theory.
PB96-123799

Simulating Device Size Effects on Magnetization Pinning Mechanisms in Spin Valves.

PB97-112593

04,158

KIMMINS, J.

Telecommunications Security Guidelines for Telecommunications Management Network. Computer Security.

PB96-139415

01,496

INARD, J.

Performance of Commercial CMOS Foundry-Compatible Multijunction Thermal Converters.

PB95-153656

02,342

INARD, J. R.

AC-DC Difference Characteristics of High-Voltage Thermal

Converters.

Development of Thin-Film Multijunction Thermal Converters at NIST

PB97-112338

02,286

Empirical Linear Prediction Applied to a NIST Calibration Service.
PB97-112353

02,287 
KING, D. S.

Dynamics of Nonthermal Reactions: Femtosecond Surtace Chemistry.

00,688

Fragment Energy and Vector Correlations in the Overtone. Pumped Dissociation of HN3X(1)A

00,802

Fragment State Correlations in the Dissociation of NO. HF $(v=1)$.

00,982

Laser-Induced Desorption of NO from Si(111): Effects of Coverage on NO Vibrational Populations.

00,959

Product Kinetic Energies, Correlations, and Scattering Ani-

Product Kinetic Energies, Correlations, and Scattering Ani-
sotropy in the Bimolecular Reactor $\mathrm{O}((1) \mathrm{D})+\mathrm{H} 2 \mathrm{O}$ yields $\mathrm{OH}$

00,838

PB94-212792

Product State Correlations in the Reaction of $O((1) D)$ and $\mathrm{H} 2 \mathrm{O}$ in Bimolecular Collisions and in $\mathrm{O} 3 . \mathrm{H} 2 \mathrm{O}$ Clusters-
Translation. PB95-153011 Vibrational Predissociation Dynamics of Overtone-Excited HN3.

PB95-125720

00,691

KING, J. B.

Configuration-Dependent AC Stark Shifts in Calcium. 04,363

KING, M.

Lean flammability limit as a fundamental refrigerant property. Phase 1, Interim technical report, 1 October 1994--31 March 1995

DE95011238

KING, M. D.

Acid Gas Production in Inhibited Diffusion Flames. PB95-180576

NGON, A.

Growth of Epitaxial KNbO3 Thin Films.

02,409

KINGSTON, H. M.

Dissolution Problems with Botanical Reference Materials PB95-126280

Hydrolysis of Proteins by Microwave Energy.

PB94-216322

03,528

KINNEY, A. L.

New High-Redshift Damped Lyman-alpha Absorption Systems and the Redshift Evolution of Damped Absorbers.

PB95-203501

00,083

KINNISON, D. E.

Environmental Aspects of Halon Replacements: Considerations for Advanced Agents and the Ozone Depletion Po. tential of CF3I.

PB97-122261

KINRA, V. K.

03,301

Dynamic Shear Modulus Measurements with Four Independent Techniques in Nickel-Based Alloys.

PB94-198900

03,320

KINSMAN, R.

Precision Oscillators: Dependence of Frequency on Temperature, Humidity and Pressure.

PB94-198306

02,031

KIRCHMANN, N.

SUSAN: SUperconducting Systems ANalysis by Low Tem. perature Scanning Electron Microscopy (LTSEM).

PB96-112065

KIRCHMAYR, $\mathrm{H}$

V-6: Effects of Temperature Variation.

PB96-148143

04.728

KIRKLAND, J.

Surface Geometry of BaO on W(100): A Surface-Extended X-Ray-Absorption Fine-Structure Sludy.

KIRSCHENBAUM, L. S.

Bias Current Dependent Resistance Peaks in NiFe/Ag Giant Magnetoresistance Multilayers.

PB97-112346 04,153

Telegraph Noise in Silver-Permalloy Giant

$\begin{array}{ll}\text { Magnetoresistance Test Structures. } & \\ \text { PB96-146717 } & 04,763\end{array}$

PB96-146717

KISELEV, S. B.

Physical Limit to the Stability of Superheated and Stretched Water

01,083

KISER, C.

Accuracy Comparisons of Josephson Array Systems.
PB95-164687

02,047

Voltage Ratio Measurements of a Zener Reference Using a Digital Voltmeter

KISHORE, $N$.

01,906

Apparent Molar Heat Capacities and Apparent Molar Volumes of Aqueous Glucose at Temperatures from 298.15 K to $327.01 \mathrm{~K}$.

PB94-212800

03,459

Thermochemistry of the Hydrolysis of L-arginine to (Lcitrulline + Ammonia) and of the Hydrolysis of L-arginine to (L-ornithine + Urea)

03,463
Thermodynamics of the Hydrolysis of Penicillin $G$ and AmpiPB94-172467

03,596

KISLIUK, P.

Molecular Microwave Specira Tables.

AD-A296 498/9

00,720

KITAGAWA, T.

Direct Dispersion Measurement of Highly-Erbium-Doped Optical Amplitiers Using a Low Coherence Reflectomete Coupled with Dispersive Fourier Spectroscopy.

PB95-150702

04,263

KITTEL, $P$.

Design Equations and Scaling Laws for Linear Compres sors with Flexure Springs.

PB95-168902

02,948

KIYOTSUKURI, T.

Thermal Stability of Intemal Electric Field and Polarization Distribution in Blend of Polyvinylidene Fluoride and Polymethylmethacrylate.

PB95-151072

01,24

SHUS, A.

Neutron Powder Diffraction Study of the Nuclear and Mag. netic Structures of the Oxygen-Deficient Perovskite PBa5-161097

00,954

KLASK, U.

Application of the Taylor Dispersion Method in Supercritical Fluids.

PB95-164323

00,977

KLASSEN, $M$.

Estimate of Flame Radiance via a Single Location Measurement in Liquid Pool Flames.

02,476

Measurement of Radiative Feedback to the Fuel Surface of a Pool Fire

PB94-211604

02,477

Structure and Radiation Properties of Pool Fires.

PB94-193802

02,473

KLAUS, E. E.

Mechano-Chemical Model: Reaction Temperatures in a Concentrated Contact.

$-11946$

03,227

KLAVINS, $P$.

Crystal Structure and Magnetic Ordering of the Rare-Earth and $\mathrm{Cu}$ Moments in $\mathrm{RBa} \mathrm{Cu} 2 \mathrm{NbO}(\mathrm{R}=\mathrm{Nd}, \mathrm{Pr})$.

PB95-140554

04,546

KLEAR, R. $M$.

Second Census Optical Character Recognition Systems Conference.

01,832

KLEBANOFF, P. S.

Characteristics of Turbulence in a Boundary Layer with Zero Pressure Gradient.

AD-A278 249/8

04,192

Evolution of a Turbulent Boundary Layer Induced by a Three-Dimensional Roughness Element. PB94-212818

04,200

KLEIBER, P. D

Alignment Probing of Rydberg States by Stimulated EmisPB96-200316

04,124

KLEIN, C. A.

Workshop on Characterizing Diamond Films (3rd). Held in Gaithersburg, Maryland on February 23-24, 1994. PB94-187663

04,456

KLEIN, M. L.

Molecular Dynamics Investigation of the Surface/Bulk Equilibrium in an Ethanol-Water Solution.

PB97-113112

01,183

\section{KLEIN, R.}

Reaction Rate Determinations of Vinyl Radical Reactions with Vinyl, Methyl, and Hydrogen Atoms.

PB94-211398

00,815

KLEIN, $W$.

Long-Lived Structures in Fragile Glass-Forming Liquids.

KLEINER, 1.

Fundamental Torsion Band in Acetaldehyde. PB94-212834

00,840

Molecular-Beam Optothermal Spectrum of the $\mathrm{OH}$ Stretching Band of Methanol.

4-212826

00,839

KLEMM, R. $\mathrm{B}$.

Experimental Determination of the Ionization Energy of $O(X$ (sup 2) $)($ sub $3 / 2)$ ) and Estimations of Delta(sub $f$ ) $H$ (sup deg)(sub O) (IO(sup - ) ) and PA(IO)

PB96-146899

00,694

Ionization Energies, Appearance Energies and Thermochemistry of CF2O and FCO.

PB97-111538

01,178

KLEMPERER, $w$.

Microwave and Submillimeter Spectroscopy of Ar-NH3 States Correlating with $\mathrm{Ar}+\mathrm{NH} 3(j=1, k=1)$.

00,942

KLEVNOY, B. B.

Precision High Temperature Blackbodies.

PB95-140059

03.885

KLEYN, M. F.

Cellular Automaton Simulations of Cement Hydration and Cevelopment.

01,320

KLIEGER, $P$.

Highway Concrete (HWYCON) Expen System User Reference and Enhancement Guide.

PB94-215670

01,316

KLINE, M. C.

Enhanced Detection of PCR Products Through Use of TOTO and YOYO Intercalating Dyes with Laser Induced Fluorescence - Capillary Electrophoresis.

PB95-164653

00,599

Intercomparison of DNA Sizing Ladders in Electrophoretic Separation Matrices and Their Potential for Accurate Typing of the D1S80 Locus.

03,485

Overview of Reference Materials Prepared for Standardization of DNA Typing Procedures.

KLINE, R. W.

Use of a Radiochromic Detector for the Determination of Stereotactic Radiosurgery Dose Characteristics. PB94-185642

03,514

KLINE, S. W.

Group 1 for the Plant Spatial Configuration STEP Applica.

tion Protocol.
PB96-165402

Group 1 for the Process Engineering Data STEP Application Protocol.

KLINEDINST, D.

Sources of Urban Contemporary Carbon Aerosol. PB95-175659

KLINEDINST, D. B.

Comparative Study of Fe.C Bead and Graphite Target Performance with the National Ocean Sciences AMS (NOSAMS) Facility Recombinator Ion Source.

PB95-175790

00,693

Distinguishing the Contributions of Residential Wood Combustion and Mobile Source Emissions Using Relative Concentrations of Dimethylphenanthrene Isomers.

02,563

KLONZ, $M$.

Intercomparison of NIST, NPL, PTB, and VSL Thermal Voltage Converters from $100 \mathrm{kHz}$ to $1 \mathrm{MHz}$.

PB94-172442

02,026

KLOPPING, $F$

Intracomparison Tests of the FG5 Absolute Gravity Meters.
PB96-102991

KLOSE, J. Z.

Atomic Branching Ratio Data for Carbon-Like lons.

PB94-212842

03,855

Atomic Branching Ratio Data for Nitrogen-Like Species.

PB96-190152

Atomic Branching Ratio Data for Oxygen-Like Species. PB95-180436

03,963 


\section{PERSONAL AUTHOR INDEX}

KORTE, B. J.

Sources of Urban Contemporary Carbon Aerosol. PB95-175659

02,551

KLUKEN, A. 0.

Cryogenic Toughness of Austenitic Stainless Steel Weld Metals: Effect of Inclusions.

PB95-161261

03,214

Effects of Copper, Nickel and Boron on Mechanical Properties of Low-Alloy Steel Weld Metals Deposited at High Heat Input.

PB96-135231

03,363

KNAB, L. I.

National Voluntary Laboratory Accreditation Program: Carpet and Carpet Cushion.

00,295

National Voluntary Laboratory Accreditation Program (NVLAP): Commercial Products Testing.

PB95-267944 02,671

National Voluntary Laboratory Accreditation Program (NVLAP): Wood Based Products.

03,405

National Voluntary Laboratory Accreditation Program: Thermal insulation Materials.

PB95-267985

02,977

KNAPP, F. F.

Assay of the Eluent from the Alumina-Based Tungsten-188Rhenium-188 Generator.

PB94-200482

03,829

KNAPPE, S.

Fabrication Issues in Optimizing YBa2Cu307-x Flux Transformers for Low $1 / f$ Noise.

PB95-175857

02,059

KNEELAND, D. $\mathbf{R}$.

Real Time Monitoring of Electron Processors. PB96-135306

03,719

KNELL, $U$.

Hydrogen in YBa2Cu3Ox: A Neutron Spectroscopy and a Nuclear Magnetic Resonance Study.

PB95-161279

04,601

Neutron-Spectroscopy Study of the Hydrogen Vibrations in Hydrogen-Doped YBa2Cu3Ox.

PB94-172475

04,441

KNIGHT, A. E. W.

High-Resolution Infrared Overtone Spectroscopy of ArHF via Nd:YAG/Dye Laser Difference Frequency Generation.

KNIGHT, R. B. D.

00,816

Intercomparison of NIST, NPL, PTB, and VSL Thermal Voltage Converters from $100 \mathrm{kHz}$ to $1 \mathrm{MHz}$. PB94-17244

02,026

KNIO, O. $M$.

Dispersion and Deposition of Smoke Plumes Generated in Massive Fires

KNOLL, $W$.

Surface Plasmon Microscopy of Biotin-Streptavidin Binding Reactions on UV-Photopatterned Alkanethiol Self-Assembled Monolayers.

PB96-176771

KNUDSEN, F.P.

01,158

High Temperature Reactions of Uranium Dioxide with Various Metal Oxides.

00,717

KNUTILLA, A.

Unified Process Specification Language: Requirements for Modeling Process.

KO, $\mathrm{M}$.

02,850

Low Temperature $\mathrm{H}($ sub 2)S Separation Using Membrane Reactor with Redox Catalyst.

DE94008991

02,471

KO, M. K.

Characterization of the Adsorption-Fouling Layer Using PB94-212909

00,842

Determination of Osmotic Pressure and Fouling Resistances and Their Effects on Performance of Ultrafittration Membranes.

PB94-212891

00,841

Determination of Total Protein Adsorbed on Solid (Mem-

brane) Surface by a Hydrolysis Technique: Single Protein

Adsorption.

KOBAYASHI, $R$.

03,552

Exponentially Rapid Coarsening and Buckling in Coherently Seli-Stressed Thin Plates.

PB95-202347

KOCAOGLU, D. F.

04,821

Learning to Change: Opportunities to Improve the Performance of Smaller Manufacturers.

PB94-166212

00,010

$\mathrm{KOCH}, \mathrm{H}$.

Fabrication Issues in Optimizing YBa2Cu307-x Flux Transformers for Low $1 / 4$ Noise.

PB95-175857

02,059

KOCH, J. A

Optimization of ECR-Based PECVD Oxide Films for Superconducting integrated Circuit Fabrication.

02,051
Superconducting Integrated Circuit Fabrication with Low Temperature ECR-Based PECVD SiO2 Dielectric Films.
PB96-103015

04,719

KOCH, W.

Frozen Human Serum Reference Material for Standardiza tion of Sodium and Potassium Measurements in Serum or Plasma by lon-Selective Electrode Analyzers.

PB94-185337

00,532

KOCH, W. F.

Absolute Determination of Electrolytic Conductivity for Primary Standard $\mathrm{KC} 1$ Solutions from 0 to $50 \mathrm{C}$

CSTL Technical Activities, 1995

00,765

6-214630

00,647

dc Method for the Absolute Determination of Conductivities of the Primary Standard $\mathrm{KCl}$ Solutions from $\mathrm{OC}$ to $50 \mathrm{C}$ PB94-219342

02,644

Development of a Standard Reference Material for ISE Deasurements of Sodium and Potassium.

PB96-159785

03,507

Faraday Constan

PB96-159793

01,955

Mass and Density Determinations.

PB94-200672

00,504

KOEDAM, J. C

Molar Absorptivities of Bilirubin (NIST SRM 916A) and Its Neutral and Alkaline Azopigments.

PB94-211042

03,456

KOELLE, D.

Fabrication Issues in Optimizing YBa2Cu3O7-x Flux Trans formers for Low $1 / 4$ Noise.

PB95-175857

02,059

Low Noise YBa2Cu3O7-x-SrTiO3-YBa2Cu3O7-x Mulitlayers for Improved Superconducting Magnetometers.

04,747

KOENIG, A

NIST ATM Network Simulator: Operation and Programming, Version 1.0 .

KOENIG, J.

01,487

KOENIG, J.

Examination Procedure Outlines: Keys to Solving the Hand book 44 Puzzle.
PB97-110217

KOENIG, J. A.

Uniform Laws and Regulations in the Areas of Legal Metrology and Motor Fuel Ouality as Adopted by the 80th $\mathrm{Na}$ tional Conterence on Weights and Measures 1995. 1996

PB96-172309

02,927

Uniform Laws and Regulations in the Areas of Legal Metrology and Motor Fuel Quality, 1994 as Adopted by the 79th .

PB95-174470

02,909

\section{KOEPKE, G.}

Screened-Room Measurements on the NIST SphericalDipole Standard Radiator.

PB96-113568 01926

Standard Source Method for Reducing Antenna Factor Errors in Shielded Room Measurements.

PB96-183157

02,013

KOEPKE, G. H.

Radiated Emissions and Immunity of Microstrip Transmission Lines: Theory and Measurements.

02,238

KOEYLUE, U. O.

Mixing and Radiation Properties of Buoyant Turbulent Diffusion Flames.

KOFFMAN, A. D.

Binary versus Decade Inductive Voltage Divider Comparison and Error Decomposition.

B96-112263

Empirical Linear Prediction Applied to a NIST Calibration

Service.
PB97-112353 02,287

Modeling and Test Point Selection for a Thermal Transfer Standard.

NIST Strategies for Reducing Testing Requirements.

PB95-180444

01,909

KOGA, K.

Structural and Magnetic Properties of $\mathrm{CuCl} 2$ Graphite Intercalation Compounds.

03,020

KOHL, M. L.

Measurement and Interpretation of Tidal Tilts in a Small Array.

PB96-102611

03,686

\section{KOHLER, B.}

Phase Shifts and Intensity Dependence in Frequency-Modulation Spectroscopy.

PB96-103205

01,071

KOHLER, B. E.

Location of a (1)A(sub g) State in Bithiophene.

01.025

Lowest Excited Singlet State of Isolated 1-phenyl-1,3-butadiene and 1-phenyl-1,3,5-hexatriene.

PB95-202339

01,026

JIMA, $H$.

High-Energy Phonon Dispersion in La1.85Sro.15CuO4. 04,748 PB96-138458

KOJIMA, T.

Investigation of Applicability of Alanine and Radiochromic Detectors to Dosimetry of Proton Clinical Beams.

PB96-146782

03,636

Orientation Effects on ESR Analysis of Alanine-Polymer Dosimeters.

KOJIMA, T. M

03,720

Resonance Structure and Absolute Cross Sections in NearThreshold Electron-Impact Excitation of the $4 \mathrm{~s}(2)$ (1)S yields $4 s 4 \mathrm{p}$ (3) P Intercombination Transition in $\mathrm{Kr}(6+)$.

YBies-202271

KOLASINSKI, K. W.

Dynamics of Hydrogen Interactions with $\mathrm{Si}(100)$ and $\mathrm{Si}(111)$ PB 96-159801

KOLESKE, D. D.

04,082

Temperature Dependence and Anharmonicity of Phonons on Ni(110) and Cu(110) Using Molecular Dynamics SimulaPB94-18

KOMITOV, L.

04,449

Studies of the Higher Order Smectic Phase of the Large Electroclinic Effect Material W317.

PB95-151601

00,935

KONJEVIC, $\mathrm{N}$.

New Critical Review of Experimental Stark Widths and Shifts

PB94-172830

03,790

KOPANSKI, J. J.

Boron-Implanted 6H-SiC Diodes.

PB96-159678 04,081

Characterization of Two-Dimensional Dopant Profiles: Status and Review.
PB96-119300

02,400

Characterization of Two-Dimensional Dopant Profiles: Status and Review.

02,451

Development and Characterization of Insulating Layers on Silicon Carbide: Annual Report for February 14, 1988 to February 14, 1989

02,295

High-Spatial-Resolution Resistivity Mapping Applied to Mercury Cadmium Telluride.

02,131

Oxidation of $\mathrm{SiC}$

PB96-119516

02,401

Review of Serniconductor Microelectronic Test Structures with Applications to Infrared Detector Materials and Processes.

PB94-212925

02,132 


\section{PERSONAL AUTHOR INDEX}

KOS, A. B.

Dependence of Contrast on Probe/Sample Spacing with the Magneto-Optic Kerr-Effect Scanning Near-Field Optical Microscope (MOKE-SNOM)
PB96-138557

04,750

Improved Eddy-Current Decay Method for Resistivity CharPB95-180451

Micromagnetic Scanning Microprobe System.

PB95-176178

02,265

Offset Susceptibility of Superconductors PB94-212263

02,224

KOSEKI, H.

Airborne Smoke Sampling Package for Field Measurements

PB95-150041 01,381

In situ Burning of Oil Spills: Mesoscale Experiments and

PB95-163747

02,587

Smoke Emission from Burning Crude Oil.

PB96-122890

01,407

KOSOBUKIN, V. A.

Theory of the Magneto-Optic Kerr Effect in the Near Field PB96-141387

KOSTER, B.

Current Activities Within the National Biomonitoring Specimen Bank.

02,516

Development of Frozen Whale Blubber and Liver Reference Materials for the Measurement of Organic and Inorganic Contaminants.

PB95-151676

00,587

National Status and Trends Program Specimen Bank: Sampling Protocols. Analytical Methods, Results, and Archive

KOSTER, B. J.

02,598

Alaska Marine Mammal Tissue Archival Project: Specimen Inventory.

02,589

Certification of Polychlorinated Biphenyl Congeners and Chlorinated Pesticides in a Whale Blubber Standard Reference Material.

PB96-103023

03,745

Concentrations of Chlorinated Hydrocarbons, Heavy Metals and Other Elements in Tissues Banked by the Alaska Ma. and Other Elements in Tissues Banked by the Alaska Marine Mammal

02,590

Considerations in the Design of an Environmental Specimen Bank: Experiences of the National Biomonitoring Specimen Bank Program.

PB96-112370

02,527

Determination of Inorganic Constituents in Marine Mammal Tissues.

PB95-152047 00,589

Determination of PCBs and Chlorinated Hydrocarbons in Marine Mammal Tissues.

PB95-162640

03,744

Relationship of Silver with Selenium and Mercury in the Liver of Two Species of Toothed Whales (Odontocetes). PB96-167275

02,596

Standard Reference Materials for the Determination of Polycyclic Aromatic Hydrocarbons in Environmental Sam. ples - Current Activities.

PB95-151668

00.586

Trace Element Concentrations in Cetacean Liver Tissues Archived in the National Marine Mammal Tissue Bank.
PB96-167127
02,595

KOSTIJUCHENKO, $\mathrm{V}$.

Investigation of Applicability of Alanine and Radiochromic Detectors to Dosimetry of Proton Clinical Beams.

PB96-14678

03,636

KOSTREVA, M. M.

Mathematical Modeling of Human Egress from Fires in Res. idential Buildings.

00,337

Sensitivily Analysis for Mathematical Modeling of Fires in Residential Buildings.

PB96-154968

00,215

KOTELES, E. S

Use of Pressure for Quantum-Well Band-Structure Characterization.

PB96-164058

04,779

KOTRAPPA, P.

Calibration of Electret-Based Integral Radon Monitors Using NIST Polyethylene-Encapsulated (226)Ra/(222)Rn Emanation (PERE) Standards.

01,950

Response Comparison of Electret Ion Chambers, LiF TLD, and HPIC

PB96-190103

02,578

KOTSUJI, $\mathrm{H}$.

Molecular Weight Dependence of the Lamellar Domain Spacing of ABC Triblock Copolymers and Their Chain Conformation in Lamellar Domains.

PB95-161691

01,254
KOVAC, A.

Radiation-Chemical Reaction of 2,3,5-Triphenyl-Tetrazolium Chloride in Liquid and Solid State

PB96-146733

01,124

KOVACS, A.

Alcohol Solutions of Triphenyl-Tetrazolium Chloride as High-Dose Radiochromic Dosimeters.

PB96-135249

03,716

Pavel Radiochromic Films for Clinical Dosimetry.

03,641

Oscillometric and Conductometric Analysis of Aqueous and Organic Dosimeter Solutions.

PB96-135256

04,054

Radiochromic Solid-State Polymerization Reaction PB95.180683

01,271

Radiochromic Solid-State Polymerization Reaction PB96-180146

01,290

KOVAL, C.

Gas Transport Properties of Solution-Cast Perfluorosulfonic Acid lonomer Films Containing lonic Surfactants.

PB95-175998

01,267

KOWALAK, J.

beta-D-Glucosyl-Hydroxymethyluracil: A Novel Modified Base Present in the DNA of the Parastic Protozoan T.

PB94-172319

03,524

KOYLU, U. U.

Radiation and Mixing Properties of Buoyant Turbulent Diffusion Flames.
PB94-165974

KOZIN, I. N.

Tunneling-Rotation Spectrum of the Hydrogen Fluoride Dimer.

PB94-198678

00,793

KRAFT, K. M.

Performance of Tape-Bonded Seams of EPDM Membranes: Comparison of the Peel Creep-Rupture Response of Tape Bonded and Liquid-Adhesive-Bonded Seams.

PB96-183249

03,012

KRAJEWSKI, J. J.

Observation of Oscillatory Magnetic Order in the Antiferromagnetic

04,679

PB95-180303

KRAMAR, J. A

Measurement of Patterned Film Linewidth for Interconnect Characterization.

02,420

Metrology Standards for Advanced Semiconductor Lithography Referenced to Atomic Spacings and Geometry.
PB96-160718

02,424

KRAMER, $C$.

In situ Observation of Surface Morphology of InP Grown on Singular and Vicinal (001) Substrates.

PB95.168431

04,636

KRAMER, E. J.

Grazing Incidence Prompt Gamma Emissions and Resonance-Enhanced Neutron Standing Waves in a Thin-Film.

PB95-150470

03,892

KRAMER, G. H.

Summary of the Proceedings of the Workshop on Standard Phantoms for In-vivo Radioactivity Measurement.

PB94-212933

03,622

KRAMER, G. $W$.

Integrating Automated Systems with Modular Architecture. PB95-150231

00,577

Minutes of the CAALS Workshop on modularity and communications standards.

02,621

KRAMER, T.

NIST Support to the Next Generation Controller Program: 1991 Final Technical Report.

02,808

KRAMER, T. R

Feasibility Study: Reference Architecture for Machine Control Systems Integration.

02,804

Issues and Recommendations for a STEP Application Proocol Framework. National PDES Testbed.

02,770

Issues Concerning Material Removal Shape Element Volumes (MRSEVs).

02,882

Issues Concerning Material Removal Shape Element Volumes (MRSEVS).

NIST RS274KT Interpreter.

PB96-147954

NIST RS274/NGC Interpreter Version 1

02,885

PB94-187788

02,835

Reference Architecture for Machine Control Systems Integration: Interim Report

Structural EXPRESS Editor.

02,820

PB94-159795

KRANBUEHL, D. E.

Effects of Variable Excluded Volume on the Dimensions of Oft-Lattice Polymer Chains.

PB94-212941

KRANZ, H. G.

Standardised Computer Data File Format for Storage, Transport, and Off-Line Processing of Partial Discharge

PB96-122486

01,930

KRAUSE, $\boldsymbol{R}$.

Fracture Mechanism Maps: Their Applicability to Silicon

B96-204532

03,094

High Temperature Structural Reliability of Silicon Nitride.

PB97-110456

03,104

Tensile Creep Testing of Structural Ceramics.

PB97-110464

03,105

KRAUSE, R. F.

Cavitation Contributes Substantially to Tensile Creep in Silicon Nitride.

PB96-122577

03,171

Flat and Rising R-Curves for Elliptical Surface Cracks from Indentation and Superposed Flexure.

PB95-161295

03,156

Observed and Theoretical Creep Rates for an Alumina Ce. ramic and a Silicon Nitride Ceramic in Flexure. PB94-212958

03,040

Tensile Creep of a Silicon Nitride Ceramic.

PB95-161303

03,049

KRAUSE, S

Defect Formation Mechanism Causing Increasing Defect Density during Decreasing Implant Dose in Low-Dose

PB96-119524

02,402

KRAUSE, S. J.

Defect of Thermal Ramping and Annealing Conditions on Defect Formation in Oxygen Implanted Silicon-On-Insulator Materia.

PB94-212966

02,318

Defect Pair Formation by Implantation-Induced Stresses in High-Dose Oxygen Implanted Silicon-on-Insulator Material.

PB95-175824

02,364

Etfect of Annealing Ambient on the Removal of Oxide Precipitates in High-Dose Oxygen Implanted Silicon.
PB95-164356

Effect of Single versus Multiple Implan

PB96-160353

01,957

Mechanism of Defect Formation in Low-Dose Oxygen Implanted Silicon-on-Insulator Material.

PB97- 111462

02,453

Stacking Fault Pyramid Formation and Energetics in Siliconon-Insulator Material Formed by Multiple Cycles of Oxygen Implantation and Annealing.

PB96-160221

04,083 
KUNZ, A. K.

Thin Film Thermocouples for Measurement of Wall Temperatures in Internal Combustion Engines.

PB94-172103

KREIDER, K. G.

High Temperature Silicide Thin-Film Thermocouples.
PB94-185501 NIST/NCMS Program on Electronic Packaging: First Update.

03,008

Sputtered High Temperature Thin Film Thermocouples.

PB95-161311 02,259

Thin-Film Ruthenium Oxide - Iridium Oxide Thermocouples.

PB97-110225 00,520

Thin Film Thermocouple Research at NIST.

02,283

Thin Film Transparent Thermocouples.

02,283

PB94-185519

02,253

KREMER, D.

Planar Near-Field Alignment.

PB94-172491

KREMER, D. P.

Dual-Frequency Millimeter-Wave Radiometer Antenna for Airborne Remote Sensing of Atmosphere and Ocean.

PB96-112289

Dual Frequency mm-Wave Radiometer Antenna for Airborne Remote Sensing of Atmosphere and Ocean.

PB95-180378

02,006

New Extrapolation/Spherical/Cylindrical Measurement Facil-

ity at the National Institute of Standards and Technology.
PB95-153755

02,004

KRESIN, V. $Z$.

From Superconductivity to Supernovae: The Ginzburg Symposium. Report on the Symposium Held in Honor of Vitaly L. Ginzburg. Held in Gaithersburg, Maryland on May 22, PB95-1

KRETCHMER, $J$

04,649

Boron-Implanted $6 \mathrm{H}$-SiC Diodes.

PB96-159678

04,081

KRISHNAMOORTI, R.

Thermodynamic Interactions and Correlations in Mixtures of Two Homopolymers and a Block Copolymers by Small Angle Neutron Scattering.

01,247

Thermodynamic Interactions in Model Polyolefin Blends Obtained by Small-Angle Neutron Scattering.

01,208

KRISHNAN, $\mathbf{S}$.

Adaptive, Predictive 2-D Feature Tracking Algorithm for Finding the Focus of Expansion.

01,588

Simultaneous Measurement of Normal Spectral Emissivity by Spectral Radiometry and Laser Polarimetry at High Temperatures in Pulse-Heating Experiments: Application to Moperatures in Pulse-Heating

PB97-118376

KRISHNAN, S. K.

Mixing and Radiation Properties of Buoyant Luminous Flame Environments.

PB96-202254

02,694

KRISHNASASTRY, $M$.

Genetically Engineered Pore as a Metal Ion Biosensor.

PB96-161658

Genetically Engineered Pores as Metal Ion Biosensors.

03,553

Genetically Engineered Pores for New Materials

PB96-161641

03,550

Pore-Forming Protein with a Metal-Actuated Switch

03,554

KRIZ, R. D.

Generalized Plane Strain Analysis of a Bimaterial Composite Containing a Free Surface Normal to the Interface. PB95-163341

\section{KROKAN, H. E.}

Novel Activities of Human Uracil DNA N-glycosylase for $\mathrm{Cy}$ tosine-Derived Products of Oxidative DNA Damage.

PB96-164132

03,479

KROLL, $M$.

Liposome-Based Flow-Injection Immunoassay for Determining Theophylline in Serum.

KRUEGER, $\mathrm{S}$.

Anisotropy of the Surfaces of Pores in Plasma Sprayed Alumina Deposits.

03,126

PB96-123211

Characterization of the Binding of Gallium, Platinum, and
Uranium to Pseudomonas Fluorescens by Small-Angle $X-$ ray Scattering and Transmission Electron Microscopy.

03,453

Characterization of the Densification of Alumina by Multiple Small-Angle Neutron Scattering. (Reannouncement with New Availability Information)

AD-A249 179/3

03,024

Effect of Green Density and the Role of Magnesium Oxide

Additive on the Densification of Alumina Measured by
Small-Angle Neutron Scattering. (Reannouncement with New Availability Information)

03,022

Evolution of the Pore Size Distribution in Final-Stage SinterEvolution of the Pore Size Distribution in Final-Stage Sinter-
ing of Alumina Measured by Small-Angle $X$-ray Scattering. (Reannouncement with New Availability Intormation).
AD-A249 178/5

03,023

Extending the Angular Range of Neutron Reflectivity Measurements from Planar Lipid Bilayers: Applications to a Model Biological Membrane.

03,476

Small-Angle Neutron Scattering Characterization of Processing/Microstructure Relationships in the Sintering of Crystalline and Glassy Ceramics. (Reannouncernent with New Availability Information)

AD-A249 $510 / 9$

03,025

Small Angle Neutron Scattering Studies of Structural Characteristics of Argarose Gels.

PB96-112305

03,475

\section{KRULLE, C. A.}

SUSAN: SUperconducting Systems ANalysis by Low Temperature Scanning Electron Microscopy (LTSEM).

PB96-112065

04,728

KRUMRINE, J. R.

Comment On: Two-Photon Absorption Series of Calcium. PB96-157979

04,074

KRUPKA, J.

Dielectric Properties of Single Crystals of $\mathrm{Al}_{2} \mathrm{O} 3, \mathrm{LaAlO}$, $\mathrm{SrTiO3}$, and $\mathrm{MgO}$ at Cryogenic Temperatures.

02,266 PB95-180477

Influence of Films' Thickness and Air Gaps in Surface Impedance Measurements of High Temperature Superconductors Using the Dielectric Resonator Technique.
01,946 Measurements of Permittivity and the Dielectric Loss Tangent of Low Loss Dielectric Materials with a Dielectric Resonator Operating on the Higher Order Te(sub 0 gamma delta) Modes.
PB96-111869

02,273

Microwave Dielectric Properties of Anisotropic Materials at Cryogenic Temperatures.

KRUPNOV, A. F

Tunneling-Rotation Spectrum of the Hydrogen Fluoride

PB94-198678

00,793

KUANG, J.

Transient Errors in a Precision Resistive Divider.

PB97-111512

01,983

KUBIAK, G. D.

XUV Characterization Comparison of Mo/Si Multilayer Coatings.

04,278

KUBOTA, T.

Experimental and Numerical Studies on Two-Dimensional Gravity Currents in a Horizontal Channel.

PB94-165941

01,359

KUDCHADKER, $\mathbf{S}$.

Thermodynamic and Thermophysical Properties of Organic Nitrogen Compounds. Part II. 1- and 2-Butanamine, 2-Methyl-1-Propanamine, 2-Methyl-2-Propanamine, Pyrole, 1-, 2-, and 3-Methylpyrrole, Pyridine, 2-, 3, and 4-Methylpyridine, Pyrrolidine, Piperidine, Indole, Ouinoline, Isoquinoline. Acridine, Carbazole, Phenanthridine, 1- and 2Naphthalenamine, and 9-Methylcarbazole.

00,741

PB94-162294

\section{KUDRITZKI, R.P.}

Radiation-Driven Winds of Hot Luminous Stars $X$. The Determination of Stellar Masses Radii and Distances from Terminal Velocities and Mass-Loss Rates.

PB94-213022

00,060

KUFFEL, J.

Dig tal Techniques in HV Tests - Summary of 1989 Panel Session

PB94-216702

02,035

KUHN, D. R.

Analysis of Selected Software Safety Standards

PB95-151262

01,708

Analysis of Standards for the Assurance of High Integrity Soltware.

03,735

Control and Instrumentation: Standards for High-Integrity

Software.

03,736

Formal Specification and Verification of Control Software for Cryptc graphic Equipment.

High Integrity Software Standards Activities at NIST
PB96-112214

01,585

IEEE's POSIX: Making Progress

01,744

$\begin{array}{ll}01,757 & \end{array}$

Open Systems Software Standards in Concurrent Engineer-

PBg6-160932

01,758

Predicate Differences and the Analysis of Dependencies in Formal Specifications.

01,759

Report of a Workshop on the Assurance of High Integrity PB96-161377

Report on the Advanced Software Technology Workshop. Held on February 1, 1994.

01,707

PB95-136610

Formal

Specifications.

KUHN, M.

Dielectric Properties of Single Crystals of $\mathrm{Al}_{2} \mathrm{O} 3, \mathrm{LaAlO} 3$, $\mathrm{SrTiO3}$, and $\mathrm{MgO}$ at Cryogenic Temperatures.

PB95-180477

02,266

KUHN, $R$

Security in Open Systems.

PB95-105383

01,473

Intensity-Dependent Scattering Rings in High Order AboveThreshold lonization

PB96-110739

KULKARNI, A. K.

Turbulent Upward Flame Spread on a Vertical Wall under External Radiation.

PB94-207388

00,341

KUMAR, A.

Bending-Induced Loss in Dual-Mode Rectangular Waveguides.

04,288

Bending-Induced Phase Shitts in Dual-Mode Planar Optical Waveguides.

Bent Rectangular Core Waveguides: An Accurate Perturbation Approach
PB95-168803

04,289

Fibre Splice Loss: A Simple Method of Calculation.

PB95-175519 04,299

Modal Characteristics of Bent Dual Mode Planar Optical Waveguide

PB95-180485

04,311

Mode Coupling and Loss on Tapered Optical Waveguides.

PB95-168571 04,282

Symbolic Programming with Series Expansions: Applications to Optical Waveguides.

PB95-168589

04,283

KUMAR, $\mathbf{S}$

Evidence of Crosslinking in Methyl Pendent PBZT Fiber

PB97-112486

03,393

PB96-160635

01,501

KUMP, $M$.

Characterization of Two-Dimensional Dopant Profiles: Status and Review
PB96-119300

KUMP, M. R.

Characterization of Two-Dimensional Dopant Profiles: Status and Review.
PB97-110134

KUNDU, M. $\mathbf{R}$.

Observing Stellar Coronae with the Goddard High Resolu-

tion Spectrograph. I. The dMe Star AU Microscopii.
PB96-102777

KUNDUR, S. R

Novel Active-Vision-Based Motion Cues for Local Naviga-

PB96-193727

02,941

Scale-Space-Based Visual-Motion-Cue for Autonomous 


\section{PERSONAL AUTHOR INDEX}

KUNZE, H. J.

Investigation of LS Coupling in Boronlike Ions. PB94-185295

KUo, S. C.

03,797

Experimental Determination of the Ionization Energy of $\mathrm{IO}(X$ (sup 2)ll(sub 3/2)) and Estimations of Delta(sub f) $\mathrm{H}$ (sup deg)(sub 0)(IO(sup - )) and $P A(1 O)$.

PB96.146899

00,694

Ionization Energies, Appearance Energies and $\begin{array}{ll}\text { Thermochemistry of CF2O and FCO. } & 01,178\end{array}$

KUPPUSAMY, $P$.

Research and Development Activities in Electron $\begin{array}{ll}\text { Paramagnetic Resonance Dosimetry. } & 03,635\end{array}$

KURFESS, J. D.

From Superconductivity to Supernovae: The Ginzburg Symposium. Report on the Symposium Held in Honor of Vitaly $L$. Ginzburg. Held in Gaithersburg, Maryland on May 22, 1992

(171963

04,649

KURITSYN, Y. A.

High-Resolution Measurements of the nu2 and 2nu2-nu2 Bands of (34)S(16)O2.

PB94-216223

00,855

KURIYAMA, $M$.

Correlation of $\mathrm{HgCdTe}$ Epilayer Defects with Underlying Substrate Defects by Synchrotron X-Ray Topography.
PB94-200714 129

02,129

Diffraction Imaging of Polycrystalline Materials.

02,971

High Resolution Hard X-Ray Microscope.

03,856

Materials Science with SR Using $X$-Ray Imaging: SpatialResolution/Source Size.

PB94-213048

04,510

KURMOO, $M$

Crystal Structure and Magnetic Properties of $\mathrm{CuGeO} 3$

PB95-18028

04,678

Critical-Current Degradation in Nb3 Al Wires Due to Axial and Transverse Stress.

PB95-202784

KUROKAWA, $\mathrm{H}$.

02,226

Small-Angle Neutron Scattering of Poly(vinyl alcohol) Gels.
PB95-164117

Small Angle Neutron Scattering Studies on Chain Asymmetry of Coextruded Poly(Vinyl Alcohol) Film. PB95.164372

KURSTER, $M$.

Rotational Modulation and Flares on RS Canum Venaticorum and BY Draconis Stars. XVIII. Coordinated VLA, ROSAT, and IUE Observations of RS CVn Binaries. PB96-102322

00,089

KURTZ, C. A.

Hyperfine-Structure Studies of $\mathrm{Zr}$ II: Experimental and Relativistic Configuration-Interaction Results.

PB95-20382

04,011

KURTZ, R. L.

Photoelectron Spectroscopic Study of the Valence and Core-Level Electronic Structure of $\mathrm{BaTiO}$.

Resonant-Photoemission Investigation of the Heusler Alloys $\mathrm{Ni2MnSb}$ and NiMnSb.

PB95-162384

04,612

Surface Core-Level Shifts of Barium Observed in Photoemission of Vacuum-Fractured BaTiO3 (100)

PB94-212156

04,501

KURYLO, M. J.

Atmospheric Lifetimes of HFC.143a and HFC-245fa: Flash Photolysis Resonance Fluorescence Measurements of the $\mathrm{OH}$ Reaction Rate Constants.

PB97-112577

00,118

Atmospheric Reactivity of alpha-Methyl-Tetrahydrofuran.

PB95-163705 02,548

Gas Phase Reactivity Study of $\mathrm{OH}$ Radicals with 1,1Dichloroethene and cis-1,1-Dichloroethene and Trans-1,2 Dichloroethene over the Temperature Range 240-400 K.

Rate Constants for the Gas Phase Reactions of the $\mathrm{OH}$ Radical with CF3CF2CHCl2 (HCFC-225ca) and CF2CICF2CHCIF (HCFC.225cb)

PB95-152153

00,940

Temperature Dependence of the Gas and Liquid Phase UItraviolet Absorption Cross Sections of HCFC-123 (CF3CHCl2) and $\mathrm{HCFC} \cdot 142 \mathrm{~b}$ (CH3CF2Cl). (C)

03,298

UV Absorption Cross Sections of Methylchloroform: Temperature-Dependent Gas and Liquid Phase Measurements.
PB96-201041
00,643 KURZ, J.

00,643

Comparison of Methods for Gas Chromatographic Determination of PCBs and Chlorinated Pesticides in Marine Reterence Materials.

RURZBAN, S. A.

02,584

ZBAN, S. A

Computer Virus Attacks.

01,715
KUS, D.

Frozen Human Serum Reference Material for Standardization of Sodium and Potassium Measurements in Serum or Plasma by Ion-Selective Electrode Analyzers.

00,532

KUSHIDA, G.

Ignition and Transition to Flame Spread Over a Thermally Thin Cellulosic Sheet in a Microgravity Environment.

PB96-160288

04,837

KUSHNER, M. J.

Evidence for Inelastic Processes for $\mathrm{N}(+) 3$ and $\mathrm{N}(+) 4$ from Ion Energy Distributions in He/N2 Radio Frequency Glow PB96-14668

04,059

Gaseous Electronics Conference Radio-Frequency Reference Cell: A Defined Parallel-Plate Radio-Frequency System for Experimental and Theoretical Studies of PlasmaProcessing Discharges.

PB94-172327

04,404

KUSTERS, J.

Precision Oscillators: Dependence of Frequency on Temperature, Humidity and Pressure.

PB94-198306

02,031

KUWAYAMA, $\mathrm{N}$.

Influence of Tempering Method on Residual Stress in Dental Porcelain.
PB94-172012

KUYATT, C. E.

00,138

Guidelines for Evaluating and Expressing the Uncertainty of NIST Measurem

02,649

KUZIAK, $\mathbf{R}$.

Prediction of Strengthening Due to $\mathrm{V}$ Additions in DirectCooled Ferrite-Pearlite Forging Steels.

PB96-190251

03,220

KWANSA, $H$.

Positive and Negative Cooperativities at Subsequent Steps of Oxygenation Regulate the Allosteric Behavior Multistate Sebacylhemoglobin.

PB97-119374

03,486

KWEI, G. H.

Colossal Magnetoresistance without $\mathrm{Mn}(3+) / \mathrm{Mn}(4-)$ Double Exchange in the Stoichiometric Pyrochlore T/2Mn2O7. PB97-113070

04,160

KWOK, S

IRAS Spectroscopic Observations of Young Planetary Neb-

PB95-152070

00,072

LACEY, P. I.

Mechano-Chemical Model: Reaction Temperatures in a Concentrated Contact.

PB96-119466

03,227

PB94-172368

03,229

Wear Transitions in Monolithic Alumina and Zirconia-Alumina Composites.

ACHENMANN,

03,168

Frequency Dependence of the Emission from 2D Array Jo$\begin{array}{ll}\text { sephson Oscillators. } & 02,056 \\ \text { PB95-175147 }\end{array}$

SUSAN: Superconducting Systems ANalysis by Low Temperature Scanning Electron Microscopy (LTSEM). PB96-112065

04,728

LACHENMANN, S. G.

Direct Observation of Vortex Dynamics in Two-Dimensional Josephson-Junction Arrays

02,067

Novel Vortex Dynamics in Two-Dimensional Josephson Ar-

rays.

02,091

Observation of Vortex Dynamics in Two-Dimensional Josephson-Junction Arrays.

PB95-168811

02,050

LADBURY, J. M.

Rapid Evaluation of Mode-Stirred Chambers Using Impulsive Waveforms
PB96-210026

01,979

LADNER, J.

Biological Macromolecule Crystallization Database and NASA Protein Crystal Growth Archive.

PB97-109136

01,171

LAESECKE, A.

Correlation of the Ideal Gas Properties of Five Aromatic Hydrocarbons.

01,002

Measurements of the Viscosities of Saturated and Compressed Liquid 1,1,1,2-Tetrafluoroethane (R134a), 2,2Dichloro-1,1,1-Trifluoroethane (R123) and 1,1-Dichloro-1Fluoroethane (R141b).

PB95-175386

03,273

Polarized Transient Hot Wire Thermal Conductivity Measurements.
PB95-108817

00,886

Thermal Conductivity of R134a.

03,857

Viscosity of 1,1,1,2,3,3-Hexafluoropropane and 1,1,1,3,3,3Hexafluoropropane at Saturated-Liquid Conditions from 262 $\mathrm{K}$ to $353 \mathrm{~K}$.

PB96-176680

LAFFERTY, W. J.

03,292

Infrared Spectra of van der Waals Complexes of Importance in Planetary Atmospheres.

PB95-125738

00,071

Infrared Spectrum of $\mathrm{OCIO}$ in the $2000 / \mathrm{cm}(-1)$ Region: The 2(nu sub 1) and (nu sub $1+$ nu sub 3) Bands.
PB95-141032 00,908 Intensities and Dipole Moment Derivatives of the Fun-
damental Bands of (35) $\mathrm{ClO} 2$ and an Intensity Analysis of the nu1 Band

00,909

Reanalysis of the (010), (020), (100), and (001) Rotational Levels of (32)S(16)O2

00,887

Spectroscopic Constants for the 2.5 and 3.0 micrometer Bands of Acetylene.

PB94-213071

00,847

Tunneling-Rotation Spectrum of the Hydrogen Fluoride Dimer.

PB94-198678

00,793

ALANTE, A. F

High-Pressure Equilibrium Cell for Solubility Measurements in Supercritical Fluids.

00,998

Solubilities of Copper(II) and Chromium(III) betaDiketonates in Supercritical Carbon Dioxide.

PB96-164215

01,147

LAGERGREN, E.

Statistical Analysis of Parameters Affecting the Measurement of Particle-Size Distribution of Silicon Nitride Powders by Sedigraph (Trade Name)

PB94-216249

03,042

AGERGREN, E. S.

Composition and Solubility Product of a Synthetic Calcium Hydroxyapatite.

02,995

Effects of Testing Variables on the Strength of HighStrength (90 Mpa) Concrete Cylinders.

PB96-112198

00,456

LAI, J. S

Cascading Surge-Protective Devices: Options for Effective Implementation.

PB94-216488

02,464

Coordinating Cascaded Surge Protection Devices: High-

Low versus Low-High

02,463

Coordinating Cascaded Surge-Protective Devices: An Elusive Goal.

PB94-216496

02,465

HTAKIA, A.

Two Numerical Techniques for Light Scattering by Dielectric Agglomerated Structures.

PBS4-1

04,225 Analysis of Boron in CVD Diamond Surfaces Using Neutron

PB94-213089

04,511

Etfect of Stoichiometry on the Phases Present in Boron Nitride Thin Films. 
LANCASTER, R. A.

Electrical Characterization of Narrow Gap n-Type Bulk HgCdTe Single Crystals by Variable-Magnetic-Field Hall Measurements and Reduced-Conductivity-Tensor Analyses.
PB96-164199

LAND, J. L.

Interım Testing Artifact (ITA): A Performance Evaluation System for Coordinate Measuring Machines (CMMs). User PBos

02,914

LANDBERG, A. T.

Electronic Implementors' Workshop.

PB95-210936

01,484

LANDEE, C. P.

Quasielastic and Inelastic Neutron-Scattering Studies of ((CD3)3ND)FeCl3.2D2O: A One-Dimensional Ising PBromagnet.

04,547

LANDSMAN, $w$.

Goddard High-Resolution Spectrograph Observations of the Local Interstellar Medium and the Deuterium/Hydrogen Ratio along the Line of Sight Toward Capella.

00,066

LANG, S. M.

High Temperature Reactions of Uranium Dioxide with Various Metal Oxides.

AD-A286 648/1

00,717

LANGER, S. A.

Nonlinear Dynamics of Stiff Polymers
PB96-122478

LANGLAND $J$

01,278

Current Activities Within the National Biomonitoring Specimen Bank.
PB94-172806

LANGLAND, J. K.

02,516

New NIST Rapid Pneumatic Tube System.

PB96-167259

03,738

LAOR, U.

Influence of Lattice Mismatch on Indium Phosphide Based High Electron Mobility Transistor (HEMT) Structures Observed in High Resolution Monochromatic Synchrotron XRadiation Diffraction Imaging.

PB95- 164679

02,357

LARACUENTE, A.

Atomic-scale characterization of hydrogenated amorphoussilicon films and devices. Annual subcontract report, 14 February 1994--14 April 1995.

02,294

Growth and Nucleation of Hydrogenated Amorphous Silicon on Silicon (100) Surfaces.

02,991

Nanoscale Study of the Hydrogenated Amorphous Silicon Surface.

PB96-103056

04,720

LARAMEE, $S$.

Analysis of High Bay Hangar Facilities for Detector Sensitivity and Placem

LARASON, T. C.

01,429

Developing Quality System Documentation Based on ANSII NCSL 2540-1-1994: The Optical Technology Division's EfPB96-202122

01,869

NIST Detector-Based Luminous Intensity Scale.
PB96-179114

$\begin{array}{lr}\text { PB96-179114 } & 01,864\end{array}$ NIST High Accuracy Scale for Absolute Spectral Response
from $406 \mathrm{~nm}$ to $920 \mathrm{~nm}$. PB96-179122

LARBALESTIER, D. C.

01,865

Crossover in the Pinning Mechanism of Anisotropic Fluxon Cores

PB95-180170

04,673

Electromagnetic Coupling Character of (001) Twist Boundaries in Sintered Bi2Sr2CaCu2O8+x Bicrystals.

01,963

Weak-Link-Free Behavior of High Angle YBa2Cu3O7-x Grain Boundaries in High Magnetic Fields.

PB94-198421

04,459

LARRABEE, R. D.

Normal-Incidence Complex-Index Refractometry.
PB94-213097

Precision, Accuracy, Uncertainty and Traceability and Their Application to Submicrometer Dimensional Metrology.
PB94-213105 319 SEM Linewidth Metrology of X-ray Lithography Masks. PB96-201108 02,447

X-ray Mask Metrology: The Development of Linewidth Standards for X-ray Lithography.

PB95-162129

02,348

LARSEN, B. $\mathrm{H}$.

Stable Phase Locking in a Two-Cell Ladder Array of Josephson Junctions.

PB96-111679

04,722

Ultrasonic Sensing of GMAW: Laser/EMAT Defect Detection System.

02,878
LARSEN, N. W.

Second Census Optical Character Recognition Systems Conference.

LARSEN, R. J.

01,832

International Marine-Atmospheric (222)Rn Measurement Intercomparison in Bermuda. Part 2. Results for the Participating Laboratories.

00,115

LARSON, D. C.

Measurement of the $(10) \mathrm{B}(\mathrm{n}$, alpha1gamma) $(7) \mathrm{Li}$ Cross Section in the 0.3 to $4 \mathrm{MeV}$ Neutron Energy Interval. PB96-161799

04,098

LARSON, D. R.

Electrically Calibrated Pyroelectric Detector-Refinements for Improved Optical Power Measurements.

PB95-169066

02,164

Integrated Optical Polarization-Discriminating Receiver in PBlass.

02,206

Linewidth Narrowing in an Imbalanced Y-Branch Waveguide

PB95-140844

04,258

Nd:LiTaO3 Waveguide Laser.

PB95-140851

04,259

Rare-Earth-Doped Waveguide Devices: The Potential for Compact Blue-Green Lasers.

04,257

Waveguide Polarizers Processed by Localized Plasma Etching.

PB95-169264

02,171

LARSON, J. W.

Thermochemistry of the Reactions between Adenosine Adenosine $5^{\prime}$-monophosphate, Inosine, and Inosine $5^{\prime}$ monophosphate; the Conversion of L-histidine to (Urocanic Acid+Ammonia)

PB94-213113

03,460

LARSSON, $M$

Inner-Valence States $\mathrm{CO}(+)$ between $22 \mathrm{eV}$ and $46 \mathrm{eV}$ Studied by High Resolution Photoelectron Spectroscopy and ab Initio $\mathrm{Cl}$ Calculations.

PB95-180055

03,961

LAS, T.

Influence of Surface Charge on the Stochastic Behavior of Partial Discharge in Dielectrics.

PB96-122767

01,931

Modification of Cast Epoxy Resin Surfaces during Exposure to Partial Discharges.

01,086

Nonstationary Behavior of Partial Discharge during Insulation Aging.
PB95-163580

01,902

Nonstationary Behaviour of Partial Discharge during Discharge Induced Ageing of Dielectrics.
PB96-103114

Partial Discharge: Induced Aging of Cast Epoxies and Related Nonstationary Behavior of the Discharge Statistics. PB95-163598

01,903

LASCOLA, $R$.

High Resolution, Jet-Cooled Infrared Spectroscopy of (HCl)2: Analysis of nu 1 and nu2 $\mathrm{HCl}$ Stretching Fundamentals, Interconversion Tunneling, and Mode-Specific Predissociation Lifetimes.

PB95-203196

01,046

Slit-Jet Near-Infrared Spectroscopy and Internal Rotor Dynamics of the ArH2O van der Waals Complex: An Angular Potential-Energy Surface for Internal H2O Rotation.

PB95-202792 01,033

Vibration, Rotation, and Parity Specific Predissociation Dynamics in Asymmetric $\mathrm{OH}$ Stretch Excited ArH2O: A Hal Collision Study of Resonant V-V Energy Transfer in a Weakly Bound Complex.

PB95-107140

00,872

LASHMORE, D. S.

Ambient Temperature Synthesis of Bulk Intermetallics.
PB95-169074 00,168

PB95-169074
Co, 168 Multilayers.

PB96-163696 02,989

Electrochemical Synthesis of Metal and Intermetallic Com.

posites.
AD-A294 088/0 03,304

Electrodeposited Cobalt-Tungsten as a Diffusion Barrier between Graphite Fibers and Nickel.

PB96-146881

03,176

Electrodeposition.

PB94-172517

00,760

Magnetic and Structural Properties of Electrodeposited Cop-

per-Nickel Microlayered A:loys.

PB94-213121

04,512

LASHOF, T. W

Precision Laboratory Standards of Mass and Laboratory

AD-A280 562/0

02,618

ASKOWSKI, S. J. Electronic Access to Standards on the Information Highway.
PB96-131578

Usability Engineering: Industry-Government Collaboration for System Effectiveness and Efficiency.

01,514

LASKY, J.

Nano-Defects in Commercial Bonded SOI and SIMOX PB96-123674

LATTIMER, B. Y

Compartment Fire Combustion Dynamics. Annual Report September 1, 1993-September 1, 1994

PB95-217162

LATUSEK, J. P.

00,203

Evaluation of Wear Resistant Ceramic Valve Seats in Gas Fueled Power Generation Engines. Topical Report, December 1991-April 1994.

LAU, $K$.

02,466

Two New Probes for a Coordinate Measuring Machine

PB95-163093

02,653

LAUBER, $W$

Catalogue of Electromagnetic Environment Measurements, $30-300 \mathrm{~Hz}$.

01,943

Condensed Catalogue of Electromagnetic Environment Measurements, $30-300 \mathrm{~Hz}$

PB95-162210

01,899

LAUENSTEIN, C. P.

Laser-Induced Fluorescence Measurements of Rotationally Resolved Velocity Distributions for $\mathrm{CO}(+)$ Drifted in $\mathrm{He}$

PB94-213139

LAUENSTEIN, $G$

National Status and Trends Program Specimen Bank: Sampling Protocols, Analytical Methods, Results, and Archive Samples.

PB97-119226

AUENSTEIN, G. G.

Certification of Standard Reference Material (SRM) 1941 a Organics in Marine Sediment.

PB96-123690

02,593

UFER, A.

Reaction Rate Determinations of Vinyl Radical Reactions with Vinyl, Methyl, and Hydrogen Atoms.

00,815

LAUFER, A. H.

Deuterium Isotope Effect in Vinyl Radical Combination/ Dispropor tionationReactions.

01,167

Experimental Determination of the Rate Constant for the Reaction of $\mathrm{C}_{2} \mathrm{H} 3$ with $\mathrm{H} 2$ and Implications for the Partition ing of Hydrocarbons in Atmospheres of the Outer Planets.
PB97-122295

LAUG, $O$. $B$.

$100 \mathrm{~A}, 100 \mathrm{KHz}$ Transconductance Amplifier.

100 Ampere, $100 \mathrm{kHZ}$ Transconductance Amplifier.

PB96-102629

02,098

A Sampling Applications.

Wideband Sampling Voltmeter

PB97-113039

LAURITZEN, J. I.

Reference Tables for Thermocouples.

AD-A279 948/4

LAUTER, H. J.

Hydrogen in YBa2Cu3Ox: A Neutron Spectroscopy and a Nuclear Magnetic Resonance Study.

04,601

Neutron-Spectroscopy Study of the Hydrogen Vibrations in Hydrogen-Doped YBa2Cu3Ox.

PB94-172475

04,441

LAVAL, J.

Substrate Specificity of the Escherichia coli Endonuclease 


\section{PERSONAL AUTHOR INDEX}

LAWN, B.

Friction Processes in Brittle Fracture.

PB96-161765

03,076

LAWN, B. R.

Correlations between Flaw Tolerance and Reliability in Zirconia

PB96-161922

02.986

Deformation and Fracture of Mica-Containing Glass-Ceramics in Hertzian Contacts.

03,080

PB96-179452

Diffusion of Water along 'Closed' Mica Interfaces. 02,993
PB96-180039

Effect of Chemical Interaction on Barenblatt Crack Profiles in Brittle Solids.

02,996

Effect of Grain Size on Hertzian Contact Damage in Alu-

PB96-17960

03,083

Flaw-Insensitive Ceramics.

PB97-110027 03,095

Flaw Tolerance and Toughness Curves in Two-Phase Particulate Composites: SiC/Glass System.

PB96-179460

03,081

Indentation Fatigue: A Simple Cyclic Hertzian Test for Measuring Damage Accumulation in Polycrystalline Ceramics.

03,084

Model for Microcrack Initiation and Propagation beneath Hertzian Contacts in Polycrystalline Ceramics.

03.077

Model for Toughness Curves in Two-Phase Ceramics. 1. Model for Toughness Curves in Two-Phase Ceramics. 1.
Basic Fracture Mechanics.
PB96-180088
03,085

03,085

Model for Toughness Curv

03,078

PB96-163795

Objective Evaluation of Short-Crack Toughness Curves Using Indentation Flaws: Case Study on Alumina-Based Ceramics.

PB96-179429

03,079

Pressurized Internal Lenticular Cracks at Healed Mica Inter-

PB96-180252

02,997

LAWSON, A. C.

Crystallographic and Magnetic Properties of UAuSn.

PB95-140521

04,543

Incommensurate Magnetic Order in UPtGe.

PB95-140513

04,542

LAWSON, J.

Smoke Emission from Burning Crude Oił

01,407

LAWSON, J. R.

Alrborne Smoke Sampling Package for Field Measurements of Fires.

PB95-150041

01,381

Fire Performance of an Interstitial Space Construction Sys.

PB95. 188918

00,390

Ouantifying the Ignitıon Propensity of Cigarettes.

PB96-15541

00,306

LAYER, H. P.

Elastic Deformation of a Monolithic Perfect Crystal Interferometer: Implications for Gravitational Phase Shift Experiments.

PB94-213154

03,858

\section{LE, F. G.}

EPR Dosimetry of Cortical Bone and Tooth Enamel Irradiated with $X$ and Gamma Rays: Study of Energy Depend-

03,639

PB97-110373

Estimation of the Absorbed Dose in Radiation-Processed Food. 3. The Effect of Time of Evaluation on the Accuracy of the Estimate.

00.037

Estimation of the Absorbed Dose in Radiation-Processed Food. 4. EPA Measurements on Eggshell.

00,038

Inter-Laboratory Trials of the EPR Method for the Detection of Irradiated Meats Containing Bone.

\section{LEACH, $M$.}

Directory of Law Enforcement and Criminal Justice Associations and Research Centers.

PB96-178918

04,872

LEAKE, $\mathrm{S}$

Hierarchical Ada Robot Programming System (HARPS): A Complete and Working Telerobot Control System Based on the NASREM Model.

PB94-213162

02,934

LEBLANC, L.

Measured Stopping Powers of Hydrogen and Helium in Polystyrene Near Their Maximum Values.

04,729

LEBRUN, T.

Electron-Ion-X-ray Spectrometer System.

PB95-176137
Evolution of X-ray Resonance Raman Scattering into X-ray Excitation of Xenon Near the $L_{3}$ Edge

B96-10275

04,025

\section{LEBURTON, J. P}

Determination of the Complex Refractive Index of Individual Ouantum Wells from Distributed Reflectance.

PB95-175642

02,176

LECHNER, J.

Characteristics of Adhesive-Bonded Seams Sampled from EPDM Roof Membranes.

PB95-162491

00,377

Extreme Value Theory and Applications: Proceedings of the Conference on Extreme Value Theory and Applications, Volume 3. Held in Gaithersburg, Maryland in May 1993.

PB95.104956 03.432

Assessment of 'Peaks Over Threshold' Methods for Estimating Extreme Value Distribution Tails.

00,441

Degradation of Powder Epoxy Coated Panels Immersed in Saturated Calcium Hydroxide Solution Containing Sodium Chloride. PB96-101050

Extreme Wind Estimates by the Conditional Mean Exceedance Procedure.

00,120

Extreme Winds Estimation by 'Peaks Over Threshold' and Epochal Methods.

Epochal Methods.
PB96-159686

00,468

Modeling of Extreme Loading by 'Peaks Over Threshold' Methods.

PB96-159694

00,469

Recent Approaches to Extreme Value Estimation with Application to Wind Speeds. Part 1. The Pickands Method.

PB94-213170

LECHTMAN, H.

Fabrication of Platinum-Gold Alloys in Pre-Hispanic South America: Issues of Temperature and Microstructure Control. PB94-211646

LECKRONE, D. S.

Observations of $3 \mathrm{C} 273$ with the Goddard High Resolution Spectrograph on the Hubble Space Telescope. PB95-202321

\section{LEDBETTER, $\mathbf{H}$.}

Accurate Modeling of Size and Strain Broadening in the Rietveld Refinement: The 'Double-Voigt' Approach.

00,664

Artificial Crack in Steel: An Ultrasonic-Resonance-Spectros

copy and Modeling Study.
PB96-141395

Asymmetry between Flux Penetration and Flux Expulsion in Tl-2212 Superconductors.

PB95-125647 04,527

Cast-Iron Elastic Constants: Effect of Graphite Aspect Ratio.

PB94-213212

03,211

Compressibility of Polycrystal and Monocrystal Copper: Acoustic-Reso

02,990

Correlation between TC and Elastic Constants of (LaM) $2 \mathrm{CuO} 4$.

PB94-213220

04,514

Crystal Structure and Compressibility of 3:2 Mullite.

03.682

Dependence of Tc on Debye Temperature Theta(sub D) for Various Cuprates

PB95-180493

Dynamics vs. Static Young's Moduli: A Case Study.

Elastic Constants and Debye Temperature of $\mathrm{Y} 1 \mathrm{Ba} 2 \mathrm{Cu} 3 \mathrm{O} x$

Effect of Oxygen Content.
PB94-213352

Elastic Constants and Internal Friction of Polycrystalline Copper

Elastic Constants and Microcracks in YBa2Cu3O7.

PB96-200761

03,005

Elastic Constants of a Material with Orthorhombic Symmery: An Alternative Measurement Approach.

04,684

Elastic Constants of Isotropic Cylinders Using Resonant Uitrasound.

PB94-211919

Elastic Constants of Polycrystalline $\mathrm{Y} 1 \mathrm{Ba} 2 \mathrm{Cu} 3 \mathrm{Ox}$. 04,513

Elastic Properties of Al2O3/AI Composites: Measurements and Modeling.

03,157

Elastic Properties of Uniaxial-Fiber Reinforced Composites: General Features.

03,140

Enhanced Flux Pinning via Chemical Substitution in Bulk Superconducting T1-2212.

04,647

Estimation of the Orientation Distribution of Short-Fiber Composites Using Ultrasonic Velocities.

03,178

Low-Temperature Elastic Constants of $\mathrm{Y} 1 \mathrm{Ba} 2 \mathrm{Cu} 3 \mathrm{O}$

04,642 Magnetic Susceptibllity of $\mathrm{Pr} 2-x \mathrm{CexCuO} 4$ Monocrystals and Polycrystals.

04,677

Microstrains and Domain Sizes in Bi-Cu-O Superconductors: An X-Ray Diffraction Peak-Broadening PB94-198520

04,461

Nitrogen Effect on Elastic Constants of f.c.c. Fe-18Cr-19Mn

Alloys.
PB94-172541

03,203

Orthotropic Elastic Constants of a Boron-Aluminum FiberReinforced Composite: An Acoustic-Resonance-Spectroscopy Study.
PB96-200175

03,182

Relationshıp between Bulk-Modulus Temperature Dependence and Thermal Expansivity.

PB95-168829

04,641

Residual Stresses in Aluminum-Mullite (alpha-Alumina) Composites.

Thermal Expansion of an SiC Particle-Reinforced Aluminum Composite.

03,144

Torsion Modulus and Internal Friction of a Fiber-Reinforced Composite.

03,070

Ultrasonic-Resonance Spectroscopy of Bulk and Layered Solids.

PB96-141338

04,759

Void Shape in Sintered Titanium.

PB96-141023

02,705

Voigt-Function Modeling in Fourier Analysis of Size- and Strain-Broadened X-Ray Diffraction Peaks.

PB94-198538

04,462

LEDFORD, A. E.

Assessing the Credibility of the Calorific Content of Muncipal Solid Waste.

LEE, A.

02,581

Aging in Glasses Subjected to Large Stresses and Deformations.

PB95-107041

03,235

Multi-Agency Certification and Accreditation ( $\mathrm{C}$ and $\mathrm{A}$ ) Process: A Worked Example.

LE, D. H.

01,601

High-Energy Behavior of the Double Photoionization of Helium from 2 to $12 \mathrm{keV}$

PB94-213279

03,860

LEE, J. D.

Defect Formation Mechanism Causing Increasıng Defect Density during Decreasing Implant Dose in Low-Dose

PB96-119524

02,402

Defect of Thermal Ramping and Annealing Conditions on Defect Formation in Oxygen Implanted Silicon-On-Insulator 
Ouantum Hall Effect-Based Resistance Standard (Ouantum Hall Res)

Sources of Uncertainty in a DVM-Based Measurement System for a Ouantized Hall Resistance Standard.

01,884

PB94-219334

Conversion of a 2-Terminal-Pair Bridge to a 4-Terminal-Pair

Conversion of a 2-Terminal-Pair Bridge to a 4-Terminal-Pair
Bridge for Increased Range and Precision in Impedance Measurements.

$\begin{array}{ll}\text { PB97-119176 02,103 } & \end{array}$

NIST Comparison of the Ouantized Hall Resistance and the Realization of the SI Ohm Through the Calculable CapaciPB97-119184

02,291

NIST Comparison of the Quantized Hall Resistance and the Realization of the SI Ohm Through the Calculable Capacitor. Conference Proceedings, June 17-20, 1996

PB97-119192

02,292

LEE, M. L.

Comparison of Selectivities for PCBs in Gas Chroma-

tography for a Series of Cyanobiphenyl Stationary Phases.

PB96-119458

00,618

LEE, $S$.

Asymmetric Tip Morphology of Creep Microcracks Growing Along Bimaterial Interfaces.

PB94-200243

03,138

Diffusive Crack Growth at a Bimaterial Interface.

PB96-204110 03,090

Unified Telerobotic Architecture Project (UTAP) Standard Interface Environment (SIE), May 1995.

PB95-242350

02,938

LEE, S. C.

Intercomparison Study of (237) Np Determination in Artificial Urine Samples
PB96- 102645

03,633

LEE, S. $H$.

Electrolytes Constrained on Fractal Structures: DebyeHuckel Theory.

01,174

Isolated Spin Pairs and Two-Dimensional Magnetism in SrCr(sub 9p)Ga(sub 12-9p)O19.

PB97-112387

04,154

LEE, S. W.

Wear Mechanism Maps of Ceramics.

PB94-172368

03,229

LEE, S. Y.

Correlations of Modulation Noise with Magnetic Microstructure and Intergranular Interactions for CoCrTa
and CoNi Thin Film Media.
PB94-212768

LEE, V. J.

Portsmouth Fastener Manufacturing Workstation. User's Manual.

02,860

LEE, W. D.

Hybrid Digital/Analog Servo for the NIST-7 Frequency Standard.

01,544

Microwave Leakage as a Source of Frequency Error and Long-Term Instability in Cesium Atomic-Beam Frequency Standards. $\begin{array}{ll}\text { PB95-180501 } & 01,541 \\ \text { Velocity Distribution of Atomic Beams by Gated Optical }\end{array}$ PB95-180519

01,542

LEE, W. Y.

Fault Diagnosis of an Air-Handling Unit Using Artificial Neural Networks.

LEE, Y. T.

00,283

Bibliography on Apparel Sizing and Related Issues.

PB94-161924 02,806

Extensions of the Prototype Application Protocol of Readyto-Wear Apparel Pattern Making

PB96-128194

03,198

EE, Y. T. T.

Body Dimensions for Apparel.

PB94-187739

02,813

Estimation of System Damping at the Lotung Site by Application of System Identification.

Preliminary Processing of the Lotung LSST Data. PB96-165972

03,690

LEEDY, T.

Advanced Components for Electric and Hybrid Electric Vehicles. Workshop Proceedings. Held in Gaithersburg, Maryland on October 27-28, 1993.
lates.

PB94-177060

04,858

LEGG, K. 0 .

Opportunities for Innovation: Advanced Surface Engineering.

02,697

LEGOWIK, S. A.

Control System Architecture for a Remotely Operated Unmanned Land Vehicle.

03,759
Ground Vehicle Control at NIST: From Teleoperation to Aulonomy.

N94-34037/9

03,758

\section{LEGROS, $\mathrm{H}$.}

Calibration of a Superconducting Gravimeter Using Absoute Gravity Measurements.

03.684

B95-20265

Beyond the Technology Roadmaps: An Assessment of Electronic Materials Research and Development. PB96-165998

01,961

LEHMAN, J. H.

Electrically Calibrated Pyroelectric Detector-Refinements for Improved Optical Power Measurements.

PB95-169066

02,164

LEHMANN, $K$

Approach to Setting Performance Requirements for Automated Evaluation of the Parameters of High-Voltage Impulses.

$4-185634$

01,878

LEHMANN, K. K

Sub-Doppler, Infrared Laser Spectroscopy of the Propyne Band: Evidence $\mathrm{z}$-Axis Coriolis Dominate Intramolecular State Mixing in the Acetylenic $\mathrm{CH}$ Stretch Overtone.

LEI, $M$.

01,037

Elastic Constants and Debye Temperature of $\mathrm{Y} 1 \mathrm{Ba} 2 \mathrm{Cu} 3 \mathrm{OX}$

Effect of Oxygen Content.

04.515

PB94-213352

Elastic Constants of a Material with Orthorhombic Symme-

try: An Alternative Measurement Approach.

04,68

Elastic Properties of Uniaxial-Fiber Reinforced Composites: General Features.

PB94-200649

03,140

Low-Temperature Elastic Constants of $\mathrm{Y} 1 \mathrm{Ba} 2 \mathrm{Cu} 3 \mathrm{O} 7$

PB95-168837

04,642

Torsion Modulus and Internal Friction of a Fiber-Reinforced Composite.

\section{LEIGH, $\mathbf{S}$.}

03,070

Graphical Analysis of the CCRL Portland Cement Proficiency Sample Database (Samples 1-72). (Part 1. Univariant Analysis of Portland Cement).

PB94-196557

01,308

\section{LEIGH, S. D.}

Airborne Asbestos Method: Bootstrap Method for Determining the Uncertainty of Asbestos Concentration. Version 1.0.

Interlaboratory Comparison of Autoradiographic DNA Profiling Measurements. 1. Data and Summary Statistics.
PB95-175923
03,542

03,542

Interlaboratory Comparison of Autoradiographic DNA Profiling Measurements. 2. Measurement Uncertainty an Its Propagation.

03,545

Recent Approaches to Extreme Value Estimation with Application to Wind Speeds. Part 1. The Pickands Method.
PB94-213170 0019

LEIGHT, $W$.

Proceedings of the Open Forum on Laboratory Accreditation at the National Institute of Standards and Technology, October 13, 1995

LEIGHT, W. G.

U.S. Government Accreditation and Conformity Assessment System Evaluation

PB96-160239

02,678

LEISURE, R. G.

Elastic Constants of Isotropic Cylinders Using Resonant Ultrasound.

\section{LELENTAL, $M$.}

04,497

Alternating-Field Susceptometry and Magnetic Susceptibility of Supercondoctors. Presented at Office of Naval Research Workshop on Magnetic Susceptibility of Superconductors and Other Spin Systems. Held in Berkeley Springs, West Virginia on 20 May 1991.

PB94-145984

04,435

Alternating-Field Susceptometry and Magnetic Susceptibility of Superconductors.

04,638

\section{LENAHAN, P. M.}

Electron Traps. Structural Change, and Hydrogen Related SIMOX Defects.

02,312

Evidence for a Deep Electron Trap and Charge Compensation in Separation by Implanted Oxygen Oxides.

PB95-175337

02,362

LENNON, E.

NIST POSIX Testing Program.

PB96-160973

01,82

LENNON, E. B.

Computer Systems Laboratory Annual Report, 1993.
PB94-162518

Computer Systems Laboratory Annual Report 1994 01,629 Computer Systems Laboratory Computıng and Applied Mathematics Laboratory Technical Accomplishments, October 1994-March 1996

PB96-193768

01,638

LEONE, S. R.

Alignment Probing of Rydberg States by Stimulated EmisPB96-200316

04,124

Charge Transfer and Collision-Induced Dissociation Reactions of $\mathrm{CF}(2+)$ and CF2(2+) with the Rare Gases at a Laboratory Collision Energy of $49 \mathrm{eV}$

PB94-185584

00,775

Collision-Induced Neutral Loss Reactions of Molecular Dications.

00,780

Direct Detection of Atomic Arsenic Desorption from Si $(100)$ PB95-202230

01,024

Efficient $\mathrm{Br}\left({ }^{*}\right)$ Laser Pumped by Frequency-Doubled $\mathrm{Nd}$ YAG and Electronic-to-Vibrational Transfer-Pumped $\mathrm{CO} 2$ and HCN Lasers.

04,248

In-situ Monitoring of Molecular Beam Epitaxial Growth

Using Single Photon Ionization
PB95-202222

01,023

Initial and Final Orbital Alignment Probing of the Fine-Struc ture-Changing Collisions among the $\mathrm{Ca}(4 s)(1)(4 p)(1)$, (3)PJ States with He: Determination of Coherence and Conventional Cross-Sections.

PB95-203279

04,004

Investigating the 3.3 Micron Infrared Fluorescence from

Naphthalene Following Ultraviolet Excitation.
N95-15839/0

00,724

PB96-200613

00,665

Kinetics and Dynamics of Vibrationally State Resolved IonMolecule Reactions: (14) N2 $(+)(v=1$ and 2$)$ and $(15) \mathrm{N} 2(+)(v=0,1$ and 2$)$ with $(14) \mathrm{N} 2$
$\mathrm{~PB} 96-102348$

04,023

Kinetics of the Reaction $\mathrm{C} 2 \mathrm{H}+\mathrm{O} 2$ from 193 to $350 \mathrm{~K} \mathrm{Using}$

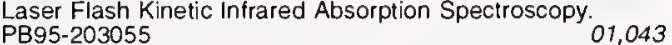
Laboratory Studies of Low-Temperature Reactions of $\mathrm{C} 2 \mathrm{H}$ with $\mathrm{C}_{2} \mathrm{H}_{2}$ and Implications for Atmospheric Models of With

PB95-108726

00,690

Laser Double Resonance Measurements of the Quenching Rates of $\mathrm{Br}((2) \mathrm{P} 1 / 2)$ with $\mathrm{H} 2 \mathrm{O}, \mathrm{D} 2 \mathrm{O}, \mathrm{HDO}$, and $\mathrm{O} 2$.

PB95-150694

00,921

Laser Flash Photolysis, Time-Resolved Fourier Transform Infrared Emission Study of the Reaction $\mathrm{Cl}+\mathrm{C} 2 \mathrm{H} 5$ yields $\mathrm{HCl}(\mathrm{v})+\mathrm{C} 2 \mathrm{H} 4$

01,049

Laser Flash Photolysis/Time-Resolved FTIR Emıssion Study of a New Channel in the Reaction of $\mathrm{CH}_{3}+\mathrm{O}$ : Production of $\mathrm{CO}(\mathrm{v})$

PB95-164281

00,974

Laser Gas Ionization Technique Monitors MEB Crystal

Growth. 112172

01.076

Laser-Induced Desorption of In and Ga from Si(100) and Adsorbate Enhanced Surface Damage.

PB95-203311

01,050 
Selected Ion Flow Tube-Laser Induced Fluorescence Instrument for Vibrationally State-Specific Ion-Molecule Reac-

PB94-185444

00,774

Single-Photon Ionization and Detection of $\mathrm{Ga}, \mathrm{In}$, and As(sub n) Species in GaAs Growth.

00,591

Single Photon Ionization, Laser Optical Probe Technique for Semiconductor Growth.

01,032

Single Photon Laser Ionization as an In-situ Diagnostic for $\mathrm{MBE}$ growth.
PB96-102025 Single-Photon Laser lonization Time-ot-Flight Mass Spectroscopy Detection in Molecular-Beam Epitaxy: Application 10 As4, As2, and Ga.

PB95-203337

01.052

Three-Vector Correlation Study of Orientation and Coherence Eftects in $\mathrm{Na}(3 \mathrm{p},(2) \mathrm{P} 1 / 2$ inversely maps (2) P3/2) $+\mathrm{He}$ Semiclassical and Quantum Calculations.

PB95-202453

03,979

Vibrational Distributions of As2 in the Cracking of As4 on Si(100) and Si(111)

PB94-198314

00.784

LEONG, $S$.

Computer-Aided Manufacturing Engineering Forum (1st) Technical Meeting Proceedings. Held in Gaithersburg, Maryland on March 21-22, 1995.

PB96-136965

02,834

Computer-Aided Manufacturing Engıneering Forum (2nd) Technical Meeting Proceedings. Held in Gaithersburg. Maryland on August 22-23, 1995.

PB96-195334

02,845

\section{LEONHARDT, R. W.}

Deep-UV ExcImer Laser Measurements at NIST.

04,355

LEONOWICZ, M. E.

Small Angle Neutron Scattering Study of the Structure and Formation of MCM-41 Mesoporous Molecular Sieves.

PB97-122337

03,110

LEOPOLD, K. R

Laboratory Measurements for the Astrophysical Identification of $\mathrm{MgH}$.

PB95-152195

00,073

Cross-Correlation Analysis Improves Time Domain Measurements.

LERIDON, B.

01,543

Autocorrelation Functions from Optical Scattering for OneDimensionally Rough Surfaces.

PB94-216538

04,244

LESAGE, $R$.

Physical Characterization of Herparin by Light Scattering PB96.119383

03.598

LETELLIER, $\mathrm{C}$.

Generalized Optical Theorem for On-Axis Gaussian Beams.
PB97-122345

LETT, P.

Hyperfine Effects and Associative Ionization of Ultracold Sodium

PB95-151221

03,903

Optical Molasses: The Coldest Atoms Ever.

03.878

Ultracold Collisions: Associative Ionization in a Laser Trap.

PB94-213238 03,859

LETT, P. D.

Atoms in Optical Molasses.

PB95-108874

03,875

Atoms in Optical Molasses: Applications to Frequency Standards

PB95-108882

03,876

Heterodyne Measurement of the Fluorescence Spectrum of Optical Molasses.

03,873

Laser Cooling and the Recoil Limit.

Laser Modificalic PB96-157987 04,075 Localization of Atoms in a Three-Dimensional Standing Wave.

PB95-163887

03,944

Measurement of the Atomic $\mathrm{Na}(3 \mathrm{P})$ Lifetime and of Retardation in the Interaction between Two Atoms Bound in a

PB97-122360

04.178

Measurements of Fluorescence from Cold Atoms: Localization in Three-Dimensional Standing Waves.

PB95-163879

03,943

Optical Molasses: Cold Atoms for Precision Measurements. PB95-108890 03,877

LETTIERI, T.

Optoelectronics and Optomechanics Manufacturing: An ATP Focused Program Development. Workshop Proceeding Held in Gaithersburg. Maryland on February 15, 1995.
02,204

\section{LETTIERI, T. R.}

Autocorrelation Functions from Optical Scattering for One. Dimensionally Rough Surfaces.

PB94-216538

04,244

Light Scattered by Coated Paper.

04,245

Light Scattering by Sinusoidal Surfaces: Illumination Win

dows and Harmonics in Standards.

PB97-110548

04,387

Light Scattering from Glossy Coatings on Paper.

04,242

Model of an Optical Roughness-Measuring Instrument.

PB97-110209 04,384

Optical Scattering from Moderately Rough Surfaces.
PB97-110415

PB97-110415 04,385
Present and Future Standard Specimens for Surtace Finish

PB97-110423

02,928

Regimes of Surface Roughness Measurable with Light

Scattering.
PB95-151213

04,265

Sinusoidal Su

04,388

LEVANDER, O. A.

Nutritional Status and Growth in Juvenile Rheumatoid Arthritis

PB94-198470

03,515

LEVELT SENGERS, J. M. H

Database for the Static Dielectric Constant of Water and

Steam.

PB96-145586

01,103

Formulation of

00,900

Measurements of the Relative Permittivity of Liquid Water at

Frequencies in the Range of $0.1 \mathrm{to} 10 \mathrm{kHz}$ and at TemperaFrequencies in the Range of 0.1 to $10 \mathrm{kHz}$ and at Temperatures between 273.1 and 373.2 K at Ambiert Pressure.
PB96-119375

Physical Limit to the Stability of Superheated and Stretched

PB96-122551

01,083

Significant Contributions of IAPWS to the Power Industry, Science and Technology

01,088

Statıc Dielectric Constant of Water and Steam.

01,090

Susceptibility Critical Exponent for a Nonaqueous Ionic BiSusceptibility Critical Exponent for a Nonaqueous Ionic Bi-
nary Mixture Near a Consolute Point.

PBY5-152112

LEVENSON, M. S.

Performance Measures for Geometric Fitting in the NIST Algorithm Testing and Evaluation Program for Coordinate Measurement Systems.

PB96-122122

01,745

LEVIN, B. C.

Development of a New Small-Scale Smoke Toxicity Tes Method and Its Comparison with Real-Scale Fire Tests. PB94-213253

00,350

Further Development of the N-Gas Mathematical Model: An Approach for Predicting the Toxic Potency of Complex Combustion Mixtures.

PB96-123260

03,650

New Approach for Reducing the Toxicity of the Combustion Products from Flexible Polyurethane Foam.

PB96-123625

01,41

Relating Bench-Scale and Full-Scale Toxicity Data. PB95-125977

00,361

EVIN, B. M.

EXITT: A Simulation Model of Occupant Decisions and ACExiT in Residential Fires.

PB94-213261

Feasibility and Design Considerations of Emergency Evacu-

$\begin{array}{ll}\text { ation by Elevators. } & 00,287 \\ \text { PB94-163441 } & \end{array}$

Feasibility of Fire Evacuation by Elevators at FAA Control Towers.

04,844

Human Factors Considerations for the Potential Use of Elevators for Fire Evacuation of FAA Air Traffic Control Tow ers.

PB94-217163

01,300

LEVIN, J. C.

Evolution of X-ray Resonance Raman Scattering into X-ray

Fluorescence from the Excitation of Xenon Near the L3

Edge.

PB96-102751

04,025

High-Energy Behavior of the Double Photoionization of $\mathrm{He}$ lium from 2 to $12 \mathrm{keV}$.

PB94-213279

03,860

Noninvasive High-Voltage Measurement in Mammography by Crystal Diffraction Spectroscopy.

00,160

LEVINE, J.

Analytical Estimation of Carrier Multipath Bias on GPS Position Measurements.

PB94-215712

04,845

Future of Time and Frequency Dissemination.

AD-P009 138/9

Future of Time and Frequency Dissemination. N94-30684/2

01,524

Measurement and Interpretation of Tidal Tilts in a Small

$\begin{array}{ll}\text { PB96-102611 03,686 } & \end{array}$

Measurement Methods and Algorithms for Comparison of Local and Remote Clocks.

PB96-102652

01,549

Measurement of Very Low Frequency Vibrations. PB96-102660

NIST Internet Time Service.

AD-P009 132/2

03,687

Smart Clock: A New Time.

PB95-151445

01,519

Time and Frequency Metrology.

PB96-190319

Time Generation and Distribution.

PB96-103049

Utc Dissemination to the Real-Time User.

N19960042622

01,530

01,556

01,550

LEVINE, J. P.

Measurement of Boron at Silicon Wafer Surfaces by Neutron Depth Profiling

PB94-211059

04,487

LEVINE, Z. H.

Large Local-Field Corrections in Optical Rotatory Power of Quartz and Selenium.

PB97-122378

04,400

LEW, H. S.

Model Precast Concrete Beam-to-Column Connections Subject to Cyclic Loading.

PB95-153094

00,438

Northridge Earthquake, 1994. Performance of Structures,

Lifelines and Fire Protection Systems.

PB94-161114

00,421

Northridge Earthquake 1994: Performance of Structures, Lifelines, and Fire Protection Systems.

04,825

Partially Prestressed and Debonded Precast Concrete Beam-Column Joints.

PB95-153102

00,439

Proceedings of a Workshop on Developing and Adopting Seismic Design and Construction Standards for Lifelines. Held in Denver, Colorado on September 25-27, 1991. PB97-115794

01,302

Seismic Performance Behavior of Precast Concrete BeamColumn Joints.

PB95-153110

00,440

Seismic Strengthening of Reinforced Concrete Frame Build-

PB95-108841

00,430

Strengthening Methodology for Lightly Reinforced Concrete 
LEWIS, M. A.

Cryogenic Flow Calibration in NIST. PB96-161930

Flow Conditioner Tests lor Thie On PB95-105540 04,201 Thermal Hydraulic Tests of a Liquid Hydrogen Cold Neutron Source.

PB95- 135570

03,884 Uncertainty Analysis of the NIST Nitrogen Flow Facility.
PB95-128906

Vortex Shedding Flowmeters for SSME Ducts.

PB95-169215

01,453

LEWIS, R. L.

Polarimetric Calibration of Reciprocal-Antenna Radars

PB95-216925 01,8

Polarimetric Calibration of Reciprocal-Antenna Radars.
PB96-200696

Proposed Analysis of RCS Measurement Uncertainty

PB95-203568

01,871

RangeCAD and the NIST RCS Uncertainty Analysis.

PB94-218591 01,870

Spherical-Wave Source-Scattering Matrix Analysis of Antennas and Antenna-Antenna Interactions.

PB96-111166

02,008

LEWIS, $S$.

Proposed International Interactive Courseware Standard

PB96-123161

00,137

LEWIS, S. P.

Total-Dielectric-Function Approach to Electron and Phonon Response in Solids.

LEWIS, V. E.

01,067

Neutron Measurement Intercomparisons Sponsored by CCEMRI, Section 3 (Neutron Measurements).

PB94-199916

03,819

LEWITUS, A. J.

High-Performance Liquid Chromatography of Phytoplankton

Pigments Using a Polymeric Reversed-Phase C18 Column.
PB95-151130

LGROSSHANDLER, W. L.

In Search of Alternative Fire Suppressants.

PB96-164165

LI, $\mathbf{Q}$.

03,285

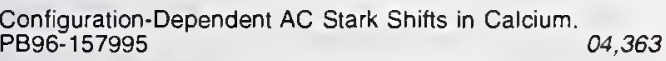

Effect of Axial Strain on the Critical Current of Ag-Sheathed Bi-Based Superconductors in Magnetic Fields Up to $25 \mathrm{~T}$. PB94-211315

04,493

RIS Studies of Autoionization in Calcium.

PB94-213295 00,849

Thermally Activated Hopping of a Single Abrikosov Vortex.

PB95-140810

04,548

LI, R. S.

Ouadratic Response of a Chemical Reaction to External Oscillations.

01,138

I, S. H.

Effect of Three Sterilization Techniques on Finger Pluggers. PB94-216090 00,150 LI, $x$.

Accurate Measurement of Optical Detector Nonlinearity.
PB95.203576 Automated Measurement of Nonlinearity of Optical Fiber Power Meters.

PB96-176540 04,110

Optical Detector Nonlinearity: A Comparison of Five MethPBs. 169355

04,291

Optical Detector Nonlinearity: Simulation.

PB96-165378

02,199

Optical Power Meter Calibration Using Tunable Laser Dioces.

04,290

Spatial Uniformity of Optical Detector Responsivity.
PB95-168845

LI, Z. Y.

Field Dependence of the Magnetic Ordering of $\mathrm{Cu}$ in $\mathrm{R} 2 \mathrm{CuO} 4(\mathrm{R}=\mathrm{Nd}, \mathrm{Sm})$.

04,633

Observation of Noncollinear Magnetic Structure for the $\mathrm{Cu}$ Spins in Nd2CuO4-Type Systems.

PB95-164539

04,634

LIAO, Y.

Transient Cooling of a Hot Surface by Droplets Evaporation.
PB94-156957
03.783

LIBBRECHT, $K$.

Low-Noise High-Speed Diode Laser Current Controller. PB95.202826

02,178

LIBES, D.

Concepts of the NIST EXPRESS Server.

PB95-180543

Debugger for Tcl Applications.

02,781

01,695
Handling Passwords with Security and Reliability in Background Processes.

PB95-180550 01,722

Kibitz-Connecting Multiple Interactive Programs Together.

PB94-213311

01,696

Object-Oriented Tel/Tk Binding for Interpreted Control of the

NIST EXPRESS Toolkit in the NIST STEP Application Pro.

tocol Development Environment.
PB96-141049

02,785

Ouch Those Programs are Painful.

01,739

PB6.102678

Using Expect to Automate System Administration Tasks.
PB94-213329

LIBURDY, $K$.

Testers Open Dialogue at Inaugural NIST Workshop.

ICHT, S. J.

High Frequency Magnetic Field Sensors Based on the Faraday Effect in Garnet Thick Films.

PB96-190384

LICHTENWALNER, D. J.

02,282

Growth of Epitaxial KNbO3 Thin Films.

PB96-135181

Use of lon Scattering Spectroscopy to Monitor the Nb Tar-

get Nitridation during Reactive Sputtering

LIDE, D. $\mathbf{R}$.

Molecular Spectroscopy.
PB94-213337

00,761

LIDGARD, A.

Annealing of Bragg Gratings in Hydrogen-Loaded Optical

PB96-155437 04,361

Decrease of Fluorescence in Optical Fiber during Exposure

to Pulsed or Continuous-Wave Ultraviolet Light

PB95-203071

04,320

IEBERMAN, A. G.

Rechargeable Batteries for Personal/Portable.

PB96-164231

LIEBERMAN, G.

Model Minimum Performance Specifications for Lidar Speed Measurement Devices.

PB95-197455

LIEBERMAN, R. A.

High Frequency Magnetic Field Sensors Based on the Faraday Effect in Gamet Thick Films

PB96-190384

LIEBMAN, J. F.

Experimental Determination of the Ionization Energy of $10(X($ sup 2) $\mid($ (sub 3/2)) and Estimations of Delta(sub f) $H$ (sup deg)(sub o) (IO (sup - )) and PA(IO).

00,694

LIFSCHITZ, M.

How Far Is Far from Critical Point in Polymer Blends. Lattice Cluster Theory Computations for Structured Monomer Compressible Systems.

PB94.211141

01,217

\section{FSHITZ, A.}

Shock Tube Techniques in Chemical Kinetics. PB95-163465

LIGGETT, $W$.

Developing Measurement for Experimentation.

PB97-118707 03,450

Discussion: Statistical Signal Processing of

Ouasiperiodicities.
PB96-119532

Replicate Measurements for Data Ouality and Environmental Modeling

PB94-172533 02,515

Scientific Protocols in Statistical Standards for Environmental Studies

LIGGETT, W. $S$

02,517

Experimental Optimization of Peak Shape with Application

to Aerosol Generation.
PB94. 185535

00,501

Materials-Science Based Approach to Phenol Emissions

from a Flooring Material in an Office Building.

PB97-118749

02,572

Pilot Studies for Improving Sampling Protocols.

PB97-118715

02,530

Source of Phenol Emissions Affecting the Indoor Air of an Office Building

LIGHT, G. M.

03,600

Assessment of Technology for Detection of Stress Corrosion Cracking in Gas Pipelines. Final Report, July 1993-

MBarch 1994.206646

02,475

Gas-Coupled Pulse-Echo Ultrasonic Crack Detection and Thickness Gaging.

B96-147129

04,847

ILLESTOLEN, T.

Development of Frozen Whale Blubber and Liver Reference

Materials for the Measurement of Organic and Inorganic

Contaminants.

00,587 Development of the National Marine Mammal Tissue Bank.
PB95-161402
02,586

LILLY, W. D.

Behavior of Charring Materials in Simulated Fire Environments.

01,368

LIN, C.

Diffusion of Cations Beneath Organic Coatings on Steel Substrate.

PB94.215704

03,119

Effect of Ethanol on the Solubility of Hydroxyapatite in the System $\mathrm{Ca}(\mathrm{OH}) 2 \cdot \mathrm{H} 3 \mathrm{PO} 4-\mathrm{H} 2 \mathrm{O}$ at $25 \mathrm{C}$ and $33 \mathrm{C}$. PB95-16923

00,169

In situ Measurements of Chloride Ion and Corrosion Potential at the Coating/Metal Interface.

03,122

Relation between AC Impedance Data and Degradation of Coated Steel. 1. Effects of Surface Roughness and Con tamination on the Corrosion Behavior of Epoxy Coated Stee

PB94-213345

03,189

LIN, C. C.

Thermodynamic Interactions and Correlations in Mixtures of Two Homopolymers and a Block Copolymers by Small Angle Neutron Scattering

PB95-152872

01,247

LIN, H. B.

Photonic Band-Structure Effects for Low-Index-Contrast Two-Dimensional Lattices in the Near Infrared.

PB97.122469

04,401

LIN, I. H.

Fracture in Multilayers.

PB96-163613

02,988

Thermodynamic Assessment and Calculation of the Ti-A

System. System.

LIN, J. H.

Comparisons of Some NIST Fixed-Point Cells with Similar Cells of Other Standards Laboratories.

PB97-119242

00,655

LIN, K. F.

Fluorescence Monitoring of Polarity Change and Gelation during Epoxy Cure.

PB94-185543

01,204

LIN, M. Y.

Small-Angle Neutron Scattering (SANS) Study of WormLike Micelles Under Shear.

LIN, S.

Elastic Constants and Debye Temperature of $\mathrm{Y}_{1} \mathrm{Ba}_{2} \mathrm{Cu} 3 \mathrm{OX}$ Effect of Oxygen Content.

PB94-213352

04,515

Nitrogen Effect on Elastic Constants of f.c.c. Fe-18Cr-19Mn Alloys

PB94-17254

03,203

LIN, Z.

Tert-Butyl Hydroperoxide-Mediated DNA Base Damage in Cultured Mammalian Cells.

LIN, Z. C.

Rare-Earth Isotopes as Tracers of Particulate Emissions: An Urban Scale Test.

PB94-161635

LIN, Z. Y.

Phonon Density of States in R2CuO4 and Superconducting $\mathrm{R} 1.85 \mathrm{Ce} 0.15 \mathrm{CuO} 4(\mathrm{R}=\mathrm{Nd}, \mathrm{Pr})$.

PB95. 150686

04,574

LIND, D. M.

Structural and Magnetic Ordering in Iron Oxide/Nickel Oxide Multilayers by $X$-ray and Neutron Diffraction (Invited) PB94-172558 
Polarized X-Ray Emıssion Spectroscopy. PB94-213360

03,862

LINDLER, D. J.

Observations of 3C 273 with the Goddard High Resolution Spectrograph on the Hubble Space Telescope.

00,076

B95-202321

LINDNER, $P$

Partial Scattered Intensities from a Binary Suspension of Polystyrene and Silica.

PB95-175618

00,996

LINDSAY, C. G.

Multimedia Tutorial on Phase Equilibria Diagrams.

PB96-200829

03,088

LINDSTROM, E. R.

National Voluntary Laboratory Accreditation Program: Electromagnetic Compatibility and Telecommunications. FCC Methods.

PB95-242376

02,664

NIST-NRL Free-Electron Laser Facility

PB94-212511

04,237

LINDSTROM, R. M.

Analytical Applications of Guided Neutron Beams.

04.041

Cold Neutron Prompt Gamma Activation Analysis at NIST A Progress Report.

PB95-175964

00,602

Dead Time, Pileup, and Accurate Gamma-Ray Spectrom-

PB96-167101

00,697

Determination of Hydrogen in Titanium Alloy Jet Engine Compressor Blades by Cold Neutron Capture Prompt Gamma-ray Activation Analysis.

01,448

Effects of Target Shape and Neutron Scattering on Elemen Effects of Target Shape and Neutron Scattering on Element
Sensitivities for Neutron-Capture Prompt Gamma-ray Activation Analysis.

00,558

Grazing Incidence Prompt Gamma Emissions and Resonance-Enhanced Neutron Standing Waves in a Thin-Film.
PB95-150470

High-Sensitivity Determination of lodine Isotopic Ratios by Thermal and Fast Neutron Activation.

00,555

Inelastic Neutron Scattering Studies of Rotational Excithe Orientational Potential in $\mathrm{C} 60$ and $\mathrm{A} 3 \mathrm{C} 60$ Compounds.

B94-172673

00.763

Local Area Networks in NAA: Advantages and Pitfalls. PB94-172095

00,527

oss-Free Counting at IRI and NIST.

04,105

Measuring Hydrogen by Cold-Neutron Prompt-Gamma Activation Analysis.

PB96-111877 00,612

Neutron Capture Prompt Gamma-Ray Activation Analysis at

the NIST Cold Neutron Research Facility.

PB94-213394

00,556

New NIST Rapid Pneumatic Tube System

PB96-167259

03,738

SUM and MEAN: Standard Programs for Activation Analy-

PBis. 112149

00,617

Use of Neutron Beams for Chemical Analysis at NIST.

PB97-112437

00,652

LINEBERGER, W. C.

Photoelectron Spectroscopy of Negatively Charged Bismuth Clusters: $\mathrm{Bi}(-) 2, \mathrm{Bi}(-) 3$, and $\mathrm{Bi}(-) 4$

PB95-108494

00,880

Photoelectron Spectroscopy of Small Antimony Cluster Anions: $\mathrm{Sb}(-)$, $\mathrm{Sb} 2(-), \mathrm{Sb} 3(-)$, and $\mathrm{Sb} 4(-)$

PB95-203139

01,045

LINHOLM, L. W.

Application of the Modified Voltage-Dividing Potentiometer to Overlay Metrology in a CMOS/Bulk Process.

Comparisons of Measured Linewidths of Sub-Micrometer Lines Using Optical, Electrical, and SEM Metrologies.

PB95-152807 02,338

Design Guide for CMOS-On-SIMOX. Test Chips NIST3 and

02,297

Electrical Test Structure for Overlay Metrology Referenced to Absolute Length Standards.

02,336

Electrical Test Structures Replicated in Silicon-on-Insulator Material.

PB97-11 1827
Enhanced Voltage-Dividing Potentiometer for High-Precision Feature Placement Metrology.

PB96-164025

02,428

Hybrid Optical-Electrical Overlay Test Structure.
PB96-204136

Measurement of Patterned Film Linewidth for Interconnect Characterization.

PB96-14!'168

02,420
Metrology Standards for Advanced Semiconductor Lithography Referenced to Atomic Spacings and Geometry. 02,424

Microelectronic Test Structures for Feature Placement and

$\begin{array}{ll}\text { Electrical Linewidth Metrology. } & \\ \text { PB95-180568 } & 02,367\end{array}$

Microelectronic Test Structures for Overlay Metrology. New Test Structure for Nanometer-Level Overlay and Fea-

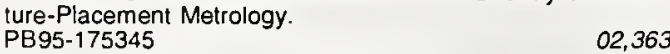

Test Structures for Determining Design Rules for Microelectromechanical-Based Sensors and Actuators.

Test Structures for the In-Plane Locations of Projected Fea fures with Nanometer-Level Accuracy Traceable to a Coordinate Measurement System.

PB94-200565

02,313

LINK, A. N.

Evaluation of the Economic Impacts Associated with the NIST Power and Energy Calibration Services.

LINKS, J. M.

EPR Dosimetry of Cortical Bone and Tooth Enamel Irradiated with $X$ and Gamma Rays: Study of Energy Depend-

PB97-110373

03,639

Exposure-to-Absorbed-Dose Conversion for Human Adult Cortical Bone.

PB97-110381

Conformance Testing for OSI Protocols.
PB96-102686

03,640

01,631

Copyright and Information Services in the Context of the National Research and Education Network.

PB96-160536

02,736

Formal Multi-Layer Test Methodology and Its Application to

PB94-172194

02,718

Using Technology to Manage and Protect Intellectual PropPBty.

01,513

LINNARTZ, $H$.

Microwave and Submillimeter Spectroscopy of Ar-NH3 States Correlating with $\mathrm{Ar}+\mathrm{NH} 3(j=1, k=1)$.

00,942

LINSKY, J.

High Sensitivity Survey of Radio Continuum Emission in Herbig Ae/Be Stars.

00,051

Sleuthing the Dynamo: HST/FOS Observations of UV EmisSleuthing the Dynamo: HST/FOS Observations of UV Emis-
sions of Solar-Type Stars in Young Clusters.
PB96-122817

LINSKY, J. E.

Dynamic Phenomena on the RS Canum Venaticorum Binary II Pegasi in August 1989. 1. Observational Data.

LINSKY, J. L.

Accurate Measurements of the Local Deuterium Abundance from HST Spectra.

$\begin{array}{ll}\text { PB96-200621 } & 00,110\end{array}$

Atomic Data Needed for Far Ultraviolet Astronomy with HUT and FUSE.

PB94-213402 00,062

Class of Radio-Emitting Magnetic B Stars and a Wind-Fed Magnetosphere Model.

Deuterium and the Local Interstellar Medium: Properties for the Procyon and Capella Lines of Sight.

PB96-200639

00,111

Deuterium in the Local Interstellar Medium: Its

Cosmological Significance.

00,081

Developments in Stellar Coronae.

PB96-176706

00,107

Distant Future of Solar Activity: A Case Study of Beta Hydri.

3. Transition $\mathrm{A}$

00,049

Distant Future of Solar Activity: A Case Study of beta Hydri.

3. Transition Region, Corona, and Stellar Wind.

00,074

Efficient Way of Identifying New Active Stars: A VLA Survey

of $X$-ray Selected Active Stellar Candidates.

PB96-122882

00,099

Far-Ultraviolet Flare on a Pleiades G Dwart.

00,086

First Results from a Coordinated ROSAT, IUE, and VLA First Results from a Coordinated ROSAT, IUE, and VLA
PB94-213477
PB Systems.

00,069

First Results from the Goddard High-Resolution Spectrograph: The Chromosphere of Tauri.

00,054

Four Years of Monitoring alpha Orionis with the VLA:

Where Have All the Flares Gone.

PB94-185212

00,048

FUSE: The Far

00,063

GHRS Observations of Cool, Low-Gravity Stars. 1. The FarUltraviolet Spectrum of alpha Orions (M2 lab).

00,094

Goddard High Resolution Spectrograph: Instrument, Goals, and Science Results.

PB96-123278

00,044

Goddard High-Resolution Spectrograph Observations of the Local Interstellar Medium and the Deuterium/Hydrogen Ratio along the Line of Sight Toward Capella.

00,066

High Velocity Plasm in the Transition Region of Au Mic: A Stellar Analog of Solar Explosive Events.

PB96-123294

00,102

High-Velocity Plasma in the Transition Region of AU Microscopii: Evidence for Magnetic Reconnection and Saturated Heating during Quiescent and Flaring Conditions.

PB96-102694

Hydrogen Lyman
PB95-202263

00,091

IUE Observations of Solar-Type Stars in the Pleiades and

the Hyades.
PB94-199437 00,053

Observations of $3 \mathrm{C} 273$ with the Goddard High Resolution Spectrograph on the Hubble Space Telescope.

00,076

Observing Stellar Coronae with the Goddard High Resolu-

tion Spectrograph. I. The dMe Star AU Microscopii.
PB96-102777

00,092

Peeking Through the Picket Fence: What Astrophysical Surprises May Be Present in the 100-1200 Angstrom Region.

Radio and $X$-ray Emissions from Chemically Peculiar $B$ and A-Type Stars: Observations and a Model.

PB96-123302

00,103

Radio Continuum and X-Ray Properties of the Coronae of RS Canum Venaticorum and Related Active Binary SysPB94-211083

00.057

Radio Emission from Chemically Peculiar Stars.

PB94-213469

00,068

Recalibration for the Final Archive of the International Ultraviolet Explorer (IUE) Satellite.

PB96-135264

00,106

Redshifts in Stellar Transition Regions.

00,104

Relationship between Radiative and Magnetic Fluxes for Three Active Solar-Type Dwarts. PB96-119540

00,097

Riass Coronathon: Joint $X$-ray and Ultraviolet Observations of Normal F-K Stars.

PB96-200217

00,109

ROSAT All-Sky Survey of Active Binary Coronae. 1. Quiescent Fluxes for the RS Canum Venaticorum Systems.
PB95-202479

ROSAT All-Sky Survey of Active Binary Coronae. 2. Coronal Temperatures of the RS Canum Venaticorum Systems. Rotational Modulation and Flares on RS Canum Venaticorum and BY Draconis Stars. XVI. IUE Spectroscopy and VLA Observations of $C 1182(=V 1005$ Orionis) in October 1983

PB94-185626

00,050

Rotational Modulation and Flares on RS Canum Venaticorum and BY Draconis Stars. XVIII. Coordinated VLA, ROSAT, and IUE Observations of RS CVn Binaries. PB96-102322

Scientific Rationale and Present Implementation Strategy for the Far Ultraviolet Spectrograph Explorer (FUSE). 00,045

Search for Radio Emission from the 'Non-Magnetic' Chemically Peculiar Stars. 
LINTERIS, G. T.

Asymptotic and Numerical Analysis of a Premixed Lamina Nitrogen Dioxide-Hydrogen Flame.

01,422

Burning Rate of Premixed Methane-Air Flames Inhibited by Fluorinated Hydrocarbons.

01,391

Effect of CF3H and CF3Br on Laminar Diffusion Flames in Normal and Microgravity.
PB96-161831

01,420

Effect of $\mathrm{CF}_{3} \mathrm{H}$ and $\mathrm{CF}_{3} \mathrm{Br}$ on Laminar Diffusion Flames in Normal and Microgravity.

01,421

Experimental and Numerical Burning Rates of Premixed Methane-Air Inhibited by Fluoromethanes.

01,392 Inhibition of Premixed Methane-Air Flames by Halon Alternatives.

PB96-146741 01,414

Inhibition of Premixed Methane-Air Flames by Iron Pentacarbonyl.
PB96-163712

LINZ, A.

Scalability Test for Parallel Code.

PB96-146758

00,513

LIPE, T. E.

01.749

AC-DC Difference Characteristıcs of High-Voltage Thermal Converters.

02,083

Development of Thin-Film Multijunction Thermal Converters at NIST

PB97-112338

02,286

Empirical Linear Prediction Applied to a NIST Calibration PB 97.112353

02,287

Frequency Extension of the NIST AC-DC Difference Calibration Service for Current.

01.895

High-Current Thin Film Multijunction Thermal Converters and Multi-Converter Modules.

PB97-112379

01.989

Nutritional Status and Growth in Juvenile Rheumatoid Arthritis.

03,515

LIPPIATT, B. C.

Evaluating Investments in Law Enforcement Equipment: An Annotated Bibliography.

PB95-151379

LISOWSKI, P. W.

Intermediate Structure in the Neutron-Induced Fission Cross Section of 236

03,802

Measurements of the $(235) \cup(n, f)$ Cross Section in the 3 to $30 \mathrm{MeV}$ Neutron Energy Region.

PB97-119051

04,172

Measurements of the $(237) \mathrm{Np}(\mathrm{n}, \mathrm{f})$ Cross Section

PB97-119069

04,173

LITTLE, W. A.

From Superconductivity to Supernovae: The Ginzburg Symposium. Report on the Symposium Held in Honor of Vitaly 1992 .

PB95-171963

04,649

LITTLER, C. L.

Heavily Accumulated Surfaces of Mercury Cadmium Tellu.

ride Detectors: Theory and Experiment.
PB94-216074

02,134

Investigation of Mercury Interstitials in $\mathrm{Hg}(1 \cdot x) \mathrm{CdxTe}$ Alloys
Using Resonant Impact-Ionization Spectroscopy.

PB94-213485

02,133

RII Spectroscopy of Trap Levels in Bulk and LPE Hg1. $x$ CdxTe.

PB96-160247

04,084

LITTRELL, $K$.

Multi-Stage, Position Stabilized Vibration Isolation System for Neutron Interferometry.

03,955

LIU, A.

Acid Gas Production in Inhibited Diffusion Flames. PB95-180576

LIU, C.

Variationally Stable Treatment of Two- and Three-Photon Detachment of $\mathrm{H}(-)$ Including Electron-Correlation Effects. PB95-202867

03,992

Preparation and Characterization of Cyclopolymerizable Resin Formulations.

PB96-146840

01,285

LIU, $\mathrm{H}$.

Existence and Nonexistence Theorems of Finite Diamete Sequential Confidence Regions for Errors-in-Variables ModPB95-180352

03,441

General Motion Model and Spatio-Temporal Filters for Com. puting Optical Flow

01,847
General Motion Model and Spatio-Temporal Filters for 3-D

Motion Interpretations.
PB96-210703

01,861

Image Gradient Evolution: A Visual Cue for Danger.

PB96-154562

02.939

Motion-Model-Based Boundary Extraction.

PB95-189502

01.849

Reliable Optical Flow Algorithm Using 3-D Hermite Poly-

PB94-145620

01,829

LIU, H. K.

Critical Current Density, Irreversibility Line, and Flux Creep

Activation Energy in Silver-Sheathed Bi2Sr2Ca2Cu2Ox

Superconducting Tapes.

PB95-162749

04,616

Interlaboratory Comparison of Autoradiographic DNA

Profiling Measurements. 1. Data and Summary Statistics.

03,542

Interlaboratory Comparison of Autoradiographic DNA Profiling Measurements. 2. Measurement Uncertainty and Its Propagation.

PB96-112123

03,545

LIU, L.

Observations of $3 \mathrm{C} 273$ with the Goddard High Resolution Spectrograph on the Hubble Space Telescope.

00.076

LIU, $R$.

Atmospheric Reactivity of alpha-Methyl-Tetrahydrofuran.

PB95-163705 02,548

Gas Phase Reactivity Study of $\mathrm{OH}$ Radicals with 1,1Dichloroethene and cis-1,1-Dichloroethene and Trans-1,2Dichloroethene over the Temperature Range 240-400 K

PB95-152146 00,939

Rate Constants for the Gas Phase Reactions of the $\mathrm{OH}$ Radical with CF3CF2CHCl2 (HCFC-225ca) and CF2CICF2CHClF (HCFC-225cb)

LIU, S.

00,940

Droplet Transfer Modes for a MIL 100S-1 GMAW Electrode,
PB95-209300

Mapping the Droplet Transter Modes for an ER100S-1 GMAW Electrode.
PB96-190095

03,295

LIU, S. T.

Performance Testing of a Family of Type I Combination Ap-

$\begin{array}{ll}\text { pliance. } & \\ \text { PB95-220521 } & 02,505\end{array}$

Predicting the Energy Pertormance Ratings of a Family of Type I Combination Appliances.

PB95-105524

02,504

LIU, W.

Tensile Creep of Whisker Reinforced Silicon Nitride. PB94-211984

LIU, $X$

Electron Beam Crosslinking of Poly(vinylmethyl ether) PB94-185550

01,205

LIU, Y.

Comparisons of Some NIST Fixed-Point Cells with Similar Cells of Other Standards Laboratories.

00,655

LIVIGNI, D.

Spatial Unitormity of Optical Detector Responsivity. 02,162

LIVIGNI, D. J.

Thermal Modeling and Analysis of Laser Calorimeters. PB96-140405

LIVINGSTON, C. A.

Intrinsic Stress in DC Sputtered Niobium PB94-19903

LLOYD, R. S.

Novel DNA N-Glycosylase Activity of $E$. coli T4 Endonuclease $v$ That Excises 4,6-Diamino-5. Formamidopyrimidine from DNA, a UV-Radiation- and Hydroxyl Radical-Induced Product of Adenine. PB96-160478

03,549

LOBB, C. J.

Application of Single Electron Tunneling: Precision Capacitance Ratio Measurements.

PB96-102157

04,703

Diffusion Studies in a Digital-/mage-Based Cement Paste Microstructural Model.

PB94-198801

01,312

Physical Basis for Half-Integral Shapiro Steps in a DC SQUID

PB96-102264

04,704

LOBO, C.

Study on the Reuse of Plastic Concrete Using Extended Set-Retarding
PB96-122130

00,402

LOCASCIO-BROWN, L.

Flow Immunoassay Using Solid-Phase Entrapment.
PB96-200951

Liposome-Based Flow-Injection Immunoassay for Determining Theophylline in Serum

03,494

Planar Waveguide Optical Sensors

PB94-200185

03,586

LOEVINGER, $R$.

Needs for Brachytherapy Source Calibrations in the United States

97-110092

LOEZOS, J. M.

Magnetic Dielectric Oxides: Subsolidus Phase Relations in the $\mathrm{BaO}: \mathrm{Fe} 2 \mathrm{O} 3: \mathrm{TiO} 2$ System.

PB96-176524

01,156

LOGSDON, B. W.

Membrane Gas Separation for a Fluidized-Bed Incinerator PB95-169041

LOH, H. T.

Concurrent Flow Flame Spread Study

PB94-156866

02,550

LOHSE, D. J.

Thermodynamic Interactions in Model Polyolefin Blends Obtained by Small-Angle Neutron Scattering

PB94-19

LOMAKIN, S. M.

Investigation of the Thermal Stability and Char-Forming Tendency of Cross-linked Poly(methyl methacrylate).

PB94-213501

LOMBARDI, $M$.

Introduction to Frequency Calibration. Part 1

PB96-200654

Preliminary Comparison of Time Transfers via LASSO, GPS and Two-Way Satellite

Using LORAN-C Broadcasts for Automated Frequency Cali-

PB94-216017

01,526

LOMBARDI, M. A

High Resolution Time Interval Counter

PB96-138607

01,495

How to Get NIST-Traceable Time on Your Computer.

PB96-200647

Keeping Time on Your PC

PB95-161410

01,559

NIST Frequency Measurement Service.

01,537

Satellite Two-Way Time Transfer: Fundamentals and RePB95-161089

LONBERGER, S. T.

Reference Tables for Thermocouples.

AD-A279 948/4

01,536

ONG, F. G.

PC-Based Spinning Rotor Gage Controller

PB95-175832

02,614

LONG, G. G.

Anisotropy of the Surfaces of Pores in Plasma Sprayed Alu-

mina Deposits.

Cavitation Contributes Substantially to Tensile Creep in Silicon Nitride.

PB96-122577

03,171

Cavity Evolution during Tensil Creep of Si3N4

03,376

Characterization of the Densification of Alumina by Multiple Small-Angle Neutron Scattering. (Reannouncement with New Availability Information)

AD-A249 179/3

03,024

Effect of Green Density and the Role of Magnesium Oxide Additive on the Densification of Alumina Measured by 
LOONEY, J.P.

Decoupling in the Line Mixing of Acetylene Infrared O Branches.
PB95-108452

00,877

Influence of the Filament Potential Wave Form on the Sensitivity of Glass-Envelope Bayard-Alpert Gages.

02,657

Laser Bandwidth Effects in Ouantitative Cavity Ring-Down Ppectroscopy.

04,394

Laser Photoionization Measurements of Pressure in VacuUB. 5 -180600

03,964

Measurement of Co Pressures in the Ultrahigh Vacuum Regime Using Resonance-Enhanced Multiphoton-Ionization Regime Using Resonance-Enhanced
Time-of-Flight Mass Spectroscopy.

Time-of-Flight

03,864

PC-Based Spinning Rotor Gage Controller.

02,609 \begin{tabular}{l} 
Simultaneous Forward-Backward Raman Scattering Studies \\
of D2 Broadened by D2, He, and Ar. \\
PB95-162459 \\
\hline
\end{tabular} PB95-16245

LOONG, C. K.

Phonon Density of States in R2CuO4 and Superconducting $\mathrm{R} 1.85 \mathrm{Ce} 0.15 \mathrm{CuO4}(\mathrm{R}=\mathrm{Nd}, \mathrm{Pr})$.

PB95-15068

LOPES, V. C.

Comparison of Techniques for Nondestructive Composition Measurements in CdZnTe Substrates.

PB96-103098

02,703

LORENTZ, S. R.

Determination of Complex Scattering Amplitudes in Low-Energy Elastic Electron-Sodium Scattering

03,869

Spin-Resolved Elastic Scattering of Electrons from Sodium. PB95-161774 03,933 Thermal Modeling of Absolute Cryogenic Radiometers PB95-181236

LORENZEN, T. J.

Taguchi's Parameter Design: A Panel Discussion. PB96-111802

LOS ARCOS, J. M.

Standardizatıon and Decay Scheme of Rhenium-186

LOUGHAY, T. A.

Band-Limited, White Gaussian Noise Excitation for Reverberation Chambers and Applications to Radiated SusceptiPB96-165410

01,960

LOVAS, F. J.

Experimental Studies of Line Shapes from a Balle-Flygare Spectrometer.

00,796

Infrared and Microwave Spectroscopy of the Argon Propyne Dimer.

00,794

Microwave Spectra of van der Waals Complexes of Importance in Planetary Atmospheres.

00,919

Microwave Spectrum and Structure of $\mathrm{CH} 2 \mathrm{O}-\mathrm{H} 2 \mathrm{O}$

PB97-118723

04,168

Microwave Spectrum and Structure of $\mathrm{CH} 3 \mathrm{NO}_{2}-\mathrm{H} 2 \mathrm{O}$

AD-A296 $377 / 5$ 00, 719

Rotational Spectrum and Structure of a Weakly Bound Complex of Ketene and Acetylene.

PB95-126140

00.896

Thermal Decomposition Pathways in Nitramine Propellants. AD-A295 896/5

03,753

LOVEJOY, C. M.

High Resolution, Jet-Cooled Intrared Spectroscopy of ( $\mathrm{HCl}$ 2: Analysis of nu1 and nu2 $\mathrm{HCl}$ Stretching Fundamentals, Interconversion Tunneling, and Mode-Specific Predissociation Lifetimes.

01.046

Laser Double Resonance Measurements of the Ouenching

Rates of $\mathrm{Br}((2) \mathrm{P} 1 / 2)$ with $\mathrm{H} 2 \mathrm{O}, \mathrm{D} 2 \mathrm{O}, \mathrm{HDO}$, and $\mathrm{O} 2$.
$\mathrm{PB} 95-150694$

00,921

Preferential In-Plane Rotational Excitation of $\mathrm{H} 2 \mathrm{O}(001)$ by Translational-to-Vibrational Transfer from $2.2 \mathrm{eV} H$ Atoms. PB95-202875 01,035

Rigid Bender Analysis of van der Waals Complexes: The Intermolecular Bending Potential of a Hydrogen Bond:

PB95-203022

01,042

Slit Jet Infrared Spectroscopy of Hydrogen Bonded N2HF Isotopomers: Rotational Rydberg-Klein-Rees Analysis and
$H / D$ Dependent Vibrational Predissociation Rates. PB95-16 1873

00,956

Spectroscopic Puzzle in ArHF Solved: The Test of a New Potential.

PB94-216058

00,850

Optical Fiber Sensors: Accelerating Applications in Navy

Ships.
PB94-186848 02,632

LOVETT, C. D.

Machine Pertormance Standard Provides Opportunity to $1 \mathrm{~m}$ prove Ouality and Productivity.

02,837
Standards Promote Credibility and Technology Transter: The Need for Greater Industry Support of Technical Committees

LoW, S. R.

02,961

Fracture Behavior of Large-Scale Thin-Sheet Aluminum Alloy.

N95-19494/0

03,311

Fracture Testing of Large-Scale Thin-Sheet Aluminum Alloy. AD-A306 625/5 03,30 Fracture Testing of Large-Scale Thin-Sheet Aluminum Alloy.
PB95-242368 Review and Upgrading of Military Fastener Test Standard $\begin{array}{ll}\text { MIL-STD-1312. } & 02,947\end{array}$ LOWE, D.

Analysis of High Bay Hangar Facilities for Detector Sensitivity and Placement.

PB96-190210

01,429

Assessing Halon Alternatives for Aircraft Engine Nacelle Fire Suppression

01,401

Protection of Data Processing Equipment with Fine Wate Sprays

02,610

Suppression Effectiveness of Extinguishing Agents under Highly Dynamic Conditions.

00.020

Turbulent Spray Burner for Assessing Halon Alternative Fire Suppressants.

01,385

Validation of a Turbulent Spray Flame Facility for the Assessment of Halon Alternatives.

PB96-159728

03,283

LOWE, J.

Ultra-High Stability Synthesizer for Diode Laser Pumped PB94-216066

Ultralinear Small-Angle Phase Modulator.

01,527

PB95-168852

02,261

LOWE, J. L.

Error Analysis of the NIST Optically Pumped Primary Frequency Standard.

01,535

NIST Optically Pumped Cesium Frequency Standard

PB94-211117

01,535

LWE, J.P.

Diode-Laser Pumped, Rubidium Cell Frequency Standards.

Hybrid Digital/Analog Servo for the NIST-7 Frequency Standard.

PB95-180618

01,544

Microwave Leakage as a Source of Frequency Error and Long-Term Instability in Cesium Atomic-Beam Frequency Standards.

NIST-7, the New US Primary Frequency Standard.

PB95.153458

01,541

Reducing the Effect of Local Oscillator Phase Noise on the Frequency Stability of Passive Frequency Standards.

LOWNEY, J. R.

Band-to-Band Photoluminescence and Luminescence Excitation in Extremely Heavily Carbon-Doped Epitaxial GaAs.

04,570

Characterization of Interface Defects in Oxygen-Implanted Silicon Films.

PB94-216629 02,322

Effects of Heavy Doping on Numerical Simulations of Gallium Arsenide Bipolar Transistors.

02334

Heavily Accumulated Surfaces of Mercury Cadmium Tellu-

ride Detectors: Theory and Experiment.
PB94-216074

02,134

Hg1-xCdxTe Characterization Measurements: Current Practice and Future Needs.

PB95-164299

02,157

High-Spatial-Resolution Resistivity Mapping Applied to Mer cury Cadmium Telluride.

PB94-212917

02,131

Investigation of Mercury Interstitials in $\mathrm{Hg}(1-\mathbf{x}) \mathrm{CdxTe}$ Alloys Using Resonant Impact-Ionization Spectroscopy. 4-213485

02,133

Junction Locations by Scanning Tunneling Microscopy: In Air-Ambient Investigation of Passivated GaAs pn Junctions.
PB94-185964 Magneto-Transport Properties of HgCdTe.

04,601

PB95-175840
04,661 Device Simulations.

PB94-198728

02,311

Model for Determining the Density and Mobility of Carriers in Thin Semiconducting Layers with Only Two Contacts. B996-102702

02,378

MONSEL-II: Monte Carlo Simulation of SEM Signals for Linewidth Metrology.

02,379

Monte Carlo Model for SEM Linewidth Metrology.

02,331

Monte Carlo Simulation of Scanning Electron Microscope

PBgals.

02,444

Physics for Device Simulations and Its Verification by Measurements.

02,327

Physics for Device Simulations and Its Verification by Meas-

PB95-152914

02,339

RII Spectroscopy of Trap Levels in Bulk and LPE Hg1xCdxTe.

04,084

Scanning Capacitance Microscopy Measurements and Modeling for Dopant Profiling of Silicon

PB96-164207

04,781

Scanning Capacitance Microscopy Measurements and Modeling: Progress Towards Dopant Protiling of Silicon.

P96-148150

04,773

Scanning Capacitance Microscopy Measurements and Mod-

eling: Progress Towards Dopant Profiling of Silicon.

01,964

SEM Linewidth

02,447

Semiconductor Measurement Technology: Improved Characterization and Evaluation Measurements for $\mathrm{HgCdTe} \mathrm{De}$ tector Materials, Processes, and Devices Used on the GOES and TIROS Satellites.

PB94-188810

02,122

Transverse Magnetoresistance: A Novel Two-Terminal Method for Measuring the Carrier Density and Mobility of a Semiconductor Layer.

02,332

Use of Monte Carlo Modeling for Interpreting Scanning Electron Microscope Linewidth Measurements.

02,413

User's Manual for the Program MONSEL-1: Monte Carlo Simulation of SEM Signals for Linewidth Metrology.

PB95-111522

02,325

$X$-ray Mask Metrology: The Development of Linewidth Standards for X-ray Lithography

PB95-162129
LOWRY, R. E.

02,348

Fluorescence Anisotropy Measurements on a Polymer Melt as a Function of Applied Shear Stress.

01,209

Observations of Shear Induced Molecular Orientation in a Polymer Melt Using Fluorescence Anisotropy Measure-

$\begin{array}{ll}\text { PB94-199304 01,210 } & \end{array}$

Observations of Shear Stress and Molecular Orientation Using Fluorescence Anisotropy Measurements.

PB94-199312

01,211

\section{LOZEV, M. G.}

Application of Electromagnetic-Acoustic Transducers for Nondestructive Evaluation of Stresses in Steel Bridge Structures.

01,301

Determination of Sheet Steel Formability Using Wide Band Electromagnetic-Acoustic Transducers.

Sensor System for Intelligent Processing of Hot-Rolled Steel. 186069

03,373

LOZIER, D. W.

Basic Linear Algebra Operations in SLI Arithmetic.

PB96-165931

Error-Bounding in Level-Index Computer Arithmetic. 
LUBELL, J.

Application Protocol Information Base World Wide Web

PB96-202320

02,791

SC4 Short Names Registry

PB97-122410

SGML Environment for STEP.

02,799

PB95-143103

02,778

LUCAS, J. $M$

Taguchi's Parameter Design: A Panel Discussion.

PB96-111802

Soft X-ray Reflectometry Program at the National Institute of Standards and Technology.

PB96-160395

04,368

LUCATORTO, T. B.

Comment On: Two-Photon Absorption Series of Calcium.

PB96-157979 04,074

Configuration-Dependent AC Stark Shifts in Calcium.
PB96-157995

Improved Dose Metrology in Optical Lithography.

PB96-179510

02,439

Improved Reflectometry Facility at the National Institute of Standards and Technology.

04,087

Laser Ablation of Thin Films as a Free Atom Source for Pulsed RIMS.
PB94-198710
Measurement

Measurement of $\mathrm{CO}$ Pressures in the Ultrahigh Vacuum Regime Using Resonance-Enhanced Multiphoton-Ionization Time-of-Flight Mass Spectroscopy.

PB94.216041

03,864

New NIST/ARPA National Soft X-ray Reflectometry Facility. PB96-158092

04,080

NIST Metrology for Soft X-ray Multilayer Optics.

PB96-160379

04,088

RIS Measurement of AC Stark Shifts and Photoionization Cross Sections in Calcium.

PB96-157953

04,073

RIS Studies of Autoionization in Calcium.

00.849

Study of Laser Resonance Ionization Mass Spectrometry Using a Glow Discharge Source.

03,308

DE94018566 03,308 Using a Glow Discharge Source.

PB96-123203 Upgraded Facility for Multilayer Mirror Characterization at

NIST.

04,367

Verification of the Ponderomotive Approximation for the ac Stark Shift in Xe Rydberg Levels.

PB94-185709

03,801

LUCE, M.

NIST SIMA Interactive Management Workshop. Held in For Belvoir, Virginaa on November 14-16, 1994

PB96-154877

02,838

LUCE, M. E.

Systems Integration for Manufacturing Applications Program 1995 Annual Report.

PB96-1937

02,844

Evaluation and Oualification Standards for an X-Ray

Laminography System.
PB94-172954

LUCK, $R$.

02,029

Real Time Differential Range Estimation Based on TimeSpace Imagery Using PIPE.

PB95-161808

01,844

LUDWIG, $B$.

Isolation and Structural Elucidation of the Predominant Geometrical Isomers of alpha-Carotene.

PB96-190061

00,640

LUDWIG, F.

Fabricatıon Issues in Optimizing YBa2Cu307-x Flux Transformers for Low $1 / /$ Noise.

PB95-175857

02,059

Low Noise YBa2Cu3O7-x-SrTiO3-YBa2Cu3O7-x Mulitlayers for Improved Superconducting Magnetometers.

04,747

PB96-138417

Documentation for Immediately Dangerous to Life or Health

Concentrations (IDLHs)
PB94-195047

03,602

UDWIG, K. F.

Coexistence of Grains with Differing Orthorhombicity in High Ouality YBa2Cu307-delta Thin Films.

04,742

Increased Transition Temperature in In situ Coevaporated YBa2Cu307-delta Thin Films by Low Temperature Post-Annealing.

PB95-180071

04,672

LUECKE, $W$.

Cavity Evolution during Tensil Creep of Si3N4

PB96-204193

03,376
LUECKE, W. E.

Cavitation Contributes Substantially to Tensile Creep in Silicon Nitride.

03,171

Sources of Strain-Measurement Error in Flag-Based Extensometry.

03,108

Tension/Compression Creep Asymmetry in Si3N4.

PB97-110258

03,096

LUGEZ, C. L.

Matrix Isolation Study of the Interaction of Excited Neon Atoms with O3: Infrared Spectrum of $\mathrm{O}(($ sub 3)(-)) and Evi dence for the Stabilization of $\mathrm{O} 2 \ldots \mathrm{O}(($ sub 4$)(+))$

PB97-112403

04,155

PB97-118723

04,168

Vibrational Spectra of Molecular Ions isolated in Solid Neon. 13. Ions Derived from $\mathrm{HBr}$ and $\mathrm{HI}$

01,188

LUJAN, A.

Gaseous Electronics Conference Radio-Frequency Reference Cell: A Defined Parallel-Plate Radio-Frequency System for Experimental and Theoretical Studies of PlasmaProcessing Discharges.

PB94-172327

04,404

LUKASHEV, E. P.

Bacteriorhodopsin Retains Its Light-Induced Proton-Pumping Function After Being Heated to $140 \mathrm{C}$

03,471

Rapid pH Change Due to Bacteriorhodopsin Measured with a Tin-Oxide Electrode.

PB96-112081

03,544

LUM, L. S. H.

Ceramic Powders Characterization: Results of an International Laboratory Study

PB95-270039

02,672

Characterization of Phase and Surface Composition of Silicon Carbide Platelets.

PB94-216264

03,043

Statıstical Analysis of Parameters Affecting the Measurement of Particle-Size Distribution of Silicon Nitride Powders by Sedigraph (Trade Name).

PB94-216249

03,042

LUMIA, $R$.

Evolution of the Flight Telerobotic Servicer

04,832

Overview of NASREM: The NASANBS Standard Reference Model for Telerobot Control System Architecture.

PB94-194560

04,831

Unified Telerobotic Architecture Project (UTAP) Standard Interface Environment (SIE), May 1995.

PB95-242350 02,938

World Model Registration for Effective Off-Line Programming of Robots.

LUMPKIN, J.

02,933

Tert-Butyl Hydroperoxide-Mediated DNA Base Damage in Cultured Mammalian Cells.

PB94-182003

LUMSDAINE, A.

03.644

ML++ v.1.2 Iterative Methods Library Reference Guide.

PB96-195219 01,776

SparseLib++ v. 1.5 Sparse Matrix Class Library. Reference Guide

PB96-193636

01.775

EGARD, R. J.

02,708

PB94-199064

LUNDQVIST, $M$.

Inner-Valence States $\mathrm{CO}(+)$ between $22 \mathrm{eV}$ and $46 \mathrm{eV}$ Studied by High Resolution Photoelectron Spectroscopy and ab initio Cl Calculations.

PB95-180055

03,961

LUO, J.

Grain Alignment and Transport Properties of Bi2Sr2CaCu2O 8 Grown by Laser Heated Float Zone Method.

PB95-16145

04.602

LUPER, W. D.

Effect of Three Sterilization Techniques on Finger Pluggers.

LUPO, J.

Real Time Differential Range Estimation Based on TimeSpace Imagery Using PIPE.

PB95-161808

01,844

Real-Time Imp

01,839

PB95-108650

LUTGEN, C. L.

Electromechanical properties of superconductors for DOE fusion applications.

04,432

Electromechanical Properties of Superconductors for DOE Fusion Applications.

PB94-139672

02,250

LUTTERMOSER, D. G.

Peeking Through the Picket Fence: What Astrophysical Surprises May Be Present in the 100-1200 Angstrom Region.

PB95-202859 00,082

LUTZ, G. J

High-Sensitivity Determination of Iodine Isotopic Ratios by Thermal and Fast Neutron Activation.

PB94-213386

YELL, M. J.

Internal Waves in Xenon Near the Critical Point

PB97-111504

04,221

YKE, J.

Overview of U.S. Government Advanced Packaging Programs.

02,443

LYLE, J. R.

Application of the Pointer State Subgraph 10 Static Program Slicing.

PB96-167838

01,768

Assessing Functional Diversity by Program Slicing.

PB96-160890

03,734

Program Slicing

PB96.160981

01,761

PB95-125894

01,703

Unravel: A CASE Tool to Assist Evaluation of High Integrity Software. Volume 1. Requirements and Design.
PB95-267886 $\begin{array}{ll}\text { PB95-267886 } & 01,736\end{array}$ Unravel: A CASE Toftware. Volume 2. User Manual.
Solution of High Integrity PB95-267894

LYLES, $\mathrm{S}$

Directory of Law Enforcement and Criminal Justice Associations and Research Centers.

PB96-178918

04,872

LYMBEROPOULOS, D. P.

Two-Dimensional Self-Consistent Radio Frequency Plasma Simulations Relevant to the Gaseous Electronics ConPerence RF Reference Cell.

PB96-113436

02,398

YNCH, J. J.

Modified Leung-Griffiths Model of Vapor-Liquid Equilibrium: Extended Scaling and Binary Mixtures of Dissimilar Fluids. PB94-216108 00,851

Nonlinear Correlation of High-Pressure Vapor-Liquid Equilibrium Data for Ethylene + n-Butane Showing Inconsistencies in Experimental Compositions.

01,141

LYNCH, T.P.

External Gamma-ray Counting of Selected Tissues from a Thorotrast Patient.

PB96-160254

03,637

LYNN, J. E.

Intermediate Structure in the Neutron-Induced Fission Cross Section of $236 \mathrm{U}$

PB94-185741

03,802

YNN, J. W.

Analytic Calculation of Polarized Neutron Reflectivity from Superconductors. 
Observation of Noncollinear Magnetic Structure for the $\mathrm{Cu}$ Spins in Nd2CuO4-Type Systems.

04,634

Observation of Oscillatory Magnetic Order in the Antiferromagnetic Superconductor HoNi2B2C. PB95-180303

04,679

Oxygen Dependance of the Crystal Structure of PB96-102512 and Its Relation to Superconductivity. 04,711

Phonon Density of States in R2CuO4 and Superconducting R1.85Ce0.15CuO4 ( $\mathrm{R}=\mathrm{Nd}, \mathrm{Pr}$ )

PB95-150686

04,574

Polarization Analysis of the Magnetic Excitations in Fe65Ni35 Invar.

04,558

Polarization Analysis of the Magnetic Excitations in Invar and Non-Invar Amorphous Alloys.

PB94-216116

04,516

Ouasielastic and Inelastic Neutron-Scattering Studies of ((CD3)3ND)FeCl3.2D2O: A One-Dimensional Ising Ferromagnet.
PB95-140562

04,547

Temperature Dependence of the Magnetic Excitations in Ordered and Disordered Fe72Pt28.

PB95-150223

04,563

Unconventional Ferromagnetic Transition in $\mathrm{La}$ (sub 1 . x) $\mathrm{Ca}$ (sub x) $\mathrm{MnO}$

PB97-112429

04,156

Vortex Dynamics

02,073

LYON, G.

Computing Eflects and Error for Large Synthetic Perturba-

tion Screenings.
PB94-139623

Scalability Test for Parallet Code.

Scalability Test
PB96-146758

01,675

Simple Scalability Test for MIMD Code.

PB94-193638

01,749

Synthetic-Perturbation Tuning of MIMD Programs.

01,688

Time-Perturbation Tuning of MIMD Programs.

PB94-164399

01,687

Time-Perturbation Tuning of MIMD Programs.

PB94-172566

01,681

Using Synthetic-Perturbation Techniques for Tuning Shared Memory Programs (Extended Abstract).

01,685

Using Synthetic Perturbations and Statistical Screening to Assay Shared-Memory Programs.

LYON, $R$.

Materials and Fire Threat.

PB97-122311

01,740

YONS, K. W.

01,442

Design Engineering Research at NIST

PB95-267860

02,784

Product Realization Process Modeling: A Study of Requirements, Methods and Research Issues.

PB96-147962

02,836

LYONS, R. M.

Bibliography of the NIST Electromagnetic Fields Division Publications.
PB94-165990

01,875

Bibliography of the NIST Electromagnetic Fields Division Publications.

01,886

Bibliography of the NIST Electromagnetic Fields Division Publications.

LYZAK, W. A.

Diagnosis and Treatment of an Oral Base-Metal Contact Lesion Following Negative Dermatologic Patch Tests. PB95-180626

00,172

MA, D. 1.

Interaction of Stoichiometry, Mechanical Stress, and Interface Trap Density in LPCVD Si-rich SiNx-Si Structures. PB95-176301

02,366

MA, L. M.

Mechanical Properties and Warm Prestress of Ultra-Low Carbon Steel at $4 \mathrm{~K}$

PB96-190350

03,224

MA, L. S.

Delivering the Same Optical Frequency at Two Places: Accurate Cancellation of Phase Noise Introduced by an Optical Fiber or Other Time-Varying Path.

MA, M. T.

04,332

Aperture Excitation of Electrically Large, Lossy Cavities

PB94-145711 00,029

Aperture Excitation of Electrically Large, Lossy Cavities.

PB95-175675 00,031

Assessment of Data by a Second-Order Transfer Function.

PB95-182390 03,760

Characterization of Unknown Linear Systems Based on Measured CW Amplitude.

01,897
Data Evaluation of a Linear System by a Second-Order Transfer Function

PB96-200282

01,970

MACADAM, K. B.

Alignment in Two-Step Pulsed Laser Excitation of Rydberg Levels in Light Atoms: The Example of Sodium.

03,993

Low-Energy-Electron Coltisions with Sodium: Elastic and Inelastic Scattering from the Ground State. PBa6-103106

04,030

Narrow-Band Tunable Diode Laser System with Grating Feedback, and a Saturated Absorption Spectrometer for Cs

PB95-20289 1

MACCREHAN, W. A.

04,319

Determination of Vitamin $\mathrm{K} 1$ in Serum Using Catalytic-Reduction Liquid Chromatography with Fluorescence DetecPB96-138425

03,506

Separation and Identification of Organic Gunshot and Explosive Constituents by Micellar Electrokinetic Capillary Electrophoresis.

MACDONALD, M. A

Electron-Ion-X-ray Spectrometer System.

00,566

PB95-176137

03,958

Evolution of $X$-ray Resonance Raman Scattering into $X$-ray Fluorescence from the Excitation of Xenon Near the L3 Edge.

04,025

MACDONALD, M. E.

Niobium Microbolometers for Far-Infrared Detection.

PB96-111729

02,184

MACDONALD, R. A.

Analytical Method for Determining Thermal Conductivity from Dynamic Experiments.

04,024

Analytical Method of Determining the Heat Capacity at High Temperatures from the Surface Temperature of a Cooling Sphere.

PB94-216124

03,865

MACHIN, G.

Intercomparison of the ITS-90 Radiance Temperature Scales of the National Physical Laboratory (U.K.) and the National Institute of Standards and Technology. PB96-113550

MACKAY, D. R.

Performance Standards: The Pro's and Con's. PB94-216132

02,674

MACKAY, J.

Taguchi's Parameter Design: A Panel Discussion. PB96. 111802

02,896

MACKEY, E.

Development of Frozen Whale Blubber and Liver Reference Materials for the Measurement of Organic and Inorganic Contaminants.

MACKEY, E. A.

00,587

Analytical Applications of Guided Neutron Beams.

04,041

Concentrations of Chlorinated Hydrocarbons, Heavy Metals and Other Elements in Tissues Banked by the Alaska Marine Mammal Tissue Archival Project.

02,590

Ettects of Target Shape and Neutron Scattering on Element Effects of Target Shape and Neutron Scattering on Element Sensitivities for Neutron-Capture Prompt Gamma-ray Acli-
vation Analysis. vation Analysis
PB94-216157

00,558

Effects of Target Temperature on Analytical Sensitivities of Cold-Neutron Capture Prompt gamma-ray Activation Analysis.

00,616

Neutron Capture Prompt Gamma-Ray Activation Analysis at the NIST Cold Neutron Research Facility.

00,556

Neutron Scattering by Hydrogen in Cold Neutron Prompt Gamma-Activation Analysis.

PB95-175972

00,603

Relationship of Silver with Selenium and Mercury in the Liver of Two Species of Toothed Whales (Odontocetes).
PB96-167275

Scattering and Absorption Effects in Neutron Beam Activation Analysis Experiments.

tion Analysis

00,557

Trace Element Concentrations in Cetacean Liver Tissues Archived in the National Marine Mammal Tissue Bank PB96-167127 02,595

Use of Neutron Beams for Chemical Analysis at NIST. PB97-112437

00,652

MACKIE, N.

High-Sensitivity Spectroscopy with Diode Lasers. PB95-175477

04,297

MACREYNOLDS, $K$.

Planar Near-Field Measurements and Microwave Holography for Measuring Aperture Distribution on a $60 \mathrm{GHz}$ Active Array Antenna.

03,762

MACURDY, L. B

Precision Laboratory Standards of Mass and Laboratory Weights.

02,618

MADEY, T. E.

Desorption Induced by Electronic Transitions.

PB94-216173

00,853

Influence of Coadsorbed Potassium on the Electron-Stimulated Desorption of $F(+), F(-)$, and $F\left({ }^{*}\right)$ from PF3 on Pu(0001).

04,072

Role of Adsorbed Alkalis in Desorption Induced by Electronic Transitions.

00,762

Structure of Molecules on Surfaces as Determined Using Electron-Stimulated Desorption.

PB94-21616
MADIGAN, B.

00,852

Through-the-Arc Sensing for Measuring Gas Metal Arc Weld Ouality in Real Time.

PB95-164463

02,908

MADIGAN, R. B.

Computers in Welding: A Primer.

PB95-162863

02,862

Contact Tube Wear Detection in Gas Metal Arc Welding.
PB96-135330

Control of Gas-Metal-Arc Welding Using Arc-Light Sensing.
PB96-131461

Droplet Transfer Modes for a MIL 100S-1 GMAW Electrode.

PB95-209300 02,867

Electrode Extension Model for Gas Metal Arc Welding.

PB96-135074

02,871

Mapping the Droplet Transfer Modes for an ER100S-1 PB96. 190095

03,295

Power Characteristics in GMAW: Experimental and Numerical Investigation.
PB96-190145

03,296

Sensing Droplet Detachment and Electrode Extension for

Control of Gas Metal Arc Welding.

Through-the-Arc Sensing for Monitoring Arc Welding

PB94-185899

02,858

Through-the-Arc Sensing for Real-Time Measurement of Gas Metal Arc Weld Ouality.

02,863

ADIGOSKY, W. M.

Electro-Optical Sensor for Surface Displacement Measurements of Compliant Coatings.

MADISON, D.

02,123

Connection between Superelastic and Inelastic ElectronAtom Collisions Involving Polarized Collision Partners.

03,974

MADRZYKOWSKI, D.

Evaluation of Sprinkler Activation Prediction Methods.

PB96-141056

Sciences: A Winning Combination.

Flame Heights and Heat Release Rates of 1991 Kuwait Oil

Field Fires.

PB96-119342

01,404

In situ Burning of Oil Spills: Mesoscale Experiments and Analysis.
PB95-163747 
Thermophysical Properties of $\mathrm{CO} 2$ and CO2-Rich Mixtures. PB94-216199 00,854 Vapour Pressure Measurements on 1,1,1,2Tetrafluoroethane (R134a) from 180 to $350 \mathrm{~K}$. 03,265 PB95-16888

MAGYAR, J.

High-Sensitivity Spectroscopy with Diode Lasers. PB95-175477

MAIENSCHEIN, F. C.

Measurement of Absorbed Dose of Neutrons, and of Mix. tures of Neutrons and gamma rays.

AD.A286 647/3

03,710

MAITZ, A. H.

Use of a Radiochromic Detector for the Determination of Stereotactic Radiosurgery Dose Characteristics.

PB94-185642

MAJKRZAK, C. F.

Antiferromagnetic Interlayer Correlations in Annealed Ni80Fe20/Ag Multilayers.

PB97-122220

03,109

Extending the Angular Range of Neutron Reflectivity Meas. urements from Planar Lipid Bilayers: Applications to a Model Biological Membrane.

PB96-122569

03,476

In-situ Neutron Reflectivity of MBE Grown and Chemically Processed Surfaces and Interfaces.

02,416

Magnetic Dead Layer in Fe/Si Multilayer: Profile Refinement of Polarized Neutron Reflectivity Data.

04,458

PB94-198363

$80 \mathrm{Fe} 20 /$

Magnetic Structure Determination for Annealed

PB96-176615

03,739

Morphology of Symmetric Diblock Copolymers as Revealed

by Neutron Reflectivity.
PB95-140075

01,234

Neutron Reflecto

Neutron Scattering Studies of Surfaces and Interfaces.

PB94-216207 04,517

Neutron-Scattering Studies of the Two Magnetic Correlation Lengths in Terbium.

PB95-152328

04,586

Observation of Two Length Scales Above ( $T$ sub $N$ ) in a Holmium Thin Film

04,151

Origin of the Second Length Scale Above the Magnetic Spiral Phase of $\mathrm{Tb}$.

04,596

Supermirror Transmission Polarizers for Neutrons.

PB94-216215

03,866

Temperature Dependence of the Morphology of Thin Diblock Copolymer Films as Revealed by Neutron Reflectiv-

PB94-172756

01,199

MAJKRZAK, C. J.

Water Adsorption at a Polyimide/Silicon Wafer Interface.
PB96-103197

MAJURSKI, W. Framework for National Information Intrastructure Services.
PB95-103719

MAJURSKI, W. J.

Open Systems Software Standards in Concurrent Engineer-

ing.

01,758

Prototyping a Graphical User Interface for DHCP

PB96-160544

02,599

MAKEPEACE, J. L.

Intercomparison of Internal Proportional Gas Counting of $(85) \mathrm{Kr}$ and $(3) \mathrm{H}$
$\mathrm{PB94}-185576$

03,800

MAKI, A. G.

FTS Infrared Measurements of the Rotational and Vibrational Spectrum of $\mathrm{LiH}$ and $\mathrm{LiD}$.

00,856

High-Resolution Measurements of the nu2 and 2nu2-nu2 Bands of (34)S(16)O2.

PB94-216223

00,855

Improved Molecular Constants and Frequencies for the $\mathrm{CO} 2$ Laser from New High-J Regular and Hot-Band Frequency Measurements.

quency Measuren
PB95-180634

04,312

Sub-Doppler Frequency Measurements on OCS at $87 \mathrm{Thz}$ (3.4 micrometers) with the CO Overtone Laser: Considerations and Details.

PB95-128633

04,255

Sub-Doppler Frequency Measurements on OCS at $87 \mathrm{THz}$ $(3.4 \mathrm{mu} \mathrm{m}$ ) with the CO Overtone Laser.

PB96-102215

04,330

MAKINEN, J.

Calibration of a Superconducting Gravimeter Using Absolute Gravity Measurements.

03,684

PB95-202651

Test of Newton's Inverse Square Law of Gravitation Using the 300-m Tower at Erie, Colorado.

03,978

\section{MALDONADO, E}

Investigation of Mercury Interstitials in $\mathrm{Hg}(1-\mathrm{x}) \mathrm{CdxTe}$ Alloys Using Resonant Impact-Ionization Spectroscopy.

PB94-213485

02,133

MALENFANT, A.

Frozen Human Serum Reference Material for Standardization of Sodium and Potassium Measurements in Serum or Plasma by lon-Selective Electrode Analyzers.

PB94-185337

00,532

MALEWSKI, R.

Digital Techniques in HV Tests - Summary of 1989 Panel Session.

PB94-216702

02,035

MALGHAN, S. G.

Analysis of Physical Properties of Ceramic Powders in an International Interlaboratory Comparison Program. PB5-161501

03,050

Ceramic Powders Characterization: Results of an Inter-

national Laboratory Study.
PB95-270039

Characterization and Processing of Spray-Dried Zirconia Powders for Plasma Spray Application.

04,419

Characterization of Phase and Surtace Composition of Silicon Carbide Platelets.

PB94-216264

03,043

Coating of Fibers by Colloidal Techniques in Ceramic Composites.
PB94-216256

03,196

Densification of Nano-Size Powders. 1994 Report.

DE94013486

03,027

Deposition of Colloidal Sintering-Aid Particles on Silicon

PBitride. 216272

03,044

Effects of Soxhlet Extraction on the Surface Oxide Layer of Silicon Nitride Powders.

PB95-175584 03,057

Electroacoustic Characterization of Particle Size and Zeta Potential in Moderately Concentrated Suspensions. PB96-119425

01,079

Electroacoustics for Characterization of Particulates and Suspensions. Proceedings of a Workshop. Held in Gaithersburg, Maryland on February 3-4, 1993.

PB94-112695

00,725

Electrokinetic Sonic Analysis of Silicon Nitride Suspensions. PB96-123575 03,073

Equipment for Investigation of Cryogenic Compaction of Nanosize Silicon Nitride Powders. 1993 Report.

DE94013593

03,028

Interface Modification and Characterization of Silicon Carbide Platelets Coated with Alumina Particles. PB95-108734

03,12

NMR Characterization of Injection-Moulded Alumina Green Compacts. Part 2. T2-Weighted Proton Imaging.

PB96-201181

01,165

Polyelectrolytes as Dispersants in Colloidal Processing of Silicon Nitride Ceramics.

PB95-175568

03,055

Standard Reference Material for the Measurement of Particle Mobility by Electrophoretic Light Scattering.

PB96-102488

00,609

Statistical Analysis of Parameters Affecting the Measurement of Particle-Size Distribution of Silicon Nitride Powders by Sedigraph (Trade Name).

03,042

Surface Chemical Interactions of Si3N4 with Polyelectrolyte Deflocculants.

03,056

Surface Chemistry of Silicon Nitride Powder in the Presence of Dissolved lons.

PB96- 111760

01,073

Variances in the Measurement of Ceramic Powder Properties

PB97-110316

03,100

MALIK, A.

Comparison of Selectivities for PCBs in Gas Chromatography for a Series of Cyanobiphenyl Stationary Phases. PB96-119458

MALLARD, G.

Progress in the Development of a Chemical Kinetic Database for Combustion Chemistry.

PB95-151056

01,384

MALLEY, J. O.

Evaluation and Strengthening Guidelines for Federal Build ings: Identification of Current Federal Agency Programs

PB94-176278

00,424

MALONE, K.

Using Secondary Ion Mass Spectrometry (SIMS) to Characterize Optical Waveguide Materials

04,340

MALONE, K. J.

Distributed Feedback Lasers in Rare-Earth-Doped Phos-

phate Glass.

04,740

Glasses for Waveguide Lasers.

PB96-111950

04,335

Integrated Optic Laser Emitting at 905, 1057, $1356 \mathrm{~nm}$ PB94-216298 02,136 Integrated Optic Laser Emitting at 906, 1057, and $1358 \mathrm{~nm}$. Integrated-Optical Devices in Rare-Earth-Doped Glass. Integrated-Optical Devices In Rare-Earth-Doped Glass.
PB95-202909 179 Linewidth Narrowing in an Imbalanced $Y$-Branch Waveguide

PB95-140844

Nd:LiTaO3 Waveguide Laser

PB95-140851.

04,259

Passively Q-Switched Nd-Doped Waveguide Laser.

04,308

Rare-Earth-Doped Waveguide Devices: The Potential for Compact Blue-Green Lasers.

PB95-140836

04,257

Determination of Oltipraz in Serum by High-Performanace

Determination of Oltipraz in Serum by High-Performanace
Liquid Chromatography with Optical Absorbance and Mass Spectrometric Detection

Spectrometric

MALONEY, D. L.

Prototyping a Graphical User Interface for DHCP

PB96-160544

03,493

MALSHE, A. P.

Workshop on Characterızing Diamond Films (4th). Held in Gaithersburg, Maryland on March 4-5, 1996.

PB96- 183090

MAMILETI, L.

Optical Fiber Geometry by Gray-Scale Analysis with Robust Regression.
PB95-161519

MANDEL, J.

Models and Interactions.

PB94-216306

MANDELKERN, L.

SANS Study of the Plastic Deformation Mechanism in Polyethylene.

PB95-151841

01,242

MANGAMELLI, A.

Chemical Aspects of Tool Wear in Single Point Diamond Turning.

PB97-11260

MANGIN, J. F.

Prelımınary Comparison of Time Transfers via LASSO, GPS and Two-Way Satellite.

MANGUM, B. $W$.

Comparisons of Some NIST Fixed-Point Cells with Similar Cells of Other Standards Laboratories.

PB97-119242

00,655

Current Status and Trends in Temperature Measurements at NIST, Cooperative Projects and New Mutual Agreemen between NIST and IMGC

PB97-11026

02,691

Direct Comparison of Three PTB Silver Fixed-Point Cells with the NIST Silver Fixed-Point Cell.

PB96-161286 00,628

Investigation of High-Temperature Platinum Resistance Thermometers at Temperatures Up to $962 \mathrm{C}$, and. in Some Cases, $1064 \mathrm{C}$

PB96-161294 00,629

NIST Measurement Assurance of SPRT Calibrations on the 
MANN, W. B.

NBS/NIST Peltier-Effect Microcalorimeter: A Four-Decade

Review.

01,064

MANNEY, C. H

High-Resolution Atomic Spectroscopy of Laser-Cooled lons.

Recent Experiments on Trapped Ions at the National Inst:ute of Standards and Technology.

PB95-169322

MANNING, J. R.

Electronics Packaging Materials Research at NIST.

PB96-122692

NIST/NCMS Program on Electronic Packaging: First UP PBate. 204086

03,008

MANNING, $M$

Charge Transfer and Collision-Induced Dissociation Reactions of $\mathrm{CF}(2+)$ and $\mathrm{CF}_{2}(2+)$ with the Rare Gases at a Laboratory Collision Energy of $49 \mathrm{eV}$.

PB94-185584

00,775

Collision-Induced Neutral Loss Reactions of Molecula Dications.

MANNING, N. O

00,780

Protein Data Bank: Current Status and Future Challenges.

PB97-109060

00.517

MANOHAR, S.

Turbulent Upward Flame Spread on a Vertical Wall under External Radiation.

PB94-207388

00,341

MANSOOR, A.

Surging the Upside-Down House: Measurements and Modeling Results.

MANSURIPUR, $M$

Micromagnetic Structure of Domains in Co/Pt Multilayers. 1 Investigations of Wall Structure.

PB95-162111

MANTESE, $J$.

Effective Medium Theory for Ferrite-Loaded Materials PB95-154662

MANTESE, $J$.

Applicability of Effective Medium Theory to Ferroelectric/ Ferrimagnetic Composites with Composition and Frequency-Dependent Complex Permittivities and Permeabilities.

PB96-157854

01,945

MANTL, $S$.

Silicon Surface Chemistry by IR Spectroscopy in the Mid- to Far-IR Region: $\mathrm{H} 2 \mathrm{O}$ and Ethanol on $\mathrm{Si}(100)$

PB96-138565

01,097

MANTOOTH, H. A Electro-Thermal Simulation of an IGBT PWM Inverter.
PB94-185592

MAO, G.

Inelastic-Neutron-Scattering Studies of Poly( $\mathrm{p}$-phenylene viPylene).

01,014

MARAN, S. P.

Observations of $3 \mathrm{C} 273$ with the Goddard High Resolution Spectrograph on the Hubble Space Telescope. Observing Stellar Coronae with the Goddard High Resolu tion Spectrograph. 1. The dMe Star AU Microscopii. PB96-102777

MARCARINO, $P$.

Comparisons of Some NIST Fixed-Point Cells with Simila Cells of Other Standards Laboratories.

PB97-119242

00,655

MARCHIANDO, J. F.

Application of the Collocation Method in Three Dimensions to a Model Semiconductor Problem

PB97-122428

02,457

Scanning Capacitance Microscopy Measurements and Modeling for Dopant Profiling of Silicon.

PB96-164207

04,78

Scannıng Capacitance Microscopy Measurements and Modeling: Progress Towards Dopant Profiling of Silicon.

PB96-148150

04.773

Scanning Capacitance Microscopy Measurements and Modeling: Progress Towards Dopant Profiling of Silicon.

PB96-180070 01,964

Using Collocation in Three Dimensions and Solving a Mode Semiconductor Problem.

PB96-159249

01,952

MAREZIO, M.

Crystal Structure of Pb2Sr2YCu3O8+delta with delta $=1.32$ $1.46,1.61,1.71$, by Powder Neutron Diffraction.

PB94-216314

MARGOLESE, D.

Small Angle Neutron Scattering Study of the Structure and

Formation of MCM-41 Mesoporous Molecular Sieves.
PB97-122337

\section{MARGOLESE, D. I.}

Small Angle Neutron Scattering Study of the Structure and Formation of Ordered Mesopores in Silica.

03,069

PB96-111919.

Nutritional Status and Growth in Juvenile Rheumatoid Arthritis.

PB94-198470

03,515

MARGOLIS, S. A.

Amperometric Measurement of Moisture in Transformer Oil Using Karl Fischer Reagents.

PB96-146766

00,623

Ascorbic and Dehydroascorbic Acids Measured in Plasma Preserved with Dithiothrettol or Metaphorphoric Acid.

PB94-216330

03,495

Hydrolysis of Proteins by Microwave Energy.

03,528

Measurement of Ascorbic Acid in Human Plasma and Serum: Stability, Intralaboratory Repeatability, and Interlaboratory Reproducibility Interlaboratory
PB97-112445

03,511

Thermochemistry of the Hydrolysis of L-arginine to (Lcitrulline + Ammonia) and of the Hydrolysis of L-arginine to (L-ornithine + Urea)

PB95-150801

03,463

MARGRAVE, J. L.

Corrected Optical Pyrometer Readings.

AD-A279 949/2

02,615

Disilicides of Tungsten, Molybdenum, Tantalum, Titanium, Cobalt, and Nickel, and Platinum Monosilicide: A Survey of Their Thermodynamic Properties.

- 168580

00,752

MARINENKO, $R$.

Fabrication of Platinum-Gold Alloys in Pre-Hispanic South America: Issues

MARINENKO, R. B.

Apparent Bias in the X-Ray Fluorescence Determination of Titanium in Selected NIST SRM Low Alloy Steels.

PB95-108759

03,212

Compositional Mapping of the Microstructure of Materials.

PB95-107199 00,565

Design of a Protocol for an Electron Probe Microanalyzer kValue Round Robin.

PB95-107181

00,564

Epitaxial Growth of $\mathrm{BaTiO}_{3}$ Thin Films at $600 \mathrm{C}$ by Metalorganic Chemical Vapor Deposition.

03,071

Study of Diffusion Zones with Electron Microprobe Compositional Mapping.

00,559

MARINKOVIC, B.

Threshold Electron Excitation of $\mathrm{Na}$.

PB95-202917

03,994

MARJENHOFF, W. A.

Adhesion of Composites to Dentin and Enamel. PB94-199049

Development of an Adhesive Bonding System. PB94-19905

00,144

Paffenbarger Research Center: The Cutting Edge of Dental Sclence.

PB94-216355

00,151

Posterior Restor

03,582

Publication and Presentation Abstracts, 1993. (Published by Paffenbarger Research Center and Center of Excellence for Materials Science Research.

PB95-153052

03,562

Publication and Presentation Abstracts, 1994

PB96-176623

03,577

Publication and Presentation Abstracts, 1995

PB96-164082

03,576

Publication and Presentation Abstracts, 1996

PB97-122238

03,585

Publications and Presentation Abstracts, 1995. (Published by Paffenbarger Research Center and Center of Excellence for Materials Science Research).

PB96-119250

03,568

MARKIN, V.S

Energy Transduction between a Concentration Gradient and an Alternating Electric Field

PB94-216363

03,461

MARKO, $P$.

Characterization of the Adsorption-Fouling Layer Using Globular Proteins on Ultrafiltration Membranes.
PB94-212909

Self Broadening in the nu 1 Band of $\mathrm{NH}_{3}$.

PB94-216371

00,857

MARKOVIC, $M$.

Composition and Solubility Product of a Synthetic Calcium Pydroxyapatite. 02,995

Crystal Structure of Calcium Succinate Monohydrate. PB95-168928 00,167

Octacalcium Phosphate Carboxylates IV. Kinetics of Formation and Solubility of Octacalcium Phosphate Succinate. PB94-185600

00,776

Octacalcium Phosphate Carboxylates. 1. Preparation and Identification.

00,660

Octacalcium Phosphate Carboxylates. 2. Characterization and Structural Consideration.

PB95-161543

00,955

Octacalcium Phosphate Carboxylates. 5. Incorporation of Excess Succinate and Ammonium lons in the Octacalcium Phosphate Succinate Structure.

PB95-168894

00,166

Octacalcium Phosphate. 3. Infrared and Raman Vibrational Spectra

MARKOVITS, $G$.

Learning to Change: Opportunities io Improve the Pertormance of Smaller Manufacturers.

ance of Small

MARKOVITZ, $P$.

Guidelines

PB96-172325

Security in Open Systems.

PB95-105383

MARKS, R. $B$.

00,756

Accuracy and Repeatability in Time Domain Network Analy-

PBis. 202644

Accuracy in Time Domain Transmission Line Measure-

PB96-148069 04,060

Accurate Electrical Characterization of High-Speed Inter-

connections.

02,240

Accurate Experimental Characterization of Interconnects: A Discussion of 'Experimental Electrical Characterization of Interconnects and Discontinuities in High-Speed Digital Sysems

PB94-216389

02,217

Accurate Transmission Line Characterization.

PB95-151593

02,220

Calibrating On-Water Probes to the Probe Tips.

PB95-163945

Coaxial Line-Reflect-Match Calibration.

02,352

02,246

Comments on 'Conversions between $S, Z, Y, h, A B C D$, and $T$ Parameters Which Are Valid for Complex Source and Load Impedances

PB96-102785

02,069

Comments on 'Protecting EFIE-Based Scattering Computations from Effects of Internal Resonances'

01,898

PB95-161568

Tip Cali-

Compen

PB95-203519

01,915

Electrical Measurements of Microwave Flip-Chip Interconnections.

02,436 


\section{PERSONAL AUTHOR INDEX}

Verification of Scatterıng Parameter Measurements. PB95-163960

MARKS, R. F.

Combined Low- and High-Angle $X$-Ray Structural Refinement of a Co/Pt(111) Multilayer Exhibiting Perpendicular Magnetic Anisotropy.

PB94-198355

04,457

MARKS, T. J.

Suitability of Metalorganic Chemical Vapor Deposition-Derived PrGaO3 Films as Buffer Layers for YBa2Cu307-x Pulsed Laser Deposition.

PB95-168670

04,640

MARLOW, A.

Apparent Bias in the X-Ray Fluorescence Determination of Titanium in Selected NIST SRM Low Alloy Steels.

PB95-108759

MARQUARDT, E.

Design Equations and Scaling Laws for Linear Compressors with Flexure Springs

02,948

High-Sensitivity Spectroscopy with Diode Lasers.

PB95-175477

04,297

MARQUARDT, J. H

Optical Probing of Cold Trapped Atoms.

PB95-175469

MARR, G. V.

High Resolution Angle Resolved Photoelectron Spectroscopy Study of N2.

PB95-151494

03,907

MARSCHALL, E.

Comparison of Elastic and Plastic Contact Models for the Prediction of Thermal Contact Conductance.

04,605

MARSH, K. N.

Thermodynamic and Thermophysical Froperties of Organic Nitrogen Compounds. Part II. 1- and 2-Butanamine, 2-Methyl-1-Propanamine, 2-Methyl-2-Propanamine, Pyrrole, 1-2 2and 3-Methylpyrrole, Pyridine, 2-, 3-, and 4-Methylpyridine Pyrrolidine, Piperidine, Indole, Ouinoline, Isoquinoline, Acri-
dine, Carbazole, Phenanthridine, t- and 2Naphthalenamine, and 9-Methylcarbazole. PB94-162294

00,741

MARSHAK, $\mathrm{H}$.

Precision Nuclear Orientation Measurements for Determining Mixed Magnetic Dipole/Electric Ouadrupole Hyperfine Interactions.

PB94-199080

MARSHALL, H. E.

03,810

Economic Methods and Risk Analysis Techniques for Evaluating Building Investments: A Survey.

00,323

Economics of New-Technology Materials: A Case Siudy of FRP Bridge Decking.
PB96-202353

01,349

Least-Cost Energy Decisions for Buildings: Part 2. Uncertainty and Risk Video Training Workbook

00,240

Least-Cost Energy Decisions for Buildings. Part 3. Choosing Economic Evaluation Methods. Video Training Work-

PB95-253597

00.265

Multiattribute Decision Analysis Method for Evaluating Buildings and Building Systems.

00,325

Standards in Building Economics: Why We Need Them and How to Write Them.

PB94-216405

MARSHALL, $J$.

Realizing Suspended Structures on Chips Fabricated by CMOS Foundry Processes Through the MOSIS Service. PB94-193984

MARSHALL, J. A.

Low Thermal Guarded Scanner for High Resistance MeasPBrement Systems.

MARSHALL, 5 .

02,288

Color Supplement to NIST Special Publication 400-93: Semiconductor Measurement Technology: Design and Testing Guides for the CMOS and Lateral Bipolar-on-SOI Test

Library.
PB94-164316 02,298

Design Guide for CMOS-On-SIMOX. Test Chips NIST3 and NIST4.

02,297

High-Level CAD Melds Micromachined Devices with FoundPBies.

02,321

Semiconductor Measurement Technology: Design and Testing Guides for the CMOS and Lateral Bipolar-on-SOI Test

Library
PB94-178019

MARSHALL, R.

Some Notable Hurricanes Revisited.
PB96-122601

02,301

MARSHALL, R. D.

Comparison of Responses of a Select Number of Buildings

to the $10 / 17 / 1989$ Loma Prieta (California) Earthquake and

Low-Level Amplitude Test Results.

PB96-159645

00,467
Dynamic Characteristics of Five Tall Buildings during Strong and Low-Amplitude Motions.

00,427

Gust Factors Applied to Hurricane Winds.

PB95-180469

00,446

PB94-216421

Manufactured Homes: Probability of Failure and the Need for Better Windstorm Protection through Improved Anchoring Systems.

00,432

Proceedings: Workshop on Research Needs in Wind Engineering. Held in Gaithersburg, Maryland on September 12

00,448

Recommended Performance-Based Criteria for the Design of Manufactured Home Foundation Systems to Resist Wind and Seismic Loads.

PB96-128285

00,460

Response of Buildings to Ambient Vibration and the Loma Priela Earthquake: A Comparison.

00,457

Wind Load Provisions of the Manufactured Home Construction and Safety Standards: A Review and Recommendations for Improvement.

PB94-206125

00,428

MARSHALL, T. A.

Low Thermal Guarded Scanner for High Resistance Measurement Systems.

PB97-112452

02,288

MARSHALL, W. J.

Colossal Magnetoresistance without $\mathrm{Mn}(3+) / \mathrm{Mn}(4-)$ Double Exchange in the Stoichiometric Pyrochlore T/2Mn2O7.

04,160

\section{MARTE, P.}

Atomic Beam Splitters and Mirrors by Adiabatic Passage in Multilevel Systems.

PB94-216439

MARTENS, J. S

Effects of Etching on the Morphology and Surface Resistance of YBa2Cu3O7-delta Films.

PB96-135355

MARTIN, A.

Assessing MOS Gate Oxide Reliability on Wafer Level with Ramped/Constant Voltage and Current Stress.

04,115

New Oxide Degradation Mechanism for Stresses in the Fowler-Nordheim Tunneling Regime.

PB96-200985

04,128

MARTIN-CASALLO, M. T. Standardization and Decay Scheme of Rhenium-186.
PB94-200490 03,830

MARTIN, J.

Non-Osmotic, Defect-Controlled Cathodic Disbondment of a Coating from a Steel Substrate.

PB94-21

03,120

MARTIN, J. W.

Degradation of Powder Epoxy Coated Panels Immersed in a Saturated Calcium Hydroxide Solution Containing Sodium

PB96-101050

01,344

Materials-Science Based Approach to Phenol Emissions from a Flooring Material in an Office Building. 02,572
Methodologies for Predicting the Service Lives of Coating Systems.

PB95-146387

03,124

Performance Approach to the Development of Criteria for Low-Sloped Roof Membranes.

00,329

Sorption of Moisture on Epoxy and Alkyd Free Films and Coated Steel Panels.

PB95-162475

03,192

Source of Phenol Emissions Affecting the Indoor Air of an Office Building.

03,600

MARTIN, $P$

Intercomparison of NIST, NPL, PTB, and VSL Thermal Voltage Converters from $100 \mathrm{kHz}$ to $1 \mathrm{MHz}$

PB94-172442

\section{MARTIN, P. R.}

National Voluntary Laboratory Accreditation Program Acoustical Testing Services.

PB95-182234

04,188

National Voluntary Laboratory Accreditation Program: Construction Materials Testing.

01,319

National Voluntary Laboratory Accreditation Program: loniz ing Radiation Dosimetry.

PB95-128658

03,623

MARTIN, R. J.

Perspective on Software Engineering Standards.
PB95-171377

PB95-171377

01,811

Compilation of Energy Levels and Wavelengths for the Spectrum of Singly-lonized Oxygen (O II).

00,746

MARTYS, N. S

Designations of ds(2)p Energy Levels in Neutral Zirconium Hafnium, and Rutherfordium ( $Z=104)$.

PB96-180120

04,116

Spectroscopic Data Tables for Highly-lonized Atoms.
PB95-151585

Wavelengths and Energy Level Classifications for the Spectra of Sulfur ( $S$ I through $S X V I$ ).

PB94-162229

00,734

PBINEZ, R. I.

Kinetics and Mechanism of the Collision-Activated Dissociation of the Acetone Cation.
PB94-216462

Precision and Accuracy in Tandem Mass Spectrometry Measurements: A Kinetics-Based Protocol for InstrumentIndependent Measurements of Collision-Activated Dissociation in RF-Only Quadrupoles.

PB94-216454

00,858

ARTINIS, J. M.

Accuracy of the Electron Pump.

Combined Josephson and Charging. Behavior of the Supercurrent in the Superconducting Single-Electron TranPB95-168522

04,637

Electronic Microrefrigerator Based on a Normal-InsulatorSuperconductor Tunnel Junction.

PB96-10282

04,718

Even-Odd Asymmetry of a Superconductor Revealed by the Coulomb Blockade of Andreev Reflection.

PB95-153540

04,593

Hot-Electron Microcalorimeter for X-ray Detection Using a Superconducting Transition Edge Sensor with PBg6 200399 reedback.

04,792

Hot-Electron Microcalorimeters as High-Resolution X-ray PB96-12364

04,739

Hot-Electron Microcalorimeters for X-ray and Phonon Detection.

B95-168993

04,644

Hot-Electron-Microcalorimeters with $0.25 \mathrm{~mm}(2)$ Area.

PB96-200670 04,793

Measurement of the Weak-Localization Complex Conductivity at $1 \mathrm{Ghz}$ in Disordered Ag Wires.

04,731

Metrological Accuracy of the Electron Pump.

PB95-168910

03,951

Noise in the Coulomb Blockade Electrometer.

PB95-176327

04,670

Novel Hot-Electron Microbolometer.

PB96-201025

01,977

Observation of Hot-Electron Shot Noise in a Metallic Resis-

PB97-112007

01,988

Performance of the Electron Pump with Stray Capacitances. PB96-200902 01,976

Self-Biasing Cryogenic Particle Detector Utilizing Electrothermal Feedback and a SOUID Readout. $\quad 04,712$
PB96-102538

Self-Heating in the Coulomb-Blockade Electrometer.
PB94-212685 Series Array of DC SOUIDs. 
Hydraulic Radius and Transport in Reconstructed Model Three-Dimensional Porous Media.

00,403

Survey of Concrete Transport Properties and Their Meas-

urement.
PB95-220489

MARTZLOFF, F. D.

Cascading Surge-Protective Devices: Options for Effective

Implementation
PB94-216488

02,464

Classified Bibliography: Insulation Condition Monitoring Methods, 1989-1995.

PB96-131586

02,232

Coordinating Cascaded Surge Protection Devices: HighLow versus Low-High

02,463

Coordinating Cascaded Surge-Protective Devices: An Elusive Goal.

PB94-216496

02,465

Guarding Against Transients.

01,623

Important Link in Entire-House Protection: Surge Reference

$\begin{array}{ll}\text { Equalizers. } & 02,219\end{array}$

Keeping Up with the Reality of Today's Surge Environment.

02,231

Surging the Upside-Down House: Looking into Upsetting Reference Voltages

02,385

Surging the Upside-Down House: Measurements and Modeling Results.

02,243

Technical Impact of the NIST Calibration Service for Electrical Power and Energy.

PB96-147913

02,462

MARUTHAMUTHU, P.

Ferric Ion Assisted Photooxidation of Halocetates.
PB97-112460

Solvent Effects in the Reactions of Peroxyl Radicals with Organic Reductants. Evidence for Proton Transfer Mediated Electron Transfer

PB95-107157

00,873

Alternative Single Integral Equation for Scattering by a Di-

Alternative Single Integral Equation for Scattering by a Di-
electric.

04,422

Autocorrelation Functions from Optical Scattering for OneDimensionally Rough Surfaces.

PB94-216538

04,244

Causality and Maxwell's Equations.

04,429

Determination of Surface Roughness from Scattered Light.
PB94-216520

Electromagnetic Scattering from a Dielectric Wedge and the

Single Hypersingular Integral Equation.

PB97-110530 04,430

Hypersingular Single Integral Equation and the Dielectric

Wedge.
PB97-110274

04,428

Light Scattered by Coated Paper.

04,245

Light Scattering by Sinusoidal Surfaces: Illumination Win. dows and Harmonics in Standards.

PB97-110548

04,387

Light Scattering from Glossy Coatings on Paper.

04,242

Numerical Evaluation of Hypersingular Integrals for Scattering by a Dielectric Wedge.
PBg7- 110555

02,017

Numerical Reference Models for Optical Metrology Simula-

tion.

04,392

Optical Scattering from Moderately Rough Surfaces

PB97-110415 04,385

Point Charges, Radiation Reaction, and Ouantum Mechan-

ics.

04,139

Positronium in Relativistic Ouantum Mechanics

04,140

Present and Future Standard Specimens for Surface Finish

$\begin{array}{ll}\text { Metrology } & 02,928\end{array}$

Regimes of Surface Roughness Measurable with Light

Scattering.

Relativistic Quantum Mechanics of Interacting Particles.
PB97-110589

SEM Linewidth Metrology of X-ray Lithography Masks.

PB96-201108 02,447

Sinusoidal Surfaces as Standards for BRDF Instruments.

PB97-110597 04.388

Spinor Equations in Relativistic Quantum Mechanics.

PB97-110605 04,142

User's Manual for the Program MONSEL-1: Monte Carlo Simulation of SEM Signals for Linewidth Metrology.

PB95-111522

02,325
Windowing Effects on Light Scattering by Sinusoidal Sur-

PB97-111215

04,389

$X$-ray Mask Metrology: The Development of Linewidth

Standards for $X$-ray Lithography.

02,348

MARYOTT, A. A.

Table of Dielectric Constants of Pure Liquids.

AD-A278 956/8

00,712

ASCHHOFF, B.

Influence of Coadsorbed Potassium on the Electron-Stimulated Desorption of $F(+), F(-)$, and $F(*)$ from $P F 3$ on Ru(0001)

PB96-157946

04,072

MASON, J.

Low-Frequency, Active Vibration Isolation System.

PB95-203303

02,710

ASON, J. L.

Cryogenic Materials Data Handbook

AD-A286 $675 / 4$
MASTERS, L.

03,303

Suggestions for a Logically-Consistent Structure for Service Life Prediction Standards.

PB95-125795

00,358

\section{MASTERS, L. W.}

Performance Approach to the Development of Criteria for Low-Sloped Roof Membranes.

PB94-160751

00,329

Technical Program of the Factory Automation Systems Division 1993

PB94-160819

02,805

ATERSON, K. D.

Optically Sensed EM-Field Probes for Pulsed Fields.

MASTOWSKI, J.

Suppression of Ionization in One- and Two-Dimensional Model Calculations.

PB95-203600

04,009

MASUDA-JINDO, $\mathrm{K}$

Atomic Theory of Fracture of Brittle Materials: Application to Covalent Semiconductors.

PB94-216553

04,519

ASZARA, W. P.

Electron and Hole Trapping in Irradiated SIMOX, ZMR and BESOI Buried Oxides.

PB96-160320

01,956

MATAR, $O$.

Viscosity of the Saturated Liquid Phase of Six Halogenated Compounds and Three Mixtures.

MATERSON, K. D.

00,960

Optically Linked Three-Loop Antenna System for Determining the Radiation Characteristics of an Electrically Small Source.

PB95-161915

02,005

MATHENY, A.

Enhanced Curie Temperatures and Magnetoelastic Domains in Dy/Lu Super Lattices and Films.

PB94-172665

04,443

MATHEW, $M$.

Crystal Structure of a New Monoclinic Form of Potassium Dihydrogen Phosphate Containing Orthophosphacidium Ion $(\mathrm{H} 4 \mathrm{PO} 4)(\mathrm{sup}+1)$

PB96-111794

04,725

Crystal Structurt

00,153

Crystal Structure of Calcium Glutarate Monohydrate.

PB96-111893

01,074

Crystal Structure of Calcium Succinate Monohydrate.

00,167

Crystal Structure of Decacalcium Tetrapotassium Hexakis (Pyrophosphate) Nonahydrate.

01,099

Crystal Structure of Dicalcium Potassium Trihydrogen Bis(pyrophosphate) Trihydrate.

00,152

MATHEWSON, A.

Assessing MOS Gate Oxide Reliability on Wafer Level with Ramped/Constant Voltage and Current Stress.

New Oxide Degradation Mechanism for Stresses in the Fowler-Nordheim Tunneling Regime.

PB96-200985

MATHEY, R. G. Corrosion Resistant Epoxy-Coated Reinforcing Steel.
PB94-185618

Earthquake Resistant Construction of Gas and Liquid Fue

Pipeline Systems Serving, or Regulated By, the Federal
Government. PB94-161999

MATHIOUDAKIS, M.

Rotational Modulation and Flares on RS Canum

Venaticorum and BY Draconis Stars. XVI. IUE Spectroscopy and VLA Observations of C1182(=V 1005 Orionis) in October 1983

PB94-185626

00,050

MATLOCK, D.

Ultrasonic Measurement of Sheet Anisotropy and Formability.

\section{MATLOCK, D. K}

Ductile Fractur
4140 Steel.

PB96-190285

emperature-Induced Transition in Ductile Fracture Appearance of a Nitrogen-Strengthened Austenitic Stainless Steel. PB96-19026

Second Census Optical Character Recognition Systems Conference.

MATSON, D. W.

Magnetometer Calibration Services.

PB97-113252

01,832

MATSUBUCHI, $\mathrm{K}$.

In-Space Welding: Visions and Realities

PB95-16323

MATSUDA, $M$

High-Energy Phonon Dispersion in La1.85Sr0.15CuO4

MATSUMOTO, Y.

Evaluation of a Tapered Roller Bearing Spindle for HighPrecision Hard Turning Applications.

PB96-160494

MATSUSHIMA, $F$.

Absolute Frequency Measurements of Methanol from 1.5 to 6.5 $\mathrm{THz}$

PB95-175881 04,300

Atomic Oxygen Fine Structure Splittings with Tunable Far Infrared Spectroscopy

PB95-152203

03,915

MATSUSHITA, $Y$

Localization of a Homopolymer Dissolved in a Lamellar Structure of a Block Copolymer Studied by Small-Angle Neutron Scattering

- 161592

01,253

Molecular Weight Dependence of the Lamellar Domain Spacing of ABC Triblock Copolymers and Their Chain Conormation in Lamellar Domains.

PB95-161691

01,254

\section{ATSUYA, S.}

Formation of Hydroxyapatite in a Polymeric Calcium Phosphate Cement.

PB95-180642

00,173

Polymeric Calcium Phosphate Cements Derived from Poly(methyl vinyl ether-maleic acid)

MATSUYA, Y.

00,180

Formation of Hydroxyapatite in a Polyreeric Calcium Phos phate Cement.

PB95-180642

00,173

Polymeric Calcium Phosphate Cements Derived from Poly(methyl vinyl ether-maleic acid).

00,180

Properties and Interactions of Oral Structures and Restorative Materials. Annual Report for Period October 1, 1990 to September 30, 1991.

PB94-160843

MATTHEWS, M. D.

Rapid Hot Pressing of Ultra-Fine PSZ Powders.

03,558

MATTHIESEN, M. M.

Effects of Anneal Time and Cooling Rate on the Formation and Texture of $\mathrm{Bi} 2 \mathrm{Sr} 2 \mathrm{CaCu} 2 \mathrm{O} 8$ Films.

PB95-161600

MATTINGLY, G. E. 
MAUREY, J.R.

Certification of the Standard Reference Material 1473a, a Low Density Polyethylene Resin.
PB96-128251 01,282 Determination of the Weight Average Molecular Weight of Two Poly(Ethylene Oxides), SRM 1923 and SRM 1924.

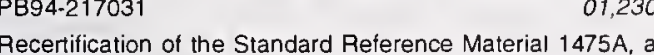

Linear Polyethylene Resin
PB94-161932

02,628

MAUTNER, M.

Carbon Acidities of Aromatic Compounds. 1. Effects of InRing Aza and External Electron-Withdrawing Groups.

MAVRIDIS, L. N.

Rotational Modulation and Flares on RS Canum copy and VLA

PB94-185626.

MAVRODINEANU, $R$.

00,050

Standard Reference Materials: Glass Filters as a Standard Reference Material for Spectrophotometry - Selection, Preparation, Certification, and Use of SRM 930 and SRM 1930 PB94-188844

MAXIMON, L. C.

Theoretical Aspects of Tagged Photon Facilities.

PB94-21661

00.536

MAY, A. D.

Simultaneous Forward-Backward Raman Scattering Studies of $\mathrm{D} 2$ Broadened by $\mathrm{D} 2, \mathrm{He}$, and $\mathrm{Ar}$.

PB95-162459

00.961

MAY, W. B.

Using Emulators to Evaluate the Performance of Building Energy Management Systems.

PB95-175774

00,259

MAY, W. E.

Determination of Polycyclic Aromatic Hydrocarbons by Liquid Chromatography.

PB95-151650

00,585

Standard Reference Materials for the Determination of Polycyclic Aromatic Hydrocarbons in Environmental Samples - Current Activities

MAYER, $F$.

00,586

Proceedings of the Workshop on the Federal Criteria for Information Technology Security. Held in Ellicot City, Mary land on June 2-3, 1993

PB94-162583

01,575

MAYER, $R$.

Anisotropy of Polarized X-ray Emission from Atoms and Molecules.

MAYERGOYZ, I.

04,62

Efficient Method to Compute the Maximum Transient Drain

Current Overshoot in Silicon on Insulator Devices.
PB94-172483

02,300

MAYES, A. M.

Observed Frustration in Confined Block Copolymers.

AYFIELD, $T$.

Functional Security Criteria for Distributed Systems. PB96-123492

01,607

Proceedings of the Workshop on the Federal Criteria for Information Technology Security. Held in Ellicot City, Maryland on June 2-3, 1993

PB94-162583

01,575

MAYO, S.

Characterization of Interface Defects in Oxygen-Implanted Silicon Films.

PB94-216629

Charge Trapping and Breakdown Mechanism in SIMOX

02,323

Electrical Characterization of Integrated Circuit Metal Line Thickness.

PB96-138433

02,414

Electrical Method for Determining the Thickness of Metal Films and the Cross-Sectional Area of Metal Lines. 02,370

Hg1-xCdxTe Characterization Measurements: Current Practice and Future Needs.

02,157

Measurement of Patterned Film Linewidth for Interconnect

Measurement of Patterned Film Linewidth for Interconnec
Characterization. Characterization

02,420

MAYS, C. W.

External Gamma-ray Counting of Selected Tissues from a Thorotrast Patien

MAYS, J.W.

03,637

Flow-Induced Structure in Polymers: Chapter 16. Shear-Induced Changes in the Order-Disorder Transition Temperature and the Morphology of a Triblock Copolymer. PB96-123237

03,127

Influence of Shear on the Ordering Temperature of a

Triblock Copolymer Melt.

01,288
Shear-Excited Morphological States in a Triblock Copoly$\begin{array}{ll}\text { mer. } & 01,196\end{array}$ Shear-Induced Changes in the Order-Disorder Transition Temperature and the Morphology of a Triblock Copolymer. PB97-118772

03,130

Shear-Induced Martensitic-Like Transformation in a Block Copolymer Melt.

01,277

Time Dependent Small Angle Neutron Scattering Behavior in Triblock Copolymers Under Steady Shear.

01,198

MCBRIDE,

Approach to Setting Performance Requirements for Automated Evaluation of the Parameters of High-Voltage Impulses.

PB94-185634

01,878

MCCABE, R. M.

NIST-Coordinated Standard for Fingerprint Data InterPhange.

01,808

Specification for Interoperability between Ballistic Imaging Systems. Part 1. Cartridge Cases.

01,860

CCABE, T. J.

Structured Testing: A Testing Methodology Using the Cyclomatic Complexity Metric.

PB97-114169

MCCALLEN, C. L.

Correlations between Flaw Tolerance and Reliability in Zirconia.

MCCALLUM, D. S.

Scaling of the Nonlinear Optical Cross Sections of GaAsAlGaAs Multiple Quantum-Well Hetero $n-i-p-i$ 's.

PB96-102793

02,183

MCCARTNEY, $R$.

Using a Multj-Layered Approach to Representing Tort Law Cases for Case-Based Reasoning.

PB96-160874

00.135

CCAULEY, J. P.

Discontinuous Volume Change at the Orientational-Ordering Transition in Solid $\mathrm{C} 60$

MCCELLAND, J.

00,821

Uncertainty Intervals for Polarized Beam Scattering Asym. metry Statistics.

MCCLELLAND, J. J.

Atom-Optical Properties of a Standing-Wave Light Field.

Determination of Complex Scattering Amplitudes in Low-En.

ergy Elastic Electron-Sodium Scattering.

03,869

Laser-Focused Alomic Deposition.

PB95-161618

04,604

Laser Focused Atomic Deposition.

PBg5-180

04,685

Laser Focusing of Atoms: A Particle Optics Approach

$\begin{array}{ll}\text { PB94-216660 } & 03,870\end{array}$

Microlithography by Using Neutral Metastable Atoms and Self-Assembled Monolayers.

PB96-190038

02,441

Nanofabrication of a Two-Dimensional Array Using Laser-

Focused Atomic Deposition.

PB96-119417

04,732

Nanostructure Fabrication via Direct Writing with Atoms Focused in Laser Fields.

PB95-150272

04,564

Nanostructure

sition (Invited)

04,132

Simple. Compact, High-Purity $\mathrm{Cr}$ Evaporator for Ultrahigh Vacuum

PB94-216678

04,520

Spin-Resolved

Using Atom Optics to Fabricate Nanostructures.

PB96-141247

03.933

MCCLELLAND, M. R.

Research and Development Activities in Electron Paramagnetic

03.635

MCCLOREY, M. J.

Microwave Properties of YBa2Cu3O7-x Films at $35 \mathrm{GHz}$ from Magnetotransmission and Magnetoreflection Measurements.

PB95-168977

04,643

MCCLURE, J. L.

Measurement of the Heat of Fusion of Tungsten by a Microsecond-Resolution Transient Technique.

03,400

Measurements of Thermophysical Properties of Nickel Near Measurements of Thermophysical Properties of Nickel Near
Its Melting Temperature by a Microsecond-Resolution Tran-

Its Melting Temper
sient Technique.
PB96-102579

04,210

Radiance Temperatures (in the Wavelength Range 523-907 m) of Group IV B Transition Metals Titanium, Zirconium, Technique. Technique.
PB96-102207

03,356

Radiance Temperatures (in the Wavelength Range 523-907 $\mathrm{nm}$ ) of Group IVB Transition Metals Titanium, Zirconium, and Hafnium at Their Melting Points by a Pulse-Heating Pechnique.

02,677

Simultaneous Measurement of Normal Spectral Emissivity by Spectral Radiometry and Laser Polarimetry at High Temby Spectral Radiometry and Laser Polarimetry at High Temperatures in Pulse-Heating

PB97-118376

02,694

MCCOLLUM, M. J.

Correlation of Optical, X.ray, and Electron Microscopy Measurements of Semiconductor Multilayer Structures. PB95. 175279

MCCOLSKEY, G. W.

Recommended Changes in ASTM Test Methods D2512-82 and G86-84 for Oxygen-Compatıbility Mechanical Impact Tests on Metals

PB94-216694

03,338

MCCOLSKEY, J. D.

Absorption of Sound in Gases between 10 and $25 \mathrm{MHz}$

PB94-199015

04,183

Alumınum-Lithium Alloys: Evaluation of Fracture Toughness by Two Test Standards, ASTM Method E 813 and E 1304. B96-19023

Composite Struts for SMES Plants.

Gas-Coupled, Pulse-Echo Ultrasonic Crack Detection and Thickness Gaging

Influence of Specimen Absorbed Energy in LOX Mechanical-Impact Tests.

PB95-107355

Macro- and Microreactions in Mechanical-Impact Tests of Aluminum Alloys

Reaction Sensitivities of Al-Li Alloys and Alloy 2219 in Mechanical-Impact Tests

MCCOMB, R. B.

Molar Absorptivities of Bilirubin (NIST SRM 916A) and Its Neutral and Alkaline Azopigments.

PB94-211042

03,456

MCСОMB, T. R

Approach to Setting Performance Requirements for Automated Evaluation of the Parameters of High-Voltage Impulses.

PB94-185634

01,878

Comparative Measurements of High-Voltage Impulses Using a Kerr Cell and a Resistor Divider. Digital Techniques in HV Tests - Summary of 1989 Panel

Session.
PB94-216702

02,035

Investigation of the Effects of Aging on the Calibration of a Kerr-Cell Measuring System for High Voltage Impulses.

PB94-172384

MCCORMICK, M. J.

Lake Erie Water Temperature Data, Put-in-Bay, Ohio, 19181992.

PB96-202452

MCCOWAN, C. N.

Cryogenic Toughness of Austenitic Stainless Steel Weld Metals: Eftect of Inclusions.

PB95-161261

03,214

Macro- and Microreactions in Mechanical-Impact Tests of Aluminum Alloys.

B95-107348

03,340 


\section{PERSONAL AUTHOR INDEX}

MCCURLEY, M. F

Optical Biosensor Using a Fluorescent, Swelling Sensing PBement. 175899

03,541

Optical Control of Enzymatic Conversion of Sucrose to Glu cose by Bacteriorhodopsin Incorporated into Self-Assem-

bled Phosphatidylcholine Vesicles.
PB96-123344

03,477

MCDEVITT, S. C.

Correlation of $\mathrm{HgCdTe}$ Epilayer Defects with Underlying

Substrate Defects by Synchrotron X-Ray Topography.

PB94-200714

02,129

MCDONALD, D. G.

Far-Infrared Kinetic Inductance Detector

PB95-126348

02,142

Optical Pertormance of Photoinductive Mixers at Terahertz

Frequencies.

02,161

Partially Coherent Transmittance of Dielectric Lamellae.

PB96-148028

04,359

Proposed High-Accuracy Superconducting Power Meter for

Millimeter Waves.

PB94-212669

Superconducting Kinetic Inductance Radiometer.

02,034

PB95-140083

02,144

Terahertz Detectors Based on Superconducting Kinetic Inductance.

MCDONALD, R. C.

02,160

Business and Manufacturing Motivations for the Developing

of Analytical Technology and Metrology for Semiconductors.
PB96-161948
04,778

MCDONOUGH, W.

Micromechanics of Fracture in Rubber-Toughened Epoxies.

PB94-212222

03,011

MCDONOUGH, W. G.

Review of Cure Monitoring Techniques for On-Line Process

PB94-216728

03,145

MCEACHRAN, R. P.

Polarization of Light Emitted After Positron Impact Excitation of Alkali Atoms.

PB94-199734

03,816

MCELROY, D. L.

Comparison of Heat-Flow-Meter Tests from Four Labora-

tories.

00,365

MCELROY, J.

Smoke Plume Trajectory from In situ Burning of Crude Oil in Alaska: Field Experiments.

PB96-131560

02,594

MCFADDEN, G. B

Anisotropy of Interfaces in an Ordered Alloy: A Multiple-

Order-Parameter Model.

04,741

Asymptotic Behavior of Modulated Taylor-Couette Flows with a Crystalline Inner Cylinder.

04,469

Boundary Integral Method for the Simulation of Two-Dimensional Particle Coarsening.

PB94-216744

03,411

Convection and Morphological Stability During Directional Solidification

03,310

Convective Stability in the Rayleigh-Benard and Directional

Solidification Problems: High-Frequency Gravity Modulation

PB95-181145 04,208

Diffuse-Interface Description of Fluid Systems.

PB96-210711

01,170

Effect of Modulated Taylor-Couette Flows on Crystal-Melt Interfaces: Theory and Initial Experiments.

PB94-216736

04,521

Effects of Crystalline Anisotropy and Buoyancy-Driven Convection on Morphological Stability.

03,328

Effects of Elastic Stress on the Stability of a Solid-Liquid Interlace.

PB95-163028

03,350

Internal Waves in Xenon Near the Critical Point.

04,221

PB97-111504

in Mul-

Laplace's Equation and the Dirichlet-Neumann Map in Mul-
tiply Connected Domains. (NIST Reprint).

PB95-180295

Laser Melting of Thin Silicon Films

PB94-199239

04,471

Lubrication Theory for Reactive Spreading of a Thin Drop.

02,865

Lubrication Theory for Reactive Spreading of a Thin Drop.
PB96-123427

04,214

Morphological Development

PB95-181111

03,355

Morphological Stability

04,591
Notion of a xi-Vector and a Stress Tensor for a General Class of Anisotropic Diffuse Interface Models.

03,345

Rayleigh Instability for a Cylindrical Crystal-Melt Interface.

Stagnant Film Model of the Effect of Natural Convection on he Dendrite Operating State.

04,765

xi-Vector Formulation of Anisotropic Phase-Field Models: 3D Asymptotics.

04,536

Xi-Vector Formulation of Anisotropic Phase-Field Models: 3. D Asymptotics.

MCGARAGHAN, 5 .

04,816

Summary of Federal Construction and Building $R$ and $D$ in PB97-114250

MCGHIE, A. R

00,234

Phase Transitions in Solid C7O: Supercooling, Metastable Phases, and Impurity Effect.

PB95-150090

00,914

MCGINNIES, R. T.

X-Ray Attenuation Coefficients from 10 kev to $100 \mathrm{Mev}$.
AD-A279 289/3

03,767

MCGLAUFLIN, M. L.

Chemical Aspects of Tool Wear in Single Point Diamond Turning.

7-112601

03,021

MCGRATTAN, $K$.

Ignition and Subsequent Transition to Flame Spread in a Microgravity Environment.

N96-15584/1

04,828

MCGRATTAN, K. B.

Computing the Effect of Sprinkler Sprays on Fire Induced

Gas Flow.

PB96-147111

00,404

Gravity-Current Transport in Building Fires.

01,415

Ignition and Transition to Flame Spread Over a Thermally Thin Cellulosic Sheet in a Microgravity Environment.

PB96-160288

04,837

In situ Burning of Oil Spills: Mesoscale Experiments and Analysis.
PB95- 163747

02,587

Large Eddy Simulations of Smoke Movement in Three Dimensions.

01,426

Mathematical Modeling and Computer Simulation of Fire Phenomena.

00,384

Numerical Simulation of Rapid Combustion in an Underground Enclosure

PB96-183132

01,424

Smoke Plume Trajectory from In situ Burning of Crude Oil: Field Experiments.

$\begin{array}{ll}\text { PB96-200993 02,597 } & \end{array}$

Smoke Plume Trajectory from In situ Burning of Crude Oil in Alaska: Field Experiments.

02,594

Transition from Localized Ignition to Flame Spread Over a Thin Cellulosic Material in Microgravity.

PB96-155809

Transport by Gravity Currents in Building Fires.

PB97.119325

04,835

MCGUCKIN, R. S.

01,441

Diagnosis and Treatment of an Oral Base-Metal Contact Lesion Following Negative Dermatologic Patch Tests.
PB95-180626

00,172

MCGUIRE, E. G.

SOA Standards and Total Ouality Management.

PB96-111844

01,743

MCGUIRE, T. J.

Noncontact Ultrasonic Inspection of Train Rails for Stress.

04,851

Ultrasonic Measurement of Residual Stress in Railroad Wheel Rims.

PB95-140430

04,849

Hot-Deformation Apparatus for Thermomechanical Processing Simulation.

03,207

Materials Reliability. Technical Activities, 1995

PB96-183082

02,999

Test of Newton's Inverse Square Law of Gravitation Using Test of Newton's Inverse Square Law of Gravitation Using
the 300-m Tower at Erie, Colorado.
PB95-202446

MCILRATH, T. J.

Configuration-Dependent AC Stark Shifts in Calcium.
PB96-157995

RIS Measurement of AC Stark Shifts and Photoionization Cross Sections in Calcium.

04,073

RIS Studies of Autoionization in Calcium.

00,849

Verification of the Ponderomotive Approximation for the ac Stark Shift in Xe Rydberg Levels.

PB94-185709

03,801

MCILROY, A.

High-Resolution Infrared Overtone Spectroscopy of ArHF

via Nd:YAG/Dye Laser Difference Frequency Generation.

High-Resolution IR Laser-Driven Vibrational Dynamics in

Supersonic Jets: Weakly Bound Complexes and Intramolecular Energy Flow.

PB94-216751

00,862

Intermolecular HF Motion in Ar(sub n)HF Micromatrices

$(n=1,2,3,4)$ : Classical and Ouantum Calculations on a

Pairwise Additive Potential Surface.

03,871

Large Amplitude Skeletal Isomerization as a Promoter of Intramolecular Vibrational Relaxation in $\mathrm{CH}$ Stretch Excited Hydrocarbons.

01,036

Sub-Doppler, Infrared Laser Spectroscopy of the Propyne 2nu1 Band: Evidence of Z-Axis Coriolis Dominated Intramolecular State Mixing in the Acetylenic $\mathrm{CH}$ Stretch

PB95-202941

MCINERNEY, $M$.

Condensed Catalogue of Electromagnetic Environment Measurements, $30-300 \mathrm{~Hz}$.

PB95-162210

MCINERNEY, $\boldsymbol{W}$.

Catalogue of Electromagnetic Environment Measurements, $30.300 \mathrm{~Hz}$.

PB96-155452

MCINTOSH, M.

Sinusoidal Surfaces as Standards for BRDF Instruments.

MCINTYRE, P. C.

Effects of Substrate Surface Steps on the Microstructure of Epitaxial Ba2YCu3O7-x Thin Films on (001) LaAlO3.

04,774

Epitaxial Nucleation and Growth of Chemically Derived Ba2YCu3O7-x Thın Films on (001) SrTiO3.

PB96-190186

MCKEE, C. F.

Magnetic Fields in Star-Forming Regions: Observations.

PB96-123005

04,787

MCKELLAR, A. R. W

Fundamental Torsion Band in Acetaldehyde.

PB94-212834

CKENNA, G.

Using Torsional Dilatometry to Measure the Eflects of Deformations on Physical Aging.

PB95-140901

MCKENNA, G. B.

Aging in

PB95-107041

Anomalous Freezing and Melting of Solvent Crystals in

Swollen Gels of Natural Rubber.

PB94-212321

01,223

Effect of Swelling on the Elasticity of Rubber: Localization

Model Description.

01,216 
MCKENNEY, $K$.

Deletion Analysis of the Mini-P1 Plasmid Origin of Replication and the Role of E.coli DnaA Protein.

03,539

DnaJ, DnaK, and GrpE Heat Shock Proteins are Required in 'ori'P1 DNA Replication Solely at the RepA Monomerization Step.

03,557

Escherichis coli Cyclic AMP Receptor Protein Mutants Provide Evidence for Ligand Contacts Important in Activation. PB96-201017 03,592
0.5ide Function of DnaJ and DnaK as Chaperones in Origin-Specific DNA Binding by RepA.

03,533

Mapping Domains in Proteins: Dissection and Expression of 'Escherichia coli' Adenylyl Cyclase.

PB96-155460 03,478

Small genomes: New initiatives in mapping and sequencing. Workshop summary report.

DE96014476

03,451

MCKINNEY, J. E.

Wear of Human Enamel against a Commercial Castable Ceramic Restorative Material.

MCKITTERICK, B.

Electron and Hole Trapping in Irradiated SIMOX, ZMR and BESOl Buried Oxides.
PB96- 160320

MCKNIGHT, M.

01,956

Relation between AC Impedance Data and Degradation of Coated Steel. 1. Effects of Surface Roughness and Contamination on the Corrosion Behavior of Epoxy Coated

PB94-213345

03,189

MCKNIGHT, M. E.

Development of a Test Method for Leaching of Lead from Lead-Based Paints Through Encapsulants.

03,128

Lead Abatement in Buildings and Related Structures.

Lead Abatement in Buildings and Related Structures.
PB,601

\section{MCLAUGHLIN, W. L.}

Alcohol Solutions of Triphenyl-Tetrazolium Chloride as PBh-Dose Radiochromic Dosimeters.

03,716

Anionic Triphenylmethane Dye Solutions for Low-Dose

Food Irradiation Dosimetry.

03,715

Calibration and Performance of GafChromic DM-100 Radiochromic Dosemeters.

00,703

Calibration of Dosimeters for the Cryogenic Irradiation of Composite Materials Using an Electron Beam.
PB95-180964 03,968

03,968

Calibration of High-Energy Electron Beams by Use of Graphite Calorimeters.

04,598

Calorimeters for Calibration of High-Dose Dosimeters in High-Energy Electron Beams.

04,055

Colour Centres in LiF for Measurement of Absorbed Doses Up to $100 \mathrm{MGy}$

04,169

Dosimetry Systems for Radiation Processing.

03,717

Effect of Formulation Changes on the Response to lonizing Radiation of Radiochromic Dye Films.

PB97-119028

04,171

Electron and Proton Dosimetry with Custom-Developed Radiochromic Dye Films

PB95-151106

ESR-Based Analysis in Radiation Processing.

PB95-161634

03,713

Food Irradiation Dosimetry.

03,931

PB95-180675

00,041

Inter-Laboratory Trials of the EPR Method for the Detection of Irradiated Meats Containing Bone.

PB96-161690

00,042

Investigation of Applicability of Alanine and Radiochromic Detectors to Dosimetry of Proton Clinical Beams.

PB96-146782

03,636

Measurement of Radial Dose Distributions Around Small Beta Particle Emitters Using High Resolution Radiochromic Foil Dosimetry.

03,518

Needs for Brachytherapy Source Calibrations in the United States.

PB97-110092

03,521

New EPR Dosimeter Based on Polyvinylalcohol.

03,619

New Method for Shielding Electron Beams Used for Head and Neck Cancer Treatmen

PB94-211430

03,621

Novel Radiochromic Films for Clinical Dosimetry.

PB97-119259 03,641

Orientation Effects on ESR Analysis of Alanine-Polyme Dosimeters.
PB96-146725

03,720
Oscillometric and Conductometric Analysis of Aqueous and Organic Dosimeter Solutions.

04,054

Overview of a Radiation Accident at an Industrial Accelera-

tor Facility.

02,612

Radiation Accident at an Industrial Accelerator Facility.

PB95-140117 02,575

Radiation-Chemical Reaction of 2,3,5-Triphenyl-Tetrazolium

Chloride in Liquid and Solid State.

PB96-146733 01,124

Radiation Process Data: Collection, Analysis, and Interpre-

tation

PB95-162632

03,628

Radiochromic

01,271

Radiochromic Solid-State Polymerization Reaction.

PB96-180146

01,290

Real Time Monitoring of Electron Processors

PB96-135306

03,719

Research and Development Activities in Electron Paramagnetic Resonance Dosimetry.

03,635

Temperature and Relative Humidity Dependence of Radiochromic Film Dosimeter Response to Gamma and Electron Radiation

PB96-135298

03,718

Thin Dyed-Plastic Dosimeter for Large Radiation Doses.
PB95-107363

Use of a Radiochromic Detector for the Determınation of Stereotactic Radiosurgery Dose Characteristics.

PB94-185642

03,514

CLAY, $M$

Validation Testing System Requirements. National PDES

Testbed Report Series.

MCLEAN, C. R.

Using Grafcet to Design Generic Controllers.
PB95-150827

MCLINDEN, $M$

Need for, and Availability of, Working Fluid Property Data:

Results from Annexes XIII and XVIII.

CLINDEN, M. O.

Annex 18: An International Study of Refrigerant Properties.
PB95-168936

Development of a Dual-Sinker Densimeter for High-Accu-

racy Fluid P-V-T Measurements.

03,267

Development of a Dual-Sinker Densimeter for High-Accuracy Fluid P.V-T Measurements. Appendix A

02,620

International Standard Equation of State for the Thermo-

dynamic Properties of Refrigerant 123 (2,2-Dichloro- 1,1,1

Tynamic Properties of Refrigerant 123 (2,2-Dichloro- 1,1,1-
Trifluane).

Physical Properties of Alternatives 10 the Fully Halogenated Chlorofluorocarbons.

PB96-119573

03,279

REFPROP Refrigerant Properties Database: Capabilities,

Limitations, and Future Directions.

PB96-167150

01,149

Report of the Refrigeration, Air Conditioning and Heat

Pumps Technical Options Committee.

03,293

Simplified Cycle Simulation Model for the Performance Rat-

ing of Refrigerants and Refrigerant Mixtures.

PB94-199890

Thermodynamic Properties of R134a(1,1,1,2-

Tetrafluoroethane)
PB95-168704

MCMAHON, B.

00,988

Role of Journals in Maintaining Data Integrity: Checking of Crystal Structure Data in 'Acta Crystallographica'.

\section{MCMICHAEL, R. D.}

Analytical Expressions for Barkhausen Jump Size Distribu-

PB95-180345

04,680

Enhanced Magnetocaloric Effect in Gd3Ga5-xFexO12.
PB94-185659

Langevin Approach to Hysteresis and Barkhausen Modeling in Steel.

PB94-185675

03,206

Magnetocaloric Effect in Rapidly Solidified Nd-Fe-Al-B Ma-

terials.

PB94-185667

04,451

Method for Determining Both Magnetostriction and Elastic Modulus by Ferromagnetic Resonance.

PB95-150108

02,974

Monte Carlo and Mean-Field Calculations of the Magnetocaloric Effect of Ferromagnetically Interactıng Clus. ter's.

MCMILLAN, C. S.

03,201

$X$-Ray Diffraction from Anodic TiO2 Films: In situ and Ex situ Comparison of the Ti(0001) Face.

PB94-185972

00,782

MCMILLIN, B. K.

In situ Characterization of Vapor Phase Growth of Iron Oxide-Silica Nanocomposites: Part 1.2-D Planar Laser-Induced Fluorescence and Mie Imaging

PB97-112478

03,185

MCMORROW, D. F

Observation of Two Length Scales Above ( $\mathrm{T}$ sub $\mathrm{N}$ ) in a

PB97-111942

MCMULLEN, W. E

Segmental Concentration Profiles of End-Tethered Polymers with Excluded-Volume and Surface Interactions.

PB97-119002

MCMURDIE, H. F.

Improved Crystallographic Data for Aluminum Niobate (AINbO4).

04,523

Powder $X$-ray Diffraction Data for $\mathrm{Ca} 2 \mathrm{Bi2O}$ and C4Bi6013.

MCNAMARA, P. D.

Correlations between Flaw Tolerance and Reliability in Zirconia.

PB96-161922

02,986

MCNICHOL, A. P.

Comparative Study of Fe-C Bead and Graphite Target Performance with the National Ocean Sciences AMS (NOSAMS) Facility Recombinator Ion Source.

PB95-175790

00,693

MCWAID, T.

Progress Toward Accurate Metrology Using Atomic Force Microscopy.

MCWAID, T. H.

02,417

Comparison of Elastic and Plastic Contact Models for the Prediction of Thermal Contact Conductance. PB95-161659

Development of a Calibrated Atomic Force Microscope. PB94-185683

02,894

Increasing the Value of Atomic Force Microscopy Process Metrology Using a High-Accuracy Scanner, Tip Characterization, and Morphological Image Analysis.

PB96-190293

02.758

MCWILLIAMS, F. F.

Dose Mapping of Radioactive Hot Particles Using PB95-162954

MEASE, N. E.

Electro-Optical Sensor for Surface Displacement Measurements of Compliant Coatings.

MECHELS, S. E.

Optical Fiber Geometry: Accurate Measurement of Cladding Diameter.

PB95-151940

04,266

Precise Laser-Based Measurements of Zero-Dispersion Wavelength in Single-Mode Fibers.

PB96-201124

01,511

MECHOLSKY, J. J.

Study of Diffusion Zones with Electron Microprobe Compositional Mapping.

MEDENDORP, N.

Texture Measurement of Sintered Alumina Using the March-Dollase Function

PB96-179494

04,784

MEDINA, L. T. 


\section{PERSONAL AUTHOR INDEX}

MEGGERS, $\boldsymbol{W}$. $F$.

Arc Spectra of Gallium, Indium, and Thallium. AD-A295 411/3

MEHL, J. B.

Greenspan Acoustic Viscometer for Gases.

PB96-204417

04,220

MEHTA, V. $R$.

Evidence of Crosslinking in Methyl Pendent PBZT Fiber

PB97-112486

03,393

MEIERS, J. C.

Surface Roughness of Glass-Ceramic Insert. Composite Restorations: Assessing Several Polishing Technıques. PB97-119010

03,583

MEIGS, B. M.

Voluntary Product Standard PS 1.95 Construction and Industrial Plywood

MEIJER, P. H. E.

03,406

Effect of Electrode-Polymer Interfacial Layers on Polymer Conduction. Part 2. Device Summary.

PB95-151155

02,335

Simulation of C60 Through the Plastic Transition Temperatures

PB96-102546

04,713

MEIRON, D. I.

Boundary Integral Method for the Simulation of Two-Dimensional Particle Coarsening.

PB94-216744

03,411

MEKHONTSEV, S. N.

Results of a NISTNNIIOFI Comparison of Spectral-Radiance Measurements.

MEL'CUK, A.

Long-Lived Structures in Fragile Glass-Forming Liquids.

PB96-119565

MELMED, A. J.

Performance of a Reflectron Energy Compensating Mirror.

PB94-199460

MELROSE, J.

Structure and Rheology of Hard-Sphere Systems.

PB96-167333

MELROY, O. R

$X$-Ray Diffraction from Anodic TiO2 Films: In situ and EX situ Comparison of the Ti(0001) Face.

PB94-185972

\section{MELVILLE, R. S.}

History of NIST's Contributions to Development of Standard Reference Materials and Reference and Definitive Methods for Clinical Chemistry.

MENACHE, M. G.

03,503

Microdosimetry and Cellular Radiation Effects of Radon

Progency in Human Bronchial Airways.

03,625

MENG, R. L.

Crystal Structure of Annealed and As-Prepared $\mathrm{HgBa} 2 \mathrm{CaCu} 2 \mathrm{O6}+$ delta Superconductors.

MENG, W. G.

03,927

Experimental Assessment of Crack-Tip Dislocation Emis sion Models for an Al67Cr8Ti25 Intermetallic Alloy.

PB96-204466

MEOT-NER, M.

03,377

Proton Affinity Ladders from Variable-Temperature Equilibrium Measurements. 1. A Re-Evaluation of the Upper Proton Affinity Range.

MERKLE, G.

00.861

Relaxation After a Temperature Jump Within the One Phase Region of a Polymer Mixture. PB97-112494 03,394 Slow Dynamics of Segregation in Hydrogen-Bonded Polymer Blends.

PB96-123591

MERMELSTEIN, L. E.

Reinforcement of Cancellous Bone Screws with Calcium Phosphate Cement.

MESSICK, C.

Assessing MOS Gate Oxide Reliability on Wafer Level with Ramped/Constant Voltage and Current Stress.

PB96-180112

04,115

Electric Field Dependent Dielectric Breakdown of Intrinsic $\mathrm{SiO} 2$ Films Under Dynamic Stress.

PB96-204102

02,449

Experimental Investigation of the Validity of TDDB Voltage Acceleration Models.

PB94-185949

02,304

Field and Temperature Acceleration of Time-Dependent Dielectric Breakdown in Intrinsic Thin SiO2.

PB94-185956

02,305

High Temperature Reliability of Thin Film $\mathrm{SiO} 2$

02,333

New Physics-Based Model for Time-Dependent- DielectricBreakdown.

02,440
New Physics-Based Model for Time-Dependent- DielectricBreakdown.

02,448

Time-Dependent Dielectric Breakdown of Intrinsic $\mathrm{SiO}_{2}$ Films under Dynamic Stress.

02,438

MESSIER, $R$.

Effect of Stoichiometry on the Phases Present in Boron Nitride Thin Films

MESSINA, C. A.

Information Retrieval Using Key Words and a Structured

PB95-161121

03,724

MESSMAN, J. D.

Standard Reference Materials: Glass Filters as a Standard Reference Material for Spectrophotometry - Selection, Preparation, Certification, and Use of SRM 930 and SRM 1930.
PB94-188844

MEYER, E. S.

Observation and Visible and uv Magnetic Dipole Transitions in Highly Charged Xenon and Barium.

-138441

04,056

Polarization Measurements on a Magnetic Quadrupole Line in Ne-Like Barium.

PBY7-113104

04,161

Joint DoD/NIST Workshop on International Manufacturing Systems Research and Development. Held in Rockville, Maryland on November 3-5, 1992. Proceedings.

PB96-109491

02,931

Proceedings of the Manufacturing Technology Needs and Issues: Establishing National Priorities and Strategies ConIerence. Held in Gaithersburg. Maryland on April 26.28,

PB95-206181

02,930

MEYER, J. L.

Selective Inhibition of Crystal Growth on Octacalcium Phosphate and Nonstoichiometric Hydroxyapatite by Pyrophosphate at Physiological Concentration. PB94-211257

00,147

\section{MEYER, $W$.}

Bonding in Doubly Charged Diatomics.

PB95-164315

00,976

MEYYAPPAN, $M$

One-Dimensional Modeling Studies of the Gaseous Electronics Conference RF Reference Cell.

PB96-113428

MICHAEL, J. R.

Phase Identitication in a Scanning Electron Microscope Using Backscattered Electron Kikuchi Patterns.

PB97-109128

MICHALOSKI, J.

Configuration and Performance Evaluation of a Real-Time Robot Control System: A Skeleton Approach.

PB95-163895

01,598

Enhanced Mach

02,802

NIST Support to the Next Generation Controller Program: 1991 Final Technical Report

02,808

Reference Architecture for Machine Control Systems Integration: Interim Report

PB95-144549

02,820

Unified Telerobotic Architecture Project (UTAP) Standard Interface Environment (SIE), May 1995.

PB95-242350

02,938

MICHALOSKI, J. L.

NIST RS274/NGC Interpreter Version 1

PB94-187788
ICHEL, K. H.

Neutron and $X$-Ray Scattering Cross Sections of Orientationally Disordered Soild C60. Orientational Fluctuations, Diffuse Scattering, and OrientaOrientational Fluctuations,
tional Order in Solid $\mathrm{C} 60$.

PB97-119275

04,176

MICHELI, A. L.

Applicability of Effective Medium Theory to Ferroelectric/ Ferrimagnetic Composites with Composition and Frequency-Dependent Complex Permittivities and PB96-157854

01,945

MIDURA, R. J.

Proteoglycan Inhibition of Calcium Phosphate Precipitation in Liposomal Suspensions.

PBO-211208

00,658

Comments on the Paper 'Wolf Shifts and Their Physical Interpretation under Laboratory Conditions'

04,246

PB94-219391

Realization of New NIST Radiation Temperature Scales for
the $1000 \mathrm{~K}$ to $3000 \mathrm{~K}$ Region, Using Absolute Radiometric Techniques.

PBechniques.

03,794

Reply to Protessor Wolf's Comments on My Paper on Wolf Shifts.

PB94-219409

04,247

Results of a NISTNNIIOFI Comparison of Spectral-Radiance Measurements.

PB97-113021

MIES, F. H.

Intersystem Crossing in Collisions of Aligned $\mathrm{Ca}(4 s 5 \mathrm{p}$ (1)P) + He: A Half Collision Analysis Using Multichannel Ouantum Defect Theory.

PB94-211133

MIGDALL, A. L.

00,813

Absolute Response Calibration of a Transfer Standard Cryogenic Bolometer.

PB96-147103

Filter Transmittance Measurements in the Infrared.

PB94-140589

04,358

MIGHELL, A

Workshop Highlights

PB97-109227
MIGHELL, A. D.

Neutron Powder Diffraction Study of the Nuclear and Magnetic Structures of the Oxygen-Deficient Perovskite

PB95-161097

00,954

NIST Crystallographic Databases for Research and Analy-

PB97-109094

04,801

\section{ER, A. P.}

Dynamic Technique for Measuring Normal Spectral Emissivity of Electrically Conducting Solids at High Temperatures with a High-Speed Spatial Scanning Pyrometer.

PB95-153045

High-Speed Spatıal Scanning Pyrometer

03,921

PB94-200003

02,636

Measurement of Surface Tension of Tantalum by a Dynamic Technique in a Microgravity Environment.

PB95-161667

03,932

Radiance Temperature (in the Wavelength Range 519-906 nm) of Tungsten at Its Melting Point by a Pulse-Heati Technique.

PB94-172590

03,397

Radiance Temperatures (in the Wavelength Range 523-907 nm) of Group IVB Transition Melals Titanium, Zirconium, and Hafnium at Their Melting Points by a Pulse-Heating Technique.

PB96-135025

02,677

Wavelength Dependence of Normal Spectral Emissivity of

High-Temperature Metals at Their Melting Point.

PB94-200011

MIKLICH, A. H.

03,398

Fabrication Issues in Optimizing YBa2Cu3O7-x Flux Transformers for Low $1 / 4$ Noise.

PB95-175857

02,059

Low Noise YBa2Cu307-x-SrTiO3-YBa2Cu3O7-x Mulitlayers for Improved Superconducting Magnetometers

PB96-138417

MILANOVIC, $V$.

Micromachined Coplanar Waveguides in CMOS Technology.
PB97-119283

MILDNER, D. F. R.

Neutron Focusing Lens Using Polycapillary Fibers

PB95-141206 
MILLER, A. P.

Radiance Temperatures (in the Wavelength Range 523-907 $\mathrm{nm}$ ) of Group IV B Transition Metals Titanium, Zirconium and Hafnium at Their Melting Points by a Pulse-Heating Technique.

MILLER, B. R.

Expression Formatter for MACSYMA

PB95-267829

03,356

MILLER, C. A.

Self-Calibrating Fiber Optic Sensors: Potential Design Methods.

Self Calibrating Fiber Optic Sensors: Potential Design Methods.

PB95-169306

02,173

Collisional Energy Transfer between Excited-State Strontium and Noble-Gas Atoms. PB95-202958

03,995

MILLER, H. C.

Etficient $\operatorname{Br}\left({ }^{*}\right)$ Laser Pumped by Frequency-Doubled Nd: YAG and Electronic-to-Vibrational Transfer-Pumped $\mathrm{CO} 2$ and HCN Lasers.

MILLER, J. $\mathbf{H}$.

04,248

Comparison of Experimental and Computed Species Concentration and Temperature Profiles in Laminar, Two-Dimensional Methane/Air Diffusion Flames.

PB95-140919

01,379

MILLER, P. A.

Gaseous Electronics Conference Radio-Frequency Reference Cell: A Defined Parallel-Plate Radio-Frequency Sysem for Experimental and Theoretical Studies of PlasmaProcessing Disch

04,404

Inductively Coupled Plasma Source for the Gaseous Electronics Conference RF Reterence Cell.

PB96-113394

02.394

Reactive Ion Etching in the Gaseous Electronics Conference RF Reference Cell.

02,395

MILLER, $\mathbf{R}$.

Frozen Human Serum Reference Material for Standardization of Sodium and Potassium Measurements in Serum or Plasma by Ion-Selective Electrode Analyzers.

PB94-185337

00,532

MILLER, R. D.

High-Energy Behavior of the Double Photoionization of Helium from 2 to $12 \mathrm{keV}$.

PB94-213279

03,860

Comparison of Heat-Flow-Meter Tests from Four Labora-

tories.
PB95-126264 PB95-126264

MILLER, T. A.

Improved Uniaxial Strain Tolerance of the Critical Current Measured in Ag-Sheathed Bi2Sr2Ca1Cu2O $+x$ Superconductors.

MILLER, W. M.

04,594

Experimental Investigation of the Validity of TDDB Voltage Acceleration Models.

02,304

Field and Temperature Acceleration of Time-Dependent Dielectric Breakdown in Intrinsic Thin SiO2

PB94-185956

02,305

MILLER, W. R.

Interface Roughness, Composition, and Alloying of LowOrder AIAs/GaAs Superlattices Studies by X-ray Diffraction.

NIST Traceable Reference Material Program for Gas Standards: Standard Reference Materials. ards: Standard

00,644

Stability of Compressed Gas Mixtures Containing Low Level Volatile Organic Compounds in Aluminum Cylinders. PB96-111968

MILLS, K. L.

Experimental Evaluation of Specification Techniques for Improving Functional Testing.

PB96-201009

01,779

Knowledge-Based Approach for Automating a Design Method tor Concurrent and Real-Time Systems.

97.112502

01,780

U.S. GOSIP Testing Program.

PB94-211455

01,807

Neutron Reflectivity of End-Grafted Polymers: Concentration and Solvent Ouality Dependence in Equilibrium Conditions. and Solvent

01,206

MILOSAVLJEVIC, I.

Behavior of Charring Materials in Simulated Fire Environ-

PB94-196045

01,368

MINCHER, B. J.

Calibration and Performance of GafChromic DM-100 Radiochromic Dosemeters

00,703
MINK, A.

Hardware Measurement Techniques for High-Speed Net-

PB96-160551

01,500

Operating Principles of MultiKron II Performance Instrumentation for MIMD Computers.

01,628

Operating Principles of Multikron Virtual Counter Pertormance Instrumentation for MIMD Computers.

01,632

Operating Principles of the SBus MultiKron Interfece Board.
PB95-231783 MINOR, D.

Evolution of the Pore Size Distribution in Final-Stage Sintering of Alumina Measured by Small-Angle X-ray Scattering. (Reannouncement with New Availability Information). AD-A249 178/5

03,023

MINOR, D. B.

Coating of Fibers by Colloidal Techniques in Ceramic Composites.
PB94-216256

MIRZADEH, $S$.

Assay of the Eluent from the Alumina-Based Tungsten-188Rhenium-188 Generator

PB94-200482

03,829

MISAKIAN, $M$.

Distributions of Measurement Error for Three-Axis Magnetic Field Meters during Measurements Near Appliances. PB96-180153

02,110

ELF Electric and Magnetic Field Measurement Methods. PB95-161675

04,423

Three-Axis Coil Probe Dimensions and Uncertainties during Measurement of Magnetic Fields from Appliances.

MISRA, D. N.

01,885

Adsorption of Low-Molecular-Weight Sodium Polyacrylate on Hydroxyapatite.

00,139

Adsorption of Polyacrylic Acids and Their Sodium Salts on Hydroxyapatite: Effect of Relative Molar Mass.

03,581

Adsorption of Potassium N-phenylglycinate on Hydroxyapatite: Role of Solvents and lonic Charge.
PB96.180161

Interaction of Chlorhexidine Digluconate with and Adsorption of Chlorhexidine on Hydroxyapatite.

PB95-175907

03,566

Interaction of Citric Acid with Hydroxyapatite: Surface Ex change of lons and Precipitation of Calcium Citrate.

Interaction of Some Coupling Agents and Organic Com-
pounds with Hydroxyapatte: Hydrogen Bonding. Adsorption pounds with Hydroxyapatite: Hydrogen Bonding, Adsorption
and Adhesion.
PB94-172616
Po, 140

00,140

MSRA, M.

Nickel(II)-Mediated Oxidative DNA Base Damage in Renal and Hepatic Chromatin of Pregnant Rats and Their Fetuses. Possible Relevance to Carcinogenesis. PB94-212628

03,646

MISSEN, N.

Phase Locking in Two-Junction Systems of High-Temperature Superconductor-Normal Metal-Superconductor JuncPBos.1

(176053

02,060

MISSERT, N.

Critical Current and Normal Resistance of High-Tc StepEdge SNS Junctions.

04.724

High-Tc Multilayer Step-Edge Josephson Junctions and SOUIDs

PB96-200183

04,790

Insulating Nanoparticles on YBa2Cu3O7-delta Thin Films Revealed by Comparison of Atomic Force and Scanning Tunneling Microscopy.

PB95-150843

04,575

Mutual Phase Locking in Systems of High-Tc Superconductor-Normal Metal-Superconductor Junctions. 04,744

Observation of Insulating Nanoparticles on YBCO ThinFilms by Atomic Force Microscopy.
PB95-163358

04,622

Temperature Dependence and Magnetic Field Modulation of Critical Currents in Step-Edge SNS YBCO/Au Junctions.

Critical Currens

MISSERT, N. A.

04,723

Thermal Noise in High-Temperature Superconducting-Normal-Superconducting Step-Edge Josephson Junctions.

PB95-175089

MITCHELL, J. S. B.

Point Probe Decision Trees for Geometric Concept Classes. PB96-160817

MITCHELL, M. J.

Capabilities for Product Data Exchange.

PB97-118764

Challenges to the National Information Infrastructure: The Barriers to Product Data Sharing. National PDES Testbed Report Series.

02,776

Initial NIST Testing Policy for STEP: Beta Testing Program for AP 203 Implementations. National PDES Testbed Report Series.

MITCHELL, P. J.

02,779

Structure and Rheology of Hard-Sphere Systems.

PB96-167333

00,662

MITLER, H. E.

Algorithm to Describe the Spread of a Wall Fire under a

PB95-182259

00,261

Comparison of Wall-Fire Behavior With and Without a Ceil-

PB94-207404

00,342

Heights of Wall-Fire Flames.

PB96-148192

00,212

MITRAKOVIC, D. $V$

Methods to Improve the Accuracy of On-Line Ultrasonic

Measurement of Steel Sheet Formability.

02,281

Noncontact Ultrasonic Inspection of Train Rails for Stress.

PB95-162673 04,851

MITTAL, J.P.

Fluoride Elimination Upon Reaction of Pentafluoroaniline with e (sub eq')(sup -), $\mathrm{H}$, and $\mathrm{OH}$ Radicals in Aqueous SoJution.

PB97.111314

01,177

MIYANO, K. E.

Barium Contributions to the Valence Electronic Structure of

YBa2Cu3O7-delta, PrBa2Cu3O7-delta, and Other BariumContaining Compounds.

PB96-158019

04,076

Phonon Relaxation in Soft-X-ray Emission of Insulators. PB96-160296

04,085

MIZE, J. H.

Learning to Change: Opportunities to Improve the Performance of Smaller Manufacturers.

PB94-166212

00,010

MIZIOLEK, A. W.

Environmental Aspects of Halon Replacements: Considerations for Advanced Agents and the Ozone Depletion Potential of CF3l.

PB97-122261

MIZUKI, J.

Rotational Dynamics of $\mathrm{C} 60$ in Na2RbC60

PB95-153201

00,948

MIZUMACHI, $\mathrm{H}$.

Micromechanics of Fracture in Rubber-Toughened Epoxies.

MOCK, J. L.

Gaseous Electronics Conference Radio-Frequency Reference Cell: A Defined Parallel-Plate Radio-Frequency System for Experimental and Theoretical Studies of PlasmaProcessing Discharges.

PB94-172327

04,404

MODELSKI, J.

Influence of Films' Thickness and Air Gaps in Surtace Impedance Measurements of High Temperature Superconductors Using the Dielectric Resonator Technique.
PB96-157862

MOEN, W. E.

Z39.50 Implementation Experiences.

PB96. 114939

01,816

MOERMAN, $\mathrm{R}$.

Coexistence of Grains with Differing Orthorhombicity in High 
Evaluation of Two-Photon Exchange Graphs for HighlyCharged Heliumlike lons.
PB94-198918

03,808

\section{MOHRAZ, B.}

Method of Estimating the Parameters of Tuned Mass Dampers for Seismic Applications.

PB96-167820

00,473

Modified Optimal Algorithm for Active Structural Control

PB96-165949

Post-Earthquake Fire and Lifelines Workshop. Held in Long Beach, California on January 30-31, 1995. Proceedings.
PB96-117916

Proceedings of a Workshop on Developing and Adopting Seismic Design and Construction Standards for Litef

PB97-1 15794

01,302

MOIROUX, J.

Oxidation of 10-Methylacridan, a Synthetic Analogue of NADH and Deprotonation of Its Cation Radical. Convergen Application of Laser Flash Photolysis and Direct and Redox Catalyzed Electrochemistry to the Kinetics of Deprotonation of the Cation Radical.

PB94-198371

00,785

\section{MOISEEVA, N. P.}

Investigation of High-Temperature Platinum Resistance Thermometers at Temperatures Up to $962 \mathrm{C}$, and, in Some Cases, $1064 \mathrm{C}$

MOLDOVER, $M . R$.

00,629

Ab initio Calculations for Helium: A Standard for Transport

Property Measurements.

Dielectric Studies of Fluids with Reentrant Resonators.

PB95-153730

Dielectric Studies of Fluids with Reentrant Resonators. Ap. pendix B.
DE93019683

Electric Field Effects on a Near-Critical Fluid in Microgravity.

Greenspan Acoustic Viscometer for Gases.

PB96-204417

04,220

High-Temperature High-Pressure Oscillating Tube Densim-

eter.

01,123

Properties of Working Fluids for Thermoacoustic Refrigerators

04,864

Structure of the Vapor-Liquid Interface Near the Critical Point.

PB95-140174 00,902
Thermodynamic Properties of CF3-CHF-CHF2, 1,1,1,2,3,3Hexafluoropropan

PB97-118384

03,299

Thermodynamic Properties of CHF2.CF2-CHF, 1,1,2,2,3-

Pentafluoropropane.

03,300

Thermodynamic Properties of CHF2-O

CHF2,Bis(difluoromethyl) Ether.
PB94-199569

MOLINAR, G.

00,798

Pressure Measurements with the Mercury Melting Line Referred to ITS-90

MOLINE, J.

01,136

Electronic Access: Blueprint for the National Archives and Records Administration.

PB95-219218

User Protile for Researchers Studying Objects: Implications for Computer Systems

PB94-188463

00,133

User Study: Informational Needs of Remote National Ar-

chives and Records Administration Customers.

$\begin{array}{lr}\text { PB95-154738 } & 02,725 \\ \text { Virtual Environments for Health Care. A White Paper for the }\end{array}$ Advanced Technology Program (ATP), the National Institute of Standards and Technology

PB96-147814

\section{MOLMER, $K$.}

03,59

Temperature of Optical Molasses for Two Different Atomic Angular Momenta.

MONCARZ, H. T.

03,881

Automated Optical Roughness Inspection.
PB95-152179

Information Technologies Make Business Sense for the Custom Therapeutic Footwear Industry.

PB95-251708

02,829

Program Requirements to Advance the Technology of Custom Footwear Manufacturing

02,883

Visualization Applications for Manufacturing: A State-ot-the Art Survey. Final Report.

PB94-194552

02,816

MONDAIN-MONVAL, $P$.

Open Issues in OSI Protocol Development and Conform. ance Testing.

01,818
MONKS, P. S.

Experimental Determination of the Ionization Energy of $1 O(X$ (sup 2)川(sub 3/2)) and Estimations of Delta(sub f) $\mathrm{H}$ (sup deg) (sub 0)(IO(sup -)) and PA(IO)

00,694

PB96-146899

Experimental Determination of the Rate Constant for the
Reaction of $\mathrm{C} 2 \mathrm{H} 3$ with $\mathrm{H} 2$ and Implications for the PartitionReaction of $\mathrm{C}_{2} \mathrm{H} 3$ with $\mathrm{H} 2$ and Implications for the Partitioning of Hydrocarbons in Atmospheres of the Outer Planets.
PB97-122295 MONROE, C. R.

Trapped Ions and Laser Cooling 4: Selected Publications of the Ion Storage Group of the Time and Frequency Division, NIST, Boulder, Colorado.

04,108

\section{MONSINGNORI-FOSSI, B.}

Distant Future of Solar Activity: A Case Study of Beta Hydri. 3. Transition Reg

00,049

Distant Future of Solar Activity: A Case Study of beta Hydri. 3. Transition Region, Corona, and Stellar Wind.

00,074

MONTESALVO, M

Estimation of the Absorbed Dose in Radiation-Processed

Food. 4. EPR Measurements on Eggshell.

Food. 4. EPR
PB94-199692

00,038

MONTGOMERY, D.

NIST Cooperative Laboratory for OSI Routing Technology.
01,791

DENTI, C.

National Voluntary Laboratory Accreditation Program 1995 Directory.

PB95-174454

00,483

MONTRESS, G. K

Surface Transverse Wave Oscillators with Extremely Low Thermal Noise Floors.

PB96-186010

01,967

MOODY, J. R.

Development of the Ion Exchange-Gravimetric Method for Sodium in Serum as a Definitive Method.

PB96-179148

01,867

MOON, B. M.

Novel YBa2Cu3O7-x and YBa2Cu3O7-x/4Ba3O9 Multilayer Films by Bias-Masked 'On-Axis' Magnetron Sputtering.
PB95-181186

\section{MOORE, C. E.}

Atomic Energy Levels As Derived from the Analyses of Optical Spectra Volume 1. Section 1. The Spectra of Hydrogen, Deuterium, Helium, Lithium Beryllium, Boron, Carbon, Nitrogen, Oxygen, and Fluorine.

AD-A278 130/0

03,764

Atomic Energy Levels. As Derived From the Analyses of Optical Spectra. Volume 3

AD-A280 279/1

00,714

Ultraviolet Multiplet Table.

00,710

Ultraviolet Multiplet Table. Finding List for Spectra of the Elements Molybdenum to Lanthanum ( $Z=42$ to 57 ); Hafnium to Radium ( $Z=72$ to 88 )

AD-A278 $131 / 8$

00,709

MOORE, $F$.

Electrostatic Modes of Ion-Trap Plasmas.

PB95-152963

03,920

Experimental Results on Normal Modes in Cold, Pure Ion Plasmas.

03,956

Lattice Position of Si in GaAs Determined by X-Ray Standing Wave Measurements.

PB95-164406

04,632

MOORE, F. L.

Electrostatic Modes as a Diagnostic in Penning-Trap Experiments.

PB95-176244

03,959

High-Order Mutil

04,044

High-Resolution Atomic Spectroscopy of Laser-Cooled lons. PB95-169330 03,953

Precise Spectroscopy for Fundamental Physics.

PB96-112164

04,040

Ouantum Measurements of Trapped Ions

PB95-161147

03,928

Ouantum Projection Noise: Population Fluctuations in TwoLevel Systems.

03.850

Recent Experiments on Trapped Ions at the National Institute of Standards and Technology.

03,952

Spin Squeezing and Reduced Ouantum Noise in Spectroscopy.

MOORE, $J$.

03,912

Escherichis coli Cyclic AMP Receptor Protein Mutants Pro-

vide Evidence for Ligand Contacts Important in Activation.
PB96-201017 PB96-201017

MOORE, J. H.

Associative Electron Attachment to S2F10, S2OF10, and $\mathrm{S} 2 \mathrm{O} 2 \mathrm{~F} 10$.

00,907

Electron Scattering and Dissociative Attachment by SF6 and Its Electrical-Discharge By-Products.

PB95-151288

02,256

MOORE, L. J.

Comparative Strategies for Correction of Interferences in Isotope Dilution Mass Spectrometric Determination of Vanadium.

MOPSIK, F. I.

00,531

Advances in the Measurement of Polymer CTE: Micrometer- to Atomic-Scale Measurements. 03,390 Dielectric Behavior of a Polycarbonate/Polyester Mixture Upon Transesterification

PB96-179551

04,785

Electronics Packaging Materials Research at NIST.

PB96-122692 02,405

Review of Cure Monitoring Techniques for On-Line Process Control.

PB94-216728

03,145

ORAN, B.

Model Minimum Performance Specifications for Lidar Speed Measurement Devices.

PB95-197455

04,861

Fracture Behavior of Large-Scale Thin-Sheet Aluminum

N95-19494/0

MOREA, G. F.

03,311

Operating Procedures and Life Cycle Documentation for the Initial Graphics Exchange Specification.

PB95.242285

MORELAND, $\mathrm{J}$.

02,782

Comparison of Magnetic Fields of Thin-Film Heads and Their Corresponding Bit Patterns Using Magnetıc Force Microscopy.

03,763

Critical Current Behavior of Ag-Coated YBa2Cu307-x Thin

Films.

PB95-141016

dc Magnetic Force Microscopy Imaging of Thin-Film Recording Head

04,665

Development of Highly Conductive Cantilevers for Atomic Force Microscopy Point Contact Measurements.

PB96-138573

04,751

Epitaxial Growth and Characterization of the Ordered Vacancy Compound Culn3Ses on GaAs (100) Fabricated by Molecular Beam Epitaxy.

PB95-180725

04,687

Flexible-Diaphragm Force Microscope.

PB95-180915

03,966

Growth of Laser Ablated YBa2Cu307-delta Films as Examined by Rheed and Scanning Tunneling Microscopy

04,614

High Critical Temperature Superconductor Tunneling Spectroscopy Using Squeezable Electron Tunneling Junctions. 
YBa2Cu3O7-x to $\mathrm{Si}$ Interconnection for Hybrid

Superconductor/Semiconductor Integration.
PB94-211711 PB94-211711
MORGAN, C. A.

Beam Line for Highly Charged lons.

Beam

04,143

Observation and Visible and uv Magnetic Dipole Transitions in Highly Charged Xenon and Barium.

PB96-138441

04,056

MORGAN, G. L.

Intermediate Structure in the Neutron-Induced Fission Cross Section of $236 \mathrm{U}$

PB94-185741

03,802

Measurements of

04,173

MORGAN, N.

Planar Resistors for Probe Station Calibration

PB95-163697

MORGAN, T. J.

Wear Mechanism Maps of $440 \mathrm{C}$ Martensitic Stainless Steel.

PB96-111810 04,834

MORI, $K$.

Molecular Weight Dependence of the Lamellar Domain Spacing of ABC Triblock Copolymers and Their Chain Contormation in Lamellar Domains.

PB95-161691

01,254

MORI, T.

DNA Base Damage Generated In vivo in Hepatic Chromatin of Mice upon Whole Body y-Irradiation.

03,627

DNA Base Modifications in Renal Chromatin of Wistar Rats Treated with a Renal Carcinogen, Ferric Nitrilotriacetate.

PB95-150363 03,648

Ionizing Radiation Causes Greater DNA Base Damage in Radiation-Sensitive Mutant M10 Cells That in Parent Mouse Lymphoma L5178Y Cells.

PB95-175915

03,632

Treatment of Wistar Rats with a Renal Carcinogen, Ferric Nitrilotriacetate, Causes DNA-Protein Cross-Linking be-
tween Thymine and Tyrosine in Their Renal Chromatin.

PB96-112115

03,649

MORNIS, M.

Through-the-Arc Sensing for Measuring Gas Metal Arc Weld Quality in Real Time.

PB95-164463

02,908

MORNIS, M. A.

Contact Tube Wear Detection in Gas Metal Arc Welding.
PB96-135330

Through-the-Arc Sensing for Monitoring Arc Welding.

PB94-185899 02,858

Through-the-Arc Sensing for Real-Time Measurement of Gas Metal Arc Weld Quality.

PB95-162871

02,863

MORRA, M. M.

Charpy Impact Test as an Evaluation of $4 \mathrm{~K}$ Fracture Toughness.

PB96-190194

MORRIS, D. E.

Coupled-Bilayer Two-Dimensional Magnetic Order of the Dy lons in Dy2Ba4Cu7O15.

PB95-152104

04,584

MRRIS, K. C.

Structural EXPRESS Editor

02,769

Validation Testing System Requirements. National PDES Testbed Report Series.

02,771

MORRIS, P. A.

Growth of Epitaxial KNbO3 Thin Films.

PB96-13518

02,409

MORRIS, R. E.

Determination of Complex Structures from Powder Diffraction Data: The Crystal Structure of La3Ti5Al15O37.

PB95-202966

01.038

MORRIS, S. C.

Enhanced Detection of PCR Products Through Use of TOTO and YOYO Intercalating Dyes with Laser Induced Fluorescence - Capillary Electrophoresis.

PB95-164653

00,599

MORRIS, S. L.

Observations of $3 \mathrm{C} 273$ with the Goddard High Resolution Spectrograph on the Hubble Space Telescope.

PB95-20232

00.076

MORRISON, F. A.

Flow-Induced Structure in Polymers: Chapter 16. Shear-Induced Changes in the Order-Disorder Transition Tempera-
ture and the Morphology of a Triblock Copolymer. PBre and the $\mathrm{M}$.

03,127

Influence of Shear on the Ordering Temperature of a Triblock Copolymer Met.

PB96-163753

01,288

Shear-Excited Morphological States in a Triblock Copoly-

01,196
Shear-Induced Changes in the Order-Disorder Transition Temperature and the Morphology of a Triblock Copolymer.

Shear-Induced Martensitic-Like Transformation in a Block Copolymer Melt

PB96-119508

01,277

Time Dependent Small Angle Neutron Scattering Behavior in Triblock Copolymers Under Steady Shear.

PB94-172632

01,198

PORRISON, G.

Compressed Liquid Densities, Saturated Liquid Densities, PB97-110118

01,173

Interaction Coefficients for 15 Mixtures of Flammable and Non-Flammable Components.

03,281

REFPROP Refrigerant Properties Database: Capabilities, Limitations, and Future Directions.

PB96-167150

01,149

Shape of the Temperature-Entropy Saturation Boundary.

Study to Determine the Existence of an Azeotropic R-22 'Drop-In' Substitute.

PB96-167812

02,568

Thermodynamic Properties of CHF2-O

CHF2,Bis(difluoromethyl) Ether.

B B4-199569

00,798

Thermodynamic Properties of Difluoromethane

PB94-185204

00,772

Vapor Pressure of 1,1-dichloro-2,2,2-trifluoroethane (R123).

PB95-126231

00.899

\section{MORRISON, H. D.}

Decomposition of SF6 and Production of S2F10 in Power PB96-122619

01,084

Investigation of S2F10 Production and Mitigation in Com-

pressed SF6-Insulated Power Systems.
PB94-212388

02,467

Investigation of $\mathrm{S} 2 \mathrm{~F} 10$ Production and Mitigation in Compressed SF6- Insulated Power Systems.

MORRISON, J. $F$.

02,468

Instrument for Evaluating Phase Behavior of Mixtures for Supercritical Fluid Experiments.

00,606

Supercritical Fluid Extraction-Immunoassay for the Rapid Screening of Cocaine in Hair.

PB96-167168

00,637

MORRISON, M. A.

Alignment in Two-Step Pulsed Laser Excitation of Rydberg

Levels in Light Atoms: The Example of Sodium.

03,993

Analyses of Recent Experimental and Theoretical Determinations of e-H2 Vibrational Excitation Cross Sections: Assessing a Long-Standing Controversy.

PB95-202438

03,977

Importance of Bound-Free Correlation Effects for Vibrational Excitation of Molecules by Electron Impact: A Sensitivity

Analysis.

03,996

Low-Energy-Electron Collisions with Sodium: Elastic and Inelastic Scattering from the Ground State.

04,030

Modified Effective Range Theory as an Alternative to LowEnergy Close-Coupling Calculations.

PB95-202701

03,988

OSER, E. K

Microwave Properties of YBa2Cu3O7-x Films at $35 \mathrm{GHz}$ from Magnetotransmission and Magnetoreflection Measurements.

MOSER, M.

04,643

Laser-Induced Fluorescence Measurements of $\mathrm{OH}$. Concentrations in the Oxidation Region of Laminar, Hydrocarbon Diffusion Flames.

PB95-162160

01,387

Laser-Induced Fluorescence Measurements of $\mathrm{OH}$ in Laminar Diffusion Flames in the Presence of Soot Particles. PB96-123120

Acceleration of Intramolecular Vibrational Redistribution by methyl Internal Rotation. A Chemical Timing Study of $p$ morotoluene and p-fluorotoluene-d3.

PB95-202982

01,039

Moss, S. C.

Epitaxial Growth of Sb/GaSb Structures: An Example of V/ III-V Heteroepitaxy.

PB95-202560

04,693

Epitaxial Integration of Single Crystal C60 PB95-153490

04,592

MOSS, W. F.

Analyzing and Exploiting Numerical Characteristics of Zone

Fire Models.
PB96-102314

01,400

Numerical Analysis Support for Compartment Fire Modeling and Incorporation of Heat Conduction into a Zone Fire

01,357

MOSSMAN, K. L.

External Gamma-ray Counting of Selected Tissues from a Thorotrast Patient.

PB96-160254

MOSTOWSKI, J.

03,637

Cone Emission from Laser-Pumped Two-Level Atoms. 1

Quantum Theory of Resonant Light Propagation.

Cone Emission from Laser-Pumped Two-Level Atoms. 2. Analytical Model Studies.

PB95-203592

04,326

U. $\mathrm{J}$.

Integrated Inspection System for Improved Machine PerPB96-160569

MOUDDEN, A. H.

High-Energy Phonon Dispersion in La1.85Sro. 15CuO4

PB96-138458

02,959

MOUDDEN,

Inelastic Neutron Scattering Measurements of Phonons in cosahedral Al-Li-Cu.

PB95-12621

MOULD, $J$.

04,532

Numerical Reference Models for Optical Metrology Simulation

PB97-111330

04,392

MOULT, J.

Crystal Packing Interactions of Two Different Crystal Forms Covine Aibonuclease A.

PB95-152823

00,943

Positive and Negative Cooperativities at Subsequent Steps of Oxygenation Regulate the Allosteric Behavior of Multistate Sebacylhemoglobin.

03,486

MOUNT, D.

Point Probe Decision Trees for Geometric Concept Classes. PB96-160817

MOUNTAIN, R. D.

01.612

Activated Dynamics, Loss of Ergodicity, and Transport in Supercooled Liquids.

PB95-150819

00,925

Comparison of a Fixed-Charge and a Polarizable Water Model

PB96-111620

01,072

Length Scales for Fragile Glass-Forming Liquids.

Long-Lived Structures in Fragile Glass-Forming Liquids.
PB96-119565

Neighbor Tables for Molecular Dynamics Simulations.

PB95-171948

Quantitative Measure of Efficiency of Monte Carlo Simula-

tions.
PB95-18069 
MUELLER, D. R.

Barium Contributions to the Valence Electronic Structure of YBa2Cu3O7-delta, PrBa2Cu3O7-delta, and Other BariumContaining Compounds.

PB96-158019

04,076

Charge-Transter-Induced Multiplet Structure in the $\mathrm{N} 4,5 \mathrm{O} 2,3$ Soft-X-ray Emission Spectrum of Lanthanum.

Cooper M(sub II,III) X-ray-Emission Spectra of Copper Oxides and the Bismuth Cuprate Superconductor.

PB96-158027

04,077

Laser-Synchrotron Hybrid Experiments: A Photon to Tickle, A Photon to Poke.

03,704

Local Partial Densities of States in $\mathrm{Ni}$ and $\mathrm{Co}$ Silicides Studied by Soft X-Ray Emission Spectroscopy.
PB94-212412

Phonon Relaxation in Soft-X-ray Emission of Insulators.

PB96-160296 04,085

Simple Variable Line Space Grating Monochromator for Synchrotron Light Source Beamlines.

PB96-156203

04,065

Soft-X-ray-Emission Investigation of Cobalt Implanted Silicon Crystals.

04,069

Soft-X-ray-Emission Spectra of Solid $\mathrm{Kr}$ and $\mathrm{Xe}$

04,070

Soft-X-ray-Emission Studies of Bulk Fe3Si, FeSi, and FeSi2, and Implanted Iron Silicides.

PB96-157938

04,071

MUELLER, W. H

Phase Transitions in Solid C70: Supercooling. Metastable Phases, and Impurity Effect.

00.914

MUIR, D. C. G.

Concentrations of Chlorinated Hydrocarbons, Heavy Merals and Other Elements in Tissues Banked by the Alaska Marine Mammal Tissue Archival Project.

PB95-209870

02,590

MULAZZANI, Q. G

Critical Review of Rate Constants for Reactions of Transients from Metal lons and Metal Complexes in Aqueous Solution

PB96-145859

01,109

MULEV, $Y$.

Database for the Static Dielectric Constant of Water and

PB96. 145586

01,103

Static Dielectric Constant of Water and Steam.

PB96-123559

01,090

MULHOLLAND, G.

Smoke Emission from Burning Crude Oil

PB96-122890

01,407

Working Conference on Global Growth of Technology: Is America Prepared. Held in Gaithersburg. Maryland on December 7, 1995

PB96-210059

00,018

MULHOLLAND, G. W.

Airborne Smoke Sampling Package for Field Measurements of Fires.

PB95-150041

01,381

Generation and Characterization of Acetylene Smokes.

PB94-200292 01,372

Global Climatic Effects of Aerosols: The AAAR Symposium.

PB95-108791 00,122

Radiometric Model of the Transmission Cell-Reciprocal Nephelometer

00,124

Two Numerical Techniques for Light Scattering by Dielectric Agglomerated Structures.

PBg $94-140597$

04,225

Ultratine Combustion Aerosol Generator.

PB94-200300

01,373

MULLEN, J. L.

Electrochemical Synthesis of Metal and Intermetallic Composites.

AD.A294 088/0

03,304

MULLER, A.

Dielectronic Capture Processes in the Electron-Impact Ionization of $\mathrm{Sc}(2+)$

04,000

Merged-Beams Energy-Loss Technique for Electron-Ion Excitation: Absolute Total Cross Sections for $\mathrm{O}(5+)$ (2s yields

2p). 102058

04,017

MULLINGS, G. M.

Effects of Testing Variables on the Strength of HighStrength (90 Mpa) Concrete Cylinders.

00,456

MULROY, W. J.

Study to Determine the Existence of an Azeotropic R-22 'Drop-in' Substitute.

PB96-167812

02,568

MULVENNA, G.

IGOSS-Industry/Government Open Systems Specification.

PB94-207453

01,806
MULVENNA, $J$

Data Communications Strategy

PB96-167846

02,738

RO, R. G.

Ceramic Powders Characterization: Results of an International Laboratory Study.

02,672

Characterizing Materials Properties for Ceramic Matrix Composites.

Corrosion Characteristics of Silicon Carbide and Silicon Nitride.

PB96-169081

03,372

Database Development and Management (Project A.2.2) The Annual Report for 1992-1993.

03,098

Reference Relations for the Evaluation of the Materials Properties of Orthorhombic YBa2Cu3Ox Superconductors.
PB96-176763
04,782

Review of Corrosion Behavior of Ceramic Heat Exchanger Materals: Corrosion Characteristics of Silicon Carbide and Silicon Nitride. Final Report, September 11, 1992--March 11.1993

DÉ 93041307

03,228

Role of Corrosion in a Material Selector Expert System fo Advanced Structural Ceramics.

03,099

Structural Ceramics Database. Topical Report, June 1989

May 1991.

PB95-203758 03,060

Variances in the Measurement of Ceramic Powder Properties.

PB97-110316

03,100

Wear Model for Alumina Sliding Wear

PB95-163796

03,239

\section{MURAKAWA, $M$.}

Applications of Diamond Films and Related Materials: International Conference (3rd). Held in Gaithersburg, Maryland on August 21-24, 1995. Supplement to NIST Special Publication 885 .

PBation 885.

03,063

Proceedings of the Applied Diamond Conference 1995: Ap plications of Diamond Films and Related Materials International Conference (3rd). Held in Gaithersburg, Maryland on August 21-24, 1995

PB95-255204

MURALIDHAR, $\mathrm{K}$.

Rapıd Method for the Isolation of Genomic DNA from 'Aspergillus fumigatus

MURE, T.

Characterization of the Interaction of Hydrogen with Iridium Clusters in Zeolites by Inelastic Neutron Scattering Spectroscopy.

MURPHY, D. W

Neutron-Scattering Study of $\mathrm{C6O}(\mathrm{n}-)(\mathrm{n}=3,6)$ Librations in Al

kali-Metal Fullerides
PB94-200219

\section{MURPHY, E.}

TDDB Characterization of Thin $\mathrm{SiO} 2$ Films with Bimoda Fallure Populations.

PB96-102926

02,381

MURPHY, K.

Control System Architecture for a Remotely Operated Unmanned Land Vehicle.

63200

03,759

\section{MURPHY, K. E.}

Determination of Sulfur in Fossil Fuels by Isotope Dilution Thermal lonization Mass Spectrometry

PB96-141379

02,495

Ground Vehicle Control at NIST: From Teleoperation to Autonomy.

N94-34037/9

MURPHY, R. J.

Arc Spectra of Gallium, Indium, and Thallium

AD-A295 411/3

03,758

MURRAY, A.

Examination of Objects Made of Wood Using Air-Coupled Ultrasound.

PB95-125712

MURRAY, B. T

Asymptotic Behavior of Modulated Taylor-Couette Flows with a Crystalline Inner Cylinder.

PB94-199072

04,469

Computation of Dendrites Using a Phase Field Model.

PB94-160744 04,436

Convection and Morphological Stability During Directional Solidification
N95-14548/8

03,310

Convective Stability in the Rayleigh-Benard and Directiona Solidification Problems: High-Frequency Gravity Modulation.
PB95-181145

04,208

Effect of Modulated Taylor-Couette Flows on Crystal-Mel Interfaces: Theory and Initial Experiments.

04,521

Lubrication Theory for Reactive Spreading of a Thin Drop. Lubrication Theory for Reactive Spreading of a Thin Drop.
PB96-123427 Rayleigh Instability for a Cylindrical Crystal-Melt Interface. PB95-180667 PB.

Hydration in Semicrystalline Polymers: Small-Angle Neutron Scattering Studies of the Effect of Drawing in Nylon-6 Fi-

PB95-202990

03,385

PRT,

Extension of Heterodyne Frequency Measurements on OCS to $87 \mathrm{THz}(2900 \mathrm{~cm}(-1))$

Optically Stabilized Tunable Diode-Laser System for Saturation Spectroscopy.

PB96-102819

04,717

Stabilization of 3.3 and $5.1 \mathrm{~m}$ Lead-Salt Diode Lasers by Optical Feedback

MUSGROVE, A.

Comment On: Two-Photon Absorption Series of Calcium.

Compilation of Energy Levels and Wavelengths for the
Spectrum of Singly-lonized Oxygen (O II). Spectrum of Singly-lonized Oxygen (O II)

Energy Levels of Germanium, Ge I through Ge XXXII. PB94-162351

Energy Levels of Zinc, Zn I through Zn XXX.

01,122

Spectroscopic Data Tables for Highly-lonized Atoms. PB95-151585

MUSIELOK, $J$

Atomic Transition Probabilities and Tests of the Spectroscopic Coupling Scheme for N I.

Investigation of LS Coupling in Boronlike Ions. PB94-185295

04,057

MUTH, L. A.

03,797

Accurate Computations of Radar Cross Sections of Simple

Objects.
PB96-138474

04,426

General Order ' $N$ ' Analytic Correction of Probe-Position Errors in Planar Near-Field Measurements.

PB96-200688

01,562

Polarimetric Calibration of Reciprocal-Antenna Radars. PB95-216925 01,872

Polarimetric Calibration of Reciprocal-Antenna Radars

PB96-200696

Proposed Analysis of RCS Measurement Uncertainty.

PB95-203568

RangeCAD and the NIST RCS Uncertainty Analysis. PB94-218591

MUTHUKUMAR, M

Flow-Induced Structure in Polymers: Chapter 16. Shear-Induced Changes in the Order-Disorder Transition Temperature and the Morphology of a Triblock Copolymer.

PB96-123237

03,127

Influence of Shear on the Ordering Temperature of a Triblock Copolymer Melt.

PB96-163753

01,288

Shear-Excited Morphological States in a Triblock Copoly-

mer.

01,196

Shear-Induced Changes in the Order-Disorder Transition Temperature and the Morphology of a Triblock Copolymer. 
Development and Application of an Indoor Air Quality Commissioning Program in a New Office Building.

PB96-155601

00,275

Environmental Evaluation of a New Federal Otfice Building. PB94-199874 02,538 Indoor Air Quality PB95-182309 uilding. Indoor Air Quality Commissioning of a New Office Building. PB97-110142 00,279

Measurements of Indoor Pollutant Emissions from EPA Phase II Wood Stoves.

PB95-198735

02.556

Study of Ventilation and Carbon Dioxide in an OHice Building. $95-150140$

02,542

Ventilation Effectiveness Measurements in Two Modern Of-

fice Buildings.
PB95-176012

02,553

NABLO, S. V.

Real Time Monitoring of Electron Processors.

PB96-135306

03,719

NACHT, G. G.

Operating Principles of the SBus MultiKron Interface Board.

PB95-231783

01,630

NACLERIO, N. J.

Overview of U.S. Govemment Advanced Packaging Programs.

02,443

NADLER, $W$.

International Marine-Almospheric (222)Rn Measuremen Intercomparison in Bermuda. Part 2. Results for the Participating Laboratories.

pating Laborat

00,115

NAGARAJAN, $R$.

Neutron Scattering Study of Antiferromagnetic Order in the Magnetic Superconductors ANi2B2C.

PB97-112411

04,812

NAGASAKA, $Y$.

Standard Reference Data for the Thermal Conductivity of Water

PB96-145875

01,111

Status of the Round Robin on the Transport Properties of

01,152

NAGASHIMA, A.

Standard Reference Data for the Thermal Conductivity of Water.

PB96-145875

01,111

NAGI, $M$.

Optimization of Highway Concrete Technology.

PB94-182995

01,333

NAGUSRINIVAS, $N$

Effect of DC Tests on Induced Space Charge. PB94-172350

02,212

Complex Time Dependence of the EPR Signal of Irradiated L-alpha-alanine. L-alpha-alaning.

NAHOR, G. S.

04,180

Changes in the Redox State of Iridium Oxide Clusters and Their Relation to Catalytic Water Oxidation: Radiolytic and Electrochemical Studies.

PB95-107017 00,864

Metalloporphyrin Sensitized Photooxidation of Water to Oxygen on the Surface of Colloidal Iridium Oxides - Photochemical and Pulse Radiolytic Studies.

PB95-107082

00,868

One-Electron Oxidation of Nickel Porphyrins. Effect of Structure and Medium on Formation of Nickel(III) Porphyrin or Nickel(II) Porphyrin pi-Radical Cation.

PB95-107058

00,865

Reduction of Dinitrogen to Ammonia in Aqueous Solution Mediated by Colloidal Metals.

PB95-107074

00,867

Site of One-Electron Reduction of Ni(II) Porphyrins. Formation of Ni(I) Porphyrin of Ni(II) Porphyrin pi-Radical Anion. PB95-107066

00,866

NAHUM, $M$.

Electronic Microrefrigerator Based on a Normal-InsulatorSuperconductor Tunnel Junction.

04,718

Hot-Electron Microcalorimeters as High-Resolution X-ray Detectors.

$\begin{array}{ll}\text { PB96-123641 04,739 } & \end{array}$

Hot-Electron Microcalorimeters for X-ray and Phonon Deection.

PB95-168993

04,644

Metrological Accuracy of the Electron Pump.

PB95-168910

03,951

Novel Hot-Electro

01,977

Testing for Metrological Accuracy of the Electron Pump.

PB95-175873

04,663

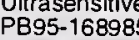

02,163
NAIR, $R$.

Turbulent Upward Flame Spread on a Vertical Wall under External Radiation.

AJARRO, F.

00,341

Radiation-Driven Winds of Hot Luminous Stars $\mathbf{X}$. The Deermination of Stellar Masses Radii and Distances from Terminal Velocities and Mass-Loss Rates.

PB94-213022

00,060

NAKABE, $K$.

Ignition and Subsequent Flame Spread Over a Thin Cellulosic Material.

PB96-160270 04,836

Ignition and Transition to Flame Spread Over a Thermally Thin Cellulosic Sheet in a Microgravity Environment.

PB96-160288

04,837

Transition from Localized Ignition to Flame Spread Over a Thin Cellulosic Material in Microgravity.

PB96-155809

NAKAGAKI, T.

Spectral Data and Grotrian Diagrams for Highly Ionized Chromium, CrV through Cr XXIV.

PB94-16236.
IAKAI, $Y$.

00,748

Spectral Data and Grotrian Diagrams for Highly lonized

PB94-162369

NAKAJIMA, $\mathrm{H}$.

Charpy Impact Test as an Evaluation of $4 \mathrm{~K}$ Fracture Toughness.

PB96-190194

03,219

NAKAKI, S. D.

Simplified Design Procedure for Hybrid Precast Concrete Connections.
PB96-154836

NAKANTANI, A. I.

Flow-Induced Structure in Polymer. Chapter 1. An Introduction to Flow-Induced Structures in Polymers.

PB96-123369

03,387

NAKASSIS, A

Security in Open Systems

PB95-105383

01,473

NAKATANI, A.

Dynamic Light-Scattering Study of a Diluted Polymer Blend Near Its Critical Point.

PB95-151890

01,245

AKATANI, A. I.

Anisotropic Phase Separation Kinetics in a Polymer Blend Solution Following Cessation of Shear Studied by Light Scattering.

01,241

Crossover to Strong Shear in a Low-Molecular-Weight Critical Polymer Blend

01,222

Flow.Induced Structure in Polymers: Chapter 16. Shear-Induced Changes in the Order-Disorder Transition Temperature and the Morphology of a Triblock Copolymer. PB96-123237

03,127

Flow-Induced Structure in Polymers. Chapter 17. PhaseSeparation Kinetics of a Polymer Blend Solution Studied by a Two-Step Shear Quench

03,388

Influence of Shear on the Ordering Temperature of a Triblock Copolymer Melt.

01,288

Neutron Scattering Study of Shear Induced Turbidity in Polystyrene/Dioctyl Phthalate Solutions at High Shear Rates. Neutron Scattering Study of Shear Induced Turbidity in Polystyrene Dissolved in Dioctyl Phthalate.

01,256

SANS and LS Studies of Polymer Mixtures Under Shear

PB95-107090

01,231

Shear Dependence of Critical Fluctuations in Binary Polymer Mixtures by Small Angle Neutron Scattering.
PB94-211612

Shear-Excited Morphological States in a Triblock Copoly-

PB94-172392

01,196

Shear-Induced Changes in the Order-Disorder Transition Temperature and the Morphology of a Triblock Copolymer.
PB97-118772

Shear-Induced Martensitic-Like Transformation in a Block Copolymer Melt.

PB96-119508

01,277

Shear-Induced Mixing in Polymer Blends.

PB96-148085

01,287

Shear Suppression of Critical Fluctuations in a Diluted Polymer Blend.

04,418

Synthesis of Hybrid Organic-Inorganic Materials from Interpenetrating Polymer Network Chemistry.
PB96-180054

Time Dependent Small Angle Neutron Scattering Behavior in Triblock Copolymers Under Steady Shear.

01,198

NAKOTTE, $H$.

Crystallographic and Magnetic Properties of UAuSn.
PB95-140521

Magnetic Properties of Single-Crystalline UCu3A12.

PB95-180717

04,686

S. $\mathrm{W}$

Self-Biasing Cryogenic Particle Detector Utilizing Electrothermal Feedback and a SOUID Readout.
PB96-102538

NAMAVAR, $F$.

Soft-X-ray-Emission Investigation of Cobalt Implanted Silicon Crystals.

NANCOLLAS, G. H.

Influence of Natural and Synthetic Inhibitors on the Crystallization of Calcium Oxalate Hydrates.

PB95-150967

03,560

NARAYAN, A.

Density Dependence of Fluid Properties and Non-Newtonian Flows: The Weisenberg Etfect.

PB96-161898

01,140

NARAYAN, A. P.

Non-Newtonian Flow between Concentric Cylinders Calculated from Thermophysical Properties Obtained from Sim-

Ulations.
PB96-163761

04,103

NASH, S. G.

Guidelines for Reporting Results of Computational Experiments. Report of the Ad hoc Committee.

PB94-212347

03,427

Ground Vehicle Control at NIST: From Teleoperation to AUtonomy.

03,758

Integrated Vision Touch-Probe System for Dimensional Inspection Tasks.

Real-Time Obstacle Avoidance Using Central Flow Divergence and Peripheral Flow. PB95-107108 01,78

Visual Road Following without 3-D Reconstruction PB95-161030

NASSIMBENE, $R$.

Distributed measurements of tracer response on packed bed flows using a fiberoptic probe array. Final report DE95013079

00,667

NASSIMBENE, R. D.

Characterization of the Adsorption-Fouling Layer Using Globular Proteins on Ultrafiltration Membranes.

NATER, $R$.

Determination of Density of Mass Standards: Requirement and Method.

NAVARRO, $M$.

Further Development of the N-Gas Mathematical Model: An Approach for Predicting the Toxic Potency of Complex Combustion Mixtures.

PB96-123260

Study of Multiple Scattering Background in Compton Scatter Imaging.

NAYAK, A.

Temperature Dependent Ultraviolet Absorption Cross Sections of Propylene, Methylacetylene and Vinylacetylene PB96-20417

NAYAK, A. $K$

Temperature Dependence of the Gas and Liquid Phase Ultraviolet Absorption Cross Sections of HCFC-123 (CF3CHCl2) and HCFC-142b (CH3CF2Cl). PB96-201033

Temperature Dependence of the Ultraviolet Absorption Cross Section of CF31. 
First Results from a Coordinated ROSAT, IUE, and VLA Study of RS CVn Systems.

00,069

Rotational Modulation and Flares on RS Canum Venaticorum and BY Draconis Stars. XVIII. Coordinated VLA, ROSAT, and IUE Observations of RS CVn Binaries. PB96-102322

00,089

NEGIZ, A.

Stochastic Modeling of a New Spectrometer.

PB96- 157870
NELDER, J. A.

04.068

Taguchi's Parameter Design: A Panel Discussion.

PB96-111802

NELIS, T.

Atomic Oxygen Fine Structure Splittings with Tunable Far Infrared Spectroscopy.

PB95-152203

03,915

Determination of the Molecular Parameters of $\mathrm{NiH}$ in Its (2)Delta Ground State by Laser Magnetic Resonance.
PB95-107116 869 NELSON, A. J.

Enhanced Flux Pinning via Chemical Substitution in Bulk

Superconducting T1-2212.

04,647

Epitaxial Growth and Characterization of the Ordered Vacancy Compound Culn3Se5 on GaAs (100) Fabricated by Molecular Beam Epitaxy.
PB95-180725

NELSON, C. W.

04,687

Design Criteria for BJT Amplifiers with Low I/f AM and PM Noise.

02,442

Frequency Synthesis and Metrology at $10(-17)$ and Beyond.
PB97-113187

New 5 and $10 \mathrm{MHz}$ High Isolation Distribution Amplifier.

PB96-190202 01,510

Relationship of AM to PM Noise in Selected RF Oscillators.

PB95-169009

02,262

NELSON, D. D.

Microwave and Submillimeter Spectroscopy of Ar-NH3 States Correlating with $\mathrm{Ar}+\mathrm{NH} 3(\mathrm{j}=1, \mathrm{k}=1)$.

00.942

Slit-Jet Near-Infrared Diode Laser Spectroscopy of (DCI)2 nu1, nu2 DCl Stretching Fundamentals, Tunneling Dynamics, and the Influence of Large Amplitude 'Geared' ins, and the Influence

PB95-203204

NELSON, H. E.

01,047

Fire Growth Analysis of the Fire of March 20, 1990. Pulask Building, 20 Massachusetts Avenue, N.W., Washington, PB94-205952

00,194

FPETOOL: Fire Protection Tools for Hazard Estimation. An Overview of Features.

NELSON, L. M.

00,367

Frequency Synthesis and Metrology at 10(-17) and Beyond.

Relationship of AM to PM Noise in Selected RF Oscillators. PB95-169009

NELSON, R. D.

Learning to Change: Opportunities to Improve the Performance of Smaller Manufacturers.

NEMETH, D. T.

00,010

Fabrication Issues in Optimizing $\mathrm{YBa}$ Cu3O7-x Flux Transformers for Low $1 / 4$ Noise.

PB95-175857

02,059

Low Noise YBa2Cu3O7-x-SrTiO3-YBa2Cu3O7-x Mulitlayers for Improved Superconducting Magnetometers

PB96-138417

04,747

NEMIROVSKY, A.

Hypercubic Lattice SAW Exponents nu and gamma : 3.99 Dimensions Revisited.

NEMIROVSKY, A. M.

Examination of the $1 / d$ Expansion Method from Exact Enumeration for a Self-Interacting Self-Avoiding Walk.

PB95-175733

01,266

NENOFF, T. M.

Crystal Structure of a New Sodium Zinc Arsenate Phase Solved by 'Simulated Annealing'.

PB95-107124

00,870

Structural and Chemical Investigations of

$\mathrm{Na3}(\mathrm{ABO} 4) 3.4 \mathrm{H} 2 \mathrm{O}-\mathrm{Type}$ Sodalite Phases.
$\mathrm{PB95}-180733$ PESB- 180733

Collisional Alignment of $\mathrm{CO} 2$ Rotational Angular Momentum States in a Supersonic Expansion.

PB96-103171

01,069

High-Resolution, Direct Intrared Laser Absorption Spectros copy in Slit Supersonic Jets: Intermolecular Forces and Unimolecular Vibrational Dynamics in Clusters.

PB95-203006

01,040

High-Resolution Infrared Overtone Spectroscopy of ArHF via Nd:YAG/Dye Laser Difference Frequency Generation. PB94-211448
High-Resolution Infrared Overtone Spectroscopy of N2-HF: Vibrational Red Shifts and Predissociation Rate as a Function of HF Stretching Quanta.

01,061

High-Resolution Infrared Spectroscopy of DF Trimer: A Cyclic Ground State Structure and DF Stretch Induced Intramolecular Vibrational Coupling.

00,920

High-Resolution IR Laser-Driven Vibrational Dynamics in Supersonic Jets: Weakly Bound Complexes and Supersonic Jets: Weakly Bound Complexes and Intramolecular Energy Flow.
PB94-216751

00,862

High Resolution IR Studies of Polymolecular Clusters: Micromatrices and Unimolecular Ring Opening.

00,777

High Resolution, Jet-Cooled Infrared Spectroscopy of $(\mathrm{HCl}) 2$ : Analysis of nu1 and nu2 $\mathrm{HCl}$ Stretching Fundamentals, Interconversion Tunneling, and Mode-Specific Predissociation Lifetimes.

PB95-203196

01,046

High Resolution Near Infrared Spectroscopy of $\mathrm{HCl}-\mathrm{DCl}$ and $\mathrm{DCl}-\mathrm{HCl}$ : Relative Binding Energies, Isomer Interconversion Rates, and Mode Specific Vibrational Predissociation.

PB95-203212

01,048

Intermolecular HF Motion in $\mathrm{Ar}($ sub $n) \mathrm{HF}$ Micromatrices $(n=1,2,3,4)$ : Classical and Ouantum Calculations on a Painwise Additive Potential Surface.

PB95-107025

03,871

Large Amplitude Skeletal Isomerization as a Promoter of Intramolecular Vibrational Relaxation in $\mathrm{CH}$ Stretch Excited Hydrocarbons.

01,036

Mode Specific Vibrational Predissociation Dynamics in Fragile Molecules.

00.871

Painwise and Nonpainwise Additive Forces in Weakly Bound Pairwise and Nonpairwise Additive Forces in Weakly Bound ArnDF $(n=1,2,3)$.

PB96-200357 04,125

Photodissociation Dynamics in Ouantum State-Selected

Clusters: A Test of the One-Atom Cage Effect in Ar-H2O.
PB95-203121

Potential Surfaces and Dynamics of Weakly Bound Trimers: Perspectives from High Resolution IR Spectroscopy.
PB96-176508

ution IR

Laser Spectroscopy.

PB96-102835 01,066

Rigid Bender Analysis of van der Waals Complexes: The Intermolecular Bending Potential of a Hydrogen Bond. 01,042

Rotational-RKR Inversion of Intermolecular Stretching Potentials: Extension to Linear Hydrogen Bonded Complexes.

Signatures of Large Amplitude Motion in a Weakly Bound Complex: High-Resolution IR Spectroscopy and Quantum Calculations for $\mathrm{HeCO} 2$

PB95-203485

01.054

Slit Jet Infrared Spectroscopy of Hydrogen Bonded N2MF Isotopomers: Rotational Rydberg-Klein-Rees Analysis and H/D Dependent Vibrational Predissociation Rates. Slit-Jet Near-Infrared Diode Laser Spectroscopy of (DC1)2: nu1, nu2 DCl Stretching Fundamentals, Tunneling Dynam ics, and the Influence of Large Amplitude 'Geared' Intermolecular Rotation.

PB95-203204

01,047

Slit-Jet Near-Infrared Spectroscopy and Internal Rotor Dy. namics of the ArH2O van der Waals Complex: An Angular Potential-Energy Surface for Internal H2O Rotation.

Spectroscopic Puzzle in ArHF Solved: The Test of a New Potential.
PB94-216058

00,850

Stabilization and Precise Calibration of a Continuous-Wave

Difference Frequency Spectrometer by Use of a Simple Transfer Cavity.

State-Resolved Rotational Energy Transfer in Open Shell Collisions: $\mathrm{Cl}((2) \mathrm{P} 3 / 2)+\mathrm{HCl}$.

PB96-176607

01,157

Sub-Doppler, Infrared Laser Spectroscopy of the Propyne 2nu1 Band: Evidence of Z-Axis Coriolis Dominated Intramolecular State Mixing in the Acetylenic $\mathrm{CH}$ Stretch PB95-202941

01,037 Vibration, Rotation, and Parity Specific Predissociation Dynamics in Asymmetric OH Stretch Excited ArH2O: A Half Weak'y Bound Complex.

PB95-107140

00,872

\section{NETA, P.}

Changes in the Redox State of Iridium Oxide Clusters and Their Relation to Catalytic Water Oxidation: Radiolytic and Electrochemical Studies.

00,864

Electron Transfer Reaction Rates and Equilibria of the Carbonate and Sulfate Radical Anions.

00,829

Fluoride Elimination Upon Reaction of Pentafluoroaniline with e (sub eq')(sup -), $\mathrm{H}$, and $\mathrm{OH}$ Radicals in Aqueous Solution.

PB97-111314

01,177

lodine Atoms and lodomethane Radical Cations: Their Formation in the Pulse Radiolysis of lodomethane in Organic Solvents, Their Complexes, and Their Reactivity with Organic Reductants.

PB95-162764

00,965

Metalloporphyrin Sensitized Photooxidation of Water to Oxygen on the Surface of Colloidal Iridium Oxides - Photochemical and Pulse Radiolytic Studies.

PB95-107082

00,868

One-Electron Oxidation of Metalloporphycenes as Studied by Radiolytic Methods.

01,179

One-Electron Oxidation of Nickel Porphyrins. Effect of Structure and Medium on Formation of Nickel(III) Porphyrin or Nickel(II) Porphyrin pi-Radical Cation.

PB95-107058

00,865

Oxidation of Cafteic Acid and Related Hydroxycinnamic Acids.

00.651

Oxidation of Ferrous and Ferrocyanide Ions by Peroxyl Radicals.

PB97-122402

01,191

Oxidation of 10-Methylacridan, a Synthetic Analogue of NADH and Deprotonation of Its Cation Radical. Convergent Application of Laser Flash Photolysis and Direct and Redox Aatalyzed Electrochemistry to the Kinetics of Deprotonation

of the Cation Radical.
PB94-198371

00,785

Radiation Chemistry of Cyanine Dyes: Oxidation and Reduction of Merocyanine 540 .

PB94-211661

00.818

Reduction of Dinitrogen to Ammonia in Aqueous Solution

Mediated by Colloidal Metals.

00,867

Site of One-Electron Reduction of Ni(II) Porphyrins. Formation of $\mathrm{Ni}(\mathrm{I})$ Porphyrin of Ni(II) Porphyrin pi-Radical Anion.

PB95-107066

00,866

Solvent Effects in the Reactions of Peroxyl Radicals with Organic Reductants. Evidence for Proton Transfer Mediated Electron Transfer.

PB95-107157

00,873

Temperature Dependence of the Rate Constants for Reaction of Dihalide and Azide Radicals with Inorganic Reductants.

PB95-162756

00,964

Temperature Dependence of the Rate Constants for Reaction of Inorganic Radicals with Organic Reductants

PB94-198280

00,783

Temperature Dependence of the Rate Constants for Reactions of the

Reductants.

NETTLETON, D. H.

International Intercomparison of Detector Responsivity at 1300 and $1550 \mathrm{~nm}$.

PB95-125928

International Intercomparison of Detector Responsivity at 1300 and $1550 \mathrm{~nm}$.

NEUBAUER, G.

2D-Scanning Capacitance Microscopy Measurements of Cross-Sectioned VLSI Teststructures.

PB96-163779

NEUDECK, A

Oxidation of Caffeic Acid and Related Hydroxycinnamic Acids.

PB97-111975

00,651

NEUGEBAUER, G. T.

Correlation of $\mathrm{HgCdTe}$ Epilayer Defects with Underlying 

Lattice Dynamics of Semiconducting, Metallic, and
Superconducting Ba 1-xKXBiO3 Studied by Inelastic Neutron Scattering.

04,708

Maximum Entropy as a Tool for the Determination of the CAxis Profile of Layered Compounds.

PB94-199619

00,800

Methyi Torsional Levels of Solid Acetonitrile $(\mathrm{CH} 3 \mathrm{CN})$ : A Neutron Scattering Study.

00,926

Neutron-Scattering Study of $\mathrm{C} 60(n-)(n=3,6)$ Librations in Alkali-Metal Fullerides.

PB94-200219

00,806

Neutron-Scattering Study of Librations and Intramolecular Phonons in Rb2.6K0.4C60.

00,958

Phase Transitions in Solid C70: Supercooling, Metastable Phases, and Impurity Etfect.

PB95-150090

00,914

Phonon Density of States in R2CuO4 and Superconducting R1.85Ce0.15CuO4 ( $R=$ Nd, $\mathrm{Pr})$.

04,574

Rotational Dynamics of $\mathrm{C} 60$ in Na2RbC60

00,948

Rotational Dynamics of Solid C70: A Neutron-Scattering PBty. 172178

00,755

Rotational Dynamics of Solid C70: A Neutron-Scattering Study. 153219

00,949

Structure and Dynamics of Buckyballs.

PB95-153292

00,950

$X$-ray Powder Diffraction from Carbon Nanotubes and Nanoparticles.

NEUMAYER, D. A.

03.064

Suitability of Metalorganic Chemical Vapor Deposition-Derived PrGaO3 Films as Buffer Layers for YBa2Cu3O7-x Pulsed Laser Deposition.

PB95-168670

04,640

NEUSSER, H. J.

Doppler-Free Spectroscopy of Large Polyatomic Molecules

and van der Waals Complexes.

04,339

Frequency Shitting of Pulsed Narrow-Band Laser Light in a Multipass Raman Cell.

04,32

Van der Waals Bond Lengths and Electronic Spectral Shifts of the Benzene- $\mathrm{Kr}$ and Benzene-Xe Complexes.

PB95-151387

00,932

NEWBURY, D.

Fabrication of Platinum-Gold Alloys in Pre-Hispanic South America: Issues of Temperature and Microstructure Control. PB94-211646

NEWBURY, D. E.

Compositional Mapping of the Microstructure of Materials. PB95-107199 00,565 Concentration Histogram Imaging: A Scatter Diagram Technique for Viewing Two or Three Related Images.

PB94-199114

00,542

Design of a Protocol for an Electron Probe Microanalyzer kValue Round Robin

PB95-107181

00,564

Electron Probe X-Ray Microanalysis.

00,562

Microanalysis to Nanoanalysis: Measuring Composition at High Spatial Resolution.

PB95-107173

00,563

Monte Carlo Electron Trajectory Simulation of X-Ray Emission from Filims Supported on Substrates.

PB95-107207

04,522

NEWBURY, N. R. Gravitational Sisyphus Cooling of (87)Rb in a Magnetic
Trap.

PB96-200704

NEWELL, A.

Planar Near-Field Alignment.

NEWELL, A. C.

Comparison of Ultralow-Sidelobe-Antenna Far-Field Patterns Using the Planar-Near-Field Method and the Far-Field Method.

PB96-200373

02,015

Planar Near-Field Measurements of Low-Sidelobe Antennas.

02,001

NEWELL, B. L.

Fabrication Issues for the Prototype National Institute of Standards and Technology SRM $2090 \mathrm{~A}$ Scanning Electron Microscope Magnification Calibration Standard.

PB96-160585

01,131

NEWELL, D.

Low-Frequency, Active Vibration Isolation System. PB95-203303

Measurement and Reduction of Alignment Errors of the NIST Watt Experiment

01,987
NIST Watt Balance: Progress Toward Monitoring the Kilogram.

01,991

NEWELL, $K$.

National Type Evaluation Program: Index of Device Evalua. tions by Company. NCWM Publication 5 Part A (Second

PB94-160835

02,889

NEWHALL

Lunar Laser Ranging: A Continuing Legacy of the Apollo Program.

NEWSAM, J. M.

03,683

Crystal Structure of a New Sodium Zinc Arsenate Phase Solved by 'Simulated Annealing'.

NEWTON, J.

00,870

Application of Metadata Standards.

PB96-180187

01.771

MTON, J. J.

Standards and Linkages: What Data Sharing Needs.

NG, B.

Hybrid Undulator for the NIST-NRL Free-Electron Laser. PB94-212529

04,238

NGUYEN, N. V.

Characterization of the ZnSe/GaAs Interface Layer by TEM

and Spectroscopic Ellipsometry.
PB95-175360

04,655

Determination of the Optical Constants of ZnSe Films by Spectroscopic Ellipsometry.

04,656

High-Accuracy Principal-Angle Scanning Spectroscopic Ellipsometry of Semiconductor Interfaces.

02,427

Interface Roughness-Induced Changes in the Near-E(sub 0) Spectroscopic Behavior of Short-Period AIAs/GaAs Spectroscopic

PB94-185154

02,118

Interface Roughness of Short-Period AIAs/GaAs Superlattices Studied by Spectroscopic Ellipsometry
PB95-107215

02,137

Interface Sharpness during the Initial Stages of Growth of Thin, Short-Period III-V Superlattices.

PB95-108783

02,139

Interface Sharpness in Low-Order III-V Superlattices.

PB95-108775

02,138

Spectroscopic Ellipsometry Determination of the Properties of the Thin Underlying Strained Si Layer and the Roughness at SiO2/Si Interface.

04,560

NGUYEN, T.

Degradation of Powder Epoxy Coated Panels Immersed in a Saturated Calcium Hydroxide Solution Containing Sodium Chloride.

PB96-101050

01,344

Development of a Method for Measuring Water-Stripping Resistance of Asphalt/Siliceous Aggregate Mixtures. PB96-197249

Development of a Method for Measuring Water-Stripping Resistance of Asphalt/Siliceous Aggregate Mixtures.
PB96-202296

Development of a Test Method for Leaching of Lead from Lead-Based Paints Through Encapsulants.

03,128 PB96-154984

Diffusion of Cations Beneath Organic Coatings on Steel Substrate.

PB94-215704

03,119

Effect of Environmentally Exposures on the Properties of Polyisocyanurate Foam Insulation: Thermal Conductivity

$\begin{array}{ll}\text { MBg5-181210. } & 00,388\end{array}$

Effects of Humidity and Elevated Temperature on the Density and Thermal Conductivity of a Rigid Polyisocyanurate Foam

PB95-152021

00,373

Effects of Humidity and Elevated Temperature on the Density and Thermal Conductivity of a Rigid Polyisocyanurate Foam Co-Blown with $\mathrm{CCl} 3 \mathrm{~F}$ and $\mathrm{CO} 2$

PB95-150462

00,371

In situ Measurements of Chloride Ion and Corrosion Potential at the Coating/Metal Interface.

03,122

PB95-140893

Materials-Science Based Approach to Phenol Emissions from a Flooring Material in an Otfice Building

02,572

Relation between AC Impedance Data and Degradation of Coated Steel. 1. Effects of Surface Roughness and Contamination on the Corrosion Behavior of Epoxy Coated Steel.

PB94-213345

03, 189

Source of Phenol Emissions Aftecting the Indoor Air of an Otfice Building
PB94-154382

NICHIPOROV, D.

03,600

Investigation of Applicability of Alanine and Radiochromic Detectors to Dosimetry of Proton Clinical Beams.

03,636

NICOL, J.

Inelastic Neutron Scattering Studies of Nonlinear Optica Materials: p-Nitroaniline Adsorbed in ALPO-5.

00,874

NICOL, J. M.

Characterization of the Interaction of Hydrogen with Iridium Clusters in Zeolites by Inelastic Neutron Scattering Spectroscopy.

01,013

Crystal Structure of a New Sodium Zinc Arsenate Phase Solved by 'Simulated Annealing'.

00,870

Neutron and Raman Spectroscopies of 134 and 134a Hydrofluorocarbons Encaged in Na-X Zeolite.

03,001

Powder Neutron Diffraction Investigation of Structure and Cation Ordering in $\mathrm{Ba} 2+x \mathrm{Bi} 2 \cdot x \mathrm{O} 6-\mathrm{y}$. Powder

01,015

Small Angle Neutron Scattering Study of the Structure and Formation of MCM-41 Mesoporous Molecular Sieves.

PB97-122337

03,110

Small Angle Neutron Scattering Study of the Structure and Formation of Ordered Mesopores in Silica.

03,069

Structural and Chemical Investigations of $\mathrm{Na} 3(\mathrm{ABO})$ ) 3.4H2O-Type Sodalite Phases.

PB95-180733

01,012

Tetrahedral-Framework Lithium Zinc Phosphate Phases: Location of Light-Atom Positions in LiZnPO4 H2O by Powder Neutron Diffraction and Structure Determination of LiZnPO 4 by ab Initio Methods.

NIEBAUER, T. M

01,129

Continuous Gravity Observations Using Joint Institute for Laboratory Astrophysics Absolute Gravimeters.

PB95-203048

03,685

Intracomparison Tests of the FG5 Absolute Gravity Meters.

03,688

Test of Newton's Inverse Square Law of Gravitation Using the 300-m Tower at Erie, Colorado.

PB95-202446

03,978

NIELSEN, L.

Precision Comparison of the Lattice Parameters of Silicon Monocrystals.

04,438

SUSAN: SUperconducting Systems ANalysis by Low Temperature Scanning Electron Microscopy (LTSEM).

PB96-112065

NIESEN, V. G.

Coexisting Densities, Vapor Pressures and Critical Densities of Refrigerants R-32 and R-152a, at $300-385 \mathrm{~K}$

03,274

Experimental Method for Obtaining Critical Densities of $\mathrm{Bi}$ nary Mixtures: Application to Ethane + n-Butane.
PB95-151148

NIETO DE CASTRO, C. A.

Polarized Transient Hot Wire Thermal Conductivity Meas- 
NOBLE, $R$.

Gas Transport Properties of Solution-Cast Perfluorosulfonic Acid Ionomer Films Containing lonic Surfactants.

PB95-175998

01,267

NOCHOLS-BOHLIN, J.

Recalibration for the Final Archive of the International Ultraviolet Explorer (IUE) Satellite.

PB96-135264

00,106

NODA, I.

Localization of a Homopolymer Dissolved in a Lamellar Structure of a Block Copolymer Studied by Small-Angle Neutron Scattering.

PB95-16159

01,253

Molecular Weight Dependence of the Lamellar Domain Spacing of ABC Triblock Copolymers and Their Chain Conformation in Lamellar Domains.

PB95.161691

01,254

NOESTERER, $M$.

Microdosimetry and Cellular Radiation Effects of Radon Progency in Human Bronchial Airways.

PB95-152344

03,625

NOMURA, $\mathrm{S}$

Small Angle Neutron Scattering Studies on Chain Asymmetry of Coextruded Poly(Vinyl Alcohol) Film.

PB95-164372

01,262

NORCROSS, D. W.

Angle-Differential and Momentum-Transfer Cross Sections for Low-Energy Electron-Cs Scattering

04,005

Characteristics of Light Emission After Low-Energy Electron Impact Excitation of Caesium Atoms. Impact Excitation of Caesium Atoms.

03,806

High-Precision Calculations of Cross Sections for Low-Energy Electron Scattering by Ground and Excited State of Sodium

03,914

Low-Energy-Electron Collisions with Sodium: Elastic and InLow-Energy-Electron Collisions with Sodium: Elastic and In-
elastic Scattering from the Ground State.
PB96-103106

Relativistic Eftects in Spin-Polarization Parameters for Low-
Energy Electron-Cs Scattering. PB95-150868

Relativistic R-Matrix Calculations for Electron - Alkali-MetalAtom Scattering: Cs as a Test Case.

04,006

Slow-Electron Collisions with CO Molecules in an Exact-Exchange Plus Parameter-Free Polarization Model.

PB95-202719

03,989

NORCROSS, R. J.

AMRF Composite Fabrication Workstation.

PB94-172681

02,810

NORDLANDER, $P$

Influence of Coadsorbed Potassium on the Electron-Stimulated Desorption of $F(+), F(-)$, and $F\left({ }^{*}\right)$ from $P F 3$ on

PB96-157946

04,072

Photoluminescence Spectra of Epitaxial Single Crystal C60.

PB96-141205

04,754

NORMAN, B. $\mathrm{A}$.

High-Sensitivity Determination of lodine Isotopic Ratios by Thermal and Fast Neutron Activation.

PB94-213386

00,555

NORMAN, G. R.

Regulation of Lithium and Boron Levels in Normal Human Blood: Environmental and Genetic Considerations.

PB94-198579

03,491

NORRIS, G. A

Multiattribute Decision Analysis Method for Evaluating Buildings and Building Systems.

PB96-158670

00,325

NORRIS, J.A.

Apparent Bias in the X-Ray Fluorescence Determination of Titanium in Selected NIST SRM Low Alloy Steels.

PB95-108759

03,212

NORTH, S

Oxidative DNA Base Damage in Renal, Hepatic, and Pul-

monary Chromatin of Rats After Intraperitoneal Injection of Cobalt (II) Acetate.

PB95-150025

03,647

NORTHROP, D. M.

Separation and Identification of Organic Gunshot and Explosive Constituents by Micellar Electrokinetic Capillary Electrophoresis
PB95-107249

NORTON, M.

Optical Conductivity of Single Crystals of Ba1$\mathrm{XMXBiO3}(\mathrm{M}=\mathrm{K}, \mathrm{Rb}, \mathrm{x}=0.04,0.37)$.

PB94-185329

00,566

NORTON, P. W.

Comparison of Techniques for Nondestructive Composition Measurements in CdZnTe Substrates.

NORTON, $\mathrm{s}$.

02,703

Analysis of Thermal Wave Propagation in Diamond Film

PB94-211471
Thermal Wave Propagation in Diamond Films. PB94-211489

03,015

NORTON, S. J.

Reconstructing Stratified Fluid Flow from Reciprocal Scattering Measurements.

PB95-107256

04,202

Ultrasonic Method for Reconstructing the Two-Dimensional Liquid-Solid Interface in Solidifying Bodies.

03,349

Ultrasonic Spectroscopy of Metallic Spheres Using Electromagnetic-Acoustic Transduction.

04,185

\section{NORTON, T. S.}

Comparison of Experimental and Computed Species Concentration and Temperature Profiles in Laminar, Two-Dimensional Methane/Air Diffusion Flames.

PB95-140919

01,379

NOSSAL, $R$.

Small Angle Neutron Scattering Studies of Structural Characteristics of Argarose Gels.

PB96-112305

03,475

NOTARIANNI, $K$

Protection of Data Processing Equipment with Fine Wate Sprays.

PB95-174975

02,610

NOTARIANNI, K. A.

Analysis of High Bay Hangar Facilities for Detector Sensitiv.

ity and Placement.
PB96-190210 01,429

Comparison of Fire Sprinkler Piping Materials: Steel, Copper, Chlorinated Polyvinyl Chloride and Polybutylene, in Residential and Light Hazard Installations.

00,299

PB95-182267

Measurement of Room Conditions and Response of Sprin-
klers and Smoke Detectors during a Simulated Two-Bed klers and Smoke Detectors during a Simulated Two-Bed
Hospital Patient Room Fire.
00,292

PB94-213717

00,292

PASA Fire Detector Study.

01,423

Use of Computer Models to Predict Temperature and Smoke Movement in High Bay Spaces.

PB94-145976

00,19

Water Mist Fire Suppression Workshop Summary.

PB95-161907

02,853

NOVAK, S. W.

Using Secondary lon Mass Spectrometry (SIMS) to Characterize Optical Waveguide Materials.

PB96-119599

04,340

NOVICK, A

Diode-Laser Pumped, Rubidium Cell Frequency Standards.
PB95-163218

Reducing the Effect of Local Oscillator Phase Noise on the

Frequency Stability of Passive Frequency Standards. Frequency Stabi

NOVICK-COHEN, A.

Evolution Equations for Phase Separation and Ordering in Binary Alloys.

NOVOTNY, D.

Realizing Suspended Structures on Chips Fabricated by CMOS Foundry Processes Through the MOSIS Service.

PB94-193984

NOVOTNY, D. B.

Development and Characterization of Insulating Layers on Silicon Carbide: Annual Report for February 14, 1988 to

February 14,1989
PB94-155579

02,295

Development of Thin-Film Multijunction Thermal Converters at NIST

$\begin{array}{ll}\mathrm{PB} 97-112338 & 02,286\end{array}$

High-Current Thin Film Multijunction Thermal Converters and Multi-Converter Modules.

01,989

High-Spatial-Resolution Resistivity Mapping Applied to Mercury Cadmium Telluride.

02,131

Integrated Thin-Film Micropotentiometers.

02,109

Performance of Multilayer Thin-Film Multijunction Thermal

Performance of Multilayer Thin-Film Multijunction Therma
Converters. Converters.

NOVOTNY, D. R.

Optically Linked Three-Loop Antenna System for Determining the Radiation Characteristics of an Electrically Small Source.

PB95-161915

02,005

NOZAKI, $H$.

Elastic Constants and Microcracks in YBa2Cu3O7. PB96-200761

03,005

NUBBEMEYER, $\mathrm{H}$. G.

Comparisons of Some NIST Fixed-Point Cells with Similar Cells of Other Standards Laboratories

00,655

Direct Comparison of Three PTB Silver Fixed-Point Cells with the NIST Silver Fixed-Point Cell.

with the NIST
PB96-161286

00,628

NUNEZ, $V$

Crystallographic and Magnetic Properties of UAuSn PB95-14052

Supermirror Transmission Polarizers for Neutrons

03,866

Use of Building Emulators to Evaluate the Performance of Building Energy Management Systems.

PB96-111901

Lowest Excited Singlet State of Isolated 1-phenyl-1,3-butadiene and 1-phenyl-1,3,5-hexatriene.

01,026

NYBERG, G. L.

Spot-Profile-Analyzing LEED Study of the Epitaxial Growth of Fe, Co, and $\mathrm{Cu}$ on $\mathrm{Cu}(100)$

NYDEN, M.

Agent Screening for Halon 1301 Aviation Replacement

PB96-159710

03,282

NYDEN, M. R.

Computer-Aided Molecular Design of Fire Resistant Aircraft Materials.

PB96-160601

00,025

Halon Thermochemistry: 'Ab Initio' Calculations of the Enthalpies of Formation of Fluoromethanes.

03,289

Investigation into the Flammability Properties of Honeycomb Composites.

03,152

Investigation of the Thermal Stability and Char-Forming Tendency of Cross-linked Poly(methyl methacrylate).
PB94-213501

New Generation of Fire Resistant Polymers. Part 1. Computer-Aided Molecular Design.

01,419

Tomographic Reconstruction of the Moments of Local Probability Density Functions in Turbulent Flow Fields.

PBפ 180195

O'BRIAN, T.

RIS Measurements of the AC Stark Shift.

PB96-158035

Configuration-Dependent AC Stark Shifts in Calcium

PB96-157995

PB96-179510

04,078

Measurement of CO Pressures in the Ultrahigh Vacuum Regime Using Resonance-Enhanced Multiphoton-Ionization Time-of-Flight Mass Spectroscopy

PB94-216041

03,864 Cross Sections in Calcium.

04,073

Verification of the Ponderomotive Approximation for the ac Stark Shift in Xe Rydberg Levels.

PB94-185709

03,801

O'BAIEN, W. L.

Al L2,3 Core Excitons in AlxGa1-x as Studied by Soft-X-ray Reflection and Emission.

04,067

Barium Contributions to the Valence Electronic Structure of YBa2Cu3O7-delta, PrBa2Cu307-delta, and Other Barium- 
O'CONNOR, C. L.

NIST Reactor: Summary of Activities October 1992 through September 1993

PB94-161502

04,437

NIST Reactor: Summary of Activities, October 1993 through September 1994

O'FARRELL, T. J.

04,700

Effect of Ethanol on the Solubility of Dicalcium Phosphate Dihydrate in the System $\mathrm{Ca}(\mathrm{OH}) 2-\mathrm{H} 3 \mathrm{PO} 4-\mathrm{H} 2 \mathrm{O}$ at $37 \mathrm{C}$ PB95-163507

O'HARE, P. A. G.

Conference Report: Calorimetry Conference (50th). PB97-122279

03,722

Disilicides of Tungsten, Molybdenum, Tantalum, Titanium, Cobalt, and Nickel, and Platinum Monosilicide: A Survey of Their Thermodynamic Properties.

00,752

Thermochemical Studies of Inorganic Chalocogenides by Fluorine-Combustion Calorimetry: Binary Compounds of Germanium and Silicon with Sulfur, Selenium and Tellu-

PB97-112528

01,181

Thermodynamic Properties of Gaseous Silicon Monoteiluride and the Bond Dissociation Enthalpy $D$ (sub m)(SiTe) at $T$ approaches 0 .

00,751

Thermodynamic Properties of Silicides. 5. Standard Molar Enthalpy of Formation at the Temperature $298.15 \mathrm{~K}$ of Trimolybdenum Monosilicide Mo3Si Determined by Fluorine-Combustion Calorimetry.

PB97-119358

01,190

Thermodynamics of (Germanium + Selenium): A Review and Critical Assessment.

01,182

O'HAVER, T. C.

Liquid Chromatography: Laser-Enhanced Ionization Spectrometry for the Speciation of Organolead Compounds. PB94-185253

O'MALLEY, M. L.

Increased Transition Temperature in In situ Coevaporated YBa2Cu307-delta Thin Films by Low Temperature Post-An-

PB95-180071

04,672

O'REILLY, J. W.

Magnetic Ordering of the $\mathrm{Cu}$ Spins in $\mathrm{PrBa} 2 \mathrm{Cu} 3 \mathrm{O} 6+\mathrm{x}$ PB95.140547

04.545

O'SHANNESSY, D. J.

Phospholipid/Alkanethiol Bilayers for Cell-Surface Receptor Studies by Surface Plasmon Resonance.

PB96-102900

03,472

O'SULLIVAN, $P$.

Assessing MOS Gate Oxide Reliability on Wafer Level with Ramped/Constant Voltage and Current Stress.

04,115

New Oxide Degradation Mechanism for Stresses in the Fowler-Nordheim Tunneling Regime.

PB96-200985

04,128

OAKLEY, E.

Enthalpy increment Measurements from $4.5 \mathrm{~K}$ to $350 \mathrm{~K}$ and the Thermodynamic Properties of the Titanium Silicide TisSi3(cr).

PB96-204037

00,679

OAKLEY, L.

Materials-Science Based Approach to Phenol Emissions

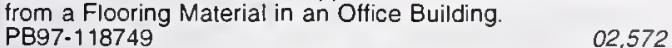

Source of Phenol Emissions Affecting the Indoor Air of an Office Building

PAKLEY, L. M.

03,600 Certification of Polychlorinated Biphenyl Congeners and
Chlorinated Pesticides in a Whale Blubber Standard Reference Material.

PBence Materia

03,745

OATES, C. W.

High-Resolution Optical Multiplex Spectroscopy.

PB95-203543

04,323

Improved Hyperfine Measurements of the Na NP Excited State Through Frequency-Controlled Dopplerless Spectroscopy in a Zeeman Magneto-Optic Laser Trap.

PB95-203840

04,012

OBERLE, R. $R$.

Magnetic and Structural Properties of Electrodeposited Copper-Nickel Microlayered Alloys.

PB94-213121

04,512

OBERNDORF, P.

Next Generation Computer Resources: Reference Model for Project Support Environments (Version 2.0)

01,677

OELKERS, E. H.

Summary of the Apparent Standard Partial Molal Gibbs Free Energies of Formation of Aqueous Species, Minerals, and Gases at Pressures 1 to 5000 Bars and Temperatures 25 to $1000 \mathrm{C}$

01,113
OGBURN, $\mathbf{F}$.

Aging Effects on XRF Measurements of Solder Coatings.
PB95-140927 PB95-140927

One-and Two-Sided Burning of Thermally Thin Materials. PB95-140935

03,151

OHLEMILLER, T. J.

Behavior of Mock-Ups in the California Technical Bulletin 133 Test Protocol: Fabric and Barrier Effects. PB95-231585

00,301

Effect of Suppressants on Metal Fires.

PB96-109574

01,402

Examination of the Correlation between Cone Calorimeter Data and Full-Scale Furniture Mock-Up Fires.

01,417

Influence of Ignition Source on the Flaming Fire Hazard of Upholstered Furniture. (NIST Reprint).

PB95-180162

00,297

Low Heat-Flux Measurements: Some Precautions.

6-201116

02,685

Quantifying the Ignition Propensity of Cigarettes.

PB96-155411

00,306

OHNO, Y.

Characterization of Modified FEL Quartz-Halogen Lamps for Photometric Standards

PB97-112544

00,282

Detector-Based Candela Scale and Related Photometric Calibration Procedures at NIST

04,273 Integrating Sphere Simulation: Application to Total Flux
Scale Realization.

PB95-150173 04,261

Intercomparison of Photometric Units Maintained at NIST (USA) and PTB (Germany), 1993

04,329

Interface-Filter Characterization of Spectraradiometers and Colorimeters.
PB97-122212

04,399

New Method for Realizing a Luminous Flux Scale Using an Integrating Sphere with an External Source.

04,333

NIST Detector-Based Luminous Intensity Scale.

PB96-179114

01,864

Realization of NIST 1995 Luminous Flux Scale Using the Integrating Sphere Method.

04,374

OISHI, Y.

Preparation of 2-Dimensional Ultra Thin Polystyrene Film by Water Casting Method

OKAMOTO, P.

Optimization of Highway Concrete Technology PB94-182995

04,619

OKAMOTO, T.

Standardised Computer Data File Format for Storage, Transport, and Off-Line Processing of Partial Discharge Data.

PB96-122486

01,930

OKAYASU, S.

Harmonic and Static Susceptibilities of $\mathrm{YBa} 2 \mathrm{Cu} 3 \mathrm{O} 7$

PB95-161139

04,599

OKAZAKI, K.

Spectral Data for Highly lonized Krypton, $\mathrm{Kr} V$ through $\mathrm{Kr}$ XXXVI

PB96-145917

01,115

OKORODUDU, A.

Frozen Human Serum Reference Material for Standardization of Sodium and Potassium Measurements in Serum o Plasma by Ion-Selective Electrode Analyzers.

PB94-185337

OLDEHOEFT, A. E.

Assessment of the DOD Goal Security Architecture (DGSA) for Non-Military Use.

PB95-189510

03,653

Report of the NIST Workshop on Key Escrow Encryption Held in Gaithersburg. Maryland on June 8-10, 1994. PB94-209459

01.584

OLDHAM, $\mathbf{N}$.

Exploring the Low-Frequency Performance of Thermal Converters Using Circuit Models and a Digitally Synthesized Source

PB97-112551

02,848

OLDHAM, N. M.

Automatic Calibration of Inductive Voltage Dividers for the NASA Zeno Experiment.

PB95-152849

02,041

Automatic Inductive Voltage Divider Bridge Operates from $10 \mathrm{~Hz}$ to $100 \mathrm{kHz}$

PB94-1984 13
Binary versus Decade Inductive Voltage Divider Comparison and Error Decomposition.

PB96-112263

02,07

$\mathrm{DC} \cdot \mathrm{MHz}$ Wattmeter Based on RMS Voltage Measurements. PB97.113211

Digital Impedance Bridge.

PB96-103155 02.272

Inductive Voltage Divider Calibration for the NASA Flight

Experiment.

02,042

Low Voltage Standards in the $10 \mathrm{~Hz}$ to $1 \mathrm{MHz}$ Range.

PB97-112569

02,100

Overview of Bioelectrical Impedance Analyzers

PB96-122635

00,176

Overview of Bioelectrical Impedance Analyzers.

PB97-118780

OLINGER, T. M.

CCD Mosaic Images of the Supernova Remnant 3C 400.2

PB95-203527

00,084

OLINSKI, R.

DNA Base Damage in Lymphocytes of Cancer Patients Undergoing Radiation Therapy.

PB97-122444

03,643

Modification of DNA Bases in Chromatin of Intact Target Human Cells by Activated Human Polymorphonuclear Leukocytes.

03,526

Nickel(II)-Mediated Oxidative DNA Base Damage in Rena and Hepatic Chromatin of Pregnant Rats and Their Fetuses. Possible Relevance to Carcinogenesis.

PB94-212628

03,646

LK, C. H.

$X$-ray Powder Diffraction from Carbon Nanotubes and Nanoparticles

OLSEN, K.

03,064

Impact of the FCC's Open Network Architecture on NS/NP Telecommunications Security.

PB95-189445

01,483

Introduction to the P1003.1g and CPI-C Network Application Programming Interfaces.

PB95.231726

01,731

Security in Open Systems.

OLSEN, P. T.

01,473

Measurement and Reduction of Alignment Errors of the NIST Watt Experimen

PB97-111959

01,987

Methods for Aligning the NIST Watt-Balance.

PB96-123153

01,934

NIST Watt Balance: Progress Toward Monitoring the Kilo-

gram. 113062

OLSON, D. A.

Heat Transfer in Thin, Compact Heat Exchangers with Circular, Rectangular, or Pin-Fin Flow Passages.

PB95-140943

02,751

Thermal Hydraulic Tests of a Liquid Hydrogen Cold Neutron Source.

03,884

OLSON, G. J.

Atmospheric and Marine Trace Chemistry: Interfacia Biomediation and Monitoring.

PB94-199122

03,752

Bioleaching of Cobalt from Smelter Wastes by 'Thiobacillus 
OLTHOFF, J. K.

Appearance Potentials of Ions Produced by Electron-Impact Induced Dissociative Ionization of SF6, SF4, SF5Cl, S2F10, $\mathrm{SO} 2, \mathrm{SO} 2 \mathrm{~F} 2$, SOF2, and SOF4.

01,080

Associative Electron Attachment to S2F10, S2OF10, and $\mathrm{S} 2 \mathrm{O} 2 \mathrm{~F} 10$.

PB95-140992

00,907

Decomposition of Sulfur Hexafluoride by $X$-rays.

01,095

Effect of Electrode Material on Measured Ion Energy Distributions in Radio-Frequency Discharges.

PB96-102850

04,026

Electrical Sensors for Monitoring if Plasma Sheaths.

PB95-162962

04,412

Electron Scattering and Dissociative Attachment by SF6 and Its Electrical-Discharge By-Products.

02,256

Evidence for Inelastic Processes for $\mathrm{N}(+) 3$ and $\mathrm{N}(+) 4$ from Ion Energy Distributions in He/N2 Radio Frequency Glow Ion Energy Distributions in He/N2 Radio Frequency Glow Discharges.
PB96-146683

Fundamental Processes in Gas Discharges.

04,059

PB96-123450

01,089

Gaseous Electronics Conference Radio-Frequency Reference Cell: A Defined Parallel-Plate Radio-Frequency System for Experimental and Theoretical Studies of PlasmaProcessing Discharges.

04,404

Gaseous Electronics Conterence RF Reference Cell: An Introduction.

PB96-113329

02,387

Influence of Electrode Material on Measured Ion Kinetic-Energy Distributions in Radio-Frequency Discharges.

PB96-123179

01,935

Investigation of S2F10 Production and Mitigation in Compressed SF6-Insulated Power Systems.

$\begin{array}{ll}\text { PB94-212388 } & 02,467\end{array}$

Investigation of S2F10 Production and Mitigation in Compressed SF6- Insulated Power Systems.

PB96-155528

02,468

Ion Kinetic-Energy Distributions and Balmer-alpha (Halpha) Excitation in Ar-H2 Radio-Frequency Discharges.

04,029

Ion Kinetic-Energy Distributions in Argon $\mathrm{A}$ Glow Discharges.

04,409

Ion Kinetics and Symmetric Charge-Transfer Collisions in Low-Current, Diffuse (Townsend) Discharges in Argon and Nitrogen

PB96-123658

04,051

Kinetic Energy Distribution of lons Produced from Townsend Discharges in Neon and Argon.

04,413

Kinetic Energy Distributions of $\mathrm{H}(+), \mathrm{H} 2(+)$, and $\mathrm{H}_{3}(+)$ from Kinetic Energy Distributions of $\mathrm{H}(+), \mathrm{H} 2(+)$, and $\mathrm{H} 3(+)$ from
a Diffuse Townsend Discharge in $\mathrm{H} 2$ at High $\mathrm{EN}$.
PB96-123351
04,415

04,415

Kinetic-Energy Distributions of lons Sampled from Argon Plasma

PB95-161964

03,935

Kinetic-Energy Distributions of lons Sampled from RadioFrequency Discharges in Helium, Nitrogen, and Oxygen. PB96-123732 01,092

Kinetic-Energy Distributions of $\mathrm{K}(+)$ in Argon and Neon in Uniform Electric Fields.

03,902

Measurement of S2OF10, and S2O2F10 Production Rates from Spark and Negative Glow Corona Discharge in SF6/ O2 Gas Mixtures.

PB96-123740

01.093

Metrology Requirements of Future Space Power Systems.

PB95-140984 04,840

Optical and Mass Spectrometric Investigations of lons and Neutral Species in SF6 Radio-Frequency Discharges.

PB97-111918

01,985

Procedure for Measuring Trace Quantities of S2F10, S2OF10, and S2O2F10 in SF6 Using a Gas Chromatograph-Mass Spectrometer.

02,513

Studies of Ion Kinetic-Energy Distributions in the Gaseous Electronics Conference RF Reference Cell.

02,391

Time-Resolved Balmer-Alpha Emission from Fast Hydrogen Atoms in Low Pressure, Radio-Frequency Discharges in Atoms in Low Pressure, Radio-Frequency Discharges in
Hydrogen. Hydrogen.

04,028

Use of an Ion Energy Analyzer: Mass Spectrometer to Measure Ion Kinetic-Energy Distributions from RF Discharges in Argon-Helium Gas Mixtures.

PB94-185717

04,406

OLVER, F. W. J.

Numerical Evaluation of Special Functions.

PB96- 119557

03,417

Review of Mathematical Function Library for MicrosottFORTRAN, John Wiley and Sons, 1989.

01,679
OMIDVAR, O. M.

Effect of Training Dynamics on Neural Network PerformPB95-267845

01,852

Improving Neural Network Performance for Character and Fingerprint Classification by Altering Network Dynamics.
PB95-267803

Improving Neural Network Performance for Character and Fingerprint Classification by Altering Network Dynamics. PB96-123195

01,856

OMORI, A.

Effects of Molecular Weight and Thermal Stability on Polymer Gasification.

PB94-212610

OMRON, R. M.

Rotational Spectra of $\mathrm{CH} 3 \mathrm{CCH}-\mathrm{NH} 3, \mathrm{NCCCH}-\mathrm{NH} 3$, and NCCCH-OH2.

\section{ONDOV, J. M.}

Rare-Earth Isotopes as Tracers of Particulate Emissions: An Urban Scale Test.

ONDREJKA, A.

Time-Domain Measurements of the Electromagnetic Backscatter of Pyramidal Absorbers and Metallic Plates.

PB94-185410

ONDREJKA, A. R.

Aperture Excitation of Electrically Large, Lossy Cavities.

PB94-145711 00,029

Aperture Excitation of Electrically Large, Lossy Cavities.

PB95-175675

00,031

Bistatic Scattering of Absorbing Materials from 30 to 1000 $\mathrm{MHz}$.

01,891

Measurements of Shielding Effectiveness and Cavity Characteristics of Airplanes.

00,030

Rapid Evaluation of Mode-Stirred Chambers Using Impulsive Waveforms

PB96-210026

Time-Domain Antenna Characterizations.

PB95-152781

01,979

ONEIL, $\mathbf{S}$.

Bonding in Doubly Charged Diatomics.

PB95-164315

ONO, R. H.

Characterization of a Tunable Thin Film Microwave

YBa2Cu3O7-x/SrTiO3 Coplanar Capacitor.
PB95-175527

02,264

Coexistence of Grains with Differing Orthorhombicity in High Cuality YBa2Cu3O7-delta Thin Films.

04,742

Controlling the Critical Current Density of High-Temperature

SNS Josephson Junctions.
PB96-200712

Critical Current and Normal Resistance of High-Tc StepEdge SNS Junctions.

Critical Current Behavior of Ag-Coated YBa2Cu3O7-x Thin Films.

PB95-141016 04,549

Effect of Thermal Noise on Shapiro Steps in High-Tc Josephson Weak Links.

PB94-212677

04,506

High Critical Temperature Superconductor Tunneling Spectroscopy Using Squeezable Electron Tunneling Junctions.

PB95-163721 04,627

High-Tc Multilayer Step-Edge Josephson Junctions and SQUIDs.

PB96-200183

04,790

High Temperature Superconductor-Normal MetalSuperconductor Josephson Junctions with High Characteristic Voltages.

PB95-176079

04,666

Increased Transition Temperature in In situ Coevaporated YBa2Cu3O7-delta Thin Films by Low Temperature Post-Annealing.
PB95-180071

04,672

Magnetic Field Dependence of the Critical Current Anisotropy in Normal Metal-YBa2Cu3O7-delta Thin Film Bilayers.
PB95-141024

Mutual Phase Locking in Systems of High-Tc Superconductor-Normal Metal-Superconductor Junctions.
PB96-135348

Phase Locking in Two-Junction Systems of High-Temperature Superconductor-Normal Metal-Superconductor Junctions.

PB95-176053

02,060

Stacked Series Arrays of High-Tc Trilayer Josephson Junctions.

Step-Edge and Stacked-Heterostructure High-Tc Josephson Junctions for Voltage-Standard Arrays.

Temperature Dependence and Magnetic Field Modulation of Critical Currents in Step-Edge SNS YBCO/Au Junctions.
PB96-111745

Thermal Noise in High-Temperature Superconducting-Normal-Superconducting Step-Edge Josephson Junctions.

PB95-175089 04,650

Tunable High Temperature Superconductor Microstrip Res-

B95-168423

02,048

Tunneling Measurement of the Zero-Bias Conductance Peak and the Bi-Sr-Ca-Cu-O Thin-Film Energy Gap.

PB95-163739 Superconductor/Semiconductor Integration.

PB94-211711

02,315

Standardization of Testing Methods for Optical Disk Media Characteristics and Related Activities at NiST.

PB95-108486

OP DE BEEK, S. S.

Alignment Probing of Rydberg States by Stimulated Emis-

Sion.

OPANSKY, B. J.

04,124

Kinetics of the Reaction $\mathrm{C} 2 \mathrm{H}+\mathrm{O} 2$ from 193 to $350 \mathrm{~K}$ Using Laser Flash Kinetic Infrared Absorption Spectroscopy.
PB95-203055

Laboratory Studies of Low-Temperature Reactions of $\mathrm{C}_{2} \mathrm{H}$ with $\mathrm{C}_{2} \mathrm{H}_{2}$ and Implications for Atmospheric Models of

PB95-108726

00,690

Laser Double Resonance Measurements of the Quenching Rates of $\mathrm{Br}((2) \mathrm{P} 1 / 2)$ with $\mathrm{H} 2 \mathrm{O}, \mathrm{D} 2 \mathrm{O}, \mathrm{HDO}$, and $\mathrm{O} 2$.

PB95-150694

00,921

OPPERMANN, $\mathrm{H}$.

Metrology and Regional Trade Pacts.

PB96-155429

02,923

PPERMANN, H. V.

NIST Handbook 44, 1994: Specifications, Tolerances and Other Technical Requirements for Weighing and Measuring Devices as Adopted by the 78th National Conference on Weights and Measures 1993

PB94-136009

02,888

NIST Handbook 44, 1995: Specifications, Tolerances and Other Technical Requirements for Weighing and Measuring Devices as Adopted by the 79th National Conference on Weights and Measures 1994

PB95-146379

02,903

OREN, A. L.

Design and Characterization of X-Ray Multilayer Analyzers for the $50-1000$ eV Region.

PB94-211851

03,848

ORKIN, $V$. L.

Atmospheric Litetimes of HFC-143a and HFC-245fa: Flash Photolysis Resonance Fluorescence Measurements of the OH Reaction Rate Constants.

PB97-112577

00,118

ANDINI, $E$.

Self-Avoiding Surfaces, Topology, and Lattice Animals.

PB95-150512

Effects of Anneal Time and Cooling Rate on the Formation and Texture of Bi2Sr2CaCu2O8 Films.

PB95-161600

04,603

Grain Alignment and Transport Properties of Bi2Sr2CaCu2O8 Grown by Laser Heated Float Zone Meth-

PB95-16145

04,602

Increased Pinning Energies and Critical Current Densities in Heavy-lon-Irradiated $\mathrm{B}$ i2Sr2CaCu2O8 Single Crystals.

PB95-175352

04,654 
Water Adsorption at a Polyimide/Silicon Water Intertace. PB96-103197

OSBURN. J. E.

LMTO/CVM and LAPW/CVM Calculations of the Nicke Aluminide/Nickel Titanium Pseudobinary Phase Diagram. PB94-199353

03,323

OSELLA, S. A.

Development of Adaptive Control Strategies for Inert Gas Atomization.

Expert Control System Shell Version 1.0 User's Guide. PB95-198859 01,790 Intelligent Control of an Inert Gas Atomization Process. PB95-141057 03,344 Process Modeling and Control of Inert Gas Atomization. PB95.162343 02,824 OSTADAN, F.

Energy-Based Method for Liquefaction Potential Evaluation. Phase 1. Feasibility Study.

PB96-214747

OSTAPCZUK, P.

Determination of Inorganic Constituents in Marine Mammal Tissues.

PB95-152047

00,589

Development of Frozen Whale Blubber and Liver Reierence Materials for the Measurement of Organic and Inorganic Contaminants.

PB95-151676

00,587

OSTERTAG, $\mathrm{C}$

Tensile Creep of Silicide Composites

STERTAG, C. P.

Coating of Fibers by Colloidal Techniques in Ceramic Com-

posites.

OSTROM, R. M.

Construction of Silicon Nanocolumns with the Scanning Tunneling Microscope.

04.696

Nanoscale Study of the As-Grown Hydrogenated Amorphous Silicon Surface.

phous Silicon

04,573

OTI, J.

Experimental Verification of a Vector Preisach Model.

PB95-163564

04,626

OTI, J. 0.

Experimental Verification of a Micromagnetic Model of Dual-

Layer Magnetic Films.
PB95-141081

Magnetic and Magnetoresistive Properties of Inhomogeneous Magnetic Dual-Layer Films.

Magnetoresistance of Thin-Film NiFe Devices Exhıbiting Single-Domain Behavior.

04,766

PB96-147087

ecording

Micromagn

PB95-141065

04,55

Micromagnetic Simulations of Tunneling Stabilized Magnetic Force Microscopy.

PB95-141073

04,552

Modeling Effects of Temperature Annealing on Giant Magnetoresistive Response in Discontinuous Multilayer $\mathrm{NiFe} / \mathrm{Ag}$ Films.

04,157

Models of Granualr Giant Magnetoresistance Multilayer Thin Film

PB96-190228

01,968

Numerical Micromagnetic Techniques and Their Applications to Magnetic Force Microscopy Calculations.

Proposed Antiferromagnetically Coupled Dual-Layer Mag netic Force Microscope Tips

PB95-169017

04,645

Simulating Device Size Effects on Magnetization Pinning Mechanisms in Spin Valves.

04,158

Size Effects and Giant Magnetoresistance in Unannealed NiFe/Ag Multilayer Stripes.

PB97-111306

Size Effects in Submicron NiFe/Ag GMR Devices.

PB96-155510

OTTEWILL, R. H.

Structure and Rheology of Hard-Sphere Systems. PB96.167333

04,145

02,237

OTTO, R

Hyperfine Structure Investigations and Identification of New Energy Levels in the Ionic Spectrum of (147) Pm.

PB96-180203

04,117

Electronic Access: Blueprint for the National Archives and Records Administration

02,731

User Study: Informational Needs of Remote National Archives and Records Administration Customers.

PB95-154738

02,725
OUELLETTE, M. J.

Performance of Compact Fluorescent Lamps at Different Ambient Temperatures.

PB95-175329

00,258

OUTCALT, S. L.

Coexisting Densities, Vapor Pressures and Critical Densities of Refrigerants R-32 and R-152a, at $300-385 \mathrm{~K}$

PB95-175691 03,274

Fugacity Coefficients of Hydrogen in (Hydrogen + Butane).

02,491

Process Gas Chromatography Detector for Hydrocarbons Based on Catalytic Cracking.

PB95-141099

02,485

YANG, $R$.

Displacement Method for Machine Geometry Calibration PB95-152088

02,946

OVER, P.

Z39.50 Implementation Experiences.

PB96-114939

01,816

OVERETT, T.

Calibration of Dosimeters for the Cryogenic Irradiation of Composite Materials Using an Electron Beam.

PB95-180964

03,968

OVERMAN, J.

National Center for Standards and Certification Information: Service and Programs.

02,717

OVERMAN, J. R.

GATT Standards Code Activities of the National Institute of Standards and Technology 1994.

PB96-106935

00.497

TBT Agreement Activities of the National Institute of Standards and Technology, 1995

OVERZET, L. J.

00,499

Microwave Diagnostic Results from the Gaseous Electronics Conference RF Reference Cell.

PB96-113378

02,392

OWEN, J. C.

Application of the Modified Voltage-Dividing Potentiometer to Overlay Metrology in a CMOS/Bulk Process.

02,302

Comparisons of Measured Linewidths of Sub-Micrometer Lines Using Optical, Electrical, and SEM Metrologies. PB95-152807

02,338

OWEN, J. J.

Determination of Complex Structures from Powder Diffrac. tion Data: The Crystal Structure of La3Ti5Al15O37.

OZERY, N.

01,038

Visual-Motion Fixation Invariant

PB94-20628

01,836

OZIER, I.

Use of Extended Permutation-Inversion Groups in Constructing Hyperfine Hamiltonians for Symmetrical-Top Internal Rotor Molecules Like $\mathrm{H}_{3} \mathrm{C}-\mathrm{SiH} 3$.

PB94-212032

00,823

PAABO, M.

Further Development of the N-Gas Mathematical Model: An Approach for Predicting the Toxic Potency of Complex Combustion Mixtures.

PB96-123260

03,650

New Approach for Reducing the Toxicity of the Combustion Products from Flexible Polyurethane Foam.

PB96-123625

01.411

PAABO, $\mathrm{S}$.

DNA Damage and DNA Sequence Retrieval from Ancient

PB97-111983

03,556

PACE, M. 0.

Observations of Partial Discharges in Hexane Under High Magnification.
PB95-163127

PADMAJA, S.

01,900

Reaction of Nitric Oxide with Organic Peroxyl Radicals

PB95-141107

00,910

Reaction of NO with Superoxide.

PB94-212198

00,830

PBTURE, N.P.

Effect of Grain Size on Hertzian Contact Damage in Alumina.

03,083

Flaw-Insensitive Ceramics.

PB97-110027

03,095

Flaw-Tolerance and Crack-Resistance Properties of Alumina-Aluminum Titanate Composites with Tailored Microstructures.

Microstructures.
PB97-110324

03, 101

Flaw Tolerance and Toughness Curves in Two-Phase Particulate Composites: SiC/Glass System.

PB96-179460

03,081

In situ-Toughened Silicon Carbide.

03,102 PB97.110332

Indentation Fatigue: A Simple Cyclic Hertzian Test for
Measuring Damage Accumulation in Polycrystalline Ceramics.

PB96-180013

03,084

Model for Microcrack Initiation and Propagation beneath Hertzian Contacts in Polycrystalline Ceramics.

PB96-163704

03,077

Model for Toughness Curves in Two-Phase Ceramics. 1

Basic Fracture Mechanics.

Model for Toughness Curves in Two-Phase Ceramics. 2

Microstructural Variables.

PB96-163795

03,078

Postfailure Subsidiary Cracking from Indentation Flaws in

Brittle Materials

PAGANO, I.

First Results from a Coordinated ROSAT, IUE, and VLA Study of RS CVn Systems.

PB94.213477

00,069

Rotational Modulation and Flares on RS Canum Venaticorum and BY Draconis Stars. XVIII. Coordinated VLA, ROSAT, and IUE Observations of RS CVn Binaries.
PB96-102322

\section{PAGE, R. A}

Characterization of the Densification of Alumina by Multiple Small-Angle Neutron Scattering. (Reannouncement with New Availability Information)

03,024

Effect of Green Density and the Role of Magnesium Oxide Additive on the Densification of Alumina Measured by Small-Angle Neutron Scattering. (Reannouncement with New Availability Information)

AD-A244 582/3

03.022

Small-Angle Neutron Scattering Characterization of Processing/Microstructure Relationships in the Sintering of Crys talline and Glassy Ceramics. (Reannouncement with New Availability Information

AD-A249 510/9

03,025

PAGE, S. H.

Instrument for Evaluating Phase Behavior of Mixtures for Supercritical Fluid Experiments.

PB95-180758

00,606

Selectivity Trends in Packed Column Supercritical Fluid Chromatography with $\mathrm{C} 18$ Stationary Phases.

PB96-138581

00,622

PAGEL, C.

Overview of U.S. Govemment Advanced Packaging Pro-

grams.

02,443

PAGNI, P. J.

Fire Induced Thermal Fields in Window Glass I: Theory

PB94-139722

00.328

AINTER, R. B.

Modification of Deoxyribose-Phosphate Residues by Ex tracts of Ataxia Telangiectasia Cells.

PB94-212602

03,458

PAL, B. P.

LP11-Mode Leakage Loss in Coated Depressed Clad Fi- 
PAN, L.

Perturbative Calculation of the AC Stark Effect by the Com. plex Rotation Method.

PB95-141123

04,260

PANDEY, P. C.

Amperometric Flow-Injection Analysis Biosensor for Glu$\cos \theta$ Based on Graphite Paste Modified with Tetracyanoquinodimethane.

PB95-161980

03,498

Application of Photochemical Reaction in Electrochemical Detection of DNA Intercalation.

00,686

Detection of Aromatic Compounds Based on DNA Intercalation Using an Evanescent Wave Biosensor.

PB96-111976

03,473

Thermodynamics of the Hydrolysis of N-Acetyl-Lphenylalanine Ethyl Ester in Water and in Organic Solvents. PB95-203386

01,053

PANDIT, A.

Two Phase Aqueous Extraction: Rheological Properties of Dextran, Polyethylene Glycol, Bovine Serum Albumin and Their Mixtures.

PB95-161998

00,676

PAO, C. K.

Electrical Measurements of Microwave Flip-Chip Interconnections.

02,436

Microwave Characterization of Flip-Chip MMIC Components.
PB96-176722

PB96-176722
Microwave Characterization of Flip-Chip MMIC Interconnec-

PB96-176730

02,435

PAPANEK, $P$.

Inelastic-Neutron-Scattering Studies of Poly(p-phenylene vinylene).

PB95-180766

01,014

PAPANYAN, V. $O$.

Photoluminescence Spectra of Epitaxial Single Crystal C60. PB96-141205

04,754

PAPILLO, $M$.

Report on the Workshop on Advanced Digital Video in the National Information Infrastructure. Held in Washington, D.C. On May 10-11, 1994.

PB95-103677

01,472

Summary Report on the Workshop on Advanced Digital Video in the National Information Infrastructure.

01,497

PARAMESWARAN, $M$.

High-Level CAD Melds Micromachined Devices with Foundries.

02,321

PARANTHAMAN, M.

Asymmetry between Flux Penetration and Flux Expulsion in Tl-2212 Superconductors.

04,527

Enhanced Flux Pinning via Chemical Substitution in Bulk

Superconducting T1-2212.

PB95-169033

04,647

PARDAVI-HORVATH, $M$.

Experimental Verification of a Vector Preisach Model.

PB95-163564

04,626

PARETZKIN, B.

Crystal Chemistry and Phase Equilibria Studies of the $\mathrm{BaO}(\mathrm{BaCO}$ )-R2O3-CuO Systems. 4. Crystal Chemistry and Subsolidus Phase Relationship Studies of the CuORich Region of the Ternary Diagrams, R=Lanthanides.

PB95-151759

00,936

Crystal Chemistry and Phase Equilibrium Studies of the $\mathrm{BaO}(\mathrm{BaCO} 3)-\mathrm{R} 2 \mathrm{O}$ 3.CuO Systems. 5. Melting Relations in $\mathrm{Ba} 2(\mathrm{Y}, \mathrm{Nd}, \mathrm{Eu}) \mathrm{Cu} 3 \mathrm{O} 6+\mathrm{x}$

04,580

Crystal Chemistry and Phase Equilibrium Studies of the $\mathrm{BaO}-\mathrm{R} 2 \mathrm{O} 3-\mathrm{CuO}$ Systems. 2. X-Ray Characterization and Standard Patterns of BaR2CuO4, R=Lanthanides.

PB95-151734

04,582

PARISE, J. B.

Ca4Bi6013: A Compound Containing an Unusually Low Bismuth Coordination Number and Short Bi Bi Contacts. PB95-141131

PARK, C.

Fault Diagnosis of an Air-Handling Unit Using Artificial Neural Networks

00,283

Reproducibility of Tests on Energy Management and Control Systems Using Building Emulators.

00,260

Using Emulator/Testers for Commissioning EMCS Software, Operator Training, Algorithm Development, and Tuning Local Control Loops.

PB94-212735

00.245

Using Emulators to Evaluate the Performance of Building Energy Management Systems.

00,259

PARK, G. S.

Noise Reduction in Low-Frequency SOUID Measurements with Laser-Driven Switching

02,081
Trapped Vortices in a Superconducting Microbridge. PB95-141149

04,554

PARK, J. C.

Defect Formation Mechanism Causing Increasing Defect Density during Decreasing Implant Dose in Low-Dose

B96-119524

02,402

Defect of Thermal Ramping and Annealing Conditions on Defect Formation in Oxygen Implanted Silicon-On-Insulator Materia

PB94-212966

02,318

Defect Pair Formation by Implantation-Induced Stresses in High-Dose Oxygen Implanted Silicon-on-Insulator Material. PB95-175824

02,364

Effect of Intermediate Thermal Processing on Microstructural Changes of Oxygen Implanted Silicon-on-In sulator Material.

02,982

Effect of Single versus Multiple Implant Processing on Defect Types and Densities in SIMOX

01,957

Stacking Fautt Pyramid Formation and Energetics in Siliconon-Insulator Material Formed by Multiple Cycles of Oxygen Implantation and Annealing.

Implantation and

PARK, S. G.

Self-Biasing Cryogenic Particle Detector Utilizing

Electrothermal Feedback and a SOUID Readout

PB96-102538

PARKER, A. J.

Characterization of Polyquinoline Blends Using Small Angle Scattering

PB95-164125

01,261

Characterization of Polyquinoline Block Copolymer Using Small Angle Scattering

PB95-151882

01,244

PARKER, F.

Precision of Marchall Stability and Flow Test Using 6-in. (152.4-mm) Diameter Specimens

\section{PARKER, $K$.}

New Method for Achieving Accurate Thickness Control for Uniform and Graded Multilayer Coatings on Large Flat SubStrates.

PB96-159744

04,366

\section{PARKER, $M$. A}

Antiferromagnetic Interlayer Correlations in Annealed Ni80Fe20/Ag Multilayers.

PB97-122220

03,109

Magnetic Structure Determination for Annealed Ni80Fe20 Ag Multilayers Using Polarized-Neutron Reflectivity. PB96-176615

03,739

PARKER, M. E.

Exploring the Low-Frequency Performance of Thermal Con verters Using Circuit Models and a Digitally Synthesized Source.

PB97-112551

Low Voltage Standards in the $10 \mathrm{~Hz}$ to $1 \mathrm{MHz}$ Range

PB97-112569

02,100

ARKER, M. R

Size and Self-Field Effects in Giant Magnetoresistive ThinFilm Devices.

PB95-180188

PARKER, T. E.

Surface Transverse Wave Oscillators with Extremely Low Thermal Noise Floors.

PB96-186010

01,967

PARKER, V. $B$.

Thermodynamic Properties of the Aqueous Ba(sup 2+) Ion and the Key Compounds of Barlum

01,107

Thermodynamic Properties of the Aqueous lons $(2+$ and Thermodynamic Properties of the Aqueous lons $(2+$ and
$3+$ ) of Iron and the Key Compounds of Iron.
PB96-145958

PARKER, W. E.

Intermediate Structure in the Neutron-Induced Fission Cross Section of $236 \mathrm{U}$

PB94-185741

Measurements of the $(237) \mathrm{Np}(n, f)$ Cross Section.

PB97-119069

03,802

PARKER, W. J.

Effects of Specimen Edge Conditions on Heat Release PB95-152864

00,375

PARKS, C.

Operating Procedures and Life Cycle Documentation for the Initial Graphics Exchange Specification.

PB95-242285

02,782

PARKS, E. J.

Bioleaching of Cobalt from Smelter Wastes by 'Thiobacillus terrooxidans'.

PARKS, J. E.

02,582

Anomalous Odd- to Even-Mass Isotope Ratios in Resonance lonization with Broad-Band Lasers.

03,839

PARKS, N. J.

Experimental Validation of Radiopharmaceutical Absorbed Dose to Mineralized Bone Tissue.

PB94-199668

03,617

Radiation Doses.

PB94-19967

03,618

Rapid Post-Polishing of Diamond-Turned Optics.

PB95-175949 $\quad 04,301$

Test of a Slow OH-Axis Parabola at Its Center of Curvature. PB96-138482 04,352 Visualization of Surface Figure by the Use of Zernike Poly-

6-137757

04,351

PARMENTER, C. $S$.

Acceleration of Intramolecular Vibrational Redistribution by methyl Internal Rotation. A Chemical Timing Study of $p$ fluorotoluene and p-fluorotoluene-d3.

01,039

Vibrational Energy Transfer in S1 p-Difluorobenzene. A Comparison of Low and Room Temperature Collisions. PB95-108619

00,883

Vibrations of S1((1)B2u) p-Difluorobenzene - d4. S1-So Fluorescence Spectroscopy and ab Initio Calculations.

PB95-202586

01,028

PARNAS, R. S.

Comparison of the Unidirectional and Radial In-Plane Flow of Fluids Through Woven Composite Reinforcements.

PB95-162004 02,698

Effect of Heterogeneous Porous Media on Mold Filling in Resin Transfer Molding

PB95-108676

03, 197

Effect of Hydrodynamic Interactions on a Terminally Anchored Bead-Rod Model Chain.

Interaction between Micro and Macroscopic Flow in RTM Preforms.

PB95-162012

03,159

Report on the Workshop on Manufacturing Polymer Composites by Liquid Molding. Held in Gaithersburg, Maryland on September 20-22, 1993

03,131

Response of a Terminally Anchored Polymer Chain to Simple Shear Flow
PB95-108668

01,233

PARR A.

Rayleigh Scattering Limits for Low-Level Bidirectional Reflectance Distribution Function Measurements.

PB95-180030

04,307

PARR, A. C.

Cryogenic Blackbody Calibrations at the National Institute of Standards and Technology Low Background Infrared Calibration Facility.

PB94-169802

02,117

High Resolution Angle Resolved Photoelectron Spectroscopy Study of N2.
PB95-151494

03,907

Inner-Valence States $\mathrm{CO}(+)$ between $22 \mathrm{eV}$ and $46 \mathrm{eV}$ Studied by High Resolution Photoelectron Spectroscopy and ab Initio $\mathrm{Cl}$ Calculations. 
PARRIS, $N$.

Structure of a Triglyceride Microemulsion: A Small Angle Neutron Scattering Study.

PB96-11225

01,077

Comparison of Methods for Gas Chromatographic Determination of PCBs and Chlorinated Pesticides in Marine Reterence Materials.

PB95.140091

PARRIS, R. $M$.

NIST Standard Reference Materials (SRMs) for Poly chlorinated Biphenyl (PCB) Determinations and Their Applicability to Toxaphene Measurements.

PB95-140109

02,585

Standard Reference Materials for the Determination of Polycyclic Aromatic Hydrocarbons in Environmental Samples - Current Activities.

00,586

PARRY, E. E.

Dental Materials.

$\begin{array}{ll}\text { PB94-172871 } & 00,142\end{array}$

Tapered Cross-Pin Attachments for Fixed Bridges.
PB94-185238

00,142

00,185

PARSONS, C. A.

Characterization of Vertical-Cavity Semiconductor Struc-

$\begin{array}{ll}\text { tures. } & 02,126\end{array}$

Vertical-Cavity Optoelectronic Structures: CAD, Growth, and Structural Characterization.

PB95-153177 02,148

Vertical-Cavity Semiconductor Lasers: Structural Characterization, CAD, and DFB Structures
PB95-153193

02,150

PASHLEY, D. H.

Effects of Aluminum Oxalate/Glycine Pretreatment Solutions on Dentin Permeability.

03,565

Effects of Calcium Phosphate Solutions on Dentin Permeability.

00,157

PASSOW, M.

Gaseous Electronics Conference Radio-Frequency Reference Cell: A Defined Parallel-Plate Radio-Frequency System for Experimental and Theoretical Studies of PlasmaProcessing Discharges.

PB94-172327

04,404

PASTEL, R. L.

Efficient $\mathrm{Br}\left({ }^{*}\right)$ Laser Pumped by Frequency-Doubled $\mathrm{Nd}$ YAG and Electronic-to-Vibrational Transfer-Pumped $\mathrm{CO}$ and HCN Lase

PASTUREL, A

04,248

LMTO/CVM and LAPW/CVM Calculations of the Nickel Aluminide/Nickel Titanium Pseudobinary Phase Diagram. PB94-199353

03,323

PATE, B. $\mathrm{H}$.

Sub-Doppler, Intrared Laser Spectroscopy of the Propyne Intramolecular State Mixing in the Acetylenic $\mathrm{CH}$ Stretch Overtone.

PB95-202941

01,037

PATIL, T. A.

Continuous Counter-Current Two Phase Aqueous ExtracPB95-161212

00,675

PATRICK, $\mathrm{H}$.

Annealing of Bragg Gratings in Hydrogen-Loaded Optical Fiber.

$\begin{array}{ll}\text { PB96-155437 04,361 } & \end{array}$

Bragg Gratings in Optical Fibers Produced by a Continuous-Wave Ultraviolet Source.

PB95-162020

04,274

Comparison of UV-Induced Fluorescence and Bragg Grating Growth in Optical Fiber.

04,284

Comparison of UV Photosensitivity and Fluorescence during Fiber Grating Formation.

04,362

Decay of Bragg Gratings in Hydrogen-Loaded Optical FiPB96-122643

04,345

Decrease of Fluorescence in Optical Fiber during Exposure to Pulsed or Continuous-Wave Ultraviolet Light. 04,320

Electronically Tunable Fiber Laser for Optical Pumping of (3) He and (4) He.

PB96-201165

04,381

Growth Characteristics of Fiber Grating

04,346

Growth of Bragg Gratings Produced by Continuous-Wave Ultraviolet Light in Optical Fiber.

PB95-162038

04,275

PATTEN, K. O.

Environmental Aspects of Halon Replacements: Considerations for Advanced Agents and the Ozone Depletion Potential of CF3I.

03,301
PAUL, A. E.

Time-Resolved Measurements of the Polarization State of Four-Wave Mixing Signals from GaAs Multiple Quantum

PB96-201058

04,796

PAUL, E.

Chemical Aspects of Tool Wear in Single Point Diamond Turning.

03,021

PAUL, R. L.

Analytical Applications of Guided Neutron Beams.

PB96-1 12347

04,041

Cold Neutron Prompt Gamma Activation Analysis at NIST: A Progress Report

PB95-175964

00,602

Determination of Hydrogen in Titanium Alloy Jet Engine Compressor Blades by Cold Neutron Capture Prompt Gamma-ray Activation Analysis.

01,448

PB95-175956

Grazing Incidence Prompt Gamma Emissions and Reso-
nance-Enhanced Neutron Standing Waves in a Thin-Film. nance-Enhanced Neutron Standing Waves in a Thin-Film.
PB95-150470

Inelastic Neutron Scattering Studies of Rotational Excitations and the Orientational Potential in C60 and A3C60 Compounds. PB94- $\uparrow 72673$

Measuring Hydrogen by Cold-Neutron Prompt-Gamma Activation Analysis
PB96-111877

00,612

Neutron Scattering by Hydrogen in Cold Neutron Prompt Gamma-Activation Analysis.

$95-175972$

00,603

Use of Neutron Beams for Chemical Analysis at NIST

PB97-112437

00,652

PAUL, $W$.

Use of Pressure for Ouantum-Well Band-Structure Characterization.

PB96-164058

04,779

PAULDRACH, A. W. A

Radiation-Driven Winds of Hot Luminous Stars $X$. The Determination of Stellar Masses Radii and Distances from Terminal Velocities and Mass-Loss Rates.

PB94-213022

00,060

PAULE, $\mathbf{R}$.

Frozen Human Serum Reference Material for Standardiza. tion of Sodium and Potassium Measurements in Serum or Plasma by Ion-Selective Electrode Analyzers.

PB94-185337

00,532

PAULE, R. C.

Ascorbic and Dehydroascorbic Acids Measured in Plasma Preserved with Dithiothreitol or Metaphorphoric Acid.

PB94-216330 03,495

Assessing the Credibility of the Calorific Content of

Muncipal Solid Waste

02,581

Certification of Morphine and Codeine in a Human Urine Standard Reference Material.

PB95-176160

03,499

Determination of 3-Ouinuclidinyl Benzilate (Onb) and Its Major Metabolites in Urine by Isotope Dilution Gas Chromatography Mass Spectrometry.

PB94-199379

03,492

Development of a Standard Reference Material for ISE Measurements of Sodium and Potassium.

03,507

Molar Absorptivities of Bitirubin (NIST SAM 916A) and Its Neutral and Alkaline Azopigments.

PB94-211042

03,456

PAULSEN, P. J.

Determination of Sulfur in Fossil Fuels by Isotope Dilution Thermal Ionization Mass Spectrometry

PB96-141379

02,495

Rare-Earth Isotopes as Tracers of Particulate Emissions: An Urban Scale Test.

PB94-161635

02,535

PAULTER, N. G.

Casual Regularizing Deconvolution Filter for Optimal Waveform Reconstruction.

PB95-203089

01,603

Electro-Optic-Based RMS Voltage Measurement Technique. PB96- 138490

02,194

PAUWELS, J.

Preparation and Characterization of (6) LiF and (10)B Reference Deposits for the Measurement of the Neutron Life-

PB95-108692

03,874

Problems Related to the Determination of Mass Densities of Evaporated Reference Deposits.

PB95-163226

03,941

AVONE, F. S.

Rotational Far Infrared Spectrum of (13) CO

PB95-152187

00,941

PAWLAK, C. G.

Survey of Standards for the U.S. Fiber/Textile/Apparel Industry.
PB96-193792

03,199

PAYNE, B. F.

Vibration Laboratory Automation at NIST with Personal Computers.

PEACOCK, R. D.

Fire Satety of Passenger Trains: A Review of Current ApFroaches and of New Concepts.

PB94-152006

04,848

New Concepts for Fire Protection of Passenger Rail Trans. portation Vehicles.

PB95-162046

04,850

New Concepts for Fire Protection of Passenger Rail Transortation Vehicles. (NIST Reprint).

04,863

Standardization of Formats and Presentation of Fire Data The FDMS

PB94-198462

01,371

Verification of a Model of Fire and Smoke Transpont

PB95-108718

00,357

PEARSON, J.

Resonance Enhanced Multiphoton Ionization Spectroscopy

PB97-111223

01,176

PEARSON, J. C.

Millimeter- and Submillimeter-Wave Spectrum of trans-Ethyl Alcohol.

PB96-145578

01,102

PECHENIK, A.

Densification of Nano-Size Powders. 1994 Report

DE94013486

03,027

Equipment for Investigation of Cryogenic Compaction of Nanosize Silicon Nitride Powders. 1993 Report.

E94013593

03,028

Fabrication of Transparent gamma-Al2O3 from Nanosize

Particles.

03,054

Rapid Hot Pressing of Ultra-Fine PSZ Powders.

PB94-216587

03,045

PECK, W. F.

Observation of Oscillatory Magnetic Order in the Antiferromagn

04,679

PECKER, $C$.

Papers on the Symposium on Collision Phenomena in Astrophysics, Geophysics, and Masers.

PECKERAR, $M$. C.

Interaction of Stoichiometry, Mechanical Stress, and Interface Trap Density in LPCVD Si-rich SiNx-Si Structures. PB95-176301

02,366

PEDERSEN, J. O. P.

Kinetics of the Reaction $\mathrm{C} 2 \mathrm{H}+\mathrm{O} 2$ from 193 to $350 \mathrm{~K}$ Using Laser Flash Kinetic Infrared Absorption Spectroscopy.

01,043

Laboratory Studies of Low-Temperature Reactions of $\mathrm{C} 2 \mathrm{H}$ with $\mathrm{C} 2 \mathrm{H} 2$ and Implications for Atmospheric Models of

PB95-108726

00,690

PEDERSEN, J. S. 
Windows NT, Version 1.0. Intel Deskside Server with Inte $80486 \mathrm{D} \times 266=>$ Intel Deskside Server with Intel $80486 \mathrm{D} \times 266$

AD-A288 $571 / 3$

01,658

PEIFFER, D. G.

Compatibilization of Polymer Blends by Complexation. 2. Kinetics of Interfacial Mixing.

01,295

PB97-111900

Small-Angle Neutron Scattering (SANS) Study of Worm-
Like Micelles Under Shear.
PB96-176698
P PB96-176698

04.171

New Values for Silicon Reference Materials, Certified for Isotope Abundance Ratios. Letter to the Editor.

00,863

Reference Materials by Isotope Dilution Mass Spectrometry. PB95-153383 00,592

PEITSMAN, H. C.

Reproducibility of Tests on Energy Management and Control Systems Using Building Emulators.

PB95-175980

00,260

PELLA, P. A.

Addition of $\mathrm{M}$ and L-Series Lines to NIST Algorithm for Calculation of X-Ray Tube Output Spectral Distributions.

PB95-108742

00,569

Apparent Bias in the X-Ray Fluorescence Determination of Titanium in Selected NIST SRM Low Alloy Steels.

PB95-108759

03,212

Secondary Target $X$-Ray Excitation for In vivo Measurement of Lead in Bone.

PB95-108767

03,496

\section{PELLEGRINO, J.}

$\mathrm{CO} 2 / \mathrm{CH} 4$ Transport in Polyperfluorosulfonate lonomers: Effects of Polar Solvents on Permeation and Solubility.

PB96-163803

01,145

Determination of Total Protein Adsorbed on Solid (Membrane) Surface by a Hydrolysis Technique: Single Protein Adsorption.

PB96-167093

03,552

Gas Transport Properties of Solution-Cast Perfluorosulfonic Acid lonomer Films Containing lonic Surfactants.

PB95-175998

01,267

Interface Sharpness in Low-Order III-V Superlattices.

PB95-108775

02,138

PELLEGRINO, J. C. Mesoscopic Conductance Fluctuations in Large Devices.
PB96-119656

04,735

PELLEGRINO, J. G.

Buffer Layer Modulation-Doped Field-Etfect-Transistor Interactions in the Al0.33Ga0.67As/GaAs Superlattice System. PB96-102876 Characterization of Vertical-Cavity Semiconductor Structures.

02,126

Comparative Photoluminescence Measurement and Simulation of Vertical-Cavity Semiconductor Laser Structures.

PB95-169173

02, 169

Correlation of Optical, X-ray, and Electron Microscopy Measurements of Semiconductor Multilayer Structures.

PB95-175279

02,174

Determination of the Complex Refractive Index of Individual Quantum Wells from Distributed Reflectance.

PB95-175642

02,176

Double-Modulation and Selective Excitation Photoreflectance for Wafer-Level Characterization of Quantum-Well Laser Structures.

04,372

High-Accuracy Principal-Angle Scanning Spectroscopic $\begin{array}{lr}\text { Ellipsometry of Semiconductor Interfaces. } \\ \text { PB96-163787 } & 02,427\end{array}$ Interface Roughness, Composition, and Alloying of LowOrder AIAs/GaAs Superlattices Studies by X-ray Diffraction. Order AlAs/GaAs Superlattices Studies by X-ray Diffraction.
PB96-160262 Interface Roughness-Induced Changes in the Near-E(sub 0 ) Spectroscopic Behavior of Short-Period AIAs/GaAs Superlattices.

PB94-185154

02,118

Interface Roughness of Short-Period AlAs/GaAs Superlattices Studied by Spectroscopic Ellipsometry.
02,137 02,137
Intertace Sharpness during the Initial Stages of Growth of Thin, Short-Period III-V Superlattices.

PB95-108783

02,139

Novel Magnetic Field Characterization Techniques for Compound Semiconductor Materials and Devices.
PB96-176458
02,433

02,433

Quantum Cond

PB97-111264

04,144

Scaling of the Nonlinear Optical Cross Sections of GaAs AlGaAs Multiple Quantum-Well Hetero $n-j-p-i$ 's.

PB96-102793

02,183

Spectroscopic Ellipsometry Determination of the Properties of the Thin Underlying Strained Si Layer and the Roughness at SiO2/Si Intertace.

04,560 Time-Resolved Measurements of the Polarization State of
Four-Wave Mixing Signals from GaAs Multiple Quantum Wells. Vertical-Cavity Optoelectronic Structures: CAD, Growth, and Structural Characterization.

PB95-153177

02,148

Vertical-Cavity Semiconductor Lasers: Structural Characterization, CAD, and DFB Structures.

02,150

Vertical-Cavity Semiconductor Structures: Materials Characterization.

PB95-153185

02,149

$X$-ray Reflectivity Determination of Interface Roughness Correlated with Transport Properties of (AlGa)As/GaAs High Electron Mobility Transistor Devices.

PB97-111868

04,149

PELLEGRINo, J. J.

Characterization of the Adsorption-Fouling Layer Using

Globular Proteins on Ultrafiltration Membranes.

Determination of Osmotic Pressure and Fouling Resistances and Their Effects on Performance of Ultrafiltration Membranes.

00,841

Low Temperature H(sub 2)S Separation Using Membrane Reactor with Redox Catalyst.

02,471

Membrane Gas Separation for a Fluidized-Bed Incinerator. PB95-169041

02,550

PELLEU, G. B.

Wear of Human Enamel against a Commercial Castable Ceramic Restorative Material.

PB95-16197

00,161

PENDER, J.

Gaseous Electronics Conference Radio-Frequency Reference Cell: A Defined Parallel-Plate Radio-Frequency System for Experimental and Theoretical Studies of PlasmaProcessing Discharges.

04,404

PENDER, J. T. P.

Reactive Ion Etching in the Gaseous Electronics Conference RF Reference Cell.

PB96-113402

02,395

PENFOLD, J.

Variation in Magnetic Properties of Cu/Fcc (001) Sandwich Structures.

PB95-141164

04,555

PENG, J. L.

Field Dependence of the Magnetic Ordering of $\mathrm{Cu}$ in PB95-164521

04,633

Magnetic Structure and Spin Dynamics of the $\mathrm{Pr}$ and $\mathrm{Cu}$ in $\mathrm{Pr} 2 \mathrm{CuO} 4$.

PB96-111836

04,036

Observation of Noncollinear Magnetic Structure for the $\mathrm{Cu}$ Spins in Nd2CuO4-Type Systems.

PB95-164539 04,634

Phonon Density of States in $\mathrm{R} 2 \mathrm{CuO} 4$ and Superconducting R1.85Ce0.15CuO4 (R = Nd, Pr)

PENN, D. R.

04,574

Anharmonic Phonons and the Isotope Effect in Superconductivity.
PB94-200557

04,486

Calculations of Electron Inelastic Mean Free Paths (IMFPs). 4. Evaluation of Calculated IMFPs and of the Predictive IMFP Formula TPP-2 for Electron Energies between 50 and 2000 eV

PB95-150728

00,922

Calculations of Electron Inelastic Mean Free Paths. 5. Data for 14 Organic Compounds over the 50-2000 eV Range.
PB95-150355

0,916

Effects of Interfacial Roughnes

PB95-150017

04,556

Electronic Correlations and Satellites in Superconducting

Oxides.

04,477

Experimental Constraints on Some Mechanisms for HighTemperature Superconductivity.

PB94-198553

04,463

Total-Dielectric-Function Approach to Electron and Phonon Response in Solids.

PB96-102884

01,067

Use of Sum Rules on the Energy-Loss Function for the Evaluation of Experimental Optical Data.

PB95-150736

04,264

PENN, S. $M$

Laser-Induced Fluorescence Measurements of Rotationally Resolved Velocity Distributions for $\mathrm{CO}(+)$ Drifted in $\mathrm{He}$. PB94-213139

00,848

PENNER, J. E.

Global Climatic Effects of Aerosols: The AAAR Symposium. PB95-108791
PENNER, $\mathbf{S}$.

00,122

Hybrid Undulator for the NIST-NRL Free-Electron Laser.

PB94-212529

NIST-NRL Free-Electron Laser Facility.

04,237

PENONCELLO, S. G.

Static Dielectric Constant of Water and Steam.

PB96-123559

PENUMATCHU, D.

01,090

New Method for the Detection and Measurement of Polyaromatic Carcinogens and Related Compounds by DNA Intercalation.

PB96-167382

PENZES, W. $B$.

03,481

Electrical Test Structure for Overlay Metrology Referenced to Absolute Length Standards.

PB95-152278

02,336

Evolution of Automatic Line Scale Measurement at the $\mathrm{Na}$ tional Institute of Standards and Technology.

PB95-108809

02,897

Metrology Standards for Advanced Semiconductor Lithography Referenced to Atomic Spacings and Geometry.
PB96-160718

New Test Structure for Nanometer-Level Overlay and Feaure-Placement Metrology.

PB95-175345

02,363

Test Structures for the In-Plane Locations of Projected Fea ures with Nanometer-Level Accuracy Traceable to a Coordinate Measurement System.

PB94-200565

PEPPIN, R. J.

National Voluntary Laboratory Accreditation Program Acoustical Testing Services.

PB95-182234

04,188

PERAHIA, D.

Neutron Reflectivity of End-Grafted Polymers: Concentration and Solvent Quality Dependence in Equilibrium Conditions. PB94-185758

PERCIVAL, D. B.

Wavelet Analysis for Synchronization and Timekeeping.

WB96-200381

PB96-190111

01,509

PEREIRA, N. R.

Electrical Breakdown in Transformer Oil in Large Gaps.

PB95-150579

PERERA, $\mathbf{S}$.

Broadband Mismatch Error in Noise Measurement Systems. PB95-162061

PEREZ, J. $M$.

Deposit Forming Tendencies of Diesel Engine Oils-Correlaion between the Two-Peak Method and Engine Tests.

PB95-152138

New Method to Evaluate Deposit Forming Te Liquid Lubricants by Differential Scanning Calorimetry.

PERKINS, R. A

Measurement of the Thermal Properties of Electrically Conducting Fluids Using Coated Transient Hot Wires.

(17816

00,722 


\section{PERSONAL AUTHOR INDEX}

PHELPS, J. M.

PERSILY, A.

Ventilation Rates in Office Buildings.

PB95-108825

PERSILY, A. K

Air Change Effectiveness Measurements in Two Modern Qffice Buildings.

Application of a Multizone Airflow and Contaminant Dispersal Model to Indoor Air Quality Control in Residential BuildPBg5-180238

02,555

Assessing Ventilation Effectiveness in Mechanically Ventilated Qffice Buildings.

PB95-162079

00,255

Carbon Monoxide Dispersion in Residential Buildings: Literature Review and Technical Analysis.

02,571

Computer Simulations of Airflow and Radon Transport in Four Large Buildings.

02,557

PB95-220422

CQNTAM88 Building Input Files for Multi-Zone Airflow and
Contaminant Dispersal Modeling.
PB94-194388
02,537

PB94-194388

Development and Application of an Indoor Air Quality Com-
missioning Program in a New Qffice Building.

missioning Program in a New Qffice Building.
PB96-155601

Effectiveness of a Heat Recovery Ventilator, an Qutdoor Air

Intake Damper and an Electrostatic Particulate Filter at

PB96-146642

02,564

Environmental Evaluation of a New Federal Qffice Building.
PB94-199874

Field Measurements of Ventilation and Ventilation Effective-

ness in an Office/Library Building.

PB95-108833

00,247

Improving the Evaluation of Building Ventilation.

00,271

Indoor Air Quality Commissioning of a New Qffice Building.
PB95-182309

Indoor Air Quality Commissioning of a New Qffice Building.
PB97-110142

Indoor Air Quality Impacts of Residential HVAC Systems

Phase II.B Report: IAQ Control Retrofit Simulations and

Analysis.
PB96-106877

02,559

Indoor Air Quality Impacts of Residential HVAC Systems,

Phase 1 Report: Computer Simulation Plan.

00,249

Indoor Air Quality Impacts of Residential HVAC Systems.

Phase 2.A Report: Baseline and Preliminary Simulations.

PB95-178893

02,554

Issues in the Field Measurement of VQC Emission Rates.

PB97-118806 02,573

Limits of $\mathrm{CQ} 2$ Monitoring in Determining Ventilation Rates.

PB95-176004

02,552

Manual for Ventilation Assessment in Mechanically Ventilated Commercial Buildings.

00,239

Materials-Science Based Approach to Phenol Emissions from a Flooring Material in an Office Building.

Measurements of Indoor Pollutant Emissions from EPA Phase II Wood Stoves.

EPA

PB95-198735

Build-

Meas

PB95-210944

00,264

Modeling Radon Transport in Multistory Residential Buildings.

00,256

Multizone Modeling of Three Residential Indoor Air Quality Control Qptions

02,565

Multizone Modeling of Three Residential Indoor Air Quality

Control Options.

PB96-165782

02,567

Relationship between Indoor Air Quality and Carbon Diox-

PB97-111249

02,569

Study of Ventilation and Carbon Dioxide in an Office Build-

ing.

02,542

Study of Ventilation Measurement in an Qffice Building.

Ventilation, Carbon Dioxide and ASHRAE Standard 62-

PB95-162095

02,544

Ventilation Effectiveness Measurements in Two Modern Of-

fice Buildings.

02,553

Workplan to Analyze the Energy Impacts of Envelope

Airtightness in Qffice Buildings.

PB96-154463

00,273

Few Caveats on Carbon Dioxide Monitoring

02,562

PB96-122650
Controlling Indoor Air Quality in Residential Buildings.

PERSON, D. C

Magnetic Properties of $\mathrm{Pd} / \mathrm{Co}$ Multilayers.

PB94-19875

PESHKIN, D.

Optimization of Highway Concrete Technology.

PB94-182995

PETERS, C. J.

Principle of Congruence and Its Application to Compressible States.

PB96-102892

PETERSEN, S. R.

BLCC: The NIST 'Building Life-Cycle Cost' Program, Version 4.21. User's Guide and Reference Manual.

PB95-190682

00,263

EMISS: A Program for Estimating Local Air Pollution Emission Factors Related to Energy Use in Buildings: User's Guide and Reference Manual.

PB96-109566

02,560 Energy Price Indices and Discount Factors for Life-Cycle
Cost Analysis 1995. Annual Supplement to NIST Handbook 135 and NBS Special Publication 709. (Revised)

PB95-105011

02,509

Energy Price Indices and Discount Factors for Life-Cycle Cost Analysis 1996. Annual Supplement to NIST Handbook 135 and NBS Special Publication 709. (Revised).

PB96-162441

02,510

Energy Price Indices and Discount Factors for Life-Cycle Cost Analysis 1997. Annual Supplement to NIST Handbook 135 and NBS Special Publication 709. (Revised).

PB96-210745

02,512

Energy Prices and Discount Factors for Life-Cycle Cost Analysis 1994. Annual Supplement to NIST Handbook 135 and NBS Special Publication 709 .

PB94-206018

02,508

Life-Cycle Costing Manual for the Federal Energy Management Program. 1995 Edition.

02,511

Life-Cycle Costing Workshop for Energy Conservation in Buildings: Student Manual.

PB95-175006

00,257

Present Worth Factors for Life-Cycle Cost Studies in the Department of Defense (1995)

03,664

Present Worth Factors for Life-Cycle Cost Studies in the Department of Defense (1996).
PB96-106869

PB96-106869

PETERSON, M. B.

Wear of Selected Materials and Composites Sliding against MOS2 Films.

PETERSON, S. M.

03,231 Measurements of the Viscosities of Saturated and Com-
pressed Fluid 1-chloro-1,2,2,2-tetrafluoroethane (R124) and pressed Fluid 1-chloro-1,2,2,2-tetrafluoroethane (R124) and
Pentafluoroethane (R125) at Temperatures between 120 and $420 \mathrm{~K}$

PB94-199791

PETERSONS, 0 .

Active High Voltage Divider with 20-PPM Uncertainty.
PB97-119317 104

PETIETTE-HALL, C. L.

Microwave Properties of YBa2Cu3O7-x Films at $35 \mathrm{GHz}$ from Magnetotransmission and Magnetoreflection Measure-

ments. 168977

04,643

PETIT, G.

Comparison of GPS Broadcast and DMA Precise

AD-P009 114/0 01,518

Comparison of GPS Broadcast and DMA Precise Ephemerides

01,523

PETRELla, E. C

Phospholipid/Alkanethiol Bilayers for Cell-Surface Receptor Studies by Surface Plasmon Resonance.

PB96-102900

03,472

PETRICH, W.

Behavior of Atoms in a Compressed Magneto-Optical Trap.
PB95-203105

Reduction of Light-Assisted Collisional Loss Rate from a Low-Pressure Vapor-Cell Trap.

03,971

Stable, Tightly Confining Magnetic Trap for Evaporative Cooling of Neutral Atoms.

PB96-200720

PETRILLO, $\mathrm{K}$

Nano-Defects in Commercial Bonded SQI and SIMQX

PB96-123674

PETROVIC, J. J.

Creep Rupture of MoSi2/SiCp Composites.

PB95-152294

Tensile Creep of Silicide Composites.

PB96-200803

04,126

.

Excitation of Balmer Lines in Low-Current Discharges of Hydrogen and Deuterium

02,407

PETTIT, K.

Enhanced Curie Temperatures and Magnetoelastic Domains in Dy/Lu Super Lattices and Films.

PB94-172665

PFEIFER, M. J.

04,443

Effects of Elastic Stress on Phase Equilibrium in the Ni-V System.

03,313

\section{PFEIFFER, E. $R$.}

Comparisons of Some NIST Fixed-Point Cells with Similar Cells of Qther Standards Laboratories.

PB97-119242

00,655

Practical Applications of the ITS-90: Inherent Uncertainties.

PB95-161527

03,930

PFUFF, $M$.

Potential Drop in the Center-Cracked Panel with Asymmetric Crack Extension

PHADKE, M. S.

04,819

Taguchi's Parameter Design: A Panel Discussion.

PB96-111802

03,445

PHALIPPOU, $M$.

Formal Methods in Conformance Testing: Result and Perspectives.

PHAN, L.

01,710

Performance of HUD-Affiliated Properties during the January 17, 1994 Northridge Earthquake.

PHAN,

00,443

Comparison of Responses of a Select Number of Buildings to the 10/17/1989 Loma Prieta (California) Earthquake and Low-Level Amplitude Test Results.

PB96-159645

00,467

Dynamic Characteristics of Five Tall Buildings during Strong and Low-Amplitude Motions.

00,427

Response of Buildings to Ambient Vibration and the Loma Prieta Earthquake: A Comparison.

PB96-119607

00,457

Seismic Instrumentation of Existing Buildings.

PB94-159779

00,420

Seismic Strengthening of Reinforced Concrete Frame Build-

PB95-108841

00,430

State of the Art Report on Seismic Design Requirements for Nonstructural Building Components.

PB96-193800

00,308

Strengthening Methodology for Lightly Reinforced Concrete PBa6-1580

Strengthening Methodology for Lightly Reinforced Concrete Frames-II. Recommended Calculation Techniques for the

Design of Infill Walls.
PB94-187648

00,426

Strengthening Methodology for Lightly Reinforced Concrete Frames: Recommend

ing with Infill Walls.

PHANEUF, R. A.

Absolute Cross Sections for Electron-Impact Single Ionization of $\mathrm{Si}(+)$ and $\mathrm{Si}(2+)$.

PB95-202529

03,982

Backscattering in Electron-Impact Excitation of Multiply Charged Ions

03,799

Electron-Impact Excitation of $\mathrm{Si}(3+)(3 \mathrm{~S}$ yields $3 \mathrm{P})$ Using a Merged-Beam Electron-Energy-Loss Technique.

03,904

Evidence for Significant Backscattering in Near-Threshold Electron-Impact Excitation of $\operatorname{Ar}(7+)(3 s$ yields $3 p)$. PB95-126405

03,883 


\section{PERSONAL AUTHOR INDEX}

Scanning Electron Microscopy Observations of Misfit Dislocations in Epitaxial In0.25Ga0.75As on GaAs(001). PB96-200159

Tensile Deformation-Induced Microstructures in Free-Standing Copper Thin Films.

PB96-102595

04,715

PHILLIPS, L.

SGML Environment for STEP.

PB95-143103

02,778

PHILLIPS, L, C.

Development in the Theory and Analysis of Eddy Current Sensing of Velocity in Liquid Metals.

PB94-212586

03,335

PHILLIPS, M.P.

Stranding Experiments on Double Hull Tanker Structures. PB96-123112

PHILLIPS, S.

Automated Inspection: The Integration of National Standards and Commercial Products at NIST.

02,906

PB95-163077

02,653

PB95-163093

PHILLIPS, S. D.

Development of an Automated Part Inspection System

Using the DMIS Standard.

02,899

Estimation of Measurement Uncertainty of Small Circular Features Measured by CMMs.

PB95-267928

02,918

Interim Testing Artifact (ITA): A Performance Evaluation System for Coordinate Measuring Machines (CMMs). User

PB95-210589

02,914

NIST SRM 9983 High-Rigidity Ball-Bar Stand. User Manual. PB95-255840

02,669

Some Considerations for Interim Testing of Coordinate Measuring Machine Performance Using a Specific Artifact. PB95-108858 02,898

User's Guide to NIST SRM 2084: CMM Probe Performance

PBandard.

02,709

PHILLIPS, $W$.

Hyperfine Effects and Associative Ionization of Ultracold Sodium.

PB95-151221

03,903

Optical Molasses: The Coldest Atoms Ever.

03,878

PB95-108908

Temperature of Optica! Molasses for Two Different Atomic Angular Momenta.

03,881

Ultracold Collisions: Associative Ionization in a Laser Trap.

PB94-213238

03859

PHILLIPS, W. D.

Atoms in Optical Molasses.

PB95-108874

03,875

Atoms in Optical Molasses: Applications to Frequency

PB95- 108882

03,876

Heterodyne Measurement of the Fluorescence Spectrum of Optical Molasses.

PB95-108411 03,873

Laser-Cooled Neutral Atom Frequency Standards.

PB96-160312

Laser Cooling

04,086

PB95-151502

03,908

Laser Cooling and the Recoil Limit.

PB97-111280

04,391

Laser Modification of Ultracold Collisions: Experiment.
PB96-157987

Localization of Atoms in a Three-Dimensional Standing

WBave. 163887

03,944

Measurement of the Atomic $\mathrm{Na}(3 \mathrm{P})$ Lifetime and of Retar-

dation in the Interaction between Two Atoms Bound in a Molecule.

PB97-122360

04,178

Measurements of Fluorescence from Cold Atoms: Localiza. tion in Three-Dimensional Standing Waves.

03,943

Microlithography by Using Neutral Metastable Atoms and Self-Assembled Monolayers.

PB96-190038

02,441

New Mechanisms for Laser Cooling

03.923

Optical Molasses: Cold Aroms for Precision Measurements. PB95-108890 03,877 sigma+-sigma- Optical Molasses in a Longitudinal Magnetic Field.

- 161840

03,934

HINNEY, C. S.

Analysis by a Combination of Gas Chromatography and Tandem Mass Spectrometry: Development of Quantitative Tandem-in-Time Ion Trap Mass Spectrometry: Isotope Dilution Ouantification of 11-Nor-Delta-9-Tetrahydro cannabinol9-Carboxylic Acid.

PB96-117221

02,561
NIST Reference Materials to Support Accuracy in Drug PB96-123807

03,505

PHIPPS, K. O.

Surging the Upside-Down House: Measurements and Modeling Results.

02,243

PI, J. I.

Realizing Suspended Structures on Chips Fabricated by CMOS Foundry Processes Through the MOSIS Service. CMOS Foundry 01,881 PIATKO, C. D. Point Probe Decision Trees for Geometric Concept Classes.
PB96-160817

PICARD, A.

Measurement and Reduction of Alignment Errors of the NIST Watt Experiment.

01,987

NIST Watt Balance: Progress Toward Monitoring the Kilogram.

3062

01,991

\section{ICOLO, J. L.}

Intercomparison of Internal Proportional Gas Counting of $(85) \mathrm{Kr}$ and (3) $\mathrm{H}$

PB94- 185576
PIELERT, J. H.

03,800

Graphical Analysis of the CCRL Portland Cement Proficiency Sample Database (Samples 1-72). (Part 1. Univariant Analysis of Portland Cement).

Proficiency Testing as a Component of Ouality Assurance in Construction Materials Laboratories.
PB94-185774
00,334

Contrast Matched Studies of a Sheared Binary Colloidal
Suspension. Suspension.

PIEPER, J. B.

00.918

Measurement of the Weak-Localization Complex Conductivity at $1 \mathrm{Ghz}$ in Disordered $\mathrm{Ag}$ Wires.

PB96-117239

04,731

Coarsening of Unstable Surface Features during $\mathrm{Fe}(001)$ Homoepitaxy

04,121

Growth of Iron on Iron Whiskers.

04,567

Homoepitaxial Growth of Iron and a Real Space View of Reflection-High-Energy-Electron Diffraction.
PB94-173069 PB94-173069 04,445 Influence of $\mathrm{Cr}$ Growth on Exchange Coupling in $\mathrm{Fe} / \mathrm{Cr} /$
$\mathrm{Fe}(100)$.
$\mathrm{PB} 95-150181$ Influence of Thickness Fluctuations on Exchange Coupling in $\mathrm{Fe} / \mathrm{Cr} / \mathrm{Fe}$ Structures.

PB96-135371

04,745

Magnetic Momer

04,525

Oscillatory Exchange Coupling in Fe/Au/Fe(100).

PB95-15037

04,569

Scaling of Diffusion-Mediated Island Growth in Iron-on-Iron Homoepitaxy

04,455

Scanning Tunneling Microscopy Study of the Growth of $\mathrm{Cr} /$ Fe(001): Correlation with Exchange Coupling of Magnetic

LB95-150330

04,568

SEMPA Studies of Exchange Coupling in Magnetic Multilayers.

SEMPA Studies of Oscillatory Exchange Coupling

PB95-163556

04,780

Simple, Compact, High-Purity Cr Evaporator for Ultrahigh PB94-216678

04,520

Surface Magnetic Microstructural Analysis Using Scanning Electron Microscopy with Polarization Analysis (SEMPA). PB95-162657

Tunneling Spectroscopy of bcc(001) Surtace States. B96-155585

PIERMARINI, G.

Densification of Nano-Size Powders. 1994 Report.

DE94013486

PIERMARINI, G. J.

1,4-Dinitrocubane and Cubane under High Pressure.

PB95-108437

03,938

Alvin Van Valkenburg and the Diamond Anvil Cell. PB96-204474 gamma-Alumina PB96-204185

04,775

Equipment for Investigation of Cryogenic Compaction of Nanosize Silicon Nitride Powders. 1993 Report. 4013593

03,028

Fabrication of Transparent gamma-Al2O3 from Nanosize Particles.

PB95-175493

03,054

Low temperature fabrication from nano-size ceramic pow-

03,029

NIST/NCMS Program on Electronic Packaging: First Update.

PB96-20408

03,008

PIKIN, A. I.

Beam Line for Highly Charged Ions.

PB97-111256

04,143

PILIONE, L.

Analysis of Boron in CVD Diamond Surfaces Using Neutron PB94-213089

PILIONE, L. J.

04,511

Effect of Stoichiometry on the Phases Present in Boron Nitride Thin Films.

PB96-102470

04,710

PINA, C.

Realizing Suspended Structures on Chips Fabricated by CMOS Foundry Processes Through the MOSIS Service.

PB94.193984

PINDZOLA, M. S.

Absolute Cross Sections for Electron-Impact Single Ionization of $\mathrm{Si}(+)$ and $\mathrm{Si}(2+)$

PB95-202529

03,982

Dielectronic Capture Processes in the Electron-Impact Ionization of $\mathrm{Sc}(2+)$

04,000

PINE, A. S.

Decoupling in the Line Mixing of Acetylene Infrared $O$ PB95-108452

00,877

Infrared Spectra of van der Waals Complexes of Importance in Planetary Atmospheres.

00,071

Molecular-Beam Optothermal Spectrum of the $\mathrm{OH}$ Stretch-

ing Band of Methanol. ing Band of Methanol.

P-Type Doubling in the Infrared Spectrum of NO-HF.

PB94-211463

00,817

Perpendicular C-H Stretching Band nug/nu13 and the Torsional Potential of Dimethylacetylene.

BB97.122451

01,192

Self Broadening in the nu1 Band of $\mathrm{NH}_{3}$

00,857

Self-, N2- and Ar-Broadening and Line Mixing in HCN and $\mathrm{C} 2 \mathrm{H} 2$

PB95-108445

00,876

Spectroscopic Constants for the 2.5 and 3.0 micrometer Bands of Acetylene.

PB94-21307

00,847

PINEO, V. C.

Comparison of Meteor Activity with Occurrence of Sporadic AD-A292 039/

00,116 
Carbon Monoxide Production in Compartment Fires: Reduced-Scale Enclosure Test Facility.

PB95-231700

01,394

Chemical Stability of Upper-Layer Fire Gases.

01,410

Evaluation of Altemative in-Flight Fire Suppressants for Full-Scale Testing in Simulated Aircraft Engine Nacelles and Dry Bays.

00,023

Experimental Study of the Stabilization Region of Litted Turbulent-Jet Diffusion Flames.

PB96-122676

01,405

Global Equivalence Ratio Concept and the Formation Mechanisms of Carbon Monoxide in Enclosure Fires.
PB96-146790

00,210 Carbon Monoxide Formation in Enclosure Fires.

00,313

Greatly Enhanced Soot Scattering in Flickering Ch4/Air Diffusion Flames.

01,361

Reactivity of Product Gases Generated in Idealized Enclosure Fire Environments.

PB95-161790

01,386

Scaling Compartment Fires: Reduced- and Full-Scale Enclosure Burns.

00,224

PLANT, A.

Liposome-Based Flow-Injection Immunoassay for Determin ing Theophylline in Serum.
PB94-213493

PLANT, A. L.

03,49

Fokker-Planck Description of Multivalent Interactions.

Phospholipid/Alkanethiol Bilayers for Cell-Surface Receptor Studies by Surface Plasmon Resonance.

03,472

PB96-102900

Phospholipid/Alkanethiol Biomimetic

Self-Assembled

PB95. 108460

00,878

Supported Phospholipid/Alkanthiol Biomimetic Membranes: Insulating Properties.
PB95-180782

PLESS, $R$.

03,470

Ultrasonic Spectroscopy of Metallic Spheres Using Electromagnetic-Acoustic Transduction.

PB9 4-212537

04.185

PLIVA, J.

Perpendicular $\mathrm{C}-\mathrm{H}$ Stretching Band nu9/nu13 and the Torsional Potential of Dimethylacetylene.

01,192

PLOESSL, R.

Micromagnetic Structure of Domains in Co/Pt Multilayers. 1. Investigations of Wall Structure.

PB95-16211

04.610

PLOTT, $M$.

Evaluation and Oualification Standards for an X-Ray Laminography System

02,029

SPC Artifact for Automated Solder Joint Inspection.

PB96-161716

04,095

PLUMB, O. A.

Development of an Economical Video Based Fire Detection and Location System.

PB96-193743

PLUSQUELLIC, D.

Photodissociation Dynamics in Ouantum State-Selected
Clusters: A Test of the One-Atom Cage Effect in Ar-H2O.

Clusters: A Test

POCHAN, P. D.

Inductively Coupled Plasma Source for the Gaseous Elec-

tronics Conference RF Reference Cell.

PB96-113394

02,394

Reactive Ion Etching in the Gaseous Electronics Conference RF Reference Cell.

PB96-11340

02,395

NIST Program for Investigating Error Reporting Capabilities of Optical Disk Drives.
PB96-160627

01,635

Optical Storage Media Data Integrity Studies.

01,620

Research on Methods for Determining Optical Disk Media Life Expectancy Estimates.

PB96-160304

01,633

Standardization of Testing Methods for Optical Disk Media Characteristics and Related Activities at NIST.

01,624

PB95-108486

Status of Emerging Standards for Removable Computer Storage Media and Related Contributions of NIST.

PB96-160619

01,634

POITZSCH, M. E.

Progress on a Cryogenic Linear Trap for $(199) \mathrm{Hg}(+)$ lons.

POKHO-180790

lons.

POKHODUN, A. I.

Investigation of High-Temperature Platinum Resistance

Thermometers at Temperatures Up to $962 \mathrm{C}$, and, in Some Cases, $1064 \mathrm{C}$.
PB96-161294

00,629

\section{POKROVSKII, V. A.}

Summary of the Apparent Standard Partial Molal Gibbs Free Energies of Formation of Aqueous Species, Minerals, and Gases at Pressures 1105000 Bars and Temperatures

PB96-145891

01,113

POLAK, M. J.

Display-Measurement Round-Robin.

PB96-1 19227

02,186

POLAK, M. L.

Photoelectron Spectroscopy of Negatively Charged Bismuth Clusters: $\mathrm{Bi}(-) 2, \mathrm{Bi}(-) 3$, and $\mathrm{Bi}(-) 4$.

00,880

Photoelectron Spectroscopy of Small Antimony Cluster Anions: $\mathrm{Sb}(-), \mathrm{Sb} 2(-), \mathrm{Sb} 3(-)$, and $\mathrm{Sb} 4(-)$

PB95.203139

01,045

POLAKOS, P. A.

Increased Transition Temperature in in situ Coevaporated YBa2Cu3O7-delta Thin Films by Low' Temperature Post-Annealing.

POLAND, C. D.

04,672

Evaluation and Strengthening Guidelines for Federal Buildings: Assessment of Current Federal Agency Evaluation Programs and Rehabilitation Criteria and Development of Typical Costs for Seismic Rehabilitation.

PB94-181856

00,425

Evaluation and Strengthening Guidelines for Federal Buildings: Identification of Current Federal Agency Programs.
PB94-176278

POLAND, D. E.

Corrected Optical Pyrometer Readings.

POLIAN, G.

02,615

International Marine-Atmospheric (222)Rn Measurement Intercomparison in Bermuda. Part 2. Results for the Participating Laboratories.

PB96-175682

00,115

POLK, M. B

Evidence of Crosslinking in Methyl Pendent PBZT Fiber. PB97-112486

POLK, W. T.

Precise Identification of Computer Viruses.

PB96-160825

01,613

Security Considerations for SOL-Based Implementations of PB94-139649

02,766

POLLAND, J. F.

Analysis of Autocorrelations in Dynamic Processes.
PB95-181228

POLVANI, R. S.

Chemical Aspects of Tool Wear in Single Point Diamond PB97-112601

03,021

POLYANSKY, O. L.

Tunneling-Rotation Spectrum of the Hydrogen Fluoride Dimer.

POMMERSHEIM, J.

00,793

Diffusion of Cations Beneath Organic Coatings on Steel Substrate.
PB94-215704

POMMERSHEIN, J. M.

Long-Term Performance of Engineered Concrete Barriers.
PB95-260816
03,727

Sulfate Attack of Cementitious Materials: Volumetric Relations and Expansions.

03,232

POMMERSHEIM, J. R.

Expansion of Cementitious Materials Exposed to Sulfate Solutions.

PB94-185782

02,577

POMPE, $\mathbf{R}$.

Analysis of Physical Properties of Ceramic Powders in an International interlaboratory Comparison Program.

PB95-161501

03,050

POOLE, J.

Distributed Communication Methods and Role-Based Access Control for Use in Health Care Applications.

PB96-183165

01,508

Method to Determine a Basis Set of Paths to Perform Program Testing.
PB96-131503

01,747

POOLE, J.P.

Unravel: A CASE Tool to Assist Evaluation of High integrity Sottware. Volume 1. Requirements and Design. PB95-267886

01,736

Unravel: A CASE Tool to Assist Evaluation of High integrity Software. Volume 2. User Manual.

PB95-267894

01,737

POPEL, R.

SUSAN: SUperconducting Systems ANalysis by Low Temperature Scanning Electron Microscopy (LTSEM).

04,728

POPESCU, G.

Scattered Fractions of Dose from 18 and 25 MV X-ray Ra. diotherapy Linear Accelerators.

PORTIER, R. W.

Fire Data Management System, FDMS 2.0, Technical Documentation.

POSTEK, M. T.

01,358

Critical Issues in Scanning Electron Microscope Metrology.

Fabrication Issues for the Prototype National Institute of Fabrication Issues for the Prototype National Institute of
Standards and Technology SRM 2090A Scanning Electron Microscope Magnification Calibration Standard.

PB96-160585

01,131

Modification of a Commercial SEM with a Computer Controlled Cathode Stabilized Power Supply.

PB96-201066

04,129

Monte Carlo Model for SEM Linewidth Metrology.

PB95-150058

02,331

Precision, Accuracy, Uncertainty and Traceability and Their

Application to Submicrometer Dimensional Metrology
PB94-213105

02,319

Report on the NIST Low Accelerating Voltage SEM Mag-

nification Standard Interlaboratory Study.

02,445

Scanning Electron Microscope Magnification Calibration Interlaboratory Study.

Scanning Electron Microscope Metrology.

01,164

PB96-201090

SEM Linewidth Metrology of X-ray Lithography Masks.

PB96-201108

02.446

X-ray Mask Metrology: The Development of Linewidth Standards for $\mathrm{X}$-ray Lithography.

PB95-162129
POTTMEYER, J.

Framework for National Information Infrastructure Services. PB95-103719

POTZICK, J.

Comparisons of Measured Linewidths of Sub-Micrometer Lines Using Optical, Electrical, and SEM Metrologies.
PB95-152807 Metrology Model for Submicrometer Dimensional Measure-

PB95-108502

02.647

Practical Photomask Linewidth Measurements

PB95-108510

02,324

Accuracy in Integrated Circuit Dimensional Measurements.

02,368

Improving Photomask Linewidth Measurement Accuracy via Emulated Stepper Aerial Image Measurement.

PB95-180816
POWELL, C. J.

Activities of ISO Technical Committee 201 on Surface Chemical Analysis

Activities of the ASTM Committee E.42 on Surface AnalyPBis. 108528

00,881

Calculations of Electron Inelastic Mean Free Paths (IMFPs) 4. Evaluation of Calculated IMFPs and of the Predictive IMFP Formula TPP-2 for Electron Energies between 50 and $2000 \mathrm{eV}$.

PB95-150728

00,922

Calculations of Electron Inelastic Mean Free Paths. 5. Data 


\section{PERSONAL AUTHOR INDEX}

\section{POWELL, R. M.} Electronics and Electrical Engineering Laboratory: 1994
Strategic Plan. Supporting Technology for U.S. Competitiveness in Electronics.

PB94-161320

01,874

POWELL, $S$.

Guidelines for Reporting Results of Computational Experiments. Report of the Ad hoc Committee.

PB94-212347

POZO, R.

IML++ V.1.2 Iterative Methods Library Reference Guide.
PB96-195219

MV++ v. 1.5a Matrix Vector Class Reference Guide.

PB96-195326 01,777

Performance Characteristics of Fast Elliptic Solvers on Parallel Platforms.

01,723

SparseLib++ v. 1.5 Sparse Matrix Class Library. Reference Guide.

01,775

PRASK, H. J.

Determination of the Residual Stresses Near the Ends of Skip Welds Using Neutron Diffraction and X-ray Diffraction .

02,868

PB95-253589

Neutron Techniques in Materials Science and Related Disciplines.

PB96-119698

Texture Study of Two Molybdenum Shaped Charge Liners

by Neutron Diffraction.

03,754

Textures of Tantalum Metal Sheets by Neutron Diffraction. PB94-200169

03,399

PRASSIDES, $K$.

Lattice Dynamics of $\mathrm{Ba} 1-\mathrm{xKxBiO}$

PB96-10242

04,706

Lattice Dynamics of Semiconducting, Metallic, and Superconducting $\mathrm{Ba} 1-\times \mathrm{K} \times \mathrm{BiO} 3$ Studied by Inelastic Neutron Scattering.

04,708

Neutron-Scattering Study of $\mathrm{C} 60(n-)(n=3,6)$ Librations in 41 kali-Metal Fullerides.

PB94-200219

00,806

Rotational Dynamics of $\mathrm{C} 60$ in Na2RbC60.

00,948

Rotational Dynamics of Solid C70: A Neutron-Scattering

Study.

00,755

Rotational Dynamics of Solid C70: A Neutron-Scattering
Study.

Study.

Structure and Conductivity of Layered Oxides

$(\mathrm{Ba}, \mathrm{Sr}) \mathrm{n}+1(\mathrm{Sn}, \mathrm{Sb}) \mathrm{nO} 3 \mathrm{n}+1$

PB96-102439

04,707

PRATHER, J. L.

Atomic Energy Levels in Crystals.

AD-A280 150/4

Automated, High-Precision Coulometric Titrimetry. Part 1. Engineering and Implementation

00,575

Automated, High Precision Coulometric Titrimetry. Part 2.

Strong and Weak Acids and Bases.

PB95-150207

00,576

Dissolution Problems with Botanical Reference Materials.

PB95-126280

03,487

\section{PRATT, M. J.}

Product Models and Virtual Prototypes in Mechanical Engi-

neering.

02,783

Requisite Elements, Rationale, and Technology Overview for the Systems Intergration for Manufacturing Applications (SIMA) Program. Background Study.

PB96-112685

02,831

PREISEGGER, E

Report of the Refrigeration, Alr Conditioning and Heat Pumps Technical Options Committee.

PB96-176755

03,293

PREMACHANDRAN, $R$

Surface Chemical Interactions of Si3N4 with Polyelectrolyte Deflocculants.

PREMACHANDRAN, R. S

03,056

Electrokinetic Sonic Analysis of Silicon Nitride Suspensions.

Standard Reference Material for the Measurement of Particle Mobility by Electrophoretic Light Scattering PB96-102488

00,609

PRENDERGAST, J.

TDDB Characterization of Thin $\mathrm{SiO} 2$ Films with Bimodal Failure Populations.

PB96-102926

02,381

PRENTISS, $M$.

Microlithography by Using Neutral Metastable Atoms and Self-Assembled Monolayers.

PB96-190038

02,441
PRESSER, C.

Analysis of Droplet Arrival Statistics in a Pressure-Atomized Spray Flame.

01,352

Assessing Halon Alternatives for Aircraft Engine Nacelle Fire Suppression.
PB96-102454

01,401

Combustion of Methanol and Methanol/Dodecanol Spray Flames.

02,478
EBffect of Dodecanol Content on the Combustion of Meth-

$\begin{array}{ll}\text { anol Spray Flames. } & \\ \text { PB95-176020 } & 01,389\end{array}$

Effect of Finite Beam Width on Elastic Light Scattering from Droplets.

01,144

Effectiveness of Halon Alternatives in Suppressing Dynamic

$\begin{array}{ll}\text { Combustion Process. } & 03,288\end{array}$

Elastic Scattering from Spheres under Non Plane-Wave Illu-

mination.

04,370

Forward Scattering of a Gaussian Beam by a Nonabsorbing

Sphere.

PB97-112288

04,395

In-situ Fume Particle Size and Number Density Measure-

ments from a Synthetic Smelt.

PB94-212040

03,334

Internal Droplet Circulation Induced by Surface-Driven Rota-

$\begin{array}{ll}\text { tion. } & 02,500\end{array}$

Parametric Investigation of Metal Powder Atomization Using

Laser Diffraction.

03,342

Role of Combustion on Droplet Transport in Pressure-Atom-

$\begin{array}{ll}\text { ized Spray Flames. } & \text { 01,434 } \\ \text { PB96-204433 }\end{array}$

Structure of a Swirl-Stabilized Kerosene Spray Flame.

Prict-108569

Study of Droplet Transport in Alcohol-Based Spray Flames Using Phase/Doppler Interferometry.

PB95-108551

02,479

Suppression of Elevated Temperature Hydraulic Fluid and JP-8 Spray Flames.

Suppression of Ignition Over a Heated Metal Surface.

PB96-176425

03,291

Turbulent Spray Burner for Assessing Halon Alternative Fire Suppressants.

01,385

Validation of a Turbulent Spray Flame Facility for the Assessment of Halon Alternatives.

PB96-159728

03,283

PRESSESKY, J. L.

Correlations of Modulation Noise with Magnetic Microstructure and Intergranular Interactions for $\mathrm{CoCrTa}$ and $\mathrm{CoNi}$ Thin Film Media.

04,509

PREVEDELLI, $M$.

High-Order Harmonic Mixing with GaAs Schottky Diodes.

Rotational Far Inirared Spectrum of (13)CO.

PRIBANIC, J. A.

Binder Characterization and Evaluation by Nuclear Magnetic Resonance Spectroscopy

PB94-193471

PRICE, J.

Dielectric Properties of Thin Film SrTiO3 Grown on LaAlO3 with YBa2Cu3O7-x Electodes.

PB95-181160

PRICE, J. C.

Characterization of a Tunable Thin Film Microwave Ba2Cu307-x/SrTiO3 Coplanar Capacitor.

PB95-175527 02,264

Ferroelectric Thin Film Characterization Using Superconducting Microstrip Resonators

PB96-102389

02,270

Measurement of the Weak-Localization Complex Conductivity at $1 \mathrm{Ghz}$ in Disordered Ag Wires.

PB96-117239

04,731

Tunable High Temperature Superconductor Microstrip ResOnators.

PRICE, S. D.

02,048

Charge Transfer and Collision-Induced Dissociation Reactions of $\mathrm{CF}(2+)$ and $\mathrm{CF} 2(2+)$ with the Rare Gases at a Laboratory Collision Energy of $49 \mathrm{eV}$.

00,775

Collision-Induced Neutral Loss Reactions of Molecular

PBications.

00,780

Precision Lifetime Measurements of Cs $6 p(2) P_{1} 1 / 2$ and $6 p$ (2) $\mathrm{P} 3 / 2$ Levels by Single-Photon Counting

PRILUSKY, J.

Protein Data Bank: Current Status and Future Challenges.

PRINCE, E.

Construction of Maximum-Entropy Density Maps, and Their in Phase Determination and Extension.

B95-108593

00,882

Fast-lon Conducting Y2(ZryTi1-y)2O7 Pyrochlores: Neutron Rietveld Analysis of Disorder Induced by $\mathrm{Zr}$ Substitution.

Fast-Ion Conduction and Disorder in Cation and Anion Arrays in Y2(ZryTi(1-y))2O7 Pyrochlores Induced by $\mathrm{Zr}$ Substitution: A Neutron Rietveld Analysis.

04,496

Mathematical Aspects of Rietveld Refinement.

PB95-108601

04,526

Neutron Powder Diffraction Study of a $\mathrm{Na}, \mathrm{Cs}$-Rho Zeolite.

PB94-198629

00,791

Rietveld Analysis of $\mathrm{NaxWO}+x / 2 . y \mathrm{H} 2 \mathrm{O}$, Which Has the Prongonal Tungsten Bronzture.

04,524

Statistical Descriptors in Crystallography. 2. Report of a Working Group on Expression of Uncertainty in Measure.

PB96-146824

04,764

PRITZKER, R. A

Learning to Change: Opportunities to Improve the Performance of Smaller Manufacturers.

PROCHOROV, A. V

Precision High Temperature Blackbodies.

ROCTOR, F.

00,010

NIST RS274KT Interpreter.

PB96-147954

03,885

1991 Final Technical Report.

PB94-163490

02,808

PROCTOR, F. M.

Enhanced Machine Controller Architecture Overview.

PB94-142460

NIST RS274/NGC Interpreter Version 1.

PB94-187788

Open Architect

PROKES, $K$.

Magnetic Properties of Single-Crystalline UCu3AI2 PB95-180717

PROVENCHER, D.

Evaluation and Strengthening Guidelines for Federal Build-

ings: Identificatior

PROVENCHER, D. L

Evaluation and Strengthening Guidelines for Federal Buildings: Assessment of Current Federal Agency Evaluation Programs and Rehabilitation Criteria and Development of Typical Costs for Seismic Rehabilitation.

PB94-181856

00,425

\section{PRUSS, A.}

International Equations for the Saturation Properties of Ordinary Water Substance. Revised According to the International Temperature Scale of 1990 . Addendum to Journa of Physical and Chemical Reterence Data 16, 893 (1987).
PB94-162302
00,742

PUCIC, S. P.

Derivation of the System Equation for Null-Balanced TotalPower Radiometer System NCS 1.

PB94-169786

02,022

Diffusion of Copper into Gold Plating. 
Radiation Accident at an Industrial Accelerator Facility. PB95.140i17 02,575

Temperature and Relative Humidity Dependence of Radiochromic Film Dosimeter Response to Gamma and Electron Radiation

PB96-135298

03,718

PULS, J.

Radiation-Driven Winds of Hot Luminous Stars $X$. The Determination of Stellar Masses Radii and Distances from Terminal Velocities and Mass-Loss Rates.

PB94-213022

00,060

PUPPO, E.

Data-Parallel Algorithm for Three-Dimensional Delaunay Triangulation and Its Implementation.

01,714

PURI, K. D.

Thermodynamics of the Binding of Galactopyranoside Derivatives to the Basic Lectin from Winged Bean (Psophocarpus Tetrogonolobus)

PB95-162715

03,465

PURI, R.

Laser-Induced Fluorescence Measurements of $\mathrm{OH}$. Concentrations in the Oxidation Region of Laminar, Hydrocarbon Diffusion Flames.

01,387

Laser-Induced Fluorescence Measurements of $\mathrm{OH}$ in Laminar Diffusion Flames in the Presence of Soot Particles.

Oxidation of Soot and Carbon Monoxide in Hydrocarbon Diffusion Flames.

PB95-150215
PURSELL, C. J.

01,382

Vibrational Energy Transfer in S1 p-Difluorobenzene. A Comparison of Low and Room Temperature Collisions.

PB95-1086t9

PURTSCHER, P. T.

Aluminum-Lithium Alloys: Evaluation of Fracture Toughness by Two Test Standards, ASTM Method E 813 and E 1304 . Ductile Fracture and Tempered Martensite Embrittlement of 4140 Steel.

PB96-190285

03,222

Light-Weight Alloys for Aerospace Applications II.

03,375

Microstructure and Tensile Properties of Microalloyed Steel Forgings.
PB94-172715

03,204

Noncontact Ultrasonic Inspection of Train Rails for Stress.

PB95-162673

04,851

Prediction of Strengthening Due to $V$ Additions in DirectCooled Ferrite-Pearlite Forging Steels.

03,220

Prediction of the Strength Properties for Plain-Carbon and Vanadium Micro-Alloyed Ferrite-Pearlite Steel.

03,216

PB96-123393

Temperature-Induced Transition in Ductile Fracture Appear-
ance of a Nitrogen-Strengthened Austenitic Stainless Steel.

ance of a Nitrogen-Strengthened Austenitic Stainless Steel.
PB96-190269
03,221

PURWANTO, A.

Magnetic Properties of Single-Crystalline UCu3Al2.
PB95-180717

PB95-180717
PUTORTI, A. D.

In situ Burning of Oil Spills: Mesoscale Experiments and AB95-163747

02,587

Santa Ana Fire Department Experiment at 1315 South Bristol. July 14, 1994
PB95-188868

00,389

Santa Ana Fire Department Experiment at 1315 South Bristol. July 14, 1994. (Reprint)

PB96-102934

00,207

Santa Ana Fire Departmerit Experiments at South Bristol

PBg6-154810

00,305

Smoke Plume Trajectory from In situ Burning of Crude Oil in Alaska: Field Experiments.

02,594

PYE, J. $P$.

Volume-Limited ROSAT Survey of Extreme Ultraviolet

Emission from all Nondegenerate Stars within 10 Parsecs.
PB96-103189 00.093

PB96-103189

PYKA, N.

Inelastic Neutron Scattering Measurements of Phonons in lcosahedral Al-Li-Cu.

PB95-126215

04,532

QADRI, S. B.

Buffer Layer Modulation-Doped Field-Effect-Transistor Interactions in the AlO.33Ga0.67As/GaAs Superlattice System.
PB96-102876 Interface Roughness-Induced Changes in the Near-E(sub 0 ) Spectroscopic Behavior of Short-Period AlAs/GaAs Superlattices

PB94-185154 02,118

Interface Roughness of Short-Period AIAs/GaAs Superlattices Studied by Spectroscopic Ellipsometry. PB95-107215
Interface Sharpness during the Initial Stages of Growth of Thin, Short-Period III-V Superlattices.

PB95- 108783

Interface Sharpness in Low-Order III-V Superlattices. PB95-108775

02,139

QIAN, C.

Turbulent Flame Spread on Vertical Corner Walls. PB96-1 14764

Upward Flame Spread along the Vertical Corner Walls (October 1993).

00,340

QIAN, $x$.

Comparison of FDDI Asynchronous Mode and DQDB Queue Arbitrated Mode Data Transmission for Metropolitan Area Network Applications.

AB96-160452

Provision of Isochronous Service on IEEE 802.6. PB96-160635

01,498

QIU, $Y$

Structural Stabilization of Phase Separating PC/Polyester Blends through Interfacial Modification by Transesterification

PB95-150454

01,239

QUANG, E.

Neutron Leakage Benchmark for Criticality Safety ReSearch.

03,723

QUEEN, Y. H.

Advanced Angle Metrology System

PB94-211364

02,637

Tilt Effects in Optical Angle Measurements.

04,294

QUENARD, D. A

Modelling Drying Shrinkage of Cement Paste and Mortar Part 1. Structural Models from Angstroms to Millimeters. PB96-135132

03,074

QUENTER, D.

SUSAN: SUperconducting Systems ANalysis by Low Temperature Scanning Electron Microscopy (LTSEM) PB96-112065

04,728

QUINN, G.

Fracture Toughness of Advanced Ceramics at Room Temperature: A Vamas Round-Robin.

162194

03,160

QUINN, G. D.

Fracture Mechanism Maps: Their Applicability to Silicon Nitride.

03,094 High Temperature Structural Reliability of Silicon Nitride.

03,104

Room-Temperature Flexure Fixture for Advanced Ceramics. PB95-210498

03,061

QUINN, T.

Through-the-Arc Sensing for Measuring Gas Metal Arc Weld Quality in Real Time.

QUINN, T. P.

Computers in Welding: A Primer.

PB95-162863

02,908

02,872
$0896-135330$

Control of Gas-Metal-Arc Welding Using Arc-Light Sensing.
PB96-131461

Electrode Extension Model for Gas Metal Arc Welding.
PB96-135074

02,87
Mathematical Models of Transport Phenomena Associated with Arc-Welding Processes: A Survey.

02,870

Power Characteristics in GMAW: Experimental and Numerical Investigation.

03,296

Sensing Droplet Detachment and Electrode Extension fo Control of Gas Metal Arc Welding.

PB96-190160

03,297

Through-the-Arc Sensing for Monitoring Arc Welding. 02,858

PB94-185899 02,858 Through-the-Arc Sensing for

PB95-162871

QUINTERO, R.

NIST Support to the Next Generation Controller Program 1991 Final Technical Report.

PB94-163490

02,808

Overview of NASREM: The NASANBS Standard Reference Model for Telerobot Control System Architecture. PB94-194560

04,831

Reference Architecture for Machine Control Systems Integration: Interim Report.

PB95-144549

02,820

Submarine Automation: Demonstration No. 5.

03,748

PB95-251633

Task Decomposition Methodology for the Design of a Coa Mining Automation Hierarchical Real-Time Control System.
PB94-185386

Toward a:Reterence Model Architecture for Real-Time Intelligent Control Systems (ARTICS).

QUINTIERE, J. G

02,932

Fire Growth Models for Materials.

PB94-195856

01,367

Heat Flux from Flames to Vertical Surfaces.

PB97-110357

01,438

Wall Flame Heights with External Radiation

PB95-16348

00,380

RABAGO, R.

Gas Transport Properties of Solution-Cast Perfluorosulfonic Acid lonomer Films Containing lonic Surfactants.

PB95-175998

RABY, T. M.

Reactor Radiation Technical Activities, 1994. NAS-NRC Assessment Panel, April 6-7, 1995

PB95-209888

03,732

RADACK, $S$. $M$.

Computer Systems Laboratory Annual Report, 1993.
PB94- 162518

Computer Systems Laboratory Annual Report 1994.

PB95-209920

01,629

Conference Report: International Conference on the Application of Standards for Open Systems (6th).

PB96-161211 01,762

Federal Government and Information Technology Standards: Building the National Information Infrastructure. PB95-180840

GOSIP Testing Program.

PB96-161229 01,504

Guidance of the Legality of Keystroke Monitoring.

PB96-161237

00,005

Industry/Government Open Systems Specification: The Development of GOS
PB96-161245

01,505

Information Technology Standards in Federal Acquisitions.

PB96-161252 01,636

Using Information Technology Standards in Federal AcquisiPB96-161260

RADEBAUGH, $R$.

Building a Better Crycooler.

PB96-119615

01,637

Design Equations and Scaling Laws for Linear Compressors with Flexure Springs.

Energy Flows in an Orifice Pulse Tube Refrigerator.

PB95-169082

Graded and Nongraded Regenerator Performance. PB95-169090

02,752

Resistance Thermometers with Fast Response for Use in Rapidly Oscillating Gas Flows.

PB95-107298

Thermal Anemonetry for Mass Flow Measurement in Oscillating Cryogenic Gas Flows.

PB96-176789

04,218

RADERMACHER, $K$.

Silicon Surface Chemistry by IR Spectroscopy in the Mid- to Far-IR Region: $\mathrm{H} 2 \mathrm{O}$ and Ethanol on $\mathrm{Si}(100)$.

PB96-138565

01,097

RADFORD, H. E.

Far Infrared Laser Frequencies of $\mathrm{CH} 3 \mathrm{OD}$ and $\mathrm{N}_{2} \mathrm{H}_{4}$. PB96-119623

RADOUSKY, H. B.

Crystal Structure and Magnetic Ordering of the Rare-Earth and $\mathrm{Cu}$ Moments in RBa2Cu2NbO8(R=Nd, $\mathrm{Pr}$ ).

PB95-140554

RADOVANOV, $\mathrm{s}$.

Ion Kinetic-Energy Distributions in Argon if Glow Discharges. 
Kinetic-Energy Distributions of lons Sampled from Argon Plasmas in a Parallel-Plate, Radio-Frequency Reference Call. 03,935 Studies of Ion Kinetic-Energy Distributions in the Gaseous Electronics Conference RF Reference Cell.

02,391

Time-Resolved Balmer-Alpha Emission from Fast Hydrogen Atoms in Low Pressure, Radio-Frequency Discharges in Hydrogen.

04,028

Use of an Ion Energy Analyzer: Mass Spectrometer to Measure Ion Kinetic-Energy Distributions from RF Discharges in Argon-Helium Gas Mixtures.

04,406

RADZIEMSKI, L. J.

Measurements of the Resonance Lines of (6) Li and (7)Li by Doppler-Free Frequency-Modulation Spectroscopy. PB96-180211

RAEKER, A.

Charge Cloud Distribution of Heavy Atoms After Excitation by Polarized Electrons.

PB95-203147

04,001

RAFELSKI, J.

Strangeness Flow Difference in Nuclear Collisions at $15 \mathrm{~A}$ and $200 \mathrm{~A} \mathrm{GeV}$

04,042

RAGHAV RAO, K. S. M. S.

Protein Extraction in a Spray Column Using a Polyethylene Glycol Maltodextrin Two-Phase Polymer System.

PB95-162228

00,595

RAGHAVA RAO, K. S. M. S

Continuous Counter-Current Two Phase Aqueous Extrac-

PB95. 161212

00,675

RAGHAVACHARI, $K$.

Silicon Surface Chemistry by IR Spectroscopy in the Mid- to Far-IR Region: $\mathrm{H} 2 \mathrm{O}$ and Ethanol on Si(100)

PB96-138565

01,097

RAHN, L. A.

Measurement of the Self Broadening of the H2Q(0-5) Raman Transitions from 295 to $1000 \mathrm{~K}$

PB95-108627

00,884

RAI, R. S.

Characterization of Vertical-Cavity Semiconductor Structures.

02,126

Correlation of Optical, X-ray, and Electron Microscopy Measurements of Semiconductor Multilayer Structures.

PB95-175279 02,174

Vertical-Cavity Optoelectronic Structures: CAD, Growth, and Structural Characterization.

PB95-153177

02,148

Vertical-Cavity Semiconductor Lasers: Structural Characterization, CAD, and DFB Structures.

PB95-153193

02,150

RAINWATER, J. C.

Calculation of Enthalpy and Entropy Differences of NearCritical Binary Mixtures with the Modified Leung-Griffiths

PB95-108635

00,885

Composition Dependence of a Field Variable Along the Binary Fluid Mixture Critical Locus.

PB95.176038

01,003

Critical Properties and Vapor-Liquid Equilibria of the Binary System Propane + Neopentane.

00,999

Density Dependence of Fluid Properties and Non-Newtonian Flows: The Weisenberg Effect.

Equilıbrium Parr Distribution Function of a Gas: Aspects Associated with the Presence of Bound States.

01,004

Experimental Method for Obtaining Critical Densities of Binary Mixtures: Application to Ethane + n-Butane.
PB95-151148

00,930

Modified Leung-Griffiths Model of Vapor-Liquid Equilibrium: Extended Scaling and Binary Mixtures of Dissimilar Fluids.
00,85 Non-Newtonian Flow between Concentric Cylinders Cal-
culated from Thermophysical Properties Obtained from Simculated from Thermophysical Properties Obtained from Simulations.

PB96-163761 04,103

Nonlinear Correlation of High-Pressure Vapor-Liquid Equilibrium Data for Ethylene $+n$-Butane Showing Inconsistencies in Experimental Compositions.

01,141

Quantum Collisional Transfer Contributions to the Density Dependence of Gaseous Viscosity

01,142

Vapor-Liquid Equilibria of Mıxtures of Propane and Isomeric Hexanes.

00,995

Vapor-Liquid Equilibria of Ternary Mixtures in the Critical Vapor-Liquid Equilibria of Ternary Mixtures in the Critical
Region on Paths of Constant Temperature and Overall Composition.
PB96-161856

01,139
RAITEN, D. J.

Nutritional Status and Growth in Juvenile Rheumatoid Arthritis.

PB94-198470

03,515

RAIZEN, M. G.

High-Resolution Atomic Spectroscopy of Laser-Cooled lons.
PB95-169330

Interference in the Resonance Fluorescence of Two Trapped Atoms.

03,948

Light Scattered from Two Atoms.

PB95-168753

Precise Spectroscopy for Fundamental Physics.

Ouantum Measurements of Trapped lons.

04,286

PB95-161147

04,040

Quantum Projection Noise: Population Fluctuations in TwoLevel Systems
PB94-212271

03,850

Recent Experiments on Trapped lons at the National Institute of Standards and Technology.

PB95.169322

03,952

RAJA, J.

Dimensional Characterization of Small Bores: A Survey.
PB95-162202

Residual Error Compensation of a Vision-Based Coordinate Measuring Machine.

-161617

RAJAPPAN, $G$.

2nu9 Band of Propyne-d3.

PB95-164513

04,091

RAKOSKI, B.

Standard Source Method for Reducing Antenna Factor Errors in Shielded Room Measurements.

PB96-183157

RAMAN, R.

NMR Characterization of Injection-Moulded Alumina Green Compacts. Part 2. T2-Weighted Proton Imaging.

PB96-201181

01,165

RAMATY, $R$.

From Superconductivity to Supernovae: The Ginzburg Sym-

posium. Report on the Symposium Held in Honor of Vitaly

L. Ginzburg. Held in Gaithersburg. Maryland on May 22, 1992

PB95-171963
RAMAYYA, V. V.

04,649

Electronic Access to Standards on the Information Highway.
PB96-131578

PAMBOZ J. D.

Technical Impact of the NIST Calibration Service for Electrical Power and Energy.

PB96-147913

02,462

RAMIRES, M. L. V.

Standard Reference Data for the Thermal Conductivity of Water.

01,111

AMIREZ, A. P.

Colossal Magnetoresistance without $\mathrm{Mn}(3+) / \mathrm{Mn}(4-)$ Double Exchange in the Stoichiometric Pyrochlore T12Mn2O7.

PB97-113070

04,160

RAMLI, E.

Small Angle Neutron Scattering Study of the Structure and Formation of MCM-41 Mesoporous Molecular Sieves.

PB97-122337

03,110

Small Angle Neutron Scattering Study of the Structure and Formation of Ordered Mesopores in Silica.

03,069

PB96-111919

Crystallographic Characterization of Some Intermetallic Compounds in the Al-Cr System.

PB94-198702

RAMOS, B. L

Embossable Grating Couplers for Planar Waveguide Optical Sensors.

PB96-190277
RAMOS, R. A.

Long-Lived Structures in Fragile Glass-Forming Liquids. PB96-119565

RAMSEY, J.

High-Spatial-Resolution Resistivity Mapping Applied to Mercury Cadmium Telluride.

PB94-212917

02,131

RAMSEY, L.

Sleuthing the Dynamo: HST/FOS Observations of UV Emissions of Solar-Type Stars in Young Clusters.

PB96-122817

RAMSEY, L. $W$.

Far-Ultraviolet Flare on a Pleiades G Dwarf.

RAMSEY, N. F.

Accurate Measurement of Time.

PB96-119482

RANCOURT, C. F.

Opportunities for Innovation: Advanced Manufacturing Tech-

nology.

02,801

RANDA, J.

Catalogue of Electromagnetic Environment Measurements, $30-300 \mathrm{~Hz}$.

PB96-155452

01,943

Condensed Catalogue of Electromagnetic Environment Measurements, $30-300 \mathrm{~Hz}$

PB95-162210

01,899

Correction Factor for Nonplanar Incident Field in Monopole

Calibrations.

Low-Frequency Model for Radio-Frequency Absorbers.

PB95-261939 04,424

Screened-Room Measurements on the NIST Spherical-

Dipole Standard Radiator.

PB96-113568

01,926

RANDALL, C. E.

Observations of 3C 273 with the Goddard High Resolution

Spectrograph on the Hubble Space Telescope.

PB95-202321

00,076

RANDERS-EICHHORN, L.

Formation of DNA-Protein Cross-Links in Cultured Mammalian Cells Upon Treatment with Iron lons.

PB96-137724

03,651

RANDERS, L.

Tert-Butyl Hydroperoxide-Mediated DNA Base Damage in Cultured Mammalian Cells.

PB94-182003

03,644

RANDOVANOV, S. B.

Kinetic-Energy Distributions of lons Sampled from RadioFrequency Discharges in Helium, Nitrogen, and Oxygen

Frequency Discharges in Helium, Nitrogen, and Oxygen.

RANGACHAR, $R$.

Real Time Differential Range Estimation Based on TimeSpace Imagery Using PIPE

PB95-161808

01,844

Real-Time Implementation of a Differential Range Finder.

PB95-108650

RANSOM, $M$.

Security in Open Systems

RAO, G.

Formation of DNA-Protein Cross-Links in Cultured Mammalian Cells Upon Treatment with Iron lons.

PB96-137724

03,651

Tert-Butyl Hydroperoxide-Mediated DNA Base Damage in Cultured Mammalian Cells.

PB94-182003

03,644

RAO, K. V.

Novel YBa2Cu3O7-x and YBa2Cu3O7-x/Y4Ba3O9 Multilayer Films by Bias-Masked 'On-Axis' Magnetron Sputtering.

RAO, M. V.

Range Statistics and Rutherford Backscattering Studies on Fe-Implanted In $0.53 \mathrm{GaO} .47 \mathrm{As}$.

PB95-126397

04,535

Transition Metal Implants in In0.53GA0.47As.

PB95-126389

04,534

RAO, M. V. V.S.

Kinetic Energy Distribution of lons Produced from Townsend Discharges in Neon and Argon.

04,413

Kinetic Energy Distributions of $\mathrm{H}(+), \mathrm{H} 2(+)$, and $\mathrm{H} 3(+)$ from a Diffuse Townsend Discharge in $\mathrm{H} 2$ at High $\mathrm{E} / \mathrm{N}$.

PB96-12335

04,415

RAPPAPORT, A. G.

Studies of the Higher Order Smectic Phase of the Large Electroclinic Effect Material W317.

00,935

$X$-ray Observation of Electroclinic Layer Constriction and 
RAUFASTE, N. J

Project Summaries 1994: NIST Building and Fire Research Laboratory.

00,343

Project Summaries 1995: NIST Building and Fire Research Laboratory.

00,400

Wind and Seismic Effects: Proceedings of the Joint Meeting of the U.S.-Japan Cooperative Program in Natural Resources Panel on Wind and Seismic Effects (28th). Held in Gaithersburg, Maryland on May 14-17, 1996.

00,476

Wind and Seismic Effects. Proceedings of the U.S.-Japan Cooperative Program in Natural Resources Panel on Wind and Seismic Effects (26th). Held in Gaithersburg, Maryland on May $17-20,1994$.

PB95-147385

00,433

RAVIV, D.

Adaptive, Predictive 2-D Feature Tracking Algorithm for Finding the Focus of Expansion.
PB94-218575 Integrated Mobile Robot System for Testing Vision Algorithms.

02,936

New Method to Calculate Looming for Autonomous Obsta$\begin{array}{ll}\text { cle Avoidance. } & \\ \text { PB95-171435 } & 01,600\end{array}$ Novel Active-Vision-Based Motion Cues for Local Navigation.

PB96-193727

02,941

Reconstruction during Camera Fixation.

01,593

Scale-Space-Based Visual-Motion-Cue for Autonomous Navigation.
PB96-183173

02,940

Texture-Independent Vision-Based Closed-Loop Fuzzy Con-

trollers for Navigation Tasks.
PB95-220505

00,183

Unified Approach to Camera Fixation and Vision-Based Road Following.
PB95-162244

01,594

Visual-Motion Fixation Invariant

PB94-206281

Visual Road Following without 3-D Reconstruction.

PB95-161030

01,836

RAWLINS, W.

Energy Flows in an Orifice Pulse Tube Refrigerator.
PB95-169082

Graded and Nongraded Regenerator Performance.
PB95-169090

Resistance Thermometers with Fast Response for Use in

Rapidly Oscillating Gas Flows.

PB95-107298

03,261

Thermal Anemonetry for Mass Flow Measurement in Oscil-

lating Cryogenic Gas Flows.

RAWN, C. J.

$\mathrm{Ca} 1-\mathrm{XCuO} 2$, a NaCuO2-Type Related Structure.
PB95-162822

04,218 Bismuth Coordination Number and Shor Bi Bi Contally Low PB95-141131 00,911
PBrion Number and Shor Bi Bi Contacts. Improved Crystallographic Data for Aluminum Niobate (AINbO4)

PB95-107306 04,523

Phase Equilibria in the Systems $\mathrm{CaO}-\mathrm{CuO}$ and $\mathrm{CaO}-\mathrm{Bi} 2 \mathrm{O} 3$.

Powder X-ray Diffraction Data for $\mathrm{Ca} 2 \mathrm{Bi2O} 5$ and C4Bi6O13.
PB96-161278

RAY, S.

Control Entity Interface Specification
PB94-191715

04,777

02,815

Unified Process Specification Language: Requirements for Modeling Process

RAY, S. R.

02,850

Production Management Information Model for Discrete Manufacturing.
PB96-112008

02,830

Reference Architecture for Machine Control Systems Integration: Interim Report

PB95-144549

02,820

Summary and Notes of the Joint ISO/IGES/PDES Organization Technical Committee Meeting. Held in Albuquerque, New Mexico on October 15-20, 1989.

PB95-107314

02,774

RAYNES, A. S.

Electric Field Effects on Crack Growth in a Lead Magnesium Niobate.
PB95-107322

03,339

Moisture and Water-Induced Crack Growth in Optical Materials.

RAZYNSKA A.

04,267

Positive and Negative Cooperativities at Subsequent Steps of Oxygenation Regulate the Allosteric Behavior of Multistate Sebacylhemoglobin.

03,486
READ, D. T.

Electronics Packaging Materials Research at NIST.
PB96-122692 PB96-122692
NIST/NCMS Program on Electronic Packaging: First date.

03,008

Potential Drop in the Center-Cracked Panel with Asymmetric Crack Extension.

PB95-107330

04,819

Tensile Deformation-Induced Microstructures in Free-Standing Copper Thin Films.

PB96-102595

04,715

Theory of Electron Beam Moire.

PB96-175690

04,373

Apparel Manufacturing Glossary for Application Protocol Development.
PB95-198750

PB95- 198750
READER, J.

Hyperfine Structure Investigations and Identification of New Energy Levels in the lonic Spectrum of (147) Pm

PB96-180203

04,117

Irradiances of Spectral Lines in Mercury Pencil Lamps.
PB96-176466

04,375

Laser-Produced and Tokamak Spectra of Lithiumlike Iron, $\mathrm{Fe}(23+)$.

PB95-180857

04,314

Measurements of quantum electrodynamic sensitive transi tions in Na-like and $\mathrm{Cu}$-like ions: Final report, 1 October 1993--29 September 1994

DE95011593

04,403

Spectrum and Energy Levels of Five-Times-Ionized Niobium (Nb VI)

04,226
SB94-185246

IV).

03,888

Wavelengths and Isotope Shitts for Lines of Astrophysical Interest in the Spectrum of Doubly lonized Mercury ( $\mathrm{Hg} \mathrm{III)}$.

Wavelengths of Spectral Lines in Mercury Pencil Lamps.

PB96-176474

04,376

READEY, M. J.

Correlations between Flaw Tolerance and Reliability in Zirconia.

PB96-161922

REBBERT, R. E.

02,986

Standard Reference Materials for the Determination of Polycyclic Aromatic Hydrocarbons in Environmental Samples - Current Activities.

ples - Current

REBULDELA, G.

00,586

Intercomparison of Thermal Converters at NIM, NIST, PTB, SIRI and VSL from 10 to $100 \mathrm{MHz}$

PB94-172459

02,027

REDDY, $P$.

Glucose Permease of Bacillus Subtilis is a Single Polypeptide Chain That Functions to Energize the Sucrose Permease.

03,466

Mapping Domains in Proteins: Dissection and Expression of 'Escherichia coli' Adenylyl Cyclase.

PB96-155460

03,478

Rapid Method for the Isolation of Genomic DNA from 'Aspergillus fumigatus'.
PB96-147061

REDMAN, J. W.

Intercomparison of DNA Sizing Ladders in Electrophoretic Separation Matrices and Their Potential for Accurate Typing of the D1S80 Locus.

PB96-200928

03,485

REED, $B$.

Ring-Opening Dental Resin Systems Based on Cyclic Acetals.

PB95-16225

00,162

REED, K. A.

Development of Computer-Based Models of Standards and Attendant Knowledge-Base and Procedural Systems.

PB96-155783

Distributed Architecture for Standards Processing. PB96-16418

00,464

REED, R. P.

Composite Struts for SMES Plants.

PB95-155586 cal-Impact Tests.

PB95-107355

Mechani-

Macro- and Microreactions in Mechanical-Impact Tests of Aluminum Alloys.

PB95-107348 03,340

Mechanical Properties and Warm Prestress of Ultra-Low Carbon Steel at $4 \mathrm{~K}$

C 190350

Reaction Sensitivities of Al-Li Alloys and Alloy 2219 in Mechanical-Impact Tests.

03,314

Recommended Changes in ASTM Test Methods D2512-82 and G86-84 for Oxygen-Compatibility Mechanical Impact Tests on Meta's.

PB94-216694

03,338

Temperature Increases in Aluminum Alloys during Mechanical-Impact Tests for Oxygen Compatibility.

03,316

REED, $W . P$. Reference Materials by Isotope Dilution Mass Spectrometry.
PB95-153383 00,592

REEDER, D.

Resolution of DNA in the Presence of Mobility Modifying Polar and Nonpolar Compounds by Discontinuous Electrophoresis on Rehydratable Polyacrylamide Gels.

PB95-152799

REEDER, D. J.

Enhanced Detection of PCR Products Through Use of TOTO and YOYO Intercalating Dyes with Laser Induced Fluorescence - Capillary Electrophoresis.

00,599

Intercomparison of DNA Sizing Ladders in Electrophoretic

Separation Matrices and Their Potential for Accurate Typing of the D1S80 Locus.

PB96-200928

03,485

Interlaboratory Comparison of Autoradiographic DNA Profiling Measurements. 1. Data and Summary Statistics. PB95-175923 03,542

Interlaboratory Comparison of Autoradiographic DNA Profiling Measurements. 2. Measurement Uncertainty and Its Propagation

03,545

L-threo-beta-Hydroxyhistidine, an Unprecedented Iron(III) Ion-Binding Amino Acid in a Pyoverdine-type Siderophore from Pseudomonas fluorescens 244

PB94-211620

00,553

Overview of Reference Materials Prepared for Standardization of DNA Typing Procedures.

\section{REES, J. A.}

Kinetic-Energy Distributions of lons Sampled from Argon Plasmas in a Parallel-Plate, Radio-Frequency Reference Call.

PB95-161964

03,935

Use of an Ion Energy Analyzer: Mass Spectrometer to Measure Ion Kinetic-Energy Distributions from RF Discharges in Argon-Helium Gas Mixtures.

PEEVE G. R.

NIST and the Navy: Past, Present and Future.

PB96-119649

04,406

REHM, R.

Dispersion and Deposition of Smoke Plumes Generated in Massive Fires.

PB95-126066

02,540

REHM, R. G

Gravity-Current Transport in Building Fires.

PB96-147046

01,415

In situ Burning of Oil Spills: Mesoscale Experiments and Analysis.

PB95-163747

02,587

Internal Waves

04,221

Large Eddy Simulations of Smoke Movement in Three Di-

mensions.
PB96-190012

01,426

Mathematical Modeling and Computer Simulation of Fire PBenomena.

Pressure Equations in Zone-Fire Modeling.

PB96-102967

00,384

Transport by 
REINTSEMA, C.

High Critical Temperature Superconductor Tunneling Spectroscopy Using Squeezable Electron Tunneling Junctions. PB95-163721

REINTSEMA, C. D.

Controlling the Critical Current Density of High-Temperature SNS Josephson Junctions.

PB96-200712

04,794

Critical Current and Normal Resistance of High-Tc StepEdge SNS Junctions.

04,724

Critical Current Behavior of Ag-Coated YBa2Cu307-x Thin Films.

04,549

Effect of Thermal Noise on Shapiro Steps in High-TC Josephson Weak Links.
PB94-212677 High-Tc Multilayer Step-Edge Josephson Junctions and SOUIDs.

PB96-200183

04,790

Large-Amplitude Shapiro Steps and Self-Field Effects in High-Tc Josephson Weak Links.

PB95-180410

04,682

Mutual Phase Locking in Systems of High-TC Superconductor-Normal Metal-Superconductor Junctions.

Phase Locking in Two-Junction Systems of High-Tempera. ture Superconductor-Normal Metal-Superconductor Junc

PB95-176053

02,060

Step-Edge and Stacked-Heterostructure High-Tc Josephson
Junctions for Voltage-Standard Arrays. Junctions for Voltage-Standard Arrays.

04,702

Temperature Dependence and Magnetic Field Modulation of Critical Currents in Step-Edge SNS YBCO/Au Junctions.

04,723

REINTSEMA, C. R.

Magnetic Field Dependence of the Critical Current Anisotropy in Normal Metal-YBa2Cu3O7-delta Thin Film Bilayers.
PB95-141024 REIPA, V.

Conformational Alterations of Bovine Insulin Adsorbed on a Silver Electrode.

PB96-161773

00,510

Non-Perturbative Relation between the Mutual Diffusion Coefficient, Suspension Viscosity, and Osmotic Compressibility: Application to Concentrated Protein Solutions.

PB96-102355 01,062

Physicochemical Characterization of Low Molecular Weight Heparin.

PB96-112040

03,474

Structural Analys

03,480

REIPURTH, B.

G203.2-12.3: A New Optical Supernova Remnant in Orion. PB95.203535

00,085

REIS, K. P

Powder Neutron Diffraction Investigation of Structure and Cation Ordering in Ba2+xBi2-xO6-y.

PB95-180865

01,015

Rietveld Analysis of NaxWO3+x/2.yH2O, Which Has the

Hexagonal Tungsten Bronze Structure.
PB95-107371

04,524

REITMEIER, G.

Report on the Workshop on Advanced Digital Video in the National Information Infrastructure. Held in Washington D.C. on May 10-11, 1994.

01,472

Summary Report on the Workshop on Advanced Digital Video in the National Intormation Intrastructure.

01,497

REIZER, J.

Glucose Permease of Bacillus Subtilis Is a Single Polypeptide Chain That Functions to Energize the Sucrose Permease.

REKHARSKY, M. V.

03,466

Thermodynamic and NMR Study of the interactions of

Cyclodextrins with Cyclohexane Derivatives.

PB94-185816

00,781

Thermodynamic Study of the Reactions of Cyclodextrins with Primary and Secondary Aliphatic Alcohols, with D- and with Primary and Secondary Aliphatic Alcohols, with D- and PB95-180873

01,016

Thermodynamics of the Hydrolysis of N-Acetyl-Lphenylalanine Ethyl Ester in Water and in Organic Solvents.
01.053

Thermodynamics of the Hydrolysis of 3,4,5 Trihydroxybenzoic Acid Propyl Ester (n-Propylgallate) to 3,4,5-Trihydroxybenzoic Acid (Gallic Acid) and Propan-I-ol in Aqueous Media and in Toluene.

01,160

REMACLE, J.

Tert-Butyl Hydroperoxide-Mediated DNA Base Damage in Cultured Mammalian Cells.

03,644
REMINGTON, K. A.

IML++ V.1.2 Iterative Methods Library Reterence Guide.
PB96-195219

SparseLib++ v. 1.5 Sparse Matrix Class Library. Reference Guide.

01.775

REN, Z. B.

Improved Annealing Technique tor Optical Fiber.

PB96-119680

04,343

RENEKE, P. A.

Fire Safety of Passenger Trains: A Review of Current Approaches and of New Concepts.

04,848

New Concepts for Fire Protection of Passenger Rail Trans. portation Vehicles.

PB95-162046

04,850

New Concepts for Fire Protection of Passenger Rail Transportation Vehicles. (NIST Reprint).

portation Vehicle
PB95-180774

04,863

RENEKER, D. $\mathrm{H}$.

Polyethylene Crystallized from an Entangled Solution Observed by Scant

01,232

RENKEN, M. C.

Determination of Sheet Steel Formability Using Wide Band

Electromagnetic-Acoustic Transducers.

PB96-186036

02,279

Examination of Objects Made of Wood Using Air-Coupled

Ultrasound.

PB95-125712

03,404

Gas-Coupled, Pulse-Echo Ultrasonic Crack Detection and Thickness Gaging

\section{RENNEX, $\mathrm{B}$.}

04,847

Standard Reference Materials: Certification of a Standard Reference Material for the Determination of Interstitial Oxygen Concentration in Semiconductor Silicon by Infrared Spectrophotometry.

PB95-125076

02,326

RENNEX, B. G.

Development of a Standard Reference Material for Meas. urement of Interstitial Oxygen Concentration in Semiconductor Silicon by Intrared Absorption.

PB96-122668

02,404

Methodology for the Certification of Reference Specimens for Determination of Oxygen Concentration in Semiconductor Silicon by Infrared Spectrophotometry.

02,421

RENNIE A. R.

Structure and Rheology of Hard-Sphere Systems. PB96-167333

00,662

RENO, R. C.

Ultrasonic Measurement of Sheet Anisotropy and Formability.

03,213

RENSBERGER, R. A.

Standards Setting in the European Union: Standards Organization and Officials in EU Standards Activities.

REPJAR, A.

Planar Near-Field Alignment.

PB94-172491

02,919

RESNICK, R.

Two New Probes for a Coordinate Measuring Machine. PB95-163093

01,998

RESSLER, S.

Applying Virtual Environments to Manufacturing

PB94-142502

Approaches Using Virtual Environments with Mosaic. PB95-169108

01,599

RETTIG, J. B.

Accuracy and Repeatability in Time Domain Network AnalySis.

02,064

REYMANN, $D$.

Accuracy Comparisons of Josephson Array Systems.
02, 047 REZNIK, D.

High Resolution inelastic Neutron Scattering Study of Phonon Self-Ene

04,688

Inelastic Neutron Scattering Studies of Rotational Excitations and the Orientational Potential in $\mathrm{C} 60$ and $\mathrm{A} 3 \mathrm{C} 60$ Compounds.
PB94-172673

00,763

Neutron-Scattering Study of Librations and Intramolecular Phonons in Rb2.6K0.4C60.

00,958

9 Dependence ot Self-Energy Effects of the Plane Oxygen Vibration in $\mathrm{YBa} 2 \mathrm{Cu} 307$.

01,096

$X$-ray Powder Diffraction from Carbon Nanotubes and Nanoparticles.

RHINE, W. E

$X$-Ray Powder Diffraction Data for $\mathrm{BaCu}\left(\mathrm{C}_{2} \mathrm{O} 4\right) 2.6 \mathrm{H} 2 \mathrm{O}$.

PB95-151767

04,583

DODRICK, G. C.

Development of a Gas Standard Reterence Material CoI taining Eighteen Volatile Organic Compounds.

PB95-162277

$02,54:$

Development of Gas Standards from Solid 1,4 dichlorobenzen

02,496

Measurement of Atmospheric Methyl Bromide Using Gravimetric Gas Standards.

PB96-155494

02,497

NiST Traceable Reference Material Program for Gas Standards: Standard Reference Materials.

PB96-210786

00,644

Radiocarbon Measurements of Atmospheric Volatile Organic Compounds: Ouantifying the Biogenic Contribution.
PB97.122352

Stability/Instability of Gas Mixtures Containing 1,3-Butadiene in Treated Aluminum Gas Cylinders.

PB95-162285

02,546

Stability of Compressed Gas Mixtures Containing Low Levei Volatile Organic Compounds in Aluminum Cylinders.

PB96-111968

00,613

RHODES, $B$.

Fire Growth Models for Materials.

PB94-195856

01,367

HODES, B. T.

Burning Rate and Flame Heat Flux For PMMA in the Cone Calorimeter.

PB95-216990

00,393

Report on the Advanced Software Technology Workshop. Held on February 1, 1994

PB95-136610

01,707

RHORER, R. L

Fabrication of Optics by Diamond Turning

02,954

RHYNE, J. J.

Enhanced Curie Temperatures and Magnetoelastic Domains in Dy/Lu Super Lattices and Films.

04,443

Incorporation of Gold into YBa2Cu307: Structure and Tc Enhancement.

PB94-200276

Magnetic Rare Earth Artificial Metallic Superlattices.

PB95-162293

04,481

Magnetoelasticity in Rare-Earth Multilayers and Films.
PB94-211356

Unexpected Effects of Gold on the Structure, Superconductivity, and Normal State of $\mathrm{YBa} \mathrm{Cu} 3 \mathrm{O} 7$.

PB94-200284

RIBOT, J. J.

Approach to Setting Performance Requirements for Automated Evaluation of the Parameters of High-Voltage $1 \mathrm{~m}$ -

Pulses.

01,878

ICCl, A.

Reactive ion Etching in the Gaseous Electronics Conference RF Reterence Cell.

PB96-113402

02,395

RICE, J.

Nickel(II)-Mediated Oxidative DNA Base Damage in Renal and Hepatic Chromatin of Pregnant Rats and Their Fetuses. Possibl

Oxidative DNA Base Damage in Renal, Hepatic and Pulmonary Chromatin of Rats After Intraperitoneal Injection of Cobalt (II) Acetate.

PB95-150025

03,647

RICE, J.P.

Antenna-Coupled High-Tc Air-Bridge Microbolometer on Sil- 
dc Magnetıc Force Microscopy Imaging of Thin-Film Recording Head.

Flexible-Diaphragm Force Microscope.

03,966

Magnetic Force Microscopy Images of Magnetic Garnet with

PB95-176210

Magnetic Force Microscopy of Flux in Superconductors.

PB95-161733

04,608

Micromagnetic Simulations of Tunneling Stabilized Magnetic Force Microscopy

04,552

Proposed Antiferromagnetically Coupled Dual-Layer Magnetic Force Microscope Tips.

04,645

Recent Results in Magnetic Force Microscopy

PB96-103130

04,721

RICHARDS, C

Global Density Effects on the Self-Preservation Behavior of Turbulent Free Jets.

PB95-162301

04.207

RICHARDS, C. D.

Experimental Study of the Stabilization Region of Lifted Turbulent-Jet Diffusion Flames.

PB96-122676

01,405

RICHARDS, N. D.

Effect of Two Initiator/Stabilizer Concentrations in a Metal Primer on Bond Strengths of a Composite to a Base Metal Alloy.

PB94-172723 00,147

Reduction of Marginal Gaps in Composite Restorations by Use of Glass-Ceramic Inserts.

PB96-102405

00,174

RICHARDS, R. F.

Development of an Economical Video Based Fire Detection and Location System.

00,228

Measurements of Moisture Diffusivity for Porous Building Materials.

PB95-107397

00,356

RICHARDS, R. J.

Survey of the Literature on Heat Transfer from Solid Surfaces to Cryogenic Fluids.

AD-A286 680/4

04,193

RICHIE, K. L.

Overview of Reference Materials Prepared for Standardization of DNA Typing Procedures

PB96-111653

00,611

RICHMAN, $\mathrm{S}$.

Low-Frequency, Active Vibration Isolation System

PB95.203303

02,710

RICHMOND, H. M.

NVLAP Procedures U.S. Code of Federal Regulations. Title 15, Subtitle A, Chapter 2, Part 7. (Effective December 1984 15, Subtitle A, Chapter 2, Part 7. (Effective December 1984,
Amended September 1990). Amended Sep
PB94-160850

02,627

Proficiency Tests for the NIST Airborne Asbestos Program,

1993

00,610

AICHOU, B.

Measurements of the Resonance Lines of (6) Li and (7) L $\mathrm{L}$ by Doppler-Free Frequency-Modulation Spectroscopy.

PB96-180211

RICHTER, C. A.

Buffer Layer Modulation-Doped Field-Eftect-Transistor Interactions in the Al0.33Ga0.67As/GaAs Superlattice System.

PB96-102876

02,380

Mesoscopic C
PB96- $\uparrow 19656$

04,735

Novel Magnetic Field Characterization Techniques for Compound Semiconductor Materials and Devices.
PB96-176458 Quantum Conductance Fluctuations in the Larger-SizeScale Regime

04,144

X-ray Reflectivity Determination of Interface Roughness Correlated with Transport Properties of (AlGa)As/GaAs High Electron Mobility Transistor Devices

PB97-111868

04,149

RICHTER, D.

Low-Energy Vibrations and Octahedral Site Occupation in Nb95V5H(D)y
PB96-160734

RICHTER, L. J.

01,133

Laser-Induced Desorption of NO from Si(111): Etfects of Coverage on
PB95-162319

Photodecomposition Dynamics of Mo(CO)6/Si(111) $7 \times 7: \mathrm{CO}$ Internal State and Translational Energy Distributions---Trans.

lation.

PB94-199288

00,687

Photodecomposition of Mo(CO)6/Si(111) 7x7: CO State-Resolved Evidence for Excited State Relaxation and Ouench-

PBg5-180154

01,009
Photodesorption Dynamics of CO from Si(111): The Role of Surface Defects.

AICHTER, $R$.

03,066

Federal Labs Have Key Role in Metrication.

PB96-12340

Analysis of Failed Dry Pipe Fire Suppression System Couplings from the Filene Center at Wolf Trap Farm Park for the Performing Arts

00,331

Characterization of the Hydrogen Induced Cold Cracking Susceptibility at Simulated Weld Zones in HSLA-100 Steel.
AD-A279 759/5
03,200

Characterization of the Hydrogen Induced Cold Cracking Susceptibility at Simulated Weld Zones in HSLA-100 Steel. PB94-174505

03,746

Comparison of the Corrosion Rates of $\mathrm{FeAl}, \mathrm{Fe}$ (sub 3)A and Steel in Distilled Water and $0.5 \mathrm{M}$ Sodium Chloride. Technical Repor

03,186

Corrosion Resistance of Materials for Renovation of the United States Botanic Garden Conservatory.

00,032

Evaluation of the Electrochemical Behavior of Ductile Nicke Evaluation of the Electrochemical Behavior of Ductile Nicke
Aluminide and Nickel in a pH 7.9 Solution. Technical Repor Aluminide and Nickel in a pH 7.9 Solution. Technical Repon
Number 3. April-June 1991. Number 3, April-June 199

03,307

Evaluation of the Environmentally Induced Fracture Resistance of Ductile Nickel Aluminide. Technical Report Number 1, Final report. October-December 1990.

DE94017331

03,306

Evidence of Film-Induced Cleavage by Electrodeposited PB95-162327

03,191

Modeling Polarization Curves and Impedance Spectra fo Simple Electrode Systems

AICKLEFS, $R$. L.

03,188

Lunar Laser Ranging: A Continuing Legacy of the Apollo Program.

RIDDER, S. D.

Beneficial Effects of Nitrogen Atomization on an Austenitic Stainless Steel.
PB94-212396

03,209

Development of Adaptive Control Strategies for Inert Gas Atomization.

02,823

Effect of Backfill and Atomizıng Gas on the Powder Porosity and Mechanical Properties of 304L Staintess Steel.
PB94-185394 Intelligent Control of an Inert Gas Atomization Process.

PB95-141057 03,344 Optimization of Inert Gas Atomızation.

PB95-107405

Parametric Investigation of Metal Powder Atomization Using

Laser Diffraction
PB95-108577 Process Modelin

RIDDLE, B. F.

02,824

Aperture Excitation of Electrically Large, Lossy Cavities

00,029

Aperture Excitation of Electrically Large, Lossy Cavities

PB95-175675 00,031

TEM/Reverberating Chamber Electromagnetic Radiation Test Facility at Rome Laboratory.

03,675

RIEDLE, E.

Doppler-Free Spectroscopy of Large Polyatomic Molecules and van der Waals Complexes.

04,339

Frequency Shifting of Pulsed Narrow-Band Laser Light in a Multipass Raman Cell.

PB95-203352

04,321

High-Efficiency, High-Power Difference-Frequency Generation of $0.9-1.5 \mathrm{mu} \mathrm{m}$ Light in $\mathrm{BBO}$

PB95-202255

04,317

Stabilization and Precise Calibration of a Continuous-Wave Difference Frequency Spectrometer by Use of a Simple Transfer Cavity.

04,276

Van der Waals Bond Lengths and Electronic Spectral Shifts of the Benzene-Kr and Benzene-Xe Complexes. PB95-15138

00,932

AIES, J. G.

Lunar Laser Ranging: A Continuing Legacy of the Apollo Program.

RIGGAN, W. B.

Cyclic Polyamine Ionophore for Use in a Dibasic Phosphate-Selective Electrode.

PB95-180121

RIGGS, C.

01,008

Oxidative DNA Base Damage in Renal, Hepatic, and Pulmonary Chromatın of Rats After Intraperitoneal Injection of Cobalt (II) Acetate

Cobalt (II) Aceta

03,647

RIGHINI, F.

Issues in High-Speed Pyrometry.

PB97-118368 02,693

Wavelength Dependence of Normal Spectral Emissivity of High-Temperature Metals at Their Melting Point

PB94-200011

03,398

RILEY, M. E.

Gaseous Electronics Conference Radio-Frequency Reference Cell: A Defined Parallel-Plate Radio-Frequency System for Experimental and Theoretical Studies of Plasma. Processing Discharges.

PB94-172327

04,404

RILEY, W. J.

Aging, Warm-Up Time and Retrace; Important Characteristics of Standard Frequency Generators

PB96-103122

04,031

RIMINGTON, P. D.

Learning to Change: Opportunities to Improve the Performance of Smaller Manufacturers.

PB94-166212

00,010

\section{DOT, G. R.}

Requisite Elements, Rationale, and Technology Overview for the Systems Intergration for Manufacturing Applications (SIMA) Program. Background Study.

02,831

STEP On-Line Information Service (SOLIS). The IGES/ PDES Organization.

PB95-137790

02,777

Summary and Notes of the Joint ISO/IGES/PDES Organization Technical Committee Meeting. Held in Albuquerque, New Mexico on October 15-20, 1989

PB95-10731

02,774

RINKINEN, W.

Assessing Halon Alternatives for Aircraft Engine Nacelle Fire Suppression

01,401

Protection of Data Processing Equipment with Fine Water Sprays.

02,610

Suppression of Elevated Temperature Hydraulic Fluid and JP-8 Spray Flames.

Turbulent Spray Burner for Assessing Halon Alternative Fire Suppressants.
PB95-153748

01,385

RINN, K.

Merged-Beams Energy-Loss Technique for Electron-Ion Excitation: Absolute Total Cross Sections for $\mathrm{O}(5+)$ (2s yields 2p)

PB96-102058

04,017

RIPPEY, $W$.

Integrated Vision Touch-Probe System for Dimensional Inspection Tasks
PB95-255832

AIPPEY, W. G.

02,917

Proceedings of NIST Workshop: Industry Needs in Welding Research and Standards Development. Held on August 15-

$\begin{array}{ll}\text { PB96-183124 02,877 } & \end{array}$

Reference Architecture for Machine Control Systems Integration: Interim Report.

RIPPLE, D.

02,820

Assessment of Uncertaintıes of Thermocouple Calibrations at NIST

PB94-152691

03,782

Flow of Microemulsions through Microscopic Pores.
PB95-140463 00,905

$\begin{array}{rr}\text { PB95-140463 } & 00,905 \\ \text { VIScosity of the Saturated Liquid Phase of Six Halogenated }\end{array}$ 
RIZZI, G.

Approach to Setting Performance Requirements for Automated Evaluation of the Parameters of High-Voltage Im-

pulses.

01,878

ROACH, R. K.

Computer Systems Laboratory Annual Report, 1993.
PB94-162518 PB94-162518 PB95-209920

ROADARMEL, G

Fire Performance of an Interstitial Space Construction System.

ROBACK, E.

Data Encryption Standard.

PB95-162376

Security Program Managemen

PB96-15611

ROBACK, E. A.

Computer Security: An Introduction to Computer Security. The NIST Handbook

PB96-131610

01,608

ROBB, $F$

Small genomes: New initiatives in mapping and sequencing Workshop summary report

DE96014476

ROBERTS, D. E.

Tensile Creep of Silicide Composites.

03,451

ROBERTS, J. R.

Gaseous Electronics Conference Radio-Frequency Reference Cell: A Defined Parallel-Plate Radio.Frequency System for Experimental and Theoretical Studies of PlasmaProcessing Discharges.

04,404

Hydrogen Balmer Alpha Line Shapes for Hydrogen-Argon Mixtures in a Low-Pressure of Discharge.

03,924

Observation and Visible and uv Magnetic Dipole Transitions in Highly Charged Xenon and Barium.

04,056

Optical and Mass Spectrometric Investigations of lons and Neutral Species in SF6 Radio-Frequency Discharges.
PB97.111918 PB97-111918

Optical Emission Spectroscopy on the Gaseous Electronics
Conference RF Reference Cell.
PB96-113345

Polarization Measurements on a Magnetic Ouadrupole Line in Ne-Like Barium

04,161

Time-Resolved Balmer-Alpha Emission from Fast Hydrogen Atoms in Low Pressure, Radio-Frequency Discharges in Hydrogen.

ROBERTS, J. W.

04,028 Displays. Held in San Jose, California on January 13.14

PB95-136388

ROBERTS, $K$.

Application Profile for ISDN.

PB95-163689

01,625

North American Agreements on ISDN.

01,503

ROBERTSON, A. F.

Low Heat-Flux Measurements: Some Precautions.

PB96-201116

ROBERTSON, B.

Bacteriorhodopsin Immobilized in Sol-Gel Glass.

PB95-151429 ing Function After Being Heated to $140 \mathrm{C}$.

Determining Mobility from Homodyne ac Electrophoretic Light Scattering.
PB95-140497

03,462

Energy Transduction between a Concentration Gradient and an Alternating Electric Field.

03,461

Imposed Oscillations of Kinetic Barriers Can Cause an En zyme to Drive a Chemical Reaction Away from Equilibrium.
PB96-161625 Michaelis-Menten Equation for an Enzyme in an Oscillating Electric Field.

00,906

Nonequilibrium Statistical Mechanics.

PB96-161781

04,097

Quadratic Response of a Chemical Reaction to External Oscillations.

01,138

Rapid $\mathrm{pH}$ Change Due to Bacteriorhodopsin Measured with a Tin-Oxide Electrode.

PB96-11208

ROBEY, S. W.

03,544

Photoetectron Spectroscopic Study of the Valence and Core-Level Electronic Structure of $\mathrm{BaTiO}$.

04,500
Reactive Coevaporation of DyBaCuO Superconducting Films: The Segregation of Bulk Impurities on Annealed .

PB95-164562

04,635

Resonant-Photoemission Investigation of the Heusler Alloys $\mathrm{Ni2MnSb}$ and NiMnSb.

04,612

PB95-162384

Substitution-Induced Midgap States in the Mixed Oxides
RxBa1-ChiTiO3-Delta, with R=Y, La, and Nd.
PB95-140505 PB95-140505

Surface Core-Level Shifts of Barium Observed in Photo-
emission of Vacuum-Fractured BaTiO3 (100). PB94-212156

04,501

\section{ROBIN HUTCHINSON, J. M.}

Intercomparison Study of (237) Np Determination in Artificial Urine Samples.

ROBINS, J. R.

03,633

Investigation of $\mathrm{S} 2 \mathrm{~F} 10$ Production and Mitigation in Compressed SF6-Insulated Power Systems.

PB94-212388

02,467

ROBINS, L. H.

Lineshape Analysis of the Raman Spectrum of Diamond Films Grown by Hot-Filament and Microwave-Plasma Chemical-Vapor Deposition.

PB95-162392

03,016

Studies of Defects in Diamond Films and Particles by Raman and Luminescence Spectroscopies.

03,017

Surface Roughness Evaluation of Diamond Films Grown on Surface Roughness Evaluation of Diamond Films Grown on
Substraies with a High Density of Nucleation Sites.

ROBINSON, H. G.

High-Sensitivity Spectroscopy with Diode Lasers.

PB95-175477

Optical Probing of Cold Trapped Atoms

PB95-175469

04,297 quency Measurements with Diode Lasers.

ROBINSON, L.

One-Electron Oxidation of Nickel Porphyrins. Effect of Structure and Medium on Formation of Nickel(III) Porphyrin or Nickel(II) Porphyrin pi-Radical Cation.

PB95-107058

00,865

Site of One-Electron Reduction of Ni(II) Porphyrins. Formation of Ni(I) Porphyrin of Ni(II) Porphyrin pi-Radical Anion. tion of Ni(I) Porphyrin of Ni(II) Porphyrin pi-Radical Anion.
PB95-107066

\section{ROBINSON, $\mathbf{R}$.}

Atomic Oxygen Fine Structure Splittings with Tunable Far Infrared Spectroscopy.

PB95-152203

03,915

Crystallographic and Magnetic Properties of UAuSn PB95-140521

Incommensurate Magnetic Order in UPIGe.

PB95-140513 Magnetic Properties of Single-Crystalline UCu3AI2.
PB95-180717

ROBINSON, R. D.

First Results from the Goddard High-Resolution Spectrograph: The Chromosphere of Tauri.

04,543

GHRS Observations of Cool, Low-Gravity Stars. 1. The FarUltraviolet Spectrum of alpha Orions (M2 lab).

PB96-112016

00,094

Observations of $3 \mathrm{C} 273$ with the Goddard High Resolution Spectrograph on the Hubble Space Telescope. PB95-202321

00,076

Observing Stellar Coronae with the Goddard High Resolution Spectrograph. I. The dMe Star AU Microscopii. PB96-102777

00,092

ROBY, R. J.

Compartment Fire Combustion Dynamics. Annual Report, September 1, 1993-September 1, 1994.

PB95-217162

00,203

Dynamics, Transport and Chemical Kinetics of Compartment Fire Exhaust Gases.

PB96-195508

00,229

ROCHFORO, K. B.

Effect of Semiconductor Laser Characteristics on Optical Fiber Sensor Performance.

PB95-169132

02,167

Faraday Effect Current Sensor with Improved SensitivityBandwidth Product

Faraday Effect Sensors for Magnet Field and Electric CurFent

PB96-119664

04,736

Fundamentals and Problems of Fiber Current Sensors.

PB97.111835

02,205

Magneto-Optic Rotation Sensor Using a Laser Diode as Both Source and Detector.

Polarization Dependence of Response Functions in $3 \times 3$ Sagnac Optical Fiber Current Sensors.

02,154

Polarization Dependence of Response Functions in $3 \times 3$ Sagnac Optical Fiber Current Sensors.

02,189

Polarization Insensitive $3 \times 3$ Sagnac Current Sensor Using Polarizing Spun High-Birefringence Fiber. 02,187

Simultaneous Laser-Diode Emission and Detection for Fiber-Optic Sensor Applications.

PB96-155502

Standard Polarization Components: Progress Toward an Optical Retardance Standard.

Wideband Current and Magnetic Field Sensors Based on PBon Garnets.

ROCKWELL, R. J.

Optical Density Measurements of Laser Eye Protection Ma-

PB96-19030

00,190

ROD, B. J.

Microstructure and Ferroelectric Properties of Lead Zirconate-Titanate Films Produced by Laser Evaporation. PB94-199148

04,470

RODD, J. L.

Stranding Experiments on Double Hull Tanker Structures.

RODE, C.

Empirical Validation of a Transient Computer Model for Combined Heat and Moisture Transter.

PB97-111991

00,416

RODEL, J.

Loading Device for Fracture Testing of Compact Tension Specimens in the Scanning Electron Microscope.

PB95-162434

RODENBUSH, A. $J$.

Effect of Magnetic Field Orientation on the Critical Current of HTS Conductor and Coils

PB96-141189

RODER, H. M.

Transient Methods for Thermal Conductivity.

RODGERS, A. S.

Thermodynamic and Thermophysical Properties of Organic Nitrogen Compounds. Part II. 1- and 2-Butanamine, 2-Methyl-1-Propanamine, 2-Methyl-2-Propanamine, Pyrrole, 1-2. 2 and 3-Methylpyrrole, Pyridine, 2-, 3-, and 4-Methylpyridine, Pyrrolidine, Piperidine, Indole, Ouinoline, Isoquinoline, AcriNaphthalenamine, and 9-Methylcarbazole.

PB94-162294

RODGERS, J. E.

00,741

Scattered Fractions of Dose from 18 and 25 MV X-ray Radiotherapy Linear Accelerators.

RODGERS, J. $P$.

04,120

\section{CRYSTMET: The}

PB97-109029

\section{RODONO, M.}

First Results from a Coordinated ROSAT, IUE, and VLA Study of RS CVn Systems.

PB94-213477

00,069

Rotational Modulation and Flares on RS Canum Venaticorum and BY Draconis Stars. XVI. IUE Spectroscopy and VLA Observations of C1182(=V 1005 Orionis) in
October 1983 . PB94-185626

00,050

Rotational Modulation and Flares on RS Canum 
Electronics and Electrical Engineering Laboratory Technical Progress Bulletin Covering Laboratory Programs, April to June 1996 with 1996-1998 EEEL Events Calendar.

PB97-113880

01,994

Electronics and Electrical Engineering Laboratory Technical Progress Bulletin Covering Laboratory Programs, January to March 1994 with 1994/1995 EEEL Events Calendar.
PB94-193810 308

Electronics and Electrical Engineering Laboratory Technica Progress Bulletin Covering Laboratory Programs, January to March 1996, with 1996 EEEL Events Calendar.

PB96-191390

01.969

Electronics and Electrical Engineering Laboratory Technica Progress Bulletin Covering Laboratory Programs, July to September 1993 with 1994 EEEL Events Calendar.
PB94-194354

02,309

Electronics and Electrical Engineering Laboratory Technica Progress Bulletin Covering Laboratory Programs, July PB95-170395

02,360

Electronics and Electrical Engineering Laboratory Technical Progress Bulletin Covering Laboratory Programs, July to September 1995 with 1996 EEEL Events Calendar.
PB96-147905 Electronics and Electrical Engıneering Laboratory Technica Progress Bulletin Covering Laboratory Programs, October to December 1992, with 1992/1993 EEEL Events Calendar. PB94-165958

02,299

Electronics and Electrical Engineering Laboratory Technical Progress Bulletin Covering Laboratory Programs, Octobe to December 1994 with 1995 EEEL Events Calendar.
PB95-208724 372

Electronics and Electrical Engineering Laboratory Technical Progress Bulletin Covering Laboratory Programs, October to December 1995 with 1996 EEEL Events Calendar

PB96-183116

01,966

Electronics and Electrical Engineering Laboratory Technical Progress Bulletin Covering Programs, October to December 1993, with 1994/1995 EEEL Events Calendar.

02,115

Electronics and Electrical Engineering Laboratory Technica Publication Announcements Covering Laboratory Programs, April to June 1995 with 1995 EEEL Events Calendar

PB96-137187

01,94

Electronics and Electrical Engıneerıng Laboratory Technical Publication Announcements Covering Laboratory Programs, January to March 1994 with 1994/1995 EEEL Events CalPB94-213774

01,883

Electronics and Electrical Engineering Laboratory Technical Publication Announcements Covering Laboratory Programs, January to March 1995 with 1995 EEEl Events Calendar. PB95-242277

02,373

Electronics and Electrical Engineering Laboratory Technical Publication Announcements Covering Laboratory Programs, January to March 1996, with 1996 EEEL Events Calendar.
PB96-214622

01,981

Electronics and Electrical Engineering Laboratory Technica Publication Announcements Covering Laboratory Programs, July to September 1994 with 1995 EEEL Events Calendar.

Electronics and Electrical Engineering Laboratory Technical Publication Announcements Covering Laboratory Programs, Jublication Announcements Covering Laboratory Programs, July to September 1995 with 1996 EEEL Events Calendar.
PB96-183066

Electronics and Electrical Engineering Laboratory Technica Publication Announcements Covering Laboratory Programs, October to December 1993 with 1994/1995 EEEL Events

PB94-193752

02,307

Electronics and Electrical Engineering Laboratory Technical Publication Announcements Covering Laboratory Programs, October to December 1994 with 1995 EEEL Events Cal-

PB95-231841

01,918

Electronics and Electrical Engineering Laboratory Technica Publication Announcements Covering Laboratory Programs,
October to December 1995, with 1996 EEEL Events Cal-

PB96-202346

01,978

ROITBURD, A.

Temperature Dependence of Vortex Twin Boundary Interaction in Yttrium Barium Copper Oxide (YBa2Cu3O6+X).

04,613

ROITMAN, P.

Characterization of Interface Defects in Oxygen-Implanted SBilicon Films.

Charge Trapping and Breakdown Mechanism in SIMOX

B2,323

Defect Formation Mechanism Causing Increasing Defect Density during Decreasing Implant Dose in Low-Dose PBimox.

Defect of Thermal Ramping and Annealing Conditions on Defect Formation in Oxygen Implanted Silicon-On-Insulator

PB94-212966

02,318

Defect Pair Formation by Implantation-Induced Stresses in PB95-175824

02,364
Design Guide for CMOS-On-SIMOX. Test Chips NIST3 and PB94-163458

02,297

Effect of Annealing Ambient on the Removal of Oxide Precipitates in High-Dose Oxygen Implanted Silicon.
PB95-164356 Effect of Intermediate Thermal Processing on sulator Materia.

PB96-160213

02,982

Effect of Single versus Multiple Implant Processing on Defect Types and Densities in SIMOX

01,957

Electron and Hole Trapping in Irradiated SIMOX, ZMR and BESOI Buried Oxides.

PB96-160320

01,956

Electron Traps, Structural Change, and Hydrogen Related

SIMOX Defects.
PB94-200391 02,312

Evidence for a Deep Electron Trap and Charge Compensation in Separation by Implanted Oxygen Oxides.

Mechanism of Defect Formation in Low-Dose Oxygen Im planted Silicon-on-Insulator Material.

PB97-111462

Nano-Defects in Commercial Bonded SOI and SIMOX PB96-12367

02,453

02,407

Stacking Fault Pyramid Formation and Energetics in Silicon-

on-Insulator Material Formed by Multiple Cycles of Oxygen Implantation and Annealing.

PB96-160221

04,083

ROLANDO, C.

Oxidation of Caffeic Acid and Related Hydroxycinnamic Acids.

PB97-111975

00,651

ROLSTON, S.

Hyperfine Effects and Associative Ionization of Ultracold Sodium.

PB95-15122

03,903

Optical Molasses: The Coldest Atoms Ever.

03,878

Ultracold Collisions: Associative Ionization in a Laser Trap. PB94-213238

ROLSTON, S. L.

Atoms in Optical Molasses.

PB95-108874 03,859

03,875

Atoms in Optical Molasses: Applications to Frequency Standards.

PB95-108882

03,876

Heterodyne Measurement of the Fluorescence Spectrum of Optical Molasses.

03,873

Laser-Cooled Neutral Atom Frequency Standards.

PB96-160312

04,086

Laser Cooling and the Recoil Limit.

PB97-111280 04,391

Laser Modification of Ultracold Collisions: Experiment

PB96-157987

04,075

Localization of Atoms in a Three-Dimensional Standing PBave. 163887

03,944

Magneto-Optical Trapping of Metastable Xenon: IsotopeShitt Measurements.

PB95-151254

03,905

Measurements of Fluorescence from Cold Atoms: Localization in Three-Dimensional Standing Waves.

03,943 PB5-163879

Microlithography by Using Neutral Metastable Atoms and
Self-Assembled Monolayers.
PB96-190038
02,441 PB96-190038

Optical Molasses: Cold Atoms for Precision Measurements.
PB95-108890
03,877

03,877
sigma+-sigma- Optical Molasses in a Longitudinal Magnetic

PB95-161840

03,934

ROMAN, P.

Precision Nuclear Orientation Measurements for Determining Mixed Magnetic Dipole/Electric Ouadrupole Hyperfine

PB94-199080

03,810

ROMANKIW, L

Magnetic and Structural Properties of Electrodeposited Cop per-Nickel Microlayered Alloys.

PB94-21312

04,512

ROMANOVSKY, A.

Precise Optical Frequency References and Difference Frequency Measu
PB95-176228

04,305

RONNING, $C$.

Modeling Detector Response for Neutron Depth Profiling.
PB96-157813 ROOK, H. L.

1994

Materials Science and Engineering Laboratory Annual $R$

port, 1993. NAS

Materials Science and Engineerıng Laboratory Annual Report, 1995. Technical Activities.

03,009

RoOS, C.

Parametric Study of Wall Moisture Contents Using a Revised Variable Indoor Relative Humidity Version of the 'Moist' Transient Heat and Moisture Transfer Model.

PB97-122535

00,419

ROOS, M

Problem of Convection in the Water Absorbed Dose Calorimeter.

PB97-110159

03,523

OOVERS, J.

Thermodynamic Properties of Dilute and Semidilute Solutions of Regular Star Polymers.

RORRER, D. E.

Videoconferencing Procurement and Usage Guide.

PB94-217023

01,284

ROSASCO, G. J.

Development of the NIST Transient Pressure and Tempera. ture Calibration Facility

00,626

Executive Summary: Proceedings of the Workshop on the Measurement of Transient Pressure and Temperature.

PB96-160841

02,679

Measurement of the Self Broadening of the $\mathrm{H} 2 \mathrm{O}(0-5)$ Raman Transitions from 295 to $1000 \mathrm{~K}$

00,884

O Branch Lineshape Functions for CARS Thermometry

PB96-160643 01,132

Simultaneous Forward-Backward Raman Scattering Studies of D2 Broadened by $\mathrm{D} 2, \mathrm{He}$, and $\mathrm{Ar}$.

PB95-162459

00,961

ROSE, A. H

Effect of Semiconductor Laser Characteristics on Optica! Fiber Sensor Performance.

02,167

Faraday Effect Current Sensor with Improved SensitivityBandwidth Product.

PB95-203154

02,180

PB94-200698

Faraday Effect Sensors: A Review of Recent Progress.

PB94-200706

02,128

Faraday Effect Sensors for Magnet Field and Electric CurPB96-119664

04,736

Fundamentals and Problems of Fiber Current Sensors.

PB97-111835

02,205

Improved Anneal

04,343

Magneto-Optic Rotation Sensor Using a Laser Diode as Both Source and Detector.

PB97-111272

Magnetooptic Effects.

04,390

96-119292

04,338

Optical Current Transducer for Calibration Studies

PB94-185907

02,121 
ROSE, K. J.

Intensive Swimming: Can It Affect Your Patients' Smiles. PB96-123666

03,570

ROSEN, B.

Preliminary Functional Specifications of a Prototype Electronic Research Notebook for NIST.

PB94-207

00,012

ROSEN, H. N.

Sorption of Moisture on Epoxy and Alkyd Free Films and Coated Steel Panels.

PB95-162475

03,192

ROSENBERG, A.

Photonic Band-Structure Effects for Low-Index-Contrast Two-Dimensional Lattices in the Near Infrared. PB97.122469

04,401

ROSENBLATT, J. R.

Churchill Eisenhart, 1913-1994.

PB96-137740

03,447

ROSENFELD, A. H.

National Planning for Construction and Building $R$ and $D$.

PB96-137104 00,324

Program of the Subcommittee on Construction and Building.

PB94-193646

00,319

Program of the Subcommittee on Construction and Building (July 1994)

PB95-122537

00,321

Rationale and Preliminary Plan for Federal Research for Construction and Building.

00,322

ROSENFELD, D. A.

Procedure for Product Data Exchange Using STEP Developed in the AutoSTEP Pillot.

PB96-183058

02,843

Reference Manual for the Algorithm Testing System Version 2.0 .

PB96-128244

02,922

User's Guide for the Algorithm Testing System Version 20

PB95-251666

02916

ROSENTHAL, L. S.

Computer Graphics Metafile (CGM): Procedures for NIST CGM Validation Test Service.

PB94-161809

01,804

Initial Graphics Exchange Specification (IGES): Procedures for the NIST IGES Validation Test Service.

02,780

STandard for the Exchange of Product Model Data (STEP):

Procedures for NIST STEP Validation.

Procedures tor

02,787

ROSENTHAL, P. A.

Critical Current and Normal Resistance of High-Tc StepEdge SNS Junctions.

PB96-111752 04,724

High Temperature Superconductor-Normal MetalSuperconductor Josephson Junctions with High Char-

acteristic Voltages.
PB95-176079

04,666

Terahertz Shapiro Steps in High Temperature SNS Josephson Junctions.

02,168

Thermal Noise in High-Temperature Superconducting-Normal-Superconducting Step-Edge Josephson Junctions

PB95-175089

04,650

ROSHKO, A.

Coexistence of Grains with Differing Orthorhombicity in High Ouality YBa2Cu307-delta Thin Films.

PB96-135033

04,742

Correlation between $\mathrm{Tc}$ and Elastic Constants of (LaM)2CuO4.

04,514

Critical Current Behavior of Ag-Coated YBa2Cu307-x Thin Films.

PB95-141016

04,549

Distributed Feedback Lasers in Rare-Earth-Doped Phosphate Glass.

04,740

Effects of Etching on the Morphology and Surface Resistance of YBa2Cu307-delta Films.

PB96-135355

02,410

Effects of Substrate Surface Steps on the Microstructure of Epitaxial Ba2YCu3O7-x Thin Films on (001) LaAlO3. PB96-148184

04,774

Epitaxial Nucleation and Growth of Chemically Derived Ba2YCu307-x Thin Films on (001) $\mathrm{SrTiO}$.

PB96-190186

04,787

Growth of Epitaxial KNbO3 Thin Films.

PB96-13518

02,409

Growth of Laser Ablated YBa2Cu307-delta Films as Examined by Rheed and Scanning Tunneling Microscopy. 04,614 High Current Pressure Contacts $10 \mathrm{Ag}$ Pads on Thin Film Superconductors.

04,639

Influence of Deposition Parameters on Properties of Laser Ablated YBa2Cu307-Delta Films.

04,544
Magnetic Flux Pinning in Epitaxial YBa2Cu3O7-delta Thin

04,795

PB96-200746

04,259

PB.LiTaO3 Waveguide Laser.

Passively O-Switched Nd-Doped Waveguide Laser.

PB95-180048 04,308

Size Effects and Giant Magnetoresistance in Unannealed NiFe/Ag Multilayer Stripes.

PB97.111306

04,145

Surface Degradation of Superconducting YBa2Cu3O7-delta Thin Films.

04,667

Surface Modification of YBa2Cu307-delta Thin Films Using the Scanning Tunneling Microscope: Five Methods

PB95-203394

04,699

ROSOV, N.

Crystal Structure and Magnetic Ordering of the Rare-Earth and $\mathrm{Cu}$ Moments in $\mathrm{RBa} 2 \mathrm{Cu} 2 \mathrm{NbO}$ ( $\mathrm{R}=\mathrm{Nd}, \mathrm{Pr}$ ).

04,546

Determination of Anomalous Superexchange in $\mathrm{MnCl} 2$ and Its Graphite Intercalation Compound. Its Graphite Intercalation Compound.

Magnetic Ordering of the Cu Spins in PrBa2Cu306+x PB95-140547

04.545

Neutron Powder Diffraction Study of the Nuclear and Magnetic Structures of the Oxygen-Deficient Perovskite YBaCuCoO5

00,954

Polarization Analysis of the Magnetic Excitations in Fe65Ni35 Invar.

04,558

Polarization Analysis of the Magnetic Excitations in Invar and Non-Invar Amorphous Alloys

PB94-216116

04.516

Ouasielastic and Inelastic Neutron-Scattering Studies of ((CD3)3ND)FeCl3.2D2O: A One-Dimensional Ising Ferromagnet.

04,547

Structural and Magnetic Properties of $\mathrm{CuCl} 2$ Graphite Intercalation Compounds.

PB96-119748

03,020

Temperature Dependence of the Magnetic Excitations in

Ordered and Disordered Fe72Pt28.

PB95-150223

04,563

Vortex Dynamics and Melting in Niobium

02,073

\section{Ross, A. B.}

Critical Review of Rate Constants for Reactions of Transients from Metal Ions and Metal Complexes in Aqueous Solution.

PB96. 145859

01,109

Ouantum Yields for the Photosensitized Formation of the Lowest Electronically Excited Singlet State of Molecular Ox-

ygen in Solution
PB94-161007

00,732

Rate Constants for the Decay and Reactions of the Lowest Electronically Excited Singlet State of Molecular Oxygen in Solution. An Expanded and Revised Compilation.

PB96-145826

01,106

ROSS, G. G.

Measured Stopping Powers of Hydrogen and Helium in Polystyrene Nea
PB96-112321

04,729

Ross, J.

Ouadratic Response of a Chemical Reaction to External Oscillations.

ROSSEINSKY, M. J.

01,138

Neutron-Scattering Study of $\mathrm{C} 60(n-)(n=3,6)$ Librations in Alkali-Metal Fullerides.

PB94-200219

\section{ROSSI, H. H.}

Measurement of Absorbed Dose of Neutrons, and of Mixtures of Neutrons and gamma rays.

AD-A286 647/3

03,710

ROSSITER, W.

Characteristics of Adhesive-Bonded Seams Sampled from EPDM Roof Membranes.

Laboratory Accreditation for Testing Energy Efficient Lighting.

00,270

ROSSITER, W. J.

Application of Thermal Analysis Techniques to the Characterization of EPDM Roofing Membrane Materials.
PB95-125845

00,359

Effects of Adhesive Thickness, Open Time, and Surface Seams of EPDM Rubber Roofing Membrane

PB95-151338

00,372

Efficiency of Electric Motors. National Voluntary Lab. ACcreditation Program (NVLAP)

02,107

National Voluntary Laboratory Accreditation Program: Car-

pet and Carpet Cushion.

00,295

National Voluntary Laboratory Accreditation Program: Energy Efficient Lighting Products.

PB94-219060

02,642

National Voluntary Laboratory Accreditation Program (NVLAP): Wood Based Products.

03,405

Performance Approach to the Development of Criteria for ow-Sloped Roof Membranes.

PB94-160751

00,329

Performance of Tape-Bonded Seams of EPDM Membranes: Comparison of the Peel Creep-Rupture Response of TapeBonded and Liquid-Adhesive-Bonded Seams.

PB96-183249

03,012

Pulse-Echo Ultrasonic Evaluation of the Integrity of Seams of Single-Ply Roof Membranes.

00,381

Use of Thermal Mechanical Analysis to Characterize Ethylene-Propylene-Diene Terpolymer (EPDM) Roofing Membrane Materials.

PB95-125852

00,360

Rosso, A

Wavelength Dependence of Normal Spectral Emissivity of High-Temperature Metals at Their Melting Point.

PB94-200011

03,398

OSSO, C.

X-ray Emission from Chemically Peculiar Stars.

PB96-102256

00,088

Continuous Counter-Current Two Phase Aqueous Extraction.

PB95-161212

00,675

ROTH, R. S.

$\mathrm{Ca} 1-\mathrm{xCuO} 2$, a NaCuO2-Type Related Structure.

PB95-162822

04,620

Ca4Bi6013: A Compound Containing an Unusually Low Bismuth Coordination Number and Short Bi Bi Contacts.
PB95-141131

Crystallographic Characterization of Some Intermetallic Compounds in the Al-Cr System.

PB94-198702 03,318

High Temperature Reactions of Uranium Dioxide with Varlous Metal Oxides.

AD-A286 648/1

00,717

(AlNbO4).

PB95-107306

04,523

Magnetic Dielectric Oxides: Subsolidus Phase Relations in he BaO: Fe203: $\mathrm{TiO} 2$ System.

01,156

Phase Equilibria in the Systems $\mathrm{CaO}-\mathrm{CuO}$ and $\mathrm{CaO}-\mathrm{Bi} 2 \mathrm{O} 3$

03,048

Powder $X$-ray Diffraction Data for $\mathrm{Ca} 2 \mathrm{Bi} 2 \mathrm{O} 5$ and C4Bi6013.

PB96-161278

04,777

Preparation and Crystal Structure of Sr5TiNb4017.

PB96-167341 04,107

Preparation, Crystal Structure, Dielectric Properties, and 
ROUSE, W. B.

Learning to Change: Opportunities to Improve the Perform-

ance of Smaller Manufacturers.
PB94-166212

00,010

Electric Field Dependent Dielectric Breakdown of Intrinsic $\mathrm{SiO} 2$ Films Under Dynamic Stress.

02,449 PB96-204102

Time-Dependent Dielectric Breakdown of Intrinsic $\mathrm{SIO}$
Films under Dynamic Stress.
PB96-179478

PB96-179478

ROWAN, W. L.

Improved Wavelengths for Prominent Lines of $\mathrm{Cr} X V I$ to $\mathrm{Cr}$

03,895

PB95-150629

$X$ to $\mathrm{Fe}$

Improv.

PB96-111638
Observation of Pd-Like Resonance Lines Through Pt(32+) and $Z n$-Like Resonance Lines of $\operatorname{Er}(38+)$ and $\operatorname{Hf}(42+)$.

PB95-150637

03,896

Rb-Like Spectra: Pd X to Nd XXIV

03,897

PB95-150645

(23+) to

Pt (33+).

03,898

PB95-150652

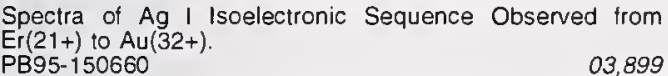

PB95-1506

ROWE, J. E.

Local Partial Densities of States in $\mathrm{Ni}$ and $\mathrm{Co}$ Silicides Studied by Soft X-Ray Emission Spectroscopy.

04,504

ROWE, J.

Cold Neutron Gain Calculations for the NBSR Using MCNP.
PB 935163978

Liquid-Hydrogen Cold Neutron Source for the NBSR.

03,729

Neutron Techniques in Materials Science and Related Disciplines

02,980

Nuclear Heat Load Calculations for the NBSR Cold Neutron Nuclear Heat Load Calculations for the NBSR Cold Neutron
PB95-152955 MCNP.

03,730

Reactor Radiation Technical Activities, 1994. NAS-NRC Assessment Panel, April 6.7, 1995

03,732

Thermal Hydraulic Tests of a Liquid Hydrogen Cold Neutron Source

03,884

ROWE, P.

Recommendations on Selection of Vehicle-to-Roadside Communications Standards for Commercial Vehicle Operations.

PB94-195914

04,859

Vehicle-to-Roadside Communications for Commercial Vehicle Operations: Requirements and Approaches

04,860

ROWE, P. S.

Sources of Uncertainty in a DVM-Based Measurement System for a Ouantized Hall Resistance Standard.

01,884

ROY, $\mathbf{P .}$

Vibronic Coupling and Other Many-Body Effects in the 4 sigmag(-1) Photoionization Channel of $\mathrm{CO} 2$.

ROY, S.

Anharmonic Oscillator Analysis Using Modified Airy Func-

PB94-185311

03,798

Small-Angle Neutron Scattering of Poly(vinyl alcohol) Gels.

PB95-164117 01,260

Small Angle Neutron Scattering Studies on Chain Asymmetry of Coextruded Poly(Vinyl Alcohol) Film.

PB95-164372

01,262

ROYTBURD, A.

Coherent Precipitates in the BCC/Orthorhombic Two Phase Field of the Ti-Al-Nb System

03,317

Transformation of $\mathrm{BCC}$ and $\mathrm{B} 2$ High Temperature Phases to HCP and Orthorhombic Structures in the Ti-Al-Nb System. Part 1. Microstructural Predictions Based on a Subgroup Relation between Phases.

group Relation
PB96-169065

03,370

ROYTBURD, A. L.

Determination of Thermoactivation Parameters of Vortex Mobility in YBa2Cu3O7 Using Only Magnetic Measurements.

PB95-163499

04,624

ROZSA, K.

Deposition Rates in Direct Current Diode Sputtering.

PB95-203345

RUBENSSON, J. E.

Intermediate Coupling in L2-L3 Core Excitons of MgO, $\mathrm{Al} 2 \mathrm{O} 3$, and $\mathrm{SiO} 2$

04,079
Laser-Synchrotron Hybrid Experiments: A Photon to Tickle, A Photon to Poke.

03,704

Local Partial Densities of States in $\mathrm{Ni}$ and Co Silicides Studied by Soft X-Ray Emission Spectroscopy.
PB94-212412 Soft-X-ray-Emission Investigation of Cobalt Implanted Silicon Crystals.

04,069 Sott-X-ray-Emission Spectra of Solid $\mathrm{Kr}$ and $\mathrm{Xe}$
PB96-157920

04,070

Fluctuation Dominated Recombination Kinetics with Traps. PB95-107264

\section{QUDDER, F. F.}

Geometric Characterization of Rockwell Diamond Indenters.
PB95-203287

Microform Calibration Uncertainties of Rockwell Diamond Indenters.
PB96-122114

03,280

Microform Calibrations in Surface Metrology.

02,951

(203295

Post-Process Control of Machine Tools.

PB95-203451

02,952

Static Structural Analysis of a Reconfigurable Rigid Platform Supported by Elastic Legs.

PB97-113898

02,960

Stylus Technique for the Direct Verification of Rockwell Diamond Intenders.

RUDELL, $M$.

02,958

Liposome-Based Flow-Injection Immunoassay for Determining Theophylline in Serum.

PB94-213493

03,494

\section{RUDMAN, D. A}

$30 \mathrm{THz}$ Mixing Experiments on High Temperature Superconducting Josephson Junctions.
PB96-102462 Antenna-Coupled High-Tc Air-Bridge Microbolometer on Sil-

PB95-180899

04,315

Coexistence of Grains with Differing Orthorhombicity in High Ouality YBa2Cu307-delta Thin Films.

04,742

Critical Current and Normal Resistance of High-Tc StepEdge SNS Junctions.

PB96-111752

04,724

Effect of Microstructure on Phase Formation in the Reaction of $\mathrm{Nb} / \mathrm{Al}$ Multilayer Thin Films.

PB95-168415

03,352

Effects of Anneal Time and Cooling Rate on the Formation and Texture of $\mathrm{Bi2Sr} 2 \mathrm{CaCu} 2 \mathrm{O} 8$ Films.

PB95-161600

04,603

First Phase Formation Kinetics in the Reaction of $\mathrm{Nb} / \mathrm{Al}$.

PB95-168456

03,353

Grain Alignment and Transport Properties of Bi2Sr2CaCu2O8 Grown by Laser Heated Float Zone MethPB95-161451

04,602

Growth of Laser Ablated YBa2Cu3O7-delta Films as Examined by Rheed and Scanning Tunneling Microscopy.

PB95-162541

04,614

High-Tc Superconducting Antenna-Coupled Microbolometer on Silicon.

Increased Pinning Energies and Critical Current Densities in Heavy-Ion-Irradiated Bi2Sr2CaCu2O8 Single Crystals.

PB95-175352

04,654

Increased Transition Temperature in In situ Coevaporated

YBa2Cu3O7-delta Thin Films by Low Temperature Post-An-

PB95-180071

04,672

Influence of Deposition Parameters on Properties of Laser Ablated YBa2Cu3O7-Delta Films.

PB95-140539

04,544

Insulating Nanoparticles on YBa2Cu307-delta Thin Films Revealed by Comparison of Atomic Force and Scanning

Tunneling Microscopy.

04,575

Magnetic Flux Pinning in Epitaxial YBa2Cu3O7-delta Thin

PBilms.

04,795

Microwave Noise in High-Tc Josephson Junctions.

PB96-148010

04,771

Microwave Properties of Voltage-Tunable YBa2Cu3O7. delta/SrTiO3 Coplanar Waveguide Transmission Lines.

PB96-141262 02,235

Observation of Insulating Nanoparticles on YBCO ThinFilms by Atomic Force Microscopy.

PB95-163358

04,622

Suitability of Metalorganic Chemical Vapor Deposition-Derived PrGaO3 Films as Buffer Layers for YBa2Cu3O7-X Pulsed Laser Deposition.

PB95-168670

04,640

Temperature Dependence and Magnetic Field Modulation of Critical Currents in Step-Edge SNS YBCO/Au Junctions. PB96-111745

Thermal Isolation of High-Temperature Superconducting Micromachining. Silicon Wafer Bonding and PB96-135017

02,408

Thin Film Reaction Kinetics of Niobium/Aluminum

PB95-175295

04,651

Use of Ion Scattering Spectroscopy to Monitor the Nb Target Nitridation during Reactive Sputtering

00,761

Vortex Images in Thin Films of $\mathrm{YBa2Cu3O}$ (sub 7-x) and Bi2Sr2Ca1Cu2O(sub $8+x$ ) Obtained by Low-Temperature Magnetic Force Microscopy.

PB97-119408

04,815

YBa2Cu3O7-x to Si Interconnection for Hybrid Superconductor/Semiconductor Integration

PB94-211711

02,315

RUFF, A. W.

Considerations on Data Requirements for Tribological Modeling.

Critical Factors in Non-Lubricated, Non-Abrasive Wear Testing. 140588

03,236

Damage Processes in Ceramics Resulting from Diamond Tool Indentation and Scratching in Various Environments. PB96-102983 03,065

Hybrid Method for Determining Material Properties from In strumented Micro-Indentation Experiments.

PB95-152229

03,348

Modified Surface Layers and Coatings.

B95-176087

03,125

Nanoindentation and Instrumented Scratching Measurements on Hard Coatings.

P97-122477

03,111

Tribological Data: Needs and Opportunities.

PB95-140596

03,237

Wear of Selected Materials and Composites Sliding agains PB94-172749

RUHL, M. K.

Findings and Recommendations from a Software Reengineering Case Study.

PB96-15579

01,752

E, D.

Thermal Conductivity of Polypyromellitimide Film with Alumina Filler Particles from 4.2 to $300 \mathrm{~K}$

PB96-200753

01,292

RUMBLE, J.

Access Paths for Materials Databases: Approaches for Large Databases and Systems.

PB95-162525

02,975

RUNKLES, $R$.

Laboratory Accreditation for Testing Energy Efficient Lighting.

RUNYAN, J. L

Fabrication of Flaw-Tolerant Aluminum-Titanate-Reinforced Alumina.

PB95-162533

03,161

Flaw-Insensitive Ceramics.

PB97. 110027 
Inelastic Neutron Scattering Studies of Nonlinear Optical Materials: $p$-Nitroaniline Adsorbed in ALPO-5.

00,874

Inelastic Neutron Scattering Studies of Rotational Excitations and the Orientational Potential in $\mathrm{C} 60$ and $\mathrm{A} 3 \mathrm{C} 60$ Compounds.

PB94-172673

00,763

Local-Mode Dynamics in $\mathrm{YH} 2$ and $\mathrm{YD} 2$ by Isotope-Dilution Neutron Spectroscopy.

01.017

Low-Energy Vibrations and Octahedral Site Occupation in $\mathrm{Nb95V} 5 \mathrm{H}(\mathrm{D}) \mathrm{y}$.
$\mathrm{PB} 96-160734$

01,133

Neutron and Raman Spectroscopies of 134 and 134a Hydrofluorocarbons Encaged in $\mathrm{Na}-\mathrm{X}$ Zeolite. PB96-186168

03,001

Neutron-Powder-Diffraction Study of the Long-Range Order in the Octahedral Sublattice of LaD2.25.

PB96-141155

04,753

Neutron-Scattering Study of $\mathrm{C} 60(\mathrm{n}-)(\mathrm{n}=3,6)$ Librations in Alkali-Metal Fullerides.

PB94-200219

00,806

Neutron Scattering Study of the Lattice Modes of Solid Cubane.

PB96-147152

01,126

Neutron Spectroscopic Comparison of beta-Phase Rare

Earth Hydrides.
PB96-160742

Neutron Spectroscopic Comparison of Rare-Earth/Hydrogen alpha-Phase Systems.

00,970

Neutron Spectroscopic Evidence of Concentration-Dependent Hydrogen Ordering in the Octahedral Sublattice of beta-

PB95-181020

01,018

Neutron-Spectroscopy Study of the Hydrogen Vibrations in Hydrogen-Doped

04,441

Phonon Density of States in $\mathrm{R} 2 \mathrm{CuO} 4$ and Superconducting $\mathrm{R} 1.85 \mathrm{CeO} .15 \mathrm{CuO} 4$ ( $\mathrm{R}=\mathrm{Nd}, \mathrm{Pr}$ )

PB95-150686

04,574

Vibrations of Hydrogen and Deuterium in Solid Solution with Lutetium.

PB95-181038

01,019

RUSHMEIER, $\mathrm{H}$.

Simultaneous Optical Measurement of Soot Volume Fraction, Temperature, and $\mathrm{CO} 2$ in Heptane Pool Fire.

PB96-102132

01,397

RUSSEK, $\mathbf{S}$

Proposed Antiferromagnetically Coupled Dual-Layer Magnetic Force Microscope Tips.

PB95-169017

RUSSEK, S. E.

04,645

Bias Current Dependent Resıstance Peaks in NiFe/Ag Giant Magnetoresistance Multilayers.

04,153

Effects of Etching on the Morphology and Surface Resistance of YBa2Cu3O7-delta Films.

PB96-135355

02,410

Evidence for Tunneling and Magnetic Scattering at 'In situ' YBCO/Noble-Metal Interfaces.

PB96-141098

04,752

Experimental Verification of a Micromagnetic Model of DualLayer Magnetic Films

PB95-141081 04,553

Growth of Laser Ablated YBa2Cu3O7-delta Films as Exam-

ined by Rheed and Scanning Tunneling Microscopy.

04,614

In situ Noble Metal YBa2Cu3O7 Thin-Film Contacts. PB94-211323

04,494

Insulating Boundary Layer and Magnetic Scattering in YBa2Cu307-delta/Ag Interfaces Over a Contact Resistivity Range of $10(-8)-10(-3)$ Omega $\mathrm{cm}(2)$

PB95-169157

04,648

Low Magnetostriction in Annealed NiFe/Ag Giant Magnetoresistive Multilayers. Magnetic and Magnetoresistive Properties of Inhomogeneous Magnetic Dual-Layer Films.
PB95-169025 Magnetoresistance of Thin-Film NiFe Devices Exhibiting Single-Domain Behavior.

Single-Domain
PB $96-147087$

04,766

Models of Granualr Giant Magnetoresistance Multilayer Thin Films.

PB96-190228

01,968

Oxygen Annealing of Ex-situ YBCO/Ag Thin-Film Interfaces.

Simulating Device Size Effects on Magnetization Pinning Mechanisms in Spin Valves.

PB97-112593

04,158

Size and Self-Field Effects in Giant Magnetoresistive ThinFilm Devices.

Size Effects and Giant Magnetoresistance in Unannealed NiFe/Ag Multilayer Stripes.

04,145
Size Elfects in Submicron NiFe/Ag GMR Devices. PB96-155510

Surface Degradation of Superconducting YBa2Cu3O7-delta Thin Films.

$\begin{array}{ll}\text { PB95-176095 } & 04,667\end{array}$

Telegraph Noise in Silver-Permalloy Giant

Magnetoresistance Test Structures.

04,763

RUSSELL, C. S.

Standard Generalized Markup Language Test Suite Evaluation Report.

PB96-154992

RUSSELL, D. L. F.

01,751

Publications of the Intelligent Systems Division (Previously Robot Systems Division) Covering the Period January 1971-April 1994

PB94-217098

02,935

RUSSELL, J. B.

Measurement of Boron at Silicon Wafer Surfaces by Neutron Depth Profiling.

PB94-211059

\section{RUSSELL, $R$.}

Unified Telerobotic Architecture Project (UTAP) Standard Interface Environment (SIE), May 1995

PB95-242350

02,938

RUSSELL, T. J.

Beyond the Technology Roadmaps: An Assessment of Electronic Materials Research and Development. PB96-165998

RUSSELL, T. P.

Grazing Incidence Prompt Gamma Emissions and Reso nance-Enhanced Neutron Standing Waves in a Thin-Film. PB95-150470

03,892

Morphology of Symmetric Diblock Copolymers as Revealed by Neutron Reflectivity.

PB95-140075 01,234

Observed Frustration in Confined Block Copolymers.

PB95-150033 01,238

Temperature Dependence of the Morphology of Thin Diblock Copolymer Films as Revealed by Neutron Reflectiv. ity.

01,199

RUST, B. W.

Modulation of Fossil Fuel Production by Global Temperature Variations, 2

PB94-1466

02,533

New Extrapolation/Spherical/Cylindrical Measurement Facility at the National Institute of Standards and Technology. ity at the Nationa

RUZICKA, V.

Estimation of the Heat Capacities of Organic Liquids as a Function of Temperature Using Group Additivity. I. Hydrocarbon Compounds.

PB94-162278

00,739

Estimation of the Heat Capacities of Organic Liquids as a Function of Temperature Using Group Additivity. II. Compounds of Carbon, Hydrogen, Halogens, Nitrogen, Oxygen

PB94-162286

00,740

RYAN, H. M.

Ultrasonic Measurements of Surface Roughness.

PB94-172137

04,181

RYBICKI, G. B.

Sobolev Approximation for Line Formation with Partial Frequency Redistribution.

PB95-202669

00,078

RYTZ, D.

Anomalous Dispersion and Thermal Expansion in LightlyDoped $\mathrm{KTa} 1-\mathrm{xNb} \times \mathrm{O} 3$

PB95-152302

RZAZEWSKI, $K$.

Appearance Intensities for Multiply Charged lons in a Strong Laser Field.

PAAR S. H.

04,089

Relationship between Radiative and Magnetic Fluxes for Three Active Solar-Type Dwarfs.

PB96-119540

00,097

SABATINI, R. L.

Transverse stress effect on the critical current of internal tin and bronze process $\mathrm{Nb}$ (sub 3 ) $\mathrm{Sn}$ superconductors.

DE95016659

04,434

Enthalpy Increment Measurements from 4.5 to $350 \mathrm{~K}$ and the Thermodynamic Properties of Titanium Disilicide(cr) to

$1700 \mathrm{~K}$.

00,678

SACCHET, L. L.

Electronics and Electrical Engineering Laboratory: 1996 Program Plan. Supporting Technology for U.S. Competitiveness in Electronics.

PB96-175237

01,962

SACKS, J.

Taguchi's Parameter Design: A Panel Discussion.

PB96-111802

SADANA, D. K.

Nano-Defects in Commercial Bonded SOI and SIMOX PB96-123674

02,407

SADEK, $F$.

Method of Estimating the Parameters of Tuned Mass

Dampers for Seismic Applications.

PB96-167820

00,473

Modified Optimal Algorithm for Active Structural Control. PB96-165949

00,472

SADIQ, T. A. K.

Interaction between Micro and Macroscopic Flow in RTM Preforms.

SAGERGREN, E. S

03,159

Efficient Experiment to Study Superconducting Ceramics. PB94.212578

SAIER, M. H.

04,505

Glucose Permease of Bacillus Subtilis is a Single Polypeptide Chain That Functions to Energize the Sucrose Polypeptice

PB95-163192

03,466

SAITO, $K$.

Upward Flame Spread along the Vertical Corner Walls (October 1993).

SAKAI, C. K.

Bioleaching of Cobalt from Smelter Wastes by 'Thiobacillus ferrooxidans'.

PB95-140968

02,582

SALAH, L. M.

Reanalysis of the (010), (020), (100), and (001) Rotational Levels of (32) $\mathrm{S}(16) \mathrm{O} 2$

PB95-125621

00,887

SALAMANCA-RIBA, L.

Characterization of the ZnSe/GaAs Interface Layer by TEM and Spectroscopic Ellipsometry.

04,655

Determination of the Optical Constants of ZnSe Films by Spectroscopic Ellipsometry.

04,656

\section{SALAMON, M. B.}

Enhanced Curie Temperatures and Magnetoelastic Domains in Dy/Lu Super Lattices and Films.

PB94-172665

04,443

Magnetic Rare

Magnetoelasticity in Rare-Earth Multilayers and Films. PB94-211356

04,611

SALAMON, $W$.

Distributed Communication Methods and Role-Based ACcess Control for
PB96-183165

SALAMON, W. J.

Object-Oriented Technology Research Areas.

PB95-199329 01,726

Ouality Characteristics and Metrics for Reusable Sottware (Preliminary Report).

SALEM, A. J.

Comparison of the Unidirectional and Radial In-Plane Flow of Fluids Through Woven Composite Reinforcements.

Interaction between Micro and Macroscopic Flow in RTM Preforms. 
RIS Studies of Autoionization in Calcium.

PB94-213295

SALOMON, C.

Atoms in Optical Molasses.

PB95-108874

03,875

Atoms in Optical Molasses: Applications to Frequency PBtandards.

03,876

Frequency-Stabilized LNA Laser at 1.083 mum: Application to the Manipulation of Helium 4 Atoms.

04,304

Optical Molasses: Cold Atoms for Precision Measurements.
PB95-108890

Optical Molasses: The Coldest Atoms Ever.

03,878

ALSBURY, J. G.

Development of an Automated Part Inspection System Using the DMIS Standard.

02,899

SALTMAN, $R$.

Good Security Practices for Electronic Commerce, Including Electronic Data Interchange.

PB94-139045

01,463

Planning the Infrastructure for Global Electronic Commerce.

PB94-185832

00,494

SALZBORN, E.

Dielectronic Capture Processes in the Electron-Impact Ionization of $\mathrm{Sc}(2+)$.

04,000

SAMI, $\mathbf{S}$.

Nutritional Status and Growth in Juvenile Rheumatoid Arthritis.

PB94-198470

03,515

SAMOTYJ, M.

Important Link in Entire-House Protection: Surge Reference Equalizers.

PB94-216504

02,219

SAMS, R. L.

Radiocarbon Measurements of Atmospheric Volatile Organic Compounds: Ouantifying the Biogenic Contribution.

PB97-122352

02,574

SAMUELSON, $S$.

Novel Bulk Iron Garnets for Magneto-Optic Magnetic Field Sensing.

04,675

SANBORN, B. A.

Correction to the Decay Rate of Nonequilibrium Carrier Distributions Due to Scattering-in Processes.

PB94-185840

04,452

Cryogenic Precision Capacitance Bridge Using a Single Electron Tunneling Electrometer

02,072

Electron-electron Interactions, Coupled-Plasmon-Phonon Modes, and Mobility in n-Type GaAs.

PB96-138524

04,749

Nonequilibrium Total-Dielectric-Function Approach to the Electron Boltzmann Equation for Inelastic Scattering in Doped Polar Semiconductors.

PB96-138532

04,416

SANCHEZ, A.

Effects of Critical Current Density, Equilibrium Magnetization and Surface Barrier on Magnetization of High Temperaure Superconductors.

PB94-185162

04,446

Effects of Critical Current Density, Equilibrium Magnetization and Surface Barrier on Magnetization of High Temperature Superconductors.

04,588 Surface Barrier and Lower Critical Field in YBa2Cu3O7. delta Superconductors.

PB94-200128

04,478

SANDER, L.

Comparison of the Liquid Chromatographic Behavior of Selected Steroid Isomers Using Different Reversed-Phase Materials and Mobile Phase Compositions.

PB95-140976

00,574

Development of Engineered Stationary Phases for the Separation of Carotenoid Isomers.

PB95-150249

00,578

SANDER, L. C.

Analysis of SANS from Controlled Pore Glasses.

PB94-198843

03,035

Certification of Morphine and Codeine in a Human Urine Standard Reference Material.

PB95-176160

03,499

Characterization of Chemically Modified Pore Surfaces by

Small Angle Neutron Scattering.

00,898

Determination of Polycyclic Aromatic Hydrocarbons by Liquid Chromatography.

00,585

Determination of 3-Ouinuclidinyl Benzilate (Onb) and Its Major Metabolites in Urine by Isotope Dilution Gas Chromatography Mass Spectrometry.

03,492
Device for Subambient Temperature Control in Liquid Chro-

PB95-140604

00,573

Influence of Stationary Phase Chemistry on Shape Recognition in Liquid Chromatography.

00,621

Isolation and Structural Elucidation of the Predominant Geometrical Isomers of alpha-Carotene.

PB96-190061

00,640

Liquid Chromatographic Determination of Carotenoids in Human Serum Using an Engineered C30 and a C18 Stationary Phase. ( 302 in Environmental Standard Reference Materials.
PB95-164042 NIST Reference Materials to Support Accuracy in Drug Testing.
PB96-123807

03,505

Selectivity Trends in Packed Column Supercritical Fluid Chromatography with $\mathrm{C} 18$ Stationary Phases.

PB96-138581

00,622

Shape Selectivity Assessment of Stationary Phases in Gas Chromatography.

PB95-150256

00,579

Shape Selectivity in Reversed-Phase Liquid Chromalography for the Separation of Planar and Non-Planar Solutes

00,596

Standard Reference Materials for the Determination of Polycyclic Aromatic Hydrocarbons in Environmental Samples - Current Activities.

PB95-151668

00,586

Use of a Naphthylethylcarbamoylated- beta-Cyclodextrin Chiral Stationary Phase for the Separation of Drug Supercritical Fluid Chromatography.

00,653

SANDERS, J. A

Ductile Fracture and Tempered Martensite Embrittlement of 4140 Steel.

PB96-190285

SANDERS, P. A.

Post-Occupancy Evaluation of the Forrestal Building.
PB97-111298
00,280

SANDERS, P. G.

Small Angle Neutrons Scattering from Nanocrystalline Palladium as a Function of Annealing.

PB95-176103

SANDERS, R. E.

Fire Service and Fire Sciences: A Winning Combınation. PB95-150264

03,354

SANDERS, $\mathbf{S}$.

Insulating Nanoparticles on YBa2Cu3O7-delta Thin Films Revealed by Comparison of Alomic Force and Scanning Tunneling Microscopy.

PB95-150843

ANDERS, $S$. A.

NIST Serial Holdings, 1994

PB94-178068

04,575

NIST Serial Holdings, 1995

PB95-188926

02,745

NIST Serial Holdings, 1996

PB96-172523

02,746

SANDERS, $S . C$

Bias Current Dependent Resistance Peaks in NiFe/Ag Giant Magnetoresistance Multilayers.

Magnetoresistan

04,153

Effects of Etching on the Morphology and Surface Resist-

ance of YBa2Cu3O7-delta Films.
PB96-135355

02,410

Evidence for Tunneling and Magnetic Scattering at 'In situ'

YBCO/Noble-Metal Interfaces.

Growth of Laser Ablated YBa2Cu307-delta Films as Examined by Rheed and Scanning Tunneling Microscopy.

PB95-162541

04,614

Insulating Boundary Layer and Magnetic Scattering in YBa2Cu3O7-delta/Ag Interfaces Over a Contact Resistivity Range of $10(-8)-10(-3)$ Omega $\mathrm{cm}(2)$

04,648

Low Magnetostriction in Annealed NiFe/Ag Giant Magnetoresistive Multilayers.

04,762

Magnetic and Magnetoresistive Properties of Inhomogeneous Magnetic Dual-Layer Films. PB95-169025

04,646

Magnetostriction and Giant Magnetoresistance in Annealed NiFe/Ag Multilayers.

04,716

Models of Granualr Giant Magnetoresistance Multilayer Thin Films.

01,968

Observation of Insulating Nanoparticles on YBCO ThinFilms by Atomic Force Microscopy.

04,622

Oxygen Annealing of Ex-situ YBCO/Ag Thin-Film Interfaces.
04,758 Size and Self-Field Effects in Giant Magnetoresistive ThinFilm Devices.

04,674

Size Effects and Giant Magnetoresistance in Unannealed NiFe/Ag Multilayer Stripes.

PB97-111306

04,145

Size Effects in Submicron NiFe/Ag GMR Devices.

PB96-155510

02,237

Surface Degradation of Superconducting YBa2Cu3O7-delta Thin Films. Telegraph Noise in Silver-Permalloy Giant Magnetoresistance Test Structures.

PB96-146717

04,763

PB95-140810

SANDLIN, A. C.

Surface Energy Reduction in Fibrous Monotectic Structures PB95-140828

SANFORD, N. A.

Distributed Feedback Lasers in Rare-Earth-Doped Phosphate Glass.

Glasses for Waveguide Lasers.

PB96-111950

04,740 Integrated Optic Laser Emitting at 905, 1057, $1356 \mathrm{~nm}$ PB94-216298 02,136 Integrated Optic Laser Emitting at 906, 1057, and $1358 \mathrm{~nm}$ PB94-216280 02, 135 Linewidth Narrowing in an Imbalanced Y-Branch Waveguide Laser.

PB95-140844

04,258

Nd:LiTaO3 Waveguide Laser.

PB95-140851

Passively O-Switched Nd-Doped Waveguide Laser PB95-180048

04,308

Rare-Earth-Doped Waveguide Devices: The Potential for Compact Blue-Green Lasers.

PB95-140836

SANGRAS, $R$.

Mixing and Radiation Properties of Buoyant Luminous Flame Environments.

PB96-202254

SANSALONE, $M$.

Detection of Voids in Grouted Ducts Using the Impact-Echo Metho

PB94-185121

SANSONETTI, C. J.

Irradiances of Spectral Lines in Mercury Pencil Lamps. PB96-176466

Measurements of the Resonance Lines of $(6) \mathrm{Li}$ and $(7) \mathrm{Li}$ by Doppler-Free Frequency-Modulation Spectroscopy.
PB96-180211 Spectrum and Energy Levels of Triply Ionized Barium (Ba PB95-140877

03,888

Wavelengths and Isotope Shifts for Lines of Astrophysical Interest in the Spectrum of Doubly lonized Mercury $(\mathrm{Hg} \mathrm{III)}$.
PB95-140869

Wavelengths of Spectral Lines in Mercury Pencil Lamps. PB96-176474

SANTORE, $M$.

04,376

Using Torsional Dilatometry to Measure the Effects of Deformations on Physical Aging.

PB95-140901 
Neutron Powder Diffraction Study of the Structures of La1.9Ca1.1Cu2O6 and La1.9Sr1.1Cu2O6+Delta. PB95-140042

Observation of Oscillatory Magnetic Order in the Antiferromagnetic Superconductor HoNi2B2C. 04,679

Preparation, Crystal Structure, Dielectric Properties, and Magnetic Behavior of Ba2Fe2Ti4O13.

PB96-186176

01,162

Unconventional Ferromagnetic Transition in La(sub 1 . x) $\mathrm{Ca}$ (sub x) $\mathrm{MnO} 3$.

04,156

\section{SANTORO, R. J.}

Fundamental Mechanisms for $\mathrm{CO}$ and Soot Formation.

PB95-143160

01,380

Laser-Induced Fluorescence Measurements of $\mathrm{OH}$ Concentrations in the Oxidation Region of Laminar, Hydrocarbon Diffusion Flames.

Carbon Diffusion

01,387

Laser-Induced Fluorescence Measurements of $\mathrm{OH}$ in Laminar Diffusion Flames in the Presence of Soot Particles. Oxidation of Soot and Carbon Monoxide in Hydrocarbon Oxidation of Soot and Carbon Monoxide in Hydrocarbon
Diffusion Flames.

SAPAK, D. L.

Integrated Optic Laser Emitting at 905, 1057, $1356 \mathrm{~nm}$. PB94-216298

SAPIRSTEIN, J.

Evaluation of Two-Photon Exchange Graphs for HighlyCharged Heliumlike Ions.

PB94-198918

03,808

SAPRITSKY, V.

Comparison of Regular Transmittance Scales of Four National Standardizing Laboratories.

PB94-211232 04,233

Method of Realizing Spectral Irradiance Based on an Absolute Cryogenic Radiometer.

PB95-161204

04,270

Realization of New NIST Radiation Temperature Scales for the $1000 \mathrm{~K}$ to $3000 \mathrm{~K}$ Region, Using Absolute Radiometric Techniques.

SAPRITSKY, $V$. I

03,794

Precision H.

Precision High Temperature Blackbodies

PB95-140059 03,885

Results of a NIST/VNIIOFI Comparison of Spectral-Radiance Measurements.

04,159

Multiwavelength Birefringent-Cavity Mode-Locked Fibre Laser.

SASAGAWA, G. S.

04,262

Intracomparison Tests of the FG5 Absolute Gravity Meters.

PB96-102991

03,688

SASLOW, W. M.

Normal Modes and Structure Factor for a Canted Spin System: The Generalized Villain Model.

PB95-14006

04,539

SASSI, M. P

Precise Optical Frequency References and Difference Frequency Measurements with Diode Lasers.

176228

04,305

SASTRY, K. V. L. N.

Millimeter- and Submillimeter-Wave Spectrum of trans-Ethyl Alcohol.

PB96. 145578

01,102

SATHYAMURTHY, N.

Energy Dependence of Collision Characteristics in Molecule-Surface Collisions.

SATIJA, S. K.

00,786

Diffraction of Neutron Standing Waves in Thin Films with Resonance Enhancement.

PB97-113278

04,164

Grazing Incidence Prompt Gamma Emissions and Resonance-Enhanced Neutron Standing Waves in a Thin-Film. PB95-150470

03,892

Morphology of Symmetric Diblock Copolymers as Revealed by Neutron Reflectivity.

01,234

Neutron Reflectivity of End-Grafted Polymers: Concentration and Solvent Ouality Dependence in Equilibrium Conditions.
PB94-185758

Neutron Reflectivity Study of the Density Profile of a Mode! End-Grafted Polymer Brush: Influence of Solvent Ouality. (1)

Observed Frustration in Confined Block Copolymers.
PB95-150033 PB95-150033 01,238 Temperature Dependence of the Morphology of Thin
Diblock Copolymer Films as Revealed by Neutron Reflectivity.

01,199

SAUDER, D. A.

Challenges to the National Information Infrastructure: The Barriers to Product Data Sharing. National PDES Testbed Report Series.
PB95-136347

02,776
Structural EXPRESS Editor. PB94-159795

02,769

SAUDER, D. G.

Product Kinetic Energies, Correlations, and Scattering Anjsotropy in the Bimolecular Reactor $\mathrm{O}((1) \mathrm{D})+\mathrm{H} 2 \mathrm{O}$ yields

00,838

Product State Correlations in the Reaction of $O((1) D)$ and $\mathrm{H} 2 \mathrm{O}$ in Bimolecular Collisions and in O3.H2O ClustersTranslation.

Translation,

SAUERS, I.

Associative Electron Attachment to $\mathrm{S}_{2} \mathrm{~F}_{10}, \mathrm{~S} 2 \mathrm{OF} 10$, and $\mathrm{S} 2 \mathrm{O} 2 \mathrm{~F} 10$.

PB95-140992

00,907

Decomposition of SF6 and Production of S2F10 in Power

PB96-122619 01,084

Investigation of S2F10 Production and Mitigation in Compressed SF6-Insulated Power Systems.

PB94-212388 02.467

Investigation of S2F10 Production and Mitigation in Compressed SF6- Insulated Power Systems.

02,468

PB96-155528 02,468

Measurement of S2OFive Glow Corona Discharge in SF6 22 Gas Mixtures.

PB96- 123740

SAUERWEIN, J. C.

NIST Standard Reference Data Products Catalog, 1994.

PB94-151842

NIST Standard Reference Data Products Catalog, 1995-96. Achieve with Standard Reference Data.

PB95-260808

01,057

SAUERWEIN, T

Hierarchical Ada Robot Programming System (HARPS): A Complete and Working Telerobot Control System Based on the NASREM Mode

PB94-213162

02,934

SAUNDERS, B. $V$.

Boundary Conforming Grid Generation System for Interface Pracking.

03,312

Boundary Conforming Grid Generation System for Interface

Tracking.
PB96-103007

Portable Vectorized Software for Bessel Function Evalua PBion. 198975

SAUNDERS, C. A.

Manufactured Housing Walls That Provide Satisfactory

Moisture Performance in All Climates.

00,383

Test Procedures for Advanced Insulation Panels.

PB97-111892

00,415

SAUNDERS, M. H.

International Challenges in Defining the Public and Private

Interest in Standards.
PB96-160361 00,498

ISO Environmental Management Standardization Efforts.

PB95-220513 02,524

ISO Environmental Management Standardization Efforts.

PB96-158662

SAUNDERS, $R$.

Method of Realizing Spectral Irradiance Based on an Absolute Cryogenic Radiometer.

04,270

\section{SAUNDERS, R. D.}

Comparison of Filter Radiometer Spectral Responsivity with the NIST Spectral-Irradiance and Illuminance Scales.

B97.113161

04,162

Precision High Temperature Blackbodies.

PB95-140059

03,885

Realization of New NIST Radiation Temperature Scales for the $1000 \mathrm{~K}$ to $3000 \mathrm{~K}$ Region, Using Absolute Radiometric Techniques.

03,794

Results of a NIST/VNIIOFI Comparison of Spectral-Radiance Measurements.

PB97-113021

04,159

SAUNDERS, S. C

Methodologies for Predicting the Service Lives of Coating Systems.

03,124

SAUTER, G.

Intercomparison of Photometric Units Maintained at NIST USA) and PTB (Germany), 1993.

PB95-261913

04,329

SAUVAGEAU, J. E.

Far-Infrared Kinetic Inductance Detector.
PB95-126348

PB95-126348 02,142

PB95-168662

02,161

Optimization of ECR-Based PECVD Oxide Films for Superconducting Integrated Circuit Fabrication.
PB95-169165

Superconducting Integrated Circuit Fabrication with Low Temperature ECR-Based PECVD SiO2 Dielectric Films. PB96-103015

04,719

Superconducting Kinetic Inductance Radiometer PB95-140083

02,144

Terahertz Detectors Based on Superconducting Kinetic Inductance.

PB95-168647

02,160

SAUVAJOL, J. L.

Inelastic-Neutron-Scattering Studies of Poly(p-phenylene vi-

PBlene).

01,014

SAVAGE, B.

Deuterium in the Local Interstellar Medium: Its Cosmological Significance.

PB95-202842

00,081

Deuterium and the Local Interstellar Medium: Properties for the Procyon and Capella Lines of Sight.

PB96-200639

00,111

Goddard High-Resolution Spectrograph Observations of the Local Interstellar Medium and the Deuterium/Hydrogen Ratio along the Line of Sight Toward Capella.

PB94-213444

00,066

Observations of $3 \mathrm{C} 273$ with the Goddard High Resolution Spectrograph on the Hubble Space Telescope.

PB95-202321

00,076

SAVAGE, D. A

System for Calibration of the Marshall Compaction Hammer.

PB94-145661

01,303

SAVEANT, J. M.

Oxidation of 10-Methylacridan, a Synthetic Analogue of NADH and Deprotonation of Its Cation Radical. Convergent Application of Laser Flash Photolysis and Direct and Redox Catalyzed Electrochemistry to the Kinetics of Deprotonation of the Cation Radical.

PB94-198371

00,785

SAWANO, $K$.

Determination of Thermoactivation Parameters of Vortex Mobility in YBa2Cu3O7 Using Only Magnetic Measure-

ments.

SAWANT, S. B.

04,624

Continuous Counter-Current Two Phase Aqueous Extraction.

00.675

Enzyme and Protein Mass Transfer Coefficient in Aqueous Two Phase Systems. 1. Spray Extraction Columns.

Protein Extraction in a Spray Column Using a Polyethylene Glycol Maltodextrin Two-Phase Polymer System.

PB95-162228

00,595

Two Phase Aqueous Extraction: Rheological Properties of Dextran, Polyethylene Glycol, Bovine Serum Albumin and Their Mixtures.

PB95-161998

00,676

SAWIN, H. H.

Gaseous Electronics Conference Radio-Frequency Reference Cell: A Defined Parallel-Plate Radio-Frequency Sys- 
SCACE, R. I.

Development of a Standard Reference Material for Meas urement of Interstitial Oxygen Concentration in Semiconductor Silicon by Infrared Absorption.

02,404

Methodology for the Certification of Reference Specimens Methodology for the Certification of Reference Specimens
for Determination of Oxygen Concentration in Semiconducfor Determination of Oxygen Concentration

tor Silicon by 1 .
PB96.155478

SCANNELL, M. J.

Dose Mapping of Radioactive Hot Particles Using PB95-162954

SCAWTHORN, C.

Earthquake and Fire in Japan: When the Threat Became a

Reality.

00,20

SCHAAFSMA, D. T.

Comparative Photoluminescence Measurement and Simulation of Vertical-Cavity Semiconductor Laser Structures.
PB95-169173 02,169

02,169

Correlation of Optical, X-ray, and Electron Microscopy Measurements of Semiconductor Multilayer Structures.
PB95-175279 Cross-Sectional Photoluminescence and Its Application to Buried-Layer Semiconductor Structures.

PB96-141106

02,415

Measurement and Simulation of Photoluminescence Spectra from Vertical-Cavity Ouantum-Well Laser Structures.

Vertical-Cavity Semiconductor Lasers: Structural Characterization, CAD, and DFB Structures.

02,150

PB95-153193

Charac-

Vertical-Cav

PB95-153185

02,149

SCHABANEL, N.

Basic Linear Algebra Operations in SLI Arithmetic.

PB96-165931

03,42

SCHAEFER, $M$.

Optically Stabilized Tunable Diode-Laser System for Saturation Spectroscopy

04.717

Stabilization of 3.3 and $5.1 \mathrm{~m}$ Lead-Salt Diode Lasers by Optical Feedback.

PB95.180709

04,313

SCHAEFER, R. J.

Computation of Dendrites Using a Phase Field Model.
PB94-160744

Intelligent Processing of Hot Isostatic Pressing.

PB94-172913

03,315

Micromechanics of Densification and Distortion.

03.327

Surface Energy Reduction in Fibrous Monotectic Structures.

PB95-140828 03,150

SCHAFER, K. J.

Intensity-Dependent Scattering Rings in High Order Above. Threshold lonization.

PB96-110739

04,032

SCHAFFER, $R$

History of NIST's Contributions to Development of Standard Reference Materials and Reference and Definitive Methods for Clinical Chemistry.

PB96-119706

SCHAFFT, H. A.

03,503

Electrical Characterization of Integrated Circuit Metal Line Phickness.

02,414

Electrical Method for Determining the Thickness of Metal Films and the Cross-Sectional Area of Metal Lines.

PB95-203170

02,370

JEDEC 'TCR' Interlaboratory Experiment: Lessons Learned.

02,371

Measurement of Patterned Film Linewidth for Interconnect Characterization

PB96-148168

SCHANTZ, $M$.

02,420

Comparison of Methods for Gas Chromatographic Determination of PCBs and Chlorinated Pesticides in Marine Reference Materials.

02,584

Development of Frozen Whale Blubber and Liver Reference Materials for the Measurement of Organic and Inorganic Contaminants.

00,587

National Status and Trends Program Specimen Bank: Sampling Protocols, Analytical Methods, Results, and Archive PB97.119226

SCHANTZ, M. $M$.

02,598

Certification of Polychlorinated Biphenyl Congeners and Chlorinated Pesticides in a Whale Blubber Standard Reference Material

erence Material.

03,745

Certification of Polycyclic Aromatic Hydrocarbons in a Marine Sediment Standard Reference Material.

02,592
Certification of Standard Reference Material (SRM) 1941a, Organics in Marine Sediment.

02,593

Comparison of Selectivities for PCBs in Gas Chroma. tography for a Series of Cyanobiphenyl Stationary Phases.
00,618

Concentrations of Chlorinated Hydrocarbons, Heavy Metals and Other Elements in Tissues Banked by the Alaska Ma. rine Mammal Tissue Archival Project.

PB95-209870

02,590

Determination of PCBs and Chlorinated Hydrocarbons in

Marine Mammal Tissues.
PB95-162640

Investigations of Sulfur Interferences in the Extraction of Methylmercury from Marine Tissues.

03,482

NIST Standard Reference Materials (SRMs) for Polychlorinated Biphenyl (PCB) Determinations and Their Applicability to Toxaphene Measurements.

02,585

Ouantitative Analysis of Selected PCB Congeners in Marine

Ouantitative Analysis of Selected PCB Congeners in Marine
Matrix Reference Materials Using a Novel Cyanobiphenyl Matrix Reference

02,591

Standard Reference Materials for the Determination of Polycyclic Aromatic Hydrocarbons in Environmental Samples - Current Activities.

00,586

Thermodynamics of the Hydrolysis of N-Acetyl-L-
phenylalanine Ethyl Ester in Water and in Organic Solvents.
PB95-203386

Thermodynamics of the Hydrolysis of $3,4,5$ -

Trihydroxybenzoic Acid Propyl Ester (n-Propylgallate) to

3,4,5-Trihydroxybenzoic Acid (Gallic Acid) and Propan-I-ol in Aqueous Media and in Toluene.

01,160

SCHAPPACHER, J.B.

Line-Reflect-Match Calibrations with Nonideal Microstrip Standards.

SCHAPS, S. R.

02,242

Determination of Sheet Steel Formability Using Wide Band Electromagnetic-Acoustic Transducers.

02,279

Methods to Improve the Accuracy of On-Line Ultrasonic

Methods to Improve the Accuracy of On-Line Ultrasonic
Measurement of Steel Sheet Formability. Measurement of Steel Sheet Formability.

02,281

Safety Assessment of Railroad Wheels Through Roll-by Detection of Tread Cracks.

04,856

Well-Shielded EMAT for On-Line Ultrasonic Monitoring of GMA Welding.

SCHAUER, D. A.

02,879

EPR Dosimetry of Cortical Bone and Tooth Enamel Irradiated with $X$ and Gamma Rays: Study of Energy Depend-

PB97-110373

03,639

Experimental Validation of Radiopharmaceutical Absorbed Dose to Mineralized Bone Tissue.

PB94-199668

03,617

Exposure-to-Absorbed-Dose Conversion for Human Adult Cortical Bone.

03,640

Overview of a Radiation Accident at an Industrial Accelerator Facility.

Radiation Accident at an Industrial Accelerator Facility
PB95-140117

02,612

SCHAUER, $W$.

Harmonic and Static Susceptibilities of YBa2Cu3O7.

PB95-161139

SCHEFER, J.

Characterization of the Structure of TbD2.25 at $70 \mathrm{~K}$ by Neutron Powder Dittraction.

PB96-160528

Neutron-Powder-Diffraction Study of the Long-Range Order in the Octahedral Sublattice of LaD2.25.

04,753

SCHEIFLER, $R$. $\mathbf{W}$.

$X$ Window Sys
PB96-169099

01,769

SCHEINFEIN, M. R.

Correlations of Modulation Noise with Magnetic Microstructure and Intergranular Interactions for CoCrTa and CoNi Thin Film Media.

PB94-212768

04,509

Laser Focusing of Atoms: A Particle Optics Approach

: A Particle Oplics Approach. 03,870

Micromagnetic Structure of Domains in Co/Pt Multilayers. 1.

Investigations of Wall Structure.

04,610

Surface Magnetic Microstructural Analysis Using Scanning

Electron Microscopy with Polarization Analysis (SEMPA).

PB95-162657

SCHEN, $M$.

Advances in the Measurement of Polymer CTE:

Micrometer- to Atomic-Scale Measurements.
PB96-180229

SCHEN, M. A.

Beyond the Technology Roadmaps: An Assessment of Electronic Materials Research and Development.

PB96-165998

01,961

Electronics Packaging Materials Research at NIST.

PB96-122692

02,405

Metrology and Data for Microelectronic Packaging and Interconnection: Results of a Joint Workshop on Materials Metrology and Data for Commercial Electrical and Optical Packaging and Interconnection Technologies. Held in Gaithersburg, Maryland on May 5-6, 1994. Volume 1. Re-

PB95-143111

02,328

Metrology and Data for Microelectronic Packaging and Interconnection: Results of a Joint Workshop on Materials Metrology and Data for Commercial Electrical and Optica Gaithersburg, Maryland on May 5-6, 1994 . Volume 2. PresGaithersburg, Maryla

entation Mate

Novel Polydiacetylenes Derived from Liquid Crystallıne Monomers.

PB95-140125

01,235

SCHENCK, A.

Dynamics of $\mathrm{Mu}(+)$ in $\mathrm{Sc}$ and $\mathrm{ScH}$

PB96-180021

04,112

SCHENCK, P. $K$.

Microstructure and Ferroelectric Properties of Lead Zirconate-Titanate Films Produced by Laser Evaporation.

PB94-199148

SCHIEBENER, $P$.

Formulation of the Refractive Index of Water and Steam.

PB95-140133

04,470

SCHIESSL, U.

Stabilization of 3.3 and $5.1 \mathrm{~m}$ Lead-Salt Diode Lasers by Optical Feedback

PB95. 180709

SCHILLER, $\mathbf{S}$.

Combining Data from Independent Chemical Analysis Meth-

PB95-140141

00,572

SCHILLER, S. $B$.

Certification of Polychlorinated Biphenyl Congeners and Chlorinated Pesticides in a Whale Blubber Standard Ret

PB96-103023

03,745

Certification of Polycyclic Aromatic Hydrocarbons in a $\mathrm{Ma}$ rine Sediment Standard Reference Material.

02,592

Certification of Standard Reference Material (SRM) 1941a Organics in Marine Sediment.

PB96-123690

02,593

Introduction of a NIST Instrument Sensitivity Standard Reference Material for X-Ray Powder Diffraction.

Preparation and Monitoring of Lead Acetate Containing Drinking Water Solutions for Toxicity Studies.

PB94-193885

00.538

Standard Reference Material for the Measurement of Particle Mobility by Electrophoretic Light Scattering. PB96-102488

Statistical Aspects of the Certification of Chemical Batch SRMs. Standard Reference Materials.

210877

00,645

CHIMA, F. J.

Alpha-Particle and Electron Capture Decay of (209)Po.
PB96-186085

Assay of the Eluent from the Alumina-Based Tungsten-188 Rhenium-188 Generator.

PB94-200482

03,829 
SCHLAGER, J. B.

High-Sensitivity Optical Sampling Using an Erbium-Doped Fiber Laser Strobe

PB95-176111

04,302

Metrology Applications of Mode-Locked Erbium Fiber La-

Sers.

04,256

Millimeter-Resolution Optical Time-Domain Reflectometry Using a Four-Wave Mixing Sampling Gate.

02,190

Multiwavelength Birefringent-Cavity Mode-Locked Fibre Laser.

PB95-150496 04,262

Optical Sampling Using Nondegenerate Four-Wave Mixing in a Semiconductor Laser Amplifier.

02,076

Optical Sampling Using Nondegenerate Four-Wave Mixing in a Semiconductor Laser Amplifier.

PB96-123609

04,348

Precise Laser-Based Measurements of Zero-Dispersion Wavelength in Single-Mode Fibers.

01,511

\section{SCHLENOFF, $\mathrm{C}$.}

Unified Process Specification Language: Requirements for Modeling Process.

PB97.116123

SCHLENOFF, C. I.

02,850

World Wide Web and Mosaic: User's Guide.

PB94-20735

02,722

SCHLUND, B.

New Physics-Based Model for Time-Dependent- DielectricBreakdown.

02,440

New Physics-Based Model for Time-Dependent- Dielectric Breakdown

PB96-201132

02,448

SCHMIDT, J.

Frequency Dependence of the Emission from 2D Array Josephson Oscillators.

PB95-175147

02,056

SUSAN: SUperconducting Systems ANalysis by Low Temperature Scanning Electron Microscopy (LTSEM)

PB96-112065

04,728

CHMIDT, J. W.

Near Critical Fluid Interfaces: A Comparison of Theory and Experiment.

00,901

Operational Mode and Gas Species Effects on Rotational Drag in Pneumatic Dead Weight Pressure Gages.

Structure of the Vapor-Liquid Intertace Near the Critical Point

00,902

PB95-140174

Thermodynamic Properties of CF3-CHF-CHF2, 1,1,1,2,3,3-
Hexafluoropropane.

Hexafluoropropane.

03,299

Thermodynamic Properties of CHF2-CF2-CHF, 1,1,2,2,3Pentafluoropropane.

03,300

Thermodynamic Properties
CHF2,Bis(difluoromethyl) Ether.

SCHMIDT, M. A.

Thermal Isolation of High-Temperature Superconducting Thin Films Using Silicon Wafer Bonding and Micromachining

SCHMIDT, $V$

Vibronic Coupling and Other Many-Body Effects in the 4 sigmag(-1) Photoionization Channel of $\mathrm{CO} 2$. PB95-16250

00,962

SCHMITT, C.

First Results from a Coordinated ROSAT, IUE, and VLA Study of RS CVn Systems.

SCHMITT, J. H. M. M.

00,069

\section{SCHMIT, J. H. M. M.}

First Results from a Coordinated ROSAT, IUE, and VLA Study of RS CVn Systems.

00,069

ROSAT All-Sky Survey of Active Binary Coronae. 1. QuiesCent Fluxes for the RS Canum Venaticorum Systems.
PB95-202479 00,077

ROSAT All-Sky Survey of Active Binary Coronae. 2. Coronal Temperatures of the RS Canum Venaticorum Systems.
PB94-199601

Rotational Modulation and Flares on RS Canum Venaticorum and BY Draconis Stars. XVIII. Coordinated VLA, ROSAT, and IUE Observations of RS CVn Binaries.

PB96-102322

00,089

PB-ray Emission

00,088

SCHNATTERLY, S. E.

Soft-X-ray Damage to p-terphenyl Coatings for Detectors.
PB96-159611

SCHNEEMAN, $\mathbf{R}$.

4,364

Framework for National Information Infrastructure Services.
SCHNEEMAN, R. D. Distributed Supercomputing Software: Experiences with the
Parallel Virtual Machine - PVM.

01.680

Porting Multimedia Applications to the Open System Environment. PBO4-172921

02,721

SCHNEEMEYER, L. F.

Increased Pinning Energies and Critical Current Densities in Heavy-Ion-Irradiated $\mathrm{Bi} 2 \mathrm{Sr} 2 \mathrm{CaCu} 2 \mathrm{O} 8$ Single Crystals.

PB95-175352

SCHNEIDER, G. $M$.

Application of the Taylor Dispersion Method in Supercritica Fluids.

PB95-164323

00,977

SCHNEIDER, H. A.

Glass Temperature of Polymer Blends: Comparison of Both the Free Volume and the Entropy Predictions with Data.
PB95-140190

SCHNEIDER, J. A

Computer Graphics Metafile (CGM): Procedures for NIST CGM Validation Test Service.

PB94-161809

01,804

Initial Graphics Exchange Specification (IGES): Procedures for the NIST IGES Validation Test Service.

PB95-171427

SCHNEIDER, $M$.

02,780

Extension of Heterodyne Frequency Measurements on OCS

to $87 \mathrm{THz}(2900 \mathrm{~cm}(-1))$

00,811

Shape Selectivity Assessment of Stationary Phases in Gas Chromatography

PB95-150256

00,579

Stabilizatıon of 3.3 and $5.1 \mathrm{~m}$ Lead-Salt Diode Lasers by Optical Feedback

SCHNEIDER, R. J

04,313

Comparative Study of Fe-C Bead and Graphite Target Performance with the National Ocean Sciences AMS (NOSAMS) Facility Recombinator Ion Source.

PB95-175790

00,693

CHNEIDER, $S$.

Classification of Advanced Technical Ceramics

N94-35335/6

New Materials,

SCHNEIDERMAN, $\mathrm{H}$

Ground Vehicle Control at NIST: From Teleoperation to Autonomy

N94-34037/9

03,758

Visual Road Following without 3-D Reconstruction.

PB95-161030

SCHNEIR, J.

Development of a Calibrated Alomic Force Microscope.

02,894

Increasing the Value of Atomic Force Microscopy Process Metrology Using a High-Accuracy Scanner, Tip Character ization, and Morphological Image Analysis.

PB96-190293

02,758

Integration of Scanning Tunneling Microscope Nanolithography and Electronics Device Processing.

Polyethylene Crystallized from an Entangled Solution Observed by Scanning Tunneling Microscopy.
PB95-107389

01,232

Progress Toward Accurate Metrology Using Atomic Force Microscopy.

Scanning Tunneling Microscopy and Fabrication of Nanometer Scale Structures at the Liquid-Gold Interface.
PB95-140414

SCHOEN, F, J.

Physicochemical Characterization of Natural and Bioprosthetic Heart Valve Calcific Deposits: Implications for Prevention.

Physicochemical Properties of Calcific Deposits Isolated from Porcine Bioprosthetic Heart Valves Removed from Patients Following 2-13 Years Function.

PB94-172863

SCHOLTEN, R. E

Determination of Complex Scattering Amplitudes in Low-Energy Elastic Electron-Sodium Scattering

PB94-216652

aser-Focused Atomic Deposition.

PB95-161618

03,869

Laser Focused Atomic Deposition

PB95-180659

04,604 cused in Laser Fields.

PB95-150272 04,564

Nanostructure Fabrication via Laser-Focused Atomic Deposition (Invited)

04,132

Simple, Compact, High-Purity $\mathrm{Cr}$ Evaporator for Ultrahıgh

Vacuum.

04,520

Spin-Resolved Elastic Scattering of Electrons from Sodium.

PB95-161774

Using Atom Optics to Fabricate Nanostructures.

03,933

- 141247

04,757

CHON, K.

Digital Techniques in HV Tests - Summary of 1989 Panel Session.

PB94-216702

02,035

CHONBERGER, E.

Determination of Vitamin $\mathrm{K} 1$ in Serum Using Catalytic-Reduction Liquid Chromatography with Fluorescence DetecPB96-138425

SCHOONOVER, R. $M$.

Application of the Electronic Balance in High Precision PB94-18756

00,534

Determination of Density of Mass Standards: Requirement and Method.

03,787

Electronic Balance and Some Gravimetric Applications.

(The Density of Solids and Liquids, Pycnometry and Mass).
PB94-163052

Examination of Parameters That Can Cause Error in Mass Determinations

PB94-163037

03,784

Mass Unit Disseminated to Surrogated Laboratories Using the NIST Portable Mass Calibration Package.

03,781

Piggyback Balance Experiment: An Illustration of Archimedes' Principles and Newton's Third Law.

03,786

Use of the Electronic Balance for Highly Accurate Direct Mass Measurements Without the Use of External Mass Standards

PB94-187713

03,803

RACK, R. A

Measurement of the $(10) \mathrm{B}(\mathrm{n}$, alpha 1gamma)(7)Li Cross Section in the 0.3 to $4 \mathrm{MeV}$ Neutron Energy Interval.

PB96-161799

04,098 $\mathrm{keV}$

PB95-140422

03,886

CHAAMM, R. E.

Dynamometer-Induced Residual Stress in Railroad Wheels: Ultrasonic and Saw Cut Measurements. Report No. 30.

PB96-183199

04,857

Gas-Coupled, Pulse-Echo Ultrasonic Crack Detection and Thickness Gaging.

Noncontact Ultrasonic Inspection of Train Rails for Stress. PB95-162673

04,851

Residual Stress in Induction-Heated Railroad Wheels: Ultrasonic and Saw Cut Measurements. Report No. 28

PB96-106992

04,854

Safety Assessment of Railroad Wheels by Residual Stress Measurements.
PB96-141114

04,855

Safety Assessment of Railroad Wheels Through Roll-by Detection of Tread Cracks.

04,856

Ultrasonic Measurement of Residual Stress in Cast Steel Railroad Wheels

PB95-169199

04,852

Ultrasonic Measurement of Residual Stress in Railroad Wheel Rims.

SCHRANK, H. E. 
Slit-Jet Near-Infrared Diode Laser Spectroscopy of $(D C l) 2$ nu1, nu2 DCl Stretching Fundamentals, Tunneling Dynam ics, and the Influence of Large Amplitude 'Geared'

01,047

SCHULTZ, A. E.

Performance of HUD-Affiliated Properties during the January 17, 1994 Northridge Earthquake.

00,443

\section{SCHULZ, F.}

Open System Environment (OSE): Architectural Framework for Information Infrastructure.

PB96-146360

00,002

Open System Environments.

01,674

Open Systems Software Standards in Concurrent Engineering.

PB96-160932

01,758

SCHULZE, D. W.

Interfaces in Mo/Si Multilayers.

PB96-160668

02,423

SCHUMACHER, G. E.

Effects of Surface-Active Resins on Dentin/Composite

Bonds.

00,156

SCHUSTER, C. E.

Electrical Test Structure for Improved Measurement of Feature Placement and Overlay in Integrated Circuit Fabrication Processes.
PB95-164273

02,355

Review of Semiconductor Microelectronic Test Structures with Applications to Infrared Detector Materials and Processes.

PB94-212925

02,132

Semiconductor Measurement Technology: Test Structure Implementation Document: DC Parametric Test Structures and Test Methods for Monolithic Microwave Integrated Circuits (MMICs)

SCHUTTE, C. L.

Environmental Durability of Glass-Fiber Composites.

PB95-203220

02,399

SCHWARTZ, L. H.

Glimpse of Materials Research in China: A Report from an Interagency Study Team on Materials Visiting China from June 19,1995 to June 30,1995

PB96-11267?

02,978

Industry and Government-Laboratory Cooperative $R$ and $D$ An Idea Whose Time Has Come.

02,970

Materials Science and Engineering Laboratory Annual Report, 1993. NAS-NRC Assessment Panel, April 21-22, 1994.
PB94-162534

Materials Science and Engineering Laboratory Annual Report, 1994. NAS-NRC Assessment Panel, April 6-7, 1995.

Materials Science and Engineering Laboratory Annual Report, 1995. Technical Activities.

PB96-214754

03,009

Nondestructive Evaluation and Materials Processing

B95-140455

02,902

Recent VAMAS Activity in Ceramics.

PB95-162681

03,051

Cross-Property Relations and Permeability Estimation in Model Porous Media.

PB95-150280 04,205

Interfacial Transport in Porous Media: Application to do Electrical Conductivity of Mortars.

PB96-146816

01,326

Transport and Diffusion in Three-Dimensional Composite Media.

SCHWARTZ, $M$.

04,668

Halon Thermochemistry: 'Ab Initio' Calculations of the Enthalpies of Formation of Fluoromethanes.

PB96-175740

03,289

SCHWARTZ, S. J.

Isolation and Structural Elucidation of the Predominant Geometrical isomers of alpha-Carotene.

PB96-190061

00,640

SCHWARZ, F. P.

Biological Thermodynamic Data for the Calibration of Differential Scanning Calorimeters: Dynamic Temperature Data on the Gel to Liquid Crystal Phase Transition of Dialkylphosphatidylcholine in Water Suspensions.

Thermodynamic Analysis of Heparin Binding to Human Antithrombin.

Antithrombin.

03,455

Thermodynamic and NMR Study of the Interactions of Cyclodextrins with Cyclohexane Derivatives.

00,781

Thermodynamic Study of the Reactions of Cyclodextrins with Primary and Secondary Aliphatic Alcohols, with D- and

L-Phenylalanine, and with L-Phenylalanineamide.
PB95-180873

01,016
Thermodynamics of the Binding of Galactopyranoside Derivatives to the Basic Lectin from Winged Bean (Psophocarpus Tetrogonolobus).

PB95-162715

03,465

SCHWARZACHER, W.

Concurrent Enhancement of Kerr Rotation and Antiferromagnetic Coupling in Epitaxial Fe/Cu/Fe Structures.
PB94-198769

Variation in Magnetic Properties of Cu/Fcc (001) Sandwich Structures.

SCHWARZBEK, S. M.

04,555

Microwave Properties of YBa2Cu307-x Films at $35 \mathrm{GHz}$ from Magnetotransmission and Magnetoreflection Measure-

ments.

04,643

SCHWARZENBACH, D.

Statistical Descriptors in Crystallography. 2. Report of a Working Group on Expression of Uncertainty in Measurement.

PB96-146824

04,764

SCHWENDIMAN, G. L.

Calibration and Performance of GafChromic DM-100 Radiochromic Dosemeters.

00,703

SCIRE, F. E.

Metrology Standards for Advanced Semiconductor Lithography Referenced to Atomic Spacings and Geometry. 20,424 SCOLES, G.

Sub-Doppler, Infrared Laser Spectroscopy of the Propyne 2nu1 Band: Evidence of Z-Axis Coriolis Dominated Intramolecular State Mixing in the Acetylenic $\mathrm{CH}$ Stretch Overtone.

PB95-20294

01,037

SCOTT, $\mathrm{H}$.

Recommendations on Selection of Vehicle-to-Roadside Communications Standards for Commercial Vehicle Oper-

PB94-195914

04,859

Vehicle-to-Roadside Communications for Commercial Vehicle Operations: Requirements and Approaches.

SCOTT, H. A.

04,860

Control System Architecture for a Remotely Operated Unmanned Land Vehicle.

PB95-163200

03,759

Ground Vehicle Control at NIST: From Teleoperation to AuN94-340

03,758

Vehicle-Command Center Communications in a Robotic Ve hicle System.
PB95-162723

SCOTT, J. L.

03,665

Comparing NIST-B $50 \mathrm{~mm}$ Orifice Meter Gas Data to the ANSI Equation

02,949

Cryogenic Flow Calibration in NIST

PB96-161930

01,143

Flow Conditioner Tests for Three Orifice Flowmeter Sizes.

04,201 Uncertainty Analysis of the NIST Nitrogen Flow Facility.
PB95-128906

02,608 Preparation and Characterization of $(6) \mathrm{LiF}$ and $(10) \mathrm{B}$ Ret-
erence Deposits for the Measurement of the Neutron Lifetime.

PB95-108692

03,874

SCOTT, R. D.

Problems Related to the Determınation of Mass Densities of Evaporated Reference Deposits.

PB95-163226

03,941

SCOTT, T. R.

Accurate Measurement of Optical Detector Nonlinearity.
PB95-203576

Automated Measurement of Nonlinearity of Optical Fiber Power Meters.

PB96-176540

Beam Analysis Round Robin

PB94-212545

04,110

Deep-UV Excimer Laser Measurements at NIST.

PB96-141031

04,239

Error Propagation in Laser Beam Spatial Parameters.

PB95-180394

04,310

Laser-Beam Analysis Pinpoints Critical Parameters.

PB94-212552

04,240

Optical Density Measurements of Laser Eye Protection Ma

terials.

00,190

Optical Detector Nonlinearity: A Comparison of Five Meth-

PB95-169355

04,291

Optical Detector Nonlinearity: Simulation.

02,199

Optical Power Meter Calibratıon Using Tunable Laser Di-

odes

PB95-169256

04,290

Thermal Modeling and Analysis of Laser Calorimeters.

PB96-140405 04.354

Widths and Propagation of a Truncated Gaussian Beam

PB95-168779

04,287

CROGER, M. G.

Assessment of Uncertainties of Thermocouple Calibrations at NIST

SEAKINS, P. W

03,782

Kinetics of the Reaction $\mathrm{C} 2 \mathrm{H}+\mathrm{O} 2$ from 193 to $350 \mathrm{~K} \mathrm{Using}$

Laser Flash Kinetic Infrared Absorption Spectroscopy.
PB95-203055 043

Laser Flash Photolysis, Time-Resolved Fourier Transform

Infrared Emission Study of the Reaction $\mathrm{Cl}+\mathrm{C} 2 \mathrm{H} 5$ yields

$\mathrm{HCl}(v)+\mathrm{C}_{2} \mathrm{H} 4$

PB95-203238

01,049

Laser Flash Photolysis/Time-Resolved FTIR Emission Study

of a New Channel in the Reaction of $\mathrm{CH} 3+\mathrm{O}$ : Production of

SEARS, T. J.

Far Infrared Laser Frequencies of $\mathrm{CH} 3 \mathrm{OO}$ and N2H4.

PB96-119623

SEATON, M. J.

Papers on the Symposium on Collision Phenomena in Astrophysics, Geophysics, and Masers

AD-A280 291/6

SECHOVSKY, $V$.

Magnetic Properties of Single-Crystalline UCu3AI2

00,974

ROM, S. J.

Measurements of the $(237) N p(n, f)$ Cross Section.

PB97-119069

SEETULA, J. A.

Kinetics of the Reaction of $\mathrm{CCl} 3-\mathrm{Br}-2$ and the

PB94-212115

00,824

SEILA, R. L.

Radiocarbon Measurements of Atmospheric Volatile Organic Compounds: Ouantifying the Biogenic Contribution

PB97-122352

SEILER, D. G.

Business and Manufacturing Motivations for the Developing of Analytical Technology and Metrology for Semiconductors.

04,778

Characterization of Liquid-Phase Epitaxially Grown $\mathrm{HgCdTe}$ Films by Magnetoresistance Measurements.

04,738

Characterization of LPE $\mathrm{HgCdTe}$ Film by Magnetoresistance.
PB96-157961

02,197

Characterization of Two-Dimensional Dopant Profites: Status and Review.

PB96-119300

02,400

Characterization of Two-Dimensional Dopant Profiles: Status and Review.

Electrical Characterization of Liquid-Phase Epitaxially Grown Single-Crystal Films of Mercury Cadmium Telluride by Variable-Magnetic-Field Hall Measurements.

02,177

Electrical Characterization of Narrow Gap n-Type Bulk $\mathrm{HgCdTe}$ Single Crystals by Variable-Magnetic-Field Hall Measurements and Reduced-Conductivity-Tensor Analyses. PB96-164199 01,146

Heavily Accumulated Surfaces of Mercury Cadmium Telluride Detectors: Theory and Experiment.

02,134

Hg1-xCdxTe Characterization Measurements: Current Prac- 
Ouantum Conductance Fluctuations in the Larger-SizeScale Regime

04,144 RII Spectroscopy of Trap Levels in Bulk and LPE Hg1$x$ CdxTe.

PB96-160247

04,084

Semiconductor Measurement Technology Improved Characterization and Evaluation Measurements for $\mathrm{HgCdTe} \mathrm{De}$ tector Materials, Processes, and Devices Used on the GOES and TIROS Satellites.

PB94-188810

02,122

Semiconductor Measurement Technology: Survey of Optical Characterization Methods for Materials, Processing, and Manufacturing in the Semiconductor Industry. PB96-154596

02,706

Transverse Magnetoresistance: A Novel Two-Terminal Method for Measuring the Carrier Density and Mobility of a Semiconductor Layer.

PB95-150066

02,332

SEILER, J

Characteristics of Adhesive-Bonded Seams Sampled from EPDM Roof Membranes.

PB95-162491

00,377

Degradation of Powder Epoxy Coated Panels Immersed in a Saturated Calcium Hydroxide Solution Containing Sodium Chloride.

PB96-101050

01,344

Development of a Method for Measuring Water-Stripping Resistance of Asphalt/Siliceous Aggregate Mixtures

PB96-197249

01,348

Development of a Method for Measuring Water-Stripping Resistance of Asphalt/Siliceous Aggregate Mixtures.

PB96-202296

SEILER, J. F.

Performance of Tape-Bonded Seams of EPDM Membranes: Comparison of the Peel Creep-Rupture Response of TapeBonded and Liquid-Adhesive-Bonded Seams.

PB96-183249

03,012

SEKERKA, R $F$.

Effects of Crystalline Anisotropy and Buoyancy-Driven Convection on Morphological Stability.

PB94-200441

03,328

Stagnant Film Model of the Effect of Natural Convection on the Dendrite Operating State.

PB96-146832

04,765

SELIM, M. S

Brownian Diffusion of Hard Spheres at Finite Concentra-

PBO5-164307

00,975

SELLECK, M. E.

Asymptotic Behavior of Modulated Taylor-Couette Flows with a Crystalline Inner Cylinder.

PB94-199072

04,469

Effect of Modulated Taylor-Couette Flows on Crystal-Melt Interfaces: Theory and Inttial Experiments.

PB94-216736

04,521

\section{SELLIN, I. A.}

High-Energy Behavior of the Double Photoionization of Helium from 2 to $12 \mathrm{keV}$.

PB94-213279

03,860

SELTZER, S. $M$.

Calculation of Photon Mass Energy-Transfer and Mass Energy-Absorption Coefficients.

PB97-110399

04,137

Electron-Photon Monte Carlo Calculations: The ETRAN Code

04,138

Electron transport calculations with biomedical and environ. mental applications. Final report, December 23, 1992--January 31,1994
DE95007065

03,613

EPR Dosimetry of Cortical Bone and Tooth Enamel Irradiated with $X$ and Gamma Rays: Study of Energy Depend. PBce. 110373

03,639

Exposure-to-Absorbed-Dose Conversion for Human Adult Cortical Bone.

03,640

Monte Carlo and Analytic Methods in the Transport of Electrons, Neutrons, and Alpha Particles.

PB96-111612

04,033

Pattern-Recognition Analysis of Low-Resolution X-Ray Fluorescence Spectra.

Study of Multiple Scattering Background in Compton Scatter Imaging

PB96-112222

04,425

Tables of X-ray Mass Attenuation Coefficients and Mass Energy-Absorption Coefficients $1 \mathrm{keV}$ to $20 \mathrm{MeV}$ for Elements $Z=1$ to 92 and 48 Additional Substances of

PB95-220539

04,013

Tomographic Decoding Algorithm for a Nonoverlapping Redundant Array.

PB95-151932

01,842

Updated Calculations for Routine Space-Shieldıng Radiation PB95-171039

04,838
SELWYN, G.

Gaseous Electronics Conference Radio-Frequency Reference Cell: A Defined Parallel-Plate Radio-Frequency System for Experimental and Theoretical Studies of PlasmaProcessing Discharges.

PB94-172327

04,404 Conductance Response of Pd/SnO2(110) Model Gas Sensors to $\mathrm{H} 2$ and $\mathrm{O} 2$

00,892

Fundamental Studies of Gas Sensor Response Mechanisms: Palladium on $\mathrm{SnO} 2(110)$.

00,963

NIST Workshop on Gas Sensors: Strategies for Future Technologies. Proceedings of a Workshop. Held in Gaithersburg, Maryland on September $8 \cdot 9,1993$. Held B95-210225

00507

Reactivity of Pd and Sn Adsorbates on Plasma and Thermally Oxidized $\mathrm{SnO} 2(110)$

00,804

Tin Oxide Gas Sensor Fabricated Using CMOS MicroHotplates and In-situ Processing.

B95-150603

00,580

SEMERJIAN, H. G.

Combustion of Methanol and Methanol/Dodecanol Spray Flames.

PB95-108544

CSTL Technical Activities, 1994.

CSTL Technical Activities, 1995.

02,478

CSTL Technical

00,608

Cothanol Spray Flames.

Elastic Scattering from Spheres under Non Plane-Wave Illu-

PB96-163688

04,370

Experimental and Numerical Studies of Refractory Particle Formation in Flames: Application to Silica Growth.

PB95-152005

00,673

Parametric Investigation of Metal Powder Atomization Using Laser Diffraction.

PB95-108577

03,342

Simulation of Ceramic Particle Formation: Comparison with In-situ Measurements.

PB95-152013

00.674

Structure of a Swirl-Stabilized Kerosene Spray Flam PB95-108569

Study of Droplet Transport in Alcohol-Based Spray Flames Using Phase/Doppler Interferometry.

PB95-108551

02,479

SENEHI, M. K.

Control Entity Interface Specification.
PB94-191715

02,815

Feasibility Study: Reference Architecture for Machine Control Systems Integration.

Reference Architecture for Machine Control Systems Integration: Interim Report

PB95-144549

02,820

ENEKOWITSCH, J.

Bonding in Doubly Charged Diatomics.

PB95-164315

00,976

SENGERS, J. M. H

Principle of Congruence and Its Application to Compressible States.

PB96-102892

SENGERS, J. M. H. L

Application of the Taylor Dispersion Method in Supercritical

Fluids.

Critıcal Behavior of Ionıc Fluids.

PB95-164331

00,977

Critical Lines for Type-III Aqueous Mixtures by Generalized Corresponding-States Models.

01,063

Standard States, Reference States and Finite-Concentration Effects in Near-Critical Mixtures with Applications to Aqueous Solutions.

PB95-164349

00,979

Thermodynamic Behavior of the $\mathrm{CO} 2-\mathrm{H} 2 \mathrm{O}$ System from 400 to $1000 \mathrm{~K}$, up to $100 \mathrm{MPa}$ and $30 \%$ Mole Fraction of $\mathrm{CO} 2$

PB94-162245

00,736

SENGERS, J. V.

Critical Scaling Laws and a Classical Equation of State.
PB95-169249

Global Thermodynamic Behavior of Fluid Mixtures in the Critical Region.

SENGUPTA, $s$.

04,199

Critical Current Density, Irreversibility Line, and Flux Creep Activation Energy in Silver-Sheathed $\mathrm{Bi} 2 \mathrm{Sr} 2 \mathrm{Ca} 2 \mathrm{Cu} 2 \mathrm{O}$ Superconducting Tapes.

PB95-162749

04,616

Correlation of $\mathrm{HgCdTe}$ Epilayer Defects with Underlying Substrate Defects by Synchrotron X-Ray Topography.

SERAPHIN, $\mathrm{S}$

Effect of Annealing Ambient on the Removal of Oxide Precipitates in High-Dose Oxygen Implanted Silicon.

PB95-164356

SERPA, F. G.

Observation and Visible and uv Magnetic Dipole Transitions in Highly Charged Xenon and Barium.

PB96-138441

SESHADRI, $K$

Chemical Inhibition of Methane-Air Diffusion Flame.

PB96-195532

SETTLE-RASKIN, A. D.

National Semiconductor Metrology Program, Project Portfolio FY 1996.
PB96-195268

SHADDIX, C. R

04,789

Computations of Enhanced Soot Production in Time-Varying $\mathrm{CH} 4 /$ Air Diffusion Flames.

B97-119218

01,440

Laser Imaging of Chemistry-Flowfield Interactions: Enhanced Soot Formation in Time-Varying Diffusion Flames. Ouantitative Measurements of Enhanced Soot Production in a Flickering Methane/Air Diffusion Flame.

PB95-203246

01,393

SHAFFNER, T. $J$.

Business and Manufacturing Motivations for the Developing of Analytical Technology and Metrology for Semiconductors.

PB96-161948

04,778

SHALER, T. A.

Lowest Excited Singlet State of Isolated 1-phenyl-1,3-butadiene and 1-phenyl-1,3,5-hexatriene.

PB95-202339

01,026

SHANNON, R. D.

Neutron Powder Diffraction Study of a $\mathrm{Na}$, Cs-Rho Zeolite. PB94-198629

00,791

SHAO, L.

Test of a Slow Off-Axis Parabola at Its Center of Curvature. PB96-138482

SHAPIRO, S. M

Anomalous Dispersion and Thermal Expansion in LightlyDoped KTa $1-\mathrm{xNbxO3}$

04,585

Low-Frequency Excitations of Oriented DNA

PB96-137799

03,548

SHAPOVAL, V. 1.

Precision High Temperature Blackbodies.

PB95-140059

03,885

SHARMA, J. K. N.

Intercomparison between NPL (India) and NIST (USA) Pressure Standards in the Hydraulic Pressure Region Up to $26 \mathrm{MPa}$ 
SHECHTMAN, D.

Crystallographic Characterization of Some Intermetallic Compounds in the Al-Cr System.

PB94-198702

SHEEHAN, M. E.

Influence of Natural and Synthetic Inhibitors on the CrysIallization of Calcium Oxalate Hydrates.

PB95-150967

03,560

SHELTON, D. B.

Helium Refrigeration and Liquefaction Using a Liquid Hydrogen Refrigerator for Precooling.
$A D-A 286683 / 8$

SHELTON, R. N.

02,749

Crystal Structure and Magnetic Ordering of the Rare-Earth and $\mathrm{Cu}$ Moments in RBa2Cu2NbO8(R=Nd,Pr).

PB95-140554

SHELUS, P. J.

Lunar Laser Ranging: A Continuing Legacy of the Apollo

Program.

03,683

SHEN, M. C.

Evaluation of Wear Resistant Ceramic Valve Seats in GasFueled Power Generation Engines. Topical Report, Decem ber 1991-April 1994.

PB95-200218

02,466

Mechano-Chemical Model: Reaction Temperatures in a Concentrated Contact.

PB96-119466

Wear Modeling of Si-Based Ceramics.

03,227

PB97-122501

03,112

Low-Temperature Elastic Constants of $\mathrm{Y} 1 \mathrm{Ba} 2 \mathrm{Cu} 3 \mathrm{O} 7$

PB95-168837

04,642

SHENKER, $\mathrm{H}$.

Reference Tables for Thermocouples.

SHENTON, H, W.

Draft Guideline for Testing and Evaluation of Seismic Isolation Systems

00,423

Draft Guidelines for Pre-Oualification and Prototype Testing of Seismic Isolation Systems.

01,331

Draft Guidelines for Quality Control Testing of Elastomeric Seismic Isolation Systems.

00,422

Draft Guidelines for Ouality Control Testing of Sliding Seismic Isolation Systems.

01,332

Field Evaluation of the System for Calibration of the Marshall Compaction Hammer.

01,323

Guidelines for Pre-Oualification, Prototype and Ouality Control Testing of Seismic Isolation Systems.

01,347

Pertormance of HUD-Aftiliated Properties during the January 17, 1994 Northridge Earthquake.

00,443

Summary and Results of the NIST Workshop on Proposed Guidelines for Testing and Evaluation of Seismic Isolation Systems. Held in San Francisco, California on July 25,

00,463

System for Calibration of the Marshall Compaction Hammer.

PB94-14566

SHERN, R. J.

01,303

Effects on Whole Saliva of Chewing Gums Containing Calcium Phosphates

SHERWOOD, G. V.

03,563

Dimensional Characterization of Precision Coaxial Transmission Line Standards.

PB96-176482

02,24

SHI, $D$.

Critical Current Density, Irreversibility Line, and Flux Creep Activation Energy in "Silver-Sheathed Bi2Sr2Ca2Cu2O Superconducting Tapes.

(9) 162749

04,616

SHIBATANI, T.

Thermochemistry of the Hydrolysis of L-arginine to (Lcitrulline + Ammonia) and of the Hydrolysis of L-arginine to (L-ornithine + Urea)

PB95-150801

03,463

SHIBAYAMA, $M$.

Small Angle Neutron Scattering Studies on Chain Asymmetry of Coextruded Poly(Vinyl Alcohol) Film.

01,262

Small Angle Neutron Scattering Study on Poly(N-Isopropyl Acrylamide) Gels Near Their Volume-Phase Transition Temperature.

01,263

Small-Angle Neutron Scattering Study on Weakly Charged Temperature Sensitive Polymer Gels.

PB95-164398

01,264

SHIELDS, J.

One- and Two-Sided Burning of Thermally Thin Materials.

PB95-140935
SHIELDS, J. 0.

Conversion of a 2-Terminal-Pair Bridge to a 4-Terminal-Pair Bridge for Increased Range and Precision in Impedance Measurements.

PB97-119176 02,103

NIST Comparison of the Ouantized Hall Resistance and the Realization of the SI Onm Through the Calculable CapaciPB. 97.119184

02,291

NIST Comparison of the Ouantized Hall Resistance and the Realization of the SI Ohm Through the Calculable Capacitor. Conference Proceedings, June 17-20, 1996.

PB97-119192

02,292

SHIELDS, J. R.

Behavior of Mock-Ups in the California Technical Bulletin 133 Test Protocol: Fabric and Barrier Effects.

PB95-231585

00,301

Effect of Suppressants on Metal Fires.

01,402

SHIH, A.

Lattice Position of $\mathrm{Si}$ in GaAs Determined by X-Ray Standing Wave Measurements

04,632

Surface Geometry of $\mathrm{BaO}$ on W(100): A Surface-Extended X-Ray-Absorption Fine-Structure Study.

PB95-164414

00,980

SHIH, C. K.

Summary Report: Workshop on Industrial Applications of Scanned Probe Microscopy (2nd). A Workshop Co-Sponsored by NIST, SEMATECH, ASTM E42.14, and the American Vacuum Society. Held in Gaithersburg, Maryland on MBgy 2-3, 1995 .

00,509

Workshop Summary Report: Industrial Applications of Scanned Probe Microscopy. A Workshop Co-sponsored by NIST, SEMATECH ASTM. E42.14, and the American Vacuum Society. Held in Gaithersburg, Maryland on March 24Uum PB95-170387

00,506

SHIMIZU, $M$

Pump-Induced Dispersion of Erbium.Doped Fiber Measured by Fourier-Transtorm Spectroscopy.

PB94-211935

04,236

SHIMIZU, $R$

Activities of ISO Technical Committee 201 on Surface Chemical Analysis.

00,607

Formation of Technical Committee 201 an Surtace Chemical Analysis by the International Organization for Standardcal Analys

PB95-108536

00,568

SHIN, $\mathrm{H}$.

Damage Processes in Ceramics Resulting from Diamond Tool Indentation and Scratching in Various Environments. PB96-102983

03,065

SHINDO, Y.

Elastic Constants and Microcracks in $\mathrm{YBa} 2 \mathrm{Cu} 3 \mathrm{O} 7$ PB96-200761

03,005

HINN, N. D.

Observation of a Stable Methoxy Intermediate on $\operatorname{Cr}(110)$. SHIRAI, T.

Spectral Data and Grotrian Diagrams for Highly lonized Chromium, Cr V through Cr XXIV.

PB94-162369

00,748

Spectral Data for Highly lonized Krypton, $\mathrm{Kr} V$ through $\mathrm{Kr}$

XXXVI.
PB96-145917

01,115

SHIRANE, G.

High-Energy Phonon Dispersion in La 1.85Sro.15CuO 4.
PB96-138458 PB96-138458
Neutron-Scattering Studies of the Two Magnetic Correlation Lengths in Terbium.

PB95-152328

04,586

Origin of the Second Length Scale Above the Magnetic Spiral Phase of $T$

PB95-153698

04,596

Photonic Band-Structure Effects for Low-Index-Contrast Two-Dimensional Lattices in the Near Infrared. PB97-122469

04,401

Self-Consistent 'GW' and Higher-Order Calculations of Electron States in Metals.

PB97-119341

01,189

SHIRLEY, J.

NIST-7, the New US Primary Frequency Standard.

PB95-15345

SHIRLEY, J. H.

Error Analysis of the NIST Optically Pumped Primary Frequency Standard. Microwave Leakage as a Source of Frequency Error and Long-Term Instability in Cesium Atomic-Beam Frequency Standards.

PB95-180501

01,541

NIST Optically 03,835

Velocity Distribution of Atomic Beams by Gated Optical Pumping.

01,542

SHOBE, J.

Scattered Fractions of Dose from 18 and 25 MV X-ray Radiotherapy Linear Accelerators.

PB96. 18610

04,120

SHOCK, E. L.

Summary of the Apparent Standard Partial Molal Gibbs

Free Energies of Formation of Aqueous Species. Minerals,

and Gases at Pressures 1 to 5000 Bars and Temperatures 25 to $1000 \mathrm{C}$

SHOEMAKER, A. C.

Taguchi's Parameter Design: A Panel Discussion

SHORE, S. N.

01,113

Goddard High-Resolution Spectrograph Observations of the Local Interstellar Medium and the Deuterium/Hydrogen Ratio along the Line of Sight Toward Capella.

PB94-213444

00,066

Observations of 3C 273 with the Goddard High Resolution Spectrograph on the Hubble Space Telescope.

PB95-202321

00,076

Observing Stellar Coronae with the Goddard High Resolution Spectrograph. I. The dMe Star AU Microscopii.

Fragment State Correlations in the Dissociation of NO.HF $(v=1)$.

PB95-164430

SHOUTE, L. C. T.

Fluoride Elimination Upon Reaction of Pentafluoroaniline with e (sub eq')(sup -), $\mathrm{H}$, and $\mathrm{OH}$ Radicals in Aqueous So-

PB97-111314

01,177

lodine Atoms and lodomethane Radical Cations: Their Formation in the Pulse Radiolysis of lodomethane in Organic Solvents, Their Complexes, and Their Reactivity with Organic Reductants.

PBg5-162764

Reduction of Dinitrogen to Ammonia in Aqueous Solution

$\begin{array}{ll}\text { Mediated by Colloidal Metals. } & 00,867\end{array}$

Temperature Dependence of the Rate Constants for Reaction of Dihalide and Azide Radicals with Inorganic Reduc-

00964

Temperature Dependence of the Rate Constants for Reaction of Inorganic Radicals with Organic Reductants

PB94-198280

00,783

Temperature Dependence of the Rate Constants for Reactions of the Carbonate Radical with Organic and Inorganic Peductants.

SHUH, D. K.

00,831

Influence of Coadsorbed Potassium on the Electron-Stimulated Desorption of $F(+), F(-)$, and $F(*)$ from PF3 on Ru(0001).

PB96-157946

04,072

SHUKER, $R$

Laser-Synchrotron

A Photon to Poke

SHULL, $K$.

Neutron Scattering by Multiblock Copolymers of Structure

(A-B)N-A

PB94-211547

SHULL, R. D.

Enhanced Magnetocaloric Effect in Gd3Ga5-xFexO12.

PB94-185659

04,618

PB95-162798 
SHY, J. T.

Improved Molecular Constants and Frequencies for the $\mathrm{CO} 2$ Laser from New High-J Regular and Hot-Band Frequency Measurements.

SIBENER, S. J.

Precision Lifetime Measurements of Cs $6 p(2) P_{1 / 2}$ and $6 p$ (2)P3/2 Levels by Single-Photon Counting.

04,010

Temperature Dependence and Anharmonicity of Phonons on Ni(110) and Cu(110) Using Molecular Dynamics Simula(tions.

PB94-185477

04,449

SICARRDI, $M$

New 5 and $10 \mathrm{MHz}$ High Isolation Distribution Amplitier.

PB96-190202

07,510

SIECK, B. A.

Deposition of Loosely Bound and Firmly Bound Fluorides on Tooth Enamel by an Acidic Gel Containing Fluorosilicate and Monocalcium Phosphate Monohydrate.

03,559

Effects on Whole Saliva of Chewing Gums Containing Cal clum Phosphates.
PB95-153169

SIECK, L. W.

03,563

Ionization Energy of Sulfur Pentafluoride and the Sulfur Pentafluoride-Fluorine Atom Bond Dissociation Energy
PB95-162814

Proton Affinity Ladders from Variable-Temperature Equilibrium Measurements. 1. A Re-Evaluation of the Upper Proton Affinity Range.

PB94-216603

00,861

SIEGAL, W. $O$.

Atmospheric Reactivity of alpha-Methyl-Tetrahydrofuran PB95-163705

SIEGEL, R. W.

Small Angle Neutrons Scattering from Nanocrystalline Palladium as a Function of Annealing.

PB95-176103

03,354

SIEGRIST, T.

$\mathrm{Ca} 1-\mathrm{xCuO} 2$, a NaCuO2-Type Related Structure.

SIEGWARTH, J. D

04,620

Experimental plan to determine the performance of the Oak Ridge National Laboratory Cold Neutron Moderator. Final report, September 1, 1993-November 30, 1993. DE95011352

Friction and Oxidative Wear of $440 \mathrm{C}$ Ball Bearing Steels Under High Load and Extreme Bulk Temperatures.

PB95-175253

03,215

SSME LOX Duct Flowmeter Design and Test Results.

PB96-161955 04,826

Thermal Hydraulic Tests of a Liquid Hydrogen Cold Neutron Source.

PB95-135570

03,884

Tribometer for Measurements in Hostile Environments. PB95.180949

Vortex Shedding Flowmeters for SSME Ducts

PB95-169215

01,453

SIES, $R$.

Manager's Guide for Monitoring Data Integrity in Financial Systems.

PB96-165915

00,003

SIES, R. F.

Self Monitoring Accounting Systems.

00,007

SIEVERS, R. E.

High-Pressure Equilibrium Cell for Solubility Measurements in Supercritical Fluids.

PB95-175634 00.998

Solubilities of Copper(II) and Chromium(III) betaDiketonates in Supercritical Carbon Dioxide.

PB96-164215

01,147

SIEW, C.

Procedure for the Study of Acidic Calcium Phosphate Precursor Phases in Enamel Mineral Formation.

CUIS0 16444

03.564

SIEWERT, T.

In-Space Welding: Visions and Realities.

PB95-163234

International Institute of Welding: Report on 1992 Actions.
PB94-185873 02,856

International Institute of Welding: Report on 1995 Actions.

PB96-158076 02,874

Through-the-Arc Sensing for Measuring Gas Metal Arc Weld Quality in Real Time.

PB95-164463

02,908

SIEWERT, T. A.

Computers in Welding: A Primer

PB95-162863

02,862

PB96. 135330

02,872

Contributions of Out-of-Plane Material to a Scanned-Beam Laminography Image.

02,704
Control of Gas-Metal-Arc Welding Using Arc-Light Sensing.
PB96-131461

Cryogenic Toughness of Austenitic Stainless Steel Weld Metals: Effect of Inclusions.

03,214

Droplet Transfer Modes for a MIL 100S-1 GMAW Electrode. PB95-209300

02,867

Etfect of Charpy V-Notch Striker Radii on the Absorbed EnPBg6-141122

03,365

Effects of Copper, Nickel and Boron on Mechanical Properties of Low-Alloy Steel Weld Metals Deposited at High Heat Input.

PB96-135231

03,363

Electrode Extension Model for Gas Metal Arc Welding.

PB96.135074 02,871

Evaluation and Qualification Standards for an X-Ray Laminography System.

02,029

IIW Commission V Quality Control and Quality Assurance of Welded Products Annual Report 1994/95.

PB95-198743

02,866

IIW Commission V Quality Control and Quality Assurance of

Welded Products, Annual Report 1995/96.

PB96-191366

02,880

International Institute of Welding: Report on 1993 Actions.

PB94-185881

02,857

Mapping the Droplet Transter Modes for an ER100S-1 GMAW Electrode.

03,295

Materials Reliability. Technical Activities, 1995

PB96-183082

02,999

Report on 1994 Actions of the International Institute of Welding.

PB96-138540

02,873

Sensing Droplet Detachment and Electrode Extension for Control of Gas Metal Arc Welding.

03,297

Status Report: AWS Standards for Identifying Arc Welds Status Report: AWS Standards for Identifying Arc Welds
(A91.1) and Recording Weld Data (A9.2).
PB95-162855

Through-the-Arc Sensing for Monitoring Arc Welding.

02,858

Through-the-Arc Sensing for Real-Time Measurement of Gas Metal Arc Weld Quality.

PB95-162871

Welding for Cryogenic Service.

PB95-162889

02,863

What's Available in Welding Software.

PB96-158084

02,852

ator Designed for Easy Align-

PB95-164455

02,907

SIGGEL, M. R. F.

Inner-Valence States $\mathrm{CO}(+)$ between $22 \mathrm{eV}$ and $46 \mathrm{eV}$ Studied by High Resolution Photoelectron Spectroscopy

and ab Initio Cl Calculations.

03.961

Photoelectron Study of Electronic Autoionization in Rolationally Cooled N2: The $n=6$ Member of the Hoptield Series.

PB95-163531

00.971

Shape-Resonance-Enhanced Continuum-Continuum Coupling in Photoionization of $\mathrm{CO} 2$

00,983

Vibrational Autoionization in $\mathrm{H} 2$. Vibrational Branching $\mathrm{Ba}-$ tios and Photoelectron Angular Distributions Near the $v(+)=3$ Threshold

PB94-199577

00,799

Vibrationally Resolved Photoelectron Angular Distributions and Branching Ratios for the Carbon Dioxide Molecule in

the Wavelength Region 685.795 Angstrom
PB96-201207

04,131

SIJELMASSI, R.

PET and DINGO Tools for Deriving Distributed Implementations from Estelle.

PB95-203253

01,727

SIKDAR, S. K.

Continuous Counter-Current Two Phase Aqueous Extrac-

PB95-161212

00,675

Enzyme and Protein Mass Transter Coefficient in Aqueous Two Phase Systems. 1. Spray Extraction Columns. PB95-161162

00.594

Protein Extraction in a Spray Column Using a Polyethylene Glycol Maltodextrin Two.Phase Polymer System. PB95-162228

00,595

Two Phase Aqueous Extraction: Rheological Properties of Dextran, Polyethylene Glycol, Bovine Serum Albumin and Their Mixtures

00,676

SILBERSTEIN, $S$.

Proficiency Tests for the NIST Airborne Asbestos Program,

PB96-106463

00,610

SILVA, A. M.

Design of a High-Pressure Ebulliometer, with Vapor-Liquid Equilibrium Results for the Systems CHF2Cl + CF3.CH3 and $\mathrm{CF}_{3}-\mathrm{CH}_{2} \mathrm{~F}+\mathrm{CH}_{2} \mathrm{~F}_{2}$.

PB97-113229

04,163

Ebulliometers for Measuring the Thermodynamic Properties of Fluids and Fluid Mixtures.

04,795

Ebulliometric Measurement of the Vapor Pressure of 1 Chloro-1,1-Difluoroethane and 1,1-Difluoroethane. PB95-164489

00,984

Measurements of the Vapor Pressures of Difluoromethane, 1-Chloro-1,2,2,2-Tetrafluoroethane, and Pentafluoroethane. PB95-169272

03,270

SILVA, T.

Magnetoresistance of Thin-Film NiFe Devices Exhibiting Single-Domain Behavior

PB96-147087

04,766

SILVA, T. J.

Dependence of Contrast on Probe/Sample Spacing with the Magneto-Optic Kerr-Effect Scanning Near-Field Optical Microscope (MOKE-SNOM)

PB96-138557

04,750

Observation of the Transverse Second Harmonic MagnetoOptic Kerr Effect from Ni81Fe19 Thin Film Structures.

PB96-200332

01,971

SILVER, J. D.

Photographic Response to X-Ray Irradiation. 3. Photographic Linearization of Beam-Foil Spectra.

03,823

SILVER, R. M.

Junction Locations by Scanning Tunneling Microscopy: InAir-Ambient Investigation of Passivated Gäis pn Junctions. PB94-185964

SIMAKHODSKIY, I.

Mapping Integration Definition for Information Modeling (IDEF1X) Model into CASE Data Interchange Format (CDIF) Transfer File.

SIMAKHODSKIY, I. V

Mapping Integration Definition for Function Modeling (IDEFO) Model into CASE Data Interchange Format (CDIF) Transfer File.

SIMIC, M. G.

Standard Reference Materials (SRM's) for Measuring Genetic Damage.

03,516

SIMIU, E.

Algebraic Approximation of Attractors for Galloping OscillaPBS. 162897

04,820

Assessment of 'Peaks Over Threshold' Methods for Estimating Extreme Value Distribution Tails.

PB95-161360

00,441

Chaotic Motions of Coupled Galloping Oscillators and Their Modeling as Diffusion Progresses.

PB96-122718

04,823

Deterministic and Stochastic Chaos.

De-156138

Development of Computer-Based Models of Standards and 
Noise-Induced Chaos and Phase Space Flux. PB95-125761

Noise-Induced Transitions to Chaos.

03,433

00,217

Noise Modeling and Reliability of Behavior Prediction for Multi-Stable Hydroelastic Systems.

PB96-111943

04,822

Non-Gaussian Noise Effects on Reliability of Multistable

PB96-122726

04,213

Recent Approaches to Extreme Value Estimation with Application to Wind Speeds. Part 1. The Pickands Method.

PB94-213170 00,019

Spectrum of the Stochastically Forced Duffing-Holmes Os. cillator.

00,216

Transitions to Chaos Induced by Additive and Multiplicative

Noise.
PB96-155759

03,750

SIMMON, E.

Active High Voltage Divider with 20-PPM Uncertainty.
PB97-119317 104 PB97-119317

SIMMON, E. D.

Optical Current Transducer for Calibration Studies.

10

SIMMONDS, M. B.

$24 \mathrm{GHz}$ Josephson Array Voltage Standard.

PB94-211588

SIMMONS, A.

02,033

High-Spatial-Resolution Resistivity Mapping Applied to Mercury Cadmium Telluride.

PB94-212917

SIMMONS, J. A.

Leady Axisymmetric Modes in Infinite Clad Rods. Part 1.

PB95-162905

SIMMONS, J. D.

Should NIST Accredit U.S. Calibration Laboratories. PB95-107280

SIMON, H.

Beyond the Technology Roadmaps: An Assessment of Electronic Materials Research and Development.

PB96-165998

01,961

SIMON, L. N.

U.S. Green Building Conference, 1994

PB94-206364

02,519

SIMON, N. J.

Cryogenic Properties of Inorganic Insulation Materials for ITER Magnets: A Review.

03,706

Influence of Specimen Absorbed Energy in LOX Mechanical-Impact Tests.

PB95-107355

03,341

Irradiation Damage in Inorganic Insulation Materials for ITER Magnets: A Review.

03,705

Macro- and Microreactions in Mechanical-impact Tests of Aluminum Alloys.

Aluminum Alloys.

03,340

Reaction Sensitivities of Al-Li Alloys and Alloy 2219 in Mechanical-Impact Tests

PB94-172764

03,314

Recommended Changes in ASTM Test Methods D2512-82 and G86.84 for Oxygen-Compatibility Mechanical Impact Tests on Metals.

03,338

Temperature Increases in Aluminum Alloys during Mechanical-Impact Tests for Oxygen Compatibility.

PB94-172962

03,316

SIMON, T.

Distant Future of Solar Activity: A Case Study of Beta Hydri 3. Transition Region, Corona, and Stellar Wind

00,049

Distant Future of Solar Activity: A Case Study of beta Hydri. 3. Transition Region, Corona, and Stellar Wind.

Efficient Way of Identifying New Active Stars: A VLA Survey of X-ray Selected Active Stellar Candidates.

PB96-122882

00,099

Far-Ultraviolet Flare on a Pleiades G Dwarf.

00,086

Four Years of Monitoring alpha Orionis with the VLA: Where Have All the Flares Gone.

PB94-185212

00,048

Radio Continuum and $X$-Ray Properties of the Coronae of RS Canum Venaticorum and Related Active Binary Sys. ems.

00,057

Sleuthing the Dynamo: HST/FOS Observations of UV Emis-

sions of Solar-Type Stars in Young Clusters.
PB96-122817

00,098

SIMONI, F. V

Effect of Two Initiator/Stabilizer Concentrations in a Metal Primer on Bond Strengths of a Composite to a Base Metal Alloy.

00,141
SIMONS, D.

Relative Sensitivity Factors and Useful Yields for a Microfocused Gallium Ion Beam and Time-of-Flight Secondary lon Mass Spectrometer.

00,541

SIMONS, D. S.

Effect of Annealing Ambient on the Removal of Oxide Precipitates in High-Dose Oxygen Implanted Silicon.
PB95-164356

Molecular Ion Imaging and Dynamic Secondary Ion Mass Spectrometry of Organic Compounds.

00,571

Range Statistics and Rutherford Backscattering Studies on Fe-Implanted In $0.53 \mathrm{Ga0} .47 \mathrm{As}$

PB95-126397

Transition Metal Implants in In0.53GA0.47As.

PB95-126389

04,535

SIMONS, G. R.

04,534

Opportunities for Innovation: Advanced Manufacturing TechPB94-100278

02,801

SIMPSON, J. A.

Metrology.

PB95-164497

03,946

Effects of Aluminum Oxalate/Glycine Pretreatment Solutions on Dentin Permeability.

03,565

SINGER, A. T

Interim Testing Artifact (ITA): A Performance Evaluation System for Coordinate Measuring Machines (CMMs). Use Manual.

PB95-210589

02,914

SINGH, $K$.

2nu9 Band of Propyne-d3

PB95-164513

00,985

SINGH, $\mathbf{S}$.

Resonance Fluorescence with Squeezed-Light Excitation PB95-203469

04,322

SINHA, K.

Epitaxial Growth and Characterization of the Ordered Vacancy Compound Culn3Ses on GaAs (100) Fabricated by Molecular Beam Epitaxy.

PB95-180725

04,687

SINHA, S. K.

Neutron Reflectivity of End-Grafted Polymers: Concentration and Solvent Quality Dependence in Equilibrium Conditions. PB94-185758 01,206

Neutron Scattering Study of Antiferromagnetic Order in the Magnetic Superconductors RNi2B2C.

PB97-112411

04,812

Small-Angle Neutron Scattering (SANS) Study of WormLike Micelles Under Shear.

PB96-176698

Human and Machine Recognition of Faces: A Survey.

SIROHEY, S. A.

Face Recognition Technology for Law Enforcement Applications.

SIVAKUMAR

Deposition of Colloidal Sintering-Aid Particles on Silicon

Nitride.

03,044

SIVATHANU, Y. R.

Exits in Multistable Systems Excited by Coin-Toss Square Wave Dichotomous Noise: A Chaotic Dynamics Approach. PB96-160650

04,824

Investigation

03,695

Tomographic Reconstruction of the Moments of Local Probability Density Functions in Turbulent Flow Fields.

PB96-18019

04,219

SJOLIN, L.

Crystal Packing Interactions of Two Different Crystal Forms PB95-152823

SKAMSER, D. J.

Calculation of the Thermal Conductivity and Gas Permeability in a Uniaxial Bundle of Fibers.

PB95-180931

03,058

SKANTHAKUMAR, $S$.

Field Dependence of the Magnetic Ordering of $\mathrm{Cu}$ in $\mathrm{R} 2 \mathrm{CuO} 4(\mathrm{R}=\mathrm{Nd}, \mathrm{Sm})$.

04,633

Observation of Noncollinear Magnetic Structure for the $\mathrm{Cu}$ Spins in Nd2CuO4-Type Systems.

PBins in 164539

04,634

SKEELS, J.

Group 1 for the Plant Spatial Configuration STEP Application Protocol.

02,789

SKIENA, S. $S$.

Point Probe Decision Trees for Geometric Concept Classes. PB96-160817

SKINNER, S. L.

High Sensitivity Survey of Radio Continuum Emission in

PB94-185915

SKOCPOL, W. J.

Coexistence of Grains with Differing Orthorhombicity in High Quality YBa2Cu307-delta Thin Films.

04,742

Increased Transition Temperature in In situ Coevaporated

YBa2Cu307-delta Thin Films by Low Temperature Post-An-

PB95-180071

04,672

Thermal Noise in High-Temperature Superconducting-Nor-

mal-Superconducting Step-Edge Josephson Junction

SKOWYRA, D.

DnaJ, DnaK, and GrpE Heat Shock Proteins are Required

in 'ori'P1 DNA Replication Solely at the RepA Monomerization Step.

PB97-119382

SKRTIC, D.

Bioactive Polymeric Dental Materials Based on Amorphous Calcium Phosphate.

03,572

Effect of 1-Hydroxyethylidene-1,1-Bisphosphonate on Membrane-Mediated Calcium Phosphate Formation in Model Liposomal Suspensions.

03,469

In vitro Inhibition of Membrane-Mediated Calcification by Novel Phosphonates.

03,595

Membrane-Mediated Precipitation of Calcium Phosphate in Model Liposomes with Matrix Vesicle-Like Lipid Composi-

PB95-164547

03,468

Polymeric Calcium Phosphate Composites with Remineralization Potential.
PB96-155544 03,575

Remineralizing Dental Composites Based on Amorphous Calcium Phosphate.

PB96-147020

03,573

SLABACK, L. A.

Characterization of a Health Physics Instrument Calibration

Range.

PB95-164554

03,629

Germanium Detector Optimization of MDA for Efficiency vs. Low Intrinsic Background

SLABACK, L. S.

00,543

External Gamma-ray Counting of Selected Tissues from a Thorotrast Patient.

PB96-160254

SLAUGHTER, J. M.

Interfaces in Mo/Si Multilayers.

PB96-160668

SLEIGHT, A. W.

Colossal Magnetoresistance without $\mathrm{Mn}(3+) / \mathrm{Mn}(4-)$ Double Exchange in the Stoichiometric Pyrochlore T12Mn2O7. PB97-113070

SLEZSAK, I.

Oscillometric and Conductometric Analysis of Aqueous and Organic Dosimeter Solutions.

PB96-135256

04,054

SLIFKA, A. J.

Friction and Oxidative Wear of $440 \mathrm{C}$ Ball Bearing Steels Under High Load and Extreme Bulk Temperatures. 
SMATHERS, D.

Transverse stress effect on the critical current of internal tin and bronze process $\mathrm{Nb}$ (sub 3 ) $\mathrm{Sn}$ superconductors. DE95016659

SMID, M. E.

Response to Comments on the NIST Proposed Digital Sig-

nature Standard
PB96-161815

01,615

SMILGYS, R. V.

Laser Gas lonization Technique Monitors MEB Crystal

PB96-112172

01,076

Reactive Coevaporation of DyBaCuO Superconducting Films: The Segregation of Bulk Impurities on Annealed $\mathrm{MgO}(100)$ Substrates.

PB95-164562

04,635

Single-Photon Laser Ionization Time-of-Flight Mass Spectroscopy Detection in Molecular-Beam Epitaxy: Application to As4, As2, and Ga.

PB95-203337

Vibrational Distributions of As2 in the Cracking of As4 on Si(100) and $\mathrm{Si}(111)$

00,784

SMIRL, A. L

Interdigitated Stacked P-I-N Multiple Quantum Well Modula-

PB97-112296

02,455

Scaling of the Nonlinear Optical Cross Sections of GaAsAlGaAs Multiple Quantum-Well Hetero n-i-p-i's.

PB96-102793

02,183

Time-Resolved Measurements of the Polarization State of Four-Wave Mixing Signals from GaAs Multiple Quantum

201058

04,796

SMITH, A. B.

Discontinuous Volume Change at the Orientational-Ordering Transition in Solid C60.

00,821

Neutron-Scattering Siudy of Librations and Intramolecular Phonons in Rb2.6Ko.4C60.

PB95-162269

00,958

SMITH, A. C. H.

Backscattering in Electron-Impact Excitation of Multiply Charged lons

PB94-185345

03,799

Collisions of Electrons with Highly-Charged lons.

PB96-200340

04,791

Electron-Impact Excitation of Si(3+)(3S yields 3P) Using a Merged-Beam Eleciron-Energy-Loss Technique.

Evidence for Significant Backscattering in Near-Threshold Electron-Impact Excitation of $\operatorname{Ar}(7+)(3 s$ yields $3 p)$. PB95-126405

03,883

Merged-Beams Energy-Loss Technique for Electron-Ion Excitation: Absolute Total Cross Sections for $\mathrm{O}(5+)$ (2s yields

PB96-102058

04,017

SMITH, A. J.

Bibliography of the NIST Optoelectronics Division

PB96-128210

02,193

Bibliography of the NIST Optoelectronics Division.

PB97-116040

02,207

Metrology for Electromagnetic Technology: A Bibliography of NIST Publications.

PB95-135588

02,143

SMITH, A. M.

Observations of $3 \mathrm{C} 273$ with the Goddard High Resolution

Spectrograph on the Hubble Space Telescope.

PB95-202321

00,076

SMITH, B. M.

ISO TC 184/SC4 Reference Manual.

PB95-242293

02,663

SMITH, C.

Electronic Balance and Some Gravimetric Applications. (The Density of Solids and Liquids, Pycnometry and Mass).
PB94-163052

SMITH, C. J.

Initial and Final Orbital Alignment Probing of the Fine-Struciure-Changing Collisions among the $\mathrm{Ca}(4 \mathrm{~s})(1)(4 \mathrm{p})(1)$, (3)PJ States with He: Determination of Coherence and Conventional Cross-Sections.

PB95-203279

04,004

Laser Preparation and Probing of Initial and Final Orbital Alignment in Collision-Induced Energy Transfer $\mathrm{Ca}\left(4 s 5 p_{1}(1) \mathrm{P} 1\right)+\mathrm{He}$ yields $\mathrm{Ca}\left(4 s 5 p_{,}(3) \mathrm{P} 2\right)+\mathrm{He}$

PB95-203261

04,003

Orbital Alignment and Vector Correlations in Inelastic Alomic Collisions.

SMITH, D. K.

Powder Diffraction File: Past, Present, and Future. PB97-109086

04,047

PBITH, D. R.

Cryogenic Properties of Silver.

PB94-203593
Low-Temperature Properties of Silver.

PB96-126198 mina Filler Particles from 4.2 to $300 \mathrm{~K}$.

B96-200753

01,292

MITH, D. T.

Adhesion, Contact Electrification, and Acid-Base Properties of Surfaces.

PB96-204425 03,693

Conference Proceedings: International Workshop on Instrumented Indentation. Held in San Diego, California on April 22-23, 1995.

PB96-158688 01,948

Contact Electrification Induced by Monolayer Modification of a Surface and Relation to Acid-Base Interactions.

PB94-185378

03,034

Effect of Beam Voltage on the Properties of Aluminum Nitride Prepared by lon Beam Assisted Deposition. $\begin{array}{ll}\text { PB97-118616 } & 01,995\end{array}$ Measuring Contact Charge Transfer at Interfaces: A New
Experimental Technique.
03,053 PB95-164570

Surface Forces and Adhesion between Dissimilar Materials Measured in Various Environments.

PB94-172970

03,033

SMITH, E. R.

Table of Dielectric Constants of Pure Liquids.

AD-A278 956/8

00,712

SMITH, H. G.

Structures of Sodium Metal.

PB94-198850

03,319

Guide to Instrumentation Literature

02,617

MITH, J. H.

Ashland Tank Collapse Investigation

PB95-126314

02,481

Ashland Tank-Collapse Investigation: Closure by Authors.

02,482

Geometric Characterization of Rockwell Diamond Indenters.

PB95-203287 02,950

Meirology Approach 10 Unifying Rockwell C Hardness Scales.

PB96-155551

02,957 Indenters.

$\begin{array}{ll}\text { PB96-122114 03,280 } & \end{array}$

Microform Calibrations in Surface Metrology.

PB95-203295

02,951

Stylus Technique for the Direct Verification of Rockwell Diamond Intenders

PB96-155569

02,958

MITH $\mathrm{J}$ L.

Superconducting Energy Gap of Bulk UBe13. PB95-150116

04,559

Optimization of Highway Concrete Technology

SMITH, L. C.

Determination of the Prior-Austenitic Grain Size of Selected Steels Using a Molten Glass Etch.

PB94-211927

03,208

SMITH, L. E.

Polymers Technical Activities 1994. NAC-NRC Assessment Panel, April 6-7, 1995

PB95-209896

01,275

Polymers Technical Activities, 1995.

01,291

SMITH, M.

Computer-Aided Manufacturing Engineering Forum (1st) Technical Meeting Proceedings. Held in Gaithersburg, Maryland on March 21-22, 1995.

PB96-136965

02,834

Computer-Aided Manufacturing Engineering Forum (2nd). Technical Meeting Proceedings. Held in Gaithersburg. Maryland on August 22-23, 1995.

PB96-195334

02,845

Critical Current Density, Irreversibility Line, and Flux Creep Activation Energy in Silver-Sheathed Bi2Sr2Ca2Cu2Ox Superconducting Tapes.

PB95-162749

04,616

SMITH, M. V.

Determination of the Transmittance Uniformity of Optical Filter Standard Reference Materials.

PB95-26192

02,182

Standard Reference Materials: Glass Filters as a Standard Reference Material for Spectrophotometry - Selection, Preparation, Certification, and Use of SRM 930 and SRM 1930 PB94-188844

00,536

SMITH, $R$.

Effects of Copper, Nickel and Boron on Mechanical Properties of Low-Alloy Steel Weld Metals Deposited at High

Heat Input.
PB96-135231 03,363

Standard Source Method for Reducing Antenna Factor Errors in Shielded Room Measurements.

PB96-183157

02,013

SMITH, R. L.

Exposure: An Expert System Fire Code.

PB95-162913

04,868

Performance Parameters of Fire Detection Systems.

PB94-194339

00,288

Risk Analysis for the Fire Safety of Airline Passengers.

PB94-194065

MITH, R. W.

Federal Basis for Weights and Measures: A Historical Review of Federal Legislative Effort, Statutes, and Administrative Action in the Field of Weights and Measures in the United States.

AD-A280 086/0

02,616

MITH, S. B.

Annual Conference on Fire Research: Book of Abstracts, October $17-20,1994$

SMITH, S. M.

01,376

Application of a Novel Slurry Furnace AAS Protocol for Rapid Assessment of Lead Environmental Contamination.

PB96-112354

02,526

SMITH, T. B.

In vitro Fracture Behavior of Ceramic and Metal-Ceramic Restorations.

SMITH, T. F.

Optimal Control of Building and HVAC Systems.

PB96-141353

SMOOKE, M. D

Comparison of Experimental and Computed Species Concentration and Temperature Profiles in Laminar, Two-Dimensional Methane/Air Diffusion Flames.

PB95-140919

01,379

$X$-Ray Diffraction from Anodic TiO2 Films: In situ and Ex situ Comparison of the Ti(0001) Face.

PB94-185972

00,782

SMYTH, D. C.

Laser-Induced Fluorescence Measurements of Formaldehyde in a Methane/Air Diffusion Flame.

PB94-211679

01,374

SMYTH, K. C.

Comparison of Experimental and Computed Species Concentration and Temperature Profiles in Laminar, Two-Dimensional Methane/Air Diffusion Flames.

PB95-140919

01,379

Computations of Enhanced Soot Production in Time-Varying $\mathrm{CH} 4 /$ Air Diffusion Flames.

01,440

Gordon Research Conference on the Physics and Chemistry of Laser Diagnostics in Combustion Held in Plymouth, 
SNELICK, R.

Synthetic-Perturbation Tuning of MIMD Programs PB94-185568

Time-Perturbation Tuning of MIMD Programs.

PB94-164399

Time-Perturbation Tuning of MIMD Programs. PB94-172566

Using Synthetic-Perturbation Techniques for Tuning Shared Memory Programs (Extended Abstract)

PB94-172657

01,685

Using Synthetic Perturbations and Statistical Screening to Assay Shared-Memory Programs.

SNELICK, R. D.

Using S.Check, Alpha Release 1.0
PB96-165964

01,740

01,767

SNELL, J. E.

Elements of a Framework for Fire Safety Engineering PB96-151402 00,214

Fire Hazard and Risk: Evaluating Alternative Technologies. PB94-173077 00,242 Fire Safety Engineering Research in the United States. Fire Safety Engineering Research in the United States.
PB96-151394

Forum for International Cooperation on Fire Research PB95-162939

04,869

Fresh Look at Strategies for Fire Safety.

04,870

Internationalization of Fire Safety Engineering Research and Strategy. and Strategy.
PB96-156153

00,220

Quantitative Evaluation of Building Fire Safety: New Tools

for Assessing Fire and Building Code Provisions.
PB95-164588

00,199

SNIDER, R. F.

Equilibrium Pair Distribution Function of a Gas: Aspects Associated with the Presence of Bound States.

PB95-176046

SNIEGOSKI, L.

01,004

Hair Analysis for Drugs of Abuse: Evaluation of Analytical Methods, Environmental Issues, and Development of Reference Materials.

PB95-176269

03,501

SNIEGOSKI, L. T.

Determination of 3-Quinuclidinyl Benzilate $(\mathrm{Qnb})$ and Its Major Metabolites in Urine by Isotope Dilution Gas Chromatography Mass Spectrometry.

03,492 Interlaboratory Comparison Studies on the Analysis of Hair

for Drugs of Ábuse
PB95-176251

03,500

Interlaboratory Studies on the Analysis of Hair for Drugs of Abuse: Results from the Fifth Exercise.

PB97-110449

03,509

Interlaboratory Studies on the Analysis of Hair for Drugs of Abuse: Results from the Fourth Exercise.

03,510

Isotope Dilution Mass Spectrometry as a Candidate Definitive Method for Determining Total Glycerides and

Triglycerides in Serum.
PB96-102280

03,519

NIST Reference Materials to Support Accuracy in Drug Testing.

SNIEGOWSKI, J. J.

03,505

Electrical Test Structures Replicated in Silicon-on-Insulator Material.

PB97-111827

02,454

SNOOTS, $P$.

Interim Testing Artifact (ITA): A Performance Evaluation System for Coordinate Measuring Machines (CMMs). User Manual.

PB95-210589

02,914

Measuring the Stability of Three Copper Alloys.

PB94-199866

03,326

NIST SRM 9983 High-Rigidity Ball-Bar Stand. User Manual. PB95-255840

02,669

SNOW, M.

Observations of $3 \mathrm{C} 273$ with the Goddard High Resolution Spectrograph on the Hubble Space Telescope.

SNOW, W. M.

00,076

Measurement of the Neutron Lifetime.

PB96-161708

SNYDER, $K$.

04,094

Highway Concrete (HWYCON) Expert System User Ref-

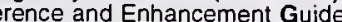

PB94-215670

01,316

Long-Term Performance of Engineered Concrete Barriers.

PB95-260816

03,727

Percolation and Pore Structure in Mortars and Concrete. PB95-150439

00,370

SNYDER, K. A

4SIGHT Manual: A Computer Program for Modelling Degradation of Underground Low Level Waste Concrete Vaults.
Geometrical Percolation Threshold of Overlapping Ellipsoids.

PB96-102397

03,167

\section{SNYDER, S. M.}

Phase Composition, Viscosities, and Densities for Aqueous Two-Phase Systems Composed of Polyethylene Glycol and Various Salts at $25 \mathrm{C}$

PB95-164596

00,986

, C. G.

Angular Variation of the Personal Dose Equivalent, $\mathrm{Hp}(0.07)$, for Beta Radiation and Nearly Monoenergetic PBectron Beams:

03,630

Comparison of NIST and ISO Filtered Bremsstrahlung Calibration Beams.

PB95-180956

03,967

Comparison of NIST and Manufacturer Calibrations of (90) $\mathrm{Sr}+(90)$ Y Ophthalmic Applicators.

03,634

Dose Mapping of Radioactive Hot Particles Using

Radiochromic Film.
PB95-162954

Extrapolation Chamber Measurements on (90) $\mathrm{S} r+(90) \mathrm{Y}$ Beta-Particle Ophthalmic Applicator Dose Rates.
PB95-153375

Measurement of Radial Dose Distributions Around Small Beta Particle Emitters Using High Resolution Radiochromic

PB95-164604

03,518

Needs for Brachytherapy Source Calibrations in the United

PB97-110092

03,521

Secondary Target X-Ray Excitation for In vivo Measurement of Lead in Bone.

PB95-108767

03,496

Use of a Radiochromic Detector for the Determination of Stereotactic Radiosurgery Dose Characteristics.

PB94-185642

03,514

Individual Carotenoid Content of SRM 1548 Total Diet and Influence of Storage Temperature, Lyophilization, and Irradiation on Dietary Carotenoids.

PB94-200524

00,033

\section{SOBOLEWSKI, M. A.}

Current and Voltage Measurements in the Gaseous Electronics Conference RF Reterence Cell.

PB96-113337

02,388

Electrical Characteristics of Argon Radio Frequency Glow Discharges in an Asymmetric Cell.

04,109

Electrical Characterization of Radio-Frequency Discharges in the Gaseous Electronics Conterence Reference Call.
PB95-164612

Electrical Measurements for Monitoring and Control of if Plasma Processing

04,369

Electrical Sensor

04,412

Gaseous Electronics Conterence Radio-Frequency Reference Cell: A Defined Parallel-Plate Radio-Frequency System for Experimental and Theoretical Studies of PlasmaProcessing Discharges

SOK, J.

Thermally Activated Hopping of a Single Abrikosov Vortex. PB95-140810 04,548

\section{SOLOMON, O. M.}

Bounds on Least-Squares Four-Parameter Sine-Fit Errors Due to Harmonic Distortion and Noise.

PB96-141304

01,609

SOLT, G.

Dynamics of $\mathrm{Mu}(+)$ in $\mathrm{Sc}$ and $\mathrm{ScHx}$.

PB96-180021

04,112

OMASUNDARAN, $P$.

Deposition of Colloidal Sintering-Aid Particles on Silicon

03,044

\section{SOMERS, T. A.}

Room Temperature Thermal Conductivity of Fumed-Silica Insulation tor a Standard Reference Material.

SOMMER, $F$.

00,374

Extrapolation of the Heat Capacity in Liquid and Amorphous Phases.

PB97-1

Application of Single Electron Tunneling: Precision Capacitance Ratio Measurements.

SONG, J. F.

Autocorrelation Functions from Optical Scattering for OneDimensionally Rough Surfaces.

PB94-216538

04,244

Geometric Characterization of Rockwell Diamond Indenters. PB95-203287 02,950

Light Scattered by Coated Paper

04,245

Light Scattering from Glossy Coatings on Paper.

PB94-213246 04,242

Metrology Approach to Unifying Rockwell C Hardness Scales.

02,957

Microform Calibration Uncertainties of Rockwell Diamond

PB96-122114

03,280

Microform Calibrations in Surface Metrology.

02,951

Present and Future Standard Specimens for Surface Finish Metrology.
PB97-110423

Stylus Flight in Surface Profiling.

02,928

02,675

Stylus Technique for the Direct Verification of Rockwell Diamond Intenders.

PB96-155569

Surface Texture

SONG, $K$.

Lowest Excited Singlet State of Isolated 1-phenyl-1,3-butadiene and 1-phenyl-1,3,5-hexatriene.

PB95-202339

SONG, $S$.

Characterization of Cytochrome c/Alkanethiolate Structures Prepared by Self-Assembly on Gold

PB95-164638

SONG, $X, N$.

Heavily Accumulated Surfaces of Mercury Cadmium Telluride Detectors: Theory and Experiment.

PB94-216074

02,134

Investigation of Mercury Interstitials in $\mathrm{Hg}(1-\mathrm{x}) \mathrm{CdxTe}$ Alloys Using Resonant Impact-Ionization Spectroscopy.

02,133

RII Spectroscopy of Trap Levels in Bulk and LPE $\mathrm{Hg}^{1-}$ XCdxTe.

PB96-160247

SONIKER, J.

04,084

Reactive Ion Etching in the Gaseous Electronics Conference RF Reference Cell.

PB96.113402

02,395

SONNENFELD, $R$.

Scanning Tunneling Microscopy and Fabrication of Nanometer Scale Structures at the Liquid-Gold Interface.

PB95-140414

SOODPRASERT, T

Interpreting the Readings of Multi-Element Personnel

Dosimeters in Terms of the Personal Dose Equivalent.
PB95-175428

SOONS, H. A.

Precision in Machining: Research Challenges.

PB95-242301

SORA, I. N.

Neutron Powder Diffraction Study of the Nuclear and Magnetic Structures of the Oxygen-Deficient Perovskite PB95-161097

SORATHIA, U.

Materials and Fire Threat.

PB97-122311

SORENSEN, C. M.

Post-Flame Soot.

PB96-193701 


\section{PERSONAL AUTHOR INDEX}

SOULAGES, J. R.

Evaluation and Strengthening Guidelines for Federal Buildings: Assessment of Current Federal Agency Evaluation Programs and Rehabilitation Criteria and Development of Typical Costs for Seismic Rehabilitation

PB94-181856

00,425

SOULEN, R. J.

Application of Single Electron Tunneling: Precision Capacitance Ratio Measurements.

04,703

Cryogenic Precision Capacitance Bridge Using a Single Electron Tunneling Electrometer.

04.529

PB95-126074

Cryogenic Precision Capacitance Bridge Using a Single

Electron Tunneling Electrometer

02,040

Development of a Temperature Scale below $0.5 \mathrm{~K}$

03,879

Superconducting Energy Gap of Bulk UBe13.

PB95-150116

04,559

Systematic Studies of the Effect of a Bandpass Filter on a

Josephson-Junction Noise Themometer.

03.939

Systematic Studies of the Effect of a Post-Detection Filter

on a Josephson-Junction Noise Thermometer.

PB95-162988

03,940

SOUTHWORTH, S

Lattice Position of Si in GaAs Determined by X-Ray Standing Wave Measurements.

PB95-164406

04.632

SOUTHWORTH, S. H.

Anisotropy of Polarized X-ray Emission from Atoms and Molecules.

Electron-Ion-X-ray Spectrometer System.

PB95-176137

04,621 Fluorescence from the Excitation of Xenon Near the L3 PB96-102751

04,025

High Resolution Angle Resolved Photoelectron Spectroscopy Study of N2

03,907

Measuring Nondipolar Asymmetries of Photoelectron Angular Distributions.

01,193

Resonance and Threshold Effects in Polarized X-Ray Emission from Atoms and Molecules.

03,891

Vibronic Coupling and Other Many-Body Effects in the 4sigmag(-1) Photoionization Channel of $\mathrm{CO} 2$.
PB95-162509

Anomalous Odd- to Even-Mass Isotope Ratios in Reso-
nance lonization with Broad-Band Lasers.

nance Ionization with Broad-Band Lasers.

PB94-211406

03,839

SPAIN, E. M.

Alignment Probing of Rydberg States by Stimulated EmisPB96-200316

04,124

Initial and Final Orbital Alignment Probing of the Fine-Structure-Changing Collisions among the Ca $(4 s)(1)(4 \mathrm{p})(1)$. (3)PJ States with $\mathrm{He}$ : Determination of Coherence and Conventional Cross-Sections.

PB95-203279

04,004

Orbital Alignment and Vector Correlations in Inelastic Atomic Collisions.

SPAL, $R$.

High Resolution Hard X-Ray Microscope.

PB94-213055

04,047

SPAL, R. D.

Diffraction Imaging of Polycrystalline Materials.

PB94-198884
Effect of a Crystal Monochromator on the Local Angular Di-

Effect of a Crystal Monochromator on the Local Angular Di-

vergence of an X-Ray Beam.

04,565

Structures of Vapor-Deposited Yttria and Zirconia Thin Films.

PB94-216025

SPANGLER, C. J.

03,041

In situ Fluorescence Cell Mass Measurements of 'Saccharomyces cerevisiae' Using Cellular Tryptophan. In situ On-Line Optical Fiber Sensor for Fluorescence Monitoring in Bioreactors.

PB94-212024

SPARKS, L. L.

Cryogenics.

03.587

02,654

Thermal Conductivity of Polypyromellitimide Film with Alumina Filler Particles from 4.2 to $300 \mathrm{~K}$.

PB96-200753

01,292

SPARKS, R. A.

Using NIST Crystal Data within Siemens Sottware for Four-

Circle and SMART CCD Diffractometers.

04,803
SPEAKE, C.

Validation of the Inverse Square Law of Gravitation Using the Tower at Erie. Colorado, USA.

PEAKE, C. C.

Test of Newton's Inverse Square Law of Gravitation Using the 300-m Tower at Erie, Colorado.

PB95-202446

SPELIOTIS, D. E.

03,978

Correlations of Modulation Noise with Magnetic Microstructure and Intergranular Interactions for $\mathrm{CoCrTa}$ and CoNi Thin Film Media.

PB94-212768

04,509

SPELLERBERG, P. A.

Precision of Marchall Stability and Flow Test Using 6-in (152.4-mm) Diameter Specimens.

03,006

System for Calibration of the Marshall Compaction Ham-

PB94-145661

SPENCER, B. J.

Effects of Elastic Stress on the Stability of a Solid-Liquid Interface.

SPENCER, L. V.

Further Calculations of X-ray Diffusion in an Infinite $\mathrm{Me}$ -

AD-A295 314/9

03,772

Polarization Effects on Multiple Scattering Gamma Trans. port.

PB95.153615

03,926

SPENCER, $W$.

Characteristics of Adhesive-Bonded Seams Sampled from EPDM Roof Membranes.

00,377

SPERHAC, J. M.

Signatures of Large Amplitude Motion in a Weakly Bound Complex: High-Resolution IR Spectroscopy and Quantum Calculations for $\mathrm{HeCO} 2$.

PB95-203485

01,054

SPETZLER, R. C.

Analysis of the Happyland Social Club Fire with HAZARD I.
PB94-199270
00,193

SPIEGEL, $V$.

Neutron Leakage Benchmark for Criticality Safety Research

PB95-126132

03,723

SPINDEL, A.

Calibration of Dosimeters for the Cryogenic Irradiation of Composite Materials Using an Electron Beam.

PB95- 180964

03,968

SPLETT, J. D.

Developing a NIST Coaxial Microwave Power Standard at 1

PB95-202412

01,914

Outlier-Resistant Methods for Estimation and Model Fitting. PB95-203436

03,444

Proposed Changes to Charpy V-Notch Machine Certification Requirements

SPLICHAL, M. P.

02,955 erence Cell: A Defined Parallel-Plate Radio-Frequency Sys tem for Experimental and Theoretical Studies of PlasmaProcessing Discharges
PB94-172327

PPOMER R L

04,404

Magnetic Characteristics and Measurements of Filamentary $\mathrm{Nb}-\mathrm{Ti}$ Wire for the Superconducting Super Collider. DE94005988

03,775

SPOTZ, M. S.

Calculation of the Thermal Conductivity and Gas Permeabil-

ity in a Uniaxial Bundle of Fibers.

SPRANGLE, $P$.

NIST-NRL Free-Electron Laser Facility.

PB94-212511

SPRING, C. B.

Graphical Analysis of the CCRL Portland Cement Proficiency Sample Database (Samples 1-72). (Part 1. Univariant Analysis of Portiand Cement)

01,308

SPRING, J. D.

Electronic Publishing and the Journals of the American Chemical Society.

SPRINGMANN, J. L.

Virtual Software Repository System

PB94-198983

04,807

SPROUL, W. D.

Opportunities for Innovation: Advanced Surface Engineering.

SRDANOV, V.I.

Structural and Chemical Investigations of $\mathrm{Na} 3(\mathrm{ABO} 4) 3,4 \mathrm{H} 2 \mathrm{O}-\mathrm{Type}$ Sodalite Phases.
PB95-180733

SRINIVASAN, $K$

Electrophoretic Separations of Polymerase Chain Reaction: Amplified DNA Fragments in DNA Typing Using a Capillary Electrophoresis-Lased Induced Fluorescence System. PB95-163036 03,536

Enhanced Detection of PCR Products Through Use of TOTO and YOYO Intercalating Dyes with Laser Induced Fluorescence - Capillary Electrophoresis

PB95-16

00.599

SRIVASTAVA, A. N.

Anomalous Switching Phenomenon in Critical-Current Measurements When Using Conductive Mandrels.

PB96-137781

02,233

Comparing the Accuracy of Critical-Current Measurements Using the Voltage-Current Simulator.

PB96-119219

02,227

First VAMAS USA Interlaboratory Comparison of High Temperature Superconductor Critical Current Measurements. PB96-147178 04,768

High Current Pressure Contacts to Ag Pads on Thin Film Superconductors.

PB95-168621

04,639

11-3: Critical Current Measurement Methods: Quantitative Evaluation

PB96-147160

04,767

I1-5: Thermal Contraction of Materials Used in Nb3Sn Critical Current Measurements.

$\begin{array}{ll}\text { PB96-147186 } & 04,769\end{array}$

$\mathrm{n}$-Value and Second Derivative of the Superconductor Voltage-Current Characteristic

PB95-126223

04,533

Simple and Repeatable Technique for Measuring the Criti-

cal Current of Nb3Sn Wires.

PB96-119409

02,229

Standard Reference Devices for High Temperature Superconductor Critical Current Measurements.

PB95-175543

04,659

Superconductor Critical Current Standards for Fusion Applications. Final Progress Report, October 1993-July 1994. PB95-169538

USA Interlaboratory Comparison of Superconductor Simulator Critical Current Measurements.

PB96-147194

04,770

SROLOVITZ, D. J.

Microstructural Evolution in Two-Dimensional Two-Phase Polycrystals.

04,498

ST. PIERRE, J. A.

Conformance Testing and Specification Management. PB97-113781

Roadmap for the Computer Integrated Manufacturing (CIM) Application Framework.

PB96-122759

02,832

STACEY, G. J.

Extended $\mathrm{CO}(7$ yields 6) Emission from Warm Gas in

Orion. 102504

00.090

STACKHOUSE, W

Report on the Workshop on Advanced Digital Video in the National Information Infrastructure. Held in Washington, D.C. on May 10-11, 1994

PB95-103677

01,472

Summary Report on the Workshop on Advanced Digital Video in the National Information Infrastructure.

PB96-141320 
STALICK, J. K.

Determination of Complex Structures from Powder Diffraction Data: The Crystal Structure of La3Ti5Al15037.

PB95-202966

01,038

Fast-lon Conducting Y2(ZryTi1-y)2O7 Pyrochlores: Neutron Rietveld Analysis of Disorder Induced by $\mathrm{Zr}$ Substitution.
PB96-156104

Fast-Ion Conduction and Disorder in Cation and Anion Arrays in $\mathrm{Y} 2(\mathrm{ZryTi}(1-\mathrm{y})) 2 \mathrm{O} 7$ Pyrochlores Induced by $\mathrm{Zr}$ Substitution: A Neutron Rietveld Analysis.

PB94-211869 04,496

Incorporation of Gold into YBa2Cu3O7: Structure and Tc Enhancement.

PB94-200276 04,481

Structure and Conductivity of Layered Oxides $(\mathrm{Ba}, \mathrm{Sr}) \mathrm{n}+1(\mathrm{Sn}, \mathrm{Sb}) \mathrm{nO} 3 \mathrm{n}+1$.

PB96-102439

04,707

Unexpected Effects of Gold on the Structure, Superconductivity, and Normal State of YBa2Cu3O7.
PB94-200284

STAMPF, D. R.

Protein Data Bank: Current Status and Future Challenges. PB97-109060

STANSBURY, J.

Ring-Opening Dental Resin Systems Based on Cyclic AB $95-162251$

00,162

STANSBURY, J. W.

Dental Materials
PB94-172871

00,142

Evaluation of Methylene Lactone Monomers in Dental Res-

PB95-164661

00,164

Facile Synthesis of Novel Fluorinated Multifunctional

Acrylates.

01,207

Preparation and Characterization of Cyclopolymerizable Resin Formulations.

PB96-146840

01,285

Properties and Interactions of Oral Structures and Restorative Materials. Annual Report for Period October 1, 1990 to September 30, 1991.

PB94-160843

03,558

Ring-Opening Polymerication of a 2-Methylene Spiro Orthocarbonate Bearing a Pendant Methacrylate Group.
PB95-176145

Synthesis and Polymerization of Difunctional and Multifunctional Monomers Capable of Cyclopolymerization. PB95-163044

01,257

STARACE, A. F.

Angular Distributions for Near-Threshold $(e, 2 e)$ Processes for $\mathrm{Li}$ and $\mathrm{Mg}$.

00,778

Relative Photoionization and Photodetachment Cross Sections for Particular Fine-Structure Transitions with Applica. tion to $\mathrm{Cl} 3 \mathrm{~s}$-subshell Photoionization.

03,998

PB95-203097

Resonant Two-Color Detachment of $\mathrm{H}(-)$ with Excitation of
$\mathrm{H}(\mathrm{n}=2)$.
PB95-202552
03,984

Short-Pulse Detachment of $\mathrm{H}(-)$ in the Presence of a Static

Electric Field.

04,007

Variationally Stable Treatment of Two- and Three-Photon Detachment of $\mathrm{H}(-)$ Including Electron-Correlation Effects.
PB95-202867
0392

STARNER, K. K.

Proficiency Tests for the NIST Airborne Asbestos Program,

PB94-188836

00,535

TARZYK, J. A.

Diakoptic and Large Change Sensitivity Analysis. 02,038

STASSIS, $C$.

Inelastic Neutron Scattering Measurements of Phonons in Icosahedral Al-Li-Cu.

PB95-126215

04,532

STAUDENMANN, J. L.

Energy Dependences of Absorption in Beryllium Windows and Argon Gas.

STAUFFER, A. D.

Polarization of Light Emitted After Positron Impact Excitation of Alkali Atoms.

PB94-199734

03,816

STAUFFER, J.

Sleuthing the Dynamo: HST/FOS Observations of UV Emissions of Solar-Type Stars in Young Clusters

STAUFFER, J. R.

Far-Uitraviolet Flare on a Pleiades G Dwarf.

PB96-102033

00,098

STAUFFER, T. C

Critical Current Behavior of Ag-Coated YBa2Cu3O7-x Thin Films.

PB95-141016
First VAMAS USA Interlaboratory Comparison of High Temperature Superconductor Critical Current Measurements.
PB96-147178 High Current Pressure Contacts to Ag Pads on Thin Film Superconductors.

PB95-168621

Standard Reference Devices for High Temperature Superconductor Critical Current Measurements.
PB95-175543

Superconductor Critical Current Standards for Fusion Applications. Final Progress Report, October 1993-July 1994.

PB95-169538 02,222

USA Interlaboratory Comparison of Superconductor Simula tor Critical Current Measurements.
PB96-147194

TEBBINS,

Low-Frequency, Active Vibration Isolation System.

PB95-203303

04,770

STECKLER, K. D.

Comparison of Wall-Fire Behavior With and Without a CeilPB94-207404

00,342

Methodology for Developing and Implementing Alternative Temperature-Time Curves for Testing the Fire Resistance of Barriers for Nuclear Power Plant Applications.

PB96-193784

03,742

STEEL, E.

Measurement of the Uniformity of Particle Deposition of Fil. ter Cassette Sampling in a Low Velocity Wind Tunnel.

PB95.163754

02,549

STEEL, E. B.

Airborne Asbestos Analysis: National Voluntary Laboratory Accreditation Program

02,566

Airborne Asbestos Method: Bootstrap Method for Determining the Uncertainty of Asbestos Concentration. Version 1.0. ing the Uncertainty of Asbestos Concentration. Version 1.0.
00,646 Airborne Asbestos Method: Standard Practice for Recording Transmission Electron Microscopy Data for the Analysis of Asbestos Collected onto Filters. Version 1.0.

PB94-210168

00,552

Airborne Asbestos Method: Standard Test Method for High Precision Counting of Asbestos Collected on Filters. Version 1.0.

PB94-163003

00,525

Airborne Asbestos Method: Standard Test Method for Verified Analysis of Asbestos by Transmission Electron Microscopy. Version 2.0

PB94-163045

00,526

Guidelines for Refractive Index Measurements of Asbestos.

PB95-151189 02,543

Monte Carlo Electron Trajectory Simulation of X-Ray Emission from Films Supported on Substrates.

04,522

National Voluntary Laboratory Accreditation Program: Bulk Asbestos Analysis.

02,541

Proficiency Tests for the NIST Airborne Asbestos Program,

1990.
PB94- 188836

00,535

Proficiency Tests for the NIST Airborne Asbestos Program PB94-193828

00,537

Proficiency Tests for the NIST Airborne Asbestos Program -

1992.
PB94-194362

00,539

Proficiency Tests for the NIST Airborne Asbestos Program, PB96-106463

00,610

STEELE, R. D.

Unified Telerobotic Architecture Project (UTAP) Standard Interface Environment (SIE), May 1995

PB95-242350

STEELE, W. V.

Thermodynamic Properties of Alkenes (Mono-Olefins Larger Than C4).

PB94-162237

00,735

STEENKEN, $\mathrm{S}$.

Solvent Effects in the Reactions of Peroxyl Radicals with Organic Reductants. Evidence for Proton Transfer Mediated Electron Transfer
PB95-107157

STEFFENS, K. L

00,873

In-situ Studies of a Novel Sodium Flame Process for Synthesis of Fine Particles.

00,681

Optical and Modeling Studies of Sodium/Halide Reactions

for the Formation of Titanium and Boron Nanoparticles.

for the Formation
PB97.113054

STEHLE, S.

00,682

SUSAN: SUperconducting Systems ANalysis by Low Temperature Scanning Electron Microscopy (LTSEM).

PB96-112065

04,728

STEIN, R. S.

Small-Angle Neutron Scattering of Poly(vinyl alcohol) Gels.

Small Angle Neutron Scattering Studies on Chain Asymmetry of Coextruded Poly(Vinyl Alcohol) Film.

01,262

STEIN, S. E.

Diamond and Graphite Precursors: Comments.

PB95-163051

00,967

International Conference on Chemical Kinetics (2nd). Held in Gaithersburg, Maryland on July 24-27, 1989.

00,822

Thermal Decomposition of Hydroxy- and Methoxy-Substituted Anisoles.

STEINBACH, A.

Narrow-Band Tunable Diode Laser System with Grating Feedback, and a Saturated Absorption Spectrometer for Cs and $\mathrm{Rb}$.

PB95-202891

04,319

STEINBACH, A. H.

Observation of Hot-Electron Shot Noise in a Metallic ResisPB. $97-112007$

STEINER, $B$.

01,988

Influence of Lattice Mismatch on Indium Phosphide Based High Electron Mobility Transistor (HEMT) Structures Observed in High Resolution Monochromatic Synchrotron $X$ Radiation Diffraction Imaging.

PB95-164679

02,357

Recent VAMAS Activity in Ceramics.

PB95-162681

03,051

TEINER, $R$.

Measurement and Reduction of Alignment Errors of the NIST Watt Experiment.

PB97-111959

01,987

Methods for Aligning the NIST Watt-Balance.

01,934

NIST Watt Balance: Progress Toward Monitoring the Kilogram.

01,991

STEINER, R. L. Accuracy Comparisons of Josephson Array Systems.
PB95-164687

Evidence for Parallel Junctions Within High-Tc GrainBoundary Junctions.

PB95-175410

04,657

New Refractometer by Combining a Variable Length Vacuum Cell and a Double-Pass Michelson Interferometer. PB97-111926 01,986

Noise Characteristics Below $1 \mathrm{~Hz}$ of Zener Diode-Based Voltage Reference.

PB96-123476

04,049

Voltage Ratio Measurements of a Zener Reference Using a Digital Voltmeter.

STEINER, T. W.

Comparison of Techniques for Nondestructive Composition Measurements in CdZnTe Substrates.

PB96-103098

02,703

STELLA, A. L.

Self-Avoiding Surtaces, Topology, and Lattice Animals. PB95-150512

STENBAKKEN, G.

Measurement and Reduction of Alignment Errors of the NIST Watt Experiment.

PB97-111959 01,987

NIST Watt Balance: Progress Toward Monitoring the Kilogram.

STENBAKKEN, G. N.

01,991

Binary versus Decade Inductive Voltage Divider Comparison and Error Decomposition.

PB96-112263

02,071

Developing Linear Error Models for Analog Devices.

Diakoptic and Large Change Sensitivity Analysis.

EB Effects of Nonmedel Errors on Model-Based Testing

02,037

PB96-155577 
STEPHENS, E. F.

Electronically Tunable Fiber Laser for Optical Pumping of

PB96-201165

04,381

STEPHENS, P. W.

Phase Transitions in Solid C70: Supercooling. Metastable Phases, and Impurity Effect.

PB95-150090

00,914

STEPHENSON, G. B.

Real-Time Small-Angle X-Ray Scattering Study of the Early Stage of Phase Separation in the SiO2-BaO-K20 System.
PB95-163069 STEPHENSON, J. C.

Dynamics of Nonthermal Reactions: Femrosecond Surface Chemistry.

00,688

Fragment Energy and Vector Correlations in the OventonePumped Dissociation of $H N 3 X(1) A$

00,802

Hot Carrier Excitation of Adiayers: Time-Resolved Measurement of Adsorbate-Lattice Coupling. $\quad 00.758$

00.758

Picosecond Measurement of Substrate-to-Adsorbate Energy

PB lation. 126041

00,895

Time-Resolved Measurements of Energy Transfer at Surfeces. 14198

00,913

Time-Resolved Measurements of Energy Transfer at SupPB95-153037

00,947

Time-Resolved Probes of Surface Dynamics.

PB94-199957

00,803

Uliratast Time-Resolved Inftared Probing of Energy Transfer at Surfaces

$\infty, 620$

Vibrational Predissociation Dynamics of Overtone-Excited HN3.

$\infty, 691$

Vibrational Relaxation Measurements of Carbon Monoxide on Meial Clusters.

STEPHENSON, M

TDDB Characterization of Thin SiO2 Films with Bimodal Failure Pcpulations.

STERN, R.

02.381

Sleuthing the Dynamo: HST/FOS Observations of UV Emissions of Solar-Type Stars in Young Clusters.

00,098

STERN, R.A.

Fer-Ultravio'et Flare on a Pleiades G Dwart

BB96-102033

$\infty, 086$

STEVENS, R. K.

Distinguishing the Contributions of Residential Wood Combustion and Mobile Source Emissions Using Relative Concentrations of Dimethylphenanthrene Isomers.

PB96-13512

02,563

Radiccarbon Measurements of Almospheric Volatile Organic Compounds: Quentifying the Biogenic Contribution.
PB $97-122352$

STEVENS, $W . J$.

Analysis of Protein Metal Binding Selectivity in a Cluster Mode

PB94-212990

$\infty, 845$

Cs Cluster Binding to a GaAs Surface.

00,846

Strong Hydrogen Bond in the Formic Acid-Formate Anion PBg 940 - 198595

00.788

Siructure of Glycine-Water H-Bonded Complexes.

PB94-198603

$\infty 0,789$

STEWARD, W. G

Survey of the Literature on Heat Transfer from Solid Surtaces to Cryogenic rluids.

$A D-A 286680 / 4$

04,193

STEWART, H. D.

Application of the Modified Voltage-Dividing Potentiometer to Overlay Metrolcgy in a CMOS/Bulk Process.

02,302

STEWART, R. T

High Sensitivity Survey of Radio Continuum Emission in Herbig $A \in / B e$ Stars.

STEWART, S. L

00,051

Conformance Testing and Specification Management.

PB97-11378

02.849

Roadmep for the Computer Integrated Manufacturing (CIM) Application Framework.

PB96-122759

02,832

STIEF, L J.

Excerimental Determination of the Ionization Energy of O (X) (sup 2)ll(sub 3/2)) and Estimations of Delita(sub f) H(sup deg) (sub o) (IO (sup - $)$ ) and PA(IO).

0,694
Experimental Determination of the Rate Constant for the Reaction of $\mathrm{C} 2 \mathrm{H} 3$ with $\mathrm{H} 2$ and Implications for the Partition-

ing of Hydrocartons in Atmospheres of the Outer Planets.
PB97-122295 00,112

STIEFEL, S. W.

Implementation of the Fastener Quality Act.

PB96-160676

STIEG, $M$.

$24 \mathrm{GHz}$ Josephson Array Voltage Standard

02,876

PB94-211588

02,033

STIEREN, D. C.

Automated Inspection: The Integration of National Stand-

ards and Commercial Products at NIST.

02,906

Automated Manufacturing Research Facility 1994 Annual Report.

PB95-209854

$\infty, 015$

Development of an Automated Part Inspection System Using the DMIS Standard

02,899

U.S. Navy Coordinate Measuring Machines: A Study of Needs.

PB94-162831

02,807

STILES, M. D.

Coarsening of Unstable Surface Features during Fe(001)

PB96-186127

04,121

Exchange Coupling in Magnetic Heterostructures.
PB95-150314

Spin-Dependent Interface Transmission and Reflection in Magnetic Multilayers (Invited)

PB96-201173

04,130

STILLE, J. K.

Characterization of Polyquinoline Blends Using Small Angle

Scattering.

01,261

Characterization of Polyquinoline Block Copolymer Using Small Angle Scattering

PB95-151882

01,244

STILLMAN, S. E

Enthalpy Increment Measurements from 4.5 to $350 \mathrm{~K}$ and the Thermodynamic Properties of Titanium Disilicide(cr) to $1700 \mathrm{~K}$

PB96-204029

00,678

STINSON, $F$

Further Calculations of X-ray Diffusion in an Infinite $\mathrm{Me}$ dium.

AD-A295 314/9

03,772

STOCK, K. D.

Intemational Intercomparison of Detector Responsivity at 1300 and $1550 \mathrm{~nm}$.

PB95-125928

Intemational Intercomparison of Detector Responsivity at 1300 and $1550 \mathrm{~nm}$

PB95-126017

02,14

STOCKBAUER, R. L.

Photoelectron Spectroscopic Study of the Valence and Core-Level Electronic Structure of BaTiO3.
PB94-212149

PB94-212149 04,500

Surface Core-Level

PB94-212156

STOJANOVIC, $V$.

Excitation of Balmer Lines in Low-Current Discharges of Hydrogen and Deutenium.

50546

03,893

STOKESBERRY, D.

ISDN in North America.

PB96-160767

01,502

STOKESBERRY, D.P.

Application Software Interface: ISDN Services for an Open Systems Environment.

PB96-131487

01.492

Integrated Network Management.

01,583

North American ISDN Users" Forum Agreements on Integrated Services Digital Network.

01,466

STOKIC, $z$.

Excitation of Balmer Lines in Low-Current Discharges of Hydrogen and Deuterium.

PB95-150546

03,893

STONE, C. A

Neutron Capture Prompt Gamma-Ray Activation Analysis at the NIST Cold Neutron Research Facility.

00,556

STONE, J. Two New Probes for a Coordinate Measuring Machine.
PB95-163093 02,653

STONE, $\$$.

National Status and Trends Progrem Specimen Bank: Sampling Protocols, Analytical Methods, Results, and Archive Samples.

02,598

STONE, S. F

Trace Elements Associated with Proteins. Neutron Activa. tion Analysis Combined with Biological Isolation Techniques.

PB95-163101

00,597

STONE, W. C.

Evaluating the Seismic Performance of Lightly-Reinforced Circular Concrete Bridge Columns.

01,335

Jacket Thickness Requirements for Seismic Retrofitting of Circular Bridge Columns.

PBrcular Bridge

01,336

NIST Construction Automation Program Repon No. 2. Proceedings of the NIST Construction Automation Workshop. Held in Gaithersburg, Maryland on March 30-31, 1995.

PB96-202239

00,413

Partially Prestressed and Debonded Precast Concrete Beam-Column Join's.

00,439

Performance of $1 / 3$-Scale Model Precast Concrete BeamColumn Connections Subjected to Cyclic Inelastic Loads. Report No. 4

PB95-179024

00,444

Seismic Performance Behavior of Precast Concrete Beam. Column Joints.

Seismic Performance of Circular Bridge Columns Designed in Accordance with AASHTO/CALTRANS Standards. Simplified Design Procedure for Hybrid Precast Concrete Connections.

PB96-154836

00,405

Fast Computer Evaluation of Radiative Properties of Hydrogenic Systems.

03,894

Recombination Line Intensities for Hydrogenic lons-III. Effects of Finite Optical Depth and Dust.

PB95-202677

00,079

TTOT, H. L.

Modeling and Test Point Selection for a Thermal Transfer

PB95-161287

01,896

STOUDT, M. R

Analysis of Failed Dry Pipe Fire Suppression System Couplings from the Filene Center at Wolf Trap Farm Park for he Performing Arts.

PB94-164407

00,331

Characterization of the Hydrogen Induced Cold Cracking Susceptibility at Simulated Weld Zones in HSLA-100 Steel-

Characterization of the Hydrogen Inducad Cold Cracking Susceptibility at Simulated Weld Zones in HSLA-100 Steel.
PB94-174505

Evaluation of the Environmentally Induced Fracture Resistance of Ductile Nickel Aluminide. Technical Report Number 1. Final report. October-December 1990. DE94017331

03,306

Loading Device for Fracture Testing of Compact Tension Specimens in the Scanning Electron Microscope.

PB95-16243

02,652

STOUFFER, $K$.

Recommendations on Selection of Vehicle-to-Roadside Communications Standards for Commercial Vehicle Oper-

04.859
VB94-195914 cle Operations: Requirements and Approaches. PB95-188827

STOUP, J.

Measuring the Stability of Three Copper Alloys PB94-19986

04,860

STOVEL, L.

Z39.50 Implementation Experiences.

PB96-114939

03,326 
Small-Angle Neutron-Scattering Study of Dense Sheared Silica Gels.

PB96-167184

01,151

Structure and Rheology of Hard-Sphere Systems. PB96-167333

00,662

STRAUB, J.

Formulation of the Refractive Index of Water and Steam.

PB95-140133

STRAUSSER, $B$

PET and DINGO Tools for Deriving Distributed Implementations from Estelle.

01,727

STRAWBRIDGE, M. L.

Virtual Software Repository System

PB94-198983

01,691

STRICKLETT, K. L.

Advanced Components for Electric and Hybrid Electric Vehicles. Workshop Proceedings. Held in Gaithersburg, Maryland on October 27.28, 1993.

PB94-177060

04,858

Appearance Potentials of Ions Produced by Electron-Impact Induced Dissociative Ionization of SF6, SF4, SF5Cl, S2F10, $\mathrm{SO} 2$, SO2F2, SOF2, and SOF4

01,080

Associative Electron Altachment to S2F10, S2OF10, and $\mathrm{S} 2 \mathrm{O} 2 \mathrm{~F} 10$.

00,907

Correlations between Electrical and Acoustic Detection of Partial Discharge in Liquids and Implications for Continuous Data Recording.
PB96-204490

02,248 Electrical Breakdown in Transformer Oil in Large Gaps.
PB95-150579

Electrohydrodynamic Instability and Electrical Discharge Initiation in Hexane.

02,244

Investigation of S2F10 Production and Mitigation in ComInvestigation of S2F10 Production and Mitigation in Compressed SF6-Insulated Power Systems.
PB94-212388

02,467

Investigation of S2F10 Production and Mitigation in Compressed SF6- Insulated Power Systems.

02,468

Observations of Partial Discharges in Hexane Under High Magnification

01,900

Procedure for Measuring Trace Ouantities of $52 \mathrm{~F} 10$, $\mathrm{S} 2 \mathrm{OF} 10$, and $\mathrm{S} 2 \mathrm{O} 2 \mathrm{~F}_{10}$ in SF6 Using a Gas Chromatograph-Mass Spectrometer

PB96-119755

02,513

Refraction of Light by Graded Birefringent Media. PB96-123716

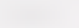

STROBRIDGE, T. R.

02,192

Helium Refrigeration and Liquefaction Using a Liquid Hydrogen Refrigerator for Precooling.

STROM, K.

02,749

Status of the Round Robin on the Transport Properties of $\mathrm{R} 134 \mathrm{a}$

PB96-167218

01,152

STRONGIN, R. M.

Neutron-Scattering Study of Librations and Intramolecular Phonons in Rb2.6K0.4C60.

PB95-162269

00,958

STROSCIO, J. A

Atomic Manipulation of Polarizable Atoms by Electric Field

Directional Diffusion.
PB95-150587

Closed Loop Controller for Electron-Beam Evaporators.

PB97-111470 04,393

Coarsening of Unstable Surface Features during Fe(001) Homoepitaxy

PB96-186127

04,121

PB95.150322

04,567

Homoepitaxial Growth of Iron and a Real Space View of Reflection-High-Energy-Electron Diffraction.
PB94-173069

04,445

Influence of $\mathrm{Cr}$ Growth on Exchange Coupling in $\mathrm{Fe} / \mathrm{Cr}$ / $\mathrm{Fe}(100)$

PB95-150181

04,562

Influence of Thickness Fluctuations on Exchange Coupling in $\mathrm{Fe} / \mathrm{Cr} / \mathrm{Fe}$ Structures.

04,745

Manipulation of Adsorbed Atoms and Creation of New Structures on Room-Temperature Surfaces with a Scanning Tunneling Microscope.

PB95-151536

04,578

Scaling of Diffusion-Mediated Island Growth in Iron-on-Iron Homoepitaxy,

04,455

Scanning Tunneling Microscopy Siudy of the Growth of $\mathrm{Cr} /$ Fe(001): Correlation with Exchange Coupling of Magnetic Layers.

PB95-150330

04,568

SEMPA Studies of Oscillatory Exchange Coupling

PB95-163556

04,625
Tunneling Spectroscopy of bcc(001) Surface States. PB96-155585

04,775

STROUSE, G. F.

Assessment of Uncertainties of Calibration of Resistance

Thermometers at the National Institute of Standards and

Thernomets

PB94-142478

02,624

Comparisons of Some NIST Fixed-Point Cells with Similar

Cells of Other Standards Laboratories.

00,655

Direct Comparison of Three PTB Silver Fixed-Point Cells

with the NIST Silver Fixed-Point Cell.

00,628

Investigation of High-Temperature Platinum Resistance

Thermometers at Temperatures Up to $962 \mathrm{C}$, and, in Some Cases, $1064 \mathrm{C}$

PB96-161294

00,629

Investigation of the ITS-90 Subrange Inconsistencies for

25.5 Omega SPRTs.

PB96-161302

00,630

ITS-90 Calibration Facility

00,627

NIST Assessment of ITS-90 Non-Uniqueness for $25.5 \mathrm{Ohm}$

SPRTs at Gallium, Indium and Cadmium Fixed Points.

PB96-161310 00,631

NIST Implementation and Realization of the ITS-90 Over

the Range $83 \mathrm{~K}$ to $1235 \mathrm{~K}$ : Reproducibility, Stability, and

Uncertainties.

PB96-161328

00,632

NIST Measurement Assurance of SPRT Calibrations on the

ITS-90: A Ouantitative Approach.

00,633

Practical Applications of the ITS-90: Inherent Uncertainties.

PB95-161527
03,930

Preliminary Results of a Comparison of Water Triple-Point

Cells Prepared by Different Methods.

00,634

Standard Reference Material 1744: Aluminum Freezing. Point Standard
PB95-251732

STRUBLE, L.

01,055

Interaction between Naphthalene Sulfonate and Silica Fume

in Portland Cement Pastes.

PB94-199759

01,315

Rheology of Fresh Cement Paste.

PB95-163150

STRUCK, L. M.

Silicon Surface Chemistry by IR Spectroscopy in the Mid- to

Far-IR Region: $\mathrm{H} 2 \mathrm{O}$ and Ethanol on Si(100)

STRUPP, P. G.

01,097

Direct Detection of Atomic Arsenic Desorption from Si(100).
01,024

Laser Gas Ionization Technique Monitors MEB Crystal Growth.

PB96-112172

01,076

Laser-Induced Desorption of In and Ga from Si(100) and Adsorbate Enhanced Surface Damage.

01,050

Pulsed Laser Irradiation at $532 \mathrm{~nm}$ of In and Ga Adsorbed on Si(100): Desorption, Incorporation, and Damage.
PB95-203329

Single-Photon Ionization and Detection of $\mathrm{Ga}, \ln$, and As(sub n) Species in GaAs Growth.

PB95-152815

00,591

Single Photon Laser Ionization as an In-situ Diagnostic for MBE growth

01,059

Single-Photon Laser Ionization Time-of-Flight Mass Spectroscopy Detection in Molecular-Beam Epitaxy: Application to As4, As2, and Ga.

PB95-203337

01,052

Vibrational Distributions of As2 in the Cracking of As4 on Si(100) and Si(111).

STUART DOLS, W.

00,784

Development and Application of an Indoor Air Ouality Com-

PB96-155601

00,275

Study of Ventilation Measurement in an Office Building. PB96-155593

STUART, J. W.

Sliding Vane Flow Conditioner Tests in a 100 Diameter

Long 10 inch Natural Gas Orifice Meter at Pacitic Gas and

Electric. Topical Report, 1990-1992.

PB95-256335

02,493

STUBENRAUCH, C. F.

Development of Near-Field Test Procedures for Communication Satellite Antennas.

PB96-135082

02,010

Crystal Structure of a New Sodium Zinc Arsenate Phase

Solved by 'Simulated Annealing'.

00,870

PB95-107124

\begin{tabular}{l} 
Inelastic Neutron Scattering Studies of Nonlinear Optical \\
Materials: p-Nitroaniline Adsorbed in ALPO-5. \\
PB95-107223 \\
\hline
\end{tabular}

Small Angle Neutron Scattering Study of the Structure and Formation of MCM-41 Mesoporous Molecular Sieves. PB97-122337

Small Angle Neutron Scattering Study of the Structure and Formation of Ordered Mesopores in Silica.

PB96.111919

03.069

Structural and Chemical Investigations of $\mathrm{Na3}(\mathrm{ABO} 4) 3.4 \mathrm{H} 2 \mathrm{O}$-Type Sodalite Phases

PB95-180733

01,012

Tetrahedral-Framework Lithium Zinc Phosphate Phases: Location of Light-Atom Positions in LiZnPO4 H2O by Pow. der Neutron Diffraction and Structure Determination of LiZnPO 4 by ab Initio Methods.

PB96- 1605

01,129

STUHR, U.

Inelastic Neutron Scattering Study of Hydrogen in PBnocrystalline Pd.

Vibrational Excitations and the Position of Hydrogen in Nanocrystalline Palladium

STULL, D. M.

Membrane Gas Separation for a Fluidized-Bed Incinerator.

PB95-169041

02,550

STUTZIN, G. C.

Deposition Rates in Direct Current Diode Sputtering

PB95-203345

04,697 phous Silicon Surface.

50595

04,573

UTZMAN, $P$

Characteristics of Adhesive-Bonded Seams Sampled from EPDM Roof Membranes.

PB95-162491

00,377

Warping of Terrace Pavers at the U.S. Capitol Building

PB96-193651

STUTZMAN, P. E.

Cellular Automaton Simulations of Cement Hydration and Microstructure Development.

PB95-175055

01,320

Cement and Concrete Characterization by Scanning Electron Microscopy.

Compositional Analysis of Beneficiated Fly Ashes.
PB95-220497

00,379

00,397

Diagnosis of Causes of Concrete Deterioration in the MLP. 7A Parking Garage.

01,318

Evolution of Porosity and Calcium Hydroxide in Laboratory Concretes Containing Silica Fume.

PB95-175063

01,321

Microstructural Features of Some Low Water/Solids, Silica Fume Mortars Cured at Different Temperatures.

PB94-160777

00,330

Ouantitative Phase Abundance Analysis of Three Cemen Clinker Reference Materials by Scanning Electron MicrosPB94 Ouantitative X-Ray Powder Diffraction Methods for Clinker and Cement.

Serial Sectioning of Hardened Cement Paste for Scanning 
New Physics-Based Model for Time-Dependent- DielectricPreakdown.

02,440

SUEHLE, J. S.

Characterization of Time-Dependent Dielectric Breakdown in Intrinsic Thin $\mathrm{SiO}_{2}$

PB97-122527

02,458

Charge Trapping and Breakdown Mechanisn in SIMOX.

02,323

Electric Field Dependent Dielectric Breakdown of Intrinsic $\mathrm{SiO} 2$ Films Under Dynamic Stress.

PB96-204102

02,449

Electrical Method for Determining the Thickness of Metal Films and the Cross-Sectional Area of Metal Lines.

02,370
Experimental Investigation of the Validity of TDDB Voltage $\begin{array}{ll}\text { Acceleration Models. } & \\ \text { PB9 } & 185949\end{array}$ Field and Temperature Acceleration of Time-Dependent Dielectric Breakdown in Intrinsic Thin SiO2.

PB94-185956

02,305

High Temperature Reliability of Thin Film SiO2.

02,333

Interaction of Stoichiometry, Mechanical Stress, and Interface Trap Density in LPCVD Si-rich SiNx-Si Structures

PB95-176301 02,366

JEDEC 'TCR' Interlaboratory Experiment: Lessons Learned.

New Oxide Degradation Mechanism for Stresses in the Fowler-Nordheim Tunneling Regime.

04,128

New Physics-Based Model for Time-Dependent- DielectricBreakdown.

PB96-201132

02,448

Reproducibility of JEDEC Standard Current and Voltage Ramp Test Procedures for Thin-Dielectric Breakdown Characterization.

PB94-185931

01,879

TDDB Characterization of Thin $\mathrm{SiO} 2$ Films with Bimodal Failure Populations.

PB96-102926

02,381

Time-Dependent Dielectric Breakdown of Intrinsic $\mathrm{SiO} 2$ Films under Dynamic Stress.

PB96-179478

02,438

Tin Oxide Gas Sensor Fabricated Using CMOS MicroHotplates and In-situ Processing

00,580

\section{SUENRAM, R. D.}

Microwave Spectra of van der Waals Complexes of Importance in Planetary Atmospheres.

PB95-150611

00,919 Microwave Spectrum and Structure of $\mathrm{CH}_{3} \mathrm{NO} 2-\mathrm{H}_{2} \mathrm{O}$

00,719

Rotational Spectra of $\mathrm{CH} 3 \mathrm{CCH}-\mathrm{NH} 3, \mathrm{NCCCH}-\mathrm{NH} 3$, and $\mathrm{NCCCH}-\mathrm{OH} 2$

04,170

Rotational Spectrum and Structure of a Weakly Bound Complex of Ketene and Acetylene.

PB95-126140

00,896

Thermal Decomposition Pathways in Nitramine Propellants.

AD-A295 896/5 03,753

Tunneling-Rotation Spectrum of the Hydrogen Fluoride

Dimer.

$\infty 0,793$

SUGAR, J.

Analysis of the $(5 d(2)+5 d 6 s)-5 d 6 p$ Transition Arrays of Os VII and Ir VIII, and the 6s (2)S-6p (2)P Transitions of Ir IX.
PB96-159264

01,954

Analysis of the $5 s(2) 5 p(2)-(5 s 5 p(3)+5 s(2) 5 p 5 d+5 s(2) 5 p 6 s)$ Transitions of Four-Times lonized Xenon ( $X e \mathrm{~V}$ ).

03,900

Designations of ds(2)p Energy Levels in Neutral Zirconium, Hatnium, and Rutheriordium $(Z=104)$

PB96-180120

04,116

Energy Levels of Germanium, Ge I through Ge XXXII.

PB94-162351

Energy Levels of Zinc, Zn I through Zn XXX.

01,122

Improved Wavelengths for Prominent Lines of $\mathrm{Cr} X \mathrm{VI}$ to $\mathrm{Cr}$

PB95-150629 03,895

Improved Wavelengths for Prominent Lines of $\mathrm{Fe} X X$ to $\mathrm{Fe}$ XXIII.

04,334

Laser-Produced and Tokamak Spectra of Lithiumlike Iron, $\mathrm{Fe}(23+)$

PB95-180857

04,314

Magnetic Dipole Line from $U$ LXXI Ground-Term Levels Predicted at 3200 Angstroms.

04,407

Observation and Visible and uv Magnetic Dipole Transitions

in Highly Charged Xenon and Barium.

Observation of Pd-Like Resonance Lines Through $\mathrm{Pt}(32+)$ and $\mathrm{Zn}$-Like Resonance Lines of $\operatorname{Er}(38+)$ and $\mathrm{HF}(42+)$

PB95-150637

03,896
Rb-Like Spectra: Pd X to Nd XXIV. PB95-150645

Rh I Isoelectronic Sequence Observed from $\operatorname{Er}(23+)$ to Pt(33+).

03,898

Spectra of $\mathrm{Ag}$ I Isoelectronic Sequence Observed from Er(21+) to $\mathrm{Au}(32+)$.

03,899

Spectral Data and Grotrian Diagrams for Highly lonized Chromium, Cr V through Cr XXIV

PB94-162369

00,748

Spectral Data for Highly Ionized Krypton, $\mathrm{Kr} V$ through $\mathrm{Kr}_{\mathrm{r}}$ XXXVI

01,115

Spectroscopic Data Tables for Highly-lonized Atoms PB95-151585

03,910

SUHM, M. A. High-Resolution Infrared Spectroscopy of DF Trimer: A Cy-
clic Ground State Structure and DF Stretch Induced clic Ground Stramolecular Vibrational Coupling.
PB95-150678

00,920

Potential Surfaces and Dynamics of Weakly Bound Trimers: Perspectives from High Resolution IR Spectroscopy.
PB96-176508

01,155

SUI, P. C.

Evaluation of Wear Resistant Ceramic Valve Seats in Gas Fueled Power Generation Engines. Topical Report, Decem ber 1991-April 1994.

ber 1991-April
PB95-200218

SUKHDEV, $R$

Modified Airy Function Method for the Analysis of Tunneling Problems in Optical Waveguides and Quantum Well Structures.

PB94-185824

02,120

SULLIVAN, D. B.

Time and Frequency: Bibliography of NIST Publications, March 1995.

PB95-220463

Time and Frequency Metrology

PB96-190319

01,548

01,556

Time and Frequency Technology at NIST

N94-30641/2

Time Generation and Distribution

PB96-103049

01,522

SULLIVAN, $F$.

Computing S(alpha) Using Symbolic Monte Carlo.
PB94-198660
03,410

Data-Parallel Algorithm for Three-Dımensional Delaunay Tri-

angulation and lis Implementation.

PB95-163309

Faster BKL Monte Carlo Simulations.

PB95-136370

Faster Monte Carlo Simulations.

PB96-102074

Heap of Data.

PB97-111488

Making Connections.

PB97-119044

01,714

Measuring Perto

port.

01,706

Measuring Performance of Parallel Computers. Progress Report, 1989.

DE94014587

Self-Avoiding Surfaces, Topology, and Lattice Animals.

PB95-150512

04,571

Submissions to a Planned Encyclopedia of Operations Research on Computational Geometry and the Voronoi/ Delaunay Construct.

PB94-152709

03,425

Tree-Lookup for Partial Sums Or: How Can I Find This Stuff Quickly

PB96-179411

SULLIVAN, $P$. J.

01,770

Visualization of Surface Figure by the Use of Zernike Polynomials.

SUMARLIN, I. W.

Dispersions of Magnetic Excitations of the $\mathrm{Pr}$ lons in $\mathrm{Pr} 2 \mathrm{CuO} 4$.

PB94-173044

04,444

Magnetic Structure and Spin Dynamics of the Pr and $\mathrm{Cu}$ in

PB96-111836

04,036

Phonon Density of States in $\mathrm{R} 2 \mathrm{CuO} 4$ and Superconducting R1.85Ce0.15CuO4 (R = Nd, Pr).

PB95-150686

04,574

SUNG, L.

Dimensional Crossover in the Phase Separation Kinetics of Thin Polymer Blend Films

PB97-113088

03,395

Light-Scattering Studies on Phase Separation in a Binary Blend with Addition of Diblock Copolymers.

01,286

Morphology and Phase Separation Kinetics of a Compatibilized Blend.

01,297

Phase Separation in Thin Film Polymer Blends With and Without Block Copolymer Additives.

PB96-204482

01,294

SUNG, $P$.

Effect of Ethanol on the Solubility of Hydroxyapatite in the System $\mathrm{Ca}(\mathrm{OH}) 2-\mathrm{H} 3 \mathrm{PO} 4-\mathrm{H} 2 \mathrm{O}$ at $25 \mathrm{C}$ and $33 \mathrm{C}$.

PB95-169231

SUNSHINE, S. A.

Neutron Powder Diffraction Study of the Crystal Structure of YSr2AlCu2O7.

PB94-212073

Refraction

Refraction of Light by Graded Birefringent Media.

PB96-123716

SUPERCYNSKI, M. J.

Quench Energy and Fatigue Degradation Properties of $\mathrm{Cu}$ and Al/Cu-Stablized Nb-Ti Epoxy-Impregnated Superconductor Coils.

SURETTE, J.

04,755

Electronics and Electrical Engineering Laboratory 1995 Technical Accomplishments: Advancing Metrology for Electrotechnology to Support the U.S. Economy. PB96-164520

SURETTE, J. $M$.

01,959

Electronics and Electrical Engineering Laboratory 1994 Program Plan: Supporting Technology for U.S. Competitiveness in Electronics.

PB94-126901

01,873

SUROLIA, A.

Thermodynamics of the Binding of Galactopyranoside Derivatives to the Basic Lectin from Winged Bean (Psophocarpus Tetrogonolobus)

03,465

SUROWIEC, $R$.

Kinetic-Energy Distributions of Ions Sampled from Argon Plasmas in a Parallel-Plate, Radio-Frequency Reference Call.

PB95-161964

03,935

SURYAN, M. M.

Thermal Decomposition of Hydroxy- and Methoxy-Substituted Anisoles.

PB94-173036

00,767

SUSHKOV, D, $P$

Fine Structure of Negative lons of Alkaline-Earth-Metal Atoms.

03,837

SUSHKOV, O. P.

Hole Dispersion and Enhancement of Antiferromagnetic Interaction of Localized Spins in High-Tc Superconductors.

PB95-202602

SUSSMAN, J. L.

Protein Data Bank: Current Status and Future Challenges. PB97-109060

SUSSMANN, $R$.

Frequency Shifting of Pulsed Narrow-Band Laser Light in a Multipass Raman Cell.

PB95-203352

04,321

SUTRINA, S. L. 
TANAKA, T.

and Gases at Pressures 1 to 5000 Bars and Temperatures 25 to $1000 \mathrm{C}$.

PB96-145891

01,113

SVINCEK, P. R.

International Green Building Conference and Exposition (3rd). Held in San Diego, California on November 17.19, 1996. (Reannouncement with new abstract).

PB97-121826

02,531

SWAFFIELD, J. A.

Water Efficient Plumbing Fixtures through Standards and Test Methods.

PB95-12595

00,248

SWANSON, $M$.

Computer Security: Generally Accepted Principles and Practices for Securing Information Technology Systems. PB97-110811

01,619

SWARTZ, S.

Phase Shifts and Intensity Dependence in Frequency-Modulation Spectroscopy

PB96-103205

01,071

SWARTZENDRUBER, L.

Magnetic and Structural Properties of Electrodeposited Copper-Nickel Microlayered Alloys.

B94-21312

04,512

SWARTZENDRUBER, L. J.

Determination of Thermoactivation Parameters of Vortex Mobility in YBa2Cu3O7 Using Only Magnetic Measurements

PB95-163499

04,624

Experimental Verification of a Vector Preisach Model.

PB95-163564

04,626

Langevin Approach to Hysteresis and Barkhausen Modeling

PB94-185675

03,206

Magnetocaloric Effect in Nanocomposites.

PB95-162798

04,618

Meissner, Shielding, and Flux Loss Behavior in Single-Crys. tal YBa2Cu3O6+x.

PB94-198744 04,464

Monte Carlo and Mean-Field Calculations of the Magnetocaloric Effect of Ferromagnetically Interacting Clus. ers.

03,201

Temperature Dependence of Vortex Twin Boundary InterTemperature Dependence of Vortex Twin Boundary Interaction in Yttrium Barium Copper Oxide (YBa2Cu3O6+x).
PB95- 162442
04,613 SWENSON, D. $R$

Merged-Beams Energy-Loss Technique for Electron-Ion Excitation: Absolute Total Cross Sections for $\mathrm{O}(5+)(2 \mathrm{~s}$ yields 2p).

2P).

04,017

SWIDERSKY, $P$

Application of the Taylor Dispersion Method in Supercritical Fluids.

PB95-164323

00,977

SWIFT, R. P.

Intrared and Microwave Spectroscopy of the Argon Propyne Dimer.

SWYDEN, T. A.

Planar Lenses for Field-Emitter Arrays
PB96-103064

00,794

SWYT, D. A.

Design, Specification and Tolerancing of Micrometer-Tolerance Assemblies

PB95-209862

02,913

Investing in Education to Meet a National Need for a Technical-Professional Workforce in a Post-Industrial Economy.

New Concepts of Precision Dimensional Measurement for Modern Manufacturing.

PB96-160684

02,924

Uncertainties in Dimensional Measurements Made at Nonstandard Temperatures.

PB94-169760

SYLLAIOS, A. J.

Comparison of Techniques for Nondestructive Composition

Measurements in CdZnTe Substrates.
PB96-103098

02,703

SZABO, S.

Control System Architecture for a Remotely Operated Unmanned Land Vehicle.

PB95-163200

03,759

Ground Vehicle Control at NIST: From Teleoperation to AuN94-34037/9

03,758

SZEKELY, C.

Diode-Laser Pumped, Rubidium Cell Frequency Standards. PB95-163218

01,538

Improved Rubidium Frequency Standards Using Diode Lasers with $A M$ and FM Noise Control.

04,303

Reducing the Effect of Local Oscillator Phase Noise on the Frequency Stability of Passive Frequency Standards.
PB95-180972
SZEKELY, J.

Mathematical Models of Transport Phenomena Associated with Arc-Welding Processes: A Survey.

02,870

Power Characteristics in GMAW: Experimental and NumeriCal Investigation.

SZELAZEK, J.

03,296

Dynamometer-Induced Residual Stress in Railroad Wheels: Uttrasonic and Saw Cut Measurements. Report No. 30

PB96-183199 04,857

Residual Stress in Induction-Heated Railroad Wheels: Ultrasonic and Saw Cut Measurements. Report No. 28.
PB96-106992

04,854
Satety Assessment of Railroad Wheels by Residual Stress Measurements.

PB96-141114

04,855

Ultrasonic Measurement of Residual Stress in Cast Steel Railroad Wheels.

SZLAG, D. C.

04,852

Phase Composition, Viscosities, and Densities for Aqueous Two-Phase Systems Composed of Polyethylene Glycol and Various Salts at $25 \mathrm{C}$

00,986

Statistical Thermodynamics of Phase Separation and Ion Partitioning in Aqueous Two-Phase Systems.

01,212

SZLAG, D. S.

Protein Extraction in a Spray Column Using a Polyethylene Glycol Maltodextrin Two-Phase Polymer System. PB95-162228

00,595

SZYKMAN, $S$.

Combining Interactive Exploration and Optimization for AsSembly Design

02,794

HVAC CAD Layout Tools: A Case Study of University/lndustry Collaboration.

PB97-112221

00,281

Improving the Design Process by Predicting Downstream Values of Design Attributes.

PB97-113096

02,795

TAATJES, C. A.

Laser Double Resonance Measurements of the Ouenching Rates of $\mathrm{Br}((2) \mathrm{P} 1 / 2)$ with $\mathrm{H} 20, \mathrm{D} 20, \mathrm{HDO}$, and $\mathrm{O} 2$.
$\mathrm{PB} 95-150694$

TACHIBANA, $\mathrm{H}$.

Orientation Effects on ESR Analysis of Alanine-Polymer Dosimeters.
PB96-1 46725

03,720

TACHIKAWA

VAMAS Intercomparison of Critical Current Measurements on NB3Sn Superconductors: A Summary Report.

PB96-119763

04,043

TACKE, $M$.

Stabilization of 3.3 and $5.1 \mathrm{~m}$ Lead-Salt Diode Lasers by Optical Feedback

PB95-180709

TAGUCHI, S.

Taguchi's Parameter Design: A Panel Discussion.

PB96-111802

04,313

TAGZIRIA, $\mathrm{H}$.

Preparation and Characterization of (6)LiF and (10)B Reference Deposits for the Measurement of the Neutron Life-

PB95-108692

03,874

Problems Related to the Determination of Mass Densities of Evaporated Reference Deposits.

Evaporated Re

03,941

TAI, G.

Efficient Method to Compute the Maximum Transient Drain Current Overshoot in Silicon on Insulator Devices. PB94-172483

02,300

TAI, S. S. C.

Certification of Morphine and Codeine in a Human Urine Standard Reference Material.

PB95-176160

03,499

Certification of Phencyclidine in Lyophilized Human Urine Reference Materials.

PB96-160692

03,508

NIST Reference Materials to Support Accuracy in Drug PB96-123807

03,505

TAKACS, E.

Observation and Visible and uv Magnetic Dipole Transitions in Highly Charged Xenon and Barium.

PB96-138441

04,056

Polarization Measurements on a Magnetic Ouadrupole Line in Ne-Like Barium

PB97-11310

04,161

DA, K.

Direct Dispersion Measurement of Highly-Erbium-Doped Optical Amplifiers Using a Low Coherence Reflectometer Coupled with Dispersive Fourier Spectroscop

04,263

Pump-Induced Dispersion of Erbium-Doped Fiber Measured by Fourier-Transtorm Spectroscopy.

PB94-211935

04,236

TAKAGI, K. K.

Effects on Whole Saliva of Chewing Gums Containing Calclum Phosphates

TAKAGI, S.

Behavior of a Calcium Phosphate Cement in Simulated Blood Plasma in vitro.

PB95-168712

00,165

Calcium Phosphate Cements.

PB97-111595

03,580

Crystal Structure of Calcium Adipate Monohydrate.

PB94-216579

00,153

Crystal Structure of Calcium Glutarate Monohydrate.

01,074

Crystal Structure of Calcium Succinate Monohydrate.

PB95-168928

00,167

Deposition of Loosely Bound and Firmly Bound Fluorides on Tooth Enamel by an Acidic Gel Containing Fluorosilicate and Monocalcium Phosphate Monohydrate.

PB95- 150710

03,559

Effects on Whole Saliva of Chewing Gums Containing Calcium Phosphates.

PB95-153169

03,563

Formation of Hydroxyapatite in a Polymeric Calcium Phosphate Cemen

Formation of Hydroxyapatite in Cement Systems.

00,173

PB95-175261

00,170

Physical and Chemical Properties of Resin-Reinforced Calcium Phosphate Cements.

B95-180212

00,17

Polymeric Calcium Phosphate Cements Derived from Poly(methyl vinyl ether-maleic acid).

PB96-164264

00,180

Properties and Mechanisms of Fast-Setting Calcium Phos phate Cements.

B 96-123229

00,178

Remineralization of Root Lesions with Concentrated Calcium and Phosphate Solutions.

PB96-102140

03,567

TAKARA, $\mathrm{H}$.

Multiwavelength Birefringent-Cavity Mode-Locked Fibre

PB95-150496

04,262

TAMIR, D.

In-Space Welding: Visions and Realities.

PB95-163234

04,830

TAMURA, D.

Planar Near-Field Measurements and Microwave Holog raphy for Measuring Aperture Distribution on a $60 \mathrm{GHz} A C$ tive Array Antenna.

PB96-167366

03,762

TAMURA, G. T.

Smoke Control Systems for Elevator Fire Evacuation. B94-212883

00,291

TAN, $Z$. 


\section{PERSONAL AUTHOR INDEX}

TANENBAUM, D

Atomic-scale characterization of hydrogenated amorphoussilicon films and devices. Annual subcontract report, 14 February 1994--14 April 1995

DE9500928

02,294

TANENBAUM, D. M.

Construction of Silicon Nanocolumns with the Scanning Tunneling Microscope.

04,696

Growth and Nucleation of Hydrogenated Amorphous Silicon on Silicon (100) Surfaces.

PB96-176516

02,991

Nanoscale Study of the As-Grown Hydrogenated Amorphous Silicon Surface.

04,573

Nanoscale Study of the Hydrogenated Amorphous Silicon

PB96-103056

TANG, C. M.

NIST-NRL Free-Electron Laser Facility

PB94-212511

Planar Lenses for Field-Emitter Arrays.

02,112

TANG, D.

Interlaboratory Comparison of Polarization-Holding Parameter Measurements on High-Birefringence Optical Fiber.

TANG, H. C

Thermal Modeling of Absolute Cryogenic Radiometers.
PB95-181236

TANG, J.

Neutron Leakage Benchmark for Criticality Safety Research.
PB95-126132

03,723

Properties and Interactions of Oral Structures and Restorative Materials. Annual Report for Period October 1, 1990 to

September 30, 1991.

03,558

TANG, J. T.

Application of a Simple Technique for Estimating Errors of

Finite-Element Solutions Using a General-Purpose Code.
PB94-200250

Global Thermodynamic Behavior of Fluid Mixtures in the Critical Region
PB94-212420

04,199

TANG, $W$.

Neutron Scattering by Multiblock Copolymers of Structure (A-B)N-A.

PB94-211547

01,219

TANIGAK, $\mathrm{K}$.

Rotational Dynamics of $\mathrm{C} 60$ in Na2RbC60

PB95-153201

00,948

ANK, R. C.

Maturity Functions for Concrete Made with Various Cements and Admixtures.

PB94-199502

01,314

Measurement and Determination of Radon Source Potential: A Literature Review.

PB94-165602

TANNER, B. K.

02,576

Comparison of Techniques for Nondestructive Composition Measurements in CdZnTe Substrates.

PB96-103098

02,703

TANNER, $\mathrm{C}$.

Optical Molasses: The Coldest Atoms Ever

PB95-108908

TANNER, C. E.

Atoms in Optical Molasses

03,875

Atoms in Optical Molasses: Applications to Frequency Standards.
PB95-108882

03,876

Heterodyne Measurement of the Fluorescence Spectrum of Optical Molasses.

03,873

Localization of Atoms in a Three-Dimensional Standing Wave.

03,944

Measurements of Fluorescence from Cold Atoms: Localiza-

tion in Three-Dimensional Standing Waves.

03,943

Optical Molasses: Cold Atoms for Precision Measurements.

$\begin{array}{ll}\text { PB95-108890 } & \text { 03,877 }\end{array}$

Precision Lifetime Measurements of $\mathrm{Cs} 6 p(2) \mathrm{P} 1 / 2$ and $6 p$ (2) P3/2 Levels by Single-Photon Counting.

PB95-203816

Laser-Synchrotron Hybrid Experiments: A Photon to Tickle, A Photon to Poke.

PB96- 157847
TANNER, J. T.

Mixed Diet Reference Materials for Nutrient Analysis of Foods: Preparation of SRM-1548 Total Diet.

03,593
TANUMA, $\mathbf{S}$.

Calculations of Electron Inelastic Mean Free Paths (IMFPs) 4. Evaluation of Calculated IMFPS and of the Predictive IMFP Formula TPP-2 for Electron Energies between 50 and

PB95-150728 00,922

Calculations of Electron Inelastic Mean Free Paths. 5. Data for 14 Organic Compounds over the 50-2000 eV Range. Use of Sum Rules on the Energy-Loss Function for the Evaluation of Experimental Optical Data.

PB95-150736

04,264

TAO, H. S.

Influence of Coadsorbed Potassium on the Electron-Stimulated Desorption of $F(+), F(-)$, and $F\left({ }^{*}\right)$ from PF3 on PB96-157946

04,072

\section{TAREK, $M$}

Molecular Dynamics Investigation of the Surface/Bulk Equilibrium in an Ethanol-Water Solution.

PB97-113112

01,183

TARLOV, M. J.

Characterization of Cytochrome c/Alkanethiolate Structures Prepared by Self-Assembly on Gold.

00,987

Silver Metalization of Octadecanethiol Monolayers Self-Assembled on Gold.

PB95-150744

00,923

Surface Plasmon Microscopy of Biotin-Streptavidin Binding Reactions on UV-Photopatterned Alkanethiol Self-Assem-

bled Monolayers.

01,158

UV-Photopatterning of Alkylthiolate Monolayers Self-Assem-

bled on Gold and Silver
PB95-150751

00,924

TARNOFF, N.

NIST Support to the Next Generation Controller Program 1991 Final Technical Report.

PB94-163490

02,808

World Model Registration for Effective Off-Line Programming of Robots.

02,933

TARPEY, T.

Principal Points and Self-Consistent Points of Symmetric Multivariate Distributions.

PB96-135090

03,446

Representing a Large Collection of Curves: A Case for Principal Points.

PB95-152286 03,438

Two Principal Points of Symmetric, Strongly Unimodal Dis-

tributions.
PB95-203360

03,443

TARRINI, $O$.

Self Broadening in the nut Band of $\mathrm{NH3}$.

PB94-216371

00,857

TARAIO, C.

Improved Reflectometry Facility at the National Institute of Standards and Technology.

PB96-160338

04.087

Influence of Electrical Isolation on the Structure and Reflectivity of Multilayer Coatings Deposited on Dielectric Sub-

66-159736

04,365

New Method for Achieving Accurate Thickness Control for Uniform and Graded Multilayer Coatings on Large Flat SubStrates.

PB96-159744

04,366

New NIST/ARPA National Soft X-ray Reflectometry Facility.

PB96-158092 04,080

Soft-X-ray Dam

04,364

Soft X-ray Reflectometry Program at the National Institute of Standards and Technology.

PB96-160395

04,368

Transient Cooling of a Hot Surface by Droplets Evaporation.

Transient Cooli

03,783

TASSEY, G.

Functions of Technology Infrastructure in a Competitive Economy.

00,478

TAUHEED, A.

Analysis of the $5 s(2) 5 p(2)-(5 s 5 p(3)+5 s(2) 5 p 5 d+5 s(2) 5 p 6 s)$ Transitions of Four-Times lonized Xenon (Xe V).

PB95-150769

03,900

Spectrum and Energy Levels of Triply Ionized Barıum (Ba IV).

03,888

TAY, S. P.

Structural and Magnetic Ordering in Iron Oxide/Nickel Oxide Multilayers by $X$-ray and Neutron Diffraction (Invited).

Multilayers by $X-1$

04,442

TAYLOR, A.

Isolated Spin Pairs and Two-Dimensional Magnetism in $\mathrm{SrCr}($ sub $9 \mathrm{p}) \mathrm{Ga}$ (sub 12-9p)O19.

PB97-112387

04,154

Performance of HUD-Affiliated Properties during the January 17, 1994 Northridge Earthquake.

AYLOP, A

00,443

Evaluating the Seismic Performance of Lightly-Reinforced Circular Concrete Bridge Columns.

01,335

Jacket Thickness Requirements for Seismic Retrofitting of Circular Bridge Columns.

PB95-163267

01,336

Method of Estimating the Parameters of Tuned Mass Dampers for Seismic Applications.

PB96-167820

00,473

Northridge Earthquake, 1994. Performance of Structures

Lifelines and Fire Protection Systems.

PBg4-161114

00,421

Northridge Earthquake 1994: Performance of Structures, Lifelines, and Fire Protection Systems.

PB94-207461

04,825

Report of a Workshop on Requalification of Tubular Stee Joints in Offshore Structures. Held in Houston, Texas on September 5-6, 1995

03,699

Seismic Performance of Circular Bridge Columns Designed in Accordance with AASHTO/CALTRANS Standards.

PB96-146352

State of the Art Report on Seismic Design Requirements for

Nonstructural Building Components.

PB96-193800

00,308

TAYLOR, B. N.

Fine-Structure Constant.

B94-172996

03.795

Guide for the Use of the International System of Units (SI).

PB95-226692

(SI).
02,747

Guidelines for Evaluating and Expressing the Uncertainty of NIST Measurement Results. 1994 Edition.

02,649

How Accurate Are the Josephson and Ouantum Hall Effects and OED

PB95-163283

03,942

Journal of Research of the National Institute of Standards and Technology. March/April 1994. Volume 99, Number 2. PB94-219219

02,000

Journal of Research of the National Institute of Standards and Technology. May/June 1994. Volume 99, Number 3

PB94-219326

02,643

Journal of Research of the National institute of Standards

Number 5 .

PB96-134954

03,362

New International Representations of the Voit and Ohm Ef

fective January 1,1990
PB95-150777

01,890

Report on the Meeting of the CCU (10th) (of the International Committee of Weights and Measures). Held on July $10-11,1990$

TAYLOR, J.

03,792

Electronic Balance and Some Gravimetric Applications (The Density of Solids and Liquids, Pycnometry and Mass).

PB94-163052 03,785 
TEAGUE, E. C.

Comparison of Finite Element and Analytic Calculations of the Resonant Modes and Frequencies of a Thick Shell Sphere.

02,626

Electrical Test Structure for Overlay Metrology Referenced PB95-152278 Length Standards.
to Absolute

02,336

Force Calibrations in the Nanonewton Regime.

03,949

Generating and Measuring Displacements Up to $0.1 \mathrm{~m}$ to an Accuracy of $0.1 \mathrm{~nm}$ : Is it Possible.

PB96-160700

04,090

Measurement of Patterned Film Linewidth for Interconnect Characterization.

PB96-148168

02,420

Metrology Standards for Advanced Semiconductor Lithography Referenced to Atomic Spacings and Geometry.
PB96-160718

Nanometrology

02,425

New Test Structure for Nanometer-Level Overlay and Feature-Placement Metrology.

02,363

Scanned Probe Microscopies: Opportunities and Issues in Metrology.

MB96-160783

02,426

Test Structures for the In-Plane Locations of Projected Features with Nanometer-Level Accuracy Traceable to a Coordinate Measurement System.

PB94-200565

02,313

TEBBUTT, J.

Guidelines for the Evaluation of X.500 Directory Products.

PB95-231908 02,732

Impact of the FCC's Open Network Architecture on NS/NP Telecommunications Security.

PB95-189445

X.500 Directory Schema Design Handbook.

01,483

PB96-183041

02,739

TEKLE, E.

Electropermeabilization of Cell Membranes: Effect of the Resting Membrane Potential.

PB95-163291

03,537

TELLER, C. M.

Assessment of Technology for Detection of Stress Corrosion Cracking in Gas Pipelines. Final Report, July 1993March 1994.

02,475

Gas-Coupled, Pulse-Echo Ultrascnic Crack Detection and Thickness Gaging

PB96-147129

04,847

TEMPLE, D.

Photoelectron Spectroscopic Study of the Valence and Core-Level Electronic Structure of BaTiO3.

04,500

Surface Core-Level Shifts of Barium Observed in Photoemission of Vacuum-Fractured BaTiO3 (100).

PB94-212156

04.501

TENBRINK, J.

Effect of Axial Strain on the Critical Current of Ag-Sheathed Bi-Based Superconductors in Magnetic Fields Up to $25 \mathrm{~T}$. PB94-211315 04,493 Improved Uniaxial Strain Tolerance of the Critical Current Measured in Ag-Sheathed $\mathrm{Bi} 2 \mathrm{Sr} 2 \mathrm{Ca} 1 \mathrm{Cu} 2 \mathrm{O} 8+x$ Measured in

PB 95-153565

04.594

TENG, Y. A.

Data-Parallel Algorithm for Three-Dimensional Delaunay Triangulation and is Implementation.

PB95-163309

01,714

Parallel Monte Carlo Simulation of MBE Growth.

PB96-122841

02,406

TENNYSON, E. J.

In situ Burning of Oil Spills: Mesoscale Experiments and Analysis.

02,587

TENWOLDE, A.

Controlling Moisture in the Walls of Manufactured Housing.
PB95-105136

Manufactured Housing Walls That Provide Satisfactory Moisture Performance in All Climates. Moisture Pert

TER MEER, H. U.

00,383

Phase Transitions in Solid C70: Supercooling, Metastable Phases, and Impurity Effect.

PB95-150090

00,914

TERLIZZI, C. P.

Performance Testing of a Family of Type I Combination Appliance.

PB95-220521

TERRANOVA, $P$.

02,505

Dynamic Shear Modulus Measurements with Four Independent Techniques in Nickel-Based Alloys.

03,320

TESI, M. C.

Self-Avoiding Surfaces, Topology, and Lattice Animals.

PB95-150512

04,571
TESK, $\mathbf{J}$

Intluence of Tempering Method on Residual Stress in Dental Porcelain

PB94-172012

00,138

SK, J. A.

Dental Materials.

00,142

Effect of Transformation of Alloy on Transient and Residual Stresses in a Porcelain-Metal Strip.

00,143

Error Propagation Biases in the Calculation of Indentation Fracture Toughness for Ceramics.

03,032

Evaluation of Fracture Toughness and Residual Stress in Dental Porcelain by Indentation-Microfracture Method. PB95-125613

00,154

Evaluation of Fracture Toughness and Residual Stress in Dental Porcelain by Indentation-Microfracture Method.

00,159

Failure of All-Ceramic Fixed Partial Dentures 'In vitro' and 'In vivo': Analysis and Modeling.

PB96-122536

00,175

In vitro Fracture Behavior of Ceramic and Metal-Ceramic Restorations

03,569

International Standards and Reference Materials. PB97-113120

00,188

Properties and Interactions of Oral Structures and Restorative Materials. Annual Report for Period October 1, 1990 to September 30, 1991.

SBptember 30,

03,558

What Is a 'Standard Reference Material' - What Is Any Reference Material
PB96-186135

TESORIERO, $R$.

Measurement of Process Complexity.

\section{TEW, W. L.}

03,000

Assessment of Uncertainties of Calibration of Resistance Thermometers at the National Institute of Standards and Technology.

02,624

Flux-Locked Current Source Reference.

PB95-15078

02,039

TEWARI, Y. B.

Apparent Molar Heat Capacities and Apparent Molar Volumes of Aqueous Glucose at Temperatures from $298.15 \mathrm{~K}$ to $327.01 \mathrm{~K}$

PB94-212800

03,459

Application of Thermodynamics to Biotechnology.

PB95-150793

03,529

Equilibrium and Calorimetric Investigation of the Hydrolysis of L-Tryptophan to (Indole + Pyruvate + Ammonia).

Thermochemistry of the Hydrolysis of L-arginine to (L-
citrulline + Ar, 10 monia) and of the Hydrolysis of L-arginine to (L-ornithine + Urea)

PB95-150801

03,463

Thermochemistry of the Reactions between Adenosine, Adenosine 5'-monophosphate, Inosine, and Inosine 5'monophosphate; the Conversion of L-histidine to (Urocanic Acid+Ammonia)

Thermodynamic and NMR Study of the Interactions of Cyclodextrins with Cyclohexane Derivatives.

00,781

Thermodynamic Study of the Reactions of Cyclodextrins with Primary and Secondary Aliphatic Alcohols, with D- and with Primary and Secondary Aliphatic Alcohols, with D- and L-Phenylalanine, and with L-Phenylalanineamide.
PB95-180873

Thermodynamics of Enzyme-Catalyzed Reactions: Part 1. Oxidoreductases.

PB94-162252

00,737

Thermodynamics of Enzyme-Catalyzed Reactions. Part 4. Lyases.
PB96-145941

01,118

Thermodynamics of Enzyme-Catalyzed Reactions. Part 5 Isomerases and Ligases.

PB96-145974

01,121

Thermodynamics of the Hydrolysis of N-Acetyl-Lphenylalanine Ethyl Ester in Water and in Organic Solvents.
PB95-203386 Thermodynamics of the Hydrolysis of Penicillin G and Ampi-

PB94-172467

03,596

Thermodynamics of the Hydrolysis of 3,4,5Trihydroxybenzoic Acid Propyl Ester (n-Propylgallate) to 3,4,5-Trihydroxybenzoic Acid (Gallic Acid) and Propan-I-ol in Aqueous Media and in Toluene.

PB96-186143

01,160

TEWARY, $V$.

Lattice Imperfections Studied by Use of Lattice Green's Functions.

TEWARY, V. K.

04,576

Atomic Theory of Fracture of Brittle Materials: Application to Covalent Semiconductors.

04,519

Elastic Green's Function for a Bimaterial Composite Solid Containing a Free Surface Normal to the Interface.
PB95-163325

Generalized Plane Strain Analysis of a Bimaterial Compos-

ite Containing a Free Surface Normal to the Interface.
PB95-163341

Green's Function for Generalized Hilbert Problem for Cracks and Free Surfaces in Composite Materials. -163333

03,163

Lattice Statics of Interfaces and Interfacial Cracks in Bimaterial Solids.

PB96-161823

THALWEISER, $R$.

Femtosecond Time-Resolved Molecular Multiphoton Ionization: The $\mathrm{Na} 2$ System.

PB95-202305

03,975

Femtosecond Time-Resolved Wave Packet Motion in Mo-

lecular Multiphoton Ionization and Fragmentation.

00,790

THEIN, $M$.

Intercomparison Study of (237)Np Determination in Artificial Urine Samples.

PB96-102645

THEN, S. $S$.

Loss-Free Counting at IRI and NIST.

03,633

THEODOSIOU, C. E.

Oscillator Strengths and Radiative Branching Ratios in

Atomic Sr.

PB95-203493

Activated Dynamics, Loss of Ergodicity, and Transport in Supercooled Liquids.

PB95-150819

00,925

Quantitative Measure of Efficiency of Monte Carlo Simula-

tions.

PB95-18069

01,011

HODE, W. F.

Proposed International Interactive Courseware Standard. PB96-123161

THOMAS, B. $\mathrm{H}$.

Generic Manufacturing Controllers.

PB94-199940

Using Grafcet

PB95-150827

02,818

HOMAS, B. N.

Studies of the Higher Order Smectic Phase of the Large Electroclinic Effect Material W317.

PB95-151601

00,935

$X$-ray Observation of Electroclinic Layer Constriction and Rearrangement in a Chiral Smectic-A Liquid Crystal.

PB96-141080

01,100

THOMAS, C.

Implementation of a Standard Format for GPS Common View Data.

N95-32323/4

03.779

Implementation of a Standard Format for GPS Common 
THOMPSON, A

Beamcon III, a Linearity Measurement Instrument for OptiCal Detectors

04.337

Improved Automated Current Control for Standard Lamps.

PB94-219367 00,246

Irradiance of Horizontal Ouartz-Halogen Standard Lamps.

PB96-179130

01,866

Report on USDA Ultraviolet Spectroradiometers. PB96-214648

Standards for Corrected Fluorescence Spectra.

00,125

PB95-150835

00,581

THOMPSON, A. N.

Metalloporphyrin Sensitized Photooxidation of Water to Oxygen on the Surface of Colloidal Iridium Oxides - Photochemical and Pulse Radiolytic Studies.

PB95-107082

00,868

THOMPSON, C

Report on Application Integration Architectures (AIA) Workshop. Held in Dallas, Texas on February 8-12, 1993

PB94-142536

01,803

THOMPSON, C. A.

Alternating-Field Susceptometry and Magnetic Susceptibility Workshop on Magnetic Susceptibility of Superconductors and Other Spin Systems Held in Berkeley Springs. West Virginia on 20 May 1991.

PB94-145984

04,435

Apparatus for Resistance Measurement of Short, Small-Diameter Conductors

PB96-141130

04,417

Micromagnetic Scanning Microprobe System.

02,224

THOMPSON, G.

FTS Infrared Measurements of the Rotational and Vibrational Spectrum of LiH and LiD.

PB94-216231

THOMPSON, J. S

Backscattering in Electron-Impact Excitation of Multiply PB $94-185345$

Electron-Impact Excitation of Si( $3+)(3 S$ yields $3 \mathrm{P})$ Using a Merged-Beam Electron-Energy-Loss Technique.
PB95-151239

03,904

Electron-lmpact Excitation of $\operatorname{Ar}(7+)(3 s$ yields $3 p)$. PB95-126405

Merged-Beams Energy-Loss Technique for Electron-Ion Ex citation: Absolute Total Cross Sections for $\mathrm{O}(5+)(2 \mathrm{~s}$ yields 2p). $96-102058$

04,017

THOMPSON, R.

Fracture in Multilayers.

HOMPSON, R. B.

Ultrasonic Measurement of Sheet Anisotropy and PB95-153235

Ultrasonic Methods

PB96-190327

THOMPSON, W. E.

Infrared and Near-Infrared Spectra of HCC and DCC Trapped in Solid Neon.

-A295 578/9

03,773

Matrix Isolation Study of the Interaction of Excited Neon Atoms with BCl3: Infrared Spectra of $\mathrm{BCl}$ (sub 3, sup +). $\mathrm{BCl}($ sub 2, sup + ), and $\mathrm{BCl}$ (sub 3 , sup - ).

01,187

Matrix Isolation Study of the Interaction of Excited Neon Matrix Isolation Study of the Interaction of Excited Neon
Atoms with 03: Infrared Spectrum of $\mathrm{O}(($ sub 3$)(-))$ and EviAtoms with 03: Infrared Spectrum of $\mathrm{O}(($ sub 3$)(-))$ and Evi-

04,155

Mid-and Near-Infrared Spectra of Water and Water Dimer solated in Solid Neon.

PB95-125662

00,888

Production and Spectroscopy of Small Polyatomic Molecular Ions Isolated in Solid Neon. (Reannouncement with New Availability Intormation)

AD-A234 043/8

00,704

Vibrational Spectra of Molecular Ions Isolated in Solio Neon: $\mathrm{HCCH}+$ and HCC-. (Reannouncement with New Availability Information).

00,707

Vibrational Spectra of Molecular Ions Isolated in Solio Neon. X. $\mathrm{H} 2 \mathrm{O}(+), \mathrm{HDO}(+)$, and $\mathrm{D} 2 \mathrm{O}(+)$

00.889

Vibrational Spectra of Molecular Ions Isolated in Solid Neon. $\mathrm{XI}$. NO2(+), NO2(-), and NO3(-).

00,890

Vibrational Spectra of Molecular Ions Isolated in Solid Neon. 6. CO4(-). (Reannouncement with New Availability Information).

00,705

Vibrational Spectra of Molecular Ions Isolated in Solid Neon. 7. $\mathrm{CO}(+), \mathrm{C} 2 \mathrm{O} 2(+)$, and $\mathrm{C} 2 \mathrm{O} 2(-)$. (Reannouncement with New Availability Information)

with New Avail

00,706
Vibrational Spectra of Molecular lons Isolated in Solio Neon. 11. NO2(+), NO2(-), and NO3 $(-)$

00,708

Vibrational Spectra of Molecular Ions Isolated in Solid Neon. 13. Ions Derived from $\mathrm{HBr}$ and $\mathrm{HI}$

PB97-119234

01,188

\section{THOMSON, $R$.}

Atomic Theory of Fracture of Brittle Materials: Application to Covalent Semiconductors.

PB94-216553

04,519

Cracks and

PB96-163696

02,989

Dislocation Core-Core Interaction and Peierls Stress in a Model Hexagonal Lattice.

PB96-162003

04,10

PB $95-164240$

04,630

Fundamentals of Fracture: A 1993 Prologue, and Other Comments.

03,218

Interaction between Dislocations and Intergranular Cracks.

PB95-152096

03,190

Interfacial Crack in a Two-Dimensional Hexagonal Lattice.
PB96-161989

PB96-161989 04,100

Lattice Imperfections Siudied by Use of Lattice Green's Functions.

04,576

Lattice Statics of Interfaces and Interfacial Cracks in

Bimaterial Soliós.
PB96-161823

Nonlocal Effects of Existing Dislocations on Crack-Tip Emission and Cleavage.

03,367

Shielding of Cracks in a Plastically Polarizable Material.
PB95-164257

Whither Computational Materials Science. Some Thoughts from the Mechanical Properties Front.

PB96-161997

02,987

THOMSON, R. E.

Development of Highly Conductive Cantilevers for Atomic Force Microscopy Point Contact Measurements.

PB96-138573

04,751

PBg6-138573 formers for Low l/f Noise.

02,059

Insulating Nanoparticles on YBa2Cu3O7-delta Thin Films

Revealed by Comparison of Atomic Force and Scanning Tunneling Microscopy.

PB95-150843

04.575

Low Noise YBa2Cu307-x-SrTiO3-YBa2Cu307-x Mulitlayers for Improved Superconducting Magnetometers.

Observation of Insulating Nanoparticles on YBCO ThinFilms by Atomic Force Microscopy

04,622

Scanning Tunneling Microscopy of the Charge-DensityWave Siructure in 1 T-TaS2.

Surface Modification of YBa2Cu307-delta Thin Films Using

the Scanning Tunneling Microscope: Five Methods.

04,699

Temperature Dependence and Magnetic Field Modulation of Critical Currents in Step-Eoge SNS YBCO/Au Junctions.

THORNE, B. B

Airbome Asbestos Method: Bootstrap Method for Determining the Uncertainty of Asbestos Concentration. Version 1.0 .
PB96-214614. THORPE, M. F.

Elastic Properties of Central-Force Networks with BondLength Mismatch.

THORPE, M. R.

Geometrical Percolation Threshold of Overlapping

Ellipsoids.

THUDIUM, R. N

03,167

Microstructure Effect on the Phase Behavior of Blends of Deuterated Polybutadiene and Protonated Polyisopene.
PB97-113146 THUMM, $\mathrm{U}$.

Angle-Differential and Momentum-Transter Cross Sections for Low-Energy Electron.Cs Scattering.

PB95-203402 04,005

Characteristics of Light Emission Atter Low-Energy Electron Impact Excitation of Caesium Atoms.

03.806

Relativistic Effects in Spin-Polarization Parameters for LowEnergy Electron-Cs Scattering.

03,901

Relativistic R-Matrix Calculations for Electron - Alkali-MetalAtom Scattering: Cs as a Test Case.

THURBER, W. R.

04.006

Heavily Accumulated Surfaces of Mercury Cadmium Tellu-

ride Detectors: Theory and Experiment.

02,134

Novel Magnetic Field Characterization Techniques for Compound Semiconductor Materials and Devices.
PB96-176458 Semiconductor Measurement Technology: Improved Characterization and Evaluation Measurements for $\mathrm{HgCoTe} \mathrm{De}$ tector Materials, Processes, and Devices Used on the GOES and TIROS Satellites.

02,122

Transverse Magnetoresistance: A Novel Two-Terminal Method for Measuring the Carrier Density and Mobility of a Semiconductor Layer.

PB95-150066

02,332

RGATE, $S$

Grazing-Incidence X-Ray Photoemission Spectroscopy Investigation of Oxidized GaAs(100): A Novel Approach to Nondestructive Depth Profiling.

PB94-200151

04,480

URGATE, S. M.

Grazing Angle X-Ray Photoemission System for Depth-Dependent Analysis.

Grazing Incidence X-ray Photoemission and Its Implementation on Synchrotron Light Source X-ray Beamlines.

B95-175766

01,001

IDSTROM, K. D.

Evolution of a Turbulent Boundary Layer Induced by a PB94-212818

04,200

TIERNEY, E. J.

Total Surface Areas of Group IVA Organometallic Compounds: Predictors of Toxicity to Algae and Bacteria.

PB94-211331

00,814

TIESINGA, E.

Measurement of the Atomic $\mathrm{Na}(3 \mathrm{P})$ Lifetime and of Retardation in the Interaction between Two Atoms Bound in a

PB97-122360

04,178

TIETZ, L. A.

Fiber Coating Diameter: Toward a Glass Artifact Standard.
PB96-140389

TILFORD, C. R

Characteristics of Partial Pressure Analyzers.

PB95-150876 00,582

Comments on the Stability of Bayard-Alpert Ionization Gages.

Measurement of Very-Low Partial Pressures.

PB95-180998

Partial Pressure Analysis in Space Testing.

95.14084/4

Partial Pressure Analysis in Space Testing.

PB96-103072

PC-Based Spi

Limiting Factors.

PB95-181004

Vacuum Gauges ano Partial Pressure Analyzers.

PB95-150884

02,673

TILLNER-ROTH, $R$

Report of the Refrigeration. Air Conditioning and Heat Pumps Technical Options Committee.

PB96-176755

02,659

04,827

04,829

02,609

TILSTRA, L

Microbial Degradation of Polysulfides and Insights into Their Possible Occurrence in Coa

PB95-163374

02,488

TIMMER, C. A.

Merged-Beams Energy-Loss Technique for Electron-Ion Excitation: Absolute Total Cross Sections for $\mathrm{O}(5+)$ (2s yields 2p). 02058

04,017

TIMMERHAUS, $K$

Energy Flows in an Orifice Pulse Tube Refrigerato
PB95-169082

02,752

PMERHAUS, K. D. 
Water Droplet Evaporation from Radiantly Heated Solids PB95-217147

00,394

TINO, G. M.

High-Resolution Diode-Laser Spectroscopy of Calcium PB95-181244

TINSCHERT, $K$

Dielectronic Capture Processes in the Electron-Impact IonIzation of $\mathrm{Sc}(2+)$

PB95-203113

04,000

TIRUMALAI, V. C

Solid State (13)C NMR and Raman Studies of Cellulose Triacetate: Oligomers, Polymorphism, and Inferences about Chain Polarity.

TISON, S. A.

01,289

Commercial Helium Permeation Leak Standards: Their Properties and Reliability.

PB97-111413

04,146 Critical Evaluation of Thermal Mass Flow Meters.
PB97-113153

Experimental Data and Theoretical Modeling of Gas Flows Through Metal Capillary Leaks.

PB95-150892

04,206

Improved Gas Flow Measurements for Next-Generation Processes.

PB96-156013

04,216

TJOSSEM, P. J. H.

Optical Measurements of Atomic Hydrogen, Hydroxyl, and Carbon Monoxide in Hydrocarbon Diffusion Flames.

TBS

02,487

TOBIAS, D. J.

Molecular Dynamics Investigation of the Surface/Bulk Equilibrium in an Ethanol-Water Solution.

TOBIN, S. P.

Comparison of Techniques for Nondestructive Composition Measurements in CdZnTe Substrates.

PB96-103098

02,703

TOBLER, R. L.

Charpy Impact Test as an Evaluation of $4 \mathrm{~K}$ Fracture

Toughness.

03,219

Charpy Specimen Tests at $4 \mathrm{~K}$.

03,002

Fatigue Crack Thresholds of a Nickel-Iron Alloy for Superconductor Sheaths at $4 \mathrm{~K}$.
PB96-190343

Mechanical Properties and Warm Prestress of Ultra-Low Carbon Steel at $4 \mathrm{~K}$.

PB96-190350

03,224

TOBY, B. H.

Colossal Magnetoresistance without $\mathrm{Mn}(3+) / \mathrm{Mn}(4-)$ Double Exchange in the Stoichiometric Pyrochlore Ti2Mn2O7.

TODD, D.

De Facto Microzonation through the Use of Soils Factors in Design Triggers.

00,462

Evaluation and Retrofit Standards for Existing Federally Owned and Leased Buildings.

PB95-150918

00,434

Executive Order 12941. Seismic Safety of Existing Feder. ally Owned or Leased Buildings: It's History, Content and PBjectives.

00,465

How-To Suggestions for Implementing Executive Order 12941 on Seismic Safety of Existing Federal Buildings, A

PB96-131552

00,461

ICSSC Guidance on Implementing Executive Order 12941 on Seismic Safety of Existing Federally Owned or Leased Buildings.

00,459

Northridge Earthquake, 1994. Performance of Structures, Lifelines and Fire Protection Systems.

PB94-161114

00,421

Northridge Earthquake 1994: Performance of Structures,

Lifelines, and Fire Protection Systems.

04,825

PB94-207461

Performance of Federal Buildings in the January 17, 1994 Northridge Earthquake

00,453

Performance of HUD-Affiliated Properties during the January 17, 1994 Northridge Earthquake.

ary 17,1994 Nort
PB 95.174488

00,443

Some Basics on Who's Who and What's What in Seismic

PBafety. 150926

00,435

World of Building Codes.

PB95-203428

00,449

TODD, D. $R$.

Seismic Safety of Federal Buildings. Initial Program: How

Much Will It Cost

Seismic Strengthening of Reinforced Concrete Frame BuildPB95. 108841

00,430
Standards of Seismic Safety for Existing Federally Owned or Leased Buildings and Commentary.

00,431

Strengthening Methodology for Lightly Reinforced Concrete

PB96-158050

00,466

Strengthening Methodology for Lightly Reinforced Concrete Frames-II. Recommended Calculation Techniques for the Design of Infill Walls.

00,426

Strengthening Methodology for Lightly Reinforced Concrete Frames: Recommended Design Guidelines for Strengthening with infill Walls.

PB95-260725

00,454

\section{TODD, F. G.}

Vibrations of $S 1((1) B 2 u) p$-Difluorobenzene - d4. S1.So Fluorescence Spectroscopy and ab Initio Calculations. PB95-20258

TODD, J. H.

Measurement of the $(10) \mathrm{B}(\mathrm{n}$, alpha1gamma)(7) Li Cross Section in the 0.3 to $4 \mathrm{MeV}$ Neutron Energy Interval.

PB96-161799

TODD, $P$.

Gravity Dependent Processes and Intracellular Motion. PB95-163382

TOFANI, $\mathrm{S}$.

Bistatic Scattering of Absorbing Materials from 30 to 1000

01,891

Time-Domain Measurements of the Electromagnetic

Backscatter of Pyramidal Absorbers and Metallic Plates.
PB94-185410
01,877 TOLLE, J. W.

Convergence Properties of a Class of Rank-Two Updates. (NIST Reprint)
PB95-180097

03,430

\section{TOM, $\mathrm{H}$.}

Geographic Information Systems Standards: A Federal Per-

PB95-163390

03,678

Spatial Information and Technology Standards Evolving.
PB96-135108

PB96-135108
Standards: A Cardinal Direction for Geographic Information Systems.

PB95-150942

TOMASCH, W. J.

03,677

Microwave Properties of YBa2Cu307-x Films at $35 \mathrm{GHz}$ from Magnetotransmission and Magnetoreflection Measurements.
PB95-168977

TOMASZKIEWICZ, I.

04,643

Thermodynamic Properties of Silicides. 5. Standard Mola Enthalpy of Formation at the Temperature $298.15 \mathrm{~K}$ of Trimolybdenum Monosilicide Mo3Si Determined by Fluo rine-Combustion Calorimetry.

PB97-119358

01,190

TOMAZIC, B. B.

Critical Evaluation of the Purification of Biominerals by $\mathrm{Hy}$ pochlorite Treatmen

PB95-150959

00,186

Influence of Natural and Synthetic Inhibitors on the Crystallization of Calcium Oxalate Hydrates.

03,560

Physicochemical Characterization of Natural and Bioprosthetic Heart Valve Calcific Deposits: Implications for Prevention.

PB96-156039

00,187

Physicochemical Properties of Calcific Deposits Isolated from Porcine Bioprosthetic Heart Valves Removed from Patients Following 2-13 Years Function.

PB94.172863

00,184

TOMEK, A

dc Method for the Absolute Determination of Conductivities of the Primary Standard $\mathrm{KCl}$ Solutions from $\mathrm{OC}$ to $50 \mathrm{C}$. PB94-219342

02,644

TOMIZAWA, M.

Effects of Heavy Doping on Numerical Simulations of Gallium Arsenide Bipolar Transistors.

PB95-150975

02,334

TONER, $M$.

Crystal Packing Interactions of Two Different Crystal Forms of Bovine Ribonuclease A

PB95-152823

00,943

$X$-Ray Diffraction from Anodic $\mathrm{TiO} 2$ Films: In situ and Ex situ Comparison of the Ti(0001) Face.

PB94-185972

TONG, G.

Compensation of Markov Estimator Errors in Time-Jittered Sampling of Nonmonotonic Signals.

PB95-150983

TONUCCl, R. J.

Photonic Band-Structure Effects for Low-Index-Contrast PBo-Dimensiona 122469

TOPOROWSKI, P. M.

Thermodynamic Properties of Dilute and Semidilute Solutions of Regular Star Polymers.

01,284

TORARDI, C. C.

Ca4Bi6013: A Compound Containing an Unusually Low Bismuth Coordination Number and Short Bi Bi Contacts.
PB95-141131

TORCZYNSKI, J.

Gaseous Electronics Conference Radio-Frequency Reference Cell: A Defined Parallel-Plate Radio-Frequency Sys em for Experimental and Theoretical Studies of PlasmaProcessing Discharges.

TORIKAI, N.

04,404

Localization of a Homopolymer Dissolved in a Lamellar Structure of a Block Copolymer Studied by Small-Angle Neutron Scattering

PB95-161592

01,253

TORQUATO, $\mathrm{s}$

Cross-Property Relations and Permeability Estimation in

Model Porous Media.
PB95-150280

Transport and Diffusion in Three-Dimensional Composite Media.

PB95-176129

04,668

TORRE, J. $M$.

Preliminary Comparison of Time Transfers via LASSO, GPS and Two-Way Satellite.

TORRENCE, $S$.

Development and Validation of Multicriteria Ratings: A Case Study.

TORTONESE, $M$

Vortex Images in Thin Films of $\mathrm{YBa} C \mathrm{Cu} 3 \mathrm{O}$ (sub 7-x) and Bi2Sr2Ca1Cu2O(sub $8+x$ ) Obtained by Low-Temperature Magnetic Force Microscopy.

TOSSELL, J. A.

Associative Electron Attachment to $\mathrm{S} 2 \mathrm{~F} 10, \mathrm{~S} 2 \mathrm{OF} 10$, and $\mathrm{S} 2 \mathrm{O} 2 \mathrm{~F} 10$.

TOTH, P.

Proceedings of the Workshop on the Federal Criteria for In formation Technology Security. Held in Ellicot City, Mary land on June 2-3, 1993

PB94-162583

01,575

Proceedings Report of the International Invitation Workshop on Development Assurance. Held in Ellicott City, Maryland on June 16-17, 1994

PB95-18949

02,912

Concept Paper: An Overview of the Proposed Trust Technology Assessment Program.

PB96-160882

01,614

Head Start on Assurance: Proceedings of an Invitational Workshop on Information Technology (IT) Assurance and Trustworthiness. Held in Williamsburg, Virginia on March $21-23,1994$.

TOTH, R. $\mathrm{B}$.

Standards Activities of Organizations in the United States. PB97-124135

TOULOUSE, J.

Anomalous Dispersion and Thermal Expansion in LightlyDoped KTa1-xNbxO3.

PB95-152302

04,585

URDE, $\boldsymbol{A}$

Preliminary Comparison of Time Transfers via LASSO, GPS and Two-Way Satellite.

TOWER, J. P. 
TRAHEY, N. M.

NIST Standard Reterence Materials (Trade Name) Catalog 1995-1996.

PB95-232518

00,508

TRAIBER, A. J. S.

Electronic Structure and Phase Equilibria in Ternary Substitutional Alloys

TRAIL, W. K.

03,378

Importance of Bound-Free Correlation Effects for Vibrationa Excitation of Molecules by Electron Impact: A Sensitivity

PB95-202974

03,996

Low-Energy-Electron Collisions with Sodium: Elastic and Inelastic Scattering from the Ground State.

PB96-103106

04,030

TRAUGOTT, A. E.

U.S. Green Building Conterence, 1994

PB94-20636

02,519

TRAVIS, J.

Laser Ablation of Thin Films as a Free Atom Source for Pulsed RIMS.

TRAVIS, J. C.

00,540

Determination of the Transmittance Uniformity of Optical Filter Standard Reference Materials.

PB95-261921

02,182

Fourier Transform Atomic Emission Studies Using a Glow Discharge as the Emission Source.

00,533

Standard Reference Materials: Glass Filters as a Standard Reference Material for Spectrophotometry - Selection. Preparation, Certification, and Use of SRM 930 and SRM 1930. aration, Certification, and Use of SRM 930 and SRM 1930.
PB94-188844

Trace Detection in Conducting Solids Using Laser-Induced Fluorescence in a Cathodic Sputtering Cell.

PB95-163424

TREADO, S. J.

Lighting and HVAC.

PB95-150991

NIST Lighting and HVAC Interaction Test Facility.

Optical Performance of Commercial Windows.

PB95-208757

00,598 Ambient Temperatures.

PB95-175329

00,250

00,251

TREE, D. R.

Role of R22 in Refrigerating and Air Conditioning Equipment.

PB94-199783

03,253

TRELA, W. J.

Vibronic Coupling and Other Many-Body Effects in the

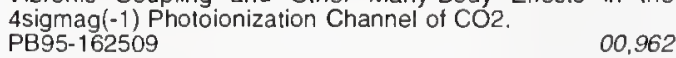

TRETYAKOV, M. Y

Tunneling-Rotation Spectrum of the Hydrogen Fluoride

Dimer.
PB94-198678

TREVINO, S. F.

00,793

Maximum Entropy as a Tool for the Determination of the $\mathrm{C}$ Axis Profile of Layered Compounds.

PB94-199619

00,800

Methyl Torsional Levels of Solıd Acetonitrile $(\mathrm{CH} 3 \mathrm{CN})$ : A Neutron Scattering Study.

PB95-151015

00,926

Structure of a Triglyceride Microemulsion: A Small Angle Neutron Scattering Study

01,077

TRIPP, G. A.

Surface Roughness of Glass-Ceramic Insert. Composite Restorations: Assessing Several Polishing Technıques.

PB97-119010

TRIVISONNO, $\mathbf{J}$

Structures of Sodium Metal.

PB94-198850

TROCCOLO, P.

Comparisons of Measured Linewidths of Sub-Micrometer Lines Using Optical, Electrical, and SEM Metrologies. PB95-152807

02,338

TROTT, K. A.

Effects of Etching on the Morphology and Surface Resistance of $\mathrm{YBa} 2 \mathrm{Cu} 3 \mathrm{O} 7$-delta Films.

02,410

TROY, E. F.

Computer Security Management and Planning in the U.S Federal Government.

PB95-163432 01.596

Guidance to Federal Agencies on the Use of Trusted Sys-

lems.

01,597

TROY, G.

Proceedings of the Workshop on the Federal Criteria for Information Technology Security. Held in Ellicot City, Mary. land on June 2-3, 1993.

01,575
TRUETT, L.

Burning Rate of Premixed Methane-Air Flames Inhibited by Fluorinated Hydrocarbons.

01,391

Experimental and Numerical Burning Rates of Premixed Methane-Air Inhibited by Fluoromethanes. PB95-180592

Inhibition of Premixed Methane-Air Flames by Halon Alter-

PB96-146741

01,414

RUS, $S$.

Analyzing Electronic Commerce.

PB94-219102

00,480

TSAI, $B$.

Resonance Enhanced Multiphoton Ionization Detection of $\mathrm{GeF}$ and $\mathrm{GeCl}$ Radicals.

PB94-212123

00,825

Comparison of Filter Radiometer Spectral Responsivity with the NIST Spectral-Irradiance and Illuminance Scales. the NIST Spect

04,162

TSAI, B. P.

Electronic Spectra of $\mathrm{CF} 2 \mathrm{Cl}$ and $\mathrm{CFCl} 2$ Radicals Observed by Resonance Enhanced Multiphoton Ionization.

00,927 B95-151023

Experimental and Abinitio Studies of Electronic Structures
of the CCI3 Radical and Cation.
PB94-212131
00,826 PB94-212131

Multiphoton Ionization of $\mathrm{SiH} 3$ and $\mathrm{SiD} 3$ Radicals: Elec-
tronic Spectra, Vibrational Analyses of the Ground and tronic Spectra, Vibrational Analyses of the Ground and
Rydberg States, and lonization Potential. Rydberg States
PB94-212503

00,837

Resonance Enhanced Multiphoton Ionization Spectroscopy of 2-Butene-1-yl $(\mathrm{C} 4 \mathrm{H} 7)$ between $455-485 \mathrm{~nm}$. PB95-151031

00,670

TSAI, T. M.

Certainty Grid to Object Boundary Algorithm.

01,835

\section{TSAI, V W.}

Increasing the Value of Atomic Force Microscopy Process Metrology Using a High-Accuracy Scanner, Tip Characterization, and Morphological Image Analysis.

PB96-190293

02,758

Progress Toward Accurate Metrology Using Atomic Force Microscopy.

02,417

TSANG, J. $\mathbf{W}$.

Characterization of Polyquinoline Blends Using Small Angle Scattering.

01,261

Characterization of Polyquinoline Block Copolymer Using Small Angle Scattering.

PB95-151882

01.244

TSANG, $W$.

Fluorinated Hydrocarbon Flame Suppression Chemistry,
PB94-185113

Homogeneous Gas Phase Decyclization of Tetralin and Benzocyclobutene.

PB95-151049

Hydrogen Atom Altack on Perchloroethylene.

00,928

PB95-163473

00,969

Incinerability of Perchloroethylene and Chlorobenzene.

PB95-163457

01,388

International Conference on Chemical Kinetics (2nd). Held in Gaithersburg, Maryland on July 24-27, 1989. PB94-211901

00,822

Mechanism and Rate Constants for the Reactions of Hydrogen Atoms with Isobutene at High Temperatures.

PBg-151064

Progress in the Development of a Chemical Kinetic Database for Combustion Chemistry.

01,384

Rate Constants for Hydrogem Alom Altack on Some Chlorinated Benzenes at High Temperature.

PB94.20058

00,810

Shock Tube Techniques in Chemical Kinetics.

PB95-163465

00,968

Thermochemical and Chemical Kinetic Data for Fluorinated Hydrocarbons.

01,056

TSAO, W.

Non-Osmotic, Defect-Controlled Cathodic Disbondment of a Coating from a Steel Substrate.

PB94-216447

03,120

TSENG, L. K.

Mixıng and Radiation Properties of Buoyant Turbulent DiffuSion Flames.

01,396

Radiation and Mixing Properties of Buoyant Turbulent Diffusion Flames.

PB94- 165974

01,360

Infrared and Microwave Spectroscopy of the Argon Propyne Dimer.

00,794

TSENG, $W$.

Influence of Lattice Mismatch on Indium Phosphide Based High Electron Mobility Transistor (HEMT) Structures Observed in High Resolution Monochromatic Synchrotron $X$ Radiation Diffraction Imaging.

PB95-164679

02,357

Integration of Scanning Tunneling Microscope Nanolithography and Electronics Device Processing.

PB95-153359

SENG, W. F.

Interdigitated Stacked P-I-N Multiple Quantum Well Modula-

PB97-112296

02,455

Junction Locations by Scanning Tunneling Microscopy: InAir-Ambient Investigation of Passivated GaAs pn Junctions. PB94-185964 02,306

Multicarrier Characterization Method for Extracting Mobilities and Carner Densities of Semiconductors from Variable Magnetic Field Measurements.

PB94-212776

02,317

Novel Magnetic Field Characterization Techniques for Compound Semiconductor Materials and Devices.

PB96-176458

Scaling of the Nonlinear Optical Cross Sections of GaAsAIGaAs Multiple Quantum-Well Hetero $n$-i-p-i's.

02,183

Vertical-Cavity Optoelectronic Structures: CAD, Growth, and Structural Characterization.

PB95-153177

02,148

Vertical-Cavity Semiconductor Structures: Materials Characterization.

TSONG, T. Y.

02,149

Energy Transduction between a Concentration Gradient and an Alternating Electric Field.

PB94-216363

03,461

TSONGAS, $\mathrm{G}$

Parametric Study of Wall Moisture Contents Using a Re-

vised Variable Indoor Relative Humidity Version of the 'Moist' Transient Heat and Moisture Transfer Model.

PB97-122535

TSONGAS, G. A.

Mathematical Analysis of Practices to Control Moisture in the Roof Cavities of Manufactured Houses.

PB97-106843

00,278

SUI, K. L.

Taguchi's Parameter Design: A Panel Discussion.

TSUJI, $H$.

Charpy Impact Test as an Evaluation of $4 \mathrm{~K}$ Fracture

Toughness.

TSUTSUMI, $N$.

Thermal Stability of Internal Electric Field and Polarization Distribution in Blend of Polyvinylidene Fluoride and Polymethylmethacrylate.

PB95-151072

01,240

TSVETKOV, $F$.

Small Angle Neutron Scattering Study of a Clay Suspension Under Shear.
PB96-167374

00,663

TU, K. M.

Wall Flame Heights with External Radiation. 
URIBE, R. M.

TURGEL, R. S.

$10 \mathrm{kV}$ DC Resistive Divider Calibration. PB95-198685

TURK, G. C.

Diode Laser as a Spectroscopic Tool

PB95-175485

Liquid Chromatography: Laser-Enhanced Ionization Spectrometry for the Speciation of Organolead Compounds.
PB94-185253

Spectral Interference in the Determination of Arsenic in High-Purity Lead and Lead-Base Alloys Using Electrothermal Atomic Absorption Spectrometry and Zeeman-Effect Background Correction.

Trace Detection in Conducting Solids Using Laser-Induced Fluorescence in a Cathodic Sputtering Cell.

TURNER, A. H.

Report of the National Conference on Weight and Measures (78th). Held in Kansas City, MO. on July 18-22, 1993. PB94-138989

02,623

Report of the National Conference on Weights and Measures (79th). Held in San Diego, California on July 17-21, 1994

02,656

Report of the National Conference on Weights and Meas. ures (80th) as Adopted by the 80th National Conference on Weights and Measures, 1995. Held in Portland, Maine on July 16-20, 1995.

PB96-165840

Basic Linear Algebra Operations in SLI Arithmetic.

Error-Bounding in Level-Index Computer Arithmetic

02,681

PB96-109582

03,421

MasPar MP.1 as a Computer Arithmetic Laboratory

MB95-189437

PB96-179155

01,742

Parallel and Serial Implementations of SLI Arithmetic. PB95-242335

TURNER, $\mathrm{S}$.

Airborne Asbestos Analysis: National Voluntary Laboratory Accreditation Program.

PB96-147392

02,566

Airbome Asbestos Method: Bootstrap Method for Determining the Uncertainty of Asbestos Concentration. Version 1.0. PB96-214614

00,646

Airborne Asbestos Method: Standard Practice for Recording Transmission Electron Microscopy Data for the Analysis of Asbestos Collected onto Filters. Version 1.0.

00,552

PB94-210168

Airborne Asbestos Method: Standard Test Method for High
Precision Counting of Asbestos Collected on Filters. VerPrecision Counting of Asbestos Collected on Filters. Ver-
sion 1.0 .
PB94-163003
00,525

Airborne Asbestos Method: Standard Test Method for Verified Analysis of Asbestos by Transmission Electron Microscopy. Version 2.0

00,526

Proficiency Tests for the NIST Airborne Asbestos Program, 1990.

00,535

PB94-188836

Proficiency Tests for the NIST Airborne Asbestos Program -

PB94-193828

Proficiency Tests for the NIST Airborne Asbestos Program 1992.

00,539

Proficiency Tests for the NIST Airborne Asbestos Program, 1993.

00,610

TURNER, T. R.

Gaseous Electronics Conference Radio-Frequency Reterence Cell: A Defined Parallel-Plate Radio-Frequency System for Experimental and Theoretical Studies of PlasmaProcessing Discharges.

PB94-172327

04,404

TWILLEY, W. H.

Effects of Specimen Edge Conditions on Heat Release Rate.

PB95-152864

00.375

In situ Burning of Oil Spills: Mesoscale Experiments and Analysis.

02,587

Santa Ana Fire Department Experiment at 1315 South Bristol, July 14, 1994

PB95-188868

00,389

Santa Ana Fire Department Experiment at 1315 South Bristol, July 14, 1994. (Reprint).

00,207

Santa Ana Fire Department Experiments at South Bristol

Sitreet.

00,305

Smoke Plume Trajectory from In situ Burning of Crude Oil in Alaska: Field Experiments.

02,594
TYLER, J. E.

Models, Managing Models, Quality Models: An Example of Quality Management PB94-163466

TYREE, $V$.

Realizing Suspended Structures on Chips Fabricated by CMOS Foundry Processes Through the MOSIS Service.

PB94-193984 Processes Through the MOSIS Service.
081

TZENG, Y.

Applications of Diamond Films and Related Materials: International Conference (3rd). Held in Gaithersburg, Maryland on August 21-24, 1995. Supplement to NIST Special Publi-

PB95-256053

03,063

Proceedings of the Applied Diamond Conference 1995: Applications of Diamond Films and Related Materials International Conterence (3rd). Held in Gaithersburg, Maryland on August 21-24, 1995

UDEN, P. C.

04,701

Investigations of Sulfur Interferences in the Extraction of Methylmercury from Marine Tissues.

PB96-190020

03,482

UDOVIC, T. J.

Characterization of the Interaction of Hydrogen with Iridium Clusters in Zeolites by Inelastic Neutron Scattering Spectroscopy.

01,013

Characterization of the Structure of LaD2.50 by Neutron

Powder Diffraction.

04,783

Characterization of the Structure of TbD2.25 at $70 \mathrm{~K}$ by Neutron Powder Diffraction.

PB96-160528

01,130

Characterization of the Structure of YD3 by Neutron Powder

PB96-186150

01,161

01,091

dral Sublattices of LaD2.25 and LaH2.25.

PB96- 123724

Dynamics of $\mathrm{Mu}(+)$ in $\mathrm{Sc}$ and $\mathrm{ScHx}$.

PB96-180021

04,112

Hydrogen in YBa2Cu3OX: A Neutron Spectroscopy and a Nuclear Magnetic Resonance Study.

04,601

Inelastic Neutron Scattering Studies of Nonlinear Optical Materials: p-Nitroaniline Adsorbed in ALPO-5.
PB95-107223 00,874

gen in

Nelastic Neutron

PB96-146857

03,366

Local-Mode Dynamics in YH2 and YD2 by Isotope-Dilution Neutron Spectroscopy.

PB95-181012

01,017

Low-Energy Vibrations and Octahedral Site Occupation in Nb95V5H(D)y.

01,133

Neutron and Raman Spectroscopies of 134 and 134a Hydrofluorocarbons Encaged in Na-X Zeolite.

03,001

Neutron-Powder-Diffraction Study of the Long-Range Order in the Octahedral Sublattice of LaD2.25.

04,753

PB96-141155

Neutron Spectroscopic Comparison of beta-Phase Rare Earth Hydrides.
PB96-160742

01,134

Neutron Spectroscopic Comparison of Rare-Earth/Hydrogen alpha-Phase Systems.

00,970

Neutron Spectroscopic Evidence of Concentration-Dependent Hydrogen Ordering in the Octahedral Sublattice of beta-

PB95-181020

01,018

Neutron-Spectroscopy Study of the Hydrogen Vibrations in Hydrogen-Doped

04,441

Vibrational Excitations and the Position of Hydrogen in Nanocrystalline Palladium.

04,035

Vibrations of Hydrogen and Deuterium in Solid Solution with

$\begin{array}{ll}\text { Lutetium. } & 01,019\end{array}$

UEDA, $K$.

Photoelectron Study of Electronic Autoionization in Rotationally Cooled N2: The $n=6$ Member of the Hopfield Series.

PB95-163531

00,971

UEDA, Y.

Thermal Stability of Intemal Electric Field and Polarization

Distribution in Blend of Polyvinylidene Fluoride and Polymethylmethacrylate.

PB95-151072

01,240

ANSKY, G. M.

Report of the National Conference on Weights and Measures (80th) as Adopted by the 80th National Conference on Weights and Measures, 1995. Held in Portland, Maine on July 16-20, 1995 .

02,681

UHAICH, P.

Preliminary Comparison of Time Transfers via LASSO, GPS and Two-Way Satellite.

ULBRECHT, J. J.

Metrology.
PB96.160759

ULLMANN, J. L.

Measurements of the $(235) \mathrm{U}(\mathrm{n}, \mathrm{f})$ Cross Section in the 3 to $30 \mathrm{MeV}$ Neutron Energy Region

PB97-11905

Comparison of Regular Transmittance Scales of Four Na-

tional Standardizing Laboratories.
PB94-211232

04,233

UNGER, $R$.

Positive and Negative Cooperativities at Subsequent Steps of Oxygenation Regulate the Allosteric Behavior of PB97-119374

UNGURIS, $J$

03,486 Influence of $\mathrm{Cr}$ Growth on Exchange Coupling in $\mathrm{Fe} / \mathrm{Cr}$ /

$\mathrm{Fe}(100)$
$\mathrm{PB} 95-150181$

04,562

Influence of Thickness Fluctuations on Exchange Coupling in $\mathrm{Fe} / \mathrm{Cr} / \mathrm{Fe}$ Structures.

PB96-135371

04,745

Magnetic Moments in $\mathrm{Cr}$ Thin Films on $\mathrm{Fe}(100)$

04,525

Oscillatory Exchange Coupling in $\mathrm{Fe} / \mathrm{Au} / \mathrm{Fe}(100)$
PB95-150371

04,569

Scanning Tunneling Microscopy Study of the Growth of $\mathrm{Cr} /$

$\mathrm{Fe}(001)$ : Correlation with Exchange Coupling of Magnetic Layers.

04,568

SEMPA Studies of Exchange Coupling in Magnetic Multilayers.
PB96-164074

04,780

SEMPA Studies of Oscillatory Exchange Coupling

PB95- 163556

04,625

Simple, Compact, High-Purity $\mathrm{Cr}$ Evaporator for Ultrahigh PBa4-216678

04,520

Surface Magnetic Microstructural Analysis Using Scanning

Electron Microscopy with Polarization Analysis (SEMPA).

PB95- 162657

03,938

UNTERWEGER, M. P.

Assay of the Eluent from the Alumina-Based Tungsten-188Rhen ium-188 Generator.

PB94-200482

03,829

Intercomparison of Internal Proportional Gas Counting of (85) $\mathrm{Kr}$ and (3) $\mathrm{H}$

International Marine-Atmospheric (222)Rn Measurement

Intercomparison in Bermuda. Part 1. NIST Calibration and

Methodology for Standardized Sample Additions.
PB96-175674

00,114

International Marine-Atmospheric (222)Rn Measurement Intercomparison in Bermuda. Part 2. Results for the Participating Laboratories.

Liquid-Scintillation Counting Techniques for the Standardization of Radionuclides Used in Therapy.

03,709 
USHA SARMA, P.

Rapid Method for the Isolation of Genomic DNA from 'Aspergillus fumigatus'

UTHE, E. E.

03,488

Smoke Plume Trajectory from In situ Buming of Crude Oil: Field Experiments.

PB96-200993

02.597

VADER, H. L.

Molar Absorptivities of Bilirubin (NIST SRM 916A) and Its Neutrał and Alkaline Azopigments.

PB94-211042

VAESSEN, $P$.

Approach to Setting Performance Requirements for Automated Evaluation of the Parameters of High-Voltage Impulses.

PB94-185634

01,878

VAEZI-NEJAD, H

Use of Building Emulators to Evaluate the Performance of Building Energy Management Systems.

VAJDA, F.

00,269

Experimental Verification of a Vector Preisach Model.

PB95-163564

04,626

VAJDA, J. N.

Vibrations of Hydrogen and Deuterium in Solid Solution with Lutetium.

01,019

VAJDA, P.

Neutron Spectroscopic Comparison of Rare-Earth/Hydrogen

alpha-Phase Systems

00,970

VALE, $L$.

Coexistence of Grains with Differing Orthorhombicity in High Ouality YBa2Cu307-delta Thin Films.

PB96-135033

04,742

VALE, L. R.

$30 \mathrm{THz}$ Mixing Experiments on High Temperature Superconducting Josephson Junctions.

Controlling the Critical Current Density of High-Temperature SNS Josephson Junctions.

04,794

Critical Current and Normal Resistance of High-Tc StepEdge SNS Junctions

04,724

Heterodyne Mixing and Direct Detection in High Temperature Josephson Junctions.

PB96-147202

High Current Pressure Contacis to $\mathrm{Ag}$ Pads on Thin Film Superconductors.

PB95-168621 04,639

High Temperature Superconductor-Normal MetalSuperconductor Josephson Junctions with High Characteristic Voltages.

04.666

Increased Transition Temperature in In situ Coevaporated YBa2Cu307-delta Thin Films by Low Temperature Post-An-

nealing.

04,672

Influence of Deposition Parameters on Properties of Laser Ablated YBa2Cu307-Delta Films.

04,544

Magnetic Flux Pinning in Epitaxial YBa2Cu3O7-delta Thin Film

PB96-200746

04,795

Microwave Noise in High-Tc Josephson Junctions.
PB96-148010

Mutual Phase Locking in Systems of High-Tc Superconductor-Normal Metal-Superconductor Junctions.
PB96-135348

Phase Locking in Two-Junction Systems of High-Temperature Superconductor-Norma! Metal-Superconductor Junctions.

02,060

Temperature Dependence and Magnetic Field Modulation of Critical Currents in Step-Edge SNS YBCO/Au Junctions.

PB96-111745 04,723

Thermal Noise in High-Temperature Superconducting-Normal-Superconducting Step-Edge Josephson Junctions. PB95-175089

VALENCIA-RODRIGUEZ, J.

04,650

Comparisons of Some NIST Fixed-Point Cells with Similar Cells of Other Standards Laboratories.

00,655

VALKIERS, $\mathbf{S}$.

New Values for Silicon Reference Materials, Certified for Isotope Abundance Ratios. Letter to the Editor. PB94-219268

VALLIKUL, P.

Tomographic Reconstruction of the Moments of Local Probability Density Functions in Turbulent Flow Fields. PB96-180195

VAMAN, D.

04,219

Comparison of FDDI Asynchronous Mode and DODB Oueue Arbitrated Mode Data Transmission for Metropolitan Area Nerwork Applications.

PB96-160452

01,498
Error Protecting Characteristics of CDMA and Impacts on Speech. PB96-122452

01,491

Provision of Isochronous Service on IEEE 802.6.

PB96-160635

01,501

VAMLING, L.

Need for, and Availability of, Working Fluid Property Data: Results from Annexes XIII and XVIII.

PB95-168969

VAN BRUNT, R. J.

03,268 Appearance Potentials of lons Produced by Electron-Impact
Induced Dissociative Ionization of SF6, SF4, SF5Cl, S2F10, $\mathrm{SO} 2, \mathrm{SO}_{2} 2$, SOF2, and SOF4.

01,080

Associative Electron Altachment to S2F10, S2OF10, and $\mathrm{S} 2 \mathrm{O} 2 \mathrm{~F} 10$.

00,907

PB95-140992

Behavior of Suriace Partial Discharge on Aluminum Oxide PB96-12378

01,937 Continuous Recording and Stochastic Analysis of PD.
PB $96-112156$

Correlations between Electrical and Acoustic Detection of
Partial Discharge in Liquids and Implications for Continuous Data Recording

02,248

Decomposition of SF6 and Production of S2F10 in Power Arcs.

PB96-122619

01,084

Decomposition

01,095

Effect of Electrode Material on Measured Ion Energy Distributions in Radio-Frequency Discharges.

PB96-102850

04,026

Electron Scattering and Dissociative Attachment by SF6 and Its Electrical-Discharge By-Products.

02,256

Evidence for Inelastic Processes for $\mathrm{N}(+) 3$ and $\mathrm{N}(+) 4$ from Ion Energy Distributions in He/N2 Radio Frequency Glow Discharges.
PB96-146683

04,059

Fundamental Processes in Gas Discharges.

01,089

PB96-123450

Gaseous Dielectrics Research: Possible SF6 Substitutes.
PB96-119268

Gaseous Electronics Conference Radio-Frequency Reference Cell: A Defined Parallel-Plate Radio-Frequency System for Experimental and Theoretical Studies of Plasma-
Processing Discharges. Processing Discharges.

Importance of Unraveling Memory Propagation Effects in Interpreting Data on Partial Discharge Statistics.

01,901

Influence of Electrode Material on Measured Ion Kinetic-Energy Distributions in Radio-Frequency Discharges.
PB96-123179

Influence of Surface Charge on the Stochastic Behavior of

Partial Discharge in Dielectrics.
PB96-122767

01,931

Investigation of $\mathrm{S}_{2} \mathrm{~F}_{10}$ Production and Mitigation in Compressed SF6-In

02,467

Investigation of $\mathrm{S}_{2} \mathrm{~F}_{10}$ Production and Mitigation in Com. pressed SF6- Insulated Power Systems.

PB96-155528

02,468

Ion Kinetic-Energy Distributions and Balmer-alpha (Halpha) Excitation in Ar-H2 Radio-Frequency Discharges.

Ion Kinetic-Energy Distributions in Argon if Glow Dis-
charges.
PB95-141008
04,409

PB95-141008

Ion Kinetics and Symmetric Charge-Transfer Collisions in Low-Current, Diffuse (Townsend) Discharges in Argon and PB96-123658

04,051

Kinetic Energy Distribution of lons Produced from TownSend Discharges in Neon and Argon.

04,413

Kinetic Energy Distributions of $\mathrm{H}(+), \mathrm{H} 2(+)$, and $\mathrm{H} 3(+)$ from a Diffuse Town

04,415

Kinetic-Energy Distributions of Ions Sampled from Argon Plasmas in a Parallel-Plate, Radio-Frequency Reference

PB95-161964

03,935

Kinetic-Energy Distributions of Ions Sampled from RadioFrequency Discharges in Helium, Nitrogen, and Oxygen.
PB96-123732

01,092 Kinetic-Energy Distributio

PB95-151122

03,902

Measurement of S2OF10, and S2O2F10 Production Rates from Spark and Negative Glow Corona Discharge in SF6. $\mathrm{O} 2$ Gas Mixtures.

PB96-123740

01,093

Modification of Cast Epoxy Resin Surfaces during Exposure to Partial Discharges

01,086

Nonstationary Behavior of Partial Discharge dunng Insulation Aging.

01,902

Nonstationary Behaviour of Partial Discharge during Discharge Induced Ageing of Dielectrics.

PB96-103114

01,922

Optical and Mass Spectrometric Investigations of lons and Neutral Species in SF6 Radio-Frequency Discharges.
PB97-111918 01,985 Partial Discharge: Induced Aging of Cast Epoxies and Related Nonstationary Behavior of the Discharge Statistics. PB95-163598

Physics and Chemistry of Partial Discharge and Corona: Recent Advances and Future Challenges.

01,910

Physics and Chemistry of Partial Discharge and Corona Recent Advances and Future Challenges.

PB96-123757

01,936

Plasma Chemical Model for Decomposition of SF6 in a Negative Glow Corona Discharge.

PB95-181053

01,020

Procedure for Measuring Trace Quantities of S2F10, S2OF10, and S2O2F10 in SF6 Using a Gas Chromatograph-Mass Spectrometer.

PB96-119755

02,513

Recent Developments at NIST on Optical Current Sensors and Partial Discharge Diagnostics.

PB95-151114

02,147

SF6 Insulation: Possible Greenhouse Problems and Solu-

lions.

02,269

SF6/N2 Mixtures: Basic and High-Voltage-Insulation Prop-

PB96-123468

02,230

Studies of Ion Kinetic-Energy Distributions in the Gaseous Electronics Conference RF Reference Cell.

02,391

Use of an Ion Energy Analyzer: Mass Spectrometer to Measure Ion Kinetic-Energy Distributions from RF Discharges in Argon-Helium Gas Mixtures.

PB94-185717

04,406

VAN CLARK, A.

Noncontact Ultrasonic Inspection of Train Rails for Stress. PB95-162673 04,851

列 ion System.

VAN DE ZANDE, $R$.

Standards Setting in the European Union: Standards Orga-

PB96-115019

02,919

VAN DEGRIFT, $\mathrm{C}$

Report on the Workshop on Advanced Digital Video in the National Information Intrastructure. Held in Washington, D.C. on May 10-11, 1994.

PB95-103677

01,472

Summary Report on the Workshop on Advanced Digital Video in the National Information Infrastructure. PB96-141320

01,497

VAN DEGRIFT, C. T.

Anomalous Behavior of a Ouantized Hall Plateau in a HighMobility Si Metal-Oxide-Semiconductor Field-Effect Transistor. 
VAN LEEUWEN, $F$.

beta-D-Glucosyl-Hydroxymethyluracil: A Novel Modified Base Present in the DNA of the Parastic Protozoan T. PBucei.

03,524

VAN PELT, A.

Critical Scaling Laws and a Classical Equation of State PB95-169249

VAN POOLEN, L. J.

Coexisting Densities, Vapor Pressures and Critical Densities of Refrigerants R-32 and R-152a, at $300-385 \mathrm{~K}$. PB95-175691 03,274

Experimental Method for Obtaining Critical Densities of Binary Mixtures: Application to Ethane $+n$-Butane

00,930

Vapor-Liquid Equilibria of Ternary Mixtures in the Critical Region on Paths of Constant Temperature and Overall

CBmposition.

01,139

VAN ROGGEN, A.

Effect of Electrode-Polymer Interfacial Layers on Polymer Conduction. Part 2. Device Summary.

PB95-151155

02,335

VAN ZANTEN, J. $\mathbf{H}$.

Influence of an Impenetrable Interface on a Polymer GlassTransition Temperature.

03,175

Terminally Anchored Chain Interphases: The Effect of Multicomponent, Polydisperse Solvents on Their Equilibrium Properties

PB95-181079

01,273

Terminally Anchored Chain Interphases: Their Chromatographic Properties.
PB95-181061 01,272

Zimm Plot and Its Analogs as Indicators of Vesicle and Micelle Size Polydispersity.

PB96-123765

01,094

Laser Bandwidth Effects in Quantitative Cavity Ring-Down Spectroscopy.

VANBRONKHORST, D. A.

Workplan to Analyze the Energy Impacts of Envelope Airtightness in Office Buildings.

PB96-154463

00,273

VANBRUNT, R. J.

Performance Evaluation of a New Digital Partial Discharge Recording and Analysis System.

PB95-150389

01,888

VANDER SANDE, J. $B$.

Effects of Anneal Time and Cooling Rate on the Formation and Texture of $\mathrm{Bi} 2 \mathrm{Sr} 2 \mathrm{CaCu} 2 \mathrm{O} 8$ Films.

PB95-161600

04,603

VANDERAH, T. A.

Magnetic Dielectric Oxides: Subsolidus Phase Relations in the BaO: Fe2O3: TiO2 System.

PB96-176524

01,156

Preparation and Crystal Structure of Sr5TiNb4017.
PB96-167341 PB96-167341 04,107 Preparation, Crystal Structure, Dielectric Properties, and
Magnetic Behavior of Ba2Fe2Ti4O13.

PB96-186176

01,162

VANDERHART, D. L.

Binder Characterization and Evaluation by Nuclear Magnetic Resonance Spectroscopy.

PB94.193471

01,334 Evidence of Crosslinking in Methyl Pendent PBZT Fiber.
PB97-112486

03,393

Solid State (13)C NMR and Raman Studies of Cellulose

Triacetate: Oligomers, Polymorphism, and Inferences about Chain Polarity

VANDERWEGE, B. A.

01,289

Effect of CF3H and $\mathrm{CF} 3 \mathrm{Br}$ on Laminar Diffusion Flames in Normal and Microgravity.

01,420

Effect of CF3H and CF3Br on Laminar Diffusion Flames in Normal and Microgravity.

PB96-161849

01,421

VANDERZWAAG, $D$.

Escherichis coli Cyclic AMP Receptor Protein Mutants Provide Evidence for Ligand Contacts Important in Activation. PB96-201017

03,592

VANDSBURGER, $U$.

Compartment Fire Combustion Dynamics. Annual Report, September 1, 1993-September 1, 1994

00,203

Dynamics, Transport and Chemical Kinetics of Compartment Fire Exhaust Gases.

PB96-195508

00,229

VANGEL, M. G.

Anova Estimates of Variance Components for a Class of Mixed Models.
PB96-141163

One-Sided beta-Content Tolerance Intervals for Mixed Models.

03,449
Performance of Tape-Bonded Seams of EPDM Membranes: Comparison of the Peel Creep-Rupture Response of TapeBonded and Liquid-Adhesive-Bonded Seams.

PB96-183249

03,012

VANIER, $\mathrm{J}$.

Aging, Warm-Up Time and Retrace; Important Characteristics of Standard Frequency Generators.

PB96-103122

04,031

VANMEURS, D. P.

Neutron Scattering Study of the Lattice Modes of Solid

Cubane.
PB96-147152

01,126

VANSTEENKISTE, $N$.

Frequency-Stabilized LNA Laser at 1.083 mum: Application to the Manipulation of Helium 4 Aloms.

PB95-176186

04,304

VANZURA, E. J.

NIST 60-Millimeter Diameter Cylindrical Cavity Resonator Performance Evaluation for Permittivity Measurements.
PB94-151776 251

VARBERG, T. D.

Detection of $\mathrm{OH}+$ in Its a(1)Delta State by Far Infrared Laser Magnetic Resonance.

PB95-181087

01,021

Laser Spectroscopy of Carbon Monoxide: A Frequency Reference for the Far Infrared.

PB95-163606

04,277

Pure Rotational Spectra of CuH and CuD in Their Ground States Measured by Tunable Far-infrared Spectroscopy.
PB95-176194

01,005

Rotational Spectrum of $\mathrm{OH}$ in the $\mathrm{v}=0-3$ Levels of its Ground State.

01,006

VARGAS-ABURTO, $\mathrm{C}$

Electron and Proton Dosimetry with Custom-Developed Radiochromic Dye Films.

PB95-151106

03,713

VARMA, D. S.

Copolymerization of N-Phenyl Maleimide and gamma-

Methacryloxypropyl Trimethoxysilane.

01,248

Thermal Behavior of 4-Maleimidophenyl Glycidyl Ether Res. PB95-153151

01,249

Thermal Behaviour of Methyl Methacrylate and N-Pheny Maleimide Copolymers.

PB95-152237

01,246

VARMA, I. K.

Copolymerization of N-Phenyl Maleimide and gammaMethacryloxypropyl Trimethoxysilane.

01,248

Thermal Behavior of 4-Maleimidophenyl Glycidyl Ether Res-

PB95-153151

01,249

Thermal Behaviour of Methyl Methacrylate and N-Phenyl Maleimide Copolymers.

01,246

VARVOGLIS, $P$.

Rotational Modulation and Flares on RS Canum Venaticorum and BY Draconis Stars. XVI. IUE Spectros copy and VLA Observations of C1182( $=\mathrm{V} 1005$ Orionis) in PB94-185626

VASCONCELLOS, E. C. C.

Far Infrared Laser Frequencies of (13) $\mathrm{CD} 3 \mathrm{OH}$. B95-169363

00,050

ASILIU-DOLOC,

High-Energy Phonon Dispersion in La 1.85Sro.15CuO4. PB96-138458

VAUDIN, $M$

Characterization of Phase and Surface Composition of Silicon Carbide Platelets.

PB94.216264

VAUDIN, M. D.

Ceramic Characterization

DE94013170

03,043

Electromagnetic Coupling Character of (001) Twist Bound aries in Sintered Bi2Sr2CaCu2O8+x Bicrystals.

Epitaxial Growth of $\mathrm{BaTiO} 3$ Thin Films at $600 \mathrm{C}$ by Metalorganic Chemical Vapor Deposition.

03,071

Experimental Assessment of Crack-Tip Dislocation Emission Models for an Al67Cr8Ti25 Intermetallic Alloy.

Sion Models for

03,377

Texture Measurement of Sintered Alumina Using the March-Dollase Function.

PB96-179494

04,784

VAUGHAN, G. B. M.

Discontinuous Volume Change at the Orientational-Ordering Transition in Solid $\mathrm{C} 60$.

PB94-211828

00,821

VAYSHENKER, I.

Accurate Measurement of Optical Detector Nonlinearity.
PB95-203576

Automated Measurement of Nonlinearity of Optical Fiber Power Meters

04,110

Optical Detector Nonlinearity: A Comparison of Five Methods.

PB95-169355

04,291

Optical Detector Nonlinearity: Simulation.

Optical Power Meter Calibration Using Tunable Laser Di-

PBes. 169256

04,290

AZQUEZ, I.

Suppression of Elevated Temperature Hydraulic Fluid and JP-8 Spray Flames

PB95-181095

00,021

ALE, A.

Rotational Modulation and Flares on RS Canum Venaticorum and BY Draconis Stars. XVIII. Coordinated VLA, ROSAT, and IUE Observations of RS CVn Binaries.
PB96-102322

VEALE, $R$.

Dimensional Characterization of Small Bores: A Survey.
PB95-162202 Displacement Method for Machine Geometry Calibration. PB95-152088 02,946 Variations in Size Measurements by Indicating Gaging Systems.

B95.16361

02,864

VEALE, R. C.

U.S. Navy Coordinate Measuring Machines: A Study of Needs.

PB94-162831

02,807

VEASEY, D. L

Distributed Feedback Lasers in Rare-Earth-Doped PhosPhate Glass.

Glasses for Waveguide Lasers.

PB96-111950

04,740 Glass.

PB97.113179

Passively Q-Switched Nd-Doped Waveguide Laser.
PB95-180048

Waveguide Polarizers Processed by Localized Plasma Etching.

PB95-169264

02,171

VECCHIA, D. F

Moments of the Quartic Assignment Statistic with an Application to Multiple Regression.

PB95-181103

03,442

Optical Fiber Geometry by Gray-Scale Analysis with Robust Regression.
PB95.161519

04,272

Outlier-Resistant Methods for Estimation and Model Fitting PB95-203436

VEERAPANDIAN, $B$

Crystal Packing Interactions of Two Different Crystal Forms of Bovine Ribonuclease A

PB95-152823

00,943

EEIGL, I.

Waveguide Polarizers Processed by Localized Plasma Etching.

PB95-169264 
VENTURELLI, J.

Regulation of Lithium and Boron Levels in Normal Human Blood: Environmental and Genetic Considerations.

PB94-198579

03,491

VENZ, $S$.

Modified Surface-Active Monomers for Adhesive Bonding to

PB95-151163

00,158

NIR-Spectroscopic Investigation of Water Sorption Characteristics of Dental Resins and Composites.

00,189

VERDEYEN, J. T.

Gaseous Electronics Conference Radio-Frequency Reference Cell: A Defined Parallel-Plate Radio-Frequency System for Experimental and Theoretical Studies of Plasma. Processing Discharges.

PB94-172327

04,404

VERDIER, P. $\mathbf{H}$.

Effects of Variable Excluded Volume on the Dimensions of Off-Lattice Polymer Chains.

PB94-21294

01,229

VERDONK, $J$

Problems Related to the Determination of Mass Densities of Evaporated Reference Deposits.

PB95-163226

03,941

VERES, G.

Atomic Transition Probabilities and Tests of the Spectroscopic Coupling Scheme for NI.

04,057

VERKOUTEREN, J.

National Voluntary Laboratory Accreditation Program: Bulk Asbestos Analysis.

PB95-138129

02,541

VERKOUTEREN, J. R.

Guidelines for Refractive Index Measurements of Asbestos. PB95-151189

02,543

VERKOUTEREN, R. $M$

Comparative Study of $\mathrm{Fe}-\mathrm{C}$ Bead and Graphite Target Performance with the National Ocean Sciences AMS (NOSAMS) Facility Recombinator Ion Source.

PB95-175790

00,693

Factorial Design Techniques Applied to Optimization of AMS Graphite Target Preparation.

VESOVIC, $V$.

Viscosity of Ammonia.

PB96-145933

00,584

EST, R. E.

Stable Silicon Photodiodes for Absolute Intensity Measurements in the VUV and Soft X-ray Regions.

PB97-110175

04,135

VETTER, T. W

Development of the Ion Exchange-Gravimetric Method for Sodium in Serum as a Definitive Method.

PB96-179148

01,867

VETTORI, R. L.

Sprinkler Fire Suppression Algorithm

PB94-216181

00,293

VIADAR, A. E.

$X$-ray Mask Metrology: The Development of Linewidth Standards for X-ray Lithography.

PB95-162129

02,348

VIEHLAND, L. A.

Absence of Ouantum-Mechanical Effects on the Mobility of Argon Ions in Helium Gas at $4.35 \mathrm{~K}$

PB97-122543

01,194

VIG, J. R.

Fundamental Limits on the Frequency Stabilities of Crystal Oscillators.
PB96-176565

02,277

VIGLIANTE, A.

Epitaxial Growth of $\mathrm{Sb} / \mathrm{GaSb}$ Structures: An Example of V/ III-V Heteroepitaxy.

PB95.202560

04,693

Observation of Two Length Scales Above ( $T$ sub $N$ ) in a Holmium Thin Film

PB97-111942

04,151

VIGLIOTTI, D. P.

Effect of Charpy V-Notch Striker Radii on the Absorbed EnPrgy. 141122

03,365

VIGUE, J.

Laser Assisted Collisions at Ultracold Temperatures.
PB95-161220

Theory of Atomic Collisions at Ultracold Temperatures.

PB94-212560

VILGIS, T. A.

Electrostatic Rigidity of Polyelectrolytes from Reparametrization Invariance.

PB96-180062

04,113

VILHU, 0 .

IUE Observations of Solar-Type Stars in the Pleiades and the Hyades.
PB94-199437

00.053
VILLA, $K$.

Influence of Ignition Source on the Flaming Fire Hazard of Upholstered Furniture. (NIST Reprint).

PB95-180162

00,297

VILLA, K. M

Quantifying the Ignition Propensity of Cigarettes

PB96-155411

VILLAGRAN, E. S.

Standardization of Testing Methods for Optical Disk Media Characteristics and Related Activities at NIST.

PB95-108486

01,624

LARRUBIA, J. S.

Electrical Test Structures Replicated in Silicon-on-Insulator

7-111827

02,454

Increasing the Value of Atomic Force Microscopy Process

Metrology Using a High-Accuracy Scanner, Tip Characterization, and Morphological Image Analysis.

02,758

Metrology Standards for Advanced Semiconductor Lithography Referenced to Atomic Spacings and Geometry.
PB96-160718 424

Morphological Estimation of Tip Geometry for Scanned Probe Microscopy.

PB95-203444

02,662

Progress Toward Accurate Metrology Using Atomic Force Microscopy.

02,417

Scanned Probe Microscope Tip Characterization Without Calibrated Tip Characterizers.

PB96-190368

02,759

VILLARS, $P$.

CRYSTMET: The NRCC Metals Crystallographic Data File.
PB97-109029 VINCENT, D. H.

Cold Neutron Prompt Gamma Activation Analysis at NIST A Progress Report.

PB95-175964

00,602

Measuring Hydrogen by Cold-Neutron Prompt-Gamma Activation Analysis.

00,612

Neutron Capture Prompt Gamma-Ray Activation Analysis at the NIST Cold Neutron Research Facility.

PB94-213394

00,556

VINING, G. G.

Taguchi's Parameter Design: A Panel Discussion.

PB96-111802

03,445

VINK, K. L. J.

Molar Absorptivities of Bilirubin (NIST SRM 916A) and Its Neutral and A

VIRSHUP, G. F

Stacked Series Arrays of High-Tc Trilayer Josephson Junctions.

PB96-102272

04,705

Step-Edge and Stacked-Heterostructure High-Tc Josephson Junctions for Voltage-Standard Arrays.

PB96-102066

04,702

Trace Elements Associated with Proteins. Neutron Activa-

Trace Elements Associated with Proteins. Neutron Activa-
tion Analysis Combined with Biological Isolation Techniques. PB95-163101

VLADAR, A. E.

00,597

Modification of a Commercial SEM with a Computer Conrolled Cathode Stabilized Power Supply.

PB96-201066

04,129 Monte Carlo Model for SEM Linewidth Metrology.
PB95-150058

Report on the NIST Low Accelerating Voltage SEM Mag-
nification Standard Interlaboratory Study.

PB96-201074

Scanning Electron Microscope Magnification Calibration Interlaboratory Study.

PB96-201082

01,164

SEM Linewidth Metrology of X-ray Lithography Masks. PB96-201108

VLASOV, L. V.

Precision High Temperature Blackbodies.

PB95-140059

02,447

VLIEGENTHART, J.

03,885

beta-D-Glucosyl-Hydroxymethyluracil: A Novel Modified Base Present in the DNA of the Parastic Protozoan T.

PB94-172319

VOCCIO, J.P.

Effect of Magnetic Field Orientation on the Critical Current of HTS Conductor and Coils.

PB96-141189

02,956

Certification of Standard Reference Material (SRM) 1941a, Organics in Marine Sediment.

PB96-123690

02,593

Determination of Sulfur in Fossil Fuels by Isotope Dilution Thermal lonization Mass Spectrometry.

02,495

VOGEL, $\mathrm{E}$.

One-Electron Oxidation of Metalloporphycenes as Studied by Radiolytic Methods.

PB97-111967

Status of the Round Robin on the Transport Properties of

PB96- 167218

01,152

Viscosity of Ammonia

PB96-145933

01,117

VOGEL, G.

Fluoride Analytical Methods

PB96-180237

03,578

VOGEL, G. L.

Distribution of Fluoride in Saliva and Plaque Fluid After a $0.048 \mathrm{~mol} / \mathrm{L} \mathrm{NaF}$ Rinse.

PB95-151205

VOGL, T.P.

Second Census Optical Character Recognition Systems Conterence.
PB94-188711

01,832

VOLK, C. $M$.

Analytıcal Estimation of Carrier Multipath Bias on GPS Position Measurements.

VOLK, S. R.

04,845

Application of the Modified Voltage-Dividing Potentiometer to Overlay Metrology in a CMOS/Bulk Process.

PB94-181997

VON GLAHN, $P$.

Behavior of Surface Partial Discharge on Aluminum Oxide Dielectrics.

PB96-123781

01,937

Comment and Discussion on Digital Processing of PD

Pulses.

PB96-122775

01,932

Continuous R

01,925

Correlations between Electrical and Acoustic Detection of

Partial Discharge in Liquids and Implications for Continuous Data Recording.

PB96-204490

02,248

Importance of Unraveling Memory Propagation Effects in In-

terpreting Data on Partial Discharge Statistics.

PB95-163572

01,901

Influence of Surface Charge on the Stochastic Behavior of Partial Discharge in Dielectrics.

PB96-122767

01,931

Nonstationary Behavior of Partial Discharge during Insula-

tion Aging.

01,902

Nonstationary Behaviour of Partial Discharge during Discharge Induced Ageing of Dielectrics.

PB96-103114

01,922

Partial Discharge: Induced Aging of Cast Epoxies and Related Nonstationary Behavior of the Discharge Statistics. PB95-163598

01,903

Performance Evaluation of a New Digital Partial Discharge Recording and Analysis System.

01,888

Standardised Computer Data File Format for Storage.

Transport, and Off-Line Processing of Partial Discharge Data. 
Light Scattered by Coated Paper. PB94-216546

04,245

Light Scattering by Sinusoidal Surfaces: Illumination Windows and Harmonics in Standards.

04,387

04,242

Light Scattering

Measurement and Uncertainty of a Calibration Standard for
the Scanning Electron Microscope.
PB94-219250
PB,560

PB94-219250 00,560

Metrology Approach to Unifying Rockwell C Hardness
Scales.

02,957

Microform Calibration Uncertainties of Rockwell Diamond Indenters.

PB96-122114

03,280

Microform Calibrations in Surface Metrology

02,951

Model of an Optical Roughness-Measuring Instrument.

PB97-110209 04,384

Optical Scattering from Moderately Rough Surfaces.
PB97-110415

Post-Process Control of Machine Tools

PB95-203451

02,952

Present and Future Standard Specimens for Surface Finish

Metrology.
PB97-110423

02,928

Regimes of Surlace Roughness Measurable with Light

Scattering.
PB95-151213

04,265

Sinusoidal Surfaces as Standards for BRDF instruments.

PB97-110597 04,388

Stylus Flight in Surface Profiling.

02,675

Stylus Technique for the Direct Verification of Rockwell Diamond Intenders.

PB96-155569

02,958

Surface Texture

03,351

Upgraded Facility for Multilayer Mirror Characterization at NIST.

PB96-160387

04,367

Windowing Effects on Light Scattering by Sinusoidal Sur-

taces.

04,389

VORIS, P. G.

Coaxial Reference Standard for Microwave Power.
PB94-193786

VOTAVA, 0 .

High-Efficiency, High-Power Difference-Frequency Generation of $0.9-1.5 \mathrm{mu} \mathrm{m}$ Light in BBO.

04,317

Photodissociation Dynamics in Ouantum State-Selected Clusters: A Test of the One-Atom Cage Effect in Ar-H2O.

PB95-203121

01,044

VSEVOLODOV, N. N.

Holographic Properties of Triton X-100-Treated Bacteriorhodopsin Embedded in Gelatin Films.
PB96-119284 Optical Properties of Triton X-100-Treated Purple Membranes Embedded in Gelatin Films. PB96-123500

03,546

Retinal-Protein Complexes as Optoelectronic Components.
PB95-150397

02,146

VYAS, R.

Resonance Fluorescence with Squeezed-Light Excitation

PB95-203469

04,322

VYDYANATH, H. R.

Correlation of $\mathrm{HgCdTe}$ Epilayer Defects with Underlying Substrate Defects by Synchrotron X-Ray Topography.
PB94-200714 129

02,129

Security in Open Systems.
PB95-105383

WACK, J. P.

Computer Virus Attacks.

PB95-163655

01,715

Keeping Your Site Comfortably Secure: An Introduction to Internet Firewalis

PB95-182275

02,730

WADA, $\mathrm{H}$.

Critical-Current Degradation in Nb3 Al Wires Due to Axial

and Transverse Stress.
PB95-202784

$\mathrm{n}-\mathrm{Value}$ and Second Derivative of the Superconductor Volt-

age-Current Characteristic

PB95.126223

04,533

VAMAS Intercomparison of Critical Current Measurements on NB3Sn Superconductors: A Summary Report.

PB96-119763

04,043

WADAS, A.

dc Magnetic Force Microscopy Imaging of Thin-Film Recording Head

04,665
Magnetic Force Microscopy Images of Magnetic Garnet with Thin-Film Magnetic Tip.

PB95-176210

04,669

Magnetic Force Microscopy of Flux in Superconductors.

PB95-161733

Recent Results in Magnetic Force Microscopy.

PB96-103130

04,608

WADLEY, H. N. G.

Leady Axisymmetric Modes in Infinite Clad Rods. Part 1

PB95-162905

04,187

Ultrasonic Method for Reconstructing the Two-Dimensional Liquid-Solid Interface in Solidifying Bodies.

PB95-161782

03,349

WAGNER, R. $P$.

Determination of Acoustic Center Correction for Type LS2aP Condensor Microphones.

04,190

\section{WAGNER, W.}

International Equations for the Saturation Properties of Ordinary Water Substance. Revised According to the International Temperature Scale of 1990 . Addendum to Journal of Physical and Chemical Reference Data 16, 893 (1987).

of Physical an

WAGSHUL, $M$.

Hypertine Effects and Associative lonization of Ultracold So-

PB95-151221

03,903

WAHL, D. C.

Standards and Linkages: What Data Sharing Needs.

PB95-161881

01,713

WAHLGREN, G. $M$.

First Results from the Goddard High-Resolution Spectrograph: The Chromosphere of Taur

00,054

GHAS Observations of Cool, Low-Gravity Stars. 1. The FarUltraviolet Spectrum of alpha Orions (M2 lab).

PB96-112016

00,094

\section{WAHLIN, E. K.}

Electron-Impact Excitation of Si(3+)(3S yields 3P) Using a Merged-Beam

03,904

Merged-Beams Energy-Loss Technique for Electron-Ion Excitation: Absolute Total Cross Sections for $O(5+)(2 s$ yields 2p).

PB96-102058

04,017

WAIT, D. F.

Comparison of Three Techniques for the Precision Measurement of Amplifier Noise.

PB95-163663

02,349

Measurement Accuracies for Various Techniques for Measuring Amplifier Noise.

PB95-163671

02,350

Radiometer Equation for Noise Comparison Radiometers.

PB96-140363 02,195

Relative Accuracy of Isolated and Unisolated Noise Comparison Radiometers.

PB96-111851

01,924

Terminal Invariant Description of Amplifier Noise. PB95-153573

02,043

WAKEHAM, W. A.

Standard Reference Data for the Thermal Conductivity of Water.
PB96-145875

01,111

Status of the Round Robin on the Transport Properties of R134a.

PB96-167218

01,152

Transient Methods for Thermal Conductivity

PB94-198405

04,197

iscosity of Ammonia.

96-145933

01,117

VAKID, S.

Application Profile for ISDN

PB95-163689

01,479

Comparison of FDDI Asynchronous Mode and DODB Oueve Arbitrated Mode Data Transmission for Metropolitan Area Network Applications.

PB96-160452

01,498

Error Protecting Characteristics of CDMA and Impacts on

PB96-122452

01,491

Hardware Measurement Techniques for High-Speed Net-

works.

PB96-160551

01,500

Introduction to

PB94-142494

SDN.

ISDN in North America.

PB96-160767

01,464

Provision of Isoc

01502

PB96-160635

01,501

Standardization for ATM and Related B-ISDN Technologies. PB96-160460

01,499

WAKID, S. A.

North American Agreements on ISDN

PB96-160775

WALBA, D. M.

Studies of the Higher Order Smectic Phase of the Large Electroclinic Effect Material W317.

PB95-151601

00,935

$X$-ray Observation of Electroclinic Layer Constriction and Rearrangement in a Chiral Smectic-A Liquid Crystal.

PB96-141080

Bacteriorhodopsin Immobilized in Sol-Gel Glass. PB95-151429

WALDOW, D. A.

Anisotropic Phase Separation Kinetics in a Polymer Blend Solution Following Cessation of Shear Studied by Light PB95-151247

WALHOUT, $M$.

Improved Dose Metrology in Optical Lithography.

PB96-179510

01,241

Magneto-Optical Trapping of Metastable Xenon: IsotopeShith Measurements

PB95-151254

03,905

sigmat

PB95-161840

03,934

WALKER, $B$.

Genetically Engineered Pore as a Metal Ion Biosensor.

PB96-161658

03,551

Genetically Engineered Pores as Metal Ion Biosensors.

Genetically Engineered Pores for New Materıals.

03,553

PB96-161641

03,550

Intensity-Dependent Scattering Rings in High Order AboveThreshold lonization.

6-110739

Pore-Forming Protein with a Metal-Actuated Switch.

WALKER, D. K.

04,032

Planar Resistors for Probe Station Calibration PB95-163697

Proposed High-Acc

Millimeter Waves

WALKER, J. A

Hydrogen Atom Attack on Perchloroethylene

PB95-163473

Mechanism and Rate Constants for the Reactions of gen Atoms with Isobutene at High Temperatures. PB95-151064

WALKER, J. H.

Improved Autom

WALKER, M. D.

Noninvasive High-Voltage Measurement in Mammography by Crystal Diffraction Spectroscopy

PB95-153417

00,160

Role of the Office of Radiation Measurement in Ouality Assurance.

PB94-212255

00,689

WALKER, M. L.

Alcohol Solutions of Triphenyl-Tetrazolium Chloride as 


\section{PERSONAL AUTHOR INDEX}

Experimental Models for Software Diagnosis. PB97-113906

01,783

Framework for the Development and Assurance of High Integrity Software.

01,716

High Integrity Software Standards Activities at NIST

PB96-112214

01,744

Object-Oriented Technology Research Areas.

PB95-199329

01,726

Perspective on Software Engineering Standards.

PB95. 171377

01,811

Proceedings of the Digital Systems Reliability and Nuclear Safety Workshop. Held in Rockville, Maryland on SeptemDURE 14, 1993.

03,728

Ouality Characteristics and Metrics for Reusable Software (Preliminary Report).

PB94-203437

01,693

Reference Information for the Software Veritication and Validation Process.

PB96-188164 01,773 Report of a Workshop on the Assurance of High Integrity
Software.

PB96-161377

01,763

Report on the Advanced Sottware Technology Workshop. Held on February 1, 1994

PB95-136610

01,707

SOA Standards

01,743

Standards for High integrity Software.

PB96-161385

01,764

Structured Testing: A Testing Methodology Using the

Cyclomatic Complexity Metric.
PB97-114169

Study on Hazard Analysis in High Integrity Sottware Standards and Guidelines.

PB95-198727

01,725

Unravel: A CASE Tool to Assist Evaluation of High Integrity Software. Volume 1. Requirements and Design.

01,736

Unravel: A CASE Tool to Assist Evaluation of High Integrity Software. Volume 2. User Manual

PB95-267894

01,737

Verification and Validation.

PB96-161393

01,765

Verification and Validation of Reengineered Software.

PB96-161401

01,766

WALLACE, E. K

Contral Entity Interface Specification.

PB94-191715

02,815

WALLACE, J.

Cooper M(sub II,III) X-ray-Emission Spectra of Copper OXides and the Bismuth Cuprate Superconductor.

PB96-158027

04,077

WALLACE, J. S.

Barium Contributions to the Valence Electronic Structure of YBa2Cu307-delta, PrBa2Cu3O7-delta, and Other BariumContaining Compounds.

PB96-158019 04,076

Effect of Microstructure on the Wear Transition of ZirconiaToughened Alumina.

PB94-211778

03,141

mage Depth Profiling SIMS: An Evaluation for the Analysis of Light Element Difiusion in YBa2Cu307-x Single Crystal Superconductors.

PB95-126116

04,530

WALLACE, M. A.

Videoconferencing Procurement and Usage Guide.

PB94-217023

01,470

WALLACE, $\mathrm{S}$

Control Entity Interface Specification

PB94-191715

02,815

Production Management Information Model for Discrete Manufacturing

02,830

Reference Architecture for Machine Control Systems Integration: Interim Report.

PB95-144549

02,820

WALLACE, W. E.

Advances in the Measurement of Polymer CTE: Micrometer- to Atomic-Scale Measurements.
PB96-180229 03,390

Electronics Packaging Materials Research at NIST.

PB96-122692

02,405

Gas Absorption during Ion-Irradiation of a Polymer Target. PB96-161864 04,099 Influence of an Impenetrable Interface on a Polymer GlassTransition Temperature.

03,175

Measured Stopping Powers of Hydrogen and Helium in Polystyrene Near Their Maximum Values.

04,729

Novel Method for Determining Thin Film Density by Energy-

Dispersive X-ray Reflectivity.
PB96-122783
WALLINDER, $F$.

Distant Future of Solar Activity: A Case Study of Beta Hydri. 3. Transition Region, Corona, and Stellar Wind. PB94-185220

00,049

Distant Future of Solar Activity: A Case Study of beta Hydri. 3. Transition Region, Corona, and Stellar Wind.

00,074

\section{WALLINGTON, T. J.}

Atmospheric Reactivity of alpha-Methyl-Tetrahydrofuran

PB95-163705 02,548

Collection of Results for the SPC/E Water Model.

PB96-147889

01,127

WALLS, F. L.

Aging, Warm-Up Time and Retrace; Important Characteristics of Standard Frequency Generators

PB96-103122

04,031

Confidence on the Modified Allan Variance and the Time Variance.

01,557

Cross-Correlation Analysis Improves Time Domain Measurements.

PB95-180535

01,543

Design Criteria for BJT Amplifiers with Low V 4 AM and PM PB96-200365 02,442
Diode-Laser Pumped, Rubidium Cell Frequency Standards.
01,538

Effect of Harmonic Distortion on Phase Errors in Frequency Distribution and Synthesis.

01,563

Environmental Sensitivities of Ouartz Oscillators.

PB96-103148

02,271

Frequency Synthesis and Metrology at 10(-17) and Beyond

02,101
Fundamental Limits on the Frequency Stabilities of Crystal Osciliators.

PB96-176565

02,277

High-Order Harmonic Mixing with GaAs Schottky Diodes.

PB95-108585

01.528

PB95-163713

02,045

Hybrid Digital/Analog Servo for the NIST-7 Frequency Standard

PB95-180618 01,544

Investigations of $\mathrm{AM}$ and PM Noise in X-Band Devices.

PB95-180022

02,062

Local Oscillator Requirements and Strategies for the Next Generation of High-Stability Frequency Standards.

PB96-112230

01,551

New Model of $1 / F$ Noise in Baw Ouartz Resonators.

New 5 and $10 \mathrm{MHz}$ High Isolation Distribution Amplifie

PB96-190202 01.510

Origin of $\mathrm{V} / \mathrm{PM}$ and AM Noise in Bipolar Junction Transistor Amplifiers.

PB96-200787

02,096

Practical Standards for PM and AM Noise at 5, 10 and 100

PB95-181129

01.546

Precision Oscillators: Dependence of Frequency on Temperature, Humidity and Pressure.

02,031

Ouest to Understand and Reduce V/ Noise in Amplitiers and Baw Ouartz Oscillators.

PB96-200795

02,097

Reducing Errors, Complexity, and Measurement Time of PM Noise Measurements.

02,075

Reducing the Effect of Local Oscillator Phase Noise on the Frequency Stability of Passive Frequency Standards. PB95-180972

01,545

Reducing the $1 / f$ AM and PM Noise in Electronics for Precision Frequency Metrology.

02,102

Relationship of AM to PM Noise in Selected RF Oscillators. PB95-169009

02,262

Secondary Standard for PM and AM Noise at 5, 10, and $100 \mathrm{MHz}$. PB96-123187
Surface Transverse Wave Oscillators with Extremely Low
Thermal Noise Floors.

PB96-186010

01,967

Ultra-High Stability Synthesizer for Diode Laser Pumped Rubidium

PB94-216066

Ultralinear Small-Angle Phase Modulator

PB95-168852

01,527

WALSH, T.

02,261

High Critical Temperature Superconductor Tunneling Spectroscopy Using Squeezable Electron Tunneling Junctions.
PB95-163721

Tunneling Measurement of the Zero-Bias Conductance Peak and the Bi-Sr-Ca-Cu-O Thin-Film Energy Gap.

WALTER, F

Sleuthing the Dynamo: HST/FOS Observations of UV Emissions of Solar-Type Stars in Young Clusters.

PB96-122817

00,098

WALTER, F. $M$.

Far-Ultraviolet Flare on a Pleiades G Dwart

PB96-102033

00,086

First Results from the Goddard High-Resolution Spectrograph: The Chromosphere of Tauri.

199528

00,054

Observations of $3 \mathrm{C} 273$ with the Goddard High Resolution Spectrograph on the Hubble Space Telescope.

PB95-202321

00,076

SALTER, $S$.

Slant Path Atmospheric Refraction Calibrator: An Instrument to Measure the Microwave Propagation Delays Induced by Atmospheric Water Vapor.

PB95-151270

01,476

WALTER, T.

Confidence on the Modified Allan Variance and the Time Variance.

PB96-190376

WALTERS, C. R.

VAMAS Intercomparison of Critical Current Measurements on NB3Sn Superconductors: A Summary Report.

PB96-119763

04,043

WALTERS, $D$.

Telecommunications Security Guidelines for Telecommunications Management Network. Computer Security.

PB96-139415

01,496

WALTERS, J.

NIST List of Publications, LP 103, March 1996. National Semiconductor Metrology Program.

PB96-175856

02,432

WALTMAN, D. J.

Ouench Energy and Fatigue Degradation Properties of Cuand AVCu-Stablized

PB96-141213

04,755

WALTMAN, $\mathbf{S}$.

Precise Optical Frequency References and Difference Frequency Measurements with Diode Lasers.

PB95-176228

04,305

WALTON, G. N.

Application of a Multizone Airflow and Contaminant Disper-

sal Model to Indoor Air Ouality Control in Residential Build-

ings.

02.555

Computer Programs for Simulation of Lighting/HVAC Interactions.

PB94-140407

02.501

CONTAM93 User Manual.

PB94-164381

02,536

CONTAM94: A Multizone Airfow and Contaminant Disper- 
Digital Impedance Bridge.

PB96-103155

Low Voltage Standards in the $10 \mathrm{~Hz}$ to $1 \mathrm{MHz}$ Range.

PB97-112569

02,100

Wideband Sampling Voltmeter.

PB97.113039

01,990

WAN, H. X.

Electron Scattering and Dissociative Attachment by SF6 and Its Electrical-Discharge By-Products.

02,256

WAN, K. T.

Diffusion of Water along 'Closed' Mica Interfaces. $\quad 02,993$
PB96-180039

Effect of Chemical Interaction on Barenblatt Crack Profiles in Brittle Solids.

PB96-180245

02,996

Pressurized Internal Lenticular Cracks at Healed Mica Intertaces.

02,997

\section{WANG, C. C.}

Measurement of the Uniformity of Particle Deposition of Filter Cassette Sampling in a Low Velocity Wind Tunnel.

PB95-163754

02,549

WANG, C. L.

Elastic Constants of Isotropic Cylinders Using Resonant Ultrasound 19

WANG, C. M.

04,497

Approximate Confidence Intervals on Linear Combinations of Expected Mean Squares.

PB95-151296

03,435

Approximate Confidence Intervals on Positive Linear Com. binations of Expected Mean Squares.

03,436

Ranges of Confidence Coefficients for Confiedence Intervals on Variance Components.

vB95-151312

03,437

Tolerance Intervals for the Distribution of True Values in the Presence of Measurement Errors.

PB95.150405

WANG, C. M. J.

03,434

Optical Fiber Geometry by Gray-Scale Analysis with Robust RBgression.

04,272

WANG, $D$

Gas Transport Properties of Solution-Cast Perfluorosulfonic Acid lonomer Films Containing lonic Surfactants.

PB95-175998

01,267

WANG, F. W.

Applications of Fluorescence Spectroscopy in Polymer Science and Technology.

01,258

Copolymerization of N-Phenyl Maleimide and gammaMethacryloxypropyl Trimethoxysilane.

01,248

Fluorescence Anisotropy Measurements on a Polymer Melt as a Function of Applied Shear Stress

01,209

Fluorescence Monitoring of Polarity Change and Gelation during Epoxy Cure.

01,204

In-Line Optical Monitoring of Injection Molding.

01,201

PB94-185105

Observations of Shear Induced Molecular Orientation in a
Polymer Melt Using Fluorescence Anisotropy Measurements.

$\begin{array}{lr}\text { PB94-199304 } & 01,210 \\ \text { Observations of Shear Stress and Molecular Orientation }\end{array}$ Using Fluorescence Anisotropy Measurements.

01,211

PB94-199312

ontaining

Preparation and Monitoring of Lead Acet

00,538

Drinking Water
PB94-193885

Thermal Behavior of 4-Maleimidophenyl Glycidyl Ether Res-

ins.

01,249

Thermal Behaviour of Methyl Methacrylate and N-Phenyl Maleimide Copolymers.

PB95-152237

01,246

WANG, $H$. P.

Interaction between Micro and Macroscopic Flow in RTM

Preforms.

03,159

NANG, $J$

Determination of the Complex Refractive Index of Individual Quantum Wells from Distributed Reflectance.
PB95-175642 WANG, J. C.

Chemically Assisted Machining of Si3N4

PB96-122999

03,072

WANG, J. C. $M$.

Proposed Changes to Charpy V-Notch Machine Certification Requirements.

PB96-135363

02,955

Technique to Evaluate Benchmarks: A Case Study Using the Livermore Loops.

PB95-151320

04,577
WANG, J. L.

Electromagnetic Coupling Character of (001) Twist Boundaries in Sintered Bi2Sr2CaCu2O8+x Bicrystals. PB96.176573

WANG, J. T.

01,963

Periapical Tissue Reactions to a Calcium Phosphate Cement in the Teeth of Monkeys.

PB94-212008

00,149

WANG, L

Band-to-Band Photoluminescence and Luminescence Excitation in Extremely Heavily Carbon-Doped Epitaxial GaAs.
PB95-150413

Standard Reference Materials: Polystyrene Films for Calibrating the Wavelength Scale of Infrared

brating the Wavelength
Spectrophotometers - SRM 1921. Scale of Intrared
PB95-226866 PB95-226866

WANG, $M$.

Theoretical Analysis of the Coherence-Induced Spectral Shift Experiments of Kandpal, Vaishya, and Joshi.

PB94-219383

00,56

WANG, $P$.

Threshold Electron Excitation of $\mathrm{Na}$

WANG, P. S.

Characterization of Phase and Surface Composition of Silicon Carbide Platelets.

Coating of Fibers by Colloidal Techniques in Ceramic Composites.

$\begin{array}{ll}\text { PB94-216256 03,196 } & \end{array}$

Deposition of Colloidal Sintering-Aid Particles on Silicon Nitride.

03,044

Effects of Soxhlet Extraction on the Surface Oxide Layer of Silicon Nitride Powders.

03,057

NMR Characterization of Injection-Moulded Alumina Green Compacts. Part 2. T2-Weighted Proton Imaging.

Tribochemical Reaction of Stearic Acid on Copper Surface Studied by Surface Enhanced Raman Spectroscopy. PB94-212057

02,964

WANG, $Q$.

Short-Pulse Detachment of $\mathrm{H}(-)$ in the Presence of a Static Electric Field

04,007

User's Guide for the PHIGS Validation Tests (Version 2.1). PB94-165206

01,682

WANG, $\mathrm{S}$.

Reproducibility of Tests on Energy Management and Control Systems Using Building Emulators.

Use of Building Emulators to Evaluate the Performance of Building Energy Management Systems.

WANG, S. S.

Composite Materials for Offshore Operations: Proceedings of the International Workshop (1st). Held in Houston, Texas of the International Worksh
on October 26-28, 1993.

109509

WANG, S. Y.

L-threo-beta-Hydroxyhistidine, an Unprecedented Iron(III) lon-Binding Amino Acid in a Pyoverdine-type Siderophore from Pseudomonas fluorescens 244

PB94-211620

00,553

WANG, Y. S.

Effect of Microstructure on the Wear Transition of Zirconia. Toughened Alumina.

PB94-211778

Wear Mechanism Maps of Ceramics

PB94-172368

03,141

Wear Model for Alumina Sliding Wear

03,229

PB95-163796

03,239

Wear Transitions in Monolithic Alumina and Zirconia-Alumina Composites.

WANG, $Z$.

03,168

Critical Current Density, Irreversibility Line, and Flux Creep Activation Energy in Silver-Sheathed $\mathrm{Bi} 2 \mathrm{Sr} 2 \mathrm{Ca} 2 \mathrm{Cu} 2 \mathrm{OX}$ Superconducting Tapes.

WANG, $Z$ D.

04,616

Analysis of Creep in a Si-SiC C-Ring by Finite Element

PB94-200268

03,037

WANG, $\mathbf{Z}$. $\mathbf{L}$.

Epitaxial Growth of BaTiO3 Thin Films at $600 \mathrm{C}$ by Metalorganic Chemical Vapor Deposition.

PB96-122510

03,071

WANNBERG, $B$.

Inner-Valence States $\mathrm{CO}(+)$ between $22 \mathrm{eV}$ and $46 \mathrm{eV}$ Studied by High Resolution Photoelectron Spectroscopy and $\mathrm{ab}$ Initio $\mathrm{Cl}$ Calculations.

PB95-180055

03,961

WARBURTON, $W . K$.

Real-Time Small-Angle X-Ray Scattering Study of the Early Stage of Phase Separation in the SiO2-BaO-K20 System.

WARD, $\mathrm{B}$.

Digital Techniques in HV Tests - Summary of 1989 Panel SB94-216702

02,035

WARD, D.

Interim Testing Artifact (ITA): A Performance Evaluation System for Coordinate Measuring Machines (CMMs). User

02,914

NIST SRM 9983 High-Rigidity Ball-Bar Stand. User Manual. PB95-255840

02,669

WARD, D. B.

Intercomparison between NPL (India) and NIST (USA) Pressure Standards in the Hydraulic Pressure Region Up to $26 \mathrm{MPa}$.

PB96-113543

04,211

WARD, D. E.

User's Guide 10 NIST SRM 2084: CMM Probe Performance

Standard.

02,709

WARD, R. C.

Variation in Magnetic Properties of Cu/Fcc (001) Sandwich Structures.

WARD, R. C. C.

04,555

Observation of Two Length Scales Above ( $T$ sub $N$ ) in a Holmium Thin Film.

PB97.111942

04,151

WARDLE, C. E.

SQA and TQM in Software Quality Improvement

PB96- 160791

SQA Standards and Total Quality Management.

PB96-111844

WARNAR, C. A.

X.500 Directory Schema Design Handbook.

PB96- 183041

International Challenges in Defining the Public and Private Interest in Standards.

96-16036

00,498

WASKIEWICZ, W. $K$.

XUV Characterization Comparison of Mo/Si Multilayer Coat

ings.

04,278

WASSERMANN, E. F

Temperature Dependence of the Magnetic Excitations in Ordered and Disordered Fe72Pt28.

PB95-150223

04,563

WASSON, O. A.

Measurement of the $(10) \mathrm{B}(\mathrm{n}$, alpha1gamma)(7)Li Cross Section in the 0.3 to $4 \mathrm{MeV}$ Neutron Energy Interval.

PB96-161799

04,098

Measurements of the $(235) \mathrm{U}(\mathrm{n}, \mathrm{f})$ Cross Section in the 3 to $30 \mathrm{MeV}$ Neutron Energy Region.

PB97-119051

04,172

WASZCZAK, J. V.

Increased Pinning Energies and Critical Current Densities in Heavy-Ion-Irradiated Bi2Sr2CaCu2O8 Single Crystals.

PB95-175352

\section{WATANABE, $H$.}

Effects of Adhesive Thickness, Open Time, and Surface Cleanness on the Peel Strength of Adhesive-Bonded Seams of EPDM Rubber Roofing Membrane.

PB95-151338 
WATSON C I

Comparison of FFT Fingerprint Filtering Methods for Neural Network Classification.

01,840

PCASYS: A Pattern-Level Classification Automation System for Fingerprints.

WATSON, D. G.

Cambridge Structural Database (CSD): Current Activities and Future Plans.

PB97-109052

00.516

How the Cambridge Crystallographic Data Centre Obtains Its Information

WATSON, J. T. R.

Viscosity of Ammonia.

PB96-145933

04,808

ATSON, R. E.

01,117

Monte Carlo and Mean-Field Calculations of the Magnetocaloric Effect of Ferromagnetically Interacting Clus-

PB94-172087

03,201

WATTERS, R. L

Dissolution Problems with Botanical Reference Materials.

$\begin{array}{ll}\text { PB95-126280 03,487 } & \end{array}$

Preparation and Certification of a Rhodium Standard Reference Material Solution.

PB94-185071

00,529

Trace Detection in Conducting Solids Using Laser-Induced Fluorescence in a Cathodic Sputtering Cell.

00,598

Traceability to the Mole: A New Initiative by CIPM

PB95-180246

00,605

WATTS, L

Low Temperature H(sub 2)S Separation Using Membrane Reactor with Redox Catalys

DE9400899

02,471

Atoms in Optical Molasses.

PB95-108874

03,875

Influence of Electrical isolation on the Structure and Reflectivity of Multilayer Coatings Deposited on Dielectric Sub-

PB96-159736

04,365

New Method for Achieving Accurate Thickness Control for Uniform and Graded Multilayer Coatings on Large Flat Substrates.

PB96-159744

04,366

Optical Molasses: The Coldest Atoms Ever.

03,878

PB95-108908

Institute

Soft X-ray Reflectometry Prog

of Standards and Technology.

PB96-160395

04,368

WATTS, R. N.

Atoms in Optical Molasses: Applications to Frequency Standards

PB95-108882

03,876

Heterodyne Measurement of the Fluorescence Spectrum of Optical Molasses.

$\begin{array}{ll}\text { PB95-108411 03,873 } & \end{array}$

Improved Reflectometry Facility at the National Institute of

Standards and Technology.

PB96-160338

04,087

Interlaces in $\mathrm{Mo} / \mathrm{Si}$ Multilayers.

PB96-160668 02,423

Localization of Atoms in a Three-Dimensional Standing

PBave. 163887

03,944

Measurements of Fluorescence from Coid Atoms: Localiza-

tion in Three-Dimensional Standing Waves.

PB95-163879 03,943

New NIST/ARPA National Soft $X$-ray Reflectometry Facility.

PB96-158092

04,080

NIST Metrology for Soft X-ray Multilayer Optics.

04,088

Optical Molasses: Cold Atoms for Precision Measurements.

PB95-108890 03,877

Simple Variable Line Space Grating Monochromator for

$\begin{array}{ll}\text { Synchrotron Light Source Beamlines. } & \\ \text { PB96-156203 } & 04,065\end{array}$

Upgraded Facility for Multilayer Mirror Characterization at

PB96- 160387

04.367

XUV Characterization Comparison of Mo/Si Multilayer Coatings.

WATTS, R. 0 .

04,278

Infrared and Microwave Spectroscopy of the Argon Propyne Dimer

WAVERING, A. J.

00,794

Visual Pursuit Systems
PB95-143285

WEAVER, J. T.

Needs for Brachytherapy Source Calibrations in the United States.

PB97-110092

03,521
WEBER, A.

2nu9 Band of Propyne-d3.

PB95-164513

Molecular Spectroscopy.

PB94-213337

Criteria for Establishing Accurate Vapor Pressure Curves.

PB95-163812

Design of a High-Pressure Ebulliometer, with Vapor-Liquid Equilibrium Results for the Systems CHF2Cl + $\mathrm{CF} 3-\mathrm{CH}_{3}$ and $\mathrm{CF} 3-\mathrm{CH} 2 \mathrm{~F}+\mathrm{CH} 2 \mathrm{~F} 2$

PBg7-113229

04,163

Ebulliometers for Measuring the Thermodynamic Properties of Fluids and Fluid Mixtures.

DE94017817

04,195

Ebulliometric Measurement of the Vapor Pressure of Difluoromethane.

PB95-151361

00,931

Ebulliometric Measurement of the Vapor Pressure of 1 Chloro-1,1-Difluoroethane and 1,1-Difluoroethane.

PB95-164489

00,984

Estimating the Virial Coefficients of Small Polar Molecules. PB95-176236 03,276

Measurements of the Vapor Pressures of Difluoromethane, 1-Chloro-1,2,2,2-Tetrafluoroethane, and Pentafluoroethane. PB95-169272 03,270

Measurements of the Virial Coefticients and Equation of State of the Carbon Dioxide + Ethane System in the Supercritical Region

PB95-151353

03,906

Model for Calculating Virial Coefficients of Natural Gas Hydrocarbons with Impurities.

04,064

Thermodynamic Properties of CF3-CHF-CHF2, 1,1,1,2,3,3 Hexafluoropropane.

PB97-118384

03,299

Thermodynamic Properties of CHF2-CF2-CHF, 1,1,2,2,3 Pentafluoropropane.

PB97-118392

Thermodynamic Properties of Difluoromethane.

PB94-185204

Vapor Pressure of Pentafluorodimethyl Ether PB96-201199

Vapor Pressure of 1,1,1,2,2-Pentafluoropropane.

PB97-113237

of 1,1-dichloro-2,2,2-trifluoroethane (R123).

00,899

Vapor Pressures and Gas-Phase PVT Data for 1-Chloro$1,2,2,2$-Tetrafluoroethane (R124)

03,271

Vapour Pressures and Gas-Phase ( $p$, rho $n, T)$ Values for CF3CHF2(R125).

PB96-102090

Virial Coefficients of Five Binary Mixtures of Fluorinated Methanes and Ethanes.

PB96-156054

01,128

PER, S. F.

AutoBid 2.0: The Microcomputer System for Police Patrol Vehicle Selection

04,871

Benefits and Costs of Research: A Case Study of the Fire Safety Evaluation System.

PB96-202288

00,232

Evaluating Investments in Law Enforcement Equipment: An Annotated Bibliography.

PB95-15

Doppler-Free Spectroscopy of Large Polyatomic Molecules and van der Waals Complexes.

PB96-119581

04,339

Frequency Shitting of Pulsed Narrow-Band Laser Light in a

Multipass Raman Cell.

04,321

Van der Waals Bond Lengths and Electronic Spectral Shifts of the Benzene-Kr and Benzene-Xe Complexes.

PB95-15 1387

00,932

WEDDING, A. B.

N2(a'(sup 1)Sigma(sub g)(sup +)) Metastable Collisional Destruction and Rotational Excitation Transfer by $\mathrm{N} 2$. PB95-151395

\section{WEERTMAN, J. $R$.}

Small Angle Neutrons Scattering from Nanocrystalline Palladium as a Function of Annealing.

PB95-176103

03,354

Bacteriorhodopsin Immobilized in Sol-Gel Glass.

PB95-151429

03,532

Atfinity Chromatography on Inorganic Support Materials.

PB95-163820 03,467

Aggregation Kinetics of Colloidal Particles Destabilized by

Enzymes.

00,894

Amperometric Flow-Injection Analysis Biosensor for Glu-

cose Based on Graphite Paste Modified with

Tetracyanoquinodimethane.

03,498

Application of Photochemical Reaction in Electrochemical Detection of DNA Intercalation
PB94-185733

00,686

Autofluorescence Detection of 'Escherichia coli' on Silver Membrane Filters.

PB96- 163639

03,590

Bacterial Enumeration in Storage Water.

PB96-163647

03,591

Detection of Aromatic Compounds Based on DNA Intercalation Using an Evanescent Wave Biosensor.

PB96-111976

03,473

Method for the Assay of Hydrolytic Enzymes Using Dynamic Light Scattering.

03,531

New Method for the Detection and Measurement of Polyaromatic Carcinogens and Related Compounds by DNA intercalation.

PB96- 167382

03,481

Novel Amperometric Immunosensor for Procainamide Em-

ploying Light Activated Labels
PB96-163662

00,512

Optical Properties of Triton X-100-Treated Purple Mem-

branes Embedded in Gelatin Films.

03,546

Preparation of Immobilized Proteins Covalently Coupled Through Silane Coupling Agents to Inorganic Supports.

PB95-151403

03,530

WEI, L

Evaluation of Thermal Wave Imaging for Detection of Machining Damage in Ceramics.

03,062

Thermal Wave NDE of Advanced Materials Using Mirage Effect Detection.

PB96-204516

04,191

WEI, R.

Tribological Behavior of 440/Diamond-Like-Carbon Film Couples.

PB96-119714

03,019

WEIDA, M. J.

Collisional Alignment of $\mathrm{CO} 2$ Rotational Angular Momentum States in a Supersonic Expansion.

01,069

Signatures of Large Amplitude Motion in a Weakly Bound Complex: High-Resolution IR Spectroscopy and Quantum Calculations for $\mathrm{HeCO} 2$.

PB95-203485

01,054

WEIDE, $K$

Nonadiabatic Effects in the Photoassociation of H2S. PB95-151437

00,934

WEIDENHEIMER, D. $M$.

Electrical Breakdown in Transformer Oil in Large Gaps. PB95-150579

01,889

WEIDER, $T$

Shear-Induced Melting of Two-Dimensional Solids. PB96-112057 
WHALEN, J. J.

Comparison of GPS Broadcast and DMA Precise AD-P009 114/0 01,518 Comparison of GPS Broadcast and DMA Precise Ephemerides

01,523 N94-30660/2

Time

Variance

PB96-190376

01,557

Confidence on the Second Difference Estimation of Frequency Drift.
PB95-151460

01,532

Confidence on the Three-Point Estimator of Frequency Drift. PB95-163838 01539 Implementation of a Standard Format for GPS Common View Data.

N95-32323/4 03,779

Implementation of a Standard Format for GPS Common

View Data.

01,555

Promise into Practice: Implementing TA2 on Real Clocks at NIST.

PB95-151478 01,533

Sitting Through Nine Years of NIST Clock Data with TA2.

PB95-181137 01,547

Smart Clock: A New Time.

PB95-151445

01,530

Time Scale Algorithm for Post-Processing: AT1 Plus Frequency Variance.

WEISS, $\mathrm{P}$.

01,525

Report of the Refrigeration, Air Conditioning and Heat Pumps Technical Options Committee.

PB96-176755

03,293

WEISS, R. A.

Compatibilization of Polymer Blends by Complexation. 2. Kinetics of Interfacial Mixing.

WEISS, $V$.

Femtosecond Time-Resolved Wave Packet Motion in Molecular Multiphoton Ionization and Fragmentation.

PB94-198611

00,790

WEISSBECKER, $B$.

Dielectronic Capture Processes in the Electron-Impact Ionization of $\mathrm{Sc}(2+$

WEISSER, P.

04.000

HVAC CAD Layout Tools: A Case Study of University/lndustry Collaboration.

PB97-112221

WEISSERT, T.

Promise into Practice: Implementing TA2 on Real Clocks at
NIST. PB95-151478

01,533

Time Scale Algorithm for Post-Processing: AT1 Plus Frequency Variance.
PB94-172772

Use of Ionospheric Data in GPS Time Transfer.

PB95-163853

01,525

WEISSERT, T. P.

Sifting Through Nine Years of NIST Clock Data with TA2.
PB95-181137

WEISSHAAR, A.

Fibre Splice Loss: A Simple Method of Calculation.
PB95-175519 04,299

Mode Coupling and Loss on Tapered Optical Waveguides.
PB95-168571

Symbolic Programming with Series Expansions: Applications to Optical Waveguides.
PB95-168589

04,283

Vector and Ouasi-Vector Solutions for Optical Waveguide Modes Using Efficient Galerkin's Method with HermiteGauss Basis Functions.

PB96-141197

04,357

WEISSMULLER, $\mathbf{J}$.

Inelastic Neutron Scattering Study of Hydrogen in Nanocrystalline Pd

PB96-146857

03,366

Vibrational Excitations and the Position of Hydrogen in Nanocrystalline Palladium.

PB96-111828

04,035

Pressure Measurements with the Mercury Melting Line Referred to ITS-90

PB96-161005

01,136

WELCH, B. E.

Operational Mode and Gas Species Effects on Rotational Drag in Pneumatic Dead Weight Pressure Gages.
PB95-140182

WELCH, M.

How to Verify Reference Materials.

PB95-151486

WELCH, M. J.

Analysis by a Combination of Gas Chromatography and Tandem Mass Spectrometry: Development of Ouantitative
Tandem-in-Time Ion Trap Mass Spectrometry: Isotope Dilution Ouantification of 11-Nor-Delta-9-Tetrahydro cannabinol-

9-Carboxylic Acid.
PB96-117221

02,561

Certification of Morphine and Codeine in a Human Urine Standard Reference Material.

PB95-176160

03,499

Certification of Phencyclidine in Lyophilized Human Urine Reference Materials.

PB96-160692

03,508

Determination of Amphetamine and Methamphetamine in a Lyophilized Human Urine Reference Material.

PB95-175444

03,597

Hair Analysis for Drugs of Abuse: Evaluation of Analytica Methods, Environmental Issues, and Development of Reference Materials.

PB95-176269

03,501

Hair Testing for Drugs of Abuse: International Research on Standards and Technology.

PB96-120555

03,504

Interlaboratory Comparison Sidudies on the Analysis of Hair for Drugs of Ábuse.

PB95-176251

03,500

Interlaboratory Siudies on the Analysis of Hair for Drugs of Abuse: Results from the Fith Exercise.

PB97-110449

03,509

Interlaboratory Studies on the Analysis of Hair for Drugs of Abuse: Results from the Fourth Exercise.

03,510

Isotope Dilution Mass Spectrometry as a Candidate Definitive Method for Determining Total Glycerides and Triglycerides in Serum.

PB96-102280

03,519

NIST Reference Materials to Support Accuracy in Drug Testing.
PB96-123807

03,505

WELCH, W. J.

Taguchi's Parameter Design: A Panel Discussion.

PB96-111802

WELLINGTON, J. D.

ISO TC 184/SC4 Reference Manual.

PB95-242293

02,663

Precise Optical Frequency References and Difference Fre-

Precise Optical Frequency References and Difference Fre-
quency Measurements with Diode Lasers.

quency Measurer

04,305

WELLS, J. S.

Extension of Heterodyne Frequency Measurements on OCS $1087 \mathrm{THz}(2900 \mathrm{~cm}(-1))$.

PB94-200680

00,811

Optically Stabilized Tunable Diode-Laser System for Satura-

tion Spectroscopy.
PB96-102819

Stabilization of 3.3 and $5.1 \mathrm{~m}$ Lead-Salt Diode Lasers by

Optical Feedback.
PB95-180709

04,313

Sub-Doppler Frequency Measurements on OCS at $87 \mathrm{Thz}$ (3.4 micrometers) with the CO Overtone Laser: Considerations and Details.

PB95-128633

04,255

Sub-Doppler Frequency Measurements on OCS at $87 \mathrm{THz}$ (3.4 mu $\mathrm{m}$ ) with the CO Overtone Laser.

PB96-102215

04,330

WELLS, M. R.

Observation of Two Length Scales Above ( $T$ sub $N$ ) in a Holmium Thin Film.

PB97-111942

WELLSTOOD, F. C.

Application of Single Electron Tunneling: Precision Capacitance Ratio Measurements.

PB96-102157

04,703

WELSCH, L. A.

Proposed International Interactive Courseware Standard.

PB96-12316

00,137

WELTY, R. P.

Self-Biasing Cryogenic Particle Detector Utilizing Electrothermal Feedback and a SOUID Readout.

96-102538

04,712

Series Array of DC SOUIDs

02,046

Two-Stage Integrated SOUID Amplifier with Series Array

PB95-176277

02,061

WEN, C. P

Electrical Measurements of Microwave Flip-Chip Interconnections.
PB96-176748

02,436

Microwave Characterization of Flip-Chip MMIC Compo-

PB96-176722

02,434

Microwave Characterization of Flip-Chip MMIC Interconnections

PB96-176730

WERIJ, H. G. C.

02,435

Oscillator Strengths and Radiative Branching Ratios in Aromic Sr.

04,008

WERT, J. A.

Experimental Assessment of Crack-Tip Disiocation Emission Models for an Al67Cr8Ti25 Intermetallic Alloy. PB96-204466

03,377

\section{WERTZ, K. H.}

Retention of Halocarbons on a Hexafluoropropylene Epoxide-Modified Graphitized Carbon Black. 4. Propane-Based Compounds.

WEST, J. A.

Disorder Trapping in Ni2TiA

PB94-198942

03,284

WEST, J. B.

High Resolution Angle Resolved Photoelectron Spectroscopy Study of N2.

PB95-151494

03,907

Inner-Valence States $\mathrm{CO}(+)$ between $22 \mathrm{eV}$ and $46 \mathrm{eV}$ Studied by High Resolution Photoelectron Spectroscopy and $a b$ Initio Cl Calculations.

PB95-180055

03,961

Photoelectron Situdy of Electronic Autoionization in Rotationally Cooled N2: The $n=6$ Member of the Hopfield Series.

00,971

Shape-Resonance-Enhanced Continuum-Continuum Coupling in Photoionization of $\mathrm{CO} 2$.

00,983

Vibrational Autoionization in $\mathrm{H} 2$ : Vibrational Branching Ratios and Photoelectron Angular Distributions Near the $v(+)=3$ Threshold

PB94-199577

00,799

Vibrationally Resolved Photoelectron Angular Distributions and Branching Ratios for the Carbon Dioxide Molecule in the Wavelength Region 685-795 Angstrom.

PB96-201207

04,131

WESTBROOK,

Atoms in Optical Molasses.

PB95-108874

03,875

Optical Molas

03,878

Temperature of Optical Molasses for Two Different Atomic Angular Momenta.

PB95-126058

03,881

Uttracoid Collisior

WESTBROOK, C. 1.

Atoms in Optical Molasses: Applications to Frequency PB95-108882

03,876

Heterodyne Measurement of the Fluorescence Spectrum of Optical Molasses.

PB95-108411

Laser Cooling
PB95.151502

03,873

Laser Modification of Ultracold Collisions: Experiment.
PB96- 157987

Localization of Atoms in a Three-Dimensional Standing Wave.

PB95-163887

03,944

Measurements of Fluorescence from Cold Atoms: Localization in Three-Dimensional Standing Waves

03,943

Optical Molasses: Cold Atoms for Precision Measurements. PB95-108890

WESTBROOK, J.

Nucleic Acid Database: Present and Future.

PB97-109078 
WHALEN, T.

Estimates of Hurricane Wind Speeds by the 'Peaks Ove Threshold' Method.

WHALEN, T.

Dynamics of Multi-DOF Stochastic Nonlinear Systems. PB97-113245 00,477 Probabilistic Estimates of Design Load Factors for WindSensitive Structures Using the 'Peaks Over Threshold' Approach.

-183223

00,474

WHANGBO, M. H.

Ca4Bi6013: A Compound Containing an Unusually Low Bismuth Coordination Number and Short Bi Bi Contacts. PB95-141131

00,911

WHEATLEY, T.

Configuration and Performance Evaluation of a Real-Time Robot Control System: A Skeleton Approach.

01,598 PB95-163895

\section{WHEATLEY, T. E.}

Mapping Processes to Processors for Space-Based Robot

$\begin{array}{ll}\text { Systems. } & 04,833\end{array}$

Unified Telerobotic Architecture Project (UTAP) Standard Interface Environment (SIE), May 1995

PB95-242350

02,938

\section{WHEELER, A. A}

Anisotropy of Intertaces in an Ordered Alloy: A MultipleOrder-Parameter Model.

PB96-131594

04,741

Computation of Dendrites Using a Phase Field Model. PB94-160744 04.436

Convective Stability in the Rayleigh-Benard and Directional Solidification Problems: High-Frequency Gravity Modulation. PB95-181145 04,208 Notion of a xi-Vector and a Stress Tensor for a General Class of Anisotropic Diffuse Interface Models.

PB96-193776 04,788

Phase-Field Model for Solidification of a Eutectic Alloy.

03,345 xi-Vector Formulation of Anisotropic Phase-Field Models: 3D Asymptotics.
PB95- 136628

04,536

Xi-Vector Formulation of Anisotropic Phase-Field Models: 3D Asymptotics.

WHEELER, D. J.

Procedure for Measuring Trace Quantities of S2F10, S2OF10, and S2O2F10 in SF6 Using a Gas Chro. matograph-Mass Spectrometer.

PB96-119755

02,513

WHEELER, N. S.

Electrodeposited Cobalt-Tungsten as a Diffusion Barrier between Graphite Fibers and Nicke

PB96-146881

03,176

Microstructural Characterization of Cobalt-Tungsten Coated Graphite Fibers.

WHETSTONE, J. R.

01.951

Electrical Measurements for Monitoring and Control of if Plasma Processing.

04,369

Gaseous Electronics Conference Radio-Frequency Reference Cell: A Defined Parallel-Plate Radio-Frequency System for Experimental and Theoretical Studies of PlasmaProcessing Discharges.

04,404

New Expressions of Uncertainties for Humidity Calibrations at the National Institute of Standards and Technology.

WHIPPLE, A. L.

Lunar Laser Ranging: A Continuing Legacy of the Apollo PBogram.

03,683

WHITE, A. E.

Increased Pinning Energies and Critical Current Densities in Heavy-Ion-Irradiated Bi2Sr2CaCu2O8 Single Crystals.

PB95-175352 04,654

Silicon Surface Chemistry by IR Spectroscopy in the Mid- to Far-IR Region: $\mathrm{H} 2 \mathrm{O}$ and Ethanol on $\mathrm{Si}(100)$.

PB96-138565

01,097

WHITE, E.

Determination of 3-Quinuclidinyl Benzilate (Onb) and Its Major Metabolites in Urine by Isotope Dilution Gas Chromatography Mass Spectrometry.

WHITE, G.

03,492

Transient Cooling of a Hot Surface by Droplets Evaporation. PB95-143194

03,890

WHITE, G. S.

Electric Field Effects on Crack Growth in a Lead MagneSium Niobate.

03,339

Moisture and Water-Induced Crack Growth in Optical MatePBials.

04.267
Molecular Orbital Calculations of Bond Rupture in Brittle

Solids.

00,973

Molecular Orbital Study of Water Enhanced Crack Growth

Process.

PB95-164067

03,240

Thermal Wave NDE of Advanced Materials Using Mirage Effect Detection
PB96-204516

04,191

WHITE, N. E.

Efticient Way of Identifying New Active Stars: A VLA Survey

of X-ray Selected Active Stellar Candidates.

00,099

WHITE, P. H.

Nutritional Status and Growth in Juvenile Rheumatoid Arthritis.

PB94-198470

03,515

WHITE, R. L

Discovery of an X-Ray Selected, Radio-Loud Ouasar at $z=3.9$.

PB94-198652

00,052

New High-Redshift Damped Lyman-alpha Absorption Systems and the Redshift Evolution of Damped Absorbers.
PB95-203501
O0,083

WHITE, S.

Observing Stellar Coronae with the Goddard High Resolution Spectrog

00,092

WHITE, V. E.

L-threo-beta-Hydroxyhistidine, an Unprecedented Iron(III) lon-Binding Amino Acid in a Pyoverdine-type Siderophore from Pseudomonas fluorescens 244.

PB94-211620

00,553

WHITE, V. R.

National Voluntary Laboratory Accreditation Program: Procedures and General Requirements.

02,630

National Voluntary Laboratory Accreditation Program 1994 Directory.

PB94-178969 00,482

National Voluntary Laboratory Accreditation Program 1995

Directory.

00,483

National Voluntary Laboratory Accreditation Program 1996 Directory.
PB96-162714

00,485

WHITENTON, E. P.

Measuring Matching Wear Scars on Balls and Flats.
PB95-151528

Surface Roughness Evaluation of Diamond Films Grown on Substrates with a High Density of Nucleation Sites.

PB95-162418

03,018

WHITESEL, H. K.

Optical Fiber Sensors: Accelerating Applications in Navy Ships.
PB94-186848

02,632

Self-Calibrating Fiber Optic Sensors: Potential Design Meth-

Ods.

Self Calibrating Fiber Optic Sensors: Potential Design MethPBS. 169306

02,173

WHITESIDES, G. M.

Microlithography by Using Neutral Metastable Atoms and Self-Assembled Monolayers.

PB96-190038

02,441

ING, D.

Optimization of Highway Concrete Technology.

WHITMAN, L. J.

Atomic Manipulation of Polarizable Atoms by Electric Field Directional Diffusion.

PB95-150587

Manipulation of Adsorbed Atoms and Creation of New Structures on Room-Temperature Surfaces with a Scanning Tunneling Microscope.

PB95-151536

04,578

WHITTAKER, H. L.

Calibration of Dosimeters for the Cryogenic Irradiation of Composite Materials Using an Electron Beam.

03,968

WHITTAKER, J. K.

NIST-NRL Free-Electron Laser Facility.

PB94-212511

04,237

\section{WHITTEN, B. L}

High-Precision Calculations of Cross Sections for Low-Energy

PB95-152161

03,914

Low-Energy-Electron Collisions with Sodium: Elastic and Inelastic Scattering from the Ground State.

04,030

WHITTER, K. M

International Green Building Conference and Exposition (2nd). Held in Big Sky, Montana on August 13-15, 1995.

U.S. Green Building Conference, 1994.

02,519

\section{WHITINGHAM, M. S.}

Rietveld Analysis of NaxWO3 $+x / 2 . y \mathrm{H} 2 \mathrm{O}$, Which Has the Hexagonal Tungsten Bronze Structure.

04,524

WHITTLESTONE, S

International Marine-Atmospheric (222)Rn Measurement Intercomparison in Bermuda. Part 2. Results for the Participating Laboratories.

PB96-175682

00,115

WIANT, J. R.

Lunar Laser Ranging: A Continuing Legacy of the Apollo PB95-202495

03,683

\section{WICKNER, $\mathrm{S}$.}

Deletion Analysis of the Mini-P1 Plasmid Origin of Replication and the Role of E.coli DnaA Protein.

PB95-163911

03,539

DnaJ, DnaK, and GrpE Heat Shock Proteins are Required in 'ori'P1 DNA Replication Solely at the RepA Monomerization Step

PB97- 119382

03,557

Function of DnaJ and DnaK as Chaperones in Origin-Specific DNA Binding by RepA.

PB95-151544

03,533

WIEDENMANN, E.

Femtosecond Time-Resolved Wave Packet Motion in Molecular Multiphoton Ionization and Fragmentation.

PB94-198611

00,790

EERHORN, S.M.

Cavitation Contributes Substantially to Tensile Creep in Silicon Nitride.
PB96-122577

03,171

Cavitation Damage During Flexural Creep of SiAION-YAG Ceramics.

PB94-200110

03,036

Cavity Evolution during Tensil Creep of Si3N4

PB96-204193

03,376

Creep and Creep Rupture of Ceramic Matrix Composites.

03,165

Creep and Creep Rupture of Structural Ceramics.

PB96-204524

03,093

Creep Rupture of MoSi2/SiCp Composites.

03,154

Fracture Mechanism Maps: Their Applicability to Silicon Nitride.

PB96-204532

03,094

Fracture of Silicon Nitride and Silicon Carbide at Elevated

Temperatures

03,179

High Temperature Degradation of Structural Composites.

PB94-172848

03,132

High Temperature Structural Reliability of Silicon Nitride.

PB97-110456 03,104

Stability and Surface Energies of Wetted Grain Boundaries in Aluminum Oxide.

PB95-202750

03,059

Tensile Creep of a Silicon Nitride Ceramic.

Tensile Creep of Silicide Composites.

PB96-200803

03,049

Tensile Creep of Whisker Reinforced Silicon Nitride.

PB94-211984

Tensile Creep

Tension/Compression Creep Asymmetry in Si3N4 
Laser Cooling and Trapping for the Masses. PB95-126090

04,253

Precision Lifetime Measurements of Cs $6 p(2) P 1 / 2$ and $6 p$ (2) $\mathrm{P} 3 / 2$ Levels by Single-Photon Counting

PB95-203816

04,010

WIESE, W. L.

Atomic Branching Ratio Data for Carbon-Like Ions.

PB94-212842

Atomic Branching Ratio Data for Nitrogen-Like Species.

PB96-190152 04,122

Atomic Branching Ratio Data for Oxygen-Like Species.

PB95-180436 03,963

Atomic Transition Probabilities and Tests of the Spectroscopic Coupling Scheme for $\mathrm{N} 1$. PB96-138466

04,057

Atomic Transition Probability Ratios between Some Ar I 4s$4 p$ and $4 s-5 p$ Transitions.

03,842

Comprehensive Spectroscopic Data Tabulations and Progress in the Compilation of Alomic Transition Probabilities

PB95-151551

03,909

Investigation of LS Coupling in Boronlike lons.

PB94-185295 03.797

New Critical Review of Experimental Stark Widths and

PB94-172830

03,790

Spectral Data and Grotrian Diagrams for Highly lonized

Chromium, Cr V through Cr XXIV.

Spectroscopic Data for Fusion Edge Plasmas.

00,748

04,410

Spectroscopic Data Tables for Highly-lonized Atoms.

PB95-151585 03,910

Spectroscopic Diagnostics of Low Temperature Plasmas: Techniques and Required Data.

PB95-151577

04,411

WIESENFELD, $K$.

Phase-Locked Oscillator Optimization for Arrays of Josephson Junctions

PB95-169314

02,052

WIESLER, D. G.

Determination of Anomalous Superexchange in $\mathrm{MnCl} 2$ and

Its Graphite Intercalation Compound
PB97.122568

00,666

Neutron Reflectivity of End-Grafted Polymers: Concentration and Solvent Quality Dependence in Equilibrium Conditions.

Neutron Reflectometry Studies of Surface Oxidation.

PB95-150421 00,917

Structural and Magnetic Properties of $\mathrm{CuCl} 2$ Graphite Intercalation Compounds.

PB96-119748 03,020

$X$-Ray Ditfraction from Anodic TiO2 Films: In situ and EX situ Comparison of the Ti(0001) Face.
PB94-185972

WIETING, T.

Optical Conductivity of Single Crystals of Ba1$x M \times B i O 3(M=K, R b, x=0.04,0.37)$.

WIGHT, S. A.

Environmental Scanning Electron Microscope Imaging Examples Related to Particle Analysis.

PB94-172822

00,766

Rare-Earth Isotopes as Tracers of Particulate Emissions: An Urban Scale Test.

PB94-161635

02,535

WIGNALL, G. D.

SANS Study of the Plastic Deformation Mechanism in Polyethylene.

01,242

WILBUR, J. L

Microlithography by Using Neutral Metastable Atoms and Self-Assembled Monolayers.

WILBUR, $P$.

02,441

Tribological Behavior of 440/Diamond-Like-Carbon Film Couples.

WILHOIT, R. C.

03,019

Thermodynamic and Thermophysical Properties of Organic Nitrogen Compounds. Part II. 1- and 2-Butanamine, 2-Methyl-1-Propanamine, 2-Methyl-2-Propanamine, Pyrrole, 1-, 2-, and 3-Methylpyrrole, Pyridine, 2-, 3-, and 4-Methylpyridine, Pyrrolidine, Piperidine, Indole, Ouinoline, Isoquinoline, Acridine, Carbazole, Phenanthridine, 1- and 2Naphthalenamine, and 9-Methylcarbazole.

PB94-162294

WILKERSON, $T$.

00,741

Collision-Induced Emission in the Fundamental VibrationRotation Band of $\mathrm{H} 2$.

PB94-199445

03,811

WILKIN, N. D.

NIST-NRL Free-Electron Laser Facility

PB94-212511

04,237
WILKINS, J. W.

Large Local-Field Corrections in Optical Rotatory Power of Ouartz and Selenium.

PB97-122378

04,400

WILKINSON, F.

Ouantum Yields for the Photosensitized Formation of the Lowest Electronically Excited Singlet State of Molecular Ox-

ygen in Solution
PB94-161007

00,732

Rate Constants for the Decay and Reactions of the Lowest Electronically Excited Singlet State of Molecular Oxygen in Solution. An Expanded and Revised Compilation.

PB96-145826

WILKINSON, R. A.

01,106

Analysis of a Biologically Motivated Neural Network for Character Recognition

Electric Field Effects on a Near-Critical Fluid in Microgravity. PB96-161880 04,217

PCASYS: A Pattern-Level Classification Automation System Por Fingerprints
PB95-267936

01,853

Second Census Optical Character Recognition Systems Conference.

PB94-188711

Self-Organizing Neural Network Character Recognition on a Massively Parallel Computer.

01,845

Sell-Organizing Neural Network Character Recognition Using Adaptive Filtering and Feature Extraction.

PB96-119797

01,855

WILLIAMS, C. J.

Measurement of the Atomic Na(3P) Lifetime and of Retardation in the Interaction between Two Atoms Bound in a Molecule

PB97-122360

04,178

WILLIAMS, D.

Proposed High-Accuracy Superconducting Power Meter for Millimeter Waves.

PB94-212669

WILLIAMS, D. F.

02,034

Accurate Electrical Characterization of High-Speed InterConnections.

02,240

Accurate Experimental Characterization of Interconnects: A Discussion of 'Experimental Electrical Characterization of Interconnects and Discontinuities in High-Speed Digital Systems

PB94-216389

02,217

Accurate Transmission Line Characterization

02,217

PB95-151593

02,220

Calibrating On-W

02,352

Coaxial Line-Reflect-Match Calibration.

PB96-200118

02,246

Comments on 'Conversions between $\mathrm{S}, \mathrm{Z}, \mathrm{Y}, \mathrm{h}, \mathrm{ABCD}$, and $T$ Parameters Which Are Valid for Complex Source and Load Impedances'.

PB96-102785

02,069

Compensation for Substrate Permittivity in Probe-Tip Cali-

bration.

01,915

Interconnection Transmission Line Parameter Characterization.

PB94-216397 02,218

Line-Reflect-Match Calibrations with Nonideal Microstrip Standards.

PB96-176599

LRM Probe-Tip Calibrations Using Nonideal Standards.

PB96-135389 02,411

LRM Probe-Tip Calibrations with Imperfect Resistors and Lossy Lines.

PB95-163952

02,353

Microwave Characterization of Printed Circuit Transmission Lines.

PB96-122585

02,077

On-Wafer Impedance Measurement on Lossy Substrates.

PB95-176285

Planar Resistors for Probe Station Calibration.

PB95-163697

02,365

Reciprocity Relations in Waveguide Junctions

PB94-172814

02,351

Verification of Commercial Probe-Tip Calibrations.

PB95-161576

Verification of Scattering Parameter Measurements. PB95-163960

WILLIAMS, D. H.

Fiber Coating Diameter: Toward a Glass Artifact Standard. PB96-14038

WILLIAMS, E.

Measurement and Reduction of Alignment Errors of the NIST Watt Experiment

01,987

NIST Watt Balance: Progress Toward Monitoring the Kilo-

gram. 113062

01,991

WILLIAMS, E. R

Application of Single Electron Tunneling: Precision Capacitance Ratio Measurements.

PB96-102157

04,703

Cryogenic Precision Capacitance Bridge Using a Single Electron Tunneling Electromerer

04,529

Cryogenic Precision Capacitance Bridge Using a Single Electron Tunneling Electrometer.

02,040

Cryogenic Precision Capacitance Bridge Using a Single Electron Tunneling Electrometer.

PB96-112271

02,072

Flux-Locked Cur

02,039

Magnetometer Calibration Services.

PB97-113252

01,993

Plits for Aligning the NIST Watt-Balance.

01,934

New Refractometer by Combining a Variable Length Vacuum Cell and a Double-Pass Michelson Interferometer

PB97-111926

01,986

Results of Capacitance Ratio Measurements for the Single Electron Pump-Capacitor Charging Experiment.

PB97-113286

04,813

WILLIAMS, F.

Time Domain Network Analysis Using the Multiline TRL Calibration.

WILLIAMS, F. A

Asymptotic and Numerical Analysis of a Premixed Laminar Nitrogen Dioxide-Hydrogen Flame.

PB96-164256

01,422

WILLIAMS, G. P.

Laser-Synchrotron Hybrid Experiments: A Photon to Tickle, A Photon to Poke.

PB96-157847

03,704

Silicon Surface Chemistry by IR Spectroscopy in the Mid- to

Far-IR Region: $\mathrm{H} 2 \mathrm{O}$ and Ethanol on $\mathrm{Si}(100)$.

01,097

WILLIAMS, J.

Thermodyriamic Analysis of Heparin Binding to Human Antithrombin.

WILLIAMS, J. G.

Lunar Laser Ranging: A Continuing Legacy of the Apollo Program.

03,683

WILLIAMS, J. J.M.

Quasielastic and Inelastic Neutron-Scattering Studies of ((CD3)3ND)FeCl3.2D2O: A One-Dimensional Ising FBerromagnet. 140562

WILLIAMS, K. L

Selectivity Trends in Packed Column Supercritical Fluid Chromatography with C18 Stationary Phases.

PB96-138581

00,622

Use of a Naphthylethylcarbamoylated- beta-Cyclodextrin Chiral Stationary Phase for the Separation of Drug Supercritical Fluid Chromatography.

PB97-113260

WILLIAMS, P.

Molecular Ion Imaging and Dynamic Secondary Ion Mass Spectrometry of Organic Compounds.

PB95-126124

WILLIAMS, P. A. 
MCNP Model of the National Bureau of Standards Reactor (NBSR) Core.

03,733

Nuclear Heat Load Calculations for the NBSR Cold Neutron Source Using MCNP.

PB95-152955

03,730

Thermal Hydraulic Tests of a Liquid Hydrogen Cold Neutron

Source.

03,884

Upgrade and Modernization Projects at the NBSA

03,737

WILLIAMS, R. M.

Investigating the 3.3 Micron Infrared Fluorescence from Naphthalene Following Ultraviolet Excitation.

N95-15839/0

00,724

WILLIAMSON, C. K.

Environmental Aspects of Halon Replacements: Considerations for Advanced Agents and the Ozone Depletion Potential of CF31.

PB97-122261

WILLIAMSON, M. P.

03,301

NIST Workshop on the Computer Interface to Flat Panel Displays. Held in San Jose, California on January 13-14, PB95-136388

01,625

WILLIAMSON, T G.

Measurement of the $(93) \mathrm{Nb}(\mathrm{n}, 2 \mathrm{n})(92 \mathrm{~m}) \mathrm{Nb}$ Cross Section in a (235) U Fission Spectrum.

03,945

WILLIS, R. F.

Variation in Magnetic Properties of Cu/Fcc (001) Sandwich Structures.

PB95-141164

04,555

WILLMAN, $N$.

Design and Development of an Information Retrieval System for the EAMATE Data. Volume 2 of 2 . Appendices.

PB94-168390

00,487

WILLMAN, N. E.

Prototype Information Retrieval System to Perform a BestMatch Search for Names.

PB95-181152

02,740

WILSON, A. J. C.

Statistical Descriptors in Crystallography. 2. Report of a Working Group on Expression of Uncertainty in Measure-

PB96-146824

04,764

\section{WILSON, C. L.}

Analysis of a Biologically Motivated Neural Network for Character Recognition.

00,182

Binary Decision Clustering for Neural Network Based Optical Character Recognition.

01,848

Binary Decision Clustering for Neural-Network-Based Optical Character Recognition.

01,857

Effect of Training Dynamics on Neural Network Performance.

01,852

Face Recognition Technology for Law Enforcement Applica. tions.

01,837

Human and Machine Recognition of Faces: A Survey.

PB96-111687 01,854

Improving Neural Network Performance for Character and Fingerprint Classification by Altering Network Dynamics. PB95-267803 01,851

Improving Neural Network Performance for Character and Fingerprint Classification by Altering Network Dynamics.
PB96-123195 NIST Form-Based Handprint Recognition System. PB94-217106

01,838

PCASYS: A Pattern-Level Classification Automation System for Fingerprints.

01,853

Second Census Optical Character Recognition Systems Conference.
PB94-188711

01,832

Self-Organizing Neural Network Character Recognition on a Massively Parallel Computer.

01,845

Self-Organizing Neural Network Character Recognition Using Adaptive Filtering and Feature Extraction.

PB96-119797

01,855

WILSON, C. R.

Economic, Energy, and Environmental Impacts of the Energy-Related Inventions Program.

00,008

WILSON, G. L

Estimation of the Absorbed Dose in Radiation-Processed Food. 1. Test of the EPR Response Function by a Linear Regression Analysis.

PB94-199718

00,039

WILSON, M. A.

Hybrid Undulator for the NIST-NRL Free-Electron Laser.
NIST-NRL Free-Electron Laser Facility.

PB94-21251

04,237

Time Dependent Vector Dynamic Programming Algorithm for the Path Planning Problem.

for the Path Pla

03,428

WILSON, R. B.

SGML Parser Validation Procedures.

PB95-174959

WINCHESTER, M. R.

Fourier Transform Atomic Emission Studies Using a Glow Discharge as the Emission Source.

00,533

WINCHESTER, N. K.

Determination of the Transmittance Uniformity of Optical Filer Standard Reference Materials.

PB95-261921

02,182

WINDORBSKA, $W$

DNA Base Damage in Lymphocytes of Cancer Patients Undergoing Radiation Therapy.
PB97-122444

WINDSOR, E. S.

03,643

Airborne Asbestos Method: Standard Practice for Recording Transmission Electron Microscopy Data for the Analysis of Asbestos Collected onto Filters. Version 10 .

PB94-210168

00,552

Proficiency Tests for the NIST Airborne Asbestos Program,

PB94-188836

00,535

WINDT, D. L.

XUV Characterization Comparison of Mo/Si Multilayer Coat ings.

PB95-164000

WINEBURG, J. P

04,278

Methodologies for Predicting the Service Lives of Coating Systems.

03,124

WINELAND, D.

Electrostatic Modes of Ion-Trap Plasmas.

PB95-152963

03,920

Experimental Results on Normal Modes in Cold, Pure Ion Plasmas.
PB95-17510

WINELAND, D. J.

Electrostatic Modes as a Diagnostic in Penning-Trap Experiments.

High-Order Multipole Excitation of a Bound Electron.

High-Resolution Atomic Spectroscopy of Laser-Cooled lons.

Interference in the Resonance Fluorescence of Two Trapped Atoms

PB95-168514

Laser-Cooled Positron Source.

PB95-169348

03,948

Laser Cooling of Trapped Ions.

03,954

PB95-168746

03,950

Light Scattered from Two Atoms.

04,286

Liquid and Solid Atomic lon Plasmas.

03,809

Non-Neutral Ion Plasmas and Crystals, Laser Cooling, and $\begin{array}{ll}\text { Atomic Clocks. } & \\ \text { PB95-175113 } & \end{array}$

03,957

Precise Spectroscopy for Fundamental Physics.

PB96-112164

04,040

Progress on a Cryogenic Linear Trap for $(199) \mathrm{Hg}(+)$ lons.

PB95-180790 03,965

Ouantum-Limited Cooling and Detection of Radio-Frequency Oscillations by Laser-Cooled Ions.

PB96-112073

04,039

Quantum Measurements of Trapped lons.

Quantum Projection Noise: Population Fluctuations in TwoLevel Systems.

03,850

Recent Experiments on Trapped Ions at the National Insti-

tute of Standards and Technolog;

03,952

Spin Squeezing and Reduced Ouantum Noise in Spectroscopy.

03,912

Squeezed Atomic States and Projection Noise in Spectroscopy.

03,960

Trapped Atoms and Laser Cooling

03,911

PB95-151627

Trapped lons and Laser Cooling 4: Selected Publications of
the Ion Storage Group of the Time and Frequency Division, the lon Storage Group of the Time and Frequency Division,
NIST, Boulder, Colorado.
PB PB96-172358

04,708

Unusual Spin-Trap Chemistry for the Reaction of Hydroxyl

Radical with the Carcinogen N-Nitrosodimethylamine. 00,692

WINKLER, P. F.

CCD Mosaic Images of the Supernova Remnant $3 C 400.2$. G203 2-12.3: A New Optical Supernova Remnant in Orion. PB95-203535 00,085 Rapıd Decline in the Optical Emission from SN 1957 in M83.

PB94-216033

00,070

WINNE WISSER, $M$

Millimeter- and Submillimeter-Wave Spectrum of trans-Ethyl Alcohol.

PB96-145578

01,102

WINOKUR, M. J.

Inelastic-Neutron-Scattering Studies of Poly(p-phenylene vi-

nylene).

WINSLOW, D.

01,014

Percolation and Pore Structure in Mortars and Concrete.

PB95-150439

WINTENBURG, A. L

Observations of Partial Discharges in Hexane Under High Magnification.

WINTERS, M. P.

High-Resolution Optical Multiplex Spectroscopy.

PB95-203543

01,900

WIPF, $\mathrm{H}$.

Hydrogen in YBa2Cu3Ox: A Neutron Spectroscopy and a Nuclear Magnetic Resonance Study.

PB95-161279

04,601

Inelastic Neutron Scattering Study of Hydrogen in Nanocrystalline Po.

PB96-146857

03,366

Neutron-Spectroscopy Study of the Hydrogen Vibrations in Hydrogen-Doped YBa2Cu3Ox

PB94-172475

04,447

Vibrational Excitations and the Position of Hydrogen in Nanocrystalline Palladium.

PB96-111828

04,035

WIPF, S. L.

Temperature and Field Dependence of Flux Pinning in $\mathrm{NbTi}$ with Aritificial Pinning Centers.

PB96-112024

04,726

WISE, G.

Analysis of Transverse Flow in Aligned Fibrous Porous Media.

03,177

WISE, J. A.

Assessment of Uncertainties of Liquid-in-Glass Thermometer Calibrations at the National Institute of Standards and Technology.

02,625

WISE, $S$.

Comparison of Methods for Gas Chromatographic Determination of PCBs and Chlorinated Pesticides in Marine Reference Materials

PB95-140091

02,584

Comparison of the Liquid Chromatographic Behavior of Selected Steroid Isomers Using Different Reversed-Phase Materials and Mobile Phase Compositions.

00,574

Development of Engineered Stationary Phases for the Separation of Carotenoid Isomers.

00,578

Development of Frozen Whale Blubber and Liver Reference Materials for the Measurement of Organic and Inorganic Materials for the Measurement of Organic and Inorganic Contaminants. 
Considerations in the Design of an Environmental Specimen Bank: Experiences of the National Biomonitoring Specimen Bank Program.

02,527

PB96-112370

Current Activities Within the National Biomonitoring Speci-
men Bank.
PB94-172806
02,516

PB94-172806

Mammal

Tissues.

Tissues.
PB95-152047

00,589

Determination of PCBs and Chlorinated Hydrocarbons in Marine Mammal Tissues.

03,744

Determination of Polycyclic Aromatic Hydrocarbons by Liquid Chromatography.

00,585

Distinguishing the Contributions of Residential Wood Combustion and Mobile Source Emissions Using Relative Concentrations of Dimethylphenanthrene Isomers.

PB96-135124

02,563

Individual Carotenoid Content of SRM 1548 Total Diet and Influence of Storage Temperature, Lyophilization, and Irradiation on Dietary Carotenoids.

diation on Dietar
PB94-200524

00,033

Influence of Stationary Phase Chemistry on Shape Recognition in Liquid Chromatography.

00,621

Liquid Chromatographic Determination of Carotenoids in Human Serum Using an Engineered $\mathrm{C} 30$ and a C18 Stationary Phase.

03,512

Liquid Chromatographic Determination of Polycyclic Aromatic Hydrocarbon Isomers of Molecular Weight 278 and 302 in Environmental Standard Reference Materials

PB95-164042

02,523

Measurements of Indoor Pollutant Emissions from EPA Phase II Wood Stoves.

02,556

NIST Standard Reference Materials (SRMs) for Polychlorinated Bipheny! (PCB) Determinations and Their Applicability to Toxaphene Measurements.

PB95-140109

02,585

Quality Assurance of Contaminant Measurements in Marine Mammal Tissues.

02,588

Quantitative Analysis of Selected PCB Congeners in Marine Matrix Reference Materials Using a Novel Cyanobiphenyl Stationary Phase.

PB96-111737

02,591

Relationship of Silver with Selenium and Mercury in the Liver of Two Species of Toothed Whales (Odontocetes).

Selectivity Trends in Packed Column Supercritical Fluid Chromatography with $\mathrm{C} 18$ Stationary Phases.

00,622

Shape Selectivity Assessment of Stationary Phases in Gas Chromatography

00,579

Shape Selectivity in Reversed-Phase Liquid Chromatography for the Separation of Planar and Non-Planar PB95-162608

00,596

Standard Reference Materials for the Determination of Polycyclic Aromatic Hydrocarbons in Environmental Samples - Current Activities.

00,586

Standard Reference Materials for the Determination of Trace Organic Constituents in Environmental Samples.

PB95-164026

02,522

Trace Element Concentrations in Cetacean Liver Tissues Archived in the National Marine Mammal Tissue Bank.
PB96-167127 Use of a Naphthylethylcarbamoylated- beta-Cyclodextrin Chiral Stationary Phase for the Separation of Drug Enantiomers and Related Compounds by Sub- and Supercritical Fluid Chromatography.

PB97-113260
WITCZAK, S. C.

00,653

Interaction of Stoichiometry, Mechanical Stress, and Interface Trap Density in LPCVD Si-rich SiNx-Si Structures. PB95-176301

02,366

WITT, T. J.

Accuracy Comparisons of Josephson Array Systems. PB95-164687

02,047

WITTE, A.

Magneto-Optical Trapping of Metastable Xenon: IsotopeShit Measurements.

WITTMAN, R. C.

03,905

Vector Theory of Diffraction by a Single-Mode Fiber: Application to Mode-Field Diameter Measurements.

PB95-164182

WITTMANN, R. C.

Integral Occurring in Coherence Theory

04,324

PB95-216925

Polarimetric Calibration of Reciprocal-Antenna Radars.
Proposed Analysis of RCS Measurement Uncertainty.

RangeCAD and the NIST RCS Uncertainty Analysis.

01,871

PB94-21859

01,870

WITZGALL, C.

Algorithmic Enhancements to the Method of Centers for Lin-

ear Programming Problems.
PB94-198959

03,426

Interior-Point Method for Linear and Ouadratic Programming Problems. (NIST Reprint).

03,429

Submissions to a Planned Encyclopedia of Operations Research on Computational Geometry and the Voronoi Delaunay Construct.

PB94-152709

03,425

LASSICH, J. J.

Investigation into a Practical Grain Growth Model for Hot Isostatic Pressing

PB95-151684

Crystal Packing Interactions of Two Different Crystal Forms of Bovine Ribonuclease A

PB95-15282

00,943

Numerical Reference Models for Optical Metrology Simula-

PBon.

04,392

WOJNAROVITS, L.

Alcohol Solutions of Triphenyl-Tetrazolium Chloride as High-Dose Radiochromic Dosimeters.

PB96-135249

03,716

Novel Radiochromic Films for Clinical Dosimetry.

PB97-119259

03,641

Radiation-Chemical Reaction of 2,3,5-Triphenyl-Tetrazolium Chloride in Liquid and Solid State.

01,124

Radiochromic Solid-State Polymerization Reaction.

PB95-180683

01,271

Radiochromic Solid-State Polymerization Reaction.

PB96-180146

01,290

WOLCOTT, D.

Multi-Agency Certification and Accreditation ( $\mathrm{C}$ and $\mathrm{A})$ Process: A Worked Example.

PB95-171955

01,601

WOLF, W. R.

Mixed Diet Reference Materials for Nutrient Analysis of Foods: Preparation of SRM-1548 Total Diet.

03,593

WOLFE, J.

Distributed measurements of tracer response on packed bed flows using a fiberoptic probe array. Final report.
DE 95013079

00,667

NOLFE, R.

High Frequency Magnetic Field Sensors Based on the Far-

aday Effect in Garnet Thick Films.

02,282

Magneto-Optic Magnetic Field Sensors Based on Uniaxial Iron Garnet Films in Optical Waveguide Geometry.

PB95-153409 Iron Garnet Films in Optical Waveguide Geometry.

PB95-168498

WOLFENDEN, A.

Dynamic Shear Modulus Measurements with Four Inde-

pendent Techniques in Nickel-Based Alloys
PB94-198900

03,320

WOLTZ, L. A.

Ion Broadening Parameters for Several Argon and Carbon

Lines.

03,843

WOMELDOAF, C

Acid Gas Production in Inhibited Diffusion Flames.

PB95-180576

01,390

Lean flammability limit as a fundamental retrigerant property. Phase 1, Interim technical report, 1 October 1994--31 March 1995.

WONG-NG, W.

03,248

Crystal Chemistry and Phase Equilibria Studies of the $\mathrm{BaO}(\mathrm{BaCO}$ )-R2O3-CuO Systems. 4. Crystal Chemistry and Subsolidus Phase Relationship Studies of the CuORich Region of the Ternary Diagrams, R=Lanthanides. PB95-151759

00,936

Crystal Chemistry and Phase Equilibrium Studies of the $\mathrm{BaO}(\mathrm{BaCO}) \cdot \mathrm{R} 2 \mathrm{O} 3-\mathrm{CuO}$ Systems. 5. Melting Relations in $\mathrm{Ba2}$ (Y,Nd,Eu)Cu3O6+X

PB95- 151718

04,580

Crystal Chemistry and Phase Equilibrium Studies of the BaO-R2O3-CuO Systems. 2. X-Ray Characterization and Standard Patterns of BaR2CuO4, R=Lanthanides. PB95-151734

04,582

Crystal Structure of a New Monoclinic Form of Potassium Dihydrogen Phosphate Containing Orthophosphacidium Ion, (H4PO4)(sup+1).

04,725

Efficient Experiment to Study Superconducting Ceramics PB94-212578 04,505 Microstructure and Ferroelectric Properties of Lead Zirconate-Titanate Films Produced by Laser Evaporation

PB94-199148 04,470

Molecular Orbital Calculations of Bond Rupture in Brittle Solids.

PB95-164059 00,973

Molecular Orbital Study of Water Enhanced Crack Growth Process.

PB95-164067

03,240

Preparation and Crystal Structure of Sr5TiNb4017.

04,107

Preparation, Crystal Structure, Dielectric Properties, and Magnetic Behavior of Ba2Fe2Ti4O13.

PB96-186176

01,162

Raman and Fluorescence Spectra Observed in Laser Microprobe Measurements of Several Compositions in the Ln-Ba-Cu-O System

$\mathrm{X}$-Ray Characterization of the Crystallization Process of High-Tc Superconducting Oxides in the Sr-Bi-Pb-Ca-Cu-O System.

04,579

$X$-Ray-Diffraction Study of a Thermomechanically Detwinned Single Crystal of $\mathrm{YBa} 2 \mathrm{Cu} 3 \mathrm{O} 6+x$

04,581

$X$-Ray Powder Diffraction Data for $\mathrm{BaCu}(\mathrm{C} 2 \mathrm{O} 4) 2.6 \mathrm{H} 2 \mathrm{O}$.

PB95-151767

04,583

WOOD, B. E.

Deuterium and the Local Interstellar Medium: Properties for the Procyon and Capella Lines of Sight.

00,111

High Velocity Plasm in the Transition Region of Au Mic: A Stellar Analog of Solar Explosive Events.

00,102

High-Velocity Plasma in the Transition Region of $\mathrm{AU}$ Microscopii: Evidence for Magnetic Reconnection and Satu. rated Heating during Quiescent and Flaring Conditions.

PB96-102694

00,091

Redshifts in Stellar Transition Regions.

00.104

Transition Regions of Capella.

PB96-123336

00,105

Transition Regions of Capella (1995)

PB96-176714

00,108

Volume-Limited ROSAT Survey of Extreme Ultraviolet

WOOD,

00,093

D. $\mathrm{H}$

CRYSTMET: The NRCC Metals Crystallographic Data File.
PB97-109029

WOOD, J.

Influence of Electrical Isolation on the Structure and Reflectivity of Multilayer Coatings Deposited on Dielectric Sub-

PB96-159736

04,365

WOOD, J. L.

New Method for Achieving Accurate Thickness Control for Uniform and Graded Multilayer Coatings on Large Flat Sub.

PB96-159744 
Spectrally Smooth Reflectances That Match.

PB95-176319

WORTHINGTON, J. L.

Design and Machining of Copper Specimens with Micro Holes for Accurate Heat Transfer Measurements. PB95-180428

02,658

WORTHY, J.

Model Minimum Performance Specifications for Lidar Speed Measurement Devices.

PB95-197455

04,861

WRIGHT, J.

Polymer Composites Workshop. Held in Winona, Minnesota on April 29-30, 1992 (Video).

PB94-780129

WRIGHT, R. N.

Ashland Tank Collapse Investigation

PB95-126314

Ashland Tank-Collapse Investigation: Closure by Authors.

PB95-126322 02,482

Implementation of Executive Order 12699: Seismic Safety of Federal and Federally Assisted or Regulated New Build

ing Construction
PBg5-151809

Infratechnologies: Tools for Innovation.

PB94-185998

00,436

Lessons from the Loma Prieta Earthquake.

PB95-164091

00,317

Planning for Construction and Building $R$ and $D$

PB96-137104

00,324

Proceedings of a Workshop on Developing and Adopting Seismic Design and Construction Standards for Lifelines. Held in Denver, Colorado on September 25-27, 1991.

Program of the Subcommittee on Construction and Building PB94-193646 00.319 Program of the Subcommittee on Construction and Building (July 1994).

Rationale and Preliminary Plan for Federal Research for Construction and Building.

PB95-154704

00,322

Status of Construction and Construction Technologies.

PB94-186004

00,318

Structural Analysis in Contex.

PB95-151817

00,437

Think Metric

PB95-151825

01,298

NU, A.

Use of a Radiochromic Detector for the Determination of

Stereotactic Radiosurgery Dose Characteristics.

PB94-185642

03,514

WU, C. F.

Taguchi's Parameter Design: A Panel Discussion.

03.445

WU, D.

Analysis of Creep in a Si-SiC C-Ring by Finite Element Method

PB94-200268

03,037

Wu, D. I.

Aperture Coupling to a Coaxial Air Line: Theory and Experi-

PB94-211968

02,216

WU, $\mathrm{H}$.

Dielectric Properties of Thin Film $\mathrm{SrTiO} 3$ Grown on LaAlO3 with YBa2Cu3O7-x Electodes.

PB95-181160

02,267

WU, J.S.

Mixing and Radiation Properties of Buoyant Luminous Flame Environments.

PB96-202254

01.432

WU, T. G.

Potentiometric Enzyme-Amplified Flow Injection Analysis Detection System: Behavior of Free and Liposome-Reased Peroxidas

PB95-151833

03,534

WU, W.

Advances in the Measurement of Polymer CTE Micrometer- to Atomic-Scale Measurements. PB96-180229 03,390 SANS Study of the Plastic Deformation Mechanism in Polyethylene.

PB95-151841

01.242

Small-Angle Neutron Scattering of Poly(vinyl alcohol) Gels. PB95-164117

01,260

WU, W. L.

Characterization of Molecular Network of Thermosets Using Neutron Scattering.

01,259

Characterization of Polyquinoline Blends Using Small Angle Scattering.
PB95-164125

01,261

Characterization of Polyquinoline Block Copolymer Using Small Angle Scattering.
Elastic Scattering of Polymer Networks

Electronics Packaging Materials Research at NIST

PB96-122692

01,255

Influence of an Impenetrable Interface on a Polymer GlassTransition Temperature.

PB96-146873

03, 175

Novel Method for Determining Thin Film Density by EnergyDispersive X-ray Reflectivity.

04,737

Small Angle Neutron Scattering Studies on Chain Asym. metry of Coextruded Poly(Vinyl Alcohol) Film.

01.262

Structural Heterogeneity in Epoxies.

01,243

Thermoacoustic Technique for Determining the Interface and/or Interply Strength in Polymeric Composites.

PB95-161824

03,158

Water Adsorption at a Polyimide/Silicon Wafer Interface.

PB96-103197 01,070

Water Adsorotion at Polymer/Silicon Wafer Interfaces. PB95-181178

01,022

WU, Y.

Precision of Marchall Stability and Flow Test Using 6-in. (152.4-mm) Diameter Specimens.

PB96-200910

03,006

Y. C

Absolute Determination of Electrolytic Conductivity for Primary Standard KC 1 Solutions from 0 to $50 \mathrm{C}$.

PB94-172798

00,765

dc Method for the Absolute Determination of Conductivities of the Primary Standard KCI Solutions from OC to $50 \mathrm{C}$

PB94-219342

Low Electrolytic Conductivity Standards.

PB96-122098

02,644

WUEBBLES, D. J.

Environmental Aspects of Halon Replacements: Considerations for Advanced Agents and the Ozone Deplation Poiential of CF3l.

PB97-122261

WUENSCH, B. J.

03,301

Fast-Ion Conducting Y2(ZryTi1-y)2O7 Pyrochlores: Neutron

Rietveld Analysis of Disorder Induced by Zr Substitution.
PB96-156104

04.776

Fast-Ion Conduction and Disorder in Cation and Anion Arrays in Y2(ZryTi(1-y))2O7 Pyrochlores Induced by Zr Substitution: A Neutron Rietveld Analysis.

PB94-211869

04,496

WYART, J. F.

Analysis of the $(5 d(2)+5 d 6 s)-5 d 6 p$ Transition Arrays of Os VII and Ir VIII, and the 6s (2)S-6p (2)P Transitions of Ir IX.

PB96-159264

Hyperfine Structure Investigations and Identification of New Energy Levels in the Ionic Spectrum of (147) Pm.

WYCKOFF, H. O.

$X$-ray Protection Design

AD-A279 181/2

04,117

WYSS, J. C

Self-Calibrated Intelligent Optical Sensors and Systems.

XIA, H. R.

Phase Shitts and Intensity Dependence in Frequency-Modulation Spectroscopy.

XIANG, D.

01,071

Design, Construction and Application of a Large Aperture Lens-Less Line-Focus PVDF Transducer

02,765

Material Characterization By a Time-Resolved and Polarization-Sensitive Ultrasonic Technique. tion-Sensitive
PB97-122576

02,764

Transient Analysis of a Line-Focus Transducer Probing a Liquid/Solid Intertace.

PB97-118681

02,763

$X I A O, G$

Incorporation of Gold into YBa2Cu307: Structure and Tc Enhancement.

04,481

Unexpected Effects of Gold on the Structure,
Superconductivity, and Normal State of YBa2Cu307. PB94-200284

04,482

XIAO, J. $Q$.

Domain Structures in Magnetoresistive Granular Metals.

PB96-141346

04,760

XIAO, Q. F.

Neutron Focusing Lens Using Polycapillary Fibers

03,889

Neutron Focusing Lens Using Polycapillary Fibers PB95-153078

03,922

Elastic Constants of a Material with Orthorhombic Symmetry: An Alternative Measurement Approach.

04,684

XIN, J.

Internal Droplet Circulation Induced by Surlace-Driven RotaPB97-119267

02,500

XIONG, $Q$.

Oxygen Dependance of the Crystal Structure of $\mathrm{HgBa} 2 \mathrm{CuO} 4+$ and its Relation to Superconductivity. PB96-102512

04,711

XIONG, $x$.

Comparative Strategies for Correction of Interferences in sotope Dilution Mass Spectrometric Determination of Vanadium.

PB94-185261

00.531 Configuration-Dependent AC Stark Shitts in Calcium.
PB96-157995

RIS Measurement of AC Stark Shifts and Photoionization Cross Sections in Calcium.

PB96-157953

04,073

Study of Laser Resonance Ionization Mass Spectrometry Using a Glow Discharge Source.

DE94018566

03,308

Study of Laser Resonance Ionization Mass Spectrometry Using a Glow Discharge Source.

PB96-123203

XIONG, $X . Z$.

Epitaxial Integration of Single Crystal $\mathrm{C} 60$

PB95-153490

03,360

XIU-YE, $X$.

Wide Band Active Current Transformer and Shunt.

PB95-126371

04,592

XU, H. H. K.

Evaluation of Thermal Wave Imaging for Detection of Machining Damage in Ceramics.

PB95-220547

$\mathrm{XU}, \mathrm{J} . \mathrm{H}$.

Novel YBa2Cu3O7-x and YBa2Cu307-x/Y4Ba3O9 Multilayer Films by

PB95-181186

$\mathrm{XU}, \mathrm{Y}$.

of Finite Element and Analytic Calculations of

the Resonant Modes and Frequencies of a Thick Shell Sphere.

$\mathrm{Xu}, \mathrm{z}$.

02,626

Characterization of the Interaction of Hydrogen with Iridium Clusters in Zeolites by Inelastic Neutron Scattering Spectroscopy.

YACZKO, D.

Commentary on 'Optimization of Experimental Parameters or the EPR Detection of the Cellulosic Radical in Irradiated Foodstuff':

PB96-164124

YACZKO, D. M.

Activities in Electron PB96-141288

03.635

YAJIMA, $H$.

Dynamic Light-Scattering Study of a Diluted Polymer Blend NBo5-151890 
YANG, A.

Beyond the Technology Roadmaps: An Assessment of Electronic Materials Research and Development PB96-165998

01,961

YANG, A. J. M.

Mixing Plate-Like and Rod-Like Molecules with Solvent: A Test of Flory-Huggins Lattice Statistics.

03,173

YANG, $B$.

Intensity-Dependent Scattering Rings in High Order AboveThreshold lonization.

PB96-110739

04,032

YANG, $C$.

Integration of Real-Time Process Planning for Small-Batch Flexible Manufacturing.

PB95-151908

02,822

Open Architectures for Machine Control.

02,942

YANG, D.

Interoperability Experiments with CORBA and Persistent Object Base Systems.

PB96-183140

01,772

YANG, J.

Agent Screening for Halon 1301 Aviation Replacement

PB96-159710 03,282

Ultrasonic Technique for Sizing Voids Using Area Func-

tions. 151916

02,904

YANG, J.C.

Combustion of a Polymer (PMMA) Sphere in Microgravity. N96-15569/2

01,354

Estimate of the Effect of Scale on Radiative Heat Loss Fraction and Combustion Efficiency.

PB95-150447

02,486

Flow of Alternative Agents in Piping

00,022

Minimum Mass Flux Requirements to Suppress Burning Surfaces with Water Sprays.

PB96-183181

01,425

Solid Propellant Gas Generators: Proceedings of the 1995 Workshop. Held in Gaithersburg, Maryland on June 28-29, 1995.

PB96-131479

01,412

YANG, $S$.

Accurate Measurement of Optical Detector Nonlinearity.
PB95-203576 Automated Measurement of Nonlinearity of Optical Fiber Power Meters.

04,110

Optical Detector Nonlinearity: A Comparison of Five Meth. ods.

PB95-169355

Optical Detector Nonlinearity: Simulation.

04,291

PB96-165378

02,199

YANIV, S. L.

Precision in Machining: Research Challenges. PB95-242301

02953

YAP, W.

Supported Phospholipid/Alkanthiol Biomimetic Membranes: Insulating Properties.

YAP, W. T.

Thermodynamics of the Hydrolysis of Penicillin $G$ and Ampicillin.

PB94-172467

03,596

YARBROUGH, D. W.

Comparison of Heat-Flow-Meter Tests from Four Laboraories.

126264

00,365

YARBROUGH, W. A.

Applications of Diamond Films and Related Materials: International Conference (3rd). Held in Gaithersburg, Maryland on August 21-24, 1995. Supplement to NIST Special Publication 885 .

PB95-256053

03,063

Proceedings of the Applied Diamond Conference 1995: Applications of Diamond Films and Related Materials international Conference (3rd). Held in Gaithersburg, Maryland on August 21-24, 1995.

PB95-255204

04,701

PBMOFF, J. A.

Desorption Induced by Electronic Transitions.

B94-21617

00,853

Influence of Coadsorbed Potassium on the Electron-Stimulated Desorption of $F(+), F(-)$, and $F(*)$ from $P F 3$ on Ru(0001).

YE, J.

04,072

Delivering the Same Optical Frequency at Two Places: Accurate Cancellation of Phase Noise Introduced by an Optical Fiber or Other Time-Varying Path.

PB96-102736

04,332

Transient Creep Behaviour of Hot Isostatically Pressed Sili-

Transient Creep Behaviour of Hot Isostatically Pressed Sil-
con Nitride. con Nitride.
PB96-180278

03,086
YEE, K. W.

Post-Process Control of Machine Toots. PB95-203451

02,952

YEH, T. I.

Comparisons of Some NIST Fixed-Point Cells with Similar Cells of Other Standards Laboratories.

00,655

YEH, T. T.

Effects of Pipe Elbows and Tube Bundles on Selected Types of Flowmeters.

PB96-160999

01,135

Flowmeter Installation Effects Due to a Generic Header.

PB96-210893 02,606

Laser Doppler Velocimeter Studies of the Pipeflow Produced by a Generic Header.

PB95-226916 02,602

Summary Report of NIST's Industry-Government Consortium Research Program on Flowmeter Installation Effects: The 45 Degree Elbow.

04,204

Summary Report of NIST's Industry-Government Consortium Research Program on Flowmeter Installation Effects with Emphasis on the Research Period, January-September 1991: The Reducer.

PB94-160736

04,196

YENTIS, $\boldsymbol{R}$.

Micromachined Display Output for a Cellular Neural Net-

Work. 156070

02,422

YESHA, Y.

Channel Coding for Code Excited Linear Prediction (CELP) Encoded Speech in Mobile Radio Applications.

PB95-143178

01.475

Coping with Different Retrieval Methods in Next Generation Networks.

PB95-168555

02,726

YIN, L. I.

Pattern-Recognition Analysis of Low-Resolution X-Ray Fluorescence Spectra.

00,588

Tomographic Decoding Algorithm for a Nonoverlapping Redundant Array

01,842

YING, T. N.

Asperity-Asperity Contact Mechanisms Simulated by a TwoBall Collision Apparatus.

PB95-164158

02,966

Chemical Effect in Ceramics Grinding.

PB97-122592

03,113

YODER, C. F.

Lunar Laser Ranging: A Continuing Legacy of the Apollo Program.

YOKEL, $F$. Y.

03,683

Earthquake Resistant Construction of Electric Transmission and Telecommunication Facilities Serving the Federal Government Report.

PB94-161817

02,460

Earthquake Resistant Construction of Gas and Liquid Fuel Pipeline Systems Serving, or Regulated By, the Federal Government.

04,846

Recommended Performance-Based Criteria for the Design of Manufactured Home Foundation Systems to Resist Wind and Seismic Loads.

PB96-128285

00,460

YOLKEN, H. T.

Intelligent Processing of Materials

YOON, $\mathrm{H}$.

02,811

Structural Stabilization of Phase Separating PC/Polyester Blends through Interfacial Modification by Transesterification Reaction.

PB95-150454

01,239

YOSHIDA, $\mathrm{K}$

Charpy Impact Test as an Evaluation of $4 \mathrm{~K}$ Fracture Toughness.

PB96-190194

SHIHIRO, $K$.

Anomalous Behavior of a Quantized Hall Plateau in a HighMobility Si Metal-Oxide-Semiconductor Field-Effect Transis-

PB95-164174

02,354

YOSHIKAWA, $M$.

Applications of Diamond Films and Related Materials: International Conference (3rd). Held in Gaithersburg, Maryland on August 21-24, 1995 . Supplement to NIST Special Publication 885

PB95-256053

03,063

Proceedings of the Applied Diamond Conference 1995: Applications of Diamond Films and Related Materials International Conference (3rd). Held in Gaithersburg, Maryland, on August 21-24, 1995.

PB95-255204

04,701

YOU, L

Collisional Energy Transfer between Excited-State Strontium and Noble-Gas Atoms.

PB95-202958

03,995

Cone Emission from Laser-Pumped Two-Level Atoms. 1 Quantum Theory of Resonant Light Propagation.

04,325
Cone Emission from Laser-Pumped Two-Level Atoms. 2. Analytical Model Studies.

PB95-203592

04,326

Failures of the Four-Wave Mixing Model for Cone Emission

PB95-202636 04,318

Probing Bose-Einstein Condensed Atoms with Short Laser Pulses.

PB95-202818

03,991

Suppression of Ionization in One- and Two-Dimensional Model Calculation

YOUNG, G.

04,009

Histopathology, Blood Chemistry, and Physiological Status of Normal and Moribund Striped Bass ('Morone saxatilis') (nvolved in Summer Mortality ('Die-Off') in the SacramentoSan Joaquin Delta of California.

PB94-198157

00,034

YOUNG, $\mathbf{K}$.

Submarine Automation: Demonstration No. 5

PB95-251633

03,748

YOUNG, L.

Hyperfine-Structure Studies of $\mathrm{Zr}$ II: Experimental and Relativistic Configuration-Interaction Results.

04,011

Precision Lifetime Measurements of Cs $6 p(2) P 1 / 2$ and $6 p$ (2) $P 3 / 2$ Levels by Single-Photon Counting

PB95-203816

04,010

YOUNG, $M$

Fiber Coating Diameter: Toward a Glass Artifact Standard. PB96-140389 02,234

Optical Fiber, Fiber Coating, and Connector Ferrule Geometry: Results of Interlaboratory Measurement Comparisons.

PB96-154422 04,360

Optical Fiber Geometry: Accurate Measurement of Cladding Diameter

PB95-151940

04,266

Optical Fiber Geometry by Gray-Scale Analysis with Robust Regression.

Standard Reference Materials for Optical Fibers and Connectors

PB96-119805

04,344

Vector Theory of Diffraction by a Single-Mode Fiber: Application to Mode-Field Diameter Measurements.

PB95-164182 04,279

Video Microscopy Applied to Optical Fiber Geometry Meas-

urements.
PB95-173068

04,295

YOUNGER, F. C.

Hybrid Undulator for the NIST-NRL Free-Electron Laser.

PB94-212529

04,238 
YUST, $M$

Thin Film Thermocouples for Measurement of Wall Temperatures in Internal Combustion Engines.

01,449

YUYAMA, M.

$\mathrm{n}$-Value and Second Derivative of the Superconductor Voltage-Current Characteristic.

PB95-126223

Agent Screening for Halon 1301 Aviation Replacement.

Agent Screening 159710

04,533

ZACHARIAH, M. R.

Controlled Nucleation in Aerosol Reactors for Supression of Agglomerate Formation

PB95-151973

00,672

Experimental and Numerical Studies of Refractory Particle Formation in Flames: Application to Silica Growth.

PB95-152005

00,673

Flame Synthesis of High Tc Superconductors.

PB95-151981

00,659

Fluorinated Hydrocarbon Flame Suppression Chemistry.
PB94-185113

Gas Phase Reactions Relevant to Chemical Vapor Deposition: Numerical Modeling.

PB94-199346

03.117

Halon Thermochemistry: 'Ab Initio' Calculations of the Enthalpies of Formation of Fluoromethanes.

PB96-175740

03,289

In situ Characterization of Vapor Phase Growth of Iron Oxide-Silica Nanocomposites: Part 1. 2-D Planar Laser-Induced Fluorescence and Mie Imaging.

03,185

In-situ Studies of a Novel Sodium Flame Process for Synthesis of Fine Particles.

00.681

PB97-113047

Modeling Ceramic Sub-Micron Particle Formation from the Vapor Using Detailed Chemical Kinetics: Comparison with In-situ Laser Diagnostics.

00.671

Multiphoton Ionization Spectroscopy Measurements of Silicon Atoms during Vapor Phase Synthesis of Ceramic Par-

PB95-151999

03.913

Optical and Modeling Studies of Sodium/Halide Reactions

for the Formation of Titanium and Boron Nanoparticles.
PB97-113054 00.682

Simulation of Ceramic Particle Formation: Comparison with In-situ Measurements.

PB95-152013

00,674

Thermochemical and Chemical Kinetic Data for Fluorinated Hydrocarbons.

ZAENGL, $W$

01,056

Approach to Setting Performance Requirements for Automated Evaluation of the Parameters of High-Voltage Im-

pulses.
PB94-185634

01,878

ZAGHLOUL, M

High-Level CAD Melds Micromachined Devices with Foundries.

PB94-216413

02,321

Realizing Suspended Structures on Chips Fabricated by CMOS Foundry Processes Through the MOSIS Service. PB94-193984

01,881

ZAGHLOUL, M. E

Color Supplement to NIST Special Publication 400-93: Semiconductor Measurement Technology: Design and Testing Guides for the CMOS and Lateral Bipolar-on-SOI Test Library.

PB94-164316

02,298

Design Guide for CMOS-On-SIMOX. Test Chips NIST3 and PB94-163458

02,297

Micromachined Coplanar Waveguides in CMOS TechPB97-119283

02,456

Micromachined Display Output for a Cellular Neural NetPBO6-156070

02,422

Semiconductor Measurement Technology: Design and Testing Guides for the CMOS and Lateral Bipolar-on-SOI Test Library.

PB94-178019

02,301

Test Structures for Determining Design Rules for Microelectromechanical-Based Sensors and Actuators.
PB95-150488

PB95-150488
ZAGLOUL, M. E.

CMOS Circuit Design for Controlling Temperature in Micromachined Devices.

PB96. 156088

02,196

ZAIDI, M. K.

Calibration and Performance of GafChromic DM-100 Radiochromic Dosemeters.

PB97-119291

ZAJCHOWSKI, P. H.

00,703

Characterization and Processing of Spray-Dried Zirconia Powders for Plasma Spray Application

04,419
ZANDER, $M$.

Optical Detector Nonlinearity: Simulation

PB96-165378

02,199

Coarsening of Unstable Surface Features during $\mathrm{Fe}(001)$ Homoepitaxy

ZAPAS, L. J.

Performance of Plastic Packaging for Hazardous Materials Transportation. Part 1. Mechanical Properties.

AD-A301 258/0

02,580

ZARDECKI, C.

Nucleic Acid Database: Present and Future.

PB97-109078

ZAREMBA, C. M.

Structural and Chemical Investigations of Na3(ABO4)3.4H2O-Type Sodalite Phases. 01,012

\section{ZARR, R. R.}

Comparison of Heat-Flow-Meter Tests from Four Labora-

PB95-126264

00.365

Control Stability of a Heat-Flow-Meter Appartus

BB95-181194

00,386

Effect of Environmentally Exposures on the Properties of Polyisocyanurate Foam Insulation: Thermal Conductivity Measurements.

PB95-181210

00,388

Effects of Humidity and Elevated Temperature on the Density and Thermal Conductivity of a Rigid Polyisocyanurate

PB95-152021

00,373

Effects of Humidity and Elevated Temperature on the Density and Thermal Conductivity of a Rigid Polyisocyanurate Foam Co-Blown with $\mathrm{CCl} 3 \mathrm{~F}$ and $\mathrm{CO} 2$.

B95-150462

00.371

Experimental Verification of a Moisture and Heat Transte Model in the Hygroscopic Regime.

PB97-111546

00,309

Heat and Moisture Transfer in Wood-Based Wall Construction: Measured versus Predicted.

PB95-200655

00,391

Intra-Laboratory Comparison of a Line-Heat-Source Guarded Hot Plate and Heat-Flow-Meter Apparatus.

00,387

5-181202

Line-Heat-Source Guarded-Hot-Plate Apparatus. $\quad 00,417$

Room-Temperature Thermal Conductivity of Expanded Polystyrene Board for a Standard Reference Material.

PB96-193693

00,412

Room Temperature Thermal Conductivity of Fumed-Silica Insulation for a Standard Reference Material.

B95-152039

ZASTAWNY, T.

Novel Activity of E. coli uracil DNA N-glycosylase Excision of Isodialuric Acid (5,6-dihydroxyuracil), a Major Product of Oxidative DNA Damage, from DNA

PB96-110747

03,543

Oxidative DNA Base Damage in Renal, Hepatic, and Pulmonary Chromatin of Rats After Intraperitoneal Injection of Cobalt (II) Acetate.

PB95-150025

03,647

Tert-Butyl Hydroperoxide-Mediated DNA Base Damage in Cultured Mammalian Cells.

PB94-182003

03.644

ZASTAWNY, T. H.

DNA Base Damage in Lymphocyles of Cancer Patients Un dergoing Radiation Therapy.

PB97-122444

03,643

DNA Damage and DNA Sequence Retrieval from Ancient Tissues.

PB97-111983

03,556

Formation of DNA.Protein Cross-Links in Cultured Mammalian Cells Upon Treatment with Iron lons.

03,651 Novel DNA $\mathrm{N}$-Glycosylase Activity of $\mathrm{E}$. coli T4
Endonuclease $\mathrm{V}$ That Excises Endonuclease $V$ That Excises 4,6-Diamino-5Formamidopyrimidine from DNA, a UV-Rad Hydroxyl Radical
PB96-160478

03,549

ZAVADA, J. M.

Using Secondary Ion Mass Spectrometry (SIMS) to Characterize Optical Waveguide Materials.

PB96-119599

04,340

ZEISLER, $R$.

Certifying the Chemical Composition of a Biological Material: A Case Study.

00,636

Current Activities Within the National Biomonitoring Speci-

men Bank.
PB94-172806

02,516

Determination of Inorganic Constituents in Marine Mammal

Pissues.

00,589

National Status and Trends Program Specimen Bank: Sampling Protocols, Analytical Methods, Results, and Archive Samples.

02,598

Neutron Capture Prompt Gamma-Ray Activation Analysis at the NIST Cold Neutron Research Facility.

00,556

Trace Elements Associated with Proteins. Neutron Activation Analysis Combined with Biological Isolation Techniques.

PB95-163101

00,597

ZEISSLER, $\mathrm{C} . \mathrm{J}$

Environmental Scanning Electron Microscope Imaging Examples Related to Particle Analysis.

PB94-172822

00,766

ZELKOWITZ, M.

Center for High Integrity Software System Assurance: Initial Goals and Activities.

PB95-251674

01,734

Defining Envir

02,733

Information Technology Engineering and Measurement Model: Adding Lane Markings to the Intormation Superhighway

PB95-143145

01,474

Next Generation Computer Resources: Reference Model for Project Support Environments (Version 2.0)

PB94-143401

01,677

ZELKOWITZ, M. V.

Experimental Models for Software Diagnosis.

PB97-113906

01,783

Measurement of Process Complexity.

PB97-113138

Use of an Environment Classification Model.

PB95-152062

01.781

ZEMKE, W. T.

01,709

Spectroscopy and Structure of the Lithium Hydride Diatomic Molecules and lons.

PB94-160991

00,737

NNER, G. P.

Measurement of Boron at Silicon Wafer Surfaces by Neutron Depth Profiling

PB94-211059

04,487

Scanning Tunneling Microscopy of the Charge-DensityPB95-180980

04,689

ZHANG, C. Y.

IRAS Spectroscopic Observations of Young Planetary NebUlae.

ZHANG, D.

Effects of Etching on the Morphology and Surface Resistance of YBa2Cu307-delta Films

PB96-135355

02,410

ZHANG, G.

Displacement Method for Machine Geometry Calibration PB95-152088

02,946

ZHANG, $\mathrm{H}$. 
Dispersion and Deposition of Smoke Plumes Generated in Massive Fires

ZHANG, $Y$.

02,540

Deposit Forming Tendencies of Diesel Engine Oils-Correlation between the Two-Peak Method and Engine Tests.
PB95-152138

01,452

New Method to Evaluate Deposit Forming Tendencies of Liquid Lubricants by Differential Scanning Calorimetry.

ZHANG, $Y . X$

Digital Techniques in HV Tests - Summary of 1989 Panel Session.

ZHANG, $\mathbf{Z}$.

02,035

Almospheric Reactivity of alpha-Methyl-Tetrahydrofuran.

PB95-163705 02,548

Diffusion of Cations Beneath Organic Coatings on Steel Substrate.

03,119

Experimental Determination of the Ionization Energy of $I O(X($ sup 2) $) \mid($ sub $3 / 2)$ ) and Estimations of Delta(sub f) $\mathrm{H}$ (sup deg)(sub 0$)(10$ (sup - -1$)$ and PA(IO).
PB96-146899

00,694

Gas Phase Reactivity Study of $\mathrm{OH}$ Radicals with 1,1Dichloroethene and cis-1,1-Dichloroethene and Trans-1,2Dichloroethene over the Temperature Range 240-400 K.
PB95-152146 Ionization Energies, Appearance Energies and

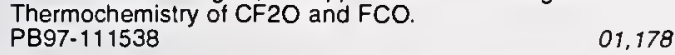
Rate Constants for the Gas Phase Reactions of the $\mathrm{OH}$ Radical with $\mathrm{CF} 3 \mathrm{CF} 2 \mathrm{CHCl} 2$ (HCFC-225ca) and CF2CICF2CHCIF (HCFC-225cb).

PB95-152153

00,940

Structures of Vapor-Deposited Yttria and Zirconia Thin Films.

ZHANG, Z. $M$.

Broadband High-Optical-Density Filters in the Infrared.
PB95-180261 309

Development of Neutral-Density Infrared Filters Using Me-

tallic Thin Films.
PB96-180286

Liquid-Nitrogen-Cooled High Tc Electrical Substitution Radiometer as a Broadband IR Transter Standard. 02,198

Thermal and Nonequilibrium Responses of Superconductors for Radiation Detectors.
PB95-164232

Thermal Modeling and Analysis of Laser Calorimeters.

PB96-140405 04,354

Thermal Modeling of Absolute Cryogenic Radiometers.

PB95-181236

04,316

Comparisons of Some NIST Fixed-Point Cells with Similar

Cells of Other Standards Laboratories.
PB97-119242

00,655

ZHAO, Z. Q.

State-Resolved Rotational Energy Transfer in Open Shell

Collisions: $\mathrm{Cl}((2) \mathrm{P} 3 / 2)+\mathrm{HCl}$.
PB96-176607

01,157

ZHEN, $\mathbf{Z}$.

Intercomparison of Thermal Converters at NIM, NIST, PTB, SIRI and VSL from 10 to $100 \mathrm{MHz}$

PB94-172459

02,027

ZHENG, G. G.

Novel $Y B a 2 C u 3 O 7-x$ and $Y B a 2 C u 3 O 7-x / 48 B a 3 O 9$ Multi-

layer Films by Bias-Masked 'On-Axis' Magnetron Sputtering.

ZHENG, $\mathrm{O}$.

Physical Limit to the Stability of Superheated and Stretched

PB96-122551

01,083

ZHENG, $Z$.

Vortex Images in Thin Films of $\mathrm{YBa} C \mathrm{Cu} 3 \mathrm{O}$ (sub $7-\mathrm{x}$ ) and Bi2Sr2Ca1Cu2O(sub $8+x$ ) Obtained by Low-Temperature Magnetic Force Microscopy.

PB97-119408

04,815

ZHIGUNOV, D. I.

Magnetic Structure and Spin Dynamics of the Pr and $\mathrm{Cu}$ in PB96-111836

ZHOU, H. L.

04,036

High-Precision Calculations of Cross Sections for Low-Energy Electron Scattering by Ground and Excited State of Sodium.

PB95-152161

03,914

Low-Energy-Electron Collisions with Sodium: Elastic and Inelastic Scattering from the Ground State.

ZHOU, $\mathrm{S}$.

04,030

Lattice Imperfections Studied by Use of Lattice Green's Functions.
ZHOU, S. J.

Dislocation Core-Core Interaction and Peierls Stress in a Model Hexagonal Lattice.

PB96-162003

04,101

Dislocation Emission at Ledges on Cracks.

PB95-164240

04,630

Interfacial Crack in a Two-Dimensional Hexagonal Lattice.

PB96-161989 04,100

Shielding of Cracks in a Plastically Polarizable Material

PB95-164257 04,631

ZHU, J.

Fluctuation Dominated Recombination Kinetics with Traps. PB95-107264

00,875

ZHU, J. G.

Surface Topography and Ordering-Variant Segregation in GalnP2.

04,595

ZHU, M.

Dreams About the Next Generation of Super-Stable Lasers. 04,235

Improved Hyperfine Measurements of the Na NP Excited State Through Frequency-Controlled Dopplerless Spectroscopy in a Zeeman Magneto-Optic Laser Trap.

PB95-203840

04,012

Introduction to Phase-Stable Optical Sources.

04,347

Stabilization of Optical Phase/Frequency of a Laser System: Application to a Commercial Dye Laser with an Externa Stabilizer.

PB95-203832

04,327

ZIBROV, A. S.

High-Resolution Diode-Laser Spectroscopy of Calcium.

PB95-181244

03,969

High-Sensitivity Spectroscopy with Diode Lasers.

PB95-175477

04,297

\section{ZIEGLER, R. G.}

Ascorbic and Dehydroascorbic Acids Measured in Plasma Preserved with Dithiothreitol or Metaphorphoric Acid.

PB94-216330

Liquid Chromatographic Method for the Determination of Carotenoids, Retinoids, and Tocopherols in Human Serum and in Food.

PB95-153599

00,593

\section{ZIMMERLI, G.} Electric Field Etfects on a Near-Critical Fluid in Microgravity.
PB96-161880

Noise in the Coulomb Blockade Electrometer.

Self-Heating in the Coulomb-Blockade Electrometer. Self-Heating in Junctions.

Voltage Gain in the Single-Electron Transistor.

PB95-176335

MERMAN, B. E.

63Ni Half-Life: A New Experimental Determination and Critical Review.
PB97-1 1603

Nickel-63 Standardization: 1968-1995

PB97-111819

00,700

ZIMMERMAN, J.

Residual Error Compensation of a Vision-Based Coordinate Measuring Machine.

PB96-161617

ZIMMERMAN, J. H

Automated Optical Roughness Inspection.

PB95-152179

ZIMMERMAN, N. M.

Application of Single Electron Tunneling: Precision Capaciance Ratio Measurements.

PB96-102157

04,703

Capacitors with Very Low Loss: Cryogenic Vacuum-Gap Capacitors.

Proposed Tests to Evaluate the Frequency-Dependent Capacitor Ratio for Single Electron Tunneling Experiment.

PB97-111454

01,982

Results of Capacitance Ratio Measurements for the Single Electron Pump-Capacitor Charging Experiment.

PB97-113286

04,813

ZINCKE, C

CMOS Circuit Design for Controlling Temperature in Micromachined Devices.

PB96-156088 02,196

Micromachined Display Output for a Cellutar Neural Net-

work.
PB96-156070 02,422

Test Structures for Determining Design Rules for Microelectromechanical-Based Sensors and Actuators.
PB95-150488

ZINK, L.

Absolute Frequency Measurements of Methanol from 1.510 $6.5 \mathrm{THz}$.

PB95-175881

04,300

ZINK, L. $R$.

$30 \mathrm{THz}$ Mixing Experiments on High Temperature Superconducting Josephson Junctions.

04,709
Atomic Oxygen Fine Structure Splittings with Tunable Far Infrared Spectroscopy.

PB95-152203

03,915

Atomic Sulfur: Frequency Measurement of the $\mathrm{J}=0$ inversely maps 1 Fine-Structure Transition at 56.3 Microns by Laser Magnetic Resonance.

01,007

Far Intrared Laser Frequencies of $\mathrm{CH} 3 \mathrm{OD}$ and $\mathrm{N}_{2} \mathrm{H} 4$.

PB96-199623

04,341

Far Infrared Laser Frequencies of (13) $\mathrm{CD} 3 \mathrm{OH}$.

PB95-169363 04,292

Improved Molecular Constants and Frequencies for the $\mathrm{CO} 2$ Laser from New High-J Regular and Hot-Band Frequency Measurements

PB95-180634

04,312

Laboratory Measurements for the Astrophysical Identification of $\mathrm{MgH}$.

aser Magnetic-Resonance Measurement of the (3) $\mathrm{P} 1$ 3)P2 Fine-Structure Splittings in (17) O and (18)O

PB95-175170

00,994

Measurement of the $J=2$ less than 1 Fine-Structure Interval for (28)Si and (29) Si in the Ground (3)P State.

PB94-185097

00,771

PB95-152187

00,941

ZINK, S.

Investigation of Applicability of Alanine and Radiochromic Detectors to Dosimetry of Proton Clinical Beams.

PB96-146782

03,636

\section{ZOBOV, N.}

Microwave Spectrum and Structure of $\mathrm{CH} 3 \mathrm{NO} 2 \cdot \mathrm{H} 2 \mathrm{O}$

AD-A296 $377 / 5$

00,719

ZOBOV, N. F.

Tunneling-Rotation Spectrum of the Hydrogen Fluoride PB94-198678

00,793

ZOLLER, $P$.

Atomic Beam Splitters and Mirrors by Adiabatic Passage in Multilevel Systems.

Phase Shifts and Intensity Dependence in Frequency-Modulation Spectroscopy.

ZUKOSKI, E. E.

Experimental and Numerical Studies on Two-Dimensional Gravity Currents in a Horizontal Channel.

PB94-165941

01,359

Review of Flows Driven By Natural Convection in Adiabatic Shafts.

PB96-147897

01,416

ZULFUGARZADE, E. E.

Proceedings of the Meeting of the Intergovernmental U.S. Russian Business Development Committee's Standard Working Group (4th). Held in New York City, New York on March 27-29, 1995 and in Northbrook, Illinois on March 30 31.1995

PB95-25588

00,496

ZWART, E.

Microwave and Submillimeter Spectroscopy of Ar-NH3 States Correlating with $\mathrm{Ar}+\mathrm{NH} 3(j=1, k=1)$.

00,942

ZWEIBEL, E. G.

Magnetic Fields in Star-Forming Regions: Observation PB96-123005

00,100

ZWEIDINGER, $\mathrm{R}$. $\mathrm{B}$.

Distinguishing the Contributions of Residential Wood Combustion and Mobile Source Emissions Using Relative Concentrations of Dimethylphenanthrene Isomers.

PB96-135124 



\title{
SAMPLE ENTRY
}

\author{
FIRE DETECTORS \\ Performance Parameters of Fire Detection Systems \\ PB94-194339
}

00,123

Keyword term

Title

NTIS order number
Abstract number
$1,1,1,2,3,3$-HEXAFLUOROPROPANE Dielectric Studies of Fluids with Reentrant Resonators. PB95-153730

00,952

1,1,1,2-TETRAFLUOROETHANE Viscosity of the Saturated Liquid Phase of Six Halogenated Compounds and Three Mixtures.

PB95-162368

00,960

Thermodynamic Properties of R134a(1,1,1,2Tetrafluoroethane)

00,988

Measurements of Molar Heat Capacity at Constant Volume (Cv) for 1,1,1,2-Tetrafluoroethane (R134a).

PB95-168878

03,264

Vapour Pressure Measurements on 1,1,1,2Tetrafluoroethane (R134a) from 180 to $350 \mathrm{~K}$. 03,265 3.

03,265

Stability/Instability of Gas Mixtures Containing 1,3-Butadiene in Treated Aluminum Gas Cylinders. PB95-162285

02,546

1-CHLORO-1

Polarized Transient Hot Wire Thermal Conductivity MeasUrements.

PB95-108817
CHLORO-1,2,2,2-TETRAFLUOROETHANE

00,886

Viscosity of the Saturated Liquid Phase of Six Halogenated Compounds and Three Mixtures.

PB95-162368

00,960

10-METHYLACRIDAN

Oxidation of 10-Methylacridan, a Syntheric Analogue of NADH and Deprotonation of Its Cation Radical. Convergent Application of Laser Flash Photolysis and Direct and Redox Catalyzed Electrochemistry PB94-198371

00,785

2-DIFLUOROMETHOXY-1,1,1-TRIFLUOROETHANE Viscosity of the Saturated Liquid Phase of Six Halogenated Compounds and Three Mixtures.

00,960

2-METHYLPROPENE

Mechanism and Rate Constants for the Reactions of Hydrogen Atoms with Isobutene at High Temperatures. PB95-151064
2D ARRAYS

SUSAN: SUperconducting Systems ANalysis by Low Temperature

High-Frequency Oscillators Using Phase-Locked Arrays of Josephson Junctions.

PB96-135157

02,080

3-QUINUCLIDINOL

Determination of 3-Quinuclidinyl Benzilate (Qnb) and its Major Metabolites in Urine by Isotope Dilution Gas Chromatography Mass Spectrometry. matography Mas

03,492

3C 273 RADIO SOURCE

Observations of $3 \mathrm{C} 273$ with the Goddard High Resolution Spectrograph on the Hubble Space Telescope. PB95-202321

00,076

A STARS

A-type and Chemically Peculiar Stars.

PB96-123286

00,10

Radio and X-ray Emissions from Chemically Peculiar Band A-Type Stars: Observations and a Model.

PB96-123302

AAMACS SYSTEM

Advanced Angle Metrology System. PB94-211364

00,103

\section{B INITIO CALCULATION}

Ab initio Calculations for Helium: A Standard for Transport Property Measurements.

PB96-102041

ABRASION

Abrasive Wear by Diesel Engine Coal-Fuel and Related PB95-104915

ABRIKOSOV VORTICES

Thermally Activated Hopping of a Single Abrikosov Vor

PB95-140810

04,548

ABSOLUTE GRAVITY

Intracomparison Tests of the FG5 Absolute Gravity Meters.

03,688

ABSOLUTE SPECTRAL RESPONSE

NIST. High Accuracy Scale for Absolute Spectral Response from $406 \mathrm{~nm}$ to $920 \mathrm{~nm}$.

01,865 Developing Quality System Documentation Based on
ANSI/NCSL Z540-1-1994: The Optical Technology Division's Effort.

01,869 ABSORBATES

Uitrafast Time-Resolved Infrared Probing of Energy Transfer at Surfaces.

PB96-123443

00,620

ABSORBED DOSE

Problem of Convection in the Water Absorbed Dose Calorimeter

110159

03,523

ABSORBERS (EQUIPMENT)

Low-Frequency Model for Radio-Frequency Absorbers.
PB95-261939

ABSORBERS (MATERIALS)

Bistatic Scattering of Absorbing Materials from 30 to 1000

PB95-150934

01,891

ABSORPTANCE

Optical Performance of Commercial Windows. $\quad 00,392$
PB95-208757 ABSORPTION

NiR-Spectroscopic Investigation of Water Sorption Characteristics of Dental Resins and Composites. acteristics of Den

00,189

Energy Dependences of Absorption in Beryllium Windows and Argon Gas.

04,020

ABSORPTION CROSS SECTIONS

Scaling of the Nonlinear Optical Cross Sections of GaAsAIGaAs Multiple Quantum-Well Hetero $n$-i-p-i's.

PB96-102793

02,183

Temperature Dependence of the Gas and Liquid Phase Ultraviolet Absorption Cross Sections of HCFC-123 (CF3CHCl2) and $\mathrm{HCFC}-142 \mathrm{~b}$ (CH3CF2Cl)

PB96-201033

03,298

Temperature Dependent Ultraviolet Absorption Cross Sections of Propylene, Methylacetylene and Vinylacetylene

PB96-204177

01,169

ABSORPTION SPECTRA

Memory Function Approach to the Shape of Pressure Broadened Molecular Bands.

PB95-152930

00,944 
Intermediate Coupling in L2-L3 Core Excitons of $\mathrm{MgO}$ $\mathrm{Al} 2 \mathrm{O} 3$, and $\mathrm{SiO} 2$
PB96-158043

ABSORPTION SPECTROMETRY

Spectral Interference in the Determination of Arsenic in High-Purity Lead and Lead-Base Alloys Using Electrothermal Atomic Absorption Spectrometry and PB96-112099

ABSORPTION SPECTROSCOPY

$0.0,614$

Application of a Novel Slurry Fumace AAS Protocol for Rapid Assessment of Lead Environmental Contamination. PB96-112354

02,526

ABSORPTIVITY

Molar Absorptivities of Bilirubin (NIST SRM 916A) and lis Neutral and Alkaline Azopigments.

03,456

ABSTRACTS

Abstract and Index Collection in the Research Information Center of the National Institute of Standards and Technology.

PB94-152204 02,744

Annual Conference on Fire Research: Book of Abstracts, October 17-20, 1994

PB95-104964

01,376

Publication and Presentation Abstracts, 1993. (Published by Paffenbarger Research Center and Center of Excellence for Materials Science Research).

PB95-153052

03,562

Abstract and Index Collection in the Research Information Center of the National Institute of Standards and Technology.

02,741

Publications and Presentation Abstracts, 1995. (Published by Paffenbarger Research Center and Center of Excellence for Materials Science Research).

9250

03,568

Publication and Presentation Abstracts, 1995

PB96-164082

03,576

Publication and Presentation Abstracts, 1994

PB96-176623

03,577

Publication and Presentation Abstracts, 1996 PB97-122238

03,585

ABUNDANCE

New IUPAC Guidelines for the Reporting of Stable Hydrogen, Carbon, and Oxygen Isotope-Ratio Data. Letter to

PB95-261962 01,058

Accurate Measurements of the Local Deuterium Abundance from HST Spectra.

00,110

AC STARK EFFECT

Configuration-Dependent AC Stark Shifts in Calcium.
PB96-157995

AC STARK SHIFT

RIS Measurements of the AC Stark Shift.

PB96-158035

04,078

AC STARK SHIFTS

RIS Measurement of AC Stark Shifts and Photoionization

Cross Sections in Calcium.

PB96-157953

04,073

ACCELERATED LIFE TESTS

Macro- and Microreactions in Mechanical-Impact Tests of Aluminum Alloys.

PB95-107348

03,340

ACCELERATED TESTING

Assessing MOS Gate Oxide Reliability on Wafer Level with Ramped/Constant Voltage and Current Stress.

PB96-180112

Modification of a Commercial SEM with a Computer Con-

trolled Cathode Stabilized Power Supply.

PB96-201066

04,129

ACCELERATOR FACILITIES

Overview of a Radiation Accident at an Industrial Accelerator Facility.

ACCELERATOR MASS SPECTROMETRY

Factorial Design Techniques Applied to Optimization of AMS Graphite Target Preparation.

PB95-151197

00,584

Comparative Study of Fe-C Bead and Graphite Target Periormance with the National Ocean Sciences AMS (NOSAMS) Facility Recombinator Ion Source.

00,693

ACCELERATOR MASS SPECTROSCOPY

$36 \mathrm{CVCl}$ Accelerator-Mass-Spectrometry Standards: Verification of Their Serial-Dilution-Solution Preparations by Radio activity Measurements.
PB94-140563

00,524

\section{ACCELEROMETERS}

Testing the Sensitivity of Accelerometers Using Mechanical Shock Pulses Under NIST Special Publication 250 Test No. 24040 .

02,683

ACCEPTANCE DIAGRAMS

Joy of Acceptance Diagrams.

03,828
Transmission Properties of Short Curved Neutron Guides. Part 1. Acceptance Diagram Analysis and Calculations.

04,021

ACCESS CONTROL

Computer Security Management and Planning in the U.S Fieral Government.

01,596

Guidance to Federal Agencies on the Use of Trusted Systems.

01,597

Functional Security Criteria for Distributed Systems

PB96-123492

01,607

Distributed Communication Methods and Role-Based ACcess Control for Use in Health Care Applications.

PB96-183165

01,508

TMACH Experiment Phase 1. Preliminary Developmenta Evaluation.

01,618

ACCESS TO INFORMATION

Global Information Infrastructure: Agenda for CooperaPB95-178604

01,482

ACCESSIBILITY

Electronic Access to Standards on the Information HighWay. 131578

ACCIDENT INVESTIGATIONS

Ashland Tank Collapse Investigation

02,481

Ashland Tank-Collapse Investigation: Closure by Authors.
PB95-126322

Radiation Accident at an Industrial Accelerator Facility.

02,575

Overview of a Radiation Accident at an Industrial Accelerator Facility

ACCOUNTING

Manager's Guide for Monitoring Data Integrity in Financial

PB96-165915

ACCREDITATION

NVLAP Procedures U.S. Code of Federal Regulations. Title 15, Subtitle A, Chapter 2, Part 7. (Effective December 1984; Amended September 1990).

PB94-160850

02,627

Program Handbook: Requirements for Ootaining NIST Approval/Recognition of a Laboratory Accreditation Body Under P.L. 101-592. The Fastener Ouality Act.

PB94-210143

02,859

Survey on the Implementation of ISO/IEC Guide 25 by National Laboratory Accreditation Programs.

00,479

National Voluntary Laboratory Accreditation Program: Energy Efficient Lighting Products.

PB94-219060

02,642

Should NIST Accredit U.S. Calibration Laboratories

PB95-107280

02,646

National Voluntary Laboratory Accreditation Program

(NVLAP): Commercial Products Testing.

02,671

Laboratory Accreditation for Testing Energy Efficient Lighting.

00,270

Proceedings of the Open Forum on Laboratory Accredita. tion at the National Institute of Standards and Technology, October 13, 1995

02,686

State Weights and Measures Laboratories: Program Handbook.

ACCURACY

Numeric Data Distribution: The Vital Role of Data Exchange in Today's World.

N95-15937/2

02,622

Utc Dissemination to the Real-Time User.

N19960042622

01,521

Detector-Based Candela Scale and Related Photometric Calibration Procedures at NIST.

PB95-161949

04,273

Estimation of Measurement Uncertainty of Small Circular Features Measured by CMMs.

PB95-267928

02,918

Error-Bounding in Level-Index Computer Arithmetic

PB96-109582 01,742

Progress Toward Accurate Metrology Using Atomic Force Microscopy.

02,417

New Concepts of Precision Dimensional Measurement for Modem Manufacturing.

PB96-160684

02,924

NIST Frequency Measurement Service.

01,561

ACETALDEHYDE

Fundamental Torsion Band in Acetaldehyde.

PB94-212834

00,840

CETALS

Ring-Opening Dental Resin Systems Based on Cyclic

PB95-16225

00,162

ACETONE CATIONS

Kinetics and Mechanism of the Collision-Activated Dissociation of the Acetone Cation.

00,859

ACETONITRILE

Methyl Torsional Levels of Solid Acetonitrile $(\mathrm{CH} 3 \mathrm{CN})$ : A Neutron Scattering Study.

PB95-151015

00,926

\section{ACETYLENE}

Spectroscopic Constants for the 2.5 and 3.0 micrometer Bands of Acetylene.

PB94-213071

00,847

Seli-, N2- and Ar-Broadening and Line Mixing in $\mathrm{HCN}$

2

00.876

Decoupling in the Line Mixing of Acetylene Infrared 0

PB95-108452

00,877

Laboratory Studies of Low-Temperature Reactions of

$\mathrm{C} 2 \mathrm{H}$ with $\mathrm{C} 2 \mathrm{H} 2$ and implications for Atmospheric Models

00,690

Novel Polydiacetylenes Derived from Liquid Crystalline Monomers.

PB95-140125

01,235

ACETYLENE COMPLEXES

Rotational Spectrum and Structure of a Weakly Bound

Complex of Ketene and Acetylene.

00,896

\section{ACETYLENE RADICALS}

Kinetics of the Reaction $\mathrm{C} 2 \mathrm{H}+\mathrm{O} 2$ from 193 to $350 \mathrm{~K}$ Using Laser Flash Kinetic Infrared Absorption SpectrosCOpY. 203055

01,043

ACETYLENES

Vibrational Spectra of Molecular Ions Isolated in Solid Neon: HCCH+ and HCC-. (Reannouncement with New Availability Information

AD-A253 551/6

00,707

ACID-BASE EQUILIBRIUM

Contact Electrification Induced by Monolayer Modification of a Surface and Relation to Acid-Base Interactions.

PB94-185378

03,034

ACID BONDED REACTION CEMENTS

Development of an Adhesive Bonding System.
PB94-199056

00,145

Polymeric Calcium Phosphate Cements Derived from

Poly(methyl vinyl ether-maleic acid)

PB96-164264

ACID GAS PRODUCTION

Acid Gas Production in Inhibited Diffusion Flames.

PB95-180576

01,390

ACIDIFICATION

Procedure for the Study of Acidic Calcium Phosphate Precursor Phases in Enamel Mineral Formation.

03,564

\section{ACIDITY}

Carbon Acidities of Aromatic Compounds. 1. Effects of InRing Aza and

ACOUSTIC ABSORPTION

Absorption of Ultrasonic Waves in Air at High Frequencies $(10-20 \mathrm{MHz})$

04,182

Absorption of Sound in Gases between 10 and $25 \mathrm{MHz}$ : Argon

ACOUSTIC 
ACOUSTIC MEASUREMENTS

Speed-of-Sound Measurements in Liquid and Gaseous PB95-151957

04,186

ACOUSTIC RESONATORS

Speed-ot-Sound Measurements in Liquid and Gaseous PB95-151957

04,186

ACOUSTIC SCATTERING

Reconstructing Stratified Fluid Flow from Reciprocal Scattering Measurements.

$$
\begin{aligned}
& \text { tering Measurem } \\
& \text { PB95-107256 }
\end{aligned}
$$

04,202

ACOUSTIC TESTING

National Voluntary Laboratory Accreditation Program Acoustical Testin

04,188

ACOUSTIC VELOCITY

Speed-ot-Sound Measurements in Liquid and Gaseous PB95-151957

04,186

Thermodynamic Properties of Two Gaseous Halogenated Ethers from Speed-of-Sound Measurements: Difluoromethoxy-Difluoromethane and 2-Difluoromethoxy$1,1,1$-Trifluoroethane.

PB96-102413

04,189

ACOUSTICAL INSTRUMENTS

Determination of Acoustic Center Correction for Type

LS2aP Condensor Microphones.
PB96-204508

04,190

ACOUSTICS

Determination of Sheet Steel Formability Using Wide Band Electrom

02,279

Effect of Liftoft on Accuracu of Phase Velocity Measurements Made with Electromagnetic-Acoustic Transducers.
PB96-186044 Sensor System for Intelligent Processing of Hot-Rolled Steel.

03,373

Well-Shielded EMAT for On-Line Ultrasonic Monitoring of Well-Shielded EMAT for On-Line Ultrasonic Monitoring of
GMA Welding.
PB96-186077
02,879

\section{ACOUSTICS \& SOUND}

Electroacoustics for Characterization of Particulates and Suspensions. Proceedings of a Workshop. Held in Gaithersburg, Maryland on February 3-4, 1993.

00,725

Absorption of Sound in Gases between 10 and $25 \mathrm{MHz}$

Argon.

PB94-199015

04,183

Interaction of Rayleigh Waves with a Rib Attached to a Plate.

PB94-199023 04,184

Benchmarks for the Evaluation of Speech Recognizers.

PB94-211539 01,566

Elastic Constants of Isotropic Cylinders Using Resonant

Uitrasound.
PB94-211919 04,497

Examination of Objects Made of Wood Using Air-Coupled Uitrasound.

03,404

Ultrasonic Measurement of Sheet Anisotropy and Formability.

03,213

Electroacoustic Characterization of Particle Size and Zeta Potential in Moderately Concentrated Suspensions.

PB96-119425

01,079

Electrokinetic Sonic Analysis of Silicon Nitride Suspen-

PBo6-123575

03,073

Band-Limited, White Gaussian Noise Excitation for Reverberation Chambers and Applications to Radiated Susceptibility Testing.

Ultrasound Power Measurement Techniques at NIST.

PB96-179569

01,960

Ultrasonic ND

02,684

02,761

Determination of Acoustic Center Correction for Type LS2aP Condensor Microphones.

04,190

Transient Analysis of a Line-Focus Transducer Probing a Liquid/Solid Interface.

02,763

Material Characterization By a Time-Resolved and PolarMaterial Characterization By a Time-Resolved and Polar-
ization-Sensitive Ultrasonic Technique.
PB97-122576
02,764

ACQUISITION

AutoBid 2.0: The Microcomputer System for Police Patrol Vehicle Selection
PB96-154570

CQUISITIONS

04,871

Federal Implementation Guideline for Electronic Data Interchange: ASC X12 003040 Transaction Set 838 Trading Partner Profile (Vendor Registration), Implementation PBg6-112651

03,674

ACRONYMS

Some Basics on Who's Who and What's What in Seismic Safety.

00,435

\section{ACRYLATE}

Synthesis of Hybrid Organic-Inorganic Materials from Interpenetrating Polymer Network Chemistry.

02,994 PB96-18005

Facile Synthesis of Novel Fluorinated Multifunctional Acrylates.
PB94-198389

01,207

ACRYLIC RESINS

Preparation and Characterization of Cyclopolymerizable Resin Formulations.

PB96- 146840

01,285

\section{ACRYLONITRILE POLYMERS}

Acrylonitrile-Butadiene-Styrene (ABS) Plastic Drain, Waste, and Vent Pipe and Fittings.

ACTIS DATA BASE

Tribological Data: Needs and Opportunities.

PB95-140596

03,237

ACTIVATION

Evaluation of Sprinkler Activation Prediction Methods.
PB96-141056

ACTIVATION ANALYSIS

Local Area Networks in NAA: Advantages and Pitfalls.
PB94-172095

High-Sensitivity Determination of lodine Isotopic Ratios by

Thermal and Fast Neutron Activation.

00,555

Neutron Capture Prompt Gamma-Ray Activation Analysis at the NIST Cold Neutron Research Facility. 00,556

Scattering and Absorption Effects in Neutron Beam Activation Analysis Experiments.

PB94-216140

00,557

Etfects of Target Shape and Neutron Scattering on Element Sensitivities for Neutron-Capture Prompt Gammaray Activation Analysis. NIST: A Progress Report.

00,558 PB95-175964

00,602

Neutron Scattering by Hydrogen in Cold Neutron Prompt Gamma-Activation Analysis.

PB95-175972

00,603

Measuring Hydrogen by Cold-Neutron Prompt-Gamma Activation Analysis

PB96-111877 00,612

Effects of Target Temperature on Analytical Sensitivities of Cold-Neutron Capture Prompt gamma-ray Activation Analysis.

PB96-112131

00,616

SUM and MEAN: Standard Programs for Activation Analysis.

00,617

ACTIVE CONTROL

Modified Optimal Algorithm for Active Structural Control. PB96-165949

00,472

ACTIVE SITE IONICITY

Active Site Ionicity and the Mechanism of Carbonic Anhydrase.

00,843

ACTIVITY COEFFICIENT

Model for Calculating Virial Coefficients of Natural Gas Hydrocarbons with Impurities.

\section{ACUTE EXPOSURE}

Documentation for Immediately Dangerous to Life or Health Concentrations (IDLHs).

PB94-195047

03,602

ADA PROGRAMMING LANGUAGE

Ada Compiler Validation Summary Report: Certificate Number: 931029S1.11330, Digital Equipment Corporation, DEC Ada for DEC OSF/1 AXP Systems, Version 3.1, DEC 3000 Model 400 AXP Workstation, DEC 3000 Model 400 AXP Workstation.

AD-A274 872/1

01,639

Ada Compiler Validation Summary Report: Certificate Number: 931217S1.11336 Control Data Systems, Inc. NOSNE Ada, Version 1.4 Cyber 180-930-31 $\Rightarrow$ Cyber 180-930-31.

AD-A275 $977 / 7$

01,640

Ada Compiler Validation Summary Report. Certificate Number: 931119S1.11332, DDC-I, Inc. DACS MIPS R3000 Bare Ada Cross Compiler System, Version 4.7.1 Sun SPARCstation IPX $=>$ DACS Sun SPARC/SunOS to MIPS R3000 Bare Instruction Set Architecture Simulator. Version 4.7.1.

AD-A276 181/5 01,641

Ada Compiler Validation Summary Report: Certificate Number: 931119S1.11331 DDC-I, Inc. DACS Sun SPARC/SunOS to 80386 PM Bare Ada Cross Compiler System, Version 4.6.4 Sun Sparcstation $1+\Rightarrow$ Bare Board iSBC $386 / 116$.

AD-A276 283/9

01,642

Ada Compiler Validation Summary Report. Certificate Number: 930927\$1.11328 Green Hills Sottware C Ada, Version 1.1 ZENY $386 \Rightarrow$ ZENY 386

01,643 Ada Compiler Validation Summary Report: Certificate Solaris to 80386 PM Bare Ada Cross Compiler System,
Version 4.6.4 Sun SPARCclassic $\Rightarrow$ Intel iSBC $386 / 116$ AD-A279 642/3

01,644

Ada Compiler Validation Summary Report: Certificate umber: 940325 S1.11341 DDC-I. DACS Sun SPARC/ SunOS to 80186 Bare Ada Cross Compiler System, Ver(Bare Machine) (Bare Machine)

Ada Compiler Validation Summary Report: Certiticat Number: 940325 S1.11349 DDC-I, DACS Sun SPARC/ Solaris to 80386 PM Bare Ada Cross Compiler System with Rate Monotonic Scheduling, Version 4.6.4 Sun SPARCclassic $=>$ Intel iSBC 386/116 (Bare Machine). AD-A279 644/9

Ada Compiler Validation Summary Report: Certiticate Number: 940325S1.11354 DDC-I, DACS Sun SPARC/ Solaris Native Ada Compiler System, Version 4.6.2 Sun SPARCClassic $\Rightarrow>$ Sun SPARCclassic

Ada Compiler Validation Summary Report: Certificate Number: $940325 S 1.11346$ DDC-I, DACS Sun SPARC SunOS to $680 \times 0$ Bare Ada Cross Compiler System
(BASIC MODE), Version 4.6.9 Sun SPARCstation IPX $=>$

Lynwood j435TU (68030) (Bare Machine)

01,648

Ada Compiler Validation Summary Report: Certificate Number: 940325S1.11343 DDC-I, DACS Sun SPARC/ sion 4.6.4 Sun SPARCclassic $\Rightarrow$ Intel iSBC 186/100 (Bare Machine)

Ada Compiler Validation Summary Report: Certificate Number: 940325S1.11344 DDC-I, DACS Sun SPARC/ Solaris to 80186 Bare Ada Cross Compiler System with Rate Monotonic Scheduling, Version 4.6.4 Su SPARCclassic $=>$ Intel iSBC $186 / 100$ (Bare Machine).
AD-A279 $758 / 7$

Ada Compiler Validation Summary Report: Certificate Number: $940325 S 1.11347$ DDC-I, DACS Sun SPARC SunOS to $680 \times 0$ Bare Ada Cross Compiler System (SE CURE MODE), Version 4.6.9 Sun SPARCstation IPX $=$ Lynwood j435TU (68030) (Bare Machine).

01,651

Ada Compiler Validation Summary Report: Certificate Number: 940325S1.11342 DDC-I. DACS Sun SPARC/ SunOS to 80186 Bare Ada Cross Compiler System with SPARCstation IPX => Intel iSBC 186/100 (Bare Ma-

AD-A279 779/3

01,652

Ada Compiler Validation Summary Report: Certficate Number: 940325 S1.11351 DDC-I, DACS Sun SPARC/ SunOS to Pentium PM Bare Ada Cross Compiler System with Rate Monotonic Scheduling, Version 4.6.4 Sun SPARCstation IPX $=>$ Intel Pentium (operated as Bare Machine) based in Xpress Desktop (Intel product number XBASE6E4F-B

AD-A279 804/9

01,653

Ada Compiler Validation Summary Report: Certificate Number: 940325S1.11353 DDC-I. DACS Sun SPARC Solaris to Pentium PM Bare Ada Cross Compiler System with Rate Monotonic Scheduling, Version 4.6.4 Sun PPARClassic $\Rightarrow$ Intel Pentium (operated as Bare Ma chine) based in Xpress Desktop (Intel product number XBASE6E4F-B

Ada Compiler Validation Summary Report: Certificate Number: 940325S1.11350 DDC-I, DACS Sun SPARC/ SunOS to Pentium PM Bare Ada Cross Compiler System Version 4.6.4 Sun SPARCstation IPX $\Rightarrow$ Intel Pentium (Operated as Bare Machine) Based in Xpress Desktop (Intel Product Number: XBASE6E4F-B).

AD-A279 864/3 


\section{KEYWORD INDEX}

Ada Complier Validation Summary Report: Certificate Number: 940929S1.11378. Digital Equipment Corporation DEC Ada for DEC OSF $/ 1$ AXP Systems, Version 3.2 DEC 3000 Model 400 AXP Workstation $\Rightarrow$ DEC 3000 Model 400 AXP Workstation.

AD-A288 574/7

01,661

Ada Compiler Validation Summary Report. Certificate Number $941117 \$ 1.11380$. Electronic Data Systems Corp. Compiler: OC Systems Legacy Ada/370, Release 1.4 .1 (without optimization).

AD-A289 895/5

01662

ADA Compiler Validation Summary Report, VC Number 950303S1.11381. Digital Equipment Corporation - Compiler Name: DEC Ada for OpenVMS Alpha Systems, Ver sion 3.2

AD-A293 709/2

01,663

Ada Compiler Validation Summary Report, VC No. 950609S1.11390 Digital Equipment Corporation - Compiler Name: DEC Ada Version 3.2 for OpenVMS VAX
Systerns. AD-A296 794/1

01,664

ADA; Category: Software Standard; Subcategory: Programming Language.

01,667

Validated Products List (Cobol, Fortran, ADA, Pascal, MUMPS, SOL

01,700

Validated Products List (Cobol, Fortran, ADA, Pascal, MUMPS, SOL

PB95-937300

01,738

ADAPTERS

Single-Port Technique for Adaptor Efficiency Evaluation.

ADENOSINE MONOPHOSPHATES

Thermochemistry of the Reactions between Adenosine, Adenosine $5^{\prime}$-monophosphate, Inosine, and Inosine 5' monophosphate; the Conversion of L-histidine to (Urocanic Acid+Ammonia).

PB94-213113

03,460

ADHESION

Interaction of Some Coupling Agents and Organic Compounds with Hydroxyapatite: Hydrogen Bonding. Adsorption and Adhesion.

PB94-172616 00,140

Surface Forces and Adhesion between Dissimilar Mate. rials Measured in Various Environments.

PB94-172970

03,033

Peeling a Polymer from a Surface or from a Line.
PB94-199809

Effects of Adhesive Thickness, Open Time, and Surface Cleanness on the Peel Strength of Adhesive-Bonded Seams of EPDM Rubber Roofing Membrane.

PB95-151338

00,372

Diffusion of Water along 'Closed' Mica Interfaces.
PB96-180039

Pressurized Internal Lenticular Cracks at Healed Mica Interfaces.

PB96-180252

02,997

Adhesion, Contact Electrification, and Acid-Base Properties of Surfaces.

ADHESIVE BONDING

Clinical Perspective on Dentin Adhesives.

PB94-211240

03.693

Micromechanics of Fracture

Epoxies.

03,011

Modified Surface-Active Monomers for Adhesive Bonding

PB95-151163

00,158

Characteristics of Adhesive-Bonded Seams Sampled from EPDM Roof Membranes.

00,377

Pulse-Echo Ultrasonic Evaluation of the Integrity of Seams of Single-Ply Roof Membranes.

00,381

PB95-163804

Polymerization Initiation by $\mathrm{N}$-p-Tolylglycine: Free-Radical

Reactions Studied by Pulse and Steady-State Radiolysis.
PB95-180014

New Surface-Active Comonomer for Adhesive Bonding.

PB96-204052

03,579

ADHESIVE TAPES

Performance of Tape-Bonded Seams of EPDM Membranes: Comparison of the Peel Creep-Rupture Response of Tape-Bonded and Liquid-Adhesive-Bonded

PB96-183249

03,012

ADHESIVES

Adhesion of Composites to Dentin and Enamel. PB94-199049

Development of an Adhesive Bonding System. PB94-199056

Modified Sur

PB95-151163

Bonding

AABATIC CALORIMETERS

High-Temperature Adiabatic Calorimeter for Constant-Volume Heat Capacity Measurements of Compressed Gases and Liquids.

00,989
ADIABATIC CONDITIONS

Review of Flows Driven By Natural Convection in Adia-

PB96-147897

01.416

ADMIXTURES

Interaction between Naphthalene Sulfonate and Silica Fume in Portland Cement Pastes.

01,315

Study on the Reuse of Plastic Concrete Using Extended Set-Retarding Admixtures.

00,402

Guide to a Format for Data on Chemical Admixtures in a Materials Property Database.

01,327

Guide 10 Format for Data on Chemical Admixtures in a Guide to a Format for Data on Chemical Admixtures in a
Materials Property Database. (Reannouncement with new abstract).

\section{ADSORBATES}

01,328

Vibrational Relaxation Measurements of Carbon Monoxide on Metal Clusters.

00,820

Picosecond Measurement of Substrate-to-Adsorbate Energy Transfer: The Frustrated Translation of CO/Pt(111)-Translation.

Time-Resolved Measurements of Energy Transfer at Sur-

PB95-141198

00,913

Time-Resolved Measurements of Energy Transfer at Sur-

PB95-153037

00,947

ADSORPTION

Adsorption of Low-Molecular-Weight Sodium Polyacrylate

on Hydroxyapatite.
PB94-172608

00,139

Interaction of Some Coupling Agents and Organic Compounds with Hydroxyapatite: Hydrogen Bonding. Adsorption and Adhesion.

PB94-172616

00,140

Topological Influences on Polymer Adsorption and Desorption Dynamics.

PB94-212479

01,227

Characterization of the Adsorption-Fouling Layer Using Globular Proteins on Ultrafiltration Membranes. Characterization of Cytochrome c/Alkanethiolate Structures Prepared by Self-Assembly on Gold.

00,987

Interaction of Chlorhexidine Digluconate with and Adsorption of Chlorhexidine on Hydroxyapatite.

PB95-175907

03.566

Water Adsorption at Polymer/Silicon Wafer Interfaces.

PB95-181178 01,022

Determination of Total Protein Adsorbed on Solid (Membrane) Surface by a Hydrolysis Technique: Single Protein Adsorption.

03,552

Adsorption of Potassium $\mathrm{N}$-phenylglycinate on Hydroxyapatite: Role of Solvents and lonic Charge. PB96-180161

ADV (ADVANCED DIGITAL VIDEO)

Report on the Workshop on Advanced Digital Video in the National Information Infrastructure. Held in Washing ton, D.C. on May 10-11, 1994. PB95-103677

01,472

ADVANCED DIGITAL VIDEO

Report on the Workshop on Advanced Digital Video in the National Information Infrastructure. Held in Washingon, D.C. on May 10-11, 1994

01,472

\section{ADVANCED MATERIALS}

NIST Workshop on Nanostructured Material (1st): Report of an Industrial Workshop Conducted by the National Institute of Standards and Technology. Held in Gaithersburg, Maryland on May 14-15, 1992

02,973

Control of Friction and Wear of Alpha-Alumina with a Composite Solid-Lubricant Coating.

PB95.125969

03,225

New Materials, Advanced Ceramics and Standards. PB95-140208

Recent VAMAS Activity in Ceramics.

03,047

PB95-16268

03,051

Structural Ceramics Database. Topical Report, June 1989-May 1991.

PB95-203758 03,060

Room-Temperature Flexure Fixture for Advanced Ceram-

PB95-210498

03,061

ADVANCED TECHNOLOGY

Opportunities for Innovation: Advanced Surface Engineering.

02,697

ADVANCED TECHNOLOGY LABORATORY

Design of Technically Complex Facilities.

PB97-119101

02,695

AERODYNAMICS

Aerodynamic Phenomena in Stellar Atmospheres - A Bibliography.

00,046

AEROSOL GENERATORS

Experimental Optimization of Peak Shape with Application to Aerosol Generation.

PB94-185535

00,501

Ultrafine Combustion Aerosol Generator.

PB94-200300

01,373

AEROSOL SPECTROMETERS

Stochastic Modeling of a New Spectrometer.

PB96-157870

04,068

AEROSOLS

Global Climatic Effects of Aerosols: The AAAR Sympo-

PB95-108791

00,122

Radiometric Model of the Transmission Cell-Reciprocal Nephelometer.

00,124

Controlled Nucleation in Aerosol Reactors for Supression of Agglomerate Formation.

PB95-151973

00,672

Measurement of the Uniformity of Particle Deposition of Filter Cassette Sampling in a Low Velocity Wind Tunnel.

PB95-163754

Sources of Urban Contemporary Carbon Aerosol. PB95-175659

02,549

AEROSPACE

Light-Weight Alloys for Aerospace Applications II.

AEROSPACE ENVIRONMENTS

In-Space Welding: Visions and Realities.

PB95-163234

02,551

AEROSPACE VEHICLES

Combustion of a Polymer (PMMA) Sphere in Microgravity.

03,375

AFFINITY CHROMATOGRAPHY

Affinity Chromatography on Inorganic Support Materials. PB95-163820

03,467

AGAROSE

Small Angle Neutron Scattering Studies of Structural Characteristics of Argarose Gels.

03,475

AGGLOMERATION

Controlled Nucleation in Aerosol Reactors for Supression of Agglomerate Formation.

PB95-151973

00,672

\section{AGGREGATES}

Digital Simulation of the Aggregate-Cement Paste PB95-125993

00,363

AGING (MATERIALS)

Aging in Glasses Subjected to Large Stresses and Defor-

Suggestions for a Logically-Consistent Structure for Serv-

ice Life Prediction Standards.
PB95-125795 
AIR

Absorption of Ultrasonic Waves in Air at High Frequencies (10-20 MHz).
PB94-199007

04,182

Speed-of-Sound Measurements in Liquid and Gaseous

04,186

04,186
Molar Heat Capacity at Constant Volume for Air from 67 Molar Heat Capacity at Constant Volume for Air from 67
to $300 \mathrm{~K}$ at Pressures to $35 \mathrm{MPa}$.
PB96- 163738

AIR CHANGE EFFECTIVENESS

Air Change Effectiveness Measurements in Two Modern PB94-185766

AIR CIRCULATION

Assessing Ventilation Effectiveness in Mechanically Venti-

lated Otfice Buildings.
PB95-162079 00,255

Measurements of Outdoor Air Distribution in an Office

PB95-210944

00,264

Improving the Evaluation of Building Ventilation

PB96-138508

00,271

AIR CONDITIONING

Theoretical Evaluation of the Vapor Compression Cycle with a Liquid-Line/Suction-Line Heat Exchanger, Economizer, and Ejector.

\section{R CONDITIONING EQUIPMENT}

Role of R22 in Refrigerating and Air Conditioning Equip.

PB94-199783

03,253

Simple Method of Composition Shifting with a Distillation Column for a Heat Pump Employing a Zeotropic Retrigerant Mixture.

02,603

AIR FLOW

Air Flow in the Boundary Layer of an Elliptic Cylinder.
AD-A297 $391 / 5$

02,536

CONTAM93 User Manual.

CONTAM88 Building Input Files for Multi-Zone Airflow

and Contaminant Dispersal Modeling.

PB94-194388

02,537

Combined Buoyancy- and Pressure-Driven Flow Through Shallow, Horizontal, Circular Vent.

PB94-210077 00,344

Field Measurements of Ventilation and Ventilation Effec-

tiveness in an Otfice/Library Building
PB95-108833

00,247

Simulating Smoke Movement through Long Vertical Shafts in Zone-Type Compartment Fire Models. Method of Predicting Smoke Movement in Atria with Application to Smoke Management.

PB95-154746

00,376

Fire and Smoke Control: An Historical Perspective.

PB95-175808

00,202

Application of a Multizone Airflow and Contaminant Dispersal Model to Indoor Air Ouality Control in Residential

B95-180238

02,555

Computer Simulations of Airflow and Radon Transport in Four Large Buildings.

PB95-220422

02,557

New Mass Transport Elements and Components for the NIST IAO Model.

02,558

Indoor Air Ouality Impacts of Residential HVAC Systems

Phase II.B Report: IAO Control Retrofit Simulations and

PB96-106877

02,559

Study of Ventilation Measurement in an Office Building.

PB96-155593 00,274

Calculating Combined Buoyancy- and Pressure-Driven Flow Through a Shallow, Horizontal, Circular Vent; Application to Problem of Steady Burning in a Ceiling-Vented Enclosure.

PB96-164108

00,409

Combined Buoyancy and Pressure-Driven Flow Through a Shallow, Horizontal, Circular Vent

a Shallow, Horiz

00,410

NASA Fire Detector Study

PB96-183108

01,423

CONTAM94: A Multizone Airflow and Contaminant Dispersal Model with a Graphic User Interface.

PB97-113203

02,570

AIR FORCE TRAINING

Robotics Application to Highway Transportation. Volume 1. Final Report

PB95-203790

03,654

AIR INFILTRATION

Ventilation Effectiveness Measurements in Two Modern

Office Buildings.

PB95-176012

02,553

AIR POLLUTION

Modulation of Fossil Fuel Production by Global Temperature Variations, 2

02,533
Rare-Earth Isotopes as Tracers of Particulate Emissions

$\begin{array}{ll}\text { An Urban Scale Test. } & \\ \text { PB94-161635 } & 02,535\end{array}$

Global Climatic Effects of Aerosols: The AAAR Sympo-

sium.

00,122

Atmospheric Reactivity of alpha-Methyl-Tetrahydrofuran. PB95-163705

02,548

Airborne Asbestos Analysis: National Voluntary Laboracory Accreditation Program.

PB96-147392

02,566

AIR POLLUTION ABATEMENT

Simultaneous Visual and Calorimetric Measurements of R11, R123, and R123/Alkylbenzene Nucleate Flow Boiling.

03,251

Chemical and Microbiological Problems Associated with Research on the Biodesulfurization of Coal.

02,484

PB95-140950

Microbial Degradation of Polysulfides and Insights into Their Possible Occurrence in Coal

02,488

AIR POLLUTION CONTROL

Sulfur Dioxide Capture in the Combustion of Mixtures of Lime, Refuse-Derived Fuel, and Coal.

Lime, Refuse-Der

02534

Membrane Gas Separation for a Fluidized-Bed IncinerPB95-169041

02,550

Multizone Modeling of Three Residential Indoor Air Ouality Control Options.

AIR POLLUTION DETECTION

Proficiency Tests for the NIST Airborne Asbestos Program, 1990.

00,535

Proficiency Tests for the NIST Airborne Asbestos Program - 1991.

00,537

Proficiency Tests for the NIST Airborne Asbestos Program - 1992.

AIR POLLUTION DISPERSION

New Mass Transport Elements and Components for the NIST IAO Model.

PB95-255899

02,558

Carbon Monoxide Dispersion in Residential Buildings: Literature Review and Technical Analysis.

PB97.114227

02,571

AIR POLLUTION MONITORING

Environmental Evaluation of a New Federal Office Build PB94-199874

02,538

Limits of $\mathrm{CO} 2$ Monttoring in Determining Ventilation PBa5-176004

02,552

ndoor Air Ouality Impacts of Residential HVAC Systems. Phase 2.A Report: Baseline and Preliminary Simulations. PB95-178893

02,554

NIST Workshop on Gas Sensors: Strategies for Future Technologies. Proceedings of a Workshop. Held in Gaithersburg Maryland on September 8-9, 1993.

Gaithersburg,

00,507

Indoor Air Ouality Impacts of Residential HVAC Systems Phase II.B Report: IAO Control Retrofit Simulations and Analysis.

PB96-106877

02,559

EMISS: A Program for Estimating Local Air Pollution Emission Factors Related to Energy Use in Buildings: User's Guide and Reference Manual.

PB96-109566

02,560

Few Caveats on Carbon Dioxide Monitoring

02,562

Relationship between Indoor Air Ouality and Carbon Dioxide.

02,569

Issues in the Field Measurement of VOC Emission Rates. Issues in the Field Measurement of VOC Emission Rates.
PB97-118806 AIR POLLUTION SAMPLING

Ground-Based Smoke Sampling Techniques Training Course and Collaborative Local Smoke Sampling in Saudi Arabia.

PB94.143542

Standards for Atmospheric Measurements.

02,532

PB95-163622

02,547

Measurement of the Uniformity of Particle Deposition of Filter Cassette Sampling in a Low Velocity Wind Tunnel. PB95-163754 02,549 Measurements of Indoor Pollutant Emissions from EPA Phase II Wood Stoves.

PB95-198735

02,556

Radiocarbon Measurements of Atmospheric Volatile Organic Compounds: Ouantifying the Biogenic Contribution.

PB97-122352

02,574

AIR POLLUTION SOURCES

Measurement and Determination of Radon Source Potential: A Literature Review.

02,576

Sources of Urban Contemporary Carbon Aeroso PB95-175659

02,551

Distinguishing the Contributions of Residential Wood Combustion and Mobile Source Emissions Using Relative Concentrations of Dimethylphenanthrene Isomers.

PB96-135124

AIR QUALITY

Manual for Ventilation Assessment in Mechanically Ventilated Commercial Buildings

PB94-145653

00,239

Environmental Evaluation of a New Federal Office Build-

PB94-199874

02,538

Ventilation Rates in Office Buildings.

PB95-108825

02,539

Application of a Multizone Airflow and Contaminant Dispersal Model to Indoor Air Ouality Control in Residential Buildings.

PB95-180238

02,555

AIR QUALITY STANDARDS

Ventilation, Carbon Dioxide and ASHRAE Standard 62. 1989

PB95-162095

02,544

AIR SAMPLING

International Radon-in-Air Measurement Intercomparison Using a New Transfer Standard.

AIR TIGHTNESS

03.708

Workplan to Analyze the Energy Impacts of Envelope Airtightness in Office Buildings.

PB96-154463

00,273

AIR TRAFFIC CONTROL

Feasibility of Fire Evacuation by Elevators at FAA Control PBg $4-213$

AIR-WATER INTERFACES

Atmospheric and Marine Trace Chemistry: Interfacial Biomediation and Monitoring.

PB94-199122

03,752

AIRBORNE EQUIPMENT

Airborne Smoke Sampling Package for Field Measurements of Fires.

Dual-Frequency Millimeter-Wave Radiometer Antenna for Airborne Remote Sensing of Atmosphere and Ocean.

PB96-112289

02,009

AIRCRAFT

Fracture Testing of Large-Scale Thin-Sheet Aluminum Alloy.

00,024

AIRCRAFT CONSTRUCTION MATERIALS

Investigation into the Flammability Properties of Honey. comb Composites.

PB95-143293

03,152

Computer-Aided Molecular Design of Fire Resıstant Aircraft Materials

AIRCRAFT ELECTRONICS

Aperture Excitation of Electrically Large, Lossy Cavities. 


\section{KEYWORD INDEX}

In Search of Alternative Fire Suppressants.

PB96-164165

03,285

Effectiveness of Halon Alternatives in Suppressing Dynamic Combustion Process.

PB96-175732 03,288

Interaction of HFC-125, FC-218 and CF3I with High Speed Combustion Waves

03,290

AIRLINES

Risk Analysis for the Fire Safety of Airline Passengers.
PB94- 194065
04,862 AIRPORT TOWERS

Feasibility of Fire Evacuation by Elevators at FAA Control

Towers.

Human Factors Considerations for the Potential Use of Elevators for Fire Evacuation of FAA Air Traffic Control

Towers.
PB94-217163

01,300

ALANINE

Research and Development Activities in Electron

Paramagnetic Resonance Dosimetry.

03,635

Orientation Effects on ESR Analysis of Alanine-Polymer Dosimeters.

PB96-146725

03,720

Investigation of Applicability of Alanine and Radiochromic

Detectors to Dosimetry of Proton Clinical Beams.

PB96-14678? 03,636

Complex Time Dependence of the EPR Signal of Irradiated L-alpha-alanine.

PB97-122436

04,180

ALCOHOL FUELS

Study of Droplet Transport in Alcohol-Based Spray

Flames Using Phase/Doppler Interferometry.

02,479

ALCOHOLS Thermodynamic Study of the Reactions of Cyclodextrins
with Primary and Secondary Aliphatic Alcohols, with Dand L-Phenylalanine, and with L-Phenylalanineamide.

PB95-180873

01,016

Silicon Nitride Boundary Lubrication: Lubrication Mechanism of Alcohols.

ALGAE

03,067

Total Surface Areas of Group IVA Organometallic Compounds: Predictors of Toxicity to Algae and Bacteria.

PB94-211331

00,814

ALGORITHMS

Secure Hash Standard. Category: Computer Security,
FIPS PUB 180-1

Calculating Flame Spread on Horizontal and Vertical Sur-

PB94-187283

00,335

VENTCF2: An Algorithm and Associated FORTRAN 77 Subroutine for Calculating Flow through a Horizontal Ceil-
ing/Floor Vent in a Zone-Type Compartment Fire Model. ing/Floor Vent in a Zone-Type Compartment Fire Model.
PB94-210127

Sprinkler Fire Suppression Algorithm.

00,293

PB94-216181

Algorithm to Describe the Spread of a Wall Fire under a

Ceiling

00,261

Computing Radiative Heat Transter Occurring in a Zone Fire Model.

01,399

PB96-102306

tics of

Analyzing and Exp

Zone Fire Model

01,400

Measurement Methods and Algorithms for Comparison of Local and Remote Clocks.

PB96-102652

01,549

Performance Measures for Geometric Fitting in the NIST Algorithm Testing and Evaluation Program for Coordinate Measurement Systems.

PB96-122122

01,745

Modified Optimal Algorithm for Active Structural Control.

PB96-165949 00,472

IML++ V.1.2 Iterative Methods Library Reference Guide.

PB96-195219

01,776

Representation of Axes for Geometric Fitting

01,782

\section{ALIGNMENT}

Electrical Test Structure for Improved Measurement of Feature Placement and Overlay in Integrated Circuit Fabrication Processes.

02,355

Collisional Alignment of $\mathrm{CO} 2$ Rotational Angular MomenCollisional Alignment of $\mathrm{CO} 2$ Rotational Angular Momen-
tum States in a Supersonic Expansion.
PB96-103171

Microelectronic Test Structures for Overlay Metrology

IPHATIC HYDROCARBONS

Physical Properties of Some Purified Aliphatic HydroCarbons. $265 / 1$

00,657

ALKALI AGGREGATE REACTIONS

Alkali-Silica Reaction and High Performance Concrete.

01,345

\section{ALKALI METALS}

Role of Adsorbed Alkalis in Desorption Induced by Electronic Transitions.

00,762

PB94-172574

Polarization of Light Emitted After Positron Impact Exci-
tation of Alkali Atoms.
PB94-199734
03,816 PB94-199734

Atomic Manipulation of Polarizable Atoms by Electric PB95-150587

04,572

Relativistic R-Matrix Calculations for Electron - AlkaliMetal-Atom Scattering: Cs as a Test Case.

04,006 PB95-203410

ALKANE THIOLATES

Characterization of Cytochrome c/Alkanethiolate Structures Prepared by Self-Assembly on Gold.

00,987

ALKANES

Calculation of Enthalpy and Entropy Differences of NearCritical Binary Mixtures with the Modified Leung-Griffiths Model

PB95-108635

00,885

ALKANETHIOL MONOLAYERS

Supported Phospholipid/Alkanthiol Biomimetic Membranes: Insulating Properties.

PB95-180782

03,470

Phospholipid/Alkanethiol Bilayers for Cell-Surface Recepor Studies by Surface Plasmon Resonance.

03,472

ALKANETHIOLS

Self-Assembled Phospholipid/Alkanethiol Biomimetic Bilayers on Gold.

PB95-108460

00,878

ALKYD RESINS

Cylinder Wipe Air-Drying Intaglio Ink Vehicles for U.S. Currency Inks.

03,115

ALKYLTHIOLATES

UV-Photopatterning of Alkylthiolate Monolayers Self-Assembled on Gold and Silver

PB95-150751

00,924

ALLAN VARIANCE

Cross-Correlation Analysis Improves Time Domain Meas-

PB95-180535

01,543

Confidence on the Modified Allan Variance and the Time

Variance.
PB96- 190376

01,557

ALERGIC DISEASES

Diagnosis and Treatment of an Oral Base-Metal Contact Lesion Following Negative Dermatologic Patch Tests. PB95-180626

00,172

ALLOSTERIC EFFECTORS

Positive and Negative Cooperativities at Subsequent Steps of Oxygenation Regulate the Allosteric Behavior of Multistate Sebacylhemoglobin

PB97-119374

03,486

ALLOYING

Prediction of the Strength Properties for Plain-Carbon and Vanadium Micro-Alloyed Ferrite-Pearlite Steel.

PB96-123393

03,216

ALLOYS

Thermal Conductivity of Metals and Alloys at Low Temperatures. A Review of the Literature.

AD-A279 180/4

03,302

Effert of Two Initiator/Stabilizer Concentrations in a Metal Primer on Bond Strengths of a Composite to a Base MBg4-172723

00,141

Characterization of the Hydrogen Induced Cold Cracking Susceptibility at Simulated Weld Zones in HSLA-100

PB94-174505

03, 746

Effect of Transformation of Alloy on Transient and Residual Stresses in a Porcelain-Metal Strip.

PB94-198397

00,143

Comparison of Techniques for Nondestructive Composition Measurements in CdZnTe Substrates.

02,703

PB96-103098 02,703 Evolution Equations for Phase Separation and Ordering
in Binary Alloys.
PB96-119243

Anisotropy of Interfaces in an Ordered Alloy: A MultipleOrder-Parameter Mode

PB96-131594 04,741

Characterization of the Structure of YD3 by Neutron Powder Diffraction

01,161

Charpy Impact Test as an Evaluation of $4 \mathrm{~K}$ Fracture Toughness.

03,219

ALPHA ORIONIS STAR

Four Years of Monitoring alpha Orionis with the VL.A: Where Have All the Flares Gone

00,048

GHRS Observations of Cool, Low-Gravity Stars. 1. The Far-Ultraviolet Spectrum of alpha Orions (M2 lab) PB96-112016

00,094

ALPHA PARTICLES

Systematics of Alpha-Particle Energy Spectra and Lineal Energy $(Y)$ Spectra for Radon Daughters.

03,615

Monte Carlo and Analytic Methods in the Transport of Electrons, Neutrons, and Alpha Particles.

PB96-111612

04,033

ALPHA SOURCES

Measurement and Calibration of Large-Area Alpha-Particle Sources at NIST.

ALPHA TOXIN

Protonation Dynamics in an lon Channel Pore.
PB96-161757

03,791

ALTERNATING CURRENT

AC-DC Difference Characteristics of High-Voltage Thermal Converters.
PB96-148093

02,083

ALTERNATING ELECTRIC FIELDS

Energy Transduction between a Concentration Gradient and an Alternating Electric Field

\section{ALTERNATIVE REFRIGERANTS}

03,461

Permeation Tube Approach to Long-Term Use of Automatic Sampler Retention Index Standards.

PB96-167291

00,639

ALTERNATIVES

Inhibition of Premixed Methane-Air Flames by Halon AIPB96-146741

01,414

In Search of Alternative Fire Suppressants.

PB96-164165

03,285

\section{ALUMINA}

Objective Evaluation of Short-Crack Toughness Curves Using Indentation Flaws: Case Study on Alumina-Based Ceramics.

PB96-179429

03,079

Texture Measurement of Sintered Alumina Using the March-Dollase Function.

04,784

Effect of Grain Size on Hertzian Contact Damage in Alu-

mina. 179601

03,083

Indentation Fatigue: A Simple Cyclic Hertzian Test for Measuring Damage Accumulation in Polycrystalline Ce-

PB96-180013

03,084

Flaw-Tolerance and Crack-Resistance Properties of Alumina-Aluminum Titanate Composites with Tailored Microstructures

ALUMINIUM ALLOYS

Evaluation of the Environmentally Induced Fracture Resistance of Ductile Nickel Aluminide. Technical Report Number 1, Final report. October-December 1990. DE94017331

03,306 
Fracture Testing of Large-Scale Thin-Sheet Aluminum Alloy.

03,305

AD-A306 625/5

Aluminum

Alloy.

03,311

Temperature Increases in Aluminum Alloys during Mechanical-Impact Tests for Oxygen Compatibility.
PB94-172962

03,316

Thermodynamic Calculation of the Ternary Ti-Al-Nb SysPB94-212636

03,336

Thermodynamic Assessment and Calculation of the Ti-Al System.
PB94-212644

03,337

Recommended Changes in ASTM Test Methods D251282 and G86-84 for Oxygen-Compatibility Mechanical Impact Tests on Metals.

PB94-216694

03,338

Macro- and Microreactions in Mechanical-Impact Tests of Aluminum Alloys.

03,340

Influence of Specimen Absorbed Energy in LOX Mechanical-Impact Tests

03,341

Residual Stresses in Aluminum-Mullite (alpha-Alumina) Composites.

03,155

Thin Film Reaction Kinetics of Niobium/Aluminum Multilayers.
PB95-175295

04,651

Fracture Testing of Large-Scale Thin-Sheet Aluminum Alloy.

PB95-242368

00,024

Journal of Research of the National Institute of Standards and Technology. September/October 1993. Volume 98, Number 5 .

PB96-169057

03,368

Light-Weight Alloys for Aerospace Applications II.

PB96-190244

03,375

ALUMINUM ANTIMONY ALLOYS

Assessment of the AI-Sb System.

ALUMINUM BASE ALLOYS

03,329

Study of Laser Resonance lonization Mass Spectrometry Using a Glow Discharge Source.

03,360

ALUMINUM FLUORIDES

New Rydberg States of Aluminum Monofluoride Observed by Resonance-Enhanced Multiphoton Ionization SpectrosPB94-199544

00,797

ALUMINUM GALLIUM ARSENIDE

Al $L 2,3$ Core Excitons in AlXGa 1-X as Studied by Soft-Xray Reflection and Emission.

04,067

$X$-ray Reflectivity Determination of Interface Roughness Correlated with Transport Properties of (AIGa)As/GaAs High Electron Mobility Transistor Devices.
PB97-111868

04,149

04.90

Time-Resolved Measurements of the Polarization State of Time-Resolved Measurements of the Polarization State of
Four-Wave Mixing Signals from GaAs Multiple Ouantum

PB96-201058

04,796

ALUMINUM GAS CYLINDERS

Stability/Instability of Gas Mixtures Containing 1,3-Butadiene in Treated Aluminum Gas Cylinders.

PB95-162285

02,546

ALUMINUM GRAPHITE COMPOSITES

Thermal Expansion of an SiC Particle-Reinforced Aluminum Composite.

ALUMINUM INTERMETALLICS

Coherent Precipitates in the BCC/Orthorhombic Two

Phase Field of the Ti-Al-Nb System.

PB94-198694

03,317

Crystallographic Characterization of Some Intermetallic Compounds in the Al-Cr System.

PB94-198702

03,318

ALUMINUM-LITHIUM ALLOYS

Reaction Sensitivities of Al-Li Alloys and Alloy 2219 in Mechanical-Impact Tests.

PB94-172764

03.314

Alumınum-Lithium Alloys: Evaluation of Fracture Toughness by Two Test Standards, ASTM Method E 813 and E

PB96-190236

03,374

ALUMINUM MANGANESE ALLOYS

Effect of Mn Content on the Microstructure of Al-Mn Alloys Electrodep
PB95-126355

03,343

ALUMINUM MONOFLUORIDE

New Rydberg States of Aluminum Monofluoride Observed by Resonance-Enhanced Multiphoton Ionization SpectrosPB94-199544

00,797

ALUMINUM NIOBATES

Improved Crystallographic Data for Aluminum Niobate (AlNbO4).

04,523
ALUMINUM NITRIDE

Effect of Beam Voltage on the Properties of Aluminum Nitride Prepared by Ion Beam Assisted Deposition. PB97-118616

01,995

ALUMINUM OXALATE

Effects of Aluminum Oxalate/Glycine Pretreatment Soluions on Dentin Permeability.

03,565

ALUMINUM OXIDE

Characterization of the Densification of Alumina by Multiple Small-Angle Neutron Scattering. (Reannouncemen with New Avallability Information).

AD-A249 179/3

03,024

Tribological Characteristics of Alpha-Alumina at Elevated Temperatures.

02,963

Effect of Microstructure on the Wear Transition of Zirconia-Toughened Alumina.

PB94-211778

03,14

Mechanism of Mild to Severe Wear Transition in AlphaAlumina.

03,233

Observed and Theoretical Creep Rates for an Alumina

Ceramic and a Silicon Nitride Ceramic in Flexure.
PB94-212958

03,040

Interface Modification and Characterization of Silicon Carbide Platelets Coated with Alumina Particles.

PB95-108734

03,121

Control of Friction and Wear of Alpha-Alumina with a Composite Solid-Lubricant Coating.

B95-125969 03,225

Fabrication of Flaw-Tolerant Aluminum-Titanate-Reinforced Alumina.

PB95-162533

03,161

Wear Model for Alumina Sliding Wear

B95-163796

03,239

Dielectric Properties of Single Crystals of $\mathrm{Al}_{2} \mathrm{O} 3$, LaAlO3, $\mathrm{SrTiO3,} \mathrm{and} \mathrm{MgO}$ at Cryogenic Temperatures.

PB95-180477

02,266

Stability and Surface Energies of Wetted Grain Boundaries in Aluminum Oxide.

03,059

Wear Transitions in Monolithic Alumina and Zirconia-Alumina Composites.

PB96-103163

ALUMINUM OXIDES

Effect of Green Density and the Role of Magnesium

Oxide Additive on the Densification of Alumina Measured

by Small-Angle Neutron Scattering. (Reannouncemen AD-A244 582/3

03,022

Evolution of the Pore Size Distribution in Final-Stage Sintering of Alumina Measured by Small-Angle X-ray Scattering. (Reannouncement with New Availability Informa-

AD-A249 178/5

03,023

Measuring Matching Wear Scars on Balls and Flats.

PB95-151528 03,153

Residual Stresses in Aluminum-Mullite (alpha-Alumina) Composites.

PB95-152880 03,155

Flat and Rising R-Curves for Elliptical Surface Cracks from Indentation and Superposed Flexure.

03,156

Fabrication of Transparent gamma-Al2O3 from Nanosize Fabrication of Transparent gamma-Al2O3 from Nanosize
Particles.
PB95-175493

ALUMINUM TIT ANATE

03,054

Flaw-Tolerance and Crack-Pesistance Properties of Alumina-Aluminum Titanate Composites with Tailored Microstructures.

PB97-110324

03,101

ALUMINUM TITANATES

Fabrication of Flaw-Tolerant Aluminum-Titanate-ReinPB95-162533

03,161

AMALGAM

Wear of Enamel against Glass-Ceramic, Porcelain, and Amalgam.

PB96-179593

03,082

AMBIENT TEMPERATURE

Ambient Temperature Synthesis of Bulk Intermetallics.
PB95-169074

AMERICAN ASSOCIATION OF AEROSOL RESEARCH

Global Climatic Effects of Aerosols: The AAAR Sympo-

PBO5-108791

00,122

AMINE/N-NITROSODIMETHYL

Unusual Spin-Trap Chemistry for the Reaction of Hydroxy! Radical with the Carcinogen $\mathrm{N}$ Nitrosodimethylamine.

00,692

AMINES

New Surface-Active Comonomer for Adhesive Bonding. PB96-204052

03,579

AMINO ACIDS

L-threo-beta-Hydroxyhistidine, an Unprecedented Iron(III) Ion-Binding Amino Acid in a Pyoverdine-type Siderophore from Pseudomonas fluorescens 244

00,553

Protonation Dynamics of the alpha-Toxin Ion Channel from Spectral Analysis of $\mathrm{pH}$-Dependent Current Fluctua-

PB96-161740

03,652

AMMONIA

Self Broadening in the nu 1 Band of $\mathrm{NH}_{3}$.

00,857

Photodissociation of Ammonia at $193.3 \mathrm{~nm}$ : Rovibrational State Distribution of the NH2(A(2)A1) Fragment.

PB95-151775

00,937

Equilibrium and Calorimetric Investigation of the Hydrolysis of L-Tryptophan to (Indole + Pyruvate + Ammonia).
PB95-163317 00,661

Viscosity of Ammonia

PB96-145933

01,117

MMONIA COMPLEXES

Microwave and Submillimeter Spectroscopy of Ar-NH3 States Correlating with $\mathrm{Ar}+\mathrm{NH} 3(\mathrm{j}=1, \mathrm{k}=1)$

00,942

AMMONIA RADICALS

Photodissociation of Ammonia at 193.3nm: Rovibrational State Distribution of the NH2(A(2)A1) Fragment.

PB95-151775

AMMONIUM COMPOUNDS

Use of Kinetic Energy Distributions to Determine the Relative Contributions of Gas Phase and Surface Fragmentation in KeV Ion Sputtering of a Quaternary Ammonium Salt.

PB95-126108

00,570

MMONIUM IONS

Planar Waveguide Optical Sensors.

03,586

AMORPHOUS FERROMAGNETS

Polarization Analysis of the Magnetic Excitations in Invar and Non-Invar Amorphous Alloys.

AMORPHOUS SILICON

Plasma Chemistry in Disilane Discharges.

PB94-211075

04,516

Nanoscale Study of the As-Grown Hydrogenated Amorphous Silicon Surface.

AMORPHOUSE PHASES

Extrapolation of the Heat Capacity in Liquid and Amorphous Phases.

AMPEROMETRIC

Novel Amperometric Immunosensor for Procainamide Employing Light Activated Labels.

PB96-163662

00,512

AMPERTURE AMPLITUDE DISTRIBUTION

Planar Near-Field Measurements and Microwave Holography for Measuring Aperture Distribution on a $60 \mathrm{GH}$ Active Array Antenna.

PB96-16736

03.762

AMPHETAMINE

Determination of Amphetamine and Methamphetamine in a Lyophilized Human Urine Reference Material.

PB95-175444

03,597 


\section{KEYWORD INDEX}

ANALINES

Inelastic Neutron Scattering Studies of Nonlinear Optical Materials: p-Nitroaniline Adsorbed in ALPO-5.

00.874

ANALOG CIRCUITS

Developing Linear Error Models for Analog Devices

02.037

Diakoptic and Large Change Sensitivity Analysis.

PB95-150538

02,038

ANALOG TO DIGITAL CONVERTERS

Developing Linear Error Models for Analog Devices.

NALYSIS OF VARIANCE

Anova Estimates of Variance Components for a Class of Mixed Models
PB96-141163

ANALYTICAL CHEMISTRY

03,448

Airborne Asbestos Method: Standard Test Method for High Precision Counting of Asbestos Collected on Filters. Version 1.0.

00.525

Airborne Asbestos Method: Standard Test Method for Verified Analysis of Asbestos by Transmission Electron

Microscopy. Version 2.0
PB94-163045

00,526

Raman and Fluorescence Spectra Observed in Laser Microprobe Measurements of Several Compositions in the Ln-Ba-Cu-O System.

04,440

Radiance Temperature (in the Wavelength Range 519

Radiance Temperature (in the Wavelength Range 519-
$906 \mathrm{~nm}$ ) of Tungsten at Its Melting Point by a Pulse-Heat$906 \mathrm{~nm}$ ) of Tungsten at lts Melting Point by a Pulse-Heating Technique

03,397

Absolute Determination of Electrolytic Conductivity for Primary Standard KC1 Solutions from 0 to $50 \mathrm{C}$.

00,765

Classical Analysis: A Look at the Past, Present, and Fu-

PB.

00,528

Long-Term Stability of Carrier-Free Polonium Solution Standards.

PB94-185170

00,685

Liquid Chromatography: Laser-Enhanced Ionization Spec-

trometry for the Speciation of Organolead Compounds.

PB94-185253

00,530

Comparative Strategies for Correction of interferences in Isotope Dilution Mass Spectrometric Determination of Va-

PB94-185261

00,531

Relative Sensitivity Factors and Useful Yields for a Microfocused Gallium Ion Beam and Time-of-Flight Secondary Ion Mass Spectrometer.

Object Finder for Digital Images Based on Multiple Object Finder for Digital Images Based on Multiple
Thresholds, Connectivity, and Internal Structures.
01,833 PB94-199106 01,833

Concentration Histogram Imaging: A Scatter Diagram Technique for Viewing Two or Three Related Images.
PB94-199114

Atmospheric and Marine Trace Chemistry: Interfacial Biomediation and Monitoring. Determination of 3-Quinuclidinyl Benzilate (Onb) and Its Major Metabolites in Urine by Isotope Dilution Gas Chromatography Mass Spectrometry.

PB94-199379

03,492

Thermodynamic Analysis of Heparin Binding to Human Antithrombin.

03,455

Establishing Ouality Measurements for Inorganic Analysis of Biomaterials.

PB94-199726

00,548

Determination of Oltipraz in Serum by High-Performanace Liquid Chromatography with Optical Absorbance and Mass Spectrometric Detection.

PB94-200201

03,493

Importance of Chemometrics in Biomedical Measure-

PB94-200599

00,550

Metrological Measurement Accuracy: Discussion of 'Measurement Error Models' by Leon Jay Gleser.

PB94-200607

00,551

Airbome Asbestos Method: Standard Practice for Record ing Transmission Electron Microscopy Data for the Analy. sis of Asbestos Collected onto Filters. Version 1.0.
PB94-210168 00,552 PB94-210168 00,552 Matrices Using Neutron Activation - Mass Spectrometry

PB94-212289

00,554

Spectroscopic Constants for the 2.5 and 3.0 micrometer Bands of Acetylen

00,847

Measurement of CO Pressures in the Ultrahigh Vacuum Regime Using Resonance-Enhanced Multiphoton-lonization Time-of-Flight Mass Spectroscopy.

PB94-216041

03,864

Effects of Target Shape and Neutron Scattering on Element Sensitivities for Neutron-Capture Prompt Gammaray Activation Analysis.

00,558
Study of Diffusion Zones with Electron Microprobe Compositional Mapping.

00.559

Electron Probe X-Ray Microanalysis.

00,562

Microanalysis to Nanoanalysis: Measuring Composition at High Spatial Resolution.

00,563

Design of a Protocol for an Electron Probe Microanalyzer -Value Round Robin.

PB95-107181

00,564

Compositional Mapping of the Microstructure of Materials.

00,565

Separation and Identification of Organic Gunshot and Ex plosive Constituents by Micellar Electrokinetic Capillary Electrophoresis.

PB95-107249

00.566

Secondary Target X-Ray Excitation for In vivo Measurement of Lead in Bone.

03,496

Image Depth Profiling SIMS: An Evaluation for the Analysis of Light Element Diffusion in YBa2Cu3O7-x Single Crystal Superconductors.

PB95-126116

04,530

Molecular Ion Imaging and Dynamic Secondary Ion Mass Spectrometry of Organic Compounds.

00.571

$95-126124$

Transition Metal Implants in In0.53GA0.47As.
PB95-126389

Range Statistics and Rutherford Backscattering Studies on Fe-Implanted In0.53Ga0.47As.

PB95-126397

04,535

Device for Subambient Temperature Control in Liquid Chromatography.

00,573

Comparison of the Liquid Chromatographic Behavior of Selected Steroid Isomers Using Different Reversed Phase Materials and Mobile Phase Compositions.

PB95-140976

00,574

Automated, High-Precision Coulometric Titrimetry. Part 1 Engineering and Implementation.

0199

00,575

Automated, High Precision Coulometric Titrimetry. Part 2 Strong and Weak Acids and Bases.

00,576

Integrating Automated Systems with Modular Architec-

ture.

00.577

Development of Engineered Stationary Phases for the Separation of Carotenoid Isomers.

PB95-150249

00.578

Shape Selectivity Assessment of Stationary Phases in Gas Chromatography.

00,579

Guidelines for Refractive Index Measurements of Asbeslos.

PB95-151189 02,543

Factoriał Design Techniques Applied to Optimization of AMS Graphite Target Preparation.

PB95-151197

00,584

Comprehensive Spectroscopic Data Tabulations and Progress in the Compilation of Atomic Transition Probabilities.

PB95-15155

03,909

Determination of Polycyclic Aromatic Hydrocarbons by Liquid Chromatography.

PB95-151650

00,585

Potentiometric Enzyme-Amplified Flow Injection Analysis Detection System: Behavior of Free and Liposome-Fe eased Peroxidase.

PB95-151833

03,534

New Method to Evaluate Deposit Forming Tendencies of Liquid Lubricants by Differential Scanning Calorimetry.
PB95-152120 Development of a Gas Standard Reference Material Containing Eighteen Volatile Organic Compounds. $\quad 02,545$
PB95-162277 Stability/Instability of Gas Mixtures Containing 1,3-Butadiene in Treated Aluminum Gas Cylinders. PB95-162285

02,546

Shape Selectivity in Reversed-Phase Liquid Chromaography for the Separation of Planar and Non-Plana Solutes.

PB95-162608

00,596

Trace Elements Associated with Proteins. Neutron Activation Analysis Combined with Biological Isolation Tech-

PB95-163101

00,597

Trace Detection in Conducting Solids Using Laser-induced Fluorescence in a Cathodic Sputtering Cell. PB95-163424

00,598

Measurement of the Uniformity of Particle Deposition of Filter Cassette Sampling in a Low Velocity Wind Tunnel. PB95-163754 02,549

Applications of Fluorescence Spectroscopy in Polymer Science and Technology.

01,258

Localization of Atoms in a Three-Dimensional Standing Wave.

PB95-163887

03,944

Comparative Study of Fe-C Bead and Graphite Target Performance with the National Ocean Sciences AMS (NOSAMS) Facility Recombinator Ion Source.

PB95-175790

00,693

Determination of Hydrogen in Titanium Alloy Jet Engine Compressor Blades by Cold Neutron Capture Prompt Gamma-ray Activation Analysis.

PB95-175956 01,448 Interlaboratory Comparison Studies on the Analysis of Hair for Drugs of Abuse.

PB95-176251

03,500

Cyclic Polyamine lonophore for Use in a Dibasic Phosphate-Selective Electrode.

01,008

Instrument for Evaluating Phase Behavior of Mixtures for Supercritical Fluid Experiments.

PB95-180758

00,606

Methods for Analysis of Cancer Chemopreventive Agents

in Human Serum.

03,502

Concentrations of Chlorinated Hydrocarbons, Heavy Metals and Other Elements in Tissues Banked by the Alaska Marine Mammal Tissue Archival Project.

PB95-209870

02,590

Isotope Dilution Mass Spectrometry as a Candidate Definitive Method for Determining Total Glycerides and Triglycerides in Serum.

PB96-102280

03,519

Intercomparison Study of (237)Np Determination in Artificial Urine Samples.

PB96-102645

03,633

PB96-103072

04,829

Proficiency Tests for the NIST Airborne Asbestos Program, 1993.

00,610

Ouantitative Analysis of Selected PCB Congeners in Marine Matrix Reference Materials Using a Novel Cyanobiphenyl Stationary Phase.

Stability of Compressed Gas Mixtures Containing Low Level Volatile Organic Compounds in Aluminum Cyl-

PB96-111968

00,613

Spectral Interference in the Determination of Arsenic in High-Purity Lead and Lead-Base Alloys Using Electrothermal Atomic Absorption Spectrometry and Zeeman-Effect Background Correction.

00,614

SUM and MEAN: Standard Programs for Activation Analysis. 112149

00,617

Application of a Novel Slurry Furnace AAS Protocol for Rapid Assessment of Lead Environmental Contamination. PB96-112354 
Isotopic and Nuclear Analytical Techniques in Biological Systems: A Critical Study. 10. Elemental Isotopic Dilution Analysis with Radioactive and Stable Isotopes (Technical PB96-164157

00,696

Certitying the Chemical Composition of a Biological Material: A Case Study.

PB96-164272

00,636

Dead Time, Pileup, and Accurate Gamma-Ray Spectrom-

PBg6-167101

00,697

Loss-Free Counting at IRI and NIST.

04,105

Trace Element Concentrations in Cetacean Liver Tissues Archived in the National Marine Mammal Tissue Bank.

PB96-167127

02,595

Supercritical Fluid Extraction-Immunoassay for the Rapid

Screening of Cocaine in Hair.

00,637

New NIST Rapid Pneumatic Tube System.

PB96-167259

03,738

Development of the Ion Exchange-Gravimetric Method for Sodium in Serum as a Definitive Method.

PB96-179148 01,867

Preliminary Investigation of Oleoresin Capsicum.
PB96-179486
03,520

Fluoride Analytical Methods.

PB96-180237

03,578

Isolation and Structural Elucidation of the Predominant Geometrical Isomers of alpha-Carotene.

00,640

Role of Certified Reference Materials in Trace Analysis Ouality Assurance.

PB97-110019

00,650

Interlaboratory Studies on the Analysis of Hair for Drugs of Abuse: Results from the Fourth Exercise.

PB97-111322

03,510

Use of Neutron Beams for Chemical Analysis at NIST.

PB97-112437 00,652

Measurement of Ascorbic Acid in Human Plasma and Serum: Stability, Intralaboratory Repeatability, and Interlaboratory Reproducibility.

03,511

CONTAM94: A Multizone Airflow and Contaminant Dispersal Model with a Graphic User Interface.

02,570

Liquid Chromatographic Determination of Carotenoids in Human Serum Using an Engineered C30 and a C18 Sta. tionary Phase
PB97-119333

03,512

Radiocarbon Measurements of Atmospheric Volatile Organic Compounds: Ouantifying the Biogenic Contribution. ganic Compounc

02,574

ANALYTICAL METHODS

Certification of Polycyclic Aromatic Hydrocarbons in a Marine Sediment Standard Reference Material. PB96-111778

Application of a Novel Slurry Furnace AAS Protocol for Rapid Assessment of Lead Environmental Contamination.

NALYTICAL TECHNIQUES

Classical Analysis: A Look at the Past, Present, and FuPB94-185063

00,528

Dissolution Problems with Botanical Reference Materials.
PB95- 126280
03,487

Results of the ASTM Nuclear Methods Intercomparison on NIST Apple and Peach Leaves Standard Reference Materials.

03,490

ANALYZERS

Design and Characterization of X-Ray Multilayer Analyz-

ers for the $50-1000 \mathrm{eV}$ Region.
PB94-211851

03,848

ANATOMIC MODELS

Summary of the Proceedings of the Workshop on Standard Phantoms for In-vivo Radioactivity Measurement PB94-212933

ANELASTICITY

Temperature and Frequency Dependence of Anelasticity in a Nickel Oscillator.

ANEMOMETERS

03,689

Thermal Anemonetry for Mass Flow Measurement in Oscillating Cryogenic Gas Flows.

ANGE MEASUREMENT

Advanced Angle Metrology System.

PB94-211364

04,218

NGLES (GEOMETRY)

Tilt Effects in Optical Angle Measurements.

ANGLAR DISTRIBUTIONS

Measuring Nondipolar Asymmetries of Photoelectron Angular Distributions
PB97-122493

ANGULAR MOMENTUM

Collisional Alignment of $\mathrm{CO} 2$ Rotational Angular Momenum States in a Supersonic Expansion.

PB96-103171

01,069
ANHARMONIC OSCILLATORS

Anharmonic Oscillator Analysis Using Modified Airy Functions.

03,798

\section{ANIMAL FEED}

Individual Carotenoid Content of SRM 1548 Total Diet and Influence of Storage Temperature, Lyophilization, and Irradiation on Dietary Carotenoids.

00,033 ANIMAL HUSBANDRY

Preparation and Monitoring of Lead Acetate Containing Drinking Water Solutions for Toxicity Studies.

00,538

ANIMAL PHYSIOLOGY

Histopathology, Blood Chemistry, and Physiological Status of Normal and Moribund Striped Bass ("Morone saxatilis') Involved in Summer Mortality ('Die-Off') in the Sacramento-San

00,034

\section{ANIMAL TISSUES}

Development of Frozen Whale Blubber and Liver Reference Materials for the Measurement of Organic and Inorganic Contaminants.

PB95-151676

00,587

Determination of Inorganic Constituents in Marine Mammal Tissues.

Development of the National Marine Mammal Tissue Bank.

PB95-161402

02,586

Ouality Assurance of Contaminant Measurements in Ma-

rine Mammal Tissues.
PB95-164034

Alaska Marine Mammal Tissue Archival Project: Specimen Inventory.

02,589

Concentrations of Chlorinated Hydrocarbons, Heavy Metals and Other Elements in Tissues Banked by the Alaska Marine Mammal Tissue Archival Project.

PB95-209870

02,590

Certification of Polychlorinated Biphenyl Congeners and Chlorinated Pesticides in a Whale Blubber Standard Reference Material
PB96-103023

03,745

National Status and Trends Program Specimen Bank: Sampling Protocols, Analytical Methods, Results, and Archive Samples.

ANIONS

02,598

Vibrational Spectra of Molecular Ions Isolated in Solid Neon. 11. NO2(+), NO2(-), and NO3(-)

00,708

Temperature Dependence of the Rate Constants for ReTemperature Dependence of the Rate Constants for Re-
actions of the Sulfate Radical, SO4-, with Anions.

Temperature Dependence of the Rate Constants for Reactions of the Carbonate Radical with Organic and Inorganic Reductants.

00,831

ANISOLE

Thermal Decomposition of Hydroxy- and Methoxy-Substituted Anisoles.

PB94-17303

ANISOTROPHY

Ultrasonic Measurement of Sheet Anisotropy and Formability.

03,213

ANISOTROPIC MATERIALS

Microwave Dielectric Properties of Anisotropic Materials at Cryogenic Temperatures

02,412

ANISOTROPY

Convection and Morphological Stability During Directional Solidification

03,310

Anisotropic Phase Separation Kinetics in a Polymer Blend Solution Following Cessation of Shear Studied by Light Scattering.

01,241

Anisotropy of Polarized $X$-ray Emission from Atoms and

PB95-163002

04,621

ANISTROPY

Fluorescence Anisotropy Measurements on a Polymer PB94-199296

01,209

Hemispherical Test Fixture for Measuring the Wavefields Generated in an Anisotropic Solid. PB96-190087

03,181

Notion of a xi-Vector and a Stress Tensor for a General Class of Anisotropic Difluse Interface Models.

$04,78 \varepsilon$

ANNEALINC

Magnetostriction and Giant Magnetoresistance in Annealed NiFe/Ag Multilayers.

04,716

Low Magnetostriction in Annealed NiFe/Ag Giant Magnetoresistive Multilayers.

04,762

Annealing of Bragg Gratings in Hydrogen-Loaded Optical

Fiber.

04,361

Effect of Intermediate Thermal Processing on Microstructural Changes of Oxygen Implanted Silicon-on

Insulator Material.
PB96-160213 02,982

Antiferromagnetic Interlayer Correlations in Annealed Ni80Fe20/Ag Multilayers.

03,109

ANNELING

Oxygen Annealing of Ex-situ YBCO/Ag Thin-Film Interfaces.

ANNULAR FLOW

04,758

Single-Phase Heat Transfer and Pressure Drop Characteristics of an Integral-Spine-Fin Within an Annulus.

PB94-194073

Single-Phase Heat Transfer and Pressure Dror teristics of an Integral-Spine Fin Within an Annulus. PB97-122386

ANOMALOUS DISPERSIONS

Theoretical Form Factor, Attenuation and Scattering Tabulation for $Z=1-92$ from $E=1-10 \mathrm{eV}$ to $E=0.4-1.0 \mathrm{MeV}$ PB96-145594

01,104

ANSUASHRAE STANDARD 124-1991

Predicting the Energy Performance Ratings of a Family of PB95-105524

02,504

ANTENNA ARRAYS

Optically Linked Three-Loop Antenna System for Determining the Radiation Characteristics of an Electrically PB95-161915

ANTENNA MEASUREMENTS

Planar Near-Field Alignment.

01998

New Extrapolation/Spherical/Cylindrical Measurement $\mathrm{Fa}$ cility at the National Institute of Standards and Technology.
PB95- 153755

02,004

ANTENNA RADIATION PATTERNS

Correction Factor for Nonolanar Incident Field in

Monopole Calibrations.

02,002

ANTENNAS

Standard Probes for Electromagnetic Field Measure-

PB94-185436

01,999

Planar Near-Field Measurements of Low-Sidelobe Anten-

Dual Frequency mm-Wave Radiometer Antenna for Air-

bome Remote Sensing of Atmosphere and Ocean.

Standard Antennas for Electromagnetic Interference Measurements and Methods to Calibrate Them. 02,007
PB96-102561

Dual-Frequency Millimeter-Wave Radiometer Antenna for Airborne Remote Sensing of Atmosphere and Ocean. Airborne Remote Sensing of Atmosphere and Ocean.
PB96-112289 Comparison of k-Correction and Taylor-Series Correction for Probe-Position Errors in Planar Near-Field Scanning.
PB96-147137 PB96-147137
02,012 Standard Source Method for Reducing Antenna Facto
Errors in Shielded Room Measurements. PB96-183157

Methodology for Electromagnetic Interference Measure. ments.

02.014

Comparison of Ultralow-Sidelobe-Antenna Far-Field Pattems Using the Planar-Near-Field Method and the FarField Method.

P896-200373

02,015

General Order ' $N$ ' Analytic Correction of Probe-Position

Errors in Planar Near-Field Measurements.

P896-200688 
ANTIMONY

Epitaxial Growth of $\mathrm{Sb} / \mathrm{GaSb}$ Structures: An Example of V/III-V Heteroepitaxy.

PB95-202560

04,693

ANTIMONY CLUSTERS

Photoelectron Spectroscopy of Small Antimony Cluster Anions: $\mathrm{Sb}(-)$, Sb2(-), Sb3(-), and Sb4(-) PB95-203139

01,045

ANTITHROMBIN III

Thermodynamic Analysis of Heparin Binding to Human Antithrombin.

03,455

ANTIVIRAL AGENTS

Radiation Chemistry of Cyanine Dyes: Oxidation and Reduction of Merocyanine 540

PB94-211661

00,818

APATITE/HYDROXY

Effect of Ethanol on the Solubility of Hydroxyapatite in the System $\mathrm{Ca}(\mathrm{OH}) 2-\mathrm{H} 3 \mathrm{PO} 4-\mathrm{H} 2 \mathrm{O}$ at $25 \mathrm{C}$ and $33 \mathrm{C}$

00,169

APATITES

Effect of Supersaturation on Apatite Crystal Formation in Aqueous Solutions at Physiologic pH and Temperature. Aqueous Solutions at Physiologic pH and Temperature.
PB96-135215

APDE (APPLICATION PROTOCOL DEVELOPMENT

ENVIRONMENT)

APDE Demonstration System Architecture. National PDES Testbed Report Series.

PB94-154325

02,767

APERTURES

Aperture Excitation of Electrically Large, Lossy Cavities. PB94-145711 00,029

Aperture Excitation of Electrically Large, Lossy Cavities.

PB95-175675 00,031

High Accuracy Measurement of Aperture Area Relative to

$\begin{array}{ll}\text { a Standard Known Aperture. } & 01,919\end{array}$

Third Generation Water Bath Based Blackbody Source.

PB96-122148

04,046

APPAREL CLOTHING

Survey of Standards for the U.S. Fiber/Textile/Apparel Industry.
PB96-193792

03,199

APPEARANCE POTENTIAL

Appearance Potentials of Ions Produced by Electron- $1 \mathrm{~m}$ pact Induced Dissociative Ionization of SF6, SF4, SF5Cl, $\mathrm{S} 2 \mathrm{~F} 10, \mathrm{SO2}$
$\mathrm{PB} 96-119730$

01,080

APPLICATION PORTABILITY PROFILE

Application Portability Profile (APP): The U.S. Government's Open System Environment Profile Version 3.0

PB96-158712

01,753

APPLICATION PROGRAMS (COMPUTERS)

User Interface Component of the Applications Portability Profile Category: Software Standard; Subcategory: Application Program Interface.

FIPS PUB 158-1

01,793

APPLICATION PROTOCOLS

APDE Demonstration System Architecture. National

PDES Testbed Report Series.
PB94-154325

Extensions of the Prototype Application Protocol of Ready-to-Wear Apparel Pattern Making.

PB96-128194

03,198

APPLICATION SPECIFIC INTEGRATED CIRCUITS

Custom Integrated Circuit Comparator for High-Performance Sampling Applications.

02,320

APPLICATIONS PROGRAMS (COMPUTERS)

Open System Environments.

N94-36857/8

01,674

Associated Object Model for Distributed Systems

PB94-212016

01,694

Security in Open Systems.

01,473

APPLIED MATHEMATICS

Computer Systems Laboratory Computing and Applied
Mathematics Laboratory Technical Accomplishments, October 1994-March 1996

PB96-193768

01,638

APPROXIMATION

Higher-Order Approximations in Ionospheric Wave-Propagation.

04,420

APS (APPLICATION PROTOCOLS)

Issues and Fecommendations for a STEP Application

Protocol Framework. National PDES Testbed.

02,770

AQEOUS SPECIES

Summary of the Apparent Standard Partial Molal Gibbs

Free Energies of Formation of Aqueous Species, Min-

erals, and Gases at Pressures 1 to 5000 Bars and Temperatures 25 to $1000 \mathrm{C}$

PB96-145891

01,113

AOUEOUS ELECTROLYTES

Absolute Determination of Electrolytic Conductivity for Primary Standard KC1 Solutions from 0 to $50 \mathrm{C}$

00,765
AQUEOUS IRON

Thermodynamic Properties of the Aqueous lons $(2+$ and $3+)$ of Iron and the Key Compounds of Iron

01,119

AUEOUS PHASE

Free Radical Chemistry of the Atmospheric Aqueous PB96-148101

00,117

AQUEOUS SOLUTIONS

Apparent Molar Heat Capacities and Apparent Molar Volumes of Aqueous Glucose at Temperatures from 298.15 K to $327.01 \mathrm{~K}$

03,459

Two Phase Aqueous Extraction: Rheological Properties of Dextran, Polyethylene Glycol, Bovine Serum Albumin and Their Mixtures.

00,676

Standard States, Reference States and Finite-Concentration Effects in Near-Critical Mixtures with Applications to Aqueous Solutions.

PB95-164349

00,979

Critical Lines for Type-III Aqueous Mixtures by Generalized Corresponding-States Models.

01,063

Significant Contributions of IAPWS to the Power Industry, Science and Technology.

PB96-123252

01,088

Critical Review of Rate Constants for Reactions of Transients from Metal lons and Metal Complexes in Aqueous

PB96-145859

01,109

System for Intercomparing Standard Solutions of BetaParticle Emitting Radionuclides.

PB96-159637

03,707

AC DISCHARGES

Decomposition of SF6 and Production of S2F10 in Power

PB96-122619

01,084

ARC MODELS

Mathematical Models of Transport Phenomena Associated with Arc-Welding Processes: A Survey. PB96-135058

02,870

ARC SPECTRA

Arc Spectra of Gallium, Indium, and Thallium.

AD-A295 411/3

00,718

RC WELDING

Status Report: AWS Standards for Identifying Arc Welds A91.1) and Recording Weld Data (A9.2).

PB95-162855

02,861

Through-the-Arc Sensing for Measuring Gas Metal Arc Weld Oality in Real Time.

PB95-164463

02,908

ARCHIE

Using Archie to Find Files on the INTERNET.

PB95-168605

02,727

ARCHITECTURE (COMPUTERS)

Overview of NASREM: The NASANBS Standard Reference Model for Telerobot Control System Architecture. PB94-194560 04,831 AREA

High Accuracy Measurement of Aperture Area Relative to a Standard Known Aperture.

PB95-261954

01.919

\section{ARGININE}

Thermochemistry of the Hydrolysis of L-arginine to $(\mathrm{L}$ citrulline + Ammonia) and of the Hydrolysis of $\mathrm{L}$-arginine to (L-ornithine + Urea)

PB95-150801

03,463

\section{ARGON}

Measurement of the Thermal Properties of Electrically Conducting Fluids Using Coated Transient Hot Wires.

Conducling Fluids Using Coaled Transient Hot Wirs 222

Absorption of Sound in Gases between 10 and $25 \mathrm{MHz}$ : Argon.

Atomic Transition Probability Ratios between Some Ar $4 s-4 p$ and $4 s-5 p$ Transitions.

PB94-211554 03,842

Ion Broadening Parameters for Several Argon and Carbon Lines.

PB94-211562 03,843

Intermolecular HF Motion in $\operatorname{Ar}(s u b$ n)HF Micromatrices $(n=1,2,3,4$,): Classical and Ouantum Calculations on a Pairwise Additive Potential Surface.

03,871

B 95-107025

Vibration, Rotation, and Parity Specific Predissociation Dynamics in Asymmetric OH Stretch Excited ArH2O: A Half Collision Study of Resonant $V \cdot V$ Energy Transfer in a Weakly Bound Complex

PB95-107140

Interatomic Potential of Argon.

00,872

00,912

Interatomic Potential of Argon.

PB95-152989

00,945

PB95-162962

04,412

Energy Dependences of Absorption in Beryllium Windows and Argon Gas.

04,020

Ion Kinetics and Symmetric Charge-Transfer Collisions in Low-Current, Diffuse (Townsend) Discharges in Argon

PB96-123658 04,051

Absence of Ouantum-Mechanical Effects on the Mobility

of Argon lons in Helium Gas at $4.35 \mathrm{~K}$

01,194

ARGON COMPLEXES

Infrared and Microwave Spectroscopy of the Argon .

PB94-198892

00,794

High-Resolution Infrared Overtone Spectroscopy of ArHF

via Nd:YAG/Dye Laser Difference Frequency Generation.

PB94-211448 00,816

Spectroscopic Puzzle in ArHF Solved: The Test of a New Potential.

PB94-216058

00,850

Microwave and Submillimeter Spectroscopy of Ar-NH3 States Correlating with $\mathrm{Ar}+\mathrm{NH} 3(j=1, k=1)$.

00,942

Vibrational Dependence of the Anisotropic Intermolecular Potential of Ar-HCl.

PB95-202685

01,029

Vibrational Dependence of the Anisotropic Intermolecular Potential of Ar-HF

PB95-202693

01,030

Slit-Jet Near-Infrared Spectroscopy and Internal Rotor Dynamics of the ArH2O van der Waals Complex: $\mathrm{An} A n$ -

gular Potential-Energy Surface for Internal H2O Rotation.

PB95-202792 01,033

Photodissociation Dynamics in Ouantum State-Selected

Clusters: A Test of the One-Atom Cage Effect in Ar-H2O.

PB95-203121

ARGON HYDRIDES

Ion Kinetic-Energy Distributions in Argon of Glow Dis-

charges.
PB95-14 1008

04,409

ARGON IONS

Determination of Atomic Data Pertinent to the Fusion Energy Program. Progress Report for FY 92 .

04,402

Backscattering in Electron-Impact Excitation of Multiply Charged lons.

PB94-185345

03,799

Use of an Ion Energy Analyzer: Mass Spectrometer to (1) Kinetic-Energy Distributions from RF Discharges in Argon-Helium Gas Mixtures.

04,406

Evidence for Significant Backscattering in Near-Threshold Electron-Impact Excitation of $\operatorname{Ar}(7+)(3 s$ yields $3 p$ ).

PB95-126405

03,883

Ion Kinetic-Energy Distributions in Argon of Glow Dis-

charges.

04,409

Kinetic-Energy Distributions of lons Sampled from Argon Plasmas in a Parallel-Plate, Radio-Frequency Reference

PB95-161964

03,935

Ion Kinetic-Energy Distributions and Baimer-alpha (Halpha) Excitation in Ar-H2 Radio-Frequency Discharges.
PB96-102959 
Design of High-Frequency, High-Power Oscillators Using Josephson-Junction Arrays.

02,094

High-Power, High-Frequency Oscillators Using Distributed Josephson-Junction Arrays.

02,095

High Power Generation with Distributed Josephson-Junction Arrays.

ARSENIC

Vibrational Distributions of As2 in the Cracking of As4 on

$\mathrm{Si}(100)$ and $\mathrm{Si}(111)$

02,099

PB94-198314

00,784

Single-Photon lonization and Detection of Ga, In, and As(sub n) Species in GaAs Growth.

00,591

In-situ Monitoring of Molecular Beam Epitaxial Growth Using Single Photon lonization.

01,023

Direct Detection of Atomic Arsenic Desorption from Si(100).

PB95-202230

01,024

Single-Photon Laser Ionization Time-of-Flight Mass Spectroscopy Detection in Molecular-Beam Epitaxy: Application to As4, As2, and Ga.

PB95-203337

01,052

\section{ARTIFICIAL INTELLIGENCE}

Intelligent Processing of Materials, Technical Activities 1993 (NAS-NRC Assessment Panel, April 21-22, 1994). Hierarchical Interaction between Sensory Processing and World Modeling in Intelligent Systems.

PB94-198256

01,580

Role of World Modeling and Value Judgment in Percep-

PB94-198264

01,581

Theory of Intelligent Systems.

PB94-198272

01,582

Intelligent Control for Multiple Autonomous Undersea Vehicles.

03,747

Publications of the Intelligent Systems Division (Previously Robot Systems Division) Covering the Period January 1971-April 1994

PB94-217098

02,935

Intelligent Control of an Inert Gas Atomization Process.

PB95-141057 03,344

Reference Model Architecture for Intelligent Systems Design. 1 PB95-143137

01,789

Exposure: An Expert System Fire Code

04,868

Unified Telerobotic Architecture Project (UTAP) Standard Interface Environment (SIE), May 1995.

02,938

Intelligent Processing of Materials, Technical Activities 1994 (NAS-NRC Assessment Panel, April 6-7, 1995).

PB96-115050

03,359

ARTIFICIAL MEMBRANES

Determination of Osmotic Pressure and Fouling Resist-

ances and Their Effects on Performance of Ultrafittration

Membranes.

PB94-212891

00,841

Characterization of the Adsorption-Fouling Layer Using

Globular Proteins on Ultrafiltration Membranes.

$\begin{array}{ll} & 00,842 \\ \text { Supported Phospholipid/Alkanthiol Biomimetic Mem- } & \end{array}$ branes: Insulating Properties

PB95-180782

03,470

Phospholipid/Alkanethiol Bilayers for Cell-Surface Receptor Studies by Surface Plasmon Resonance. PB96-102900

03,472

ARTIFICIAL SATELLITES

Frozen Orbits for Satellites Close to an Earth-Like Planet.
PB96-102165 PB96-102165

ARTIFICIAL SUPERLATTICES

Magnetic Rare Earth Artificial Metallic Superlattices.

PB95-162293

04,611

ARYL ETHERS

Thermal Decomposition of Hydroxy- and Methoxy-SubStituted Anisoles.

00,767

ASBESTOS

Airborne Asbestos Method: Standard Test Method for High Precision Counting of Asbestos Collected on Filters. Version 1.0 .

PB94-163003

00,525

Airborne Asbestos Method: Standard Test Method for Verified Analysis of Asbestos by Transmission Electron Microscopy. Version 2.0

PB94-163045

00,526

Proficiency Tests for the NIST Airborne Asbestos Program, 1990.

00,535

Proficiency Tests for the NIST Airborne Asbestos Program -1991.

00,537
Proficiency Tests for the NIST Airborne Asbestos ProPram - 1992.

00.539

Airborne Asbestos Method: Standard Practice for Recording Transmission Electron Microscopy Data for the Analysis of Asbestos Collected onto Filters. Version 1.0.

PB94-210168

National Bulk Asbestos Analysis.

00,552

PB95-138129

02,541

Guidelines for Refractive Index Measurements of Asbes-

PB95-1

Proficiency Tests for the NIST Airbome Asbestos Program, 1993.

00,610

Airborne Asbestos Analysis: National Voluntary Laboratory Accreditation Program.

02,566

Airborne Asbestos Method: Bootstrap Method for Determining the Uncertainty of Asbestos Concentration. Version 1.0.

SCORBIC ACID

00,646

Temperature Dependence of the Rate Constants for Reaction of Inorganic Radicals with Organic Reductants.

0,783
Ascorbic and Dehydroascorbic Acids Measured in Plasma Preserved with Dithiothreitol or Metaphorphoric Acid

PB94-216330

03,495

Measurement of Ascorbic Acid in Human Plasma and Serum: Stability, Intralaboratory Repeatability, and Interlaboratory Reproducibility.

PB97-112445

ASHRAE STANDARD 62-1989

03,511

Ventilation, Carbon Dioxide and ASHRAE Standard 62 1989.

PB95-162095

02,544

ASPERGILLUS FUMIGATUS

Rapid Method for the Isolation of Genomic DNA from 'Aspergillus fumigatus'

ASPERITY

03,488

Asperity-Asperity Contact Mechanisms Simulated by a Two-Ball Collision Apparatus.

PB95-164158

02,966

ASPHALT PAVEMENTS

Development of a Method for Measuring Water-Stripping Resistance of Asphalt/Siliceous Aggregate Mixtures. PB96-202296

01,329

ASSIGNMENT MODELS

Moments of the Quartic Assignment Statistic with an Application to Multiple Regression.

ASSOCIATIVE IONIZATION

Ultracold Collisions: Associative Ionization in a Lase Trap.

ASTM STP

03,859

Introduction to ASTM 1199 'Wear Test Selection for Design and Application

PB95-162517

03,238

ASTRONOMICAL SATELLITES

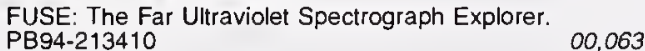

ASTROPHYSICS

Papers on the Symposium on Collision Phenomena in Astrophysics, Geophysics, and Masers.

00,047

ASYMMETRY PARAMETERS

Vibrationally Resolved Photoelectron Angular Distributions and Branching Ratios for the Carbon Dioxide Molecule in the Wavelength Region 685-795 Angstrom.

PB96-201207

04,131

ASYNCHRONOUS TRANSFER MODE

Asynchronous Transfer Mode Procurement and Usage

PB95-174967

01,481

NIST ATM Network Simulator: Operation and Programming, Version 1.0

PB96- 106851

01,487

Strategy to Support Multipoint Communication Service Over Native ATM
PB96- 176672

01.507

ATAXIA TELANGIECTASIA

Modification of Deoxyribose-Phosphate Residues by Extracts of Ataxia Telangiectasia Cells.

03,458

ATM (ASYNCHRONOUS TRANSFER MODE)

Asynchronous Transfer Mode Procurement and Usage Guide.

PB95-174967

01,481

NIST ATM Network Simulator: Operation and Programming, Version 1.0

01,487

Strategy to Support Multipoint Communication Service Over Native ATM Service.

PB96-176672

01,507

ATMOSPHERIC CHEMISTRY

Thermodynamic Properties of Gas Phase Species of Importance to Ozone Depletion.

PB94-198215

00,126

Atmospheric Reactivity of alpha-Methyl-Tetrahydrofuran.

PB95-163705 02,548

Atmospheric Lifetimes of HFC-143a and HFC-245fa: Flash Photolysis Resonance Fluorescence Measurements of the $\mathrm{OH}$ Reaction Rate Constants.

PB97-112577

00,118

Kinetics of the Reaction of the Sulfate Radical with the PB97-119127

ATMOSPHERIC DIFFUSION

Smoke Plume Trajectory from In situ Burning of Crude Oil: Field Experiments.

PB96-200993

02,597

ATMOSPHERIC SCATTERING

Radiometric Model of the Transmission Cell-Reciprocal Nephelometer.

ATMOSPHERIC TEMPERATURE

00,124

Modulation of Fossil Fuel Production by Global TemperaPure Variations, 2.

PB94-146636

02,533

ATOM-ATOM COLLISIONS

Intersystem Crossing in Collisions of Aligned $\mathrm{Ca}(4 \mathrm{~s} 5 \mathrm{p}$ (1)P) + He: A Half Collision Analysis Using Multichannel Quantum Defect Theory.

PB94-211133

00,813

Astrophysical Aspects of Neutral Atom Line Broadening. PB94-213287 00,061

Associative Ionization in Collisions of Slowed and Trapped Sodium.

PB95-125886

03,880

Hydrogen Balmer Alpha Line Shapes for Hydrogen-Argon Mixtures in a Low-Pressure if Discharge.

PB95- 153433

03,924 ence Effects in $\mathrm{Na}(3 \mathrm{p}$, (2) $\mathrm{P} 1 / 2$ inversely maps (2)P3/ 2)+He: Semiclassical and Ouantum Calculations.

95-202453

Laser Preparation and Probing of Initial and Final Orbital Alignment in Collision-Induced Energy Transfer

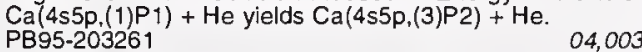
Initial and Final Orbital Alignment Probing of the Fine Structure-Changing Collisions among the $\mathrm{Ca}$ $(4 s)(1)(4 p)(1)$. (3)PJ States with He: Determination of Coherence and Conventional Cross-Sections.

PB95-203279

ATOM COOLING

sigma+-sigma- Optical Molasses in a Longitudinal Magnetic Field.

ATOM LITHOGRAPHY

Nanofabrication of a Two-Dimensional Array Using LaserFocused Atomic Deposition.

PB96-119417

04,732

Microlithography by Using Neutral Metastable Atoms and Self-Assembled Monolayers.

6-190038

02,441

ATOM-MOLECULE COLLISIONS

Collision-Induced Emission in the Fundamental VibrationRotation Band of $\mathrm{H} 2$.

PB94-199445

03,811 


\section{KEYWORD INDEX}

Behavior of Atoms in a Compressed Magneto-Optical Trap

PB95-203105

03,999

Improved Hyperfine Measurements of the Na NP Excited State Through Frequency-Controlled Dopplerless Spectroscopy in a Zeeman Magneto-Optic Laser Trap.

PB95-203840 04,012

Comment on « $\ll$ A Dynamic Electric Trap for Ground-State

PB96-200290

04,123

ATOMIC ABSOAPTION

Application of a Novel Slurry Furnace AAS Protocol for Rapid Assessment of Lead Environmental Contamination.

PB96-112354

02,526

\section{ATOMIC BEAMS}

Atomic Beam Splitters and Mirrors by Adiabatic Passage in Multilevel Systems.

PB94.216439

Laser Focusing of Atoms: A Particle Optics Approach.

PB94-216660 03,870

Velocity Distribution of Atomic Beams by Gated Optical Pumping

01,542

Nanofabrication of a Two-Dimensional Array Using LaserFocused Atomic Deposition.

PB96-119417

04,732

\section{OMIC CLOCKS}

Utc Dissemination to the Real-Time User.

N19960042622

01,521

Confidence on the Three-Point Estimator of Frequency Drift.

01,539

Non-Neutral Ion Plasmas and Crystals, Laser Cooling. $\begin{array}{ll}\text { and Atomic Clocks. } & \\ \text { PB95-175113 } & 03,957\end{array}$

03,957

Time and Frequency: Bibliography of NIST Publications, PB95-220463

01,548

Accurate Measurement of Time.

01,552

Atomic Clock
PB96-119490

01,553

ATOMIC CLUSTERS

Photoelectron Spectroscopy of Negatively Charged Bismuth Clusters: $\mathrm{Bi}(-) 2, \mathrm{Bi}(-) 3$, and $\mathrm{Bi}(-) 4$.
PB95-108494

00,880

Photoelectron Spectroscopy of Small Antimony cluster
Anions: $\mathrm{Sb}(-), \mathrm{Sb} 2(-), \mathrm{Sb} 3(-)$, and $\mathrm{Sb} 4(-)$. PB95-203139

01,045

\section{ATOMIC COLLISIONS}

Three-Vector Correlation Theory for Orientation/Alignment Studies in Atomic and Molecular Collisions.

03,834

Intersystem Crossing in Collisions of Aligned $\mathrm{Ca}(4 s 5 \mathrm{p}$ (1) P) + He: A Half Collision Analysis Using Multichannel Quantum Defect Theory.

PB94-211133

00,813

Theory of Atomic Collisions at Ultracold Temperatures.

PB94-212560 03,851

Associative Ionization in Collisions of Slowed and Trapped Sodium.

03,880
Hyperfine Effects and Associative Ionization of Ultracold Sodium

PB95-151221

03,903

Laser Assisted Collisions at Ultracold Temperatures.
PB95-161220

Three-Vector Correlation Study of Orientation and Coherence Effects in $\mathrm{Na}(3 \mathrm{p}$, (2) $\mathrm{P} 1 / 2$ inversely maps (2)P3/ 2)+He: Semiclassical and Ouantum Calculations.

PB95-202453

03,979

$\mathrm{n}$-Vector Correlations in Collision Dynamics with Atomic Orbital Alignment: The Importance of Coherence Denoting Azimuthal Structure for $n$ (greater than or equal to) 3.
PB95-202545
03,983

Collisional Energy Transfer between Excited-State Strontium and Noble-Gas Atoms.

PB95-202958

03,995

Energy-Pooling Collisions in Barium.

PB95-203030

03,997

Laser Preparation and Probing of Initial and Final Orbital Alignment in Collision-Induced Energy Transfer $\mathrm{Ca}(4 s 5 \mathrm{p},(1) \mathrm{P} 1)+\mathrm{He}$ yields $\mathrm{Ca}(4 \mathrm{~s} 5 \mathrm{p},(3) \mathrm{P} 2)+\mathrm{He}$. 04,003 Initial and Final Orbital Alignment Probing of the FineStructure-Changing Collisions among the $\mathrm{Ca}$ $(4 s)(1)(4 p)(1)$, (3)PJ States with He: Determination of Coherence and Conventional Cross-Sections.

04,004

Orbital Alignment and Vector Correlations in Inelastic Atomic Collisions.

PB96-122742

04,047

Atom Cooling and Trapping, and Collisions of Trapped

Aloms.

04,048

Laser Modification of Ultracold Collisions: Experiment.

PB96-157987
ATOMIC DATA

Spectral Data for Highly lonized Krypton, $\mathrm{Kr} V$ through $\mathrm{Kr}_{\mathrm{r}}$

01,115

PB96-145917

nization

Mass Spectrometry Data Service. V-Data Sheets for Ga

$\mathrm{Mn}, \mathrm{Sc}$, and TI.

00,625

OMIC DEPOSITION

Nanostructure Fabrication via Laser-Focused Atomic Deposition (Invited).

PB96-204094

04,132

ATOMIC ENERGY LEVELS

Atomic Energy Levels As Derived from the Analyses of Optical Spectra. Volume 1. Section 1. The Spectra of Hydrogen, Deuterium, Helium, Lithium Beryllium, Boron, Carbon, Nitrogen, Oxygen, and Fluorine.

03,764

AD-A278 130\%

04,437

Alomic Energy Levels in Crystals.

AD-A280 150/4

yses of

Atomic Energy Levels. As

AD.A280 279/1

00.714

Wavelengths and Energy Levels of Neutral $\mathrm{Kr}(84)$ and Level Shifts in All Kr Even Isotopes.

PB94-140605 03,780

Energy Levels of Germanium, Ge I through Ge XXXII

Spectrum and Energy Levels of Five-Times-Ionized Niobium ( $\mathrm{Nb}$ VI).

Pectrum and Energy Levels of Triply lonized Barium (B

IV).

03,888

Precision Lifetime Measurements of Cs $6 p$ (2)P1/2 and $6 p$ (2)P $3 / 2$ Levels by Single-Photon Counting. PB95-203816

04,010

Designations of ds(2)p Energy Levels in Neutral Zirconium, Hafnium, and Rutherfordium $(Z=104)$.

04,116

ATOMIC FLUIDS

Conditions for Existence of a Reentrant Solid Phase in a Sheared Atomic Fluid.

PB94-211380

04,198

ATOMIC FORCE MICROSCOPES

Development of a Calibrated Atomic Force Microscope.

02,894

Increasing the Value of Atomic Force Microscopy Process Metrology Using a High-Accuracy Scanner, Tip Characterization, and Morphological Image Analysis. PB96-190293

02,758

Scanned Probe Microscope Tip Characterization Without Calibrated Tip Characterizers.

PB96-190368

02,759

ATOMIC FORCE MICROSCOPY

Force Calibrations in the Nanonewton Regime.

Flexible-Diaphragm Force Microscope.

03,966

Morphological Estimation of Tip Geometry for Scanned

$\begin{array}{ll}\text { Probe Microscopy. } & 02,662 \\ \text { PB95-203444 }\end{array}$

02,662

Recent Results in Magnetic Force Microscopy

PB96-103130

04,721

Effects of Etching on the Morphology and Surface Resistance of YBa2Cu3O7-delta Films.

ance 135355

02,410

Development of Highly Conductive Cantilevers for Atomic Force Microscopy Point Contact Measurements.

PB96-138573

04,751

ATOMIC FOUNTAIN

Laser-Cooled Neutral Atom Frequency Standards. PB96-160312

04,086

\section{ATOMIC INTERACTIONS}

Measurement of the Atomic Na(3P) Lifetime and of Retardation in the Interaction between Two Atoms Bound in a Molecule.

PB97-122360

04,178

ATOMIC IONS

Early Dielectronic Recombination Measurements: Singly Charged lons.

PB94-211158

Bonding in Doubly Charged Diatomics.

03,836

B95-164315

00,976

ATOMIC MANIPULATION

Nanostructure Fabrication via Direct Writing with Atoms Focused in Laser Fields.

PB95-150272

04,564

Atomic Manipulation of Polarizable Atoms by Electric Field Directional Diffusion.

04,572

PB95-150587

Manipulation of Adsorbed Atoms and Creation of New
Structures on Room-Temperature Surfaces with a ScanStructures on Room-Temperature Surfaces with a Scan-
ning Tunneling Microscope.
PB95-151536
04,578

for

New Values for Silicon Reference Materials, Certified for Isotope Abundance Ratios. Letter to the Editor.

PB94-219268

\section{ATOMIC \& MOLECULAR STUDIES}

Production and Spectroscopy of Small Polyatomic Molecular lons Isolated in Solid Neon. (Reannouncement with New Availability Information.

AD-A234 043/8

00,704

Vibrational Spectra of Molecular Ions Isolated in Solid Neon. 6. CO4(-). (Reannouncement with New Availability (ormation).

00,705

Vibrational Spectra of Molecular Ions Isolated in Solid Neon. 7. CO(t), $\mathrm{C} 2 \mathrm{O} 2(+)$, and $\mathrm{C} 2 \mathrm{O} 2(-)$. (Reannouncement with New Availability Information).
AD-A239 729/7

Vibrational Spectra of Molecular Ions Isolated in Solid Neon: $\mathrm{HCCH}_{+}$and $\mathrm{HCC}-$. (Reannouncement with New Availability Information)

AD-A253 $551 / 6$

00,707

Vibrational Spectra of Molecular Ions Isolated in Solid

Neon. $11 . \mathrm{NO2}(+), \mathrm{NO} 2(-)$, and NO3(-)
AD-A275 828/2

00,708

Atomic Energy Levels As Derived from the Analyses of Optical Spectra. Volume 1. Section 1. The Spectra of Hydrogen, Deuterium, Helium, Lithium Beryllium, Boron, Carbon, Nitrogen, Oxygen, and Fluorine.

03,764

AD-A278 130\%

Ultraviolet Multiplet Table. Finding List for Spectra of the
Elements Molybdenum to Lanthanum ( $Z=42$ to 57 ); HafElements Molybdenum to Lanthanum ( $Z=42$ to 57 ); Hat-
nium to Radium $(Z=72$ to 88 ).
$\begin{array}{ll}\text { AD-A278 } 131 / 8 & 00,709\end{array}$

04,437

Atomic Energy Levels in Crystals.

Arc Spectra of Gallium, Indium, and Thallium.

AD-A295 411/3

00,718

Infrared and Near-Infrared Spectra of HCC and DCC

Trapped in Solid Neon.

03,773

Thermal Decomposition Pathways in Nitramine Propel-

AD-A295 896/5

03,753

Spectroscopic Study of Reaction Intermediates and Mechanisms in Nitramine Decomposition and Combus-

AD-A296 061/5

03,774

Microwave Spectrum and Structure of $\mathrm{CH} 3 \mathrm{NO} 2-\mathrm{H} 2 \mathrm{O}$

AD-A296 377/5

00,719

Molecular Microwave Spectra Tables.

AD-A296 498/9 00,720

Physical Properties of Some Purified Aliphatic Hydro-

AD-A297 265/1

00,657

Determination of Atomic Data Pertinent to the Fusion En- 


\section{KEYWORD INDEX}

\section{ATOMIC \& MOLECULAR STUDIES}

Electron-Impact Ionization of $\ln (+)$ and $X e(+)$ PB94-185089

00,770

Measurement of the $\mathrm{J}=2$ less than 1 Fine-Structure Interval for (28)Si and (29)Si in the Ground (3)P State. PB94-185097

Spectrum and Energy Levels of Five-Times-Ionized Nio. bium (Nb VI).
PB94-185246

04,226

Investigation of LS Coupling in Boronlike lons.

Ouantum Dynamics of Renner-Teller Vibronic Coupling The Predissociation $\mathrm{HCO}$.

PB94-185303

00,773

Optical Conductivity of Single Crystals of Ba1$\mathrm{M} \times \mathrm{BiO} 3(\mathrm{M}=\mathrm{K}, \mathrm{Rb}, \mathrm{x}=0.04,0.37)$

PB94-185329 Backscattering in Electron-Impact Excitation of Multiply
Charged lons.
PB94-185345
TB,799 PB94-185345

03,799

on Ni(110) and Cu(110) Using Molecular Dynamics Simulations.

PB94-185477

04,449

Charge Transfer and Collision-Induced Dissociation Reactions of $\mathrm{CF}(2+)$ and $\mathrm{CF} 2(2+)$ with the Rare Gases at a Laboratory Collision Energy of $49 \mathrm{eV}$

00,775

High Resolution IR Studies of Polymolecular Clusters: Micromatrices and Unimolecular Ring Opening. PB94-185691

Verification of the Ponderomotive Approximation for the ac Stark Shift in Xe Rydberg Levels.

03,801

Angular Distributions for Near-Threshold $(e, 2 e)$ Processes for $\mathrm{Li}$ and $\mathrm{Mg}$.

00,778

Collision-Induced Neutral Loss Reactions of Molecular Dications.

00,780

Fourier Transform Atomic Emission Studies Using a Glow Discharge as the Emission Source.

PB94-185980

00,533

Oxidation of 10-Methylacridan, a Synthetic Analogue of $\mathrm{NADH}$ and Deprotonation of Its Cation Radical. Convergent Application of Laser Flash Photolysis and Direct and Redox Catalyzed Electrochemistry to the Kinetics of Deprotonation of the Cation Radical.

PB94-198371

00,785

Energy Dependence of Collision Characteristics in Molecule-Surface Collisions.

PB94-198504

00,786

Density Matrix Calculation of Population Transfer between Vibrational Levels of $\mathrm{Na} 2$ by Stimulated Raman Scattering with Temporally Shifted Laser Beams. PB94-198546
Po, 787 Strong Hydrogen Bond in the Formic Acid-Formate Anion

PB94-198595

00,788

Structure of Glycine-Water H-Bonded Complexes.

00,789

Rotational Spectrum of Copper Hydride Using Tunable Far Infrared Radiation.

00,792

Tunneling-Rotation Spectrum of the Hydrogen Fluoride Dimer.

00.793

Laser Ablation of Thin Films as a Free Atom Source for Pulsed RIMS.

PB94-198710 00,540

Scattering Properties of the Leveled-Wave Model of Random Morphologies.

PB94-198835

03,807

Infrared and Microwave Spectroscopy of the Argon . Propyne Dimer

00,794

Evaluation of Two-Photon Exchange Graphs for HighlyCharged Heliumlike Ions.

PB94-198918

03,808

Collision-Induced Emission in the Fundamental VibrationRotation Band of $\mathrm{H} 2$

PB94-199445

03,811

New Rydberg States of Aluminum Monofluoride Observed by Resonance-Enhanced Multiphoton lonization SpectrosPB94-199544

00,797

Vibrational Autoionization in $\mathrm{H} 2$ : Vibrational Branching Ratios and Photoelectron Angular Distributions Near the $\mathrm{V}(+)=3$ Threshold

PB94-199577

00,799

Experimental Aspects and Z-Dependent Systematics in One- and Two-Electron lons and Single Vacancy SysPB9 1

03,815

Status of a Silicon Lattice Measurement and Dissemination Exercise

04,474

Fragment Energy and Vector Correlations in the Overone-Pumped Dissociation of $\mathrm{HN} 3 \times(1) \mathrm{A}$.

00,802
2-Tunneling Path Internal-Axis-Method-Like Treatment of he Microwave Spectrum of Divinyl Ether.

00,808

Two-Dimensional POMMIE Carbon-Proton Chemical Shift Correlated (13)C NMR Spectrum Editing. Correlated (13)C NMR Spectrum Editing.
PB94-200508 Anharmonic Phonons and the Isotope Effect in Superconductivity.

04,486

Extension of Heterodyne Frequency Measurements on OCS to $87 \mathrm{THz}(2900 \mathrm{~cm}(-1))$.

00,811

Multichannel Ouanturn Defect Half Collision Analysis of K2 Photodissociation Through the B1Pi(sub u) State.

PB94-211125

00,812

ntersystem Crossing in Collisions of Aligned $\mathrm{Ca}(4 \mathrm{~s} 5 \mathrm{p}$ (1) P) + He: A Half Collision Analysis Using Multichannel Ouantum Defect Theory.

PB94-211133 00,813

Early Dielectronic Recombination Measurements: Singly Charged lons.

03,836

Brillouin Light Scattering Intensities for Thin Magnetic Films with Large Perpendicular Anisotropies. PB94-211174

Reaction Rate Determinations of Vinyl Radical Reactions with Vinyl, Methyl, and Hydrogen Atoms

PB94-211398 00,81

PB94-211463 in the Infrared Spectrum of NO-HF. 00,817

Magnetic Dipole Line from U LXXI Ground-Term Levels Predicted at 3200 Angstroms.

PB94-211497

04,407

Photoionization of Small Molecules Using Synchrotron

PB94-211505

03,841

Atomic Transition Probability Ratios between Some Ar I

$\begin{array}{ll}4 s-4 p \text { and } 4 s-5 p \text { Transitions. } & 03,842\end{array}$ Ion Broadening Parameters for Several Argon and CarLon Lines.

PB94-211562

03,843

Asymptotic Wave Function Splitting Procedure for Propagating Spatially Extended Wave Functions: Application to intense Field Photodissociation of $\mathrm{H} 2(+)$.

03,847

Discontinuous Volume Change at the Orientational-Order-

ing Transition in Solid $\mathrm{C} 60$.

PB94-211828

00,821

Design and Characterization of X-Ray Multilayer Analyzers for the $50-1000 \mathrm{eV}$ Region

B 94-211851

03,848

Use of Extended Permutation-Inversion Groups in Constructing Hyperfine Hamiltonians for Symmetrical-Top ln. ernal Rotor Molecules Like $\mathrm{H} 3 \mathrm{C}$-SiH 3 .

PB94-212032

00,823

Experimental and Abinitio Studies of Electronic Structures of the $\mathrm{CCl} 3$ Radical and Cation.

B 94-212131

00,826

Photoelectron Spectroscopic Study of the Valence and Core-Level Electronic Structure of BaTiO3. 04,500 $\mathrm{K}$ alpha Transitions in Few-Electron lons and in Atorns. PB94-212248 03849 Quantum Projection Noise: Population Fluctuations in Two-Level Systerns.

PB 94-212271

03,850

Multiphoton Ionization of $\mathrm{SiH} 3$ and $\mathrm{SiD} 3$ Radicals. 2 Three Photon Resonance-Enhanced Spectra Observed between 460 and $610 \mathrm{~nm}$.

PB94-212487

00,835

New Electronic States of NH and ND Observed from 258 to $288 \mathrm{~nm}$ by Resonance Enhanced Multiphoton Ionizaion Spectroscopy.

PB94-212495

00,836

Multiphoton Ionization of $\mathrm{SiH} 3$ and $\mathrm{SiD} 3$ Radicals: Elecronic Spectra, Vibrational Analyses of the Ground and Pydoes Aydberg States,

00,837

Theory of Atomic Collisions at Ultracold Temperatures.

PB94-212560 03,851

Attempts at Extending the Unified Theory to Include Many-Body Effects.

PB94-21271

04,408

PB94-212727

03,853

Relativistic and Ouantum Electrodynamic Effects in Highy-Charged lon

03,854

Product Kinetic Energies, Correlations, and Scattering Anisotropy in the Bimolecular Reactor $\mathrm{O}((1) \mathrm{D})+\mathrm{H} 2 \mathrm{O}$ yields $20 \mathrm{H}$.

PB94-212792

00,838

Molecular-Beam Optothermal Spectrum of the $\mathrm{OH}$

Stretching Band of Methanot.

00,839

Fundamental

PB94-212834

00,840

Atomic Branching Ratio Data for Carbon-Like lons.

PB94-212842 03,855

Active Site lonicity and the Mechanism of Carbonic Anhy-

drase.

PB94-212974

00,843

Dipole Moments in Rare Gas Interactions.

PB94-212982

00,844

Ultracold Collisions: Associative Ionization in a Laser

Trap.

High-Energy Behavior of the Double Photoionization of Helium from 2 to $12 \mathrm{keV}$.

PB94-213279

03,860

RIS Studies of Autoionization in Calcium.

PB94-213295

00,849

Molecular Spectroscopy.

PB94-213337

Atomic, Molecular, and Optical Physics with X-rays.
PB94-213378

FTS Infrared Measurements of the Rotational and Vibra. ional Spectrum of $\mathrm{LiH}$ and $\mathrm{LiD}$.

PB94-216231

00,856

Self Broadening in the nu1 Band of $\mathrm{NH}_{3}$.

PB94.216371 00.857

Kinetics and Mechanism of the Collision-Activated Dissociation of the Acetone Cation.

PB94-216462

Crystal Structure of Calcium Adipate Monohydrate.

PBg 16579

00.153

Proton Affinity Ladders from Variable-Temperature Equilibrium Measurements. 1. A Re-Evaluation of the Upper Proton Affinity Range.

PB94-216603

00,861

Determination of Complex Scattering Amplitudes in Low-

Energy Elastic Electron-Sodium Scattering.

PB94-216652

03,869

Laser Focusing of Atoms: A Particle Optics Approach

PB94-216660

03,870

One-Electron Oxidation of Nickel Porphyrins. Effect of

Structure and Medium on Formation of Nickel(III) Por phyrin or Nickel(II) Porphyrin pi-Radical Cation.

5-107058

00,865

Site of One-Electron Reduction of $\mathrm{Ni}(\mathrm{II})$ Porphyrins. Formation of $\mathrm{Ni}(\mathrm{I})$ Porphyrin of Ni(II) Porphyrin pi-Radical

PB95-107066

00,866

Metalloporphyrin Sensitized Photooxidation of Water to Oxygen on the Surface of Colloidal Iridium Oxides - Photochernical and Pulse Radiolytic Studies.

PB95-107082

00,868 


\section{KEYWORD INDEX}

Single-Atom Point Source for Electrons: Field-Emission Resonance Tunneling in Scanning Tunneling Microscopy.
PB95-125860 Associative Ionization in Collisions of Slowed and Trapped Sodium.

PB95-125886
Temperature of Optical Molasses for Two Different Atomic Angular Momenta.

PB95-126058

03,881

Rotational Spectrum and Structure of a Weakly Bound Complex of Ketene and Acetylene.

00,896

Wavelengths and Isotope Shifts for Lines of Astrophysical Interest in the Spectrum of Doubly Ionized Mercury $(\mathrm{Hg}$ PB95-140869

03,887

Spectrum and Energy Levels of Triply Ionized Barium (Ba

03,888

Associative Electron Attachment to S2F10, S2OF10, and $\mathrm{S} 2 \mathrm{O} 2 \mathrm{~F} 10$.

PB95-140992

00,907

Infrared Spectrum of $\mathrm{OClO}$ in the $2000 / \mathrm{cm}(-1)$ Region: The 2(nu sub 1) and (nu sub $1+$ nu sub 3) Bands.
PB95-141032 Intensities and Dipole Moment Derivatives of the Fundamental Bands of (35) CIO2 and an Intensity Analysis of

PB95-141040

00,909

Perturbative Calculation of the AC Stark Effect by the
Complex Rotation Method.

PB95-141123

04,260

Interatomic Potential of Argon.

PB95-141180

00,912

Resonance and Threshold Effects in Polarized X-Ray Emission from Atoms and Molecules.

03,891

Self-Avoiding Surfaces, Topology, and Lattice Animals.

PB95-150512 04,571

Contrast Matched Studies of a Sheared Binary Colloidal

Suspension.

00,918

Atomic Manipulation of Polarizable Atoms by Electric Field Directional Diffusion.

04,572

Microwave Spectra of van der Waals Complexes of Importance in Planetary Atmospheres

PB95-150611

00,919

Improved Wavelengths for Prominent Lines of $\mathrm{Cr} X \mathrm{XV}$ to Cr XXII.

PB95-150629 03,895

Observation of Pd-Like Resonance Lines Through $\mathrm{Pt}(32+)$ and $\mathrm{Zn}$-Like Resonance Lines of $\mathrm{Er}(38+)$ and $\mathrm{Hf}(42+)$.

PB95-150637

Rb-Like Spectra: Pd X to Nd XXIV.

03,896

PB95-150645

03,897

Rh 1 Isoelectronic Sequence Observed from $\operatorname{Er}(23+) 10$ $\mathrm{Pt}(33+)$.

PB95-150652

03,898

Spectra of $\mathrm{Ag}$ ! Isoelectronic Sequence Observed from Er(21+) to $\mathrm{Au}(32+)$

B95-150660

03,899

High-Resolution Infrared Spectroscopy of DF Trimer: A Cyclic Ground State Structure and DF Stretch Induced Intramolecular Vibrational Coupling.

00,920

Calculations of Electron Inelastic Mean Free Paths Calculations of Electron Inelastic Mean Free Paths (IMFPs). 4. Evaluation of Calculated IMFPs and of the Predictive IMFP Formula TPP-2 for Electron Energies beween 50 and $2000 \mathrm{eV}$

00,922

$\begin{array}{lll}\text { Analysis of } & \text { the } & 5 s(2) 5 p(2)- \\ (5 s 5 p(3)+5 s(2) 5 p 5 d+5 s(2) 5 p 6 s) & \text { Transitions of Four-Times }\end{array}$ lonized Xenon (Xe V)

03,900

Relativistic Effects in Spin-Polarization Parameters for Relativistic Effects in Spin-Polarization Parameters for Low-Energy Electron-Cs Scattering

03,901

Methyl Torsional Levels of Solid Acetonitrile $(\mathrm{CH} 3 \mathrm{CN})$ : A Neutron Scattering Study.

00,926

Electronic Spectra of $\mathrm{CF} 2 \mathrm{Cl}$ and $\mathrm{CFCl} 2$ Radicals ObElectronic Spectra of $\mathrm{CF} 2 \mathrm{Cl}$ and $\mathrm{CFCl} 2$ Radicals $\mathrm{Ob}$ served by Resonance Enhanced Multiphoton Ionization.
PB95-151023

00,927
Homogeneous Gas Phase Decyclization of Tetralin and Benzocyclobutene.

PB95-151049

00,928

Hypertine Effects and Associative Ionization of Ultracold Sodium.

PB95-151221 03,903

Magneto-Optical Trapping of Metastable Xenon: IsotopeShitt Measurements.

PB95-151254

03,905

High Resolution Angle Resolved Photoelectron Spectroscopy Study of N2

Spectroscopic Data for Fusion Edge Plasmas.

03,907

PB95-151569

04,410
Spectroscopic Diagnostics of Low Temperature Plasmas: .

PB95-15 1577

04,41

Spectroscopic Data Tables for Highly-lonized Atoms.

Trapped Atoms and Laser Cooling.

03,910

PB95-151627

03,91

Spin Squeezing and Reduced Quantum Noise in Spectroscopy.

Rotational Far Infrared Spectrum of (13)CO.

03,912

PB95-152187

00,94

Laboratory Measurements for the Astrophysical Identificaion of $\mathrm{MgH}$.

00,073

Atornic Oxygen Fine Structure Splittings with Tunable Far Infrared Spectroscopy.

PB95-152203

03,915

Microwave and Submillimeter Spectroscopy of $\mathrm{Ar}-\mathrm{NH} 3$ (

00,942

Electron-Impact lonization of $\ln (+)$ and $\mathrm{Xe}(+)$

PB95-152906

03,918

Memory Function Approach to the Shape of Pressure Broadened Molecular Bands.

PB95-152930

Interatomic Potential of Argon.

PB95-152989

00,944

roduct State Correlations in the Reaction of $\mathrm{O}((1) \mathrm{D})$ and $\mathrm{H} 2 \mathrm{O}$ in Bimolecular Collisions and in $\mathrm{O} 3 . \mathrm{H} 2 \mathrm{O}$ Clustersranslation.

PB5-153011

00,946

Rotational Dynamics of $\mathrm{C} 60$ in Na2RbC60.

PB95-153201

00,948

Neutron and $X$-Ray Scattering Cross Sections of Orientationally Disordered Soild C60.

PB95.153300

00,951

Morphological Stability.

PB95-153318

04,591

Hydrogen Balmer Alpha Line Shapes for Hydrogen-Argon Mixtures in a Low-Pressure of Discharge.

03.924

Ouantum Measurements of Trapped lons.

PB95-161147

03,928

Laser Assisted

03,929

Hydrogen in YBa2Cu3Ox: A Neutron Spectroscopy and a Nuclear Magnetic Resonance Study.

PB95-161279

04,601

Spin-Resolved Elastic Scattering of Electrons from Sodium.

03,933

sigma+-sigma- Optical Molasses in a Longitudinal Magnetic Field.

PB95-161840

03,934

Slit Jet Infrared Spectroscopy of Hydrogen Bonded N2HF Isotopomers: Rotational Rydberg-Klein-Rees Analysis and H/D Dependent Vibrational Predissociation Rates. Laser-Induced Fluorescence Measurements of $\mathrm{OH}_{\text {. Con- }}$ centrations in the Oxidation Region of Laminar. Hydrocarbon Diffusion Flames.

PBo5-162160

01,387

Neutron-Scattering Study of Librations and Intramolecular Phonons in Rb2.6Ko.4C60.

PB 95-162269

00,958

Resonant-Photoemission Investigation of the Heusler Alloys Ni2MnSb and NiMnSb.

B95-162384

04,612

Simultaneous Forward-Backward Raman Scattering Studies of D2 Broadened by D2, He, and Ar.

00,961

Labeling Conventions in Isoelectronic Sequences - Reply.

03,937

lodine Atoms and lodomethane Radical Cations: Their Formation in the Pulse Radiolysis of lodomethane in Organic Solvents. Their Complexes, and Their Reactivity with Organic Reductants.

PB95-162764

00,965

Ionization Energy of Sulfur Pentafluoride and the Sulfur

Pentafluoride-Fluorine Atom Bond Dissociation Energy.
PB95-162814 00,966

ms and

Anisotrop

PB95-163002

04,621

Photoelectron Study of Electronic Autoionization in

Rotationally Cooled N2: The $n=6$ Member of the Hopfield Series.

PB95-163531

00,971

Laser Spectroscopy of Carbon Monoxide: A Frequency Reference for the Far Infrared.

04,277

Measurements of Fluorescence from Cold Atoms: Local-

ization in Three-Dimensional Standing Waves. PB95-163879

03,943

XUV Characterization Comparison of Mo/Si Multilayer

Coatings.
PB95-164000

04,278

Molecu

PB95-164059

Lattice Position of Si in GaAs Determined by X-Ray Standing Wave Mea surements.

04,632

Surface Geometry of $\mathrm{BaO}$ on W(100): A Surface-Extended X-Ray-Absorption Fine-Structure Study.

PB95-164414

00,980

Fragment State Correlations in the Dissociation of NO.HF $(v=1)$.

Shape-Resonance-Enhanced Continuum-Continuum Coupling in Photoionization of $\mathrm{CO} 2$.

2nu9 Band of Propyne-d3.

00.983

00,985

Interference in the Resonance Fluorescence of Two Trapped Atoms.

PB95-168514

03,948

New cw CO2 Laser Lines: The 9-mu $m$ Hot Band.

PB95-168548

Laser Cooling of Trapped Ions.

PB95-168746

04,281

Light Scattered from Two Atoms.

03,950

PB95-168753

Recent Experiments on Trapped lons

PB95-169322

03,952

PB95-169330

Laser-Cooled Positron Source

PB95-169348

Far Infrared Laser Frequencies of (13) CD3OH

PB95-169363

Cooled

03,954

3) Phi (3) Phi State by Far-Infrared Laser Magn

PB95-175048

Non-Neutral Ion Plasmas and Crystals, Laser Cooling, and Atomic Clocks.

03,957

Fine-Structure Intervals of (14) N(+) By Far-Infrared Laser Magnetic Resonance.

00,993

Laser Magnetic-Resonance Measurement of the (3)P1 - 
Laser-Produced and Tokamak Spectra of Lithiumlike Iron, $\mathrm{Fe}(23+)$.

04,314 Neutron Spectroscopic Evidence of Concentration-Dependent Hydrogen Ordering in the Octahedral Sublattice of beta-TbH $2+x$.

PB95-181020

01,018

Vibrations of Hydrogen and Deuterium in Solid Solution with Lutetium.

01,019

Detection of $\mathrm{OH}+$ in Its a(1)Delta State by Far Infrared Laser Magnetic Resonance.

PB95-181087

01,021

Core Potentia/s

03,970

Reduction of Light-Assisted Collisional Loss Rate from a Low-Pressure Vapor-Cell Trap

PB95-202248

03,971

High-Efficiency, High-Power Difference-Frequency Generation of 0.9-1.5 mu $\mathrm{m}$ Light in BBO.

PB95-202255

04,317

Hydrogen Lyman-alpha Emission of Capella.

00,075

Resonance Structure and Absolute Cross Sections in Near-Threshold Electron-Impact Excitation of the $4 \mathrm{~s}(2)$ (1)S yields $4 s 4 p$ (3)P Intercombination Transition in $\operatorname{Kr}(6+)$.

PB95-202271

03,972

Short-Ranye Correlation and Relaxation Effects on the (6p(2))(1) SO Autoionizing State of Atomic Barium. PB95-202289

03,973

Connection between Superelastic and Inelastic ElectronAtom Collisions Involving Polarized Collision Partners.
PB95-202297 Femtosecond Time-Resolved Molecular Multiphoton Ionization: The $\mathrm{Na} 2$ System.

PB95-202305

03,975

Location of a (1)A(sub g) State in Bithiophene.

PB95-202313

01,025

Observations of $3 \mathrm{C} 273$ with the Goddard High Resolution Spectrograph on the Hubble Space Telescope. Lowest Excited Singlet State of Isolated 1-phenyl-1,3-bu-

tadiene and 1-phenyl-1,3,5-hexatriene.
PB95-202339

01,026

Analyses of Recent Experimental and Theoretical Determinations of e-H2 Vibrational Excitation Cross Sections: Assessing a Long-Standing Controversy.

03,977

Test of Newton's Inverse Square Law of Gravitation Using the 300-m Tower at Erie, Cotorado.
PB95-202446

Three-Vector Correlation Study of Orientation and Coherence Effects in $\mathrm{Na}(3 p$, (2) $\mathrm{P} 1 / 2$ inversely maps (2)P3/ 2)+He: Semiclassical and Ouantum Calculations.

PB95-202453

03,979

ROSAT All-Sky Survey of Active Binary Coronae. 1. Ouiescent Fluxes for the RS Canum Venaticorum Systems.
PB95-202479

Absolute Cross-Section Measurements for Electron-Impact Single lonization of $\mathrm{Se}(+)$ and $\mathrm{Te}(+)$

03,980

Crossed-Beams Measurements of Absolute Cross Sections for Electron Impact Ionization of $\mathrm{S}(+)$. tions for Electron Impact Ionization of S( + ). 03,981
PB95-202511

Absolute Cross Sections for Electron-Impact Single Ionization of $\mathrm{Si}(+)$ and $\mathrm{Si}(2+)$.

03,982

Plasma Chemistry in Silane/Germane and Disilane/Germane Mixtures.

01,027

$n$-Vector Correlations in Collision Dynamics with Atomic Orbital Alignment: The Importance of Coherence Denoting Azimuthal Structure for $n$ (greater than or equal to) 3 .
PB95-202545
03,983 Resonant Two-Color Detachment of $\mathrm{H}(-)$ with Excitation of $H(n=2)$.

03,984

Numerical Solution of the Nonlinear Schroedinger Equation for Small Samples of Trapped Neutral Atoms.

PB95-202578

03,985

Vibrations of S1((1)B2u) p-Difluorobenzene - d4. S1-So Fluorescence Spectroscopy and ab Initio Calculations.
PB95-202586 Long-Range Parit

01,028 PB95-202594 03,986 Hole Dispersion and Enhancement of Antiferromagnetic Interaction of Localized Spins in High-TC Superconductors.

PB95-202602

04,694

Ouantum Mechanics of a Solid-State Bar Gravitational Antenna.

PB95-202628 03,987

Failures of the Four-Wave Mixing Model for Cone Emis-

PB95-202636

04,318

Calibration of a Superconducting Gravimeter Using Absolute Gravity Mea
PB95-202651

03,684
Sobolev Approximation for Line Formation with Partial Frequency Redistribution

00,078

Recombination Line Intensities for Hydrogenic lons-III. Ef fects of Finite Optical Depth and Dust.

PB95-202677

00,079

Vibrational Dependence of the Anisotropic Intermolecular Potential of Ar-HCl.

P95-202685

Vibrational Dependence of the Anisotropic Intermolecular Potential of Ar-HF

Moditied Effective Range Theory as an Alternative to Low-Energy Close-Coupling Calculations.

03,988

Slow-Electron Collisions with CO Molecules in an ExactExchange Plus Parameter-Free Polarization Model. Superconducting Resonator and a Cryogenic GaAs FieldEffect Transistor Amplifier as a Single-lon Detection Sys tem.

03,990

Slit-Jet Near-Infrared Spectroscopy and Internal Rotor Dynamics of the ArH2O van der Waals Complex: An An gular Potential-Energy Surface for Internal H2O Rotation. Orbital Stereochemistry: Discovering the Symmetries of Collision Processes.

01.034

Probing Bose-Einstein Condensed Atoms with Short Laser Pulses.

03,991

Deuterium in the Local Interstellar Medium: Its Cosmological Significance.

00,081

Peeking Through the Picket Fence: What Astrophysica Peeking Through the Picket Fence: What Astrophysical
Surprises May Be Present in the 100-1200 Angstrom Region.

895-202859

00,082

Variationally Stable Treatment of Two- and Three-Photon Detachment of $\mathrm{H}(-)$ Including Electron-Correlation Etfects.
PB95-202867

Preferential In-Plane Rotational Excitation of $\mathrm{H} 2 \mathrm{O}(001)$

by Translational-to-Vibrational Transfer from $2.2 \mathrm{eV} \mathrm{H}$

PB95-202875 Aydberg Levels in Light Atoms: The Example of Sodium.
PB95-202883 Narrow-Band Tunable Diode Laser System with Grating
Feedback, and a Saturated Absorption Spectrometer for $\mathrm{Cs}$ and $\mathrm{Rb}$.

PB95-202891

04,319

Threshold Electron Excitation of $\mathrm{Na}$

03,994

Large Amplitude Skeletal Isomerization as a Promoter of Intramolecular Vibrational Relaxation in $\mathrm{CH}$ Stretch Excited Hydrocarbons.

PB95-202933

01,036 Sub-Doppler, Intrared Laser Spectroscopy of the Propyne
2nu1 Band: Evidence of z-Axis Coriolis Dominated Intramolecular State Mixing in the Acetylenic CH Stretch Overtone.

PB95-202941

01,037

Collisional Energy Transfer between Excited-State Strontium and Noble-Gas Atoms.

Importance of Bound-Free Correlation Effects for Vibra-

tional Excitation of Molecules by Electron Impact: A Sen-

Sitivity Analysis.
PB95-202974

03,996

Acceleration of Intramolecular Vibrational Redistribution by methyl Internal Rotation. A Chemical Timing Study of p-fluorotoluen

01,039

High-Resolution, Direct Infrared Laser Absorption Spectroscopy in Slit Supersonic Jets: Intermolecular Forces and Unimolecular Vibrational Dynamics in Clusters. PB95-203006 01,040

Rotational-RKR Inversion of Intermolecular Stretching Potentials: Extension to Linear Hydrogen Bonded Com-

plexes.

01,041

Rigid Bender Analysis of van der Waals Complexes: The

Intermolecular Bending Potential of a Hydrogen Bond.
PB95-203022 PB95-20302

Energy-Pooling
PB95-203030

03,997

Continuous Gravity Observations Using Joint Institute for Laboratory Astrophysics Absolute Gravimeters. Kinetics of the Reaction $\mathrm{C} 2 \mathrm{H}+\mathrm{O} 2$ from 193 to $350 \mathrm{~K}$ Using Laser Flash Kinetic Infrared Absorption SpectrosPB95-203055

01,043

Construction of Silicon Nanocolumns with the Scanning Tunneling Microscope.

04,696

Relative Photoionization and Photodetachment Cross

Relative Photoionization and Photodetachment Cross
Sections for Particular Fine-Structure Transitions with ApSections for Particular Fine-Structure Transition
plication to $\mathrm{Cl} 35$-subshell Photoionization.

plication to $\mathrm{Cl}$
$\mathrm{PB} 95-203097$

03,998

Behavior of Atoms in a Compressed Magneto-Optical Trap.

Dielectronic Capture Processes in the Electron-Impac onization of $\mathrm{Sc}(2+)$

PB95-203113

04,000

Photodissociation Dynamics in Ouantum State-Selected Clusters: A Test of the One-Atom Cage Effect in Ar-H2O. PB95-203121

Photoelectron Spectroscopy of Small Antimony Cluster Anions: $\mathrm{Sb}(-)$, Sb2(-), Sb3(-), and Sb4(-)

PB95-203139

01,045

Charge Cloud Distribution of Heavy Atoms After Excilation by Polarized Electrons.

PB95-203147

Pulsed Laser Photolysis Time-Resolved FT-IR Emission Studies of Molecular Dynamics.

PB95-203162

04,002

High Resolution, Jet-Cooled Infrared Spectroscopy of (HCl)2: Analysis of nu1 and nu2 $\mathrm{HCl}$ Stretching FunPredissoc, Inter conversion

PB95-203196

01,046

Slit-Jet Near-Infrared Diode Laser Spectroscopy of (DCI): nu1, nu2 DCl Stretching Fundamentals, Tunneling Dynamics, and the Influence of Large Amplitude 'Geared' molecular Rotation.

PB95-203204

01,047

High Resolution Near Infrared Spectroscopy of $\mathrm{HCl}-\mathrm{DCl}$ and $\mathrm{DCl}-\mathrm{HCl}$ : Relative Binding Energies, Isomer Interconversion Rates, and Mode Specific Vibrational Predissociation

PB95-203212

01,048

Laser Flash Photolysis, Time-Resolved Fourier Transform Infrared Emission Study of the Reaction $\mathrm{Cl}+\mathrm{C} 2 \mathrm{H} 5$ yields $\mathrm{HCl}(v)+\mathrm{C} 2 \mathrm{H} 4$

PBg5-203238

01049

Laser Preparation and Probing of Initial and Final Orbita Alignment in Collision-Induced Energy Transfer $\mathrm{Ca}(4 s 5 p,(1) \mathrm{P} 1)+\mathrm{He}$ yields $\mathrm{Ca}(4 s 5 p,(3) \mathrm{P} 2)+\mathrm{He}$. 04,003
$\mathrm{~PB} 95-203261$ Initial and Final Orbital Alignment Probing of the FineStructure-Changing Collisions among the $\mathrm{Ca}$
$(4 s)(1)(4 p)(1)$, (3)PJ States with He: Determination of $\mathrm{Co}$ herence and Conventional Cross-Sections.

PB95-203279

Laser-Induced Desorption of In and Ga from Si(100) and Adsorbate Enhanced Surface Damage.

PB95-203311

01,050

Pulsed Laser Irradiation at $532 \mathrm{~nm}$ of In and $\mathrm{Ga} \mathrm{Ad-}$ sorbed on Si(100): Desorption, Incorporation, and DamPge.

01,051

Single-Photon Laser Ionization Time-of-Flight Mass Spectroscopy Detection in Molecular-Beam Epitaxy: Application to As4, As2, and Ga.

PB95-203337

01,052

Deposition Rate

Frequency Shifting of Pulsed Narrow-Band Laser Light in aultipass Raman Cell.

Angle-Differential and Momentum-Transfer Cross Seclions for Low-Energy Electron-Cs Scattering.

Relativistic R-Matrix Calculations for Electron - AlkaliMetal-Atom Scattering: $\mathrm{Cs}$ as a Test Case.

PB95-203410 04,006

Resonance Fluorescence with Squeezed-Light Excitation

PB95-203469 04,322

Short-Pulse Detachment of $\mathrm{H}(\cdot)$ in the Presence of a Static Electric Field 

Stabilization of Optical Phase/Frequency of a Laser Sys-
tem: Application to a Commercial Dye Laser with an ExPB95-203832

04,327

Tables of X-ray Mass Attenuation Coefficients and Mass Energy-Absorption Coefficients $1 \mathrm{keV}$ to $20 \mathrm{MeV}$ for Elements $Z=1$ to 92 and 48 Additional Substances of Dosimetric Interest.

PB95-220539

04,013

New IUPAC Guidelines for the Reporting of Stable Hydrogen, Carbon, and Oxygen Isotope-Ratio Data. Letter to he Editor.

PB95-261962

01,058

Ab initio Calculations for Helium: A Standard for Transport Property Measurements.

01,060

Merged-Beams Energy-Loss Technique for Electron-Ion Excitation: Absolute Total Cross Sections for $\mathrm{O}(5+)$ (2s yields $2 p$

PB96-102058

04,017

Energy Dependences of Absorption in Beryllium Windows and Argon Gas.

04,020

Sub-Doppler Frequency Measurements on OCS at 87 $\mathrm{THz}(3.4 \mathrm{mu} \mathrm{m})$ with the CO Overtone Laser.

PB96-102215

04,330

High-Pesolution Intrared Overtone Spectroscopy of N2 $\mathrm{HF}$ : Vibrational Red Shifts and Predissociation Rate as a Function of HF Stretching Quanta.

01,061

Kinetics and Dynamics of Vibrationally State Resolved on-Molecule Reactions: (14) N2 $(+)(v=1$ and 2$)$ and $(15) \mathrm{N} 2(+)(v=0,1$ and 2$)$ with $(14) \mathrm{N} 2$

04,023

Length Scales for Fragile Glass-Forming Liquids.

01,065

Optically Stabilized Tunable Diode-Laser System for Saturation Spectroscopy.

PB96-102819

04,717

Probing Potential-Energy Surfaces via High-Resolution IR Laser Spectroscopy.

01,056

Time-Resolved Balmer-Alpha Emission from Fast Hydrogen Aloms in Low Pressure, Radio-Frequency Dis-

charges in Hydrogen.
PB96-102942

Low-Energy-Electron Collisions with Sodium: Elastic and Lelastic Scattering from the Ground State.

04,030

Collisional Alignment of $\mathrm{CO} 2$ Rotational Angular Momentum States in a Supersonic Expansion.

PB96-103171

01,069

Improved Wavelengths for Prominent Lines of Fe $\mathrm{XX}$ to Fe XXIII.

PB96-111638

04,334

Vibrational Excitations and the Position of Hydrogen in Nanocrystalline Palladium.

04,035

Electron-Ion Collisions in the Plasma Edge.

PB96-111885

04,037

Ouantum-Limited Cooling and Detection of Radio-Fre-

quency Oscillations by Laser-Cooled Ions.

04,039

Precise Spectroscopy for Fundamental Physics

PB96-112164

04,040

Visible and UV Light from Highly Charged Ions: Exotic

Matter Advancing Technology.

04,414

Nanofabrication of a Two-Dimensional Array Using LaserFocused Atomic Deposition.

PB96-119417

Atomic Clock.

04,732

Doppler-Free Spectroscopy of Large Polyatomic Mol-

ecules and van der Waals Complexes.

04,339

Far Infrared Laser Frequencies of $\mathrm{CH} 3 \mathrm{OD}$ and $\mathrm{N} 2 \mathrm{H} 4$

PB96-119623 04,341

High-Order Multipole Excitation of a Bound Electron.

PB96-119789 04,044

Orbital Alignment and Vector Correlations in Inelastic Atomic Collisions.

PB96-122742 04,047

Atom Cooling and Trapping, and Collisions of Trapped

04,048

PB96-122916
Search for Small Violations of the Symmetrization Postu. late in an Excited State of Helium.

PB96-123518

04,050

Relativistic Modifications of Charge Expansion Theory.

Observation and Visible and uv Magnetic Dipole Transi-

tions in Highly Charged Xenon and Barium.
PB96-13844

04,056

High-Energy Phonon Dispersion in La1.85Sro.15CuO4.

PB96-138458 04,748

Atomic Transition Probabilities and Tests of the $\begin{array}{ll}\text { Spectroscopic Coupling Scheme for N I. } & 04,057 \\ \text { PB96-138466 } & \end{array}$ q Dependence of Self-Energy Effects of the Plane Oxygen Vibration in YBa2Cu3O7.

01,096

Inexpensive Laser Cooling and Trapping Experiment for $\begin{array}{ll}\text { Undergraduate Laboratories. } & \\ \text { PB96-140371 } & 04,353\end{array}$ Photoluminescence Spectra of Epitaxial Single Crystal C60.

PB96-141205

04,754

Atomic Iron in Its (5)D Ground State: A Direct Measure-

ment of the $\mathrm{J}=0$ inverted arrow 1 and $\mathrm{J}=1$ inverted

arrow 2 Fine-Structure Intervals (1.2)

04,756

Using Alom Optics to Fabricate Nanostructures

PB96-141247

04,757

Inelastic Neutron Scattering Study of Hydrogen in Nanocrystalline $\mathrm{Pd}$ tron Rietveld Analysis of Disorder Induced by Zr Substi-

PB96-156104

04,776

RIS Measurement of AC Stark Shifts and Photoionization Cross Sections in Calcium.

PB96-157953

04,073

Comment On: Two-Photon Absorption Series of Calcium. PB96-157979 04,074 Configuration-Dependent AC Stark Shifts in Calcium. PB96-157995

04,363

Barium Contributions to the Valence Electronic Structure of YBa2Cu307-delta, PrBa2Cu307-delta, and Other Barum-Containing Compounds.

PB96-158019

04,076

PB96-158035

04,078

Analysis of the $(5 d(2)+5 d 6 s)-5 d 6 p$ Transition Arrays of Os VII and Ir VIII, and the 6s (2)S-6p (2)P Transitions of Ir PB96-159264

01,954

Appearance Intensities for Multiply Charged Ions in a Strong Laser Field.

PB96-160445 04,089

Low-Energy Vibrations and Octahedral Site Occupation in Nb95V5H(D)y.

01,133

Dislocation Core-Core Interaction and Peierls Stress in a Model Hexagonal Lattice.

PB96-162003

04,101

Charge-Transfer-Induced Multiplet Structure in the $\mathrm{N} 4,5 \mathrm{O} 2,3$ Soft-X-ray Emission Spectrum of Lanthanum. PB96-163746 04,102

Population Trapping in Short-Pulse Multiphoton Ioniza-

PB96-164140

04,371

Trapped lons and Laser Cooling 4. Selected Publications of the Ion Storage Group of the Time and Frequency Division, NIST, Boulder, Colorado.

vision, NIST, Bo

04,108

Irradiances of Spectral Lines in Mercury Pencil Lamps.
PB96-176466

Wavelengths of Spectral Lines in Mercury Pencil Lamps.

PB96-176474 04,376

Potential Surfaces and Dynamics of Weakly Bound Trimers: Perspectives from High Resolution IR Spectros-

PB96-176508

01,155

Electromagnetic Coupling Character of (001) Twist Boundaries in Sintered Bi2Sr2CaCu2O8+x Bicrystals. State-Resolved Rotational Energy Transfer in Open Shell State-Resolved Rotational Energy Transter in Open Shell Collisions: $\mathrm{Cl}($

01,157

Energy Distributions of Neutrons Scattered from Solid 60 by the Beryllium Detector Method.

PB96-176631

03,740

Characterization of the Structure of LaD2.50 by Neutron Powder Diffraction.

PB96-176797 04,783

Theory for Ouantum-Dot Quantum Wells: Pair Correlation and Internal Ouantum Confinement in PB96-179445

02,437

Dynamics of $\mathrm{Mu}(+)$ in $\mathrm{Sc}$ and $\mathrm{ScHx}$

04,112

Synthesis of Hybrid Organic-Inorganic Materials from Interpenetrating Polymer Network Chemistry.

02,994

Designations of ds(2)p Energy Levels in Neutral Zirconium, Hatnium, and Rutherfordium $(\mathrm{Z}=104)$. PB96-180120

04,116

Hypertine Structure Investigations and Identification of New Energy Levels in the lonic Spectrum of (147)Pm.
PB96-180203

Measurements of the Resonance Lines of (6) $\mathrm{Li}$ and (7) $\mathrm{Li}$ by Doppler-Free Frequency-Modulation Spectroscopy. Neutron and Raman Spectroscopies of 134 and $134 a$ Hydrofluorocarbons Encaged in Na-X Zeolite.

PB96-186168

03,001

Microlithography by Using Neutral Metastable Atoms and Selt-Assembled Monolayers.

PB96-190038

02,441

Atomic Branching Ratio Data for Nitrogen-Like Species.

PB96-190152 04, 122

Excitation Transfer in Barium by Collisions with Noble Gases.

PB96-200274

01,163

Comment on $\ll$ A Dynamic Electric Trap for Ground-State PB96-200290

04,123

Alignment Probing of Rydberg States by Stimulated Emis-

PB96-200316

04,124

Collisions of Electrons with Highly-Charged Ions.

PB96-200340

04,791

Pairwise and Nonpairwise Additive Forces in Weakly Bound Complexes: High Resolution Infrared SpectrosCopy of AmDF $(n=1,2,3)$

PB96-200357

04,125

Gravitational Sisyphus Cooling of (87)Rb in a Magnetic rap.

$96-200704$

04,379

Stable, Tightly Confining Magnetic Trap for Evaporative Cooling of Neutral Atoms.

PB96-200720

04,126

UV Absorption Cross Sections of Methylchloroform: Tem perature-Dependent Gas and Liquid Phase Measure-

PB96-201041

00,643

Vibrationally Resolved Photoelectron Angular Distributions and Branching Ratios for the Carbon Dioxide Molecule in the Wavelength Region 685-795 Angstrom. PB96-201207

Fabrication via Laser-Focused Deposition (Invited)

PB96-204094

04,132

Properties of a Bose-Einstein Condensate in an Anisoropic Harmonic Potential.

PB96-204144

04,133

Temperature Dependence of the Ultraviolet Absorption Cross Section of CF3I.

PB96-204169

01,168

Positronium in Relativistic Quantum Mechanics.

B 97-110571

04,140

PB97-110589

Beam Line for Highly Charged lons.

Systematıc Correction in Bragg $\mathrm{X}$-ray Diffraction of Flat and Curved Crystals.

PB97-112239

04,152

Matrix Isolation Study of the Interaction of Excited Neon Atoms with 03: Infrared Spectrum of O((sub 3$)(-))$ and Evidence for the Stabilization of $\mathrm{O} 2 \ldots \mathrm{O}\left(\left(\right.\right.$ sub $\left.\left._{4}\right)(+)\right)$. PB97-112403

04,155

Polarization Measurements on a Magnetic Quadrupole Line in Ne-Like Barium.

PB97-113104

04,161 
AUTOMATIC \& ROBOTICS

Atomic Energy Levels. As Derived From the Analyses of Optical Spectra. Volume 3.

00,714

Relativistic and Quantum Electrodynamic Effects in Highly-Charged lons.

03,854

High-Resolution Atomic Spectroscopy of Laser-Cooled PB95-169330

03,953

Measurements of the Resonance Lines of $(6) \mathrm{Li}$ and $(7) \mathrm{Li}$ by Doppler-Free Frequency-Modulation Spectroscopy.
PB96-180211

ATOMIC SPECTROSCOPY

Atomic, Molecular, and Optical Physics with X-rays.
PB94-213378 03,863 Comprehensive Spectroscopic Data Tabulations and Progress in the Compilation of Atomic Transition Probabilities

PB95-151551

03,909

Spectroscopic Data Tables for Highly-lonized Atoms.
PB95-151585

Spin Squeezing and Reduced Quantum Noise in Spec-

troscopy.

03,912

Trace Detection in Conducting Solids Using Laser-Induced Fluorescence in a Cathodic Sputtering Cell.
PB95-163424 00,598

Squeezed Atomic States and Projection Noise in Spec-

troscopy.

03,960

Qbservation and Visible and uv Magnetic Dipole Transitions in Highly Charged Xenon and Barium. PB96-13844

04,056

ATOMIC STRUCTURE

Relativistic and Quantum Electrodynamic Effects in Highly-Charged lons.

03,854

Core Potentials for Quasi-Qne-Electron Systems.

PB95-202214

03,970

Relativistic Modifications of Charge Expansion Theor

PB96-123799

04,052

ATOMIC THEORY

Atomic Theory of Fracture of Brittle Materials: Application

to Covalent Semiconductors.

PB94-216553

04,519

ATOMIC TRANSITION PROBABILITIES

Atomic Transition Probabilities and Tests of the Spectroscopic Coupling Scheme for $N$

04,057

ATOMIC WEIGHTS

Atomic Weights of the Elements, 1993.
PB96-145909

ATOMISTIC CALCULATIONS

Dislocation Core-Core Interaction and Peierls Stress in a Model Hexagonal Lattice.

PB96-162003

04,101

ATOMIZATION

Beneficial Effects of Nitrogen Alomization on an Austenitic Stainless Steel.

PB94-212396

03,209

PB95-107405

01,377

Study of Droplet Transport in Alcohol-Based Spray Flames Using Phase/Doppler Interferometry.
PB95-108551 02,479
PB95-108551 Using Laser Diffraction.

PB95-108577 0,342

Turbulent Spray Burner for Assessing Halon Alternative Fire Suppressants.

01,385

ATOMIZERS

Development of Adaptive Control Strategies for Inert Gas Atomization.

02,823

ATOMIZING

Process Modeling and Control of Inert Gas Atomization. PB95-162343

02,824

ATOMS

Determination of Atomic Data Pertinent to the Fusion Energy Program. Progress Report for FY 92. Atomic Branching Ratio Data for Nitrogen-Like Species PB96-190152 04,122

ATOMSPHERIC CHEMISTRY

Free Radical Chemistry of the Atmospheric Aqueous PBase. 148101

00,117

ATRIA

Method of Predicting Smoke Movement in Atria with Application to Smoke Management.

00,376

ATTENUATION

Theoretical Form Factor, Attenuation and Scattering TabPB96-145594 01,104

\section{ATTENUATION CALIBRATION SYSTEM}

Revised Uncertainty Analysis for the NIST $30-\mathrm{MHz}$ Attenuation Calibration System.

PB95.168761

01,907

$30 \mathrm{MHz}$ Comparison Receiver.

PB96-200407

01,972

SITING

Self Monitoring Accounting Systems.

\section{AUGER ELECTRON SPECTROSCOPY}

Inelastic Interactions of Electrons with Surfaces: Application to Auger-Electron Spectroscopy and $X$-ray Photoelectron Spectroscopy.

00,764

$\mathrm{X}$-ray Photoelectron and Auger Electron Forward Scatter$X$-ray Photoelectron and Auger Electron Forward
ing: A Structural Diagnostic for Epitaxial Thin Films. ing: A Structural Diagnostic for Epitaxial Thin Films. 04,676 AUGMENTED REALITY

Virtual Environments for Health Care. A White Paper for the Advanced Technology Program (ATP), the National Institute of Standards and Technology.

PB96-147814

03.594

AUSTENITE

Charpy Impact Test as an Evaluation of $4 \mathrm{~K}$ Fracture Toughness.
PB96-190194

AUSTENTIC STAINLESS STEELS

Determination of the Prior-Austenitic Grain Size of Selected Steels Using a Molten Glass Etch.

03,208

PB94-211927

an Aus-

Beneficial Effects of

PB94-212396

03,209

Dynamics vs. Static Young's Moduli: A Case Study.

PB94-213188 03,210

Cryogenic Toughness of Austenitic Stainless Steel Weld Metals: Effect of Inclusions.

PB95-161261

03,214

AUSTENITIC STEELS

Charpy Specimen Tests at $4 \mathrm{~K}$.
PB96-190335

03,002

AUTENNAS

Spherical-Wave Source-Scattering Matrix Analysis of Antennas and Antenna-Antenna Interaction

02,008

AUTHENTICATION

Guideline for the Use of Advanced Authentication Technology Alternatives. Category: Computer Security. Subcategory: Access Control.

\section{AUTOFLUORESCENCE}

01,798

\section{TOFLUORESCENCE}

Autofluorescence Detection of 'Escherichia coli' on Silver Membrane Filters.

PB96-163639

03,590

AUTOIONIZATION

Autoionizing Resonances in Electric Fields.

RIS Studies of Autoionization in Calcium.

03,853

PB94-213295

00,849

Photoelectron Study of Electronic Autoionization in Rotationally Cooled N2: The $n=6$ Member of the Hopfield Serie

PB95-163531

00,971

Short-Range Correlation and Relaxation Effects on the $(6 \mathrm{p}(2))(1) \mathrm{SO}$ Autoionizing State of Atomic Barium.

PB95-202289

03,973

AUTOMATED BRIDGE

Automated Guarded Bridge for Calibration of Multimegohm Standard Resistors.

PB97.119150

02,289

AUTOMATED INSPECTION

Automated Inspection: The Integration of National Standards and Commercial Products at NIST.

ards and Com

02,906

AUTOMATED SYSTEMS

Programmable Guarded Coaxial Connector Panel. PB96-122544

02,108

AUTOMATIC CONTROL

Automated, High-Precision Coulometric Titrimetry. Part 1. Engineering and Implementation.

00,575

Automated, High Precision Coulometric Titrimetry. Part 2.

Strong and Weak Acids and Bases.

PB95-150207

00,576

Integrating Automated Systems with Modular Architec-

00,577

PB95-150231

Robotic

Vehicle-Command

03,665

Outline of a Multiple Dimensional Reference Model Architecture and a Knowledge Engineering Methodology for Intelligent Systems Control.

PB95-220414

03,703

AUTOMATIC \& ROBOTICS

Open Architectures for Machine Control.

02,942

Enhanced Machine Controller Architecture Qverview. 02,802

Feasibility Study: Reference Architecture for Machine Control Systems Integration.

PB94-14279

02,804

Reliable Optical Flow Algorithm Using 3-D Hermite PolyRomials.

PB94-145620

01,829

APDE Demonstration System P.rchitecture. National PDES Testbed Report Series.

PB94-154325

02,767

Variant Design for Mechanical Artfacts-A State of the Art Survey.

$0 . ?, 768$

Technical Program of the Factory Automation Systems Division 1993

PB94-160819 02,805

U.S. Navy Coordinate Measuring Machines: A Study of Needs.

PB94-162831

02,807

Concept tor an Algorithm Testing and Evaluation Program at NIST.

NIST Support to the Next Generation Controller Program 1991 Final Technical Report.

PB94-163490

02,808

Publications of the Manufacturing Engineering Laboratory Covering the Period January 1989-September 1992.
PB94-165966

Toward a Reference Model Architecture for Real-Time In elligent Control Systems (ARTICS).

PB94-172046

02,932

Uitrasonic Measurements of Surface Roughness.

04,181

AMRF Composite Fabrication Workstation

PB94-172681

02,810

World Model Registration for Effective OH-Line Programming of Robots

02,933

Characterization of the Hydrogen Induced Cold Crackin

Susceptibility at Simulated Weld Zones in HSLA-100

PB94-174505

03,746

Task Decomposition Methodology for the Design of Coal Mining Automation Hierarchical Real-Time Control System.

Issues Concerning Material Removal Shape Element Volumes (MRSEVs)

PB94-185493

02,882

Development of a Calibrated Atomic Force Microscope.

02,894

Prediction of Geometric-Thermal Machine Tool Errors by Artificial Neural Networks.

PB94-186673

02,943

State-of-the-Art Survey of Methodologies for Representing Manufacturing Process Capabilities.

B94-187655

02,812

NIST RS274/NGC Interpreter Version 1.

PB94-187788

02,814

Control Entity

02,815

Overview of NASREM: The NASANBS Standard Reference Model for Telerobot Control System Architecture. PB94-194560

04,831 
Advanced Angle Metrology System

PB94-211364

02,637

Intelligent Control for Multiple Autonomous Undersea Vehicles

03,747

Real-Time Vision for Autonomous and Teleoperated Control of Unmanned Vehicles.

PB94-211885

03,701

Real-Time Vision for Unmanned Vehicles.

Hierarchical Real-Time Control System for Use with Coal Mining Automation.

PB94-212065

03,698

Hierarchical Ada Robol Programming System (HARPS):

A Complete and Working Telerobot Control System Based on the NASREM Model.

02,934

Technical Program Description Systems Integration for Manufacturing (SIMA)

PB94-213758

02,819

Evolution of the Flight Telerobotic Servicer.

PB94-216082
Publications of the Intelligent Systems Division (Previously Robot Systems Division) Covering the Period January 1971-April 1994

PB94-217098

02,935

Adaptive, Predictive 2-D Feature Tracking Algorithm for Finding the Focus of Expansion.

PB94-218575

01,588

Three Dimensional Position Determination from Motion

PB95-107108 01,788

Real Time Compensation for Tool Form Errors in Turning Using Computer Vision.

02,945

Summary and Notes of the Joint ISO/IGES/PDES Organization Technical Committee Meeting. Held in Albuquerque, New Mexico on October $15-20,1989$.

PB95-107314 02,774

Real-Time Implementation of a Differential Range Finder. PB95-108650 01,839

Evolution of Automatic Line Scale Measurement at the

National Institute of Standards and Technology.
PB95-108809 PB95-108809

Some Considerations for Interim Testing of Coordinate Measuring Machine Performance Using a Specific Arti-

PBat. 108858

02,898

National PDES Testbed: An Overview.

02,775

STEP On-Line Information Service (SOLIS). The IGES/ PDES Organization.

PB95-137790

02,777

SGML Environment for STEP.

02,778

Reference Model Architecture for Intelligent Systems DePign. 143137

01,789

Visual Pursuit Systems.

01,841

Reference Architecture for Machine Control Systems Integration: Interim Report.

02,820

Program Requirements to Advance the Technology of Custom Footwear Manufacturing.

02,883

Portsmouth Fastener Manufacturing Workstation. User's Manual.

PB95-147922

02,860

Using Grafcet to Design Generic Controllers.

Mapping Processes to Processors for Space-Based Robot Systems

PB95-151510

04,833

Integration of Real-Time Process Planning for Small-

Batch Flexible Manufacturing.
PB95-151908

02,822

Displacement Method for Machine Geometry Calibration.

PB95-152088

02,946

Automated Optical Roughness Inspection.

02,905

Visual Road Following without 3-D Reconstruction.

PB95-161030

01,591

Real Time Differential Range Estimation Based on TimeSpace Imagery Using PIPE.

PB95-16180

01,844

PBeconstruction

01,593

Unified Approach to Camera Fixation and Vision-Based Road Following

01,594

Vehicle-Command Center Communications in a Robotic Vehicle System.

03,665

Automated Inspection: The Integration of National StandAutomated Inspection: The Integration of National Stand-
ards and Commercial Products at NIST.
PB95-163077
Two New Probes for a Coordinate Measuring Machine. PB95-163093 02,653

Control System Architecture for a Remotely Operated Unmanned Land Vehicle.

03,759

Variations in Size Measurements by Indicating Gaging

Systems.

02,864

Configuration and Performance Evaluation of a Real-Time

Robot Control System: A Skeleton Approach.
PB95.163895

Integrated Mobile Robot System for Testing Vision Algo-

PB95-164133

02,936

Force Calibrations in the Nanonewton Regime.

03,949

Workshop Summary Report: Industrial Applications of Scanned Probe Microscopy. A Workshop Co-sponsored by NIST, SEMATECH, ASTM, E42.14, and the American Vacuum Society. Held in Gaithersburg, Maryland on March 24-25, 1994.

PB95-170387

00,506

Robotics Application 10 Highway Transportation. Volume 2. Literature Search.

01,337

New Method to Calculate Looming for Autonomous Ob-

stacle Avoidance.
PB95-171435

Robotics Application to Highway Transportation. Volume 3. Proposed Research Topics and Cost/Benefit Evaluations by CER

01,338

Workshop on the Application of Virtual Reality to Manufacturing. Final Report. Held in Gaithersburg, Maryland on August 9,1994

PB95-173555

02,825

Dimensional Inspection Planning Based on Product Data Standards.

$\begin{array}{ll}\text { PB95-175451 02,910 } & \end{array}$

Accuracy in Integrated Circuit Dimensional Measure-

PB95-180808 02,368

Vehicle-to-Roadside Communications for Commercial Vehicle Operations: Requirements and Approaches.

PB95-188827

04,860

Program of the Manufacturing Engineering Laboratory, 1995. Infrastructural Technology, Measurements, and Standards for the U.S. Manufacturing Industries.

Standards for th

02,754

Motion-Model-Based Boundary Extraction

PB95-189502

01,849

Robotics Application to Highway Transportation. Volume 4. Proposals for Potential Research.

01,339

Real-Time Obstacle Avoidance Using Central Flow Divergence and Peripheral Flow.

PB95-198677 $\quad 02,937$

Expert Control System Shell Version 1.0 User's Guide.

01,790

Post-Process Control of Machine Tools.

1,790

PB95-203451

02,952

Proceedings of the Manufacturing Technology Needs and Issues: Establishing National Priorities and Strategies Conference. Held in Gaithersburg, Maryland on April 26 $28,1994$.

PB95-206181 02,930

Automated Manufacturing Research Facility 1994 Annual

PB95-209854

00,015

Design, Specification and Tolerancing of Micrometer-Tolerance Assemblies.

02,913

Issues Concerning Material Removal Shape Element VolUmes (MRSEVs)

PB95-210167

02,885

Calculating Time-to-Contact Using Real-Time Quantized

Optical Flow.

Interim Testing Artifact (ITA): A Performance Evaluation

System for Coordinate Measuring Machines (CMMs).

User Manual.

PB95-210589

02,914

Japan Technology Program Assessment. Simulation: State-of the-Art in Japan.

PB95-217097

02,827

Outline of a Multiple Dimensional Reference Model Architecture and a Knowledge Engineering Methodology for Intelligent Systems Control.

03,703

Texture-Independent Vision-Based Closed-Loop Fuzzy Controllers for Navigation Tasks.

00,183

Algorithm Testing and Evaluation Program for Coordinate Measuring Systems: Long Range Plan.

02,915

Conceptual Design Plan for the National Advanced Manufacturing Testbed

02,828

ISO TC 184/SC4 Reference Manual.

PB95-242293

02,663

Precision in Machining: Research Challenges.

02,953

Unified Telerobotic Architecture Project (UTAP) Standard Interface Environment (SIE), May 1995.

02,938

DETAN 95: Computer Code for Calculating Spectrum. Averaged Cross Sections and Detector Responses in Neutron Spectra.

PB95-242384

04,014

Measuring Long Gage Blocks with the NIST Line Scale Interferometer

Submarine Automation: Demonstration No. 5.

02,665

PB95-251633

03,748

Study of Potential Applications of Automation and Robotics Technology in Construction, Maintenance and Operation of Highway Systems: A Final Report. Volume 4.

01,340

Algorithm Testing and Evaluation Program for Coordinate Measuring Systems: Testing Methods.

PB95-251658

02,666 2.0 .

PB95-25166

02,916

Study of Potential Applications of Automation and Robotics Technology in Construction, Maintenance and Operation of Highway Systems: A Final Report. Volume 1

PB95-251682

01,341

Study of Potential Applications of Automation and Robotics Technology in Construction, Maintenance and Operation of Highway Systems: A Final Report. Volume 3

PB95-251690

01,342

Information Technologies Make Business Sense for the Custom Therapeutic Footwear Industry.

PB95-251708

02,829

Gage Block Handbook

02,667

Product Models and Virtual Prototypes in Mechanical En-

gineering.

02,783

Integrated Vision Touch-Probe System for Dimensional Inspection Tasks.

PB95-255832

02,917

Study of Potential Applications of Automation and Robotics Technology in Construction, Maintenance and Operation of Highway Systems: A Final Report. Volume 2

Design Engineering Research at NIST.

PB95-267860

02,784

Estimation of Measurement Uncertainty of Small Circular Features Measured by CMMs.

PB95-267928

02,918

Ouch Those Programs are Painful.

01,739

Joint DoD/NIST Workshop on International Manufacturing Joint DoD/NIST Workshop on International Manufacturing

Systems Research and Development. Held in
Maryland on November 3-5, 1992. Proceedings.

Fabrication of 0

PB96-111695

02,931 
Visualization of Surface Figure by the Use of Zernike Polynomials.
PB96-137757

04,351

Object-Oriented Tel/Tk Binding for Interpreted Control of the NIST EXPRESS Toolkıt in the NIST STEP Application Protocol Development Environment.

02,785

Progress Toward Accurate Metrology Using Atomic Force Microscopy.
PB96-146774

02,417

NIST RS274KT Interpreter.

PB96-147954

02,835

Product Realization Process Modeling: A Study of Requirements, Methods and Research Issues.
PB96-147962 02,836 Machine Performance Standard Provides Opportunity to Improve Ouality and Productivity

PB96-154521

02,837

Guidelines for the Development of Mapping Tables.

PB96-154539 02,786

Image Gradient Evolution: A Visual Cue for Danger.
PB96-154562

NIST SIMA Interactive Management Workshop. Held in

Fort Belvoir, Virginia on November 14-16, 1994 . 02,838
PB96-154877

Rockwell

Stylus Technique for
Diamond Intenders.

PB96-155569

02,958

Ouality in Automated Manufacturing.

PB96-160437

02,839

Development of a New Ouality Control Strategy for Automated Manufacturing

02,840

Integrated Inspection System for Improved Machine Per. formance.

PB96-160569

02,959

Fabrication Issues for the Prototype National Institute of Standards and Technology SRM 2090A Scanning Electron Microscope Magnification Calibration Standard

PB96-160585

01,131

Metrology Standards for Advanced Semiconductor Lithography Referenced to Atomic Spacings and Geometry.
PB96-160718

Residual Error Compensation of a Vision-Based Coordi-

nate Measuring Machine.
PB96-161617

04,091

SPC Artifact for Automated Solder Joint Inspection.

PB96-161716 04,095

Machining Process Planning Activity Model for Systems

Integration.

02,841

PIECS: A Software Program for Machine Tool ProcessIntermittent Error Compensation.

PB96-165980

02,842

Testing the Sensitivity of Accelerometers Using Mechanical Shock Pulses Under NIST Special Publication 250 Test No. $24040 \mathrm{~S}$

PB96.179544

02,683

Procedure for Product Data Exchange Using STEP Developed in the AutoSTEP Pilot.

PB96-183058

02,843

Proceedings of NIST Workshop: Industry Needs in Welding Research and Standards Development. Held on August 15-16, 1995

02,877

Scale-Space-Based Visual-Motion-Cue for Autonomous Navigation.

PB96-183173

02.940

Aspects of a Product Model Supporting Apparel Virtual Enterprises.

02,790

Dynamic Objects and Meta-Level Programming of an EXPRESS Language Environment

01,774

Increasing the Value of Atomic Force Microscopy Process Metrology Using a High-Accuracy Scanner, Tip CharacMetrology Using a High-Accuracy Scanner, T
terization, and Morphological Image Analysis.

terization, and

02,758

Scanned Probe Microscope Tip Characterization Without Calibrated Tip Characterizers.

02,759

Novel Active-Vision-Based Motion Cues for Local Navigation. 193727

02,941

Systems Integration for Manufacturing Applications Program 1995 Annual Report.

PB96-193735

02,844

Survey of Standards for the U.S. Fiber/Textile/Apparel In-

dustry.
PB96-193792

03,199

Computer-Aided Manufacturing Engineering Forum (2nd). Maryland Meeting Proceedings.

PB96-195334

02,845

Operator Experience with a Hierarchical Real-Time Control System (RCS)

03,751
Scanning Electron Microscope Magnification Calibration Interlaboratory Study.

01,164

Application Protocol Information Base World Wide Web Gateway.

02,791

General Motion Model and Spatio-Temporal Filters for 3D Motion Interpretations.

01,86

Optoelectronics and Optomechanics Manufacturing: An ings. Held in Gaithersburg, Maryland on February 15,

PB97-104186

02,204

Compensation of Errors Detected by Process-Intermittent Gauging.

02,846

Compensation for Errors Introduced by Nonzero Fringe Densities in Phase-Measuring Interferometers.

PB97-110506

04,386

Representing Designs with Logic Formulations of Spatial Relations.

02,792

Using Logic to Specify Shapes and Spatial Relations in Design Grammars.

PB97-111579

02,793

HVAC CAD Layout Tools: A Case Study of University/lndustry Collaborat
PB97-112221

00,281

Chip Morphology, Tool Wear and Cutting Mechanics in Finish Hard Turning.

PB97-112247

03,106

Combining Interactive Exploration and Optimization for Assembly Design

02,794

Chemical Aspects of Tool Wear in Single Point Diamond

Purning.

03,021

Improving the Design Process by Predicting Downstream Values of Design Attributes.

02,795

Static Structural Analysis of a Reconfigurable Rigid Plat-

form Supported by Elastic Legs.
PB97-113898

02,960

Interoperability Requirements for CAD Data Transfer in the AutoSTEP Project.

02,796

Unified Process Specification Language: Requirements for Modeling Process

PB97-116123

Capabilities for Product Data Exchange.

PB97-118764

02,850

SC4 Short Names Registry.

PB97-122410

02,798

Absence of Ouantum-Mechanical Effects on the Mobility of Argon lons in Helium Gas at $4.35 \mathrm{~K}$

$$
\text { PB97-122543 }
$$

01,194

\section{AUTOMATION}

Technical Program of the Factory Automation Systems

Division 1993.
PB94-160819

Hierarchical Real-Time Control System for Use with Coal Mining Automation.

PB94-212065

03,698

Publications of the Intelligent Systems Division (Previously Robot Systems Division) Covering the Period Jan uary 1971-April 1994.

PB94-217098

02,935

Framework for Information Technology Integration in Process Plant and Related Industries.

02,772

CALS-Markup Requirements and Generic Style Specifications for Electronic Printed Output and Exchange of Text. PB94-962200 03,659

Vibration Laboratory Automation at NIST with Personal Computers.

02,648

Development of an Automated Part Inspection System Using the DMIS Standard

PB95-108866

02,899

Pesters Open

01,718

Post-Process Control of Machine Tools.

PB95-203451

Submarine Automation: Demonstration No. 5.

02,952

(251633

03,748

Study of Potential Applications of Automation and Robotics Technology in Construction, Maıntenance and Ope ation of Highway Systems: A Final Report. Volume 4 PB95-251641

01,340

Study of Potential Applications of Automation and Robotics Technology in Construction, Maintenance and Operation of Highway Systems: A Final Report. Volume 1

PB95-251682

Study of Potential Applications of Automation and Robotics Technology in Construction, Maintenance and Operation of Highway Systems: A Final Report. Volume 3 PB95-251690

01,342

Study of Potential Applications of Automation and Robotics Technology in Construction, Maintenance and Ope ation of Highway Systems: A Final Report. Volume 2. PCASYS: A Pattern-Level Classification Automation Sys tem for Fingerprints.

PB95-267936

01,853

CALS-Markup Requirements and Generic Style Specifications for Electronic Printed Output and Exchange of Text. PB95-962200

Automated Resistance Measurements at NIST.

PB96-119326

Ouality in Automated Manufacturing.

PB96-160:437

02,274

PB96-160486

02,840

Manager's Guide for Monitoring Data Integrity in Financial Systems.
PB96-165915

00,003

PIECS: A Sottware Program for Machine Tool Process. Intermittent Error Compensation.

PB96-165980

02,842

NIST Construction Automation Program Report No. 2 Proceedings of the NIST Construction Automation Workshop. Held in Gaithersburg, Maryland on March 30-31

PB96-202239

00,413

Compensation of Errors Detected by Process-Intermittent Gauging.

02,846

Data Management for Error Compensation and Process Control.

PB97-110480

02,847

Testing Conformance and Interoperability of BACnet (Trade Name) Building Automation Products.

PB97-111553

00,310

Resistors.

PB97-111876

02,284

AUTOMOBILE CATALYST

Resolution of Discrepant Analytical Data in the Certifi-

cation of Platinum in Two Automobile Catalyst SRM

PB96-167283

AUTOMOTIVE ENGINEERING

Interoperability Requirements for CAD Data Transfer in the AutosTEP Project.

PB97-114268

02,796

AUTOMOTIVE INDUSTRY

Procedure for Product Data Exchange Using STEP Developed in the
PB96-183058

02,843

AUTONOMOUS GALLOPING OSCILLATORS

Foias-Temam Approximations of Attractors fo: Galloping Oscillators.

04,817

PB94-198298

Oscil- 


\section{KEYWORD INDEX}

Interlaboratory Comparison of Autoradiographic DNA Profiling Measurements. 2. Measurement Uncertainty and Its Propagation

AUTOREGRESSIVE PROCESSES

Analysis of Autocorrelations in Dynamic Processes.
PB95-181228
02,826 AVALANCHE BREAKDOWN

Experimental Study of Reverse-Bias Failure Mechanisms in Bipolar Mode JFET (BMFET)

PB95-152997

02,340

AVIATION FUELS

Measurement of Diffusion in Supercritical Fluid Systems: A Review.

00,795

Measurement of Diffusion in Fluid Systems: Applications to the Supercritical Fluid Region.

PB95-175188

02,490

AVIATION SAFETY

Suppression of High Speed Turbulent Flames in a Detonation/Deflagration Tube.

\section{VIONICS}

01,395

Measurements of Shielding Effectiveness and Cavity Characteristics of Airplanes.

PB94-210051

00,030

AXES OF ROTATION

Evaluation of a Tapered Roller Bearing Spindle for HighPrecision Hard Turning Applications.

PB96-160494

02,700

AXIAL STRAIN

Effect of Axial Strain on the Critical Current of $\mathrm{Ag}$ Sheathed Bi-Based Superconductors in Magnetic Fields Up to $25 \mathrm{~T}$.

PB94-211315

04,493

AZEOTROPES

Role of Refrigerant Mixtures as Alternatives to CFCs.
PB94-199775

Study to Determine the Existence of an Azeotropic R-22 'Drop-In' Substitute.

\section{PB96-167812}

Molar Absorptivities of Bilirubin (NIST SRM 916A) and Its

Neutral and Alkaline Azopigments.
PB94-211042

03,456

BACILLUS SUBTILIS

Glucose Permease of Bacillus Subtilis Is a Single Polypeptide Chain That Functions to Energize the SuCrose Permeas

BACKDRAFT PHENOMENA

Backdraft Phenomena

PB94-193927

03,466

ACKGROUND CURRENTS

Novel Amperometric Immunosensor for Procainamide Employing Light Activated Labels.

ACKSCATTERING

Evidence for Significant Backscattering in Near-Threshold Electron-Impact Excitation of $\operatorname{Ar}(7+)(3 s$ yields $3 p)$.
PB95-126405

elium in

Measured Stopping Powers of Hydrogen and Helium in Polystyrene Near Their Maximum Values.

04,729

BACKSCATTERING SPECTROMETERS

Design of a High-Flux Backscattering Spectrometer for Ultra-High Resolution Inelastic Neutron Measurements.
PB96-179577 02,992

BACTERIA

Total Surface Areas of Group IVA Organometallic Compounds: Predictors of Toxicity to Algae and Bacteria.

PB94-211331 00,814 Holographic Properties of Triton X-100-Treated Bacteriorhodopsin Embedded in Gelatin Films.

PB96-119284

03,761

Autofluorescence Detection of 'Escherichia coli' on Silver Membrane Filters.

Bacterial Enumeration in Storage Water

PB96-163647

03,590

BACTERIORHODOPSIN

Retinal-Protein Complexes as Optoelectronic Components.
PB95.150397

Bacteriorhodopsin Immobilized in Sol-Gel Glass.

PB95-151429

Bacteriorhodopsin Retains Its Light-Induced ProtonPumping Function After Being Heated to $140 \mathrm{C}$. PB96-102728

Rapid pH Change Due to Bacteriorhodopsin Measured with a Tin-Oxide Electrode.

PB96-112081

03,544

Holographic Properties of Triton X-100-Treated Bacteriorhodopsin Embedded in Gelatin Films.
PB96-119284
BALANCES

Precision Laboratory Standards of Mass and Laboratory AD-A280 562/0

02,618

\section{BALL-BAR STANDS}

NIST SRM 9983 High-Rigidity Ball-Bar Stand. User ManPB95-255840

02,669

BALLE-FLYGARE SPECTROMETERS

Experimental Studies of Line Shapes from a BalleFlygare Spectrom
PB94-199452

00,796

BALMER LINES

Excitation of Balmer Lines in Low-Current Discharges of Hydrogen and Deuterium.

03,893

Hydrogen Balmer Alpha Line Shapes for Hydrogen-Argon Mixtures in a Low-Pressure if Discharge.

03,924

\section{BAND SIZE}

Interlaboratory Comparison of Autoradiographic DNA Profiling Measurements. 2. Measurement Uncertainty and Its Propagation

BAND STRUCTURE OF SOLIDS

03,545

Band-to-Band Photoluminescence and Luminescence Excitation in Extremely Heavily Carbon-Doped Epitaxial GaA PB95-150413

04,570

BAND THEORY

Substitution-Induced Midgap States in the Mixed Oxides RxBa1-ChiTiO3-Delta, with $R=Y, L a$, and $N d$

04,541

BANDPASS FILTERS

Systematic Studies of the Effect of a Bandpass Filter on a Josephson-Junction Noise Thermometer.

03,939

BARIUM

Short-Range Correlation and Relaxation Effects on the $(6 \mathrm{p}(2))(1)$ SO Autoionizing State of Atomic Barium

Energy-Pooling Collisions in Barium.

03,973 PB95-203030

03,997

Excitation Transfer in Barium by Collisions with Noble Gases.

$$
\text { PB96-200274 }
$$

01,163

BARIUM BISMUTH OXIDES

Powder Neutron Diffraction Investigation of Structure and Cation Ordering in $\mathrm{Ba} 2+x \mathrm{Bi} 2-x \mathrm{O} 6-\mathrm{y}$.

PB95-180865

01,015

BARIUM BISMUTHATES

Powder Neutron Diffraction Investigation of Structure and Cation Ordering in $\mathrm{Ba} 2+x \mathrm{Bi} 2-x \mathrm{O} 6-\mathrm{y}$.

01,015

BARIUM BORATES

High-Efficiency, High-Power Difference-Frequency Generation of $0.9-1.5 \mathrm{mu}$ m Light in BBO.

PB95-202255

04,317

BARIUM COMPOUNDS

Epitaxial Growth of BaTiO3 Thin Films at $600 \mathrm{C}$ by Metalorganic Chemical Vapor Deposition.

03,071

BARIUM COPPER OXALATES

$X$-Ray Powder Diffraction Data for $\mathrm{BaCu}(\mathrm{C} 2 \mathrm{O} 4) 2.6 \mathrm{H} 2 \mathrm{O}$. PB95-151767

04,583

BARIUM IONS

Fine Structure of Negative Ions of Alkaline-Earth-Metal

PB94-211182

03,837

Spectrum and Energy Levels of Triply Ionized Barium (Ba

PB95-140877

03,888

Thermodynamic Properties of the Aqueous Ba(sup 2+) lon and the Key Compounds of Barium.

01,107

BARIUM IRON TITANATE

Preparation, Crystal Structure, Dielectric Properties, and Magnetic Behavior of Ba2Fe2Ti4O13.

PB96-186176

01,162

BARIUM OXIDES

Crystal Chemistry and Phase Equilibria Studies of the $\mathrm{BaO}(\mathrm{BaCO} 3)-\mathrm{R} 2 \mathrm{O} 3-\mathrm{CuO}$ Systems. 4. Crystal Chemistry and Subsolidus Phase Relationship Studies of the CuORich Region of the Ternary Diagrams, $\mathrm{R}=$ Lanthanides. PB95-151759

00,936

Surface Geometry of BaO on W(100): A Surface-Extended $X$-Ray-Absorption Fine-Structure Study.

PB95-164414 00,980

Magnetic Dielectric Oxides: Subsolidus Phase Relations in the BaO: Fe2O3: TiO2 System.

PB96-176524

01,156

BARIUM PARTIAL DENSITY

Barium Contributions to the Valence Electronic Structure of YBa2Cu3O7-delta, PrBa2Cu3O7-delta, and Other Barium-Containing Compounds.

04,076

\section{BARIUM TITANATES}

Photoelectron Spectroscopic Study of the Valence and Pore-Level Elect

04,500

Surface Core-Level Shits of Barium Observed in Photoemission of Vacuum-Fractured $\mathrm{BaTiO}_{3}(100)$

PB94-212156

04,501

BARKHAUSEN EFFECT

Langevin Approach to Hysteresis and Barkhausen Modeling in Steel.

03,206

Analytical Expressions for Barkhausen Jump Size Distributions.

BARRIERS

Helping to Reduce Technical Barriers to Trade.

PB96-190046

04,680

Methodology for Developing and Implementing Alternative Temperature-Time Curves for Testing the Fire Resistance of Barriers for Nuclear Power Plant Applications.

PB96-193784

03,742

TBT Agreement Activities of the National Institute of Standards and Technology, 1995.

00,499

Stacking the Cards in Europe: One Company's Story. PB97-110126

00,493

BARS

Degradation of Powder Epoxy Coated Panels Immersed

in a Saturated Calcium Hydroxide Solution Containing Sodium Chloride.

PB96-101050

01,344

BASE ISOLATION

Modified Optimal Algorithm for Active Structural Control. PB96-165949

00,472

BASELINE MEASUREMENTS

Indoor Air Ouality Impacts of Residential HVAC Systems. Phase 2.A Report: Baseline and Preliminary Simulations. PB95-178893

BAYARD-ALPERT GAGES

Comments on the Stability of Bayard-Alpert Ionization PB96-103080

02,673

BAYARD-ALPERT IONIZATION GAGES

Influence of the Filament Potential Wave Form on the Sensitivity of Glass-Envelope Bayard-Alpert Gages

PB95-175014

( ; 657

Long-Term Stability of Bayard-Alpert Gauge Performance: Results Obtained from Repeated Calibrations against the National Institute of Standards and Technology Primary Vacuum Standard.

PB96-123567

02,676

BAYS (STRUCTURAL UNITS)

Use of Computer Models to Predict Temperature and Smoke Movement in High Bay Spaces.

PB94-145976

BCC LATTICES

Transformation of $\mathrm{BCC}$ and $\mathrm{B} 2$ High Temperature Phases to HCP and Orthorhombic Structures in the Ti-Al-Nb System. Part 1. Microstructural Predictions Based on a Subgroup Relation between Phases.

03,370

Transformation of $\mathrm{BCC}$ and $\mathrm{B} 2$ High Temperature Phases to HCP and Orthorhombic Structures in the Ti-Al-Nb Sys. tom. Part 2 Experimental TEM Study of Microstructures. 
BEAMS

Generalized Optical Theorem for On-Axis Gaussian

PB97-122345

04,177

BEAMS (RADIATION)

Merged-Beams Energy-Loss Technique for Electron-Ion Excitation: Absolute Total Cross Sections for $\mathrm{O}(5+)$ (2s yields 2p).

04,017

Beamcon III, a Linearity Measurement Instrument for Optical Detectors.

BEAMS (STRUCTURAL)

Model Precast Concrete Beam-to-Column Connections Subject to Cyclic Loading.

PB95-153094

00,438

Partially Prestressed and Debonded Precast Concrete Beam-Column Joints.

00,439

Seismic Performance Behavior of Precast Concrete Beam-Column Joints

00,440

BEAMS (SUPPORTS)

Performance of 1/3-Scale Model Precast Concrete BeamColumn Connections Subjected to Cyclic Inelastic Loads. Report No. 4.

PB95.179024 00,444

Simplified Design Procedure for Hybrid Precast Concrete Connections.

00,405

Shear Design of High-Strength Concrete Beams: A Review of the State-of-the-An.

PB96-214713

01,330

BEAMSPLITTERS

Deep-UV Excimer Laser Measurements at NIST.
PB96-141031

BEANS

Thermodynamics of the Binding of Galactopyranoside Derivatives to the Basic Lectin from Winged Bean (Psophocarpus Tetrogonolobus).

PB95-162715

03,465

BEDS Measurement of Room Conditions and Response of Bed Hospital Patıent Room Fire.

PB94-213717

00,292

BENCH TESTS

Modern Test Methods for Flammability.

01,370

BEND STICK MODELS

Effects of Variable Excluded Volume on the Dimensions of Off-Lattice Polymer Chains.

PB94-212941

01,229

BENDING

Bending-Induced Phase Shifts in Dual-Mode Planar Optical Waveguide
PB95-161329

04,271

BENEFIT-COST ANALYSIS

Building Life Cycle Cost Computer Program (BLCC) Version 4.22-95 (for Microcomputers).

00,268

Building Life Cycle Cost Computer Program (BLCC) Version 4. 4-97 (for Microcomputers)

PB97-500342

00,284

BENZENE

Carbon Acidities of Aromatic Compounds. 1. Effects of InRing Aza and External Electron-Withdrawing Groups.
PB94-216595 Calculation of Enthalpy and Entropy Differences of NearCritical Binary Mixtures with the Modified Leung-Gritfiths

PB95-108635

00,885

Correlation of the Ideal Gas Properties of Five Aromatic Hydrocarbons.

01,002

Doppler-Free Spectroscopy of Large Polyatomic Molecules and van der Waals Complexes.

04,339

\section{BENZENE COMPLEXES}

Van der Waals Bond Lengths and Electronic Spectra Shifts of the Benzene-Kr and Benzene-Xe Complexes. PB95-151387

BENZILIC ACID Determination of 3 -Quinuclidiny! Benzilate (Qnb) and Its
Major Metabolites in Urine by Isotope Dilution Gas Chromatography Mass Spectrometry.

03,492

BENZOCYCLOBUTENE

Homogeneous Gas Phase Decyclization of Tetralin and Benzocyclobuten

PB95-151049

00,928

BENZOIC ACIDS

Automated, High Precision Coulometric Titrimetry. Part 2. Strong and Weak Acids and Bases.

00,576

BENZOYL PEROXIDE

Copper Ion-Mediated Modification of Bases in DNA in Vitro by Benzoyl Peroxide.

03,645
BERYLLIUN

Energy Dependences of Absorption in Beryllium Windows and Argon Gas

04,020

BERYLLIUM IONS

Experimental Results on Normal Modes in Cold, Pure Ion Plasmas.

03,956

Precise Spectroscopy for Fundamental Physics P96-112164

BESSEL FUNCTIONS

Portable Vectorized Software for Bessel Function Evaluation.

PB94-198975

01,690

BETA DOSIMETRY

Extrapolation Chamber Measurements on (90) $\mathrm{Sr}+(90) \mathrm{Y}$ Beta-Particle Ophthalmic Applicator Dose Rates. PB95-153375

03,626

\section{BETA HYDRI STAR}

Distant Future of Solar Activity: A Case Study of Beta Hydri. 3. Transition Region, Corona, and Stellar Wind PB94-185220

00,049

BETA PARTICLES

Measurement of Radial Dose Distributions Around Small Beta Particle Emitters Using High Resolution Radiochromic Foil Dosimetry.

03,518

Angular Variation of the Personal Dose Equivalent, $\mathrm{Hp}(0.07)$, for Beta Radiation and Nearly Monoenergetic Electron Beams: Preliminary Results.

03,630

System for Intercomparing Staridard Solutions of BetaSystem for Intercomparing Starid
Particle Emitting Radionuclides.
PB $96-159637$

03,707

GETZ-QUARTZ

Wear of Enamel against Glass-Ceramic, Porcelain, and Amalgam.

03,082

BIAS

Error Propagation Biases in the Calculation of Indentation Fracture Toughness for Ceramics.

PB94-172434

03,032

BIBLIOGRAPHIC DATABASES

Evolution of a United States Information System.
PB96-157896

BIBLIOGRAPHIES

Bibliography of Books and Published Reports on Gas Turbines, Jet Propulsion, and Rocket Power Plants.
AD-A278 138/3 Bibliography of Books and Published Reports on Gas Turbines, Jet Propulsion, and Rocket Power Plants, January 1950 through December 1953

AD-A278 213/4

01,446

Aerodynamic Phenomena in Stellar Atmospheres - A Bibliography.

00,046

Survey of the Literature on Heat Transfer from Solid Surfaces to Cryogenic Fluids.

AD-A286 $680 / 4$

04,193

Preliminary List of References Containing Compilations of Data on Properties of Materials.

03,243

Metrology for Electromagnetic Technology: A Bibliography of NIST Publications.

PB94-159761

02,116

Bibliography on Apparel Sizing and Related Issues.

BFRL Fire Publicatıons, 1993.

02,806

PB94-16419

o0, 192

Publications of the Manufacturing Engineering Laboratory Covering the Period January 1989-September 1992.

PB94-165966

02,750

Bibliography of the NIST Electromagnetic Fields Division Publications

PB94-165990

01,875

NIST Serial Holdings, 1994

02,745

Electronics and Electrical Engineering Laboratory Technical Publication Announcements Covering Laboratory Programs, October to December 1993 with $1994 / 1995$ EEEL. Events Calendar.

PB94-193752

02,307

Bibliography of Photon Total Cross Section (Attenuation Coefficient) Measurements $10 \mathrm{eV}$ to $13.5 \mathrm{GeV}, 1907$ 1993.

PB94-193760

03,804

Electronics and Electrical Engineering Laboratory Technical Progress Bulletin Covering Laboratory Programs, January to March 1994 with 1994/1995 EEEL Events Cal-

PB94-193810

02,308

Electronics and Electrical Engineering Laboratory Technical Progress Bulletin Covering Laboratory Programs,

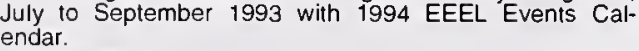

PB94-194354

02,309

Electronics and Electrical Engineering Laboratory Technical Publication Announcements Covering Laboratory

Programs, January to March 1994 with $1994 / 1995$ EEEL Events Calendar.

PB94-213774

01,883

Bibliography of Computer Security Glossaries.

01,587

Publications of the Intelligent Systems Division (Previously Robot Systems Division) Covering the Period January 1971-April 1994.

PB94-217098

02,935

Bibliography of the NIST Electromagnetic Fields Division PB95-135562

01,886

Metrology for Electromagnetic Technology: A Bibliography of NIST Publications.

PB95-135588

02,143

Electronics and Electrical Engineering Laboratory Technical Progress Bulletin Covering Laboratory Programs. April to June 1994 with 1994/1995 EEEL Events CalPBo5-14

02,329

Building and Fire Research Laboratory Publications,

PB95-143202

00,369

Evaluating Investments in Law Enforcement Equipment: An Annotated Bibliography.

04,867

95.151379

Electronics and Electrical Engineering Laboratory Tech-
nical Progress Bulletin Covering Laboratory Programs, nical Progress Bulletin Covering Laboratory Programs, July to Sept

PB95-170395

02,360

NIST Serial Holdings, 1995

02,746

Electronics and Electrical Engineering Laboratory Techical Publication Announcements Covering Laborator Programs, July to September 1994 with 1995 EEEL Events Calendar

Publications of the National Institute of Standards and Technology 1992 Catalog.

PB95-200747

00,014

Building and Fire Research Laboratory Publications,

PB95-226684

00,398

Electronics and Electrical Engineering Laboratory Technical Publication Announcements Covering Laboratory Programs, January to March 1995 with 1995 EEEl Events

PB95-242277

02,373

Bibliography of the NIST Optoelectronics Division.

02,193

Metrology for Electromagnetic Technology: A Bibliography of NIST Publications, September 1995.

01,938

Classified Bibliography: Insulation Condition Monitoring Methods, 1989-1995. 02,232

Catalogue of Electromagnetic Environment Measurements, $30-300 \mathrm{~Hz}$

PB96-155452

01,943

Publications 1995: NIST Building and Fire Research Lab-

oratory. 
BIMOLECULAR REACTION

Bimolecular Interactions in (Et)3SiOH:Base:CCl4 Hydrogen-Bonded Solutions Studied by Deactivation of the Free OH-Stretch Vibration.

PB97.118657

04,166

BINARY ALLOY SYSTEMS

Thermodynamic Assessment and Calculation of the Ti-Al System.
PB94-212644

03,337

BINARY ALLOYS

Convection and Morphological Stability During Directional Solidification.

03,310

Effects of Crystalline Anisotropy and Buoyancy-Driven Convection on Morphological Stability.

03,328

BINARY MIXTURES

Calculation of Enthalpy and Entropy Differences of NearCritical Binary Mixtures with the Modified Leung-Griffiths Model.

PB95-108635

00,885

Thermodynamic Properties of the Methane-Ethane Sys-

PB95-125779

00,891

Experimental Method for Obtaining Critical Densities of Binary Mixtures: Application to Ethane + n-Butane.

PB95-151148

00,930

Measurements of the Virial Coefficients and Equation of

State of the Carbon Dioxide + Ethane System in the

Supercritical Region.

PB95-151353 03,906

Stability/Instability of Gas Mixtures Containing 1,3-Buta-

diene in Treated Aluminum Gas Cylinders.

02,546

Viscosity of the Saturated Liquid Phase of Six Halo-

genated Compounds and Three Mixtures.

00,960

Critical Properties and Vapor-Liquid Equilibria of the Bi-

nary System Propane + Neopentane.
PB95-175683 00,999

Critical Lines for Type-III Aqueous Mixtures by Generalized Corresponding-States Models.

BINARY STARS

01,063

ROSAT All-Sky Survey of Active Binary Coronae. 2. Coronal Temperatures of the RS Canum Venaticorum SysPB94-199601

00,055

Dynamic Phenomena on the RS Canum Venaticorum $\mathrm{Bi}$ nary II Pegasi in August 1989. 1. Observational Data.
PB94-211067

Radio Continuum and $X$-Ray Properties of the Coronae of

RS Canum Venaticorum and Related Active Binary Systems.

00,057

ROSAT All-Sky Survey of Active Binary Coronae. 1. Oui-

escent Fluxes tor the RS Canum Venaticorum Systems.

PB95-202479 00,077
0

Rotational Modulation and Flares on RS Canum Venaticorum and BY Draconis Stars. XVIII. Coordinated
VLA, ROSAT, and IUE Observations of RS CVn Binaries. PB96-102322 00,089
0

Discussion: Statistical Signal Processing of Ouasiperiodicities.

PB96-119532

00,096

Transition Regior

00,105

Transition Regions of Capella (1995)

00,108

BINARY SYSTEM (MATERIALS)

Statistical Thermodynamics of Phase Separation and Ion

Partitioning in Aqueous Two-Phase Systems.
PB94-199387

01,212

BINARY SYSTEMS (MATERIALS)

Shear Dependence of Critical Fluctuations in Binary Polymer Mixtures by Small Angle Neutron Scattering. 01,220

Small-Angle X-Ray and Neutron Scattering Study of Block Copolymer/Homopolymer Mixtures.

01,221

Generalized Plane Strain Analysis of a Bimaterial Composite Containing a Free Surface Normal to the Interface.
PB95.163341

Partial Scattered Intensities from a Binary Suspension of Polystyrene and Silica.
PB95-175618

BINDERS

00,996

Binder Characterization and Evaluation by Nuclear Magnetic Resonance Spectroscopy.

Computer Simulations of Binder Removal from 2-D and 3-D Model Particulate Bodies.

PB97-121339

00,418

BINDING ENERGY

Thermal Decomposition of Hydroxy- and Methoxy-Substituted Anisoles.

PB94-173036

00,767

Energy Calibration of X-ray Photoelectron Spectrometers: Results of an Interlaboratory Comparison to Evaluate a Proposed Calibration Procedure.

PB96-102918

04,027
Theory for Ouantum-Dot Quantum Wells: Pair Correlation
and Internal Ouantum Confinement in Nanoheterostructures.

PB96-179445

02,437

BINDING SITES

Characterization of the Binding of Gallium. Platinum, and Uranium to Pseudomonas Fluorescens by Small-Angle $X$. ray Scattering and Transmission Electron Microscopy.

03,453

Fokker-Planck Description of Multivalent Interactions. PB95-108478

00,879

BIOACCUMULATION

Current Activities Within the National Biomonitoring Specimen Bank.

02,516

Determination of Inorganic Constituents in Marine Mammal Tissues.

00,589

Development of the National Marine Mammal Tissue Bank.

PB95-161402

02,586

Quality Assurance of Contaminant Measurements in Ma. rine Mammal Tissues.

PB95-164034

02,588

Certification of Polychlorinated Biphenyl Congeners and Chlorinated Pesticides in a Whale Blubber Standard Reference Materia

PB96-103023 03,745

Trace Element Concentrations in Cetacean Liver Tissues Archived in the National Marine Mammal Tissue Bank.

PB96-167127

02,595

Relationship of Silver with Selenium and Mercury in the Liver of Two Species of Toothed Whales (Odontocetes).
02,596

\section{BIOACTIVE MATERIALS}

Binactive Polymeric Dental Materials Based on Amorphous Calcium Phosphate.

PB96-147012

03,572

BIOASSAY

Alaska Marine Mammal Tissue Archival Project: Specimen Inventory

02,589

Concentrations of Chlorinated Hydrocarbons, Heavy Metals and Other Elements in Tissues Banked by the Alaska Marine Mammal Tissue Archival Project.

PB95-209870 02,590

Intercomparison Study of (237)Np Determination in Artificial Urine Samples.

PB96-102645

03,633

Considerations in the Design of an Environmental Specimen Bank: Experiences of the National Biomonitoring Specimen Bank Program.

PB96-112370

02,527

OCHEMISTRY

Aftinity Chromatography on Inorganic Support Materials. PB95-163820

03,467

BIOCOMPATIBILITY

Behavior of a Calcium Phosphate Cement in Simulated PB95-168712

00,165

BIOCOMPATIBLE MATERIALS

Establishing Ouality Measurements for Inorganic Analysis of Biomaterials
PB94-199726

00,548

Periapical Tissue Reactions to a Calcium Phosphate $\mathrm{Ce}$ ment in the Teeth of Monkeys.

4-212008

00,149

Dental Applications of Ceramics.

00,177

Posterior Restorative Materials Research.

03,582 PB97-118624

BIODETERIORATION

Microbial Degradation of Polysulfides and Insights into Their Possible Occurrence in Coal.

PB95-163374

02,488

BIOELECTRICAL IMPEDANCE ANALYZERS

Overview of Bioelectrical Impedance Analyzers.

B96-122635

Overview of Bioelectrical Impedance Analyzers.

PB97-118780

00,181

BIOFOULING

Determination of Total Protein Adsorbed on Solid (Membrane) Surface by a Hydrolysis Technique: Single Protein Adsorption.

BIOINSTRUMENTATION

Overview of Bioelectrical Impedance Analyzers. PB96-122635

03,552

00,176 PB97.118780

00,181

BIOLOGICAL ACCUMULATION

Development of Frozen Whale Blubber and Liver Reference Materials for the Measurement of Organic and Inorganic Contaminants.

PB95-151676

00,587

Concentrations of Chlorinated Hydrocarbons, Heavy Metals and Other Elements in Tissues Banked by the Alaska Marine Mammal Tissue Archiva! Project.

02,590

BIOLOGICAL EFFECTS

Periapical Tissue Reactions to a Calcium Phosphate Cement in the Teeth of Monkeys.

00,149

BIOLOGICAL MACROMOLECULES

Biological Macromolecular Crystallization Database: A Tool for Developing Crystallization Strategies.

00,897

Biological Macromolecule Crystallization Database and NASA Protein Crystal Growth Archive.

01,171

OLOGICAL MATERIALS

Certifying the Chemical Composition of a Biological Material: A Case Study

00,636

BIOLOGICAL RADIATION EFFECTS

Systematics of Alpha-Particle Energy Spectra and Lineal Energy (Y) Spectra for Radon Daughters.

03,615

Microdosimetry and Cellular Radiation Effects of Radon Progency in Human Bronchial Airways.

PB95-152344

03,625

BIOLOGICAL SYSTEMS

Isotopic and Nuclear Analytical Techniques in Biological Systems: A Critical Study. 10. Elemental Isotopic Ditution Analysis with Radioactive and Stable Isotopes (Technical PB96-1641

\section{BIOLOGICAL TREATMEN}

00,696

Chemical and Microbiological Problems Associated with Research on the Biodesulfurization of Coal.

PB95-140950

Bioleaching of Cobalt from Smelter Wastes by Thiobacillus ferro

02,582

BIOMARKERS

Hair Analysis for Drugs of Abuse: Evaluation of Analytical Methods, Environmental Issues, and Development of Reference Materials.

03,501

BIOMASS

Calculation of Higher Heating Values of Biomass Materials and Waste Components from Elemental Analyses. PB94-199254

Radiocarbon Measurements of Atmospheric Volatile Or ganic Compounds: Ouantifying the Biogenic Contribution. PB97-122352

BIOMATERIALS

Reinforcement of Cancellous Bone Screws with Calcium Phosphate Cement.

PB96-158001

BIOMEDICAL MEASUREMENT

Prototyping a Graphical User Interface for DHCP.

02,599

BIOMEDICAL MEASUREMENTS

Importance of Chemometrics in Biomedical Measure

\section{IOMEDICAL RADIOGRAPHY}

00,550

Image Information Transter Properties of X-Ray Intensifying Screens in the Energy Range from 17 to $320 \mathrm{keV}$. PB95-126173 
Opportunities for Innovation: Advanced Surface Engineering. 02,697 Tert-Butyl Hydroperoxide-Mediated DNA Base Damage in Cultured Mammalian Cells.

03,644

Application of Photochemical Reaction in Electrochemical Detection of DNA Intercalation.
PB94-185733

00,686

Copper Ion-Mediated Modification of Bases in DNA in Vitro by Benzoyl Peroxide.

03,645

Calorimetric Determination of the Standard Transformed Enthalpy of a Biochemical Reaction at Specified PH and PBg. 198249

03,454

Regulation of Lithium and Boron Levels in Normal Human Blood: Environmental and Genetic Considerations. 03,491 Standard Reference Materials (SRM's) for Measuring Genetic Damage.
PB94-198827

03,516

Oxidative Damage to DNA in Mammalian Chromatin.

PB94-199825 03,525

Modification of DNA Bases in Chromatin of Intact Target Human Cells by Activated Human Polymorphonuclear Leukocytes.

Carotenoid Reversed-Phase High-Performance Liguid Chromatography Methods: Reference Compendium. Individual Carotenoid Content of SRM 1548 Total Diet and Influence of Storage Temperature, Lyophilization, and Irradiation on Dietary Carotenoids.

PB94-200524

00,033

Mixed Phospholipid Liposome Calcification.

Proteoglycan Inhibition of Calcium Phosphate Precipitation in Liposomal Suspensions.

PB94-211208

00,658

Total Surface Areas of Group IVA Organometallic Compounds: Predictors of Toxicity 10 Algae and Bacteria. L-threo-beta-Hydroxyhistidine, an Unprecedented Iron(III) Ion-Binding Amino Acid in a Pyoverdine-type Siderophore from Pseudomonas fluorescens 244.

PB94-211620

00,553

In situ On-Line Optical Fiber Sensor for Fluorescence Monitoring in Bioreactors.

03,587

Modification of Deoxyribose-Phosphate Residues by Extracts of Ataxia Telangiectasia Cells.

PB94-212602

03,458

Nickel(11)-Mediated Oxidative DNA Base Damage in Renal and Hepatic Chromatin of Pregnant Rats and Their Feluses. Possible Relevance to Carcinogenesis.

Analysis of Protein Metal Binding Selectivity in a Cluster Model.

PB94-212990

00,845

Liposome-Based Flow-Injection Immunoassay for Determining Theophylline in Serum.

03,494

Hydrolysis of Proteins by Microwave Energy
PB94-216322

03,528

Ascorbic and Dehydroascorbic Acids Measured in Plasma Preserved with Dithiothreitol or Metaphorphoric Acid.
PB94-216330

Energy Transduction between a Concentration Gradient and an Alternating Electric Field.

PB94-216363

03,461

Self-Assembled Phospholipid/Alkanethiol Biomimetic Bilayers on Gold

Fokker-Planck Description of Multivalent Interactions.

PB95-108478

00,878

Aggregation Kinetics of Colloidal Particles Destabilized by PBg5-125878

00,894

Biological Macromolecular Crystallization Database: A Tool for Developing Crystallization Strategies.

00,897

Michaelis-Menten Equation for an Enzyme in an Oscillating Electric Field.

PB95-140489 00,906

Chemical and Microbiological Problems Associated with Research on the Biodesulfurization of Coal.

Bioleaching of Cobalt from Smelter Wastes by 'Thiobacillus ferrooxidans'. $\quad 02,582$
PB95-140968 Opportunities for Innovation: Pollution Prevention PB95-147146

Oxidative DNA Base Damage in Renal, Hepatic, and Pulmonary Chromatin of Rats After Intraperitoneal Injection of Cobalt (II) Acetate.

03,647
DNA Base Modifications in Renal Chromatin of Wistar Rats Treated with a Renal Carcinogen, Ferric Nitrilotriacetate

PB95-150363 03,648

Retinal-Protein Complexes as Optoelectronic CompoPB95-150397

02,146

Application of Thermodynamics to Biotechnology. 03,529

Critical Evaluation of the Purification of Biominerals by Hypochlorite Treatment.

PB95-150959

00,186

Influence of Natural and Synthetic Inhibitors on the Crys-

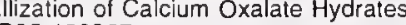

03,560

High-Performance Liquid Chromatography of High-Pertormance Liquid Chromatography of
Phytoplankton Pigments Using a Polymeric ReversedPhase C18 Column.

$51130 \quad 00,583$

Preparation of Immobilized Proteins Covalently Coupled Through Silane Coupling Agents to Inorganic Supports.
PB,530

Method for the Assay of Hydrolytic Enzymes Using Dynamic Light Scattering.

PB95.151411

03,531

Bacteriorhodopsin Immobilized in Sol-Gel Glass.
PB95-151429 03,532
PB $95-151429$
Function of DnaJ and DnaK as Chaperones in OriginSpecific DNA Binding by RepA.

PB95-151544

03,533

Development of Frozen Whale Blubber and Liver Reterence Materials for the Measurement of Organic and Inorganic Contaminants.

PB95-151676

00,587

Determination of Inorganic Constituents in Marine Mammal Tissues.
PB95-152047

00,589

Resolution of DNA in the Presence of Mobility Modifying Polar and Nonpolar Compounds by Discontinuous Electrophoresis on Rehydratable Polyacrylamide Gels.

PB95-152799

00,590

Substrate Specificity of the Escherichia coli Endonuclease III: Exicision of Thymine- and Cylosine-Derived Lesions in DNA Produced by Radiation-Generated Free Radicals.
PB95-153425

Liquid Chromatographic Method for the Determination of Carotenoids, Retinoids, and Tocopherols in Human PB95. 153599 Food.

Development of the National Marine Mammal Tissue

Bank.

Tissue

02,586

DNA Base Damage Generated In vivo in Hepatic Chromatin of Mice upon Whole Body y-Irradiation.

03,627

Amperometric Flow-Injection Analysis Biosensor for Glucose Based on Graphite Paste Modified with Tetracyanoquinodimethane.

PB95-161980

03,498

Determination of PCBs and Chlorinated Hydrocarbons in Marine Mammal Tissues.

03,744

Biological Thermodynamic Data for the Calibration of Differential Scanning Calorimeters: Dynamic Temperature Data on the Gel to Liquid Crystal Phase Transition of

Dialkylphosphatidylcholine in Water Suspensions.
PB95-162707

03,464

Thermodynamics of the Binding of Galactopyranoside Derivatives to the Basic Lectin from Winged Bean (Psophocarpus Tetrogonolobus).

03,465

Electrophe Chain ReacElectrophoretic Separations of Polymerase Chain Reaction: Amplified DNA Fragments in DNA Typing Using a

PBstem.

03,536

Glucose Permease of Bacillus Subtilis Is a Single Polypeptide Chain That Functions to Energize the Sucrose Permease
PB95-163192

03,466

Electropermeabilization of Cell Membranes: Effect of the Resting Membrane Potential.

Resting Memb
PB95.163291

03,537

Microbial Degradation of Polysulfides and Insights into Their Possible Occurrence in Coal.

PB95-163374

02,488

Affinity Chromatography on Inorganic Support Materials.

Deletion Analysis of the Mini-P1 Plasmid Origin of Replication and the Role of E.coli DnaA Protein. lication and the
PB95-163911

03,539

Membrane-Mediated Precipitation of Calcium Phosphate in Model Liposomes with Matrix Vesicle-Like Lipid Composition.

PB 95-164547

03,468

Characterization of Cytochrome c/Alkanethiolate Structures Prepared by Self-Assembly on Gold.

PB95-164638

00,987

Enhanced Detection of PCR Products Through Use of TOTO and YOYO Intercalating Dyes with Laser Induced Fluorescence - Capillary Electrophoresis.

00,599

Alaska Marine Mammal Tissue Archival Project: Specimen Inventory.

Supercritical Fluid Extraction of Biological Products

PB95-175204 00,040

Chemical Determination of Oxidative DNA Damage by Gas Chromatography-Mass Spectrometry.

Determination of Amphetamine and Methamphetamine in a Lyophilized Human Urine Reference Material.

PB95-175444

03,597

Fluorescence Measurements of Tetracycline in High Cell Mass for Fermentation Monitoring

PB95-175709

00,601

Optical Biosensor Using a Fluorescent, Swelling Sensing Element.

B95-175899

03,541

onizing Radiation Causes Greater DNA Base Damage in Radiation-Sensitive Mutant M10 Cells That in Parent Mouse Lymphoma L5178Y Cells.

03,632

Interlaboratory Comparison of Autoradiographic DNA Profiling Measurements. 1. Data and Summary Statistics.
PB95-175923

Hair Analysis for Drugs of Abuse: Evaluation of Analytical Methods, Environmental Issues, and Development of Reference Materials.

PB95-176269

03,501

Supported Phospholipid/Alkanthiol Biomimetic Membranes: Insulating Properties.

03,470

Non-Perturbative Relation between the Mutual Diffusion Coefficient, Suspension Viscosity, and Osmotic Com pressibility: Application to Concentrated Protein Solutions.
01,062

Bacteriorhodopsin Retains Its Light-Induced ProtonPumping Function After Being Heated to $140 \mathrm{C}$

PB96-102728

Phospholipid/Alkanethiol Bilayers for Cell-Surface Receptor Studies by Surface Plasmon Resonance.

PB96-102900

03,472

Novel Activity of E. coli uracil DNA N-glycosylase Excision of Isodialuric Acid (5.6-dihydroxyuracil), a Major Product of Oxidative DNA Damage, from DNA. PB96-110747

Overview of Reference Materials Prepared for Standardization of DNA Typing Procedures.

B96-111653

00,611

Detection of Aromatic Compounds Based on DNA Intercalation Usir

03,473

Physicochemical Characterization of Low Molecular Weight Heparin

Rapid pH Change Due to Bacteriorhodopsin Measured with a Tin-Oxide Electrode.

PB96-112081

03,544

Treatment of Wistar Rats with a Renal Carcinogen, Ferric Nitrilotriacetate, Causes DNA-Protein Cross-Linking between Thymine and Tyrosine in Their Renal Chromatin.

Interlaboratory Comparison of Autoradiographic DNA Profiling Measurements. 2. Measurement Uncertainty and Its Propagation.

PB96-112123

03,545

Considerations in the Design of an Environmental Specimen Bank: Experiences of the National Biomonitoring Specimen Bank Program.

02,527

Holographic Properties of Triton X-100-Treated Bacteriorhodopsin Embedded in Gelatin Films.
PB96-119284 Physical Characterization of Herparin by Light Scattering.
PB96-119383

Optical Control of Enzymatic Conversion of Sucrose to Glucose by Bacteriorhodopsin Incorporated into Self-Assembled Phosphatidylcholine Vesicles.

03,477

Optical Properties of Triton X-100-Treated Purple Mem- 


\section{KEYWORD INDEX}

Evaluation of a Tapered Roller Bearing Spindle for HighPrecision Hard Turning Applications.

Genetically Engineered Pores for New Materials.

PB96-161641

03,550

Genetically Engineered Pore as a Metal Ion Biosensor.

PB96-161658 03551

Current Noise Reveals Protonation Kinetics and Number

of Ionizable Sites in an Open Protein lon Channel.
PB96-161674

Noise Analysis of Ionization Kinetics in a Protein Ion Channel.

04,093

Current Fluctuations Reveal Protonation Dynamics and Number of lonizable Residues in the alpha-Toxin Chan-

03,588

PB96-161732

Protonation Dynamics of the alpha-Toxin Ion Channel from Spectral Analysis of $\mathrm{pH}$-Dependent Current Fluctua-

PB96-161740

03,652

Protonation Dynamics in an Ion Channel Pore.

PB96-161757

03,589

Conformational Alterations of Bovine Insulin Adsorbed on a Silver Electrode.

00,510

Autofluorescence Detection of 'Escherichia coli' on Silver Membrane Filters.

PB96-163639

03,590

Bacterial Enumeration in Storage Water.

03,591

Feasibility of Fluorescence Detection of Tetracycline in Media Mixtures Employing a Fiber Optic Probe.

PB96-163654

00,511

Novel Amperometric Immunosensor for Procainamide Employing Light Activated Labels.

00,512

Novel Activities of Human Uracil DNA N-glycosylase for Cytosine-Derived Products of Oxidative DNA Damage.

Structural Analysis of Heparin by Raman Spectroscopy.

PB96-167226 03,480

Relationship of Silver with Selenium and Mercury in the Liver of Two Species of Toothed Whales (Odontocetes).
PB96-167275

New Method for the Detection and Measurement of Polyaromatic Carcinogens and Related Compounds by

DNA Intercalation

03,481

Genetically Engineered Pores as Metal Ion Biosensors.

PB96-167408 03,553

Pore-Forming Protein with a Metal-Actuated Switch.

Investigations of Sulfur Interferences in the Extraction of Methylmercury from Marine Tissues.

PB96-190020

03,482

Repair of Products of Oxidative DNA Base Damage in Human Cells.

03,555

Quantitative Determination of Oxidative Base Damage in DNA by Stable Isotope-Dilution Mass Spectrometry.

PB96-200886

03,483

Commentary: The Measurement of Oxidative Damage to DNA by HPLC and GC/MS Techniques.

03,484

Intercomparison of DNA Sizing Ladders in Electrophoretic Separation Matrices and Their Potential for Accurate Typing of the D1580 Locus.

PB96-200928

03,485

Flow Immunoassay Using Solid-Phase Entrapment.

PB96-200951 00,642

Escherichis coli Cyclic AMP Receptor Protein Mutants Provide Evidence for Ligand Contacts Important in Activa. tion.

PB96-201017

03,592

Interlaboratory Studies on the Analysis of Hair for Drugs of Abuse: Results from the Fifth Exercise.

PB97-110449

03,509

DNA Damage and DNA Sequence Retrieval from Ancient Tissues

PB97-111983

03,556

Use of a Naphthylethylcarbamoylated- beta-Cyclodextrin Chiral Stationary Phase for the Separation of Drug Enantiomers and Related Compounds by Sub- and

Supercritical Fluid Chromatography.

00,653

National Status and Trends Program Specimen Bank: Sampling Protocols, Analytical Methods, Results, and Archive Samples.

PB97-119226

02,598

Positive and Negative Cooperativities at Subsequent Steps of Oxygenation Regulate the Allosteric Behavior of Multistate Sebacylhemoglobin

03,486

DnaJ, DnaK, and GrpE Heat Shock Proteins are Required in 'ori'P1 DNA Replication Solely at the RepA Monomerization Step.

03,557
DNA Base Damage in Lymphocytes of Cancer Patients Undergoing Radiation Therapy

03,643

\section{BIPOLAR TRANSISTORS}

Investigation of the Drive Circuit Requirements for the Power Insulated Gate Bipolar Transistor (IGBT).

02,316

Effects of Heavy Doping on Numerical Simulations of Gallium Arsenide Bipolar Transistors.

Gallium Arsenide

02,334

Experimental Study of Reverse-Bias Failure Mechanisms in Bipolar Mode JFET (BMFET).

PB95-152997

Modeling Buffer Layer IGBTs for Circuit Simulation.

PB95-153805 02,344

Modeling Buffer Layer IGBT's for Circuit Simulation.

PB96-164173

02,429

Design Criteria for BJT Amplifiers with Low $1 / 4$ AM and PM Noise.

BIREFRINGENCE

02,442

Polarization Insensitive $3 \times 3$ Sagnac Current Sensor Using Polarizing Spun High-Birefringence Fiber.

02,187

Standard Polarization Components: Progress Toward an Optical Retardance Standard.

B 96-119672

04,342

mproved Annealing Technique for Optical Fiber.

04,343

BIREFRINGENT MEDIA

Reirraction of Light by Graded Birefringent Media. PB96-123716

02,192

IS (DIFLUOROMETHYL)ETHER

Thermodynamic Properties of CHF2-O

CHF2,Bis(difluoromethyl) Ether.

PB94-199569

Viscosity of the Saturated Liquid Phase of Six Halo. genated Compounds and Three Mixtures.

00,960

BISMUTH

Enthalpy Increment Measurements from 4.5 to $318 \mathrm{~K}$ for Bismuth (cr). Thermodynamic Properties from $0 \mathrm{~K}$ to the Melting Point.

PB96-204011

01,166

BISMUTH CLUSTERS

Photoelectron Spectroscopy of Negatively Charged Bis-

muth Clusters: $\mathrm{Bi}(-) 2, \mathrm{Bi}(-) 3$, and $\mathrm{Bi}(-) 4$ PB95-108494

00,880

\section{BISMUTH LEAD STRONTIUM CALCIUM CUPRATES}

$X$-Ray Characterization of the Crystallization Process of High-Tc Superconducting Oxides in the $\mathrm{Sr}-\mathrm{Bi}-\mathrm{Pb}-\mathrm{Ca}-\mathrm{Cu}$ PB95-151700

BISMUTH OXIDES

Phase Equilibria in the Systems $\mathrm{CaO}-\mathrm{CuO}$ and $\mathrm{CaO}$ -

PB95-140570

03,048

BISMUTH STRONTIUM CALCIUM CUPRATES

Microstrains and Domain Sizes in Bi-Cu-O

Superconductors: An X-Ray Diffraction Peak-Broadening Study.

04,461

Effect of Axial Strain on the Critical Current of $\mathrm{Ag}$ Sheathed Bi-Based Superconductors in Magnetic Fields Up to $25 \mathrm{~T}$.

04,493

Flux Expulsion at Intermediate Fields in Type-II Superconductors

04,502

Improved Uniaxial Strain Tolerance of the Critical Current Measured in Ag-Sheathed Bi2Sr2Ca1Cu2O $8+x$ Superconductors

PB95-153565

04,594

Grain Alignment and Transport Properties Bi2SraCaCu2O8 Grown by Laser Heated Float Zone Method.

PB95-161451

04,602

Effects of Anneal Time and Cooling Rate on the Forma tion and Texture of Bi2Sr2CaCu2OB Films.

PB95-161600

04,603

Tunneling Measurement of the Zero-Bias Conductance Peak and the Bi-Sr-Ca-Cu-O Thin-Film Energy Gap. PB95-163739

04,628

Increased Pinning Energies and Critical Current Densities in Heavy-Ion-Irradiated Bi2Sr2CaCu2O8 Single Crystals. PB95-175352

04,654

BISPHENOL-A

Effect of Curing History on Ultimate Glass Transition Temperature and Network Structure of Crosslinking Poly.

PB94-200052

01,214

BISTATIC SCATTERING

Bistatic Scattering of Absorbing Materials from 30 to 1000 PB95-150934

01,891

BITHIOPHENES

Location of a (1)A(sub g) State in Bithiophene.

PB95-202313

BITUMINOUS CONCRETES

Binder Characterization and Evaluation by Nuclear MagPB94-193471

01,334

Development of a Method for Measuring Water-Stripping Resistance of Asphalt/Siliceous Aggregate Mixtures.
PB96-197249

BIVARIATE DISTRIBUTIONS

Population Distributions and Intralaboratory Reproducibility for Fat-Soluble Vitamin-Related Compounds in Human

00,624

BLACK BODY RADIATION

Activities of NIST (National Inst. Of Standards and Tech-

N94-23605/6

04,222

BLACK DETECTORS

Measurement of the $(10) B(n$, alpha1gamma) $(7) L i$ Cross

Section in the 0.3 to $4 \mathrm{MeV}$ Neutron Energy Interval.

PB96-161799

04,098

BLACKBODY RADIATION

Cryogenic Blackbody Calibrations at the National Institute
of Standards and Technology Low Background Infrared Calibration Facility.

PB94-169802

02,117

Precision High Temperature Blackbodies.

03,885

Third Generation Water Bath Based Blackbody Source. PB96-122148

04,046

BLCC COMPUTER PROGRAM

Life-Cycle Costing Workshop for Energy Conservation in Buildings: Student Manual.

BLENDS

00,257

Glass Temperature of Polymer Blends: Comparison of Both the Free Volume and the Entropy Predictions with Data.

BLIND RECONSTRUCTION

01,236

Dcanned Probe Microscope Tip Characterization Without Calibrated Tip Characterizers.

PB96-190368

02,759

BLISTERING

Non-Osmotic, Defect-Controlled Cathodic Disbondment of a Coating from a Steel Substrate.

03,120

Gas Absorption during Ion-Irradiation of a Polymer Tar-

PBet. 161864

04,099

BLOCK COPOLYMERS

Shear-Excited Morphological States in a Triblock CopolyPB94-172392

01,196

Time Dependent Small Angle Neutron Scattering Behavior in Triblock Copolymers Under Steady Shear.

PB94-172632

01,198

Temperature Dependence of the Morphology of Thin Diblock Copolymer Films as Revealed by Neutron Reflec- 


\section{BLOOD CHEMICAL ANALYSIS}

Histopathology, Blood Chemistry, and Physiological Status of Normal and Moribund Striped Bass ('Morone saxatilis') Invoived in Summer Mortality ('D SBacramento-S

00,034

Regulation of Lithium and Boron Levels in Normal Human Blood: Environmental and Genetic Considerations

PB94-198579

03,491

Determination of Oltipraz in Serum by High-Performanace Liquid Chromatography with Optical Absorbance and Mass Spectrometric Detection.

PB94-200201

03,493

Liposome-Based Flow-Injection Immunoassay for Determining Theophylline in Serum.

03,494

Ascorbic and Dehydroascorbic Acids Measured in Plasma Preserved with Dithiothreitol or Metaphorphoric Acid. PB94-216330

03,495 Measurement of Ascorbic Acid in Human Plasma and Serum: Stability, Intralaboratory Repeatability, and Interlaboratory Reproducibility.

03,511

BLOOD PRESERVATION

Ascorbic and Dehydroascorbic Acids Measured in Plasma Preserved with Dithiothreitol or Metaphorphoric Acid.

PB94-216330

03,495

BLOWOUTS

Development of Hazard Assessment and Suppression Technology for Oil and Gas Well Blowout and Diverter Fires.

01,408

BLUBBER

Certification of Polychlorinated Biphenyl Congeners and Chlorinated Pesticides in a Whale Blubber Standard RefPB96-103023

03,745

\section{BLUE GREEN LASERS}

Rare-Earth-Doped Waveguide Devices: The Potential for Compact Blue-Green Lasers.

PB95-140836

04,257

BODY ARMOR

Study to Determine the Most Important Parameters for Evaluating the Resistance of Soft Body Armor to Penetration by Edged Weapons.

PB94-158573

03,757

BODY COMPOSITION

Overview of Bioelectrical Impedance Analyzers

00,176

Overview of Bioelectrical Impedance Analyzers.

PB97-118780

00,181

(BIOLOGY)

Body Dimensions for Apparel.

PB94-187739

02,813

BOILING POINTS

Ebulliometers for Measuring the Thermodynamic Prop-

erties of Fluids and Fluid Mixtures
DE94017817

04,195

BOLOMETER MOUNTS

Coaxial Reference Standard for Microwave Power.
PB94-193786 01,880

BOLOMETERS

Thermal and Nonequilibrium Responses of

PBperconductors for Radiation Detectors. 02,156

Terahertz Detectors Based on Superconducting Kinetic

Inductance.
PB95-168647 02,160

Optical Performance of Photoinductive Mixers at Terahertz Frequencies

02,161

PB95-168662

$\begin{array}{ll} & 02,163 \\ \text { PB95-168985 } & \end{array}$

High-Tc Superconducting Antenna-Coupled

PB95-169124 on silicon.

02,166

Antenna-Coupled High-Tc Air-Bridge Microbolometer on

Silicon.

04,315

Niobium Microbolometers for Far-Infrared Detection.

PB96-111729 02,184

Absolute Response Calibration of a Transfer Standard Cryogenic Bolometer

PB96-147103

04,358

Novel Hot-Electr
PB96-201025

01,977

BOLTZMANN EQUATION

Quantum Collisional Transfer Contributions to the Density Dependence of Gaseous Viscosity.

PB96-161914

01,142

BOMB CALORIMETER

Precise Measurement of Heat of Combustion with a Bomb Calorimeter.

03,770

BOND-LENGTH MISMATCH

Elastic Properties of Central-Force Networks with BondLength Mismatch

04,623
BONDED PHASE

Influence of Stationary Phase Chemistry on Shape Recognition in Liquid Chromatography

00,621

BONDING

Bonding Wires to Ouantized Hall Resistors.

PB96-102637

01,921

BONE DISORDERS

In vitro Inhibition of Membrane-Mediated Calcification by Novel Phosphonates

ONE TISSUES

Experimental assessment of absorbed dose to mineralized bone tissue from internal emitters: An electron paramagnetic resonance study.

BONES

EPR Bone Dosimetry: A New Approach to Spectral Deconvolution $\mathrm{Pr}$

03,616

Experimental Validation of Radiopharmaceutical Absorbed Dose to Mineralized Bone Tissue.

PB94-199668

03,617

Estimation of the Absorbed Dose in Radiation-Processed Food. 1. Test of the EPR Response Function by a Linear Regression Analysis.

00,039

Secondary Target X-Ray Excitation for In vivo Measurement of Lead in Bone.

PB95-108767

03,496

Exposure-to-Absorbed-Dose Conversion for Human Adult Cortical Bone

03,640

BORON

Regulation of Lithium and Boron Levels in Normal Human Blood: Environmental and Genetic Considerations.

PB94-198579

03,491

Measurement of Boron at Silicon Wafer Surfaces by Neuron Depth Profiling.

PB94-211059

04,487

Determination of Boron and Lithium in Diverse Bıological Matrices Using Neutron Activation - Mass Spectrometry NA-MS)

PB94-212289 00,554

Analysis of Boron in CVD Diamond Surfaces Using Neutron Depth Profiling.

04,511

Optical and Modeling Studies of Sodium/Halide Reactions Optical and Modeling Studies of Sodium/Halide Reactions for the Formation of Titanium and Boron Nanoparticles.
PB97-113054 00,682

Preparation and Characterization of (6) LiF and (10)B Reference Deposits for the Measurement of the Neutron Life-

time.

03,874

BORON-LIKE IONS

Investigation of LS Coupling in Boronlike lons. PB94-185295

03,797

BORON NITRIDES

Effect of Stoichiometry on the Phases Present in Boron Nitride Thin Films

Chip Morphology. Tool Wear and Cutting Mechanics in Finish Hard Turning.

PB97-112247

BORON TARGETS

Mass Assay and Uniformity Test of Boron Targets by PB97-119085

04,175

BOSE CONDENSATION

Stable, Tightly Confining Magnetic Trap for Evaporative Cooling of Neutral Atoms.

PB96-200720

04,126

BOSE-EINSTEIN CONDENSATION

Properties of a Bose-Einstein Condensate in an Anisotropic Harmonic

04,133

BOTANICAL REFERENCE MATERIAL

Determination of 21 Elements by INAA for Certification of SRM 1570a, Spinach.

PB96-167242

00,698

BOTANICAL REFERENCE MATERIALS

Recent Developments in NIST Botanical SRMs. $\quad 03,489$
PB96-167267

BOTANY

Dissolution Problems with Botanical Reference Materials. PB95-126280 03,487 Results of the ASTM Nuclear Methods Intercomparison on NIST Apple and Peach Leaves Standard Reference Materials.

PB97-119036

03,490

BOTTOM SEDIMENTS

Certification of Standard Reference Material (SRM)

1941a, Organics in Marine Sediment.

02,593

BOUNDARY

Using Collocation in Three Dimensions and Solving a Model Semiconductor Problem.

PB96-159249

01,952

BOUNDARY CONDITIONS

ransport by Gravity Currents in Buildıng Fires

01,441

BOUNDARY ELEMENT METHOD

Application of Boundary Element Methods to a Transien Axis-Symmetric Heat Conduction Problem.

PB94-212693

01,375

OUNDARY INTEGRAL METHOD

Boundary Integral Method for the Simulation of Two-Dimensional Particle Coarsening

03,41

BOUNDARY LAYER

Air Flow in the Boundary Layer of an Elliptic Cylinde AD-A297 391/5

BOUNDARY LAYER TRANSITION

Evolution of a Turbulent Boundary Layer Induced by a Three-Dimensional Roughness Element.

04,200

OUNDARY LUBRICATION

Boundary Lubrication of Silicon Nitride.

03,226

Silicon Nitride Boundary Lubrication: Lubrication Mechanism of Alcohols.

PB96-111703

03,067

Silicon Nitride Boundary Lubrication: Effect of

Oxygenates.

Mechano-Chemical Model: Reaction Temperatures in Concentrated Contact.

OUNDARY VALUE PROBLEMS

Slow Evolution from the Boundary: A New Stabilizing Constraint in III-Posed Continuation Problems.

PB96-122858

BOVINE SERUM ALBUMIN

Hydrolysis of Proteins by Microwave Energy.

BRACHYTHERAPY

Needs for Brachytherapy Source Calibratıons in the United States.

PB97-110092

03,521

BAGG GRATINGS

Bragg Gratings in Optical Fibers Produced by a Continuous-Wave Ultraviolet Source.

PB95-162020

04,274

Growth of Bragg Gratings Produced by Continuous-Wave Ultraviolet Light in Optical Fiber.

04,275

Comparison of UV-Induced Fluorescence and Bragg Grating Growth in Optical Fiber.

04,284

Annealing of Bragg Gratings in Hydrogen-Loaded Optical Fiber.

PB96-155437

04,361

Comparison of UV Photosensitivity and Fluorescence during Fiber Grating Formation.

04,36

BRAGG X-RAY DIFFRACTION

Systematic Correction in Bragg $\mathrm{X}$-ray Diffraction of Flat and Curved Crystals.

PB97-112239

04,15 


\section{BRIDGE MAINTENANCE}

Optimization of Highway Concrete Technology.

BRIDGES

Conversion of a 2-Terminal-Pair Bridge to a 4-TerminalPair Bridge for Increased Range and Precision in Imped ance Measurements. ance Measureme

02,103

BRIDGES (STRUCTURES)

Draft Guidelines for Pre-Qualification and Prototype Testing of Seismic Isolation Systems.

PB94-161940

01,331

Draft Guidelines for Ouality Control Testing of Sliding Seismic Isolation Systems.

01,332

Evaluating the Seismic Performance of Lightly-Reinforced Circular Concrete Bridge Columns.

PB95-163259

01,335

Jacket Thickness Requirements for Seismic Retrofitting of Circular Bridge Columns.

01,336

Seismic Performance of Circular Bridge Columns Designed in Accordance with AASHTO/CALTRANS Stand ards.

01,346

Application of Electromagnetic-Acoustic Transducers for Nondestructive Evaluation of Stresses in Steel Bridge Structures.

PB96-167978

01,301

BRIDGING

ISDN LAN Bridging.

PB95-154696

BRIGHTNESS

Far-Ultraviolet Flare on a Pleiades $G$ Dwart

PB96-102033

01,477

BRILLOUIN EFFECT

Brillouin Light Scattering Intensities for Thin Magnetic Films with Large Perpendicular Anisotropies. $\quad 04,488$

BRITTLE FRACTURING

Analysis of Failed Dry Pipe Fire Suppression System Couplings from the Filene Center at Wolf Trap Farm Park tor the Pertorming Arts.

PB94-164407 Ars. 00,331

Atomic Theory of Fracture of Brittle Materials: Application to Covalent Semiconductors.

04,519

BRITTLE MATERIALS

rack Growth Resistance of Strain-Softening Materials under Flexural Loading.

Friction Processes in Brittle Fracture.

02.972

PB96-161765

03,076

BRITTLE SOLIDS

Effect of Chemical Interaction on Barenblatt Crack Profiles in Brittle Solids.

PB96-180245

02,996

\section{BRITTLENESS}

Ashland Tank Collapse Investigation.

Ashland Tank-Collapse Investigation: Closure by Authors.
PB95-126322

Molecular Orbital Calculations of Bond Rupture in Brittle

Solids.

00,973

BROADBAND ANTENNAS

Time-Domain Antenna Characterizations.

PB95-152781

02,003

NETWORK

Introduction to Traffic Management for Broadband ISDN. PB94-142494

BROMINE ATOMS

Laser Double Resonance Measurements of the Ouenching Rates of $\mathrm{Br}((2) \mathrm{P} 1 / 2)$ with $\mathrm{H} 2 \mathrm{O}, \mathrm{D} 2 \mathrm{O}, \mathrm{HDO}$, and $\mathrm{O} 2$. PB95-150694

\section{BROMINE LASERS}

Efficient $\mathrm{Br}\left({ }^{*}\right)$ Laser Pumped by Frequency-Doubled Nd: YAG and Electronic-to-Vibrational Transfer-Pumped $\mathrm{CO}$ and HCN Lasers.

BRONCHI

Systematics of Alpha-Particle Energy Spectra and Lineal Energy (Y) Spectra for Radon Daughters.
PB94-185139 03,615 Microdosimetry and Cellular Radiation Effects of Radon Progency in Human Bronchial Airways

03,625

OWNIAN MOTION

Hydrodynamic Friction of Arbitrarily Shaped Brownian Particles.

ROWNIAN MOVEMENT

Brownian Diffusion of Hard Spheres at Finite Concentrations.

BSCCO SUPERCONDUCTORS

Microstrains and Domain Sizes in Bi-Cu-O Superconductors: An X-Ray Diffraction Peak-Broadening Study.
PB94-198520

04,461
Etfect of Axial Strain on the Critical Current of AgSheathed $\mathrm{Bi}$-Based Superconductors in Magnetic Fields Up to $25 \mathrm{~T}$.

PB94-211315

04,493

Flux Expulsion at Intermediate Fields in Type-ll Superconductors

04,502

$X$-Ray Characterization of the Crystallization Process of High-Tc Superconducting Oxides in the $\mathrm{Sr}-\mathrm{Bi}-\mathrm{Pb}-\mathrm{Ca}-\mathrm{Cu}$ O System.
PB95-151700

04,579

Improved Uniaxial Strain Tolerance of the Critical Current Measured in Ag-Sheathed Bi2Sr2Ca1Cu2O 8+x Measured in Ag-Sheathed Bi2Sr2Ca1Cu2O $8+x$ Superconductors.

Grain Alignment and Transport Properties of Bi2Sr2CaCu2O8 Grown by Laser Heated Float Zone Method.

04,602

Effects of Anneal Time and Cooling Rate on the FormaEffects of Anneal Time and Cooling Rate on the Forma-
tion and Texture of Bi2Sr2CaCu2O8 Films.

04,603

Tunneling Measurement of the Zero-Bias Conductance Peak and the Bi-Sr-Ca-Cu-O Thin-Film Energy Gap.

PB95-163739

04,628

Increased Pinning Energies and Critical Current Densities in Heavy-Ion-Irradiated Bi2Sr2CaCu2O8 Single Crystals.
PB95-175352

BSDF (BIDIRECTIONAL SCATTERING DISTRIBUTION

FUNCTION)

Rayleigh Scattering Limits for Low-Level Bidirectional Reflectance Distribution Function Measurements.

PB95-180030

04,307

BUBBLE CHAMBERS

Design and Construction of a Liquid Hydrogen Temperature Refrigeration System.

AD-A286 618/4

02,619

BUBBLES

Heat Transfer in an Intumescent Material Using a ThreeDimensional Lagrangian Model.

PB96-164066

00,408

\section{BUCKLED COLUMNS}

Experimental and Numerical Chaos in Continuous Systems: Two Case Studies.

BUCKLING

Exponentially Rapid Coarsening and Buckling in Coherently Self-Stressed Thin Plates.

PB95-202347

04,821

BUCKMINSTERFULLERENE

Inelastic Neutron Scattering Studies of Rotational Excitations and the Orientational Potential in C60 and A3C60 Compounds.

PB94-172673

00,763

Discontinuous Volume Change at the Orientational-Ordering Transition in Solid C60.

PB94-211828

00,821

Rotational Dy

00,948

Structure and Dynamics of Buckyballs.

PB95-153292

00,950

Neutron and X-Ray Scattering Cross Sections of Orientationally

00,951

Epitaxial Integration of Single Crystal C60.

04,592

BUILDING

Project Summaries 1995: NIST Building and Fire Research Laboratory.

CFAST Output Comparison Method and Its Use in Com. paring Different CFAST Versions.

PB96-109541

00,401

BUILDING CODES

Dratt Guideline for Testing and Evaluation of Seismic Isolation Systems.

Standards Development in North America for Performance of Whole Buildings and Facilities.

PB94-185196

00,312

Lessons Learned by a Wing Engineer.

PB94-216421

00.429

Test Methods.

PB95-125951

Growing Significance of CIB.

PB95-126306

rds and

Evaluation and Retrofit Standards for Existing Federally Owned and Leased Buildings.

B95-150918

00,434

Exposure: An Expert System Fire Code.

04,868

Ouantitative Evaluation of Building Fire Safety: New Tools for Assessing Fire and Building Code Provisions. PB95-164588

00,199

Developing Rational Performance-Based Fire Safety Requirements in Model Building Codes.

00.200

World of Building Codes

PB95-203428

00,449

Comparison of the Seismic Provisions of Model Building

Codes and Standards to the 1991 NEHRP Rec-

mmended Provisions.

PB95-231858

00,315

Fire Codes for Global Practice.

PB96-102108

00,205

Recommended Performance-Based Criteria for the Design of Man sist Wind and Seismic Loads.

PB96-128285

00,460

Summary and Results of the NIST Workshop on Proposed Guidelines for Testing and Evaluation of Seismic solation Systems. Held in San Francisco. California on July 25, 1994

PB96-154901

00,463

State of the Art Report on Seismic Design Requirements or Nonstructural Building Components.

00,308

PB96-193800

Fire Safety Engineering in the Pursuit of Performance-
Based Codes: Collected Papers. Based Codes: Collected Papers.

PB97-114482

00,235

LDING DESIGN

Distributed Architecture for Standards Processing

PB96-164181

00,276

BUILDING ENERGY MANAGEMENT SYSTEMS

Using Emulators to Evaluate the Pertormance of Building Energy Management Systems.

00,259

Use of Building Emulators to Evaluate the Performance of

Building Energy Management Systems.

PB96-111901

00,269

BUILDING FIRES

BFRL Fire Publications, 1993

PB94-164191

00,192

Studies Assess Performance of Residential Detectors.

00,290

Fire Growth Analysis of the Fire of March 20, 1990, Pulaski Building, 20 Massachusetts Avenue, N.W., Washing-

PB94-205952

00,194

Overview of Smoke Control Technology.

00,348

Preview of ASHRAE's Revised Smoke Control Manual.

PB94-212875

00,349

Development of a New Small-Scale Smoke Toxicity Test

Method and Its

PB94-213253

Sprinkler Fire Suppression Algorithm.

PB94-216181

00,350

Simulating Smoke Movement through Long Vertical Shafts in Zone-Type Compartment Fire Models.

PB95-143152

00,368

Exposure: An Expert System Fire Code

PB95-162913

04,868

BUILDING MATERIALS

Percolation and Pore Structure in Mortars and Concrete. 00,370 
Earthquake Resistant Construction of Electric Transmission and Telecommunication Facilities Serving the Federal Govemment Report.

02,460

Draft Guidelines for Pre-Oualification and Prototype Testing of Seismic Isolation Systems.
PB94-161940 Draft Guidelines for Ouality Control Testing of Sliding Seismic Isolation Systems.

PB94-161957

01,332

CONTAM93 User Manual.

02,536

Measurement and Determination of Radon Source Potenial: A Literature Review

PB94-165602

02,576

Least-Cost Energy Decisions for Buildings: Part 2. Uncertainty and Risk Video Training Workbook.

PB94-165982

00,240

Psychological Aspects of Lighting: A Review of the Work

$\begin{array}{ll}\text { of ClE TC } 3.16 . & 00,241 \\ \text { PB94-172160 } & \end{array}$

Digitized Simulation of Mercury Intrusion Porosimetry.

Simultaneous Visual and Calorimetric Measurements of R11, R123, and R123/Alkylbenzene Nucleate Flow Boiling.

03,251

Serial Sectioning of Hardened Cement Paste for Scanning Electron Microscopy.

01,305

Draft Guideline for Testing and Evaluation of Seismic Isolation Systems.

00.423

Ouantitative Phase Abundance Analysis of Three Cement Clinker Reference Materials by Scanning Electron MicrosPB94-173051

00,333

Evaluation and Strengthening Guidelines for Federal Buildings: Identification of Current Federal Agency Programs.

00,424 Evaluation and Strengthening Guidelines for Federal Buildings: Assessment of Current Federal Agency Evaluation Programs and Rehabilitation Criteria and Development of Typical Costs for Seismic Rehabilitation.

PB94-181856

00,425

Optimization of Highway Concrete Technology. $\quad 01,333$
PB94-182995

Detection of Voids in Grouted Ducts Using the ImpactEcho Method.

PB94-185121

01,306

Standards Development in North America for Performance of Whole Buildings and Facilities.

PB94-185196 00,312 Corrosion Resistant Epoxy-Coated Reinforcing Steel. PB94-185618 01,307 Air Change Effectiveness Measurements in Two Modern Office Buildings.

00,243

Proficiency Testing as a Component of Ouality Assurance in Construction Materials Laboratories. Expansion of Cementitious Materials Exposed to Sulfate

Solutions.

02,577

Infratechnologies: Tools for Innovation

00,317

Status of Construction and Construction Technologies.

PB94-186004

00,318

Sulfate Attack of Cementitious Materials: Volumetric Relations and Expansions

03,232

Strengthening Methodology for Lightly Reinforced Concrete Frames-1I. Recommended Calculation Techniques for the Design of Infilt Walls.

PB94-187648

00,426

Federai Certification Authority Liability and Policy: Law and Policy of Certificate-Based Public Key and Digital Signatures.

01,578

Program of the Subcommittee on Construction and Building. $94-193646$

00,319

Single-Phase Heat Transfer and Pressure Drop Characteristics of an Integral-Spine-Fin Within an Annulus.
PB94-194073 Graphical Analysis of the CCRL Portland Cement Proficiency Sample Database (Samples 1-72). (Part 1. Univariant Analysis of Portland Cement)

PB94-196557

01,308

Foias-Temam Approximations of Attractors for Galloping

Oscillators.

04,817

Digitized Direct Simulation Model of the Microstructural Development of Cement Paste.

PB94-198777

01,309

Digitized Simulation Model for Microstructural Development.

01.310
Modelling the Leaching of Calcium Hydroxide from Cement Paste: Effects on Pore Space Percolation and Diffusivity.

01,311

Diffusion Studies in a Digital-Image-Based Cement Paste Microstructural Model.

PB94-198801

01,312

Analysis of Moisture Accumulation in a Wood-Frame Wall Subjected to Winter Climate.

PB94-199320

00,338

Maturity Method.

PB94-199494

01,313

Maturity Functions for Concrete Made with Various Cements and Admixtures.

PB94-199502

01,314

Interaction between Naphthalene Sulfonate and Silica Fume in Portland Cement Pastes.

Fume in Portland

01,315

Role of Refrigerant Mixtures as Altematives to CFCs.

PB94-199775 03,252

Role of R22 in Refrigerating and Air Conditioning Equip-

03,253

Environmental Evaluation of a New Federal Office BuildPB94-199874

02,538

Simplified Cycle Simulation Model for the Performance Rating of Refrigerants and Refrigerant Mixtures.

Rating of Refrigerants and Refrigerant Mixtures.

Dynamic Characteristics of Five Tall Buildings during Strong and Low-Amplitude Motions.

PB94-199981

00,427

Papers Presentations Shine.

00,244

Energy Prices and Discount Factors for Life-Cycle Cos Analysis 1994. Annual Supplement to NIST Handbook 135 and NBS Special Publication 709

PB94-206018

02,508

Wind Load Provisions of the Manufactured Home Construction and Safety Standards: A Review and Recommendations for Improvement.

PB94-206125

U.S. Green Building Conference, 1994.

00,428

PB94-206364

02,519

Northridge Earthquake 1994: Performance of Structures. Lifelines, and Fire Protection Systems.

04,825

PB94-207461

Project Summaries 1994: NIST Building and Fire Research Laboratory.

Causes of the Apparent Heat Transfer Degradation for Refrigerant Mixtures.

PB94-212701

03,259

Using Emulator/Testers for Commissioning EMCS Software, Operator Training, Algorithm Development, and Tuning Local Control Loops

PB94-212735

00,245

Highway Concrete (HWYCON) Expert System User Reference and Enhancement Guide.

01,316

Diffusion of Cations Beneath Organic Coatings on Stee Substrate.

PB94-215704

03,119

Standards in Building Economics: Why We Need Them and How to Write Them.

PB94-216405

00,320

Lessons Learned by a Wing Engineer

PB94-216421

00,429

Hollow Clay Tile Prism Tests for Martin Marietta Energy Systems: Task 2 Testing.

00,352

Study of Heat Pump Pertormance Using Mixtures of R32 R134a and R32/R125/R134a as 'Drop-In' Working Fluids for $R 22$ with and Without a Liquid-Suction Heat Ex-

changer.
PB94-218559

02,503

Survey of Recent Cementitious Materials Research in Western Europe

00,353

NIST Research Program on the Seismic Resistance of Partially-Grouted Masonry Shear Walls.

PB94-219052

00,354

National Voluntary Laboratory Accreditation Program: En ergy Efficient Lighting Products.

02,642

Framework for Information Technology Integration in Process Plant and Related Industries.

02,772

PB94-219086

Energy Price Indices and Discount Factors for Life-Cycle book 135 and NBS Special Publication 709. (Revised)

PB95-105011

02,509

Present Worth Factors for Life-Cycle Cost Studies in the Department of Defense (1995)

PB95-105029

03,664

Controlling Moisture in the Walls of Manufactured Hous-

PB95-105136

00,355

Measurements of Moisture Diffusivity for Porous Building Materials.

PB95-107397

00,356

Ventilation Rates in Office Buildings.

PB95-108825 02,539

Field Measurements of Ventilation and Ventilation Effectiveness in an Office/Library Building

PB95-108833

00,247

Seismic Strengthening of Reinforced Concrete Frame Buildings.

Application of Thermal Analysis Techniques to the Characterization of EPDM Roofing Membrane Materials.

PB95-125845

00,359

Use of Thermal Mechanical Analysis to Characterize Ethylene-Propylene-Diene Terpolymer (EPDM) Roofing .

00,360

Water Efficient Plumbing Fixtures through Standards and

$\begin{array}{ll}\text { Test Methods. } & 00,248\end{array}$

Computer Simulation of the Diffusivity of Cement-Based Materials.

PB95-125985

00,362

Digital Simulation of the Aggregate-Cement Paste Interfacial Zone in Concrete.

PB95-125993

00,363

Fundamental Computer Simulation Models for CementBased Materials.

00.364

Comparison of Heat-Flow-Meter Tests from Four Laboratories.

PB95-126264

00,365

Growing Signi

00,314

Ashland Tank-Collapse Investigation: Closure by Authors.

PB95-126322

02,482

Standards of Seismic Safety for Existing Federally Owned or Leased Buildings and Commentary.

00,431

Indoor Air Ouality Impacts of Residential HVAC Systems, Phase 1 Report: Computer Simulation Plan.
PB95-135596

00,249

Ouantitative X-Ray Powder Diffraction Methods for Clinker and Cement
PB95-143079

01,317

Diagnosis of Causes of Concrete Deterioration in the MLP-7A Parking Garage.

01,318

Manufactured Homes: Probability of Failure and the Need for Better Windstorm Protection through Improved Anchoring Systems.

PB95-1 43129

00,432

Visual Measurement Technique for Analysis of Nucleate Flow Boiling.

03,262

Methodologies for Predicting the Service Lives of Coating Systems.

03,124

Wind and Seismic Effects. Proceedings of the U.S.-Japan Cooperative Program in Natural Resources Panel on 

Implementation of Executive Order 12699: Seismic Safety
of Federal and Federally Assisted or Regulated New Building Construction.

PB95-151809

00,436

Structural Analysis in Context

00,437

Room Temperature Thermal Conductivity of Fumed-Silica Insulation for a Standard Reference Material.

PB95-152039

00,374

Partially Prestressed and Debonded Precast Concrete Beam-Column Joints.

PB95-153102

00,439

Seismic Performance Behavior of Precast Concrete Beam-Column Joints.

00,440

Psychological Aspects of Lighting: A Review of the Work of CIE TC 3.16.

PB95-153276 00,254

Rationale and Preliminary Plan for Federal Research for Construction and Building.

PB95-154704

00,322

Assessing Ventilation Effectiveness in Mechanically Ventilated Otfice Buildings.

PB95-162079

00,255

Modeling Radon Transport in Multistory Residential Build-

ings.

00,256

Ventilation, Carbon Dioxide and ASHRAE Standard 62 1989

PB95-162095

02,544

Characteristics of Adhesive-Bonded Seams Sampled rom EPDM Roof Membranes.

00,377

Algebraic Approximation of Attractors for Galloping Oscillators.

PB95-162897

Rheology of Fresh Cement Paste.

04,820

PB95-163150

00,378

Cement and Concrete Characterization by Scanning Eleciron Microscopy.

00,379

Evaluating the Seismic Performance of Lightly-Reinforced Circular Concrete Bridge Columns.

PB95-163259

01,335

Jacket Thickness Requirements for Seismic Retrofitting of Circular Bridge Columns.

01,336

Pulse-Echo U/trasonic Evaluation of the Integrity of Seams of Single-Ply Roof Membranes.

PB95-163804

00,381

Lessons from the Loma Prieta Earthquake.

00,442

Fire-Plume-Generated Ceiling Jet Characteristics and Convective Heat Transfer to Ceiling and Wall Surfaces in a Two-Layer Fire Environment: Uniform Temperature Ceiling and Walls.

PB95-164711

00,382

Performance of HUD-Affiliated Properties during the Janvary 17, 1994 Northridge Earthquake.

PB95-174488

00,443

Life-Cycle Costing Workshop for Energy Conservation in Buildings: Student Manual.

PB95-175006

00,257

Cellular Automaton Simulations of Cement Hydration and Microstructure Development.

01,320

Evolution of Porosity and Calcium Hydroxide in Labora tory Concretes Containing Silica Fume.

01,321

Earthquake and Fire in Japan: When the Threat Became a Reality.

PB95-175238 00,201

Performance of Compact Fluorescent Lamps at Different Ambient Temperatures.

00,258

Using Emulators to Evaluate the Performance of Building Energy Management Systems.

PB95-175774

00.259

Reproducibility of Tests on Energy Management and

Control Systems Using Building Emulators.

PB95-175980

00,260

Limiss of $\mathrm{CO} 2$ Monitoring in Determining Ventilation Rates.

02,552

Ventilation Effectiveness Measurements in Two Modern $\begin{array}{ll}\text { Office Buildings. } & \\ \text { PB95.176012 } & \end{array}$

02,553

Spectrally Smooth Reflectances That Match

04,306

Manufactured Housing Walls That Provide Satisfactory Moisture Performance in All Climates. PB95-178885

00,383

Indoor Air Ouality Impacts of Residential HVAC Systems. Phase 2.A Report: Baseline and Preliminary Simulations. PB95-178893

02,554

Performance of 1/3-Scale Model Precast Concrete BeamColumn Connections Subjected to Cyclic Inelastic Loads. Report No. 4.

00,444
Effects of Testing Variables on the Measured Compressive Strength of High-Strength (90 MPa) Concrete.
PB95-179040 Nondestructive Testing of Concrete: History and Chal-

PB95-180139

00,385

High-Performance Concrete: Research Needs to Enhance Its Use.

01,322

Application of a Multizone Airflow and Contaminant Dispersal Model to Indoor Air Quality Control in Residential Buildings.

PB95-180238

02,555

Gust Factors Applied to Hurricane Winds

00,446

Melnikov Function and Homoclinic Chaos Induced by Weak Perturbations.

PB95-180923

03,414

Control Stability of a Heat-Flow-Meter Appartus.

PB95-181194

00,386

Intra-Laboratory Comparison of a Line-Heat-Source Guarded Hot Plate and Heat-Flow-Meter Apparatus.

PB95-181202

00,387

Seismic Safety of Federal Buildings. Initial Program: How Much Will it Cost.

PB95-182291

00,447

Indoor Air Quality Commissioning of a New Office Build-

PBg5-182309

00,262

Proceedings: Workshop on Research Needs in Wind Engineering. Held in Gaithersburg, Maryland on September $12-13,1994$

PB95-189528

00,448

Field Evaluation of the System for Calibration of the Marshall Compaction Hammer.

PB95-190674

01,323

BLCC: The NIST 'Building Life-Cycle Cost' Program, Version 4.21. User's Guide and Reference Manual.

PB95-190682

00,263

Heat and Moisture Transfer in Wood-Based Wall Construction: Measured versus Predicted.

PB95-200655

00,391

Optical Performance of Commercial Windows.

PB95-208757

00,392

Measurements of Outdoor Air Distribution in an Office Building.

00,264

Survey of Steel Moment-Resisting Frame Buildings Affected by the 1994 Northridge Earthquake.

00,451

White Papers Prepared for the White House: ConstrucWhite Papers Prepared for the White House: Construction Industry Workshop on Nation
Held on December 14-16, 1994

01,299

PB95-21689

Theoretical Evaluation of the Vapor Compression Cycle with a Liquid-Line/Suction-Line Heat Exchanger, Economizer, and Ejector.

PB95-216917 02,607

Extreme Wind Distribution Tails: A 'Peaks Over Thresh. old' Approach.
PB95-219416

00,127

Computer Simulations of Airflow and Radon Transport in Four Large Buildings.

02,557

Prediction of Cracking in Reinforced Concrete Structures

PB95-220448 03,725

Testing of Selected Self-Leveling Compounds for Floors.

PB95-220455 00,395

Extreme Wind Estimates by the Conditional Mean Exceedance Procedure.

PB95-220471

00,120

Survey of Concrete Transport Properties and Their Measurement.

00,396

Compositional Aralysis of Beneficiated Fly Ashes.

PB95-220497

00,397

Performance Testing of a Family of Type I Combination

$\begin{array}{ll}\text { Appliance. } & \\ \text { PB95-220521 } & 02,505\end{array}$

4SIGHT Manual: A Computer Program for Modelling Degradation of Underground Low Level Waste Concrete Vaults.

PB95-231593

03,726

Enhancements to Program IDARC: Modeling Inelastic Behavior of Welded Connections in Steel Moment-Resisting

PB95-231601

00,452

Performance of Federal Buildings in the January 17, 1994 Northridge Earthquake.

00,453

PB95-231775

Comparison of the Seismic Provisions of Model Building
Codes and Standards to the 1991 NEHRP Recommended Provisions.

PB95-231858

00,315

Water-Vapor Measurements of Low-Slope Roofing Mate-

PB95-251617

00,399

Least-Cost Energy Decısions for Buildings. Part 3 Choosing Economic Evaluation Methods. Video Training Cing

International Green Building Conference and Exposition (2nd). Held in Big Sky, Montana on August 13-15, 1995.

PB95-253605 02,525 Simple Method of Composition Shifting with a Distillation Column for a Heat Pump Employing a Zeotropic Refrigerant Mixture.

02,603

New Mass Transport Elements and Components for the NIST IAQ Model.

PB95-255899

02,558

Strengthening Methodology for Lightly Reinforced ConDesign Guidelines for Strengthening with Infill Wals.

PB95-260725

Long-Term Performance of Engineered Concrete Bar-

PB95-260816

03,727

National Construction Sector Goals: Industry Strategies or Implementation.

00,204

Building Life Cycle Cost Computer Program (BLCC) Version 4.21-95 (for Microcomputers).

B95-502779

00,267

Building Life Cycle Cost Computer Program (BLCC) Version 4.22-95 (for Microcomputers).

PB95-503397

00,268

Geometrical Percolation Threshold of Overlapping Ellipsoids

B B96-102397

03,167

100 Ampere, $100 \mathrm{kHZ}$ Transconductance Amplifier

PB96-102629

02,068

Present Worth Factors for Life-Cycle Cost Studies in the Department of Defense (1996).

PB96-106869

03,673

Indoor Air Quality Impacts of Residential HVAC Systems Phase II.B Report: IAO Control Retrofit Simulations and Analysis.

PB96-106877

02,559

Literature Review on Seismic Pertormance of Building

EMISS: A Program for Estimating Local Air Pollution Emission Factors Related to Energy Use in Buildings: User's Guide and Reference Manual. 


\section{KEYWORD INDEX}

BUILDING TECHNOLOGY

Alkali-Silica Reaction and High Performance Concrete.
PB96-131537

How-To Suggestions for Implementing Executive Order 12941 on Seismic Safety of Existing Federal Buildings, A PB96-131552

00,461 Modelling Drying Shrinkage of Cement Paste and Mortar. Part 1. Structural Models from Angstroms to Millimeters. PB96-135132 03,074

Effect of Inclination on the Performance of a Compact Brazed Plate Condenser and Evaporator.

02,756

National Planning for Construction and Building $\mathrm{R}$ and $\mathrm{D}$.

National Planning for Construction and Building $R$ and D.
PB96-137104 00,324

Improving the Evaluation of Building Ventilation

PB96-138508

00,271

De Facto Microzonation through the Use of Soils Factors in Design Triggers

00,462

Predicting the Fire Performance of Buildings: Establishing Appropriate Calculation Methods for Regulatory Applica-

PB96-141239

00,316

Unreacted Cement Content in Macro-Defect-Free Composites: Impact on Processing-Structure-Property Rela-

PB96-141270

03,174

Optimal Control of Building and HVAC Systems.

PB96-141353

00,272

Seismic Performance of Circular Bridge Columns Designed in Accordance with AASHTO/CALTRANS Standards.

PB96-146352

01,346

Effectiveness of a Heat Recovery Ventilator, an Outdoor Air Intake Damper and an Electrostatic Particulate Filter at Controlling Indoor Air Ouality in Residential Buildings. PB96-146642

Multizone Modeling of Three Residential Indoor Air Ouality Control Options.

02,565 Interfacial Transport in Porous Media: Application to dc
Electrical Conductivity of Mortars. PBectrical Conduc

01,326

Water Permeability and Chloride Ion Diffusion in Portland Cement Mortars: Relationship to Sand Content and Critical Pore Diameter.

PB96-148036

03,193

Workplan to Analyze the Energy Impacts of Envelope Airtightness in Otfice Buildings.

00,273

AutoBid 2.0: The Microcomputer System for Police Patrol Vehicle Selection.

PB96-154570 04,871

Simplified Design Procedure for Hybrid Precast Concrete PB96-154836

00,405

Summary and Results of the NIST Workshop on Proposed Guidelines for Testing and Evaluation of Seismic Isolation Systems. Held in San Francisco, California on July 25, 1994

PB96-154901 00,463
Sensitivity Analysis for Mathematical Modeling of Fires in Residential Buildings.

00,215

PB96-154968

Development of a Test Method for Leaching of Lead from
Lead-Based Paints Through Encapsulants.

PB96-154984

03,128

Study of Ventilation Measurement in an Office Building.
PB96-155593 00,274

Development and Application of an Indoor Air Ouality Commissioning Program in a New Office Building. Transitions to Chaos Induced by Additive and Multiplicative Noise.

PB96-155759 03,750

Spectrum of the Stochastically Forced Duffing-Holmes

PB96-155767

00,216

Necessary Condition for Homoclinic Chaos Induces by Additive Noise

04,063

Executive Order 12941. Seismic Safety of Existing Feder-

ally Owned or Leased Buildings: It's History, Content and Objectives.

PB96-156021

00,465

Noise-Induced Transitions to Chaos.

00,217

Experimental and Numerical Chaos in Continuous Systems: Two Case Studies.

PB96-156146

00,219

Application of Digital-Image-Based Models to Microstructure, Transport Properties, and Degradation of Cement-Based Materials.

00,406

Intermediate Coupling in L2-L3 Core Excitons of MgO, $\mathrm{Al} 2 \mathrm{O} 3$, and $\mathrm{SiO} 2$

PB96-158043

04,079

Strengthening Methodology for Lightly Reinforced Con-

crete Frames.

00,466
Multiattribute Decision Analysis Method for Evaluating Buildings and Building Systems.

PB96.158670

00,325

Comparison of Responses of a Select Number of Buildings to the 10/17/1989 Loma Prieta (California) Earthquake and Low-Level Amplitude Test Results.

quake and Low-1

00,467

Extreme Winds Estimation by 'Peaks Over Threshold' and Epochal Methods

PB96-159686

00,468

Modeling of Extreme Loading by 'Peaks Over Threshold'

PB96-159694 00,469

Workgroup Summary Report: Plastic Hinge-Based Techniques for Advanced Analysis.

00,470

Effects of Pipe Elbows and Tube Bundles on Selected Types of Flowmeters.

PB96-160999

01,135

Energy Price Indices and Discount Factors for Life-Cycle Cost Analysis 1996. Annual Supplement to NIST Handbook 135 and NBS Special Publication 709. (Revised).

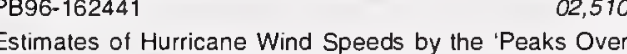
Threshold' Method.

PB96-162540

00,471

Distributed Architecture for Standards Processing.

00,276

Guide to a Format for Data on Chemical Admixtures in a Materials Property Database.

PB96-165394

01,327

Group 1 for the Plant Spatial Configuration STEP Application Protocol.

02,789

Multizone Modeling of Three Residential Indoor Air Ouality Control Options.

02,56

Modified Optimal Algorithm for Active Structural Control.

PB96-165949 00,472

Enhancement of R123 Pool Boiling by the Addition of $\mathrm{N}$ Hexane.

B96-165956

02,605

Preliminary Processing of the Lotung LSST Data.

PB96-165972

03,690

Study to Determine the Existence of an Azeotropic R-22 'Drop-In' Substitute.

PB96-167812

02,568

Method of Estimating the Parameters of Tuned Mass Dampers for Seismic Applications.

PB96-167820

00,473

Life-Cycle Costing Manual for the Federal Energy Management Program. 1995 Edition.

PB96-172317

02,511

Innovation in the Japanese Construction Industry: A 1995 Appraisal.

00,225

Probabilistic Estimates of Design Load Factors for Wind Sensitive Structures Using the 'Peaks Over Threshold' Approach

PB96-183223

00,474

Performance of Tape-Bonded Seams of EPDM Membranes: Comparison of the Peel Creep-Rupture Response of Tape-Bonded and Liquid-Adhesive-Bonded

03,012

Guide to a Format for Data on Chemical Admixtures in a Materials Property Database. (Reannouncement with new

$\begin{array}{ll}\text { abstract). } & 01,328\end{array}$

Warping of Terrace Pavers at the U.S. Capitol Building.
PB96-193651

00,411

Guidelines for Pre-Oualification, Prototype and Ouality Control Testung of Seismic Isolation Systems.
PB96-193685

01,347
PB96-193685 or Nonstructural Building Components.

00,308

PB96-193800

Precision of Marchall Stability and Flow Test Using 6-in.
$152.4-\mathrm{mm})$ Diameter Specimens. (152.4-mm) Dian

03,006

Benefits and Costs of Research: Two Case Studies in Building Technology.

PB96-202221

00,230

NIST Construction Automation Program Report No. 2. Proceedings of the NIST Construction Automation shop. Held in Gaithersburg. Maryland on March 30-31,

B96-202239

00,413

Development of a Method for Measuring Water-Strippıng Resistance of Asphalt/Siliceous Aggregate Mixtures.

PB96-202296 01,329

Measurement of Rheological Properties of High Performance Concrete: State of the Art Report.

00,414

Economics of New-Technology Materials: A Case Study of FRP Bridge Decking.

01,349

Materials Aspects of Fiber-Reinforced Polymer Composites in Infrastructure.

03,184 Energy Price Indices and Discount Factors for Life-Cycle
Cost Analysis 1997. Annual Supplement to NIST Handbook 135 and NBS Special Publication 709. (Revised).
PB96-210745

Report of a Workshop on Requalification of Tubular Steel Joints in Offshore Structures. Held in Houston, Texas on September 5-6, 1995

PB96-210760

03.699

Estimation of System Damping at the Lotung Site by Application of System Identification

PB96-214697

01,351

Shear Design of High-Strength Concrete Beams: A Review of the State-of-the-Art.

01,330

Energy-Based Method for Liquefaction Potential Evaluation. Phase 1. Feasibility Study.

PB96-214747

03,691

January 17, 1995 Hyogoken-Nanbu (Kobe) Earthquake. Performance of Structures, Lifelines and Fire Protection Systems. Executive Summary and Paper.

Wind and Seismic Effects: Proceedings of the Joint Meet ing of the U.S.-Japan Cooperative Program in Natura Resources Panel on Wind and Seismic Effects (28th). Held in Gaithersburg, Maryland on May 14-17, 1996 PB97-104376

00,476

Indoor Air Ouality Commissioning of a New Oftice Build-

PB97-110142

00,279

Relationship between Indoor Air Quality and Carbon Di-

PBide. 111249

02,569

Post-Occupancy Evaluation of the Forrestal Building.

Experimental Verification of a Moisture and Heat Transfer Model in the Hygroscopic Regime.

PB97-111546

00,309

Testing Conformance and Interoperability of BACnet (Trade Name) Building Automation Products.

PB97-111553 E

00,310

Test Procedures for Advanced Insulation Panels

PB97-111892

00,415

Empirical Validation of a Transient Computer Model for Combined Heat and Moisture Transfer.

PB97-111991 00,416

Factors Affecting the Energy Consumption of Two Refrig-

$\begin{array}{ll}\text { erator-Freezers. } & 00,311\end{array}$

Dynamics of $M$

00,477

Carbon Monoxide Dispersion in Residential Buildings: Lit-

erature Review and Technical Analysis
PB97.114227

02,571

Summary of Federal Construction and Building $R$ and $D$ in 1994.

PB97-114250

00,234

Proceedings of a Workshop on Developing and Adopting 


\section{BUILDINGS}

Computer Programs for Simulation of Lighting/HVAC Interactions.

02,501

Draft Guidelines for Ouality Control Testing of Elastomeric Seismic Isolation Systems.

PB94-161734

00,422

CONTAM93 User Manual.

02,536

Least-Cost Energy Decisions for Buildings: Part 2. Uncertainty and Risk Video Training Workbook

Lead Abatement in Buildings and Related Structures.

Lead Abatement in Buildings and Related Structures.
PB94-172038

Psychological Aspects of Lighting: A Review of the Work of CIE TC 3.16.

PB94-172160

00,241

Improvement in Predicting Smoke Movement in Compartmented Structures.

PB94-172418 00,332

Fire Hazard and Risk: Evaluating Alternative Tech-

PB94-173077

00,242

Prediction of Fire Dynamics.

PB94-193620

Performance Parameters of Fire Detection Systems.

PB94-194339

CONTAM88 Building Input Files for Multi-Zone Airflow and Contaminant Dispersal Modeling.

02,537

Analysis of the Happyland Social Club Fire with HAZARD

PB94-199270

00,193

Dynamic Characteristics of Five Tall Buildings during

Strong and Low-Amplitude Motions.

00,427

Development of the Fire Data Management System.

PB94-206091

00,339

U.S. Green Building Conference, 1994

02,519

Project Summaries 1994: NIST Building and Fire Research Laboratory.

00,343

Global Equivalence Ratio Concept and the Prediction of Carbon Monoxide Formation in Enclosure Fires.

Carbon Monox

00.313

Using Emulator/Testers for Commissioning EMCS Software, Operator Training, Algorithm Development, and Tuning Local Control Loops.

00,245

Full Scale Smoke Control Tests at the Plaza Hotel Build-

PB94-212859

00,347

Overview of Smoke Control Technology.

00,348

Preview of ASHRAE's Revised Smoke Control Manual.

PB94-212875 00,349

Sprinkler Fire Suppression Algorithm

PB94-216181

00,349

Standards in Building Economics: Why We Need Ther and How to Write Them.

PB94-216405

00,320

HAZARD I Fire Hazard Assessment Method (Version 1.2) (for Microcomputers)

PB94-501988

00,196

HAZARD I Fire Hazard Assessment Method, Version 1.2 (Upgrade Package) (for Microcomputers).

Relating Bench-Scale and Full-Scale Toxicity Data.

PB95-125977

00,361

Assessment of Technologies for Advanced Fire Detection.

00,294

Summaries of BFRL Fire Research In-House Projects and Grants, 1994.

PB95-130845

00,366

FPETOOL: Fire Protection Tools for Hazard Estimation. An Overview of Features.

PB95-140885

00,367

Building and Fire Research Laboratory Publications, 1993.

00,369

PB95-143202

Rationale and Preliminary Plan for Federal Research for
Construction and Building.

Construction and

00,322

Method of Predicting Smoke Movement in Atria with Ap-

plication to Smoke Management.

PB95-154746

00,376

Gust Factors Applied to Hurricane Winds.

00,446

Algorithm to Describe the Spread of a Wall Fire under a Ceiling.

PB95-182259

00,261

Comparison of Fire Sprinkler Piping Materlals: Steel, Copper, Chlorinated Polyvinyl Chloride and Polybutylene, in Residential and Light Hazard Installations.

00,299
BLCC: The NIST 'Building Life-Cycle Cost' Program, Version 4.21. User's Guide and Reference Manual. PB95-190682

00,263

Survey of Steel Moment-Resisting Frame Buildings Aflected by the 1994 Northridge Earthquake.

00,451

Compartment Fire Combustion Dynamics. Annual Report, September 1, 1993-September 1, 1994

00,203

Building and Fire Research Laboratory Publications,

PB95-226684

00,398

Least-Cost Energy Decisions for Buildings. Part 3. Choosing Economic Evaluation Methods. Video Training PBg5-253597

00,265

International Green Building Conference and Exposition (2nd). Held in Big Sky, Montana on August 13-15, 1995.
PB95-253605 Building Life Cycle Cost Computer Program (BLCC), Version 4.2-95 (for Microcomputers)

PB95-501953

00,266

Building Life Cycle Cost Computer Program (BLCC) Version 4.21-95 (for Microcomputers).

00,267

Building Life Cycle Cost Computer Program (BLCC) Version 4.22-95 (for Microcomputers).

00,268

How to Evaluate Alternative Designs Based on Fire Mod-

PB96-102116

00,206

Literature Review on Seismic Performance of Building

$\begin{array}{ll}\text { Cladding Systems. } & 00,455 \\ \text { PB96-106901 }\end{array}$

Economic Methods and Risk Analysis Techniques for Evaluating Building Investments: A Survey.

00,323

96-122593

Optimal Control of Building and HVAC Systems.
PB96-141353
00,272

Strengthening Methodology for Lightly Reinforced Concrete Frames

00,466

Multiattribute Decision Analysis Method for Evaluating

Buildings and Building Systems.
PB96-158670

00,325

Comparison of Responses of a Select Number of Buildings to the 10/17/1989 Loma Prieta (California) Earthquake and Low-Level Amplitude Test Results.

PB96-159645

00,467

Benefits and Costs of Research: Two Case Studies in Building Technology.

00,230

NIST Construction Automation Program Report No. 2. Proceedings of the NIST Construction Automation Workshop. Held in Gaithersburg, Maryland on March 30-31, PB96-202239

00,413

Building Life Cycle Cost Computer Program (BLCC) Version 4.22-95 (for Micrcomputers)

00,277

Testing Conformance and Interoperability of BACnet (Trade Name) Building Automation Products.

00,310

International Green Building Conference and Exposition International Green Building Conference and Exposition
(3rd). Held in San Diego, California on November 17-19,
1996 . (Reannouncement with new abstract). 1996. (Reannoun
PB97-121826

02,531

Building Life Cycle Cost Computer Program (BLCC) Version 4.4-97 (for Microcomputers)

00,284

BULK MODULUS

Relationship between Bulk-Modulus Temperature De-

pendence and Thermal Expansivity.

04,641

Compressibility of Polycrystal and Monocrystal Copper: Acoustic-Resonance Spectroscopy.

02,990

Bulk Modulus and Young's Modulus of Nanocrystalline gamma-Alumina.

PB96-204185

03,092

BUOYANCY

Computational Model for the Rise and Dispersion of Wind-Blown, Buoyancy-Driven Plumes. Part 2. Linearly Stratified Atmosphare.

PB94-143427

00,119

Piggyback Balance Experiment: An Illustration of Archimedes' Principles and Newton's Third Law.

03,786

Combined Buoyancy- and Pressure-Driven Flow Through a Shallow, Horizontal, Circular Vent.

00,344

PB94-210077

Simulating Smoke Movement through Long Vertical Shafts in Zone-Type Compartment Fire Models.
PB95-143152 Calculating Combined Buoyancy- and Pressure-Driven Flow Through a Shallow, Horizontal, Circular Vent; Application to Problem of Steady Burning in a Ceiling-Vented Enclosure.

PB96-164108

00,409

Combined Buoyancy and Pressure-Driven Flow Through Shallow, Horizontal, Circular Vent.

00,410

BUOYANT SMOKE PLUMES

Dispersion and Deposition of Smoke Plumes Generated in Massive Fire

BURIED OXIDES

Electron and Hole Trapping in Irradiated SIMOX, ZMR and BESOI Buried Oxides.

PB96-160320

01,956

BURNING RATE

Fire Growth Models for Materials

PB94-195856

01,367

Comparison of Wall-Fire Behavior With and Without a Ceiling.

00,342

In Situ Burning Oil Spill Workshop Proceedings. Held in Orlando, Florida on January 26-28, 1994 02,583

One- and Two-Sided Burning of Thermally Thin Materials.

PB95-140935 03,15

Estimate of the Effect of Scale on Radiative Heat Loss Fraction and Combustion Efficiency.

PB95-150447

02,486

Wall Flame Heights with External Radiation.

PB95-163481

00,380

In situ Buming of Oil Spills: Mesoscale Experiments and Analysis.

02,587

Burning Rate of Premixed Methane-Air Flames Inhibited

by Fluorinated Hydrocarbons.
PB95-180584

01,391

Experimental and Numerical Burning Rates of Premixed Methane-Air Inhibited by Fluoromethanes.

01,392

Burning Rate and Flame Heat Flux For PMMA in the Cone Calorimeter

PB95-216990

00,393

Turbulent Flame Spread on Vertical Corner Walls.

Smoke Emission from Burning Crude Oil.

01,403

PB96-122890

01,407

Predicting the Ignition Time and Burning Rate of Thermo-

plastics in the Cone Calorimeter.

01,418

Minimum Mass Flux Requirements to Suppress Burning Surfaces with Water Sprays.

PB96-183181

01,425

BUTADIENES

Acrylonitrile-Butadiene-Styrene (ABS) Plastic Drain,

Waste, and Vent
$\mathrm{AD}-\mathrm{A} 310724 / 0$

00,327

Stability/lnstability of Gas Mixtures Containing 1,3-Butadiene in Treated Aluminum Gas Cylinders.

PB95-162285

02,546

BUTANES

Modified Leung-Griffiths Model of Vapor-Liquid Equi- 


\section{KEYWORD INDEX}

CALCIFICATION

Mixed Phospholipid Liposome Calcification.

PB94-211190

Effect of Supersaturation on Apatie Crystal For

Aqueous Solutions at Physiologic pH and Temperature.
PB96-135215 PB96-135215

CALCIUM

Studies of Autoionization in Calcium.

PB94-213295 Audionization in Calcium.

High-Resolution Diode-Laser Spectroscopy of Calcium.
PB95-181244

$n$-Vector Correlations in Collision Dynamics with Atomic Orbital Alignment: The Importance of Coherence Denoting Azimuthal Structure for $n$ (greater than or equal to) 3 .
PBg, Laser Preparation and Probing of Initial and Final Orbital Alignment in Collision-Induced Energy Transfer $\mathrm{Ca}(4 \mathrm{~s} 5 \mathrm{p},(1) \mathrm{P} 1)+\mathrm{He}$ yields $\mathrm{Ca}(4 \mathrm{~s} 5 \mathrm{p},(3) \mathrm{P} 2)+\mathrm{He}$. PB95-203261 04,003 Initial and Final Orbital Alignment Probing of the FineStructure-Changing Collisions among the $\mathrm{Ca}$
$(4 \mathrm{~s})(1)(4 \mathrm{p})(1)$, (3) PJ States with He: Determination of $\mathrm{Co}$ herence and Conventional Cross-Sections.

04,004 PB95-203279

Inelastic

Orbital Alignment

PB96-122742
RIS Measurement of AC Stark Shifts and Photoionization RIS Measurement of AC Stark Shifts and Photoionization Cross Sections in Calcium

PB96-157953 04,073

Comment On: Two-Photon Absorption Series of Calcium. PB96-157979

04,074

Configuration-Dependent AC Stark Shifts in Calcium.
PB96-157995 Alignment Probing of Rydberg States by Stimulated Emis-

SBion. 200316

04,124

CALCIUM ADIPATE MONOHYDRATE

Crystal Structure of Calcium Adipate Monohydrate. ${ }^{00,153}$

\section{ALCIUM BISMUTH OXIDES}

Ca4Bi6013: A Compound Containing an Unusually Low Bismuth Coordination Number and Short Bi Bi Contacts.

CALIUM BISMUTHATES

Phase Equilibria in the Systems $\mathrm{CaO}-\mathrm{CuO}$ and $\mathrm{CaO}$ -

PB95-140570 03,048

Ca4Bi6013: A Compound Containing an Unusually Low Bismuth Coordination Number and Short Bi Bi Contacts.
PB95-141131

CALM CITRATE

Interaction of Citric Acid with Hydroxyapatite: Surface Exchange of lons and Precipitation of Calcium Citrate.

03.584

\section{CALCIUM DICARBOXYLATE} Crystal Structure of Calcium Adipate Monohydrate. 00,153

CALCIUM GLUTARATE MONOHYDRATE

Crystal Structure of Calcium Glutarate Monohydrate. PB96-111893

CALCIUM HYDROXIDES

Evolution of Porosity and Calcium Hydroxide in Laboratory Concretes Containing Silica Fume.

01,321

CALCIUM HYDROXYAPATITE

Composition and Solubility Product of a Synthetic Calcium Hydroxyapatite.

PB96-180104

02,995

CALCIUM IONS

Fine Structure of Negative lons of Alkaline-Earth-Metal PBO4-211

03,837

Analysis of Protein Metal Binding Selectivity in a Cluster

Model.
PB94-212990

00,845

CALCIUM METABOLISM

In vitro Inhibition of Membrane-Mediated Calcification by Novel Phosphonates.

PB96-201140

03,595

CALCIUM OXALATE HYDRATE

Influence of Natural and Synthetic Inhibitors on the Crystallization of Calcium Oxalate Hydrates.
PB95-150967

03,560

CALCIUM OXIDES

Phase Equilibria in the Systems $\mathrm{CaO}-\mathrm{CuO}$ and $\mathrm{CaO}$ PB95- 140570

03,048

CALCIUM PHOSPHATE

Behavior of a Calcium Phosphate Cement in Simulated Blood Plasma In vitro.

00,165

Polymeric Calcium Phosphate Composites with Remineralization Potential.

03,575

CALCIUM PHOSPHATE CEMENT

Reinforcement of Cancellous Bone Screws with Calcium Phosphate Cement

00,179

\section{CALCIUM PHOSPHATE CEMENTS}

Calcium Phosphate Cements.

PB97.111595

03,580

CALCIUM PHOSPHATES

Calcium Phosphate Precipitation in Liposomal Suspen-

PB94-172145

03,452

Physicochemical Properties of Calcific Deposits Isolated from Porcine Bioprosthetic Heart Valves Removed from Patients Following 2-13 Years Function.

00,184

Proteoglycan Inhibition of Calcium Phosphate Precipitaion in Liposomal Suspensions.

PB94-211208

00,658

Selective Inhibition of Crystal Growth on Octacalcium Phosphate and Nonstoichiometric Hydroxyapatite by Pyrophosphate at Physiological Concentration.

00,147

Periapical Tissue Reactions to a Calcium Phosphate Cement in the Teeth of Monkeys

00,149

Crystal Structure of Dicalcium Potassium Trihydrogen Bis(pyrophosphate) Trihydrate.

00,152

Effects of Calcium Phosphate Solutions on Dentin Permeability.

00,157

Effects on Whole Saliva of Chewing Gums Containing Calcium Phosphates.

03,563

Effect of Ethanol on the Solubility of Dicalcium Phosphate

Dihydrate in the System $\mathrm{Ca}(\mathrm{OH}) 2-\mathrm{H} 3 \mathrm{PO} 4-\mathrm{H} 2 \mathrm{O}$ at $37 \mathrm{C}$.
$\mathrm{PB} 95-163507$

Membrane-Mediated Precipitation of Calcium Phosphate in Model Liposomes with Matrix Vesicle-Like Lipid Composition.

position.

03,468

Effect of 1-Hydroxyethylidene-1,1-Bisphosphonate on Membrane-Mediated Calcium Phosphate Formation in Model Liposomal Suspensions.

PB95-169223

03,469

Physical and Chemical Properties of Resin-Reinforced Calcium Phosphate Cements.

PB95-180212

00,171

Formation of Hydroxyapatite in a Polymeric Calcium Phosphate Cement.

00,173

Remineralization of Root Lesions with Concentrated Calcium and Phosphate Solutions.

PB96-102140

03,567

Properties and Mechanisms of Fast-Setting Calcium Phosphate Cements.

00,178

Crystal Structure of Decacalcium Tetrapotassium Hexakis (Pyrophosphate) Nonahydrate.

PB96-141064

01,099

Bioactive Polymeric Dental Materials Based on Amorphous Calcium Phosphate.

03.572

Remineralizing Dental Composites Based on Amorphous Remineralizing Dental Composites Based on Amorphous
Calcium Phosphate.
PB96-147020 03,573

PBnamics of

03,574

Polymeric Calcium Phosphate Cements Derived from Poly(methyl vinyl ether-maleic acid)

00,180

\section{CALCIUM SILICATES}

Modelling Drying Shrinkage of Cement Paste and Mortar Part 1. Structural Models irom Angstroms to Millimeters.

CALCIUM SUCCINATE MONOHYDRATE Crystal Structure of Calcium Succinate Monohydrate.
PB95-168928 00,167

\section{CALCULABLE CAPACITORS}

NIST Comparison of the Quantized Hall Resistance and the Realization of the SI Onm Through the Calculable Capacitor

PB97-119184

02,291

NIST Comparison of the Quantized Hall Resistance and the Realization of the SI Ohm Through the Calculable the Realization of the SI Ohm Through the Calcula Capacitor. Conference Proceedings, June 17-20, 1996.
02,292

\section{CALCULATION METHODS}

Halon Thermochemistry: 'Ab Initio' Calculations of the Enthalpies of Formation of Fluoromethanes.

03,289

PB96-175740

Activities of NIST (National Inst. Of Standards and Technolog

N94-23605/6 04,222

NIST Calibration of ASTM E127-Type Ultrasonic Reference Blocks.

02,702

Role of the Office of Radiation Measurement in Quality Assurance.

00,689

Journa! of Research of the National Institute of Standards and Technology. May/June 1994. Volume 99, Number 3.
PB94-219326

Sources of Uncertainty in a DVM-Based Measurement System for a Quantized Hall Resistance Standard. PB94-219334 01,884 Constant-Width Calibration Intervals for Linear Regression.

03,439

Characterization of a Health Physics Instrument Calibration Range.
PB95-164554

03,629

Tilt Effects in Optical Angle Measurements

04,294

Field Evaluation of the System for Calibration of the Marshall Compaction Hammer.

PB95-190674

Gage Block Handbook

$\begin{array}{ll} & 02,667\end{array}$

Advanced Mass Calibration and Measurement Assurance Program for State Calibration Laboratories.

02,492

Radiometer Equation for Noise Comparison Radiometers. PB96-140363

02,195

\section{CALIBRATING STANDARDS}

Overview of Reference Materials Prepared for Standardization of DNA Typing Procedures.

PB96-111653

00,611

\section{CALIBRATION}

Assessment of Uncertainties of Calibration of Resistance Thermometers at the National Institute of Standards and Technology.

02,624

Mass Unit Disseminated to Surrogated Laboratories Using the NIST Portable Mass Calibration Package.
PB94-142486
0381 Assessment of Uncertainties of Liquid-in-Glass Thermom eter Calibrations at the National Institute of Standards and Technology.

PB94-142510

02,625

Assessment of Uncertainties of Thermocouple Calibrations at NIST.

03,782

NIST Measurement Services: NIST Pressure Calibration

PB94-164043

02,892

Null-Balanced Total-Power Radiometer System NCS1

PB94-169778 02,02

Cryogenic Blackbody Calibrations at the National Institute of Standards and Technology Low Background Infrared Calibration Facility.

PB94-169802

02,117

Investigation of the Effects of Aging on the Calibration of a Kerr-Cell Measuring System for High Voltage Impulses.

02,025

Measurement and Calibration of Large-Area Alpha-Particle Sources at NIST.

PB94-172855

03,791

Development and Calibration of UVNUV Radiometric Sources.

PB94-199098 04,229

Update on the Low Background IR Calibration Facility at the National Institute of Standards and Technology. Using LORAN-C Broadcasts for Automated Frequency Calibrations.

New Expressions of Uncertainties for Humidity Calibrations at the National Institute of Standards and Tech- 


\section{KEYWORD INDEX}

Stabilization and Precise Calibration of a ContinuousWave Difference Frequency Spectrometer by Use of a Simple Transfer Cavity.

PB95-162350 04,276

Calibrating On-Wafer Probes to the Probe Tips. PB95-163945

02,352

LRM Probe-Tip Calibrations with Imperfect Resistors and Lossy Lines.

Verification of Scattering Parameter Measurements. PB95-163960

01,904

Force Calibrations in the Nanonewton Regime.

03.949

Optical Power Meter Calibration Using Tunable Laser Diodes.

PB95-169256 04,290

Self-Calibrating Fiber Optic Sensors: Potential Design Methods.

PB95-169298 02,172

Self Calibrating Fiber Optic Sensors: Potential Design Methods.

PB95-169306 02,173

Calibration of Dosimeters for the Cryogenic Irradiation of Composite Materials Using an Electron Beam. $\quad 03,968$

03,968

Control Stability of a Heat-Flow-Meter Appartus.
PB95-181194

Evaluation of the Economic Impacts Associated with the NIST Power and Energy Calibration Services. 02,461

PB95-188850

2,461

$10 \mathrm{kV}$ DC Resistive Divider Calibration.
PB95-198685 02,063

Calibration of a Superconducting Gravimeter Using Absolute Gravity Measurements.

PB95-202651 03,684

Time Domain Network Analysis Using the Multiline TRL Calibration.

PB95-202925 02,065

Geometric Characterization of Rockwell Diamond Indent-

PB95-203287

02,950

Microform Calibrations in Surface Metrology.

02,951

Energy Calibration of X-ray Photoelectron Spectrometers: Results of an Interlaboratory Comparison to Evaluate a Proposed Calibration Procedure.

PB96-102918

04,027

Intracomparison Tests of the FG5 Absolute Gravity Me-

ters.
PB96-102991

03,688

Uncertainties of the NIST Coaxial Noise Calibration Sys-

tem.

02,070

Automated Resistance Measurements at NIST.

02,274

History of NIST's Contributions to Development of Standard Reference Materials and Reference and Definitive Methods for Clinical Chemistry.

PB96-119706

03,503

Noise Characteristics Below $1 \mathrm{~Hz}$ of Zener Diode-Based Voltage Reference.

04,049

Long-Term Stability of Bayard-Alpert Gauge Performance: Results Obtained from Repeated Calibrations against the National Institute of Standards and Technology Primary Vacuum Standard.

PB96-123567

02,676

Comparison of NIST and Manufacturer Calibrations of (90) Sr+(90)Y Ophthalmic Applicators.

PB96-123708

03,634

Analysis of an Open-Ended Coaxial Probe with Lift-Off for Nondestructive Testing.

PB96-135116

01,940

Recalibration for the Final Archive of the International UItraviolet Explorer (IUE) Satellite.

PB96-135264

00,106

Dosimetry Systems for Radiation Processing.

03,717

RM Probe-Tip Calibrations Using Nonideal Standards.

PB96-135389

Fiber Coating Diameter: Toward a Glass Artifact StandPB96-140389

02,234

Dielectric Measurements on Printed-Wiring and Circuit Boards, Thın Films, and Substrates: An Overview. 02,236 Absolute Response Calibration of a Transfer Standard Cryogenic Bolometer

04,358

PB96-147103
Technical Impact of the NIST Calibration Service for Electrical Power and Energy.

02,462

PB96-147913

Metrology Approach to Unifying Rockwell C Hardness Scales.
PB96-155551

02,957

Stylus Technıque for the Direct Verification of Rockwell Diamond Intenders.

02,958
Direct Comparison Transfer of Microwave Power Sensor Calibrations.

02,086

Development of the NIST Transient Pressure and Tem. perature Calibration Facility.

PB96-160833

00,626

Residual Error Compensation of a Vision-Based Coordinate Measuring Machine.

04,091

NIST Measurement Assurance Program for Capacitance Standards at $1 \mathrm{kHz}$.

PB96-172333

02,276

Journal of Research of the National Institute of Standards and Technology, January/February 1996. Volume 101 Number 1.

PB96-175666

00,113

International Marine-Atmospheric (222)Rn Measuremen Intercomparison in Bermuda. Part 1. NIST Calibration and Methodology for Standardized Sample Additions.

PB96-175674

00,114

Journal of Research of the National Institute of Standards and Technology, March/April 1996. Volume 101, Number

PB96-177381

01,863

NIST Detector-Based Luminous Intensity Scale.

PB96-179114

01,864

Organization and Implementation of Calibration in the OS Project. Part 1.

PB96-179437 04,841

Ultrasound Power Measurement Techniques at NIST

PB96-179569 02,684

Standard Source Method for Reducing Antenna Factor

Errors in Shielded Room Measurements.

02,013

Time and Frequency Metrology.

01,556

NIST Frequency Measurement Service.

01,561

Selt-Calibrated Intelligent Optical Sensors and Systems. Selt-Calibrated Intelligent Optical Sensors and Systems.
PB96-200738

04,380

ANSI/NCSL Z540-1-1994: The Optical Technology Division's Effort.

Improving Color Measurements of Displays.

01,869

PB96-204441

02,203

Evaluation and Accreditation of State Calibration Labora-

PB97-110183

00,486

Electrical Test Structures Replicated in Silicon-on-Insulator Material.

PB97-1118

PB97-111876

02,454

Calibration in the Earth Observing System (EOS) Project. Part 2. Implementation.

04,842

Empirical Linear Prediction Applied to a NIST Calibration

PB97-112353

02,287

CALIBRATION LABORATORIES

Should NIST Accredit U.S. Calibration Laboratories.
PB95-107280

\section{CALIBRATION STANDARDS}

Improving measurement quality assurance for photon irradiations at Department of Energy facilities. Final technica report.

03,711

Recertification of the Standard Reference Material 1475A a Linear Polyethylene Resin.

PB94-161932

02,628

Determination of the Weight Average Molecular Weight of Two Poly(Ethylene Oxides), SRM 1923 and SRM 1924.

Measurement and Uncertainty of a Calibration Standard for the Scanning Electron Microscope.

PB94-219250

00,560

Stability/Instability of Gas Mixtures Containing 1,3-Butadiene in Treated Aluminum Gas Cylinders.

PB95-162285 02,546

Standard Reference Material 1744: Aluminum FreezingPoint Standard.

PB95-251732 01,055

Intercomparison of Photometric Units Maintained at NIST (USA) and PTB (Germany), 1993.

PB95-261913

04,329

Determination of the Transmittance Uniformity of Optical Filter Standard Reference Materials.

PB95-261921

02,182

Standard Reference Material for the Measurement of Particle Mobility by Electrophoretic Light Scattering

PB96-102488

00,609

Journal of Research of the National Institute of Standards and Technology, September/October 1995. Volume 100, Number 5 .

Low Electrolytic Conductivity Standards.

01,927

PB96-122098

01,081

Microform Calibration Uncertainties of Rockwell Diamond

03,280

Performance Measures for Geometric Fitting in the NIST Algorithm Testıng and Evaluation Program for Coordinate Measurement Systems.

PB96-122122

01,745 Development of a Standard Reference Material for Measconductor Silicon by Infrared

PB96-122668

02,404

Certification of the Standard Reference Material 1473a, a Low Density Polyethylene Resin

01,282

High-Speed Interconnection Characterization Using Time Domain Network Analysis.

PB96-148176

04,061

Liquid-Nitrogen-Cooled High Tc Electrical Substitution Radiometer as a Broadband IR Transter Standard.

PB96-158704

02,198

Journal of Research of the National Institute of Standards and Technology, November/December 1995. Volume 100, Number 6 .

01,949

Calibration of Electret-Based Integral Radon Monitors Using NIST Polyethylene-Encapsulated (226)Ra/(222)Rn Emanation (PERE) Standards.

PB96-159223

01,950

Fabrication Issues for the Prototype National Institute of Standards and Technology SRM 2090A Scanning Electron Microscope Magnification Calibration Standard. PB96-160585

01,131

Certification of Phencyclidine in Lyophllized Human Urine Reference Materials.

PB96-160692

03,508

Calibration Service for Coaxial Reference Standards for Microwave Powe

01,958

Investigations of Sulfur Interferences in the Extraction of Methylmercury from Marine Tissues.

PB96- 190020

03,482

Coaxial Line-Reflect-Match Calibration

PB96-200118 02,246

How to Get NIST-Traceable Time on Your Computer

PB96-200647

01,559

Introduction to Frequency Calibration. Part 1

01,560

Scanning Electron Microscope Magnification Calibration Interlaboratory Study.

PB96-201082

01,164

NIST Traceable Reference Material Program for Gas

Standards: Standard Reference Materials.

00,644

PB96-210786

Statistical Aspects of the Certification of Chemical Batch
SRMs. Standard Reference Materials.

SRMs. Standard Reference Materials

PB96-210877

State Weights and Measures Laboratories: Program 
Sealed Water Calorimeter for Measuring Absorbed Dose. Effects of Specimen Edge Conditions on Heat Release

PB95-152864

00,375

New Coaxial Microwave Microcalorimeter Evaluation PB95-153227

01,892

Crystal Structure of Annealed and As-Prepared $\mathrm{HgBa2} \mathrm{CaCu} 2 \mathrm{O} 6$ +delta Superconductors.

PB95-161105

03,927

High-Temperature Adiabatic Calorimeter for Constant-Volume Heat Capacity Measurements of Compressed Gases and Liquids.

00,989

Hot-Electron Microcalorimeters for X-ray and Phonon De-

tection. 04,644

Method to Determine the Calorimetric Equivalence Correction for a Coaxial Microwave Microcalorimeter. Burning Rate and Flame Heat Flux For PMMA in the

Cone Calorimeter.
PB95-216990 00,393

Some Factors Affecting the Design of a Calorimeter Hood and Exhaust.

00,302

NBS/NIST Peltier-Effect Microcalorimeter: A Four-Decade Review.

PB96-102769

01,064

Hot-Electron Microcalorimeters as High-Resolution X-ray Detectors.

04,739

Calorimeters for Calibration of High-Dose Dosimeters in High-Energy Electron Beams.
PB96-135272

Thermal Modeling and Analysis of Laser Calorimeters.

04,055

PB96-140405

04,354

Deep-UV Excimer Laser Measurements at NIST.
PB96-141031

Examination of the Correlation between Cone Calorimeter

Data and Full-Scale Furniture Mock-Up Fires.

01,417

Molar Heat Capacity at Constant Volume for Air from 67 to $300 \mathrm{~K}$ at Pressures to $35 \mathrm{MPa}$.

00,515

Molar Heat Capacity at Constant Volume for $(x \mathrm{CO} 2+(1-$ x) C2H6) from 220 to $340 \mathrm{~K}$ at Pressures to $35 \mathrm{MPa}$.
$\mathrm{PB} 96-167135$

Office Work Station Heat Release Rate Study: Full Scale versus Bench Scale.

versus Bench Sca
PB96-190178

01,428

Hot-Electron Microcalorimeter for X-ray Detection Using a Superconducting Transition Edge Sensor with Electrothermal Feedback.

PB96-200399

04,792

Hot-Electron-Microcalorimeters with $0.25 \mathrm{~mm}(2)$ Area.

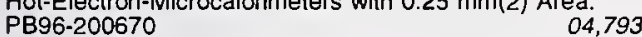

Flammability Characterization with the Lift Apparatus and the Cone Calorimeter.
PB97-110050

01,435

Problem of Convection in the Water Absorbed Dose Cal-

orimeter.

03,523

CALORIMETRY

Equilibrium and Calorimetric Investigation of the Hydrolysis of L-Tryptophan to (Indole + Pyruvate + Ammonia).
PB95-163317 Thermodynamics of the Hydrolysis of 3,4,5Trihydroxybenzoic Acid Propyl Ester (n-Propylgallate) to 3,4,5-Trihydroxybenzoic Acid (Gallic Acid) and Propan-I-ol in Aqueous Media and in Toluene. UV Absorption Cross Sections of Methylchloroform: Temperature-Dependent Gas and Liquid Phase Measurements.

PB96-201041

00,643

Conference Report: Calorimetry Conference (50th) PB97-122279

03,722

CALS

Computer Graphics Metafile (CGM): Procedures for NIST CGM Validation Test Service.

01,804

Initial Graphics Exchange Specification (IGES): Procedures for the NIST IGES Validation Test Service.
PB95-171427
02,780

T2, 780

SUPPORT)

Impact of Computer-Aided Acquisition and Logistic Support (CALS) in th

01,756

CALS (COMPUTER-AIDED ACQUISITION AND LOGISTICS

\section{CALS}

CALS-Automated Interchange of Technical Information.
PB94-962000 CALS-Digital Representation for Communication of Product Data: IGES Application Subsets.

03,658
CALS-Markup Requirements and Generic Style Specifications for Electronic Printed Output and Exchange of Text. PB94-962200 03,659
0 CALS-Raster Graphics Representation Binary Format Re-

quirements.
PB94-962300 03,660

CALS-Digital Representation for Communication of Illustration Date: CGM Application Profile.

tration Date: CGr

03,661

CALS-Department of Defense Computer Aided Acquisi-

tion Logistic Support (CALS).

03,662

CALS-Contractor Integrated Technical Information Service (CITIS), Functional Requirements.

PB94-962600

03,663

CALS (CONTINUOUS ACQUISITION AND LIFE-CYYCLE

SUPPORT)

CALS-Automated Interchange of Technical Information.
PB95- 962000

CALS-Digital Representation for Communication of Prod-

uct Data: IGES Application Subsets.
PB95-962100

CALS-Markup Requirements and Generic Style Specifica-

tions for Electronic Printed Output and Exchange of Text.

PB95-962200

03,668

CALS-Raster Graphics Representation Binary Format Requirements.

03,669

CALS-Digital Representation for Communication of Illustration Date: CGM Application Profile.

03,670

CALS-Department of Defense Computer Aided AcquisiCALS-Department of Defense Computer Aided Acquisi-
tion Logistic Support (CALS).
PB95-962500
P3,671

03,671
ServCALS-Contractor Integrated Technical Information Serv-
ice (CITIS), Functional Requirements.
PB95-962600

CAMERA FIXATION

Reconstruction during Camera Fixation

PB95-162236

01,593

Unified Approach to Camera Fixation and Vision-Based Road Following

01,594

CANCELLOUS BONE

Reinforcement of Cancellous Bone Screws with Calcium Phosphate Cement.

PB96-158001

00,179

CANDELA

NIST Detector-Based Luminous Intensity Scale.

01,864

CANDELA SCALE

Detector-Based Candela Scale and Related Photometric Calibration Procedures at NIST.

PB95-161949

04,273

CANTILEVERS

Development of Highly Conductive Cantilevers for Atomic

Force Microscopy Point Contact Measurements.
PB96-138573

04,751

CAPACITANCE

NIST Capacitance Measurement Assurance Program

PB94-200060

02,254

Application of Single Electron Tunneling: Precision Capacitance Ratio Measurements.

04,703

NIST Measurement Assurance Program for Capacitance Standards at $1 \mathrm{kHz}$
PB96-172333

02,276

Results of Capacitance Ratio Measurements for the Single Electron Pump-Capacitor Charging Experiment

PB97-113286

04,813

CAPACITANCE BRIDGES

Cryogenic Precision Capacitance Bridge Using a Single Electron Tunneling Electrometer.

PB95-126074

04,529

Cryogenic Precision Capacitance Bridge Using a Single Electron Tunneling Electrometer.

02,040

Cryogenic Precision Capacitance Bridge Using a Single Electron Tunneling Electrometer.

02,072

Database for the Static Dielectric Constant of Water and

Steam.

01,103

CAPACITANCE IMAGING

Scanning Capacitance Microscopy Measurements and Modeling: Progress Towards Dopant Profiling of Silicon.
PB96-180070

CAPACITOR RATIO

Proposed Tests to Evaluate the Frequency-Dependent Capacitor Ratio for Single Electron Tunneling Experiment.

01,982

CAPACITORS

Development and Characterization of Insulating Layers on Silicon Carbide: Annual Report for February 14, 1988 to February 14, 1989

02,295

Capacitors with Very Low Loss: Cryogenic Vacuum-Gap Capacitors.

PB97-122600

02,293

CAPELLA STAR

Ultraviolet Observations of Stellar Coronae: Early Results from HST.

PB94-213428

00,064

Hydrogen Lyman-alpha Emission of Capella

Transition Regions of Capella.

PB96-123336

00,075

Transition Regions of Capella (1995)

PB96-176714

00,105

CAPILLARY ELECTROPHORESIS

Electrophoretic Separations of Polymerase Chain Reaction: Amplified DNA Fragments in DNA Typing Using a Capillary Electrophoresis-Lased Induced Fluorescence System.

PB95-163036

03,536

Enhanced Detection of PCR Products Through Use of TOTO and YOYO Intercalating Dyes with Laser Induced Fluorescence - Capillary Electrophoresis.

PB95-164653

00,599

CAPILLARY FLOW

Lubrication Theory for Reactive Spreading of a Thin

Prop.

02,865

CAPILLARY TUBES

Experimental Data and Theoretical Modeling of Gas Flows Through Metal Capillary Leaks.

04,206

CARBON

Recommendations for the Disposal of Carbon-14 Wastes.
AD-A279 133/3

Ion Broadening Parameters for Several Argon and Carbon Lines.

03,843

Carbon Acidities of Aromatic Compounds. 1. Effects of InRing Aza and External Electron-Withdrawing Groups. PB94-216595 00,860

Microstructural Characterization of Cobalt-Tungsten Coated Graphite Fibers.

CARBON 13

Two-Dimensional POMMIE Carbon-Proton Chemical Shift Correlated (13)C NMR Spectrum Editing

PB94-200508

00,809

CARBON 14

Factorial Design Techniques Applied to Optimizatıon of AMS Graphite Target Preparation.

PB95-151197

00,584

Radiocarbon Measurements of Atmospheric Volatile Or-

ganic Compounds: Quantifying the Biogenic Contribution.

PB97-122352

CARBON 14 TARGET Comparative Study of Fe-C Bead and Graphite Target
Performance with the National Ocean Sciences AMS (NOSAMS) Facility Recombinator Ion Source.

PB95-175790

00,693

CARBON 60

Simulation of C60 Through the Plastic Transition Temperatures.

04,713

\section{CARBON ACIDITY}

Carbon Acidities of Aromatic Compounds. 1. Effects of InRing Aza and External Electron-Withdrawing Groups. PB94-216595

CARBON DIOXIDE

Photoionization of Small Molecules Using Synchrotron Radiation

PB94-211505

03,841

Modified Leung-Griffiths Model of Vapor-Liquid Equi- 
Collisional Alignment of $\mathrm{CO} 2$ Rotational Angular Momentum States in a Supersonic Expansion.

PB96-103171

Few Caveats on Carbon Dioxide Monitoring. $\quad 02,562$ 02,562
PB96-122650 Diketonates in Supercritical Carbon Dioxide. PB96-164215

CARBON DIOXIDE COMPLEXES

Infrared Spectra of van der Waals Complexes of Importance in Planetary Atmospheres.

00.071

Signatures of Large Amplitude Motion in a Weakly Bound Complex: High-Resolution IR Spectroscopy and Quantum Calculations for $\mathrm{HeCO} 2$.

PB95-203485

01,054

\section{CARBON DIOXIDE LASERS}

Efficient $\mathrm{Br}\left({ }^{*}\right)$ Laser Pumped by Frequency-Doubled $\mathrm{Nd}$ $Y A G$ and Electronic-to-Vibrational Transfer-Pumped $\mathrm{CO} 2$ and $\mathrm{HCN}$ Lasers.

PB95-108684

04,248 New cw CO2 Laser Lines: The 9-mu m Hot Band.
PB95-168548

04,281 Improved Molecular Constants and Frequencies for the

quency Measurements
PB95-180634

04,312

CARBON FLUORIDES

Charge Transfer and Collision-Induced Dissociation Reactions of CF $(2+)$ and CF2 $(2+)$ with the Rare Gases at a Laboratory Collision Energy of $49 \mathrm{eV}$.

PB94-185584

00,775

Collision-Induced Neutral Loss Reactions of Molecular Dications.

CARBON ISOTOPES

00,780

New IUPAC Guidelines for the Reporting of Stable Hydrogen, Carbon, and Oxygen Isotope-Ratio Data. Letter to the Editor

PB95-261962

01,058

CARBON-LIKE IONS

Atomic Branching Ratio Data for Carbon-Like Ions.

PB94-212842

03,855

CARBON MONOXIDE

Vibrational Spectra of Molecular Ions Isolated in Solid Neon. 7. $\mathrm{CO}(+), \quad \mathrm{C}_{2} \mathrm{O} 2(+)$, and $\mathrm{C}_{2} \mathrm{O} 2(-)$. (Reannouncement with New Availability Information).
AD-A239 729/7

Compilation of the Physical Equilibria and Related Properties of the Hydrogen-Carbon Monoxide System.

AD-A286 603/6

00,716

Hot Carrier Excitation of Adlayers: Time-Resolved Measurement of Adsorbate-Lattice Coupling

00,758

Photodecomposition Dynamics of $\mathrm{Mo}(\mathrm{CO}) 6 / \mathrm{Si}(111) 7 \times 7$ : CO Internal State and Translational Energy DistributionsTranslation.

PB94-199288

00,687

Time-Resolved Probes of Surface Dynamics.

PB94-199957

00,803

Global Equivalence Ratio Concept and the Prediction of Carbon Monoxide Formation in Enclosure Fires.

PB94-207511

00,313

Photoionization of Small Molecules Using Synchrotron

Radiation.
PB94-211505 03,841

Vibrational Relaxation Measurements of Carbon Monoxide on Metal Clusters.

PB94-211810

00,820

Laser-Induced Fluorescence Measurements of Rotationally Resolved Velocity Distributions for $\mathrm{CO}^{(+)}$ Drifted in He.

PB94-213139

00,848

Measurement of $\mathrm{CO}$ Pressures in the Ultrahigh Vacuum Regime Using Resonance-Enhanced Multiphoton-Ionization Time-of-Flight Mass Spectroscopy.

PB94-216041

03,864

Picosecond Measurement of Substrate-to-Adsorbate Energy Transfer: The Frustrated Translation of CO/Pt(111)-Translation.

00,895

Fundamental Mechanisms for $\mathrm{CO}$ and Soot Formation

PB95-143160 01,380

Oxidation of Soot and Carbon Monoxide in Hydrocarbon Diffusion Flames.

PB95-150215

01,382

Optical Measurements of Alomic Hydrogen, Hydroxyl,

and Carbon Monoxide in Hydrocarbon Diffusion Flames.

PB95-150900

02,487

Rotational Far
PB95-152187

00,941

Laser Spectroscopy of Carbon Monoxide: A Frequency Reference for the Far Infrared.

PB95.163606

04,277

Laser Flash Photolysis/Time-Resolved FTIR Emission Study of a New Channel in the Reaction of $\mathrm{CH} 3+\mathrm{O}$ : Production of $\mathrm{CO}(\mathrm{v})$.
PB95-164281

00,974
Shape-Resonance-Enhanced Continuum-Continuum Coupling in Photoionization of $\mathrm{CO} 2$.

00,983

Inner-Valence States $\mathrm{CO}(+)$ between $22 \mathrm{eV}$ and $46 \mathrm{eV}$ Studied by High Resolution Photoelectron Spectroscopy and $a b$ Initio Cl Calculations.

PB95-180055

03,961

Slow-Electron Collisions with CO Molecules in an ExactExchange Plus Parameter-Free Polarization Model. PB95-202719

Carbon Monoxide Production in Compartment Fires: Reduced-Scale Enclosure Test Facility

01,394

Photodesorption Dynamics of CO from Si(111): The Role of Surface Defects.

PB96-111646

03,066

Relationship between Indoor Air Quality and Carbon Di-

PB97-111249

02,569

Carbon Monoxide Dispersion in Residential Buildings: Literature Review and Technical Analysis.

02,571

CARBON OXYSULFIDE

Extension of Heterodyne Frequency Measurements on OCS to $87 \mathrm{THz}(2900 \mathrm{~cm}(-1))$.

00,811

Sub-Doppler Frequency Measurements on OCS at 87 Sub-Doppler Frequency Measurements on OCS at 87 Thz ( 3.4 micrometers) with
siderations and Details. siderations and
PB95-128633

04,255

\section{CARBON STEELS}

Comparison of the Corrosion Rates of FeAl, Fe(sub 3)Al and Steel in Distilled Water and 0.5 M Sodium Chloride. Technical Report Number 2, January-March 1991. DE94017332

03,186

Characterization of the Hydrogen Induced Cold Cracking Susceptibility at Simulated Weld Zones in HSLA-100

PB94-174505

03,746

Langevin Approach to Hysteresis and Barkhausen Modeling in Steel.

03,206

CARBONACEOUS MATERIALS

Sources of Urban Contemporary Carbon Aerosol.
PB95-175659

\section{CARBONATE RADICALS}

Electron Transfer Reaction Rates and Equilibria of the Carbonate and Sulfate Radical Anions.

PB94-212180

00,829

Temperature Dependence of the Rate Constants for Reactions of the Carbonate Radical with Organic and Inorganic Reductants

00,831

CARBONATES

Temperature Dependence of the Rate Constants for Reactions of the Carbonate Radical with Organic and Inorganic Reductants.

CARBONIC ANHYDRASE

Active Site lonicity and the Mechanism of Carbonic Anhy-

PB94-212974

00,843

\section{CARBONYL COMPOUNDS}

Sub-Doppler Frequency Measurements on OCS at 87 PB96-102215

04,330

CARBONYL SULFIDE

Extension of Heterodyne Frequency Measurements on OCS to $87 \mathrm{THz}(2900 \mathrm{~cm}(-1))$.

00.811

Sub-Doppler Frequency Measurements on OCS at 87 Thz (3.4 micrometers) with the CO Overtone Laser: Considerations and Details.

PB95-128633

04,255

CARCINOGENESIS

Novel Activity of E. coli uracil DNA N-glycosylase Excision of Isodialuric Acid (5,6-dihydroxyuracil), a Major Product of Oxidative DNA Damage, from DNA. PB96-110747

03,543

CARCINOGENS

DNA Base Modifications in Renal Chromatin of Wistar Rats Treated with a Renal Carcinogen, Ferric Nitrilotriacetate
PB95-150363

03,648

Treatment of Wistar Rats with a Renal Carcinogen, Ferric tween Thymine and Tyrosine in Their Renal Chromatin.
PB96-112115

03,649

CAREER DEVELOPMENT

Robotics Application to Highway Transportation. Volume 1. Final Report
PB95-203790

03,654

CAROTENE

Development of Engineered Stationary Phases for the Separation of Carotenoid Isomers.

PB95-150249

00,578

Isolation and Structural Elucidation of the Predominant Geometrical Isomers of alpha-Carotene.

PB96-190061

00,640

CAROTENOIDS

Carotenoid Reversed-Phase High-Performance Liquid Chromatography Methods: Reference Compendium. Individual Carotenoid Content of SRM 1548 Total Diet and Influence of Storage Temperature, Lyophilization, and Irradiation on Dietary Carotenoids.

PB94-200524

00,033

Liquid Chromatographic Method for the Determination of Carotenoids, Retinoids, and Tocopherols in Human Serum and in Food.

00,593

Population Distributions and Intralaboratory Reproducibility for Fat-Soluble Vitamin-Related Compounds in Human

PB96-155536

00,624

CARPETS

National Voluntary Laboratory Accreditation Program: Carpet and Carpet Cushion.

PB95-155560

00,295

CARQUINEZ STRAIT

Histopathology, Blood Chemistry, and Physiological Status of Normal and Moribund Striped Bass ('Morone saxatilis') Involved in Summer Mortality ('Die-Off') in the Sacramento-San Joaquin Delta of California.

00,034

\section{CARRIER DENSITY}

Multicarrier Characterization Method for Extracting Mobilities and Carrier Densities of Semiconductors from Variable Magnetic Field Measurements.

PB94-212776

02,317

Transverse Magnetoresistance: A Novel Two-Terminal Method for Measuring the Carrier Density and Mobility of a Semiconductor Laye

02,332

CARRIER MOBILITY

Multicarrier Characterization Method for Extracting Mobilities and Carrier Densities of Semiconductors from Variable Magnetic Field Measurements.

PB94-212776

02,317

Transverse Magnetoresistance: A Novel Two-Terminal Method for Measuring the Carrier Density and Mobility of a Semiconductor Layer.

PB95-150066

02,332

\section{CARRIERS}

Model for Determining the Density and Mobility of Carriers in Thin Semiconducting Layers with Only Two ConPB96-102702

02,378

CARTRIDGES (EXPLOSIVES)

Specification for Interoperability between Ballistic Imaging

Systems. Part 1. Cartridge Cases.

CARVON MONOXIDE

Chemical Stability of Upper-Layer Fire Gases.

PB96-123385 
Condensed Catalogue of Electromagnetic Environment Measurements, $30-300 \mathrm{~Hz}$

PB95-162210

01,899

Publications of the National Institute of Standards and Technology 1992 Catalog.

00,014

NIST Standard Reference Materials (Trade Name) Catalog $1995-1996$.

00,508

NIST Standard Reference Data Products Catalog, 1995. 6. Achieve with Standard Reference Data.

96. Achieve with

Publications of the National Institute of Standards and Technology 1993 Catalog.

00,017

CATALYSTS

Changes in the Redox State of Iridium Oxide Clusters and Their Relation to Catalytic Water Oxidation Radiolytic and Electrochemical Studies. PB95-107017

00,864

\section{CATALYTIC CRACKING}

Process Gas Chromatography Detector for Hydrocarbons Based on Catalytic Cracking.

PB95-141099

02,485

CATHODE RAY TUBE SCREENS

Display-Measurement Round-Robin

PB96-119227

02,186

CATHODE RAY TUBES

Improving Color Measurements of Displays. PB96-204441

02,203

CATHODIC DISBONDMENT MODEL

Non-Osmotic, Defect-Controlled Cathodic Disbondment of Coating from a Steel Substrate.

PB94-216447

03,120

CATIONS

Vibrational Spectra of Molecular Ions Isolated in Solid Neon. 11. $\mathrm{NO} 2(+), \mathrm{NO} 2(-)$, and $\mathrm{NO} 3(-)$

00,708

Tetrahedral-Framework Lithium Zinc Phosphate Phases: Location of Light-Atom Positions in LiZnPO4 $\mathrm{H} 2 \mathrm{O}$ by Powder Neutron Diffraction and Structure Determination of LiZnPO 4 by ab Initio Methods.

PB96-160510

01,129

CAUSALITY

Causality and Maxwell's Equations.

PBTATION

Cavitation Damage During Flexural Creep of SiAIONYAG Ceramics.

PB94-200110

03,036

Cavitation Contributes Substantially to Tensile Creep in Silicon Nitride.

Tension/Compression Creep Asymmetry in Si3N4

PB97-110258

03,171

CAVITIES

Dimensional Characterization of Small Bores: A Survey.

PB95-162202 02,65

Aperture Excitation of Electrically Large, Lossy Cavities.

PB95-175675

00,031

Cavity Evolution during Tensil Creep of Si3N4.
PB96-204193

CAVITY LINERS

Texture Study of Two Molybdenum Shaped Charge Liners by Neutron Diffraction.

PB94-200177 03,754

Microstructure Study of Molybdenum Liners by Neutron Diffraction.

CAVTYY 0

03,756

Aperture Excitation of Electrically Large, Lossy Cavities. PB94-145711

00,029

CAVITY RESONATORS

NIST 60-Millimeter Diameter Cylindrical Cavity Resonator:

Performance Evaluation for Permittivity Measurements.

PB94-151776

02,251

Dielectric Studies of Fluids with Reentrant Resonators. PB95-153730

00,952

CAVTY RINGDOWN

Laser Bandwidth Effects in Ouantitative Cavity RingDown Spectroscopy.

04,394

CDIF (CASE DATA INTERCHANGE FORMAT)

Mapping Integration Definition for Information Modeling (IDEF 1X) Model into CASE Data Interchange Forma (CDIF) Transfer File.

01,711

Mapping Integration Definition for Function Modeling (IDEFO) Model into CASE Data Interchange Forma

(CDIF) Transfer File.
PB96-109533

01,741

CEILING JETS

Prediction of Fire Dynamics.

PB94-193620

00,336

Comparison of Wall-Fire Behavior With and Without a Ceiling.

00,342
Field Modeling: Simulating the Effect of Sloped Beamed Ceilings on Detector and Sprinkler Response.

01,406

PB96-122866

State of the Art Report on Seismic Design Requirements for Nonstructural Building Components.

00,308

CEILINGS (ARCHITECTURE)

Fire-Plume-Generated Ceiling Jet Characteristics and

Convective Heat Transfer to Ceiling and Wall Surfaces in

a Two-Layer Fire Environment: Uniform Temperature

PB95-164711

00,382

Algorithm to Describe the Spread of a Wall Fire under

PBiling.

00,261

CELL DAMAGE

Microdosimetry and Cellular Radiation Effects of Radon Progency in Human Bronchial Airways.

03,625

Radon in the Lung.

PB97-110035

03,638

CELL MASS

In situ Fluorescence Cell Mass Measurements of 'Saccharomyces cerevisiae' Using Cellular Tryptophan.
PB96-135041

CELL MEMBRANE

Electropermeabilization of Cell Membranes: Effect of the Resting Membrane Potential.

PB95-16329 1

CELL MOVEMENT

Gravity Dependent Processes and Intracellular Motion PB95-163382

03,538

CELLS

Gaseous Electronics Conference RF Reference Cell: An Introduction.

PB96-113329

02,387

Current and Voltage Measurements in the Gaseous Elec-

tronics Conterence RF Reference Cell.

PB96-113337

02,388

Optical Emission Spectroscopy on the Gaseous Electronics Conference RF Reference Cell.

02,389

Optical Diagnostics in the Gaseous Electronics Conterence Reference Cell. PB96-113352

02,390

Studies of Ion Kinetic-Energy Distributions in the Gaseous Electronics Conference RF Reference Cell.

PB96-113360

02,391

Microwave Diagnostic Results from the Gaseous Elecronics Conference RF Reference Cell.

02,392

Langmuir Probe Measurements in the Gaseous Electronics Conference RF Reference Cell.

PB96-113386

02,393

Inductively Coupled Plasma Source for the Gaseous Electronics cont

02,394

Reactive Ion Etching in the Gaseous Electronics Con erence RF Reference Cell.

PB96-113402

02,395

Dusty Plasma Studies in the Gaseous Electronics Conerence Reference Cell.

PB96-113410

02,396

One-Dimensional Modeling Studies of the Gaseous Electronics Conterence RF Rejerence Cell.

PB96-113428

02,397

Two-Dimensional Self-Consistent Radio Frequency Plasma Simulations Relevant to the Gaseous Electronics Conference RF Reference Cell.

PB96-113436

02,398

CELLS (BIOLOGY)

Gravity Dependent Processes and Intracellular Motion.

03,538

Fluorescence Measurements of Tetracycline in High Cell Mass for Fermentation Monitoring

PB95-175709

00,601

Repair of Products of Oxidative DNA Base Damage in Human Cells.

03,555

CELLULOSE

Behavior of Charring Materials in Simulated Fire Environ-

PB94-196045

01,368

Transition from Localized Ignition to Flame Spread Over Thin Cellulosic Material in Microgravity.

gnition and Subsequent Flame Spread Over a Thin Cellulosic Material.

PB96-160270

04,836

Ignition and Transition to Flame Spread Over a Thermally Thin Cellulosic Sheet in a Microgravity Environment. PB96-160288

04,837

CELLULOSE TRIACETATE

Solid State (13)C NMR and Raman Studies of Cellulose Triacetate: Oligomers, Polymorphism, and Inferences about Chain Polarity.

01,289

CELP (CODE EXCITED LINEAR PREDICTION)

Channel Coding for Code Excited Linear Prediction (CELP) Encoded Speech in Mobile Radio Applications. 95-143178

CEMENT

Cellular Automaton Simulations of Cement Hydration and Microstructure Development.

PB95-175055

01,320

CEMENT PASTES

Serial Sectioning of Hardened Cement Paste for Scanning Electron Microscopy

EMENT SYSTEMS

Formation of Hydroxyapatte in Cement Systems.
PB95-175261

01,305

MENTS

Digitized Simulation of Mercury Intrusion Porosimetry.
PB94-172236

Serial Sectioning of Hardened Cement Paste for Scanning Electron Microscopy.

PB94-172640

01,305

Ouantitative Phase Abundance Analysis of Three Cement

Clinker Reference Materials by Scanning Electron Micros-

PB94-173051

00,333

Expansion of Cementitious Materials Exposed to Sulfate

Solutions.
PB94-185782

02,577

Sulfate Attack of Cem

tions and Expansions.

PB94-187317

Digitized Direct Simulation Model of the Microstructural Development of Cement Paste.

PB94-198777

01,309

ment.

PB94-198785

01,310

Modelling the Leaching of Calcium Hydroxide from $\mathrm{Ce}$ ment Paste: Effects on Pore Space Percolation and Diffusivity.

Diffusion Studies in a Digital-Image-Based Cement Paste Microstructural Model.

PB94-198801

01,312

Survey of Recent Cementitious Materials Research in Western Europe
PB94-218583

Fundamental Computer Simulation Models for CementBased Materials.

PB95-126009

00,364

Rheology of F

00,378

Unreacted Cement Content in Macro-Defect-Free Composites: Impact on Processing-Structure-Property Rela-

PB96-141270

03,174

Application of Digital-Image-Based Models to Cement-Based Materials. 
Diffusive Crack Growth at a Bimaterial Interface PB96-204110

CERAMIC FIBERS

Wear Mechanism Maps of Ceramics.

03.229

Coating of Fibers by Colloidal Techniques in Ceramic Composites.

PB94-216256 03,196

Calculation of the Thermal Conductivity and Gas Permeability in a Uniaxial Bundle of Fibers.

PB95-180931

03,058

\section{CERAMIC MATERIALS}

Small-Angle Neutron Scattering Characterization of Processing/Microstructure Relationships in the Sintering of Crystalline and Glassy Ceramics. (Reannouncement with New Availability Intormation).

03,025

Standard Materials. A Descriptive List with Prices.

Standard Materials. A Descriptive List with Prices.
AD-A278 140/9

00,500

Environmentally Enhanced Fracture of Ceramics.

PB95.125746

03,046

Tensile Creep of a Silicon Nitride Ceramic.

03, 049

Damage Processes in Ceramics Resulting from Diamond Tool Indentation and Scratching in Various Environments. PB96-102983 03,065

Fracture Mechanism Maps: Their Applicability to Silicon Nitride.

PB96-204532

03,094

CERAMIC MATRIX COMPOSITES High Temperature Degradation of Structural Composites.
PB94-172848 Matrix Grain Bridging Contribution to the Toughness of Whisker Reinforced Ceramics.

PB94-198645

03,134

Interface Properties for Ceramic Composites from a Sin. gle Fiber Pull-Out Test.

PB94-199361

03,135

Micro-Mechanical Aspects of Asperity-Controlled Friction in Fiber-Toughened Ceramic Composites.

03,136

Generic Model for Creep Rupture Lifetime Estimation on Fibrous Ceramic Composites.

PB94-200235

03,137

Assessment of Testing Methodology for Ceramic Matrix

Composites.
PB94-200532 03,139

Effect of Microstructure on the Wear Transition of Zirconia-Toughened Alumina.

03,141

Creep Rupture of MoSi2/SiCp Composites.

$\begin{array}{lr}\text { PB95-152294 } & 03,154 \\ \text { Fracture Toughness of Advanced Ceramics at Room }\end{array}$ Temperature: A Vamas Round-Robin.

03, 160

PB95-162194

Creep and Creep Rupture of Ceramic Matrix Composites.

PB95-163929 03, 165

Fracture of Silicon Nitride and Silicon Carbide at Elevated Temperatures.

CERAMIC POWDER

03,179

Variances in the Measurement of Ceramic Powder Properties

PB97-110316

03,100

CERAMIC SLURRIES

NMR Characterization of Injection-Moulded Alumina Green Compacts. Part 2. T2-Weighted Proton Imaging.

PB96-201181 01,165

\section{CERAMICS}

Classification of Advanced Technical Ceramics.

N94-35335/6

03,030

Ceramics Technical Activities, 1993 (NAS-NRC Assessment Panel April 21-22, 1994)

03,031

Fracture Mechanics Analysis of Near-Surface Cracks.

PB94-172400 03,230

Error Propagation Biases in the Calculation of Indentation Fracture Toughness for Ceramics.

03,032

Cavitation Damage During Flexural Creep of SiAIONYAG Ceramics.

PB94-200110

03,036

Transient Subcritical Crack-Growth Behavior in Trans-

formation-Toughened Ceramics.

03,038

Observed and Theoretical Creep Rates for an Alumina Ceramic and a Silicon Nitride Ceramic in Flexure.

PB94-212958

03,040

Control of Friction and Wear of Alpha-Alumina with a

Composite Solid-Lubricant Coating.

03,225

New Materials, Advanced Ceramics and Standards.

PB95-140208

03,047

Modeling Ceramic Sub-Micron Particle Formation from the Vapor Using Detailed Chemical Kinetics: Comparison with In-situ Laser Diagnostics.

PB95-151965
Multiphoton Ionization Spectroscopy Measurements of Silicon Atoms during Vapor Phase Synthesis of Ceramic Particles.

PB95-151999

03,913

Simulation of Ceramic Particle Formation: Comparison with In-situ Measurements.

00,674

B95.152013

Analysis of Physical Properties of Ceramic Powders in an
International Interlaboratory Comparison Program.
PB95-161501 03,050 PB95-161501

astable

Wear of Human Enamel against

PB95-161972

00,161

Recent VAMAS Activity in Ceramics.

03,051

Measuring Contact Charge Transfer at Interfaces: A New Experimental Technique.

03,053

B95-164570

Polyelectrolytes as Dispersants in Colloidal Processing of
Silicon Nitride Ceramics.
$\begin{array}{ll}\text { PB95-175568 } & 03,055\end{array}$ PB95-175568

Evaluation of Wear Resistant Ceramic Valve Seats in
Gas-Fueled Power Generation Engines. Topical Report,

PB95-200218 02,466

Structural Ceramics Database. Topical Report, June 1989-May 1991

PB95-203758

03,060

Room-Temperature Flexure Fixture for Advanced Ceram-

PB95-210498

03,061

Boundary Lubrication of Silicon Nitride.

03,226

Evaluation of Thermal Wave Imaging for Detection of Machining Damage in Ceramics.

PB95-220547

03,062

Ceramic Powders Characterization: Results of an International Laboratory Study

PB95-270039

02,672

Electroacoustic Characterization of Particle Size and Zeta Potential in Moderately Concentrated Suspensions

PB96-119425

Chemically Assisted Machining of Si3N4.

01,079

PB96-122999

03,072

High-Temperature Furnace for In situ Small-Angle Neuron Scattering during Ceramic Processing.

03,743

NIST Metrology Program on Electromagnetic Characterization of Materials.

PB96-156062

01,944

Model for Microcrack Initiation and Propagation beneath Hertzian Contacts in Polycrystalline Ceramics.

03,077

$6 \cdot 163704$

Preparation and Crystal Structure of Sr5TiNb4017.
PB96-167341 04,107

Reference Relations for the Evaluation of the Materials Properties of Orthorhombic YBa2Cu3OX PB96-176763

04,782

Indentation Fatigue: A Simple Cyclic Hertzian Test for Measuring Damage Accumulation in Polycrystalline Ceamics.

PB96-180013

03,084

Transient Creep Behaviour of Hot Isostatically Pressed Silicon Nitride
PB96-180278

03,086

Ceramics Technical Activities, 1995

03,087

Need for Advanced Characterization Techniques in Prod uct Manufacturing: A Case Study on Ceramic Matrix Composites.

PB96-204060

03,089

Life Prediction of a Continuous Fiber Reinforced Ceramic Composite Under Creep Conditions.

PB96-204128

Creep and Creep Rupture of Structural Ceramics.

PB96-204524

03,093

Flaw-Insensitive Ceramics.

PB97-110027 03,095

Characterizing Materials Properties for Ceramic Matrix Composites.

PB97-110282

03,097

Database Development and Management (Project A.2.2) The Annual Report for 1992-1993.

PB97-110290

03,098

Role of Corrosion in a Material Selector Expert System for Advanced Structural Ceramics.

03,099

Postfailure Subsidiary Cracking from Indentation Flaws in Brittle Materials

PB97-110340

03,103

High Temperature Structural Reliability of Silicon Nitride.

PB97-110456

03,104

Tensile Creep Testing of Structural Ceramics.

03,105

Surface Roughness of Glass-Ceramic Insert. Composite Surface Roughness of Glass-Ceramic Insert. Composite
Restorations: Assessing Several Polishing Techniques.
PB97-119010
03,583

Nanoindentation and Instrumented Scratching Measure. ments on Hard Coatings.

PB97-122477

Wear Modeling of Si-Based Ceramics.

03,112

Chemical Effect in Ceramics Grinding

PB97-122592

03,113

CERTAINTY GRID TO OBJECT BOUNDARY

Certainty Grid to Object Boundary Algorithm.

PB94-203510

01,835

CERTIFICATION

National Center for Standards and Certification Information: Service and Programs.

N95-15938/O

02,717

Recertification of the Standard Reference Material 1475A.

a Linear Polyethylene Resin.

02,628

National Voluntary Laboratory Accreditation Program: Bulk Asbestos Analysis.

PB95-138129

02,541

National Voluntary Laboratory Accreditation Program:

Construction Materials Testing.

PB95.155552

01,319

National Voluntary Laboratory Accreditation Program:

Carpet and Carpet Cushion.

PB95-155560

00,295

Standard Reference Materials for the Determination of

Trace Organic Constituents in Environmental Samples.

National Voluntary Laboratory Accreditation Program (NVLAP): Wood Based Products.

PB95-170429

03,405

National Voluntary Laboratory Accreditation Program: Electromagnetic Compatibility and Telecommunications. FCC Methods

PB95-242376 02,664

Electrical Product Requirements (Especially Quality Requirements) in the United States.

PB96-119235

01,929

Certification of the Standard Reference Material 1473a, a

Low Density Polyethylene Resin.

01,282

U.S. Government Accreditation and Conformity Assessment System Evaluation.

02,678

Statistical Aspects of the Certification of Chemical Batch SRMs. Standard Reference Materials.

PB96-210877 
NIST-7, the New US Primary Frequency Standard. PB95-153458

01,534

Microwave Leakage as a Source of Frequency Error and Long-Term Instability in Cesium Atomic-Beam Frequency

PB95-180501

01.541 Velocity Distribution of Atomic Beams by Gated Optical Pumping.

01,542 Hybrid Digital/Analog Servo for the NIST-7 Frequency Standard.

CF3I

PB95-180618 01,544

Temperature Dependence of the Ultraviolet Absorption Cross Section of $\mathrm{CF}_{3}$.

01,168

CGOB (CERTAINTY GRID TO OBJECT BOUNDARY) Certainty Grid to Object Boundary Algorithm

PB94-203510

01,835

CHAIN MOLECULES

Principle of Congruence and Its Application to Compressble States.

PB96-102892

01,068

CHAIN POLARITY

Solid State (13)C NMR and Raman Studies of Cellulose

Triacetate: Oligomers, Polymorphism, and Inferences about Chain Polarity.

PB96- 176532

01,289

\section{CHAIN SCISSION}

Gas Phase Oxygen Effect on Chain Scission and Monomer Content in Bulk Poly(methyl methacrylate) Degraded by External Thermal Radiation.

PB96-204078

01,293

CHAINS

Effect of Hydrodynamic Interactions on a Terminally Anchored Bead-Rod Model Chain.

PB95-141156

01,237

CHALCOGENIDES

Thermochemical Studies of Inorganic Chalocogenides by Fluorine-Combustion Calorimetry: Binary Compounds of Germanium and Silicon with Sulfur, Selenium and TelluPB97-112528

01,181

CHAMBERS

Spatial Correlation Function for Fields in a Reverberation Chambe

PB96-148077

04,427

CHANGES

Operating Procedures and Life Cycle Documentation for the Initial Graphics Exchange Specification.

PB95-242285

02,782

CHANNEL CAPACITY

Capacity of the Lp Norm-Constrained Poisson Channel. PB95-125753

01,515

CHANNEL FLOW

Heat Transfer in Thin, Compact Heat Exchangers with Circular, Rectangular, or Pin-Fin Flow Passages.

02,751

CHANNELS (DATA TRANSMISSION)

Channel Coding for Code Excited Linear Prediction (CELP) Encoded Speech in Mobile Radıo Applications. PB95-143178

01,475

CHAOS

Chaos in a Computer-Animated Pendulum.

PB94-212651 03,852

Fluctuations in Probability Distribution on Chaotic AttracPB96-102330

04,022

Chaotic Motions of Coupled Galloping Oscillators and Their Modeling as Diffusion Progresses.

Their Modeling
PB96-122718

04,823

Non-Gaussian Noise Effects on Reliability of Multistable

Systems.
PB96-122726

04,213

Transitions to Chaos Induced by Additive and Multiplicative Noise.

03,750

Spectrum of the Stochastically Forced Duffing-Holmes Oscillator.

00,216

Necessary Condition for Homoclinic Chaos Induces by Additive Noise.

Noise-Induced Transitions to Chaos.

04,063

PB96-156120

00.217

Deterministic and Stochastic Chaos.

PB96-156138
Experimental and Numerical Chaos in Continuous Systems: Two Case Studies.

PB96-156146

00,219

CHARACTER RECOGNITION

Analysis of a Biologically Motivated Neural Network for Character Recognition.

00,182

Self-Organizing Neural Network Character Recognition on a Massively Parallel Computer.

01,845
Self-Organizing Neural Network Character Recognition Using Adaptive Filtering and Feature Extraction. PB96-119797

CHARACTERISTIC IMPEDANCE

Measurements of the Characteristic Impedance of $\mathrm{Co}$ axial Air Line Standards.

PB95-168787

02,221

Comments on 'Conversions between $S, Z, Y, h, A B C D$, and $T$ Parameters Which Are Valid for Complex Source and Load Impedances'

PB96-102785

02,069

\section{CHARACTERIZATION}

Characteristics of Adhesive-Bonded Seams Sampled from EPDM Roof Membranes

PB95-162491

00,377

Cement and Concrete Characterization by Scanning Electron Microscopy

CHARGE CARRIERS

Correction to the Decay Rate of Nonequilibrium Carrier Distributions Due to Scattering-in Processes.

PB94-185840

04,452

CHARGE DISTRIBUTION

Influence of Surface Charge on the Stochastic Behavior of Partial Discharge in Dielectrics.

\section{CHARGE EXPANSION THEORY}

Relativistic Modifications of Charge Expansion Theory. PB96-123799

01,931

\section{CHARGE TRANSFER}

Charge Transfer and Collision-Induced Dissociation Reactions of $\mathrm{CF}(2+)$ and $\mathrm{CF} 2(2+)$ with the Rare Gases at a Laboratory Collision Energy of $49 \mathrm{eV}$

PB94-185584

00,775

Measuring Contact Charge Transfer at Interfaces: A New Experimental Technique.

PB95-164570

03,053

Kinetics and Dynamics of Vibrationally State Resolved Ion-Molecule Reactions: $(14) \mathrm{N} 2(+)(v=1$ and 2$)$ and $(15) \mathrm{N} 2(+)(v=0,1$ and 2$)$ with (14) N2

PB96-102348

04.023

Kinetic Energy Distribution of Ions Produced from Townsend Discharges in Neon and Argon.

04,413

Influence of Coadsorbed Potassium on the Electron-StimInfluence of Coadsorbed Potassium on the Electron-Stim-
ulated Desorption of $F(+), F(-)$, and $F\left({ }^{*}\right)$ from PF3 on $\mathrm{Ru}(0001)$

CHARGED IONS

04,072

Observation and Visible and uv Magnetic Dipole Transitions in Highly Charged Xenon and Barium.

PB96-138441

04,056

CHARGED PARTICLES

Visible and UV Light from Highly Charged Ions: Exotic Matter Advancing Technology.

PB96-119391

04,414

CHARPY V NOTCH MACHINES

Proposed Changes to Charpy V-Notch Machine Certification Requirem

02,955

\section{CHARRING}

Behavior of Charring Materials in Simulated Fire Environ-

PB94-196045

01,368

Investigation of the Thermal Stability and Char-Forming

Tendency of Cross-linked Poly(methyl methacrylate). PB94-213501

CHARTS

Cryogenic Properties of Silver.

PB94-203593

03,330

CHEMICAL AFFINITY

Imposed Oscillations of Kinetic Barriers Can Cause an Enzyme to Drive a Chemical Reaction Away from EquiPBos-

\section{CHEMICAL ANALYSIS}

Airborne Asbestos Method: Standard Test Method for High Precision Counting of Asbestos Collected on Filters. Version 1.0

PB94-163003

00,525

Airborne Asbestos Method: Standard Test Method for Verified Analysis of Asbestos by Transmission Electron Microscopy. Version 2.0

00,526

Classical Analysis: A Look at the Past, Present, and Fu-

fure. 185063 PB94-528

Preparation and Certification of a Rhodium Standard Reference Material Solution.

PB94-185071 00,529

Proficiency Tests for the NIST Airborne Asbestos Pro-

gram, 1990.

00,535

Proficiency Tests for the NIST Airborne Asbestos Program - 1991.

Proficiency Tests for the NIST Airborne Asbestos Pro

gram - 1992.

00,539

Standard Reference Materials for Dioxins and Other Environmental Pollutants.

PB94. 198330

02,518

Establishing Quality Measurements for Inorganic Analysis of Biomaterials.

B B 00,548

Carotenoid Reversed-Phase High-Performance Liquid Chromatography Methods: Reference Compendium.

Airborne Asbestos Method: Standard Practice for Record ing Transmission Electron Microscopy Data for the AnalyPB of Asbestos Collected onto Filters. Version 1.0. 00,552

Biological

Matrices Using Neutron Activation - Mass Spectrometry (NA-MS)

PB94-212289

00,554

Certification, Development and Use of Standard Reference Materials.

Formation of Technical Committee 201 on Surface Chemical Analysis by the International Organization for Stand-

PB95-108536

00,568

Apparent Bias in the X-Ray Fluorescence Determination of Titanium in Selected NIST SRM Low Alloy Steels.

PB95-108759 03,212

Dissolution Problems with Botanical Reference Materials.

PB95-126280 03,487

Comparison of Methods for Gas Chromatographic Deter mination of PCBs and Chlorinated Pesticides in Marine Reference Materials.

PB95-140091 02,584

NIST Standard Reference Materials (SRMs) for Poly chlorinated Biphenyl (PCB) Determinations and Their Applicability to Toxaphene Measurements.

PB95-140109

02,585

Combining Data from Independent Chemical Analysis Methods

D. 572

Automated, High-Precision Coulometric Titrimetry. Part 1. Engineering and Implementation.

PB95-150199

00,575

Automated, High Precision Coulometric Titrimetry. Part 2. Strong and Weak Acids and Bases.

PB95-150207 00,576

Integrating Automated Systems with Modular Architec-

PB95-15023

00,577

Standard Reference Materials for the Determination of Polycyclic Aromatic Hydro

PB95-151668

00,586

Mixed Diet Reference Materials for Nutrient Analysis of Foods: Preparation of SRM-1548 Total Diet.

PB95-151692 
Certification of Standard Reference Material (SRM) 1941a, Organics in Marine Sediment.

02,593

Airbome Asbestos Analysis: National Voluntary Laboratory Accreditation Program.

02,566

Isotopic and Nuclear Analytical Techniques in Biological Isotopic and Nuclear Analytical Techniques in Biological
Systems: A Critical Study. 10. Elemental Isotopic Dilution Analysis with Radioactive and Stable Isotopes (Technical

PB96-164157

00,696

Composition and Solubility Product of a Synthetic Calcium Hydroxyapatite.

02,995

Statistical Aspects of the Certification of Chemical Batch

SRMs. Standard Reference Materials.
PB96-210877

00,645

Airborne Asbestos Method: Bootstrap Method for Determining the Uncertainty of Asbestos Concentration. Version 1.0

PB96-214614

00,646

Results of the ASTM Nuclear Methods Intercomparison on NIST Apple and Peach Leaves Standard Reference Materials.

PB97.119036

03,490

CHEMICAL ASSISTED MACHINING

Chemically Assisted Machining of Si3N4

HEMICAL BONDS

Effect of Two Initiator/Stabilizer Concentrations in a Metal Primer on Bond Strengths of a Composite to a Base Metal Alloy.

00,141

Effects of Surface-Active Resins on Dentin/Composite Bonds.

00,156

PB95-140448

Van der Waals Bond Lengths and Electronic Spectral
Shifts of the Benzene-Kr and Benzene-Xe Complexes.

PB95-151387 00,932

Molecular Orbital Calculations of Bond Rupture in Brittle Solids.

PB95-164059

00,973

Bonding in Doubly Charged Diatomics.

00,976

Neutron Scattering Study of the Lattice Modes of Solid Cubane

PB96-147152

01,126

CHEMICAL COMPOSITION

Standard Materials. A Descriptive List with Prices. 00,500

Classification of Advanced Technical Ceramics.

N94-35335/6

03,030

Recently Developed NIST Food Related Standard Reference Materials.

00,035

Constituents and Physical Properties of the C6+ Fraction of Natural Gas. Topical Report, April-June 1994.

PB95-136644

02,483

Grazing-Incidence X-Ray Photoelectron Spectroscopy: A Novel Approach to Thin Film Characterization.

PB95-153128

04,589

Determinatıon of Sulfur in Fossil Fuels by Isotope Dilution Thermal lonization Mass Spectrometry.

02,495

Inorganic Crystal Structure Database (ICSD) and Standardized Data and Crystal Chemical Characterization of Inorganic Structure Types (TYPIX): Two Tools for Inorganic Chemists and Crystallographers.

Chemists and

00,648

Evaluation of Crystallographic Data with the Program DI-

AMOND.

00,649

CHEMICAL COMPOSITIONS

Certitying the Chemical Composition of a Biological Material: A Case Study.

CHEMICAL COMPOUNDS

NIOSH Pocket Guide to Chemical Hazards. PB95-100368

00,636

HEMICAL DISSOCIATION

Fragment State Correlations in the Dissociation of NO. $H F(V=1)$.
PB95.164430

00,982

CHEMICAL ENGINEERING

Application of Expert System to Select Data Sources from Chemical Information Databases.

PB95-125654

00.505

CHEMICAL EOUILIBRIUM

Compilation of the Physical Equilibria and Related Properties of the Hydrogen-Carbon Monoxide System.

AD-A286 603/6 00,716

Calorimetric Determination of the Standard Transformed Enthaipy of a Biochemical Reaction at Specified PH and PMg.

03,454

Electron Transfer Reaction Rates and Equilibria of the Carbonate and Sulfate Radical Anions.

00,829
Thermochemistry of the Reactions between Adenosine. 5'-monophosphate, Inosine, and Inosine 5' monophosphate; the Conversion of L-histidine to Urocanic Acid+Ammonia)

PB94-213113

03,460

CHEMICAL EXPLOSIVES

Separation and Identification of Organic Gunshot and Explosive Constituents by Miceliar Electrokinetic Capiliary Electrophoresis.

PB95.107249

CHEMICAL INHIBITION

Effect of CF3H and CF3Br on Laminar Diffusion Flames in Normal and Microgravity.

PB96-161831

01,420

Effect of CF3H and CF3Br on Laminar Diffusion Flames in Normal and Microgravity.

PB96-161849

01,42

Inhibition of Premixed Methane-Air Flames by Iron Pentacarbonyl.

00,513

Parametric Study of Hydrogen Fluoride Formation in Suppressed Fires.

CHEMICAL INTERACTIONS

00.514

Effect of Chemical Interaction on Barenblatt Crack Profiles in Brittle Solids.

PB96-180245

02,996

CHEMICAL KINETICS

Rate Constants for the Decay and Reactions of the Lowes: Electronically Excited Singlet State of Molecular Oxygen in Solution. An Expanded and Revised Compilation. B96-145826

01,106

Critical Review of Rate Constants for Reactions of Tran. sients from Metal Ions and Metal Complexes in Aqueous

PB96-145859

01,109

Kinetics of the Reaction of the Sulfate Radical with the Oxalate Anion.

01,186

CHEMICAL MODELS

Fokker-Planck Description of Multivalent Interactions.

PB95-108478

00,879

CHEMICAL PREPARATION

Octacalcium Phosphate Carboxylates. 1. Preparation and Identification.

00,660

\section{CHEMICAL PROPERTIES}

Classification of Advanced Technical Ceramics. $\quad 03,030$
N94-35335/6

Journal of Physical and Chemical Reference Data, Volume 22, No. 1, January/February 1993.

00,729

Journal of Physical and Chemical Reference Data, Volume 22, No. 2, March/April 1993.

PB94-162211

00,733

Joumal of Physical and Chemical Reference Data, Vol-

ume 22, No. 3, May/June 1993

00.738

Journal of Physical and Chemical Reference Data, Volume 22. No. 4, July/August 1993

PB94-162310

00,743

Journal of Physical and Chemical Reference Data, Volume 22, No. 5

00,745

Journal of Physical and Chemical Reference Data, Volume 22, No. 6, November/December 1993

PB94-168556

00,749

Physicochemical Properties of Calcific Deposits Isolated rom Porcine Bioprosthetic Heart Valves Removed from Patients Following 2-13 Years Function.

PB94-172863

00,184

Physical and Chemical Properties of Resin-Reinforced Calcium Phosphate Cements

PB95-18021

00,171

Journal of Physical and Chemical Reference Data, Volume 24, No. 1, January/February 1995.

PB96-145560

01,101

Journal of Physical and Chemical Reference Data, Volume 24, No. 2, March/April 1995

01,105

Journal of Physical and Chemical Reference Data, Volume 24, No. 3, May/June 1995.

01,108

Joumal of Physical and Chemical Reference Data, Volume 24, No. 4, July/August 1995 .
PB96-145883

01,112

Journal of Physical and Chemical Reference Data, Volume 24, No. 5, September/October 1995.

01,116

Journal of Physical and Chemical Reference Data, Volume 24 , No. 6 , November/December 1995. PB96-145966

01,120

Environmental Aspects of Halon Replacements: Consid erations for Advanced Agents and the Ozone Depletion Potential of CF3L.

PB97-122261

03,301

CHEMICAL RADICALS

Temperature Dependence of the Rate Constants for Reaction of Inorgar

00.783

Oxidation of 10-Methylacridan, a Synthetic Analogue of gent Application of Laser Flash Photolysis and Direct and Redox Catalyzed Electrochemistry to the Kinetics of Deprotonation of the Cation Radical

00,785

Temperature Dependence of the Rate Constants for Reactions of the Sulfate Radical, SO4-, with Anions. PB94-212172 Temperature Dependence of the Rate Constants for Re-
actions of the Carbonate Radical with Organic and Inoractions of the Carbonate Radical with Organic and Inor-

ganic Reductants.
PB94-212206

00,831

Resonance Enhanced Multiphoton Ionization Spectroscopy of the SnF Radical.

PB97-111223

01,176

CHEMICAL REACTION KINETICS

Progress in the Development of a Chemical Kinetic PB95-151056

01,384

Atmospheric Lifetimes of HFC-143a and HFC-245fa: Flash Photolysis Resonance Fiuorescence Measurements of the $\mathrm{OH}$ Reaction Rate Constants.

PB97-112577

00,118

CHEMICAL REACTION MECHANISMS

Fluorinated Hydrocarbon Flame Suppression Chemistry.

Unusual Spin-Trap Chemistry for the Reaction of Hydroxyl Radical with the Carcinogen $\mathrm{N}$ Nitrosodimethylamine.

PB95-151643

00,692

Polymerization Initiation by N-p-Tolylglycine: Free-Radical Reactions Studied by Puise and Steady-State Radiolysis. PB95-180014

07,269

\section{CHEMICAL REACTIONS}

Tables of Chemical Kinetics Homogeneous Reactions.

AD-A280 293/2

Facile Synthesis of Novel Fluorinated Multifunctional

$\begin{array}{ll}\text { Acrylates. } & 01,207\end{array}$

New Rydberg States of Aluminum Monofluoride Observed by Resonance-Enhanced Multiphoton Ionization Spectroscopy.

00.797

Equilibrium and Calorimetric Investigation of the Hydrolysis of L-Tryptophan to (Indole + Pyruvate + Ammonia).
PB95-163317 00,661

Thermodynamic Study of the Reactions of Cyclodextrins with Primary and Secondary Aliphatic Alcohols, with Dand L-Phenylalanine, and with L-Phenylalanineamide. PB95-180873 01,016 Ouadratic Response of a Chemical Reaction to External

Oscillations.
PB96-161633

01,138

Noise Analysis of Ionization Kinetics in a Protein Ion Channel.

PB96-161682

04,093

\section{CHEMICAL REACTORS}

Low Temperature H(sub 2)S Separation Using Membrane Redor With Redox Catalyst. 
Optimization of ECR-Based PECVD Oxide Films for Superconducting Integrated Circuit Fabrication.
PB95-169165

CHEMICALS

Standard Materials. A Descriptive List with Prices.
AD-A278 $140 / 9$

CHEMISTRY

CSTL Technical Activities 1991.

PB94- 160769
CSTL Technical Activities, 1993.

PB95-160602

00,728

CSTL Technical Activities, 1994

PB95-242319

CSTL Technical Activities, 1995

PB96-214630

00,953

00,608

CHEMISTRY WORKCELL

Integrating Automated Systems with Modular Architec-

ture.
PB95-150231

00,577

CHEMOMETRICS

Importance of Chemometrics in Biomedical Measurements.
PB94-200599

00,550

Metrological Measurement Accuracy: Discussion of 'Measurement Error Models' by Leon Jay Gleser. PB94-200607

00,551

CHEMOPREVENTIVE AGENTS

Methods for Analysis of Cancer Chemopreventive Agents in Human Serum

03,502

Liquid Chromatographic Determination of Carotenoids in Human Serum Using an Engineered C30 and a C18 Stationary Phase.

CHEWING GUM

Effects on Whole Saliva of Chewing Gums Containing Calcium Phosphates.

CHINA

03,563

Glimpse of Materials Research in China: A Report from an Interagency Study Team on Materials Visiting China from June 19, 1995 to June 30, 1995 PB96-112677

02,978

CHISSA (CENTER FOR HIGH INTEGRITY SOFTWARE

SYSTEM ASSURANCE)

Center for High Integrity Software System Assurance: Initial Goals and

01,734

CHLORHEXIDINE

Interaction of Chlorhexidine Digluconate with and Adsorption of Chlorhexidine on Hydroxyapatite.

PB95-175907

03,566

CHLORHEXIDINE DIGLUCONATE

Interaction of Chlorhexidine Digluconate with and Adsorption of Chlorhexidine on Hydroxyapatite.

tion of Chlorhexic
PB95-175907

03,566

CHLORIDES

Computer Simulation of the Diffusivity of Cement-Based Materials.

PB95-125985

00,362

In situ Measurements of Chloride Ion and Corrosion Potential at the Coating/Metal Interface.

PB95-140893

03,122

CHLORINATED AROMATIC HYDROCARBONS

Determination of PCBs and Chlorinated Hydrocarbons in Marine Mammal Tissues.

PB95-162640

03,744

Comparison of Selectivities for PCBs in Gas Chromatography for a Series of Cyanobiphenyl Stationary Phases.

00,618

CHLORINATED POLYVINYL CHLORIDE

Comparison of Fire Sprinkler Piping Materials: Steel Copper, Chlorinated Polyvinyl Chloride and Polybutylene, in Residential and Light Hazard Installations. PB95-182267

00,299

CHLORINE

Relative Photoionization and Photodetachment Cross Sections for Particular Fine-Structure Transitions with Ap-

plication to $\mathrm{Cl}$ 3s-subshell Photoionization.

03,998

Laser Flash Photolysis, Time-Resolved Fourier Transform Infrared Emission Study of the Reaction $\mathrm{Cl}+\mathrm{C} 2 \mathrm{H} 5$ yields $\mathrm{HCl}(v)+\mathrm{C}_{2} \mathrm{H} 4$

State-Resolved Rotational Energy Transfer in Open Shell Collisions: $\mathrm{Cl}((2) \mathrm{P} 3 / 2)+\mathrm{HCl}$.

PB96-176607

CHLORINE 36

01,157

$36 \mathrm{Cl} / \mathrm{Cl}$ Accelerator-Mass-Spectrometry Standards: Verification of Their Serial-Dilution-Solution Preparations by Radioactivity Measurements.

PB94-140563

00,524

CHLORINE IONS

Absolute Cross-Section Measurements for Electron-Impact lonization of $\mathrm{C} 1(+1)$

PB94-199841

03,818

CHLORINE OXIDES

Infrared Spectrum of OClO in the $2000 / \mathrm{cm}(-1)$ Region: The 2(nu sub

00,908 Intensities and Dipole Moment Derivatives of the Fun
damental Bands of (35) $\mathrm{ClO} 2$ and an Intensity Analysis of damental Ban the nu1 Band

CHLOROBENZENE

Oscillometric and Conductometric Analysis of Aqueous and Organic Dosimeter Solutions.
PB96-135256

00,909

04,054

CHLOROBENZENES

Rate Constants for Hydrogem Atom Attack on Some Chlorinated Benzenes at High Temperature. PB94-200581

00,810 Incinerability of Perchloroethylene and Chlorobenzene. PB95-163457

01,388

CHLOROFLUOROCARBONS

Role of R22 in Refrigerating and Air Conditioning Equip-

03,253

Retention of Halocarbons on a Hexafluoropropylene Epoxide Modified Graphitized Carbon Black. Part 1. Methane-Based Compounds.

PB95-175196

03,272

Physical Properties of Alternatives to the Fully Halogenated Chlorofluorocarbons.

03,279

Retention of Halocarbons on a Hexafluoropropylene EpRetention of Halocarbons on a Hexafluoropropylene EpOxide-Modified Graphitized Carbon Black. 4. Propane-
Based Compounds.
PB96-164033
03,284

03,284 REFPROP Refrigerant Properties Database: Capabilities,
Limitations, and Future Directions.
PB96-167150

Retention of Halocarbons on a Hexafluoropropylene Epoxide-Modified Graphitized Carbon Black. 3. Ethene Based Compounds.

03,286

CHLOROHYDROCARBONS

Gas Phase Reactivity Study of $\mathrm{OH}$ Radicals with 1,1Dichloroethene and cis-1,1-Dichloroethene and Trans-1,2-

Dichloroethene over the Temperature Range $240-400 \mathrm{~K}$.
PB95-152146
00,939

CHOLESTEROL

Isotope Dilution Mass Spectrometry as a Candidate Definitive Method for Determining Total Glycerides and Triglycerides in Serum

PB96- 102280
CHRIAL RECOGNITION

03.519

Use of a Naphthylethylcarbamoylated- beta-Cyclodextrin Chiral Stationary Phase for the Separation of Drug Supercritical Fluid Chromatography.

PB97-113260

00,653

CHROMATIN Oxidative Damage to DNA in Mammalian Chromatin.
PB94-199825 Modification of DNA Bases in Chromatin of Intact Target Leukocyle

PB94-199833

03,526

Nickel(II)-Mediated Oxidative DNA Base Damage in Renal and Hepatic Chromatin of Pregnant Rats and Their Fetuses. Possible Relevance to Carcinogenesis.

PB94-212628

03,646

Oxidative DNA Base Damage in Renal, Hepatic, and Pulmonary Chromatin of Rats After Intraperitoneal Injection of Cobalt (II) Acetate.

PB95-150025

03,647

DNA Base Modifications in Renal Chromatin of Wistar Rats Treated with a Renal Carcinogen, Ferric Nitrilotriacetate

03,648

DNA Base Damage Generated In vivo in Hepatic Chromatin of Mice upon Whole Body y-Irradiation.

PB95-161741

03,627

Treatment of Wistar Rats with a Renal Carcinogen, Ferric Nitrilotriacetate, Causes DNA-Protein Cross-Linking between Thymine and Tyrosine in Their Renal Chromatin. PB96-112115 03,649 Formation of DNA-Protein Cross-Links in Cultured Mammalian Cells Upon Treatment with Iron lons.

03,651

CHROMATOGRAPHIC ANALYSIS

Liquid Chromatography: Laser-Enhanced Ionization Spectrometry for the Speciation of Organolead Compounds.

PB94-185253

Measurement of Diffusion in Supercritical Fluid Systems: A Review.

PB94-199189 00,795

Comparison of the Liquid Chromatographic Behavior of Selected Steroid Isomers Using Different ReversedPhase Materials and Mobile Phase Compositions.

PB95-140976

00,574

Chromatographic Cryofocusing and Cryotrapping with the PBortex Tube.

Terminally Anchored Chain Interphases: Their Chromatographic Properties.
PB95-181061

01,272

Permeation Tube Approach to Long-Term Use of Automatic Sampler Retention Index Standards.

00,639

\section{CHROMATOGRAPHY}

Measurement of Diffusion in Fluid Systems: Applications to the Supercritical Fluid Region

02,490

Solubility Measurement by Direct Injection of Supercritical-Fluid Solutions into a HPLC System.
PB95-175626

Determination of Vitamin K1 in Serum Using Catalytic-Reduction Liquid Chromatography with Fluorescence Detection.

03,506

Selectivity Trends in Packed Column Supercritical Fluid Chromatography with C18 Stationary Phases.

00,622

Simple and Efficient Methane-Marker Devices for Chromatographic Samples. PB96-164041

00,635

\section{CHROMIUM}

Simple, Compact, High-Purity Cr Evaporator for Ultrahigh PB94-216678

04,520

Magnetic Moments in $\mathrm{Cr}$ Thin Films on Fe(100).
PB95-108429

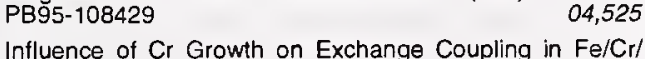
$\mathrm{Fe}(100)$.

04,562

Scanning Tunneling Microscopy Study of the Growth of Scanning Tunneling Microscopy Study of the Growth of
$\mathrm{Cr} / \mathrm{Fe}(001)$ : Correlation with Exchange Coupling of Magnetic Layers.

04,568

Nanofabrication of a Two-Dimensional Array Using LaserFocused Atomic Deposition.

PB96-119417

04,732

Influence of Thickness Fluctuations on Exchange Coupling in $\mathrm{Fe} / \mathrm{Cr} / \mathrm{Fe}$ Structures.

CHROMIUM INTERMETALLICS

Crystallographic Characterization of Some Intermetallic Compounds in the Al-Cr System.

03,318

CHROMIUM IONS

Improved Wavelengths for Prominent Lines of $\mathrm{Cr} X \mathrm{VI}$ to Cr XXII

PB95-150629

03,895

CHROMOSPHERES

High-Velocity Plasma in the Transition Region of AU Microscopii: Evidence for Magnetic Reconnection and Saturated Heating during Ouiescent and Flaring Condi-
tions. tions.

00,091

CHURCHILL EISENHART

Churchill Eisenhart, 1913-1994.

PB96-137740

03,447

CIGARETTES

Ouantifying the Ignition Propensity of Cigarettes.
PB96-155411

00,306

CIM (COMPUTER INTEGRATED MANUFACTURING)

Roadmap for the Computer Integrated Manufacturing

(CIM) Application Framework. 


\section{KEYWORD INDEX}

Metrology and Data for Commercial Electrical and Optical Packaging and Interconnection Technologies. Held in Gaithersburg, Maryland on May 5-6, 1994. Volume 1. Results.

PB95-143111 02,328

Metrology and Data for Microelectronic Packaging and Interconnection: Results of a Joint Workshop on Materials Interconnection. Results of a Joint Workshop on Materials Patrology and Data lor Commercial Electrical and Optical Gaithersburg, Maryland on May 5-6, 1994. Volume 2. Gaithersburg, Maryland

Presentation

02,330

Provision of Isochronous Service on IEEE 802.6.

PB96-160635

01,501

Accurate Electrical Characterization of High-Speed Interconnections.

CIRCUIT PROTECTION

Coordinating Cascaded Surge Protection Devices: HighLow versus Low-High.

PB94-172061

02,463

Guarding Against Transients

01,623

Cascading Surge-Protective Devices: Options for Effective Implementation.

PB94-216488

02,464

Coordinating Cascaded Surge-Protective Devices: An

Elusive Goal.

02,465

Important Link in Entire-House Protection: Surge Reference Equalizers.

PB94-216504

02,219

Surging the Upside-Down House: Looking into Upsetting Reference Voltages.

02,385

CIRCUIT SIMULATORS

Simulating the Dynamic Electro Thermal Behavior of Power Electronic Circuits and Systems.
PB95-161014

Thermal Component Models for Electro-Thermal Network Simulation.

PB95-161022

02,346

CIRCUIT TECHNOLOGY

Business and Manufacturing Motivations for the Developing of Analytical Technology and Metrology for Semiconductors.
PB96-161948

CIRCUIT TESTERS

Automated Josephson Integrated Circuit Test System.
PB95-175246

CIRCUITS

Handbook Preferred Circuits Navy Aeronautical Electronic Equipment. Supplement Number 3.

AD-A278 782/8

00,026

Handbook Preferred Circuits Navy Aeronautical Electronic Equipment. Supplement Number 2

Equipment. Supp

00.027

Handbook Preferred Circuits Navy Aeronautical Electronic Equipment. Supplement Number 1.

00,028

Wide Band Active Current Transformer and Shunt.

PB95-126371

02,036

Precision Tests of a Quantum Hall Effect Device DC Equivalent Circuit Using Double-Series and Triple-Series Connections.

PB96-159256
CIRCULAR BRIDGE COLUMNS

01,953

Jacket Thickness Requirements for Seismic Retrofitting of

Circular Bridge Columns.
PB95-163267

01,336

CITIS (CONTRACTOR INTEGRATED TECHNICAL

INFORMATION SERVICE)

CALS-Contractor Integrated Technical Information Service (CITIS), Functional Requirements.

PB94-962600

03,663

CALS-Contractor Integrated Technical Information Service (CITIS), Functional Requirements.

PB95-962600

03,672

CITRIC ACID

Interaction of Citric Acid with Hydroxyapatite: Surface Exchange of lons and Precipitation of Calcium Citrate.

PB97.1 19309

03,584

CIVIL ENGINEERING

Materials Aspects of Fiber-Reinforced Polymer Composites in Infrastructure.

AD METALS

Leady Axisymmetric Modes in Infinite Clad Rods. Part 1.

PB95-162905

03,184

CLADDING

Literature Review on Seismic Performance of Building Cladding Systems.

CLADDINGS

Optical Fiber Geometry: Accurate Measurement of Cladding Diameter
PB95-151940

LAMPING CIRCUITS

Perception of Clamp Noise in Television Receivers.
PB96-119433

\section{CLAPEYRON EQUATION}

Calculation of Enthalpy and Entropy Differences of NearCritical Binary Mixtures with the Modified Leung-Griffiths

PB95-108635

00,885

CLASSICAL ANALYSIS

Classical Analysis: A Look at the Past, Present, and Future.

PB94-185063

00,528

CLASSICAL LIMIT

Quantum Collisional Transfer Contributions to the Density Dependence of Gaseous Viscosity.

01,142

CLASSIFI

Metropolitan Areas (Including MSAs, CMSAs, PMASs, and NECMAs). Category: Data Standards and Guidelines: Subcategory: Representations and Codes.

FIPS PUB 8-6

04,873

Countries, Dependencies, Areas of Special Sovereignty, and Their Principal Administrative Divisions. Category: Data Standards and Guidelines; Subcategory: Representation and Codes.

00,128

Guideline: Codes for Named Populated Places, Primary County Divisions, and Other Locational Entities of the United States, Puerto Rico, and the Outlying Areas. Category: Data Standards and Guidelines. Subcategory: Representations and Codes.

04,865

Guideline: Codes for Named Populatd Places, Primary County Divisions, and Other Locational Entities of the County Divisions, and Other Locational Entities of the egory: Data Standards and Guidelines; Subcategory: egory: Data Slandards and Guidelines; Subcalegory. Representation and Codes.

04,866 FIPS PUB 8-6, Metropolitan Areas (for Microcomputers).
PB95-503280

Countries, Dependencies, Areas of Special Sovereignty, and Their Principal Administrative Divisions (for Microcomputers).

CLASSIFICATIONS

Classification of Advanced Technical Ceramics.

N94-35335/6

00,130

New Materials, Advanced Ceramics and Standards. PB95-140208

03,030

ABSIFYING

Description of Layered Structures: Applications to High Tc Superconductors

CLAY

Small Angle Neutron Scattering Study of a Clay Suspension Under Shear.

CLAYS

Hollow Clay Tile Prism Tests for Martin Marietta Energy Systems: Task 2 Testing.

PB94-217486

00,352

CLEANING

Effects of Soxhlet Extraction on the Surface Oxide Layer of Silicon Nitride Powders

03,057

CLEAVAGE

Evidence of Film-Induced Cleavage by Electrodeposited PB95-162327

03,191

CLIMATIC CHANGES

Global Climatic Effects of Aerosols: The AAAR Sympo-

PB95-108791

00,122

CLINICAL CHEMISTRY

History of NIST's Contributions to Development of Standard Reference Materials and Reference and Definitive Methods for Clinical Chemistry. PB96-119706

03,503

CLINKER

Quantitative X-Ray Powder Diffraction Methods for Clinker and Cement.

PB95-143079

01,317

CLOCK

Wavelet Variance, Allan Variance, and Leakage. PB96-190111

01,509

CLOCKS

Time and Frequency Technology at NIST

Future of Time and Frequency Dissemination.

N94-30684/2

01,522

Smart Clock: A New Time.

PB95- 151445 .

Keeping Time on Your PC.
PB95-161410

Nine Years of NIST Clock Data with TA2.

LOSED LOOP CONTROLLERS

Closed Loop Controller for Electron-Beam Evaporators

PB97-111470

04,393

CLOTHING

Bibliography on Apparel Sizing and Related Issues.

02,806

CLOTHING INDUSTRY

Body Dimensions for Apparel.

PB94-187739

02,813

Apparel Manufacturing Glossary for Application Protocol Development.

02,755

Extensions of the Prototype Application Protocol of Ready-to-Wear Apparel Pattern Making.

03,198

Aspects of a Product Model Supporting Apparel Virtual Enterprises.

02,790

Survey of Standards for the U.S. Fiber/Textile/Apparel In-

PB96-193792

03,199

CLUSTER ANALYSIS

Representing a Large Collection of Curves: A Case for Principal Poin

CLUSTER MODELS

03,438

Active Site lonicity and the Mechanism of Carbonic AnhyPBase. 212974

00,843

CLUSTERS

Potential Surfaces and Dynamics of Weakly Bound Trimers: Perspectives from High Resolution IR SpectrosPB96-176508

01,155

CMM (COOROINATE MEASURING MACHINES)

Two New Probes for a Coordinate Measuring Machine.

PB95-163093 02,653

User's Guide to 'SuperFit' Modeling Sottware for CMM PBob-128236

02,921

CMMS (COORDINATE MEASURING MACHINES)

Some Considerations for Interim Testing of Coordinate Measuring Machine Performance Using a Specific Arti-

PB95-108858

02,898

Sensitivity of Three-Point Circle Fitting

02,901

NIST SRM 9983 High-Rigıdity Ball-Bar Stand. User Manual.

PB95-255840

02,669

Estimation of Measurement Uncertainty of Small Circular Features Measured by CMMs.

PB95-267928

02,918

CMOS

Defect of Thermal Ramping and Annealing Conditions on Defect Formation in Oxygen Implanted Silicon-On-Insula-

PB94-212966

02,318

High-Level CAD Melds Micromachined Devices with Foundries.

02,321

MEMS in Standard CMOS VLSI Technology.

PB96-102363

02,377

Development of Thin-Film Multijunction Thermal Converters at NIST.

02,286

Micromachined Coplanar Waveguides in CMOS Technology.
PB97-119283

02,456

CMS

Process for Selecting Standard Reference Algorithms for Evaluating Coord

02,629 
COATING PROCESSES

Opportunities for Innovation: Advanced Surface Engineering. 176666

Perspective on Fiber Coating Technology. PB94-200540

02,697 Interface Modification and Characterization of Silicon Carbide Platelets Coated with Alumina Particles.

03,121

Methodologies for Predicting the Service Lives of Coating

Pystems.

03,124

UV-Photopatterning of Alkylthiolate Monolayers Self-Assembled on Gold and Silver.

00,924

COATINGS

Light Scattering from Glossy Coatings on Paper. $\quad 04,242$ Non-Osmotic, Defect-Controlled Cathodic Disbondment of a Coating from a Steel Substrate

03,120

Light Scattered by Coated Paper.

04,245

Tribological Behavior of 440/Diamond-Like-Carbon Film Couples.

03,019

Proposed Coating Technology Consortium. (National Coil Coaters Association Fall Conterence). Held in Rosemont, Illinois in September 1992.

PB97-110431

03,129

Nanoindentation and Instrumented Scratching Measurements on Hard Coatings.

COAXIAL AIR LINES

Aperture Coupling to a Coaxial Air Line: Theory and Experiment.

02,216

Measurements of the Characteristic Impedance of Coaxial Air Line Standards.

PB95-168787

02,221

COAXIAL CABLES

Programmable Guarded Coaxial Connector Panel.
PB96-122544 108

Open-Ended Coaxial Probes for Nondestructive Testing of Substrates and Circuit Boards.

PB96-122825

02,078

Dielectric Measurements on Printed-Wiring and Circuit Boards. Thin Films, and Substrates: An Overview.
PB96-147038 02,236 Dimensional Characterization of Precision Coaxial Trans-

mission Line Standards.
PB96-176482

02,241

Coaxial Line-Reflect-Match Calibration.

02,246

COAXIAL CONFIGURATIONS

Calibration Service for Coaxial Reference Standards for Microwave Power.

PB96-162722

01,958

COAXIAL PROBES

Analysis of an Open-Ended Coaxial Probe with Lift-Off for Nondestructive Testing

PB96-135116

01,940

COBALT

Combined Low- and High-Angle X-Ray Structural Refinement of a Co/Pt(111) Multilayer Exhibiting Perpendicular Magnetic Anisotropy.

PB94-198355

04,457

Magnetic Properties of $\mathrm{Pd} / \mathrm{C}$ o Multilayers.

04,465

Bioleaching of Cobalt from Smelter Wastes by 'Thiobacillus ferrooxidans'. 02,582 Spot-Profile-Analyzing LEED Study of the Epitaxial Growth of $\mathrm{Fe}_{\mathrm{e}} \mathrm{Co}$, and $\mathrm{Cu}$ on $\mathrm{Cu}(100)$.
$\mathrm{PB} 95-150165$ Microstructural Characterization of Cobalt-Tungsten Coat-

ed Graphite Fibers.
PB96-159231

01,951

COBALT 60

Protection Against Fadiations from Radium, Cobalt-60, and Cesium- 137.

03,607

Dose Mapping of Radioactive Hot Particles Using Radiochromic Film.

PB95-162954

03,714

COBALT ACETATE

Oxidative DNA Base Damage in Renal, Hepatic, and Pul. monary Chromatin of Rats After Intraperitoneal Injection of Cobalt (II) Acetate.

03,647

COBALT ALLOYS

Correlations of Modulation Noise with Magnetic Microstructure and Intergranular Interactions for CoCrTa and CoNi Thin Film Media.

PB94-212768

04,509

COBALT BASE ALLOYS

Electrodeposited Cobalt-Tungsten as a Diffusion Barrier between Graphite Fibers and Nickel.

03,176
COBALT HYDRIDES

Rotational Spectroscopy of the CoH Radical in Its Ground (3)Phi State by Far-Infrared Laser Magnetic Resonance: Determination of Molecular Parameters.

PB95-175048

00,992

COBALT SILICIDES

Local Partial Densities of States in Ni and Co Silicides Studied by Soft X-Ray Emission Spectroscopy.

04.504

COBOL

COBOL. Category: Software Standard; Subcategory: Programming Language. Includes ANSI'S X3.23-1985, X3.23A-1989 and X3.23B-1993.

FIPS PUB 21-4

01,670

COBOL. Category: Sottware Standard; Subcategory: Programming Language. Part A.

01,671

COBOL. Category: Software Standard; Subcategory: Programming Language. Part $B$.
FIPS PUB 21-4B

01,672

COBOL PROGRAMMING LANGUAGE

Validated Products List (Cobol, Fortran, ADA, Pascal, MUMPS, SOL)

01,700

Validated Products List (Cobol, Fortran, ADA, Pascal,

MUMPS, SOL).
PB95-937300.

COCAINE

Supercritical Fluid Extraction-Immunoassay for the Rapid Screening of Cocaine in Hair.

00,637

COCOMBUSTION

Sulfur Dioxide Capture in the Combustion of Mixtures of Lime, Refuse-Derived Fuel, and Coal.

02,534

CODE DIVISION MULTIPLE ACCESS

Error Protecting Characteristics of CDMA and Impacts on PB96-122452

01,491

CODE EXCITED LINEAR PREDICTION

Channel Coding for Code Excited Linear Prediction (CELP) Encoded Speech in Mobile Padio Applications. 43178

CODE SCALABILITY

Scalability Test for Parallel Code.

PB96-146

Certification of Morphine and Codeine in a Human Urine Standard Reference Material.

CODING

Channel Coding for Code Excited Linear Prediction (CELP) Encoded Speech in Mobile Radio Applications.

COEFFICIENT OF FRICTION

Mechanism of Mild to Severe Wear Transition in AlphaAlumina.

PB94-212354

03,233

COEFFICIENT OR THERMAL EXPANSION

Advances in the Measurement of Polymer CTE:

Micrometer- to At

03,390

COEXTRUDING

Small Angle Neutron Scattering Studies on Chain Asymmetry of Coexin

01,262

COHERENT INTERFACES

Cracks and Dislocations in Face-Centered Cubic Metallic Multilayers.

02,989

COHERENT LIGHT

Integral Occurring in Coherence Theory.
PB95-203550

COILS

Ouench Energy and Fatigue Degradation Properties of $\mathrm{Cu}$ - and AlCU-Stablized Nb-Ti Epoxy-Impregnated Superconductor Coils.

PB96-141213

04,755

COLD NEUTRON CAPTURE PROMPT GAMMA-RAY

ACTIVATION ANALYSIS

Determination of Hydrogen in Titanium Alloy Jet Engine Compressor Blades by Cold Neutron Capture Prompt Gamma-ray Activation Analysis.

PB95-175956

01,448

COLD NEUTRONS

Thermal Hydraulic Tests of a Liquid Hydrogen Cold Neutron Source.

Neutron Focusing Lens Using Polycapillary Fibers.

PB95-141206

03,884

Liquid-Hydrogen Cold Neutron Source for the NBSR.
PB95-151619

Neutron Focusing Lens Using Polycapillary Fibers.

PB95-153078 03,922 Cold Neutron Gain Calculations for the NBSR Using

PB95-163978

03,731

Analytical Applications of Guided Neutron Beams.
PB96-112347 04,041 MCNP Model of the National Bureau of Standards Reactor (NBSR) Core.

03,733

COLD PLASMAS

Spectroscopic Diagnostics of Low Temperature Plasmas: Techniques and Pequired Data.

PB95-151577

04,411

COLLCTION

NIST Serial Hold

02,745

COLLECTION SYSTEMS

All-Metal Collection System for Preparative-Scale Gas Chromatography: Purification of Low-Boiling-Point Com-

PBonds. 23435

00,619

COLLIMATORS

Analysis of the Effectiveness of Oscillating Padial Collimators in Neutron Scattering Applications.

PB95-152252

03,917

Tilt Effects in Optical Angle Measurements. PB95-169389

04,294

COLLISION

Alignment Probing of Rydberg States by Stimulated EmisSBion. 200316

04,124

COLLISION AVOIDANCE

Image Gradient Evolution: A Visual Cue for Danger.
PB96-154562 939

Novel Active-Vision-Based Motion Cues for Local Navigation.

OLLISIONS

State-Pesolved Rotational Energy Transfer in Open Shell Collisions: $\mathrm{Cl}((2) \mathrm{P} 3 / 2)+\mathrm{HCl}$

01,157

Using Collocation in Three Dimensions and Solving a Model Semiconductor Problem.

PB96-159249 01,952

Application of the Collocation Method in Three Dimensions to a Model Semiconductor Problem.

PB97. 122428

02,457

COLLOIDAL DISPERSION

Structure and Rheology of Hard-Sphere Systems.

00,662

\section{COLLOIDAL SUSPENSION}

Non-Newtonian Flow between Concentric Cylinders Calculated from Thermophysical Properties Obtained from Simulations.

COLLOIDAL SUSPENSIONS

Contrast Matched Studies of a Sheared Binary Colloidal Suspension.

Partial Scattered Intensities from a Binary Suspension of Polystyrene and Silica.

PB95-175618

00,996

COLLOIDING

Polyelectrolytes as Dispersants in Colloidal Processing of Silicon Nitride Ceramics.

PB95-175568

03,055

Surface Chemical Interactions of Si3N4 with PB95-175576

03,056

COLLOIDS

Electroacoustics for Characterization of Particulates and Suspensions. Proceedings of a Workshop. Held in Gaithersburg, Maryland on February 3-4, 1993.

PB94-112695

00,725

Deposition of Colloidal Sintering-Aid Particles on Silicon Nitide.

PB94-216272

03,044

Reduction of Dinitrogen to Ammonia in Aqueous Solution Mediated by Colloidal Metals.

00,867

Aggregation Kinetics of Colloidal Particles Destabilized by PB95-12587

00,894 
COLUMN BUCKLING Dynamics of Multi-DOF Stochastic Nonlinear Systerns
PB97-113245

COLUMNS (PROCESS ENGINEERING)

Continuous Counter-Current Two Phase Aqueous Extraction.

00,675

COLUMNS (SUPPORTS)

Model Precast Concrete Beam-to-Column Connections Subject to Cyclic Loading

00,438

Partially Prestressed and Debonded Precast Concrete Beam-Column Joints.

PB95.153102

00,439

Seismic Pertormance Behavior of Precast Concrete Beam-Column Joints.

00,440

Evaluating the Seismic Performance of Lightly-Reinforced Circular Concrete Bridge Columns.

PB95-163259

01,335

COMBINATION APPLIANCES

Predicting the Energy Performance Ratings of a Family of Type I Combination Appliances.

OMBUSTION

02,504

Gordon Research Conference on the Physics and Chemistry of Laser Diagnostics in Combustion Held in Plymouth, New Hampshire on 12-16 July 1993.

01,353

Spectroscopic Study of Reaction Intermediates and Mechanisms in Nitramine Decomposition and Combustion.

03,774

Combustion of a Polymer (PMMA) Sphere in Micro. gravity.

01,354

Summaries of Center for Fire Research In-House Projects and Grants: 1990.
PB94-160876

00.286

Combustion of Methanol and Methanol/Dodecanol Spray Flames.

PB95-108544 02,478

Effect of Dodecanol Content on the Combustion of Meth-

$\begin{array}{ll}\text { anol Spray Flames. } & 01,389 \\ \text { PB95-176020 } & \end{array}$

Polymer Combustion and Flammability: Role of the Condensed Phase

01,279

Further Development of the N-Gas Mathematical Model: An Approach for Predicting the Toxic Potency of Complex Combustion Mixtures.

PB96-123260

03,650

Numerical Simulation of Rapid Combustion in an Underground Enclosure.

01,424

Analysis of Droplet Arrival Statistics in a Pressure-Atomized Spray Flame.

01,352

COMBUSTION CHEMISTRY

Progress in the Development of a Chemical Kinetic Database for Combustion Chemistry. New Approach for Reducing the Toxicity of the Combustion Products from Flexible Polyurethane Foam.

PB96-123625

01,411

Asymptotic and Numerical Analysis of a Premixed Laminar Nitrogen Dioxide-Hydrogen Flame.

01,422

Scaling Compartment Fires: Reduced- and Full-Scale Enclosure Burns.

00,224

NO Production and Destruction in a Methane/Air Diffusion

Flame.
PB97-122519 01,443

COMBUSTION EFFICIENCY

Estimate of the Effect of Scale on Radiative Heat Loss

Fraction and Combustion Efficiency.
PB95-150447

02,486

COMBUSTION GASES

Reactivity of Product Gases Generated in Idealized Enclosure Fire Environments.

PB95-161790

01,386

COMBUSTION KINETICS

Fluorinated Hydrocarbon Flame Suppression Chemistry.
PB94-185113

Hydrogen Atom Attack on Perchloroethylene.

PB95-163473

00,969

Effect of Suppressants on Metal Fires.

Smoke Emission from Burning Crude Oil.

01,402

PB96-122890

01,407

Inhibition of Premixed Methane-Air Flames by Halon Alternatives.
PB96-146741

01,414

Suppression of High-Speed C2H4/Air Flames with $\mathrm{C}_{1}$ Halocarbons.
PB96-175724

03,287
Effectiveness of Halon Alternatives in Suppressing Dynamic Combustion Process. Interaction of HFC.125, FC-218 and CF31 with High Speed Combustion Waves.

03,290 PB96-176417

Dynamics, Transport and Chemical Kinetics of CompartDent Fire Exhaust Gases.

Chemical Inhibition of Methane-Air Diffusion Flame. PB96-195532

00,229

MBUSTION PHYSICS

Prediction of Fire Dynamics.

OMBUSTION PRODUCTS

Airborne Smoke Sampling Package for Field Measurements of Fires.

PB95-150041 01,381

Reactivity of Product Gases Generated in Idealized Enclosure Fire Environments.

PB95-161790

01,386

Acid Gas Production in Inhibited Diffusion Flames.

PB95-180576

01,390

Compartment Fire Combustion Dynamics. Annual Report, September 1, 1993-September 1, 1994.

00,203

Carbon Monoxide Production in Compartment Fires: Re duced-Scale Enclosure Test Facility.
PB95-231700

01,394

Distinguishing the Contributions of Residential Wood Combustion and Mobile Source Emissions Using Relative Concentrations of Dimethylphenanthrene Isomers.

PB96-135124

02,563

Application of Thermodynamic and Detailed Chemical Kinetic Modeling to Understanding Combustion Produc Generation in Enclosure Fires.

Generation in

01,413

Global Equivalence Ratio Concept and the Formation Mechanisms of Carbon Monoxide in Enclosure Fires. PB96-146790

00,210

Scaling Compartment Fires: Reduced- and Full-Scale Enclosure Burns.

Post-Flame Soot.

00,224

PB96-193701

01,430

Smoke Plume Trajectory from In situ Burning of Crude Oil: Field Experiments.

PB96-200993

COMMENSURATE LATTICES

Pinch Effect in Commensurate Vortex-Pin Lattices. 01,125

COMMERCE

Analyzing Electronic Commerce.
PB94-219102

00.480

Guidelines for the Evaluation of Electronic Data Interchange Products.

OMMERCIAL BUILDINGS

01,506

Performance Approach to the Development of Criteria for Low-Sloped Roof Membranes.

PB94-160751

Lighting and HVAC

PB95-150991

Improving the Evaluation of Building Ventilation. PB96-138508

00,329

00,250

COMMERCIAL DEVELOPMENT

Opportunities for Innovation: Biotechnology. PB94-157831

00,271

OMMERCIAL SECTOR

Assessment of the DOD Goal Security Architecture (DGSA) for No

03,653

COMMERCIAL VEHICLES

Recommendations on Selection of Vehicle-to-Roadside Communications Standards for Commercial Vehicle Operations.

PB94-195914

04,859

Vehicle-to-Roadside Communications for Commercial Vehicle Operations: Requirements and Approaches. PB95-188827

04,860

COMMERCIALIZATION

Water Mist Fire Suppression Workshop Summary.
PB95-161907

COMMODITIES

Checking the Net Contents of Packaged Goods as Adopted by the 79 th National Conference on Weights and Measures, 1994. Third Edition, Supplement 4. PB95-182226

00,484

COMMUNICATION CHANNELS

Capacity of the Lp Norm-Constrained Poisson Channel. PB95-125753

01,515

COMMUNICATION EQUIPMENT

Ground Vehicle Control at NIST: From Teleoperation to Autonomy.
N94-34037/9

03,758

COMMUNICATION NETWORKS

Integrated Services Digital Network (ISDN); Category: Telecommunications Standard; Subcategory: Integrated Services Digital Network.

01,460

Putting the Information Infrastructure to Work: Report of the Information Infrastructure Task Force Committee on Applications and Technology

N94-31228/7

02,715

Context Analysis of the Network Management Domain. Conducted as Part of the Domain Analysis Case Study.

PB94-142528

01,465

Supplement to Stable Implementation Agreements for Open Systems Interconnection Protocols Version 3, September 1990. Change Page index, Version 3, June 1990

(Stable) Change Pages Issued December 1990; Output from September 1990 OSI Workshop (NIST Special PUblication 500-177)

PB94-164035

01,467

National Voluntary Laboratory Accreditation Program. GOSIP: Government Open Systems Interconnection ProPB95-267993

01,486

NIST ATM Network Simulator: Operation and Programming, Version 1.0.

PB96-106851

01,487

Application Software Interface: ISDN Services for an Open Systems Environment.

Provision of Isochronous Service on IEEE 802.6.

PB96-160635

01,501

ISDN in North America.

PB96-160767

01,502

North American Agreements on ISDN.

PB96-160775

01,503

COMMUNICATIONS NETWORKS

Introduction to Traffic Management for Broadband ISDN.

PB94-142494 01,464

Domain Analysis of the Alarm Surveillance Domain. Version 1.0. Conducted as Part of the Domain Analysis Case Study Project.
PB95-136339

\section{COMMUNITIES}

Codes for Named Populated Places, Primary County Divisions, and Other Locational Entities of the United States (FIPS PUB 55-3) (on Magnetic Tape)

PB95-502563

00,129

COMPACT HEAT EXCHANGERS

Heat Transfer in Thin, Compact Heat Exchangers with

Circular, Rectangular, or Pin-Fin Flow Passages.

PB95-140943

02,751

COMPACTING

Rapid Hot Pressing of Ulira-Fine PSZ Powders.

PB94-216587

03,045

COMPACTORS

Field Evaluation of the System for Calıbration of the Marshall Compaction Hammer

PB95-190674

01,323

COMPARATOR CIRCUITS

Leakage Current Detection in Cryogenic Current Com-

parator Bridges.

Custom Integrated Circuit Comparator for High-Performance Sampling Applications.

PB94-213147

02,320

\section{COMPARATORS}

NIST Comparison of the Quantized Hall Resistance and the Realization of the SI Ohm Through the Calculable 
Dynamics, Transport and Chemical Kinetics of Compartment Fire Exh
PB96-195508

00,229

\section{COMPETITION}

Status of Construction and Construction Technologies.

Differences in Competitive Strategies between the United States and Japan.

PB94-2†1836

00,013

COMPETITIVENESS

Program of the Subcommittee on Construction and BuildPB94-193646 00,319 Program of the Subcommittee on Construction and Building (July 1994).

00,321

COMPILEAS

Ada Compiler Validation Summary Report: Certificate Number: 931029S1.11330, Digital Equipment Corpora(ion, DEC Ada for DEC OSF/1 AXP Systerns, Version 3.1, DEC 3000 Model 400 AXP Workstation, DEC 3000 Model 400 AXP Workstation.

01,639

Ada Compiler Validation Summary Report: Certificate Number: 93121751.11336 Control Data Systems, Inc NOSNE Ada, Version 1.4 Cyber 180-930-31 $\Rightarrow$ Cyber 180-930-31.

AD-A275 97777

01,640

Ada Compiler Validation Summary Report. Certificate Number: 931119S1.11332, DDC-1, Inc. DACS MIPS Version 4.7.1 Sun SPARCstation IPX $\Rightarrow$ DACS Sun SPARC/SunOS to MIPS R3000 Bare Instruction Set Architecture Simulator, Version 4.7.1.

01,641

Ada Compiler Validation Summary Report: Certificate Number: 931119S1.11331 DDC-I, Inc. DACS Sun SPARC/SunOS to 80386 PM Bare Ada Cross Compiler System, Version 4.6.4 Sun Sparcstation 1+ $\Rightarrow$ Bare Board iSBC 386/116.

AD-A276 283/9

01,642

Ada Compiler Validation Summary Report. Certificate Number: 930927S1.11328 Green Hills Software C Ada, Version 1.1 ZENY $386=>$ ZENY 386

01,643

AD-A277 981/7

Ada Compiler Validation Summary Report: Certificate Number: $940325 S 1.11348$ DDC-I, DACS Sun SPARC/ Solaris to 80386 PM Bare Ada Cross Compiler System,
Version 4.64 Sun SPARCclassic $\Rightarrow>$ Intel iSBC $386 / 116$ (Bare Machine).

01,644

Ada Compiler Validation Summary Report: Certificate Ada Compiler Validation Summary Report: Certificate Number: 940325 S1.11341 DDC-1, DACS Sun SPARC/ SunOS to 80186 Bare Ada Cross Compiler System, Ver-
sion 4.6.4 Sun SPARCstation IPX $\Rightarrow$ Intel iSBC 186/100 (Bare Machine)

01,645

Ada Compiler Validation Summary Report: Certificate Number: $940325 S 1.11349$ DDC-1, DACS Sun SPARC/ Solaris to 80386 PM Bare Ada Cross Compiler System with Rate Monotonic Scheduling Version 4.6.4 Sun SPARCclassic $=>$ intel iSBC 386/116 (Bare Machine)

AD-A279 644/9

01,646

Ada Compiler Validation Summary Report: Certificate un SPARC/ Solaris Native Ada Compiler System, Version 4.6.2 Sun SPARCclassic $\Rightarrow>$ Sun SPARCclassic

01,647

Ada Compiler Validation Summary Report: Certificate Number: 940325 S1.11346 DDC-I, DACS Sun SPARC/ SunOS to $680 \times 0$ Bare Ada Cross Compiler System (BASIC MODE), Version 4.6.9 Sun SPARC

AD-A279 646/4

01,648

Ada Compiler Validation Summary Report: Certificate Number: 940325 S1.11343 DDC-I, DACS Sun SPARC/ Solaris to 80186 Bare Ada Cross Compiler System, Ver-
sion 4.6.4 Sun SPARCclassic $\Rightarrow$ Intel iSBC $186 / 100$ sion 4.6.4 Sun

(Bare Machine)
AD-A279 757/9

01,649

Ada Compiler Validation Summary Report: Certificate Number: 940325 S1.11344 DDC-I, DACS Sun SPARC/ Solaris to 80186 Bare Ada Cross Compiler System with Rate Monotonic Scheduling, Version 4.6.4 Sun SPARCclassic $=>$ Intel iSBC 186/100 (Bare Machine). Ada Compiler Validation Summary Report: Certificate Number: 940325 S1.11347 DDC-I, DACS Sun SPARC/ SunOS to $680 \times 0$ Bare Ada Cross Compiler System (SE CURE MODE), Version 4.6.9 Sun SPARCstation IPX => Lynwood j435TU (68030) (Bare Machine).

01,651

Ada Compiler Validation Summary Report: Certificate Number: $940325 S 1.11342$ DDC-1, DACS Sun SPARC/ SunOS to 80186 Bare Ada Cross Compiler System with Rate Monotonic Scheduling Version 4.6.4 Sun SPARCstation IPX $\Rightarrow$ Intel iSBC 186/100 (Bare Machine).

01,652

Ada Compiler Validation Summary Report: Certificate Number: 940325S1.11351 DDC-1, DACS Sun SPARC/ SunOS to Pentium PM Bare Ada Cross Compiler System with Rate Monotonic Scheduling, Version 4.6.4 Sun
SPARCstation IPX $\Rightarrow$ Intel Pentium (operated as Bare Machine) based in Xpress Desktop (Intel product number: XBASE6E4F-B).

A279 804/9 01,653

Ada Compiler Validation Summary Report: Certificate Number: 940325S1.11353 DDC-I, DACS Sun SPARC/ Solaris to Pentium PM Bare Ada Cross Compiler System with Rate Monotonic Scheduling, Version 4.6.4 Sun SPARCclassic $=>$ Intel Pentium (operated as Bare $\mathrm{Ma}$ chine) based in Xpress Desktop (Intel product number: XBASE6E4F-B)

01,654

Ada Compiler Validation Summary Report: Certificate Number: 940325S1.11350 DDC-1, DACS Sun SPARC/ Compiler System 政 Operated as Bare Machine) Based in $X$ BASE6E4F-B)

01,655

Ada Compiler Validation Summary Report: Certificate Number $940325 S 1.11345$ DDC-I. DACS Sun SPARC SunOS to $680 \times 0$ Bare Ada Cross Compiler System, Version 4.6.9 Sun SPARCstation IPX $=>$ Mótorola MVME143 $68030 / 68882$ (Bare Machine)

01,656

Ada Compiler Validation Summary Report: Certificate Ada Compiler Validation Summary Report: Certificate Nomber: 94032551.11352 DDC-1 DACS Sun SPARC/ tem, Version 46.4 Sun SPARCclassic $\Rightarrow$ Intel Pentium Operated as Bare Machine) Based in Xpress Desktop (Intel Product Number: XBASE6E4F-B). AD-A280 295/7

01,657

Ada Compiler Validation Summary Report: Certificate Number 940902S1.11377 UNISYS Corporation. integrAda for Windows NT, Version 1.0. Intel Deskside Server with Intel 80486DX266 $\Rightarrow$ Intel Deskside Server with Intel $80486 \mathrm{D} \times 266$.

AD-A288 571/3

01,658

Ada Compiler Validation Summary Report: Certificate Number 940902S1.11376. UNISYS Corporation (1.0. Intel Deskside er with Intel Pentium $60 \mathrm{MHz}$. AD-A288 572/1

01,659

Ada Compiler Validation Summary Report: Certificate Number 94101251. 1379 TISOFT, Inc. Green Hills Optimizing Ada Compileer, Version 1.8.7 with PATCK ID 11 AD-A288 573/9

01,660

Ada Complier Validation Summary Report: Certificate Number: 940929S1.11378. Digital Equipment Corporation DEC Ada for DEC OSF/1 AXP Systems, Version 3.2 DEC 3000 Model 400 AXP

AD-A288 5747

01,66

Ada Compiler Validation Summary Report. Certificate Number $941117 \mathrm{~S} 1.11380$. Electronic Data Systems Corp. Compiler: OC Systems Legacy Ada/370, Release 1.4.1 (without optimization)

AD-A289 805/5

01,662

ADA Compiler Validation Summary Report, VC Number 950303S1.11381. Digital Equipment Corporation - Compiler Name: DEC Ada for OpenVMS Alpha Systems, Verpiler Name
sion 3.2 .

AD-A293 709/2

01,663

Ada Compiler Validation Summary Report, VC No. 950609S1.11390 Digital Equipment Corporation - Com piler Name: DEC Ada Version 3.2 for OpenVMS VAX

AD-A296 794/

01,664

FORTRAN Compiler Validation System, Version 2.1.

01,698 $M$ (also known as MUMPS) Validation Test Suite, Version 8.3 (for Microcomputers)

PB94-502077

01,699

COMPLEX RESONANCE ENERGIES

Population Trapping in Short-Pulse Multiphoton Ionization.

PB96-164140

04,371

COMPLEX TIME DEPENDENCE

Complex Time Dependence of the EPR Signal of Irradiated L-alpha-alanine.

PB97-122436

04,180

COMPLEX VARIABLE

Exact Series Solution to the Epstein-Hubbell Generalized Elliptic Type Integral Using Complex Variable Residue PB97-110167

03,423

COMPLEXES

High-Resolution IR Laser-Driven Vibrational Dynamics in Supersonic Jets: Weakly Bound Complexes and Intramolecular Energy Flow.

PB94-216751

00,862

Infrared Spectra of van der Waals Complexes of Importance in Planetary Atmospheres.

00,071

Microwave Spectra of van der Waals Complexes of Importance in Planetary Atmospheres.

00,919

COMPLEXITY

Measurement of Process Complexity.

01,781

COMPOSITE FABAICATION

Report on the Workshop on Manufacturing Polymer Composites by Liquid Molding. Held in Gaithersburg, Maryland on September 20-22, 1993

\section{PB94- 160066}

03,131

Report on the Workshop on Manufacturing Polymer Composites by Liquid Molding. Held in Gaithersburg, Maryland on September 20-22, 1993

PB94-160066
Effect of Two Initiator/Stabilizer Concentrations in a Metal Primer on Bond Strengths of a Composite to a Base Metal Alloy.

00,141

Wear of Selected Materials and Composites Sliding against MoS2 Films.

Intelligent Process

03,231

PB94-172780

02,811

Phase Behavior of a Hydrogen Bonding Molecular ComPosite.

01,202

Effect of Transformation of Alloy on Transient and Resid ual Stresses in a Porcelain-Metal Strip.

PB94-198397

00,143

Asymmetric Tip Morphology of Creep Microcracks Growing Along Bimaterial Interfaces.

Physics Required for Prediction of Long Term Performance of Polymers and Their Composites.

03,146

Polymer Composites Workshop. Held in Winona, Minnesota on April 29-30, 1992 (Video)

PB94-780129

03,147

One- and Two-Sided Burning of Thermally Thin Materials.
PB95-140935

Investigation into the Flammability Properties of Honey. comb Composites.

03,152

NIR-Spectroscopic Investigation of Water Sorption Characteristics of Dental Resins and Composites.

PB95-151171

00,189

Interaction between Micro and Macroscopic Flow in RTM Preforms.

PB95-162012

03,159

Nanocomposite Magnetic Materials.

PB95-162780

04,617

Elastic Green's Function for a Bimaterial Composite Solid Containing a Free Surface Normal to the Interface.

Green's Function for Generalized Hilbert Problem for Cracks and Free Surfaces in Composite Materials.

PB95-163333

03,163

Generalized Plane Strain Analysis of a Bimaterial Com posite Containing a Free Surface Normal to the Interface.

Polymers Technical Activities 1994. NAC-NRC Assessment Panel, April 6-7, 1995

01,275

Geometrical Percolation Threshold of Overlapping Ellipsoids.

Reduction of Marginal Gaps in Composite Restorations by Use of Glass-Ceramic Inserts.

PB96-102405

00,174

Wear Transitions in Monolithic Alumina and Zirconia-Alumina Composites. 
Applicability of Effective Medium Theory to Ferroelectric/ Ferrimagnetic Composites with Composition and Frequency-Dependent Complex Permittivities and Permeabilities.

01,945

Lattice Statics of Interfaces and Interfacial Cracks in Bimaterial Solids.

PB96-161823

02,985

Estimation of the Orientation Distribution of Short-Fiber Composites Using Ultrasonic Velocities.

PB96-176656

03,178

Flaw Tolerance and Toughness Curves in Two-Phase Particulate Composites: SiC/Glass System.

03,081

Orthotropic Elastic Constants of a Boron-Aluminum FiberOrthotropic Elastic Constants of a Boron-Aluminum Fiber-
Reinforced Composite: An Acoustic-Resonance-Spectroscopy Study.

PB96-200175

03,182

Tensile Creep of Silicide Composites.

03,183

Need for Advanced Characterization Techniques in Prod-

uct Manufacturing: A Case Study on Ceramic Matrix Composites.

03,089

Life Prediction of a Continuous Fiber Reinforced Ceramic Composite Under Creep Conditions.

PB96-204128

03,091

Letter Report on Flame Spread Testing of a Composite Material.

PB97-110068 01,436

Characterizing Materials Properties for Ceramic Matrix Composites.

PB97-110282

03,097

Surface Roughness of Glass-Ceramic Insert. Composite Restorations: Assessing Several Polishing Techniques. PB97-119010

03,583

COMPOSITION (PROPERTY)

Compositional Homogeneity in Processing Precurso Powders to the Ba2YCu307-x High Tc Superconductor. PB94-212743

04,508

\section{COMPOSITIONAL MAPPING}

Study of Diffusion Zones with Electron Microprobe Compositional
PB94-216348

00,559

\section{COMPOUND SEMICONDUCTORS}

Development and Characterization of Insulating Layers on Silicon Carbide: Annual Report for February 14, 1988 to February 14, 1989

PB94-155579

02,295

COMPRESSED GAS

Stability of Compressed Gas Mixtures Containing Low Level Volatile Organic Compounds in Aluminum CylPB96-111968

00,613

COMPRESSED GASES

Method of Sale for CNG Paves Way to Greater Public Acceptance.

COMPRESSIBILITY

Crystal Structure and Compressibility of 3:2 Mullite.
PB95-175030

PB95-175030
Compressibility of Polycrystal and Monocrystal Copper

Acoustic-Resonance Spectroscopy.

PB96-164223

02,990

COMPRESSIBLE FLUIDS

Measurements of the Viscosities of Saturated and Compressed Fluid 1-chloro-1,2,2,2-tetrafluoroethane (R124) pressed Fluid 1-chloro-1,2,2,2-tetrafluoroethane (R124)
and Pentafluoroethane $(R 125)$ at Temperatures between 120 and $420 K$

03,254

Measurements of the Viscosities of Saturated and Compressed Liquid 1,1,1,2-Tetrafluoroethane (R134a), 2 , Dichloro-1,1,1-Trifluoroethane (R123) and 1,1-Dichloro-1Fluoroethane (R141b).

PB95-175386

03,273

COMPRESSION Shape of the Temperature-Entropy Saturation Boundary.
PB96-135066

COMPRESSIVE STRENGTH

Maturity Method.

01313

Maturity Functions for Concrete Made with Various Cements and Admixtures

PB94-199502

01,314

Hollow Clay Tile Prism Tests for Martin Marietta Energy Systems: Task 2 Testing.

00,352

Effects of Testing Variables on the Measured Compres-

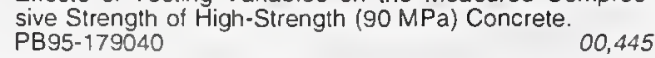
PB95- 179040

PB96-112180 Effects of Testing Variables on the Strength of High

$\begin{array}{ll}\text { Strength }(90 \mathrm{Mpa}) \text { Concrete Cylinders. } & \\ \text { PB96-112198 } & 00,456\end{array}$

PB96-112198

COMPRESSOR BLADES

Determination of Hydrogen in Titanium Alloy Jet Engine Compressor Blades by Cold Neutron Capture Prompt Gamma-ray Activation Analysis.

01,448
COMPTON EFFECT

Stud: of Multiple Scattering Background in Compton Scatter lmaging.
PB96-112222

04,425

COMPTON SCATTERING

Penetration and Diffusion of Hard X-rays through Thick Barriers. III. Studies of Spectral Distributions.

03,771

OMPUTATION

Computing S(alpha) Using Symbolic Monte Carlo. 03,410

Portable Vectorized Software for Bessel Function Evalua-

PB94-198975

01,690

Software Needs in Special Functions.

01,702

Leady Axisymmetric Modes in Infinite Clad Rods. Part 1. PB95-162905 04,187 Performance Characteristics of Fast Elliptic Solvers on Parallel Platforms.

PB95-180832

01,723

Error-Bounding in Level-Index Computer Arithmetic.

PB96-109582

01,742

COMPUTATIONAL FLUID DYNAMICS

Experimental and Numerical Studies on Two-Dimensional Gravity Currents in a Horizontal Channel.

01,359

Dispersion and Deposition of Smoke Plumes Generated in Massive Fires.

PB95-126066. 02,540

Mathematical Modeling and Computer Simulation of Fire Phenomena.

PB95-180063 00,384

Book Review: Aspects and Applications of the Random

PB96-123534

04,215

Transport by Gravity Currents in Building Fires. PB97-119325

01,441

\section{COMPUTATIONAL GEOMETRY}

Submissions to a Planned Encyclopedia of Operations Research on Computational Geometry and the Voronoi Delaunay Construct.

PB94-152709

03,425

Growth Surface for the Slopes at the Boundary of a Polygon. 152725

03,408

Data-Parallel Algorithm for Three-Dimensional Delaunay Triangulation and its Implementation.

01,714

PB95-163309

Inserting Line Segments into Triangulations and
Tetrahedralizations.
PB95-198933
03,415 PB95-198933

Whither Computational Materials Science. Some Thoughts from

02,987

\section{COMPUTER-AIDED ACQUISITION AND LOGISTIC}

SUPPORT

mpact of Computer-Aided Acquisition and Logistic Support (CALS) in the Application of Standards.

01,756

COMPUTER AIDED DESIGN

APDE Demonstration System Architecture. National PDES Testbed Repon Series.

02,767

Variant Design for Mechanical Artifacts-A State of the Art Survey.

02,768

Design Guide for CMOS-On-SIMOX. Test Chips NIST3 and NIST4.

PB94-163458

02,297

Semiconductor Measurement Technology: Design and Testing Guides for the CMOS and Lateral Bipolar-on-SOI Test Library.

02,301

High-Level CAD Melds Micromachined Devices with Foundries.

PB94-216413

02,321

CALS-Digital Representation for Communication of Product Data: IGES Application Subsets.

03.658

CALS-Raster Graphics Representation Binary Format Requirements.

03,660

CALS-Digital Representation for Communication of Illus tration Date: CGM Application Profile.

PB94-962400

03,661

Summary and Notes of the Joint ISO/IGES/PDES Organization Technical Committee Meeting Held in Albuquerque, New Mexico on October 15-20, 1989

02,774

National PDES Testbed: An Overview.

PB95-125829

02,775

Challenges to the National Information Infrastructure. The Barriers to Product Data Sharing. National PDES Testbed Report Series.

02,776

Vertical-Cavity Semiconductor Lasers: Structural Characterization, $\mathrm{CAD}$, and DFB Structures.

02,150

CALS-Digital Representation for Communication of Product Data: IGES Application Subsets

03,667

CALS-Raster Graphics Representation Binary Format Re-

quirements.
PB95-962300 03,669

CALS-Digital Representation for Communication of Illustration Date: CGM Application Profile.

PB95-962400

Characterization of Two-Dimensional Dopant Profiles:

Status and Review.
PB96-119300 02,400

Extensions of the Prototype Application Protocol of Ready-to-Wear Apparel Pattern Making.

03,198

Micromachined Display Output for a Cellular Neural Net-

$\begin{array}{ll}\text { work. } & 02,422\end{array}$

New Generation of Fire Resistant Polymers. Part 1. Computer-Aided Molecular Design.

PB96-160593

01,419

Computer-Aided Molecular Design of Fire Resistant Aircraft Materials.

PB96-160601

00,025

PB96-164181

00,276

Group 1 for the Plant Spatial Configuration STEP Application Protocol

PB96-165402 02,789

Aspects of a Product Model Supporting Apparel Virtual

PB $96-183231$

02,790

Characterization of Two-Dimensional Dopant Profiles: Status and Review.

PB97-11013

02,451

Representing Designs with Logic Formulations of Spatial Relations.

PB97-111561

02,792

Using Logic to Specify Shapes and Spatial Relations in Design Grammars.

PB97-111579

02,793

HVAC CAD Layout Toois: A Case Study of University/lndustry Collaboration.

PB97-112221

00,281

Combining Interactive Exploration and Optimization for Assembly Design

Knowledge-Based Approach for Automating a Design Method for Concurrent and Real-Time Systems.

PB97-112502

01,780

Improving the Design Process by Predicting Downstream Values of Design Attributes.

02,795

Interoperability Requirements for CAD Data Transfer in the AutoSTEP Project.

PB97-114268

02,796 
Visualization Applications for Manufacturing: A State-ofthe-Art Survey. Final Report.

02,816

Implementing a Transition Manager in the AMRF Cell Controller.

PB94-199932

02,817

Generic Manufacturing Controllers.

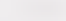

PB94-199940

02,818

Technical Program Description Systems Integration for Manufacturing (SIMA)

02,819

Summary and Notes of the Joint ISO/IGES/PDES Organization Technical Committee Meeting. Held in Albuquerque, New Mexico on October 15-20, 1989

PB95-107314

National PDES Testbed: An Overview.

02,774

PB95-125829

02,775

Challenges to the National Information Infrastructure: The Barriers to Product Data Sharing. National PDES Testbed Report Series.
PB95-136347

02,776

Reference Architecture for Machine Control Systems Integration: Interim Report.

02,820

Portsmouth Fastener Manufacturing Workstation. User's Manual.

PB95-147922

02,860

Using Grafcet to Design Generic Controllers.

PB95-150827

02,821

Integration of Real-Time Process Planning for SmallBatch Flexible Manufacturing.

02,822

Automated Optical Roughness Inspection

02,905

Workshop on the Application of Virtual Reality to Manufacturing. Final Report. Held in Gaithersburg, Maryland on August 9, 1994

02,825

Automated Manufacturing Research Facility 1994 Annual

Report.
PB95-209854

00,015

Information Technologies Make Business Sense for the Custom Therapeutic Footwear Industry.

PB95-251708

02,829

Production Management Information Model for Discrete Manufacturing.
PB96-112008

02,830

Requisite Elements, Rationale, and Technology Overview for the Systems Intergration for Manufacturing Applications (SIMA) Program. Background Study.

02,831

Overview of the Manufacturing Engıneering Toolkit Proto-

type. 128228

02,833

Computer-Aided Manufacturing Engineering Forum (1st). Technical Meeting Proceedings. Held in Gaithersburg,
Maryland on March 21-22, 1995 .

PB96-136965

02,834

NIST RS274KT Interpreter.

02,835

Product Realization Process Modeling: A Study of Requirements, Methods and Research Issues. PB96-147962

02,836

Machine Performance Standard Provides Opportunity to Improve Ouality and Productivity.

02,837

Guidelines for the Development of Mapping Tables.

PB96-154539 02,786

NIST SIMA Interactive Management Workshop. Held in Fort Belvoir, Virginia on November 14-16, 1994. PB96-154877

02,838

Ouality in Automated Manufacturing.

PB96-160437

02,839

Development of a New Ouality Control Strategy for Automated Manufacturing

02,840

Group 1 for the Plant Spatial Configuration STEP Application Protocol.
PB96-165402

02,789

Procedure for Product Data Exchange Using STEP Developed in the AutoSTEP Pilot.

02,843

Systems Integration for Manufacturing Applications Program 1995 Annual Report

02,844

Computer-Aided Manufacturing Engineering Forum (2nd). Technical Meeting Proceedings. Held in Gaithersburg, Maryland on August 22-23, 1995.

02,845

Optoelectronics and Optomechanics Manufacturing: An ATP Focused Program Development. Workshop Proceedings. Held in Gaithersburg, Maryland on February 15, PB97-10 02,204 Interoperability Requirements for CAD Data Transfer in the AutoSTEP Project

02,796
Group 1 for the Process Engineering Data STEP Application Protocol.

$\begin{array}{ll}\text { PB97-116073 02,797 } & \end{array}$

Unified Process Specification Language: Requirements for Modeling Process.

02,850

Capabilities for Product Data Exchange.

02,798

COMPUTER AIDED MAPPING

Geographic Information Systems Standards: A Federal Perspective.

03,678

COMPUTER ALGEBRA SYSTEM

Expression Formatter for MACSYMA.

PB95-267829

01,735

COMPUTER APPLICATIONS

Benchmarks for the Evaluation of Speech Recognizers.
PB94-211539

PB94-211539 01,566 Important Papers in the History of Document Preparation
Systems: Basic Sources.
PB95.125837

02,712

Standards: A Cardinal Direction for Geographic Information Systems.

Computers in Welding: A Primer.
PB95-162863

03,677

02,862

COMPUTER ARCHITECTURE

VLSI Architectures for Template Matching and Block Matching.

01,834

COMPUTER ARITHMETIC

Journal of Research of the National Institute of Standards and Technology, March/April 1996. Volume 101, Number PB96-177381

01,863

COMPUTER ARITHMETICS

MasPar MP-1 as a Computer Arithmetic Laboratory.
PB96-179155

COMPUTER ASSISTED INSTRUCTION

Proposed International Interactive Courseware Standard.

COMPUTER CIRCUITS

High-Temperature Superconductor Cryogenic Current

PB96-119334

02,074

COMPUTER CODES

Experience with MPI: 'Converting Pvmmake to Mpimake under LAM' and 'MPI and Parallel Genetic Programming'.
PB96-141296 COMPUTER COMMUNICATIONS

Comparing Remote Procedure Calls: Open Network Computing, Distributed Computing Environment and Inter-

national Organization for Standardization.

01,724

Ouch Those Programs are Painful.

PB96-102678

01,739

Contormance

01,631

Lessons from the Establishment of the U.S. GOSIP Test-

ing Program.

GOSIP Testing Program

01,817

PB96-161229

01,504

COMPUTER GENERATED ENVIRONMENTS

Approaches Using Virtual Environments with Mosaic.

PB95-169108
MPUTER GRAPHICS

Graphical Kernel System (GKS), Category: Software Standard. Subcategory: Graphics. International Standard Information Technology; Computer Graphics; Graphica Kernel System (GKS) Language Bindings. Part 4: C.
FIPS PUB 120-1C Open Document Architecture (ODA) Raster Document Application Profile (DAP). Category: Software Standard; Subcategory: Graphics.
FIPS PUB 194

01,669

Computer Graphics Metafile (CGM): Procedures for NIST CGM Validation Test Service.

PB94-161809 01,804

Visualization Applications for Manufacturing: A State-of-

the-Art Survey. Final Report.
PB94-194552

02,816

Graphical Conceptual Navigation as a Presentation Technique for a Graphics Standard.

PB94-200573

01,692

CALS-Raster Graphics Representation Binary Format Requirements.

03,660

CALS-Digital Representation for Communication of Illustration Date: CGM Application Profile.

PB94-962400

03,661

Underflow-Induced Graphics Failure Solved by SLI Arithmetic.

01,712

CALS-Raster Graphics Representation Binary Format Requirements.

03,669

CALS-Digital Representation for Communication of Illustration Date: CGM Application Profile.

PB95-962400

03,670

COMPUTER GRAPHICS METAFILE

Computer Graphics Metafile (CGM): Procedures for NIST CGM Validation

COMPUTER INFORMATION SECURITY

Standard Security Label for Information Transfer; Category: Computer Security; Subcategory: Security Labels.
FIPS PUB 188 Computer Security Management and Planning in the U.S Federal Government. PB95-163432

01,596

Guidance to Federal Agencies on the Use of Trusted Systems.

PB95-163440

01,597

Addressing U.S. Government Security Requirements for PBI. 160577

01,611

Concept Paper: An Overview of the Proposed Trust Technology Assessment Program.

COMPUTER INTEGRATED MANUFACTURING

Roadmap for the Computer Integrated Manufacturing (CIM) Application Framework

\section{COMPUTER INTERFACE}

NIST Workshop on the Computer Interface to Flat Pane Displays. Held in San Jose, California on January 13-14, 1994.

$$
\text { PB95-136388 }
$$

01,625

COMPUTER MEDIATED COMMUNICATION

Information, Infrastructure: Reaching Society's Goals. A
Report of the Information Infrastructure Task Force Committee on Applications and Technology.

ED-376 823

00,131

COMPUTER MODELS

CFAST Output Comparison Method and Its Use in Comparing Different CFAST Versions.

00,401

Empirical Validation of a Transient Computer Model for Combined Heat and Moisture Transfer.

PB97-111991

00,416

COMPUTER NETWORKS

NIST Cooperative Laboratory for OSI Routing Technology.
DE94015308

01,791

Report of the Federal Internetworking Requirements Panel.

DE95017761

03,407

Putting the Information Infrastructure to Work: Report of the Information Infrastructure Task Force Committee on Applications and Technology.

94-31228/7

02,715

Open System
N94-36858/6

02,716

Supplement to Stable Implementation Agreements for Open Systems Interconnection Protocols. Version 3, Sep tember 1990. Change Page Index, Version 3, June 1990 from September 1990 OSI Workshop (NIST Special Publication 500-177).

lication 500-177)

Conformance Assessment of Transport Layer Security PB94-164373

01,576

Local Area Networks in NAA: Advantages and Pitfalls.

PB94-172095 00,527 Conformance Testing of a Lower Layer Security Protocol.
01,57

Integrated Network Management

01,583

IGOSS-Industry/Government Open Systems Specification.

01,806 


\section{KEYWORD INDEX}

Introduction to the P1003.1g and CPI-C Network Application Programming Interfaces.

01,731 National Voluntary Laboratory Accreditation Program. National Voluntary Laboratory Accreditation Program. GOSIP: Government Open Systems Interconnection Profile.

01,486

Z39.50 Implementation Experiences.

01.816

Open System Environment (OSE): Architectural Frame-

Open System Environment (OSE):
work for Information Infrastructure.

work for Inform

00,002

Evolution of a United States Information System.

PB96-157896

02,713

Comparison of FDDI Asynchronous Mode and DODB Oueue Arbitrated Mode Data Transmission for MetropoliIan Area Network Applications.

PB96-160452

01,498

Standardization for ATM and Related B-ISDN Technologies.

01,499

Data Communications Strategy.

02,738

Federal Implementation Guideline for Electronic Data Interchange. ASC X12 003050 Transaction Set 840 Request for Ouotation. Implementation Convention.

quest for Ouotation. Impiementation Convention.

Federal Implementaion Guideline for Eletronic Data Interchange. ASC X12 003050 Transaction Set 865 Purchase Order Change Acknowledgement/Request - Seller Initiated. Implementation Convention.

01,825

Five Q's (Cues) of the U.S. GOSIP Testing Program. 01,826

Open System Environment Implementors Workshop (OIW); Standardization Role Defined.

01,828

Unpredictable Certainty. Information Infrastructure through 2000

00,016

COMPUTER PERFORMANCE EVALUATION

Hardware Measurement Techniques for High-Speed Net-

works.

01,500

Report of a Workshop on the Assurance of High Integrity Software.

PB96-161377

01,763

Standards for High Integrity Software.

Using S-Check, Alpha Release 1.0.

01,764

01,767

\section{COMPUTER PROGRAM}

Algorithm Testing and Evaluation Program for Coordinate Measuring Systems: Long Range Plan.

\section{COMPUTER PROGRAM DOCUMENTATION}

02,915

MLL++

PB96.195219

01,776

COMPUTER PROGRAM INTEGRITY

Concept for an Algorithm Testing and Evaluation Program at NIST

02,890

Study on Hazard Analysis in High integrity Sottware Standards and Guidelines.

01,725

PB95-198727

01,743

PB96-111844

Slicing in the Presence of Parameter Aliasing.

01,755

Analysis of Standards for the Assurance of High Integrity Software.

PB96-16135

03,735

COMPUTER PROGRAM MANAGEMENT

Reference Information for the Software Verification and Validation Process.

COMPUTER PROGRAM PORTABILITY

Porting Multımedia Applications to the Open System Environment.

PB94-172921

02,721

Simple Scalability Test for MIMD Code.

01,688

Application Portability Profile (APP): The U.S. Government's Open System Environment Profile Version 3.0.
PB96-158712

Open Systems Software Standards in Concurrent Engineering.

PB96-160932

NIST POSIX Testing Program.

01,821

CSL View of Applications Portability, Scalability, and Interoperability.
PB97-122303

COMPUTER PROGRAM RELIABILITY

Software Safety and Program Slicing.

01,703
Center for High Integrity Software System Assurance: Initial Goals and Activities.

PB95-251674

01,734

Unravel: A CASE Tool to Assist Evaluation of High Integrity Software. Volume 1. Requirements and Design. PB95-267886

01,736

Unravel: A CASE Tool to Assist Evaluation of High Integrity Soltware. Volume 2. User Manual.

01,737

COMPUTER PROGRAM VALIDATION

Experimental Models for Software Diagnosis.

PB97.113906

01,783

COMPUTER PROGRAM VERFICATION

Verification and Validation.

PBg6-161393

01,765

\section{COMPUTER PROGRAM VERIFICATION}

User's Guide for the PHIGS Validation Tests (Version 2.1).

01,682

Process for Selecting Standard Reference Algorithms for

Evaluating Coordinate Measurement Software.

02,629

Formal Specification and Verification of Control Software for Cryptographic Equipment

FORTRAN Compiler Validation System, Version 2.

PB94-500691

SGML Parser

01,698

Testers Open Dialogue at Inaugural NIST Workshop.

PB95-175550 01,718

Algorithm Testing and Evaluation Program for Coordinate Measuring Systems: Testing Methods.

PB95-251658 02,666

User's Guide for the Algorithm Testing System Version P.0. 2595.251666

02,916

Method to Determine a Basis Set of Paths to Perform Program Testing.

01,747

Standard Generalized Markup Language Test Suite Evaluation Report.
PB96-154992

IEEE's POSIX: Making Progress.

01,751

PB96-160924

01,757

Analysis of Standards for the Assurance of High Integrity Software.

03,735

Verification and Validation of Reengineered Software.

PB96-161401 01,766

Reference Information for the Software Verification and Validation Process

\section{COMPUTER PROGRAMMING}

01,773

World Model Registration for Effective Off-Line Programming of Robots

02,933

Introduction to the P1003.19 and CPI-C Network Application Programming Interfaces.

PB95-231726

01,731

$X$ Window System, Version 11, Release 5

01,769

Interoperability Experiments with CORBA and Persistent

$\begin{array}{ll}\text { Object Base Systems. } & 01,772 \\ \text { PB96-183140 }\end{array}$

Experimental Evaluation of Specification Techniques for Improving Functional Testing.

01,779

\section{COMPUTER PROGRAMS}

Semiconductor Measurement Technology: HOTPAC. Programs for Thermal Analysis including Version 3.0 of the TXYZ Program, TXYZ30, and the Thermal MultiLaye Program, TML Error-Bounding in Level-Index Computer Arithmetic.
PB96-109582

01,742

Federal Implementation Guideline for Electronic Data Interchange: ASC X12 003040 Transaction Sel 838 Trading Partner Profile (Confirmation of Vendor Registration). impiementation Convention.

01,813

SUM and MEAN: Standard Programs for Activation Analysis. 112149

00,617

Federal Implementation Guideline for Electronic Data interchange: ASC X12 003040 Transaction Set 838 Trading Partner Profile (Vendor Registration), Implementation Convention.

PB96-11265

03,674

User's Guide to 'SuperFit' Modeling Software for CMM PB96-128236

02,921

Fire Protection Engineering Tools. Simple Tools: The PQ96-156179

00,221

Prototyping a Graphical User interface for DHCP.
PB96-160544 02,599

Application of the Pointer State Subgraph to Static Program Slicing.

01,768

Structured Testing: A Testing Methodology Using the Cyclomatic Complexity Metric

01,784

COMPUTER SCIENCE \& TECHNOLOGY

Ada Compiler Validation Summary Report: Certificate Number: 931029S1.11330, Digital Equipment Corporation, DEC Ada for DEC OSF/1 AXP Systems, Version 3.1, DEC 3000 Model 400 AXP Workstation, DEC 3000 Model 400 AXP Workstation.

AD-A274 872/1

01,639

Ada Compiler Validation Summary Report: Certificate Number: 931217S1.11336 Control Data Systems, Inc. NOSNE Ada, Version 1.4 Cyber 180-930-31 $\Rightarrow$ Cyber $180-930-31$.

01,640

Ada Compiler Validation Summary Report. Certificate Number: 931119S1.11332, DDC-1, Inc. DACS MIPS R3000 Bare Ada Cross Compiler System, Version 4.7.1 Sun SPARCstation IPX $=>$ DACS Sun SPARC/SunOS to MIPS R3000 Bare Instruction Set Architecture Simulator. Version 4.7.1

AD-A276 $181 / 5$

01,641

Ada Compiler Validation Summary Report: Certificate Number: 931119S1.11331 DDC-1, Inc. DACS Sun SPARC/SunOS to 80386 PM Bare Ada Cross Complier System, Version 4.6.4 Sun Sparcstation 1+ $\Rightarrow$ Bare

01,642 Ada Compiler Validation Summary Report. Certificate Version 1.1 ZENY 386 => ZENY 386

AD-A277 $981 / 7$

01,643

Ada Compiler Validation Summary Report: Certificate Number: 940325 S1.11348 DDC-I, DACS Sun SPARC/ Solaris to 80386 PM Bare Ada Cross Compiler System, Version 4.6.4 Sun SPARCclassic $=>$ intel iSBC 386/116 AD-A279 642/3

01,644

Ada Compiler Validation Summary Report: Certificate Number: 940325 S1.11341 DDC-I, DACS Sun SPARC/ sion 4.6.4 Sun SPARCstation IPX $\Rightarrow$ Intel iSBC $186 / 100$ (Bare Machine)

01,645

Ada Compiler Validation Summary Report: Certificate Number: 940325S1.11349 DDC-I, DACS Sun SPARC/ Solaris to 80386 PM Bare Ada Cross Compiler System with Rate Monotonic Scheduling, Version 4.6.4 Sun AD-A279 644/9

01,646

Ada Compiler Validation Summary Report: Certificate Version 4.6.2 Sun Solaris Native Ada Compiler System

AD-A279 645/6

01,647

Ada Compiler Validation Summary Report: Certificate Number: 340325 S1.11346 DDC-I, DACS Sun SPARC/ SunOS to $680 \times 0$ Bare Ada Cross Compiler System
(BASIC MODE) Version 4.6.9 Sun SPARCstation IPX $\Rightarrow>$ Lynwood j435TU (68030) (Bare Machine).

01,648

Ada Compiler Validation Summary Report: Certificate Number: 940325S1.11343 DDC-I, DACS Sun SPA Solaris to 80186 Bare Ada Cross Compiler System, Bare Machine)

AD-A279 757/9

01,649

Ada Compiler Validation Summary Report: Certificate Number: 940325S1.11344 DDC-I, DACS Sun SPARCI Solaris io 80186 Bare Ada Cross Compiler System with Solaris to 80186 Bare Ada Cross Compiler System with
Rate Monotonic Scheduling, Version 4.6.4 Sun SPARCclassic $=>$ Intel iSBC $186 / 100$ (Bare Machine).
AD-A279 $758 / 7$ 
Ada Compiler Validation Summary Report: Certificate Number: 940325S1.11350 DDC-I, DACS Sun SPARC/ SunOS to Pentium PM Bare Ada Cross Compiler System, Version 4.6.4 Sun SPARCstation IPX $=>$ Intel Pentium (Operated as Bare Machine) Based in Xpress Desktop AD-A279 864/3

01,655

Ada Compiler Validation Summary Report: Certificate Number 940325 S1.11345 DDC-I. DACS Sun SPARC/ SunOS to $680 \times 0$ Bare Ada Cross Compiler System, Version 4.6.9 Sun SPARC station IPX $\Rightarrow$ Motorola MVME 143 $68030 / 68882$ (Bare Machine)

01,656

Ada Compiler Validation Summary Report: Certificate Number 940902S1.11377 UNISYS Corporation. IntegrAda for Windows NT, Version 1.0. Intel Deskside Server with Intel 80486DX266 $\Rightarrow>$ Intel Deskside Server with Intel 80486D X266.

AD-A288 $571 / 3$

01,658

Ada Compiler Validation Summary Report: Certificate Number 940902S1.11376. UNISYS Corporation IntegrAda for Windows NT, Version 1.0. Intel Deskside Server for Intel Pentium $60 \mathrm{MHz}=>$. Intel Deskside Server with Intel Pentium $60 \mathrm{MHz}$.

AD-A288 572/1

01,659

Ada Compiler Validation Summary Report: Certificate Number 94101251.11379 TISOFT, Inc. Green Hills Optimizing Ada Compileer, Version 1.8 .7 with PATCK ID 1 ProLiant 2000 Model $5 / 66$. Model 55/66 $=>$ COMPAO ProLiant 2000 Model 5/66.

01,660

Ada Complier Validation Summary Report: Certificate Number: 940929S1.11378. Digital Equipment Corporation DEC Ada for DEC OSF/1 AXP Systems, Version 3.2: DEC 3000 Model 400 AXP Workstation $=>$ DEC 3000 Model 400 AXP Workstation.

01,661

Ada Compiler Validation Summary Report. Certificate Ada Compiler Validation Summary Report. Certificate
Number $941117 S 1.11380$. Electronic Data Systems Corp. Number $941117 \mathrm{~S} 1.11380$. Electronic Data Systems Corp.
Compiler: OC Systems Legacy Ada/370. Release 1.4 .1 (without optimization)

(without optimiza

01,662

ADA Compiler Validation Summary Report, VC Number 950303S1.11381. Digital Equipment Corporation - Compiler Name: DEC Ada for OpenVMS Alpha Systems, Version 3.2 .

AD-A293 709/2

01,663

Ada Compiler Validation Summary Report, VC No. 950609S1.11390 Digital Equipment Corporation - Compiler Name: DEC Ada Version 3.2 for OpenVMS VAX AD-A296 794/1

01,664

Report of the Federal Internetworking Requirements Panel.

DE95017761

03,407

Metropolitan Areas (Including MSAs, CMSAs, PMASs, and NECMAs). Category: Data Standards and Guidelines: Subcategory: Representations and Codes.

04,873

Countries, Dependencies, Areas of Special Sovereignty, and Their Principal Administrative Divisions. Category: Data Standards and Guidelines; Subcategory: Representation and Codes.

00,128

COBOL. Category: Software Standard; Subcategory: Programming Language. Includes ANSI'S X3.23-1985, X3.23A-1989 and X3.23B-1993.

01,670

COBOL. Category: Suftware Standard; Subcategory: Pro-

gramming Language. Part A.
FIPS PUB 21-4A

COBOL. Category: Software Standard; Subcategory: Programming Language. Part B.
FIPS PUB 21-4B

01,672

Data Encryption Standard (DES); Category: Computer Security: Subcategory: Cryplography. Guideline: Codes for Named Populated Places, Primary County Divisions, and Other Locational Entities of the United States, Puerto Rico, and the Outlying Areas. Category: Data Standards and Guidelines. Subcategory: Representations and Codes.

04,865

Graphical Kernel System (GKS). Category: Software Standard. Subcategory: Graphics. International Standard: Information Technology; Computer Graphics; Graphical Intormation Technology; Computer Graphics; Graphical Security Requirements for Cryptographic Modules; Category: Computer Security: Subcategory: Cryptography.
FIPS PUB 140-1 User Interface Component of the Applications Portability Profile Category: Software Standard; Subcategory: Application Program Interface.

cation Program Inter
FIPS PUB $158-1$

01,793

Spatial Data Transfer Standard (SDTS): Category: Sottware Standard; Subcategory: Information Interchange.
FIPS PUB 173-1 Spatial Data Transfer Standard (SDTS). Category: Sottware Standard; Subcategory: Information Interchange. ware Standard; Subc

(FIPS PUB 173-1A)

01,795 Spatial Data Transfer Standard (SDTS). Category: Sott-
ware Standard; Subcategory: Information Interchange. (FIPS PUB 173-1B).
FIPS PUB 173-1B Secure Hash Standard. Category: Computer Security. FIPS PUB 180-1 01,568 Integrated Services Digital Network (ISDN); Category Telecommunications Standard; Subcategory: Integrated

$\begin{array}{ll}\text { Services Digital Network. } & 01,460 \\ \text { FIPS PUB } 182 & \end{array}$

Escrowed Encryption Standard (EES); Category: Computer Security; Subcategory: Cryptography.
FIPS PUB 185

Digital Signature Standard (DSS). Category: Computer Security: Subcategory: Cryptography.
FIPS PUB 186

01,570

Administration Standard for the Telecommunications In Arastructure of Federal Buildings. Category: Telecommunifrastructure of Federal Buildings. Category: Telecommuniministration.

FIPS PUB 187

01,461

Standard Security Label for Information Transfer; Category: Computer Security; Subcategory: Security Labels.
FIPS PUB 188
01,571

Portable Operating System Interface (POSIX). Part 2 Shell and Utilities. Category: Software Standard; Subcategory: Operating Systems.

01,797

Guideline for the Use of Advanced Authentication Technology Alternatives. Category: Computer Security. Subcategory: Access Contro.

01,798

Guideline for the Analysis of Local Area Network Security. Category: Computer Security; Subcategory: Risk Analysis and Contingency Planning.

FIPS PUB 191

01,799

Application Profile for the Government Information Loca or Service (GILS). Category: Software Standard; Subcalegory: Inform
FIPS PUB 192

01,800

SQL Environments. Category: Software Standard; Subcategory: Database.
FIPS PUB 193

01,801

Integration Definition for Function Modeling (IDEFO); Category: Sottware Standard; Subcategory: Modeling TechFIPSPUB 183

02,800

Integration Definition for Information Modeling (IDEF1X); Category: Software Standard; Subcategory: Modeling Techniques.

01,673

Optical Storage

01,620

Proceedings of the Digital Systems Reliability and Nuclear Safety Workshop. Held in Rockville, Maryland on eptember 13-14, 1993.

NUREG/CP-0136 03,728

Technology Trends in Telecommunications: An Overview.
PB94-123080

General Procedures for Registering Computer Security Objects.

01,573

Report of the NIST Workshop on Digital Signature Certificate Management. Held on December 10-11, 1992

PB94-135001 01,574

Planning for the Fiber Distributed Data Interiace (FDDI). 01,621
NIST Model PM2 Power Measurement System for $1 \mathrm{~mW}$ at $1 \mathrm{GHz}$.

Good Security Practices for Electronic Commerce, Including Electronic Data Interchange.

PB94-139045

01,463

Security Considerations for SQL-Based Implementations PB94-139649

02,766

Guide to Software Engineering Environment Assessment and Evaluation

01,676

Introduction to Traffic Management for Broadband ISDN.

PB94-142494 01.464

Applying Virtual Environments to Manufacturing.

PB94-142502

02,803

Context Analysis of the Network Management Domain.

Conducted as Part of the Domain Analysis Case Study.

Report on Application Integration Architectures (AIA) Workshop. Held in Dallas, Texas on February 8-12, 1993.
PB94-142536
01,803

01,803

Next Generation Computer Resources: Reference Model for Project Support Environments (Version 2.0)

01,677

Guide to Configuration Management and the Revision Control System for Testbed Users.

01,678

Structural EXPRESS Editor.

02,769

Issues and Recommendations for a STEP Application Protocol Framework. National PDES Testbed.

02,770

COMPUTER SCIENCE \& TECHNOLOGY

Computer Graphics Metafile (CGM): Procedures for NIST CGM 161809 Test

01,804

Computer Systems Laboratory Annual Report, 1993. PB94-162518 01,622

North American ISDN Users' Forum Agreements on Integrated Services Digital Network.

PB94-162559

01,466

Proceedings of the Workshop on the Federal Criteria for Information Technology Security. Held in Ellicot City, Maryland on June 2-3, 1993

PB94-162583

01,575

Distributed Supercomputing Software: Experiences with the Parallel Virtual Machine - PVM.

PB94-163086

Compatibility Analysis of the ANSI and ISO IRDS Serv-

$\begin{array}{ll}\text { ices Interfaces. } & 01,805 \\ \text { PB94-163474 } & \end{array}$

Validation Testing System Requirements. National PDES Testbed Report Series.

94-163482

Fire Data Management System, FDMS 2.0, Technical Documentation.
PB94-164019

01,358

Supplement to Stable Implementation Agreements for Open Systems Interconnection Protocols. Version 3, September 1990. Change Page Index, Version 3, June 1990 (Stable) Change Pages Issued December 1990; Outpul trom September 1990 OSI Workshop (NIST Special Pub-

B94-164035

Conformance Assessment of Transport Layer Security Implementations.

Time-Perturbation Tuning of MIMD Programs.

01,576

PB94-164399

01,681

User's Guide for the PHIGS Validation Tests (Version 2.1).

PB94-165206 01,682

Evaluating Form Designs for Optical Character Recogni-

PB94-168044

01,830

Unconstrained Handprint Recognition Using a Limited LB94-168051

01,831

Design and Development of an Information Retrieval System for the EAMATE Data. Volume 2 of 2. Appendices.
PB94-168390 00,487 Local Area Networks in NAA: Advantages and Pitfalls.

PB94-172095 00,527

Formał Multi-Layer Test Methodology and Its Application

$\begin{array}{ll}\text { PBOSI.172194 } & 02,718\end{array}$

ISO/IEC Workshop on Worldwide Recognition of OSI Test Results Regional Progress - North America.

PB94-172202

02,719

Analysis of a Biologically Motivated Neural Network for Character Recognition

RDI-SIM ECMA Inter-Domain Routing Protocol Simulation Tool

PB94-172301

01,683

Bringing Natural Language Information Retrieval Out of PB94-172335

Time-Perturbation Tuning of MIMD Programs. PB94-172566

02,720

Synthetic-Perturbation Techniques tor Using Synthetic-Perturbation Techniques for Tuning
Shared Memory Programs (Extended Abstract).
PB94-172657 Porting Multimedia Applications to the Open System Envi-

ronment.
PB94-172921 02,721

Second Text REtrieval Conference (TREC-2). Held in Gaithersburg, Maryland on August 31-September 2

01,686

Conformance Testing of a Lower Layer Security Protocol. PB94-185402 01,577 Synthetic-Perturbation Tuning of MIMD Programs. PB94-185568

User Profile for Researchers Sudying Object ons for Computer Systems. 
VLSI Architectures for Template Matching and Block Matching.

01,834

Graphical Conceptual Navigation as a Presentation Technique for a Graphics Standard.

01,692

Quality Characterıstics and Metrics for Reusable Sottware (Preliminary Report)

01,693

World Wide Web and Mosaic: User's Guide.

PB94-207354

02,722

IGOSS-Industry/Government Open Systems Specifica-

PB94-207453

01,806

Preliminary Functional Specifications of a Prototype Electronic Research Notebook for NIST

00,012

Face Recognition Technology for Law Enforcement Appli$\begin{array}{ll}\text { cations. } & \text { 01,837 }\end{array}$

PB94-207768 01,837

Report of the NIST Workshop on Key Escrow Encryption

Held in Gaithersburg, Maryland on June 8-10, 1994.
PB94-209459

U.S. GOSIP Testing Program.

01,807

Associated Object Model for Distributed Systems.

PB94-212016

01,694

Formal Specification and Verification of Control Software for Cryptographıc Equipment.

01,585

Debugger for $\mathrm{Tcl}$ Applications.

PB94-213303

01,695

Kibitz-Connecting Multiple Interactive Programs Together.

PB94-213311 01,696

Using Expect to Automate System Administration Tasks.

\begin{tabular}{l} 
PB94-213329 01,697 \\
\hline
\end{tabular}

Time Dependent Vector Dynamic Programming Algorithm for the Path Planning Problem.

03,428

Head Start on Assurance: Proceedings of an Invitational Workshop on Information Technology (IT) Assurance and Trustworthiness. Held in Williamsburg, Virginia on March 21-23, 1994

PB94-215746 01,586

NIST-Coordinated Standard for Fingerprint Data Inter-

change.

01,808

Bibliography of Computer Security Glossaries.

PB94-216710

01,587

NIST Form-B

01,838

ISDN Conformance Testing Guidelines: Guidelines for Implementors of ISDN Customer Premises Equipment to Conform to Both National ISDN-1 and North American ISDN Users' Forum Layer 3 Basic Rate Interface Basic Call Control Abstract Test Suites.

PB94-219094

01,471

Analyzing Electronic Commerce.

00.480

Industry/Government Open Systems Specification Testing Framework. Version 1.0

PB94-219110

01,809

FORTRAN Compiler Validation System, Version 2.1

PB94-500691 01,698

Validated Products List (Cobol, Fortran, ADA, Pascal,

MUMPS, SOL).
PB94-937300

CALS-Automated Interchange of Technical Information

PB94-962000 03,657

CALS-Digital Representation for Communication of Product Data: IGES Application Subsets.

PB94-962100

03,658

CALS-Markup Requirements and Generic Style Specifications for Electronic Printed Output and Exchange of Text. PB94-962200 03,659

CALS-Raster Graphics Representation Binary Format Requirements.

03,660

CALS-Digital Representation for Communication of Illus tration Date: CGM Application Profile.

03,661

CALS-Department of Defense Computer Aided Acquisi$\begin{array}{ll}\text { tion Logistic Support (CALS). } & 03,662\end{array}$ PB94-962500 03,662 CALS-Contractor Integrated Technical Information Serv-
ice (CITIS), Functional Requirements.
PB94-962600
F

03,663

Framework for National Information Infrastructure Serv-

PB95-103719

02,723

Making Sense of Software Engineering Environment Framework Standards.

PB95-105037

01,701

Software Needs in Special Functions.

01,702

Security in Open Systems.

PB95-105383

01,473
Standardization of Testing Methods for Optical Disk Media Characteristics and Related Activities at NIST.
PB95-108486

Application of Expert System to Select Data Sources from Chemical Information Databases.

PB95-125654

00,505

Important Papers in the History of Document Preparation

Systems: Basic Sources.

02,712

Software Safety and Program Slicing

01,703

Object SOL: Language Extensions for Object Data Management.
PB95-125902 Information Resource Dictionary System (IRDS): A Status

PB95-126207

01,810

Computer Security Training and Awareness Course Compendium.

Domain Analysis of the Alarm Surveillance Domain. Version 1.0. Conducted as Part of the Domain Analysis Case Study Project.
PB95-136339

01,705

Challenges to the National Information Infrastructure: The Barriers to Product Data Sharing. National PDES Testbed Report Series

02,776

Comparison of FFT Fingerprint Filtering Methods for Neural Network Classification.

PB95-136362

01,840

Faster BKL Monte Carlo Simulations.

PBO5-130

01,706

NIST Workshop on the Computer Interface to Flat Pane Displays. Held in San Jose, California on January 13-14, 1994.

PB95-136388

01,625

Report on the Advanced Software Technology Workshop.

Held on February 1, 1994

PB95-136610

01,707

Information Technology Engineering and Measurement Model: Adding Lane Markings to the Information Super-

PB95-143145

01,474

Channel Coding for Code Excited Linear Prediction (CELP) Encoded Speech in Mobile Radio Applications. 01.475

Standards: A Cardinal Direction for Geographic Informa. tion Systems.

03,677

Analysis of Selected Software Safety Standards.
PB95-151262

01,708
Formal Methods in Conformance Testing: Result and Per.

PB95-153029

01,710

Model Precast Concrete Beam-to-Column Connections Subject to Cyclic Loading.

00,438

Mapping Integration Definition for Information Modeling (IDEF1X) Model into CASE Data Interchange Format (CDIF) Transter File.

PB95-154670

01,711

Initial NIST Testing Policy for STEP: Beta Testing Program for AP 203 Implementations. National PDES Testbed Report Series.

PB95-154688

02,779

ISDN LAN Bridging

01,477

User Study: Informational Needs of Remote National Archives and Records Administration Customers.

PB95-154738

02,725

SDNS Security Management.

PB95-161170

01,592

Keeping Time on Your PC.

01,537

Standards and Linkages: What Data Sharing Needs.

PB95-161881

01,713

Data Encryption Standard

PB95-162376 01.595

Access Paths for Materials Databases: Approaches for Large Databases and Systems.

PB95-162525

02,975

ISDN Conformance Testing

PB95-163176 01,478

Geographic Information Systems Standards: A Federal Perspective.

03,678

Computer Security Management and Planning in the U.S. Federal Government.

Federal Govern

01,596

Guidance to Federal Agencies on the Use of Trusted Systems.

PB95-163440

01,597

Computer Virus Attacks.

PB95-163655

01,715

Application Pro

01,479

Self-Organizing Neural Network Character Recognition on a Massively Parallel Computer.
P95.163994

Coping with Different Retrieval Methods in Next Generation Networks.

PB95-168555

02,726

Using Archie to Find Files on the INTERNET.

02,727

Approaches Using Virtual Environments with Mosaic

PB95-169108 01,599

Guide on Open System Environment (OSE) Procure.

PB95-169496

Perspective on Sotware Engineering Standards.

PB95-171377

01,811

Initial Graphics Exchange Specification (IGES): Proce dures for the NIST IGES Validation Test Service.

PB5-171427

02,780

Binary Decision Clustering for Neural Network Based Opical Character Recognition.

PB95-171971

01,848

Framework for the Development and Assurance of High Integrity Soltware.

PB95-173084

01,716

SGML Parser Validation Procedures.

PB95-174959

01,717

Asynchronous Transfer Mode Procurement and Usage

PB95-174967

01,481

Testers Open Dialogue at Inaugural NIST Workshop

Obtaining and Installing a Public Domain TEX.

PB95-175741

01,718

Global Information Infrastructure: Agenda for Coopera-

PB95.178604

Glossary of Software Reuse Terms.

PB95-178992

01,482

Taxonomy for Security Standards.

Concepts of the NIST EXPRESS Server.

01,720

PB95-180543

01,602

andling Passwords with Security and Reliability in Background Processes.

Federal Government and Information Technology Standards: Building the National Information Infrastructure.
PB95-180840

Prototype Information Retrieval System to Perform a Best-Match Search for Names.

PB95-181152

02,740

Keeping Your Site Comfortably Secure: An Introduction to Internet Firewalls.

PB95-182275

02,730

Impact of the FCC's Open Network Architecture on NS/ NP Telecommunications Security.

PB95-189445

01,483

Operating Principles of Multikron II Performance Instrumentation for MIMD Computers.

PB95-189486

01,628

Proceedings Report of the International Invitation Work- 


\section{KEYWORD INDEX}

Analysis of ANSI ASC $\times 12$ and UN/EDIFACT Electronic

PB95-220554 01,729 Persistent Object Base System Testing and Evaluation. PB95-220588 01.730 Introduction to the P1003.1g and CPI-C Network Applica. tion Programming Interfaces.

PB95-231726

01,731

Operating Principles of the SBus Multikron Interface Board

PB95-231783

01,630

Standards Policy and information Infrastructure.

01,485 Guidelines for the Evaluation of X.500 Directory Products. PB95-231908 02,732 Testability of Object-Oriented Systems.

01,733

Center for High Integrity Sottware System Assurance: Initial Goals and Activities

01.734

Method and Evaluation of Character Stroke Preservation on Handprint Recognition.

PB95-251724

01,850

Improving Neural Network Pertormance for Character and Fingerprint Classification by Altering Network Dynamics.
PB95-267803 Effect of Training Dynamics on Neural Network Pertormance.

01,852

Unravel: A CASE Tool to Assist Evaluation of High Integrity Software. Volume 1. Requirements and Design. 01,736 Unravel: A CASE Tool to Assist Evaluation of High Integrity Software. Volume 2. User Manual.

01,737

PCASYS: A Pattern-Level Classification Automation System for Fingerprints.

PB95-267936

01,853

Building Life Cycle Cost Computer Program (BLCC), Version 4.2-95 (for Microcomputers)

PB95-501953

00,266

Validated Products List (Cobol, Fortran, ADA, Pascal, MUMPS, SOL)

01,738

CALS-Automated Interchange of Technical Intormation.

PB95-962000 03,666

CALS-Digital Representation for Communication of Product Data: IGES Application Subsets.

PB95-962100

03,667

CALS-Markup Requirements and Generic Style Specifications for Electronic Printed Output and Exchange of Text. PB95-962200 03,668

CALS-Raster Graphics Representation Binary Format Requirements.

03,669

CALS-Digital Representation for Communication of Illus.

tration Date: CGM Application Profile.

Pration Date: CG

03,670

CALS-Department of Defense Computer Aided Acquisition Logistic Support (CALS)

PB95-962500

03,671

CALS-Contractor Integrated Technical Information Serv-

ice (CITIS), Functional Requirements.
PB95-962600

03,672

Faster Monte Carlo Simulations

PB96-102074

04,018

Using Synthetic Perturbations and Statistical Screening to Assay Shared-Memory Programs.

PB96-103031

01,740

NIST ATM Network Simulator: Operation and Programming, Version 1.0

PB96-106851

01,487

Mapping Integration Definition for Function Modeling (IDEFO) Model into CASE Data Interchange Format (CDIF) Transfer File.

PB96-109533

01,741

Federal Implementation Guideline for Electronic Data Interchange: ASC $\times 12003040$ Transaction Set 838 Trading Partner Profile (Confirmation of Vendor Registration) Implementation Convention.

PB96-111190

01,813

Human and Machine Recognition of Faces: A Survey

PB96-111687

01,854

SOA Standards and Total Ouality Management.

PB96-111844 01,743

High Integrity Software Standards Activities at NIST.

PB96-112214

01,744

Federal Implementation Guideline for Electronic Data Interchange: ASC $\times 12003040$ Transaction Set 838 TradInterchange: ASC $\times 12003040$ Transaction Set 838 Trad-
ing Partner Profile (Vendor Registration), Implementation Convention.

PB96-112651

03,674

Federal Implementation Guideline for Electronic Data Interchange. ASC $\times 12003050$ Transaction Set 850 Award Instrument. Implementation Convention.

PB96-114913

01,814

Federal Implementation Guideline for Electronic Data Interchange. ASC $\times 12003050$ Transaction Set 860 Modi- fications to Award Instrument. Implementation Convention.

PB96-114921

01,815

Z39.50 Implementation Experiences

01,816

Lessons trom the Establishment of the U.S. GOSIP Test-

ing Program.
PB96-119359 01,817

Seli-Organizing Neural Network Character Recognitın Using Adaptive Filtering and Feature Extraction.

PB96-119797

01.855

Error Protecting Characteristics of CDMA and Impacts on

PB96-122452

01,491

EDI and EFT Security Standards.

PB96-122833

01,605

Open Issues in OSI Protocol Development and Conformance Testing.

01,818

Database Management Standards: Status and Applicabil-

PB96-122924

01,819

Nonlinear Color Transformations in Real Time Using a Video Supercomputer.

02,191

Proposed International Interactive Courseware Standard

$\begin{array}{ll}\text { PB96-123161 00,137 } & \end{array}$

Improving Neural Network Performance for Character and Fingerprint Classification by Altering Network Dynamics. PB96-123195 01,856

Common Criteria: On the Road to International Harmoni-

PB96-123484

01,606

Functional Security Criteria for Distributed Systems.

PB96-123492 01,607

Distributed Systems: Survey of Open Management Ap proaches.

PB96-128202

01,746

Overview of the Manufacturing Engineering Toolkit Proto-

PBPe. 128228

02,833

Application Software Interface: ISDN Services for an Open Systems Environment.

01,492

Comparison of POSIX Open System Environment (OSE) Comparison of POSIX Open System Environment (OSE) and Open Distributed Processing (ODP) Reference Models.

01,820

Method to Determine a Basis Set of Paths to Perform Program Testing.

PB96-131503

01,747

Sharing Information via the Internet: An Infoserver Case Study

PB96.131511

01.493

Operating Principles of MultiKron Virtual Counter Performance Instrumentation for MIMD Computers.

PB96-131529

01,632

Defining Environment Integration Requirements.

02,733

Computer Security: An Introduction to Computer Security. The NIST Handbook.

PB96-131610

01,608

Spatial Intormation and Technology Standards Evolving. PB96-135108 03,679 Telecommunications Security Guidelines for Telecommunications Management Network. Computer Secu-

PB96-139415

01.496

Experience with MPI: 'Converting Pvmmake to Mpimake under LAM' and 'MPI and Parallel Genetic Programming' 6-14129

01,748

Open System Environment (OSE): Architectural Framework for Information Intrastructure.

PB96-146360

00,002

Scalability Test for Parallel Code.

01,749

Virtual Environments for Health Care. A White Paper for he Advanced Technology Program (ATP) the National Institute of Standards and Technology.

PB96-147814

03,594

$\mathrm{C}_{++}$in Safety Critical Systems.

01,750

STandard for the Exchange of Product Model Data (STEP): Procedures for NIST STEP Validation. 02787 Standard Generalized Markup Language Test Suite Evaluation Report.

PB96-154992

01,751

Development of Computer-Based Models of Standards and Attendant Knowledge-Base and Procedural Systems.

Findings and Recommendations from a Software Reengineering Case Study.

PB96-155791

01,752

Security Program Management

01,610

COMPUTER SCIENCE \& TECHNOLOGY

Application Portability Protile (APP): The U.S. Government's Open System Environment Profile Version 3.0.
PB96-158712

Research on Methods for Determining Optical Disk Media Life Expectancy Estimates.

PB96-160304

01,633

Comparison of FDDI Asynchronous Mode and DODB Oueve Arbitrated Mode Data Transmission for MetropoliIan Area Notwork Applications.

PB96-160452 01,498

Standardization for ATM and Related B-ISDN Tech-

PB96-160460

01,499

Copyright and Intormation Services in the Context of the National Research and Education Network.

B96-160536

02,736

Protolyping a Graphical User Interface for DHCP.
PB96-160544

02,599

Hardware Measurement Techniques for High-Speed Net-

works.

PB96-160551 01.500

Addressing U.S. Government Security Requirements for OS

PB96-160577

01,611

Status of Emerging Standards for Removable Computer Storage Media and Related Contributions of NIST.

PB96-160619

01,634

NIST Program for Investigating Error Reporting Capabili-

ties of Optical Disk Drives.

PB96-160627

01,635

Provision of Isochronous Service on IEEE 802.6

PB96-160635

01.501

ISDN in North America

PB96-160767

01.502

North American

01,503

SOA and TOM in Software Ouality Improvement.

PB96-160791

Precise Identification of Computer Viruses.

PB96-160825

01,754

Slicing in the Presence of Parameter Aliasing

PB96-160858

01.613

Information Technology Standards in a Changing World:

B96-160866

Using a Multi-Layered Approach to Representing Tort Law Cases for Case-Based Reasoning.

00,135

Concept Paper: An Overview of the Proposed Trust Technology Assessment Program.

PB96-160882

01,614

Assessing Functional Diversity by Program Slicing.
PB96-160890 03,734 
Report of a Workshop on the Assurance of High Integrity Sottware.

01,763

Standards for High Integrity Software.

01,764

Verification and Validation.

01,765

Verification and Validation of Reengineered Soltware.

PB96-161401

01,766

Response to Comments on the NIST Proposed Digital Signature Standard.

01,615

Manager's Guide for Monitoring Data Integrity in Financia Systems.

PB96-165915

00,003

Public Key Infrastructure Invitational Workshop. Held in McLean, Virginia on September 28, 1995.

01,616

Application of the Pointer State Subgraph to Static Program Slicing.

01,768

Data Communications Strategy

PB96-167846

02,738

$X$ Window System, Version 11, Release 5

01,769

Guidelines for the Evaluation of Electronic Data InterGuidelines for the Evaluation of Electronic Data Interchange Products.

01,506

Federal Implementation Guideline for Electronic Data Interchange. ASC X12 003050 Transaction Set 855 Purchase Order Acknowledgment: Implementation Conven-

tion. 172374

01,823

Federal Implementation Guideline for Electronic Data Interchange. ASC X12 003050 Transaction Set $840 \mathrm{Re}$ quest for Quotation. Implementation Convention.

PB96-172531

01,824

Federal Implementaion Guideline for Eletronic Data Inter. change. ASC X12003050 Transaction Sel 865 Purchase Order Change Acknowledgement/Request - Seller Initiated Implementation Convention.

PB96-172549 01,825

Five Q's (Cues) of the U.S. GOSIP Testing Program.

PB96-175716 01,826

Strategy to Support Multipoint Communication Service Over Native ATM Service.

01,507

Federal Implementation Guideline for Electronic Data Interchange: ASC X12 003050 Transaction Set 836 Procurement Notices. Implementation Convention.

PB96-178892

01,827

Deformation and Fracture of Mica-Containing Glass-Ceramics in Hertzian Contacts.

03,080

Open System Environment Implementors Workshop (OIW); Standardization Role Defined.

PB96-180047

01,828

Application of Metadata Standards.

PB96-180187

X.500 Directory Schema Design Handbook.

01,771

PB96-183041

02,739

Interoperability Experiments with CORBA and Persistent Object Base Systems

01,772

Distributed Communication Methods and Role-Based Access Control for Use in Health Care Applications.

PB96-183165

01,508

Binary Decision Clustering for Neural-Network-Based Opical Character Recognition.

01,857

Reference Information for the Software Verification and Validation Process.

PB96-188164

01,773

Generalized Form Registration Using Structure-Based Techniques.

01,858

Component-Based Handprint Segmentation Using Adaptive Writing Style Model.

PB96-193669

01,859

Computer Systems Laboratory Computing and Applied Mathematics Laboratory Technical Accomplishments, Oc-

lober 1994-Marc

01,638

TMACH Experiment Phase 1. Preliminary Developmental Evaluation.

PB96-195318

01,618

Experimental Evaluation of Specification Techniques for

Improving Functional Testing.

01,779

Guide to Locating and Accessing Computerized Numeric Materials Databases.

PB96-204045

03,007

Introduction to Secure Telephone Terminals.

01,512

Computer Security: Generally Accepted Principles and Practices for Securing Information Technology Systems. PB97-110811

01,619
Using Technology to Manage and Protect Intellectual PBoperty.

01513

Knowledge-Based Approach for Automating a Design Method for Concurrent and Real-Time Systems.

PB97-112502

01.780

Peasurement of Process Complexity.

PB97-113138

Experimental Models for Software Diagnosis.
PB97-113906

01,783
PB97-113906 Cyclomatic Complexity Metric.

PB97-114169

01,784

Lab Report Special Section: Natural Language Processing and Information Retrieval Group Information Access and User Interfaces Division, National Institute of Standards and Technology

PB97-118665

02,742

Panel: Building and Using Test Collections.

02,743

Text REtrieval Conference (4th) (TREC-4). Held in Gaithersburg, Maryland on November 1-3, 1995.
PB97-121636

boration

Usability Engineering: Industry-Govern

PB97-122287

01,514

CSL View of Applications Portability, Scalability, and Interoperability
PB97-122303

01,787

\section{COMPUTER SECURITY}

Security Requirements for Cryptographic Modules: Category: Computer
FIPS PUB $140-1$

Secure Hash Standard. Category: Computer Security.

FIPS PUB 180-1 01,568

Digital Signature Standard (DSS). Category: Computer Security; Subcategory: Cryptography.

01,570

Guideline for the Use of Advanced Authentication Technology Alternatives. Category: Computer Security. Subcategory: Access Control

01,798

Guideline for the Analysis of Local Area Network Security. Category: Computer Security; Subcategory: Risk Analysis and Contingency Planning.

01,799

General Procedures for Registering Computer Security Objects

01.573

Report of the NIST Workshop on Digital Signature Certificate Management. Held on December 10-11, 1992

PB94-135001 01,574

Security Considerations for SQL-Based Implementations

02,766

Proceedings of the Workshop on the Federal Criteria for Information Technology Security. Held in Ellicot City, Maryland on June 2-3, 1993.

PB94-162583

01,575

Conformance Assessment of Transport Layer Security Implementations Conformance Testing of a Lower Layer Security Protocol. PB94-185402 01,577 Federal Certification Authority Liability and Policy: Law and Policy of Certificate-Based Public Key and Digital Signatures.

01,578

Head Start on Assurance. Proceedings of an Invitational Head Start on Assurance: Proceedings of an Invitational
Workshop on Information Technology (IT) Assurance and Workshop on Information Technology (IT) Assurance and
Trustworthiness. Held in Williamsburg. Virginia on March Trustworthin

PB 1 -23, 1994.

Bibliography of Computer Security Glossaries

01,586

PB94-216710

01,587

Security in Open Systems.

01,473

Computer Security Training and Awareness Course Com. pendium.

PB95-130985

01,589

SDNS Security Management.

01,592

Multi-Agency Certification and Accreditation ( $C$ and $A$ ) Process: A Worked Example.

PB95-17195

01,601

Taxonomy for Security Standards.

PB95-180386 01,602

Handling Passwords with Security and Reliability in Back-

$\begin{array}{ll}\text { ground Processes. } & 01,722\end{array}$

Keeping Your Site Comfortably Secure: An Introduction to Internet Firewalls.

02,730

Assessment of the DOD Goal Security Architecture Assessment of the DOD Goal Security Architecture
(DGSA) for Non-Military Use (DGSA) for Non-Military Use.
PB95-189510 03,653

Computer Security: An Introduction to Computer Security. The NIST Handbook.

01,608

Security Program Management.

PB96-156112

Response to Comments on the NIST Proposed Digital Signature Standard.

PB96-161815

01,615

Public Key Infrastructure Invitational Workshop. Held in McLean, Virginia on September 28, 1995.

01,616

TMACH Experiment Phase 1. Preliminary Developmental Evaluation.

PB96-195318

01,618

Computer Security: Generally Accepted Principles and Practices for

01,619

COMPUTER SIMULATION

Effect of Transformation of Alloy on Transient and Residual Stresses in a Porcelain-Metal Strip.

PB94-198397

00,143

COMPUTER SOFTWARE

Graphical Kernel System (GKS). Category: Software Standard. Subcategory: Graphics. International Standard: Information Technology: Computer Graphics: Graphica Kernel System (GKS)

01,792

Programmer's Hierarchical Interactive Graphics System (PHIGS). Category: Software Standard; Subcategory: Graphics.

FIPS PUB 153-1

01,668

Guide to Configuration Management and the Revision

Control System for Testbed Users.

PB94-150919

01,678

Computer Systems Laboratory Annual Report, 1993

PB94-162518

01,622

Distributed Supercomputing Sottware: Experiences with the Parallel Virtual Machine - PVM.

01,680

PB94-163086

Portable Vectorized Software for Bessel Function Evalua-

PB94-198975

01,690

Virtual Software Repository System.

01,691

Kibitz-Connecting Multiple Interactive Programs Together

PB94-21331

Software Needs in Special Functions.

01,696

PB95-105045

01,702

Report on the Advanced Software Technology Workshop. Held on February 1, 1994

PB95-136610

01,707

Analysis of Selected Software Safety Standards.

Opportunities for Innovation: Software for Manufacturing.

Obtaining and Installing a Public Domain TEX.

PB95-175741

02851

Getting Started on Mosaic.

01,719 
Operating Principles of MultiKron Virtual Counter Performance Instrumentation for MIMD Computers. PB96-131529

01,632

COMPUTER SYSTEMS LABORATORY

Computer Systems Laboratory Annual Report, 1993.

Computer Systems Laboratory Annual Report 1994.
PB95-209920

Computer Systems Laboratory Computing and Applied Mathematics Laboratory Technical Accomplishments, October 1994-March 1996.

PB96-193768

01,638

COMPUTER SYSTEMS PROGRAMS

Using Expect to Automate System Administration Tasks. PB94-21332

Locating Fire Information.

PB96-190137

01,697

00,227

COMPUTER VIRUSES

Computer Virus Attacks.

PB95-163655

01,715

Precise Ident

01,613

COMPUTER VISION

Computer Vision Based Tool Setting Station.

PB94-199858

02,944

Visual-Motion Fixation Invariant.

B94-206281

Real-Time Vision for Autonomous and Teleoperated Control of Unmanned Vehicles

03,701

Real-Time Vision for Unmanned Vehicles.

03,702

Adaptive, Predictive 2-D Feature Tracking Algorithm for Finding the Focus of Expansion.
PB94-218575

Peal Time Compensation for Tool Form Errors in Turning Using Computer Vision.

PB95-107231

02,945

Visual Pursuit Systems.

PB95-143285 01,841

Visual Road Following without 3-D Reconstruction.

PB95-161030

01,591

Reconstruction during Camera Fixation

01,593

01,593
Unified Approach to Camera Fixation and Vision-Based Road Following.

01,594

Physics-Based Vision: Principles and Practice, Shape Re-

covery (Book Review).
PB95-164075

01,846

Integrated Mobile Robot System for Testing Vision Algo rithms.

PB95-164133

02,936

Motion-Model-Based Boundary Extraction

01,849

Real-Time Obstacle Avoidance Using Central Flow Divergence and Peripheral Flow.

02,937

Calculating Time-to-Contact Using Real-Time Ouantized $\begin{array}{ll}\text { Optical Flow. } & \\ \text { PB95-210522 } & 01,604\end{array}$

Texture-Independent Vision-Based Closed-Loop Fuzzy Controllers for Navigation Tasks.

00,183

PB95-220505

Integrated Vision Touch-Probe System for Dimensional
Inspection Tasks. Inspection Tasks.
PB95-255832

Human and Machine Recognition of Faces: A Survey.

PB96-111687

Image Gradient Evolution: A Visual Cue for Danger.

PB96-154562 02,939

Point Probe Decision Trees for Geometric Concept Class-

$\begin{array}{lr}\text { PS. } & 01,612\end{array}$

Scale-Space-Based Visual-Motion-Cue for Autonomous

Navigation.

02,940

Novel Active-Vision-Based Motion Cues for Local Naviga-

tion.

PB96-193727

02,941

COMPUTERIZED CONTROL SYSTEMS

Overview of NASREM: The NASANBS Standard Reference Model for Telerobot Control System Architecture. PB94-194560

04,831

COMPUTERIZED SIMULATION

Ignition and Subsequent Transition to Flame Spread in a Microgravity Environment.

N96-15584/1

04,828

Computer Programs for Simulation of Lighting/HVAC Interactions.

02,501

Use of Computer Models to Predict Temperature and Smoke Movement in High Bay Spaces.

PB94-145976

00, 191

Digitized Simulation of Mercury Intrusion Porosimetry.

PB94-172236

01,304
RDI-SIM ECMA Inter-Domain Routing Protocol Simulation

Tool.

01,683

Digitized Direct Simulation Model of the Microstructural Development of Cement Paste.

01,309

Digitized Simulation Model for Microstructural Develop-

ment

01,310

Diffusion Studies in a Digital-Image-Based Cement Paste Microstructural Model.

PB94-198801

01,312

Simplified Cycle Simulation Model for the Performance

Rating of Refrigerants and Refrigerant Mixtures.

Rating of Rerrigerants and Refrigerant Mixtures. 03,255
PB94-199890

EXITT: A Simulation Model of Occupant Decisions and

Actions in Residential Fires

PB94-213261

00,351

Computer Simulation of the Diffusivity of Cement-Based

PB95-125

00,362

Digital Simulation of the Aggregate-Cement Paste Interfacial Zone in Concrete.

00,363

Fundamental Computer Simulation Models for CementBased Materials.

PB95-126009

00,364

Indoor Air Quality Impacts of Residential HVAC Systems,

Phase 1 Report: Computer Simulation Plan.

PB95-135596

00,249

Faster BKL M

01,706

Physics for Device Simulations and Its Verification by

$\begin{array}{ll}\text { Measurements. } & 02,327 \\ \text { PB95-141172 } & \end{array}$

Simulation of Ceramic Particle Formation: Comparison

with In-situ Measurements. $\quad 00,674$
PB95-152013

Physics for Device Simulations and its Verification by

Measurements.
PB95-152914 02,339

Incinerability of Perchloroethylene and Chlorobenzene.

$\begin{array}{ll}\text { PB95-163457 } & 01,388\end{array}$

Neighbor Tables for Molecular Dynamics Simulations.

PB95-171948 00,991

Using Emulators to Evaluate the Performance of Building Energy Management Systems.

PB95-175774

00,259

Reproducibility of Tests on Energy Management and

Control Systems Using Building Emulators.

PB95. 175980

00,260

Application of a Multizone Airflow and Contaminant Dis.

persal Model to Indoor Air Ouality Control in Residential

Buildings.

PB95-180238

02,555

Computer Simulations of Airflow and Radon Transport in

Four Large Buildings.

Enhancements to Program IDARC: Modeling Inelastic Be-

havior of Welded Connections in Steel Moment-Resisting

Frames.

PB95-231601

00,452

Length Scales for Fragile Glass-Forming Liquids.

Use of Building Emulators to Evaluate the Performance of Building Energy Management Systems.

PB96-111901

00,269

Simulation Studies of Supercooled and Glass Forming

Liquids.

01,085

PB96-122627

Fire Hazard Model Developments and Research Efforts at NIST.

PB96-159652

00,407

Large Eddy Simulations of Smoke Movement in Three Di-

mensions.

01,426

COMPUTERS

Computer Systems Laboratory Annual Report, 1993

PB94-162518

01,622

Guarding Against Transients.

PB94-216470 01,623

Mapping Processes to Processors for Space-Based Robot Systems.

PB95-151510

04,833

Computers in Welding: A Primer.

PB95-162863 02,862

Computer Systems Laboratory Annual Report 1994.

PB95-209920 01,629

Computer Systems Laboratory Computing and Applied Mathematics Laboratory Technical Accomplishments, Oc-

Mathematics Laboratory

PB96-193768

01,638

How to Get NIST-Traceable Time on Your Computer.

PB96-200647

01,559

CONCENTRATING

Simple, Inexpensive Apparatus for Sample Concentration.

CONCENTRATION (COMPOSITION)

Concentration Histogram Imaging: A Scatter Diagram Technique for Viewing Two or Three Related Images.

PB94-199114

00,542

Shear Dependence of Critical Fluctuations in Binary Polymer Mixtures by Small Angle Neutron Scattering.

B94-211612 Concentration and Temperature Profiles in Laminar, Two imensional Methane/Air Diffusion Flames.

PB95-140919

01,379

aser-Induced Fluorescence Measurements of $\mathrm{OH}$. Con Lentrations in the Oxidation Region of Laminar, Hydrocarbon Diffusion Flames.

01,387

Determination of Hydrogen in Titanium Alloy Jet Engine Compressor Blades by Cold Neutron Capture Prompt Gamma-ray Activation Analysis.

PB95-175956

01,448

CONCENTRATION HISTOGRAM IMAGING

Concentration Histogram Imaging: A Scatter Diagram

Technique for Viewing Two or Three Related Images.

PB94-199114

CONCENTRATIONS (COMPOSITION)

Development of a Standard Reference Material for Measurement of Interstitial Oxygen Concentration in Semiconductor Silicon by Infrared Absorption.

PB96-122668

02,404

CONCEPTUAL NAVIGATION

Graphical Conceptual Navigation as a Presentation Technique for a Graphics Standard.

PB94-200573

01,692

CONCRETE

Cement and Concrete Characterization by Scanning Elecron Microscopy.

00,379

Evolution of Porosity and Calcium Hydroxide in Laboratory Concretes Containing Silica Fume.

01,321

Prediction of Potential Concrete Strength at Later Ages.

PB96-112180 01,324

Recent Development in Nondestructive Testing of Con-

crete. 122445

01,325

CONCRETE CONSTRUCTION

Graphical Analysis of the CCRL Portand Cement Proticiency Sample Database (Samples 1-72). (Part 1. Univariant Analysis of Portland Cement)

PB94-196557

01,308

CONCRETE DURABILITY

Diagnosis of Causes of Concrete Deterioration in the MLP-7A Parking Garage.

PB95-143095

01,318

CONCRETE PAVEMENTS

Optimization of Highway Concrete Technology.

PB94-182995

01,333

CONCRETE STRUCTURES

Detection of Voids in Grouted Ducts Using the ImpactEcho Method.

01,306

Evaluating the Seismic Performance of Lightly-Reinforced Circular Concrete Bridge Columns. 
Survey of Concrete Transport Properties and Their Measurement.

PB95-220489

00,396

Compositional Analysis of Beneficiated Fly Ashes.

PB95-220497

00,397

4SIGHT Manual: A Computer Program for Modelling Degradation of Underground Low Level Waste Concrete Vaults.

03,726

Long-Term Pertormance of Engineered Concrete Bar-

riers.

Alkali-Silica Reaction and High Performance Concrete.

Alkali-Silica Reaction and High Performance Concrete.
PB96-131537

Measurement of Rheological Properties of High Pertorm-

ance Concrete: State of the Art Report.

00,414

Multi-Scale Picture of Concrete and Its Transport Properties: Introduction for Non-Cement Researchers.

PB97-115802

03,107

CONCURRENT ENGINEERING

Impact of Computer-Aided Acquisition and Logistic Sup-

port (CALS) in the Application of Standards.

PB96-160908 01,756

Open Systems Software Standards in Concurrent Engi-

neering.

PB96-160932 01,758

Knowledge-Based Approach for Automating a Design

Method for Concurrent and Real-Time Systems.
PB97-112502

01,780

CONCURRENT FLOW

Fire Propagation in Concurrent Flows.

PB94-193844

01,365

CONDENSED MATTER PHYSICS

Neighbor Tables for Molecular Dynamics Simulations.

PB95-171948

CONDENSED PHASE

Polymer Combustion and Flammability: Role of the Condensed Phase.

CONDUCTANCE

01,279

Mesoscopic Conductance Fluctuations in Large Devices.

Evidence for Tunneling and Magnetic Scatttering at 'In situ' YBCO/Noble-Metal Intertaces.

PB96-141098

04,752

Quantum Conductance Fluctuations in the Larger-SizeScale Regıme
PB97-111264

04,144

CONDUCTANCE FLUCTUATIONS

Protonation Dynamics of the alpha-Toxin Ion Channel
from Spectral Analysis of $\mathrm{pH}$-Dependent Current Fluctuations.

PB96-161740

03,652

CONDUCTION

Numerical Analysis Support for Compartment Fire Modeling and Incorporation of Heat Conduction into a Zone Fire

Model.

01,357

Slowly Divergent Space Marching Schemes in the Inverse Heat Conduction Problem.

PB94-199486

03,812

CONDUCTIVE HEAT TRANSFER

Application of Boundary Element Methods to a Transient Axis-Symmetric Heat Conduction Problem.
PB94-212693

CONDUCTIVITY

Absolute Determination of Electrolytic Conductivity for Primary Standard KC1 Solutions from 0 to $50 \mathrm{C}$.

$\mathrm{dc}$ Method for the Absolute Determination of Conductiv-
ittes of the Primary Standard $\mathrm{KCl}$ Solutions from OC to ities of the Primary Standard KCl Solutions from OC to

02,644

PB94-219342

01,081

LOW Electrolytic

Oscillometric and Conductometric Analysis of Aqueous and Organic Dosimeter Solutions.

04,054

Intrinsic Conductivity of Objects Having Arbitrary Shape and Conductivity.

ONDUCT

04,150

Apparatus for Resistance Measurement of Short, SmallApparatus for Resistance
Diameter Conductors.
PB96-141130 PB96-141130 04,417 Electric Dipole Excitation of a Long Conductor in a Lossy
Medium.
PB96-146675 04,058

CONE CALORIMETERS

Examination of the Correlation between Cone Calorimeter Data and Full-Scale Furniture Mock-Up Fires.

PB96-148200 01,417

Flammability Characterization with the Liff Apparatus and the Cone Calorimeter.

PB97-110050

01,435

CONE EMISSION
Failures of the Four-Wave Mixing Model for Cone EmisFailures of the Four-Wave Mixing Model for Cone Emis-
sion.
PB95-202636
04,318
Cone Emission from Laser-Pumped Two-Level Atoms. 1. Quantum Theory of Resonant Light Propagation.

04,325

Cone Emission from Laser-Pumped Two-Level Atoms. 2 Analytical Model Studies.

Analytical Model

04,326

\section{CONFIDENCE INTERVALS}

Effects of Nonmodel Errors on Model-Based Testing.
PB96-123146

Effects of Nonmedel Errors on Model-Based Testing

PB96-155577

03,420

\section{CONFIDENCE LIMITS}

Approximate Confidence Intervals on Linear Combinations of Expected Mean Squares.

PB95-151296

03,435

Approximate Confidence Intervals on Positive Linear Approximate Confidence Intervals on

Combinations of

03,436

Ranges of Confidence Coefficients for Confiedence Intervals on Variance Components.

PB95-151312

03,437

One-Sided beta-Content Tolerance Intervals for Mixed

PB96-141171 03,449

Confidence on the Modified Allan Variance and the Time Variance.

PB96-190376

01,557

CONFIGURATION MANAGEMENT

Guide to Configuration Management and the Revision PB94-150919

01,678

CONFINED SEMIFLEXIBLE POLYMERS

Influence of Surface Interaction and Chain Stiffness on Polymer-Induced Entropic Forces and the Dimensions of Confined Polymers.

PB94-185469

01,203

CONFORMANCE

Open Issues in OSI Protocol Development and Conformance Testing

CONFORMANCE TESTING

Formal Methods in Conformance Testing: Result and Perspectives.

ISDN Conformance Testing.

PB95-163176

01,710

NIST POSIX Testing Program

PB96-160973

Conformance Testing and Specification Management PB97-113781

02,849

CONFORMITY

Conformance Testing of a Lower Layer Security Protocol.

CONFORMITY ASSESSMENTS

Stacking the Cards in Europe: One Company's Story.
PB97.110126

CONGRUENCE

Principle of Congruence and Its Application to CompressPBle States.

01,068

CONNECTION MACHINE

Making Connections.

PB97-119044

01,785

CONNECTORS

Programmable Guarded Coaxial Connector Panel PB96-122544

02,108

CONSTANT POWER

CMOS Circuit Design for Controlling Temperature in Micromachined Devices.

PB96-156088

02,196

CONSTANT VOLUME HEAT CAPACITY

High-Temperature Adiabatic Calorimeter for Constant-Volume Heat Capacity Measurements of Compressed Gases and Liquids.

CONSTRUCTION

Infratechnologies: Tools for Innovation.

PB94-185998

ing.

PB94-193646

and Build-

00,319

Program of the Subcommittee on Construction and Building (July 1994)
PB95-122537

Rationale and Preliminary Plan for Federal Research for Construction and Building.

PB95-154704

00,322

Literature Review on Seismic Performance of Building Cladding Systems.

PB96-10690

00,455

NIST Construction Automation Program Report No. 2 Proceedings of the NIST Construction Automation Workshop. Held in Gaithersburg, Maryland on March 30-31, 1995.

PB96-202239

00,413

Summary of Federal Construction and Building $R$ and $D$

PB97-114250

00,234

Line-Heat-Source Guarded-Hot-Plate Apparatus. PB97-118996

CONSTRUCTION INDUSTRY

Status of Construction and Construction Technologies.

White Papers Prepared for the White House: Construction Industry Workshop on National Construction Goals. Held on December 14-16, 1994.

PB95-216891

01,299

National Construction Sector Goals: Industry Strategies for Implementation.

PB95-269817

00,204

National Planning for Construction and Building $R$ and $D$.

PB96-137104 00,324

Innovation in the Japanese Construction Industry: A 1995 Appraisal.

177373

00,225

CONSTRUCTION JOINTS

Performance of 1/3-Scale Model Precast Concrete BeamColumn Connections Subjected to Cyclic Inelastic Loads. Report No. 4

PB95-179024

00,444

Simplified Design Procedure for Hybrid Precast Concrete

Connections.
PB96-154836

Workgroup Summary Report: Plastic Hinge-Based Tech-

niques for Advanced Analysis.

PB96-159702

00,470

Economic Methods and Risk Analysis Techniques fo Evaluating Building Investments: A Survey. PB96-122593

00,323

CONSTRUCTION MATERIALS

Proficiency Testing as a Component of Quality Assurance

in Construction Materials Laboratories.

PB94-185774

00,334

Graphical Analysis of the CCRL Portland Cement Proficiency Sample Database (Samples 1-72). (Part 1. Univariant Analysis of Portland Cement)

PB94-196557

01,308

Controlling Moisture in the Walls of Manufactured Hous-

ing.

0,355

Measurements of Moisture Diffusivity for Porous Building Materials.

PB95.107397

00,356

Suggestions for a Logically-Consistent Structure for Service Life Prediction Standards.

PB95-125795

00,358

Fundamental Computer Simulation Models for CementBased Materials.

PB95-126009

00,364

Effects of Specimen Edge Conditions on Heat Release

PBa5-152864

00,375

National Voluntary Laboratory Accreditation Program: Construction Materials Testing. 
High Current Pressure Contacts to Ag Pads on Thin Film Superconductors.

04,639

Surface Degradation of Superconducting YBa2Cu3O7delta Thin Films.

04,667

Thermal Conductivity of Polypyromellitimide Film with Alu-

Thermal Conductivity of Polypyromellitimide Film with AluPBina Filler Partict

01,292

CONTAM88 COMPUTER PROGRAM

CONTAM88 Building Input Files for Multi-Zone Airflow and Contaminant Dispersal Modeling.

PB94-194388

02,537

CONTAM93 COMPUTER PROGRAM

CONTAM93 User Manual.

94-164381

02,536

CONTAM93 MODEL

Application of a Multizone Airflow and Contaminant Dispersal Model to Indoor Air Ouality Control in Residential Buildings.

PB95-180238

02,555

CONTAMINANT DISPERSAL MODELING

CONTAM88 Building Input Files for Multi-Zone Airflow and Contaminant Dispersal Modeling

02,537 PB94-194388

CONTAMINANTS

CONTAM94: A Multizone Airflow and Contaminant Dispersal Model with a Graphic User Interface.

CONTAMINATION

Partıal Pressure Analysis in Space Testıng.
N95-14084/4 N95-14084/4 04,827 of Coated Steel. 1. Effects of Surface Roughness and Contamination on the Corrosion Behavior of Epoxy Coated Steel.

PB94-213345

03,189

CONTINUOUS MINERS

Integration of Servo Control into a Large-Scale Control System Design: An Example from Coal Mining.
PB94-203429 PB94-203429 Control System.

03,700 PB94-203528

Environment Simulation for a Continuous Mining MaPB94-203536

03,697

CONTINUOUS RECORDING

Correlations between Electrical and Acoustic Detection of Partial Discharge in Liquids and Implications for Continuous Data Recording.

PB96-204490

02,248

CONTINUOUS WAVE LASERS

Stabilization of Optical Phase/Frequency of a Laser System: Application to a Commercial Dye Laser with an External Stabilizer.

CONTRACT MANAGEMENT

04,327

Federal Implementation Guideline for Electronic Data Interchange: ASC X12 003050 Transaction Set 836 Procurement Notices. Implementation Convention.

PB96-178892

01,827

CONTROL

Post-Process Control of Machine Tools.

PB95-203451

02,952

CONTROL SYSTEMS

Feasibllity Study: Reference Architecture for Machine Control Systems Integration.

PB94-142791

02,804

Toward a Reference Model Architecture for Real-Time Intelligent Control Systems (ARTICS).

02.932

Task Decomposition Methodology for the Design of a Coal Mining Automation Hierarchical Real-Time Control

PB94-185386

03,694

Control Entity Interface Specification

02,815

Hierarchical Real-Time Control System for Use with Coal Mining Automation

PB94-212065

03,698

Using Emulator/Testers for Commissioning EMCS Software, Operator Training, Algorithm Development, and Tuning Local Control Loops.

PB94-212735

00,245

Hierarchical Ada Robot Programming System (HARPS):

A Complete and Working Telerobot Control System Based on the NASREM Mode.

PB94-213162

02,934

Three Dimensional Position Determination from Motion.

01,788

Reference Architecture for Machine Control Systems Inte-

gration: Interim Report.

02,820

Control System Architecture for a Remotely Operated Unmanned Land Vehicle.

PB95-163200

03,759

Reproducibility of Tests on Energy Management and Control Systems Using Building Emulators.

00,260
CONTROL SYSTEMS DESIGN

Continuous Mining Machine Control Using the Real-Time

$\begin{array}{ll}\text { Control System. } & 03,700 \\ \text { PB94-203528 } & \end{array}$

Reference Model Architecture for Intelligent Systems De.

sign. 143137

01,789

Expert Control System Shell Version 1.0 User's Guide.

PB95-198859

01,790

Testing Conformance and Interoperability of BACnet Trade Name) Building Automation Products.

00,310

CONTROLLED NUCLEATION

Controlled Nucleation in Aerosol Reactors for Supression of Agglomerate Formation.

PB95-151973

00,672

CONTROLLERS

Open Architectures for Machine Control.

02,942

Enhanced Machine Controller Architecture Overview.
PB94-142460

PB94- 142460
NIST Support to the Next Generation Controller Program:

1991 Final Technical Report

PB94-163490

02,808

Implementing a Transition Manager in the AMRF Cell Controller.

PB94-199932

02,817

Generic Manu
PB94-199940

02,818

Intelligent Control of an Inert Gas Atomization Process.

PB95-141057 03,344

Using Grafcet to Design Generic Controllers.

02,821

Texture-Independent Vision-Based Closed-Loop Fuzzy Controllers for Navigation Tasks.

PB95-220505

00,183

Intelligent Processing of Materials, Technical Activities 1994 (NAS-NRC Assessment Panel, April 6-7, 1995).
PB96-115050

ONVECTION

Convection and Morphological Stability During Directional

Solidification

03,310

Large Eddy Simulations of Smoke Movement in Three Di-

mensions.

01,426

Problem of Convection in the Water Absorbed Dose Cal-

orimeter.

03,523

CONVECTIVE FLOW

Mathematical Modeling and Computer Simulation of Fire Phenomena

PB95-180063

00,384

CONVECTIVE HEAT TRANSFER

Fire-Plume-Generated Ceiling Jet Characteristics and

Convective Heat Transfer to Ceiling and Wall Surfaces in

a Two-Layer Fire Environment: Uniform Temperature

Ceiling and Wals.

CONVERGENCE

Convergence Properties of a Class of Rank-Two Updates. (NIST Reprint)

PB95-180097

03,430

Hybrid Gauss-Trapezoidal Ouadrature Rules.

03,422

CONVERSION TABLES

Guide for the Use of the International System of Units

PB95-226692

02,747

CONVERTERS

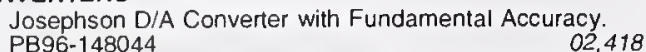

AC-DC Difference Characteristics of High-Voltage Thermal Converters.

02,083

Performance of Multilayer Thin-Film Multijunction Thermal

Converters.
PB96-148135

02,084

\section{CONVOLUTION INTEGRALS}

Data Evaluation of a Linear System by a Second-Order Transfer Function.

PB96-200282

01,970

COOKING DEVICES

Study of Technology for Detecting Pre-Ignition Conditions of Cooking-Related Fires Associated with Electric and Gas Ranges and Cooktops, Phase 1 Report.
PB96-128095

00,303

COOL STARS

GHRS Observations of Cool, Low-Gravity Stars. 1. The Far-Ultraviolet Spectrum of alpha Orions (M2 lab). PB96-112016

00,094

COOLANTS

Chemical Effect in Ceramics Grinding

PB97-122592

03,113

COOLING

Transient Cooling of a Hot Surface by Droplets Evapo-

ration. 2505

03,783

COOLING SYSTEMS

Device for Subambient Temperature Control in Liquid Chromatography.

00,573

Graded and Nongraded Regenerator Pertormance.

PB95-169090 02,753

Multizone Modeling of Three Residential Indoor Air Ouality Control Options.

PB96.165782

COOPERATION

Industry and Government-Laboratory Cooperative $\mathrm{A}$ and PB94-172939

\section{COORDINATE MEASURING MACHINES}

Comparison of Finite Element and Analytic Calculations of the Resonant Modes and Frequencies of a Thick Shell

PB94-160785

02,626

U.S. Navy Coordinate Measuring Machines: A Study of PB94-162831

02,807

User's Guide to NIST SRM 2084: CMM Probe Performance Standard.

02,709

Some Considerations for Interim Testing of Coordinate Measuring Machine Performance Using a Specific ArtiPB95-108858

02,898

Sensitivity of Three-Point Circle Fitting

PB95-136354

02,901

Displacement Method for Machine Geometry Calibration.

PB95-152088 02,946

Two New Probes for a Coordinate Measuring Machine

PB95-163093 02,653

NIST SRM 9983 High-Rigidity Ball-Bar Stand. User Man-

ual.

02,669

Estimation of Measurement Uncertainty of Small Circular Features Measured by CMMs.

PB95-267928

02,918

User's Guide to 'SuperFit' Modeling Software for CMM Probe Lobing.

COORDINATE MEASURING SYSTEMS

02,921

Process for Selecting Standard Reference Algorithms for Evaluating Coordinate Measurement Software.

PB94-173754

02,629

\section{COPLANAR WAVEGUIDES}

Microwave Properties of Voltage-Tunable YBa2Cu3O7delta/SrTiO3 Coplanar Waveguide Transmission Lines. PB96-141262

COPLANARITY

Micromachined Coplanar Waveguides in CMOS TechPB97-119283

02,456

COPOLYMERIZATION

Copolymerization of $\mathrm{N}$-Phenyl Maleimide and gammaMethacryloxypropyl Trimethoxysilane.

PB95-15314

01,248

COPOLYMERS 
Spot-Profile-Analyzing LEED Study of the Epitaxial Growth of $\mathrm{Fe}, \mathrm{Co}$, and $\mathrm{Cu}$ on $\mathrm{Cu}(100)$.
PB95-150165 Hybrid Method for Determining Material Properties from Instrumented Micro-Indentation Experiments. Design and Machining of Copper Specimens with Micro Holes for Accurate Heat Transfer Measurements.
PB95-180428

02,658 Comparison of Fire Sprinkler Piping Materials: Steef in Residential and Light Hazard Installations.

PB95-182267

00,299

Tensile Deformation-Induced Microstructures in FreeStanding Copper Thin Films.

04,715

Magnetic Structure and Spin Dynamics of the $\mathrm{Pr}$ and $\mathrm{Cu}$ in Pr2CuO4.

PB96-111836 04,036

Elastic Constants and Internal Friction of Polycrystalline Copper

PB96-141015 03,364

Solubilities of Copper(II) and Chromium(III) betaDiketonates in Supercritical Carbon Dioxide.

PB96-164215

01,147

Compressibility of Polycrystal and Monocrystal Copper: Acoustic-Resonance Spectroscopy.

02,990

COPPER ALLOYS

Study of Laser Resonance Ionization Mass Spectrometry Using a Glow Discharge Source.

DE94018566

Measuring the Stability of Three Copper Alloys.

PB94-199866

03,308

Roles of Copper in Applied Superconductivity.

PB94-211521

03,326

Diffusion of Copper into Gold Plating.

02,255

00,957

Study of Laser Resonance Ionization Mass Spectrometry Using a Glow Discharge Source.

03,360

COPPER BISMUTHATES

Phase Equilibria in the Systems $\mathrm{CaO}-\mathrm{CuO}$ and $\mathrm{CaO}$

03,048

COPPER CHLORIDES

Structural and Magnetic Properties of $\mathrm{CuCl} 2$ Graphite Intercalation Compounds.

PB96-119748

03,020

COPPER DEUTERIDES

Pure Rotational Spectra of $\mathrm{CuH}$ and $\mathrm{CuD}$ in Their Ground States Measured by Tunable Far-Infrared Spectroscopy.

01,005

COPPER GERMANATES

Crystal Structure and Magnetic Properties of $\mathrm{CuGeO}_{3}$

PB95-180287

04,678

COPPER HYDRIDES

Rolational Spectrum of Copper Hydride Using Tunable Far Infrared Radiation.

00,792

Pure Rotational Spectra of CuH and CuD in Their Ground States Measured by Tunable Far-Infrared Spectroscopy.
PB95-176194

\section{COPPER INDIUM SELENIDES}

Epitaxial Growth and Characterization of the Ordered Va cancy Compound CuIn3Se5 on GaAs (100) Fabricated by Molecular Beam Epitaxy.

PB95-180725

04,687

COPPER OXIDES

Phase Equilibria in the Systems $\mathrm{CaO}-\mathrm{CuO}$ and $\mathrm{CaO}$ Bi2O3.

PB95-140570

03,048

Crystal Chemistry and Phase Equilibria Studies of the $\mathrm{BaO}$ (BaCO3)-R2O3-CuO Systems. 4. Crystal Chemistry and Subsolidus Phase Relationship Studies of the CuORich Region of the Ternary Diagrams, R=Lanthanides.
PB95-151759 00,936

COPYRIGHTS

Copyright and Information Services in the Context of the National Research and Education Network.

PB96-160536

02,736

CORM (COUNCIL FOR OPTICAL RADIATION

NIST Response to the Fitth CORM Report on the Pressing Problems and Projected Needs in Optical Radiation MB94-188240

CORONA DISCHARGE

Measurement of $\mathrm{S} 2 \mathrm{OF} 10$, and $\mathrm{S} 2 \mathrm{O} 2 \mathrm{~F} 10$ Production Rates from Spark and Negative Glow Corona Discharge in SF6/O2 Gas Mixtures.

PB96-123740

01,093

CORONA DISCHARGES

Plasma Chemical Model for Decomposition of SF6 in a Negative Glow Corona Discharge.

01,020

CORRECTIONS

Large Local-Field Corrections in Optical Rotatory Powe of Quartz and Selenium.

PB97-122378

04,400
CORRELATION FUNCTION

Nonequilibrium Statistical Mechanics.

PB96-161781

04,097

CORRESPONDING STATES

Viscosity of Defined and Undefined Hydrocarbon Liquids Calculated Using an Extended Corresponding-States

02,498

CORROSION

Volatile Corrosion Inhibitors.

AD-A310 087/2

03,114

Evaluation of

03,187

Relation between $\mathrm{AC}$ Impedance Data and Degradation of Coated Steel. 1. Effects of Surface Roughness and Contamination on the Corrosion Behavior of Epoxy Coated Stee

PB94-213345

03,189

In situ Measurements of Chloride Ion and Corrosion Potential at the Coating/Metal Interface.

03,122
Journal of Research of the National Institute of Standards and Technology, July/August 1994. Volume 99, Number 4. Special Issue: Extreme Value Theory and Applications. Proceedings of the Conference on Extreme Value Theory and Applications. Volume 2. Held at Gaithersburg, Maryland, in May 1993.

03,440

Sorption of Moisture on Epoxy and Alkyd Free Films and Coated Steel Panels.

PB95-162475

03,192

Degradation of Powder Epoxy Coated Panels Immersed in a Saturated Calcium Hydroxide Solution Containing Sodium Chloride.

PB96-101050

01,344

Journal of Research of the National Institute of Standards and Technology, September/October 1993. Volume 98, Number 5 .

PB96-169057

03,368

Corrosion Characteristics of Silicon Carbide and Silicon

PB96-169081 03,372

Role of Corrosion in a Material Selector Expert System

for Advanced Structural Ceramics.
PB97-110308

03,099

CORROSION INHIBITION

Volatile Corrosion Inhibitors.

AD-A310 087/2

03,114

CORROSION MECHANISMS

Diffusion of Cations Beneath Organic Coatings on Steel Substrate.

03,119

CORROSION POTENTIAL

In situ Measurements of Chloride Ion and Corrosion Potential at the Coating/Metal Interface.

PB95-140893

03,122

CORROSION RESISTANCE

Review of Corrosion Behavior of Ceramic Heat Exchanger Materals: Corrosion Characteristics of Silicon Carbide and Silicon Nitride. Final Report, September 11 , 1992--March 11, 1993.

DE93041307

03,228

Corrosion Resistance of Materials for Renovation of the United States Botanic Garden Conservatory. PB94-154390

00,032

Corrosion Resistant Epoxy-Coated Reinforcing Steel.

PB94-185618

01,307

CORROSION TESTS

Comparison of the Corrosion Rates of FeAl, Fe(sub 3)Al and Steel in Distilled Water and $0.5 \mathrm{M}$ Sodium Chloride. Technical Report Number 2, January--March 199 DE94017332

03,186

CORROSIVE EFFECTS

Comparison of the Corrosion Rates of FeAl, Fe(sub 3)A and Steef in Distilled Water and $0.5 \mathrm{M}$ Sodium Chloride. and Stee in Distilled Water and O.5 M Sodium Chlo DE94017332

03,186

\section{CORTICAL BONE}

EPR Dosimetry of Cortical Bone and Tooth Enamel Irradiated with $X$ and Gamma Rays: Study of Energy DependPBce.

03,639

COSMIC DUST

IRAS Spectroscopic Observations of Young Planetary PBgulae. 152070

00,072

COST ANALYSIS

Energy Prices and Discount Factors for Life-Cycle Cost Analysis 1994. Annual Supplement to NIST Handbook 135 and NBS Special Publication 709 .

02,508 Energy Price Indices and Discount Factors for Life-Cycle
Cost Analysis 1995. Annual Supplement to NIST HandCost Analysis 1995. Annual Supplement to NIST Handbook 135 and NBS Special Publication 709. (Revised).
PB95-105011

00,250

PB95-150991

Energy Price Indices and Discount Factors for Life-Cycle Cost Analysis 1996. Annual Supplement to NIST Han

book 135 and NBS Special Publication 709. (Revised).

Economics of New-Technology Materials: A Case Study of FRP Bridge Decking.

01,349

Energy Price Indices and Discount Factors tor Life-Cycle Cost Analysis 1997. Annual Supplement to NIST Handbook 135 and NBS Special Publication 709. (Revised). PB96-210745

COST BENEFIT ANALYSIS

Robotics Application to Highway Transportation. Volume 3. Proposed Research Topics and CosvBenefit Evaluations by CERF.

PB95-171633

01,338

east-Cost Energy Decisions for Buildings. Part 3 Choosing Economic Evaluation Methods. Video Training PB95-253597

00,265

Economic Methods and Risk Analysis Techniques for Evaluating Building Investments: A Survey

PB96-122593

00,323

Multiattribute Decision Analysis Method for Evaluating Buildings and Building Systems.

PB96-158670

00,325

COST ESTIMATES

Seismic Safety of Federal Buildings. Initial Program: How .

PB95-182291

COUETTE FLOW

Non-Newtonian Flow between Concentric Cylinders Calculated from Thermophysical Properties Obtained from Simulations.

04,103

COULOMB BLOCKADE

Effect in Environmental Noise on the Accuracy of Couomb-Blockade Devices.

PB95-175865

04,662

COULOMB BLOCKADE ELECTROMETERS

Noise in the Coulomb Blockade Electrometer.

PB95-176327

04,670

COULOMETERS

Automated, High-Precision Coulometric Titrimetry. Part 1

Engineering and Implementation.

00,575

Automated, High Precision Coulometric Titrimetry. Part 2

Strong and Weak Acids and Bases.

00,576

COULOMN BLOCKAGE

Performance of the Electron Pump with Stray Capacitances

01,976

COUNSTRUCTION MATERIALS

Unreacted Cement Content in Macro-Defect-Free Composites: Impact on Processing-Structure-Property Rela-

PB96-141270

03,174

COUNTIES

Codes for Named Populated Places, Primary County Divi- 
Potential Drop in the Center-Cracked Panel with Asymmetric Crack Extension

04,819

Lattice Imperfections Studied by Use of Lattice Green's Functions.

PB95-150850

04,576

Moisture and Water-Induced Crack Growth in Optical Ma-

terials.

PB95-153334 04,267

Molecular Orbital Study of Water Enhanced Crack Growth

Process.

PB95-164067

03,240

Nonlocal Effects of Existing Dislocations on Crack-Tip

Emission and Cleavage.

PB96-161807

03,367

Interfacial Crack in a Two-Dimensional Hexagonal Lattice. PB96-161989 04,100 Model for Microcrack Initiation and Propagation beneath

Hertzian Contacts in Polycrystalline Ceramics.
PB96-163704

03,077

CRACK RESISTANCE

Characterization of the Hydrogen Induced Cold Cracking Susceptibility at Simulated Weld Zones in HSLA-100

PB94-174505 03,746

Flaw-Tolerance and Crack-Resistance Properties of Alumina-Aluminum Titanate Composites with Tailored Microstructures.

PACK TIPS

RACK TIPS

Dislocation Emission at Ledges on Cracks.

PB95-164240

04,630

Shielding of Cracks in a Plastically Polarizable Material.

PB95-164257

04,631

CRACKING

Postfailure Subsidiary Cracking from Indentation Flaws in Brittle Materials.
PB97-110340

03,103

CRACKING (FRACTURING)

Prediction of Cracking in Reinforced Concrete Structures.
PB95-220448 PB95-220448

CRACKS

Characterization of the Hydrogen Induced Cold Cracking Susceptibility at Simulated Weld Zones in HSLA-100 Stee

AD-A279 759/5

03,200

Fracture Behavior of Large-Scale Thin-Sheet Aluminum N95.1

03,311

Safety Assessment of Railroad Wheels Through Roll-by Detection of Tread Cracks.

04,856

Artificial Crack in Steel: An Ultrasonic-Resonance-Spectroscopy and Modeling Study.

PB96-141395

03,241

Diffusion of Water along 'Closed' Mica Interfaces.

PB96-180039

02,993

CRASH TESTS

Effect of Fuel Tank Rupture Mode on the Ignitability of Expelled Fuel

CREEP

Temperature and Frequency Dependence of Anelasticity in a Nickel Oscillator.

03,689

Tensile Creep of Silicide Composites.

PB96-200803

03,183

Cavity Evolution during Tensil Creep of Si3N4

Creep and Creep Rupture of Structural Ceramics.

PB96-204524

Tension/Comp
PB97-110258

03,093

03,096

CREEP BUCKLING

Analysis of Creep in a Si-SiC C-Ring by Finite Element

PB94-200268

03,037

CREEP PROPERTIES

PC-Based Prototype Expert System for Data Management and Analysis of Creep and Fatigue of Selected Materials at Elevated Temperatures.

PB94-172251

03,202

High Temperature Degradation of Structural Composites. PB94-172848 03,132 Cavitation Damage During Flexural Creep of SiAIONYAG Ceramics.

03,036

CREEP RAPTURE STRENGTH

Generic Model for Creep Rupture Lifetime Estimation on Fibrous Ceramic Composites.

PB94-200235

03,137

CREEP RATE

Observed and Theoretical Creep Rates for an Alumina Ceramic and a Silicon Nitride Ceramic in Flexure.

PB94-212958

03,040

CREEP RUPTURE

Transient Creep Behaviour of Hot Isostatically Pressed Silicon Nitride.

03,086
Life Prediction of a Continuous Fiber Reinforced Ceramic Composite Under Creep Conditions.

03,091

CREEP RUPTURE STRENGTH

Creep Rupture of MoSi2/SiCp Composites.

PB95-152294

03.154

Creep and Creep Rupture of Ceramic Matrix Composites. PB95-163929 03,165

CREEP TESTS

Tensile Creep Testing of Structural Ceramics.

RITICAL CURRENT

Critical Magnetic-Field Angle for High-Field Current Transport in $\mathrm{YBa} 2 \mathrm{Cu} 3 \mathrm{O} 7$ at $76 \mathrm{~K}$.

PB94-211281

04,490

Transport Critical Current of Aligned Polycrystalline Yttrium Barium Copper Oxide (YBa2Cu3O7-delta).

Effect of Axial Strain on the Critical Current of AgSheathed $\mathrm{Bi}$-Based Superconductors in Magnetic Fields Up to $25 \mathrm{~T}$.

PB94-211315 04,493

Magnetic Measurement of Transport Critical Current Density of Granular Superconductors.

PB95-126199

04,531

Critical Current Behavior of Ag-Coated YBa2Cu3O7-x Thin Films.

PB95-141016 04,549

Magnetic Field Dependence of the Critical Current Anisolropy in Normal Metal-YBa2Cu3O7-delta Thin Film Bilayers.

PB95-14 1024

04,550

Grain Alignment and Transport Properties of Bi2Sr2CaCu2O8 Grown by Laser Heated Float Zone

PB95-161451

04,602

Superconductor Critical Current Standards for Fusion Applications. Final Progress Report, October 1993-July 1994.

02,222

PB95-169538

Increased Pinning Energies and Critical Current Densities in Heavy-Ion-Irradiated Bi2Sr2CaCu2O8 Single Crystals.
PB95-175352

Standard Reference Devices for High Temperature Superconductor Critical Current Measurements

PB95-175543

04,659

Critical-Current Degradation in Nb3 Al Wires Due to Axial and Transverse Stress.

02,226

Temperature Dependence and Magnetic Field Modulation of Critical Currents in Step-Edge SNS YBCO/Au Junc-

PB96-111745

04,723

Critical Current and Normal Resistance of High-Tc StepEdge SNS Junctions.

PB96-111752

04,724

Comparing the Accuracy of Critical-Current Measurements Using the Voltage-Current Simulator.

PB96-119219
Simple and Repeatable Technique for Measuring the Critical Current of Nb3Sn Wires.

02,229

PB96-119409

Effect of Sm2BaCuO5 on the Properties of Sintered
(Bulk) YBa2Cu3O6+x.

(Bulk) YBa2Cu3O6+x

VAMAS Intercomparison of Critical Current Measurements on NB3Sn Superconductors: A Summary Report. PB96-119763

04,043

Anomalous Switching Phenomenon in Critical-Current Measurements When Using Conductive Mandrels.
02,233

Effect of Magnetic Field Orientation on the Critical Current of HTS Conductor and Coils.

PB96-141189

02,956

II-3: Critical Current Measurement Methods: Quantitative Evaluation.

04,767

First VAMAS USA Interlaboratory Comparison of High Temperature Superconductor Critical Current Measure-

PB96-147178 04,768

II-5: Thermal Contraction of Materials Used in Nb3Sn Critical Current Measurements.

PB96.147186

04,769

USA Interlaboratory Comparison of Superconductor Simulator Critical Current Measurements.

PB96-147194

04,770

CRITICAL CURRENT DENSITY

Electromagnetic Coupling Character of (001) Twist
Boundaries in Sintered Bi2Sr2CaCu2O 8+x Bicrystals.

PB96-176573

CRITICAL DENSITY

Experimental Method for Obtaining Critical Densities of Binary Mixtures: Application to Ethane + n-Butane.
PB95-151148

CRITICAL FLOW

Electric Field Effects on a Near-Critical Fluid in Microgravity.

PB96-161880

04,217

CRITICAL LINES

Critical Lines for Type-III Aqueous Mixtures by Generalized Corresponding-States Models.

PB96-102371

01,063

CRITICAL POINT

Supercritical Solubility of Solids from Near-Critical Dilute-

Mixture Theory.

Global Thermodynamic Behavior of Fluid Mixtures in the Critical Region
PB94-212420

04,199

Structure of the Vapor-Liquid Interface Near the Critical

Point.

PB95-140174 00,902

Dynamic Light-Scattering Study of a Diluted Polymer Blend Near Its Critical Poin

PB95-151890

01,245

Thermal Equilibration Near the Critical Point: Effects Due

to Three Dimensions and Gravity.

Standard States, Reference States and Finite-Concentra-

tion Effects in Near-Critical Mixtures with Applications to

Aqueous Solutions.

Critical Scaling Laws and a Classical Equation of State.

Coexisting Densities, Vapor Pressures and Critical Den-

sities of Refrigerants R-32 and R-152a, at $300-385 \mathrm{~K}$.

PB95-175691

03,274

Diffuse-Interface Description of Fluid Systems.

PB96-210711

01,170

CRITICAL POINTS

Internal Waves in Xenon Near the Critical Point

PB97-111504

04,221

CRITICAL REGION

Vapor-Liquid Equilibria of Ternary Mixtures in the Critical Region on Paths of Constant Temperature and Overall Composition.

Nonlinear Correlation of High-Pressure Vapor Equilibrium Data for Ethylene + n-Butane Showing sistencies in Experimental Compositions.

PB96-161906

01,141

CRITICAL STATE

Pinch Effect in Commensurate Vortex-Pin Lattices
PB96-147079

01,125

CRITICAL TEMPERATURE

Dependence of Tc on Debye Temperature Theta(sub D) for Various Cuprates.

04,683

CRITICALITY

Neutron Leakage Benchmark for Criticality Safety Research.

MICALITY CALCULATIONS

MCNP Model of the National Bureau of Standards Reactor (NBSR) Core.

PB96-138599

Low-Energy-Electron Collisions with Sodium: Elastic and PB96-103106

CROSSLINKING 
CRYOELECTRONICS

Metrology for Electromagnetic Technology: A Bibliography of NIST Publications.

PB95-135588

02,143

CRYOFOCUSING

Chromatographic Cryofocusing and Cryotrapping with the Vortex Tube.
PB95-180113

00,604

CRYOGENIC COOLING

Resistance Thermometers with Fast Response for Use in Rapidly Oscillating Gas Flows.

PB95-107298

03,261

Chromatographic Cryofocusing and Cryotrapping with the Vortex Tube.
PB95-180113

00,604

CRYOGENIC CURRENT COMPARATORS

High-Temperature Superconductor Cryogenic Current Comparator.

02,074

CRYOGENIC EQUIPMENT

Cryogenic Toughness of Austenitic Stainless Steel Weld Metals: Effect of Inclusions

PB95-161261

03,214

CRYOGENIC FLUIDS

Reference Data for the Thermophysical Properties of Cryogenic Fluids.
PB95-168688

03,263

CRYOGENIC PROPELLANTS

Cryogenic Research and Development (Ouarterly Report Number 1 for Period Ending September 30, 1960).

AD-A280 401/1 01,456 Cryogenic Research and Development (June 30, 1961). AD-A280 679/2 01,457

CRYOGENIC PROPERTIES

Temperature-Induced Transition in Ductile Fracture Appearance of a Nitrogen-Strengthened Austenitic Stainless

03,221

CRYOGENIC RADIOMETERS

Journal of Research of the National Institute of Standards and Technology, March/April 1996. Volume 101, Number PB96-177381

01,863 NIST High Accuracy Scale for Absolute Spectral ReSponse from $406 \mathrm{~nm}$ to $920 \mathrm{~nm}$

01,865

\section{CRYOGENIC TEMPERATURE}

Development of a Temperature Scale below $0.5 \mathrm{~K}$.
PB95-125639

Cryogenics

PB95-164703

CRYOGENIC TEST PROCEDURES

Charpy Specimen Tests at $4 \mathrm{~K}$.

PB96-190335

YOGENICS

03,002

Cryogenic Research and Development (Ouarterly Report Number 2 for Period Ending December 31, 1960).

AD-A280 398/9

01,454

Cryogenic Research and Development (Progress Report Number 4 for Period Ending December 31, 1961).

AD-A280 399/7 01,455

Cryogenic Research and Development (Ouarterly Report Number 1 for Period Ending September 30, 1960).

AD-A280 401/1

01,456

Cryogenic Research and Development (June 30, 1961).

AD-A280 679/2 01,457

Bibliography of the Physical Equilibria and Related Properties of Some Cryogenic Systems.

AD-A281 167/7

03,769

Progress Report to National Aeronautics and Space Administration on Cryogenic Research and Development.

AD-A286 612/7

01.458

Cryogenic Materials Data Handbook

AD-A286 675/4

03,303

Survey of the Literature on Heat Transfer from Solid Surfaces to Cryogenic Fluids.

Cryogenic Properties of Silver.

PB94-203593

04,193

Welding for Cryogenic Service.

03,330

02,852

High-Temperature Superconductor Cryogenic Current Comparator.
PB96-119334

Building a Better Crycooler.

PB96-119615

04,734

Microwave Dielectric Properties of Anisotropic Materials at Cryogenic Temperatures.

PB96-137765

02,412

Cryogenic Flow Calibration in NIST.

01,143

National Institute of Standards and Technology High-Accuracy Cryogenic Radiometer.

04,378

Charpy Impact Test as an Evaluation of $4 \mathrm{~K}$ Fracture Toughness.

03,219
Realization of a Scale of Absolute Spectral Response Using the NIST High Accuracy Cryogenic Radiometer.
PB97.118640

04,397

Anomalous Freezing and Melting of Solvent Crystals in Swollen Gels of Natural Rubber.

PB94-212321

01,223

CRYOTRAPPING

Chromatographic Cryofocusing and Cryotrapping with the

PB95-180113

00,604

CRYPTOGRAPHY

Security Requirements for Cryptographic Modules; Category: Computer Security; Subcategory: Cryptography.
FIPS PUB 140-1

Digital Signature Standard (DSS). Category: Computer Security; Subcategory: Cryplography. Report of the NIST Workshop on Digital Signature Certifi-

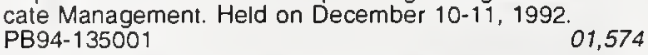

Federal Certification Authority Liability and Policy: Law and Policy of Certificate-Based Public Key and Digital Signatures.

01,578

Formal Specification and Verification of Control Software for Cryptographic Equipment.

PB94-213030

01,585

EDI and EFT

01,605

Response to Comments on the NIST Proposed Digital Signature Standard

01,615

CRYPTOLOGY

Data Encryption Standard

01,595

CRYSTAL CHEMISTRY

Crystal Chemistry and Phase Equilibrium Studies of the $\mathrm{BaO}(\mathrm{BaCO} 3)-\mathrm{R} 2 \mathrm{O} 3-\mathrm{CuO}$ Systems. 5. Melting Relations in $\mathrm{Ba} 2(\mathrm{Y}, \mathrm{Nd}, \mathrm{Eu}) \mathrm{Cu} 3 \mathrm{O} 6+\mathrm{x}$.

PB95-151718

04,580

Crystal Chemistry and Phase Equilibrium Studies of the $\mathrm{BaO}-\mathrm{R} 2 \mathrm{O} 3-\mathrm{CuO}$ Systems. 2. X-Ray Characterization and Standard Patterns of BaR2CuO4, R=Lanthanides. 04,582 Crystal Chemistry and Phase Equilibria Studies of the $\mathrm{BaO}(\mathrm{BaCO} 3)-\mathrm{R} 2 \mathrm{O} 3-\mathrm{CuO}$ Systems. 4. Crystal Chemistry and Subsolidus Phase Relationship Studies of the $\mathrm{CuO}$ Rich Region of the Ternary Diagrams, $\mathrm{R}=$ Lanthanides. PB95-151759

00,936

\section{CRYSTAL DEFECTS}

Correlation of $\mathrm{HgCdTe}$ Epilayer Defects with Underlying Substrate Defects by Synchrotron X-Ray Topography.
PB94-200714.

02,129

Defect of Thermal Ramping and Annealing Conditions on Defect Formation in Oxygen Implanted Silicon-On-Insulator Material.

PB94-212966

02,318

Characterization of Interface Defects in Oxygen-Implanted Silicon Films.
PB94-216629

02,322

Defective Structures of Barium Yttrium Copper Oxide (Ba2YCu3Ox) and Ba2YCu3-yMyOz ( $\mathrm{M}=\mathrm{Fe}, \mathrm{Co}, \mathrm{Al}, \mathrm{Ga}$ PB95-140034

04,537

Lattice Imperfections Studied by Use of Lattice Green's Functions.

PB95-150850 04,576

Lineshape Analysis of the Raman Spectrum of Diamond Films Grown by Hot-Filament and Microwave-Plasma Chemical-Vapor Deposition.

PB95-162392

03,016

Studies of Defects in Diamond Films and Particles by Raman and Luminescence Spectroscopies.

03,017

Elastic Properties of Central-Force Networks with BondLength Mismatch.

PB95-163366

04,623

Influence of Lattice Mismatch on Indium Phosphide Based High Electron Mobility Transistor (HEMT) Structures Observed in High Resolution Monochromatic Synchrotron X-Radiation Diffraction Imaging.

02,357

Small Angle Neutrons Scattering from Nanocrystalline Palladium as a Function of Annealing. Nano-Defects in Commercial Bonded SOI and SIMOX. PB96-123674

02,407

\section{CRYSTAL DISLOCATIONS}

Defect Pair Formation by Implantation-Induced Stresses in High-Dose Oxygen Implanted Silicon-on-Insulator Maerial.

PB95-175824

02,364

CRYSTAL GROWTH

Computation of Dendrites Using a Phase Field Model.
PB94-160744

Asymptotic Behavior of Modulated Taylor-Covette Flows with a Crystalline Inner Cylinder.

04,469

Selective Inhibition of Crystal Growth on Octacalcium Phosphate and Nonstoichiometric Hydroxyapatite by Pyrophosphate at Physiological Concentration. PB94-211257 00,147

Microstructural Evolution in Two-Dimensional Two-Phase Polycrystals.

04,498

Effect of Modulated Taylor-Couette Flows on Crystal-Melt Interfaces: Theory and Initial Experiments.

PB94-216736

04,521

Morphological Stability

04,591

Lineshape Analysis of the Raman Spectrum of Diamond Films Grown by Hot-Filament and Microwave-Plasma Chemical-Vapor Deposition.

PB95-162392

03,016

Studies of Defects in Diamond Films and Particles by Raman and Luminescence Spectroscopies.

03,017

Surface Roughness Evaluation of Diamond Films Grown on Substrates with a High Density of Nucleation Sites. PB95-162418 03,018

In-situ Monitoring of Molecular Beam Epitaxial Growth Using Single Photon Ionization.

01,023

Single Photon Ionization, Laser Optical Probe Technique for Semiconductor Growth.

PB95-202776

01,032

Laser $\mathrm{Gas}$

PB96-112172

01,076

Stagnant Film Model of the Effect of Natural Convection on the Dendrite Operating State.

PB96-146832 04,765

Powder X-ray Diffraction Data for $\mathrm{Ca} 2 \mathrm{Bi} 2 \mathrm{O} 5$ and PB96-161278

04,777

Preparation and Crystal Structure of Sr5TiNb4017.

PB96-167341

CRYSTAL LATTICES

Status of a Silicon Lattice Measurement and Dissemination Exercise.

04,474

Elastic Properties of Central-Force Networks with BondLength Mismatch.

PB95-163366

Lattice Dynamics of $\mathrm{Ba1} 1 \times \mathrm{K} \times \mathrm{BBO} 3$

04.706

Lattice Dynamics of Semiconducting, Metallic, and Superconducting $\mathrm{Ba} 1-\times \mathrm{K} \times \mathrm{BiO} 3$ Studied by Inelastic Neutron Scattering

04,708

CRYSTAL-MELT INTERFACE

Effect of Modulated Taylor-Couette Flows on Crystal-Melt Interfaces: Theory and Initial Experiments.

PB94-216736

04,521

Effects of Elastic Stress on the Stability of a Solid-Liquid Intertace.

03,350

Rayleigh Instability for a Cylindrical Crystal-Melt Interface. PB95-180667

01,010

CRYSTAL OSCILLATORS

Fundamental Limits on the Frequency Stabilities of Crystal Oscillators. 
Crystal Structure of $\mathrm{Pb} 2 \mathrm{Sr} Y \mathrm{YCu} 3 \mathrm{O} 8+$ delta with delta $=1.32,1.46,1.61,1.71$, by Powder Neutron Diffraction.

04,518

Crystal Structure of Dicalcium Potassium Trihydrogen Bis (pyrophosphate) Trihydrate.

PB94-216561

00,152

Crystal Structure of Calcium Adipate Monohydrate.
PB94-216579

Crystal Structure of a New Sodium Zinc Arsenate Phase Solved by 'Simulated Annealing'.

00,870

Rietveld Analysis of $\mathrm{NaxWO}^{2}+x / 2 . \mathrm{yH} 2 \mathrm{O}$, Which Has the Hexagonal Tungsten Bronze Structure.

04,524

Neutron Powder Diffraction Study of the Structures of La1.9Ca1.1 Cu2O6 and La1.9Sr1.1Cu2O6+Delta.

PB95-140042

04,538

Crystallograp

04,543

Crystal Structure and Magnetic Ordering of the RareEarth and Cu Moments in RBa2Cu2NbO8(R=Nd, $\mathrm{Pr})$. 04,546

Ca4Bi6013: A Compound Containing an Unusually Low Bismuth Coordination Number and Short Bi Bi Contacts.
PB95-141131

$X$-Ray-Diffraction Study of a Thermomechanically Detwinned Single Crystal of YBa2Cu3O6+x.
PB95-151726 Crystal Chemistry and Phase Equilibrium Studies of the BaO-R2O3-CuO Systems. 2. X-Ray Characterization and Standard Patterns of BaR2CuO4, R=Lanthanides.

PB95-151734

04,582

$X$-Ray Powder Diffraction Data for $\mathrm{BaCu}(\mathrm{C} 2 \mathrm{O} 4) 2.6 \mathrm{H} 2 \mathrm{O}$.

04,583

Neutron Powder Diffraction Study of the Nuclear and

Magnetic Structures of the Oxygen-Deficient Perovskite YBaCuCoO5

PB95-161097

00,954

Calibration of High-Energy Electron Beams by Use of Graphite Calorimeters.

04,598

$\mathrm{Ca} 1-\times \mathrm{CuO} 2$, a NaCuO2-Type Related Structure.
PB95-162822

Octacalcium Phosphate Carboxylates. 5. Incorporation of Excess Succinate and Ammonium lons in the Octacalcium Phosphate Succinate Structure. Crystal Structure of Calcium Succinate Monohydrate. PB95-168928 00,167 Crystal Structure and Compressibility of 3:2 Mullite.

Crystal Structure and Magnetic Properties of $\mathrm{CuGeO}_{04,678}$
PB95-180287

Structural and Chemical Investigations of $\mathrm{Na3}(\mathrm{ABO} 4) 3.4 \mathrm{H} 2 \mathrm{O}-$ Type Sodalite Phases.
$\mathrm{PB95}-180733$

Powder Neutron Diffraction Investigation of Structure and

Cation Ordering in Ba2+xBi2-xO6-y.

01,015

Early History and Future Outlook for the X-ray Crystal Density Method
PB95-202487

04,692

Determination of Complex Structures from Powder Diffraction Data: The Crystal Structure of La3Ti5Al15037.

PB95-202966

01,038

Structure and Conductivity of Layered Oxides $(\mathrm{Ba}, \mathrm{Sr}) \mathrm{n}+1(\mathrm{Sn}, \mathrm{Sb}) \mathrm{nO} \mathrm{O} n+1$.
PB96-102439

04,707

Oxygen Dependance of the Crystal Structure of $\mathrm{HgBa} 2 \mathrm{CuO} 4+$ and Its Relation to Superconductivity.

PB96-102512

04,711

Crystal Structure of a New Monoclinic Form of Potassium Dihydrogen Phosphate Containing Orthophosphacidium lon, (H4PO4)(sup +1)

PB96-111794

04,725

Crystal Structure of Calcium Glutarate Monohydrate

PB96-111893

01,074

Crystal Structure of Decacalcium Tetrapotassium Hexakis PBg6-141064

01,099

Neutron-Powder-Diffraction Study of the Long-Range Order in the Octahedral Sublattice of LaD2.25.
PB96-141155

Fast-Ion Conducting Y2(ZryTi1-y)2O7 Pyrochlores: Neutron Rietveld Analysis of Disorder Induced by $\mathrm{Zr}$ Substitution

04,776

Tetrahedral-Framework Lithium Zinc Phosphate Phases: Location of Light-Atom Positions in LiZnPO4 $\mathrm{H} 2 \mathrm{O}$ by Powder Neutron Diffraction and Structure Determination

of LiZnPO4 by ab Initio Methods.
PB96- 160510

01,129

Characterization of the Structure of TbD2.25 at $70 \mathrm{~K}$ by Neutron Powder Diffraction.

PB96-160528

01,130

CRYSTALLINE CERAMICS

Small-Angle Neutron Scattering Characterization of Processing/Microstructure Relationships in the Sintering of
Crystalline and Glassy Ceramics. (Reannouncement with New Availability Information). AD-A249 510/9

03,025

CRYSTALLIZATION

Polyethylene Crystallized from an Entangled Solution Observed by Scanning Tunneling Microscopy.

Biological Macromolecular Crystallization Database: A Tool for Developing Crystallization Strategies.

00,897

Influence of Natural and Synthetic Inhibitors on the Crys-

tallization of Calcium Oxalate Hydrates.

03,560

$X$-Ray Characterization of the Crystallization Process of High-Tc Superconducting Oxides in the $\mathrm{Sr}-\mathrm{Bi}-\mathrm{Pb}-\mathrm{Ca}-\mathrm{Cu}$ PB95-151700

04,579

CRYSTALLOGRAPHY

Statistical Descriptors in Crystallography. 2. Report of a Working Group on Expression of Uncertainty in Measurement. 146824

04,764

Journal of Research of the National Institute of Standards and Technology, May/June 1996. Volume 101, Number 3 Special Issue: NIST Workshop on Crystallographic Databases.

PB97-109011

04,798

CRYSTMET: The NRCC Metals Crystallographic Data File.

04,799

Inorganic Crystal Structure Database (ICSD) and Standardized Data and Crystal Chemical Characterization of Inorganic Structure Types (TYPIX): Two Tools for Inorganic Chemists and Crystallographers.

00,648

Evaluation of Crystallographic Data with the Program DI-

PB97-109045 00,649

Cambridge Structural Database (CSD): Current Activities and Future Plans.

PB97-109052

00,516

Protein Data Bank: Current Status and Future Challenges.

00,517

Nucleic Acid Dat

Powder Diffraction File: Past, Present, and Future.
PB97-109086 PB97-109086
NIST Crystallographic Databases for Research and AnalPsis. 109094

04,801

Conventional and Eccentric Uses of Crystallographic Databases in Practical Materials Identification Problems. PB97-109102

04,802

Using NIST Crystal Data within Siemens Software for our-Circle and SMART CCD Diffractometers.

04,803

Phase Identification in a Scanning Electron Microscope Using Backscattered Electron Kikuchi Patterns. PB97-109128

04,804

Biological Macromolecule Crystallization Database and NASA Protein Crystal Growth Archive.

PB97-109136

01,171

Investigations of the Systematics of Crystal Packing Using the Cambridge Structural Database. B97-109144

00,519

Troublesome Crystal Structures: Prevention, Detection, PB97-109151

01,172

CIF Crystallographic Information File: A Standard for Crystallographic Data Interchange.

PB97-109169

04,805

Role of Journals in Maintaining Data Integrity: Checking

of Crystal Structure Data in 'Acta Crystallographica'.
PB97-109177

Electronic Publishing and the Journals of the American Chemical Society.

PB97-109185 04,807

How the Cambridge Crystallographic Data Centre Obtains is Information

04,808

Data Import and Validation in the Inorganic Crystal Structure Database

PB97-109201

World Wide Web for Crystallography.

04,809

04,810

Workshop Highlights

PB97-109227

04,811

CRYSTALS

Effect of Electrode-Polymer Interfacial Layers on Polymer Conduction. Part 2. Device Summary.

PB95-151155

02,335

Crystal Packing Interactions of Two Different Crysta

Forms of Bovine Ribonuclease A

PB95-152823

00,943

Dynamics of

04,112

Systematic Correction in Bragg X-ray Diffraction of Fla and Curved Crystals.

04,152

CSOR (COMPUTER SECURITY OBJECTS REGISTER)

General Procedures for Registering Computer Security

Objects.

PB94-134897

01,573

CUBANE

1,4-Dinitrocubane and Cubane under High Pressure. PB95-108437

03,755

CUBANE/DINITRO

1,4-Dinitrocubane and Cubane under High Pressure. PBo5-108437

03,755

CUBANES

Neutron Scattering Study of the Lattice Modes of Solid

PB96-147152

01,126

CUPRIC OXIDE

Cooper M(sub II,III) X-ray-Emission Spectra of Copper Oxides and the Bismuth Cuprate Superconduct

04,077

\section{CURIE TEMPERATURE}

Monte Carlo and Mean-Field Calculations of the Clusters.

PB94-172087

03,201

CURING

Fluorescence Monitoring of Polarity Change and Gelation during Epoxy Cure

Digitized Direct Simulation Model of the Microstructural Development of Cement Paste.

PB94-198777

01,309

Digitized Simulation Model for Microstructural Develop-

Diffusion Studies in a Digital-Image-Based Cement Paste Microstructural Model.

PB94-198801

01,312

Maturity Method

PB94-199494

Maturity Functions for Concrete Made with Various Cements and Admixtures.

PB94-139502

01,314

Interaction between Naphthalene Sulfonate and Silica Fume in Portland Cement Pastes.

01,315

Effect of Curing History on Ultimate Glass Transition Effect of Curing History on Ultimate Glass Transition

PB94-200052

01,214

Monitoring Polymer Cure by Fluorescence Recovery After PB94-211422

01,218

Review of Cure Monitoring Techniques for On-Line Process Control.

Structural Heterogeneity in Epoxies.

03,145

PB95-151866

01,243

Applications of Fluorescence Spectroscopy in Polymer Science and Technology.

CURRENT

Electrical Characteristics of Argon Radio Frequency Glow Discharges in an Asymmetric Cel.

PB96-176490

04,109

CURRENT CONTROLLERS

Low-Noise High-Speed Diode Laser Current Controller. PB95-202826 
CURVE FITTING

Sensitivity of Three-Point Circle Fitting.
PB95-136354

Outlier-Resistant Methods for Estimation and Model FitPB95-203436

03,444

CURVE MOTION

Nonlinear Dynamics of Stiff Polymers.

PB96-122478

01,278

CURVED GUIDE

Transmission Properties of Short Curved Neutron Guides. Part 1. Acceptance Diagram Analysis and Calculations. PB96-102199

04,021

CYANINE DYES

Radiation Chemistry of Cyanine Dyes: Oxidation and Reduction of Merocyanine 540

PB94-211661

00,818

CYANOACETYLENE

Rotational Spectra of $\mathrm{CH} 3 \mathrm{CCH}-\mathrm{NH} 3, \mathrm{NCCCH}-\mathrm{NH} 3$, and $\mathrm{NCCCH}-\mathrm{OH} 2$

04,170

CYANOBIPHENYL PHASES

Comparison of Selectivities for PCBs in Gas Chromatography for a Series of Cyanobiphenyl Stationary Phase

PB96-119458

00,618

CYCLIC COMPOUNDS

Ring-Opening Dental Resin Systems Based on Cyclic

00,162

PB95-162251

Synthesis and Polymerization of Difunctional and Multifunctional Monomers

Capable 0

PB95-163044

01,257

CYCLIC LOADS

Model Precast Concrete Beam-to-Column Connections Subject to Cyclic Loading.

PB95-153094

00,438

Partially Prestressed and Debonded Precast Concrete Beam-Column Joints.

00.439

Seismic Performance Behavior of Precast Concrete Seismic Performance Behavior of Precast Concrete
Beam-Column Joints.
PB95-153110
00,440

Evaluating the Seismic Performance of Lightly-Reinforced Circular Concrete Bridge Columns.

01,335

Performance of 1/3-Scale Model Precast Concrete Beam. Column Connections Subjected to Cyclic Inelastic Loads. PB95-179024

00,444

CYCLOBUTENE COMPOUNDS

Homogeneous Gas Phase Decyclization of Tetralin and Benzocyclobutene.

PB95-151049

00,928

CYCLODEXTRINS

Thermodynamic and NMR Study of the Interactions of Cyclodextrins with Cyclohexane Derivatives.

00,781

Thermodynamic Study of the Reactions of Cyclodextrins with Primary and Secondary Aliphatic Alcohols, with D and L-Phenylalanine, and with L-Phenylalanineamide.

and L-Phenylalanine, and with L-Phenylalanineamide. 01.016

CYCLOHEXANOLS

Thermodynamic and NMR Study of the Interactions of

Cyclodextrins with Cyclohexane Derivatives.
PB94-185816

00,781

CYLINDRICAL BODIES

Elastic Constants of Isotropic Cylinders Using Resonant Ultrasound.

CYTOCHROME C

04,497

Characterization of Cytochrome c/Alkanethiolate Structures Prepared by Seli-Assembly on Gold.

PB95-164638

00,987

CrTOSINE

Substrate Specificity of the Escherichia coli Endonuclease III: Exicision of Thymine- and Cytosine-Derived Lesions in DNA Produced by Radiation-Generated Free Radicals. PB95-153425

03,535

DAMAGE

Repair of Products of Oxidative DNA Base Damage in Human Cells.

DAMAGE ACCUMULATION

03,555

Model for Toughness Curves in Two-Phase Ceramics. 2. Microstructural Variables.

PB96-163795

03.078

Effect of Grain Size on Hertzian Contact Damage in Alumina.

03,083

Indentation Fatigue: A Simple Cyclic Hertzian Test to Measuring Damage Accumulation in Polycrystalline $\mathrm{Ce}-$

ramics.

03,084

DAMAGE ASSESSMENT

Northridge Earthquake, 1994. Performance of Structures, Lifelines and Fire Protection Systems.

00,421
Northridge Earthquake 1994: Performance of Structures, Lifelines, and Fire Protection Systems.

04,825

Lessons Learned by a Wing Engineer.

PB94-216421 00,429

Performance of HUD-Affiliated Properties during the January 17, 1994 Northridge Earthquake.

00,443

Evaluation of Thermal Wave Imaging for Detection of Machining Damage in Ceramics.

PB95-220547

03,062

Some Notable

00,458

Comparison of Responses of a Select Number of Build. ings to the 10/17/1989 Loma Prieta (California) Earth quake and Low-Level Amplitude Test Results.

PB96-159645

00,467

January 17, 1995 Hyogoken-Nanbu (Kobe) Earthquake. Performance of Structures, Lifelines and Fire Protection Systems. Executive Summary and Paper.

00,475

\section{DAMPERS}

Method of Estimating the Parameters of Tuned Mass Dampers for Seismic Applications.

PB96-167820

00,473

DATA ACCESS

Sharing Information via the Internet: An Infoserver Case Pludy.

01,493

DATA ACQUISITION

Tabulation of Data on Receiving Tubes. Handbook 68.

AD-A285 495/8

02,111

User Study: Informational Needs of Remote National Archives and Records Administration Customers. PB95-154738

02,725

DATA ANALYSIS

Concept for an Algorithm Testing and Evaluation Program at NIST,

02,890

Analysis of Autocorrelations in Dynamic Processes.
PB95-181228

Algorithm Testing and Evaluation Program for Coordinate Measuring Systems: Long Range Plan.

02,915

Reference Manual for the Algorithm Testing System Ver sion 2.0.
s.o.

DATA BASE ADMINISTRATORS

Computer Security Management and Planning in the U.S. Federal Government.

PB95.163432

01,596

DATA BASE MANAGEMENT

Database Management Standards: Status and ApplicabilPB96-122924

01,819

Findings and Recommendations from a Software Reengineering Case Study

01,752

\section{DATA BASE MANAGEMENT SYSTEMS}

Technical Program of the Factory Automation Systems Division 1993.

02,805

Standardization of Formats and Presentation of Fire Data - The FDMS.

PB94-198462 01,371

Development of the Fire Data Management System.

00,339

Application of Expert System to Select Data Sources from Chemical Information Databases.

PB95-125654

00,505

DATA BASES

NIST Standard Reference Data Products Catalog, 1994

00,727

Fire Data Management System, FDMS 2.0, Technical Documentation.

PB94-164019

01,358

Considerations on Data Requirements for Tribological Modeling.

02,962

National Institute of Siandards and Technology Resonance Ionization Spectroscopy/Resonance Ionization Mass Spectroscopy Data Service.

PB94-172897

03,793

Thermophysical Property Computer Packages from NIST. PB95-125787 04,203

Biological Macromolecular Crystallization Database: A Tool for Developing Crystallization Strategies.

00,897

Databases Available in the Research Information Center of the National Institute of Standards and Technology.
PB95-128641

2,724

PB95-140596

Status Report: AWS Standards for Identifying Arc Welds (A91.1) and Recording Weld Data (A9.2).
PB95-162855

Structural Ceramics Database. Topical Report, June 1989-May 1991

PB95-203758

03,060

NIST Standard Reference Data Products Catalog, 199596. Achieve with Standard Reference Data.

PB95-260808

01.057

Electron-Ion Collisions in the Plasma Edge.

04.037

Databases Available in the Research Information Center of the National Institute of Standards and Technology December 1995)

PB96-139407

Information Resources for the Fire Community.

02,734

00.211

Guide to a Format for Data on Chemical Admixtures in a Materials Property Database.

PB96-165394

01,327

Guide to a Format for Data on Chemical Admixtures in a Materials Property Database. (Reannouncement with new abstract)

PB96-186192

01,328

Guide to Locating and Accessing Computerized Numeric Materials Databases.

PB96-204045

03.007

Database Development and Management (Project A.2.2): The Annual Report for 1992-1993. PB97-110290

03,098

DATA COLLECTION

Panel: Building and Using Test Collections.

PB97-118673

02,743

DATA COLLECTIONS

Lake Erie Water Temperature Data, Put-in-Bay, Ohio, PB96-202452

DATA COMMUNICATION SYSTEMS

Data Communications Strategy.

PB96.167846

DATA DICTIONARIES

Information Resource Dictionary System (IRDS): A Status

Report.

PB95-126207

DATA ELEMENTS

Application of Metadata Standards

ATA ENCRYPTION

Data Encryption Standard (DES); Category: Compute Security; Subcategory: Cryptography.

01,572

Escrowed Encryption Standard (EES); Category: Computer Security; Subcategory: Cryptography.

Report of the NIST Workshop on Key Escrow Encryption. Held in Gaithersburg. Maryland on June 8-10, 1994. PB94-209459

01,584

Data Encryption Standard.

PB95-162376

01,595

EDI and EFT Security Standards.

PB96-122833

Introduction to Secure Telephone Terminals. PB97-110498

01,605

DATA FILE 
DATA MODELS

Integration Definition for Information Modeling (IDEF1X) Category: Soltware Standard: Subcategory: Modeling FIPSPUB18

01,673

DATA PROCESSING

ADA; Category: Software Standard; Subcategory: Programming Language
FIPS PUB 119-1

01,667

SOL Environments. Category: Software Standard; Subcategory: Database.

01,801

Implementation of a Standard Format for GPS Common View Data.

N95-32323/4 03,779

Radiation Process Data: Collection, Analysis, and Inter-

pretation.

Application Profile for ISDN.

03.628

PB95-163689

01,479

Federal Implementation Guideline for Electronic Data Interchange. ASC X12 003050 Transaction Set 840 Re-

quest for Ouotation. Implementation Convention.

DATA PROCESSING EQUIPMENT

Protection of Data Processing Equipment with Fine Wate Sprays.

02,610

DATA PROCESSING SECURITY

Good Security Practices for Electronic Commerce, Including Electronic Data Interchange.

01,463

Study of Federal Agency Needs for Information Technology Security
PB94-193653

01,579

Report of the NIST Workshop on Key Escrow Encryption

Held in Gaithersburg, Maryland on June 8-10, 1994.
PB94-209459

01,584

Analysis of Selected Sottware Safety Standards.

PB95-151262 01.708

Keeping Your Site Comfortably Secure: An Introduction to Internet Firewalls

02,730

Common Criteria: On the Road to International Harmonization.

PB96-123484 01,606

Functional Security Criteria for Distributed Systems

PB96-123492

01,607

DATA PROCESSING TERMINALS

Guidance of the Legality of Keystroke Monitoring
PB96-161237

ATA QUALTY

00,005

Replicate Measurements for Data Quality and Environmental Modeling.

DATA RETRIEVAL

Retrieving Articles from the Internet (without a UNIX

Workstation). Part 1 . File Formats and Software Tools.

PB95-168720

02,728

Retrieving Articles from the Intemet (without a UNIX Workstation). Part 2. An Example.

02,729

DATA SERVICE

Resonance lonization Spectroscopy/Resonance lonization Mass Spectrometry Data Service. V-Data Sheets for Ga, $\mathrm{Mn}, \mathrm{Sc}$, and TI.

00,625

DATA SHARING Standards and Linkages: What Data Sharing Needs.
PB95-161881

ATA STORAGE

Optical Storage Media Data Integrity Studies.

Dilemma-Preservation versus Access.

01,620

PB94-198488

02,711

Preliminary Functional Specifications of a Prototype Electronic Research Notebook for NIST.

PB94-207750

00,012

Standardised Computer Data File Format for Storage, Transport, and Off-Line Processing of Partial Discharge

Data.
PB96-122486

Status of Emerging Standards for Removable Computer Storage Media

01,634

DATA STORAGE SYSTEMS

Recalibration for the Final Archive of the International UItraviolet Explorer (IUE) Satellite.

PB96-135264

00,106

DATA STRUCTURES

Standards and Linkages: What Data Sharing Needs.
PB95-161881

Tree-Lookup for Partial Sums Or: How Can I Find This Stuff Ouickly

PB96-179411

01,770

Move-10-Root Rule for Self-Organizing Trees with Markov

Dependent Requests.

03,431
Component-Based Handprint Segmentation Using Adaptive Writing Style Model.

01,859

Heap of Data.
PB97-111488

Making Connections.

03,424

01,785

DATA TRANSFER (COMPUTERS)

Spatial Data Transfer Standard (SDTS): Category: Software Standard; Subcategory: Information Interchange.
FIPS PUB 173-1

Spatial Data Transfer Standard (SDTS). Category: Sottware Standard; Subcategory: Information Interchange. (FIPS PUB 173-1A)

FIPS PUB 173-1A $\quad 01,795$

Spatial Data Transter Standard (SDTS). Category: Software Standard; Subcategory: Information Interchange. FIPS PUB $173-1 B$

01,796

Standardization of Formats and Presentation of Fire Data - The FDMS.

01,371

NIST-Coordinated Standard for Fingerprint Data Inter-

change

01,808

National PDES Testbed: An Overview.

02,775

Mapping Integration Definition for Information Modeling (IDEF1X) Model into CASE Data Interchange Format (CDIF) Transfer File.

PB95-154670

01,711

Using Archie to Find Files on the INTERNET.

01,711

PB95-168605

02,727

Mapping Integration Definition for Function Modeling (IDEFO) Model into CASE Data Interchange Format (CDIF) Transfer File.

PB96-109533

01,741

DATA TRANSMISSION

Recommendations on Selection of Vehicle-to-Roadside Communications Standards for Commercial Vehicle Oper-

ations. 04,859

Vehicle-to-Roadside Communications for Commercial Ve-

hicle Operations: Requirements and Approaches.
PB95-188827
04,860

Comparison of FDDI Asynchronous Mode and DODB Oueue Arbitrated Mode Data Transmission for Metropoli$\tan$ Area Network Applications.

01,498

Addressing U.S. Government Security Requirements for OSI

PB96-160577

01,611

DATA TRANSMISSION SYSTEMS

NIST ATM Network Simulator: Operation and Programming, Version 1.0

DATA VALIDATION

How the Cambridge Crystallographic Data Centre Obtains Its Information
PB97-109193

04,808

Data Import and Validation in the Inorganic Crystal Structure Database

04,809

DATABASES

Progress in the Development of a Chemical Kinetic

Database for Combustion Chemistry.
PB95-151056

01,384

Information Retrieval Using Key Words and a Structured

Review

PB95-161121

03,724

Locating Fire Engineering Information.

PB95-161188

00,198

Access Paths for Materials Databases: Approaches for Large Databases and Systems.

02,975

Database for the Static Dielectric Constant of Water and

Database for the Static Dielectric Constant of Water and
Steam.
PB96-145586
01,103

Locating Fire Information.

PB96-190137

00,227

Workshop Highlights

04,811

DAUGHTER PRODUCTS

Systematics of Alpha-Particle Energy Spectra and Lineal Energy (Y) Spectra for Radon Daughters.

PB94-185139

03,615

DAYLIGHT

Lighting Ouality and Light Source Size.

PB95-151783

00,252

S Presentations Shine.

PB94-200383

00,244

DEBONDING

Determination of Fiber-Matrix Interfacial Properties of Im-

portance to Ceramic Composite Toughening.

03,149

DEBUGGING (COMPUTERS)

Debugger for Tcl Applications.
PB94-213303

01,695

Method to Determine a Basis Set of Paths to Perform PBg6-131503

Program Slicing.

PB96-160981

01,747

PB96-165964

01.761

BYE-HUCKEL THEORY

Electrolytes Constrained on Fractal Structures: DebyeHuckel Theory.
PB97.110241

DEBYE TEMPERATURE

Dependence of Tc on Debye Temperature Theta(sub D) for Various Cuprates.

DEC/MASPAR MP-1 COMPUTER SYSTEMS

MasPar MP.1 as a Computer Arithmetic Laboratory.
PB95-189437

DECISION MAKING

EXITT: A Simulation Model of Occupant Decisions and PB94-213261

00,351

DECISION SUPPORT SYSTEMS

AutoBid 2.0: The Microcomputer System for Police Patrol Vehicle Selection.

PB96-154570

DECISION TREE ANALYSIS

04,871

Point Probe Decision Trees for Geometric Concept Class-

PB. 9600817

DECOMPOSITION

Thermal Decomposition Pathways in Nitramine Propellants.

AD-A295 896/5

03,753

Spectroscopic Study of Reaction Intermediates and Mechanisms in Nitramine Decomposition and Combustion.

AD-A296 061/5

03,774

Decomposition of SF6 and Production of S2F10 in Power

ArCs.

01,084

DECOMPOSITION REACTIONS

Oxidation of 10-Methylacridan, a Synthetic Analogue of NADH and Deprostion of is Calion Radical. Convergent Application of Laser Flash Photolysis and Direct and Deprotonation of the Cation Radical. Deprot-198371 the Cation Radical.

Mechanism and Rate Constants for the Reactions of $\mathrm{Hy}$ drogen Atoms with Isobutene at High Temperatures.
PB95-151064

Decomposition of Sulfur Hexafluoride by $\mathrm{X}$-rays

PB96-135314

01,095

DECYCLIZATION

Homogeneous Gas Phase Decyclization of Tetralin and Benzocyclobutene.

PB95-151049

00,928

DEFECT FORMATION

Mechanism of Defect Formation in Low-Dose Oxygen $1 \mathrm{~m}$ planted Silicon-on-Insulator Material.

DEFECTS

Fabrication of Flaw-Tolerant Aluminum-Titanate-Reintorced Alumina

PB95-162533

03,161

Defect Formation Mechanism Causing Increasing Defect Density during Decreasing Implant Dose in Low-Dose

Nano-Defects in Commercial Bonded SOI and SIMOX.

PB96-123674 02,407 


\section{KEYWORD INDEX}

DEGENERATE STATES

Accuracy-Weighted Variational Principle for Degenerate Continuum States. PB94-200615

03,831

DEGRADATION

Relation between AC impedance Data and Degradation of Coated Steel. 1. Effects of Surface Roughness and Contamination on the Corrosion Behavior of Epoxy Coated Steel

PB94-213345

03,189

DEGREES OF FREEDOM

Confidence on the Modified Allan Variance and the Time Variance.

01,557

DEHYDROASCORBIC ACID

Ascorbic and Dehydroascorbic Acids Measured in Plasma Preserved with Dithiothreitol or Metaphorphoric Acid PB94-216330

03,495

DELAUNAY TRIANGULATION

Submissions to a Planned Encyclopedia of Operations Research on Computational Geometry and the Voronoi/ Delaunay Construct. Delaunay Constru
PB94.152709

03,425

\section{DELAY LINES}

Low-Coherence Interferometric Measurement of Group Transit Times in Precision Optical Fibre Delay Lines. PB95-168480

DELAY TIME

Use of lonospheric Data in GPS Time Transfer. PB95-163853

01.540

DEMONSTRATION PROJECTS

Submarine Automation: Demonstration No. 5. PB95-251633

03,748

DENDRITIC CRYSTALS

Computation of Dendrites Using a Phase Field Model.
PB94-160744

Stagnant Film Model of the Effect of Natural Convection on the Dendrite Operating State.

DENSIFICATION

Micromechanics of Densification and Distortion.

PB94-200326

04,765

Rapid Hot Pressing of Ultra-Fine PSZ Powders PB94-216587

03,327

DENSIMETERS

Development of a Dual-Sinker Densimeter for High-Accuracy Fluid P-V-T Measurements. Appendix A.

19682

02,620

DENSITOMETERS

High-Temperature High-Pressure Oscillating Tube DenPB96-146618

01,123

DENSITY

Density of Solids and Liquids.

AD-A278 517/8

00,711

DENSITY CORAECTION

Ouantum Collisional Transfer Contributions to the Density

Dependence of Gaseous Viscosity.
PB96-161914

01,142

DENSITY (MASSNOLUME)

Neutron Reflectivity of End-Grafted Polymers: Concentra. tion and Solvent Ouality Dependence in Equilibrium Conditions. PB94-185758

01,206

Apparent Molar Heat Capacities and Apparent Molar Volumes of Aqueous Glucose at Temperatures from 298.15 $K$ to $327.01 \mathrm{~K}$
$\mathrm{~PB} 94-212800$

03,459

Etfects of Humidity and Elevated Temperature on the Density and Thermal Conductivity of a Rigid Polyisocyanurate Foam Co-Blown with CCl3F and $\mathrm{CO}_{0 .}$.
PB95-150462

Experimental Method for Obtaining Critical Densities of Binary Mixtures: Application to Ethane + n-Butane.
PB95-151148 00,930

Effects of Humidity and Elevated Temperature on the Density and Thermal Conductivity of a Rigid Polyisocyanurate Foam.
PB95-152021

Global Density Effects on the Self-Preservation Behavior of Turbulent Free Jets.

PB95-162301

04,207

Neutron Reflectivity Study of the Density Profile of a Model End-Grafted Polymer Brush: Influence of Solvent Ouality

PB95-202735

01,274

DENSITY MEASUREMENT

Electronic Balance and Some Gravimetric Applications. (The Density of Solids and Liquids. Pycnometry and Mass)

PB94-163052

03,785

Determination of Density of Mass Standards: Requirement and Method.

03,787

Report of Density Intercomparisons Undertaken by the Working Group on Density of the CCM.

PB94-200664

00,503

Mass and Density Determinations.

00,504
Lattice Dynamics of $\mathrm{Ba} 1-\mathrm{xKxBiO} 3$

PB96-102421

04,706

DENSITY PROFILES

Internal Waves in Xenon Near the Critical Point. 04,221

DENTAL

Fluoride Analytical Methods

PB96-180237

03,578

DENTAL CEMENTS

Adsorption of Low-Molecular-Weight Sodium Polyacrylate on Hydroxyapatite.

00,139

Behavior of a Calcium Phosphate Cement in Simulated

Blood Plasma In vitro.

PB95-168712

00.165

Formation of Hydroxyapatite in Cement Systems.

PB95-175261

00,170

Physical and Chemical Properties of Resin-Reinforced Calcium Phosphate Cements.

-180212

00,171

Formation of Hydroxyapatite in a Polymeric Calcium Phosphate Cement.

PB95.180642

00.173

Properties and Mechanisms of Fast-Setting Calcium Phosphate Cements.

00,178

Bioactive Polymeric Dental Materials Based on Amorphous Calcium Phosphate.

PB96-147012

03,572

Remineralizing Dental Composites Based on Amorphous Calcium Phosphate

03,573

Dynamics of Calcium Phosphate Precipitation. PB96-147095

03,574

DENTAL CERAMICS

In vitro Fracture Behavior of Ceramic and Metal-Ceramic PB96-119722

DENTAL ENAMEL

Deposition of Loosely Bound and Firmly Bound Fluorides on Tooth Enamel by an Acidic Gel Containing Fluorosilicate and Monocalcium Phosphate Monohydrate PB95-150710
O3,559

Wear of Human Enamel against a Commercial Castable Ceramic Restorative Material.

PB95-161972

00,161

Procedure for the Study of Acidic Calcium Phosphate Precursor Phases in Enamel Mineral Formation.

Precursor Phase

03.564

\section{DENTAL MATERIALS}

Properties and Interactions of Oral Structures and Restorative Materials. Annual Report for Period October 1, 1990 to September 30, 199

PB94-160843

03,558

Influence of Tempering Method on Residual Stress in Dental Porcelain

Interaction of Some Coupling Agents and Organic Compounds with Hydroxyapatite: Hydrogen Bonding, Adsorption and Adhesion.

00,140

Etfect of Two Initiator/Stabilizer Concentrations in a Metal Effect of Two Initiator/Stabilizer Concentrations in a Metal
Primer on Bond Strengths of a Composite to a Base PB94-172723

Dental Materials.

00,141

PB94-172871

00,142

Effect of Transformation of Alloy on Transient and Residual Stresses in a Porcelain-Metal Strip.

Adhesion of Composites to Dentin and Enamel PB94-199049

00,143

Development of an Adhesive Bonding System.

00,144

Clinical Perspective on Dentin Adhesives

00,145

PB94-211240

00,14

Periapical Tissue Reactions to a Calcium Phosphate Cement in the Teeth of Monkeys.

00,149

Paffenbarger Research Center: The Cutting Edge of Dental Science.

00,151

Evaluation of Fracture Toughness and Residual Stress in Dental Porcelain by Indentation-Microfracture Method

PB95-125613

Effects of Surface-Active Resins on Dentin/Composite Bonds.

PB95-140448

00,156

Effects of Calcium Phosphate Solutions on Dentin Permeability.

00,157

NIR-Spectroscopic investigation of Water Sorption Characteristics of Dental Resins and Composites.

00,189

Evaluation of Fracture Toughness and Residual Stress in Evaluat Porcelain by Indentation-Microfracture Method.
Dental Pores
PB95-152831

Publication and Presentation Abstracts, 1993. (Published by Paftenbarger Research Center and Center of Excelence for Materials Science Research).

PB95-153052

03,562

Wear of Human Enamel against a Commercial Castable Ceramic Restorative Material.

PB95-161972

00,161

Ring-Opening Dental Resin Systems Based on Cyclic

PB95-162251

00,162

Effect of Ethanol on the Solubility of Dicalcium Phosphate

Dihydrate in the System $\mathrm{Ca}(\mathrm{OH}) 2-\mathrm{H} 3 \mathrm{PO} 4-\mathrm{H} 2 \mathrm{O}$ at $37 \mathrm{C}$

PB95-163507

00.163

Evaluation of Methylene Lactone Monomers in Dental

PB95-164661

00,164

Octacalcium Phosphate Carboxylates. 5. Incorporation of Excess Succinate and Ammonium lons in the Octacalcium Phosphate Succinate Structure.

00,166

395-168894

Crystal Structure of Calcium Succinate Monohydrate.

PB95-168928 00,167

Ambient Temperature Synthesis of Bulk Intermetallics

PB95-169074 00,168

Effect of Ethanol on the Solubility of Hydroxyapatite in the System $\mathrm{Ca}(\mathrm{OH}) 2-\mathrm{H} 3 \mathrm{PO} 4-\mathrm{H} 2 \mathrm{O}$ at $25 \mathrm{C}$ and $33 \mathrm{C}$.

PB95-169231

00,169

Physical and Chemical Properties of Resin-Reinforced Calcium Phosphate Cements.

00.171

Diagnosis and Treatment of an Oral Base-Metal Contact Lesion Following Negative Dermatologic Patch Tests. PB95-180626 00,172

Polymers Technical Activities 1994. NAC-NRC Assessment Panel, April 6-7, 1995.

PB95-209896

01,275

Reduction of Marginal Gaps in Composite Restorations by Use of Glass-Ceramic Inserts.

00,174

Publications and Presentation Abstracts, 1995. (Published by Paffenbarger Research Center and Center of Excellence for Materials Science Research).

PB96-119250

03,568

Failure of All-Ceramic Fixed Partial Dentures 'In vitro' and 'In vivo': Analysis and Modeling.

00,175

Dental Applications of Ceramics.

PB96-122940

00,177

Preparation and Characterization of Cyclopolymerizable Resin Formulations.

PB 96-146840 
Effects of Aluminum Oxalate/Glycine Pretreatment Solutions on Dentin Permeability.

PB95-164505

03,565

DENTISTRY

Clinical Perspective on Dentin Adhesives

PB94-211240

00,146

Paffenbarger

PB94-216355

of Den-

Influence of Natural and Synthetic Inhibitors on the Crystallization of Calcium Oxalate Hydrates.

03,560

Interaction of Chlorhexidine Digluconate with and Adsorption of Chlorhexidine on Hydroxyapatite.

03,566 PB95-175907

DEOXYRIBONUCLEIC ACID

Resolution of DNA in the Presence of Mobility Modifying Polar and Nonpolar Compounds by Discontinuous Electrophoresis on Rehydratable Polyacrylamide Gels. PB95-152799 00,590 Repair of Products of Oxidative DNA Base Damage in Human Cells.

03,555

DEOXYRIBONUCLEIC ACIDS

Function of DnaJ and DnaK as Chaperones in OriginSpecific DNA Binding by RepA.

03,533

PB95-151544

Substrate Specificity of the Escherichia coli Endonuclease DA Produced by Radiation-Generated Free Radicals.

PB95-153425 03,535

Electrophoretic Separations of Polymerase Chain Reaction: Amplified DNA Fragments in DNA Typing Using a Capillary Electrophoresis-Lased Induced Fluorescence System.
PB95-163036

03,536

Interlaboratory Comparison of Autoradiographic DNA Profiling Measurements. 1. Data and Summary Statistics.

Detection of Aromatic Compounds Based on DNA Intercalation Using an Evanescent Wave Biosensor. PB96-111976 03,473 Interlaboratory Comparison of Autoradiographic DNA Its Propagation

03,545

Rapid Method for the Isolation of Genomic DNA from 'Aspergillus fumigatus'

Novel DNA N-Glycosylase Activity of E. coli T4 Endonuclease $V$ That Excises 4,6-Diamino-5 Formamidopyrimidine from DNA, a UV-Radiation- and Hydroxyl Radical-Induced Product of Adenine.

PB96-160478

03.549

Novel Activities of Human Uracil DNA N-glycosylase for Cytosine-Derived Products of Oxidative DNA Damage.

New Method for the Detection and Measurement of Polyaromatic Carcinogens and Related Compounds by DNA Intercalation.

PB96-167382

03,481

DnaJ, DnaK, and GrpE Heat Shock Proteins are Required in 'ori'P1 DNA Replication Solely at the RepA Monomerization Step.

PB97-119382

03,557

DEOXYRIBOSE

Modification of Deoxyribose-Phosphate Residues by Extracts of Ataxia Telangiectasia Cells.

PB94-212602

03,458

DEPOLARIZATION

Polarimetric Calibration of Reciprocal-Antenna Radars.

DEPOSITION

Perspective on Fiber Coating Technology.

PB94-200540

03,118

Coating of Fibers by Colloidal Techniques in Ceramic PB94-216256

03,196

Deposition of Colloidal Sintering-Aid Particles on Silicon Nitride.

PB94-216272

03,044

Deposition of Loosely Bound and Firmly Bound Fluorides on Tooth Enamel by an Acidic Gel Containing Fluorosilicate and Monocalcium Phosphate Monohydrate.
PB95-150710 Nanofabrication of a Two-Dimensional Array Using LaserFocused Atomic Deposition.

PB96-119417

04,732

New Method for Achieving Accurate Thickness Control for Uniform and Graded Multilayer Coatings on Large Flat Substrates.

DEPOSITS

New Method to Evaluate Deposit Forming Tendencies of iquid Lubricants by Differential Scanning Calorimetry. PB95-152120 01,451

Deposit Forming Tendencies of Diesel Engine Oils-Correlation between the Two-Peak Method and Engine Tests.

01,452
DEPROTEINATION

Critical Evaluation of the Purification of Biominerals by Hypochlorite Treatment

00,186

DESCRIBING FUNCTIONS

Electrode Extension Model for Gas Metal Arc Welding.
PB96-135074 02,871

DESIGN

Simple, Inexpensive Apparatus for Sample Concertration.

PB94-199205 00.546

Advanced Mass Calibration and Measurement Assurance

Program for State Calibration Laboratories.

02,492

Design Engineering Research at NIST

PB95-267860

02,784

Line-Heat-Source Guarded-Hot-Plate Apparatus.
PB97-118996

DESIGN ANALYSIS

How to Evaluate Alternative Designs Based on Fire Mod-

PBing.

00,206

DESIGN CRITERIA

Design Equations and Scaling Laws for Linear Compres-

sors with Flexure Springs.
PB95-168902

Can Displays Deliver a Full Measure: Manufacturing.

Recommended Performance-Based Criteria for the Design of Manufactured Home Foundation Systems to Resist Wind and Seismic Loads

PB96- 128285

00,460

Elements of a Framework for Fire Safety Engineering

PB96-151402

00,214

Distributed Architecture for Standards Processing

00,276

Design of Technically Complex Facilities.

02.695

DESIGN PROCESS

Improving the Design Process by Predicting Downstream Values of Design Attributes.

DESIGN STANDARDS

Evaluation and Strengthening Guidelines for Federal Buildings: Assessment of Current Federal Agency Evaluation Programs and Rehabilitation Criteria and Development of Typical Costs for Seismic Rehabilitation.

PB94-181856

00,425

Evaluation and Retrofit Standards for Existing Federally

Owned and Leased Buildings

PB95-150918

00,434

Lessons from the Loma Prieta Earthquake.

00,442

World of Building Codes.

PB95-203428

00,449

Fire Codes for Global Practice.

00.205

ICSSC Guidance on implementing Executive Order 12941 on Seismic Safety of Existing Federally Owned or Leased Buildings.

00,459

PB96-128103

Predicting the Fire Performance of Buildings: Establishing Appropriate Calculation Methods for Regulatory Applications.

PB96-141239

00,316

DESORPTION

Vibrational Distributions of As2 in the Cracking of As4 on Sil(100) and Si(111).

00,784

Topological Influences on Polymer Adsorption and Desorption Dynamics. 212479

01,227

Desorption Induced by Electronic Transitions.

PB94-216173

00,853

Laser-Induced Desorption of NO from Si(111): Effects of Coverage on

00,959

Direct Detection of Atomic Arsenic Desorption from Si(100)

PB95-202230

01.024

Photodesorption Dynamics of CO from Si(111): The Role of Surface Defects.

03,066

DESULFURIZATION

Low Temperature H(sub 2)S Separation Using Membrane Reactor with Redox Catalys

02,471

Chemical and Microbiological Problems Associated with Research on the Biodesulfurization of Coal.

02,484

PB95-140950

DETAN 95 COMPUTER CODE

DETAN 95: Computer Code for Calculating SpectrumAveraged Cross Sections and Detector Responses in Neutron Spectra.

PB95-242384

04,014

Hair Analysis for Drugs of Abuse: Evaluation of Analytical Methods, Environmental Issues, and Development of Reterence Materials.

PB95-176269

03,501

NIST Reference Materials to Support Accuracy in Drug Testing.
PB96-123807

03,505

Safety Assessment of Railroad Wheels by Residual Stress Measurements.

PB96-141114 04,855

Safety Assessment of Railroad Wheels Through Roll-by Detection of Tread Cracks.

Interlaboratory Studies on the Analysis of Hair for Drugs of Abuse: Results from the Fourth Exercis

03,510

DETECTOR RESPONSE

Modeling Detector Response for Neutron Depth Profiling.
PB96-157813

DETECTORS

Use of a Radiochromic Detector for the Determination of Stereotactic Radiosurgery Dose Characteristics.

Germanium Detector Optimization of MDA for Efficiency vs. Low Intrinsic Background.

PB94-199155

00,543

Process Gas Chromatography Detector for Hydrocarbons Based on Catalytic Cracking

02,485

Simultaneous Laser-Diode Emission and Detection for Fiber-Optic Sensor Applications.

PB96-155502 04,062

Soft-X-ray Damage to p-terphenyl Coatings for Detectors.
PB96-159611

Magneto-Optic Rotation Sensor Using a Laser Diode as Both Source and Detector.
PB97-111272

04,390

Fundamentals and Problems of Fiber Current Sensors.

PB97-111835

02,205

ETERIORATION

Diagnosis of Causes of Concrete Deterioration in the MLP.7A Parking Garage.

01,318

DEUTERIUM

Experimental plan to determine the performance of the Oak Ridge National Laboratory Cold Neutron Moderator. Final report, September 1, 1993--November 30, 1993.

DE95011352

03,778

Simultaneous Forward-Backward Raman Scattering Stud-

00,961

Vibrations of Hydrogen and Deuterium in Solid Solution with Lutetium.

PB95-181038 01,019

Deuterium in the Local Interstellar Medium: Its Cosmological Significance.
PB95-202842 00,081

Accurate Measurements of the Local Deuterium Abundance from HST Spectra

Deuterium and the Local Interstellar Medium: Properties for the Procyon and Capella Lines of Sight.

PB96-200639

00,111

DEUTERIUM CHLORIDE COMPLEXES

Vibrational Dependence of the Anisotropic Intermolecular

Potential of $\mathrm{Ar}-\mathrm{HCl}$

PB95-202685 01,029

High Resolution Near Infrared Spectroscopy of $\mathrm{HCl}-\mathrm{DCl}$ and $\mathrm{DCl}-\mathrm{HCl}$ : Relative Binding Energies, Isomer 
DEVICE CHARACTERIZATION

Progress on the Ouantized Hall Resistance Recommended Intrinsic/Derived Standards Practice.

PB96-122460

02,403

DEW BUBBLE CURVES

Vapor-Liquid Equilibria of Mixtures of Propane and Isomeric Hexanes.

DEXTRAN

Two Phase Aqueous Extraction: Rheological Properties of Dextran, Polyethylene Glycol, Bovine Serum Albumin and

Their Mixtures.

00,676

DGSA (DOD GOAL SECURITY ARCHITECTURE)

Assessment of the DOD Goal Security Architecture (DGSA) for Non-Military Use.

03,653

DIACETYLENES

Radiochromic Solid-State Polymerization Reaction. 01,290

DIAGRAMS

Shape of the Temperature-Entropy Saturation Boundary.
PB96-135066

DIAMOND

Tribological Behavior of 440/Diamond-Like-Carbon Film Couples.

03,019

DIAMOND ANVIL CELLS

Alvin Van Valkenburg and the Diamond Anvil Cell

PB96-204474

04,797

DIAMOND FILMS

Workshop on Characterizing Diamond Films (3rd). Held in Gaithersburg, Maryland on February 23-24, 1994. Analysis of Thermal Wave Propagation in Diamond Films. PB94-211471 03,014 Thermal Wave Propagation in Diamond Films. PB94-211489 $\quad 03,015$ Analysis of Boron in CVD Diamond Surfaces Using Neutron Depth Profiling.

PB94-213089

04,511

Lineshape Analysis of the Raman Spectrum of Diamond Films Grown by Hot-Filament and Microwave-Plasma Chemical-Vapor

PB95-162392

03,016

Studies of Defects in Diamond Films and Particles by Raman and Luminescence Spectroscopies.

03,017

Surface Roughness Evaluation of Diamond Films Grown on Substrates with a High Density of Nucleation Sites.

On Substrates with a High Density of Nucleation Sites.
PB95-162418

DIAMOND INDENTERS

Geometric Characterization of Rockwell Diamond IndentPB95-203287

02,950

Metrology Approach to Unitying Rockwell C Hardness Scales.

PB96-155551

02,957

Stylus Technique for the Direct Verification of Rockwell Diamond Intenders.

PB96-155569

02,958

DIAMOND TURNING

Fabrication of Optics by Diamond Turning.

IAMONDS

Diamond and Graphite Precursors: Comments

PB95-163051

Proceedings of the Applied Diamond Conter Applications of Diamond Films and Related Materials International Conference (3rd). Held in Gaithersburg.
Maryland, on August 21-24, 1995 . Maryland, on August 21-24, 1995

Applications of Diamond Films and Related Materials: International Conference (3rd). Held in Gaithersburg. Maryland on August 21-24, 1995. Supplement to NIST Special Publication 885

PB95-256053

03,063

Damage Processes in Ceramics Resulting from Diamond Tool Indentation and Scratching in Various Environments. Tool Indentation and Scratching in Various Environments.
PB96-102983

Fabrication of Optics by Diamond Turning.

PB96-111695

02,954

Workshop on Characterizing Diamond Films (4th). Held in Gaithersburg, Maryland on March 4-5, 1996.

04,786

Chemical Aspects of Tool Wear in Single Point Diamond Turning.
PB97-112601

03,021

DIAPHRAGMS (MECHANICS)

Flexible-Diaphragm Force Microscope.
PB95- 180915

DIAZINES

Carbon Acidities of Aromatic Compounds. 1. Effects of InRing Aza and External Electron-Withdrawing Groups.

PB94-216595
IBLOCK COPOLYMER ADDITIVES

00,860

Modification of the Phase Stability of Polymer Blends by

Diblock Copolymer Additivies.
PB96-123542
DICALCIUM PHOSPHATE DIHYDRATE

Effect of Ethanol on the Solubility of Dicalcium Phosphate Dihydrate in the System $\mathrm{Ca}(\mathrm{OH}) 2-\mathrm{H} 3 \mathrm{PO} 4-\mathrm{H} 2 \mathrm{O}$ at $37 \mathrm{C}$ PB95-163507

00,163

\section{DICTIONARIES}

Compatibility Analysis of the ANSI and ISO IRDS Services Interfaces.
PB94-163474

Bibliography of Computer Security Glossaries.
PB94-216710

01,805

01,587

Glossary of Software Reuse Terms.

PB95-178992

01,720

Apparel Manufacturing Glossary for Application Protocol Development.
PB95-198750

02,755

DIELECTRIC BREAKDOWN

Reproducibility of JEDEC Standard Current and Voltage Ramp Test Procedures for Thin-Dielectric Breakdown Characterization.

PB94-185931

01879

Experimental Investigation of the Validity of TDDB Voltage Acceleration Models.

PB94-185949

02,304

Field and Temperature Acceleration of Time-Dependent

Dielectric Breakdown in Intrinsic Thin $\mathrm{SiO} 2$.

PB94-185956

02305

Charge Trapping and Breakdown Mechanism in SIMOX. PB94-216637

02,323

High Temperature Reliability of Thin Film $\mathrm{SiO}_{2}$

B95-150348

02,333

Electrical Breakdown in Transformer Oil in Large Gaps.
PB95-150579

Observations of Partial Discharges in Hexane Under High

Magnification

01,900

TDDB Characterization of Thin SiO2 Films with Bimodal Failure Populations

PB96-102926

02,381

Time-Dependent Dielectric Breakdown of Intrinsic $\mathrm{SiO}_{2}$ Films under Dynamic Stress.

PB96-179478

02,438

New Physics-Based Model for Time-Dependent- Dielec-

$\begin{array}{ll}\text { tric-Breakdown. } & 02,448 \\ \text { PB96-201132 } & \end{array}$

Electric Field Dependent Dielectric Breakdown of Intrinsic $\mathrm{SiO} 2$ Films Under Dynamic Stress.

PB96-204102

02,449

Characterization of Time-Dependent Dielectric Breakdown in Intrinsic Thin $\mathrm{SiO} 2$

122527

02,458

\section{DIELECTRIC CONSTANT}

Preparation, Crystal Structure, Dielectric Properties, and Magnetic Behavior of Ba2Fe2Ti4O13.

PB96-186176

01,162

DIELECTRIC CONSTANTS

Analysis of an Open-Ended Coaxial Probe with Litt-Off for Nondestructive Testing.

01,940

DIELECTRIC FILMS

Novel YBa2Cu3O7-x and YBa2Cu3O7-x/Y4Ba3O9 Multilayer Films by Bias-Masked 'On-Axis' Magnetron Sputtering.

DIELECTRIC FUNCTIONS

Nonequilibrium Total-Dielectric-Function Approach to the Electron Boltzmann Equation for Inelastic Scattering in Doped Polar Semiconductors. PB96-138532

04,416

DIELECTRIC MATERIALS

Nonstationary Behaviour of Partial Discharge during Discharge Induced Ageing of Dielectrics.

PB96-103114

01,922

DIELECTRIC MEASUREMENTS

Dielectric Properties Measurements and Data PB94-172186

01,876

DIELECTRIC PROPERTIES

Table of Dielectric Constants of Pure Liquids.

0,712
Dielectric Studies of Fluids with Reentrant Resonators. Appendix B.

DE93019683

nators.

Dielectric Studies of Fluids with Reentrant Resonators.

00,952

Dielectric Properties of Single Crystals of $\mathrm{Al}_{2} \mathrm{O} 3, \mathrm{LaAlO} 3$ $\mathrm{SrTiO3}$, and $\mathrm{MgO}$ at Cryogenic Temperatures

PB95-180477

02,266

Dielectric Properties of Thin Film $\mathrm{SrTiO}_{3}$ Grown on $\mathrm{LaAlO} 3$ with $\mathrm{YBa} 2 \mathrm{Cu} 3 \mathrm{O} 7-x$ Electodes.

PB95-181160

02,267

Measurements of the Relative Permittivity of Liquid Water at Frequencies in the Range of 0.1 to $10 \mathrm{kHz}$ and at Temperatures between 273.1 and $373.2 \mathrm{~K}$ at Ambient

Pressure.
PB96-119375

01,078

PB96-123559

01,090

Dielectric and Magnetic Measurements from $-50 \mathrm{C}$ to $200 \mathrm{C}$ and in the Frequency Band $50 \mathrm{MHz}$ to $2 \mathrm{GHz}$.

DIELECTRIC RELAXATION

Distribution of Dielectric Relaxation Times and the MoPB96-112032

04,727

DIELECTRIC RESONATORS

Measurements of Permittivity and the Dielectric Loss Tangent of Low Loss Dielectric Materials with a Dielectric Resonator Operating on the Higher Order Te(sub 0 gamma delta) Modes.

02,273

Influence of Films' Thickness and Air Gaps in Surface $1 \mathrm{~m}$ pedance Measurements of High Temperature Superconductors Using the Dielectric Resonator Tech-

PB96-157862

01,946

DIELECTRIC SPECTROSCOPY

Dielectric Behavior of a Polycarbonate/Polyester Mixture Upon Transesterification

PB96-179551

04,785

DIELECTRIC WAVEGUIDES

Mode Coupling and Loss on Tapered Optical

Waveguides.

04,282

Bent Rectangular Core Waveguides: An Accurate Perturbation Approach.

PB95-168803

04,289

DIELECTRIC WEDGE

Hypersingular Single Integral Equation and the Dielectric PB97-110274

04,428

\section{DIELECTRIC WEDGES}

Numerical Evaluation of Hypersingular Integrals for Scattering by a Dielectric Wedge.

02,017

DIELECTRICS

Spatial Dependence of Electrical Fields Due to Space Charges in Films of Organic Dielectrics Used for Insulation of Power Cables.

PB94-199130

02,214

Modification of Cast Epoxy Resin Surfaces during Exposure to Partial Discharges.

01,086

Influence of Surface Charge on the Stochastic Behavior of Partial Discharge in Dielectrics.

PB96-122767

01,931

$\mathrm{SF} 6 / \mathrm{N} 2 \mathrm{M}$

PBoperties.

02,230

Behavior of Surface Partial Discharge on Aluminum Oxide Dielectrics.

PB96-123781

01,937

Partially Cohe

President's Column 'Editorial'.

PB96-164090

04,359 the Single Hyp

04,430

DIELECTRICS AND ELECTRICAL INSULATION SOCIETY

President's Column for Dielectrics and Electrical Insula- 
Antiferromagnetic Interlayer Correlations in Annealed Ni80Fe20/Ag Multilayers.

03,109

DIFFRACTION ANALYSIS

Parametric Investigation of Metal Powder Atomization Using Laser Diffraction.

PB95-108577

03,342

DIFFRACTION IMAGING

Diffraction Imaging of Polycrystalline Materials.
PB94-198884

DIFFRACTOMETERS

Introduction of a NIST Instrument Sensitivity Standard Reference Material for X-Ray Powder Diffraction. 00,807
PB94-200318

DIFFUSE INTERFACE

Diffuse-Interface Description of Fluid Systems.

PB96-210711

01,170

DIFFUSE REFLECTION

Specular and Diffuse Reflection Measurements of Electronic Displays
PB97-119200

02,208

DIFFUSE SCATTERING

Orientational Fluctuations, Diffuse Scattering, and Orientational Order in Solid $\mathrm{C} 60$

04,176

DIFFUSION

Further Calculations of $X$-ray Diffusion in an Infinite Me-

03,772

Energy and Migration of Grain Boundaries in Polycrystals.

PB94-211638
Transport and Diffusion in Three-Dimensional Composite

04,668

Chaotic Motions of Coupled Galloping Oscillators and Their Modeling as Diffusion Progresses. PB96-122718

04,823

Electrodeposited Cobalt-Tungsten as a Diffusion Barrier between Graphite Fibers and Nickel.

03,176

DIFFUSION COEFFICIENT

Measurement of Diffusion in Supercritical Fluid Systems:

A Review.

PB94-199189

00,795

Diffusion of Copper into Gold Plating.

00,957

Brownian Diffusion of Hard Spheres at Finite Concentrations.

00,975

PB95-164307

Measurement of Diffusion in Fluid Systems: Applications
to the Supercritical Fluid Region. PB95-175188

02,490

Non-Perturbative Relation between the Mutual Diffusion Coefficient, Suspension Viscosity, and Osmotic Compressibility: Application to Concentrated Protein Solutions.
PB96-102355 DIFFUSION FLAMES

Greatly Enhanced Soot Scattering in Flickering Ch4/Air Diffusion Flames.

PB94-172988 01,361

Laser Imaging of Chemistry-Flowfield Interactions: Enhanced Soot Formation in Time-Varying Diffusion Flames.
PB94-185352 Investigation of Oil and Gas Well Fires and Flares.

PB94-193976 03,695

Generation and Characterization of Acetylene Smokes.

PB94-200292

01,372

Ultrafine Combustion Aerosol Generator.

PB94-200300

01,373

Laser-Induced Fluorescence Measurements of Formaldehyde in a Methane/Air Diffusion Flame.

PB94-211679

01,374

Comparison of Experimental and Computed Species Concentration and Temperature Profiles in Laminar, TwoDimensional Methane/Air Diffusion Flames.

PB95-140919

01,379

Fundamental Mechanisms for $\mathrm{CO}$ and Soot Formation

PB95-143160

01,380

Oxidation of Soot and Carbon Monoxide in Hydrocarbon

Diffusion Flames.
PB95-150215

Optical Measurements of Atomic Hydrogen, Hydroxyl, and Carbon Monoxide in Hydrocarbon Diffusion Flames. Experimental and Numerical Studies of Refractory Particle Formation in Flames: Application to Silica Growth. PB95-152005

00,673

Laser-Induced Fluorescence Measurements of $\mathrm{OH}$. Concentrations in the Oxidation Region of Laminar, Hydrocarbon Diffusion Flames.

PB95-162160

01,387

Acid Gas Production in Inhibited Diffusion Flames. PB95-180576 01,390

Burning Rate of Premixed Methane-Air Flames Inhibited by Fluorinated Hydrocarbons.

01,391
Experimental and Numerical Buming Rates of Premixed Methane-Air Inhibited by Fluoromethanes.

01,392

Quantitative Measurements of Enhanced Soot Production in a Flickering Methane/Air Diffusion Flame.

PB95-203246

01,393

Mixing and Radiation Properties of Buoyant Turbulent Diffusion Flames.

01,396

Experimental Study of the Stabilization Region of Lifted Turbulent-Jet Diffusion Flames.

PB96-122676

01,405

Laser-Induced Fluorescence Measurements of $\mathrm{OH}$ in Laminar Diffusion Flames in the Presence of Soot Par-

PB96-123120

01,409

Chernical Inhibition of Methane-Air Diffusion Flame. PB96-195532

01,431

Mixing and Radiation Properties of Buoyant Luminous Flame Environments.

PB96-202254

01,432

Computations of Enhanced Soot Production in TimeVarying $\mathrm{CH} 4 /$ Air Diffusion Flames.

01,440

NO Production and Destruction in a Methane/Air Diffusion

Flame.

DIFFUSION THEORY

Modeling the Evolution of Structure in Unstable Solid Solution Phases by Diffusional Mechanisms.

PB94-199403

DIFFUSION ZONES

Study of Diffusion Zones with Electron Microprobe Compositional Mapping

\section{DIFFUSIVITY}

Measurements of Moisture Diffusivity for Porous Building Materials.

PB95-107397

00,356

Computer Simulation of the Diffusivity of Cement-Based Materials.

00,362

DIFLUOROBENZENE

Vibrational Energy Transfer in S1 p-Difluorobenzene. A Comparison of Low and Room Temperature Collisions.

Vibrations of $S 1((1) B 2 u) p$-Difluorobenzene - d4. S1-SO Fluorescence Spectroscopy and ab Initio Calculations.
PB95-202586

DIFLUOROETHANE Coexisting Densities, Vapor Pressures and Critical Den-
sities of Refrigerants R-32 and R-152a, at $300-385 \mathrm{~K}$. Sities of Rerriger

03,274

Compressed Liquid Densities, Saturated Liquid Densities, and Vapor Pressures of 1,1-Difluoroethane.

01,173 PB97-110118

DIFLUOROMETHANE

Ebulliometric Measurement of the Vapor Pressure of Difluoromethane.

00,931

Viscosity of the Saturated Liquid Phase of Six Halogenated Compounds and Three Mixtures. 00,960 Measurements of the Vapor Pressures of Difluoromethane, 1-Chloro-1,2,2,2-Tetrafluoroethane, and Difluoromethane, 1-Chloro-1,2,2,2-Tetrafluoroethane, and
Pentafluoroethane.
PB95-169272 03,270

03,270

Coexisting Densities, Vapor Pressures and Critical Densities of Refrigerants R-32 and R-152a, at $300-385 \mathrm{~K}$. PB95-175691

DIGITAL COMMUNICATIONS

Integrated Services Digital Network (ISDN); Category: Integrated Services Digital Network (ISDN); Category: Selecommunications Digital Network.

Services Digita
FIPS PUB 182

01,460

\section{DIGITAL DATA}

Report on the Workshop on Advanced Digital Video in the National Information Infrastructure. Held in Washington, D.C. on May 10-11, 1994.

PB95-103677

01,472

National Information Infrastructure and Advanced Digital Video.

PB96-119367

01,488

Spatial Information and Technology Standards Evolving.

PB96-135108 03,679

Summary Report on the Workshop on Advanced Digital Video in the National Information Infrastructure. Status of Emerging Standards for Removable Computer Storage Media and Related Contributions of NIST. PB96-160619

01,634

DIGITAL MULTIMETER

Quantum Hall Effect-Based Resistance Standard: Capabilities and Implementation

04,114

Quantum Hall Effect-Based Resistance Standard (Quantum Hall Res

04,127

DIGITAL RECORDING SYSTEMS

Performance Evaluation of a New Digital Partial Discharge Recording and Analysis System.
PB95-150389

Continuous Recording and Stochastic Analysis of PD. PB96-112156

01,925

DIGITAL SIGNATURES

Digital Signature Standard (DSS). Category: Computer Security; Subcategory: Cryptography.
FIPS PUB 186

Report of the NIST Workshop on Digital Signature Certificate Management. Held on December 10-11, 1992.

PB94-135001

01,574

Response to Comments on the NIST Proposed Digital Signature Standard

01,615

Public Key Infrastructure Invitational Workshop. Held in McLean, Virginia on September 28, 1995

01,616

PB96-

Proceedings of the Digital Systems Reliability and $\mathrm{Nu}$ clear Safety Workshop. Held in Rockville, Maryland on September 13-14, 1993

NUREG/CP.0136

03,728

Digital Impedance Bridge.

02,272

Comment and Discussion on Digital Processing of PD

Pulses.

01,932

DIGITAL TECHNIQUES

Digital Techniques in HV Tests - Summary of 1989 Pane PB94-216702

02,035

DILATOMETRY

Aging in Glasses Subjected to Large Stresses and Defor-

PB95-107041

03,235

DILUTED BLENDS

Shear Suppression of Critical Fluctuations in a Diluted PB96-204458

04,418

\section{DIMENSIONAL MEASUREMENT}

Numeric Data Distribution: The Vital Role of Data Exchange in Today's World.

02,622

U.S. Navy Coordinate Measuring Machines: A Study of

PBeeds.

02,807

Concept for an Algorithm Testing and Evaluation Program at NIST.

Uncertainties in Dimensional Measurements Made at Nonstandard Temperatures.

PB94-169760

02,893

User's Guide to NIST SRM 2084: CMM Probe Performance Standard.

02,709

Precision, Accuracy, Uncertainty and Traceability and Their Application to Submicrometer Dimensional MetrolPB94-213105

02,319

Metrology Model for Submicrometer Dimensional Measurements.

02,647

Practical Photomask Linewidth Measurements.
PB95-108510

PB95-108510 02,324 National Institute of Standards and Technology. 
Accuracy in Integrated Circuit Dimensional Measurements.

02,368

Improving Photomask Linewidth Measurement Accuracy via Emulated Stepper Aerial Image Measurement.

PB95-180816

Electrical Method for Determining the Thickness of Films and the Cross-Sectional Area of Metal Lines.

PB95-203170

of Metal

Interim Testing Artifact (ITA): A Performance Evaluation Systeri for Coordinate Measuring Machines (CMMs) User Manual.

PB95-210589

02,914

Algorithm Testing and Evaluation Prograin for Coordinate

Measuring Systems: Long Range Plan.

02,915

Integrated Vision Touch-Probe System for Dimensional Inspection Tasks.

PB95-255832

02,917

Estimation of Measurement Uncertainty of Small Circular Features Measured by CMMs.

PB95-267928 02,918

MONSEL-II: Monte Carlo Simulation of SEM Signals for Linewidth Metrology.

PB96-102710

02,379

User's Guide to 'SuperFit' Modeling Soltware for CMM Probe Lobing.

02,921

Reference Manual for the Algorithm Testing System Version 2.0.

PB96-128244

02,922

Uuality in Automated Manufacturing.

02839

New Concepts of Precision Dimensional Measurement for Modern Manufacturing.

PB96-16068

02,924

Dimensional Characterization of Precision Coaxial Transmission Line Standards.

02,241

Representation of Axes for Geometric Fitting

01,782

DIMENSIONAL MEASUREMENTS

Metrology and Regional Trade Pacts.

PB96-155429

02,923

Report on the NIST Low Accelerating Voltage SEM Magnification Standard Interlaboratory Study.

02,445

DIMENSIONAL MEASURING INTERFACE STANDARD

Dimensional Inspection Planning Based on Product Data PBan-17545.

DIMENSIONAL METROLOGY

02,910

Residual Error Compensation of a Vision-Based Coordinate Measuring Machin PB96-161617

DIMENSIONAL STABILITY

Measuring the Stability of Three Copper Alloys. PB94-199866

04,091

DMERS

Equilibrium Pair Distribution Function of a Gas: Aspects Associated with the Presence of Bound States. PB95-176046

DIMETHYLACETYLENE

Perpendicular C-H Stretching Band nu9/nu13 and the Torsional Potential of Dimethylacetylene.

METHYLPHENANTHRENES

Distinguishing the Contributions of Residential Wood Combustion and Mobile Source Emissions Using Relative Concentrations of Dimethylphenanthrene Isomers. PB96-135124

02,563

DIOXINS

Standard Reference Materials for Dioxins and Other Environmental Pollutants.

PB94-198330

02,518

DIPOLE ANTENNAS

Alternative Contour Technique for the Efficient Computation of the Effective Length of an Antenna.

02,011

DIPOLE MOMENT

Microwave Spectrum and Structure of $\mathrm{CH} 2 \mathrm{O}-\mathrm{H} 2 \mathrm{O}$.
PB97-118723

DIPOLE MOMENTS

Dipole Moments in Rare Gas Interactions.
PB94-212982

DIPOLES

Electric Dipole Excitation of a Long Conductor in a Lossy PB96-146675

DIRECT CURRENT

Physical Basis for Half-Integral Shapiro Steps in a DC PB96-102264

Interfacial Transport in Porous Media: Application to do Electrical Conductivity of Mortars.

01,326

AC-DC Difference Characteristics of High-Voltage Thermal Converters
PB96-148093

02,083
DIRECTIONAL SOLIDIFICATION

Effects of Crystalline Anisotropy and Buoyancy-Driven Convection on Morphological Stability.

03,328

Convective Stability in the Rayleigh-Benard and Directiona! Solidification Problems: High-Frequency Gravity Modulation.

PB95-181145

04,208

DIRECTIONAL SOLIDIFICATION (CRYSTALS)

Convection and Morphological Stability During Directiona

$\begin{array}{ll}\text { Nolidification. } & 03,310\end{array}$

Surface Energy Reduction in Fibrous Monotectic Struc-

tures.

03,150

DIRECTIVITY

Radiated Emissions and Immunity of Microstrip Transmission Lines: Theory and Measurements.

02,238

DIRECTORIES

National Voluntary Laboratory Accreditation Program 1994 Directory

State Weights and Measures Laboratories: State Standards Program Description and Directory. 1994 Edition.

PB94-207727 02,895

National Voluntary Laboratory Accreditation Program 1995 Directory

PB95-174454 00,483

National Voluntary Laboratory Accreditation Program

1996 Directory.
PB96-162714.

Directory of Law Enforcement and Criminal Justice Associations and Research Centers.

PB96-178918

04,872

X.500 Directory Schema Design Handbook

PB96-183041 02,739

Directory of U.S. Private Sector Product Certification Pro-

grams.

02,688

Standards Activities of Organizations in the United States. PB97-124135

00,006

DISCHARGE

Ion Kinetics and Symmetric Charge-Transfer Collisions in Low-Current, Diffuse (Townsend) Discharges in Argon and Nitrogen.

04,051

DISCHARGES

Fundamental Processes in Gas Discharges.

PB96-123450

01,089

Electrical Characteristics of Argon Radio Frequency Glow Discharges in an Asymmetric Cell.

PB96-176490

04,109

DISCOLORATION

Intensive Swimming: Can It Affect Your Patients' Smiles. PB96-123666

03,570

DISK SOURCE

Exact Series Solution to the Epstein-Hubbell Generalized Elliptic Type Integral Using Complex Variable Residue Theory.

03,423

DISLOCATION CORE

Dislocation Core-Core Interaction and Peierls Stress in a Model Hexagonal Lattice.

PB96-162003

04,101

DISLOCATION EMISSION

Cracks and Dislocations in Face-Centered Cubic Metallic Multilayers.

Experimental Assessment of Crack-Tip Dislocation Emis sion Models for an Al67Cr8Ti25 Intermetallic Alloy.
PB96-204466

DISLOCATIONS

Dislocation Emission at Ledges on Cracks

PB95-164240 04,630

Shielding of Cracks in a Plastically Polarizable Material.

PB95-164257 04,631

Scanning Electron Microscopy Observations of Misfit Dislocations in Epitaxial In0.25Ga0.75As on GaAs(001). PB96-200159

03,004

DISLOCATIONS (MATERIALS)

Interaction between Dislocations and Intergranular Cracks.

PB95-152096

03,190

Nonlocal Effects of Existing Dislocations on Crack-Tip Emission and Cleavage.

03,367

DISPERSANTS

Polyelectrolytes as Dispersants in Colloidal Processing of Silicon Nitride Ceramics.

PB95-175568

03,055

DISPERSIONS

Computational Model for the Rise and Dispersion of Wind-Blown, Buoyancy-Driven Plumes. Part 2. Linearly Stratified Atmosphere.

00,119

Contrast Matched Studies of a Sheared Binary Colloidal Suspension.

00,918

DISPLACEMENT

Generating and Measuring Displacements Up to $0.1 \mathrm{~m}$ to an Accuracy of $0.1 \mathrm{~nm}$ : Is It Possible.

PB96-160700

04,090

DISPLACEMENT MEASUREMENT

Electro-Optical Sensor for Surface Displacement Meas. urements of Compliant Coatings.

02,123

Displacement Method for Machine Geometry Calibration.

PB95-152088 02,946

Integrated Inspection System for Improved Machine Per-

PB96-160569

02,959

DISPLACEMENT REACTIONS

Rate Constants for Hydrogem Atom Attack on Some Chlorinated Benzenes at High Temperature.

00,810

DISPL

PLAY DEVICES

NIST Workshop on the Computer Interface to Flat Pane Displays. Held in San Jose, California on January 13-14,

PB95-136388

01,625

Can Displays Deliver a Full Measure: Manufacturing

PB96-111935

Display-Measurement Round-Robin

PB96-119227

02,185

Making Displays Deliver a Full Measure

02,186

PB96-122411 01,490

Survey of the Components of Display-Measurement Standards.

PB96-122528

02,188

Micromachined Display Output for a Cellular Neural Net-

PB96-156070

02,422

Specular and Diffuse Reflection Measurements of Elecronic Displays.

02,208

Survey of the Components of Display-Measurement Standards.

DISSIMILAR MATERIALS BONDING

Surface Forces and Adhesion between Dissimilar Materials Measured in Various Environments.

PB94-172970

03,033

DISSOCIATION

Fragment Energy and Vector Correlations in the Overtone-Pumped Dissociation of HN3X(1)A

PB94-199908

00,802

DISSOCIATIVE IONIZATION

Appearance Potentials of Ions Produced by Electron-Impact Induced Dissociative lonization of SF6, SF4, SF5Cl $\mathrm{S}_{2} \mathrm{~F} 10, \mathrm{SO} 2, \mathrm{SO} 2 \mathrm{~F} 2, \mathrm{SOF} 2$, and SOF 4 .

PB96-119730

01,080

DISSOLVING

Dissolution Prot

DISTILLATION EQUIPMENT

Development of Engineered Stationary Phases for the Separation of Carotenoid Isomers.

Shape Selectivity Assessment of Stationary Phases in Gas Chromatography

DISTORTION

Micromechanics of Densification and Distortion. PB94-200326

00.579 
Application Portability Profile (APP): The U.S. Govern ment's Open System Environment Profile Version 3.0. PB96-158712

DISTRIBUTION FUNCTIONS

Equilibrium Pair Distribution Function of a Gas: Aspects Associated with the Presence of Bound States. PB95-176046

01,004

DISTRIBUTION MOMENTS

Moments of the Quartic Assignment Statistic with an Application to Multiple Regression.

03,442

DITHIOTHREITOL

Ascorbic and Dehydroascorbic Acids Measured in Plasma Preserved with Dithiothreitol or Metaphorphoric Acid.

PB94-216330

DIVALENT CATIONS

Genetically Engineered Pore as a Metal lon Biosensor

PB96-161658

03,551

DIVERSIFICATION

Technology Trends in Telecommunications: An Overview.

DIVINYL ETHER

01,462

2-Tunneling Path Internal-Axis-Method-Like Treatment of the Microwave Spectrum of Divinyl Ether.

PB94-200466

00,808

DMIS (DIMENSIONAL MEASURING INTERFACE

Dimensional Inspection Planning Based on Product Data Standards.

PB95-17545

02,910

DNA

Novel Activity of E. coli uracil DNA N-glycosylase Excision of Isodialuric Acid (5,6-dihydroxyuracil), a Majo
Product of Oxidative DNA Damage, from DNA. PB96-110747

03,543

Overview of Reference Materials Prepared for Standardization of DNA Typing Procedures.

PB96-111653

00.611

Low-Frequency Excitations of Oriented DNA.

03,548

Quantitative Determination of Oxidative Base Damage in DNA by Stable Isotope-Dilution Mass Spectrometr. PB96-200886

03,483

Commentary: The Measurement of Oxidative Damage to DNA by HPLC and GC/MS Techniques.

03,484

Intercomparison of DNA Sizing Ladders in Electrophoretic Separation Matrices and Their Potential for Accurate Typing of the D1S80 Locus.

PB96-200928

03,485

DNA-BINDING PROTEINS

Function of DnaJ and DnaK as Chaperones in OriginSpecific DNA Binding by RepA

03,533

Formation of DNA-Protein Cross-Links in Cultured Mammalian Cells Upon Treatment with Iron Ions.

PB96-137724

03.651

DNA DAMAGE

lonizing radiation-induced DNA damage and its repair in human cells. Progress report, (April 1, 1993--February 28

03,612

DE94014709

mage in

Tert-Butyl Hydroperoxide-Medi

PB94-182003

03,644

Application of Photochemical Reaction in Electrochemical Detection of DNA Intercalation.

PB94-185733

00,686

Copper Ion-Mediated Modification of Bases in DNA in Vitro by Benzoyl Peroxide.

03,645

PB94-198231

Standard Reference Materials (SRM's) for Measuring Genetic Damage.

03,516

Oxidative Damage to DNA in Mammalian Chromatin

PB94-199825

03,525

Modification of DNA Bases in Chromatin of Intact Target Human Cells by Activated Human Polymorphonuclear Leukocytes.

PB94-199833

03,526

Nickel(II)-Mediated Oxidative DNA Base Damage in Renal and Hepatic Chromatin of Pregnant Rats and Their Fetuses. Possible Relevance to Carcinogenesis. PB94-212628

03,646

Oxidative DNA Base Damage in Renal, Hepatic, and Pulmonary Chromatin of Rats After Intraperitoneal Injection of Cobalt (II) Acetate.

PB95-150025

03,647

DNA Base Modifications in Renal Chromatin of Wistar Rats Treated with a Renal Carcinogen, Ferric Nitrilotriacetate.

PB95-150363

03,648

DNA Base Damage Generated In vivo in Hepatic Chromatin of Mice upon Whole Body y-Irradiation.

03,627

Chemical Determination of Oxidative DNA Damage by Gas Chromatography-Mass Spectrometry.

03.540 lonizing Radiation Causes Greater DNA Base Damage in Mouse Lymphoma L5178Y Cells.

PB95-175915

03,632

Treatment of Wistar Rats with a Renal Carcinogen, Ferric Nitrilotriacetate, Causes DNA-Protein Cross-Linking between Thymine and Tyrosine in Their Renal Chromatin.

Novel Activities of Human Uracil DNA N-glycosylase for Cytosine-Derived Products of Oxidative DNA Damage.
PB96-164132

DNA Damage and DNA Sequence Retrieval from Ancient Tissues.

PB97-111983

03,556

DNA Base Damage in Lymphocytes of Cancer Patients Undergoing Radiation Therapy.

03,643

DNA REPAIR

lonizing radiation-induced DNA damage and its repair in human cells. Progress report, (April 1, 1993--February 28 ,

DE94014709

03,612

DNA SEQUENCING

Small genomes: New initiatives in mapping and sequencing. Workshop summary report

03,451

\section{DNAA PROTEINS}

Deletion Analysis of the Mini-P1 Plasmid Origin of Replication and the Role of E.coli DnaA Protein

03,539 DOCUMENTS

Open Document Architecture (ODA) Raster Document Application Profile (DAP). Category: Software Standard; Subcategory: Graphics

01,669

Important Papers in the History of Document Preparation Systems: Basic Sources.

PB95-125837

02,712

SGML Environment for STEP

02,778

NIST List of Publications, LP 103, March 1996. National Semiconductor Metrology Program.

02,432

Application Protocol Information Base World Wide Web

Application Protocol Information Base World Wide Web
Gateway.
$\begin{array}{ll}\text { PB96-202320 } & 02,791\end{array}$

02,743

Panel: Building

DOD GOAL SECURITY ARCHITECTURE

Assessment of the DOD Goal Security Architecture (DGSA) for Non-Military Use.

03.653

DODECANOIC ACID

Effect of Dodecanol Content on the Combustion of Methanol Spray Flames.

PB95-176020

01,389

DODECANOL

Effect of Dodecanol Content on the Combustion of Methanol Spray Flames.

PB95-176020

01,389

DOMAIN ANALYSIS

Context Analysis of the Network Management Domain.

Conducted as Part of the Domain Analysis Case Study.
PB94-142528

\section{DOMAIN STRUCTURES}

Transformation of BCC and B2 High Temperature Phases to HCP and Orthorhombic Structures in the Ti-Al-Nb System. Part 1. Microstructural Predictions Based on a Subgroup Relation between Phases.

PB96-169065

03,370

Transformation of $\mathrm{BCC}$ and $\mathrm{B} 2$ High Temperature Phases to HCP and Orthorhombic Structures in the Ti-Al-Nb System. Part 2. Experimental TEM Study of Microstructures.

03,371

DOMAIN WALLS

Micromagnetic Structure of Domains in Co/Pt Multilayers 1. Investigations of Wall Structure.

PB95-162111

04,610

DOMAINS

Mapping Domains in Proteins: Dissection and Expression of 'Escherichia coli' Adenylyl Cyclase. PB96-155460

03,478

DOPED MATERIALS

Incorporation of Gold into YBa2Cu3O7: Structure and Tc Enhancement.

Unexpected Effects of Gold on the Structure Superconductivity, and Normal State of $\mathrm{YBa} 2 \mathrm{Cu} 3 \mathrm{O} 7$.
$\mathrm{PB} 94-200284$

DOPED POLAR SEMICONDUCTORS

Nonequilibrium Total-Dielectric-Function Approach to the Electron Boltzmann Equation for Inelastic Scattering in Electron Poltzmann Equars. Doped Polar Sem

04,416

\section{DOPPLER BROADENED EMISSION}

Time-Resolved Balmer-Alpha Emission from Fast Hydrogen Atoms in Low Pressure, Radio-Frequency Discharges in Hydrogen.

PB96-102942

04,028

DOPPLER-FREE SPECTROSCOPY

Doppler-Free Spectroscopy of Large Polyatomic Molecules and van der Waals Complexes.

PB96-119581

04,339

DOPPLER RADAR

Delivering the Same Optical Frequency at Two Places: Accurate Cancellation of Phase Noise Introduced by an Optical Fiber or Other Time-Varying Path.

PB96-102736

04,332

DOSE EQUIVALENTS

Interpreting the Readings of Multi-Element Personne DBSim-175428

DOSE RESPONSE RELATIONSHIPS

Microdosimetry and Cellular Radiation Effects of Radon Progency in Human Bronchial Airways.

03.625

Radon in the Lung.

PB97-110035

03,638

DOSEMETERS

Improving measurement quality assurance for photon irradiations at Department of Energy facilities. Final technical

6010065

03,711

Dosimetry Systems for Radiation Processing

03,717

Calibration and Performance of GafChromic DM-100 Radiochromic Dosemeters.

00,703

DOSIMETERS

Thin Dyed-Plastic Dosimeter for Large Radiation Doses. PB95-107363

03,872

Calibration of Dosimeters for the Cryogenic Irradiation of Composite Materials Using an Electron Beam.

PB95-180964

03,968

Real Time Monitoring of Electron Processors.

03,719

Orientation Effects on ESR Analysis of Alanine-Polymer

Dosimeters.
PB96-146725 03,720

Response Comparison of Electret Ion Chambers, LiF

TLD, and HPIC.

02,578

Colour Centres in LiF for Measurement of Absorbed Doses Up to $100 \mathrm{MGy}$.

\section{DOSIMETRY}

EPR Bone Dosimetry: A New Approach to Spectral PB94-199643

03.616

Estimation of the Absorbed Dose in Radiation-Processed Food. 2. Test of the EPR Response Function by an Exponential Fitting Analysis.

00,036

Experimental Validation of Radiopharmaceutical Ab-

sorbed Dose to Mineralized Bone Tissue.

PB94-199668 
Research and Development Activities in Electron Paramagnetic Resonance Dosimetry.

Investigation of Applicability of Alanine and Radiochromic Detectors to Dosimetry of Proton Clinical Beams.
PB96-146782 Needs for Brachytherapy Source Calibrations in the United States.

03.521

EPR Dosimetry of Cortical Bone and Tooth Enamel Irradiated with $X$ and Gamma Rays: Study of Energy Dependated with $X$ and Gamma Rays: Study of Energy Depend ence. 110373

Measurements of the $(237) \mathrm{Np}(n, f)$ Cross Section PB97-119069

03,639

Novel Radiochromic Films for Clinical Dosimetry. PB97-119259

04,173

DOUBLE HULLS

Stranding Experiments on Double Hull Tanker Structures.
PB96-123112

DOUBLE-MODULATION

Double Modulation and Selective Excitation Photoreflectance for Characterizing Highly Luminescen Semiconductor Structures and Samples with Poor Sur-
face Morphology. face Morphology.

02,452

DRAINAGE

Polyvinyl Chloride (PVC) Plastic Drain, Waste, and Vent Pipe and Fittings.
AD-A310 426/2

00,326

Acrylonitrile-Butadiene-Styrene (ABS) Plastic Drain, Waste, and Vent $P i p e$ and Fittings.

DRAWING

Hydration in Semicrystalline Polymers: Small-Angle Neutron Scattering Studies of the Effect of Drawing in Nylon6 Fibers.

PB95-202990

03,385

DRILLING

Design and Machining of Copper Specimens with Micro Holes for Accurat
PB95-180428

DRINKING WATER

Preparation and Monitoring of Lead Acetate Containing Drinking Water Solutions for Toxicity Sfudies. PB94-193885

00,538

DROPLET-SOLID INTERACTIONS

Transient Cooling of a Hot Surface by Droplets Evaporation

03,783

DROPLETS

Transient Cooling of a Hot Surface by Droplets Evaporation

PB95-143194

03,890

Effect of Finite Beam Width on Elastic Light Scattering from Droplets.

01,144

Internal Droplet Circulation Induced by Surface-Driven Rotation.

DROPS

Lubrication Theory for Reactive Spreading of a Thin Drop.

02,865

Droplet Transfer Modes for a MIL 100S-1 GMAW Electrode.

02,867

Analysis of Droplet Arrival Statistics in a Pressure-Atomized Spray Flame

DROPS (LIOUIDS)

Water Droplet Evaporation from Radiantly Heated Solids.
PB95-217147

DRUG ABUSE

Interlaboratory Comparison Studies on the Analysis of Hair for Drugs of Abuse.

PB95-176251

03,500

NIST Reference Materials to Support Accuracy in Drug

Testing.

03,505

Certification of Phencyclidine in Lyophilized Human Urine Reference Materials.

PB96-160692

03,508

Interlaboratory Studies on the Analysis of Hair for Drugs of Abuse: Results from the Fifth Exercise.

03,509

PB97-110449

Interlaboratory Studies on the Analysis of Hair for Drugs of Abuse: Results from the Fourth Exercise.

03,510

DRUG ANALYSIS

Analysis by a Combination of Gas Chromatography and Tandem Mass Spectrometry: Development of Quantitative Tandem-in-Time Ion Trap Mass Spectrometry: Isotope Dilution Quantification of 11-Nor-Delta-9-Tetrahydro can-

nabinol-9-Carboxylic Acid.
PB96-117221

02,561

DRUG USERS

Hair Analysis for Drugs of Abuse: Evaluation of Analytical Methods, Environmental Issues, and Development of Reterence Materials.

03,501
Hair Testing for Drugs of Abuse: International Research on Standards and Technology.

03,504

DRY PROCESSES

Kinetic-Energy-Enhanced Neutral Etching
PB $96-200613$

DRYING

Modelling Drying Shrinkage of Cement Paste and Mortar. Part 1. Structural Models from Angstroms to Millimeters.
PB96-135132

DRYING TECHNIQUES

Recent Developments in NIST Botanical SRMs. PB96-167267

DUCTED ROCKET ENGINES

Vortex Shedding Flowmeters for SSME Ducts

DUCTILE BRITTLE TRANSITION

Nonlocal Effects of Existing Dislocations on Crack-Tip Emission and Cleavage.

PB96-161807

03,367

DUCTILE FRACTURE

Ductile Fracture and Tempered Martensite Embrittlement of 4140 Steel.

PB96-190285

03,222

DUFFING DIFFERENTIAL EQUATION

Melnikov Function and Homoclinic Chaos Induced by Weak Perturbations.

03,414

\section{DURABILITY}

Evolution of Porosity and Calcium Hydroxide in Laboratory Concretes Containing Silica Fume.

Environmental Durability of Glass-Fiber Composites.

Environmental Durability of Glass-Fiber Composites.
03,166

\section{DWARF STARS}

Far-Ultraviolet Flare on a Pleiades G Dwart.

PB96-102033

00,086

Relationship between Radiative and Magnetic Fluxes for Three Active Solar-Type Dwarfs.

PB96- 119540
DYE BLEACHING

00,097

Anionic Triphenylmethane Dye Solutions for Low-Dose Food Irradiation Dosimetry.

PB96-135173

03,715

DYE LASERS

Stabilization of Optical Phase/Frequency of a Laser System: Application to a Commercial Dye Laser with an Extemal Stabilize

DYES

Ded TOTO and YOYO Intercalating Dyes with Laser Induced Fluorescence - Capillary Electrophoresis.

PB95-164653

00,599

DYNAMIC MODULUS OF ELASTICITY

Dynamics vs. Static Young's Moduli: A Case Study.
PB94-213188

03,210

DYNAMIC PROGRAMMING

Time Dependent Vector Dynamic Programming Algorithm for the Path Planning Problem.

03,428

DYNAMIC RANGE

Genetically Engineered Pores as Metal Ion Biosensors.

NAMIC RESPONSE

03,553

Dynamic Characteristics of Five Tall Buildings during Strong and Low-Amplitude Motions.

00,427

Response of a Terminally Anchored Polymer Chain to Simple Shear Flow.

PB95-108668

01,233

Response of Buildings to Ambient Vibration and the Loma Prieta Earthquake: A Comparison.

00,457 PB96-119607

from Bounds on Frequency Respon

03,419 PB96-122874 Comparison of Responses of a Select Number of Build-
ings to the 10/17/1989 Loma Prieta (California) Earthings to the 10/17/1989 Loma Prieta (Califor quake and Low

00,467

DYNAMIC SCALING

Growth and Nucleation of Hydrogenated Amorphous Siticon on Silicon (100) Surfaces.

PB96-17651

02,991

DYNAMIC STRUCTURAL ANALYSIS

Strengthening Methodology for Lightly Reinforced Concrete Frames-II. Recommended Calculation Techniques for the Design of Infill Walls.
PB94-187648

00,426

Wind and Seismic Effects. Proceedings of the U.S.-Japan Cooperative Program in Natural Resources Panel on Wind and Seismic Effects (26th). Held in Gaithersburg. Maryland on May $17-20,1994$.

00,433

PB95-147385

Seismic Performance of Circular Bridge Columns De-
signed in Accordance with AASHTO/CALTRANS Stand-

01,346

Strengthening Methodology for Lightly Reinforced ConPB96-158050

00,466

January 17, 1995 Hyogoken-Nanbu (Kobe) Earthquake Performance of Structures, Lifelines and Fire Protection Systems. Executive Summary and Paper.

00,475

Wind and Seismic Effects: Proceedings of the Joint Meeting of the U.S.-Japan Cooperative Program in Natural Resources Panel on Wind and Seismic Effects (28th). Held in Gaithersburg, Maryland on May 14-17, 1996. PB97-104376

00,476

DYNAMICAL SYSTEMS

Noise-Induced Chaos and Phase Space Flux. PB95-125761

03,433

Non-Gaussian Noise Effects on Reliability of Multistable Systems.

PB96-122726

04,213

DYNAMICS

Dynamic Light-Scattering Study of a Diluted Polymer Blend Near lits Critical Point.

PB95-151890

DYSPROSIUM

Enhanced Curie Temperatures and Magnetoelastic Do-

mains in Dy/Lu Super Lattices and Films.
PB94-172665

04,443

Magnetoelasticity in Rare-Earth Multilayers and Films.

PB94-211356

Coupled-Bilayer Two-Dimensional Magnetic Order of the Dy lons in Dy2Ba4Cu7O15.

PB95-152104

04,584

Reactive Coevaporation of DyBaCuO Superconducting Films: The Segregation of Bulk Impurities on Annealed $\mathrm{MgO}(100)$ Substrates.

DYSPROSIUM IONS

Coupled-Bilayer Two-Dimensional Magnetic Order of the Dy lons in Dy2Ba4Cu7O15.

PB95-152104

04,584

E. COLI

Novel Activity of E. coli uracil DNA N-glycosylase Excision of Isodialuric Acid (5,6-dihydroxyuracil), a Major Product of Oxidative DNA Damage, from DNA.

03.543

E134

Thermodynamic Properties of CHF2-O CHF2,Bis (difluoromethyl) Ether.

00,798

EAMATE

Design and Development of an Information Retrieval System for the EAMATE Data. Volume 2 of 2. Appendices. PB94-168390

EARTH GRAVITATION

Integrated Laser Doppler Method for Measuring Planetary Gravity Fields

03,681

EARTH OBSERVING SYSTEM (EOS)

Activities of NIST (National Inst. Of Standards and TechNology).

04,222

Calibration in the Earth Observing System (EOS) Project. Part 2. Implementation.

NIST Thermal Infrared Transfer Standard Radiometer for the Earth Observing System (EOS) Program.

04,843

EARTH TIDES

Measurement and Interpretation of Tidal Tilts in a Small Array.
PB96-102611

03,686

EARTHQUAKE DAMAGE 


\section{KEYWORD INDEX}

ELASTIC PROPERTIES

Draft Guidelines for Quality Control Testing of Elastomeric Seismic Isolation Systems.

00,422

Evaluation and Strengthening Guidelines for Federal Buildings: Identification of Current Federal Agency Programs.

00,424

Evaluation and Strengthening Guidelines for Federa Buildings: Assessment of Current Federal Agency Evaluation Programs and Rehabilitation Criteria and Development of Typical Costs for Seismic Rehabilitation.

PB94-181856

00,425

Northridge Earthquake 1994: Performance of Structures, Lifelines, and Fire Protection Systems.

04,825

Evaluation and Retrofit Standards for Existing Federally Owned and Leased Buildings.

00,434

Some Basics on Who's Who and What's What in Seismic

Safety.
PB95-150926

00,435

Implementation of Executive Order 12699: Seismic Safety of Federal and Federally Assisted or Regulated New

PB95-151809

Lessons from the Loma Prieta Earthquake.

00,436

00,442

Seismic Safety of Federal Buildings. Initial Program: How

$\begin{array}{ll}\text { Much Will lt Cost. } & 00,447 \\ \text { PB95-182291 } & \end{array}$

Enhancements to Program IDARC: Modeling Inelastic Behavior of Welded Connections in Steel Moment-Resisting Frames.

PB95-231601

00,452

How-To Suggestions for Implementing Executive Order 12941 on Seismic Safety of Existing Federal Buildings, A Handbook.

00,461

Executive Order 12941. Seismic Safety of Existing Federally Owned or Leased Buildings: It's History, Content and Objectives.

Preliminary Processing of the Lotung LSST Data. PB96-165972 03,690

State of the Art Report on Seismic Design Requirements for Nonstructural Building Components.

PB96-193800

00,308

EARTHQUAKE RESISTANCE

Literature Review on Seismic Performance of Building Cladding Systems.

Ground Improvement Techniques for Liquefaction Remediation Near Existing Lifelines.

PB96-128111

01,350

Proceedings of a Workshop on Developing and Adopting Seismic Design and Construction Standards for Lifelines.

Seismic Design and Construction Standards for Litelin PB97-115794

EARTHQUAKE RESISTANT STRUCTURES

Earthquake Resistant Construction of Electric Transmission and Telecommunication Facilities Serving the Federal Government Report.

PB94-161817

02,460

Draft Guidelines for Pre-Oualification and Prototype Testing of Seismic Isolation Systems.

B94-161940

01331

Draft Guidelines for Quality Control Testing of Sliding Seismic Isolation Systems.

PB94-161957

01,332

Earthquake Resistant Construction of Gas and Liquid Fuel Pipeline Systems Serving, or Regulated By, the Federal Government.

PB94-161999

04,846

Draft Guideline for Testing and Evaluation of Seismic Isolation Systems.

00,423

Seismic Strengthening of Reinforced Concrete Frame

Buildings.

00,430

Jacket Thickness Requirements for Seismic Retrofitting of Circular Bridge Columns.

PB95-163267

01,336

Pertormance of $1 / 3-$ Scale Model Precast Concrete BeamColumn Connections Subjected to Cyclic Inelastic Loads. Repor No. 4

PB95-179024

World of Building Codes.

00,444

PB95-203428

00,449

Comparison of the Seismic Provisions of Model Building Codes and Standards to the 1991 NEHRP Recommended Provisions.

PB95-231858

00,315

ICSSC Guidance on Implementing Executive Order 12941 on Seismic Safety of Existing Federally Owned or Leased Buildings

00,459

Seismic Performance of Circular Bridge Columns Designed in Accordance with AASHTO/CALTRANS Stand-

PB96-146352

01,346
Simplified Design Procedure for Hybrid Precast Concrete Connections.

00,405

Summary and Results of the NIST Workshop on Proposed Guidelines for Testing and Evaluation of Seismic
Isolation Systems. Held in San Francisco, California on Isolation System

July 25,1994

00,463

Guidelines for Pre-Qualification, Prototype and Quality Control Testing of Seismic Isolation Systems.

01,347 - 193685

ARTHQUAKES

Northridge Earthquake, 1994. Performance of Structures, Lifelines and Fire Protection Systems.

00,42

Earthquake and Fire in Japan: When the Threat Became a Reality.

00,201

Energy-Based Method for Liquefaction Potential Evaluation. Phase 1. Feasibility Study.

03,691

EBULLIOMETERS Design of a High-Pressure Ebulliometer, with Vapor-Liq-
uid Equilibrium Results for the Systems $\mathrm{CHF} 2 \mathrm{Cl}+\mathrm{CF} 3-$ $\mathrm{CH} 3$ and $\mathrm{CF} 3-\mathrm{CH} 2 \mathrm{~F}+\mathrm{CH} 2 \mathrm{~F} 2$

PB97-113229

04,163

EBULLIOMETRY

Model for Calculating Virial Coefficients of Natural Gas Hydrocarbons with Impurities.

04,064

ECONOMIC ANALYSIS

Least-Cost Energy Decisions for Buildings: Part 2. Uncertainty and Risk Video Training Workbook.

PB94-165982

00,240

Standards in Building Economics: Why We Need Them and How to Write Them.

PB94-216405

00,320

EONOMIC DEVELOPMENT

Aid for Smaller Businesses.

PB94-212461

00,492

CONOMIC IMPACT

Economic, Energy, and Environmental Impacts of the Energy-Related Inventions Program.

00,008

ECONOMIC IMPACTS

Evaluation of the Economic Impacts Associated with the NIST Power and Energy Calibration Services.

PB95-188850

02,461

ECONOMIC MODELS

Functions of Technology Infrastructure in a Competitive EB94-173002

00,478

EDDY CURRENTS

Development in the Theory and Analysis of Eddy Current Sensing of Velocity in Liquid Metals.

03,335

Improved Eddy-Current Decay Method for Resistivity Characterization.

PB95-180451

02,265

EDGE DETECTION

Monte Carlo Model for SEM Linewidth Metrology.

PB95-150058

02,331

Motion-Model-Based Boundary Extraction

PB95-189502

01,849

EDGES

Effects of Specimen Edge Conditions on Heat Release PB95-152864

00,375

EDI (ELECTRONIC DATA INTERCHANGE)

Federal Implementation Guideline for Electronic Data Interchange: ASC X12 003040 Transaction Set 838 Trading Partner Profile (Vendor Registration), Implementation Convention.

PB96-112651

03,674

Federal Implementation Guideline for Electronic Data Interchange ASC X12 003050 Transaction Set 850 Award Instrument. Implementation Convention.

PB96-114913

01,814

Federal Implementation Guideline for Electronic Data Interchange. ASC X12 003050 Transaction Set 860 Modifications to Award Instrument. Implementation Conven-

01,815

PB96-114921

Federal Implementation Guideline for Electronic Data Interchange. ASC X12 003050 Transaction Set 843 Response

PB96-168984

01,822

Guidelines for the Evaluation of Electronic Data Inter-

change Products

01,506

Federal Implementation Guideline for Electronic Data Interchange. ASC X12 003050 Transaction Set 855 Purchase Order Acknowledgment: Implementation Conven-

01,823

Federal Implementaion Guideline for Eletronic Data Interchange. ASC X12 003050 Transaction Set 865 Purchase

Order Change Acknowledgement/Request - Seller Initiated. Implementation Convention.

PB96-172549

01,825

EDITING

Operating Procedures and Life Cycle Documentation for the Initial Graphics Exchange Specification

PB95-242285

EDITING ROUTINES

Structural EXPRESS Editor.

PB94-159795

02,782

EDLEN EQUATION

Verification of Revised Water Vapour Correction to the Index of Refraction of Air.

PB96-161666

02,680

EDUCATION

Investing in Education to Meet a National Need for a Technical-Professional Workforce in a Post-Industrial Economy.

00,132

Tribology Education: Present Status and Future Chal-

PB94-212362

02,965

Structural Analysis in Context.

PB95-151817

00,437

PB96-179502

00,136

EEL PROGRAM

National Voluntary Laboratory Accreditation Program: Energy Efficient Lighting Products.

02,642

EFFECTIVE RANGE THEORY

Modified Effective Range Theory as an Alternative to

Low-Energy Close-Coupling Calculations.

PB95-202701

03,988

EFFICIENC

Usability Engineering: Industry-Government Collaboration tor System Effectiveness and Efficiency.

EGRESS

Mathematical Modeling of Human Egress from Fires in Residential Buildings.

PB94-193778

00,337

Accuracy of Eigenvalues: A Comparison of Two Methods. PB95-126249

03,413

EJECTORS

Theoretical Evaluation of the Vapor Compression Cycle with a Liquid-Line/Suction-Line Heat Exchanger, Economizer, and Ejector

PB95-216917

02,607

ELASTIC CONSTANTS

Compressibility of Polycrystal and Monocrystal Copper:

Acoustic-Resonance Spectroscopy 
Off-Diagonal Orthorhombic-Symmetry Elastic Constants. PB95-202768 04,695 Elastic Constants and Internal Friction of Polycrystalline Copper.

PB96-141015

03,364

ELASTIC SCATTERING

Low-Energy-Electron Collisions with Sodium: Elastic and Inelastic Scattering from the Ground State.

04,030

Effect of Finite Beam Width on Elastic Light Scattering Effect of Finite Beam width on Elasic Light Scatering
from Droplets.
PBg6-163670 Elastic Scattering from Spheres under Non Plane-Wave Illumination.

04.370

ELASTICITY

Nonequilibrium Thermodynamic Theory of Viscoplastic Materials.

PB96-111661

04,034

ELASTICITY THEORY

Whither Computational Materials Science. Some Thoughts from the Mechanical Properties Front

02,987

ELASTOMER BLENDS

Microstructure Effect on the Phase Behavior of Blends of Deuterated Polybutadiene and Protonated Polyisopene.
PB97-113146

\section{ELASTOMERS}

Synthesis of Thermally Stable Elastomers.

AD-A307 789/8

03,194

Effect of Swelling on the Elasticity of Rubber: Localization Model Description.

PB94-211034

01,216

Anomalous Freezing and Melting of Solvent Crystals in Swollen Gels of Natural Rubber.

PB94-212321

01,223

ELCETRON PARAMAGNETIC RESONANCE

Orientation Effects on ESR Analysis of Alanine-Polymer Dosimeters.

03,720

ELECRON MICROSCOPY

SUSAN: SUperconducting Systems ANalysis by Low Temperature Scanning Electron Microscopy (LTSEM).
PB96-112065

ELECTRET

Calibration of Electret-Based Integral Radon Monitors Using NIST Polyethylene-Encapsulated (226)Ra/(222)Rn Emanation (PERE) Standards.

Emanation $(P E R$

01.950

ELECTRETS

Response Comparison of Electret Ion Chambers, LiF TLD, and HPIC

02,578

ELECTRIC APPLIANCES

Three-Axis Coil Probe Dimensions and Uncertainties during Measurement of Magnetic Fields from Appliances. PB94-219359 01.885 Distributions of Measurement Error for Three-Axis Magnetic Field Meters during Measurements Near Appliances

02,110

ELECTRIC ARCS

Decomposition of SF6 and Production of S2F10 in Power

ArCs.

01,084

ELECTRIC BRIDGES

Digital Impedance Bridge.

ELECTRIC CABLES

Effect of DC Tests on Induced Space Charge.
PB94-172350

02,272

ELECTRIC CHARGE

Measuring Contact Charge Transfer at Interfaces: A New Experimental Technique.

PB95-164570

03,053

Electric Dipole Excitation of a Long Conductor in a Lossy PB96-146675

04,058

ELECTRIC CONDUCTIVITY

Critical Current and Normal Resistance of High-Tc StepEdge SNS Junctions.

ECTRIC CONTACTS

Contact Electrification Induced by Monolayer Modification of a Surface and Relation to Acid-Base Interactions. PB94-185378

In situ Noble Metal YBa2Cu3O7 Thin-Film Contacts.

YBa2Cu307-x to $\mathrm{Si}$ Interconnection for Hybrid Superconductor/Semiconductor Integration.
PB94-211711 PB94-211711 02,315 Superconductors.
PB95-153557

02,258

High Current Pressure Contacts to Ag Pads on Thin Film Superconductors.

04,639
Oxygen Annealing of Ex-situ YBCO/Ag Thin-Film Inter-

PB96-141312

04,758

ELECTRIC CONVERTERS

Comments on "Conversions between S. Z, Y, h, ABCD, and $T$ Parameters Which Are Valid for Complex Source and Load Impedances'.

PB96-102785

02.069

ELECTRIC CURRENT

Optical Current Transducer for Calibration Studies. BQ94-185907

Faraday Effect Current Sensors.

PB94-200698

02,127

Faraday Effect

02,128

Frequency Extension of the NIST AC-DC Difference Calibration Service for Current

01,895

Polarization Insensitive $3 \times 3$ Sagnac Current Sensor Using Polarizing Spun High-Birefringence Fiber.

PB96-119276

02,187

ELECTRIC CURRENT METERS

Recent Developments at NIST on Optical Current Sensors and Partial Discharge Diagnostics.

PB95-151114

02,147

ELECTRIC CURRENT SENSORS

Polarization Dependence of Response Functions in $3 \times 3$ Sagnac Optical Fiber Current Sensors.

02,154

Submicroampere-Per-Root-Hertz Current Sensor Based on the Faraday Effect in Ga: YIG.

PB95-162467

02,155

Faraday Effect Current Sensor with Improved SensitivityBandwidth Product.

B 95-203154

02,180

Faraday Effect Sensors for Magnet Field and Electric

Current.
PB96-119664 04,736

Polarization Dependence of Response Functions in $3 \times 3$ Sagnac Optical Fiber Current Sensors.

PB96-122684

02,189

Fundamentals and Problems of Fiber Current Sensors. B 97.111835

02,205

\section{ELECTRIC DEVICES}

Effect of Electrode-Polymer Interfacial Layers on Polymer Conduction. Part 2. Device Summary.

02,335

ELECTRIC DISCHARGES

Electrical Breakdown in Transformer Oil in Large Gaps.

PB95-150579

01,889

Recent Developments at NIST on Optical Current Sensors and Partial Discharge Diagnostics.

02,147

Electron Scattering and Dissociative Attachment by SF6 Electron Scattering and Dissociative Attachment by SF6 and Its Electrical-Discharge By-Products.
PB95-151288 Observations of Partial Discharges in Hexane Under High Magnification

01,900

Importance of Unraveling Memory Propagation Effects in Interpreting Data on Partial Discharge Statistics.

01,901

Nonstationary Behavior of Partial Discharge during Insulation Aging.
PB95-163580

01,902

Partial Discharge: Induced Aging of Cast Epoxies and Related Nonstationary Behavior of the Discharge Statistics.

01,903

Physics and Chemistry of Partial Discharge and Corona: Recent Advances and Future Challenges.

01,910

Nonstationary Behaviour of Partial Discharge during Discharge Induced Ageing of Dielectrics.

PB $96-103114$

01,922

Physics and Chemistry of Partial Discharge and Corona Recent Advances and Future Challenges. PB96-123757

01,936

ELECTRIC FAULTS

Keeping Up with the Reality of Today's Surge EnvironPB96-123633

ELECTRIC FIELDS

Electric-Field Strengths Measured Near Personal Transceivers.

$$
01,564
$$

Spatial Dependence of Electrical Fields Due to Space Charges in Films of Organic Dielectrics Used for Insulation of Power Cables. PB94-199130

02,214

Energy Transduction between a Concentration Gradient and an Altemating Electric Field.

PB94-216363

03,461

Electric Field Effects on Crack Growth in a Lead Magnesium Niobate.

03,339

Michaelis-Menten Equation for an Enzyme in an Oscillating Electric Field.

00,906

ELF Electric and Magnetic Field Measurement Methods. 04.423 Using Ouantized Breakdown Voltage Signals to Determine the Maximum Electric Fields in a Quantum Hall Efrect Sample.

02,375

Electric Field Effects on a Near-Critical Fluid in Microgravity. 161880

ELECTRIC GENERATORS

Evaluation of Wear Resistant Ceramic Valve Seats in Gas-Fueled Power Generation Engines. Topical Report, December 1991-April 1994.

PB95-200218

02,466

ELECTRIC MEASURING INSTRUMENTS

NIST $30 \mathrm{MHz}$ Linear Measurement System.

PB94-169752

02,020

\section{ELECTRIC MOTORS}

Efficiency of Electric Motors. National Voluntary Lab. Accreditation Progra

ELECTRIC POTENTIAL

Potential Drop in the Center-Cracked Panel with Asymmetric Crack Extension.

04.819

Step-Edge and Stacked-Heterostructure High-Tc Josephson Junctions for Voltage-Standard Arrays.

PB96-102066

04,702

Spectroscopic Study of Quantized Breakdown Voltage States of the Quantum Hall Effect.

PB96-113584

04,730

ELECTRIC POWER

Evaluation of the Economic Impacts Associated with the NIST Power and

02,461

\section{ELECTRIC POWER DISTRIBUTION}

Investigation of S2F10 Production and Mitigation in Compressed SF6-Insulated Power Systems.

PB94-212388

02,467

Investigation of S2F10 Production and Mitigation in Compressed SF6. Insulated Power Systems.

02,468

ELECTRIC POWER GENERATION

Earthquake Resistant Construction of Electric Transmission and Telecommunication Facilites Serving the Federal Govemment Report.

PB94-161817

02,460

ELECTRIC POWER METERS

Technical Impact of the NIST Calibration Service for Electrical Power and Energy.

ELECTRIC-POWERED VEHICLES

Advanced Components for Electric and Hybrid Electric Vehicles. Workshop Proceedings. Held in Gaithersburg, Maryland on October 27-28, 1993.

PB94-177060

04,858

ELECTRIC PROBES

Standard Probes for Electromagnetic Field Measure-

PB94-185436

01,999

Verification of Commercial Probe-Tip Calibrations. 
October to December 1992, with 1992/1993 EEEL Events Calendar.

PB94-165958

02,299

Electronics and Electrical Engineering Laboratory Tech. nical Progress Bulletin Covering Laboratory Programs, October to December 1994 with 1995 EEEL Events Calendar

PB95-208724

02,372

Electronics and Electrical Engineering Laboratory Technical Progress Bulletin Covering Laboratory Programs,
January to March 1992, with 1992/1993 EEEL Events PB $95-210480$

01,917

Electronics and Electrical Engineering Laboratory Technical Publication Announcements Covering Laboratory Programs, October to December 1994 with 1995 EEEL Events Calenda
PB95-231841

01,918

Electronics and Electrical Engineering Laboratory Technical Progress Bulletin Covering Laboratory Programs, April to June 1995 with 1995 EEEL Events Calendar.

Electronics and Electrical Engineerıng Laboratory Technical Publication Announcements Covering Laboratory Programs, April to June 1995 with 1995 EEEL Events Calendar.

01,941

Electronics and Electrical Engineering Laboratory Technical Progress Bulletin Covering Laboratory Programs, July to September 1995 with 1996 EEEL Events CalPB96-147905

01,942

Electronics and Electrical Engineering Laboratory 1995 Technical Accomplishments: Advancing Metrology for Electrotechnology to Support the U.S. Economy.
PB96-164520 01,959
PB96-164520 nical Publication Announcements Covering Laborator Programs, July to September 1995 with 1996 EEEL Events Calenda

PB96-183066

01,965

Electronics and Electrical Engineering Laboratory Technical Progress Bulletin Covering Laboratory Programs, October to December 1995 with 1996 EEEL Events Calendar.

$96-183116$

01,966

Electronics and Electrical Engineering Laboratory Technical Progress Bulletin Covering Laboratory Programs, endar.

PB96-191390

01,969

Electronics and Electrical Engineering Laboratory Technical Publication Announcements Covering Laboratory Events Calendar.

01,978

Electronics and Electrical Engineering Laboratory Technical Publication Announcements Covering Laboratory Programs, January to March 1996, with 1996 EEEL Events Calendar
PB96-214622

01,981

Electronics and Electrical Engineering Laboratory Technical Progress Bulletin Covering Laboratory Programs, April to June 1996 with 1996-1998 EEEL Events Cal-

PB97.113880

01,994

Bibliography of the NIST Optoelectronics Division.
PB97-116040

02,207

ELECTRICAL EQUIPMENT

Electrical Product Requirements (Especially Ouality Requirements) in the United States

01,929

Surging the Upside-Down House: Measurements and Modeling Results.

PB96-180138

02,243

ELECTRICAL FAULTS

Using Ouantized Breakdown Voltage Signals to Determine the Maximum Electric Fields in a Ouantum Hall Et. fect Sample.
PB95-261947

02,375

Spectroscopic Study of Ouantized Breakdown Voltage States of the Ouantum Hall Effect.

PB96-113584

04,730

ELECTRICAL IMPEDANCE

On-Wafer Impedance Measurement on Lossy Substrates. PB95-176285 02,365

Overview of Bioelectrical Impedance Analyzers.

PB96-122635

00,176

Overview of Bioelectrical Impedance Analyzers

PB97-118780

00,181

ELECTRICAL INSULATION

Spatial Dependence of Electrical Fields Due to Space Charges in Films of Organic Dielectrics Used for Insulation of Power Cables.

PB94-199130

02,214

Irradiation Damage in Inorganic Insulation Materials for ITER Magnets: A Review.

PB95-147351

03,705

Performance Evaluation of a New Digital Partial Discharge Recording and Analysis System.

01,888
Importance of Unraveling Memory Propagation Effects in Interpreting Data on Partial Discharge Statistics.
PB95-163572 Nonstationary Behavior of Partial Discharge during Insu-

ation Aging.
PB95.163580

01,902

Partial Discharge: Induced Aging of Cast Epoxies and Related Nonstationary Behavior of the Discharge Statis-

01,903

Cryogenic Properties of Inorganic Insulation Materials for ITER Magnets: A Review

03,706

SF6 Insulation: Possible Greenhouse Problems and Solu-

PB95-251625

02,269

Gaseous Dielectrics Research: Possible SF6 Substitutes.

02,228

Modification of Cast Epoxy Resin Surfaces during Exposure to Partial Discharges.

01,086

SF6/N2 Mixtures: Basic and High-Voltage-Insulation

PB96-123468

02,230

Classified Bibliography: Insulation Condition Monitoring Methods, 1989-1995.

PB96-131586

02,232

President's C

02,239

Adhesion, Contact Electrification, and Acid-Base Properties of Surfaces.

03,693

\section{ELECTRICAL MEASUREMENT}

Electronics and Electrical Engineering Laboratory 1994 Program Plan: Supporting Technology for U.S. Competitiveness in Electronics.

PB94-126901

01.873

Center for Electronics and Electrical Engineering Technical Progress Bulletin Covering Center Programs, October to December, with 1991 CEEE Events Calendar.
PB94-159787 O2,296 Strategic Plan. Supporting Technology for U.S. Competitiveness in Electronics.

PB94-161320

01,874

Transmission/Reflection and Short-Circuit Line Methods for Measuring Permittivity and Permeability.

Electric-Field Strengths Measured Near Personal Transceivers.

PB94-172020

01,564

Optical Current Transducer for Calibration Studies.

02,121

Reproducibility of JEDEC Standard Current and Voltage Ramp Test Procedures for Thin-Dielectric Breakdown

$\begin{array}{ll}\text { Characterization. } & 01,879\end{array}$

Electronics and Electrical Engineering Laboratory Technical Publication Announcements Covering Laboratory Programs, October to December 1993 with 1994/1995 EEEL Events Calenda

PB94-193752

02,307

Electronics and Electrical Engineering Laboratory Technical Progress Bulletin Covering Laboratory Programs, January to March 1994 with 1994/1995 EEEL Events CalPB94-193810

02,308

Electronics and Electrical Engineering Laboratory Technical Progress Bulletin Covering Laboratory Programs, July to September 1993 with 1994 EEEL Events Cal-

PB94-194354

02,309

Electronics and Electrical Engineering Laboratory Technical Progress Bulletin Covering Laboratory Programs, April to June 1994 with 1994/1995 EEEL Events Cal-

5-143186

02,329

Electronics and Electrical Engineering Laboratory 1995 Program Plan. Supporting Technology for U.S. Competiiveness in Electronics

01,894

Frequency Extension of the NIST AC-DC Difference Calirequen Service for Current.

PB95-161253

01,895

Verification of Scattering Parameter Measurements.

01,904

Through-the-Arc Sensing for Measuring Gas Metal Arc Weld Ouality in Real Time.

PB95-164463

02,908

Accuracy Comparisons of Josephson Array Systems.

PB95-164687 02,047

Voltage Ratio Measurements of a Zener Reference Using a Digital Voltmeter.

PB95-164695

01,906

Superconductor Critical Current Standards for Fusion Applications. Final Progress Report, October 1993-July 1994.

PB95-169538

02,222

Electronics and Electrical Engineering Laboratory Technical Progress Bulletin Covering Laboratory Programs.

July to September 1994 with 1994/1995 EEEL Events PB95-170395

02,360

Standard Reference Devices for High Temperature Superconductor Critical Current Measurements.
PB95.175543 04,659

mproved Eddy-Cu

PB95-180451

02,265

Accuracy and Repeatability in Time Domain Network Analysis.

02,064

Electronics and Electrical Engineering Laboratory Technical Publication Announcements Covering Laboratory Programs, January to March 1995 with 1995 EEEl Events Calenda

PB95-242277

02,373

Voltage-Standard Devices.

01,920

Electronics and Electrical Engineering Laboratory Technical Publication Announcements Covering Laboratory Programs, April to June 1995 with 1995 EEEL Events

PB96-137187

01,941

Amperometric Measurement of Moisture in Transformer Oil Using Karl Fischer Reagents.

00,623

Technical Impact of the NIST Calibration Service for Electrical Power and Energy.

PB96-147913

02,462

Electronics and Electrical Engineering Laboratory: 1996 Program Plan. Supporting Technology for U.S. Competitiveness in Electronics.

01,962

Single-Port Technique for Adaptor Efficiency Evaluation.

PB96-176441 02,088 Dielectric and Magnetic Measurements from $-50 \mathrm{C}$ PB96-191382

02,245

Electrical Test Structures Replicated in Silicon-on-Insulaor Material.

Loading Effects in Resistance Scaling.

02,454

PB97-111884

02,285

Measurement and Reduction of Alignment Errors of the NIST Watt Experiment.

01987

Low Thermal Guarded Scanner for High Resistance Measurement Systems.

PB97-112452

02,288

Exploring the Low-Frequency Performance of Thermal Converters Using Circuit Models and a Digitally Syn-

02,848

Low Voltage Standards in the $10 \mathrm{~Hz}$ to $1 \mathrm{MHz}$ Range.
02,100

\section{ELECTRICAL MEASUREMENTS}

Electrical Measurements for Monitoring and Control of $n$ Plasma Processing.

PB96-161963

Coaxial Line-Reflect-Match Calibration. 
Diffusion of Copper into Gold Plating.

PB95-162152

00,957

Improved Eddy-Current Decay Method for Resistivity Characterization.

02,265

Apparatus for Resistance Measurement of Short, SmallDiameter Conductors.

PB96-141130

04,417

Interfacial Transport in Porous Media: Application to dc Electrical Conductivity of Mortars.

01,326

Characterization of LPE HgCdTe Film by

Magnetoresistance. $\quad 02,197$

ELETRICAL SUBSTITUTION

National Institute of Standards and Technology High-Accuracy Cryogenic Radiometer.

04.378

Realization of a Scale of Absolute Spectral Response Using the NIST High Accuracy Cryogenic Radiometer. Using the NIST High Accuracy Cryogenic Radiometer.
PB97-118640

ELECTRICAL SYSTEMS

Electronics and Electrical Engineering Laboratory Technical Publication Announcements Covering Laboratory Events Calendar.

01,918

ELECTRICAL TRANSPORT

Evidence for Tunneling and Magnetic Scatttering at 'In situ' YBCO/Noble-Metal Interfaces.

PB96-141098

04,752

ELECTRICALLY CONDUCTING FLUIDS

Measurement of the Thermal Properties of Electrically Conducting Fluids Using Coated Transient Hot Wires. PB95-169058

ELECTRICALLY ISOLATED

Influence of Electrical Isolation on the Structure and Reflectivity of Multilayer Coatings Deposited on Dielectric Substrates.
PB96-159736

ELECTRIFICATION

Contact Electrification Induced by Monolayer Modification of a Surface and Relation to Acid-Base Interactions.

PB94-185378 03,034

Adhesion, Contact Electrification, and Acid-Base Properties of Surfaces

PB96-204425

03,693

ELECTRO DEPOSITED COATINGS

Chemical Aspects of Tool Wear in Single Point Diamond Purning.

03,021

ELECTRO-OPTIC CRYSTALS

Electro-Optic-Based RMS Voltage Measurement Technique.

02,194

ELECTROACOUSTIC WAVES

Electroacoustic Characterization of Particle Size and Zeta Potential in Moderately Concentrated Suspensions. PB96-119425

01,079

ELECTROACOUSTICS

Electroacoustics for Characterization of Particulates and Suspensions. Proceedings of a Workshop. Held in Gaithersburg, Maryland on February 3-4, 1993 . 00,725
Electrokinetic Sonic Analysis of Silicon Nitride Suspen-

PB96-123575

03,073

Complicated Cases and Shielded Rooms: Audiometric Booths Shielded to Attenuate Electromagnetic InterPB96-180179

02,278

ELECTROCHEMICAL CELLS

Structural and Magnetic Properties of $\mathrm{CuCl} 2$ Graphite Intercalation Compounds.

PB96-119748

ELECTROCHEMICAL CORROSION

Modeling Polarization Curves and Impedance Spectra for Simple Electrode Systems

03,188

ELECTROCHEMICAL IMMUNOASSAYS

Novel Amperometric Immunosensor for Procainamide Employing Light Activated Labels.

LECTROCHEMISTRY

Electrochemical Synthesis of Metal and Intermetallic AD-A294 088/0

03,304

Evaluation of the Electrochemical Behavior of Ductile Nickel Aluminide and Nickel in a pH 7.9 Solution. Technical Report Number 3. April-June 1991.

DE94017351

03,307

Application of Photochemical Reaction in Electrochemical Detection of DNA Intercalation.

PB94-185733

00,686

Status of Electrocomposites.

03,143

Self-Assembled Phospholipid/Alkanethiol Biomimetic Bilayers on Gold.

00,878
Lithium-Dritt Technique for Making Submicron Thick Silicon Membranes.

PB95-161386

Faraday Constant.

PB96-159793

02,260

ELECTROCLINIC EFFECT

Studies of the Higher Order Smectic Phase of the Large Electroclinic Effect Material W317.

Dielectric Spectroscopic Determination of Temperature Behavior of Electroclinic Parameters in the Liquid Crystal WB17.

01,098

$X$-ray Observation of Electroclinic Layer Constriction and Rearrangement in a Chiral Smectic-A Liquid Crystal.

PB96-141080

01,100

ELECTROCONFORMATIONAL COUPLING

Energy Transduction between a Concentration Gradient and an Alternating Electric Field.

03,461

ELECTRODE EXTENSIONS

Electrode Extension Model for Gas Metal Arc Welding.
PB96-135074

ELECTRODE SURFACE EFFECTS

Optical and Mass Spectrometric Investigations of lons and Neutral Species in SF6 Radio-Frequency Discharges.
01,985

ELECTRODEPOSITED COATINGS

Magnetic and Structural Properties of Electrodeposited Copper-Nickel Microlayered Alloys.

PB94-213121

04,512

\section{ELECTRODEPOSITION}

Electrodepositio

00,760

Effect of Mn Content on the Microstructure of Al-Mn Alloys Electrodeposited at $150 \mathrm{C}$.

03,343

Evidence of Film-Induced Cleavage by Electrodeposited $\begin{array}{ll}\text { Rhodium. } & 03,191\end{array}$

03,19

Nanocomposite Magnetic Materials.

PB95-162780

04,617

ELECTRODES

Modeling Polarization Curves and Impedance Spectra for Simple Electrode Systems.

03,188

Effect of Electrode-Polymer Interfacial Layers on Polymer Conduction. Part 2. Device Summary.

02,335

Effect of Electrode Material on Measured Ion Energy Distributions in Radio-Frequency Discharges. PB96-102850 04,026

Rapid $\mathrm{pH}$ Change Due to Bacteriorhodopsin Measured with a Tin-Oxide Electrode.

PB96-112081

03,544

Conformational Alterations of Bovine Insulin Adsorbed on a Silver Electrode.

00,510

Mapping the Droplet Transfer Modes for an ER100S-1

GMAW Electrode.
PB96-190095

Sensing Droplet Detachment and Electrode Extension for

Control of Gas Metal Arc Welding.
PB96-190160

03,297

ELECTROHYDRODYNAMICS

Electrohydrodynamic Instability and Electrical Discharge Initiation in Hexane

02,244

ELECTROLYTE SOLUTIONS

Summary of the Apparent Standard Partial Molal Gibbs Free Energies of Formation of Aqueous Species, Minerals, and Gases at Pressures 1 to 5000 Bars and Tem. peratures 25 to $1000 \mathrm{C}$

PCTROL

01,113

Critical Behavior of Ionic Fluids

PB95-164331

Low Electrolytic Conductivity Standards.
PB96-122098

00,978

01,081

Electrolytes Constrained on Fractal Structures: DebyeHuckel Theor

01.174

ELECTROLYTIC POLARIZATION

Modeling Polarization Curves and Impedance Spectra for Simple Electrode Systems. PB94-198876

03,188

ELECTROMAGNETIC ACOUSTIC TRANSDUCERS

Determination of Sheet Steel Formability Using Wide Band Electromagnetic-Acoustic Transducers.

Effect of Liftoff on Accuracu of Phase Velocity Measurements Made with Electromagnetic-Acoustic Transducers. PB96-186044

ELECTROMAGNETIC CAPABILITY

Alternative EMC Compliance Test Facilities. PB96-200324

02,247

ELECTROMAGNETIC COMPATIBILITY

Surging the Upside-Down House: Measurements and Modeling Results.

02,243

Rapid Evaluation of Mode-Stirred Chambers Using Impulsive Waveforms.

01,979

ELECTROMAGNETIC COUPLING

Electromagnetic Coupling Character of (001) Twist Boundaries in Sintered Bi2Sr2CaCu2O8+x Bicrystals.

LECTROMAGNETIC EMISSIONS

Optically Linked Three-Loop Antenna System for Determining the Radiation Characteristics of an Electrically PB95-161915

02,005

ELECTROMAGNETIC ENVIRONMENTS

Condensed Catalogue of Electromagnetic Environment PBs $30-300 \mathrm{~Hz}$

01,899

Catalogue of Electromagnetic Environment Measurements, $30-300 \mathrm{~Hz}$.

PB96-155452

01,943

ELECTROMAGNETIC FIELDS

Bibliography of the NIST Electromagnetic Fields Division PB94-165990

01,875

Bibliography of the NIST Electromagnetic Fields Division Publications.

Electro-Optic-Based RMS Voltage Measurement Tech-

PB96-138490

02,194

Standard Source Method for Reducing Antenna Factor Errors in Shielded Room Measurements.

PB96-183157

02,013

Bibliography of the NIST Electromagnetic Fields Division Publications.

01,980

Metrology for Electromagnetic Technology: A Bibliography of NIST Publications.

PB97-116057

04,396

ELECTROMAGNETIC FLOWMETERS

Development in the Theory and Analysis of Eddy Current Sensing of Velocity in Liquid Metals.

PB94-212586

03,335

ELECTROMAGNETIC INTERACTIONS

Relativistic Quantum Mechanics of Interacting Particles. PB97-110589

\section{ELECTROMAGNETIC INTERFERENCE}

Aperture Excitation of Electrically Large, Lossy Cavities.

PB94-145711

00,029

Measurements of Shielding Effectiveness and Cavity Characteristics

00,030

Assessment of Data by a Second-Order Transter Func-

tion.

03,760

Electronics and Electrical Engineering Laboratory Technical Publication Announcements Covering Laboratory Programs. October to December 1994 with 1995 EEEL Events Calendar.

Standard Antennas for Electromagnetic Interference Measurements and Methods to Calibrate Them.

PB96-102561

02,007

Screened-Room Measurements on the NIST SphericalDipole Standard Radiator.

PB96-113568

01,926

Methodology for Electromagnetic Interference Measure-

ments.

02,014 


\section{KEYWORD INDEX}

ELECTROMAGNETIC METROLOGY

Submissions to a Planned Encyclopedia of Operations Research on Computational Geometry and the Voronoi/ Delaunay Construct.

03,425

Metrology for Electromagnetic Technology: A Bibliography of NIST Publications.

PB94-159761

02,116

Transmission/Reflection and Short-Circuit Line Methods for Measuring Permittivity and Permeability.

PB94-165537

02,211

Bibliography of the NIST Electromagnetic Fields Division PB94-165990

01,875

NIST $30 \mathrm{MHz}$ Linear Measurement System.

02,020

Null-Balanced Total-Power Radiometer System NCS1.

PB94-169778 02,021

Electric-Field Strengths Measured Near Personal Transceivers.

01,564

Lead Abatement in Buildings and Related Structures.

PB94-172038 03,601

Leakage Current Detection in Cryogenic Current Comparator Bridges.

PB94-172228

02,024

Effect of DC Tests on Induced Space Charge.

02,212

Investigation of the Effects of Aging on the Calibration of a Kerr-Cell Measuring System for High Voltage Impulses. PB94-172384 02,025

Intercomparison of NIST, NPL, PTB, and VSL Thermal Voltage Converters from $100 \mathrm{kHz}$

02,026

Intercomparison of Thermal Converters at NIM, NIST, PTB, SIRI and VSL from 10 to $100 \mathrm{MHz}$.

PB94-172459

02,027

Planar Near-Field Alignment.

PB94-172491 01,998

Comparative Measurements of High-Voltage Impulses Using a Kerr Cell and a Resistor Divider.
PB94-172582

Time Scale Algorithm for Post-Processing: AT1 Plus Frequency Variance.

Reciprocity Relations in Waveguide Junctions.

01.525

PB94-172814

02,213

Advanced Components for Electric and Hybrid Electric Vehicles. Workshop Proceedings. Held in Gaithersburg. Maryland on October 27-28, 1993.

Maryland on Ocic
PB94-177060

04.858

Time-Domain Measurements of the Electromagnetic Backscatter of Pyramidal Absorbers and Metallic Plates.
01.877 Standard Probes for Electromagnetic Field Measure-

PB94-185436

01,999

Approach to Setting Performance Requirements for Automated Evaluation of the Parameters of High-Voltage Im-

PB94-185634

01,878

Coaxial Reference Standard for Microwave Power.

PB94-193786 01,880

Automatic Inductive Voltage Divider Bridge Operates from $10 \mathrm{~Hz}$ to $100 \mathrm{kHz}$.

PB94-198413

02,032

Spatial Dependence of Electrical Fields Due to Space Charges in Films of Organic Dielectrics Used for Insulation of Power Cables.

PB94-199130 02,214

PB94-199395

04,472

NIST Capacitance Measurement Assurance Program (MAP). 20060

02,254

Faraday Effect Current Sensors.

02,127

Measurements of Shielding Effectiveness and Cavity Characteristics of Airplanes.
PB94-210051 00,030 PB94-210051 00,030 Longterm Changes of Silicon Photodiodes and Their Use
for Photometric Standardization.
PB94-211349 Currents Induced on Multiconductor Transmission Lines by Radiation and Injection

PB94-211943

02,215

Electromagnetic Scattering by a Periodic Surface with a Wedge Profile.

04,421

Ultrasonic Spectroscopy of Metallic Spheres Using Electromagnetic-Acoustic Transduction

PB94-212537

04,185

Optically Sensed

02,130

Accurate Experimental Characterization of Interconnects:

A Dissussion of 'Experimental Electrical Characterization of Interconnects and Discontinuities in High-Speed Digital Systems'

02,217

Interconnection Transmission Line Parameter Character-

PB94-216397

02,218

Cascading Surge-Protective Devices: Options for Effective Implementation.

PB94-216488 02,464

Coordinating Cascaded Surge-Protective Devices: An Elusive Goal.

02,465

Important Link in Entire-House Protection: Surge Reference Equalizers.

PB94-216504

02,219

PB94-216611

03,868

Digital Techniques in HV Tests - Summary of 1989 Panel Session.
PB94-216702

02,035

RangeCAD and the NIST RCS Uncertainty Analysis.

PB94-218591

01,870

Planar Near-Field Measurements of Low-Sidelobe Anten-

PB94-219235 02,001

Three-Axis Coil Probe Dimensions and Uncertainties during Measurement of Magnetic Fields from Appliances.
PB94-219359

High-Order Harmonic Mixing with GaAs Schottky Diodes.

PB95-108585 01,528

Correction Factor for Nonplanar Incident Field in Monopole Calibrations.

PB95-108643

02,002

Vibrational Predissociation Dynamics of Overtone-Excited HN3.

PB95-125720

00,691

Sub-Doppler Frequency Measurements on OCS at 87 Thz (3.4 micrometers) with the CO Overtone Laser: Considerations and Details.

PB95.128633

04,255

Bibliography of the NIST Electromagnetic Fields Division Publications.

PB95-135562

01,886

Metrology for Electromagnetic Technology: A Bibliography of NIST Publications.

02,143

Ion Kinetic-Energy Distributions in Argon if Glow Discharges.

PB95-141008 04,409

LP11-Mode Leakage Loss in Coated Depressed Clad Fi.

LPers.

02,145

Electronics and Electrical Engineering Laboratory Technical Progress Bulletin Covering Laboratory Programs, Aprit to June 1994 with 1994/1995 EEEL Events Cal

PB95-143186

02,329

Superconducting Energy Gap of Bulk UBe13.

04,559

Electrical Breakdown in Transformer Oil in Large Gaps.
PB95-150579

Flux-Locked Current Source Reference.

1,889

PB95-150785

02,039

Bistatic Scattering of Absorbing Materials from 30 to 1000 然

01,891

Preliminary Comparison of Time Transfers via LASSO, GPS and Two-Way Satellite.

PB95-151098

01,529

Accurate Trans

02,220

Time-Domain Antenna Characterizations. PB95-152781

02,003

Automatic Calibration of Inductive Voltage Dividers for the NASA Zeno Experiment.

PB95-152849

02,041

Inductive Voltage Divider Calibration for the NASA Flight Experiment.

02,042

New Coaxial Microwave Microcalorimeter Evaluation Technique.

PB95-153227

01,892

Pressure Dependencies of Standard Resistors.

02,257 Terminal Invariant Description of Amplifier Noise.

02,043

Performance of Commercial CMOS Foundry-Compatible Multijunction Thermal Converters.

PB95-153656

02,342

New Extrapolation/Spherical/Cylindrical Measurement Facility at the National Institute of Standards and Technology.

02,004

Effective Medium Theory for Ferrite-Loaded Materials.

PB95-154662

01,893

Simulating the Dynamic Electro Thermal Behavior of

Power Electronic Circuits and Systems.

02,345

Frequency Extension of the NIST AC-DC Difference Calibration Service for Curren

PB95-161253

01,895

Modeling and Test Point Selection for a Thermal Transfer Standard.

Bending-Induced Phase Shifts in Dual-Mode Planar Opti-

cal Waveguides.
PB95-161329

Characterization of Unknown Linear Systems Based on Measured CW Amplitude.

PB95-161485

01,897

Comments on 'Protecting EFIE-Based Scattering Computations from Effects of Internal Resonances'.

PB95-161568

01,898

Verification of Commercial Probe-Tip Calibrations.

PB95-161576

02,347

ELF Electric and Magnetic Field Measurement Methods.

PB95-161675 04,423

Optically Linked Three-Loop Antenna System for Deter mining the Radiation Characteristics of an Electrically Small Source.

PB95-161915

02,005

Kinetic-Energy Distributions of lons Sampled from Argon Plasmas in a Parallel-Plate, Radio-Frequency Reference Call.

03935 Broadband Mismatch Error in Noise Measurement Sys-

PB95-162061

02,044

PB95-162152

00,957

Condensed Catalogue of Electromagnetic Environment Measurements, $30-300 \mathrm{~Hz}$

PB95-162210

01,899

Importance of Unraveling Memory Propagation Effects in Interpreting Data on Partial Discharge Statistics.

PB95-163572

01,901

Nonstationary Behavior of Partial Discharge during Insulation Aging.

Comparison of Three Techniques for the Precision Meas-

urement of Amplifier Noise
PB95-163663

02,349

Measurement Accuracies for Various Techniques for Measuring Amplifier Noise.

PB95-163671

02,350

Planar Resistors for Probe Station Calibration.

95-163697

Use of lonospheric Data in GPS Time Transfer
PB95-163853

Calibrating On-Wafer Probes to the Probe Tips.

PB95-163945

01,540

LRM Probe-Tip Calibrations with Imperfect Resistors and Lossy Lines.

PB95-163952

02,353

PB95.163960

01,904

Electrical Characterization of Radio-Frequency Dis charges in the Gaseous Electronics Conference Ref- 
On-Water Impedance Measurement on Lossy Substrates.
02,365 Electronic Mode Stirring for Reverberation Chambers. PB95-180329 01,908 Crosstalk between Microstrip Transmission Lines. (NIST Reprint).

PB95-180337

02,225

Dual Frequency mm. Wave Radiometer Antenna for Airbome Remote Sensing of Atmosphere and Ocean. 02,006 Large-Amplitude Shapiro Steps and Self-Field Effects in High-Tc Josephson Weak Links.

PB95-180410

04,682

Dielectric Properties of Single Crystals of $\mathrm{Al}_{2} \mathrm{O} 3, \mathrm{LaAlO} 3$ $\mathrm{SrTiO3}$, and $\mathrm{MgO}$ at Cryogenic Temperatures.

PB95-180477

02,266

Physics and Chemistry of Partial Discharge and Corona: Recent Advances and Future Challenges.

01,910

Practical Standards for PM and AM Noise at 5, 10 and

$100 \mathrm{MHz}$.
PB95-181129

01,546

Assessment of Data by a Second-Order Transfer Func-

PB95-182390

03,760

Evaluation of the Economic Impacts Associated with the NIST Power and Energy Calibration Services.

PB95-188850

02,461

$10 \mathrm{kV}$ DC Resistive Divider Calibration.

02,063

Electromagnetic Shielding Characterization of Gaskets.

PB95-198917 01,911

Electronics and Electrical Engineering Laboratory Technical Publication Announcements Covering Laboratory
Programs, July to September 1994 with 1995 EEEL Events Calenda

PB95-198925

01,912

Method to Determine the Calorimetric Equivalence Correction for a Coaxial Microwave Microcalorimeter

01,913

Developing a NIST Coaxial Microwave Power Standard at $1 \mathrm{~mW}$.

01,914

Dielectric Properties of Materials at Cryogenic Temperatures and Microwave Frequencies.

02,369

Casual Regularizing Deconvolution Filter for Optimal Waveform Reconstruction.

PB95-203089

01,603

Integral Occurring in Coherence Theory

04,324

Proposed Analysis of RCS Measurement Uncertainty.

PB95-203568 01,871

Accurate Measurement of Optical Detector Nonlinearity.

PB95-203576 02, 18

Droplet Transfer Modes for a MIL 100S-1 GMAW Elec-

rode.

02,867

Electronics and Electrical Engineering Laboratory Tech nical Progress Bulletin Covering Laboratory Programs, January to March 1992, with 1992/1993 EEEL Events

PB95-210480

01,917

Polarimetric Calibration of Reciprocal-Antenna Radars.

PB95-216925 01,872

Electronics and Electrical Engineering Laboratory Technical Publication Announcements Covering Laboratory Programs, January to March 1995 with 1995 EEEI Events

PB95-242277

02,373

SF6 Insulation: Possible Greenhouse Problems and Solutions.

02,269

Low-Frequency Model for Radio-Frequency Absorbers.

PB95-261939 04,424

Using Ouantized Breakdown Voltage Signals to Deter mine the Maximum Electric Fields in a Ouantum Hall Effect Sample.

PBect Sample.

02,375

Standard Antennas for Electromagnetic Interference Measurements and Methods to Calibrate Them.

PB96-10256

02,007

Comments on 'Conversions between $S, Z, Y, h, A B C D$, and $T$ Parameters Which Are Valid for Complex Source and Load Impedances'.

PB96-102785

02,069

Effect of Electrode Material on Measured Ion Energy Distributions in Radio-Frequency Discharges.

04,026

Ion Kinetic-Energy Distributions and Balmer-alpha (Halpha) Excitation in Ar-H2 Radio-Frequency Discharges.

04,029

Time Generation and Distribution

PB96-103049

01,550

Digital Impedance Bridge.

02,272
Spherical-Wave Source-Scattering Matrix Analysis of Antennas and Antenna-Antenna Interactions.

02,008

Relative Accuracy of Isolated and Unisolated Noise Comparison Radiometers.

PB96-111851

01,924

Measurements of Permittivity and the Dielectric Loss Tangent of Low Loss Dielectric Materials with a Dielectric Resonator Operating on the Higher Order Te(sub 0 gamma delia) Modes.

02,273

Kinetic Energy Distribution of lons Produced from Townsend Discharges in Neon and Argon.

PB96-111927

04,413

Uncertainties of the NIST Coaxial Noise Calibration System

PB96-111984 02,070

Distribution of Dielectric Relaxation Times and the Moment Problem

04,727 Continuous Recording and Stochastic Analysis of PD.
PB96-112156

PB96-112156
Binary versus Decade Inductive Voltage Divider Comparison and Error Decomposition.

PB96-112263

02,071

Surging the Upside-Down House: Looking into Upsetting Reference Voltages.

02,385

Gaseous Electronics Conference RF Reference Cell: An Introduction.

PB96-113329

02,387

Studies of Ion Kinetic-Energy Distributions in the Gaseous Electronics Conference RF Reference Cell.

Microwave Diagnostic Results from the Gaseous Electronics Conference RF Reference Cell.

PB96-113378

02,392

Two-Dimensional Self-Consistent Radio Frequency Plasma Simulations Relevant to the Gaseous Electronics Conference RF Relevant to th

PBo6-113436

02,398

Screened-Room Measurements on the NIST SphericalDipole Standard Radiator.

PB96-113568

01,926

PBisplay-Measur-119227

02,186

Gaseous Dielectrics Research: Possible SF6 Substitutes. PB96-119268 02,228 High-Temperature Superconductor Cryogenic Current Comparator.

PB96-119334

02,074

Perception of Clamp Noise in Television Receivers.

Progress on the Ouantized Hall Resistance Recommended Intrinsic/Derived Standards Practice.

PB96-122460

02,403

Standardised Computer Data File Format for Storage, Transport, and Otf-Line Processing of Partial Discharge

PB96-122486 01,930

Constant Temperature and Humidity Chamber for Standard Resistors

PB96-122494

Programmable Guarded Coaxial Connector Panel.

02,108

Microwave Characterization of Printed Circuit Transmission Lines.

PB96-122585 02,077

Modification of Cast Epoxy Resin Surfaces during Exposure to Partial Discharges.

PB96-122734

01,086

Influence of Surface Charge on the Stochastic Behavior of Partial Discharge in Dielectrics.

PB96-122767

01,931

Comment and Discussion on Digital Processing of PD Pulses

PB96-122775

01,932

Electromagnetic Properties of Materials: The NIST Metrology Program

01,933

Open-Ended Coaxial Probes for Nondestructive Testing of Substrates and Circuit Boards.

02,078

Bounds on Frequency Response Estimates Derived from Uncertain Step Response Data.

PB96-122874

03,419

Methods for Aligning the NIST Watt-Balance.

01,934

Influence of Electrode Material on Measured Ion Kinetic-

PB96-123179

01,935

Kinetic Energy Distributions of $\mathrm{H}(+), \mathrm{H} 2(+)$, and $\mathrm{H} 3(+)$ from a Diffuse Townsend Discharge in $\mathrm{H} 2$ at High E/N.
PB96-123351

04,415

PB96-123450

Keeping Up with the Reality of Today's Surge Environ-

ment. 23633

02,231

Ion Kinetics and Symmetric Charge-Transfer Collisions in Low-Current, Diffuse (Townsend) Discharges in Argon and Nitrogen.

Refraction of Light by Graded Birefringent Media.
PB96-123716
02,192

04,051

Kinetic-Energy Distributions of Ions Sampled from RadioFrequency Discharges in Helium, Nitrogen, and Oxygen.
PB96-123732 Development of Near-Field Test Procedures for Communication Satellite Antennas.

PB96-135082

02,010

Analysis of an Open-Ended Coaxial Probe with Lift-Off for Nondestructive Testing.

PB96-135116

01,940

RM Probe-Tip Calibrations Using Nonideal Standards. PB96-135389

02,411

Electronics and Electrical Engineering Laboratory Technical Publication Announcements Covering Laboratory Programs, April to June 1995 with 1995 EEEL Events Calendar.

PB96-137187

01,941

Microwave Dielectric Properties of Anisotropic Materials at Cryogenic Temperatures.

B96-137765

02,412

Accurate Computations of Radar Cross Sections of Simple Objects.

04,426

Alternative Contour Technique for the Efficient Computation of the Effective Length of an Antenna.

P896-141361

02,011

Electric Dipole Excitation of a Long Conductor in a Lossy (Medium.

PB96-146675

04,058

Evidence for Inelastic Processes for $N(+) 3$ and $N(+) 4$ from Ion Energy Distributions in He/N2 Radio Frequency Glow Discharges.

PB96-146683

04,059

Comparison of k-Correction and Taylor-Series Correction for Probe-Position Errors in Planar Near-Field Scanning. Electronics and Electrical Engineering Laboratory Technical Progress Bulletin Covering Laboratory Programs, July to September 1995 with 1996 EEEL Events Cal-

PB96-147905 01,942

Technical Impact of the NIST Calibration Service for Electrical Power and Energy.

PB96-147913

02,462

Partially Coherent Transmittance of Dielectric Lamellae.

PB96-148028 04,359

Accuracy in Time Domain Transmission Line Measurements.

04,060 Spatial Correlation Function for Fields in a Reverberation

PB96-148077

04,427

AC-DC Difference Characteristics of High-Voltage Thermal Converters.

02,083

Performance of Multilayer Thin-Film Multijunction Thermal Converters. 
Implementation of a Standard Format for GPS Common View Data.

PB96-176581 01,555

Line-Reflect-Match Calibrations with Nonideal Microstrip Standards.

PB96-176599 02,242

Microwave Characterization of Flip-Chip MMIC Components.

02,434

Evidence That Voltage Rather Than Resistance is Ouan. tized in Breakdown of the Quantum Hall Effect.

PB96-179163

01,868

Surging the Upside-Down House: Measurements and Modeling Results.

02,243

Complicated Cases and Shielded Rooms: Audiometric Booths Shielded to Attenuate Electromagnetic Inter-

PB96-180179

02,278

Electronics and Electrical Engineering Laboratory Technical Publication Announcernents Covering Laboratory

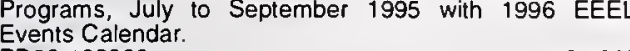
PB96-183066

01,965

Standard Source Method for Reducing Antenna Factor Errors in Shielded Room Measurements.

02,013

Electrohydrodynamic Instability and Electrical Discharge Initiation in Hexane.

PB96-186119

Dielectric and Magnetic Measurements from $-50 \mathrm{C}$ to $200 \mathrm{C}$ and in the Frequency Band $50 \mathrm{MHz}$ to $2 \mathrm{GHz}$.
PB96-191382

Electronics and Electrical Engineering Laboratory Technical Progress Bulletin Covering Laboratory Programs, January to March 1996, with 1996 EEEL Events Calendar.

PB96-191390

01,969

Coaxial Line-Peflect-Match Calibration.

02,246

Methodology for Electromagnetic Interference Measurements.

PB96-200126

02,014

Alternative EMC Compliance Test Facilities.

02,247

Comparison of Ultralow-Sidelobe-Antenna Far-Field Pat terns Using the Planar-Near-Field Method and the FarField Method.

PB96-200373

02,015

General Order ' $N$ ' Analytic Correction of Probe-Position

Errors in Planar Near-Field Measurements.

01,562

Polarimetric Calibration of Reciprocal-Antenna Radars.

PB96-200696

02,016

$100 \mathrm{~A}, 100 \mathrm{kHz}$ Transconductance Amplifier.

02,098

Quantum Hall Effect-Based Resistance Standard (Quanlum Hall Res

PB96-200944 $\quad 04,127$

Electronics and Electrical Engineering Laboratory Technical Publication Announcements Covering Laboratory

PB96-202346

01,978

Correlations between Electrical and Acoustic Detection of Partial Discharge in Liquids and Implications for Continuous Data Recording

PB96-204490

02,248

Rapid Evaluation of Mode-Stirred Chambers Using Impulsive Waveforms.

01,979

Bibliography of the NIST Electromagnetic Fields Division

Publications.
PB96-210778 01,980

Electronics and Electrical Engineering Laboratory Technical Publication Announcements Covering Laboratory Programs, January to March 1996, with 1996 EEEL Events Calendar.

NIST High-Accuracy Sampling Wattmeter.

PB97-108575

01,981

Causality and Maxwell's Equations.

02,689

Point Charges, Radiation Reaction, and Quantum MePB97-110563

04,139

Quantum Conductance Fluctuations in the Larger-SizeScale Regime.

04,144

Proposed Tests to Evaluate the Frequency-Dependent Capacitor Ratio for Single Electron Tunneling Experiment. PB97-111454

01,982

Uncertainties of Frequency Response Estimates Derived from Responses to Uncertain Step-Like Inputs.

PB97-111843

01,984

Resistors.

02,284

Loading Effects in Resistance Scaling

02,285
Low Thermal Guarded Scanner for High Resistance Measurement Systems.

02,288

Exploring the Low-Frequency Performance of Thermal Converters Using Circuit Models and a Digitally Synthesized Source.

PB97-112551 PB97-112569

02,848

Wideband S

02,100

PB97-113039

01,990

$\mathrm{DC}-\mathrm{MHz}$ Wattmeter Based on RMS Voltage Measurements.

01,992

Magnetometer Calibration Services.

01,993

PB97-113252

Results of Capacitance Ratio Measurements for the Sin-
gle Electron Pump-Capacitor Charging Experiment. gle Electron Pump-Capacitor Charging Experiment. 04,813 Electronics and Electrical Engineering Laboratory Technical Progress Bulletin Covering Laboratory Programs, April to June 1996 with 1996-1998 EEEL Events Cal-

PB97-113880

01,994

Metrology for Electromagnetic Technology: A Bibliography of NIST Publications.

04,396

Conversion of a 2-Terminal-Pair Bridge to a 4-TerminalPair Bridge for Increased Range and Precision in Impedance Measurements.

PB97-119176

02,103

NIST Comparison of the Quantized Hall Resistance and the Realization of the SI Ohm Through the Calculab Capacitor. Conference Proceedings, June 17-20, 1996. Active High Voltage Divider with 20-PPM Uncertainty. PB97-119317 02, 104 NIST Watt Experiment: Monitoring the Kilogram. PB97-12232

01,997

\section{ELECTROMAGNETIC NOISE}

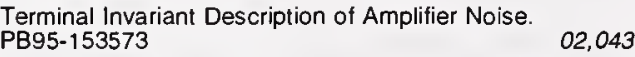

Relationship of AM to PM Noise in Selected RF Oscillaors.

PB95-169009

02,262

Investigations of $A M$ and PM Noise in X-Band Devices. PB95-180022 02,062 Secondary Standard for PM and AM Noise at 5, 10, and $100 \mathrm{MHz}$

PB96-123187

01,554

Origin of $\mathrm{V} / \mathrm{P} \mathrm{PM}$ and $\mathrm{AM}$ Noise in Bipolar Junction Transistor Amplifiers.

02,096

Reducing the $1 / / \mathrm{AM}$ and PM Noise in Electronics for Precision Frequency Metrology. PB97-113195

02,102

ELECTROMAGNETIC NOISE MEASUREMENT

Broadband Mismatch Error in Noise Measurement Sys-

PB95-162061 02,044

Comparison of Three Techniques for the Precision Measurement of Amplifier Noise.

PB95-163663

02,349

Measurement Accuracies for Various Techniques for Measuring Amplifier Noise

02,350

Radiometer Equation for Noise Comparison Radiometers. PB96-140363

02,195

ELECTROMAGNETIC PROPERTIES

Substrate and Thin Film Measurements. 02,384

Electromagnetic Properties of Materials: The NIST MetrolOgy Program.

01,933

NIST Metrology Program on Electromagnetic Characterzation of Materials.

PB96-156062

01,944

\section{ELECTROMAGNETIC SCATTERING}

Time-Domain Measurements of the Electromagnetic Backscatter of Pyramidal Absorbers and Metallic Plates. PB94-185410 01,877

Electromagnetic Scattering by a Periodic Surface with a Wedge Profile.

04,421

Alternative Single Integral Equation for Scattering by a Dielectric.

PB94-216512

04,422

Comments on 'Protecting EFIE-Based Scattering Computations from Effects of Internal Resonances'.

PB95-161568

01,898

Study of Multiple Scattering Background in Compton Scatter Imaging.

04,425

Hypersingular Single Integral Equation and the Dielectric

PBedge.

04,428

Causality and Maxwell's Equations.

04,429

Electromagnetic Scattering from a Dielectric Wedge and the Single Hypersingular Integral Equation.
PB97-110530 04,430 Numerical Evaluation of Hypersingular Integrals for Scattering by a Dielectric Wedge.

PB97-110555

02,017

Sinusoidal Surfaces as Standards for BRDF Instruments. PB97-1 10597 04,388 Numerical Reference Models for Optical Metrology Sim-

Ulation.

04,392

ELECTROMAGNETIC SHIELDING

Aperture Excitation of Electrically Large, Lossy Cavities.
PB94-145711

Measurements of Shielding Effectiveness and Cavity Characteristics of Airplanes.

Aperture Coupling to a Coaxial Air Line: Theory and Experiment.

PB94-211968

02,216

Aperture Excitation of Electrically Large, Lossy Cavities.

PB95-175675 00,031

Electromagnetic Shielding Characterization of Gaskets

PB95-198917

01,911

Band-Limited, White Gaussian Noise Excitation for Reverberation Chambers and Applications to Radiated Susceptibility Testing.

01,960

Complicated Cases and Shielded Rooms: Audiometric Booths Shielded to Attenuate Electromagnetic Inter-

PB96-180179

02,278

ELECTROMAGNETIC SUSCEPTIBILITY

Band-Limited, White Gaussian Noise Excitation for Reverberation Chambers and Applications to Radiated Susceptibility Testing.

01,960

ELECTROMAGNETIC TESTING

TEM/Reverberating Chamber Electromagnetic Radiation Test Facility at Rome Laboratory.

PB96-155023

03,675

ELECTROMAGNETIC THEORY

Effective Medium Theory for Ferrite-Loaded Materials.

PB95-154662

01,893

ELECTROMANGETIC ACOUSTIC TRANSDUCERS

Methods to Improve the Accuracy of On-Line Ultrasonic Measurement of Steel Sheet Formability.

02,281

ELECTROMECHANICAL DEVICES

Test Structures for Determining Design Rules for Microelectromechanical-Based Sensors and Actuators.

ELECTROMECHANICS

Electromechanical Properties of Superconductors for DOE Fusion Applications.

ELECTROMETERS

Cryogenic Precision Capacitance Bridge Using a Single Electron Tunneling Electrometer.

PB95-126074

04,529

Noise in the Coul
PB95-176327

Pryogenic Precisior Electron Tunneling Electrometer.

PB96-112271

Determination of Atomic Data Pertinent to the Fusion Energy Program. Progress Report for FY 92. 
High-Precision Calculations of Cross Sections for LowEnergy Electron Scattering by Ground and Excited State of Sodium.

03,914

Semiclassical Explanation of the Generalized RamsauerTownsend Minima in Electron-Atom Scattering. PB95-153532

03,925

Spin-Resolved Elastic Scattering of Electrons from So-

dium.

03,933

Connection between Superelastic and Inelastic ElectronAtom Collisions Involving Polarized Collision Partners.

PB95-202297

03,974

Threshold Electron Excitation of $\mathrm{Na}$.

PB95-202917

03,994

Charge Cloud Distribution of Heavy Atoms After Excitation by Polarized Electrons.

04,001

Angle-Differential and Momentum-Transier Cross Sections for Low-Energy Electron-Cs Scattering.
PB95-203402

04,005

Relativistic R-Matrix Calculations for Electron - Alkali-
Metal-Atom Scattering: Cs as a Test Case. PB95-203410

04,006

Low-Energy-Electron Collisions with Sodium: Elastic and Inelastic Scattering from the Ground State.

04,030

ELECTRON ATTACHMENT

Associative Electron Attachment to $\mathrm{S} 2 \mathrm{~F} 10, \mathrm{~S} 2 \mathrm{OF} 10$, and $\mathrm{S} 2 \mathrm{O} 2 \mathrm{~F} 10$.

Electron Attachment to Excited Molecules(1)

00,907

PB96-122809

01,087

Dependence of the Thermal Electron Attachment Rate Constant in Gases and Liquids on the Energy Position of the Electron Attaching State.

PB97-122253

01,996

ELECTRON-BEAM HEATING

Closed Loop Controller for Electron-Beam Evaporators.
PB97-111470

ELECTRON BEAM ION TRAP (EBIT)

Observation and Visible and uv Magnetic Dipole Transitions in Highly Charged Xenon and Barium.

04,056

Polarization Measurements on a Magnetic Quadrupole Line in $\mathrm{Ne}$-Like Barium.

PB97-113104

04,161

ELECTRON BEAMS

New Method for Shielding Electron Beams Used for Head and Neck Cancer Treatment.

03,621

Crystal Structure of Annealed and As-Prepared $\mathrm{HgBa} 2 \mathrm{CaCu} 2 \mathrm{O} 6+d$ elta Superconductors.

03,927 Angular Variation of the Personal Dose Equivalent,
$\mathrm{Hp}(0.07)$, for Beta Radiation and Nearly Monoenergetic Electron Beams: Preliminary Results. Electron Beams:

03,630

Planar Lenses for Field-Emitter Arrays.

02,112

Calorimeters for Calibration of High-Dose Dosimeters in High-Energy Electron Beams.

04,055

Journal of Research of the National Institute of Standards and Technology, January/February 1996. Volume 101,

PB96-175666

00,113

ELECTRON CAPTURE

Dielectronic Capture Processes in the Electron-Impact Ionization of $\mathrm{Sc}(2+)$

ELECTRON CAPTURE DECAY

Alpha-Particle and Electron Capture Decay of (209)Po PB96-186085

04,000

ELECTRON CHARGE

Cryogenic Precision Capacitance Bridge Using a Single Electron Tunneling Electrometer.

PB95-126074

04,529

ELECTRON CLOUDS

Electrostatic Modes as a Diagnostic in Penning-Trap Experiments.

ELECTRON COLLISIONS

03,959

Calculations of Electron Inelastic Mean Free Paths. 5. Data for 14 Organic Compounds over the $50-2000 \mathrm{eV}$ PBange.

00,916

Calculations of Electron Inelastic Mean Free Paths (IMFPS). 4. Evaluation of Calculated IMFPs and of the Predictive IMFP Formula TPP-2 for Electron Energies between 50 and $2000 \mathrm{eV}$.

PB95-150728

00,922

ELECTRON CONFIGURATION

Designations of ds(2)p Energy Levels in Neutral Zirconium, Hafnium, and Ruthertordium ( $\mathrm{Z}=104)$.

04,116

ELECTRON CORRELATION

Relativistic Modifications of Charge Expansion Theory.
PB96-123799
ELECTRON DENSITY

Cooper M(sub II,III) X-ray-Emission Spectra of Copper Oxides and the Bismuth Cuprate Superconductor

PB96-158027

04,077

ELECTRON DENSITY (CONCENTRATION)

Construction of Maximum-Entropy Density Maps, and Their Use in Phase Determination and Extension.
PB95-108593

ELECTRON DETACHMENT

Resonant Two-Color Detachment of $\mathrm{H}(-)$ with Excitation of $\mathrm{H}(\mathrm{n}=2)$.

PB95-202552 03,984

Short-Pulse Detachment of $\mathrm{H}(-)$ in the Presence of a Static Electric Field.
PB95-203477

04,007

ELECTRON DIFFUSION CONSTANT

Measurement of the Weak-Localization Complex Conductivity at $1 \mathrm{Ghz}$ in Disordered $\mathrm{Ag}$ Wires

PB96-117239

04,731

ELECTRON DOSIMETRY

Electron and Proton Dosimetry with Custom-Developed Radiochromic Dye Films.

03,713

Calibration of Dosimeters for the Cryogenic Irradiation of Composite Materials Using an Electron Beam.

03,968

ECTRON-ELECTRON INTERACTIONS

Electron-electron Interactions, Coupled-Plasmon-Phonon Modes, and Mobility in n-Type GaAs.

PB96-138524

04,749

ELECTRON-ION COLLISIONS

Determination of Atomic Data Pertinent to the Fusion Energy Program. Progress Report for FY 92

04,402

Electron-Impact Ionization of $\ln (+)$ and $\mathrm{Xe}(+)$.

PB94-185089

00,770

Backscattering in Electron-Impact Excitation of Multiply Charged lons.

03,799

Absolute Cross-Section Measurements for Electron-Impact lonization of $\mathrm{C} 1(+1)$

03,818

Bg4-199841

Evidence for Significant Backscattering in Near-Threshold
Electron-Impact Excitation of $\operatorname{Ar}(7+)(3 s$ yields $3 p)$.

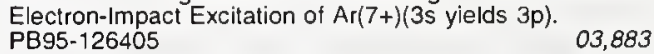

Electron-Impact Excitation of Si( $3+$ )(3S yields $3 \mathrm{P}$ ) Using a Merged-Beam Electron-Energy-Loss Technique.

PB95-151239

03,904

Electron-Impact Ionization of $\ln (+)$ and $\mathrm{Xe}(+)$

PB95-152906

03,918

Resonance Structure and Absolute Cross Sections in Near-Threshold Electron-Impact Excitation of the $4 \mathrm{~s}(2)$ (1) $S$ yields $4 s 4 p$ (3) $P$ Intercombination Transition in PB95-202271

03,972

Absolute Cross-Section Measurements for Electron-Impact Single lonization of $\mathrm{Se}(+)$ and $\mathrm{Te}(+)$

03,980

Crossed-Beams Measurements of Absolute Cross Sec-

tions for Electron Impact Ionization of $\mathrm{S}(+)$. PB95-202511

Absolute Cross Sections for Electron-Impact Single Ionization of $\mathrm{Si}(+)$ and $\mathrm{Si}(2+)$

PB95-202529

03,982

Dielectronic Capture Processes in the Electron-Impact Ionization of $\mathrm{Sc}(2+)$.

04,000

Electron-Ion Collisions in the Plasma Edge.

PB96-111885

04,037

Collisions of Electrons with Highly-Charged lons.

PB96-200340

04,791

ELECTRON-ION COUPLING

Merged-Beams Energy-Loss Technique for Electron-Ion Excitation: Absolute Total Cross Sections for $\mathrm{O}^{(5+)}$ (2s yields $2 p$ ).

ELECTRON MICROPROBE ANALYSIS

Study of Diffusion Zones with Electron Microprobe Compositional Mapping.

ELECTRON MICROSCOPES

Surtace Magnetic Microstructural Analysis Using Scanning Electron Microscopy with Polarization Analysis

PB95-162657

03,938

Fabrication Issues for the Prototype National Institute of Standards and Technology SRM 2090A Scanning Electron Microscope Magnification Calibration Standard.

PB96-160585

01,131

Scanning Electron Microscope Magnification Calibration Interlaboratory Study.

PB96-201082

01,164

ELECTRON MICROSCOPY

Characterization of the Binding of Gallium, Platinum, and ray Scattering and Transmission Electron Microscopy.
PB94-172509

Domain Structures in Magnetoresistive Granular Metals. PB96-141346

04,760

ELECTRON MOBILITY

Majority and Minority Mobilities in Heavily Doped Silicon for Device Simulations.

PB94-198728

02,311

Majority and Minority Electron and Hole Mobilities in Heavily Doped Gallium Aluminum Arsenide.

04,814

ECTRON-MOLECULE COLLISIONS

Associative Electron Attachment to S2F10, S2OF10, and PBg5-140992

00,907

Electron Scattering and Dissociative Attachment by SF6 and Its Electrical-Discharge By-Products.

02,256

Analyses of Recent Experimental and Theoretical Determinations of e-H2 Vibrational Excitation Cross Sections: Assessing a Long-Standing Controversy.

PB95-202438

03,977

Modified Effective Range Theory as an Alternative to Low-Energy Close-Coupling Calculations.

03,988

Slow-Electron Collisions with CO Molecules in an ExactExchange Plus Parameter-Free Polarization Mode

PB95-202719

03,989

mportance of Bound-Free Correlation Effects for Vibrational Excitation of Molecules by Electron Impact: A Sensitivity Analysis.

ELECTRON PARAMAGNETIC RESONANCE

03,996

Inter-Laboratory Trials of the EPR Method for the Detection of trradiated Meats Containing Bone.

PB96-161690

00,042

ELECTRON PROBE MICROANALYSIS

Design of a Protocol for an Electron Probe Microanalyzer k-Value Round Robin.

PB95-107181

00,564

ELECTRON PROBES

Electron Probe X-Ray Microanalysis.

00,562

ELECTRON PROCESSORS

Real Time Monitoring of Electron Processors.

PB96-135306

ELECTRON PUMPS

Metrological Accuracy of the Electron Pump.

PB95-168910

03,951

Testing for Metrological Accuracy of the Electron Pump. PB95-175873

Accuracy of the Electron Pump

04,663

PB96-135223

04,743

Performance of the Electron Pump with Stray Capacitances.
PB96-200902

01,976

ELECTRON SCATTERING

Inelastic Interactions of Electrons with Surfaces: Application to Auger-Electron Spectroscopy and X-ray Phoctroscopy.

00,764

Evidence for Significant Backscattering in Near-Threshold Electron-Impact Excitation of $\operatorname{Ar}(7+)$ (3s yields 3p)
PB95-126405 


\section{KEYWORD INDEX}

ELECTRONIC TECHNOLOGY

ELECTRON SPECTROMETERS Electron-Ion-X-ray Spectrometer System
PB95-176137

LECTRON SPECTROSCOPY

Compositional Analyses of Surfaces and Thin Films by Electron and Ion Spectroscopies.

ELECTRON SPIN POLARIZATION

00,779

Relativistic Effects in Spin-Polarization Parameters for Low-Energy Electron-Cs Scattering

03,901

ELECTRON SPIN RESONANCE

Experimental assessment of absorbed dose to mineralized bone tissue from internal emitters: An electron paramagnetic resonance study.

DE96007979

03,614

ESR-Based Analysis in Radiation Processing.

03,931

LECTRON STATES

Self-Consistent ' $G W$ ' and Higher-Order Calculations of Electron States in Metals.

PB97-119341

01,189

ELECTRON STRUCTURE

Soft-X-ray-Emission Spectra of Solid $\mathrm{Kr}$ and $\mathrm{Xe}$. PB96-157920

ELECTRON TEMPERATURE

Self-Heating in the Coulomb-Blockade Electrometer. PB94-212685

04,507

ELECTRON TRANSFER

Electron Transfer Reaction Rates and Equilibria of the Carbonate and Sulfate Radical Anions.

00,829

Solvent Effects in the Reactions of Peroxyl Radicals with Organic Reductants. Evidence for Proton Transfer Mediated Electron Transier.

PB95-107157

00,873

ELECTRON TRANSITIONS

Resonance Enhanced Multiphoton Ionization Detection of GeF and $\mathrm{GeCl}$ Radicals.

00,825

Experimental and Abinitio Studies of Electronic Structures of the $\mathrm{CCl} 3$ Radical and Cation.

00,826

PB94-212131

Multiphoton Ionization of $\mathrm{SiH} 3$ and $\mathrm{SiD} 3$ Radicals. 2.
Three Photon Resonance-Enhanced Spectra Observed Three Photon Resonance-Enhanced Spectra Observed between 460 and $610 \mathrm{~nm}$.

PB94-212487

00,835

New Electronic States of NH and ND Observed from 258 to $288 \mathrm{~nm}$ by Resonance Enhanced Multiphoton Ionization Spectroscopy.

PB94-212495

00,836

Multiphoton Ionization of $\mathrm{SiH} 3$ and SiD3 Radicals: Electronic Spectra, Vibrational Analyses of the Ground and Aydberg States, and Ionization Potential.

00,837

ELECTRON TRANSPORT

Buffer Layer Modulation-Doped Field-Effect-Transistor Interactions in the Al0.33Ga0.67As/GaAs Superlattice

System.
PB96-102876

02,380

ELECTRON TRAPS

Evidence for a Deep Electron Trap and Charge Compensation in Separation by Implanted Oxygen Oxides. Electron and Hole Trapping in Irradiated SIMOX, ZMR and BESOI Buried Oxides. PB96-180320

01,956

ELECTRON TUNNELING

Modified Airy Function Method for the Analysis of Tunneling Problems in Optical Waveguides and Quantum Well Sructures. Insulating Boundary Layer and Magnetic Scattering in YBa2Cu3O7-delta/Ag Interfaces Over a Contact Resistiv ity Range of $10(-8)-10(-3)$ Omega cm(2).

PB95-169157

04,648

Thermal Enhancement of Cotunneling in Ultra-Small Tunnel Junctions
PB95-175436

04,658

Application of Single Electron Tunneling: Precision $\mathrm{Ca}$ pacitance Ratio Measurements.

04,703

Results of Capacitance Ratio Measurements for the Single Electron Pump-Capacitor Charging Experiment. PB97-113286

04,813

ELECTRON TUNNELING SPECTROSCOPY

Tunneling Spectroscopy of Thallium-Based High-Temerature Superconductors.

04,606

ELECTRON WITHDRAWING GROUPS

Carbon Acidities of Aromatic Compounds. 1. Effects of InRing Aza and External Electron-Withdrawing Groups. PB94-2 16595

00,860

ELECTRONIC ACCESS PROJECT

Electronic Access: Blueprint for the National Archives and Records Administration.

PB95-219218

02,731

ELECTRONIC BALANCES

Electronic Balance and Some Gravimetric Applications. (The Density of Solids and Liquids, Pycnometry and Mass).

PB94-163052

03,785
ELECTRONIC BEAMS

Theory of Electron Beam Moire.

ECTRONIC CIRCUITS

04,373

Simulating the Dynamic Electro Thermal Behavior of Power Electronic Circuits and Systems.

02,345

Thermal Component Models for Electro-Thermal Network Simulation.

PB95-161022

02,346

ELECTRONIC COMMERCE

Planning the Infrastructure for Global Electronic ComPB94-185832

00,494

Analyzing Electro

00,480

ELECTRONIC DATA EXCHANGE

Guidelines for the Evaluation of Electronic Data Interchange Products
PB96-172325

01,506

\section{ELECTRONIC DATA INTERCHANGE}

Federal Implementation Guideline for Electronic Data Interchange: ASC $\times 12003040$ Transaction Set 838 Trading Partner Profile (Vendor Registration), Implementation Convention.

Federal Implementation Guideline for Electronic Data Interchange. ASC X12 003050 Transaction Set 850 Award Instrument. Implementation Convention.

PB96-114913

01,814

Federal Implementation Guideline for Electronic Data Interchange. ASC $\times 12003050$ Transaction Set 860 Modifications to Award Instrument. Implementation Conven-

PB96-114921

01,815

Federal Implementation Guideline for Electronic Data Interchange. ASC X12 003050 Transaction Set $843 \mathrm{Re}$ sponse to Request for Quotation. Implementation Con-

PB96-168984

01,822

Federal Implementation Guideline for Electronic Data Interchange ASC $\times 12003050$ Transaction Set 855 Purchase Order Acknowledgment: Implementation Convention

PB96-172374

01,823

Federal Implementation Guideline for Electronic Data Interchange. ASC X12 003050 Transaction Set $840 \mathrm{Re}$ quest for Quotation. Implementation Convention.

PB96-172531

01,824

Federal Implementation Guideline for Electronic Data Interchange: ASC X12 003050 Transaction Set 836 Procurement Notices. Implementation Convention.

curement Notice

01,827

\section{ELECTRONIC EQUIPMENT}

Handbook Preferred Circuits Navy Aeronautical Electronic Equipment. Supplement Number 3.

AD-A278 782/8

Handbook Preferred Circuits Navy Aeronautical Electronic Equipment. Supplement Number 2.

00,027

Handbook Preferred Circuits Navy Aeronautical Electronic Equipment. Supplement Number 1.

00,028

Application of the Electronic Balance in High Precision Application of the Electronic Balance in High Precision
Pycnometry.
PB94-187564 PB94-187564 00,534 Important Link in Entire-House Protection: Surge Re PB94-216504

02,219

NIST Strategies for Reducing Testing Requirements. PB95-180444 01,909 Surging the Upside-Down House: Looking into Upselting PB96-112313

02,385

Beyond the Technology Roadmaps: An Assessment of Electronic Materials Research and Development.

PB96-165998

01,961

Empirical Linear Prediction Applied to a NIST Calibration Service.

\section{LECTRONIC INDUSTRY}

02,287

Electronics and Electrical Engineering Laboratory: 1996 Program Plan. Supporting Technology for U.S. Competiiveness in Electronics.

PB96-175237

01,962

ELECTRONIC MAIL

Good Security Practices for Electronic Commerce, Including Electronic Data Interchange.

01,463

\section{ELECTRONIC PACKAGING}

Metrology and Data for Microelectronic Packaging and Interconnection: Results of a Joint Workshop on Materials Metrology and Data for Commercial Electrical and Optical Packaging and Interconnection Technologies. Held in Gaithersburg, Maryland on May 5-6, 1994. Volume 1. ReSults.

PB95-143111

02,328

Metrology and Data for Microelectronic Packaging and Interconnection: Results of a Joint Workshop on Materials

Metrology and Data for Commercial Electrical and Optical Packaging and Interconnection Technologies. Held in Gaithersburg, Maryland on May 5-6, 1994. Volume 2. Presentation Materia

PB95-143327

02,330

Electronics Pac

02,405

Natural Convection from an Array of Electronic Packages Mounted on a Horizontal Board in a Narrow Aspect Ratio

$\begin{array}{ll}\text { Enclosure. } & 02,087\end{array}$

Measurements of Properties of Materials in Electronic

Packaging.

01,973

Overview of U.S. Government Advanced Packaging Pro-

grams

NIST/NCMS Program on Electronic Packaging: First Up date.

03,008

ELECTRONIC SECURTYY

Computer Virus Attacks.

PB95-163655

01,715

EDI and EFT Security Standards.

PB96-122833 01,605

Computer Security: An Introduction to Computer Security. The NIST Handbook.

PB96-131610

01,608

Assessing Functional Diversity by Program Slicing.

Predicate Differences and the Analysis of Dependencies in Formal Specifications.

PB96-160940

01,759

Technique for Analyzing the Effects of Changes in Formal PBecifications.

01,760

Information Technology Standards in Federal Acquisitions.

01,636

Using Information Technology Standards in Federal ACquisitions.

PB96-161260

01,637

Control and Instrumentation: Standards for High-Integrity Sottware.

ELECTRONIC SPECTRA

03,736

New Rydberg States of Aluminum Monofluoride Observed by Resonance-Enhanced Multiphoton Ionization SpectrosPB94-199544

00,797

Resonance Enhanced Multiphoton Ionization Detection of $\mathrm{GeF}$ and $\mathrm{GeCl}$ Radicals.

00,825

Experimental and Abinitio Studies of Electronic Structures

of the CCI3 Radical and Cation.

00,826
Multiphoton Ionization of $\mathrm{SiH} 3$ and SiD3 Radicals.

Three Photon Resonance-Enhanced Spectra Observed 
Electronics and Electrical Engineering Laboratory 1994 Program Plan: Supporting Technology for U.S. Competitiveness in Electronics.

01,873

Electronics and Electrical Engineering Laboratory Technical Progress Bulletin Covering Laboratory Programs, January 10 March 1991, with 1991 EEEL Events Cal-

PB94-145968

02,113

Electronics and Electrical Engineering Laboratory Technical Progress Bulletin Covering Programs, October to December 1993, with 1994/1995 EEEL Events Calendar.
PB94-154341 115

Development and Characterization of Insulating Layers on Silicon Carbide: Annual Report for February 14, 1988 to February 14, 1989

PB94-155579

02,295

Center for Electronics and Electrical Engineering Technical Progress Bulletin Covering Center Programs, October to December, with 1991 CEEE Events Calendar.
PB94-159787 Electronics and Electrical Engineering Laboratory: 1994 Strategic Plan. Supporting Technology for U.S. Competitiveness in Electronics.

PB94-161320 01.874

Design Guide for CMOS-On-SIMOX. Test Chips NIST3 and NIST4.

02,297

Color Supplement to NIST Special Publication 400-93: Semiconductor Measurement Technology: Design and
Testing Guides for the CMOS and Lateral Bipolar-on-SOI Test Library.

02,298

Experimental and Numerical Studies on Two-Dimensional Gravity Currents in a Horizontal Channel.

01,359

Electronics and Electrical Engineering Laboratory Technical Progress Bulletin Covering Laboratory Programs,
October to December 1992, with 1992/1993 EEEL Events

PB94-165958

02,299

Coordinating Cascaded Surge Protection Devices: HighLow versus Low-High.

02,463

Gaseous Electronics Conference Radio-Frequency Reference Cell: A Defined Parallel-Plate Radio-Frequency System for Experimental and Theoretical Studies of Plasma-Processing Discharges.

PB94-172327

04,404

Efficient Method to Compute the Maximum Transient Drain Current Overshoot in Silicon on Insulator Devices. PB94-172483 02,300

Semiconductor Measurement Technology: Design and
Testing Guides for the CMOS and Lateral Bipolar-on-SOI Testing Guides

PB94-178019

02,301

Application of the Modified Voltage-Dividing Potentiometer to Overlay Metrology in a CMOS/Bulk Process. PB94-181997 02,302

Interface Roughness-Induced Changes in the Near-E(sub O) Spectroscopic Behavior of Short-Period AIAs/GaAs Superlattices.
PB94-185154

Thin Film Transparent Thermocouples.

Electro-Thermal Simulation of an IGBT PWM Inverter.

PB94-185592

Use of an Ion Energy Analyzer: Mass Spectrometer to Measure Ion Kinetic-Energy Distributions from RF Discharges in Argon-Helium Gas Mixtures.

04,406

Planning the Infrastructure for Global Electronic Com-

PB94-185832

00,494

Correction to the Decay Rate of Nonequilibrium Carrier Distributions Due to Scattering-in Processes. 04,452 Optical Current Transducer for Calibration Studies.

PB94-185907

02,121

Reproducibility of JEDEC Standard Current and Voltage Ramp Test Procedures for Thin-Dielectric Breakdown Characterization.

PB94-185931 01,879

Experimental Investigation of the Validity of TDDB Voltage Acceleration Models.

02,304

Field and Temperature Acceleration of Time-Dependent Fielectric Breakdown in Intrinsic Thin SiO2.
PB94-185956

02,305

Junction Locations by Scanning Tunneling Microscopy:

PB94-185964

02,306

Semiconductor Measurement Technology: Improved Characterization and Evaluation Measurements fo HgCdTe Detector Materials, Processes, and Devices Used on the GOES and TIROS Satellites.

02,122

Electronics and Electrical Engineering Laboratory Technical Publication Announcements Covering Laboratory
Programs, October to December 1993 with 1994/1995 EEEL Events Calendar.

02,307

Electronics and Electrical Engineering Laboratory Technical Progress Bulletin Covering Laboratory Programs, January to March 1994 with 1994/1995 EEEL Events Cal-

PB94-193810

02,308

Realizing Suspended Structures on Chips Fabricated by CMOS Foundry Processes Through the MOSIS Service

01881

Electronics and Electrical Engineering Laboratory Technica| Progress Bulletin Covering Laboratory Programs, July 10 September 1993 with 1994 EEEL Events Cal-

02,309

Numerical Simulation of Submicron Photolithographic $\begin{array}{ll}\text { Processing. } & \text { 02,310 }\end{array}$ Majority and Minority Mobilities in Heavily Doped Silicon or Device Simulations.

PB94-198728

02,311

Electron Traps, Structural Change, and Hydrogen Related

SIMOX Defects

02,312

President's Column for Dielectrics and Electrical Insulation Society Newsletter.

PB94-200409

01,882

Test Structures for the In-Plane Locations of Projected Features with Nanometer-Level Accuracy Traceable to a Coordinate Measurement System.

02,313

PB94-200565

Survey on the Implementation of ISO/IEC Guide 25 by
National Laboratory Accreditation Programs.
PB94-210150
00,479 PB94-210150

Grain

Half-Integral Constant

PB94-211216

04,489

Investigation of the Drive Circuit Requirements for the Power Insulated Gate Bipolar Transistor (IGBT).

PB94-211794

02,316

Aperture Coupling to a Coaxial Air Line: Theory and Experiment.

02,216

Investigation of S2F10 Production and Mitigation in Compressed SF6-Insulated Power Systems.

02,467

Multicarrier Characterization Method for Extracting Mobilities and Carrier Densities of Semiconductors from Variable Magnetic Field Measurements.

PB94-212776

02,317

High-Spatial-Resolution Resistivity Mapping Applied to Mercury Cadmium Telluride.

02,131

Review of Semiconductor Microelectronic Test Structures with Applications to Infrared Detector Materials and ProcPB94-212925

02,132

Defect of Thermal Ramping and Annealing Conditions on Defect Formation in Oxygen Implanted Silicon-On-Insula Detect Format

PB94-212966

02,318

Precision, Accuracy, Uncertainty and Traceability and Their Application to Submicrometer Dimensional MetrolPBg4-213105

02,319

Custom Integrated Circuit Comparator for High-Pertormance Sampling Applications.

02,320

Investigation of Mercury Interstitials in $\mathrm{Hg}(1-\mathrm{x}) \mathrm{CdxTe} \mathrm{Al}$ loys Using Resonant Impact-Ionization Spectroscopy.

Electronics and Electrical Engineering Laboratory Technical Publication Announcements Covering Laboratory Programs, January to March 1994 with 1994/1995 EEEL

Events Calendar

01,883

Heavily Accumulated Surfaces of Mercury Cadmium Telluride Detectors: Theory and Experiment.

02,134

High-Level CAD Melds Micromachined Devices with Foundries.

PBoundries.

Guarding Against Transients

02,321

PB94-216470

01,623

Characterization of Interface Defects in Oxygen-Implanted Silicon Films.

PB94-216629 02,322

Charge Trapping and Breakdown Mechanism in SIMOX.

PB94-216637 02,323

Sources of Uncertainty in a DVM-Based Measurement System for a Quantized Hall Resistance Standard

PB94-219334 01,884 Report on the Workshop on Advanced Digital Video in the National Information Infrastructure. Held in Washing ton, D.C. on May 10-11, 1994.

01,472

Interface Roughness of Short-Period AIAs/GaAs Superlattices Studied by Spectroscopic Ellipsometry.

Metrology Model for Submicrometer Dimensional MeasUrements.

02,647

Practical Photomask Linewidth Measurements.

02,324

Interface Sharpness in Low-Order III-V Superlattices.

Interface Sharpness in Low-Order III-V Superlattices.
PB95-108775 138

Interface Sharpness during the Initial Stages of Growth of Thin, Short-Period III-V Superlattices.

PB95-108783

02,139

User's Manual for the Program MONSEL-1: Monte Carlo Simulation of SEM Signals for Linewidth Metrology.

02,325

Far-Infrared Kinetic Inductance Detector

PB95-126348

02,142

Wide Band Active Current Transformer and Shunt.

PB95-12637 1

02,036

Superconducting Kinetic Inductance Radiometer

PB95-140083

02,144

Metrology Requirements of Future Space Power Sys-

tems.

04,840

Physics for Device Simulations and Its Verification by Measurements.

PB95-141172

02,327

Metrology and Data for Microelectronic Packaging and Interconnection: Results of a Joint Workshop on Materials Metrology and Dato for Commercial Electrical and Optical Packaging and Gaithersbur. sults.

PB95-143111

02,328

Metrology and Data for Microelectronic Packaging and Interconnection: Results of a Joint Workshop on Materials Metrology and Data for Commercial Electrical and Optica Packaging and Interconnection Technologies. Held in Gaithersburg, Maryland on May 5-6, 1994. Volume 2. Presentation Material.

PB95-143327

02,330

Electronics and Electrical Engineering Laboratory 1994 Technical Accomplishments Supporting Technology for U.S. Competitiveness in Electronics.

PB95. 144309

01,887

Monte Carlo Model for SEM Linewidth Metrology.

PB95-150058

02,331

Transverse Magnetoresistance: A Novel Two-Terminal Method for Measuring the Carrier Density and Mobility of a Semiconductor Laye

PB95-150066 02,332

Spectroscopic Ellipsometry Determination of the Properties of the Thin Underlying Strained Si Layer and the Roughness at $\mathrm{SiO} 2 / \mathrm{Si}$ Interface.

PB95-150157

High Temperature Reliability of Thin Film SiO2

04,560

Performance Evaluation of a New Digital Partial Discharge Recording and Analysis System.

01,888

Band-to-Band Photoluminescence and Luminescence Excitation in Extremely Heavily Carbon-Doped Epitaxia GaAs.

PB95-150413

04,570 
Physics for Device Simulations and Its Verification by Measurements.
PB95-152914

Experimental Study of Reverse-Bias Failure Mechanisms in Bipolar Mode JFET (BMFET).

02,340

PB95-152997

Integration of Scanning Tunneling Microscope
Nanolithography and Electronics Device Processing.
PB95-153359

PB95-153359 02,341

Multijunction Thermal Converters by Commercial CMOS

PBab-15366

02,343

Directions in MEMS Research Application Development.

PB95-153797 02,106

Modeling Buffer Layer IGBTs for Circuit Simulation.

PB95-153805 02,344

Electronics and Electrical Engineering Laboratory 1995 Program Plan. Supporting Technology for U.S. Competitiveness in Electronics.

PB95-159885 01,894

Thermal Component Models for Electro-Thermal Network

Simulation.
PB95-161022

02,346

Lithium-Drift Technique for Making Submicron Thick Silicon Membranes.

02,260

$X$-ray Mask Metrology: The Development of Linewidth Standards for X-ray Lithography.

PB95-162129

02,348

Effects of Elastic Stress on the Stability of a Solid-Liquid

Interface.

03,350

Partial Discharge: Induced Aging of Cast Epoxies and Related Nonstationary Behavior of the Discharge Statistics.

PB95-163598

01,903

High Spectral
PB95-163713

02,045

Series Array of DC SQUIDs.

PB95-163861

02,046

Anomalous Behavior of a Ouantized Hall Plateau in a High-Mobility Si Metal-Oxide-Semiconductor Field-Effect

Transistor.
PB95-164174

02,354

Electrical Test Structure for Improved Measurement of Feature Placement and Overlay in Integrated Circuit Fabrication Processes.

02,355

$\mathrm{Hg} 1-\mathrm{xCdxTe}$ Characterization Measurements: Current Practice and Future Needs

02,157

Effect of Annealing Ambient on the Removal of Oxide

Precipitates in High-Dose Oxygen Implanted Silicon.
PB95-164356

Measuring Contact Charge Transfer at Interfaces: A New

Experimental Technique.
PB95-164570

03,053

Accuracy Comparisons of Josephson Array Systems

02,047

Ultralinear Small-Angle Phase Modulator. $\quad 02,261$
PB95-168852

02,261
Optical Characterization in Microelectronics Manufactur-

PB.95-169397

02,358

Design Challenges in a Commercial Ouantum Hall EffectBased Resistance Standard

02,263

From Superconductivity to Supernovae: The Ginzburg Symposium. Report on the Symposium Held in Honor of Vitaly $L$. Ginzburg. Held in Gaithersburg, Maryland on May 22, 1992.

04,649

Status and Trend

02,361

Evidence for a Deep Electron Trap and Charge Compensation in Separation by Implanted Oxygen Oxides.

PB95-175337 02,362

New Test Structure for Nanometer-Level Overlay and Feature-Placement Metrology.

02,363

Evidence for Parallel Junctions Within High-Tc Grain-

Boundary Junctions.
PB95-175410

04,657

Electrical Characterization of Liquid-Phase Epitaxially Grown Single-Crystal Films of Mercury Cadmium Telluride by Variable-Magnetic-Field Hall Measurements.
PB95-175782 177 Defect Pair Formation by Implantation-Induced Stresses
in High-Dose Oxygen Implanted Silicon-on-Insulator Main High-Dose Oxygen Implanted Silicon-on-Insulator Ma-
terial.
$\begin{array}{ll}\text { PB95-175824 } & 02,364\end{array}$

02,364

MBgneto-Tran

04,661

Fabrication Issues in Optimizing YBa2Cu3O7-x Flux Transformers for Low l/f Noise

02,059

Interaction of Stoichiometry, Mechanical Stress, and Inter-

face Trap Density in LPCVD Si-rich SiNx-Si Structures.
PB95-176301
02,366
Voltage Gain in the Single-Electron Transistor PB95-176335

Design and Machining of Copper Specimens with Micro Holes for Accurate Heat Transfer Measurements.
PB95-180428 Microelectronic Test Structures for Feature Placement and Electrical Linewidth Metrology.

PB95-180568 02,367

Hybrid Digital/Analog Servo for the NIST-7 Frequency PB95-180618

01,544

Plasma Chemical Model for Decomposition of SF6 in a Negative Glow Corona Discharge.

01,020

Epitaxial Growth of $\mathrm{Sb} / \mathrm{GaSb}$ Structures: An Example of V/III-V Heteroepitaxy.

PB95-202560

04,693

Accuracy and Repeatability in Time Domain Network Analysis.
PB95-202644

02,064

Time Domain Network Analysis Using the Multiline TRL Calibration.

PB95-202925 02,065

Electrical Method for Determining the Thickness of Metal Films and the Cross-Sectional Area of Metal Lines.
PB95-203170 02,370 PB95-203170
JEDEC 'TCR' Interlaboratory Experiment: Lessons

PB95-203188

02,371

Compensation for Substrate Permittivity in Probe-Tip Cali-

95-203519

01,915

Electronics and Electrical Engineering Laboratory Technical Progress Bulletin Covering Laboratory Programs, October to December 1994 with 1995 EEEL Events Cal

PB95-208724

02,372

Electronics and Electrical Engineering Laboratory Technical Publication Announcements Covering Laboratory Eventams, Oclober

PB95-231841

01,918

Operating Procedures and Life Cycle Documentation for the Initial Graphics Exchange Specification.

PB95-242285

02,782

Semiconductor Measurement Technology: HOTPAC. Programs for Thermal Analysis Including Version 3.0 of the TXYZ Program, TXYZ30, and the Thermal MultiLayer Program, TML.

02,374

Application of Single Electron Tunneling: Precision $\mathrm{Ca}$ pacitance Ratio Measurements.

04,703

Physical Basis for Half-Integral Shapiro Steps in a DC

PB96-102264

04,704

MEMS in Standard CMOS VLSI Technology.

PB96-102363

02,377

Bonding Wires to Ouantized Hall Resistors.

01,921 Model for Determining the Density and Mobility of Car-
riers in Thin Semiconducting Layers with Only Two Conacts.
lin Semiconducting Layers with Only Two ConMONSEL-II: Monte Carlo Simulation of SEM Signals for MONSEL-II: Monte Carlo Simulation of SEM Signals for
Linewidth Metrology.
PB96-102710 02,379

2,379

Scaling of the Nonlinear Optical Cross Sections of GaAsAlGaAs Multiple Ouantum-Well Hetero n-i-p-i's. $\quad 02,183$ Butfer Layer Modulation-Doped Field-Effect-Transistor Interactions in the Al0.33Ga0.67As/GaAs Superlattice System.

PB96-102876 02,380

TDDB Characterization of Thin SiO2 Films with Bimodal Failure Populations.

PB96-102926 02,381

Comparison of Techniques for Nondestructive Composition Measurements in CdZnTe Substrates.
PB96-103098 02,703 02,703
Nonstationary Behaviour of Partial Discharge during Discharge Induced Ageing of Dielectrics.

PB96-103114

01,922

Environmental Sensitivities of Ouartz Oscillators. 02,271

Electronics and Electrical Engineering Laboratory Technical Progress Bulletin Covering Laboratory Programs, April to June 1995 with 1995 EEEL Events Calendar. PB96-106455 01,923

Can Displays Deliver a Full Measure: Manufacturing.

Effect of Anneal Temperature on Si/Buried Oxide Inter-

face Roughness on SIMOX.
PB96-112206

New Model of 1/F Noise in Baw Ouartz Resonators.

PB96-112248 02,383

Cryogenic Precision Capacitance Bridge Using a Single Electron Tunneling Electrometer.

02,072

Substrate and Thin Film Measurements.

02,384

Semiconductor Measurement Technology: Test Structure Semiconductor Measurement Technology: Test Structure Implementation Document: DC Parametric Test Strucgrated Circuits (MMICs)

grated Circuits

02,399

Opportunities for Innovation: Optoelectronics.

PB96-118039

01,928

Characterization of Two-Dimensional Dopant Profiles: Status and Review.

$6-119300$

02,400

Automated Resistance Measurements at NIST.

PB96-119326

02,274

National Information Infrastructure and Advanced Digital

PB96-119367

01,488

Oxidation of $\mathrm{SiC}$

PB96-119516

02,40

Defect Formation Mechanism Causing Increasing Defect Density during Decreasing Implant Dose in Low-Dose PB96-119524

02,402

Mesoscopic Conductance Fluctuations in Large Devices. PB96-119656

04,735

Appearance Potentials of Ions Produced by Electron-Impact Induced Dissociative lonization of SF6, SF4, SF5Cl, $\mathrm{S} 2 \mathrm{~F} 10, \mathrm{SO} 2, \mathrm{SO} 2 \mathrm{~F} 2$, SOF2, and SOF 4 .

PB96-119730

01,080

Procedure for Measuring Trace Ouantities of S2F10, S2OF10, and S2O2F10 in SF6 Using a Gas Chromatograph-Mass Spectromete

02,513

Potential and Current Distributions Calculated Across a Ouantum Hall Effect Sample at Low and High Currents.

PB96-122106

04,045

Making Displays Deliver a Full Measure.

Survey of the Components of Display-Measurement

PB96-122528

02,188

Decomposition of SF6 and Production of S2F10 in Power

Arcs.

-122619

01,084

Overview of Bioelectrical Impedance Analyzers.

PB96-122635

Electron Attachment to Excited Molecules(1)

00,176

-122809

01,087

SF6/N2 Mixtures: Basic and High-Voltage-Insulation Properties.

PB96-123468

02,230

Nolse Characteristics Below $1 \mathrm{~Hz}$ of Zener Diode-Based

Wire Bonding to Multichip Modules and Other Soft Substrates.

PB96-123583

02,079

Characterization of Liquid-Phase Epitaxially Grown $\mathrm{HgCdTe}$ Films by Magnetoresistance Measurements.
$\mathrm{PB} 96-123617$

Nano-Defects in Commercial Bonded SOI and SIMOX.

PB96-123674 02,407

Measurement of $\mathrm{S}_{2} \mathrm{OF}_{10}$, and $\mathrm{S}_{2} \mathrm{O}_{2} \mathrm{~F}_{10}$ Production Rates from Spark and Negative Glow Corona Discharge in $\mathrm{SF} 6 / \mathrm{O} 2$ Gas Mixtures.

PB96-123740

01,093 
Bounds on Least-Squares Four-Parameter Sine-Fit Errors Due to Harmonic Distortion and Noise.

01,609

Summary Report on the Workshop on Advanced Digital Video in the National Information Infrastructure.

PB96-141320

Integrated Thin-Film Micropotentiometers.

Josephson D/A Converter with Fundamental Accuracy.
PB96-148044

Performance and Reliability of NIST $10-\mathrm{V}$ Josephson Ar-

rays. 148051

02,419

Scanning Capacitance Microscopy Measurements and Modeling: Progress Towards Dopant Profiling of Silicon. PB96-148150 04,773 Measurement of Patterned Film Linewidth for Interconnect Characterization.

02,420 PB96-148168

Semiconductor Measurement Technology: Survey of Optical Characterization Methods for Materials, Processing. and Manufacturing in the Semiconductor Industry

PB96-154596

02,706

Micromachined Display Output for a Cellular Neural Net-

work.

02,422

CMOS Circuit Design for Controlling Temperature in Micromachined Devices.

PB96-156088

02,196

Optimizing Time-

02,085

Two-Tier Multiline TRL for Calibration of Low-Cost Network Analyzers

01,947

Characterization of LPE HgCdTe Film by $\begin{array}{ll}\text { Magnetoresistance. } & 02,197 \\ \text { PB96-157961 }\end{array}$

Using Collocation in Three Dimensions and Solving a Model Semiconductor Problem.

PB96-159249

01,952

Boron-Implanted 6H-SiC Diodes.

04,081

Effect of Intermediate Thermal Processing on Microstructural Changes of Oxygen Implanted Silicon-onInsulator Material.

02,982

Stacking Fault Pyramid Formation and Energetics in Silicon-on-Insulator Material Formed by Multiple Cycles of con-on-Insulator Material Formed by Multiple Cycles of Oxygen Implantation and Annealing

04,083

RII Spectroscopy of Trap Levels in Bulk and LPE Hg1$x \mathrm{CdxTe}$

PB96-160247

04,084

Interface Roughness, Composition, and Alloying of Low Order AlAs/GaAs Superlattices Studies by X-ray Diffraclion. 160262

02,983

Electron and Hole Trapping in Irradiated SIMOX, ZMR and BESOI Buried Oxides.

PB96-160320

01,956

Effect of Single versus Multiple Implant Processing on Defect Types and Densities in SIMOX

PB96-160353

01,957

Business and Manufacturing Motivations for the Developing of Analytical Technology and Metrology for Semiconductors.

PB96- 161948

04,778

Electrical Measurements for Monitoring and Control of $i$

Plasma Processing.
PB96-161963 04,369

2D-Scanning Capacitance Microscopy Measurements of Cross-Sectioned VLSI Teststructures.

PB96-163779

04,104

High-Accuracy Principal-Angle Scanning Spectroscopic Ellipsometry of Semiconductor Interfaces.

02,427

Natural Convection trom an Array of Electronic Packages Mounted on a Horizontal Board in a Narrow Aspect Ratio Enclosure.

PB96-164017

02,087

Enhanced Voltage-Dividing Potentiometer for High-Precision Feature Placement Metrology.

02,428

Use of Pressure for Quantum-Well Band-Structure Characterization.

PB96-164058

04.779

President's Column 'Editorial'

02,239

Modeling Buffer Layer IGBT's for Circuit Simulation.

PB96-164173

02,429

Electrical Characterization of Narrow Gap n-Type Bulk $\mathrm{HgCdTe}$ Single Crystals by Variable-Magnetic-Field Hal Measurements and Reduced-Conductivity-Tensor Analy PB96-164199

01,146

Scanning Capacitance Microscopy Measurements and Modeling tor Cupant Profiling of Silicon.

04,781
Microelectronic Test Structures for Overlay Metrology.

Beyond the Technology Roadmaps: An Assessment Beyond the Technology Roadmaps: An Assessment of Electronic Materials Research and Development.
PB96-165998 Optical Characterization of Materials and Devices for the Semiconductor Industry: Trends and Needs. PB96-167192

Double-Modulation and Selective Excitation Photoreflectance for Wafer-Level Characterization of Ouantum-Well Laser Structures.

04,372

Electronics and Electrical Engineering Laboratory: 1996 Program Plan. Supporting Technology for U.S. Competitiveness in Electronics.

tiveness in Electr
PB96-175237

01,962

Theory of Electron Beam Moire.

04,373

Single-Port Techniq se for Adaptor Efficiency Evaluation.

PB96-176441 02,088
0

Novel Magnetic Field Characterization Techniques for Compound Semiconductor Materials and Devices.

PB96-176458

02,433

Microwave Characterization of Flip-Chip MMIC Interconnections.

Electrical Measurements of Microwave Flip-Chip Interconnections.
PB96-176748

02,436

Time-Dependent Dielectric Breakdown of Intrinsic $\mathrm{SiO} 2$ Films under Dynamic Stress.

Films under Dyn
PB96-179478

02,438

Scanning Capacitance Microscopy Measurements and Modeling: Progress Towards Dopant Protiling of Silicon. Ouantum Hall Effect-Based Resistance Standard: Capa-

bilities and Implementation

04,114

Assessing MOS Gate Oxide Reliability on Wafer Leve with Ramped/Constant Voltage and Current Stress.

PB96-180112

Electronics and nical Progress Bulletin Covering Laboratory Programs, October to December 1995 with 1996 EEEL Events Cal-

PB96-183116 01,966

Surface Transverse Wave Oscillators with Extremely Low Thermal Noise Floors.

PB96-186010

01,967

New Physics-Based Model for Time-Dependent- Dielectric-Breakdown

PB96-186093

02,440

New 5 and $10 \mathrm{MHz}$ High Isolation Distribution Amplifie

PB96-190202

01.510

Charpy Specimen Tests at $4 \mathrm{~K}$

PB96-190335

03,002

National Semiconductor Metrology Program, Project Portfolio FY 1996

04,789

Specification for Interoperability between Ballistic Imaging Specification for Interoperability between Ballistic Imaging
Systems. Part 1. Cartridge Cases.
PB96-195524

01.860

Design Criteria for BJT Amplifiers with Low V/f AM and PM Noise.

02,442

$30 \mathrm{MHz}$ Comparison Receiver.

01,972

How to Get NIST-Traceable Time on Your Compute

PB96-200647

01.559

Origin of $1 / 4 P M$ and $A M$ Noise in Bipolar Junction Transistor Amplifiers.

PB96-200787 02,096

Ouest to Understand and Reduce I/f Noise in Amplifiers and Baw Ouartz Oscillators.

02,097

Measurements of Properties of Materials in Electronic Packaging.

PB96-200837 01,973

Monte Carlo Simulation of Scanning Electron Microscope Signals.

Software Needs in Special Functions

02,444

PB96-200977

01,778

New Oxide Degradation Mechanism for Stresses in the Fowler-Nordheim Tunneling Regime.

04,128

PB96-200985

Time-Resolved Measurements of the Polarization State of
Four-Wave Mixing Signals trom GaAs Multiple Ouantum Four-Wave Mixing Signals trom GaAs Multiple Ouantum
Wells.
PB96-201058
04,796

02,446

Scanning Electro

SEM Linewidth Metrology of X-ray Lithography Masks. PB96-201108

02,447

New Physics-Based Model for Time-Dependent- Dielectric-Breakdown

02,448

NIST/NCMS Program on Electronic Packaging: First Up-

date.

03,008

Electric Field Dependent Dielectric Breakdown of Intrinsic $\mathrm{SiO} 2$ Films Under Dynamic Stress.

PB96-204102

02,449

Hybrid Optical-Electrical Overlay Test Structure.

02,450

Characterization of Two-Dimensional Dopant Profiles: Status and Review.

PB97-110134

02,451

Double Modulation and Selective Excitation Photoreflectance for Characterizing Highly Luminescent Semiconductor Structures and Samples with Poor Surface Morphology.

02,452

Mechanism of Defect Formation in Low-Dose Oxygen Implanted Silicon-on-Insulator Material.

02,453

Transient Errors in a Precision Resistive Divider.

Pransient Errors in a Precision Resistive Divider. 01,983
PB97-11512

Electrical Test Structures Replicated in Silicon-on-Insulator Material.

PB97-111827

02,454

Optical and Mass Spectrometric Investigations of lons and Neutral Species in SF6 Radio-Frequency Discharges 7-111918

New Refractometer by Combining a Variable Length Vacuum Cell and a Double-Pass Michelson Interterometer. Measurement and Reduction of Alignment Errors of the NIST Watt Experiment.

PB97-111959

01,987

Interdigitated Stacked P-I-N Multiple Quantum Well Mod-

PB97-112296

02,455

Development of Thin-Film Multijunction Thermal Converters at NIST.

High-Current Thin Film Multijunction Thermal Converters and Multi-Converter Modules.

PB97-112379

01,989

NIST Watt Balance: Progress Toward Monitoring the Kilo-

gram.

01,991

Frequency Synthesis and Metrology at $10(-17)$ and Be-

yond

02,101

Conformance Testing and Specification Management.

PB97-113781

Majority and Minority Electron and Hole Mobilities in

PB97-118335

04.814

Automated Guarded Bridge for Calibration of Multimegohm Standard Resistors.

02,289

Resistance Measurements from $10 \mathrm{M}$ Omega to $1 \mathrm{~T}$ Omega at NIST.

PB97-119168

02,290

NIST Comparison of the Ouantized Hall Resistance and the Realization of the SI Ohm Through the Calculable Capacitor. 
Programs, April to June 1995 with 1995 EEEL Events Calendar.

01,941

Electronics and Electrical Engineering Laboratory: 1996 Program Plan. Supporting Technology for U.S. Comperitiveness in Electronics.

PB96-175237

01,962

ELECTRONICS INDUSTRY

Electronics and Electrical Engineering Laboratory 1994 Program Plan: Supporting Technology for U.S. Competitiveness in Electronics.

01,873

PB94-12690

Electronics and Electrical Engineering Laboratory 1994 U.S. Competitiveness in Electronics.

01,887

Electronics and Electrical Engineering Laboratory 1995 Program Plan. Supporting Technology for U.S. Competitiveness in Electronics.

01,894

ELECTRONS

Electron-Photon Monte Carlo Calculations: The ETRAN Code

PB97-110407

04,138

ELECTROOPTICS

Opportunities for Innovation: Optoelectronics.

PB96-118039

01.928

Optoelectronics and Optomechanics Manufacturing: An ATP Focused Program Development. Workshop Proceed-
ings. Held in Gaithersburg. Maryland on February 15, PB97-104186

02,204

Bibliography of the NIST Optoelectronics Division. PB97-116040

02,207

ELECTROPHORESIS

Separation and Identification of Organic Gunshot and Explosive Constituents by Micellar Electrokinetic Capillary Electrophoresis.

00,566

Standard Reference Material for the Measurement of Particle Mobility by Electrophoretic Light Scattering.

PB96-102488

00,609

ELECTROPHORETIC MOBILITY

Determining Mobility from Homodyne ac Electrophoretic Light Scattering
PB95-140497

ELECTROPLATING

Diffusion of Copper into Gold Plating.

\section{ECTROSTATIC MODES}

03,462

periments.

PB95-176244

00.957

ELECTROSTRICTION

Electric Field Effects on a Near-Critical Fluid in Microgravity.

04,217

ELEVATOR FIRES

Smoke Control Systems for Elevator Fire Evacuation.

PB94-212883

00,291

ELEVATORS (LIFT)

Feasibility and Design Considerations of Emergency Evacuation by Elevators.

PB94-163441

Smoke Control Systems for Elevator Fire Evacuation

PB94-212883

00,291

Human Factors Considerations for the Potential Use of Elevators for Fire Evacuation of FAA Air Traffic Control Towers.

PB94-217163

01,300

ELLIPSOIDAL MIRROR

Simulations of Neutron Focusing with Curved Mirrors.
PB96-176649

ELLIPSOMETRY

Structure of the Vapor-Liquid Interface Near the Critical

00,902

ELLIPTIC DIFFERENTIAL EQUATIONS

Performance Characteristics of Fast Elliptic Solvers on Parallel Platforms.

PB95-180832

01,723

ELLIPTIC-TYPE INTEGRAL

Exact Series Solution to the Epstein-Hubbell Generalized Elliptic Type Integral Using Complex Variable Residue

Theory.
PB97-110167

03,423

ELLIPTICAL CONFIGURATION

Near Critical Fluid Interfaces: A Comparison of Theory and Experiment.

00,901

ELLIPTICAL DISTRIBUTIONS

Principal Points and Self-Consistent Points of Symmetric Multivariate Distributions.

PB96-135090

03,446

EMATS

Well-Shielded EMAT for On-Line Ultrasonic Monitoring of GMA Welding
PB96-186077

02,879
EMBOSSABLE GRATING

Embossable Grating Couplers for Planar Waveguide OptiCal Sensors.

00,641

EMERGENCY PLANNING

Post-Earthquake Fire and Lifelines Workshop. Held in
Long Beach, Califomia on January 30-31, 1995. Proceed-

ings.

00,209

EMERGENCY PREPAREDNESS

Earthquake and Fire in Japan: When the Threat Became

a Reality.

PB95-175238

00,201

Impact of the FCC's Open Network Architecture on NS/ NP Telecommunications Security.

01,483

EMISSION

Interaction between Dislocations and Intergranular

PB95-152096

03,190

Dislocation Em

04,630

Resonances in Two-Dimensional Array Oscillator Circuits. PB96-102082

02,066

EMISSION FACTORS

EMISS: A Program for Estimating Local Air Pollution Emission Factors Related to Energy Use in Buildings:

User's Guide and Reference Manual.
PB96-109566

02,560

EMISSION SPECTROSCOPY

Fourier Transform Atomic Emission Studies Using a Glow Discharge as the Emission Source.

00,533

EMISSION SPECTRUM

Charge-Transfer-Induced Multiplet Structure in the $\mathrm{N} 4,5 \mathrm{O} 2,3$ Soft-X-ray Emission Spectrum of Lanthanum.
$\mathrm{PB} 96-163746$
04,102

04,102

EMISSIONS

Distinguishing the Contributions of Residential Wood Combustion and Mobile Source Emissions Using Relative Concentrations of Dimethylphenanthrene Isomers.

02,563

Issues in the Field Measurement of VOC Emission Rates.

02,573

Wavelength Dependence of Normal Spectral Emissivity of High-Temperature Metals at Their Melting Point. 03,398

Radiance Temperatures (in the Wavelength Range 523-
$907 \mathrm{~nm}$ ) of Group IV B Transition Metals Titanium, Zir$907 \mathrm{~nm}$ ) of Group IV B Transition Metals Titanium, Zirconium, and Hafnium at Their Melting Points by a Pulse-
Heating Technique.
PB96-102207 03,356

EMPIRICAL EQUATIONS

Correlation of the Ideal Gas Properties of Five Aromatic Hydrocarbons

01,002

ENAMELS

Adhesion of Composites to Dentin and Enamel.

PB94-199049

00,144

Remineralization of Root Lesions with Concentrated Calcium and Phosphate Solutions.

PB96-102140 03,567

Polymeric Calcium Phosphate Composites with Remineralization Potential.

03,575

ENANTIOMERIC SEPARATION

Use of a Naphthylethylcarbamoylated- beta-Cyclodextrin Chiral Stationary Phase for the Separation of Drug Enantiomers and Related Compounds by Sub- and Supercritical Fluid Chromatography.

00,653

PBg7-113260

ENCAPSULATION

Development of a Test Method for Leaching of Lead from Lead-Based Paints Through Encapsulants.

03,128

ENCLOSURE FIRES

Reactivity of Product Gases Generated in Idealized Enclosure Fire Environments.

PB95-161790

01,386

Mathematical Modeling and Computer Simulation of Fire Phenomena.
PB95-180063

00,384

END EFFECTS

Optical Fiber Geometry by Gray-Scale Analysis with Robust Regression

04,272

END GRAFTED POLYMERS

Neutron Reflectivity of End-Grafted Polymers: Concentration and Solvent Ouality Dependence in Equilibrium Con-

PB94-185758

01,206

Neutron Reflectivity Study of the Density Profile of a Model End-Grafted Polymer Brush: Influence of Solvent

PB95-202735

01,274

ENDONUCLEASE III

Substrate Specificity of the Escherichia coli Endonuclease II. Exicision of Thymine- and Cytosine-Derived Lesions in DNA
PB95-153425 by Radiation-Generated Free Radicals.
03

ENERGY ABSORPTION

Influence of Speclmen Absorbed Energy in LOX Mechani-

PB95-107355

03,341

Effect of Charpy V-Notch Striker Radii on the Absorbed

03,365

Calculation of Photon Mass Energy-Transfer and Mass Energy-Absorption Coefficients.

PB97-110399

04,137

ENERGY CONSERVATION

Least-Cost Energy Decisions for Buildings: Part 2. Uncer-

tainty and Risk

00,240

Papers Presentations Shine.

PB94-200383

00,244

Energy Prices and Discount Factors for Life-Cycle Cost Analysis 1994. Annual Supplement to NIST Handbook 135 and NBS Special Publication 709.

PB94-206018

02,508

Energy Price Indices and Discount Factors for Life-Cycle Cost Analysis 1995. Annual Supplement to NIST Handbook 135 and NBS Special Publication 709. (Revised). 02,509

Life-Cycle Costing Workshop for Energy Conservation in Buildings: Student Manual.

PB95-175006

00,257

Building Life Cycle Cost Computer Program (BLCC), Ver4.2-95 (for Microcomputers)

PB95-501953

00,266

Building Life Cycle Cost Computer Program (BLCC) Version 4.21-95 (for Microcomputers)

PB95-502779

00,267

Building Life Cycle Cost Computer Program (BLCC) Version 4.22-95 (for Microcomputers).

00,268

Energy Price Indices and Discount Factors for Life-Cycle Cost Analysis 1996. Annual Supplement to NIST Hand book 135 and NBS Special Publication 709. (Revised).
PB96-162441 02,510

Energy Price Indices and Discount Factors for Life-Cycle Cost Analysis 1997. Annual Supplement to NIST Hand book 135 and NBS Special Publication 709. (Revised).
PB96-210745 Building Life Cycle Cost Computer Program (BLCC) Ver-

sion 4.22-95 (for Micrcomputers)

00,277

Building Life Cycle Cost Computer Program (BLCC) Version 4.4-97 (for Microcomputers).

PB97-500342

ENERGY CONSERVATION \& PRODUCTION

Predicting the Energy Performance Ratings of a Family of

Type I Combination Appliances.

B95-105524

02,504

Composite Materials for Offshore Operations: Proceedings of the International Workshop (1st). Held in Houston, exas on October 26-28, 1993

03,169 


\section{KEYWORD INDEX}

Laboratory Accreditation for Testing Energy Efficient Lighting.

PB96-122932

00.270

International Green Building Conference and Exposition (3rd). Held in San Diego, California on November 17-19, 1996. (Reannouncement with new abstract).

02,531 PB97-121826

ENERGY EFFICIENT LIGHTING PROGRAM

National Voluntary Laboratory Accreditation Program: Energy Etficient Lighting Products.
PBg 919060

02,642

ENERGY FLOW

Energy Flows in an Orifice Pulse Tube Refrigerator.
PB95-169082

ENERGY GAP

Superconducting Energy Gap of Bulk UBe13.

PB95-150116

(1)

Tunneling Measurement of the Zero-Bias Co Peak and the Bi-Sr-Ca-Cu-O Thin-Film Energy Gap.
PB95-163739 PB95-163739

ENERGY-LEVEL CALCULATIONS

Designations of ds(2)p Energy Levels in Neutral Zirconium, Hatnium, and Rutherfordium $(Z=104)$.

04,116

ENERGY LEVELS

Energy Levels of Zinc. Zn I through $\mathrm{Zn} X X X$.
P896-145982

01,122

Comment On: Two-Photon Absorption Series of Calcium.

04,074

Analysis of the $(5 d(2)+5 d 65)-5 d 6 p$ Transition Arrays of Os VII and Ir VIII, and the 6s (2)S-6p (2)P Transitions of If PBg6-159264

01,954

Hyperfine Struclure Investigations and Identification of New Energy Levels in the lonic Specirum of (147) Pm.
P896-180203 Quantum Dots in Ouantum Well Structures.

P897-118350

01,185

ENERGY LOSSES

Measured Stopping Powers of Hydrogen and Helium in Polystyrene Near Their Maximum Values.

04,729

ENERGY MANAGEMENT

Using Emulator/Testers for Commissioning EMCS Sottware, Operator Training, Algorithm Development, and Tuning Local Control Loops.

00,245

Life-Cycle Costing Manual for the Federal Energy Management Program. 1995 Edition.

02,511

ENERGY MANAGEMENT SYSTEMS

Reproducibility of Tests on Energy Management and Control Systems Using Building Emulators.

00,260

\section{ENERGY POSITIONS}

Dependence of the Thermal Electron Attachment Rate Constant in Gases and Liquids on the Energy Position of the Electron Attaching State.

P897-122253

01,996

ENERGY RELATED INVENTIONS PROGRAM

Encouraging Environmentally-Aware Inventions.
P895-161394

P895-161394

02,521

ENERGY SUPPLIES

Present Worth Factors for Life-Cycle Cost Studies in the

Department of Defense (1995).

Present Worth Factors for Life-Cycle Cost Studies in the Department of Defense (1996).
P896-106869

ENERGY TRANSFER

Atom Cooling and Trapping, and Collisions of Trapped

P896-122916

04,048

Ultrafast Time-Resolved Infrared Probing of Energy Transfer at Surfaces.

00,620

P896-123443

State-Resolved Rotational Energy Transfer in Open Shell
Collisions: $\mathrm{Cl}((2) \mathrm{P} / 2)+\mathrm{HCl}$. Collisions: $\mathrm{Cl}((2) \mathrm{P} 3 / 2)+\mathrm{HC}$

01,157

Excitation Transfer in Barium by Collisions with Noble Gases.

01,163

Calculation of Photon Mass Energy-Transfer and Mass Energy-Absorption Coefficients.

PB97.110399

04,137

ENERGY USE

EMISS: A Program for Estimating Local Air Pollution Emission Factors Related to Energy Use in Buildings: User's Guide and Reference Manual.

PB96-109566

02,560

ENGINE TESTS

New Method to Evaluate Deposit Forming Tendencies of Liquid Lubricants by Differential Scanning Calorimetry.
P895-152120

Deposit Forming Tendencies of Diesel Engine Oils-Correlation between the Two-Peak Method and Engine Tests.

PB95-152138

01,452
ENGINE VALVES

Evaluation of Wear Resistant Ceramic Valve Seats in Gas-Fueled Power Generation Engines. Topical Report, December 1991-April 1994.

P895-200218

02,466

\section{ENGINE WEAR}

Evaluation of Wear Resistant Ceramic Valve Seats in Gas-Fueled Power Generation Engines. Topical Report, December 1991-April 1994. P895.200218

02,466

ENGINEERINGIPRODUCT/NFORMATION STANDARDS

Federal Basis for Weights and Measures: A Historical Review of Federal Legislative Effor, Statutes, and Administrative Action in the Field of Weights and Measures in the
United States. AD-A280 086/0

02.616

National Type Evaluation Program: Index of Device Evaluations by Company. NCWM Publication 5 Part A (Second Edition).

PB94-160835

02,889

NVLAP Procedures U.S. Code of Federal Regulations. Tittle 15, Subtitle A, Chapter 2, Part 7. (Etfective DecemTitle 15, Subilie A Chapter 2, Part 7.

PB94-160850

02,627

Voluntary Product Standard PS 20-94. American Softwood Lumber Standard.

03,402

Models, Managing Models, Ouality Models: An Example of Ouality Management.

02,891

National Voluntary Laboratory Accreditation Program: Procedures and General Requirements.

02,630

National Voluntary Laboratory Accreditation Program 1994 Directory.

00,482

International Institute of Welding: Report on 1992 Actions. PB94-185873 02,856

International Institute of Welding: Report on 1993 Actions. PB94-185881 02,857

Precision Oscillators: Dependence of Frequency on Temperature. Humidity and Pressure.

02,031

State Weights and Measures Laboratories: State Standards Program Description and Directory. 1994 Edition.
PB94-207727

Program Handbook: Requirements for Obtaining NIST Approval/Recognition of a Laboratory Accreditation Body Under P.L. 101-592. The Fastener Ouality Act. PB94-210143

02,859

Performance Standards: The Pro's and Con's.

PB94-216132

02,896

Formulation of Position on U.S. Standards Role in Enterprise Integration

02,773

Flow Conditioner Tests for Three Orifice Flowmeter Sizes.

PB95-105540 04,201

Should NIST Accredit U.S. Calibration Laboratories.

02,646

National Voluntary Laboratory Accreditation Program: Ionizing Radiation Dosimetry.

03,623

National Voluntary Laboratory Accreditation Program: Bulk Asbestos Analysis.

02,541

NIST Handbook 44, 1995: Specifications, Tolerances and Other Technical Requirements for Weighing and Measuring Devices as Adopted by the 79th National Conference on Weights and Measures 1994

PB95-146379

02,903

Review and Upgrading of Military Fastener Test Standard MIL-STD-1312.

02,947

National Voluntary Laboratory Accreditation Program: Construction Materials Testing.

01,319

National Voluntary Laboratory Accreditation Program: Carpet and Carpet Cushion.

00,295

PB95-155560 00,295 (A91.1) and Recording Weld Data (A9.2).

02,861

Report of the National Conference on Weights and Measures (79th). Held in San Diego, California on July 17-21,

PB95-169819

02,656

National Voluntary Laboratory Accreditation Program (NVLAP): Wood Based Products.

03,405

Multi-Agency Certification and Accreditation ( $C$ and $A$ ) Process: A Worked Example.

01,601

National Voluntary Laboratory Accreditation Program 1995 Directory

00,483

Uniform Laws and Regulations in the Areas of Legal Me trology and Motor Fuel Ouality, 1994 as Adopted by the

79th National Conference on Weights and Measures

PB95.174470

02,909

Checking the Net Contents of Packaged Goods as Adopted by the 79th National Conference on Weights and Measures, 1994. Third Edition, Supplement 4.

PB95-182226

00,484

National Voluntary Laboratory Accreditation Program Acoustical Testing Services.

04,188

National Voluntary Laboratory Accreditation Program:

Pation

02,661

Electronics and Electrical Engineering Laboratory Technical Progress Bulletin Covening Laboratory Programs, April to June 1991, with 1992 EEEL Events Calendar.

P895-209821 01,916

ISO Environmental Management Standardization Etforts.

PB95-220513 02,524

National Voluntary Laboratory Accreditation Program: Electromagnetic Compatibility and Telecommunications. PB95-242376

02,664

Advanced Mass Calibration and Measurement Assurance Program for State Calibration Laboratories.

02,492

Proceedings of the Meeting of the Intergovernmental U.S.-Russian Business Development Committee's Standard Working Group (4th). Held in New York City, New York on March 27-29, 1995 and in Northbrook, 1 llinois on March 30-31, 1995

PB95-255881

00,496

National Voluntary Laboratory Accreditation Program (NVLAP): Commercial Products Testing.

02,671

National Voluntary Laboratory Accreditation Program: Thermal Insulation Materials.

National Voluntary Laboratory Accreditation Program. GOSIP: Government Open Systems Interconnection Profile.

PB95-267993

01,486

Aging, Warm-Up Time and Retrace; Important Characteristics of Standard Frequency Generators.

04,031

GATT Slandards Code Activities of the National Institute of Standards and Technology 1994.

P896-106935

00,497

Standards Setting in the European Union: Standards Organization and Officials in EU Standards Activities.

Electrical Product Requirements (Especially Ouality Requirements) in the United States.

01,929

Laboratory Accreditation for Testing Energy Etficient Lighting.

00,270

Electronic Access 10 Standards on the Information High-

PB96-131578

01,494

ISO Environmental Management Standardization Efforts.

PBo6-158662

02,528

Intemational Organization for Standardization: Curren

Activities in Fire Safety Engineering.

00,223

U.S. Government Accreditation and Conformity Assessment System Evaluation.

PB96-160239

02,678

International Challenges in Defining the Public and Pri- 
ENVIRONMENTAL STUDIES: POLLUTION MEASUREMENT

IIW Commission V Quality Control and Quality Assurance of Weided Products, Annual Report 1995/96.

02,880

Proceedings of the Open Forum on Laboratory Accreditation at the National Institute of Standards and Technology, October 13, 1995

02,686

State Weights and Measures Laboratories: Program Handbook.

02,687

TBT Agreement Activities of the National Institute of Standards and Technology, 1995.

00,499 PB97-104178

Labora.

tories.

PB97-110183

00,486

Examination Procedure Outlines: Keys to Solving the Handbook 44 Puzzle

02,690

Measurement Comparability, Traceability, and Measurement Assurance Programs.

PB97-111850 Programs. 02,692

National Voluntary Laboratory Accreditation Program (NVLAP): Fasteners and Metals

02,881

Standards Promote Credibility and Technology Transfer: The Need for Greater Industry Support of Technical Com-

mittees.
PB97-116206

02,961

Standards Activities of Organizations in the United States. PB97-124135

00,006

ENTERPRISE INTEGRATION

Formulation of Position on U.S. Standards Role in Enterprise Integration.

Defining Environment Integration Requirements.
PB96-131545

02,773

02,733

ENTHALPY

Tabulation of the Thermodynamic Properties of Normal Hydrogen from Low Temperatures to $300 \mathrm{~K}$ and from 1 to AD-A279 951/8

00,713

Calibration Standards for Differential Scanning Calorimetry. 1. Zinc Absolute Calorimetric Measureme of Enthalpy of Fusion and Temperature of Fusion HM. Calculation of Enthalpy and Entropy Differences of NearCritical Binary Mixtures with the Modified Leung-Griffiths

PB95-108635 00,885
Correlation of the Ideal Gas Properties of Five Aromatic Correlation of the Ideal Gas Properties of Five Aromatic Pydrocarbons.

01,002

Thermodynamics of the Hydrolysis of 3,4,5Trihydroxybenzoic Acid Propyl Ester (n-Propylgallate) to 3,4,5-Trihydroxybenzoic Acid (Gallic Acid) and Propan-1-0! in Aqueous Media and in Toluene.

01,160

Thermodynamic Properties of Synthetic Otavite, $\mathrm{CdCO}(\mathrm{cr})$ : Enthalpy Increment Measurements from 4.5 K $10350 \mathrm{~K}$.

00,680

Thermochemical Studies of Inorganic Chalocogenides by Fluorine-Combustion Calorimetry: Binary Compounds of Germanium and Silicon with Sulfur, Selenium and Tellurium

PB97-112528

01,181

NTHALPY FLOW

Energy Flows in an Orifice Pulse Tube Refrigerator.

NTHALPY INCREMENTS

Thermodynamic Properties of Silicides. 5. Standard Molar Enthalpy of Formation at the Temperature $298.15 \mathrm{~K}$ of Trimolybdenum Monosilicide Mo3Si Determined by Fluo-

rine-Combustion Calorimetry
PB97-119358

01,190

ENTHALPY OF FORMATION

Halon Thermochemistry: 'Ab Initio' Calculations of the Enthalpies of Formation of Fluoromethanes.

03,289

NTREPRENEURSHIP

Network Brokers Handbook: An Entrepreneurial Guide to Cooperative Strategies for Manufacturing Competitive-

00,490

\section{ENTROPIC CASIMIN FORCES}

Influence of Surface Interaction and Chain Stiffness on Polymer-Induced Entropic Forces and the Dimensions of Confined Polymers

01,203

ENTROPY

Tabulation of the Thermodynamic Properties of Normal Hydrogen from Low Temperatures to $300 \mathrm{~K}$ and from 1 to 100 Almospheres.

00,713

Influence of Surface Interaction and Chain Stiffness on Polymer-Induced Entropic Forces and the Dimensions of Confined Polymers.

PB94-185469

01,203
Calculation of Enthalpy and Entropy Differences of NearCritical Binary Mixtures with the Modified Leung-Griffiths

00,885

Glass Temperature of Polymer Blends: Comparison of Both the Free Volume and the Entropy Predictions with

PB95-140190

01,236

Correlation of the Ideal Gas Properties of Five Aromatic Hydrocarbons.

01,002

Shape of the Temperature-Entropy Saturation Boundary. PB96-135066

02,506

ENUMERATION

Examination of the 1/d Expansion Method from Exact Enumeration for a Self-Interacting Self-Avoiding Walk.

Self-Avoiding-Walk Contacts and Random-Walk SelfIntersections in Variable Dimensionality.

01.276

ENVELOPES

Influence of Envelopes Geometry on the Sensitivity of 'Nude' lonization Gauges.

04,174

ENVIROMENTAL CHEMICAL SUBSTITUTES

Report of the Refrigeration, Air Conditioning and Heat Pumps Technical Options Committee.

PB96-176755

03,293

ENVIRONIMENTAL CHEMICAL SUBSTITUTES

Gaseous Dielectrics Research: Possible SF6 Substitutes

PB96-119268

02,228

ENVIRONMENT EFFECTS

Dispersion and Deposition of Smoke Plumes Generated In Massive Fires
PB95-126066

02,540

ENVIRONMENT SIMULATION

Approaches Using Virtual Environments with Mosaic.

ENVIRONMENTAL CHARACTERIZATION

Suggestions for a Logically-Consistent Structure for Service Life Prediction Standards.

PB95-125795

00,358

ENVIRONMENTAL CHEMICAL AGENTS

Elfectiveness of Halon Alternatives in Suppressing Dynamic Combu
PB96-175732

03,288

ENVIRONMENTAL CHEMICAL SUBSTITUTES

Simultaneous Visual and Calorimetric Measurements of

ing.

03,251

Annex 18: An International Study of Refrigerant Prop-

erties

03,266

Development of a Dual-Sinker Densimeter for High-Accuracy Fluid P-V.T Measurements.

03,267

Assessing Halon Alternatives for Aircraft Engine Nacelle Fire Suppression.

Fire Suppress

01,401

Fire Suppression System Performance of Alternative Agents in Aircraft Engine and Dry Bay Laboratory Simulations. SP890: Volume

PB96- 117775

03,277

Fire Suppression System Performance of Alternative Agents in Aircraft Engine and Dry Bay Laboratory Simulations. SP 890: Volume

PB96- 117783

03,278

Agent Screening for Halon 1301 Aviation Replacement.

PB96-159710 03,282

Validation of a Turbulent Spray Flame Facility for the As-

sessment of Halon Alternatives.

03,283

Study to Determine the Existence of an Azeotropic R-22

Study to Determine the Existence of an Azeotropic R-22

'Drop-In' Substitute.
PB96- 167812

Environmental Aspects of Halon Replacements: Considerations for Advanced Agents and the Ozone Depletion Potential of CF3

PB97-122261

03,301

ENVIRONMENTAL COMPENSATION

Verification of Revised Water Vapour Correction to the Index of Refraction of Air.

PB96-161666

02,680

ENVIRONMENTAL EFFECTS

Expansion of Cementitious Materials Exposed to Sulfate Solutions.

02,577

Sulfate Attack of Cementitious Materials: Volumetric Relations and Expansions.

PB94-187317

03,232

PB94-198348

03,187

Analysis of Moisture Accumulation in a Wood-Frame Wall Subjected to Winter Climate.

PB94-199320

00,338

Methodologies for Predicting the Service Lives of Coating

PBstems.

03,124

Molecular Orbital Calculations of Bond Rupture in Brittle

Solids.

00,973

Molecular Orbital Study of Water Enhanced Crack Growth

Process.
PB95-164067 03,240

Environmental Durability of Glass-Fiber Composites.

PB95-203220

03,166

Environmental Sensitivities of Quartz Oscillators.
PB96-103148

PB96-103148
Temperature and Relative Humidity Dependence

Radiochromic Film Dosimeter Response to Gamma and Electron Radiation.

PB96-135298

03,718

VIRONMENTAL ENGINEERING

U.S. Green Building Conference, 1994.

02519

ENVIRONMENTAL HEALTH

Regulation of Lithium and Boron Levels in Normal Human Blood: Environmental and Genetic Considerations. PB94-198579

ENVIRONMENTAL IMPACT ASSESSMENTS

Atmospheric and Marine Trace Chemistry: Interfacial Biomediation and Monitoring

03,752

ENVIRONMENTAL IMPACTS

Environmental Aspects of Halon Replacements: Considerations for Advanced Agents and the Ozone Depletion Potential of CF31.

IVIRONMENTAL MONITORING

03,301

Environmental Scanning Electron Microscope Imaging Examples Related to Particle Analysis

00,766

Atmospheric and Marine Trace Chemistry: Interfacial Biomediation and Monitoring.

03,752

Certification of Polycyclic Aromatic Hydrocarbons in a

Marine Sediment Standard Reference Material.

PB96-111778

02,592

Considerations in the Design of an Environmental Specimen Bank: Experiences of the National Biomonitoring Specimen Bank Program.

PB96-112370

02,527

Response Comparison of Electret Ion Chambers, Lif TLD, and HPIC

02,578

National Status and Trends Program Specimen Bank Sampling Protocols, Analytical Methods, Results, and Archive Samples
PB97-119226

ENVIRONMENTAL POLLUTION

Standard Reference Materials for Dioxins and Other EnviPB94-198330

02,518

ENVIRONMENTAL PROTECTION

Encouraging Environmentally-Aware Inventions
PB95-161394

02,521

ISO Environmental Management Standardization Efforts.

PB95-220513 02,524

ISO Environmental Management Standardization Efforts.

PB96-158662 02,528

India: Environme

Plan.

ENVIRONMENTAL RESEARCH

Replicate Measurements for Data Quality and Environmental Modeling.

PB94-172533

Pilot Studies 
Proficiency Tests for the NIST Airborne Asbestos ProPram - 1991.

00,537

Proficiency Tests for the NIST Airborne Asbestos Program - 1992.
PB94-194362

00,539

Dilemma-Preservation versus Access

02,711

PB94-198488

Precision and Accuracy in Tandem Mass Spectrometry Measurements: A Kinetics-Based Protocol for InstrumentIndependent Measurements of Collision-Activated Dissociation in RF-Only Ouadrupoles.

PB94-216454

00,858

Use of an Environment Classification Model.

PB95-152062

01,709

Encouraging Environmentally-Aware Inventions.

PB95-161394

02,521

Atmospheric Reactivity of alpha-Methyl-Tetrahydrofuran.

PB95-163705 02,548

Ouality Assurance of Contaminant Measurements in Marine Mammal Tissues.

PB95-164034

02,588

Sources of Urban Contemporary Carbon Aerosol.

PB95-175659 02,551

Effect of Environmentally Exposures on the Properties of Polyisocyanurate Foam Insulation: Thermal Conductivity Measurements.

00,388

Measurements of Indoor Pollutant Emissions from EPA Phase II Wood Stoves.

PB95-198735

02,556

Environmental Durability of Glass-Fiber Composites.

PB95-203220

03,166

NIST Workshop on Gas Sensors: Strategies for Future Technologies. Proceedings of a Workshop. Held in Gaithersburg, Maryland on September 8-9, 1993.

PB95-210225

00,507

Few Caveats on Carbon Dioxide Monitoring.

02,562

Distinguishing the Contributions of Residential Wood Combustion and Mobile Source Emissions Using Relative Concentrations of Dimethylphenanthrene Isomers.

PB96-135124 02,563

Free Radical Chemistry of the Atmospheric Aqueous

Phase.
PB96-148101

00,117

Airbome Asbestos Method: Bootstrap Method for Determining the Uncertainty of Asbestos Concentration. Ver-

sion 1.0.
PB96-214614

00,646

Proposed Coating Technology Consortium. (National Coil Coaters Association Fall Conference). Held in Rosemont, Illinois in September 1992

03, 129

Environmental Aspects of Halon Replacements: Considerations for Advanced Agents and the Ozone Depletion Potential of CF3l.

PB97-122261

03,301

ENVIRONMENTAL SURVEYS

Scientific Protocols in Statistical Standards for Environmental Studies.

02,517

NIST Standard Reference Materials (SRMs) for Polychlorinated Biphenyl (PCB) Determinations and Their APplicability to Toxaphene Measurements.

02,585

Development and Application of an Indoor Air Ouality Commissioning Program in a New Office Building. Commissioning Program in a New Office Building.
PB96-155601 Indoor Aır Ouality Commissioning of a New Office Building.

00,279

ENVIRONMENTS

Use of an Environment Classification Model.

NZYMATIC METHODS

01,709

Optical Control of Enzymatic Conversion of Sucrose to Glucose by Bacteriorhodopsin Incorporated into Self-As-

sembled Phosphatidylcholine Vesicles.
PB96-123344

03,477

ENZYME-CATALYZED REACTIONS

Thermodynamics of Enzyme-Catalyzed Reactions. Part 4. Lyases.

PB96-145941

01,118

ENZYME KINETICS

Imposed Oscillations of Kinetic Barriers Can Cause an Enzyme to Drive a Chemical Reaction Away from Equi-

PB96-161625 01,137

Novel Activities of Human Uracil DNA N-glycosylase for Cytosine-Derived Products of Oxidative DNA Damage.
PB96-164132
03,479

ENZYMES

Aggregation Kinetics of Colloidal Particles Destabilized by PB95-125878

00,894

Michaelis-Menten Equation for an Enzyme in an Oscillating Electric Field.
PB95-140489

00.906
Method for the Assay of Hydrolytic Enzymes Using Dynamic Light Scattering.

03,531

Potentiometric Enzyme-Amplified Flow Injection Analysis Detection System: Behavior of Free and Liposome-Re. leased Peroxidase.

03,534

Enzyme and Protein Mass Transfer Coefficient in AqueEus Two Phase Systems. 1. Spray Extraction Columns. ous Two Phase

00.594

\section{EPDM ROOF MEMBRANES}

Characteristics of Adhesive-Bonded Seams Sampled from EPDM Roof Membranes.

PB95-162491

00,377

EPDM RUBBER ROOFING

Application of Thermal Analysis Techniques to the Characterization of EPDM Roofing Membrane Materials.

PB95-125845

00,359

Use of Thermal Mechanical Analysis to Characterize Ethylene-Propylene-Diene Terpolymer (EPDM) Roofing Membrane Materials.

\section{EPDM RUBBER ROOFING MEMBRANE}

00,360

Effects of Adhesive Thickness, Open Time, and Surface Cleanness on the Peel Strength of Adhesive-Bonded Seams of EPDM Rubber Roofing Membrane.

PB95-151338

00,372

EPHEMERIDES

Comparison of GPS Broadcast and DMA Precise Ephemerides.

AD-P009 114/0 01,518

Comparison of GPS Broadcast and DMA Precise Ephemerides.

01,523

\section{EPIC COMPUTER PROGRAM}

Hybrid Method for Determining Material Properties from Instrumented Micro-Indentation Experiments.

PB95-152229

03,348

EPIFLUORESCENCE

Autofluorescence Detection of 'Escherichia coli' on Silver Membrane Filters

PB96-163639

03,590

EPITAXIAL FILMS

Scanning Electron Microscopy Observations of Misfit Dislocations in Epitaxial In0.25Ga0.75As on GaAs(001)

PB96-200159

03,004

EPITAXIAL GROWTH

Homoepitaxial Growth of Iron and a Real Space View of Reflection-High-Energy-Electron Diffraction.

04,445

Scaling of Diffusion-Mediated Island Growth in Iron-on-

Iron Homoepitaxy.
PB94-185923

Spot-Profile-Analyzing LEED Study of the Epitaxial Growth of $\mathrm{Fe}, \mathrm{Co}$, and $\mathrm{Cu}$ on $\mathrm{Cu}(100)$.

Influence of $\mathrm{Cr}$ Growth on Exchange Coupling in $\mathrm{Fe} / \mathrm{Cr} /$ $\mathrm{Fe}(100)$

PB95-150181

04,562

Growth of Iron on Iron Whiskers.

04,567

Scanning Tunneling Microscopy Study of the Growth of $\mathrm{Cr} / \mathrm{Fe}(001)$ : Correlation with Exchange Coupling of Magnetic Layers.
PB95-150330

Oscillatory Exchange Coupling in $\mathrm{Fe} / \mathrm{Au} / \mathrm{Fe}(100)$.

Epitaxial Integration of Single Crystal C60.

04,568

PB95-153490

04,569

$X$-ray Photoelectron and Auger El,592 ing: A Structural Diagnostic for Epitaxial Thin Films.
PB95-180220 Epitaxial Growth and Characterization of the Ordered Vacancy Compound Culn3Ses on GaAs (100) Fabricated by Molecular Beam Epitaxy.

PB95-180725

04,687

Laser Vacuum Ultraviolet Single Photon Ionization Prob. ing of III-V Semiconductor Growth

04,691

Epitaxial Growth of $\mathrm{Sb} / \mathrm{GaSb}$ Structures: An Example of V/III-V Heteroepitaxy.

PB95-202560

04,693

Epitaxial Growth of $\mathrm{BaTiO} 3$ Thin Films at $600 \mathrm{C}$ by Metalorganic Chemical Vapor Deposition.

03,071

Growth of Epitaxial KNbO3 Thin Films.

02,409

Photoluminescence Spectra of Epitaxial Single Crystal C60.

$\begin{array}{lr}\text { PB96-141205 } & 04,754 \\ \text { Coarsening of Unstable Surface Features during Fe(001) }\end{array}$

Homoepitaxy.

PB96-186127

04,121

PITAXY

Effects of Substrate Surface Steps on the Microstructure of Epitaxial Ba2YCu3O7-x Thin Films on (001) LaAlO3. PB96-148184

Epitaxial Nucleation and Growth of Chemically Derived Ba2YCu3O7-x Thin Films on (001) $\mathrm{SrTiO}$

04,787

\section{EPOXY COATINGS}

Corrosion Resistant Epoxy-Coated Reinforcing Steel.
PB94-185618

Degradation of Powder Epoxy Coated Panels Immersed in a Saturated Calcium Hydroxide Solution Containing in a Saturated Calcium Hydroxide Solution Containing Sodium Chloride.

EPOXY MATRIX COMPOSITES

Composite Struts for SMES Plants.

01,344

PB95-155586

02,507

Torsion Modulus and Internal Friction of a Fiber-Reinforced Composite.

PB96-112339

03,070

\section{OXY RESINS}

Fluorescence Monitoring of Polarity Change and Gelation during Epoxy Cure.

01,204

Torsional Dilatometer for Volume Change Measurements on Deformed Glasses: Instrument Description and Meas. urements on Equilibrated Glasses.

PB94-211166

03,379

Micromechanics of Fracture in Rubber-Toughened Epoxies.

Relation between AC Impedance Data and Degradation of Coated Steel. 1. Effects of Surface Roughness and Contamination on the Corrosion Behavior of Epoxy Coat-

PB94-213345

03,189

Influence of Physical Aging on the Yield Response of Model DGEBA + Poly(propylene oxide) Epoxy Glasses. PB95-126363 03,381

Volume Recovery in Epoxy Glasses Subjected to Torsional Deformations: The Question of Rejuvenation. Torsional Relaxation and Volume Response during Physical Aging in Epoxy Glasses Subjected to Large Torsiona

PB95-140026 03,383

Using Torsional Dilatometry to Measure the Effects of Deformations on Physical Aging.

PB95-140901

03,384

Structural Heterogeneity in Epoxies.

PB95-151866

01,243

Partial Discharge: Induced Aging of Cast Epoxies and Related Nonstationary Behavior of the Discharge Statistics.

Applications of Fluorescence Spectroscopy in Polymer Science and Technology.

PB95- 163770

01,258

Characterization of Molecular Network of Thermosets Using Neutron Scattering.

01,259

PB95-164109

Expo-

Modification of Cast Epox 
3,4,5-Trihydroxybenzoic Acid (Gallic Acid) and Propan-I-ol in Aqueous Media and in Toluene.

PB96-186143

\section{UUIPMENT AND SUPPLIES}

Dental Materials.

\section{EQUIPMENT INTERFACES}

NIST Cooperative Laboratory for OSI Routing Tech. nology.
DE 94015308

01,791

ERBIUM

Magnetoelasticity in Rare-Earth Multilayers and Films.

\section{ERBIUM GLASS LASERS}

Frequency Stabilization of a Fiber Laser to Rubidium: A High-Accuracy $1.53 \mathrm{mu} \mathrm{m}$ Wavelength Standard.
PB95-126082 Metrology Applicatıons of Mode-Locked Erbium Fiber LaSers.
PB95-140158 Multiwavelength Birefringent-Cavity Mode-Locked Fibre Lase

PB95-150496

04,262

ERBIUM IONS

Observation of Pd-Like Resonance Lines Through $\mathrm{Pt}(32+)$ and $\mathrm{Zn}$-Like Resonance Lines of $\mathrm{Er}(38+)$ and $\mathrm{Hf}(42+$

03,896

Rh I Isoelectronic Sequence Observed from $\operatorname{Er}(23+)$ to $\mathrm{Pt}(33+)$

PB95-150652

03,898

Spectra of $\mathrm{Ag}$ I Isoelectronic Sequence Observed from $\operatorname{Er}(21+)$ to $\mathrm{Au}(32+)$

ERROR ANALYSIS

Error Propagation Biases in the Calculation of Indentation Fracture Toughness for Ceramics.

PB94-172434

03,032

Application of a Simple Technique for Estimating Errors of Finite-Element Solutions Using a General-Purpose Code

04,818

RangeCAD and the NIST RCS Uncertainty Analysis.

PB94-218591
Use of lonospheric Data in GPS Time Transfer.

PB95-163853

01,870

Software Needs in Special Functions.

PB96-200977 01,778

Troublesome Crystal Structures: Prevention, Detection, and Resolution.
PB97-109151

01,540

ERROR CORRECTING CODES

Optical Storage Media Data Integrity Studies. N95-24130/3

01,172 5-24130/3

01,620

ERROR CORRECTION CODES

Prediction of Geometric-Thermal Machine Tool Errors by Artificial Neural Networks.

PB94-186673

02,943

PIECS: A Software Program for Machine Tool ProcessIntermittent Error Compensation.

PB96-165980 02,842

Compensation of Errors Detected by Process-Intermittent Gauging.

02,846

Data Management for Error Compensation and Process

Control.
PB97-110480

02,847

ERROR DETECTION CODES

NIST Program for Investigating Error Reporting Capabilities of Optical Disk Drives.

01,635

\section{ERRORS}

Real Time Compensation for Tool Form Errors in Turning Using Computer Vision

PB95-107231

02,945

ERRORS IN VARIABLES MODELS

Existence and Nonexistence Theorems of Finite Diameter Sequential Confidence Regions for Errors-in-Variables Models.

PB95-180352

03,441

Substrate Specificity of the Escherichia coli Endonuclease III: Exicision of Thymine- and Cytosine-Derived Lesions in DNA Produced by Radiation-Generated Free Radicals.

PB95-153425

Deletion Analysis of the Mini-P1 Plasmid Origin of Replication and the Role of E.coli DnaA Protein. PB95-163911

03,539

ESR-Based Analysis in Radiation Processing. PB95-161634

03,931

\section{ESTELLE TRANSLATOR}

PET and DINGO Tools for Deriving Distributed Implementations from Estelle.

PB95-203253

01,727

Structural Stabilization of Phase Separating PC/Polyester Blends through Interfacial Modification by Transesterification Reaction.

01,239
ESTIMATING

Recent Approaches to Extreme Value Estimation with Application to Wind Speeds. Part 1. The Pickands Method.
PB94-213170 00,019 Outlier-Resistant Methods for Estimation and Model FitPBg5-203436

03,444

Extreme Wind Estimates by the Conditional Mean Exceedance Procedure.

PB95-220471

00,120

Estimation of Measurement Uncertainty of Small Circular Features Measured by CMMs.

\section{ESTIMATION}

Extreme Winds Estimation by 'Peaks Over Threshold' and Epochal Methods.

PB96-159686

00,468

Estimates of Hurricane Wind Speeds by the 'Peaks Over Threshold' Method

ETCHING

Kinetic-Energy-Enhanced Neutral Etching

PB96-200613

00,471

ETHANE

Thermodynamic Properties of the Methane-Ethane Sys-

PB95-125779

Experimental Method for Obtaining Critical Densities of Binary Mixtures: Application to Ethane + n-Butane

PB95-151148

00,930

Measurements of the Virial Coefficients and Equation of State of the Carbon Dioxide + Ethane System in the Supercritical Region. Vapor-Liquid Equilibria of Ternary Mixtures in the Critical Region on Paths of Constant Temperature and Overall Composition.

PB96-161856

01,139

ETHANE/CHLORO-TETRAFLUORO

Measurements of the Viscosities of Saturated and Com pressed Fluid 1-chloro-1,2,2,2-tetrafluoroethane (R124) and Pentafluoroethane (R125) at Temperatures between 120 and $420 \mathrm{~K}$.

PB94-199791

ETHANE/DICHLORO-DIFLUORO

Measurements of the Viscosities of Saturated and Com pressed Liquid 1,1,1,2-Tetrafluoroethane (R134a), 2,2 Dichloro-1,1,1-Trifluoroethane (R123) and 1,1-Dichloro-1 Fluoroethane (R141b)

PB95-175386

03,273

ETHANE/DICHLORO-FLUORO

Measurements of the Viscosities of Saturated and Compressed Liquid 1,1,1,2-Tetrafluoroethane (R134a), 2,2 Dichloro-1,1,1-Trifluoroethane (R123) and 1,1-Dichloro-1Fluoroethane (R141b) PB95-175386

03,273

ETHANE/DICHLORO-TRIFLUORO

Vapor Pressure of 1,1-dichloro-2,2,2-trifluoroethane (R123).

THANE/PENT AFLUORO

Measurements of the Viscosities of Saturated and Com pressed Fluid 1-chloro-1,2,2,2-tetrafluoroethane (R124) and Pentafluoroethane (R125) at Temperatures between 120 and $420 \mathrm{~K}$ PB94.199791

03,254

\section{ETHANE/TETRAFLUORO}

Measurements of the VIscosities of Saturated and Compressed Liquid 1,1,1,2-Tetratluoroethane (R134a), 2,2 Dichloro-1,1,1-Trifluoroethane (R123) and 1,1-Dichloro-1Fluoroethane (R141b)

PB95-175386

03,273

ETHANOL

Effect of Ethanol on the Solubility of Dicalcium Phosphate Dihydrate in the System $\mathrm{Ca}(\mathrm{OH}) 2-\mathrm{H} 3 \mathrm{PO} 4-\mathrm{H} 2 \mathrm{O}$ at $37 \mathrm{C}$ PB95-163507 00,163

Effect of Ethanol on the Solubility of Hydroxyapatte in the System $\mathrm{Ca}(\mathrm{OH}) 2-\mathrm{H} 3 \mathrm{PO} 4-\mathrm{H} 2 \mathrm{O}$ at $25 \mathrm{C}$ and $33 \mathrm{C}$.

00,169

Millimeter- and Submillimeter-Wave Spectrum of transEthyl Alcohol. Ethyl Alcohol.

01,102

\section{ETHANOL MONOCHLOROBENZENE}

Oscillometric and Conductometric Analysis of Aqueous and Organic Dosimeter Solutions.

04,054

\section{ETHANOL WATER SOLUTION}

Molecular Dynamics Investigation of the Surface/Bulk Equilibrium in an Ethanol-Water Solution.

PB97-113112

01,183

\section{ETHER/MALEIMIDOPHENYL GLYCIDYL}

Thermal Behavior of 4-Maleimidophenyl Glycidyl Ether

Resins.
PB95-153151

01,249

ETHERS

Thermodynamic Properties of CHF2-O$\begin{array}{ll}\text { CHF2,Bis (difluoromethyl) Ether. } & \\ \text { PB94-199569 } & 00,798\end{array}$

00,798

2-Tunneling Path Internal-Axis-Method-Like Treatment of the Microwave Spectrum of Divinyl Ether.

00,808

Thermodynamic Properties of Two Gaseous Halogenated Ethers from Speed-of-Sound Measurements: Difluoromethoxy-Difluoromethane and 2-Difluoromethoxy-
1,1,1-Trifluoroethane.
PB96-102413 04,189

ETHYL RADICALS

Laser Flash Photolysis, Time-Resolved Fourier Transform Infrared Emission Study of the Reaction $\mathrm{Cl}+\mathrm{C} 2 \mathrm{H} 5$ yields $\mathrm{HCl}(\mathrm{v})+\mathrm{C} 2 \mathrm{H} 4$

\section{ETHYLENE}

01,049

Nonlinear Correlation of High-Pressure Vapor-Liquid Equilibrium Data for Ethylene $+n$-Butane Showing Inconsistencies in Experimental Compositions.

Sistencies in Exp

01,141

\section{ETHYLENE/DICHLORO}

Gas Phase Reactivity Study of $\mathrm{OH}$ Radicals with 1,1Dichloroethene and cis-1,1-Dichloroethene and Trans-1,2Dichloroethen $\Theta$ over the Temperature Range 240-400 K.

ETHYLIDENE BISPHOSPHONATE/HYDROXY

Effect of 1-Hydroxyethylidene-1,1-Bisphosphonate on Mernbrane-Mediated Calcium Phosphate Formation in Model Liposomal Suspensions.

PB95-169223

03,469

ETRAN CODE

Electron-Photon Monte Carlo Calculations: The ETRAN Code.

PB97-110407

04,138

EULER-POINSOT PROBLEM

Complete Reduction of the Euler-Poinsot Problem. PB94-172152

EUROPE

Aid for Smaller Businesses.

PB94-212461

00,492

EUROPEAN COMMUNITIES

TMACH Experiment Phase 1. Preliminary Developmental Evaluation.

EUROPEAN UNION

Standards Setting in the European Union: Standards Or. ganization and Officials in EU Standards Activities.

PB96-115019

EUTECTIC ALLOYS

Phase-Field Model for Solidification of a Eutectic Alloy PB95-147914

03,345

EVACUATING (TRANSPORTATION)

Feasibility and Design Considerations of Emergency Evacuation by Elevators
PB94-163441

00,287

Feasibility of Fire Evacuation by Elevators at FAA Control Towers.

PB94-213857

04,844

EVACUATION

Smoke Control Systems for Elevator Fire Evacuation.
PB94-212883

PB94-212883
EXITT: A Simulation Model of Occupant Decisions and Aclions in Residential Fires. 


\section{KEYWORD INDEX}

EXAMINATION PROCEDURE OUTLINES (EPOS) Examination Procedure Outlines: Keys to Solving the Handbook 44 Puzzle.

PB97-110217

02,690

EXCHANGE COUPLING

Influence of Thickness Fluctuations on Exchange Cou oling in $\mathrm{Fe} / \mathrm{Cr} / \mathrm{Fe}$ Structures.

04,745

SEMPA Studies of Exchange Coupling in Magnetic Multilayers.

PB96-164074

04,780

EXCHANGE INTERACTIONS

SEMPA Studies of Oscillatory Exchange Coupling.
PB95-163556

EXCITATION

Estimation of System Damping at the Lotung Site by Application of System Identification.

plication of Syste

01,351

EXCITATIONS

Low-Frequency Excitations of Oriented DNA

PB96-137799

03,548

EXCITED STATES

Search for Small Violations of the Symmetrization Postulate in an Excited State of Helium.

04,050

EXCITODION SPECTRA

Population Trapping in Short-Pulse Multiphoton Ionization.

04,371

EXCITON

Intermediate Coupling in L.2-L3 Core Excitons of $\mathrm{MgO}$, $\mathrm{Al} 2 \mathrm{O} 3$, and $\mathrm{SiO}_{2}$
$\mathrm{~PB} 96-158043$

04,079

EXCITONS

Excitons in Complex Ouantum Nanostructures.

01,184

EXCLUDED VOLUME

Segmental Concentration Profiles of End-Tethered Polymers with Excluded-Volume and Surface Interactions. PB97-119002

00,654

EXIMER LASERS

Deep-UV Excimer Laser Measurements at NIST.

04,355

EXIT89 COMPUTER MODEL

Enhancement of EXIT89 and Analysis of World Trade Center Data.

00,231

EXOSKELETON

Robotics Application to Highway Transportation. Volume 2. Literature Search

PB95-170551

01,337

XOTOXINS

Genetically Engineered Pores for New Materials.

03,550

EXPECT COMPUTER PROGRAM

Ouch Those Programs are Painful

PB96-102678

01,739

EXERIMENTAL DATA

Models and Interactions.

PB94-216306

02,641

EXPERIMENTAL DESIGN

Measuring the Stability of Three Copper Alloys.

Introduction to ASTM 1199 'Wear Test Selection for Design and Application

PB95-162517

03,238

Taguchi's Parameter Design: A Panel Discussion.

Developing Measurement for Experimentation.

03,445

PB97-118707

03,450

EXPERIMENTAL MECHANICS

Journal of Research of the National Institute of Standards and Technology, January/February 1996. Volume 101, Number 1 .

PB96-175666

00,113

Theory of Electron Beam Moire.

PB96-175690

04,373

EXPERIMENTATION

Guidelines for Reporting Results of Computational Experiments. Report of the Ad hoc Committee.

03,427

\section{EXPERT PROGRAMMING LANGUAGE}

Kibitz-Connecting Multiple Interactive Programs Together PB94-213311

EXPERT SYSTEMS

PC-Based Prototype Expert System for Data Management and Analysis of Creep and Fatigue of Selected Materials at Elevated Temperature

PB94-172251

03,202

Highway Concrete (HWYCON) Expert System User Reference and Enhancement Guide.

01,316

Application of Expert System to Select Data Sources from Chemical Information Databases.

PB95-125654

00,505

PB95-162913

04,868
Expert Control System Shell Version 1.0 User's Guide PB95-198859

01,790

EXPOSURE (PHYSIOLOGY)

Maximum Permissible Body Burdens and Maximum Permissible Concentrations of Radionuclides in Air and in Water for Occupational Exposure.

03,610

EXPRESS

Concepts of the NIST EXPRESS Server.

PB95-180543

02,781

EXPRESS LANGUAGE

Extensions of the Prototype Application Protocol of Ready-to-Wear Apparel Pattern Making.

03,198

XPRESS LANGUAGE ENVIRONMENT

Dynamic Objects and Meta-Level Programming of an EX-

PRESS Language Environment.

01,774

EXPRESS MODELING LANGUAGE

Structural EXPRESS Editor.

PB94-159795

02,769

EXTENDED CORRESPONDING STATES

Thermodynamic Properties of the Methane-Ethane Sys tem.
PB95-125779

00,891

EXTENSOMETERS

Torsional Dilatometer for Volume Change Measurements on Deformed Glasses: Instrument Description and Meas. urements on Equilibrated Glasses.

PB94-211166

03.379

EXTENSOMETRY

Sources of Strain-Measurement Error in Flag-Based PBtensometry.

03,108

EXTINCTION TIME

Suppression Research: Strategies.

PB94-211372

00,346

EXTRACTION

Distributed measurements of tracer response on packed bed flows using a fiberoptic probe array. Final report.

DE95013079 00,667

Thermophysical Property Data for Supercritical Fluid Extraction Design.
PB94-199221

00,668

EXTRACTION COLUMNS

Continuous Counter-Current Two Phase Aqueous Extraction.

00,675

EXTRACTIVE METALLURGY

Vapor Transport in Materials and Process Chemistry.
PB94-211745

EXTRACTORS

Influence of Envelopes Geometry on the Sensitivity of 'Nude' Ionization Gauges

PB97-119077

04,174

EXTRAPOLATION CHAMBERS

Extrapolation Chamber Measurements on (90) Sr $+(90) \mathrm{r}$

Beta-Particle Ophthalmic Applicator Dose Rates.

03,626

\section{EXTREME ULTRAVIOLET}

Improved Reflectometry Facility at the National Institute of Standards and Technology.

PB96-160338

04,087

EXTREME ULTRAVIOLET RADIATION

Volume-Limited ROSAT Survey of Extreme Ultraviole Emission from all Nondegenerate Stars within 10 Parsecs.

PB96-103189

00,093

EXTREME VALUE ESTIMATION

Recent Approaches to Extreme Value Estimation with Application to Wind Speeds. Part 1. The Pickands Method.

plication to Wind Speeds. Part 1. The Pickands Method.
PB94-213170

\section{EXTREME-VALUE PROBLEMS}

Recent Approaches to Extreme Value Estimation with Application to Wind Speeds. Part 1. The Pickands Method. PB94-213170

00,019

Journal of Research of the National Institute of Standards and Technology, July/August 1994. Volume 99, Number 4. Special Issue. Extreme Value Theory and Applications. Proceedings or ine Coniterence On Exteme Value Theory and Applications, Volume 2. Held at Gaithersburg. Mary land, in May 1993

PB95-160594

03,440

Assessment of 'Peaks Over Threshold' Methods for Estimating Extreme Value Distribution Tails.

00.441

Extreme Wind Distribution Tails: A 'Peaks Over Threshold' Approach

PB95-219416 00,127

Development of Computer-Based Models of Standards and Attendant Knowledge-Base and Procedural Systems. PB96-155783 00,464 Extreme Winds Estimation by 'Peaks Over Threshold'

and Epochal Methods.

00,468

Modeling of Extreme Loading by 'Peaks Over Threshold'$$
\text { Methods. }
$$

00,469

EXTREME VALUE THEORY

Extreme Value Theory and Applications: Proceedings of the Conference on Extreme Value Theory and Applications, Volume 3. Held in Gaithersburg, Maryland in May PB95-104956

03,432

Journal of Research of the National Institute of Standards and Technology, July/August 1994. Volume 99. Number 4. Special Issue: Extreme Value Theory and Applications Proceedings of the Conterence on Extreme Value Theory and Applications, Volume 2. Held at Gaithersburg. Maryland, in May 1993
PB95.160594

03,440

\section{EXTREMELY HIGH FREQUENCIES}

Comparison of k-Correction and Taylor-Series Correction or Probe-Position Errors in Planar Near-Field Scanning. PB96-147137

EXTREMELY LOW FREQUENCIES

ELF Electric and Magnetic Field Measurement Methods. PB95-161675

04,423

EXTREMELY LOW FREQUENCY

Condensed Catalogue of Electromagnetic Environment Measurements, $30-300 \mathrm{~Hz}$

PB95-162210

01,899

Catalogue of Electromagnetic Environment Measure. ments, 30-300 $\mathrm{Hz}$

$$
\text { PB96-155452 }
$$

EXTREMUM VALUES

Heap of Data
PB97-111488

03,424

EYE PIGMENTS

Retinal-Protein Complexes as Optoelectronic Components.

PB95-150397

02,146

YE PROTEINS

Retinal-Protein Complexes as Optoelectronic Components.

02,146

EYE SAFETY

Optical Density Measurements of Laser Eye Protection MBaterials.

F STARS

IUE Observations of Solar-Type Stars in the Pleiades and the Hyades.

PB94-199437

00,053

FABRICATION

Fabrication of Flaw-Tolerant Aluminum-Titanate-Reinorced Alumina.

PB95-162533

03,161

MEMS in Standard CMOS VLSI Technology

02,377

Superconducting Integrated Circuit Fabrication with Low Superconducting Integrated Circuit Fabrication with Low Temperature ECR-Based PECVD SiO2 Dielectric Films.
PB96-103015 Fabrication of Optics by Diamond Tuming 


\section{KEYWORD INDEX}

FEDERAL INFORMATION PROCESSING STANDARDS

4. Special Issue: Extreme Value Theory and Applications. Proceedings of the Conference on Extreme Value Theory and Applications, Volume 2. Held at Gaithersburg, Maryland, in May 1993.

PB95-160594

03,440

Characteristics of Adhesive-Bonded Seams Sampled from EPDM Roof Membranes.

00,377

Exponentially Rapid Coarsening and Bucklirig in Coherently Self-Stressed Thin Plates.

04,821

Critical-Current Degradation in Nb3 Al Wires Due to Axial and Transverse Stress.

PB95-202784

02,226

Room-Temperature Flexure Fixture for Advanced Ceram-

PB95-210498

03,061

Evaluation of Thermal Wave Imaging for Detection of Ma-

chining Damage in Ceramics.
PB95-220547

03,062

Fracture Testing of Large-Scale Thin-Sheet Aluminum

00,024

Failure of All-Ceramic Fixed Partial Dentures 'In vitro' and 'In vivo': Analysis and Modeling.

00,175

PB96-122536

Me-

Catastroph

PB96-135140

03,217

Correlations between Flaw Tolerance and Reliability in

Zirconia.
PB96-161922

Cracks and Dislocations in Face-Centered Cubic Metallic Multilayers.

PB96-163696

02,989

Model for Microcrack Initiation and Propagation beneath Hertzian Contacts in Polycrystalline Ceramics.

03,077

PB96-163704

Elevated

Fracture of Silicor

03,179

B96-180260

Diffusive Crack Growth at a Bimaterial Interface.
PB96-204110
03,090

Life Prediction of a Continuous Fiber Reinforced Ceramic Composite Under Creep Conditions.

03,091

Postfailure Subsidiary Cracking from Indentation Flaws in Britżle Materials.

PB97-110340

03,103

AILURE (MECHANICS)

Catastrophic Failures Propagate Field of Fracture Me-

chanics.
PB96-135140

03,217

AILURE MODES

Evaluating the Seismic Performance of Lightly-Reinforced Circular Concrete Bridge Columns.

PB95-163259

01,335

FAR INFRARED RADIATION

Laser Spectroscopy of Carbon Monoxide: A Frequency Reference for the Far Intrared.

PB95-163606

04,277

Niobium Micro
PB96-111729

02, 184

FAR ULTRAVIOLET RADIATION

Bibliography of Photon Total Cross Section (Attenuation Coefficient) Measurements $10 \mathrm{eV}$ to $13.5 \mathrm{GeV}, 1907$.

PB94-193760 03,804

GHRS Observations of Cool, Low-Gravity Stars. 1. The Far-Ultraviolet Spectrum of alpha Orions (M2 lab)

PB96-112016

00,094

FAR ULTRAVIOLET SPECTROGRAPH EXPLORER

Scientific Rationale and Present Implementation Strategy

for the Far Ultraviolet Spectrograph Explorer (FUSE).

PB96-123328

00,045

FARADAY CONSTANT

Faraday Constant.

PB96- 159793
ARADAY EFFECT

01,955

Submicroampere-Per-Root-Hertz Current Sensor Based on the Faraday Effect in Ga: YIG.

PB95-162467

02,155

Magnetooptic Effects

PB96-119292

04,338

Faraday Effect Sensors for Magnet Field and Electric PB96-119664

04,736

Fiber-Optic Faraday-Effect Magnetic-Field Sensor Based on Flux Concentrators.

PB96-200308 02,201

Fundamentals and Problems of Fiber Current Sensors. PB97-111835

02,205

FARMED STRUCTURES

Enhancements to Program IDARC: Modeling Inelastic Behavior of Welded Connections in Steel Moment-Resisting Frames.

PB95-231601

00,452
FAST FOURIER TRANSFORMATIONS

Comparison of FFT Fingerprint Filtering Methods for Neural Network Classification

PB95-136362

01,840

FASTENERS

Program Handbook: Requirements for Obtaining NIST

Approval/Recognition of a Laboratory Accreditation Body

Under P.L. 101-592. The Fastener Quality Act.

02,859

Portsmouth Fastener Manufacturing Workstation. User's Manual.

02,860

Review and Upgrading of Military Fastener Test Standard MIL-STD-1312.

PB95-154720

02,947

National Voluntary Laboratory Accreditation Program NVLAP): Fasteners and Metals.

02,881

FASTNER QUALITY ACT

mplementation of the Fastener Quality Act.

PB96- 160676

02,876

FATIGUE LIFE

Generic Model for Creep Rupture Lifetime Estimation on

Fibrous Ceramic Composites
PB94-200235

03,137

FATIGUE (MECHANICS)

PC-Based Prototype Expert System for Data Management and Analysis of Creep and Fatigue of Selected Ma. erials at Elevated Temperatures.

PB94-172251 03,202

Transient Subcritical Crack-Growth Behavior in Transformation-Toughened Ceramics.

03,038

FAULT DETECTION

Recent Developments at NIST on Optical Current Sensors and Partial Discharge Diagnostics.

02,147

Fault Diagnosis of an Air-Handling Unit Using Artificial Neural Networks.

PB97-121321

FAULTS

Software Needs in Special Functions.

FDDI

Planning for the Fiber Distributed Data Interface (FDDI).

FDMS COMPUTER DATA BASE

Fire Data Management System, FDMS 2.0, Technical Documentation.
PB94-164019

01,358

FEATURE EXTRACTION

Object Finder for Digital Images Based on Multiple Thresholds, Connectivity, and Internal Structures.

PB94-199106 01,833

Self-Organizing Neural Network Character Recognition Using Adaptive Filtering and Feature Extraction.
PB96-119797

FEDERAL AGENCIES

Study of Federal Agency Needs for Information Technology Security

01,579

Guidance to Federal Agencies on the Use of Trusted Systems.
PB95-163440

01,597

Multi-Agency Certification and Accreditation (C and $A$ )

Process: A Worked Example.

PB95-171955

01,601

Federal Labs Have Key Role in Metrication

PB96-123401

02,920

Security Program Management.

PB96-156112

TMACH Experiment Phase 1. Preliminary Developmental

$\begin{array}{ll}\text { Evaluation. } & 01,618\end{array}$

Science, Technology, and Competitiveness: Retrospective on a Symposium in Celebration of NIST's 90th Anniversary and the 25th Anniversary of the Gaithersburg Laboratories, November 14-15, 1991

PB97-121610

02,696

FEDERAL BUILDING

Summary of Federal Construction and Building $R$ and $D$ in 1994

PB97-114250

00,234

FEDERAL BUILDINGS

Seismic Instrumentation of Existing Buildings. $\quad 00,420$
PB9 4-159779

Evaluation and Strengthening Guidelines for Federal Buildings: Identification of Current Federal Agency Pro-

PB94-176278

00,424

Evaluation and Strengthening Guidelines for Federal Buildings: Assessment of Current Federal Agency Evaluation Programs and Rehabilitation Criteria and Development of Typical Costs for Seismic Rehabilitation. PB94-181856

00,425

Environmental Evaluation of a New Federal Office Build-

PB94-199874

02,538

Standards of Seismic Safety for Existing Federally Owned or Leased Buildings and Commentary.

PB95-130209

00,431

Evaluation and Retrofit Standards for Existing Federally Owned and Leased Buildings.

PB95-150918

00,434

(2699: Seismic Safety of Federal and Federally Assisted or Regulated New Building Construction.

Life-Cycle Costing Workshop for Energy Conservation in Buildings: Student Manual.

PB95-175006

00,257

Seismic Safety of Federal Buildings. Initial Program: How Much Will lt Cost.

00,447

Performance of Federal Buildings in the January 17, 1994 Northridge Earthquake.

PB95-231775

00,453

ICSSC Guidance on Implementing Executive Orde 12941 on Seismic Safety of Existing Federally Owned or Leased Buildings

00,459

How-To Suggestions for Implementing Executive Order 12941 on Seismic Safety of Existing Federal Buildings, A

PB96-131552

00,461

Executive Order 12941. Seismic Safety of Existing Federally Owned or Leased Buildings: It's History, Content and Objectives.

00,465

Life-Cycle Costing Manual for the Federal Energy Management Program. 1995 Edition.

\section{EDERAL COMMUNICATIONS COMMISSION}

National Voluntary Laboratory Accreditation Program: Electromagnetic Compatibility and Telecommunications. FCC Methods.

FEDERAL CRITERIA

02,664

Proceedings of the Workshop on the Federal Criteria for Information Technology Security. Held in Ellicot City, Maryland on June 2-3, 1993

PB94-162583

01,575

FEDERAL GOVERNMENT

Computer Security Management and Planning in the U.S

Federal Government.
PB95-163432 01,596

Addressing U.S. Government Security Requirements for

PB96-160577

01,611

Federal Implementation Guideline for Electronic Data Interchange: ASC X12 003050 Transaction Set 836 Procurement Notices

01,827

FEDERAL INFORMATION PROCESSING STANDARDS

Data Encryption Standard (DES); Category: Computer Security; Subcategory: Cryplography. Graphical Kernel System (GKS). Category: Software Standard. Subcategory: Graphics. International Standard Information Technology; Computer Graphics: Graphica Kernel System (GKS) Language Bindings. Part 4: C.
FIPS PUB 120-1C 
Portable Operating System Interface (POSIX). Part 2. Shell and Utilities. Category: Soltware Standard; Subcategory: Operating Systems.

01,797

Guideline for the Analysis of Local Area Network Security. Category: Computer Security; Subcategory: Risk Analysis and Contıngency Planning.

FIPS PUB 191

01,799

Applicatıon Profile for the Government Information Locator Service (GILS). Category: Software Standard; Subcategory: Information Interchange.

category: Informati
FIPS PUB 192

01,800

SOL Environments. Category: Software Standard; Subcategory: Database.

FIPS PUB 193

01,801

Open Document Architecture (ODA) Raster Document Application Profile (DAP). Category: Sottware Standard: Subcategory: Graphics.

01,669

Federal Building Grounding and Bonding Requirements for Telecommunications. Category: Telecommunications Standard; Subcategory: Grounding and Bonding.

FIPS PUB 195

01,802

Validated Products List (Cobol, Fortran, ADA, Pascal, MUMPS, SOL)

01,700

Codes for Named Populated Places, Primary County Divisions, and Other Locational Entities of the United States (FIPS PUB 55-3) (on Magnetic Tape).

00,129

Validated Products List (Cobol, Fortran, ADA, Pascal, Validated Products List (Cobol, Fortran, ADA, Pascal
MUMPS SOL). MUMPS, SOL)
PB95-937300

01,738

FEDERAL INFORMATION PROCESSING STANDARDS

CRYPTOGRAPHY

Guideline for the Use of Advanced Authentication Tech. nology Alternatives. Category: Computer Security. Subcategory: Access Control.

Category: Access

01,798

FEDERAL LAW

1950 Supplement to Screw-Thread Standards for Federal Services. 1944.
AD-A280 223/9

03,656

FEEDBACK CONTRO

Control of Gas-Metal-Arc Welding Using Arc-Light Sensing.

02,869

FERMENTATION

Feasibility of Fluorescence Detection of Tetracycline in

Media Mixtures Employing a Fiber Optic Probe.
PB96-163654

00.511

FERRIC NITRILOTRIACETATE

DNA Base Modifications in Renal Chromatin of Wista Rats Treated with a Renal Carcinogen, Ferric Nitrilotriacetate.

03,648

Treatment of Wistar Rats with a Renal Carcinogen, Ferric Nitrilotriacetate, Causes DNA-Protein Cross-Linking be tween Thymine and Tyrosine in Their Renal Chromatin.

FERRITE GARNETS

Magneto-Optic Magnetic Field Sensors Based on Uniaxial Iron Garnet Films in Optical Waveguide Geometry.

PB95-168498 02,159

Magnetic Force Microscopy Images of Magnetic Garnet with Thin-Film Magnetic Tip.

PB95-176210

04,669

Novel Bulk Iron Garnets for Magneto-Optic Magnetic Field Sensing.

04,675

Domain Effects in Faraday Effect Sensors Based on Iron

Garnets.

02,268

FERRITES

Applicability of Effective Medium Theory to Ferroelectric Ferrimagnetic Composites with Composition and Fre quency-Dependent Complex Permittivities and Permeabilities
PB96-157854

\section{FERROCENES}

High-Pressure Equilibrium Cell for Solubility Measurements in Supercr
PB95- 175634

FERROELECTRIC MATERIALS

Hysteresis Measurements of Remanent Polarization and Coercive Field in Polymers.

PB94-199767

FERROELECTRICITY

Ferroelectric Thin Film Characterization Using Superconducting Microstrip Resonators.

FERROMAGNETIC MATERIALS

Monte Carlo and Mean-Field Calculations of the Magnetocaloric Effect of Ferromagnetically Interacting Clusters.

FERROMAGNETIC RESONANCE

03,201

Method for Determining Both Magnetostriction and Elastic Modulus by Ferromagnetic Resonance.

PB95.150108

02,974
FERROUS TRIMETHYLAMMONIUM CHLORIDE

Ouasielastic and Inelastic Neutron-Scattering Studies of ((CD3)3ND)FeCl3.2D2O: A One-Dimensional Ising Ferromagnet PB95-140562

04,547

FIBER AMPLIFIERS

Pump-Induced Dispersion of Erbium-Doped Fiber Measured by Fourier-Transform Spectroscopy.

04,236 B 94-211935

FIBER ANNEALING

Improved Annealing Technique for Optical Fiber. PB96-119680

04,343

FIBER COMPOSITES

Interface Properties for Ceramic Composites from a Single Fiber Pull-Out Test.

03,135

Generic Model for Creep Rupture Lifetime Estimation on Fibrous Ceramic Composites.

PB94-200235

03,137

Elastic Properties of Uniaxial-Fiber Reinforced Compostes: General Features.

03,140

Determination of Fiber-Matrix Interfacial Properties of $1 \mathrm{~m}$ portance to Ceramic Composite Toughening.

PB95-12581

03,149

Environmental Durability of Glass-Fiber Composites.

03,166 Torsion Modulus and Internal Friction of a Fiber-Reinforced Composite.

PB96-112339

03,070

IBER DISTRIBUTED DATA INTERFACE

Planning for the Fiber Distributed Data Interface (FDDI).

PB94-135761

FIBER GRATINGS

Growth Characteristics of Fiber Gratings.

PB96-122957

01,621

PBR LASERS

Electronically Tunable Fiber Laser for Optical Pumping of (3) He and (4) He.

04,381

\section{FIBER OPTIC SENSORS}

Faraday Effect Current Sensors.

PB94-200698

02,127

Faraday Effect Sensors: A Review of Recent Progress.

PB94-200706 02,128

Recent Developments at NIST on Optical Current Sensors and Partial Discharge Diagnostics.

02,147

High Speed, High Sensitivity Magnetic Field Sensors .

02,151

PB95-153391 02,151

Polarization Dependence of Response Functions in $3 \times 3$ Sagnac Optical Fiber Current Sensors.

02,154

Effect of Semiconductor Laser Characteristics on Optical Fiber Sensor Performance.

PBg5-169132

02,167

Selt-Calibrating Fiber Optic Sensors: Potential Design Methods

PB95-169298

02,172

Self Calibrating Fiber Optic Sensors: Potentıal Design Methods.

02,173

Simultaneous Laser-Diode Emission and Detection for Fiber-Optic Sensor Applications.

PB96-155502

04,062

High Frequency Magnetic Field Sensors Based on the Faraday Effect in Garnet Thick Films.

PB96-190384

Magneto-Optic Rotation Sensor Using a Laser Diode as Both Source and Detector.

PB97-111272

FIBER OPTICS

Technical Digest: Symposium on Optical Fiber Measurements (8ih), 1994. Held in Boulder, Colorado on September $13-15,1994$

PB94-207636

04,231

Complex Propagation Constants for Nonuniform Optical Waveguides: Calculations.

PB95-125910

04,249

Fiber Spot Size: A Simple Method of Calculation.

LP11-Mode Leakage Loss in Coated Depressed Clad Fi-

PB95-141115

02,145

Opportunities for Innovation: Optoelectronics.

01,928

Polarization Dependence of Response Functions in $3 \times 3$ Sagnac Optical Fiber Current Sensors.

PB96-122684

02,189

Growth Characteristics of Fiber Gratings.

04,346

Fiber Coating Diameter: Toward a Glass Artifact Standard. 140389

02,234

Feasibility of Fluorescence Detection of Tetracycline in Media Mixtures Employing a Fiber Optic Probe.

00,511

Automated Measurement of Nonlinearity of Optical Fiber Power Meters

04,110

Technical Digest: Symposium on Optical Fiber Measurements (9th), 1996. Held in Boulder, Colorado on October $1-3,1996$

PB97-108583

04,383

Anomalous Relation between Time and Frequency DoAnain PMD Measurements.

04,398

FIBER OPTICS TRANSMISSION LINES

Optical Fiber Sensors: Accelerating Applications in Navy Ships.

PB94-186848

02,632

FIBER REINFORCED COMPOSITES

AMRF Composite Fabrication Workstation.

PB94-172681

02,810

Friction Processes in Brittle Fracture.

PB96-161765

03,076

Estimation of the Orientation Distribution of Short-Fiber Composites Using Ultrasonic Velocities.

PB96-176656

03,178

FIBERBOARD

Wall Flame Heights with External Radiation

PB95-163481

00,380

FIBERS

Fuzed-Ouartz Fibers. A Survey of Properties, Applications and Production Methods.

00,656

Perspective on Fiber Coating Technology.

03,118

Effect of Heterogeneous Porous Media on Mold Filling in Resin Transfer Molding

PB95-108676

03,197

Video Microscopy Applied to Optical Fiber Geometry Measurements.

PB95-173068

04,295

Optical Fiber, Fiber Coating, and Connector Ferrule Geometry: Results of Interlaboratory Measurement Compari-

Evidence of Crosslinking in Methyl Pendent PBZT Fiber. PB97-112486

Analysis of Transverse Flow in Aligned Fibrous Porous

PB96-167200

03,177

FIELD EFFECT TRANSISTORS

Efficient Method to Compute the Maximum Transient

Drain Current Overshoot in Silicon on Insulator Devices.

PB94-172483

Experimental Study of Reverse-Bias Failure Mechanisms

in Bipolar Mode JFET (BMFET).

PB95-152997

02,340

Anomalous Behavior of a Ouantized Hall Plateau in a High-Mobility Si Metal-Oxide-Semiconductor Field-Effect Transistor.

PB95-164174 
Planar Resistors for Probe Station Calibration. PB95-163697

Electrical Method for Determining the Thickness of Meta Films and the Cross-Sectional Area of Metal Lines.
PB95-203170 Electrical Characterization of Integrated Circuit Metal Line Thickness.
PB96-138433

FILMS

Wear of Selected Materials and Composites Sliding against MoS2 Films

against MoS2
PB94-172749

03,231

Critical Current Density, Irreversibility Line, and Flux Creep Activation Énergy in Silver-Sheathed Bi2S $2 \mathrm{Ca}$ 2Cu2Ox Superconducting Tapes.

04,616

Nanoscale Study of the Hydrogenated Amorphous Silicon Surface.

Characterization of Liquid-Phase Epitaxially Grown $\mathrm{HgCdTe}$ Films by Magnetoresistance Measurements.

PB96-123617 04,738 Stagnant Film Model of the Effect of Natural Convection on the Dendrite Operating State.

04,765

Lattice Statics of Interfaces and Interfacial Cracks in Bimaterial Solids.

PB96-161823

02,985

Workshop on Characterizing Diamond Films (4th). Held in Gaithersburg, Maryland on March 4-5, 1996.

FILTERS

Systematic Studies of the Effect of a Post-Detection Filter on a Josephson-Junction Noise Thermometer.

PB95-162988

03,940

Interface-Filter Characterization of Spectraradiometers and Colorimeters.

04,399

FINANCE

Financing Tomorrow's Infrastructure: Challenges and Issues. Proceedings of a Colloquium. Held in Washington DC. on October 20,1995. PB96-189444

00,481

FINANCIAL MANAGEMENT

Self Monitoring Accounting Systems.

PB95-216602

00,007

Manager's Guide for Monitoring Data Integrity in Financial Systems.
PB96-165915

00,003

FINDING LIST

Ultraviolet Multiplet Table. Finding List for Spectra of the Elements Molybdenum to Lanthanum ( $Z=42$ to 57 ); Hatnium to Radium ( $Z=72$ to 88 ).

AD-A278 $131 / 8$

00,709

Ultraviolet Multiplet Table

00,710

FINE STRUCTURE

Fine Structure of Negative lons of Alkaline-Earth-Metal

PBg4-211

03,837

Atomic Oxygen Fine Structure Splittings with Tunable Far Infrared Spectroscopy.

PB95-152203

03,915

FINE STRUCTURE CONSTANT

Fine-Structure Constant.

PB94-172996

03,795

FINE WATER SPRAYS

Protection of Data Processing Equipment with Fine Water

Sprays

$95-174975$

02,610

FINGER PLUGGERS

Effect of Three Sterilization Techniques on Finger Pluggers.

00,150

FINGERPRINT CLASSIFICATION

Comparison of FFT Fingerprint Filtering Methods for Neural Network Classification

PB95-136362

01,840

Improving Neural Network Performance for Character and Fingerprint Classification by Altering Network Dynamics.
PB95-267803

PCASYS: A Pattern-Level Classification Automation Sys-

tem for Fingerprints.
PB95-267936

Improving Neural Network Performance for Character and

Fingerprint Classification by Altering Network Dynamics.

PB96-123195

01,856

FINGERPRINTS

NIST-Coordinated Standard for Fingerprint Data InterChange.

01,808

FINTE ELEMENT METHOD

Application of a Simple Technique for Estimating Errors of Finite-Element Solutions Using a General-Purpose Code.

04,818
Analysis of Creep in a Si-SiC C-Ring by Finite Element

PB94-200268

03,037

Single-Phase Heat Transfer and Pressure Drop Characteristics of an Integral-Spine-Fin Within an Annulus. Single-Phase Heat Transfer and Pressure Drop Characteristics of an Integral-Spine Fin Within an Annulus.
PB97-122386

FIPS PUB 180

Secure Hash Standard. Category: Computer Security.
FIPS PUB 180-1

FIRE ALARM SYSTEMS

Assessment of Technologies for Advanced Fire Detection.

00,294

FIRE CODES

Exposure: An Expert System Fire Code.

PB95-162913

04,868

FIRE DATA MANAGEMENT SYSTEM

Fire Data Management System, FDMS 2.0, Technical Documentation

01,358

Standardization of Formats and Presentation of Fire Data - The FDMS

PB94-198462

01,371

Development of the Fire Data Management System

PB94-206091

00,339

FIRE DETECTION SYSTEMS

Proceedings of the 1995 Workshop on Fire Detector Research. Held on February 6-7, 1995.

PB95-270062

02,61

Development of an Economical Video Based Fire Detection and Location System.

IRE DETECTORS

Performance Parameters of Fire Detection Systems.

PB94-194339 00,288 Studies Assess Performance of Residential Detectors. PB94-199262 00,290 Assessment of Technologies for Advanced Fire Detection. Acoustic Emission of Structural Materials Exposed to Open Flames.

Early Detection of Room Fires Through Acoustic Emission. (NIST Reprint).

PB95-180311

00,298

Santa Ana Fire Department Experiment at 1315 South Bristol, July 14, 1994

00,389

Review of Measurements and Candidate Signatures for Early Fire Detection.

PB95-189452

00,300

Field Modeling. Simulating the Effect of Sloped Beamed Ceilings on Detector and Sprinkler Response. PB96-122866

01,406

NASA Fire Detector Study.

PB96-183108

01,423

DYNAMICS

Prediction of Fire Dynamics.

PB94-193620

00,336

FIRE ESTINGUISHING AGENTS

Minımum Mass Flux Requirements to Suppress Burning Surfaces with Water Sprays.

PB96-183181

01,425

FIRE EXTINGUISHERS

Suppression Research: Strategies.

EXTINGUISHING AGENTS

Water Mist Fire Suppression Workshop Summary.
PB95-161907

PB95-161907 Extectiveness of Extinguishing Agent Highly Dynamic Conditions.

00,020

Burning Rate of Premixed Methane-Air Flames Inhibited by Fluorinated Hydrocarbons.

PB95-180584

01,391

Experimental and Numerical Burning Rates of Premixed Expe-Air Inhibited by Fluoromethanes.

PB95-180592
Suppression of Elevated Temperature Hydraulic Fluid and Suppression of Elevated Temperature Hydraulic Fluid and
JP-8 Spray Flames.
PB95-181095 00,021

00,022

Flow of Alternative Agents in Piping.

Suppression of High Speed Turbulent Flames in a Deto. nation/Deflagration Tube.

PB95-231817

01,395

Assessing Halon Alternatives for Aircraft Engine Nacelle Fire Suppression.

01,401

Solid Propellant Gas Generators: Proceedings of the 1995 Workshop. Held in Gaithersburg, Maryland on June $28-29,1995$.

01,412

Inhibition of Premixed Methane-Air Flames by Halon Alternatives

PB96-146741 01,414

Agent Screening for Halon 1301 Aviation Replacement.

Validation of a Turbulent Spray Flame Facility for the As. sessment of Halon Alternatives.

PB96-159728

03,283

PB96-164165

03,285

Effectiveness of Halon Altematives in Suppressing Dy-

namic Combustion Process.
PB96-175732 03,288

Interaction of HFC-125, FC-218 and CF3I with High Speed Combustion Waves.

PB96-176417

03,290

RE EXTINGUISHING AGENTS. FIRE SUPPRESSION

Acid Gas Production in Inhibited Diffusion Flames.

PB95-18057

01,390

Fire Service and Fire Sciences: A Winning Combination.

PB95-150264

01,383

FIRE GASES

Effective Measurement Techniques for Heat, Smoke, and Toxic Fire Gases.

PB94-198439

01,369

Chemical Stability of Upper-Layer Fire Gases.

01,410

FIRE HAZARDS

Fire Hazard and Risk: Evaluating Alternative Tech.

PB94-173077

00,242

Modern Test Methods for Flammability.

01,370

Toxicity, Fire Hazard and Upholstered Furniture.
PB94-198454

00,289

Analysis of the Happyland Social Club Fire with HAZARD

PB94-199270

00,193

Global Equivalence Ratio Concept and the Prediction of Carbon Monoxide Formation in Enclosure Fires.

PB94-207511

00,313

HAZARD I Fire Hazard Assessment Method (Version 1.2) (for Microcomputers)

00,196

HAZARD I Fire Hazard Assessment Method, Version 1.2 (Upgrade Package) (for Microcomputers)

00,197

Annual Conference on Fire Research: Book of Abstracts, October 17-20, 1994

PB95-104964

01,376

FPETOOL: Fire Protection Tools for Hazard Estimation. An Overview of Features.

Fire Service and Fire Sciences: A Winning Combination.

PB95-150264 01,383

Quantitative Evaluation of Building Fire Safety: New Tools for Assessing Fire and Building Code Provisions.

00,199

Influence of Ignition Source on the Flaming Fire Hazard of Upholstered Furniture. (NIST Reprint)

PB95-180162

00,297

Post-Earthquake Fire and Lifelines Workshop. Held in Long Beach, California on January 30-31, 1995. Proceed-

PB96-117916 00,209

Global Equivalence Ratio Concept and the Formation Mechanisms of Carbon Monoxide in Enclosure Fires.

PB96. 146790 


\section{KEYWORD INDEX}

Generation Rate and Distribution of Products of Combustion in Two-Layer Fire Environments: A Model and Appli-

01,398

Computing Radiative Heat Transfer Occurring in a Zone

PB96-102306 01,399

Analyzing and Exploiting Numerical Characteristics of Zone Fire Models.

PB96-102314

01,400

Fire Hazard Model Developments and Research Efforts at NIST.

PB96-159652
Numerical Simulation of Rapid Combustion in an Underground Enclosure.

PB96-183132

01,424

FIRE PREVENTION

Fire and Smoke Control: An Historical Perspective. 00,202

Transition from Localized Ignition to Flame Spread Over Thin Cellulosic Material in Microgravity.

PB96-155809

gnition and Subsequent Flame Spread Over a Thin Celulosic Materia.

04,836

Ignition and Transition to Flame Spread Over a Thermally hin Cellulosic Sheet in a Microgravity Environmen PB96-160288

04,837

FIRE PROTECTION

VENTCF2: An Algorithm and Associated FORTRAN 77 Subroutine for Calculating Flow through a Horizontal Ceilng/Floor Vent in a Zone-Type Compartment Fire Model. Measurement of Room Conditions and Response of Sprinklers and Smoke Detectors during a Simulated Two Bed Hospital Patient Room Fire

PB94-213717

00,292

FPETOOL: Fire Protection Tools for Hazard Estimation. An Overview of Features.

00,367

New Concepts for Fire Protection of Passenger Rail

New Concepts for Fire Protection of Passenger Rai

Transportation Vehicles.

04,850

Protection of Data Processing Equipment with Fine Water

02,610

Santa Ana Fire Department Experiment at 1315 South Bristol, July 14, 1994. (18) 1994 -

Review of Measurements and Candidate Signatures for Early Fire Detection.

PB95-189452

00,300

Concepts for Fire Protection of Passenger Rail Transpor-

lation Vehicles: Past, Present, and Future.

PB96-102868

04.853

Protecting Your Family from Fire.

00,307

Methodology for Developing and Implementing Alternative Temperature-Time Curves for Testing the Fire Resistance of Barriers for Nuclear Power Plant Applications.

PB96-193784

Fire Protection Foam Behavior in a Radiative Environment.

00,237

FIRE RESEARCH

Gordon Research Conference on the Physics and Chemistry of Laser Diagnostics in Combustion Held in Plymouth. New Hampshire on 12-16 July 1993.

01,353

AD-Á274 609/7

Some Factors Affecting Design of a Fumiture Calorimeter Hood and Exhaust.

00,285

Fire Induced Thermal Fields in Window Glass I: Theory.
PB94-139722

Two Numerical Techniques for Light Scattering by Dielec-

IrIC Agglomerated Structures.

PB94-140597

04,225

Computational Model for the Rise and Dispersion of Wind-Blown, Buoyancy-Driven Plumes. Part 2. Linearly Stratified Atmosphere.

PB94-143427

00.119

Ground-Based Smoke Sampling Techniques Training Course and Collaborative Local Smoke Sampling in Saudi Arabia.

02,532

Use of Computer Models to Predict Temperature and Smoke Movement in High Bay Spaces.

PB94-145976

00, 191

Fire Safety of Passenger Trains: A Review of Current Approaches and of New Concepts.

Concurrent Flow Flame Spread Study.

04,848

PB94-156866

01,356

Transient Cooling of a Hot Surface by Droplets Evapo.

PB94-156957

03,783

Numerical Analysis Support for Compartment Fire Modeling and Incorporation of Heat Conduction into a Zone Fire

01,357
Summaries of Center for Fire Research In-House rojects and Grants: 1990.

PB94-160876

00,286

Feasibility and Design Considerations of Emergency Evacuation by Elevators.

BFRL Fire Publications, 1993.

00,287

PB94-164191

00,192

Radiation and Mixing Properties of Buoyant Turbulent Diffusion Flames

01,360

Improvement in Predicting Smoke Movement in Compart-

$\begin{array}{ll}\text { mented Structures. } & 00,332\end{array}$

Greatly Enhanced Soot Scattering in Flickering Ch4/Air Diftusion Flames.

01,361

Fire Hazard and Risk: Evaluating Alternative Tech-

PB94-173077

00,242

Fluorinated Hydrocarbon Flame Suppression Chemistry. PB94-185113

01,362

Flame Retardants - Overview.

01,363

Laser Imaging of Chemistry-Flowfield Interactions: En-

haseed Soot Formation in Time-Varying Diffusion Flames.

hanced Soot Formation in Time-Varying Diffusion Flames.
PB94-185352

Calculating Flame Spread on Horizontal and Vertical Surfaces.

PB94-187283

00,335

Prediction of Fire Dynamics

PB94-193620

00,336

Mathematical Modeling of Human Egress from Fires in Residential Buildings.

PB94-193778

00,337

Structure and Radiation Properties of Pool Fires.

PB94-193802

02,473

Fire Propagation in Concurrent Flows.

PB94-193844

01,365

Backdraft Phenomena

PB94-193927

01,366

Investigation of Oil and Gas Well Fires and Flares.

PB94-193976

03,695

Risk Analysis for the Fire Safety of Airline Passengers.

04,862

Performance Parameters of Fire Detection Systems.

PB94-194339

00,288

CONTAM88 Building Input Files for Multi-Zone Airflow and Contaminant Dispersal Modeling

PB94-194388

02,537

Fire Growth Models for Materials

PB94-195856

01,367

Behavior of Charring Materials in Simulated Fire Environments.

PB94-196045 01,368

Effective Measurement Techniques for Heat, Smoke, and Toxic Fire Gases.

PB94-198439

01,369

Modern Test Methods for Flammability.

01,370

Toxicity, Fire Hazard and Upholstered Furniture.

PB94-198454

00,289

Standardization of Formats and Presentation of Fire Data - The FDMS.

01,371

Studies Assess Performance of Residential Detectors. PB94-199262

00,290

Analysis of the Happyland Social Club Fire with HAZARD

PB94-199270

00,193

Generation and Characterization of Acetylene Smokes.

PB94-200292

01,372

Ultrafine Combus

01,373

Evaluation of Alternative In-Flight Fire Suppressants for Full-Scale Testing in Simulated Aircraft Engine Nacelles and Dry Bays.

00,023

Fire Growth Analysis of the Fire of March 20, 1990, Pu. laski Building, 20 Massachusetts Avenue, N.W., Washington, DC.

PB94-205952

00,194

Development of the Fire Data Management System. PB94-20609

00,339

Upward Flame Spread along the Vertical Corner Walls (October 1993)

00,340

Evaluating Small Board and Care Homes: Sprinklered vs. Nonsprinklered Fire Protection.

PB94-206356

00,195

Turbulent Upward Flame Spread on a Vertical Wall under External Radiation.

PB94-207388

00,341

Comparison of Wall-Fire Behavior With and Without a Ceiling.

00,342

Global Equivalence Ratio Concept and the Prediction of Carbon Monoxide Formation in Enclosure Fires.

PB94-207511

00,313

Combined Buoyancy- and Pressure-Driven Flow Through

a Shallow, Horizontal, Circular Vent.

00,344

VENTCF2: An Algorithm and Associated FORTRAN 77 Subroutine for Calculating Flow through a Horizontal Ceiling/Floor Vent in a Zone-Type Compartment Fire Model. PB94-210127

00,345

Suppression Research: Strategies.

00,346

Estimate of Flame Radiance via a Single Location Measurement in Liquid Pool Flames.

PB94-211596

02,476

Measurement of Radiative Feedback to the Fuel Surface of a Pool Fire.

02,477

Laser-Induced Fluorescence Measurements of Formaldehyde in a Methane/Air Diffusion Flame.

PB94-211679

01,374

Rate of Heat Release of Wood Products.

PB94-212404

03,403

Effects of Molecular Weight and Thermal Stability on mer Gasification.

01,228

Application of Boundary Element Methods to a Transient Axis-Symmetric Heat Conduction Problem.

01,375

Full Scale Smoke Control Tests at the Plaza Hotel Build-

PBg4-212859

00,347

Overview of Smoke Control Technology.

PB94-212867

00,348

PB94-212875

Smoke Control Systems for Elevator Fire Evacuation.

PB94-212883 00,291

Development of a New Small-Scale Smoke Toxicity Test Method and lts Comparison with Real-Scale Fire Tests.

PB94-213253

00,350

EXITT: A Simulation Model of Occupant Decisions and

Actions in Residential Fires.

PB94-213261

00,351

Investigation of the Thermal Stability and Char-Forming

Tendency of Cro

Measurement of Room Conditions and Response of Sprinklers and Smoke Detectors during a Simulated TwoBed Hospital Patient Room Fire.

00,292

Feasibility of Fire Evacuation by Elevators at FAA Control 


\section{KEYWORD INDEX}

One- and Two-Sided Burning of Thermally Thin Materials. PB95-140935 03,151 Simulating Smoke Movement through Long Vertical Shafts in Zone-Type Compartment Fire Models. PB95-143152 00,368 Transient Cooling of a Hot Surface by Droplets Evapo-

03,890

Building and Fire Research Laboratory Publications, 1993

00,369

PB95-143202

Investigation into the Flammability Properties of Honey-
comb Composites.

Comb Compos

03,152

Airbome Smoke Sampling Package for Field Measurements of Fires.

01,381

Oxidation of Soot and Carbon Monoxide in Hydrocarbon Diffusion Flames.

PB95-150215

01,382

Fire Service and Fire Sciences: A Winning Combination. PB95-150264 01,383

Estimate of the Effect of Scale on Radiative Heat Loss Fraction and Combustion Efficiency.

PB95-150447

02,486

Optical Measurements of Atomic Hydrogen, Hydroxyl, and Carbon Monoxide in Hydrocarbon Diffusion Flames. PB95-150900

02,487

Approximate Confidence Intervals on Linear Combinations of Expected Mean Squares.

PB95-151296

03,435

Flame Synthesis of High Tc Superconductors.

PB95-151981 00,659

Effects of Specimen Edge Conditions on Heat Release Rate.

00,375

Turbulent Spray Burner for Assessing Halon Alternative Fire Suppressants

PB95-153748
Method of Predicting Smoke Movement in Atria with Application to Smoke Management.

PB95-154746

00,376

Locating Fire Engineering Information.

00,198

Reactivity of Product Gases Generated in Idealized Enclosure Fire Environments.

PB95-161790

01,386

Water Mist Fire Suppression Workshop Summary.

New Concepts for Fire Protection of Passenger Rail Transportation Vehicles.

PB95-162046

04,850

Global Density Effects on the Self-Preservation Behavior of Turbulent Free Jets.

PB95-162301

04,207

Exposure: An Expert System Fire Code

PB95-162913 04,868

Forum for International Cooperation on Fire Research

PB95-162939

04,869

Fresh Look at Strategies for Fire Safety

04,870

Wall Flame Heights with External Radiation

PB95-163481

00,380

In situ Burning of Oil Spills: Mesoscale Experiments and

Analysis.

02,587

Quantitative Evaluation of Building Fire Safety: New Tools for Assessing Fire and Building Code Provisions.

PB95-164588

00,199

Acoustic Emission of Structural Materials Exposed to Open Flames.

00,296

Protection of Data Processing Equipment with Fine Water

Sprays.

02,610

Developing Rational Performance-Based Fire Safety Requirements in Model Building Codes

PB95-175220

00,200

Fire and Smoke Control: An Historical Perspective.
PB95-175808 00,202

Effect of Dodecanol Content on the Combustion of Methanol Spray Flames.

PB95-176020

01,389

Mathematical Modeling and Computer Simulation of Fire Phenomena.

PB95-180063

00,384

Influence of Ignition Source on the Flaming Fire Hazard of Upholstered Furniture. (NIST Reprint)

00,297

Suppression Effectiveness of Extinguishing Agents under Highly Dynamic Conditions.

00,020

00,020
PB95-180279 sion. (NIST Reprint).

PB95-180311

00,298

Acid Gas Production in Inhibited Diffusion Flames.

PB95-180576
Burning Rate of Premixed Methane-Air Flames Inhibited by Fluorinated Hydrocarbons.

01,391

Experimental and Numerical Burning Rates of Premixed Methane-Air Inhibited by Fluoromethanes.

01,392 PB95-180592

New Concepts for Fire Protection of Passenger Rail
Transportation Vehicles. (NIST Reprint). Transportation Vehicles. (NIST Reprint)

Suppression of Elevated Temperature Hydraulic Fluid and JP-8 Spray Flames.

PB95-181095

00,021

Algorithm to Describe the Spread of a Wall Fire under a Ceiling.

00,261

Comparison of Fire Sprinkler Piping Materials: Steel Copper, Chlorinated Polyvinyl Chloride and Polybutylene, in Residential and Light Hazard Installations.

PB95-182267

00,299

Santa Ana Fire Department Experiment at 1315 South Bristol, July 14, 1994

PB95-188868 00,389

Fire Performance of an Interstitial Space Construction

System.

00,390

Review of Measurements and Candidate Signatures for Early Fire Detection

PB95-189452

00,300

PB95-202420

00,022

Ouantitative Measurements of Enhanced Soot Production in a Flickering Methane/Air Diffusion Flame.

01,393

Burning Rate and Flame Heat Flux For PMMA in the Cone Calorimeter.

PB95-216990

00,393

Compartment Fire Combustion Dynamics. Annual Report, September 1, 1993-September 1, 1994.

PB95-217162

00,203

Building and Fire Research Laboratory Publications, 1994.

00,398

Behavior of Mock-Ups in the California Technical Bulletin 133 Test Protocol: Fabric and Barrier Effects.

PB95-231585

00,301

Carbon Monoxide Production in Compartment Fires: Reduced-Scale Enclosure Test Facility.

01,394

Suppression of High Speed Turbulent Flames in a Detonation/Deflagration Tube.

PB95-231817 01,395

Mixing and Radiation Properties of Buoyant Turbulent Diffusion Flames.

01,396

Proceedings of the 1995 Workshop on Fire Detector Research. Held on February 6-7, 1995.

0062

02,611

Fire Codes for Global Practice.

PB96-102108

00,205

How to Evaluate Alternative Designs Based on Fire Modeling.

00,206

Simultaneous Optical Measurement of Soot Volume Fraction, Temperature, and $\mathrm{CO} 2$ in Heptane Pool Fire.
PB96-102132 Generation Rate and Distribution of Products of Combustion in Two-Layer Fire Environments: A Model and Appli-

PB96-102173

01,398

Some Factors Affecting the Design of a Calorimeter Hood and Exhaust.

00,302

Computing Radiative Heat Transfer Occurring in a Zone Fire Model.

PB96-102306

01,399

Analyzing and Exploiting Numerical Characteristics of Zone Fire Models.

PB96-102314

01,400

Assessing Halon Alternatives for Aircraft Engine Nacelle Fire Suppression.

PB96-102454 01,401

Concepts for Fire Protection of Passenger Rail Transporation Vehicles: Past, Present, and Future.

04,853

Santa Ana Fire Department Experiment at 1315 South

Bristol, July 14, 1994. (Reprint)

Bristol, July 14,

00,207

Pressure Equations in Zone-Fire Modeling.

00,208

CFAST Output Comparison Method and Its Use in Com-

paring Different CFAST Versions.

PB96-109541

00,401

Effect of Suppressants on Metal Fires.

01,402

Turbulent Flame Spread on Vertical Corner Walls.

PB96-114764

01,403

Fire Suppression System Performance of Alternative

Agents in Aircraft Engine and Dry Bay Laboratory Simula-

tions. SP890: Volume 1.

PB96-117775

03,277

Fire Suppression System Performance of Alternative Agents in Aircraft Engine and Dry Bay Laboratory Simula tions. SP 890: Volume 2

PB96-117783

03,278

Flame Heights and Heat Release Rates of 1991 Kuwait Oil Field Fires.

01,404

Experimental Study of the Stabilization Region of Lifted Turbulent-Jet Diffusion Flames.

PB96-122676

01,405

Field Modeling: Simulating the Effect of Sloped Beamed Ceilings on Detector and Sprinkler Response.

PB96-122866

01,406

Smoke Emission from Burning Crude Oil.

PB96-122890

01,407

Development of Hazard Assessment and Suppression Technology for Oil and Gas Well Blowout and Diverter

01,408

Laser-Induced Fluorescence Measurements of $\mathrm{OH}$ in Laminar Diffusion Flames in the Presence of Soot Par-

PB96-123120

01,409

Polymer Combustion and Flammability: Role of the Condensed Phase

01,279

Further Development of the N-Gas Mathematical Model: An Approach for Predicting the Toxic Potency of Complex Combustion Mixtures.

PB96-123260

03,650

Chemical Stability of Upper-Layer Fire Gases.

01,410

New Approach for Reducing the Toxicity of the Combus tion Products from Flexible Polyurethane Foam.

PB96-123625

01,411

Solid Propellant Gas Generators: Proceedings of the 1995 Workshop. Held in Gaithersburg, Maryland on June 28-29, 1995.

01,412

Smoke Plume Trajectory from In situ Burning of Crude Oi in Alaska: Field Experiments

PB96-131560

02,594

Application of Thermodynamic and Detailed Chemical Ki netic Modeling to Understanding Combustion Product Generation in Enclosure Fires.

PB96-135322

01,413

Evaluation of Sprinkler Activation Prediction Methods PB96- 141056 00,304 Inhibition of Premixed Methane-Air Flames by Halon Alternatives.

01,414

Global Equivalence Ratio Concept and the Formation Mechanisms of Carbon Monoxide in Enclosure Fires. 
Information Transfer in the 21st Century. PB96-157904

02,714

Fire Hazard Model Developments and Research Efforts at NIST.

PB96-159652 00,407

Agent Screening for Halon 1301 Aviation Replacement.

03,282

Validation of a Turbulent Spray Flame Facility for the As. sessment of Halon Alternatives.

PB96-159728

03,283

Effects of Sample Mounting on Flammability Properties of

Intumescent Polymers.
PB96-159777

03,389

Ignition and Subsequent Flame Spread Over a Thin Cellulosic Material.
PB96-160270

04,836

Ignition and Transition to Flame Spread Over a Thermally Thin Cellulosic Sheet in a Microgravity Environment.

New Generation of Fire Resistant Polymers. Part 1. Com. puter-Aided Molecular Design.

PB96-160593

01,419

Computer-Aided Molecular Design of Fire Resistant Aircraft Materials.

00,025

EHect of CF3H and CF3Br on Laminar Diffusion Flames in Normal and Microgravity.

PB96-161831

01,420

Effect of CF3H and CF3Br on Laminar Diffusion Flames in Normal and Microgravity.

01,421

Inhibition of Premixed Methane-Air Flames by Iron Pentacarbonyl.

00,513

Parametric Study of Hydrogen Fluoride Formation in Suppressed Fires

00,514

Heat Transfer in an Intumescent Material Using a ThreeDimensional Lagrangian Model.

PB96-164066

00,408

Calculating Combined Buoyancy- and Pressure-Driven Flow Through a Shallow, Horizontal, Circular Vent; Application to Problem of Steady Burning in a Ceiling-Vented

PB96-164108 00,409

Combined Buoyancy and Pressure-Driven Flow Through a Shallow, Horizontal, Circular Vent.

PB96-164116

00,410

In Search of Altemative Fire Suppressants.

03,285

Asymptotic and Numerical Analysis of a Premixed Lam-

inar Nitrogen Dioxide-Hydrogen Flame.

Scaling Compartment Fires: Reduced- and Full-Scale En-

$\begin{array}{ll}\text { closure Burns. } & 00,224 \\ \text { PB96-175708 } & \end{array}$

Suppression of High-Speed $\mathrm{C}_{2} \mathrm{H} 4 /$ Air Flames with C1-

Halocarbons.

03,287

Effectiveness of Halon Alternatives in Suppressing Dynamic Combustion Process.

03,288

Interaction of HFC-125, FC-218 and CF3l with High

Speed Combustion Waves.
PB96-176417

Suppression of Ignition Over a Heated Metal Surface.

PB96-176425 03,291

Publications 1995: NIST Building and Fire Research Laboratory.

183074

00,226

NASA Fire Detector Study.

PB96-183108

01,423

Numerical Simulation of Rapid Combustion in an Underground Enclosure.

01,424

Minimum Mass Flux Requirements to Suppress Burning Surfaces with Water Sprays

PB96-183181

01,425

Large Eddy Simulations of Smoke Movement in Three Dimensions.

Large Fire Experiments for Fire Model Evaluations

PB96-190079

01,426

Locating Fire Information.

01,427

B96-190137

00,227

Office Work Station Heat Release Rate Study: Full Scale versus Bench Scale.
PB96-190178

01,428

Analysis of High Bay Hangar Facilities for Detector Sensitivity and Placement.

01,429

Post-Flame Soot

01,430

Development of an Economical Video Based Fire Detection and Location System.

tion and Locatio

00,228
Methodology for Developing and Implementing Alternative Temperature-Time Curves tor Testing the Fire Resistance of Barriers for Nuclear Power Plant Applications.

PB96-193784 03,742

Dynamics, Transport and Chemical Kinetics of Compartment Fire Exhaust Gases

PB96- $195508 \quad 00,229$

Chemical Inhibition of Methane-Air Diffusion Flame.

PB96-195532 01,431

Smoke Plume Trajectory from In situ Burning of Crude

Oil: Field Experiments.
PB96-200993

02,597

Low Heat-Flux Measurements: Some Precautions.

PB96-2011 116

02,685

Enhancement of EXIT89 and Analysis of World Trade Center Data.

Mixing and Radiation Properties of Buoyant Luminous Flame Environments.

PB96-202254

01,432

Benefits and Costs of Research: A Case Study of the

Fire Safety Evaluation System.

00,232

Sparse Water Sprays in Fire Protection

01,433

Gas Phase Oxygen Effect on Chain Scission and Monomer Content in Bulk Poly(methyl methacrylate) Degraded by External Thermal Radiation.

by External Ther

01,293

Role of Combustion on Droplet Transport in Pressure-

$\begin{array}{ll}\text { Atomized Spray Flames. } & 01,434\end{array}$

EHect of Fuel Tank Rupture Mode on the Ignitability of Expelled Fuel

01,444

Flammability Characterization with the Lift Apparatus and Flammability Characterization with the Lift Apparatus and
the Cone Calorimeter.
PB97-110050
L

posite

Letter Rep

PB97.1 10068

01,436

Interaction of an Isolated Sprinkler Spray and a TwoLayer Compartment Fire Environment. Phenomena and Model Simulations.

01,437

Heat Flux from Flames to Vertical Surfaces.

01,438

Analysis of Droplet Arrival Statistics in a Pressure-Atomized Spray Flame. ized Spray Flam

01,352

Survey of Fuel Loads in Contemporary Office Buildings.
PB97-114235

Fire Safety Engineering in the Pursuit of PerformanceBased Codes: Collected Papers.

00,235

Full-Scale Room Fire Experiments Conducted at the University of Maryland
PB97-116081

00,236

Fire Protection Foam Behavior in a Radiative Environ-

PB97-116131

00,237

NIST Research on Less-Flammable Materials

PB97-118632

01,439

Computations of Enhanced Soot Production in TimeVarying $\mathrm{CH} 4 /$ Air Diffusion Flames.

PB97.119218

01,440

Transport by Gravity Currents in Building Fires.

PB97-119325

01,441

Materials and Fire Threat

PB97-122311

NO Production and Destruction in a Methane/Air Diffusion Flame.

PB97-122519

01,443

FIRE RESEARCH INFORMATION SERVICES

FIREDOC Users Manual, 3rd Edition

PB95-128674

01,378

FIRE RESISTANT MATERIALS

Summaries of BFRL Fire Research in-House Projects and Grants, 1994

PB95-130845

00,366

Etfects of Sample Mounting on Flammability Properties of Intumescent Polymers.

PB96-159777

03,389

New Generation of Fire Resistant Polymers. Part 1. Computer-Aided Molecular Design

PB96-160593

01,419

Computer-Aided Molecular Design of Fire Resistant Aircraft Materials.

PB96-16060

Materials and Fire Threat

00,025

PB97-12231

01,442

FIRE SAFETY

Fire Safety of Passenger Trains: A Review of Current Approaches and of New Concepts.

PB94-152006

04,848

Mathematical Modeling of Human Egress from Fires in Residential Buildings.

00,337

Risk Analysis for the Fire Safety of Airline Passengers. PB94-194065 04,862 Studies Assess Performance of Residential Detectors. PB94-199262 00,290 Evaluating Small Board and Care Homes: Sprinklered vs. Nonsprinklered Fire Protection.

PB94-206356

00,195

Overview of Smoke Control Technology.

PB94-212867

00,348

00,349

Smoke Control Systems for Elevator Fire Evacuation

PB94-212883

00,291

Feasibility of Fire Evacuation by Elevators at FAA Control

PB94-213857

04,844

Human Factors Considerations for the Potential Use of Elevators for Fire Evacuation of FAA Air Traftic Control Towers.

PB94-217163

01,300

FIREDOC Users Manual, 3rd Edition

PB95-128674 01,378

Summaries of BFRL Fire Research in-House Projects and Grants, 1994

PB95-130845

00,366

FPETOOL: Fire Protection Tools for Hazard Estimation. An Overview of Features.

PB95-140885

00,367

New Concepts for Fire Protection of Passenger Rail Transportation Vehicles.

PB95-162046

04,850

Fresh Look at Strategies for Fire Safety.

PB95-162947

04,870

Developing Rational Performance-Based Fire Safery Requirements in Model Building Codes.

00,200

New Concepts for Fire Protection of Passenger Rail Transportation Vehicles. (NIST Reprint)

PB95-180774

04,863

Fire Codes for Global Practice.

00,205

How to Evaluate Alternative Designs Based on Fire Mod-

PBing.

00,206

Predicting the Fire Pertormance of Buildings: Establishing Appropriate Calculation Methods for Regulatory Applica-

PB96-141239

00,316

Fire Safety Engineering Research in the United States.

PB96-151394

00,213

Elements of a Framework for Fire Safety Engineering

PB96-151402 00,21 
Turbulent Spray Bumer for Assessing Halon Alternative Fire Suppressants. Water Mist Fire Suppression Workshop Summary.
PB95-161907 Suppression Effectiveness of Extinguishing Agents under

Highly Dynamic Conditions.
PB95-180279

Flow of Alternative Agents in Piping.

00,022

Fire Suppression System Performance of Alternative Agents in Aircraft Engine and Dry Bay Laboratory Simula. tions. SP890: Volume 1.

03,277

Fire Suppression System Performance of Alternative Fire Suppression in Aircraft Engine and Dry Bay Laboratory Simula(ions. SP 890: Volume 2 .

03,278

Development of Hazard Assessment and Suppression Technology for Oil and Gas Well Blowout and Diverter Fires.

PB96-122965 01,408

Computing the Effect of Sprinkler Sprays on Fire Induced

Gas Flow. 00,404

Suppression of High-Speed $\mathrm{C}_{2} \mathrm{H} 4 / \mathrm{Air}$ Flames with $\mathrm{C}_{1}$ Halocarbons.
PB96-175724

03,287

Suppression of Ignition Over a Heated Metal Surface.

PB96-176425 03,291

Analysis of High Bay Hangar Facilities for Detector Sen. sitivity and Placement.

PB96-190210

01,429

FIRE TEST DATA

Standardization of Formats and Presentation of Fire Data - The FDMS.

PB94-198462

01,371

FIRE TESTS

Some Factors Affecting Design of a Fumiture Calorimeter Hood and Exhaus.

00,285

In situ Buming of Oil Spills: Mesoscale Experiments.

PB94-142973 01,355

Use of Computer Models 10 Predict Temperature and Smoke Movement in High Bay Spaces.

00,191

Fire Data Management System, FDMS 2.0, Technical Documentation.

PB94-164019

01,358

BFRL Fire Publications, 1993

00,192

Experimental and Numerical Studies on Two-Dimensional Gravity Currents in a Horizontal Channel.

PB94-165941

01,359

Fire Propagation in Concurrent Flows.

PB94-193844 01,365

Development of the Fire Data Management Systern

PB94-206091

00,339

Suppression Research: Strategies.

00,346

Full Scale Smoke Control Tests at the Plaza Hotel Build-

ing. $94-212859$

00,347

In Situ Buming Oil Spill Workshop Proceedings. Held in

Orlando, Florida on January 26-28, 1994.

PB95-104907

02,583

One- and Two-Sided Burning of Thermally Thin Materials.
PB95-140935

Effects of Specimen Edge Conditions on Heat Release Rate

PB95- 152864

00,375

Wall Flame Heights with External Radiation.

PB95-163481

00,380

In situ Buming of Oil Spills: Mesoscale Experiments and Analysis.

02,587

Acoustic Emission of Structural Materials Exposed 10 Open Flames.

00,296

Santa Ana Fire Department Experiment at 1315 South Bristol, July 14, 1994

00,389

Fire Performance of an Interstitial Space Construction System.
PB95-188918

00,390

Carbon Monoxide Production in Compartment Fires: Reduced-Scale Enclosure Test Facility.

01,394

Some Factors Affecting the Design of a Calorimeter Hood and Exhaust.

00,302

Santa Ana Fire Department Experiment at 1315 South Bristol, July 14, 1994. (Reprint)

PB96-102934

00,207

Pressure Equatic
PB96-102967

00,208
Application of Thermodynamic and Detailed Chemical Kinetic Modeling to Understanding Combustion Produc Generation in Enclosure Fires.

PB96-135322 01,413

Review of Flows Driven By Natural Convection in Adia-

batic Shafts.
PB96-147897 01,416

Santa Ana Fire Department Experiments at South Bristol Street.

00,305

Scaling Compartment Fires: Reduced- and Full-Scale Enclosure Burns.

PB96-175708 00,224

Mixing and Radiation Properties of Buoyant Luminous Flame Environments.

PB96-202254

01,432

Sparse Water Sprays in Fire Protection

01,433

Interaction of an Isolated Sprinkler Spray and a Two Layer Compartment Fire Environment. Phenomena and Model Simulations.

PB97-110076

01,437

Full-Scale Room Fire Experiments Conducted at the University of Maryland.

00,236

NIST Research on Less-Flammable Materials.

PB97-118632

01,439

REDOC COMPUTER PROGRAM

FIREDOC Users Manual, 3rd Edition

FIRES

Fire Induced Thermal Fields in Window Glass I: Theory. PB94-139722

Ground-Based Smoke Sampling Techniques Training
Course and Collaborative Local Smoke Sampling in Course and Collaborative Local Smoke Sampling in PB94-143542

02,532

Numerical Analysis Support for Compartment Fire Modeling and Incorporation of Heat Conduction into a Zone Fire Model.

PB94-156965

01,357

Radiation and Mixing Properties of Buoyant Turbulent Diffusion Flames.

Improvement in Predicting Smoke Movement in Compartmented Structures.

PB94-172418

00,332

Mathematical Modeling of Human Egress from Fires in Residential Buildings.

00,337

Effective Measurement Techniques for Heat, Smoke, and Toxic Fire Gases

PB94-198439

01,369

Standardization of Formats and Presentation of Fire Data - The FDMS.

01.371

Fire Growth Analysis of the Fire of March 20, 1990, Pulaski Building, 20 Massachusetts Avenue, N.W., Washing

PB94-205952

00,194

Project Summaries 1994: NIST Building and Fire Research Laboratory.

PB94-207495

00.343

Rate of Heat Release of Wood Products.

03,403

Verification of a Model of Fire and Smoke Transport.

PB95-108718 00,357

Relating Bench-Scale and Full-Scale Toxicity Data.
PB95.125977

$\begin{array}{ll}\text { PB95-125977 } & 0,361 \\ \text { DIspersion and Deposition of Smoke Plumes Generated }\end{array}$ in Massive Fires.

PB95-126066

02,540

Summaries of BFRL Fire Research In-House Projects and Grants, 1994

00,366

Building and Fire Research Laboratory Publications,

00,369

Method of Predicting Smoke Movement in Atria with Application to Smoke Management.

00,376

Fire-Plume-Generated Ceiling Jet Characteristics and Convective Heat Transfer to Ceiling and Wall Surfaces in a Two-Layer Fire Environment: Uniform Temperature Ceiling and Walls.

PB95-164711

00,382

Earthquake and Fire in Japan: When the Threat Became

$\begin{array}{ll}\text { a Reality. } & 00,201\end{array}$

Compartment Fire Combustion Dynamics. Annual Report September 1, 1993-September 1, 1994.

00,203

Building and Fire Research Laboratory Publications.

00,398

PB95-226684

Project Summaries 1995: NIST Building and Fire Re-
search Laboratory.
PB95-270047
00,400

Simultaneous Optical Measurement of Soot Volume Fraction, Temperature, and $\mathrm{CO} 2$ in Heptane Pool Fire.

PB96-102132 01397

CFAST Output Comparison Method and Its Use in Comparing Different CFAST Versions.

PB96-109541 00,401

Flame Heights and Heat Release Rates of 1991 Kuwait Oil Field Fires.

01,404

Study of Technology for Detecting Pre-Ignition Conditions of Cooking-Related Fires Associated with Electric and Gas Ranges and Cooktops, Phase 1 Repor

PB96-128095

00,303

Gravity-Curren
PB96-147046

01,415

Examination of the Correlation between Cone Calorımeter

Data and Full-Scale Furniture Mock-Up Fires.

01,417

Sensitivity Analysis for Mathematical Modeling of Fires in Residential Buildings

PB96-154968

00,215

Ignition and Subsequent Flame Spread Over a Thin Celulosic Material.

04,836

Evaluation of Survey Procedures for Determining Occu pant Load Factors in Contemporary Office Buildings. PB97-116222 00,238 Transport by Gravity Currents in Building Fires.

SHER RENORMALIZATION

Shear Suppression of Critical Fluctuations in a Diluted PB96-204458

FISSION CHAMBER

Measurements of the $(235) \mathrm{U}(\mathrm{n}, \mathrm{f})$ Cross Section in the 3 to $30 \mathrm{MeV}$ Neutron Energy Region.

PB97-119051

04,172

FISSION CROSS SECTION

Measurements of the $(237) \mathrm{Np}(n, f)$ Cross Section
PB97-119069

FISSION CROSS SECTIONS

Intermediate Structure in the Neutron-Induced Fission Cross Section of 236

Measurement of the $(235) \mathrm{U}(\mathrm{n}, \mathrm{f})$ Reaction from Thermal to

1 keV.

03,886

ITING

User's Guide for the Algorithm Testing System Version 2.0.

02,916

FITTINGS

Acrylonitrile-Butadiene-Styrene (ABS) Plastic Drain, Waste, and Vent Pipe and Fittings.
$A D-A 310724 / 0$

FIXED INVESTMENT

Investing in Education to Meet a National Need for a Technical-Professional Workforce in a Post-Industrial Economy,

00,132

FIXED POINTS

NIST Assessment of ITS-90 Non-Uniqueness for 25.5 Ohm SPRTs at Gallium, Indium and Cadmium Fixed PB96-

00,631

NIST implementation and Realization of the ITS-90 Ove the Range $83 \mathrm{~K}$ to $1235 \mathrm{~K}$ : Reproducibility, Stability, and Uncertainties.

NIST Measurement Assurance of SPRT Calibrations on the ITS-90: A Ouantitative Approach.
PB96-161336

00,633

Preliminary Results of a Comparison of Water TriplePoint Cells Prepared by Different Methods.

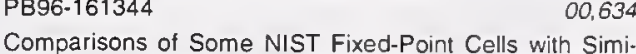

lar Cells of Other Standards Laboratories.
PB97-119242

00,655 


\section{KEYWORD INDEX}

FLAME PROPAGATION

Ignition and Subsequent Transition to Flame Spread in a Microgravity Environment.

N96-15584/1

04,828

Concurrent Flow Flame Spread Study.

01.356

Radiation and Mixing Properties of Buoyant Turbulent Ditfusion Flames.

01.360

PB94-165974

Calculating Flame Spread on Horizontal and Vertical Sur-

faces.

Fire Propagation in Concurrent Flows

PB94-193844

01,365

Backdraft Phenomena.

01.366

Upward Flame Spread along the Vertical Corner Walls (October 1993)

00.340

Turbulent Upward Flame Spread on a Vertical Wall under External Radiation.

00,341

Comparison of Wall-Fire Behavior With and Without a Ceiling.

00,342

Combined Buoyancy- and Pressure-Driven Flow Through a Shallow, Horizontal, Circular Vent.

00,344

VENTCF2: An Algorithm and Associated FORTRAN 77 Subroutine for Calculating Flow through a Horizontal Ceiling/Floor Vent in a Zone-Type Compartment Fire Model.
PB94-210127 Annual Conference on Fire Research: Book of Abstracts. October $17-20,1994$

PB95-104964

01.376

Combustion of Methanol and Methanol/Dodecanol Spray Flames.

PB95-108544

02,478

Study of Droplet Transport in Alcohol-Based Spray Flames Using Phase/Doppler Interferometry.

02,479

Algorithm to Describe the Spread of a Wall Fire under a Ceiling.

PB95-182259

00,261

Generation Rate and Distribution of Products of Combustion in Two-Layer Fire Environments: A Model and Appli-

PB96-102173 01,398

CFAST Output Comparison Method and Its Use in Com. paring Different CFAST Versions.

PB96-109541

00,401

Flame Heights and Heat Release Rates of 1991 Kuwait Oil Field Fires

Review of Internatioanl Fire Risk Predictions Methods.

PB96-156195 00,222

Ignition and Subsequent Flame Spread Over a Thin Cellulosic Materia

Calculating Combined Buoyancy- and Pressure-Driven Flow Through a Shallow, Horizontal, Circular Vent: Application to Problem of Steady Burning in a Ceiling-Vented Enclosure.

PB96-164108

00.409

Combined Buoyancy and Pressure-Driven Flow Through a Shallow, Horizontal, Circular Vent.

00.410

Dynamics, Transport and Chemical Kinetics of Compartment Fire Exhaust Gases.

PB96-195508

FLAME RETARDANTS

Flame Retardants - Overview

PB94-185287

01,363

FLAME SPREAD

Letter Report on Flame Spread Testing of a Composite Materia

PB97-110068

01,436

FLAME SPREADING

Turbulent Flame Spread on Vertical Corner Walls.
PB96-114764

FLAME SYNTHESIS

Flame Synthesis of High Tc Superconductors.
PB95-151981

FLAMES

Ignition and Subsequent Transition to Flame Spread in a Microgravity Environment.

N96-15584/1

04,828

Fluorinated Hydrocarbon Flame Suppression Chemistry.
PB94-185113

Estimate of Flame Radiance via a Single Location Measurement in Liquid Pool Flames.

PB94-211596

02,476

Structure of a Swirl-Stabilized Kerosene Spray Flame.

PB95-108569 02,480

Burning Rate and Flame Heat Flux For PMMA in the Cone Calorimeter.

00,393
Heights of Wall-Fire Flames.

00,212

Role of Combustion on Droplet Transport in PressureAtomized Spray Flames.

01,434

B96-204433

01,438

In-situ Studies of a Novel Sodium Flame Process for Synthesis of Fine Particles.

$$
\text { PB97-113047 }
$$

00,681

FLAMMABILITY

Reaction Sensitivities of Al-Li Alloys and Alloy 2219 in Mechanical-Impact Tests.

PB94-172764

03,314

Pdern Test Methods for Flammability.

01,370

Recommended Changes in ASTM Test Methods D2512 82 and G86-84 for Oxygen-Compatibility Mechanical Impact Tests on Metals.

PB94-216694

03,338

One- and Two-Sided Burning of Thermally Thin Materials.

03,151

Investigation into the Flammability Properties of Honeycomb Composites.

PB95-143293

03,152

Polymer Combustion and Flammability: Role of the Condensed Phase.

Flammability Characterization with the Lift Apparatus and the Cone Calorimeter.

PB97-110050

01,435

NIST Research on Less-Flammable Materials.

01,439

\section{FLAMMABILITY TESTING}

Behavior of Mock-Ups in the California Technical Bulletin 133 Test Protocol: Fabric and Barrier Effects.

PB95-231585

00,30

Predicting the Ignition Time and Burning Rate of Thermoplastics in the Cone Calorimeter.

01,418

PB96-155411

00,306

Effects of Sample Mounting on Flammability Properties of intumescent Polymers.

PB96-159777

03,389

Computer-Aided Molecular Design of Fire Resistant Aircraft Materials.

00,025

FLARE STARS

High-Velocity Plasma in the Transition Region of AU Microscopii: Evidence for Magnetic Reconnection and Saturated Heating during Quiescent and Flaring Conditions. PB96-102694

00,091

FLASKS

Specifications and Tolerances for Reference Standards and Field Standard Weights and Measures. 2. Specificaand Field Standard Weights and Measures. 2. Specifica-
tions and Tolerances for Field Standard Measuring tions and

PB96-178926

02,682

\section{FLAT PANEL DISPLAYS}

NIST Workshop on the Computer Interface to Flat Panel Displays. Held in San Jose, California on January 13-14, 1994.

PB95-136388

01,625

Survey of the Components of Display-Measurement Standards.
PB96-122528

FLAW INSENSITIVITY

Flaw-Insensitive Ceramics.

PB97-110027

FLAW TOLERANCE

Correlations between Flaw Tolerance and Reliability in Zirconia.

PB96-161922

02,986

Objective Evaluation of Short-Crack Toughness Curves Using Indentation Flaws: Case Study on Alumina-Based Ceramics.

PB96-179429

03,079

Flaw-Tolerance and Crack-Pesistance Properties of Alumina-Aluminum Titanate Composites with Tailored Microstructures.
PB97.110324

FLAW TOLERANCES

Flaw Tolerance and Toughness Curves in Two-Phase Particulate Composites: SiC/Glass System.

03,101 PB96-179460

03,081

\section{FLEET MANAGEMENT}

AutoBid 2.0: The Microcomputer System for Police Patrol Vehicle Selection.

04,871

\section{FLEXIBLE PAVEMENTS}

System for Calibration of the Marshall Compaction HamPB94-145661

01,303

Development of a Method for Measuring Water-Stripping Resistance of Asphalt/Siliceous Aggregate Mixtures. 01,348

FLEXING

Observed and Theoretical Creep Rates for an Alumina Ceramic and a Silicon Nitride Ceramic in Flexure. PB94-212958

Flat and Rising R-Curves for Elliptical Surface Cracks PB95-161295

03.156

\section{FLEXURAL PROPERTIES}

Crack Growth Resistance of Strain-Softening Materials under Flexural Loading.

02,972

FLEXURAL SPRINGS

Design Equations and Scaling Laws for Linear Compres. sors with Flexure Springs.

PB95-168902

FUGHT

Stylus Flight in Surface Profiling.

PB96-123138

02,675

FLIGHT TELEROBOTIC SERVICER

Evolution of the Flight Telerobotic Servicer. PB94-216082

04,832

\section{FLIP.CHIP TECHNOLOGY}

Microwave Characterization of Flip-Chip MMIC Compo-

Microwave Characterization of Flip-Chip MMIC Interconnections.

02.435

Electrical Measurements of Microwave Flip-Chip Interconnections.

02,436

FLOATING POINT ARITHMETIC

Underilow-Induced Graphics Failure Solved by SLI Arith-

PB95-161444

01.712

Parallel and Serial implementations of SLI Arithmetic. PB95-242335

FLOORS

Fire Performance of an Interstitial Space Construction System.

PB95-188918 00.390

Testing of Selected Self-Leveling Compounds for Floors. PB95-220455 00,395 Materials-Science Based Approach to Phenol Emissions from a Flooring Material in an Office Building. PB97-118749

02,572

FLOREFFE TERMINAL

Ashland Tank Collapse Investigation.

PB95-126314

02,481

Ashland Tank-Collapse Investigation: Closure by Authors PB95-126322

02,482

FLOW CALIBRATION

Cryogenic Flow

01,143

FLOW CHARACTERISTICS

Effect of Heterogeneous Porous Media on Mold Filling in 


\section{KEYWORD INDEX}

FLUORESCENCE

\section{FLOW INJECTION}

Flow Immunoassay Using Solid-Phase Entrapmen PB96-200951

00,642

\section{OW INJECTION ANALYSIS}

Liposome-Based Flow-Injection Immunoassay for Determining Theophylline in Serum.

PB94-213493

03,494

Potentiometric Enzyme-Amplified Flow Injection Analysis Detection System: Behavior of Free and Liposome-Released Peroxidase.

PB95-151833

03,534

Amperometric Flow-Injection Analysis Biosensor for Glucose Based on Graphite Paste Modified with Tetracyanoquinodimethane.

PB95-161980

03,498

FLOW MEASUREMENT

Ventilation Rates in Office Buildings. $\quad 02,539$
PB95-108825

Energy Flows in an Orifice Pulse Tube Refrigerator

PB95-169082 02,752

Laser Doppler Velocimeter Studies of the Pipeflow Produced by a Generic Header.

02,602

Sliding Vane Flow Conditioner Tests in a 100 Diamete Long 10 inch Natural Gas Orifice Meter at Pacific Gas and Electric. Topical Report, 1990-1992.

PB95.256335

02,493

\section{LOW METERS}

Summary Report of NIST's Industry-Government Consortium Research Program on Flowmeter Installation Effects with Emphasis on the Research Period, January-September 1991: The Reducer.

04,196

Summary Report of NIST's Industry-Government Consortium Research Program on Flowmeter Installation Effects: The 45 Degree Elbow.

PB95-143061

04,204

Laser Doppler Velocimeter Studies of the Pipeflow Produced by a Generic Header.

PB95-226916

02,602

Critical Evaluation of Thermal Mass Flow Meters.

PB97-113153

00,683

FLOW MODELS

Improvement in Predicting Smoke Movement in Compart-

$\begin{array}{ll}\text { mented Structures. } & 00,332 \\ \text { PB94-172418 }\end{array}$

Modeling Radon Transport in Multistory Residential Buildings.

00,256

FLOW RATE

Experimental Data and Theoretical Modeling of Gas Flows Through Metal Capillary Leaks.

PB95-150892

04,206

FLOW VELOCITY

Backdraft Phenomena.

PB94-193927

01,366

Visual Measurement Technique for Analysis of Nucleate Flow Boiling.

03,262

FLOWGRAPHS

Method to Determine a Basis Set of Paths to Perform Program Testing
PB96-131503

01,747

OWMETERS

Flow Conditioner Tests for Three Orifice Flowmeter Sizes.

04,201

PB95-105540 04,201 ANSI Equation.

02,949 Effects of Pipe Elbows and Tube Bundles on Selected
Types of Flowmeters. Types of Flowmeters.

PB96-160999 01,135

SSME LOX Duct Flowmeter Design and Test Results.

Flowmeter Installation Effects Due to a Generic Header. Flowmeter Installation Effects Due to a Generic Header.
PB96-210893 02,606

02,606

Fluctuation Dominated Recombination Kinetics with Traps.
PB95-107264 00,875 Mesoscopic Conductance Fluctuations in Large Devices. PB96-119656 04,735 Quantum Conductance Fluctuations in the Larger-SizeScale Regime.

04,144

Orientational Fluctuations, Diffuse Scattering, and Orientational Order in Solid C60.

PB97-119275

04,176

FLUID FLOW

Reconstructing Stratified Fluid Flow from Reciprocal Scattering Measurem
PB95-107256

04,202

Effect of Heterogeneous Porous Media on Mold Filling in Resin Transfer Molding.

03,197
Comparison of the Unidirectional and Radial In-Plane Flow of Fluids Through Woven Composite Reinforce ments.

PB95-162004

02,698

Flow of Alternative Agents in Piping.

00,022

\section{FLUID MIXERS}

Milliwatt Mixer for Small Fluid Samples.

PB94-198819

02,634

FLUIDELASTICITY

Chaotic Motions of Coupled Galloping Oscillators and Their Modeling as Diffusion Progresses.

04,823

FLUIDS

Survey of the Literature on Heat Transfer from Solid Sur to Cryogenic Fluids.

04,193

AD-A286 680/4

Ebulliometers for Measuring the Thermodynamic Properties of Fluids and Fluid Mixtures.

DE94017817

04,195

Thermophysical Properties of Fluids for the Gas Industry. Final Report, February 1, 1988-August 31, 1993.

PB94-14667

02,472

Transient Methods for Thermal Conductivity. PB94-198405

04,197

Measurements of the Viscosities of Saturated and Compressed Fluid 1-chloro-1,2,2,2-tetrafluoroethane (R124) and Pentafluoroethane (R125) at Temperatures between 120 and $420 \mathrm{~K}$

120 and $420 \mathrm{~K}$

03,254

Global Thermodynamic Behavior of Fluid Mixtures in the

Critical Region.
PB94-212420 04,199

Thermophysical Property Computer Packages from NIST.

04,203

Thermophysical Properties of Fluids for the Gas Industry.
PB96-122437

FLUIDS: LIQUIDS/GASES/PLASMAS

Characteristics of Turbulence in a Boundary Layer with Zero Pressure Gradient.

AD-A278 249/8 04,192

Air Flow in the Boundary Layer of an Elliptic Cylinder.

AD-A297391/5 04,194

Distributed measurements of tracer response on packed bed flows using a fiberoptic probe array. Final report.

00,667

Summary Report of NIST's Industry-Government Consortium Research Program on Flowmeter Installation Effects with Emphasis on the Research Period, January-Septem ber 1991: The Reducer

PB94-160736

04,196

Transient Methods for Thermal Conductivity.

PB94-198405

04,197

Liquid and Solid Atomic Ion Plasmas.

PB94-19899

03,809

Absorption of Ultrasonic Waves in Air at High Frequencies $(10-20 \mathrm{MHz}$.

PB94-199007

04,182

Asymptotic Behavior of Modulated Taylor-Couette Flows with a Crystalline Inner Cylinder.

PB94-199072

04,469

Summary of the Patent Literature of Supercritical Fluid Technology.

00,502

Development in the Theory and Analysis of Eddy Current Sensing of Velocity in Liquid Metals.

PB94-212586

03,335

Evolution of a Turbulent Boundary Layer Induced by a Three-Dimensional Roughness Element.

04,200

Reconstructing Stratified Fluid Flow from Reciprocal Scat-

tering Measurements.
PB95-107256

Structure of the Vapor-Liquid Interface Near the Critical

PB95-140174 00.902

Flow of Microemulsions through Microscopic Pores.
PB95-140463 00,905

PB95-140463

Summary Report of NIST's Flowmeter Installation Effects:

tium Research Program

PB95-143061 04,204

Simulations of Glass Forming Liquids: What Has Been

PB95-150124

00,915

Experimental Data and Theoretical Modeling of Gas Flows Through Metal Capillary Leaks.

04,206

Controlled Nucleation in Aerosol Reactors for Supression of Agglomerate Formation.

00,672

Gas Phase Reactivity Study of $\mathrm{OH}$ Radicals with 1,1Dichloroethene and cis-1,1-Dichloroethene and Trans-1,2Dichloroethene over the Temperature Range $240-400 \mathrm{~K}$.

Dichloroethene

Electrostatic Modes of Ion-Trap Plasmas.

PB95-152963

00,939

03,920

Two Phase Aqueous Extraction: Rheological Properties of Dextran, Polyethylene Glycol, Bovine Serum Albumin and Their Mixtures.

00,676

Comparison of the Unidirectional and Radial In-Plane Flow of Fluids Through Woven Composite Reinforce-

PB95-162004

02,698

Critical Behavior of Ionic Fluids.

00,978

First Phase Formation Kinetics in the Reaction of $\mathrm{Nb} / \mathrm{Al}$

First Phase Formation Kinetics in the Reaction of $\mathrm{Nb/Al}$.
PB95.168456

Experimental Results on Normal Modes in Cold, Pure Ion

PB95-175105

03,956

Equilibrium Pair Distribution Function of a Gas: Aspects Associated with the Presence of Bound States.

PB95-176046

01,004

Electrostatic Modes as a Diagnostic in Penning-Trap Ex-

PB95-176244

03,959

Convective Stability in the Rayleigh-Benard and Directional Solidification Problems: High-Frequency Gravity Modulation.

04,208

Laser Doppler Velocimeter Studies of the Pipeflow Pro. duced by a Generic Header.

PB95-226916

02,602

Sliding Vane Flow Conditioner Tests in a 100 Diameter Long 10 inch Natural Gas Orifice Meter at Pacific Gas and Electric Topical Report $1990-1992$.

a

02,493

Langmuir Probe Measurements in the Gaseous Electronics Conference RF Reference Cell.

PB96-113386

02,393

Inductively Coupled Plasma Source for the Gaseous Electronics Conference RF Reference Cell.

02,394

Reactive lon Etching in the Gaseous Electronics Conference RF Reference Cell.

Dusty Plasma Studies in the Gaseous Electronics Conference Reference Cell.

02,396

Measurements of the Relative Permittivity of Liquid Water at Frequencies in the Range of 0.1 to $10 \mathrm{kHz}$ and at Temperatures between 273.1 and $373.2 \mathrm{~K}$ at Ambient

PB96-119375

01,078

Improved Gas Flow Measurements for Next-Generation Processes.

Analysis of Transverse Flow in Aligned Fibrous Porous

PB96-167200

03,177

Greenspan Acoustic Viscometer for Gases.

PB96-204417 


\section{KEYWORD INDEX}

In situ Characterization of Vapor Phase Growth of Iron Oxide-Silica Nanocomposites: Part 1.2-D Planar LaserInduced Fluorescence and Mie Imaging

PB97-112478

03,185

\section{FLUORESCENCE DETECTION}

Determination of Vitamin K1 in Serum Using Catalytic-Reduction Liquid Chromatography with Fluorescence Detec-

$$
\text { PB96-138425 }
$$

03,506

FLUORESCENCE RECOVERY AFTER PHOTOBLEACHING

TECHNIQUE

Monitoring Polymer Cure by Fluorescence Recovery After Photobleaching.

01,218

FLUORESCENCE SPECTROSCOPY

In situ On-Line Optical Fiber Sensor for Fluorescence Monitoring in Bioreactors.

PB94-212024

Standards for Corrected Fluorescence Spectra.
PB95-150835

PB95-150835 00,581

Electrophoretic SNA Fragments in DNA Typing Using a Capillary Electrophoresis-Lased Induced Fluorescence System.

PB95-163036

03,536

Enhanced Detection of PCR Products Through Use of TOTO and YOYO Intercalating Dyes with Laser Induced Fluorescence - Capillary Electrophoresis.

00,599

Fluorescence Measurements of Tetracycline in High Cell Mass for Fermentation Monitoring.

00,601

LUORESCENCE STANDARDS

Standards for Corrected Fluorescence Spectra. PB95-150835

00,581

FLUORESCENT DYES

Applications of Fluorescence Spectroscopy in Polymer Science and Technology.

01,258

FLUORESCENT LAMPS

Performance of Compact Fluorescent Lamps at Different Ambient Temperatures.

PB95-175329

00,258

FLUORESCENT SCREENS

Image Information Transfer Properties of X-Ray Intensify-

ing Screens in the Energy Range from 17 to $320 \mathrm{keV}$.

FLUORIDE

Fluoride Analytical Methods.

PB96-180237

FLUORIDES

Deposition of Loosely Bound and Firmly Bound Fluorides on Tooth Enamel by an Acidic Gel Containin Fluorosilicate and Monocalcium Phosphate Monohydrate. PB95-150710

03,559

Distribution of Fluoride in Saliva and Plaque Fluid After a $0.048 \mathrm{~mol} / \mathrm{L} \mathrm{NaF}$ Rinse.

PB95-151205

03,561

FLUORINATED ACRYLATES

Facile Synthesis of Novel Fluorinated Multifunctional Acrylates.

01,207

FLUORINATED ALIPHATIC HYDROCARBONS

Measurement of the Thermal Properties of Electrically Conducting Fluids Using Coated Transient Hot Wires. DE94017816 00,722

FLUORINATED CELIPHATIC HYDROCARBONS

Vapour Pressures and Gas-Phase ( $p$, rho $n, T)$ Values for CF3CHF2(R125)

PB96-102090

04,019

FLUORINATED HYDROCARBONS

Burning Rate of Premixed Methane-Air Flames Inhibited by Fluorinated Hydrocarbons.

01,391

FLUORINATION

Thermochemical and Chemical Kinetic Data for PB95-260618

FLUORINE

Influence of Coadsorbed Potassium on the Electron-Stimulated Desorption of $F(+), F(-)$, and $F\left(^{*}\right)$ from $P F 3$ on PB96-157946

FLUORINE-COMBUSTION CALORIMETRY

Thermochemical Studies of Inorganic Chalocogenides by Fluorine-Combustion Calorimetry: Binary Compounds of Germanium and Silicon with Sulfur, Selenium and Tellu-

PB97-112528

01,181

FLUORINE ORGANIC COMPOUNDS

Facile Synthesis of Novel Fluorinated Multifunctional Acrylates.

01,207

Thermodynamic Properties of CHF2-OCHF2,Bis(difluoromethyl) Ether

(-) 94-199569

00,798

FLUOROALKANES

Electronic Spectra of $\mathrm{CF} 2 \mathrm{Cl}$ and $\mathrm{CFCl} 2$ Radicals $\mathrm{Ob}-$ served by Resonance Enhanced Multiphoton Ionization,
PB95-151023
FLUOROCARBONS

Retention of Halocarbons on a Hexafluoropropylene Epoxide Modified Graphitized Carbon Black. Part 1. Meth ane-Based Compounds.

PB95-175196

03,272

Retention of Halocarbons on a Hexafluoropropylene Epoxide-Modified Graphitized Carbon Black. 4. PropaneBased Compounds.

PB96-164033 03,284

Retention of Halocarbons on a Hexafluoropropylene Epoxide-Modified Graphitized Carbon Black. 3. EtheneBased Compounds.

PB96-167309

03,286

Halon Thermochemistry: 'Ab Initio' Calculations of the Enthalpies of Formation of Fluoromethanes.

03,289

FLUOROHYDROCARBONS

Fluorinated Hydrocarbon Flame Suppression Chemistry.
PB94-185113

Thermodynamic Properties of Difluoromethane

Thermal Conductivity of R134a.

PB94-213063

00,772

Polarized Transient Hot Wire Thermal Conductivity Measurements

Ebulliometric Measurement of the Vapor Pressure of Difluoromethane.

PB95-151361

00,931

Dielectric Studies of Fluids with Reentrant Resonators

PB95-153730 00,952

Viscosity of the Saturated Liquid Phase of Six Halogenated Compounds and Three Mixtures.

00,960

Criteria for Establishing Accurate Vapor Pressure Curves.

Criteria for Establishing Accurate Vapor Pressure Curves.
PB95-163812

00,972

Ebulliometric Measurement of the Vapor Pressure

PB95-164489

00,984

Thermodynamic Properties of R134a(1,1,1,2Tetrafluoroethane)

00,988

Measurements of Molar Heat Capacity at Constant Volume (Cv) for 1,1,1,2-Tetrafluoroethane (R134a)

PB95- 168878

Vapour Pressure Measurements on 1,1,1,2Tetrafluoroethane (R134a) from 180 to $350 \mathrm{~K}$.

03,265

Annex 18: An International Study of Refrigerant Properties.

PBries. 168936

03,266

Development of a Dual-Sinker Densimeter for High-Accuracy Fluid P-V-T Measurements.

PB95-168951

03,267

Measurement of the Thermal Properties of Electrically

Conducting Fluids Using Coated Transient Hot Wires.

PB95-169058 03,269

Measurements of the Vapor Pressures of

Difluoromethane, 1-Chloro-1,2,2,2-Tetrafluoroethane, and Pentafluoroethane.

PB95-169272

03,270

Vapor Pressures and Gas-Phase PVT Data for 1-Chloro-

1,2,2,2-Tetrafluoroethane (R124)

PB95-175154

03,271

Predictive Extended Corresponding States Model for Pure and Mixed Refrigerants Including an Equation of State for R134a

PB95-175717

03,275

FLUOROMETHANES

Experimental and Numerical Burning Rates of Premixed Methane-Air Inhibited by Fluoromethanes.

PB95-180592

01,392

FLUOROMETRY

Optical Biosensor Using a Fluorescent, Swelling Sensing

Element.

03,541

\section{FLUOROTOLUENES}

Acceleration of Intramolecular Vibrational Redistribution by methyl Internal Rotation. A Chemical Timing Study of

p-fluorotoluene and p-fluorotoluene-d3

01,039

FLUX PINNING

Temperature Dependence of Vortex Twin Boundary Interaction in Ytrium Barium Copper Oxide (YBa2Cu3O6+x). PB95-162442

04,613

Enhanced Flux Pinning via Chemical Substitution in Bulk Superconducting $\mathrm{T} 1-2212$.

04,647

Increased PInning Energies and Critical Current Densities in Heavy-lon-Irradiated $\mathrm{Bi} 2 \mathrm{Sr} 2 \mathrm{CaCu} 2 \mathrm{O} 8$ Single Crystals.

Crossover in the Pinning Mechanism of Anisotropic Fluxon Cores

04,673

Temperature and Field Dependence of Flux Pinning in $\mathrm{NbTi}$ with Aritificial Pinning Centers.

04,726

Magnetic Flux Pinning in Epitaxial YBa2Cu307-delta Thin PB96-200746

04,795

FLUX (RATE)

Single Photon Laser lonization as an In-situ Diagnostic for MBE growth

01,059

FLUX TRANSFORMERS

Fabrication Issues in Optimizing YBa2Cu3O7-x Flux Transformers for Low $1 / f$ Noise.

PB95-175857

02,059

Low Noise YBa2Cu3O7-x-SrTiO3-YBa2Cu3O7-x Mulitlayers for Improved Superconducting PB96-138417

04,747

FLUX TRAPPING

Trapped Vortices in a Superconducting Microbridge.

PB95-141149

04,554

FLUX VORTICES

Trapped Vortices in a Superconducting Microbridge PB95-141149

04,554

FLUXON OSCILLATIONS

SUSAN: SUperconducting Systems ANalysis by Low Temperature Scanning Electron Microscopy (LTSEM). PB96-112065
P4,728

FLUXONS

Deformable Superconductor Model for the Fluxon Mass. PB95-175311

04,653

FLY ASH

Compositional Analysis of Beneficiated Fly Ashes. PB95-220497

00,397

FAM

Fire Protection Foam Behavior in a Radiative Environment.

00,237

FOAMS

Effects of Humidity and Elevated Temperature on the Density and Thermal Conductivity of a Rigid Polyisocyanurate Foam Co-Blown with $\mathrm{CCl} 3 \mathrm{~F}$ and $\mathrm{CO}_{0}$.

OCUSING

Laser Focusing of Atoms: A Particle Optics Approach. PB94-216660 03,870 Neutron Focusing Lens Using Polycapillary Fibers PB95-141206

03,889

FOIAS-TEMAM APPROXIMATION

Foias-Temam Approximations of Attractors for Galloping Oscillators.

04,817

FOIAS-TEMAN APPROXIMATION

Algebraic Approximation of Attractors for Galloping Oscillators.

PB95-162897

04,820

FOKKER-PLANCK DESCRIPTIONS

Fokker-Planck Description of Multivalent Interactions.

PB95-108478

00,879

OOD

Inter-Laboratory Trials of the EPR Method for the Detection of Irradiated Meats Containing Bone.

00,042

Recently Developed NIST Food Related Standard Reference Materials

00,035 
Estimation of the Absorbed Dose in Radiation-Processed Food. 1. Test of the EPR Response Function by a Linear Regression Analysis.

00,039

Commentary on 'Optimization of Experimental Param. Commentary on 'Optimization of Experimental Parameters for the EPR Dete

radiated Foodstu

00,043

FOOD STORAGE

Individual Carotenoid Content of SRM 1548 Total Diet and Influence of Storage Temperature, Lyophilization, and Irradiation on Dietary Carotenoids.

00,033 PB94-200524

Program Requirements to Advance the Technology of
Custom Footwear Manufacturing. Custom Foolw

02,883

\section{OOTWEAR INDUSTRY}

Information Technologies Make Business Sense for the Custom Therapeutic Footwear Industry.

ORCE MEASUREMENT

Force Calibrations in the Nanonewton Regime.
PB95.168696

\section{PB95-168696
OREIGN COUNTRIES}

Countries, Dependencies, Areas of Special Sovereignty, and Their Principal Adminıstrative Divisions. Category: Data Standards and Guidelines; Subcategory: Representation and Codes.
FIPS PUB 10-4

00,128

Countries, Dependencies, Areas of Special Sovereignty. and Their Principal Administrative Divisions (for Microcomputers).

00,130

\section{OREIGN TECHNOLOGY}

Statistical Quality Control Technology in Japan.
PB94-199064

Japan Technology Program Assessment. Simulation State-of the-Art in Japan.

PB95-217097

02,827

Parallel and Serial Implementations of SLI Arithmetic.

PB95-242335 01,732

Optically Stabilized Tunable Diode-Laser System for Saturation Spectroscopy.

04.717 Energy Calibration of X-ray Photoelectron Spectrometers: Proposed Calibration Procedure.

PB96-102918

04,027

Damage Processes in Ceramics Resulting from Diamond Tool Indentation and Scratching in Various Environments.

Measurements of Permittivity and the Dielectric Loss TanMeasurements of Permittivity and the Dielectric Loss Tangent of Low Loss Dielectric Materials with a Dielectric Resonator Operating

gamma delta)

02,273

Measuring Hydrogen by Cold-Neutron Prompt-Gamma Activation Analysis.

PB96-111877

00,612

Crystal Structure of Calcium Glutarate Monohydrate.

PB96-111893 01,074

Stability of Compressed Gas Mixtures Containing Low Level Volatile Organic Compounds in Aluminum Cyl-

PB96-111968

00,613

Temperature and Field Dependence of Flux Pinning in NbTi with Aritificial Pinning Centers.

04,726

Effects of Target Temperature on Analytical Sensitivities Effects of Target Temperature on Analytical Sensitivities
of Cold-Neutron Capture Prompt gamma-ray Activation Analysis

PB96-112131 00,616

Study of Multiple Scattering Background in Compton Scatter Imaging.

04,425

Local Oscillator Requirements and Strategies for the Next Generation of High-Stability Frequency Standards. 01.551 Analytical Applications of Guided Neutron Beams.

PB96-112347

04,041

Vortex Dynamics and Melting in Niobium.

02,073

Glimpse of Materials Research in China: A Report from an Interagency Study Team on Materials Visiting China from June 19,1995 to June 30,1995

PB96-112677

02,978

Improved Annealing Technique for Optical Fiber. PB96-119680

04,343

Neutron Techniques in Materials Science and Related

Disciplines.
PB96-119698 02,980

Atom Cooling and Trapping, and Collisions of Trapped

Atoms

04,048

Introduction to Phase-Stable Optical Sources.

04,347

Effects of Spindle Dynamic Characteristics on Hard Turning.

02,699
Study of Laser Resonance Ionization Mass Spectrometry Using a Glow Discharge Source.

03,360

Hydraulic Radius and Transport in Reconstructed Model Three-Dimensional Porous Media.

00,403

Lubrication Theory for Reactive Spreading of a Thin

Drop.

04,214

Ultrafast Time-Resolved Infrared Probing of Energy

Transfer at Surfaces.

00,620

Fundamental Processes in Gas Discharges

01,089

Kinetic-Energy Distributions of lons Sampled from Radio-

Frequency Discharges in Helium, Nitrogen, and Oxygen.
PB96-123732

Measurement of S2OF10, and S2O2F10 Production

Rates from Spark and Negative Glow Corona Discharge in SF6/O2 Gas Mixtures.

01,093

Distributed Feedback Lasers in Rare-Earth-Doped Phos-

phate Glass.

04,740

Anionic Triphenylmethane Dye Solutions for Low-Dose Food Irradiation Dosimetry.

03,715

Alcohol Solutions of Triphenyl-Tetrazolium Chloride as High-Dose Radiochromic Dosimeters.

03,716

Oscillometric and Conductometric Analysis of Aqueous and Organic Dosimeter Solutions. and Organic Dosimeter Solutions.
PB96-135256 04,054

Calorimeters for Calibration of High-Dose Dosimeters in High-Energy Electron Beams.

PB96-135272

04,055

Real Time Monit

03,719

Influence of Thickness Fluctuations on Exchange Coupling in $\mathrm{Fe} / \mathrm{Cr} / \mathrm{Fe}$ Structures.

PB96-135371

04,745

Fiber Coating Diameter: Toward a Glass Artifact Stand-

PB96-140389

02,234

Research and Development Activities in Electron Paramagnetic Resonance Dosimetry.

03,635
PB96-141288 Solids.

PB96-141338

04,759

Inelastic Neutron Scattering Study of Hydrogen in Nanocrystallin

03,366

II-3: Critical Current Measurement Methods: Quantitative Evaluation

PB96-147160

04,767

11-5: Thermal Contraction of Materials Used in Nb3Sn Critical Current Measurements.

04,769

Development of Gas Standards from Solid 1,4dichlorobenzene.

PB96-155486

02,496

Transitions to Chaos Induced by Additive and Multiplicative Noise.

03,750

Spectrum of the Stochastically Forced Dutfing-Holmes Oscillator.

00,216

Necessary Condition for Homoclinic Chaos Induces by Additive Noise.
PB96-155775

04,063

Physicochemical Characterization of Natural and Bioprosthetic Heart Valve Calcific Deposits: Implications for Prevention.

00,187

Model for Calculating Virial Coefficients of Natural Gas

Hydrocarbons with Impurities.
PB96-156047

Deterministic and Stochastic Chaos.

00,218 Application of Digital-Image-Based Models to
Microstructure, Transport Properties, and Degradation of Microstructure, Transport Properties, and Degradation of

Cement-Based Materials.
PB96-156161

00.406

Modeling Detector Response for Neutron Depth Profiling.

Influence of Films' Thickness and Air Gaps in Surface Im-
pedance Measurements of High Temperature

pedance Measurements of High Temperature
Superconductors Using the Dielectric Resonator Tech-

nique.
PB96-157862

01,946

Information Transfer in the 21st Century.

02,714

Comment On: Two-Photon Absorption Series of Calcium.

Comment On: Two-Photon Absorption Series of Calcium.
PB96-157979

Resonance lonization Spectroscopy/Resonance lonization

Mass Spectrometry Data Service. V-Data Sheets for Ga,

$\mathrm{Mn}, \mathrm{Sc}$, and $\mathrm{Tl}$

00,625

Faraday Constant

01,955

Dynamics of Hydrogen Interactions with $\mathrm{Si}(100)$ and Si(111) Surfaces.

04,082

RII Spectroscopy of Trap Levels in Bulk and LPE Hg1-

PCdxTe.

04,084

Instrumental Smearing Effects in Radially Symmetric Small-Angle Neutron Scattering by Numerical and Analytical Methods.

02,984

Novel DNA N-Glycosylase Activity of $E$. coli T4 Endonuclease $V$ That Excises 4,6-Diamino-5 Formamidopyrimidine from DNA, a UV-Radiation- and Hydroxyl Radical-Induced Product of Adenine.

PB96-160478

03,549

Development of the NIST Transient Pressure and Temperature Calibration Facility.

00,626

Effects of Pipe Elbows and Tube Bundles on Selected Types of Flowmeters.

P86-160999

Pressure Measurements with the Mercury Melting Line

$\begin{array}{ll}\text { Referred to ITS-90. } & 01,136 \\ \text { PB96-161005 } & \end{array}$

Direct Comparison of Three PTB Silver Fixed-Point Cells with the NIST Silver Fixed-Point Cell.

00,628

Investigation of the ITS-90 Subrange Inconsistencies for

25.5 Omega SPRTs.
PB96- 161302
00,630

Verification of Revised Water Vapour Correction to the Index of Refraction of Air.

PB96-161666

02,680

Current Noise Reveals Protonation Kinetics and Number of lonizable Sites in an Open Protein lon Channel. PB96-161674

04,092

Inter-Laboratory Trials of the EPR Method for the Detec-

(ion of Irradiated Meats Containing Bone.

B96-161690

00,042

Protonation Dynamics in an Ion Channel Pore.

PB96-161757

03,589

PB96-161765

03,076

Nonequilibrium Statistical Mechanıcs.

PB96-161781

04,097

Measurement of the $(10) \mathrm{B}$ ( $\mathrm{n}$, alpha 1gamma)(7) Li Cross Section in the 0.3 to $4 \mathrm{MeV}$ Neutron Energy Interval. Gas Absorption during Ion-Irradiation of a Polymer TarPBet. 161864

04,099

Fundamentals of Fracture: A 1993 Prologue, and Other Comments.

Fracture in Multilayers

PB96-163613

03,218

Autuorescence

PB96.163639

Silver

Model for Microcrack Initiation and Propagation beneath Hertzian Contacts in Polycrystalline Ceramics.

PB96-163704

03.077

Non-Newtonian Flow between Concentric Cylinders Calculated from Thermophysical Properties Obtained trom Simulations.

04,103 


\section{KEYWORD INDEX}

Determination of 21 Elements by INAA for Certification of SRM $1570 a$, Spinach.

New NIST Rapid Pneumatic Tube System.

PB96-167259

03,489

Recent Develop
PB96-167267

Relationship of Silver with Selenium and Mercury in the Liver of Two Species of Toothed Whales (Odontocetes). PB96-167275 02,596

Resolution of Discrepant Analytical Data in the Certification of Platinum in Two Automobile Catalyst SRMs.

PB96-167283 00,638

Retention of Halocarbons on a Hexafluoropropylene Epoxide-Modified Graphitized Carbon Black. 3. Etheneoxide-Modified Graphi
Based Compounds.

Based Compound

Structure and Rheology of Hard-Sphere Systems.

03,286

PB96-167333

00,662

Thermophysical Property Standard Reference Data from

07,153

PB96-167358

Novel Magnetic Field Characterization Techniques for Compound Semiconductor Materials and Devices.

PB96-176458

Energy Distributions of Neutrons Scattered from Solid C60 by the Beryllium Detector Method.

03,740

Small-Angle Neutron Scattering (SANS) Study of WormLike Micelles Under Shear.

04,111

Surtace Plasmon Microscopy of Biotin-Streptavidin Binding Reactions on UV-Photopatterned Alkanethiol Self-Assembled Monolayers.

PB96-17677

01,158

Flaw Tolerance and Toughness Curves in Two-Phase Particulate Composites: SiC/Glass System. PB96- 179460 Adsorption of Potassium N-phenylglycinate on Hydroxyapatite: Role of Solvents and lonic Charge. PB96-180161

Fluoride Analytical Methods.

PB96-180237

03,578

Fracture of Silicon Nitride and Silicon Carbide at Elevated Temperatures.

PB96-180260 03,179

Alpha-Particle and Electron Capture Decay of (209)Po.

PB96-186085 04.119

Scattered Fractions of Dose from 18 and 25 MV X-ray Radiotherapy Linear Accelerators.

PB96-186101

04,120

Characterization of the Structure of YD3 by Neutron Powder Diffraction.

07,161

Comment on $\ll<$ A Dynamic Electric Trap for Ground-State

PB96-200290

04,123

Collisions of Electrons with Highly-Charged lons.

PB96-200340

04,791

Kinetic-Energy-Enhanced Neutral Etching.

PB96-200613

00,665

Thermal Conductivity of Polypyromellitimide Film with Alumina Filler Particles trom 4.2 to $300 \mathrm{~K}$

PB96-200753

01,292

Quantitative Determination of Oxidative Base Damage in DNA by Stable Isotope-Dilution Mass Spectrometry.

03,483

Commentary: The Measurement of Oxidative Damage to DNA by HPLC and GC/MS Techniques.

03,484

UV Absorption Cross Sections of Methylchioroform: Temperature-Dependent Gas and Liquid Phase Measure-

PB96-201041

00,643

Low Heat-Flux Measurements: Some Precautions.

PB96-201116

02,685

Gas Phase Oxygen Effect on Chain Scission and Monomer Content in Bulk Poly(methyl methacrylate) Degraded by External Thermal Radiatio

B96-204078

01,293

Creep and Creep Rupture of Structural Ceramics.

PB96-204524 03,093

Role of Certified Reference Materials in Trace Analysis Quality Assurance.

PB97-110019 00,650

Interaction of an Isolated Sprinkler Spray and a Two-

Layer Compartment Fire Environment. Phenomena and Model Simulations.

PB97-110076

01,437

Liquid-Scintillation Counting Techniques for the Standardization of Radionuclides Used in Therapy.

03,709

Needs for Brachytherapy Source Calibrations in the United States.

PB97-110092

Radioassays of Yterium-90 Used in Nuclear Medicine.

PB97-110100
Problem of Convection in the Water Absorbed Dose CalProblem or

PB97.110159

03,523

Stable Silicon Photodiodes for Absolute Intensity Measrements in the VUV and Soft X-ray Regions.

BQ97-110175

04,135

Tension/Compression Creep Asymmetry in Si3N4.

PB97-110258

03,096

Current Status and Trends in Temperature Measurements at NIST, Cooperative Projects and New Mutual Agreement between NIST and IMGC

PB97-110266

02691

Electron-Photon Monte Cario Calculations: The ETRAN

PB97-110407

04,138

Interlaboratory Studies on the Analysis of Hair for Drugs of Abuse: Results from the Fifth Exercise.

03,509

B97-110449

High Temperature Structural Reliability of Silicon Nitride.
PB97-110456

Magneto-Optic Rotation Sensor Using a Laser Diode as Both Source and Detector.

Both Source and
PB97-111272

04,390

Proposed Tests to Evaluate the Frequency-Dependen Capacitor Ratio for Single Electron Tunneling Experiment. New Refractometer by Combining a Variable Length Vacuum Cell and a Double-Pass Michelson Interferometer.
PB97-111926

Observation of Two Length Scales Above ( $T$ sub $N$ ) in a Holmium Thin Film.

PB97-111942

04,151

DNA Damage and DNA Sequence Retrieval from Ancient Tissues.

B97.111983

03,556

High-Current Thin Film Multijunction Thermal Converters and Multi-Converter Modules.

01,989

Ferric Ion Assisted Photooxidation of Halocetates.
PB97-112460

Wideband Sampling Voltmeter.

PB97-113039

01,990

NIST Watt Balance: Progress Toward Monitoring the Kito-

gram.

01,991

Molecular Dynamics Investigation of the Surface/Bulk Equilibrium in an Ethanol-Water Solution.

PB97-113112

01,183

Comparison of Filter Radiometer Spectral Responsivity with the NIST Spectral-Irradiance and Illuminance Scales. 04,162 $\mathrm{DC}-\mathrm{MHz}$ Wattmeter Based on RMS Voltage Measure. ments.

PB97-113211

01,992

Results of Capacitance Ratio Measurements for the Single Electron Pump-Capacitor Charging Experiment.

B97-113286

Issues in High-Speed Pyrometry.

PB97-118368

04,813

Patiance Temperatures at $1500 \mathrm{~nm}$ of Niobium and Molybdenum at Their Melting Points by a Pulse-Heating echnique.

04,167

Effect of Formulation Changes on the Response to Ioniz ing Radiation of Radiochromic Dye Films.

PB97-119028

04,17

Results of the ASTM Nuclear Methods Intercomparison on NIST Apple and Peach Leaves Standard Reference

PB97-119036

03,490

Measurements of the $(235) \cup(n, f)$ Cross Section in the 3 to $30 \mathrm{MeV}$ Neutron Energy Region.

PB97-119051

04,172

Automated Guarded Bridge for Calibration of Multimegohm Standard Resistors.

02,289

B97.119150

Conversion of a 2-Terminal-Pair Bridge to a 4-Teminal-
Pair Bridge for Increased Range and Precision in ImpedPair Bridge for Increased

ance Measurements.
PB97-119176

02,103

Novel Radiochromic Films for Clinical Dosimetry.

03,641

Orientational Fluctuations, Diffuse Scattering, and Orientational Order in Solid C60.

PB97-119275

04,176

Calibration and Performance of GafChromic DM-100 Radiochromic Dosemeters.

PB97-119291

00.703

Electronic Structure and Phase Equilibria in Ternary Substitutional Alloys.

PB97-119366

03,378

Anomalous Relation between Time and Frequency Do-

04,398

Dependence of the Thermal Electron Attachment Rate Constant in Gases and Liquids on the Energy Position of the Electron Altaching State.

01,996

Generalized Optical Theorem for On-Axis Gaussian Beams.

PB97-122345

04.177

Measurement of the Atomic $\mathrm{Na}(3 \mathrm{P})$ Lifetime and of Retar-

dation in the Interaction between Two Atoms Bound in a

PB97-122360

04.178

Complex Time Dependence of the EPR Signal of Irradiated L-alpha-alanine.

PB97-122436

04,180

DNA Base Damage in Lymphocytes of Cancer Patients

Undergoing Radiation Therapy.

03,643

Wear Modeling of SI-Based Ceramics.
PB97-122501

03,112

Characterization of Time-Dependent Dielectric Breakdown in Intrinsic Thin $\mathrm{SiO} 2$

PB97-122527

02,458

Xi-Vector Formulation of Anısotropic Phase-Field Models: 3-D Asymptotics

PB97-122550

04,816

PB97-122592

03,113

FOREIGN TECHNOLOOGY

Viscosity of Defined and Undefined Hydrocarbon Liquids Calculated Using an Extended Corresponding-States

PB96-167234

02,498

FORENSIC SCIENCE

Separation and Identification of Organic Gunshot and Ex-

Plosive Constituents by Micellar Electrokinetic Capillary

Electrophoresis.

00,566

FORENSIC SCIENCES

Face Recognition Technology for Law Enforcement Applications.

PB94-207768

01,837

FORENSICS

Specification for Interoperability between Ballistic Imaging

Systems. Part 1. Cartridge Cases.

01,860

FORGING

Microstructure and Tensile Properties of Microalloyed Steel Forgings.
PB94-172715 


\section{KEYWORD INDEX}

FORMIC ACID

Strong Hydrogen Bond in the Formic Acid-Formate Anion

Pystem.

00,788

FORMS (PAPER)

Evaluating Form Designs for Optical Character Recogni-

PB94-168044

01,830

Generalized Form Registration Using Structure-Based

Techniques.
PB96-191374

01,858

FORMYL RADICALS

Ouantum Dynamics of Renner-Teller Vibronic Coupling: The Predissociation $\mathrm{HCO}$

PB94-185303

00,773

FORTRAN

Review of Mathematical Function Library for MicrosoftFORTRAN, John Wiley and Sons, 1989

01,679

FORTRAN PROGRAMMING LANGUAGE

FORTRAN Compiler Validation System, Version 2.1.

Validated Products List (Cobol, Fortran, ADA, Pascal, MUMPS, SOL).

PB94-937300). 01,700

Validated Products List (Cobol, Fortran, ADA, Pascal, MUMPS, SOL).

01,738

ORWARD SCATTERING

$X$-ray Photoelectron and Auger Electron Forward Scattering: A Structural Diagnostic for Epitaxial Thin Films PB95-180220

04,676

FOSSIL FUELS

Modulation of Fossil Fuel Production by Global Temperature Variations, 2.

02,533

Determination of Sulfur in Fossil Fuels by Isotope Dilution Thermal lonization Mass Spectrometry.

02,495

PB96-141379

FOULING ORGANISMS

Determination of Total Protein Adsorbed on Solid (Membrane) Surface by a Hydrolysis Technique: Single Protein Adsorption.

03,552

FOULING RESISTANCE

Determination of Osmotic Pressure and Fouling Resistances and Their Effects on Performance of Ultrafiltration Membranes.

PB94-212891

00,841

Characterization of the Adsorption-Fouling Layer Using Globular Proteins on Ultrafiltration Membranes.
PB94-212909

FOUNDATIONS

Static Structural Analysis of a Reconfigurable Rigid Platform Supported by Elastic Legs.

02,960

FOUR-TERMINAL-PAIR-BRIDGE

NIST Comparison of the Ouantized Hall Resistance and the Realization of the SI Ohm Through the Calculable Capacitor.

02,291

FOURIER ANALYSIS

Exact Recursion Relation Solution for the Steady-State Surface Temperature of a General Multilayer Structure. PB96-102017

02,376

FOURIER-TRANSFORM SPECTROSCOPY

Wavelengths of Spectral Lines in Mercury Pencil Lamps.

PB96-176474 04,376

FOURIER TRANSFORMATION

Standard Reference Materials: Polystyrene Films for Calibrating the Wavelength Scale of Infrared Spectrophotometers - SRM 1921.

PB95-226866

03,386

Self-Reciprocal Fourier Functions

PB96-200852

01,974

FOWLER-NORDHEIM

New Oxide Degradation Mechanism for Stresses in the Fowler-Nordheim Tunneling Regime.

PB96-200985

04,128

FPETOOL COMPUTER PROGRAM

FPETOOL: Fire Protection Tools for Hazard Estimation. An Overview of Features.

00,367

FRACTAL AGGREGATION

Dynamic Scaling in an Aggregating 2D Lennard-Jones PBstem.

04,106

FRACTAL STRUCTURES

Electrolytes Constrained on Fractal Structures: DebyeHuckel Theory

01,174

FRACTOGRAPHY

Loading Device for Fracture Testing of Compact Tension Specimens in the Scanning Electron Microscope.
PB95-162434

02,652

of 4140 Steel.

PB 4140 -190285

03,222
FRACTURE

Lattice Statics of Interfaces and Interfacial Cracks in Bimaterial Solids.

PB96-161823

02,985

\section{FRACTURE (MATERIALS)}

Fundamentals of Fracture: A 1993 Prologue, and Other PB96-16197

FRACTURE (MECHANICS)

Fracture Testing of Large-Scale Thin-Sheet Aluminum AD-A306 625/5

03,305

Fracture Behavior of Large-Scale Thin-Sheet Aluminum Alloy.

03,311

Fracture Mechanics Analysis of Near-Surface Cracks

PB94-172400

03,230

Error Propagation Biases in the Calculation of Indentation Fracture Toughness for Ceramics.

03,032

Temperature Increases in Aluminum Alloys during $\mathrm{Me}$ chanical-Impact Tests for Oxygen Compatibility.

PB94-172962

03,316

Generic Model for Creep Rupture Lifetime Estimation on Fibrous Ceramic Composites.

PB94-200235

03,137

Application of a Simple Technique for Estimating Errors of Finite-Element Solutions Using a General-Purpose

PB94-200250

04,818

Environmentally Enhanced Fracture of Ceramics.

PB95-125746

03,046

Prediction of the Strength Properties for Plain-Carbon and Vanadium Micro-Alloyed Ferrite-Pearlite Steel.

PB96-123393

03,216

Catastrophic Failures Propagate Field of Fracture Mechanics.

03,217

Pressurized Internal Lenticular Cracks at Healed Mica Interfaces.

PB96-180252

02,997

Fracture of Silicon Nitride and Silicon Carbide at Elevated Temperatures.

03,179

Fatigue Crack Thresholds of a Nickel-iron Alloy for Superconductor Sheaths at $4 \mathrm{~K}$.
PB96-190343

03,223

Fractur

PB96-204532

03,094

FRACTURE MECHANICS MODEL

Model for Toughness Curves in Two-Phase Ceramics. 2 Microstructural Variables.

03,078

PB96-163795

Model for Toughness Curves

Basic Fracture

03,085

\section{FRACTURE PROPERTIES}

Evaluation of the Environmentally Induced Fracture Resistance of Ductile Nickel Aluminide. Technical Report Number 1, Final report. October-December 1990.

DE94017331

03,306

Charpy Impact Test as an Evaluation of $4 \mathrm{~K}$ Fracture Toughness.

03,219

\section{FRACTURE RESISTANCE}

Effects of Copper, Nickel and Boron on Mechanical Properties of Low-Alloy Steel Weld Metals Deposited at High Heat Input.

PB96-135231

03,363

FRACTURE STRENGTH

Matrix Grain Bridging Contribution to the Toughness of Whisker Reinforced Ceramics.

03,134

Potential Drop in the Center-Cracked Panel with Asym. metric Crack Extension.

PB95.107330

04,819

Evaluation of Fracture Toughness and Residual Stress in Dental Porcelain by Indentation-Microfracture Method. PB95-125613 00,154 New Alloys Show Extraordinary Resistance to Fracture and Wear.

03,346

Evaluation of Fracture Toughness and Residual Stress in Dental Porcelain by Indentation-Microfracture Method. PB95-152831 00,159

Fracture Toughness of Advanced Ceramics at Room Temperature: A Vamas Round-Robin.

Shielding of Cracks in a Plastically Polarizable Material.

Shielding of Cracks in a Plastically Polarizable Material.
PB $95-164257$

FRACTURE TESTS

Fracture Testing of Large-Scale Thin-Sheet Aluminum Alloy.

00,024

Factors Significant to Precracking of Fracture Specimens.

PB96-109558

03,358

FRACTURE TOUGHNESS

Aluminum-Lithium Alloys: Evaluation of Fracture Tough-

ness by Two Test Standards, ASTM Method E 813 and E

PB96-190236

03,374

Light-Weight Alloys for Aerospace Applications II.

PB96-190244

03,375

Charpy Specimen Tests at $4 \mathrm{~K}$

03,002

Mechanical Properties and Warm Prestress of Ultra-Low Carbon Steel at $4 \mathrm{~K}$.

PB96-190350

03,224

FRACTURES

Fracture in Mu
PB96-163613

02,988

FRACTURES (MATERIALS)

Micromechanics of Fracture in Rubber-Toughened

PB94-212222

03,011

Ashtand Tank Collapse Investigation.

02,481

Ashland Tank-Collapse Investigation: Closure by Authors.

PB95-126322 02,482

FRACTURING

Fracture Behavior of Large-Scale Thin-Sheet Aluminum Alloy.

FRAGILE GLASS

Long-Lived Structures in Fragile Glass-Forming Liquids.

PB96-119565

FRAME STRUCTURES

Survey of Steel Moment-Resisting Frame Buildings Affected by the 1994 Northridge Earthquake.

PB95-211918

00,451

FRAMES

Strengthening Methodology for Lightly Reinforced Con-

crete Frames-II. Recommended Calculation Techniques

for the Design of Infilt Walls.

Strengthening Methodology for Lightly Reinforced Concrete Frames: Recommended Design Guidelines for Strengthening with Intill Walls.

PB95-260725

00,454

Strengthening Methodology for Lightly Reinforced ConCrete Frames.

00,466

FREE ELECTRON LASERS

NIST-NRL Free-Electron Laser Facility

PB94-212511

04,237

Hybrid Undulator

04,238

FREE ENERGY

Interfacial Free Energies from Substrate Curvature Measurements of the Creep of Multilayer Thin Films.

PB94-185428

04,448

Stability and Surface Energies of Wetted Grain Bound-

aries in Aluminum Oxide.

PB95-202750

03,059

FREE ENERGY TRANSDUCTION

Imposed Oscillations of Kinetic Barriers Can Cause an

Enzyme to Drive a Chemical Reaction Away from Equi-

librium 


\section{KEYWORD INDEX}

Polymerization Initiation by N-p-Tolylglycine: Free-Radical Reactions Studied by Pulse and Steady-State Radiolysis. Reactions Studied by Pulse and Steady-State Radiolysis.
PB95-180014 Free Radical Chemistry of the Atmospheric Aqueous Phase.

00,117

FREE ROTORS

Perpendicular C.H Stretching Band nu9/nu13 and the Torsional Potential of Dimethylacetylene.

PB97-122451

01,192

FREEZERS

Factors Affecting the Energy Consumption of Two Refrigerator-Freezers
PB97-112312

FREEZING

Anomalous Freezing and Melting of Solvent Crystals in Swollen Gels of Natural Rubber.

12321

01,223

FREEZING POINT

Direct Comparison of Three PTB Silver Fixed-Point Cells with the NIST Silver Fixed-Point Cell PB96-161286

00,628

FREEZING POINT DEPRESSION

Anomalous Freezing and Melting of Solvent Crystals in Anollen Gels of Natural Rubber.

PB94-212321

01223

FREONS

Thermophysical Properties of HFC. $143 a$ and HFC-152a. Ouarterly Report, 1 July 1993--30 September 1993. DE94004236

03,245

FREOUENCIES

Improved Molecular Constants and Frequencies for the $\mathrm{CO} 2$ Laser from New High.J Regular and Hot-Band Frequency Measurements.

04,312

FREQUENCY

Aging, Warm-Up Time and Retrace; Important Characterstics of Standard Frequency Generators.

04,031

Effect of Harmonic Distortion on Phase Errors in Frequency Distribution and Synthesis.

01,563

REQUENCY DRIFT

Confidence on the Three-Point Estimator of Frequency Driti

01,539

PB95-163838

Extension of Heterodyne Frequency Measurements on PBg $1087 \mathrm{~Hz}(2900 \mathrm{~cm}(-1))$

00,811

Using LORAN-C Broadcasts for Automated Frequency Calibrations.

01,526

Sub-Doppler Frequency Measurements on OCS at 87 Thz (3.4 micrometers) with the CO Overtone Laser: Considerations and Details.

PB95-128633

04,255

ELF Electric and Magnetic Field Measurement Methods.

PB95-161675 04,423

Absolute Frequency Measurements of Methanol from 1.5 to $6.5 \mathrm{THz}$.

04,300

Precise Optical Frequency References and Difference Frequency Measurements with Diode Lasers.

04,305

Sub-Doppler Frequency Measurements on OCS at 87

$\mathrm{THz}$ (3.4 mu m) with the CO Overtone Laser.

$\mathrm{THz}(3.4 \mathrm{mu} \mathrm{m})$ with the CO Overtone Laser.
PB96-102215

Atomic Clock

01,553

Introduction to Frequency Calibration. Part 1.

01,560

NIST Frequency Measurement Service.

PB96-200662

01,561

REQUENCY MEASUREMENTS

Accurate Measurement of Time.

PB96-119482

01,552

FREQUENCY MULTIPLIERS

High-Order Harmonic Mixing with GaAs Schottky Diodes.

EQUENCY REFERENCES

High-Resolution Diode-Laser Spectroscopy of Calcium.

PB95-181244

03,969

FREQUENCY RESPONSE

Photodetector Frequency Response Measurements at NIST, US, and NPL, UK: Preliminary Results of a Stand-

ards Laboratory Comparison.

02,175

Bounds on Frequency Response Estimates Derived from Uncertain Step Response Data.

03,419

Uncertainties of Frequency Response Estimates Derived from Responses to Uncertain Step-Like Inputs. PB97-111843

01,984

FREQUENCY RESPONSES

Comparison of Photodiode Frequency Response Measurements to $40 \mathrm{GHz}$ between NPL and NIST.

04,038
FREQUENCY SHIFT

Frequency Shitting of Pulsed Narrow-Band Laser Light in Multipass Raman Cell. PB95-203352

04,321

\section{FREQUENCY STABILITY}

Precision Oscillators: Dependence of Frequency on Temperature, Humidity and Pressure.

PB94-198306

02.031

Frequency Stabilization of a Fiber Laser to Rubidium: A High-Accuracy $1.53 \mathrm{mu} \mathrm{m}$ Wavelength Standard.

PB95-126082

04,252

Confidence on the Second Difference Estimation of Fre quency Drift.

01,532 Frequency Stabilized Lasers: A Parochial Review.
PB95-153771

Reducing the Effect of Local Oscillator Phase Noise on the Frequency Stability of Passive Frequency Standards.
PB95-180972 Local Oscillator Requirements and Strategies for the Next Generation of High-Stability Frequency Standards.
PB96-112230

01,551

undamental

PB96-176565

FREQUENCY STANDARDS

Future of Time and Frequency Dissemination

AD-P009 138/9

Time and Frequency Technology at NIST.

N94-30641/2

01,522

Future of Time and Frequency Dissemination.

N94-30684/2

01,524

Utc Dissemination to the Real-Time User.

N19960042622 01521

NIST Optically Pumped Cesium Frequency Standard.

PB94-211117 03,835

Ultra-High Stability Synthesizer for Diode Laser Pumped

PB94.21606

01,527

Atoms in Optical Molasses: Applications to Frequency

Standards.

PB95-108882

03,876

Frequency Stabilization of a Fiber Laser to Rubidium: A High-Accuracy $1.53 \mathrm{mu} \mathrm{m}$ Wavelength Standard.

04,252

Confidence on the Second Difference Estimation of Frequency Dritt.

PB95-151460

01.532

NIST-7, the New US Primary Frequency Standard. 01,534
PB95-153458

Error Analysis of the NIST Optically Pumped Primary Frequency Standard.

01,535

Diode-Laser Pumped, Rubidium Cell Frequency Stand-

ards. 01,538

Confidence on the Three-Point Estimator of Frequency

Drift.

PB95-163838

01,539

Improved Rubidium Frequency Standards Using Diode Lasers with AM and FM Noise Control.

PB95-176152

04,303

Microwave Leakage as a Source of Frequency Error and Long-Term Instability in Cesium Atomic-Beam Frequency

PB95-180501

01,541

Velocity Distribution of Atomic Beams by Gated Optical Pumping.

01,542

Hybrid Digital/Analog Servo for the NIST-7 Frequency Standard.

PB95-180618

01,544

Reducing the Effect of Local Oscillator Phase Noise on the Frequency Stability of Passive Frequency Standards.

Time and Frequency: Bibliography of NIST Publications March 1995

PB95-220463

01,548

Time Generation and Distribution.

PB96-103049 01,550

Laser-Cooled Neutral A.tom Frequency Standards.

PB96-160312

04,086

Time and Frequency Metrology

\section{FEQUENCY SYNTHESIZERS}

High Spectral Purity X-Band Source.

Reducing Errors, Complexity, and Measurement Time of PM Noise Measurements.

PB96-119771

02,075

Effect of Harmonic Distortion on Phase Errors in Fre.

quency Distribution and Synthesis.

Q 200779

Frequency Synthesis and Metrology at $10(-17)$ and $B e-$ yond.

PB97-113187

02,10

Reducing the $1 / f$ AM and PM Noise in Electronics for Precision Frequency Metrology.

02,102

FRICTION

Micro-Mechanical Aspects of Asperity-Controlled Friction in Fiber-Toughened Ceramic Composites.

03,136

Asperity-Asperity Contact Mechanisms Simulated by a Two-Ball Collision Apparatus.

PB95-164158

02,966

FICTION FACTOR

Mechanism of Mild to Severe Wear Transition in AlphaAlumina.

PB94-212354

03,233

FRICTION MEASUREMENT

Tribometer for Measurements in Hostile Environments. PB95-180949

02,967

FRICTION REDUCTION

Control of Friction and Wear of Alpha-Alumina with a Composite Solid-Lubricant Coating.

03,225

TP (FILE TRANSFER PROTOCOL)

Using Archie to Find Files on the INTERNET. PB95-168605

02,727

(FLIGHT TELEROBOTIC SERVICER)

Evolution of the Flight Telerobotic Servicer. PB94-216082

04,832

FUEL ADDITIVES

Atmospheric R

FUEL COMBUSTION

Estimate of the Effect of Scale on Radiative Heat Loss Fraction and Combustion Efficiency. PB95-150447

02,486

FUEL SPRAYS

Analysis of Droplet Arrival Statistics in a Pressure-Atomized Spray Flame.

\section{UEL TANKS}

Effect of Fuel Tank Rupture Mode on the Ignitability of PB97-110043

01,444

UELS

Structure and Radiation Properties of Pool Fires.

PB94-193802

02,473 urement in Liquid Pool Flames.

02,476

Measurement of Radiative Feedback to the Fuel Surface of a Pool Fire.

$\begin{array}{ll}\text { PB94-211604 } & 02,477\end{array}$

Large Fire Experiments for Fire Model Evaluations. PB96-190079

01,427

FUGACITY

Fugacity Coefficients of Hydrogen in (Hydrogen + Bu. PB95-175212

02,491

FUGACITY COEFFICIENTS

Fugacity Coefficients of Hydrogen in (Hydrogen + Bu- 


\section{KEYWORD INDEX}

GAS ATOMIZATION

FULLERIDES

Neutron-Scattering Study of $\mathrm{C} 60(n-)(n=3,6)$ Librations in Alkali-Metal Fullerides.

PB94-200219

00,806

FUNCTIONAL TESTING

Experimental Evaluation of Specification Techniques for

Improving Functional Testing.
PB96-201009

01,779

FUNCTIONS (MATHEMATICS)

Numerical Evaluation of Special Functions.
PB96-119557

03,417

Polarization Dependence of Response Functions in $3 \times 3$ Sagnac Optical Fiber Current Sensors.

PB96-122684

02,189

FUNDAMENTAL CONSTANTS

Fine-Structure Constant

PB94-172996

03,795

FURAN/METHYL-TETRAHYDRO

Atmospheric Reactivity of alpha-Methyl-Tetrahydrofuran

FURNACE

ITS-90 Calibration Facility

PB96-160916

00,627

FURNANCES

High-Temperature Furnace for In situ Small-Angle Neutron Scattering during Ceramic Processing.

03,743

FURNITURE

Some Factors Affecting Design of a Furniture Calorimeter Hood and Exhaust

PB94-139193 00,285

Summaries of Center for Fire Research In-House Projects and Grants: 1990.

PB94-160876

00,286

Toxicity, Fire
PB94-198454

Sprinkler Fire Suppression Algorithm

00,289

PB94-21618

00,293

Summaries of BFRL Fire Research In-House Projects and Grants, 1994

PB95-130845

00,366

Behavior of Mock-Ups in the California Technical Bulletin 133 Test Protocol: Fabric and Barrier Effects. PB95.231585

00,301

Some Factors Affecting the Design of a Calorimeter Hood and Exhaust.

00,302

Examination of the Correlation between Cone Calorimeter Data and Full-Scale Furniture Mock-Up Fires.

Data and Full-Sc

01,417

NIST Research on Less-Flammable Materials.

PB97-118632

01,439

USE (FAR ULTRAVIOLET SPECTROGRAPH EXPLORER) Scientific Rationale and Present Implementation Strategy for the Far Ultraviolet Spectrograph Explorer (FUSE). PB96-123328

00,045

FUSE SATELLITE

FUSE: The Far Uitraviolet Spectrograph Explorer.
PB94-213410 00,063

FUSED SILICA

Fuzed-Ouartz Fibers. A Survey of Properties, Applications and Production Methods.

00,656

FUSION (MELTING)

Calibration Standards for Differential Scanning Calorimetry. 1. Zinc Absolute Calorimetric Measuremen of Enthalpy of Fusion and Temperature of Fusion HM PB94-199817

FUZZY LOGIC

Texture-Independent Vision-Based Closed-Loop Fuzzy Controllers for Navigation Tasks.

00,183

STARS

IUE Observations of Solar-Type Stars in the Pleiades and the Hyades. Far-Ultraviolet Flare on a Pleiades G Dwarf.
PB96-102033

00,053

00,086

GAAS/ALGAAS

Quantum Conductance Fluctuations in the Larger-SizeScale Regime.
PB97-111264

04,144

GADOLINIUM GALLIUM FERRATES

Enhanced Magnetocaloric Effect in Gd3Ga5-xFexO12.

PB94-185659

Magnetocaloric Effect of Ferromagnetic Particles. PB94-185857

GAGE BLOCKS

Gage Block Handbook.

02,667

Gage Block Standards, Measurement Capabilities and Laboratory Accreditation.

02,757

PB96-163621

Interstellar Disk-Halo Connection in Galaxies: Review of

Observational Aspects.

PB94-211802

00,058
GALACTOPYRANOSIDE

Thermodynamics of the Binding of Galactopyranoside Derivatives to the Basic Lectin from Winged Bean (Psophocarpus Tetrogonolobus) PSOph-162715

03,465

\section{GALERKIN METHOD}

Alternative Contour Technique for the Efficient Computation of the Effective Length of an Antenna. PB96-141361

02,011

GALLIUM

Arc Spectra of Gallium, Indium, and Thallium.

Arc

Characterization of the Binding of Gallium, Platinum, and Uranium to Pseudomonas Fluorescens by Small-Angle $X$ ray Scattering and Transmission Electron Microscopy.
PB94-172509

Single-Photon Ionization and Detection of $\mathrm{Ga}, \mathrm{In}$, and As(sub n) Species in GaAs Growth.

00,591

n-situ Monitoring of Molecular Beam Epitaxial Growth Using Single Photon Ionization.

PB95-202222

01,023

Laser-Induced Desorption of In and $\mathrm{Ga}$ from $\mathrm{Si}(100)$ and Adsorbate Enhanced Surface Damage.

01,050

Pulsed Laser Irradiation at $532 \mathrm{~nm}$ of $\mathrm{In}$ and $\mathrm{Ga}$ Adsorbed on Si(100): Desorption, Incorporation, and DamPB95-203329

01,051

Single-Photon Laser Ionization Time-of-Flight Mass Spectroscopy Detection in Molecular-Beam Epitaxy: Application to As4, As2, and Ga.

PB95-203337

01,052

GALLIUM ANTIMONIDES

Epitaxial Growth of Sb/GaSb Structures: An Example of VIIII-V Heteroepitaxy.

PB95-202560

04,693

GALLIUM ARSENIDE

$X$-ray Reflectivity Determination of Interface Roughness

Correlated with Transport Properties of (AIGa)As/GaAs High Electron Mobility Transistor Devices.

04,149

GALLIUM ARSENIDE TRANSISTORS

Effects of Heavy Doping on Numerical Simulations of Gallium Arsenide Bipolar Transistors.

PB95-150975

02,334

GALLIUM ARSENIDES

Junction Locations by Scanning Tunneling Microscopy n-Air-Ambient Investigation of Passivated GaAs pn Junc-

02,306

$X$-Ray Photoelectron and Auger Electron Spectroscopy Study of Ultraviolet/Ozone Oxidized P2S5/(NH4)2S Treated GaAs(100) Surfaces.

PB94-200144

04,479

Grazing-Incidence X-Ray Photoemission Spectroscopy Investigation of Oxidized GaAs(100): A Novel Approach to Nondestructive Depth Profiling. PB94-200151

04,480

Cs Cluster Binding to a GaAs Surface.

PB94-213006

00,846

Band-to-Band Photoluminescence and Luminescence Excitation in Extremely Heavily Carbon-Doped Epitaxia iaAs.

04,570

Single-Photon Ionization and Detection of $\mathrm{Ga}, \mathrm{In}$, and As(sub n) Species in GaAs Growth.

PB95-152815

00,591

Grazing Angle X-Ray Photoemission System for DepthDependent Analysis

04,600

Lattice Position of $\mathrm{Si}$ in GaAs Determined by X-Ray Standing Wave Measurements.

B 95-164406

04,632

Characterization of the $\mathrm{ZnSe} / \mathrm{GaAs}$ Interface Layer by TEM and Spectroscopic Ellipsometry.

04,655

n-situ Monitoring of Molecular Beam Epitaxial Growth Using Single Photon lonization. Using Single Photon lonization

01,023

Laser Vacuum Ultraviolet Single Photon Ionization Probing of III-V Semiconductor Growth.

PB95-202370

04,691

Epitaxial Growth of Sb/GaSb Structures: An Example of IIII-V Heteroepitaxy.

04,693

04,693
PB95-202560 or Semiconductor Growth.

PB95-202776

01,032

Single-Photon Laser Ionization Time-of-Flight Mass Spectroscopy Detection in Molecular-Beam Epitaxy: Applicaion to As4, As2, and $\mathrm{Ga}$.

PB95-203337

01,052

Single Photon Laser Ionization as an In-situ Diagnostic or MBE growth.

PB96-102025

01,059

Time-Resolved Measurements of the Polarization State of Four-Wave Mixing Signals from GaAs Multiple Ouantum Wells.

PB96-201058

04,796

Majority and Minority Electron and Hole Mobilities in Heavily Doped Gallium Aluminum Arsenide.

PB97.118335

04,814

GALLIUM INDIUM PHOSPHIDES

Surface Topography and Ordering-Variant Segregation in

PB95-153649

04,595

ALLIUM IONS

Relative Sensitivity Factors and Useful Yields for a Microfocused Gallium Ion Beam and Time-of-Flight Secondary Ion Mass Spectrometer. PB94-198736

00,541

\section{GALLOPING OSCILLATORS}

Chaotic Motions of Coupled Galloping Oscillators and Their Modeling as Diffusion Progresses.

PB96-122718

04,823

GAMMA DOSIMETRY

Measurement of Absorbed Dose of Neutrons, and of Mixtures of Neutrons and gamma rays.

AD-A286 647/3

03,710

Thin Dyed-Plastic Dosimeter for Large Radiation Doses. PB95-107363

03,872

GAMMA DRACONIS STAR

Ultraviolet Observations of Stellar Coronae: Early Results rom HST.

GAMMA RADIATION

00,064

Effect of Formulation Changes on the Response to lonizing Radiation of Radiochromic Dye Films.

04,171

GAMMA RAY ASTRONOMY

From Superconductivity to Supernovae: The Ginzburg Symposium. Report on the Symposium Held in Honor of Vitaly L. Ginzburg. Held in Gaithersburg. Maryland on May 22, 1992

GAMMA RAY SCATTERING

Polarization Effects on Multiple Scattering Gamma Transport

PB95-153615

03,926

GAMMA RAY SPECTROMETRY

Dead Time, Pileup, and Accurate Gamma-Ray Spectrom PB96-167101

00,697

GAMMA RAY SPECTROSCOPY

Local Area Networks in NAA: Advantages and Pitfalls. PB94-172095

00,527

GAMMA RAYS

X-ray Attenuation Coefficients from $10 \mathrm{Kev}$ to $100 \mathrm{Mev}$ 
GAS CHROMATOGRAPHY Comparison of Methods for Gas Chromatographic Deter-
mination of PCBs and Chlorinated Pesticides in Marine Reference Materials.

PB95-140091

02,584

Process Gas Chromatography Detector for Hydrocarbons Based on Catalytic Cracking.

02,485

Shape Selectivity Assessment of Stationary Phases in Gas Chromatography.

00,579

Comparison of Selectivities for PCBs in Gas Chroma. Comparison of Selectivities for PCBs in Gas Chroma-
tography for a Series of Cyanobiphenyl Stationary

PB96-119458

00,618

All-Metal Collection System for Preparative-Scale Gas Chromatography: Purification of Low-Boiling-Point Compounds.

00,619

Investigations of Sulfur Interferences in the Extraction of Methylmercury from Marine Tissues.
PB96-190020
Petron the Extraction of

GAS CHROMOTOGRAPHY

Procedure for Measuring Trace Ouantities of S2F10, S2OF10, and S2O2F10 in SF6 Using a Gas Chromatograph-Mass Spectrometer.

PB96-119755

GAS CYLINDERS

Stability/Instability of Gas Mixtures Containing 1,3-Butadiene in Treated Aluminum Gas Cylinders.

02,546

Stability of Compressed Gas Mixtures Containing Low Stability of Compressed Gas Mixtures Containing Low
Level Volatile Organic Compounds in Aluminum Cyl-

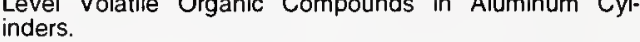
inders.

00,613

\section{GAS DETECTORS}

Conductance Response of Pd/SnO2(110) Model Gas Sensors to $\mathrm{H} 2$ and $\mathrm{O} 2$.

PB95-125803

00,892

Tin Oxide Gas Sensor Fabricated Using CMOS MicroHotplates and In-situ Processing

00,580

Fundamental Studies of Gas Sensor Response Mechanisms: Palladium on $\mathrm{SnO} 2(110)$

PB95-162731

00,963

NIST Workshop on Gas Sensors: Strategies for Future Technologies. Proceedings of a Workshop. Held in Gaithersburg, Maryland on September 8-9, 1993.

GAS DISCHARGES

Excitation of Balmer Lines in Low-Current Discharges of Hydrogen and Deuterium.

03,893

Kinetic-Energy Distributions of lons Sampled from Argon Plasmas in a Parallel-Plate, Radio-Frequency Reference Call.

03,935

Electrical Characterization of Radio-Frequency Discharges in the Gaseous Electronics Conference Reference Call.

PB95-164612

01,905

Ion Kinetic-Energy Distributions and Balmer-alpha (Halpha) Excitation in Ar-H2 Radio-Frequency Discharges.

PB96-102959 04,029

Kinetic Energy Distribution of lons Produced from Townsend Discharges in Neon and Argon.

PB96-111927

04,413

Modification of Cast Epoxy Resin Surfaces during Exposure to Partial Discharges.

01,086

Influence of Electrode Material on Measured Ion KineticEnergy Distributions in Radio-Frequency Discharges.

Kinetic Energy Distributions of $\mathrm{H}(+), \mathrm{H} 2(+)$, and $\mathrm{H} 3(+)$ from a Diffuse Townsend Discharge in $\mathrm{H} 2$ at High E/N.
PB96-123351

Fundamental Processes in Gas Discharges.

PB96-123450

01,089

Kinetic-Energy Distributions of Ions Sampled from RadioFrequency Discharges in Helium, Nitrogen, and Oxygen.
PB96-123732

GAS DISSOCIATION

Associative Electron Attachment to $\mathrm{S} 2 \mathrm{~F} 10, \mathrm{~S} 2 \mathrm{OF} 10$, and $\mathrm{S} 2 \mathrm{O} 2 \mathrm{~F} 10$.

PB95-140992

00,907

GAS DYNAMICS

Operational Mode and Gas Species Effects on Rotational Drag in Pneumatic Dead Weight Pressure Gages.

PB95-140182

00,903

GAS FLOW

Concurrent Flow Flame Spread Study.

01,356

Experimental Data and Theoretical Modeling of Gas Flows Through Metal Capillary Leaks.

04,206

Improved Gas Flow Measurements for Next-Generation Processes. PB96-156013

04,216

\section{GAS GENERATORS}

Solid Propellant Gas Generators: Proceedings of the 1995 Workshop. Held in Gaithersburg, Maryland on June 28-29, 1995.

01,412

GAS GIANT PLANETS

Experimental Determination of the Rate Constant for the Reaction of $\mathrm{C}_{2} \mathrm{H}_{3}$ with $\mathrm{H} 2$ and Implications for the Partilioning of Hydrocarbons in Atmospheres of the Outer

PB97-122295

00,112

GAS METAL ARC WELDING

Through-the-Arc Sensing for Real-Time Measurement of Gas Metal Arc Weld Ouality.

PB95-162871

02,863

Through-the-Arc Sensing for Measuring Gas Metal Arc Weld Ouality in Real Time.

PB95-164463 02,908

Droplet Transfer Modes for a MIL 100S-1 GMAW Elec-

PB95-209300

02,867

Control of Gas-Metal-Arc Welding Using Arc-Light SensPB̈96-131461

02,869

Contact Tube Wear Detection in Gas Metal Arc Welding.

Ultrasonic Sensing of GMAW: Laser/EMAT Defect Detec ion System

PB96-186028

02,878

Mapping the Droplet Transfer Modes for an ER100S-1 GMAW Electrode.

03,295

Power Characteristics in GMAW: Experimental and Nu merical Investigation.

PB96-190145

03,296

Sensing Droplet Detachment and Electrode Extension for Control of Gas Metal Arc Welding.

AS PIPELINES

03,297

Low Temperature H(sub 2)S Separation Using Membrane Reactor with Redox Catalyst.

02,471

\section{Partial Pressure}

N95-14084/4

04,827

GAS STANDARDS

Development of Gas Standards from Solid 1,4 dichlorobenzene.

02,496

GAS TRANSPORT

Gas Transport Properties of Solution-Cast
Perfluorosulfonic Acid lonomer Films Containing lonic Surfactants.

PB95-175998

GAS TURBINES

Bibliography of Books and Published Reports on Gas Turbines, Jet Propulsion, and Rocket Power Plants.
AD-A278 138/3

Bibliography of Books and Published Reports on Gas
Turbines, Jet Propulsion, and Rocket Power Plants, JanuTurbines, Jet Propulsion, and Rocket Power Plants, Janu-
ary 1950 through December 1953 .
AD-A278 213/4

GASEOUS

Studies of Ion Kinetic-Energy Distributions in the Gaseous Electronics Conference RF Reference Cell. B96-113360

02,391

\section{GASEOUS ELECTRONICS}

Journal of Research of the National Institute of Standards and Technology, July/August 1995 . Volume 100, Number 4. Special Issue. The Radio-Frequency Reference Cell.

Radio-Frequency

02,386

Gaseous Electronics Conference RF Reterence Cell: An

Introduction.
PB96-113329 02,387

Current and Voltage Measurements in the Gaseous Electronics Conference RF Reference Cell.

02,388

Optical Emission Spectroscopy on the Gaseous Elec-

tronics Conference RF Reference Cell.
PB96-113345

02,389

Microwave Diagnostic Results from the Gaseous Elecronics Conference RF Reference Cell.

02,392

Langmuir Probe Measurements in the Gaseous Electronics Conference RF Reference Cell.

PB96-113386

02,393

Inductively Coupled Plasma Source for the Gaseous Electronics Conference RF Reference Cell.

02,394

Reactive Ion Etching in the Gaseous Electronics Conference RF Reference Cell.

PB96-113402

02,395

Dusty Plasma Studies in the Gaseous Electronics Conerence Reference Cell.

02,396

One-Dimensional Modeling Studies of the Gaseous Electronics Conference RF Reference Cell.

02,397

Two-Dimensional Self-Consistent Radio Frequency Plasma Simulations Relevant to the Gaseous Electronics Conterence RF Reference Cell.

PB96-113436

02,398

Electrical Measurements for Monitoring and Control of $n$ Plasma Processing.

161963

04,369

GASEOUS ELECTRONICS CONFERENCE

Electrical Characteristics of Argon Radio Frequency Glow Discharges in an Asymmetric Cell.

GASES

Equilibrium Pair Distribution Function of a Gas: Aspects Associated with the Presence of Bound States.

PB95-176046

01,004

Vapour Pressures and Gas-Phase ( $p$, rho $n, T$ ) Values for CF3CHF2(R125)

PB96-102090

04.019

Thermodynamic Properties of Two Gaseous Halogenated Ethers from Speed-of-Sound Measurements: Difluoromethoxy-Difluoromethane and 2-Difluoromethoxy1,1,1-Trifluoroethane

PB96-102413

04,189

Gaseous Electronics Conference RF Reference Cell: An PBg6-113329

02,387

Current and Voltage Measurements in the Gaseous Electronics Conference RF Reference Cell.

PB96-113337

02,388

Optical Emission Spectroscopy on the Gaseous Elecronics Conference RF Reference Cell.

02,389

Optical Diagnostics in the Gaseous Electronics Conference Reference Cell.

02,390

Studies of Ion Kinetic-Energy Distributions in the Gaseous Electronics Conference RF Reference Cell.

PB96-113360

02,391

Microwave Diagnostic Results from the Gaseous Electronics Conference RF Reference Cell.

PB96-113378

02,392

Langmuir Probe Measurements in the Gaseous Electronics Conference RF Reference Cell.

PB96-113386

02,393

Inductively Coupled Plasma Source for the Gaseous Electronics Conference RF Reference Cell.

02.394

Reactive Ion Etching in the Gaseous Electronics Conference RF Reference Cell.

PB96-113402

02,395

Dusty Plasma Studies in the Gaseous Electronics Conference Reference Cell.

02,396

One-Dimensional Modeling Studies of the Gaseous Electronics Conference RF Reference Cell.

PB96-113428

02,397

Two-Dimensional Selt-Consistent Radio Frequency Plasma Simulations Relevant to the Gaseous Electronics Conference RF Reference Cell.

PB96-113436 
GELATON

Dynamic Scaling in an Aggregating 2D Lennard-Jones System.

04,106

GELETIN FILMS

Optical Properties of Triton X-100-Treated Purple Membranes Embedded in Gelatin Films.

PB96-123500

03,546

GELS

Imaging of Fine Porosity in a Colloidal Silica: Potassium Silicate Gel by Defocus Contrast Microscopy.

PB94-212750

03,039

Small-Angle Neutron Scattering of Poly(vinyl alcohol) Gels. 164117

01,260

Small Angle Neutron Scattering Study on Poly(N-Isopropyl Acrylamide) Gels Near Their Volume-Phase Transition Temperature.

PB95-164380 01,263

Small-Angle Neutron Scattering Study on Weakly Charged Temperature Sensitive Polymer Gels. $\quad 01,264$
PB95-164398

Optical Biosensor Using a Fluorescent, Swelling Sensing Elemen

PB95-175899

03,541

Small Angle Neutron Scattering Studies of Structural Characteristics of Argarose Gels.

PB96-112305 03,475 Silica Gels.

PB96-167184

01,151

GENERAL INTEREST

Bibliography of Books and Published Reports on Gas Turbines, Jet Propulsion, and Rocket Power Plants.

AD-A278 138/3

01,445

Standard Materials. A Descriptive List with Prices.

AD-A278 140/9 00,500

Bibliography of Books and Published Reports on Gas Turbines, Jet Propulsion, and Rocket Power Plants, January 1950 through December 1953

01,446

Screw-Thread Standards for Federal Services, 1957. Part AD-A279 121/8

02,854 Screw-Thread Standards for Federal Services, 1957. Part AD-A279 290/1

02,855

Characterization of the Hydrogen Induced Cold Cracking Susceptibility at Simulated Weld Zones in HSLA-100 Steel.

$759 / 5$

03,200

National Standard Petroleum Oil Tables.

AD-A279 952/6

02,469

Screw-Thread Standards for Federal Services, 1957. Handbook H28 (1957), Part 2. Revised.

03,599

1950 Supplement to Screw-Thread Standards for Federal Services. 1944.

AD-A280 223/9

03,656

Guide 10 Instrumentation Literature.

02,617

Aromic Energy Levels. As Derived From the Analyses of Optical Spectra. Volume 3.

00,714

Maximum Permissible Amounts of Radioisotopes in the Human Body and Maximum Permissible Concentrations in Air and Water.

03,609

Maximum Permissible Body Burdens and Maximum Permissible Concentrations of Radionuclides in Air and in Water for Occupational Exposure.

AD-A280 282/5

03,610

$X$-Ray Attenuation Coefficients from $10 \mathrm{KEV}$ to $100 \mathrm{MEV}$. AD-A280 290/8 C3.768 Papers on the Symposium on Collision Phenomena in

Astrophysics, Geophysics, and Masers.
AD-A280 291/6 00,047

Tables of Chemical Kinetics Homogeneous Reactions.

AD-A280 293/2 00,715

Ada Compiler Validation Summary Report: Certificate Number: 940325 S1.11352 DDC-I DACS Sun SPARC/ Solaries to Pentium PM Bare Ada Cross Compiler System, Version 4.6.4 Sun SPARCclassic $\Rightarrow$ Intel Pentium (Operated as Bare Machine) Based in Xpress Desktop (Intel Product Number: XBASE6E4F-B)

AD-A280 295/7

01,657

Cryogenic Research and Development (Quarterly Report Number 2 for Period Ending December 31, 1960).

AD-A280 398/9 Cryogenic Research and Development (Progress Report Number 4 for Period Ending December 31, 1961).
AD-A280 399/7

Cryogenic Research and Development (Quarterly Report Number 1 for Period Ending September 30, 1960). AD-A280 401/1

Precision Laboratory Standards of Mass and Laboratory Weights.

AD-A280 562/0

02,618
Cryogenic Research and Development (June 30, 1961)
AD-A280 679/2 Bibliography of the Physical Equllibria and Related Properties of Some Cryogenic Systems.

AD-A281 167/

03,769

Precision Resistors and Their Measurement.

02,249

AD-A284 623/6
Tabulation of Data on Receiving Tubes. Handbook 68.

AD-A285 495/8

02,111

Compilation of the Physical Equilibria and Related Propenties of the Hydrogen-Carbon Monoxide System. A286 603/6

00,716

Progress Report to National Aeronautics and Space Administration on Cryogenic Research and Development.
AD-A286 $612 / 7$

Design and Construction of a Liquid Hydrogen Temperature Refrigeration System.

AD-A286 618/4

02,619

Ionospheric Radio Propagation

AD-A286 619/2

01,459

Fuzed-Quartz Fibers. A Survey of Properties, Applications and Production Methods.

AD-A286 620/0

00,656

Measurement of Absorbed Dose of Neutrens, and of Mixtures of Neutrons and gamma rays.

03,710

High Temperature Reactions of Uranium Dioxide with Various Metal Oxides.

AD-A286 648/1

00,717

Survey of the Literature on Heat Transfer from Solid Surfaces to Cryogenic Fluids.

04,193

Performance of Plastic Packaging for Hazardous Mateials Transportation. Part 1. Mechanical Properties.

AD-A301 258/0

02,580

Weatherability of Plastic Materials.

02,968

Preliminary Subject and Authors Index to Compilations of Data on Properties of Materials.

AD-A302 669/7

03,242

Preliminary List of References Containing Compilations of Data on Properties of Materials.

AD-A302 670/5 03,243

Fracture Testing of Large-Scale Thin-Sheet Aluminum

AD-A306 625/5

03,305

AD-A307 789 /8

03,194

Volatile Corrosion Inhibitors.

AD-A310 087/2 03,114

Polyvinyl Chloride (PVC) Plastic Drain, Waste, and Vent Pipe and Fittings.

00,326

Acrylonitrile-Butadiene-Styrene (ABS) Plastic Drain, Waste, and Vent Pipe and Fittings.

Comparison of GPS Broadcast and DMA Precise Ephemerides.

AD-POO9 114/0

01,518

NIST Internet Time Service.

01,519

Future of Time and Frequency Dissemination.
AD-P009 $138 / 9$

AD-P009 138/9 01,520

ergy-Related Inventions Program.

DË94-017162

00,008

Ceramic Characterization.

DE94013170

03,026

Densification of Nano-Size Powders. 1994 Report. DE94013486

03,027

Equipment for Investigation of Cryogenic Compaction of Nanosize Silicon Nitride Powders. 1993 Report. DE94013593

03,028

Measuring Performance of Parallel Computers. Final Re-

DE94014586

01,665

Measuring Performance of Parallel Computers. Progress Report, 1989

01,666

NIST Cooperative Laboratory for OSI Routing Technology.

01,791

Evaluation of the Environmentally Induced Fracture Resistance of Ductile Nickel Aluminide. Technical Report Number 1 Final report October-December 1990.

03,306

Comparison of the Corrosion Rates of $\mathrm{FeAl}, \mathrm{Fe}$ (sub 3)A and Steel in Distilled Water and $0.5 \mathrm{M}$ Sodium Chloride. Technical Report Number 2, January--March 1991 DE94017332

03,186

Evaluation of the Electrochemical Behavior of Ductile Nickel Aluminide and Nickel in a pH 7.9 Solution. Technical Report Number 3, April-June 1991

DE94017351

03,307

Development of Measurement Capabilities for the Thermophysical Properties of Energy-Related Fluids. Annual Report, December 1, 1993--November 30, 1994.

DE94017738

Measurement of the Thermal Properties of Electrically Conducting Fluids Using Coated Transient Hot Wires. DE94017816

00,722

Dulliometers for Measuring the Thermodynamic Properties of Fluids and Fluid Mixtures.

DE94017817 04,195
Improvement of Ultrasensitive Techniques Isotopic Bias ing in the RIS Process Ionization Efficiencies and

DE94018563

00,522

Improvement of Resonance Ionization Spectroscopy (RIS) Techniques; Il: Alomic Data for RIS: III: Standards for Ultratrace Analysis. Progress Report.

DE94018565

00.523

Study of Laser Resonance Ionization Mass Spectrometry Using a Glow Discharge Source.

DE94018566

03,308

Minutes of the CAALS Workshop on modularity and communications standards.

DE95005780

02,621

Experimental assessment of absorbed dose to mineralized bone tissue from internal emitters: An electron paramagnetic resonance study.

DE96007979

03,614

mproving measurement quality assurance for photon irradiations at Department of Energy facilities. Final technica report

DE96010065

03,711

Thermophysical properties of HCFC alternatives. Quarterly report, October 1--December 31, 1995.

DE96010433

03,249

Thermophysical properties of HCFC alternatives. Quarterly report, April 1--June 30, 1995

Small genomes: New initiatives in mapping and sequencing. Workshop summary repor.

DE96014476

03,45

Information Infrastructure: Reaching Society's Goals. A Report of the Information Infrastructure Task Force Committee on Applications and Technology.

00,131

Guideline: Codes for Named Populatd Places, Primary County Divisions, and Other Locational Entities of the United States, Puerto Rico, and the Outlying Areas. Category: Data Standards and Guidelines; Subcategory: .

FIPS PUB 55-DC3

04,866

ADA; Category: Sottware Standard; Subcategory: Programming Language.

01,667

Procedures for Document Facsimile Transmission Issued by General Services Administration, April 14, 1982. Federal Standard 1063

FIPS PUB 148

01,516

Programmer's Hierarchical Interactive Graphics System (PHIGS). Category: Software Standard; Subcategory: FIPS PUB 153-1

01,668

Open Document Architecture (ODA) Raster Documen Application Profile (DAP). Category: Software Standard, Subcategory: Graphics. 
Combustion of a Polymer (PMMA) Sphere in Micro. gravity.

01,354

Ignition and Subsequent Transition to Flame Spread in a Microgravity Environment.

N96-15584/1

04,828

Utc Dissemination to the Real-Time User

01,521

In situ Burning of Oil Spills: Mesoscale Experiments.
PB94-142973

Abstract and Index Collection in the Research Information Center of the National Institute of Standards and Tech-

PB94-152204

02,744

Study to Determine the Most Important Parameters for Evaluating the Resistance of Soft Body Armor to Penetra-

$\begin{array}{ll}\text { tion by Edged Weapons. } & 03,757 \\ \text { PB94-158573 }\end{array}$

Metrology for Electromagnetic Technology: A Bibliography of NIST Publications.

02,116

Review of Mathematical Function Library for Microsoft-

Review of Mathematical Function Library for Microsoft-
FORTRAN, John Wiley and Sons, 1989 .

01,679

Bibliography on Apparel Sizing and Related Issues.

PB94-161924 02,806

Earthquake Resistant Construction of Gas and Liquid Fuel Pipeline Systems Serving, or Regulated By, the Federal Government

PB94-161999 04,846

Materials Science and Engineering Laboratory Annual Report, 1993. NAS-NRC Assessment Panel, April 21-22, 1994

PB94-162534

02,969

Ceramics Technical Activities, 1993 (NAS-NRC Assessment Panel April 21-22, 1994)

03,031

Putting the Information Infrastructure to Work: Report of the Information Infrastructure Task Force Committee on

Applications and Technology.

00,001

Intelligent Processing of Materials, Technical Activities 1993 (NAS-NRC Assessment Panel, April 21-22, 1994). PB94-164183 02,809

Catalog of National ISDN Solutions for Selected NIUF Applications.

01,468

Learning to Change: Opportunities to Improve the Performance of Smaller Manufacturers.

PB94-166212

00,010

Metrication.

$\begin{array}{ll}\text { PB94-172079 } & 03,676\end{array}$

Industry and Government-Laboratory Cooperative R and D: An ldea Whose Time Has Come.

PB94-172939

02,970

Functions of Technology Infrastructure in a Competitive Economy.

00,478

Investing in Education to Meet a National Need for a Technical-Professional Workforce in a Post-Industrial

PB94-173028

00,132

Physics Laboratory Technical Activities, 1993.

4-176088

03,796

NIST Serial Holdings, 1994

PB94-178068

02,745

Four Years of Monitoring alpha Orionis with the VLA:

Where Have All the Flares Gone.
PB94-185212

00,048

Distant Future of Solar Activity: A Case Study of Beta

PB94-185220 00,049

Rotational Modulation and Flares on RS Canum Venaticorum and BY Draconis Stars. XVI. IUE Spectroscopy and VLA Observations of $\mathrm{C} 1182(=\mathrm{V} 1005$ Orionis) in October 1983

00,050

High Sensitivity Survey of Radio Continuum Emission in Herbig Ae/Be Stars.

PB94-185915

00,051

Metric for Success

PB94-187630

02,633

Body Dimension

02,813

Optical Metrology and More. Programs and Services of the Radiometric Physics Division, Physics Laboratory.
PB94-191707

Binder Characterization and Evaluation by Nuclear Magnetic Resonance Spectroscopy.

PB94-193471

01,334

Bibliography of Photon Total Cross Section (Attenuation Coefficient) Measurements $10 \mathrm{eV}$ to $13.5 \mathrm{GeV}, 1907$.

03,804

Visualization Applications for Manufacturing: A State-ofthe-Art Survey. Final Report.

02,816
Federal Metric Progress in 1993. PB94-196029

02,600

Histopathology, Blood Chemistry, and Physiological Status of Normal and Moribund Striped Bass ('Morone saxatilis') Involved in Summer Mortality ('Die-Off') in the Sacramento-San Joaquin Delta of California.

00,034 B94-198157

First Results from the Goddard High-Resolution Spectrograph: The Chromosphere of Tauri.

PB94-199528

00,054

ROSAT All-Sky Survey of Active Binary Coronae. 2. Coronal Temperatures of the RS Canum Venaticorum Systems.

94-199601

00,055

Polarization of Light Emitted After Positron Impact Exciation of Alkali Atoms.

PB94-199734

03,816

Metric Path to Global Markets and New Jobs: A QuesMen-and-Answer and Thematic Discussion.

PB94-206307 and Thematic Discussion.

Assessment of Technology for Detection of Stress Corrosion Cracking in Gas Pipelines. Final Report, July 1993March 1994.

02,475

Dynamic Phenomena on the RS Canum Venaticorum Bi nary II Pegasi in August 1989. 1. Observational Data. PB94-211067

Differences in Competitive Strategies between the United States and Japan

PB94-211836

00,013

Importance of Measurement in Technology-Based Com-

PB94-211844 02,929

Opacity Project and the Practical Utilization of Atomic Data.

00,059

Aid for Smaller Businesses

PB94-212461

00,492

Class of Radio-Emitting Magnetic B Stars and a WindFed Magnetosphere Model.

00,067

94-213451

Information Infrastructure: Reaching Society's Goals. Re-
port of the Information Infrastructure Task Force Commit-

port of the Information Infrastructure Task Force Commit-
tee on Applications and Technology.
PB94-214756

Videoconferencing Procurement and Usage Guide.
PB94-217023

HAZARD I Fire Hazard Assessment Method (Version 1.2) (for Microcomputers).

PB94-501988

00.196

HAZARD I Fire Hazard Assessment Method, Version 1.2 (Upgrade Package) (for Microcomputers).

4-501996

00,197

$M$ (also known as MUMPS) Validation Test Suite, Version 8.3 (for Microcomputers)

PB94-502077

01,699

Polymer Composites Workshop. Held in Winona, Minnesota on April 29-30, 1992 (Video)

03,147

Questions and Answers on Ouality, the ISO 9000 Stand ard Series, Quality System Registration, and Related Is sues. More Questions and Answers on the ISO 9000 Standard Series and Related Issues.

PB95-103461

00,495

NIST Industrial Impacts: A Sampling of Successful Part-

nerships.
PB95-111514 00,488

Program of the Subcommittee on Construction and Building (July 1994).

00,32

Evidence for Significant Backscattering in Near-Threshold Electron-Impact Excitation of $\operatorname{Ar}(7+)$ (3s yields 3p).
PB95-126405

03,883

Databases Available in the Research Information Center

of the National Institute of Standards and Technology.

FIREDOC Users Manual, 3rd Edition.

02,724

PB95-128674

01,378

Constituents and Physical Properties of the C6+ Fraction of Natural Gas. Topical Report. Aprit-June 1994.

02,483
Evaluating Investments in Law Enforcement Equipment: Evaluating Investments in Law Enforcement Equipment:
An Annotated Bibliography.
PB95-151379 04,867

Promise into Practice: Implementing TA2 on Real Clocks

at NIST.

01,533

Think Metric.

PB95-151825

01,298

Transverse Thermomagnetic Effects in the Mixed State and Lower Critical Field of High-Tc Superconductors.

PB95-153250 04,590

Distant Future of Solar Activity: A Case Study of beta Hydri. 3. Transition Region, Corona, and Stellar Wind.
PB95-153441

Opportunities for Innovation: Software for Manufacturing.

PB95-155578

Information Retrieval Using Key Words and a Structured

PB95-161121

Computers in Welding: A Primer.

02,862

Gravity Dependent Processes and Intracellular Motion.

PB95-163382

03,538

Physics-Based Vision: Principles and Practice, Shape Re. covery (Book Review).

01,846

Method of Sale for CNG Paves Way to Greater Public Acceptance.
PB95-168449

02,489

Retrieving Articles from the Internet (without a UNIX

Workstation). Part 1. File Formats and Software Tools.

Workstation). Part 1. File Formats and Software Tools.
PB95-168720

Retrieving Articles from the Internet (without a UNIX

Workstation). Part 2. An Example.

PB95-168738

02,729

Getting Started on Mosaic.

PB95-180360

01,721

NIST Serial Holdings, 1995

PB95-188926

02,746

Materials Science and Engineering Laboratory Annual Report, 1994. NAS-NRC Assessment Panel, April 6.7

PB95.196697

02,976

Apparel Manufacturing Glossary for Application Protocol Development.

02,755

Publications of the National Institute of Standards and Technology 1992 Catalog.

PB95-200747

00,014

CCD Mosaic Images of the Supernova Remnant $3 \mathrm{C}$ 400.2.

PB95-203527

00,084

Robotics Application to Highway Transportation. Volume

1. Final Report.
PB95-203790 03,654

NIST Industrial Impacts: A Sampling of Successful Partnerships (Revision, March 1995)

00,489

Polymers Technical Activities 1994. NAC.NRC Assessment Panel, April 6-7, 1995

PB95-209896

01,275

Assessment of

Water Droplet Evaporation from Radiantly Heated Solids.

PB95-217147

00,394

Abstract and Index Collection in the Research Information Absiact and Index Colleclion in the Research Intormation nology

02,741

CSTL Technical Activities, 1994

PB95-242319

00,608

Materials Science and Engineering Laboratory Annual 


\section{KEYWORD INDEX}

GENERAL THEORETICAL CHEMISTRY \& PHYSICS

Monte Carlo and Analytic Methods in the Transpon of Electrons, Neutro

04,033

Effects of Target Temperature on Analytical Sensitivities of Cold-Neutron Capture Prompt gamma-ray Activation Analysis.

PB96-112131

00,616

Glimpse of Materials Research in China: A Report from an Interagency Study Team on Materials Visiting China from June 19,1995 to June 30,1995

from June 19 ,
PB96-112677

02,978

Intelligent Processing of Materials, Technical Activities 1994 (NAS-NRC Assessment Panel, April 6-7, 1995).

PB96-115050

NIST and the Navy: Past, Present and Future.

03,655

VAMAS Intercomparison of Critical Current Measurements on NB3Sn Superconductors: A Summary Report. PB96-119763

04,043

Some Notable

00,458

Magnetic Fields in Star-Forming Regions: Observations.

PB96-123005 00,100

Federal Labs Have Key Role in Metrication.

02,920

Book Review: Statistical Physics of Macromolecules.

PB96-123526 01,280

Book Review: Aspects and Applications of the Random Walk.

PB96-123534

04,215

Journal of Research of the National Institute of Standards and Technology. September-October 1993. Volume 98

PB96-134954 03,362

Metallurgy Technical Activities 1994 (NAS-NRC Assessment Panel, April 6-7, 1995).

PB96-136981

02,981

Churchill Eisenhart, 1913-1994

PB96-137740

03,447

Report on 1994 Actions of the International Institute of Welding.

02,873

Databases Available in the Research Information Center

of the National Institute of Standards and Technology

PB96-139407

02,734

Influence of an Impenetrable Interface on a Polymer

Glass-Transition Temperature.

PB96-146873

03.175

General Types

02,735

Arrborne Asbestos Analysis: National Voluntary Laboratory Accreditation Program.

PB96-147392

02,566

Metrology and
PB96-155429

02,923

Information Transfer in the 21st Century

PB96-157904

02,714

What's Available in Welding Software.

PB96-158084

02,875

Conference Proceedings: International Workshop on Instrumented indentation. Held in San Diego, Califomia on April 22-23, 1995.

April 22-23, 1995
PB96-158688

01,948

METRICATION: An Economic Wake-Up Call for Surveyors and Mappers.
PB96-159629

03,680

Montgomery Education Connection and Resource Education Awareness Partnership Making Connections between Local Schools and NIST Volunteers.

PB96-159769

00,134

Metrology.
PB96-160759

02,925

Lattice Statics of Interfaces and Interfacial Cracks in Bimaterial Solids.

02,985

Gage Block Standards, Measurement Capabilities and Laboratory Accreditation.

PB96-163621

02,757

PBblication and

03,576

Rechargeable Batteries for Personal/Portable.

PB96-164231

02,459

Electronics and Electrical Engineering Laboratory 1995 Technical Accomplishments: Advancing Metrology for Electrotechnology 10 Support the U.S. Economy.

PB96-164520

01,959

NIST Handbook 44, 1996: Specifications, Tolerances, and Other Technical Requirements for Weighing and Measuring Devices as Adopted by the 80 th National Con ference on Weights and Measures, 1995.

02,926

Application of Electromagnetic-Acoustic Transducers for Nondestructive Evaluation of Stresses in Steel Bridge

Structures.
PB96-167978

01,301

Federal Implementation Guideline for Electronic Data Interchange. ASC X12 003050 Transaction Set 843 Re- sponse to Request for Ouotation. Implementation Convention

PB96-168984

01,822

NIST Serial Holdings, 1996

02,748

NIST List of Publications, LP 103, March 1996. National Semiconductor Metrology Program.

B96-175856

02,432

Automated Measurement of Nonlinearity of Optical Fiber PB96-176540

Developments in Stellar Coronae.

00,107

Directory of Law Enforcement and Criminal Justice Associations and Research Centers.

PB96-178918

04,872

Unpredictable Certainty. Information Infrastructure through 2000

00,016

Materials Reliability. Technical Activities, 1995

PB96-183082

02,999

Publications of the National Institute of Standards and Technology 1993 Catalog.

00,017

PB96-183215

Financing Tomorrow's Infrastructure: Challenges and issues. Proceedings of a Colloquium. Held in Washington, DC. On October 20, 1995.

PB96-189444

00,481

Reactor Radiation Technical Activities, 1995.

PB96-193644

03,741

Ceramics Technical Activities, 1995

PB96-193677

03,087

PB96-193719

01,291

Program of the Manufacturing Engineering Laboratory, 1996. Infrastructural Technology, Measurements, and Standards for the U.S. Manufacturing Industries.

PB96-195276

Metallurgy. Technical Activities, 1995

02,760

PB96-195284

03,003

Development of a Method for Measuring Water-Stripping Resistance of Asphalt/Siliceous Aggregate Mixtures.
PB96-197249

NIST Frequency Measurement Service.

01,56

Overview of U.S. Government Advanced Packaging Programs.

02,443

Modification of a Commercial SEM with a Computer Controlled Cathode Stabilized Power Supply.

PB96-201066

04,129

Lake Erie Water Temperature Data, Put-in-Bay, Ohio, 1918-1992.

03,692

Improving Color Measurements of Displays.

02,203

Working Conference on Global Growth of Technology: Is America Prepared. Held in Gaithersburg. Maryland on December 7, 1995.

PB96-210059

00,018

CSTL Technical Activities, 1995

PB96-214630

00,647

Materials Science and Engineering Laboratory Annual Report, 1995. Technical Activities.

03,009

Building Life Cycle Cost Computer Program (BLCC) Version 4.22-95 (for Micrcomputers)

Stacking the Cards in Europe: One Company's Story.

PB97-110126

00,493

Sinusoidal Surfaces as Standards for BRDF Instruments. PB97-110597 04,388 Extrapolation of the Heat Capacity in Liquid and Amorphous Phases.

04,147

NIST Thermal Infrared Transfer Standard Radiometer for the Earth Observing System (EOS) Program.

B97-113013

04,843

Design of a High-Pressure Ebulliometer, with Vapor-Liquid Equilibrium Results for the Systems $\mathrm{CHF}_{2} \mathrm{Cl}+\mathrm{CF}_{3}$ $\mathrm{CH} 3$ and $\mathrm{CF} 3-\mathrm{CH} 2 \mathrm{~F}+\mathrm{CH}_{2} \mathrm{~F}_{2}$.

PB97-113229

04,163

India: Environmental Technologies Export Market Plan.

PB97-114359 02,529

Bibliography of the NIST Optoelectronics Division.

PB97-116040

02,207

Orientational Fluctuations, Diffuse Scattering, and Orientational Order in Solid C60.

04,176

B97-119275

Science, Technology, and Competitiveness: Retrospec-
tive on a Symposium in Celebration of NIST's 90th Annitive on a Symposium in Celebration of NIST's 90 th Anni-
versary and the 25 th Anniversary of the Gaithersburg Laboratories, Novermber 14-15, 1991.

PB97-121610

02,696

Photonic Band-Structure Effects for Low-Index-Contrast Two-Dimensional Lattices in the Near Infrared.

04,401

Chemical Effect in Ceramics Grinding

PB97-122592 03,113

Building Life Cycle Cost Computer Program (BLCC) Version 4.4-97 (for Microcomputers).

GENERAL THEORETICAL CHEMISTRY \& PHYSICS

Aerodynamic Phenomena in Stellar Atmospheres - A Bibliography.

00,046

Atom-counting standards and Doppler-free resonance ionization mass spectroscopy. (Progress report).

DE94018562

00,723

Time and Frequency Technology at NIST.

N94-30641/2 01,522

Implementation of a Standard Format for GPS Common View Data.

N95-32323/4

03,779

CSTL Technical Activities 1991

00,728

Diffraction of $X$-rays at the Far Tails of the Bragg Peaks. PB94-199924

High Temperature

Fluctuation Dominated Recombination Kinetics with

PBas-107264

00,875

CSTL Technical Activities, 1993

PB95-160602

Elastic Scattering of Polymer Networks.

PB95-161816

00,953

Diamond and Graphite Precursors: Comments.

PB95-16305

Hydrogen Atom Attack on Perchloroethylene.

PB95-163473

01,255

Ouasipotential and the Stability of Phase Lo, 0069

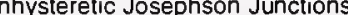

B95-180402

Generalized Stokes-Einstein Equation for Spherical Particle Suspensions.

PB95-202743

Stellar Coronal Structures.

PB95-202834

01,031

New High-Redshift Damped Lyman-alpha Absorption Systems and the Redshift Evolution of Damped AbsorbPrs. 203501

Far-Ultraviolet Flare on a Pleiades G Dwart

00,083

PB96-102033

00,086

Search for Radio Emission from the 'Non-Magnetic' Chemically Peculiar Stars.

00,087

High-Velocity Plasma in the Transition Region of $\mathrm{AU}$ 


\section{KEYWORD INDEX}

Quadratic Response of a Chemical Reaction to Extema Oscillations.

PB96-161633 01,138

Verification of Revised Water Vapour Correction to the Index of Refraction of Air.

02,680

Effect of Finite Beam Width on Elastic Light Scattering from Droplets.

PB96-163670

Transition Regions of Capella (1995)

01,144

PB96-176714

00,108

Riass Coronathon: Joint $X$-ray and Ultraviolet Observations of Normal F-K Stars.

00,109

Accurate Measurements of the Local Deuterium Abundance from HST Spectra.

$\infty 0,110$

Deuterium and the Local Interstellar Medium: Properties Deuterium and the Local Interstellar Medium: Properties for the Procyon and Capella Lines of Sight.
PB96-200639 PB96-200639 00,111 Electrolytes Con

PB97-110241

01,174

Spinor Equations in Relativistic Quantum Mechanics.

PB97-110605 04,142

GENERALLY ACCEPTED PRINCIPLES AND PRACTICES

Computer Security: Generally Accepted Principles and Practices for Securing Information Technology Systems. PB97-110811

GENETIC MAPPING

Small genomes: New initiatives in mapping and sequencing. Workshop summary report

DË96014476

03,451

GENETICS

Regulation of Lithium and Boron Levels in Normal Human Blood: Environmental and Genetic Considerations.

PB94-198579

GEOCODING

Metropolitan Areas (Including MSAs, CMSAs, PMASs, and NECMAs). Category: Data Standards and Guide

lines: Subcategory: Representations and Codes.
FIPS PUB 8-6

04,873

Countries, Dependencies, Areas of Special Sovereignty and Their Principal Administrative Divisions. Category Data Standards and Guidelines; Subcategory: Represen tation and Codes

00,128

Guideline: Codes for Named Populated Places, Primary County Divisions, and Other Locational Entities of the United States, Puerto Rico, and the Outlying Areas. Category: Data Standards and Guidelines. Subcategory: Representations and Codes.

FIPS PUB 55-3

04,865

Guideline: Codes for Named Populated Places, Primary County Divisions, and Other Locational Entities of the United States, Puerto Rico, and the Outlying Areas. Category: Data Standards and Guidelines; Subcategory: Representation and Codes.

FIPS PUB 55-DC3 04,866

FIPS PUB 8-6, Metropolitan Areas (for Microcomputers).

PB95-503280 04,874

Countries, Dependencies, Areas of Special Sovereignty, and Their Principal Administrative Divisions (for MicroComputers).

00,130

GEODYNAMICS

Lunar Laser Ranging: A Continuing Legacy of the Apollo Program.

PB95-202

03.683

GEOGRAPHIC INFORMATION SYSTEMS

Standards: A Cardinal Direction for Geographic InformaPB95-150942

03,677

Geographic Information Systems Standards: A Federal Perspective.

03,678

Spatial Information and Technology Standards Evolving. PB96-135108

03,679

\section{GEOGRAPHY}

Metropolitan Areas (Including MSAs, CMSAs, PMASs, and NECMAs). Category: Data Standards and Guidelines; Subcategory: Representations and Codes.

04,873

Countries, Dependencies, Areas of Special Sovereignty, and Their Principal Administrative Divisions. Category Data Standards and Guidelines; Subcategory: Representation and Codes.
FIPS PUB 10-4

00,128

Guideline: Codes for Named Populated Places, Primary County Divisions, and Other Locational Entities of the United States, Puerto Rico, and the Outlying Areas. Cat egory: Data Standards and Guidelines. Subcategory: Representations and Codes.

04,865

Guideline: Codes for Named Populated Places, Primary County Divisions, and Other Locational Entities of the United States, Puerto Rico, and the Outlying Areas. Cate gory: Data Standards and Guidelines; Subcategory: Representation and Codes.

04,866
FIPS PUB 8-6, Metropolitan Areas (for Microcomputers) PB95-503280

04,874

Countries, Dependencies, Areas of Special Sovereignty, and Their Principal Administrative Divisions (for Microcomputers).

00,130

\section{GEOMETRIC ABBERATIONS}

Compensation for Errors Introduced by Nonzero Fringe Densities in Phase-Measuring Interferometers.

04,386

GEOMETRIC FITTING SOFTWARE

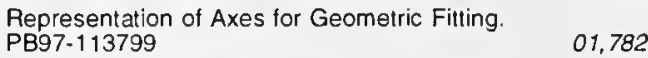

GEOMETRICAL ABERRATIONS

Test Optics Error Removal.

PB96-179536

04,377

GEOMETRY

User's Guide for the Algorithm Testing System Version

PB95-251666

02,916

Optical Fiber, Fiber Coating, and Connector Ferrule Geometry: Results of Interlaboratory Measurement Comparisons.

PB96-154422

04,360

Using Logic to Specify Shapes and Spatial Relations in Design Grammars

02,793

GERMANES

Plasma Chemistry in Silane/Germane and Disilane/Germane Mixtures.

01,027

GERMANIUM

Energy Levels of Germanium, Ge I through Ge XXXII.

PB94-162351 00,747

Germanium Detector Optimization of MDA for Efficiency vs. Low Intrinsic Background.

PB94-199155

00.543

Resonance Enhanced Multiphoton Ionization Detection of $\mathrm{GeF}$ and $\mathrm{GeCl}$ Radicals.

PB94-212123

00,825

Thermodynamics of (Germanium + Selenium): A Review and Critical Assessment.

01,182

GERMANIUM CHLORIDE RADICALS

Resonance Enhanced Multiphoton Ionization Detection of $\mathrm{GeF}$ and $\mathrm{GeCl}$ Radicals.

PB94-212123

00,825

GERMANIUM DETECTOR

Measurement of the (10)B(n, alpha1gamma)(7) Li Cross Section in the 0.3 to $4 \mathrm{MeV}$ Neutron Energy Interval. PB96-161799

04,098

GERMANIUM FLUORIDE RADICALS

Resonance Enhanced Multiphoton Ionization Detection of $\mathrm{GeF}$ and $\mathrm{GeCl}$ Radicals.

GERMANY (UNIFIED)

Ceramic Powders Characterization: Results of an International Laboratory Study. PB95-270039

02,672

GII (GLOBAL INFORMATION INFRASTRUCTURE)

Global Information Infrastructure: Agenda for Coopera-

PB95-178604

01,482

GILS (GOVERNMENT INFORMATION LOCATOR SERVICE) Application Profile for the Government Information Locator Service (GILS). Category: Software Standard; Sub. calegory: Informal
FIPS PUB 192

01,800

\section{GINZBURG SYMPOSIUM}

From Superconductivity to Supernovae: The Ginzburg Symposium. Report on the Symposium Held in Honor o Vitaly L. Ginzburg. Held in Gaithersburg. Maryland on May 22, 1992

04,649

GLASS

Fire Induced Thermal Fields in Window Glass I: Theory.

Analysis of SANS from Controlled Pore Glasses.

PB94-198843 03,035

Glass Transition of Organic Liquids Confined to Small Pores.

PB94-212305

00,833

Melting Behavior of Organic Materials Confined in Porous

PB94-212313

00,834

Aging in Glasses Subjected to Large Stresses and Deformations.

PB95-107041

Environmentally Enhanced Fracture of Ceramics.

PB95-125746

03,235

tength Scales for Fragile Glass-Forming Liquids.

PB96-102801

03,046

Glasses for Waveguide Lasers.

01,065

PB96-111950

04,335

Long-Lived St
PB96-119565

04,21

Simulation Studies of Supercooled and Glass Forming

PB96-122627

01,085

Distributed Feedback Lasers in Rare-Earth-Doped Phosphate Glass.

04,740 Integrated Optical Polarization-Discriminating Receiver in

PB97-113179

02,206

GLASS-CERAMIC

Wear of Enamel against Glass-Ceramic, Porcelain, and Amalgam.

GLASS FIBER REINFORCED PLASTICS

Composite Struts for SMES Plants.

PB95-155586

03,082 forced Composite.

PB96-112339

GLASS LASERS

PB94-216280

Integrated Optic Laser Emitting at 905, 1057, $1356 \mathrm{~nm}$ PB94-216298
ASS TRANSITION TEMPERATURE

Effect of Curing History on Ultimate Glass Transition Temperature and Network Structure of Crosslinking Poly-

PB94-200052

01,214

Glass Transition of Organic Liquids Confined to Small PB94-212305

00,833

Physics Required for Prediction of Long Term Pertormance of Polymers and Their Composites.

03,146

Glass Temperature of Polymer Blends: Comparison of Both the Free Volume and the Entropy Predictions with

PB95-140190

01,236

Influence of an Impenetrable Interface on a Polymer Glass-Transition Temperature.

03,175

Small-Angle Neutron Scattering Characterization of Processing/Microstructure Relationships in the Sintering Crystalline and Glassy Ceramics. (Reannouncement with New Availability Information)

AD-A249 $510 / 9$

03,025

GLOBAL ASPECTS

Global Climatic Effects of Aerosols: The AAAR SympoSium.

GLOBAL DENSITY RATIO

Global Density Effects on the Self-Preservation Behavior of Turbulent Free Jets.

PB95-16230

04,207

GLOBAL ECONOMY

Working Conterence on Global Growth of Technology: Is America Prepared. Held in Gaithersburg. Maryland on December 7, 1995.

PB96-210059

00,018 
Ion Kinetic-Energy Distributions in Argon if Glow Discharges.
PB95-141008

04,409

Electrical Characterization of Radio-Frequency Dis charges in the Gaseous Electronics Conference Reference Call.

PB95-164612

01,905

Plasma Chemical Model for Decomposition of SF6 in Negative Glow Corona Discharge.

01,020

Plasma Chemistry in Silane/Germane and Disilane/Germane Mixtures.

01,027

Evidence for Inelastic Processes for $\mathrm{N}(+) 3$ and $\mathrm{N}(+) 4$ from lon Energy Distributions in He/N2 Radio Frequency Glow Discharges.

GuUCOSE

Apparent Molar Heat Capacities and Apparent Molar Vol umes of Aqueous Glucose at Temperatures from 298.15 $\mathrm{K}$ to $327.01 \mathrm{~K}$.

PB94-212800

03,459

Amperometric Flow-Injection Analysis Biosensor for Glucose Based on Graphite "Paste Modified with Tetracyanoquinodimethan

PB95-161980

03,498

GLUCOSE PERMEASE

Glucose Permease of Bacillus Subtilis Is a Single Polypeptide Chain That Functions to Energize the Sucrose Permease.

03,466

GLYCEROL

Isotope Dilution Mass Spectrometry as a Candidate De finitive Method for Determining Total Glycerides and Triglycerides in Serum.

PB96-102280

03,519

GLYCIDYL ETHERS

Effect of Curing History on Ultimate Glass Transition Temperature and Network Structure of Crosslinking PolyPB94-200052

01,214

GLYCINE

Structure of Glycine-Water H-Bonded Complexes. 00,789 Effects of Aluminum Oxalate/Glycine Pretreatment Solutions on Dentin Permeability.

03,565

GLYCINE-WATER COMPLEX

Structure of Glycine-Water H-Bonded Complexes.
PB94-198603

GMR

Size Effects in Submicron NiFe/Ag GMR Devices. PB96-155510

02,237

GOALS

White Papers Prepared for the White House: Construction Industry Workshop on National Construction Goals. Held on December 14-16, 1994

PB95-216891

01,299

GODDARD HIGH RESOLUTION SPECTROGRAPH

Goddard High Resolution Spectrograph: Instrument Goals, and Science Results.

PB96-123278

00,044

GOES SATELLITES

Semiconductor Measurement Technology: Improved Characterization and Evaluation Measurements for HgCdTe Deteclor Materials, Processes,

02,122

GOLD

Incorporation of Gold into YBa2Cu3O7: Structure and Tc Enhancement.

04,481

Unexpected Effects of Gold on the Structure,

Superconductivity, and Normal State of YBa2Cu3O7.
PB94-200284

Self-Assembled Phospholipid/Alkanethiol Biomimetic Bilayers on Gold.

PB95-108460

00,878

Scanning Tunneling Microscopy and Fabrication of Nanometer Scale Structures at the Liquid-Gold Interface. PB95-140414

00,904

Oscillatory Exchange Coupling in Fe/Au/Fe(100).

Silver Metalization of Octadecanethiol Monolayers SelfAssembled on Gold.

PB95-150744

00,923

UV-Photopatterning of Alkylthiolate Monolayers Self-Assembled on Gold and Silver.

00,924

Characterization of Cytochrome c/Alkanethiolate Structures Prepared by Self-Assembly on Gold.

PB95-164638

Collisions of Electrons with Highly-Charged Ions. PB96-200340

00,987

GOLD ALLOYS

Fabrication of Platinum-Gold Alloys in Pre-Hispanic South America: Issues of Temperature and Microstructure Control.

03,333
Diffusion of Copper into Gold Plating PB95- 162152

00,957

GOLD IONS

Spectra of Ag I Isoelectronic Sequence Observed from Er(21+) to Au(32+)

GOSIP (GOVERNMENT OPEN SYSTEMS

03,899

ERCONNECTION PROFILE)

U.S. GOSIP Testing Program.

PB94-211455

01,807

National Voluntary Laboratory Accreditation Program. GOSIP: Government Open Systems Interconnection Pro-

PB95-267993

01,486

Lessons from the Establishment of the U.S. GOSIP Testing Program.

GOSIP Testing Program

01,817

PB96-161229

01,504

Industry/Government Open Systems Specification: The Development of GOSIP Version 3.

PB96-161245

01,505

Five O's (Cues) of the U.S. GOSIP Testing Program

PB96-175716

01,826

GOVERNMENT/INDUSTRY RELATIONS

Transfer of Technology from Defense to Civilian Sectors.

NIST Industrial Impacts: A Sampling of Successful PartNIST Industrial Impacts: A Sampling of Successful Pannerships.

NIST Industrial Impacts: A Sampling of Successful Partnerships (Revision, March 1995)

PB95-209193

00,489

Conceptual Design Plan for the National Advanced Manufacturing Testbed

PB95-231866

02,828

National Planning for Construction and Building $R$ and $D$.

PB96-137104 00,324

Industry/Government Open Systems Specification: The Development of GOSIP Version 3.

GOVERNMENT INFORMATION LOCATOR SERVICE Application Profile for the Government Information Loca-
tor Service (GILS). Category: Software Standard; Subtor Service (GILS). Category: Software Standard; Sub-
category: Information Interchange. category: Informat
FIPS PUB 192

01,800

GOVERNMENT OPEN SYSTEMS INTERCONNECTION

PROFILE

U.S. GOSIP Testing Program.

PB94-211455

01,807

National Voluntary Laboratory Accreditation Program. GOSIP: Government Open Systems Interconnection Pro-

PBgs-2

01,486

Lessons from the Establishment of the U.S. GOSIP Testing Program.

GOSIP Testing Program.

01,817

GOSIP Testing P

01,504

Industry/Government Open Systems Specification: The Development of GOSIP Version 3

PB96-16i245

01,505

Five O's (Cues)
PB96-175716

01,826

GOVERNMENT POLICIES

Proceedings of the Manufacturing Technology Needs and Issues: Establishing National Priorities and Strategies Conference. Held in Gaithersburg, Maryland on April 26 PB95-206181

Unpredictable Certainty. Information Infrastructure through 2000

tructure PB96-182266

00,016

GOVERNMENT PROCUREMENT

Open System Environment Procurement.
N94-36858/6

Guide on Open System Environment (OSE) Procure-

PB95-169496

01,626

Information Technology Standards in Federal Acquisi-

PB96-161252

01,636

Using Information Technology Standards in Federal Ac-

quisitions.
PB96-161260

01,637

Federal Implementation Guideline for Electronic Data Interchange: ASC X12 003050 Transaction Set 836 Procurement Notices. Implementation Convention.

B96-178892

01,827

GADIOMETERS

Gradiometer Antennas for Detection of Long Subsurface Conductors.

GRAFT POLYMERIZATION

Grafted Interpenetrating Polymer Networks.

PB94-185055

01,862

RAFTED POLYMER LAYER

Segmental Concentration Profiles of End-Tethered Polymers with Excluded-Volume and Surface Interactions. PB97-119002

GRAIN BOUNDARIES

Weak-Link-Free Behavior of High Angle YBa2Cu3O7-x Grain Boundaries in High Magnetic Fields.

04,459

Energy and Migration of Grain Boundaries in Polycrystals.

PB94-211638 03,332

Examination of Objects Made of Wood Using Air-Coupled Ultrasound.

PB95-125712

03,404

Nature of (001) Tilt Grain Boundaries in YBa2Cu3O $6+\mathrm{X}$.

04,528

Small Angle Neutrons Scattering from Nanocrystalline

Palladium as a Function of Annealing.

PB95-176103

03,354

Stability and Surface Energies of Wetted Grain Bound-

aries in Aluminum Oxide.

PB95-202750 03,059

Inelastic Neutron Scattering Study of Hydrogen in Nanocrystalline Pd

PB96-146857

03,366

Modeling Effects of Temperature Annealing on Giant Magnetoresistive Response in Discontinuous Multilayer NiFe/Ag Films.

NiFe/Ag Films.
PB97-112585

GRAIN GROWTH

Stability, Microstructural Evolution, Grain Growth, and

Coarsening in a Two-Dimensional Two-Phase Microstructure.
PB94-199429

03,325

Investigation into a Pracical Grain Growth Model for Hot Isostatic Pressing.

GRAIN SIZE

Determination of the Prior-Austenitic Grain Size of Selected Steels Using a Molten Glass Etch

PAIN STRUCTURE

Grain Alignment and Transport Properties of Bi2Sr2CaCu2O8 Grown by Laser Heated Float Zone

95-161451

04,602

GRANULAR MATERIALS

Domain Structures in Magnetoresistive Granular Metals. PB96-141346

04,760

GRAPHICAL USER INTERFACE

Prototyping a Graphical User Interiace for DHCP.
PB96-160544

Automated Guarded Bridge for Calibration of

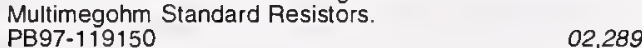

GRAPHITE

Thermal Diffusivity of POCO AXM-501 Graphite in the Range 1500 to $2500 \mathrm{~K}$ Measured by a Laser-Pulse Tech-

PB94-185022

03,013

PBiamond and

00,967

Determination of Anomalous Superexchange in $\mathrm{MnCl} 2$ and Its Graphite Intercalation Compound.

00,666

GRAPHITE CALORIMETERS

Crystal Structure of Annealed and As-Prepared $\mathrm{MgBa2CaCu2}$

03,927

GRAPHITE COMPOSITES

Thermoacoustic Technique for Determining the Interface and/or Interply Strength in Polymeric Composites.

03,158

GRAPHITE INTERCALATION COMPOUNDS

Structural and Magnetic Properties of $\mathrm{CuCl} 2$ Graphite 


\section{KEYWORD INDEX}

Continuous Gravity Observations Using Joint Institute for Laboratory Astrophysics Absolute Gravimeters.

03,685 Intracomparison Tests of the FG5 Absolute Gravity MePers. 02991

03,688

GRAVIMETRIC STANDARDS

Measurement of Atmospheric Methyl Bromide Using Gravimetric Gas Standards.

PB96-155494

02,497

GRAVIMETRY

Journal of Research of the National Institute of Standards and Technology, March/April 1996. Volume 101, Numbe PB96-177381

01,863

Development of the Ion Exchange-Gravimetric Method for Sodium in Serum as a Definitive Method.

01,867

GRAVITATION

Earth-Based Gravitational Experiments.

PB94-211414

03,840

Elastic Deformation of a Monolithic Perfect Crystal Interferometer: Implications for Gravitational Phase Shift Ex (1)

PB94-213154 03,858

Validation of the Inverse Square Law of Gravitation Using the Tower at Erie, Colorado, USA.

PB95-164646

03,947

Test of Newton's Inverse Square Law of Gravitation Using the 300-m Tower at Erie, Colorado.

03,978

GRAVITATIONAL EFFECTS

Ignition and Subsequent Transition to Flame Spread in a Microgravity Environment.

04,828

GRAVITATIONAL FIELDS

Integrated Laser Doppler Method for Measuring Planetary Gravity Fields.

03,681

GRAVITATIONAL WAVE ANTENNAS

Quantum Mechanics of a Solid-State Bar Gravitationa Antenna.

PB95-202628

03,987

GRAVITY

Gravity Dependent Processes and Intracellular Motion.
PB95-163382

Validation of the Inverse Square Law of Gravitation Using the Tower at Erie, Colorado, USA.

PB95-164646

03,947

Test of Newton's Inverse Square Law of Gravitation Using the 300-m Tower at Erie, Colorado. PB95-202446

03,978

Continuous Gravity Qbservations Using Joint Institute for Laboratory Astrophysics Absolute Gravimeters.

RAVITY CURRENTS

Gravity-Current Transport in Building Fires

PB96-147046

03,685

Transport by Gravity Currents in Building Fires. PB97-119325

01,415

01,441

GREEN'S FUNCTION

Design, Construction and Application of a Large Aperture Lens-Less Line-Focus PVDF Transducer.

REEN'S FUNCTIONS

Fracture in Multilayers.

PB96-163613

02,765

REENHOUSES

Corrosion Resistance of Materials for Renovation of the

United States Botanic Garden Conservatory.
PB94-154390

GREENS FUNCTION

Lattice Imperfections Studied by Use of Lattice Green's

Elastic Green's Function for a Bimaterial Composite Solid Containing a Free Surface Normal to the Interface.

PB95-163325 03,162

Green's Function for Generalized Hilbert Problem for Cracks and Free Surfaces in Composite Materials. PB95-163333

GROUND BASED CONTROL

Ground Vehicle Control at NIST: From Teleoperation to N $94-34037 / 9$

GROUND MOTION

Assessment of Site Response Analysis Procedures.
PB95-210928

Energy-Based Method for Liquefaction Potential Evaluation. Phase 1. Feasibility Study.

ROUND STATIONS

03,691

Satellite Two-Way Time Transfer: Fundamentals and Recent Progress

GROUND VEHICLES

Vehicle-Command Center Communications in a Robotic Vehicle System.
PB95-162723

03,665
GROUNDINGS

Stranding Experiments on Double Hull Tanker Structures. PB96-12311

03.749

\section{GROWTH ABNORMALITIES}

Nutritional Status and Growth in Juvenile Rheumatoid Ar-

PB94-198470

03,515

GUARDED HOT PLATE

Intra-Laboratory Comparison of a Line-Heat-Source Guarded Hot Plate and Heat-Flow-Meter Apparatus. PB95-181202

00,387

ine-Heat-Source Guarded-Hot-Plate Apparatus. PB97-118996

00,417

GUIDELINES

Guidelines for Reporting Results of Computational Experiments. Report of the Ad hoc Committee.

03,427

Guidelines for Refractive Index Measurements of Asbestos.

PB95-151189

02,543

Telecommunications Security Guidelines for Telecommunications Management Network. Computer SecuPB96-139415

01,496

GUIDES

Guide to Instrumentation Literature.

GUNS

S

Specification for Interoperability between Ballistic Imaging
Systems. Part 1. Cartridge Cases.

Systems. Part
PB96-195524

01,860

GUST FACTORS

Gust Factors Applied to Hurricane Winds.

PB95-180469

00,446

GUST LOADS

Gust Factors Applied to Hurricane Winds.

PB95-180469

00,446

AFNIUM IONS

Observation of Pd-Like Resonance Lines Through

$\mathrm{Pt}(32+)$ and $\mathrm{Zn}$-Like Resonance Lines of $\operatorname{Er}(38+)$ and

PB95-150637

03,896

Rh I Isoelectronic Sequence Qbserved from $\operatorname{Er}(23+)$ to $\mathrm{Pt}(33+)$

PB95-150652

03,898

Spectra of $\mathrm{Ag}$ I Isoelectronic Sequence Qbserved from $\operatorname{Er}(21+)$ to $\mathrm{Au}(32+)$

\section{HAFNIUM TO RADIUM}

Ultraviolet Multiplet Table. Finding List for Spectra of the Elements Molybdenum to Lanthanum ( $Z=42$ to 57): Hafnium to Radium ( $Z=72$ to 88 ).

AD-A278 131/8

00,709

HAIR

Interlaboratory Comparison Studies on the Analysis of Hair for Drugs of Abuse.

03,500 PB95-17625

Hair Analysis for Drugs of Abuse: Evaluation of Analytical Methods, Environmental Issues, and Development of Reference Materias.

03,501

Hair Testing for Drugs of Abuse: International Research on Standards and Technology.

03,504

Supercritical Fluid Extraction-Immunoassay for the Rapid Screening of Cocaine in Hair.

PB96-167168

00,637

Interlaboratory Studies on the Analysis of Hair for Drugs of Abuse: Results from the Fitth Exercise.

03,509

Interlaboratory Studies on the Analysis of Hair for Drugs of Abuse: Results from the Fourth Exercise.

03,510

HALF-LIFE

New and Revised Half-Life Measurement Results.
PB96-160346

63Ni Half-Life: A New Experimental Determination and Critical Review.

PB97-111603

00,700

Quantized Dissipation of the Quantum Hall Effect at High Currents.

PB94-199395

04,472

Using Quantized Breakdown Voltage Signals to Determine the Maximum Electric Fields in a Quantum Hall EF-

$\begin{array}{ll}\text { fect Sample. } & 02,375 \\ \text { PB95-261947 } & \end{array}$

Spectroscopic Study of Quantized Breakdown Voltage States of the Quantum Hall Effect.

04,730

Potential and Current Distributions Calculated Across a Quantum Hall Effect Sample at Low and High Currents. PB96-122106 04,045 Electrical Characterization of Narrow Gap n-Type Bulk HgCdTe Single Crystals by Variable-Magnetic-Field Hall Measurements and Reduced-Conductivity-Tensor AnalyPB96-164199

01,146

HALLUCINOGENS

Determination of 3-Quinuclidinyl Benzilate (Qnb) and Its Major Metabolites in Urine by Isotope Dilution Gas Chromatography Mass Spectrometry.

PB94-199379

03,492

HALOACETATES

Ferric Ion Assisted Photooxidation of Halocetates.

PB97-112460

00,521

HALOBACTERIUM

Bacteriorhodopsin Retains its Light-Induced ProtonPumping Function After Being Heated to $140 \mathrm{C}$.

03,471

HALOCARBONS

Retention of Halocarbons on a Hexafluoropropylene Epoxide Modified Graphitized Carbon Black. Part 1. Meth. ane-Based Compounds.

03,272

Retention of Halocarbons on a Hexafluoropropylene Epoxide-Modified Graphitized Carbon Black. 3. EtheneBased Compounds.

PB96-167309

03,286

\section{HALOGEN LAMPS}

Characterization of Modified FEL Quartz-Halogen Lamps Or Photometric Standards.

00,282

HALOGENATED HYDROCARBONS

Suppression of High-Speed $\mathrm{C}_{2} \mathrm{H} 4 /$ Air Flames with $\mathrm{C}_{1}$. Halocarbons.

PB96-175724

03,287

Suppression of Ignition Over a Heated Metal Surface. PB96-176425

\section{HALOHYDROCARBONS}

Thermochemical and Chemical Kinetic Data for Fluorinated Hydrocarbons.

01,056

Thermodynamic Properties of Two Gaseous Halogenated Ethers from Speed-of-Sound Measurements: Difluoromethoxy-Difluoromethane and 2-Difluoromethoxy1,1,1-Trifluoroethane.

PB96-102413

04,189

HALON 1301

Evaluation of Alternative In-Flight Fire Suppressants for Full-Scale Testing in Simulated Aircraft Engine Nacelles and Dry Bays.

00,023

\section{HALON ALTERNATIVES}

Evaluation of Alternative In-Flight Fire Suppressants for Full-Scale Testing in Simulated Aircraft Engine Nacelles and Dry Bays

00,023

HALONS

Chemical Inhibition of Methane-Air Diffusion Flame. PB96-195532

01,431

\section{HANDBOOKS}

Handbook Preferred Circuits Navy Aeronautical Electronic Equipment. Supplement Number 3.

AD-A278 782/8

00,026

Handbook Preferred Circuits Navy Aeronautical Electronic Equipment. Supplement Number 2

00,027

Handbook Preferred Circuits Navy Aeronautical Electronic Equipment. Supplement Number 1.

00,028

Report of the International Commission on Radiological 
NIST Handbook 44, 1996: Specifications, Tolerances, and Other Technical Requirements for Weighing and and Other Technical Requirements for Weighing and ference on Weights and Measures, 1995.

terence on Weic

02,926

HANDPRINT

NIST Form-Based Handprint Recognition System.
PB94-217106

Component-Based Handprint Segmentation Using Adaptive Writing Style Model.

ive Writing Style
PB96-193669

HANDWRITING

Unconstrained Handprint Recognition Using a Limited Lexicon.

PB94-168051

01,831

Second Census Optical Character Recognition Systems Conference.

NIST Form-Based Handprint Recognition System.
PB94-217106

01,832

01,838

Binary Decision Clustering for Neural Network Based Op-

tical Character Recognition

Method and Evaluation of Character Stroke Preservation on Handprint Recognition.

01,850

Binary Decision Clustering for Neural-Network-Based Optical Character Recognition.

01,857

Generalized Form Registration Using Structure-Based Techniques.
PB96-191374

01,858

Component-Based Handprint Segmentation Using Adaptive Writing Style Model.

01,859

HANGARS

Analysis of High Bay Hangar Facilities for Detector Sensitivity and Placement.

PB96-190210

01,429

HAPPYLAND SOCIAL CLUB FIRE

Analysis of the Happyland Social Club Fire with HAZARD

PB94-199270

00,193

HARD TURNING

Evaluation of a Tapered Roller Bearing Spindle for HighPrecision Hard Turning Applications.

PB96-160494

02,700

HARMONIC DISTORTION

Effect of Harmonic Distortion on Phase Errors in Frequency Distribution and Synthesis

PB96-200779

01,563

HARMONIC MIXING

High-Order Harmonic Mixing with GaAs Schottky Diodes.

HASH ALGORITHM

Secure Hash Standard. Category: Computer Security.
FIPS PUB 180-1

HAZARD ASSESSMENT

HAZARD I Fire Hazard Assessment Method (Version 1.2) (for Microcomputers)

00,196

HAZARD I Fire Hazard Assessment Method, Version 1.2 (Upgrade Package) (for Microcomputers)

00,197

ZARDOUS MATERIALS

Performance of Plastic Packaging for Hazardous Materials Transportation. Part 1. Mechanical Properties.

AD-A301 258/0

02,580

Calculation of Higher Heating Values of Biomass Mate-

rials and Waste Components from Elemental Analyses.
PB94-199254

Membrane Gas Separation for a Fluidized-Bed Incinerator.

PB95-169041

02,550

HAZARDOUS WASTES

Recommendations for the Disposal of Carbon- 14 Wastes. AD-A279 133/3 02,579

HAZARDS

Study on Hazard Analysis in High Integrity Software Standards and Guidelines.

PB95-198727

01,725

HCFC-123

Temperature Dependence of the Gas and Liquid Phase Ultraviolet Absorption Cross Sections of HCFC-123 (CF3CHCl2) and HCFC-142b (CH3CF2Cl)

03,298

HCFC-142B Temperature Dependence of the Gas and Liquid Phase
Ultraviolet Absorption Cross Sections of HCFC-123 (CF3CHCl2) and HCFC-142b (CH3CF2CI).

03,298

HEAD AND NECK NEOPLASMS

New Method for Shielding Electron Beams Used for Head and Neck Cancer Treatment.

PB94-211430

03,621

HEADERS

Flowmeter Installation Effects Due to a Generic Header. PB96-210893
HEALTH

Virtual Environments for Health Care. A White Paper for he Advanced Technology Program (ATP), the National Institute of Standards and Technology.

03,594

HEALTH CARE

Distributed Communication Methods and Role-Based ACcess Control for Use in Health Care Applications.

PB96-183165

HEALTH HAZARDS

Lead Abatement in Buildings and Related Structures.

NIOSH Pocket Guide to Chemical Hazards.

03,603

PB95-100368

Characterization of a Health Physics Instrument Calibration Range.

03,629

External Gamma-ray Counting of Selected Tissues from a Thorotrast Patien

PB96-160254

03,637

ALTH \& SAFETY

Crystal Diffraction Spectrometry for Accurate, NonInvasive KV/Spectral Measurement for Improvement of Mammographic Image Quality.

00.721

Electron transport calculations with biomedical and environmental applications. Final report, December 23, 1992-. January 31, 1994.

DE95007065

03,613

Properties and Interactions of Oral Structures and Restor ative Materials. Annual Report for Period October 1, 1990 to September 30, 1991

PB94-160843

03,558

Influence of Tempering Method on Residual Stress in Dental Porcelain.

PB94-172012

00,138

Calcium Phosphate Precipitation in Liposomal Suspensions.

03,452

Octacalcium Phosphate. 3. Infrared and Raman Vibra. tional Spectra.

00,756

Adsorption of Low-Molecular-Weight Sodium Polyacrylate on Hydroxyapatit

00,139

Interaction of Some Coupling Agents and Organic Compounds with Hydroxyapatite: Hydrogen Bonding, Adsorption and Adhesion.

00,140

Effect of Two Initiator/Stabilizer Concentrations in a Metal

Effect of Two Initiator/Stabilizer Concentrations in a Metal
Primer on Bond Strengths of a Composite 10 a Base Primer on Bond Strengths of a Composite 10 a Base Metal Alloy.

00,141

Physicochemical Properties of Calcific Deposits Isolated from Porcine Bioprosthetic Heart Valves Removed from Patients Following 2-13 Years Function.

PB94-172863

00,184

Dental Materials.

PB94-172871

00,142

Tapered Cross-P

00,185

Octacalcium Phosphate Carboxylates IV. Kinetics of Formation and Solubility of Octacalcium Phosphate Succi-

PB94-185600

00,776

Preparation and Monitoring of Lead Acetate Containing Drinking Water Solutions for Toxicity Studies.

PB94-193885

00,538

Documentation for Immediately Dangerous to Life or

Health Concentrations (IDLHs).
PB94-195047

03,602

Nutritional Status and Growth in Juvenile Rheumatoid Arthritis.

PB94-198470

03,515

Adhesion of Composites to Dentin and Enamel

PB94-199049

00,144

Development of an Adhesive Bonding System. PB94-199056

00,145

Clinical Perspective on Dentin Adhesives

00,146

Selective Inhibition of Crystal Growth on Octacalcium Phosphate and Nonstoichiometric Hydroxyapatite by Pyrophosphate at Physiological Concentration. 00,147
PB94-211257 New Method for Shielding Electron Beams Used for Head and Neck Cancer Treatment.

PB94-211430

03,621

Periapical Tissue Reactions to a Calcium Phosphate $\mathrm{Ce}$ ment in the Teeth of Monkeys.

00,149

Effect of Three Sterilization Techniques on Finger Pluggers.

00,150

Paffenbarger Research Center: The Cutting Edge of Den-

tal Science. $\quad 00,151$

NIOSH Pocket Guide to Chemical Hazards.

PB95-100368

03,603

Evaluation of Fracture Toughness and Residual Stress in Dental Porcelain by Indentation-Microfracture Method.

PB95-125613 O0,154

Image Intormation Transfer Properties of X-Ray Intensifying Screens in the Energy Range from 17 to $320 \mathrm{keV}$.

PB95-126173

00,155

Effects of Surface-Active Resins on Dentin/Composite Bonds.

PB95-140448

00,156

Deposition of Loosely Bound and Firmly Bound Fluorides on Tooth Enamel by an Acidic Gel Containing Fluorosilicate and Monocalcium Phosphate Monohydrate. 03,559 Effects of Calcium Phosphate Solutions on Dentin Permeability.

00,157

Modified Surface-Active Monomers for Adhesive Bonding to Dentin

PB95.151163

00.158

NIR-Spectroscopic Investigation of Water Sorption Char-

acteristics of Dental Resins and Composites.
PB95-151171

00,189

Distribution of Fluoride in Saliva and Plaque Fluid After a $0.048 \mathrm{~mol} / \mathrm{L} \mathrm{NaF}$ Rinse.

03,56

.

Evaluation of Fracture Toughness and Residual Stress in Dental Porcelain by Indentation-Microfracture Method.
PB95-152831

Publication and Presentation Abstracts, 1993. (Published by Paffenbarger Research Center and Center of Excellence for Materials Science Research).

PB95-153052

03,562

Effects on Whole Saliva of Chewing Gums Containing Calcium Phosphates.

PB95-153169

03,563

Noninvasive High-Voltage Measurement in Mammography by Crys
PB95-153417

00,160

Octacalcium Phosphate Carboxylates. 1. Preparation and Identification.

PB95-161535

Octacalcium Phosphate Carboxylates. 2. Characterization and Structural Consideration.

PB95-161543

00,955

Wear of Human Enamel against a Commercial Castable Ceramic Restorative Material.

00,161

Ring-Opening Dental Resin Systems Based on Cyclic Acetals.

Effect of Ethanol on the Solubility of Dicalcium Phosphate Dihydrate in the System $\mathrm{Ca}(\mathrm{OH}) 2-\mathrm{H} 3 \mathrm{PO} 4-\mathrm{H} 2 \mathrm{O}$ at 37C.
PB95-163507

00,163

Procedure for the Study of Acidic Calcium Phosphat Precursol Phases in Enamel Mineral Formation. $\quad 03,564$
PB95-164448

Effects of Aluminum Oxalate/Glycine Pretreatment Solutions on Dentin Permeability.

Evaluation of Methylene Lactone Monomers in Dental Resins.

PB95-164661

00,164

Behavior of a Calcium Phosphate Cement in Simulated Blood Plasma In vitro.

00,165 
Remineralization of Root Lesions with Concentrated Calcium and Phosphate Solutions.

03,567

Reduction of Marginal Gaps in Composite Restorations by Use of Glass-Ceramic Inserts.

PB96-102405

00,174

Crystal Structure of a New Monoclinic Form of Potassium Containing Orthophosphacidium lon, $(\mathrm{H} 4 \mathrm{PO} 4)($ sup +1$)$.

04,725

Publications and Presentation Abstracts, 1995. (Published by Paffenbarger Research Center and Center of Excelence for Materials Science Research).

03,568

In vitro Fracture Behavior of Ceramic and Metal-Ceramic Restoratior

03,569

PB96-119722

Failure of All-Ceramic Fixed Partial Dentures 'In vitro' and
'In vivo': Analysis and Modeling.
PB96-122536
00,175

PB96-122536

00,177

PB96-122940

intensive Swimming: Can It Affect Your Patients' Smiles.

PB96-123666

03,570

Effect of Supersaturation on Apatite Crystal Formation in Aqueous Solutions at Physiologic $\mathrm{pH}$ and Temperature.

PB96-135215

03,571

Bioactive Polymeric Dental Materials Based on Amor-

phous Calcium Phosphate.

03.572

Remineralizing Dental Composites Based on Amorphous Calcium Phosphate.

03.573

Polymeric Calcium Phosphate Composites with

Remineralization Potential.

PB96-155544 03,575

Physicochemical Characterization of Natural and Bioprosthetic Heart Valve Calcific Deposits: Implications for Prevention.

00,187

Reinforcement of Cancellous Bone Screws with Calcium Phosphate Cement.

PB96-158001

00,179

Commentary on 'Optimization of Experimental Parameters for the EPR Detection of the Cellulosic Radical in Irradiated Foodstuffs

00,043

Polymeric Calcium Phosphate Cements Derived from Poly(methyl vinyl ether-maleic acid)

PB96-164264

00,180

Publication and Presentation Abstracts, 1994

03,577

Wear of Enamel against Glass-Ceramic, Porcelain, and

Amalgam.

03,082

Composition and Solubility Product of a Synthetic Calcium Hydroxyapatite.

Adsorption of Potassium N-phenylglycınate on Hydroxyapatite: Role of Solvents and Ionic Charge.
PB96-180161

In vitro Inhibition of Membrane-Mediated Calcification by Novel Phosphonates.

PB96-201140

03,595

New Surface-Active Comonomer for Adhesive Bonding PB96-204

03,579

Calcium Phosphate Cements.

PB97-111595

03,580

Adsorption of Polyacrylic Acids and Their Sodium Salts on Hydroxyapatite: Effect of Relative Molar Mass.

Posterior Restorative Materials Research.

PB97-118624

03,581

Overview of Bioelectrical Impedance Analyzers.

03,582

PB97-118780

00,181

Surface Roughness of Glass-Ceramic Insert. Composite Restorations: Assessing Several Polishing Techniques. 03,583 Interaction of Citric Acid with Hydroxyapatite: Surlace Ex change of lons and Precipitation of Calcium Citrate.

PB97-119309

Publication and Presentation Abstracts, 1996

03,584

03,585

HEART VALVE PROSTHESIS

Physicochemical Properties of Calcific Deposits Isolated from Porcine Bioprosthetic Heart Valves Removed from Patients Following 2-13 Years Function.

00,184

Critical Evaluation of the Purification of Biominerals by

Hypochlorite Treatment.
PB95-150959

00,186

HEART VALVE PROTHESIS

Physicochemical Characterization of Natural and Bioprosthetic Heart Valve Calcific Deposits: Implications for Prevention

00,187

HEAT

Effective Measurement Techniques for Heat, Smoke, and Toxic Fire Gases

01,369
Effects of Copper, Nickel and Boron on Mechanical Properties of Low-Alloy Steel Weld Metals Deposited at High PB96-135231

03,363

\section{HEAT CAPACITIES}

Thermodynamic Properties of Silicides. 5. Standard Molar Enthalpy of Formation at the Temperature $298.15 \mathrm{~K}$ of Trimolybdenum Monosilicide Mo3Si Determined by Fluorine-Combustion Calorimetry.

PB97-119358

01,190

HEAT CAPACITY

Molar Heat Capacity at Constant Volume for Air from 67 to $300 \mathrm{~K}$ at Pressures to $35 \mathrm{MPa}$.

PB96-163738

00,515

Molar Heat Capacity at Constant Volume for (xCO2 + (1x) $\mathrm{C} 2 \mathrm{H6}$ ) from 220 to $340 \mathrm{~K}$ at Pressures to $35 \mathrm{MPa}$

PB96-167135

Extrapolation of the Heat Capacity in Liquid and Amorphous Phases
PB97-111421

HEAT DETECTORS

Studies Assess Performance of Residential Detectors.
PB94-199262 00,290

\section{HEAT EXCHANGERS}

Review of Corrosion Behavior of Ceramic Heat Exchanger Materals: Corrosion Characteristics of Silicon 1992--March 11, 1993.

DE93041307

03,228

Theoretical Evaluation of the Vapor Compression Cycle with a Liquid-Line/Suction-Line Heat Exchanger, Economizer, and Ejector

PB95-216917

02,607

Upgrade and
PB96-161872

03,737

HEAT EXCHANGES

Effect of Inclination on the Performance of a Compact Brazed Plate Condenser and Evaporator.

02.756

HEAT FEEDBACK

Measurement of Radiative Feedback to the Fuel Surface of a Pool Fire.

of a Pool Fire.
PB94-211604

02,477

HEAT FLOWMETERS

Comparison of Heat-Flow-Meter Tests from Four Labora-

PB95-126264

00,365

HEAT FLUX

Ignition and Subsequent Transition to Flame Spread in a Microgravity Envi

04,828

Effects of Specimen Edge Conditions on Heat Release Rate

PB95-152864

00,375

Burning Rate and Flame Heat Flux For PMMA in the Cone Calorimeter.

00,393

Heat Flux from Flames to Vertical Surfaces.

01,438

HEAT FLUXES

Low Heat-Flux Measurements: Some Precautions.

PB96-201116

02,685

HEAT LOSS

Estimate of Flame Radiance via a Single Location Meas. Urement in Liquid Pool Flames.

PB94-211596

02,476

Estimate of the Effect of Scale on Radiative Heat Loss Fraction and Combustion Efficiency.

PB95-150447

02,486

\section{HEAT MEASUREMENT}

Assessing the Credibility of the Calorific Content of Muncipal Solid Waste.

02,581

Comparison of Heat-Flow-Meter Tests from Four Labora-

Comparison of Heat-Flow-Meter Tests from Four Labora-
tories.
PB95-126264
The0,365

Thermochemistry of the Hydrolysis of L-arginine to (L citrulline + Ammonia) and of the Hydrolysis of L-arginine to (L-ornithine + Urea)

PB95-150801

03,463

Biological Thermodynamic Data for the Calibration of Dif ferential Scanning Calorimeters: Dynamic Temperature Data on the Gel to Liquid Crystal Phase Transition of Dialkylphosphatidylcholine in Water Suspensions. PB95-162707

03,464

Thermodynamics of the Binding of Galactopyranoside Derivatives to the Basic Lectin from Winged Bean (Psophocarpus Tetrogonolobus).

03,465

\section{HEAT METERS}

Control Stability of a Heat-Flow-Meter Appartus.

PB95-181194

00,386

Intra-Laboratory Comparison of a Line-Heat-Source

Guarded Hot Plate and Heat-Flow-Meter Apparatus.

PB95-181202

00,387

\section{HEAT OF COMBUSTION}

Precise Measurement of Heat of Combustion with a Bomb Calorimeter.

03,770

Assessing the Credibility of the Calorific Content of Muncipal Solid Waste.

PB94-199882

02,581

HEAT OF FUSION Calibration Standards for Differential Scanning
Calorimetry. 1. Zinc Absolute Calorimetric Measurement of Enthalpy of Fusion and Temperature of Fusion HM. PB94-199817

Measurement of the Heat of Fusion of Tungsten by a Microsecond-

03,400

\section{HEAT OF REACTION}

Thermodynamic and NMR Study of the interactions of Cyclodextrins with Cyclohexane Derivatives. PB94-185816

00,781

Calorimetric Determination of the Standard Transformed Enthalpy of a Biochemical Reaction at Specified PH and pMg. 198249

03,454

\section{HEAT PUMPS}

Study of Heat Pump Performance Using Mixtures of R32/ R134a and R32/R125/R134a as 'Drop-In' Working Fluids for R22 with and Without a Liquid-Suction Heat ExPB94-218559

02,503

Simple Method of Composition Shifting with a Distillation Column for a Heat Pump Employing a Zeotropic Refrigerant Mixture.

02,603

HVAC CAD Layout Tools: A Case Study of University/ln. dustry Collaboration

00,281

HEAT RELEASE RATE

New Concepts for Fire Protection of Passenger Rail

04,863

Effects of Sample Mounting on Flammability Properties of Effects of Sample Mounting on Flammability Properties of
Intumescent Polymers.
PB96-159777
03,389

HEAT SHOCK PROTEINS

DnaJ, DnaK, and GrpE Heat Shock Proteins are Required in 'ori'P1 DNA

PB97-119382

03,557

HEAT-SHOCK PROTEINS 60

Function of DnaJ and DnaK as Chaperones in OriginSpecific DNA Binding by RepA PB95-151544

03,533 
HEATING SYSTEMS

Multizone Modeling of Three Residential Indoor Air Quality Control Options. PB96-165782

02,567

HEAVY METALS

Genetically Engineered Pore as a Metal Ion Biosensor.
PB96-161658

HELIUM

Helium Refrigeration and Liquefaction Using a Liquid $\mathrm{Hy}$ drogen Refrigerator for Precooling.

AD-A286 683/8 02749

High-Energy Behavior of the Double Photoionization of Helium from 2 to $12 \mathrm{keV}$

PB94-213279

03,860

Qperational Mode and Gas Species Effects on Rotational

Drag in Pneumatic Dead Weight Pressure Gages.

Ab initio Calculations for Helium: A Standard for Trans. port Property Measurements.

PB96-102041

01,060

Search for Small Violations of the Symmetrization Postulate in an Excited State of Helium.

04,050

Kinetic-Energy Distributions of Ions Sampled from RadioFrequency Discharges in Helium, Nitrogen, and Qxygen.
PB96-123732

Electronically Tunable Fiber Laser for Qptical Pumping of (3) $\mathrm{He}$ and (4) $\mathrm{He}$

(3) He and (4) He.
PB96-201165

Commercial Helium Permeation Leak Standards: Their Properties and Reliability

04,146

Absence of Quantum-Mechanical Effects on the Mobility of Argon lons in Helium Gas at $4.35 \mathrm{~K}$.

PB97-122543

01,194

HELIUM COMPLEXES

Signatures of Large Amplitude Motion in a Weakly Bound Complex: High-Resolution IR Spectroscopy and Quantum Calculations for $\mathrm{HeCO} 2$.

PB95-203485

01,054

HELIUM IONS

Use of an Ion Energy Analyzer: Mass Spectrometer to Measure Ion Kinetic-Energy Distributions from RF Dis charges in Argon-Helium Gas Mixtures.

04,406

HELIUM-LIKE IONS

Evaluation of Two-Photon Exchange Graphs for HighlyCharged Heliumlike Ions.

03,808

Experimental Aspects and Z-Dependent Systematics in Experimental Aspects and Z-Dependent Systematics in
Qne- and Two-Electron lons and Single Vacancy Systems

PB94-199627

03,815

HELIUM SPECTROSCOPY

Frequency-Stabilized LNA Laser at 1.083 mum: Application to the Manipulation of Helium 4 Atoms.

04,304 PB95-176186

HELMET MOUNTED DISPLAYS

NIST Construction Automation Program Report No. 2. Proceedings of the NIST Construction Automation Workshop. Held in Gaithersburg, Maryland on March 30-31,

PB96-202239

00,413

HELMHOLTZ RESONATORS

Greenspan Acoustic Viscometer for Gases.
PB96-204417

HEMOGLOBIN

Positive and Negative Cooperativities at Subsequent Steps of Qxygenation Regulate the Allosteric Behavior of Multistate Sebacylhemoglobin.

MBltistate Sebac

03,486

HEMOLYSIN Genetically Engineered Pores as Metal Ion Biosensors.
PB96-167408

HEMOLYSIS

Genetically Engineered Pores for New Materials.
PB96-161641

HENDERSON METHOD

Anova Estimates of Variance Components for a Class of PB96-141163

03,448

HEPARIN

Thermodynamic Analysis of Heparin Binding to Human Antithrombin

03,455

Physicochemical Characterization of Low Molecular

03,474

PB96-112040

copy.

Structural Analy

03,480

HEPARINS

Physical Characterization of Herparin by Light Scattering.
PB.56-119383

HEPTANE

Simultaneous Optical Measurement of Soot Volume Fraction, Temperature, and CQ2 in Heptane Pool Fire.

PB96-102132
HERTZIAN CONTACT

Deformation and Fracture of Mica-Containing Glass-Ceramics in Hertzian Contacts.

03,080

Effect of Grain Size on Hertzian Contact Damage in Alu-

mina. 179601

03,083

HETERODYNE DETECTION

Heterodyne Mixing and Direct Detection in High Tern-

perature Josephson Junctions.
PB96-147202

01,565

HETERODYNE MIXERS

Terahertz Detectors Based on Superconducting Kinetic Inductance.

HETERODYNING

$30 \mathrm{THz}$ Mixing Experiments on High Temperature Superconducting Josephson Junctions.

04,709

HETEROGENEITY

Structural Heterogeneity in Epoxies.

PB95-151866

01,243

PETEROJUNCTIONS

Scaling of the Nonlinear Optical Cross Sections of GaAsAlGaAs Multiple Quantum-Well Hetero $n-i-p-i$ 's. PB96-102793

02,183

HEURISTIC METHODS

Move-to-Root Rule for Self-Qrganizing Trees with Markov Dependent Requests.

(179528

03,431

HEXACARBONYLMOLYBDENUM

Photodecomposition of $\mathrm{Mo}(\mathrm{CO}) 6 / \mathrm{Si}(111) 7 \times 7$ : CO StateResolved Evidence for Excited State Relaxation and Ouenching.

HEXAFLUOROPROPANE

Thermodynamic Properties of CF3-CHF-CHF2, $1,1,1,2,3,3-$ Hexafluoropropane.

PB97-118384

03,299

HEXANES

Observations of Partial Discharges in Hexane Under High Magnification

01,900

Vapor-Liquid Equilibria of Mixtures of Propane and Isomeric Hexanes.
PB95-175287

00,995

Electrohydrodynamic Instability and Electrical Discharge Initiation in Hexane.

PB96-186119

02,244

HFC-236EA

Thermodynamic Properties of CF3-CHF-CHF2, $1,1,1,2,3,3$-Hexafluoropropane.

PB97-118384

03,299

HFC-245CA

Thermodynamic Properties of CHF2-CF2-CHF, 1,1,2,2,3Pentafluorooropane

03,300

HIERARCHIES

Hierarchical Real-Time Control System for Use with Coal Mining Automation.

PB94-212065

03,698

\section{HIGH ELECTRON MOBILITY TRANSISTORS}

Multicarrier Characterization Method for Extracting Mobilities and Carrier Densities of Semiconductors from Variable Magnetic Field Measurements.

PB94-212776

02,317

Influence of Lattice Mismatch on Indium Phosphide Based High Electron Mobility Transistor (HEMT) Structures Observed in High Resolution Monochromatic Synchrotron X-Radiation Diffraction Imaging.

02,357

HIGH INTEGRITY

High Integrity Software Standards Activities at NIST. 01,744

Report of a Workshop on the Assurance of High Integrity Software.

PB96-161377

01,763

Standards fo

01,764

HIGH INTEGRITY SOFTWARE

C++ in Safety Critical Systems.

PB96-154588

01.750

IIGH LEVEL RADIOACTIVE WASTES

Information Retrieval Using Key Words and a Structured

PB95-161121

03,724

HIGH-PERFORMANCE CONCRETES

High-Performance Concrete: Research Needs to Enhance Its Use.

01,322

HIGH PRESSURE

Molar Heat Capacity at Constant Volume for $(x \mathrm{CO} 2+<1$ x) $\mathrm{C} 2 \mathrm{H} 6$ ) from 220 to $340 \mathrm{~K}$ at Pressures to $35 \mathrm{MPa}$ PB96-167135

01,148

Isochoric ( $p-p-T)$ Measurements on Liquid and Gaseous Air from 67 to $400 \mathrm{~K}$ at Pressures to $35 \mathrm{MPa}$.

01,154

Alvin Van Valkenburg and the Diamond Anvil Cell. PB96-204474

04,797

HIGH PRESSURE CELLS

High-Pressure Equilibrium Cell for Solubility Measurements in Supercritical Fluids.

PB95-175634

00,998

HIGH PRESSURE TESTS

1,4-Dinitrocubane and Cubane under High Pressure.

PB95-108437

03,755

HIGH RESOLUTION

Intermolecular HF Motion in $\operatorname{Ar}$ (sub n)HF Micromatrices $(n=1,2,3,4$,$) : Classical and Quantum Calculations on a$ Pairwise Additive Potential Surface.

PB95-107025

03,871

Mode Specific Vibrational Predissociation Dynamics in Fragile Molecules.

PB95-107132

00,871

Vibration, Rotation, and Parity Specific Predissociation Dynamics in Asymmetric $\mathrm{OH}$ Stretch Excited ArH2Q: A Half Collision Study of Resonant V-V Energy Transfer in a Weakly Bound Complex.

Making Displays Deliver a Full Measure.

00,872

PB96-12241

01,490

Potential Surfaces and Dynamics of Weakly Bound Trimers: Perspectives from High Resolution IR SpectrosPB96-176508

01,155

HIGH-SPEED MEASUREMENTS

Simultaneous Measurement of Normal Spectral Emissivity by Spectral Radiometry and Laser Polarimetry at High Temperatures in Pulse-Heating Experiments: Application to Molybdenum and Tungsten.

HIGH-SPEED TECHNIQUES

Issues in High-Speed Pyrometry.

GH STRENGTH CONCRETE

Effects of Testing Variables on the Measured Compressive Strength of High-Strength (90 MPa) Concrete. PB95-179040

00,445

Effects of Testing Variables on the Strength of HighStrength (90 Mpa) Concrete Cylinders.

HIGH SULFUR COAL

Sulfur Dioxide Capture in the Combustion of Mixtures of Lime, Refuse-Derived Fuel, and Coal.

PB94-155587

02,534

HIGH-TC SUPERCONDUCTORS

Flame Synthesis of High Tc Superconductors

00,659

Effects of Critical Current Density, Equilibrium Magnetization and Surface Barrier on Magnetization of High Temperature Superconductors. Superconductors.

Dependence of Tc on Debye Temperature Theta(sub D) PB95-180493rates.

Hole Dispersion and Enhancement of Antiferromagnetic Interaction of Localized Spins in High-T Superconductors.

Effect of Magnetic Field Qrientation on the Critical Cur rent of HTS Conductor and Coils.

PB96-141189

02,956

First VAMAS USA Interlaboratory Comparison of High Temperature Superconductor Critical Current Measurements. 
HIGH TEMPERATURE SUPERCONDUCTING

Thermal Isolation of High-Temperature Superconducting Thin Films Using Silicon Wafer Bonding and Micromachining. PB96-135017

HIGH TEMPERATURE SUPERCONDUCTOR

Growth of Laser Ablated YBa2Cu3O7-delta Films as Examined by Rheed and Scanning Tunneling Microscopy.
PB95-162541 IGH TEMPERATURE SUPERCONDUCTORS

Effects of Critical Current Density, Equilibrium Magnetization and Surface Barrier on Magnetization of High Tem. perature Superconductors.

PB94-185162

04,446

Experimental Constraints on Some Mechanisms for High-

Temperature Superconductivity.
PB94-198553

Anharmonic Phonons and the Isotope Effect in Superconductivity.

PB94-200557 04,486

YBa2Cu3O7-x to Si Interconnection for Hybrid Superconductor/Semiconductor Integration.

02,315

Offset Susceptibility of Superconductors.

PB94-212263 04,503

Efficient Experiment to Study Superconducting Ceramics.
PB94-212578

Asymmetry between Flux Penetration and Flux Expulsion in Tl-2212 Superconductors.

PB95-125647

04,527

Magnetic Measurement of Transport Critical Current Density of Granular Superconductors.

PB95-126199

04,531

Insulating Nanoparticles on $\mathrm{YBa} C \mathrm{Cu} 3 \mathrm{O} 7$-delta Thin Films Revealed by Comparison of Atomic Force and Scanning Tunneling Microscopy.

PB95-150843

04,575

$X$-Ray Characterization of the Crystallization Process of High-Tc Superconducting Oxides in the Sr-Bi-Pb-Ca-CuO System.

04,579

Crystal Chemistry and Phase Equilibrium Studies of the $\mathrm{BaO}(\mathrm{BaCO})-\mathrm{R} 2 \mathrm{O} 3-\mathrm{CuO}$ Systems. 5. Melting Relations in $\mathrm{Ba2}(\mathrm{Y}, \mathrm{Nd}, \mathrm{Eu}) \mathrm{Cu} 3 \mathrm{O} 6+\mathrm{x}$

PB95-151718

04,580

$X$-Ray-Diffraction Study of a Thermomechanically Detwinned Single Crystal of $\mathrm{YBa} 2 \mathrm{Cu} 3 \mathrm{O} 6+\mathrm{X}$

PB95-151726

04.581

Flame Synthesis of High Tc Superconductors.

PB95-151981

00,659

Coupled-Bilayer Two-Dimensional Magnetic Order of the Dy lons in Dy2Ba4Cu7015.

PB95-152104

04,584

Effects of Critical Current Density, Equilibrium Magnetization and Surface Barrier on Magnetization of High Temperature Superconductors.

B95-153060

04.588

Transverse Thermomagnetic Effects in the Mixed State and Lower Critical Field of High-Tc Superconductors.

PB95.153250 04.590

Preparation of Low Resistivity Contacts for High-Tc Superconductor

02,258

Improved Uniaxial Strain Tolerance of the Critical Curren Measured in Ag-Sheathed Bi2Sr2Ca1Cu2O8+x Superconductors.

PB95-153565

04,594

Calibration of High-Energy Electron Beams by Use of

Graphite Calorimeters.

04,598

Harmonic and Static Susceptibilities of $\mathrm{YBa} 2 \mathrm{Cu} 3 \mathrm{O} 7$.

PB95-161139 04,599

Hydrogen in YBa2Cu3OX: A Neutron Spectroscopy and a Nuclear Magnetic Resonance Study.

04,601

Graın Alignment and Transport Properties of Bi2Sr2CaCu2O8 Grown by Laser Heated Floa: Zone Method.

PB95-161451

04,602

Effects of Anneal Time and Cooling Rate on the Forma-

tion and Texture of $\mathrm{Bi} 2 \mathrm{Sr} 2 \mathrm{CaCu} 2 \mathrm{O} 8$ Films.

PB95-161600 04,603

Tunneling Spectroscopy of Thallium-Based High-Temperature Superconductors.

04,606

Temperature Dependence of Vortex Twin Boundary Interaction in Yttrium Barium Copper Oxide (YBa2Cu3O6+x).
PB95-162442

Observation of Insulating Nanoparticles on YBCO Thin-

Films by Atomic Force Microscopy.

PB95-163358

04,622

Determination of Thermoactivation Parameters of Vortex Mobility in YBa2Cu3O7 Using Only Magnetic MeasurePBg5-

04,624

High Critical Temperature Superconductor Tunneling Spectroscopy Using Squeezable Electron Tunneling Junctions.

04,627
Tunneling Measurement of the Zero-Bias Conductance Peak and the Bi-Sr-Ca-Cu-O Thin-Film Energy Gap. Thermal and Nonequilibrium Responses of Thermal and Nonequilibrium Responses of Superconductors for Radiation Detectors.
PB95-164232 PB95-164232 Superconductors.

PB95-168621

04,639

From Superconductivity to Supernovae: The Ginzburg Symposium. Report on the Symposium Held in Honor of Vitaly L. Ginzburg. Held in Gaithersburg, Maryland on

$\begin{array}{ll}\text { May 22, } 1992 . & 04,649\end{array}$

Standard Reterence Devices for High Temperature Superconductor Critical Current Measurements.

Hents. 059

Mutual Phase Locking in Systems of High-To Superconductor-Nomal Metal-Superconductor Junctions.
04,744

Influence of Films' Thickness and Air Gaps in Surface Impedance Measurements of High Temperature nique.
01,946

High-Tc Multilayer Step-Edge Josephson Junctions and SQUIDS

PB96-200183

04,790

\section{PIETTS}

Recent Experimental and Modeling Developments in High Temperature Thermochemistry.

00,759

High-Temperature Laser-Pulse Thermal Diffusivity AppaPB9 1

02,631

Millisecond-Resolution Pulse Heating System for SpecificHeat Measurements at High Temperatures.

02,635

Effects of Humidity and Elevated Temperature on the Density and Thermal Conductivity of a Rigid Polyisocyanurate Foam Co-Blown with CCI3F and $\mathrm{CO} 2$. PB95-150462

Effects of Humidity and Elevated Temperature on the Density and Thermal Conductivity of a Rigid Polyisocyanurate Foam

00,373

HIGH TEMPERATURE THERMOMETRY

Investigation of High-Temperature Platinum Resistance Thermometers at Temperatures Up to $962 \mathrm{C}$, and, in Some Cases, $1064 \mathrm{C}$

PB96-161294

00,629

HIGH VOLTAGE

Investigation of the Effects of Aging on the Calibration of a Kerr-Cell Measuring System for High Voltage Impulses. Comparative Measurements of High-Voltage Impulses Using a Kerr Cell and a Resistor Divider. PB94-172582

02,028

Approach to Setting Periormance Requirements for Automated Evaluation of the Parameters of High-Voltage Impulses.

01,878

Digital Techniques in HV Tests - Summary of 1989 Panel Session.

PB94-216702

02,035

HILY CHARGED IONS

Beam Line for Highly Charged lons.

\section{HIGHWAY COMMUNICATION}

Recommendations on Selection of Vehicle-to-Roadside Communications Standards for Commercial Vehicle Oper-

PB94-195914

04,859

Vehicle-to-Roadside Communications for Commercial Vehicle Operations: Requirements and Approaches.
PB95-188827

04,860

HIGHWAY CONSTRUCTION

National Voluntary Laboratory Accreditation Program:

Construction

01,319

Robotics Application to Highway Transportation. Volume 4. Proposals for Potential Research.

01,339

Study of Potential Applications of Automation and Robotics Technology in Construction, Maintenance and Operics Technology in Construction, Maintenance and Op ation of Highway Systems: A Final Report. Volume 4.
PB95-251641 Study of Potential Applications of Automation and Robotics Technology in Construction, Maintenance and Operation of Highway Systems: A Final Report. Volume PB95-251682

01,341

Study of Potential Applications of Automation and Robotics Technology in Construction. Maintenance and Operation of Highway Systems: A Final Report. Volume 3 PB95-251690 01,342

Study of Potential Applications of Automation and Robotics Technology in Construction, Maintenance and Oper-

ation of Highway Systems: A Final Report. Volume 2.

01,343

HIGHWAY MAINTENANCE

Highway Concrete (HWYCON) Expert System User Reference and Enhancement Guide.

01,316

Robotics Application to Highway Transportation. Volume 4. Proposals for Potential Research.

01,339

Study of Potential Applications of Automation and Robotics Technology in Construction, Maintenance and Operation of Highway Systems: A Final Report. Volume 4.

Study of Potential Applications of Automation and Robotics Technology in Construction, Maintenance and Operation of Highway Systems: A Final Report. Volume 1. PB95-251682 01,341

Study of Potential Applications of Automation and Robotics Technology in Construction, Maintenance and Operation of Highway Systems: A Final Report. Volume 3.

PB95-251690 01,342

Study of Potential Applications of Automation and Robotics Technology in Construction, Maintenance and Ope ation of Highway Systems: A Final Report. Volume 2.

PB95-255865

Robotics Application to Highway Transportation. Volume 4. Proposals for Potential Research

\section{GHWAY TRANSPORTATION}

Robotics Application to Highway Transportation. Volume 2. Literature Search.

PB95-170551

01,337

Robotics Application to Highway Transportation. Volume 3. Proposed Research Topics and CostBenefit Evaluations by CERF

HIGHWAYS

Binder Characterization and Evaluation by Nuclear Magnetic Resonance Spectroscopy.

PB94-193471

01,334

HILBERT PROBLEMS

Green's Function for Generalized Hilbert Problem for Cracks and Free Surfaces in Composite Materials. PB95-163333

03,163

HILSCH TUBES

Applications of the Vortex Tube in Chemical Analysis.

Chromatographic Cryofocusing and Cryotrapping with the Vortex Tube

00,604

Applications of the Vortex Tube in Chemical Analysis. Part 2. Applications.

HISTIDINE

Pore-Forming Protein with a Metal-Actuated Switch

ISTOGRAMS

Concentration Histogram Imaging: A Scatter Diagram PBChnique for

HISTORICAL ASPECTS

Fire and Smoke Control: An Historical Perspective PB95-175808

HOLD-UP TIMES

Simple and Efficient Methane-Marker Devices for Chromatographic Samples.

00,635

HOLE MOBILTY

Majority and Minority Mobilities in Heavily Doped Silicon

for Device Simulations.
PB94-198728

Hole Dispersion and Enhancement of Antiferromagnetic Interaction of

PB95-202602

04,694

Majority and Minority Electron and Hole Mobilities in 
HOME FIRES

Influence of Ignition Source on the Flaming Fire Hazard of Upholstered Furniture. (NIST Reprint).

PB95-180162

00,297

HOMOGENEITY

Recent Developments in NIST Botanical SRMs.

HOMOSCEDASTICITY Developing Measurement for Experimentation.
PB97-118707

HONEYCOMB COMPOSITES

Investigation into the Flammability Properties of Honeycomb Composites.

HOSPITAL FIRES

Measurement of Room Conditions and Response of Sprinklers and Smoke Detectors during a Simulated TwoBed Hospital Patient Room Fire.

PB94-213717

HOT ELECTRONS

$30 \mathrm{THz}$ Mixing Experiments on High Temperature Superconducting Josephson Junctions.

00,292

04,709

HOT ISOSTATIC PRESSING

Intelligent Processing of Hot Isostatic Pressing

PB94-172913

03,315

Micromechanics of Densification and Distortion

PB94-200326 Investigation into a Practical Grain Growth Model for Hot Isostatic Pressing

HOT MIX PAVING MIXTURES

03,347

System for Calibration of the Marshall Compaction HamPB94.

01,303

Field Evaluation of the System for Calibration of the Marshall Compaction Hammer.

PB95-190674

Intelligent Processing of Hot Isostatic Pressing.
PB94-172913

01,323

Applicability to Silicon

03,094

HOT STARS

Radiation-Driven Winds of Hot Luminous Stars $X$. The Determination of Stellar Masses Radii and Distances from Terminal Velocities and Mass-Loss Rates.

PB94-213022

00,060

HOT SURFACES

Transient Cooling of a Hot Surface by Droplets Evaporation.

03,890

HOT WIRE

Transient Methods for Thermal Conductivity. PB94-198405

04,197

HOT WORKING

Hot-Deformation Apparatus for Thermomechanical Processing Simulation.

PB94-200136

03,207

HOTEL FIRES

Full Scale Smoke Control Tests at the Plaza Hotel Bulding.

00,347

HOUSING (DWELLINGS)

Controlling Moisture in the Walls of Manufactured Hous-

ing.

00,355

Predicting the Energy Performance Ratings of a Family of Type I Combin
PB95-105524

02,504

HPC (HIGH PERFORMANCE CONCRETE)

Microstructural Features of Some Low Water/Solids, Silica Fume Mortars Cured at Different Temperatures.

A-100

00,330

Characterization of the Hydrogen Induced Cold Cracking
SLA STEEL Susceptibility at Simulated Weld Zones in HSLA-100

AD-A279 759/5

03,200

HUMAN BEHAVIOR

Mathematical Modeling of Human Egress from Fires in Residential Buildings.

00,337

Enhancement of EXIT89 and Analysis of World Trade Center Data

PB96-202247

00,231

Maximum Permissible Amounts of Radioisotopes in the Human Body and Maximum Permissible Concentrations in Air and Water.

03,609 Maximum Permissible Body Burdens and Maximum Per-
missible Concentrations of Radionuclides in Air and in missible Concentrations of Radionuclides in Air and in
Water for Occupational Exposure. WD-A280 282/5

03,610

HUMAN FACTORS

Human Factors Considerations for the Potential Use of Elevators for Fire Evacuation of FAA Air Trattic Control Towers.

PB94-217163

01,300
HUMAN FACTORS ENGINEERING

Evaluating Form Designs for Optical Character RecogniPB94-168044

01,830

Psychological Aspects of Lighting: A Review of the Work of CIE TC 3.16.

00,241

Workshop on the Application of Virtual Reality to Manufacturing. Final Report. Held in Gaithersburg, Maryland on August 9, 1994.
PB95-173555

HUMAN SERUM

Development of the Ion Exchange-Gravimetric Method for Sodium in Serum as a Definitive Methoc. 96-179148

01,867

HUMANITIES

User Profile for Researchers Studying Objects: Implica. tions for Computer Systems.

PB94-188463

00,133

HUMANS

Human and Machine Recognition of Faces: A Survey.

HUMIDITY

Methods of Measuring Humidity and Testing AD-A278 851/1

00,123

Effects of Humidity and Elevated Temperature on the Density and Thermal Conductivity of a Rigid Polyisocyanurate Foam Co-Blown with CCl3F and $\mathrm{CO}_{0.37}$.
PB95-150462 Effects of Humidity and Elevated Temperature on the Density and Thermal Conductivity of a Rigid Polyisocyanurate Foam.

PB95-15202

00,373

HUMIDITY MEASUREMENT

New Expressions of Uncertainties for Humidity Calibrations at the National Institute of Standards and Technology.

02,645

HURRICANES

Gust Factors Applied to Hurricane Winds.

PB95-180469

00,446

Some Notab

00,458

Estimates of Hurricane Wind Speeds by the 'Peaks Over Threshold' Method.

PB96-162540

00,47

Probabilistic Estimates of Design Load Factors for WindSensitive Structures Using the 'Peaks Over Threshold' Approach.

PB96-183223

00.474

HY-100 STEEL

Characterization of the Hydrogen Induced Cold Cracking Susceptibility at Simulated Weld Zones in HSLA-100

03,200

HYADES CLUSTER

IUE Observations of Solar-Type Stars in the Pleiades and the Hyades.

HYBRID ELECTRIC-POWERED VEHICLES Advanced Components for Electric and Hybrid Electric
Vehicles. Workshop Proceedings. Held in Gaithersburg, Maryland on October 27-28, 1993 . Held in Gaithersburg. Maryland on Ock

04,858

HYBRID METHODS

Hybrid Method for Determining Material Properties from Instrumented Micro-Indentation Experiments.

PB95-152229

03,348

HYDRATES

Modelling Drying Shrinkage of Cement Paste and Mortar. Part 1. Structural Models from Angstroms to Millimeters. PB96-135132

03,074

HYDRATION

Digitized Direct Simulation Model of the Microstructural Development of Cement Paste.

PB94-198777

01,309

Digitized Simulation Model for Microstructural Develop-

PB94-198785

Modelling the Leaching of Calcium Hydroxide from $\mathrm{Ce}$ ment Paste: Effects on Pore Space Percolation and Diffusivity.

PB94-198793

01,311

Diffusion Studies in a Digital-Image-Based Cement Paste Microstructural Model.

PB94-198801

01,312

Survey of Recent Cementitious Materials Research in Western Europe.

PB94-218583 00,353

Resolution of DNA in the Presence of Mobility Modifying Polar and Nonpolar Compounds by Discontinuous Electrophoresis on Rehydratable Polyacrylamide Gels.
PB95-152799 Cellular Automaton Simulations of Cement Hydration and Microstructure Development.

01,320

Hydration in Semicrystalline Polymers: Small-Angle Neutron Scattering Studies of the Effect of Drawing in Nylon6 Fibers.

PB95-202990

03,385

HYDRAULIC FLUIDS

Suppression of Elevated Temperature Hydraulic Fluid and JP-8 Spray Flames.

00,021

Intercomparison between NPL (India) and NIST (USA) Pressure Standards in the Hydraulic Pressure Region Up PBg6-113543

HYDRAULIC RADIUS

Hydraulic Radius and Transport in Reconstructed Model Three-Dimensional Porous Media.

PB96-123419

00,403

YYRAZINE

Far Infrared Laser Frequencies of $\mathrm{CH} 3 \mathrm{OD}$ and N2H4

PB96-119623

04,341

HYDRAZINE TREATMENT

Critical Evaluation of the Purification of Biominerals by Hypochlorite Treatment.

00,186

HYDRAZOIC ACID

Fragment Energy and Vector Correlations in the Overtone-Pumped Dissociation of $\mathrm{HN} 3 \mathrm{X}(1) \mathrm{A}^{\prime}$.

00,802

HYDRIDES

Neutron Spectroscopic Comparison of beta-Phase Rare Earth Hydrides.

HYDROAZOIC ACID

Vibrational Predissociation Dynamics of Overtone-Excited PBos-1

HYDROCARBON DETECTORS

Process Gas Chromatography Detector for Hydrocarbons Based on Catalytic Cracking.
PB95-141099

HYDROCARBONS

Standard Materials. A Descriptive List with Prices.
AD-A278 140/9 00,500

Process Gas Chromatography Detector for Hydrocarbons Based on Catalytic Cracking.

PB95-141099

02,485

Optical Measurements of Atomic Hydrogen, Hydroxyl, and Carbon Monoxide in Hydrocarbon Diffusion Flames.

02,487

Large Fire Experiments for Fire Model Evaluations PB96-190079

01,427

Experimental Determination of the Rate Constant for the Reaction of $\mathrm{C}_{2} \mathrm{H}_{3}$ with $\mathrm{H} 2$ and Implications for the Partitioning of Hydrocarbons in Atmospheres of the Outer PB97-122295

HYDROCHLORIC ACID

Automated, High Precision Coulometric Titrimetry. Part 2. Strong and $W$

00,576

HYDROCHLOROFLUOROCARBONS

Rate Constants for the Gas Phase Reactions of the $\mathrm{OH}$ Radical with $\mathrm{CF} 3 \mathrm{CF} 2 \mathrm{CHCl} 2$ (HCFC-225ca) and CF2CICF2CHCIF (HCFC-225cb)

PB95-152153

00,940

Annex 18: An International Study of Refrigerant Properties.

PB95-168936

03,266

HYDRODYNAMIC FRICTION

Hydrodynamic Friction of Arbitrarily Shaped Brownian Particles.

PB97-110191

04,136 
HYDROFLUOROCARBON

Neutron and Raman Spectroscopies of 134 and 134a Hydrofluorocarbons Encaged in Na-X Zeolite. PB96-186168

03,001

HYDROFLUOROCARBONS

Development of a Dual-Sinker Densimeter for High-Accuracy Fluid P-V-T Measurements.

PB95-168951

03,267

Atmospheric Lifetimes of HFC-143a and HFC-245fa: Flash Photolysis Resonance Fluorescence Measurements of the $\mathrm{OH}$ Reaction Rate Constants.

PB97-112577

00,118

HYDROFLUROROCARBONS

Virial Coefficients of Five Binary Mixtures of Fluorinated Methanes and Ethanes.

PB96-156054

01,128

HYDROGEN

Tabulation of the Thermodynamic Properties of Norma Hydrogen from Low Temperatures to $300 \mathrm{~K}$ and from 1 to 100 Aimospheres.

00,713

Compilation of the Physical Equilibria and Related Properties of the Hydrogen-Carbon Monoxide System.
AD-A286 603/6
00,716 Collision-Induced Emission in the Fundamental VibrationRotation Band of $\mathrm{H} 2$

PB94-199445

03,811

Vibrational Autoionization in $\mathrm{H} 2$ : Vibrational Branching Ratios and Photoelectron Angular Distributions Near the $\mathrm{v}(+)=3$ Threshold

00,799

Rate Constants for Hydrogem Atom Attack on Some Chlorinated Benzenes at High Temperature.

00,810

Measurement of the Self Broadening of the $\mathrm{H} 2 \mathrm{O}(0-5)$ Raman Transitions from 295 to $1000 \mathrm{~K}$

PB95-108627

00,884

Conductance Response of $\mathrm{Pd} / \mathrm{SnO} 2(110)$ Model Gas Sensors to $\mathrm{H} 2$ and $\mathrm{O} 2$
PB95.125803

00,892

Operational Mode and Gas Species Effects on Rotational Drag in Pneumatic Dead Weight Pressure Gages.

PB95-140182

00,903

Optical Measurements of Atomic Hydrogen, Hydroxyl and Carbon Monoxide in Hydrocarbon Diffusion Flames.

Mechanism and Rate Constants for the Reactions of $\mathrm{Hy}$ drogen Atoms with Isobutene at High Temperatures.

PB95-151064 00,929

Simultaneous Forward-Backward Raman Scattering Studies of D2 Broadened by D2, $\mathrm{He}$, and $\mathrm{Ar}$.

PB95-162459

00.961

Hydrogen Atom Attack on Perchloroethylene.

00,969

Fugacity Coefficients of Hydrogen in (Hydrogen $+\mathrm{Bu}$

PB95-175212

02,491

Neutron Scattering by Hydrogen in Cold Neutron Prompt

Gamma-Activation Analysis.

00,603

Characterization of the Interaction of Hydrogen with Iridium Clusters in Zeolites by Inelastic Neutron Scattering Spectroscopy.

01,013

Vibrations of Hydrogen and Deuterium in Solid Solution with Lutetium.

01.019

Analyses of Recent Experimental and Theoretical Determinations of e-H2 Vibrational Excitation Cross Sections Assessing a Long-Standing Controversy.

03,977

Recombination Line Intensities for Hydrogenic Ions-III. Eftects of Finite Optical Depth and Dust.

00,079

Modified Effective Range Theory as an Alternative to

Low-Energy Close-Coupling Calculations. Low-Energy Close-Coupling Calculations.

PB95-20270

03,988

Deuterium in the Local Interstellar Medium: Its Cosmological Significance.

PB95-202842

00,081

Importance of Bound-Free Correlation Effects for Vibra-

tional Excitation of Molecules by Electron Impact: A Sensitivity Analysis.

Time-Resolved Balmer-Alpha Emission from Fast Hydrogen Atoms in Low Pressure, Radio-Frequency Discharges in Hydrogen.

PB96-102942

04,028

Measuring Hydrogen by Cold-Neutron Prompt-Gamma Activation Analysis.

Decay of Bragg Gratings in Hydrogen-Loaded Optical Fi-

PB96-122643

04,345

Asymptotic and Numerical Analysis of a Premixed Laminar Nitrogen Dioxide-Hydrogen Flame.

PB96-164256

01,422

HYDROGEN ABSORPTION

Vibrational Excitations and the Position of Hydrogen in Nanocryslalline
PB96-111828

04,035
HYDROGEN ADDITIONS

Hydrogen in YBa2Cu3Ox: A Neutron Spectroscopy and a Nuclear Magnetic Resonance Study.

04,601

HYDROGEN ATOMS

Rate Constants for Hydrogem Atom Altack on Some

Chlorinated Benzenes at High Temperature.

394-200581

00,810

Perturbative Calculation of the $A C$ Stark Effect by the Complex Rotation Method.

04,260

Mechanism and Rate Constants for the Reactions of Hy-

drogen Atoms with isobutene at High Temperatures.
PB95-151064

Hydrogen Atom Attack on Perchloroethylene.

PB95-163473

00,969

Vibrational Excitations and the Position of Hydrogen in Nanocrystalline Palladium.

HYDROGEN BONDING

Interaction of Some Coupling Agents and Organic Compounds with Hydroxyapatite: Hydrogen Bonding. Adsorption and Adhesion.

PB94-172616

00,140

Lattice Model of a Hydrogen-Bonded Polymer Blend.

03,391

Relaxation After a Temperature Jump Within the One Phase Region of a Polymer Mixture.

03,394

Bimolecular Interactions in (Et) $3 \mathrm{SiOH}: \mathrm{Base}: \mathrm{CCl} 4 \mathrm{Hydro}-$ gen-Bonded Solutions Studied by Deactivation of the Free $\mathrm{OH}-S t r e t c h$ Vibration.

PB97-118657

04,166

Rotational Spectra of $\mathrm{CH} 3 \mathrm{CCH}-\mathrm{NH} 3, \mathrm{NCCCH}-\mathrm{NH} 3$, and $\mathrm{NCCCH}-\mathrm{OH} 2$.
PB97-118798

HYDROGEN BONDS

04,170

Phase Behavior of a Hydrogen Bonding Molecular Com-

PBIte.

01,202

Strong Hydrogen Bond in the Formic Acid-Formate Anion PBstem.

00,788

Structure of Glycine-Water H-Bonded Complexes.

PB94-198603

00,789

P-Type Doubling in the Infrared Spectrum of NO-HF.

00,817

Mode Specific Vibrational Predissociation Dynamics in Fragile Molecules.

PB95-107132

00,871

Rigid Bender Analysis of van der Waals Complexes: The Intermolecular Bending Potential of a Hydrogen Bond.

Comparison of a Fixed-Charge and a Polarizable Water Model

PB96-111620

01,072

State-Resolved Rotational Energy Transfer in Open Shell Collisions: $\mathrm{Cl}((2) \mathrm{P} 3 / 2)+\mathrm{HCl}$

PB96-176607

01,157

HYDROGEN CHLORIDE

Slit-Jet Near-Infrared Diode Laser Spectroscopy of (DCI)2: nu1, nu2 DCl Stretching Fundamentals, Tunneling Dynamics, and the Influence of Large Amplitude 'Geared' PB95-203204

01,047

\section{HYDROGEN CHLORIDE COMPLEXES}

Vibrational Dependence of the Anisotropic Intermolecular Potential of Ar-HCl.

PB95-202685

01,029

High Resolution Near Infrared Spectroscopy of $\mathrm{HCl}-\mathrm{DCl}$ and $\mathrm{DCl}-\mathrm{HCl}$ : Relative Binding Energies, Isomer Interconversion Rates, and Mode Specific Vibrational Predissociation

HYDROGEN CHLORIDE DIMERS

01,048

High Resolution, Jet-Cooled Infrared Spectroscopy of ( $\mathrm{HCl}$ 2: Analysis of nu1 and nu2 $\mathrm{HCl}$ Stretching Fundamentals Interconversion Tunneling and Mode-Specific Predissociation Lifetimes.

PB95-203196

01,046

HYDROGEN CYANIDE

Self-, N2. and Ar-Broadening and Line Mixing in HCN

PB95-108445 00,876

New Approach for Reducing the Toxicity of the Combus. tion Products from Flexible Polyurethane Foam. PB96-123625

01,411

HYDROGEN CYANIDE LASERS

Efficient $\mathrm{Br}\left({ }^{*}\right)$ Laser Pumped by Frequency-Doubled Nd: $Y A G$ and Electronic-to-Vibrational Transfer-Pumped $\mathrm{CO} 2$ and HCN Lasers.

HYDROGEN EMBRITTLEMENT

04,248

Determination of Hydrogen in Titanium Alloy Jet Engine Compressor Blades by Cold Neutron Capture Prompt Gamma-ray Activation Analysis.

PB95-175956

01,448

HYDROGEN FLUORIDE

Tunneling-Rotation Spectrum of the Hydrogen Fluoride Dimer.
PB94-198678

00,793

P-Type Doubling in the Infrared Spectrum of NO-HF

PB94-211463 00,817 Intermolecular HF Motion in Ar(sub n)HF Micromatrices $(n=1,2,3,4$, : Classical and Quantum Calculations on a Pairwise Additive Potential Surface.

PB95-107025

03,871

High-Resolution Infrared Spectroscopy of DF Trimer: A Cyclic Ground State Structure and DF Stretch Induced intramolecular Vibrational Coupling.

PB95-150678

00,920

HYROGEN FLUORIDE COMPLEXES

High-Resolution Infrared Overtone Spectroscopy of ArHF via Nd:YAG/Dye Laser Difference Frequency Generation. PB94-211448

00,816

Spectroscopic Puzzle in ArHF Solved: The Test of a New Potential.

PB94-216058

00,850

Slit Jet Infrared Spectroscopy of Hydrogen Bonded N2HF Isotopomers: Rotational Rydberg-Klein-Rees Analysis and H/D Dependent Vibrational Predissociation Rates.

PB95-161873

00,956

Fragment State Correlations in the Dissociation of NO. $H F(v=1)$.
PB95-164430

00,982

Vibrational Dependence of the Anisotropic Intermolecular Potential of Ar-HF.

PB95-202693

01,030

Rotational-RKR Inversion of Intermolecular Stretching Potentials: Extension to Linear Hydrogen Bonded Complexes.

PB95-203014

01,041

Rigid Bender Analysis of van der Waals Complexes: The Intermolecular Bending Potential of a Hydrogen Bond. PB95-203022

HYDROGEN IONS

Resonant Two-Color Detachment of $\mathrm{H}(-)$ with Excitation of $\mathrm{H}(\mathrm{n}=2)$.
PB95-20255

03,984

Variationally Stable Treatment of Two- and Three-Photon Detachment of $H(-)$ Including Electron-Correlation Effects. PB95-202867

03,992

Short-Pulse Detachment of $\mathrm{H}(-)$ in the Presence of a Static Electric Field.

PB95-203477

04,007

Kinetic Energy Distributions of $\mathrm{H}(+), \mathrm{H} 2(+)$, and $\mathrm{H} 3(+)$ from a Diffuse

HYDROGEN IONS 2 PLUS

Asymptotic Wave Function Splitting Procedure for Propagating Spatially Extended Wave Functions: Application to intense Field Photodissociation of $\mathrm{H} 2(+)$.

PB94-211786

03,847

HYDROGEN ISOTOPES

New IUPAC Guidelines for the Reporting of Stable Hydrogen, Carbon, and Oxygen Isotope-Ratio Data. Letter to PB95-261962

HYOROGEN-LIKE IONS 

Thermodynamics of the Hydrolysis of N-Acetyl-L-
phenylalanine Ethyl Ester in Water and in Organic Solvents.

PB95-203386

01,053

HYDROXYANISOLES

Thermal Decomposition of Hydroxy- and Methoxy-Substituted Anisoles.

PB94-173036

00,767

HYDROXYAPATITE

Formation of Hydroxyapatite in Cement Systems.
PB95-175261 00,170

Adsorption of Polassium N-phenylglycinate on Hydroxyapatite: Role of Solvents and lonic Charge.
PB96-180161 In vitro Inhibition of Membrane-Mediated Calcification by Novel Phosphonates.

PB96-201140 03,595

Interaction of Citric Acid with Hydroxyapatite: Surface Exchange of lons and Precipitation of Calcium Citrate.

PB97-119309

03,584

HYDROXYAPATITES

Adsorption of Low-Molecular-Weight Sodium Polyacrylate on Hydroxyapatite.

00,139

Interaction of Some Coupling Agents and Organic Compounds with Hydroxyapatite: Hydrogen Bonding, Adsorption and Adhesion.

00,140

Interaction of Chlorhexidine Digluconate with and Adsorption of Chlorhexidine on Hydroxyapatite. tion of Chlorhexidine on Hydroxyapatite.

PB95-175907

03,566

Formation of Hydroxyapatite in a Polymeric Calcium Phosphate Cement

PB95-180642

00,173

Adsorption of Polyacrylic Acids and Their Sodium Salts on Hydroxyapatite: Effect of Relative Molar Mass.

03,581

HYDROXYL EMISSION

Rotational Spectrum of $\mathrm{OH}$ in the $\mathrm{v}=0-3$ Levels of its Ground State.

01,006

HYDROXYL RADICAL

Commentary: The Measurement of Oxidative Damage to DNA by HPLC and GC/MS Techniques. PB96-200894

03,484

\section{HYDROXYL RADICALS}

Product Kinetic Energies, Correlations, and Scattering Anisotropy in the Bimolecular Reactor $\mathrm{O}((1) \mathrm{D})+\mathrm{H} 2 \mathrm{O}$ yields $2 \mathrm{OH}$.

00,838

Optical Measurements of Atomic Hydrogen, Hydroxyl, and Carbon Monoxide in Hydrocarbon Diffusion Flames. PB95-150900

02,487

Unusual Spın-Trap Chemistry for the Reaction of Hydroxyl Radical with the Carcinogen N. Nitrosodimethylamine.
PB95-151643 00,692

Gas Phase Reactivity Study of $\mathrm{OH}$ Radicals with 1,1Dichloroethene and cis-1,1-Dichloroethene and Trans-1,2Dichloroethene and cis-1,1-Dichloroethene and Trans-1,2-
Dichloroethene over the Temperature Range $240-400 \mathrm{~K}$. Dichloroethene over the Temperature Range 240-400 K.
PB95-152146 Rate Constants for the Gas Phase Reactions of the $\mathrm{OH}$ Radical with CF3CF2CHCl2 (HCFC-225ca) and CF2CICF2CHCIF (HCFC-225cb)

PB95-152153

00,940

Rotational Spectrum of $\mathrm{OH}$ in the $\mathrm{v}=0.3$ Levels of Its Ground State.

01,006

Detection of $\mathrm{OH}+$ in Its a(1)Delta State by Far Infrared Laser Magnetic Resonance.

PB95-181087

01,021

Laser-Induced Fluorescence Measurements of $\mathrm{OH}$ in Laminar Diffusion Flames in the Presence of Soot Particles.

01,409

Alm- 123120 . Atmospheric Lifetimes of HFC-143a and HFC-245ta: Flash Photolysis Resonance Fluorescence Measurements
of the $\mathrm{OH}$ Reaction Rate Constants.

PB97-112577

00,118

HYDROXYMETHYL PEROXY RADICALS

Kinetics of the Self-Reaction of Hydroxymethylperoxyl Radicals.

PB94-212164

00,827

HYGROMETERS

Methods of Measuring Humidity and Testing Hygrometers.
AD-A278 851/1

00,123

Low-Temperature Performance of Radiosonde Electric Hygrometer Elements.

AD-A295 319/8

00,121

HYGROTHERMAL EFFECT

Hygrothermal Effects on the Performance of Polymers Gaithersburg, Maryland on September 21-22, 1995. PB96-183207

HYOGOKEN-NANBU EARTHQUAKE

January 17, 1995 Hyogoken-Nanbu (Kobe) Earthquake. Performance of Structures, Lifelines and Fire Protection Systems. Execurive Summary and Paper.

00,475
HYPERFINE STRUCTURE

Hyperfine-Structure Studies of $\mathrm{Zr}$ 1I: Experimental and Relativistic Configuration-Interaction Results.

04.011

HYPERFINE STRUCTURES

Hyperfine Structure Investigations and Identification of

New Energy Levels in the lonic Spectrum of (147) Pm.

PB96-180203

HYPOCHLORITE TREATMENT

Critical Evaluation of the Purification of Biominerals by Hypochlorite Treatment.

00,186

HYSTERESIS

Langevin Approach to Hysteresis and Barkhausen Modeling in Steel.

03,206

Hysteresis Measurements of Remanent Polarization and Coercive Field in Polymers.

PB94-199767

04,475

HYSTERETIC FAILURE MODEL

Evaluating the Seismic Performance of Lightly-Reinforced Circular Concrete Bridge Columns.

PB95-163259

01,335

ICE POINT

Reproducibility of the Temperature of the Ice Point in Routine Meas
PB95-255923

04,015

IDEAL GAS

Correlation of the Ideal Gas Properties of Five Aromatic Hydrocarbons

01,002

IDEFO (INTEGRATION DEFINITION FOR FUNCTION

MODELING)

Integration Definition for Function Modeling (IDEFO); Category: Software Standard; Subcategory: Modeling TechFIPSPUB183

02,800

IDEF1X (INFORMATION DEFINITION FOR INFORMATION

MODELING)

Integration Definition for Information Modeling (IDEF1X) Category: Software Standard; Subcategory: Modeling FIPSPUB184

01,673

IDEF1X (INTEGRATION DEFINITION FOR INFORMATION

MODELING)

Mapping Integration Definition for Information Modeling (IDEF1X) Model into CASE Data Interchange Format CDIF) Transfer File.

PB95-154670

01,711

IDEFO (INTEGRATION DEFINITION FOR FUNCTION

MODELING)

Mapping Integration Definition for Function Modeling IDEFO) Model into CASE Data Interchange Forma CDIF) Transfer File

PB96-109533

01.741

IDENTIFICATION

Precise Identification of Computer Viruses.

01,613

Specification for Interoperability between Ballistic Imaging Systems. Part 1. Cartridge Cases.

01,860

IDLH (IMMEDIATELY DANGEROUS TO LIFE OR HEALTH)

Documentation for Immediately Dangerous to Life or Health Concentrations (IDLHs).

PB94-195047

03,602

IGES (INITIAL GRAPHICS EXCHANGE SPECIFICATION)

CALS-Digital Representation for Communication of Prod-

CAct Data: IGES Application Subsets.

Uct Data. IGES

03,658

Summary and Notes of the Joint ISO/IGES/PDES Organization Technical Committee Meeting. Held in Albuquerque, New Mexico on October 15-20, 1989.

02,774

Initial Graphics Exchange Soecification (IGES): Procedures for the NIST IGES Validation Test Service.
PB95-171427

Operating Procedures and Life Cycle Documentation for the Initial Graphics Exchange Specification.

02,782 PB95-242285

CALS-Digital Representation for Communication of Prod-
uct Data: IGES Application Subsets.
PB95-962100

IGNITION

Ignition and Subsequent Transition to Flame Spread in a Microgravity Environment.

N96-15584/1 04,828

Influence of Ignition Source on the Flaming Fire Hazard of Upholstered Furniture. (NIST Reprint)

00,297

Study of Technology for Detecting Pre-Ignition Conditions of Cooking-Related Fires Associated with Electric and Gas Ranges and Cooktops, Phase 1 Report

Gas Ranges

00,303

Ouantitying the Ignition Propensity of Cigarettes.

PB96-155411

00,306

Transition from Localized Ignition to Flame Spread Over

Thin Cellulosic Material in Microgravity.

04,835

gntion and Subsequent Flame Spread Over a Thin Celulosic Material.

04,836

Ignition and Transition to Flame Spread Over a Thermally Thin Cellulosic Sheet in a Microgravity Environment. PB96-160288

IGNITION TIME

Predicting the Ignition Time and Burning Rate of Thermo-

lastics in the Cone Calorimeter.

PB96-154794

01,418

IGOSS (INDUSTRY/GOVERNMENT OPEN SYSTEMS

PECIFICATION

Industry/Government Open Systems Specification Testing Framework. Version 1.0.

PB94-219110

01,809

ILL.POSED CONTINUATION

Slow Evolution from the Boundary: A New Stabilizing Constraint in III-Posed Continuation Problems.

PB96-122858

03,418

MAGE ANALYSIS

Reliable Optıcal Flow Algorithm Using 3-D Hermite Poly-

Ouantitative Phase Abundance Analysis of Three Cement Clinker Reference Materials by Scanning Electron MicrosPB94-173051

00,333

Visual Measurement Technique for Analysis of Nucleate Flow Boiling.

03,262

Optical Fiber Geometry by Gray-Scale Analysis with Robust Regression.

AGE DEBLURRING

Overcoming Hoelder Continuity in $111-\mathrm{Posed}$ Continuation

PB95-202354

IMAGE DECODING

Tomographic Decoding Algorithm for a Nonoverlapping Redundant Array.

MAGE GRADIENT

mage Gradient Evolution: A Visual Cue for Danger. PB96-154562

01,842

MAGE MOTION ANALYSIS

General Motion Model and Spatio-Temporal Filters for Computing Optical Flow.

B95-171096

01,847

General Motion Model and Spat10-Temporal Filters for 3DBotion Interpretations.

01,861

IMAGE MOTION COMPENSATION

General Motion Model and Spatio-Temporal Filters for 3 D Motion Interpretations.

PB96-210703

IMAGE PROCESSING

Object Finder for Digital Images Based on Multiple Thresholds, Connectivity, and Internal Structures.

PB94-199106 01,833

Concentration Histogram Imaging: A Scatter Diagram Technique for Viewing Two or Three Related Images.
PB94-199114 VLSI Architectures for Template Matching and Block

PB94-200029

01,834

Certainty Grid to Object Boundary Algorithm.

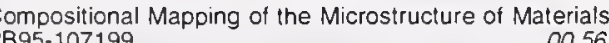


IMAGE RESOLUTION

Contributions of Out-of-Plane Material to a Scanned-

Beam Laminography Image.
PB96-111786

02,704

IMAGE RESTORATION

Image Restoration and Diffusion Processes. PB95-153003

01,843

IMAGES

Metrology Standards for Advanced Semiconductor Lithography Referenced to Atomic Spacings and Geometry.
PB96-160718

IMAGING TECHNIQUES

Diffraction Imaging of Polycrystalline Materials.

PB94-198884

02,971

Molecular Ion Imaging and Dynamic Secondary Ion Mass Spectrometry of Organic Compounds.

dc Magnetic Force Microscopy Imaging of Thin-Film Recording Head

04,665

Magnetic Force Microscopy Images of Magnetic Garnet with Thin-Film Magnetic Tip.

PB95-1762 10

04,669

Evaluation of Thermal Wave Imaging for Detection of Ma. chining Damage in Ceramics.

MIDOGEN RADICALS

03,062

New Electronic States of NH and ND Observed from 258 to $288 \mathrm{~nm}$ by Resonance Enhanced Multiphoton Ionization Spectroscopy.
PB94-212495

00,836

IMMEDIATELY DANGEROUS TO LIFE OR HEALTH

Documentation for Immediately Dangerous to Life or Health Concentrations (IDLHs)

03,602

IMMERSION TEST (CORROSION)

Degradation of Powder Epoxy Coated Panels Immersed in a Saturated Calcium Hydroxide Solution Containing Sodium Chloride

IMMUNOASSAY

Liposome-Based Flow-Injection Immunoassay for Determining Theophylline in Serum.

Flow Immunoassay Using Solid-Phase Entrapment.
PB96-200951

IMMUNOASSAYS

Novel Amperometric Immunosensor for Procainamide Employing Light Activated Labels.

01,344

\section{MMUNOLOGIC DISEASES}

Diagnosis and Treatment of an Oral Base-Metal Contact Lesion Following Negative Dermatologic Patch Tests.

PB95-180626

00,172

IMPACT-ECHO METHOD

Detection of Voids in Grouted Ducts Using the ImpactEcho Method.
PB94-185121

01,306

IMPACT TESTS

Reaction Sensitivities of Al-Li Alloys and Alloy 2219 in Mechanical-Impact Tests.

PB94-172764

03,314

Temperature Increases in Aluminum Alloys during Mechanical-Impact Tests for Oxygen Compatibility.

PB94-172962

03,316

Recommended Changes in ASTM Test Methods D2512 82 and G86-84 for Oxygen-Compatibility Mechanical Impact Tests on Metals.

pact Tests on

03,338

Macro- and Microreactions in Mechanical-Impact Tests of Aluminum Alloys

PB95-107348

03,340

Influence of Specimen Absorbed Energy in LOX Mechanical-Impact Tests.

PB95-107355

03,341

Hybrid Method for Determining Material Properties from Instrumented Micro-Indentation Experiments.

PB95-152229

03,348

Factors Significant to Precracking of Fracture Specimens.

PB96.109558 03,358

Effect of Charpy V-Notch Striker Radii on the Absorbed

Energy.

03,365

Charpy Impact Test as an Evaluation of $4 \mathrm{~K}$ Fracture

Toughness.

PB96-190194

03,219

IMPEDANCE

Modeling Polarization Curves and Impedance Spectra for Simple Electrode Systems.

MPEDANCE BRIDGES

Automatic Inductive Voltage Divider Bridge Operates from

$10 \mathrm{~Hz}$ to $100 \mathrm{kH}$
PB94-198413

03,188

02,032

IMPEDANCE GENERATORS

Multi-State Two-Port: An Altemative Transfer Standard.

PB95-168530 02,049
IMPEDANCE MEASUREMENT

Measurements of the Characteristic Impedance of COaxial Air Line Standards.

02,221

On-Wafer Impedance Measurement on Lossy Substrates.
PB95-176285

IMPEDENCE MEASUREMENT

Digital Impedance Bridge.

PB96-103155

02,272

MPLEMENTATION

Implementation of the Fastener Ouality Act

PROVEM

02,876

PROVEMENTS

Testability of Object-Oriented Systems

PB95-242418
SITU COMBUSTION

In situ Burning of Oil Spills: Mesoscale Experiments.

PB94-142973

In Situ Buming Oil Spill Workshop Proceedings. Held in Orlando. Florida on January 26-28, 1994.

02,583

In situ Burning of Oil Spills: Mesoscale Experiments and Analysis.

PB95-163747

02,587

Smoke Emission

01,407

Smoke Plume Trajectory from In situ Burning of Crude Oil in Alaska: Field Experiments.

PB96.131560

02,594

Smoke Plume Trajectory from In situ Burning of Crude Oil: Field Experiments.

PB96-200993

02,597

IN SITU MEASUREMENT

In situ Measurements of Chloride lon and Corrosion Potential at the Coating/Metal Interface.

PB95-140893

03,122

N VIVO ANALYSIS

Secondary Target $X$-Ray Excitation for In vivo Measurement of Lead in Bone.

PB95-108767

03,496

INCANDESCENT LAMPS

Characterization of Modified FEL Ouartz-Halogen Lamps for Photometric Standards.

00,282

INCINERATORS

Incinerability of Perchloroethylene and Chlorobenzene.

Membrane Gas Separation for a Fluidized-Bed Incinerator.

02,550

INCLUSIONS

Cryogenic Toughness of Austenitic Stainless Steel Weld PB95-161261

03,214

INDENTATION

Hybrid Method for Determining Material Properties from Instrumented Micro-Indentation Experiments.

PB95-152229

03,348

Flat and Rising R-Curves for Elliptical Surface Cracks from Indentation and Superposed Flexure.

03,156

Postfailure Subsidiary Cracking from Indentation Flaws in Brittle Materials.

$$
\text { PB97-110340 }
$$

03,103

\section{INDENTATION HARDNESS TESTS}

Conference Proceedings: International Workshop on Instrumented Indentation. Held in San Diego, California on April 22-23, 1995

$$
\text { PB96-158688 }
$$

01,948

INDENTATION-MICROFRACTURE METHOD

Evaluation of Fracture Toughness and Residual Stress in Dental Porcelain by Indentation-Microfracture Method. PB95-125613

00,154

Evaluation of Fracture Toughness and Residual Stress in Dental Porcelain by Indentation-Microfracture Method. PB95-152831

00,159

INDENTATION STRENGTH

Objective Evaluation of Short-Crack Toughness Curves Using Indentation Flaws: Case Study on Alumina-Based Ceramics.

PB96-179429

03,079

INDEXES (DOCUMENTATION)

Abstract and Index Collection in the Research Information Center of the National Institute of Standards and TechPB94-152204

02,744

Abstract and Index Collection in the Research Information Center of the National Institute of Standards and TechPB95-232633

02,741

INDIA

India: Environmental Technologies Export Market Plan

INDIUM

02,529

Arc Spectra of Gallium, Indium, and Thallium.

00,718

Single-Photon Ionization and Detection of $\mathrm{Ga}, \mathrm{In}$, and As(sub $n$ ) Species in GaAs Growth

00,591

Laser-Induced Desorption of In and $\mathrm{Ga}$ from $\mathrm{Si}(100)$ and Adsorbate Enhanced Surface Damage.

PB95-203311
Pulsed Laser Irradiation at $532 \mathrm{~nm}$ of $\mathrm{In}$ and $\mathrm{Ga}$ Adsorbed on Si(100): Desorption, Incorporation, and Dam. PBe.

01,051

INDIUM GALLIUM ARSENIDES

Transition Metal Implants in In0.53GA0.47As.

04,534

Range Statistics and Rutherford Backscattering Studies on Fe-Implanted In $0.53 \mathrm{Ga0} 0.47 \mathrm{As}$.

PB95-126397

04,535

INDIUM IONS

Electron-Impact Ionization of $\ln (+)$ and $\mathrm{Xe}(+)$

Electron-Impact Ionization of $\ln (+)$ and $\mathrm{Xe}(+)$

PB95-152906

INDIUM OXIDES

Thin Film Thermocouple Research at NIST.

PB97-110233

00,770

03,918

INDIUM PHOSPHIDES

In situ Observation of Surface Morphology of $\ln P$ Grown on Singular and Vicinal (001) Substrates.

PB95-168431

04,636

INDOLES

Equilibrium and Calorımetric Investigation of the Hydrolysis of L-Tryptophan to (Indole + Pyruvate + Ammonia).

\section{INDOOR AIR POLLUTION}

Source of Phenol Emissions Affecting the Indoor Air of an PB94-154382

CONTAM93 User Manual.

02,536

Measurement and Determination of Radon Source Potential: A Literature Review.

PB94-165602

02,576

CONTAM88 Building Input Files for Multi-Zone Airflow and Contaminant Dispersal Modeling.

02,537

Environmental Evaluation of a New Federal Office Build-

PB94-199874

02,538

Ventilation Rates in Office Buildings.

PB95-108825

02,539

Field Measurements of Ventilation and Ventilation Effectiveness in an Office/Library Building.

PB95-108833

00,247

Indoor Air Ouality Impacts of Residential HVAC Systems,

Phase 1 Report: Computer Simulation Plan.

Limits of $\mathrm{CO} 2$ Monitoring in Determining Ventilation

PB95-176004

02,552

Ventilation Effectiveness Measurements in Two Modern

Office Buildings.

02,553

Indoor Air Quality Impacts of Residential HVAC Systems.

Phase 2.A Report: Baseline and Preliminary Simulations.
PB95-178893

02,554 
Issues in the Field Measurement of VOC Emission Rates. PB97-118806

02,573

INDOOR AIR QUALITY

Air Change Effectiveness Measurements in Two Modern Office Buildings.

00,243

Study of Ventilation and Carbon Dioxide in an Office Building.

02,542

Assessing Ventilation Effectiveness in Mechanically Ventilated Office Buildings.

PB95-162079

00,255

Ventilation, Carbon Dioxide and ASHRAE Standard 62.

PB95-162095

02.544

Indoor Air Quality Commissioning of a New Office Build-

PB̈95-182309

00,262

Measurements of Outdoor Air Distribution in an Office Buildin

PB95-210944

00,264

Computer Simulations of Airflow and Radon Transport in Four Large Buildings.

PB95-220422

02,557

Development and Application of an Indoor Air Quality Commissioning Program in a New Office Building.
PB96-155601

0,275
Air Oual-

Multizone Modeling of Three Residential Indoor Air OualMy Control Options.

02,567

Indoor Air Quality Commissioning of a New Office Build-

Indoor Air Quality Commissioning of a New Otfice Build-
PBg7-110142
00,279

INDOOR ENVIRONMENTS

Parametric Study of Wall Moisture Contents Using a Revased Variable Indoor Relative Humidity Version of the 'Moist' Transient Heat and Moisture Transfer Model.

PB97-122535

00,419

INDUCED CURRENT

Currents Induced on Multiconductor Transmission Lines by Radiation and Injection

PB94-211943

02,215

NDUSTRIAL BUILDINGS

Performance Approach to the Development of Criteria for Low-Sloped Roof Membranes.

PB94-160751

00,329

INDUSTRIAL MANAGEMENT

Agile Manufacturing from a Statistical Perspective. 02,886

INDUSTRIAL MEDICINE

Radiation Accident at an Industrial Accelerator Facility.
PB
$02,575-140117$

NDUSTRIAL PLANTS

Technical Program of the Factory Automation Systems Division 1993.

02,805

B94-160819

03,187

of Corrosion Data: A Review.

PB94-198348

ation in

Framework for Information Technology Integr
Process Plant and Related Industries.

PB94-219086

Summaries of BFRL Fire Research In-House Projects and Grants, 1994

00,366

Group 1 for the Plant Spatial Configuration STEP Application Protocol.

02,789

PB96-165402

Opportunities for Innovation: Pollution Prevention.
PB95-147146

NDUSTRIES

NIST Industrial Impacts: A Sampling of Successful Partnerships.

PB95-111514 00,488

Workshop Summary Report: Industrial Applications of Scanned Probe Microscopy. A Workshop Co-sponsored by NIST, SEMATECH, ASTM, E42.14, and the American March 24-25, 1994

PB95-170387

00,506

NIST Industrial Impacts: A Sampling of Successful Partnerships (Revision, March 1995)

PB95-209193

00,489

Summary Report: Workshop on Industrial Applications of Scanned Probe Microscopy (2nd). A Workshop Co-Sponsored by NIST, SEMATECH, ASTM E42.14, and the American Vacuum Societ

PB96-131602

00,509

INDUSTRY/GOVERNMENT OPEN SYSTEMS

SPECIFICATION

Industry/Government Open Systems Specification Testing Framework. Version 1.0

PB94-219110

01,809

INELASTIC SCATTERING

Low-Energy-Electron Collisions with Sodium: Elastic and Inelastic Scattering from the Ground State.

04,030
Orbital Alignment and Vector Correlations in Inelastic Atomic Collisions.

INERTIAL CONFINEMENT FUSION

04,047

Physics and Prospects of Inertial Confinement Fusion. PB94-185048

04,405

NERTIAL NAVIGATION

Ground Vehicle Control at NIST: From Teleoperation to N9utonomy.

INFILLED WALLS

03,758

Strengthening Methodology for Lightly Reinforced Concrete Frames: Recommended Design Guidelines for Strengthening with Infill Walls.

PB95-260725

00,454

NFORMATION CAPACITY

Capacity of the Lp Norm-Constrained Poisson Channel PB95-125753

01,515

IFORMATION CENTERS

NIST Serial Holdings, 1994

02,745

Databases Available in the Research Information Center
of the National Institute of Standards and Technology of the National Institute of Standards and Technology
(December 1995).
PB96-139407
PB, 734

02,734

NIST Crystallographic Databases for Research and Anal-

YSis. 109094

04,801

\section{NFORMATION DISSEMINATION}

Numeric Data Distribution: The Vital Role of Data Exchange in Today's World.

02,622

National Center for Standards and Certification Information: Service and Programs.

N95-15938/0

02,717

Sharing Information via the Internet: An Infoserver Case Study.

01,493

INFORMATION EXCHANGE

Federal Implementation Guideline for Electronic Data Interchange: ASC $\times 12003040$ Transaction Set 838 Trading Partner Profile (Confirmation of Vendor Registration). Implementation Convention.

PB96-111190

01,813

Federal Implementation Guideline for Electronic Data Interchange: ASC X12 003040 Transaction Set 838 Trading Partner Profile (Vendor Registration), Implementation Convention.

PB96-112651
Procedure for Product Data Exchange Using STEP Developed in the AutoSTEP Pilot.

02,843

FORMATION HIGHWAYS

Electronic Access to Standards on the Information High Way.

01,494

INFORMATION MANAGEMENT

Dilemma-Preservation versus Access.

PB94-198488

Preliminary Functional Specifications of a Prototype Electronic Research Notebook for NIST.

00,012

INFORMATION PROCESSING

User Profile for Researchers Studying Objects: Implications for Computer Systems.

00,133

PB94-188463

Stand

Federal Government and Information Technology

ards: Building the National Information Infrastructure.
PB95-180840

INFORMATION RESOURCE DICTIONARY SYSTEM

Information Resource Dictionary System (IRDS): A Status

Report

01,810

INFORMATION RESOURCES

Montgomery Education Connection and Resource Education Awareness Partnership Making Connections be tween Local Schools and NIST Volunteers.

00,134 PB96-159769

INFORMATION RETRIEVAL

Design and Development of an Information Retrieval System tor the EAMATE Data. Volume 2 of 2. Appendices. PB94-168390

Bringing Natural Language Information Retrieval Out of PB94-172335

02,720

Second Text REtrieval Conference (TREC-2). Held in Gaithersburg, Maryland on August 31-September 2

PB94-178407

01,686

World Wide Web and Mosaic: User's Guide.

PB94-207354

02,722

FIREDOC Users Manual, 3rd Edition

01,378

PB95-128674

uctured

PB95-161121

Access Paths for Materials Databases: Approaches for

Large Databases and Systems.

02,975

Coping with Different Retrieval Methods in Next Generation Networks.

02,726

Retrieving Articles from the Internet (without a UNIX Workstation). Part 1. File Formats and Software Tools.

PB95-168720 02,728

Retrieving Articles from the Internet (without a UNIX

Workstation). Part 2. An Example.

02,729

Prototype Information Retrieval System to Perform a Best-Match Search for Names.

PB95-181152

02,740

Overview of the Text REtrieval Conference (3rd) (TREC 3). Held in Gaithersburg, Maryland on November 2-4,

PB95-216883

01,728

Electronic Access: Blueprint for the National Archives and Records Administration

PB95-219218

02,731

PB96-114939

Information Resources for the Fire Community.

00,211

Using a Multi-Layered Approach to Representing Tort Law Cases for Case-Based Reasoning.

PB96-160874

00,135

Lab Report Special Section: Natural Language Process. ing and Information Retrieval Group Information Access and User Interfaces Division, National Institute of Standards and Technology.

$7-118665$

02,742

Panel: Building and Using Test Collections.

PB97-118673

02,743

Text REtrieval Conference (4th) (TREC-4). Held in Gaithersburg, Maryland on November 1-3, 1995.

\section{IFORMATION SERVICES}

Information Infrastructure: Reaching Society's Goals. Report of the Information Intrastructure Task Force Committee on Applications and Technology.

PB94-214756

01,469

CALS-Contractor Integrated Technical Information Service (CITIS), Functional Requirements.

03,663

Databases Available in the Research Information Center of the National Institute of Standards and Technology.
PB95-128641 PB95- 128641
STEP On-Line Information Service (SOLIS). The IGES PDES Organization.

PB95-137790

02,777

User Study: Informational Needs of Remote National Archives and Records Administration Customers.

PB95-154738

02,725

CALS-Contractor Integrated Technical Information Service (CITIS), Functional Requirements.

PB95-962600

03,672

National Information Infrastructure and Advanced Digital 
FIREDOC Users Manual, 3rd Edition

PB95-128674

01,378

Tribological Dai
PB95-140596

03,237

Information Technology Engineering and Measurement Model: Adding Lane Markings to the Information Superhighway.

01,474

Locating Fire Engineering Information.

00,198

Global Information Infrastructure: Agenda for Cooperation.

PB95-178604

01,482

Prototype Information Retrieval System to Perform a Best-Match Search for Names.

02,740

PB95-181152

Structural Ceramics Database. Topical Report, June 1989-May 1991

PB95-203758 03,06

PB95-209920 01,629

Analysis of ANSI ASC X12 and UN/EDIFACT Electronic

Data Interchange (EDI) Standards.
PB95-220554

Guidelines for the Evaluation of X.500 Directory Products.

Federal Implementation Guideline for Electronic Data Interchange. ASC $\times 12003050$ Transaction Set 850 Award Instrument. Implementation Convention.

PB96-114913

01,814

Federal Implementation Guideline for Electronic Data Interchange. ASC X12 003050 Transaction Set 860 Modifications to Award Instrument. Implementation Convention.

01,815

Defining Environment Integration Requirements.
PB96-131545

PB96-131545 02,733

Open System Environment (OSE): Architectural Frame-
work for Intormation Infrastructure.

PB96-146360

00,002

Security Program Management

01,610

Federal Implementation Guideline for Electronic Data Interchange. ASC X12 003050 Transaction Set $843 \mathrm{Re}-$ sponse to Request for Ouotation. Implementation Con. sponse

PB96-168984

01,822

Guidelines for the Evaluation of Electronic Data Interchange Products.

PB96-172325

01,506

Federal Implementation Guideline for Electronic Data Interchange. ASC X12 003050 Transaction Set 855 Purchase Order Acknowledgment: Implementation Conven-

PB96-172374

01,823

Federal Implementaion Guideline for Eletronic Data Inter change. ASC X12 003050 Transaction Set 865 Purchase Order Change Acknowledgement/Request - Seller Initiated. Implementation Convention.

PB96-172549

01,825

X.500 Directory Schema Design Handbook.

02,739

Computer Systems Laboratory Computing and Applied Mathematics Laboratory Technical Accomplishments, October 1994-March 1996.

PB96-193768

01,638

Journal of Research of the National Institute of Standards and Technology, May/June 1996. Volume 101, Number 3 Special Issue: NIST Workshop on Crystallographic PB97-109011

04,798

CRYSTMET: The NRCC Metals Crystallographic Data FBile.

04,799

Inorganic Crystal Structure Database (ICSD) and Standardized Data and Crystal Chemical Characterization of In ardized Data and Crystal Chemical Characterization of InChemists and Crystallographers.

Chemists and

00,648

Evaluation of Crystallographic Data with the Program DIAMOND.

PB97-109045

00,649

Cambridge Structural Database (CSD): Current Activities and Future Plans

00,516

Protein Data Bank: Current Status and Future Challenges.

00,517

Nucleic Acid Database: Present and Future

00,518

Powder Diffraction File: Past, Present, and Future.

PB97-109086 04,800

NIST Crystallographic Databases for Research and Anal-

ysis.

04,801

Conventional and Eccentric Uses of Crystallographic Databases in Practical Materials Identification Problems.
Using NIST Crystal Data within Siemens Software for Four-Circle and SMART CCD Diffractometers.

04,803 PB97-109110

Using the Cambridge Structural Database.

00,519

Troublesome Crystal Structures: Prevention, Detection, and Resolution.

01,172

CIF Crystallographic Information File: A Standard for Crystallographic Data Interchange.
PB97-109169

04,805

How the Cambridge Crystallographic Data Centre Obtains Is Information.

Data Import and Validation in the Inorganic Crystal Struc-

ture Database.

World Wide Web for Crystallography

04,809

PB97-109219

04,810

Workshop Highlights.

PB97-109227

04,811

Experimental Models for Software Diagnosis.

01,783

\section{INFORMATION TECHNOLOGY}

Report on Application Integration Architectures (AIA) Workshop. Held in Dallas, Texas on February 8-12, 1993. PB94-142536

01,803

Study of Federal Agency Needs for Information Technology Security.

01,579

Framework for National Information Infrastructure Serv-

PB95-103719

02,723

Computer Systems Laboratory Annual Report 1994.

PB95-209920

01,629

Electronic Implem

01,484

Information Technologies Make Business Sense for the

Custom Therapeutic Footwear Industry.

02,829

Common Criteria: On the Road to International Harmonization.

PB96-123484

01,606

Functional Security Criteria for Distributed Systems.

01,607

Information Technology Standards in a Changing World: The Rose of the Users.

PB96-160866

02,737

Concept Paper: An Overview of the Proposed Trust Technology Assessment Program.

01,614

Information Technology Standards in Federal Acquisi-

PB96-161252

01,636

Using Information Technology Standards in Federal ACPuisitions.

01,637

Computer Systems Laboratory Computing and Applied Mathematics Laboratory Technical Accomplishments, October 1994-March 1996.

PB96-193768

01,638

INFORMATION TRANSFER

Standards and Linkages: What Data Sharing Needs.

PB95-161881

01,713

PB96-157904

02,714

INFRARED ABSORPTION

Laser-Synchrotron Hybrid Experiments: A Photon to Tickle, A Photon to Poke.

PB96-157847

03,704

RRARED BOLOMETERS

Far-Intrared Kinetic Inductance Detector.

Superconducting Kinetic Inductance Radiometer.

PB95-140083

02,142

NFRARED DETECTORS

Semiconductor Measurement Technology: Improved Characterization and Evaluation Measurements for $\mathrm{HgCdTe}$ Detector Materials, Processes, and Devices Used on the GOES and TIROS Satellites.

02,122

Review of Semiconductor Microelectronic Test Structures with Applications to Infrared Detector Materials and Processes.

PB94-212925

02,132

Heavily Accumulated Surfaces of Mercury Cadmium Telluride Detectors: Theory and Experiment.

02,134

International Intercomparison of Detector Responsivity at 1300 and $1550 \mathrm{~nm}$

PB95-125928

02,140

International Intercomparison of Detector Responsivity at 1300 and $1550 \mathrm{~nm}$

PB95-126017

02,141

Far-Infrared
PB95-12634

02,142

Thermal and Nonequilibrium Responses of Superconductors for Radiation Detectors.
PB95-164232

Hg1-XCdxTe Characterization Measurements: Current Practice and Future Needs.

PB95-164299 02,157

Terahertz Detectors Based on Superconducting Kinetic Inductance.

02,160

Optical Performance of Photoinductive Mixers at Terahertz Frequencies.

PB95-168662

02,161

Spatial Uniformity of Optical Detector Responsivity B95-168845

Ultrasensitive-Hot-Electron Microbolometer

02,162

PB95-168985

02,163

Kinetic-Inductance Intrared Detector Based on an Anenna-Coupled High-Tc SOUID.

02,165

Optical Detector Nonlinearity: A Comparison of Five Methods.

PB95-169355

04,291

Optical Detector Nonlinearity: Simulation.

B96-165378

02,199

Novel Hot-Electron Microbolometer.

PB96-201025

01,977

PRARED FILTERS

Filter Transmittance Measurements in the Intrared.

PBo4-140589

-180261

Development of Neutral-Density Infrared Filters Using Metallic Thin Films.

PB96-180286

02,998

NFRARED RADIOMETERS

Activities of NIST (National Inst. Of Standards and Tech-

Nology).

04,222

Superconducting Kinetic Inductance Radiometer.

PB95-140083

02,144

Terahertz Detectors Based on Superconducting $K^{\prime}$ ' vetic Inductance.

02,160

Thermal Modeling of Absolute Cryogenic Radiometers.

PB95-181236 04,316

Liquid-Nitrogen-Cooled High Tc Electrical Substitution Radiometer as a Broadband IR Transfer Standard.

PB96-158704

02,198

NIST Thermal Infrared Transfer Standard Radiometer for the Earth Observing System (EOS) Program. PB97-113013

04,843

INFRARED REFLECTANCE

Optica! Conductivity of Single Crystals of Ba1XMxBiO3(M=K

04,447

INFRARED SIGNATURES

Review of Measurements and Candidate Signatures for Early Fire Detection.

PB95-189452

00,300

INFRARED SOURCES 
Rotational Far Infrared Spectrum of (13)CO.

PB95-152187

00,941

2nu9 Band of Propyne-d3.

New cw CO2 Laser Lines: The 9-mu $m$ Hot Band.

PB95-168548 04,28

Absolute Frequency Measurements of Methanol from 1.5 to $6.5 \mathrm{THz}$.

PB95-175881

04,300

Pure Rotational Spectra of $\mathrm{CuH}$ and $\mathrm{CuD}$ in Their Ground States Measured by Tunable Far-Infrared Spectroscopy.

Rotational Spectrum of $\mathrm{OH}$ in the $\mathrm{v}=0-3$ Levels of Its Ground State.

PB95-176202 01,006

Atomic Sulfur: Frequency Measurement of the $\mathrm{J}=0$ in versely maps 1 Fine-Structure Transition at 56.3 Microns by Laser Magnetic Resonance.

01,007

FRARED SPECTROMETRY

Ultrafast Time-Resolved Infrared Probing of Energy Transfer at Surfaces.

00,620

NFRARED SPECTROPHOTOMETERS

Simple and Efficient Low-Temperature Sample Cell for Infrared Spectrophotometry.

PB94-199197

00,545

Standard Reference Materials: Polystyrene Films for Calibrating the Wavelength Scale of Infrared Spectrophotometers - SRM 1921

03,386

INFRARED SPECTROSCOPY

High-Resolution Infrared Overtone Spectroscopy of ArHF via Nd:YAG/Dye Laser Difference Frequency Generation. PB94-211448 Laser Difere Frequency Gention. Mode Specific Vibrational Predissociation Dynamics in Fragile Molecules

00,871

Vibration, Rotation, and Parity Specific Predissociation Dynamics in Asymmetric OH Stretch Excited ArH2O: A Half Collision Study of Resonant V-V Energy Transter in a Weakly Bound Complex.

00,872

High-Resolution Infrared Spectroscopy of DF Trimer: A High-Resolution Infrared Spectroscopy of DF Trimer: A
Cyclic Ground State Structure and DF Stretch Induced Cyclic Ground State Structure and DF Stretch Induced
Intramolecular Vibrational Coupling.
PB95-150678

00,920

Slit Jet Infrared Spectroscopy of Hydrogen Bonded N2HF sotopomers: Rotational Rydberg-Klein-Rees Analysis and H/D Dependent Vibrational Predissociation Rates.
PB95-161873 00,956 High-Resolution, Direct Infrared Laser Absorption Specroscopy in Slit Supersonic Jets: Intermolecular Forces and Unimolecular Vibrational Dynamics in Clusters. PB95-203006 01,040 High-Resolution Infrared Overtone Spectroscopy of N2$\mathrm{HF}$ : Vibrational Red Shifts and Predissociation Rate as a Function of HF Stretching Ouanta.

PB96-102298

01,061

Silicon Surface Chemistry by IR Spectroscopy in the Midto Far-IR Region: $\mathrm{H} 2 \mathrm{O}$ and Ethanol on Si(100). PB96-138565

01,097

INFRARED SPECTRUMS

Perpendicular C-H Stretching Band nu9/nu13 and the Torsional Potential of Dimethylacetylene.

PB97-122451

01,192

INFRASTRUCTURE

Functions of Technology Intrastructure in a Competitive

Economy.

00,478

Planning the Infrastructure for Global Electronic Com. merce.
PB94-185832

00,494

Financing Tomorrow's Infrastructure: Challenges and Issues. Proceedings of a Colloquium. Held in Washington, DC. on October 20, 1995 . PB96-189444

00,48

INHIBITORS

Selective Inhibition of Crystal Growth on Octacalcium Pyrophosphate at Physiological Concentration. PB94-211257

00,147

INITIAL GRAPHICS EXCHANGE SPECIFICATION

Summary and Notes of the Joint ISO/IGES/PDES Organization Technical Committee Meeting. Held in Albuquerque, New Mexico on October 15-20, 1989. Initial Graphics Exchange Specification (IGES): Procedures for the NIST IGES Validation Test Service.

PB95-171427

02,780

Operating Procedures and Life Cycle Documentation for the Initial Graphics Exchange Specification. PB95-242285

02,782

NJECTION MOLDING

In-Line Optical Monitoring of Injection Molding.

NKS

Cylinder Wipe Air-Drying Intaglio Ink Vehicles for U.S Currency Inks.
PB94-160801

03,115
INORGANIC COMPOUNDS

Establishing Ouality Measurements for Inorganic Analysis of Biomaterials.

00,548

Determination of Inorganic Constituents in Marine Mammal Tissues.

PB95-152047

00,589

Affinity Chromatography on Inorganic Support Materials.

PB95-163820 03,467

Characterization of the Structure of YD3 by Neutron Powder Diffraction.

01,161

Inorganic Crystal Structure Database (ICSD) and Standardized Data and Crystal Chemical Characterization of Inorganic Structure Types (TYPIX): Two Tools for Inorganic rganic Structure Types (TYPIX): Two Tools for Inorganic Chemists and Crystallographers.

00,648

Evaluation of Crystallographic Data with the Program DI-

AMOND.

00,649

Data Import and Validation in the Inorganic Crystal Structure Database.

INORGANIC SALTS

Phase Composition, Viscosities, and Densities for Aque ous Two-Phase Systems Composed of Polyethylene Glycol and Various Salts at $25 \mathrm{C}$

PB95-164596

00,986

INORGANIC SUPPORTS

Preparation of Immobilized Proteins Covalently Coupled Through Silane Coupling Agents to Inorganic Supports. PB95-151403

03,530

NOSINE MONOPHOSPHATE

Thermochemistry of the Reactions between Adenosine, Adenosine 5'-monophosphate, Inosine, and Inosine 5'monophosphate; the Conversion of L-histidine to (Urocanic Acid+Ammonia).

PB94-213113

03,460

INSERTS

Reduction of Marginal Gaps in Composite Restorations by Use of Glass-Ceramic Inserts.

00,174

INSOLATORS

Phonon Relaxation in Soft-X-ray Emission of Insulators.

SPECTION

Development of an Automated Part Inspection System Using the DMIS Standard.

02,899

Sensitivity of Three-Point Circle Fitting

PB95-136354

02,901

Automated Optical Roughness Inspection.

02,905

Dimensional Inspection Planning Based on Product Data

Standards.
PB95-175451 02,910

Interim Testing Artifact (ITA): A Performance Evaluation System for Coordinate Measuring Machines (CMMs). User Manual.

PB95-210589

02,914

International Institute of Welding: Report on 1995 Actions. PB96-158076 02,874 Integrated Inspection System for Improved Machine Per-

PB96-160569

02,959

NSPECTIONS

SPC Artifact for Automated Solder Joint Inspection. 04,095

INSTALLATION

Flowmeter Installation Effects Due to a Generic Header. PB96-210893

02,606

INSTRUMENT ERRORS

Partial Pressure Analysis in Space Testing.

Test Optics Error Removal.

PB96-179536

04,827

Nonzero Fringe Densities in Phase-Measuring Interferometers. PB97-110506

04,386

INSTRUMENT SETTINGS

Optimizing Time-Domain Network Analysis.

PB96-157821

02,085

NSTRUMENTAL NEUTRON ACTIVATION ANALYSIS

Relationship of Silver with Selenium and Mercury in the Liver of Two Species of Toothed Whales (Odontocetes). 02,596

Resolution of Discrepant Analytical Data in the Certification of Platinum in Two Automobile Catalyst SRMs. PB96-167283

00,638

INSTRUMENTATION

Guide to Instrumentation Literature. $\quad 02,617$

Instrument for Evaluating Phase Behavior of Mixtures for Supercritical Fluid Experiments.

PB95-180758

00,606

INSTRUMENTATION \& EXPERIMENTAL METHODS

Development of a Dual-Sinker Densimeter for High-Accu-

racy Fluid P-V-T Measurements. Appendix A.

02,620

Low Temperature H(sub 2) S Separation Using Membrane Reactor with Redox Catalyst.

02,47

Thin Film Thermocouples for Measurement of Wall Temperatures in Internal Combustion Engines.

01,449

Evaluation and Qualification Standards for an X-Ray Evaluation and Qualification Standards for an X-Ray
Laminography System. Laminography System.
PB94-172954

02,029

Physics and Prospects of Inertial Confinement Fusion.

PB94-185048

04,405

High-Temperature Laser-Pulse Thermal Diffusivity Apparatus.

02,631

Selection of Appropriate Ultrasonic System Components for NDE of Thick Polymer-Composites.

03,133

Electro-Optical Sensor for Surface Displacement Measurements of Compliant Coatings.

PB94-198223

02,123

Voigt-Function Modeling in Fourier Analysis of Size- and Strain-Broadened X-Ray Diffraction Peaks.

PB94-198538

Milliwatt Mixer for Small Fluid Samples.

04,462

B94-198819

02,634

Applications of the Vortex Tube in Chemical Analysis.

00,544

Simple and Efficient Low-Temperature Sample Cell for Inrared Spectrophotometry.

00.545

Simple, Inexpensive Apparatus for Sample Concentration PB94-199205 00,54

Experimental Studies of Line Shapes from a BalleFlygare Spectrometer

PB94-199452

00,796

Millisecond-Resolution Pulse Hearing System for SpecificHeat Measurements at High Temperatures.

PB94-199999

High-Speed Spatial Scanning Pyrometer.

PB94-200003

02,635

Planar Waveguide Optical Sensors.

02,636

94-200185

03,586

Salt-PEG Two-Phase Aqueous Systems to Purify Pro-

teins and Nucleic Acid Mixtures.
PB94-200375

03,527

Torsional Dilatometer for Volume Change Measurements on Deformed Glasses: Instrument Description and Measurements on Equilibrated Glasses.

PBQ4-211166

03,379

Update on the Low Background IR Calibration Facility at the National Institute of Standards and Technology.

NIST Power Reference Source.

PB94-211513

00,148

ntercomparison of the Effective Areas of a Pneumatic Piston Gauge Determined by Different Techniques.

P94-212370 ances and Their Effects on Performance of Ultrafiltration Merouras.

Characterization of the Adsorption-Fouling Layer Using

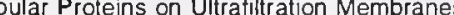


Uncertainty Analysis of the NIST Nitrogen Flow Facility. PB95-128906 02,608 Thermal Hydraulic Tests of a Liquid Hydrogen Cold Neutron Source.

03,884

Heat Transfer in Thin, Compact Heat Exchangers with Circular, Rectangular, or Pin-Fin Flow Passages.

PB95-140943 02,75

Intelligent Control of an Inert Gas Aromization Process.

PB95-141057 03,344

Process Gas Chromatography Detector for Hydrocarbons Based on Catalytic Cracking.

PB95-141099 02,485

Radiometric Model of the Transmission Cell-Reciprocal Nephelometer

00,124

Integrating Sphere Simulation: Application to Total Flux Scale Realization.

PB95-150173

04,261

Characteristics of Partial Pressure Analyzers.

00,582 Experimental and Numerical Studies of Refractory Particle Formation in Flames: Application to Silica Growth.
PB95-152005
00,673

Simulation of Ceramic Particle Formation: Comparison with In-situ Measurements.

PB95-152013

00,674

Thermal Behaviour of Methyl Methacrylate and N-Phenyl Maleimide Copolymers.

01,246

Dynamic Technique for Measuring Normal Spectral Emissivity of Electrically Conducting Solids at High Temperatures with a High-Speed Spatial Scanning Pyrometer.

PB95-153045 03,921

Grazing Angle X-Ray Photoemission System for Depth-

Dependent Analysis.

04,600

Enzyme and Protein Mass Transfer Coefficient in Aqueous Two Phase Systems. 1. Spray Extraction Columns. PB95-161162 00,594 Continuous Counter-Current Two Phase Aqueous Extraction.

PB95-161212
Fracture Toughness of Advanced Ceramics at Room Temperature: A Vamas Round-Robin.

PB95-162194

03,160

Protein Extraction in a Spray Column Using a Polyethylene Glycol Maltodextrin Two-Phase Polymer System. Development of Adaptive Control Strategies for Inert Gas

Atomization.
PB95-162335 02,823

Loading Device for Fracture Testing of Compact Tension Specimens in the Scanning Electron Microscope.

PB95-162434

Noncontact Ultrasonic Inspection of Train Rails for Stress.

PB95-162673 04,851

Preparation of 2-Dimensional Ultra Thin Polystyrene Film

by Water Casting Method.

PB95-162806

04,619

Electrical Sensors for Monitoring of Plasma Sheaths.
PB95-162962

Brownian Diffusion of Hard Spheres at Finite Concentra.

PB95-164307

00,975

Through-the-Arc Sensing for Measuring Gas Metal Arc Weld Ouality in Real Time.

02,908

Cryogenics.
PB95-164703

02,654

Annex 18: An International Study of Refrigerant Properties.

03,266

PB95-168936

Proposed Antiferromagnetically Coupled Dual-Layer Magnetic Force Microscope Tips.

PB95-169017

04,645

Membrane Gas Separation for a Fluidized-Bed Inciner-

ator.

02,550

Energy Flows in an Orifice Pulse Tube Ref́rigerator

PB95-169082

Graded and Nongraded Regenerator Performance. 02,753

Optimization of ECR-Based PECVD Oxide Films for Superconducting Integrated Circuit Fabrication.

PB95-169165

02,051

Comparative Photoluminescence Measurement and Simulation of Vertical-Cavity Semiconductor Laser Structures.
PB95-169173 Measurement and Simulation of Photoluminescence Spectra from Vertical-Cavity Ouantum-Well Laser Structures.

B95-169181

02,170

Vortex Shedding Flowmeters for SSME Ducts.

01,453

Phase-Locked Oscillator Optimization for Arrays of 30 . sephson Junctions.

02,052
Critical Issues in Scanning Electron Microscope MetrolPBy.

02,359

Video Microscopy Applied to Optical Fiber Geometry Measurements.

04,295

Influence of the Filament Potential Wave Form on the Influence of the Filament Potential Wave Form on
Sensitivity of Glass-Envelope Bayard-Alpert Gages.

PB95-175014

02,657

Retention of Halocarbons on a Hexafluoropropylene Epoxide Moditied Graphitized Carbon Black. Part 1. Methane-Based Compounds.

PB95-175196

03,272

Automated Josephson Integrated Circuit Test System. PB95-175246

02,057

Friction and Oxidative Wear of $440 \mathrm{C}$ Ball Bearing Steels Under High Load and Extreme Bulk Temperatures.

03,215

Formation of Hydroxyapatite in Cement Systems. PB95-175261

00,170

Correlation of Optical, X-ray, and Electron Microscopy Measurements of Semiconductor Multilayer Structures.
PB95-175279

High-Pressure Equilibrium Cell for Solubility Measurements in Supercritical Fluids.

00,998

Determination of the Complex Refractive Index of Individual Ouantum Wells from Distributed Reflectance. ual Ouantum

PC-Based Spinning Rotor Gage Controller.

02,176

PB95-175832

02,609

Investigations of $\mathrm{AM}$ and $\mathrm{PM}$ Noise in X-Band Devices.

PB95-180022 02,062

Passively O-Switched Nd-Doped Waveguide Laser

PB95-180048 04,308

Chromatographic Cryofocusing and Cryotrapping with the PB95-180113

00,604

Tribometer for Measurements in Hostile Environments.

PB95-180949 02,967

Reducing the Effect of Local Oscillator Phase Noise on the Frequency Stability of Passive Frequency Standards. PB95-180972 01,545

Low-Noise High-Speed Diode Laser Current Controller.

PB95-202826 02,178

Decrease of Fluorescence in Optical Fiber during Exposure to Pulsed or Continuous-Wave Ultraviolet Light.

PB95-20307

04,320

Low-Frequency, Active Vibration Isolation System.

02,710

Surface Modification of YBa2Cu3O7-delta Thin Films Using the Scanning Tunneling Microscope: Five Methods. PB95-203394 04,699

Single Photon Laser Ionization as an In-situ Diagnostic or MBE growth.

01,059

Resonances in Two-Dimensional Array Oscillator Circuits. PB96-102082 02,066 Direct Observation of Vortex Dynamics in Two-Dimensional Josephson-Junction Arrays.

02,067

Stacked Series Arrays of High-Tc Trilayer Josephson Junctions.

PB96-102272

04,705

Self-Biasing Cryogenic Particle Detector Utilizing Electrothermal Feedback and a SOUID Readout. 96-102538

04,712

Measurement of Very Low Frequency Vibrations.

PB96-102660

03,687

Electronic Microrefrigerator Based on a Normal-InsulatorSuperconductor Tunnel Junction.

PB96-102827

04,718

New Method for Realizing a Luminous Flux Scale Using

an Integrating Sphere with an External Source.
PB96-102843

04,333

Superconducting Integrated Circuit Fabrication with Low Temperature ECR-Based PECVD SiO2 Dielectric Films. PB96-103015

04,719

Comments on the Stability of Bayard-Alpert Ionization

PBg6-103080

02,673

Niobium Microbolometers for Far-infrared Detection.

PB96-111729

02,184

Temperature Dependence and Magnetic Field Modulation of Critical Currents in Step-Edge SNS YBCO/Au Junc PBo6.

04,723

Critical Current and Normal Resistance of High-Tc StepEdge SNS Junctions.

04,724

GHRS Observations of Cool, Low-Gravity Stars. 1. The Far-Ultraviolet Spectrum of alpha Orions (M2 lab).

PB96-112016

Applications of the Vortex Part 2. Applications

Analysis. PB96-112107

Dual-Frequency Millimeter-Wave Radiometer Antenna for Airborne Remote Sensing of Atmosphere and Ocean. PB96-112289 02,009

Measured Stopping Powers of Hydrogen and Helium in PBg6- 112321

04,729

Intercomparison between NPL (India) and NIST (USA) Pressure Standards in the Hydraulic Pressure Region Up to $26 \mathrm{MPa}$.

Intercomparison of the ITS-90 Radiance Temperature

Scales of the National Physical Laboratory (U.K.) and the National Institute of Standards and Technology.

PB96-113550

02,674

Beamcon III, a Linearity Measurement Instrument for Optical Detectors

Comparing the Accuracy of Critical-Current Measure. ments Using the Voltage-Current Simulator.

PB96-119219

02,227

Polarization Insensitive $3 \times 3$ Sagnac Current Sensor Using

Polarizing Spun High-Birefringence Fiber.

02,187

Simple and Repeatable Technique for Measuring the Critical Current of Nb3Sn Wires.

PB96-119409

02,229

Faraday Effect Sensors for Magnet Field and Electric

PB96-119664

04,736

Standard Polarization Components: Progress Toward an Optical Retardance Standard.

PB96-119672

04,342

Improved Annealing Technique for Optical Fiber.

PB96-119680

04,343

Reducing Errors, Complexity, and Measurement Time of

PM Noise Measurements.

PB96-119771

02,075

Standard Reference Materials for Optical Fibers and Con-

PB96-1 19805

04,344

Third Genera

Hydrodynamic Similarity in an Oscillating-Body Viscom-

PBer. 122429 01,082

Thermophysical Properties of Fluids for the Gas Industry.

PB96-122437 02,494.

Polarization Dependence of Response Functions in $3 \times 3$

Sagnac Optical Fiber Current Sensors.

PB96-122684

02,189

Novel Method for Determining Thin Film Density by En-

ergy-Dispersive $X$-ray Reflectivity.

Secondary Stand

$100 \mathrm{MHz}$.

PB96-123187

0 , and

Goals, and Science Results.

PB96-123278

A-type and Chem

00,044 


\section{KEYWORD INDEX}

INTEGRATED CIRCUITS

Dependence of Contrast on Probe/Sample Spacing with the Magneto-Optic Kerr-Effect Scanning Near-Field Optical Microscope (MOKE-SNOM)

PB96-138557

04,750

Development of Highly Conductive Cantilevers for Atomic Force Microscopy Point Contact Measurements. Radiometer Equation for Noise Comparison Radiometers. PB96-140363 02,195 Cross-Sectional Photoluminescence and Its Application to Buried-Layer Semiconductor Structures.

PB96-141106 02,415

Safety Assessment of Railroad Wheels by Residual PB96-141114

04,855

Effect of Charpy V-Notch Striker Radii on the Absorbed Energy

PB96-141122

03,365

Safety Assessment of Railroad Wheels Through Roll-by Detection of Tread Cracks.

04,856

Microwave Properties of Voltage-Tunable YBa2Cu3O7delta/SrTiO3 Coplanar Waveguide Transmission Lines. High-Temperature High-Pressure Oscillating Tube Densimeter.

01,123

Low Magnetostriction in Annealed NiFe/Ag Giant Magnetoresistive Multilayers.

PB96-146691 04,762

Telegraph Noise in Silver-Permalioy Giant Magnetoresistance Test Structures.

Magnetoresistance of Thin-Film NiFe Devices Exhibiting Single-Domain Behavior.

PB96-147087

04,766

Gas-Coupled, Pulse-Echo Ultrasonic Crack Detection and Thickness Gaging

04,847

Simultaneous Laser-Diode Emission and Detection for Fiber-Optic Sensor Applications.

PB96-155502

04,062

Simple Variable Line Space Grating Monochromator for Synchrotron Light Source Beamlines.

PB96-156203

04,065

Laser-Synchrotron Hybrid Experiments: A Photon to Tickle, A Photon to Poke.

PB96-157847

03,704

Improved Reflectometry Facility at the National Institute of Standards and Technology.

PB96-160338

04,087

Cryogenic Flow Calibration in NIST

PB96-161930

01,143

PB96-161955

its.

Retention of Halocarbons on a Hexafluoropropylene Ep. oxide-Modified Graphitized Carbon Black. 4. PropaneBased Compounds.

03,284

Simple and Efficient Methane-Marker Devices for Chromatographic Samples.

PB96-164041

00,635

Compressibility of Polycrystal and Monocrystal Copper: Acoustic-Resonance Spectroscopy.

02,990

Determination of Total Protein Adsorbed on Solid (Membrane) Surface by a Hydrolysis Technique: Single Protein Adsorption.

03,552

Permeation Tube Approach 10 Long-Term Use of Automatic Sampler Retention Index Standards.

B96-167291

00,639

Structure and Rheology of Hard-Sphere Systems.

PB96-167333

00,662

Fundamental Limits on the Frequency Stabilities of Crystal Oscillators.

02,277

Thermal Anemonetry for Mass Flow Measurement in Oscillating Cryogenic Gas Flows.

PB96-176789

04,218

NIST Detector-Based Luminous Intensity Scale.

01,864

Development of Neutral-Density Infrared Filters Using Metallic Thin Films.

PB96-180286

02,998

Dynamometer-Induced Residual Stress in Railroad Wheels: Ultrasonic and Saw Cut Measurements. Report No. 30 .

PB96-183199

04,857

Sensor System for Intelligent Processing of Hot-Rolled

Sieel.

03,373

oring of

Well-Shielded

PB96-186077

02,879

Response Comparison of Electret Ion Chambers, LiF TLD, and HPIC

02,578
Epitaxial Nucleation and Growth of Chemically Derived Ba2YCu3O7-x Thin Films on (001) SrTiO3.

04,787

Charpy Impact Test as an Evaluation of $4 \mathrm{~K}$ Fracture Toughness.

PB96-190194

03,219

Prediction of Strengthening Due to $\mathrm{V}$ Additions in DirectCooled Ferrite-Pearlite Forging Steels.

PB96-190251

03,220

Uttrasonic Methods.

PB96-190327

Scanning Electron Microscopy Observations of Misfit Dislocations in Epitaxial Ino.25Ga0.75As on GaAs(001).

PB96-200159 03,004

Arrays.

PB96-200167

02,091

Superconductor- Normal-Superconductor Junctions for Digital/Analog Converters.

02,092

Superconductor- Normal-Superconductor Junctions for Programmable Voltage Standards.

PB96-200241

02,093

Design of High-Frequency, High-Power Oscillators Using

Josephson-Junction Arrays.
PB96-200258

02,094

High-Power, High-Frequency Oscillators Using Distributed Josephson-Junction Arrays.

PB96-200266

02,095

Fiber-Optic Faraday-Effect Magnetic-Field Sensor Based on Flux Concentrators.

PB96-200308

02,201

Hot-Electron Microcalorimeter for X-ray Detection Using a Superconducting Transition Edge Sensor with Electrothermal Feedback.

PB96-200399

04,792

Hot-Electron-Microcalorimeters with $0.25 \mathrm{~mm}(2)$ Area.

PB96-200670

04,793

Controlling the Critical Current Density of High-Temperature SNS Josephson Junctions.

PB96-200712

04,794

Self-Calibrated Intelligent Optical Sensors and Systems.

PB96-200738 04,380

Effect of Harmonic Distortion on Phase Errors in Fre. quency Distribution and Synthesis.

01,563

Precise Laser-Based Measurements of Zero-Dispersion Wavelength in Single-Mode Fibers.

01,511

Electronically Tunable Fiber Laser for Optical Pumping of (3) $\mathrm{He}$ and (4) $\mathrm{He}$.

PB96-201165

Report on USDA Uitraviolet Spectroradiometers.

PB96-214648

Thin Film Thermocouple Research at NIST.

04,381

00,125

PB97-110233

02,283

Magneto-Optic Rotation Sensor Using a Laser Diode as Both Source and Detector.

PB97-111272 04,390

Closed Loop Controller for Electron-Beam Evaporators.

04,393

Pulse-Driven Programmable Josephson Voltage Stand-

ard.

04,148

High Power Generation with Distributed Josephson-Junction Arrays.

02,099

Fundamentals and Problems of Fiber Current Sensors.

PB97-111835

02,205

Integrated Optical Polarization-Discriminating Receiver in

02,206

Reducing the $1 / f$ AM and PM Noise in Electronics for Precision Frequency Metrology.

PB97-113195

02,102

Micromachined Coplanar Waveguides in CMOS Tech-

PBology. 119283

02,456

Anomalous Relation between Time and Frequency Domain PMD Measurements.

PB97-119390

04,398

Design, Construction and Application of a Large Aperture Lens-Less Line-Focus PVDF Transducer.

PB97-122584

02,765

INSTRUMENTS

Conference Proceedings: International Workshop on Instrumented Indentation. Held in San Diego, California on April 22-23, 1995 .

PB96-158688

01,948

INSULATED GATE BIPOLAR TRANSISTORS

Investigation of the Drive Circuit Requirements for the Power Insulated Gate Bipolar Transistor (IGBT).

PB94-211794

02,316

Modeling Butfer

02,344

Modeling Buffer Layer IGBT's for Circuit Simulation

PB96-164173

INSULATING GAS

SF6 Insulation: Possible Greenhouse Problems and Solu-

PB95-251625

02,269

INSULATING MATERIALS

National Voluntary Laboratory Accreditation Program: Thermal Insulation Materials.

PB95-267985

02,977

\section{INSULATION}

Investigation of S2F10 Production and Mitigation in Com-

pressed SF6-In

02,467

Investigation of S2F10 Production and Mitigation in Com. pressed SF6- Insulated Power Systems.

02,468

INSULIN

Conformational Alterations of Bovine Insulin Adsorbed on a Silver Electrode.

PB96-161773

Cylinder Wipe Air-Drying Intaglio Ink Vehicles for U.S. Currency Inks.

INTEGRAL EQUATIONS

Alternative Single Integral Equation for Scattering by a $\mathrm{Di}$ -

PB94-216512

04,422

Causality and Maxwell's Equations.

PB97-110522

04,429

INTEGRALS

Integral Occur

04,324

INTEGRATED CIRCUITS

Design Guide for CMOS-On-SIMOX. Test Chips NIST3 PB94-163458

02,297

Color Supplement to NIST Special Publication 400-93: Semiconductor Measurement Technology: Design and Test Library.

02,298

Semiconductor Measurement Technology: Design and Testing Guides for the CMOS and Lateral Bipolar-on-SOI Test Library.

02,301

Application of the Modified Voltage-Dividing Potentiom-

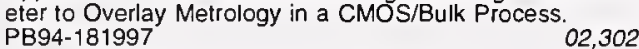
YBa2Cu3O7-x to Si Interconnection for Hybrid Superconductor/Semiconductor Integration.
PB94-211711

Precision, Accuracy, Uncertainty and Traceability and Their Application to Submicrometer Dimensional MetrolPBg.

02,319

Practical Photomask Linewidth Measurements.

PB95-108510

02,324

User's Manual for the Program MONSEL-1: Monte Carlo Simulation of SEM Signals for Linewidth Metrology. Metrology and Data for Microelectronic Packaging and Interconnection: Results of a Joint Workshop on Materials Metrology and Data for Commercial Electrical and Optical 
Microelectronic Test Structures for Feature Placement and Electrical Linewidth Metrology.

02,367

Accuracy in Integrated Circuit Dimensional Measure-

PB95-180808

02,368

Electrical Method for Determining the Thickness of Metal

Films and the Cross-Sectional Area of Metal Lines.
PB95-203170 02,370

JEDEC 'TCR' Interlaboratory Experiment: Lessons Learned.

02,371

Semiconductor Measurement Technology: HOTPAC. Programs for Thermal Analysis Including Version 3.0 of the TXYZ Program, TXYZ30, and the Thermal MultiLayer Program, TML
PB95-260766

MEMS in Standard CMOS VLSI Technology.

02,374

MEMS in Stan

02,377

MONSEL-II: Monte Carlo Simulation of SEM Signals for Linewidth Metrology.

PB96-102710 02,379

Superconducting Integrated Circult Fabrication with Low Temperature ECR-Based PECVD SiO2 Dielectric Films.

Semiconductor Measurement Technology: Test Structure Implementation Document: DC Parametric Test StrucImplementation Document: DC Parametric Test Struc-
tures and Test Methods for Monolithic Microwave Intetures and Test Methods

grated Circuits

02,399

LRM Probe-Tip Calibrations Using Nonideal Standards.

PB96-135389 02,411

Electrical Characterization of Integrated Circuit Metal Line Thickness.

02,414

Measurement of Patterned Film Linewidth for Inter-

$\begin{array}{ll}\text { connect Characterization. } & 02,420 \\ \text { PB96-148168 }\end{array}$

Scanned Probe Microscopies: Opportunities and Issues in

Metrology.

Scanning Electron Microscope Metrology

PB96-201090

02,426

TEGRATED OPTICS

Integrated-Optical Devices in Rare-Earth-Doped Glass.

PB95-202909

02,446

NTEGRATED SERVICES DIGITAL NETWORK

Integrated Services Digital Network (ISDN); Category:

Telecommunications Standard; Subcategory: Integrated Services Digital Network.

FIPS PUB 182

01,460

Introduction to Traffic Management for Broadband ISDN.

PB94-142494 01.464

North American ISDN Users' Forum Agreements on Integrated Services Digital Network.

PB94-162559

01,466

ISDN Conformance Testing Guidelines: Guidelines for Implementors of ISDN Customer Premises Equipment to Conform to Both National ISDN-1 and North American ISDN Users' Forum Layer 3 Basic Rate Interface Basic Call Control Abstract Test Suites.

PB94-219094

ISDN LAN Bridging.

PB95-154696

01,471

Application Protil

01,477

01,479

Application Software Interface: ISDN Services for an Open Systems Environment

PB96-131487

01,492

ISDN in North America.

P96-160767

01,502

North American Agreements on ISDN

PB96-160775

01,503

Journal of Research of the National Institute of Standards and Technology, September/October 1993. Volume 98, Number 5 .

PB96-169057

INTEGRATED SERVICES DIGITAL NETWORKS

ISDN Conformance Testing.

PB95- 163176

Feasibility Study: Reference Architecture for Machine Control Systems Integration.

PB94-142791

02,804

Integrated Network Management.

PB94-199247 01,583

Technical Program Description Systems Integration for Manufacturing (SIMA).

PB94-213758

02,819

Reference Architecture for Machine Control Systems Integration: Interim Report

02,820

Automated Inspection: The Integration of National Standards and Commercial Products at NIST.

PB95-163077

02,906

Requisite Elements, Rationale, and Technology Overview for the Systems Intergration for Manufacturing Applications (SIMA) Program. Background Study.

02,831
NIST SIMA Interactive Management Workshop. Held in Fort Belvoir, Virginia on November 14-16, 1994.

PB96-154877

02,838

Template-Driven Systems Development with IDEF: Enterprise Standards for Reuse.

PB96-160965

02,788

Systems Integration for Manufacturing Applications Program 1995 Annual Report.

02,844

INTEGRATING SPHERES

Integrating Sphere Simulation: Application to Total Flux Scale Realization

04,261

INTEGRATION DEFINITION FOR FUNCTION MODELING

Mapping Integration Definition for Function Modeling (IDEFO) Model into CASE Data Interchange Format (CDIF) Transfer File. PB96-109533

01,741

\section{MODELING} Mapping Integration Definition for Information Modeling
(IDEF1X) Model into CASE Data Interchange Format (CDIF) Transfer File.

PB95-154670

01,711

INTELLECTUAL PROPERTY

Standards Policy and Information Infrastructure.

PB95-231882

Using Technology to Manage and Protect Intellectua PB97-112395

INTELLIGENT PROCESSING

Intelligent Processing of Materials.

PB94-172780

Ptelligent Processing of Hot Isostatic Pressing.

02,811 Steet.

PB96-186069

-Rolled

PELLIGENT PROCESSING OF MATERIALS

03,373

PB95-140455 Evaluation and Materials Processing. 02,902

INTENSITY

Phase Shitts and Intensity Dependence in FrequencyModulation Spectroscopy.

PB96-103205

01,071

INTERACTION COEFFICIENTS

Interaction Coefficients for 15 Mixtures of Flammable and Non-Flammable Components.

PB96-146626

03,281

INTERACTION PARAMETERS

Microstructure Effect on the Phase Behavior of Blends of Deuterated Polybutadiene and Protonated Polyisopene.
PB97-113146

PACTIVE GRAPHICS

Programmer's Hierarchical Interactive Graphics System PHIGS). Category Software Standard; Subcategory: FIPS PUB 153-1

01,668

User's Guide for the PHIGS Validation Tests (Version 2.1).

PB94-165206

01,682

Approaches Using Virtual Environments with Mosaic

PB95-169108

01,599

INTERACTIVE SYSTEMS

Kibitz-Connecting Multiple Interactive Programs Together.
PB94-213311 PB94-213311 01,696 Handling Passwords with Security and Reliability in Back-
ground Processes. PB95-180550

Ouch Those Programs are Painful.

01,722

TERAGENCY COOPERATION

NIST and the Navy: Past, Present and Future. PB96-119649

01,739

TERAGENCY COORDINATION

Overview of U.S. Government Advanced Packaging Programs.

PB96-200845

02,443

NTERATOMIC POTENTIALS

Interatomic Potential of Argon.

Interatomic Potential of Argon.

PB95-152989

00,912

\section{INTERBAND ABSORPTION}

Band-to-Band Photoluminescence and Luminescence Excitation in Extremely Heavily Carbon-Doped Epitaxial GaAs.

PB95-150413

04,570

INTERCALATING AGENTS

Application of Photochemical Reaction in Electrochemical Detection of DNA Intercalation.

00,686

Enhanced Detection of PCR Products Through Use of TOTO and YOYO Intercalating Dyes with Laser Induced Fluorescence - Capillary Electrophoresis.

00,599

INTERCALATION

Detection of Aromatic Compounds Based on DNA Intercalation Using an Evanescent Wave Biosensor.

03,473

INTERCALATION COMPOUND

Determination of Anomalous Superexchange in $\mathrm{MnCl} 2$ and Its Graphite Intercalation Compound

00,666

TERFACE CRACKS

Pressurized Internal Lenticular Cracks at Healed Mica PB96-180252

02,997

INTERFACE FILTERS

Interface-Filter Characterization of Spectraradiometers and Colorimeters.

PB97-122212

INTERFACE ROUGHNESS

Interface Roughness-Induced Changes in the Near-E(sub

0) Spectroscopic Behavior of Short-Period AIAs/GaAs Superlattices

02,118

Effects of Interfacial Roughness on the Magnetoresistance of Magnetic Metallic Multilayers.
PB95-150017 04,556

Spectroscopic Ellipsometry Determination of the Propenties of the Thin Underlying Strained Si Layer and the Roughness at SiO2/Si Interface.

Roughness at
PB95-150157

04,560

Interface Roughness, Composition, and Alloying of LowOrder AIAs/GaAs Superlattices Studies by X-ray Diffrac-

PB96-160262

02,983

INTERFACE STABILITY

Convection and Morphological Stability During Directional Solidification.

03,310

INTERFACE TRANSMISSION

Spin-Dependent Interface Transmission and Reflection in Magnetic Multilayers (Invited).

PB96-201173

INTERFACES

Control Entity Interface Specification.

02,815

Interface Properties for Ceramic Composites from a Sin-

gle Fiber Pull-Out Test
PB94-199361

Asymmetric Tip Morphology of Creep Microcracks Growing Along Bimaterial Interfaces.

03,138

Neutron Scattering Studies of Surfaces and Interfaces

PB94-216207

04,517

Interface Roughness of Short-Period AIAs/GaAs Superlattices Studied by Spectroscopic Ellipsometry. Interface Modification and Characterization of Silicon Carbide Platelets Coated with Alumina Particles.

PB95-108734

03,121

Interface Shar

02,138

Interface Sharpness during the Initial Stages of Growth of Thin, Short-Period III-V Superlattices.

PB95-108783 
INTERFACIAL FRACTURE

Cracks and Dislocations in Face-Centered Cubic Metallic PB96-163696

INTERFACIAL REACTIONS

Effect of Microstructure on Phase Formation in the Reac(ion of $\mathrm{Nb} / \mathrm{Al}$ Multilayer Thin Films.

PB95-168415 03352

First Phase Formation Kinetics in the Reaction of Nb/A PB95-168456

03,353

INTERFACIAL TENSION

Measurement of Surface Tension of Tantalum by a Dynamic Technique in a Microgravity Environment.

PB95-161667

03,932

INTERFACIAL ZONE

Digital Simulation of the Aggregate-Cement Paste Interfacial Zone in Concrete.

PB95-125993

00,363

INTERFEROMETERS

Measuring Long Gage Blocks with the NIST Line Scale Interterometer.

02,665

Visualization of Surface Figure by the Use of Zernike Polynomials.

PB96-137757 04,351

New Refractometer by Combining a Variable Length Vacuum Cell and a Double-Pass Michelson Interferometer.

PB97-111926

01,986

INTERGRANULAR CORROSION

Interaction between Dislocations and Intergranular

Cracks.

03,190

INTERIOR POINT METHODS

Interior-Point Method for Linear and Ouadratic Programming Problems. (NIST Reprint).

PB95-180089

03,429

INTERLABORATORY COMPARISONS

Proficiency Testing as a Component of Ouality Assurance in Construction Materials Laboratories.

00,334

Analysis of Physical Properties of Ceramic Powders in an International Interlaboratory Comparison Program. PB95-161501

03,050

Interlaboratory Comparison of Autoradiographic DNA Profiling Measurements. 1. Data and Summary Statistics. PB95-175923 03,542

Interlaboratory Comparison Studies on the Analysis of Hair for Drugs of Abuse.

03,500

Intercomparison of Photometric Units Maintained at NIST (USA) and PTB (Germany), 1993

PB95-261913

04,329

Ceramic Powders Characterization: Results of an International Laboratory Study.

PB95-270039

02,672

Interlaboratory Comparison of Autoradiographic DNA Profiling Measurements. 2. Measurement Uncertainty and Its Propagation.

Intercomparison of the ITS-90 Radiance Temperature

Scales of the National Physical Laboratory (U.K.) and the National Institute of Standards and Technology.

PB96-113550

02,674

V-6: Effects of Temperature Variation

PB96-148143 04,772

Optical Fiber, Fiber Coating, and Connector Ferrule Geometry: Results of Intertaboratory Measurement Compari-

PB96-154422

04,360

parison

International Radon-in-Air Meas

03,708

PB96-159751

Interlaboratory Studies on the Analysis of Hair for Drugs
of Abuse: Pesults from the Fifth Exercise.
PB97-110449

of Abuse: Results from the Fifth Exercise.

03,509

Measurement Comparability. Traceability, and Measurement Assurance Programs.

02,692

Comparisons of Some NIST Fixed-Point Cells with SimiComparisons of Some NIST Fixed-Point Cells with Simi-
lar Cells of Other Standards Laboratories.
PB97-119242

00,655

Deference Material for ISE Measurements of Sodium and Potassium. PB96-159785

03,507

INTERLABORATORY TESTS

Gage Block Standards, Measurement Capabilities and Laboratory Accreditation.

PB96-163621

02,757

INTERMETALLIC ALLOYS

Experimental Assessment of Crack-Tip Dislocation Emission Models for an Al67Cr8Ti25 Intermetallic Alloy. PB96-204466

03,377

INTERMETALLIC COMPOUNDS

Intelligent Processing of Hot isostatic Pressing. 03,315
PB94-172913

Thermodynamic Constraints on Non-Equilibrium Solidi-

fication of Ordered Intermetallic Compounds.

PB94-198934
Ambient Temperature Synthesis of Bulk Intermetallics. PB95-169074 00,168

INTERMETALLICS

Status of Electrocomposites.

PB94-212453

03,143

INTERMOLECULAR FORCES

Pairwise and Nonpairwise Additive Forces in Weakly Bound Complexes: High Resolution Infrared Spectroscopy of AmDF $(n=1,2,3)$

04,125

INTERMOLECULAR POTENTIALS

Vibrational Dependence of the Anisotropic Intermolecula Potential of $\mathrm{Ar}-\mathrm{HCl}$

01,029

Vibrational Dependence of the Anisotropic Intermolecular Potential of Ar-HF.

PB95-202693

01,030

INTERNAL COMBUSTION ENGINES

Thin Film Thermocouples for Measurement of Wall Temperatures in Internal Combustion Engines.

01,449

INTERNAL CONVERSION COEFFICIENTS

Alpha-Particle and Electron Capture Decay of (209)Po. PB96-186085

04,119

INTERNAL ELECTRIC FIELDS

Thermal Stability of Internal Electric Field and Polarization Distribution in Blend of Polyvinylidene Fluoride and

Polymethylmethacrylate.
PB95-151072

01,240

NTERNAL STATE DISTRIBUTION

Dynamics of Hydrogen Interactions with $\mathrm{Si}(100)$ and Si(111) Surfaces.
PB96-159801

04,082

INTERNAL WAVES

Internal Waves in Xenon Near the Critical Point PB97-111504

04,221

NTERNATIONAL AGREEMENTS

International Challenges in Defining the Public and Private Interest in Standards.

PB96-160361

00,498

INTERNATIONAL COOPERATION

National Center for Standards and Certification Information: Service and Programs.

02717

Utc Dissemination to the Real-Time User.

01,521

Forum for International Cooperation on Fire Research.
PB95-162939

INTERNATIONAL INSTITUTE OF WELDING

IIW Commission V Ouality Control and Ouality Assurance of Welded Products Annual Report 1994/95.

PB95-198743

02,866

INTERNATIONAL ORGANIZATION FOR

TANDARDIZATION

Ouestions and Answers on Ouality, the ISO 9000 Stand ard Series, Ouality System Registration, and Related Is sues. More Ouestions and Answers on the ISO 9000 Standard Series and Related Issues.

00,495

TTERNATIONAL SYSTEM OF UNITS

Guide for the Use of the International System of Units (S1).

02,747

INTERNATIONAL TEMPERATURE SCALE OF 1990

Practical Applications of the ITS-90: Inherent Uncertainties.

03,930

Current Status and Trends in Temperature Measurements at NIST, Cooperative Projects and New Mutua Agreement between NIST and IMGC

PB97-110266

02,691

INTERNATIONAL TRADE

Planning the Infrastructure for Global Electronic Commerce.

00,494

Ouestions and Answers on Ouality, the ISO 9000 Standard Series, Ouality System Registration, and Related Issues. More Ouestions and Answers on the ISO 9000 Standard Series and Related Issues.

PB95-103461

00,495

GATT Standards Code Activities of the National Institute of Standards and Technology 1994.

00,497

PB96-106935

02,920

PB96-123401

02,923

Metrology and Regional Trade Pacts

TBT Agreement Activities of the National Institute of Standards and Technology, 1995.

PB97-104178

00,499

NTERNET

Retrieving Articles from the Internet (without a UNIX Workstation). Part 1. File Formats and Software Tools.
PB95-168720

Retrieving Articles from the Intemet (without a UNIX Workstation). Part 2. An Example.

PB95-168738

02,729

Keeping Your Site Comfortably Secure: An Introduction to Internet Firewalls.

02,730

Sharing Information via the Internet: An Infoserver Case

PB96-131511

01,493

World Wide Web for Crystallography.

PB97-109219

04,810

INTEROPERABILITY

Procedures for Document Facsimile Transmission Issued by General Services

eral Standard 1063

FIPS PUB 148

Electronic Implementors' Workshop

01,516

PB95-210936

01,484

Open Issues in OSI Protocol Development and Conform ance Testing.

01,818

Application Portability Profile (APP): The U.S. Government's Open System Environment Profile Version 3.0 .
PB96-158712

Information Technology Standards in a Changing World: The Rose of the Users.

PB96-160866

02,737

Data Communications Strategy.

PB96-167846

02,738

Five O's (Cues)

01,826

Open System Environment Implementors Workshop (OIW); Standardization Role Defined.

PB96-180047

01,828

CSL View of Applications Portability, Scalability, and Interoperability

01,787

INTERPENETRATING POLYMER NETWORK

Synthesis of Hybrid Organic-Inorganic Materials from Interpenetrating Polymer Network Chemistry.

PB96-180054

02,994

INTERENETRATING POLYMER NETWORKS

Grafted Interpenetrating Polymer Networks.

PB94-185055

01,200

INTERPRETERS

NIST RS274/NGC Interpreter Version 1

PB94-187788

NIST RS274KT Interpreter.

PB96-147954

02,814

INTERPROCESSOR COMMUNICATION

KBibitz-Connectin

02,835

ERSTELLAR GAS

Extended $\mathrm{CO}(7$ yields 6) Emission from Warm Gas in

PB96-102504

00,090

INTERSTELLAR MATTER

Goddard High-Resolution Spectrograph Observations of the Local Interstellar Medium and the Deuterium/Hydrogen Ratio along the Line of Sight Toward Capella. 


\section{KEYWORD INDEX}

INVERTERS

Electro-Thermal Simulation of an IGBT PWM Inverter. PB94-185592 INVESTMENTS

Least-Cost Energy Decisions for Buildings: Part 2. Uncertainty and Risk Video Training Workbook.
PB94-165982 Evaluating Investments in Law Enforcement Equipment: An Annotated Bibliography. PB95-151379

04,867

IODINE

lodine Atoms and lodomethane Radical Cations: Their Formation in the Pulse Radiolysis of lodomethane in Organic Solvents, Their Complexes, and Their Reactivity with Organic Reductants.

PB95-162764

00,965

IODINE 127

High-Sensitivity Determination of lodine Isotopic Ratios by mal and Fast Neutron Activation.

ODINE 129

00,555

High-Sensitivity Determination of lodine Isotopic Ratios by Thermal and Fast Neutron Activation PB94-213386

00,555

IODINE MONOXIDE Absorption Cross Sections, Kinetics of Formation, and
Self-Reaction of the IO Radical Produced via the Laser Photolysis of N2O/2/2/N2 Mixtures.

PB97-112361

01,180

IODINE ORGANIC COMPOUNDS

lodine Atoms and lodomethane Radical Cations: Their Formation in the Pulse Radiolysis of lodomethane in Organic Solvents, Their Complexes, and Their Reactivity with Organic Reductants.

PB95-162764

00,965

IODINE OXIDE

Experimental Determination of the Ionization Energy of $\operatorname{IO}(X$ (sup 2) II(sub 3/2)) and Estimations of Delta(sub f) $H$ (sup deg) (sub

00,694

ION-ATOM COLLISIONS

Charge Transfer and Collision-Induced Dissociation Reactions of $\mathrm{CF}(2+)$ and $\mathrm{CF} 2(2+)$ with the Rare Gases at a Laboratory Collision Energy of $49 \mathrm{eV}$.

00,775

Collision-Induced Neutral Loss Reactions of Molecular Collision-Induced Neutral Loss Reactions of Molecular
Dications. Dications.
PB94-185808

00,780

Hydrogen Balmer Alpha Line Shapes for Hydrogen-Argon Mixtures in a Low-Pressure rf Discharge.

PB95-153433

03,924

Collisions of Elec

04,791

ION BEAM ASSISTED DEPOSITION

Effect of Beam Voltage on the Properties of Aluminum Nitride Prepared by lon Beam Assisted Deposition.
PB97-118616

01,995

ION BEAM MODIFICATION

Gas Absorption during Ion-Irradiation of a Polymer Target.

04,099

ION BEAMS

Growth of Epitaxial KNbO3 Thin Films

PB96-135181

02,409

ION CHANNEL

Noise Analysis of Ionization Kinetics in a Protein Ion Channel.

04,093

ION CHANNELS

Genetically Engineered Pores for New Materials.
PB96-161641 03,550

Current Fluctuations Reveal Protonation Dynamics and Number of lonizable Residues in the alpha-Toxin ChanNumber of lonizable Residues in the alpha-Toxin Chan-
nel. PB96-161732

03,588

Protonation Dynamics in an Ion Channel Pore.

PB96-161757

03,589

ON DETECTION

Superconducting Resonator and a Cryogenic GaAs FieldEffect Transistor Amplifier as a Single-Ion Detection SysPB95.202727

ION DISTRIBUTION

03,990

Evidence for Inelastic Processes for $\mathrm{N}(+) 3$ and $\mathrm{N}(+) 4$ from Ion Energy Distributions in He/N2 Radio Frequency Glow Discharges.

ION EXCHANGE RESINS

Tetrahedral-Framework Lithium Zinc Phosphate Phases: Location of Light-Atom Positions in LiZnPO4 $\mathrm{H}_{2} \mathrm{O}$ by Powder Neutron Diffraction and Structure Determination of LiZnPO4 by ab Initio Methods.

ION FILMS

01,129

Variation in Magnetic Properties of Cu/Fcc (001) Sandwich Structures

ION IMAGING

Molecular Ion Imaging and Dynamic Secondary Ion Mass Spectrometry of Organic Compounds
PB95.126124

04,555

00,571
ION IMPLANTATION

ransition Metal Implants in In0.53GA0.47As. PB95-126389

Boron-Implanted $6 \mathrm{H}-\mathrm{SiC}$ Diodes. PB96-159678

04,534

\section{ON IRRADIATION}

ncreased Pinning Energies and Critical Current Densities

ION MOLECULE INTERACTIONS

Vibrational Spectra of Molecular lons Isolated in Solid Neon. 7. $\mathrm{CO}(+), \mathrm{C}_{2} \mathrm{O} 2(+)$, and $\mathrm{C}_{2} \mathrm{O} 2(-)$. (Reannouncement with New Availability Information).
AD-A239 $729 / 7$

Selected Ion Flow Tube-Laser Induced Fluorescence In strument for Vibrationally State-Specific Ion-Molecule Re-

actions.

00,774

ION OPTICS

Beam Line for Highly Charged lons. PB97-111256

PLASMAS

iquid and Solid Atomic Ion Plasmas.

PB94-198991

03,809

ION SELECTIVE ELECTRODES

Development of a Standard Reference Material for ISE Mea surements of Sodium and Potassium.

PB96-159785

03,507

\section{ION SOURCES}

Relative Sensitivity Factors and Useful Yields for Microfocused Gallium Ion Beam and Time-of-Flight Secndary Ion Mass Spectrometer. PB94-198736

00,541

ION SPECTROSCOPY

Compositional Analyses of Surfaces and Thin Films by Electron and lon Spectroscopies.

00,779

ION STORAGE

Ouantum Projection Noise: Population Fluctuations in Two-Level Systems

PB94-21227

Electrostatic Modes of Ion-Trap Plasmas.

03,850

03,920

Ouantum Measurements of Trapped lons.

03,928

Laser Cooling of Trapped Ions.

PB95-168746

03,950

Recent Experiments on Trapped lons at the National In

stitute of Standards and Technology.

03,952

High-Resolution Atomic Spectroscopy of Laser-Cooled

PB95-169330

03,953

Experimental Results on Normal Modes in Cold, Pure Ion Plasmas.

03,956

Non-Neutral Ion Plasmas and Crystals, Laser Cooling, and Atomic Clocks.

PB95-175113

03,957

Progress on a Cryogenic Linear Trap for $(199) \mathrm{Hg}(+)$ lons.

PB95-180790 03,965

Precise Spectroscopy for Fundamental Physics.

PB96-112164

04,040

Trapped Ions and Laser Cooling 4: Selected Publications of the Ion Storage Group of the Time and Frequency Division, NIST, Boulder, Colorado.

PB96-172358

04,108

ION TRANSPORT

Current Fluctuations Reveal Protonation Dynamics and Number of Ionizable Residues in the alpha-Toxin Chan-

PB96-161732

03,588

ION TRAPS

Progress on a Cryogenic Linear Trap for $(199) \mathrm{Hg}(+)$ lons.
PB95-180790

Cooling and Detection of Radio-Frequency Oscillations by Laser-Cooled lons.

PB96-112073

04,039

IONIC CONDUCTIVITY

Fast-Ion Conduction and Disorder in Cation and Anion Arrays in Y2(ZryTi(1-y))207 Pyrochlores Induced by Z Substitution: A Neutron Rietveld Analysis.

04,496

Fast-Ion Conducting Y2(ZryTi1-y)2O7 Pyrochlores: Neutron Rietveld Analysis of Disorder Induced by Zr Substitution.

PB96-156104

04,776

IONIZATION

Multiphoton Ionization Spectroscopy Measurements of Silicon Atoms during Vapor Phase Synthesis of Ceramic

PB95-151999

03,913

Current Noise Reveals Protonation Kinetics and Number of Ionizable Sites in an Open Protein Ion Channel. PB96-161674

04,092

IONIZATION CHAMBERS

Mass Assay and Uniformity Test of Boron Targets by Neutron Beam Methods.

04,175

IONIZATION ENERGY

Experimental Determination of the Ionization Energy of $1 O(X$ (sup 2) II(sub 3/2)) and Estimations of Delta(sub f) $\mathrm{H}$ (sup deg)(sub 0)(IO(sup -)) and $\mathrm{PA}(\mathrm{IO})$. Ionization Energies, Appearance Energies and Thermochemistry of CF2O and FCO

01,178

PB97-111538

Influence of Envelopes Geometry on the Sensitivity of 'Nude' lonization Gauges.

NIZATION POTENTIALS

04,174

Ionization Energy of Sulfur Pentafluoride and the Sulfur Pentafluoride-Fluorine Atom Bond Dissociation Energy. NIZATION SPECTROSCOPY

Resonance Enhanced Multiphoton Ionization Spectroscopy of the PF Radical.

PB97-119119

00,702

IONIZATION SUPPRESSION

Suppression of Ionization in One- and Two-Dimensional Model Calculations

IONIZATION TRAILS

04,009

Comparison of Meteor Activity with Occurrence of Sporadic E Reflections.

AD-A292 039/5

00,116

IONIZING RADIATION

Permissible Dose from External Sources of lonizing Radi-

AD-A279 281/0

03,608

lonizing radiation-induced DNA damage and its repair in human cells. Progress report, (April 1, 1993--February 28,

DE94014709

03,612

Estimation of the Absorbed Dose in Radiation-Processed Food. 2. Test of the EPR Response Function by an Exponential Fitting Analysis.

PB94-199650

00,036

Estimation of the Absorbed Dose in Radiation-Processed Food. 3. The Effect of Time of Evaluation on the Accuracy of the Estimate.

00,037

Estimation of the Absorbed Dose in Radiation-Processed Food. 4. EPR Measurements on Eggshell.

00,038

Role of the Office of Radiation Measurement in Ouality

Assurance.
PB94-212255 00,689

Substrate Specificity of the Escherichia coli Endonuclease III: Exicision of Thymine- and Cytosine-Derived Lesions in PB95-153425 Ionizing Radiation Causes Greater DNA Base Damage in Radiation-Sensitive Mutant M10 Cells That in Parent

Mouse Lymphoma L5178Y Cells.
PB95-175915

03,632

IONIZING RADIATION DOSIMETRY 
IR LASER SPECTROSCOPY

Probing Potential-Energy Surlaces via High-Resolution If PB96-102835

01,066 RDS (INFORMATION RESOURCE DICTIONARY SYSTEM) Information Resource Dictionary System (IRDS): A Status

PB95-126207

01,810

BIDIUM

Use of Sum Rules on the Energy-Loss Function for the Evaluation of Experimental Optical Data.

PB95-150736 04,264

Characterization of the Interaction of Hydrogen with Iridium Clusters in Zeolites by Inelastic Neutron Scattering PBectroscopy

01,013

Analysis of the $(5 d(2)+5 d 6 s)-5 d 6 p$ Transition Arrays of Os VII and Ir VIII, and the 6S (2)S.6P (2)P Transitions of If PBg6-159264

01,954

RIDIUM OXIDE

Thin-Film Ruthenium Oxide - Iridium Oxide Thermocouples.

00,520

RIDIUM OXIDES

Iridium Oxide Thin-Film Stability in High-Temperature Corrosive Solutions.

PB94-213014

03,234

Changes in the Redox State of Iridium Oxide Clusters and Their Relation to Catalytic Water Oxidation: Radiolytic and Electrochemical Studies. PB95-107017

00,864 IRON

Homoepitaxial Growth of Iron and a Real Space View of Reflection-High-Energy-Electron Diffraction.

04,445

Scaling of Diffusion-Mediated Island Growth in Iron-onIron Homoepitaxy. 04,455 Magnetic Dead Layer in $\mathrm{Fe} / \mathrm{Si}$ Multilayer: Profile Refinement of Polarized Neutron Reflectivity Data.
PB94-198363 Concurrent Enhancement of Kerr Rotation and Antiferromagnetic Coupling in Epitaxial $\mathrm{Fe} / \mathrm{Cu} / \mathrm{Fe}$ Structures.

04,466

Spot-Profile-Analyzing LEED Study of the Epitaxial Growth of $\mathrm{Fe}, \mathrm{Co}$, and $\mathrm{Cu}$ on $\mathrm{Cu}(100)$.
$\mathrm{PB} 95-150165$ PB95-150165 04,561 $\mathrm{Fe}(100)$.

Growth of Iron on Iron Whiskers.

04,562

Growth of Iron

04,567

Oscillatory Exchange Coupling in $\mathrm{Fe} / \mathrm{Au} / \mathrm{Fe}(100)$

PB95-150371

04,569

Improved Wavelengths for Prominent Lines of $\mathrm{Fe} X X$ to $\mathrm{Fe}$ XXIII.

04,334

Formation of DNA-Protein Cross-Links in Cultured Mammalian Cells Upon Treatment with Iron lons.

PB96-137724

03,651

Atomic Iron in Its (5)D Ground State: A Direct Measurement of the $J=0$ inverted arrow 1 and $J=1$ inverted arrow 2 Fine-Structure Intervals (1.2)

04,756

IRON ALLOY 18CR 19MN

Nitrogen Effect on Elastic Constants of f.c.c. Fe-18Cr19Mn Alloys.

03,203

IRON ALLOYS

Study of Laser Resonance Ionization Mass Spectrometry Using a Glow Discharge Source.

Temperature Dependence of the Magnetic Excitations in Ordered and Disordered Fe72Pt28. B95-150223

Study of Laser Resonance Ionization Mass Spectrometry Using a Glow Discharge Source.

PB96-123203

03,360

Electronic Structure and Phase Equilibria in Ternary Sub. stitutional Alloys.

IRON BASE ALLOYS

03,378

Comparison of the Corrosion Rates of $\mathrm{FeAl}, \mathrm{Fe}(\mathrm{sub}$ 3) A and Steel in Distilled Water and $0.5 \mathrm{M}$ Sodium Chloride. Technical Report Number 2, January-March 1991. DE94017332

03,186

ON COMPOUNDS

Thermodynamic Properties of the Aqueous lons $(2+$ and $3+$ ) of Iron and the Key Compounds of Iron.

PB96-145958

01,119

IRON GARNETS

Wideband Current and Magnetic Field Sensors Based on Iron Garnets.

01,975

RON IONS

Measurements of quantum electrodynamic sensitive transitions in Na-like and Cu-like ions: Final report, 1 October 1993--29 September 1994.

DE95011593

04,403
Laser-Produced and Tokamak Spectra of Lithiumlike Iron, $\mathrm{Fe}(23+)$.

04,314

Oxidation of Ferrous and Ferrocyanide Ions by Peroxyl Radicals.

PB97-122402

01,191

RON OXIDES

Structural and Magnetic Ordering in Iron Oxide/Nickel

Oxide Multilayers by $X$-ray and Neutron Diffraction (In-

PB94-172558

04,442

IRON SILICIDES

Soft-X-ray-Emission Studies of Bulk Fe3Si, FeSi, and FeSi2, and Implanted Iron Silicides

PB96-157938

04,071

IRRADIÁNCE MEASUREMENT

Method of Realizing Spectral Irradiance Based on an Absolute Cryogenic Radiometer

04,270

IRRADIANCES

Irradiances of Spectral Lines in Mercury Pencil Lamos.

PB96-176466

04,375

IRRADIATION

Research and Development Activities in Electron Paramagnetic Resonance Dosimetry.

03,635

Radiated Emissions and Immunity of Microstrip Trans. mission Lines: Theory and Measurements.

PB96-162649

02,238

ISDN (INTEGRATED SERVICES DIGITAL NETWORK)

Catalog of National ISDN Solutions for Selected NIUF Applications.

PB94-166006

01,468

ISDN Conformance Testing Guidelines: Guidelines for Implementors of ISDN Customer Premises Equipment to Conform to Both National ISDN-1 and North American ISDN Users' Forum Layer 3 Basic Rate Interface Basic
Call Control Abstract Test Suites. PB94-219094

01,471

ISDN LAN Bridging.

PB95-154696

01,477

Application $P$

01,479

Application Software Interface: ISDN Services for an Open Systems Environment.

PB96-131487

01,492

SDN in North America.

01,502

North American Agreements on ISDN

PB96-160775

01,503

ISDN (INTEGRATED SERVICES DIGITAL NETWORKS)

ISDN Conformance Testing.

01,478

ISO

ISO Environmental Management Standardization Etrorts.
PB95-220513

Conformance Testing for OSI Protocols.

PB96-102686

ISO Environmental Management Standardization Efforts. PB96-158662

02,528

SO/IEC GUIDE 25

Survey on the Implementation of ISO/IEC Guide 25 by National Laboratory Accreditation Programs.

PB94-210150

00,479

ISO (INTERNATIONAL ORGANIZATION FOR

STANDARDIZATION)

Questions and Answers on Quality, the ISO 9000 Standard Series, Quality System Registration, and Related Issues. More Questions and Answers on the ISO 9000 Standard Series and Related Issues.

00,495 PB95.10346

ISOBUTENE

Mechanism and Rate Constants for the Reactions of Hydrogen Atoms with Isobutene at High Temperatures.
PBg

\section{ISOCHORIC}

Isochoric (p-p-T) Measurements on Liquid and Gaseous Air from $6710400 \mathrm{~K}$ at Pressures to $35 \mathrm{MPa}$.

01,154 PB96-167390

Labeling Conventions in Isoelectronic Sequences - Reply.
PB95-162574 PLECTRONIC SEQUENCE

ISOLATION AMPLIFIERS

New 5 and $10 \mathrm{MHz}$ High Isolation Distribution Amplifier. PB96-190202

01,510

ISOMERASES

Thermodynamics of Enzyme-Catalyzed Reactions. Part 5. Isomerases and Ligases.

01,121

145974

Development of Engineered Stationary Phases for the
Separation of Carotenoid Isomers.
PB95-150249
O0,578 Separation of
PB95-150249

00,578

ISOMERS

Comparison of the Liquid Chromatographic Behavior of Selected Steroid Isomers Using Different ReversedPhase Materials and Mobile Phase Compositions. PB95-140976

00,574

Isolation and Structural Elucidation of the Predominant Geometrical Isomers of alpha-Carotene.

PB96-190061

00,640

ISOTHERMS

Water-Vapor Measurements of Low-Slope Roofing Mate-

PB95-25 1617

00,399

ISOTOPE DILUTION

Isotope Dilution Mass Spectrometry as a Candidate Definitive Method for Determining Total Glycerides and Triglycerides in Serum.

03,519

Isotoplc and Nuclear Analytical Techniques in Biological Systems: A Critical Study. 10. Elemental Isotopic Dilution Analysis with Radioactive and Stable Isotopes (Technical

PB96-164157

00,696

ISOTOPE DILUTION MASS SPECTROSCOPY

Comparative Strategies for Correction of Interferences in Isotope Dilution Mass Spectrometric Determination of Vanadium.

PB94-185261

00,531

ISOTOPE EFFECT

Wavelengths and Energy Levels of Neutral $\operatorname{Kr}(84)$ and Level Shitts in All Kr Even Isolopes.

03,780

Isotope Shifts and Hypertine Splittings of the $398.8-\mathrm{nm}$ Isotope Shit
Yb I Line.

PB94-199585

03,814

Wavelengths and Isotope Shifts for Lines of Astrophysical Interest in the Spectrum of Doubly lonized Mercury $(\mathrm{Hg}$ PB95-140869

03,887

Magneto-Optical Trapping of Metastable Xenon: IsotopeShift Measurements.

PB95-151254

03905

Deuterium Isotope Effect in Vinyl Radical Combination/ Dispropor tionationReactions.

PB96-204:51

Laser Magnetic-Resonance Measurement of the (3) P1 (3)P2 Fine-Structure Splittings in (17) $\mathrm{O}$ and (18) $\mathrm{O}$

PB95- 175170

ISOTOPE RATIO

Deuterium in the Local Interstellar Medium: Its Cosmological Significance.

PB95-202842

00,081

New IUPAC Guidelines for the Reporting of Stable Hydrogen, Carbon, and Oxygen Isotope-Ratio Data Letter to

PB95-261962

01,058

SOTOPE RATIOS

New Values for Silicon Reference Materials, Certified for Isotope Abundance Ratios. Letter to the Editor. PB94-219268

00,863

ISOTOPE SHIFT

Measurements of the Resonance Lines of (6) $\mathrm{Li}$ and (7) $\mathrm{Li}$

by Doppler-Free Frequency-Modulation Spectroscopy. 


\section{KEYWORD INDEX}

JAPAN

Statistical Ouality Control Technology in Japan

PB94-199064

02,708

Differences in Competitive Strategies between the United States and Japan

211836

00,013

Calibration of GPS Equipment in Japan

01.531

Japan Technology Program Assessment: Precision Engineering/Precision Optics in Japan

02,884

Earthquake and Fire in Japan: When the Threat Became a Reality.

PB95-175238 00.201

Japan Technology Program Assessment. Simulation: State-of the-Art in Japan.

PB95-217097 02,827

Innovation in the Japanese Construction Industry: A 1995 Appraisal.

JET ENGINES

00,225

Bibliography of Books and Published Reports on Gas

Turbines, Jet Propulsion, and Rocket Power Plants.

AD-A278 138/3

01,445

Bibliography of Books and Published Reports on Gas Turbines, Jet Propulsion, and Rocket Power Plants, January 1950 through December 1953

$213 / 4$

01,446

Determination of Hydrogen in Titanium Alloy Jet Engine Compressor Blades by Cold Neutron Capture Prompt Gamma-ray Activation Analysis.

PB95-175956

01,448

JET FLOW

Experimental Study of the Stabilization Region of Lifted Turbulent-Jet Diffusion Flames

PB96-122676

01,405

Global Density Effects on the Self-Preservation Behavior of Turbulent Free Jets.

PB95-162301

04,207

JOB CREATION

Metric Path to Global Markets and New Jobs: A Ouestion-and-Answer and Thematic Discussion.

PB94-206307

02,601

JOINTS (JUNCTIONS)

Model Precast Concrete Beam-to-Column Connections Subject to Cyclic Loading.

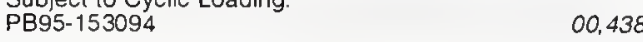

Partially Prestressed and Debonded Precast Concrete

$\begin{array}{ll}\text { Beam-Column Joints. } & 00,439 \\ \text { PB95-153102 }\end{array}$

Seismic Performance Behavior of Precast Concrete Beam-Column Joints.

00,440

Report of a Workshop on Requalification of Tubular Stee Joints in Offshore Structures. Held in Houston, Texas on September 5-6, 1995 .

PB96-210760

03,699

JOSEPHSON ARRAYS

Accuracy Comparisons of Josephson Array Systems.
PB95-164687 02,047

JOSEPHSON EFFECT

How Accurate Are the Josephson and Ouantum Hall Effects and OED

JOSEPHSON JUNCTIONS

Effect of Thermal Noise on Shapiro Steps in High-Tc Josephson Weak Links.

PB94-212677

04,506

Combined Josephson and Charging Behavior of the Supercurrent in the Superconducting Single-Electron

PB95-168522 04,637

Observation of Vortex Dynamics in Two-Dimensional Josephson-Junction Arrays.

PB95-168811 02,050

Terahertz Shapiro Steps in High Temperature SNS Jo-

sephson Junctions.
PB95-169140

Phase-Locked Oscillator Optimization for Arrays of Josephson Junctions.

PB95-169314 02,052

Thermal Noise in High-Temperature SuperconductingNormal-Superconducting Step-Edge Josephson Junctions.
PB95-175089

04,650

Characterization of the Emission from 2D Array Josephson Oscillators.

02,054

Emission Linewidth Measurements of Two-Dimensional Array Josephson Oscillators.

02,055

Frequency Dependence of the Emission from 2D Array

PB95-175147 02,056

Evidence for Parallel Junctions Within High-Tc GrainBoundary Junctions.

04,657
Josephson Voltage Standard Based on Single-Flux-Quantum Voltage Multipliers.

02,058

Phase Locking in Two-Junction Systems of High-Temperature Superconductor-Normal Metal-Superconductor perature

PB95-176053

02,060

High Temperature Superconductor-Normal MetalSuperconductor Josephson Junctions with High Characteristic Voltages.

PB95-176079

04,666

Ouasipotential and the Stability of Phase Lock in Nonhysteretic

04,681

Large-Amplitude Shapiro Steps and Self-Field Effects in High-Tc Josephson Weak Links.

PB95-1804 10

04,682

Step-Edge and Stacked-Heterostructure High-Tc Josephson Junctions for Voltage-Standard Arrays.

SB $96-102066$

04,702

Resonances in Two-Dimensional Array Oscillator Circuits. PB96-102082 02,066
0 Direct Observation of Vortex Dynamics in Two-Dimensional Josephson-Junction Arrays.

PB96-102223 02,067

Physical Basis for Half-Integral Shapiro Steps in a DC

PB96-102264

04,704

Stacked Series Arrays of High-Tc Trilayer Josephson

Junctions.

04,705

$30 \mathrm{THz}$ Mixing Experiments on High Temperature Superconducting Josephson Junctions.

04,709

Phase Locking in Two-Dimensional Arrays of Josephson Junctions: Effect of Critical-Current Nonuniformity. PB96-102587

Superconducting Integrated Circuit Fabrication with Low Temperature ECR-Based PECVD SiO2 Dielectric Films.
PB96-103015

04,719

Stable Phase Locking in a Two-Cell Ladder Array of Josephson Junctions.

04,722 Temperature Dependence and Magnetic Field Modulation
of Critical Currents in Step-Edge SNS YBCO/Au Juncof Critical Currents in Step-Edge SNS YBCO/Au Junc-

Critical Current and Normal Resistance of High-Tc StepEdge SNS Junctions.

PB96-111752

04,724

SUSAN: SUperconducting Systems ANalysis by Low Temperature Scanning Electron Microscopy (LTSEM). PB96-112065 04,728 High-Frequency Oscillators Using Phase-Locked Arrays of Josephson Junctions.

PB96-135157

02,080

Heterodyne Mixing and Direct Detection in High Tem. perature Josephson Junctions.

perature Josep

01,565

Microwave Noise in High-Tc Josephson Junctions.

PB96-14801

04,771

Josephson D/A Converter with Fundamental Accuracy.

Performance and Reliability of NIST $10-\mathrm{V}$ Josephson Arrays.

02,419

Metallic-Barrier Junctions for Programmable Josephson Voltage Standards.

PB96-200134

02,089

Shapiro Steps in Large-Area Metallic-Barrier Josephson Junctions.

PB96-200142 02,090

Novel Vortex Dynamics in Two-Dimensional Josephson Arrays.

PB96-200167

02,091

High-Tc Multilayer Step-Edge Josephson Junctions and SOUIDs.

PB96-200183

04,790

Superconductor- Normal-Superconductor Junctions for Digital/Analog Converters.

PB96-200233

02,092

Superconductor- Normal-Superconductor Junctions for Programmable Voltage Standards.

PB96-20024

02,093

Design of High-Frequency, High-Power Oscillators Using Josephson-Junction Arrays.

PB96-200258

02,094

High-Power, High-Frequency Oscillators Using Distributed Josephson-Junction Arrays.

02,095

Controlling the Critical Current Density of High-Temperature SNS Josephson Junctions.

PB96-200712

04,794

Pulse-Driven Programmable Josephson Voltage Standard.

04,148

High Power Generation with Distributed Josephson-Junction Arrays

02,099

\section{JOSEPHSON OSCILLATORS}

Phase-Locked Oscillator Optımizatıon for Arrays of Josephson Junctions.

PB95- 169314

02,052

Characterization of the Emission from 2D Array Josephson Oscillators

02,054

Emission Linewidth Measurements of Two-Dimensional Array Josephson Oscillators.

02,055

Frequency Dependence of the Emission from 2D Array

$\begin{array}{ll}\text { Josephson Oscillators. } & 02,056 \\ \text { PB95-175147 }\end{array}$

Design of High-Frequency. High-Power Oscillators Using Josephson-Junction Arrays.

02,094

High-Power, High-Frequency Oscillators Using Distributed

$\begin{array}{ll}\text { Josephson-Junction Arrays. } & \\ \text { PB96-200266 } & 02,095\end{array}$

High Power Generation with Distributed Josephson-Junction Arrays.

02,099

JOURNALS

Role of Journals in Maintaining Data Integrity: Checking of Crystal Structure Data in 'Acta Crystallographica'

PB97-109177

04,806

Electronic Publishing and the Journals of the American Chemical Society.
PB97-109185

How the Cambridge Crystallographic Data Centre Obtains Its Information.
PB97-109193

04,808

JP-8 JET FUEL

Suppression of Elevated Temperature Hydraulic Fiuid and JP-8 Spray Flames

PB95-181095

00,021

JUVENILE RHEUMATOID ARTHRITIS

Nutritional Status and Growth in Juvenile Rheumatoid Ar-

thritis

PB94-198470

03,515

K LINES

$\mathrm{K}$ alpha Transitions in Few-Electron lons and in Atoms.

K-VALUE

Design of a Protocol for an Electron Probe Microanalyzer k-Value Round Robin.

PB95-107181

KALMAN FILTERS

Comparison of GPS Broadcast and DMA Precise Ephemerides.

01,523

KEROSENE

Struclure of a Swirl-Stabilized Kerosene Spray Flame.
PB95-108569

KERR CELLS

Investigation of the Effects of Aging on the Calibration of a Kerr-Cell Measuring System for High Voltage Impulses. PB94-172384

KERR EFFECT

Observation of the Transverse Second Harmonic Magneto-

PB96-200332

01971

KERR MAGNETOOPTICAL EFFECT

Concurrent Enhancement of Kerr Rotation and Antiferromagnetic Coupling in Epitaxial Fe/Cu/Fe Structures. 
KILOGRAMS

NIST Watt Experiment: Monitoring the Kilogram. PB97-122329

KINEMATIC VISCOSITY

Viscosity of the Saturated Liquid Phase of Six Halogenated Compounds and Three Mixtures.
PB95-162368

00,960

KINETIC ENERGY

Kinetic Energy Distribution of lons Produced from Townsend Discharges in Neon and Argon.

04,413

\section{KINETICS}

Fokker-Planck Description of Multivalent Interactions.

PB95-108478 00,879

Aggregation Kinetics of Colloidal Particles Destabilized by Enzymes.

00,894

Anisotropic Phase Separation Kinetics in a Polymer Blend Solution Following Cessation of Shear Studied by Ligh Scattering.

01,241

Current Noise Reveals Protonation Kinetics and Number of lonizable Sites in an Open Protein Ion Channel.
PB96-161674

\section{KNOWLEDGE-BASED SYSTEMS}

Development of Computer-Based Models of Standards and Attendant Knowledge-Base and Procedural Systems. KRYPTON

Intensity-Dependent Scattering Rings in High Order Above-Threshold Ionization.

04,032

Spectral Data for Highly Ionized Krypton, $\mathrm{Kr} V$ through $\mathrm{Kr}$ XXXVI.

$$
\text { PB96-145917 }
$$

01,115

KAYPTON 84

Wavelengths and Energy Levels of Neutral $\operatorname{Kr}(84)$ and Level Shifts in All Kr Even Isotopes.

PB94-140605

03,780

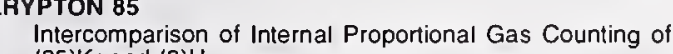
Intercomparison of Internal Proportional Gas Counting of
(85) $\mathrm{Kr}$ and (3)H.
PB94-185576

\section{KRYPTON COMPLEXES}

Van der Waals Bond Lengths and Electronic Spectral Shitts of the Benzene- $\mathrm{Kr}$ and Benzene-Xe Complexes.

\section{KAYPTON IONS}

Resonance Structure and Absolute Cross Sections in Near-Threshold Electron-Impact Excitation of the $4 \mathrm{~s}(2)$ (1) $S$ yields $4 S 4 p$ (3) $P$ Intercombination Transition in

PB95-202271

03,972

-S COUPLING

Investigation of LS Coupling in Boronlike Ions. PBg4-185295

03,797

L-THREO-BETA-HYDROXYHISTIDINE

L-threo-beta-Hydroxyhistidine, an Unprecedented Iron(III) lon-Binding Amino Acid in a Pyoverdine-type Siderophore from Pseudomonas fluorescens 244 . PB94-211620

00,553

ABELUNG COMPLIANCE

Inter-Laboratory Trials of the EPR Method for the Detection of Irradiated Meats Containing Bone.

00,042

LABELS

Checking the Net Contents of Packaged Goods as Adopted by the 79th National Conference on Weights and Measures, 1994, Third Edition, Supplement 4.

PB95-182226

00,484

ABOR FORCE

Investing in Education to Meet a National Need for a Technical-Professional Workforce in a Post-Industria Economy

PB94-173028

00,132

BORATORIES Title 15, Subtitle A, Chapter 2, Part 7. (Effective December 1984; Amended September 1990). Der 1984; Amended September 1990).
PB94-160850 National Voluntary Laboratory Accreditation Program: Procedures and General Requirements.
PB94-178225 National Voluntary Laboratory Accreditation Program 1994 Directory. Proficiency Testing as a Component of Quality Assurance in Construction Materials Laboratories. State Weights and Measures Laboratories: State Standards Program Description and Directory. 1994 Edition. ards Program Description and Directory. 1994 Edition.
PB94-207727 Program Handbook: Requirements for Obtaining NIST Approval/Recognition of a Laboratory Accreditation Body Under P.L. 101-592. The Fastener Quality Act. PB94-210143

National Voluntary Laboratory Accreditation Program: En ergy Efficient Lighting Products.

02,642
Comparison of Heat-Flow-Meter Tests from Four Laboratories.

PB95-126264

00,365

National Voluntary Laboratory Accreditation Program: Ionizing Radiation Dosimetry.

PB95-128658

03,623

National Voluntary Laboratory Accreditation Program: Bulk Asbestos Analysis.

02,541

National Voluntary Laboratory Accreditation Program: Construction Materials Testing.

PB95-155552

01,319

National Voluntary Laboratory Accreditation Program: Carpet and Carpet Cushion.

PB95-155560

00,295

National Voluntary Laboratory Accreditation Program (NVLAP): Wood Based Products

PB95-170429

03,405

National Voluntary Laboratory Accreditation Program 1995 Directory

00,483

National Voluntary Laboratory Accreditation Program Acoustical Testing Services.

PB95-182234

04,188

National Voluntary Laboratory Accreditation Program: POSIX. Portable Operating System Interface.

02,661

National Voluntary Laboratory Accreditation Program: Electromagnetic Compatibility and Telecommunications. FCC Methods.

PB95-242376

02,664

Materials Science and Engineering Laboratory Annual Report, December 1993

PB95-254439 02,668

National Voluntary Laboratory Accreditation Program (NVLAP): Commercial Products Testing

02,671

National Voluntary Laboratory Accreditation Program:

Thermal Insulation Materials.

Proficiency Tests for the NIST Airborne Asbestos Pro-

gram, 1993.
PB96-106463

00,610

Laboratory Accreditation for Testing Energy Efficient Lighting.

00,270

Inexpensive Laser Cooling and Trapping Experiment for Undergraduate Laboratories.

04,353

U.S. Government Accreditation and Conformity Assess-

$\begin{array}{ll}\text { ment System Evaluation. } & \\ \text { PB96-160239 } & 02,678\end{array}$

Program

National Voluntary Laboratory Accreditation Program 1996 Directory
PB96-162714

00,485

State Weights and Measures Laboratories: Program Handbook.

02,687

\section{LABORATORY ACCREDITATION}

Survey on the Implementation of ISO/IEC Guide 25 by National Laboratory Accreditation Programs.

PB94-210150

00,479

Should NIST Accredit U.S. Calibration Laboratories

PB95-107280 02,646

Gage Block Standards, Measurement Capabilities and Laboratory Accreditation.

PB96-163621

02,757

Proceedings of the Open Forum on Laboratory Accreditation at the National Institute of Standards and Technology, October 13,1995.

02,686

Evaluation and Accreditation of State Calibration Laboratories.

\section{LABORATORY AUTOMATION}

Integrating Automated Systems with Modular Architec ture.

00,577

LABORATORY EQUIPMENT

Precision Laboratory Standards of Mass and Laboratory

02,618

AD-Â280 562/0 Electroacoustics for Characterization of Particulates and Gaithersburg, Maryland on February 3-4, 1993.

PB94-112695

00,725

Simple, Inexpensive Apparatus for Sample Concentration. PB94-199205 00.546 NIST Power Reference Source.

00,148 PB94-211513

Anisotropic Phase Separation Kinetics in a Polymer Blend Solution Following Cessation of Shear Studied by Light

Scattering.
PB95-151247

01,241

Constant Temperature and Humidity Chamber for Standard Resistors.

02,275

LAKE ERIE

Lake Erie Water Temperature Data, Put-in-Bay, Ohio, PB96-202452

03.692

LAMELLA

Partially Coherent Transmittance of Dielectric Lamellae.
PB96-148028

AMINAR FLOW

Probabilistic Computation of Poiseuille Flow Velocity Fields.

996-102520

04,209

\section{LAMINATED GLASS}

Influence of Physical Aging on the Yield Response of Model DGEBA + Poly(propylene oxide) Epoxy Glasses.

Volume Recovery in Epoxy Glasses Subjected to Torsional Deformations: The Question of Rejuvenation. PB95.140018 03,382 Torsional Relaxation and Volume Response during Physical Aging in Epoxy Glasses Subjected to Large Torsional Deformations.

03,383

Using Torsional Dilatometry to Measure the Effects of De.

formations on Physical Aging. formations on

LAMINOGRAPHY

Evaluation and Qualification Standards for an X-Ray Laminography System.

02,029

Contributions of Out-of-Plane Material to a ScannedBeam Laminography Image.

PB96-111786

02,704

LAMP ORIENTATION

Irradiance of Horizontal Quartz-Halogen Standard Lamps.
PB96-179130

LAMPS

Improved Automated Current Control for Standard Lamps.

00,246

\section{LANDSAT SATELLITES}

Organization and Implementation of Calibration in the EOS Project. Part 1.

04,841

LANTHANUM

Charge-Transfer-Induced Multiplet Structure in the $\mathrm{N} 4,5 \mathrm{O} 2,3$ Sott-X-ray Emission Spectrum of Lanthanum.
PB96-163746

LANTHANUM ALUMINATES

Dielectric Properties of Single Crystals of $\mathrm{Al}_{2} \mathrm{O} 3$, LaAlO3, $\mathrm{SrTiO}_{3}$ and $\mathrm{MgO}$ at Cryogenic Temperatures.

LANTHANUM BARIUM CUPRATES

Correlation between TC and Elastic Constants of (LaM)2CuO4.

\section{LANTHANUM BARIUM TITANATES}

Substitution-Induced Midgap States in the Mixed Oxides $\mathrm{R} \times \mathrm{Ba} 1-\mathrm{ChiTiO3-Delta}$, with $\mathrm{R}=\mathrm{Y}, \mathrm{La}$, and $\mathrm{Nd}$. PB95-140505

04,541

LANTHANUM CALCIUM CUPRATES

Correlation between TC and Elastic Constants of (La$\mathrm{M}) 2 \mathrm{CuO} 4$

PB94-213220

04,514

Neutron Powder Diffraction Study of the Structures of La1.9Ca1.1Cu2O6 and La1.9Sr1.1Cu2O6+Delta. PB95-140042

\section{LANTHANUM DEUTERIDE}


LASER ABLATION

Influence of Deposition Parameters on Properties of Laser Ablated YBa2Cu3O7-Delta Films.

04,544

\section{ASER AMPLIFIERS}

Optical Sampling Using Nondegenerate Four-Wave Mixing in a Semiconductor Laser Amplifier.

02,076

Optical Sampling Using Nondegenerate Four-Wave Mixing in a Semiconductor Laser Amplifier.

$$
\text { PB96-123609 }
$$

LASER ANNEALING Improved Dose Metrology in Optical Lithography.
PB96-179510

ASER APPLICATIONS

Optically Stabilized Tunable Diode-Laser System for Saturation Spectroscopy.

PB96-102819

04,717

LASER BEAMS

Beam Analysis Round Robin.

PB94-212545

04,239

Laser-Beam Analysis Pinpoints Critical Parameters.
PB94-212552

Widths and Propagation of a Truncated Gaussian Beam.

PB95-168779 04,287

Error Propagation in Laser Beam Spatial Parameters.

PB95-180394

Characterization of a Clipped Gaussian Beam.
PB96-102553

LASER COOLING

Laser Cooling and Trapping for the Masses.

Laser Cooling.

Trapped Atoms and Laser Cooling.

PB95-151627

New Mechanisms for Laser Cooling

PB95-153268

Laser Cooling of Trapped Ions

0

04,253

03,908

03,911

PB95-168746

03,923

Ouantum-Limited Cooling and Detection of Radio-Fre-

quency Oscillations by Laser-Cooled Ions.

Inexpensive Laser Cooling and Trapping Experiment for Undergraduate Laboratories.

PB96-140371

04,353

Laser Modification of Ultracold Collisions: Experiment.

Laser-Cooled Neutral Atom Frequency Standards.

PB96-160312

04,075

Gravitational Sisyphus Cooling of (87)Rb in a Magnetic

Trap.

04,379

Properties of a Bose-Einstein Condensate in an Anisotropic Harmonic Potential.

PB96-204144

04,133

Laser Cooling and the Recoil Limit.

111280

04,391

ASER DEPOSITION

Laser-Focused Atomic Deposition.

PB95-161618

04,604

LASER DIAGNOSTICS

Modeling Ceramic Sub-Micron Particle Formation from the Vapor Using Detailed Chemical Kinetics: Comparison with In-situ Laser Diagnostıcs.

$$
\begin{aligned}
& \text { with In-Situ Lase } \\
& \text { PB95-151965 }
\end{aligned}
$$

00,671

LASER DIFFRACTION

Parametric Investigation of Metal Powder Atomization Using Laser Diffraction.

PB95-108577

03,342

LASER DIODES

Simultaneous Laser-Diode Emission and Detection for Fiber-Optic Sensor Applications.

PB96-155502 04,062

Magneto-Optic Rotation Sensor Using a Laser Diode as Both Source and Detector.

PB97-111272

LASER FOCUSING

Laser Focused Atomic Deposition

PB95-180659

Nanostructure Fabrication via Laser-Focused Atomic Deposition (Invited)

PB96-204094

04,132

LASER HAZARDS

Optical Density Measurements of Laser Eye Protection PB96-190301

00,190

LASER INDUCED DESORPTION

Laser-Induced Desorption of NO from Si(111): Etfects of Coverage on NO Vibrational Populations.

00,959

PB95-162319

100) and

Adsorbate Enhanced Surface Damage. PB95-203311

01,050

Pulsed Laser Irradiation at $532 \mathrm{~nm}$ of In and $\mathrm{Ga} \mathrm{Ad-}$ sorbed on Si(100): Desorption, Incorporation, and DamPBg. 203329

01,051

\section{LASER INDUCED FLUORESCENCE}

Greatly Enhanced Soot Scattering in Flickering Ch4/Air Diffusion Flames.

01,361

Laser Imaging of Chemistry-Flowfield Interactions: Enhanced Soot Formation in Time-Varying Diffusion Flames. PB94-185352 01,364

Laser-Induced Fluorescence Measurements of Formaldehyde in a Methane/Air Diffusion Flame.

01,374

Optical Measurements of Atomic Hydrogen, Hydroxyl, and Carbon Monoxide in Hydrocarbon Diffusion Flames. PB95-150900

02,487

Laser-Induced Fluorescence Measurements of $\mathrm{OH}$. Concentrations in the Oxidation Region of Laminar, Hydrocarbon Diffusion Flames.

PB95-162160 01,387

Trace Detection in Conducting Solids Using Laser-Induced Fluorescence in a Cathodic Sputtering Cell.

PB95-163424

00,598

ASER MAGNETIC RESONANCE

Determination of the Molecular Parameters of $\mathrm{NiH}$ in its (2)Delta Ground State by Laser Magnetic Resonance. PB95-107116

00,869

\section{LASER MATERIALS}

Vertical-Cavity Semiconductor Structures: Materials Characterization.

02,149

LASER MODE LOCKING

Millimeter-Resolution Optical Time-Domain Reflectometry Using a Four-Wave Mixing Sampling Gate

02,190

LASER PHOTOIONIZATION

Laser Photoionization Measurements of Pressure in VacPBum. 180600

03,964

LASER PHOTOLYSIS

Absorption Cross Sections, Kinetics of Formation, and Self-Reaction of the IO Radical Produced via the Laser Photolysis of N2O/2/N2 Mixtures.

PB97-112361

01,180

\section{LASER POLARIMETRY}

Simultaneous Measurement of Normal Spectral Emissivity by Spectral Radiometry and Laser Polarimetry at High Temperatures in Pulse-Heating Experiments: Application to Molybdenum and Tungsten.

PB97.118376

02,694

LASER-PRODUCED PLASMA

Measurements of quantum electrodynamic sensitive transitions in $\mathrm{Na}$-like and $\mathrm{Cu}$-like ions: Final report, 1 October 1993--29 September 1994.

04,403

LASER PULSE METHOD

Thermal Diffusivity of POCO AXM-501 Graphite in the Range 1500 to $2500 \mathrm{~K}$ Measured by a Laser-Pulse Technique

03,013

High-Temperature Laser-Puise Thermal Diflusivity Appa-

ratus. 185147

02,631

LASER RADIATION

Far Infrared Laser Frequencies of (13)CD3OH.
PB95-169363

Frequency Shifting of Pulsed Narrow-Band Laser Light in a Multipass Raman Cell.

PB95-203352

04,321

Nanofabrication of a Two-Dimensional Array Using LaserFocused Atomic Deposition.

PB96-119417 04.732

Thermal Modeling and Analysis of Laser Calorimeters

PB96-140405

04,354

LASER RANGE FINDERS

Preliminary Comparison of Time Transfers via LASSO, GPS and Two-Way Satellite.

01,529

LASER SCATTERING

In situ Characterization of Vapor Phase Growth of Iron Oxide-Silica Nanocomposites: Part 1. 2-D Planar LaserInduced Fluorescence and Mie Imaging.

PB97-112478

03,185

LASER SPECTROSCOPY

Dreams About the Next Generation of Super-Stable La-

PB94-211570

04,235

High-Resolution Spectroscopy of Laser-Cooled Rubidium in a Vapor-Cell Trap.

04,268

Laser Spectroscopy of Carbon Monoxide: A Frequency Reference for the Far Infrared.

PB95-163606

04,277

High-Sensitivity Spectroscopy with Diode Lasers.

Diode Laser as a Spectroscopic Tool.

04,297

PB95-175485

00,600

High-Resolution Diode-Laser Spectroscopy of Calcium

03,969

High-Resolution Optical Multiplex Spectroscopy.

PB95-203543

04,323

Doppler-Free Spectroscopy of Large Polyatomic Molecules and van der Waals Complexes.

04,339

Search for Small Violations of the Symmetrization Postu-

late in an Excited State of Helium.
PB96-123518

04,050

Frequency-Stabilized Lasers: A Driving Force for New PB96-135199

04,350

Comment On: Two-Photon Absorption Series of Calcium. PB96-157979 04,074 Configuration-Dependent AC Stark Shifts in Calcium. PB96-157995

04,363

Measurements of the Resonance Lines of $(6) \mathrm{Li}$ and $(7) \mathrm{Li}$ by Doppler-Free Frequency-Modulation Spectroscopy.
PB96-180211 PB 04,118 (3) He and (4) He.

PB96-201165

LASER STABILITY

Dreams About the Next Generation of Super-Stable La-

PB94-211570 04,235

Frequency Stabilized Lasers: A Parochial Review.

PB95-153771

04,269

Stabilization of 3.3 and $5.1 \mathrm{~m}$ Lead-Salt Diode Lasers by Optical Feedback

PB95-180709

04,313

Stabilization of Optical Phase/Frequency of a Laser Sysem: Application to a Commercial Dye Laser with an External Stabilizer.

04,327

Frequency-Stabilized Lasers: A Driving Force for New Spectroscopies.
PB96-135199

LASER STRUCTURES Double-Modulation and Selective Excitation
Photoreflectance for Wafer-Level Characterization of Ouantum-Well Laser Structures.
PB96-167325

04,372

LASER SYNCHROTRON COINCIDENCE

Laser-Synchrotron Hybrid Experiments: A Photon to Tickle, A Photon to Poke.

ASER TRAPPING

Laser Cooling and Trapping for the Masses
PB95-126090

03,704 PB96-140371

04,353

ASER TRIGGERED BREAKDOWN

Electrohydrodynamic Instability and Electrical Discharge nitiation in Hexane.

PB96-186119

02,244

LASERS 


\section{KEYWORD INDEX}

LEAD (METAL)

Magneto-Optic Magnetic Field Sensor with $1.4 \mathrm{pT} /$ square root of $1(\mathrm{~Hz})$ Minimum Detectable Field at $1 \mathrm{kHz}$.

PB94-199551

02,125

Pump-Induced Dispersion of Erbium-Doped Fiber Measured by Fourier-Transform Spectroscopy.

PB94-211935

04,236

Beam Analysis Round Robin

04,239

Laser-Beam Analysis Pinpoints Critical Parameters.

PB94-212552 04,240 Integrated Optic Laser Emitting at 906, 1057, and 1358 PB94-216280

Integrated Optic Laser Emitting at 905, 1057, $1356 \mathrm{~nm}$. PB94-216298 02,136 Laser Cooling and Trapping for the Masses.

PB95-126090

Metrology Applications of Mode-Locked Erbium Fiber La. PBrs. 140158

04,256

Rare-Earth-Doped Waveguide Devices: The Potential for

PB95-140836 04,257

Linewidth Narrowing in an Imbalanced $Y$-Branch Waveguide Laser.

04,258

Nd:LiTaO3 Waveguide Laser.

04,259

Nanostructure Fabrication via Direct Writing with Atoms Nanostructure Fabrication via Direct Writing with Atoms
Focused in Laser Fields.
PB95-150272

Multiwavelength Birefringent-Cavity Mode-Locked Fibre
Laser. Direct Dispersion Measurement of Highly-Erbium-Doped
Optical Amplifiers Using a Low Coherence Reflectometer

Coupled with Dispersive Fourier Spectroscopy.

04,263

Laser Cooling

03,908

Multiphoton Ionization Spectroscopy Measurements of Silicon Atoms during Vapor Phase Synthesis of Ceramic Particles.

03,913

Vertical-Cavity Optoelectronic Structures: CAD, Growth, and Structural Characterization.

02,148

Vertical-Cavity Semiconductor Structures: Materials Characterization.

02,149

Vertical-Cavity Semiconductor Lasers: Structural Characerization, CAD, and DFB Structures.

PB95-153193

02,150

New Mechanisms for Laser Cooling.

03,923

High Speed, High Sensitivity Magnetic Field Sensors Based on the Faraday Effect in Iron Garnets.

02,151

PB95-153391

Magneto-Optic Magnetic Field Sensors Based on Uniaxial
ron Garnet Films in Optical Waveguide Geometry. Iron Garnet Films in Optical Waveguide Geometry.
PB95-153409 High-Resolution Spectroscopy of Laser-Cooled Rubidium in a Vapor-Cell Trap.

04,268

Accurate Characterization of High Speed Photodectors.

PB95-153763 02,153

Frequency Stabilized Lasers: A Parochial Review.
PB95-153771 04,269

Bragg Gratings in Optical Fibers Produced by a Continu-

ous-Wave Ultraviolet Source.

PB95-162020

04,274

Growth of Bragg Gratings Produced by Continuous-Wave Ultraviolet Light in Optical Fiber.

PB95-162038

04,275

Stabilization and Precise Calibration of a Continuous Wave Difference Frequency Spectrometer by Use of a

Simple Transfer Cavity
PB95-162350

04,276

Polarization Dependence of Response Functions in $3 \times 3$ Sagnac Optical Fiber Current Sensors.

02,154

Vector Theory of Diffraction by a Single-Mode Fiber: Application to Mode-Field Diameter Measurements.

plication to Mod

04,279

In situ Observation of Surface Morphology of InP Grown on Singular and Vicinal (001) Substrates.

PB95-168431

04,636

Interlaboratory Comparison of Polarization-Holding $\mathrm{Pa}$ rameter Measurements on High-Birefringence Optical Fiber.

PB95-168464

04,280

Low-Coherence Interferometric Measurement of Group Transit Times in Precision Optical Fibre Delay Lines.
PB95-168480

Wroved Variational Analysis of

04,285
Spatial Uniformity of Optical Detector Responsivity. 02,162 Electrically Calibrated Pyroelectric Detector-Pefinements or Improved Optical Power Measurements.

02,164

Optical Power Meter Calibration Using Tunable Laser Di-

PBes. $169256 \quad 04,290$

Waveguide Polarizers Processed by Localized Plasma PBtching.

02,171

Self-Calibrating Fiber Optic Sensors: Potential Design Methods.

PB95-169298

02,172 High-Sensitivity Spectroscopy with Diode Lasers.
PB95-175477
04,297

Diode Laser as a Spectroscopic Tool.

PB95-175485

00,600

Calculated Fiber Attenuation: A General Method Yielding Stationary Values.

04,298

High-Sensitivity Optical Sampling Using an Erbium-Doped Fiber Laser Strobe.

PB95-176111

04,302

Precise Optical Frequency References and Difference Frequency Meas

04,305

Error Propagation in Laser Beam Spatial Parameters.

PB95-180394 04,310

Modal Characteristics of Bent Dual Mode Planar Optica Waveguide.

04,31

Laser Photoionization Measurements of Pressure in Vacuum.

PB95-180600

03,964

Stabilization of 3.3 and $5.1 \mathrm{~m}$ Lead-Salt Diode Lasers by Optical Feedback

High-Resolution Diode-Laser Spectroscopy of Cal

03,969 Speed Measurement Devices.

PB95-197455

04,861

Lunar Laser Ranging: A Continuing Legacy of the Apollo Program.

03,683

Improved Hyperfine Measurements of the Na NP Excited State Through Frequency-Controlled Dopplerless Specroscopy in a Zeeman Magneto-Optic Laser Trap.

PB95-203840

04,012

Delivering the Same Optical Frequency at Two Places: Accurate Cancellation of Phase Noise Introduced by an Optical Fiber or Other Time-Varying Path.

PB96-102736 04,332

Phase Shifts and Intensity Dependence in FrequencyModulation Spectroscopy.

PB96-103205

01,071

Glasses for Waveguide Lasers.

04,335

Laser Gas Ionization Technique Monitors MEB Crystal

Laser Gas Ionization Technique Monitors MEB Crystal
Growth.
PB96-112172

PB96-122973

04,347

Frequency-Stabilized Lasers: A Driving Force for New Spectroscopies

04,350

Laser Modification of Ultracold Collisions: Experiment.

PB96-157987 04,075

Optical Density Measurements of Laser Eye Protection Materials.

00,190

Laser Cooling and the Recoil Limit.

PB97-111280

04,391

Laser Bandwidth Effects in Quantitative Cavity RingDown Spectroscopy.

\section{LATE STARS}

Volume-Limited ROSAT Survey of Extreme Ultraviole Emission from all Nondegenerate Stars within 10

PB96-103189

00,093

Sleuthing the Dynamo: HST/FOS Observations of UV Emissions of Solar-Type Stars in Young Clusters.

PBissions of 122817

High Velocity Plasm in the Transition Region of Au Mic: A Stellar Analog of Solar Explosive Events.

00,102

LATHES

Effects of Spindle Dynamic Characteristics on Hard Turn-

ing.

02,699

LATTICE MISMATCH

Influence of Lattice Mismatch on Indium Phosphide Based High Electron Mobility Transistor (HEMT) Structures Observed in High Resolution Monochromatic Synchrotron X-Radiation Diffraction Imaging.

02,357

LATTICE MODELS

Lattice Model of a Hydrogen-Bonded Polymer Blend.

PB97-112262

03,391

\section{LATTICE PARAMETERS}

Precision Comparison of the Lattice Parameters of Silicon Monocrystals.
PB94-169745

04,438

Improved Crystallographic Data for Aluminum Niobate (AINbO4).

LATTICE SITES

Lattice Position of $\mathrm{Si}$ in GaAs Determined by X-Ray Standing Wave Measurements.

PB95-164406

LATTICE STRAIN

Accurate Modeling of Size and Strain Broadening in the Rietveld Refinement: The 'Double-Voigt' Approach PB96-200225

00,664

LATTICES

Interfacial Crack in a Two-Dimensional Hexagonal Lattice.

PB96-161989 04,100

Dislocation Core-Core Interaction and Peierls Stress in a MBdel Hexagonal Lattice.

04,101

Photonic Band-Structure Effects for Low-Index-Contrast Two-Dimensional Lattices in the Near Infrared.
PB97-122469

04,401

\section{LAW ENFORCEMENT}

Face Recognition Technology for Law Enforcement Applications.

Evaluating Investments in Law Enforcement Equipment:

An Annotated Bibliography.
PB95-151379 04,867

Directory of Law Enforcement and Criminal Justice Associations and Research Ceniers.

PB96-178918

04,872

LAW FREQUENCIES

Low-Frequency Model for Radio-Frequency Absorbers.
PB95-261939

LAW (JURISPRUDENCE)

Federal Certification Authority Liability and Policy: Law and Policy of Certificate-Based Public Key and Digital PB94-191202

01.578

Uniform Laws and Regulations in the Areas of Legal Metrology and Motor Fuel Quality as Adopted by the 80th National Conference on Weights and Measures 1995. 1996 Edition.

LAYER CONSTRICTION

$X$-ray Observation of Electroclinic Layer Constriction and

Rearrangement in a Chiral Smectic-A Liquid Crysta
PB96-141080

01,100

LAYERED COMPOUNDS

Maximum Entropy as a Tool for the Determination of the C-Axis Profile of Layered Compounds.

PB94-199619

00,800

LAYERS

Contact Electrification Induced by Monolayer Modification of a Surface and Relation to Acid-Base Interactions. PB94-185378

Modified Surface Layers and Coatings.

PB95-176087

03,034

LEACHING

Modelling the Leaching of Calcium Hydroxide from $\mathrm{Ce}$ ment Paste: Effects on Pore Space Percolation and Diffusivity.

01,311

Bioleaching of Cobalt from Smelter Wastes by 


\section{KEYWORD INDEX}

Secondary Target X-Ray Excitation for In vivo Measurement of Lead in Bone.

03,496

Spectral Interference in the Determination of Arsenic in High-Purity Lead and Lead-Base Alloys Using Electrothermal Atomic Absorption Spectrometry an Zeeman-Effect Background Correction

00,614

Application of a Novel Slurry Furnace AAS Protocol for Rapid Assessment of Lead Environmental Contamination. PB96-l12354 02,526

Development of a Test Method for Leaching of Lead from Lead-Based Paints Through Encapsulants.

03,128

LEAD POISONING

Lead Abatement in Buildings and Related Structures.

PB94-172038

03,601

LEAD SALT DIODE LASERS

Optically Stabilized Tunable Diode-Laser System for Saturation Spectroscopy

04,717

LEAD STRONTIUM YTTRIUM CUPRATES

Crystal Structure of Pb2Sr2YCu3O8+delta with delta $=1.32,1.46,1.61,1.71$, by Powder Neutron Diffraction.

04,518

LEAD ZIRCONATE TITANATES

Microstructure and Ferroelectric Properties of Lead Zirconate-Titanate Films Produced by Laser Evaporation. PB94-199148

04,470

LEAK DETECTORS

Experimental Data and Theoretical Modeling of Gas Flows Through Metal Capillary Leaks.

04,206

EAKAGE CURRENT

Leakage Current Detection in Cryogenic Current Comparator Bridges.

02,024

LEAST SOUARE FIT

Bounds on Least-Squares Four-Parameter Sine-Fit Errors Due to Harmonic Distortion and Noise.

01,609

LEDGES

Dislocation Emission at Ledges on Cracks.

PB95-164240

04,630

LEGALITY

Guidance of the Legality of Keystroke Monitoring.
PB96-161237 LENGTH

Uncertainties in Dimensional Measurements Made at Nonstandard Temperatures.

PB94-169760 02,893

Metrology Model for Submicrometer Dimensional Meas-

urements.
PB95-108502 02,647

Evolution of Automatic Line Scale Measurement at the National Institute of Standards and Technology.

PB95-108809

02,897

Alternative Contour Technique for the Efficient Computation of the Effective Length of an Antenna. PB96-141361

02,011

LENNARD-JONES POTENTIAL

Long-Lived Structures in Fragile Glass-Forming Liquids.
PB96-119565

ENSES

Planar Lenses for Field-Emitter Arrays.

PB96-103064

\section{EUKOCYTES}

Modification of DNA Bases in Chromatin of Intact Target Human Cells by Activated Human Polymorphonuclea Leukocytes.

LEUNG-GRIFFITHS MODEL

Calculation of Enthalpy and Entropy Differences of NearCritical Binary Mixtures with the Modified Leung-Griffiths Model.

PB95-108635

00,885

LEUNG-GRIFFITHS THEORY

Vapor-Liquid Equilibria of Mixtures of Propane and Isomeric Hexanes.

00,995

Critical Properties and Vapor-Liquid Equilibria of the Binary System Propane + Neopentane.

00,999

LIBRARIES

National Center for Standards and Certification Information: Service and Programs.

N95-15938/0

02,717

Field Measurements of Ventilation and Ventilation Effectiveness in an Office/Library Building.

\section{BRATIONAL MOTION}

Intermolecular HF Motion in $\operatorname{Ar}($ sub n)HF Micromatrices $(n=1,2,3,4$,$) : Classical and Quantum Calculations on a$ Pairwise Additive Potential Surface. PB95-107025

03,871

LIOAR (LIGHT DETECTION AND RANGING)

Model Minimum Performance Specifications for Lidar Speed Measurement Devices.

04,861
LIFE APPARATUS

Flammability Characterization with the Lit Apparatus and the Cone Calorimeter

PB97-110050

01,435

LIFE CYCLE ANALYSIS

ISO Environmental Management Standardization Efforts. PB95-220513 02,524 ISO Environmental Management Standardization Efforts. PB96-158662

02,528

\section{LIFE CYCLE COSTS}

Energy Prices and Discount Factors for Life-Cycle Cost Analysis 1994. Annual Supplement to NIST Handbook 135 and NBS Special Publication 709.

PB94-206018

02,508

Energy Price Indices and Discount Factors for Life-Cycle Cost Analysis 1995. Annual Supplement to NIST Handbook 135 and NBS Special Publication 709. (Revised).

PB95-105011

02,509

Present Worth Factors for Life-Cycle Cost Studies in the Department of Defense (1995)

PB95-105029

03,664

Life-Cycle Costing Workshop for Energy Conservation in Buildings: Student Manual.

00,257

BI CC. The NIST 'Building Life-Cycle Cost' Program, VerBLCC: The NIST 'Building Life-Cycle Cost' Program, Ver-
sion 4.21. User's Guide and Reterence Manual. sion 4.21. User's Guide and Reference Manual. 00,263
PB95-190682 Building Life Cycle Cost Computer Program (BLCC), Version 4.2-95 (for Microcomputers).

PB95-501953

00,266

Building Life Cycle Cost Computer Program (BLCC) Version 4.21-95 (for Microcomputers).

00,267

Building Life Cycle Cost Computer Program (BLCC) Ver-

sion $4.22-95$ (for Microcomputers).
PB95-503397

00,268

Present Worth Factors for Life-Cycle Cost Studies in the
Department of Defense (1996).

Department of Defense (1996)
PB96-106869

03,673

Economic Methods and Risk Analysis Techniques for Evaluating Building Investments: A Survey.

00,323

Multiattribute Decision Analysis Method for Evaluating Buildings and Building Systems.

00,325

Energy Price Indices and Discount Factors for Life-Cycle Cost Analysis 1996. Annual Supplement to NIST Handbook 135 and NBS Special Publication 709. (Revised). PB96-162441

Life-Cycle Costing Manual for the Federal Energy Management Program. 1995 Edition.

02,511

Energy Price Indices and Discount Factors for Life-Cycle Cost Analysis 1997. Annual Supplement to NIST Handbook 135 and NBS Special Publication 709. (Revised). PB96-210745

02,512

Building Life Cycle Cost Computer Program (BLCC) Version 4.22-95 (for Micrcomputers).

00,277

Building Life Cycle Cost Computer Program (BLCC) Version 4.4-97 (for Microcomputers)

PB97-500342

00,284

LIFE (DURABILITY)

Research on Methods for Determining Optical Disk Media Life Expectancy Estimates.

PB96-160304

01,633

LIFE TESTS

Methodologies for Predicting the Service Lives of Coating Systems.

PB95-146387

03,124

LIFELINE SYSTEMS

Ground Improvement Techniques for Liquefaction Reme-

diation Near Existing Lifelines.

diation Near Exist

01,350

LIFELINES

Proceedings of a Workshop on Developing and Adopting Seismic Design and Construction Standards for Lifelines. Held in Denver, Colorado on September 25-27, 1991. PB97-115794

01,302

LIFETIMES

Measurement of the Atomic Na(3P) Lifetime and of Retardation in the Interaction between Two Aloms Bound in a PB97-122360

04,178

ㄴIF

Experimental Study of the Stabilization Region of Lifted Turbulent-Jet Diffusion Flames.

01,405

PB96-122676

IGAND CONTACTS Escherichis coli Cyclic AMP Receptor Protein Mutants
Provide Evidence for Ligand Contacts Important in ActivaProvide Evidence for Ligand Contacts Important in Activation.

03,592

LIGAND ENVIRONMENT

Isolated Spin Pairs and Two-Dimensional Magnetism in $\mathrm{SrCr}($ sub 9p) Ga(sub 12-9p)Ot9.

04,154

LIGANDS

Aftinity Chromatography on Inorganic Support Materials. PB95-163820 03,467

LIGASES

Thermodynamics of Enzyme-Catalyzed Reactions. Part 5. somerases and Ligases.

GHT AMPLIFIERS

01,121

Direct Dispersion Measurement of Highly-Erbium-Doped Optical Amplifiers Using a Low Coherence Reflectometer Coupled with Dispersive Fourier Spectroscopy

PB95-150702

04,263

Millimeter-Resolution Optical Time-Domain Reflectometry

Using a Four-Wave Mixing Sampling Gate.

GHT REFRACTION

Refraction of Light by Graded Birefringent Media

PB96-123716

02,190

LIGHT SCATTERING

Two Numerical Techniques for Light Scattering by Dielec-

ric Agglomerated Structures.

04,225

Brillouin Light Scattering Intensities for Thin Magnetic Films with Large Perpendicular Anisotropies.

PB94-211174

04,488

Light Scattering from Glossy Coatings on Paper.

04,242

Determination of Surface Roughness Irom Scattered

PB94-216520

Autocorrelation Functions from Optical Scattering for One-Dimensionally Rough Surfaces

PB94-216538

04,244

Light Scattered by Coated Paper

PB94-216546

04,245

SANS and LS Studies of Polymer Mixtures Under Shear

PB95-107090 01,231

Radiometric Model of the Transmission Cell-Reciprocal Nephelometer.

PB95-150†32

00,124

Regimes of Surface Roughness Measurable with Light

PB95-151213

04,265

Method for the Assay of Hydrolytic Enzymes Using Dynamic Light Scattering

03,539

Dynamic Light-Scattering Study of a Diluted Polymer Blend Near lts Critical Point.

PB95-151890

01,245

Light Scattered from Two Atoms.

PB95-168753

04,286

Rayleigh Scattering Limits for Low-Level Bidirectional Reflectance Distribution Function Measurements.

04,307

Standard Reference Material for the Measurement of Particle Mobility by Electrophoretic Light Scattering.

PB96-102488

Physicochemical Characterization of Low Molecular 
Psychological Aspects of Lighting: A Review of the Work

of CIE TC 3.16
PB94-172160

00,241

Papers Presentations Shine.

PB94-200383

00,244

Lighting and HVAC.

PB95-150991

NIST Lighting and HVAC Interaction Test Facility.

Lighting Research and Theory Can Create Business Prospects.
PB95-151791

00,253

LIGHTWEIGHT CONCRETES

Testing of Selected Self-Leveling Compounds for Floors.

INE BROADENING

Profile Fitting of $X$-Ray Diffraction Lines and Fourier Analysis of Broadening.

PB94-198512

04,460

Ion Broadening Parameters for Several Argon and Carbon Lines.

Astrophysical Aspects of Neutral Atom Line Broadening. PB94-213287

03,843

NE-FOCUS TRANSDUCER

Transient Analysis of a Line-Focus Transducer Probing a Liquid/Solid Interface.

02,763

Material Characterization By a Time-Resolved and Polar-

ization-Sensitive Ultrasonic Technique.

02,764

INE-FOCUS TRANSDUCERS

Design, Construction and Application of a Large Aperture Lens-Less Line-Focus PVDF Transducer.

02,765

INE HEAT SOURCES

Intra-Laboratory Comparison of a Line-Heat-Source Guarded Hot Plate and Heat-Flow-Meter Apparatus.

PB95-181202

00,387

Line-Heat-Source Guarded-Hot-Plate Apparatus.

PB97-118996

00,417

Linewidth Narrowing in an Imbalanced Y-Branch Waveguide Laser.

PB95-140844

04,258

UINE OF SIGHT

Deuterium and the Local Interstellar Medium: Properties for the Procyon and Capella Lines of Sight.

00,111 PB96-200639

LINE REFLECT MATCH CALIBRATIONS

Line-Reflect-Match Calibrations with Nonideal Microstrip Standards.

02,242

LINE SHAPE

Experimental Studies of Line Shapes from a BalleFlygare Spectrometer.
PBg 4-199452

00,796

LINE SPECTRA

Improved Wavelengths for Prominent Lines of $\mathrm{Fe} X X$ to Fe XXIII.

PB96-111638

04,334

LINE WIDTH

Practical Photomask Linewidth Measurements.

PB95-108510 02,324

User's Manual for the Program MONSEL-1: Monte Carlo Simulation of SEM Signals for Linewidth Metrology.
PB95-111522

Linewidth Narrowing in an Imbalanced Y-Branch Waveguide Laser.

PB95-140844

04,258 Monte Carlo Model for SEM Linewidth Metrology.
PB95-150058

cometer

Comparisons of Measured Linewidths of Sub-Microm

Lines Using Optical, Electrical, and SEM Metrologies.

$X$-ray Mask Metrology: The Development of Linewidth Standards for X-ray Lithography

PB95-162129

02,348

Microelectronic Test Structures for Feature Placement and Electrical Linewidth Metrology.

PB95-180568

02,367

Improving Photomask Linewidth Measurement Accuracy via Emulated Stepper Aerial Image Measurement. PB95-180816

02,911

MONSEL-II: Monte Carlo Simulation of SEM Signals for Linewidth Metrology.

02,379

Use of Monte Carlo Modeling for Interpreting Scanning Electron Microscope Linewidth Measurements.
PB96-137807

02,413

LINEAR ACCELERATOR SCATTER

Scattered Fractions of Dose from 18 and 25 MV X-ray Radiotherapy Linear Accelerators.

PB96-186101

04,120

LINEAR ALGEBRA

Basic Linear Algebra Operations in SLI Arithmetic.
PB96-165931

\section{LINEAR PROGRAMMING}

Algorithmic Enhancements to the Method of Centers for Linear Programming Problems.

LINEAR REGRESSION

Constant-Width Calibration Intervals for Linear Regression.

03,439

LINEAR SYSTEMS

Characterization of Unknown Linear Systems Based on Measured CW Amplitude.

PB95-161485 01,897

SparseLib++ v. 1.5 Sparse Matrix Class Library. Reference Guide.
PB96-193636

LINEARITY

Beamcon III, a Linearity Measurement Instrument for Optical Detectors.

04,337

LINKAGES

Standards and Linkages: What Data Sharing Needs. PB95-161881

01,713

LIPID BILAYERS

Self-Assembled Phospholipid/Alkanethiol Biomimetic Bilayers on Gold.

00,878

Supported Phospholipid/Alkanthiol Biomimetic Mernbranes: Insulating Properties.

PB95-180782

03,470

Phospholipid/Alkanethiol Bilayers for Cell-Surface Receptor Studies by Surface Plasmon Resonance.

03,472

LIPIDS

Biological Thermodynamic Data for the Calibration of Differential Scanning Calorimeters: Dynamic Temperature Dialkylphosphatidylcholine in Water Suspensions.

PB95-162707

03,464

Extending the Angular Range of Neutron Reflectivity Measurements from Planar Lipid Bilayers: Applications to a Model Biological Membran

PB96-122569

03,476

LIPOSOMES

Calcium Phosphate Precipitation in Liposomal SuspenSions.

Mixed Phospholipid Liposome Calcification. PB94-211190 03,457 Proteoglycan Inhibition of Calcium Phosphate Precipitation in Liposomal Suspensions.

PB94-211208

00,658

Liposome-Based Flow-Injection Immunoassay for Derermining Theophylline in Serum

03,494

Potentiometric Enzyme-Amplified Flow Injection Analysis Detection System: Behavior of Free and Liposome-Reeased Peroxidase.

PB95-151833

03,534

Membrane-Mediated Precipitation of Calcium Phosphate in Model Liposomes with Matrix Vesicle-Like Lipid Composition

03,468

-164547

Effect of 1-Hydroxyethylidene-1,1-Bisphosphonate on
Membrane-Mediated Calcium Phosphate Formation in Model Liposomal Suspensions.

PB95-169223

03,469

Zimm Plot and Its Analogs as Indicators of Vesicle and Micelle Size Polydispersity

01,094

\section{LIOUEFACTION}

Helium Refrigeration and Liquefaction Using a Liquid $\mathrm{Hy}$ drogen Refrigerator for Precooling.

AD-A286 683/8

02,749

Ground Improvement Techniques for Liquetaction Remediation Near Existing Lifelines.

01,350

Energy-Based Method for Liquefaction Potential Evaluation. Phase 1. Feasibility Study.

03,69

LIQUID ALLOYS

Dynamic Measurements of Thermophysical Properties of Metals and Alloys at High Temperatures by Subsecond Pulse Heating Techniques. N94-25124/6

03,309

\section{LIQUID CHROMATOGRAPHY}

Liquid Chromatography: Laser-Enhanced Ionization Spectrometry for the Speciation of Organolead Compounds.

PB94-185253

00,530

Liquid Chromatographic Method for the Determination of Carotenoids, Retinoids, and Tocopherols in Human Serum and in Food

PB95-153599

00,593

Shape Selectivity in Reversed-Phase Liquid Chromatography for the Separation of Planar and Non-Planar Solutes.

PB95-162608

00,596

Influence of Stationary Phase Chemistry on Shape Recognition in Liquid Chromatography.

00,621

LIOUID CIRCULATION

Internal Droplet Circulation Induced by Surface-Driven Rotation

PB97-119267

02,500

UOUID COLUMN CHROMATOGRAPHY

Device for Subambient Temperature Control in Liquid Chromatography.

00,573

Comparison of the Liquid Chromatographic Behavior of Selected Steroid Isomers Using Different ReversedPhase Materials and Mobile Phase Compositions.

PB95-140976

00,574

Determination of Polycyclic Aromatic Hydrocarbons by Liquid Chromatography.

00,585

Shape Selectivity in Reversed-Phase Liquid Chroma lography for the Separation of Planar and Non-Plana Solutes.

PB95-162608

00,596

Liquid Chromatographic Determination of Polycyclic Aro. matic Hydrocarbon Isomers of Molecular Weight 278 and 302 in Environmental Standard Reference Materials PB95-164042

LIOUID COMPOSITE MOLDING

Report on the Workshop on Manufacturing Polymer Com posites by Liquid Molding. Held in Gaithersburg, Maryland on September 20-22, 1993.

PB94-160066

03,131

UOUID CRYSTALLINE POLYMERS

Phase Behavior of a Hydrogen Bonding Molecular Composite.

PB94-185188

01,202

Neutron Scattering Study of the Orientation of a Liquid Crystalline Polymer by Shear Flow.

PB95-180196

01,270

OUID CRYSTALS

Polymer Liquid Crystalline Materials.

Novel Polydiacetylenes Derived from Liquid Crystalline

MBonomers.

01,235

Studies of the Higher Order Smectic Phase of the Large Electroclinic Effect Material W317.

PB95-151601

00,935

Dielectric Spectroscopic Determination of Temperature Behavior of Electroclinic Parameters in the Liquid Crysta

B96-140397

01,098

$X$-ray Observation of Electroclinic Layer Constriction and PB96-141080

LIOUID DENSITIES

Compressed Liquid Densities, Saturated Liquid Densities, and Vapor Pressures of 1,1-Difluoroethane.

LOUID FUELS

Development of Measurement Capabilities for the Thermophysical Properties of Energy-Related Fluids. Annual Report, December 1, 1990--November 30, 1991

DE94004399

02,470

Combustion of a Polymer (PMMA) Sphere in Microgravity. 


\section{KEYWORD INDEX}

Wear Mechanism Maps of $440 \mathrm{C}$ Martensitic Stainless Steel.

04,834

LIQUID PHASE EPITAXY

RII Spectroscopy of Trap Levels in Bulk and LPE Hg1$\mathrm{xCdxTe}$

PB96-160247

04,084

LIQUID PHASES

New Data and Correlations for the Custody Transfer of Natural Gas Liquids.

PB96-176664

02,499

UIQUID-SOLID INTERFACE

Effects of Elastic Stress on the Stability of a Solid-Liquid

PB95-163028

03,350

LIQUID-SOLID INTERFACES

Boundary Conforming Grid Generation System for Interface Tracking.

03,312

Scanning Tunneling Microscopy and Fabrication of Nanometer Scale Structures at the Liquid-Gold Interface.
PB95-140414 00,904

00,904

sional Liquid-Solid Interface in Solidifying Bodies.

PB95-161782

03,349

Boundary Conforming Grid Generation System for Interface Tracking.
PB96-103007

03,357

LIQUID-VAPOR EQUILIBRIUM

Modified Leung-Gritfiths Model of Vapor-Liquid Equilibrium: Extended Scaling and Binary Mixtures of Dissimilar Fluids.

PB94-216108

00,851

Calculation of Enthalpy and Entropy Differences of NearCritical Binary Mixtures with the Modified Leung-Grittiths

00,885

PB95-108635

Vapor-Liquid Equilibria of Mixtures of Propane and Isomeric Hexanes.
PB95-175287

00,995

Critical Properties and Vapor-Liquid Equilibria of the Binary System Propane + Neopentane.

00,999

LIQUID-VAPOR INTERFACES

Structure of the Vapor-Liquid Interface Near the Critical

00,902

Thermal Equilibration Near the Critical Point: Effects Due

to Three Dimensions and Gravity

03,919

LIQUIDS

Density of Solids and Liquids.

AD-A278 517/8

00,711

Able of Dielectric Constants of Pure Liquids.

AD-A278 956/8 00,712

Near Critical Fluid Interfaces: A Comparison of Theory and Experiment.

B95-140166

00,901

Length Scales for Fragile Glass-Forming Liquids.

(102801

Intrinsic Viscosity and the Polarizability of Particles Hav-

ing a Wide Range of Shapes.

03,170

Long-Lived Structures in Fragile Glass-Forming Liquids.

PB96-119565

04,212

Simulation Studies of Supercooled and Glass Forming

PB96-122627

01,085

LIQUIFIED AIA

Speed-of-Sound Measurements in Liquid and Gaseous PB95-151957

04,186

LITERATURE SURVEYS

Guide to Instrumentation Literature.
AD-A280 278/3

02,617
AD Irradiation Damage in Inorganic Insulation Materials for
ITER Magnets: A Review.
PB95-147351

Volume

Robotics Application

2. Literature Search.

PB95-170551 01,337

Literature Review on Seismic Performance of Building Cladding Systems.

PB96-106901

00,455

LTHHIUM

Angular Distributions for Near-Threshold $(e, 2 e)$ Processes tor $\mathrm{Li}$ and $\mathrm{Mg}$

00,778

Regulation of Lithium and Boron Levels in Normal Human

Blood: Environmental and Genetic Considerations.
PB94-198579

03,491

Determination of Boron and Lithium in Diverse Biological Matrices Using Neutron Activation - Mass Spectrometry (NA-MS)

PB94-212289

00.554

LITHIUM 6

Preparation and Characterization of (6) LiF and (10)B Reference Deposits for the Measurement of the Neutron Lifetime.

03,874

LITHIUM ALLOYS

Recommended Changes in ASTM Test Methods D2512. 82 and G86-84 for Oxygen-Compatibility Mechanical Impact Tests on Metals.

PB94-216694

Macro- and Microreactions in Mechanical-Impact Tests of

Aluminum Alloys.
PB95-107348

Influence of Specimen Absorbed Energy in LOX Mechani-

cal-Impact Tests.

ITHIUM DEUTERIDES

FTS Intrared Measurements of the Rotational and Vibrational Spectrum of $\mathrm{LiH}$ and $\mathrm{LiD}$.

PB94-216231

00,856

LTHIUM FLUORIDE

Colour Centres in LiF for Measurement of Absorbed Doses Up to 100 MGy.

04,169

LITHIUM FLUORIDES

Preparation and Characterization of (6) LiF and (10)B Reference Deposits for the Measurement of the Neutron Lifetime.

PB95-108692

03,874

LTHHIUM HYDRIDES

FTS Infrared Measurements of the Rotational and Vibrational Spectrum of $\mathrm{LiH}$ and $\mathrm{LiD}$.

PB94-216231

00,856

LTHHIUM-LIKE IONS

Laser-Produced and Tokamak Spectra of Lithiumlike Iron Fe(23+).

04,314

LITHOGRAPHY

Test Structures for the In-Plane Locations of Projected Features with Nanometer-Level Accuracy Traceable to a Coordinate Measurement System.

$\begin{array}{ll}\text { PB94-200565 } & 02,313\end{array}$

Practical Photomask Linewidth Measurements.

PB95-108510

02,324

X-ray Mask Metrology: The Development of Linewidth Standards for $\mathrm{X}$-ray Lithography.

PB95-162129

02,348

New Test Structure for Nanometer-Level Overlay and Feature-Placement Metrology.

02,363

Microelectronic Test Structures for Feature Placement and Electrical Linewidth Metrology.

and Electrical

02,367

Nanofabrication of a Two-Dimensional Array Using LaserFocused Atomic Deposition.

PB96-119417

04,732

Use of Monte Carlo Modeling for Interpreting Scanning Electron Microscope Linewidth Measurements. 02,413

Atom-Optical Properties of a Standing-Wave Light Field.
PB96-141072 New NIST/ARPA National Soft X-ray Reflectometry Facility. $96-158092$

04,080

Improved Reflectometry Facility at the National Institute of Standards and Technology.

PB96-160338

NIST Metrology for Soft X-ray Multilayer Optics. PB96-160379

04,087

Microlithography by Using Neutral Metastable Atoms and Self-Assembled Monolayers.

PB96-190038

02,441

ITHOGAAPHY MASKS

SEM Linewidth Metrology of X-ray Lithography Masks. PB96-201108

\section{LTIGATION}

Using a Multi-Layered Approach to Representing Tor Law Cases for Case-Based Reasoning.

PB96-160874

00,135

LIVER

Nickel(II)-Mediated Oxidative DNA Base Damage in Renal and Hepatic Chromatin of Pregnant Rats and Their Fetuses. Possible Relevance to Carcinogenesis.

PB94-212628

03,646

Oxidative DNA Base Damage in Renal, Hepatic, and Pulmonary Chromatin of Rats After Intraperitoneal Injection of Cobalt (II) Acetate.

03,647

B95-150025

DNA Base Damage Generated In vivo in Hepatic Chro matin of Mice upon Whole Body y-Irradiation.

PB95-161741

03,627

LIVERMORE LOOPS

Technique to Evaluate Benchmarks: A Case Study Using the Livermore

04.577

LMR (LASER MAGNETIC RESONANCE)

Guideline for the Analysis of Local Area Network Security. Category: Computer Security.

Analysis and Contingency Planning.
FIPS PUB 191

Planning for the Fiber Distributed Data Interface (FDDI).

PB94-135761

01,621

PB94-172095

ISDN LAN Bridging

PB95-154696

00,527

CAL OSCILLATORS

Ulitra-High

PB94-216066

01,477

LCALIZATION MODEL

Effect of Swelling on the Elasticity of Rubber: Localization Model Description.

(1)

Experimental Results on Single Flux Quantum Logic

95-17507

LOGIC PROGRAMMING

Representing

PB97-111561

LOGISTICS MANAGEMENT

CALS-Department of Defense Computer Aided Acquisition Logistic Support (CALS)

PB94-962500

03,662

CALS-Department of Defense

tion Logistic Support (CALS)

03,671

Federal Implementation Guideline for Electronic Data Interchange: ASC X12 003040 Transaction Set 838 Trading Partner Profile (Confirmation of Vendor Registration). Implementation Convention.

PB96-111190

01,813

PA PRIETA EARTHOUAKE

Assessment of Site Response Analysis Procedures.

$5-210928$

00,450

LORAN C

Using LORAN-C Broadcasts for Automated Frequency PB94-216017

LSS FREE COUNTING

Loss-Free Counting at IRI and NIST.

PB96-167119

04,105

LOSSY MEDIA

Aperture Excitati

(ties.

LOW ALLOY STEELS

Characterization of the Hydrogen Induced Cold Cracking

Susceptibility at Simulated Weld Zones in HSLA-100

AD-A279 759/5

03,200

Apparent Bias in the X-Ray Fluorescence Determination of Titanium in Selected NIST SRM Low Alloy Steels. PB95-108759

LOW FLOW PLUMBING FIXTURES

Water Efficient Plumbing Fixtures through Standards and Test Methods

LOW FREQUENCY

Noise Reduction in Low-Frequency SQUID Measurements with Laser-Driven Switching. 
LOW-LOSS CAPACITORS

Capacitors with Very Low Loss: Cryogenic Vacuum-Gap Capacitors.

PB $97-122600$

02,293

Thermal Conductivity of Metals and Alloys at Low Temperatures. A Review of the Literature.

peratures. A Revie

03,302

Design and Construction of a Liquid Hydrogen Temperature Refrigeration System

AD-A286 $618 / 4$

02,619

Survey of the Literature on Heat Transfer from Solid Surfaces to Cryogenic Fluids.

OW TEMPERATURE CELL

Simple and Efficient Low-Temperature Sample Cell for Intrared Spectrophotometry.

PB94-199197

04,193

LOW TEMPERATURE SCIENCE \& ENGINEERING Cryogenic Materials Data Handbook.

AD-A286 675/4

00,545 drogen Refrigerator for Precooling.

02,749

Cryogenic Blackbody Calibrations at the National Institute of Standards and Technology Low Background Infrared Calibration Facility

PB94-169802

02,117

Effects of Critical Current Density, Equilibrium Magnetization and Surface Barrier on Magnetization of High Temperature Superconductors.

04,446

Surface Barrier and Lower Critical Field in YBa2Cu3O7delta Superconductors.

PB94-200128

04,478

High-Frequency Linear Response of Anisotropic Type-11 Superconductors in the Mixed State.

04,483

Nonlinear Response of Type-ll Superconductors in the Mixed State in Slab Geometry.

PB94-200367

04,484

Cryogenic Properties of Silver

03,330

Critical Magnetic-Field Angle for High-Field Current Transport in $\mathrm{YBa} 2 \mathrm{Cu} 3 \mathrm{O} 7$ at $76 \mathrm{~K}$.

PB94-211281

04,490

Superconducting Materials: Specification.

04,491

Effect of Axial Strain on the Critical Current of AgSheathed Bi-Based Superconductors in Magnetic Fields Up to $25 \mathrm{~T}$.

PB94-211315

04,493

In situ Noble Metal YBa2Cu3O7 Thin-Film Contacts.

04,494

YBa2Cu307-x to $\mathrm{Si}$ Interconnection for Hybrid Superconductor/Semiconductor Integration.
PB94-211711

Flux Expulsion at Intermediate Fields in Type-II Superconductors.

04,502

Proposed High-Accuracy Superconducting Power Meter

for Millimeter Waves.
PB94-212669 02,034

Effect of Thermal Noise on Shapiro Steps in High-Tc Josephson Weak Links.

PB94-212677

04,506

Self-Heating in the Coulomb-Blockade Electrometer.
PB94-212685

Cryogenic Precision Capacitance Bridge Using a Single Electrometer.

PB95-126074 04,529

Magnetic Measurement of Transport Critical Current Den-

sity of Granular Superconductors.
PB95-126199

04,531

$n$-Value and Second Derivative of the Superconductor Voltage-Current Characteristic.

Voltage-Current
PB95-126223

04,533

Thermally Activated Hopping of a Single Abrikosov Vor-

PB95-140810

04,548

Micromagnetic Simulations of Tunneling Stabilized Magnetic Force Microscopy.

PB95-141073

04,552

Experimental Verification of a Micromagnetic Model of Dual-Layer Magnetic Films.

PB95-141081

04,553

Insulating Nanoparticles on YBa2Cu307-delta Thin Films Revealed by Comparison of Atomic Force and Scanning
Tunneling Microscopy. PB95-150843

04,575

Effects of Critical Current Density, Equilibrium Magnetization and Surface Barrier on Magnetization of High Temperature Superconductors.

04,588

Even-Odd Asymmetry of a Superconductor Revealed by

the Coulomb Blockade of Andreev Reflection.

04,593
Preparation of Low Resistivity Contacts for High-Tc Superconductor

02,258

Improved Uniaxial Strain Tolerance of the Critical Current Measured in Ag-Sheathed Bi2Sr2Ca1Cu2O8+x Superconductors. Volume Magnetic Hysteresis Loss of Nb3Sn Superconductors as a Function of Wire Length.
PB95-153722 Tunneling Spectroscopy of Thallium-Based High-TemTuperconductors.

PB95-161709

04,606

Magnetic Force Microscopy of Flux in Superconductors.

04,608

Growth of Laser Ablated YBa2Cu3O7-delta Films as Examined by Rheed and Scanning Tunneling Microscopy.
PB95-162541

Critical Current Density, Irreversibility Line, and Flux Creep Activation Energy in Silver-Sheathed Bi2Sr2Ca2Cu2Ox Superconducting Tapes. PB95-162749

4,616

Welding for Cryogenic Service.

PB95-162889

02,852

Combined Josephson and Charging Behavior of the Supercurrent in the Superconducting Single-Electron Transistor.

04,637

High Current Pressure Contacts to Ag Pads on Thin Film

Superconductors.
PB95-168621

Terahertz Detectors Based on Superconducting Kinetic Inductance.

02,160

Optical Periormance of Photoinductive Mixers at $\begin{array}{ll}\text { Terahertz Frequencies. } & 02,161 \\ \text { PB95-168662 }\end{array}$ Suitability of Metalorganic Chemical Vapor Deposition-Derived $\mathrm{PrGaO} 3$ Films as Buffer Layers for YBa2Cu3O7-x Pulsed Laser Deposition.

04,640 Microwave Properties of YBazCu3O7-x Films at $35 \mathrm{GHz}$
from Magnetotransmission and Magnetoreflection Measfrom Magnetotransmission and Magnetoreflection Measurements.
PB95-168977

04,643

Ultrasensitive-Hot-Electron Microbolometer.

02,163

Hot-Electron Microcalorimeters for $X$-ray and Phonon Detection.
PB95-168993 04,644

04,644
B95-168993 enna-Coupled High-Tc SOUID.

PB95-169116

02,165

High-Tc Superconducting Antenna-Coupled

Microbolometer on Silicon.

Terahertz Shapiro Steps in High Temperature SNS Jo-

PB95-169140 $\quad 02,168$

Optical Detector Nonlinearity: A Comparison of Five

PB95-169355

04,291

Experimental Results on Single Flux Ouantum Logic.

02,053

Characterization of the Emission from 2D Array Joseph-

son Oscillators.

02,054

Emission Linewidth Measurements of Two-Dimensional Array Josephson Oscillators.

02,055

Frequency Dependence of the Emission from 2D Array

JBse5-175147 02,056

Aspects of a Deformable Superconductor Model for the Vortex Mass.

04,652

Deformable Superconductor Model for the Fluxon Mass.

Thermal Enhancement of Cotunneling in Ultra-Small Tunnel Junctions.

B95-175436

04,658

Characterization of a Tunable Thin Film Microwave YBa2Cu3O7-x/SrTiO3 Coplanar Capacitor.

PB95-175527

02,264

Standard Reference Devices for High Temperature Superconductor Critical Current Measurements.

Josephson Voltage Standard Based on Single-Flux-Ouanum Voltage Multipliers.

PB95-175600

02,058

Experimental Aspects of Flux Expulsion in Type-II Superconductors.

04,660

Effect in Environmental Noise on the Accuracy of Couomb-Blockade Devices.

PB95-175865

04,662

Testing for Metro
PB95-175873

04,663

dc Magnetic Force Microscopy Imaging of Thin-Film Recording Head.
PB95-176061

04,665

High Temperature Superconductor-Normal MetalSuperconductor Josephson Junctions with High Characteristic Voltages

04,666

Two-Stage Integrated SOUID Amplifier with Series Array Output

PB95-176277

02,061

PB95-176327

04,670

Increased Transition Temperature in In situ Coevaporated YBa2Cu307-delta Thin Films by Low Temperature PostAnnealing.

Improved Eddy-Current Decay Method for Resistivity Characterization.

PB95-180451

02,265

Antenna-Coupled High-Tc Air-Bridge Microbolometer on Silicon

04,315

Comparison of Magnetic Fields of Thin-Film Heads and Their Corresponding Bit Patterns Using Magnetic Force

Microscopy.
PB95-180907

03,763

Flexible-Diaphragm Force Microscope

PB95-180915

Faraday Effect Current Sensor with Improved Sensitivity Bandwidth Produc

PB95-203154

02,180

Step-Edge and Stacked-Heterostructure High-Tc Josephson Junctions for Voltage-Standard Arrays.

04.702

Ferroelectric Thin Film Characterization Using

Superconducting Microstrip Resonators.
PB96-102389

02,270

SUSAN: SUperconducting Systems ANalysis by Low Temperature Scar

04,728

Thermal Isolation of High-Temperature Superconducting Thin Films Using Silicon Wafer Bonding and Micromachining
PB96-135017

02,408

Noise Reduction in Low-Frequency SOUID Measurements with Laser-Driven Switching.

02,081

Accuracy of the Electron Pump.

B96-135223

04,743

Deep-UV Excimer Laser Measurements at NIST

PB96-141031

04,355

I-3: Critical Current Measurement Methods: Ouantitative Evaluation.

04,767

II-5: Thermal Contraction of Materials Used in Nb3Sn Critical Current Measurements.

PB96-147186

04,769

Microwave Noise in High-Tc Josephson Junctions. PB96-148010

04,771

V-6: Effects of Temperature Variation.

PB96-148143

04,772

National Institute of Standards and Technology High-Accuracy Cryogenic Radiometer.

Cy585 179585

High-Tc Multilayer Step-Edge Josephson Junctions and

PBg6-200183

Performance of the Election Pump with Stray Capacitances

Novel Hot-Electron Microbolometer.

PB96-201025

01,976 
Silicon Nitride Boundary Lubrication: Lubrication Mechanism of Alcohols.

03,067

Silicon Nitride Boundary Lubrication: Effect of Oxygenates.

03,068

LUBRICATION THEORY

Lubrication Theory for Reactive Spreading of a Thin Drop.

PB96-123427

04,214

LUMBER

Voluntary Product Standard PS 20-94. American Softwood Lumber Standard

03,402

LUMINAIRES

Papers Presentations Shine.

PB94-200383

00,244

LUMINOSITY

Detector-Based Candela Scale and Related Photometric Calibration Procedures at NIST.

PB95-161949

04,273

LUMINOUS FLUX

Integrating Sphere Simulation: Application to Total Flux Scale Realization.

04,261

New Method for Realizing a Luminous Flux Scale Using an Integrating Sphere with an External Source. 04,333 Realization of NIST 1995 Luminous Flux Scale Using the Integrating Sphere Method.

PB96-176433

04,374

Performance of Compact Fluorescent Lamps at Different Ambient Temperatures.

PB95-175329 00,258

Intercomparison of Photometric Units Maintained at NIST (USA) and PTB (Germany), 1993

PB95-261913

In-Space Welding: Visions and Realities.

PB95-163234

04,329

UNAR GRAVITATION

Integrated Laser Doppler Method for Measuring Planetary Gravity Fields.
PB94-198686

LUNAR RANGEFINDING

Lunar Laser Ranging: A Continuing Legacy of the Apollo Program.
PB95-202495

03,683

LUNG

Oxidative DNA Base Damage in Renal, Hepatic, and Pulmonary Chromatin of Rats After Intraperitoneal Injection of Cobalt (II) Acetate.

PB95-150025

03,647

LUNGS

Radon in the Lung

PB97-110035

03,638

LUTETIUM

Enhanced Curie Temperatures and Magnetoelastic Domains in Dy/Lu Super Lattices and Films.

04,443

Vibrations of Hydrogen and Deuterium in Solid Solution with Lutetium.

LUTETIUM HYDRIDES

Neutron Spectroscopic Comparison of Rare-Earth/Hydrogen alpha-Phase Systerns.

LYASES

Thermodynamics of Enzyme-Catalyzed Reactions. Part 4.

Lyases.

LYMAN ALPHA RADIATION

Hydrogen Lyman-alpha Emission of Capella.

PB95-202263

New High-Redshift Damped Lyman-aipha Absorption Systems and the Redshift Evolution of Damped AbsorbPB95-203501

00,083

LYMPHOCYTES

DNA Base Damage in Lymphocytes of Cancer Patients Undergoing Radiation Therapy.
PB97-122444

03,643

M PROGRAMMING LANGUAGE

$M$ (also known as MUMPS) Validation Test Suite, Version 8.3 (for Microcomputers)

PB94-502077

Magnetic Dipole Line from U LXXI Ground-Term Levels Predicted at 3200 Angstroms.

PB94-211497

04,407

MACHINE LEARNING

Effect of Training Dynamics on Neural Network Performance.

01,852

MACHINE TOOLS

Open Architectures for Machine Control.

PB94-135621
Prediction of Geometric-Thermal Machine Tool Errors by Artificial Neural Networks.

PB94-186673

02,943

Displacement Method for Machine Geometry Calibration. PB95-152088

02,946

Post-Process Control of Machine Tools.

PB95-203451

02,952

Machine Pertormance Standard Provides Opportunity to Improve Quality and Productivity.

02,837

Development of a New Quality Control Strategy for Automated Manufacturing.

PB96-160486

02,840

Integrated Inspection System for Improved Machine Perormance.

02,959

New Concepts of Precision Dimensional Measurement for Modern Manufacturing.

PB96-160684

02,924

PIECS: A Software Program for Machine Tool ProcessIntermittent Error Compensation.

PB96-165980

02,842

Compensation of Errors Detected by Process-Intermittent GBuging.

02,846

Data Management for Error Compensation and Process Control.

PB97-110480

02,847

Static Structural Analysis of a Reconfigurable Rigid Platorm Supported by Elastic Legs.

PB97-113898

02,960

MACHINE VISION

Hierarchical Interaction between Sensory Processing and World Modeling in Intelligent Systems.

01,580

Physics-Based Vision: Principles and Practice, Shape Re-

Physics-Based Vision: Principles and Practice, Shape Re-
covery (Book Review).
PB95-164075
01,846 PB95-164075

MACHING

Evaluation of a Tapered Roller Bearing Spindle for HighPrecision Hard Turning Applications.

PB96-160494

02,700

MACHINING

Issues Concerning Material Removal Shape Element Volumes (MRSEVs)

PB94-185493 02,882

Japan Technology Program Assessment: Precision Engineering/Precision Optics in Japan.

02,884

Issues Concerning Material Removal Shape Element Volumes (MRSEVs).

02,885

Evaluation of Thermal Wave Imaging for Detection of $\mathrm{Ma}$ chining Damage in Ceramics.

PB95-220547

03,062

Precision in Machining: Research Challenges.

Effects of Spindle Dynamic Characteristics on Hard Turn-

PB96-122981

02,699

Machining Process Planning Activity Model for Systems Integration.

02,84

Chip Morphology, Tool Wear and Cutting Mechanics in Finish Hard Turning

PB97-112247

03,106

Standards Promote Credibility and Technology Transfer: The Need for Greater Industry Support of Technical Com-

PB97-116206

02,961

MACHINING RATE

Chemical Effect in Ceramics Grinding

PB97-122592

03,113

MACROMOLECULES

Book Review: Statistical Physics of Macromolecules

PB96-123526

01,280

MAGENETIC PROPERTIES

Determining the Magnetic Properties of $1 \mathrm{~kg}$ Mass Stand-

PB95-261905

04,016

MAGNESIUM

Angular Distributions for Near-Threshold $(e, 2 e)$ Processes for $\mathrm{Li}$ and $\mathrm{Mg}$

00,778

Effect of Suppressants on Metal Fires.

01,402

PB96-109574

Electric Field Effects on Crack Growth in a Lead MagneSium Niobate.

03,339

MAGNESIUM HYDRIDES

Laboratory Measurements for the Astrophysical Identification of $\mathrm{MgH}$.

MAGNESIUM OXIDES

Reactive Coevaporation of DyBaCuO Superconducting of Bulk Impurities on Annealed $\mathrm{MgO}(100)$ Substrates.

04,635

Dielectric Properties of Single Crystals of Al2O3, LaAlO3, $\mathrm{SrTiO3}$, and $\mathrm{MgO}$ at Cryogenic Temperatures.

PB95-180477

02,266

MAGNETIC CORRELATION LENGTHS

Neutron-Scattering Studies of the Two Magnetic Correlation Lengths in Terbium.

PB95-152328

04,586

MAGNETIC DETECTION

Gradiometer Antennas for Detection of Long Subsurface Conductors.

01,862

MAGNETIC DEVICES

Size and Self-Field Effects in Giant Magnetoresistive Thin-Film Devices.

PB95-180188

04,674

MAGNETIC DOMAINS

Micromagnetic Structure of Domains in Co/Pt Multilayers.

1. Investigations of Wall Structure.

04,610

Magnetic Force Microscopy Images of Magnetic Garnet with Thin-Film Magnetic Tip.

PB95-176210

04,669

MAGNETIC ENERGY STORAGE EQUIPMENT

Composite Struts for SMES Plants.

02,507

MAGNETIC EXCITATIONS

Polarization Analysis of the Magnetic Excitations in Fe65Ni35 Invar.

04,558

Temperature Dependence of the Magnetic Excitations in Ordered and Disordered Fe72P:28.

PB95-150223

04,563

MAGNETIC FIELD

NIST Watt Experiment: Monitoring the Kilogram. PB97-122329

01,997

MAGNETIC FIELD SENSORS

Magneto-Optic Magnetic Field Sensor with $1.4 \mathrm{pT} / \mathrm{square}$ root of $1(\mathrm{~Hz})$ Minimum Detectable Field at $1 \mathrm{kHz}$.

PB94-199551

02,125

High Speed, High Sensitivity Magnetic Field Sensors Based on the Faraday Effect in Iron Garnets. PB95-153391

02,151

Magneto-Optic Magnetic Field Sensors Based on Uniaxial Iron Garnet Films in Optical Waveguide Gøometry. PB95-153409

02,152

Magneto-Optic Magnetic Field Sensors Based on Uniaxial Iron Garnet Films in Optical Waveguide Geometry.

PB95-168498 02,159

Novel Bulk Iron Garnets for Magneto-Optic Magnetic Field Sensing.

PB95-180204

04,675

Domain

PB95-202461

High Frequency Magnetic Field Sensors Based on the Faraday Effect in Garnet Thick Films.

PB96-190384

02,282

Wideband Current and Magnetic Field Sensors Based on Iron Garnets.

01,975

MAGNETIC FIELDS

Faraday Effect Sensors: A Review of Recent Progress.

PB94-200706

02,128

Three-Axis Coil Probe Dimensions and Uncertainties dur- 
Experimental Verification of a Micromagnetic Model of Dual-Layer Magnetic Films.

04,553

Magnetic and Magnetoresistive Properties of Inhomogeneous Magnetic Dual-Layer Films.
PB95-169025 $X$-ray Photoelectron and Auger Electron Forward Scattering: A Structural Diagnostic for Epitaxial Thin Films. PB95-180220

04,676

MAGNETIC FLUX

Magnetic Force Microscopy of Flux in Superconductors. PB95-161733 04,608 Relationship between Radiative and Magnetic Fluxes for Three Active Solar-Type Dwarts

PB96-119540

00,097

MAGNETIC FORCE MICROSCOPY

Micromagnetic Simulations of Tunneling Stabilized Mag. netic Force Microscopy

04,552

Tunneling Stabilized Magnetic-Force Microscopy.

PB95-161717 04,607

Magnetic Force Microscopy of Flux in Superconductors.

PB95-161733 04,608

Proposed Antiferromagnetically Coupled Dual-Layer Magnetic Force Microscope Tips.

04,645

Vortex Images in Thin Films of $\mathrm{YBa} 2 \mathrm{Cu} 3 \mathrm{O}$ (sub $7-\mathrm{x}$ ) and Bi2Sr2Ca1Cu2O(sub $8+x$ ) Obtained by Low-Temperature Magnetic Force Microscopy.

PB97-119408

04,815

MAGNETIC FORCES

Methods for Aligning the NIST Watt-Balance.

PB96- 123153

01,934

NIST Watt Balance: Progress Toward Monitoring the Kilogram.

01,991

MAGNETIC HETEROSTRUCTURES

Exchange Coupling in Magnetic Heterostructures.
PB95-150314

AGNETIC HYSTERESIS

Volume Magnetic Hysteresis Loss of Nb3Sn

Superconductors as a Function of Wire Length.

PB95-153722

04,597

MAGNETIC MATERIALS

Surface Magnetic Microstructural Analysis Using Scan-

ning Electron Microscopy with Polarization Analysis PB95-162657

03,938

Nanocomposite Magnetic Materials. PB95-162780

04,617

MAGNETIC MEASUREMENT

Metrology for Electromagnetic Technology: A Bibliography of NIST Publications.

02,143

Domain Effects in Faraday Effect Sensors Based on Iron Domain Effects in Faraday Effect Sensors Based on Iron
Garnets.
PB95-202461
02,268

Distributions of Measurement Error for Three-Axis Magances.

PB96-180153 02,110

High Frequency Magnetic Field Sensors Based on the Faraday Effect in Garnet Thick Films.

PB96-190384

02,282

AGNETIC MOMENTS

Magnetic Moments in $\mathrm{Cr}$ Thin Films on $\mathrm{Fe}(100)$

PB95-108429

04,525

MAGNETIC MULTILAYERS

SEMPA Studies of Exchange Coupling in Magnetic

Multilayers.
PB96-164074

04,780

Spin-Dependent Interface Transmission and Reflection in Magnetic Multilayers (Invited)

04,130

MAGNETIC ORDER

Neutron Scattering Study of Antiferromagnetic Order in the Magnetic Superconductors RNi2B2C.

PB97-112411

04,812

MAGNETIC ORDERING

Incommensurate Magnetic Order in UPtGe.

PB95-1405 13

04,542

Magnetic Ordering of the Cu Spins in PrBa2Cu3O6+x.

Crysta Structure and Magnetic Ordering of the Rare

Earth and $\mathrm{Cu}$ Moments in $\mathrm{RBa2Cu} 2 \mathrm{NbO8}(\mathrm{R}=\mathrm{Nd}, \mathrm{Pr})$

PB95-140554

04,546

Coupled-Bilayer Two-Dimensional Magnetic Order of the Dy lons in Dy2Ba4Cu7O15.

04,584

Neutron Powder Diffraction Study of the Nuclear and Magnetic Structures of the Oxygen-Deficient Perovskite YBaCuCoO5.

Field Dependence of the Magnetic Ordering of $\mathrm{Cu}$ in $\mathrm{R} 2 \mathrm{CuO} 4(\mathrm{R}=\mathrm{Nd}, \mathrm{Sm})$

04,633
Observation of Oscillatory Magnetic Order in the Antiferromagnetic Superconductor HoNi2B2C

04,679

\section{MAGNETIC PERMEABILITY}

Transmission/Reflection and Short-Circuit Line Methods for Measuring Permittivity and Permeability.

02,211

MAGNETIC PROPERTIES

Monte Carlo and Mean-Field Calculations of the Magnetocaloric Effect of Ferromagnetically Interacting Clusters.

PB94-172087

03,201

Magnetocaloric Effect in Nanocomposites.

04,618

Recent Results in Magnetic Force Microscopy. PB96-103130

04,721

Magnetic Structure and Spın Dynamics of the Pr and Cu in Pr2CuO4.

04,036

Preparation, Crystal Structure, Dielectric Properties, and Magnetic Behavior of Ba2Fe2Ti4O13

01,162

Dielectric and Magnetic Measurements from $-50 \mathrm{C}$ to $200 \mathrm{C}$ and in the Frequency Band $50 \mathrm{MHz}$ to $2 \mathrm{GHz}$. PB96-191382

02,245

MAGNETIC RECORDING

Experimental Verification of a Vector Preisach Model PB95-163564

04,626

MAGNETIC RESONANCE

Atomic Iron in Its (5)D Ground State: A Direct Measurement of the $\mathrm{J}=0$ inverted arrow 1 and $\mathrm{J}=1$ inverted arrow 2 Fine-Structure intervals (1.2). PB96-141221

04,756

MAGNETIC SPECTROSCOPY

Binder Characterization and Evaluation by Nuclear Magnetic Resonance Spectroscopy.

01,334

AAGNETIC STARS

Class of Radio-Emitting Magnetic B Stars and a WindFed Magnetosphere Model.

PB94-213451

00,067

Radio Emission from Chemically Peculiar Stars.

PB94-213469

00,068

MAGNETIC STRUCTURE

Colossal Magnetoresistance without $\mathrm{Mn}(3+) / \mathrm{Mn}(4-)$ Double Exchange in the Stoichiometric Pyrochlore TI2Mn2O7.
PB97-113070

MAGNETIC SUSCEPTIBILITY

Alternating-Field Susceptometry and Magnetic Susceptibility of Supercondoctors. Presented at Office of Naval Research Workshop on Magnetic Susceptibility of Superconductors and Other Spin Systems.
ley Springs, West Virginia on 20 May 1991.

PB94-145984

04,435

Harmonic and Static Susceptibilities of YBa2Cu3O7.

04,599

Alternating-Field Susceptometry and Magnetic Susceptibility of Superconductors.

04,638

Magnetic Susceptibility of $\mathrm{Pr} 2-x \mathrm{CexCuO} 4$ Monocrystals Magnetic Susceptibility of $\mathrm{Pr}-\mathrm{xCexCuO} 4$ Monocrystals
and Polycrystals.
PB95-180253

MAGNETIC TRAPS

Gravitational Sisyphus Cooling of (87)Rb in a Magnetic Trap.

04,379

Stable, Tightly Confining Magnetic Trap for Evaporative Cooling of Neutral Atoms.

04,126

MAGNETIORESISTANCE

Domain Structures in Magnetoresistive Granular Metals. PB96-141346

04,760

MAGNETISM

Magnetic Neutron Scattering (Invited).
PB95-150074

Critical Current Density, Irreversibility Line, and Flux Creep Activation Energy in Silver-Sheathed Bi2Sr2Ca2Cu2Ox Superconducting Tapes.

PB95-162749

04,616

SEMPA Studies of Oscillatory Exchange Coupling. PB95-163556 04,625 Influence of Thickness pling in $\mathrm{Fe} / \mathrm{Cr} / \mathrm{Fe}$ Structures.

04,745

Panel Discussion on Units in Magnetism

PB96-137773 04,746 Isolated Spin Pairs and Two-D
SrCr(sub $9 p) \mathrm{Ga}($ sub 12-9p)O19.

PB97-112387

04,154

MAGNETIZATION

Effects of Critical Current Density, Equilibrium Magnetization and Surface Barrier on Magnetization of High Tem perature Superconductors.

PB94-185162

04,446

Effects of Critical Current Density, Equilibrium Magnetization and Surface Barrier on Magnetization of High Temperature Superconductors.

PB95-153060

04,588

Simulating Device Size Effects on Magnetization Pinning Mechanisms in Spin Valves.

PB97-112593

04,158

MAGNETO-OPTIC

Dependence of Contrast on Probe/Sample Spacing with the Magneto-Optic Kerr-Effect Scanning Near-Field Optical Microscope (MOKE-SNOM)

AGNETO-OPTICAL EFFECTS

Theory of the Magneto-Optic Kerr Effect in the Near

PB96-141387

04,761

MAGNETO OPTICS

Fiber-Optic Faraday-Effect Magnetic-Field Sensor Based on Flux Concentrators.

AGNETOABSORPTION RII Spectroscopy of Trap Levels in Bulk and LPE Hg1-
XCdxTe.

PB96-160247

04,084

IAGNETOCALORIC EFFECT

Monte Carlo and Mean-Field Calculations of the Magnetocaloric Effect of Ferromagnetically Interacting

Enhanced Magnetocaloric Effect in Gd3Ga5-xFexO12

PB94-185659

04,450

Magnetocaloric Effect in Rapidly Solidified Nd-Fe-Al-B PBg4-185667

Magnetocaloric Effect of Ferromagnetic Particles.
PB94-185857

04,451

MAGNETOCONDUCTANCE

Measurement of the Weak-Localization Complex Conductivity at $1 \mathrm{Ghz}$ in Disordered Ag Wires.

MAGNETOMETERS

Faraday Effect Sensors: A Review of Recent Progress.

PB94-200706

02,128

Low Noise YBa2Cu3O7-x-SrTiO3-YBa2Cu3O7-x Mulitlayers for Improved Superconducting Magnetometers.

Magnetometer Calibration Services.

04,747

PB97-113252

01,993

MAGNETOOPTICS

Magneto-Optical Trapping of Metastable Xenon: IsotopeShift Measurements.

15125

Magnetooptic Effects.

PB96-119292

03,905

AGNETORESISTANCE

Effects of Interfacial Roughness on the Magnetoresistance of Magnetic Metallic Multilayers.
PB95-150017 04,556 Transverse Magnetoresistance: A Novel Two-Terminal Method for Measuring the Carrier Density and Mobility of a Semiconductor Layer.

PB95-150066

02,332

Magnetic and Magnetoresistive Properties of Inhomogeneous Magnetic Dual-Layer Films. Micromagnetic Scanning Microprobe System. PB95-176178

02,224

Size and Self-Field Effects in Giant Magnetoresistive Thin-Film Devices.

Model for Determining the Density and Mobility of Carriers in Thin Semiconducting Layers with Only Two Con

PB96-102702

02,378

Characterization of Liquid-Phase Epitaxially Grown $\mathrm{HgCdTe}$ Films by Magnetoresistance Measurements. 


\section{KEYWORD INDEX}

MAGNETORESISTIVITY

Magnetostriction and Giant Magnetoresistance in Annealed NiFe/Ag Multilayers.

04,716

Low Magnetostriction in Annealed NiFe/Ag Giant

Magnetoresistive Multilayers.
PB96-146691

Telegraph Noise in Silver-Permalloy Giant

Magnetoresistance Test Structures.

Magnetoresistance of Thin-Film NiFe Devices Exhibiting Single-Domain Behavior.

PB96-147087

04,766

MAGNETORESTRICTION

Low Magnetostriction in Annealed NiFe/Ag Giant Magnetoresistive Multilayers.
PB96-146691

MAGNETOSTATICS

Size Effects and Giant Magnetoresistance in Unannealed NiFe/Ag Multilayer Stripes.

NiFe/Ag Multilaye

04,145

MAGNETOSTRICTION

Magnetoelasticity in Rare-Earth Multilayers and Films.
PB94-211356

Method for Determining Both Magnetostriction and Elastic

Modulus by Ferromagnetic Resonance.

02,974

Magnetostriction and Giant Magnetoresistance in Annealed NiFe/Ag Multilayers.

PB96-102603

04,716

MAGNIFICATION STANDARDS

Report on the NIST Low Accelerating Voltage SEM Magnification Standard Interlaboratory Study.

PB96-201074

02,445

MALEIMIDEN-PHENYL

Thermal Behaviour of Methyl Methacrylate and N-Phenyl Maleimide Copolymers.

01,246

Copolymerization of N-Phenyl Maleimide and gammaMethacryloxypropyl Trimethoxysilane.

01,248 PB95-153144

MALTODEXTRIN

Protein Extraction in a Spray Column Using a Polyethylene Glycol Maltodextrin Two-Phase Polymer System.
PB95-162228

MAMMOGRAPHY

Noninvasive High-Voltage Measurement in Mammography by Crystal Diffraction Spectroscopy.

Flat and Curved Crystal Spectrography for Mammographic X-ray Sources.

03,642

MAN

Electron transport calculations with biomedical and environmental applications. Final report, December 23, 1992January 31, 1994.

JE95007065

03,613

MAN COMPUTER INTERFACE User Interface Component of the Applications Portability
Profile Category: Software Standard; Subcategory: Application Program Intertace.

01,793

Experimental Evaluation of Specification Techniques for Improving Functional Testing. Improving Functid
PB96-201009

MANAGEMENT

01,779

Models, Managing Models, Quality Models: An Example of Quality Management.

PB94-163466

02,891

Domain Analysis of the Alarm Surveillance Domain. Version 1.0. Conducted as Part of the Domain Analysis Case Study Project.

01,705

Distributed Systems: Survey of Open Management Ap-

PB96-128202

01,746

MANAGEMENT INFORMATION SYSTEMS

Integration Definition for Function Modeling (IDEFO); Category: Software Standard; Subcategory: Modeling Tech-

FIPSPUB183

02,800

Fire Data Management System, FDMS 2.0, Technical Documentation

01,358

Production Management Information Model for Discrete Production Management Information Model for Discrete
Manufacturing.
PB96-112008
02,830

02,830

Effect of Mn Content on the Microstructure of Al-Mn Al-

loys Electrodeposited at 150C.

ANGANESE ALLOYS

Electrochemical Synthesis of Metal and Intermetallic Composites.

MANIPULATORS

Evolution of the Flight Telerobotic Servicer.
PB94-21608?
Three Dimensional Position Determination from Motion PB95-107108

01,788

MANOMETERS

Intercomparison of the Effective Areas of a Pneumatic Piston Gauge Determined by Different Techniques. PB94-212370

02,640

IANUALS

ISO TC 184/SC4 Reference Manual.

B95-242293

NIST ATM Network Simulator: Operation and Programming, Version 1.0

MANUFACTURED HOUSING

Controlling Moisture in the Walls of Manufactured Housing.

00,355

MANUFACTURERS

Network Brokers Handbook: An Entrepreneurial Guide to Cooperative Strategies for Manufacturing Competitive.

PB95-219325

00,490

MANUFACTURING

Opportunities for Innovation: Advanced Manufacturing PBechnology.

02,801

Applying Virtual Environments to Manufacturing. 02,803
PB94-142502

Learning to Change: Opportunities to Improve the Perormance of Smaller Manufacturers.

PB94-166212

00,010

World Model Registration for Effective Off-Line Programming of Robots.

Importance of Measurement in Technology-Based Com-

petition.

02,929

Program Requirements to Advance the Technology of Custom Footwear Manufacturing

02,883

Opportunities for Innovation: Software for Manufacturing.
PB95-155578

Optical Characterization in Microelectronics Manufactur-

$\begin{array}{ll}\text { PBg. } & \text { 02-169397 } 358\end{array}$

Critical Issues in Scanning Electron Microscope Metrol-

PB95-169405

02,359

Apparel Manufacturing Glossary for Application Protocol Development

02,755

Proceedings of the Manufacturing Technology Needs and Issues: Establishing National Priorities and Strategies Conference. Held in Gaithersburg, Maryland on April 2628, 1994.

PBं95-206181

02,930

Network Brokers Handbook: An Entrepreneurial Guide to Cooperative Strategies for Manufacturing Competitive-

ness.

00,490

Conceptual Design Plan for the National Advanced Manufacturing Testbed.

PB95-231866

02,828

Joint DoD/NIST Workshop on International Manufacturing Systems Research and Development. Held in Rockville. Maryland on November $3-5,1992$. Proceedings.

PB96-109491

02,931

Agile Manufacturing from a Statistical Perspective.

PB96-109525

02,886

Can Displays Deliver a Full Measure: Manufacturing.
PB96-111935

Proceedings of the Annual Manufacturing Technology Conference (2nd): Toward a Common Agenda. Held in

Gaithersburg, Maryland on April 18-20, 1995.
PB96-112693

02,887

Scanning Electron Microscope Metrology. PB96-201090

MANUFACTURING ENGINEERING LABORATORY

Publications of the Manufacturing Engineering Laboratory Covering the Period January 1989-September 1992.

PB94-165966

Program of 995. Infrastructural Technology Measurements, and Standards for the U.S. Manufacturing Industries. B95-188835

02.754

MAPPING

Metrication.

METRICATION: An Economic Wake-Up Call for SurveyOrs and Mappers.

ARCH-DOLLASE

03,680

Texture Measurement of Sintered Alumina Using the March-Dollase Function.

PB96-179494

04,784

MARINE ANIMALS

Determination of PCBs and Chlorinated Hydrocarbons in Marine Mammal Tissues.

03,744

MARINE ATMOSPHERES

Joumal of Research of the National Institute of Standards and Technology, January/February 1996. Volume 101 Number 1.

International Marine-Atmospheric (222)Rn Measurement Intercomparison in Bermuda. Part 1. NIST Calibration and Methodology for Standardized Sample Additions.

PB96-175674 Intercomparison in Bermuda. Part 2. Results for the Participating Laboratories.

PB96-17568?

00,115

MARINE CHEMISTRY

Atmospheric and Marine Trace Chemistry: Interfacial Biomediation and Monitoring.

03,752

MARINE ENVIRONMENTS

Comparison of Methods for Gas Chromatographic Determination of PCBs and Chlorinated Pesticides in Marine Reference Materials.

PB95-140091

02,584

MARINE MAMMALS

Current Activities Within the National Biomonitoring Specimen Bank.

Determination of Inorganic Constituents in Marine Mam-

mal Tissues.

00,589

Development of the National Marine Mammal Tissue

PB95-161402

02,586

Quality Assurance of Contaminant Measurements in $\mathrm{Ma}$ rine Mammal Tissues.

PB95-164034

02,588

Alaska Marine Mammal Tissue Archival Project: Specimen Inventory.

02,589

Concentrations of Chlorinated Hydrocarbons, Heavy Metals and Other Elements in Tissues Banked by the Alaska Marine Mammal Tissue Archival Project.

MBa5-209870

02,590

Quantitative Analysis of Selected PCB Congeners in $\mathrm{Ma}$ rine Matrix Reference Materials Using a Novel Cyanobiphenyl Stationary Phase.

PB96-111737

02,591

Trace Element Concentrations in Cetacean Liver Tissues

Archived in the National Marine Mammal Tissue Bank.
PB96-167127
02,595

Relationship of Silver with Selenium and Mercury in the Liver of Two Species of Toothed Whales (Odontocetes). PB96-167275

MARINE SEDIMENTS

Certification of Polycyclic Aromatic Hydrocarbons in a Marine Sediment Standard Reference Materia! PB96-111778

02,592

MARKET RESEARCH

India: Environmental Technologies Export Market Plan PB97-114359

MARKETING

Method of Sale for CNG Paves Way to Greater Public

PB95-168449

02,489

MARKOV ESTIMATORS

Compensation of Markov Estimator Errors in Time-Jittered Sampling

01,590

MARSHALL COMPACTION HAMMER

Field Evaluation of the System for Calibration of the Marshall Compaction Hammer.

PB95-190674

01,323 
Advanced Mass Calibration and Measurement Assurance Program for State Calibration Laboratories.

Determining the Magnetic Properties of $1 \mathrm{~kg}$ Mass Standards.

PB95-261905

04,016

NIST Watt Experi
PB97-122329

01,997

MASS ATTENUATION COEFFICIENTS

Tables of X-ray Mass Attenuation Coefficients and Mass Energy-Absorption Coefficients $1 \mathrm{keV}$ to $20 \mathrm{MeV}$ for Elements $Z=1$ to 92 and 48 Additional Substances of Dosimetric Interest.

PB95-220539

04,013

MASS ENERGY-ABSORPTION COEFFICIENTS

Tables of X-ray Mass Attenuation Coefficients and Mass Energy-Absorption Coefficients $1 \mathrm{keV}$ to $20 \mathrm{MeV}$ for Elements $Z=1$ to 92 and 48 Additional Substances of PB95-220539

04,013

\section{MASS FLOW}

Energy Flows in an Orifice Pulse Tube Refrigerator. PB95-169082

ASS FLOW MEASUREMENT

Cryogenic Flow Calibration in NIST

PB96-161930

01,143

ASS FRAGMENTOGRAPHY

Chemical Determination of Oxidative DNA Damage by Gas Chromatography-Mass Spectrometry.

03,540

MASS MEASUREMENT

Use of the Electronic Balance for Highly Accurate Direct Mass Measurements Without the Use of External Mass Standards.

PB94-187713

03,803

Mass and Density Determinations.

00,504

PB94-200672

MASS SPECTROMETERS

Compositional Mapping of the Microstructure of Materials.

00,565

Characteristics of Partial Pressure Analyzers.

00,582

Electron-Ion-X-ray Spectrometer System.

03,958

Isotope Dilution Mass Spectrometry as a Candidate Definitive Method for Determining Total Glycerides and Triglycerides in Serum.

PB96-102280

03,519

Analysis by a Combination of Gas Chromatography and Tandem Mass Spectrometry: Development of Quantitative Tandem-in-Time Ion Trap Mass Spectrometry: Isotope Dilution Quantification of 11-Nor-Delta-9-Tetrahydro cannabinol-9-Carboxylic Acid.

PB96-117221

02,561 Procedure for Measuring Trace Ouantities of S2F10, $\mathrm{S} 2 \mathrm{OF} 10$, and $\mathrm{S}_{2} \mathrm{O} 2 \mathrm{~F}_{10}$ in SF6 Using a Gas Chromatograph-Mass Spectrometer.

PB96-119755

02,513

MASS SPECTROMETRY

$36 \mathrm{Cl} / \mathrm{Cl}$ Accelerator-Mass-Spectrometry Standards: Verification of Their Serial-Dilution-Solution Preparations by Radioactivity Measurements. L-threo-beta-Hydroxyhistidine, an Unprecedented Iron(III) lon-Binding Amino Acid in a Pyoverdine-type Siderophore from Pseudomonas fluorescens 244.

00,553

Determination of Boron and Lithium in Diverse Biologica Matrices Using Neutron Activation - Mass Spectrometry Matrices

PB94-212289

00,554

MASS SPECTROSCOPY

National Institute of Standards and Technology Resonance lonization Spectroscopy/Resonance Ionization Mass Spectroscopy Data Service.

03,793 Comparative Strategies for Correction of Interferences in
Isotope Dilution Mass Spectrometric Determination of VaIsotope

PB94-185261

00,531

Precision and Accuracy in Tandem Mass Spectrometry Measurements: A Kinetics-Based Protocol for Instrument Independent Measurements of Collision-Activated Dissociation in RF-Only Ouadrupoles.

PB94-216454

00,858

Use of Kinetic Energy Distributions to Determine the Relative Contributions of Gas Phase and Surface Fragmentation in $\mathrm{KeV}$ lon Sputtering of a Quaternary Ammonium Salt.

PB95-126108

00,570

Molecular Ion Imaging and Dynamic Secondary ion Mass Spectrometry of Organic Compounds.

00,571 Factorial Design Techniques Applied to Optimization of
AMS Graphite Target Preparation. PB95-151197

00,584

Single-Photon Ionization and Detection of $\mathrm{Ga}$, In, and As(sub $n$ ) Species in GaAs Growth.

00,591
Reference Materials by Isotope Dilution Mass SpectromPBg onization Energy of Sulfur Pentafluoride and the Sulfur Pentafluoride-Fluorine Atom Bond Dissociation Energy.
PB95-162814 Using Secondary Ion Mass Spectrometry (SIMS) to Charcterize Optical Waveguide Materials.

PB96-119599

04,340

MASS STANDARDS

Mass Unit Disseminated to Surrogated Laboratories Using the NIST Portable Mass Calibration Package.

PB94-142486

Examination of Parameters That Can Cause Error in

03,784

Advanced Mass Calibration and Measurement Assurance Program for State Calibration Laboratories.

02,492

MASS TRANSFER

Enzyme and Protein Mass Transfer Coefficient in Aqueous Two Phase Systems. 1. Spray Extraction Columns.
PB95-161162 Droplet Transfer Modes for a MIL 100S-1 GMAW Electrode. 209300

02,867

MASSIVELY PARALLEL PROCESSORS

Self-Organizıng Neural Network Character Recognition on a Massively Parallel Computer.

PB95-163994

01,845

MATERIAL MODULES

Wire Bonding to Multichip Modules and Other Sott SubStrates.

02,079

Wire Bonding to Multichip Modules and Other Sott Sub-

PB96-135207

02,082

MATERIAL REMOVAL SHAPE ELEMENT VOLUMES

Issues Concerning Material Removal Shape Element Volumes (MRSEVs)

Issues Concerning Material Removal Shape Element Volumes (MRSEVS)
PB95-210167

MATERIAL STRENGTH

02,885

One-Sided beta-Content Tolerance Intervals for Mixed Models

03,449

MATERIALS

Preliminary Subject and Authors Index to Compilations of Data on Properties of Materials.

AD-A302 669/7

03,242

Preliminary List of References Containing Compilations of Data on Properties of Materials.

Data on Propertie

03,243

Intelligent Processing of Materials, Technical Activities 1993 (NAS-NAC Assessment Panel, April 21-22, 1994). PB94-164183 02,809

Industry and Government-Laboratory Cooperative $\mathrm{B}$ and D. An Idea Whose Time Has Come.

PB94-172939

02,970

Fire Growth Models for Materials.

PB94-195856

01,367

Evaluation of Corrosion Data: A Review.

03,187

Access Paths for Materials Databases: Approaches for Large Databases and Systems. PB95-162525

02,975

Glimpse of Materials Research in China: A Report from an Interagency Study Team on Materials Visiting China from June 19,1995 to June 30,1995

PB96-112677

02,978

Intelligent Processing of Materials, Technical Activities 1994 (NAS-NRC Assessment Panel, April 6-7, 1995).

PB96-115050

03,359

Beyond the Technology Roadmaps: An Assessment of Electronic Materials Research and Development.

PB96-165998

01,961

Materials Reliability. Technical Activities, 1995

PB96-183082

02,999

Guide to Locating and Accessing Computerized Numeric Materials Databases.

PB96-204045

03,007

Conventional and Eccentric Uses of Crystallographic Databases in Practical Materials Identification Problems. PB97-109102

04,802

MATERIALS CHARACTERIZATION

Design, Construction and Application of a Large Aperture Lens-Less Line-Focus PVDF Transducer.

02,765

MATERIALS CHEMISTRY

Vapor Transport in Materials and Process Chemistry.
PB94-211745

MATERIALS RECOVERY

Vapor Transport in Materials and Process Chemistry.
PB94-211745

Bioleaching of Cobalt from Smelter Wastes by 'Thiobacillus ferrooxidans'.

02,582

MATERIALS SCIENCE

Materials Science with SR Using X-Ray Imaging: SpatialPB94-213048

04,510

New Materials, Advanced Ceramics and Standards.

PB95-140208

03,047

Neutron Techniques in Materials Science and Related Disciplines.

02,980

MATERIALS SCIENCE AND ENGINEERING LABORATORY Materials Science and Engineering Laboratory Annua Report, 1993. NAS-NRC Assessment Panel, April 21-22,

PB94-162534

02,969 Materials Science and Engineering Laboratory Annual
Report, 1994. NAS-NRC Assessment Panel, April 6-7, PB95-196697

02,976

MATERIALS SCIENCE AND ENGINEERING LABORATORY MSEL)

Materials Science and Engineering Laboratory Annual Report, 1995.

03,009

\section{MATERIALS SUBSTITUTION}

Fire Suppression System Performance of Alternative Agents in Aircratt Engine and Dry Bay Laboratory Simula. Agns. SP890: Volume 1.

PB96-117775

03,277

Fire Suppression System Performance of Alternative Agents in Aircratt Engine and Dry Bay Laboratory SimulaPB96-117783

03,278

MATERIALS TESTING

Assessment of Testing Methodology for Ceramic Matrix Composites.

PB94-200532

03,139

Proficiency Tests for the NIST Airborne Asbestos Program, 1993.

MATHEMATICAL MODELS

00,610

Numerical Analysis Support for Compartment Fire Modeling and Incorporation of Heat Conduction into a Zone Fire

$\begin{array}{ll}\text { PB94-156965 01,357 } & \end{array}$

Replicate Measurements for Data Ouality and Environmental Modeling.

02,515

Scientific Protocols in Statistical Standards for Environmental Studies.

02517

Sulfate Attack of Cementitious Materials: Volumetric Relations and Expansions.

PB94-187317

03,232

Mathematical Modeling of Human Egress from Fires in Residential Buildings.

00,337

Analysis of Moisture Accumulation in a Wood-Frame Wall Anter Climate.

PB94-199320 
MATHEMATICAL SOFTWARE

Software Libraries, Numerical and Statistical.

PB94-198967 01,689

Portable Vectorized Software for Bessel Function Evaluation.

01,690

01,691

Virtual Software
PB94-198983

MATHEMATICAL \& STATISTICAL METHODS

Computing Effects and Error for Large Synthetic Perturbation Screenings.

01,675

Assessment of Uncertainties of Calibration of Resistance Thermometers at the National Institute of Standards and Technology.

02,624

Growth Surface for the Slopes at the Boundary of a Poly-

gBon. 152725

03,408

Boundary Conforming Grid Generation System for Interface Tracking.

03,312

Computation of Dendrites Using a Phase Field Model.

PB94-160744

04,436

Comparison of Finite Element and Analytic Calculations of the Resonant Modes and Frequencies of a Thick Shell

PB94-160785

02,626

Uncertainties in Dimensional Measurements Made at Nonstandard Temperatures.

PB94-169760

02,893

Derivation of the System Equation for Null-Balanced Total-Power Radiometer System NCS1.

02,022

Evaluation of Uncertaintıes of the Null-Balanced TotalPower Radiometer System NCS1.

PB94-16979

02,023

Monte Carlo Approach to the Approximation of Invariant Measures.

PB94-172053

03,409

Infinite Divisibility and the Identification of Singular Waveforms

Complete Reduction of the Euler-Poinsot Problem.

PB94-172152

02,701

PC-Based Prototype Expert System for Data Management and Analysis of Creep and Fatigue of Selected Materials at Elevated Temperatures.

PB94-172251

03,202

Error Propagation Biases in the Calculation of Indentation Fracture Toughness for Ceramics.

03,032

Replicate Measurements for Data Quality and Environmental Modeling.

PB94-172533 02,515

Process for Selecting Standard Reference Algorithms for Evaluating Coordinate Measurement Software.

PB94-173754

02,629

Anharmonic Oscillator Analysis Using Modified Airy Func-

03,798

Scientific Protocols in Statistical Standards for Environmental Studies.

02,517

Experimental Optimization of Peak Shape with Application to Aerosol Generation.

00,501

Modified Airy Function Method for the Analysis of Tunneling Problems in Optical Waveguides and Quantum Well Structures.

PB94-185824

Computıng S(alpha) Using Symbolic Monte Carlo.

PB94-198660

03,410

Modeling Polarization Curves and Impedance Spectra for Simple Electrode Systems.

PB94-198876

03,188

Algorithmic Enhancements to the Method of Centers for Linear Programming Problems.

03,426

Portable Vectorized Software for Bessel Function Evalua-

PB94-198975

01,690

Statistical Quality Control Technology in Japan.
PB94-199064

Slowly Divergent Space Marching Schemes in the Inverse Heat Conduction Problem.

PB94-199486

03,812

Application of a Simple Technique for Estimating Errors of Finite-Element Solutions Using a General-Purpose PB94-200250

04,818

Analysis of Scattering Asymmetry Statistics When Background Corrected Counts Are Negative.

03,824

Uncertainty Intervals for Polarized Beam Scattering Asymmetry Statistics.
PB94-200342

03,825
Hypercubic Lattice SAW Exponents nu and gamma : 3.99 Dimensions Revisited.

01.215

Guidelines for Reporting Results of Computational Experiments. Report of the Ad hoc Committee

03,427

Chaos in a Computer-Animated Pendulum.

03.852

PB94-212651

Recent Approaches to Extreme Value Estimation with Application to Wind Speeds. Part 1. The Pickands Method. PB94-213170

00,019

Statistical Analysis of Parameters Affecting the Measurement of Particle-Size Distribution of Silicon Nitride Powders by Sedigraph (Trade Name).

03,042

Models and Interactions.

PB94-216306

02,641

Alternative Single Integral Equation for Scattering by a DiAlectric.

PB94-2165:2

04,422

Autocorrelation Functions from Optical Scattering for One-Dimensionally Rough Surtaces

PB94-216538

04,244

Boundary Integral Method for the Simulation of Two-Dimensional Particle Coarsening.

03.411

PB94-216744

Measurement and Uncertainty of a Calibration Standard for the Scanning Electron Microscope.

00,560

New Expressions of Uncertainties for Humidity Calibra. tions at the National Institute of Standards and Technology.

02,645

Extreme Value Theory and Applications: Proceedings of the Conference on Extreme Value Theory and Applications, Volume 3 . Held in Gaithersburg. Maryland in May 993.

PB95-104956

03,432

Capacity of the Lp Norm-Constrained Poisson Channel.

PB95-125753

01,515

Noise-Induced Chaos and Phase Space Flux.

03,433

Complex Propagation Constants for Nonuniform Optical Waveguides: Calculations.

PB95-125910

04,249

Fiber Spot Size: A Simple Method of Calculation.

PB95-125936

04,250

Modal Properties of Circular and Noncircular Optical Waveguides.

04,251

Development and Validation of Multicriteria Ratings: $A$ Case Study.

PB95-126025

00,004

Constructing Invariant Tori for Two Weakly Coupled van der Pol Oscillators.

PB95-126165

03,412

Accuracy of Eigenvalues: A Comparison of Two Methods. PB95-126249 03,413

Approximate Solution to the Scalar Wave Equation for Optical Waveguides.

PB95-126256

04,254

Sensitivity of Three-Point Circle Fitting.

02,901

xi-Vector Formulation of Anisotropic Phase-Field Models: 3-D Asymptotics.

PB95-136628

04,536

Combining Data from Independent Chemical Analysis Methods.

PB95-140141

00,572

Micromagnetic Model of Dual-Layer Magnetic-Recording Thin Films.

04,551

Guidelines for Evaluating and Expressing the Uncertainty of NIST Measurement Results. 1994 Edition.

PB95-143087

02,649

Phase-Field Model for Solidification of a Eutectic Alloy.

P3,345

Tolerance Intervals for the Distribution of True Values in the Presence of Measurement Errors.

PB95-150405

03,434

Approximate Confidence Intervals on Positive Linear Combinations of Expected Mean Squares.

03,436

Ranges of Confidence Coefficients for Confiedence Intervals on Variance Components.

03,437

Technique to Evaluate Benchmarks: A Case Study Using the Livermore Loops.

PB95-151320

04,577

Smart Clock: A New Time.

01,530

Confidence on the Second Difference Estimation of Fre quency Drift.

01,532

Effect of Spluting on Estimation of Emission Rate Profiles rom Neutron Depth Profiling Spectra.

03.916

Representing a Large Collection of Curves: A Case for Principal Points.

PB95-152286

03,438

Image Restoration and Diffusion Processes.

PB95-153003

01,843

Constant-Width Calibration Intervals for Linear Regres-

PB95-153524

03,439

Assessment of 'Peaks Over Threshold' Methods for Estimating Extreme Value Distribution Tails

PB95-161360

00.441

Underflow-Induced Graphics Falure Solved by SLI Arith-

PB95-161444

01,712

Comparison of Elastic and Plastic Contact Models for the Prediction of Thermal Contact Conductance. PB95-161659

04,605

New Exact Solution of the One-Dimensional Schrotinger Equation and Its Application to Polarized Neutron Reflectometry

PB95-161832

04,609

Data-Parallel Algorithm for Three-Dimensional Delaunay Triangulation and Its Implementation.

PB95-163309

01,714

Confidence on the Three-Point Estimator of Frequency

01,539

Look at Uncertainties over Twenty Decades of Pressure Measurement.

Mode Coupling and Loss on Tapered Optical PB95-168571

04,282

Symbolic Programming with Series Expansions: Applications to Optical Waveguides.

PB95-168589

04,283

Bending-Induced Loss in Dual-Mode Rectangular Paveguides.

04,288

Bent Rectangular Core Waveguides: An Accurate Perturbation Approach

PB95-168803

04,289

Design Equations and Scaling Laws for Linear Compressors with Flexure Springs.

PB95-168902

02,948

General Motion Model and Spatio-Temporal Filters for

Computing Optical Flow.

01,847

Fibre Splice Loss: A Simple Method of Calculation

PB95-175519

04,299

Examination of the $1 / d$ Expansion Method from Exact Enumeration for a Self-Interacting Self-Avoiding Walk. 
MasPar MP-1 as a Computer Arithmetic Laboratory.
PB95-189437 Lubrication Theory for Reactive Spreading of a Thin Drop. 02,865 Inserting Line Segments into Triangulations and Tetrahedralizations.
PB95-198933 03,415 Overcoming Hoelder Continuity in III-Posed Continuation Problems.

PB95-202354

03,416

Two Principal Points of Symmetric, Strongly Unimodal Distributions.

03,443

Linking Anisotropic Sharp and Diffuse Surface Motion Laws via Gradient Flows.

PB95-203378

04,698

Outlier-Resistant Methods for Estimation and Model Fit-

ting.

03,444

Morphological Estimation of Tip Geometry for Scanned Probe Microscopy.
PB95-203444

Parallel and Serial Implementations of SLI Arithmetic.

PB95-242335

02,662

PB95-242335

01,732

PB95-267829

01,735

Ceramic Powders Characterization: Results of an International Laboratory Study.

02,672

FB95-270039

Frozen Orbits for Satellites Close to an Earth-Like Planet.
PB96-102165

Self-Avoiding-Walk Contacts and Random-Walk Selfintersections in Variable Dimensionality.

PB96-102231 01,276

Fluctuations in Probability Distribution on Chaotic Attrac-

tors.

PB96-102330 04,022

Probabilistic Computation of Poiseuille Flow Velocity

Fields.

04,209

Phase Locking in Two-Dimensional Arrays of Josephson

Junctions: Effect of Critical-Current Nonuniformity.

Measurement Methods and Algorithms for Comparison of Local and Remote Clocks.

PB96-102652

01,549

Conformance Testing for OSI Protocols.

01,631

Analytical Method for Determining Thermal Conductivity from Dynamic Experiments.

PB96-102744 04,024

Boundary Conforming Grid Generation System for Interface Tracking.

03,357

Agile Manufacturing from a Statistical Perspective.
PB96-109525 PB96-109525

Error-Bounding in Level-Index Computer Arithmetic.
PB96-109582 Stable Phase Locking in a Two-Cell Ladder Array of Jo-

sephson Junctions.
PB96-111679

Taguchi's Parameter Design: A Panel Discussion.

PB96-111802

03,445

One-Dimensional Modeling Studies of the Gaseous Elec-

tronics Conference RF Reference Cell.

PB96-113428

02,397

Evolution Equations for Phase Separation and Ordering in Binary Alloys.
PB96-1 19243

02,979

Discussion: Statistical Signal Processing of

$\begin{array}{ll}\text { Quasiperiodicities. } & 00,096 \\ \text { PB96-119532 }\end{array}$

Numerical Evaluation of Special Functions.

00,096

PB96-119557 03,417

Performance Measures for Geometric Fitting in the NIST

Algorithm Testing and Evaluation Program for Coordinate

Measurement Systems.

PB96-122122

01,745

Nonlinear Dynamics of Stiff Polymers
PB96-122478

01,278

Parallel Monte Carlo Simulation of MBE Growth

PB96-122841 02,406

Slow Evolution from the Boundary: A New Stabilizing Constraint in Ill-Posed Continuation Problems.
PB96-122858
03,418

Effects of Nonmodel Errors on Model-Based Testing.

PB96-123146 02,604

Lubrication Theory for Reactive Spreading of a Thin

Drop. 123427

04,214

Anisotropy of Interfaces in an Ordered Alloy: A MultipleOrder-Parameter Model.

04,741

Principal Points and Self-Consistent Points of Symmetric Multivariate Distributions.

03,446
Proposed Changes to Charpy V-Notch Machine Certification Requirements.

02,955

Test of a Slow Off-Axis Parabola at Its Center of Cur-

vature.
PB96-138482

04,352

Thermal Modeling and Analysis of Laser Calorimeters.

PB96-140405 04,354

Anova Estimates of Variance Components for a Class of

Mixed Models.
PB96-141163

03,448

One-Sided beta-Content Tolerance Intervals for Mixed

Models.

PB96-141171 03,449

Response to 'Draining in Dilute Polymer Solutions and Renormalization

PB96-146667

01,283

Statistical Descriptors in Crystallography. 2. Report of a Working Group on Expression of Uncertainty in Measure-

ment.
PB96-146824

04,764

Stagnant Film Model of the Effect of Natural Convection

on the Dendrite Operating State.
PB96-146832

Collection of Results for the SPC/E Water Model.

PB96-147889

01,127

Effects of Nonmedel Errors on Model-Based Testing.
PB96-155577

Deterministic and Stochastic Chaos.

PB96-156138

03,420

PBder

PB96-157813 04.066

Stochastic Modeling of a New Spectrometer.

PB96-157870

04,066

Exits in Multistable Systems Excited by Coin-Toss Square-Wave Dichotomous Noise: A Chaotic Dynamics Approach.

PB96-160650

04,824

Point Probe Decision Trees for Geometric Concept Class-

PB. $96-160817$

01,612

Nonequilibrium Statistical Mechanics.

PB96-161781

Optical Detector Nonlinearity: Simulation.

04,097

BB96-165378

02, 199

PB96-165931

03,421

Using S-Check, Alpha Release 1.0

$\begin{array}{ll}\text { PB96-165964 } & 01,767\end{array}$

MasPar MP-1 as a Computer Arithmetic Laboratory

PB96-179155 01,617

Tree-Lookup for Partial Sums Or: How Can I Find This

Stuff Ouickly.

01,770

Move-to-Root Rule for Self-Organizing Trees with Markov Dependent Requests.

PB96-179528

03,431

Distributions of Measurement Error for Three-Axis Magnetic Field Meters during Measurements Near Appliances.

02,110

Tomographic Reconstruction of the Moments of Local Probability Density Functions in Turbulent Flow Fields.
PB96-180195

Wavelet Variance, Allan Variance, and Leakage.

PB96-190111

01,509

Confidence on the Modified Allan Variance and the Time Variance.

01,557

Sparselib++ v. 1.5 Sparse Matrix Class Library. Reference Guide.

01,775

Hybrid Gauss-

03,422

Notion of a xi-Vector and a Stress Tensor for a General

Class of Anisotropic Diffuse Interface Models.

PB96-193776

04,788

IML++ V.1.2 I

01,776

$\mathrm{MV}++$ v. 1.5a Matrix/Nector Class Reference Guide.

PB96-195326 01,777

Shapiro Steps in Large-Area Metallic-Barrier Josephson Junctions.

Junctions.

02,090

Accurate Modeling of Size and Strain Broadening in the Rietveld Refinement: The 'Double-Voigt' Approach.

PB96-200225

00,664

Data Evaluation of a Linear System by a Second-Order Transfer Function.

PB96-200282

01,970

Wavelet Analysis for Synchronization and Timekeeping.

PB96-200381

Self-Reciprocal Fourier Functions.

01,558

PB96-200852

01,974

Statistical Aspects of the Certification of Chemical Batch SRMs. Standard Reference Materials.

PB96-210877

00,645

Exact Series Solution to the Epstein-Hubbell Generalized Elliptic Type Integral Using Complex Variable Residue Theory.
PB97-110167

03,423

Hydrodynamic Friction of Arbitrarily Shaped Brownian

PB97-11019.

04,136

Hypersingular Single Integral Equation and the Dielectric

Wedge.
PB97-110274 04,428

Variances in the Measurement of Ceramic Powder Prop-

Prties.

03,100

Electron-Photon Monte Carlo Calculations: The ETRAN

Code.
PB97-110407

04,138

Electromagnetic Scattering from a Dielectric Wedge and the Single Hypersingular Integral Equation.

04,430

Numerical Evaluation of Hypersingular Integrals for Scat-

tering by a Dielectric Wedge.
PB97-1 10555

02,017

Numerical Reference Models for Optical Metrology Sim-

PB97-111330

04,392

Heap of Data.

PB97-111488

Representation of Axes for Geometric Fitting

PB97-113799

03,424

Developing Measurement for Experimentation.

PB97-118707

01,782

Pilot Studies for Improving Sampling Protocols.

03,450

PB97-118715

02,530

Sources of Strain-Measurement Error in Flag-Based Extensometry.
PB97-118731

Making Connections.

03,108

PB97-119044

01,785

Application of the Collocation Method in Three Dimensions to a Model Semiconductor Problem.

02,457

Xi-Vector Formulation of Anisotropic Phase-Field Models: 3-D Asymptotics.

04,816

MATRICES (MATHEMATICS)

SparseLib++ v. 1.5 Sparse Matrix Class Library. Reference Guide.
PB96-193636

MATRIX ALGEBRA

MV++ v. 1.5a Matrix/Vector Class Reference Guide

PB96-195326

MATRIX ISOLATION

Production and Spectroscopy of Small Polyatomic Molecular lons Isolated in Solid Neon. (Reannouncement with New Availability Information).

AD-A234 043/8

00,704

Matrix Isolation Study of the Interaction of Excited Neon Atoms with O3: Infrared Spectrum of $\mathrm{O}(($ sub 3$)(-))$ and Evidence for the Stabilization of O2...O((sub 4)(+)). B97-112403

04,155

Matrix Isolation Study of the Interaction of Excited Neon Atoms with $\mathrm{BCl}$ : Infrared Spectra of $\mathrm{BCl}(\mathrm{sub} 3$, sup + ) $\mathrm{BCl}$ (sub 2, sup + ), and $\mathrm{BCl}$ (sub 3, sup -). 


\section{KEYWORD INDEX}

\section{MEASUREMENT}

Report of the International Commission on Radiological Units and Measurements (ICRU), 1956.

03,513

Federal Basis for Weights and Measures: A Historical ReFederal Basis for Weights and Measures: A Historical Re-
view of Federal Legislative Effort, Statutes, and Adminisview of Federal Legislative Effort, Statutes, and Adminis-
trative Action in the Field of Weights and Measures in the trative Action in the Field of Weights and Measures in the
United States.
AD-A280 086/0
02,616

02,616 Voluntary Product Standard PS 20-94. American
Softwood Lumber Standard. Importance of Measurement in Technology-Based Competition.

02,929

Survey of the Components of Display-Measurement Standards

PB96-122528

02,188

Accurate Computations of Radar Cross Sections of Simple Objects.
PB96- 138474

04,426

New and Revised Half-Life Measurement Results.

PB96-160346

00,695

Methodology for Electromagnetic Interference Measure-

ments.

02,014

Comparison of Uitralow-Sidelobe-Antenna Far-Field Patterns Using the Planar-Near-Field Method and the FarField Method.

PB96-200373

02.015

Measurements of Properties of Materials in Electronic Packaging.

01,973

Developing Measurement for Experimentation.

PB97-118707

03,450

Pilot Studies for Improving Sampling Protocols.

PBg7-118715

Specular and Diffuse Reflection Measurements of Electronic Displays.
PB97.119200

02,208

Survey of the Components of Display-Measurement Standards.

MEASUREMENT ACCURACY

Effects of Pipe Elbows and Tube Bundles on Selected Types of Flowmeters.

01,135

MEASUREMENT ASSURANCE PROGRAM

NIST Measurement Assurance Program for Capacitance Standards at $1 \mathrm{kHz}$

PB96-172333

02,276

MEASUREMENT ERRORS

Low Heat-Flux Measurements: Some Precautions

PB96-201116

02,685

\section{MEASUREMENT SCIENCE \& TECHNOLOGY}

ALIBRATION

Mass Unit Disseminated to Surrogated Laboratories Using the NIST Portable Mass Calibration Package.

Assessment of Uncertainties of Liquid-in-Glass Thermometer Calibrations at the National Institute of Standards and Technology.

02,625

Assessment of Uncertainties of Thermocouple Calibrations at NIST.

03,782

NIST Measurement Services: NIST Pressure Calibration Service.

02,892

NIST Calibration of ASTM E127-Type Ultrasonic Reference Block

02,702

PB94-191640

diometric

Sovelopment and Calibration of UVNUV Radiometric PB94-199098

04,229

Ensuring Accuracy and Traceability of Weighing Instruments.
PB94-211687

02,638

Summary of the Proceedings of the Workshop on Standard Phantoms for In-vivo Radioactivity Measurement.
PB94-212933 dc Method for the Absolute Determination of Conductivities of the Primary Standard $\mathrm{KCl}$ Solutions from $\mathrm{OC}$ to $50 \mathrm{C}$.

02,644

Review of the USCEAVNIST Measurement Assurance Program for the Nuclear Power Industry.

Calibration of GPS Equipment in Japan.

03,712

PB95-151452 01,531

Characterization of a Health Physics Instrument Calibration Range.

03,629

Geometric Characterization of Rockwell Diamond Indent-

PB.r. 203287

02,950

Standard Reference Materials: Polystyrene Films for Calibrating the Wavelength Scale of Infrared

$\begin{array}{lr}\text { Spectrophotometers - SRM } 1921 . & \\ \text { PB95-226866 } & 03,386\end{array}$
Energy Calibration of $X$-ray Photoelectron Spectrometers: Results of an Interlaboratory Comparison to Evaluate a Proposed Calibration Procedure.

PB96-102918

04,027

Long-Term Stability of Bayard-Alpert Gauge Performance: Results Obtained from Repeated Calibrations against the National Institute of Standards and Technology Primary Vacuum Standard

02,678

Comparison of NIST and Manufacturer Calibrations of (90) $\mathrm{Sr}+(90)$ Y Ophthalmic Applicators.

03,634

Calorimeters for Calibration of High-Dose Dosimeters in High-Energy Electron Beams.
PB96-135272

Absolute Response Calibration of a Transfer Standard ic Bolometer.

04,358

Direct Comparison Transfer of Microwave Power Sensor Calibrations.

02,086

Calibration of Electret-Based Integral Radon Monitors Using NIST Polyethylene-Encapsulated (226)Ra/(222)Rn Emanation (PERE) Standards.

PB96. 159223

01.950

Development of the NIST Transient Pressure and Temperature Calibration Facility.

00,626

NIST Measurement Assurance of SPRT Calibrations on the ITS-90: A Ouantitative Approach.

PB96-161336

00,633

Irradiance of Horizontal Ouartz-Halogen Standard Lamps.

PB96-179130 01,866

Organization and Implementation of Calibration in the EOS Project. Part 1

PB96-179437 04,841

Report on the NIST Low Accelerating Voltage SEM Magnification Standard Interlaboratory Study.
PB96-201074

PB96-201074
Calibration in the Earth Observing System (EOS) Project. Part 2. Implementation.

PB97-112213 04,842

Empirical Linear Prediction Applied to a NIST Calibration Service.

02,287

PB97-112353

Realization of a Scale of Absolute Spectral Response

Using the NIST High Accuracy Cryogenic Radiometer.
PB97-118640

\section{MEASUREMENT SCIENCE \& TECHNOLOGY: PHYSICAL}

\section{TANDARDS \& FUNDAMENTAL CONSTANTS}

NIST Handbook 44, 1994: Specifications, Toierances and Other Technical Requirements for Weighing and Measuring Devices as Adopted by the 78 th National Conference

on Weights and Measures 1993

02,888

Report of the National Conference on Weight and Measures (78th). Held in Kansas City, MO. On July 18-22, 1993.

PB94-138989

02,623

Examination of Parameters That Can Cause Error in Mass Determinations.

$\begin{array}{ll}\text { PB94-163037 03,784 } & \end{array}$

Electronic Balance and Some Gravimetric Applications. (The Density of Solids and Liquids, Pycnometry and

Mass).

03,785

Piggyback Balance Experiment: An Illustration of Archimedes' Principles and Newton's Third Law

PB94-163060

03,786

Determination of Density of Mass Standards: Require ment and Method.

PB94-163078

03,787

Realization of New NIST Radiation Temperature Scales for the $1000 \mathrm{~K}$ to $3000 \mathrm{~K}$ Region, Using Absolute Radio-

PB94-172905

03,794

Fine-Structure Constant.

03,795

Design and Operation of Series-Array Josephson Voltage Standards.
PB94-185451 02,030

02,030

Effect of Curing History on Ultimate Glass Transition Temperature and Network Structure of Crosslinking Poly-

PB94-200052

01,214

Report of Density Intercomparisons Undertaken by the Working Group on Density of the CCM.

00,503

NIST Optically Pumped Cesium Frequency Standard.

PB94-211117

03,835

$24 \mathrm{GHz}$ Josephson Array Voltage Standard.

02,033

Particle Size Standards and Their Certification at NIST.

PB94-211695

02,639

Development of a Temperature Scale below $0.5 \mathrm{~K}$.
PB95-125639

International Intercomparison of Detector Responsivity at

1300 and $1550 \mathrm{~nm}$

02,141

Frequency Stabilization of a Fiber Laser to Rubidium: A High-Accuracy $1.53 \mathrm{mu} \mathrm{m}$ Wavelength Standard.

PB95-126082

04,252

Precision High Temperature Blackbodies.

PB95-140059

03,885

Operational Mode and Gas Species Effects on Rotational Drag in Pneumatic Dead Weight Pressure Gages.

PB95-140182

00,903

New International Representations of the Volt and Ohm Effective January 1, 1990

PB95-150777

01,890

Piezoelectric and Pyroelectric Polymers.

NIST-7, the New US Primary Frequency Standard.

PB95-153458

Error Analysis of the NIST Optically Pumped Primary Frequency Standard

01,535

Practical Applications of the ITS-90: Inherent Uncertain-

PB95-161527

03,930

Systematic Studies of the Effect of a Post-Detection Filter on a Josephson-Junction Noise Thermometer.

PB95-162988

03,940

Diode-Laser Pumped, Rubidium Cell Frequency Standards.

PB95-163218

01,538

How Accurate Are the Josephson and Ouantum Hall Effects and OED

03,942

Criteria for Establishing Accurate Vapor Pressure Curves. PB95-163812

00,972

Metrology.
PB95-164497

03,946

Superconductor Critical Current Standards for Fusion Applications. Final Progress Report, October 1993-July 1994.

PB95-169538

02,222

Improved Rubidium Frequency Standards Using Diode Lasers with $A M$ and FM Noise Control.

B95-176152

04,303

Traceability to the Mole: A New Initiative by CIPM.

PB95-180246

00,605

Early History and Future Outlook for the X-ray Crystal Density Metho

Voltage-Standard Devices.

04,692

PB96-102496

01,920

Local Oscillator Requirements and Strategies for the Next Generation of High-Stability Frequency Standards.

PB96-112230

01,551

Measurement of the Weak-Localization Complex Conductivity at $1 \mathrm{Ghz}$ in Disordered Ag Wires.

PB96-117239

04,731 
Investigation of the ITS-90 Subrange Inconsistencies for 25.5 Omega SPRTs.

00,630

NIST Assessment of ITS-90 Non-Uniqueness for 25.5 Ohm SPRTs at Gallium, Indium and Cadmium Fixed Points.

PB96-161310

00,631

NIST Implementation and Realization of the ITS-90 Ove the Range $83 \mathrm{~K}$ to $1235 \mathrm{~K}$ : Reproducibility, Stability, and Uncertainties.
PB96-161328

00,632

Length Metrology of Complimentary Small Plastic Rulers.

PB96-161724 04,096

Calibration Service for Coaxial Reference Standards for Microwave Power

01,958

Advances in the Measurement of Polymer CTE: Micrometer- to Atomic-Scale Measurements.

PB96-180229

03,390

Transient Creep Behaviour of Hot Isostatically Pressed

Silicon Nitride.
PB96-180278

Introduction to Frequency Calibration. Part 1.

01,560

Tensile Creep of Silicide Composites.

PB96-200803

03,183

National Measurement System for Radiometry, Photom etry, and Pyrometry Base Upon Absolute Detectors.

PB97-108559

04,382

Current Status and Trends in Temperature Measurements at NIST, Cooperative Projects and New Mutual Agreement between NIST and IMGC.

02,691

Comparisons of Some NIST Fixed-Point Cells with Simiar Cells of Other Standards Laboratories.

00,655

PB97-119242

\section{MEASUREMENT SCIENCE \& TECHNOLOGY: POLICY \&}

ATE-OF-THE-ART SURVEYS

Dielectric Properties Measurements and Data.

PB94-172186

01,876

Report on the Meeting of the CCU (10th) (of the International Committee of Weights and Measures). Held on

$\begin{array}{ll}\text { July } 10-11,1990 . & 03,792 \\ \text { PB94-172889 }\end{array}$

Optical Fiber Sensors: Accelerating Applications in Navy Ships.

02,632

Measurement of Diffusion in Supercritical Fluid Systems: A Review.

PB94-199189

00,795

Mass and Density Determinations.

PB94-200672

00,504

Comparison of Regular Transmittance Scales of Four $\mathrm{Na}$ tjonal Standardizing Laboratories.

PB94-211232 04,233

National Quality Assurance Program for Personnel Radiation Dosimetry: A Case History.

PB94-211273

03,620

Lightwave Stand

01,480

Effect of Semiconductor Laser Characteristics on Optical Fiber Sensor Performance.

02,167

PB95-169132

02,167

Comparing NIST-B $50 \mathrm{~mm}$ Orifice Meter Gas Data to the PB95-169207

02,949

Japan Technology Program Assessment: Precision Engineering/Precision Optics in Japan.

02,884

Integrated-Optical Devices in Rare-Earth-Doped Glass.

PB95-202909

02,179

Guide for the Use of the International System of Units

PB95-226692

02,747

NBS/NIST Peltier-Effect Microcalorimeter: A Four-Decade

Review.

01,064

Metrology for Electromagnetic Technology: A Bibliography

of NIST Publications. September 1995.
PB96-128269

Panel Discussion on Units in Magnetısm.

04,746

Optoelectronics at NIST

02,202

USCEANIST Measurement Assurance Programs for the Radiopharmaceutical and Nuclear Power Industries.

PB97-110514

03,721

MEASUREMENT SYSTEMS

Length Metrology of Complimentary Small Plastic Rulers. PB96-161724

04,096

MEASUREMENT UNCERTAINTY

Assessment of Uncertainties of Calibration of Resistance Thermometers at the National Institute of Standards and Technology.

02,624

Uncertainty Analysis of the NIST Nitrogen Flow Facility.

PB95-128906

02,608
Guidelines for Evaluating and Expressing the Uncertainty of NIST Measurement Results. 1994 Edition.

02,649

MEASURING INSTRUMENTS

Methods of Measuring Humidity and Testing ADgrometers.

00,123

Technologic Papers of the Bureau of Standards: Number 170. Pyrometric Practice.

AD-A279 282/8

03,766

AD-A280 278/3

02,617

Development of Measurement Capabilities for the Thermophysical Properties of Energy-Related Fluids. Annual Report, December 1, 1990--November 30, 1991. nual Report, December 1, 1990--November 30, 1991.
DE94004399 Development of Measurement Capabilities for the Thermophysical Properties of Energy-Related Fluids. An nual Report, December 1, 1993--November 30, 1994

DE94017738

03,246

Minutes of the CAALS Workshop on modularity and communications standards.

Partial Pressure Analysis in Space Testing.
N95-14084/4

NIST Handbook 44, 1994: Specifications, Tolerances and Other Technical Requirements for Weighing and Measuring Devices as Adopted by the 78th National Conference on Weights and Measures 1993.

PB94-136009

02,888

Transient Methods for Thermal Conductivity.

04,197

Microanalysis to Nanoanalysis: Measuring Composition at High Spatial Resolution.

PB95-107173

00,563

NIST Handbook 44, 1995: Specifications, Tolerances and Other Technical Requirements for Weighing and Measur ing Devices as Adopted by the 79th National Conference on Weights and Measures 1994

on Weights and

02,903

Airborne Smoke Sampling Package for Field Measurements of Fire

Two New Probes for a Coordinate Measuring Machine

PB95-163093

02,653

Characterization of a Health Physics Instrument Calibration Range.

High-Pressure Equilibrium Cell for Solubility Measurements in Supercritical Fluids.

PB95-175634

00,998

Interim Testing Artifact (ITA): A Performance Evaluation System for Coordinate Measuring Machines (CMMs). User Manual.

PB95-210589

Gage Block Handbook

02,914

PB95-251716

02,667

NIST Handbook 44, 1996: Specifications, Tolerances and Other Technical Requirements for Weighing and Measuring Devices as Adopted by the 80th National Conference on Weights and Measures, 1995. PB96-166616

02,926

MEATS

Inter-Laboratory Trials of the EPR Method for the Detection of Irradia

MECC (MICELLAR ELECTROKINETIC CAPILLARY

CHROMATOGRAPHY)

Separation and Identification of Organic Gunshot and Ex plosive Constituents by Micellar Electrokinetic Capillary Electrophoresis.

00,566

MECHANICAL ENGINEERING

Tribology Education: Present Status and Future Chal-

02,965

Product Models and Virtual Prototypes in Mechanical Engineering

PB95-253563

02,783

MECHANICAL PROPERTIES

AD.A286 675/4

Effect of Backfill and Atomizing Gas on the Powder Porosity and Mechanical Properties of $304 \mathrm{~L}$ Stainless Steel.

Micro-Mechanical Aspects of Asperity-Controlled Friction in Fiber-Toughened Ceramic Composites.

03,136

Assessment of Testing Methodology for Ceramic Matrix Composites.

03,139

PB94-200532

Tribological Characteristics of Alpha-Alumina at Elevated Temperatures
PB94-211018

02,963

Fundamental Computer Simulation Models for Cemer :Based Materials.

00,364

Comparison of Fire Sprinkler Piping Materials: Steel,

Comparison of Fire Sprinkler Piping Materials: Steel, in Residential and Light Hazard Installations.

in Residential

00,299

Low-Temperature Properties of Silve

PB96-126198

03,36

MECHANICAL TESTS

Recent VAMAS Activity in Ceramics.

PB95-162681

03,051

MECHANICAL VIBRATION

Testing the Sensitivity of Accelerometers Using MechaniTest No. 24040 S.

PB96-179544

02,683

MECHANICS: DESIGNTTESTING/MEASUREMENT

Recommended Changes in ASTM Test Methods D2512 82 and G86-84 for Oxygen-Compatibility Mechanical Impact Tests on Metals.

B94-21669

03,338

Ultrasonic Measurement of Residual Stress in Railroad Wheel Rims.

PB95-140430

04,849

Fundamental Mechanisms for $\mathrm{CO}$ and Soot Formation.

Ultrasonic Technique for Sizing Voids Using Area Func-

PB95-151916

02,904

Leady Axisymmetric Modes in Infinite Clad Rods. Part 1.

Elastic Green's Function for a Bimaterial Composite Solid Containing a Free Surface Normal to the Interface.

PB95-163325

03,162

Green's Function for Generalized Hilbert Problem for Cracks and Free Surfaces in Composite Materials.

PB95.163333

03,163

Generalized Plane Strain Analysis of a Bimaterial Com posite Containing a Free Surface Normal to the Interface.
03,164 Stylus Flight in Surface Profiling.

Ultrasonic Sensing of GMAW: Laser/EMAT Defect Detec-

$\begin{array}{ll}\text { tion System. } & 02,878\end{array}$

Determination of Sheet Steel Formability Using Wide Band Electromas

Ettect of Littoft ments Made with Electromagnetic-Acoustic Transducers.
PB96-186044

02,280

Methods to Improve the Accuracy of On-Line Ultrasonic PB96-186051

02,281

Hemispherical Test Fixture for Measuring the Wavefields Generated in an Anisotropic Solid.

03,181

Sensing Droplet Detachment and Electrode Extension for Control of Gas Metal Arc Welding.

PB96-190160

03,297

Dilemma-Preservation versus Access. 
Electroacoustics for Characterization of Particulates and Suspensions. Proceedings of a Workshop. Held in Gaithersburg, Maryland on February 3-4, 1993 PB94-112695

00,725

Report of the NIST Workshop on Digital Signature Certificate Management. Held on December 10-11, 1992. Report of the National Conference on Weight and Measures (78th). Held in Kansas City, MO. On July 18-22,

PB94-138989

02,623

Report on Application Integration Architectures (AIA) Workshop. Held in Dallas, Texas on February 8-12, 1993. Photonic Materials: A Report on the Results of a Workshop. Held in Gaithersburg, Maryland on August 26-27, 1992, Volume

PB94-152733

02,114

Report on the Workshop on Manufacturing Polymer Composites by Liquid Molding. Held in Gaithersburg, Maryland on September 20-22, 1993.

03,131

Proceedings of the Workshop on the Federal Criteria for Information Technology Security. Held in Ellicot City, Maryland on June 2-3, 1993

PB94-162583

01,575

Advanced Components for Electric and Hybrid Electric Vehicles. Workshop Proceedings. Held in Gaithersburg, Maryland on October 27-28, 1993.

PB94-177060

04.858

Second Text REtrieval Conference (TREC-2). Held in Gaithersburg, Maryland on August 31-September 2,

PB94-178407

01.686

International Institute of Welding: Report on 1992 Actions.

PB94-185873 02,856

International Institute of Welding: Report on 1993 Action

$\begin{array}{lr}\text { PB94-185881 02,857 } & \end{array}$

Workshop on Characterizing Diamond Films (3rd). Held in Gaithersburg, Maryland on February 23-24, 1994.
PB94-187663 PB94-187663 Conference.

PB94-188711

01.832

U.S. Green Building Conference, 1994.

02.519

Technical Digest: Symposium on Optical Fiber Measurements (8th), 1994. Held in Boulder, Colorado on September 13-15, 1994

PB94-207636

04,231

International Conference on Chemical Kinetics (2nd). Held in Gaithersburg, Maryland on July 24-27, 1989.
PB94-211901 00,822 Summary of the Proceedings of the Workshop on Standard Phantoms for In-vivo Radioactivity Measurement.

PB94-212933 03,622 Head Start on Assurance: Proceedings of an Invitational Workshop on Information Technology (IT) Assurance and Trustworthiness. Held in Williamsburg, Virginia on March 21-23, 1994.
PB94-215746

01,586

NIST Workshop on Nanostructured Material (1st): Report of an Industrial Workshop Conducted by the National In-
stitute of Standards and Technology. Held in Gaithersburg, Maryland on May 14-15, 1992. Held in Polymer Composites Workshop. Held in Winona, Min nesota on April 29-30, 1992 (Video).

PB94-780129

03,147

Report on the Workshop on Advanced Digital Video in the National Information Infrastructure. Held in Washington, D.C. on May 10-11, 1994

03677

01,472

In Situ Burning Oil Spill Workshop Proceedings. Held in Orlando, Florida on January 26-28, 1994

02,583

Extreme Value Theory and Applications: Proceedings of Extreme Value Theory and Applications: Proceedings of the Conference on Extreme Value Theory and Applica-
tions, Volume 3. Held in Gaithersburg, Maryland in May

PB95-104956

03,432

Annual Conference on Fire Research: Book of Abstracts, October 17-20, 1994.

PB95-104964

01,376

Global Climatic Effects of Aerosols: The AAAR Symposium.

00,122

NIST Workshop on the Computer interface to Flat Panel Displays. Held in San Jose, California on January 13-14, Disp

PB95-136388

01,625

Summary Report of NIST's Industry-Government Consortium Research Program on Flowmeter Installation Effects: The 45 Degree Elbow.

PB95-14306

04,204

Metrology and Data for Microelectronic Packaging and Interconnection: Results of a Joint Workshop on Materials Packaging and Interconnection Technologies. Held in
Gaithersburg, Maryland on May 5-6, 1994. Volume 1. Results.

02,328

Metrology and Data for Microelectronic Packaging and Interconnection: Results of a Joint Workshop on Materials Metrology and Data for Commercial Electrical and Optical Packaging and Interconnection Technologies. Held in Gaithersburg Maryland on May 5-6, 1994. Volume 2. Presentation Material.

PB95-143327

02,330

Wind and Seismic Effects. Proceedings of the U S.Japan Cooperative Program in Natural Resources Panel on Wind and Seismic Effects (26th). Held in Gaithersburg. Maryland on May 17-20, 1994

00,433

Program Requirements to Advance the Technology of Custom Footwear Manufacturing.

02,883

Journal of Research of the National Institute of Standards and Technology, July/August 1994. Volume 99, Number and Technology. July/August 1994 . Volume 99, Number Proceedings of the Conference on Extreme Value Theor and Applications, Volume 2. Held at Gaithersburg, Maryand Applications,

land, in May 1993

Forum for International Cooperation on Fire Research.

PB95-162939

04,869

Journal of Research of the National Institute of Standards and Technology, September/October 1994. Volume 99 Number 5

PB95-169371

04,293

Report of the National Conference on Weights and Measures (79th). Held in San Diego, California on July 17-21,

PB95-169819

02,656

Workshop Summary Report: Industrial Applications of Scanned Probe Microscopy. A Workshop Co-sponsored by NIST, SEMATECH, ASTM, E42.14, and the American Vacuum Society. Held in Gaithersburg, Maryland on March 24-25, 1994

From Superconductivity to Supernovae: The Ginzburg Symposium. Report on the Symposium Held in Honor of Vitaly $L$. Ginzburg. Held in Gaithersburg, Maryland on May 22, 1992.
PB95-171963

04,649

Proceedings: Workshop on Research Needs in Wind Engineering. Held in Gaithersburg, Maryland on September 12-13, 1994.

00,448

Proceedings of the Manufacturing Technology Needs and Issues: Establishing National Priorities and Strategie Conference. Held in Gaithersburg. Maryland on April 26

PB95-206181 02,930

NIST Workshop on Gas Sensors: Strategies for Future Technologies. Proceedings of a Workshop. Held in Gaithersburg, Maryland on September 8-9, 1993. Overview of the Text REtrieval Conference (3rd) (TREC3). Held in Gaithersburg, Maryland on November 2-4

PB95-216883

01,728

Study of Potential Applications of Automation and Robotics Technology in Construction, Maintenance and Operation of Highway Systems: A Final Report. Volume 3.

PB95-251690 International Green Building Conference and Exposition (2nd). Held in Big Sky. Montana on August 13-15, 1995.
PB95-253605

PB95-253605 02,525

Proceedings of the Applied Diamond Conference 1995 : Applications of Diamond Films and Related Materials International Conference (3rd). Held in Gaithersburg, Maryland, on August 21-24, 1995.

04,701

Applications of Diamond Films and Related Materials: Applications of Diamond Films and Related Materials: International Conference (3rd). Held in Gaithersburg.
Maryland on August 21-24, 1995. Supplement to NIST Maryland on August 21

Special Publicatio
PB95-256053

03,063

Proceedings of the 1995 Workshop on Fire Detector Research. Held on February 6-7, 1995.

PB95-270062

02,611

Joint DOD/NIST Workshop on International Manufacturing Systems Research and Development. Held in Rockville, Maryland on November 3-5, 1992. Proceedings. PB96-109491

02,931

Composite Materials for Offshore Operations: Proceedings of the International Workshop (1st). Held in Houston Texas on October 26-28, 1993.

PB96-109509

03,169

Proceedings of the Annual Manufacturing Technology Conference (2nd): Toward a Common Agenda. Held in Gaithersburg, Maryland on April 18-20, 1995.

02,887

Journal of Research of the National Institute of Standard and Technology, July/August 1995. Volume 100, Number 4. Special Issue: The Gaseous Electronics Conterence Radio-Frequency Reference Cell.

Current and Voltage Measurements in the Gaseous Electronics Conference RF Reference Cell.

02,388

Optical Emission Spectroscopy on the Gaseous Elec. ronics Conference RF Reference Cell.

02,389

Optical Diagnostics in the Gaseous Electronics Conerence Reference Cell.

02,390

Studies of Ion Kinetic-Energy Distributions in the Gaseous Electronics Conference RF Reference Cell.

PB96-113360

02,391

Microwave Diagnostic Results from the Gaseous Elecronics Conference RF Reference Cell.

02,392

Langmuir Probe Measurements in the Gaseous Electronics Conference RF Reference Cell.

02,393

Inductively Coupled Plasma Source for the Gaseous Electronics Conference RF Reference Cell.

02,394

B 96-113394

Con-

Reactive Ion Etching in th

ference RF Re
PB96-113402

02,395

Dusty Plasma Studies in the Gaseous Electronics Conference Reference Cell.

02,396

PB96-113410

Elec-

tronics Conference RF Reference Cell.

PB96-113428

02,397

Two-Dimensional Self-Consistent Radio Frequency Plasma Simulations Relevant to the Gaseous Electronics Conference RF Reference Cell.

PB96-113436

02,398

Post-Earthquake Fire and Lifelines Workshop. Held in Long Beach, California on January 30-31, 1995. Proceedings.

00,209

Journal of Research of the National Institute of Standards and Technology, March/April 1995. Volume 100, Number PB96-126180

04,349

Metrology Issues in Terahertz Physics and Technology.
PB96-128277

Solid Propellant Gas Generators: Proceedings of the 1995 Workshop. Held in Gaithersburg, Maryland on June 28-29, 1995.
PB96-131479

01,412

Summary Report: Workshop on Industrial Applications of Scanned Probe Microscopy (2nd). A Workshop Co-Spon. sored by NIST, SEMATECH, ASTM E42.14, and the American Vacuum Society. Held in Gaithersburg, Maryland on May 2-3, 1995.

00,509

Computer-Aided Manufacturing Engineering Forum (1st) Technical Meeting Proceedings. Held in Gaithersburg, Maryland on March 21-22, 1995.

PB96-136965

02,834

Report on 1994 Actions of the International Institute of PB96-138540

02,873

Summary and Results of the NIST Workshop on Proposed Guidelines for Testing and Evaluation of Seismic Isolation Systems. Held in San Francisco, California on July 25, 1994

00,463

Conference Proceedings: International Workshop on Instrumented Indentation. Held in San Diego. California on 
Computer-Aided Manufacturing Engineering Forum (2nd). Technical Meeting Proceedings. Held in Gaithersburg,

Maryland on August 22-23, 1995

02,845

NIST Construction Automation Program Report No. 2. Proceedings of the NIST Construction Automation Work shop. Held in Gaithersburg, Maryland on March 30-31, PB96-202239

Phase Separation in Thin Film Polymer Blends With and Without Block Copolymer Additives.

Without Block

01,294

Thermal Wave NDE of Advanced Materials Using Mirage Effect Detection

PB96-204516

04,191

Report of a Workshop on Requalification of Tubular Steel Joints in Offshore Structures. Held in Houston, Texas on September 5-6, 1995

PB96-210760

03,699

Wind and Seismic Effects: Proceedings of the Joint Meet ing of the U.S.-Japan Cooperative Program in Natura Resources Panel on Wind and Seismic Effects (28th)

Held in Gaithersburg, Maryland on May 14-17, 1996.
PB97-104376

00,476

Technical Digest: Symposium on Optical Fiber Measurements (9th), 1996. Held in Boulder, Colorado on October 1-3, 1996

04,383

Proceedings of a Workshop on Developing and Adopting Seismic Design and Construction Standards for Lifelines. Held in Denver, Colorado on September 25-27, 1991

PB97-115794

01,302

Science, Technology, and Competitiveness: Retrospec. tive on a Symposium in Celebration of NIST's 90th Anniversary and the 25th Anniversary of the Gaithersburg Laboratories, November $14-15,1991$

PB97-121610

02,696

Text REtrieval Conference (4th) (TREC-4). Held in Gaithersburg, Maryland on November 1-3, 1995.
PB97.121636 PB97-121636
International Green Building Conterence and Exposition (3rd). Held in San Diego, California on November 17-19, 1996. (Reannouncement with new abstract).

PB97-121826

02,531

Conference Report: Calorimetry Conference (50th). PB97-122279

03,722

MEISSNER EFFECT

Meissner, Shielding, and Flux Loss Behavior in SingleCrystal YBa2Cu3O6+x

PB94-198744

04,464

MELNIKOV'S METHOD

Dynamics of Multi-DOF Stochastic Nonlinear Systems.
PB97.113245

MELTING

Shear-Induced Melting of Two-Dimensional Solids.
PB96-112057

MELTING LINE

Pressure Measurements with the Mercury Melting Line Referred to ITS-90.

PB96-161005

01,136

MELTING POINT

Radiance Temperature (in the Wavelength Range 519 . $906 \mathrm{~nm}$ ) of Tungsten at Its Melting Point by a Pulse-Heat ing Technique.

03.397

Wavelength Dependence of Normal Spectral Emissivity of High-Temperature Metals at Their Melting Point. High-Temperat

03,398

MELTING POINT DEPRESSION

Melting Behavior of Organic Materials Confined in Porous

PB94-212313

00,834

MELTING POINTS

Melting Behavior of Organic Materials Confined in Porous

PB94-212313

00,834

reezing-

Standard Referen

01,055

PB95-251732

Radiance Temperatures (in the Wavelength Range 523(V) B Transition Metals Titanium, Zirconium, and Hafnium at Their Melting Points by a PulseHeating Technique.

03,356

Measurements of Thermophysical Properties of Nickel Near Its Melting Temperature by a Microsecond-Resolution Transient Technique.

04,210

PB96-102579

Radiance Temperatures (in the Wavelength Range 523$907 \mathrm{~nm}$ ) of Group IVB Transition Metals Titanium, Zirconium, and Hafnium

Heating Techniqu
PB96-135025

02,677

MELTS

Influence of Shear on the Ordering Temperature of a Triblock Copolymer Melt.

PB96-163753

01,288

MELTS (CRYSTAL GROWTH)

Fluorescence Anisotropy Measurements on a Polymer

Melt as a Function of Applied Shear Stress.

01,209
Observations of Shear Induced Molecular Orientation in a Polymer Melt Using Fluorescence Anisotropy Measure-

PB94-199304 01,210

Observations of Shear Stress and Molecular Orientation Using Fluorescence Anisotropy Measurements.

PB94-199312

01,211

Shear-Induced Martensitic-Like Transformation in a Block Copolymer Melt.

EMBRANE LIPIDS

01,277

Membrane-Mediated Precipitation of Calcium Phosphate in Model Liposomes with Matrix Vesicle-Like Lipid Composition.

PB95-164547

03,468

Effect of 1-Hydroxyethylidene-1,1-Bisphosphonate on Membrane-Mediated Calcium Phosphate Formation in Model Liposomal Suspensions.

03,469

MEMBRANE POTENTIAL

Electropermeabilization of Cell Membranes: Effect of the Resting Membrane Potential.

PB95-163291

03,537

MEMBRANES

Performance Approach to the Development of Criteria for Low-Sloped Roof Membranes.

PB94-160751

00,329

Application of Thermal Analysis Techniques to the Characterization of EPDM Roofing Membrane Materials. -125845

00,359

Use of Thermal Mechanical Analysis to Characterize Ethylene-Propylene-Diene Terpolymer (EPDM) Roofing Membrane Materials.

PB95-125852

00,360

Gas Transport Properties of Solution-Cast Perfluorosulfonic Acid lonomer Films Containing lonic Surfactants.

PBractants.

01,267

Extending the Angular Range of Neutron Reflectivity Measurements from Planar Lipid Bilayers: Applications to a Model Biological Membrane.

PB96-122569

03,476

Swelling and Growth of Polymers, Membranes and PBonges.

03,396

MEMORY FUNCTION

Memory Function Approach to the Shape of Pressure Broadened Molecular Bands.

PB95-152930

00,944

MEMS SYSTEMS

Directions in MEMS Research Application Development

PB95-153797

02,106

MERCURY

Pressure Measurements with the Mercury Melting Line Referred to ITS-90.

PB96-161005

01,136

MERCURY BARIUM CALCIUM CUPRATES

Calibration of High-Energy Electron Beams by Use of GB95-161113

04,598

\section{MERCURY CADMIUM TELLURIDES}

Correlation of $\mathrm{HgCdTe}$ Epilayer Defects with Underlying Substrate Defects by Synchrotron X-Ray Topography.

PB94-200714

02,129

High-Spatial-Resolution Resistivity Mapping Applied to Mercury Cadmium Telluride.

02,131

Review of Semiconductor Microelectronic Test Structures with Applications to Infrared Detector Materials and Proc-

PB94-212925

02,132

Investigation of Mercury Interstitials in $\mathrm{Hg}(1-\mathrm{x}) \mathrm{CdxTe} \mathrm{Al}$ loys Using Resonant Impact-Ionization Spectroscopy.

Heavily Accumulated Surfaces of Mercury Cadmium Telluride Detectors: Theory and Experiment.

02,134

$\mathrm{Hg} 1 \cdot \times \mathrm{CdxTe}$ Characterization Measurements: Current Practice and Future Needs.

PB95-164299

02,157

Electrical Characterization of Liquid-Phase Epitaxially Grown Single-Crystal Films of Mercury Cadmium Telluride by Variable-Magnetic-Field Hall Measurements.

PB95-175782

02,177

Magneto-Transport Properties of $\mathrm{HgCdTe}$

04,661

Characterization of LPE HgCdTe Film by Magnetoresistance.

02,197

MERCURY INTRUSION POROSIMETRY

Digitized Simulation of Mercury Intrusion Porosimetry.
PB94-172236 ERCURY IONS

Wavelengths and Isotope Shifts for Lines of Astrophysical Interest in the Spectrum of Doubly lonized Mercury ( $\mathrm{Hg}$ III). PB95-140869 03,887

Progress on a Cryogenic Linear Trap for (199) Hg(t) Ions. PB95-180790

MERCURY OXIDES

Oxygen Dependance of the Crystal Structure of $\mathrm{HgBa}_{2} \mathrm{CuO} 4+$ and its Relation to Superconductivity. PB96-102512

04,711

MERCURY PENCIL LAMP

Wavelengths of Spectral Lines in Mercury Pencil Lamps.

\section{MERCURY PENCIL LAMPS}

Irradiances of Spectral Lines in Mercury Pencil Lamps.

ERGED BEAM!

Merged-Beams Energy-Loss Technique for Electron-lon Excitation: Absolute Total Cross Sections for $\mathrm{O}(5+)(2 \mathrm{~s}$ yields $2 p$ ).
PB96.102058

04,017

MEROCYANINE 540

Radiation Chemistry of Cyanine Dyes: Oxidation and $\mathrm{Re}$ duction of Merocyanine 540

PB94-211661

00,818

MESOSCOPIC

Mesoscopic Conductance Fluctuations in Large Devices.
PB96-119656

MESOSCOPICS

Observation of Hot-Electron Shot Noise in a Metallic Resistor.

PB97-112007

01,988

\section{MESSAGE-PASSING INTERFACE}

Experience with MPI: 'Converting Pvmmake to Mpimake PB96-141296

MESSAGE PROCESSING

Analysis of ANSI ASC X12 and UN/EDIFACT Electronic Data Interchange (EDI) Standards.

01,729

National Voluntary Laboratory Accreditation Program. GOSIP: Government Open Systems Interconnection Profile.

01,486

\section{METABOLITES}

Determination of 3-Ouinuclidinyl Benzilate (Qnb) and Its Major Metabolites in Urine by Isotope Dilution Gas Chromatography Mass Spectrometry.

METADATA

Application of Metadata Standards.

PB96-180187

03,492

METAL CARBONYLS

Photodecomposition of $\mathrm{Mo}(\mathrm{CO}) 6 / \mathrm{Si}(111) 7 \times 7$ : CO StateResolved Evidence for Excited State Relaxation and Ouenching.
PB95-180154

METAL CLUSTERS

01,009

Characterization of the Interaction of Hydrogen with Iridium Clusters in Zeolites by Inelastic Neutron Scattering Spectroscopy

METAL COATINGS

In situ Measurements of Chloride Ion and Corrosion Potential at the Coating/Metal Interface. 
Intelligent Control of an Inert Gas Atomization Process. PB95-141057

METAL POWDERS

Development of Adaptive Control Strategies for Inert Gas Atomization.

METAL SHEETS

Fracture Behavior of Large-Scale Thin-Sheet Aluminum Alloy.

03,311

Ultrasonic Measurement of Sheet Anisotropy and Formability.

03,213

METAL SURFACES

Scanning Tunneling Microscopy and Fabrication of Nanometer Scale Structures at the Liquid-Gold Interface.
PB95-140414

METAL VAPORS

Vapor Transport in Materials and Process Chemistry. 00,669

METALLIC IONS

Calorimetric Determination of the Standard Transformed Enthalpy of a Biochemical Reaction at Specified PH and PM94-198249

03,454

METALLOIDS

Atmospheric and Marine Trace Chemistry: Interfacial Biomediation and
PB94-199122

03,752

METALLOPORPHYCENES

One-Electron Oxidation of Metalloporphycenes as Studied by Radiolytic Methods.

PB97-111967

01,179

METALLURGY

Fabrication of Platinum-Gold Alloys in Pre-Hispanic South America: Issues of Temperature and Microstructure Control.

PB94-211646

03,333

Metallurgy Technical Activities 1994 (NAS-NRC Assessment Panel, April 6-7, 1995)

PB96-136981

02,981

Metallurgy. Technical Activities, 1995

03,003

METALS

Standard Materials. A Descriptive List with Prices.
AD-A278 $140 / 9$

Thermal Conductivity of Metals and Alloys at Low Temperatures. A Review of the Literature.

AD-A279 180/4 03,302

High Temperature Reactions of Uranium Dioxide with Various Metal Oxides.

AD-A286 648/1

Intelligent Processing of Hot Isostatic Pressing.

PB94-172913

00,717

Calorimetric Determination of the Standard Transformed Enthalpy of a Biochemical Reaction at Specified $\mathrm{PH}$ and PMg.

03,454

Atmospheric and Marine Trace Chemistry: Interfacial Biomediation and Monitoring. Investigation into a Practical Grain Growth Model for Hot Isostatic Pressing

PB95-151684

03,347

Radiance Temperatures (in the Wavelength Range $523-$ $907 \mathrm{~nm})$ of Group IVB Transition Metals Titanium, Zirconium, and Hafnium at Their Melting Points by a PulseHeating Technique.

Suppression of Ignition Over a Heated Metal Surface. PB96-176425 03,291 Characterization of the Structure of YD3 by Neutron Powder Diffraction

01,161

CRYSTMET: The NRCC Metals Crystallographic Data

File.

04,799

Self-Consistent 'GW' and Higher-Order Calculations of Electron States in Metals.

PB97-119341

01,189

METAPHOSPHORIC ACID

Ascorbic and Dehydroascorbic Acids Measured in Plasma Preserved with Dithiothreitol or Metaphorphoric Acid

PB94-216330 03,495

Measurement of Ascorbic Acid in Human Plasma and Serum: Stability, Intralaboratory Repeatability, and Interlaboratory Reproducibility.

ASTABLE ATOMS

03,511

Microlithography by Using Neutral Metastable Atoms and Self-Assembled Monolayers.

PB96-190038

METEOR BURST COMMUNICATIONS

02,441

Comparison of Meteor Activity with Occurrence of Sporadic E Reflections.

METEORS

00,116

Comparison of Meteor Activity with Occurrence of Sporadic E Reflections.

00,116
METHACRYLATE/HYDROXYETHYL

Effects of Surface-Active Resins on Dentin/Composite

PB95-140448

00,156

METHACRYLATES

Ring-Opening Polymerication of a 2-Methylene Spiro Orthocarbonate Bearing a Pendant Methacrylate Group. PB95-176145

01,268

METHAMPHETAMINE

Determination of Amphetamine and Methamphetamine in a Lyophilized Human Urine Reference Material. PB95-175444

03,597

METHANE

Thermodynamic Properties of the Methane-Ethane Sys-

PB95-125779 00,891

Chemical Inhibition of Methane-Air Diffusion Flame.

PB96-195532

01,431

Computations of Enhanced Soot Production in TimeVarying $\mathrm{CH} 4 /$ Air Diffusion Flames.

PB97-119218

01,440

NO Production and Destruction in a Methane/Air Diffusion Flame.

01,443

METHANE/DIFLUORO

Thermodynamic Properties of Difluoromethane.

PB94-185204

00,772

METHANE-MARKER DEVICES

Simple and Efficient Methane-Marker Devices for Chromatographic Samples.

00,635

METHANOL

Molecular-Beam Optothermal Spectrum of the $\mathrm{OH}$ Stretching Band of Methanol.

00,839

Combustion of Methanol and Methanol/Dodecanol Spray Flames.

PB95-108544

02,478

Far Infrared Lase

04,292

Absolute Frequency Measurements of Methanol from 1.5 to $6.5 \mathrm{THz}$.

PB95-175881 Kinetics of the Self-Reaction of Hydroxymethylperoxyl

PB94-212164

00,827

METHLACETYLENE

Rotational Spectra of $\mathrm{CH} 3 \mathrm{CCH}-\mathrm{NH} 3, \mathrm{NCCCH}-\mathrm{NH} 3$, and $\mathrm{NCCCH}-\mathrm{OH} 2$.
$\mathrm{PB} 97-118798$

04,170

METHOD OF CENTERS

Algorithmic Enhancements to the Method of Centers for Linear Programming Problems.

03,426

METHOXY RADICALS

Observation of a Stable Methoxy Intermediate on Cr(110).

00,981

METHOXYANISOLES

Thermal Decomposition of Hydroxy- and Methoxy-Substituted Anisoles.

PB94-173036

00,767

METHYL ALCOHOL

Far Infrared Laser Frequencies of (13)CD3OH.
PB95-169363

Effect of Dodecanol Content on the Combustion of Methanol Spray Flames.

PB95-176020

01,389

Far Infrared Laser Frequencies of $\mathrm{CH} 3 \mathrm{OD}$ and $\mathrm{N} 2 \mathrm{H} 4$ PB96-119623

METHYL BROMIDE

Measurement of Atmospheric Methyl Bromide Using Gravimetric Gas Standards.

PB96-155494

02,497

METHYL METHACRYLATE

Thermal Behaviour of Methyl Methacrylate and N-Pheny Maleimide Copolymers.

PB95-152237

01,246

METHYL PENDANT PBZT

Evidence of Crosslinking in Methyl Pendent PBZT Fiber. PB97-112486

03,393

METHYL RADICALS

Laser Flash Photolysis/Time-Resolved FTIR Emission Study of a New Channel in the Reaction of $\mathrm{CH} 3+\mathrm{O}$ : Production of $\mathrm{CO}(\mathrm{v})$

00,974

METHYLENE LACTONES

Evaluation of Methylene Lactone Monomers in Dental

PB95-164661

00,164

METRIC SYSTEM

Report on the Meeting of the CCU (10th) (of the International Committee of Weights and Measures). Held on July 10-11, 1990.

PB94-172889

Metric for Success

03,792

PB94-187630

02,633

Metric Path to Global Markets and New Jobs: A Quesion-and-Answer and Thematic Discussion

02,601

Think Metric.

PB95-151825

01,298

Guide for the Use of the International System of Units (SI).

B95-226692

02,747

METRICATION: An Economic Wake-Up Call for Surveyors and Mappers.

METRIC SYSTEMS

Federal Metric Progress in 1993.

PB94-196029

03,680

METRICATION

PB94-172079

Metric for Success.

PB94-187630

Federal Labs Have Key Role in Metrication.

PB96-123401

02,600

\section{ETROLOGY}

NIST Handbook 44, 1994: Specifications, Tolerances and Other Technical Requirements for Weighing and Measuring Devices as Adopted by the 78th National Conference on Weights and Measures 1993.

PB94-136009

02,888

Report of the National Conference on Weight and Measures (78th). Held in Kansas City, MO. on July 18-22, PB94-138989

02,623

Electronics and Electrical Engineering Laboratory Technical Progress Bulletin Covering Laboratory Programs, January to March 1991, with 1991 EEEL Events Cal-

PB94-145968

02,113

Electronics and Electrical Engineering Laboratory Technical Progress Bulletin Covering Programs, October 10 December 1993, with 1994/1995 EEEL Events Calendar. PB94-154341

02,115

Metrology for Electromagnetic Technology: A Bibliography of NIST Publications.

PB94-159761

02,116

Center for Electronics and Electrical Engineering Technical Progress Bulletin Covering Center Programs, October to December, with 1991 CEEE Events Calendar. PB94-159787

02,296

National Type Evaluation Program: Index of Device Evaluations by Companv. NCWM Publication 5 Part A (Second Edition)

PB94-160835

02,889

U.S. Navy Coordinate Measuring Machines: A Study of Needs.

02,807

Electronics and Electrical Engineering Laboratory Technical Progress Bulletin Covering Laboratory Programs, October to December 1992, with 1992/1993 EEEL Events Calendar.

PB94-165958

02,299

Process for Selecting Standard Reference Algorithms for Evaluating Coordinate Measurement Software.

PB94-173754

02,629

NIST Response to the Fifth CORM Report on the Pressing Problems and Projected Needs in Optical Radiation Measure 
Sensitivity of Three-Point Circle Fitting

02,901 Metrology Requirements of Future Space Power Systems. 140984

04,840

Metrology and Data for Microelectronic Packaging and Interconnection: Results of a Joint Workshop on Materials Metrology and Data for Commercial Electrical and Optical Gaithersburg, Maryland on May 5-6, 1994. Volume 1. ReGaith
sults.
PB95-

PB95-143111

02,328

Electronics and Electrical Engineering Laboratory Tech. nical Progress Bulletin Covering Laboratory Programs,
April to June 1994 with 1994/1995 EEEL Events Calendar.

PB95-143186

02,329

Electronics and Electrical Engineering Laboratory 1994 Technical Accomplishments Supporting Technology for U.S. Competitiveness in Electronics.

PB95-144309

01,887

Electronics and Electrical Engineering Laboratory 1995 Program Plan. Supporting Technology for U.S. Competiiveness in Electronics.

PB95-15988

Metrology.

01,894

Report of the National Conference on Weights and Measures (79th). Held in San Diego, California on July 17-21, PB95-169819

02,656

Electronics and Electrical Engineering Laboratory Technical Progress Bulletin Covering Laboratory Programs, July to September 1994 with 1994/1995 EEEL Events PB95-170395

02,360

Program of the Manufacturing Engineering Laboratory. 1995. Infrastructural Technology Measurements, and Standards for the U.S. Manufacturing Industries. PB95-188835

02,754

Electronics and Electrical Engineering Laboratory Technical Publication Announcements Covering Laboratory Programs, July to September 1994 with 1995 EEE PB95-198925

01,912

Electronics and Electrical Engineering Laboratory Technical Progress Bulletin Covering Laboratory Programs, endar.

PB95-208724

02,372

Electronics and Electrical Engineering Laboratory Tech nical Progress Bulletin Covering Laboratory Programs, April to June 1991, with 1992 EEEL Events Calendar PB95-209821 01,916

Design, Specification and Tolerancing of Micrometer-Tolerance Assemblies.

02,913

Electronics and Electrical Engineering Laboratory Technical Progress Bulletin Covering Laboratory Programs, January to March 1992, with 1992/1993 EEEL Events

PB95-210480

01,917

Electronics and Electrical Engineering Laboratory Technical Publication Announcements Covering Laboratory Programs. January to March 1995 with 1995 EEEl Events Calendar. Algorithm Testing and Evaluation Program for Coordinate Measuring Systems: Testing Methods

PB95-251658

02,666

PB95-251716

02,667

Journal of Research of the National Institute of Standards and Technology, May/June 1995. Volume 100, Number 3. Determining the Magnetic Properties of $1 \mathrm{~kg}$ Mass StandPBds. 261905

04,016

High Accuracy Measurement of Aperture Area Relative to Standard Known Aperture.

01,919

Application of Single Electron Tunneling: Precision $\mathrm{Ca}$ pacitance Ratio Measurements.

04,703

Journal of Research of the National Institute of Standards and Technology, July/August 1995. Volume 100, Number 4. Special Issue: The Gaseous Electronics Conference 4. Special Issue: The Gaseous

Radio-Frequency

02,386

Journal of Research of the National Institute of Standards and Technology, November/December 1994. Volume 99 Number 6 .

PB96-113535

04,336

Semiconductor Measurement Technology: Test Structure Implementation Document: DC Parametric Test Structures and Test Methods for Monolithic Microwave Integrated Circuits (MMICs)

02,399

Journal of Research of the National Institute of Standards and Technology, September/October 1995. Volume 100, Number 5.

01,927
Electronics Packaging Materials Research at NIST. 02,405 Electromagnetic Properties of Materials: The NIST MetrolOgy Program.

01,933

Bibliography of the NIST Optoelectronics Division.

Metrology for Electromagnetic Technology: A Bibliography of NIST Publications, September 1995.
PB96- 128269 Metrology Issues in Terahertz Physics and Technology. Metallurgy Technical Activities 1994 (NAS-NRC Assessment Panel, April 6-7, 1995)

PB96-13698

02,981

Progress Toward Accurate Metrology Using Atomic Force MBicroscopy.

02,417

New NIST/ARPA National Soft X-ray Reflectometry FacilPBं.96-158092

04,080

Journal of Research of the National Institute of Standards and Technology. November/December 1995. Volume 100, Number 6 .
PB96-159215

01.949

Calibration of Electret-Based Integral Radon Monitors Using NIST Polyethylene-Encapsulated (226)Ra/(222)Rn Emanation (PERE) Standards.

01,950

Generating and Measuring Displacements Up to $0.1 \mathrm{~m}$ to an Accuracy of $0.1 \mathrm{~nm}$ : Is it Possible.

PB96-160700

04,090

Metrology Standards for Advanced Semiconductor Lithography Referenced to Atomic Spacings and Geometry.
PB96-160718 Nanometrology.

PB96-160726

02,425

Metrology.

02,925

Scanned Probe Microscopies: Opportunities and Issues in Metrology.

02.426

Residual Error Compensation of a Vision-Based Coordinate Measuring Machine.

PB96-161617

04,091

Length Metrology of Complimentary Small Plastic Rulers.

PB96-161724 04,096 Business and Manutacturing Motivations tor the Develop-
ing of Analytical Technology and Metrology for SemiPB96-161948

04.778

Enhanced Voltage-Dividing Potentiometer for High-Precision Feature Placement Metrology.

02,428

Electronics and Electrical Engineering Laboratory 1995 Technical Accomplishments: Advancing Metrology for Electrotechnology to Support the U.S. Economy.
PB96-164520

Report of the National Conference on Weights and Measures (80th) as Adopted by the 80th National Conference on Weights and Measures, 1995. Held in Portland, Maine on July 16-20, 1995.

PB96-165840

02,681

Electronics and Electrical Engineering Laboratory: 1996 Program Plan. Supporting Technology for U.S. Competitiveness in Electronics.

PB96-175237

01,962

Improved Dose Metrology in Optical Lithography.

PB96-179510

02,439

Increasing the Value of Atomic Force Microscopy Process Metrology Using a High-Accuracy Scanner. Tip Characterization and Morphological Image Analysis.

terization, and

02,758

Scanned Probe Microscope Tip Characterization Without Calibrated Tip Characterizers.

PB96-190368

02,759

National Semiconductor Metrology Program, Project Portfolio FY 1996

Program of the Manufacturing Engineering Laboratory. 1996. Intrastructural Technology. Measurements, and Standards for the U.S. Manufacturing Industries.

PB96-195276

02,760

Metallurgy. Technical Activities, 1995

03,003

Performance of the Electron Pump with Stray Capacitances.

01,976

Monte Carlo Simulation of Scanning Electron Microscope

Signals. 0969

02,444

SEM Linewidth Metrology of X-ray Lithography Masks.

PB96-201108
NIST/NCMS Program on Electronic Packaging: First Up-

PB96-204086

03,008

Present and Future Standard Specimens for Surface Finish Metrology.

02,928

Results of Capacitance Ratio Measurements for the Single Electron Pump-Capacitor Charging Experiment. 04,813

\section{METROLOGY: PHYSICAL MEASUREMENTS}

Density of Solids and Liquids.

AD-A278 517/8

AD-A278 851/1 00,123

Technologic Papers of the Bureau of Standards: Number 170. Pyrometric Practice.

AD-A279 282/8

03,766

Journal of Research of the National Institute of Standards and Technology, November-December 1993. Volume 98 Number 6 .

Journal of Research of the National Institute of Standards and Technology. January/February 1994. Volume 99. PB94.169737

02,019

Application of the Electronic Balance in High Precision Pycnometry.

00,534

Use of the Electronic Balance for Highly Accurate Direct Mass Measurements Without the Use of External Mass

PB94-187713

03,803

Overview of Radiometric Program of the NIST Thermal Imaging Laboratory.

Measuring the Stability of Three Copper Alloys.
PB94-199866

04,230

03,326

Characterization of Vertical-Cavity Semiconductor Struc-

PBres. 200193

02,126

Faraday Effect Sensors: A Review of Recent Progress

PB94-200706 02, 128

Technical Digest: Symposium on Optical Fiber Measurements (8th), 1994. Held in Boulder, Colorado on September 13-15, 1994

PB94-207636

Hybrid Undulator for the NIST-NRL Free-Electron Laser.
PB94-212529

Using LORAN-C Broadcasts for Automated Frequency

Calibrations.

Light Scattered by Coated Paper

PB94-216546

01,526

Journal of Research of the National Institute or and Technology. March/April 1994. Volume 99 , PB94-219219

02,000 Journal of Research of the National Institute of Standards and Technology. May/June 1994. Volume 99. Number 3. PB94-219326 02,643

Development of an Automated Part Inspection System Using the DMIS Standard.

02,899

International Intercomparison of Detector Responsivity at 1300 and $1550 \mathrm{~nm}$

PB95-125928

02,140

PB95-150884

Oplical Fiber ding Diameter.

04,266

Journal of Research of the National Institute of Standards and Technology, July/August 1994. Volume 99, Number 4. Special Issue: Extreme Value Theory and Applications. Proceedings of the Conference on Extreme Value Theory and Applications, Volume 2. Held at Gaithersburg, Mary. 
Reduction of Interfilament Contact Loss in Nb3Sn Superconductor Wires.

Photodetector Frequency Response Measurements at Photodetector Frequency Response Measurements at
NIST, US, and NPL, UK: Preliminary Results of a Standards Laboratory Comparison.

PB95-175592 02,175

Time and Frequency: Bibliography of NIST Publications, March 1995.

Reproducibility of the Temperature of the Ice Point in Routine Measurements.

PB95-255923

04,015

Journal of Research of the National Institute of Standards and Technology, May/June 1995. Volume 100, Number 3. High Accuracy Measurement of Aperture Area Relative to a Standard Known Aperture.

PB95-261954

01,919

Intracomparison Tests of the FG5 Absolute Gravity Me-

Contributions of Out-of-Plane Material to a ScannedBeam Laminography Image.

PB96-111786

02,704

Comparison of Photodiode Frequency Response Measurements to $40 \mathrm{GHz}$ between NPL and NIST.

04,038

Journal of Research of the National Institute of Standards Journal of Research of the National Institute of Standards 4. Special Issue: The Gaseous Electronics Conference Radio-Frequency Reference Cell.

PB96-113311

02,386

Journal of Research of the National Institute of Standards and Technology, November/December 1994. Volume 99, Number 6.

PB96-113535

04,336

Journal of Research of the National Institute of Standards and Technology, September/October 1995. Volume 100,

$\begin{array}{ll}\text { Number } 5 . & 01,927 \\ \text { PB96-117767 } & 01,90\end{array}$

Journal of Research of the National Institute of Standards and Technology, March/April 1995. Volume 100, Number

PB96-126180

04,349

Apparatus for Resistance Measurement of Short, Small-

Diameter Conductors.

04,417

Metrology Approach to Unifying Rockwell C Hardness Scales.

PB96-155551

02,957

Liquid-Nitrogen-Cooled High Tc Electrical Substitution Ra-

diometer as a Broadband IR Transfer Standard.

02,198

Journal of Research of the National Institute of Standards and Technology. November/December 1995. Volume PB96-159215

01,949

New Method for Achieving Accurate Thickness Control for

Uniform and Graded Multilayer Coatings on Large Flat

Substrates.

04,366

New Concepts of Precision Dimensional Measurement for Modern Manufacturing.

PB96-160684

02,924

Nanometrology.

02,425

Scanned Probe Microscopies: Opportunities and Issues in MBtrology.

02,426

Journal of Research of the National Institute of Standards and Technology, September/October 1993. Volume 98 Number 5 .

03,368

PB96-169057

Transformation of BCC and B2 High Temperature Phases to HCP and Orthorhombic Structures in the Ti-Al-Nb Sys-
tem. Part 1. Microstructural Predictions Based on a Subgroup Relation between Phases.

03,370

Transformation of BCC and B2 High Temperature Phases to HCP and Orthorhombic Structures in the Ti-Al-Nb Systo HCP and Orthorhombic Structures in the Ti-Al-Nb System. Part 2. Experimental TEM Study of Microstructures.
PB96-169073 Corrosion Characteristics of Silicon Carbide and Silicon Nitride.

PB96-169081

03,372

Journal of Research of the National Institute of Standards and Technology, January/February 1996. Volume 101 Number 1. Journal of Research of the National Institute of Standards and Technology, March/April 1996. Volume 101, Number PB96-177381

NIST High Accuracy Scale for Absolute Spectral Response from $406 \mathrm{~nm}$ to $920 \mathrm{~nm}$.

01,865

Texture Measurement of Sintered Alumina Using the March-Dollase Function.

04,784
Time and Frequency Metrology.

01,556

Journal of Research of the National Institute of Standards and Technology May/June 1996. Volume 101. Number 3. and Technology, May/June 1996. Volume 101, Number 3 .
Special Issue: NIST Workshop on Crystallographic PB97-109011

04,798

Model of an ent.

PB97-110209

04,384

Optical Scattering from Moderately Rough Surfaces.

04,385 Present and Future Standard Specimens for Surface Finish Metrology.

02,928

Light Scattering by Sinusoidal Surfaces: Illumination Windows and Harmonics in Standards.

dows and Harm

04,387

Characterization of Modified FEL Quartz-Halogen Lamps for Photometric Standards.

PB97-112544

00,282

ssues in High-Speed Pyrometry

02,693

\section{METROPOLITAN AREAS}

Metropolitan Areas (Including MSAs, CMSAs, PMASs, and NECMAs). Category: Data Standards and Guidelines; Subcategory: Representations and Codes.

FIPS PUB 8-6

04,873

FIPS PUB 8-6, Metropolitan Areas (for Microcomputers). MICA

Diffusion of Water along 'Closed' Mica Interfaces. PB96-180039

02,993

\section{MICELLAR ELECTROKINETIC CAPILLARY}

Separation and Identification of Organic Gunshot and ExSeparation and Identification of Organic Gunshot and Ex-
plosive Constituents by Micellar Electrokinetic Capillary Electrophoresis.

\section{MICHAELIS-MENTEN EQUATION}

00,566

Michaelis-Menten Equation for an Enzyme in an Oscillating Electric Field.

MICHELSON INTERFEROMETERS

New Refractometer by Combining a Variable Length Vacuum Cell and a Double-Pass Michelson Interferometer.
PB97-111926

MICRO CALORIMETERS

Hot-Electron Microcalorimeter for X-ray Detection Using a Superconducting Transition Edge Sensor with Electrothermal Feedback.

04,792

MICROACTUATORS

Test Structures for Determining Design Rules for Microelectromechanical-Based Sensors and Actuators.
PB95-150488

MICROALLOYING

Microstructure and Tensile Properties of Microalloyed Steel Forgings

MICROANALYSIS

Concentration Histogram Imaging: A Scatter Diagram Technique for Viewing Two or Three Related Images.
PB94-199114 Electron Probe X-Ray Microanalysis.

00,562

Microanalysis to Nanoanalysis: Measuring Composition at High Spatial Resolution

00,563 PB95-107173

03,561

Distribution of Fluoride

PB95-151205

Micromagnetic Scanning Microprobe System

03,561 PB95-176178

02,224

MICROBIAL DEGRADATION

Microbial Degradation of Polysulfides and Insights into Their Possible Occurrence in Coal.

PB95.163374

02,488

MICROBOLOMETER

Niobium Microbolometers for Far-Infrared Detection. PB96-111729

02,184

MICROBOLOMETERS

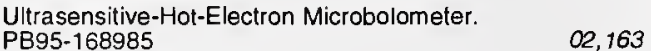

High-Tc Superconducting Antenna-Coupled Microbolometer on Silicon.

02,166

Antenna-Coupled High-Tc Air-Bridge Microbolometer on Silicon.

PB95-180899

MICROCALORIMETERS

New Coaxial Microwave Microcalorimeter Evaluation PB95-15322

01,892

Hot-Electron Microcalorimeters for $X$-ray and Phonon Detection.

04,644

Method to Determine the Calorimetric Equivalence Correction for a Coaxial Microwave Microcalorimeter.

01,913

Hot-Electron Microcalorimeters as High-Resolution X-ray Detectors.

PB96-123641

04,739

Hot-Electron-Microcalorimeters with $0.25 \mathrm{~mm}(2)$ Area PB96-200670

MICROCRACK

Model for Microcrack Initiation and Propagation beneath Hertzian Contacts in Polycrystalline Ceramics.

03,077

MICROCRACKING

Asymmetric Tip Morphology of Creep Microcracks Growing Along Bimaterial Interfaces.

PB94-200243

03,138

MICROCRACKS

Elastic Constants and Microcracks in $\mathrm{YBa} 2 \mathrm{Cu} 3 \mathrm{O} 7$.

PB96-200761

03,005

MICRODOSIMETRY

Microdosimetry and Cellular Radiation Effects of Radon Progency in Hum
PB95-152344

MICROELECTROMECHANICS

Directions in MEMS Research Application Development.

PB95-153797

02,106

MEMS in Standard CMOS VLSI Technology.

PB96-102363

02,377

MICROELECTRONICS

Electronics and Electrical Engineering Laboratory Technical Progress Bulletin Covering Laboratory Programs, January to March 1991, with 1991 EEEL Events Cal-

PB94-145968

02,113

Electronics and Electrical Engineering Laboratory Technical Progress Bulletin Covering Programs, October to
December 1993, with 1994/1995 EEEL Events Calendar. PB94-154341
02,115 Electronics and Electrical Engineering Laboratory Technical Publication Announcements Covering Laboratory Programs, October to December 1993 with 1994/1995 EEEL Events Calenda

PB94-193752

02,307

Electronics and Electrical Engineering Laboratory Technical Progress Bulletin Covering Laboratory Programs, January to March 1994 with 1994/1995 EEEL Events Cal-

endar

02,308

Electronics and Electrical Engineering Laboratory Technical Progress Bulletin Covering Laboratory Programs, July to September 1993 with 1994 EEEL Events Cal-

PB94-194354 02,309

Test Structures for the In-Plane Locations of Projected Features with Nanometer-Level Accuracy Traceablo to a Coordinate Measurement System.

PB94-200565

02,313

Wire Bond Testing

PB94-211653

02,314

Electronics and Electrical Engineering Laboratory Technical Publication Announcements Covering Laboratory Programs, January

Events Calendar.

01,883

Metrology and Data for Microelectronic Packaging and Interconnection: Results of a Joint Workshop on Materials 
Current and Voltage Measurements in the Gaseous Electronics Conference RF Reference Cell.

PB96-113337

02,388

Optical Emission Spectroscopy on the Gaseous Electronics Conference RF Reference Cell.

PB96-113345

02,389

Optical Diagnostics in the Gaseous Electronics Conference Reference Cell.

PB96-113352

02,390

Studies of Ion Kinetic-Energy Distributions in the Gaseous Electronics Conference RF Reference Cell. PB96-113360

02,391

Microwave Diagnostic Results from the Gaseous Electronics Conference RF Reference Cell.

02,392

Langmuir Probe Measurements in the Gaseous Electronics Conference RF Reference Cell.

PB96-113386 02,393

Inductively Coupled Plasma Source for the Gaseous Electronics Conterence RF Reference Cell.
PB96-113394 Reactive Ion Elching in the Gaseous Electronics Conference RF Reference Cell.

PB96-113402

02,395

Dusty Plasma Studies in the Gaseous Electronics Conference Reference Cell.

PB96-113410

02,396

One-Dimensional Modeling Studies of the Gaseous Elec-

tronics Conference RF Reference Cell.

PB96-113428

02,397

Two-Dimensional Self-Consistent Radio Frequency Plasma Simulations Relevant to the Gaseous Electronics Conference RF Reference Cell.

PB96-113436

02,398

Measurement of Patterned Film Linewidth for Interconnect Characterization.

02,420

High-Speed Interconnection Characterization Using Time

High-Speed Interconnection Characterization Using Time
Domain Network Analysis.
PB96-148176

Microelectronic Test Structures for Overlay Metrology.
PB96-164249

Beyond the Technology Roadmaps: An Assessment of Electronic Materials Research and Development.

Electronic Mater

01,961

MICROEMULSIONS

Flow of Microemulsions through Microscopic Pores.
PB95-140463 00,905

Structure of a Triglyceride Microemulsion: A Small Angle

Neutron Scattering Study.

PB96-112255

01.077

MICROFORM

Microform Calibrations in Surface Metrology.

PB95-203295

02,951

MICROGRAVITY

Combustion of a Polymer (PMMA) Sphere in Micro-

Nravity.

01,354

Ignition and Subsequent Transition to Flame Spread in a Microgravity Environment.

N96-15584/1

04,828

Electric Field Effects on a Near-Critical Fluid in Micro-

gravity.

MICROINSTRUMENTATION

04,217

Integrated Thin-Film Micropotentiometers.

PB96-146709

02,109

MICROMACHINING

Realizing Suspended Structures on Chips Fabricated by CMOS Foundry Processes Through the MOSIS Service.

01,881 High-Level CAD Melds Micromachined Devices with

$\begin{array}{ll}\text { Foundries. } & 02,321\end{array}$

Design and Machining of Copper Specimens with Micro Holes for Accurate Heat Transfer Measurements. 02,658 Thermal Isolation of High-Temperature Superconducting
Thin Films Using Silicon Wafer Bonding and Micromachining.

PB96-135017

02,408

Micromachined Display Output for a Cellular Neural Network.

02,422

Micromachined Coplanar Waveguides in CMOS Technology.

PB97-119283

02,456

MICROMAGNETIC MODELS

Experimental Verification of a Micromagnetic Model of Dual-Layer Magnetic Films.

PB95-141081

MICROMAGNETICS

Numerical Micromagnetic Techniques and Their Applications to Magnetic Force Microscopy Calculations. PB95-175931

MICROMECHANICS

Micromechanics of Fracture in Rubber-Toughened

Epoxies.

03,011
Hybrid Method for Determining Material Properties from Instrumented Micro-Indentation Experiments.

03,348 PB95-152229

MICROMETERS

Optical Fiber Geometry: Accurate Measurement of Cladding Diameter.
PB95-151940

04,266

MICROMINIATURIZATION

Directions in MEMS Research Application Development.

PB95-153797

02,106

MICROPHONES

Determination of Acoustic Center Correction for Type LS2aP Condensor Microphones.

04,190

Proposed Antiferromagnetically Coupled Dual-Layer Magnetic Force Microscope Tips.

04,645

MICROSCOPES

Development of a Calibrated Atomic Force Microscope.
02,894

Video Microscopy Applied to Optical Fiber Geometry Measurements.

PB95-173068

04,295

Flexible-Diaphragm Force Microscope.

03,966

Standard Reference Materials for Optical Fibers and Con-

nectors. neclors.

MICROSCOPY

National Voluntary Laboratory Accreditation Program: Bulk Asbestos Analysis.

PB95-138129

02,541

Workshop Summary Report: Industrial Applications of Scanned Probe Microscopy. A Workshop Co-sponsored by NIST, SEMATECH, ASTM, E42.14, and the American Vacuum Society. Held in Gailhersburg, Maryland on March 24-25, 1994

PB95-17038

00,506

PB96-103130

04,721

Summary Report: Workshop on Industrial Applications of Scanned Probe Microscopy (2nd). A Workshop Co-SponAmerican Vacuum Society. Held in Gaithersburg, Maryland on May 2-3, 1995.

00,509

Progress Toward Accurate Metrology Using Atomic Force Microscopy.

02,417

Scanning Capacitance Microscopy Measurements and Modeling: Progress Towards Dopant Profiling of Silicon. PB96-148150 04,773

2D-Scanning Capacitance Microscopy Measurements of Cross-Sectioned VLSI Tesistructures.

04,104

Scanning Capacitance Microscopy Measurements and Modeling for Dopant Profiling of Silicon, 04,781 Morphology and Phase Separation Kinetics of a Compatibilized Blend.

01,297 PB97-119135

Vortex Images in Thin Films of $\mathrm{YBa} C \mathrm{Cu} 3 \mathrm{O}$ (sub 7-x) and Bi2Sr2Ca1Cu2O (sub 8+x) Oblained by Low-Temperalure Magnetic Force Microscopy.

04,815

MICROSENSORS

Test Structures for Determining Design Rules for Microelectromechanical-Based Sensors and Acluators.
02,105

ICROSTRIP TRANSMISSION LINES

Crosstalk between Microstrip Transmission Lines.
PB94-135639 Crosstalk between Microstrip Transmission Lines. (NIST Reprint)

PB95-180337

02,225

Radiated Emissions and Immunity of Microstrip Transmission Lines: Theory and Measurements. PB96-162649

02,238

MICROSTRIPS

Line-Reflect-Match Calibrations with Nonideal Microstrip Standards.

02,242

MICROSTRUCTURAL DESIGN

Model for Toughness Curves in Two-Phase Ceramics. 2. Microstructural Variables.

PB96-163795

03,078

MICROSTRUCTURE

Microstructural Features of Some Low Water/Solids, Silica Fume Mortars Cured et Different Temperetures.

Digitized Simulation of Mercury Intrusion Porosimetry,
PB94-172236 Microstructure and Tensile Properties of Microalloyed Steel Forgings

03,204

Energy and Migration of Grain Boundaries in Polycrystals.

Energy and Migration of Grain Boundaries in Polycrystals.
PB94-211638

Fabrication of Platinum-Gold Alloys in Pre-Hispanic South America: Issues of Temperature and Microstructure Con

PB94-211646

03,333

Effect of Microstructure on the Wear Transition of Zirconia-Toughened Alumina. Microstructural Evolution in Two-Dimensional Two-Phase Polyerystals.

04,498

Compositional Mapping of the Microstructure of Materials.

Compositional Mapping of the Microstructure of Matenals.
00,565

Computer Simulation of the Diffusivity of Cement-Based Materials.

PB95-125985

00,362

Fundamental Computer Simulation Models for CementBased Materials.

00,364

Effect of Mn Content on the Microstructure of Al-Mn Alloys Electrodeposited at $150 \mathrm{C}$.

PB95-126355

03,343

Rheology of Fresh Cement Paste.

B95-163150

00,378

Wear Model for Alumina Sliding Wear

PB95-163796

03,239 Microstructure Development.

01,320

Tensile Deformation-Induced Microstructures in Free Slanding Copper Thin Films.

04,715

Low-Energy Vibrations and Octahedral Site Occupation in PB96-160734

01,133

Neutron Spectroscopic Comparison of beta-Phase Rare Earth Hydrides.

01,134

Phase Identification in a Scanning Electron Microscope Using Backscattered Electron Kikuchi Patterns.

PB97-109128

04,804

Multi-Scale Picture of Concrete and its Transport Properties: Introduction for Non-Cement Researchers.

03,107

\section{MICROSTRUCTURE EFFECTS}

Microstructure Effect on the Phase Behavior of Blends of Deuterated Poly

MICROSTRUCTURES

Microlithography by Using Neutral Metastable Aloms and Selí-Assembled

02,441

MICROWAVE ABSORBERS

Bistatic Scattering of Absorbing Materials from 30 to 1000

01,891

MICROWAVE AMPLIFERS

Comparison of Three Techniques for the Precision Measurement of Amplifier Noise.

Measurement Accuracies for Various Techniques for Measuring Amplifier Noise.

PB95-163671

02,350

Investigations of AM and PM Noise in X-Band Devices.

PB95-180022 02,062

Origin of Uf PM and AM Noise in Bipolar Junction Transistor Amplifiers

02,096

Quest to Understand and Reduce It Noise in Amplifiers 


\section{KEYWORD INDEX}

NIST Model PM2 Power Measurement System for $1 \mathrm{~mW}$ at $1 \mathrm{GHz}$.
$\mathrm{PB} 94.135803$

02,018

Direct Comparison Transfer of Microwave Power Sensor Calibrations.

02,086

MICROWAVE FREQUENCIES

Ferroelectric Thin Film Characterization Using Superconducting Microstrip Resonators.

02,270

MICROWAVE OSCILLATORS

Investigations of AM and PM Noise in X-Band Devices.

PB95-180022 02,062

Phase Locking in Two-Dimensional Arrays of Josephson Junctions: Effect of Critical-Current Nonuniformity.

PB96-102587 04,714

Quest to Understand and Reduce $1 / f$ Noise in Amplifiers and Baw Quartz Oscillators.

\section{MICROWAVE POWER STANDARDS}

02,097

New Coaxial Microwave Microcalorimeter Evaluation

PB95.153227

01,892

Method to Determine the Calorimetric Equivalence Correction for a Coaxial Microwave Microcalorimeter.
PB95-202404

Developing a NIST Coaxial Microwave Power Standard at $1 \mathrm{~mW}$

PB95-202412

01914

Direct Comparison Transfer of Microwave Power Sensor Calibrations.

PB96- 158654

Performance of Commercial CMOS Foundry-Compatible

Multijunction Thermal Converters.

Multijunction

MICROWAVE SPECTRA

Tunneling-Rotation Spectrum of the Hydrogen Fluoride

PB94-198678

00,793

2-Tunneling Path Internal-Axis-Method-Like Treatment of

the Microwave Spectrum of Divinyl Ether.

00,808

Microwave Spectra of van der Waals Complexes of Im-

portance in Planetary Atmospheres.
PB95-150611

00,919

MICROWAVE SPECTROMETERS

Experimental Studies of Line Shapes from a Balle. Flygare Spectrometer.

00,796

MICROWAVE SPECTROSCOPY

Microwave and Submillimeter Spectroscopy of Ar-NH3

States Correlating with $\mathrm{Ar}+\mathrm{NH} 3(\mathrm{j}=1, k=1)$.

MICROWAVE SPECTRUM

Microwave Spectrum and Structure of $\mathrm{CH} 2 \mathrm{O}-\mathrm{H} 2 \mathrm{O}$.
PB97-118723 168

MICROWAVE SUBSTRATES

Wire Bonding to Multichip Modules and Other Soft Substrates.

02,079

Wire Bonding to Multichip Modules and Other Sott Sub. strates.

ICROWAVE TRANSMISSION

02,082

Slant Path Atmospheric Refraction Calibrator: An Instrument to Measure the Microwave Propagation Delays In duced by Atmospheric Water Vapor.

PB95-151270

01,476

Microwave Diagnostic Results from the Gaseous Elec-

tronics Conference RF Reference Cell.

PB96.113378

02,392

MICROWAVES

Microwave Spectrum and Structure of $\mathrm{CH} 3 \mathrm{NO} 2-\mathrm{H} 2 \mathrm{O}$.

AD-A296 377/5

00,719

Molecular Microwave Spectra Tables.

00,720

AD-A296 498/9

PB94-193786

Hydrolysis of Proteins by Microwave Energy.

01,880

94-216322

03,528

Diffusion of Copper into Gold Plating

PB95-162152

00,957

Microwave Characterization of Printed Circuit Transmission Lines

02,077

Calibration Service for Coaxial Reference Standards for

Microwave Power.
PB96-162722

01,958

MICROWAVES POWER TRANSMISSION

Planar Near-Field Measurements of Low-Sidelobe AntenPBas. 219235

02,001

MICROZONATION

De Facto Microzonation through the Use of Soils Factors in Design Triggers.

00,462
MIL-STD-1312

Review and Upgrading of Military Fastener Test Standard PB95-154720

02,947

MILITARY FACILITIES

Present Worth Factors for Life-Cycle Cost Studies in the Department of Defense (1995).

03,664

ILITARY STANDARDS

Review and Upgrading of Military Fastener Test Standard MIL-STD-1312.

PB95-154720

02,947

LITARY VEHICLES

Ground Vehicle Control at NIST: From Teleoperation to Autonomy.
N94-34037/9

03,758

\section{MILLIMETER WAVE EQUIPMENT}

Dual-Frequency Millimeter-Wave Radiometer Antenna for Airbome Remote Sensing of Almosphere and Ocean. PB96-112289

02,009

MILUMMETER WAVES

Proposed High-Accuracy Superconducting Power Meter for Millimeter Waves.

02,034

MIMD

Time-Perturbation Tuning of MIMD Programs.

PB94-164399

01,681

Time-Perturbation Tuning of MIMD Programs.

PB94-172566

01,684

Using Synthetic-Perturbation Techniques for Tuning Shared Memory Programs (Extended Abstract). PB94-172657

01,685

MIMD (COMPUTERS)

Operating Principles of Multikron II Performance Instrumentation for MIMD Computers.

PB95-189486

01,628

Scalability Test for Parallel Code.

01,749

MIMD (MULTIPLE INSTRUCTION MULTIPLE DATA)

Synthetic-Perturbation Tuning of MIMD Programs.

PB94-185568

01,687

Simple Scalability Test for MIMD Code.

01,688

MINERALIZATION

Calcium Phosphate Precipitation in Liposomal Suspensions.

PB94-172145

03.452

Critical Evaluation of the Purification of Biominerals by Hypochlorite Treatment.

PB95.150959

00,186

Remineralizing Dental Composites Based on Amorphous Calcium Phosphate.

03.573

MINERALS

Procedure for the Study of Acidic Calcium Phosphate Precursor Phases in Enamel Mineral Formation.

03,564

CRYSTMET: The NRCC Metals Crystallographic Data

File.
PB97-109029

04,799

MINIMAL SURFACES

Singularities in Minimum Surface Energy Problems and Their Influence in Surface Motion.

04,473

INIMUM DETECTABLE ACTIVITY

Germanium Detector Optimization of MDA for Efficiency vs. Low Intrinsic Background.

00.543

MINING EQUIPMENT

Environment Simulation for a Continuous Mining Machine

03,697

MIRAGE DETECTION

Thermal Wave NDE of Advanced Materials Using Mirage Etfect Detection.

$\begin{array}{ll}\text { PB96-204516 } & 04,191\end{array}$

MIRRORS

Simulations of Neutron Focusing with Curved Mirrors.

PB96-176649

02,200

MIS CAPACITORS

Development and Characterization of Insulating Layers on Silicon Carbide: Annual Report for February 14, 1988 to February 14, 1989.

PB94-155579

02,295

MIXERS

Milliwatt Mixer for Small Fluid Samples.

PB94-198819

02,634

MIXERS (ELECTRONICS)

Investigations of $A M$ and $P M$ Noise in X-Band Devices. PB95-180022 02,062 Frequency Synthesis and Metrology at $10(-17)$ and BeyBond. 113187

02,101

MIXING

Neutron Scattering Study of Shear Induced Turbidity in Polystyrene/Dioctyl Phthalate Solutions at High Shear

01,197

MIXTURES

Prediction of the Thermal Conductivity of Refrigerants and Refrigerant Mixtures.

03,258

Global Thermodynamic Behavior of Fluid Mixtures in the Critical Region.

04,199

SANS and LS Studies of Polymer Mixtures Under Shear

PB95-107090 01,231

Thermophysical Property Computer Packages from NIST.

04,203 Intrinsic Viscosity and the Polarizability of Particles Having a Wide Range of Shapes.

PB96-119318

03,170

MMIC (MONOLITHIC MICROWAVE INTEGRATED

Microwave Characterization of Flip-Chip MMIC Compo-

PB96-176722

02,434

Microwave Characterization of Flip-Chip MMIC Interconnections.

PB96-176730

02,435

Electrical Measurements of Microwave Flip-Chip Interconnections.

02,436

MOBILE COMMUNICATION SYSTEMS

Channel Coding for Code Excited Linear Prediction CELP) Encoded Speech in Mobile Radio Applications.

PB95-143178 01.475

Error Protecting Characteristics of CDMA and Impacts on Speech

PB96-122452

01,491

MOBILE HOMES

Wind Load Provisions of the Manufactured Home Construction and Safety Standards: A Review and Recommendations for Improvement.

00,428

Manufactured Homes: Probability of Failure and the Need for Better Windstorm Protection through Improved Anchoring Systems
PB95-143129

00,432

MOBILE PHASE COMPOSITIONS

Comparison of the Liquid Chromatographic Behavior of Selected Steroid Isomers Using Different ReversedPhase Materials and Mobile Phase Compositions. PB95-140976

00,574

MOBILE ROBOTS

Control System Architecture for a Remotely Operated Unmanned Land Vehicle.

PB95-163200

03.759

MODE COUPLING

Orientational Fluctuations, Diffuse Scattering, and Orientational Order in Solid C60.

PB97-119275

04,176

MODE LOCKED LASERS

Metrology Applications of Mode-Locked Erbium Fiber Lasers.

PB95-140158 04,256

Multiwavelength Birefringent-Cavity Mode-Locked Fibre Laser.

04,262

MDEL

Model Precast Concrete Beam-to-Column Connections Subject to Cyclic Loading.

00,438

PB95-153094

Partially Prestressed and Debonded Precast Concrete 


\section{KEYWORD INDEX}

MOLECULAR ORBITALS

Comparison of POSIX Open System Environment (OSE) and Open Distributed Processing (ODP) Reference ModPB96-131495

01,820

Machining Process Planning Activity Model for Systems integration.

PB96-165428 02,841

Characterization of Two-Dimensional Dopant Profiles: Status and Review.

02,451

MODEMS

High Resolution Time Interval Counter.

PB96-138607

01,495

MODERATORS

Experimental plan to determine the performance of the Oak Ridge National Laboratory Cold Neutron Moderator Final report, September 1, 1993 -November 30, 1993. DE95011352 03,778 Thermal Hydraulic Tests of a Liquid Hydrogen Cold Neutron Source.
PB95-135570

03,884

Nuclear Heat Load Calculations for the NBSR Cold Neutron Source Using MCNP.

PB95-152955

03,730

MODERNIZATION

Learning to Change: Opportunities 10 Improve the Performance of Smaller Manufacturers.

PB94-166212

00,010

ODULATOR

Interdigitated Stacked P-I-N Multiple Quantum Well Modulator.

02,455

MODULES

Integrating Automated Systems with Modular ArchitecPB95-150231

00,577

MODULES OF ELASTICITY

Ultrasonic Measurement of Sheet Anisotropy and Formability.

PB95-153235

03,213

Dynamic Shear Modulus Measurements with Four Independent Techniques in Nickel-Based Alloys

PB94-198900

03,320

Dynamics vs. Static Young's Moduli: A Case Study.

PB94-213188 03,210

Hollow Clay Tile Prism Tests for Martin Marietta Energy Systems: Task 2 Testing

00,352

Method for Determining Both Magnetostriction and Elastic Modulus by Ferromagnetic Resonance.

02,974

PB95-150108

Controlling Moisture in the Walls of Manufactured Hous-

ing.

00,355

Measurements of Moisture Diffusivity for Porous Building Materials.

00,356

Moisture and Water-Induced Crack Growth in Optical Materials.

04,267

Sorption of Moisture on Epoxy and Alkyd Free Films and Coated Steel Panels.

PB95-162475

03,192

Water-Vapor Measurements of Low-Slope Roofing Materials

PB95-251617

00,399

Water Adsorption at a Polyimide/Silicon Wafer Interface.

01,070

Mathematical Analysis of Practices to Control Moisture in the Root Cavities of Manufactured Houses.

00,278

Experimental Verification of a Moisture and Heat Transfer Model in the Hygroscopic Regime.

PB97-111546

00,309

\section{MOISTURE CONTENT}

Analysis of Moisture Accumulation in a Wood-Frame Wall Subjected to Winter Climate

PB94-199320

00,338

Heat and Moisture Transfer in Wood-Based Wall Construction: Measured versus Predicted

PB95-200655

00,391

Amperometric Measurement of Moisture in Transformer Oil Using Karl Fischer Reagents.

00,623

Empirical Validation of a Transient Computer Model for Combined Heat and Moisture Transfer

00,416 PB97-111991 Parametric Study of Wall Moisture Contents Using a Re'Moist' Transient Heat and Moisture Transter Mode.

PB97-122535

00,419

\section{MOISTURE TRANSFER}

Manufactured Housing Walls That Provide Satisfactory Moisture Performance in All Climates.

00,383
MOISTUREPROOFING

Manufactured Housing Walls That Provide Satisfactory Moisture Performance in All Climates.

00,383

178885

Thermochemistry of the Hydrolysis of L-arginine to (Lcitrulline + Ammonia) and of the Hydrolysis of L-arginine (L-ornithine + Urea)

03,463

MOLAR MASS

Adsorption of Polyacrylic Acids and Their Sodium Salts on Hydroxyapatite: Effect of Relative Molar Mass.

PB97-112510

MOLAR VOLUME

Apparent Molar Hea: Capacities and Apparent Molar Volumes of Aqueous Glucose at Temperatures from 298.15 $\mathrm{K}$ to $327.01 \mathrm{~K}$
$\mathrm{~PB} 94-212800$

MOLDING TECHNIQUES

Report on the Workshop on Manufacturing Polymer Composites by Liquid Molding. Held in Gaithersburg, Maryland on September 20-22, 1993

03,131

Effect of Heterogeneous Porous Media on Mold Filling in Resin Transfer Molding.

PB95-108676

03,197

MOLE FRACTION ERRORS

Nonlinear Correlation of High-Pressure Vapor-Liquid

Equilibrium Data for Ethylene $+n$-Butane Showing Inconsistencies in Experimental Compositions.

01,141

MOLECULAR ABSORPTION

Memory Function Approach to the Shape of Pressure

Broadened Mo
PB95-152930

00,944

\section{MOLECULAR BEAM EPITAXY}

Single-Photon Ionization and Detection of $\mathrm{Ga}$, In, and As(sub n) Species in GaAs Growth.

00.591

In-situ Monitoring of Molecular Beam Epitaxial Growth Using Single Photon Ionization.

PB95-202222

01.023

Laser Vacuum Ultraviolet Single Photon Ionization Probing of III-V Semiconductor Growth.

04,691

Epitaxial Growth of Sb/GaSb Structures: An Example of

V/III-V Heteroepitaxy.
PB95-202560

Single Photon Ionization, Laser Optical Probe Technique for Semiconductor Growth.

PB95-202776

01,032

Single-Photon Laser Ionization Time-of-Flight Mass Spectroscopy Detection in Molecular-Beam Epitaxy. Application to As4, As2, and Ga

tion to As4, Ast
PB95-203337

01,052

Single Photon Laser Ionization as an in-situ Diagnostic for MBE growth

PB96-102025

01,059

Stacked Series Arrays of High-Tc Trilayer Josephson Junctions.

04,705

Buffer Layer Modulation-Doped Field-Effect-Transistor

Interactions in the Al0.33Ga0.67As/GaAs Superlattice

System.
PB96.102876

02,380

Laser Gas Ionization Technique Monitors MEB Crystal

Growth.

01,076

02,406

PB96-122841

In-situ Neutron Reflectivity of MBE Grown and Chemically
Processed Surfaces and Interfaces. Processed Surtac
PB96-146634

02,416

MOLECULAR BEAM SCATTERING

Dynamics of Hydrogen Interactions with $S i(100)$ and Si(111) Surfaces.

MOLECULAR BEAMS

04,082

Use of Extended Permutation-Inversion Groups in Constructing Hypertine Hamitonians for Symmetrical-Top Internal Rotor Molecules Like H3C-SiH3.

PB94-212032

00,823

MOLECULAR CHAINS

Molecular Weight Dependence of the Lamellar Domain Spacing of ABC Triblock Copolymers and Their Chain Conformation in Lamellar Domains.

PB95-161691

01,254

Small Angle Neutron Scattering Studies on Chain Asymmetry of Coextruded Poly(Vinyl Alcohol) Film.

PB95-164372

01,262

MOLECULAR CLOUDS

Extended $\mathrm{CO}(7$ yields 6) Emission from Warm Gas in Orion.

PB96-102504

00,090

MOLECULAR CLUSTERS

High Resolution IR Studies of Polymolecular Clusters: Micromatrices and Unimolecular Ring Opening.

PB94-185691

High-Resolution Intrared Spectroscopy of DF Trimer. A Cyclic Ground State Structure and DF Stretch Induced Intramolecular Vibrational Coupling.

PB95-150678

00,920

Product State Correlations in the Reaction of $O((1) D)$ and $\mathrm{H} 2 \mathrm{O}$ in Bimolecular Collisions and in $\mathrm{O} 3 . \mathrm{H} 2 \mathrm{O}$ ClustersPB95-15301

MOLECULAĂ COLLISIONS

Three-Vector Correlation Theory for Orientation/Alignment Studies in Atomic and Molecular Collisions.
PB94-211109

03,834

N2(a'(sup 1) Sigma(sub g)(sup +)) Metastable Collisiona Destruction and Rotational Excitation Transter by N2.

PB95-151395 Rotational Excitation Transter by N2. 00,933

Product State Correlations in the Reaction of $\mathrm{O}((1) \mathrm{D})$ and $\mathrm{H}_{2} \mathrm{O}$ in Bimolecular Collisions and in $\mathrm{O}_{3} \mathrm{H} 2 \mathrm{O}$ Clusters-

Translation.
PB95-153011 00,946

Collisional Alignment of $\mathrm{CO} 2$ Rotational Angular Momentum States in a Supersonic Expansion.

OLECULAR COMPOSITES

Phase Behavior of a Hydrogen Bonding Molecular Com-

posite.
PB94-185188

01.202

MOLECULAR CONFORMATION

Molecular Weight Dependence of the Lamellar Domain Spacing of $A B C$ Triblock Copolymers and Their Chain Contormation in Lamellar Domains.

161691

Small Angle Neutron Scattering Studies on Chain Asym. metry of Coextruded Poly(Vinyl Alcohol) Film. PB95-164372

01,262

MOLECULAR DYNAMICS

Femtosecond Time-Resolved Wave Packet Motion in Moecular Multiphoton Ionization and Fragmentation.

00,790

Neighbor Tables for Molecular Dynamics Simulations

00,991

Pulsed Laser Photolysis Time-Resolved FT-IR Emission Studies of Molecular Dynamics.

PB95-203162

04,002

Comparison of a Fixed-Charge and a Polarizable Water Model.

PB96-111620 01,072

Long-Lived Structures in Fragile Glass-Forming Liquids.

PB96-119565 04,212

Molecular Dynamics Investigation of the Surface/Bulk Equilibrium in an Ethanol-Water Solution.

01,183

MOLECULAR EXCITATION

Electron Attachment to Excited Molecules(1)

PB96-122809

01,087

LECULAR IONS

Production and Spectroscopy of Small Polyatomic Molecular Ions Isolated in Solid Neon. (Reannouncement with New Availability Information)

00,704

Vibrational Spectra of Molecular lons Isolated in Solid Neon. 6. CO4(-). (Reannouncement with New Availability Information).

AD-A238 $415 / 4$

00,705

Vibrational Spectra of Molecular lons Isolated in Solid Neon. 7. $\mathrm{CO}(+), \quad \mathrm{C}_{2} \mathrm{O} 2(+)$, and $\mathrm{C}_{2} \mathrm{O} 2(-)$. 
Analysis of Protein Metal Binding Selectivity in a Cluster Mode 00,845

Molecular Orbital Calculations of Bond Rupture in Brittle Solids.

PB95-164059

00,973

Molecular Orbıtal Study of Water Enhanced Crack Growth Process.
PB95.164067

03,240

MOLECULAR PROPERTIES

Molecular Microwave Spectra Tables.

Collection of Results for the SPC/E Water Model.

PB96-147889

00,720

MOLECULAR SIEVES

Small Angle Neutron Scattering Study of the Structure and Formation of MCM-41 Mesoporous Molecular Sieves.

MOLECULAR SPECTROSCOPY

Molecular Spectroscopy

PB94-213337

03,861

Atomic, Molecular, and Optical Physics with X-rays. PB94-213378

03,863

\section{MOLECULAR STRUCTURE}

Complementary Molecular Information on Phthalocyanine Compounds Derived from Laser Microprobe Mass Spec. trometry and Micro-Raman Spectroscopy

PB94-172269

00,757

Structure of Glycine-Water H-Bonded Complexes.

PB94-198603

00.789

Fluorescence Anisotropy Measurements on a Polymer Melt as a Function of Applied Shear Stress.

PB94-199296

01,209

Observations of Shear Induced Molecular Orientation in a Polymer Melt Using Fluorescence Anisotropy Measure-

ments.
PB94-199304 01,210

Observations of Shear Stress Using Fluorescence Anisotropy Measurements. 01.211 Neutron Scattering by Multiblock Copolymers of Structure

(A-B)N-A.
PB94-211547

01,219

Structure of Molecules on Surfaces as Determined Using Electron-Stimulated Desorption.

00,852

Determination of the Molecular Parameters of $\mathrm{NiH}$ in its (2)Delta Ground State by Laser Magnetic Resonance.
PB95-107116

00,869

Construction of Maximum-Entropy Density Maps, and
Their Use in Phase Determination and Extension. PB95-108593

0,882

Characterization of Chemically Modified Pore Surfaces by Small Angle Neutron Scattering.

PB95-126181

00,898

Structural Stabilization of Phase Separating PC/Polyester Blends through interfacial Modification by Transesterification Reaction.

PB95-150454

01,239

Structural Heterogeneity in Epoxies.

PB95-151866

01,243

SANS Studies of Space-Time Organization of Structure in Polymer Blends.

01,251

Octacalcium Phosphate Carboxylates. 2. Characterization and Structural Consideration.

00,955

Localization of a Homopolymer Dissolved in a Lamellar Structure of a Block Copolymer Studied by Small-Angle Neutron Scattering.

PB95.161592

01,253

Elastic Scattering of Polymer Networks

PB95-161816

01,255

Characterization of Molecular Network of Thermosets Using Neutron Scattering.

PB95-164109 01,259

Small-Angle Neutron Scattering of Poly(vinyl alcohol) Gels.

01,260

Competition between Hydrodynamic Screening ('Draining') and Excluded Volume Interactions in an Isolated

Polymer Chain

01,265

Comparison of a Fixed-Charge and a Polarizable Water Model.

PB96-111620

01,072

Long-Lived Structures in Fragile Glass-Forming Liquids.

PB96-119565 04,212

Slow Dynamics of Segregation in Hydrogen-Bonded Poly. mer Blends.

01,281

Neutron Scattering Study of the Lattice Modes of Solid Cubane

01,126

New Generation of Fire Resistant Polymers. Part 1. Com. puter-Aided Molecular Design.

01,419 nvestigations of the Systematics of Crystal Packing Using the Cambridge Structural Database.

00,519

\section{MOLECULAR VIBRATION}

Vibrational Spectra of Molecular Ions Isolated in Solid Neon. 6. $\mathrm{CO} 4(-)$. (Reannouncement with New Availability Information)

AD-A238 415/4

00,705

High-Resolution IR Laser-Driven Vibrational Dynamics in Supersonic Jets: Weakly Bound Complexes and Intramolecular Energy Flow.

PB94-216751

00,862

Acceleration of Intramolecular Vibrational Redistribution by methyl Internal Rotation. A Chemical Timing Study of -fluorotoluene and p-fluorotoluene-d3.

01.039

\section{MOLECULAR WEIGHT}

Adsorption of Low-Molecular-Weight Sodium Polyacrylate on Hydroxyapatite.

00,139

Effects of Molecular Weight and Thermal Stability on Polymer Gasification.

PB94-212610

01,228

Determination of the Weight Average Molecular Weight of Two Poly(Ethylene Oxides). SRM 1923 and SRM 1924. PB94-217031

01,230

Molecular Weight Dependence of the Lamellar Domain Spacing of ABC Triblock Copolymers and Their Chain Conformation in Lamellar Domains.

01,254

MOLECULE COLLISIONS

Energy Dependence of Collision Characteristics in Molecule-Surface Collisions.

PB94-198504

00,786

OLECULE-MOLECULE COLLISIONS

Excitation Transfer in Barium by Collisions with Noble

01,163

\section{MOLYBDENUM}

Texture Study of Two Molybdenum Shaped Charge Liners by Neution Diffraction.

PB94-200177

03.754

Anomalous Odd- to Even-Mass Isotope Ratios in Resonance Ionization with Broad-Band Lasers.

PB94-211406

03,839

Analytical Method of Determining the Heat Capacity at High Temperatures from the Surface Temperature of a Cooling Sphere.
PB94-216124

03,865

Use of Sum Rules on the Energy-Loss Function for the Evaluation of Experimental Optical Data.

PB95.150736

04,264

Dynamic Technique for Measuring Normal Spectral Emissivity of Electrically Conducting Solids at High Temperatures with a High-Speed Spatial Scanning Pyrometer PB95-153045

Interfaces in Mo/Si Multilayers.

03,921

PB96-160668

02,423

MLYBDENUM DISILICIDE

Creep Rupture of MoSi2/SiCp Composites.

03,154

MOLYBDENUM DISULFIDE

Wear of Selected Materials and Composites Sliding

against MoS2 Films.

03,231

MOLYBDENUM-LANTHANUM

Atomic Energy Levels. As Derived From the Analyses of Optical Spectra. Volume

AD-A280 279/

00,714

MOLYBDENUM TO LANTHANUM

Ultraviolet Multiplet Table. Finding List for Spectra of the Elements Molybdenum to Lanthar

nium to Radium ( $Z=72$ to 88 )

AD-A278 131/8

00.709

MOMENT PROBLEM

Distribution of Dielectric Relaxation Times and the Moment Problem

MONITORING

04,727

Through-the-Arc Sensing for Monitoring Arc Welding.

PB94-185899 02,858

Review of Cure Monitoring Techniques for On-Line Process Control.

PB94-216728 03,145

Guidance of the Legality of Keystroke Monitoring

PB96-161237

00,005

Manager's Guide for Monitoring Data Integrity in Financial Systems.

MONITORS

Monitoring Polymer Cure by Fluorescence Recovery After Photobleaching

MONOCHROMATOR

Sott $X$-ray Reflectometry Program at the National Institute of Standards and Technology. PB96-160395

04,368

MONOCHROMATORS

Simple Variable Line Space Grating Monochromator for PBnchrotron Ligh 156203

04,065

MONOLITHIC MICROWAVE INTEGRATED CIRCUITS

Microwave Characterization of Flip-Chip MMIC Compo nents.

02,434

Microwave Characterization of Flip-Chip MMIC Interconnections.

02,435

Electrical Measurements of Microwave Flip-Chip Interconnections

\section{MONOMERIZATION}

02,436

DnaJ, DnaK and GrpE Heat Shock Proteins are Required in 'ori'P1 DNA Replication Solely at the RepA Monomerization Step.

PB97-119382

03,557

MONOMERS

Neutron Reflectivity of End-Gratted Polymers: Concentration and Solvent Ouality Dependence in Equilibrium Con-

PB94-185758 01,206

Modified Surface-Active Monomers for Adhesive Bonding

to Dentin.

00,158

Ring-Opening Dental Resin Systems Based on Cyclic Acetais

Synthesis and Polymerization of Difunctional and Multifunctional

Cyclopolymerizati

Pon.

Difunctional
Capable

of

Evaluation of Methylene Lactone Monomers in Dental Resins.

Ring-Opening Polymerication of a 2-Methylene Spiro

Orthocarbonate Bearing a Pendant Methacrylate Group.

PB95-176145

MONOMOLECULAR FILMS

Silver Metalization of Octadecanethiol Monolayers SelfAssembled on Gold

00,923

UV-Photopatterning of Alkylthiolate Monolayers Self-As.

sembled on Gold and Silver.

C.924

Surtace Geometry of BaO on W(100): A Surface-Extended X-Ray-Absorption Fine-Structure Study.

PB95-164414

00.980

Characterization of Cytochrome c/Alkanethiolate Struc-

tures Prepared by Self-Assembly on Gold.

ONOPOLE ANTENNAS

Correction Factor for Nonplanar Incident Field in Monopole Calibrations.

PB95-108643

02.002

MONOTECTIC ALLOYS

Surface Energy Reduction in Fibrous Monotectic Struc- 


\section{KEYWORD INDEX}

MORPHOLOGY

Shear-Excited Morphological States in a Triblock Copoly-

PB94-172392

01,196

Temperature Dependence of the Morphology of Thin Diblock Copolymer Films as Revealed by Neutron Reflec

PBivity. 172756

01,199

Morphology of Symmetric Diblock Copolymers as Revealed by Neutron Reflectivity.

01,234

Morphological Estimation of Tip Geometry for Scanned Probe Microscopy.

PB95-20344

02,662

MORTALITY

Histopathology, Blood Chemistry, and Physiological Status of Normal and Moribund Striped Bass ('Morone saxatilis') Involved in Summer Mortality ('Die-Off') in the Sacramento-San Joaquin Delta of California.

PB94-198157

00,034

MORTARS (MATERIAL)

Water Permeability and Chloride Ion Diffusion in Portland Cement Mortars: Relationship to Sand Content and Critical Pore Diamet

MORTARS (MATERIALS)

03,193

Microstructural Features of Some Low Water/Solids, Sili-

ca Fume Mortars Cured at Different Temperatures.
PB94-160777 00,330 Interfacial Transport in Porous Media: Application to do Electrical Conductivity of Mortars.

Electrical Con

01,326

MOS CAPACITORS

Development and Characterization of Insulating Layers on Silicon Carbide: Annual Report for February 14, 1988 to February 14, 1989.

PB94-155579

02,295

MOS GATE OXIDES

Assessing MOS Gate Oxide Reliability on Wafer Leve with Ramped/Constant Voltage and Current Stress. PB96-180112

MOSAIC Approaches Using Virtual Environments with Mosaic
PB95-169108

01,599

SAIC COMPUTER PROGRAM

Getting Started on Mosaic. PB95-180360

01,721

MOSAIC SOFTWARE PACKAGE

World Wide Web and Mosaic: User's Guide.
PB94-207354

MOSFET

Numerical Simulation of Submicron Photolithographic Processing.

$0,2,310$

Anomalous Behavior of a Ouantized Hall Plateau in a High-Mobility Si Metal-Oxide-Semiconductor Field-Etfect Transistor.

PB95-164174

02,354

MOTARS

Percolation and Pore Structure in Mortars and Concrete PB95-150439

00,370

MOTION

Adaptive, Predictive 2-D Feature Tracking Algorithm for Finding the Focus of Expansion.

PB94-218575

01,588

Three Dimensional Position Determination from Motion

PB95-107108

01,788

Motion-Model-Based Boundary Extraction

PB95-189502

01,849

MOTION SIMULATORS

Integration of Servo Control into a Large-Scale Control System Design: An Example from Coal Mining.

PB94-203429

03,696

Continuous Mining Machine Control Using the Real-Time Control System

Environment Simulation for a Continuous Mining Ma-

PB94-203536

03,697

MOUTHWASHES

Deposition of Loosely Bound and Firmly Bound Fluorides on Tooth Enamel by an Acidic Gel Containing Fluorosilicate and Monocalcium Phosphate Monohydrate Interaction of Chlorhexidine Digluconate with and Adsorption of Chlorhexidine on Hydroxyapatite. PB95-175907

03,566

MPI (MESSAGE-PASSING INTERFACE)

Experience with MPI: 'Converting Pvmmake to Mpimake under LAM' and 'MPI and Parallel Genetic Programming'. PB96-141296

MRSEVS (MATERIAL REMOVAL SHAPE ELEMENT

VOLUMES)

Issues Concerning Material Removal Shape Element Volumes (MRSEVs)
PB94-185493

02,882

MSEL

Materials Science and Engineering Laboratory Annual Report, 1993. NAS-NRC Assessment Panel, April 21-22 1994

PB94-162534

02,969
MSEL (MATERIALS SCIENCE AND ENGINEERING

(MATORY)

Materials Science and Engineering Laboratory Annual Report, 1994. NAS-NRC Assessment Panel, April 6-7,

02,976

PB95-196697

03,009

Report, 1995. Technical Activities.

PB96-214754

MULLITE

Crystal Structure and Compressibility of 3:2 Mullite.

03,682

MULTI-ELEMENT ANALYSIS

Interpreting the Readings of Multi-Element Personne Dosimeters in
PB95-175428

MULTI-PHOTON PAOCESSES

Resonance Enhanced Multiphoton Ionization Spectroscopy of the SnF Radical.

MULTI-ZONE AIR FLOW

CONTAM88 Building Input Files for Multi-Zone Airflow and Contaminant Dispersal Modeling

02,537

MULTICHARGED IONS

Evaluation of Two-Photon Exchange Graphs for HighlyCharged Heliumlike Ions

03,808

Experimental Aspects and Z-Dependent Systematics in One- and Two-Electron lons and Single Vacancy SysPB94-199627

03,815

Relativistic and Ouantum Electrodynamic Effects in Highly-Charged lons.

Atomic Branching Ratio Data for Carbon-Like lons.

PB94-212842

Spectroscopic Data Tables for Highly-lonized Atoms.

Collisions of Electrons with Highly-Charged Ions. PB96-200340

03,910

MULTICHIP MODULES

Wire Bonding to Multichip Modules and Other Soft Sub-

02,079

Wire Bonding to Multichip Modules and Other Soft Substrates.

PB96-135207

02,082

MULTICAITERIA

Development and Validation of Multicriteria Ratings: A Case Study.

00,004

MULTIDIMENSIONAL SYSTEMS

Dynamics of Multi-DOF Stochastic Nonlinear Systems
PB97-113245

00,477

MULTIJUNCTION THERMAL CONVERTERS

Development of Thin-Film Multijunction Thermal ConvertPBg7-112338

MULTIKAON

Operating Principles of MultiKron II Periormance Instrumentation for MIMD Computers.

PB95-189486

01,628

MULTILATERAL AGREEMENTS

GATT Standards Code Activities of the National Institute of Standards and Technology 1994.

00,497

TBT Agreement Activities of the National Institute of Standards and Technology, 1995. PB97-104178

00,499

MULTILAYER FILMS

Modeling Effects of Temperature Annealing on Giant Magnetoresistive Response in Discontinuous Multilayer NiFe/Ag Films. PB97-112585

04,157

\section{MULTILAYERS}

Fracture in Multilayers.

PB96-163613

02,988

MULTIMEDIA

Porting Multimedia Applications to the Open System Environment.
PB94-172921

02,721

Preliminary Functional Specifications of a Prototype Electronic Research Notebook for NIST.

00,012

Multimedia Tutorial on Phase Equilibria Diagrams. Mullimed

03,088

\section{MULTIPATH TRANSMISSION}

Analytical Estimation of Carrier Multipath Bias on GPS Position Measurements.

PB94-215712

04,845

MULTIPHOTON

Resonance Enhanced Multiphoton Ionization Spectroscopy of the PF
PB97-119119

00,702

MULTIPHOTON IONIZATION

Femtosecond Time-Resolved Wave Packet Motion in Mo. lecular Multiphoton Ionization and Fragmentation. PB94-198611

Electronic Spectra of $\mathrm{CF} 2 \mathrm{Cl}$ and $\mathrm{CFCl} 2$ Radicals Observed by Resonance Enhanced Multiphoton Ionization.
PB95-151023

Femtosecond Time-Resolved Molecular Multiphoton Ionization: The Na2 System.

PB95-202305

03,975

Population Trapping in Short-Pulse Multiphoton Ioniza-

PBg

04,371

Resonance Enhanced Multiphoton Ionization Spectroscopy of the SnF Radical.

01,176

MULTIPLE AUTONOMOUS UNDERSEA VEHICLES

MAJECT

Intelligent Control for Multiple Autonomous Undersea Ve-

PB94-211877

03,747

MULTIPLE-INSTRUCTION MULTIPLE-DATA

Time-Perturbation Tuning of MIMD Programs.

PB94-164399

01,681

Time-Perturbation Tuning of MIMD Programs.

01,684

Using Synthetic-Perturbation Techniques for Tuning Shared Memory Programs (Extended Abstract).

PB94-172657

01,685

Synthetic-Perturbation Tuning of MIMD Programs. PB94-185568

MULTIPLE REFLECTION THEORY

Observation of the Transverse Second Harmonic Magneto-Optic Kerr Effect from Ni81Fe19 Thin Film Struc-

B96-200332

01,971

MULTIPLE SCATTERING

Study of Multiple Scattering Background in Compton Scatter Imaging.

MULTIPLET STAUCTURES

Charge-Transfer-Induced Multiplet Structure in the

$\mathrm{N} 4,5 \mathrm{O} 2,3$ Soft-X-ray Emission Spectrum of Lanthanum.

PB'96-163746

04,102

MULTIPLET TABLE

Ultraviolet Multiplet Table. Finding List for Spectra of the

Elements Molybdenum to Lanthanum $(Z=42$ to 57$)$; Hafnium to Radium ( $Z=72$ to 88 )

AD-A278 131/8

00,709

Ultraviolet Multiplet Table

AD-A278 446/0

00,710

MULTIPOINT COMMUNICATION

Strategy to Support Multipoint Communication Service PB96-176672 Service.

ULTIPOLE EXCITATION

High-Order Multipole Excitation of a Bound Electron.

PB96-119789

MULTIPROCESSORS

Operating Principles of MultiKron Virtual Counter Performance Instrum

01,632

MULTIRANGE INSTRUMENTS

Modeling and Test Point Selection for a Thermal Transfer 

Pore-Forming Protein with a Metal-Actuated Switch.
PB96-176557

MUTAGENS

Tert-Butyl Hydroperoxide-Mediated DNA Base Damage in Cultured Mammalian Cells.

03,644

PB94-18200

DNA in

Copper Ion-Mediated Moditic

03,645

Oxidative DNA Base Damage in Renal, Hepatic, and PulOxidative DNA Base Damage in Renal, Hepatic, and Pul-
monary Chromatin of Rats After Intraperitoneal Injection of Cobalt (II) Acetate.

PB95-150025

03,647

MUTATION

Ionizing Radiation Causes Greater DNA Base Damage in Radiation-Sensitive Mutant M10 Cells That in Parent Mouse Lymphoma L5178Y Cells.

Mouse Lymphon

03,632

\section{N-GAS MODEL}

Further Development of the N-Gas Mathematical Model: An Approach for Predicting the Toxic Potency of Complex Combustion Mixtures.

PB96-123260

03,650

N-HEXANE

Enhancement of R123 Pool Boiling by the Addition of NHexane.

PB96-165956

02,605

NACELLES

Flow of Alternative Agents in Piping.

PB95-202420

00,022

NANOANALYSIS

Microanalysis to Nanoanalysis: Measuring Composition at High Spatial Resolution.

PB95-107173

00,563

NANOCOMPOSITES

In situ Characterization of Vapor Phase Growth of Iron Oxide-Silica Nanocomposites: Part 1. 2-D Planar LaserInduced Fluorescence and Mie Imaging.

PB97-112478

03,185

NANOCRYSTALLINE CERAMIC

Bulk Modulus and Young's Modulus of Nanocrystalline gamma-Alumina.

NANOCRYSTALLINE MATERIALS

03,092

Small Angle Neutrons Scattering from Nanocrystalline Palladium as a Function of Annealing.

03,354 PB95-176103

Inelastic Neutron Scattering Study of Hydrogen in
Nanocrystalline Pd. Nanocrystalline

03,366

NANOCRYSTALS

Vibrational Excitations and the Position of Hydrogen in Nanocrystalline Palladium.

PB96-111828

04,035

NANOELECTRONICS

Integration of Scanning Tunneling Microscope Nanolithography and Electronics Device Processing.
PB95-153359

NANOHARDNESS

Force Calibrations in the Nanonewton Regime.

PB95-168696

NANOLITHOGRAPHY

Integration of Scanning Tunneling Microscope Nanolithography and Electronics Device Processing.
PB95-153359

NANOMATERIALS

Viewpoint: Nanocrystalline and Nanophase Materials.
PB94-185865

NIST Workshop on Nanostructured Material (1st): Report of an Industrial Workshop Conducted by the National Institute of Standards and Technology. Held in Gaithersburg, Maryland on May 14-15, 1992

02,973

$\mathrm{X}$-ray Powder Diffraction from Carbon Nanotubes and Nanoparticles. Nanoparticles

03,064

\section{NANOPARTICULATES}

Growth and Nucleation of Hydrogenated Amorphous Sili-

Con on Silicon (100) Surfaces.
PB96-176516

02,991

NANOSCALE PORES

Vitritication and Crystallization of Organic Liquids Confined to Nanoscale Pores.

PB97-112304

03,392

NANOSTRUCTURE

NIST Metrology for Soft X-ray Multilayer Optics. $\quad 04,088$

Nanostructure Fabrication via Laser-Focused Atomic Deposition (Invited)

PB96-204094

04,132

NANOSTRUCTURES

NIST Workshop on Nanostructured Material (1st): Report

an Industrial Workshop Conducted by the National In-

stitute of Standards and Technology. Held in Gaithersburg. Maryland on May 14-15, 1992.

02,973

Nanostructure Fabrication via Direct Writing with Atoms Focused in Laser Fields.

04,564
Laser-Focused Atomic Deposition.

PB95-161618

04,604

aser Focused Atomic Deposition.

04,685

Construction of Silicon Nanocolumns with the Scanning Tunneling Microscope.

PB95-203063

Using Alom Optics to Fabricate Nanostructures.

Excitons in Complex Ouantum Nanostructures.

PB97-118343

04,757

NAPHTHALENE

nvestigating the 3.3 Micron Infrared Fluorescence from aphthalene Following Ultraviolet Excitation. N95.15839/0

00,724

NASA STANDARD REFERENCE MODEL

Hierarchical Ada Robot Programming System (HARPS) A Complete and Working Tele

PB94-213162

02,934

NASREM (NASA STANDARD REFERENCE MODEL)

Hierarchical Ada Robot Programming System (HARPS) A Complete and Working Telerobot Control System Based on the NASREM Model.

PB94-213162

02,934

NATIONAL ARCHIVES AND RECORD

Electronic Access: Blueprint for the National Archives and Records Administration.

02,731

NATIONAL ARCHIVES AND RECORDS ADMINISTRATION ser Study: Informational Needs of Remote National Archives and Records Administration Customers. PB95-154738

02,725

NATIONAL BUREAU OF STANDARDS

Standard Materials. A Descriptive List with Prices. AD-A278 140/9

00,500

NATIONAL GOVERNMENT

Self Monitoring Accounting Systems.

6602

00,007

NATIONAL INFORMATION INFRASTRUCTURE

Information Infrastructure: Reaching Society's Goals. A Report of the Information Infrastructure Task Force Committee on Applications and Technology.

ED-376 823

00,131

Information Infrastructure: Reaching Society's Goals. Report of the Information Infrastructure Task Force Commitee on Applications and Technology.

01.469

Report on the Workshop on Advanced Digital Video in the National Information Infrastructure. Held in Washington, D.C. on May 10-11, 1994.

PB95-103677

01,472

Framework for National Information Infrastructure Serv-

PB95-103719

02,723

nformation Technology Engineering and Measuremen Model: Adding Lane Markings to the Information Superhighway.

01,474

Standards Policy and Information Infrastructure.

01,485

Electronic Access to Standards on the Information High-

Way. 131578

01,494

NATIONAL INSTITUTE FOR STANDARDS AND

TECHNOLOGY

Publications of the National Institute of Standards and Technology 1992 Catalog.

00.014

PB95-200747

NATIONAL INSTITUTE OF STANDARDS AND

ECHNOLOGY

Abstract and Index Collection in the Research Information Center of the National Institute of Standards and Technology.

02,744

Ceramics Technical Activities, 1993 (NAS-NRC Assessment Panel April 21-22, 1994).

03,031

PB94-162591

Program:

NIST Support to the Next Ge

PB94-163490

02,808

NIST Serial Holdings, 1994

PB94-178068 02,745

Study of Federal Agency Needs for Information Tech. nology Security.

01,579

Graphical Analysis of the CCRL Portland Cement Proficiency Sample Database (Samples 1-72). (Par 1. ficiency Sample Database (Samples 1-72). (Part 1. Univanant Analy

01,308

National Voluntary Laboratory Accreditation Program 1995 Directory.
PB95-174454

00,483

Abstract and Index Collection in the Research Information Center of the National Institute of Standards and Technology.

02,741

Electronics and Electrical Engineering Laboratory Technical Publication Announcements Covering Laboratory

Programs, April to June 1995 with 1995 EEEL Events PB96-137187

01,941

Publications of the National Institute of Standards and Technology 1993 Catalog.

00,017

Proposed Coating Technology Consortium. (National Coil Coaters Association Fall Conference). Held in Rosemont, Illinois in September 1992

PB97-110431

03,129

\section{NATIONAL INSTITUTE OF STANDARDS AND} Journal of Research of the National Institute of Standards
and Technology. March/April 1995. Volume 100, Number and

PB96-126180

04,349

\section{NATIONAL INSTITUTE OF STANDARDS AND}

TECHNOLOGY PRODUCT TESTING

National Voluntary Laboratory Accreditation Program 1996 Directory.

00,485

NATIONAL INTERESTS

Putting the Information Infrastructure to Work: Report of the Information Infrastructure Task Force Committee on Applications and Technology.

PB94-163383

00,001

White Papers Prepared for the White House: ConstrucConstruction Goals. Held on December 14-16, 1994.

PB95-21689

01,299

NATIONAL MARINE MAMMAL TISSUE BANK

Development of the National Marine Mammal Tissue

PB95-161402

02,586

NATIONAL SECURTY

Impact of the FCC's Open Network Architecture on NS/ NP Telecommunications Security. PB95-189445

01,483

NATIONAL TYPE EVALUATION PROGRAM

National Type Evaluation Program: Index of Device Evaluations by Company. NCWM Publication 5 Part A (SecOnd Edition).

Proficiency Tests for the NIST Airborne Asbestos Program, 1990.

National Voluntary Laboratory Accreditation Program 1995 Directory.

00,483

NATIONAL VOLUNTARY LABORATORY ACCREDITATION

ROGRAM

NVLAP Procedures U.S. Code of Federal Regulations. Title 15, Subtitle A, Chapter 2, Part 7. (Effective December 1984; Amended September 1990)

PB94-160850

02,627

National Voluntary Laboratory Accreditation Program: Procedures and General Requirements.

02,630 


\section{KEYWORD INDEX}

NEUTRON ACTIVATION ANALYSIS

Sliding Vane Flow Conditioner Tests in a 100 Diameter Long 10 inch Natural Gas Orifice Meter at Pacific Gas and Electric. Topical Report, 1990-1992.

PB95-256335

02,493

Thermophysical Properties of Fluids for the Gas Industry. PB96-122437 02,494 Development of Gas Standards from Solid 1,4. dichlorobenzene

02,496

NATURAL GAS LIQUIDS

New Data and Correlations for the Custody Transfer of Natural Gas Liquids.

02,499

NATURAL GAS PIPELINES

Assessment of Technology for Detection of Stress Corrosion Cracking in Gas Pipelines. Final Report, July 1993. March 1994.

PB94-206646

02,475

NATURAL GAS WELLS

Investigation of Oil and Gas Well Fires and Flares.
PB94-193976

Development of Hazard Assessment and Suppression Technology for OIl and Gas Well Blowout and Diverter Fires.

PB96-122965

01,408

NATURAL LANGUAGE PROCESSING

Bringing Natural Language Information Retrieval Out of the Closet.

PB94-172335

02,720

Lab Report Special Section: Natural Language Processing and Information Retrieval Group Information Access and User Interfaces Division, National Institute of Standards and Technology.

PB97-118665

02,742

NATURAL RUBBER

Localization Model of Rubber Elasticity: Comparison with Torsional Data for Natural Rubber Networks in the Dry

Ptate. 107033

03,195

NAVAL VESSELS

Characterization of the Hydrogen Induced Cold Cracking Susceptibility at Simulated Weld Zones in HSLA-100 PB94-174505

03,746

Optical Fiber Sensors: Accelerating Applications in Navy PBips. 186848

02,632

NAVIGATION SATELLITES

Preliminary Comparison of Time Transfers via LASSO GPS and Two-Way Satellite.
PB95-151098

NAVY

NIST and the Navy: Past, Present and Future

PB96-119649

0

NBSR REACTOR

NIST Reactor: Summary of Activities October 1992 through September 1993

PB94-161502

04,437

Thermal Hydraulic Tests of a Liquid Hydrogen Cold Neu-

tron Source.

03,884

Liquid-Hydrogen Cold Neutron Source for the NBSR.

$\begin{array}{ll}\text { PB95-151619 } & 03,729\end{array}$

Nuclear Heat Load Calculations for the NBSR Cold Neutron Source Using MCNP.

PB95-152955

03,730

Cold Neutron Gain Calculations for the NBSR Using PB95-163978

03,731

NIST Reactor: Summary of Activities, October 1993 through September 1994.

04,700

MCNP Model of the National Bureau of Standards Reactor (NBSR) Core.
PB96-138599

03,733

NCS1 SYSTEM

Journal of Research of the National Institute of Standards and Technology. January/February 1994. Volume 99, Number 1.

Null-Balanced Total-Power Radiometer System NCS1.

PB94-169778 02,021

Derivation of the System Equation for Null-Balanced Total-Power Radiometer System NCS1.

02,022

Evaluation of Uncertainties of the Null-Balanced TotalEvaluation of Uncertainties of the Null-Balanced Total-
Power Radiometer System NCS1.
PB94-169794 02,023

NEAR-AZEOTROPES

Role of Refrigerant Mixtures as Alternatives $10 \mathrm{CFCs}$.

PB94-199775

03,252

NEAR-CRITICAL DILUTE MIXTURE THEORY

Supercritical Solubility of Solids from Near-Critical DiluteMixture Theory.
PB94-211703

00,819

NEAR FIELD

Spherical-Wave Source-Scattering Matrix Analysis of Anennas and Antenna-Antenna Interactions.

PB96-111166

02,008
NEAR FIELDS

Development of Near-Field Test Procedures for Communication Satellite Antennas.

\section{NEAR INFRARED RADIATION}

Infrared and Near-Infrared Spectra of HCC and DCC Trapped in Solid Neon

03,773

High-Efficiency, High-Power Difference-Frequency Generation of $0.9-1.5 \mathrm{mu}$ m Light in $\mathrm{BBO}$.

04,317

NEAR INFRARED SPECTROSCOPY

NIR-Spectroscopic Investigation of Water Sorption Characteristics of Dental Resins and Composites.

00,189

NEIGHBOR TABLES

Neighbor Tables for Molecular Dynamics Simulations.
PB95.171948

NEODYMIUM BARIUM COPPER NIOBATES

Crystal Structure and Magnetic Ordering of the RareEarth and $\mathrm{Cu}$ Moments in $\mathrm{BBa} 2 \mathrm{Cu} 2 \mathrm{NbO8}(\mathrm{R}=\mathrm{Nd}, \mathrm{Pr})$.
$\mathrm{PB} 95-140554$

\section{NEODYMIUM BARIUM TITANATES}

Substitution-Induced Midgap States in the Mixed Oxides RxBa1-ChiTiO3-Delta, with $R=Y, L a$, and Nd. PB95-140505

04,541

NEODYMIUM CERIUM CUPRATES

Phonon Density of States in $\mathrm{R}_{2} \mathrm{CuO} 4$ and Superconducting $\mathrm{R} 1.85 \mathrm{Ce} 0.15 \mathrm{CuO} 4(\mathrm{R}=\mathrm{Nd}, \mathrm{Pr})$.
PB95-150686

NEODYMIUM CUPRATES

Phonon Density of States in $\mathrm{R}_{2} \mathrm{CuO} 4$ and Superconducting $\mathrm{R} 1.85 \mathrm{Ce} 0.15 \mathrm{CuO} 4(\mathrm{R}=\mathrm{Nd}, \mathrm{Pr})$.
PB95-150686 Field Dependence of the Magnetic Ordering of $\mathrm{Cu}$ in $\mathrm{R} 2 \mathrm{CuO} 4(\mathrm{R}=\mathrm{Nd}, \mathrm{Sm})$

PB95-164521

04,633

Observation of Noncollinear Magnetic Structure for the $\mathrm{Cu}$ Spins in Nd2CuO4-Type Systems.

04,634

NEODYMIUM GALLATES

Dielectric Properties of Single Crystals of $\mathrm{Al2O}$, LaAlO3, $\mathrm{SrTiO} 3$, and $\mathrm{MgO}$ at Cryogenic Temperatures.

PB95-180477

02,266

NEODYMIUM IONS

Rb-Like Spectra: Pd X to Nd XXIV.

PB95-150645

03,897

NEODYMIUM LASERS

Integrated Optic Laser Emitting at 906, 1057, and 1358

PB. $94-216280$

02,135

Integrated Optic Laser Emitting at 905, 1057, $1356 \mathrm{~nm}$.

PB94-216298

02,136

Linewidth Narrowing in an Imbalanced Y-Branch Waveguide Laser.

PB95-140844

04,258

PB95-140851

04,259

Passively O-Switched Nd-Doped Waveguide Laser.
PB95.180048 NEON

Vibrational Spectra of Molecular Ions Isolated in Solid

Vibrational Spectra of Molecular lons Isolated in Solid
Neon. 7 . CO $(+), \quad \mathrm{C} 2 \mathrm{O} 2(+)$ and $\mathrm{C} 2 \mathrm{O} 2(\cdot)$.

Neon. 7 . $\mathrm{CO}(+)$, $\mathrm{C2O} 2(+)$ and $\mathrm{C} 2 \mathrm{O} 2(\cdot)$.
(Reannouncement with New Avaliability Information).
O0, 706

Vibrational Spectra of Molecular Ions Isolated in Solid

Neon: HCCH+ and HCC-. (Reannouncement with New Availability Information)

AD-A253 551/6

00,707

Vibrational Spectra of Molecular lons Isolated in Solid Neon. 11. NO2(+), NO2(-), and NO3(-)

00,708

Infrared and Near-Intrared Spectra of HCC and DCC Trapped in Solid Neon.

AD-A295 578/9

03,773

Matrix Isolation Study of the Interaction of Excited Neon Atoms with BCl3: Infrared Spectra of $\mathrm{BCl}$ (sub 3, sup +), $\mathrm{BCl}($ sub 2 , sup + ), and $\mathrm{BCl}($ sub 3 , sup -)

BCI (sub 2, sup
PB97-119143

01,187

NEON ATOMS

Matrix Isolation Study of the Interaction of Excited Neon Atoms with 03: Intrared Spectrum of $\mathrm{O}(($ sub 3)(-)) and Evidence for the Stabilization of O2...O((sub 4) $(+)$.

PB97-112403

04,155

NEON MATRICES

Vibrational Spectra of Molecular lons Isolated in Solid Neon. $6 . \mathrm{CO} 4(-)$. (Reannouncement with New Availability Information).

AD-A238 415/4

00,705

NEPHELOMETERS

Radiometric Model of the Transmission Cell-Reciprocal Nephelometer

PB95-150132
PTUNIUM 237

00,124

Intercomparison Study of (237)Np Determination in Artificial Urine Samples.

PB96-102645

03,633

NETWORK ANALYSIS

Accuracy and Repeatability in Time Domain Network Analysis.
PB95-202644

02,064

Hardware Measurement Techniques for High-Speed Net-

PB96-160551

01,500

NETWORK ANALYZERS

Multi-State Two-Port: An Alternative Transfer Standard.

PB95-168530 02,049

Time Domain Network Analysis Using the Multiline TRL Calibration.

PB95-202925

02,065

Two-Tier Multiline TRL for Calibration of Low-Cost Network Analyzers.

NETWORK CONTROL

01947

Context Analysis of the Network Managemen! Domain. Conducted as

NETWORK MANAGEMENT

Integrated Network Management

PB94-199247

NETWORKS

Network Brokers Handbook: An Entrepreneurial Guide to Cooperative Strategies for Manufacturing Competitiveness.

PB95-219325

00,490

NEURAL NETS

Analysis of a Biologically Motivated Neural Network for Character Recognition.

PB94-172277

00,182

Prediction of Geometric-Thermal Machine Tool Errors by Artificial Neural Networks.

02,943

Comparison of FFT Fingerprint Filtering Methods for Neural Network Classification.

PB95-136362

01,840

Self-Organizing Neural Network Character Recognition on a Massively Parallel Computer.

01,845

Binary Decision Clustering for Neural Network Based Op ical Character Recognition.

PB95-171971

01,848

Improving Neural Network Performance for Character and Fingerprint Classification by Altering Network Dynamics.

Effect of Training Dynamics on Neural Network Performance.

01,852

Improving Neural Network Performance for Character and Fingerprint Classification by Altering Network Dynamics. Binary Decision Clustering for Neural-Network-Based OpBal Character Recognition.

01,857

Fault Diagnosis of an Air-Handling Unit Using Artificial Neural Networks.

PB97-121321

NEURAL NETWORKS

Self-Organizing Neural Network Character Recognition Using Adaptive Filtering and Feature Extraction.

Net-

Micromachined Display Output for a Cellular Neural NetPB96-156070

02,422

NEURON DIFFRACTION

Structure and Conductivity of Layered Oxides $(\mathrm{Ba}, \mathrm{Sr}) \mathrm{n}+1(\mathrm{Sn}, \mathrm{Sb}) \mathrm{nO} 3 \mathrm{n}+1$

04,707

\section{NEUTRON ACTIVATION ANALYSIS}




\section{KEYWORD INDEX}

Unique Ouality Assurance Aspects of INAA for Reference Material Homogeneity and Certification.

00,699

NEUTRON BEAMS

Neutron Focusing Lens Using Polycapillary Fibers.
PB95.141206

Neutron Focusing Lens Using Polycapillary Fibers.

PB95-153078

03,922

Mass Assay and Uniformity Test of Boron Targets by Neutron Beam Methods.

PB97-119085

04,175

NEUTRON CAPTURE GAMMA RAYS

Grazing Incidence Prompt Gamma Emissions and Resonance-Enhanced Neutron Standing Waves in a Thin-Film.
PB95-150470 Use of Neutron Beams for Chemical Analysis at NIST. PB97-112437 NEUTRON CROSS SECTICNS

Neutron Standard Cross Sections in Reactor Physics: Need and Status.

PB94-199510

03,813

Measurement of the $(93) \mathrm{Nb}(n, 2 n)(92 \mathrm{~m}) \mathrm{Nb}$ Cross Section in a (235) U Fission Spectrum.

PB95.163986

03.945

DETAN 95: Computer Code for Calculating Specirum Averaged Cross Sections and Detector Responses in Neutron Spectra.

PB95-242384

04,014

NEUTRON DECAY

Current Results and Future Prospects for a Neutron Life lime Determination Using Trapped Protons.

03,817

EUTRON DEPTH PROFILING

Effect of Splitting on Estimation of Emission Rate Profiles trom Neutron Depth Profiling Spectra.

PB95-152245 03,916

Modeling Detector Response for Neutron Depth Profiting. PB96-157813

04,066

NEUTRON DIFFRACTION

Determination of the Residual Stresses Near the Ends of Skip Welds Using Neutron Diffraction and X-ray Diffraction Procedures.

PB95-253589 02,868

Characterization of the Structure of LaD2.50 by Neutron Powder Diffraction.

PB96-176797

04,783

Neutron Diffraction Texture Study of Deformed Uranium

03,010

Colossal Magnetoresistance without $\mathrm{Mn}(3+) / \mathrm{Mn}(4-) \mathrm{Dou}$ ble Exchange in the Stoichiometric Pyrochlore TI2Mn2O7.
PB97-113070

NEUTRON DIFFRACTIONS

Determination of Anomalous Superexchange in $\mathrm{MnCl} 2$ and lis Graphite Intercalation Compound. and lis Graphite

00.666

\section{NEUTRON DOSIMETRY}

Measurement of Absorbed Dose of Neutrons, and of Mixtures of Neutrons and gamma rays.

Neutron Energy Deposition on the Nanometer Sca

PB95-152260
UTRON ENERGY

Measurements of the (235) U(n,f) Cross Section in the 3 to $30 \mathrm{MeV}$ Neutron Energy Region.

PB97-119051

NEUTRON FOCUSING

Neutron Focusing Lens Using Polycapillary Fibers
PB95-141206

03,889

NEUTRON FOCUSING LENSES

Neutron Focusing Lens Using Polycapillary Fibers. 03,922

EUTRON GUIDES

Transmission Properties of Short Curved Neutron Guides. Part 1. Acceptance Diagram Analysis and Calculations.

PB96-102199

Analytical Applications of Guided Neutron Beams. PB96-112347

04,041

\section{NEUTRON INTERFEROMETRY}

Multi-Stage, Position Stabilized Vibration Isolation System or Neutron Interferometry.

03,955

EUTRON LEAKAGE

Neutron Leakage Benchmark for Criticality Safety Re-

PB95-126132

03,723

NEUTRON LIFETIME

Current Results and Future Prospects for a Neutron Lifeime Determination Using Trapped Protons.

03,817

Preparation and Characterization of (6) LiF and (10)B Reference Deposits for the Measurement of the Neutron LifePB95-108692

03,874

NEUTRON MEASUREMENT

Neutron Measurement Intercomparisons Sponsored by

CCEMRI, Section 3 (Neutron Measurements).

03.819
NEUTRON MONOCHROMATORS

Acceptance Diagram Analysis of the Performance of Vertically Curved Neutron Monochromators.

03,827

NEUTRON PHYSICS

Some Aspects of Fundamental Neutron Physics.
PB95-126298

NEUTRON POLARIZERS

Supermirror Transmission Polarizers for Neutrons.
PB94-216215

NEUTRON RADIOGRAPHY

Protection Against Neutron Radiation Up to 30 Million Electron Volts.

03,611

NEUTRON REACTIONS

Intermediate Structure in the Neutron-Induced Fission Cross Section of $236 \mathrm{U}$

03,802

Neutron Standard Cross Sections in Reactor Physics: Need and Status.

03,813

Measurement of the $(235) \cup(n, f)$ Reaction from Thermal to $1 \mathrm{keV}$

PB95-140422

03,886

Preparation of 2-Dimensional Ultra Thin Polystyrene Film by Water Casting Method

04,619

Measurement of the $(93) \mathrm{Nb}(n, 2 n)(92 m) \mathrm{Nb}$ Cross Section in a (235) U Fission Spectrum.

PB95-163986

03,945

NEUTRON REFLECTION

Analytic Calculation of Polarized Neutron Reflectivity from Superconductors.

PB95-164224

04,629

Simulations of Neutron Focusing with Curved Mirrors. PB96-176649

02,200

NEUTRON REFLECTIVITY

Neutron Reflectivity Study of the Density Profile of a Model End-Grafted Polymer Brush: Influence of Solvent PB $95-202735$

01,274

Extending the Angular Range of Neutron Reflectivity Measurements from Planar Lipid Bilayers: Applications to Measurements from Planar Lipid PB96-122569

03,476

Magnetic Structure Determination for Annealed Ni80Fe20/ Ag Multilayers Using Polarized-Neutron Reflectivity.

PB96-176615

03,739

Compatibilization of Polymer Blends by Complexation. 2 . Kinetics of Interfacial Mixing.

01,295

\section{NEUTRON SCATTERING}

Small-Angle Neutron Scattering Characterization of Processing/Microstructure Relationships in the Sintering of Crystalline and Glassy Ceramics. (Reannouncement with New Availability Information).

AD-A249 $510 / 9$

03.025

Neutron Scattering Study of Shear Induced Turbidity in Polystyrene/Dioctyl Phthalate Solutions at High Shear

$\begin{array}{ll}\text { PB94-172624 } & 01,197\end{array}$

Time Dependent Small Angle Neutron Scattering Behavior in Triblock Copolymers Under Steady Shear. $\quad 01,198$ Neutron Scattering by Multiblock Copolymers of Structure

PB94-211547 01,219

Small-Angle X-Ray and Neutron Scattering Study of Block Copolymer/Homopolymer Mixtures. 01,221

Crossover to Strong Shear in a Low-Molecular-Weight Critical Polymer Blend.

PB94-211976

01,222

Magnetic Neutron Scattering (Invited).

04,557

SANS Study of the Plastic Deformation Mechanism in Polyethylene.

PB95-151841 01,242

Analysis of the Effectiveness of Oscillating Radial Collimators in Neutron Scattering Applications.

PB95-152252

03,917

Thermodynamic Interactions and Correlations in Mixtures of Two Homopolymers and a Block Copolymers by Small Angle Neutron Scattering.

PB95-152872

01.247

SANS Studies of Space-Time Organization of Structure in Polymer Blends.

01,251 Time-Resolved Small-Angle Neutron Scattering Study of
Spinodal Decomposition in Deuterated and Protonated Spinodal Decomposition in Deuterated and Protonated
Polybutadiene Blends. 1. Etiect of Initial Thermal FluctuaPolyb

PB95-161196

01,252

Localization of a Homopolymer Dissolved in a Lamellar Structure of a Block Copolymer Studied by Small-Angle Neutron Scattering.

PB95-161592

01,253

Elastic Scattering of Polymer Networks.

01,255

Neutron Scattering Study of Shear Induced Turbidity in Polystyrene Dissolved in Dioctyl Phthalate.

01,256

Characterization of Molecular Network of Thermosets Using Neutron Scattering.

01.259

B95-164109

Small-Angle Neutron Scattering of Poly(vinyl alcohol)

PB95-164117 01,260

Characterization of Polyquinoline Blends Using Small Angle Scattering.

01,261

Small Angle Neutron Scattering Studies on Chain Asymmetry of Coextruded Poly(Vinyl Alcohol) Film.

PB95-164372

01,262

Small Angle Neutron Scattering Study on Poly(N-IsoDropyl Acrylamide) Gels Near Their Volume-Phase Tran.

PB95-164380

01,263

Small-Angle Neutron Scattering Study on Weakly Charged Temperature Sensitive Polymer Gels.

PB95-164398

01,264

Partial Scattered Intensities from a Binary Suspension of Polystyrene and Silica.

00,996

Neutron Scattering by Hydrogen in Cold Neutron Prompt Gamma-Activation Analysis.

PB95-175972

00,603

Small Angle Neutrons Scattering from Nanocrystalline Palladium as a Function of Annealing.

03,354

B95-176103

a Liquid

Neutron Scattering Study of the O

01,270

Water Adsorption at Polymer/Silicon Wafer Interfaces.

PB95-181178 01,022

Lattice Dynamics of Semiconducting, Metallic, and Superconduc'ing $\mathrm{Ba} 1-\times K \times \mathrm{BiO} 3$ Studied by Inelastic Neutron Scattering.

PB96-102447

04,708

Small Angle Neutron Scattering Study of the Structure and Formation of Ordered Mesopores in Silica.

03,069

Effects of Target Temperature on Analytical Sensitivities of Cold-Neutron Capture Prompt gamma-ray Activation

Analysis.

00,616

Structure of a Triglyceride Microemulsion: A Small Angle Neutron Scattering Study.

01.077

PB6-112255

Small Angle Neutron Scattering Studies of Structural
Characteristics of Argarose Gels. Characteristics of Argarose Gels

PB96-112305

03,475

Vortex Dynamics and Melting in Niobium.

02,073

Anisotropy of the Surfaces of Pores in Plasma Sprayed Alumina Deposits.

03,126

Slow Dynamics of Segregation in Hydrogen-Bonded Polymer Blends.

PB96-123591 
Liquid-Hydrogen Cold Neutron Source for the NBSR.

03,729

Nuclear Heat Load Calculations for the NBSR Cold Neuron Source Using MCNP.

PB95-152955

03,730

Cold Neutron Gain Calculations for the NBSR Using PB95-163978

03,731

In-situ Neutron Reflectivity of MBE Grown and Chemically Processed Surfaces and Interfaces.

PB96-146634

02,416

NEUTHON SPECTRA

DETAN 95: Computer Code for Calculating SpectrumAveraged Cross Sections and Detector Responses in PB95-242384

NEUTAON SPECTROSCOPY

Neutron and Raman Spectroscopies of 134 and $134 a$ Hydrofluorocarbons Encaged in Na-X Zeolite.
PB96-186168

03,001

NEUTAON STANDARDS

Measurement of the (10)B(n, alpha1gamma) $(7) \mathrm{Li}$ Cross Section in the 0.3 to $4 \mathrm{MeV}$ Neutron Energy Interval.
PB96-161799

\section{NEUTRON STANDING WAVES}

Grazing Incidence Prompt Gamma Emissions and Resonance-Enhanced Neutron Standing Waves in a Thin-Film. UTRON TRANSPORT

Slab Transmission and Reflection for Point Source and Point Detector.

PB94-211265 03,838

Neutron Focusing Lens Using Polycapillary Fibers. PB95. 153078

03,922

NEUTRON VELOCITY SELECTORS

Acceptance Diagram Analysis of the Performance of Multidisk Neutron Velocity Selectors.

03,826

NEUTRONS

Polarizability of the Nucleon.

PB94-211760

03,846

Measurement of the Neutron Lifetime.

04,094

Diffraction of Neutron Standing Waves in Thin Films with Resonance Enhancement.

PB97-113278

04,164

NICKEL

Temperature Dependence and Anharmonicity of Phonons on $\mathrm{Ni}(110)$ and $\mathrm{Cu}(110)$ Using Molecular Dynamics SimPBlations.

04,449

Nickel(II)-Mediated Oxidative DNA Base Damage in Renal and Hepatic Chromatin of Pregnant Rats and Their Fetuses. Possible Relevance to Carcinogenesis.

PB94-212628

03,646

Measurements of Thermophysical Properties of Nickel Near Its Melting Temperature by a Microsecond-Resolution Transient Technique.

PB96-102579

04,210

NICKEL 63

63Ni Half-Life: A New Experimental Determination and Critical Review.
PB97.111603

00,700

Nickel-63 Standardization: 1968-1995.

PB97-111819

00,701

NICKEL ALLOYS

Dynamic Shear Modulus Measurements with Four Independent Techniques in Nickel-Based Alloys.

PB94-198900

03,320

Diagnosis and Treatment of an Oral Base-Metal Contact Lesion Following Negative Derm atologic Patch Tests. 00,172 Chemical Aspects of Tool Wear in Single Point Diamond Turning.

03,021

ICKEL ALUMINIDES

LMTO/CVM and LAPW/CVM Calculations of the Nicke Aluminide/Nickel Titanium Pseudobinary Phase Diagram. PB94-199353

\section{NICKEL ALUMINUM TITANIUM}

LMTO/CVM and LAPW/CVM Calculations of the Nicke Aluminide/Nickel Titanium Pseudobinary Phase Diagram. CKEL BASE ALLOYS

Evaluation of the Environmentally Induced Fracture $\mathrm{Re}$ sistance of Ductile Nickel Aluminide. Technical Report Number 1, Final report. October-December 1990.

DE94017331

03,306

Evaluation of the Electrochemical Behavior of Ductile Nickel Aluminide and Nickel in a pH 7.9 Solution. Technical Report Number 3, April-June 1991.

03,307 DE94017351

NICKEL BASE SUPERALLOY

Fatigue Crack Thresholds of a Nickel-Iron Alloy for Superconductor Sheaths at $4 \mathrm{~K}$

03,223

NICKEL-CADMIUM BATTEAIES

Rechargeable Batteries for Personal/Portable.

PB96-164231

\section{NICKEL COMPOUNDS}

One-Electron Oxidation of Nickel Porphyrins. Effect of Structure and Medium on Formation of Nickel(III) Por phyrin or Nickel(II) Porphyrin pi-Radical Cation.

00,865

Site of One-Electron Reduction of Ni(II) Porphyrins. For mation of $\mathrm{Ni}(\mathrm{I})$ Porphyrin of $\mathrm{Ni}(11)$ Porphyrin pi-Radical

PB95-107066

00,866

NICKEL HYDRIDES

Determination of the Molecular Parameters of $\mathrm{NiH}$ in Its 2) Delta Ground State by Laser Magnetic Resonance.

00,869

NICKEL INTERMETALLICS

Disorder Trapping in Ni2TiAl.

PB94-198942

03,322

NICKEL MANGANESE ANTIMONIDES

Resonant-Photoemission Investigation of the Heusler Alloys Ni2MnSb and NiMnSb.

PB95-16238

04,612

NICKEL OXIDES

Structural and Magnetic Ordering in Iron Oxide/Nicke Oxjde Multilayers by X-ray and Neutron Diftraction (In-

PB94-172558

04,442

NICKEL SILICIDES

Local Partial Densities of States in $\mathrm{Ni}$ and $\mathrm{Co}$ Silicides Studied by Soft X-Ray Emission Spectroscopy.

04,504

CKEL TITANIUM

LMTO/CVM and LAPW/CVM Calculations of the Nickel Aluminide/Nickel Titanium Pseudobinary Phase Diagram. Aluminide/Nickel Titanium Pseudobinary Phase Diagram.
PB94-199353

NICKEL VANADIUM

Effects of Elastic Stress on Phase Equilibrium in the Ni-V System.

PB94-172707

03,313

NII (NATIONAL INFORMATION INFRASTRUCTURE)

Putting the Information Infrastructure to Work: Report of the Information Infrastructure Task Force Committee on Applications and Technology.

00,001

Information Infrastructure: Reaching Society's Goals. Report of the Information Infrastructure Task Force Commitee on Applications and Technology.

PB94-214756

01,469

Report on the Workshop on Advanced Digital Video in the National Information Infrastructure. Held in Washington D.C on May 10-11, 1994.

PB95-103677

01,472

Unpredictable Certainty. Information Infrastructure through 2000

00,016

NIOBATES

Electric Field Effects on Crack Growth in a Lead Magnesium Niobate

03,339

NIOBIUM

Intrinsic Stress in DC Sputtered Niobium.

PB94-199031

Effect of Microstructure on Phase Formation in the Reac tion of $\mathrm{Nb} / \mathrm{Al}$ Multilayer Thin Films.

PB95-168415

03,352

First Phase Formation Kinetics in the Reaction of $\mathrm{Nb} / \mathrm{Al}$.

PB95-168456

03,353

Niobium Microbc

02,184

Vortex Dynamics and Melting in Niobium.

02,073

Metallic-Barrier Junctions for Programmable Josephson Voltage Standards.

PB96-200134

02,089

Superconductor- Normal-Superconductor Junctions for Digital/Analog Converters.

02,092

Superconductor- Normal-Superconductor Junctions fo Programmable Voltage Standards.

02,093 PB96-200241

Radiance Temperatures at $1500 \mathrm{~nm}$ of Niobium and Molybdenum at Their Melting Points by a Pulse-Heating Technique.

04,167

\section{NIOBIUM 93 TARGE}

Measurement of the $(93) \mathrm{Nb}(n, 2 n)(92 \mathrm{~m}) \mathrm{Nb}$ Cross Section in a (235) U Fission Spectrum.

PB95-163986

03,945

NIOBIUM ALLOYS

Magnetic Characteristics and Measurements of Filamentary Nb-Ti Wire for the Superconducting Super Collider. DE94005988

Transverse stress effect on the critical current of internal tin and bronze process $\mathrm{Nb}$ (sub 3 ) $\mathrm{Sn}$ superconductors. DE95016659

04,434

Thermodynamic Calculation of the Ternary Ti-Al-Nb Sys.

PB94-212636

03,336

Thin Film Reaction Kinetics of Niobium/Aluminum Multilayers.
PB95-175295

04,65

Journal of Research of the National Institute of Standards and Technology, September/October 1993. Volume 98 5.

PB96-169057

03,368

NIOBIUM ALUMINIDES

Thin Film Reaction Kinetics of Niobium/Aluminum PB95-175295

04,651

NIOBIUM BASE ALLOYS

VAMAS interlaboratory comparisons of critical current vs. strain in $\mathrm{Nb}$ (s
DE95016656

04,433

NIOBIUM COMPOUNDS

ow-Energy Vibrations and Octahedral Site Occupation in Nb95V5H(D)y
PB96-160734

NIOBIUM INTERMETALLICS

Coherent Precipitates in the BCC/Orthorhombic Two Phase Field of the Ti-Al-Nb System.

PB94-19869

03,317

NIOBIUM IONS

Spectrum and Energy Levels of Five-Times-Ionized Niobium (Nb Vl).

NIOBIUM NITRIDES

04,226

Use of Ion Scattering Spectroscopy to Monitor the Nb Target Nitridation during Reactive Sputtering.

B94-172525

00,76

NIOBIUM STANNIDES

Electromechanical Properties of Superconductors for DOE Fusion Applications.

Flux Expulsion at Intermediate Fields in Type-II Superconductors

PB94-212230

04,502

Volume Magnetic Hysteresis Loss of Nb3Sn Superconductors as a Function of Wire Length.

Superconductor Critical Current Standards for Fusion Applications. Final Progress Report, October 1993-July

PB95-169538 02,222

Reduction of Interfilament Contact Loss in Nb3Sn Superconductor Wires.

02,223

Experimental Aspects of Flux Expulsion in Type-1 Superconductors

04,660

Simple and Repeatable Technique for Measuring the Critical Current of Nb3Sn Wires.

PB96-119409

02,229

VAMAS Intercomparison of Critical Current Measurements on NB3Sn Superconductors: A Summary Report.

PB96-119763

04,043

NIOBIUM STANNOIDES

11-5: Thermal Contraction of Materials Used in Nb3S Critical Current Measurements.

PB96-147186

04,769 
NITRIC OXIDE COMPLEXES

Fragment State Correlations in the Dissociation of NO. HF (v=1).

00,982

NITRITE IONS

Vibrational Spectra of Molecular Ions Isolated in Solid Neon. XI. NO2(+), NO2(-), and NO3(-)

PB95-125688

00,890

NITROANILINES

Inelastic Neutron Scattering Studies of Nonlinear Optical Materials: p-Nitroaniline Adsorbed in ALPO-5. PB95-107223

00,874

\section{NITROGEN}

Beneficial Effects of Nitrogen Atomization on an Austenitic Stainless Steel.

Reduction of Dinitrogen to Ammonia in Aqueous Solution Mediated by Colloidal Metals.

PB95-107074

00,867

Operational Mode and Gas Species EHects on Rotational

Drag in Pneumatic Dead Weight Pressure Gages. 00,903

N2(a'(sup 1) Sigma(sub g)(sup +)) Metastable Collisional Destruction and Rotational Excitation Transfer by N2. PB95-151395 00,933

High Resolution Angle Resolved Photoelectron Spectroscopy Study of N2.

03,907

Photoelectron Study of Electronic Autoionization in Rotationally Cooled N2: The $n=6$ Member of the Hopfield Series.

00,971

Modified Effective Range Theory as an Alternative to Low-Energy Close-Coupling Calculations. PB95-202701

03,988

Ion Kinetics and Symmetric Charge-Transfer Collisions in Low-Current, Diffuse (Townsend) Discharges in Argon and Nitrogen.

04,051

NITROGEN COMPLEXES

Slit Jet Infrared Spectroscopy of Hydrogen Bonded N2HF sotopomers: Rotational Rydberg-Klein-Rees Analysis and $H / D$ Dependent Vibrational Predissociation Rates.

PB95-161873

00,956

Rotational-RKR Inversion of Intermolecular Stretching Potentials: Extension to Linear Hydrogen Bonded Complexes.

01,041

\section{NITROGEN COMPOUNDS}

High-Resolution Infrared Overtone Spectroscopy of N2HF: Vibrational Red Shifts and Predissociation Rate as a Function of HF Stretching Ouanta.

PB96-102298

01,061

NITROGEN DIOXIDE

Vibrational Spectra of Molecular Ions Isolated in Solid

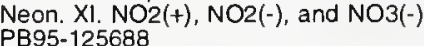

00,890

Asymptotic and Numerical Analysis of a Premixed Lam. inar Nitrogen Dioxide-Hydrogen Flame.

PB96-164256

01,422

NITROGEN FLOW FACILITY

Uncertainty Analysis of the NIST Nitrogen Flow Facility. PB95-128906

02,608

Oxidation of 10-Methylacridan, a Synthetic Analogue of Cation Radical. Converent Application of Laser Flash Photolysis and Direct and Redox Catalyzed Electrochemistry to the Kinetics of Deprotonation of the Cation Radical.

Deprotonation

00,785

NITROGEN IONS

Selected Ion Flow Tube-Laser Induced Fluorescence Instrument for Vibrationally State-Specific Ion-Molecule Reactions.

PB94-185444

00,774

Fine-Structure Intervals of $(14) \mathrm{N}(+)$ By Far-Infrared Laser Magnetic Resonance.

PB95-175162

00,993

NITROGEN-LIKE IONS

Atomic Branching Ratio Data for Nitrogen-Like Species. PB96-190152

04,122

NITROGEN OXIDES

P-Type Doubling in the Infrared Spectrum of NO-HF.
PB94-211463

NO Production and Destruction in a Methane/Air Diffusion Flame. 22519

01,443

NITROMETHANE

Microwave Spectrum and Structure of $\mathrm{CH} 3 \mathrm{NO}_{2}-\mathrm{H} 2 \mathrm{O}$

AD-A296 $377 / 5$

NOBLE GASES

Excitation Transfer in Barium by Collisions with Noble Gases.

PB96-200274

01,163

NODULAR IRON

Application of ODF to the Rietveld Profile Refinement of Polycrystalline Solid.

03,401
NOISE

New Model of 1/F Noise in Baw Quartz Resonators.
PB96-112248

Non-Gaussian Noise Effects on Reliability of Multistable Systems.

04,213

Telegraph Noise in Silver-Permalloy Giant

Magnetoresistance Test Structures.
PB96-146717 04,763

Transitions to Chaos Induced by Additive and Multiplica-

tive Noise

03,750

Spectrum of the Stochastically Forced Duffing-Holmes

PB96-155767 00,216

Necessary Condition for Homoclinic Chaos Induces by

Additive Noise.

PB96-155775

04,063

Noise-Induced Transitions to Chaos.

PB96-156120

00,217

Deterministic

00,218

Exits in Multistable Systems Excited by Coin-Toss Square-Wave Dichotomous Noise: A Chaotic Dynamics Approach.

PB96-160650

04,824

Observation of Hot-Electron Shot Noise in a Metallic Re-

PB97-112007

01,988

NOISE ANALYSIS

Current Noise Reveals Protonation Kinetics and Number of Ionizable Sites in an Open Protein Ion Channel.

BB96-161674

04,092

Noise Analysis of Ionization Kinetics in a Protein Ion Channel.

PB96-161682

04,093

Current Fluctuations Reveal Protonation Dynamics and Number of Ionizable Residues in the alpha-Toxin Channel.

B96-161732

03,588

Protonation Dynamics of the alpha-Toxin Ion Channe from Spectral Analysis of $\mathrm{pH}$-Dependent Current Fluctua-

PB96-161740

03,652

rotonation Dyn

03,589

\section{NOISE CALIBRATION SYSTEM}

Journal of Research of the National Institute of Standards and Technology. January/February 1994. Volume 99 Number 1

PB94-169737

02,019

Null-Balanced Total-Power Radiometer System NCS1.

Derivation of the System Equation for Null-Balanced Total-Power Radiometer System NCS1. PB94-169786

02,022

Evaluation of Uncertainties of the Null-Balanced TotalPower Radiometer System NCS1.

PB94-169794

02,023

NOISE CALIBRATION SYSTEMS

Uncertainties of the NIST Coaxial Noise Calibration Sys ter.

02,070

NOISE MEASUREMENT

Relative Accuracy of Isolated and Unisolated Noise Comparison Radiometers.

PB96-111851

01,924

NOISE REDUCTION

Slow Evolution from the Boundary: A New Stabilizing Constraint in III-Posed Continuation Problems.
PB96-122858 03,418
NB96-122858 ments with Laser-Driven Switching.

PB96-135165

02,081

NOISE STANDARDS

Practical Standards for PM and AM Noise at 5, 10 and $100 \mathrm{MHz}$

\section{PB95- 181129}

Systematic Studies of the Effect of a Bandpass Filter on a Josephson-Junction Noise Thermometer.

PB95-162970 03,939

Systematic Studies of the Effect of a Post-Detection Filter on a Josephson-Junction Noise Thermometer. on a Josephs

03,940

\section{NOISE THERMOMETERS}

Systematic Studies of the Effect of a Bandpass Filter on a Josephson-Junction Noise Thermometer.

PB95- 162970

03,939

Systematic Studies of the Effect of a Post-Detection Filter J Josephson-Junction Noise Thermometer. PB95-162988

03,940

\section{NON-ABRASIVE WEAR TESTING}

Critical Factors in Non-Lubricated, Non-Abrasive Wear

Testing.
PB95-140588

03,236

NON-GAUSSIAN EFFECTS

Non-Gaussian Noise Effects on Reliability of Multistable Systems.

\section{NON-NEWTONIAN FLUIDS}

Density Dependence of Fluid Properties and Non-Newtonian Flows. The Weisenberg Effect

PB96-161898

NONAQUEOUS ELECTROLYTES

Susceptibility Critical Exponent for a Nonaqueous lonic Binary Mixture Near a Consolute Point.

PB95-152112

00,938

NONDEGENERATE STARS Volume-Limited ROSAT Survey of Extreme Ultraviolet
Emission from all Nondegenerate Stars within 10 Parsecs.

PB96-103189

00,093

NONDESTRUCTIVE EVALUATION

Ultrasonic NDE of Sprayed Ceramic Coatings.

02,761

NONDESTRUCTIVE TESTING

Intelligent Processing of Materials.

994-172780

Recent Development in Nondestructive Testing of Con-

Classified Bibliography: Insulation Condition Monitoring Methods, 1989-1995.

PB96-131586

02,232

NONDESTRUCTIVE TESTS

Detection of Voids in Grouted Ducts Using the ImpactEcho Method.

01,306

International Institute of Welding: Report on 1992 Actions. PB94-185873

02,856

International Institute of Welding: Report on 1993 Actions.

PB94-185881 02,857

Dynamic Shear Modulus Measurements with Four Independent Techniques in Nickel-Based Alloys.

PB94-198900

03,320

Assessment of Technology for Detection of Stress Corrosion Cracking in Gas Pipelines. Final Report, July 1993. March 1994.

02,475

PB94-206646

Proc-

Review of C

PB94-216728

03,145

Potential Drop in the Center-Cracked Panel with Asym-

metric Crack Extension.

04,819
PB95-107330 Wheel Rims.

PB95-140430

Nondestructive Evaluation and Materials Processing.

02,902 


\section{KEYWORD INDEX}

NUCLEAR PHYSICS \& RADIATION TECHNOLOGY

Development of a Method for Measuring Water-Stripping Resistance of Asphalt/Siliceous Aggregate Mixtures.
PB96-202296

NONEQUILIBRIUM ENTROPY

Nonequilibrium Statistical Mechanics.
PB96-161781

NONEQUILIBRIUM MOLECULAR DYNAMICS

Simulation and SANS Studies of Gelation Under Shear.

PB96-167176

01,150

NONIONIZING RADIATION

Bibliography of the NIST Electromagnetic Fields Division

PB94-165990

01,875

NONLINEAR ALGEBRAIC EQUATIONS

Algebraic Approximation of Attractors for Galloping Oscillators

04,820

PB95-162897

Melnikov Function and Homoclinic Chaos Induced by
Weak Perturbations.

NONLINEAR DYNAMICS

Nonlinear Dynamics of Stiff Polymers.
PB96-122478

NONLINEAR EQUATIONS

Foias-Temam Approximations of Attractors for Galloping Oscillators.
PB94-198298

04,817

NONLINEAR OPTICS

Scaling of the Nonlinear Optical Cross Sections of GaAsAIGaAs Multiple Quantum-Well Hetero n-i-p-i's.

PB96-102793

02,183

Appearance Intensities for Multiply Charged lons in a Strong Laser Field

PB96-160445

04,089

NONLINEAR SYSTEMS

Exponentially Rapid Coarsening and Buckling in Coherently Self-Stressed Thin Plates.

PB95-202347

04,821

NONMODEL ERRORS

Effects of Nonmodel Errors on Model-Based Testing.
PB96-123146

NONPAIRWISE ADDITIVE FORCES

Pairwise and Nonpairwise Additive Forces in Weakly Bound Complexes: High Resolution Infrared Spectroscopy of ArnDF $(n=1,2,3)$.

04,125

NORDIC COUNTRIES

Forum for International Cooperation on Fire Research.
PB95-162939

NORTH AMERICA

ISO/IEC Workshop on Worldwide Recognition of OSI Test Results Regional Progress - North America.
PB94-172202
02,719 Status of Construction and Construction Technologies. PB94-186004

NORTHERN HEMISPHERE

Modulation of Fossil Fuel Production by Global Temperature Variations, 2

02,533

NORTHRIDGE (CALIFORNIA)

Northridge Earthquake 1994: Performance of Structures, Lifelines, and Fire Protection Systems.

PB94-207461

04,825

NORTHRIDGE EARTHQUAKE

Performance of HUD-Affiliated Properties during the January 17,1994 Northridge Earthquake.
PB95.174488

00,443

Survey of Steel Moment-Resisting Frame Buildings Affected by the 1994 Northridge Earthquake.

00,451

Performance of Federat Buildings in the January 17, 1994 Northridge Earthquake.

PB95-231775

00,453

NUCLEAR COLLISIONS

Strangeness Flow Difference in Nuclear Collisions at $15 \mathrm{~A}$ and $200 \mathrm{~A} \mathrm{GeV}$

NUCLEAR MAGNETIC RESONANCE

Binder Characterization and Evaluation by Nuclear Magnetic Resonance Spectroscopy.

01,334

Two-Dimensional POMMIE Carbon-Proton Chemical Shift Correlated (13)C NMR Spectrum Editing.

00,809

L-threo-beta-Hydroxyhistidine, an Unprecedented Iron(III) Ion-Binding Amino Acid in a Pyoverdine-type Siderophore from Pseudomonas fluorescens 244.

00.553

PB94-211620

NMR Characterization of Injection-Moulded Alumina

Green Compacts. Part 2. T2-Weighted Proton Imaging.

NUCLEAR MEDICINE

Liquid-Scintillation Counting Techniques for the Standardization of Radionuclides Used in Therapy.

PB $97-\uparrow 10084$

03,709

Radioassays of Yttrium-90 Used in Nuclear Medicine.

PB97-110100
NUCLEAR PHYSICS \& RADIATION TECHNOLOGY

Evolution of the Pore Size Distribution in Final-Stage Sinlering of Alumina Measured by Small-Angle $X$-ray Scatering. (Reannouncement with Now Availability InformaAD-A249 178/5

03,023

Characterization of the Densification of Alumina by $\mathrm{Mul}$. tiple Small-Angle Neutron Scattering. (Reannouncement with New Availability Information)

03,024

Small-Angle Neutron Scattering Characterization of ProcSmall-Angle Neutron Scattering Characterization of ProcCrystalline and Glassy Ceramics. (Reannouncement with New Availability Information).

A249 510/9 03,025

X-ray Attenuation Coefficients from $10 \mathrm{Kev}$ to $100 \mathrm{Mev}$.

Ultraviolet Multiplet Table

03,765

00,710

Report of the International Commission on Radiological Units and Measurements (ICRU), 1956.

03,513

03,605

X-ray Protection.

Recommendations for the Disposal of Carbon-14 Wastes. AD-A279 133/3

$X \cdot r a y$ Protection Design.

AD-A279 181/2

03,606

Protection Against Radiations from Radium, Cobalt-60, and Cesium-137.

AD-A279 261/2 03,607

Permissible Dose from External Sources of Ionizing Radi-

AD-A279 281/0

03,608

X-Ray Attenuation Coefficients from $10 \mathrm{kev}$ to $100 \mathrm{Mev}$

AD-A279 289/3 03,767

Protection Against Neutron Radiation Up to 30 Million Electron Volts.

03,611

Penetration and Diffusion of Hard $X$-rays through Thick Barriers. III. Studies of Spectral Distributions. Barriers. III. Studies of Spectral Distributions.
AD-A292 502/2

Further Calculations of X-ray Diffusion in an Infinite Me-

AD-A295 314/9

03,772

Experimental plan to determine the performance of the Oak Ridge National Laboratory Cold Neutron Moderator. Final report, September 1, 1993--November 30, 1993.

FE 95011352
03,778

$36 \mathrm{CV} / \mathrm{Cl}$ Accelerator-Mass-Spectrometry Standards: Verification of Their Serial-Dilution-Solution Preparations by Padioactivity Measurements.

PB94-140563

00,524

NIST Reactor: Summary of Activities October 1992 hrough September 1993.

04,437

Characterization of the Binding of Gallium, Platinum, and

Characterization of the Binding of Gallium, Platinum, and Uranium to Pseudomonas Fluorescens by Small-Angle $X$ ray Scattering and Transmission Electron Microscopy.
PB94-172509 Neutron Scattering Study of Shear Induced Turbidity in Polystyrene/Dioctyl Phthalate Solutions at High Shear

PB94-172624

01,197

Time Dependent Small Angle Neutron Scattering Behavior in Triblock Copolymers Under Steady Shear. 01,198 Measurement and Calibration of Large-Area Alpha-Particle Sources at NIST.

PB94-172855

03,791

Systematics of Alpha-Particle Energy Spectra and Lineal Energy $(Y)$ Spectra for Radon Daughters.

03,615

Intercomparison of Internal Proportional Gas Counting of $(85) \mathrm{Kr}$ and $(3) \mathrm{H}$.
PB94-185576

03,800

Use of a Radiochromic Detector for the Determination of Stereotactic Radiosurgery Dose Characteristics. Intermediate Structure in the Neutron-Induced Fission Cross Section of $236 \mathrm{U}$

PB94-185741

03,802

Neutron Reflectivity of End-Grafted Polymers: Concentration and Solvent Quality Dependence in Equilibrium Conditions.

01,206

Vibrational Distributions of As2 in the Cracking of As4 on Si(100) and Si( 111$)$.

PB94-198314

00,784

Combined Low- and High-Angle X-Ray Structural Refinement of a Co/Pt(111) Multilayer Exhibiting Perpendicular Magnetic Anisotr

PB94-198355

04,457

Characteristics of Light Emission After Low-Energy ElecImpact Excitation of Caesium Atoms.

PB94-198587

03,806

Femtosecond Time-Resolved Wave Packet Motion in Molecular Multiphoton Ionization and Fragmentation.

PB94-198611

00,790

Discovery of an X-Ray Selected, Radio-Loud Quasar at $z=3.9$.
PB94-198652

00,052

Integrated Laser Doppler Method for Measuring Planetary PBa4-198686

03,681

Germanium Detector Optimization of MDA for Efficiency v. Low Intrinsic Background.

IUE Observations of Solar-Type Stars in the Pleiades and the Hyades.

00,053

Silicon Photodiodes Optimized for EUV and Sott X-Ray Reglons.

02,124

Neutron Standard Cross Sections in Reactor Physics Need and Status.

PB94-199510

03,813

Bone Dosimetry: A New Approach to Spectral Deconvolution Problems.

03,616

Estimation of the Absorbed Dose in Radiation-Processed Food. 2. Test of the EPR Response Function by an Exponential Fitting Analysis.

PB94-199650

00.036

Experimental Validation of Radiopharmaceutical Absorbed Dose to Mineralized Bone Tissue.

B94-199668

03,617

Radiation Doses.

PB94-199676

03,618

Estimation of the Absorbed Dose in Radiation-Processed Food. 3. The Effect of Time of Evaluation on the Accuracy of the Estimate.

PB94-199684

00,037

Estimation of the Absorbed Dose in Radiation-Processed Food. 4. EPR Measurements on Eggshell. New EPR Dosimeter Based on Polyvinylalcohol. PB94-199700

03,619

Estimation of the Absorbed Dose in Radiation-Processed Food. 1. Test of the EPR Response Function by a Linea Regression Analysis.

PB94-199718

00,039

Current Results and Future Prospects for a Neutron Lifeime Determination Using Trapped Protons.

PB94-199742

03,817

Absolute Cross-Section Measurements for Electron-Impact Ionization of $\mathrm{C}_{1}(+1)$

PB94-199841 03,818

Neutron Measurement Intercomparisons Sponsored by CCEMRI, Section 3 (Neutron Measurements).

PB94-199916

03,819

Provements in Computation of Form Factors.

Inprovements in Computation of Form Fac

Photographic Response to X-Ray Irradiation. 1. Estimation of the Photographic Error Statistic and Development of Analytic Density-Intensity Equations.

PB94-200086

03,821

Photographic Response to X-Ray Irradiation. 2. CorPB94-200094

03.822

Photographic Response to X-Ray Irradiation. 3. Photographic Linearization of Beam-Foil Spectra.

03,823

Textures of Tantalum Metal Sheets by Neutron Diffrac-

PB94-200169

03,399

Texture Study of Two Molybdenum Shaped Charge Liners by Neutron Diffraction 


\section{KEYWORD INDEX}

Plasma Chemistry in Disilane Discharges. PB94-211075

02,514

Radıo Continuum and X-Ray Properties of the Coronae of RS Canum Venaticorum and Related Active Binary Sys-

00,057

B 94-211083

Three-Vector Correlation Theory for Orientation/Alignment
Studies in Atomic and Molecular Collisions.
PB94-211109 Studies in Atomic and Molecular Collisions.

Fine Structure of Negative Ions of Alkaline-Earth-Metal
Atoms.

PB94-211182

Slab Transmission and Reflection for Point Source and Point Detecto

03,838

Anomalous Odd- to Even-Mass Isotope Ratios in Resonance Ionization with Broad-Band Lasers.

PB94-211406

03,839

Earth-Based Gravitational Experiments.

03.840

PB94-211414

High-Pesolution Infrared Overtone Spectroscopy of ArHF via Nd:YAG/Dye Laser Difference Frequency Generation. Dreams About the Next Generation of Super-Stable LaDers. PB94-211570

04,235

Radiation Chemistry of Cyanine Dyes: Oxidation and $\mathrm{Re}$ duction of Merocyanine 540.

PB94-211661

00,818

Drill-Hearn-Gerasimov Sum Rule.

PB94-211752

03,845

Polarizability of the Nucleon.

PB94-211760

03,846

nterstellar Disk-Halo Connection in Galaxies: Review of Observational Aspects.

PB94-211802 00,058

Role of the Office of Radiation Measurement in Ouality Assurance.

PB94-212255 00,689

Local Partial Densities of States in $\mathrm{Ni}$ and Co Silicides

Studied by Soft X-Ray Emission Spectroscopy.

NIST-NRL Free-Electron Laser Facility.

04,504

PB94-212511

04,237

Radiation-Driven Winds of Hot Luminous Stars $X$. The Determination of Stellar Masses Radii and Distances from Terminal Velocites and Mass-Loss Rates.

PB94-213022 00,060

Materials Science with SR Using X-Ray Imaging: SpatialResolution/Source Size.

04,510

Laser-Induced Fluorescence Measurements of Rotationally Resolved Velocity Distributions for $\mathrm{CO}(+)$ PB94-213139

00,848

Astrophysical Aspects of Neutral Atom Line Broadening.

Polarized X-Ray Emission Spectroscopy.

PB94-213360

00,06

High-Sensitivity Determination of lodine Isotopic Rat Thermal and Fast Neutron Activation.

PB94-213386 00,555

Neutron Capture Prompt Gamma-Ray Activation Analysis at the NIST Cold Neutron Research Facility.

PB94-213394

00,556

Atomic Data Needed for Far Ultraviolet Astronomy with HUT and FUSE

FUSE: The Far Ultraviolet Spectrograph Explorer.
PB94-213410 00,063

Ultraviolet Observations of Stellar Coronae: Early Results from HST

PB94-213428

00,064

$X$-rays from Stellar Flares.

PB94-213436

00,065

Goddard High-Resolution Spectrograph Observations of the Local Interstellar Medium and the Deuterium/Hydrogen Ratio along the Line of Sight Toward Capella.

Radio Emission from Chemically Peculiar Stars.
PB94-213469

First Results from a Coordinated ROSAT, IUE, and VLA

Study of RS CVn Systems
PB94-213477

00,069

Rapid Decline in the Optical Emission from SN 1957D in M83.

00,070

Spectroscopic Puzzle in ArHF Solved: The Test of a New Potentia.

PB94-216058

00,850

Scattering and Absorption Effects in Neutron Beam Activation Analysis Experiments.

00,557

Supermirror Transmission Polarizers for Neutrons.

PB94-216215

03,866

High-Resolution Measurements of the nu2 and 2nu2-nu2 Bands of (34) S(16)O2

00,855
Atomic Beam Splitters and Mirrors by Adiabatic Passage in Multilevel Systems.

03,867

High-Resolution IR Laser-Driven Vibrational Dynamics in Supersonic Jets: Weakly Bound Complexes and Intramolecular Energy Flow.

PB94-216751

00,862

Sealed Water Calorimeter for Measuring Absorbed Dose. PB94-219227

03,517

Intermolecular HF Motion in $\mathrm{Ar}$ (sub n)HF Micromatrices $(n=1,2,3,4$, : Classical and Ouantum Calculations on a Pairwise Additive Potential Surface.

03,871

SANS and LS Studies of Polymer Mixtures Under Shear

PB95-107090

01.231

Vibration, Rotation, and Parity Specific Predissociation Dynamics in Asymmetric OH Stretch Excited ArH2O: A Half Collision Study of Resonant V.V Energy Transfer in Weakly Bound Complex.

PB95-107140 00,872

Monte Carlo Electron Trajectory Simulation of X-Ray Emission from Films Supported on Substrates.
PB95-107207

04,872
03,872

PB95-107363

03,872

Photoelectron Spectroscopy of Negatively Charged Bismuth Clusters: $\mathrm{Bi}(-) 2, \mathrm{Bi}(-) 3$, and $\mathrm{Bi}(-) 4$

00,880

Efficient $\mathrm{Br}\left({ }^{*}\right)$ Laser Pumped by Frequency-Doubled Nd: YAG and Electronic-to-Vibrational Transier-Pumped $\mathrm{CO} 2$ and HCN Lasers.

PB95-108684

04,248

Preparation and Characterization of (6)LiF and (10)B Ref. erence Deposits for the Measurement of the Neutron Life-

PB95-108692

03,874

Neutron Leakage Benchmark for Criticality Safety Re-

PB95-126132

03,723

Some Aspects of Fundamental Neutron Physics.
PB95-126298

Radiation Accident at an Industrial Accelerator Facility.

PB95-140117 02.575

Measurement of the $(235) \cup(n, f)$ Reaction from Thermal to

PB95-140422 03.886

Neutron Focusing Lens Using Polycapillary Fibers.

PB95-141206

03,889

Grazing Incidence Prompt Gamma Emissions and Resonance-Enhanced Neutron Standing Waves in a Thin-Film. Excitation of Balmer Lines in Low-Current Discharges of Hydrogen and Deuterium.

PB95-150546

03,893

Fast Computer Evaluation of Radiative Properties of Hydrogenic Systems.

03,894

Nanoscale Study of the As-Grown Hydrogenated Amorphous Silicon Surface.

04.573

Laser Double Resonance Measurements of the Ouenching Rates of $\mathrm{Br}((2) \mathrm{P} 1 / 2)$ with $\mathrm{H} 2 \mathrm{O}, \mathrm{D} 2 \mathrm{O}, \mathrm{HDO}$, and $\mathrm{O} 2$.

PB95-150694

00.921

Standards for Corrected Fluorescence Spectra.

00,581

Electron and Proton Dosimetry with Custom-Developed Radiochromic Dye Films.

PB95-151106

03,713

Electron-Impact Excitation of $\mathrm{Si}(3+)$ (3S yields $3 \mathrm{P})$ Using a Merged-Beam Electron-Energy-Loss Technique.

Slant Path Atmospheric Refraction Calibrator: An Instrument to Measure the Microwave Propagation Delays in. duced by Atmospheric Water Vapor.

PB95-151270

01.476

Van der Waals Bond Lengths and Electronic Spectral Shitts of the Benzene-Kr and Benzene-Xe Complexes. PB95-151387

N2(a'(sup 1)Sigma(sub g)(sup +)) Metastable Collisional Destruction and Rotational Excitation Transfer by N2. PBo5-151305

Nonadiabatic Effects in the Photoassociation of H2S.

PB95-151437 00.934

Liquid-Hydrogen Cold Neutron Source for the NBSR.

PB95-151619

03, 729

Unusual Spin-Trap Chemistry for the Reaction of Hydroxyl Radical with the Carcinogen PBg5-15164hylamine.

00,692

Photodissociation of Ammonia at $193.3 \mathrm{~nm}$ : Rovibrational State Distribution of the NH2(A(2)A1) Fragment.

00,937

Pattern-Recognition Analysis of Low-Resolution X-Ray Fluorescence Spectra.

PB95-151924

00,588

Tomographic Decoding Algorithm for a Nonoverlapping Redundant Array.

01,842

IRAS Spectroscopic Observations of Young Planetary

PB95-152070

00,072

High-Precision Calculations of Cross Sections for LowScattering by Ground and Excited

B95-152161

03,914

Analysis of the Effectiveness of Oscillating Radial Collimators in Neutron Scattering Applications.

PB95-152252

03.917

Neutron Energy Deposition on the Nanometer Scale.

03,624

Anomalous Dispersion and Thermal Expansion in LightlyDoped KTa1-xNbxO3

PB95-152302

04,585

Microdosimetry and Cellular Radiation Effects of Radon Progency in Human Bronchial Airways.

03,625

Nuclear Heat Load Calculations for the NBSR Cold Neuron Source Using MCNP.

PB95-152955

03,730

Neutron Focusing Lens Using Polycapillary Fibers.

03,922

Extrapolation Chamber Measurements on $(90) \mathrm{Sr}+(90) \mathrm{Y}$ Beta-Particle Ophthalmic Applicator Dose Rates.

PB95-153375

03,626

Polarization Effects on Multiple Scattering Gamma Transport.

03,926

Origin of the Second Length Scale Above the Magnetic Spiral Phase of Tb.

PB95-153698

04,596

Calibration of High-Energy Electron Beams by Use of Graphite Calorimeters.

04,598

Time-Resolved Small-Angle Neutron Scattering Study of Spinodal Decomposition in Deuterated and Protonated Polybutadiene Blends. 1. Effect of Initial Thermal Fluctua-

PB95-161196

01,252

Method of Realizing Spectral Irradiance Based on an Absolute Cryogenic Radiometer.

ESR-Based Analysis in Radiation Processing

04,270

PB95-161634

03,931

Detector-Based Candela Scale and Related Photometric Calibration Procedures at NIST.

PB95-161949

04,273

Vibronic Coupling and Other Many-Body Effects in the 4sigmag(-1) Photoionization Channel of CO2.

00,962

Radiation Process Data: Collection, Analysis, and Interpretation.

PB95-162632 03,628 
NUCLEAR PHYSICS \& RADIATION TECHNOLOGY

Interpreting the Readings of Multi-Element Personnel Dosimeters in Terms of the Personal Dose Equivalent. Grazing Incidence X-ray Photoemission and Its Implementation on Synchrotron Light Source X-ray Beamlines.
PB95.175766 Cold Neutron Prompt Gamma Activation Analysis at NIST: A Progress Report.

00,602

Neutron Scattering by Hydrogen in Cold Neutron Prompt Gamma-Activation Analysis.

00,603

Small Angle Neutrons Scattering from Nanocrystalline Palladium as a Function of Annealing.

PB95-176103

03,354

Electron-Ion-X-ray Spectrometer System

03,958

Polymerization Initiation by $\mathrm{N}$-p-Tolylglycine: Free-Radical Reactions Studied by Pulse and Steady-State Radiolysis.
PB95-180014

Rayleigh Scattering Limits for Low-Level Bidirectional Reflectance Distribution Function Measurements.

PB95-180030

04,307

Neutron Scattering Study of the Orientation of a Liquid Crystalline Polymer by Shear Flow.

PB95-180196

01,270

Food Irradiation Dosimetry.

PB95-180675 00,041

Inelastic-Neutron-Scattering Studies of Poly(p-phenylene

$\begin{array}{ll}\text { vinylene). } & \text { 01,014 } \\ \text { PB95-180766 } & \end{array}$

High Resolution Inelastic Neutron Scattering Study of

Phonon Self-Energy Effects in YBCO.

Comparison of NIST and ISO Filtered Bremsstrahlung Calibration Beams.

PB95-180956

03,967

Calibration of Dosimeters for the Cryogenic Irradiation of Composite Materials Using an Electron Beam.

PB95-180964

03,968

Local-Mode Dynamics in YH2 and YD2 by Isotope-Dilution Neutron Spectroscopy.

PB95-181012

01,017

Roles of Local Classical Acceleration and Spatial Separation in the Neutral Particle Analogs of the AharonovBohm Phases.

03.976

Neutron Reflectivity Study of the Density Profile of a Model End-Grafted Polymer Brush: Influence of Solvent Quality

PB95-202735

01,274

Hydration in Semicrystalline Polymers: Small-Angle Neutron Scattering Studies of the Effect of Drawing in Nylon6 Fibers.

03,385

Reactor Radiation Technical Activities, 1994. NAS-NRC Assessment Panel, April 6-7, 1995

03,732

NIST Reactor: Summary of Activities, October 1993 through September 1994

04,700

45 deg/0 deg Reflectance Factors of Pressed Polytetrafluoroethylene (PTFE) Powder.

04,328

Intercomparison of Photometric Units Maintained at NIST (USA) and PTB (Germany), 1993.

04,329

Transmission Properties of Short Curved Neutron Guides.

Transmission Properties of Short Curved Neutron Guides.
Part 1. Acceptance Diagram Analysis and Calculations.
PB96-102199 04,021

Evolution of $X$-ray Resonance Raman Scattering into $X$ ray Fluorescence from the Excitation of Xenon Near the L3 Edge.

04,025

Measuring Hydrogen by Cold-Neutron Prompt-Gamma Activation Analysis.

00,612

Study of Multiple Scattering Background in Compton Scatter Imaging.

04,425

Small Angle Neutron Scattering Studies of Structural Characteristics of Argarose Gels.

PB96-112305

03,475

Analytical App

04,041

Strangeness Flow Difference in Nuclear Collisions at $15 \mathrm{~A}$ and $200 \mathrm{~A} \mathrm{GeV}$

PB96-119631 04,042

Neutron Techniques in Materials Science and Related Disciplines.

PB96-119698

02,980

Extending the Angular Range of Neutron Reflectivity

Measurements from Planar Lipid Bilayers: Applications to

a Model Biological Membrane.

PB96-122569

03,476

Study of Laser Resonance Ionization Mass Spectrometry

Using a Glow Discharge Source.

03,360
Anionic Triphenylmethane Dye Solutions for Low-Dose Food Irradiation Dosimetry.

03.715

Alcohol Solutions of Triphenyl-Tetrazolium Chloride as High-Dose Radiochromic Dosimeters.

03,716 PB96-135249

Aqueous

Oscillometric and Conductometric

PB96-135256

04,054

Dosimetry Systems for Radiation Processing.

03,717

Temperature and Relative Humidity Dependence of Radiochromic Film Dosimeter Response to Gamma and Electron Radiation.

PB96-135298

03,718

Real Time Monitoring of Electron Processors.

PB96-135306

03,718

Low-Frequency Excitations of Oriented DNA.

03,719

PB96-137799

03,548

MCNP Model of the National Bureau of Standards Reactor (NBSR) Core.

PB96-138599 03,733

Research and Development Activities in Electron Paramagnetic Resonance Dosimetry.

PB96-141288

03,635

Orientation Effects on ESR Analysis of Alanine-Polymer

Dosimeters.
PB96-146725 03,720

Radiation-Chemical Reaction of 2,3,5-Triphenyl-Tetra-

zolium Chloride in Liquid and Solid State.
PB96-146733

Investigation of Applicability of Alanine and Radiochromic Detectors to Dosimetry of Proton Clinical Beams.
PB96-146782 03,636

AI L2,3 Core Excitons in AlxGa 1-x as Studied by Sott-Xray Retlection and Emission.

PB96-157839

04,067

Soft-X-ray-Emission Investigation of Cobalt Implanted Silicon Crystals.

PB96-157912 04,069

Soft-X-ray-Emission Spectra of Solid $\mathrm{Kr}$ and $\mathrm{Xe}$

PB96-157920

04,070

Soft-X-ray-Emission Studies of Bulk Fe3Si, FeSi, and FeSi2, and Implanted Iron Silicides.

PB96-157938

04,071

Cooper M(sub II,III) X-ray-Emission Spectra of Copper Oxides and the Bismuth Cuprate Superconductor. New NIST/ARPA National Soft X-ray Reflectometry Facility.

04,080

Soft-X-ray Damage to $\mathrm{p}$-terphenyl Coatings for Detectors.

System for Intercomparing Standard Solutions of BetaParticle Emitting Radionuclides.

PB96-159637

03,707

International Radon-in-Air Measurement Intercomparison Using a New Transfer Standard.

03,708

External Gamma-ray Counting of Selected Tissues from a Thorotrast Patient.

PB96-160254

03,637

PB96-160296

04,085 New and Revised Half-Lite Measurement Results.
PB96-160346
00,695

NIST Metrology for Soft X-ray Multilayer Optics.

PB96-160379

04,088

Soft $X$-ray Reflectometry Program at the National Institute of Standards and Technology.

PB96-160395

04,368

Instrumental Smearing Effects in Radially Symmetric Small-Angle Neutron Scattering by Numerical and Analytical Methods.

ical Methods.

02,984

Inter-Laboratory Trials of the EPR Method for the Detection of Irradiated Meats Containing Bone.

PB96-161690

00,042

PB96-161708

04,094

Measurement of the (10)B(n, alpha1gamma)(7)Li Cross Section in the 0.3 to $4 \mathrm{MeV}$ Neutron Energy Interval. PB96-161799 04,098 Gas Absorption during Ion-Irradiation of a Polymer Target.

04,099

Upgrade and Modernization Projects at the NBSR. PB96-161872

03,737

International Marine-Atmospheric (222)Rn Measurement Intercomparison in Bermuda. Part 1 . NIST Calibration and

Methodology for Standardized Sample Additions.
PB96-175674

00,114

International Marine-Atmospheric (222)Rn Measurement Intercomparison in Bermuda. Part 2. Results for the Participating Laboratories.

00.115

Realization of NIST 1995 Luminous Flux Scale Using the Integrating Sphere Method.

PB96-176433

04,374

Simulations of Neutron Focusing with Curved Mirrors

PB96-176649 02,200

Design of a High-Flux Backscattering Spectrometer for Ultra-High Resolution Inelastic Neutron Measurements.

Radiochromic Solid-State Polymerization Reaction. PB96-180146 01,290

Alpha-Particle and Electron Capture Decay of (209)Po.

PB96-186085 04,119

Scattered Fractions of Dose from 18 and $25 \mathrm{MV}$ X-ray Radiotherapy Linear Accelerators.

PB96-18610

04,120

PB96-204201

Radon in the Lung

PB97-110035

04,134

Liquid-Scintillation Counting Techniques for the StandardPB97-110084

03,709

Needs for Brachytherapy Source Calibrations in the United States.
PB97-110092

Radioassays of Yttrium-90 Used in Nuclear Medicine

PB97-110100 03,522

Problem of Convection in the Water Absorbed Dose Cal-

Orimeter.

03,523

Stable Silicon Photodiodes for Absolute Intensity Measurements in the VUV and Soft X-ray Regions.

04,135

EPR Dosimetry of Cortical Bone and Tooth Enamel Irradiated with $X$ and Gamma Rays: Study of Energy DependPBC. 110373

03,639

Exposure-to-Absorbed-Dose Conversion for Human Adult Cortical Bone
PB97-110381

03,640

Calculation of Photon Mass Energy-Transier and Mass Energy-Absorption Coefficients.

04,137

Neutron Diffraction Texture Study of Deformed Uranium

PB97-111587

03,010

63Ni Half-Life: A New Experimental Determination and Critical Review

Nickel-63 Standardization: 1968-1995

00,700

PB97-111819

00,701

$X$-ray Reflectivity Determination of Interface Roughness Correlated with Transport Properties of (AIGa)As/GaAs High Electron Mobility Transistor Devices.

PB97-111868

04,149

Results of a NISTNNIIOFI Comparison of Spectral-Radiance Measurements.

PB97-113021

04,159

Comparison of Filter Radiometer Spectral Responsivity with the NIST Spectral-Irradiance and Illuminance Scales.

04,162

Diffraction of Neutron Standing Waves in Thin Films with Resonance Enhancement.

PB97-113278

04,164

Experimentally Measured Total X-ray Attenuation Coefficients Extracted from Previously Unprocessed Documents Held by the NIST Photon and Charged Particle Data Center. 
Flat and Curved Crystal Spectrography for Mammo-

graphic X-ray Sources.
PB97-122246

03,642

Small Angle Neutron Scattering Study of the Structure and Formation of MCM-41 Mesoporous Molecular Sieves. PB97-122337 03,110 Complex Time Dependence of the EPR Signal of Irradiated L-alpha-alanine.

PB97-122436

04,180

Overview of a Radiation Accident at an Industrial Accelerator Facility

02,612

Measuring Nondipolar Asymmetries of Photoelectron Angular Distributions.

UCLEAR POWER

01,193

Review of the USCEANIIST Measurement Assurance Program for the Nuclear Power Industry.

03,712

NUCLEAR POWER PLANTS

Proceedings of the Digital Systems Reliability and Nu. clear Safety Workshop. Held in Rockville, Maryland on September 13-14, 1993

03,728

Assessing Functional Diversity by Program Slicing.

PB96-160890
Analysis of Standards for the Assurance of High Integrity Sottware.

PB96-161351

03,735

Control and Instrumentation: Standards for High-Integrity

SBtware.

03,736

Preliminary Processing of the Lotung LSST Data.
PB96-165972

03,690

Methodology for Developing and Implementing Alternative Temperature-Time Curves for Testing the Fire Resistance of Barriers for Nuclear Power Plant Applications.

of Barriers for Nuclear Power Plant Applications.

Estimation of System Damping at the Lotung Site by Application of System Identification.

PB96-214697

01,351

USCEAINIST Measurement Assurance Programs for the Radiopharmaceutical and Nuclear Power Industries. 03,721

NUCLEAR REACTORS

Reactor Radiation Technical Activities, 1994. NAS-NRC Assessment Panel, April 6-7, 1995

PB95-209888

03,732

Upgrade and Modernization Projects at the NBSR.

03,737

Reactor Radiation Technical Activities, 1995

03,741

NUCLEATE BOILING

Simultaneous Visual and Calorimetric Measurements of R11, R123, and R123/Alkylbenzene Nucleate Flow BoilPB94-172426

03,251

Visual Measurement Technique for Analysis of Nucleate Flow Boiling.

03,262

NUCLEATION

Controlted Nucleation in Aerosol Reactors for Supression of Agglomerate Formation

00,672

Surtace Roughness Evaluation of Diamond Films Grown on Substrates with a High Density of Nucleation Sites.

PB95-162418 03,018

Growth and Nucleation of Hydrogenated Amorphous Silicon on Silicon (100) Surtaces

PB96-176516 02,991

Epitaxial Nucleation and Growth of Chemically Derived $\mathrm{Ba2YCu} 3 \mathrm{O} 7-x$ Thin Films on (001) $\mathrm{SrTiO}_{3}$

PB96-190186

04,787

NUCLEIC ACIDS

Salt-PEG Two-Phase Aqueous Systems to Purify Proteins and Nucleic Acid Mixtures.

PB94-200375

03,527

Nucleic Acid

00,518

NUCLEOTIDES

beta-D-Glucosyl-Hydroxymethyluracil: A Novel Modified Base Present in the DNA of the Parastic Protozoan T. brucei.

PB94-172319

03,524

NUETRON ATOMS

Gravitational Sisyphus Cooling of (87)Rb in a Magnetic PB96-200704

04,379

NUETRON MEASUREMENTS

Design of a High-Flux Backscattering Spectrometer for Ultra-High Resolution Inelastic Neutron Measurements. PB96-179577

NUMERICAL ANALYSIS

Convergence Properties of a Class of Rank-Two Updates. (NIST Reprint)

03,430

Numerical Evalua

03,417
NUMERICAL CONTROL

NIST RS274/NGC Interpreter Version 1.

PB94-187788

02,814

Issues Concerning Material Removal Shape Element Vol-

umes (MRSEVs)
PB95-210167

02,885

NIST RS274KT Interpreter

02,835

NUMERICAL DATA BASES

Numeric Data Distribution: The Vital Role of Data Exchange in Tod
N95-15937/2

02,622

NUMERICAL SIMULATION

Gravity-Current Transport in Building Fires.

PB96-147046

01,415

NURSING HOMES

Evaluating Small Board and Care Homes: Sprinklered vs. Nonsprinklered Fire Protection.

PB94-206356

00.195

NUTRIENTS

Recently Developed NIST Food Related Standard Reference Materials.

NUTRITION

00,035

Nutritional Status and Growth in Juvenile Rheumatoid Arthritis.

PB94-198470

03,515

NUTRITIVE VALUE

Mixed Diet Reference Materials for Nutrient Analysis of Foods: Preparation of SRM-1548 Total Diet. PB95-151692

03,593

NVLAP (NATIONAL VOLUNTARY LABORATORY

ACCREDITATION PROGRAM)

National Voluntary Laboratory Accreditation Program: Energy Efficient Lighting Products.

02,642

National Voluntary Laboratory Accreditation Program: POSIX. Portable Operating System Interiace.

PB95-189478

02,661

NVLAP (NATIONAL VOLUNTARY LABORATORY

CCREDITION PROGRAM)

National Voluntary Laboratory Accreditation Program 1996 Directory
PB96-162714

00,485

NVLAP PROGRAM

National Voluntary Laboratory Accreditation Program: Ionizing Radiation Dosimetry.

03,623

NVLAP SYSTEM

NVLAP Procedures U.S. Code of Federal Regulations. Title 15, Subtitle A Chapter 2, Part 7. (Effective December 1984; Amended September 1990).

02,627 LON 6

Hydration in Semicrystalline Polymers: Small-Angle Neutron Scattering Studies of the Effect of Drawing in Nylon6 Fibers.

PB95-202990

03,385

NYLON FIBERS

Hydration in Semicrystalline Polymers: Small-Angle Neutron Scattering Studies of the Effect of Drawing in Nylon(1) Fibs.

OBITUARIES

Churchill Eisenhart, 1913-1994.

PB96-137740

03,385

OBJECT DATABASE MANAGEMENT

Persistent Object Base System Testing and Evaluation.
PB95-220588

01,730

Interoperability Experiments with CORBA and Persistent Object Base Systems.

01,772

\section{OBJECT-ORIENTED PROGRAMMING}

Object SOL: Language Extensions for Object Data Man-

PBg5-125902

01,704

Object-Oriented Technology Research Areas.

PB95-199329

01,726

Testability of

01,733

Object-Oriented Tel/Tk Binding for Interpreted Control of the NIST EXPRESS Toolkit in the NIST STEP Application Protocol Development Environment.

PB96-141049

02,785

OBJECT ORIENTED PROGRAMS

Conformance Testing and Specification Management. PB97-113781

OBJECT ORIENTED SYSTEMS

Roadmap for the Computer Integrated Manufacturing (CIM) Application Framework

02,832

OBJECT-ORIENTED TECHNOLOGY

Object-Oriented Technology Research Areas.

01,726

OBJECT PROGRAMS

Associated Object Model for Distributed Systems.

01,694

OBSERVATION

Developments in Stellar Coronae.

PB96-176706

00,107

OBSTACLE AVOIDANCE

New Method to Calculate Looming for Autonomous Ob. stacle Avoidance.

Real-Time Obstacle Avoidance Using Central Flow Divergence and Peripheral Flow.

02,937

OCCUPATIONAL EXPOSURE

Interpreting the Readings of Multi-Element Personnel Dosimeters in Terms of the Personal Dose Equivalent. PB95-175428

03,631

CUPATIONAL SAFETY AND HEALTH

Documentation for Immediately Dangerous to Life or Health Concentrations (IDLHS).

NIOSH Pocket Guide to Chemical Hazards

03,602

PB95-100368

03,603

Radiation Accident at an Industrial Accelerator Facility.

PB95-140117 02,575

Characterization of a Health Physics Instrument Calibra-

PB95-164554

03,629

$\mathrm{NIOSH}$ Comments to DOL on Risk Estimates from the Cadmium Cohort Study by L. Stayner, February 7, 1992. PB95-267779

03,604

Overview of a Radiation Accident at an Industrial Accelerator Facility.

02,612

OCEAN ENGINEERING

Non-Gaussian Noise Effects on Reliability of Multistable

Systems.
PB96-122726

04,213

OCTACALCIUM PHOSPHATE

Octacalcium Phosphate. 3. Infrared and Raman Vibrational Spectra

00,756

Procedure for the Study of Acidic Calcium Phosphate Precursor Phases in Enamel Mineral Formation.

PB95-164448

03,564

OCTACALCIUM PHOSPHATE CARBOXYLATES

Octacalcium Phosphate Carboxylates. 1. Preparation and Identification.

00,660

Octacalcium Phosphate Carboxylates. 2. Characterization and Structural Consideration.

00,955

\section{CTACALCIUM PHOSPHATE SUCCINATE}

Octacalcium Phosphate Carboxylates IV. Kinetics of Formation and Solubility of Octacalcium Phosphate Succi-

PB94-185600

00,776

Octacalcium Phosphate Carboxylates. 5. Incorporation of Excess Succinate and Ammonium lons in the Octacalcium Phosphate Succinate Structure.

PB95-168894

00,166

OCTADECANETHIOL

Silver Metalization of Octadecanethiol Monolayers SelfAssembled on Gold.

00,923 


\section{KEYWORD INDEX}

\section{OPTICAL DENSITY}

Study of Ventilation and Carbon Dioxide in an Otfice Puilding.

02,542

Assessing Ventilation Effectiveness in Mechanically Ventilated Office Buildings.

PB95-162079

00,255

Ventilation Effectiveness Measurements in Two Modern Office Buildings.

02,553

Indoor Air Ouality Commissioning of a New Office BuildPB́.

00,262

Measurements of Outdoor Air Distribution in an Office

Building.

00,264

Workplan to Analyze the Energy Impacts of Envelope Airtightness in Office Buildings.

PB96-154463

00,273

Study of Ventilation Measurement in an Office Building.

PB96-155593 00,274

Development and Application of an Indoor Air Ouality Commissioning Program in a New Office Building.
PB96-155601 Indoor Air Quality Commissioning of a New Office Building.

00,279

Post-Occupancy Evaluation of the Forrestal Building.
PB97-111298

Survey of Fuel Loads in Contemporary Office Buildings

Survey of Fuel Loads in Contemporary Office Buildings.
PB97-114235 00,233

Evaluation of Survey Procedures for Determining Occupant Load Factors in Contemporary Office Buildings.
PB97-116222

00,238

Materials-Science Based Approach to Phenol Emissions from a Flooring Material in an Office Building.

PB97-118749

02,572

FFICE EQUIPMENT

Office Work Station Heat Release Rate Study: Full Scale versus Bench Scale.

FFSET SUSCEPTIBILITY

01,428

Offset Susceptibility of Superconductors.

PB94-212263

04,503

FFSHORE DRILLING

Composite Materials for Offshore Operations: Proceed ings of the International Workshop (1st). Held in Houston, Texas on October 26-28, 1993.

PB96-109509

03,169

\section{OFFSHORE STRUCTURES}

Report of a Workshop on Requalification of Tubular Steel Joints in Offshore Structures. Held in Houston, Texas on September 5-6, 1995.

03,699

New International Representations of the Volt and Ohm Effective January 1, 1990

PB95-150777

01,890

fn situ Burning of Oil Spills: Mesoscale Experiments.

In Situ Burning Oil Spill Workshop Proceedings. Held in Orlando, Florida on January 26-28, 1994.

02,583

In situ Buming of Oil Spills: Mesoscale Experiments and

Analysis.

02,587

Smoke Emission from Burning Crude Oil.

01,407

Smoke Plume Trajectory from In situ Burning of Crude Oil in Alaska: Field Experiments.

02,594

Smoke Plume Trajectory from In situ Burning of Crude Smoke Plume Trajectory from In situ Burning of Crude
Oil: Field Experiments. Oil: Field Experiments.

02,597

OIL TANKERS

Stranding Experiments on Double Hull Tanker Structures.

IL WELLS

Ground-Based Smoke Sampling Techniques Training Course and Collaborative Local Smoke Sampling in Saudi Arabia

PB94-143542

02,532

Investigation of Oil and Gas Well Fires and Flares.

PB94.193976 03,695 Flame Heights and Heat Release Rates of 1991 Kuwait

$\begin{array}{ll}\text { Oil Field Fires. } & 01,404 \\ \text { PB96-119342 } & \end{array}$

Development of Hazard Assessment and Suppression Technology for Oil and Gas Well Blowout and Diverter Fires.

01,408

PB96-122965

OLEORESINS Preliminary Investigation of Oleoresin Capsicum.
PB96-179486

03,520

OLIGOMERS

Solid State (13)C NMR and Raman Studies of Cellulose Triacetate: Oligomers, Polymorphism, and Interences

about Chain Polarity.

01,289

OLTIPAAZ

Determination of Oltipraz in Serum by High-Performanace Liquid Chromatography with Optical Ábsorbance and Mass Spectrometric Detection.

PB94-200201

03,493

OMEGA PHASE

Ordered omega-Derivatives in a Ti-37.5Al-2ONb at\% Alloy. 211091

ON-LINE SYSTEMS

STEP On-Line Information Service (SOLIS). The IGES/ PDES Organization.

PB95-137790 02,777

Electronic Access to Standards on the Information HighPBay. 131578

01,494

ONLINE SYSTEMS

Global Information Infrastructure: Agenda for CooperaPB95-178604

01,482

OPEN CHANNEL FLOW

Probabilistic Computation of Poiseuille Flow Velocity Fields.

04,209

OPEN DISTRIBUTED PROCESSING

Comparison of POSIX Open System Environment (OSE) and Open Distributed Processing (ODP) Reference Models.

01,820

OPEN-ENDED PROBES

Open-Ended Coaxial Probes for Nondestructive Testing of Substrates and Circuit Boards.

\section{PEN SYSTEM ENVIRONMENT}

Porting Multimedia Applications to the Open System Envi-

PB94-172921

02,721

Guide on Open System Environment (OSE) Procure-

PB95-169496

01,626

Comparison of POSIX Open System Environment (OSE)
and Open Distributed Processing (ODP) Reference Mod-

els.

PB96-131495

01,820

Open System Environment (OSE): Architectural Framework for Information Infrastructure.

PB96-146360

00,002

Application Portability Profile (APP): The U.S. Government's Open System Environment Profile Version 3.0.

PB96-158712 01,753

OPEN SYSTEMS

Conference Report: International Conference on the Application of Standards for Open Systems (6th).

01,762

PB96-16121

OPEN SYSTEMS ENVIRONMENT

Open System Environment Implementors Workshop (OIW); Standar

OPEN SYSTEMS INTERCONNECTION

Formal Multi-Layer Test Methodology and Its Application to OSI.

PB94-172194

02,718

ISO/IEC Workshop on Worldwide Recognition of OSI Test Results Regional Progress - North America.
PB94-172202 pecifica-

PB94-207453

01,806

Conformance Testing for OSI Protocols.

01,631

01,631
Lessons from the Establishment of the U.S. GOSIP Test-

ing Program.

01,817

Open Issues in OSI Protocol Development and Conformance Testing.

01,818

Data Communications Strategy.

02,738

OPEN SYSTEMS INTERCONNECTIONS

Supplement to Stable Implementation Agreements for Open Systems Interconnection Protocols. Version 3, SepIndex, Version 3, June 1990 (Stable) Change Pages Issued December 1990; Output from September 1990 OSI Workshop (NIST Special PubPB94-164035

01,467

Physical Properties of Some Purified Aliphatic Hydrocarbons

00,657

ILS

National Standard Petroleum Oil Tables.

AD-A279 952/6

Open Systems Sottware Standards in Concurrent Engineering.

01,758

NIST POSIX Testing Program.

PB96-160973

01,821

OPERATIONS RESEARCH

Time Dependent Vector Dynamic Programming Algorithm or the Path Planning Problem.

PB94-215688

03,428

OPHTHALMIC APPLICATIONS

Comparison of NIST and Manufacturer Calibrations of $(90) \mathrm{Sr}+(90)$ Y Ophthalmic Applicators.

PHTHALMIC APPLICATORS

Extrapolation Chamber Measurements on $(90) \mathrm{Sr}+(90) \mathrm{Y}$ Beta-Particle Ophthalmic Applicator Dose Rates.

PB95-153375

03,626

\section{OPHTHALMOLOGY}

Extrapolation Chamber Measurements on $(90) \mathrm{Sr}+(90) \mathrm{Y}$ Beta-Particle Ophthalmic Applicator Dose Rates.
PB95-153375

Measurement of Radial Dose Distributions Around Small Beta Particle Emitters Using High Resolution Radiochromic Foil Dosimetry

TICAL ABSORPTION

Interdigitated Stacked P-I-N Multiple Ouantum Well ModPBator- 112296

02,455

OPTICAL BIOSENSORS

Optical Biosensor Using a Fluorescent, Swelling Sensing Element.

OPTICAL CHARACTER RECOGNITION

03,541

Evaluating Form Designs for Optical Character Recogni-

PB94-168044 01.830

Second Census Optical Character Recognition Systems Conference.

01,832

NIST Form-Based Handprint Recognition System

PB94-217106 01,838

Binary Decision Clustering for Neural Network Based Optical Character Recognition.

PB95-171971 01,848

Method and Evaluation of Character Stroke Preservation on Handprint Recognition.

Improving Neural Network Performance for Character and Fingerprint Classification by Altering Network Dynamics.
PB95-267803

Effect of Training Dynamics on Neural Network Perform.

ance.

Improving Neural Network Performance for Character and Fingerprint Classification by Altering Network Dynamics.
PB96-123195

Binary Decision Clustering for Neural-Network-Based Op-

tical Character Recognition.
PB96-186184

Generalized Form Registration Using Structure-Based Techniques.

Component-Based Handprint Segmentation Using Adapt-

ive Writing Style Model.
PB96-193669

01,859

OPTICAL CHARACTERIZATION

Optical Characterization of Materials and Devices for the Semiconductor Industry: Trends and Needs.

PB96-167192

02,431

OPTICAL COATINGS

XUV Characterization Comparison of $\mathrm{Mo} / \mathrm{Si}$ Multilayer

Coatings.

04,278

OPTICAL COMMUNICATION

Lightwave Standards Development at NIST

PB95-168563

01,480

Precise Laser-Based Measurements of Zero-Dispersion

Wavelength in Single-Mode Fibers.

PB96-201124

01.511 
OPTICAL DETECTION

Beamcon III, a Linearity Measurement Instrument for Optical Detectors.
PB96-1 13576

04,337

OPTICAL DETECTORS

Lightwave Standards Development at NIST. $\quad 01,480$
PB95-168563

Spatial Uniformity of Optical Detector Responsivity.
PB95-168845 Optica! Detector Nonlinearity: A Comparison of Five

04.291

Accurate Measurement of Optical Detecis Nonlinearity. PB95-203576 02, 181

Optical Detector Nonlinearity: Simulation.

02,199

Precise Laser-Based Measurements of Zero-Dispersion Wavelength in Single-Mode Fibers

PB96-201124

01,511

OPTICAL DISKS

Optical Storage Media Data Integrity Studies. $\quad 01,620$

Design and Development of an Information Retrieval System tor the EAMATE Data. Volume 2 of 2. Appendices.
PB94- 168390 Standardization of Testing Methods for Optical Disk Media Characteristics and Related Activities at NIST.
PB95-108486 NIST Program for Investigating Error Reporting Capabilities of Optical Disk Drives.

01,635

OPTICAL EQUIPMENT

Simple and Efficient Low-Temperature Sample Cell for In-

frared Spectrophotometry.
PB94-199197 00,545

Video Microscopy Applied to Optical Fiber Geometry Measurements.

OPTICAL FIBER SENSORS

Optical Fiber Sensors: Accelerating Applications in Navy Ships.

02,632

Polarization Dependence of Response Functions in $3 \times 3$ Sagnac Optical Fiber Current Sensors.

02,154

Effect of Semiconductor Laser Characteristics on Optical Fiber Sensor Performance.

PB95-169132

02,167

Self-Calibrating Fiber Optic Sensors: Potential Design Methods.

02,172

Self Calibrating Fiber Optic Sensors: Potential Design Methods.

PB95-169306

02,173

Polarization Dependence of Response Functions in $3 \times 3$ Sagnac Optical Fiber Current Sensors.

OPTICAL FIBERS

02,189

Technical Digest: Symposium on Optical Fiber Measurements (8th), 1994. Held in Boulder, Colorado on September 13-15, 1994.
PB94-207636

04,231

In situ On-Line Optical Fiber Sensor for Fluorescence Monitoring in Bioreactors. Complex Propagation Constants for Nonuniform Optical Waveguides: Calculations.

PB95-125910

04,249

Fiber Spot Size: A Simple Method of Calculation.
PB95-125936 LP11-Mode Leakage Loss in Coated Depressed Clad FiPBS5-141115

02,145

Optical Fiber Geometry: Accurate Measurement of Cladding Diameter.

04,266

Optical Fiber Geometry by Gray-Scale Analysis with Robust Regression.
PB95-161519

04,272

Bragg Gratings in Optical Fibers Produced by a Continuous-Wave Ultraviolet Source.

04,274

Growth of Bragg Gratings Produced by Continuous-Wave

Ultraviolet Light in Optical Fiber.
PB95-162038

04,275

Vector Theory of Diffraction by a Single-Mode Fiber: Application to Mode-Field Diameter Measurements. 04,279 Interlaboratory Comparison of Polarization-Holding $\mathrm{Pa}$ rameter Measurements on High-Birefringence Optical Fiber.

PB95-168464

04,280

Low-Coherence Interferometric Measurement of Group Transit Times in Precision Optical Fibre Delay Lines.

Lightwave Standards Development at NIST.

02,158 PB95-168563

01,480
Symbolic Programming with Series Expansions: Applications to Optical Waveguides

04,283

Comparison of UV-Induced Fluorescence and Bragg Grating Growth in Optical Fiber.

PB95-168597

04,284

Bent Rectangular Core Waveguides: An Accurate Perturbation Approach

04,289

Calculated Fiber Attenuation: A General Method Yielding Stationary Values.

PB95-175501

04,298

Fibre Splice Loss: A Simple Method of Calculation.

PB95-175519 04,299

Decrease of Fluorescence in Optical Fiber during Exposure to Pulsed or Continuous-Wave Ultraviolet Light.

PB95-203071 04,320

Polarization Insensitive $3 \times 3$ Sagnac Current Sensor Using Polarizing Spun High-Birefringence Fiber.

02,187

Standard Reference Materials for Optical Fibers and Con-

nectors.
PB96- 119805

04,344

Decay of Bragg Gratings in Hydrogen-Loaded Optical Fi-

PB96-122643

04,345

Fiber Coating Diameter: Toward a Glass Artifact Stand-

ard.

02,234

Annealing of Bragg Gratings in Hydrogen-Loaded Optical

04,361

Comparison of UV Photosensitivity and Fluorescence during Fiber Grating Formation.

PB96-155445

04.362

Automated Measurement of Nonlinearity of Optical Fiber Power Meters.

04,110

Precise Laser-Based Measurements of Zero-Dispersion Wavelength in Single-Mode Fibers.

PB96-201124

01,511

Technical Digest: Symposium on Optical Fiber Measurements (9th), 1996. Held in Boulder, Colorado on October $1-3,1996$.

PB97-108583

04,383

Anomalous Relation between Time and Frequency Domain PMD Measurements.

PB97-119390

04,398

Standard Reference Materials: Glass Filters as a Standard Reference Material for Spectrophotometry - Selection, Preparation, Certification, and Use of SRM 930 and SRM 1930.

PB94-188844

00,536

Determination of the Transmittance Uniformity of Optical Filter Standard Reference Materials.

02,182

OPTICAL FLOW

Reliable Optical Flow Algorithm Using 3-D Hermite Poly-

nomials.
PB94-145620 01,829

General Motion Model and Spatio-Temporal Filters for

Computing Optical Flow.
PB95-171096

01,847

General Motion Model and Spatio-Temporal Filters for 3D Motion Interpretations.

OPTICAL FLOW (IMAGE ANALYSIS)

01,861

Visual-Motion Fixation Invariant.

PB94-206281

01,836

Real-Time Implementation of a Differential Range Finder.

PB95-108650 01,839

Real Time Differential Range Estimation Based on TimeSpace Imagery Using PIPE.

01,844

Calculating Time-to-Contact Using Real-Time Ouantized Optical Flow.
PB95-210522

01,604

OPTICAL IMAGES

Greatly Enhanced Soot Scattering in Flickering Ch4/Air Diffusion Flames.
PB94-172988

01,361

OPTICAL INTERCONNECTIONS

Metrology and Data for Microelectronic Packaging and Interconnection: Results of a Joint Workshop on Materials Metrology and Data for Commercial Electrical and Optical Packaging and Interconnection Technologies. Held in Gaithersburg, Maryland on May 5-6, 1994. Volume 1. Results.

02,328

OPTICAL INTERFERENCE

Interference in the Resonance Fluorescence of Two Trapped Atoms.

PB95-168514

Light Scattered from Two Atoms.

OPTICAL INTERFEROMETERS

Test Optics Error Removal.
PB96-179536

03,948

04,286

04,377

Compensation for Errors Introduced by Nonzero Fringe Densities in Phase-Measuring Interferometers.

04,386

OPTICAL LITHOGRAPHY

Improved Dose Metrology in Optical Lithography.

PTICAL MATERIALS

Moisture and Water-Induced Crack Growth in Optical Materials

PB95-153334

04,267

OPTICAL MEASUREMENT

NIST Response to the Fitth CORM Report on the Pressing Problems and Projected Needs in Optical Radiation Measurements.

Optical Metrology and More. Programs and Services of the Radiometric Physics Division, Physics Laboratory. 04,228 Technical Digest: Symposium on Optical Fiber Measurements (8th), 1994. Held in Boulder, Colorado on September $13-15,1994$

PB94-207636

04,231

PB94-213097

04,241

Integrating Sphere Simulation: Application to Total Flux

Scale Realization
PB95-150173

04.261

Direct Dispersion Measurement of Highly-Erbium-Doped Optical Amplifiers Using a Low Coherence Reflectometer Coupled with Dispersive Fourier Spectroscopy.

PB95-150702

04,263

Guidelines for Retractive Index Measurements of Asbes10s.

PB95-151189

02.543

Regimes of Surface Roughness Measurable with Light Scattering.

04,265

Optical Fiber Geometry: Accurate Measurement of Cladding Diameter.

04,266

Electrically Calibrated Pyroelectric Detector-Refinements for Improved Optical Power Measurements.

02,164

PB95-169066

Optical Power Meter Calibration Using Tunable Laser Di-

PB95-169256

04,290

New Method for Realizing a Luminous Flux Scale Using an Integrating Sphere with an External Source.

04,333

Semiconductor Measurement Technology: Survey of Optical Characterization Methods for Materials, Processing, and Manufacturing in the Semiconductor Industry.
PB96-154596 Realization of NIST 1995 Luminous Flux Scale Using the Integrating Sphere Method.

Integrating Sphe
PB96-176433

04,374

Technical Digest: Symposium on Optical Fiber Measurements (9th), 1996. Held in Boulder, Colorado on October $1-3,1996$.

PB97-108583

04,383

Numerical Reference Models for Optical Metrology Simulation.

PTICAL MEASURING INSTRUMENTS

Automated Optical Roughness Inspection

PB95-152179

04,392

OPTICAL MEMORY (DATA STORAGE)

Optical Storage Media Data Integrity Studies. N95-24130/3

02,905

OPTICAL METROLOGY

Model of an Optical Roughness-Measuring Instrument.

PB97-1 10209

04,384

OPTICAL MICROSCOPES

Optical Fiber Geometry: Accurate Measurement of CladPB95-151940

OPTICAL MOLASSES 
Optical Power Meter Calibration Using Tunable Laser DiOdes. 169256

\section{OPTICAL PROPERTIES}

Lighting Quality and Light Source Size.

00,252

Simultaneous Optical Measurement of Soot Volume FracSimultaneous Optical Measurement of Soot Volume Frac-
tion, Temperature, and CO2 in Heptane Pool Fire.
PB96-102132

Mixing and Radiation Properties of Buoyant Luminous
Flame Environments.
PB96-202254 PB96-202254

OPTICAL PUMPING
Electronically Tunable Fiber Laser for Optical Pumping of (3) $\mathrm{He}$ and (4) $\mathrm{He}$.

04,381

PTICAL PYROMETERS

Technologic Papers of the Bureau of Standards: Number 170. Pyrometric Practice.

AD-A279 282/8

03,766

\section{OPTICAL RADAR}

Model Minimum Performance Specifications for Lidar Speed Measurement Devices.

04,861

Delivering the Same Optical Frequency at Two Places: Delivering the Same Optical Frequency at Two Places:
Accurate Cancellation of Phase Noise Introduced by an Accurate Cancellation of Phase Noise Introduced by an
Optical Fiber or Other Time-Varying Path.

04,332

\section{OPTICAL RANGE FINDEAS}

Real-Time Implementation of a Differential Range Finder. PB95-108650

\section{OPTICAL RETARDANCE}

Standard Polarization Components: Progress Toward an Optical Retardance Standard.

PB96-1 19672

04,342

PTICAL SAMPLING

High-Sensitivity Optical Sampling Using an Erbium-Doped Fiber Laser Strobe.

Optical Sampling Using Nondegenerate Four-Wave Mix. ing in a Semiconductor Laser Amplifier.

PB96-122502

02,076

Millimeter-Resolution Optical Time-Domain Reflectometry Using a Four-Wave Mixing Sampling Gate.

02,190

Optical Sampling Using Nondegenerate Four-Wave Mixing in a Semiconductor Laser Amplifier.

PB96-123609

04.348

\section{OPTICAL SCATTERING}

Optical Scattering from Moderately Rough Surfaces.
PB97-1 10415

OPTICAL SENSORS

Embossable Grating Couplers for Planar Waveguide Optical Sensors.
PB96-190277

00,641

OPTICAL STORAGE

Retinal-Protein Complexes as Optoelectronic Compo-

nents. 150397

02,146

OPTICAL SURFACES

Rapid Post-Polishing of Diamond-Turned Optics.

PB95-175949

04,301

PICAL SYSTEMS

Glasses for Waveguide Lasers.

PB96- 111950

Visualization of Surtace Figure by the Use of Zernike Polynomials.

Test of a Slow Off-Axis Parabola at its Center of Curvature. 138482

04,352

PTICAL TESTS

Gas Phase Reactions Relevant to Chemical Vapor Deposition: Optical Diagnostics.

OPTICAL THEOREMS

03,116

Generalized Optical Theorem for On-Axis Gaussian Beams.

\section{OPTICAL THERMOMETERS}

Self-Calibrated Intelligent Optical Sensors and Systems.
PB96-200738 OPTICAL TRACKING

Adaptive, Predictive 2-D Feature Tracking Algorithm for Finding the Focus of Expansion.

PB94-218575

01,588

Visual Pursuit Systems.

01,841

OPTICAL WAVEGUIDES

Modified Airy Function Method for the Analysis of Tunneling Problems in Optical Waveguides and Quantum Well

PB94-185824

02,120

Planar Waveg

03,586
Complex Propagation Constants for Nonuniform Optical Waveguides: Calculations.

PB95-125910

04,249

Modal Properties of Circular and Noncircular Optical Waveguides.

Approximate Solution to the Scalar Wave Equation for

Optical Waveguides.
PB95-126256 04,254

Bending-Induced Phase Shifts in Dual-Mode Planar Optical Waveguides.

PB95-161329 04,271

Mode Coupling and Loss on Tapered Optical

Waveguides.

04,282

Symbolic Programming with Series Expansions: Applications to Optical Waveguides.

PB95-168589

04,283

Improved Variational Analysis of Inhomogeneous Optical

Waveguides Using Airy Functions.

PB95-168639

04,285

Bending-Induced Loss in Dual-Mode Rectangular Waveguides.

PB95-168795 04,288

Bent Rectangular Core Waveguides: An Accurate Pertur-

bation Approach.

04,289

Waveguide Polarizers Processed by Localized Plasma

$\begin{array}{ll}\text { Etching. } & \\ \text { PB95-169264 } & 02,171\end{array}$

Modal Characteristics of Bent Dual Mode Planar Optical

Waveguide.

04,311

Using Secondary Ion Mass Spectrometry (SIMS) to Characterize Optical Waveguide Materials.

PB96-119599

04,340

Vector and Quasi-Vector Solutions for Optical Waveguide Modes Using Efficient Galerkin's Method with HermiteGauss Basis Functions.

OPTICS

Atomic Energy Levels. As Derived From the Analyses of Optical Spectra. Volume 3.

04,357

AD-A280 279/1

00,714

Japan Technology Program Assessment: Precision Engineering/Precision Optics in Japan.

PB95-171112

02,884

Fabrication of Optics by Diamond Turning.

02,954

Sott X-ray Reflectometry Program at the National Institute of Standards and Technology.

PB96-160395

04,368

OPTICSINON-LINEAR OPTICS

Corrected Optical Pyrometer Readings

AD-A279 949/2

Characterization of a Clipped Gaussian Beam.

PB96-102553

02,615

Planar Lenses for Field-Emitter Arrays

PB96-103064

04,331

PB96-113345

02,389

Optical Diagnostics in the Gaseous Electronics Conference Reference Cell.

PB96-113352

02,390

Millimeter-Resolution Optical Time-Domain Reflectometry

Using a Four-Wave Mixing Sampling Gate.

PB96-122700

02,190

Growth Characteristics of Fiber Gratings.

04,346

Optical Sampling Using Nondegenerate Four-Wave Mixing in a Semiconductor Laser Amplifier.

PB96-123609

04,348

Atom-Optical Properties of a Standing-Wave Light Field.

PB96-141072 04,356

Vector and Quasi-Vector Solutions for Optical Waveguide Modes Using Efficient Galerkin's Method with HermiteGauss Basis Functions.

PB96-141197

04,357

Theory of the Magneto-Optic Kerr Effect in the Near

Field.

04,761

Optical Fiber, Fiber Coating, and Connector Ferrule Geometry: Results of Interlaboratory Measurement Compari-

sons.

PB96-154422

04,360

Improved Dose Metrology in Optical Lithography.

PB96-179510

02,439

Test Optics Error Removal

04,377

Embossable Grating Couplers for Planar Waveguide Optical Sensors.

PB96-190277

00,641

Developing Quality System Documentation Based on ANSI/NCSL 2540-1-1994: The Optical Technology Division's Eftort.

01,869

Technical Digest: Symposium on Optical Fiber Measurements (9th), 1996. Held in Boulder, Colorado on October $1-3,1996$

PB97-108583

04,383

Optical and Modeling Studies of Sodium/Halide Reactions for the Formation of Titanium and Boron Nanoparticles. PB97-11305

00,682
aussian

Generalized Optical Theorem for On-Axis Gaussian

PB97-122345

04,177

OPTIMAL CONTROL

Optimal Control of Building and HVAC Systems.

PB96-141353

00,272

Modified Optimal Algorithm for Active Structural Control. PB96-165949

OPTIMIZATION

Convergence Properties of a Class of Rank-Two Updates. (NIST Reprint)

PB95-180097

03,430

OPTOELECTRONIC DEVICES

Photonic Materials: A Report on the Results of a Workshop. Held in Gaithersburg, Maryland on August 26-27. 1992, Volume 1

02,114

Electronics and Electrical Engineering Laboratory Technical Progress Bulletin Covering Programs, October to December 1993, with 1994/1995 EEEL Events Calendar. PB94-154341

02,115

Metrology for Electromagnetic Technology: A Bibliography of NIST Publications.

02,143

Metrology and Data for Microelectronic Packaging and Interconnection: Results of a Joint Workshop on Materials Metrology and Data for Commercial Electrical and Optical Gackaging and Interconnection Technologies. Held in suith

Sults.

02,328

Electronics and Electrical Engineering Laboratory Technical Progress Bulletin Covering Laboratory Programs, Aprilar.

endar.

02,329

Metrology and Data for Microelectronic Packaging and Interconnection: Results of a Joint Workshop on Materials Metrology and Data for Commercial Electrical and Optical Packaging and Interconnection Technologies. Held in
Gaithersburg, Maryland on May 5-6, 1994. Volume 2. Presentation Material

PB95-143327

02,330

PB95-202909

Optoelectronics at NIST.

PB96-200860

OPTOELECTRONICS

Opportunities for Innovation: Optoelectronics.

PB96-118039

Optoelectronics and Optomechanics Manufacturing: An ATP Focused Program Development. Workshop Proceedings. Held in Gaithersburg, Maryland on February 15, PB97-104186

02,204

Bibliography of the NIST Optoelectronics Division.

PB97-116040

02,207

ORDER-DISORDER TRANSFORMATIONS 
ORGANIC IONS

Temperature Dependence of the Rate Constants for Reactions of the Carbonate Radical with Organic and Inorganic Reductants.

ORGANIC LIQUIDS

00,831

Glass Transition of Organic Liquids Confined to Small

PB94-212305

00,833

Melting Behavior of Organic Materials Confined in Porous Solids

00,834

Vitrification and Crystallization of Organic Liquids Confined to Nanoscale Pores. fined to Nanoscal
PB97-112304

ORGANIZATIONS

Directory of Law Enforcement and Criminal Justice Associations and Research Centers.

PB96-178918 04,872

Standards Activities of Organizations in the United States. PB97-124135 00,006

ORGANOMETALLIC COMPOUNDS

Liquid Chromatography: Laser-Enhanced Ionization Spectrometry for the Speciation of Organolead Compounds.

PB94-185253 00,530

Maximum Entropy as a Tool for the Determination of the C-Axis Profile of Layered Compounds.

PB94-199619

00,800

Total Surface Areas of Group IVA Organometaltic Compounds: Predictors of Toxicity to Algae and Bacteria.
PB94-211331 Cambridge Structural Database (CSD): Current Activities

and Future Plans
PB97-109052

00,516

ORIENTATION

Fluorescence Anisotropy Measurements on a Polymer Melt as a Function of Applied Shear Stress.

01.209

Observations of Shear Induced Molecular Orientation in a Polymer Melt Using Fluorescence Anisotropy Measure-

PB94-199304 01,210

Observations of Shear Stress and Molecular Orientation Using Fluorescence Anisotropy Measurements. PB94-199312

01,211

Neutron Scattering Study of the Orientation of a Liquid Crystalline Polymer by Shear Flow.

ORIENTATIONAL DISORDER

Orientational Fluctuations, Diffuse Scattering, and Orientational Order in Solid C60.

PB97-119275

04,176

ORIFICE FLOW

Flow Conditioner Tests for Three Orifice Flowmeter Sizes

04,201

Flowmeter Installation Effects Due to a Generic Header.

Flowmeter Installation Effects Due to a Generic Header.
02,606

ORIFICE METERS

Comparing NIST.B $50 \mathrm{~mm}$ Orifice Meter Gas Data to the ANSI Equation.

02,949

Sliding Vane Flow Conditioner Tests in a 100 Diameter Long 10 inch Natural Gas Orifice Meter at Pacific Gas and Electric. Topical Report, 1990-1992.

PB95-256335

02,493

ORIFICE PULSE TUBE REFRIGERATORS

Energy Flows in an Orifice Pulse Tube Refrigerator.
PB95-169082

ORIGIN OF REPLICATION

Function of DnaJ and DnaK as Chaperones in OriginSpecific DNA Binding by RepA.

03,533

Deletion Analysis of the Mini-P1 Plasmid Origin of Replication and the Role of E.coli DnaA Protein.

PB95-163911

03,539

ORION NEBULA

Extended $\mathrm{CO}(7$ yields 6) Emission from Warm Gas in

PB96-102504

00,090

Magnetic Fields in Star-Forming Regions: Observations. PB96-123005

00,100

ORTHOPEDIC EQUIPMENT

Program Requirements to Advance the Technology of Custom Footwear Manufacturing.

PB95-147906

02,883

ORTHOPHOSPHACIDIUM ION

Crystal Structure of a New Monoclinic Form of Potassium Dihydrogen Phosphate Containing Orthophosphacidium Ion, (H4PO4)(sup+1)

PB96-111794

ORTHORHOMBIC LATTICES

Transformation of BCC and B2 High Temperature Phases to HCP and Orthorhombic Structures in the Ti-Al-Nb Sys-
tem. Part 1. Microstructural Predictions Based on a Subgroup Relation between Phases.

03,370
Transformation of $\mathrm{BCC}$ and $\mathrm{B} 2$ High Temperature Phases to HCP and Orthorhombic Structures in the Ti-Al-Nb Sys(em. Part 2. Experimental TEM Study of Microstructures. (em. Part 2. Experimental TEM Study of Microstructures.
PB96-169073

\section{OSCILLATING FLOW}

Resistance Thermometers with Fast Response for Use in Rapidly Oscillating Gas Flows.

PB95-107298

03,261

Thermal Anemonetry for Mass Flow Measurement in Oscillating Cryogenic Gas Flows.

PB96-176789

04,218

OSCILLATIONS

Foias-Temam Approximations of Attractors for Galloping

$\begin{array}{ll}\text { Oscillators. } & 04,817\end{array}$

High-Temperature High-Pressure Oscillating Tube Densimeter.

01,123

Ouadratic Response of a Chemical Reaction to Extemal Oscillations.

SCILLATOR STRENGTH

Theory for Ouantum-Dot Quantum Wells: Pair Correlation and Internal Ouantum Confinement in

PB96-179445

02,437

OSCILLATOR STRENGTHS

Oscillator Strengths and Radiative Branching Ratios in PB95-203493

04,008

OSCILLATORS

Precision Oscillators: Dependence of Frequency on Temperature. Humidity and Pressure.

02,031

Algebraic Approximation of Attractors for Galloping Oscillator

PB95-162897

04,820

Environmental Sensitivities of Ouartz Oscillators.

PB96-103148

02,271

Noise Modeling and Reliability of Behavior Prediction for Multi-Stable Hydroelastic Systems.

PB96-111943

04,822

Local Oscillator Requirements and Strategies for the Next Generation of High-Stability Frequency Standards.
PB96-112230

01,551

Reducing Errors, Complexity, and Measurement Time of PM Noise Measurements.

02,075

Chaotic Motions of Coupled Galloping Oscillators and Their Modeling as Diffusion Progresses. PB96-122718

04,823

OSE

Porting Multimedia Applications to the Open System Envi-

PB94-17292

02,721

OSE (OPEN SYSTEM ENVIRONMENT)

Guide on Open System Environment (OSE) Procure-

ments.

01,626

Comparison of POSIX Open System Environment (OSE) and Open Distributed Processing (ODP) Reference Models.

OSI PB96-131495

01,820

Formal Multi-Layer Test Methodology and Its Application

to OSI.

02,718

ISO/IEC Workshop on Worldwide Recognition of OS Test Results Regional Progress - North America. PB94-172202

02,719

OSI (OPEN SYSTEMS INTERCONNECTION)

GOSS-Industry/Government Open Systems SpecificaPB94-207453

01,806

Conformance Testing for OSI Protocols.

01,631

Lessons from the Establishment of the U.S. GOSIP Testing Program.

01,817

Open Issues in OSI Protocol Development and Conform-

ance Testing.
PB96-122908

01.818

OSMIUM

Analysis of the $(5 d(2)+5 d 6 s)-5 d 6 p$ Transition Arrays of O VII and Ir VIII, and the 6s (2)S-6p (2)P Transitions of II IX.

01,954

osMosis

Non-Perturbative Relation between the Mutual Diffusion Coefficient, Suspension Viscosity, and Osmotic Compressibility: Application to Concentrated Protein Solutions.

SMOTIC PRESSURE

Determination of Osmotic Pressure and Fouling Resistances and Their Effects on Performance of Ultrafiltration PB94-212891

00,841

OTAVITE

Thermodynamic Properties of Synthetic Otavite, $\mathrm{CdCO} 3(\mathrm{cr})$ : Enthalpy Increment Measurements from 4.5 K to $350 \mathrm{~K}$.
PB97.111447

00,680

OUT-OF-PLANE

Contributions of Out-of-Plane Material to a ScannedBeam Laminography Image.

02,704

OUTLIERS (STATISTICS)

Outlier-Resistant Methods for Estimation and Model Fit-

ting.

03,444

OVERCURRENT

Surging the Upside-Down House: Measurements and Modeling Results.

PB96-180138

02,243

OVERLAYS

Application of the Modified Voltage-Dividing Potentiom-

eter to Overlay Metrology in a CMOS/Bulk Process.
PB94-181997

Electrical Test Structure for Overlay Metrology Referenced to Absolute Length Standards.

02,336

New Test Structure for Nanometer-Level Overlay and Feature-Placement Metrology

PB95-175345

02,363

Microelectronic Test Structures for Overlay Metrology. PB96-164249

OVERVOLTAGE

Keeping Up with the Reality of Today's Surge Environ-

PB96-123633

02,231

OXAZOLE ORANGE

Enhanced Detection of PCR Products Through Use of TOTO and YOYO Intercalating Dyes with Laser Induced Fluorescence - Capillary Electrophoresis.

PB95-164653

00,599

OXIDATION

Oxidation of 10-Methylacridan, a Synthetic Analogue of NADH and Deprotonation of Its Cation Radical. Convergent Application of Laser Flash Photolysis and Direct and Redox Catalyzed Electrochemistry to the Kinetics of Deprotonation of the Cation Radical.

00,785

$\mathrm{X}$-Ray Photoelectron and Auger Electron Spectroscopy Study of Ultraviolet/Ozone Oxidized P2S5/(NH4)2S Treated GaAs $(100)$ Surfaces.

PB94-200144

04,479

Changes in the Redox State of Iridium Oxide Clusters and Their Relation to Catalytic Water Oxidation: Radiolytic and Electrochemical Studies.

PB95-107017

00,864

One-Electron Oxidation of Nickel Porphyrins. Effect of Structure and Medium on Formation of Nickel(III) Porphyrin or Nickel(II) Porphyrin pi-Radical Cation.

PB95-107058

00,865

Metalioporphyrin Sensitized Photooxidation of Water to Oxygen on the Surface of Colloidal Iridium Oxides - Photochemical and Pulse Radiolytic Studies.

PB95-107082

00,868

Oxidation of Soot and Carbon Monoxide in Hydrocarbon Ditfusion Flames.

PB95-150215

01,382

Neutron Reflectometry Studies of Surface Oxidation.

PB95-150421

00,917

Laser-Induced Fluorescence Measurements of $\mathrm{OH}$. Concentrations in the Oxidation Region of Laminar, Hydrocarbon Diffusion Flames.

PB95-162160 
Optimization of ECR-Based PECVD Oxide Films for Superconducting Integrated Circuit Fabrication. 02,051 Effects of Soxhlet Extraction on the Surface Oxide Layer of Silicon Nitride Powders. of Silicon Nitride

03,057

OXIDE REMOVAL

Effect of Annealing Ambient on the Removal of Oxide Precipitates in High-Dose Oxygen Implanted Silicon.
PB95-164356

OXIDES

High Temperature Reactions of Uranium Dioxide with Various Metal Oxides.

00,717

Description of Layered Structures: Applications to High Tc Superconductors
PB95-162624

04,615

New Oxide Degradation Mechanism for Stresses in the Fowler-Nordheim Tunneling Regime.

PB96-200985

04,128

New Physics-Based Model for Time-Dependent- Dielectric-Breakdown.

02,448

OXYGEN

Conductance Response of Pd/SnO2(110) Model Gas Sensors to $\mathrm{H}_{2}$ and $\mathrm{O} 2$

PB95.125803

00,892

Product State Correlations in the Reaction of $O((1) D)$ and $\mathrm{H} 2 \mathrm{O}$ in Bimolecular Collisions and in O3. $\mathrm{H} 2 \mathrm{O}$ Clusters-Translation.

00,946

Development of a Standard Reference Material for Measurement of Interstitial Oxygen Concentration in Semiconductor Silicon by Infrared Absorption

02,404

Measurement of $\mathrm{S}_{2} \mathrm{OF} 10$, and $\mathrm{S} 2 \mathrm{O} 2 \mathrm{~F}_{10}$ Production Measurement of $\mathrm{S} 2 \mathrm{OF} 10$, and $\mathrm{S} 2 \mathrm{O} 2 \mathrm{~F} 10$ Production
Rates from Spark and Negative Glow Corona Discharge in $\mathrm{SF} 6 / \mathrm{O} 2 \mathrm{Gas}$ Mixtures.

01,093

OXYGEN 17

Laser Magnetic-Resonance Measurement of the (3)P1 (3)P2 Fine-Structure Splittings in (17)O and (18)O.
PB95-175170

00,994

OXYGEN 18

Laser Magnetic-Resonance Measurement of the (3)P1 -

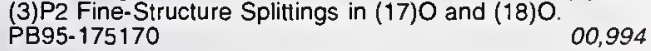
OXYGEN ATOMS

Atomic Oxygen Fine Structure Splittings with Tunable Far Infrared Spectroscopy.

03,915

Laser Flash Photolysis/Time-Resolved FTIR Emission Study of a New Channel in the Reaction of $\mathrm{CH} 3+\mathrm{O}$ : Production of $\mathrm{CO}(\mathrm{v})$ Laser Magnetic-Resonance Measurement of the (3)P1 (3) P2 Fine-Structure Splittings in (17)O and (18)O. PB95-175170

00,994

OXYGEN COMPATIBILITY

Reaction Sensitivities of Al-Li Alloys and Alloy 2219 in PB94-172764

OXYGEN CONCENTRATION

Standard Reference Materials: Certification of a Standard $\quad$ Measuring Performance of Parallel Computers. Final ReReference Material for the Determination of Interstitial Oxygen Concentration in Semiconductor Silicon by Infrared Spectrophotometry.
PB95-125076

OXYGEN IONS Determination of Atomic Data Pertinent to the Fusion En-
ergy Program. Progress Report for FY 92 .

04,402

\section{OXYGEN ISOTOPES}

New IUPAC Guidelines for the Reporting of Stable Hydrogen, Carbon, and Oxygen Isotope-Ratio Data. Letter to the Editor.

OXYGEN-LIKE IONS

Atomic Branching Ratio Data for Oxygen-Like Species.

OZONE

Product State Correlations in the Reaction of $O((1) D)$ and $\mathrm{H} 2 \mathrm{O}$ in Bimolecular Collisions and in O3. $\mathrm{H} 2 \mathrm{O}$ ClustersTranslation.

00,946

OZONE DEPLETION

Thermodynamic Properties of Gas Phase Species of $1 \mathrm{~m}$. portance to Ozone Depletion.

INVARIANCE

Long-Range Parity-Nonconserving Interaction
PB95-202594

00,126

N JUNCTIONS

03,986

Junction Locations by Scanning Tunneling Microscopy: In-Air-Ambient Investigation of Passivated GaAs pn Junctions.

02,306

PACKAGING

Performance of Plastic Packaging for Hazardous Mate-

rials Transportation. Part 1. Mechanical Properties.
AD-A301 258/0
Checking the Net Contents of Packaged Goods as Adopted by the 79th National Conference on Weights and Measures, 1994, Third Edition, Supplement 4.

00,484

High-Speed Interconnection Characterization Using Time Domain Network Analysis.

ACKED BEDS

Distributed measurements of tracer response on packed bed flows using a fiberoptic probe array. Final report.

DE95013079

00,667

PACKING MATERIALS

Volatile Corrosion Inhibitors.

AD-A310 087/2

03,114

AFFENBARGER RESEARCH CENTER

Patfenbarger Research Center: The Cutting Edge of Dental Science.
PB94-216355

00,151

PALLADIUM

Magnetic Properties of $\mathrm{Pd} / \mathrm{Co}$ Multilayers.

PB9 4-198751

04,465

Conductance Response of $\mathrm{Pd} / \mathrm{SnO} 2(110)$ Model Gas

00,892

Fundamental Studies of Gas Sensor Response Mechanisms: Palladium on $\mathrm{SnO} 2(110)$.

00,963

Small Angle Neutrons Scattering from Nanocrystalline Palladium as a Function of Annealing

PB95-176103

03,354

Vibrational Excitations and the Position of Hydrogen in Nanocrystalline Palladium.

$\begin{array}{ll}\text { PB96-111828 } & 04,035\end{array}$

Inelastic Neutron Scattering Study of Hydrogen in Nanocrystalline Pd. PB96-146857

03,366

ALLADIUM ALLOYS

New Alloys Show Extraordinary Resistance to Fracture and 15134

03,346

PALLADIUM IONS

Rb-Like Spectra: Pd X to Nd XXIV.

PB95-150645

03,897

PALLADIUM-LIKE IONS

Observation of Pd-Like Resonance Lines Through $\mathrm{Pt}(32+)$ and $\mathrm{Zn}$-Like Resonance Lines of $\mathrm{Er}(38+)$ and PB95-150637

03,896

PANCREATIC RIBONUCLEASE

Crystal Packing Interactions of Two Different Crystal Forms of Bovine Ribonuclease A.
PB95-152823

PANELS

Test Procedures for Advanced Insulation Panels PB97-111892

00,415

PARAFFIN HYDROCARBONS

Physical Properties of Some Purified Aliphatic Hydro carbons.

00,657

Measuring Performance of Parallel Computers. Progress

Report, 1989

DE94014587

01,666

Distributed Supercomputing Software: Experiences with

PB94-163086

01,680

Using Synthetic Perturbations and Statistical Screening to Assay Shared-Memory Programs.

PB96-103031

01,740 Parallel Monte Carlo Simulation of MBE Growth.
PB96-122841

02,406

Experience with MPI: 'Converting Pvmmake to Mpimake under LAM' and

01,748

Using S-Check, Alpha Release 1.0

01,767

PB96-16596

Find This

Tree-Lookup

PBg6-179411

Making Connections

PB97-119044

01,770

PALLEL PROCESSING (COMPUTERS)

Scalability Test for Parallel Code.

PB96-146758

01,785

ARALLEL PROGRAMMING bation Screenings.

PB94-139623

01,675

Time-Perturbation Tuning of MIMD Programs. PB94-164399

01,681

Using Synthetic-Perturbation Techniques for Tuning Shared Memory Programs (Extended Abstract). PB94-172657

01,685 Sensors to $\mathrm{H}_{2}$ and $\mathrm{O} 2$

AD-A297 265/9

Measuring Performance of Parallel Computers. Final Re
port. the Parallel Virtual Machine - PVM

Computing Effects and Error for Large Synthetic Pertur-

Synthetic-Perturbation Tuning of MIMD Programs. PB94-185568

Simple Scalability Test for MIMD Code.

01,688

PARALLEL VIRTUAL MACHINE

Pertormance Characteristics of Fast Elliptic Solvers on Parallel Platforms.

PB95-180832

01,723

RAMETERS

Taguchi's Parameter Design: A Panel Discussion.

03,445

PARAMETRIC PROCESSES

High-Order Multipole Excitation of a Bound Electron. PB96-119789

PARTY

Long-Range Parity-Nonconserving Interaction.

PB95-202594

03,986

PARKING GARAGES

Diagnosis of Causes of Concrete Deterioration in the MLP-7A Parking Garage.

PARSERS

SGML Parser Validation Procedures

PB95-174959

01,318 uation Report

PARTIAL DIFFERENTIAL EQUATIONS

Overcoming Hoelder Continuity in III-Posed Continuation Problems.

PB95-202354

03,416

PARTIAL DISCHARGE

Behavior of Surface Partial Discharge on Aluminum Oxide Dielectrics.

PARTIAL DISCHARGES

Performance Evaluation of a New Digital Partial Discharge Recording and Analysis System.

PB95-150389

01,889

Recent Developments at NIST on Optical Current Sensors and Partial Discharge Diagnostics.

02,147

Observations of Partial Discharges in Hexane Under High

Magnification.
PB95-163127

01,900

Importance of Unraveling Memory Propagation Effects in Interpreting Data on Partial Discharge Statistics.

PB95-163572

01,901

Nonstationary Behavior of Partial Discharge during Insulation Aging.
PB95-163580

01,902

Partial Discharge: Induced Aging of Cast Epoxies and Related Nonstationary Behavior of the Discharge Statis-

01,903

Physics and Chemistry of Partial Discharge and Corona: Recent Advances and Future Challenges

PB95-181046

01,910

Continuous Recording and Stochastic Analysis of PD

PB96-112156

01,925

Standardised Computer Data File Format for Storage, Transport, and Off-Line Processing of Partial Discharge

PB96-122486

01,930

Physics and Chemistry of Partial Discharge and Corona Recent Advances and Future Challenges.

PB96-123757

01,936

PATIAL PRESSURE 
Simulation of Ceramic Particle Formation: Comparison with In-situ Measurements.

00,674

\section{PARTICLE SHAPE}

Morphological Development of Second-Phase Particles in Elasticaliy-Stressed Solids.

PB95-181111

03,355

PARTICLE SIZE

Particle Size Standards and Their Certification at NIST. PB94-211695 02,639 Development of Adaptive Control Strategies for Inert Gas Atomization.

PB95-162335

02,823

Process Modeling and Control of Inert Gas Atomization. PB95-162343

02,824

PARTICLE SIZE DISTRIBUTION

Experimental Optimization of Peak Shape with Application to Aerosol Generation.

PB94-185535

00,501

In-situ Fume Particle Size and Number Density Measurements from a Synthetic Smelt.

03,334

Statistical Analysis of Parameters Affecting the Measurement of Particle-Size Distribution of Silicon Nitride Powders by Sedigraph (Trade Name).

PB94-216249

03,042

Parametric Investigation of Metal Powder Atomization Using Laser Diffraction.

PB95-108577

03,342

Modeling Ceramic Sub-Micron Particle Formation from the Vapor Using Detailed Chemical Kinetics: Comparison

with In-situ Laser Diagnostics.

00,671

PARTICLES

Airborne Asbestos Method: Standard Test Method for High Precision Counting of Asbestos Collected on Filters. Version 1.0 .

PB94-163003

00,525

Environmental Scanning Electron Microscope Imaging Examples Related to Particle Analysis.

PB94-172822 00,766

Generation and Characterization of Acetylene Smokes.

PB94-200292 01,372

Characterization of Chemically Modified Pore Surfaces by

Small Angle Neutron Scattering. $\quad 00,898$

Relativistic Quantum Mechanics of Interacting Particles. PB97-110589 04,141
0

In-situ Studies of a Novel Sodium Flame Process for Synthesis of Fine Particles.

PB97-113047

00,681

PARTICULATE REINFORCED COMPOSITES

Residual Stresses in Aluminum-Mullite (alpha-Alumina) Composites.

$\begin{array}{ll}\text { PB95-152880 03,155 } & \end{array}$

Elastic Properties of $\mathrm{Al} 2 \mathrm{O} 3 / \mathrm{Al}$ Composites: Measurements and Modeling.

PB95-161378

03,157

Fabrication of Flaw-Tolerant Aluminum-Titanate-Reinforced Alumina

03,161

PARTICULATES

Electroacoustics for Characterization of Particulates and Suspensions. Proceedings of a Workshop. Held in Gaithersburg, Maryland on February 3-4, 1993. PB94-112695 00,725 Rare-Earth Isotopes as Tracers of Particulate Emissions An Urban Scale Test.

PB94-161635

02,535

Computer Simulations of Binder Removal from 2-D and 3.D Model Particulate Bodies.

PB97-121339

00,418

PASCAL PROGRAMMING LANGUAGE

Validated Products List (Cobol, Fortran, ADA, Pascal, MUMPS, SQL)

01,700

Validated Products List (Cobol, Fortran, ADA, Pascal, MUMPS, SQL). MUMPS, SOL

01,738

PASSENGERS

Fire Safety of Passenger Trains: A Review of Current Approaches and of New Concepts.

PB94-152006

04,848

PASSIVATION

Heavily Accumulated Surfaces of Mercury Cadmium Teluride Detectors: Theory and Experiment. PB94-216074

PASSIVE CONTROL

02,134

Method of Estimating the Parameters of Tuned Mass Dampers for Seismic Applications.

PB96-167820

00,473

PATHS

Time Dependent Vector Dynamic Programming Algorithm for the Path Planning Problem.

PB94-215688

03,428

PATTERN RECOGNITION

Face Recognition Technology for Law Enforcement Applications.

01.837
Pattern-Recognition Analysis of Low-Resolution X-Ray Fluorescence Spectra.

00,588

PCASYS: A Pattern-Level Classification Automation System for Fingerprints.

PB95-267936 01,853

Human and Machine Recognition of Faces: A Survey.

Using Logic to Specify Shapes and Spatial Relations in Design Grammars.
PB97-111579

PAVERS

Warping of Terrace Pavers at the U.S. Capitol Building

PDES (PRODUCT DATA EXCHANGE SPECIFICATION)

National PDES Testbed: An Overview.

PB95-125829

02,775

Capabilities for Product Data Exchange.

02,798

PDES (PRODUCT DATA EXCHANGE USING STEP)

Security Considerations for SQL-Based Implementations

PB94-139649

02,766

Guide to Configuration Management and the Revision

Control System for Testbed Users.

PB94-150919 01,678

Summary and Notes of the Joint ISO/IGES/PDES Organi-

zation Technical Committee Meeting. Held in Albuquer-

que, New Mexico on October 15-20, 1989

02,774

Group 1 for the Process Engineering Data STEP Applica-

tion Protocol.

02,797

PEAKS OVER THRESHOLD METHOD

Estimates of Hurricane Wind Speeds by the 'Peaks Over

Threshold' Method

PB96-162540

00,471

Probabilistic Estimates of Design Load Factors for WindSensitive Structures Using the 'Peaks Over Threshold' Approach.

PEAKS OVER THRESHOLD METHODS

Extreme Wind Distribution Tails: A 'Peaks Over Threshold' Approach
PB95-219416

PECULIAR STARS

Wavelengths and Isotope Shifts for Lines of Astrophysica Interest in the Spectrum of Doubly lonized Mercury $(\mathrm{Hg}$ III).

PB95-140869

03,887

Search for Radio Emission from the 'Non-Magnetic' Chemically Peculiar Stars.

PB96-102249

00,087

X-ray Emission

00,088

A-type and Chemically Peculiar Stars.

PB96-123286

00,101

Radio and $X$-ray Emissions from Chemically Peculiar Band A-Type Stars: Observations and a Model.

00,103

PEEL STRENGTH

Effects of Adhesive Thickness, Open Time, and Surface Cleanness on the Peel Strength of Adhesive-Bonded Seams of EPDM Rubber Roofing Membrane.

00.372

PELTIER EFFECTS

NBS/NIST Peltier-Effect Microcalorimeter: A Four-Decade Review.

PB96-102769

01,064

PENDULUM IMPACT MACHINES

Proposed Changes to Charpy V-Notch Machine Certification Requirements.

02,955

PENDULUMS

Chaos in a Computer-Animated Pendulum.

PB94-212651

03,852

PENICILLIN G

Thermodynamics of the Hydrolysis of Penicillin G and Ampicillin.

03,596

PENS

Stylus Technique for the Direct Verification of Rockwell Diamond Intenders.

PB96-155569

02,958

PENTAFLUOROANILINE

Fluoride Elimination Upon Reaction of Pentafluoroaniline with e (sub eq')(sup -), $\mathrm{H}$, and $\mathrm{OH}$ Radicals in Aqueous

PB97-111314

01,177

PENTAFLUORODIMETHYL ETHER

Vapor Pressure of Pentafluorodimethyl Ether. PB96-201199

00,677

\section{PENTAFLUOROETHANE}

Viscosity of the Saturated Liquid Phase of Six Halogenated Compounds and Three Mixtures.

00,960 Measurements of the Vapor Pressures of
Difluoromethane, 1-Chloro-1,2,2,2-Tetrafluoroethane, and Pentafluoroethane.

Pentafluoroethan

03,270

Vapour Pressures and Gas-Phase ( $p$, rho $n, T)$ Values for CF3CHF2(R125)

PENTAFLUOROPROPANE

04,019

Vapor Pressure of 1,1,1,2,2-Pentafluoropropane.

PB97-113237 00,684

Thermodynamic Properties of CHF2-CF2-CHF, 1,1,2,2,3. Pentafluoropropane

PB97-118392

03,300

PENTANES

Critical Properties and Vapor-Liquid Equilibria of the Binary System Propane + Neopentane.

PEPERS

Preliminary Investigation of Oleoresin Capsicum PB96-179486

00,999

PETIDES

Glucose Permease of Bacillus Subtilis is a Single Polypeptide Chain That Functions to Energize the Sucrose Permease.

PB95-163192

03,466

Incinerability of Perchloroethylene and Chlorobenzene PB95-163457

Hydrogen Atom Attack on Perchloroethylene.

PB95-163473

01,388

PERCOLATION

Percolation and Pore Structure in Mortars and Concrete. PB95-150439 00,370 Geometrical Percolation Threshold of Overlapping Ellipsoids.

PB96-102397

03,167

PERFORMANCE EVALUATION

Configuration and Performance Evaluation of a Real-Time Robot Control System: A Skeleton Approach.

PB95- 163895

01,598

Performance of Compact Fluorescent Lamps at Different Ambient Temperatures.

00,258

Interim Testing Artifact (ITA): A Performance Evaluation System for Coordinate Measuring Machines (CMMs). User Manual.

PB95-210589

02,914

Performance

PB95-220521

02,505

4SIGHT Manual: A Computer Program for Modelling Degradation of Underground Low Level Waste Concrete

PB95-231593

03,726

NIST SRM 9983 High-Rigidity Ball-Bar Stand. User Manual.

PB95-255840 02,669

Factors Affecting the Energy Consumption of Two Refrig erator-Freezers

PB97-112312 00,311

Survey of the Components of Display-Measurement Standards.

PB97-122394

02,209

PERFORMANCE MEASUREMENT

Display-Measurement Round-Robin.

PB96-119227 
Joumal of Research of the National Institute of Standards and Technology, March/April 1995. Volume 100, Number PB96-126180

NIST Serial Holdings, 1996

04,349

PB96-172523

02,748

PERMALLOYS (TRADEMARK)

Telegraph Noise in Silver-Permalloy Giant Magnetoresistance Test Structures.
PB96-146717

04,763

PERMANENT DENTAL RESTORATION

Properties and Interactions of Oral Structures and Restorative Materials. Annual Report for Period October 1, 1990 to September 30, 1991

PB94-160843

03,558

PERMEABILITY

Controlling Moisture in the Walls of Manufactured Housing.

00,355

Cross-Property Relations and Permeability Estimation in Model Porous Media.

PB95-150280

04,205

Calculation of the Thermal Conductivity and Gas Permeability in a Uniaxial Bundle of Fibers.

PB95-180931

03,058

Water-Vapor Measurements of Low-Slope Roofing Materials.

PB95-251617

00,399

PERMEATION

$\mathrm{CO} 2 / \mathrm{CH} 4$ Transport in Polyperfluorosulfonate lonomers: Effects of Polar Solvents on Permeation and Solubility PB96-163803 of 145

PERMEATION LEAKS

Commercial Helium Permeation Leak Standards: Their Properties and Reliability

PB97-111413

04,146

PERMITTIVITY

Transmission/Reflection and Short-Circuit Line Methods for Measuring Permittivity and Permeability.

02,211

Distribution of Dielectric Relaxation Times and the Moment Problem

04,727

PERMITTIVITY MEASUREMENTS

NIST 60-Millimeter Diameter Cylindrical Cavity Resonator: Performance Evaluation for Permittivity Measurements. PB94-151776

PEROXIDASES

Potentiometric Enzyme-Amplified Flow Injection Analysis Detection System: Behavior of Free and Liposome-Released Peroxidase

03,534

PEROXY RADICALS

Kinetics of the Self-Reaction of Hydroxymethylperoxyl PB94-212164

00,827

PEROXYL RADICALS

Solvent Effects in the Reactions of Peroxyl Radicals with Organic Reductants. Evidence for Proton Transfer Mediated Electron Transfer.

00,873

Reaction of Nitric Oxide with Organic Peroxyl Radicals

PB95-141107 00,910

Oxidation of Ferrous and Ferrocyanide lons by Peroxyl Radicals

PEROXYNITRITE RADICALS

01,191 Reaction of NO with Superoxide.

PB94-212198

00,830

ERSISTENCE LENGTH

Electrostatic Rigidity of Polyelectrolytes from Reparametrization Invariance.

04,113

PERSONAL COMPUTERS

Vibration Laboratory Automation at NIST with Personal Computers.

Keeping Time on Your PC.

02,648

PB95-161410

01,537

Precise Identification of Computer Viruses.

01,613 PB96-160825

PERSONAL DOSE EQUIVALENT

Angular Variation of the Personal Dose Equivalent, $H p(0.07)$, for Beta Radiation and Nearly Monoenergetic Electron Beams: Preliminary Results.

PB95-168472

03,630

PERSONNEL DOSIMETRY

National Quality Assurance Program for Personnel Radiation Dosimetry: A Case History.

03,620

National Voluntary Laboratory Accreditation Program: Ionizing Radiation Dosimetry.

03,623

Interpreting the Readings of Multi-Element Personnel Dosimeters in Terms of the Personal Dose Equivalent. PBo5-175428

03,631

PESTICIDES

Determination of Oltipraz in Serum by High-Performanace Liquid Chromatography with Optical Absorbance and Mass Spectrometric Detection.

03,493
Comparison of Methods for Gas Chromatographic Determination of PCBs and Chlorinated Pesticides in Marine Reference Materials.

PB95-140091

02,584

NIST Standard Reference Materials (SRMs) for Polychlorinated Biphenyl (PCB) Determinations and Their Applicability to Toxaphene Measurements.

PB95-140109

02,585

Determination of PCBs and Chlorinated Hydrocarbons in Marine Mammal Tissues.

03,744

Certification of Polychlorinated Biphenyl Congeners and Chlorinated Pesticides in a Whale Blubber Standard Reterence Material.

PETROCHEMISTRY

Framework for Information Technology Integration in Process Plant and Related Industries.

02,772

PETROLEUM FRACTIONS

iscosity of Defined and Undefined Hydrocarbon Liquids Calculated Using an Extended Corresponding-States Model.

PB96-167234

02,498

PF RADICALS

Resonance Enhanced Multiphoton Ionization Spectros. copy of the PF Radical

PH

00,702

Calorimetric Determination of the Standard Transformed Enthalpy of a Biochemical Reaction at Specified PH and PMg.

03,454

PHARMACOLOGY

Modification of DNA Bases in Chromatin of Intact Target

Human Cells by Activated Human Polymorphonuclea

Leukocytes.

PB94-199833

03,526

Supercritical

00,040

PHASE DIAGRAMS

Effects of Elastic Stress on Phase Equilibrium in the Ni-V System

PB94-172707

03,313

Statistical Thermodynamics of Phase Separation and Ion

Partitioning in Aqueous Two-Phase Systems.

PB94-199387

Assessment of the Al-Sb System.

01,212

PB94-200474

03,329

Supercritical Solubility of Solids from Near-Critical DiluteMixture Theory.

PB94-211703

00,819

Inversion of the Phase Diagram from UCST to LCST in Deuterated Polybutadiene and Protonated Polybutadiene Blends.

PB94-212446

01,226

Crystal Chemistry and Phase Equilibria Studies of the BaO(BaCO3)-R2O3-CuO Systems. 4. Crystal Chemistry and Subsolidus Phase Relationship Studies of the CuORich Region of the Ternary Diagrams, $\mathrm{R}=$ Lanthanides.

PB95-151759

00,936

Phase Composition, Viscosities, and Densities for Aqueous Two-Phase Systems Composed of Polyethylene Gly col and Various Salts at $25 \mathrm{C}$

PB95-164596

00,986

Vapor-Liquid Equilibria of Mixtures of Propane and Isomeric Hexanes.
PB95-175287

00,995

Critical Properties and Vapor-Liquid Equilibria of the Binary System Propane + Neopentane.

nary System

00,999

Critical Lines for Type-III Aqueous Mixtures by Generalized Corresponding-States Models.

PB96-10237

01,063

Magnetic Dielectric Oxides: Subsolidus Phase Relations in the BaO: Fe2O3: $\mathrm{TiO}_{2}$ System.

PB96-176524

01,156

Multimedia Tutorial on Phase Equilibria Diagrams

PB96-200829

03,088

PHASE EQUILIBRIA

Multimedia Tutorial on Phase Equilibria Diagrams. PB96-200829

03,088

PHASE EQUILIBRIUM Design of a High-Pressure Ebulliometer, with Vapor-Liq-
uid Equilibrium Results for the Systems CHF2CI + CF3$\mathrm{CH}_{3}$ and $\mathrm{CF} 3-\mathrm{CH} 2 \mathrm{~F}+\mathrm{CH}_{2} \mathrm{~F} 2$

PB97-113229

04,163

PHASE ERROR

Effect of Harmonic Distortion on Phase Errors in Frequency Distributi

01,563

\section{PHASE FIELD MODELS}

xi-Vector Formulation of Anisotropic Phase-Field Models: 3-D Asymptotics.

PB95-136628

04,536

Phase-Field Model for Solidification of a Eutectic Alloy.

$\mathrm{Xi}$-Vector Formulation of Anisotropic Phase-Field Models:

3-D Asymptotics.

04,816

PHASE LOCKED SYSTEMS

Phase Locking in Two-Junction Systems of High-Temperature Superconductor-Normal Metal-Superconductor Junctions.

PB95-176053

02,060

Phase Locking in Two-Dimensional Arrays of Josephson Junctions: Effect of Critical-Current Nonuniformity. PB96-102587

PHASE LOCKING

Stable Phase Locking in a Two-Cell Ladder Array of Josephson Junctions.

PB96-111679

04,722

Mutual Phase Locking in Systems of High-T Superconductor-Nomal Metal-Superconductor Junctions.

PHASE MODULATION

Investigations of AM and PM Noise in X-Band Devices.

PB95-180022

02,062

Practical Standards for PM and AM Noise at 5, 10 and $100 \mathrm{MHz}$

PB95-181129 01,546

Secondary Standard for PM and AM Noise at 5, 10, and $100 \mathrm{MHz}$

PB96-123187

01,554

Origin of $1 / f \mathrm{PM}$ and AM Noise in Bipolar Junction Transistor Amplifiers.

Reducing the $1 /$ AM and PM Noise in Electronics for Precision Frequency Metrology.

PB97-113195

02,102

PSE MODULATORS

Ultralinear Small-Angle Phase Modulator.

PHASE NOISE

Delivering the Same Optical Frequency at Two Places: Accurate Cancellation of Phase Noise Introduced by an Optical Fiber or Other Time-Varying Path.

PB96-102736

04,332

Reducing Errors, Complexity, and Measurement Time of PM Noise Measurements.

02,075

Surface Transverse Wave Oscillators with Extremely Low Thermal Noise Floors.

PB96-186010

01,967

PHASE ORDERING

Evolution Equations for Phase Separation and Ordering in Binary Alloys.

02979

PHASE SEPARATION

Evolution Equations for Phase Separation and Ordering 
PHASE TRANSFORMATIONS

Phase Behavior of a Hydrogen Bonding Molecular Com-

Posite.

01,202

Effect of Transformation of Alloy on Transient and Residual Stresses in a Porcelain-Metal Strip.

PB94-198397

00,143

Phase-Field Mod

03,345

Phase Transitions in Solid C70: Supercooling, Metastable Phases, and Impurity Effect.

PB95-150090

00.914

Comment on 'Phase Transitions in Antiferromagnetic Superlattices'.

04.587

Small Angle Neutron Scattering Study on Poly(N-Isopropyl Acrylamide) Gels Near Their Volume-Phase Transition Temperature.

PB95-164380 01,263

New Data and Correlations for the Custody Transfer of Natural Gas Liquids.

PB96-176664

02,499

PHASE VELOCITY

Effect of Liftoff on Accuracu of Phase Velocity Measurements Made with Electromagnetic-Acoustic Transducers. PB96-186044

02,280

PHENOLS

Source of Phenol Emissions Affecting the Indoor Air of an Office Building.

03,600

Temperature Dependence of the Rate Constants for Reaction of Inorganic Radicals with Organic Reductants. PBg4-198280

Materials-Science Based Approach to Phenol Emissions from a Flooring Material in an Office Building. PB97-118749

02,572

PHENYLALANINE/N-ACETYL- (ETHYL-ESTER)

Thermodynamics of the Hydrolysis of N-Acetyl-Lphenylalanine Ethyl Ester in Water and in Organic Sol-

PB95-203386

01,053

\section{PHENYLBUTADIENE}

owest Excited Singlet State of Isolated 1-phenyl-1,3-butadiene and 1-phenyl-1,3,5-hexatriene.

PHENYLHEXATRIENE

Lowest Excited Singlet State of Isolated 1-phenyl-1,3-butadiene and 1-phenyl-1,3,5-hexatriene. PB95-202339

01,026

PHONETICS

Photonic Materials: A Report on the Results of a Workshop. Held in Gaithersburg, Maryland on August 26-27, 1992, Volume

02,114

PHONON DETECTORS

Hot-Electron Microcalorimeters for X-ray and Phonon Deection.

PB95-168993

04,644

Hot-Electron Microcalorimeters as High-Resolution X-ray Detectors.

04,739

Hot-Electron Microcalorimeter for X-ray Detection Using a Superconducting Transition Edge Sensor with Electrothermal Feedback.

PB96-200399

04.792

PHONON DISPERSION

High-Energy Phonon Dispersion in La1.85Sro.15CuO4.

PB96-138458

04.748

PHONON RESPONSE

Total-Dielectric-Function Approach to Electron and Phonon Response in Solids.

PB96-102884

01,067

PHONONS

Temperature Dependence and Anharmonicity of Phonons on Ni(110) and $\mathrm{Cu}(110)$ Using Molecular Dynamics Simulations.

PB94-185477 04.449

Anharmonic Phonons and the Isotope Effect in

Superconductivity.
PB94-200557 04,486

Phonon Density of States in $\mathrm{R}_{2} \mathrm{CuO} 4$ and

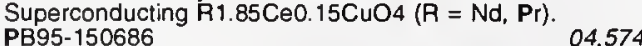
High Resolution Inelastic Neutron Scattering Study of Phonon Self-Energy Effects in YBCO

PB95-180881

04,688

Self-Biasing Cryogenic Particle Detector Utilizing Electrothermal Feedback and a SOUID Readout.

PB96-102538 04,712

Phonon Relaxation in Soft-X-ray Emission of Insulators. PB96-160296

04,085

PHOSPHATE INORGANIC COMPOUNDS

Crystal Structure of a New Monoclinic Form of Potassium Dihydrogen Phosphate Containing Orthophosphacidium lon, (H4PO4)(sup+1).

PHOSPHATES

Modification of Deoxyribose-Phosphate Residues by Extracts of Ataxia Telangiectasia Cells.

04,725 PB94-212602

03,458
Cyclic Polyamine Ionophore for Use in a Dibasic Phosphate-Selective Electrode.

01,008

PHOSPHOLIPIDS

Mixed Phospholipid Liposome Calcification.

PB94-211190

03,457

Self-Assembled Phospholipid/Alkanethiol Biomimetic Bilayers on Gold.

00,878

Supported Phospholipid/Alkanthiol Biomimetic Membranes: Insulating Properties.

03,470

Phospholipid/Alkanethiol Bilayers for Cell-Surtace Receptor Studies by Surface Plasmon Resonance.

03,472

\section{PHOSPHOPROTEINS}

Trace Elements Associated with Proteins. Neutron Activation Analysis Combined with Biological Isolation Techniques.

PB95-163101

00,597

PHOTOASSOCIATION

Nonadiabatic Effects in the Photoassociation of H2S.

PB95-151437

00,934

PHOTOCHEMICAL REACTIONS

Fragment Energy and Vector Correlations in the Overtone-Pumped Dissociation of HN3X(1)A'

00,802

Changes in the Redox State of Iridium Oxide Clusters and Their Relation to Catalytic Water Oxidation: Radiolytic and Electrochemical Studies.

PB95-107017

00,864

Bacteriorhodopsin Retains Its Light-Induced Proton-

Pumping Function After Being Heated to $140 \mathrm{C}$
PB96-102728

03,471

PHOTOCHEMISTRY

Application of Photochemical Reaction in Electrochemical Detection of D
PB94-185733

00,686

\section{PHOTODECOMPOSITION}

Photodecomposition of Mo(CO)6/Si(111) 7x7: CO StateResolved Evidence for Excited State Relaxation and Ouenching.

PHOTODESORPTION

Photodesorption Dynamics of CO from Si(111): The Role of Surtace Defects.

PB96-111646

03,066

PHOTODETACHMENT

Resonant Two-Color Detachment of $H(-)$ with Excitation of $\mathrm{H}(\mathrm{n}=2)$.

PB95-202552

03,984

Relative Photoionization and Photodetachment Cross Sections for Particular Fine-Structure Transitions with Application to $\mathrm{Cl}$ 3s-subshell Photoionization.

PB95-203097 03,998

Short-Pulse Detachment of $H(-)$ in the Presence of a Static Electric Field.

PB95-203477

04,007

PHOTODETECTORS

Accurate Characterization of High Speed Photodectors.
PB95-153763

Electrically Calibrated Pyroelectric Detector-Refinements for Improved Optical Power Measurements.

PB95-169066

02,164

Photodetector Frequency Response Measurements at NIST, US, and NPL, UK: Preliminary Results of a Standards Laboratory Comparison.

02,175

PHOTODIODES

One Gigarad Passivating Nitrided Oxides for $100 \%$ Inter-

nal Ouantum Efficiency Silicon Photodiodes.

02,119

Silicon Photodiodes Optimized for EUV and Soft X-Ray

Regions.
PB94-199478

02,124

Longterm Changes of Silicon Photodiodes and Their Use for Photometric Standardization.

PB94-211349 04,234

International Intercomparison of Detector Responsivity at 1300 and $1550 \mathrm{~nm}$.

PB95-126017

02,141

Accurate Characterization of High Speed Photodectors.

PB95-153763

02,153

Photodetector Frequency Pesponse Measurements at NIST, US, and NPL, UK: Preliminary Results of a Standards Laboratory Comparison.

02,175

Comparison of Photodiode Frequency Response MeasComparison of Photodiode Frequency Response Meas-
urements to $40 \mathrm{GHz}$ between NPL and NIST.
PB96-111992

04,038

Stable Silicon Photodiodes for Absolute Intensity Measurements in the VUV and Soft X-ray Regions.

PB97-110175

04,135

PHOTODISSOCIATION

Multichannel Ouantum Defect Half Collision Analysis of K2 Photodissociation Through the B1Pi(sub u) State.
PB94-211125

Asymptotic Wave Function Splitting Procedure for Propagating Spatially Extended Wave Functions: Application to Intense Field Photodissociation of $\mathrm{H} 2(+)$

03,847

Vibrational Predissociation Dynamics of Overtone-Excited VN3 PB95-125720 00,691 Nonadiabatic Effects in the Photoassociation of H2S. PB95-151437

00,934

Photodissociation of Ammonia at $193.3 \mathrm{~nm}$ : Rovibrational State Distribution of the $\mathrm{NH}$ (A(2)A 1) Fragment. PB95-151775

PHOTOELECTRON SPECTROSCOPY

Photoelectron Spectroscopy of Negatively Charged Bismuth Clusters: $\mathrm{Bi}(-) 2, \mathrm{Bi}(-) 3$, and $\mathrm{Bi}(-) 4$.

PB95-108494

00,880

High Resolution Angle Resolved Photoelectron Spectroscopy Study of N2.

03,907

Photoelectron Spectroscopy of Small Antimony Cluster Anions: $\mathrm{Sb}(-)$, Sb2(-), Sb3(-), and $\mathrm{Sb} 4(-)$

PB95-203139

01,045

PHOTOELECTRONS

Measuring Nondipolar Asymmetries of Photoelectron Angular Distributions

01,193

HOTOEMISSION

Resonant-Photoemission Investigation of the Heusler Aloys $\mathrm{Ni} 2 \mathrm{MnSb}$ and $\mathrm{NiMnSb}$.

PB95-162384

04,612

Influence of Coadsorbed Potassium on the Electron-Stimulated Desc

PB(0001).

PHOTOGRAPHIC ANALYSIS

Photographic Response to X-Ray Irradiation. 1. Estimation of the Pholographic Error Statistic and Development of Analytic Density-Intensity Equations.

PB94-200086

03,821

Photographic Response to X-Ray Irradiation. 2. Correlated Models.

03,822

Photographic Response to X-Ray Irradiation. 3. Photographic Linearization of Beam-Foil Spectra. PB94-200102

03,823

\section{PHOTOGRAPHIC IMAGES}

Human and Machine Recognition of Faces: A Survey.

HOTOIONIZATION

Vibrational Autoionization in $\mathrm{H} 2$ : Vibrational Branching Ratios and Photoelectron Angular Distributions Near the $v(+)=3$ Threshold

PB 4-199577

00,799

Photoionization of Small Molecules Using Synchrotron Radiation

PB94-211505 


\section{KEYWORD INDEX}

PHYSICOCHEMICAL PROPERTIES

Comparison of Techniques for Nondestructive Composition Measurements in CdZnTe Substrates.

Cross-Sectional Photoluminescence and Its Application to Cross-Sectional Photoluminescence and Its Application to
Buried-Layer Semiconductor Structures.
PB96-141106
P2.415 Photoluminescence Spectra of Epitaxial Single Crystal PB96-141205

04,754

PHOTOLYSIS

Metalloporphyrin Sensitized Photooxidation of Water to Oxygen on the Surface of Colloidal Iridium Oxides - Phoochemical and Pulse Radiolytic Studies.

00,868

PHOTOMASKS

Practical Photomask Linewidth Measurements. $\quad 02.32$

Accuracy in Integrated Circuit Dimensional Measurements.

02,368

PB95-180808

Accuracy

Improving Photomask Linewidth Measurement Accu

PB95-180816

02,911

PHOTOMETERS

Detector-Based Candela Scale and Related Photometric Calibration Procedures at NIST.

PB95-161949

04.273

Intercomparison of Photometric Units Maintained at NIST (USA) and PTB (Germany), 1993.

B95-261913

04,329

Comparison of Filter Radiometer Spectral Responsivity with the NIST Spectral-Irradiance and Illuminance Scales. PB97-113161

04,162

PHOTOMETRY

Longterm Changes of Silicon Photodiodes and Their Use or Photometric Standardization.

PB94-211349

04,234

Peasurement System for Radiometry, Photometry, and Pyrometry Base Upon Absolute Detectors. Characterization of Modified FEL Ouartz-Halogen Lamps for Photometric Standards.

PB97-112544

00,282

PHOTON-ATOM COLLISIONS

Laser Assisted Collisions at Ultracold Temperatures.

PHOTON-ATOM INTERACTIONS

Laser Focused Atomic Deposition.

PHOTON BEAM EXCITATION

04,685

Barium Contributions to the Valence Electronic Structure of YBa2Cu3O7-delta, PrBa2Cu3O7-delta, and Other Barium-Containing Compounds.

PB96-158019

04,076

PHOTON CROSS SECTIONS

Bibliography of Photon Total Cross Section (Attenuation Coefficient) Measurements $10 \mathrm{eV}$ to $13.5 \mathrm{GeV}, 1907$

B94-193760

03,804

Experimentally Measured Total X-ray Attenuation Coefficients Extracted from Previously Unprocessed Documents Held by the NIST Photon and Charged Particle Data Center.

04,165

HOTON-ELECTRON COLLISIONS

Polarization Effects on Multiple Scattering Gamma Trans

PB95-153615

03,926

PHOTON TRANSPORT

Slab Transmission and Reflection for Point Source and PB94-211265

03,838

PHOTONIC BAND STRUCTURE

Photonic Band-Structure Effects for Low-Index-Contrast Two-Dimensional Lattices in the Near Infrared. PB97-122469

04.401

PHOTONIC PROBES

Optically Linked Three-Loop Antenna System for Determining the Radiation Characteristics of an Electrically Small Source.

02,005

PHOTONICS

Optically Sensed EM-Field Probes for Pulsed Fields.

B94-212594

02,130

PHOTONS

Exposure-to-Absorbed-Dose Conversion for Human Adult Cortical Bone.

Calculation of Photon Mass Energy-Transter and Mass Energy-Absorption Coefficients.

04,137

Electron-Photon Monte Carlo Calculations: The ETRAN

PB97.

04,138

PHOTOOXIDATION

Ferric Ion Assisted Photooxidation of Halocetates.
PB97-112460

00.52
PHOTOPATTERNING

UV-Photopatterning of Alkylthiolate Monolayers Selt-AsPB95-150751

00,924

PHOTOREFLECTANCE

Double-Modulation and Selective Excitation Photoreflectance for Wafer-Level Characterization o Ouantum-Well Laser Structures.

04,372

PB96-167325 Double Modulation and Selective Excitation Semiconductor Structures and Samples with Poor Surtace Morphology

PB97.111439

02,452

PHOTORESISTORS

Characterization of Interface Defects in Oxygen-Implanted Silicon Films

PB94-216629

02,322

PHTHALIC ACID/ (DIOCTYL-ESTER)

Neutron Scattering Study of Shear Induced Turbidity in Polystyrene/Dioctyl Phthalate Solutions at High Shea

PB94-172624

01,197

\section{PHTHALOCYANINES}

Complementary Molecular Information on Phthalocyanine Compounds Derived from Laser Microprobe Mass Spectrometry and Micro-Raman Spectroscopy.

PB94-172269

00,757

PHYSICAL CHEMISTRY

Journal of Physical and Chemical Reference Data, Volume 22, No. 1. January/February 1993.

00,729

Thermodynamic Properties of the Group IIA Elements.

PB94-160983

Spectroscopy and Structure of the Lithium Hydride

Diatomic Molecules and lons.
PB94-160991

00,731

Ouantum Yields for the Photosensitized Formation of the Lowest Electronically Excited Singlet State of Molecular

Journal of Physical and Chemical Reference Data, Volume 22, No. 2, March/April 1993.

PB94-162211

00,733 Spectra of Sulfur (S I through S XVI)

PB94-162229

00,734

Thermodynamic Properties of Alkenes (Mono-Olefins Larger Than C

Thermodynamic Behavior of the $\mathrm{CO} 2-\mathrm{H} 2 \mathrm{O}$ System from 400 to $1000 \mathrm{~K}$, up to $100 \mathrm{MPa}$ and $30 \%$ Mole Fraction of $2 \mathrm{~B} 2$

PB94-162245

00,736

Thermodynamics of Enzyme-Catalyzed Reactions: Part 1 Oxidoreductases.

00,737

Journal of Physical and Chemical Reference Data, Volume 22, No. 3, May/June 1993.

PB94-162260

00,738

Estimation of the Heat Capacities of Organic Liquids as a Function of Temperature Using Group Additivity. I. Hydrocarbon Compounds.

PB94-162278

00,739

Estimation of the Heat Capacities of Organic Liquids as a Function of Temperature Using Group Additivity. II. Compounds of Carbon, Hydrogen, Halogens, Nitrogen, Oxygen, and Sulfur.

00,740

Thermodynamic and Thermophysical Properties of Or-

ganic Nitrogen Compounds. Part II. 1- and 2-Butanamine, ganic Nitrogen Compounds. Part 2-Methyl-2-Propanamine, Pyrrole, 1-, 2-, and 3-Methylpyrrole, Pyridine, 2-, 3-, and 4-Methylpyridine, Pyrrolidine, Piperidine, Indole, Ouinoline, Isoquinoline, Acridine, Carbazole. Phenanthridine, 1 and 2-Naphthalenamine, and 9-Methylcarbazole.

and 2-Naphth

00,741

International Equations for the Saturation Properties of Ordinary Water Substance. Revised According to the International Temperature Scale of 1990. Addendum to Journal of Physical and Chemical Reference Data 16

PB94-162302

00,742

Journal of Physical and Chemical Reterence Data, Volume 22, No. 4, July/August 1993.

00,743

Estimation of the Thermodynamic Properties of $\mathrm{C}-\mathrm{H} \cdot \mathrm{N}-\mathrm{O}$ S-Halogen Compounds at $298.15 \mathrm{~K}$.

00,744

Joumal of Physical and Chemical Reference Data, Volume 22, No. 5 , September/October 1993.

ume 22, No.

00,745

Compilation of Energy Levels and Wavelengths for the Spectrum of Singly-lonized Oxygen (O II).

PB94-162344

00,746

Energy Levels of Germanium, $\mathrm{Ge}$ I through $\mathrm{Ge} X X X \mid$.

PB,747

Spectral Data and Grotrian Diagrams for Highly Ionized Chromium, Cr V through Cr XXIV.

00,748

Journal of Physical and Chemical Reference Data, Volume 22, No. 6, November/December 1993.

00,749

Thermodynamic Properties of Synthetic Sapphire (alphaAl2O3), Standard Reference Material 720 and the Effect of Temperature-Scale Differences on Thermodynamic Properties.
PBg-Scale Differences on Thermodynamic $\begin{array}{lll}\text { PB94-168564 } & 00,750 \\ \text { Thermodynamic Properties of Gaseous Silicon }\end{array}$ Monotelluride and the Bond Dissociation Enthalpy $D$ (sub

m) (SiTe) at T approaches 0.
PB94-168572

00,751

Disilicides of Tungsten, Molybdenum, Tantalum, Titanium, Cobalt, and Nickel, and Platinum Monosilicide: A Survey of Their Thermodynamic Properties. Evaluated Bimolecular Ion-Molecule Gas Phase Kinetics of Positive Ions for Use in Modeling Planetary Atmospheres, Cometary Comae, and Interstellar Clouds.
PB94-168598
00,753

Atomic Weights of the Elements 1991.

PB94-168606

00,754

PHYSICAL EQUILIBRIUM

Bibliography of the Physical Equilibria and Related Properties of Some Cryogenic Systems.

AD-A281 $167 / 7$

03,769

PHYSICAL PROPERTIES

Physical Properties of Some Purified Aliphatic Hydrocarbons.

AD-A297 265/1 00,657

Journal of Physical and Chemical Reference Data, Volume 22, No. 1, January/February 1993

PB94-160975

00,729

Journal of Physical and Chemical Reference Data, Volume 22, No. 2, March/April 1993.

PB94-162211 00.733

Journal of Physical and Chemical Reference Data, Volume 22, No. 3, May/June 1993.

PB94-162260

00,738

Journal of Physical and Chemical Reterence Data, Volume 22, No. 4, July/August 1993.

00,743

Journal of Physical and Chemical Reference Data, Volume 22, No. 5, September/October 1993.

PB94-162336

00,745

Journal of Physical and Chemical Reference Data, Volume 22, No. 6. November/December 1993.

Physicochemical Properties of Calcific Deposits Isolated from Porcine Bioprosthetic Heart Valves Removed from Patients Following 2-13 Years Function.

Energy and Migration of Grain Boundaries in Polycrystals.
PB94-211638

Constituents and Physical Properties of the $\mathrm{C} 6+$ Fraction of Natural Gas. Topical Report, April-June 1994. Analysis of Physical Properties of Ceramic Powders in an International Interlaboratory Comparison Program. 
PHYSICS

Physics Laboratory Technical Activities, 1993.

PB94-176088 03,796

Metrology Issues in Terahertz Physics and Technology.

PB96-128277 01,939

PHYSIOLOGIC CALCIFICATION

Experimental Validation of Radiopharmaceutical Absorbed Dose to Mineralized Bone Tissue.

03,617

PHVTOPLANKTON

High-Performance Liquid Chromatography of Phase $\mathrm{C} 18$ Column

PB95-151130

00,583

PIEZOELECTRIC CERAMICS

High-Sensitivity Acoustic Emission Sensor/Preamplifier Subsystems.

PIEZOELECTRICITY

Piezoelectric and Pyroelectric Polymers.

PB95-153367

02,900

GMENTS

01,250

Molar Absorptivities of Bilirubin (NIST SRM 916A) and Its Neutral and Alkaline Azopigments.

03,456

High-Performance Liquid Chromatography of Phytoplankton Pigments Using a Polymeric Reversed. Phase C18 Column.

PIPE BENDS

Summary Report of NIST's Industry-Government Consortium Research Program on Flowmeter Installation Effects: The 45 Degree Elbow.

PB95-143061

04,204

PIPE ELBOWS

Effects of Pipe Elbows and Tube Bundles on Selected Types of Flowmeters.

PIPE FLOW

01,135

Summary Report of NIST's Industry-Government Consortium Research Program on Flowmeter Installation Effects with Emphasis on the Research Period, January-September 1991: The Reducer

04,196

Summary Report of NIST's Industry-Government ConsorSummary Report of NIST's Industry-Government Consor-
tium Research Program on Flowmeter Installation Effects tium Research Program on Flowmeter Installation Effects The 45 Degree Elbow.

PB95-143061

04,204

PIPE JOINTS

Analysis of Failed Dry Pipe Fire Suppression System Couplings from the Filene Center at Wolf Trap Farm Park for the Performing Arts.

PB94-164407

00,331

PIPELINES

Earthquake Resistant Construction of Gas and Liquid Fuel Pipeline Systems Serving, $\bullet$ r Regulated By, the Federal Government.

PES

04,846

Polyvinyl Chloride (PVC) Plastic Drain, Waste, and Vent Pipe and Fittings.

Pipe and Fittings.

00,326

Acrylonıtrile-Butadiene-Styrene (ABS) Plastic Drain, Waste, and Vent Pipe and Fittings. AD-A $310724 / 0$

00,327

PIPING SYSTEMS

Water Efticient Plumbing Fixtures through Standards and Test Methods

Comparison of Fire Sprinkler Piping Materials: Steel Copper, Chlorinated Polyvinyl Chloride and Polybutylene, in Residential and Light Hazard Installations.

00,299

PISTON GAGES

NIST Measurement Services: NIST Pressure Calibration Service.

02,892

Intercomparison of the Effective Areas of a Pneumatic Piston Gauge Determined by Different Techniques. PB94-212370

PLANAR NEAR-FIELD METHOD

Planar Near-Field Measurements and Microwave Holography for Measuring Aperture Distribution on a $60 \mathrm{GHz}$ Active Array Antenna. Aperture Distribution on a $60 \mathrm{GHz}$ Active Array Ante
PB96-167366

03.762

PLANAR STRUCTURES

Planar Lenses for Field-Emitter Arrays. PB96-103064

02,112

PLANE OXYGEN VIBRATION

q Dependence of Self-Energy Effects of the Plane Oxygen Vibration in $\mathrm{YBa} 2 \mathrm{Cu} 3 \mathrm{O}$.

01,096

PLANE WAVES

Spatial Correlation Function for Fields in a Reverberation Chamber

PB96-148077

04,427

PLANETARY ATMOSPHERES

Infrared Spectra of van der Waals Complexes of Importance in Planetary Atmospheres.

00,071
Microwave Spectra of van der Waals Complexes of $\mathrm{Im}$ portance in Planetary Atmospheres.

00,919

Experimental Determination of the Rate Constant for the Experimental Determination of the Pate Constant for the tioning of Hydrocarbons in Atmospheres of the Outer

PB97-122295

00,112

PLANETARY GRAVITATION

Integrated Laser Doppler Method for Measuring Planetary Gravity Fields.

03,681

PLANETARY NEBULAE

IRAS Spectroscopic Observations of Young Planetary

PB $95-152070$

00,072

PLANNING

Integration of Real-Time Process Planning for Small-

PB95-151908

02,822

Computer-Aided Manufacturing Engineering Forum (1st).

Technical Meeting Proceedings. Held in Gaithersburg,

Maryland on March 21-22, 1995.

PB96-136965

02,834

Machining Process Planning Activity Model for Systems

Integration.
PB96-165428

02,841

PLASMA

Electrical Measurements for Monitoring and Control of $n$ Plasma Processing.

PB96-161963

04,369

PLASMA CHEMISTRY

Plasma Chemistry in Disilane Discharges.
PB94-211075

Plasma Chemical Model for Decomposition of SF6 in a Negative Glow Corona Discharge.

01,020

Plasma Chemistry in Silane/Germane and Disilane/GerPlasma Chemistry in Silane/Germane and Disilane/Ger-
mane Mixtures.
PB95-202537
01,027

(1)

Spectroscopic Data for Fusion Edge Plasmas.

PB95-151569

04,410

Spectroscopic Diagnostics of Low Temperature Plasmas:

Techniques and Required Data

04,411

Visible and UV Light from Highly Charged lons: Exotic Matter Advancing Technology.

PB96-119391

04,414

PLASMA EDGE

Electron-Ion Collisions in the Plasma Edge.

PB96- 111885

04,037

Waveguide Polarizers Processed by Localized Plasma

PB95-169264

02,171

PLASMA SHEATH

Electrical Sensors for Monitoring if Plasma Sheaths. PB95-162962

04,412

PLASMA SPRAY

Ultrasonic NDE of Sprayed Ceramic Coatings.

PB96-201157 02761

Characterization and Processing of Spray-Dried Zirconia Powders for Plasma Spray Application. PB97-111231

04,419

PLASMA SPRAYED ALUMINA

Anisotropy of the Surfaces of Pores in Plasma Sprayed Alumina Deposits.

PB96-123211

03,126

PLASMAS (PHYSICS)

Two-Dimensional Self-Consistent Radio Frequency Plasma Simulations Relevant to the Gaseous Electronics Conference RF Reference Cell.

PB96-113436

02,398

PLASMIDS

Deletion Analysis of the Mini-P1 Plasmid Origin of Replication and the Role of $E$ coli DnaA Protein. PB95-163911

03,539

\section{PLASTIC CONCRETE}

Study on the Reuse of Plastic Concrete Using Exiended Set-Retarding Admixtures. PB96-122130

00,402

PLASTIC DEFORMATION

SANS Study of the Plastic Deformation Mechanism in PB95-151841

PLASTIC PROPERTIES

01,242

Simulation of 660 Through the Plastic Transition Temperatures.

peratures.

04,713

PLASTICALLY POLARIZABLE MATERIALS

Shielding of Cracks in a Plastically Polarizable Material. PB95-164257

04,631

PLASTICS

Weatherability of Plastic Materials.

AD-A301 675/5 02,968

Polyvinyl Chloride (PVC) Plastic Drain, Waste, and Vent Pipe and Fittings.

00,326

PLATELETS (MATERIALS)

Characterization of Phase and Surtace Composition of Silicon Carbide Platelets.

PB94-216264

03,043

PLATFORM INTEROPERABILITY

Interoperability Experiments with CORBA and Persistent Object Base Systems

PLATINUM

Characterization of the Binding of Gallium, Platinum, and Uranium to Pseudomonas Fluorescens by Small-Angle $X$ ray Scattering and Transmission Electron Microscopy.
PB94-172509 Combined Low- and High-Angle X-Ray Structural Refinement of a Co/Pt(111) Multilayer Exhibiting Perpendicular Magnetic Anisotropy.

PB94-198355

04,457

PLATINUM ALLOYS

Fabrication of Platinum-Gold Alloys in Pre-Hispanic South America: Issues of Temperature and Microstructure Con-

PB94-211646

03,333

PLATINUM CONTAINING ALLOYS

Temperature Dependence of the Magnetic Excitations in Ordered and Disordered Fe72Pt28.

PB95-150223

04,563

PLATINUM IONS

Observation of Pd-Like Resonance Lines Through $\mathrm{Pt}(32+)$ and $\mathrm{Zn}$-Like Resonance Lines of $\operatorname{Er}(38+)$ and PB95-150637

03,896

$\mathrm{Rh} I$ Isoelectronic Sequence Observed from $\operatorname{Er}(23+)$ to $\mathrm{Pt}(33+)$.

PB95-150652

03,898

Spectra of $\mathrm{Ag}$ I Isoelectronic Sequence Observed from $\mathrm{Er}(21+)$ to $\mathrm{Au}(32+)$.

PLEIADES CLUSTER

03,899

IUE Observations of Solar-Type Stars in the Pleiades and the Hyades.

00,053

PLUMES

Computational Model for the Rise and Dispersion of Wind-Blown, Buoyancy-Driven Plumes. Part 2. Linearly Stratified Atmosphere.

PB94-143427

$\infty 0,119$

Dispersion and Deposition of Smoke Plumes Generated in Massive Fires.

PB95-126066 02,540

Airborne Smoke Sampling Package for Field Measurements of Fires.

PB95-150041

01,381

Fire-Plume-Generated Ceiling Jet Characteristics and Convective Heat Transfer to Ceiling and Wall Surfaces in a Two-Layer Fire Environment: Uniform Temperature iling and Walls.

PB95-164711

00,382

Some Factors Affecting the Design of a Calorimeter Hood and Exhaust. 
POISSON RATIO

Hollow Clay Tile Prism Tests for Martin Marietta Energy Systems: Task 2 Testing.

00,352

POISSON'S EQUATION

Scanning Capacitance Microscopy Measurements and Modeling: Progress Towards Dopant Profiling of Silicon. PB96-180070

01,964

POLAR GASES

Virial Coefficients of Five Binary Mixtures of Fluorinated Methanes and Ethanes.

01,128

OLARIMETRY

Polarimetric Calibration of Reciprocal-Antenna Radars. PB96-200696

02,016

POLARIZATION

Thermal Pulse Study of the Polarization Distributions Produced in Polyvinylidene Fluoride by Corona Poling at Constant Curren

PB94-172293

01,195

Surface Magnetic Microstructural Analysis Using Scanning Electron Microscopy with Polarization Analysis (SEMPA).

03,938

Anisotropy of Polarized $\mathrm{X}$-ray Emission from Atoms and Molecules.

PB95-163002 04,621

Vector and Quasi-Vector Solutions for Optical Waveguide Modes Using Efficient Galerkin's Method with HermiteGauss Basis Functions.

PB96-141197

04,357

Time-Resolved Measurements of the Polarization State of Four-Wave Mixing Signals from GaAs Multiple Quantum PB96-201058

04,796

Polarization Measurements on a Magnetic Quadrupole Line in Ne-Like Barium.

04,161 Integrated Optical Polarization-Discriminating Receiver in $\begin{array}{ll}\text { Glass. } & \\ \text { PB97.113179 } & 02,206\end{array}$

02,206 OLARIZATION (CHARGE SEPARATION)

Hysteresis Measurements of Remanent Polarization and Coercive Field in Polymers.

PB94-199767

04,475

Thermal Stability of Intemal Electric Field and Polarization Distribution in Blend of Polyvinylidene Fluoride and Polymethylmethacrylate.

PB95-151072

01,240

Piezoelectric and Pyroelectric Polymers.

01,250

OLARIZATION MODE DISPERSION

Anomalous Relation between Time and Frequency Domain PMD Measurements.

PB97-119390

04,398

POLARIZATION PROFILES

Thermal Pulse Study of the Polarization Distributions Produced in Polyvinylidene Fluoride by Corona Poling at PBontant Curre

01.195

OLARIZATION (WAVES)

Polarization Effects on Multiple Scattering Gamma Transport.

03,926

OLARIZED BEAMS

Uncertainty Intervals for Polarized Beam Scattering Asymmetry Statistics.

03,825

Connection between Superelastic and Inelastic ElectronAtom Collisions Involving Polarized Collision Partners.
PB95-202297

POLARIZED ELECTROMAGNETIC RADIATION Resonance and Threshold Effects in Polarized X-Ray Emission from Atoms and Molecules.

PB95-150298

03,891

POLARIZED INTERFEROMETER

New Refractometer by Combining a Variable Length Vacuum Cell and a Double-Pass Michelson Interferometer.
PB97-111926

POLARIZED LIGHT

Characteristics of Light Emission After Low-Energy Electron Impact Excitation of Caesium Atoms.

03,806

Polarization of Light Emitted After Positron Impact Exci-

tation of Alkali Atoms.
PB94-199734

03,816

National Voluntary Laboratory Accreditation Program: Bulk Asbestos Analysis.

PB95-138129

02,541

POLARIZED NEUTRON REFLECTOMETRY

New Exact Solution of the One-Dimensional Schrodinger Equation and Its Application to Polarized Neutron Reflec-

$\begin{array}{ll}\text { tometry. } & \\ \text { PB95-161832 } & 04,609\end{array}$ Analytic Calculation of Polarized Neutron Reflectivity from Superconductors.

PB95-164224

04,629

POLARIZERS

Supermirror Transmission Polarizers for Neutrons.
PB94-216215
Waveguide Polarizers Processed by Localized Plasma Erching. 9264

02,171

POLE FIGURES

Texture Measurement of Sintered Alumina Using the March-Dollase Function.

PB96-179494

04,784

POLICE PATROL CARS

AutoBid 2.0: The Microcomputer System for Police Patro Vehicle Selection

PB96-154570

04,871

Evaluating Investments in Law Enforcement Equipment: An Annotated Bibliography.

PB95-151379

04,867

POLICY FORMATION

Information Infrastructure: Reaching Society's Goals. A Report of the Information Infrastructure Task Force Committee on Applications and Technology.

00,131

POLICY MAKING

Standards Policy and Information Infrastructure. $\quad 01,485$

POLISHING

Rapid Post-Polishing of Diamond-Turned Optics. PB95-175949

04,301

POLLUTANTS

Standard Reference Materials for Dioxins and Other Environmental Pollutants.

PB94-198330

02,518

POLLUTION ABATEMENT

Opportunities for Innovation: Pollution Prevention.
PB95-147146

International Green Building Conference and Exposition (2nd). Held in Big Sky, Montana on August 13-15, 1995. PB95-253605

02,525

International Green Building Conference and Exposition (3rd). Held in San Diego, California on November 17-19,

1996. (Reannouncement with new abstract).

02.531

POLLUTION CONTROL EOUIPMENT

India: Environmental Technologies Export Market Plan.
PB97-114359

POLLUTION SAMPLING

Standard Reference Materials for the Determination of Polycyclic Aromatic Hydrocarbons in Environmental Samples - Current Activities.

POLONIUM 208

Long-Term Stability of Carrier-Free Polonium Solution

PB94-185170

00.685

POLY (AMIDE/N-PROPYLACRYL)

Small Angle Neutron Scattering Study on Poly(N-Isopropyl Acrylamide) Gels Near Their Volume-Phase Transition Temperature

PB95-164380

01,263

Small-Angle Neutron Scattering Study on Weakly PBarged Temper

01.264

POLY (P.PHENYLENE VINYLENE)

Inelastic-Neutron-Scattering Studies of Poly(p-phenylene vinylene).

PB95-180766

01,014

POLY (SULFONIC ACID/TETRAFLUORO)

Gas Transport Properties of Solution-Cas Pertluorosulfonic Acid lonomer Films Containing Ionic Surtactants.

01,267

POLYACRYLAMIDE GEL ELECTROPHORESIS

Resolution of DNA in the Presence of Mobility Modifying
Polar and Nonpolar Compounds by Discontinuous

Electrophoresis on Rehydratable Polyacrylamide Gels.

POLYACRYLATES

Adsorption of Low-Molecular-Weight Sodium Polyacrylate on Hydroxyap

00,139

POLYACRYLIC ACID

Small-Angle Neutron Scattering Study on Weakly Charged Tempera

01,264

POLYACRYLICS

Adsorption of Polyacrylic Acids and Their Sodium Salts on Hydroxyapatite: Effect of Relative Molar Mass.
PB97-112510

03,581

POLYAMINES

Cyclic Polyamine lonophore for Use in a Dibasic PhosPB95-180121

01,008

POLYAROMATIC CARCINOGENS

New Method for the Detection and Measurement of Polyaromatic Carcinogens and Related Compounds by DNA Intercalation.

PB96-167382

03,481

POLYATOMIC MOLECULES

Doppler-Free Spectroscopy of Large Polyatomic Molecules and van der Waals Complexes.

04,339

POLYBASIC ORGANIC ACIDS

Polymeric Calcium Phosphate Cements Derived from Poly(methyl vinyl ether-maleic acid)

\section{POLYBUTADIENE}

00,180

Shear-Excited Morphological States in a Triblock Copoly-

PB94-172392 01,196

Time Dependent Small Angle Neutron Scattering Behavior in Triblock Copolymers Under Steady Shear.

PB94-172632

01,198

Effect of Cross-Links on the Miscibility of a Deuterated Polybutadiene and Protonated Polybutadiene Blend. 01,225 Inversion of the Phase Diagram from UCST to LCST in Deuterated Polybutadiene and Protonated Polybutadiene

PB94-212446

01,226

Dynamic Light-Scattering Study of a Diluted Polymer PB95-151890 Critical Point

01,245

SANS Studies of Space-Time Orgarization of Structure in Polymer Blends.

01,251

Time-Resolved Small-Angle Neutron Scattering Study of Spinodal Decomposition in Deuterated and Protonated Polybutadiene Blends. 1. Effect of Initial Thermal FluctuaPlions.

OLYBUTYLENE

01,252

Comparison of Fire Sprinkler Piping Materials: Steel, Copper, Chlorinated Polyvinyl Chloride and Polybutylene, in Residential and Light Hazard Installations.

PB95-182267

POLYCHLORINATED BIPHENYLS

00.299

Comparison of Methods for Gas Chromatographic Determination of PCBs and Chlorinated Pesticides in Marine Reference Materials.

PB95-140091

02,584

NIST Standard Reference Materials (SRMs) for Polychlorinated Biphenyl (PCB) Determinations and Their APplicability to Toxaphene Measurements.

plicability to Toxa

02,585

Certification of Polychlorinated Biphenyl Congeners and Chlorinated Pesticides in a Whale Blubber Standard Reference Material.

03,745

Quantitative Analysis of Selected PCB Congeners in Marine Matrix Reference Materials Using a Novel Cyanobiphenyl Stationary Phase.

02,591

POLYCRYSTALLINE

Unconventional Ferromagnetic Transition in La(sub 1. x) $\mathrm{Ca}(\mathrm{sub} x) \mathrm{MnO} 3$

PB97-112429

04,156

POLYCRYSTALS

Energy and Migration of Grain Boundaries in Polycrystals.
PB94-211638

Microstructural Evolution in Two-Dimensional Two-Phase Polycrystals.

04,498

POLYCYCLIC AROMATIC HYDROCARBONS

Determination of Polycyclic Aromatic Hydrocarbons by Liquid Chromatography.

PB95-151650

00,585

Standard Reference Materials for the Determination of Polycyclic Aromatic Hydrocarbons in Environmental SamPles - Current Activities.

B $95-151668$

00,586

Liquid Chromatographic Determination of Polycyclic Aro- 


\section{KEYWORD INDEX}

POLYETHYLENE

Polyethylene Crystallized from an Entangled Solution ObServed by Scanning Tunneling Microscopy. SANS Study of the Plastic Deformation Mechanism in Polyethylene.

01,242

POLYETHYLENE GLYCOL

Two Phase Aqueous Extraction: Rheological Properties of Dextran, Polyethylene Glycol, Bovine Serum Albumin and Their Mixtures.

00,676

Protein Extraction in a Spray Column Using a Polyethylene Glycol Maltodextrin Two-Phase Polymer System.
PB95-162228

Phase Composition, Viscosities, and Densities for Aqueous Two-Phase Systems Composed of Polyethylene Gly$\mathrm{col}$ and Various Salts at $25 \mathrm{C}$

PB95-164596

00,986

POLYETHYLENE PLASTICS

Performance of Plastic Packaging for Hazardous Mate rials Transportation. Part 1. Mechanical Properties. AD-A301 258/0

02,580

POLYETHYLENES

Recertification of the Standard Reference Material 1475A, a Linear Polyethylene Resin.

PB94-161932

02,628

POLYIMIDE

Gas Absorption during Ion-Irradiation of a Polymer Target. 6 - 161864

04,099

POLYIMIDES

Water Adsorption at a Polyimide/Silicon Wafer Interface. PB96-103197

01,070

POLYISOCYANURATE

Effect of Environmentally Exposures on the Properties of Polyisocyanurate Foam Insulation: Thermal Conductivity Measurements.

POLYISOPRENE

SANS Studies of Space-Time Organization of Structure in Polymer Blends.

POLYMER BLENDS

Thermodynamic Interactions in Model Polyolefin Blends Obtained by Small-Angle Neutron Scattering.

Statistical Thermodynamics of Phase Separation and Ion Partitioning in Aqueous Two-Phase Systems.

01,212

How Far is Far from Critical Point in Polymer Blends. Lattice Cluster Theory Computations for Structured Monomer, Compressible Systems.

01,217

Crossover to Strong Shear in a Low-Molecular-Weight Critical Polymer Blend. PB94-211976

Effect of Cross-Links on the Miscibility of a Deuterated Polybutadiene and Protonated Polybutadiene Blend. PB94-212438 01,225

Inversion of the Phase Diagram from UCST 10 LCST in Deuterated Polybutadiene and Protonated Polybutadiene

01,226

Glass Temperature of Polymer Blends: Comparison of Both the Free Volume and the Entropy Predictions with

PB95-140190 01,236

Structural Stabilization of Phase Separating PC/Polyester Blends through Interfacial Modification by Transesterification Reaction. Thermal Stability of Internal Electric Field and Polarization Distribution in Blend of Polyvinylidene Fluoride and Polymethylmethacrylate.

01,240

Anisotropic Phase Separation Kinetics in a Polymer Blend Solution Following Cessation of Shear Studied by Light Scattering.

PB95.151247

01,241

SANS Study of the Plastic Deformation Mechanism in Polyethylene.
PB95-151841

01,242

Dynamic Light-Scattering Study of a Diluted Polymer Blend Near lis Critical Point.

01,245

Thermodynamic Interactions and Correlations in Mixtures
of Two Homopolymers and a Block Copolymers by Small of Two Homopolymers and a Block Copolymers by Small Angle Neutron Scattering.

01,247

SANS Studies of Space-Time Organization of Structure in Polymer Blends.
PB95-153789

01,251

Time-Resolved Small-Angle Neutron Scattering Study of Spinodal Decomposition in Deuterated and Protonated Polybutadiene Blends. 1. Effect of Initial Thermal Fluctua-

01,252

Characterization of Polyquinoline Blends Using Small Angle Scattering.

01,261
Polymers Technical Activities 1994. NAC-NRC Assessment Panel, April 6-7, 1995.

01,275

Modification of the Phase Stability of Polymer Blends by Diblock Copolymer Additivies.

03,172

Slow Dynamics of Segregation in Hydrogen-Bonded Poly mer Blends.

PB96-123591

01,281

Light-Scattering Studies on Phase Separation in a Binary Blend with Addition of Diblock Copolymers.

01,286

Phase Separation in Thin Film Polymer Blends With and Without Block Copolymer Additives.

PB96-204482

01,294

Lattice Model of a Hydrogen-Bonded Polymer Blend

PB97.112262

03,391

Relaxation After a Temperature Jump Within the One Phase Region of a Polymer Mixture.

PB97-112494 03,394

Morphology and Phase Separation Kinetics of a Compatibilized Blend.

PB97-119135

01,297

POLYMER BRUSH

Neutron Reflectivity Study of the Density Profile of a Model End-Grafted Polymer Brush: Influence of Solven Quality.

PB95-202735

01,274

\section{POLYMER BRUSHES}

Terminally Anchored Chain Interphases: Their Chromatographic Properties.

01,272

Terminally Anchored Chain Interphases: The Effect of Multicomponent, Polydisperse Solvents on Their Equilibrium Properties.

POLYMER CHEMISTRY

01,273

Fluorescence Anisotropy Measurements on a Polymer Melt as a Function of Applied Shear Stress.

01,209 PB94-199296

Observations of Shear Induced Molecular Orientation in a
Polymer Melt Using Fluorescence Anisotropy Measurements.

01,210

PB94-199304

Observations of Shear Stress and Molecular Orientation
Using Fluorescence Anisotropy Measurements. PB94.199312

POLYMER COMPOSITES

Materials Aspects of Fiber-Reinforced Polymer Compostes in infrastructure.

PB96-210695

03,184

POLYMER FILMS

Advances in the Measurement of Polymer CTE: Micrometer- to Atomic-Scale Measurements.

PB96- 180229

03,390

POLYMER INTERFACE

Compatibilization of Polymer Blends by Complexation. 2 . Kinetics of Interfacial Mixing.

PB97-111900

01,295

\section{POLYMER MATRIX COMPOSITES}

Selection of Appropriate Uitrasonic System Components PB94-185279

03,133

Review of Cure Monitoring Techniques for On-Line Process Control.

Polymer Composites Workshop. Held in Winona, Minnesota on April 29-30, 1992 (Video)

03,147

Appropriate Ultrasonic System Components for NDE of

Thick Polymer Composites.

03,148

Thermoacoustic Technique for Determining the Interface and/or Interply Strength in Polymeric Composites.

PB95-161824

03,158

Environmental Durability of Glass-Fiber Composites. PB95-203220

03, 166

POLYMER NETWORKS

Synthesis of Hybrid Organic-Inorganic Materials from Interpenetrating Polymer Network Chemistry.

02,994

POLYMER PHYSICS

Book Review: Statistical Physics of Macromolecules. PB96-123526

01,280

POLYMERASE CHAIN REACTION

Electrophoretic Separations of Polymerase Chain Reaction: Amplified DNA Fragments in DNA Typing Using a Capillary Electrophoresis-Lased Induced Fluorescence

P95-163036

03,536

Petection of PCR Products Through Use of FOTO and YOYO Intercalating Dyes with Laser Induced Fluorescence - Capillary Electrophoresis.

00,599

POLYMERIC FILMS

Diffusion of Cations Beneath Organic Coatings on Steel Substrate.

PB94-215704

03,119

Grazing Incidence Prompt Gamma Emissions and Resonance-Enhanced Neutron Standing Waves in a Thin-Film.

Gas Transport Properties of Solution-Cast Perfluorosulfonic Acid lonomer Films Containing lonic Surtactants.

Thermal Conductivity of Polypyromellitimide Film with Alumina Filler Particles from 4.2 to $300 \mathrm{~K}$

PB96-200753

01,292

POLYMERIZATION

Fluorescence Monitoring of Polarity Change and Gelation during Epoxy Cure.

PB94-185543

01,204

Effect of Curing History on Ultimate Glass Transition Temperature and Network Structure of Crosslinking Poly-

01,214

Thermal Behavior of 4-Maleimidophenyl Glycidyl Ether

PB95-153151

01,249

Ring-Opening Dental Resin Systems Based on Cyclic

Acelals.

00,162

Synthesis and Polymerization of Difunctional and
Multifunctional
Monomers

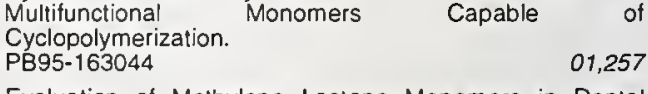

Evaluation

PB95-164661

00.164

Ring-Opening Polymerication of a 2-Methylene Spiro Orthocarbonate Bearing a Pendant Methacrylate Group.
PB95-176145

Polymerization Initiation by N-p-Tolylglycine: Free-Radical

Reactions Studied by Pulse and Steady-State Radiolysis.

Reactions Studied by Pulse and Steady-State Radiolysis.
01,269

Radiochromic Solid-State Polymerization Reaction.

PB95-180683

01,271

YMERS

Weatherability of Plastic Materials.

02,968

Combustion of a Polymer (PMMA) Sphere in Micro-

N96-15569/2

01,354

In-Line Optical Monitoring of Injection Molding

01,201

Influence of Surface Interaction and Chain Stiffness on Polymer-Induced Entropic Forces and the Dimensions of

Confined Polymers.
PB94-185469

01,203

Neutron Reflectivity of End-Gratted Polymers: Concentra-

tion and Solvent Quality Dependence in Equilibrium Con-

ditions.

01,206

Hysteresis Measurements of Remanent Polarization and Coercive Field in Polymers.

PB94-199767

04,475

Peeling a Polymer from a Surface or from a Line.

PB94-199809 01,213

Hypercubic Lattice SAW Exponents nu and gamma : 3.99 Dimensions Revisited.

PB94-211026

01,215

Clinical Perspective on Dentin Adhesives. 
Effect of Electrode-Polymer Interfacial Layers on Polymer Conduction. Part 2. Device Summary.

02,335

Localization of a Homopolymer Dissolved in a Lamellar Structure of a Block Copolymer Studied by Small-Angle Neutron Scattering.

PB95-161592

Elastic Scattering of Polymer Networks.

01,255

Applications of Fluorescence Spectroscopy in Polymer Science and Technology

01,258

Small Angle Neutron Scaltering Study on Poly(N-Isopropyl Acrylamide) Gels Near Their Volume-Phase Tran-

PB95-164380

01,263

Small-Angle Neutron Scattering Study on Weakly PB95-164398

01,264

Competition between Hydrodynamic Screening ('Draining') and Excluded Volume Interactions in an Isolated Polymer Chain.

01,265

Examination of the $1 / d$ Expansion Method from Exac Enumeration for a Self-Interacting Self-Avoiding Walk.
PB95-175733 Optical Biosensor Using a Fluorescent, Swelling Sensing

PB95-175899

03,541

Neutron Scattering Study of the Orientation of a Liquid Crystalline Polymer by Shear Flow.

Hydroxyapatite in a Polymeric Calcium Phosphate Cement

Terminally Anchored Chain Interphases: Their

Chromatographic Properties.
PB95-181061

01,272

Terminally Anchored Chain Interphases: The Effect of Multicomponent, Polydisperse Solvents on Their Equilibrium Properties.

PB95-181079

01,273

Polymers Technical Activities 1994. NAC-NRC Assessment Panel, April 6-7, 1995

PB95-209896

01,275

Selt-Avoiding-Walk Contacts and Random-Walk SelfIntersections in Variable Dimensionality.

PB96-102231

01,276

PB96-122478

01,278

Polymer Combustion and Flammability: Role of the Condensed Phase.

Flow-Induced Structure in Polymer. Chapter 1. An Introduction to Flow-Induced Structures in Polymers.

PB96-123369

03,387

Flow-Induced Structure in Polymers. Chapter 17. PhaseSeparation Kinetics of a Polymer Blend Solution Studied by a Two-Step Shear Quench.

PB96-123377

03,388

Response to 'Draining in Dilute Polymer Solutions and Renormalization'.

PB96-146667

01,283

Orientation Effects on ESR Analysis of Alanine-Polymer Dosimeters.

03,720

Thermodynamic Properties of Dilute and Semidilute Solu(ions of Regular Star Polymers.

PB96-146808

01,284

Preparation and Characterization of Cyclopolymerizable Resin Formulations.

01,285

Influence of an Impenetrable Interface on a Polyme Glass-Transition Temperature.

PB96-146873

03,175

Shear-Induced Mixing in Polymer Blends.

01,287

NIST Metrology Program on Electromagnetic Characterzation of Materials.

PB96-156062

01,944

Effects of Sample Mounting on Flammability Properties of Intumescent Polymers.

03,389

Hygrothermal Effects on the Performance of Polymers and Polymeric Composites: A Workshop Report. Held in Gaithersburg, Maryland on September 21-22, 1995

PB96-183207

03,180

Polymers Technical Activities, 1995

PB96-193719 01,291

Gas Phase Oxygen Effect on Chain Scission and Mono mer Content in Bulk Poly(methyl methacrylate) Degraded by External Thermal Radiation.

PB96-204078

01,293

Swelling and Growth of Polymers, Membranes and Sponges.

03,396

Materials and Fire Threat

01,442
POLYMETHYL METHACRYLATE

Combustion of a Polymer (PMMA) Sphere in Micro. gravity.

01,354

Grafted Interpen

01,200

Investigation of the Thermal Stability and Char-Forming Tendency of Cross-linked Poly(methyl methacrylate). Miblock Copolymers as Re Morphology of Symmetric Dibl

PB95-140075

01,234

POLYOLEFINS

Thermodynamic Interactions in Model Polyolefin Blends Obtained by Small-Angle Neutron Scattering.

01,208

Thermodynamic Interactions and Correlations in Mixtures of Two Homopolymers and a Block Copolymers by Small Angle Neutron Scattering.

PB95-152872

01,247

POLYOXYETHYLENE

Determination of the Weight Average Molecular Weight of Two Poly(Ethylene Oxides), SRM 1923 and SRM 1924. Two Poly(Ethylene Oxides), SRM 1923 and SRM 1924.
01,230 Two Phase Aqueous Extraction: Rheological Properties of Dextran, Polyethylene Glycol, Bovine Serum Albumin and Their Mixtures.

PB95-161998

00,676

Phase Composition, Viscosities, and Densities for Aqueous Two-Phase Systems Composed of Polyethylene Gly$\mathrm{col}$ and Various Salts at $25 \mathrm{C}$

PB95-164596

00,986

POLYOXYPROPYLENE

Influence of Physical Aging on the Yield Response of Model DGEBA + Poly(propylene oxide) Epoxy Glasses. PB95-126363

03,381

POLYPERFLUOROSULFONATE IONOMER

$\mathrm{CO} 2 / \mathrm{CH}_{4}$ Transport in Polyperfluorosulfonate lonomers: Effects of Polar

01, 145

POLYOUINOLINES

Characterization of Polyquinoline Block Copolymer Using Small Angle Scattering.

01,244

Characterization of Polyquinoline Blends Using Small Angle Scattering.
PB95-164125

POLYSTYRENE

01,261

Shear-Excited Morphological States in a Triblock Copoly-

PB94-172392

01,196

Neutron Scattering Study of Shear Induced Turbidity in Polystyrene/Dioctyl Phthalate Solutions at High Shear Rates.

PB94-172624

01,197

Time Dependent Small Angle Neutron Scattering Behavior in Triblock Copolymers Under Steady Shear. Shear Dependence of Critical Fluctuations in Binary Polymer Mixtures by Small Angle Neutron Scattering. PB94-211612

01,220

Small-Angle $X$-Ray and Neutron Scattering Study of Block Copolymer/Homopolymer Mixtures.

01,221

Morphology of Symmetric Diblock Copolymers as Revealed by Neutron Reflectivity.

01,234

Dynamic Light-Scattering Study of a Diluted Polymer Blend Near Its Critical Point.

01,245

Neutron Scattering Study of Shear Induced Turbidity in Polystyrene Dissolved in Dioctyl Phthalate.
PB95-161865

Preparation of 2-Dimensional Ultra Thin Polystyrene Film by Water Casting Method.

PB95-162806

04,619

Partial Scattered Intensities from a Binary Suspension of Polystyrene and Silica.

00,996

Dielectric Properties of Materials at Cryogenic Temperaures and Microwave Frequencies.

PB95-202610

02,369

Standard Reference Materials: Polystyrene Films for Calibrating the Wavelength Scale of Infrared Spectrophotometers - SRM 1921.

PB95-226866

03,386

Room-Temperature Thermal Conductivity of Expanded Polystyrene Board for a Standard Reference Material. Polystyrene Board for a Standard Reference Material.
PB96-193693

POLYSULFIDES

Microbial Degradation of Polysulfides and Insights into Their Possible Occurrence in Coal.

PB95-163374

02,488

POLYTETRAFLUOROETHYLENE

$45 \mathrm{deg} / 0$ deg Reflectance Factors of Pressed Polytetrafluoroethylene (PTFE) Powder. PB95-260758

04,328

POLYURETHANE FOAM

New Approach for Reducing the Toxicity of the Combustion Products from Flexible Polyurethane Foam.

PB96-123625

01,411

POLYVINYL ALCOHOL

New EPR Dosimeter Based on Polyvinylalcoho

PB94-199700

3,619

Gels. 164117

Small Angle Neutron Scattering Studies on Chain Asymmetry of Coextruded Poly(Vinyl Alcohol) Film.

01,262

POLYVINYL CHLORIDE

Polyvinyl Chioride (PVC) Plastic Drain, Waste, and Vent Pipe and Fittings.
$\mathrm{AD}-\mathrm{A} 310426 / 2$

00,326

POLYVINYLIDENE FLUORIDE

Thermal Pulse Study of the Polarization Distributions Produced in Polyvinylidene Fluoride by Corona Poling at Constant Current.

PB94-172293

01,195

POLYVINYLIDENE FLUORIDES

Thermal Stability of Intemal Electric Field and Polarization

Distribution in Blend of Polyvinylidene Fluoride and Polymethylmethacrylate.

POOL BOILING

01,240

Enhancement of R123 Pool Boiling by the Addition of N-

PB96-165956

02,605

POOL BUILDING

Calorimetric and Visual Measurements of R123 Pool Boil-

ing on Four Enhanced Surfaces.

PB96-128129

Structure and Radiation Properties of Pool Fires.

PB94-193802 02,473

Estimate of Flame Radiance via a Single Location Measurement in Liquid Pool Flames.

PB94-211596

02,476

Measurement of Radiative Feedback to the Fuel Surface of a Pool Fire.

02,477

Estimate of the Effect of Scale on Radiative Heat Loss Fraction and Combustion Efficiency.

02,486

PORCELAIN

Influence of Tempering Method on Residual Stress in Dental Porcelain.

PB94-172012

00,138

Evaluation of Fracture Toughness and Residual Stress in Dental Porcelain by Indentation-Microfracture Method

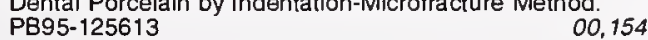

Evaluation of Fracture Toughness and Residual Stress in Dental Porcelain by Indentation-Microfracture Method.
PB95-152831

\section{PORE SIZE DISTRIBUTION}

Evolution of the Pore Size Distribution in Final-Stage Sintering of Alumina Measured by Small-Angle $X$-ray Scattering. (Reannouncement with New Availability InformaAD-A249 178/5

PORE STRUCTURE 


\section{KEYWORD INDEX}

Interaction between Micro and Macroscopic Flow in RTM Preforms.

PB95-162012

03,159

Transport and Diffusion in Three-Dimensional Composite

04,668

PB95-176129

Structure

and Formation of Ordered Mesopores in Silica.

PB96-111919

Small Angle Neutron Scattering Study of the Structure and Formation of MCM-41 Mesoporous Molecular Sieves.
PB97-122337

POROUS MEDIA

Characterization of Chemically Modified Pore Surfaces by Small Angle Neutron Scattering.

PB95-126181

00,898

Hydraulic Radius and Transport in Reconstructed Model Three-Dimensional Porous Media.

00,403

Analysis of Transverse Flow in Aligned Fibrous Porous Media.

PB96-167200

03,177

PORPHYRINS

One-Electron Oxidation of Nickel Porphyrins. Effect of Structure and Medium on Formation of Nickel(III) Porphyrin or Nickel(II) Porphyrin pi-Radical Cation.

00,865

Site of One-Electron Reduction of Ni(II) Porphyrins. ForSite of One-Electron Reduction of $\mathrm{Ni}(\mathrm{II})$ Porphyrins. For-
mation of $\mathrm{Ni}(\mathrm{I})$ Porphyrin of $\mathrm{Ni}(\mathrm{II})$ Porphyrin pi-Radical

PB95-107066 00,866

Metalloporphyrin Sensitized Photooxidation of Water to Oxygen on the Surface of Colloidal Iridium Oxides - Photochemical and Pulse Radiolytic Studies.

00,868 PB95-107082

National Voluntary Laboratory Accreditation Program: POSIX. Portable Operating System Interface.

02,661

PRTABLE OPERATING SYSTEM INTERFACE FOR

COMPUTING ENVIRONMENTS

Comparison of POSIX Open System Environment (OSE) and Open Distributed Processing (ODP) Reference Models.

01,820

PORTABLE OPERATING SYSTEMS INTERFACE FOR

COMPUTER ENVIRONMENTS

IEEE's POSIX: Making Progress.

PB96-160924

01,757

NIST POSIX Testing Program

PB96-160973

01,821

PORTLAND CEMENT

Interaction between Naphthalene Sulionate and Silica Fume in Portland Cement Pastes.

01,315

Computer Simulation of the Diffusivity of Cement-Based Materials.

PB95-125985

00,362

Cement and Concrete Characterization by Scanning Electron Microscopy
PB95-163168

PORTLAND CEMENT CLINKER

00,379

Ouantitative Phase Abundance Analysis of Three Cement Clinker Reference Materials by Scanning Electron Micros. PB94-173051

00,333

PORTLAND CEMENTS

Optimization of Highway Concrete Technology. PB94-182995

01,333

Graphical Analysis of the CCRL Portland Cement Pro. ficiency Sample Database (Samples 1-72). (Part 1. Univariant Analysis of Portland Cement)

PB94-196557

01,308

Ouantitative X-Ray Powder Diffraction Methods for Clinker and Cement.

01,317

Water Permeability and Chloride Ion Diffusion in Portland Cement Mortars: Relationship to Sand Content and Critical Pore Diameter. cal Pore Diameter.

POSITION FINDING

03,193

Analytical Estimation of Carrier Multipath Bias on GPS Position Measurements.

PB94-215712 04,845

Three Dimensional Position Determination from Motion. PB95-107108

01, 788

POSITION (LOCATION)

Spatial Data Transfer Standard (SDTS). Category: Software Standard; Subcategory: Information Interchange. (FIPS PUB 173-1A)

01,795

Spatial Data Transfer Standard (SDTS). Category: Software Standard: Subcategory: Information Interchange. (FIPS PUB 173-1B)
FIPS PUB 173-1B

POSITION SENSING

Image Gradient Evolution: A Visual Cue for Danger.
PB96-154562
POSITIONING

Microelectronic Test Structures for Overlay Metrology.

4249

POSITRON-ATOM COLLISIONS

Polarization of Light Emitted After Positron Impact Excitation of Alkali Atoms.

PB94-199734

03,816

PBON SOURCES

Laser-Cooled Positron Source.

PB95-169348

03,954

POSITRONIUM

Positronium in Relativistic Ouantum Mechanics.

PB97-110571

04,140

POSIX (PORTABLE OPERATING SYSTEM INTERFACE)

National Voluntary Laboratory Accreditation Program:

POSIX. Porta
PB95-189478

02,661

POSIX (PORTABLE OPERATING SYSTEM INTERFACE

FOR COMPUTING ENVIRONMENTS)

Comparison of POSIX Open System Environment (OSE) and Open Distributed Processing (ODP) Reference Mod-

els.

01,820

POSIX (PORTABLE OPERATING SYSTEMS INTERFACE

FOR COMPUTER ENVIRONMENTS)

IEEE's POSIX: Making Progress.

PB96-160924

01,757

NIST POSIX Testing Program.

01,821

POTASSIUM

Frozen Human Serum Reference Material for Standard. ization of Sodium and Potassium Measurements in Serum or Plasma by Ion-Selective Electrode Analyzers.

PB94-185337

00,532

Multichannel Ouantum Defect Half Collision Analysis of K2 Photodissociation Through the B1Pi(sub u) State.

00,812

POTASSIUM BARIUM BISMUTHATES

Optical Conductivity of Single Crystals of Ba1$\mathrm{XMXBIO} 3(\mathrm{M}=\mathrm{K}$
$\mathrm{PB} 94.185329$

04,447

POTASSIUM CHLORIDE

Absolute Determination of Electrolytic Conductivity for Primary Standard KC1 Solutions from 0 to $50 \mathrm{C}$

00,765

dc Method for the Absolute Determination of Conductivities of the Primary Standard $\mathrm{KCl}$ Solutions from $\mathrm{OC}$ to $50 \mathrm{C}$.

02,644

POTASSIUM DIHYDROGEN PHOSPHATE

Crystal Structure of a New Monoclinic Form of Potassium Dihydrogen Phosphate Containing Orthophosphacidium lon, $(\mathrm{H} 4 \mathrm{PO} 4)($ sup +1$)$

PB96-111794

04,725

POTASSIUM INORGANIC COMPOUNDS

Crystal Structure of a New Monoclinic Form of Potassium Dihydrogen Phosphate Containing Orthophosphacidium Ion, (H4PO4)(sup+1)

POTASSIUM IONS

Kinetic-Energy Distributions of $\mathrm{K}(+)$ in Argon and Neon in Uniform Electric Fields.

PB95-151122

03.902

POTASSIUM PHOSPHATES

Crystal Structure of Dicalcium Potassium Trihydrogen Bis(pyrophosphate) Trihydrate.

PB94-216561

00,152

POTASSIUM SILICATES

Imaging of Fine Porosity in a Colloidal Silica: Potassıum Silicate Gel by Defocus Contrast Microscopy.

03,039

\section{POTASSIUM TANTALATE NIOBATES}

Anomalous Dispersion and Thermal Expansion in Lightly Doped KTa 1-xNbxO3.

PB95-152302

04,585

POTASSIUM TANTALATES

Anomalous Dispersion and Thermal Expansion in Lightly Doped KTa1-xNbxO3.

POTENTIAL ENERGY

Equilibrium Pair Distribution Function of a Gas: Aspects Associated with the Presence of Bound States. PB95-176046

01,004

POTENTIAL FUNCTIONS

Equilibrium Pair Distribution Function of a Gas: Aspects Associated with the Presence of Bound States. PB95-176046

01,004

POTENTIOMETER

Enhanced Voltage-Dividing Potentiometer for High-Precision Feature Placement Metrology.

02,428

POTENTIOMETERS

Integrated Thin

02,109

POTENTIOMETRIC ANALYSIS

Potentiometric Enzyme-Amplified Flow Injection Analysis Detection System: Behavior of Free and Liposome-Re leased Peroxidase

03,534

POWDER METALLURGY

Effect of Backfill and Atomizing Gas on the Powder Porosity and Mechanical Properties of $304 \mathrm{~L}$ Stainless Steel. PB94-185394

POWDER METALS

Crystal Siructure of a New Sodium Zinc Arsenate Phase Solved by 'Simulated Annealing'

PB95-107124

00,870

Parametric Investigation of Metal Powder Atomization Using Laser Diffraction.

POWDER (PARTICLES)

03,342

Introduction of a NIST Instrument Sensitivity Standard Reference Material for X-Ray Powder Difiaction.

PB94-200318

00,807

Statistical Analysis of Parameters Affecting the Measurement of Particle-Size Distribution of Silicon Nitride Powders by Sedigraph (Trade Name).

Rapid Hot Pressing of Ultra-Fine PSZ Powders.

PB94-216587

03,042 ders in an PB95-161501

03,050

Effects of Soxhlet Extraction on the Surface Oxide Layer of Silicon Nitride Powders.

03,057

$45 \mathrm{deg} / 0$ deg Reflectance Factors of Pressed Polytetrafluoroethylene (PTFE) Powder

PB95-260758

04,328

Ceramic Powders Characterization: Results of an International Laboratory Study.

PB95-270039

02,672

POWDERS

$X$-ray Powder Diffraction from Carbon Nanotubes and Nanoparticles.

03,064

POWDERS (PARTICLES)

Surface Chemistry of Silicon Nitride Powder in the Pres. ence of Dissolved lons.

PB96-111760

01,073

Electroacoustic Characterization of Particle Size and Zeta

Potential in Moderately Concentrated Suspensions. PB96-119425

01,079

POWER

Power Characteristics in GMAW: Experimental and Numerical Investigation.

03,296

$\mathrm{DC}-\mathrm{MHz}$ Wattmeter Based on RMS Voltage Measure-

$\begin{array}{ll}\text { ments. } & \\ \text { PB97-113211 } & 01,992\end{array}$

POWER AMPLIFIERS

100 Ampere, $100 \mathrm{kHZ}$ Transconductance Amplifier.

PB96-102629

$100 \mathrm{~A}, 100 \mathrm{kHz}$ Transconductance Amplifier.

02,068 PB96-200936

02,098

POWER MEASUREMENT

NIST Model PM2 Power Measurement System for $1 \mathrm{~mW}$ PB94-135803

Coaxial Reference Standard for Microwave Power.

PB94-193786 


\section{KEYWORD INDEX}

PROBABILITY DENSITY FUNCTIONS

PRASEODYMIUM

Magnetic Structure and Spin Dynamics of the $\mathrm{Pr}$ and $\mathrm{Cu}$ in $\mathrm{Pr} 2 \mathrm{CuO} 4$.

04,036

RASEODYMIUM BARIUM COPPER NIOBATES

Crystal Structure and Magnetic Ordering of the RareEarth and $\mathrm{Cu}$ Moments in $\mathrm{BBa} C \mathrm{Cu} 2 \mathrm{NbOB}(\mathrm{R}=\mathrm{Nd}, \mathrm{Pr})$. PB95-140554

04,546

PRASEODYMIUM BARIUM CUPRATES

Magnetic Ordering of the Cu Spins in $\mathrm{PrBa} 2 \mathrm{Cu} 3 \mathrm{O} 6+\mathrm{x}$.

PB95-140547 04,545

PRASEODYMIUM CERIUM CUPRATES

Phonon Density of States in R2CuO4 and Superconducting R1.85Ce0.15CuO4 $(\mathrm{R}=\mathrm{Nd}, \mathrm{Pr})$.
PB95-150686 Magnetic Susceptibility of $\mathrm{Pr} 2-x \mathrm{CexCuO} 4$ Monocrystals and Polycrystals.

and Polycrysta

PRASEODYMIUM CUPRATES

Dispersions of Magnetic Excitations of the $\mathrm{Pr}$ lons in $\mathrm{Pr} 2 \mathrm{CuO} 4$

PB94-173044

04,444

Phonon Density of States in $\mathrm{R}_{2} \mathrm{CuO} 4$ and Superconducting R1.85CeO.15CuO4 ( $\mathrm{R}=\mathrm{Nd}$. Pr) PB95-150686

04,574

PRASEODYMIUM GALLATES

Suitability of Metalorganic Chemical Vapor Deposition-Derived PrGaO3 Films as Buffer Layers for YBa2Cu3O7-x Pulsed Laser Deposition

PB95-168670

04,640

PRE-MAIN SEQUENCE STARS

High Sensitivity Survey of Radio Continuum Emission in Herbig Ae/Be
PB94-185915

00,051

PRECAST CONCRETE

Model Precast Concrete Beam-to-Column Connections Subject to Cyclic Loading.

00,438

Partially Prestressed and Debonded Precast Concrete Beam-Column Joints.

00,439

Seismic Performance Behavior of Precast Concrete Beam-Column Joints.

PB95-153110

00,440

Performance of $1 / 3-S c a l e$ Model Precast Concrete BeamColumn Connections Subjected to Cyclic Inelastic Loads.

Report No. 4.

00,444

Simplified Design Procedure for Hybrid Precast Concrete Connections.
PB96-154836

PRECIPITATION (CHEMISTRY)

Study of the Hydroxycarbonate Precursor Route to the YBa2Cu307-x High Tc Superconductor.

PB95-140471

04,540

Membrane-Mediated Precipitation of Calcium Phosphate in Model Liposomes with Matrix Vesicle-Like Lipid Com-

PB95-164547

03,468

Dynamics of $C$ a

03,574

PRECIPITATION HARDENING

Effects of Spindle Dynamic Characteristics on Hard Turning.

02,699

PRECISION

Critical Issues in Scanning Electron Microscope Metrol-

PB95-169405

02,359

Japan Technology Program Assessment: Precision Engi-

neering/Precision Optics in Japan

02,884

Precision in Machining: Research Challenges

PB95-242301

02,953

Integrated inspection System for Improved Machine Per-

formance.
PB96-160569 02,959

New Concepts of Precision Dimensional Measurement for Modern Manufacturing.

PB96-160684

02,924

Scanning Electron Microscope Metrology.

02,446

PRECOOUNG

Helium Refrigeration and Liquefaction Using a Liquid $\mathrm{Hy}$ drogen Refrigerator for Precooling.

AD-A286 683/8

02,749

RECURSORS

Study of the Hydroxycarbonate Precursor Route to the YBa2Cu3O7-x High Tc Superconductor.

PB95-140471

Diamond and Graphite Precursors: Comments.

PB95-163051

04,540

PREDICATE LOGIC

00,967

Predicate Differences and the Analysis of Dependencies in Formal Specifications.

PB96-160940

01,759

Technique for Analyzing the Effects of Changes in Formal Specifications.
PB96-160957

01,760
PREDICTIONS

Prediction of Cracking in Reinforced Concrete Structures PB95-220448

03,725

\section{PREFABRICATED BUILDINGS}

Wind Load Provisions of the Manufactured Home Construction and Safety Standards: A Review and Recommendations for Improvement.

PB94-206125

00,428

Manufactured Homes: Probability of Failure and the Need for Better Windstarm Protection through Improved An for Better Windstorm Protection through Improved Anchoring Systems

Manufactured Housing Walls That Provide Satisfactory Moisture Performance in All Climates.

PB95-178885

00,383

Recommended Performance-Based Criteria for the Design of Manufactured Home Foundation Systems to Resist Wind and Seismic Loads.

PB96-128285

00,460

Mathematical Analysis of Practices to Control Moisture in the Roof Cavities of Manufactured Houses.

PB97-106843

00,278

PREFERRED CIRCUITS

Handbook Preferred Circuits Navy Aeronautical Electronic Equipment. Supplement Number 3.

00,026

Handbook Preferred Circuits Navy Aeronautical Electronic Equipment. Supplement Number 2.

00,027

A-A278 783/6

Handbook Preferred Circuits Navy Aeronautical Electronic
Equipment. Supplement Number 1 .
AD-A278 784/4 00,028 AD-A278 784/4

BTM

Interaction between Micro and Macroscopic Flow in RTM PB95-162012

03,159

PREISACH MODEL

Experimental Verification of a Vector Preisach Model.

PB95-163564

04,626

PRESENT WORTH

Present Worth Factors for Life-Cycle Cost Studies in the Department of Defense (1995)

PB95-105029

03,664

PRESSES

Equipment for Investigation of Cryogenic Compaction of Nanosize Silicon Nitride Powders. 1993 Report

03,028

PRESSURE

Pressure Dependencies of Standard Resistors.

PB95.153516

02,257

Intercomparison between NPL (India) and NIST (USA) Pressure Standards in the Hydraulic Pressure Region Up to $26 \mathrm{MPa}$.

PB96-113543

04,211

PRESSURE BROADENING

Memory Function Approach to the Shape of Pressure Broadened Molecular Bands.

PB95-152930

00,944

PRESSURE CALIBRATION SERVICE

NIST Measurement Services: NIST Pressure Calibration PB94-164043

02,892

PRESSURE CONTACTS

High Current Pressure Contacts to Ag Pads on Thin Film Superconductors.

PB95-168621

04,639

PRESSURE DEPENDENCE

Pressure Equations in Zone-Fire Modeling

PRESSURE GAGES

Intercomparison of the Effective Areas of a Pneumatic Piston Gauge Determined by Different Techniques. PB94-212370

Operational Mode and Gas Species Effects on Rotationa Drag in Pneumatic Dead Weight Pressure Gages. PB95.140182

00,903

PRESSURE GRADIENTS

Characteristics of Turbulence in a Boundary Layer with Zero Pressure Gradient

Single-Phase Heat Transter and Pressure Drop Charac eristics of an integral-Spine-Fin Within an Annulus. PB94-194073

03,805

Heat Transfer in Thin, Compact Heat Exchangers with Circular, Rectangular, or Pin-Fin Flow Passages. PB95-140943

02,751

Single-Phase Heat Transfer and Pressure Drop Characteristics of an Integral-Spine Fin Within an Annulus. PB97-122386

04,179

PRESSURE MEASUREMENT

NIST Measurement Services: NIST Pressure Calibration

SBrvice. 04043

02,892

Measurement of CO Pressures in the Ultrahigh Vacuum Regime Using Resonance-Enhanced Multiphoton-Ionization Time-of-Flight Mass Spectroscopy.

03,864

Look at Uncertainties over Twenty Decades of Pressure Measurement.

02,655

PC-Based Spinning Rotor Gage Controller.

02,609

Laser Photoionization Measurements of Pressure in Vacuum.

PB95-180600

03,964

Measurement of Very-Low Partial Pressures.

02,659

Process Monitoring with Residual Gas Analyzers (RGAs): Limiting Factors.

PB95-181004

02,660

Executive Summary: Proceedings of the Workshop on the Measurement of Transient Pressure and Temperature. PB96-160841

02,679

PRESSURE VAPOR TEMPERATURE

Virial Coefficients of Five Binary Mixtures of Fluorinated Methanes and Ethanes.

PB96-156054

PRESTRESSING

Partially Prestressed and Debonded Precast Concrete Beam-Column Joints.

PB95-153102

00,439

Seismic Performance Behavior of Precast Concrete Beam-Column Joints.

00,440

\section{PRETREATMENTS}

Effects of Aluminum Oxalate/Glycine Pretreatment Solutions on Dentin Permeability.

PRICES

Energy Prices and Discount Factors for Life-Cycle Cost Analysis 1994. Annual Supplement to NIST Handbook 135 and NBS Special Publication 709.

PB94-206018

02,508

Energy Price Indices and Discount Factors for Life-Cycle Cost Analysis 1995. Annual Supplement to NIST Handbook 135 and NBS Special Publication 709. (Revised).

PB95-105011

Energy Price Indices and Discount Factors for Life-Cycle Cost Analysis 1996. Annual Supplement to NIST Handbook 135 and NBS Special Publication 709. (Revised).

PB96.162441

02,510

Energy Price Indices and Discount Factors for Life-Cycle Cost Analysis 1997. Annual Supplement to NIST Handbook 135 and NBS Special Publication 709. (Revised).

PBMARY STANDARDS

NIST Measurement Services: NIST Pressure Calibration

PB94-164043

02,892

Absolute Determination of Electrolytic Conductivity for Primary Standard KC1 Solutions from 0 to $50 \mathrm{C}$.

PB94-172798

00,765

Automated, High-Precision Coulometric Titrimetry. Part 1 Engineering and Implementation. 
PROBABILITY DISTRIBUTION FUNCTIONS

Fluctuations in Probability Distribution on Chaotic Attractors.

PB96-102330

04,022

PROBABILITY THEORY

Three-Axis Coil Probe Dimensions and Uncertainties during Measurement of Magnetic Fields from Appliances.

Bounds on Frequency Response Estimates Derived from Uncertain Step Response Data.

PB96-122874

03,419

PROBE-POSITION ERRORS

Comparison of k-Correction and Taylor-Series Correction for Probe-Position Errors in Planar Near-Field Scanning. PB96-147137

02,012

PROBE TIP CALIBRATION

Verification of Commercial Probe-Tip Calibrations.
PB95-161576

Calibrating On-Wafer Probes to the Probe Tips.
PB95-163945

LRM Probe-Tip Calibrations with Imperfect Resistors and Lossy Lines.

02,353

Compensation for Substrate Permittivity in Probe-Tip Calibration.

PB95-203519

01,915

LRM Probe-Tip Calibrations Using Nonideal Standards.

PB96-135389 02,411
O

\section{PROBES}

Measurement of the Thermal Properties of Electrically Conducting Fluids Using Coated Transient Hot Wires.

DE94017816 00,722

User's Guide to NIST SRM 2084: CMM Probe Performance Standard

02,709

Two New Probes for a Coordinate Measuring Machine.

PB95-163093 02,653

PROBES (ELECTROMAGNETIC)

Standard Probes for Electromagnetic Field Measure-

PB94-185436

01,999

Optically Sensed EM-Field Probes for Pulsed Fields.
PB94-212594 130

General Order 'N' Analytic Correction of Probe-Position Errors in Planar Near-Field Measurements.

PB96-200688

01,562

PROCEEDINGS

Proceedings of the Annual Manufacturing Technology Conference (2nd): Toward a Common Agenda. Held in Gaithersburg, Maryland on April 18-20, 1995. PB96-112693

02,887

PROCESS CONTROL

NIST Support to the Next Generation Controller Program: 1991 Final Technical Report

02,808

Intelligent Processing of Materials, Technical Activities 1993 (NAS-NRC Assessment Panel, April 21-22, 1994). PB94-164183

Intelligent Processing of Materials.

PB94-172780

02.811

Intelligent Processing of Hot Isostatic Pressing.

PB94-172913

03,315

Integration of Real-Time Process Planning for SmallBatch Flexible Manufacturing

02,822

Development of Adaptive Control Strategies for Inert Gas Atomization.

PB95-162335

02,823

Process Modeling and Control of Inert Gas Atomization.

PB95-162343 02,824

Analysis of Autocorrelations in Dynamic Processes.

PB95-181228 02,826

Machine Performance Standard Provides Opportunity to Improve Ouality and Productivity.

PB96-154521

02,837

Unified Process Specification Language: Requirements for Modeling Process

ROCESS CONTROL (INDUSTRY)

02,850

Review of Cure Monitoring Techniques for On-Line Process Control.
PB94-216728

03,145

PROCESS MODELS

Product Realization Process Modeling: A Study of Requirements, M

02,836

PROCESSES

State-of-the-Art Survey of Methodologies for Represent-

ing Manufacturing Process Capabilities.

PB94-187655

02,812

PROCESSING \& PERFORMANCE OF MATERIALS

Reference Tables for Thermocouples

AD-A279 948/4

02,614

Low-Temperature Performance of Radiosonde Electric Hygrometer Elements.

00,121
Electromechanical properties of superconductors for DOE (U)

VAMAS interlaboratory comparisons of critical current vs. strain in $\mathrm{Nb}$ (sub 3) Sn.

DE95016656

04,433

Transverse stress effect on the critical current of internal in and bronze process $\mathrm{Nb}$ (sub 3 ) $\mathrm{Sn}$ superconductors. DE95016659

Measurement Methods and Standards for Processing and Application of Thermal Barrier Coatings.

01,447

Report on the Workshop on Manufacturing Polymer Com posites by Liquid Molding. Held in Gaithersburg, Maryland n September 20-22, 1993

03,131

Cylinder Wipe Air-Drying Intaglio Ink Vehicles for U.S. Currency Inks

03,115

Electrodeposition

PB94-172517

00,760

Intelligent Proce

02,811

Thermophysical Property Data for Supercritical Fluid Extraction Design
PB94-199221

00,668

Stability, Microstructural Evolution, Grain Growth, and Coarsening in a Two-Dimensional Two-Phase Microstructure

03,325

Hot-Deformation Apparatus for Thermomechanical Processing Simulation.

PB94-200136 03,207

Effects of Crystalline Anisotropy and Buoyancy-Driven

Convection on Morphological Stability.

03,328

Status of Electrocomposites.

03,143

Coating of Fibers by Colloidal Techniques in Ceramic Composites.

PB94-216256 03,196

Rapid Hot Pressing of Ultra-Fine PSZ Powders.

03,045

Optimization of Inert Gas Atomization.

PB95-107405

01,377

Parametric Investigation of Metal Powder Atomization Using Laser Diffraction.

03,342

Effect of Heterogeneous Porous Media on Mold Filling in Resin Transter Molding.

c3, 197

Novel Polydiacetylenes Derived from Liquid Crystalline $\begin{array}{ll}\text { Monomers. } & 01,235\end{array}$

New Materials, Advanced Ceramics and Standards.

PB95-140208 03,047

Nondestructive Evaluation and Materials Processing.
PB95-140455

Study of the Hydroxycarbonate Precursor Route to the YBa2Cu3O7-x High Tc Superconductor

04,540

Influence of Deposition Parameters on Properties of Laser Ablated YBa2Cu3O7-Delta Films. Laser Ablated YBa2Cu3O7-Delta Films.
PB95-140539 UV-Photopatterning of Alkylthiolate Monolayers Self-Assembled on Gold and Silver.

PB95-150751

00,924

Regimes of Surface Roughness Measurable with Light Scattering.
PB95-151213

04,265

Investigation into a Practical Grain Growth Model for Hot Isostatic Pressing.

PB95-151684

03,347

Deposit Forming Tendencies of Diesel Engine Oils-Correlation between the Two-Peak Method and Engine

PB95-152138

01,452

Ultrasonic Method for Reconstructing the Two-Dimensional Liquid-Solid Interface in Solidifying Bodies.

PB95-161782 03,349

Process Modeling and Control of Inert Gas Atomization.

PB95-162343 02,824

Fabrication of Flaw-Tolerant Aluminum-Titanate-Reinforced Alumina

03,161

Through-the-Arc Sensing for Real-Time Measurement of Gas Metal Arc Weld Ouatity.

PB95-162871

02,863

In-Space Welding: Visions and Realities.

04,830

Polyelectrolytes as Dispersants in Colloidal Processing of Silicon Nitride Ceramics.

PB95-175568

03,055

Process Monitoring with Residual Gas Analyzers (RGAs): Limiting Factors.

02,660

Novel YBa2Cu3O7-x and YBa2Cu3O7-x/Y4Ba3O9 Multilayer Films by Bias-Masked 'On-Axis' Magnetron Sputtering. 181186

04,690

IIW Commission V Ouality Control and Ouality Assurance of Welded Products Annual Report 1994/95.

02,866

Structural Ceramics Database. Topical Report, June 1989-May 1991.
PB95-203758 Database. Topical Report, June

03,060

Determination of the Residual Stresses Near the Ends of Skip Welds Using Neutron Diffraction and X-ray Diffraction Procedures.

PB95-253589

02,868

Simulation of $\mathrm{C} 60$ Through the Plastic Transition Temperatures.

Electronics Packaging Materials Research at NIST.

PB96-122692

02,405

Radiance Temperatures (in the Wavelength Range 523-

$907 \mathrm{~nm}$ ) of Group IVB Transition Metals Titanium, Zir-

conium, and Hafnium at Their Melting Points by a Pulse-

Heating Technique.

PB96-135025

02,677

High-Temperature Furnace for In situ Small-Angle Neutron Scattering during Ceramic Processing

03,743

Hygrothermal Effects on the Performance of Polymers and Polymeric Composites: A Workshop Report. Held in Gaithersburg, Maryland on September 21-22, 1995.

PB96-183207 003,180

Mapping the Droplet Transfer Modes for an ER100S-1 GMAW Electrode.

PB96-190095

Kinetic-Energy-Enhanced Neutral Etching

03,295

00,665

Need for Advanced Characterization Techniques in Product Manufacturing: A Case Study on Ceramic Matrix Composites.

Data Management for Error Compensation and Process Control.

PB97-110480

02,847

Characterization and Processing of Spray-Dried Zirconia

Powders for Plasma Spray Application.
PB97-111231

04,419

Vitrification and Crystallization of Organic Liquids Confined to Nanoscale Pores.

PB97-112304

03,392

In situ Characterization of Vapor Phase Growth of Iron Oxide-Silica Nanocomposites: Part 1. 2-D Planar LaserInduced Fluorescence and Mie Imaging.

PB97-112478

03,185

In-situ Studies of a Novel Sodium Flame Process for Synthesis of Fine Particles.

PB97-113047

00,681

Segmental Concentration Profiles of End-Tethered Polymers with Excluded-Volume and Surface Interactions.

00,654

PROCUREMENT

Videoconferencing Procurement and Usage Guide.

PB94-217023

01,470

Asynchronous Transfer Mode Procurement and Usage

Guide.

Federal Implementation Guideline for Electronic Data Interchange. ASC $\times 12 \quad 003050$ Transaction Set 850 Award Instrument. Implementation Convention. 


\section{KEYWORD INDEX}

Formulation of Position on U.S. Standards Role in Enterprise Integration

02,773

Proceedings Report of the International Invitation Work shop on Development Assurance. Held in Ellicott City, Maryland on June 16-17, 1994

02,912

PB95-189494

02,912

Product Models and Virtual Prototypes in Mechanical En
gineering.
PB95-253563 02,783

Product Realization Process Modeling: A Study of Requirements, M

02,836

Improving the Design Process by Predicting Downstream Values of Design Altributes.

PB97-113096

02,795

PRODUCT INSPECTION

Directory of U.S. Private Sector Product Certification Programs.

02,688

PRODUCT MODELS

Procedure for Product Data Exchange Using STEP Developed in the AutoSTEP Pilot.

PB96-183058

02,843

RODUCTION ENGINEERING

Design Engineering Research at NIST.

PB95-267860

02,784

PRODUCTION MANAGEMENT

Production Management Information Model for Discrete Manufacturing.

02,830

Roadmap for the Computer Integrated Manufacturing CIM) Application Framework.

PB96-122759 02,832

Conformance Testing and Specification Management.

PB97-113781

02,849

Proficiency Testing as a Component of Ouality Assurance in Construction Materials Laboratories.

in Construction

00,334

PROFICIENCY TESTS

Proficiency Tests for the NIST Airborne Asbestos Program, 1990.

00,535

Proficiency Tests for the NIST Airborne Asbestos Pro-

$\begin{array}{ll}\text { gram - } 1991 . & \\ \text { PB94-193828 } & 00,537\end{array}$

Proficiency Tests for the NIST Airborne Asbestos Pro-

gram - 1992.

00,539

Proficiency Tests for the NIST Airborne Asbestos Program, 1993.

00,610

ROFILE

Stylus Flight in Surface Protiling.

POFILES

02,675

Thermal Pulse Siudy of the Polarization Distributions Produced in Polyvinylidene Fluoride by Corona Poling at Constant Current

PB94-172293

01,195

PROFILOMETERS

Ultrasonic Measurements of Surface Roughness.
PB94-172137

Measuring Matching Wear Scars on Balls and Flats.

PB95-151528

03,153

PROGRAM SLICING

Software Safety and Program Slicing

PB95-125894

01,703

Unravel: A CASE Tool to Assist Evaluation of High Integrity Software. Volume 1. Requirements and Design.
PB95-267886 Unravel: A CASE Tool to Assist Evaluation of High Integ rity Software. Volume 2. User Manual.

PB95-267894

01,737

Slicing in the Presence of Parameter Aliasing.

Assessing Functional Diversity by Program Slicing

PB96-160890

03,734

Program Slicing

PB96-160981

01,761

PROGRAMMING ENVIRONMENTS

SOL Environments. Category: Software Standard; Subcategory: Database.

01,801

Introduction to the P1003.1g and CPI.C Network Application Programming Interfaces.

01,731

\section{OGRAMMING LANGUAGES}

COBOL. Category: Software Standard; Subcategory: Programming Language. Includes ANSI'S X3.23-1985, X3.23A-1989 and X3.23B-1993.

01,670

COBOL. Category: Software Standard; Subcategory: Programming Language. Part A.

01,671
COBOL. Category: Software Standard; Subcategory: Programming Language. Part $B$

01,672

Dynamic Objects and Meta-Level Programming of an EX. PRESS Language Environment.

01,774

\section{PROGRAMMING MANUALS}

DETAN 95: Computer Code for Calculating SpectrumAveraged Cross Sections and Detector Responses in Neutron Spectra.

PB95-242384

04,014

PROJECT MANAGEMENT

Framework for the Development and Assurance of High Integrity Softwar

01,716

PROMETHIUM

Hypertine Structure Investigations and Identification of New Energy Levels in the lonic Spectrum of (147) Pm.

PB96-180203

04,117

PROMPT GAMMA RADIATION

Grazing Incidence Prompt Gamma Emissions and Resonance-Enhanced Neutron Standing Waves in a Thin-Film. PB95-150470 03,892

PROPANE

Critical Properties and Vapor-Liquid Equilibria of the Binary System Propane + Neopentane

00,999

PROPANES

Vapor-Liquid Equilibria of Mixtures of Propane and Isomeric Hexanes.

00,995

Viscosity of 1,1,1,2,3,3-Hexafluoropropane and $1,1,1,3,3,3$-Hexafluoropropane at Saturated-Liquid Conditions from $262 \mathrm{~K}$ to $353 \mathrm{~K}$

03,292

PROPELLANTS

Thermal Decomposition Pathways in Nitramine Propellants.

03,753

\section{PROPER NAMES}

Prototype Information Retrieval System to Perform a Best-Match Search for Names.

PB95-181152

02,740

PROPERTIES

Metallurgy Technical Activities 1994 (NAS-NRC Assessment Panel, April 6-7, 1995

02,981

Guide to a Format for Data on Chemical Admixtures in a Materials Property Database.

01,327

Guide to a Format for Data on Chemical Admixtures in a Guide to a Format for Data on Chemical Admixtures in a
Materials Property Database. (Reannouncement with new

PB96-186192

01,328

PROPERTIES OF MATERIALS: ELECTRONIC/MAGNETIC/ OPTICAL

Magnetic Characteristics and Measurements of Filamentary Nb-Ti Wire for the Superconducting Super Collider.

DE94005988

03, 775

Filter Transmittance Measurements in the Infrared.

PB94-140589

04,224

Alternating-Field Susceptometry and Magnetic Susceptibility of Supercondoctors. Presented at Office of Naval Research Workshop on Magnetic Susceptibility of Superconductors and Other Spin Systems. Held in Berkeley Springs, West Virginia on 20 May 1991.

04,435

Photonic Materials: A Report on the Results of a WorkPhotonic Materials: A Report on the Results of a Work-
shop. Held in Gaithersburg, Maryland on August 26-27, 1992, Volume

PB94-152733 02,114

Monte Carlo and Mean-Field Calculations of the Magnetocaloric Effect of Ferromagnetically Interacting Clusters.

PB94-172087

03,201

Thermal Pulse Study of the Polarization Distributions Produced in Polyvinylidene Fluoride by Corona Poling at

PB94-172293 01,195

Structural and Magnetic Ordering in Iron Oxide/Nickel Oxide Multilayers by $\mathrm{X}$-ray and Neutron Diffraction (Invited)

PB94-172558

04,442

Dispersions of Magnetic Excitations of the Pr lons in

PB94-173044 04,444

One Gigarad Passivating Nitrided Oxides for $100 \%$ Internal Ouantum Efficiency Silicon Photodiodes.

PB94-185485

02,119

Enhanced Magnetocaloric Effect in Gd3Ga5-xFexO12.

PB94-185659 04,450

Magnetocaloric Effect in Rapidly Solidified Nd-Fe-Al-B Materials

PB94-185667

04,451

Langevin Approach to Hysteresis and Barkhausen Modeling in Steel.
PB94-185675

Magnetocaloric Effect of Ferromagnetic Particles.

03,206

PB94-185857

04,453

PROPERTIES OF MATERIALS: ELECTRONIC/

\section{MAGNETICIOPTICAL}

NIST Response to the Fifth CORM Report on the Pressing Problems and Projected Needs in Optical Radiation Measurements
PB94-188240

04,227

Magnetic Dead Layer in $\mathrm{Fe} / \mathrm{Si}$ Multilayer: Profile Refinement of Polarized Neutron Reflectivity Data.

PB94-198363 04,458

Profile Fitting of $X$-Ray Diffraction Lines and Fourier Analysis of Broadening

PB94-198512

04,460

Meissner, Shielding, and Flux Loss Behavior in Single-

Crystal YBa2Cu3O6+X

PB94-198744

04,464

Magnetic Properties of Pd/Co Multilayers.

PB94-198751

04,465

Concurrent Enhancement of Kerr Rotation and Antiferromagnetic Coupling in Epitaxial $\mathrm{Fe} / \mathrm{Cu} / \mathrm{Fo}$ StrucAntiteric

PB94-198769

04,466

Precision Nuclear Orientation Measurements for Determining Mixed Magnetic Dipole/Electric Ouadrupole Hyperine Interactions.

PB94-199080

03,810

Isotope Shifts and Hyperfine Splittings of the 398.8-nm Yb I Line.

03,814

Hysteresis Measurements of Remanent Polarization and Coercive Field in Polymers.

PB94-199767

04,475

PB94-200045

04,477

Correlation of $\mathrm{HgCdTe}$ Epilayer Defects with Underlyıng Substrate Defects by Synchrotron X-Ray Topography.
PB94-200714

O2, 129 rium Barium Copper Oxide (YBa2Cu3O7-delta).

PB94-211307

04,492

Magnetoelasticity in Rare-Earth Multilayers and Films.

PB94-211356 04,495

Roles of Copper in Applied Superconductivity.

PB94-211521

02,255

Wire Bond Testing

PB94-211653 02,314

Neutron Powder Diffraction Study of the Crystal Structure of YSr2AICu2O7.

PB94-212073

04,499

Offse: Susceptibility of Superconductors.

04,503

Correlations of Modulation Noise with Magnetic Microstructure and Intergranular Interactions for CoCrTa and $\mathrm{CoNi}$ Thin Film Media.

PB94-212768

Normal-Incidence Complex-Index Refractometry.

04,509

P

04,241

Magnetic and Structural Properties of Electrodeposited Copper-Nickel Microlayered Alloys.

PB94-213121 
Trapped Vortices in a Superconducting Microbridge. PB95-141149 04,554 Variation in Magnetic Properties of Cu/Fcc (001) Sandwich Structures.

PB95-141164. 04,555

Effects of Interfacial Roughness on the Magnetoresistance of Magnetic Metallic Multilayers.
PB95-150017 04,556 Magnetic Neutron Scattering (Invited)

04,557

PB95-150074

Polarization Analysis of the Magnetic Excitations in

Fe65Ni35 Invar.
PB95-150082

Method for Determining Both Magnetostriction and Elastic Modulus by Ferromagnetic Resonance.

PB95-150108

02,974

Temperature Dependence of the Magnetic Excitations in Ordered and Disordered Fe72Pt28.

PB95-150223

04,563

Use of Sum Rules on the Energy-Loss Function for the Evaluation of Experimental Optical Data.

04,264

Studies of the Higher Order Smectic Phase of the Large Electroclinic Effect Material W317.
PB95-151601 00,935 Coupled-Bilayer Two-Dimensional Magnetic Order of the Dy lons in Dy2Ba4Cu7O 15.
PB95-152104

04,584

Neutron-Scattering Studies of the Two Magnetic Correlation Lengths in Terbium.

PB95-152328

04,586

Comment on 'Phase Transitions in Antiferromagnetic Superlattices

04,587

Moisture and Water-Induced Crack Growth in Optical Materials.

PB95-153334

04,267

Neutron Powder Diffraction Study of the Nuclear and Magnetic Structures of the Oxygen-Deficient Perovskite YBaCuCoO5

PB95-161097

00,954

Harmonic and Static Susceptibilities of YBa2Cu3O7.

PB95-161139

04,599

Micromagnetic Structure of Domains in Co/Pt Multilayers. 1. Investigations of Wall Structure.

04,610

Magnetic Rare Earth Artificial Metallic Superlattices.

PB95-162293

04,611

Submicroampere-Per-Root-Hertz Current Sensor Based

on the Faraday Effect in Ga: YIG.

PB95-162467

02,155

Surface Magnetic Microstructural Analysis Using Scanning Electron Microscopy with Polarization Analysis PB95-162657

03.938

Nanocomposite Magnetic Materials.

PB95-162780

Magnetocaloric Effect in Nanocomposites.

04,617

PB95-162798

04,618

Systematic Studies of the Effect of a Bandpass Filter on a Josephson-Junction Noise Tnermometer.

PB95-162970

03,939

Observations of Partial Discharges in Hexane Under High Magnification.
PB95-163127

01,900

Determination of Thermoactivation Parameters of Vortex Mobility in YBa2Cu3O7 Using Only Magnetic Measure ments

PB95. 163499

04.624

SEMPA Studies of Oscillatory Exchange Coupling.

B95-163556 04,625

Experimental Verification of a Vector Preisach Model.

PB95-163564 04,626

High Critical Temperature Superconductor Tunneling Spectroscopy Using Squeezable Electron Tunneling Junctions.

04,627

Tunneling Measurement of the Zero-Bias Conductance Peak and the Bi-Sr-Ca-Cu-O Thin-Film Energy Gap.

PB95-163739

04,628

Field Dependence of the Magnetic Ordering of $\mathrm{Cu}$ in $\mathrm{R} 2 \mathrm{CuO} 4$ ( $\mathrm{R}=\mathrm{Nd}, \mathrm{Sm}$ ).

04,633

Observation of Noncollinear Magnetic Structure for the Cu Spins in Nd2CuO4-Type Systems.

PB95-164539

04,634

Magneto-Optic Magnetic Field Sensors Based on Uniaxial ron Gamet Films in Optical Waveguide Geometry. 02,159
Alternating-Field Susceptometry and Magnetic Susceptibility of Superconductors.

PB95-168613

04,638

Magnetic and Magnetoresistive Properties of Inhomogeneous Magnetic Dual-Layer Films. Insulating Boundary Layer and Magnetic Scattering in YBa2Cu307-delta/Ag Interfaces Over a Contact Resistivity Range of $10(-8)-10(-3)$ Omega cm(2)

04,648 Tilt Effects in Optical Angle Measurements.
PB95-169389

04,294

Thermal Noise in High-Temperature SuperconductingNormal-Superconducting Step-Edge Josephson JuncNormat

95-175089

04,650

Increased Pinning Energies and Critical Current Densities in Heavy-Ion-Irradiated Bi2Sr2CaCu2O8 Single Crystals.
PB95-175352

Determination of the Optical Constants of ZnSe Films by Spectroscopic Ellipsometry.

04,656

Surface Degradation of Superconducting YBa2Cu307. delta Thin Films.

PB95-176095

04,667

Magnetic Force Microscopy Images of Magnetic Garnet with Thin-Film Magnetic Tip.

04,669

Crossover in the Pinning Mechanism of Anisotropic Fluxon Cores.

PB95-180170

04,673

Size and Self-Field Effects in Giant Magnetoresistive Thin-Film Devices.

PB95-180188 04,674

Novel Bulk Iron Garnets for Magneto-Optic Magnetic Field Sensing.

PB95-180204

04,675

Magnetic Susceptibility of $\mathrm{Prz}-\mathrm{xCexCuO} 4$ Monocrystals and Polycrystals.

Broadband High-Optical-Density Filters in the Infrared.

PB95-180261 04,309

Crystal Structure and Magnetic Properties of $\mathrm{CuGeO}$

04,678

Observation of Oscillatory Magnetic Order in the Antiferromagnetic Superconductor HoNi2B2C.

PB95-180303

04,679

Dependence of Tc on Debye Temperature Theta(sub D) for Various Cuprates.

Magnetic Properties of Single-Crystalline UCu3AI2.

PB95-180717

04,683 Molecular Beam Epitaxy.

PB95-180725 Eplaxy.

Terminally Anchored Chain Interphases: Their Chromatographic Properties.
PB95.181061

07,272

Dielectric Properties of Thin Film SrTiO3 Grown on $\mathrm{LaAlO} 3$ with YBa2Cu3O7-x Electodes.

02.267

Domain Effects in Faraday Effect Sensors Based on Iron Domain Effects in Faraday Effect Sensors Based on Iron
Garnets.
PB95-202461
02,268

02,268 Single Photon Ionization, Laser Optical Probe Technique
for Semiconductor Growth.

01,032

Determining the Magnetic Properties of $1 \mathrm{~kg}$ Mass Stand. ards.

04,016

Determination of the Transmittance Uniformity of Optical Filter Standard Reference Materials.

02,182

$30 \mathrm{THz}$ Mixing Experiments on High Temperature

Superconducting Josephson Junctions.

04,709

Magnetostriction and Giant Magnetoresistance in Annealed NiFe/Ag Multilayers.

PB96-102603

04,716

Total-Dielectric-Function Approach to Electron and Phonon Response in Solids.

01,067

Nanoscale Study of the Hydrogenated Amorphous Silicon Surtace.

PB96-103056

04,720

Recent Results in Magnetic Force Microscopy.
PB96-103130

Magnetic Structure and Spin Dynamics of the $\mathrm{Pr}$ and $\mathrm{Cu}$ in Pr2CuO4.

PB96-111836

04,036

Temperature and Field Dependence of Flux Pinning in NbTi with Aritificial Pinning Centers.

04,726

Current and Voltage Measurements in the Gaseous Electronics Conference RF Reference Cell.

PB96-113337

02,388

Magnetooptic Effects.

PB96-119292 04,338

Using Secondary Ion Mass Spectrometry (SIMS) to Char acterize Optical Waveguide Materials.

PB96-119599

04,340

Structural and Magnetic Properties of $\mathrm{CuCl} 2$ Graphite Intercalation Compounds.

03,020

Decay of Bragg Gratings in Hydrogen-Loaded Optical Fi-

PB96-122643

04,345

Zimm Plot and Its Analogs as Indicators of Vesicle and

Micelle Size Polydispersity.
PB96-123765

01,094

Low-Temperature Properties of Silver.

PB96-126198 03,361

Coexistence of Grains with Differing Orthorhombicity in High Quality YBa2Cu3O7-delta Thin Films.

PB96-135033

Growth of Epitaxial KNbO3 Thin Films.

PB96-13518

02,409

Effects of Etching on the Morphology and Surface Resistance of YBa2Cu3O7-delta Films.

PB96-135355

02,410

Dielectric Spectroscopic Determination of Temperature Behavior of Electroclinic Parameters in the Liquid Crystal

01,098

$X$-ray Observation of Electroclinic Layer Constriction and Rearrangement in a Chiral Smectic-A Liquid Crystal.

PB96-141080

Evidence for Tunneling and Magnetic Scatttering at 'In Evidencer

04.752

Effect of Magnetic Field Orientation on the Critical Current of HTS Conductor and Coils.

PB96-141189

02,956

Quench Energy and Fatigue Degradation Properties of $\mathrm{Cu}$ - and Al/Cu-Stablized $\mathrm{Nb}-\mathrm{Ti}$ Epoxy-Impregnated Suls.

04,755

Oxygen Annealing of Ex-situ YBCO/Ag Thin-Film Inter-

PB96.141312 04,758

Domain Structures in Magnetoresistive Granular Metals.

PB96-141346 04,760

Dielectric Measurements on Printed-Wiring and Circuit Boards, Thin Films, and Substrates: An Overview.

PB96-147038

02,236

Inch Effect in

01,125

Heterodyne Mixing and Direct Detection in High Tem.

perature Josephson Junctions.
PB96-147202

Annealing of Bragg Gratings in Hydrogen-Loaded Optical

Comparison of UV Photosensitivity and Fluorescence during Fiber Grating Formation.

PB96-155445

04,362

PB96-155510

02,237

NIST Metrology Program on Electromagnetic Characterization of Mate
PB96-156062

01,944

Applicability of Effective Medium Theory to Ferroelectric/ Ferrimagnetic Composites with Composition and Frequency-Dependent Complex Permittivities and Permeabilities.

PB96-157854

01,945 


\section{KEYWORD INDEX}

PROPERTIES OF MATERIALS: STRUCTURAL/MECHANICAL

High Frequency Magnetic Field Sensors Based on the Faraday Effect in Garnet Thick Films.

PB96-190384

02,282

Metallic-Barrier Junctions for Programmable Josephson Voltage Standards

02,089

Observation of the Transverse Second Harmonic Magneto-Optic Kerr Effect from Ni81Fe19 Thin Film Structures.

PB96-200332

01,971

Magnetic Flux Pinning in Epitaxial YBa2Cu307-delta Thin Films.

04,795

Wideband Current and Magnetic Field Sensors Based on Iron Garnets.

01,975

Spin-Dependent Interface Transmission and Reflection in

Magnetic Multilayers (Invited).
PB96-201173

04,130

Windowing Effects on Light Scattering by Sinusoidal SurPB97.

04,389 Size Effects and Giant Magnetöresistance in Unannealed
NiFe/Ag Multilayer Stripes. NiFe/Ag Multilayer Stripes.
PB97-111306

Intrinsic Conductivity of Objects Having Arbitrary Shape and Conductivity

04,150

Observation of Two Length Scales Above ( $T$ sub $N$ ) in a Holmium Thin F

PB97-111942

04,151

Observation of Hot-Electron Shot Noise in a Metallic Re-

sistor.

01,988

Forward Scattering of a Gaussian Beam by a Nonabsorbing Sphere.

Bias Current Dependent Resistance Peaks in NiFe/Ag Giant Magnetoresistance Multilayers.

04,153 PB97-112346

tism in

Isolated Spin Pairs and Two-Dirn

PB97.112387

04,154

Neutron Scattering Study of Antiferromagnetic Order in the Magnetic Superconductors RNi2B2C. 04,812
PB97-112411 Unconventional Ferr
x) Ca(sub x) MnO3.

PB97-112429

04,156

Modeling Effects of Temperature Annealing on Giant Magnetoresistive Response in Discontinuous Multilayer NiFe/Ag Films Simulating Device Size Effects on Magnetization Pinnıng Mechanisms in Spin Valves.

PB97-112593

04,158

Colossal Magnetoresistance without $\mathrm{Mn}(3+) / \mathrm{Mn}(4-)$ Double Exchange in the Stoichiometric Pyrochlore TI2Mn2O7.

Radiance Temperatures at $1500 \mathrm{~nm}$ of Niobium and Molybdenum at Their Melting Points by a Pulse-Heating Technique.

PB97-118699

04,167

Vortex Images in Thin Films of $\mathrm{YBa} 2 \mathrm{Cu} 3 \mathrm{O}$ (sub $7-\mathrm{x}$ ) and Bi2Sr2Ca1Cu2O(sub $8+x$ ) Obtained by Low-Temperature Magnetic Force Microscopy.

PB97-119408

04.815

Antiferromagnetic Interlayer Correlations in Annealed Ni80Fe20/Ag Multilayers.

PB97-122220

03,109

Large Local-Field Corrections in Optical Rotatory Power of Ouartz and Selenium.

04,400

Determination of Anomalous Superexchange in $\mathrm{MnCl} 2$ and Its Graphite Intercalation Compound.

00,666

\section{PROPERTIES OF MATERIALS: STRUCTURAU}

ECHANICAL

Effect of Green Density and the Role of Magnesium Oxide Additive on the Densification of Alumina Measured by Small-Angle Neutron Scattering. (Reannouncement with New Availability Information)

AD-A244 582/3

03,022

Electrochemical Synthesis of Metal and Intermetallic Composites.

AD-A294 088/0 03,304

Review of Corrosion Behavior of Ceramic Heat Exchanger Materals: Corrosion Characteristics of Silicon Carbide and Silicon Nitride. Final Report, September 11 1992--March 11, 1993.

DE93041307

03,228

Low temperature fabrication from nano-size ceramic powders.

03,029

DE95013505

02250

DOE Fusion Applications.

PB94-139672

Wavelengths and Energy Levels of Neutral $\mathrm{Kr}(84)$ and Level Shifts in All Kr Even Isotopes.

03,780
Corrosion Resistance of Materials for Renovation of the United States Botanic Garden Conservatory.

PB94-154390

00,032

Precision Comparison of the Lattice Parameters of Silicon Monocrystals.

04,438

RDFs and $\mathrm{Fe}-\mathrm{Fe}$ Pair Correlations in an AlCuFe Icosahedral Alloy by Double Isotopic Substitution.

PB94-172129

04,439

Wear Mechanism Maps of Ceramics.

PB94-172368 03,229

Shear-Excited Morphological States in a Triblock Copoly-

PB94-172392

01,196

Fracture Mechanics Analysis of Near-Surface Cracks.

PB94-172400 03,230

Nitrogen Effect on Elastic Constants of f.c.c. Fe-18Cr-

PB94-172541

03,203

Enhanced Curie Temperafures and Magnetoelastic Do-

mains in Dy/Lu Super Lattices and Films.

04,443

Effects of Elastic Stress on Phase Equilibrium in the $\mathrm{Ni}-\mathrm{V}$

System,
PB94-172707

03,313

Microstructure and Tensile Properties of Microalloyed PB94-172715

03,204

Considerations on Data Requirements for Tribological

Modeling.
PB94-172731 02,962

Wear of Selected Materials and Composites Sliding gainst MoS2 Films.

03,237

Temperature Dependence of the Morphology of Thin Diblock Copolymer Films as Revealed by Neutron Reflec PBivity.

01,199

Reaction Sensitivities of Al-Li Alloys and Alloy 2219 in Mechanical-Impact Tests.

PB94-172764

03,314

High Temperature Degradation of Structural Composites.

PB94-172848 03,132

Intelligent Processing of Hot Isostatic Pressing.

03,315

Temperature Increases in Aluminum Alloys during $\mathrm{Me}$ -

chanical-Impact Tests for Oxygen Compatibility.
03,316

Grafted Interpenetrating Polymer Networks.

PB94-185055

01,200

-Line Optical Monitoring of Injection Molding.

01201

Phase Behavior of a Hydrogen Bonding Molecular Com-

posite.

01,202

Effect of Backfill and Alomizing Gas on the Powder Porosity and Mechanical Properties of 304L Stainless Steel.

Influence of Surface Interaction and Chain Stiffness on Polymer-Induced Entropic Forces and the Dimensions of Confined Polymer

01,203

Fluorescence Monitoring of Polarity Change and Gelation during Epoxy Cure.

Electron Beam Crosslinking of Poly(vinylmethyl ether).
01,205

Viewpoint: Nanocrystalline and Nanophase Materials.

PB94-185865 04,454

Through-the-Arc Sensing for Monitoring Arc Welding.

Evaluation of Corrosion Data: A Review.

02,858

PB94-198348

03,187

Facile Synthesis of Novel Fluorinated Multifunctional Acrylates.

01,207

Effect of Transformation of Alloy on Transient and Residual Stresses in a Porcelain-Metal Strip.

PB94-198397

00,143

Weak-Link-Free Behavior of High Angle YBa2Cu3O7-x Grain Boundaries in High Magnetic Fields.

04,459

Microstrains and Domain Sizes in Bi-Cu-O Superconductors: An X-Ray Diffraction Peak-Broadening

Study.

04,461

Experimental Constraints on Some Mechanisms for HighTemperature Superconductivity.

04,463

Neutron Powder Diffraction Study of a Na, Cs-Rho Zeolite.

PB94-198629

00,791

Matrix Grain Bridging Contribution to the Toughness of Whisker Reinforced Ceramics.

03,134

Coherent Preciptates in the BCC/Orthorhombic Two Phase Field of the Ti-Al-Nb System.

03,317

Crystallographic Characterization of Some Intermetallic

Compounds in the Al-Cr System
PB94-198702

03,318

Analysis of SANS from Controlled Pore Glasses.

PB94-198843

03,035

Structures of Sodium Metal.

PB94-198850

Diffraction Imaging of Polycrystalline Materials.

PB94-198884

03,319

Pynamic Shear Modulus Measurements with Four Inde-

pendent Techniques in Nickel-Based Alloys.

PB94-198900

Disorder Trapping in Ni2TiAl

PB94-198942

Intrinsic Stress in DC Sputtered Niobium.

PB94-199031

Fluorescence Anisotropy Measurements on a Polymer Melt as a Function of Applied Shear Stress.

PB94-199296

01.209

Observations of Shear Induced Molecular Orientation in a Polymer Melt Using Fluorescence Anisotropy Measure-

Observations of Shear Siress and Molecular Orientation Using Fluorescence Anisotropy Measurements.

PB94-199312

01,211

LMTO/CVM and LAPW/CVM Calculations of the Nicke Aluminide/Nickel Titanium Pseudobinary Phase Diagram. Interface Properties for Ceramir Composites from a Single Fiber Pull-Out Test.

PB94-199361

03.135

Micro-Mechanical Aspects of Asperity-Controlled Friction in Fiber-Toughened Ceramic Composites.

(199536

03,136

Peeling a Polymer from a Surface or from a Line. PB94-199809

01,213

Cavitation Damage During Flexural Creep of SIAION. YAG Ceramics.

03,036

Crack Growth Resistance of Strain-Sottening Materials under Flexural Loading.

02,972

Generic Model for Creep Rupture Lifetime Estimation on Fibrous Ceramic Composites.

PB94-200235

03,137

Asymmetric Tip Morphology of Creep Microcracks Growing Along Bimaterial Interfaces.

PB94-200243 03,138

Analysis of Creep in a Si-SiC C-Ring by Finite Element Method.

PB94-200268

Incorporation of Gold into YBa2Cu3O7: Structure and Tc Enhancemen

Unexpected Effects of Gold on the Structure, Superconductivity, and Normal State of $\mathrm{YBa}_{\mathrm{B}} \mathrm{Cu} 3 \mathrm{O} 7$.

PB94-200284 04,482

Introduction of a NIST Instrument Sensitivity Standard Reference Material for X-Ray Powder Diffraction.
PB94-200318
00,807

Micromechanics of Densification and Distortion. 
Energy and Migration of Grain Boundaries in Polycrystals.
PB94-211638
03,332

Fabrication of Platinum-Gold Alloys in Pre-Hispanic South Fabrication of Platinum-Gold Alloys in Pre-Hispanic South Americ

trol.

03,333

Small-Angle $X$-Ray and Neutron Scattering Study of Block Copolymer/Homopolymer Mixtures.

01,221

Effect of Microstructure on the Wear Transition of Zirconia-Toughened Alumina.

03,141

Fast-Ion Conduction and Disorder in Cation and Anion Arrays in Y2(ZryTi(1-y))2O7 Pyrochlores Induced by $\mathrm{Zr}$ Substitution: A Neutron Rietveld Analysis.

04,496

Determination of the Prior-Austenitic Grain Size of Selected Steels Using a Molten Glass Etch.
PB94-211927

Crossover to Strong Shear in a Low-Molecular-Weight Critical Polymer Blend.

PB94-211976

01,222

Tensile Creep of Whisker Reinforced Silicon Nitride.
PB94-211984

Microstructural Evolution in Two-Dimensional Two-Phase Polycrystals.

04,498

Micromechanics of Fracture in Rubber-Toughened Epoxies.

03.011

Glass Transition of Organic Liquids Confined to Small Pores.

PB94-212305

00,833

Melting Behavior of Organic Materials Confined in Porous Solids.

00.834

Polymer Liquid Crystalline Materials.

01,224

Mechanism of Mild to Severe Wear Transition in AlphaAlumina

PB94-212354

03.233

Tribology Education: Present Status and Future Challenges.
PB94-212362

02,965

Beneficial Effects of Nitrogen Atomization on an Austenitic Stainless Steel

03,209

Effect of Cross-Links on the Miscibility of a Deuterated Polybutadiene and Protonated Polybutadiene Blend.
PB94-212438 Inversion of the Phase Diagram from UCST to LCST in Inversion of the Phase Diagram from UCST to LCST in
Deuterated Polybutadiene and Protonated Polybutadiene PBends.

01,226

Topological Influences on Polymer Adsorption and Desorption Dynamics.

01,227

Efficient Experiment to Study Superconducting Ceramics. PB94-212578 04,505 Thermodynamic Assessment and Calculation of the Ti-Al System.

PB94-212644

03,337

Compositional Homogeneity in Processing Precursor Powders to the Ba2YCu3O7-x High Tc Superconductor. 04,508

Imaging of Fine Porosity in a Colloidal Silica: Potassium Silicate Gel by Defocus Contrast Microscopy.

03,039

Effects of Variable Excluded Volume on the Dimensions of Off-Lattice Polymer Chains. of Off-Lattice Pol
PB94-212941

01,229

Observed and Theoretical Creep Rates for an Alumina Ceramic and a Silicon Nitride Ceramic in Flexure.

PB94-212958

03,040

Elastic Deformation of a Monolithic Perfect Crystal Interferometer: Implications for Gravitational Phase Shift Ex-

periments.

03,858

Dynamics vs. Static Young's Moduli: A Case Study.

PB94-213188

PB94-213196 04,513 Cast-Iron Elastic Constants: Effect of Graphite Aspect
Ratio.

PB94-213212

03,211

Correlation between Tc and Elastic Constants of (La$\mathrm{M}) 2 \mathrm{CuO} 4$.

04,514

Elastic Constants and Debye Temperature of Y1Ba2Cu3Ox: Effect of Oxygen Content. PB94-213352

04,515

Characterization of Phase and Surface Composition of Silicon Carbide Platelets.

03,043

Crystal Structure of Pb2Sr2YCu3O8+delta with delta=1.32, 1.46, 1.61, 1.71, by Powder Neutron Diffrac-

PB94-216314

04,518
Non-Osmotic, Defect-Controlled Cathodic Disbondment of a Coating from a Steel Substrate.

PB94-216447

03,120

Atomic Theory of Fracture of Brittle Materials: Application to Covalent Semiconductors.

PB94-216553

04.519

Crystal Structure of Dicalcium Potassium Trihydrogen Bis(pyrophosphate) Trihydrate.
PB94-216561

00,152

Review of Cure Monitoring Techniques for On-Line Process Control.

03,145

NIST Workshop on Nanostructured Material (1st): Report of an Industrial Workshop Conducted by the National Institute of Standards and Technology. Held in Gaithersburg, Maryland on May 14-15, 1992.

02,973

Physics Required for Prediction of Long Term Performance of Polymers and Their Composites. ance of Polym

03,146

Abrasive Wear by Diesel Engine Coal-Fuel and Related Particles.

PB95-104915

01,450

Localization Model of Rubber Elasticity: Comparison with Torsional Data for Natural Rubber Networks in the Dry

03,195

Aging in Glasses Subjected to Large Stresses and Defor-

mations.

PB95-107041

03,235

Crystal Structure of a New Sodium Zinc Arsenate Phase Solved by 'Simulated Annealing'.

00,870

Improved Crystallographic Data for Aluminum Niobate (AlNbO4)

PB95-107306 04,523

Electric Field Effects on Crack Growth in a Lead Magne-

Sium Niobate.

03,339

Macro- and Microreactions in Mechanical-Impact Tests of Aluminum Alloys.

PB95-107348

03,340

Influence of Specimen Absorbed Energy in LOX Mechanical-Impact Tests.

03,341

Rietveld Analysis of $\mathrm{NaxWO} 3+x / 2 . y \mathrm{H} 2 \mathrm{O}$, which Has the Hexagonal Tungsten Bronze Structure.

PB95-107371

04,524

Polyethylene Crystallized from an Entangled Solution $\mathrm{Ob}$ served by Scanning Tunneling Microscopy.

01,232

1.4-Dinitrocubane and Cubane under High Pressure.

03,755

Mathematical Aspects of Rietveld Refinement

PB95-108601

04,526

Response of a Terminally Anchored Polymer Chain to Simple Shear Flow.

01,233

Environmentally Enhanced Fracture of Ceramics.

PB95-125746

01,233

Determination of Fiber-Matrix Interfacial Properties of

portance to Ceramic Composite Toughening.

03,149

Control of Friction and Wear of Alpha-Alumina with a Composite Solid-Lubricant Coating.

03,225

Nature of (001) Tilt Grain Boundaries in YBa2Cu3O6+x.

PB95-126033 04,528

Characterization of Chemically Modified Pore Surfaces by Small Angle Neutron Scattering

PB95-126181

00,898

Inelastic Neutron Scattering Measurements of Phonons in Icosahedral Al-Li-Cu.

PB95-126215

04,532

Ashland Tank Collapse Investigation

PB95-126314

02,481

Effect of Mn Content on the Microstructure of Al-Mn Alloys Electrodeposited at $150 \mathrm{C}$.

PB95-126355

03,343

Influence of Physical Aging on the Yield Response of

Model DGEBA + Poly(propylene oxide) Epoxy Glasses.
PB95-126363
03,381

03,381

Volume Recovery in Epoxy Glasses Subjected

PB95-140018

03,382

Torsional Relaxation and Volume Response during Physical Aging in Epoxy Glasses Subjected to Large Torsional Deformations.

Defective Structures of Barium Yttrium Copper Oxide (Ba2YCu3Ox) and Ba2YCu3-yMyOz (M=Fe, Co, Al, Ga, PB' $95-140034$

04,537

Neutron Powder Diffraction Study of the Structures of

La1.9Ca1.1Cu2O6 and La1.9Sr1.1Cu2O6+Delta. PB95-140042

Normal Modes and Structure Factor for a Canted Spin
System: The Generalized Villain Model.
PB95-140067

Morphology of Symmetric Diblock Copolymers as Revealed by Neutron Reflectivity.

01,234

Determining Mobility from Homodyne ac Electrophoretic ight Scattering Light Scattering

Critical Factors in Non-Lubricated, Non-Abrasive Wear

Pesting.

03,236

Tribological Data: Needs and Opportunities.

PB95-140596

03,237

In situ Measurements of Chloride Ion and Corrosion Poential at the Coating/Metal Interface.

PB95-140893

03,122

Using Torsional Dilatometry to Measure the Effects of Deformations on Physical Aging.

PB95-140901

03,384

Aging Effects on XRF Measurements of Solder Coatings.

PB95-140927

03,123

Ca4Bi6013: A Compound Containing an Unusually Low Bismuth Coordination Number and Short Bi Bi Contacts.
00,911

00,911

Iradiation Damage in In

PB95-147351

03,705

Observed Frustration in Confined Block Copolymers.

PB95-150033

01,238

Phase Transitions in Solid C70: Supercooling, Metastable Phases, and Impurity Effect.

PB95-150090

00,914

Effect of a Crystal Monochromator on the Local Angular Divergence of an X-Ray Beam.

04,565

Structural Stabilization of Phase Separating PC/Polyester Blends through Interfacial Modification by Transesterification Reaction.

PB95-150454

01,239

Phonon Density of States in $\mathrm{R}_{2} \mathrm{CuO} 4$ and

Superconducting R1.85Ce0.15CuO4 ( $R=\mathrm{Nd}, \mathrm{Pr}$ ). PB95-150686

Lattice Imperfections Studied by Use of Lattice Green's Functions.

PB95-150850

04,576

Thermal Stability of Internal Electric Field and Polarization Distribution in Blend of Polyvinylidene Fluoride and Polymethylmethacrylate.

PB95-151072

01,240

New Alloys Show Extraordinary Resistance to Fracture and Wear.

03,346

Measuring Matching Wear Scars on Balls and Flats.

03,153

$X$-Ray Characterization of the Crystallization Process of High-Tc Superconducting Oxides in the $\mathrm{Sr}-\mathrm{Bi}-\mathrm{Pb}-\mathrm{Ca}-\mathrm{Cu}-$ O System.

04,579

$X$-Ray-Diffraction Study of a Thermomechanically Detwinned Single Crystal of $\mathrm{YBa2Cu} 3 \mathrm{O} 6+\mathrm{x}$.

PB95-151726

04,581 
PROPERTIES OF MATERIALS: STRUCTURALMECHANICAL

Surface Topography and Ordering-Variant Segregation in GalnP2.

PB95-153649

04,595

SANS Studies of Space-Time Organization of Structure in Polymer Blends.

PB95-153789

01,251

Composite Strut

02,507

Crystal Structure of Annealed and As-Prepared $\mathrm{HgBa} 2 \mathrm{CaCu} 2 \mathrm{O} 6+$ delta Superconductors.
PB95-161105

Cryogenic Toughness of Austenitic Stainless Steel Weld Metals: Effect of Inclusions.

03,214

Flat and Rising R-Curves for Elliptical Surface Cracks from Indentation and Superposed Flexure.

PB95-161295

03,156

Tensile Creep of a Silicon Nitride Ceramic.

03,049

Sputtered High Temperature Thin Film Thermocouples.

PB95-161311 02,259

Elastic Properties of Al2O3/Al Composites: Measurements and Modeling.

03,157

Analysis of Physical Properties of Ceramic Powders in an International Interlaboratory Comparison Program.

PB95-161501

03,050

Localization of a Homopolymer Dissolved in a Lamellar Structure of a Block Copolymer Studied by Small-Angle Neutron Scattering.

01,253

Effects of Anneal Time and Cooling Rate on the Formation and Texture of $\mathrm{Bi} 2 \mathrm{Sr} 2 \mathrm{CaCu} 2 \mathrm{O} 8$ Films.

PB95-161600

04,603

Molecular Weight Dependence of the Lamellar Domain Spacing of ABC Triblock Copolymers and Their Chain Conformation in Lamellar Domains.

01,254

PB95-161691

Neutron Scattering Study of Shear Induced Turbidity in
Polystyrene Dissolved in Dioctyl Phthalate.
PB95-161865

PB95-161865

Interaction between Micro and Macroscopic Flow in RTM
Preforms.

PB95-162012

03,159

Evidence of Film-Induced Cleavage by Electrodeposited Rhodium.
PB95-162327

03,191

Surface Roughness Evaluation of Diamond Films Grown on Substrates with a High Density of Nucleation Sites.
PB95-162418

Temperature Dependence of Vortex Twin Boundary Interaction in Yttrium Barium Copper Oxide (YBa2Cu3O6+x).
PB95-162442 Introduction to ASTM 1199 'Wear Test Selection for Design and Application'

PB95-162517 03,238

Description of Layered Structures: Applications to High Tc Superconductors

Recent VAMAS Activity in Ceramics.

04,615

Ca1-xCuO2, a NaCuO2-Type Related Structure.

03,051

Synthesis and Polymerization of Difunctional and Multifunctional Monomers Capable of $\begin{array}{ll}\text { Cyclopolymerization. } & 01,257\end{array}$

Elastic Properties of Central-Force Networks with BondLength Mismatch.

PB95-163366

Wear Model for Alumina Sliding Wear.

04,623

PB95-163796 03,239

Creep and Creep Rupture of Ceramic Matrix Composites.

Molecular Orbital Study of Water Enhanced Crack Growth Process

PB95-164067 03,240

Characterization of Molecular Network of Thermosets Using Neutron Scattering.

PB95-164109 01,259

Small-Angle Neutron Scattering of Poly(vinyl alcohol)

PB95-164117 01,260

Characterization of Polyquinoline Blends Using Small Angle Scattering.

01,261

Asperity-Asperity Contact Mechanisms Simulated by a Two-Ball Collision Apparatus.

PB95- 164158

02,966

Dislocation Emission at Ledges on Cracks.

04,630

Shielding of Cracks in a Plastically Polarizable Material.

PB95-164257 04,631

Small Angle Neutron Scattering Studies on Chain Asym-

metry of Coextru

01,262
Small Angle Neutron Scattering Study on Poly(N-Isopropyl Acrylamide) Gels Near Their Volume-Phase Transition Temperatur

PB95.164380 01,263

Small-Angle Neutron Scattering Study on Weakly Charged Temperature Sensitive Polymer Gels. $\quad 01,264$
PB95-164398

Influence of Lattice Mismatch on Indium Phosphide Based High Electron Mobility Transistor (HEMT) Structures Observed in High Resolution Monochromatic Synchrotron X-Radiation Diffraction Imaging.

PB95-164679

02,357

Low-Temperature Elastic Constants of $\mathrm{Y} 1 \mathrm{Ba} 2 \mathrm{Cu} 3 \mathrm{O} 7$.

PB95-168837 04,642

Crystal Structure of Calcium Succinate Monohydrat

PB95-168928 00,167

Ultrasonic Measurement of Residual Stress in Cast Steel Railroad Wheels.

Crystal Structure and Compressibility of 3:2 Mullite. PB95-175030 03,682

Competition between Hydrodynamic Screening ("Draining') and Excluded Volume Interactions in an Isolated Polymer Chain

01,265

Fabrication of Transparent gamma-Al2O3 from Nanosize

Particles.
PB95-175493

03,054

Rapid Post-Polist

04,301

Modified Surface Layers and Coatings.

PB95-176087

03,125

Elastic Constants of a Material with Orthorhombic Symmetry: An Alternative Measurement Approach.

PB95-180527

04,684

Structural and Chemical Investigations of $\mathrm{Na3}(\mathrm{ABO}$ 4)3.4H2O-Type Sodalite Phases.

PB95-180733 01,012

Powder Neutron Diffraction Investigation of Structure and Cation Ordering in Ba2+xBi2-xO6-y

Scanning Tunneling Microscopy of the Charge-DensityWave Structure in 1T-TaS2.

PB95-180980 04,689

Morphological Development of Second-Phase Particles in Elastically-Stressed Solids.

PB95-181111

03,355

Evaluation of Wear Resistant Ceramic Valve Seats in Gas-Fueled Power Generation Engines. Topical Report

December 1991-April 1994.

02,466

Application of ODF to the Rietveld Profile Refinement of Polycrystalline Solid

PB95-202388

03,401

Microstructure Study of Molybdenum Liners by Neutron Diftraction.

Off-Diagonal Orthorhombic-Symmetry Elastic Constants. PB95-202768

04,695

Determination of Complex Structures from Powder Diffraction Data: The Crystal Structure of La3Ti5Al15037.

rraction Data: The Crystal Siructure of La3Ti5Al15037.
PB95-202966

Boundary Lubrication of Silicon Nitride.

PB95-213583

03,226

Proceedings of the Applied Diamond Conference 1995: Applications of Diamond Films and Related Materials International Conference (3rd). Held in Gaithersburg, Maryland, on August 21-24, 1995.

PB95-255204

04,701

Applications of Diamond Films and Related Materials: International Conference (3rd). Held in Gaithersburg, Maryland on August 21-24, 1995. Supplement to NIST Special Publication 885

PB95-256053

03,063

Degradation of Powder Epoxy Coated Panels Immersed in a Saturated Calcium Hydroxide Solution Containing Sodium Chloride.

PB96-101050

01,344

Lattice Dynamics of $\mathrm{Ba} 1-\mathrm{xK} \times \mathrm{BiO} 3$

04,706

Structure and Conductivity of Layered Oxides $(\mathrm{Ba}, \mathrm{Sr}) \mathrm{n}+1(\mathrm{Sn}, \mathrm{Sb}) \mathrm{nO} 3 \mathrm{n}+1$

04,707

Lattice Dynamics of Semiconducting, Metallic, and Lattice Dynamics of Semiconducting, Metallic, and
Superconducting Ba1-xKxBiO3 Studied by Inelastic NeuSuperconducting Ba1-xKxBiO3 Studied by Inelastic Neu-
tron Scattering.
PB96-102447
O4,708

04,708
0

Oxygen Dependance of the Crystal Structure of
HgBa2CuO4+ and Its Relation to Superconductivity. Tensile Deformation-Induced Microstructures in FreeStanding Copper Thin Films.

04,715

$X$-ray Powder Diffraction from Carbon Nanotubes and Nanoparticles
PB96-102975

03,064

Damage Processes in Ceramics Resulting from Diamond

Toot Indentation and Scratching in Various Environments.
PB96-102983

Wear Transitions in Monolithic Alumina and Zirconia-Alumina Composites

03,168

Residual Stress in Induction-Heated Railroad Wheels: UItrasonic and Saw Cut Measurements. Report No. 28. PB96-106992 04,854 Factors Significant to Precracking of Fracture Specimens. Silicon Nitride Boundary Lubrication: Lubrication Mecha-

nism of Alcohols.
PB96-111703 03,067

Silicon Nitride Boundary Lubrication: Effect of PB96-111711

03,068

Wear Mechanism Maps of $440 \mathrm{C}$ Martensitic Stainless Steel.

Crystal Structure of Calcium Glutarate Monohydrate.

PB96-111893

04,834

Small Angle Neutron Scattering Study of the Structure and Formation of Ordered Mesopores in Silica. 03,069
PB96-111919

Structure of a Triglyceride Microemulsion: A Small Angle Neutron Scattering Study.

PB96-112255

01,077

Torsion Modulus and Internal Friction of a Fiber-Reinforced Composite.

PB96-112339

03,070

PB96-112362

02,073

ntrinsic Viscosity and the Polarizability of Particles Having a Wide Range of Shapes

03,170

Effect of $\mathrm{Sm} 2 \mathrm{BaCuO5}$ on the Properties of Sintered PB96-119441

04,733

Mechano-Chemical Model: Reaction Temperatures in a Concentrated Contact.

PB96-119466

03,227

Shear-Induced Martensitic-Like Transformation in a Block PB96-119508

01,277

Long-Lived Structures in Fragile Glass-Forming Liquids.

PB96-119565

Tribological Behavior of 440/Diamond-Like-Carbon Film

PB96-119714

03,019

Optical Sampling Using Nondegenerate Four-Wave Mixing in a Semiconductor Laser Amplifier.

Cavitation Contributes Substantially to Tensile Creep in Silicon Nitride
PB96-122577

03,171

Chemically Assisted Machining of Si3N4

PB96-122999

03,072

Properties and Mechanisms of Fast-Setting Calcium PBo6-1232 9 ments.

Flow-Induced Structure in Polymers: Chapter 16. ShearInduced Changes in the Order-Disorder Transition Temperature and the Morphology of a Triblock Copolymer.

Flow-Induced Structure in Polymer. Chapter 1. An Introduction to Flow-Induced Structures in Polymers.

PB96-123369

03,387

Characterization of the Vibrational Dynamics in the Octahedral Sublattices of LaD2.25 and LaH2.25.

01,091

Effects of Copper, Nickel and Boron on Mechanical Prop erties of Low-Alloy Steel Weld Metals Deposited at High Heat Input.

PB96-135231

03,363

Contact Tube Wear Detection in Gas Metal Arc Welding.

PB96-135330 02,872

Elastic Constants and Internal Friction of Polycrystalline Copper.

PB96-141015 
Electrodeposited Cobalt-Tungsten as a Diffusion Barrier between Graphite Fibers and Nickel.

03.176

Neutron Scattering Study of the Lattice Modes of Solid Cubane.

PB96-147152

01,126

Shear-Induced Mixing in Polymer Blends

01,287

Effects of Substrate Surface Steps on the Microstructure of Epitaxial Ba2YCu3O7-x Thin Films on (001) LaAlO3. 04,774
PB96-148184 International Institute of Welding: Report on 1995 Actions. PB96-158076 02,874 Microstructural Characterization of Cobalt-Tungsten Coated Graphite Fibers.

PB96-159231

01,951

Analysis of Small-Angle Scattering Data Dominated by Multiple Scattering for Systems Containing Eccentrically Shaped Particles or Pores.

PB96-160411

03,075

Tetrahedral-Framework Lithium Zinc Phosphate Phases: Location of Light-Atom Positions in $\mathrm{LiZnPO} 4 \mathrm{H} 2 \mathrm{O}$ by Powder Neutron Diffraction and Structure Determination
of LiZnPO4 by ab Initio Methods.

Characterization of the Structure of TbD2.25 at $70 \mathrm{~K}$ by Neutron Powder Diffraction.

PB96-160528

01.130

Neutron Spectroscopic Comparison of beta-Phase Rare

Earth Hydrides.

01,134

Powder X-ray Diffraction Data for $\mathrm{Ca} 2 \mathrm{Bi} 2 \mathrm{OS}$ and $\mathrm{C} 4 \mathrm{Bi6013.}$

PB96- 161278

04,777

Friction Processes in Brittle Fracture.

03.076

Nonlocal Effects of Existing Dislocations on Crack-Tip Emission and Cleavage.

03,367

Fundamentals of Fracture: A 1993 Prologue, and Other Comments.

PB96-161971

03,218

Interfacial Crack in a Two-Dimensional Hexagonal Lattice. PB96-161989 04,100

Whither Computational Materials Science. Some Thoughts from the Mechanical Properties Front.
02,987

02,987

Fracture in Multilayers.

PB96-163613

02,988

Influence of Shear on the Ordering Temperature of a Triblock Copolymer Melt.

Model for Toughness Curves in Two-Phase Ceramics. 2. Microstructural Variables.

PB96-163795

03,078

REFPROP Refrigerant Properties Database: Capabilities, Limitations, and Future Directions.

PB96-167150

01,149

Preparation and Crystal Structure of Sr5TiNb4017.

PB96-167341

04,107

Solid State (13)C NMR and Raman Studies of Cellulose Triacetate: Oligomers, Polymorphism, and Inferences

about Chain Polarity

01.289

Estimation of the Orientation Distribution of Short-Fiber Composites Using Ultrasonic Velocities.

PB96-176656

03,178

Objective Evaluation of Short-Crack Toughness Curves Using Indentation Flaws: Case Study on Alumina-Based Ceramics.

03,079

Flaw Tolerance and Toughness Curves in Two-Phase Particulate Composites: SiC/Glass System

03,081

Effect of Grain Size on Hertzian Contact Damage in Alumina.

PB96-179601

03.083

Indentation Fatigue: A Simple Cyclic Hertzian Test for Measuring Damage Accumulation in Polycrystalline $\mathrm{Ce}$ ramics.

PB96-180013

03,084

Diffusion of Water along 'Closed' Mica Interfaces.

PB96-180039

02.993

Model for Toughness Curves in Two-Phase Ceramics. 1.

Basic Fracture Mechanics.
PB96- 180088

03,085

Effect of Chemical Interaction on Barenblatt Crack Profiles in Brittle Solids.

PB96-180245

02.996

Pressurized Internal Lenticular Cracks at Healed Mica

Interfaces.
PB96-180252 02,997

Characterization of the Structure of YD3 by Neutron Powder Diffraction

01,161

Power Characteristics in GMAW: Experimental and Numerical Investigation

03,296
Aluminum-Lithium Alloys: Evaluation of Fracture Toughness by Two Test Standards, ASTM Method E 813 and E 1304.

PB96-190236 03,374 Light-Weight 03,375 Temperature-Induced Transition in Ductile Fracture Appearance of a Nitrogen-Strengthened Austenitic Stainless Steel.

PB96-190269

03,221

Ductile Fracture and Tempered Martensite Embrittlement of 4140 Steel.

03,222

Fatigue Crack Thresholds of a Nickel-Iron Alloy for Superconductor Sheaths at $4 \mathrm{~K}$. PB96-190343 03,223 Mechanical Properties and Warm Prestress of Ultra-Low
Carbon Steel at $4 \mathrm{~K}$.
PB96-190350
03,224

Orthotropic Elastic Constants of a Boron-Aluminum FiberReinforced Composite: An Acoustic-Resonance-Spectroscopy Study.

Elastic Constants and Microcracks in YBa2Cu3O7.

PB96-200761

03,182

Multimedia Tutorial on Phase Equilibria Diagrams.
PB96-200829 03,005 NMR Characterization of Injection-Moulded Alumina Green Compacts. Part 2. T2-Weighted Proton Imaging.
PB96-201181 165

Bulk Modulus and Young's Modulus of Nanocrystalline gamma-Alumina.

Cavity Evolution during Tensil Creep of Si3N4.
PB96-204193

Shear Suppression of Critical Fluctuations in a Diluted Polymer Blend.

04,418

Experimental Assessment of Crack-Tip Dislocation EmisExperimental Assessment of Crack-Tip Dislocation Emis-
sion Models for an Al67Cr8Ti25 Intermetallic Alloy.
PB96-204466

04,797 Alvin Van Valken

Phase Separation in Thin Film Polymer Blends With and Without Block Copolymer Additives.

PB96-204482

01,294

Thermal Wave NDE of Advanced Materials Using Mirage Effect Detection
PB96-204516

04,191

Creep and Creep Rupture of Structural Ceramics. 03,093

Fracture Mechanism Maps: Their Applicability to Silicon Nitride.

PB96-204532

03.094

Mathematical Analysis of Practices to Control Moisture in the Roof Cavities of Manufactured Houses.

00,278

CRYSTMET: The NRCC Metals Crystallographic Data CRYSTMET: The NRCC Metals Crystallographic Data
File.
PB97-109029
04,799 Inorganic Crystal Structure Database (ICSD) and Standorganic Structure Types (TYP|X): Two Tools for Inorganic Chemists and Crystallographers.

PB97-109037

00,648

Evaluation of Crystallographic Data with the Program DI-

PB97-109045 00,649

Cambridge Structural Database (CSD): Current Activities and Future Plans.

PB97-109052

00,516

Protein Data Bank: Current Status and Future Challenges.

00.517

Nucleic Acid Database: Present and Future.

00,518

Powder Diffraction File: Past, Present, and Future.

NIST Crystallographic Databases for Research and Anal-

PBsis. 109094

04,801

Conventional and Eccentric Uses of Crystallographic Databases in Practical Materials Identification Problems.
PB97-109102

04.802

Using NIST Crystal Data within Siemens Software for Four-Circle and SMART CCD Diffractometers.

PB97-109110

04,803

Phase Identification in a Scanning Electron Microscope Using Backscattered Electron Kikuchi Patterns. 04804 Biological Macromolecule Crystallization Database and NASA Protein Crystal Growth Archive.

PB97-109136

01,171

Investigations of the Systematics of Crystal Packing Using the Cambridge Structural Database.

00.519

Troublesome Crystal Structures: Prevention, Detection. and Resolution

01,172

CIF Crystallographic Information File: A Standard for Crystallographic Data Interchange.

PB97-109169

04,805

Role of Journals in Maintaining Data Integrity: Checking of Crystal Structure Data in 'Acta Crystallographica'.
PB97-109177 04,806 Electronic Publishing and the Journals of the American Chemical Society.

PB97-109185

04,807

How the Cambridge Crystallographic Data Centre Obtains Its Information.

04,808

Data Import and Validation in the Inorganic Crystal Structure Database.

PB97-109201

World Wide Web for Crystallography.

PB97-109219

04,809

Workshop Highlights

PB97-109227

04,810

Flaw-Insensitive Ceramics.

04,811

P97-110027

03,095

Tension/Compression Creep Asymmetry in Si3N4.

PB97-110258

03,096

Composites.

03,097

Database Development and Management (Project A.2.2)

The Annual Report for 1992-1993.

PB97-110290

03,098

Role of Corrosion in a Material Selector Expert System

for Advanced Structural Ceramics.

03,099

Flaw-Tolerance and Crack-Resistance Properties of Alumina-Aluminum Titanate Composites with Tailored Microstructures.

PB97-110324

03,101

In situ-Toughened Silicon Carbide.

03,102

High Temperature Structural Reliability of Siticon Nitride.

PB97-110456 03,104
O

Tensile Creep Testing of Structural Ceramics.

Lattice Model of a Hydrogen-Bonded Polymer Blend.

PB97-112262 03,391

Evidence of Crosslinking in Methyl Pendent PBZT Fiber.

PB97-112486 03,393

Relaxation After a Temperature Jump Within the One Phase Region of a Polymer Mixture.

PB97-112494

03,394

Microstructure Effect on the Phase Behavior of Blends of Deuterated Polybutadiene and Protonated Polyisopene.

01,296

Excitons in Complex Quantum Nanostructures.

PB97-118343

01,184

Swelling

PB97-118400

and

Effect of Beam Voltage on the Properties of Aluminum Nitride Prepared by Ion Beam Assisted Deposition. PB97-118616

01,995 
Assessment of the Al-Sb System

PB94-200474

03,329

Conditions for Existence of a Reentrant Solid Phase in a Sheared Atomic Fluid.

04,198

Analysis of Thermal Wave Propagation in Diamond Films.

Analysis of Thermal Wave Propagation in Diamond Films.
PB94-211471

03,014

PB94-211489

03,015

Equation of State Formulation of the Thermodynamic Properties of R134a (1,1,1,2-Tetrafluoroethane). $\quad 03,256$
PB94-212081

Prediction of Viscosity of Refrigerants and Refrigerant Mixtures.

PB94-212099

03,257

Prediction of the Thermal Conductivity of Refrigerants and Refrigerant Mixtures.

03,258

Anomalous Freezing and Melting of Solvent Crystals in Swollen Gels of Natural Rubber

PB94-212321

01,223

Thermal Conductivity of R134a.

03,857

Modified Leung-Griffiths Model of Vapor-Liquid Equilibrium: Extended Scaling and Binary Mixtures of Dissimilar Fluids.

PB94-216108

00,851

Calculation of Enthalpy and Entropy Differences of NearCritical Binary Mixtures with the Modified Leung-Griffiths

$\begin{array}{ll}\text { PB95-108635 00,885 } & 00 \\ \text { Polarized Transient Hot Wire Thermal Conductivity Meas- }\end{array}$ urements.

PB95-108817

00,886

Thermodynamic Properties of the Methane-Ethane Sys-

tem.

00,891

Thermophysical Property Computer Packages from NIST.

PB95-125787 04,203

Effect of Hydrodynamic Interactions on a Terminally Anchored Bead-Rod Model Chain.

01,237

01,237
Crystal Chemistry and Phase Equilibrium Studies of the $\mathrm{BaO}(\mathrm{BaCO}$ )-R2O3-CuO Systems. 5. Melting Relations in $\mathrm{Ba} 2(\mathrm{Y}, \mathrm{Nd}, \mathrm{Eu}) \mathrm{Cu} 3 \mathrm{O} 6+\mathrm{x}$

PB95-151718

04,580

Speed-of-Sound Measurements in Liquid and Gaseous

PB95-151957

04,186

Single-Photon Ionization and Detection of $\mathrm{Ga}$, In, and As(sub n) Species in GaAs Growth.

00,591

Thermal Behavior of 4-Maleimidophenyl Glycidyl Ether Resins.

PB95-153151 01,249

Rotational Dynamics of Solid C70: A Neutron-Scattering Study

00,949

Grain Alignment and Transport Properties of Bi2Sr2CaCu2O8 Grown by Laser Heated Float Zone

PB95-161451

04,602

Real-Time Small-Angle X-Ray Scattering Study of the Early Stage of Phase Separation in the SiO2-BaO-K20

Pystem.

03,052

Phase Composition, Viscosities, and Densities for Aqueous Two-Phase Systems Composed of Polyethylene Gly$\mathrm{col}$ and Various Salts at $25 \mathrm{C}$

PB95-164596

00,986

Reference Data for the Thermophysical Properties of Cryogenic Fluids.

03,263

Thermodynamic Properties of R134a(1,1,1,2-

Tetrafluoroethane).

PB95-168704

Relationship between Bulk-Modulus Temperature Dependence and Thermal Expansivity.

PB95-168829

04,641

High-Temperature Adiabatic Calorimeter for Constant-Volume Heat Capacity Measurements of Compressed Gases and Liquids.

00,989

Measurements of Molar Heat Capacity at Constant Volume (Cv) for 1,1,1,2-Tetrafluoroethane (R134a).
PB95-168878 Development of a Dual-Sinker Densimeter for High-Accuracy Fluid P-V-T Measurements.

PB95-168951

03,267

Need for, and Availability of, Working Fluid Property Data: Results from Annexes XIII and XVIII.

03,268

Enhanced Flux Pinning via Chemical Substitution in Bulk Superconducting $\mathrm{T} 1-2212$

PB95-169033

04,647

Measurement of the Thermal Properties of Electrically

Conducting Fluids Using Coated Transient Hot Wires.

PB95-169058

03,269
Measurement of Diffusion in Fluid Systems: Applications o the Supercritical Fluid Region.

02,490

Supercritical Fluid Extraction of Biological Products.

PB95-17520400,040

Fugacity Coefficients of Hydrogen in (Hydrogen + BuFane)

02,491

Vapor-Liquid Equilibria of Mixtures of Propane and Iso-

meric Hexanes.
PB95-175287 00,995

Thin Film Reaction Kinetics of Niobium/Aluminum Multilayers.

04,651

Measurements of the Viscosities of Saturated and ComMeasurements of the Viscosities of Saturated and Compressed Liquid 1,1,1,2-Tetrafluoroethane (R134a), 2,2Dichloro-1,1,1-Trifluoro
Fluoroethane (R141b).

Fluoroethane

03,273

Solubility Measurement by Direct Injection of Supercritical-Fluid Solutions into a HPLC System. PB95-175626

0,997

Critical Properties and Vapor-Liquid Equilibria of the $\mathrm{Bi}$ nary System Propane + Neopentane.

$175683 \quad 00,999$

Coexisting Densities, Vapor Pressures and Critical Densities of Refrigerants R-32 and R-152a, at $300-385 \mathrm{~K}$.

Predictive Extended Corresponding States Model for Pure and Mixed Refrigerants Including an Equation of State for

PB95-175717

03,275

Correlation of the Ideal Gas Properties of Five Aromatic Hydrocarbons.

PB95-175816 01,002

Gas Transport Properties of Solution-Cast Perfluorosulfonic Acid lonomer Films Containing lonic Surfactants.

Composition Dependence of a Field Variable Along the Binary Fluid Mixture Critical Locus.

PB95-176038 01,003

Transport and Diffusion in Three-Dimensional Composite

PB95-176129

04,668

In-situ Monitoring of Molecular Beam Epitaxial Growth Using Single Photon Ionization.

01,023

Direct Detection of Atomic Arsenic Desorption from Si( 100$)$

PB95-202230

01,024

aser Vacuum Ultraviolet Single Photon Ionization Prob-

ing of III-V Semiconductor Growth.

B̈95-202370

04,691

Shear-Induced Melting of Two-Dimensional Solids.

PB96-112057

01,075

Physical Properties of Alternatives to the Fully HaloPhysical Chlorofluorocarbons.

PB96-119573

03,279

Mathematical Models of Transport Phenomena Associated with Arc-Welding Processes: A Survey.

B 96-135058

02,870

Vapor-Liquid Equilibria of Ternary Mixtures in the Critical Region on Paths of Constant Temperature and Overall Composition.

PB96-161856

01,139

Density Dependence of Fluid Properties and Non-Newto-

nian Flows: The Weisenberg Effect.

01,140

Nonlinear Correlation of High-Pressure Vapor-Liquid Equilibrium Data for Ethylene + n-Butane Showing Inconsistencies in Experimental Compositions.

PB96-161906

01,141

Quantum Collisional Transfer Contributions to the Density Dependence of Gaseous Viscosity.

01,142

from 67

Molar Heat Capacity at Constan

to $300 \mathrm{~K}$ at Pressures to $35 \mathrm{MPa}$.
$\mathrm{PB} 96-163738$

00,515

Non-Newtonian Flow between Concentric Cylinders Calculated from Thermophysical Properties Obtained from Simulations

PB96-163761

04,103

$\mathrm{CO} 2 / \mathrm{CH}_{4}$ Transport in Polyperfluorosulfonate lonomers:

Effects of Polar Solvents on Permeation and Solubility.
PB96-163803

01,145

Solubilities of Copper(II) and Chromium(III) betaPB96-164215

01,147

Molar Heat Capacity at Constant Volume for (xCO2 + (1x) $\mathrm{C} 2 \mathrm{H6}$ ) from 220 to $340 \mathrm{~K}$ at Pressures to $35 \mathrm{MPa}$.
PB96-167135 148 PB96-167135 01,148

PB96-167176 01,150

Small-Angle Neutron-Scattering Study of Dense Sheared Silica Gels.

Status of the Round Robin on the Transport Properties of R134a.

PB96-167218

01,152

Viscosity of Defined and Undefined Hydrocarbon Liquids Calculated Using an Extended Corresponding-States

PB96-167234

02,498

Retention of Halocarbons on a Hexafluoropropylene Epoxide-Modified Graphitized Carbon Black. 3. EtheneBased Compounds.

PB96-167309

03.286

Thermophysical Property Standard Reference Data from NIST.

PB96-167358

01,153

Small Angle Neutron Scattering Study of a Clay Suspension Under Shear.

PB96-167374

00,663

sochoric (p-p-T) Measurements on Liquid and Gaseous Air from 67 to $400 \mathrm{~K}$ at Pressures $1035 \mathrm{MPa}$.

A

01,154

New Data and Correlations for the Custody Transfer of Natural Gas Liquids.

PB96-176664

02,499

iscosity of $1,1,1,2,3,3$-Hexafluoropropane and $1,1,1,3,3,3$-Hexafluoropropane at Saturated-Liquid Condtions from $262 \mathrm{~K}$ to $353 \mathrm{~K}$.

PB96-176680

03,292

Small-Angle Neutron Scattering (SANS) Study of WormLike Micelles Under Shear

PB96-176698

04,11

Report of the Refrigeration, Air Conditioning and Hea Pumps Technical Options Committee.

03,293

International Standard Equation of State for the ThermoInnamic Properties of Refrigerant 123 (2,2-Dichloro1,1,1-Trifluoroethane)

P'B96-176805

03,294

Thermal Conductivity of Polypyromellitimide Film with Alumina Filler Particles from 4.2 to $300 \mathrm{~K}$

PB96-200753

01,292

Internal Waves in Xenon Near the Critical Point. PB97-111504

04,221

\section{PROPERTY RIGHTS}

Copyright and Information Services in the Context of the National Research and Education Network.

02,736

Using Technology to Manage and Protect Intellectua Property.
PB97-112395

PROPULSION

Combustion of a Polymer (PMMA) Sphere in Microgravity.

PROPYNE

2nu9 Band of Propyne-d3

PB95-164513

Sub-Doppler, Infrared Laser Spectroscopy of the Propyne 
Characterization of the Adsorption-Fouling Layer Using Globular Proteins on Ultrafiltration Membranes.
PB94-212909 Energy Transduction between a Concentration Gradient and an Alternating Electric Field.

03,461

Preparation of Immobilized Proteins Covalently Coupled Preparation of Immobilized Proteins Covalently Coupled
Through Silane Coupling Agents to Inorganic Supports. PB95-151403 03,530

Enzyme and Protein Mass Transter Coefficient in Aqueous Two Phase Systems. 1. Spray Extraction Columns.

PB95-161162 00,594

Continuous Counter-Current Two Phase Aqueous Extraction.

PB95-161212

00,675

Protein Extraction in a Spray Column Using a Polyethylene Glycol Maltodextrin Two-Phase Polymer System.

Non-Perturbative Relation between the Mutual Diffusion Coefficient, Suspension Viscosity, and Osmotic Compressibility: Application to Concentrated Protein Solutions.
PB96-102355 Determination of Total Protein Adsorbed on Solid (Mem. brane) Surface by a Hydrolysis Technique: Single Protein Adsorption.

Protein Data Bank: Current Status and Future Chal-

PB97-109060

00,517

PROTEOGLYCANS

Proteoglycan Inhibition of Calcium Phosphate Precipitation in Liposomal Suspensions.

PB94-211208

00,658

PROTHESIS

Physicochemical Characterization of Natural and Bioprosthetic Heart Valve Calcitic Deposits: Implications for Prevention
PB96-156039

00,187

\section{PROTOCOLS}

Conformance Assessment of Transport Layer Security Implementations.

RDI-SIM ECMA Inter-Domain Routing Protocol Simulation Tool.

01,683

Conformance Testing of a Lower Layer Security Protocol.

PB94-185402 01,577

Scientific Protocols in Statıstical Standards for Environmental Studies.

PB94-185527 02,517

ISDN Conformance Testing Guidelines: Guidelines for Implementors of ISDN Customer Premises Equipment to Conform to Both National ISDN-1 and North American ISDN Users' Forum Layer 3 Basic Rate Interface Basic Call Control Abstract Test Suites.

Comparing Remote Procedure Calls: Open Network Computing, Distributed Computing Environment and International Organization for Standardization.

PB95-194205

01,724

Conformance Testing for OSI Protocols.

PBO6-102686

01,631

Lessons from the Establishment of the U.S. GOSIP Testing Program.

01,817

Open Issues in OSI Protocol Development and Conform. ance Testing.

$X$ Window System, Version 11, Release 5 .

01,818

PB96-169099

01,769

Strategy to Support Multipoint Communication Service Over Native ATM Service.

PB96-176672

01,507

PROTON ACCELERATORS

Investigation of Applicability of Alanine and Radiochromic Detectors to Dosimetry of Proton Clinical Beams.

PB96-146782

PROTON AFFINITY

Proton Aftinity Ladders from Variable-Temperature Equilibrium Measurements. 1. A Re-Evaluation of the Upper Proton Aftinity Range.

PB94-216603

00,861

PROTON DOSIMETRY

Electron and Proton Dosimetry with Custom-Developed Radiochromic Dye Films

PB95-151106

03,713

NMR Characterization of Injection-Moulded Alumina

Green Compacts. Part 2. T2-Weighted Proton Imaging.

Green Compacts. Part 2. T2-Weighted Proton Imaging.
PB96-201181

PROTON TRANSPORT

Proton Affinity Ladders from Variable-Temperature Equilibrium Measurements. 1. A Re-Evaluation of the Upper Proton Aftinity Range.

PROTONS

00,861

Polarizability of the Nucleon.

PB94-211760

03,846
Bacteriorhodopsin Retains Its Light-Induced ProtonPumping Function After Being Heated to $140 \mathrm{C}$

03,471

PROTOTYPES

Dratt Guidelines for Pre-Oualification and Prototype Testing of Seismic Isolation Systems.

01,331

Product Models and Virtual Prototypes in Mechanical Engineering.

PB95-253563 02,783

Overview of the Manufacturing Engineering Toolkit Proto-

PB96-128228

02,833

PROTOZOAN DNA

beta-D-Glucosyl-Hydroxymethyluracil: A Novel Modified Base Present in the DNA of the Parastic Protozoan T. bruce

PSES (PROJECT SUPPORT ENVIRONMENTS)

03,524

Next Generation Computer Resources: Reference Model for Project Support Environments (Version 2.0).

PB94-143401

01,677

PSEUDOMONAS FLUORESCENS

Characterization of the Binding of Gallium, Platinum, and Uranium to Pseudomonas Fluorescens by Small-Angle $X$ ray Scattering and Transmission Electron Microscopy.
PB94-172509 L-threo-beta-Hydroxyhistidine, an Unprecedented Iron(III) on-Binding Amino Acid in a Pyoverdine-type Siderophore from Pseudomonas fluorescens 244

PB94-211620

00,553

PSN (PUELIC SWITCHED NETWORK)

Telecommunications Security Guidelines for Telecommunications Management Network. Computer Security.

01,496

PSYCHOLOGICAL EFFECTS

Psychological Aspects of Lighting: A Review of the Work of CIE TC 3.16

00,241

Psychological Aspects of Lighting: A Review of the Work of CIE TC 3.16
PB $95-153276$

PUBLIC BUILDINGS

00,254

Admınistration Standard for the Telecommunications Infrastructure of Federal Buildings. Category: Telecommunications Standard; Subcategory: Telecommunications Administration.

01,461

Federal Building Grounding and Bonding Requirements for Telecommunications. Category: Telecommunications Standard; Subcategory: Grounding and Bonding. FIPS PUB 195

01,802

Implementation of Executive Order 12699: Seismic Safety of Federal and Federally Assisted or Regulated New Building Construction

PB95-151809

00.436

PUBLIC DOMAIN

Obtaining and Installing a Public Domain TEX

01.719

PUBLIC KEY CERTIFICATES

Public Key Infrastructure Invitational Workshop. Held in McLean, Virginia on September 28, 1995.

01,616

PUBUIC POUICIES

Learning to Change: Opportunities to Improve the Performance of Smaller Manufacturers.

PB94-166212

00,010

PUBLIC POLICY

Information Infrastructure: Reaching Society's Goals. A Report of the Information Infrastructure Task Force Committee on Applications and Technology ED-376 823

00,131

PUBLIC-PRIVATE PARTNERSHIPS

Montgomery Education Connection and Resource Education Awareness Partnership Making Connections be cation Awareness Partnership Making Connections beween Local Schools and

00,134

PUBLIC SWITCHED NETWORK

Telecommunications Security Guidelines for Telecommunications Management Network. Computer Security.

01,496

PUBLIC WORKS

National Construction Sector Goals: Industry Strategies for Implementation.

00,204

PUBLISHING

CALS-Markup Requirements and Generic Style Specifications for Electronic Printed Output and Exchange of Text. PB94-962200

CALS-Markup Requirements and Generic Style Specifica. tions for Electronic Printed Output and Exchange of Text. PB95-962200

03,668

PULL-OUT TEST

Interface Properties for Ceramic Composites from a Single Fiber Pull-Out Test.

PB94-199361

03,135

PULSE-ECHO ULTRASONIC EVALUATION

Pulse-Echo Ultrasonic Evaluation of the Integrity of Seams of Single-Ply Roof Membranes.

00.381

PULSE GENERATORS

Aging. Warm-Up Time and Retrace; Important Characteristics of Standard Frequency Generators.

PB96-103122

04,031

PULSE HEATING

Dynamic Measurements of Thermophysical Properties of

Metals and Alloys at High Temperatures by Subsecond

Pulse Heating Techniques.

N94-25124/6

03,309

Millisecond-Resolution Pulse Heating System for SpecificHeat Measurements at High Temperatures.

PB94-199999

02,635

Measurement of the Heat of Fusion of Tungsten by a Microsecond-

03,400

Radiance Temperatures (in the Wavelength Range 523$907 \mathrm{~nm}$ ) of Group IV B Transition Metals Titanium, Zirconium, and Hafnium at Their Melting Points by a PulseHeating Technique.

PB96-102207

03,356

Radiance Temperatures (in the Wavelength Range 523$907 \mathrm{~nm}$ ) of Group IVB Transition Metals Titanium, Zirconium, and Hafnium at Their Melting Points by a PulseHeating Technique.

PB96-135025

02,677

PULSE PILEUP

Dead Time, Pileup, and Accurate Gamma-Ray SpectromPBY. 167101

00,697

PULSE RADIOLYSIS

Fluoride Elimination Upon Reaction of Pentafluoroaniline with e (sub eq')(sup -), H, and $\mathrm{OH}$ Radicals in Aqueous Solution

01,177

PULSE SYNCHRONIZATION

Laser-Synchrotron Hybrid Experiments: A Photon to Tickle, A Photon to Poke.

PB96-157847

03,704

PULSE TRANSFORMERS

Electrical Breakdown in Transformer Oil in Large Gaps. PB95-150579

01,889

PULSE TUBE REFRIGERATORS

Graded and Nongraded Regenerator Performance. PB95-169090

02,753

PULSED GAS METAL ARC WELDING

Through-the-Arc Sensing for Monitoring Arc Welding.
02,858

PULSED LASER DEPOSITION

Coexistence of Grains with Differing Orthorhombicity in High Ouality YBa2Cu3O7-delta Thin Films.

PULSES

Comment and Discussion on Digital Processing of PD

PBS6-122775

01,932

Radiation-Chemical Reaction of 2,3,5-Triphenyl-Tetrazolium Chloride in Liquid and Solid State.

PB96-146733

01,124

PUNCTURE RESISTANCE

Study to Determine the Most Important Parameters for Evaluating the Resistance of Soft Body Armor to Penetration by Edged Weapons.

PB94-158573

03.757

PURIFICATION

Salt-PEG Two-Phase Aqueous Systems to Purify ProPB94-200375

Critical Evaluation of the Purification of Biominerals by Hypochlorite Treatment.

00.186 


\section{KEYWORD INDEX}

\section{QUANTUM WELL LASERS}

PYROELECTRICITY

Piezoelectric and Pyroelectric Polymers. PB95-153367

01,250

PYROLYSIS

Thermal Decomposition of Hydroxy- and Methoxy-Substituted Anisoles.

00.767

Behavior of Charring Materials in Simulated Fire Environments.

PB94-196045

01,368

Upward Flame Spread along the Vertical Corner Walls (October 1993)
PB94-206299

00,340

PYROMELLITIC DIANHYDRIDE

Effects of Surface-Active Resins on Dentin/Composite Bonds.

PB95-140448

00,156

PROMETERS

Corrected Qptical Pyrometer Readings.
AD-A279 949/2

02,615

High-Speed Spatial Scanning Pyrometer.

02,636

\section{PYROMETRY}

National Measurement System for Radiometry, Photometry, and Pyrometry Base Upon Absolute Detectors. PB97-108559

Issues in High-Speed Pyrometry

02,693

PYROPHOSPHATES

Selective Inhibition of Crystal Growth on Octacalcium Phosphate and Nonstoichiometric Hydroxyapatite by PBg $94-211257$

00,147

Crystal Structure of Dicalcium Potassium Trihydrogen Bis(pyrophosphate) Trihydrate.

00,152

Crystal Structure of Decacalcium Tetrapotassium Hexakis (Pyrophosphate) Nonahydrate.

PB96-141064

01,099

PYRUVATES

Equilibrium and Calorimetric Investigation of the Hydroly sis of L-Tryptophan to (Indole + Pyruvate + Ammonia).
PB95-163317

Microstructure and Ferroelectric Properties of Lead Zirconate-Titanate Films Produced by Laser Evaporation. PB94-199148

04,470

QUADRATIC PROGRAMMING

Interior-Point Method for Linear and Ouadratic Programming Problems. (NIST Reprint).

PB95-180089

03,429

AADRATIC RESPONSE

Ouadratic Response of a Chemical Reaction to External PB96-161633

01,138

QUALITY

Models, Managing Models, Ouality Models: An Example of Quality Management.

PB94-163466

02,891

QUALITY ASSURANCE

Proficiency Testing as a Component of Ouality Assurance in Construction Materials Laboratories.

00,334

International Institute of Welding: Report on 1992 Actions. PB94-185873 02,856

International Institute of Welding: Report on 1993 Actions. $\begin{array}{ll}\text { PB94-185881 02,857 } & \end{array}$ Infratechnologies: Tools for Innovation

00,317

National Quality Assurance Program for Personnel RadiPB94-211273 A Case History. Importance of Measurement in Technology-Based Com-

PB94-211844

02,929

Role of the Office of Radiation Measurement in Ouality Assurance.

00,689

Questions and Answers on Ouality, the ISO 9000 Standard Series, Quality System Registration, and Related Is. sues. More Ouestions and Answers on the ISQ 9000 Standard Series and Related Issues.

00,495

Certification, Development and Use of Standard Ref-

$\begin{array}{ll}\text { erence Materials. } & 00,567\end{array}$ Software Safety and Program Slicing.

PB95-125894

01,703

Review of the USCEANIST Measurement Assurance Program for the Nuclear Power Industry.

03,712

B 95.126272

Quality Assurance of Contaminant Measurements in Ma-
rine Mammal Tissues.
PB95-164034 PB95-164034

Proceedings Report of the International Invitation Work-
shop on Development Assurance. Held in Ellicott City. Maryland on June 16-17, 1994 .

PB95-189494

02,912
IIW Commission V Quality Control and Quality Assurance of Welded Products Annual Report 1994/95.

02,866

Overview of Reference Materials Prepared for Standard. Overview of Reference Materials Prepared for Standard. ization of DNA Typing Procedures.

00,611

Electrical Product Requirements (Especially Quality Requirements) in the United States.

01,929

Dosimetry Systems for Radiation Processing.

03,717

Report on 1994 Actions of the International Institute of

Welding.

02,873

Unique Ouality Assurance Aspects of INAA for Reference Material Homogeneity and Certification.

Material Homoge

00,699

Developing Quality System Documentation Based on ANSI/NCSL Z540-1-1994: The Qptical Technology Division's Effort.
PB96-202122

QUALITY CONTROL

Optical Storage Media Data Integrity Studies. $\quad 01,620$

Draft Guidelines for Quality Control Testing of Sliding Seismic Isolation Systems.

PB94-161957

01,332

Through-the-Arc Sensing for Monitoring Arc Welding.

02,858

Statistical Ouality Control Technology in Japan.
PB94-199064

PB94-199064 02,708
Program

Program Handbook: Requirements for Qbtaining NIST
Approval/Recognition of a Laboratory Accreditation Body

Under P.L. 101-592. The Fastener Quality Act. 02,859
PB94-210143

Through-the-Arc Sensing for Real-Time Measurement of Gas Metal Arc Weld Quality.

PB95-16287

02,863

PB95-16362

02,547

Through-the-Arc Sensing for Measuring Gas Metal Arc Weld Quality in Real Time.

PB95-164463 02,908

Optical Characterization in Microelectronics Manufactur-

PB95-169397

02,358

IIW Commission V Quality Control and Quality Assurance of Welded Products Annual Report 1994/95.

$\begin{array}{lr}\text { PB95-198743 } & 02,866 \\ \text { Defect Formation Mechanism Causing Increasing Defect }\end{array}$ Density during Decreasing Implant Dose in Low-Dose

Simox.
PB96-119524

02,402

Development of a New Quality Control Strategy for Automated Manufacturing.

PB96-160486

02,840

Implementation of the Fastener Ouality Act

PB96-160676
IIW Commission V Ouality Control and Ouality Assurance of Welded Products, Annual Report 1995/96.

PB96-191366 02,880

NIST/NCMS Program on Electronic Packaging: First Update.

$\begin{array}{ll}\text { PB96-204086 } & 03,008\end{array}$

National Voluntary Laboratory Accreditation Program

(NVLAP): Fasteners and Metals.

PB97-114185

02,881

QUANTITATIVE ANALYSIS

Methods for Analysis of Cancer Chemopreventive Agents in Human Serum.

PB95-200648 03,502

Liquid Chromatographic Determination of Carotenoids in Human Serum Using an Engineered C30 and a C18 Stationary Phase.

QUANTITATIVE CHEMICAL ANALYSIS

03,512

How to Verify Reference Materials.

03,497

Determination of Polycyclic Aromatic Hydrocarbons by Liquid Chromatography

00,585

Development of a Gas Standard Reference Material ConDevelopment of a Gas Standard Reference Material Containing Eighteen Volatile Qrganic Compounds.
PB95-162277

02,545

Liquid Chromatographic Determination of Polycyclic Aromatic Hydrocarbon Isomers of Molecular Weight 278 and 302 in Environmental Standard Reference Materials.

PB95-164042

02,523

Interlaboratory Comparison Studies on the Analysis of Hair for Drugs of Abuse.

03,500 Hair Analysis for Drugs of Abuse: Evaluation of Analytical
Methods. Environmental Issues, and Development of Reference Materials.

PB95-176269

03,501

Ouantitative Analysis of Selected PCB Congeners in Marine Matrix Reference Materials Using a Novel Cyanobiphenyl Stationary Phase.

02,591

Hair Testing for Drugs of Abuse: International Research on Standards and Technology.

03,504

NIST Reference Materials to Support Accuracy in Drug Testing.

PB96-123807

03,505

Certification of Phencyclidine in Lyophilized Human Urine Reference Materials.

03,508

Interlaboratory Studies on the Analysis of Hair for Drugs of Abuse: Results from the Fifth Exercise.

03,509

Interlaboratory Studies on the Analysis of Hair for Drugs of Abuse: Results from the Fourth Exercise.

PB97.111322

03,510

Use of Neutron Beams for Chemical Analysis at NIST. PB97-112437

00,652

QUANTIZED DISSIPATION

Journal of Fesearch of the National Institute of Standards and Technology. March/April 1996. Volume 101, Number PB96-177381

01,863

Evidence That Voltage Rather Than Resistance is Quan-

tized in Breakdown of the Quantum Hall Effect.
PB96-179163

QUANTUM DOTS

Quantum Dots in Ouantum Well Structures.

JANTUM ELECTRODYNAMICS

How Accurate Are the Josephson and Quantum Hall Effects and QED

QUANTUM ELECTRONICS

Using Ouantized Breakdown Voltage Signals to Determine the Maximum Electric Fields in a Ouantum Hall Ef fect Sample.
PB95-261947

QUANTUM HALL EFFECT

Quantized Dissipation of the Ouantum Hall Effect at High Currents.

How Accurate Are the Josephson and Quantum Hall Effects and QED.

Anomalous Behavior of a Ouantized Hall Plateau in a High-Mobility Si Metal-Oxide-Semiconductor Field-Effect Transistor.

Design Challenges in a Commercial Quantum Hall EffectBased Resistance Standard.

PB95-171419

02,263

Progress on the Quantized Hall Resistance Recommended Intrinsic/Derived Standards Practice. PB96-122460

Precision Tests of a Quantum Hall Effect Device DC
Equivalent Circuit Using Double-Series and Triple-Series Connections.

PB96-159256

01,953

Quantum Hall Effect-Based Resistance Standard: Capa-

bilities and Implementation.

PB96-180096

04,114

Quantum Hall Effect-Based Resistance Standard (Quantum Hall Res)

QUANTUM MEASUREMENTS

Quantum Measurements of Trapped lons. 


\section{KEYWORD INDEX}

Measurement and Simulation of Photoluminescence Spectra from Vertical-Cavity Quantum-Well Laser Structures.

02,170

OUANTUM WELLS

Modified Airy Function Method for the Analysis of Tunneling Problems in Optical Waveguides and Quantum Well

$\begin{array}{ll}\text { Siructures. } & 02,120\end{array}$

Determination of the Complex Refractive Index of Individual Quantum Wells from Distributed Reflectance. 02,176 Scaling of the Nonlinear Optical Cross Sections of GaAsAlGaAs Multiple Quantum-Well Hetero n-i-p-i's.
PB96-102793

02,183

Theory for Quantum-Dot Quantum Wells: Pair Correlation $\begin{array}{ll}\text { and Internal Quantum Confinement in } \\ \text { Nanoheterostructures. } \\ \text { PB96-179445 } & 02,437\end{array}$

$02,4,37$

Time-Resolved Measurements of the Polarization State of Four-Wave Mixing Signals from GaAs Multiple Quantum

PB96-201058 04,796

Interdigitated Stacked P-I-N Multiple Quantum Well Modwlator.

02,455

Excitons in Complex Quantum Nanostructures.

01,184

PBAT7

Fuzed-Quartz Fibers. A Survey of Properties, Applications and Production Methods.

AD-A286 620/0

00,656

Dielectric Properties of Materials at Cryogenic Temperatures and Microwave Frequencies.

02.369

P95-202610

02,271

Environmental Sensitivities of Quartz Qscillators.

PB96-103148

Power

Large Local-Field Correcti

PB97-122378

04.400

OUARTZ-HALOGEN LAMPS

Joumal of Research of the National Institute of Standards and Technology, March/April 1996. Volume 101, Number PB96-177381

01.863

Irradiance of Horizontal Quartz-Halogen Standard Lamps. - 179130

OUARTZ RESONATORS

Ultralinear Small-Angle Phase Modulator.

02,261

Relationship of AM to PM Noise in Selected RF QscillaPB95-169009

02,262

New Model of 1/F Noise in Baw Quartz Resonators.
PB96-112248

Quest to Understand and Reduce I/f Noise in Amplifiers

and Baw Quartz Qscillators.
PB96-200795

02,097

OUASARS

Discovery of an X-Ray Selected, Radio-Loud Quasar at

$z=3.9$.
PB $94-198652$

00.052

Qbservations of 3C 273 with the Goddard High Resolution Spectrograph on the Hubble Space Telescope. PB95-202321

00,076

QUASICRYSTALS

RDFs and Fe-Fe Pair Correlations in an AlCuFe Icosahedral Alloy by Double Isotopic Substitution. PB94-172129 Neutron Scattering Structural Study of AICuFe Quasicrystals Using Double Isotopic Substitution.
PB94-200458 Inelastic Neutron Scattering Measurements of Phonons in cosahedral Al-Li-Cu.

PB95-126215

OUASIELASTIC COMPONENT

0.532

Unconventional Ferromagnetic Transition in La(sub 1. x) $\mathrm{Ca}$ (sub x) MnO3.

OUATERNARY AMMONIUM SALTS

Use of Kinetic Energy Distributions to Determine the Relative Contributions of Gas Phase and Surface Fragmentation in KeV lon Sputtering of a Quaternary Ammonium Salt.

OUENCHING (COOLING)

00,570

Hot-Deformation Apparatus for Thermomechanical Processing Simulation

PB94-200136

03,207

Object SQL- Language Extensions for Object Data Management.

01,704

Coping with Different Retrieval Methods in Next Generation Networks.

02,726

OUINUCLIDINYL BENZILATE

Determination of 3-Quinuclidinyl Benzilate (Qnb) and lis Major Metabolites in Urine by Isotope Dilution Gas Chromatography Mass Spectrometry.

PB94-199379

03,492
R-123 REFRIGERANT

Enhancement of R123 Pool Boiling by the Addition of NHexane.
PB96-165956

02,605

R 22

Role of R22 in Refrigerating and Air Conditioning Equip-

03,253

R-CURVES

Flat and Rising R-Curves for Elliptical Surface Cracks from Indentation and Superposed Flexure.

03,156

R123

Criteria for Establishing Accurate Vapor Pressure Curves. R124

Viscosity of the Saturated Liquid Phase of Six Halogenated Compounds and Three Mixtures.

00,960

R125

Viscosity of the Saturated Liquid Phase of Six Halogenated Compounds and Three Mixtures.

00,960

R134A

Equation of State Formulation of the Thermodynamic Properties of R134a (1,1,1,2-Tetrafluoroethane).

PB94-212081

03,256

Thermal Conductivity of R134a.

PB94-213063

03,857

Polarized Transient Hot Wire Thermal Conductivity Meas. urements.

00,886

Viscosity of the Saturated Liquid Phase of Six Halogenated Compounds and Three Mixtures. 00,960 Criteria for Establishing Accurate Vapor Pressure Curves.
PB95-163812 Thermodynamic Properties of R134a(1,1,1,2 Tetrafluoroethane)

00,988

Measurements of Molar Heat Capacity at Constant Volume (Cv) for 1,1,1,2-Tetrafluoroethane (R134a)

Vapour Pressure Measurements on 1,1,1,2. Tetrafluoroethane (R134a) from 180 to $350 \mathrm{~K}$. PB95-168886

03,265

Measurement of the Thermal Properties of Electrically Conducting Fluids Using Coated Transient Hot Wires.

Predictive Extended Corresponding States Model for Pure and Mixed Refrigerants Including an Equation of State for R134a.

PB95-175717

03,275

R142B

Polarized Transient Hot Wire Thermal Conductivity Measurements.
PB95-108817

00,886

R236EA

Dielecric Studies of Fluids with Reentrant Resonators. 32

Ebulliomeiric Measurement of the Vapor Pressure of Difluoromethane.

00,931

Viscosity of the Saturated Liquid Phase of Six Halogenated Compounds and Three Mixtures.

00.960

RADAR CROSS SECTIONS

RangeCAD and the NIST RCS Uncertainty Analysis.

Proposed Analysis of RCS Measurement Uncertainty.

PB95-203568 01,871

Polarimetric Calibration of Reciprocal-Antenna Radars.

PB95-216925 01,872

Accurate Computations of Radar Cross Sections of Simple Qbjects.
PB96-138474

04,426

Polarimetric Calibration of Reciprocal-Antenna Radars.

PB96-200696

02,016

RADAR POLARIMETRY

Polarimetric Calibration of Reciprocal-Antenna Radars.

PB95-216925

01,872

RADIANCE

Improved Automated Current Control for Standard PB94-219367

00,246

Intercomparison of the ITS-90 Radiance Temperature

Scales of the National Physical Laboratory (U.K.) and the

National Instilute of Standards and Technology.
P396-113550

02,674

\section{RADIANCE TEMPERATURES}

Radiance Temperaiures (in the Wavelength Range 523$907 \mathrm{~nm}$ ) of Group IVB Transition Metals Titanium, Zirconium, and Hafnium at Their Melting Points by a PulseHeating Technique.

02,677

Radiance Temperatures at $1500 \mathrm{~nm}$ of Niobium and Molybdenum at Their Melting Points by a Pulse-Heating

PB97-118699

04,167

\section{RADIANT COOLING}

Analytical Method of Determining the Heat Capacity at High Temperatures from the Surface Temperature of a Cooling Sphere.

03,865

RADIANT HEATING

Water Droplet Evaporation from Radiantly Heated Solids. PB95-217147

00,394

RADIATION

Protection Against Neutron Radiation Up to 30 Million Electron Volts.

03,611

RADIATION ACCIDENTS

Radiation Accident at an Industrial Accelerator Facility.

DIATION ATTENUATION

$X$-ray Attenuation Coefficients from $10 \mathrm{Kev}$ to $100 \mathrm{Mev}$ AD-A278 139/1

RADIATION COUNTERS

Self-Biasing Cryogenic Particle Detector Utilizing Electrothermal Feedback and a SQUID Readout.

04,712

RADIATION DAMAGE

Irradiation Damage in Inorganic Insulation Materials for ITER Magnets: A Review.

03,705

Quantitative Determination of Qxidative Base Damage in

DNA by Stable Isotope-Dilution Mass Spectrometry.
PB96-200886

03,483

Colour Centres in LiF for Measurement of Absorbed Doses Up to 100 MGy.

PB97-118756

04,169

\section{RADIATION DETECTION}

Unique Quality Assurance Aspects of INAA for Reference PB96-200811

00,699

RADIATION DETECTORS

Thermal and Nonequilibrium Responses of Superconductors for Radiation Detectors

PB95-164232

02,156

Report of the International Commission on Radiological

Units and Measurements (ICRU), 1956

AD-A279 120/0

03,513

Radiation Doses.

PB94-199676

03,618

National Quality Assurance Program for Personnel Radiation Dosimetry: A Case History.

03.620

Updated Calculations for Routine Space-Shielding Radiation Dose Estimates: SHIELDQSE-2.

PB95-171039

04.838

Food Irradiation Dosimetry.

PB95-180675

00,041

\section{RADIATION DOSES}

Joumal of Research of the National Institute of Standards and Technology. March/April 1994. Volume 99. Number PB94-219219 02,000 Sealed Water Calorimeter for Measuring Absorbed Dose PB94-219227 03,517 Extrapolation Chamber Measurements on $(90) \mathrm{Sr}+(90) \mathrm{Y}$ Beta-Particle Ophthalmic Applicator Dose Rates. 03.626 Crystal Structure of Annealed and As-Prepared

$\mathrm{HgBa} 2 \mathrm{CaCu} 2 \mathrm{O} 6+d e l t a$ Superconductors.
PB95-161105

03,927

Measurement of Radial Dose Distributions Around Small Beta Particle Emitters Using High Resolution Radiochromic Foil Dosimetry. 
USCEANIST Measurement Assurance Programs for the Radiopharmace
PB97-1 10514

\section{RADIATION MEASURING INSTRUMENTS}

Report of the International Commission on Radiological Units and Measurements (ICRU), 1956.

AD-A279 120/0

RADIATION MONITORS

Dosimetry Systems for Radiation Processing

03,717

RADIATION PROCESSING

ESR-Based Analysis in Radiation Processing.

PB95-161634

03,931

RADIATION PROTECTION

X-ray Protection.
$A D \cdot A 279132 / 5$

$X$-ray Protection Design.

AD-A279 181/2

Protection Against Radiations from Radium, Cobalt-60, and Cesium-137.

03,607

Maximum Permissible Body Burdens and Maximum Permissible Concentrations of Radionuclides in Air and in Water for Occupational Exposure.

AD-A280 282/5

03,610

Protection Against Neutron Radiation Up to 30 Million Electron Volts.

AD-A286 681/2

03,611

RADIATION PYROMETERS

Radiance Temperature (in the Wavelength Range 519 . $906 \mathrm{~nm}$ ) of Tungsten at its Melting Point by a Pulse-Heating Technique

03,397

RADIATION REACTIONS

Point Charges, Radiation Reaction, and Quantum Mechanics.

04,139

RADIATION SCATTERING

Characterization of the Binding of Gallium, Platinum, and Uranium to Pseudomonas Fluorescens by Small-Angle $X$ ray Scattering and Transmission Electron Microscopy.
PB94-172509

RADIATION SHIELDING

New Method for Shielding Electron Beams Used for Head and Neck Cancer Treatmen

PB94-211430

03,621

RADIATION SOURCES

Precision High Temperature Blackbodies.
PB95-140059

RADIATION THERAPY

DNA Base Damage in Lymphocytes of Cancer Patients Undergoing Radiation Therapy.

\section{ADIATION TOLERANCE}

Ionizing Radiation Causes Greater DNA Base Damage in Radiation-Sensitive Mutant M10 Cells That in Parent Mouse Lymphoma L5178Y Cells.

PB95-175915

03,632

RADIATION TRANSPORT

Electron transport calculations with biomedical and environmental applications. Final report, December 23, 1992-January 31, 1994

03,613

RADIATIVE HEAT TRANSFER

Structure and Radiation Properties of Pool Fires. $\quad 02,473$
PB94-193802

investigation of Oil and Gas Well Fires and Flares.

PB94-193976 03,695

Estimate of Flame Radiance via a Single Location Meas. urement in Liquid Pool Flames.

PB94-211596 02,476

Measurement of Radiative Feedback to the Fuel Surface

of a Pool Fire.
PB94-211604 02,477

Computing Radiative Heat Transfer Occurring in a Zone

PB96-102306

01,399

Flame Heights and Heat Release Rates of 1991 Kuwait Oil Field Fires.
PB96-119342

RADIATIVE TRANSFER

01,404

Opacity Project and the Practical Utilization of Atomic

PB94-212214

00,059

Stars, Atmospheres, Radiative Transter.

00,095

Relationship between Radiative and Magnetic Fluxes for Three Active Solar-Type Dwarts.

PB96-119540

00,097

RADIATORS

Screened-Room Measurements on the NIST SphericalDipole Standard Radiator.

PB96-113568

01,926

RADICALS

Solvent Effects in the Reactions of Peroxyl Radicals with

Organic Reductants. Evidence for Proton Transfer Medi-

ated Electron Transfer.

PB95-107157

$\infty 0,873$
Temperature Dependence of the Rate Constants for Reaction of Dihalide and Azide Radicals with Inorganic Reaction of

PB95-162756

00,964

RADIO EMISSION

Optically Linked Three-Loop Antenna System for Determining the Radiation Characteristics of an Electrically Small Source.

02,005

Search for Radio Emission from the 'Non-Magnetic' Chemically Peculiar Stars.

PB96-102249

00,087

RADIO EQUIPMENT

Rechargeable Batteries for Personal/Portable. PB96-164231

02,459

RADIO FREQUENCIES

Low-Frequency Model for Radio-Frequency Absorbers. PB95-261939

04,424

RADIO FREQUENCY DETECTION

Quantum-Limited Cooling and Detection of Radio-Frequency Oscillations by Laser-Cooled Ions.

PB96-112073

04,039

RADIO FREQUENCY DISCHARGE

Gaseous Electronics Conference Radio-Frequency Refrence Cell: A Defined Parallel-Plate Radio-Frequency System for Experimental and Theoretical Studies of Plas-
ma-Processing Discharges. ma-Processing Discharges.

Plasma Chernistry in Disilane Discharges.

04,404

PB94-21 1075

02,514

Kinetic-Energy Distributions of lons Sampled from Argon Plasmas in a Parallel-Plate, Radio-Frequency Reference Call.

03,935

Electrical Characterization of Radio-Frequency Dis. charges in the Gaseous Electronics Conference Reference Call

PB95-164612 01,905

Effect of Electrode Material on Measured Ion Energy Distributions in Radio-Frequency Discharges.

04.026

Evidence for Inelastic Processes for $\mathrm{N}(+) 3$ and $\mathrm{N}(+) 4$ Evidence for Inelastic Processes for $\mathrm{N}(+) 3$ and $\mathrm{N}(+) 4$
from Ion Energy Distributions in He/N2 Radio Frequency from Ion Energy Dist

Glow Discharg
PB96-146683

RADIO FREQUENCY DISCHARGES

Influence of Electrode Material on Measured Ion KineticEnergy Distributions in Radio-Frequency Discharges.

PB96-123179

01,935

ptical and Mass Spectrometric Investigations of lons and Neutral Species in SF6 Radio-Frequency Discharges. PB97-111918

01,985

RADIO SOURCES (ASTRONOMY)

Rotational Modulation and Flares on RS Canum Venaticorum and BY Draconis Stars. XVIII. Coordinated VLA, ROSAT, and IUE Observations of RS CVn Binaries.

00,089

Developments in Stellar Coronae.

00,107

RADIO STAF

Class of Radio-Emitting Magnetic B Stars and a WindFed Magnetosphere Model

PB94-213451

Radio Emıssion from Chemically Peculiar Stars. PB94-213469

00,067

RADIO WAVES

onospheric Radio Propagation

AD-A286 619/2

00,068

RADIOACTIVE AIR POLLUTANTS

Maximum Permissible Amounts of Radioisotopes in the Human Body and

in Air and Water.

Maximum Permissible Body Burdens and Maximum Permissible Concentrations of Radionuclides in Air and in Water for Occupational Exposure.

AD-A280 282/5

03,610

RADIOACTIVE WASTE DISPOSAL

Expansion of Cementitious Materials Exposed to Sulfate Solutions.

02,577

4SIGHT Manual: A Computer Program for Modelling Degradation of Underground Low Level Waste Concrete

PB95-231593

03,726

Long-Term Performance of Engineered Concrete Barriers.

03,727

RADIOACTIVE WASTE STORAGE

Information Retrieval Using Key Words and a Structured

03,724

RADIOACTIVE WATER POLLUTANTS

Maximum Permissible Amounts of Radioisotopes in the Human Body and Maximum Permissible Concentrations in Air and Water.

03,609 Maximum Permissible Body Burdens and Maximum Per-
missible Concentrations of Radionuclides in Air and in Water for Occupational Exposure.

WD-A280 282/5

03,610

RADIOACTIVITY

Summary of the Proceedings of the Workshop on Standard Phantoms for In-vivo Radioactivity Measurement. of Radioactive Hot Particles Using Dose Mapping of Radioactive Hot Particles Using
Radiochromic Film.
PB95-162954 NBS/NIST Peltier-Effect Microcalorimeter: A Four-Decade PB96-102769

01,064

RADIOASSAY

Intercomparison Study of (237)Np Determination in Artificial Urine Samples.

PB96-102645

03,633

RADIOASSAYS

Radioassays of Yttrium-90 Used in Nuclear Medicine.

PB97.110100

03,522

PIOCHROMIC DOSIMETERS

Electron and Proton Dosimetry with Custom-Developed Radiochromic Dye Films.

PB95-151106

03,713

Alcohol Solutions of Triphenyl-Tetrazolium Chloride as PB96-135249

03,716

RADIOCHROMIC DYES

Electron and Proton Dosimetry with Custom-Developed Radiochromic Dye Films.

PB95-151106

03,713

PIOCHROMIC FILMS

Use of a Radiochromic Detector for the Determination of Stereotactic Radiosurgery Dose Characteristics.

PB94-185642

03,514

Radiochromic Solid-State Polymerization Reaction.

PB95-180683

01,271

Effect of Formulation Changes on the Response to loniz ing Radiation of Radiochromic Dye Films.

B97-119028

04,171

Novel Radiochromic Films for Clinical Dosimetry.

PB97-119259

03,641

Calibration and Performance of GafChromic DM-100 Radiochromic Dosemeters.

PB97-11929

00,703

RADIOCHROMIC FOILS

Measurement of Radial Dose Distributions Around Small Beta Particle Emitters Using High Resolution Radiochromic Foil Dosimetry.

03,518

RADIOFREQUENCIES

Electrical Sensors for Monitoring if Plasma Sheaths.

PB95-162962

04,412

PDIOFREQUENCY CHARGES

Ion Kinetic-Energy Distributions and Balmer-alpha (Halpha) Excitation in Ar-H2 Radio-Frequency Dis-

PB96-102959

04,029

RADIOFREQUENCY DISCHARGES

Time-Resolved Balmer-Alpha Emission from Fast Hydrogen Atoms in Low Pressure, Radio-Frequency Dis- 
Solvent Effects in the Reactions of Peroxyl Radicals with Organic Reductants. Evidence for Proton Transfer Mediated Electron Transfer.

PB95-107157

00,873

Unusual Spin-Trap Chemistry for the Reaction of Hydroxyl Radical with the Carcinogen NNitrosodimethylarnine.

Temperature Dependence of the Rate Constants for Reaction of Dihalide and Azide Radicals with Inorganic $R \theta-$

ductants.
PB95-162756

00,964

lodine Atoms and lodomethane Radical Cations: Their Formation in the Pulse Radiolysis of lodomethane in Organic Solvents, Their Complexes, and Their Reactivity with Organic Reductants.

Radiation-Chemical Reaction of 2,3,5-Triphenyl-Tetrazolium Chloride in Liquid and Solid State.

01,124

One-Electron Oxidation of Metalloporphycenes as Studied by Radiolytic Methods.

by Radiolytic Met
PB97-111967

01,179

RADIOMETERS

Dual Frequency mm-Wave Radiometer Antenna for Airbome Remote Sensing of Atmosphere and Ocean.

PB95-180378

02,006

Thermal Modeling of Absolute Cryogenic Radiometers.

PB95-181236 0.4,316

Relative Accuracy of Isolated and Unisolated Noise Comparison Radiometers.

PB96-111851

01,924

Dual-Frequency Millimeter-Wave Radiometer Antenna for Airborne Remote Sensing of Atmosphere and Ocean. PB96-112289

02,009

Radiometer Equation for Noise Comparison Radiometers.
PB96-140363

Single-Port Technique for Adaptor Efficiency Evaluation.

02,088

Organization and Implementation of Calibration in the EOS Project. Part 1.

PB96-179437

04,841

National Measurement System for Radiometry, Photometry, and Pyrometry Base Upon Absolute Detectors. 04,38 Calibration in the Earth Observing System (EOS) Project. Part 2. Implementation

PB97-112213

04,842

Comparison of Filter Radiometer Spectral Responsivity with the NIST Spectral-Irradiance and Illuminance Scales. PB97-113161

RADIOMETRIC CORRECTION

Radiometric Model of the Transmission Cell-Reciprocal Nephelomeler
PB95-150132

ADIOMETRIC PHYSICS DIVISION

Optical Metrology and More. Programs and Services of he Radiometric Physics Division, Physics Laboratory. PB94-191707

04,228

RADIOMETRY

NIST Response to the Fifth CORM Report on the Pressing Problems and Projected Needs in Optical Radiation Measurements.

04,227

Optical Metrology and More. Programs and Services of the Radiometric Physics Division, Physics Laboratory.
PB94-191707

04,228

Development and Calibration of UVNUV Radiometric

PB94-199098

04,229

Overview of Radiometric Program of the NIST Thermal Imaging Laboratory.

04,230

Theoretical Analysis of the Coherence-Induced Spectral Shitt Experiments of Kandpal, Vaishya, and Joshi.

PB94-219383

00,561

National Institute of Standards and Technology High-Accuracy Cryogenic Radiometer.

04,378

Mode-Locked Lasers for High-Accuracy Radiometry.

PB96-204201 04, 134

Results of a NISTNNIIOFI Comparison of Spectral-Radiance Measurements.

PB97-113021

04,159

Realization of a Scale of Absolute Spectral Response Using the NIST High Accuracy Cryogenic Radiometer.
PB97.118640

ADIONUCLIDE MIGRATION

Modeling Radon Transport in Multistory Residential Buildings.

00,256

RADIONUCLIDES

Liquid-Scintillation Counting Techniques for the Standardization of Radionuclides Used in Therapy.

PB97-110084

03,709

RADIOPHARMACEUTICALS

Experimental Vatidation of Radiopharmaceutical Absorbed Dose to Mineralized Bone Tissue.

03,617
Radiation Doses

PB94-199676

03,618

USCEANIST Measurement Assurance Programs for the Radiopharmaceutical and Nuclear Power Industries.

PB97-110514 03,721

RADIOSONDES

Low-Temperature Performance of Radiosonde Electric Hygrometer Elements.

ADIOSURGERY

Use of a Radiochromic Detector for the Determination of Stereotactic Radiosurgery Dose Characteristics. PB94-185642

03,514

\section{RADIOTHERAPY}

Standard Reference Materials (SRM's) for Measuring Genetic Damage.

03,516

New Method for Shielding Electron Beams Used for Head and Neck Cancer Treatment.

PB94-211430

03,621

Scattered Fractions of Dose from 18 and 25 MV X-ray Radiotherapy Linear Accelerators.

04,120

RADIOTHERAPY DOSAGE

Use of a Radiochromic Detector for the Determination of Stereotactic Radiosurgery Dose Characteristics. PB94-185642

03,514

RADIUM

Protection Against Radiations from Radium, Cobalt-60 and Cesium-137.

03,607

RADIUM IONS

Fine Structure of Negative Ions of Alkaline-Earth-Meta Atoms.

RADON

B94-211182

03,837

Measurement and Determination of Radon Source Potential: A Literature Review.

Systematics of Alpha-Particle Energy Spectra and Lineal Energy (Y) Spectra for Radon Daughters.

03,615

Microdosimetry and Cellular Radiation Effects of Radon Progency in Human Bronchial Airways.

03,625

Modeling Radon Transport in Multistory Residential Build

ings. 162087

00,256

Computer Simulations of Airflow and Radon Transport in

Four Large Buildings
PB95-220422

02,557

Radon in the Lung.

PB97-110035

03,638

RADON 222

International Radon-in-Air Measurement Intercomparison Using a New Transter Standard

PB96-159751

03,708

RAIL TRANSPORTATION

New Concepts for Fire Protection of Passenger Rail Transportation Vehicles.

04,850

PB95-162046

New Concepts for Fire Protection of Passenger Rail
Transportation Vehicles. (NIST Reprint).
PB95-180774 PB95-180774

RAILROAD CARS

Ultrasonic Measurement of Residual Stress in Cast Steel Railroad Wheels
PB95-169199

04,852

RAILROAD PASSENGER SERVICE

Concepts for Fire Protection of Passenger Rail Transportation Vehicles: Past, Present, and Future.

PB96-102868

04,853

RAILROAD TRACKS

Noncontact Ultrasonic Inspection of Train Rails for Stress PB95-162673

RAILROAD TRAINS

Residual Stress in Induction-Heated Railroad Wheels: UItrasonic and Saw Cut Measurements. Report No. 28.

PB96-106992

04.854

Dynamometer-Induced Residual Stress in Railroad Wheels: Ultrasonic and Saw Cut Measurements. Report No. 30 .

04,857

- 183199

RAILROAD TRUCKS

Ultrasonic Measurement of Residual Stress in Railroad Wheel Rims.

04,849

Safety Assessment of Railroad Wheels by Residual Stress Measurements.

04,855

PB96-141114

Safety Assessment of Railroad Wheels Through Roll-by
Detection of Tread Cracks.
PB96-141254
04,856

PB96-141254

RAMAN EFFECT

Frequency Shifting of Pulsed Narrow-Band Laser Light in

a Multipass Raman Cell.

04,321

RAMAN SPECTRA

Octacalcium Phosphate. 3. Infrared and Raman Vibra-

PB94-172244

00,756

Simultaneous Forward-Backward Raman Scattering Studies of D2 Broadened by D2, He, and Ar.

00,961

Evolution of $X$-ray Resonance Raman Scattering into $X$ ray Fluorescence from the Excitation of Xenon Near the

PB96-102751

04,025

RAMAN SPECTROSCOPY

Measurement of the Self Broadening of the $\mathrm{H} 2 \mathrm{Q}(0-5)$

Raman Transitio

00,884

Lineshape Analysis of the Raman Spectrum of Diamond Films Grown by Hot-Filament and Microwave-Plasma Chemical-Vapor Deposition.

PB95-162392

03,016

Studies of Defects in Diamond Films and Particles by Raman and Luminescence Spectroscopies.

PB95-162400

03,017

Q Branch Lineshape Functions for CARS Thermometry.

PB96-160643

01,132

Development of the NIST Transient Pressure and Temperature Calibration Facility.

PB96-160833

00,626

Structural Analysis of Heparin by Raman Spectroscopy.

PB96-167226

03,480

Neutron and Raman Spectroscopies of 134 and $134 a$ Hydrofluorocarbons Encaged in $\mathrm{Na}-\mathrm{X}$ Zeolite.

03,001

\section{RAMSAUER EFFECT}

Semiclassical Explanation of the Generalized RamsauerTownsend Minima in Electron-Atom Scattering.

PB95-153532

03,925

RANDOM WALK

Book Review: Aspects and Applications of the Random Walk

B96-123534

04,215

RANDOM WALKS

Self-Avoiding-Walk Contacts and Random-Walk SelfIntersections in

01,276

RAPID RESPONSE MANUFACTURING

Visualization Applications for Manufacturing: A State-ofhe-Art Survey. Final Report

PB94-194552

02,816

RARE EARTH COMPOUNDS

Crystal Chemistry and Phase Equilibrium Studies of the BaO-R2O3-CuO Systems. 2. X-Ray Characterization and Standard Patterns of BaR2CuO4, R=Lanthanides. Crystal Chemistry and Phase Equilibria Studies of the $\mathrm{BaO}(\mathrm{BaCO}$ )-R2O3-CuO Systems. 4. Crystal Chemistry and Subsolidus Phase Relationship Studies of the CuORich Region of the Ternary Diagrams, R=Lanthanides. PB95-151759

RARE EARTH ELEMENTS

Rare-Earth Isotopes as Tracers of Particulate Emissions: An Urban Scale Test.

PB94-161635

02,535

Magnetic Rare Earth Artificial Metallic Superlattices. 


\section{KEYWORD INDEX}

RCS (REVISION CONTROL SYSTEM) Guide to Configuration Management and the Revision
Control System for Testbed Users.

PB94-150919

01,678

RE134

Viscosity of the Saturated Liquid Phase of Six Halogenated Compounds and Three Mixtures.

00,960

RE245

Viscosity of the Saturated Liquid Phase of Six Halogenated Compounds and Three Mixtures.

00,960

EACTION KINETICS

Tables of Chemical Kinetics Homogeneous Reactions. AD-A280 293/2 00,715

Thermal Decomposition of Hydroxy- and Methoxy-Substituted Anisoles.

00,767

Octacalcium Phosphate Carboxylates IV. Kinetics of ForOctacalcium Phosphate Carboxylates IV. Kinetics of For-
mation and Solubility of Octacalcium Phosphate Succimate. PBate. 185600

00.776

Temperature Dependence of the Rate Constants for Reaction of Inorganic Radicals with Organic Reductants.

Oxidation of 10-Methylacridan, a Synthetic Analogue of $\mathrm{NADH}$ and Deprotonation of its Cation Radical. Convergent Application of Laser Flash Photolysis and Direct and Redox Catalyzed Electrochemistry to the Kinetics of Redox Catalyzed Electrochemistry

Deprotonation

00,785

Gas Phase Reactions Relevant to Chemical Vapor Deposition: Optical Diagnostics.

03,116

Gas Phase Reactions Relevant to Chemical Vapor Deposition: Numerical Modeling. PB94-199346

03,117

Rate Constants for Hydrogem Atom Attack on Some Chlorinated Benzenes at High Temperature.

PB94-200581

00,810

Reaction Rate Determinations of Vinyl Radical Reactions with Vinyl, Methyl, and Hydrogen Atoms.

00,815

Radiation Chemistry of Cyanine Dyes: Oxidation and Reduction of Merocyanine 540

PB94-211661

00,818

International Conference on Chemical Kinetics (2nd).

Held in Gaithersburg, Maryland on Ju'y 24-27, 1989.

PB94-211901

00,822

Kinetics of the Reaction of $\mathrm{CCl} 3-\mathrm{Br}-2$ and the Thermochemistry of $\mathrm{CCl} 3$ Radical and Cation.

00,824

Kinetics of the Self-Reaction of Hydroxymethylperoxyl

00,827

Temperature Dependence of the Rate Constants for Re-

actions of the Sulfate Radical, SO4-, with Anions.
PB94-212172

00,828

Electron Transfer Reaction Rates and Equilibria of the Carbonate and Sulfate Radical Anions.

PB94-212180

00,829

Reaction of NO with Superoxide.

PB94-212198

00,830

Temperature Dependence of the Rate Constants for Reactions of the Carbonate Radical with Organic and Inorganic Reductants.

PB94-212206

00,831

Reaction of Nitric Oxide with Organic Peroxyl Radicals.

PB95-141107 00,910

Influence of Natural and Synthetic Inhibitors on the Crystallization of Calcium Oxalate Hydrates.

PB95-150967

03,560

Homogeneous Gas Phase Decyclization of Tetralin and Benzocyclobutene.

PB95-151049

00,928

Mechanism and Rate Constants for the Reactions of Hydrogen Atoms with Isobutene at High Temperatures. Modeling Ceramic Sub-Micron Particle Formation from the Vapor Using Detailed Chemical Kinetics: Comparison with In-situ Laser Diagnostics.

PB95-151965

00,671

Gas Phase Reactivity Study of $\mathrm{OH}$ Radicals with 1,1Dichloroethene and cis-1,1-Dichloroethene and Trans-1,2Dichloroethene over the Temperature Range 240-400 K. PB95-152146 00,939 Rate Constants for the Gas Phase Reactions of the $\mathrm{OH}$ Radical with CF3CF2CHCl2 (HCFC-225ca) and CF2CICF2CHCIF (HCFC-225cb)

00,940

Temperature Dependence of the Rate Constants for Reaction of Dihalide and Azide Radicals with Inorganic Reductants.

PB95-162756

00,964

lodine Atoms and lodomethane Radical Cations: Their Formation in the Pulse Radiolysis of lodomethane in Organic Solvents, Their Complexes, and Their Reactivity with Organic Reductants.

00,965
Shock Tube Techniques in Chemical Kinetics.

B95-163465

Hydrogen Atom Attack on Perchloroethylene. PB95-163473

00,968

Interaction of Chlorhexidine Digluconate with and Adsorpion of Chlorhexidine on Hydroxyapatite.

03,566

Kinetics of the Reaction $\mathrm{C} 2 \mathrm{H}+\mathrm{O} 2$ from 193 to $350 \mathrm{~K}$ Using Laser Flash Kinetic Infrared Absorption SpectrosPB95-203055

01,043

Thermochemical and Chemical Kinetic Data for Fluorinated Hydrocarbons.

01,056

Kinetics and Dynamics of Vibrationally State Resolved Ion-Molecule Reactions: (14) N2 $(+)(v=1$ and 2) and (15) N2 $(+)(v=0,1$ and 2$)$ with (14) N2

04,023

Radiation-Chemical Reaction of 2,3,5-Triphenyl-Tetrazolium Chloride in Liquid and Solid State.

zolium Chloride

01,124

REACTION-TO-FIRE TESTS

Effects of Specimen Edge Conditions on Heat Release

PB95-152864

00,375

REACTIVITY

Reactivity of Product Gases Generated in Idealized Enclosure Fire Environments

PB95-161790

REACTOR PHYSICS

Neutron Standard Cross Sections in Reactor Physics: Need and Status.

PB94-199510

REACTOR SAFETY

Proceedings of the Digital Systems Reliability and $\mathrm{Nu}$ clear Safety Workshop. Held in Rockville, Maryland on September 13-14, 1993

NUREG/CP-0136

03,728

REACTORS

MCNP Model of the National Bureau of Standards Reactor (NBSR) Core.

03,733

REAL TIME

Real-Time Implementation of a Differential Range Finder. PB95-108650

01,839

Through-the-Arc Sensing for Measuring Gas Metal Arc Weld Quality in Real Time.

02,908

REAL TIME CONTROL

Operator Experience with a Hierarchical Real-Time Control System (RCS)

PB96-195516

03,751

REAL-TIME CONTROL SYSTEMS

Integration of Servo Control into a Large-Scale Contro System Design: An Example from Coal Mining. PB94-203429

03,696

Continuous Mining Machine Control Using the Real-Time Control System

03,700

REAL TIME OPERATION

Ground Vehicle Control at NIST: From Teleoperation to Autonomy.
N94-34037/9

03,758

Utc Dissemination to the Real-Time User.

01,521
Continuous Mining Machine Control Using the Real-Time Continuous Mining Machine Control Using the Real-Time
Control System.
PB94-203528
03,700

Real-Time Vision for Autonomous and Teleoperated Control of Unmanned Vehicles.

PB94-211885

03,701

Real-Time Vision for Unmanned Vehicles.

03,702

Hierarchical Real-Time Control System for Use with Coal Mining Automation. Mining Automatio
PB94-212065

03,698

Knowledge-Based Approach for Automating a Design

Method for Concurrent and Real-Time Systems.
PB97-1 12502

01,780

REAL TIME OPERATIONS

Through-the-ArC Sensing for Real-Time Measurement of Gas Metal Arc Weld Ouality.

02,863

Outline of a Multiple Dimensional Reference Model Architecture and a Knowledge Engineering Methodology for Inelligent Systems Control.

PB95-220414

03,703

REAL TIME SYSTEMS

Toward a Reference Model Architecture for Real-Time Intelligent Control Systems (ARTICS)

02,932

Reference Model Architecture for Intelligent Systems Design. 143137

01,789

RECEIVERS

Integrated Optical Polarization-Discriminating Receiver in

PB97-113179

02,206

REFERENCE MATERIALS

RECEIVING TUBES

Tabulation of Data on Receiving Tubes. Handbook 68. AD-A285 495/8

02,111

RECEPTOR PROTEINS

Escherichis coli Cyclic AMP Receptor Protein Mutants Provide Evidence for Ligand Contacts Important in Activation.

RECHARGEABLE BATTERIES

Rechargeable Batteries for Personal/Portable. PB96-164231

03,592

RECOIL LIMITS

Laser Cooling and the Recoil Limit

B 97-111280

02,459

COMBINATION REACTIONS

Fluctuation Dominated Recombination Kinetics with Traps.

AECONSTRUCTION

PB96-122940

00,177

AECORDING HEADS

dc Magnetic Force Microscopy Imaging of Thin-Film Recording Head.

PB95-176061

04.665

Comparison of Magnetic Fields of Thin-Film Heads and Their Corresponding Bit Patterns Using Magnetic Force Microscopy.

RECORDS MANAGEMENT

Electronic Access: Blueprint for the National Archives and Records Administration.

PB95-219218

02,731

RECTILINEAR DIAMETER

Experimental Method for Obtaining Critical Densities of Binary Mixiures: Application to Ethane + n-Butane. PB95-15114

RED GIANT STARS

Four Years of Monitoring alpha Orionis with the VLA Where Have All the Flares Gone.

PB94-185212

00,048

RED SHIF

Redshifts in Stellar Transition Regions.

PB96-123310

00,104

REDUCED CONDUCTIVITY TENSOR

Characterization of LPE HgCdTe Film by

Magnetoresistand

02,197

REDUCED GRAVITY

Combustion of a Polymer (PMMA) Sphere in Microgravity.

01,354

Ignition and Subsequent Flame Spread Over a Thin Cellulosic Material.
PB96-160270

REDUCTION (CHEMISTRY)

Site of One-Electron Reduction of Ni(II) Porphyrins. For mation of $\mathrm{Ni}(\mathrm{I})$ Porphyrin of $\mathrm{Ni}(\mathrm{II})$ Porphyrin pi-Radica

PB95-107066

00,866 
Reference Materials by Isotope Dilution Mass SpectromPBg5. 153383

00,592 Reference Data for the Thermophysical Properties of Cryogenic Fluids.

PB95-168688

03,263

NIST Standard Reference Materials (Trade Name) Cata$\log 1995-1996$

00,508

Journal of Physical and Chemical Reference Data, Volume 24, No. 1, January/February 1995

01,101

Journal of Physical and Chemical Reference Data, Volume 24, No. 2, March/April 1995.

PB96-145818

01,105

Journal of Physical and Chemical Reference Data, Volume 24, No. 4, July/August 1995

PB96-145883

01,112

Journal of Physical and Chemical Reference Data, Volume 24, No. 5, September/October 1995.

01,116

Journal of Physical and Chemical Reference Data, Volume 24. No. 6, November/December 1995.

01,120

Interlaboratory Studies on the Analysis of Hair for Drugs of Abuse: Results from the Fourth Exercise.
PB97-111322 REERENCE MODELS

Next Generation Computer Resources: Reference Model for Project Support Environments (Version 2.0).

01,677

REFERENCE STANDARDS

Standard Samples and Reference Standards Issued by the National Bureau of Standards.

AD-A279 240/6

Coaxial Reference Standard for Microwave Power. PB94-193786

01,880

How to Verify Reference Materials.

PB95-151486

03,497

Development of a Gas Standard Reference Material Containing Eighteen Volatile Organic Compounds.

PB95-162277

Standards for Atmospheric Measurements.

02,545

PB95-163622

02,547

Standard Reference Materials for the Determination of Trace Organic Constituents in Environmental Samples.

Liquid Chromatographic Determination of Polycyclic Aromatic Hydrocarbon Isomers of Molecular Weight 278 and 302 in Environmental Standard Reference Materials. PB95-164042 02,523 Alaska Marine Mammal Tissue Archival Project: Specimen Inventory.

02,589

Determination of Amphetamine and Methamphetamine in a Lyophilized Human Urine Reference Material. $\quad 03,597$ Certification of Morphine and Codeine in a Human Urine Certification of Morphine and Codeine in a Human Urine
Standard Reference Material.

03,499

Hair Analysis for Drugs of Abuse: Evaluation of Analytical Methods, Environmental Issues, and Development of Reterence Materials.

PB95-176269

03,501

Ouantitative Analysis of Selected PCB Congeners in Marine Matrix Reference Materials Using a Novel Cyanobiphenyl Stationary Phase.

02,591

Certification of Polycyclic Aromatic Hydrocarbons in a Marine Sediment Standard Reference Material.

PB96-111778

02,592

Considerations in the Design of an Environmental Specimen Bank: Experiences of the National Biomonitoring Specimen Bank Program.

PB96-112370

02,527

Long-Term Stability of Bayard-Alpert Gauge Performance: Results Obtained from Repeated Calibrations against the National institute of Standards and Technology Primary Vacuum Standard.

PB96-123567

02,676

Certification of Standard Reference Material (SRM) 1941a, Organics in Marine Sediment.

02,593

NIST Reference Materials to Support Accuracy in Drug Testing.

03,505

Measurement Comparability, Traceability, and Measurement Assurance Programs.

PB97-111850

02,692

National Status and Trends Program Specimen Bank:

Sampling Protocols, Analytical Methods, Results, and Archive Samples.

REFERENCE SYSTEMS

02,598

Numeric Data Distribution: The Vital Role of Data Exchange in Today's World.

N95-15937/2

02,622

REFERENCES (STANDARDS)

Recertification of the Standard Reference Material 1475A

a Linear Polyethylene Resin.

a Linear Polyeth

02,628
Standard Reference Materials: Glass Filters as a Standrd Reference Material for Spectrophotometry - Selection Preparation Certification, and Use of SRM 930 and SRM

PB94-188844

00,536

REFLECTANCE

XUV Characterization Comparison of $\mathrm{Mo} / \mathrm{Si}$ Multilayer Coatings.

04,278

45 deg/0 deg Reflectance Factors of Pressed Polytetrafluoroethylene (PTFE) Powder.

PB95-260758

04,328

REFLECTANCE FACTORS

$45 \mathrm{deg} / 0$ deg Reflectance Factors of Pressed Polytetraluoroethylene (PTFE) Powder.

04,328

\section{REFLECTION SPECTRA}

Intermediate Coupling in L2-L3 Core Excitons of $\mathrm{MgO}$ $\mathrm{Al} 2 \mathrm{O} 3$, and $\mathrm{SiO}_{2}$

REFLECTIVE COATINGS

Interfaces in Mo/Si Multilayers.

PB96-160668

02,423

REFLECTIVITY

Near Critical Fluid Interfaces: A Comparison of Theory PB95-140166

00,90

In-situ Neutron Reflectivity of MBE Grown and Chemically Processed Surfaces and Interfaces.

PB96-146634

\section{REFLECTOMETERS}

Improved Reflectometry Facility at the National Institute of Standards and Technology.

04,087

Soft X-ray Reflectometry Program at the National Institute of Standards and Technology.

04,368

REFLECTOMETRY

Upgraded Facility for Multilayer Mirror Characterization at PB96-160387

REFRACTIVE INDEX

Physical Properties of Some Purified Aliphatic HydroPhysical $P$

AD-A297 265/1

00,657

Normal-Incidence Complex-Index Refractometry.

PB94-213097

04,241

Formulation of the Refractive Index of Water and Steam.

00,900

Guidelines for Refractive Index Measurements of Asbes-

PB95-151189 02,543

Calculated Fiber Attenuation: A General Method Yielding Stationary Values.

04,298

Determination of the Complex Refractive Index of Individ-

ual Ouantum Wells from Distributed Reflectance.
PB95-175642

02,176

\section{REFRACTIVE INDEXES}

Verification of Revised Water Vapour Correction to the ndex of Refraction of Air.

02,680

\section{REFRACTIVITY}

Normal-Incidence Complex-Index Refractometry. PB94-213097

04,241

Refractive Indices of Fluids Related to Alternative Refrig. erants.

\section{REFRACTOMETERS}

03,260

New Refractometer by Combining a Variable Length Vacuum Cell and a Double-Pass Michelson Interferometer. PB97-111926

01,986

\section{REFRACTORY METALS}

Wavelength Dependence of Normal Spectral Emissivity of High-Temperature Metals at Their Melting Point.

PB94-200011

03,398

Measurement of the Heat of Fusion of Tungsten by a Microsecond-Resolution Transient Technique. PB94-216686

03,400

\section{REFRIGERANT COMPRESSORS}

Design Equations and Scaling Laws for Linear Compres. sor's with Flexure Springs.

\section{PRIGERANT MIXTURES}

Model for Calculating Virial Coefficients of Natural Gas Hydrocarbons with Impurities.

PB96-156047

04,064

Design of a High-Pressure Ebulliometer, with Vapor-Liquid Equilibrium Results for the Systems $\mathrm{CHF}_{2} \mathrm{Cl}+\mathrm{CF}_{3}$ $\mathrm{CH} 3$ and $\mathrm{CF}_{3}-\mathrm{CH} 2 \mathrm{~F}+\mathrm{CH} 2 \mathrm{~F} 2$

PB97-113229

04,163

\section{REFRIGERANTS}

Properties of Working Fluids for Thermoacoustic Refrig-

AD-A297 420/2

04,864

Dielectric Studies of Fluids with Reentrant Resonators. Appendix B.

03,244

Thermophysical Properties of HFC-143a and HFC-152a. Ouarterly Report, 1 July 1993--30 September 1993. DE9400423 03,245

Development of Measurement Capabilities for the Thermophysical Properties of Energy-Related Fluids. Annual Report, December 1, 1990--November 30, 1991.

DE94004399 Development of Measurement Capabilities for the Thermophysical Properties of Energy-Related Fluids. An nual Report, December 1, 1993--November 30, 1994.

DE94017738 03,246

Thermophysical properties of HCFC alternatives. Ouarterly report, 1 July 1994--30 September 1994.

03,247

Lean flammability limit as a fundamental refrigerant property. Phase 1, Interim technical report, 1 October 1994-DE95011238

DE95011238

03,248

Thermophysical properties of HCFC alternatives. Ouarterly report, October 1.-December 31, 1995.

03,249

Thermophysical properties of HCFC alternatives. Quarterly report, April 1--June 30, 1995

DE96010579

03,250

Evaluation of GSA Maintenance Practices of Large Centrifugal Chillers and Review of GSA Refrigerant Management Practices.

02,502

Simultaneous Visual and Calorimetric Measurements of R11, R123, and R123/Alkylbenzene Nucleate Flow Boiling. 172426

03,251

Thermodynamic

of

CHF2-O-

PB94. 199569

00,798

Role of Refrigerant Mixtures as Alternatives to CFCs.

PB94-199775 03,252

Role of R22 in Refrigerating and Air Conditioning Equip-

PB94-199783

03,253

Measurements of the Viscosities of Saturated and Compressed Fluid 1-chloro-1,2,2,2-tetrafluoroethane (R124) and Pentafluoroethane (R125) at Temperatures between 120 and $420 \mathrm{~K}$

Simplified Cycle Simulation Model for the Performance Rating of Refrigerants and Refrigerant Mixtures.

PB94-199890

03,255 
Development of a Dual-Sinker Densimeter for High-Accuracy Fluid P-V-T Measurements.

PB95-168951

03,267

Measurements of the Vapor Pressures of Pentafluoroethane.

PB95-169272

03,270

Vapor Pressures and Gas-Phase PVT Data for 1-Chloro$1,2,2,2$-Tetrafluoroethane (R124)

03,271

Retention of Halocarbons on a Hexafluoropropylene Epoxide Modified Graphitized Carbon Black. Part 1. Methane-Based Compounds.

PB95-175196

03,272

Measurements of the Viscosities of Saturated and Compressed Liquid 1,1,1,2-Tetrafluoroethane (R134a), 2,2Dichloro-1,1,1-Trifluoroethane (R123) and 1,1-Dichloro-1Fluoroethane (R141b)

Fluoroethane

03,273

Coexisting Densities, Vapor Pressures and Critical Densities of Refrigerants $R-32$ and R-152a, at $300-385 \mathrm{~K}$.

PB95-175691

03,274

Predictive Extended Corresponding States Model for Pure and Mixed

PB95-175717

03,275

Estimating the Virial Coefficients of Small Polar Molecules.

PB95-176236

03,276

Simpie Method of Composition Shifting with a Distillation Column for a Heat Pump Employing a Zeotropic RefrigPBant Mixture.

02,603

Interaction Coefficients for 15 Mixtures of Flammable and Non-Flammable Components.

03,281

PB96-146626

Retention of Halocarbons on a Hexafluoropropylene Ep-
oxide-Modified Graphitized Carbon Black. 4. Propane-

oxide-Modified Graphitized Carbon Black. 4. Propane-
Based Compounds.
PB96-164033
03,284

REFPROP Refrigerant Properties Database: Capabilities,

Limitations, and Future Directions.

PB96-167150 01,149

Status of the Round Robin on the Transport Properties of R134a.

PB96-167218

01,152

Retention of Halocarbons on a Hexafluoropropylene Epoxide-Modified Graphitized Carbon Black. 3. EtheneBased Compounds.

PB96-167309

03,286

Thermophysical Property Standard Reference Data from PB96-167358

01,153

Study to Determine the Existence of an Azeotropic R-22 'Drop-In' Substitute.

PB96-167812

02,568

Viscosity of 1,1,1,2,3,3-Hexafluoropropane and $1,1,1,3,3,3-$ Hexafluoropropane at Saturated-Liquid Conditions from $262 \mathrm{~K}$ to $353 \mathrm{~K}$

03,292

Report of the Refrigeration, Air Conditioning and Heat Pumps Technical Options Committee.

PB96-176755

03,293

International Standard Equation of State for the Thermodynamic Properties of Refrigerant 123 (2,2-Dichloro1,1,1-Trifluoroethane).

PB'96-176805

03,294

REFRIGERATING MACHINERY

Evaluation of GSA Maintenance Practices of Large Centrifugal Chillers and Review of GSA Refrigerant Management Practices.

PB94-143344

02,502

Role of R22 in Refrigerating and Air Conditioning Equip-

mB94. 199783

03,253

Effect of Inclination on the Performance of a Compact Brazed Plate Condenser and Evaporator.

02,756

REFRIGERATION SYSTEMS

Design and Construction of a Liquid Hydrogen Temperature Refrigeration System.

02,619

Helium Refrigeration and Liquefaction Using a Liquid $\mathrm{Hy}$ drogen Refrigerator for Precoling.

AD-A286 $683 / 8$

02,749

Properties of Working Fluids for Thermoacoustic Refrig-

AD-A297 420/2

04,864

REFRIGERATORS

Resistance Thermometers with Fast Response for Use in Rapidly Oscillating Gas Flows.

03,261

Electronic Microrefrigerator Based on a Normal-Insulator-

Superconductor Tunnel Junction.

PB96-102827

04,718

Factors Affecting the Energy Consumption of Two Refrigerator-Freezers.

AEFUELING SYSTEM

Upgrade and Modernization Projects at the NBSR

PB96-161872

00,311

03,737
REFUSE DERIVED FUELS

Sulfur Dioxide Capture in the Combustion of Mixtures of Lime, Refuse-Derived Fuel, and Coal

PB94-155587

02,534

EGENERATORS

Graded and Nongraded Regenerator Performance.

02,753

REGIONAL DEVELOPMENT

Aid for Smaller Businesses.

PB94-212461

00,492

TRIES

SC4 Short Names Registry.

PB97-122410

02,799

REGULATIONS

National Center for Standards and Certification Information: Service and Programs.

N95-15938/0

02,717

Uniform Laws and Regulations in the Areas of Legal Metrology and Motor Fuel Quality, 1994 as Adopted by the 79th National Conference on Weights and Measures

PB95. 174470

02,909

International Challenges in Defining the Public and Private Interest in Standards.

PB96-160361

00,498

REINFORCED CONCRETE

Corrosion Resistant Epoxy-Coated Reinforcing Steel.
PB94-185618

Strengthening Methodology for Lightly Reinforced Concrete Frames-II. Recommended Calculation Techniques for the Design of Infill Walls.

PB94-187648

00,426

Seismic Strengthening of Reinforced Concrete Frame Buildings.

00,430

Enhancements to Program IDARC: Modeling Inelastic Behavior of Welded Connections in Steel Moment-Resisting

PBames. 231601

00,452

Strengthening Methodology for Lightly Reinforced Concrete Frames: Recommended Design Guidelines for Strengthening with Infill Walls. Prediction of Potential Concrete Strength at Later Ages. PB96-112180

01,324

REINFORCEMENT (STRUCTURAL)

Evaluation and Retrofit Standards for Existing Federally Owned and Leased Buildings.

REINFORCEMENT (STRUCTURES)

Strengthening Methodology for Lightly Reinforced Concrete Frames-II. Recommended Calculation Techniques for the Design of Infill Walls.

PB94-187648

00,426

Seismic Strengthening of Reinforced Concrete Frame Buildings.

00,430

Jacket Thickness Requirements for Seismic Retrofitting of Circular Bridge Columns.

B95-163267

01,336

Seismic Safety of Federal Buildings. Initial Program: How Much Will it Cost.
PB95-182291

00,447

Strengthening Methodology for Lightly Reinforced Concrete Frames: Recommended Design Guidelines for Strengthening with Infill Walls.

Strengthening Methodology for Lightly Reinforced Con Crete Frames.
PB96-158050

\section{REINFORCING FIBERS}

Micro-Mechanical Aspects of Asperity-Controlled Friction in Fiber-Toughened Ceramic Composites.

PB94-199536

03,136

Elastic Properties of Uniaxial-Fiber Reinforced Compos tes: General Features.

PB94-200649

REINFORCING STEELS

Corrosion Resistant Epoxy-Coated Reinforcing Steel.

01,307

Degradation of Powder Epoxy Coated Panels Immersed in a Saturated Calcium Hydroxide Solution Containing Sodium Chloride.
PB96-10 1050

REJUVENATION

Volume Recovery in Epoxy Glasses Subjected to Torsional Deformations: The Question of Rejuvenation. PB95-140018

03,382

Torsional Relaxation and Volume Response during Physical Aging in Epoxy Glasses Subjected to Large Torsiona

PB95-140026

03,383

Using Torsional Dilatometry to Measure the Effects of Deformations on Physical Aging.

PB95-140901

03,384

RELATIVE HUMIDITY

Temperature and Relative Humidity Dependence of Radiochromic Film Dosimeter Response to Gamma and Electron Radiation.

PB96-135298

03,718

Parametric Study of Wall Moisture Contents Using a Revised Variable Indoor Relative Humidity Version of the 'Moist' Transient Heat and Moisture Transfer Mode.

PB97-122535

00,419

RELAXATION (MECHANICS)

Operational Mode and Gas Species Effects on Rotational Drag in Pneumatic Dead Weight Pressure Gages. PB95-140182

\section{RELIABILITY}

Numeric Data Distribution: The Vital Role of Data Exchange in Today's World.

N95-15937/2 02,622

Performance and Reliabitity of NIST $10-\mathrm{V}$ Josephson Ar-

rays.

02,419

High Temperature Structural Reliability of Silicon Nitride.

PB97-110456

03,104

RELIABILITY ANALYSIS

Measurements of Properties of Materials in Electronic Packaging.
PB96-200837

01,973

REMANENT POLARIZATION

Hysteresis Measurements of Remanent Polarization and Coercive Field in Polymers.

PB94-199767

04,475

\section{REMINERALIZATION}

Remineralization of Root Lesions with Concentrated Calcium and Phosphate Solutions.

Polymeric Calcium Phosphate Composites with Remineralization Potentia

03,575

REMOTE CONSOLES

Concepts of the NIST EXPRESS Server.

PB95-180543

02,781

REMOTE PROCEDURE CALLS

Comparing Remote Procedure Calls: Open Network Computing, Distributed Computing Environment and InterPB95-194205

01,724

REMOTE SENSORS

Activities of NIST (National Inst. Of Standards and Technology).

N94-23605/6 04,222

Dual-Frequency Millimeter-Wave Radiometer Antenna for Airborne Remote Sensing of Atmosphere and Ocean PB96-112289

02,009

REMOVAL

Computer Simulations of Binder Removal from 2-D and 3-D Model Particulate Bodies.

PB97-121339

00,418

RENOVATING 
Addressing U.S. Government Security Requirements for OSI.

Rechargeable Batteries for Personal/Portable.

02,459

RESEARCH

Journal of Research of the National Institute of Standards and Technology, November-December 1993. Volume 98 Number 6 .

PB94-140555

04,223

Thermodynamic Properties of the Group IIA Elements.

PB94-160983 00,730

Spectroscopy and Structure of the Lithium Hydride Diatomic Molecules and lons.

00,731

Ouantum Yields for the Photosensitized Formation of the Lowest Electronically Excited Singlet State of Molecular Oxygen in Solution.

PB94-161007

00,732

Wavelengths and Energy Level Classifications for the Spectra of Sulfur (SI through $S$ XVI).

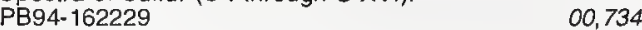

Thermodynamic Properties of Alkenes (Mono-Olefins Larger Than C4).
PB94-162237

00,735

Thermodynamic Behavior of the $\mathrm{CO} 2 \cdot \mathrm{H} 2 \mathrm{O}$ System from 400 to $1000 \mathrm{~K}$, up to $100 \mathrm{MPa}$ and $30 \%$ Mole Fraction of

00,736

PB94-162245

Part 1.

Thermodynamics

PB94-162252

00,737

Estimation of the Heat Capacities of Organic Liquids as a Function of Temperature Using Group Additivity. I. Hydrocarbon Compounds.

00,739

Estimation of the Heat Capacities of Organic Liquids as a Function of Temperature Using Group Additivity. II. Compounds of Carbon, Hydrogen, Halogens, Nitrogen, Oxygen, and Sulfur

00,740

Thermodynamic and Thermophysical Properties of Organic Nitrogen Compounds. Part II. 1- and 2-Butanamine, 2-Methyl-1-Propanamine, 2-Methyl-2-Propanamine Pyrrole, 1-, 2-, and 3-Methylpyrrole, Pyridine, 2-, 3-, and 4-Methylpyridine, Pyrrolidine, Piperidine, Indole, Ouinoline, Isoquinoline, Acridine, Carbazole, Phenanthridine, 1 and 2-Naphthalenamine, and 9-Methylcarbazole. PB94-162294

00,741

International Equations for the Saturation Properties of Ordinary Water Substance. Revised According to the International Temperature Scale of 1990. Addendum to Journal of Physical and Chemical Reference Data 16. $893(1987)$

PB94-162302

00,742

Estimation of the Thermodynamic Properties of C-H-N-OS-Halogen Compounds at $298.15 \mathrm{~K}$

00,744

Compilation of Energy Levels and Wavelengths for the Spectrum of Singly-lonized Oxygen (O II).

PBectrum of Sing

00,746

Energy Levels of Germanium, Ge I through Ge XXXII

PB94-162351

00,747

Spectral Data and Grotrian Diagrams for Highly lonized PB94-162369

00,748

Thermodynamic Properties of Synthetic Sapphire (alpha$\mathrm{Al}$ 203), Standard Reference Material 720 and the Effect of Temperature-Scale Differences on Thermodynamic Properties.

PB94-16856

00,750

Thermodynamic Properties of Gaseous Silicon Monotelluride and the Bond Dissociation Enthalpy D(sub $\mathrm{m})(\mathrm{SiTe})$ at $\mathrm{T}$ approaches 0 .

00,751

Disilicides of Tungsten, Molybdenum, Tantalum, Titanium, Cobalt, and Nickel, and Platinum Monosilicide: A Survey of Their Thermodynamic Properties.

PB94-168580

00,752

Evaluated Bimolecular Ion-Molecule Gas Phase Kinetics of Positive Ions for Use in Modeling Planetary Atmospheres, Cometary Comae, and Interstellar Clouds.

Atomic Weights of the Elements 1991.

PB94-168606

00,754

Growing Significance of $\mathrm{CIB}$

PB95-126306

00,314

PB95-143202

cations

Rationale and Preliminary Plan for Federal Research for Construction and Building.

00,322

Building and Fire Research Laboratory Publications,

PB95-226684

00,398

SESEACH AND DEVELOPMENT

CSTL Technical Activities 1991

PB94-160769

0,728
Industry and Government-Laboratory Cooperative $R$ and D: An ldea Whose Time Has Come.

02,970

Preliminary Functional Specifications of a Prototype Elecronic Research Notebook for NIST. tronic Research

00,012

Program Requirements to Advance the Technology of Custom Footwear Manufacturing

PB95-147906

02,883

CSTL Technical Activities, 1993.

00,953

Encouraging Environmentally-Aware Inventions PB95-161394

02,521

Journal of Research of the National Institute of Standards and Technology, September/October 1994. Volume 99 Number 5 .

PB95-169371

04,293

Materials Science and Engineering Laboratory Annual Report, 1994. NAS-NRC Assessment Panel, April 6-7,

PB95-196697

02,976

CSTL Technical Activities, 1994

00,608

Proceedings of the 1995 Workshop on Fire Detector $R e$ Proceedings of the 1995 Workshop
search. Held on February 6-7, 1995

PB95-270062

02,611

Joint DoD/NIST Workshop on International Manufacturing Systems Research and Development. Held in Rockville, Maryland on November 3-5, 1992. Proceedings.

PB96-109491

02,931

Journal of Research of the National Institute of Standards and Technology, July/August 1995. Volume 100, Number 4. Special Issue: The Gaseous Electronics Conference Radio-Frequency Reference Cell.

PB96-113311

02,386

Journal of Research of the National Institute of Standards and Technolc

PB96-113535

04,336

Journal of Research of the National Institute of Standards and Technology, September/October 1995. Volume 100 Number 5 .

PB96-117767

01,927

Journal of Research of the National Institute of Standards and Technology, March/April 1995. Volume 100, Number

PBg6-126180

04,349

National Planning for Construction and Building $R$ and $D$.

PB96-137104 00,324

High-Temperature Furnace for In situ Small-Angle NeuPB96-148127

03,743

Journal of Research of the National Institute of Standards and Technology, November/December 1995. Volume 100 , Number 6 .

01,949

Electronics and Electrical Engineering Laboratory 1995 Technical Accomplishments: Advancing Metrology for Electrotechnology to Support the U.S. Economy.

PB96-164520

01,959

Innovation in the Japanese Construction Industry: A 1995 Appraisal.

CSTL Technical Activities, 1995

00,225

PB96-214630

00,647

Materials Science and Engineering Laboratory Annual Report, 1995. Technical Activities.

03,009

PB96-214754

Summary of Federal Construction and Building $R$ and $D$

PB97-114250

00,234

Fire Safety Engineering in the Pursuit of PerformanceBased Codes: Collected Papers.

00,235

Science, Technology, and Competitiveness: RetrospecScience, Technology, and Competiveness. Retrospective on a Symposium in Celebration of NIST's 90 th Ann-
versary and the 25th Anniversary of the Gaithersburg Laboratories, November $14-15,1991$.

PB97-121610

02,696

RESEARCH COMMITTEE ON TRIBOLOGY

Tribology Education: Present Status and Future Challenges.

02,965

RESEARCH FACILITIES

NIST-NRL Free-Electron Laser Facility.

PB94-212511

04,237

Automated Manufacturing Research Facility 1994 Annual Report.

PB95-209854

00,015

RESEARCH LABORATORIES

Publications 1995: NIST Building and Fire Research Lab-

PBatory.

00,226

RESEARCH MANAGEMENT

Project Summaries 1994: NIST Building and Fire Research Laboratory.

00,343

Survey of Recent Cementitious Materials Research in Western Europe
PB94-218583

00,353

Project Summaries 1995: NIST Building and Fire Re-

00,400

Electronics and Electrical Engineering Laboratory TechElectronics and Electrical Engineering Laboratory Technical Publication Announcements Covering Laboratory
Programs, April to June 1995 with 1995 EEEL Events

PB96-137187

01,941

Benefits and Costs of Research: Two Case Studies in Building Technology

PB96-202221

00,230

Benefits and Costs of Research: A Case Study of the Fire Safety Evaluation System.

00,232

RESEARCH PROGRAMS

NIST Lighting and HVAC Interaction Test Facility Glimpse of Materials Research in China: A Report from an Interagency Study Team on Materials Visiting China from June 19, 1995 to June 30, 1995.

PB96-112677

02,978

Electronics Packaging Materials Research at NIST.

02,405 Internationalization of Fire Safety Engineering Research

PB96-156153. 00,220

NIST Program for Investigating Error Reporting Capabili-

ties of Optical Disk Drives.

01,635

Optoelectronics at NIST.

PB96-200860

02,202

Optoelectronics and Optomechanics Manufacturing: An ATP Focused Program Development. Workshop Proceedings. Held in Gaithersburg, Maryland on February 15, PB97-104186

02,204

RESEARCH PROJECTS

Ceramics Technical Activities, 1993 (NAS-NRC Assessment Panel April 21-22, 1994

PB94-162591

03,031

Lighting Research and Theory Can Create Business

PB95-15179

00,253

Publication and Presentation Abstracts, 1993. (Published by Paffenbarger Research Center and Center of Excellence for Materials Science Research)

PB95-153052

03,562

Structural Ceramics Database. Topical Report, June 1989-May 1991

PB95-203758 03,060

Reactor Radiation Technical Activities, 1994. NAS-NRC Assessment Panel, April 6-7, 1995.

PB95-209888

03,732 
Electronics and Electrical Engineering Laboratory Technical Publication Announcements Covering Laboratory Programs, October to December 1995, with 1996 EEEL Events Calendar.

PB96-202346

01,978

Electronics and Electrical Engineering Laboratory Technical Publication Announcements Covering Laboratory
Programs, January to March 1996, with 1996 EEEL Events , January

PB96-214622

01,981

Workshop Highlights.

PB97-10922

04,811

Electronics and Electrical Engineering Laboratory Technical Progress Bulletin Covering Laboratory Programs, April

PB97-113880

01,994

Publication and Presentation Abstracts, 1996. PB97-122238

03,585

RESIDENTIAL BUILDING

Carbon Monoxide Dispersion in Residential Buildings: Literature Review and Technical Analysis.

PB97-114227

02,571

RESIDENTIAL BUILDING FIRES

EXITT: A Simulation Model of Occupant Decisions and Actions in Residential Fires.

PB94-213261

00,351

RESIDENTIAL BUILDINGS

Mathematical Modeling of Human Egress from Fires in Residential Buildings.

PB94-193778

00,337

Studies Assess Performance of Residential Detectors.

PB94-199262 00,290

EXITT: A Simulation Model of Occupant Decisions and Actions in Residential Fires.

PB94-213261

00,351

Indoor Air Quality Impacts of Residential HVAC Systems, Phase 1 Report: Computer Simulation Plan.
PB95-135596
00,249 Modeling Radon Transport in Multistory Residential Buildings.

00,256

Performance of HUD-Affiliated Properties during the January 17, 1994 Northridge Earthquake.

00,443

Application of a Multizone Airflow and Contaminant Dispersal Model to Indoor Air Quality Control in Residential Buildings.

PB95-180238

02,555

Santa Ana Fire Department Experiment at 1315 South Bristol, July 14, 1994

00,389

Measurements of Indoor Pollutant Emissions from EPA Phase II Wood Stoves.

PB95-198735

02,556

National Construction Sector Goals: Industry Strategies for Implementation.

00,204

Santa Ana Fire Department Experiment at 1315 South Bristol, July 14, 1994. (Reprint)

00,207

Surging the Upside-Down House: Looking into Upsetting Reference Voltages.

02,385

Effectiveness of a Heat Recovery Ventilator, an Outdoo Air Intake Damper and an Electrostatic Particulate Filte at Controlling Indoor Air Quality in Residential Buildings.
PB96-146642

Santa Ana Fire Department Experiments at South Bristol

PB96-154810
Sensitivity Analysis for Mathematical Modeling of Fires in Residential Buildings.

PB96-154968

00,215

Protecting Your Family from Fire.

00,307

Multizone Modeling of Three Residential Indoor Air Quality Control Options.

PB96-165782

02,567

Surging the Upside-Down House: Measurements and Modeling Results.

02,243

Distributions of Measurement Error for Three-Axis Magnetic Field Meters during Measurements Near Appli-

ances.
PB96-180153 02,110

Full-Scale Room Fire Experiments Conducted at the University of Maryland.

00,236

RESIDUAL GAS

Measurement of Very-Low Partial Pressures.

PB95-180998 02,659

Process Monitoring with Residual Gas Analyzers (RGAs): Limiting Factors.

02,660

RESIDUAL GAS ANALYZERS

Process Monitoring with Residual Gas Analyzers (RGAs): Limiting Factors.

02,660
RESIDUAL STRENGTH

Fracture Behavior of Large-Scale Thin-Sheet Aluminum Alloy.

03,311

RESIDUAL STRESS

Influence of Tempering Method on Residual Stress in Dental Porcelain.

PB94-172012 00,138

Evaluation of Fracture Toughness and Residual Stress in Dental Porcelain by Indentation-Microfracture Method.

PB95-125613

00.154

Evaluation of Fracture Toughness and Residual Stress in Dental Porcelain by Indentation-Microfracture Method.
PB95-152831 00,159

Residual Stresses in Aluminum-Mullite (alpha-Alumina) Pomposites.

03,155

Ultrasonic Measurement of Residual Stress in Cast Steel Railroad Wheels.

Determination of the Residual Stresses Near the Ends of Skip Welds Using Neutron Diffraction and X-ray Diffraction Procedures.

PB95-253589

02,868

Ultrasonic Methods

PB96-190327

02,707

\section{RESIN TRANSFER MOLDING}

Effect of Heterogeneous Porous Media on Mold Filling in PB95-10857er Molding.

Comparison of the Unidirectional and Radial In-Plane

Flow of Fluids Through Woven Composite Reinforce-

PB95-162004 02,698

Interaction between Micro and Macroscopic Flow in RTM Preforms.

PB95-162012

03,159

RESIST ANCE

Electrical Characterization of Narrow Gap n-Type Bulk HgCdTe Single Crystals by Variable-Magnetic-Field Hall Measurements and Reduced-Conductivity-Tensor AnalyPB96-164199

01,146

RESISTANCE BRIDGES

Leakage Current Detection in Cryogenic Current Comparator Bridges

RESISTANCE MEASUREMENTS

Resistance Measurements from $10 \mathrm{M}$ Omega $10 \mathrm{TT}$ Omega at NIST
PB97-119168

02,290

RESISTANCE STANDARD

Quantum Hall Effect-Based Resistance Standard: Capabilities and Implementation.

PB96-180096

04,114

Quantum Hall Effect-Based Resistance Standard (QuanIum Hall Res).

04,127

RESISTANCE STANDARDS

New International Representations of the Volt and Ohm

Effective January 1, 1990

Design Challenges in a Commercial Quantum Hall EffectBased Resistance Standard.

PB95-171419

02,263

RESISTANCE THERMOMETERS

Assessment of Uncertainties of Calibration of Resistance Thermometers at the National Institute of Standards and Technology.

02,624

RESISTIVE DIVIDERS

Transient Errors in a Precision Resistive Divider. PB97-111512

01,983

RESISTIVITY MAPPING

High-Spatial-Resolution Resistivity Mapping Applied to Mercury Cadmium Telluride.

PB94-212917

02,131

RESISTORS

Joumal of Research of the National Institute of Standards and Technology. May/June 1994. Volume 99, Number 3.

PB94-219326 02,643

Sources of Uncertainty in a DVM-Based Measurement System for a Quantized Hall Resistance Standard.

PB94-219334

01,884

Pressure Dependencies of Standard Resistors.

PB95-153516

02,257

Bonding Wires to Quantized Hall Resistors.

PB96-102637

01,921

Automated Resistance Measurements at NIST.

PB96-119326 02274

Constant Temperature and Humidity Chamber for Stand-

ard Resistors.

PB96-122494

02,275

Resistors.

PB97-111876

Observation of Hot-Electron Shot Noise in a Metallic Re-

PB97-112007

01,988

Automated Guarded Bridge for Calibration of Multimegohm Standard Resistors.

02,289

Resistance Measurements from $10 \mathrm{M}$ Omega to $1 \mathrm{~T}$ Omega at NIST.

Omega at NIST.

02,290

RESOI

Electron and Hole Trapping in Irradiated SIMOX, ZMR and BESOI Buried Oxides.

PB96-160320

01,956

RESOLUTION FUNCTIONS

Instrumental Smearing Effects in Radially Symmetric Small-Angle Neutron Scattering by Numerical and AnalytPB96-160429

02,984

RESONANCE

Resonances in Two-Dimensional Array Oscillator Circuits.
PB96-102082

ESONANCE FLUORESCENCE

Heterodyne Measurement of the Fluorescence Spectrum f Optical Molasses.

03,873

Measurements of Fluorescence from Cold Atoms: Localization in Three-Dimensional Standing Waves.

PB95-163879

03,943

Interference in the Resonance Fluorescence of Two Trapped Atoms

03,948

Resonance Fluorescence with Squeezed-Light Excitation. PB95-203469

RESONANCE IONIZATION MASS SPECTROSCOPY

Atom-counting standards and Doppler-free resonance ionization mass spectroscopy. (Progress report). Improvement of Ultrasensitive Techniques Isotopic Biasing in the RIS Process Ionization Efficiencies and

DE94018563 00,522

I: Improvement of Resonance Ionization Spectroscopy (RIS) Techniques; II: Atomic Data for RIS; III: Standards for Ultratrace Analysis. Progress Report

DE94018565

00,523

National Institute of Standards and Technology Resonance lonization Spectroscopy/Resonance lonization Mass Spectroscopy Data Service.

03,793

Comparative Strategies for Correction of Interferences in Isotope Dilution Mass Spectrometric Determination of Va-

PB94-185261

00,531

Laser Ablation of Thin Films as a Free Atom Source for Pulsed RIMS

RESONANCE IONIZATION SPECTROSCOPY

00,540

National Institute of Standards and Technology Resonance Ionization Spectroscopy/Resonance Ionization Mass Spectroscopy Data Service.

PB94- 772897

03,793

RESONANCE LINES

Observation of Pd-Like Resonance Lines Through $\mathrm{Pt}(32+)$ and Zn-Like Resonance Lines of $\operatorname{Er}(38+)$ and PB95-150637

03,896

RESONANT FREQUENCY

Elastic Constants of Isotropic Cylinders Using Resonant 
RETARDING

Controlled Nucleation in Aerosol Reactors for Supression of Agglomerate Formation

00,672

RETENTION

Comparison of the Liquid Chromatographic Behavior of Selected Steroid Isomers Using Different ReversedPhase Materials and Mobile Phase Compositions. PB95-140976

00,574

\section{RETINOIDS}

iquid Chromatographic Method for the Determination of Carotenoids, Retinoids, and Tocopherols in Human Serum and in Food

(15359

00,593

RETROFITING

How-To Suggestions for Implementing Executive Order 12941 on Seismic Safety of Existing Federal Buildings, A PBndook.

PB96-131552

00,461

Spatial Correlation Function for Fields in a Reverberation Chamber.

PB96-148077 04.427

Rapid Evaluation of Mode-Stirred Chambers Using Impulsive Waveforms.
PB96-210026

01.979

REVERBERATION CHAMBERS

Electronic Mode Stirring for Reverberation Chambers.
PB95-180329

TEM/Reverberating Chamber Electromagnetic Radiation

Test Facility at Rome Laboratory.
PB96-155023

03,675

Band-Limited. White Gaussian Noise Excitation for Reverberation Chambers and Applications to Radiated Susceptibility Testing.

01,960

REVIEWS

Thermal Conductivity of Metals and Alloys at Low Temperatures. A Review of the Literature.

03.302

Shock Tube Techniques in Chemical Kinetics. PB95-163465

00,968

RHENIUN 186

Standardization and Decay Scheme of Rhenium-186.

PB94-200490

03,830

RHENIUN 188

Assay of the Eluent from the Alumina-Based TungstenPB94-200482

03,829

RHEOLOGICAL PROPERTIES

Measurement of Rheological Properties of High Pertormance Concrete: State of the Art Report.

00,414

RHEOLOGY

Rheology of Fresh Cement Paste.

Non-Newtonian Flow between Concentric Cylinders Calculated from Thermophysical Properties Obtained from Simulations.

PB96-163761

04,103

Structure and Rheology of Hard-Sphere Systems. PB96-167333

00,662

RHODIUM

Preparation and Certification of a Rhodium Standard Reference Material Solution.

PB94-185071

00,529

Evidence of Film-Induced Cleavage by Electrodeposited Rhodium.

PB95-162327

03,191

RHODIUM-LIKE IONS

$\mathrm{Rh}$ I Isoelectronic Sequence Observed from $\mathrm{Er}(23+)$ to

$\mathrm{Pt}(33+)$.
$\mathrm{PB} 95-150652$

03,898

RHODOPSIN

Retinal-Protein Complexes as Optoelectronic Compo-

02,146

RIETVELD METHOD

Mathematical Aspects of Rietveld Refinement

PB95-108601

04,526

Application of ODF to the Rietveld Profile Refinement of Polycrystalline

03,401

RIETVELD REFINEMENT

Accurate Modeling of Size and Strain Broadening in the Rietveld Refinement: The 'Double-Voigt' Approach.

PB96-200225

00,664

RIGID POLYISOCYANURATE FOAM

Effects of Humidity and Elevated Temperature on the Density and Thermal Conductivity of a Rigid Polyisocyanurate Foam Co-Blown with $\mathrm{CCl} 3 \mathrm{~F}$ and $\mathrm{CO} 2$.
PB95-150462

Effects of Humidity and Elevated Temperature on the Density and Thermal Conductivity of a Rigid Polyisocyanurate Foam.

00,373

RING LASERS

Multiwavelength Birefringent-Cavity Mode-Locked Fibre

Laser. 150496

04,262
Frequency-Stabilized LNA Laser at 1.083 mum: Applicaion to the Manipulation of Helium 4 Atoms.

04,304

RISK

east-Cost Energy Decisions for Buildings: Part 2. Uncer-

ainty and Risk Video Training Workbook. tainty and Risk

00,240

RISK ANALYSIS

Computer Virus Attacks

PB95.163655

01,715

Earthquake and Fire in Japan: When the Threat Became a Reality.

00,201

\section{RISK ASSESSMENT}

Fire Hazard and Risk: Evaluating Alternative Technologies.

Fire Service and Fire Sciences: A Winning Combination. PB95-150264 01,383

Quantitative Evaluation of Building Fire Safety: New Tools for Assessing Fire and Building Code Provisions.

PB95-164588

00,199

New Concepts for Fire Protection of Passenger Rail Transportation Vehicles. (NIST Reprint)

04,363

NIOSH Comments to DOL on Risk Estimates from the Cadmium Cohort Study by L. Stayner, February 7, 1992. Concepts for Fire Protection of Passenger Rail Transpor-

lation Vehicles: Past, Present, and Future.

04,853

Review of Internatioanl Fire Risk Predictions Methods.

PB96-156195

00,222

RMS VOLTAGE

Electro-Optic-Based RMS Voltage Measurement Tech-

PB96-138490

02,194

ROAD FOLLOWING

Visual Road Following without 3-D Reconstruction.
PB95-161030

Unified Approach to Camera Fixation and Vision-Based Road Following.

ROBOT CONTROL

Overview of NASREM: The NASANBS Standard Refrence Model for Telerobot Control System Architecture. erence Model for Telerobot Control System Architecture.
PB94-194560

Configuration and Performance Evaluation of a Real-Time Robot Control System: A Skeleton Approach.

01,598 PB95-163895

Unified Telerobotic Architecture Project (UTAP) Standard Interface Envir
PB95-242350

ROBOT DYNAMICS

Integrated Mobile Robot System for Testing Vision Algo-

PB95-164133

02,936

Real-Time Obstacle Avoidance Using Central Flow Divergence and Peripheral Flow.

02937

\section{ROBOTICS}

Toward a Reference Model Architecture for Real-Time Inelligent Control Systems (ARTICS).

PB94-172046

02,932

Integration of Servo Control into a Large-Scale Control System Design: An Example from Coal Mining.

PB94-203429

Certainty Grid to Object Boundary Algorithm

03,696

01,835

Continuous Mining Machine Control Using the Real-Time Control System

Environment Simulation for a Continuous Mining $\mathrm{Ma}$

PB94-203536

03,697

Publications of the Intelligent Systems Division (Pre viously Robot Systems Division) Covering the Period Jan1971-April 1994.

PB94-217098

02,935

Unified Approach to Camera Fixation and Vision-Based Road Following

01594 Vehicle-Command Center Communications in a Robotic Vehicle System

03,665

Robotics Application to Highway Transportation. Volume . Literature Search

01,337

Robotics Application to Highway Transportation Volume

Robotics Application to Highway Transportation. Volume 3. Proposed Research Topics and Cost/Benefit Evaluations by CERF

01,338

Robotics Application to Highway Transportation. Volume 4. Proposals for Potential Research.

01,339

Unified Telerobotic Architecture Project (UTAP) Standard Interface Environment (SIE), May 1995.
PB95-242350

Study of Potential Applications of Automation and Robotics Technology in Construction, Maintenance and Operation of Highway Systems: A Final Report. Volume 4. PB95-25164 01,340

Study of Potential Applications of Automation and Robotics Technology in Construction, Maintenance and Operation of Highway Systems: A Final Report. Volume 1. PB95-251682

01,341

Study of Potential Applications of Automation and Robotics Jechnology in Construction, Maintenance and Operation of Highway Systems: A Final Report. Volume 3.

PB95-251690 01,342

Study of Potential Applications of Automation and Robotics Technology in Construction, Maintenance and Ope ation of Highway Systems: A Final Report. Volume 2.

01,343

Scale-Space-Based Visual-Motion-Cue for Autonomous Navigation

PB96-183173

02,940

NIST Construction Automation Program Report No. 2. Proceedings of the NIST Construction Automation Workshop. Held in Gaithersburg. Maryland on March 30-31.

ROBOTS

00,413

AMRF Composite Fabrication Workstation.

PB94-172681 02,810

World Model Registration for Effective Off-Line Programming of Robots.

PB94-173010

02,933

Mapping Processes to Processors for Space-Based Robot Systems.

04,833

Visual Road Following without 3-D Reconstruction.

PB95-161030

01,591

Reconstruction during Camera Fixation.

PB95-162236 01,593

Novel Active-Vision-Based Motion Cues for Local Naviga-

PB96-193727

02,941

ROCK MECHANICS

Temperature and Frequency Dependence of Anelasticity in a Nickel Oscillator

PB96-137732

03,689

Bibliography of Books and Published Reports on Gas Turbines, Jet Propulsion, and Rocket Power Plants. 01,445 Bibliography of Books and Published Reports on Gas Turbines, Jet Propulsion, and Rocket Power Plants, January 1950 through December 1953.

AD-A278 213/4

01,446

ROCKWELL HARDNESS

Microform Calibration Uncertainties of Rockwell Diamond Indenters.

Metrology Approach to Unifying Rockwell C Hardness Scales.

PB96-155551 


\section{KEYWORD INDEX}

SATELLITE ORBITS

Mathematical Analysis of Practices to Control Moisture in the Roof Cavities of Manufactured Houses.

00,278 PB97-106843

ROOM FIRES

Verification of a Model of Fire and Smoke Transport.
PB95-108718 ROOMS

Application of Thermodynamic and Detailed Chemical Kinetic Modeling to Understanding Combustion Produc Generation in Enclosure Fires.

PB96-135322

01,413

ROOT CANAL FILLING MATERIALS

Periapical Tissue Reactions to a Calcium Phosphate $\mathrm{Ce}$ ment in the Teeth of Monkeys.

PB94-212008

00,149

ROTARY POWERS

Large Local-Field Corrections in Optical Rotatory Power PB97-122378

04,400

ROTATING CYLINDERS

Density Dependence of Fluid Properties and Non-Newtonian Flows: The Weisenberg Effect.

PB96-161898

01,140

ROTATION

Operational Mode and Gas Species Effects on Rotational Drag in Pneumatic Dead Weight Pressure Gages.

PB95-140182

00,903 Collisions: $\mathrm{Cl}((2) \mathrm{P} 3 / 2)+\mathrm{HCl}$

01,157

Internal Droplet Circulation Induced by Surface-Driven Rotation

PB97-119267

02,500

ROTATIONAL DRAG

Operational Mode and Gas Species Effects on Rotational Drag in Pneumatic Dead Weight Pressure Gages. PB95-140182

00,903

ROTATIONAL SPECTRA

Rotational Spectrum of Copper Hydride Using Tunable Far Infrared Radiation.

PB94-198637

00,792

Rotational Far Infrared Spectrum of (13)CO

00,941

Laboratory Measurements for the Astrophysical Identification of $\mathrm{MgH}$.

PB95-152195

00,073

Rotational Spectroscopy of the $\mathrm{CoH}$ Radical in Its Ground (3)Phi State by Far-Infrared Laser Magnetic Resonance: Determination of Molecular Parameters.

PB95-175048

00,992

Pure Rotational Spectra of CuH and CuD in Their Ground States Measured by Tunable Far-Infrared Spectroscopy.
PB95-176194 Rotational Spectrum of $\mathrm{OH}$ in the $\mathrm{v}=0-3$ Levels of Its Ground State.

PB95-176202

01,006

ROTATIONAL STATES

Reanalysis of the (010), (020), (100), and (001) Rotational Levels of (32) $\mathrm{S}(16) \mathrm{O} 2$

PB95-125621

00,887

ROTOR DYNAMICS

PC-Based Spinning Rotor Gage Controller.

PB95-175832

02,609

ROUGHNESS

Evolution of a Turbulent Boundary Layer Induced by a Three-Dimensional Roughness Element.

PB94-212818

04,200

ROUTING

RDI-SIM ECMA Inter-Domain Routing Protocol Simulation Tool.

PB94-172301

01,683

RPC (REMOTE PROCEDURE CALLS)

Comparing Remote Procedure Calls: Open Network Computing, Distributed Computing Environment and International Organization for Standardization. PB95-194205

01,724

RS CVN STARS

First Results from a Coordinated ROSAT, IUE, and VLA Study of RS CVn Systems.

RUBIDIUM

High-Resolution Spectroscopy of Laser-Cooled Rubidium in a Vapor-Cell Trap.

PB95-153714

04,268

Optical Probing of Cold Trapped Atoms.

04,296

RUBIDIUM 87

Reduction of Light-Assisted Collisional Loss Rate from a Low-Pressure Vapor-Cell Trap.

PB95-202248

RUBIDIUM ATOMS

Temperature of Optical Molasses for Two Different Atomc Angular Momenta. PB95-126058

03,881

RUBIDIUM BARIUM BISMUTHATES

Optical Conductivity of Single Crystals of Ba1. $x \mathrm{MxBiO} 3(\mathrm{M}=\mathrm{K}, \mathrm{Rb}, \mathrm{x}=0.04,0.37)$

PB94-185329

04,447
RUBIDIUM FREQUENCY STANDARDS

Ultra-High Stability Synthesizer for Diode Laser Pumped

PB94-216066 01,527

Diode-Laser Pumped, Rubidium Cell Frequency Stand-

ards.

01,538

Improved Rubidium Frequency Standards Using Diode

Lasers with $A M$ and FM Noise Control.

04,303

RUBIDIUM-LIKE IONS

Rb-Like Spectra: Pd X to Nd XXIV.

RUBIDIUM POTASSIUM FULLERIDES

Neutron-Scattering Study of Librations and Intramolecular Phonons in Rb2.6K0.4C60.

PB95-162269

00,958

RULERS

Length Metrology of Complimentary Small Plastic Rulers.

04,096

RUN TIME (COMPUTERS)

Faster BKL Monte Carlo Simulations.

01,706

RUSSIAN FEDERATION

Proceedings of the Meeting of the Intergovernmental U.S.-Russian Business Development Committee's Standard Working Group (4th). Held in New York City, New York on March 27-29, 1995 and in Northbrook, Itlinois on March 30-31, 1995

PB95-255881

00,496

RUTHENIUM ALLOYS

New Alloys Show Extraordinary Resistance to Fracture and Wear.

03,346

RUTHENIUM OXIDE

Thin-Film Ruthenium Oxide - Iridium Oxide Thermocouples
PB97-110225

RYDBERG ATOMS

RIS Measurements of the AC Stark Shift.

PB96-158035

00,520

RYDBERG STATES

Verification of the Ponderomotive Approximation for the ac Stark Shift in Xe Rydberg Levels.

PB94-185709

03,801

New Rydberg States of Aluminum Monofluoride Observed by Resonance-Enhanced Multiphoton Ionization Spectroscopy.

00,797

Resonance Enhanced Multiphoton Ionization Detection of $\mathrm{GeF}$ and $\mathrm{GeCl}$ Radicals.

PB94-212123

00,825

Experimental and Abinitio Studies of Electronic Structures of the $\mathrm{CCl} 3$ Radical and Cation.

PB94-212131

00,826

Multiphoton Ionization of $\mathrm{SiH} 3$ and $\mathrm{SiD} 3$ Radicals. 2.

Three Photon Resonance-Enhanced Spectra Observed

between 460 and $610 \mathrm{~nm}$.

PB94-212487

00,835

New Electronic States of NH and ND Obsenved from 258 to $288 \mathrm{~nm}$ by Resonance Enhanced Multiphoton Ionization Spectroscopy.

PB94-212495

00,836

Multiphoton Ionization of SiH3 and SiD3 Radicals: Electronic Spectra, Vibrational Analyses of the Ground and Rydberg States, and Ionization Potential.

00,837

Electronic Spectra of $\mathrm{CF} 2 \mathrm{Cl}$ and $\mathrm{CFCl} 2$ Radicals $\mathrm{Ob}$ Served by Resonance Enhanced Multiphoton lonization. Alignment in Two-Step Pulsed Laser Excitation of Alignment in Two-Step Pulsed Laser Excitation of Rydberg Levels in Light Atoms: The Example of Sodium.
PB95-202883 Alignment Probing of Rydberg States by Stimulated EmisPB96-200316

04,124

SACRAMENTO (CALIFORNIA)

Histopathology, Blood Chemistry, and Physiological Sta. Striped Bass ('Morone in Summer Mortality ('Die-Off') in the Sacramento-San Joaquin Delta of California. PB94-198157

00,034

SAFETY

Software Safety and Program Slicing

PB95-125894

NIST Workshop on Gas Sensors: Strategies for Future Technologies. Proceedings of a Workshop. Held in Gaithersburg, Maryland on September 8-9, 1993 PB95-210225

00,507

SAFETY ENGINEERING

Some Basics on Who's Who and What's What in Seismic Saf́ty.

00,435 Implementation of Executive Order 12699: Seismic Safety
of Federal and Federally Assisted or Regulated New of Federal and Fed

Building Const
PB95-151809

00,436

Concepts for Fire Protection of Passenger Rail Transportation Vehicles: Past, Present, and Future.

04,853

Fire Safety Engineering Research in the United States.

PB96-151394

Elements of a Framework for Fire Safety Engineering. $\mathrm{C}_{++}$in Safety Critical Systems.

PB96- 154588

01,750

Internationalization of Fire Safety Engineering Research and Strategy.

00,220

International Organization for Standardization: Current Activities in Fire Safely Engineering.

PB96-159660

00,223

SAFETY FACTORS

Predicting the Fire Performance of Buildings: Establishing Appropriate Calculation Methods for Regulatory Applica-

PB96-141239

00,316

SAFETY PROGRAMS

Control and Instrumentation: Standards for High-Integrity Software.

SAGNAC CURRENT SENSOR

03,736

Polarization Insensitive $3 \times 3$ Sagnac Current Sensor Using Polarizing Spun High-Birefringence Fiber.

PB96-119276

02,187

SAGNAC EFFECT

Polarization Dependence of Response Functions in $3 \times 3$ Sagnac Optical Fiber Current Sensors.

02,189

SALES

Method of Sale for CNG Paves Way to Greater Public Acceptance.
PB95-168449

02,489

SALIVA

Distribution of Fluoride in Saliva and Plaque Fluid After a $0.048 \mathrm{~mol} / \mathrm{L} \mathrm{NaF}$ Rinse.

PB95-151205

03,561

SALIVATION

Effects on Whole Saliva of Chewing Gums Containing Calcium Phosphates.

MAMIUM CUPRATES

Field Dependence of the Magnetic Ordering of $\mathrm{Cu}$ in $\mathrm{R} 2 \mathrm{CuO} 4(\mathrm{R}=\mathrm{Nd}, \mathrm{Sm})$

PB95-164521

04,633

Observation of Noncollinear Magnetic Structure for the Cu Spins in Nd2CuO4-Type Systems.

04,634

AMPLE CONCENTRATORS

Simple, Inexpensive Apparatus for Sample Concentration PB94-199205

00,546

SAMPLE PREPARATION

Introduction of a NIST Instrument Sensitivity Standard Reference Material for X-Ray Powder Diffraction. $4-200318$

00,807

SAMPLING

Wideband Sampling Voltmeter.

PB97-113039

Pilot Studies for Improving Sampling Protocols. 
SATELLITE THEORY

Frozen Orbits for Satellites Close to an Earth-Like Plane PB96-102165

04,839

SATELLITE TRACKING

Comparison of GPS Broadcast and DMA Precise Ephemerides,

01.523

SATURATION (CHEMISTRY)

Measurements of the Viscosities of Saturated and Compressed Fluid 1-chloro-1,2,2,2-tetrafluoroethane (R124) and Pentafluoroethane (R125) at Temperatures between 120 and $420 \mathrm{~K}$

03,254

Measurements of the Viscosities of Saturated and Compressed Liquid 1,1,1,2-Tetrafluoroethane (R134a), 2,2Dichloro-1,1,1-Tritluoroethane (R123) and 1,1-Dichloro-1Fluoroethane (R141b).

$$
\text { PB95-175386 }
$$

03,273

SATURATION SPECTROSCOPY

Optically Stabilized Tunable Diode-Laser System for Saturation Sp

04,717

SCALAR APPROXIMATION

Vector and Quasi-Vector Solutions for Optical Waveguide Modes Using Efficient Galerkin's Method with HermiteGauss Basis Functions.

PB96-141197

04,357

SCALE ERROR STANDARDS

Length Metrology of Complimentary Small Plastic Rulers.
PB96-161724

SCALE MODELS

Scaling Compartment Fires: Reduced- and Full-Scale Enclosure Burns
PB96-175708

00,224

SCALES

Comparison of Filter Radiometer Spectral Responsivity with the NIST Spectral-Irradiance and Illuminance Scales. PB97-113161

04,162

SCALING

Simple Scalability Test for MIMD Code.

CALING FACTOR

Estimate of the Effect of Scale on Radiative Heat Loss Fraction and Combustion Efficiency.

PB95-150447

02,486

SCALING LAWS

Design Equations and Scaling Laws for Linear Compressors with Flexure Springs.

PB95-168902 02,948

Critical Scaling Laws and a Classical Equation of State. PB95-169249

00,990

SCANDIUM HYDRIDES

Neutron Spectroscopic Comparison of Rare-Earth/Hydrogen alpha-Phase Systems.

00,970

SCANDIUM IONS

Dielectronic Capture Processes in the Electron-Impact lonization of $\mathrm{Sc}(2+)$.

04,000

SCANNED PROBE MICROSCOPY

Summary Report: Workshop on Industrial Applications of Scanned Probe Microscopy (2nd). A Workshop Co-Sponsored by NIST, SEMATECH, ASTM E42.14, and the American Vacuum Society. Held in Gaithersburg, Maryland on May 2-3, 1995.

00,509

SCANNING

Scanning Capacitance Microscopy Measurements and Modeling: Progress Towards Dopant Profiling of Silicon.
PB96-148150

04,773

Fabrication Issues for the Prototype National Institute of
Standards and Technology SRM $2090 \mathrm{~A}$ Scanning Electron Microscope Magnification Calibration Standard

PB96-160585 Modeling for Dopant Profiling of Silicon.
PB96-164207 Scanning Electron Microscope Magnification Calibration Interlaboratory Study.

01,164

SCANNING CAPACITANCE MICROSCOPY

2D-Scanning Capacitance Microscopy Measurements of Cross-Sectioned VLSI Teststructures.
PB96-163779

04,104

Scanning Capacitance Microscopy Measurements and Modeling: Progress Towards Dopant Profiling of Silicon. PB96-180070 01,964

SCANNING ELECTON MICROSCOPY

Phase Identification in a Scanning Electron Microscope

Using Backscattered Electron Kikuchi Patterns.
PB97-109128

04,804

SCANNING ELECTRON MICROSCOPES

Scanning Electron Microscopy Observations of Misfit Dislocations in Epitaxial InO.25Ga0.75As on GaAs(001).
PB96-200159

Monte Carlo Simulation of Scanning Electron Microscope

PBg6-200969

02,444
Modification of a Commercial SEM with a Computer Controlled Cathode Stabilized Power Supply.

96-201066

04,129

SCANNING ELECTRON MICROSCOPY

Environmental Scanning Electron Microscope Imaging

Examples Related to Particle Analysis.

00,766

Ouantitative Phase Abundance Analysis of Three Cement

Clinker Reference Materials by Scanning Electron Micros-

PB94-173051

00,333

Measurement and Uncertainty of a Calibration Standard

for the Scanning Electron Microscope.

00,560

Monte Carlo Model for SEM Linewidth Metrology.

PB95-150058

02,331

Loading Device for Fracture Testing of Compact Tension Specimens in the Scanning Electron Microscope.
PB95-162434

Cement and Concrete Characterization by Scanning Electron Microscopy.

00,379

Micromagnetic Scanning Microprobe System. 02,224

PB95-176178 02,224 Electron Microscope Linewidth Measurements. PB96-137807

SEMPA Studies of Exchange Coupling in Magnetic Multilayers.

04,780

Report on the NIST Low Accelerating Voltage SEM Magnification Standard Interlaboratory Study.

02,445

SEM Linewidth Metrology of X-ray Lithography Masks.

PB96-201108

02,447

SCANNING PROBE METHODS

Characterization of Two-Dimensional Dopant Profiles: Status and Revien
PB96-119300

02,400

SCANNING TUNNELING MICROSCOPY

Polyethylene Crystallized from an Entangled Solution $\mathrm{Ob}$ served by Scanning Tunneling Microscopy

01,232

Single-Atom Point Source for Electrons: Field-Emission Resonance Tunneling in Scanning Tunneling Microscopy.
PB95-125860

00,893

Scanning Tunneling Microscopy and Fabrication of PB95-140414

Morphological Estimation of Tip Geometry for Scanned Probe Microscopy.

02,662

SCATTERED FRACTIONS OF DOSE

Scattered Fractions of Dose from 18 and $25 \mathrm{MV} X$-ray Radiotherapy Linear Accelerators.
PB96-186101

04,120

SCATTERING

Scattering Properties of the Leveled-Wave Model of Random Morphologies.

PB94-198835

03,807

SCATTERING-PARAMETER MEASUREMENT

Line-Reflect-Match Calibrations with Nonideal Microstrip Standards.

PB96-176599

02,242

SCATTERING PARAMETERS

Verification of Scattering Parameter Measurements.
PB95-163960

Comments on 'Conversions between $S, Z, Y, h, A B C D$, and $T$ Parameters which Are Valid for Complex Source and Load Impedances

PB96-102785

02,069

Two-Tier Multiline TRL for Calibration of Low-Cost Network Analyzers.
PB96-157888

\section{SCHRODINGER EQUATION}

01,947

New Exact Solution of the One-Dimensional Schrodinger Equation and Its Application to Polarized Neutron Reflectometry.

04,609

Numerical Solution of the Nonlinear Schroedinger Equation for Small Samples of Trapped Neutral Atoms.
PB95-202578

03,985

SCIENTIFIC LITERATURE

Standard Samples and Reference Standards Issued by the National Bureau of Standards.

02613

Pretiminary List of References Containing Compilations of Data on Properties of Materials.

AD-A302 670/5

03,243

SCIENTIFIC VISUALIZATION

Visualization Applications for Manufacturing: A State-ofthe-Art Survey.

02,816

SCINTILLATION COUNTERS

Measurement of Absorbed Dose of Neutrons, and of Mixtures of Neutrons and gamma rays.

03,710

SCINTILLATION COUNTING

Liquid-Scintillation Counting Techniques for the Standardization of Radionuclides Used in Therapy.

SCREW FIXATION

Reinforcement of Cancellous Bone Screws with Calcium Phosphate Cement.

PB96-158001

00,179

SCREW THREADS

Screw-Thread Standards for Federal Services, 1957. Part AD-A279 121/8

02,854

Screw-Thread Standards for Federal Services, 1957. Part AD-A279 290/1

02,855

Screw-Thread Standards for Federal Services, 1957. Handbook H28 (1957), Part 2. Revised.

03,599

1950 Supplement to Screw-Thread Standards for Federal AD-A280 $223 / 9$

03,656

Variations in Size Measurements by Indicating Gaging Systems.

PB95-163614

02,864

SONS (SECURE DATA NETWORK SYSTEM)

SDNS Security Management.

PB95-161170

01,592

SEALING COMPOUNDS

Formation of Hydroxyapatite in Cement Systems.

PB95-175261

00,170

EAMS (JOINTS)

Effects of Adhesive Thickness, Open Time, and Surface Cleanness on the Peel Strength of Adhesive-Bonded Seams of EPDM Rubber Roofing Membrane.

00,372

Characteristics of Adhesive-Bonded Seams Sampled from EPDM Roof Membranes.

00,377

Pulse-Echo Uttrasonic Evaluation of the Integrity of Seams of Single-Ply Roof Membranes.

00,381

SEARCH PROFILES

Coping with Different Retrieval Methods in Next Generation Networks

02,726

Move-to-Root Rule for Self-Organizing Trees with Markov Dependent Requests.

\section{EARCHING}

Tree-Lookup for Partial Sums Or: How Can I Find This Stuff Ouickly.

01,770

SECONDARY ION MASS SPECTROSCOPY

Use of Kinetic Energy Distributions to Determine the Relative Contributions of Gas Phase and Surface Frag. mentation in $\mathrm{KeV}$ lon Sputtering of a Ouaternary Ammonium Salt.

00,570

Molecular Ion Imaging and Dynamic Secondary Ion Mass Spectrometry of Organic Compounds.

00,571

SECTORAL ANALYSIS

Information Infrastructure: Reaching Society's Goals. Report of the Information Infrastructure Task Force Committee on Applications and Technology. PB94-214756

SECURE COMMUNICATIONS

Data Encryption Standard.

PB95-162376

01,469

01,595 communications Management Network. Computer SecuPBity.

Introduction to Secure Telephone Terminals. PB97-110498 
SEES (SOFTWARE ENGINEERING ENVIRONMENTS) Making Sense of Software Engineering Environment Framework Standards.

PB95-105037

01,701

SEGMENT INSERTION

Inserting Line Segments into Triangulations and Tetrahedralizations.

PB95-198933

03,415

SEISMIC DESIGN

Standards of Seismic Safety for Existing Federally Owned Or Leased Buildir

00,431

Implementation of Executive Order 12699: Seismic Safety of Federal and Federally Assisted or Regulated New Building Construction.

PB95-15 1809

00.436

Worid of Building Codes.

00,449

ICSSC Guidance on Implementing Executive Order

ICSSC Guidance on Implementing Executive Order
12941 on Seismic Safety of Existing Federally Owned or Leased Buildings.

00,459

De Facto Microzonation through the Use of Soils Factors in Design Triggers
PB96-141148

00,462

Executive Order 12941. Seismic Safety of Existing Federally Owned or Leased Buildings: It's History, Content and Objectives.

00,465

Proceedings of a Workshop on Developing and Adopting Seismic Design and Construction Standards for Lifelines. Held in Denver, Colorado on September 25-27, 1991

PB97-115794

01,302

SEISMIC EFFECTS

Dynamic Characteristics of Five Tall Buildings during

Strong and Low-Amplitude Motions.

00,427

Wind and Seismic Effects. Proceedings of the U.S.-Japan Cooperative Program in Natural Resources Panel on Wind and Seismic Effects (26th). Held in Gaithersburg, Maryland on May 17-20, 1994

PB95-147385

00,433

Performance of Federal Buildings in the January 17, 1994 Northridge Earthquake.

00,453

Recommended Performance-Based Criteria for the Design of Manufactured Home Foundation Systems to Resist Wind and Seismic Loads.

PB96-128285

00,460

January 17, 1995 Hyogoken-Nanbu (Kobe) Earthquake.

Systems. Executive Summary and Paper.
PB97-104160

00,475

Wind and Seismic Effects: Proceedings of the Joint Meeting of the U.S.-Japan Cooperative Program in Natura Resources Panel on Wind and Seismic Effects (28th) Held in Gaithersburg, Maryland on May 14-17, 1996.
PB97-104376

\section{EEISMIC ISOLATION SYSTEMS}

Draft Guideline for Testing and Evaluation of Seismic Isolation Systems.
PB94-172947

00,423

Summary and Results of the NIST Workshop on Proposed Guidelines for Testing and Evaluation of Seismic posed Guidelines for Testing and Evaluation of Seismic
Isolation Systems. Held in San Francisco, California on July 25,1994 .

July 25, 1994.
PB96-154901

00,463

SEISMIC RETROFITTING

Jacket Thickness Requirements for Seismic Retrofitting of Circular Bridge Columns.
PB95-163267

EISMIC SAFETY

Evaluation and Retrofit Standards for Existing Federally Owned and Leased Buildings.

00,434 Some Basics on Who's Who and What's What in Seismic

Safety.

00,435

Lessons from the Loma Prieta Earthquake.

00,442

Seismic Safety of Federal Buildings. Initial Program: How Much Will It Cost.

PB95-182291

00,447

SEISMIC WAVES

Assessment of Site Response Analysis Procedures.
PB95-210928

Response of Buildings to Ambient Vibration and the Loma Prieta Earthquake: A Comparison.

PB96-119607

00,457

Estimation of System Damping at the Lotung Site by Application of System Identification.

01,351

SELECTION RULES

Comparison of the Liquid Chromatographic Behavior of Selected Steroid Isomers Using Different ReversedPhase Materials and Mobile Phase Compositions. PB95-140976

00,574

SELECTIVE DISSEMINATION OF INFORMATION

Computer Security Management and Planning in the U.S.

01,596
Guidance to Federal Agencies on the Use of Trusted

Systems.
PB95-163440

01,597

SELECTIVE ELECTRODES

Cyclic Polyamine Ionophore for Use in a Dibasic Phosphate-Selective Electrode.

PB95-180121

01,008

SELECTIVITY

Developing Measurement for Experimentation

SELENIUM

Thermodynamics of (Germanium + Selenium): A Review and Critical Assessment.

PB97-112536

01,182

Large Local-Field Corrections in Optical Rotatory Power of Quartz and Selenium

04,400

SELENIUM IONS

Absolute Cross-Section Measurements for Electron-Impact Single lonization of $\mathrm{Se}(+)$ and $\mathrm{Te}(+)$

PB95-202503

03,980

\section{SELF ASSEMBLED MONOLAYERS}

Silver Metalization of Octadecanethiol Monolayers SelfAssembled on Gold.
PB95-150744

00,923

UV-Photopatterning of Alkylthiolate Monolayers Self-Assembled on Gold and Silver.

00,924

Surface Plasmon Microscopy of Biotin-Streptavidin Binding Reactions on UV-Photopatterned Alkanethiol Self-Assembled Monolayers.

01,158

SELF-AVOIDING SURFACES

Self-Avoiding Surfaces, Topology, and Lattice Animals. PB95-150512

04,571

SELF-AVOIDING WALKS

Examination of the $1 / d$ Expansion Method from Exact Enumeration for a Self-Interacting Self-Avoiding Walk.
PB95-175733

Self-Avoiding-Walk Contacts and Random-Walk SelfIntersections in Variable Dimensionality.

PB96-102231

01,276

SELF CALIBRATION

Self-Calibrating Fiber Optic Sensors: Potential Design Methods.

02,172

Self Calibrating Fiber Optic Sensors: Potential Design Methods.

PB95-169306

02,173

Self-Calibrated Intelligent Optical Sensors and Systems.

PB96-200738

04,380

SELF ORGANIZING SYSTEMS

Move-to-Root Rule for Self-Organizing Trees with Markov Dependent Requests.

SEF-PRESERVATION BEHAVIOR

03,431

Global Density Effects on the Self-Preservation Behavior of Turbulent Free Jets.

PB95-162301

04,207

PEREIPROCAL FUNCTION

Self-Reciprocal Fourier Functions.

01,974

SELF-SETTING

Physical and Chemical Properties of Resin-Reinforced Calcium Phosphate Cements.

PB95-180212

00,171

SELF TESTS

Self-Calibrating Fiber Optic Sensors: Potential Design Methods.

PB95-169298

02,172

Self Calibrating Fiber Optic Sensors: Potential Design PB95-169306

02,173

\section{SEMICONDUCTOR DEVICES}

Development and Characterization of Insulating Layers on Silicon Carbide: Annual Report for February 14, 1988 to February 14, 1989.

PB94-155579

02,295

Wire Bond Testing

PB94-211653

02,314

Precision, Accuracy, Uncertainty and Traceability and

PBy 4 -213105

02,319

Physics for Device Simulations and Its Verification by Measurements
PB95-141172

02,327

Exact Solution of the Steady-State Surface Temperature for a General Multilayer Structure.

02,337

Electrical Test Siructure for Improved Measurement of Electrical Test Structure for Improved Measurement of
Feature Placement and Overlay in Integrated Circuit Fabication Processes.

PB95-164273 02,355

Optical Characterization in Microelectronics Manufactur-

PB95-169397

02,358

Status and Trends in Power Semiconductor Devices. (175097 361 Correlation of Optical, X-ray, and Electron Microscopy Measurements of Semiconductor Multilayer Structures. PB95-175279

02,174

Electronics and Electrical Engineering Laboratory Technical Progress Bulletin Covering Laboratory Programs, October to December 1994 with 1995 EEEL Events Cal-

endar.

02,372

Semiconductor Measurement Technology: HOTPAC. Programs for Thermal Analysis Including Version 3.0 of the TXYZ Program, TXYZ30, and the Thermal MultiLayer Program, TML.

PB95-260766

02,374

Exact Recursion Relation Solution for the Steady-State Surface Temperature of a General Multilayer Structure.

PBriace Temperature of a General Multilayer Structure.
02,376

Semiconductor Measurement Technology: Test Structure Implementation Document: DC Parametric Test Structures and Test Methods for Monolithic Microwave Inte. grated Circuits (MMICs)

PB96-117692

02,399

Millimeter-Resolution Optical Time-Domain Reflectometry Using a Four-Wave Mixing Sampling Gate.

02,190

Scanning Capacitance Microscopy Measurements and Modeling: Progress Towards Dopant Profiling of Silicon.
PB96-148150

Semiconductor Measurement Technology: Survey of Optical Characterization Methods for Materials, Processing, and Manufacturing in the Semiconductor Industry.
PB96-154596

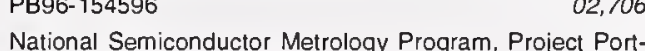
folio FY 1996

PB96-195268

SEMICONDUCTOR INTERFACES

04,789

High-Accuracy Principal-Angle Scanning Spectroscopic Ellipsometry of Semiconductor Interfaces.

PB96-163787

02,427

SEMICONDUCTOR JUNCTIONS

Junction Locations by Scanning Tunneling Microscopy

In-Air-Ambient Investigation of Passivated GaAs pn Junc-

PBg -1

SEMICONDUCTOR LASERS

02,306

Vertical-Cavity Optoelectronic Structures: CAD, Growth, and Structural Characterization.

PB95-153177

02,148

Vertical-Cavity Semiconductor Structures: Materials Characterization.

02,149

Vertical-Cavity Semiconductor Lasers: Structural Characterization, CAD, and DFB Structures.

02,150

Effect of Semiconductor Laser Characteristics on Optical

Effect of Semiconductor Laser

Fiber Sensor Per
PB95-169132

02,167

High-Sensitivity Spectroscopy with Diode Lasers.

PB95-175477

04,297

Diode Laser as a Spectroscopic Tool.

PB95- 175485

00,600 


\section{KEYWORD INDEX}

Business and Manufacturing Motivations for the Developing of Analytical Technology and Metrology for Semiconductors.

PB96-161948

04,778

President's Column 'Editorial',

02,239

Optical Characterization of Materials and Devices for the Semiconductor Industry: Trends and Needs.

02,431

Novel Magnetic Field Characterization Techniques for Compound Semiconductor Materials and Devices.

PB96-176458

02,433

Hybrid Optical-Electrical Overlay Test Structure.

PB96-204136

02,450

Double Modulation and Selective Excitation Photoreflectance for Characterizing Highly Luminescen Semiconductor Structures and Samples with Poor Surface Morphology.

PB97-111439
lace

Application of the Collocation Method in Three Dimensions to a Model Semiconductor Problem.

02,457

SEMICONDUCTORS (MATERIALS)

Lattice Dynamics of Semiconducting, Metallic, and Superconducting Ba 1-xKxBiO3 Studied by Inelastic Neutron Scattering.

Development of a Standard Reference Material for Measurement of Interstitial Oxygen Concentration in Semiconductor Silicon by Infrared Absorption.

PB96-122668

02,404

SENICONDUCTOR DEVICES

Scanning Capacitance Microscopy Measurements and Modeling for Dopant Profiling of Silicon.

PB96-164207

04,781

SENSITIVITY

Influence of the Filament Potential Wave Form on the Sensitivity of Glass-Envelope Bayard-Alpert Gages.

PB95-175014

02,657

Environmental Sensitivities of Quartz Oscillators.

PB96-103148

Developing Measurement for Experimentation. PB97-118707

02,271

ENSITIVITY ANALYSIS

Sensitivity Analysis for Mathernatical Modeling of Fires in Residential Buildings.

PB96-154968

00,215

SENSORS

Intelligent Processing of Hot Isostatic Pressing.

03,315

In-Line Optical Monitoring of Injection Molding.

01,201

Polarization Insensitive $3 \times 3$ Sagnac Current Sensor Using Polarizing Spun High-Birefringence Fiber. Contact Tube Wear Detection in Gas Metal Arc Welding PB96-135330

02,872

\section{SEPARATION MATRICES}

Intercomparison of DNA Sizing Ladders in Electrophoretic Separation Matrices and Their Potential for Accurate Typing of the D1S80 Locus.

SEPARATION PROCESSES

Low Temperature H(sub 2)S Separation Using Membrane Reactor with Redox Catalys

DE94008991

02,471

Development of Engineered Stationary Phases for the Separation of Carotenoid Isomers.

00,578

Shape Selectivity Assessment of Stationary Phases in Gas Chromatography.

00,579

Supercritical Fluid Extraction of Biological Products. PB95-175204

00,040

SEQUENCE DELETION

Deletion Analysis of the Mini-P1 Plasmid Origin of Rep-

lication and the Role of E.coli DnaA Protein.

PB95-163911

SERIAL SECTIONING

Serial Sectioning of Hardened Cement Paste for Scanning Electron Microscopy.

03,539 RIES EXPANSION

Examination of the V/d Expansion Method from Exact Enumeration for a Self-Interacting Self-Avoiding Walk. PB95-175733 01,266 Self-Avoiding-Walk Contacts and Random-Walk SelfIntersections in Variable Dimensionality. PB96-102231

01,276

SERUM ALBUMIN

Two Phase Aqueous Extraction: Rheological Properties of Dextran, Polyethylene Glycol. Bovine Serum Albumin and PBeir-161998

00,676

SERUMS

Methods for Analysis of Cancer Chemopreventive Agents in Human Serum.

03,502
Liquid Chromatographic Determination of Carotenoids in Human Serum Using an Engineered C30 and a C18 Stationary Phase.

PB97-119333

03,512

SERVICE LIFE

Suggestions for a Logically-Consistent Structure for ServCife Prediction Standards.

PB95-125795

00,358

Methodologies for Predicting the Service Lives of Coating Systems.

PB95-146387

03,124

Survey of Concrete Transport Properties and Their Measurement.

PB95-220489

00,396

SERVOCONTROL

Integration of Servo Control into a Large-Scale Control PBstem Design:

03,696

SEWAGE

Water Efficient Plumbing Fixtures through Standards and Test Methods.

PB95-125951 00,248

SGML (STANDARD GENERALIZED MARKUP LANGUAGE)

SGML Environment for STEP.

PB95-143103

02,778

SGML Parser Validation Procedures.

01,717

Standard Generalized Markup Language Test Suite Evaluation Report

01,751

SHAPE

Using Logic to Specify Shapes and Spatial Relations in Design Grammars.

PB97-111579

02,793

SHAPE RECOVERY

Physics-Based Vision: Principles and Practice, Shape Re. covery (Book Review)

01,846

SHAPE SELECTIVITY

Shape Selectivity Assessment of Stationary Phases in Gas Chromatography.

00,579

Selectivity Trends in Packed Column Supercritical Fluid Selectivity Trends in Packed Column Supercritical Fluid Chromatography with C18 Stationary Phases

00,622

SHAPED CHARGES

Texture Study of Two Molybdenum Shaped Charge Liners by Neutron Diffraction.

PB94-200177

03,754

Microstructure Study of Molybdenum Liners by Neutron Diftraction.

PB95-202396

03,756

SHAPIRO STEPS

Effect of Thermal Noise on Shapiro Steps in High-Tc Josephson Weak Links.

04,506

Terahertz Shapiro Steps in High Temperature SNS Josephson Junctions.

02,168

Large-Amplitude Shapiro Steps and Self-Field Effects in High-Tc Josephson Weak Links.

PB95-180410

04,682

SHARED MEMORY PROGRAMS

Using Synthetic Perturbations and Statistical Screening to Assay Shared-Memory Programs.

PB96-103031

01,740

SHEAR

Shear-Induced Martensitic-Like Transformation in a Block Copolymer Mell

PB96-119508

01,277

Simulation and SANS Studies of Gelation Under Shea

PB96-167176 01,150

Small-Angle Neutron-Scattering Study of Dense Sheared Silica Gels.

01,151

SHEAR ALIGNMENT

Small-Angle Neutron Scattering (SANS) Study of WormLike Micelles Under Shear.

PB96-176698

04,111

SHEAR FLOW

Crossover to Strong Shear in a Low-Molecular-Weight Critical Polymer Blend.

PB94-211976

01,222

SANS and LS Studies of Polymer Mixtures Under Shear

PB95-107090

01,231

Response of a Terminally Anchored Pclyrner Chain to Simple Shear Flow.

PB95-108668

01,233

Anisotropic Phase Separation Kinetics in a Polymer Blend Solution Following Cessation of Shear Studied by Light Scattering.

01,241

Neutron Scattering Study of the Orientation of a Liquid Crystalline Polymer by Shear Flow.

PB95-180196

01,270

Shear-Induced Mixing in Polymer Blends.

01,287

SHEAR PROPERTIES

Dynamic Shear Modulus Measurements with Four Independent Techniques in Nickel-Based Alloys.

SHEAR RATE

Shear-Excited Morphological States in a Triblock CopolyPB94-172392

01,196

Shear Dependence of Critical Fluctuations in Binary Polymer Mixtures by Small Angle Neutron Scattering.

01,220

SHEAR STRESS

Fluorescence Anisotropy Measurements on a Polymer Melt as a Function of Applied Shear Stress.

01,209

Observations of Shear Induced Molecular Orientation in a Polymer Melt Using Fluorescence Anisotropy Measurements.

PB94-199304

01,210

Observations of Shear Stress and Molecular Orientation Using Fluorescence Anisotropy Measurements.

PB94-199312

01,211

SHEAR THINNING

Structure and Rheology of Hard-Sphere Systems.

SHEET METAL

Determination of Sheet Steel Formability Using Wide Band Electromagnetic-Acoustic Transducers.

02,279

SHEETS

Fracture Testing of Large-Scale Thin-Sheet Aluminum Alloy.

SHIELDING

Shielding of Cracks in a Plastically Polarizable Material.

\section{PB95- 164257}

Updated Calculations for Routine Space-Shielding Radiation Dose Estimates: SHIELDOSE-2.

PB95-171039

04,838

SHIELDS

Standard Source Method for Reducing Antenna Factor Errors in Shielded Room Measurements.

PB96-183157

02,013

SHIP FIRES

Materials and Fire Threat

7-12231

01,442

SHOCK TESTS

Testing the Sensitivity of Accelerometers Using Mechanical Snock Pulses Under NIST Special Publication 250 Test No. $24040 \mathrm{~S}$

PB96-179544

02,683

SHOCK TUBES

Shock Tube Techniques in Chemical Kinetics. PB95-163465

00,968

SHOT NOISE

Observation of Hot-Electron Shot Noise in a Metallic Re-

sistor.

PB97-112007

01,988 
Uncertainty Intervals for Polarized Beam Scattering Asymmetry Statistics. Satellite Two-Way Time Transfer: Fundamentals and Recent Progress.

PB95-161089 01,536

Comment and Discussion on Digital Processing of PD Pulses.

PB96-122775

01,932

SIGNAL RECONSTRUCTION

Casual Regularizing Deconvolution Filter for Optimal Waveform Reconstruction.

01,603

SIGNAL-TO-NOISE RATIO

Perception of Clamp Noise in Television Receivers. PB96-119433

01.489

SIGNAL TRANSDUCTION

Energy Transduction between a Concentration Gradien and an Alternating Electric Field.

PB94-216363

03,461

SIGNAL TRANSMISSION

Preliminary Comparison of Time Transfers via LASSO GPS and Two-Way Satellite.

01,529

SIGNALS

Procedures for Document Facsimile Transmission Issued by General Services Administration, April 14, 1982. Federal Standard 1063.

01,516

Electronics and Electrical Engineering Laboratory Technical Publication Announcements Covering Laborator Programs, October to December 1994 with 1995 EEEL Events Calendar.

01,918

Accuracy in Time Domain Transmission Line Measurements.

ments.

04,060

SILANE

Preparation of Immobilized Proteins Covalently Coupled

Through Silane Coupling Agents to Inorganic Supports.
PB95-151403

03,530

SILANE/METHACRYLOXYPROPHYL TRIMETHOXY

Copolymerization of N-Phenyl Maleimide and gammaMethacryloxypropyl Trimethoxysilane.

01.248

SILANES

Plasma Chemistry in Disilane Discharges.

PB94-211075

02.514

Plasma Chemistry in Silane/Germane and Disilane/Germane Mixtures.

SILCON DIOXIDE

01,027

New Physics-Based Model for Time-Dependent- Dielectric-Breakdown

02,440

SILICA

Small Angle Neutron Scattering Study of the Structure and Formation of Ordered Mesopores in Silica. PB96-111919

03,069

SILICA FUME

Evolution of Porosity and Calcium Hydroxide in Laboratory Concretes Containing Silica Fume.

01,321

SILICATES Real-Time Small-Angle X-Ray Scattering Study of the
Early Stage of Phase Separation in the SiO2-BaO-K20 System.

03,052

SILICIDES

Tensile Creep of Silicide Composites.

PB96-200803

03,183

SILICON

Precision Comparison of the Lattice Parameters of Silicon Monocrystals.

04,438

Vibrational Distributions of As2 in the Cracking of As4 on Si( 100$)$ and Si(111)

00,784

Magnetic Dead Layer in Fe/Si Multilayer: Profile Refinement of Polarized Neutron Reflectivity Data.

04.458

PB94-198363

semina-

tion Exercise

04,474

Measurement of Boron at Silicon Wafer Surfaces by Neutron Depth Profiling

04,487

Atomic Theory of Fracture of Brittle Materials: Application to Covalent Semiconductors.

04,519

New Values for Silicon Reference Materials, Certified for New Values for Silicon Reference Materials, Certified for
Isotope Abundance Ratios. Letter to the Editor.
PB94-219268

00.863

Standard Reference Materials: Certification of a Standard ygen Concentration in Semiconductor Silicon by Infrared Spectrophotometry.

02,326
Nanoscale Study of the As-Grown Hydrogenated Amorphous Silicon Surface.

PB95-150595

04,573

Use of Sum Rules on the Energy-Loss Function for the Evaluation of Experimental Optical Data.

PB95-150736

04,264

Multiphoton Ionization Spectroscopy Measurements of Silicon Atoms during Vapor Phase Synthesis of Ceramic Particles.

03,913

Direct Detection of Atomic Arsenic Desorption from

Si(100).
PB95-202230 01,024

Nanoscale Study of the Hydrogenated Amorphous Silicon

PB96-103056 04,720

Photodesorption Dynamics of Co from Si(111): The Role of Surface Defects.

03,066

Effect of Anneal Temperature on Si/Buried Oxide Interface Roughness on SIMOX.

02,382

Silicon Surface Chemistry by IR Spectroscopy in the Midto Far-IR Region: $\mathrm{H} 2 \mathrm{O}$ and Ethanol on Si(100).

01,097

Scanning Capacitance Microscopy Measurements and Modeling: Progress Towards Dopant Profiling of Silicon. PB96-148150

Semiconductor Measurement Technology: Survey of Opti-
cal Characterization Methods for Materials, Processing. cal Characterization Methods for Materials, Processing, and Manufacturing in the Semiconductor Industry.
PB96-154596

Methodology for the Certification of Reference Specimens for Determination of Oxygen Concentration in Semiconductor Silicon by Infrared Spectrophotometr.

PB96-155478 02,421

Effect of Single versus Multiple Implant Processing on Defect Types and Densities in SIMOX

PB96-160353

01.957

PB96-160668

02,423

Scanning Capacitance Microscopy Measurements and Modeling for Dopant Profiling of Silicon.

04,781

Kinetic-Energy-Enhanced Neutral Etching

00.665

Electrical Test Structures Replicated in Silicon-on-InsulaIor Material.
PB97-111827

02,454

\section{SILICON 28}

Measurement of the $\mathrm{J}=2$ less than 1 Fine-Structure Inter-

val for (28)Si and (29)Si in the Ground (3)P State.
PB94-185097

00,771

SILICON 29

Measurement of the $\mathrm{J}=2$ less than 1 Fine-Structure Interval for (28)Si and (29)Si in the Ground (3)P State
PB94-185097

00,771

SILICON ADDITIONS

Lattice Position of Si in GaAs Determined by X-Ray Standing Wave Measurements.

PB95-164406

04,632

SILICON ATOMS

Multiphoton Ionization Spectroscopy Measurements of Silicon Atoms during Vapor Phase Synthesis of Ceramic

Particles.

03,913

SILICON CARBIDE

In situ-Toughened Silicon Carbide.

SILICON CARBIDES

Review of Corrosion Behavior of Ceramic Heat Exchanger Materals: Corrosion Characteristics of Silicon Carbide and Silicon Nitride. Final Report, September 11. 1992--March 11, 1993

DE93041307

03,228

Ceramic Characterization

DE94013170

03,026

Development and Characterization of Insulating Layers on Silicon Carbide: Annual Report for February 14, 1988 10 February 14, 1989

PB94-155579

02,295

Analysis of Creep in a Si-SiC C-Ring by Finite Element MBhod.

03,037

Coating of Fibers by Colloidal Techniques in Ceramic Composites.

03.196

Characterization of Phase and Surface Composition of Characterization of Phase and Surface Composition of
Silicon Carbide Platelets.
PB94-216264 03,043

03,043

Interface Modification and Characterization of Silicon Carbide Platelets Coated with Alumina Particles.

03,121

Creep Rupture of MoSi2/SiCp Composites.

PB95-152294

03,154

Flat and Rising R-Curves for Elliptical Surface Cracks from Indentation and Superposed Flexure.

03,156

Oxidation of $\mathrm{SiC}$

02,401

Journal of Research of the National Institute of Standards Journal of Research of the National Institute of Standards Number 5 .

PB96-169057

03,368

Corrosion Characteristics of Silicon Carbide and Silicon Nitride.

PB96-169081

03,372

SILICON COMPOUNDS

Wear Modeling of Si-Based Ceramics.

PB97-122501

03,112

SILICON DEFECTS

Stacking Fault Pyramid Formation and Energetics in Silicon-on-Insulator Material Formed by Multiple Cycles of Oxygen Implantation and Annealing.

ICON DIODES

04,083

One Gigarad Passivating Nitrided Oxides for $100 \%$ Internal Quantum Efficiency Silicon Photodiodes.

PB94-185485

02,119

Silicon Photodiodes Optimized for EUV and Soft X-Ray Regions.

ILICON DIOXIDE

Contact Electrification Induced by Monolayer Modification of a Surface and Relation to Acid-Base Interactions.

PB94-185378

03.034

Experimental Investigation of the Validity of TDDB Voliacceleration Models.

PB94-185949

02,304

Field and Temperature Acceleration of Time-Dependent Dielectric Breakdown in Intrinsic Thin SiO2.
PB94-185956

Imaging of Fine Porosity in a Colloidal Silica: Potassium Silicate Gel by Defocus Contrast Microscopy.

PB94-212750

03,039

Characterization of Chemically Modified Pore Surfaces by Small Angle Neutron Scattering.

00.898

Spectroscopic Ellipsometry Determination of the Prop erties of the Thin Underlying Strained Si Layer and the Roughness at $\mathrm{SiO} 2 / \mathrm{Si}$ Interface.

PB95-150157

04,560

High Temperature Reliability of Thin Film SiO2.

PB95-150348

Modeling Ceramic Sub-Micron Particle Formation from the Vapor Using Detailed Chemical Kinetics: Comparison with In-situ Laser Diagnostics.

PB95-151965

00,671

Experimental and Numerical Studies of Refractory Particle Formation in Flames: Application to Silica Growth.

Simulation of Ceramic Particle Formation: Comparison with In-situ Measurements.

PB95-152013

00,674

Room Temperature Thermal Conductivity of Fumed-Silica Insulation for a Standard Reference Material.

PB95-152039

00,374

Molecular Orbital Study of Water Enhanced Crack Growth Process.

Optimization of ECR-Based PECVD Oxide Films for Superconducting Integrated Circuit Fabrication

PB95-169165

02,051 
SILICON MEMBRANES

Lithium-Drift Technique for Making Submicron Thick Siljcon Membranes.

PB95.161386

02,260

SILICON NANOCOLUMNS

Construction of Silicon Nanocolumns with the Scanning Tunneling Microscope.

04,696

SILICON NITRIDE

Cavitation Contributes Substantially to Tensile Creep in Silicon Nitride.
PB96-122577

03,171

Chemically Assisted Machining of Si3N4

03,072

Transient Creep Behaviour of Hot Isostatically Pressed Silicon Nitride.

PB96-180278

03,086

Cavity Evolution

03,376

Tension/Compression Creep Asymmetry in Si3N4.
PB97.110258 PB97-110258
High Temperature Structural Reliability of Silicon Nitride. PB97-110456

03, 104

SILICON NITRIDES

Review of Corrosion Behavior of Ceramic Heat Exchanger Materals: Corrosion Characteristics of Silicon Carbide and Silicon Nitride. Final Report, September 11. 1992--March 11, 1993.

DE93041307 03,228

Densification of Nano-Size Powders. 1994 Report.

DE94013486 03,027

Equipment for Investigation of Cryogenic Compaction of Nanosize Silicon Nitride Powders. 1993 Report. Low temperature fabrication from nano-size ceramic powLow temperature fabrication from nano-size ceramic pow-
ders.
DE95013505
03,029

Tensile Creep of Whisker Reinforced Silicon Nitride.
PB94-211984 142

Observed and Theoretical Creep Rates for an Alumina Ceramic and a Silicon Nitride Ceramic in Flexure. Ceramic and a Silicon Nitride Ceramic in Flexure.
PB94-212958 040 Statistical Analysis of Parameters Affecting the Measurement of Particle-Size Distribution of Silicon Nitride Powders by Sedigraph (Trade Name)

03,042

Deposition of Colloidal Sintering-Aid Particles on Silicon Nitride.

PB94-216272

03,044

Tensile Creep of a Silicon Nitride Ceramic.
PB95-161303

Polyelectrolytes as Dispersants in Colloidal Processing of Silicon Nitride Ceramics.

PB95-175568 03,055

Surface Chemical Interactions of Si3N4 with Polyelectrolyte Deflocculants.

03,056
Etfects of Soxhlet Extraction on the Surface Oxide Layer of Silicon Nitride Powders.

PB95-175584

03,057

Interaction of Stoichiometry, Mechanical Stress, and Interface Trap Density in LPCVD Si-rich SiNx-Si Structures. PB95-176301
P2,366

Boundary Lubrication of Silicon Nitride.

PB95-213583

03,226

Silicon Nitride Boundary Lubrication: Lubrication Mechanism of Alcohols.

PB96-111703

03,067

Silicon Nitride Boundary Lubrication: Effect of Oxygenates.
PB96-111711

03,068

Surface Chemistry of Silicon Nitride Powder in the Presence of Dissolved Ions.

PB96-111760

01,073

Electrokinetic Sonic Analysis of Silicon Nitride Suspensions.

03,073

Joumal of Research of the National Institute of Standards and Technology. September/October 1993. Volume 98 , Number 5.

PB96-169057 03,368

Corrosion Characteristics of Silicon Carbide and Silicon Nitride.

PB96-169081 03,372

Fracture Mechanism Maps: Their Applicability to Silicon Nitride.

03,094

SILICON ON INSULATOR

Effect of Intermediate Thermal Processing on Microstructural Changes of Oxygen Implanted Silicon-onInsulator Material.

PB96-160213

02,982

Stacking Fault Pyramid Formation and Energetics in Silicon-on-Insulator Material Formed by Multiple Cycles of Oxygen Implantation and Annealing. PB96-160221

04,083

SILICON SOLAR CELLS

Atomic-scale characterization of hydrogenated amorphous-silicon fitms and devices. Annual subcontract report, 14 February 1994--14 April 1995.

02,294
SILVER

Cryogenic Properties of Silver.

PB94-203593

03,330

Silver Metalization of Octadecanethiol Monolayers Self-

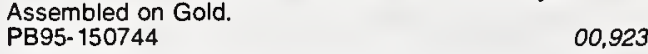

UV-Photopatterning of Alkylthiolate Monolayers Self-AsSembled on Gold and Silver.

00,924

Low-Temperature Properties of Silver.

03,361

Direct Comparison of Three PTB Silver Fixed-Point Cells with the NIST Silver Fixed-Point Cell.

PB96-161286

00,628

SILVER DEPOSITION

Silver Metalization of Octadecanethiol Monolayers SelfAssembled on Gold

SILVER ELECTRODES

Conformational Alterations of Bovine Insulin Adsorbed on a Silver Electrode.

PB96-161773

00,510

SILVER-LIKE IONS

Spectra of $\mathrm{Ag}$ I Isoelectronic Sequence Observed from $\operatorname{Er}(21+)$ to $\mathrm{Au}(32+)$

03,899

SILVER SPRING (MARYLAND)

Source of Phenol Emissions Affecting the Indoor Air of an Office Building

SILYL RADICALS

03,600

Multiphoton lonization of $\mathrm{SiH} 3$ and $\mathrm{SiD} 3$ Radicals. 2. Three Photon Resonance-Enhanced Spectra Observed between 460 and $610 \mathrm{~nm}$.

PB94-212487

00,835

Multiphoton Ionization of $\mathrm{SiH} 3$ and $\mathrm{SiD} 3$ Radicals: Electronic Spectra, Vibrational Analyses of the Ground and Rydberg States, and Ionization Potential.

PB94-212503

00,837

SIMA (SYSTEMS INTEGRATED FOR MANUFACTURING

APPLICATIONS

NIST SIMA Interactive Management Workshop. Held in Fort Belvoir, Virginia on November 14-16, 1994 PB96-154877

02,838

SIMA (SYSTEMS INTEGRATION FOR MANUFACTURING

APPLICATIONS)

Technical Program Description Systems Integration for Manufacturing (SIMA)

02,819

Requisite Elements, Rationale, and Technology Overview for the Systems Intergration for Manufacturing Applications (SIMA) Program. Background Study.

02,831

Systems Integration for Manufacturing Applications Program 1995 Annual Report.

PBram-193735

02,844

SIMD (COMPUTERS)

MasPar MP-1 as a Computer Arithmetic Laboratory.
PB95-189437

SIMOX

Electron Traps, Structural Change, and Hydrogen Related

SIMOX Defects.

Charge Trapping and Breakdown Mechanism in SIMOX.
PB94-216637

Effect of Annealing Ambient on the Removal of Oxide Precipitates in High-Dose Oxygen Implanted Silicon.

Evidence for a Deep Electron Trap and Charge Compensation in Separation by Implanted Oxygen Oxides.
PB95-175337

Defect Pair Formation by Implantation-Induced Stresses in High-Dose Oxygen Implanted Silicon-on-Insulator Material.

PB95-175824

02,364

Effect of Anneal Temperature on Si/Buried Oxide Interface Roughness on SIMOX

02,382

Nano-Defects in Commercial Bonded SOI and SIMOX

PB96-123674 02,407

Effect of Intermediate Thermal Processing on Microstructural Changes of Oxygen Implanted Silicon-on-

Insulator Material.
PB96-160213

Effect of Single versus Multiple Implant Processing on

Defect Types and Densities in SIMOX.

01,957

SIMULATION

Hot-Deformation Apparatus for Thermomechanical Processing Simulation

03,207

Cellular Automaton Simulations of Cement Hydration and Microstructure Development.

PB95-175055

01,320

Faster Monte Carlo Simulations.

04,018

Two-Dimensional Self-Consistent Radio Frequency Plasma Simulations Relevant to the Gaseous Electronics Conference RF Reference Cell.

02,398

Collection of Results for the SPC/E Water Model. PB96-147889

01,127

SINGLE CRYSTALS

Environmentally Enhanced Fracture of Ceramics.
PB95-125746

03,046

Epitaxial Integration of Single Crystal $\mathrm{C} 60$

04,592

Photoluminescence Spectra of Epitaxial Single Crystal 660.

PB96-141205

04,754

SINGLE ELECTRON TRANSISTORS

Combined Josephson and Charging Behavior of the Supercurrent in the Superconducting Single-Electron Transistor.

Voltage Gain in the Single-Electron Transistor PB95-176335

04,637

NGLE ELECTRON TUNNELING

Proposed Tests to Evaluate the Frequency-Dependent Capacitor Ratio for Single Electron Tunneling Experiment.

01,982

SINGLE FIBER PULL-OUT TEST

Determination of Fiber-Matrix Interfacial Properties of $1 \mathrm{~m}$ portance to Ceramic Composite Toughening

03,149

SINGLE ION DETECTORS

Superconducting Resonator and a Cryogenic GaAs FieldEffect Transistor Amplifier as a Single-Ion Detection Sys.

PB95-202727

03,990

SINGLE-PLY ROOF MEMBRANES

Pulse-Echo Ultrasonic Evaluation of the Integrity of Seams of Single-Ply Roof Membranes.

00,381

\section{SINTERING}

Evolution of the Pore Size Distribution in Final-Stage Sintering. (Reannina Measured by Small-Angle X-ray Scat-

AD-Á249 178/5

03,023

Small-Angle Neutron Scattering Characterization of Processing/Microstructure Relationships in the Sintering of Crystalline and Glassy Ceramics. (Reannouncement with New Availability Information).

03,025

Controlled Nucleation in Aerosol Reactors for Supression of Agglomerate Formation.

00,672

Effect of Sm2BaCuO5 on the Properties of Sintered (Bulk) YBa2Cu3O6+x

PB96-11944

04,733

SINUSOIDAL SURFACES

Light Scattering by Sinusoidal Surfaces: Illumination Windows and Harmonics in Standards.

04,387

Sinusoidal Surfaces as Standards for BRDF Instruments. PB97-110597 04,388 Windowing Effects on Light Scattering by Sinusoidal Surlaces.

ITE CHARACTERIZATION

Assessment of Site Response Analysis Procedures. PB95-210928

04,389

SIZE DETERMINATION

Bibliography on Apparel Sizing and Related Issues.

PB94-161924 0,450 Using Laser Diffraction.

PB95-108577

mization

PBo5-151916

03,342

02,904 
Friction and Oxidative Wear of $440 \mathrm{C}$ Ball Bearing Steels Under High Load and Extreme Bulk Temperatures. PB95-175253

SLIDING VANES

Sliding Vane Flow Conditioner Tests in a 100 Diameter Long 10 inch Natural Gas Orifice Meter at Pacific Gas and Electric. Topical Report, 1990-1992.

PB95-256335

02,493

SLOW ELECTRONS

Electron Attachment to Excited Molecules(1).

PB96-122809

01,087

SMALL ANGLE NEUTRON SCATTERING

High-Temperature Furnace for In situ Small-Angle Neutron Scattering during Ceramic Processing.

03,743

Instrumental Smearing Effects in Radially Symmetric Instrumental Smearing Effects in Radially Symmetric
Small-Angle Neutron Scattering by Numerical and Analytical Methods.

PB96-160429 02,984

Simulation and SANS Studies of Gelation Under Shear

PB96-167176 01,150

Small-Angle Neutron-Scattering Study of Dense Sheared Silica Gels.

Structure and Rheology of Hard-Sphere Systems.

01,151

PB96-167333

00,662

Small Angle Neutron Scattering Study of a Clay Suspension Under Shear.

00,663

Small-Angle Neutron Scattering (SANS) Study of WormLike Micelles Under Shear.

PB96-176698

04,111

MALL ANGLE SCATTERING

Neutron Scattering by Multiblock Copolymers of Structure (A-B)N-A.

Shear Dependence of Critical Fluctuations in Binary Polymer Mixtures by Small Angle Neutron Scattering. PB94-211612

01,220

Small-Angle $X$-Ray and Neutron Scattering Study of Block Copolymer/Homopolymer Mixtures.

01,221

PB94-211729

Crossover to Strong Shear in a Low-Molecular-Weight Critical Polymer Blend.

01,222

Characterization of Chemically Modified Pore Surfaces by Small Angle Neutron Scattering.

Small Angle Neut

00,898

Characterization of Polyquinoline Block Copolymer Using Small Angle Scattering.

01,244

Hydration in Semicrystalline Polymers: Small-Angle Neutron Scattering Studies of the Effect of Drawing in Nylon6 Fibers.

PB95-202990

03,385

SMALL BUSINESSES

Learning to Change: Opportunities to Improve the Performance of Smaller Manufacturers.

PB94-166212

00,010

Opportunities for Innovation: Pollution Prevention.

PB95-147146 02,520

Network Brokers Handbook: An Entrepreneurial Guide to Cooperative Strategies for Manufacturing Competitive-

PB95-219325

00,490

SMALL POLAR MOLECULES

Estimating the Virial Coefficients of Small Polar Mol-

03,276

MART CLOCK

Smart Clock: A New Time.
PB95-151445

SMELTING

In-situ Fume Particle Size and Number Density Measurements from a Synthetic Smelt

03,334

SMOKE

Two Numerical Techniques for Light Scattering by Dielec-

tric Agglomerated Structures.
PB94-140597

04,225

Ground-Based Smoke Sampling Techniques Training Course and Collaborative Local Smoke Sampling in Saudi Arabia.

02,532
EB94-143542 Toxic Fire Gases.

PB94-198439

01,369

Ultrafine Combustion Aerosol Generator

PB94-200300

01,373

Overview of Smoke Control Technology.

00,348

Preview of ASHRAE's Revised Smoke Control Manual.
PB94-212875

Smoke Control Systems for Elevator Fire Evacuation.

PB94-212883 00,291

Development of a New Smali-Scale Smoke Toxicity Test

Method and Its Comparison with Real-Scale Fire Tests.

PB94-213253

00,350
Verification of a Model of Fire and Smoke Transport.

00,357

Dispersion and Deposition of Smoke Plumes Generated in Massive Fires.

PB95-126066

02,540

Simulating Smoke Movement through Long Vertical Shafts in Zone-Type Compartment Fire Models.

00,368 B95-143152

Method of Predicting Smoke Movement in Atria with Application to Smoke Managemen.

00,376

Smoke Plume Trajectory from In situ Buming of Crude Oil Alaska: Field Experiments.

PB96-131560

02,594

Large Eddy Simulations of Smoke Movement in Three Di.

PB96-190012

01,426

SMOKE CONTROL

Full Scale Smoke Control Tests at the Plaza Hotel Build-

PBg. 212859

00,347

Overview of Smoke Control Technology

00,348

Preview of ASHRAE's Revised Smoke Control Manua.

PB94-212875 00,349

Smoke Control Systems for Elevator Fire Evacuation.

PB94-212883 00,291

Fire and Smoke Control: An Historical Perspective.

PB95-175808

00,202

SMOKE DETECTORS

Measurement of Room Conditions and Response of

Sprinklers and Smoke Detectors during a Simulated TwoBed Hospital Patient Room Fire.

PB94-213717

00,292

Analysis of High Bay Hangar Facilities for Detector Sensitivity and Placement.

01,429

SMOKE GENERATORS

Generation and Characterization of Acetylene Smokes.
PB94-200292

OKE MANAGEMENT

Method of Predicting Smoke Movement in Atria with Application to Smoke Management.

\section{SMOKE PROPAGATION}

Improvement in Predicting Smoke Movement in Compartmented Structures.

SMOKE TRANSPORT Verification of a Model of Fire and Smoke Transport.
PB95-108718

00,332

SMOKE YIELD

Airborne Smoke Sampling Package for Field Measurements of Fires.

PB95-150041

01,381

SNS JUNCTIONS

Mutual Phase Locking in Systems of High-Tc SBperconducto
PB 135348

SODIUM

Electron-atom collision studies using optically state selected beams. Progress report, May 15, 1987--May 14 1988. DE95004446

03,776

Electron-atom collision studies using optically state seected beams. Progress report, May 15, 1988--May 14 1991

03,777

DE95004447

Can Quantum Mechanical Description of Electron-Sodium Collisions Be Considered Complete. Present Status and Future Prospects for $3 s<->3 p$ Transitions.

00,768

PB94-185014

Frozen Human Serum Reference Material for Standardization of Sodium and Potassium Measurements in Serum or Plasma by Ion-Selective Electrode Analyzers.
PB94-185337

Density Matrix Calculation of Population Transfer between Vibrational Levels of $\mathrm{Na} 2$ by Stimulated Raman Scattering with Temporally Shifted Laser Beams. Scattering with Temporally Shitted Laser Beams. $\quad 00,787$
PB94-198546

Femtosecond Time-Resolved Wave Packet Motion in Molecular Multiphoton lonization and Fragmentation.

PB94-198611

00,790

Structures of Sodium Metal.

PB94-198850 03,319

Determination of Complex Scattering Amplitudes in Low-

Energy Elastic Electron-Sodium Scattering.
PB94-216652

03,869

Hyperfine Effects and Associative Ionization of Ultracold (lium.

03,903

High-Precision Calculations of Cross Sections for LowEnergy Electron Scattering by Ground and Excited State of Sodium.

PB95-152161

03,914

Spin-Resolved Elastic Scattering of Electrons from So.

dium.

03,933

Connection between Superelastic and Inelastic ElectronAtom Collisions Involving Polarized Collision Partners.
PB95-202297

Ferntosecond Time-Resolved Molecular Multiphoton Ionzation: The Na2 System.

PB95-202305

03,975

Three-Vector Correlation Study of Orientation and Coherence Effects in $\mathrm{Na}(3 \mathrm{p}$, (2) P $1 / 2$ inversely maps (2)P3/ 2)+He: Semiclassical and Quantum Calculations. B95-202453

03,979

Alignment in Two-Step Pulsed Laser Excitation of Rydberg Levels in Light Atoms: The Example of Sodium.
Py95-202883

Threshold Electron Excitation of $\mathrm{Na}$

PB95-202917

03,994

Improved Hyperfine Measurements of the Na NP Excited State Through Frequency-Controlled Dopplerless Spectroscopy in a Zeeman Magneto-Optic Laser Trap.
PB95-203840

In-situ Studies of a Novel Sodium Flame Process for Synthesis of Fine Particles.

PB97-113047

00,681

Optical and Modeling Studies of Sodium/Halide Reactions or the Formation of Titanium and Boron Nanoparticles.

PB97-11305

SODIUM ATOMS

Associative Ionization in Collisions of Slowed and Trapped Sodium

03,880

Localization of Atoms in a Three-Dimensional Standing Wave.

SODIUM BROMIDE

sopiestic Investigation of the Osmotic and Activity Coefficients of Aqueous $\mathrm{NaBr}$ and the Solubility of $\mathrm{NaBr} 2 \mathrm{H} 2 \mathrm{O}$ (cr) at 298.15 K: Thermodynamic Properties of the $\mathrm{NaBr}+\mathrm{H} 2 \mathrm{O}$ System over Wide Ranges of Temperature and Pressure.

PB97-110365

SODIUM CARBONATES

Automated, High Precision Coulometric Titrimetry. Part 2. Strong and Weak Acids and Bases.

PB95-150207

00,576

SODIUM CHLORIDES

Comparison of the Corrosion Rates of $\mathrm{FeAl}, \mathrm{Fe}$ (sub 3)A and Steel in Distilled Water and $0.5 \mathrm{M}$ Sodium Chloride. Technical Report Number 2, January--March 1991. DE94017332

SODIUM RUBIDIUM FULLERIDES

Rotational Dynamics of $\mathrm{C} 60$ in Na2RbC60

03,186

SODIUM TUNGSTATES

Rietveld Analysis of $\mathrm{NaxWO} 3+x / 2 . y \mathrm{H} 2 \mathrm{O}$, Which Has the

Hexagonal Tungs
PB95-107371

00,948

DIUM ZINC ARSENATE

Crystal Structure of a New Sodium Zinc Arsenate Phase Solved by 'Simulated Annealing'.

PB95-107124

New NIST/ARPA National Soft X-ray Reflectometry Facility.

04,080

Soft-X-ray Damage to $\mathrm{p}$-terphenyl Coatings for Detectors. PB96-15961

Influence of Electrical Isolation on the Structure and $R \theta$ 


\section{KEYWORD INDEX}

Building Life Cycle Cost Computer Program (BLCC) Version 4.22-95 (for Microcomputers).

00,268

Building Life Cycle Cost Computer Program (BLCC) Version 4.22-95 (for Micrcomputers)

00,277 PB96-502794

C) Ver-

Building Life Cycle Cost Computer

PB97-500342

00,284

SOFTWARE DEVELOPMENT

IEEE's POSIX: Making Progress.

PB96-160924

01,757

\section{SOFTWARE ENGINEERING}

User Interface Component of the Applications Portability Profile Category: Software Standard; Subcategory: Application Program Interface.

01,793

Guide to Software Engineering Environment Assessment and Evaluation

01,676

Next Generation Computer Resources: Reference Model for Project Support Environments (Version 2.0). $\begin{array}{ll}\text { PB94-143401 01,677 } & \end{array}$

Validation Testing System Requirements. National PDES Testbed Report Series.

PB94-163482

02,771

Time-Perturbation Tuning of MIMD Programs.

01,684

Report on the Advanced Software Technology Workshop. Held on February 1, 1994

PB95-136610

01,707

Information Technology Engineering and Measurement Model: Adding Lane Markings to the Information Superhighway.

01,474

Use of an Environment Classification Model.

01,709

PB95-152062

01,811

Perspective on Sottware Engineering Standards.
PB95-171377

of High

Integrity Software.
Intovelopment and Assurance of High

$\begin{array}{ll}\text { PB95-173084 } & 01,716\end{array}$

Analysis of ANSI ASC $\times 12$ and UN/EDIFACT Electron

Data Interchange (EDI) Standards.

PB95-220554

01,729

Persistent Object Base System Testing and Evaluation.

PB95-220588

01,730

Unravel: A CASE Tool to Assist Evaluation of High Integ

rity Software. Volume 1. Requirements and Design.
PB95-267886

Unravel: A CASE Tool to Assist Evaluation of High Integ-

rity Software. Volume 2. User Manual.

01,737

Defining Environment Integration Requirements.

PB96-131545

02,733

Findings and Recommendations from a Software Reengineering Case Study

PB96-155791

01,752

Slicing in the Presence of Parameter Aliasing

01,755

Assessing Functional Diversity by Program Slicing PB96-160890

Program Slicing

03,734

PB96-160981

01,761

Verification and Validation.

01,765

Interoperability Experiments with CORBA and Persistent Object Base Systems.

PB96-183140

01,772

Reference Information for the Software Verification and Validation Process.

PB96-188164

01,773

Measurement of Process Complexity.

PB97.113138 Process Complexily.

Experimental Models for Software Diagnosis.

01,781

PB97-1 13906

01,783

CSL View of Applications Portability, Scalability, and Interoperability

01,787

SOFTWARE ENGINEERING ENVIRONMENTS

Making Sense of Software Engineering Environment Framework Standards.

PB95-105037

01,701

SOFTWARE QUALITY ASSURANCE

SOA Standards and Total Ouality Management

PB96-111844

01,743

SOA and TQM in Software Ouality Improvement PB96-160791

SOFTWARE REENGINEERING

Verification and Validation of Reengineered Software.

PB96-161401

01,766

SOFTWARE RELIABILITY

Ouality Characteristics and Metrics for Reusable Sottware (Preliminary Report)

01,693
Analysis of Selected Sottware Safety Standards. PB95-151262

01,708

High Integrity Software Standards Activities at NIST.

PB96-112214 01,744

Control and Instrumentation: Standards for High-Integrity

PB96-161369 03,736

Report of a Workshop on the Assurance of High Integrity

Sotware.

Standards for High Integrity Software.

01,763

PB96-161385

01,764

SOFTWARE REUSE

Context Analysis of the Network Management Domain

Conducted as Part of the Domain Analysis Case Siudy.

01,465

Generic Manufacturing Controllers.

02,818

Ouality Characteristics and Metrics for Reusable Software (Preliminary Report)

PB94-203437

01,693

Domain Analysis of the Alarm Surveillance Domain. Ver-

sion 1.0. Conducted as Part of the Domain Analysis Case

Study Project.

01,705

Glossary of Software Reuse Terms.

PB95-178992

01,720

Template-Driven Systems Development with IDEF: Enterprise Standards for Reuse.

PB96-160965

02,788

SOFTWARE TOOLS

RDI-SIM ECMA Inter-Domain Routing Protocol Simulation

PB94-172301

01,683

Retrieving Articles from the Internet (without a UNIX

Workstation). Part 1. File Formats and Sottware Tools.
PB95-168720

Expert Control System Shell Version 1.0 User's Guide.
PB95-198859

PET and DINGO Tools for Deriving Distributed Implementations from Estelle.

PB95-203253 01.727

Unravel: A CASE Tool to Assist Evaluation of High Integrity Sottware. Volume 1. Requirements and Design.

PB95-267886

01,736

Unravel: A CASE Tool to Assist Evaluation of High Integ-

rity Software. Volume 2. User Manual.

01,737

Overview of the Manufacturing Engineering Toolkit Proto-

PB96-128228

02,833

Method to Determine a Basis Set of Paths to Perform Program Testing

01,747

Object-Oriented Tel/Tk Binding for Interpreted Control of the NIST EXPRESS Toolkit in the NIST STEP Application Protocol Development Environment.

PB96-141049

02,785

Fire Protection Engineering Tools. Simple Tools: The Equations.

Distributed Communication Methods and Role-Based ACcess Control for Use in Health Care Applications.

PB96-183165

01,508

Unified Process Specification Language: Requirements

for Modeling Process.

PB97-116123

02,850

SC4 Short Names Registry.

02,799

SOFTWEAR ENGINEERING

Making Sense of Software Engineering Environment Framework Standards.

PB95-105037

01,701

SOFTWOODS

Voluntary Product Standard PS 20-94. American

Softwood Lum

03,402

\section{SOI (SEMICONDUCTORS)}

Efficient Method to Compute the Maximum Transient Drain Current Overshoot in Silicon on Insulator Devices.

Electron Traps, Structural Change, and Hydrogen Related SIMOX Defects.

PB94-200391 02,312

Defect of Thermal Ramping and Annealing Conditions on Defect Formation in Oxygen Implanted Silicon-On-Insula. tor Material.

02,318

Evidence for a Deep Electron Trap and Charge ComEvidence for a Deep Electron Trap and Charge ComPB95-175337 02,362

Defect Pair Formation by Implantation-Induced Stresses in High-Dose Oxygen Implanted Silicon-on-Insulator Ma-

PB95-175824

02,364

Nano-Defects in Commercial Bonded SOI and SIMOX

PB96-123674

SOIL MECHANICS

Preliminary Processing of the Lotung LSST Data.

03,690

Estimation of System Damping at the Lotung Site by Application of System Identification.

01,351

SOIL STABILIZATION

Ground Improvement Techniques for Liquefaction Remediation Near Existing Lifelines.

PB96-128111

01,350

SOIL-STRUCTURE INTERACTIONS

Dynamic Characteristics of Five Tall Buildings during Strong and Low-Amplitude Motions.

Assessment of Site Response Analysis Procedures. PB95-210928 00,450 De Facto Microzonation through the Use of Soils Factors in Design Triggers.

Preliminary Processing of the Lotung LSST Data PB96-165972

03,690

January 17, 1995 Hyogoken-Nanbu (Kobe) Earthquake. Pertormance of Structures, Lifelines and Fire Protection Systems. Executive Summary and Paper.

PB97-104160

00,475

SOL-GEL GLASS

Bacteriorhodopsin Immobilized in Sol-Gel Glass.

PB95-151429

03,532

SOLAR ACTIVITY

Distant Future of Solar Activity: A Case Study of Beta Hydri. 3. Transition Region, Corona, and Stellar Win

PB94-185220

00,049

Distant Future of Solar Activity: A Case Study of beta Hydri. 3. Transition Region, Corona, and Stellar Wind.

PB95-153441

00,074

SOLDER JOINTS

SPC Artifact for Automated Solder Joint Inspection.

PB96-161716

04,095

SOLDERING

Lubrication Theory for Reactive Spreading of a Thin Drop.

02,865

SOLDERS

Aging Effects on XRF Measurements of Solder Coatings.

SOLID CLUSTERS

Vibrational Relaxation Measurements of Carbon Monoxide on Metal Clusters.

PB94-211810

00,820

Cs Cluster Binding to a GaAs Surface.

PB94-213006

00,846

SOLID FUELS

Minimum Mass Flux Requirements to Suppress Burning 
Disorder Trapping in Ni2TiAl.

PB94-198942

03,322

Asymptotic Behavior of Modulated Taylor-Couette Flows with a Crystalline Inner Cylinder.

PB94-199072

04,469

Effect of Modulated Taylor-Couette Flows on Crystal-Melt Interfaces: Theory and Initial Experiments. 04,521
PB94-216736 xi-Vector Formulation of Anisotropic Phase-Field Models: 3-D Asymptotics.

PB95-136628

04,536

Phase-Field Model for Solidification of a Eutectic Alloy.

PB95-147914

Morphological Stability

03,345

Ultrasonic Method for Reconstructing the Two-Dimen-

sional Liquid-Solid Interface in Solidifying Bodies.

PB95-161782 03,349

Xi-Vector Formulation of Anisotropic Phase-Field Models: 3-D Asymptotics.
PB97-122550

04,816

SOLIDS

Vibrational Spectra of Molecular Ions Isolated in Solid Neon: HCCH+ and HCC-. (Reannouncement with New Availability Information).

AD-A253 $551 / 6$

00,707

Vibrational Spectra of Molecular lons Isolated in Solid

AD-A275 828/2 2 , NO2(-), and NO3(-).

Density of Solids and Liquids.

00,708

AD-A278 $517 / 8$

00,711

Survey of the Literature on Heat Transfer from Solid Surtaces to Cryogenic Fluids.

AD-A286 680/4 04, 193

Infrared and Near-Infrared Spectra of HCC and DCC Trapped in Solid Neon.

AD-A295 578/9

03,773

Surface Forces and Adhesion between Dissimilar Materials Measured in Various Environments.

PB94-172970

03,033

Supercritical Solubility of Solids from Near-Critical DiluteMixture Theory.
PB94-211703

Observed Frustration in Confined Block Copolymers.

PB95-150033 01,238 Molecular Orbital Calculations of Bond Rupture in Brittle Solids.

PB95-164059 00,973

Water Droplet Evaporation from Radiantly Heated Solids.

Total-Dielectric-Function Approach to Electron and Phonon Response in Solids. Shear-Induced Melting of Two-Dimensional Solids.

PB96-112057 01,075 Intrinsic Viscosity and the Polarizability of Particles Having a Wide Range of Shapes.

Hemispherical Test Fixture for Measuring the Wavefields Generated in an Anisotropic Solid.

PB96-190087

03,181

SOLUBILITY

Octacalcium Phosphate Carboxylates IV. Kinetics of Formation and Solubility of Octacalcium Phosphate Succi-

PB94-185600 00,776 Supercritical Solubility of Solids from Near-Critical DiluteMixture Theory.
PB94-211703

00,819

Effect of Ethanol on the Solubility of Dicalcium Phosphate Dihydrate in the System $\mathrm{Ca}(\mathrm{OH}) 2-\mathrm{H} 3 \mathrm{PO} 4-\mathrm{H} 2 \mathrm{O}$ at $37 \mathrm{C}$. PB95-163507 00,163 Effect of Ethanol on the Solubility of Hydroxyapatite in the

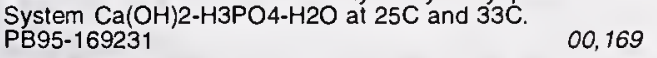
Solubility Measurement by Direct Injection of Supercritical-Fluid Solutions into a HPLC System. 00,997 High-Pressure Equilibrium Cell for Solubility Measurements in Supercritical Fluids. $\mathrm{CO} 2 / \mathrm{CH} 4$ Transport in Polyperfluorosulfonate Ionomers: Effects of Polar Solvents on Permeation and Solubility.
PB96-163803

Solubilities of Copper(II) and Chromium(III) betaDiketonates in Supercritical Carbon Dioxide.

01,147

SOLUBILITY PRODUCTS

Composition and Solubility Product of a Synthetic Calcium Hydroxyapatite.

02,995

SOLUTES

Shape Selectivity in Reversed-Phase Liquid Chromalography for the Separation of Planar and Non-Planar PB95-162608

00,596

Solubility Measurement by Direct Injection of

Supercritical-Fluid Solutions into a HPLC System.
PB95-175626
SOLUTIONS

Neutron Scattering Study of Shear Induced Turbidity in Polystyrene/Dioctyl Phthalate Solutions at High Shear

01,197

Long-Term Stability of Carrier-Free Polonium Solution PB94-185170

00,685

Neutron Scattering Study of Shear Induced Turbidity in

01,256

Effects of Aluminum Oxalate/Glycine Pretreatment Solutions on Dentin Permeability. tions on Dentin Permeability.

03,565

Competition between Hydrodynamic Screening ('Draining') and Excluded Volume Interactions in an Isolated Polymer Chain
PB95-175402

01,265

SOLVENTS

Solvent Effects in the Reactions of Peroxyl Radicals with Organic Reductants. Evidence for Proton Transfer Mediated Electron Transfer.

00,873

Terminally Anchored Chain Interphases: Their Chromatographic Properties.

01,272

Terminally Anchored Chain Interphases: The Effect of Multicomponent, Polydisperse Solvents on Their Equilibrium Properties.

01,273

Mixing Plate-Like and Rod-Like Molecules with Solvent: A Test of Flory-Huggins Lattice Statistics.

PB96-126206

03,173

SOMMERFELD CONSTANT

Fine-Structure Constant

SOOT

Greatly Enhanced Soot Scattering in Flickering Ch4/Air Diffusion Flames.

01,361

Laser Imaging of Chemistry-Flowtield Interactions: Enhanced Soot Formation in Time-Varying Diffusion Flames. PB94-185352 01,36

Fundamental Mechanisms for $\mathrm{CO}$ and Soot Formation

PB95-143160 01,380

Oxidation of Soot and Carbon Monoxide in Hydrocarbon

Diffusion Flames.
PB95-150215

Laser-Induced Fluorescence Measurements of $\mathrm{OH}$. Concentrations in the Oxidation Region of Laminar, Hydrocarbon Diffusion Flames.

PB95-162160

01,387

Ouantitative Measurements of Enhanced Soot Production in a Flickering Methane/Air Diffusion Flame. PB95-203246

01,393

Simultaneous Optical Measurement of Soot Volume Fraction, Temperature, and $\mathrm{CO} 2$ in Heptane Pool Fire.

PB96-102132

01,397

Laser-Induced Fluorescence Measurements of $\mathrm{OH}$ in Laminar Diffusion Flames in the Presence of Soot Par-

PB96-123120

01,409

Post-Flame Soot

PB96-193701

01,430

Computations of Enhanced Soot Production in TimeVarying $\mathrm{CH} 4 /$ Air Diffusion Flames.

01,440

SORPTION

Sorption of Moisture on Epoxy and Alkyd Free Films and Coated Steel Panels.

SOUND VELOCITIES

03,192

Void Shape in Sintered Titanium.

PB96-141023

02,705

SOURCE LIST

Guide to Instrume

02,617

SOXHLET EXTRACTION

Effects of Soxhlet Extraction on the Surface Oxide Layer of Silicon Nitride Powders.

PB95-175584

03,057

SPACE

Partial Pressure Analysis in Space Testing.

PB96-103072

04,829

SPACE CHARGE

Effect of DC Tests on Induced Space Charge

Spatial Dependence of Electrical Fields Due to Space Charges in Films of Organic Dielectrics Used for Insulation of Power Cables.

PB94-199130

02,214

PACE ENVIRONMENT SIMULATION

Partial Pressure Analysis in Space Testing.

N95-14084/4

04,827

SPACE GROUP SYMMETRY

Powder X-ray Diffraction Data for $\mathrm{Ca} 2 \mathrm{Bi} 2 \mathrm{O} 5$ and C4Bi6O13.

PB96-161278

04,777

SPACE HEATERS

Predicting the Energy Performance Ratings of a Family of Type 1 Combination Appliances.

PB95-105524

02,504

SPACE HVAC SYSTEMS

Computer Programs for Simulation of Lighting/HVAC

PB94-140407

Lighting and HVAC.

PB95.150991

02,501

NIST Lighting and HVAC Interaction Test Facility.

Using Emulators to Evaluate the Performance of Building Energy Management Systems.

PB95-175774

00,259

Reproducibility of Tests on Energy Management and Control Systems Using Building Emulators.

00,260

Indoor Air Ouality Impacts of Residential HVAC Systems.

Phase 2.A Report: Baseline and Preliminary Simulations.

PB95-178893

02,554

Performan

Appliance.

02,505

Indoor Air Ouality Impacts of Residential HVAC Systems

Phase II.B Report: IAO Control Retrofit Simulations and Analysis.

PB96-106877

02,559

Use of Building Emulators to Evaluate the Performance of

Building Energy Management Systems.

PB96-111901

00,269

Optimal Control of Building and HVAC Systems

PB96-141353

00,272

Multizone Modeling of Three Residential Indoor Air Oual-

ity Control Options

02,565

Workplan to Analyze the Energy Impacts of Envelope Airtightness in Office Buildings.

PB96-154463 00,273

HVAC CAD Layout Tools: A Case Study of University/lnPB97-11220

an Air-Handling Unit Us Neural Networks.

-121321

Artificial

PACE INDUSTRIALIZATION

In-Space Welding: Visions and Realities.

BB95-163234

04,830

SSME LOX Duct Flowmeter Design and Test Results.

PB96-161955

04,826

SPACE SHUTTLE MAIN ENGINE

Vortex Shedding Flowmeters for SSME Ducts.

PB95-169215

01,453

Wear Mechanism Maps of $440 \mathrm{C}$ Martensitic Stainless

PB96-111810

04,834

SPACE STATION FREEDOM

Overview of NASREM: The NASANBS Standard Ref-

erence Model for Telerobot Control System Architecture.

Evolution of the Flight Telerobotic Servicer.

PB94-216082

SPACE STATIONS

Mapping Processes to Processors for Space-Based

Robot Systems

SPACE TESTINGS

Partial Pressure Analysis in Space Testing
PB96-103072 
Elastic Scattering of Polymer Networks.

PB95-161816

01,255

SPATIAL RELATIONS

Representing Designs with Logic Formulations of Spatial Relations.

02,792

SPATIAL RESOLUTION

Microanalysis to Nanoanalysis: Measuring Composition at High Spatial Resolution.

PECIAL FUNCTIONS

Software Needs in Special Functions.

PB95-105045

00,563

01,702

Numerical Evaluation of Special Functions

03,417

SPECIAL FUNCTIONS (MATHEMATICS)

Software Needs in Special Functions.

PB96-200977

01,778

SPECIFIC HEAT

Millisecond-Resolution Pulse Heating System for SpecificHeat Measurements at High Temperatures.

PB94-199999

02,635

Apparent Molar Heat Capacities and Apparent Molar Volumes of Aqueous Glucose at Temperatures from 298.15 $\mathrm{K}$ to $327.01 \mathrm{~K}$

Analytical Method of Determining the Heat Capacity at High Temperatures from the Surface Temperature of a Cooling Sphere.

03,865

High-Temperature Adiabatic Calorimeter for Constant-Volume Heat Capacity Measurements of Compressed Gases and Liquids.

Measurements of Molar Heat Capacity at Constant Volume (Cv) for 1,1,1,2-Tetrafluoroethane (R134a).

PB95-168878 , 03,264

Correlation of the Ideal Gas Properties of Five Aromatic Hydrocarbons.
PB95-175816

SPECIFICATIONS

01,002

Formal Multi-Layer Test Methodology and Its Application

to OSI.

PB94-172194

02,718

Control Entity Interface Specification

PB94-191715

02,815

Formal Specification and Verification of Control Software for Cryptographic Equipment.

01,585

CALS-Automated Interchange of Technical Information.

PB94-962000 03,657

Model Minimum Performance Specifications for Lidar

Speed Measurement Devices.

04,861

CALS-Automated Interchange of Technical Information.

PB95-962000 03,666

Application Software Interface: ISDN Services for an

Open Systems Environment

01,492

Development of Near-Field Test Procedures for Commu-

nication Satellite Antennas.

02,010

Predicate Differences and the Analysis of Dependencies

in Formal Specifications.

01,759

Technique for Analyzing the Effects of Changes in Formal

$\begin{array}{ll}\text { Specifications. } & \\ \text { PB96-160957 } & 01,760\end{array}$

Industry/Government Open Systems Specification: The

Development of GQSIP Version 3.

01,505

Experimental Evaluation of Specification Techniques for Improving Functional Testing.

PB96-201009

01,779

SPECTRA

Atomic Energy Levels. As Derived From the Analyses of

Qptical Spectra. Volume 3

00,714

Microwave Spectrum and Structure of $\mathrm{CH} 3 \mathrm{NO} 2-\mathrm{H} 2 \mathrm{O}$.

Microwave Spectrum and Structure of CH3NO2-H20. 00,719
AD-A296 377/5

Molecular Microwave Spectra Tables.

00,720

Energy Levels of Germanium, Ge I through Ge XXXII

PB94-162351

00,747

SPECTRAL COMPARATOR

Comparison of Filter Radiometer Spectral Responsivity with the NIST Spectral-Irradiance and Illuminance Scales.
PB97-113161

SPECTRAL EMISSION

High-Speed Spatial Scanning Pyrometer.

PB94-200003

02,636

Standards for Corrected Fluorescence Spectra. PB95-150835

00,581

Dynamic Technique for Measuring Normal Spectral Emissivity of Electrically Conducting Solids at High Temperatures with a High-Speed Spatial Scanning Pyrometer. PB95-153045

03,921
SPECTRAL EMITTANCE

Wavelength Dependence of Normal Spectral Emissivity of High-Temper

03,398

\section{SPECTRAL ENERGY DISTRIBUTION}

Penetration and Diffusion of Hard X-rays through Thick Barriers. III. Studies of Spectral Distributions.

03,771

PECTRAL INTERFERENCE

Spectral Interference in the Determination of Arsenic in High-Purity Lead and Lead-Base Alloys Using Electrothermal Atomic Absorption Spectrometry and Zeeman-Effect Background Correction.

00,614

SPECTRAL LINES

Isotope Shifts and Hyperfine Splittings of the 398.8-nm PB94-199585

03,814

Sobolev Approximation for Line Formation with Partial Frequency Redistribution.

PB95-202669

00,078

Magnetic Fields in Star-Forming Regions: Observations. PB96-123005 00,100 Irradiances of Spectral Lines in Mercury Pencil Lamps. PB96-176466

04,375

SPECTRAL RADIANCE

Results of a NISTNNIIQFI Comparison of Spectral-Radiance Measurements.

PB97-113021

04,759

SPECTRAL RADIOMETRY

Simultaneous Measurement of Normal Spectral Emissivity by Spectral Radiometry and Laser Polarimetry at High Temperatures in Pulse-Heating Experiments: Application o Molybdenum and Tungsten.

PB97-118376

02,694

SPECTRAL REFLECTANCE

PB95-176319

04,306

SPECTRAL RESOLUTION

Qbserving Stellar Coronae with the Goddard High Resolution Spectrograph. 1. The dMe Star AU Microscopi

PB96-102777

Redshifts in Stellar Transition Regions.

PB96-123310

00,092

PECTRAL SHIFT

00,104

Theoretical Analysis of the Coherence-Induced Spectral Shift Experiments of Kandpal, Vaishya, and Joshi.

PB94-219383

00,561

Comments on the Paper 'Wolf Shifts and Their Physical Interpretation under Laboratory Conditions'.

04,246

Reply to Professor Wolf's Comments on My Paper on Wolf Shifts.

PB94-219409

04,247

Wavelengths and Isotope Shifts for Lines of Astrophysical Interest in the Spectrum of Doubly Ionized Mercury $(\mathrm{Hg}$ III).

PBं95-140869

03,887

Van der Waals Bond Lengths and Electronic Spectral Shitts of the Benzene- $\mathrm{Kr}$ and Benzene-Xe Complexes.

ECTROCHEMISTRY ANALYSIS

Microanalysis to Nanoanalysis: Measuring Composition at High Spatial Resolution.

PB95-107173

00,563

SPECTROGRAPHS

Scientific Rationale and Present Implementation Strategy for the Far Ultraviolet Spectrograph Explorer (FUSE)

PB96-123328

00,045

SPECTROGRAPHY

Flat and Curved Crystal Spectrography for Mammographic X-ray Sources.

03,642

SPECTROMETERS

Stabilization and Precise Calibration of a ContinuousWave Difference Frequency Spectrometer by Use of a Simple Transfer Cavity.

PB95-162350

04,276

Electron-Ion-X-ray Spectrometer System.

03,958

PB95-176137

Narrow-Band Tunable Diode Laser System with Grating
Feedback, and a Saturated Absorption Spectrometer for Feedback, and a Saturated Absorption Spectrometer for PB95-20289

04,319

Energy Calibration of $\mathrm{X}$-ray Photoelectron Spectrometers: Results of an Interlaboratory Comparison to Evaluate a Proposed Calibration Procedure.

Proposed Caliti

04,027

Simple Variable Line Space Grating Monochromator for Synchrotron Light Source Beamlines.

04,065

SPECTROMETRY

Crystal Diffraction Spectrometry for Accurate, NonInvasive kV/Spectral Measurement for Improvement of

Mammographic Image Quality
AD-A297 943/3

00,721

Spectral Interference in the Determination of Arsenic in

Electrothermal Atomic Absorption Spectrometry and Eackground Correction.

00,614

Quantitative Determination of Oxidative Base Damage in DNA by Stable Isotope-Dilution Mass Spectrometry.

PB96-200886

SPECTROPHOTOMETERS

Standard Reference Materials: Glass Filters as a Stand-

ard Reference Material for Spectrophotometry - Selection,

Preparation, Certification, and Use of SRM 930 and SRM 1930.

PB94-188844

00,536

SPECTRORADIOMETERS

Report on USDA Ultraviolet Spectroradiometers.

PBg6-214648

00,125

Interface-Filter Characterization of Spectraradiometers and Colorimeters.

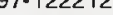

04,399

PPECTROSCOPIC ANALYSIS

Spectroscopic Data for Fusion Edge Plasmas.

04,410

Spectroscopic Diagnostics of Low Temperature Plasmas:

Techniques and Required Data.

PB95-151577

04,411

Diode Laser as a Spectroscopic Tool.

PB95-175485

00,600

Spectroscopic Study of Quantized Breakdown Voltage

States of the Quantum Hall Effect.

PB96-113584

04,730

SPECTROSCOPIC COUPLING

Atomic Transition Probabilities and Tests of the

PBectroscopic

04,057

SPECTROSCOPIC DATA

Comprehensive Spectroscopic Data Tabulations and

Progress in the Compilation of Atomic Transition Prob-

abilities.

PB95-151551

03,909

Spectroscopic
PB95-151585

SPECTROSCOPIC ELLIPSOMETRY

High-Accuracy Principal-Angle Scanning Spectroscopic PB96-163787

02,427

SPECTROSCOPY

Spectroscopic Study of Reaction Intermediates and Mechanisms in Nitramine Decomposition and Combustion.

AD-A296 061/5

03,774

Theoretical Analysis of the Coherence-Induced Spectral Shift Experiments of Kandpal, Vaishya, and Joshi.

Shift Experiment

00,561

Phase Shifts and Intensity Dependence in FrequencyModulation Spectroscopy.

01,071

In situ Fluorescence Cell Mass Measurements of 'Saccharomyces cerevisiae' Using Cellular Tryptophan.
PB96-135041

Artificial Crack in Steel: An Ultrasonic-Resonance-Spectroscopy and Modeling Study.

PB96-141395

03,241 


\section{KEYWORD INDEX}

STANDARD FOR THE EXCHANGE OF PRODUCT MODEL DATA

Two-Dimensional POMMIE Carbon-Proton Chemical Shift Corelated (13)C NMR Spectrum Editing

00,809

Resonance Enhanced Multiphoton Ionization Spectroscopy of 2-Butene-1-yl (C4H7) between 455-485 nm.
PB95-151031 00,670 Applications of Fluorescence Spectroscopy in Polymer Science and Technology.

PB95-163770

01,258

SPECULAR REFLECTION

Specular and Diffuse Reflection Measurements of Elec. tronic Displays

02,208

SPEECH

Error Protecting Characteristics of CDMA and Impacts on Speech.

01,491

PB96-122452

Benchmarks for the Evaluation of Speech Recognizers.
PB94-211539
01,566 PB94-211539

Lidar

Model Minimum Performance Specifications for Lidar Speed Measurement Devices.

PB95-197455

04,861

SPHERES

Analytical Method of Determining the Heat Capacity at High Temperatures from the Surtace Temperature of a Cooling Sphere.

PB94-216124 03,865

Brownian Diffusion of Hard Spheres at Finite Concentra-

tions.

PB95-164307 00,975

Forward Scattering of a Gaussian Beam by a Nonabsorbing Sphere.
PB97-112288

SPI

Using Synthetic-Perturbation Techniques for Tuning Shared Memory Programs (Extended Abstract). PB94-172657

01,685

SPIN DYNAMICS

Magnetic Structure and Spin Dynamics of the $\mathrm{Pr}$ and $\mathrm{Cu}$ in $\mathrm{Pr} 2 \mathrm{CuO} 4$.

$\begin{array}{ll}\text { PB96-111836 } & 04,036\end{array}$

SPIN TRAP

Unusual Spin-Trap Chemistry for the Reaction of Hydroxyl Radical with the Carcinogen NPB95-151643

00,692

SPIN VALVES

Simulating Device Size Effects on Magnetization Pinning Mechanisms in Spin Valves.

PB97-112593

04,158

SPINACH

Determination of 21 Elements by INAA for Certification of SRM $1570 a$, Spinach

SPINDLES

Evaluation of a Tapered Roller Bearing Spindle for HighPrecision Hard Turning Applications.

PB96-160494

02,700

SPINDT CATHODES

Planar Lenses for Field-Emitter Arrays.

02,112

SPINNING ROTOR GAGE CONTROLLERS

PC-Based Spinning Rotor Gage Controller.

PB95-175832

02,609

SPINODAL

Physical Limit to the Stability of Superheated and PB96-122551

01,083

SPINODAL DECOMPOSITION

Time-Resolved Small-Angle Neutron Scattering Study of Spinodal Decomposition in Deuterated and Protonated Polybutadiene Blends. 1. Effect of Initial Thermal Fluctuations.

PB95-161196

01,252

Relaxation After a Temperature Jump Within the One Phase Region of a Polymer Mixture.

03,394

Dimensional Crossover in the Phase Separation Kinetics of Thin Polymer Blend Films.

PB97-113088

03,395

SPINOR EQUATIONS

Spinor Equations in Relativistic Quantum Mechanics.
PB97-110605

SPIRALLY-REINFORCED BRIDGE COLUMNS

Evaluating the Seismic Performance of Lightly-Reinforced Circular Concrete Bridge Columns.

01,335

LICES

Fibre Splice Loss: A Simple Method of Calculation. PB95-175519

04,299

SPONGES

Swelling and Growth ot Polymers, Membranes and Sponges.

03,396

SPRAY COMBUSTION

Role of Combustion on Droplet Transport in PressureAtomized Spray Flames.

01,434
SPRAY COOLING

Transient Cooling of a Hot Surface by Droplets Evapo-

03,783

SPRAY DRIED POWDER

Characterization and Processing of Spray-Dried Zirconia Powders for Plasma Spray Application.

PB97-111231

SPRAY EXTRACTION COLUMNS

Enzyme and Protein Mass Transfer Coefficient in Aqueous Two Phase Systems. 1. Spray Extraction Columns.

SPRAYING

Measurement Methods and Standards for Processing and Application of Thermal Barrier Coalings.

N95.26123/6 01,447

Combustion of Methanol and Methanol/Dodecanol Spray Flames.

02,478

Study of Droplet Transport in Alcohol-Based Spray Flames Using Phase/Doppler Interferometry.

02,479

Structure of a Swirl-Stabilized Kerosene Spray Flame

B B95-108569 02,480

SPRAYS

Turbulent Spray Bumer for Assessing Halon Alternative Fire Suppressants.

PB95-153748

01,385

Water Mist Fire Suppression Workshop Summary. PB95-161907

02,853

SPRINKLER SYSTEMS

Analysis of Failed Dry Pipe Fire Suppression System Couplings from the Filene Center at Wolf Trap Farm Park for the Performing Arts.

PB94-164407

00,331

Field Modeling: Simulating the Effect of Sloped Beamed Ceilings on Detector and Sprinkler Response.

01,406

Analysis of High Bay Hangar Facilities tor Detector Sensitivity and Placement.

PB96-190210

01,429

State of the Art Report on Seismic Design Requirements tor Nonstructural Building Components.

PB96-193800

00,308

Interaction of an Isolated Sprinkler Spray and a TwoLayer Compartment Fire Environment. Phenomena and Model Simulations.

PB97-110076

01,437

SPRINKLERS

Application of Boundary Element Methods to a Transient Axis-Symmetric Heat Conduction Problem.

01,375

Measurement of Room Conditions and Response of Measurement of Room Conditions and Response of
Sprinklers and Smoke Detectors during a Simulated TwoBed Hospital Patient Room Fire.

00,292

Sprinkler Fire Suppression Algorithm.

00,293

Comparison of Fire Sprinkler Piping Materials: Steel, Copper, Chlorinated Polyvinyl Chloride and Polybutylene, in Residential and Light Hazard Installations.

00.299

Evaluation of Sprinkler Activation Prediction Methods.

PB96-141056 00,304

Computing the Effect of Sprinkler Sprays on Fire Induced Gas Flow.

PB96-147111

00,404

Protection of Data Processing Equipment with Fine Water

Sprays.

Sprays.
PB95-174975

02,610

SPT (SYNTHETIC PERTURBATION TUNING)

Synthetic-Perturbation Tuning of MIMD Programs. PB94-185568

01,687

SPUTTERING

Pure Element Sputtering Yield Data: Appendix 4.
PB94-200037 00,805

Deposition Rates in Direct Current Diode Sp sttering

04,697

SQA (SOFTWARE QUALITY ASSURANCE)

SQA Standards and Total Quality Management.

PB96-111844

01,743

SQA and TQM in Software Quality Improvement. PB96-160791

01,754

SQIDS (DETECTORS)

Self-Biasing Cryogenic Particle Detector Utilizing Electrothermal Feedback and a SQUID Readout. PB96-102538

04,712

SQL DATABASE LANGUAGE

Object SQL: Language Extensions for Object Data Man-

PB95-125902

01,704

SQL (STRUCTURED QUERY LANGUAGE)

SOL Environments. Category: Sottware Standard; Subcategory: Database.

01,801

SQUEEZED STATES (QUANTUM THEORY)

Squeezed Atomic States and Projection Noise in SpecIroscopy.

03,960

SQUID AMPLIFIERS

Two-Stage Integrated SQUID Amplifier with Series Array Output.

PB95-176277

02,061

SQUID (DETECTORS)

Physical Basis for Half-Integral Shapiro Steps in a DC PB96-102264

04,704

SQUID DEVICES

Series Array of DC SQUIDs.
PB95-163861

02,046

Kinetic-Inductance Infrared Detector Based on an Antenna-Coupled High-Tc SQUID.

02,165

Two-Stage Integrated SQUID Amplifier with Series Array

PBtput.

02,061

Noise Reduction in Low-Frequency SQUID Measurements with Laser-Driven Switching

02,081

SRA

Process for Selecting Standard Reference Algorithms to Evaluating Coordinate Measurement Software. PB94-173754

02,629

SRM 1165

Apparent Bias in the X-Ray Fluorescence Determination of Titanium in Selected NIST SRM Low Alloy Steels. PB95-108759

03,212

RM 1264

Apparent Bias in the X-Ray Fluorescence Determination Of Titanium in Selected NIST SRM Low Alloy Steels.

SRM 1548

Mixed Diet Reference Materials for Nutrient Analysis of Foods: Preparation of SRM-1548 Total Diet.

03,593

STABILITY

Long-Term Stability of Carrier-Free Polonium Solution Standards.

PB94-185170

00,685

Stability/Instability of Gas Mixtures Containing 1,3-Buta-

diene in Treated Aluminum Gas Cylinders.

PB95-162285

02546

Stability and Surface Energies of Wetted Grain Boundaries in Aluminum Oxide.

PB95-202750

03,059

Comments on the Stability of Bayard-Alpert Ionization Gages.

STADARDS

International Institute of Welding: Report on 1995 Actions. 158076

TTAINLESS STEEL

PC-Based Prototype Expert System tor Data Management and Analysis of Creep and Fatigue of Selected Materials at Elevated Temperatures.

PB94-172251

03,202

Temperature-Induced Transition in Ductile Fracture Appearance of a Nitrogen-Strengthened Austenitic Stainless

STAINLESS STEEL-304L

03,221 
Capabilities for Product Data Exchange. PB97-118764

02,798 STANDARD FOR THE EXCHANGE OF PRODUCT MODEL DATA (STEP)

Extensions of the Prototype Application Protocol of Ready-to-Wear Apparel Pattern Making

03,198

STANDARD GENERALIZED MARKUP LANGUAGE

SGML Environment for STEP.

PB95-143103

02,778

SGML Parser Validation Procedures.

01,717

STANDARD RADIATORS

Screened-Room Measurements on the NIST SphericalDipole Standard Radiator.

01,926

STANDARD REFERENCE ALGORTHM

Process for Selecting Standard Reference Algorithms for Evaluating Coordinate Measurement Software. PB94-173754

02,629

STANDARD REFERENCE DATA

Numeric Data Distribution: The Vital Role of Data Exchange in Today's World

N95-15937/2

02,622

Journal of Physical and Chemical Reference Data, Vol-

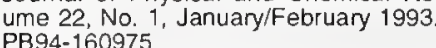

00,729

Thermodynamic Properties of the Group IIA Elements.

PB94-160983 00,730

Spectroscopy and Structure of the Lithium Hydride Diatomic Molecules and Ions.

00,731

Ouantum Yields for the Photosensitized Formation of the Lowest Electronically Excited Singlet State of Molecular Oxygen in Solution.

PB94-161007

00,732

Journal of Physical and Chemical Reference Data, Volume 22, No. 2, March/April 1993.

PB94-162211

00,733

Wavelengths and Energy Level Classifications for the Spectra of Sulfur (S I through S XVI).

00,734

Thermodynamic Properties of Alkenes (Mono-Olefins Larger Than C4).
PB94-162237

00,735

Thermodynamic Behavior of the $\mathrm{CO} 2-\mathrm{H} 2 \mathrm{O}$ System from 400 to $1000 \mathrm{~K}$, up to $100 \mathrm{MPa}$ and $30 \%$ Mole Fraction of $\mathrm{CO} 2$

B94-162245

00,736

Thermodynamics of Enzyme-Catalyzed Reactions: Part 1. Oxidoreductases

00,737

Journal of Physical and Chemical Reference Data, Volume 22, No. 3, May/June 1993.

00,738

Estimation of the Heat Capacities of Organic Liquids as a Function of Temperature Using Group Additivity. I. Hydrocarbon Compounds.

PB94-162278

00,739

Estimation of the Heat Capacities of Organic Liquids as a Function of Temperature Using Group Additivity. II. Com-
pounds of Carbon, Hydrogen, Halogens, Nitrogen, Oxypounds of Carbon, Hydrogen, Halogens, Nitrogen, Oxy gen, and Sulfur.

00,740

Thermodynamic and Thermophysical Properties of Organic Nitrogen Compounds. Part II. 1- and 2-Butanamine, 2-Methyl-1-Propanamine, 2-Methyl-2-Propanamine, Pyrrole, 1-, 2-, and 3-Methylpyrrole, Pyridine, 2-, 3-, and 4-Methylpyridine, Pyrrolidine, Piperidine, Indole, Ouinoline, Isoquinoline, Acridine, Carbazole, Phenanthridine, 1. and 2-Naphthalenamine, and 9-Methylcarbazole.

PB94-162294

00,741

International Equations for the Saturation Properties of Ordinary Water Substance. Revised According to the International Temperature Scale of 1990 . Addendum to Journal of Physical and Chemical Reference Data 16. $893(1987)$

00,742

Journal of Physical and Chemical Reference Data, Volume 22, No. 4, July/August 1993.

Estimation of the Thermodynamic Properties of $\mathrm{C}-\mathrm{H}-\mathrm{N}-\mathrm{O}$

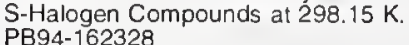

00,744

Journal of Physical and Chemical Reference Data, Volume 22, No. 5. September/October 1993 . 00,745

PB94-162336 00,745 Spectrum of Singly-lonized Oxygen (O II).

PB94-162344

00,746

Energy Levels of Germanium, Ge I through Ge XXXII.

PB94-162351 00,747

Spectral Data and Grotrian Diagrams for Highly lonized Chromium, Cr V through Cr XXIV.

00,748

Journal of Physical and Chemical Reference Data, Volume 22. No. 6. November/December 1993 . ume 22, No. 6 ,

00,749
Thermodynamic Properties of Synthetic Sapphire (alphaAl2O3), Standard Reference Material 720 and the Effect of Temperature-Scale Differences on Thermodynamic Propenties.

PB94-168564

00,750

Thermodynamic Properties of Gaseous Silicon (t) of Gaseous silicon M) (SiTe) at $T$ approaches 0

00,751

Disilicides of Tungsten, Molybdenum, Tantalum, Titanium, Cobalt, and Nickel, and Platinum Monosilicide: A Survey of Their Thermodynamic Properties.

00,752

Evaluated Bimolecular Ion-Molecule Gas Phase Kinetics of Positive lons for Use in Modeling Planetary at Positive lons tor Use in Modeling Planetary Atmospheres, Cometary Comae, and Interstellar Clouds.
PB94-168598

Atomic Weights of the Elements 1991.

00,754

PB94-168606

Theoretical Analysis of the Coherence-Induced Spectral Shift Experiments of Kandpal, Vaishya, and Joshi.

PB94-219383

00,561

NIST Standard Reference Data Products Catalog, 199596. Achieve with Standard Reference Data.

01,057

B95-260808

04,734

Building a Better Crycooler.

Journal of Physical and Chemical Reference Data, Volume 24, No. 1, January/February 1995

PB96-145560

01,101

Millimeter- and Submillimeter-Wave Spectrum of transEthyl Alcohol.

Database for the Static Dielectric Constant of Water and Siear

PB96-145586

01,103

Theoretical Form Factor, Attenuation and Scattering Tabulation for $Z=1-92$ from $E=1-10 \mathrm{eV}$ to $E=0.4-1.0 \mathrm{MeV}$.
PB96- 145594 01,104

Journal of Physical and Chemical Reference Data, Volume 24, No. 2, March/April 1995.

01,105 Rate Constants for the Decay and Reactions of the Low-
est Electronically Excited Singlet State of Molecular Oxygen in Solution. An Expanded and Revised Compilation. PB96-145826

01,106

Thermodynamic Properties of the Aqueous Ba(sup 2+) Ion and the Key Compounds of Barium.

01,107

Journal of Physical and Chemical Reference Data, VolJournal of Physical and Chemical Reference Data, Vol-
ume 24, No.3, May/June 1995 .
PB96-145842

01,108

Critical Review of Rate Constants for Reactions of Transients from Metal lons and Metal Complexes in Aqueous

01,109

Ideal Gas Thermodynamic Properties of Sulphur HeteroIdeal Gas Thermodynamic Properties of Sulphur Hetero-
cyclic Compounds.
PB96-145867

tivity of

Standarc

PB96-145875

01,111

Journal of Physical and Chemical Reference Data, Vol-

ume 24, No. 4, July/August 1995.
PB96-145883

01,112

Summary of the Apparent Standard Partial Molal Gibbs Free Energies of Formation of Aqueous Species, Minerals, and Gases at Pressures 1 to 5000 Bars and Temperatures 25 to $1000 \mathrm{C}$.

PB96-145891

01,113

Atomic Weights of the Elements, 1993

PB96-145909

01,114

Spectral Data for Highly lonized Krypton, $\mathrm{Kr}$ V through $\mathrm{Kr}$ XXXVI.

01,115

Journal of Physical and Chemical Reference Data, Volume 24, No. 5, September/October 1995.

PB96-145925

01,116

Viscosity of Ammonia

01,117

PB96-145933

Thermodynamics of Enzyme-Catalyzed Reactions. Part 4.

Lyases.

Thermodynamic Properties of the Aqueous lons $(2+$ and

$3+$ ) of Iron and the Key Compounds of Iron.

01,119

Journal of Physical and Chemical Reference Data, Volume 24, No. 6, November/December 1995.

01,120

Thermodynamics of Enzyme-Catalyzed Reactions. Part 5. Isomerases and Ligases.

01,121

Energy Levels of Zinc, $\mathrm{Zn} I$ through $\mathrm{Zn} X X X$

01,122

STANDARD REFERENCE DEVICES

Standard Reference Devices for High Temperature Superconductor Critical Current Measurements.

04,659

STANDARD REFERENCE MATERIAL

What Is a 'Standard Reference Material' - What is Any RBerence Material.

TANDARD REFERENCE MATERIAL 1473A

Certification of the Standard Reference Material 1473a, a Low Density Polyethylene Resin

PB96-12825

01,282

STANDARD REFERENCE MATERIAL 1475A

Recertification of the Standard Reference Material 1475A, a Linear Polyethylene Resin.

STANDARD REFERENCE MATERIAL 1744

02,628

Standard Reference Material 1744: Aluminum FreezingPoint Standard

01,055

\section{STANDARD REFERENCE MATERIALS}

Standard Samples and Reference Standards Issued by the National Bureau of Standards.

02,613

NIST Standard Reference Data Products Catalog, 1994.

PB94-151842 00,727

Recertification of the Standard Reference Material 1475A, a Linear Polyethylene Resin.

02,628

Preparation and Certification of a Rhodium Standard Reference Material Solution

00,529

Frozen Human Serum Reference Material for Standardization of Sodium and Potassium Measurements in Serum or Plasma by Ion-Selective Electrode Analyzers.
00,532 Standard Reference Materials: Glass Filters as a Standard Reference Material for Spectrophotometry - Selection, PB94-188844

00,536

Recently Developed NIST Food Related Standard Reference Materials.

PB94-198322

00,035

Standard Reference Materials for Dioxins and Other Environmental Pollutants.

02,518

Standard Reference Materials (SRM's) for Measuring Ge-

netic Damage.

03,516

Introduction of a NIST Instrument Sensitivity Standard Reference Material for X-Ray Powder Diffraction.
PB94-200318
00,807

al Diet

Corotenoid Content of SRM 1548 and Influence of Storage Temperature,

and Irradiation on Dietary Carotenoids.

00,033

User's Guide to NIST SRM 2084: CMM Probe Performance Standard.

PB94-206109 02,709

Molar Absorptivities of Bilirubin (NIST SRM 916A) and Its Neutral and Alkaline Azopigments.

03,456

Determination of the Weight Average Molecular Weight of Two Poly(Ethylene Oxides). SRM 1923 and SRM 1924.
PB94-217031

New Values for Silicon Reference Materials, Certified for Isotope Abundance Ratios. Letter to the Editor. 00,863 Certification, Development and Use of Standard Reference Materials

PB95-107272

00,567

Apparent Bias in the X-Ray Fluorescence Determination 
Mixed Diet Reference Materials for Nutrient Analysis of Foods: Preparation of SRM-1548 Total Diet.
PB95-151692 03,593 Room Temperature Thermal Conductivity of Fumed-Silica Insulation for a Standard Reference Material.

00,374 Reference Materials by Isotope Dilution Mass SpectromReference Materials by Isotope Dilution Mass Spectrom-
etry.
PB95-153383 00,592

02,547 Standards for Atmospheric Measurements
PB95-163622 Standard Reference Materials for the Determination of Trace Organic Constituents in Environmental Samples.
PB95-164026 Liquid Chromatographic Determination of Polycyclic Aromatic Hydrocarbon Isomers of Molecular Weight 278 and 302 in Environmental Standard Reference Materials. PB95-164042 02,523 Reference Data for the Thermophysical Properties of Cryogenic Fluids

03,263 Alaska Marine Mammal Tissue Archival Project: Specimen Inventory.

02,589

Certification of Morphine and Codeine in a Human Urine Standard Reference Material.

PB95-176160

03,499

Hair Analysis for Drugs of Abuse: Evaluation of Analytical Methods, Environmental Issues, and Development of Reference Materials.

PB95-176269

03,501

NIST Standard Reference Materials (Trade Name) Catalog 1995-1996.

PB95-232518

00,508

Standard Reference Material 1744: Aluminum Freezing-

Point Standard.

PB95-251732 01,055

NIST SRM 9983 High-Rigidity Ball-Bar Stand. User Manual.

PB95-255840 02,669

Standard Reference Material for the Measurement of Particle Mobility by Electrophoretic Light Scattering. 00,609

Certification of Polychlorinated Biphenyl Congeners and Chlorinated Pesticides in a Whale Blubber Standard RefChlorinated Pesticides in a Whale Blubber Standard Ret-
erence Material.
PB96-103023
03,745

03,745

Certification of Polycyclic Aromatic Hydrocarbons in a Marine Sediment Standard Reference Material.
PB96-111778 History of NIST's Contributions to Development of Stand. ard Reference Materials and Reference and Definitive
Methods for Clinical Chemistry. PB96-119706

03,503

Hair Testing for Drugs of Abuse: International Research On Standards and Technology

03,504

Development of a Standard Reierence Material for Measurement of Interstitial Oxygen Concentration in Semiconductor Silicon by Intrared Absorption.

PB96-122668 02,404

Certification of Standard Reference Material (SRM) 1941a, Organics in Marine Sediment.

PB96-123690

02,593

NIST Reference Materials to Support Accuracy in Drug PB96-123807

03,505

Certification of the Standard Reference Material 1473a, a

Low Density Polyethylene Resin
PB96-128251

01,282

Methodology for the Certification of Reference Specimens for Determination of Oxygen Concentration in Semiconductor Silicon by Infrared Spectrophotometry.
PB96-155478 System for Intercomparing Standard Solutions of BetaParticle Emitting Radionuclides.

03,707

Development of a Standard Reference Material for ISE Measurements of Sodium and Potassium.

PB96-159785

03,507

Certification of Phencyclidine in Lyophilized Human Urine Reference Materials.

03,508

Determination of 21 Elements by INAA for Certification of Determination of 21 Elements by INAA for Certification of
SRM $1570 \mathrm{a}$, Spinach.
PB96-167242 00,698

03,489

Recent Develop

Resolution of Discrepant Analytical Data in the Certification of Platinum in Two Automobile Catalyst SRMs.
PB96-167283 00,638

00,638
Is Any

Reference Material.

PB96-186135

03,000

Room-Temperature Thermal Conductivity of Expanded Polystyrene Board for a Standard Reference Material.

PB96-193693 00,412

Unique Ouality Assurance Aspects of INAA for Reference Material Homogeneity and Certification.

00,699
NIST Traceable Reference Material Program for Gas Standards: Standard Reference Materials.
PB96-210786 Statistical Aspects of the Certification of Chemical Batch RMs. Standard Reference Materials.

PB96-210877 00,645

Present and Future Standard Specimens for Surface Finsh Metrology

$\begin{array}{ll}\text { B B97-110423 } & 02,928\end{array}$

International Standards and Reference Materials.

PB97-113120

00,188

STANDARD RESISTORS

Automated Guarded Bridge for Calibration of Multimegohm Standard Resistors.
PB97-119150 02,289 Resistance Measurements from $10 \mathrm{M}$ Omega to $1 \mathrm{~T}$ Omega at NIST

PB97.119168

02,290

STANDARD SAMPLES

Standard Samples and Reference Standards Issued by the National Bureau of Standards.

02,613

STANDARDIZATION

Standard Materials. A Descriptive List with Prices. 00,500

Activities of NIST (National Inst. Of Standards and Tech-

N94-23605/6 04,222

Implementation of a Standard Format for GPS Common View Data.

03,779

(19) Actions.

International Institute of Welding: Report on 1992 Actions.
PB94-185873

02,856
PB94-185873

International Institute of Welding: Report on 1993 Actions.
02,857

Standardization of Formats and Presentation of Fire Data - The FDMS.

01,371

Standardization of Testing Methods for Optical Disk

Media Characteristics and Related Activities at NIST.

PB95-108486

01,624

Critical Factors in Non-Lubricated, Non-Abrasive Wear Testing.

5-140588

03,236

Think Metric.

PB95-151825 01,298

SO Environmental Management Standardization Efforts.

02,524

SO TC 184/SC4 Reference Manual.

PB95-242293

02,663

Can Displays

02,185

Comparison of POSIX Open System Environment (OSE) and Open Distributed Processing (ODP) Reference Models.

01,820

Report on 1994 Actions of the International Institute of Welding.

02,873

SO Environmental Management Standardization Efforts.

PB96-158662 02,528

Implementation of a Standard Format for GPS Common View Data.

PB96-176581

01,555

Open System Environment Implementors Workshop OIW); Standardization Role Defined.

01,828

$63 \mathrm{Ni}$ Half-Life: A New Experimental Determination and Critical Review.

PB97-111603

00,700

Nickel-63 Standardization: 1968-1995.

PB97-111819

00,701

SC4 Short Names Registry.

PB97-122410

02,799

Standards Acivi

States. ANDARDS

Screw-Thread Standards for Federal Services, 1957. Part AD-A279 121/8

02,854

Standard Samples and Reference Standards Issued by the National Bureau of Standards.

02,613

AD-A279 240/6

Technologic Papers of the Bureau of Standards: Number
170. Pyrometric Practice.
AD-A279 282/8 AD-A279 282/8

Screw-Thread Standards for Federal Services, 1957. Part

National Standard Petroleum Oil Tables.

AD-A279 952/6

02,469

Screw-Thread Standards for Federal Services, 1957. Handbook H28 (1957), Part 2. Revised.

03,599

Federal

1950 Supplement

Services. 1944.

03,656

Precision Laboratory Standards of Mass and Laboratory

Weights.
AD-A280 562/0 02,618

Standard of Attenuation for Microwave Measurements.

AD-A297 905/2 01,517

NIST Cooperative Laboratory for OSI Routing Technology.

01,791

COBOL. Category: Software Standard; Subcategory: Programming Language. Includes ANSI'S X3.23-1985 X3.23A-1989 and X3.23B-1993. COBOL. Category: Software Standard; Subcategory: Programming Language. Part A

COBOL. Category: Software Standard; Subcategory: Programming Language. Part B

01,672

ADA; Category: Software Standard; Subcategory: Programming Language

01,667

User Interface Component of the Applications Portability Profile Category: Software Standard; Subcategory: Application Program Interface.

FIPS PUB 158-1

01,793

Time and Frequency Technology at NIST

N94-30641/2

01,522

Open System

01,674

Open System Environment Procurement.

02,716

Numeric Data Distribution: The Vital Role of Data Exchange in Today's World.

N95.15937/2

02,622

National Center for Standards and Certification Information: Service and Programs.

N95-15938/0

02,717

General Procedures for Registering Computer Security Objects.

PB94-134897

01,573

Open Architectures for Machine Control.

PB94-13562

02,942

Planning for the Fiber Distributed Data Interface (FDDI)

PB94-135761 01,621

$36 \mathrm{Cl} / \mathrm{Cl}$ Accelerator-Mass-Spectrometry Standards: Verification of Their Serial-Dilution-Solution Preparations by Radioactivity Measurements.

PB94-140563

00,524

Report on Application Integration Architectures (AIA) Workshop. Held in Dallas, Texas on February 8-12, 1993. Technical Program of the Factory Automation Systems Division 1993

PB94-160819 02,805

Computer Graphics Metafile (CGM): Procedures for NIST CGM Validation Test Service.

PB94-161809

01,804

Voluntary Product Standard PS 20-94. American Softwood Lumber Standard.

PB94-162500

03,402

Models, Managing Models, Quality Models: An Example of Quality Management.

Compatibility Analysis of the ANSI and ISO IRDS Services Interfaces.
PB94-163474

User's Guide for the PHIGS Validation Tests (Version 2.1) 
Graphical Conceptual Navigation as a Presentation Technique for a Graphics Standard.

01,692

User's Guide to NIST SRM 2084: CMM Probe Performance Standard

02,709

IGOSS-Industry/Govemment Open Systems Specifica-

tion. 207453

01,806

State Weights and Measures Laboratories: State Standards Program Description and Directory. 1994 Edition.

PB94-207727

$24 \mathrm{GHz}$ Josephson Array Voltage Standard.

02,033

Summary of the Proceedings of the Workshop on Standard Phantoms for In-vivo Radioactivity Measuremen

PB94-212933

Technical Program Description Systems Integration for Manufacturing (SIMA)

02.819

Performance Standards: The Pro's and Con's.

PB94-216132

02,896

Standards in Building Economics: Why We Need Them and How to Write Them.

PB94-216405 00,320

NIST-Coordinated Standard for Fingerprint Data Inter-

PB94-216645

01,808

Joumal of Research of the National Institute of Standards and Technology. May/June 1994. Volume 99, Number 3. 02,643

Sources of Uncertainty in a DVM-Based Measurement

PB94-219334 O 01,884 dc Method for the Absolute Determination of Conductiv. ities of the Primary Standard $\mathrm{KCl}$ Solutions from $O \mathrm{C}$ to PB94-219342

02,644

Improved Automated Current Control for Standard

00,246

CALS-Autometed Interchange of Technical Information.

CALS-Automated Interchange of Technical Information.
PB94-962000

Ouestions and Answers on Ouality, the ISO 9000 Standard Series, Ouality System Registration, and Related Issues. More Ouestions and Answers on the ISO 9000 Standard Series and Related Issues.

PB95-103461

00,495

Making Sense of Software Engineering Environment Framework Standards.

PB95-105037

01,701

Formulation of Position on U.S. Standards Role in Enterprise Integration.

PB95-105052

02,773

Certification, Develooment and Use of Standard Reference Materials.

00,567

Summary and Notes of the Joint ISO/IGES/PDES Organization Technical Committee Meeting. Held in Albuquerque, New Mexico on October 15-20, 1989. Activities of the ASTM Committee E-42 on Surface Analysis.

00,881

Suggestions for a Logicaliy-Consistent Structure for Service Life Prediction Standards.

PB95-125795

00,358

National PDES Testbed: An Overview.

PB95-125829 02.775

Water Efficient Plumbing Fixtures through Standards and Test Methods.

PB95-125951

00,248

Information Resource Dictionary System (IRDS): A Status

01,810

Summaries of BFRL Fire Research In-House Projects and Grants, 1994

PB95-130845

00,366

Challenges to the National Information Infrastructure: The Barriers to Product Data Sharing. National PDES Testbed Repor Series.

02,776

NIST Workshop on the Computer Interface to Flat Pane Displays. Held in San Jose. California on January 13-14, 1994

PB95-136388

01,625

New Materials, Advanced Ceramics and Standards.

PB95-140208

03,047

SGML Environment for STEP

PB95-143103

02,778

New International Representations of the Volt and Ohm

Effective January 1., 1990

Standards for Corrected Fluorescence Spectra.

PB95-150835

01.890

PB95-150942

03,677
Analysis of Selected Software Safety Standards.

$5-151262$

01,708

New Coaxial Microwave Microcalorimeter Evaluation PB95-153227

01,892

Mapping Integration Definition for Information Modeling IDEF1X) Model into CASE Data Interchange Forma CDIF) Transfer File.

PB95-154670

01,71

Frequency Extension of the NIST AC-DC Difference Calibration Service for Current.

PB95-161253

01,895

X-ray Mask Metrology: The Development of Linewidth Standards for X-ray Lithography.

02,348

Introduction to ASTM 1199 'Wear Test Selection for De sign and Application

PB95-162517

03,238

Recent VAMAS Activity in Ceramics.

03.051

Geographic Information Systems Standards: A Federa Perspective.

PB95-163390

03,678

Accuracy Comparisons of Josephson Array Systems

Lightwave Standards Development at NIST. 02,047

PB95-168563

01,480

Measurements of the Characteristic Impedance of Coaxial Air Line Standards.

PB95-168787

02,221

Perspective on Software Engineering Standards

PB95-171377

01.811

Design Challenges in a Commercial Quantum Hall EffectBased Resistance Standard.

PB95.171419

02,263

Dimensional Inspection Planning Based on Product Data Standards.

PB95-175451

02,910

Taxonomy for Security Standards.

01,602

Federal Government and Information Technology Standards: Building the National Information Infrastructure.

PB95-180840 01,812

Practical Standards for PM and AM Noise at 5, 10 and $100 \mathrm{MHz}$

01.546

Method to Determine the Calorimetric Equivalence Correction for a Coaxial Microwave Microcalorimeter.

PB95-202404

01,913

Developing a NIST Coaxial Microwave Power Standard at $1 \mathrm{~mW}$.

01,914

Survey of Concrete Transpor Properties and Their Measurement

PB95-220489

00,396

Analysis of ANSI ASC X12 and UNEDIFACT Electronic Data Interchange (EDI) Standards.

PB95-220554

01,729

Standards Policy and Information Infrastructure. $\quad 01.485$

01.485
PIST Standard Reference Materials (Trade Name) Cataog 1995-1996.

PB95-232518

00,508

Operating Procedures and Life Cycle Documentation for the Initial Graphics Exchange Specification.

PB95-242285

02,782

Advanced Mass Calibration and Measurement Assurance Program for State Calibration Laboratories.

02,492

Proceedings of the Meeting of the Intergovemmenta U.S.-Russian Business Development Committee's StandU.S.-Russian Business Development Committee's StandYro Working Group (4th). Held in New York Cily. New March $30-31,1995$.

March $30-31$,
PB95-255881

00,496

$45 \mathrm{deg} / 0$ deg Reflectance Factors of Pressed Polytetrafluoroethylene (PTFE) Powder.

PB95-260758

04,328

Joumal of Research of the National Institute of Standards and Technology, May/June 1995. Volume 100, Number 3. 02,670 CALS-Automated Interchange of Technical Inlormation. PB95-962000 03,666 A.b initio Calculations for Helium: A Standard for Transport Property Measurements.

Voltage-Standard Devices.

01,060

PB96-102496

01,920

Aging, Warm-Up Time and Retrace; Important Characterstics of Standard Frequency Generators.

04,031

GATT Standards Code Activities of the National Institute of Standards and Technology 1994.

00,497 Mapping Integration Definition for Function Modeling
(IDEFO) Model into CASE Data Interchange Format (CDIF) Transfer File.

01,741

Efficiency of Electric Motors. National Voluntary Lab. Accreditation Program (NVLAP)

02,107

High Integrity Software Standards Activities a: NIST.

PB96-112214 01,744

Local Oscillator Requirements and Strategies for the Next Generation of High-Stability Frequency Standards. B96-112230

01.551

Joumal of Research of the National Institute of Standards and Technology, July/August 1995. Volume 100, Number 4. Special Issue: The Gaseous Electronics Conference Radio-Frequency Reference Cell.

PB96-113311

02,386

Gaseous Electronics Conference RF Reference Cell: An Introduction.

02,387

Current and Voltage Measurements in the Gaseous Electronics Conference RF Reference Cell.

PB96-113337

02,388

Optical Emission Spectroscopy on the Gaseous Electronics Conference RF Reference Cell.

PB96-113345

02,389

Optical Diagnostics in the Gaseous Electronics Conference Reference Cell.

PB96-113352

02,390

Studies of Ion Kinetic-Energy Distributions in the GaseElectronics Conference RF Reference Cell.

PB96- 113360

02,391

Langmuir Probe Measurements in the Gaseous Electronics Conference RF Reference Cell.

PB96-113386

02,393

Inductively Coupled Plasma Source for the Gaseous Electronics Conference RF Reference Cell.

PB96-113394

02,394

Reactive Ion Etching in the Gaseous Electronics Conference RF Reference Cell.

PB96-113402

02,395

Dusty Plasma Studies in the Gaseous Electronics ConTerence Reference Cell.

PB96-113410

02,396

One-Dimensional Modeling Studies of the Gaseous Electronics Conference RF Reference Cell.

PB96-113428

02,397

Two-Dimensional Self-Consistent Radio Frequency Plasma Simulations Relevant to the Gaseous Electronics Conference RF Reference Cell.

PB96-113436

02,398

Joumal of Research of the National Institute of Standards and Technology, November/December 1994. Volume 99,

Number 6.
PB96-113535 


\section{KEYWORD INDEX}

STEELS

Object-Oriented Tel/Tk Binding for Interpreted Control of the NIST EXPRESS Toolkit in the NIST STEP Application Protocol Development Environment.

PB96-141049

02,785

Summary Report on the Workshop on Advanced Digital Video in the National Information Infrastructure.
PB96-141320 01,497 Guidelines for the Development of Mapping Tables PB96-154539

Metrology and Regional Trade Pacts.

02,923

Development of Computer-Based Models of Standards and Attendant Knowledge-Base and Procedural Systems.
00,464 International Organization for Standardization: Current Activities in Fire Safety Engineering. U.S. Government Accreditation and Conformity Assess. ment System Evaluation.

02,678 PB96-160239

International Challenges in Defining the Public and Pr vate Interest in Standards.

00,498

Standardization for ATM and Related B-ISDN Tech. nologies.
PB96-1604

01,499

ISDN in North America.

01,502

North American Agreements on ISDN.
PB96-160775

01,503

Impact of Computer-Aided Acquisition and Logistic Support (CALS) in the Application of Standards.

Conference Report: International Conference on the Application of Standards for Open Systems (6th).

PB96-161211

01,762

GOSIP Testing Program.

01,504

Information Technology Standards in Federal Acquisiions.

01,636

Using Information Technology Standards in Federal AC-

quisitions

01,637

Report of a Workshop on the Assurance of High Integrity Software

PB96-161377

01,763

Standards for High Integrity Software.

01764

Group 1 for the Plant Spatial Configuration STEP Application Protocol.

PB96-165402

02.789

Federa! Implementation Guideline for Electronic Data Interchange. ASC X12 003050 Transaction Set 843 Response to Request for Quotation. Implementation Convention

PB96-168984

01,822

Federal Implementation Guideline for Electronic Data Interchange. ASC $\times 12003050$ Transaction Set 855 Purchase Order Acknowledgment: Implementation Convenion.

01,823

PB96-172374

ic Data

Federal Implementation Guideline for Electronic Data Interchange. ASC X12 003050 Transaction Set $840 \mathrm{Re}$ quest for Quotat

01,824

Federal Implementaion Guideline for Eletronic Data Interchange. ASC X12003050 Transaction Set 865 Purchase Order Change Acknowledgement/Request - Seller Initiated. Implementation Convention.

01,825

Voluntary Product Standard PS 1-95 Construction and Industria! Plywood.

03,406

Specifications and Tolerances for Reference Standards Specifications and Tolerances for Reference Standards tions and Tolerances for Field Standard Measuring Flasks.

PB96-178926

02,682

Consensus Process in Standards Development.

B96-179502

00,136

Application of Metadata Standards.

PB96-180187

01,771

Proceedings of NIST Workshop: Industry Needs in Welding Research and Standards Development. Held on August 15-16, 1995

02,877

What Is a 'Standard Reference Material' - What is Any Reference Material.

PB96-186135

03,000

Helping to Reduce Technical Barriers to Trade.

00,491

Room-Temperature Thermal Conductivity of Expanded Polystyrene Board for a Standard Reference Materia

PB96-193693

00,412

Systems Integration for Manufacturing Applications Pro-

gram 1995 Annual Report.

02,844
Report on the NIST Low Accelerating Voltage SEM Magnification Standard Interlaboratory Study.

02,445

Precise Laser-Based Measurements of Zero-Dispersion Wavelength in Single-Mode Fibers.

PB96-201124

01,511

Application Protocol Information Base World Wide Web Gateway.

02,791

Airbome Asbestos Method: Bootstrap Method for Determining the Uncertainty of Asbestos Concentration. Ver-

PB96-214614

00,646

TBT Agreement Activities of the National institute of Standards and Technology, 1995.

PB97-104178

00,499

CIF Crystallographic Information Flle: A Standard for Crystallographic Data Interchange.

04,805

Role of Certified Reference Materials in Trace Analysis Quality Assurance.

PB97-110019

00,650

Examination Procedure Outlines: Keys to Solving the Handbook 44 Puzzle.

02,690

Current Status and Trends in Temperature Measurements at NIST, Cooperative Projects and New Mutual Agreement between NIST and IMGC.

02,691

Characterization of Modified FEL Quartz-Halogen Lamps Characterization of Modified FEL Quartz-Halogen Lamps
for Photometric Standards.
PB97-112544

00,28

International Standards and Reference Materials.

PB97-113120

00,188

Standards Promote Credibility and Technology Transfer: The Need for Greater Industry Support of Technical Com

PB97-116206

02,961

STANDARDS REFERENCE MATERIALS

Role of Certified Reference Materials in Trace Analysis

PB97-110019

00,650

STANDING WAVES

Diffraction of Neutron Standing Waves in Thin Films with Resonance Enhancement.

PB97-113278

04,164

STAR PLOYMERS

Thermodynamic Properties of Dilute and Semidilute Solutions of Regular Star Polymers.

PB96-146808

01,284

STARK EFFECT

New Critical Review of Experimental Stark Widths and

PB94-172830

03,790

Perturbative Calculation of the AC Stark Effect by the Complex Rotation Method

04,260

STATE SERVICES

State Weights and Measures Laboratories: State Standards Program Description and Directory. 1994 Edition. PB94-207727

02,895

STATES (UNITED STATES)

State Weights and Measures Laboratories: Program PB96-214705

02,687

STATIC ANALYSIS

Application of the Pointer State Subgraph to Static Program Slicing.

01,768

STATIC DIELECTRIC CONSTANTS

Database for the Static Dielectric Constant of Water and PB96-

01,103

STATIC ELECTRICITY

Adhesion, Contact Electrification, and Acid-Base Properties of Surfaces.

PB96-204425

03,693

TATICS

Smali-Angle Neutron Scattering Study on Weakly Charged Temperature Sensitive Polymer Gels. 01,264

\section{STATISTICAL ANALYSIS}

Utc Dissemination to the Real-Time User

N19960042622

01,521

Computing Effects and Error for Large Synthetic Perturbation Screenings

Statistical Analysis of Parameters Affecting the Measure ment of Particle-Size Distribution of Silicon Nitride Powders by Sedigraph (Trade Name).

03,042

Extreme Value Theory and Applications: Proceedings of the Conference on Extreme Value Theory and Applications, Volume 3. Held in Gaithersburg, Maryland in May

PB95-104956

03,432

Taguchi's Param

03,445

Book Review: Statistical Physics of Macromolecules.
PB96-123526

Modeling of Extreme Loading by 'Peaks Over Threshold'

PB96-159694 00,469

Statistical Aspects of the Certification of Chemical Batch SRMs. Standard Reference Materials.

PB96-210877

00,645

Airborne Asbestos Method: Bootstrap Method for Determining the Uncertainty of Asbestos Concentration. Ver-

$6-214614$

00,646

\section{STATISTICAL DATA}

Guide to Locating and Accessing Computerized Numeric Materials Databases.

PB96-204045

03,007

STATISTICAL DISTRIBUTIONS

Tolerance Intervals for the Distribution of True Values in the Presence of Measurement Errors.

PB95-150405 03,434

Two Principal Points of Symmetric, Strongly Unimodal Distributions.
PB95-203360

03,443

STATISTICAL INFERENCE

Scientific Protocols in Statistical Standards for Environmental Studies.

PB94-185527

STATISTICAL MECHANICS

Nonequilibrium Statistical Mechanics.

PB96-161781

02,517

STATISTICAL QUALTY CONTROL

Statistical Quality Control Technology in Japan.

PB94-199064

04,097

STATISTICS

Fracture of Silicon Nitride and Silicon Carbide at Elevated Temperatures.
PB96-180260

03,179

STEAM

Formulation of the Refractive Index of Water and Steam. PB95-140133 00,900 Physical Limit to the Stability of Superheated and Stretched Water.

PB96-122551

01,083

Static Dielectric Constant of Water and Steam.

01,090

Database for the Static Dielectric Constant of Water and Steam.

PB96-145586

01,103

STEARIC ACID

Tribochemical Reaction of Stearic Acid on Copper Surface Studied by Surface Enhanced Raman Spectroscopy. STEEL

Sensor System for Intelligent Processing of Hot-Rolled

PB96-186069 03,373

Ductile Fracture and Tempered Martensite Embrittlement 
Diffusion of Cations Beneath Organic Coatings on Steel Substrate.

PB94-215704

Ashland Tank Collapse Investigation.

Ashland Tank-Collapse Investigation: Closure by Authors. PB95-126322 02,482 In situ Measurements of Chloride lon and Corrosion Po-

tential at the Coating/Metal Interface.

03,122

Ultrasonic Measurement of Sheet Anisotropy and Formability.

PB95-153235

03,213

Sorption of Moisture on Epoxy and Alkyd Free Films and Coated Steel Panels.

03,192

Friction and Oxidative Wear of $440 \mathrm{C}$ Ball Bearing Steels Under High Load and Extreme Bulk Temperatures.

PB95-175253

03,215

Comparison of Fire Sprinkler Piping Materials: Steel, Copper, Chlorinated Polyvinyl Chloride and Polybutylene in Residential and Light Hazard Installations.

PB95-182267

00,299

Factors Significant to Precracking of Fracture Specimens.

PB96-109558 03.358

Prediction of the Strength Properties for Plain-Carbon and Vanadium Micro-Alloyed Ferrite-Pearlite Sieel.

PB96-123393

03,216

Artificial Crack in Steel: An Ultrasonic-Resonance-Spec-

troscopy and Modeling Study.

PB96-141395

03,241

STELLAR ATMOSPHERES

Aerodynamic Phenomena in Stellar Atmospheres - A Bibliography.

00,046

Opacity Project and the Practical Utilization of Atomic

Data.

PB94-212214

00,059

Stars, Atmospheres, Radiative Transfe

00,095

Riass Coronathon: Joint $\mathrm{X}$-ray and Ultraviolet Observations of Normal F-K Stars.

tions of Normalt

00,109

STELLAR CHROMOSPHERES

First Results from the Goddard High-Resolution Spectrograph: The Chromosphere of Tauri.

PB94-199528

Transition Regions of Capella.

PB96-123336

Transition Regio

STELLAR CORONAE

Radio Continuum and X-Ray Properties of the Coronae of RS Canum Venaticorum and Related Active Binary Sys-

00.057

PB94-211083

Results

Ultraviolet

PB94-213428

00,064

Distant Future of Solar Activity: A Case Study of beta Hydri. 3. Transition Region, Corona, and Stellar Wind.
PB95-153441

ROSAT All-Sky Survey of Active Binary Coronae. 1. Ouiescent Fluxes for the RS Canum Venaticorum Systems.
PB95-202479

Siellar Coronal Structures.

PB95-202834

00,080

Observing Stellar Coronae with the Goddard High Resolution Spectrograph. I. The dMe Star AU Microscopii.

PB96-102777

00,092

STELLAR CORONAL

ROSAT All-Sky Survey of Active Binary Coronae. 2. Coronal Temperatures of the RS Canum Venaticorum Sys-

00,055

STELLAR CORONAS

Developments in Stellar Coronae.

PB96-176706

00.107

STELLAR DISTANCES

Radiation-Driven Winds of Hot Luminous Stars $X$. The Determination of Stellar Masses Radii and Distances from Terminal Velocities and Mass-Loss Rates. PB94-213022

00,060

STELLAR ENVELOPES

Opacity Project and the Practical Utilization of Atomic Data.

PB94-212214

00,059

STELLAR EVOLUTION

Distant Future of Solar Activity: A Case Study of Beta Hydri. 3. Transition Region, Corona, and Stellar Wind.

00,049

Distant Future of Solar Activity: A Case Study of beta Hydri. 3. Transition Region, Corona, and Stellar Wind. PB95-153441

00,074

STELLAR FLARES

Rotational Modulation and Flares on RS Canum Venaticorum and BY Draconis Stars. XVI. IUE Spectros- copy and VLA Observations of C1182(=V 1005 Orionis) in October 1983.

00,050

Dynamic Phenomena on the RS Canum Venaticorum Binary II Pegasi in August 1989. 1. Observational Data.
PB94-211067

$X$-rays from Stellar Flares.

PB94-213436

00,065

Far-Ultraviolet Flare on a Pleiades G Dwart

00,086

STELLAR MAGNETIC FIELDS

Search for Radio Emission from the 'Non-Magnetic' Chemically Peculiar Stars.

PB96-102249

00,087

$X$-ray Emission from Chemically Peculiar Stars.

00,088

Relationship between Radiative and Magnetic Fluxes for Three Active Solar-Type Dwarfs

PB96-119540

00,097

STELLAR MASS

Radiation-Driven Winds of Hot Luminous Stars X. The Determination of Stellar Masses Radii and Distances from Terminal Velocities and Mass-Loss Rates. PB94-213022

00,060

\section{STELLAR MASS ACCRETION}

Discussion: Statistical Signal Processing of Ouasiperiodicities. PB96-119532

00,096

STELLAR MASS EJECTA

High Velocity Plasm in the Transition Region of Au Mic: A Stellar Analog of Solar Explosive Events.

00,102

STELLAR RADIATION

Efficient Way of Identifying New Active Stars: A VLA Survey of X-ray Selected Active Stellar Candidates.

00,099

STELLAR RADII

Radiation-Driven Winds of $\mathrm{Hot}$ Luminous Stars $X$. The Determination of Stellar Masses Radii and Distances from Terminal Velocities and Mass-Loss Rates. PB94-213022

00,060

\section{STELLAR SPECTRA}

Observing Stellar Coronae with the Goddard High Reso-

lution Spectrograph. I. The dMe Star AU Microscopil.
PB96-102777

00,092

GHAS Observations of Cool, Low-Gravity Stars. 1. The Far-Uitraviolet Spectrum of alpha Orions (M2 lab). $\begin{array}{ll} & 00,094 \\ \text { Sleuthing the Dynamo: HST/FOS Observations of UV }\end{array}$ Emissions of Solar-Type Stars in Young Clusters. PB96-122817

Redshitts in Stellar Transition Regions.

00.098 PB96-123310

00,104

\section{STELLAR TEMPERATURE}

ROSAT All-Sky Survey of Active Binary Coronae. 2. Coronal Temperatures of the RS Canum Venaticorum Sys-

PB94-199601

00,055

A-type and Chen

00,101

Radio and $X$-ray Emissions from Chemically Peculiar Band A-Type Stars: Observations and a Model.

00,103

STELLAR WINDS

Radiation-Driven Winds of Hot Luminous Stars X. The Determination of Stellar Masses Radii and Distances from Terminal Velocities and Mass-Loss Rates.

PB94-213022

00,060

STELLAR X-RAYS

$X$-rays from Stellar Flares.

PB94-213436

00,065

STELLER FLARES

Rotational Modulation and Flares on RS Canum Venaticorum and BY Draconis Stars. XVIII. Coordinated VLA, ROSAT, and IUE Observations of RS CVn Binaries.

\section{STEP RESPONSE}

Uncertainties of Frequency Response Estimates Derived rom Responses to Uncertain Step-Like Inputs. PB97-111843

01,984

STEP (STANDARD FOR THE EXCHANGE OF PRODUCT

\section{DEL DATA}

Structural EXPRESS Editor.

PB94-159795

02,769

Issues and Recommendations for a STEP Application Protocol Framework. National PDES Testbed.

02,770

Challenges to the National Information Infrastructure: The Challenges to the National Information Infrastruclure: The
Barriers to Product Data Sharing. National PDES Testbed Barriers to Prod

Report Series.
PB95-136347

02,776

STEP On-Line Information Service (SOLIS). The IGES/ PDES Organization.
PB95-137790

SGML Environment for STEP.

02,777

02,778

Initial NIST Testing Policy for STEP: Beta Testing Program for AP 203 Implementations. National PDES Testbed Report Series.

02,779

Extensions of the Prototype Application Protocol of Ready-lo-Wear Apparel Pattern Making.

Guidelines for the Development of Mapping Tables.

PB96-154539 02,786

STandard for the Exchange of Product Model Data (STEP): Procedures for NIST STEP Validation.

PB96-154976

02,787

Group 1 for the Plant Spatial Configuration STEP Appli-

cation Protocol.

02,789

Machining Process Planning Activity Model for Systems

PB96-165428 02,841

Procedure for Product Data Exchange Using STEP Developed in the AutoSTEP Pilot.

PB96-183058

02,843

Application Protocol Intormation Base World Wide Web

Gateway.

$96-202320$

02,791

Interoperability Requirements for CAD Data Transfer in the AutoSTEP Project.

PB97-114268

02,796

Capabilities for Product Data Exchange.

02,798

STEREOCHEMISTRY

Orbital Stereochemistry: Discovering the Symmetries of Collision Processes.

PB95-202800

01,034

Isolation and Structural Elucidation of the Predominant

Geometrical Isomers of alpha-Carotene.

PB96-190061

00,640

STERILIZATION

Effect of Three Sterilization Techniques on Finger Plug.

PBers. 216090

00,150

Radiation Process Data: Collection, Analysis, and Inter-

pretation.

STIFFNESS

Polymer-Induced Entropic Forces and the Dimensions of Congued Polymers.

TOCHASTIC ANALYSIS

Continuous Recording and Stochastic Analysis of PD.

PB96-112156

01,203

TOCHASTIC DIFFERENTIAL

Stochastic Modeling of a New Spectrometer.

PB96-157870

01,925

TTOCHASTIC PROCESSES 
STRANDINGS

Stranding Experiments on Double Hull Tanker Structures. PB96-123112

STRANGE ATTRACTORS

Fluctuations in Probability Distribution on Chaotic Attractors.

PB96-102330

04,022

STRANGENESS FLOW

Strangeness Flow Difference in Nuclear Collisions at $15 \mathrm{~A}$ and $200 \mathrm{~A} \mathrm{GeV}$

04,042

STRATEGIC PLANNING

Electronic Access: Blueprint for the National Archives and Records Administration.

PB95-219218

02,731

STRATIFICATION

Reconstructing Stratified Fluid Flow from Reciprocal Scattering Measurements.

PB95-107256

04,202

TTRESS ANALYSIS

Effects of Elastic Stress on Phase Equilibrium in the Ni-V System.
PB94-172707

03,313

Effect of Transformation of Alloy on Transient and Residual Stresses in a Porcelain-Metal Strip.

PB94-198397

00,143

Application of a Simple Technique for Estimating Errors of Finite-Element Solutions Using a General-Purpose

PB94-200250

04,818

Generalized Plane Strain Analysis of a Bimaterial Com-

posite Containing a Free Surface Normal to the Intertace.

Critical-Current Degradation in Nb3 Al Wires Due to Axial and Transverse Stress.

02,226

Safety Assessment of Railroad Wheels by Residual Stress Measurements.

PB96-141114

04,855

TRESS CORROSION

Assessment of Technology for Detection of Stress Corrosion Cracking in Gas Pipelines. Final Report, July 1993March 1994.

PB94-206646

02,475

Environmentally

03,046

STRESS CORROSION CRACKING

Evidence of Film-Induced Cleavage by Electrodeposited Rhodium.

PB95-162327

03,191

STRESS MEASUREMENT

Ultrasonic Measurement of Residual Stress in Railroad Wheel Rims.

PB95-140430

04,849

Ultrasonic Methods.

02,707

TRESS TENSORS

Notion of a xi-Vector and a Stress Tensor for a General Class of Anisotropic Diffuse Interface Models.

04,788

TRESSES

Catastrophic Failures Propagate Field of Fracture MePBanics. 135140

03,217

STRIPED BASS

Histopathology, Blood Chemistry, and Physiological Status of Normal and Moribund Striped Bass ('Morone saxatilis') Involved in Summer Mortality ('Die-Off') in the Sacramento-San Joaquin Delta of California.

00,034

STRONTIUM

Collisional Energy Transter between Excited-State Strontium and Noble-Gas Atoms.

03,995

Oscillator Strengths and Radiative Branching Ratios in Atomic Sr.

04,008

STRONTIUM IONS

Fine Structure of Negative Ions of Alkaline-Earth-Metal Atoms.

PB94-211182

03,837

STRONTIUM TITANATES

Dielectric Properties of Single Crystals of $\mathrm{Al} 2 \mathrm{O} 3, \mathrm{LaAlO} 3$, $\mathrm{SrTiO3}$, and $\mathrm{MgO}$ at Cryogenic Temperatures.

PB95-180477

02,266

Dielectric Properties of Thin Film $\mathrm{SrTiO}_{3}$ Grown on $\mathrm{LaAlO}$ with $\mathrm{YBa}$ CU3O7-x Electodes.

02,267 PB95-181160

Foias-Temam Approximations of Attractors for Galloping Oscillators.

04,817

Structural Analysis in Context.

PB95-151817

00,437

Model Precast Concrete Beam-to-Column Connections Subject to Cyclic Loading

00,438
Partially Prestressed and Debonded Precast Concrete Par-Column Joints.

00,439

Seismic Performance Behavior of Precast Concrete Beam-Column Joints.

00,440

Assessment of 'Peaks Over Threshold' Methods for Estimating Extreme Value Distribution Tails.

PB95-161360

00,441

Gust Factors Applied to Hurricane Winds.

00,446

Workgroup Summary Report: Plastic Hinge-Based Techniques for Advanced Analysis.

PB96-159702

00,470

STRUCTURAL CERAMICS

Database Development and Management (Project A.2.2):

The Annual Report for 1992-1993.

Role of Corrosion in a Material Selector Expert System for Advanced Structural Ceramics.

03,099

TRUCTURAL CERAMICS DATABASE SYSTEM

Structural Ceramics Database. Topical Report, June 1989-May 1991

STRUCTURAL CHEMICAL ANALYSIS

03,060

Carbon Acidities of Aromatic Compounds. 1. Effects of InRing Aza and External Electron-Withdrawing Groups.
PB94-216595

00,860

STRUCTURAL COMPONENTS

High Temperature Degradation of Structural Composites.

03,732

STRUCTURAL DESIGN

Strengthening Methodology for Lightly Reinforced Concrete Frames: Recommended Design Guidelines for Strengthening with Infill Walls.

PB95-260725

00,454

STRUCTURAL ENGINEERING

Wind and Seismic Effects. Proceedings of the U.S.-Japan Cooperative Program in Natural Resources Panel on Wind and Seismic Effects (26th). Held in Gaithersburg Maryland on May 17-20, 1994

PB95-147385

Think Metric.

00,433

PB95-151825

01,298

Algebraic Approximation of Attractors for Galloping Oscillators.

PB95-162897

04,820

Lessons from

00,442

Structural Ceramics Database. Topical Report, June 1989-May 1991

03,060

Exits in Multistable Systems Excited by Coin-Toss Square-Wave Dichotomous Noise: A Chaotic Dynamics

Approach.
PB96-160650

04,824

Wind and Seismic Effects: Proceedings of the Joint Meeting of the U.S.-Japan Cooperative Program in Natural Resources Panel on Wind and Seismic Effects (28th). Held in Gaithersburg, Maryland on May 14-17, 1996. PB97-104376

00,476

STRUCTURAL FAILURE

Ashland Tank Collapse Investigation.

PB95-126314

02,481

Ashland Tank-Collapse Investigation: Closure by Authors.

PB95-126322 02,482

Proceedings: Workshop on Research Needs in Wind En-

gineering. Held in Gaithersburg, Maryland on September $2-13,1994$

PB95-189528

00,448

STRUCTURAL PROPERTIES

Microwave Spectrum and Structure of $\mathrm{CH}_{3} \mathrm{NO} 2-\mathrm{H} 2 \mathrm{O}$. AD-A296 $377 / 5$

00,719

STRUCTURAL RESPONSE

Seismic Strengthening of Reinforced Concrete Frame Buildings.
PB95-10884

00,430

STRUCTURAL VIBRATION

Dynamic Characteristics of Five Tall Buildings during Strong and Low-Amplitude Motions.

00,427

Response of Buildings to Ambient Vibration and the Loma Prieta Earthquake: A Comparison.

00,457

Method of Estimating the Parameters of Tuned Mass Dampers for Seismic Applications.

00,473

STRUCTURED QUERY LANGUAGE

SOL Environments. Category: Software Standard; Subcategory: Database.

STRUTS

01,801

Composite Struts for SMES Plants.

STYRENES

Acrylonitrile-Butadiene-Styrene (ABS) Plastic Drain, Waste, and Vent Pipe and Fittings.

$A D-A 310724 / 0$

00,327

SUB-DOPPLER FREQUENCIES

Sub-Doppler Frequency Measurements on OCS at 87 $\mathrm{THz}(3.4 \mathrm{mu} \mathrm{m})$ with the CO Overtone Laser.

04,330

SUBAMBIENT TEMPERATURE CONTROL

Device for Subambient Temperature Control in Liquid Chromatography
PB95-140604

00,573

SUBCRITICAL CRACK GROWTH

Transient Subcritical Crack-Growth Behavior in Trans-

ormation-Toughened Ceramics.

PB94-200656

SUBJECT INDEXING

Preliminary Subject and Authors Index to Compilations of Data on Properties of Materials.

AD-A302 669/7

03,242

SUBMARINES

Outline of a Multiple Dimensiona! Reference Model Archiecture and a Knowledge Engineering Methodology for Inlelligent Systems Control.

PB95-220414

03,703

PB95-251633

03,748

Operator Experience with a Hierarchical Real-Time Control System (RCS)

SUBMERGED ARC WELDING

Effects of Copper, Nickel and Boron on Mechanical Properties of Low-Alloy Steel Weld Metals Deposited at High Heat Input.

PB96-135231

SUBROUTINE LIBRARIES

Review of Mathematical Function Library for Microsoft FORTRAN, John Wiley and Sons, 1989

PB94-160793

01,679

Software Libraries, Numerical and Statistical.

01,689

VENTCF2: An Algorithm and Associated FORTRAN 77 Subroutine for Calculating Flow through a Horizontal Ceiling/Floor Vent in a Zone-Type Compartment Fire Model. PB94-210127

SUBSTRATE SPECIFICITY

Substrate Specificity of the Escherichia coli Endonuclease III: Exicision of Thymine- and Cytosine-Derived Lesions in DNA Produced by Radiation-Generated Free Radicals PB95-153425

SUBSTRATES

Substrate and Thin Film Measurements.

PB96-112297

03,535

SUBSURFACE CONDUCTORS

Gradiometer Antennas for Detection of Long Subsurface Conductors.

SUBTILISINS

Analysis of Protein Metal Binding Selectivity in a Cluster

PB94-212990

00,845

SUCROSE PERMEASE

Glucose Permease of Bacillus Subtilis Is a Single Polypeptide Chain That Functions to Energize the Sucrose Permease.

PB95-163192 
SULFUR DIOXIDE

Sulfur Dioxide Capture in the Combustion of Mixtures of Lime, Refuse-Derived Fuel, and Coal.

PB94-155587

02,534

High-Resolution Measurements of the nu2 and 2nu2-nu2 Bands of (34)S(16)O2.

PB94-216223

00,855

Reanalysis of the $(010),(020),(100)$, and (001) Rotational Levels of (32)S(16)O2

PB95-12562

00.887

SULFUR FLUORIDES

Collision-Induced Neutral Loss Reactions of Molecular Dications.

00,780

Investigation of S2F10 Production and Mitigation in Compressed SF6-Insulated Power Systems.

02,467

Associative Electron Attachment to S2F10, S2OF10, and $\mathrm{S} 2 \mathrm{O} 2 \mathrm{~F} 10$

00,907

Electron Scattering and Dissociative Attachment by SF6 and Its Electrical-Discharge By-Products.

02,256

Investigation of S2F10 Production and Mitigation in Compressed SF6- Insulated Power Systems.

PB96-155528

02,468

SULFUR HEXAFLUORIDE

Operational Mode and Gas Species Effects on Rotational Drag in Pneumatic Dead Weight Pressure Gages.

PB95-140182

00,903

Plasma Chemical Model for Decomposition of SF6 in Negative Glow Corona Discharge.

01,020

SF6 Insulation: Possible Greenhouse Problems and Solu

PB95-251625

02,269

Gaseous Dielectrics Research: Possible SF6 Substitutes.

PB96-119268 02,228

Decomposition of SF6 and Production of S2F10 in Power

01,084

Decomposition of Sulfur Hexafluoride by $X$-rays.

01.095

PB96-135314

Chemical and Microbiological Problems Associated with Research on the Biodesulfurization of Coal

PB95-140950

02,484

SULFUR IONS

Crossed-Beams Measurements of Absolute Cross Secions for Electron Impact Ionization of S(+)

PB95-2025 11

03,981

SULFUR-LIKE IONS

Labeling Conventions in Isoelectronic Sequences - Reply.
PB95-162574

SULFUR ORGANIC COMPOUNDS

Chemical and Microbiological Problems Associated with Research on the Biodesulfurization of Coal.

$$
\text { PB95-140950 }
$$

SULFUR OXOFLUORIDES

Associative Electron Attachment to S2F10, S2OF10, and $\mathrm{S} 2 \mathrm{O} 2 \mathrm{~F} 10$

PB95-140992

00,907

SULPHUR HETEROCYCLIC COMPOUNDS

Ideal Gas Thermodynamic Properties of Sulphur Heterocyclic Compounds.

01,110

SUM RULES

Drill-Hearn-Gerasimov Sum Rule.

PB94-211752

03,845

Use of Sum Rules on the Energy-Loss Function for the Evaluation of Experimental Optical Data.

PB95-150736

04,264

SUPERCOMPUTERS

Distributed Supercomputing Software: Experiences with the Parallel Virtual Machine - PVM.

01,680

PB94-163086

Nonlinear Color Transformations in Real Time Using a
Video Supercomputer.
PB96-123021
02,191 PB96-123021

02,19

SUPERCONDICTIVITY

Electrical Characterization of Narrow Gap n-Type Bulk $\mathrm{HgCdTe}$ Single Crystals by Variable-Magnetic-Field Hall Measurements and Reduced-Conductivity-Tensor AnalyPB96-164199

01,146

SUPERCONDUCTERS

Temperature and Field Dependence of Flux Pinning in NbTi with Arititicial Pinning Centers.

04,726

SUPERCONDUCTING

High-Energy Phonon Dispersion in La 1.85Sro.15CuO 4.
PB96-138458

SUPERCONDUCTING DEVICES

Roles of Copper in Applied Superconductivity.
PB94-211521

02,255

Far-Intrared Kine
PB95-126348

02,142
Superconducting Kinetic Inductance Radiometer.
PB95-140083

Thermal and Nonequilibrium Responses of

Superconductors for Radiation Detectors.

PB95-164232 02,156

Terahertz Detectors Based on Superconducting Kinetic Inductance.

PB95-168647 02,160

Optical Performance of Photoinductive Mixers at Terahertz Frequencies.

02,161

Ultrasensitive-Hot-Electron Microbolometer.

02,163

Electronics and Electrical Engineering Laboratory TechElectronics and Electrical Engineering Laboratory Technical Progress Bulletin Covering Laboratory Programs,
July to September 1994 with 1994/1995 EEEL Events

PB95-170395

02,360

Experimental Results on Single Flux Quantum Logic

02,053

Characterization of a Tunable Thin Film Microwave YBa2Cu3O7-x/SrTiO3 Coplanar Capacitor.

PB95-175527

02,264

Electronics and Electrical Engineering Laboratory Technical Publication Announcements Covering Laboratory Programs, January to March 1995 with 1995 EEEI Events Calendar.

PB95-242277

02,373

\section{UPERCONDUCTING FILMS}

YBa2Cu3O7-x to Si Interconnection for Hybrid Superconductor/Semiconductor Integration

Influence of Deposition Parameters on Properties of Laser Ablated YBa2Cu3O7-Delta Films. PB95-140539 04,544 Critical Current Behavior of Ag-Coated YBa2Cu3O7-x Thin Films.

PB95-141016 04,549

Magnetic Field Dependence of the Critical Current Anisotropy in Normal Metal-YBa2Cu3O7-delta Thin Film

04,550

Insulating Nanoparticles on YBa2Cu3O7-delta Thin Films Insulating Nanoparticles on YBa2Cu3O7-delta Thin Films
Revealed by Comparison of Atomic Force and Scanning Tunneling Microscopy.

PB95-150843

04,575

Effects of Anneal Time and Cooling Rate on the Formation and Texture of Bi2Sr2CaCu2O8 Films.

04,603

Growth of Laser Ablated YBa2Cu3O7-delta Films as ExGrowth of Laser Ablated YBa2Cu3O7-delta Films as Examined by Rheed and Scanning Tunneling Microscopy.
PB95-162541 PB95-162541 04,614 Films by Atomic Force Microscopy.

PB95-163358

04,622

Tunneling Measurement of the Zero-Bias Conductance Peak and the Bi-Sr-Ca-Cu-O Thin-Film Energy Gap. PB95-163739

04,628

Analytic Calculation of Polarized Neutron Reflectivity from Superconductors.

PB95-164224

04,629

Reactive Coevaporation of DyBaCuO Superconducting Films: The Segregation of Buik Impurities on Annealed $\mathrm{MgO}(100)$ Substrates.

04.635

High Current Pressure Contacts to Ag Pads on Thin Film Superconductors.

PB95-168621

04,639

Microwave Properties of YBa2Cu307-x Films at $35 \mathrm{GHz}$ from Magnetotransmission and Magnetoreflection Measurements.

Surface Degradation of Superconducting $\mathrm{YBa} 2 \mathrm{Cu} 3 \mathrm{O} 7$ delta Thin Films.

PB95-176095

04,667

Increased Transition Temperature in In situ Coevaporated YBa2Cu3O7-delta Thin Films by Low Temperature PostAnnealing.

PB95-18007

04,672

Novel YBa2Cu3O7-x and YBa2Cu3O7-x/4Ba3O9 Multilayer Films by Bias-Masked 'On-Axis' Magnetron SputterPBg5-181186

04,690

Surface Modification of YBa2Cu3O7-delta Thin Films Using the Scanning Tunneling Microscope: Five Methods. PB95-203394

04,699

UPERCONDUCTING GRAVIMETERS

Calibration of a Superconducting Gravimeter Using Absolute Gravity Measurements.

PB95-20265

03,684

SUPERCONDUCTING JUNCTIONS

Half-Integral Constant Voltage Steps in High-Tc Grain

Boundary Junctions

04,489

Tunneling Measurement of the Zero-Bias Conductance Peak and the Bi-Sr-Ca-Cu-O Thin-Film Energy Gap. PB95-163739 04,628 Hot-Electron Microcalorimeters for $\mathrm{X}$-ray and Phonon De-

PB95-168993

04,644

Hot-Electron Microcalorimeters as High-Resolution X-ray PBectors.

Hot-Electron-Microcalorimeters with $0.25 \mathrm{~mm}(2)$ Area.

PB96-200670

04,793

SUPERCONDUCTING MAGNETIC ENERGY STORAGE

Composite Struts for SMES Plants.

PB95-155586

SUPERCONDUCTING MAGNETS

02,507

Electromechanical properties of superconductors for DOE fusion applications.

DE95015476

04,432

Electromechanical Properties of Superconductors for DOE Fusion Applications.

PB94-139672

02,250

Irradiation Damage in Inorganic Insulation Materials for TER Magnets: A Review.

03,705

Cryogenic Properties of Inorganic Insulation Materials for

ITER Magnets: A Review.
PB95-198768

03,706

SUSAN: SUperconducting Systems ANalysis by LOW PB96-112065

04,728

SUPERCONDUCTING NETWORKS Automated Josephson Integrated Circuit Test System.
PB95-175246

02,057

SUPERCONDUCTING SUPER COLLIDER

Magnetic Characteristics and Measurements of Filamentary Nb-Ti Wire for the Superconducting Super Collider. DE94005988

03,775

SUPERCONDUCTING WIRES

Magnetic Characteristics and Measurements of Filamenlary Nb-Ti Wire for the Superconducting Super Collider. DE94005988

03,775

Volume Magnetic Hysteresis Loss of Nb3Sn Superconductors

04,597

Superconductor Critical Current Standards for Fusion Applications. Final Progress Report, October 1993-July 1994

PB95-169538

02,222

Reduction of Interfilament Contact Loss in Nb3Sn Superconductor Wires.

02,223

Critical-Current Degradation in Nb3 Al Wires Due to Axial and Transverse Stress.

02,226

VAMAS Intercomparison of Critical Current Measurements on NB3Sn Superconductors: A Summary Report. ments on NB3Sn Superconductors: A Summary Report.
PB96-119763 04,043

Anomalous Switching Phenomenon in Critical-Current Measurements When Using Conductive Mandrels. PB96-137781

02,233

SUPERCONDUCTIVITY

Roles of Copper in Applied Superconductivity. 


\section{KEYWORD INDEX}

SURFACE ANALYSIS

Research Workshop on Magnetic Susceptibility of Superconductors and Other Spin Systems.

04,435

PB94-145984

Raman and Fluorescence Spectra Observed in Laser Microprobe Measurements of Several Compositions in the Ln-Ba-Cu-O System.

04,440

Dispersions of Magnetic Excitations of the $\mathrm{Pr}$ lons in Pr2CuO4.

PB94-173044

04,444

Neutron-Scattering Study of $\mathrm{C} 60(\mathrm{n}-)(\mathrm{n}=3,6)$ Librations in Alkali-Metal Fullerides.

00,806

PB94-200219

Type-II

Superconductors in the Mixed State.

PB94-200359

04,483

Nonlinear Response of Type-II Superconductors in the

Mixed State in Slab Geometry.

04,484

Superconducting Materials: Specification

PB94-211299

04,491

Flux Expulsion at Intermediate Fields in Type-II Superconductors.

04,502

Offset Susceptibility of Superconductors

PB94-212263

04,503

Correlation between TC and Elastic Constants of (LaM) $2 \mathrm{CuO} 4$.

04,514

$n-V a l u e$ and Second Derivative of the Superconductor Voltage-Current Characteristic.

PB95-126223

Superconducting Energy Gap of Bulk UBe13.

Phonon Density of States in $\mathrm{R}_{2} \mathrm{CuO}_{4}$ and Superconducting R1.85Ce0.15CuO4 (R = Nd, Pr).
PB95-150686

Even-Odd Asymmetry of a Superconductor Revealed by the Coulomb Blockade of Andreev Reflection.

PB95-153540

04,593

Magnetic Force Microscopy of Flux in Superconductors

PB95-161733 04,608

New Exact Solution of the One-Dimensional Schrodinger Equation and Its Application to Polarized Neutron Reflectometry.

04,609

Description of Layered Structures: Applications to High Tc Superconductors.

04,615

Critical Current Density, Irreversibility Line, and Flux Creep Activation Energy in Silver-Sheathed PB95-162749

04,616

Magnetocaloric Effect in Nanocomposites.

04,618

Ca1-xCuO2, a NaCuO2-Type Related Structure.

PB95-162822

04,620

Analytic Calculation of Polarized Neutron Reflectivity from Superconductors.

04,629

Alternating-Field Susceptometry and Magnetic Susceptibility of Superconductors.

PB95-168613

04,638

Crossover in the Pinning Mechanism of Anisotropic PBluxon Cores.

Magnetic Susceptibility of $\mathrm{Pr} 2-x \mathrm{CexCuO} 4$ Monocrystals and Polycrystals.

04,677

Observation of Oscillatory Magnetic Order in the
Antiferromagnetic Superconductor HoNi2B2C.

Lattice Dynamics of Semiconducting, Metallic, and Superconducting Ba1-xKxBiO3 Studied by Inelastic Neutron Scattering.
PB96-102447

04,708

$30 \mathrm{THz}$ Mixing Experiments on High Temperature Superconducting Josephson Junctions. Electronic Microrefrigerator Based on a Normal-Insulator-

Superconductor Tunnel Junction.
PB96-102827

04,718

Superconducting Integrated Circuit Fabrication with Low Temperature ECR-Based PECVD SiO2 Dielectric Films.

PB96-103015 04,719

Comparing the Accuracy of Critical-Current Measurements Using the Voltage-Current Simulator.

PB96-119219

02,227

High-Temperature Superconductor Cryogenic Current Comparator.

02,074

Simple and Repeatable Technique for Measuring the Critical Current of Nb3Sn Wires.

02,229

Effect of Sm2BaCuO5 on the Properties of Sintered (Bulk) YBa2Cu3O6+x.

04,733
Thermal Isolation of High-Temperature Superconducting Thin Films Using Silicon Wafer Bonding and Micromachining.
PB96-135017

02,408

Mutual Phase Locking in Systems of High-Tc Superconductor-Normal Metal-Superconductor Junctions.
PB96-135348

rties of

Quench Energy and Fatigue Degradation Properties of

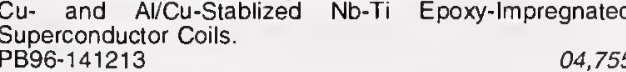

II-3: Critical Current Measurement Methods: Quantitative
Evaluation. Evaluation.

04,767

II-5: Thermal Contraction of Materials Used in Nb3Sn Critical Current Measurements.

04,769

USA Interlaboratory Comparison of Superconductor Simulator Critical Current Measurements. ulator Critical Current Measurements.

V-6: Effects of Temperature Variation

04,770

PB96-148143

04,772

Metallic-Barrier Junctions for Programmable Josephson Voltage Standards.

PB96-200134 02,089

Superconductor- Normal-Superconductor Junctions for Digital/Analog Converters

PB96-200233

02,092

Elastic Constants and Microcracks in $\mathrm{YBa} 2 \mathrm{Cu} 3 \mathrm{O} 7$.

PB96-200761,005

Neutron Scattering Study of Antiferromagnetic Order in the Magnetic Superconductors RNi2B2C.

PB97-112411

04,812

Vortex Images in Thin Films of YBa2Cu3O(sub 7-x) and Bi2Sr2Ca1Cu2O(sub $8+x$ ) Obtained by Low-Temperature Magnetic Force Microscopy.

UPERCOOLED LIQUIDS

Simulations of Glass Forming Liquids: What Has Been

PB95-150124

00,915

Activated Dynamics, Loss of Ergodicity, and Transport in Supercooled Liquids.

00,925

Simulation Studies of Supercooled and Glass Formıng

PB96-122627

01,085

SUPERCOOLING

Length Scales for Fragile Glass-Forming Liquids. 01,065
PB96-102801

Long-Lived Structures in Fragile Glass-Forming Liquids.

PB96-119565 04,212

Simulation Studies of Supercooled and Glass Forming

Liquids.

01,085

SUPERCRITICAL FLUID EXTRACTION

Thermophysical Property Data for Supercritical Fluid Ex-

PB94-199221

00,668

Supercritical Fluid Extraction of Biological Products. PB95-175204

00,040

SUPERCRITICAL FLUID SOLUTIONS

Measurement of Diffusion in Supercritical Fluid Systems: A Review.

PB94-199189

00,795

SUPERCRITICAL FLUIDS

Summary of the Patent Literature of Supercritical Fluid PB94-199213

00,502

Supercritical Solubility of Solids from Near-Critical DiluteMixture Theory
PB94-211703

00,819

Application of the Taylor Dispersion Method in Supercritical Fluids.

00,977

Measurement of Diffusion in Fluid Systems: Applications to the Supercritical Fluid Region.

02,490 Solubility Measurement by Direct Injection of
Supercritical-Fluid Solutions into a HPLC System. Supercritical-Fluid Solutions into a HPLC System. 00,997
PB95-175626

High-Pressure Equilibrium Cell for Solubility Measurements in Supercritical Fluids.

PB95-175634

00,998

Instrument for Evaluating Phase Behavior of Mixtures for Supercritical Fluid Experiments.

PB95-180758

00,606

\section{SUPERCRITICAL STATE}

Measurement of Diffusion in Supercritical Fluid Systems: A Review.

PB94-199189

00,795

Thermophysical Property Data for Supercritical Fluid Extraction Design
PB94-199221

\section{SUPERGIANT STARS}

Four Years of Monitoring alpha Orionis with the VLA: Where Have All the Flares Gone.

PB94-185212

00,048

SUPERLATTICES

Structural and Magnetic Ordering in Iron Oxide/Nickel Oxide Multilayers by $\mathrm{X}$-ray and Neutron Diffraction (In-

PB94-172558

04,442

Enhanced Curie Temperatures and Magnetoelastic Domains in Dy/Lu Super Lattices and Films.

PB94-172665

04,443

Interface Roughness-Induced Changes in the Near-E(sub 0) Spectroscopic Behavior of Short-Period AlAs/GaAs Superlattices.

02,118

Magnetic and Structural Properties of Electrodeposited Copper-Nickel Microlayered Alloys

PB94-213121

04,512

Interface Roughness of Short-Period AlAs/GaAs Superlattices Studied by Spectroscopic Ellipsometry. PB95-107215

Interface Sharpness in Low-Order III-V Superlattices.
PB95-108775

PB95-108775 02,138 Thin Short-Period III-V Superlattices.

PB95-108783

02,139

Effects of Interfacial Roughness on the Magnetoresistance of Magnetic Metallic Multilayers.
PB95-150017

Comment on 'Phase Transitions in Antiferromagnetic Superlattices

PB95-152971

Magnetic Rare Earth Artificial Metallic Superlattices.

PB95-162293

04,587

SUPERMIRRORS

Supermirror Transmission Polarizers for Neutrons.

SUPERNOVA 1957D

Rapid Decline in the Optical Emission from SN 1957D in

PB94-216033

00,070

SUPERNOVA REMNANTS

Rapid Decline in the Optical Emission from SN $1957 D$ in

PB94-216033

00,070

CCD Mosaic Images of the Supernova Remnant $3 \mathrm{C}$

PB95-203527 00,084

G203.2-12.3: A New Optical Supernova Remnant in Orion

PB95-203535

00,085

SUPERONDUCTORS

Reference Relations for the Evaluation of the Materials Properties of Orthorhombic YBa2Cu3OX Superconductors.
PB96-176763

SUPEROXIDE RADICALS

Reaction of NO with Superoxide.

PB94-212198

04,782

SUPERPOSED FLEXURE

Flat and Rising R-Curves for Elliptical Surface Cracks from Indentation and Superposed Flexure.

PB95-161295

03,156

SUPERSTABLE LASERS

Introduction to Phase-Stable Optical Sources.

PB96-122973

04,347

SUPPORTS 
Formation of Technical Committee 201 on Surface Chemical Analysis by the International Organization for Standardization

PB95-108536

00,568

Nanoscale Study of the As-Grown Hydrogenated Amorphous Silicon Surface.

PB95-150595

04,573

Grazing Angle X-Ray Photoemission System for DepthDependent Analysis.

PB95-161154

04,600

Observation of Insulating Nanoparticles on YBCO ThinFilms by Atomic Force Microscopy

PB95-163358

04,622

Reactive Coevaporation of DyBaCuO Superconducting Films: The Segregation of Bulk Impurities on Annealed

$\mathrm{MgO}(100)$ Substrates
PB95-164562

04,635

SURFACE AREA

Total Surface Areas of Group IVA Organometallic Compounds: Predictors of Toxicity to Algae and Bacteria.

PB94-211331

00,814

SURFACE CHEMISTRY

Role of Adsorbed Alkalis in Desorption Induced by Electronic Transitions.

00,762

Surface Forces and Adhesion between Dissimilar Mate-

rials Measured in Various Environments.
PB94-172970

03,033

Contact Electrification Induced by Monolayer Modification of a Surface and Relation to Acid-Base Interactions. PB94-185378

03,034

Peeling a Polymer from a Surface or from a Line.

01,213

Tribochemical Reaction of Stearic Acid on Copper Surface Studied by Surface Enhanced Raman Spectroscopy.
PB94-212057

Structure of Molecules on Surfaces as Determined Using Electron-Stimulated Desorption.

00,852

Deposition of Colloidal Sintering-Aid Particles on Silicon Nitride.

PB94-216272

03,044

Interface Modification and Characterization of Silicon Carbide Platelets Coated with Alumina Particles.

PB95-108734

03,121

Characterization of Chemically Modified Pore Surfaces by Small Angle Neutron Scattering.

PB95-126181

00,898

Silver Metalization of Octadecanethiol Monolayers SelfAssembled on Gold.

PB95-150744

00,923

Time-Resolved Measurements of Energy Transfer at Surfaces.

PB95-153037 00,947

Fundamental Studies of Gas Sensor Response Mechanisms: Palladium on $\mathrm{SnO} 2(110)$

PB95-162731

00,963

Observation of a Stable Methoxy Intermediate on $\mathrm{Cr}(110)$.

00,981

Surface Chemical Interactions of Si3N4 with Polyelectrolyte Deflocculants.
PB95-175576 03,056 Effects of Soxhlet Extraction on the Surface Oxide Layer of Silicon Nitride Powders.

PB Silicon Nit 17584

03,057

Activities of ISO Technical Committee 201 on Surtace Chemical Analysis.

PB95-180824

00,607

Surface Chemistry of Silicon Nitride Powder in the Presence of Dissolved lons.

ence of Dissolved lons.
PB96-111760

Mechano-Chemical Model: Reaction Temperatures in a Concentrated Contact.

PB96-119466 03,227

Silicon Surface Chemistry by IR Spectroscopy in the Midto Far-IR Region: $\mathrm{H} 2 \mathrm{O}$ and Ethanol on Si(100).

PB96-138565

01,097

SURFACE CRACKS

Flat and Rising R-Curves for Elliptical Surface Cracks from Indentation and Superposed Flexure. PB95-161295

03,156

SURFACE DEFECTS

Non-Osmotic, Defect-Controlled Cathodic Disbondment of a Coating from a Steel Substrate.

PB94-216447

03,120

Photodesorption Dynamics of Co from Si(111): The Role of Surface Defects

UAFACE DIFFUSION

Linking Anisotropic Sharp and Diffuse Surface Motion Laws via Gradient Flows.

PB95-203378

04,698

Notion of a xi-Vector and a Stress Tensor for a General Class of Anisotropic Diffuse Interface Models. PB96-193776

04,788

SURFACE EMITTING LASERS

Characterization of Vertical-Cavity Semiconductor Structures.

PB94-200193

02,126
Comparative Photoluminescence Measurement and Simulation of Vertical-Cavity Semiconductor Laser Structures. PB95-169173

Measurement and Simulation of Photoluminescence Spectra from Vertical-Cavity Quantum-Well Laser Struc-

PB95-169181

02,170

SURFACE ENERGY

Singularities in Minimum Surface Energy Problems and Their Influence in Surtace Motion

04,473

Surface Energy Reduction in Fibrous Monotectic Struc-

PB95-140828

03,150

Measuring Contact Charge Transfer at Interfaces: A New Experimental Technique.

03,053

\section{SURFACE EXCHANGE}

Interaction of Citric Acid with Hydroxyapatite: Surface Exchange of lons and Precipitation of Calcium Citrate.

PB97-119309

03,584

SURFACE FIGURE

Upgraded Facility for Multilayer Mirror Characterization at

PB96-160387

04,367

SURFACE FINISHING

Opportunities for Innovation: Advanced Surface Engineering. 176666

02,697

SURFACE INTERACTION

Segmental Concentration Profiles of End-Tethered Polymers with Excluded-Volume and Surface Interactions. PB97-119002

00,654

SURFACE MAGNETISM

Magnetic Moments in $\mathrm{Cr}$ Thin Films on $\mathrm{Fe}(100)$. 04,525

SURFACE METROLOGY

Microform Calibrations in Surface Metrology. PB95-203295

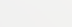

SURFACE MODIFICATION

Surface Modification of YBa2Cu3O7-delta Thin Films Using the Scanning Tunneling Microscope: Five Methods.
04,699

SURFACE MORPHOLOGY

Coarsening of Unstable Surface Features during Fe(001) PB96-186127

04,121

\section{SURFACE PLASMON RESONANCE}

Surface Plasmon Microscopy of Biotin-Streptavidin Binding Reactions on UV-Photopatterned Alkanethiol Self-Assembled Monolayers.

$$
\text { PB96-176771 }
$$

SURFACE PROFILING

Present and Future Standard Specimens for Surface Finish Metrology.

SURFACE PROPERTIES

Temperature Dependence of the Morphology of Thin Diblock Copolymer Films as Revealed by Neutron Reflec-

94-172756

01,199

Interface Properties for Ceramic Composites from a Single Fiber Pull-Out Test.

PB94-199361

03,135

Active Site Ionicity and the Mechanism of Carbonic Anhydrase. 212974

00,843

Characterization of Phase and Surface Composition of Silicon Carbide Platelets.

PB94-216264

03,043

Observed Frustration in Confined Block Copolymers.

PB95-150033

01,238

Modified Surface Layers and Coatings.

03,125

Influence of Surface Charge on the Stochastic Behavior of Partial Discharge in Dielectrics.

Stylus Flight in Surface Profiling.

01,931

PB96-123138

02,675

SURFACE REACTIONS

Dynamics of Nonthermal Reactions: Femtosecond Surtace Chemistry.

00,688

Surface Degradation of Superconducting $\mathrm{YBa} 2 \mathrm{Cu} 307$. delta Thin Films.

04,667

Single Photon Ionization Laser Optical Probe Technique Single Photon lonization, Laser Optical Probe Technique
for Semiconductor Growth.
PB95-202776

01,032

Influence of Films' Thickness and Air Gaps in Surface Impedance Measurements of High Temperature Superconductors Using the Dielectric Resonator Technique.
PB96-157862

\section{SURFACE ROUGHNESS}

Ultrasonic Measurements of Surface Roughness.

PB94-172137

Light Scattering from Glossy Coatings on Paper. PB94-213246 Relation between $A C$ Impedance Data and Degradation of Coated Steel. 1. Effects of Surface Roughness and Contamination on the Corrosion Behavior of Epoxy Coated Stee

PB94-213345

03,189

Determination of Surface Roughness from Scattered

PB94-216520 04,243

Autocorrelation Functions from Optical Scattering for One-Dimensionally Rough Surfaces.

PB94-216538

04,244

Light Scattered by Coated Paper.

04,245

Regimes of Surface Roughness Measurable with Light Scattering.

PB95-151213

04,265

Automated Optical Roughness Inspection.

02,905

Surface Roughness Evaluation of Diamond Films Grown on Substrates with a High Density of Nucleation Sites. PB95-162418

Surface Texture.

PB95-164620

03,351

Nanoscale Study of the Hydrogenated Amorphous Silicon Surtace.

PB96-103056

04,720

Optical Scattering from Moderately Rough Surfaces.

PB97-110415 04,385

SURFACE ROUGHNESS INSTRUMENT CONTROLLERS

Automated Optical Roughness Inspection.

PB95-152179

SURFACE STATES

Tunneling Spectroscopy of bcc(001) Surface States.
PB96-155585

02,905

04,775

SURFACE TEMPERATURE

Analytical Method of Determining the Heat Capacity at High Temperatures from the Surface Temperature of a

Cooling Sphere.
PB94-216124 865

Transient Cooling of a Hot Surface by Droplets Evaporation.

PB95-143194

03,890

Exact Solution of the Steady-State Surface Temperature for a General Multilayer Structure.

PB95-152773

02,337

SURFACE TENSION

Molecular Dynamics Investigation of the Surface/Bulk Equilibrium in an Ethanol-Water Solution.

EQuilibrium in

01,183 


\section{SURFACES \& INTERFACES}

\section{SURFACES \& INTERFACES}

Use of Ion Scattering Spectroscopy to Monitor the Nb Target Nitridation during Reactive Sputtering.

Inelastic Interactions of Electrons with Surfaces: Application to Auger-Electron Spectroscopy and X-ray Photoelectron Spectroscopy

00,764 PB94-172699

Environmental Scanning Electron Microscope Imaging
Examples Related to Particle Analysis. PB94-172822

Surface Forces and Adhesion between Dissimilar Mate-
rials Measured in Various Environments. rials Measured in Various Environments.

03,033

Contact Electrification Induced by Monolayer Modification of a Surface and Relation to Acid-Base Interactions.
PB94-185378 Interfacial Free Energies from Substrate Curvature Measurements of the Creep of Multilayer Thin Films.

PB94-185428

04,448 PBg4-185501

02,252

Compositional Analyses of Surfaces and Thin Films by

Electron and Ion Spectroscopies.
PB94-185790

00,779

Scaling of Diffusion-Mediated Island Growth in Iron-onIron Homoepitaxy
PB94-185923

04,455

$X$-Ray Diffraction from Anodic TiO2 Films: In situ and Ex situ Comparison of the Ti(0001) Face.

00,782

Workshop on Characterizing Diamond Films (3rd). Held in Gaithersburg, Maryland on February 23-24, 1994

PB94-187663

04,456

Microstructure and Ferroelectric Properties of Lead Zirconate-Titanate Films Produced by Laser Evaporation.

PB94-199148

Laser Melting of Thin Silicon Films.

PB94-199239

04,470

Photodecomposition Dynamics of Mo(CO)6/Si(111) $7 x 7$ : CO Internal State and Translational Energy DistributionsPBranslation.

00,687

Singularities in Minimum Surface Energy Problems and Their Influence in Surface Motion

PB94-199411

04,473

Performance of a Reflectron Energy Compensating Mirror.

Time-Resolved Probes of Surface Dynamics.

00,547

PB94-199957

00,803

Dynamics of Nonthermal Reactions: Femtosecond SurPB94-199965

00,688

Reactivity of Pd and Sn Adsorbates on Plasma and Thermally Oxidized $\mathrm{SnO}$ (110)

PB94-199973

00,804

Pure Element Sputtering Yield Data: Appendix 4

PBre-200037

$X$-Ray Photoelectron and Auger Electron Spectroscopy Study of UltravioleVOzone Oxidized P2S5/(NH4)2S Treat ed GaAs (100) Surfaces.

PB94-200144

04,479

Grazing-Incidence X-Ray Photoemission Spectroscopy Investigation of Oxidized GaAs(100): A Novel Approach to Nondestructive Depth Profiling

Perspective on Fiber Coating Technology

04,480

PB94-200540

03,118

Vibrational Relaxation Measurements of Carbon Monoxide on Metal Clusters

PB94-211810

00,820

Tribochemical Reaction of Stearic Acid on Copper Surtace Studied by Surface Enhanced Raman Spectroscopy.
PB94-212057

Surface Core-Level Shifts of Barium Observed in Photoemission of Vacuum-Fractured BaTiO3 (100).

PB94-212156

04,501

Formalism and Parameters for Ouantitative Surface Analysis by Auger Electron Spectroscopy and X-Ray Photoelectron Spectroscopy

PB94-212297

00,832

S Cluster Binding to a GaAs Surface.

00,846

Iridium Oxide Thin-Film Stability in High-Temperature Corrosive Solutions

03,234

Analysis of Boron in CVD Diamond Surfaces Using Neutron Depth Profiling.

04,511

Relation between AC Impedance Data and Degradation of Coated Steel. 1. Effects of Surface Roughness and Contamination on the Corrosion Behavior of Epoxy Coat-

PB94-213345 03,189

Structures of Vapor-Deposited Yttria and Zirconia Thin

03,041
Structure of Molecules on Surfaces as Determined Using Electron-Stimulated Desorption.

00,852

Desorption Induced by Electronic Transitions

00,853

Neutron Scattering Studies of Surfaces and Interfaces

PB94-216207 04,517

Deposition of Colloidal Sintering-Aid Particles on Silicon

03,044

Effect of Modulated Taylor-Couette Flows on Crystal-Melt Interfaces: Theory and Initial Experiments.
PB94-216736 Activities of the ASTM Committee E-42 on Surface AnalyPBis. 108528

00,881

Formation of Technical Committee 201 on Surface Chem. ical Analysis by the International Organization for Standardization.

PB95-108536 00,568

Interface Modification and Characterization of Silicon Carbide Platelets Coated with Alumina Particles.

03,121

Picosecond Measurement of Substrate-to-Adsorbate En. ergy Transfer: The Frustrated Translation of CO/Pt(111)-Translation

PB95-126041

00,895

Use of Kinetic Energy Distributions to Determine the Relative Contributions of Gas Phase and Surface Fragmentation in KeV lon Sputtering of a Ouaternary Ammonium Salt.

Near Critical Fluid Interfaces: A Comparison of Theory and Experiment.

PB95-140166

00,901

Scanning Tunneling Microscopy and Fabrication of Nanometer Scale Structures at the Liquid-Gold Interface.
PB95-140414 00,904

Substitution-Induced Midgap States in the Mixed Oxides RxBa1-ChiTiO3-Delta, with $R=Y, L a$, and Nd.

PB95-140505

04,541

Surface Energy Reduction in Fibrous Monotectic Struc-

PB95-140828 03,150

Time-Resolved Measurements of Energy Transfer at Sur-

PB95-141198

00,913

Spot-Profile-Analyzing LEED Study of the Epitaxial Growth of $\mathrm{Fe}, \mathrm{Co}$, and $\mathrm{Cu}$ on $\mathrm{Cu}(100)$. 04,561
PB95-150165 Influence of $\mathrm{Cr}$ Growth on Exchange Coupling in $\mathrm{Fe} / \mathrm{Cr}$ /
$\mathrm{Fe}(100)$. PB95-150181

04,566
Exchange Coupling in Magnetic Heterostructures.
PB95-150314

04,567

PB95-150322

Scanning Tunneling Microscopy Study of the Growth of
$\mathrm{Cr} / \mathrm{Fe}(001)$ : Correlation with Exchange Coupling of Mag$\mathrm{Cr} / \mathrm{Fe}(001)$ : Correlation with Exchange Coupling of Mag-
netic Layers.
PB95-150330 Calculations of Electron Inelastic Mean Free Paths. 5 .
Data for 14 Organic Compounds over the $50-2000 \mathrm{eV}$ PB95-150355

00,916

Oscillatory Exchange Coupling in Fe/Au/Fe(100).
PB95-150371 Neutron Reflectometry Studies of Surface Oxidation.

PB95-150421 00,917

Silver Metalization of Octadecanethiol Monolayers SelfAssembled on Gold

PB95-150744

00,923

Manipulation of Adsorbed Atoms and Creation of New Structures on Room-Temperature Surfaces with a Scanning Tunneling Microscope.

PB95-151536

04,578

Time-Resolved Measurements of Energy Transfer at SurPBaces. 153037

00,947

Grazing-Incidence X-Ray Photoelectron Spectroscopy: A Novel Approach to Thin Film Characterization.

PB95-153128

Epitaxial Integration of Single Crystal C60.

PB95-153490 04,592

Semiclassical Explanation of the Generalized RamsauerTownsend Minima in Electron-Atom Scattering.

PB95-153532

03,925

Laser-Focused Atomic Deposition.

PB95-161618 04,604

Tunneling Stabilized Magnetic-Force Microscopy.

PB95-161717

04,607

Thermoacoustic Technique for Determining the Interface and/or Interply Strength in Polymeric Composites.
PB95-161824 Production and Characterization of Ion Beam Sputtered

Multilayers.
PB95-162053

03,936

Laser-Induced Desorption of NO from Si(111): Effects of Coverage on NO Vibrational Populations.

00,959

Lineshape Analysis of the Raman Spectrum of Diamond Films Grown by Hot-Filament and Microwave-Plasma Chemical-Vapor Deposition

PB95-162392

03,016

Studies of Defects in Diamond Films and Particles by

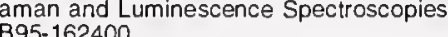

03,017

Sorption of Moisture on Epoxy and Alkyd Free Films and Coated Steel Panels.

03,192

Fundamental Studies of Gas Sensor Response Mechanisms: Palladium on $\mathrm{SnO} 2(110)$

PB95-162731

00,963

Observation of Insulating Nanoparticles on YBCO Thin-

Films by Atomic Force Microscopy.
PB95. 163358

04,622

Observation of a Stable Methoxy Intermediate on

Cr(110).
PB95-164422

00,981

Reactive Coevaporation of $\mathrm{DyBaCuO}$ Superconducting Films: The Segregation of Bulk Impurities on Annealed $\mathrm{MgO}(100)$ Substrates.

04,635

Surface Texture.

PB95-164620

03,351

Characterization of the ZnSe/GaAs Interface Layer by PB95-175360

04,655

Surface Chemical Interactions of Si3N4 with Polyelectrolyte Deflocculants.

03,056

PB95-175576

Layer

Extraction

PB95-175584

03,057

Micromagnetic Scanning Microprobe System

PB95-176178

02,224

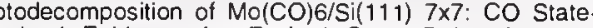
Resolved Evidence for Excited State Relaxation and Quenching.

01,009

$X$-ray Photoelectron and Auger Electron Forward Scattering: A Structural Diagnostic for Epitaxial Thin Films.

Rayleigh Instability for a Cylindrical Crystal-Melt Interface.

PB95-180667
01,010

Activities of ISO Technical Committee 201 on Surface Chemical Analysis.

Water Adsorption at Polymer/Silicon Wafer Interfaces

PB95-181178 01,022

Stability and Surface Energies of Wetted Grain Bound-

aries in Aluminum Oxide.

Microform Calibrations in Surface Metrology

03,059

Water Adsorption at a Polyimide/Silicon Wafer Interace

PB96-103197

Photodesorption Dynamics of CO from Si(111): The Role

of Surface Defects.
PB96-111646 03,066

Surface Chemistry of Silicon Nitride Powder in the Presence of Dissolved lons. Metalorganic Chemical Vapor Deposition. Anisotropy of the Surfaces of Pores in Plasma Sprayed Alumina Deposits.

PB96-123211 


\section{KEYWORD INDEX}

Surface Plasmon Microscopy of Biotin-Streptavidin Binding Reactions on UV-Photopatterned Alkanethio Self-As sembled Monolayer

PB96-17677

01,158

Workshop on Characterizing Diamond Films (4th). Held in Gaithersburg, Maryland on March 4-5, 1996

$\mathrm{Fe}(001)$

Homoeptaxy

PB96-186127

04,121

Adhesion, Contact Electrification, and Acid-Base Properties of Surfaces.

03,693

Thin-Film Ruthenium Oxide - Iridium Oxide Thermocouples.

00,520

Molecular Dynamics Investigation of the Surface/Bulk Equilibrium in an Ethanol-Water Solution.

PB97-113112

01,183

SURFACTANTS

Effects of Surface-Active Resins on Dentin/Composite Bonds

PB95-140448

00,156

SURGES

Coordinating Cascaded Surge Protection Devices: HighLow versus Low-High

02,463

Guarding Against Transients,

01,623

Cascading Surge-Protective Devices: Options for Effective Implementation.

PB94-216488 02,464

Coordinating Cascaded Surge-Protective Devices: An

PB94-216496

02,465

Important Link in Entire-House Protection: Surge Reference Equalizers.

02,219

Surging the Upside-Down House: Looking into Upsetting Reference Voltages.

PB96-112313 02,385

Keeping Up with the Reality of Today's Surge Environment.

02,231

Surging the Upside-Down House: Measurements and Modeling Results

URVEYING

METRICATION: An Economic Wake-Up Call for Surveyors and Mappers.

PBVEYING (GEOGRAPHIC)

Metrication.

PB94-172079

03,680

SURVEYS

Survey of Standards for the U.S. Fiber/Textile/Apparel Industiy.

PB96-193792

03, 199

Post-Occupancy Evaluation of the Forrestal Building

PB97-111298

00,280

SUSCEPTOMETERS

Alternating-Field Susceptometry and Magnetic Susceptibility of Supercondoctors. Presented at Office of Naval Research Workshop on Magnetic Susceptibility of Superconductors and Other Spin Systems. Held in Berkeley Springs, West Virginia on 20 May 1991.

PB94-145984

04,435

SUSPENSIONS

Contrast Matched Studies of a Sheared Binary Colloidal Suspension.

00,918

Partial Scattered Intensities from a Binary Suspension of Polystyrene and Silica.

PB95-175618

00,996

Generalized Stokes-Einstein Equation for Spherical Particle Suspensions.

PB95-202743

01,031

SWEDEN

Ceramic Powders Characterization: Results of an International Laboratory Study.

national Laborat

02,672

SWIMMING

Intensive Swimming: Can It Affect Your Patients' Smiles. PB96-123666

03,570

SWIRLING

Structure of a Swirl-Stabilized Kerosene Spray Flame. PB95-108569

02,480

SWITCHES

Noise Reduction in Low-Frequency SOUID Measurements with Laser-Driven Switching.

02,081

SYMBOLIC PROGRAMMING

Symbolic Programming with Series Expansions: Applications to Optical Waveguides.

PB95-168589

SYNCHRONISM

Smart Clock: A New Time.
PB95-151445

04,283
Measurement Methods and Algorithms for Comparison of Local and Remote Clocks.

PB96-102652

01.549

Time Generation and Distribution.

01,550

Wavelet Analysis for Synchronization and Timekeeping.

PB96-20038

01,558

SYNCHRONOUS SATELLITES

Satellite Two-Way Time Transfer: Fundamentals and Recent Progress.
PB95-161089

SYNCHROTRON RADIATION

01,536

Materials Science with SR Using X-Ray Imaging: Spatial-

Resolution/Source Size

Resolution/Source Size.
PB94-213048

Atomic, Molecular, and Optical Physics with X-rays.
PB94-213378

03,863

SYNTHESIS (CHEMISTRY)

Cylinder Wipe Air-Drying Intaglio Ink Vehicles for U.S.

PB 94 -160801

03,115

Thermal Behaviour of Methyl Methacrylate and N-Phenyl Maleimide Copolymers

PB95-152237

01,246

Synthesis and Polymerization of Difunctional and Multifunctional Monomers Capable of Cyclopolymerizat

01,257

PB95-163044

Ambient Temperature Synthesis of Bulk Intermetallics.

PB95-169074 00,168

Ring-Opening Polymerication of a 2-Methylene Spiro

Orthocarbonate Bearing a Pendant Methacrylate Group.

SYNTHETIC PERTURBATION SCREENING

Computing Effects and Error for Large Synthetic Perturbation Screenings.

PB94-139623 01,675

Using Synthetic Perturbations and Statistical Screenıng to Assay Shared-Memory Programs.

PB96-103031

01,740

PYTHETIC PERTURBATION TUNING

Using Synthetic-Perturbation Techniques for Tuning

Shared Memory Programs (Extended Abstract).

PB94-172657 01,685

Synthetic-Perturbation Tuning of MIMD Programs
PB94-185568

01,687

SYSTEM EFFECTIVENESS

Usability Engineering: Industry-Government Collaboration for System Effectiveness and Efficiency.

STEMATIC CORRECTIONS

Systematic Correction in Bragg $X$-ray Diffraction of Flat and Curved Crystals

PB97-112239

SYSTEMS ANALYSIS

Configuration and Performance Evaluation of a Real-Time Robot Control System: A Skeleton Approach.

PB95-163895

01,598

Agile Manufacturing from a Statistical Perspective. PB96-109525

02,886

SYSTEMS APPROACH

Template-Driven Systems Development with IDEF: Enterprise Standards for Reuse.

PB96-160965

02,788

SYSTEMS ARCHITECTURE

Control System Architecture for a Remotely Operated Unmanned Land

03,759

SYSTEMS ENGINEERING

Integration Definition for Information Modeling (IDEF1X); Category: Software Standard; Subcategory: Modeling Techniques.

FIPSPUB184

01,673

Information Technology Engineering and Measurement

Model: Adding Lane Markings to the Information Superhighway.

143145

01,474

Using Grafcet
PB95-150827

02,821

Roadmap for the Computer Integrated Manufacturing

(CIM) Application Framework.

PB96-122759

02,832

C++ in Safety Critical Systems.

PB96-154588

01,750

Usability Engineering: Industry-Government Collaboration for System Effectiveness and Efficiency.

01,514

SYSTEMS INTEGRATED FOR MANUFACTURING

LICATIONS

NIST SIMA Interactive Management Workshop. Held in Fort Belvoir, Virginia on November 14-16, 1994 PB96-154877

02,838

SYSTEMS INTEGRATION

Integration of Servo Control into a Large-Scale Control System Design: An Example from Coal Mining.
PB94-203429

03,696

SYSTEMS INTEGRATION FOR MANUFACTURING

Technical Program Description Systems Integration for Manufacturing (SIMA)

02,819

Requisite Elements, Rationale, and Technology Overview or the Systems Intergration for Manufacturing Applicaions (SIMA) Program. Background Study.

PB96-112685

02,831

APPLICATIONS (SIMA)

Systems Integration for Manufacturing Applications Program 1995 Annual Report.

PB96-193735

02,844

SYSTEMS MANAGEMENT

Using Expect to Automate System Administration Tasks.

PB94-213329 01,697

Common Criteria: On the Road to International Harmoni-

zation.

PB96-123484 01,606

Functional Security Criteria for Distributed Systems

PB96-123492

01,607

Open System Environment (OSE): Architectural Framework for Information Infrastructure.

00,002

SYSTEMS SIMULATION

NIST ATM Network Simulator: Operation and Programming, Version 1.0

PB96-106851

01,487

TA2 ALGORITHM

Promise into Practice: Implementing TA2 on Real Clocks at NIST.

PB95-151478

01,533

TABLES (DATA)

Tables of Chemical Kinetics Homogeneous Reactions.

AD-A280 293/2 00,715
0

Energy Levels of Germanium, $\mathrm{Ge} I$ through $\mathrm{Ge}$ XXXII.

PB94-162351

TABULATION PROCESSES

Tabulation of Data on Receiving Tubes. Handbook 68 AD.A285 495/8

TACTILE SENSORS (ROBOTICS)

Integrated Vision Touch-Probe System for Dimensiona Inspection Tasks.

TAGGED PHOTON METHOD Theoretical Aspects of Tagged Photon Facilities.
PB94-216611

TANTALUM

Textures of Tantalum Metal Sheets by Neutron Diffrac-

tion.

PB94-200169 03,399

Measurement of Surface Tension of Tantalum by a Dynamic Technique in a Microgravity Environment PB95-161667

03,932

TANTALUM SULFIDES

Scanning Tunneling Microscopy of the Charge-DensityWave Structure in $1 \mathrm{~T}$-TaS2.

PB95-180980

04,689

TASK DECOMPOSITION

Task Decomposition Methodology for the Design of a Coal Mining Automation Hierarchical Real-Time Control 


\section{KEYWORD INDEX}

TEMPERATURE DEPENDENCE

TECHNOLOGICAL INNOVATIONS

Technology Trends in Telecommunications: An Overview. PB94-123080

01,462

TECHNOLOGY ASSESSMENT

Opportunities for Innovation: Advanced Manufacturing Technology.
PB94-100278

02,801

Visualization Applications for Manufacturing: A State-ofthe-Art Survey. Final Report.

02,816

Statistical Quatity Control Technology in Japan.

PB94-199064 02,70

Benchmarks for the Evaluation of Speech Recognizers.

PB94-211539 01,566

Assessment of Technologies for Advanced Fire Detec-

tion.

00,294

Report on the Advanced Software Technology Workshop.

Held on February 1, 1994.

PB95-136610

01,707

Rationale and Preliminary Plan for Federal Research for Construction and Building.

PB95-154704

00,322

Robotics Application to Highway Transportation. Volume

2. Literature Search

01,337

Robotics Application to Highway Transportation. Volume 3. Proposed Research Topics and Cost/Benefit Evaluations by CERF.

PB95-171633 01,338

Robotics Application to Highway Transportation. Volume

4. Proposals for Potential Research

PB95-193173

01,339

Object-Oriented Technology Research Areas.

PB95-199329

01,726

Proceedings of the Manufacturing Technology Needs and Issues: Establishing National Priorities and Strategies Conference. Held in Gaithersburg, Maryland on April 2628, 1994.

206181

02,930

TECHNOLOGY INCENTIVES

Opportunities for Innovation: Advanced Manufacturing Technology.
PB94-100278

02,801

Transfer of Technology from Defense to Civilian Sectors.

PB94-185360

00.011

Network Brokers Mandbook: An Entrepreneurial Guide to Cooperative Strategies for Manufacturing Competitiveness.

PB95-219325

00,490

TECHNOLOGY INNOVATION

Materials Science and Engineering Laboratory Annual Report, 1993. NAS-NRC Assessment Panel, April 21-22, 1994.

$94-162534$

02,969

Functions of Technology Infrastructure in a Competitive Economy.

00,478

Opportunities for Innovation: Advanced Surface Engineer-

PB94-176666

02,697

Infratechnologies: Tools for Innovation.

00,317

Program of the Subcommittee on Construction and Build-

PB94-193646

00,319

Differences in Competitive Strategies between the United

States and Japan.
PB94-211836
O0,013

Importance of Measurement in Technology-Based Com-

petition

02,929

Paffenbarger Research Center: The Cutting Edge of Dental Science.

00,151

Program of the Subcommittee on Construction and Building (July 1994)

00,321

Publication and Presentation Abstracts, 1993. (Published by Paffenbarger Research Center and Center of Excelby Paffenbarger Research Center and
lence for Materials Science Research).

lence for Mate

03,562

Fresh Look at Strategies for Fire Safely.

PB95-162947

04,870

Precision in Machining: Research Challenges.

02,953

Publications and Presentation Abstracts, 1995. (Published by Paffenbarger Research Center and Center of Excellence for Materials Science Research).

PB96-119250

03,568

Publication and Presentation Abstracts, 1995

PB96-164082

03,576

Publication and Presentation Abstracts, 1994.

PB96-176623

03,577

Working Conference on Global Growth of Technology: Is America Prepared. Held in Gaithersburg. Maryland on

December 7, 1995.

PB96-210059

00,018

Publication and Presentation Abstracts, 1996 PB97-122238

03,585

TECHNOLOGY INNOVATIONS

Opportunities for Innovation: Biotechnology.

CHNOLOGY TRANSFER

Transfer of Technology from Defense to Civilian Sectors.

PB94-185360

Lighting Research and Theory Can Create Business PB95-15179

00,253

Electronics and Electrical Engineering Laboratory 1995 Program Plan. Supporting Technology for U.S. Competi-

PB95-159885

01,894

Encouraging Environmentally-Aware Inventions.

PB95-161394

02,521

Japan Technology Program Assessment. Simulation: State-of the-Art in Japan.

B95-217097

02,827

Electronics and Electrical Engineering Laboratory: 1996 Program Plan. Supporting Technology for U.S. Competitiveness in Electronics.

PB96-175237

01,962

Measurement of Process Complexity.

PB97-113138

01,781

Standards Promote Credibility and Technology Transfer: The Need for Greater Industry Support of Technical Com mittees.

PB97-116206

02,961

TECHNOLOGY UTILIZATION

Opportunities for Innovation: Advanced Manufacturing PB94-100278

02,80

Catalog of National ISDN Solutions for Selected NIUF Applications.

PB94-166006

01,468

Workshop Summary Report: Industrial Applications of Scanned Probe Microscopy. A Workshop Co-sponsored by NIST, SEMATECH, ASTM, E42.14, and the American Vacuum Society. Held in Gaithersburg, Maryland on March 24-25, 1994.

PB95-170387

00,506

Applications of Diamond Films and Related Materials: International Conference (3rd). Held in Gaithersburg, Maryland on August 21-24, 1995. Supplement to NIST Special Publication 885

PB95-256053

03,063

Opportunities for Innovation: Optoelectronics.

01,928

Summary Report: Workshop on Industrial Applications of Scanned Probe Microscopy (2nd). A Workshop Co-Sponsored by NIST, SEMATECH. ASTM E42.14, and the American Vacuum Society. Held in Gaithersburg, Maryland on May 2-3, 1995.

00,509

B96-131602

Survey of Standards for the U.S. Fiber/Textile/Apparel In-
dustry.
PB96-193792

TEETH

Remineralization of Root Lesions with Concentrated Cal-

Remineralization of Root Lesions

Cium and Phosph-102140

03,567

Intensive Swimming: Can It Affect Your Patients' Smiles.

PB96-123666 03,570

Polymeric Calcium Phosphate Composites with Remineralization Potential.

03,575

Posterior Restorative Materials Research.

PB97-118624

03,582

TEFLON

Dielectric Properties of Materials at Cryogenic Temperaures and Microwave Frequencies. PB95-202610

02,369

\section{TELECOMMUNICATION}

Administration Standard for the Telecommunications Infrastructure of Federal Buildings. Category: Telecommunications Standard; Subcategory: Telecommunications Ad-

FIPS PUB 187

01,461

Federal Building Grounding and Bonding Requirements for Telecommunications. Category: Telecommunications Standard; Subcategory: Grounding and Bonding.

FIPS PUB 195

01,802

Technology Trends in Telecommunications: An Overview.

01,462

Earthquake Resistant Construction of Electric Transmission and Telecommunication Facilities Serving the Federal Government Report

PB94-161817

02,460

Catalog of National ISDN Solutions for Selected NIUF Applications.

01,468

Planning the Infrastructure for Global Electronic Com merce. 185832

00,494

ISDN Conformance Testing Guidelines: Guidelines for Implementors of ISDN Customer Premises Equipment to

Conform to Both National ISDN-1 and North American ISDN Users' Forum Layer 3 Basic Rate Interface Basic Call Control Abstract Test Suites.

PB94-219094

01,471

Analyzing Electronic Commerce.

PB94-219102

00,480

Security in Open Systems

01,473

Impact of the FCC's Open Network Architecture on NS/ NP Telecommunications Security

PB95-189445

01,483

Electronic Impler

01,484

Summary Report on the Workshop on Advanced Digital Video in the National Information Infrastructure.

PB96-141320

01,497

Guidelines for the Evaluation of Electronic Data Interchange Products.

01,506

Federal Implementation Guideline for Electronic Data Interchange: ASC X12 003050 Transaction Set 836 Procurement Notices. Implementation Convention.

PB96-178892

Unpredictable Certainty. Information Infrastructure through 2000.

TELECONFERENCING

Videoconferencing Procurement and Usage Guide. PB94-217023

\section{TELEMEDICINE}

Virtual Environments for Health Care. A White Paper for the Advanced Technology Program (ATP), the National Institute of Standards and Technology.

$6-147814$

03,594

TELEOPERATORS

Ground Vehicle Control at NIST: From Teleoperation to Autonomy.

03,758

Unified Telerobotic Architecture Project (UTAP) Standard Interface Environment (SIE), May 1995

02,938

TELEPHONE SYSTEMS

Introduction to Secure Telephone Terminals. PB97-110498

01.512

TELEROBOTICS

Overview of NASREM: The NASANNBS Standard Reference Model for Telerobot Control System Architecture. Real-Time Vision for Autonomous and Teleoperated Control of Unmanned Vehicles.

PB94-211885

03,701

PB94-211893

03,702

Hierarchical Ada Robot Programming System (HARPS) 
Temperature Dependence of the Rate Constants for Reaction of Inorganic Radicals with Organic Reductants. PB94-198280 00,783 Kinetics of the Reaction of $\mathrm{CCl} 3-\mathrm{Br}-2$ and the Thermochemistry of $\mathrm{CCl} 3$ Radical and Cation

00,824

Temperature Dependence of the Rate Constants for Reactions of the Carbonate Radical with Organic and Inorganic Reductants.

PB94-212206

00,831

Small Angle Neutron Scattering Study on Poly(N-Isopropyl Acrylamide) Gels Near Their Volume-Phase Transition Temperature.

PB95-164380

01,263

Small-Angle Neutron Scattering Study on Weakly Charged Temperature Sensitive Polymer Gels.

01,264

Pry

02,654

PB95-164703 Temperature Dependence and Magnetic Field Modulation
of Critical Currents in Step-Edge SNS YBCO/Au Junctions.

04,723

Effect of Anneal Temperature on Si/Buried Oxide Interface Roughness on SIMOX

PB96-112206

02,382

Temperature Dependence of the Gas and Liquid Phase Ultraviolet Absorption Cross Sections of HCFC-123 ( $\mathrm{CF} 3 \mathrm{CHCl} 2$ ) and $\mathrm{HCFC}-142 \mathrm{~b}$ (CH3CF2Cl).

03,298

Temperature Dependence of the Ultraviolet Absorption Cross Section of CF3

PB96-204169

01,168

Temperature Dependent Ultraviolet Absorption Cross Sections of Propylene, Methylacetylene and VBinylacetylene.

01,169

TEMPERATURE DISTRIBUTION

Comparison of Experimental and Computed Species Concentration and Temperature Profiles in Laminar, TwoDimensional Methane/Air Diffusion Flames.

01,379

TEMPERATURE EFFECTS

Performance of Compact Fluorescent Lamps at Different Ambient Temperatures

PB95-175329 00,258

Room-Temperature Flexure Fixture for Advanced Ceramics.

03,061

Temperature and Relative Humidity Dependence of Radiochromic Film Dosimeter Response 10 Gamma and Electron Radiation.

TEMPERATURE FUNCTIONS

Correlation of the Ideal Gas Properties of Five Aromatic Hydrocarbons.
PB95-175816

03,718

\section{PB95-175816}

01,002

TEMPERATURE GRADIENTS

Temperature Increases in Aluminum Alloys during Mechanical-Impact Tests for Oxygen Compatibility.

03,316

TEMPERATURE MEASUREMENT

Technologic Papers of the Bureau of Standards: Number 170. Pyrometric Practice.

AD-A279 282/8

03,766

Corrected Optical Pyrometer Readings.

02,615

Ebulliometers for Measuring the Thermodynamic Properties of Fluids and Fluid Mixtures.

DE94017817

04,195

Assessment of Uncertainties of Liquid-in-Glass Thermometer Calibrations at the National Institute of Standards and Technology.

High-Speed Spatial Scanning Pyrometer.

02,625

PB94-200003

02,636

Dynamic Technique for Measuring Normal Spectral Emissivity of Electrically Conducting Solids at High Tempera. tures with a High-Speed Spatial Scanning Pyrometer. PB95-153045 03.921 Practical Applications of the ITS-90: Inherent UncertainPB95-161527

03,930

Design and Machining of Copper Specimens with Micro Holes for Accurate Heat Transfer Measurements. Reproducibility of the Temperature of the Ice Point in Routine Measurements.

PB95-255923

04,015

Radiance Temperatures (in the Wavelength Range 523 $907 \mathrm{~nm}$ ) of Group IVB Transition Metals Titanium, Zirconium, and Hafnium at Their Melting Points by a PulseHeating Technique.

PB96-135025

02,677

Q Branch Lineshape Functions for CARS Thermometry.
PB96-160643

Radiance Temperatures at $1500 \mathrm{~nm}$ of Niobium and Molybdenum at Their Melting Points by a Pulse-Heating Technique.
PB97-118699

04,167
Comparisons of Some NIST Fixed-Point Cells with Similar Cells of Other Standards Laboratories. lar Celis of Other
PB97-119242

00,655

\section{TEMPERATURE MEASUREMENTS}

Realization of New NIST Radiation Temperature Scales for the $1000 \mathrm{~K}$ to $3000 \mathrm{~K}$ Region, Using Absolute Radiometric Techniques.

PB94-172905

03,794

Current Status and Trends in Temperature Measurements at NIST, Cooperative Projects and New Mutual Agreement between NIST and IMGC

PB97-110266

02,691

ssues in High-Speed Pyrometry.

PB97-118368

02,693

TEMPERATURE MEASURING INSTRUMENTS

Thermal Diffusivity of POCO AXM-5Q1 Graphite in the Range 1500 to $2500 \mathrm{~K}$ Measured by a Laser-Pulse Technique.

03,013

High-Temperature Laser-Pulse Thermal Diffusivity Appaatus.

PB94-185147

02,631

TEMPERATURE SCALES

Realization of New NIST Radiation Temperature Scales for the $1000 \mathrm{~K}$ to $3000 \mathrm{~K}$ Region, Using Absolute Radiometric Techniques.

PB94-172905

03,794

Development of a Temperature Scale below $0.5 \mathrm{~K}$.
PB95-125639
03,879 Intercomparison of the ITS-90 Radiance Temperature Scales of the National Physical Laboratory (U.K.) and the National Institute of Standards and Technology. PB96-113550

02,674

TEMPERATURE SENSORS

Self-Calibrated Intelligent Optical Sensors and Systems. PB96-200738

04,380

TEMPLATES

VLSI Architectures for Template Matching and Block MBatching.

01,834

TENSILE CREEP

Tensile Creep of Whisker Reinforced Silicon Nitride.
PB94-211984 142 Tensile Creep of a Silicon Nitride Ceramic

03,049

PB95-161303

Cavitation Contributes Substantially to Tensile Creep in
Sificon Nitride. Silicon Nitride
PB96-122577

TENSILE DEFORMATION

Tensile Deformation-Induced Microstructures in FreeStanding Copper Thin Films.

PB96-102595

04,715

TENSILE PROPERTIES

Microstructure and Tensile Properties of Microalloyed Steel Forgings.
PB94-172715

TERAHERTZ PHYSICS

Metrology Issues in Terahertz Physics and Technology.

TERATOGENS

Nickel(II)-Mediated Oxidative DNA Base Damage in Renal and Hepatic Chromatin of Pregnant Rats and Thei Fetuses. Possible Relevance to Carcinogenesis.

TERBIUM

Neutron-Scattering Studies of the Two Magnetic Correlation Lengths in Terbium.

PB95-152328

04,586

Origin of the Second Length Scale Above the Magnetic Spiral Phase of Tb.

04,596

TERBIUM 160

Precision Nuclear Orientation Measurements for Determining Mixed Magnetic Dipole/Electric Quadrupole Hyperfine Interactions.

PB94-199080

03,810

TERBIUM COMPOUNDS

Characterization of the Structure of TbD2.25 at $70 \mathrm{~K}$ by Neutron Powder Diffraction.

01,130

TERBIUM HYDRIDES

Neutron Spectroscopic Evidence of Concentration-Dependent Hydrogen Ordering in the Octahedral Sublattice of beta-TbH2+x.

PB95-181020

01,018

TERMINOLOGY

Statistical Descriptors in Crystallography. 2. Report of a Working Group on Expression of Uncertainty in Measurement

PB96-146824

04,764

TERNARY ALLOY SYSTEMS

Thermodynamic Calculation of the Ternary Ti-Al-Nb Sys-

tem.

03,336

Eiectronic Structure and Phase Equilibria in Temary Substitutional Alloys
PB97-119366

TERNARY MIXTURES

03,378

Vapor-Liquid Equilibria of Mixtures of Propane and Isomeric Hexanes.
PB95-175287

00,995

TERT-BUTYL HYDROPEROXIDE

Tert-Butyl Hydroperoxide-Mediated DNA Base Damage in Cultured Mammal
PB94-182003

03,644

TEST AND EVALUATION

Indoor Air Quality Commissioning of a New Office Build. PB95-182309

00,262

TEST CHAMBERS

Constant Temperature and Humidity Chamber for Standard Resistors.

PB96-122494

02,275

Complicated Cases and Shielded Rooms: Audiometric Booths Shielded to Attenuate Electromagnetic InterPB96-180179

02,278

TEST FACILITIES

Uncertainty Analysis of the NIST Nitrogen Flow Facility.

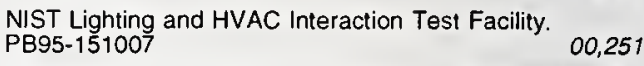

02,608

New Extrapolation/Spherical/Cylindrical Measurement Facility at the National Institute of Standards and TechPB95-153755

02,004

Characterization of a Health Physics Instrument Calibration Range.

03,629

Room-Temperature Flexure Fixture for Advanced Ceram-

PB95-210498

03,061

National Voluntary Laboratory Accreditation Program (NVLAP): Commercial Products Testing.

Alternative EMC Compliance Test Facilities.

02,671

PB96-200324

02,247

ST METHODS

Draft Guidelines for Pre-Qualification and Prototype Testing of Seismic Isolation Systems.

01,331

Draft Guidelines for Quality Control Testing of Sliding Seismic Isolation Systems.

PB94-161957

01,332

Draft Guideline for Testing and Evaluation of Seismic Isolation Systems
PB94-172947

00,423

Selection of Appropriate Ultrasonic System Components for NDE of Thick Polymer-Composites.

03,133

Graphical Analysis of the CCRL Portland Cement ProGraphical Analysis of the CCRL Portland Cement Pro-
ficiency Sample Database (Samples 1-72). (Part 1. Univariant Analysis of Portland Cement).

01,308

Effective Measurement Techniques for Heat, Smoke, and Toxic Fire Gases.

PB94-198439

01,369

Modern Test Methods for Flammability.

PB94-198447

01,370

Millisecond-Resolution Pulse Heating System for SpecificHeat Measurements at High Temperatures.

02,635

Airbome Asbestos Method: Standard Practice for RecordAirbome Asbestos Method: Standard Practice for Recordsis of Asbestos Collected onto Filters. Version 1.0.
PB94-210168 00,552 

Asperity-Asperity Contact Mechanisms Simulated by a
Two-Ball Collision Apparatus.

PBos-164158

02,966

Interlaboratory Comparison Studies on the Analysis of Hair for Drugs of Abuse.
PB95-176251

03,500

Effects of Testing Variables on the Measured Compressive Strength of High-Strength (90 MPa) Concrete. NIST Strategies for Reducing Testing Requirements.

PB95-180444 01,909

Effect of Environmentally Exposures on the Properties of Polyisocyanurate Foam Insulation: Thermal Conductivity Measurements.

PB95-181210 00,388

Effects of Testing Variables on the Strength of HighStrength $(90 \mathrm{Mpa})$ Concrete Cylinders.

PB96-112198

00,456

Summary and Results of the NIST Workshop on Proposed Guidelines for Testing and Evaluation of Seismic Isolation Systems. Held in San Francisco, California on July 25, 1994

00,463

Development of a Test Method for Leaching of Lead from Lead-Based Paints Through Encapsulants.
PB96-154984

Rechargeable Batteries for Personal/Portable.

PB96-164231

02,459

Guidelines for Pre-Qualification, Prototype and Quality Control Testing of Seismic Isolation Systems.
PB96-193685

01,347 Airbome Asbestos Method: Bootstrap Method for Detersion 1.0 .

PB96-214614

00,646

Interlaboratory Studies on the Analysis of Hair for Drugs of Abuse: Results from the Fifth Exercise.

PB97-110449

03,509

Test Procedures for Advanced Insulation Panels.

PB97-111892

00,415

Structured Testing: A Testing Methodology Using the Cyclomatic Complexity Metric

01,784

TEST STRUCTURE

Hybrid Optical-Electrical Overlay Test Structure.

PB96-204136

02,450

TEST STRUCTURES

Review of Semiconductor Microelectronic Test Structures with Applications to Infrared Detector Materials and Processes.

PB94-212925

Test Structures for Determining Design Rules for Microelectromechanical-Based Sensors and Actuators. 02,105
PB95-150488

Electrical Test Structure for Overlay Metrology Reterenced to Absolute Length Standards.

02,336

Electrical Test Structure for Improved Measurement of Feature Placement and Overlay in Integrated Circuit Fabrication Processes.

PB95-164273

02,355

Microelectronic Test Structures for Feature Placement and Electrical Linewidth Metrology.

Microelectronic Test Structures for Overlay Metrology.

PB96-164249 02,430

TESTIMONY

NIOSH Comments to DOL on Risk Estimates from the Cadmium Cohort Study by L. Stayner, February 7, 1992. PB95-267779

03,604

GOSIP Testing Program.

PB96-161229

01,504

TESTING LABORATORIES

Project Summaries 1994: NIST Building and Fire Research Laboratory

PB94-207495 00,343

Project Summaries 1995: NIST Building and Fire ReSearch Laboratory.

00,400

Efficiency of Electric Motors. National Voluntary Lab. Accreditation Program (NVLAP).
PB96-111174

02,107

TESTS

Formal Multi-Layer Test Methodology and Its Application to OSI.

02,718

ISO/IEC Workshop on Worldwide Recognition of OSI Test Results Regional Progress - North America.
PB94-172202

U.S. GOSIP Testing Program.

PB94-211455 01,807

Digital Techniques in HV Tests - Summary of 1989 Panel Session

PB94-216702

02,035

National Voluntary Laboratory Accreditation Program: Energy Efficient Lighting Products.

02,642
ISDN Conformance Testing Guidelines: Guidelines for Implementors of ISDN Customer Premises Equipment to Conform to Both National ISDN-1 and North American Call Control Abstract Test Suites.
Cate Interface Basic PB94-219094

01,471

Industry/Government Open Systems Specification Testing Framework. Version 1.0

01,809

$\mathrm{M}$ (also known as MUMPS) Validation Test Suite, Version 8.3 (for Microcomputers)

01,699

B94-502077

Standardization of Testing Methods for Optical Disk Media Characteristics and Related Activities at NIST.
PB95-108486

Some Considerations for Interim Testing of Coordinate Measuring Machine Performance Using a Specific Artifact.

PB95-108858

02,898

Developing Linear Error Models for Analog Devices. PB95-150520

02,037

Initial NIST Testing Policy for STEP: Beta Testing Program for AP 203 Implementations. National PDES gram tor AP 203 Impl

PB95-154688

02,779

Initial Graphics Exchange Specification (IGES): Procedures for the NIST IGES Validation Test Service.

PB95-171427

02,780

Testers Open Dialogue at Inaugural NIST Workshop.

PB95-175550

01,718

Testability of Object-Oriented Systems.

PB95-242418

01,733

Algorithm Testing and Evaluation Program for Coordinate Measuring Systems: Testing Methods.

02,666

Partial Pressure Analysis in Space Testing

04,829

PB96-103072

04829

Development of Near-Field

nication Satellite

02,010

STandard for the Exchange of Product Model Data (STEP): Procedures for NIST STEP Validation.

PB96-154976

02,787

TETRACHLOROETHYLENE

Incinerability of Perchloroethylene and Chiorobenzene.

$\begin{array}{ll}\text { PB95-163457 } & 01,388\end{array}$

Hydrogen Atom Attack on Perchloroethylene.

01,388

PB95-163473

00,969

TETRACYANOQUINODIMETHANE

Amperometric Flow-Injection Analysis Biosensor for Glucose Based on Graphite Paste Modified with PB95-161980

03,498

\section{TETRACYCLINE}

Fluorescence Measurements of Tetracycline in High Cell Mass for Fermentation Monitoring

PB95-175709

00,601

Feasibility of Fluorescence Detection of Tetracycline in Media Mixtures Employing a Fiber Optic Probe. PB96-163654

00,511

\section{TETRADECANOYLPHORBOL ACETATE}

Modification of DNA Bases in Chromatin of Intact Targe Human Cells by Activated Human Polymorphonuclear Leukocytes.

TETRAFLUOROETHANE

Equation of State Formulation of the Thermodynamic Properties of R134a (1,1,1,2-Tetrafluoroethane).

PB94-212081

03,256

Measurements of the Vapor Pressures of Difluoromethane, 1-Chloro-1,2,2,2-Tetrafluoroethane, and Pentafluoroethane.

03,270

Predictive Extended Corresponding States Model for Pure and Mixed Refrigerants Including an Equation of State for R134a.

PB95-175717

03,275

TETRAFLUOROETHYLENE RESINS

Dielectric Properties of Materials at Cryogenic Temperaures and Microwave Frequencies.

PB95-202610

02,369

TETRAHEDRAL SITE VIBRATIONS

Characterization of the Vibrational Dynamics in the Octahedral Sublattices of LaD2.25 and LaH2.25.

PB96-123724

01,091

TETRAHEDRONS

Inserting Line Segments into Triangulations and Tetrahedralizations.

PB95-198933

03,415

TETRALIN

Homogeneous Gas Phase Decyclization of Tetralin and Benzocyclobuten

XT PROCESSING

00,928

Teld in Gaithersburg, Maryland on August 31-September 2 1993.

PB94-178407

01,686

Important Papers in the History of Document Preparation Systems: Basic Sources.

Obtaining and Installing a Public Domain TEX.

PB95-175741

02,712

3). Held in Gaithersburg, Maryland on November 2-4

PB95-216883

01,728

Lab Report Special Section: Natural Language Process ing and Information Retrieval Group Information Acces and User Interfaces Division, National Institute of Standards and Technology.

PB97-118665

02,742

Panel: Building and Using Test Collections.

02,743

Text REtrieval Conference (4th) (TREC-4). Held in Gaithersburg, Maryland on November 1-3, 1995

PB97-121636

01,786

TEXTILE INDUSTRY

Survey of Standards for the U.S. Fiber/Textile/Apparel Industry.

03,199

THALLIUM

Arc Spectra of Gallium, Indium, and Thallium. AD-A2.95 411/3

00,718

THALLIUM BARIUM CALCIUM CUPRATES

Asymmetry between Flux Penetration and Flux Expulsion in Tl-2212 Superconductors

04,527

Tunneling Spectroscopy of Thallium-Based High-Temperature Superconductors.

PB95-161709

04,606

Enhanced Flux Pinning via Chemical Substitution in Bulk Superconducting T1-2212. PB95-169033

04,647

\section{THALLIUM COMPOUNDS}

Tunneling Spectroscopy of Thallium-Based High-Temperature Superconductors.

B95-161709

04,606

EOPHYLLINE

Planar Waveguide Optical Sensors

PB94-200185

Liposome-Based Flow-Injection Immunoassay for Determining Theophylline in Serum.

THEORIES

Lighting Research and Theory Can Create Business PB95.15179

00,253

THERMAL ANALYSIS

Fire Induced Thermal Fields in Window Glass I: Theory. Application of Thermal Analysis Techniques to the Characterization of EPDM Roofing Membrane Materials. PB95-125845

00,359

Simulating the Dynamic Electro Thermal Behavior of Power Electronic Circuits and Systems.

02,345

Thermal Component Models for Electro-Thermal Network Simulation. 
Effects of Humidity and Elevated Temperature on the Density and Thermal Conductivity of a Rigid Polyisocyanurate Foam Co-Blown with $\mathrm{CCl} 3 \mathrm{~F}$ and $\mathrm{CO} 2$.
PB95-150462 Effects of Humidity and Elevated Temperature on the Density and Thermal Conductivity of a Rigid Polyisocyanurate Foam

00,373

Room Temperature Thermal Conductivity of Fumed-Silica Insulation for a Standard Reference Material.

00,374

Measurement of the Thermal Properties of Electrically Conducting Fluids Using Coated Transient Hot Wires. Conducting Fluids Using Coated Transient Hot Wires.
PB95-169058 03,269 Calculation of the Thermal Conductivity and Gas Permeability in a Uniaxial Bundle of Fibers.

03,058 Intra-Laboratory Comparison of a Line-Heat-Source Intra-Laboratory Comparison of a Line-Heat-Source
Guarded Hot Plate and Heat-Flow-Meter Apparatus.
PB95-181202 00,387 Effect of Environmentally Exposures on the Properties of
Polyisocyanurate Foam Insulation: Thermal Conductivity Measurements.

00,388

Analytical Method for Determining Thermal Conductivity from Dynamic Experiments.

04,024

Standard Reference Data for the Thermal Conductivity of Water.

PB96-145875

01,111

Status of the Round Robin on the Transport Properties of R134a.

01,152

Thermal Conductivity of Polypyromellitimide Film with Alumina Filler Particles from 4.2 to $300 \mathrm{~K}$.

PB96-200753

01,292

THERMAL CONDUCTORS

Comparison of Elastic and Plastic Contact Models for the Prediction of Thermal Contact Conductance.

PB95-161659

04,605

THERMAL CONFORT

Ventilation Rates in Office Buildings.

THERMAL CONTROL COATINGS

02,539

Measurement Methods and Standards for Processing and Application of Thermal Barrier Coatings.

01,447

HERMAL CONVERTERS

Intercomparison of NIST, NPL, PTB, and VSL Thermal Voltage Converters from $100 \mathrm{kHz}$ to $1 \mathrm{MHz}$.

02,026

Intercomparison of Thermal Converters at NIM, NIST, PTB, SIRI and VSL from 10 to $100 \mathrm{MHz}$. Performance of Commercial CMOS Foundry-Compatible Multijunction Thermal Converters.

02,342

PB95-153656

CMOS

Multijunction

02,343

High-Current Thin Film Multijunction Thermal Converters High-Current Thin Film Multijunction Thermal Converters
and Multi-Converter Modules.
PB97-112379

1.989

THERMAL DEGRADATION

High Temperature Degradation of Structural Composites.
PB94-172848

Causes of the Apparent Heat Transfer Degradation for Refrigerant Mixtures.

03,259 Gas Phase Oxygen Effect on Chain Scission and Mono-
mer Content in Bulk Poly(methyl methacrylate) Degraded by External Thermal Radiation. PB External Thern

01,293

THERMAL DIFFUSIVITY

Thermal Diffusivity of POCO AXM-5Q1 Graphite in the Range 1500 to $2500 \mathrm{~K}$ Measured by a Laser-Pulse TechPB94-185022

03,013

High-Temperature Laser-Pulse Thermal Diffusivity Apparatus.
PB94-185147

02,631

Analysis of Thermal Wave Propagation in Diamond Films.

PB94-211471

03,014

Thermal Wave Propagation in Diamond Films.

PB94-211489

03,015

THERMAL ELECTRONS

Dependence of the Thermal Electron Attachment Rate Constant in Gases and Liquids on the Energy Position of the Electron Attaching State.

PB97-122253

01,996

THERMAL EQUILIBRIUM

Thermal Equilibration Near the Critical Point: Effects Due to Three Dimensions and Gravity.

PB95-152922

03.919

THERMAL EXPANSION

Thermal Expansion of an SiC Particle-Reinforced Aluminum Composit

03,144
Anomalous Dispersion and Thermal Expansion in LightlyDoped KTa1-xNbxO3.

04,585

Relationship between Bulk-Modulus Temperature Dependence and Thermal Expansivity.

pendence and
PB95-168829

04,641

THERMAL INSULATION

Comparison of Heat-Flow-Meter Tests from Four Labora-

PB95-126264

00,365

Effects of Humidity and Elevated Temperature on the Density and Thermal Conductivity of a Rigid Polyisocyanurate Foam Co-Blown with CCI3F and $\mathrm{CO}_{0 .}$.
PB95-150462 Effects of Humidity and Elevated Temperature on the Density and Thermal Conductivity of a Rigid Polyisocyanurate Foam.

PB95-152021

00,373

Room Temperature Thermal Conductivity of Fumed-Silica Insulation for a Standard Reference Material.

PB95-152039

00,374

Control Stability of a Heat-Flow-Meter Appartus.

PB95-181194

00,386

Intra-Laboratory Comparison of a Line-Heat-Source Guarded Hot Plate and Heat-Flow-Meter Apparatus. 5-181202

00,387

Effect of Environmentally Exposures on the Properties of Polyisocyanurate Foam Insulation: Thermal Conductivity Measurements.
PB95-181210

00,388

National Voluntary Laboratory Accreditation Program: Thermal Insulation Materials.

02,977

Room-Temperature Thermal Conductivity of Expanded Polystyrene Board for a Standard Reference Material. PB96-193693

Test Procedures for Advanced Insulation Panels. PB97-111892

00,412

HERMAL IONIZATION MASS SPECTROSCOPY

Comparative Strategies for Correction of Interferences in Isotope Dilution Mass Spectrometric Determination of $\mathrm{Va}$ -

PB94-185261

00,531

THERMAL MASS FLOW METERS

Critical Evaluation of Thermal Mass Flow Meters. PB97-113153

00,683

THERMAL MECHANICAL ANALYSIS

Use of Thermal Mechanical Analysis to Characterize Ethylene-Propylene-Diene Terpolymer (EPDM) Roofing Membrane Materials.

00,360

Null-Balanced Total-Power Radiometer System NCS1.

PB94-169778

02,021

Thermal Noise in High-Temperature SuperconductingNormal-Superconducting Step-Edge Josephson Junc-

PB95-175089

04,650

THERMAL PROPERTIES

Cryogenic Materials Data Handbook

AD-A286 $675 / 4$

03,303

Thermal Decomposition Pathways in Nitramine Propellants.

Temperature Dependence of the Gas and Liquid Phase Ultraviolet Absorption Cross Sections of HCFC-123 (CF3CHCl2) and HCFC-142b (CH3CF2Cl)

03,298

\section{THERMAL RADIATION}

Estimate of the Effect of Scale on Radiative Heat Loss Fraction and Combustion Efficiency.

$$
\text { PB95-150447 }
$$

THERMAL STABILTYY

Synthesis of Thermally Stable Elastomers.

03,194

Effects of Molecular Weight and Thermal Stability on Polymer Gasification

01,228

Investigation of the Thermal Stability and Char-Forming Tendency of Cross-linked Poly(methyl methacrylate). PB94-213501

03,380

Thermal Stability of Internal Electric Field and Polarization Distribution in Blend of Polyvinylidene Fluoride and Polymethylmethacrylate.

01,240

Thermal Behaviour of Methyl Methacrylate and N-Phenyl Maleimide Copolymers.

PB95-152237

01,246

THERMAL TRANSFER STANDARDS

Modeling and Test Point Selection for a Thermal Transfer Standard.

01,896

THERMAL VOLTAGE CONVERTERS

Intercomparison of NIST, NPL, PTB, and VSL Thermal Voltage Converters from $100 \mathrm{kHz}$ to $1 \mathrm{MHz}$

02,026

Intercomparison of Thermal Converters at NIM, NIST, PTB, SIRI and VSL from 10 to $100 \mathrm{MHz}$. 02,027

Exploring the Low-Frequency Performance of Thermal Converters Using Circuit Models and a Digitally Synthesized Source.

PB97-112551

02,848

Low Voltage Standards in the $10 \mathrm{~Hz}$ to $1 \mathrm{MHz}$ Range.

PB97-112569

02,100

THERMAL WAVE IMAGING

Evaluation of Thermal Wave Imaging for Detection of MaChining Damage in Ceramics.

03,062

THERMAL WAVES

Thermal Wave NDE of Advanced Materials Using Mirage Effect Detection

THERMISTOR MOUNTS

Coaxial Reference Standard for Microwave Power PB94-193786

THERMOACOUSTICS

Properties of Working Fluids for Thermoacoustic Refrig-

AD-A297 420/2

04,864

THERMOCHEMISTRY

Recent Experimental and Modeling Developments in High Temperature Thermochemistry.

00,759

Fluorinated Hydrocarbon Flame Suppression Chemistry. PB94-185113

Assessment of the Al-Sb System.

PB94-200474

Thermochemistry of the Reactions between Adenosine, Adenosine 5'-monophosphate, Inosine, and Inosine 5'. monophosphate; the Conversion of L-histidine to (Urocanic Acid+Ammonia).

(U) 213113

Thermochemistry of the Hydrolysis of L-arginine 10 (Lcitrulline + Ammonia) and of the Hydrolysis of L-arginine to (L-ornithine + Urea)

PB95-15080

03,463

Thermal Behavior of 4-Maleimidophenyl Glycidyl Ether Resins.

PB95-153151

01,249

Ionization Energy of Sulfur Pentafluoride and the Sulfur Pentafluoride-Fluorine Atom Bond Dissociation Energy.
PB95-162814 00,966

00,966

Thermochemical and Chemical Kinetic Data for Fluorinated Hydrocarbons.

PB95-260618

01,056

Halon Thermochemistry: 'Ab Initio' Calculations of the

Enthalpies of Formation of Fluoromethanes.

PB96-175740

03,289

Chemical Inhibition of Methane-Air Diffusion Flame.

PB96-195532

01,431

Thermochemical Siudies of Inorganic Chalocogenides by Fluorine-Combustion Calorimetry: Binary Compounds of Germanium and Silicon with Sultur. Selenium and Tellu-

PB97-112528

01,181

Thermodynamic Properties of Silicides. 5. Standard Molar Enthalpy of Formation at the Temperature $298.15 \mathrm{~K}$ of Trimolybdenum Monosilicide Mo3Si Determined by Fluorine-Combustion Calorimetry.

PB97-119358

01,190 
Ebulliometers for Measuring the Thermodynamic Properties of Fluids and Fluid Mixtures.

DE94017817

Pressure-Volume-Temperature Relations in Liquid and Solid Tritium.

Thermodynamic Properties of Difluoromethane.
PB94-185204
Thermodynamic Properties of Gas Phase Species of $1 \mathrm{~m}$ -

Thermodynamic Properties of Difluoromethane.
PB94-185204
Thermodynamic Properties of Gas Phase Species of $1 \mathrm{~m}$ Thermodynamic Properties of Gas Phase Species of $1 \mathrm{~m}-$
portance to Ozone Depletion.

Thermodynamic Properties of CHF2-O CHF2,Bis(difluoromethyl) Ether. $\quad 00,798$
PB94-199569

Simplified Cycle Simulation Model for the Pertormance Rating of Refrigerants and Refrigerant Mixtures.

PB94-199890

03,255

Assessment of the Al-Sb System.

03,329

Equation of State Formulation of the Thermodynamic Properties of R134a (1,1,1,2-Tetrafluoroethane).
PB94-212081 03,256 Kinetics of the Reaction of $\mathrm{CCl} 3-\mathrm{Br}-2$ and the Thermochemistry of $\mathrm{CCl} 3$ Radical and Cation. Global Thermodynamic Behavior of Fluid Mixtures in the Critical Region.

04,199

Thermochemistry of the Reactions between Adenosine, Adenosine 5'-monophosphate, Inosine, and Inosine 5 monophosphate; the Conversion of L-histidine to Urocanic Acid+Ammonia)

PB94-213113

03,460

Progress in the Development of a Chemical Kinetic Database for
PB95-151056

01,384

Thermal Behavior of 4-Maleimidophenyl Glycidyl Ether Resins.

PB95-153151

01,249

Thermodynamic Properties of R134a(1,1,1,2 Tetrafluoroethane).
PB95-168704 00,988

Need for, and Availability of, Working Fluid Property Data: Results from Annexes XIII and XVIII.

03,268

PB95-168969

Chloro-

Vapor Pressures and Gas-Phase

PB'2,-175154

03,271

Retention of Halocarbons on a Hexafluoropropylene Ep oxide Modified Graphitized Carbon Black. Part 1. Methane-Based Compounds.

PB95-175196

03,272

Coexisting Densities, Vapor Pressures and Critical Densities of Refrigerants R-32 and R-152a, at $300-385 \mathrm{~K}$.

Thermodynamic Study of the Reactions of Cyclodextrins with Primary and Secondary Aliphatic Alcohols, with Dand L-Phenylalanine, and with L-Phenylalanineamide.
PB95-180873 01,016

01,016

Thermodynamic Properties of Two Gaseous Halogenated Ethers from Speed-of-Sound Measurements:

Difluoromethoxy-Difluoromethane and 2-Difluoromethoxy-
1,1,1-Trifluoroethane.
PB96-102413 04,189 PB96-102413

New Approach for Reducing the Toxicity of the Combustion Products from Flexible Polyurethane Foam.
PB96-123625

Ideal Gas Thermodynamic Properties of Sulphur Heterocyclic Compounds.

PB96-145867

01,110

Thermodynamics of Enzyme-Catalyzed Reactions. Part 4.

Lyases.

Thermodynamic Properties of the Aqueous lons $(2+$ and $3+)$ of Iron and the Key Compounds of Iron

01,119

Interaction Coefficients for 15 Mixtures of Flammable and Non-Flammable Components.

03,281

Thermodynamic Properties of Dilute and Semidilute SoluThermodynamic Properties of

tions of Regular

01,284

Retention of Halocarbons on a Hexafluoropropylene Epoxide-Modified Graphitized Carbon Black. 4. PropaneBased Compounds.

PB96-164033

03,284

Retention of Halocarbons on a Hexafluoropropylene Epoxide-Modified Graphitized Carbon Black. 3. EtheneBased Compounds.

03,286

Thermophysical Property Standard Reference Data from NIST.

01,153

International Standard Equation of State for the ThermoInternational Standard Equation of State for the Thermo-
dynamic Properties of Refrigerant 123 (2,2-Dichlorodynamic Properties of Refrigerant 123 (2,2-Dichloro1,1,1-Trifluoroet
PB96-176805

03,294

Numerical Simulation of Rapid Combustion in an Underground Enclosure.

01,424
Enthalpy Increment Measurements from 4.5 to $318 \mathrm{~K}$ for Bismuth(cr). Thermodynamic Properties from $0 \mathrm{~K}$ to the Melting Point

01,166

Enthalpy Increment Measurements from 4.5 to $350 \mathrm{~K}$ and the Thermodynamic Properties of Titanium Disilicide(cr) to $1700 \mathrm{~K}$

PB96-204029

00,678

Enthalpy Increment Measurements from $4.5 \mathrm{~K}$ to $350 \mathrm{~K}$ and the Thermodynamic Properties of the Titanium Sili-

cide TisSi3( $\mathrm{Cr}$ )
PB96-204037

00,679

Thermodynamics of (Germanium + Selenium): A Review and Critical Assessment.

PB97-112536

01,182

Thermodynamic Properties of CF3-CHF-CHF2, $1,1,1,2,3,3$-Hexafluoropropane.

03,299

Thermodynamic Properties of CHF2-CF2-CHF, 1,1,2,2,3Pentafluoropropane.

PB97-118392

03,300

THERMODYNAMICS

Thermodynamics of the Hydrolysis of Penicillin $G$ and Ampicillin.

03,596

Octacalcium Phosphate Carboxylates IV. Kinetics of Formation and Solubility of Octacalcium Phosphate Succinate.

PB94-185600

00,776

Statistical Thermodynamics of Phase Separation and Ion

Partitioning in Aqueous Two-Phase Systems.
PB94-199387

01,212

Thermodynamic Analysis of Heparin Binding to Human Antithrombin.

03,455

Application of Thermodynamics to Biotechnology

PB95-150793

03,529

Thermodynamic Interactions and Correlations in Mixtures of Two Homopolymers and a Block Copolymers by Small Angle Neutron Scattering.

01,247

Biological Thermodynamic Data for the Calibration of Differential Scanning Calorimeters: Dynamic Temperature Data on the Gel to Liquid Crystal Phase Transition of

Dialkylphosphatidylcholine in Water Suspensions
PB95-162707

03,464

Thermodynamics of the Binding of Galactopyranoside Derivatives to the Basic Lectin from Winged Bean (Psophocarpus Tetrogonolobus).

03,465

Thermodynamics of the Hydrolysis of N-Acetyl-L Thermodynamics of the Hydrolysis of N-Acetyl-L-
phenylalanine Ethyl Ester in Water and in Organic Solvents.

01,053

Generation Pate and Distribution of Products of Combustion in Two-Layer Fire Environments: A Model and Applcations.

01,398

Application of Thermodynamic and Detailed Chemical Ki-

Application of Thermodynamic and Detailed Chemical Kinetic Modeling to Understandir

Generation in
PB96-135322

Nonequilibrium Statistical Mechanics.

01,413

PB96-16178

04,097

Dynamics, Transport and Chemical Kinetics of Compartment Fire Exhaust Gases.

00,229

Isopiestic Investigation of the Osmotic and Activity Coefficients of Aqueous $\mathrm{NaBr}$ and the Solubility of $\mathrm{NaBr} 2 \mathrm{H} 2 \mathrm{O}$ (cr) at 298.15 K. Thermodynamic Properties of the $\mathrm{NaBr}+\mathrm{H} 2 \mathrm{O}$ System over Wide Ranges of Temperaure and Pressure.

PB97-110365

01,175

Thermodynamic Properties of Synthetic Otavite $\mathrm{CdCO} 3(\mathrm{cr})$ : Enthalpy Increment Measurements from 4.5 PB97-111447

Conference Report: Calorimetry Conference (50th)

00,680 PB97-122279

03,722

THERMODYNAMICS \& CHEMICAL KINETICS

Tabulation of the Thermodynamic Properties of Normal Hydrogen from Low Temperatures to $300 \mathrm{~K}$ and from 1 to 100 Atmospheres.

AD-A279 951/8

00,713

Precise Measurement of Heat of Combustion with a Bomb Calorimeter.

AD-A286 701/8

03,770

Properties of Working Fluids for Thermoacoustic RefrigAD-A297 420/2

04,864

Thermophysical Properties of HFC-143a and HFC-152a

Ouarterly Report, 1 July 1993--30 September 1993

DE94004236

03,245

Development of Measurement Capabilities for the Thermophysical Properties of Energy-Related Fluids. An nual Report, December 1, 1990--November 30, 1991. DE94004399

02,470

Thermophysical properties of HCFC alternatives. Ouarterly report, 1 July 1994--30 September 1994.

03,247

Dynamic Measurements of Thermophysical Properties of Metals and Alloys at High Temperatures by Subsecond Pulse Heating Techniques.

N94-25124/6

03,309

Sulfur Dioxide Capture in the Combustion of Mixtures of Lime, Refuse-Derived Fuel, and Coa.

PB94-155587 02,534

Recent Experimental and Modeling Developments in High Temperature Thermochemistry.

PB94-172343

00,759

Thermodyna

PB94-172467

3,596

Thermal Decomposition of Hydroxy- and Methoxy-Substituted Anisoles.

PB94-173036

Thermodynamic Properties of Difluoromethane.

00,767

00,772

Thermodynamic and NMR Study of the Interactions of Cyclodextrins with Cyclohexane Derivatives.

PB94-185816 00,781

Thermodynamic Properties of Gas Phase Species of Importance to Ozone Depletion.

PB94-198215 00,126

Temperature Dependence of the Rate Constants for Reaction of Inorganic Radicals with Organic Reductants.
PB94-198280
D0,783

PB94-198280 00,783 Obtained by Small-Angle Neutron Scattering.

PB94-198496

01,208

Non-Equilibrium Thermodynamic Theory of Viscoplastic Materials.

Thermodynamic Constraints on Non-Equilibrium Solidification of Ordered Intermetallic Compounds.

PB94-198934

03,321

Calculation of Higher Heating Values of Biomass Materials and Waste Components trom Elemental Analyses.
PB94-199254

PB94-199254 02,474 sition: Optical Diagnostics.

PB94-199338

03,116

Gas Phase Reactions Relevant to Chemical Vapor Deposition: Numerical Modeling

Statistical Thermodynamics of Phase Separation and Ion

Partitioning in Aqueous Two-Phase Systems.
PB94-199387

01,212

Modeling the Evolution of Structure in Unstable Solid Solution Phases by Diffusional Mechanisms.

03,324

Thermodynamic Properties of CHF2-OCHF2,Bis(difluoromethyl) Ether

00,798

Maximum Entropy as a Tool for the Determination of the C-Axis Profile of Layered Compounds.

PB94-199619 Standards for Differential Scanning Calorimetry. 1. Zinc Absolute Calorimetric Measurement of Enthalpy of Fusion and Temperature of Fusion HM. PB94-199817 00,801

Assessing the Credibility of the Calorific Content of Muncipal Solid Waste. 
Temperature Dependence of the Rate Constants for Reactions of the Carbonate Radical with Organic and Inorganic Reductant

PB94-212206 00,831 Global Thermodynamic Behavior of Fluid Mixtures in the PB94-212420

04,199

Thermodynamic Calculation of the Ternary Ti-Al-Nb System.

03,336

PB94-212636

Apparent Molar Heat Capacities and Apparent Molar Volumes of Aqueous Glucose at Temperatures from 298.15 $\mathrm{K}$ to $327.01 \mathrm{~K}$.

03,459

Thermochemistry of the Reactions between Adenosine, Adenosine 5'-monophosphate, Inosine, and Inosine 5'. monophosphate; the Conversion of L-histidine to (Urocanic Acid+Ammonia).

PB94-213113

03,460

Thermal Expansion of an SiC Particle-Reinforced Aluminum Composite.

03,144

Analytical Method of Determining the Heat Capacity at High Temperatures from the Surface Temperature of a Cooling Sphere.
PB94-216124

03,865

Thermophysical Properties of $\mathrm{CO} 2$ and CO2-Rich Mixures.

PB94-216199

00,854

Carbon Acidities of Aromatic Compounds. 1. Effects of InRing Aza and External Electron-Withdrawing Groups.

PB94-216595 00,860

Measurement of the Heat of Fusion of Tungsten by a Microsecond-Resolution Transient Technique.
PB94-216686

03,400

Changes in the Redox State of Iridium Oxide Clusters Radiolytic and Electrochemical Studies. PB95-107017

Reduction of Dinitrogen to Ammonia in Aqueous Solution Mediated by Colloidal Metals.

00,867

Solvent Effects in the Reactions of Peroxyl Radicals with Organic Reductants. Evidence for Proton Transfer Mediated Electron Transfer.

PB95-107157

00,873

Combustion of Methanol and Methanol/Dodecanol Spray

PBames. 108544

02,478

Construction of Maximum-Entropy Density Maps, and
Their Use in Phase Determination and Extension. PB95-108593

00,882

Vibrational Energy Transfer in S1 p-Difluorobenzene. A Comparison of Low and Room Temperature Collisions.
PB95-108619

Laboratory Studies of Low-Temperature Reactions of $\mathrm{C} 2 \mathrm{H}$ with $\mathrm{C} 2 \mathrm{H} 2$ and Implications for Atmospheric Models of Titan,
PB95-108726

00,690

Vapor Pressure of 1,1-dichloro-2,2,2-trifluoroethane (R123).

PB95-126231

00,899

Formulation of the Refractive Index of Water and Steam.

PB95-140133 00,900

Glass Temperature of Polymer Blends: Comparison of
Both the Free Volume and the Entropy Predictions with

PB95-140190

01,236

Phase Equilibria in the Systems $\mathrm{CaO}-\mathrm{CuO}$ and $\mathrm{CaO}$ -

PB95-140570

03,048

Reaction of Nitric Oxide with Organic Peroxyl Radicals.

PB95-141107

Application of Thermodynamics to Biotechnology.

PB95-150793

03,529

Thermochemistry of the Hydrolysis of L-arginine to (Lcitrulline + Ammonia) and of the Hydrolysis of L-arginine to (L-ornithine + Urea)

PB95-150801

03,463

Activated Dynamics, Loss of Ergodicity, and Transport in Supercooled Liquids.

PB95-150819

00,925

Resonance Enhanced Multiphoton lonization Spectroscopy of 2-Butene-1-yl (C4H7) between $455-485 \mathrm{~nm}$.

PB95-151031 00,670

Progress in the Development of a Chemical Kinetic

Database for Combustion Chemistry.
PB95-151056

01,384

Mechanism and Rate Constants for the Reactions of Hydrogen Atoms with Isobutene at High Temperatures.

Experimental Method for Obtaining Critical Densities of Binary Mixtures: Application to Ethane + n-Butane. PB95-151148

00,930

Anisotropic Phase Separation Kinetics in a Polymer Blend Solution Following Cessation of Shear Studied by Light Scattering.

01,241
Measurements of the Virial Coefficients and Equation of State of the Carbon Dioxide + Ethane System in the Supercritical Region.

PB95-151353

03,906

Ebulliometric Measurement of the Vapor Pressure of Difluoromethane.

00,931

Crystal Chemistry and Phase Equilibrium Studies of the BaO-R2O3-CuO Systems. 2. X-Ray Characterization and Standard Patterns of BaR2CuO4, R=Lanthanides.

95-151734

04,582

Modeling Ceramic Sub-Micron Particle Formation from the Vapor Using Detailed Chemical Kinetics: Comparison with In-situ Laser Diagnostics.

00,671

Susceptibility Critical Exponent for a Nonaqueous Ionic Binary Mixture Near a Consolute Point Binary Mixture Near a Consolute Point.
PB95-152112

00,938

Rate Constants for the Gas Phase Reactions of the OH Radical with CF3CF2CHCl2 (HCFC-225ca) and CF2CICF2CHCIF (HCFC-225cb)

PB95-152153

00,940

Thermodynamic Interactions and Correlations in Mixtures of Two Homopolymers and a Block Copolymers by Small Angle Neutron Scattering

01,247

Thermal Equilibration Near the Critical Point: Effects Due Three Dimensions and Gravity.

PB95-152922

03,919

Dielectric Studies of Fluids with Reentrant Resonators.

00,952

Viscosity of the Saturated Liquid Phase of Six Halogenated Compounds and Three Mixtures.

PB95-162368

00,960

Temperature Dependence of the Rate Constants for Reductants.

PB95-162756

00,964

Equilibrium and Calorimetric Investigation of the Hydrolysis of L-Tryptophan to (Indole + Pyruvate + Ammonia).
PB95-163317 00,661 Incinerability of Perchloroethylene and Chlorobenzene PB95-163457

01,388

Shock Tube Techniques in Chemical Kinetics.

00,968 PB95-163465

Application of the Taylor Dispersion Method in
Supercritical Fluids.
PB95-164323
00,977 PB95-164323

Standard States, Reference States and Finite-Concentraion Effects in Near-Critical Mixtures with Applications to Aqueous Solutions.

00,979

Ebulliometric Measurement of the Vapor Pressure of 1 . Chloro-1,1-Difluoroethane and 1,1-Difluoroethane.

PB95-164489

00,984

Effect of Microstructure on Phase Formation in the Reaction of $\mathrm{Nb} / \mathrm{Al}$ Multilayer Thin Films.

$\begin{array}{ll}\text { PB95-168415 } & 03,352\end{array}$

Vapour Pressure Measurements on 1,1,1,2Tetrafluoroethane (R134a) from 180 to $350 \mathrm{~K}$.
PB95-168886

03,265

Critical Scaling Laws and a Classical Equation of State

PB95-169249 00,990

Measurements of the Vapor Pressures of Difluoromethane, 1-Chloro-1,2,2,2-Tetrafluoroethane, and Pentafluoroethane

PB95-169272

03,270

PB95-171948

00,991

Vapor Pressures and Gas-Phase PVT Data for 1-Chloro1,2,2,2-Tetrafluoroethane ( $\mathrm{R} 124)$

PB95-175154

03,271

Partial Scattered Intensities from a Binary Suspension of Polystyrene and Silica.

PB95-175618

00,996

Estimating the Virial Coefficients of Small Polar Molecules.

PB95-176236 03,276

Radiochromic Solid-State Polymerization Reaction.

PB95-180683

01,271

Thermodynamic Study of the Reactions of Cyclodextrins with Primary and Secondary Aliphatic Alcohols, with Dand L-Phenylalanine, and with L-Phenylalanineamide.

PB95-180873

01,016

Measurement of Very-Low Partial Pressures.

02,659

Terminally Anchored Chain Interphases: The Effect of Multicomponent, Polydisperse Solvents on Their Equiibrium Propert

PB95-181079

01,273

Thermodynamics of the Hydrolysis of N-Acetyl-Lphenylalanine Ethyl Ester in Water and in Organic Solvents.

PB95-203386

01,053

Thermochemical and Chemical Kinetic Data for Fluorinated Hydrocarbons.

01,056

Vapour Pressures and Gas-Phase ( $p$, rho $n, T)$ Values for CF3CHF2(R125)

04,019

Radiance Temperatures (in the Wavelength Range 523$907 \mathrm{~nm}$ ) of Group IV B Transition Metals Titanium, Zirconium, and Hafnium at Their Melting Points by a PulseHeating Technique.

PB96-102207

03,356

Critical Lines for Type-III Aqueous Mixtures by GeneralCorresponding-States Models.

01,063

Measurements of Thermophysical Properties of Nickel Near Its Melting Temperature by a Microsecond-Resolution Transient Technique.

PB96-102579

04,210

Principle of Congruence and Its Application to Compressible States.

01,068

Comparison of a Fixed-Charge and a Polarizable Water

PB96-111620

01,072

Nonequilibrium Thermodynamic Theory of Viscoplastic Materials.

PB96-111661 04,034

Physical Limit to the Stability of Superheated and Stretched Water.

01,083

Flow-Induced Structure in Polymers Chapter 17. PhaseSeparation Kinetics of a Polymer Blend Solution Studied by a Two-Step Shear Ouench

03,388

All-Metal Collection System for Preparative-Scale Gas All-Metal Collection System for Preparative-Scale Gas
Chromatography: Purification of Low-Boiling-Point Compounds.

PB96-123435

00,619

Static Dielectric

Slow Dynamics of Segregation in Hydrogen-Bonded Polymer Blends.

Shape of the Temperature-Entropy Saturation Boundary.

PB96-135066

02,506

Interaction Coefficients for 15 Mixtures of Flammable and Non-Flammable Components.

PB96-146626

03,281

Thermodynamic Properties of Dilute and Semidilute Solutions of Regular Star Polymers.

PB96-146808

01,284

Experimental Determination of the Ionization Energy of Estimations of Delta(sub f) $\mathrm{H}$ (sup deg)(sub 0)(IO)(sup - 1$)$ and PA(IO).

PB96-146899

00,694

Model for Calculating Virial Coefficients of Natural Gas Hydrocarbons with Impurities.

PB96-156047

04,064

Virial Coefficients of Five Binary Mixtures of Fluorinated Methanes and Ethanes. 
Isopiestic Investigation of the Osmotic and Activity Coefficients of Aqueous $\mathrm{NaBr}$ and the Solubility of the $\mathrm{NaBr}+\mathrm{H} 2 \mathrm{O}$ System over Wide Ranges of Tempera-

ture and Pressur

01,175

Resonance Enhanced Multiphoton Ionization Spectroscopy of the SnF Radical.

01,176

Fluoride Elimination Upon Reaction of Pentafluoroaniline with e (sub eq')(sup -), $\mathrm{H}$, and $\mathrm{OH}$ Radicals in Aqueous Solution.

PB97-111314

01,177

Thermodynamic Properties of Synthetic Otavite, $\mathrm{CdCO}(\mathrm{cr})$ : Enthalpy Increment Measurements from 4.5 $\mathrm{K}$ to $350 \mathrm{~K}$.

PB97-111447 00,680

Ionization Energies, Appearance Energies and Thermochemistry of CF2O and FCO. 01,178

Compatibilization of Polymer Blends by Complexation. 2 . Kinetics of Interiacial Mixing.

01,295

One-Electron Oxidation of Metalloporphycenes as Studied by Radiolytic Methods.

PB97-111967

01,179

Oxidation of Caffeic Acid and Related Hydroxycinnamic Acids.

00,651

Absorption Cross Sections, Kinetics of Formation, and Self-Peaction of the 10 Radical Produced via the Laser Photolysis of N2O/2/N2 Mixtures.

PB97-112361

01,180

Ferric lon Assisted Photooxidation of Halocetates.

PB97-112460 00,521

Thermochemical Studies of Inorganic Chalocogenides by Fluorine-Combustion Calorimetry: Binary Compounds of Germanium and Silicon with Sultur, Selenium and Tellu-

PB97-112528

01,181

Thermodynamics of (Germanium + Selenium): A Review and Critical Assessment.

01,182

Atmospheric Lifetimes of HFC-143a and HFC-245fa: Flash Photolysis Resonance Fluorescence Measurements of the $\mathrm{OH}$ Reaction Rate Constants.

00,118

Dimensional Crossover in the Phase Separation Kinetics of Thin Polymer Blend Films.

PB97-113088

03,395

Vapor Pressure of 1,1,1,2,2-Pentafluoropropane. 00,684
PB97-113237

Thermodynamic Properties of CF3-CHF-CHF2,

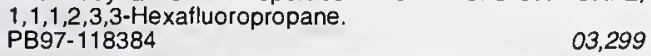

Thermodynamic Properties of CHF2-CF2-CHF, 1,1,2,2,3-

Pentafluoropropane.
PB97-118392

03,300

Kinetics of the Reaction of the Sulfate Radical with the Oxaiate Anion.

01,186

Thermodynamic Properties of Silicides. 5. Standard Molar Enthalpy of Formation at the Temperature $298.15 \mathrm{~K}$ of Trimolybdenum Monosilicide Mo3Si Determined by Fluorine-Combustion Calorimetry.

PB97-119358

01,190

Conference Report: Calorimetry Conference (50th).

03,722

Experimental Determination of the Rate Constant for the Reaction of $\mathrm{C}_{2} \mathrm{H} 3$ with $\mathrm{H} 2$ and Implications for the Partitioning of Hydrocarbons in Atmospheres of the Outer PB97-122295

00,112

Oxidation of Ferrous and Ferrocyanide lons by Peroxyl

Padicals. 202

01,191

HERMOELECTRIC COOLERS

Device for Subambient Temperature Control in Liquid Chromatography.
PB95-140604

00,573

HERMOMAGNETIC EFFECTS

Transverse Thermomagnetic Effects in the Mixed State Transverse Thitmomagnetic Effects in the Mixed
and Lower Critical Field of High-Tc Superconductors.

and Lower Critical Field of High-Tc Superconductors.
PB95-153250

\section{THERMOMECHANICAL ANALYZER}

Advances in the Measurement of Polymer CTE:

Micrometer- to Atomic-Scale Measurements

03,390

HERMOMECHANICAL PROCESSING

Hot-Deformation Apparatus for Thermomechanical Processing Simulation.

200136

03,207

THERMOMECHANICAL TREATMENTS

Hot-Deformation Apparatus for Thermomechanical Processing Simulation.

HERMOMETERS

03,207

Assessment of Uncertainties of Liquid-in-Glass Thermometer Calibrations at the National Institute of Standards and Technology.

02,625
Resistance Thermometers with Fast Response for Use in Aapidly Oscillating Gas Flows.

03,261

Systematic Studies of the Effect of a Post-Detection Filter on a Josephson-Junction Noise Thermometer.

PB95-162988

ITS-90 Calibration Facility.

03,940

PB-96-160916

00,627

Direct Comparison of Three PTB Silver Fixed-Point Cells with the NIST Silver Fixed-Point Cell.

PB96-161286

00,628

Investigation of High-Temperature Platinum Resistance Thermometers at Temperatures Up to $962 \mathrm{C}$, and, in Some Cases, 1064C.

$\infty 0,629$

\section{ERMOMETRY}

Investigation of the ITS-90 Subrange Inconsistencies for 25.5 Omega SPRT

25.5 Omega SP

00,630

NIST Assessment of ITS-90 Non-Uniqueness for 25.5 Ohm SPRTs at Gallium, Indium and Cadmium Fixed

PB96-161310

00,631

NIST Implementation and Realization of the ITS-90 Over the Range $83 \mathrm{~K}$ to $1235 \mathrm{~K}$ : Reproducibility, Stability, and Uncertainties

00,632

NIST Measurement Assurance of SPRT Calibrations on the ITS-90: A Quantitative Approach

PB96-161336

$\infty, 633$

Preliminary Results of a Comparison of Water TriplePoint Cells Prepared by Different Methods.

00,634

THERMONUCLEAR REACTOR MATERIALS

Electromechanical Properties of Superconductors fo DOE Fusion Applications.

B 94-139672

02,250

rradiation Damage in Inorganic Insulation Materials for ITER Magnets: A Review.

03,705

Superconductor Critical Current Standards for Fusion ApSuperconductor Critical Current Standards for Fusion Ap-
plications. Final Progress Report, October 1993-July

PB95-169538

02,222

THERMONUCLEAR REACTORS

Determination of Atomic Data Pertinent to the Fusion Energy Program. Progress Report for FY 92.

04,402

THERMOPHYSICAL PROPERTIES

Properties of Working Fluids for Thermoacoustic RefrigAD-A297 420/2

04,864

Thermophysical Properties of HFC-143a and HFC-152a Ouarterly Report, 1 July 1993--30 September 1993. DE94004236 03,245 Development of Measurement Capabilities for the Thermophysical Properties of Energy-Related Fluids. Annual Report, December 1, 1990--November 30, 1991. Development of Measurement Capabilities for the Thermophysical Properties of Energy-Related Fluids. AnThermophysical Properties of Energy-Related Fluids. Annual Report, December 1, 1993--November 30, 1994.
DE94017738 Thermophysical properties of HCFC alternatives. Quarterly report, 1 July 1994--30 September 1994. DE95002261

03,247

Dynamic Measurements of Thermophysical Properties of Metals and Alloys at High Temperatures by Subsecond Pulse Heating Techniques.

N94-25124/6

03,309

Thermophysical Properties of Fluids for the Gas Industry Final Report, February 1, 1988-August 31, 1993. PB94-146677

02,472

Thermophysical Property Data for Supercritical Fluid Extraction Design

00,668

Thermophysical Properties of $\mathrm{CO} 2$ and $\mathrm{CO} 2$-Rich Mixtures.

00,854

Thermodynamic Properties of the Methane-Ethane Sys-

PB95-125779

00,891

Thermophysical Property Computer Packages from NIST.

04,203

Reference Data for the Thermophysical Properties of Cryogenic Fluids.

PB95-168688 03,263

Annex 18: An Intemational Study of Refrigerant Prop-

PB95-168936

03,266

Development of a Dual-Sinker Densimeter for High-Accuracy Fluid P-V-T Measurements.

$\begin{array}{ll}\text { PB95-168951 03,267 } & \end{array}$ Measurements of the Vapor Pressures of
Difluoromethane, 1-Chloro-1,2,2,2-Tetrafluoroethane, and PB95

Predictive Extended Corresponding States Model for Pure and Mixed Refrigerants Including an Equation of State for and 134 a.

03,275

Estimating the Virial Coefficients of Small Polar Molecules.

PB95-176236

03,276

Measurements of Thermophysical Properties of Nicke Near Its Melting Temperature by a Microsecond-Resolution Transient Technique.

PB96-102579

04,210

Physical Properties of Alternatives to the Fully Halo genated Chlorofluorocarbons.

PB96-119573 03,279

Thermophysical Properties of Fluids for the Gas Industry.
PB96-122437

New Data and Correlations for the Custody Transfer of Natural Gas Liquids.

PB96-176664

02,499

Report of the Refrigeration, Air Conditioning and Heat Pumps Technical Options Committee.

PB96-176755

03,293

ERMOPILES

Process Gas Chromatography Detector for Hydrocarbons PB95-141099

THERMOPLASTIC RESINS

Predicting the Ignition Time and Burning Rate of Thermoplastics in the Cone Calorimeter

PB96-154794

01,418

Computer Simulations of Binder Removal from 2-D and 3-D Model Particulate Bodies.

PB97-121339

00,418

THERMOSETTING PLASTICS

AMRF Composite Fabrication Workstation

PB94-172681

02,810

THERMOSETTING RESINS

Characterization of Molecular Network of Thermosets Using Neutron Scattering.

THIAZOLE ORANGE

Enhanced Detection of PCR Products Through Use of TOTO and YOYO Intercalating Dyes with Laser Induced Fluorescence - Capillary Electrophoresis.

THICKNESS

Jacket Thickness Requirements for Seismic Retrofitting of Circular Bridge Columns.

THICKNESS GAGES

Gas-Coupled, Pulse-Echo Ultrasonic Crack Detection and Thickness Gaging

THIN DIELECTRIC RELIABILITY

New Physics-Based Model for Time-Dependent- Dielec tric-Breakdown
PB96-186093

THIN FILMS

Temperature Dependence of the Morphology of Thin Diblock Copolymer Films as Revealed by Neutron Reflectivity.

01,199

Morphology of Symmetric Diblock Copolymers as Re-

vealed by Neutron Reflectivity.
PB95-140075

01,234

Grazing-Incidence X-Ray Photoelectron Spectroscopy: A Novel Approach to Thin Film Characterization.

PB95-153128 04,589

Preparation of 2-Dimensional Ultra Thin Polystyrene Film by Water Casting Method.

PB95-162806 


\section{KEYWORD INDEX}

Coexistence of Grains with Differing Orthorhombicity in High Ouality YBa2Cu3O7-delta Thin Films.

PB96-135033

04,742

Growth of Epitaxial $\mathrm{KNbO} 3$ Thin Films

02,409

Effects of Etching on the Morphology and Surface Resistance of YBa2Cu3O7-delta Films.

PB96-135355

02,410

Low Magnetostriction in Annealed NiFe/Ag Giant Magnetoresistive Multilayers.

04,762

Integrated Thin-Film Micropotentiometers.

02,109

PB96-146709

Magnetoresistance of Thin-Film NiFe Devices Exhibiting Single-Domain Behavior.

04,766

Performance of Multilayer Thin-Film Multijunction Thermal Converters.

02,084

Effects of Substrate Surface Steps on the Microstructure of Epitaxial Ba2YCu3O7-x Thin Films on (001) LaAlO3. PB96-148184 04,774

Epitaxial Nucleation and Growth of Chemically Derived Ba2YCu307-x Thin Films on (001) SrTiO3.

04,787

Models of Granualr Giant Magnetoresistance Multilayer Thin Films

PB96-190228 01,968

Observation of the Transverse Second Harmonic Magneto-Optic Kerr Effect from Ni81Fe19 Thin Film Structures.

01,971

Magnetic Flux Pinning in Epitaxial YBa2Cu307-delta Thin

PB96-200746 04,795

Phase Separation in Thin Film Polymer Blends With and

Without Block Copolymer Additives.
PB96-204482

Thin-Film Ruthenium Oxide - Iridium Oxide Thermocouples.

PB97-110225

Thin Film Thermocouple Research at NIST

00,520

PB97-110233

02,283

Observation of Two Length Scales Above ( $T$ sub $N$ ) in a

Holmium Thin Film.
PB97-111942

Development of Thin-Film Multijunction Thermal Converters at NIST.

PB97-112338 02,286

Bias Current Dependent Resistance Peaks in NiFe/Ag Giant Magnetoresistance Multilayers

04,153

High-Current Thin Film Multijunction Thermal Converters and Multi-Converter Modules.

01,989

PB97-112379

Dimensional Crossover in the Phase Separation Kinetics of Thin Polymer Blend Films.

03,395

Diffraction of Neutron Standing Waves in Thin Films with Resonance Enhancement.

04,164

Vortex Images in Thin Films of YBa2Cu3O(sub 7-x) and Bi2Sr2Ca1Cu2O(sub $8+x$ ) Obtained by Low-Temperature Magnetic Force Microscopy.

PB97-119408

04,815

THIN PLATES

Exponentially Rapid Coarsening and Buckling in Coherently Self-Stressed Thin Plates.

PB95-202347

04,821

THINNESS

Fracture Testing of Large-Scale Thin-Sheet Aluminum Alloy.

03,305

THIOLS

Silver Metalization of Octadecanethiol Monolayers SelfAssembled on Gold.

PB95-150744 00,923

UV-Photopatterning of Alkylthiolate Monolayers Self-Assembled on Gold and Silver.

THIOPHENES

Location of a (1)A(sub g) State in Bithiophene.

PB95-202313

00,924

THOMAS-FERMI MODEL

Appearance Intensities for Multiply Charged Ions in a Strong Laser Field

PB96-16044

01,025

THOROTRAST

External Gamma-ray Counting of Selected Tissues from a Thorotrast Patien

PB96-160254

03,637

THREAT EVALUATION

Computer Virus Altacks.

01,715

THREE DIMENSIONAL MODELS

Data-Parallel Algorithm for Three-Dimensional Delaunay Triangulation and Its Implementation.

01,714
THREE DIMENSIONAL MOTION

Visual-Motion Fixation Invariant.

PB9 4-206281

01,836

THROUGH-THE-ARC SENSING

Through-the-Arc Sensing for Monitoring Arc Welding.
PB94-185899

Through-the-Arc Sensing for Measuring Gas Metal Arc Weld Ouality in Real Time.

THYMINE

Substrate Specificity of the Escherichia coli Endonuclease III: Exicision of Thymine- and Cytosine-Derived Lesions in DNA Produced by Radiation-Generated Free Radicals. PB95-153425

03,535

TILES

Hollow Clay Tile Prism Tests for Martin Marietta Energy Systems: Task 2 Testing

00,352

\section{TILTMETERS}

Measurement and Interpretation of Tidal Tilts in a Small Array.

03,686

Measurement of Very Low Frequency Vibrations. PB96-102660

03,687

Accuracy in Time Domain Transmission Line Measurements.

PB96-148069

04,060

High-Speed Interconnection Characterization Using Time Domain Network Analysis.

04,061

TIME-DEPENDENT DIELECTRIC BREAKDOWN

New Physics-Based Model for Time-Dependent- Dielectric-Breakdown.

PB96-186093

02,440

TIME DOMAIN

Accuracy in Time Domain Transmission Line Measure-

ments.
PB96-148069

04,060

High-Speed Interconnection Characterization Using Time Domain Network Analysis.

PB96-148176

04,061

TIME DOMAIN NETWORK ANALYSIS

Optimizing Time-Domain Network Analysis.

PB96-157821

02,085

INTERVAL ANALYZERS

High Resolution Time Interval Counter.

TIME KEEPING

How to Get NIST-Traceable Time on Your Compute

PB96-200647

TIME LAPSE

Evaluation of Sprinkler Activation Prediction Methods.
PB96-141056

Computing the Effect of Sprinkler Sprays on Fire Induced Gas Flow

PB96-147111

TIME MEASUREMENT

Time and Frequency Technology at NIST.

01,522

Future of Time and Frequency Dissemination.

N94-30684/2

01,524

Utc Dissemination to the Real-Time User

N19960042622

01,521

Time Scale Algorithm for Post-Processing: AT1 Plus Frequency Variance.
PB94-172772

TIME MEASUREMENTS

Accurate Measurement of Time.

PB96-119482

Atomic Clock

PB96-119490

TIME MEASURING INSTRUMENTS

Calibration of GPS Equipment in Japan

PB95-151452

01,525

Measurement Methods and Algorithms for Comparison of Local and Remote Clocks.

PB96-102652

01,549

TIME OF FLIGHT SPECTROMETERS

Speed-of-Sound Measurements in Liquid and Gaseous PBir. 151957

04,186

TIME-PERTURBATION TUNING

Time-Perturbation Tuning of MIMD Programs.

PB94-164399

Time-Perturbation Tuning of MIMD Programs PB94-172566

01,681

TIME SCALE ALGORITHMS

Promise into Practice: Implementing TA2 on Real Clocks at NIST.

PB95-151478

01,533

TIME SERIES ANALYSIS

Wavelet Variance, Allan Variance, and Leakage. 01,509

TIME SIGNALS

Implementation of a Standard Format for GPS Common View Data.

N95-32323/4

03,779

Utc Dissemination to the Real-Time User

N19960042622 01,521

Preliminary Comparison of Time Transfers via LASSO GPS and Two-Way Satellite.

PB95-151098

01,529

How to Get

01,559

TIME STANDARDS

NIST Internet Time Service

AD-P009 132/2

Future of Time and Frequency Dissemination.

01,519

AD-P009 138/9

01,520

Atoms in Optical Molasses: Applications to Frequency PB95-108882

03,876

Time and Frequency: Bibliography of NIST Publications, March 1995

PB95-220463

Time Generation and Distribution.

PB96-103049

Time and Frequency Metrology.

PB96-190319

TIME-TO-CONTACT

Calculating Time-to-Contact Using Real-Time Ouantized

Optical Flow.

PB95-210522

01,548

E TRANSFER

Future of Time and Frequency Dissemination

AD-P009 138/9

GPS and Two-Way Satellite.

PB95-151098

Smart Clock: A New Time.

PB95-151445

Calibration of GPS Equipment in Japan

PB95-151

cent Progress.

PB95-161089

Use of lonosph

Sermer GPS Co

View Data.

PB96-17658

TIMEKEEPING

Wavelet Analysis for Synchronization and Timekeeping

PB96-200381

TIMING DEVICES

Time and Frequency Technology at NIST.

N94-30641/2

TIMING ERRORS

yond.

PB97-113187

TIN

Anomalous Odd- to Even-Mass Isotope Ratios in Reso-

nance Ionization with Broad-Band Lasers.
PB94-211406

03,839

TIN ALLOYS

VAMAS interlaboratory comparisons of critical current vs. strain in $\mathrm{Nb}$ (sub 3) $\mathrm{Sn}$.

DE95016656

04,433

Transverse stress effect on the critical current of intemal tin and bronze process $\mathrm{Nb}$ (sub 3 ) Sn superconductors. DE95016659 
Development of the National Marine Mammal Tissue Bank.

02,586

Alaska Marine Mammal Tissue Archival Project: Specimen Inventory. PB95-171344 02,589 Considerations in the Design of an Environmental Specimen Bank: Experiences of the National Biomonitoring Specimen Bank Program.

PB96-112370

02,527

National Status and Trends Program Specimen Bank: Sampling Protocols, Analytical Methods, Results, and Archive Samples.$$
\text { TTAN }
$$

02,598

Laboratory Studies of Low-Temperature Reactions of $\mathrm{C} 2 \mathrm{H}$ with $\mathrm{C} 2 \mathrm{H} 2$ and Implications for Atmospheric Models of Titan

PB95-108726

00,690

TITANIUM

Apparent Bias in the X-Ray Fluorescence Determination of Titanium in Selected NIST SRM Low Alloy Steels.

PB95-108759 03,212

Use of Sum Rules on the Energy-Loss Function tor the Evaluation of Experimental Optical Data.

PB95-150736 04,264

Factors Significant to Precracking of Fracture Specimens. PB96-109558 03,358 Effect of Suppressants on Metal Fires.

01,402

PB96-10957

01,402

Void Shape in Sintered Titanium.

02,705

Optical and Modeling Studies of Sodium/Hatide Reactions for the Formation of Titanium and Boron Nanoparticles.
PB97-113054 00,682

Magnetic Characteristics and Measurements of Filamentary Nb-Ti Wire for the Superconducting Super Collider. Thermodynamic Calculation of the Ternary Ti-Al-Nb System.

03,336

Thermodynamic Assessment and Calculation of the Ti-Al Pystem.

03,337

Defermination of Hydrogen in Titanium Alloy Jet Engine Compressor Blades by Cold Neutron Capture Prompt Gamma-ray Activation Analysis. PB95-175956

01,448 Journal of Research of the National Institute of Standards and Technology. September/October 1993. Volume 98 , Number 5.

PB96-169057

03,368

Electronic Structure and Phase Equilibria in Ternary SubStitutional Alloys.

03,378

TITANIUM ALUMINUM NIOBIUM

Ordered omega-Derivatives in a Ti-37.5Al-2ONb at\% Alloy.

03,331

TITANIUM CONTAINING ALLOYS

Ordered omega-Derivatives in a Ti-37.5Al-2ONb at \% Alloy. 211091

03,331

TITANIUM DIOXIDE

$X$-Ray Diffraction from Anodic TiO2 Films: In situ and Ex situ Comparison of the Ti(0001) Face.

PB94-185972

00,782

TITANIUM DISILICIDE

Enthalpy Increment Measurements from 4.5 to $350 \mathrm{~K}$ and the Thermodynamic Properties of Titanium Disilicide(cr) to $1700 \mathrm{~K}$

PBNIUM INTERMETALLICS

Coherent Precipitates in the BCC/Orthorhombic Two Phase Field of the Ti-Al-Nb System.

PB94-198694

03,317

Ordered omega-Derivatives in a Ti-37.5Al-2ONb at \% Alloy.

03,331

TTANIUM OXIDES

Neutron Reflectometry Studies of Surface Oxidation.
PB95-150421

TITANIUM SILICIDES

Enthalpy Increment Measurements from $4.5 \mathrm{~K}$ to $350 \mathrm{~K}$ and the Thermodynamic Properties of the Titanium Silicide Ti5Si3(cr).

COPHEROL

00,679

Liquid Chromatographic Method for the Determination of Carotenoids, Retinoids, and Tocopherols in Human Serum and in Food
PB95-153599

00,593

TOLERANCE INTERVALS

Tolerance Intervals for the Distribution of True Values in the Presence of Measurement Errors.

PB95-150405

03,434

TOLERANCES (MECHANICS)

NIST Handbook 44, 1994: Specifications, Tolerances and Other Technical Requirements for Weighing and Measur- ing Devices as Adopted by the 78th National Conference on Weights and Measures 1993.

02,888

Design, Specification and Tolerancing of Micrometer-Tolerance Assemblies.

erance Assem
PB95-209862

02,913

TOLUENE

Correlation of the Ideal Gas Properties of Five Aromatic Hydrocarbons.
PB95-175816

TOMOGRAPHY

Tomographic Decoding Algorithm for a Nonoverlapping Redundant Array.

TOOL COMMAND LANGUAGE

Debugger for Tcl Applications.

TOOLS

Computer Vision Based Tool Setting Station.

PB94-199858

02,944

Fabrication of Optics by Diamond Turning.

PB96-111695

02,954

Combining Interactive Exploration and Optimization for Assembly Design

Chemical Aspects of Tool Wear in Single Point Diamond Turning.
PB97-112601

TOOTH ENAMEL

03,021

EPR Dosimetry of Cortical Bone and Tooth Enamel Irradiated with $X$ and Gamma Rays: Study of Energy Dependence.

03,639

TOOTH REMINERALIZATION

Effects on Whole Saliva of Chewing Gums Containing Calcium Phosphates.

TORPEDOES

03,563

Assessment of Data by a Second-Order Transfer Function. 182390

03.760

TORSION

Torsional Dilatometer for Volume Change Measurements on Deformed Glasses: Instrument Description and Measurements on Equilibrated Glasses.

21116

03,379

SOA Standards and Total Ouality Management.

PB96-111844

01,743

SOA and TQM in Software Ouality Improvement. PB96-160791

01,754

TOUGHNESS

Cryogenic Toughness of Austenitic Stainless Steel Weld Metals: Effect of Inclusions.

PB95-161261

03,214

TOXAPHENE

NIST Standard Reference Materials (SRMs) for Polychlorinated Biphenyl (PCB) Determinations and Their APplicability to Toxaphene Measurements.

PB95-140109

02,585

TOXIC SUBSTANCES

Recently Developed NIST Food Related Standard Reference Materials.

PB94-198322

00,035

Investigation of S2F10 Production and Mitigation in Compressed SF6-Insulated Power Systems.

Pressed 12388

02,467

Secondary Target X-Ray Excitation for In vivo Measurement of Lead in Bone.

PB95-108767

03,496

Analysis by a Combination of Gas Chromatography and Tandem Mass Spectrometry: Development of Quantitative Tandem-in-Time Ion Trap Mass Spectrometry: Isotope DiIution Ouantification of 11-Nor-Delta-9-Tetrahydro cannabinol-9-Carboxylic Acid.

PB96-117221

02,561

TOXICITY

Documentation for Immediately Dangerous to Life or Health Concentrations (IDLHS).

PB94-195047

03,602

Effective Measurement Techniques for Heat, Smoke, and Toxic Fire Gases.

PB94-198439

01,369

Toxicity, Fire Hazard and Upholstered Furniture.

00,289

Total Surface Areas of Group IVA Organometallic Compounds: Predictors of Toxicity to Algae and Bacteria.

PB94-211331

00,814

Development of a New Small-Scale Smoke Toxicity Test Method and Its Comparison with Real-Scale Fire Tests. PB94-213253

00,350

Relating Bench-Scale and Full-Scale Toxicity Data.

TPT PB95-125977

00,361

ime-Perturbation Tuning of MIMD Programs. PB94-172566

01,684

TRACE ELEMENTS

Long-Term Stability of Carrier-Free Polonium Solution

PB94-185170

00,685

Establishing Ouality Measurements for Inorganic Analysis of Biomaterials.
PB94-199726

00,548

Mixed Diet Reference Materials for Nutrient Analysis of Foods: Preparation of SRM-1548 Total Diet.

PB95-151692

03,593

Trace Elements Associated with Proteins. Neutron Activation Analysis Combined with Biological Isolation Tech.

PB95-163101

00.597

Methods for Analysis of Cancer Chemopreventive Agents in Human Serum.

03,502

Certitying the Chemical Composition of a Biological Mate rial: A Case Study.

PB96-164272

00,636

race Element Concentrations in Cetacean Liver Tissues Archived in the National Marine Mammal Tissue Bank.

Liquid Chromatographic Determination of Carotenoids in Human Serum Using an Engineered C30 and a C18 Stationary Phase.

TRACE QUANTITIES Procedure for Measuring Trace Ouantities of S2F10,
S2OF10, and S2O2F10 in SF6 Using a Gas Chromatograph-Mass Spectrometer.

PB96-119755

TRACER TECHNIQUES

Rare-Earth Isotopes as Tracers of Particulate Emissions: An Urban Scale Test.

PB94-161635

02,535

Air Change Effectiveness Measurements in Two Modern Office Buildings.

00,243

Monitoring Polymer Cure by Fluorescence Recovery After Photobleaching

PB94-211422

01,218

NIST Traceable Reference Material Program for Gas Standards: Standard Reference Materials.

PB96-210786

00,644

Measurement Comparability, Traceability, and Measurement Assurance Programs.

TRADE

Helping to Reduce Technical Barriers to Trade. PB96-190046

00,49

TRAFFIC MANAGEMENT

Introduction to Traffic Management for Broadband ISDN.
PB94-142494

TRAINING

Computer Security Training and Awareness Course ComPendium.

01,589

Life-Cycle Costing Workshop for Energy Conservation in Buildings: Student Manual

RAINING AIDS

Proposed International Interactive Courseware Standard. PB96-123161

Fire Safety of Passenger Trains: A Review of Current Approaches and of New Concepts.

PB94-152006

04,848

TRAJECTORIES 


\section{KEYWORD INDEX}

TRANSIENTS

Transient Subcritical Crack-Growth Behavior in Trans formation-Toughened Ceramics.

PB94-200656

03,038

TRANSISTORS

Handbook Preferred Circuits Navy Aeronautical Electronic Equipment. Supplement Number 2.

AD-A278 783/6

00,027

Physics for Device Simulations and Its Verification by Measurements.

02,339

Characterization of Two-Dimensional Dopant Profiles: Status and Review.

PB96-119300 02,400

Characterization of Two-Dimensional Dopant Profiles: Status and Review.

02,451

TRANSIT TIME

Utc Dissemination to the Real-Time User. N19960042622

01,521

TRANSITION MANAGEMENT

Implementing a Transition Manager in the AMRF Cell Controller.

PB94-199932

02,817

TRANSITION METALS

Ultraviolet Multiplet Table. Finding List for Spectra of the Elements Molybdenum to Lanthanum $(Z=42$ to 57$)$; Haf-

nium to Radium $(Z=72$ to 88$)$.

00,709

Radiance Temperatures (in the Wavelength Range 523. $907 \mathrm{~nm}$ ) of Group IV B Transition Metals Titanium, Zirconium, and Hafnium at Their Melting Points by a PulseHeating Technique.

03,356

RANSITION PROBABILITIES

Comprehensive Spectroscopic Data Tabulations and Progress in the Compilation of Atomic Transition Prob-

PB95-151551

03,909

Stars. Atmospheres, Radiative Transfer.

00,095

Transition Regions of Capella.

PB96-123336

00,105

Transition Regions of Capelia (1995)

00,108

TRANSITION TEMPERATURE

Siep-Edge and Stacked-Heterostructure High-TC Josephson Junctions for Voltage-Standard Arrays.
PB96-102066 Simulation of C60 Through the Plastic Transition Temperatures.

RANSMISSION ELECTRON MICROSCOPY

04,713

Proficiency Tests for the NIST Airborne Asbestos Program - 1991.

00,537

Proficiency Tests for the NIST Airbome Asbestos Program - 1992.

00,539

Airbome Asbestos Method: Bootstrap Method for Determining the Uncertainty of Asbestos Concentration. VerSion 1.0

TRANSMISSION LINES

00,646

Transmission/Aeflection and Short-Circuit Line Methods for Measuring Permittivity and Permeability.

02,211

Currents Induced on Multiconductor Transmission Lines Currents Induced on Multiconductor Transmission Lines by Radiation and Injection.
PB94-211943

02,215

Accurate Experimental Characterization of Interconnects: A Discussion of 'Experimental Electrical Characterization of Interconnects and Discontinuities in High-Speed Digital Systems

PBStems.

02,217

Interconnection Transmission Line Parameter Characterization.

PB94-216397

02,218

Accurate Transmission Line Characterization.

02,220

Accuracy in Time Domain Transmission Line Measure-

PB96-148069

04,060

Accurate Electrical Characterization of High-Speed Inter-

Connections.

02.240

Dimensional Characterization of Precision Coaxial Transmission Line Standards.

PB96-176482

02,241

Dielectric and Magnetic Measurements from -50C 10 $200 \mathrm{C}$ and in the Frequency $\mathrm{B}$ and $50 \mathrm{MHz} 102 \mathrm{GHz}$ PB96-191382

02,245

TRANSMISSION LOSS

LP11-Mode Leakage Loss in Coated Depressed Clad Fi-

02,145

PB95-141115

Yielding

Stationary Values.

PB95-175501

04,298
Fibre Splice Loss: A Simple Method of Calculation. PB95-175519

04,299

TRANSMITTANCE

Comparison of Regular Transmittance Scales of Four National Standardizing Laboratories.

04,233

Determination of the Transmittance Uniformity of Optical Filter Standard Reference Materials. Filter Standard

02,182

Transmission Properties of Short Curved Neutron Guides. Part 1. Acceptance Diagram Analysis and Calculations. PB96-102199 04,02

Partially Coherent Transmittance of Dielectric Lamellae. PBg6-14

04,359

TRANSMITTER RECEIVERS

Electric-Field Strengths Measured Near Personal Transceivers.
PB94-172020

TRANSPORT

Study of Droplet Transport in Alcohol-Based Spray Flames Using Phase/Doppler Interferometry. Large Eddy Simulations of Smoke Movement in Three Di-

PB96-190012

01,426

TRANSPORT CROSS SECTIONS

Absence of Quantum-Mechanical Effects on the Mobility

of Argon lons in Helium Gas at $4.35 \mathrm{~K}$

PB97-122543

01,194

TRANSPORT PROPERTIES

Transient Methods for Thermal Conductivity.

PB94-198405

04,197

Need for, and Availability of, Working Fluid Property Data:

Results from Annexes XIII and XVIII.

PB95-168969

03.268

Transport and Diffusion in Three-Dimensional Composite

PB95-176129

04,668

Mathematical Modeling and Computer Simulation of Fire Phenomena.

00,384

Calculation of the Thermal Conductivity and Gas Permeability in a Uniaxial Bundle of Fibers.

03,058

Survey of Concrete Transport Properties and Their Meas.$$
\text { urement. }
$$

PB95-220489

00,396

Ab initio Calculations for Helium: A Standard for Transport Property Measurements.

01,060

Physical Characterization of Herparin by Light Scattering.

PB96-119383

03,598

Thermophysical Property Standard Reference Data from

NIST

PB96-167358

01,153

Greenspan Acoustic Viscometer for Gases.

04,220

TRANSPORTATION SAFETY

New Concepts for Fire Protection of Passenger Rail

Transportation Vehicles. (NIST Reprint).

04,863

TRAPPED PARTICLES

Magne10-Optical Trapping of Metastable Xenon: IsotopeShift Measurements.

PB95-151254

03,905

Trapped Atoms and Laser Cooling

PB95-151627

03,911

Electrostatic Modes of Ion-Trap Plasmas.

03,920

Trapped Ions and Laser Cooling 4: Selected Publications of the lon Storage Group of the Time and Frequency $\mathrm{D}$ vision, NIST, Boulder, Colorado.

Vision, NIST, Bou 172358

04,108

TRAPPING (CHARGED PARTICLES)

Infrared and Near-Infrared Spectra of HCC and DCC Trapped in Solid Neon.

AD-A295 578/9

Disorder Trapping in Ni2TiAl.

03,773

B94-198942

03,322

Comment on $<A$ A Dynamic Electric Trap for Ground-State Atoms $>>$.

PB96-200290

04,123

TREASURE ISLAND NAVAL STATION

Assessment of Site Response Analysis Procedures. PB95-210928

00,450

TRENDS

Technology Trends in Telecommunications: An Overview. PB94-123080 01,462 Scanned Probe Microscopies: Opportunities and Issues in Metrology.

02,426

TRIACETYLOLEANDOMYCIN

Two-Dimensional POMMIE Carbon-Proton Chemical Shift Correlated (13)C NMR Spectrum Editing.

00,809

RIANGULATION

Data-Parallel Algorithm for Three-Dimensional Delaunay Triangulation and Its Implementation.

01,714

Inserting Line Segments into Triangulations and PB95-198933

03,415

\section{TRIBLOCK COPOLYMER}

Flow-Induced Structure in Polymers: Chapter 16. ShearInduced Changes in the Order-Disorder Transition Temperature and the Morphology of a Triblock Copolymer.

PB96-123237

03,127

Shear-Induced Changes in the Order-Disorder Transition Temperature and the Morphology of a Triblock Copoly-

PB97-118772

03,130

TRIBOLOGY

Wear Mechanism Maps of Ceramics.

PB94-172368 $\quad 03,229$

Considerations on Data Requirements for Tribological Modeling.

PB94-172731

02,962

Wear of Selected Materials and Composites Sliding against Mos2 Films.

03,231

Tribological Characteristics of Alpha-Alumina at Elevated Temperatures.

02.963

Tribochemical Reaction of Stearic Acid on Copper Surface Studied by Surtace Enhanced Raman Spectroscopy. PB94-212057 02,964

Tribology Education: Present Status and Future ChatPB94-212362

02,965

Tribological Data: Needs and Opportunities.

PB95-140596

Modified Surtace

PB95-176087

Boundary Lubrication of Silicon Nitride.

03,237

PB95-213583

03,125

mina Composites.

PB96-103163

03,226

03,168

Wear Mechanism Maps of $440 \mathrm{C}$ Martensitic Stainless

PB96-111810

04,834

Mechano-Chemical Model. Reaction Temperatures in a Concentrated Contact.

Tribological Behavior of 440/Diamond-Like-Carbon Film Couples

PB96-119714

03,019

TRIBOMETERS

Tribometer for Measurements in Hostile Environments. 
TRYPANOSOMA BRUCE

beta-D-Glucosyl-Hydroxymethyluracil: A Novel Modified Base Present in the DNA of the Parastic Protozoan T. PB94.172319

03,524

TRYPTOPHAN

Equilibrium and Calorimetric Investigation of the Hydroly-

sis of L-Tryptophan to (Indole + Pyruvate + Ammonia).
PB95-163317

TUNABLE LASERS

Narrow-Band Tunable Diode Laser System with Grating Feedback, and a Saturated Absorption Spectrometer for Cs and $\mathrm{Rb}$.

04,319

Doppler-Free Spectroscopy of Large Polyatomic Mol-

ecules and van der Waals Complexes.
PB96-119581

04,339

TUNGSTEN

Radiance Temperature (in the Wavelength Range 519 $906 \mathrm{~nm}$ ) of Tungsten at lis Melting Point by a Pulse-Heating Technique.

03,397

Measurement of the Heat of Fusion of Tungsten by a Microsecond-Resolution Transient Technique.

03,400

Use of Sum Rules on the Energy-Loss Function for the Use of Sum Rules on the Energy-Loss Function tor the PB95-150736

04,264

Application of ODF to the Rietveld Profile Refinement of Polycrystalline Solid.

03,401

Analytical Method for Determining Thermal Conductivity from Dynamic Experiments.

04,024

Microstructural Characterization of Cobalt-Tungsten Coated Graphite Fibers.
PB96-159231

01,951

TUNGSTEN IONS

Observation of Pd-Like Resonance Lines Through $\mathrm{Pr}(32+)$ and $\mathrm{Zn}$-Like Resonance Lines of $\operatorname{Er}(38+)$ and PBo5-15065

03,896

Rh 1 Isoelectronic Sequence Observed from $\operatorname{Er}(23+)$ to $P t(33+)$.

PB95-150652

03,898

Spectra of Ag I Isoelectronic Sequence Observed from $\operatorname{Er}(21+)$ to $\mathrm{Au}(32+)$.

TUNGSTEN SILICIDE

03,899

UV Absorption Cross Sections of Methylchloroform: Temperature-Dependent Gas and Liquid Phase Measure-

PB96-201041

00,643

TUNNEL JUNCTIONS

High Critical Temperature Superconductor Tunneling Spectroscopy Using Squeezable Electron Tunneling Junctions.

TUNNELING

New Oxide Degradation Mechanism for Stresses in the Fowler-Nordheim Tunneling Regime.

PB96-200985

04,128

UNNELING (ELECTRONICS)

Electronic Microrefrigerator Based on a Normal-InsulatorSuperconductor Tunnel Junction.

UNNELING SPECTROSCOPY

Tunneling Spectroscopy of Thallium-Based High-Temperature Superconductors.

PB95-161709

04,606

Tunneling Spectroscopy of bcc(001) Surface States. PB96-155585

04,775

TURBIDITY

Neutron Scattering Study of Shear Induced Turbidity in

Polystyrene/Dioctyl Phthalate Solutions at High Shear Rates.

01,197

Neutron Scattering Study of Shear Induced Turbidity in Polystyrene Dissolved in Dioctyl Phthalate. PB95-161865

01,256

TURBINE PUMPS

Wear Mechanism Maps of $440 \mathrm{C}$ Martensitic Stainless Steel.

PB96-111810

04,834

URBULENCE

Fire Propagation in Concurrent Flows.

01,365

Mixing and Radiation Properties of Buoyant Turbulent Diffusion Flames.

TURBULENT BOUNDARY LAYER

Characteristics of Turbulence in a Boundary Layer with Zero Pressure Gradient.

04,192

Evolution of a Turbulent Boundary Layer Induced by a Three-Dimensional Roughness Element.

04,200

RBULENT FLAMES

Turbulent Flame Spread on Vertical Corner Walls. 01,403
PB96-114764
Interaction of HFC-125, FC-218 and CF3I with High Speed Combustion Waves.

PB96-1764 17

03,290

Turbulent Upward Flame Spread on a Vertical Wall under External Radiation.

PB94-207388 00,341

Experimental Study of the Stabilization Region of Lifted

$\begin{array}{ll}\text { Turbulent-Jet Diffusion Flames. } & 01,405 \\ \text { PB96-122676 }\end{array}$

Review of Flows Driven By Natural Convection in Adiabatic Shafts.

01,416

Tomographic Reconstruction of the Moments of Local Probability Density Functions in Turbulent Flow Fields. PB96-180195

04,219

TURBULENT JETS

Global Density Effects on the Self-Preservation Behavior of Turbulent Free Jets.

\section{TURNING (MACHINING)}

Real Time Compensation for Tool Form Errors in Turning Using Computer Vision.

04,207$$
\text { (107231 }
$$

TWO-BALL COLLISION TEST APPARATUS

Asperity-Asperity Contact Mechanisms Simulated by a Two-Ball Collision Apparatus.

02,966

TWO DIMENSIONAL FLOW

Adaptive, Predictive 2-D Feature Tracking Algorithm for Finding the Focus of Expansion

\section{TWO-PEAK METHOD}

New Method to Evaluate Deposit Forming Tendencies of Liquid Lubricants by Differential Scanning Calorimetry. Deposit Forming Tendencies of Diesel Engine Oils-Correlation between the Two-Peak Method and Engine Tests.

01,452

TWO PHASE AQUEOUS EXTRACTION SYSTEMS

Continuous Counter-Current Two Phase Aqueous Extrac-

tion. 9 -161212

00,675

TWO PHASE FLOW

Simulating Smoke Movement through Long Vertical Shafts in Zone-Type Compartment Fire Models. 00,368
PB95-143152 Generation Rate and Distribution of Products of CombusGeneration Rate and Distribution of Products of Combus-
tion in Two-Layer Fire Environments: A Model and Applition in Two-Layer Fire Environments: A Model and AppliCations. 02173

01,398

\section{TYPE 2 SUPERCONDUCTORS}

High-Frequency Linear Response of Anisotropic Type-II Superconductors in the Mixed State.

04,483

Nonlinear Response of Type-ll Superconductors in the Mixed State in Slab Geometry.

PB94-200367

04,484

TYPE-III MIXTURES

Critical Lines for Type-III Aqueous Mixtures by Generalized Corresponding-States Models.

ized Correspondi

01,063

\section{ULTRACOLD ATOMS}

Measurements of Fluorescence from Cold Atoms: Localization in Three-Dimensional Standing Waves.

PB95-163879

03,943

Laser Modification of Ultracold Collisions: Experiment. PB96-157987

04,075

\section{ULTRAFILTRATION}

Determination of Osmotic Pressure and Fouling Resistances and Their Effects on Performance of Ultrafiltration

00,841

Characterization of the Adsorption-Fouling Layer Using Globular Proteins on Ultrafiltration Membranes. PB94-212909

00,842

ULTRAHIGH VACUUM

Laser Photoionization Measurements of Pressure in Vac-

PB95-180600

03,964

Measurement of Very-Low Partial Pressures.

02,659

ULTRASONIC FLAW DETECTION

Selection of Appropriate Ultrasonic System Components for NDE of Thick Polymer-Composites. PB94-185279

03,133

ULTRASONIC FLOW DETECTION

Appropriate Ultrasonic System Components for NDE of Thick Polymer Composites.

03,148

Examination of Objects Made of Wood Using Air-Coupled Ultrasound.

03,404

Ultrasonic Measurement of Residual Stress in Railroad Wheel Rims.

04,849

ULTRASONIC MICROSCOPY

Material Characterization By a Time-Resolved and Polarzation-Sensitive Ultrasonic Technique.

PB97-122576

02,764

ULTRASONIC RADIATION

Absorption of Ultrasonic Waves in Air at High Frequencies (10-20 MHz).

04,182

Absorption of Sound in Gases between 10 and $25 \mathrm{MHz}$ :

PB94-199015

04,783

ULTRASONIC REFERENCE BLOCKS

NIST Calibration of ASTM E127-Type Ulitrasonic RefPB94-191640

02,702

ULTRASONIC-RESONANCE SPECTROSCOPY

Ultrasonic-Resonance Spectroscopy of Bulk and Layered

141338

04,759

ULTRASONIC SPECTROSCOPY

Ultrasonic Spectroscopy of Metallic Spheres Using ElecPB94-212537

ULTRASONIC TESTS

Infinite Divisibility and the Identification of Singular Wave-

PB94-172111

02,701

Ultrasonic Measurements of Surface Roughness.

PB94-172137

04,181

NIST Power Reference Source.

PB94-211513 00,148

Appropriate Ultrasonic System Components for NDE of

Thick Polymer Composites
PB95-125696

03,148

Ultrasonic Technique for Sizing Voids Using Area Func-

PB95-151916

02,904

Ultrasonic Measurement of Sheet Anisotropy and Formability.

Ultrasonic Method for Reconstructing the Two-Dimensional Liquid-Solid Interface in Solidifying Bodies. PB95-161782

03,349

Pulse-Echo Ultrasonic Evaluation of the Integrity of Seams of Single-Ply Roof Membranes.

Uitrasonic Measurement of Residual Stress in Cast Stee Railroad Wheels.

PB95-169199

Ultrasonic Methods.

PB96-190327

04,852

ULTRASONIC VELOCITIES

Estimation of the Orientation Distribution of Short-Fiber Composites Using Ultrasonic Velocities.

PB96-176656

03,178

ULTRASONIC WAVE TRANSDUCERS

High-Sensitivity Acoustic Emission Sensor/Preamplifier Subsystems.

Ultrasound Power Measurement Techniques at NIST PB96-179569

02,684

ULTRASONICS

Well-Shielded EMAT for On-Line Ultrasonic Monitoring of PMA Welding.

ULTRASONOGRAPHY

Ultrasonic Sensing of GMAW: Laser/EMAT Detect Detection System. 


\section{KEYWORD INDEX}

Silicon Photodiodes Optimized for EUV and Soft X-Ray PB94-199478

02,124

ULTRAVIOLET RADIATION

Development and Calibration of UVNUV Radiometric

PB94-199098 04,229

UV-Photopatterning of Alkylthiolate Monolayers Self-Assembled on Gold and Silver.

PB95-150751

00,924

Improved Dose Metrology in Optical Lithography PB96-179510

02,439

ULTRAVIOLET SPECTRA

Ultraviolet Multiplet Table. Finding List for Spectra of the Elements Molybdenum to Lanthanum ( $Z=42$ to 57$)$; Hafnium to Radium ( $Z=72$ to 88 ).

AD-A278 131/8

00,709

Ultraviolet Multiplet Table.

AD-A278 446/0

00,710

Spectrum and Energy Levels of Five-Times-lonized Niobium (Nb VI)

04,226

Ultraviolet Observations of Stellar Coronae: Early Results from HST.

PB94-213428

00,064

Wavelengths and Isotope Shitts for Lines of Astrophysical Interest in the Spectrum of Doubly lonized Mercury $(\mathrm{Hg}$ III).

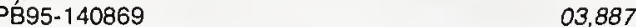

Spectrum and Energy Levels of Triply lonized Barium (Ba IV).

03,888

Improved Wavelengths for Prominent Lines of $\mathrm{Cr} X \mathrm{XV}$ to

Cr XXII.
PB95-150629

03,895

Rb-Like Spectra: Pd X to Nd XXIV

PB95-150645

03,897

Spectra of $\mathrm{Ag}$ I Isoelectronic Sequence Observed from Er(21+) 10 Au(32+). Analysis of the $\left.\begin{array}{l}5 s(2) 5 p(2)- \\ (5 s 5 p(3)+5 s(2) 5 p 5 d+5 s(2) 5 p 6 s)\end{array}\right)$ Transitions of Four-Times
lonized Xenon ( $\mathrm{Xe} \mathrm{V)}$. PB95-150769 (Xe V)

03,900

Laser-Produced and Tokamak Spectra of Lithiumlike Iron, $\mathrm{Fe}(23+)$

PB95-180857

04,314

High-Velocity Plasma in the Transition Region of AU Microscopii: Evidence for Magnetic Reconnection and Saturated Heating during Quiescent and Flaring Condi-

00.091

GHRS Observations of Cool, Low-Gravity Stars. 1. The Far-Ultraviolet Spectrum of alpha Orions (M2 lab). $\begin{array}{ll}\text { PB96-112016 } & 00,094 \\ \text { Sleuthing the Dynamo: HST/FOS Observations of UV }\end{array}$ Sleuthing the Dynamo: HST/FOS Observations of UV Emissions of Solar-Type Stars in Young Clusters.
PB96-122817 00,098 High Velocity Plasm in the Transition Region of Au Mic: A Stellar Analog of Solar Explosive Events. PB96-123294

00,102

Transition Regions of Capella

PB96-123336

00,105

Recalibration for the Final Archive of the International UItraviolet Explorer (IUE) Satellite.

PB96-135264

00,106

Transition Regions of Capella (1995)

PB96-176714

00,108

Temperature Dependence of the Ultraviolet Absorption Cross Section of CF3I.

PB96-204169

01,168

ULTRAVIOLET SPECTRORADIOMETERS

Report on USDA Ultraviolet Spectroradiometers.

PB96-214648

00,125

ULTRAVIOLET STAR

Deuterium and the Local Interstellar Medium: Properties for the Procyon and Capella Lines of Sight.

00,111

ULTRAVIOLET STARS

Accurate Measurements of the Local Deuterium Abundance from HST Spectra.

PB96-200621

00,110

UNCERTAINITY

Look at Uncertainties over Twenty Decades of Pressure MB95-168506

02,655

UNCERTAINTY

RangeCAD and the NIST RCS Uncertainty Analysis.
PB94-218591

New Expressions of Uncertainties for Humidity Calibrations at the National Institute of Standards and Technology.

02,645

UNCERTAINTY ANALYSIS

Statistical Descriptors in Crystallography. 2. Report of a Working Group on Expression of Uncertainty in Measure-

04,764
UNCERTAINTY BOUNDS

Bounds on Frequency Response Estimates Derived from Uncertain Step Response Data

PB96-122874

03,419

UNCLASSIFIED SENSITIVE INFORMATION

Guidance to Federal Agencies on the Use of Trusted Pystems.

01,597

UNDERGROUND DISPOSAL

4SIGHT Manual: A Computer Program for Modelling Degradation of Underground Low Level Waste Concrete

PB95-231593

03,726

UNDERGROUND SPACE

Numerical Simulation of Rapid Combustion in an Underground Enclosure

\section{UNDERWATER NAVIGATION}

Outline of a Multiple Dimensional Reference Model Archiecture and a Knowledge Engineering Methodology for In telligent Systems Control.

PB95-220414

03,703

UNDERWATER VEHICLES

Intelligent Control for Multiple Autonomous Undersea Ve

PB94-211877

03,747

UNITED STATES

Differences in Competitive Strategies between the United States and Japan

00,013

Earthquake and Fire in Japan: When the Threat Became

PB95-175238

00,201

Japan Technology Program Assessment. Simulation: State-of the-Art in Japan

02,827

Ceramic Powders Characterization: Results of an International Laboratory Study.

PB95-270039

02,672

Electrical Product Requirements (Especially Quality Requirements) in the United States.

01,929

Working Conference on Global Growth of Technology: Is America Prepared. Held in Gaithersburg, Maryland on December 7, 1995

PB96-210059

00,018

Directory of U.S. Private Sector Product Cerification Pro-

grams.

02,688

Standards Activities of Organizations in the United States. PB97-124135

00,006

UNITED STATES AIR FORCE ACADEMY

Robotics Application to Highway Transportation. Volume 1. Final Report.
PB95-203790

03,654

UNITED STATES BOTANIC GARDEN

Corrosion Resistance of Materials for Renovation of the United States Botanic Garden Conservatory. PB94-154390

00,032

UNITED STATES CAPITOL.

Warping of Terrace Pavers at the U.S. Capitol Building. PB96-193651

00411

UNIVERSAL TIME

Utc Dissemination to the Real-Time User.

N19960042622

01,521

UNIX (OPERATING SYSTEM)

Using Expect to Automate System Administration Tasks. PB94-213329

01,697

\section{UNMANNED VEHICLES}

Real-Time Vision for Autonomous and Teleoperated Control of Unmanned Vehicles.

Real-Time Vision for Unmanned Vehicles.

03,702 PB94-211893

\section{UPHOLSTERY}

Toxicity, Fire Hazard and Upholstered Fumiture. 00,289
PB94-198454

Influence of Ignition Source on the Flaming Fire Hazard of Upholstered Furniture. (NIST Reprint).

PB95-180162

00,297

UPWARD FLAME SPREAD

Wall Flame Heights with External Radiation. PB95-163481

00,380

URACIU/5- ( (BETA-D-GLYCOPYRANOSYL OXY)-METHYL)

beta-D-Glucosyl-Hydroxymethyluracil: A Novel Modified Base Present in the DNA of the Parastic Protozoan T. Base

PB94-172319

03,524

URANIUM

Characterization of the Binding of Gallium, Platinum, and Uranium to Pseudomonas Fluorescens by Small-Angle $X$ ray Scattering and Transmission Electron Microscopy.

URANIUM 235

Measurement of the $(235) \mathrm{U}(\mathrm{n}, \mathrm{f})$ Reaction from Thermal to keV.

PB95-140422

03,886

URANIUM 236 TARGET

Intermediate Structure in the Neutron-Induced Fission Cross Section of $236 \mathrm{U}$

PB94-185741

03,802

URANIUM BERYLLIUM

Superconducting Energy Gap of Bulk UBe13.

PB95-150116

04,559

URANIUM COPPER ALUMINIDES

Magnetic Properties of Single-Crystalline UCu3Al2. PBg5-180717

04,686

URANIUM GOLD STANNIDES

Crystallographic and Magnetic Properties of UAuSn

PB95-140521

RANIUM IONS

Magnetic Dipole Line from U LXXI Ground-Term Levels Predicted at 3200 Angstroms.

URANIUM OXIDES

High Temperature Reactions of Uranium Dioxide with Various Metal Oxides.

AD-A286 648/1

00,717

URANIUM PLATES

Neutron Diffraction Texture Study of Deformed Uranium Plates.

PB97-111587

03,010

URANIUM PLATINUM GERMANIDES

Incommensurate Magnetic Order in UPtGe.

PB95-140513

04,542

URBAN AREAS

Sources of Urban Contemporary Carbon Aerosol. PB95-175659

02,551

URINALYSIS

Determination of 3-Quinuclidinyl Benzilate (Qnb) and Its Maior Metabolites in Urine by Isotope Dilution Gas Chromatography Mass Spectrometry.

PB94-199379

03,492

How to Verify Reference Materials

PB95-151486

03,497 Standard Reference Material.

03,499

URINE

Determination of Amphetamine and Methamphetamine in a Lyophilized Human Urine Reference Material.
PB95-175444

03,597

Intercomparison Study of (237)Np Determination in Artificial Urine Samples.

03,633

Certification of Phencyclidine in Lyophilized Human Urine Reference Materials.

03,508

US COUNCIL FOR ENERGY AWARENESS

Review of the USCEANIST Measurement Assurance Program for the Nuclear Power Industry.

03,712 
Review of the USCEANIST Measurement Assurance Program for th
PB95-126272

03,712

Databases Available in the Research Information Center of the National Institute of Standards and Technology.
PB95-128641 Initial NIST Testing Policy for STEP: Beta Testing Program for AP 203 Implementations. National PDES PB95-154688

02,779

Automated Inspection: The Integration of National Standards and Commercial Products at NIST.

PB95-163077

02,906

Joumal of Research of the National Institute of Standards and Technology, September/October 1994. Volume 99 Number 5.

04,293

Concepts of the NIST EXPRESS Sener.

02,781

NIST Serial Holdings, 1995.

PB95-188926

02,746

Materials Science and Engineering Laboratory Annual Report, 1994. NAS-NRC Assessment Panel, April 6-7. 1995.

02,976

NIST Industrial Impacts: A Sampling of Successful Partnerships (Revision, March 1995)

PB95-209193

00,489

Electronics and Electrical Engineering Laboratory Technical Progress Bulletin Covering Laboratory Programs, April to June 1991, with 1992 EEEL Events Calendar.
PB95-209821 Design, Specification and Tolerancing of Micrometer-Tolerance Assemblies.

02,913

Electronics and Electrical Engineering Laboratory Technical Progress Bulletin Covering Laboratory Programs, January to March 1992, with 1992/1993 EEEL Events PB95-210480

01,917

Materials Science and Engineering Laboratory Annual

Report, December 1993.

02,668

Design Engineering Research at NIST

02,784

NIST and the Navy: Past, Present and Future

0,655

Databases Available in the Research Information Center of the National Institute of Standards and Technology (December 1995)

02,734

Fire Hazard Model Developments and Research Efforts at NIST.

PB96-159652

00,407

Montgomery Education Connection and Resource Education Awareness Partnership Making Connections between Local Schools and NIST Volunteers.

00,134

Uniform Laws and Regulations in the Areas of Legal Metrology and Motor Fuel Ouality as Adopted by the 80th National Conference on Weights and Measures 1995 1996 Edition.
PB96-172309

NIST Serial Holdings, 1996.
PB96- 172523

02,927

02,748

NIST List of Publications, LP 103, March 1996. National Semiconductor Metrology Program. Publications 1995: NIST Building and Fire Research Laboratory.

PB96-183074

00,226

NIST Frequency Measurement Service.

01,561

Optoelectronics at NIST.

02,202

Bibliography of the NIST Electromagnetic Fields Division Publications.
PB96-210778

01,980

Materials Science and Engineering Laboratory Annual Report, 1995. Technical Activities.

Report, 1995.
PB96-214754

03,009

Science, Technology, and Competitiveness: Retrospective on a Symposium in Celebration of NIST's 90th Anniversary and the 25th Anniversary of the Gaithersburg Laboratories, November 14-15, 1991. PB97-121610

USABILITY

Usability Engineering: Industry-Government Collaboration for System Effectiveness and Efficiency.

SCEA (US COUNCIL FOR ENERGY AWARENESS)

Review of the USCEANNIST Measurement Assurance Program for the Nuclear Power Industry.
PB95-126272

USER GUIDE

MV++ v. 1.5a MatrixNector Class Reference Guide.

Fire Data Management System, FDMS 2.0, Technical
Fin MANUA

01,777 Documentation.

01,358
Reference Manual for the Algorithm Testing System Version 2.0.
PB96-128244

02,922

USER MANUALS (COMPUTER PROGRAMS)

CONTAM93 User Manual.

PB94-164381

FIREDOC Users Manual, 3rd Edition.

PB95-128674

02,536

USER NEEDS

User Profile for Researchers Studying Objects: Implications for Computer Systems.

188463

User Study: Informational Needs of Remote National Archives and Records Administration Customers.

PB95-154738

02,725

USER REQUIREMENTS

Information Technology Standards in a Changing World: The Rose of the
PB96-160866

02,737

UTILIZATION

Asynchronous Transfer Mode Procurement and Usage Guide

VACUUM COATING

Wear of Selected Materials and Composites Sliding against MoS2 Films

PB94-172749

03,231

ACUUM GAGES

Vacuum Gauges and Partial Pressure Analyzers.

PC-Based Spinning Rotor Gage Controller.

02,650

PB95-175832

02,609

VACUUM-GAP CAPACITORS

Capacitors with Very Low Loss: Cryogenic Vacuum-Gap Capacitors.
PB97-122600

VACUUM ULTRAVIOLET RADIATION

Development and Calibration of UVIVUV Radiometric Sources.

04,229

Photographic Response to X-Ray Irradiation. 1. Estimation of the Photographic Error Statistic and Development of Analytic Density-Intensity Equations.

PB94-200086

03,821

Photographic Response to X-Ray Irradiation. 2. Correlated Models.

03,822

VALIDATION

Ada Compiler Validation Summary Report: Certificate Number 940902S1.11377 UNISYS Corporation. IntegrAda for Windows NT, Version 1.0. Intel Deskside Server with Intel 80486DX266 => Intel Deskside Server with Intel 80486DX266.

AD-A288 571/3

01,658

Ada Compiler Validation Summary Report: Certificate Number 940902S1.11376. UNISYS Corporation IntegrAda for Windows $\mathrm{NT}$, Version 1.0. Intel Deskside
Server for Intel Pentium $60 \mathrm{MHz} \Rightarrow$. Intel Deskside ServServer for Intel Pentium $60 \mathrm{MH}$ er with Intel Pentu

01,659

Ada Compiler Validation Summary Report: Certificate Number 9410125111379 TISOFT, Inc. Green Hills Optimizing Ada Compileer, Version 1.8.7 with PATCK ID 1 ProLiant 2000 Model $5 / 66$

AD-A288 573/9

01,660

Ada Complier Validation Summary Report: Certificate Number: 940929S1.11378. Digital Equipment Corporation DEC Ada for DEC OSF/1 AXP Systems, Version 3.2 DEC 3000 Model 400 AXP Workstation $=>$ DEC 3000 Model 400 AXP Workstation.

AD-A288 574/7

01,661

ADA Compiler Validation Summary Report, VC Number 950303S1.11381. Digital Equipment Corporation - Compiler Name: DEC Ada for OpenVMS Alpha Systems, Version 3.2 .

01,663

Analysis of Standards for the Assurance of High Integrity Software.

03,735

Verification and Validation

01,765

Verification and Validation of Reengineered Soffware. PB96-161401

01,766

VALIDATION SUMMARY REPORTS

Ada Compiler Validation Summary Report: Certificate Number. 93102951.11330 . Digital Equipment Corporation, DEC Ada for DEC OSF/1 AXP Systems, Version 3.1, DEC 3000 Model 400 AX

AD-A274 872/1

01,639

Ada Compiler Validation Summary Report: Certificate Number: 931217S1.11336 Control Data Systems, Inc NOS/VE Ada, Version 1.4 Cyber 180-930-31 $\Rightarrow$ Cybe 180-930-31

AD-A275 $977 / 7$

01,640

Ada Compiler Validation Summary Report. Certificate Number: 931119S1.11332, DDC-I, Inc. DACS MIPS

Sun SPARCstation IPX $=>$ DACS Sun SPARC/SunOS to MIPS R3000 Bare Instruction Set Architecture Simulator, Version 4.7.1.

Ada Compiler Validation Summary Report: Certificate Number: 931119S1.11331 DDC-I, Inc. DACS Sun SPARC/SunOS to 80386 PM Bare Ada Cross Compile System, Version 4.6.4

Ada Compiler Validation Summary Report. Certificate Number: $930927 \mathrm{~S} 1.11328$ Green Hills Software C Ada Version 1.1 ZENY $386 \Rightarrow$ ZENY 386

AD-A277 $981 / 7$

01,643

Ada Compiler Validation Summary Report: Certificate Number: 94032551.11341 DDC-I, DACS Sun SPARC/ SunOS to 80186 Bare Ada Cross Compiler System, Version 4.6.4 Sun SPARCstation IPX $=>$ Intel iSBC $186 / 100$

AD-A279 643/1

01,645

Ada Compiler Validation Summary Report: Certificate Number: 940325S1.11349 DDC-I, DACS Sun SPARC/ Solaris to 80386 PM Bare Ada Cross Compiler System with Rate Monotonic Scheduling. Version 4.6.4 Sun SPARCclassic $\Rightarrow$ Intel iSBC 386/116 (Bare Machine). AD-A279 644/9 01,646 Number: $940325 S 1.11354$ DDC-I, DACS Sun SPARC Solaris Native Ada Compiler System, Version 4.6.2 Sun SPARCclassic $=>$ Sun SPARCclassic.

AD-A279 645/6

01,647

Ada Compiler Validation Summary Report: Certificate Uum SunOS to $680 \times 0$ Bare Ada Cross Compiler System Lynwood j435TU (68030) (Bare Machine). Lynwood j435T

01,648

Ada Compiler Validation Summary Report: Certificate Number: 94032551.11343 DDC-1, DACS Sun SPARC/ Solaris to 80186 Bare Ada Cross Compler System, Version 4.6.4 Sun SPARCclassic $\Rightarrow$ Intel ISBC 186/100 Bare Machine

AD-A279 757/9

01,649

Ada Compiler Validation Summary Report: Certificate Number: 940325S1.11344 DDC-I, DACS Sun SPARC Solaris to 80186 Bare Ada Cross Compiler System with Rate Monotonic Scheduling, Version 4.6.4 Sun SPARCclassic $=>$ Intel iSBC 186/100 (Bare Machine).

01.650

Ada Compiler Validation Summary Report: Certificate Number: $940325 S 1.11347$ DDC-I, DACS Sun SPARC/ SunOS to $680 \times 0$ Bare Ada Cross Compiler System (SELynwood j435TU (68030) (Bare Machine).

AD-A279 778/5

01,651

Ada Compiler Validation Summary Report: Certificate Number: 940325S1.11342 DDC-I, DACS Sun SPARC SunOS to 80186 Bare Ada Cross Compiler System with Rate Monotonic Scheduling Version 4.6.4 Sun AD-A279 779/3

01,652

Ada Compiler Validation Summary Report: Certificate Number: 940325S1.11351 DDC-I, DACS Sun SPARC/ SunOS to Pentium PM Bare Ada Cross Compiler System with Rate Monotonic Scheduling, Version 4.6.4 Sun SPARCstation IPX $\Rightarrow$ Intel Pentium (operated as Bare Machine) based in Xpress Desktop (Intel product number: XBASE6E $4 \mathrm{~F} \cdot \mathrm{B}$

AD-A279 804/9

01,653

Ada Compiler Validation Summary Report: Certificate Number: $940325 S 1.11350$ DDC-I, DACS Sun SPARC SunOS to Pentium PM Bare Ada Cross Compiler System, 4.6.4 Sun SPARCstation IPX $=>$ Intel Pentiu (Intel Product Number: XBASE6E4F-B).

Ada Compiler Validation Summary Report: Certificat 


\section{KEYWORD INDEX}

VAN DER WAALS COMPLEX

P-Type Doubling in the Infrared Spectrum of NO-HF PB94-211463

00,817

\section{VANADIUM}

Comparative Strategies for Correction of Interferences in Isotope Dilution Mass Spectrometric Determination of Vanadium.

VANADIUM ALLOYS

Electronic Structure and Phase Equilibria in Ternary Substitutional Alloys
PB97-119366

03,378

VAPOR COMPRESSION CYCLE

Theoretical Evaluation of the Vapor Compression Cycle with a Liquid-Line/Suction-Line Heat Exchanger, Economizer, and Ejector.

APOR COMPRESSION REFRIGERATION CYCLE

02,607

Intracycle Evaporative Cooling in a Vapor Compression PB97-116107

VAPOR DEPOSITION

02,762

Measurement Methods and Standards for Processing and Application of Thermal Barrier Coatings.

01,447

Development and Characterization of Insulating Layers on Siticon Carbide: Annual Report for February 14, 1988

$\begin{array}{ll}\text { to February 14, } 1989 . & \\ \text { PB94-155579 } & 02,295\end{array}$

Silver Metalization of Octadecanethiol Monolayers SelfAssembled on Gold

00,923

VAPOR LIOUID EOUILIBRIUM

Vapor-Liquid Equilibria of Ternary Mixtures in the Critical Region on Paths of Constant Temperature and Overall Composition.

VAPOR PHASE

01,139

In situ Characterization of Vapor Phase Growth of Iron Oxide-Silica Nanocomposites: Part 1. 2-D Planar Laser-

Induced Fluorescence and Mie Imaging.
PB97-112478

03,185

VAPOR PHASE EPITAXY

In situ Observation of Surface Morphology of InP Grown on Singular and Vicinal (001) Substrates.

04,636

AAPOR PHASES

Thermodynamic Properties of Gas Phase Species of $1 \mathrm{~m}$ portance to Ozone Depletion.

PB94-198215

00,126

Gas Phase Reactions Relevant to Chemical Vapor Deposition: Optical Diagnostics.

03,116

Gas Phase Reactions Relevant to Chemical Vapor Deposition: Numerical Modeling.
PB94-199346

03,117

Homogeneous Gas Phase Decyclization of Tetralin and Benzocyclobutene.

00,928

Multiphoton Ionization Spectroscopy Measurements of Silicon Atoms during Vapor Phase Synthesis of Ceramic Particles.

PB95-151999

03,913

Simulation of Ceramic Particle Formation: Comparison with In-situ Measurements.

PB95-152013

00,674

Gas Phase Reactivity Study of $\mathrm{OH}$ Radicals with 1,1. Dichloroethene and cis-1,1-Dichloroethene and Trans-1,2Dichloroethene over the Temperature Range 240-400 K.
PB95-152146 Rate Constants for the Gas Phase Reactions of the $\mathrm{OH}$ Radical with $\mathrm{CF} 3 \mathrm{CF} 2 \mathrm{CHCl} 2$ (HCFC-225ca) and CF2CICF2CHCIF (HCFC-225cb)

PB95-152153

00,940

VAPOR PRESSURE

Vapor Pressure of 1,1-dichloro-2,2,2-trifluoroethane PB95-126231

00,899

Ebulliometric Measurement of the Vapor Pressure of Difluoromethane.
PB95-151361

00,931

Criteria for Establishing Accurate Vapor Pressure Curves. PB95-163812 00,972

Ebulliometric Measurement of the Vapor Pressure of 1 . Chloro-1,1-Difluoroethane and 1,1-Diffuoroethane.

PB95-164489

00,984

Vapour Pressure Measurements on 1,1,1,2Tetrafluoroethane (R134a) from 180 to $350 \mathrm{~K}$.

Coexisting Densities, Vapor Pressures and Critical Densities of Refrigerants R-32 and R-152a, at $300-385 \mathrm{~K}$ PB95-175691 03,274 Vapour Pressures and Gas-Phase ( $p$, rho $n, T)$ Values for CF3CHF2(R125)

Vapor Pressure of Pentafluorodimethyl Ether.

PB96-201199

04,019

Vapor Pressure of 1,1,1,2,2-Pentafluoropropane.

00,677

PB97-113237
VAPOR PRESSURES

Compressed Liquid Densities, Saturated Liquid Densities and Vapor Pressures of 1,1-Difluoroethane.

01,173

VAPOR TRANSPORT

Vapor Transport in Materials and Process Chemistry. PB94-211745

VARIANCE ESTIMATES

Anova Estimates of Variance Components for a Class of PB96-141163

03,448

VARIANCE (STATISTICS)

Approximate Confidence Intervals on Linear Combinations of Expected Mean Squares.

03,435

Approximate Confidence Intervals on Positive Linear Combinations of Expected Mean Squares.

03,436

VARIANCES

Variances in the Measurement of Ceramic Powder PropPB97-110316

03,100

VARIANT DESIGN

Variant Design for Mechanical Artifacts-A State of the Art PB94-154358

02,768

VARIATIOINS

Fluctuations in Probability Distribution on Chaotic AttracPB96-102330

04,022

VECTOR FIELDS

MV++ V. 1.5a Matrix/Vector Class Reference Guide.
PB96-195326

VELOCITY DISTRIBUTION

Velocity Distribution of Alomic Beams by Gated Optical Pumping.

01,542

VELOCITY MEASUREMENT

Development in the Theory and Analysis of Eddy Current Sensing of Velocity in Liquid Metals.

03,335

VENTCF2 COMPUTER PROGRAM

VENTCF2: An Algorithm and Associated FORTRAN 77 Subroutine for Calculating Flow through a Horizontal Ceiling/Floor Vent in a Zone-Type Compartment Fire Model.
PB94-210127

\section{VENTILATION}

Manual for Ventilation Assessment in Mechanically Ventilated Commercial Buildings.

00,239

PB94-145653

Modern

Air Change Etfecti

Office Buildings.
PB94-185766

Ventilation Rates in Office Buildings.

PB95-108825

02539

Field Measurements of Ventilation and Ventilation Effectiveness in an Office/Library Building

PB95-108833

00,247

Study of Ventilation and Carbon Dioxide in an Office

PBilding.

02542

Ventilation, Carbon Dioxide and ASHRAE Standard 62PB95-162095

Fire and Smoke Control: An Historical Perspective.

PB95-175808

02,544

Limits of $\mathrm{CO} 2$ Monitoring in Determining Ventilation PB95-176004

02,552

Ventilation Effectiveness Measurements in Two Modern Office Buildings.

02,553

Measurements of Outdoor Air Distribution in an Office Building.
PB95-210944 00,264

00,264

Study of Ventilation Measurement in an Office Building.
PB96-155593

NASA Fire Detector Study.

PB96-183108

01,423

VENTILATION SYSTEMS

Assessing Ventilation Effectiveness in Mechanically Ventilated Office Buildings.

PB95-162079

00,255

Indoor Air Ouality Commissioning of a New Office Build-

ing.

00,262

Improving the Evaluation of Building Ventilation

PB96-138508

00,271

Etfectiveness of a Heat Recovery Ventilator, an Outdoor Air Intake Damper and an Electrostatic Particulate Filter at Controlling Indoor Air Ouality in Residential Buildings. at Controlling Indoor Air Ouality in Residential Buildings.
02,564

Workplan to Analyze the Energy Impacts of Envelope Airtightness in Oftice Buildings.

VENTS

Polyvinyl Chloride (PVC) Plastic Drain, Waste, and Vent Pipe and Fittings.

00,326

Acrylonitrile-Butadiene-Styrene (ABS) Plastic Drain Waste, and Vent Pipe and Fittings.

00,327

Combined Buoyancy- and Pressure-Driven Flow Through a Shallow, Horizontal, Circular Vent.

00,344

VENTCF2: An Algorithm and Associated FORTRAN 77 Subroutine for Calculating Flow through a Horizontal Ceiling/Floor Vent in a Zone-Type Compartment Fire Model. PB94-210127

00,345

Calculating Combined Buoyancy- and Pressure-Driven Flow Through a Shallow, Horizontal, Circular Vent; Appl cation to Problem of Steady Burning in a Ceiling-Vented Enclosure.

PB96-164108

00,409

Combined Buoyancy and Pressure-Driven Flow Through a Shallow, Horizontal, Circular Vent

00,410

VERIFICATION

How to Verify Reference Materials.

\section{ERIFICATION INSPECTION}

User's Guide for the Algorithm Testing System Version 2.0. Integrated Vision Touch-Probe System for Dimensional Inspection Tasks.

PB95-255832

02,917

VERIFIED ANALYSIS

Airborne Asbestos Method: Standard Test Method for Verified Analysis of Asbestos by Transmission Electron Microscopy. Version 2.0

PB94-163045

00,526

VERTICAL SURFACES

Heat Flux from Flames to Vertical Surfaces.

PB97-110357

01,438

VERY LARGE SCALE INTEGRATION

Design Guide for CMOS-On-SIMOX. Test Chips NIST3 and NIST4.

PB94-163458

02,297

Color Supplement to NIST Special Publication 400-93. Semiconductor Measurement Technology: Design and Testing Guides for the CMOS and Lateral Bipolar-on-SOI PB94-164316

02,298

Semiconductor Measurement Technology: Design and Testing Guides for the CMOS and Lateral Bipolar-on-SO Test Library.
PB94-178019

02,301

Application of the Modified Voltage-Dividing Potentiometer to Overlay Metrology in a CMOS/Bulk Process. 94-181997

02,302

Electrical Test Structure for Overlay Metrology Referenced to Absolute Length Standards

02,336

Comparisons of Measured Linewidths of Sub-Micrometer Lines Using Optical, Electrical, and SEM Metrologies. Lines Using Optical, Electrical, and SEM Metrologies.
PB95-152807 338 Operating Principles of MultiKron II Performance Instrumentation for MIMD Computers.

PB95-189486

01,628

Operating Principles of the SBus MultiKron Interface

PB95-231783

01,630

MEMS in Standard CMOS VLSI Technology.

Hardware Measurement Techniques for High-Speed Networks.

01,500

VESICLES

Optical Control of Enzymatic Conversion of Sucrose to 


\section{KEYWORD INDEX}

Multi-Stage, Position Stabilized Vibration Isolation System for Neutron Interferometry.

PB95-175022

03,955

Low-Frequency, Active Vibration Isolation System.

02,710

Summary and Results of the NIST Workshop on ProSummary and Results of the NIST Workshop on Proposed Guidelines for Testing and Evaluation of Seismic
Isolation Systems. Held in San Francisco, California on Isolation Syst

PBly 25, 1994

00,463

Guidelines for Pre-Oualification, Prototype and Ouality Control Testing of Seismic Isolation Systems. 01,347 RATION MEASUREMENT

Electro-Optical Sensor for Surface Displacement Measurements of Compliant Coatings

PB94-198223

02,123

Vibration Laboratory Automation at NIST with Personal Computers.

02,648

IBRATION TESTS

Dynamic Shear Modulus Measurements with Four Independent Techniques in Nickel-Based Alloys.

03,320

RATIONAL PREDISSOCIATION

Fragment State Correlations in the Dissociation of NO.HF $(V=1)$.
PB95-164430

00,982

IBRATIONAL SPECTRA

Vibrational Spectra of Molecular lons Isolated in Solid Neon. 6. $\operatorname{CO4}(-)$. (Reannouncement with New Availability Information).

Vibrational Spectra of Molecular lons isolated in Solid Neon. 7. $\mathrm{CO}(+), \quad \mathrm{C} 2 \mathrm{O} 2(+)$ and $\mathrm{C} 2 \mathrm{O} 2(-)$. (Reannouncement with New Availability Information).
AD-A239 729/7 00,706

Vibrational Spectra of Molecular Ions Isolated in Solid Neon: $\mathrm{HCCH}+$ and HCC-. (Reannouncement with New Availability Information)

AD-A253 $551 / 6$

00,707

Vibrational Spectra of Molecular Ions Isolated in Solid Neon. 11. NO2(+), NO2(-), and NO3(-)

00,708

Octacalcium Phosphate. 3. Infrared and Raman Vibrational Spectra.

00,756

New Rydberg States of Aluminum Monofluoride Observed by Resonance-Enhanced Multiphoton lonization SpectrosCopy.

00,797

Inelastic Neutron Scattering Studies of Nonlinear Optical Materials: p-Nitroaniline Adsorbed in ALPO-5. Vibrational Spectra of Molecular Ions Isolated in Solid Neon. $X . \mathrm{H} 2 \mathrm{O}(+), \mathrm{HDO}(+)$, and $\mathrm{D} 2 \mathrm{O}(+)$.

PB95-125670

00,889

Vibrational Spectra of Molecular lons Isolated in Solid Neon. XI. NO2(+), NO2(-), and $\mathrm{NO} 3(-)$

00,890

Neutron Spectroscopic Comparison of Rare-Earth/Hydrogen alpha-Phase Systems.

00,970

Vibrations of Hydrogen and Deuterium in Solid Solution with Lutetium.

Vibrations of $S 1((1) B 2 u)$ p-Difluorobenzene - d4. S1-SO Fluorescence Spectroscopy and ab Initio Calculations.
PB95-202586

Matrix Isolation Study of the Interaction of Excited Neon Atoms with BCl3: Infrared Spectra of BCl(sub 3, sup +) $\mathrm{BCl}($ sub 2, sup + ), and $\mathrm{BCl}($ sub 3 , sup - ).

Vibrational Spectra of Molecular Ions Isolated in Solid Neon. 13. Ions Derived from $\mathrm{HBr}$ and $\mathrm{HI}$.

PB97-119234

01,188

VIBRATIONAL SPECTROSCOPY

Vibrational Excitations and the Position of Hydrogen in Nanocrystalline Palladium.

04,035

Characterization of the Vibrational Dynamics in the Octahedral Sublattices of LaD2.25 and LaH2.25.

01,091

PB96-123724

Electronic Spectra of $\mathrm{CF} 2 \mathrm{Cl}$ and $\mathrm{CFCl} 2$ Radicals Ob-
served by Resonance Enhanced Multiphoton Ionization.

served by Resonance Enhanced Multiphoton Ionization.
PB95-151023 00,927

Oxy-

q Dependence of Self-Energy Effects of the Plane Oxygen Vibration in
PB96-138516

01,096

VIDEO COMMUNICATION

Videoconferencing Procurement and Usage Guide.
PB94-217023

VIDEO COMPRESSION

Nonlinear Color Transformations in Real Time Using a Video Supercomputer.

02,191
VIDEO DATA

Report on the Workshop on Advanced Digital Video in the National Information Infrastructure. Held in Washing on, D.C. on May 10-11, 199

PB95-103677 01,472

National Information Infrastructure and Advanced Digital

01,488

Summary Report on the Workshop on Advanced Digital Video in the National Information Infrastructure.

PB96-141320

01,497

VIDEO MICROSCOPE

Video Microscopy Applied to Optical Fiber Geometry PB95-173068

VIDEO RECORDING

Proposed International Interactive Courseware Standard.
PB96-123161

Development of an Economical Video Based Fire Detection and Location System.

tion and Loca

00,228

\section{VILLAIN MODEL}

Normal Modes and Structure Factor for a Canted Spin System: The Generalized Villain Model.

PB95-140067

04.539

VINYL COPOLYMERS

Piezoelectric and Pyroelectric Polymers

PB95-153367

01,250

VINYL ETHER RESINS

Electron Beam Crosslinking of Poly(vinylmethyl ether) PB94-185550

VINYL PLASTICS

Comparison of Fire Sprinkler Piping Materials: Steel, Copper, Chlorinated Polyvinyl Chloride and Polybutylene, in Residential and Light Hazard Installations.

00,299 PB95-182267

VINYL RADICAL

Deuterium Isotope Effect in Vinyl Radical Combination/ Dispropor tionationfeactions.

01,167

VINYL RADICALS

Reaction Rate Determinations of Vinyl Radical Reactions with Vinyl, Methyl, and Hydrogen Atoms.

00,815

Thermal Pulse Study of the Polarization Distributions Produced in Polyvinylidene Fluoride by Corona Poling at Constant Current.

PB94-172293

01,195

VIRIAL COEFFICIENTS

Estimating the Virial Coefficients of Small Polar Mol.

03,276

Thermodynamic Properties of Dilute and Semidilute Solutions of Regular Star Polymers.

01,284

VIRIAL EQUATION

Measurements of the Virial Coefficients and Equation of State of the Carbon Dioxide + Ethane System in the Supercritical Region.

PB95-151353

03,906

VIRTUAL ENVIRONMENTS

Applying Virtual Environments to Manufacturing.

PB94-142502

02,803

VIRTUAL REALITY

Approaches Using Virtual Environments with Mosaic.

PB95-169108

01,599

Workshop on the Application of Virtual Reality to Manufacturing. Final Report. Held in Gaithersburg, Maryland on August 9 , 1994

02,825

Virtual Environments for Health Care. A White Paper for the Advanced Technology Program (ATP), the National Institute of Standards and Technology.

PB96-147814

03,594

VISCOELASTICITY

Physics Required for Prediction of Long Term Performance of Polym

03,146

VISCOMETERS

Hydrodynamic Similarity in an Oscillating-Body ViscomPBer. 122429

01,082

Greenspan Acoustic Viscometer for Gases. PB96-204417

04,220

VISCOPLASTICITY

Non-Equilibrium Thermodynamic Theory of Viscoplastic Materials.

VISCOSITY

Measurements of the Viscosities of Saturated and Compressed Fluid 1-chloro-1,2,2,2-tetrafluoroethane (R124) and Pentafluoroethane (R125) at Temperatures between 120 and $420 \mathrm{~K}$

PB94-199791

03,254

Prediction of Viscosity of Refrigerants and Refrigerant

Mixtures.
PB94-212099

03,257

Flow of Microemulsions through Microscopic Pores. Two Phase Aqueous Extraction: Rheological Properties of Dextran, Polyethylene Glycol, Bovine Serum Albumin and PB95-161998

00,676

Brownian Diffusion of Hard Spheres at Finite Concentrations.

PB95-164307 00,975

Measurements of the Viscosities of Saturated and Compressed Liquid 1,1,1,2-Tetrafluoroethane (R134a), 2,2 Dichloro-1,1,1-Trifluoroethane (R123) and 1,1-Dichloro-1Fluoroethane (R141b)

PB95-175386

03,273

Non-Perturbative Relation between the Mutual Diffusion Coefficient, Suspension Viscosity, and Osmotic Compressibility: Application to Concentrated Protein Solutions. PB96-102355

Viscosity of Ammonia

PB96-145933

01,062

iscosity of 1,1,1,2,3,3-Hexafluoropropane and tions from $262 \mathrm{~K}$ to $353 \mathrm{~K}$

PB96-176680

03,292

VISCOSITY PREDICTION

Viscosity of Defined and Undefined Hydrocarbon Liquids Calculated Using an Extended Corresponding-States

PB96-167234

02,498

VISUAL DISCRIMINATION

Nonlinear Color Transformations in Real Time Using a Video Supercomputer

PB96-123021

02,191

VISUAL LOOMING

New Method to Calculate Looming for Autonomous Obstacle Avoidance.

PB95-171435

01,600

VISUAL MEASUREMENT

Visual Measurement Technique for Analysis of Nucleate Flow Boiling

VISUAL PERCEPTION

Human and Machine Recognition of Faces: A Survey,
PB96-111687

VISUAL PURSUIT SYSTEMS

Visual Pursuit Systems.

IT AMIN K duction Liquid of Vitamin $\mathrm{K} 1$ in Serum Using Catalytic-Re-

PB96-138425

03,506

VITAMINS

Population Distributions and Intralaboratory Reproducibility for Fat-Soluble Vitamin-Related Compounds in Human

PB96-155536

00,624

VOICE COMMUNICATIONS

Telecommunications Security Guidelines for TelePBity.

01,496

VOID SHAPES

Void Shape in Sintered Titanium

OIDS

Detection of Voids in Grouted Ducts Using the ImpactEcho Method.

PB94-185121

01,306

Ultrasonic Technique for Sizing Voids Using Area Func tions.

PB95-151916

02,904

VOIGHT FUNCTION

Accurate Modeling of Size and Strain Broadening in the

Rietveld Refinement: The 'Double-Voigt' Approach.
PB96-200225

00,664

VOIGHT FUNCTIONS

Voigt-Function Modeling in Fourier Analysis of Size- and 


\section{KEYWORD INDEX}

VOLTAGE NIST Watt Experiment: Monitoring the Kilogram.
PB97-122329

VOLTAGE AMPLIFIERS

100 Ampere, $100 \mathrm{kHZ}$ Transconductance Amplifier. PB96-102629

Comparing the Accuracy of Critical-Current Measurements Using the Voltage-Current Simulator.

02,227

VOLTAGE DIVIDERS

Automatic Inductive Voltage Divider Bridge Operates from $10 \mathrm{~Hz}$ to $100 \mathrm{kHz}$

02,032

Automatic Calibration of Inductive Voltage Dividers for the NASA Zeno Experiment.

02,041

Inductive Voltage Divider Calibration for the NASA Flight Experiment.

02,042

PB95-152856

02,063

TO

PB95-198685

ompari-

Binary versus Decade Inductive Voltage Divider
son and Error Decomposition.

02,071
Active High Voltage Divider with 20-PPM Uncertainty

PB97-119317 02, 104
O Uncertainty.

VOLTAGE MEASUREMENT

Voltage Ratio Measurements of a Zener Reference Using a Digital Voltmeter.

01,906

Electro-Optic-Based RMS Voltage Measurement TechPique. 138490

02,194

VOLTAGE MEASURING INSTRUMENTS

Performance of Commercial CMOS Foundry-Compatible Multijunction Thermal Converters.

02,342

\section{VOLTAGE REFERENCE}

Noise Characteristics Below $1 \mathrm{~Hz}$ of Zener Diode-Based Voltage Reference.

OLTAGE REGULATIONS

04,049

VOLTAGE REGULATIONS

Surging the Upside-Down House: Looking into Upsetting Reference Voltages.

PB96- 112313
OLTAGE STANDARDS

Design and Operation of Series-Array Josephson Voltage Standards.

02,030

$24 \mathrm{GHz}$ Josephson Array Voltage Standard.

$24 \mathrm{GHz}$ Josephson Array Voltage Standard.
$\mathrm{PB94}-211588$ New International Representations of the Volt and Ohm
Etfective January 1, 1990 .

Accuracy Comparisons of Josephson Array Systems. PB95-164687 02,047 Josephson Voltage Standard Based on Single-Flux-Ouantum Voltage Multipliers.

PB95-175600 02,058

Pulse-Driven Programmable Josephson Voltage Standard.

04,148

PB97-111496 04,

Low Voltage Standards in the $10 \mathrm{~Hz}$ to $1 \mathrm{MHz}$ Range.
PB97-112569

VOLTMETERS

Wideband Sampling Voltmeter

PB97-113039

01,990

VOLUME

Torsional Dilatometer for Volume Change Measurements on Deformed Glasses: Instrument Description and Measurements on Equilibrated Glasses.

PB94-211166

03.379

Apparent Molar Heat Capacities and Apparent Molar Volumes of Aqueous Glucose at Temperatures from 298.15 $\mathrm{K}$ to $327.01 \mathrm{~K}$.

PB94-212800

03,459

Glass Temperature of Polymer Blends: Comparison of Both the Free Volume and the Entropy Predictions with Data.

PB95-140190

01,236

VORONOI DIAGRAMS

Submissions to a Planned Encyclopedia of Operations Research on Computational Geometry and the Voronoi/ Delaunay Construct.

PB94-152709

03,425

VORTEX

SSME LOX Duct Flowmeter Design and Test Results.

PB96-161955

04,826

Evolution of a Turbulent Boundary Layer Induced by a Three-Dimensional Roughness Element.

PB94-212818

04,200

VORTEX SHEDDING FLOWMETERS

Vortex Shedding Flowmeters for SSME Ducts

PB95-169215
VORTEX TUBES

Applications of the Vortex Tube in Chemical Analysis. PB94-199171 00,544 Applications of the Vortex Tube in Chemical Analysis. Part 2. Applications

00,615

VORTICES

Direct Observation of Vortex Dynamics in Two-Dimensional Josephson-Junction Arrays.

PB96-102223

02,067

Novel Vortex Dynamics in Two-Dimensional Josephson

Arrays.

02,091

VTS (VALIDATION TESTING SYSTEM)

Validation Testing System Requirements. National PDES PB94-163482

02,771

W317 LIQUID CRYSTAL

Studies of the Higher Order Smectic Phase of the Large Electroclinic Effect Material W317.

PB95-151601

00,935

WAFER BONDING

Thermal Isolation of High-Temperature Superconducting Thin Films Using Silicon Wafer Bonding and Micromachining.
PB96-135017

02,408

WAFER PROBE STATIONS

Verification of Commercial Probe-Tip Calibrations. 02,347

Planar Resistors for Probe Station Calibration.

PB95-163697

02,351

Calibrating On-Water Probes to the Probe Tips.

PB95-163945

02,352

LRM Probe-Tip Calibrations with Imperfect Resistors and Lossy Lines.

02,353

LRM Probe-Tip Calibrations Using Nonideal Standards. PB96-135389

02,411

WAFERS

Water Adsorption at Polymer/Silicon Wafer Interfaces.

PB95-181178 01,022

Water Adsorption at a Polyimide/Silicon Wafer Interface.

PB96-103197

01,070

WAGES

Design and Development of an Information Retrieval Sysem for the EAMATE Data. Volume 2 of 2. Appendices. PB94-168390

00,487

WALL FIRES

Algorithm to Describe the Spread of a Wall Fire under a Ceiling

00,261

WALLBOARDS

Empirical Validation of a Transient Computer Model for Combined Heat and Moisture Transfer.

PB97-111991

00,416

WALLS

Analysis of Moisture Accumulation in a Wood-Frame Wall Subjected to Winter Climate.

4-199320

00,338

Upward Flame Spread along the Vertical Corner Walls (October 1993).

00,340

Turbulent Upward Flame Spread on a Vertical Wall under

External Radiation

00,34

Comparison of Wall-Fire Behavior With and Without a

PB94-207404

00,342

NIST Research Program on the Seismic Resistance of Partially-Grouted Masonry Shear Walls.

00,354

Controlling Moisture in the Walls of Manufactured HousPB95-105136

00,355

Wall Flame Heights with External Radiation.

00,380

Fire-Plume-Generated Ceiling Jet Characteristics and
Convective Heat Transter to Ceiling and Wall Surfaces in Convective Heat Transfer to Ceiling and Wall Surfaces in

a Two-Layer Fire Environment: Uniform Temperature
Ceiling and Walls.
PB95-164711 PB95-164711

Manufactured Housing Walls That Provide Satisfactory

Moisture Performance in All Climates.

00,383

Heat and Moisture Transfer in Wood-Based Wall Construction: Measured versus Predicted.

00,391

Turbulent Flame Spread on Vertical Corner Walls.

ThBo

01,403

Heights of Wall-Fire Flames.

PB96-148192

00,212

Parametric Study of Wall Moisture Contents Using a Revised Variable Indoor Relative Humidity Version of the 'Moist' Transient Heat and Moisture Transfer Model.

PB97-122535

00,419

WARPING

Warping of Terrace Pavers at the U.S. Capitol Building.
PB96-193651 00,41

WASTE CONSUMPTION

Water Efficient Plumbing Fixtures through Standards and Test Methods.

WASTE DISPOSAL Recommendations for the Disposal of Carbon-14 Wastes.
AD-A279 133/3
02,579

02,579

Opportunities

02,520

WASTE RECYCLING

Opportunities for Innovation: Pollution Prevention.
PB95-147146

02,520

WASTE TREATMENT

Bioleaching of Cobalt from Smelter Wastes by PB95-140968

02,582

WASTE UTILIZATION

Calculation of Higher Heating Values of Biomass Materials and Waste Components from Elemental Analyses.

WASTES

Acrylonitrile-Butadiene-Styrene (ABS) Plastic Drain,

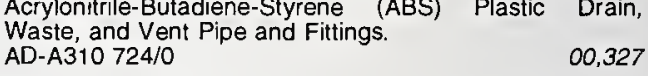

WATER

Structure of Glycine-Water H-Bonded Complexes. PB94-198603

00,789 Journal of Research of the National Institute of Standards and Technology. March/April 1994. Volume 99, Number 2 .

PB94-219219

02,000

Sealed Water Calorimeter for Measuring Absorbed Dose.

PB94-219227

Changes in the Redox State of Iridium Oxide Clusters and Their Relation to Catalytic Water Oxidation: PB95-107017

00,864

Metalloporphyrin Sensitized Photooxidation of Water to Oxygen on the Surface of Colloidal Iridium Oxides - Photochemical and Pulse Radiolytic Studies.

PB95-107082

00,868

Vibration, Rotation, and Parity Specific Predissociation Dynamics in Asymmetric OH Stretch Excited ArH2O: A Half Collision Study of Resonant $V-V$ Energy Transfer in a Weakly Bound Complex.

B95-107140

00,872

Mid- and Near-Infrared Spectra of Water and Water Dimer Isolated in Solid Neon.

00,888

Vibrational Spectra of Molecular Ions Isolated in Solid Neon. X. $\mathrm{H} 2 \mathrm{O}(+), \mathrm{HDO}(+)$, and $\mathrm{D} 2 \mathrm{O}(+)$

Neon. X. H2O

00,889

Formulation of the Refractive Index of Water and Steam.

PB95-140133

00,900

NIR-Spectroscopic Investigation of Water Sorption Characteristics of

00,189

Product State Correlations in the Reaction of $O((1) D)$ and $\mathrm{H} 2 \mathrm{O}$ in Bimolecular Collisions and in $\mathrm{O} 3 . \mathrm{H} 2 \mathrm{O}$ Clusters.Translation.

PB95-153011 


\section{KEYWORD INDEX}

WEIGHT

WATER DISTRIBUTION

NMR Characterization of Injection-Moulded Alumina Green Compacts. Part 2. T2-Weighted Proton Imaging.
PB96-201181 WATER EFFECTS

Development of a Method for Measuring Water-Stripping Resistance of Asphalt/Siliceous Aggregate Mixtures. PB96-202296

WATER EROSION

Moisture and Water-Induced Crack Growth in Optical Materials.

04,267

PB95-153334

WATER HEATERS

Predicting the Energy Performance Ratings of a Family of Type I Combination Appliances.

02,504

WATER IMMERSION

Molecular Orbital Study of Water Enhanced Crack Growth Process.

PB95-164067

03,240

WATER MIST

Water Mist Fire Suppression Workshop Summary.
PB95-161907

WATER POLLUTION CONTROL

In Situ Buming Oil Spill Workshop Proceedings. Held in Orlando, Florida on January 26-28, 1994

02583

WATER POLLUTION DETECTION

Comparison of Methods for Gas Chromatographic Determination of PCBs and Chlorinated Pesticides in Marine Reference Materials.

PB95-140091 02,584 NIST Standard Reference Materials (SRMs) for Polychlorinated Biphenyl (PCB) Determinations and Their Applicability to Toxaphene Measurements.

02,585

WATER POLLUTION EFFECTS

Certification of Standard Reference Material (SRM)

1941a, Organics in Marine Sediment.

02,593

PB96-123690

WATER POLLUTION EFFECTS (ANIMALS)

Current Activities Within the National Biomonitoring Specimen Bank.
PB94-172806

02,516

Development of Frozen Whale Blubber and Liver Reference Materials for the Measurement of Organic and Inorganic Contaminants.

00,587

Determination of Inorganic Constituents in Marine Mammal Tissues.
PB95-152047

00.589

Alaska Marine Mammal Tissue Archival Project: Specimen Inventory.

02,589

Relationship of Silver with Selenium and Mercury in the Liver of Two Species of Toothed Whales (Odontocetes). ATER RESISTANCE

Development of a Method for Measuring Water-Stripping Resistance of Asphalt/Siliceous Aggregate Mixtures. PB96-197249

01,348

WATER STRUCTURE

Physical Limit to the Stability of Superheated and PB96-122551

01,083

WATER TEMPERATURE

Lake Erie Water Temperature Data, Put-in-Bay, Ohio, $1918-1992$.

PB96-202452

03,692

WATER TRIPLE POINT CELLS

Investigation of the ITS-90 Subrange Inconsistencies for 25.5 Omega SPRTs

PB96-161302

00,630

WATER VAPOR

Formulation of the Refractive Index of Water and Steam.

00,900

Slant Path Atmospheric Refraction Calibrator: An Instrument to Measure the Microwave Propagation Delays In-

duced by Atmospheric Water Vapor.
PB95-151270

01,476

WATERPROOFING

Performance Approach to the Development of Criteria for Low-Sloped Roof Membranes.

PB94-160751

00,329

WATT BALANCE METHOD

Measurement and Reduction of Alignment Errors of the NIST Watt Experiment.

PB97-111959

01,987

WATT MEASUREMENT

Methods for Aligning the NIST Watt-Balance.

PB96-123153

01,934

NIST Watt Balance: Progress Toward Monitoring the Kilo-

gram.

01,991

WATTMETERS

NIST High-Accuracy Sampling Wattmeter.

PB97.108575

02,689
DC-MHz Wattmeter Based on RMS Voltage Measure-

01,992

113211

WAVE DIFFRACTION

Vector Theory of Diffraction by a Single-Mode Fiber: Application to Mode-Field Diameter Measurements.

PB95-164182

04,279

WAVE EQUATIONS

Approximate Solution to the Scalar Wave Equation for Optical Waveguides.

PB95-126256

04,254

WAVE PROPAGATION

Interaction of Rayleigh Waves with a Rib Attached to a

04,184

Hemispherical Test Fixture for Measuring the Wavefields Generated in an Anisotropic Solid.

03,181

PB96-190087

WAVE SPECTRUMS

Millimeter- and Submillimeter-Wave Spectrum of transEthyl Alcohol.
PB96-145578

01,102

WAVEFORM RECONSTRUCTION

Casual Regularizing Deconvolution Filter for Optimal Waveform Reconstruction.

01,603

5-203089

AVEFORMS

Approach to Setting Performance Requirements for Automated Evaluation of the Parameters of High-Voltage $1 \mathrm{~m}$ -

PB94-185634

01,878

ifluence of the Filament Potential Wave Form on the Sensitivity of Glass-Envelope Bayard-Alpert Gages. 02,657

WAVEGUIDE BENDS

Modal Characteristics of Bent Dual Mode Planar Optical Waveguide.

04,311

WAVEGUIDE JUNCTIONS

Reciprocity Relations in Waveguide Junctions.
PB94-172814

WAVEGUIDE LASERS

Rare-Earth-Doped Waveguide Devices: The Potential for Compact Blue-Green Lasers.

04,25

Linewidth Narrowing in an Imbalanced $Y$-Branch Waveguide Laser.

PB95-140844

04,258

Nd:LiTaO3 Waveguide Laser.

PB95-140851 04,259

Passively O-Switched Nd-Doped Waveguide Laser

PB95-180048

04,308

WAVEGUIDE POLARIZERS

Waveguide Polarizers Processed by Localized Plasma Etching.

WAVEGUIDES

Microwave Properties of Voltage-Tunable YBa2Cu3O7delta/SrTiO3 Coplanar Waveguide Transmission Lines.

PB96-141262 02,235

Micromachined Coplanar Waveguides in CMOS TechPBology- 119283

02,456

WAVELENGTH STANDARDS

Frequency Stabilization of a Fiber Laser to Rubidium: A High-Accuracy $1.53 \mathrm{mu} \mathrm{m}$ Wavelength Standard. PB95-126082

04,252

\section{WAVELENGTHS}

Wavelength Dependence of Normal Spectral Emissivity of High-Temperature Metals at Their Melting Point. 03,398 Improved Wavelengths for Prominent Lines of $\mathrm{Fe} X X$ to

PB96-111638 04,334

Wavelengths of Spectral Lines in Mercury Pencil Lamps.

PB96-176474

WAVELET ANALYSIS

Wavelet Analysis for Synchronization and Timekeeping.
PB96-200381

WAVES

Leady Axisymmetric Modes in Infinite Clad Rods. Part 1.

PB95-162905 04,187

Wavelet Variance, Allan Variance, and Leakage. PB96-190111

01,509

WEAK LOCALIZATION

Measurement of the Weak-Localization Complex Conductivity at $1 \mathrm{Ghz}$ in Disordered Ag Wires.
PB96-117239

WEAPON SYSTEMS

U.S. Navy Coordinate Measuring Machines: A Study of

02,807

WEAPONS SYSTEM

CALS-Department of Defense Computer Aided Acquisi-

tion Logistic Support (CALS)
PB94-962500
03,662

CALS-Department of Defense Computer Aided Acquisition Logistic Support (CALS)

WEAR

Mechanism of Mild to Severe Wear Transition in AlphaAlumina.

PB94-212354 03,233

Asperity-Asperity Contact Mechanisms Simulated by a Two-Ball Collision Apparatus.

PB95-164158

02,966

Friction and Oxidative Wear of $440 \mathrm{C}$ Ball Bearing Steels Under High Load and Extreme Bulk Temperatures. 03,215 Wear Mechanism Maps of $440 \mathrm{C}$ Martensitic Stainless

04,834

Wear of Enamel against Glass-Ceramic, Porcelain, and AB $96-179593$

03,082

Chip Morphology, Tool Wear and Cutting Mechanics in Finish Hard Turning

PB97-112247 03,106

Chemical Aspects of Tool Wear in Single Point Diamond

PB97-112601

WEAR MECHANICS

Wear Modeling of Si-Based Ceramics.

PB97-12250 1

03,021

WEAR RESISTANCE

Control of Friction and Wear of Alpha-Alumina with a Composite Solid-Lubricant Coating.

03,225

New Alloys Show Extraordinary Resistance to Fracture and Wear.

PB95-151346

03,346

Evaluation of Wear Resistant Ceramic Valve Seats in Gas-Fueled Power Generation Engines. Topical Report December 1991-April 1994.

PB95-200218

02,466

WEAR SCARS

Measuring Matching Wear Scars on Balls and Flats. PB95-151528

03,153

WEAR TESTS

Wear Mechanism Maps of Ceramics.

PB94-172368

03,229

Fracture Mechar

03,230

Wear of Selected Materials and Composites Sliding against MoS2 Films

B94-172749

03,231

Effect of Microstructure on the Wear Transition of Zirconia-Toughened Alumina.

03,141

Abrasive Wear by Diesel Engine Coal-Fuel and Related

PBrticles. 01,450

Critical Factors in Non-Lubricated, Non-Abrasive Wear PB95-140

Measuring Matching Wear Scars on Balls and Flats. PB95-151528 03,153 Wear of Human Enamel against a Commercial Castable Ceramic Restorative Material.

PB95-161972

00,161

Introduction to ASTM 1199 'Wear Test Selection for Design and Application

03,238

Wear Model for Alumina Sliding Wear.

03,239

Tribometer for Measurements in Hostile Environments.

PB95-180949 02,967

Silicon Nitride Boundary Lubrication: Lubrication Mechanism of Alcohols. 
WEIGHT INDICATORS

Application of the Electronic Balance in High Precision Pycnometry.
PBg4-187564

00,534

Ensuring Accuracy and Traceability of Weighing Instruments.

02,638

\section{WEIGHT MEASUREMENT}

NIST Handbook 44, 1994: Specifications, Tolerances and Other Technical Requirements for Weighing and Measuring Devices as Adopted by the 78th National Conference on Weights and Measures 1993.

PB94-136009

02,888

Report of the National Conference on Weight and Meas ures (78th). Held in Kansas City, MO. on July 18-22,

PB94-138989 02,623

NIST Handbook 44, 1995: Specifications, Tolerances and Other Technical Requirements for Weighing and Measuring Devices as Adopted by the 79th National Conference on Weights and Measures 1994.

PB95-146379

02,903

Report of the National Conference on Weights and Measures (79th). Held in San Diego, California on July 17-21,

PB95-169819

02,656

Uniform Laws and Regulations in the Areas of Legal Metrology and Motor Fuel Ouality, 1994 as Adopted by the 79th National Conference on Weights and Measures 1994.

PB95-174470

02,909

Report of the National Conference on Weights and Measures (80th) as Adopted by the 80th National Conference on Weights and Measures, 1995. Held in Portland, Maine on July $16 \cdot 20,1995$

$\begin{array}{ll}\text { PB96-165840 02,681 } & \end{array}$

NIST Handbook 44, 1996: Specifications, Tolerances, and Other Technical Requirements for Weighing and 列 ference on Weights and Measures, 1995.

02,926

Unitorm Laws and Regulations in the Areas of Legal Me Uniform Laws and Regulations in the Areas of Legal Metrology and Motor Fuel Ouality as Adopted by the 80 th
National Conference on Weights and Measures 1995. 1996 Edition.

$\begin{array}{ll}\text { PB96-172309 02,927 } & \end{array}$

Examination Procedure Outlines: Keys to Solving the Handbook 44 Puzzle.

02,690

WEIGHTLESSNESS SIMULATION

Measurement of Surface Tension of Tantalum by a Dy namic Technique in a Microgravity Environment. PB95.161667

03,932

WEIGHTS AND MEASURES

Federal Basis for Weights and Measures: A Historical Review of Federal Legislative Effort, Statutes, and Administrative Action in the Field of Weights and Measures in the AD-A280 086/0

02,616

State Weights and Measures Laboratories: State Standards Program Description and Directory. 1994 Edition.
PB94-207727

WEISSENBERG EFFECT

Density Dependence of Fluid Properties and Non-Newtonian Flows: The Weisenberg Effect.

nian Flows: The

01,140

WELD METAL

Effects of Copper, Nickel and Boron on Mechanical Properties of Low-Alloy Steel Weld Metals Deposited at High Heat input.

PB96-135231

03,363

WELD TESTS

IIW Commission V Ouality Control and Ouality Assurance of Welded Products Annual Report 1994/95

02,866

WELDED JOINTS

Cryogenic Toughness of Austenitic Stainless Steel Weld Metals: Effect of Inclusions.

PB95-161261

03,214

X-Ray Image Ouality Indicator Designed for Easy Alignment. 164455

02,907

IIW Commission V Ouality Control and Ouality Assurance of Welded Products Annual Report 1994/95.

PB95-198743

02,866

WELDED STEEL TANKS

Ashland Tank Collapse Investigation.

PB95-126314

02,481

Ashland Tank-Collapse Investigation: Closure by Authors. PB95-126322

02.482

WELDING

Characterization of the Hydrogen Induced Cold Cracking Susceptibility at Simulated Weld Zones in HSLA-100

AD-A279 759/5

03,200

Characterization of the Hydrogen Induced Cold Cracking Susceptibility at Simulated Weld Zones in HSLA.100 SB94-17

Internationa! Institute of Welding: Report on 1992 Actions. PB94-185873
International Institute of Welding: Report on 1993 Actions. PB94-185881

02,857

Computers in Welding: A Primer

02,862

Welding for Cryogenic Service.

02,852

In-Space Welding: Visions and Realities.

$\begin{array}{ll}\text { PB95-163234 } & 04,830 \\ \text { Mathematical Models of Transport Phenomena Associ- }\end{array}$ ated with Arc-Welding Processes: A Survey.

PB96-135058

02,870

Electrode Extension Model for Gas Metal Arc Welding

PB96-135074 02,871

Report on 1994 Actions of the International Institute of Welding.

PB96-138540

02,873

International Institute of Welding: Report on 1995 Actions.

PB96-158076 02,874

What's Available in Welding Software.

02,874

PB96-158084

02,875

Proceedings of NIST Workshop: Industry Needs in Welding Research and Standards Development. Held on August $15-16,1995$

02,877

Well-Shielded EMAT for On-Line Ultrasonic Monitoring of GMA Welding.
PB96-186077

02,879 IIW Commission V Ouality Control and Quality Assurance
of Welded Products, Annual Report 1995/96. of Welded Pro

02,880

\section{WELDING CURRENT}

Contact Tube Wear Detection in Gas Metal Arc Welding.

WELDING ÉLECTRODES

Droplet Transfer Modes for a MIL 100S-1 GMAW Elecrode.

PB95-209300

02,867

WELL FIRES

Investigation of Oil and Gas Well Fires and Flares.

\section{WELL FLARES}

Investigation of Oil and Gas Well Fires and Flares.

PB94-193976

03,695

WHALES

Development of Frozen Whale Blubber and Liver Reference Materials for the Measurement of Organic and Inorganic Contaminants.

PB95-151676

00.587

Certification of Polychlorinated Biphenyl Congeners and Chlorinated Pesticides in a Whale Blubber Standard Reference Material.

03,745

WHEELS

Ultrasonic Measurement of Residual Stress in Railroad Wheel Rims.

PB95-140430

04,849

Ultrasonic Measurement of Residual Stress in Cast Steel Railroad Wheels.

04,852

Residual Stress in Induction-Heated Railroad Wheels: UItrasonic and Saw Cut Measurements. Report No. 28.

PB96-106992 04,854

Safety Assessment of Railroad Wheels by Residual

04,855

Safety Assessment of Railroad Wheels Through Roll-by Detection of Tread Cracks.

04,856

PB96-141254

Dynamometer-Induced Residual Stress in Railroad Wheels: Ultrasonic and Saw Cut Measurements. Report

04,857

\section{WHISKER COMPOSITES}

Matrix Grain Bridging Contribution to the Toughness of Whisker Reinforced Ceramics.

PB94-198645

03,134

Tensile Creep of Whisker Reinforced Silicon Nitride.

$\begin{array}{ll}\text { PB94-211984 } & 3,142 \\ & \end{array}$

Flat and Rising R-Curves for Elliptical Surface Cracks PB95-161295

03,156

WIDE AREA NETWORKS

Comparison of FDDI Asynchronous Mode and DODB Oueue Arbitrated Mode Data Transmission for Metropolitan Area Network Applications.

PB96-160452

01,498

WIDEBAND

Wideband Sampling Voltmeter.

PB97-113039

01,990

WIGGLER MAGNETS

Hybrid Undulator for the NIST-NRL Free-Electron Laser.
PB94-212529

WIND DIRECTION

Computational Model for the Rise and Dispersion of Wind-Blown, Buoyancy-Driven Plumes. Part 2. Linearly Stratified Atmosphere.

00,119

\section{WIND EFFECTS}

Wind and Seismic Effects. Proceedings of the U.S.-Japan Cooperative Program in Natural Resources Panel on Wind and Seismic Effects (26th). Held in Gaithersburg. Maryland on May $17-20,1994$.

PB95-147385 00,433

Assessment of 'Peaks Over Threshold' Methods for Estimating Extreme Value Distribution Tails.

PB95-161360

00,441

Proceedings: Workshop on Research Needs in Wind Engineering. Held in Gaithersburg, Maryland on September $12-13,1994$.

PB95-189528

00,448

Recommended Performance-Based Criteria for the Design of Manufactured Home Foundation Systems to Resist Wind and Seismic Loads.

PB96-128285

00,460

Wind and Seismic Effects: Proceedings of the Joint Meeting of the U.S.-Japan Cooperative Program in Natural Resources Panel on Wind and Seismic Effects (28th). Held in Gaithersburg, Maryland on May 14-17, 1996. PB97-104376

00,476

WIND LOADS

Wind Load Provisions of the Manufaclured Home Construction and Safety Standards: A Review and Recommendationsi for Improvement.

PB94-206125

00,428

Manufactured Homes: Probability of Failure and the Need for Better Windstorm Protection through Improved Anchoring Systems.

Gust Factors Applied to Hurricane Winds.

00,432

PB95-180469

00,446

Extreme Winds Estimation by 'Peaks Over Threshold' and Epochal Methods.

PB96.159686

00,468

Modeling of Extreme Loading by 'Peaks Over Threshold' Methods.

PB96-159694

WIND PRESSURE

Recent Approaches to Extreme Value Estimation with Application to Wind Speeds. Part 1. The Pickands Method.

PB94-213170

00,019

Lessons Learned by a Wing Engineer.

00,429

Proceedings: Workshop on Research Needs in Wind Engineering. Held in Gaithersburg, Maryland on September gineering. He. PB95-189528

00,448

\section{WIND TUNNELS}

Measurement of the Uniformity of Particle Deposition of Filter Cassette Sampling in a Low Velocity Wind Tunnel. PB95-163754

02,549

WIND VELOCITY

Recent Approaches to Extreme Value Estimation with Ap- 


\section{KEYWORD INDEX}

WIRE BONDS Wire Bond Testing

WIREWOUND RESISTORS

Precision Resistors and Their Measurement.
AD-A284 623/6

WOLF SHIFTS

Comments on the Paper 'Wolf Shifts and Their Physical Interpretation under Laboratory Conditions'.

PB94-219391 04,246

Reply to Professor Wolf's Comments on My Paper on Wolf Shifts.

PB94-219409 04,247

WOOD

Rate of Heat Release of Wood Products.

PB94-212404

03,403

Examination of Objects Made of Wood Using Air-Coupled Ultrasound.

PB95-125712

03,404

Heat and Moisture Transfer in Wood-Based Wall Construction: Measured versus Predicted.

00,391

WOOD BURNING APPLIANCES

Measurements of Indoor Pollutant Emissions from EPA Phase 11 Wood Stoves.

PB95-198735

02,556

WOOD PRODUCTS

Analysis of Moisture Accumulation in a Wood-Frame Wall Subjected to Winter Climate

00,338

National Voluntary Laboratory Accreditation Program (NVLAP): Wood Based Products.

03,405

PB95-170429

WORD RECOGNITION

Unconstrained Handprint Recognition Using a Limited Lexicon.

PB94-168051

01,831

WORK ENVIRONMENTS

Post-Occupancy Evaluation of the Forrestal Building.
PB97-111298

WORKING FLUIDS

Development of Measurement Capabilities for the Thermophysical Properties of Energy-Related Fluids. Annual Report, December 1, 1990--November 30, 1991.

Role of Retrigerant Mixtures as Alternatives to CFCs.

Role of Retrigerant Mixtures as Alternatives to CFCs.
PB94-199775

PB94-199775 Pump Performance Using Mixtures of R32 R134a and R32/R125/R134a as 'Drop-In' Working Fluids for R22 with and Without a Liquid-Suction Heat Exchange

PB94-218559

02,503

Resistance Thermometers with Fast Response for Use in Rapidly Oscillating Gas Flows.

03,261

Need for, and Availability of, Working Fluid Property Data: Results from Annexes XIII and XVIII.

PB95-168969

03,268

WORKPLACE LAYOUT

Psychological Aspects of Lighting: A Review of the Work of CIE TC 3.16 .

PB95-15327

00,254

NIST SIMA Interactive Management Workshop. Held in Fort Belvoir, Virginia on November 14-16, 1994.
PB96-154877

02,838

WORKSTATIONS

AMRF Composite Fabrication Workstation.

02,810

Portsmouth Fastener Manufacturing Workstation. User's Manual.

PB95-147922

02,860

WORLD MODELS

World Model Registration for Effective Off-Line Programming of Robots

02,933

Role of World Modeling and Value Judgment in Perception.

01,581

PB94-198264

02,722

World Wide Web and Mosaic: User's Guide.

WOVEN COMPOSITES

Comparison of the Unidirectional and Radial In-Plane Flow of Fluids Through Woven Composite Reinforcements.

02,698

WWW (WORLD WIDE WEB)

World Wide Web and Mosaic: User's Guide.

PB94-20735

02,722

High Spectral Purity X-Band Source.

$X$ RAY

Flat and Curved Crystal Spectrography for Mammographic X-ray Sources.

03,642
$X$-RAY ABSORPTION ANALYSIS

X-Ray Attenuation Coefficients from $10 \mathrm{KEV}$ to $100 \mathrm{MEV}$ AD-A280 290/8

03,768

$X$-RAY ANALYSIS

Design of a Protocol for an Electron Probe Microanalyzer k-Value Round Robin.

PB95-107181

00,564

Monte Carlo Electron Trajectory Simulation of X-Ray

Emission from Films Supported on Substrates.

PB95-107207 04,522

Secondary Target $X$-Ray Excitation for in vivo Measurement of Lead in Bone.

PB95-108767

03,496

Electron Probe X-Ray Microanalysis.

\section{RAY ASTRONOMY}

Riass Coronathon: Joint $X$-ray and Ultraviolet Observa. tions of Normal F-K Stars.

PB96-200217

00,109

$X$ RAY DENSITY MEASUREMENT

Novel Method for Determining Thin Film Density by Energy-Dispersive $\mathrm{X}$-ray Reflectivity.

PB96-122783

04,737

Silicon Photodiodes Optimized for EUV and Soft X-Ray PBg 4-199478

02,124

$X$ RAY DETECTORS

Hot-Electron Microcalorimeters for $\mathrm{X}$-ray and Phonon Detection.

PB95-168993

04,644

Hot-Electron Microcalorimeters as High-Resolution $\mathrm{X}$-ray Detectors.

Hot-Electron Microcalorimeter for X-ray Detection Using a Superconducting Transition Edge Sensor with PB96-200399

Hot-Electron-Microcalorimeters with $0.25 \mathrm{~mm}$ (2) Area

PB96-200670

04,792

-RAY DIFFRACTION

Profile Fitting of X-Ray Diffraction Lines and Fourier Analysis of Broadening.

04,793

Voigt-Function Modeling in Fourier Analysis of Size- and Strain-Broadened X-Ray Diffraction Peaks.

PB94-198538

04,462

Diffraction Imaging of Polycrystalline Materials

PB94-198884

02,971

Diffraction of $\mathrm{X}$-rays at the Far Tails of the Bragg Peaks

PB94-199924 04,476

Introduction of a NIST Instrument Sensitivity Standard Reference Material for X-Ray Powder Diffraction.

PB94-200318

00,807

$X$-Ray Powder Diffraction Data for $\mathrm{BaCu}(\mathrm{C} 2 \mathrm{O} 4) 2.6 \mathrm{H} 2 \mathrm{O}$.

PB95-151767 04,583

Residual Stresses in Aluminum-Mullite (alpha-Alumina) Composites.

PB95-152880

03,155

Determination of the Residual Stresses Near the Ends of Skip Welds Using Neutron Diffraction and X-ray Diffraction Procedures.

PB95-253589 02,868

Crystal Structure of a New Monoclinic Form of Potassium Dihydrogen Phosphate Containing Orthophosphacidium lon, (H4PO4)(sup +1$)$.

PB96-111794

04,725

Coexistence of Grains with Differing Orthorhombicity in High Ouality YBa2Cu3O7-delta Thin Films.

Powder X-ray Diffraction Data for Ca2Bi2O5 and C4Bi6013.

PB96-161278

04,777

Preparation and Crystal Structure of Sr5TiNb4O17.

PB96-167341 04, 107

Accurate Modeling of Size and Strain Broadening in the

Rietveld Refinement: The 'Double-Voigt' Approach

PB96-200225

00,664

Biological Macromolecule Crystallization Database and NASA Protein Crystal Growth Archive.

01,171

$X$-RAY DIFFRACTOMETERS

Using NIST Crystal Data within Siemens Software for Four-Circle and SMART CCD Diffractometers. PB97-109110

04,803

$X$ RAY DIFFRACTOMETRY

Interiace Roughness, Composition, and Alloying of Low-

Order AIAS/GaAs Superlattices Studies by X-ray Diffrac-

tion. 160262

02,983

$X$-RAY EMISSION

Resonance and Threshold Effects in Polarized X-Ray

Emission from Atoms and Molecules.

PB95-150298

03,891

$X$-ray Emission
PB96-102256

00,088

X RAY PHOTOELECTRON SPECTROSCOPY

Al L2,3 Core Excitons in AlxGa1-x as Studied by Soft-Xray Reflection and Emission.

RAY EMISSIONS

Soft-X-ray-Emission Investigation of Cobalt Implanted SiliPB96-157912

Soft-X-ray-Emission Spectra of Solid $K_{r}$ and $X e$.

PB96-157920

04,069

Poft-X-ray-Emission Studies of Bulk Fe3Si, FeSi, and FeSi2, and Implanted Iron Silicides.

PB96-157938

04,071

X-RAY FLUORESCENCE

Polarized X-Ray Emission Spectroscopy.

PB94-213360

Apparent Bias in the X-Ray Fluorescence Determination of Titanium in Selected NIST SRM Low Alloy Steels.

PB95-108759 03,212

Image Information Transfer Properties of X-Ray Intensifying Screens in the Energy Range from 17 to $320 \mathrm{keV}$.
PB95-126173 Aging Effects on XRF Measurements of Solder Coatings. PB95-140927 03,123

Evolution of X-ray Resonance Raman Scattering into $X$ ray Fluorescence from the Excitation of Xenon Near the La Edge.

Cooper M(sub II,III) X-ray-Emission Spectra of Copper Oxides and the Bismuth Cuprate Superconductor. PB96-158027

$X$-RAY FLUORESCENCE ANALYSIS

Addition of $M$ and L-Series Lines to NIST Algorithm for Calculation of X-Ray Tube Output Spectral Distributions. PB95-108742 00,569 Pattern-Recognition Analysis of Low-Resolution X-Ray Fluorescence Spectra.

PB95-151924

$X$-RAY IMAGERY

Materials Science with SR Using X-Ray Imaging: Spatial-

Resolution/Source Size.

04510

$X$-Ray Image Ouality Indicator Designed for Easy Align-

ment. 164455

02,907

$X$-RAY INSPECTION

Evaluation and Oualification Standards for an X-Ray Laminography System.

$X$-Ray Image Ouality Indicator Designed for Easy Alignment.

02,907

Contributions of Out-of-Plane Material to a ScannedBeam Laminography Image.

RAY LAMINOGRAPHY

Evaluation and Oualification Standards for an X-Ray Laminography System.

Contributions of Out-of-Plane Material to a ScannedBeam Laminography Image.

PB96-111786

02,704

$X$ RAY LITHOGRAPHY

$X$-ray Mask Metrology: The Development of Linewidth Standards for X-ray Lithography.

Use of Monte Carlo Modeling for Interpreting Scanning Electron Microscope Linewidth Measurements.
PB96-137807

$X$ RAY MASKS

$X$-ray Mask Metrology: The Development of Linewidth Standards for X-ray Lithography.

PB95-162129

02,348

$X$-RAY MICROSCOPES

High Resolution Hard X-Ray Microscope.

PB94-213055 


\section{KEYWORD INDEX}

Grazing-Incidence X-Ray Photoelectron Spectroscopy: A Novel Approach to Thin Film Characterization.

04,589

Grazing Angle X-Ray Photoemission System for DepthDependent Analysis.

04,600

Elastic-Electron-Scattering Effects on Angular Distributions in X-ray Photoelectron Spectroscopy.

PB95-175758

01,000

Grazing Incidence $X$-ray Photoemission and Its Implementation on Synchrotron Light Source $X$-ray Beamlines. PB95-175766

01,001

$X$-ray Photoelectron and Auger Electron Forward Scattering: A Structural Diagnostic for Epitaxial Thin Films. $X$-RAY POWDER DIFFRACTION

Powder Diffraction File: Past, Present, and Future. PB97-109086

04,800

$X$ RAY REFLECTIVITY

$X$-ray Reflectivity Determination of Interface Roughness Correlated with Transport Properties of (AlGa)As/GaAs High Electron Mobility Transistor Devices.

04,149 PB97-111868

\section{$X$ RAY SCATTERING}

Evolution of the Pore Size Distribution in Final-Stage Sintering of Alumina Measured by Small-Angle X-ray Scattering. (Reannouncement with New Availability Informa. AD-A249 178/5

03,023

Further Calculations of X-ray Diffusion in an Infinite Medium.

03,772

AD-A295 314/9

Small-Angle X-Ray and Neutron Scattering Study of
Block Copolymer/Homopolymer Mixtures.

Block Copolym

01,221

Polarization Effects on Multiple Scattering Gamma Transport.

03.926

Molecular Weight Dependence of the Lamellar Domain Spacing of ABC Triblock Copolymers and Their Chain Conformation in Lamellar Domains.

PB95-161691

01,254

Real-Time Small-Angle X-Ray Scattering Study of the Early Stage of Phase Separation in the SiO2-BaO-K2O PBstem. 163069

03,052

Characterization of Polyquinoline Blends Using Small Angle Scattering

01,261

Analysis of Small-Angle Scattering Data Dominated by Multiple Scattering for Systems Containing Eccentrically Shaped Particles or Pores.

PB96-160411

03,075

$X$-RAY SOURCES

Discovery of an X-Ray Selected, Radio-Loud Quasar at Z=3.9.
PB94-198652

00,052

Rotational Modulation and Flares on RS Canum Venaticorum and BY Draconis Stars. XVIII. Coordinated VLA, ROSAT, and IUE Observations of RS CVn Binaries.

Efficient Way of Identifying New Active Stars: A VLA SurEfficient Way of Identifying New Active Stars: A VLA Sur-
vey of X-ray Selected Active Stellar Candidates.

Developments in Stellar Coronae.

00,099

PB96-176706

00,107

X-RAY SPECTRA

Experimental Aspects and Z-Dependent Systematics in One- and Two-Electron lons and Single Vacancy Systems.

03,815

Resonance and Threshold Effects in Polarized X-Ray

Emission from Atoms and Molecules.

03,891

Improved Wavelengths for Prominent Lines of $\mathrm{Cr} X \mathrm{VI}$ to $\mathrm{Cr}$ XXII

PB95-150629

03,895

$\mathrm{Rh}$ I Isoelectronic Sequence Observed from $\operatorname{Er}(23+)$ to

$\operatorname{Pt}(33+)$.

Spectra of $\mathrm{Ag}$ I Isoelectronic Sequence Observed from

$\operatorname{Er}(21+)$ to $\mathrm{Au}(32+)$.
PB95-150660

03,899

Discussion: Statistical Signal Processing of Quasiperiodicities.
PB96-119532

00,096

\section{$X$-RAY SPECTROMETERS}

Noninvasive High-Voltage Measurement in Mammography by Crystal Diffraction Spectroscopy. Grazing Angle X-Ray Photoemission System for DepthDependent Analysis.

PB95-161154

04,600

Electron-lon-X-ra

03,958

X-RAY SPECTROSCOPY

Polarized X-Ray Emission Spectroscopy.

PB94-213360

03,862
Design of a Protocol for an Electron Probe Microanalyzer $\mathrm{k}$-Value Round Robin.

PB95-107181

00,564

Polarization Measurements on a Magnetic Ouadrupole Line in Ne-Like Barium.

04,161

\section{$X$-RAY TUBES}

Addition of $\mathrm{M}$ and L-Series Lines to NIST Algorithm for Calculation of X-Ray Tube Output Spectral Distributions. PB95-108742

00,569

\section{RAYS}

X-ray Attenuation Coefficients from $10 \mathrm{Kev}$ to $100 \mathrm{Mev}$ AD-A278 139/1

03,765

$X$-ray Protection.

03,605

$X$-ray Protection Design

03,606

$X$-Ray Attenuation Coefficients from 10 kev to $100 \mathrm{Mev}$

AD-A279 289/3 03,767

Bibliography of Photon Total Cross Section (Attenuation Coefficient) Measurements $10 \mathrm{eV}$ to $13.5 \mathrm{GeV}$. 1907-

PB94-193760

03,804

Photographic Response to X-Ray Irradiation. 1. Estimation of the Photographic Error Statistic and Development of Analytic Density-Intensity Equations.

03,821

B94-200086

2. Cor-

Photographic Res

related Models.
PB94-200094

03,822

Photographic Response to X-Ray Irradiation. 3. Photographic Linearization of Beam-Foil Spectra.

03,823

94-200102

Anisotropy of Polarized X-ray Emission from Atoms and
Molecules.
PB95-163002
04,621

PB95-163002

Tables of X-ray Mass Attenuation Coefficients and Mass ments $Z=1$ to 92 and 48 Additional Substances of Dosimetric Interest.

PB95-220539

04,013

Decomposition of Sulfur Hexafluoride by $\mathrm{X}$-rays.

PB96-135314

01.095

PBable Silicon Photodiodes for Absolute Intensity Measurements in the VUV and Soft X-ray Regions.

04,135

EPR Dosimetry of Cortical Bone and Tooth Enamel Irradiated with $X$ and Gamma Rays: Study of Energy DependPB97-110373

03,639 Experimentally Measured Total X-ray Attenuation Coeffi-
cients Extracted from Previously Unprocessed Documents Held by the NIST Photon and Charged Particle Data Center. Data Center.

04,165

Measuring Nondipolar Asymmetries of Photoelectron Angular Distributions

$X$ WINDOW SYSTEM

$X$ Window System, Version 11, Release 5

01.193

PB96-169099

01,769

X500 DIRECTORY

Guidelines for the Evaluation of X.500 Directory Products.

X.500 Directory Schema Design Handbook.

02,732 PB96-18304

02,739

XENON

Veritication of the Ponderomotive Approximation for the ac Stark Shift in Xe Rydberg Levels.

03,801

4-185709

Evolution of $X$-ray Resonance Raman Scattering into $X$ -
ray Fluorescence from the Excitation of Xenon Near the ray Fluorescence from the Excitation of Xenon Near the
L3 Edge.
PB96-102751
In

Order Above-Threshold Ionization

04.032

XENON COMPLEXES

Van der Waals Bond Lengths and Electronic Spectral Shitts of the Benzene-Kr and Benzene-Xe Complexes.
PB95-151387

XENON IONS

Electron-Impact Ionization of $\mathrm{In}(+)$ and $\mathrm{Xe}(+)$.

PB94-185089

00,770

Analysis of the $5 s(2) 5 p(2)$ $(5 s 5 p(3)+5 s(2) 5 p 5 d+5 s(2) 5 p 6 s)$ Transitions of Four-Times lonized Xenon (Xe V)

03,900

Electron-Impact Ionization of $\ln (+)$ and $\mathrm{Xe}(+)$.

03,918

XENON ISOTOPES

Magneto-Optical Trapping of Metastable Xenon: IsotopeShift Measurements.

PB95-151254

03,905

XYLENES

Correlation of the Ideal Gas Properties of Five Aromatic PBdrocarbons

01,002

Y SPECTRA

Systematics of Alpha-Particle Energy Spectra and Linea Energy $(Y)$ Spectra for Radon Daughters.

03,615

YBCO

q Dependence of Self-Energy Effects of the Plane Oxygen Vibration in $\mathrm{YBa} 2 \mathrm{Cu} 3 \mathrm{O} 7$.

01,096

YBCO SUPERCONDUCTORS

Neutron-Spectroscopy Study of the Hydrogen Vibrations in Hydrogen-Doped YBa2Cu3Ox

04,441

Weak-Link-Free Behavior of High Angle YBa2Cu3O7-x Grain Boundaries in High Magnetic Fields.

PB94-198421

04,459

Electronic Correlations and Satellites in Superconducting

Surface Barrier and Lower Critical Field in YBa2Cu307. delta Superconductors

PB94-200128

04,478

Incorporation of Gold into YBa2Cu3O7: Structure and TC Enhancement

Unexpected Effects of Gold on the Structure, Superconductivity, and Normal State of YBa2Cu3O7.

PB94-200284

04,482

Critical Magnetic-Field Angle for High-Field Current Transport in $\mathrm{YBa} 2 \mathrm{Cu} 3 \mathrm{O} 7$ at $76 \mathrm{~K}$

PB94-211281

04,490

Transport Critical Current of Aligned Polycrystalline Yttrium Barium Copper Oxide (YBa2Cu3O7-delta).

PB94-211307

04,492

In situ Noble Metal YBa2Cu3O7 Thin-Film Contacts.

PB94-211323 04,494

Flux Expulsion at Intermediate Fields in Type-II

PB94-212230

04,502

Offset Susceptibility of Superconductors

PB94-212263

04,503

Compositional Homogeneity in Processing Precursor Powders to the Ba2YCu307-x High Tc Superconductor

PB94-212743

Elastic Constants of Polycrystalline Y1Ba2Cu3OX

04,508

P94-213196

04,513

Elastic Constants and Debye Temperature of PB94-213352

04,515

Nature of (001) Tilt Grain Boundaries in YBa2Cu3O6 $+x$.

PB95-126033 04,528

Image Depth Profiling SIMS: An Evaluation for the Analy-

sis of Light Element Diffusion in YBa2Cu3O7-x Single 
Determination of Thermoactivation Parameters of Vortex Mobility in YBa2Cu3O7 Using Only Magnetic Measure-

PB95-163499 04,624 High Current Pressure Contacts to Ag Pads on Thin Film Superconductors.

PB95-168621 04,639

Suitability of Metalorganic Chemical Vapor Deposition-Derived PrGaO3 Films as Buffer Layers for YBa2Cu307-X Pulsed Laser Deposition.

PB95- 168670

04,640

Low-Temperature Elastic Constants of $\mathrm{Y} 1 \mathrm{Ba} 2 \mathrm{Cu} 3 \mathrm{O} 7$.

PB95-168837 04,642

Microwave Properties of YBa2Cu307-x Films at $35 \mathrm{GH}$ from Magnetotransmission and Magnetoreflection Measurements.

PB95-168977

04,643

Insulating Boundary Layer and Magnetic Scattering in YBa2Cu3O7-delta/Ag Interfaces Over a Contact Resistiv ity Range of $10(-8)-10(-3)$ Omega $\mathrm{cm}(2)$

ity Range of

04,648

Experimental Aspects of Flux Expulsion in Type-1 Superconductors.

04,660

Surface Degradation of Superconducting YBa2Cu3O7-

della Thin Film

PB95-176095

04,667

Increased Transition Temperature in In situ Coevaporated YBa2Cu307-delta Thin Films by Low Temperature PostAnnealing.

PB95-18007

04,672

High Resolution Inelastic Neutron Scattering Study of PB95-180881

04,688

Novel YBa2Cu3O7-x and YBa2Cu3O7-x/YBa3O9 Multilayer Films by Bias-Masked 'On-Axis' Magnetron Sputtering.

04,690

Surface Modification of YBa2Cu3O7-delta Thin Films Using the Scanning Tunneling Microscope: Five Methods. Effect of Sm2BaCuO5 on the Properties of Sintered Effect of Sm2BaCuO5 on the Properties of Sintered
(Bulk) YBa2Cu3O6+x.
PB96-119441
04,733

04,733

Evidence for Tunneling and Magnetic Scattering at "In situ' YBCO/Noble-Metal Interfaces.

PB96-141098

04,752

Oxygen Annealing of Ex-situ YBCO/Ag Thin-Film Interfaces.

04,758

Microwave Noise in High-Tc Josephson Junctions.

PB96-148010

04,771

Effects of Substrate Surface Steps on the Microstructure of Epitaxial Ba2YCu3O7-x Thin Films on (001) LaAlO3.

04,774

Epitaxial Nucleation and Growth of Chemically Derived Ba2YCu3O7-x Thin Films on (001) $\mathrm{SrTiO} 3$.

04,787

YOUNG'S MODULUS

Bulk Modulus and Young's Modulus of Nanocrystalline

gamma-Alumina

03,092

TERBIUM

Isotope Shifts and Hypertine Splittings of the $398.8-\mathrm{nm}$ Yb I Line.

PB94-199585

03,814

YTTERBIUM COMPOUNDS

Meissner, Shielding, and Flux Loss Behavior in SingleCrystal YBa2Cu3O6+x

PB94-198744

YTTERBIUM IONS

Observation of Pd-Like Resonance Lines Through $\mathrm{Pt}(32+)$ and $\mathrm{Zn}$-Like Resonance Lines of $\mathrm{Er}(38+)$ and $\mathrm{Hf}(42+)$

PB95-150637

03,896

Rh I Isoelectronic Sequence Observed from $\operatorname{Er}(23+)$ to $\mathrm{Pt}(33+)$.

03,898

B95-150652 Spectra of $\mathrm{Ag}$ I Isoelectronic Sequence Observed from
Er(21+) to Au(32+).
PB95-150660

VTTRIUM

Magnetoelasticity in Rare-Earth Multilayers and Films.

PB94-211356 04,495

YTTRIUM-90 Radioassays of Yttrium-90 Used in Nuclear Medicine
PB97-110100

03,522

VTTRIUM BARIUM COPPER OXIDES

High-Tc Multilayer Step-Edge Josephson Junctions and PB96-200183

04,790

TTRIUM BARIUM CUPRATE COBALTATES

Neutron Powder Diffraction Study of the Nuclear and Magnetic Structures of the Oxygen-Deficient Perovskite YBaCuCoO5.
PB95-161097

00.954

TTRIUM BARIUM CLIPRATES

Weak-Link-Free Behavior of High Angle YBa2Cu3O7-x

Grain Boundaries in High Magnetic Fields.

04,459
Electronic Correlations and Satellites in Superconducting Oxides.

04,477

Surface Barrier and Lower Critical Field in YBa2Cu3O7delta Superconductors

PB94-200128

04,478

Incorporation of Gold into YBa2Cu307: Structure and TC Enhancement.

04,481

Unexpected Effects of Gold on the Structure, Superconductivity, and Normal State of YBa2Cu3O7. PB94-200284 04,482

Critical Magnetic-Field Angle for High-Field Current Transport in $\mathrm{YBa2Cu} 3 \mathrm{O} 7$ at $76 \mathrm{~K}$. 04,490 In situ Noble Metal YBa2Cu3O7 Thin-Film Contacts. PB94-211323 04,494 Flux Expulsion at Intermediate Fields in Type-ll Superconductors.

PB94-212230

04,502

Oftset Susceptibility of Superconductors.

04,503

Compositional Homogeneity in Processing Precursor Powders to the Ba2YCu3O7-x High Tc Superconductor. PB94-212743

04,508

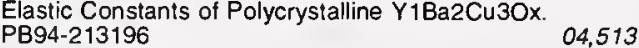
Elastic Constants and Debye Temperature of Y1Ba2Cu3Ox: Effect of Oxygen Content.

PB94-213352

04.515

Nature of (001) Tilt Grain Boundaries in YBa2Cu3O6+x

04.528

Image Depth Profiling SIMS: An Evaluation for the Analysis of Light Element Diffusion in YBa2Cu307-x Single Crystal Superconductors.

PB95- 126116

04,530

Defective Structures of Barium Yttrium Copper Oxide (Ba2YCu3Ox) and Ba2YCu3-yMyOz (M=Fe, Co, Al, Ga, PB95-140034

04,537

Siudy of the Hydroxycarbonate Precursor Route to the YBa2Cu3O7-x High Tc Superconductor.

04,540
Influence Laser Ablated YBa2Cu307-Delta Films.

PB95-140539

04,544

Critical Current Behavior of Ag-Coated YBa2Cu3O7-x PB95-141016

Magnetic Field Dependence of the Critical Current Anisotropy in Normal Metal-YBa2Cu3O7-delta Thin Film

PB95-141024

04,550

Insulating Nanoparticles on YBa2Cu3O7-delta Thin Films Revealed by Comparison of Atomic Force and Scanning Tunneling Microscopy.

PB95-150843

04,575

$X$-Ray-Diffraction Study of a Thermomechanically Detwinned Single Crystal of YBa2Cu3O6+x

PB95-151726

Flame Synthesis of High Tc Superconductors.

PB95-151981

04,581

Harmonic and

00,659

PB95-161139 04,599

Hydrogen in YBa2Cu3Ox: A Neutron Spectroscopy and a Nuclear Magnetic Resonance Study.

PB95-161279

04,601

Temperature Dependence of Vortex Twin Boundary Interaction in Yttrium Barium Copper Oxide (YBa2Cu306+x).
PB95-162442

Growth of Laser Ablated YBa2Cu3O7-delta Films as Examined by Rheed and Scanning Tunneling Microscopy.

PB95-162541

04,614

Observation of Insulating Nanoparticles on YBCO ThinFilms by Atomic Force Microscopy.

PB95-163358

04,622

Determination of Thermoactivation Parameters of Vortex Mobility in YBa2Cu307 Using Only Magnetic Measure-

PB95-163499

04,624

High Current Pressure Contacts to Ag Pads on Thin Film Superconductors.

Suitability of Metalorganic Chemical Vapor Deposition-Derived PrGaO3 Films as Buffer Layers for YBa2Cu3O7-x Pulsed Laser Depositio

PB95-168670

Low-Temperature Elastic Constants of $\mathrm{Y} 1 \mathrm{Ba2} \mathrm{Cu} 3 \mathrm{O} 7$

PB95-168837

04,640

Insulating Boundary Layer and Magnetic Scattering in YBa2Cu3O7-delta/Ag Interfaces Over a Contact Resistivity Range of $101-1$
PB95-169157

04,648

Experimental Aspects of Flux Expulsion in Type-ll Superconductors.

PB95-175725

04,660

Surface Degradation of Superconducting YBa2Cu307.

delta Thin Films.

04,667

Increased Transition Temperature in In situ Coevaporated Ba2Cu307-delta Thin Films by Low Temperature PostAnnealing.

04,672

High Resolution Inelastic Neutron Scattering Study of Phonon Self-Energy Effects in YBCO.

04,688

Novel YBa2Cu307-x and YBa2Cu307-x/Y4Ba3O9 Mulkilayer Films by Bias-Masked 'On-Axis' Magnetron Sputter-

PB95-181186

04,690

Surface Modification of YBa2Cu307-delta Thin Films Using the Scanning Tunneling Microscope: Five Methods. PB95-203394

04,699

VTTRIUM BARIUM TITANATES

Substitution-Induced Midgap States in the Mixed Oxides

RxBa 1-ChiTiO3-Delta, with $\mathrm{R}=\mathrm{Y}, \mathrm{La}$, and Nd.

PB95-140505

04,541

VTTRIUM DEUTERIOES

Local-Mode Dynamics in YH2 and YD2 by Isotope-Dilution Neutron Spectroscopy

PB95-181012

01,017

VTTRIUM HYDRIDES

Neutron Spectroscopic Comparison of Rare-Earth/Hydro-

gen alpha-Phase Systems.

Local-Mode Dynamics in $\mathrm{YH} 2$ and $\mathrm{YD} 2$ by Isotope-Dilution Neutron Spectroscopy

PB95-181012

01,017

VTTRIUM IRON GARNETS

Submicroampere-Per-Root-Hertz Current Sensor Based

on the Faraday Effect in Ga: YIG.

02,155

YTTRIUM OXIDES

Structures of Vapor-Deposited Yttria and Zirconia Thin

Films.

PB94-216025

03,041

Rapid Hot Press

03,045

Crystal Chemistry and Phase Equilibria Studies of the BaO(BaCO3)-H2O3-CuO Systems. 4. Crystal Chemistry and Subsolidus Phase Relationship Studies of the CuORich Region of the Ternary Diagrams, R=Lanthanides.
PB95-151759
00,936

Effects of Etching on the Morphology and Surface Resistance of YBa2Cu3O7-delta Films.

PB96-135355

02,410

VTTRIUM STRONTIUM ALUMINUM CUPRATES

Neutron Powder Diffraction Study of the Crystal Structure of YSr2AlCu2O
PB94-212073

04,499

ZEOLITES

Neutron Powder Diffraction Study of a Na, Cs-Rho Zeo-

lite.

00,791

ZEOTROPES

Role of Refrigerant Mixtures as Alternatives to CFCs.
PB94-199775

03,252

ZERNIKE POLYNOMIALS

Test of a Slow Otf-Axis Parabola at Its Center of Curvature.

ZERO-FIELD ORDERED MOMENT 


\section{KEYWORD INDEX}

Structures of Vapor-Deposited Yttria and Zirconia Thin

PB94-216025

03,041

PB94-216587
Wear Transitions in Monolithic Alumina and Zirconia-Alumina Composites.

PB96-103163

03,168

\section{ZONE-TYPE COMPARTMENT FIRE MODELS}

Simulating Smoke Movement through Long Vertical Shafts in Zone-Type Compartment Fire Models.

PB95-143152

$\infty 0,36$ 


\section{SAMPLE ENTRY}

Title

NTIS order number
Abstract number

Availability

Price Code
1,4-Dinitrocubane and Cubane under High Pressure. PB95-108437 03,755 Not available NTIS 2D-Scanning Capacitance Microscopy Measurements of Cross-Sectioned VLSI Teststructures.
PB96-163779 04,104 Not available NTIS 2nu9 Band of Propyne-d3. PB95- 164513 00,985 Not available NTIS 2-Tunneling Path Internal-Axis-Method-Like Treatment of the Microwave Spectrum of Divinyl Ether. PB94-200466

00,808 Not available NTIS

4SIGHT Manual: A Computer Program for Modelling Degradation of Underground Low Level Waste Concrete Vaults. PB95-231593 03,726 PC A05/MF A01

$10 \mathrm{kV}$ DC Resistive Divider Calibration PB95-198685

02,063 PC A04/MF A01

$24 \mathrm{GHz}$ Josephson Array Voltage Standard

PB94-211588 02,033 Not available NTIS

$30 \mathrm{MHz}$ Comparison Receiver. PB96-200407

01,972 Not available NTIS

$30 \mathrm{THz}$ Mixing Experiments on High Temperature Superconducting Josephson Junctions.
PB96-102462 PB96-102462 04,709 Not available NTIS 36Cl/CI Accelerator-Mass-Spectrometry Standards: Verification of Their Serial-Dilution-Solution Preparations by Radioactivity Measurements.

activity Measurer

00,524

(Order as PB94-140555, PC A06/MF A02)

$45 \mathrm{deg} / 0 \mathrm{deg}$ Reflectance Factors of Pressed Polytetrafluoroethylene (PTFE) Powder.

PB95-260758

04,328 PC A06/MF A02

63Ni Half-Life: A New Experimental Determination and Critical Review.

00,700 Not available NTIS

$100 \mathrm{~A}, 100 \mathrm{kHz}$ Transconductance Amplifier.

PB96-200936

02,098 Not available NTIS
100 Ampere, $100 \mathrm{kHZ}$ Transconductance Amplifier. $\begin{array}{ll}\text { PB96-102629 } & 02,068 \text { Not available NTIS }\end{array}$ 1950 Supplement to Screw-Thread Standards for Federal Services. 1944

AD-A280 223/9

$03,656 \mathrm{PC} \mathrm{A06/MF} \mathrm{A02}$

Ab initio Calculations for Helium: A Standard for Transport Property Measurements.

PB96-102041

01,060 Not available NTIS

Abrasive Wear by Diesel Engine Coal-Fuel and Related Particles.

PB95-104915

$01,450 \mathrm{PC} \mathrm{A04/MF} \mathrm{A01}$

Absence of Quantum-Mechanical Effects on the Mobility of Argon lons in Helium Gas at $4.35 \mathrm{~K}$.

PB97-122543

01,194 Not available NTIS

Absolute Cross-Section Measurements for Electron-Impact Ionization of $\mathrm{C} 1(+1)$

PB94-199841

03,818 Not available NTIS

Absolute Cross-Section Measurements for Electron-Impact Single lonization of $\mathrm{Se}(+)$ and $\mathrm{Te}(+)$.
PB95-202503
03,980 Not available NTIS Absolute Cross Sections for Electron-Impact Single Ionization of $\mathrm{Si}(t)$ and $\mathrm{Si}(2+)$. PB95-202529

03,982 Not available NTIS

Absolute Determination of Electrolytic Conductivity for Primary Standard KC 1 Solutions from 0 to $50 \mathrm{C}$.

PB94-172798 00,765 Not available NTIS

Absolute Frequency Measurements of Methanol from 1.5 to $6.5 \mathrm{THz}$.

PB95-175881

04,300 Not available NTIS

Absolute Response Calibration of a Transfer Standard Cryogenic Bolometer.

PB96-147103

04,358 Not available NTIS

Absorption Cross Sections, Kinetics of Formation, and SelfReaction of the 10 Radical Produced via the Laser Photolysis of N2O/12/N2 Mixtures.

PB97-112361

01,180 Not available NTIS
Absorption of Sound in Gases between 10 and $25 \mathrm{MHz}$ Argon.

PB94-199015 04,183 Not available NTIS

Absorption of Ultrasonic Waves in Air at High Frequencies $(10-20 \mathrm{MHz})$.

PB94-199007

04, 182 Not available NTIS

Abstract and Index Collection in the Research Information Center of the National Institute of Standards and Tech-

nology.

$02,744 \mathrm{PC} \mathrm{A03/MF} \mathrm{A01}$

Abstract and Index Collection in the Research Information Center of the National Institute of Standards and Tech.

nology.
PB95-232633

$02,741 \mathrm{PC} \mathrm{A03/MF} \mathrm{AO1}$

AC-DC Difference Characteristics of High-Voltage Thermal Converters.

PB96-148093

02,083 Not available NTIS

Acceleration of Intramolecular Vibrational Redistribution by methyl Internal Rotation. A Chemical Timing Study of pfluorotoluene and p-fluorotoluene-d3.

PB95-202982 01,039 Not available NTIS

Acceptance Diagram Analysis of the Performance of Multidisk Neutron Velocity Selectors.

PB94-200417

03,826 Not available NTIS

Acceptance Diagram Analysis of the Performance of Vertically Curved Neutron Monochromators.

PB94-200425

03,827 Not available NTIS

Access Paths for Materials Databases: Approaches for Large Databases and Systems. PB95-162525

02,975 Not available NTIS

Accuracy and Repeatability in Time Domain Network Analysis. 202644

02,064 Not available NTIS

Accuracy Comparisons of Josephson Array Systems.

PB95-164687 02,047 Not available NTIS

Accuracy in Integrated Circuit Dimensional Measurements. PB95-180808 02,368 Not available NTIS 


\section{TITLE INDEX}

Accuracy in Time Domain Transmission Line Measurements.
PB96-148069

04,060 Not available NTIS

Accuracy of Eigenvalues: A Comparison of Two Methods.

Accuracy of the Electron Pump

04,743 Not available NTIS

Accuracy-Weighted Variational Principle for Degenerate Continuum States.

03,831 Not available NTIS

Accurate Characterization of High Speed Photodectors.

O2,153 Not available NTIS

Accurate Computations of Radar Cross Sections of Simple Objects.

04,426 Not available NTIS

Accurate Electrical Characterization of High-Speed Interconnections.

02,240 Not available NTIS

Accurate Experimental Characterization of Interconnects: A Discussion of 'Experimental Electrical Characterization of Interconnects and Discontinuities in High-Speed Digital Systems.

02,217 Not available NTIS

Accurate Measurement of Optical Detector Nonlinearity.

PB95-203576

02,181 Not available NTIS

Accurate Measurement of Time.

96-119482

01,552 Not available NTIS

Accurate Measurements of the Local Deuterium Abundance from HST Spectra.

PB96-200621

00,110 Not available NTIS

Accurate Modeling of Size and Strain Broadening in the

Rietveld Refinement: The 'Double-Voigt' Approach.
PB96-200225
00,664 Not available NTIS

Accurate Transmission Line Characterization.

PB95-151593 02,220 Not available NTIS

Acid Gas Production in Inhibited Diffusion Flames.

PB95-180576 01,390 Not available NTIS

Acoustic Emission of Structural Materials Exposed to Open Flames.

PB95-164810

00,296 Not available NTIS

Acrylonitrile-Butadiene-Styrene (ABS) Plastic Drain, Waste, and Vent Pipe and Fittings.

$00,327 \mathrm{PC} \mathrm{A03/MF} \mathrm{A01}$

Activated Dynamics, Loss of Ergodicity, and Transport in

Supercooled Liquids.
PB95-150819

00,925 Not available NTIS

Active High Voltage Divider with 20-PPM Uncertainty

02,104 Not available NTIS

Active Site lonicity and the Mechanism of Carbonic Anhydrase.
PB94-212974

00,843 Not available NTIS

Activities of ISO Technical Committee 201 on Surface Chemical Analysis.

00,607 Not available NTIS

Activities of NIST (National Inst. Of Standards and Technology).

04,222

(Order as N94-23595/9, PC A21/MF A04)

Activities of the ASTM Committee E-42 on Surface AnalyPB95-108528

00,881 Not available NTIS

ADA; Category: Software Standard; Subcategory: Programming Language.

01,667 PC $\$ 278.00$

Ada Compiler Validation Summary Report. Certificate Number: 930927 S1.11328 Green Hills Software C Ada, Version 1.1 ZENY $386 \Rightarrow$ ZENY 386

AD-A277 $981 / 7$

01,643 PC A03/MF A01

Ada Compiler Validation Summary Report: Certificate Number: 931029S1.11330, Digital Equipment Corporation, DEC Ada for DEC OSF/1 AXP Systems, Version 3.1, DEC 3000 Model 400 AXP Workstation, DEC 3000 Model 400 AXP Workstation.

AD-A274 872/1

01,639 PC A04/MF A01

Ada Compiler Validation Summary Report: Certificate Number: 931119S1.11331 DDC-I, Inc. DACS Sun SPARC/ Sunos to 80386 PM Bare Ada Cross Compiler System SunOS to 80386 PM Bare Ada Cross Compiler System,
Version 4.6.4 Sun Sparcstation $1+=>$ Bare Board iSBC

AD-A276 283/9

$01,642 \mathrm{PC} \mathrm{A08/MF} \mathrm{A02}$

Ada Compiler Validation Summary Report. Certificate Number: 931119S1.11332, DDC-I, Inc. DACS MIPS R3000 Bare Ada Cross Compiler System, Version 4.7.1 Sun SPARCstation IPX $\Rightarrow$ DACS Sun SPARC/SunOS to MIPS
R3000 Bare Instruction Set Architecture Simulator, Version 4.7.1.
AD-A275 181/5 01,641 PC A04/MF A01
Ada Compiler Validation Summary Report: Certificate NumAda, Version 1.4 Cyber $180-930-31=>$ Cyber $180-930-31$.
AD-A275 $977 / 7$
01,640 PC AO3/MF AO 01,640 PC A03/MF A01

Ada Compiler Validation Summary Report: Certificate Num(1.1341 DDC-1, DACS Sun SPARC/SunOS to 80186 Bare Ada Cross Compiler System, Version 4.6.4 Sun SPARCstation IPX $=>$ Intel iSBC $\begin{array}{r}186 / 100 \\ \text { AD-A279 643/1 (Bare Machine). } \\ 01,645\end{array}$ PC AO6/MF A02

Ada Compiler Validation Summary Report: Certificate NumAda Compiler Validation Summary Report: Certificate Number: 940325 S1.11342 DDC-1, DACS Sun SPARC/SunOS to 80186 Bare Ada Cross Compiler System with Rate
Monotonic Scheduling Version 4.6.4 Sun SPARCstation IPX => Intel isBC

01,652 PC A06/MF A02

Ada Compiler Validation Summary Report: Certificate Number: $940325 S 1.11343$ DDC-1, DACS Sun SPARC/Solaris to 80186 Bare Ada Cross Compiler System, Version 4.6.4 Sun SPARC classic $\Rightarrow$ Intel iSBC $186 / 100$ (Bare Machine).

$01,649 \mathrm{PC} \mathrm{AO6/MF} \mathrm{A02}$

Ada Compiler Validation Summary Report: Certificate Number: 940325 S1.11344 DDC-I, DACS Sun SPARC/Solaris to 80186 Bare Ada Cross Compiler System with Rate Monotonic Scheduling, Version 4.6.4 Sun SPARCclassic $\Rightarrow$ Intel iSBC 186/100 (Bare Machine) AD-A279 $758 / 7$

01,650 PC A06/MF A02

Ada Compiler Validation Summary Report: Certificate Number 940325S1.11345 DDC-I. DACS Sun SPARC/SunOS to 680x0 Bare Ada Cross Compiler System, Version 4.6.9 Sun SPARCstation IPX $\Rightarrow$ Motorola MVME143 68030/68882 (Bare Machine)
AD-A280 145/4

01,656 PC A05/MF A02

Ada Compiler Validation Summary Report: Certificate Number: 940325 S1.11346 DDC-I, DACS Sun SPARC/SunOS to $680 \times 0$ Bare Ada Cross Compiler System (BASIC MODE). Version 4.6.9 Sun SPARCstation IPX $\Rightarrow$ Lynwood j435TU (68030) (Bare Machine)

AD-A279 646/4

$01,648 \mathrm{PC} \mathrm{A05/MF} \mathrm{A02}$

Ada Compiler Validation Summary Report: Certificate Number: 940325 S1.11347 DDC-I, DACS Sun SPARC/SunOS to $680 \times 0$ Bare Ada Cross Compiler System (SECURE MODE), Version 4.6.9 Sun SPARCstation IPX $\Rightarrow$ Lynwood j435TU (68030) (Bare Machine)

AD-A279 778/5

01,651 PC A06/MF A02

Ada Compiler Validation Summary Report: Certificate Number: 94032551.11348 DDC-I, DACS Sun SPARC/Solaris to 80386 PM Bare Ada Cross Compiler System, Version 4.6.4 Sun SPARCclassic $=>$ Intel iSBC $386 / 116$ (Bare Machine).
AD-A279 642/3

Ada Compiler Validation Summary Report: Certificate Number: $940325 S 1.11349$ DDC-I, DACS Sun SPARC/Solaris to 80386 PM Bare Ada Cross Compiler System with Rate Monotonic Scheduling, Version 4.6.4 Sun SPARCclassic $=>$ Intel iSBC $386 / 116$ (Bare Machine).

AD-A279 644/9

$01,646 \mathrm{PC} \mathrm{A06/MF} \mathrm{A02}$

Ada Compiler Validation Summary Report: Certificate Number: 940325 S1.11350 DDC-I, DACS Sun SPARC/SunOS to Pentium PM Bare Ada Cross Compiler System, Version 4.6.4 Sun SPARCstation IPX $\Rightarrow>$ Intel Pentium (Operated as Bare Machine) Based in Xpress Desktop (Intel Product Number. XBASE6E4F-B)

AD-A279 864/3

01,655 PC A06/MF A02

Ada Compiler Validation Summary Report: Certificate Number: 940325 S1.11351 DDC-I, DACS Sun SPARC/SunOS to Pentium PM Bare Ada Cross Compiler System with Rate Monotonic Scheduling, Version 4.6.4 Sun SPARCstation IPX $\Rightarrow$ Intel Pentium (operated as Bare Machine) based in Xpress Desktop (Intel product number: XBASE6E4F-B).
AD-A279 804/9 01,653 PC A06/MF A

$01,653 \mathrm{PC}$ A06/MF A02

Ada Compiler Validation Summary Report: Certificate Number: 940325 S1.11352 DDC-I DACS Sun SPARC/Solaries to 4.6.4 Sun SPARC Adassic $=>$ Intel Pentium 4.6.4 Sun SPARCclassic $=>$ Intel Pentium (Operated as Number: XBASE6E4F-B).

AD-A280 295/7

01,657 PC A06/MF A02

Ada Compiler Validation Summary Report: Certificate Number: 940325 S1.11353 DDC-I, DACS Sun SPARC/Solaris to Pentium PM Bare Ada Cross Compiler System with Rate Monotonic Scheduling, Version 4.6.4 Sun SPARCclassic $=>$ Intel Pentium (operated as Bare Machine) based in Xpress
Desktop (Intel product number: XBASE6E4F-B). Desktop (Intel product number: XBASE6E4F-B)
AD-A279 805/6 01,654 PC A06/MF A02 Ada Compiler Validation Summary Report: Certificate Number: $940325 S 1.11354$ DDC-I, DACS Sun SPARC/Solaris
Native Ada Compiler System, Version 4.6.2 Sun Native Ada Compiler System, Version 4.6.2 Sun
SPARCclassic $\Rightarrow$ Sun SPARCclassic. AD-A279 645/6

01,647 PC A04/MF A01

Ada Compiler Validation Summary Report: Certificate Number 940902S1.11376. UNISYS Corporation IntegrAda for Windows NT, Version 1.0. Intel Deskside Server for Intel Pentium $60 \mathrm{MHz} \Rightarrow$. Intel Deskside Server with Intel Pentium $60 \mathrm{MHz}$

01,659 PC A03/MF A01

Ada Compiler Validation Summary Report: Certificate Number 940902S1.11377 UNISYS Corporation. IntegrAda for
$80486 \mathrm{D} \times 266 \Rightarrow$ Intel Deskside Server with Intel AD-A288 $571 / 3$

$01,658 \mathrm{PC} \mathrm{A03/MF} \mathrm{A01}$

Ada Compiler Validation Summary Report. Certificate Number $941117 \mathrm{~S} 1.11380$. Electronic Data Systems Corp. Com piler: OC Systems Legacy Ada/370, Release 1.4.1 (without AD-A289 895/5

$01,662 \mathrm{PC} \mathrm{A05/MF} \mathrm{A01}$

Ada Compiler Validation Summary Report: Certificate Number 94101251.11379 TISOFT, Inc. Green Hills Optimizing Ada Compileer, Version 18.7 with PATCK ID 1 COMPAO ProLiiant 2000 Model $55 / 66 \Rightarrow$ COMPAO ProLiant 2000 ADdel $5 / 66$.

01,660 PC A03/MF A01

Ada Compiler Validation Summary Report, VC No 950609S1.11390 Digital Equipment Corporation - Compile Name: DEC Ada Version 3.2 for OpenVMS VAX Systems.
AD-A296 794/1

ADA Compiler Validation Summary Report, vC Number 950303S1.11381. Digital Equipment Corporation - Compile Name: DEC Ada for OpenVMS Alpha Systems, Version 3.2.
AD-A293 709/2 01,663 PC A05/MF A01

Ada Complier Validation Summary Report: Certificate Number: 940929S1.11378. Digital Equipment Corporation DEC Ada for DEC OSF/1 AXP Systems, Version 3.2; DEC 3000 Model 400 AXP Workstation => DEC 3000 Model 400 AXP Workstation

D-A288 $574 / 7$

01,661 PC A05/MF A01

Adaptive, Predictive 2-D Feature Tracking Algorithm for

Finding the Focus of Expansion.
PB94-218575

$01,588 \mathrm{PC}$ A03/MF A01

Addition of $M$ and L-Series Lines to NIST Algorithm for Cal-

culation of X-Ray Tube Output Spectral Distributions.
PB95-108742 00,569 Not available NTIS

Addressing U.S. Government Security Requirements for PBI. 01,611 Not available NTIS

Adhesion, Contact Electrification, and Acid-Base Properties of Surfaces.
PB96-204425 03,693 Not available NTIS

Adhesion of Composites to Dentin and Enamel.

00.144 Not available NTIS

Administration Standard for the Telecommunications Infrastructure of Federal Buildings. Category: Telecommunications Standard; Subcategory: Telecommunications Administration.
FIPS PUB 187

$01,461 \mathrm{PC} \mathrm{A02/MF} \mathrm{A01}$

Adsorption of Low-Molecular-Weight Sodium Polyacrylate on Hydroxyapatite
PB94-172608

00,139 Not available NTIS

Adsorption of Polyacrylic Acids and Their Sodium Salts on Hydroxyapatite: Effect of Relative Molar Mass.

03,581 Not available NTIS

Adsorption of Potassium $\mathrm{N}$-phenylglycinate on Hydroxyapatite: Role of Solvents and lonic Charge.
PB96-180161.

Advanced Angle Metrology System.

Advanced Angl

02,637 Not available NTIS

Advanced Components for Electric and Hybrid Electric Vehicles. Workshop Proceedings. Held in Gaithersburg, Maryhicles. Workshop Proceeding

land on Octob
PB94-177060

04,858 PC A1O/MF A03

Advanced Mass Calibration and Measurement Assurance Program for State Calibration Laboratories.
PB95-253571 02,492 PC A03/MF A01

Advances in the Measurement of Polymer CTE: Micrometer- to Atomic-Scale Measurements. PB96-180229

03,390 Not available NTIS

Aerodynamic Phenomena in Stellar Atmospheres - A Bibliography.
AD-A278 $521 / 0$

00,046 PC A05/MF AO2

Affinity Chromatography on Inorganic Support Materials.

Agent Screening for Halon 1301 Aviation Replacement.

Agent Screening for Halon 1301 Aviation Replacement.
PB96-159710 03,282 Not available NTIS

Aggregation Kinetics of Colloidal Particles Destabilized by Enzymes.
PB95-125878

00,894 Not available NTIS

Agile Manufacturing from a Statistical Perspective.

Aging Effects on XRF Measurements of Solder Coatings.

PB95-140927 03,123 Not available NTIS

Aging in Glasses Subjected to Large Stresses and Deformations.
PB95-107041 03,235 Not available NTIS

Aging, Warm-Up Time and Retrace; Important Characteristics of Standard Frequency Generators.
PB96-103122 


\section{TITLE INDEX}

Aid for Smaller Businesses. PB94-212461

00,492 Not available NTIS

Air Change Effectiveness Measurements in Two Modern Office Buildings.
PB94-185766

00,243 Not available NTIS

Air Flow in the Boundary Layer of an Elliptic Cylinder.

AD-A297391/5 04,194 PC A03/MF A01

Airbome Asbestos Analysis: National Voluntary Laboratory Accreditation Program.

02,566 PC A05/MF A01

Airbome Asbestos Method: Bootstrap Method for Determining the Uncertainty of Asbestos Concentration. Version 1.0 .
PB96-214614 00,646 PC A04/MF A01

Airbome Asbestos Method: Standard Practice for Recording Transmission Electron Microscopy Data for the Analysis of Asbestos Coilected onto Filters. Version 1.0.

PB94-210168 00,552 PC A03/MF AO3

Airborne Asbestos Method: Standard Test Method for High Precision Counting of Asbestos Collected on Filters. Version 1.0

PB94-163003

00,525 PC A02/MF A01

Airbome Asbestos Method: Standard Test Method for Verified Analysis of Asbestos by Transmission Electron Microscopy. Version 2.0

$00,526 \mathrm{PC} \mathrm{A03/MF} \mathrm{A01}$

Airbome Smoke Sampling Package for Field Measurements of Fires.

PB95-150041

01,381 Not available NTIS

Al L2,3 Core Excitons in AlxGa 1-x as Studied by Soft-X-ray Reflection and Emission.

PB96-157839

04,067 Not available NTIS

Alaska Marine Mammal Tissue Archival Project: Specimen Inventory.
PB95-171344

02,589 PC A05/MF A01

Alcohol Solutions of Triphenyl-Tetrazolium Chloride as High-Dose Radiochromic Dosimeters.
PB96-135249 Not available NTIS Algebraic Approximation of Attractors for Galloping Oscillators. 04,820 Not available NTIS

Algorithm Testing and Evaluation Program for Coordinate Measuring Systems: Long Range Plan.
PB95-231833 PC A03/MF A01 Algorithm Testing and Evaluation Program for Coordinate Measuring Systems: Testing Methods.
PB95-251659 PC A03/MF A01 Algorithm to Describe the Spread of a Wall Fire under a Ceiling. $00,261 \mathrm{PC} \mathrm{A04/MF} \mathrm{A01}$

Algorithmic Enhancements to the Method of Centers for Linear Programming Problems.

PB94-198959

03,426 Not available NTIS

Alignment in Two-Step Pulsed Laser Excitation of Rydberg

Levels in Light Atoms: The Example of Sodium.
PB95-202883
03,993 Not available NTIS

Alignment Probing of Rydberg States by Stimulated Emis-

04,124 Not available NTIS

Alkali-Silica Reaction and High Performance Concrete.

PB96-131537 01,345 PC A03/MF A01

All-Metal Collection System for Preparative-Scale Gas Chromatography: Purification of Low-Boiling-Point Compounds.

00,619 Not available NTIS

Alpha-Particle and Electron Capture Decay of (209)Po.

PB96-186085 04,119 Not available NTIS

Alternating-Field Susceptometry and Magnetic Susceptibility of Supercondoctors. Presented at Office of Naval Research Workshop on Magnetic Susceptibility of Superconductors and Other Spin Systems. Held in Berkeley Springs, West Virginia on 20 May 1991.

04,435 PC A01/MF A01

Alternating-Field Susceptometry and Magnetic Susceptibility

of Superconductors.
PB95-168613

04,638 Not available NTIS

Alternative Contour Technique for the Efficient Computation of the Effective Length of an Antenna.
PB96-141361 02,011 Not available NTIS

Alternative EMC Compliance Test Facilities.

PB96-200324 02,247 Not available NTIS

Alternative Single Integral Equation for Scattering by a Di-

PB94-216512

04,422 Not available NTIS

Aluminum-Lithium Alloys: Evaluation of Fracture Toughness by Two Test Standards, ASTM Method E 813 and E 1304.
PB96-190236 03,374 Not available NTIS
Alvin Van Valkenburg and the Diamond Anvil Cell. PB96-204474 04,797 Not available NTIS Ambient Temperature Synthesis of Bulk Intermetallics PB95-169074 00,168 Not available NTIS Amperometric Flow-Injection Analysis Biosensor for Glucose Based on Graphite Paste Modified with Tetracyanoquinodimethane. $\quad 03,498$ Not available NTIS
PB95-161980 Amperometric Measurement of Moisture in Transformer Oil Using Karl Fischer Reagents.
PB96-146766
00,623 Not available NTIS AMRF Composite Fabrication Workstation.

PB94-172681 02,810 Not available NTIS Analyses of Recent Experimental and Theoretical Determinations of e-H2 Vibrational Excitation Cross Sections: Assessing a Long-Standing Controversy.

PB95-202438 03,977 Not available NTIS

Analysis by a Combination of Gas Chromatography and Tandem Mass Spectrometry: Development of Quantitative Tandem-in-Time lon Trap Mass Spectrometry: Isotope Dllution Quantification of 11-Nor-Delta-9-Tetrahydro cannabinol9-Carboxylic Acid

PB96-117221

02,561 Not available NTIS

Analysis of a Biologically Motivated Neural Network for Character Recognition.

PB94-172277

00,182 Not available NTIS

Analysis of an Open-Ended Coaxial Probe with Litt-Off for

Nondestructive Testing.
PB96-135116

Analysis of ANSI ASC $\times 12$ and UN/EDIFACT Electronic Data Interchange (EDI) Standards. PB95-220554

$01,729 \mathrm{PC} \mathrm{AO3/MF} \mathrm{AO1}$

Analysis of Autocorrelations in Dynamic Processes.
PB95-181228

Analysis of Boron in CVD Diamond Surfaces Using Neutron Depth Profiling
PB94-213089

04,511 Not available NTIS

Analysis of Creep in a Si-SiC C-Ring by Finite Element Method

03,037 Not available NTIS

PB94-200268

Analysis of Droplet Arrival Statistics in a.Pressure-Atomized
Spray Flame.
PB97-112270
01,352 Not available NTIS

Analysis of Failed Dry Pipe Fire Suppression System Couplings from the Filene Center at Wolf Trap Farm Park for the Pertorming Arts.

$00,331 \mathrm{PC} \mathrm{A03/MF} \mathrm{A01}$

Analysis of High Bay Hangar Facilities for Detector Sensitivity and Placement.

PB96-1902 10

01,429 Not available NTIS

Analysis of Moisture Accumulation in a Wood-Frame Wall Subjected to Winter Climate.

PB94-199320

00,338 Not available NTIS

Analysis of Physical Properties of Ceramic Powders in an International Interlaboratory Comparison Program.

PB95-161501 03,050 Not available NTIS Analysis of Protein Metal Binding Selectivity in a Cluster
Model.

PB94-212990

00,845 Not available NTIS

Analysis of SANS from Controlled Pore Glasses.

PB94-198843 03,035 Not available NTIS

Analysis of Scattering Asymmetry Statistics When Background Corrected Counts Are Negative.
PB94-200334 Not available NTIS

Analysis of Selected Software Safety Standards.

PB95-151262 01,708 Not available NTIS

Analysis of Small-Angle Scattering Data Dominated by Multiple Scattering for Systems Containing Eccentrically Shaped Particles or Pores. PB96-160411

03,075 Not available NTIS

Analysis of Standards for the Assurance of High Integrity Software.

PB96-161351

03,735 Not available NTIS

Analysis of the Effectiveness of Oscillating Radial Collima-

tors in Neutron Scattering Applications.
PB95-152252 Not available NTIS

PB94-199270

00,193 Not available NTIS

Analysis of the $(5 d(2)+5 d 6 s)-5 d 6 p$ Transition Arrays of Os VII and Ir VIII, and the 6s (2)S-6p (2)P Transitions of Ir IX.

PB96-159264 01,954

(Order as PB96-159215, PC A07/MF A02)

Analysis of the $5 s(2) 5 p(2)-(5 s 5 p(3)+5 s(2) 5 p 5 d+5 s(2) 5 p 6 s)$

Transitions of Four-Times lonized Xenon (Xe V).
PB95-150769 03,900 Not aval

03,900 Not available NTIS
Analysis of Thermal Wave Propagation in Diamond Films. PB94-211471 03,014 Not available NTIS Analysis of Transverse Flow in Aligned Fibrous Porous Medía.

PB96-167200

03,177 Nol available NTIS

Analytic Calculation of Polarized Neutron Reflectivity from Superconductors.

04,629 Not available NTIS

Analytical Applications of Guided Neutron Beams

PB96-112347

04,041 Not available NTIS

Analytical Estimation of Carrier Multipath Bias on GPS Position Measurements.

PB94-215712

$04,845 \mathrm{PC} \mathrm{A04/MF} \mathrm{AO}$

Analytical Expressions for Barkhausen Jump Size DistribuPB95-180345

04,680 Not available NTIS

Analytical Method for Determining Thermal Conductivity from Dynamic Experiments.

PB96-102744

04,024 Not available NTIS

Analytical Method of Determining the Heat Capacity at High Temperatures from the Surface Temperature of a Cooling Sphere.

PB94-216124

03,865 Not available NTIS

Analyzing and Exploiting Numerical Characteristics of Zone PB96-102314

01,400 Not available NTIS

Analyzing Electronic Commerce.

PB94-219102

00,480 PC A03/MF AO

Angle-Differential and Momentum-Transfer Cross Sections for Low-Energy Electron-Cs Scattering.
PB95-203402 04,005 Not available NTIS

Angular Distributions for Near-Threshold $(e, 2 \theta)$ Processes for $\mathrm{Li}$ and $\mathrm{Mg}$.

PB94-185725

00,778 Not available NTIS

Angular Variation of the Personal Dose Equivalent $\mathrm{Hp}(0.07)$, for Bela Radiation and Nearly Monoenergetic Electron Beams: Preliminary Results.

PB95-168472

03,630 Not available NTIS

Anharmonic Oscillator Analysis Using Modified Airy Func-

PB94-18531

03,798 Not available NTIS

Anharmonic Phonons and the Isotope Effect in Superconductivity.

04,486 Not available NTIS

Anionic Triphenylmethane Dye Solutions for Low-Dose Food Irradiation Dosimetry.

PB96-135173

03,715 Not available NTIS

Anisotropic Phase Separation Kinetics in a Polymer Blend Solution Following Cessation of Shear Studied by Light Scattering.

01,241 Not available NTIS

Anisotropy of Interfaces in an Ordered Alloy: A Multiple Order-Parameter Model.

PB96-131594

$04,741 \mathrm{PC}$ A04/MF AO

Anisotropy of Polarized X-ray Emission from Atoms and Molecules.

04,621 Not available NTIS

Anisotropy of the Surfaces of Pores in Plasma Sprayed Alumina Deposits.

03,126 Not available NTIS

Annealing of Bragg Gratings in Hydrogen-Loaded Optical

Fiber.

04,361 Not available NTIS

Annex 18: An Intemational Study of Refrigerant Properties. PB95-168936

03,266 Not available NTIS

Annual Conference on Fire Research: Book of Abstracts,

October 17-20, 1994.

$01,376 \mathrm{PC} \mathrm{A09/MF} \mathrm{A02}$

Anomalous Behavior of a Quantized Hall Plateau in a HighMobility Si Metal-Oxide-Semiconductor Field-Effect TransisPor. 95.164174

02.354 Not available NTIS

Anomalous Dispersion and Thermal Expansion in LightlyDoped KTa1-xNbxO3.

PB95-152302

04,585 Not available NTIS

Anomalous Freezing and Melting of Solvent Crystals in Swollen Gels of Natural Rubber.
PB94-212321 01,223 Not available NTIS Anomalous Odd- to Even-Mass Isotope Ratios in Reso-

nance lonization with Broad-Band Lasers.
PB94-211406 03,839 Not available NTIS

Anomalous Relation between Time and Frequency Domain PMD Measurements.

PB97-119390

04,398 Not available NTIS 
Anomalous Switching Phenomenon in Critical-Current Measurements When Using Conductive Mandrels.
PB96-137781

Anova Estimates of Variance Components for a Class of Mixed Models.

03,448 Not available NTIS

Antenna-Coupled High-Tc Air-Bridge Microbolometer on Silicon.

04,315 Not available NTIS

Antiferromagnetic Interlayer Correlations in Annealed Ni80Fe20/Ag Multilayers

03,109 Not available NTIS

APDE Demonstration System Architecture. National PDES Testbed Report Series.

02,767 PC A03/MF A01

Aperture Coupling to a Coaxial Air Line: Theory and Experiment.

PB94-211968

02,216 Not available NTIS

Aperture Excitation of Electrically Large, Lossy Cavities. PB94-145711 00,029 PC A05/MF AO

Aperture Excitation of Electrically Large, Lossy Cavities.

Aperture Excitation of Electrically Large, Lossy Cavities.
PB95-175675 00,031 Not available NTIS

Apparatus for Resistance Measurement of Short, Small-Diameter Conductors.

ameter Conductors
PB96-141130

04,417 Not available NTIS

Apparel Manufacturing Glossary for Application Protocol Development.

PB95-198750

02,755 PC A04/MF A01

Apparent Bias in the X-Ray Fluorescence Determination of Titanium in Selected NIST SRM Low Alloy Steels.

PB95-108759 03,212 Not available NTIS

Apparent Molar Heat Capacities and Apparent Molar Volumes of Aqueous Glucose at Temperatures from $298.15 \mathrm{~K}$ to $327.01 \mathrm{~K}$.

PB94-212800

03,459 Not available NTIS

Appearance Intensities for Multiply Charged lons in a Strong Laser Field.

04,089 Not available NTIS

Appearance Potentials of Ions Produced by Electron-Impact Appearance Potentials of lons Produced by Electron-Impac $\mathrm{SO} 2, \mathrm{SO} 2 \mathrm{~F} 2$, SOF2, and SOF 4 .

PB96-119730

01,080 Not available NTIS

Applicability of Effective Medium Theory to Ferroelectric/ Ferrimagnetic Composites with Composition and Frequency-Dependent Complex Permittivities and Permeabilities.

01,945 Not available NTIS

Application of a Multizone Airflow and Contaminant Dispersal Model to Indoor Air Quality Control in Residential Buildings.
PB95-180238

02,555 Not available NTIS

Application of a Novel Slurry Furnace AAS Protocol for Rapid Assessment of Lead Environmental Contamination. Rapid Assessment of Lead Environmental Contamination.
PB96-112354 Not available NTIS

Application of a Simple Technique for Estimating Errors of Application of a Simple Technique for Estimating Errors of Finite-Element Solutions Using a General-Purpose Code.
PB94-200250 04,818 Not available NTIS

Application of Boundary Element Methods to a Transient

Axis-Symmetric Heat Conduction Problem.
PB94-212693 01,375 Not available NTIS

Application of Digital-Image-Based Models to Microstructure, Transport Properties, and Degradation of Cement-Based Materials.

PB96-156161

00,406 Not available NTIS

Application of Electromagnetic-Acoustic Transducers for Nondestructive Evaluation of Stresses in Steel Bridge Structures.

PB96-167978

$01,301 \mathrm{PC} \mathrm{A04/MF} \mathrm{A01}$

Application of Expert System to Select Data Sources from Chemical Information Databases.

PB95-125654

00,505 Not available NTIS

Application of Metadata Standards.

PB96-180187 01,771 Not available NTIS

Application of ODF to the Rietveld Profile Refinement of Polycrystalline Solic.

PB95-202388

03,401 Not available NTIS

Application of Photochemical Reaction in Electrochemical Detection of DNA Intercalation.

PB94-185733

00,686 Not available NTIS

Application of Single Electron Tunneling: Precision Capacitance Ratio Measurements.

PB96-102157

04,703 Not available NTIS

Application of the Collocation Method in Three Dimensions O a Model Semiconductor Problem.

PB97-122428
Application of the Electronic Balance in High Precision Pycnometry.
PB94-187564

00,534 PC A03/MF A01

Application of the Modified Voltage-Dividing Potentiometer

to Overlay Metrology in a CMOS/Bulk Process. 81997

02,302 Not available NTIS

Application of the Pointer State Subgraph to Static Program Slicing.

01,768 PC A03/MF A01

PB96-167838 Application of the Taylor Dispersion Method in Supercritical
Fluids.
PB95-164323 00,977 Not available NTIS Application of Thermal Analysis Techniques to the Characterization of EPDM Roofing Membrane Materials.

PB95-125845 00,359 Not available NTIS

Application of Thermodynamic and Detailed Chemical Kinetic Modeling to Understanding Combustion Product Generation in Enclosure Fires.

01,413 Not available NTIS

Application of Thermodynamics to Biotechnology.

PB95-150793 03,529 Not available NTIS

Application Portability Profile (APP): The U.S. Government's Open System Environment Profile Version 3.0.

PB96-158712

01,753 PC A07/MF A02

Application Profile for ISDN PB95-163689

01,479 Not available NTIS

Application Profile for the Government Information Locator Service (GILS). Category: Software Standard; Subcategory: Information Interchange.

Information Interch
FIPS PUB 192

01,800 PC A03/MF A01

Application Protocol Information Base World Wide Web Gateway.
PB96-202320

02,791 PC A03/MF A01

Application Software Interface: ISDN Services for an Open Systems Environment.

01,492 PC A03/MF A01

Applications of Diamond Films and Related Materials: InterAptional Conference (3rd). Held in Gaithersburg. Maryland national Conference (3rd). Held in Gaithersburg, Maryland
on August 21-24, 1995. Supplement to NIST Special Publion August

PB95-256053

03,063 PC A04/MF A01

Applications of Fluorescence Spectroscopy in Polymer Science and Technology.

01,258 Not available NTIS

Applications of the Vortex Tube in Chemical Analysis.

PB94-199171 00,544 Not available NTIS

Applications of the Vortex Tube in Chemical Analysis. Part 2. Applications

00,615 Not available NTIS

Applying Virtual Environments to Manufacturing.

PB94-142502 02,803 PC A03/MF A01

Approach to Setting Performance Requirements for Automated Evaluation of the Parameters of High-Voltage Im-

pulses.

01,878 Not available NTIS

Approaches Using Virtual Environments with Mosaic

PB95-169108 01,599 Not available NTIS

Appropriate Ultrasonic System Components for NDE of Thick Polymer Composites.

03,148 Not available NTIS

Approximate Confidence Intervals on Linear Combinations of Expected Mean Squares.

PB95-151296

03,435 Not available NTIS

Approximate Confidence Intervals on Positive Linear Combinations of Expected Mean Squares.

PB95-151304

03,436 Not available NTIS

Approximate Solution to the Scalar Wave Equation for Optical Waveguides.

04,254 Not available NTIS

Arc Spectra of Gallium, Indium, and Thallium. AD-A295 $411 / 3$

00,718 PC A03/MF A01

Artificial Crack in Steel: An Ultrasonic-Resonance-Spectroscopy and Modeling Study.

PB96-141395

03,241 Not available NTIS

Ascorbic and Dehydroascorbic Acids Measured in Plasma Preserved with Dithiothreitol or Melaphorphoric Acid.

PB94-216330

03,495 Not available NTIS

Ashland Tank Collapse Investigation.

PB95-126314 02,481 Not available NTIS

Ashland Tank-Collapse Investigation: Closure by Authors.

PB95-126322 02,482 Not available NTIS

Aspects of a Deformable Superconductor Model for the Vor-

tex Mass.
PB95-175303

04,652 Not available NTIS

Aspects of a Product Model Supporting Apparel Virtual Enterprises.

02,790 PC A03/MF A01

Asperity-Asperity Contact Mechanisms Simulated by a TwoBall Collision Apparatus.

PB95-164158

02,966 Not available NTIS

Assay of the Eluent from the Alumina-Based Tungsten-188Rhenium-188 Generator.

PB94-200482

03,829 Not available NTIS

Assessing Functional Diversity by Program Slicing.

PB96-160890 03,734 Not available NTIS

Assessing Halon Alternatives for Aircraft Engine Nacelle Fire Suppression

PB96-102454

01,401 Not available NTIS

Assessing MOS Gate Oxide Reliability on Wafer Level with Ramped/Constant Voltage and Current Stress.

PB96-180112

04,115 Not available NTIS

Assessing the Credibility of the Calorific Content of Muncipal Solid Waste.

02,581 Not available NTIS

Assessing Ventilation Effectiveness in Mechanically Ventilated Otfice Buildings.

PB95-162079

00,255 Not available NTIS

Assessment of Data by a Second-Order Transfer Function. PB95-182390

03,760 PC A05/MF A01

Assessment of 'Peaks Over Threshold' Methods for Esti-

mating Extreme Value Distribution Tails.
PB95-161360 00,441 Not available NTIS

Assessment of Site Response Analysis Procedures

PB95-210928

00,450 PC A07/MF A02
PC A

Assessment of Technologies for Advanced Fire Detection. PB95-126330

Advanced Fire Detection.
00,294 Not available NTIS

Assessment of Technology for Detection of Stress Corrosion Cracking in Gas Pipelines. Final Report, July 1993March 1994.

02,475 PC A05/MF AO2

Assessment of Testing Methodology for Ceramic Matrix Composites.

03,139 Not available NTIS

Assessment of the Al-Sb System

PB94-200474

03,329 Not available NTIS

Assessment of the DOD Goal Security Architecture (DGSA) for Non-Military Use.

PB95-189510

03,653 PC A03/MF A01

Assessment of Uncertainties of Calibration of Resistance Thermometers at the National Institute of Standards and Technology.
PB94-142478

02,624 PC A03/MF A01

Assessment of Uncertainties of Liquid-in-Glass Thermometer Calibrations at the National Institute of Standards and Technology.

02,625 PC A03/MF A01

Assessment of Uncertainties of Thermocouple Calibrations at NIST

PB94-15269

03,782 PC A03/MF A01

Associated Object Model for Distributed Systems. PB94-212016 01,694 Not available NTIS Associative Electron Attachment to S2F10, S2OF10, and S2O2F 10.

00,907 Not available NTIS

Associative Ionization in Collisions of Slowed and Trapped Sodium.
PB95-125886

03,880 Not available NTIS

Astrophysical Aspects of Neutral Atom Line Broadening

PB94-213287 00,061 Not available NTIS

Asymmetric Tip Morphology of Creep Microcracks Growing Along Bimaterial Interfaces.

PB94-200243

03,138 Not available NTIS

Asymmetry between Flux Penetration and Flux Expulsion in Tl-2212 Superconductors.

PB95-125647

04,527 Not available NTIS

Asymptotic and Numerical Analysis of a Premixed Laminar Nitrogen Dioxide-Hydrogen Flame.

PB96-164256

01,422 Not available NTIS

Asymptotic Behavior of Modulated Taylor-Couette Flows with a Crystalline Inner Cylinder.

PB94-199072

04,469 Not available NTIS

Asymptotic Wave Function Splitting Procedure for Propagating Spatially Extended Wave Functions: Application to Intense Field Photodissociation of $\mathrm{H} 2(+)$.

PB94-211786

03,847 Not available NTIS

Asynchronous Transfer Mode Procurement and Usage PB95-174967

01,481 PC A03/MF A01 


\section{TITLE INDEX}

Atmospheric and Marine Trace Chemistry: Interfacial Biomediation and Monitoring.
PB94-199122 Atmospheric Lifetimes of HFC-143a and HFC-245fa: Flash Atmospheric Lifetimes of HFC-143a Mesonance Fluorescence Measurements of the PHotolysion Rate Constants.
PB97-112577 00,118 Not available NTIS Atmospheric Reactivity of alpha-Methyl-Tetrahydrofuran.
PB95-163705 02,548 Not available NTIS Atom Cooling and Trapping, and Collisions of Trapped Aloms.
PB96-122916

04,048 Not available NTIS Atom-counting standards and Doppler-free resonance ionization mass spectroscopy. (Progress report). 00,723 PC A02/MF A01
DE94018562

Atom-Optical Properties of a Standing-Wave Light Field.
PB96-141072 04,356 Not available NTIS

Atomic Beam Splitters and Mirrors by Adiabatic Passage in Multilevel Systems.

03,867 Not available NTIS

Atomic Branching Ratio Data for Carbon-Like Ions.

PB94-212842 03,855 Not available NTIS

Atomic Branching Ratio Data for Nitrogen-Like Species.

PB96-190152 04,122 Not available NTIS

Atomic Branching Ratio Data for Oxygen-Like Species.

PB95-180436 03,963 Not available NTIS

Atomic Clock

O3,963 Not avallable NTIS

01,553 Not available NTIS

Atomic Data Needed for Far Ultraviolet Astronomy with HUT and FUSE.

PB94-213402

00,062 Not available NTIS

Atomic Energy Levels As Derived from the Analyses of Optical Spectra. Volume 1. Section 1. The Spectra of Hydrogen, Deuterium, Helium, Lithium Beryllium, Boron, Carbon, Nitrogen, Oxygen, and Fluorine.
AD-A278 $130 \% 0$

Atomic Energy Levels. As Derived From the Analyses of Optical Spectra. Volume 3.

00,714 PC A13/MF A03

Atomic Energy Levels in Crystals.

04,431 PC A05/MF A01

Atomic Iron in Its (5)D Ground State: A Direct Measurement of the $\mathrm{J}=0$ inverted arrow 1 and $\mathrm{J}=1$ inverted arrow 2 Fine-Structure Intervals (1.2).

PB96-141221

04,756 Not available NTIS

Atomic Manipulation of Polarizable Atoms by Electric Field Directional Diffusion.
PB95-150587

04,572 Not available NTIS

Atomic, Molecular, and Optical Physics with $X$-rays.

Atomic, Molecular, and Optical Physics with X-rays.
PB94-213378 03,863 Not available NTIS

Atomic Oxygen Fine Structure Splittings with Tunable Far Infrared Spectroscopy.

PB95-152203

03,915 Not available NTIS

Atomic-scale characterization of hydrogenated amorphoussilicon films and devices. Annual subcontract report, 14 February 1994--14 April 1995

DE95009287

02,294 PC A03/MF A01

Atomic Sulfur: Frequency Measurement of the $\mathrm{J}=0$ inversely maps 1 Fine-Structure Transition at 56.3 Microns by Laser Magnetic Resonance.

PB95-180105

01,007 Not available NTIS

Atomic Theory of Fracture of Brittle Materials: Application 10 Covalent Semiconductors.

PB94-216553

04,519 Not available NTIS

Atomic Transition Probabilities and Tests of the Spectroscopic Coupling Scheme for N I.
PB96-138466 04,057 Not available NTIS Atomic Transition Probability Ratios between Some Ar 145 $4 p$ and $4 \mathrm{~s}-5 \mathrm{p}$ Transitions.

PB94-211554

03,842 Not available NTIS

Atomic Weights of the Elements 1991.

PB94-168606 00,754 Not available NTIS

Atomic Weights of the Elements, 1993

PB96-145909 01,114 Not available NTIS

Atoms in Optical Molasses.

PB95-108874

03,875 Not available NTIS

Atoms in Optical Molasses: Applications to Frequency Standards.

PB95-108882

03,876 Not available NTIS

Attempts at Extending the Unified Theory to Include ManyBody Effects.
PB94-212719

04.408 Not available NTIS

AutoBid 2.0: The Microcomputer System for Police Patrol Vehicle Selection.

04,871 PC A03/MF A01
Autocorrelation Functions from Optical Scattering for OneDimensionally Rough Surfaces

PB94-216538 04,244 Not available NTIS

Autofluorescence Detection of 'Escherichia coli' on Silver Membrane Filters

PB96-163639

03,590 Not available NTIS

Autoionizing Resonances in Electric Fields.

PB94-212727 03,853 Not available NTIS

Automated Guarded Bridge for Calibration of Multimegohm Standard Resistors.

PB97-119150

02,289 Not available NTIS

Automated, High-Precision Coulometric Titrimetry. Part 1 Engineering and Implementation.

PB95-150199

00,575 Not available NTIS

Automated, High Precision Coulometric Titrimetry. Part 2 Strong and Weak Acids and Bases.

PB95-150207

00,576 Not available NTIS

Automated Inspection: The Integration of National Standards and Commercial Products at NIST.

PB95-163077 02,906 Not available NTIS

Automated Josephson Integrated Circuit Test System.

PB95-175246

02,057 Not available NTIS

Automated Manufacturing Research Facility 1994 Annual Report

PB95-209854

00,015 PC A05/MF A01

Automated Measurement of Nonlinearity of Optical Fiber Power Meters.

04,110 Not available NTIS

Automated Optical Roughness Inspection.

PB95-152179 02,905 Not available NTIS

Automated Resistance Measurements at NIST.

PB96-119326 02,274 Not available NTIS

Automatic Calibration of Inductive Voltage Dividers for the NASA Zeno Experiment.

PB95-152849

02,041 Not available NTIS

Automatic Inductive Voltage Divider Bridge Operates from $10 \mathrm{~Hz}$ to $100 \mathrm{kHz}$

02,032 Not available NTIS

Backdratt Phenomena.

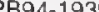

$01,366 \mathrm{PC} \mathrm{A} 11 / \mathrm{MF} \mathrm{A03}$

Backscattering in Electron-Impact Excitation of Multiply Charged lons.

03,799 Not available NTIS

Bacterial Enumeration in Storage Water.

PB96-163647

03,591 Not available NTIS

Bacteriorhodopsin Immobilized in Sol-Gel Glass.

PB95-151429 03,532 Not available NTIS

Bacteriorhodopsin Retains Its Light-Induced Proton-Pump-

ing Function After Being Heated to $140 \mathrm{C}$
PB96-102728
03,471 Not available NTIS

Band-Limited, White Gaussian Noise Excitation for Reverberation Chambers and Applications to Radiated Susceptibility Testing.

PB96-165410

$01,960 \mathrm{PC} \mathrm{A07/MF} \mathrm{A02}$

Band-10-Band Photoluminescence and Luminescence Excitation in Extremely Heavily Carbon-Doped Epitaxial GaAs. PB95-150413 04,570 Not available NTIS

Barium Contributions to the Valence Electronic Structure of YBa2Cu3O7-delta, PrBa2Cu307-delta, and Other Barium Containing Compounds.

PB96-158019

04,076 Not available NTIS

Basic Linear Algebra Operations in SLI Arithmetic.

PB96-165931 03,421 PC A03/MF A01

Beam Analysis Round Robin.

PB94-212545

04,239 Not available NTIS

Beam Line for Highly Charged lons.

PB97-111256 04,143 Not available NTIS

Beamcon III, a Linearity Measurement Instrument for Optical Detectors
PB96-113576

04,337

(Order as PB96-113535, PC A05/MF A01)

Behavior of a Calcium Phosphate Cement in Simulated Blood Plasma In vitro.

00,165 Not available NTIS

Behavior of Atoms in a Compressed Magneto-Optical Trap.

Behavior of Atoms in a Compressed Magneto-Optical Trap.
PB95-203105

Behavior of Charring Materials in Simulated Fire Environments.

PB94-196045

01,368 PC A99/MF A06

Behavior of Mock-Ups in the California Technical Bulletin 133 Test Protocol: Fabric and Barrier Effects.
PB95-231585 00,301 PC A05/MF A01
Behavior of Surface Partial Discharge on Aluminum Oxide Dielectrics.

01.937 Not available NTIS

Benchmarks for the Evaluation of Speech Recognizers. PB94-211539 01,566 Not available NTIS

Bending-Induced Loss in Dual-Mode Rectangular Waveguides.

PB95-168795

04,288 Not available NTIS

Bending-Induced Phase Shifts in Dual-Mode Planar Optica Waveguides.

PB95-161329

04,271 Not available NTIS

Beneficial Effects of Nitrogen Atomization on an Austenitic Stainless Steel
PB94-212396

03,209 Not availabie NTIS

Benefits and Costs of Research: A Case Study of the Fire Safety Evaluation System

PB96-202288

00,232 PC A06/MF A01

Benefits and Costs of Research: Two Case Studies in Building Technology.

PB96-202221

00,230 PC A07/MF A02

Bent Rectangular Core Waveguides: An Accurate Perturbafion Approach

04,289 Not available NTIS

beta-D-Glucosyl-Hydroxymethyluracil: A Novel Modified Base Present in the DNA of the Parastic Protozoan T. brucei.

PB94-172319

03,524 Not available NTIS

Beyond the Technology Roadmaps: An Assessment of Electronic Ma

01,961 PC A05/MF A01

BFRL Fire Publications, 1993

PB94-164191

$00,192 \mathrm{PC} \mathrm{A03/MF} \mathrm{A01}$

Bias Current Dependent Resistance Peaks in NiFe/Ag Giant Magnetoresistance Multilayers.

PB97-112346

04,153 Not available NTIS

Bibliography of Books and Published Reports on Gas Turbines, Jet Propulsion, and Rocket Power Plants.

Bibliography of Books and Published Reports on Gas Turbines, Jet Propulsion, and Rocket Power Plants, January 1950 through December 1953.

AD-A278 213/4

01,446 PC A06/MF A02

Bibliography of Computer Security Glossaries.
PB94-216710 01,587 Not available NTIS

Bibliography of Photon Total Cross Section (Attenuation Co-

efficient) Measurements $10 \mathrm{eV} 1013.5 \mathrm{GeV}, 1907-1993$.

$03,804 \mathrm{PC} \mathrm{A06/MF} \mathrm{A02}$

Bibliography of the NIST Electromagnetic Fields Division PB94-165990

01,875 PC A06/MF A02

Bibliography of the NIST Electromagnetic Fields Division Publications.

01,886 PC A06/MF A02

Bibliography of the NIST Electromagnetic Fields Division Publications

01,980 PC A08/MF A02

Bibliography of the NIST Optoelectronics Division

Bibliography of the NIST Optoelectronics Division.
PB96-128210

Bibliography of the NIST Optoelectronics Division.

BB97-116040
P2,207 PC A06/MF A01

Bibliography of the Physical Equilibria and Related Properties of Some Cryogenic Systems.

$03,769 \mathrm{PC}$ A07/MF A02

Bibliography on Apparel Sizing and Related Issues.

PB94-161924

02,806 PC A03/MF A01

Bimolecular Interactions in (Et)3SiOH:Base: $\mathrm{CCl} 4$ HydrogenBonded Solutions Studied by Deactivation of the Free $\mathrm{OH}$ Stretch Vibration.

Binary Decision Clustering for Neural Network Based Optical Character
PB95-171971

01,848 PC A03/MF A01

Binary Decision Clustering for Neural-Network-Based Optical Character Recognition.

01,857 Not available NTIS

Binary versus Decade Inductive Voltage Divider CompariPB96-112263 Decomposition. 02,071 Not available NTIS Binder Characterization and Evaluation by Nuclear Magnetic Resonance Spectroscopy.
PB94-193471 Bioactive Polymeric Dental Materials Based on Amorphous Calcium Phosphate.

03,572 Not available NTIS 


\section{TITLE INDEX}

Bioleaching of Cobalt from Smelter Wastes by 'Thiobacillus ferrooxidans'.

02,582 Not available NTIS

Biological Macromolecular Crystallization Database: A Tool

for Developing Crystallization Strategies.
PB95-126157 00,897 Not available NTIS

Biological Macromolecule Crystallization Database and NASA Protein Crystal Growth Archive.

01,171

(Order as PB97-109011, PC A11/MF A03)

Biological Thermodynamic Data for the Calibration of Differential Scanning Calorimeters: Dynamic Temperature Data on the Gel to Liquid Crystal Phase Transition of Dialkylphosphatidylcholine in Water Suspensions.

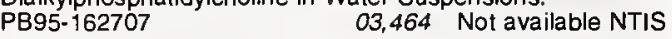

Bistatic Scattering of Absorbing Materials from 30 to 1000 $\mathrm{MHz}$

01,891 Not available NTIS

BLCC: The NIST 'Building Life-Cycle Cost' Program, Version 4.21. User's Guide and Reference Manual.

$\begin{array}{ll}\text { PB95-190682 } & 00,263 \text { PC A06/MF A02 }\end{array}$

Body Dimensions for Apparel.

PB94-187739

$02,813 \mathrm{PC} \mathrm{A03/MF} \mathrm{A01}$

Bonding in Doubly Charged Diatomics.

PB95-164315

00,976 Not available NTIS

Bonding Wires to Quantized Hall Resistors.

PB96-102637 01,921 Not available NTIS

Book Review: Aspects and Applications of the Random Walk.

04,215 Not available NTIS

Book Review: Statistical Physics of Macromolecules

PB96-123526 01,280 Not available NTIS

Boron-Implanted 6H-SiC Diodes.

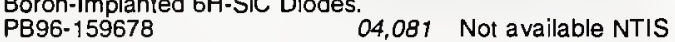

Boundary Conforming Grid Generation System for Interface Tracking.
PB94-158268

03,312 PC A03/MF A01

Boundary Conforming Grid Generation System for Interface Tracking.

03,357 Not available NTIS

Boundary Integral Method for the Simulation of Two-Dimensional Particle Coarsening

03,411 Not available NTIS

Boundary Lubrication of Silicon Nitride.

PB95-213583 03,226 PC A18/MF A04

Bounds on Frequency Response Estimates Derived from Uncertain Step Response Data.

PB96-122874

03,419 Not available NTIS

Bounds on Least-Squares Four-Parameter Sine-Fit Errors

Due to Harmonic Distortion and Noise.
PB96-141304 01,609 Not available NTIS

Bragg Gratings in Optical Fibers Produced by a Continuous-Wave Ultraviolet Source.

PB95-162020

04,274 Not available NTIS

Brillouin Light Scattering Intensities for Thin Magnetic Films with Large Perpendicular Anisotropies.

PB94-211174 04,488 Not available NTIS

Bringing Natural Language Information Retrieval Out of the PB94-172335

02,720 Not available NTIS

Broadband High-Optical-Density Filters in the Infrared.

PB95-180261 04,309 Not available NTIS

Broadband Mismatch Error in Noise Measurement Systems PB95-162061 02,044 Not available NTIS

Brownian Diffusion of Hard Spheres at Finite Concentrations.

00,975 Not available NTIS

Buffer Layer Modulation-Doped Field-Effect-Transistor Interactions in the Al0.33Ga0.67As/GaAs Superlattice System.

PB96-102876

02,380 Not available NTIS

Building a Better Crycooler.

PB96-119615

04,734 Not available NTIS

Building and Fire Research Laboratory Publications, 1993. PB95- 14320200,369 PC A06/MF A02

Building and Fire Research Laboratory Publications, 1994. PB95-226684 00,398 PC A07/MF A02

Building Life Cycle Cost Computer Program (BLCC), Version 4.2-95 (for Microcomputers)

00,266 CP D02

Building Life Cycle Cost Computer Program (BLCC) Version 4.4-97 (for Microcomputers)

00,284 CP D02
Building Life Cycle Cost Computer Program (BLCC) Version 4.21-95 (for Microcomputers).

00,267 CP D02

Building Life Cycle Cost Computer Program (BLCC) Version 4.22-95 (for Micrcomputers)

00,277 CP D02

Building Life Cycle Cost Computer Program (BLCC) Version 4.22-95 (for Microcomputers).

00,268 CP D02

Bulk Modulus and Young's Modulus of Nanocrystalline gamma-Alumina.

03,092 Not available NTIS

Burning Rate and Flame Heat Flux For PMMA in the Cone Calorimeter.
PB95-216990

00,393 PC A07/MF A02

Burning Rate of Premixed Methane-Air Flames Inhibited by Fluorinated Hydrocarbons.

PB95-180584

01,391 Not available NTIS

Business and Manufacturing Motivations for the Developing of Analytical Technology and Metrology for Semiconductors.

$C_{++}$in Safety Critical Systems.

$01,750 \mathrm{PC} \mathrm{A04/MF} \mathrm{A01}$

Ca1-xCuO2, a NaCuO2-Type Related Structure.

PB95-162822 04,620 Not available NTIS

Ca4Bi6013: A Compound Containing an Unusually Low Bismuth Coordination Number and Short Bi Bi Contacts.

PB95-141131

00,911 Not available NTIS

Calcium Phosphate Cements.

PB97-111595

03,580 Not available NTIS Calcium Phosphate Precipitation in Lipospmal Suspensions.
PB94-172145

Calculated Fiber Attenuation: A General Method Yielding Stationary Values.

04,298 Not available NTIS Calculating Combined Buoyancy- and Pressure-Driven Flow
Through a Shallow, Horizontal, Circular Vent; Application to Through a Shallow, Horizontal, Circular Vent; Application to Problem of Steady Burning in a Ceiling-Vented Enclosure.
PB96-164108 00,409 Not available NTIS

Calculating Flame Spread on Horizontal and Vertical Surfaces.

PB94-187283

00,335 PC A04/MF A01

Calculating Time-to-Contact Using Real-Time Quantized Optical Flow.

01,604 PC A03/MF A01 Calculation of Enthalpy and Entropy Differences of Near-
Critical Binary Mixtures with the Modified Leung-Griffiths Critical Binary Mixtures with the Modified Leung-Griffiths
Model.
PB95-108635 00,885 Not available NTIS

Calculation of Higher Heating Values of Biomass Materials

and Waste Components from Elemental Analyses.
PB94-199254 02,474 Not available NTIS

Calculation of Photon Mass Energy-Transfer and Mass Energy-Absorption Coefficients.

PB97-110399

04,137 Not available NTIS

Calculation of the Thermal Conductivity and Gas Permeability in a Uniaxial Bundle of Fibers.

PB95-180931

03,058 Not available NTIS

Calculations of Electron Inelastic Mean Free Paths (IMFPs) 4. Evaluation of Calculated IMFPs and of the Predictive IMFP Formula TPP-2 for Electron Energies between 50 and $2000 \mathrm{eV}$.

PB95-150728

00,922 Not available NTIS

Calculations of Electron Inelastic Mean Free Paths. 5. Data for 14 Organic Compounds over the $50-2000$ eV Range.

PB95-150355 00,916 Not available NTIS

Calibrating On-Wafer Probes to the Probe Tips.

PB95-163945 02,352 Not available NTIS

Calibration and Performance of GafChromic DM-100 Radiochromic Dosemeters.

00.703 Not available NTIS

Calibration in the Earth Observing System (EOS) Project. Part 2. Implementation.

04,842 Not available NTIS

Calibration of a Superconducting Gravimeter Using Absolute Gravity Measurements.

PB95-202651

03,684 Not available NTIS

Calibration of Dosimeters for the Cryogenic Irradiation of PB95-180964

3.968 Not available NTIS

Calibration of Electret-Based Integral Radon Monitors Using NIST Polyethylene-Encapsulated (226)Ra/(222)Rn Emanation (PERE) Standards.

PB96-159223

01,950

(Order as PB96-159215, PC A07/MF A02)
Calibration of GPS Equipment in Japan

PB95-151452 01,531 Not available NTIS Calibration of High-Energy Electron Beams by Use of Graphite Calorimeters.

04,598 Not available NTIS

Calibration Service for Coaxial Reference Standards for Microwave Power.

01,958 PC A07/MF A02

Calibration Standards for Differential Scanning Calorimetry. 1. Zinc Absolute Calorimetric Measurement of Enthalpy of Fusion and Temperature of Fusion HM.

PB94-199817 00,801 Not available NTIS

Calorimeters for Calibration of High-Dose Dosimeters in High-Energy Electron Beams.
PB96-135272

4,055 Not available NTIS

Calorimetric and Visual Measurements of R123 Pool Boiling on Four Enhanced Surtaces.

04,053 PC A04/MF A01

Calorimetric Determination of the Standard Transformed Enthalpy of a Biochemical Reaction at Specified PH and PB94-198249

03,454 Not available NTIS

CALS-Automated Interchange of Technical Information. PB94-962000 03,657 Standing Order CALS-Automated Interchange of Technical Information. PB95-962000 03,666 Standing Order CALS-Contractor Integrated Technical Information Service (CITIS), Functional Requirements.

PB94-962600

03,663 Standing Order

CALS-Contractor Integrated Technical Information Service (CITIS), Functional Requirements.

03,672 Standing Order

CALS-Department of Defense Computer Aided Acquisition Logistic Support (CALS)

03,662 Standing Order

CALS-Department of Defense Computer Aided Acquisition Logistic Support (CALS)

03,671 Standing Order

CALS-Digital Representation for Communication of Illustration Date: CGM Application Profile.

PB94-962400

03,661 Standing Order

CALS-Digital Representation for Communication of Illustration Date: CGM Application Profile.

PB95-962400

03,670 Standing Order

CALS-Digital Representation for Communication of Product Data: IGES Application Subsets.

PB94-962100

03,658 Standing Order

CALS-Digital Representation for Communication of Product Data: IGES Application Subsets.

PB95-962100

03,667 Standing Order

CALS-Markup Requirements and Generic Style Specifications for Electronic Printed Output and Exchange of Text. PB94-962200 03,659 Standing Order

CALS-Markup Requirements and Generic Style Specificaions for Electronic Printed Output and Exchange of Text. PB95-962200 03,668 Standing Order CALS-Raster Graphics Representation Binary Format Requirements.

03,660 Standing Order

CALS-Raster Graphics Representation Binary Format Requirements.

03,669 Standing Order

Cambridge Structural Database (CSD): Current Activities and Future Plans

00,516

(Order as PB97-109011, PC A11/MF A03)

Can Displays Deliver a Full Measure: Manufacturing.
PB96-111935
02,185 Not available NTIS

Can Quantum Mechanical Description of Electron-Sodium Collisions Be Considered Complete. Present Status and Fu-
ture Prospects for $3 s<->3 p$ Transitions. ture Prospects for $3 s<->3 p$ Transitions.

00,768 Not available NTIS

Capabilities for Product Data Exchange.
PB97-118764 Not available NTIS PB97-118764 02,798 Not available NTIS

Capacitors with Very Low Loss: Cryogenic Vacuum-Gap Capacitors.
PB97-122600 02,293 Not available NTIS

Capacity of the Lp Norm-Constrained Poisson Channel. PB95-125753 01,515 Not available NTIS

Carbon Acidities of Aromatic Compounds. 1. Effects of InRing Aza and External Electron-Withdrawing Groups.
PB94-216595 NTIS

Carbon Monoxide Dispersion in Residential Buildings: Literature Review and Technical Analysis.
PB97-114227 


\section{TITLE INDEX}

Carbon Monoxide Production in Compartment Fires: Reduced-Scale Enclosure Test Facility.
PB95-231700 Carotenoid Reversed-Phase High-Performance Liquid Chromatography Methods: Reference Compendium.

PB94-200516 00,549 Not available NTIS

Cascading Surge-Protective Devices: Options for Effective Implementation.

PB94-216488

02,464 Not available NTIS

Cast-Iron Elastic Constants: Effect of Graphite Aspect

PB94-213212

03,211 Not available NTIS

Casual Regularizing Deconvolution Filter for Optimal Wave-

PB95-203089

01,603 Not available NTIS

Catalog of National ISDN Solutions for Selected NIUF Applications.

01,468 PC A99/MF A06

Catalogue of Electromagnetic Environment Measurements PB96- $300 \mathrm{~Hz}$.

01,943 Not available NTIS

Catastrophic Failures Propagate Field of Fracture Mechan ics. 1396140 03,217 Not available NTIS Causality and Maxwell's Equations.

PB97-110522 04,429 Not available NTIS

Causes of the Apparent Heat Transfer Degradation for Refrigerant Mixtures.

PB94-212701

03,259 Not available NTIS

Cavitation Contributes Substantially to Tensile Creep in Silicon Nitride.

PB96-122577

03,171 Not available NTIS

Cavitation Damage During Flexural Creep of SiAION-YAG Ceramics.

PB94-200110

03,036 Not available NTIS

Cavity Evolution during Tensil Creep of $\mathrm{Si} 3 \mathrm{~N} 4$

PB96-204193 03,376 Not available NTIS

CCD Mosaic Images of the Supernova Remnant 3C 400.2 . PB95-203527 00,084 Not available NTIS

Cellular Automaton Simulations of Cement Hydration and Microstructure Development. PB95-17505

01,320 Not available NTIS

Cement and Concrete Characterization by Scanning Elecron Microscopy

00,379 Not available NTIS

Center for Electronics and Electrical Engineering Technical Progress Bulletin Covering Center Programs, October to December, with 1991 CEEE Events Calendar. $\begin{array}{ll}\text { December, with } 1991 \text { CEEE Events Calendar. } \\ \text { PB94-159787 } & 02,296 \text { PC A03/MF A01 }\end{array}$

Center for High Integrity Software System Assurance: Initial Goals and Activities.

PB95-251674

01,734 PC A03/MF A01

Ceramic Characterization

DE94013170

03,026 PC A02/MF A01

Ceramic Powders Characterization: Results of an International Laboratory Study.

02,672 PC A22/MF A04

Ceramics Technical Activities, 1993 (NAS-NRC Assessment

Panel April 21-22, 1994)
PB94-162591

$03,031 \mathrm{PC} \mathrm{A1O/MF} \mathrm{A03}$

Ceramics Technical Activities, 1995

PB96-193677

03,087 PC A11/MF A03

Certainty Grid to Object Boundary Algorithm

PB94-203510 01,835 PC A03/MF A01

Certification, Development and Use of Standard Reference Materials

PB95-107272

00,567 Not available NTIS

Certification of Morphine and Codeine in a Human Urine Standard Reference Material

PB95-176160

03.499 Not available NTIS

Certification of Phencyclidine in Lyophilized Human Urine Reference Materials.

03,508 Not available NTIS

PB96-160692 Certification of Polychlorinated Biphenyl Congeners and
Chlorinated Pesticides in a Whale Blubber Standard Reference Material.

03,745 Not available NTIS

PB96-103023

atic Hydrocarbons in a $\mathrm{Ma}$ Certification of Polycyclic Arornatio Hydrial.

PB96-111778 02,592 Not available NTIS

Certification of Standard Reference Material (SRM) 1941a, Organics in Marine Sediment.

PB96-123690
Certification of the Standard Reference Material 1473a, a Low Density Polyethylene Resin.

PB96-128251

$01,282 \mathrm{PC} \mathrm{A03/MF} \mathrm{A01}$

Certitying the Chemical Composition of a Biological Material: A Case Study.

00,636 Not available NTIS

CFAST Output Comparison Method and Its Use in Comparing Different CFAST Versions.

PBg6-109541

00,401 PC A04/MF A01

Challenges to the National Information Infrastructure: The Barriers to Product Data Sharing. National PDES Testbed Report Series.

PB95-136347

$02,776 \mathrm{PC}$ A03/MF A01

Changes in the Redox State of Iridium Oxide Clusters and Their Relation 10 Catalytic Water Oxidation: Radiolytic and Electrochemical Studies. Electrochemical Studies.

00,864 Not available NTIS

Channel Coding for Code Excited Linear Prediction (CELP) Encoded Speech in Mobile Radio Applications.

PB95-143178 01,475 PC A03/MF A01

Chaos in a Computer-Animated Pendulum.

PB94-212651 03,852 Not available NTIS

Chaotic Motions of Coupled Galloping Oscillators and Their

Modeling as Diffusion Progresses.
PB96-122718 04,823 Not available NTIS

Characteristics of Adhesive-Bonded Seams Sampled from EPDM Roof Membranes.

PB95-162491

00,377 Not available NTIS

Characteristics of Light Emission Atter Low-Energy Electron Impact Excitation of Caesium Atoms.

PB94-198587 03,806 Not available NTIS

Characteristics of Partial Pressure Analyzers.

PB95-150876 00,582 Not available NTIS

Characteristics of Turbulence in a Boundary Layer with Zero Pressure Gradient.

AD-A278 249/8

04,192 PC A03/MF A01

Characterization and Processing of Spray-Dried Zirconia

Powders for Plasma Spray Application.
PB97-111231 04,419 Not available NTIS PB97-111231

Characterization of a Clipped Gaussian Beam.

PB96-102553 04,331 Not available NTIS

Characterization of a Health Physics Instrument Calibration Range.

03,629 Not available NTIS

Characterization of a Tunable Thin Film Microwave YBa2Cu3O $7-x / \mathrm{SrTiO} 3$ Coplanar Capacitor.

PB95-175527

Characterization of Chemically Modified Pore Surfaces by Small Angle Neutron Scattering.

00,898 Not available NTIS

Characterization of Cytochrome c/Alkanethiolate Structures Prepared by Self-Assembly on Gold

Prepared by Self-Assembly on Gold.
PB95-164638 Not available NTIS

Characterization of Interface Defects in Oxygen-Implanted Silicon Films.

02,322 Not available NTIS

Characterization of Liquid-Phase Epitaxially Grown $\mathrm{HgCdTe}$ Films by Magnetoresistance Measurements.

PB96-123617 04,738 Not available NTIS
0

Characterization of LPE $\mathrm{HgCdTe}$ Film by Magnetoresistance.
PB96-157961

Characterization of Modified FEL Quartz-Halogen Lamps for Photometric Standards

00,282 Not available NTIS

Characterization of Molecular Network of Thermosets Using Neutron Scattering.

PB95-164109

01,259 Not available NTIS

Characterization of Phase and Surface Composition of Silicon Carbide Platelets

PB94-216264

03,043 Not available NTIS

Characterization of Polyquinoline Blends Using Smalł Angle Scattering.

PB95-164125

01,261 Not available NTIS

Characterization of Polyquinoline Block Copolymer Using Small Angle Scattering

PB95-151882

01,244 Not available NTIS

Characterization of the Adsorption-Fouling Layer Using Globular Proteins on Ultrafiltration Membranes.

PB94-212909 00,842 Not available NTIS

Characterization of the Binding of Gallium, Platinum, and Uranium to Pseudomonas Fluorescens by Small-Angle $X$ ray Scattering and Transmission Electron Microscopy.
PB94-172509
03,453 Not available NTIS
Characterization of the Densification of Alumina by Multiple Small-Angle Neutron Scattering. (Reannouncement with New Availability Information

03,024 PC A02/MF A01

Characterization of the Emission from 2D Array Josephson Oscillators.

02,054 Not available NTIS

Characterization of the Hydrogen Induced Cold Cracking Susceptibility at Simulated Weld Zones in HSLA-100 Steel.
AD-A279 759/5
03,200 PC A04/MF A01

Characterization of the Hydrogen Induced Cold Cracking Susceptibility at Simulated Weld Zones in HSLA-100 Steel.
PB94-174505
03,746 PC A04/MF A01

Characterization of the Interaction of Hydrogen with Iridium Clusters in Zeolites by Inelastic Neutron Scattering Spectroscopy.
PB95-180741

01,013 Not available NTIS

Characterization of the Structure of LaD2.50 by Neutron Powder Diffraction.

PB96-176797

04,783 Not available NTIS

Characterization of the Structure of $\mathrm{TbD} 2.25$ at $70 \mathrm{~K}$ by Neutron Powder Diffraction.

PB96-160528

01,130 Not available NTIS

Characterization of the Structure of YD3 by Neutron Powder Diffraction.

PB96-186150

01,161 Not available NTIS

Characterization of the Vibrational Dynamics in the Octahedral Sublattices of LaD2.25 and LaH2.25.

PB96-123724 01,091 Not available NTIS

Characterization of the $\mathrm{ZnSe} / \mathrm{GaAs}$ Interface Layer by TEM and Spectroscopic Ellipsometry.

PB95-175360

04,655 Not available NTIS

Characterization of Time-Dependent Dielectric Breakdown

in Intrinsic Thin $\mathrm{SiO} 2$
PB97-122527

02, 458 Not available NT1S

Characterization of Two-Dimensional Dopant Profiles: Stalus and Review
PB96-119300

02,400 Not available NTIS

Characterization of Two-Dimensional Dopant Profiles: Stalus and Review.

Characterization of Unknown Linear Systems Based on

Measured CW Amplitude
PB95-161485

01,897 Not available NTIS

Characterization of Vertical-Cavity Semiconductor StrucPB94-200193

02,126 Not available NTIS

Characterizing Materials Properties for Ceramic Matrix Composites.

03,097 Not available NTIS

Charge Cloud Distribution of Heavy Atoms After Excitation by Polarized Electrons.

PB95-203147

04,001 Not available NTIS

Charge Transier and Collision-Induced Dissociation Reactions of $\mathrm{CF}(2+)$ and $\mathrm{CF} 2(2+)$ with the Rare Gases at a Laboratory Collision Energy of $49 \mathrm{eV}$.

Spectrum of Lanthanum.

Charge-Transfer-Induced Multiplet Structure in the PB96-163746
PB

Charge Trapping and Breakdown Mechanism in SIMOX

PB94-216637 02,323 Not available NTIS

Charpy Impact Test as an Evaluation of $4 \mathrm{~K}$ Fracture Toughness. 03,219 Not available NTIS

Charpy Specimen Tests at $4 \mathrm{~K}$

PB96-190335

03,002 Not available NTIS

Checking the Net Contents of Packaged Goods as Adopted by the 79th National Conference on Weights and Measures, 1994, Third Edition, Supplement 4.

PB95-182226

$00.484 \mathrm{PC} \mathrm{A04/MF} \mathrm{A01}$

Chemical and Microbiological Problems Associated with Re-

search on the Biodesulfurization of Coal.
PB95-140950

Chemical Aspects of Tool Wear in Single Point Diamond Turning.
PB97-112601

03,021 Not available NTIS

Chemical Determination of Oxidative DNA Damage by Gas Chromatography-Mass Spectrometry.
PB95-175394 Not available NTIS Chemical Effect in Ceramics Grinding.
PB97-122592 Chemical Inhibition of Methane-Air Diffusion Flame.

$\begin{array}{lll}\text { PB96-195532 01,431 PC A04/MF A01 } & 0\end{array}$

Chemical Stability of Upper-Layer Fire Gases.
PB96-123385 01,410 Nat available NTIS 


\section{TITLE INDEX}

Chemically Assisted Machining of Si3N4 PB96-122999

03,072 Not available NTIS

Chip Morphology, Tool Wear and Cutting Mechanics in Finish Hard Turning.

03,106 Not available NTIS

Chromatographic Cryofocusing and Cryotrapping with the Vortex Tube.

00,604 Not available NTIS

Churchill Eisenhart, 1913-1994.

PB96-137740 03,447 Not available NTIS

CIF Crystallographic Information File: A Standard for Crystallographic Data Interchange.

04,805

(Order as PB97-109011, PC A11/MF A03)

Class of Radio-Emitting Magnetic B Stars and a Wind-Fed Magnetosphere Model.

00,067 Not available NTIS

Classical Analysis: A Look at the Past, Present, and Future, PB94-185063 00,528 Not available NTIS

Classification of Advanced Technical Ceramics.

N94-35335/6 03,030 PC A07/MF A02

Classified Bibliography: Insulation Condition Monitoring Methods, 1989-1995.

02,232 PC A05/MF A01

Clinical Perspective on Dentin Adhesives.

PBical Perspective on Dentin Adhesives.
Po, 146 Not available NTIS

Closed Loop Controller for Electron-Beam Evaporators

PB97-111470 04,393 Not available NTIS

CMOS Circuit Design for Controlling Temperature in Micromachined Devices.

02,196 Not available NTIS

$\mathrm{CO} 2 / \mathrm{CH} 4$ Transport in Polyperfluorosulfonate lonomers: Effects of Polar Solvents on Permeation and Solubility.
PB96-163803 01,145 Not available NTIS

Coarsening of Unstable Surface Features during Fe(001) Homoepitaxy.
PB96- 186127

04,121 Not available NTIS

Coating of Fibers by Colloidal Techniques in Ceramic Com-

posites.
PB94-216256

03,196 Not available NTIS

Coaxial Line-Reflect-Match Calibration

PB96-200118 02,246 Not available NTIS

Coaxial Reference Standard for Microwave Power

PB94-193786 01,880 PC A04/MF A01

COBOL. Category: Software Standard; Subcategory: Programming Language. Includes ANSI'S X3.23-1985, X3.23A1989 and $\times 3.23 B-1993$

FIPS PUB 21-4

01,670 PC E99

COBOL. Category: Software Standard; Subcategory: Programming Language. Part A

01,671 PC E99

COBOL. Caregory: Software Standard; Subcategory: Programming Language. Part B.

01,672 PC E99

Codes for Named Populated Places, Primary County Divisions, and Other Locational Entities of the United States (FIPS PUB 55-3) (on Magnetic Tape)

00,129 СР T05

Coexistence of Grains with Differing Orthorhombicity in High Ouality YBa2Cu307-delta Thin Films.
PB96-135033 Not available NTIS

Coexisting Densities, Vapor Pressures and Critical Densities of Refrigerants R-32 and R-152a, at $300-385 \mathrm{~K}$. Sities of Refrigerants R-32 and R-152a, at $300-385 \mathrm{~K}$.
PB95-175691 NTIS

Coherent Precipitates in the BCC/Orthorhombic Two Phase Field of the Ti-Al-Nb System.

PB94-198694

03,317 Not available NTIS

Cold Neutron Gain Calculations for the NBSR Using MCNP. PB95-163978 03,731 Not available NTIS

Cold Neutron Prompt Gamma Activation Analysis at NIST: A Progress Report

00,602 Not available NTIS

Collection of Results for the SPC/E Water Model.
PB96-147889 01,127 PC A03/MF A01

Collision-Induced Emission in the Fundamental VibrationRotation Band of $\mathrm{H} 2$.

PB94-199445

03,811 Not available NTIS

Collision-Induced Neutral Loss Reactions of Molecular Dications.
PB94-185808

00,780 Not available NTIS

Collisional Alignment of $\mathrm{CO} 2$ Rotational Angular Momentum States in a Supersonic Expansion.
PB96-103171 01,069 Not available NTIS
Collisional Energy Transfer between Excited-State Strontium and Noble-Gas Atoms.

03,995 Not available NTIS 95-202958

hly-Charged lons.

PB96-200340

4,791 Not available NTIS

Color Supplement to NIST Special Publication 400-93 Semiconductor Measurement Technology: Design and Testing Guides for the CMOS and Lateral Bipolar-on-SOI Test Library.

$02,298 \mathrm{PC} \mathrm{A03/MF} \mathrm{A01}$

Colossal Magnetoresistance without $\mathrm{Mn}(3+) / \mathrm{Mn}(4-)$ Double Exchange in the Stoichiometric Pyrochlore TI2Mn2O

PB97-113070

04,160 Not available NTIS

Colour Centres in LiF for Measurement of Absorbed Doses Up to 100 MGy

04,169 Not available NTIS

Combined Buoyancy-and Pressure-Driven Flow Through a Shallow, Horizontal, Circular Vent.

PB94-210077

$00,344 \mathrm{PC} \mathrm{A03/MF} \mathrm{A01}$

Combined Buoyancy and Pressure-Driven Flow Through a Shallow, Horizontal, Circular Vent.

PB96-164116

00,410 Not available NTIS

Combined Josephson and Charging Behavior of the Supercurrent in the Superconducting Single-Electron Transistor.

PB95-168522

04,637 Not available NTIS

Combined Low- and High-Angle X-Ray Structural Refinement of a Co/Pt(111) Multilayer Exhibiting Perpendicular Magnetic Anisotropy.

PB94-198355

04,457 Not available NTIS

Combining Data from Independent Chemical Analysis Meth PB95-140141

00,572 Not available NTIS

Combining Interactive Exploration and Optimization for As. sembly Design.

02,794 Not available NTIS

Combustion of a Polymer (PMMA) Sphere in Microgravity. (Order as N96-15552, PC A2O/MF A04)

Combustion of Methanol and Methanol/Dodecanol Spray Flames.

PB95-108544

02,478 Not available NTIS

Comment and Discussion on Digital Processing of $P D$ Pulses.

PB96-122775

01,932 Not available NTIS

Comment on $\ll A$ Dynamic Electric Trap for Ground-State Atoms $>>$.

PB96-200290

04,123 Not available NTIS

Comment on 'Phase Transitions in Antiferromagnetic Superlattices'
PB95-152971

04,587 Not available NTIS

Comment On: Two-Photon Absorption Series of Calcium

PB96-157979 04,074 Not available NTIS

Commentary on 'Optimization of Experimental Parameters for the EPR Detection of the Cellulosic Radical in Irradiated Foodstutfs'

PB96-164124

00,043 Not available NTIS

Commentary: The Measurement of Oxidative Damage to DNA by HPLC and GC/MS Techniques.

PB96-200894 03,484. Not available NTIS

Comments on 'Conversions between $S, Z, Y, h, A B C D$, and $T$ Parameters Which Are Valid for Complex Source and Load Impedances'

02,069 Not available NTIS

Comments on 'Protecting EFIE-Based Scattering Computations from Effects of Internal Resonances

PB95-161568

01,898 Not available NTIS

Comments on the Paper 'Wolf Shifts and Their Physical Interpretation under Laboratory Conditions'.

04,246

(Order as PB94-219326, PC A05/MF A02)

Comments on the Stability of Bayard-Alpert Ionization Gages.
PB96-103080

02,673 Not available NTIS

Commercial Helium Permeation Leak Standards: Their Properties and Reliability.

PB97-111413

04,146 Not available NTIS

Common Criteria: On the Road to International Harmoni-

zation.

01,606 Not available NTIS

Comparative Measurements of High-Voltage Impulses

Using a Kerr Cell and a Resistor Divider.
PB94-172582 02,028 Not available NTIS

Comparative Photoluminescence Measurement and Simula-

tion of Vertical-Cavity Semiconductor Laser Structures.
PB95-169173 02,169 Not available NTIS
Comparative Strategies for Correction of Interferences in Isotope Dilution Mass Spectrometric Determination of Vanadium.
PB94.185261 00,531 Not available NTIS

Comparative Study of Fe-C Bead and Graphite Target Performance with the National Ocean Sciences AMS (NOSAMS) Facility Recombinator Ion Source.

PB95.175790 00,693 Not available NTIS

Comparing NIST-B $50 \mathrm{~mm}$ Orifice Meter Gas Data to the ANSI Equation

02,949 Not available NTIS

Comparing Remote Procedure Calls: Open Network Computing, Distributed Computing Environment and International Organization for Standardization.

PB95-194205

01,724 PC A03/MF A01

Comparing the Accuracy of Critical-Current Measurements Using the Voltage-Current Simulator

PB96-119219

02,227 Not available NTIS

Comparison of a Fixed-Charge and a Polarizable Water

Model.
PB96-111620

01,072 Not available NTIS

Comparison of Elastic and Plastic Contact Models for the Prediction of Thermal Contact Conductance.

PB95-161659

04,605 Not available NTIS

Comparison of Experimental and Computed Species Concentration and Temperature Profiles in Laminar, Two-Dimensional Methane/Air Diffusion Flames.

PB95-140919

01,379 Not available NTIS

Comparison of FDDI Asynchronous Mode and DQDB Queue Arbitrated Mode Data Transmission for Metropolitan Area Network Applications.

01,498 Not available NTIS

Comparison of FFT Fingerprint Filtering Methods for Neural Network Classification.

PB95-136362

01,840 PC A03/MF A01

Comparison of Filter Radiometer Spectral Responsivity with the NIST Spectral-Irradiance and Illuminance Scales.
PB97-113161 162 Not available NTIS

Comparison of Finite Element and Analytic Calculations of the Resonant Modes and Frequencies of a Thick Shell Sphere. 02,626 PC A03/MF A01

Comparison of Fire Sprinkler Piping Materials: Steel, Copper, Chlorinated Polyvinyl Chloride and Polybutylene, in Residential and Light Hazard Installations.

PB95-182267 00,299 PC A03/MF A01

Comparison of GPS Broadcast and DMA Precise Ephemerides.

$01,518 \mathrm{PC} \mathrm{A03/MF} \mathrm{A01}$

Comparison of GPS Broadcast and DMA Precise Ephemerides

01,523

(Order as N94-30639/6, PC A25/MF A06)

Comparison of Heat-Flow-Meter Tests from Four LaboraPBies.

00,365 Not available NTIS Comparison of k-Correction and Taylor-Series Correction
for Probe-Position Errors in Planar Near-Field Scanning.
PB96-147137
02,012 Not available NTIS Comparison of Magnetic Fields of Thin-Film Heads and Their Corresponding Bit Patterns Using Magnetic Force Microscopy.
PB95-180907

03,763 Not available NTIS

Comparison of Meteor Activity with Occurrence of Sporadic E Reflections.
AD-A292 039/5

00,116 PC A01/MF A01

Comparison of Methods for Gas Chromatographic Determination of PCBs and Chlorinated Pesticides in Marine Reference Materials.

02,584 Not available NTIS

Comparison of NIST and ISO Filtered Bremsstrahlung Calibration Beams.
PB95-180956

03,967 Not available NTIS

Comparison of NIST and Manufacturer Calibrations of

(90)Sr+(90)Y Ophthalmic Applicators.
PB96-123708 03,634 Not available NTIS

Comparison of Photodiode Frequency Response Measure-

ments to $40 \mathrm{GHz}$ between NPL and NIST
PB96-111992 04,038 Not available NTIS

Comparison of POSIX Open System Environment (OSE) and Open Distributed Processing (ODP) Reference Models. PB96-131495

01,820 PC A03/MF A01

Comparison of Regular Transmittance Scales of Four National Standardizing Laboratories.

PB94-211232

04,233 Not available NTIS

Comparison of Responses of a Select Number of Buildings to the 10/17/1989 Loma Prieta (California) Earthquake and Low-Level Amplitude Test Results.

PB96-159645

00,467 Not available NTIS 


\section{TITLE INDEX}

Comparıson of Selectivities for PCBs in Gas Chromatography for a Series of Cyanobiphenyl Stationary Phases. PB96-119458

00,618 Not available NTIS

Comparison of Techniques for Nondes

Measurements in CdZnTe Substrates. Not available NTIS
PB96-103098

Comparison of the Corrosion Rates of FeAl, Fe(sub 3)Al and Steel in Distilled Water and 0.5 M Sodium Chloride. and Steal in Dist Number 2 January--March 1991.

DE94017332

03, 186 PC A02/MF A01

Comparison of the Liquid Chromatographic Behavior of Selected Steroid Isomers Using Different Reversed.Phase Ma. erials and Mobile Phase Compositions.

PB95-140976 00,574. Not available NTIS

Comparison of the Seismic Provisions of Model Building Codes and Standards to the 1991 NEHRP Recommended Provisions.

PB95-231858

00,315 PC A05/MF AO2

Comparison of the Unidirectional and Radial In-Plane Flow of Fluids Through Woven Composite Reinforcements. PB95-162004 02,698 Not available NTIS Comparison of Three Techniques for the Precision Measurement of Amplifier Noise. PB95-163663

02,349 Not available NTIS

Comparison of Ultralow-Sidelobe-Antenna Far-Field Patterns Using the Planar-Near-Field Method and the Far-Field

02,015 Not available NTIS

Comparison of UV-Induced Fluorescence and Bragg Grating Growth in Optical Fiber.

04,284 Not available NTIS

Comparison of UV Photosensitivity and Fluorescence during Fiber Grating Formation.

04,362 Not available NTIS

Comparison of Wall-Fire Behavior With and Without a Ceiling.

00,342 PC A03/MF A01

Comparisons of Measured Linewidths of Sub-Micrometer Lines Using Optical. Electrical, and SEM Metrologies.

PB95-152807 02,338 Nol available NTIS

Comparisons of Some NIST Fixed-Point Cells with Similar

Cells of Other Standards Laboratories.
PB97-119242 00,655 Not available NTIS

Compartment Fire Combustion Dynamics. Annual Report, September 1, 1993-September 1, 1994

PB95-217162 00,203 PC A04/MF A01

Compatibility Analysis of the ANSI and ISO IRDS Services Interfaces.

PB94-163474

01,805 PC A06/MF A02

Compatibilization of Polymer Blends by Complexation. 2. Kinetics of Interfacial Mixing.

01,295 Not available NTIS

Compensation for Errors Introduced by Nonzero Fringe Densities in Phase-Measuring Interferometers.

PB97.110506 04,386 Not avaiłable NTIS

Compensation for Substrate Permittivity in Probe-Tip Calibration.

PB95-203519

01.915 Not available NTIS

Compensation of Errors Detected by Process-Intermittent Gauging.
PB97-110472

02,846 Not available NTIS

Compensation of Markov Estimator Errors in Time-Jittered Sampling of Nonmonotonic Signals.

PB95-150983 01,590 Not available NTIS

Competition between Hydrodynamic Screening ('Draining') and Excluded Volume Interactions in an Isolated Polymer

PB95-175402

01,265 Not available NTIS

Compilation of Energy Levels and Wavelengths for the

PBectrum of Singly-lonized Oxygen (O II). 00.746 Not available NTIS

Compilation of the Physical Equilibria and Related Propenties of the Hydrogen-Carbon Monoxide System. AD-A286 603/6 00,716 PC A05/MF A01

Complementary Molecular Information on Phthalocyanine Compounds Derived from Laser Microprobe Mass Spectrometry and Micro-Raman Spectroscopy
PB94-172269 00.757 Not available NTIS Complete Reduction of the Euler-Poinsot Problem. PB94-172152 03,788 Not available NTIS Complex Propagation Constants for Nonuniform Opticał Waveguides: Calculations.

PB95-125910

04,249 Not available NTIS

Complex Time Dependence of the EPR Signal of Irradiated L-alpha-alanine.

04,180 Not available NTIS
Complicated Cases and Shielded Rooms: Audiometric Booths Shielded to Attenuate Electromagnetic Interterence. PB96-180179

02,278 Not available NTIS

Component-Based Handprint Segmentation Using Adaptive Writing Style Model.

$01,859 \mathrm{PC}$ A03/MF A01

Composite Materials for Offshore Operations: Proceedings of the International Workshop (1st). Held in Houston. Texas on October 26-28, 1993

PB96-109509

$03,169 \mathrm{PC} \mathrm{A17/MF} \mathrm{A04}$

Composite Struts for SMES Plants.

PB95-155586 02,507 PC A99/MF A06

Composition and Solubility Product of a Synthetic Calcium Hydroxyapatite.

02,995 Not available NTIS

Composition Dependence of a Field Variable Along the Binary Fluid Mixture Critical Locus.

95-176038

01,003 Not available NTIS

Compositional Analyses of Surfaces and Thin Films by Electron and Ion Spectroscopies.

PB94-185790

00,779 Not available NTIS

Compositional Analysis of Beneficiated Fly Ashes.

PB95-220497 00,397 PC A03/MF A01

Compositional Homogeneity in Processing Precursor Powders to the $\mathrm{Ba}$ YCu3O7-x High Tc Superconductor.

PB94-212743 04,508 Not available NTIS

Compositional Mapping of the Microstructure of Materials.

PB95-107199 00,565 Not available NTIS

Comprehensive Spectroscopic Data Tabulations and Progress in the Compilation of Atomic Transition Prob-

03,909 Not available NTIS

Comprehensive Theory of Nuclear Effects on the Intrinsic Sticking Probability. 1.

03,832 Not available NTIS

Comprehensive Theory of Nuclear Effects on the Intrinsic Sticking Probability. 2.

PB94-200631

03,833 Not available NTIS

Compressed Liquid Densities, Saturated Liquid Densities,

and Vapor Pressures of 1,1-Difluoroethane.
PB97-110118 01,173 Not available NTIS

Compressibility of Polycrystal and Monocrystal Copper: Acoustic-Resonance Spectroscopy.
PB96-164223 Not available NTIS

Computation of Dendrites Using a Phase Field Model. PB94-160744 04,436 PC A03/MF A01

Computational Model for the Rise and Dispersion of WindBlown, Buoyancy-Driven Plumes. Part 2. Linearly Stratitied Atmosphere.

PB94-143427

$00,119 \mathrm{PC} \mathrm{A03/MF} \mathrm{A01}$

Computations of Enhanced Soot Production in Time-Varying $\mathrm{CH} 4 /$ Air Diffusion Flames

01,440 Not available NTIS

Computer-Aided Manutacturing Engineering Forum (1st) Technical Meeting Proceedings. Held in Gaithersburg. Maryland on March 21.22, 1995.

PB96-136965

$02,834 \mathrm{PC} \mathrm{A10/MF} \mathrm{A03}$

Computer-Aided Manufacturing Engıneering Forum (2nd) Technical Meeting Proceedings. Held in Gaithersburg, Maryland on August 22-23, 1995.

PB96-195334

02,845 PC A11/MF A03

Computer-Aided Molecular Design of Fire Resistant Aircraft Materials

PB96-160601

00,025 Not available NTIS

Computer Graphics Metafile (CGM): Procedures for NIST CGM Validation Test Service.

PB94-161809

01,804 PC A03/MF A01

Computer Programs for Simulation of Lighting/HVAC Interactions.

PB94-140407

02,501 PC A08/MF A02

Computer Security: An Introduction to Computer Security The NIST Handbook

PB96-131610

$01,608 \mathrm{PC} \mathrm{A13/MF} \mathrm{A03}$

Computer Security: Generally Accepted Principles and Practices for Securing Information Technology Systerns.
PB97-110811 01,619 PC A05/MF A01

Computer Security Management and Planning in the U.S. Federal Government.

PB95-163432

01,596 Not available NTIS

Computer Security Training and Awareness Course Com-

PB95-130985

$01,589 \mathrm{PC} \mathrm{A09/MF} \mathrm{A02}$

Computer Simulation of the Diffusivity of Cement-Based Materials.

PB95-125985

00,362 Not available NTIS
Computer Simulations of Airtlow and Radon Transport in Four Large Buildings.

$02,557 \mathrm{PC} \mathrm{A03/MF} \mathrm{A01}$

PB95-220422

Momputer Simulations of

PB97-121339

00,418 Not available NTIS

Computer Systems Laboratory Annual Report 1993.

PB94-162518

Computer Systems Laboratory Annual Report 1994.

PB95-209920

$01,629 \mathrm{PC} \mathrm{A07/MF} \mathrm{A02}$

Computer Systems Laboratory Computing and Applied Mathematics Laboratory Technical Accomplishments, Octo ber 1994-March 1996.

PB96-193768

01,638 PC A05/MF A01

Computer Virus Attacks.

PB95-163655

01,715 Not available NTIS

Computer Vision Based Tool Setting Station.

PB94-199858

02,944 Not available NTIS

Computers in Welding: A Primer.

PB95-162863

02,862 Not available NTIS

Computing Effects and Error for Large Synthetic Perturbation Screenings.

$01,675 \mathrm{PC} \mathrm{A03/MF} \mathrm{A01}$

Computing Radiative Heat Transter Occurring in a Zone PB96-102306

01,399 No: available NTIS

Computing S(alpha) Using Symbolic Monte Carlo.

PB94-198660 03,410 Not available NTIS

Computing the Effect of Sprinkler Sprays on Fire Induced Gas Flow.

00,404 Not available NTIS

Concentration Histogram Imaging: A Scatter Diagram Tech-

nique for Viewing Two or Three Related Images.
PB94-199114 00,542 Not available NTIS

Concentrations of Chlorınated Hydrocarbons, Heavy Metals and Other Elements in Tissues Banked by the Alaska Marine Mammal Tissue Archival Project.

PB95-209870

02,590 PC A06/MF A02

Concept for an Algorithm Testing and Evaluation Program

PB94-163029

02,890 PC A03/MF A0

Concept Paper: An Overview of the Proposed Trust Tech-

nology Assessment Program.
PB96-160882

01.614 Not available NTIS

Concepts for Fire Protection of Passenger Rail Transportation Vehicles: Past, Present, and Future.

PB96-102868 04,853 Not available NTIS

Concepts of the NIST EXPRESS Server.

PB95-180543 02,781 Not available NTIS

Conceptual Design Plan for the National Advanced Manufacturing Testbed.

PB95-231866

02,828 PC A05/MF A01

Concurrent Enhancement of Kerr Rotation and Antiterromagnetic Coupling in Epitaxial $\mathrm{Fe} / \mathrm{Cu} / \mathrm{Fe}$ Structures.
$\mathrm{PB} 94-198769$

Concurrent Flow Flame Spread Study.

PB94-156866

01,356 PC A08/MF A02

Condensed Catalogue of Electromagnetic Environmen Measurements, $30-300 \mathrm{~Hz}$

PB95-162210

01,899 No: available NTIS

Conditions for Existence of a Reentrant Solid Phase in a Sheared Atomic Fluid.

04,198 Not available NTIS

Conductance Response of $\mathrm{Pd} / \mathrm{SnO} 2(110)$ Model Gas Sensors to $\mathrm{H}_{2}$ and $\mathrm{O} 2$.

PB95-125803

00,892 Not available NTIS

Cone Emission from Laser-Pumped Two-Level Atoms. 1 Ouantum Theory of Resonant Light Propagation.

PB95-203584 04,325 Not available NTIS

Cone Emission from Laser-Pumped Two-Level Atoms. 2 Analytical Model Studies

PB95-203592

04,326 Not available NTIS

Conference Proceedings: International Workshop on Instrumented Indentation. Held in San Diego, California on April 22-23, 1995.

01,948 PC A06/MF A01

Conference Report: Calorimetry Conference (50th)

PB97-122279

03,722 Not available NTIS

Conference Report: International Conference on the Application of Standards for Open Systems (6th).

PB96-161211

01,762 Not available NTIS

Confidence on the Modified Allan Variance and the Time

PBariance.
PB96-190376

01,557 Not available NTIS 


\section{TITLE INDEX}

Confidence on the Second Difference Estimation of Frequency Drift.

01,532 Not available NTIS

Confidence on the Three-Point Estimator of Frequency Drift. PB95-163838 01,539 Not available NTIS

Configuration and Performance Evaluation of a Real-Time Robot Control System: A Skeleton Approach.
PB95-163895 01,598 Not available NTIS Contiguration-Dependent AC Stark Shifts in Calcium. PB96-157995 04,363 Not available NTIS

Conformance Assessment of Transport Layer Security Implementations.

$01,576 \mathrm{PC}$ A03/MF A01

Conformance Testing and Specitication Management. PB97-113781 02,849 PC A03/MF A01

Conformance Testing for OSI Protocols.
PB96-102686

Conformance Testing of a Lower Layer Security Protocol.

PB94-185402 01,577 Not available NTIS

Conformational Alterations of Bovine Insulin Adsorbed on a Silver Electrode.

00,510 Not available NTIS

PB96-161773

stic and Inelastic ElectronConnection between Superision Partners.

PB95-202297 03,974 Not available NTIS

Consensus Process in Standards Development.

PB96-179502 00,136 Not available NTIS

Considerations in the Design of an Environmental Specimen Bank: Experiences of the National Biomonitoring Specimen Bank Program.

PB96-112370

02,527 Not available NTIS

Considerations on Data Requirements for Tribological ModPBing.

02,962 Not available NTIS

Constant Temperature and Humidity Chamber for Standard Resistors.

PB96-122494

02,275 Not available NTIS

Constant-Width

als for Linear Regression.

Constituents and Physical Properties of the C6+ Fraction of

Natural Gas. Topical Report, April-June 1994.
PB95-136644

Constructing Invariant Tori for Two Weakly Coupled van der Pol Oscillators.

03,412 Not available NTIS

Construction of Maximum-Entropy Density Maps, and Their Use in Phase Determination and Extension.

PB95-108593 00,882 Not available NTIS

Construction of Silicon Nanocolumns with the Scanning Tunneling Microscope.

04,696 Not available NTIS

PB95-203063

Contact Electrification Induced by Monolayer Modification of

a Surtace and Relation to Acid-Base Interactions.
PB94-185378 03,034 Not available NTIS

Contact Tube Wear Detection in Gas Metal Arc Welding.
PB96-135330
02,872 Not available NTIS PB96-135330

CONTAM88 Building Input Files for Multi-Zone Airflow and Contaminant Dispersal Modeling. 02,537 PC A04/MF AO CONTAM93 User Manual.

PB94-16438

$02,536 \mathrm{PC} \mathrm{A05/MF} \mathrm{A01}$

CONTAM94: A Multizone Airflow and Contaminant Disper-

sal Model with a Graphic User Interface.
PB97-113203 Not available NTIS

Context Analysis of the Network Management Domain. Conducted as Part of the Domain Analysis Case Study.

Continuous Counter-Current Two Phase Aqueous Extrac-

tion.

00,675 Not available NTIS

Continuous Gravity Observations Using Joint Institute for Laboratory Astrophysics Absolute Gravimeters.

(2) Gravimeters.

Continuous Mining Machine Control Using the Real-Time Control System

$03,700 \mathrm{PC} \mathrm{A02/MF} \mathrm{A01}$

Continuous Recording and Stochastıc Analysis of PD.

PB96-112156 01,925 Not available NTIS

Contrast Matched Studies of a Sheared Binary Colloidal Suspension.

00,918 Not available NTIS

Contributions of Out-of-Plane Material to a Scanned-Beam Laminography Image.

02,704 Not available NTIS
Control and Instrumentation: Standards for High-Integrity

PB96-161369

03,736 Not available NTIS

Control Entity Interface Specification.

PB94-191715 02,815 PC A06/MF A02

Control of Friction and Wear of Alpha-Alumina with a Composite Solid-Lubricant Coating. 03,225 Not available NTIS

Control of Gas-Metal-Arc Welding Using Arc-Light Sensing. PB96-131461 02,869 PC A06/MF A02

Control Stability of a Heat-Flow-Meter Appartus.

PB95-181194 of a Heat-Flow-Meter Appartus.
P0,386 Not available NTIS

Control System Architecture for a Remotely Operated Unmanned Land Vehicle.

03.759 Not available NTIS

Controlled Nucleation in Aerosol Reactors for Supression of Agglomerate Formation.

00,672 Not available NTIS

Controlling Moisture in the Walls of Manufactured Housing.

$00,355 \mathrm{PC}$ A03/MF A01

Controlling the Critical Current Density of High-Temperature SNS Josephson Junctions.

04,794 Not available NTIS

Convection and Morphological Stability During Directional Solidification.

03,310

(Order as N95-14522/3, PC A2O/MF A04)

Convective Stability in the Rayleigh-Benard and Directional Solidification Problems: High-Frequency Gravity Modulation.

04,208 Not available NTIS

Conventional and Eccentric Uses of Crystallographic Databases in Practical Materials Identification Problems. PB97-109102

(Order as PB97-109011, PC A11/MF A03)

Convergence Properties of a Class of Rank-Two Updates. (NIST Reprint)

03,430 Not available NTIS

Conversion of a 2-Terminal-Pair Bridge to a 4-Termınal-Pair Bridge for Increased Range and Precision in Impedance Measurements.

02,103 Not available NTIS

Cooper M(sub II,III) X-ray-Emission Spectra of Copper OXides and the Bismuth Cuprate Superconductor.

PB96-158027 04,077 Not available NTIS

Coordinating Cascaded Surge Protection Devices: HighLow versus Low-High.

02,463 Not available NTIS

Coordinating Cascaded Surge-Protective Devices: An Elusive Goal.

PB94-216496

02,465 Not available NTIS

Coping with Different Retrieval Methods in Next Generation Networks.

PB95-168555

02,726 Not available NTIS

Copolymerization of N-Phenyl Maleimide and gammaMethacryloxypropyl Trimethoxysilane.
PB95-153144 Not available NTIS

Copper Ion-Mediated Modification of Bases in DNA in Vitro

by Benzoyl Peroxide
PB94-198231

03,645 Not available NTIS

Copyright and Information Services in the Context of the National Research and Education Network.

$\begin{array}{ll}\text { PB96-160536 02,736 Not available NTIS } & \end{array}$

Core Potentials for Quasi-One-Electron Systerns.

PB95-202214 03,970 Not available NTIS

Corrected Optical Pyrometer Readings

AD-A279 949/2 02,615 PC A05/MF A01

Correction Factor for Nonplanar Incident Field in Monopole Calibrations.

02,002 Not available NTIS

Correction to the Decay Rate of Nonequilibrium Carrier Distributions Due to Scattering-in Processes.

PB94-185840 04,452 Not available NTIS

Correlation between Tc and Elastic Constants of (LaM) $2 \mathrm{CUO} 4$.

PB94-213220

04,514 Not available NTIS

Correlation of $\mathrm{HgCdTe}$ Epilayer Defects with Underlying Substrate Defects by Synchrotron X-Ray Topography.

PB94-2007t4

02,129 Not available NTIS

Correlation of Optical, X-ray, and Electron Microscopy Measurements of Semiconductor Multilayer Structures. PB95-175279

02.174 Not available NTIS

Correlation of the Ideal Gas Properties of Five Aromatic Hydrocarbons.

01,002 Not available NTIS
Correlations between Electrical and Acoustic Detection of Partial Discharge in Liquids and Implications for Continuous Data Recording.

PB96-204490

02,248 Not available NTIS

Correlations between Flaw Tolerance and Reliability in PB96-161922

02,986 Not available NTIS

Correlations of Modulation Noise with Magnetic Microstructure and Intergranular Interactions for CoCrTa and CoNi Thin Film Media. PB94-212768 04,509 Not available NTIS Corrosion Characteristics of Silicon Carbide and Silicon Nitride.

PB96-169081

03,372

(Order as PB96-169057, PC A05/MF A01)

Corrosion Resistance of Materials for Renovation of the

United States Botanic Garden Conservatory.
PB94-154390 00,032 PC A03/MF A01

Corrosion Resistant Epoxy-Coated Reinforcing Steel.

PB94-185618 01,307 Not available NTIS

Countries, Dependencies, Areas of Special Sovereignty, and Their Principal Administrative Divisions. Category: Dala Standards and Guidelines; Subcategory: Representation
and Codes.
FIPS PUB 10-4 00,128 PC E05 Countries, Dependencies, Areas of Special Sovereignty,
and Their Principal Administrative Divisions (for Microcomputers).

00,130 Diskette $\$ 30.00$

Coupled-Bilayer Two-Dimensional Magnetic Order of the Dy lons in Dy2Ba4Cu7015.

PB95-152104

04,584 Not available NTIS

Crack Growth Resistance of Strain-Softening Materials under Flexural Loading.

02,972 Not available NTIS

Cracks and Dislocations in Face-Centered Cubic Metallic Multilayers.

02,989 Not available NTIS

Creep and Creep Rupture of Ceramic Matrix Composites.

PB95-163929 03,165 Not available NTIS

Creep and Creep Rupture of Structural Ceramics.

03,093 Not available NTIS

Creep Rupture of $\mathrm{MoSi} / \mathrm{SiC} p$ Composites.

PB95-152294 03,154 Not available NTIS

Criteria for Establishing Accurate Vapor Pressure Curves.
PB95-163812 00,972 Not available NTIS

Critical Behavior of lonic Fluids PB95-164331

00,978 Not available NTIS

Critical Current and Normal Resistance of High-Tc StepEdge SNS Junctions.

04,724 Not available NTIS

Critical Current Behavior of Ag-Coated YBa2Cu3O7-x Thin

Films.

04,549 Not available NTIS

Critical-Current Degradation in Nb3 Al Wires Due to Axial and Transverse Stress.

PB95-202784

02,226 Not available NTIS

Critical Current Density, Irreversibility Line, and Flux Creep Activation Energy in Silver-Sheathed Bi2Sr2Ca2Cu2Ox Superconducting Tapes.

PB95-162749

04,616 Not available NTIS

Critical Evaluation of the Purification of Biominerals by $\mathrm{Hy}$ pochlorite Treatment.

PB95-150959

00,186 Not available NTIS

Critical Evaluation of Thermal Mass Flow Meters.

PB97-113153 00,683 Not available NTIS

Critical Factors in Non-Lubricated, Non-Abrasive Wear TestPB95-140588 03,236 Not available NTIS

Critical Issues in Scanning Electron Microscope Metrology. (Order as PB95-169371, PC A07/MF A02)

Critical Lines for Type-III Aqueous Mixtures by Generalized Corresponding-States Models.

PB96-102371

01,063 Not available NTIS

Critical Magnetic-Field Angle for High-Field Current Transport in $\mathrm{YBa} 2 \mathrm{Cu} 3 \mathrm{O} 7$ at $76 \mathrm{~K}$.

PB94-211281

04,490 Not available NTIS

Critical Properties and Vapor-Liquid Equilibria of the Binary System Propane + Neopentane.
PB95-175683 00,999 Not available NTIS

Critical Review of Rate Constants for Reactions of Transients from Metal lons and Metal Complexes in Aqueous Solution.

PB96-145859

01,109 Not available NTIS 


\section{TITLE INDEX}

Critical Scaling Laws and a Classical Equation of State 00,990 Not available NTIS Cross-Correlation Analysis Improves Time Domain MeasPB95-180535

01.543 Not available NTIS

Cross-Property Relations and Permeability Estimation in

04,205 Not available NTIS

Cross-Sectional Photoluminescence and Its Application to Buried-Layer Semiconductor Structures. PB96-141106

02,415 Not available NTIS

Crossed-Beams Measurements of Absolute Cross Sections for Electron Impact Ionization of $\mathrm{S}(+)$.

PB95-202511 03,981 Not available NTIS

Crossover in the Pinning Mechanism of Anisotropic Fluxon PB95-180170

04,673 Not available NTIS

Crossover to Strong Shear in a Low-Molecular-Weight CritiCal Polymer Biend.

01.222 Not available NTIS

Crosstalk between Microstrip Transmission Lines.
PB94-135639

Crosstalk between Microstrip Transmission Lines. (NIST PB95-180337

02,225 Not available NTIS

Cryogenic Blackbody Calibrations at the National Institute of Cryogenic Blackbody Calibrations at the National Institute of
Standards and Technology Low Background Infrared Calibration Facility. bration Facility

02,117 Not available NTIS

Cryogenic Flow Calibration in NIST.
PB96-161930

Cryogenic Materials Data Handbook

Cryogenic Materials Data Handbook.
AD-A286 675/4 03,303 PC A04/MF A01

Cryogenic Precision Capacitance Bridge Using a Single Electron Tunneling Electrometer.
PB95-126074,529 Not available NTIS

Cryogenic Precision Capacitance Bridge Using a Single Electron Tunneling Electrometer.
PB95-152310 040 Not available NTIS

Cryogenic Precision Capacitance Bridge Using a Single

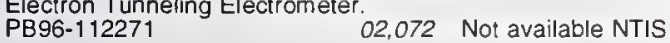
Cryogenic Properties of Inorganic Insulation Materials for TTER Magnets: A Review.

$03,706 \mathrm{PC} \mathrm{A10/MF} \mathrm{A03}$

Cryogenic Properties of Silver.

$03,330 \quad \mathrm{PC} \mathrm{A03}$

PB94-203593

Cryogenic Res

01.457 PC A04/MF A01
Mont (June 30, 1961).

Cryogenic Research and Development (Progress Report Number 4 for Period Ending December 31, 1961)

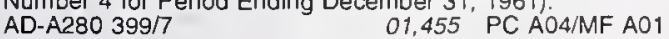

Cryogenic Research and Development (Ouarterly Report Number 1 for Period Ending September 30, 1960).
AD-A280 $401 / 1$
01,456

01,456 PC A04/MF A01

Cryogenic Research and Development (Ouarterly Report Number 2 for $P$ eri

01,454 PC A05/MF A01

Cryogenic Toughness of Austenitic Stainless Steel Weld

Metals: Effect of Inclusions.
PB95-161261 PB95-161261

Cryogenics.

02,654 Not available NTIS

Crystal Chemistry and Phase Equilibria Studies of the $\mathrm{BaO}(\mathrm{BaCO} 3)-\mathrm{R} 2 \mathrm{O} 3-\mathrm{CuO}$ Systems. 4. Crystal Chemistry and Subsolidus Phase Relationship Studies of the CuC

Rich Region of the Ternary Diagrams, $R=$ Lanthanides.
PB95-151759 00,936 Not available NTIS

Crystal Chemistry and Phase Equilibrium Studies of the Crystal Chemistry and Phase Equilibrium Studies of the
$\mathrm{BaO}(\mathrm{BaCO} 3)-\mathrm{R} 2 \mathrm{O} 3-\mathrm{CuO}$ Systems. 5. Melting Relations in Ba2(Y,Nd.Eu)Cu3O6+X.
PB95-151718 04,580 Not available NTIS

Crystal Chemistry and Phase Equilibrium Studies of the BaO-R2O3-CuO Systems. 2. X-Ray Characterization and Standard Patterns of BaR2CuO4, R=Lanthanides

04,582 Not available NTIS

Crystal Diffraction Spectrometry for Accurate, Non-Invasive $\mathrm{kV} / \mathrm{Spectral}$ Measurement for Improvement of Mammographic Image Quality.

00,721 PC A03/MF A01

Crystal Packing Interactions of Two Different Crystal Forms of Bovine Ribonuclease A

PB95-152823

00,943 Not available NTIS

Crystal Structure and Compressibility of 3:2 Mullite.

PB95-175030

03,682 Not available NTIS
Crystal Structure and Magnetic Ordering of the Rare-Earth and $\mathrm{Cu}$ Moments in $\mathrm{RBa} 2 \mathrm{Cu} 2 \mathrm{NbO}(\mathrm{R}=\mathrm{Nd}, \mathrm{Pr})$. Crystal Structure and Magnetic Properties of CuGeO3.
PB95-180287 04,678 Not available NTIS Crystal Structure of a New Monoclinic Form of Potassium Dihydrogen Phosphate Containing Orthophosphacidium Ion

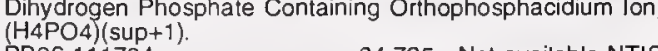
PB96-111794

04,725 Not available NTIS

Crystal Structure of a New Sodium Zinc Arsenate Phase Solved by 'Simulated Annealing'.
PB95-107124

Crystal Structure of Annealed and As-Prepared $\mathrm{HgBa} C \mathrm{CaCu} 2 \mathrm{O} 6+$ delta Superconductors.
$\mathrm{PB} 95-927$
Not

Crystal Structure of Calcium Adipate Monohydrate.
PB94-216579 00,153 Not available NTIS

Crystal Structure of Calcium Glutarate Monohydrate

PB96-111893 01,074 Not available NTIS

Crystal Structure of Calcium Succinate Monohydrate.

PB95-168928 00,167 Not available NTIS

Crystal Structure of Decacalcium Tetrapotassium Hexakis

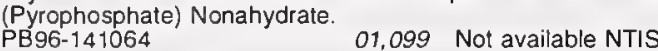

Crystal Structure of Dicalcium Potassium Trihydrogen Bis(pyrophosphate) Trihydrate.
PB94-216561 00,152 Not available NTIS Crystal Structure of Pb2Sr2YCu3O8+delta with delta=1.32, $1.46,1.61,1.71$. by Powder Neutron Diffraction.
04,518 Not available NTIS

Crystallographic and Magnetic Properties of UAuSn.
PB95-140521 04,543 Not available NTIS

Crystallographic Characterization of Some Intermetallic Compounds in the Al-Cr System. CRPT) CRYSTMET: The NRCC Metals Crystallographic Data File.
PB97-109029 (Order as PB97-109011, PC A11/MF A03) Cs Cluster Binding to a GaAs Surface.

PB94-213006 00,846 Not available NTIS CSL View of Applications Portability, Scalability, and Interoperability.
PB97-122303

01,787 Not available NTIS

CSTL Technical Activities 1991

PB94-160769 A0,728 PC A16/MF A03

CSTL Technical Activities, $1993.00,953$ PC A17/MF A04
PB95-160602

CSTL Technical Activities, 1994

PB95-242319

00,608 PC A16/MF A03

CSTL Technical Activities, 1995

PB96-214630

00,647 PC A10/MF A03

Current Activities Within the National Biomonitoring Specimen Bank.

PB94-172806

02,516 Not available NTIS

Current and Voltage Measurements in the Gaseous Elec-

tronics Conference RF Reference Cell.
PB96-113337

Order as PB96-113311, PC A09/MF A03)

Current Fluctuations Reveal Protonation Dynamics and Number of Ionizable Residues in the alpha-Toxin Channel. PB96-161732

03,588 Not available NTIS

Current Noise Reveals Protonation Kinetics and Number of Ionizable Sites in an Open Protein Ion Channel.

PB96-161674 04,092 Not available NTIS

Current Results and Future Prospects for a Neutron Life-

time Determination Using Trapped Protons.
PB94-199742 03,817 Not available NTIS

Current Status and Trends in Temperature Measurements at NIST, Cooperative Projects and New Mutual Agreement between NIST and IMGC.

PB97-110266 and IMGC. 02,691 Not available NTIS

Currents Induced on Multiconductor Transmission Lines by Radiation and Injection

PB94-211943

02,215 Not available NTIS

Custom Integrated Circuit Comparator for High-Performance Sampling Applications.

02,320 Not available NTIS

Cyclic Polyamine Ionophore for Use in a Dibasic Phosphate-Selective Electrode. 01,008 Not available NTIS

Cylinder Wipe Air-Drying Intaglio Ink Vehicles for U.S. Currency Inks.

$03,115 \mathrm{PC} \mathrm{A03/MF} \mathrm{A01}$
Damage Processes in Ceramics Resulting from Diamond Tool Indentation and Scratching in Various Environments.
PB96-102983 Data Communications Strategy

Data Encryption Standard.

$02,738 \mathrm{PC} \mathrm{A05/MF} \mathrm{A01}$

Data Encryption Standard.
PB95-162376

01,595 Not available NTIS

Data Encryption Standard (DES); Category: Computer Security: Subcategory: Cryptography.
FIPS PUB 46-2

01,572 PC E04

Data Evaluation of a Linear System by a Second-Order Transfer Function.

PB96-200282

01,970 Not available NTIS

Data Import and Validation in the Inorganic Crystal Structure Database.
PB97-109201

04,809

(Order as PB97-109011, PC A11/MF A03)

Data Management for Error Compensation and Process Control

PB97-110480

02,847 Not available NTIS

Data-Parallel Algorithm for Three-Dimensional Delaunay Triangulation and Its Implementation. 01,714 Not available NTIS

Database Development and Management (Project A.2.2) The Annual Report for 1992-1993.
PB97-110290 03,098 Not available NTIS

ctric Constant of Water and Database for the Static Dielectric Constant of Water and
Steam.
PB96-145586 01,103 Not available NTIS

Database Management Standards: Status and Applicability.
PB96-122924

Databases Available in the Research Information Center of the National Institute of Standards and Technology.
PB95-128641
02,724 PC A08/MF A01

Databases Available in the Research Information Center of the National Institute of Standards and Technology (December 1995).

02,734 PC A07/MF A02

dc Magnetic Force Microscopy Imaging of Thin-Film Recording Head

04,665 Not available NTIS

dc Method for the Absolute Determination of Conductivities of the Primary Standard KCl Solutions from OC to $50 \mathrm{C}$. (Order as PB94-219326, PC A05/MF A02) $\mathrm{DC}-\mathrm{MHz}$ Wattmeter Based on RMS Voltage Measurements. PB97-113211 01,992 Not available NTIS

De Facto Microzonation through the Use of Soils Factors in Design Triggers
PB96- 141148

00,462 Not available NTIS

Dead Time, Pileup, and Accurate Gamma-Ray Spectrom$\begin{array}{ll}\text { etry. } & 00,697 \text { Not available NTIS }\end{array}$

Debugger for Tcl Applications.

01,695 Not available NTIS Decay of Bragg Gratings in Hydrogen-Loaded Optical Fibers.

04,345 Not available NTIS

Decomposition of SF6 and Production of S2F10 in Power Arcs.
PB96-122619 01,084 Not available NTIS

Decomposition of Sulfur Hexafluoride by X-rays.
PB96-135314 01,095 Not available NTIS

Decoupling in the Line Mixing of Acetylene Infrared $O$ Branches.

00,877 Not available NTIS

Decrease of Fluorescence in Optical Fiber during Exposure to Puised or Continuous-Wave Ultraviolet Light.

to Puised or Continuous-Wave Ultraviolet Light.

Deep-UV Excimer Laser Measurements at NIST.

PB96-141031 04,355 Not available NTIS

Defect Formation Mechanism Causing Increasing Defect Density during Decreasing Implant Dose in Low-Dose Simox.
PB96-119524

02,402 Not available NTIS

Defect of Thermal Ramping and Annealing Conditions on Defect Formation in Oxygen Implanted Silicon-On-Insulator PB94-212966

02,318 Not available NTIS

Defect Pair Formation by Implantation-Induced Stresses in High-Dose Oxygen Implanted Silicon-on-Insulator Material.
PB95-175824

Defective Structures of Barium Yttrium Copper Oxide

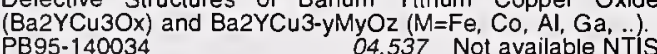




\section{TITLE INDEX}

Defining Environment Integration Requirements.
PB96-131545 02,733 PC A03/MF A01 Deformable Superconductor Model for the Fluxon Mass. PB95-175311 04,653 Not availabie NTIS

Deformation and Fracture of Mica-Containing Glass-Ceramics in Hertzian Contacts.

03,080 Not available NTIS

Degradation of Powder Epoxy Coated Panels Immersed in a Saturated Calcium Hydroxide Solution Containing Sodium Chloride.

PB96-101050

$01,344 \mathrm{PC}$ A03/MF AO

Deletion Analysis of the Mini-P1 Plasmid Origin of Replication and the Role of E.coli DnaA Protein.

PB95-163911 03,539 Not available NTIS

Delivering the Same Optical Frequency at Two Places: Accurate Cancellation of Phase Noise Introduced by an Optical Fiber or Other Time-Varying Path. Not available NTIS Densification of Nano-Size Powders. 1994 Report DE94013486 03,027 PC A03/MF A0

Density Dependence of Fluid Properties and Non-Newto-

nian Flows: The Weisenberg Effect.
PB96-161898

Density Matrix Calculation of Population Transfer between Vibrational Levels of $\mathrm{Na} 2$ by Stimulated Raman Scattering

PB94-198546 Shifted Laser Beams.

Density of Solids and Liquids.

AD-A278 517/8

$00,711 \mathrm{PC}$ A03/MF A01

Dental Applications of Ceramics.

PB96-122940

00,177 Not available NTIS

Dental Materials.

PB94-172871

00,142 Not available NTIS

Dependence of Contrast on Probe/Sample Spacing with the Magneto-Optic Kerr-Effect Scanning Near-Field Optical Microscope (MOKE-SNOM).

04,750 Not available NTIS

Dependence of Tc on Debye Temperature Theta(sub D) for Various Cuprates

04,683 Not available NTIS

Dependence of the Thermal Electron Attachment Rate Constant in Gases and Liquids on the Energy Position of the Electron Attaching State.

01,996 Not available NTIS

Deposit Forming Tendencies of Diesel Engine Ois-Correlation between the Two-Peak Method and Engine Tests.

PB95-152138 01,452 Not available NTIS

Deposition of Colloidal Sintering-Aid Particles on Silicon Nitride.

PB94-216272

03,044 Not available NTIS

Deposition of Loosely Bound and Firmly Bound Fluorides on Tooth Enamel by an Acidic Gel Containing Fluorosilicate and Monocalcium Phosphate Monohydrate.
PB,559-150710 Not available NTIS

Deposition Rates in Direct Current Diode Sputtering.

PB95-203345 04,697 Not available NTIS

Derivation of the System Equation for Null-Balanced Total Power Radiometer System NCS1.

PB94-169786 02,022 Not available NTIS

Description of Layered Structures: Applications to High Tc Superconductors
PB95-162624

04,615 Not available NTIS

Design and Characterization of X-Ray Multilayer Analyzers for the $50-1000 \mathrm{eV}$ Region.

PB94-211851

03,848 Not available NTIS

Design and Construction of a Liquid Hydrogen Temperature Refrigeration System
AD-A286 $618 / 4$

02,619 PC A03/MF A01

Design and Development of an Information Retrieval System for the EAMATE Data. Volume 2 of 2 . Appendices.
PB94-168390 00,487 PC A19/MF A04

Design and Machining of Copper Specimens with Micro Holes for Accurate Heat Transfer Measurements.

PB95-180428 02,658 Not available NTIS

Design and Operation of Series-Array Josephson Voltage Standards.

02,030 Not available NTIS

Design Challenges in a Commercial Quantum Hall EffectBased Resistance Standard.

PB95-171419

02,263 PC A03/MF A01

Design, Construction and Application of a Large Aperture Lens-Less Line-Focus PVDF Transducer.

PB97-122584-Focus PVDF Trablable NTIS

Design Criteria for BJT Amplifiers with Low $1 / f$ AM and PM Noise.
PB96-200365

02,442 Not available NTIS
Design Engineering Research at NIST. PB95-267860

02,784 PC A03/MF A01

Design Equations and Scaling Laws for Linear Compresrs with Flexure Springs.

02,948 Not available NTIS

Design Guide for CMOS-On-SIMOX. Test Chips NIST3 and NIST4.

PB94-163458

02,297 PC A05/MF A01

Design of a High-Flux Backscattering Spectrometer for PB96-179577

02,992 Not available NTIS

Design of a High-Pressure Ebulliometer, with Vapor-Liquid Equilibrium Results for the Systems CHF2Cl + CF3-CH3 and $\mathrm{CF} 3-\mathrm{CH} 2 \mathrm{~F}+\mathrm{CH} 2 \mathrm{~F} 2$.

$\mathrm{PB} 97-113229$

04,163 Not available NTIS

Design of a Protocol for an Electron Probe Microanalyzer kValue Round Robin.

PB95-107181

00,564 Not available NTIS

Design of High-Frequency, High-Power Oscillators Using Josephson-Junction Arrays.

02,094 Not available NTIS

Design of Technically Complex Facilities

PB97-119101 02,695 Not available NTIS

Design, Specification and Tolerancing of Micrometer-Tolerance Assemblies.

02,913 PC A03/MF A01

Designations of ds(2)p Energy Levels in Neutral Zirconium, Hafnium, and Rutherfordium $(Z=104)$.
PB96-180120

Desorption Induced by Electronic Transitions.

$\quad 00.853$ Not available NTIS

DETAN 95: Computer Code for Calculating Spectrum-Averaged Cross Sections and Detector Responses in Neutron

PB95-242384

04,014 PC A04/MF A01

Detection of Aromatic Compounds Based on DNA Intercalation Using an Evanescent Wave Biosensor.
PB96-111976 03,473 Not available NTIS Detection of $\mathrm{OH}_{+}$in Its a(1)Delta State by Far Infrared
Laser Magnetic Resonance.
PB95-181087 01,021 Not available NTIS PB95-181087

01,021 Not available NTIS

Detection of Voids in Grouted Ducts Using the Impact-Echo Method.
PB94-185121

01,306 Not available NTIS

Detector-Based Candela Scale and Related Photometric Calibration Procedures at NIST.
PB95-161949 04,273 Not available NTIS

04,273 Not avalable NTIS

Determination of Acoustic Center Correction for Type LS2aP Condensor Microphones.
PB96-204508 04,190 Not available NTIS Determination of Amphetamine and Methamphetamine in a

Lyophilized Human Urine Reference Material.
PB95-175444

Determination of Anomalous Superexchange in $\mathrm{MnCl} 2$ and Its Graphite Intercalation Compound PB97-122568

00,666 Not available NTIS

Determination of Atomic Data Pertinent to the Fusion Energy Program. Progress Report for FY 92.
DE94004400
04,402

Determination of Boron and Lithium in Diverse Biological Matrices Using Neutron Activation - Mass Spectrometry

PB94-212289

00,554 Not available NTIS

Determination of Complex Scattering Amplitudes in Low-Energy Elastic Electron-Sodium Scattering.
PB94-216652

Determination of Complex Structures from Powder Diffraction Data: The Crystal Structure of La3Ti5Al15O37.

PB95-202966 01,038 Not available NTIS

Determination of Density of Mass Standards: Requirement and Method.

03,787 PC A03/MF A01

Determination of Fiber-Matrix Interfacial Properties of Importance to Ceramic Composite Toughening.
PB95-125811

Determination of Hydrogen in Titanium Alioy Jet Engine Compressor Blades by Cold Neutron Capture Prompt Gamma-ray Activation Analysis.

PB95-175956

01,448 Not available NTIS

Determination of Inorganic Constituents in Marine Marmmal Tissues.

PB95-152047

00,589 Not available NTIS

Determination of Oltipraz in Serum by High-Performanace Liquid Chromatography with Optical Absorbance and Mass Spectrometric Detection.

03,493 Not available NTIS
Determination of Osmotic Pressure and Fouling Resistances and Their Effects on Performance of Ultrafiltration Membranes.

00,841 Not available NTIS

Determination of PCBs and Chlorinated Hydrocarbons in Marine Mammal Tissues.

PB95-162640

03,744 Not available NTIS

Determination of Polycyclic Aromatic Hydrocarbons by Liquid Chromatography.

00,585 Not available NTIS

Determination of Sheet Steel Formability Using Wide Band Electromagnetic-Acoustic Transducers.

PB96-186036

02,279 Not available NTIS

Determination of Sulfur in Fossil Fuels by Isotope Dilution

Thermal Ionization Mass Spectrometry.
PB96-141379 Not available NTIS

Determination of Surface Roughness from Scattered Light.

PB94-216520 04,243 Not available NTIS

Determination of the Complex Refractive Index of Individual Quantum Wells from Distributed Reflectance.

PB95-175642 02,176 Not available NTIS

Determination of the Molecular Parameters of $\mathrm{NiH}$ in its (2)Delta Ground State by Laser Magnetic Resonance.
PB95-107116 00,869 Not available NTIS

Determination of the Optical Constants of ZnSe Films by Spectroscopic Ellipsometry.

04,656 Not available NTIS

Determination of the Prior-Austenitic Grain Size of Selected Steels Using a Molten Glass Etch.

PB94-211927

03,208 Not available NTIS

Determination of the Residual Stresses Near the Ends of Skip Welds Using Neutron Diffraction and X-ray Diffraction Procedures.

PB95-253589

02,868 PC A03/MF A01

Determination of the Transmittance Uniformity of Optical Filter Standard Reference Materials.

02,182

PB95-261921

(Order as PB95-261897, PC A07/MF A02)

Determination of the Weight Average Molecular Weight of Two Poly(Ethylene Oxides), SRM 1923 and SRM 1924.
01,230 PC A03/MF A01
PB94-217031

Determination of Thermoactivation Parameters of Vortex Mobility in $\mathrm{YBa} 2 \mathrm{Cu} 307$ Using Only Magnetic Measurements.

PB95-163499

04,624 Not available NTIS

Determination of Total Protein Adsorbed on Solid (Membrane) Surface by a Hydrolysis Technique: Single Protein Adsorption.

03,552 Not available NTIS

Determination of Vitamin $\mathrm{K} 1$ in Serum Using Catalytic-Reduction Liquid Chromatography with Fluorescence Detec-

PB96-138425

03,506 Not available NTIS

Determination of 3-Quinuclidinyl Benzilate (Qnb) and Its Major Metabolites in Urine by Isotope Dilution Gas Chromatography Mass Spectrometry.

PB94-199379

03,492 Not available NTIS

Determination of 21 Elements by INAA for Certification of SRM 1570a, Spinach.

00,698 Not available NTIS

Determining Mobility from Homodyne ac Electrophoretic Light Scattering.

03,462 Not available NTIS

Determining the Magnetic Properties of $1 \mathrm{~kg}$ Mass Standards.

04,016

Order as PB95-261897, PC A07/MF A02)

Deterministic and Stochastic Chaos.

PB96-156138 00,218 Not available NTIS

Deuterium and the Local Interstellar Medium: Properties for the Procyon and Capella Lines of Sight

PB96-200639 00,111 Not available NTIS
O

Deuterium in the Local Interstellar Medium: Its Cosmological Significance.
PB95-202842

Deuterium Isotope Effect in Vinyl Radical Combination/ Dispropor tionationReactions.

PB96-204151

01,167 Not available NTIS

Developing a NIST Coaxial Microwave Power Standard at 1 PBV5-202412

01,914 Not available NTIS

Developing Linear Error Models for Analog Devices.

PB95-150520 02,037 Not available NTIS

Developing Measurement for Experimentation.

PB97-118707

03,450 Not available NTIS 


\section{TITLE INDEX} Developing Quality System Documentation Based on ANSI/
NCSL Z540-1-1994: The Optical Technology Division's EFfort.

01,869 PC A05/MF A01

Developing Rational Performance-Based Fire Safety Requirements in Model Building Codes. Development and Application of an Indoor Air Quality Com.

missioning Program in a New Office Building.
PB96-155601 00,275 Not available NTIS

Development and Calibration of UV/VUV Radiometric Sources.

PB94-199098

04,229 Not available NTIS

Development and Characterization of Insulating Layers on Silicon Carbide: Annual Report for February 14, 1988 to February 14, 1989

PB94-155579

02,295 PC A03/MF A01

Development and Validation of Multicriteria Ratings: A Case Study.
PB95-126025

00,004 Not available NTIS

Development in the Theory and Analysis of Eddy Current Sensing of Velocity in Liquid Metals.
PB94-212586 03,335 Not available NTIS Development of a Calibrated Atomic Force Microscope. Development of a Calibrated Atomic Force Microscope.
PB94-185683 02,894 Not available NTIS Development of a Dual-Sinker Densimeter for High-Accuracy Fluid P.V.T Measurements.
PB95-168951 03,267 Not available NTIS Development of a Dual-Sinker Densimeter for High-Accuracy Fluid P-V-T Measurements. Appendix A.
DE93019682

02,620 PC A02/MF A01

Development of a Gas Standard Reference Material Con-

taining Eighteen Volatile Organic Compounds.
PB95-162277 02,545 Not available NTIS

Development of a Method for Measuring Water-Stripping Resistance of AsphaltSiliceous Aggregate Mixtures.
PB96-197249

Development of a Method for Measuring Water-Stripping Resistance of Asphalt/Siliceous Aggregate Mixtures.
PB96-202296 Development of a New Quality Control Strategy for Automated Manufacturing.
PB96-160486 $02,840 \quad$ Not available NTIS Development of a New Small-Scale Smoke Toxicity Test Development of a New Small-Scale Smoke Toxicity Test
Method and Its Comparison with Real-Scale Fire Tests.
PB94-213253 Development of a Standard Reference Material for ISE Measurements of Sodium and Potassium.
PB96-159785 03,507 Not available NTIS Development of a Standard Reference Material for Measurement of Interstitial Oxygen Concentration in Semiconductor Silicon by Infrared Absorption.
PB96-122668 Development of a Temperature Scale below $0.5 \mathrm{~K}$. Development of a Temperature Scale below $0.5 \mathrm{~K}$.
PB95-125639 Development of a Test Method for Leaching of Lead from Lead-Based Paints Through Encapsulants.

PB96-154984 03,128 PC A04/MF A01
O

Development of Adaptive Control Strategies for Inert Gas Atomization.
PB95-162335

02,823 Not available NTIS

Development of an Adhesive Bonding System.

PB94-199056 00,145 Not available NTIS
Poverive Bonding System.

Development of an Automated Part Inspection System Using the DMIS Standard.

PB95.108866

02,899 Not available NTIS

Development of an Economical Video Based Fire Detection and Location System
PB96-193743

00,228 PC A05/MF A01 Development of Computer-Based Models of Standards and
Attendant Knowledge-Base and Procedural Systems. PB96-155783 00,464 Not available NTIS
N Development of Engineered Stationary Phases for the Separation of Carotenoid Isomers.

PB95-150249

00,578 Not available NTIS

Development of Frozen Whale Blubber and Liver Reference Materials for the Measurement of Organic and Inorganic Contaminants.

00,587 Not available NTIS

Development of Gas Standards from Solid 1,4dichlorobenzene.
PB96-155486 Development of Hazard Assessment and Suppression Technology for Oil and Gas Well Blowout and Diverter Fires. 01,408 Not available NTIS
Development of Highly Conductive Cantilevers for Atomic Force Microscopy Point Contact Measurements.
PB96-138573 04,751 Not available NTIS

Development of Measurement Capabilities for the Thermophysical Properties of Energy-Related Fluids. An$\begin{array}{cc}\text { nual Report, December } 1,1990--N o v e m b e r & 30,1991 . \\ \text { DE94004399 } & 02,470 \\ \text { PC A02/MF A01 }\end{array}$

Development of Measurement Capabilities for the Thermophysical Properties of Energy-Related Fluids. AnThermophysical Properties of Energy-Related Fluids. An-
nual Report, December 1, 1993--November 30, 1994. .
DE94017738
03,246 PC A03/MF A01

Development of Near-Field Test Procedures for Communication Satellite Antennas.

02,010 Not available NT1S

Development of Neutral-Density Infrared Filters Using Metallic Thin Films.

02,998 Not available NTIS

Development of the Fire Data Management System.

PB94-206091 00,339 PC A03/MF A01

Development of the Ion Exchange-Gravimetric Method for Sodium in Serum as a Definitive Method.

(Order as PB96-177381, PC A07/MF A02)

Development of the National Marine Mammal Tissue Bank. PB95-161402 02,586 Not available NTIS

Development of the NIST Transient Pressure and Temperature Calibration Facility.

PB96-160833

00,626 Not available NTIS

Development of Thin-Film Multijunction Thermal Converters

at NIST.

02,286 Not available NTIS

Developments in Stellar Coronae.

PB96-176706 00,107 Not available NTIS

Device for Subambient Temperature Control in Liquid Chromatography.

00,573 Not available NTIS

Diagnosis and Treatment of an Oral Base-Metal Contact Lesion Following Negative Dermatologic Patch Tests.
PB95-180626 00,172 Not available NTIS

Diagnosis of Causes of Concrete Deterioration in the MLP. 7A Parking Garage.

01,318 PC A03/MF A01

Diakoptic and Large Change Sensitivity Analysis.

PB95-150538 02,038 Not available NTIS

Diamond and Graphite Precursors: Comments.

PB95.163051 00,967 Not available NTIS

Dielectric and Magnetic Measurements from $-50 \mathrm{C}$ to $200 \mathrm{C}$ and in the Frequency Band $50 \mathrm{MHz}$ to $2 \mathrm{GHz}$. PB96-191382

02,245 PC A03/MF A01

Dielectric Behavior of a Polycarbonate/Polyester Mixture Upon Transesterification

04,785 Not available NTIS

Dielectric Measurements on Printed-Wiring and Circuit Boards, Thin Films, and Substrates: An Overview.
PB96-147038 02,236 Not available NTIS

Dielectric Properties Measurements and Data.

PB94-172186 01,876 Not available NTIS

Dielectric Properties of Materials at Cryogenic Temperatures and Microwave Frequencies.
PB95-202610 02,369 Not available NTIS

Dielectric Properties of Single Crystals of $\mathrm{Al} 2 \mathrm{O} 3, \mathrm{LaAlO} 3$, $\mathrm{SrTiO3}$, and $\mathrm{MgO}$ at Cryogenic Temperatures.

PB95-180477 02,266 Not available NTIS

Dielectric Properties of Thin Film $\mathrm{SrTiO}_{3}$ Grown on $\mathrm{LaAlO} 3$ with YBa2Cu3O7-x Electodes.

PB95-181160

02,267 Not available NTIS

Dielectric Spectroscopic Determination of Temperature Behavior of Electroclinic Parameters in the Liquid Crystal

W317.
PB96-140397

01,098 Not available NTIS

Dielectric Studies of Fluids with Reentrant Resonators

PB95-153730 00,952 Not available NTIS

Dielectric Studies of Fluids with Reentrant Resonators. Appendix B.
DE93019683

03,244 PC A02/MF A01

Dielectronic Capture Processes in the Electron-Impact Ionization of $\mathrm{Sc}(2+)$

PB95-203113

04,000 Not available NTIS

Differences in Competitive Strategies between the United States and Japan
PB94-211836

00,013 Not available NTIS

Diffraction Imaging of Polycrystalline Materials

PB94-198884 02,971 Not available NTIS
Diffraction of Neutron Standing Waves in Thın Films with Resonance Enhancement.

PB97-113278

04,164 Not available NTIS

Diffraction of X-rays at the Far Tails of the Bragg Peaks.

PB94-199924 04,476 Not available NTIS

Diffuse-Interface Description of Fluid Systems

PB96-210711 01,170 PC A04/MF A01

Diffusion of Cations Beneath Organic Coatings on Steel Substrate.

03,119 PC A03/MF AO

Diffusion of Copper into Gold Plating.

PB95-162152 00,957 Not available NTIS

Diffusion of Water along 'Closed' Mica Interfaces.

PB96-180039 02,993 Not available NTIS

Diffusion Studies in a Digital-Image-Based Cement Paste Microstructural Model.

PB94-198801

01,312 Not available NTIS

Diffusive Crack Growth at a Bimaterial Interface.

PB96-204110

03,090 Not available NTIS

Digital Impedance Bridge.

PB96-103155

02,272 Not available NTIS

Digital Signature Standard (DSS). Category: Computer Security; Subcategory: Cryptography.

$01,570 \quad P C E 04$

Digital Simulation of the Aggregate-Cement Paste Interfacia Zone in Concrete.

00,363 Not available NTIS

Digital Techniques in HV Tests - Summary of 1989 Panel

PB94-216702

02,035 Not available NTIS

Digitized Direct Simulation Model of the Microstructural Development of Cement Paste.

PB94-198777

01,309 Not available NTIS

Digitized Simulation Model for Microstructural Development.

Digitized Simulation of Mercury Intrusion Porosimetry.

PB94-172236

01,304 Not available NT1S

Dilemma-Preservation versus Access

PB94-198488 02,711 Not available NTIS

Dimensional Characterization of Precision Coaxial Trans mission Line Standards.

PB96-176482

02,241 Not available NTIS

Dimensional Characterization of Small Bores: A Survey.

PB95-162202

02,651 Not available NTIS

Dimensional Crossover in the Phase Separation Kinetics of Thin Polymer Blend Films.

PB97-113088

03,395 Not available NTIS

Dimensional Inspection Planning Based on Product Data Standards.

02,910 Not available NTIS

Diode Laser as a Spectroscopic Tool

PB95-175485 00,600 Not available NTIS

Diode-Laser Pumped, Rubidium Cell Frequency Standards.
PB95-163218 01,538 Not available NTIS

Dipole Moments in Rare Gas Interactions.

PB94-212982 00,844 Not available NTIS

Direct Comparison of Three PTB Silver Fixed-Point Cells with the NIST Silver Fixed-Point Cell.

PB96-161286 00,628 Not available NTIS

Direct Comparison Transter of Microwave Power Sensor Calibrations.

PB96-158654

02,086 PC A03/MF A0

Direct Detection of Atomic Arsenıc Desorption from Si(100). PB95-202230 01,024 Not available NTIS

Direct Dispersion Measurement of Highly-Erbium-Doped Optical Amplifiers Using a Low Coherence Reflectometer Coupled with Dispersive Fourier Spectroscopy.

PB95-150702 04,263 Not available NTIS

Direct Observation of Vortex Dynamics in Two-Dimensional Josephson-Junction Arrays.

PB96-102223

02,067 Not available NTIS

Directions in MEMS Research Application Development.

PB95-153797 02,106 Not available NTIS

Directory of Law Enforcement and Criminal Justice Associations and Research Centers.

PB96-178918

04,872 PC A05/MF A0

Directory of U.S. Private Sector Product Certification Programs.

02,688 PC A2O/MF AO4

Discontinuous Volume Change at the Orientational-Ordering Transition in Solid C60.

PB94-211828

00,821 Not available NTIS 


\section{TITLE INDEX}

Discovery of an X-Ray Selected, Radio-Loud Quasar at $z=3.9$.
PB94-198652

00,052 Not available NTIS

Discussion: Statistical Signal Processing of Quasiperiodicities.
PB96-119532

Disilicides of Tungsten, Molybdenum, Tantalum, Titanium Cobalt, and Nickel, and Platinum Monosilicide: A Survey of Their Thermodynamic Properties.

PB94-168580

$\infty, 752$ Not available NTIS

Dislocation Core-Core Interaction and Peierls Stress in a Model Hexagonal Lattice.

04,101 Not available NTIS

Dislocation Emission at Ledges on Cracks.
PB95-164240 04,630 Not available NTIS

Disorder Trapping in Ni2TiAI.

PB94-198942

03,322 Not available NTIS

Dispersion and Deposition of Smoke Plumes Generated in
Massive Fires. Massive Fires.

02,540 Not available NTIS

Dispersions of Magnetic Excitations of the Pr lons in PB94-173044

04,444 Not available NTIS

Displacement Method for Machine Geometry Calibration

PB95-152088

02,946 Not available NTIS

Display-Measurement Round-Robin

PB96-119227

02,186 Not available NTIS

Dissolution Problems with Botanical Reference Materials.

PB95.126280 03,487 Not available NTIS

Distant Future of Solar Activity: A Case Study of Beta Hydri. 3. Transition Region, Corona, and Stellar Wind.

PB94-185220 00,049 Not available NTIS

Distant Future of Solar Activity: A Case Study of beta Hydri.

3. Transition Region, Corona, and Stellar Wind.
PB95-153441 00,074 Not available NTIS

Distinguishing the Contributions of Residential Wood Com-

Distinguishing the Contributions of Residential Wood Com-
bustion and Mobile Source Emissions Using Relative Con-

bustion and Mobile Source Emissions Using Relative Con-
centrations of Dimethylphenanthrene Isomers.
PB96-135124 02,563 Not available NTIS

Distributed Architecture for Standards Processing

PB96-164181 00,276 Not available NTIS

Distributed Communication Methods and Role-Based ACcess Control for Use in Health Care Applications.
PB96-183165 01,508 PC A05/MF A01

Distributed Feedback Lasers in Rare-Earth-Doped Phosphate Glass.

04,740 Not available NTIS

Distributed measurements of tracer response on packed bed flows using a fiberoptic probe array. Final report.
DE95013079 00,667 PC A03/MF A01

Distributed Supercomputing Software: Experiences with the Parallel Virtual Machine - PVM.

PB94-163086

01,680 PC A03/MF A01

Distributed Systems: Survey of Open Management Approaches.

$01,746 \mathrm{PCA03/MF} \mathrm{A01}$

Distribution of Dielectric Relaxation Times and the Moment Problem

PB96-112032

04,727 Not available NTIS

Distribution of Fluoride in Saliva and Plaque Fluid After a 0.048 moll NaF Rinse.
PB95-151205

03,561 Not available NTIS

Distributions of Measurement Error for Three-Axis Magnetic Field Meters during Measurements Near Appliances.

PB96-180153 02,110 Not available NTIS
O N

DNA Base Damage Generated In vivo in Hepatic Chromatin

of Mice upon Whole Booy y-irradiation.
PB95-161741
03,627 Not available NTIS

DNA Base Damage in Lymphocytes of Cancer Patients Undergoing Radiation Therapy.

03,643 Not available NTIS

DNA Base Modifications in Renal Chromatin of Wistar Rats Treated with a Renal Carcinogen, Ferric Nitrilotriacetate.

DNA Damage and DNA Sequence Retrieval from Ancient PB97-111983

03,556 Not available NTIS

DnaJ, DnaK, and GrpE Heat Shock Proteins are Required in 'or'P1 DNA Replication Solely at the RepA Monomerization Step.

03,557 Not available NTIS

Documentation for Immediately Dangerous to Life or Health Concentrations (IDLHs)

$03,602 \mathrm{PC}$ A22/MF $\dot{A 0} 4$
Domain Analysis of the Alarm Surveillance Domain. Version 1.0. Conducted as Part of the Domain Analysis Case Study Project

01,705 PC A10/MF A03 Domain Etfects in Faraday Effect Sensors Based on Iron
Gamets.
PB95-202461 02,268 Not available NTIS

Domain Structures in Magnetoresistive Granular Metals

PB96-141346 04,760 Not available NTIS

Doppler-Free Spectroscopy of Large Polyatomic Molecules and van der Waals Complexes. 04,339 Not available NTIS

Dose Mapping of Radioactive Hot Particles Using PB95-162954

03,714 Not available NTIS

Dosimetry Systems for Radiation Processing.
PB96-135280 03,717 Not available NTIS Double Modulation and Selective Excitation Semiconductor Structures and Samples with Poor Surface Morphology. PB97-111439

2,452 Not available NTIS

Double-Modulation and Selective Excitation Photoreflectance for Wafer-Level Characterization of Quan-
tum-Well Laser Structures. tum-Well Laser Structures.
PB96-167325

Draft Guideline for Testing and Evaluation of Seismic Isolation Systems.
PB94-172947

00,423 Not available NTIS

Draft Guidelines for Pre-Qualification and Prototype Testing of Seismic Isolation Systems.

01,331 PC A06/MF A01

Draft Guidelines for Quality Control Testing of Elastomeric Seismic Isolation Systems.

00,422 PC A03/MF A01

Draft Guidelines for Quality Control Testing of Sliding Seismic Isolation Systems.

01,332 PC A03/MF A01

Dreams About the Next Generation of Super-Stable Lasers.
PB94-211570 04,235 Not available NTIS

Drill-Hearn-Gerasimov Sum Rule.

03,845 Not available NTIS

Droplet Transfer Modes for a MIL 100S-1 GMAW Electrode
PB95-209300

Dual-Frequency Millimeter-Wave Radiometer Antenna for Airborne Remote Sensing of Atmosphere and Ocean

PB96-112289

02,009 Not available NTIS

Dual Frequency mm-Wave Radiometer Antenna for Airbome Remote Sensing of Atmosphere and Ocean

PB95-180378

02,006 Not available NTIS

Ductile Fracture and Tempered Martensite Embrittlement of 4140 Steel.

03,222 Not available NTIS

Dusty Plasma Studies in the Gaseous Electronics Conerence Reference Cell.

PB96-113410

02,396

(Order as PB96-113311, PC A09/MF A03)

Dynamic Characteristics of Five Tall Buildings during Strong and Low-Amplitude Motions.

00,427 Not available NTIS

Dynamic Light-Scattering Study of a Diluted Polymer Blend Near Its Critical Point

01,245 Not available NTIS

Dynamic Measurements of Thermophysıcal Properties of Metals and Alloys at High Temperatures by Subsecond Pulse Heating Techniques.
N94-25124/6

03,309

(Order as N94-25120/4, PC A11/MF A03)

Dynamic Objects and Meta-Level Programming of an EXPRESS Language Environment. 01,774 Not available NTIS

Dynamic Phenomena on the RS Canum Venaticorum Bi-

nary II Pegasi in August 1989. 1. Observational Data.
PB94-211067

Dynamic Scaling in an Aggregating 2D Lennard-Jones SysPB96-167317

04,106 Not available NTIS

Dynamic Shear Modulus Measurements with Four Inde pendent Techniques in Nickel-Based Alloys.

03,320 Not available NTIS

Dynamic Technique for Measuring Normal Spectral Emissivity of Electrically Conducting Solids at High Temperatures with a High-Speed Spatial Scanning Pyrometer.

PB95-153045 03,921 Not available NTIS

Dynamics of Calcium Phosphate Precipitation.
P896-147095 03,574 Not available NTIS
Dynamics of Hydrogen Interactions with $\mathrm{Si}(100)$ and Si(111) Surfaces.
PB96-159801

04,082 Not available NTIS

Dynamics of $\mathrm{Mu}(+)$ in $\mathrm{Sc}$ and $\mathrm{ScHx}$

B96-180021 04,112 Not available NTIS

Dynamics of Multi-DOF Stochastic Nonlinear Systems.

B97-113245 00,477 Not available NTIS

Dynamics of Nonthermal Reactions: Femtosecond Surface

PB94-199965

00,688 Not available NTIS

Dynamics, Transport and Chemical Kinetics of Compartment Fire Exhaust Gases.

PB96-195508

$00,229 \mathrm{PC} \mathrm{A06/MF} \mathrm{A01}$

Dynamics vs. Static Young's Moduli: A Case Study.

PB94-213188 03,210 Not available NTIS

Dynamometer-Induced Residual Stress in Railroad Wheels: Uitrasonic and Saw Cut Measurements. Report No. 30.

PB96-183199 04,857 PC A04/MF A01

Early Detection of Room Fires Through Acoustic Emission. (NIST Reprint)

00,298 Not available NTIS

Early Dielectronic Recombination Measurements: Singly PBa4-211158

03,836 Not available NTIS

Early History and Future Outlook for the X-ray Crystal DenPB95-202487

04,692 Not available NTIS

Earth-Based Gravitational Experiments

PB94-211414

03,840 Not available NTIS

Earthquake and Fire in Japan: When the Threat Became a

Reality.

00,201 Not available NTIS

Earthquake Resistant Construction of Electric Transmission and Telecommunication Facilities Serving the Federal Govemment Report

02,460 PC A03/MF A01

Earthquake Resistant Construction of Gas and Liquid Fuel Pipeline Systems Serving, or Regulated By, the Federal Govemment.

04.846 PC A05/MF A01

Ebulliometers for Measuring the Thermodynamic Properties of Fluids and Fluid Mixtures.

04,195 PC A02/MF A01

Ebulliometric Measurement of the Vapor Pressure of

Difluoromethane
PB95-151361

00,931 Not available NTIS

Ebulliometric Measurement of the Vapor Pressure of 1Chloro-1,1-Difluoroethane and 1,1-Difluoroethane

PB95-164489 00,984 Not available NTIS

Economic, Energy, and Environmental Impacts of the En-

ergy-Related Inventions Program.
DE94-017162

00,008 PC A05/MF A02

Economic Methods and Risk Analysis Techniques for EvalUating Building Investments: A Survey.
PB96-122593 Not available NTIS Economics of New-Technology Materials: A Case Study of FRP Bridge Decking

PB96-202353

01,349 PC A06/MF A01

EDI and EFT Security Standards.

PB96-122833

01,605 Not available NTIS

Effect in Environmental Noise on the Accuracy of CoulombBlockade Devices.

PB95-175865

04,662 Not available NTIS

Effect of a Crystal Monochromator on the Local Angular Di-

vergence of an X-Ray Beam.
PB95.150306

04,565 Not available NTIS

Effect of Anneal Temperature on Si/Buried Oxide Interface Roughness on SIMOX

02,382 Not available NTIS

Effect of Annealing Ambient on the Removal of Oxide Precipitates in High-Dose Oxygen Implanted Silicon.
PB95-164356

Effect of Axial Strain on the Critical Current of Ag-Sheathed Bi-Based Superconductors in Magnetic Fields Up to 25 T.
PB94-211315 04,493 Not available NTIS

Effect of Backfill and Atomizing Gas on the Powder Porosity and Mechanical Properties of 304L Stainless Steel.
PB9 205 Not available NTIS

Effect of Beam Voltage on the Properties of Aluminum Nitride Prepared by lon Beam Assisted Deposition.
01,995 Not available NTIS Effect of $\mathrm{CF}_{3} \mathrm{H}$ and $\mathrm{CF} 3 \mathrm{Br}$ on Laminar Diffusion Flames in Normal and Microgravity.

PB96-161831

01,420 Not available NTIS 


\section{TITLE INDEX}

Etlect of CF3H and CF3Br on Laminar Diffusion Flames in Normal and Microgravity.

01.421 Not available NTIS

Effect of Charpy V-Notch Striker Radii on the Absorbed EnPrgy. 141122 03,365 Not available NTIS

Effect of Chemical Interaction on Barenblatt Crack Profiles in Brittle Solids.

02,996 Not available NTIS

Effect of Cross-Links on the Miscibility of a Deuterated Polybutadiene and Protonated Polybutadiene Blend. PB94-212438 01,225 Not available NTIS Effect of Curing History on Ultimate Glass Transition Temperature and Network Structure of Crosslinking Polymers.
01,214 Not available NTIS

PB94-200052

Effect of DC Tests on induced Space Charge.
PB94-172350 Effect of Dodecanol Content on the Combustion of Methanol Spray Flames.

01,389 Not available NTIS

Effect of Electrode Material on Measúred Ion Energy Distributions in Radio-Frequency Discharges.
04,026 Not available NTIS 04,026 Not available NTIS Effect of Electrode-Polymer Interfacial Layers on Polymer Conduction. Part 2. Device Summary,
PB95-151155 Not available NTIS

Effect of Environmentally Exposures on the Properties of Effect of Environmentally Exposures on the Properties of
Polyisocyanurate Foam Insulation: Thermal Conductivity Measurements.

00,388 Not available NTIS

Effect of Ethanol on the Solubility of Dicalcium Phosphate Dihydrate in the System Ca(OH)2-H3PO4-H2O at 37C.
PB95-163507 163 Not available NTIS

Effect of Ethanol on the Solubility of Hydroxyapatite in the System $\mathrm{Ca}(\mathrm{OH}) 2-\mathrm{H} 3 \mathrm{PO} 4-\mathrm{H} 2 \mathrm{O}$ at $25 \mathrm{C}$ and $33 \mathrm{C}$.
P 00,169 . Not available NTIS

Effect of Finite Beam Width on Elastic Light Scattering from Droplets.

01,144 Not available NTIS

Effect of Formulation Changes on the Response to lonizing Radiation of Radiochromic Dye Films.
PB97-119028 Not available NTIS

Effect of Fuel Tank Rupture Mode on the Ignitability of ExPelled Fuel. $\quad 01,444$ Not available NTIS Effect of Grain Size on Hertzian Contact Damage in Alumina. $179601 \quad 03,083$ Not available NTIS

Effect of Green Density and the Role of Magnesium Oxide Additive on the Densification of Alumina Measured by Additive on heutron Scattering (Reannouncement with New Availability Information).

Effect of Harmonic Distortion on Phase Errors in Frequency Distribution and Synthesis. 01,563 Not available NTIS
PB96-200779

Effect of Helerogeneous Porous Media on Mold Filling in Resin Transfer Molding. $\quad 03,197$ Not available NTIS
PB95-108676

Effect of Hydrodynamic Interactions on a Terminally Anchored Bead-Rod Model Chain. 01,237 Not available NTIS

Effect of Inclination on the Perlormance of a Compact Brazed Plate Condenser and Evaporator.
PB 2,756 PC A03/MF A01 Effect of Intermediate Thermal Processing on sulator Material.

PB96-160213

02,982 Not available NTIS

Effect of Liftoff on Accuracu of Phase Velocity Measurements Made with Electromagnetic-Acoustic Transducers.
PB96-186044 Not available NTIS

Effect of Magnetic Field Orientation on the Critical Current of HTS Conductor and Coils.

PB96-141189

02,956 Not available NTIS

Effect of Microstructure on Phase Formation in the Reaction of Nb/Al Multilayer Thin Films.

PB95-168415

03,352 Not available NTIS

Effect of Microstructure on the Wear Transition of ZirconiaToughened Alumina.

PB94-211778

03,141 Not available NTIS

Effect of Mn Content on the Microstructure of Al-Mn Alloys Electrodeposited at $150 \mathrm{C}$.

PB95-126355

03,343 Not available NTIS

Effect of Modulated Taylor-Couette Flows on Crystal-Mell Intertaces: Theory and Initial Experiments.
PB94-216736 Not available NTIS
Effect of Semiconductor Laser Characteristics on Optical Fiber Sensor Performance.

02,167 Not available NTIS

Effect of Single versus Multiple Implant Processing on Defect Types and Densities in SIMOX

PB96-160353 01,957 Not available NTIS

Effect of Sm2BaCuO5 on the Properties of Sintered (Bulk) YBa2Cu3O6+x

04,733 Not available NTIS

PB96-11944

on of Emission Rate Profiles Effect of Splitting on Estimation

03,916 Not available NTIS

Effect of Stoichiometry on the Phases Present in Boron Nitride Thin Films

PB96-102470

04,710 Not available NTIS

Effect of Supersaturation on Apatite Crystal Formation in Aqueous Solutions at Physiologic $\mathrm{pH}$ and Temperature.

Aqueous Solutions at Physiologic pH and Temperature.
PB96-135215

Effect of Suppressants on Metal Fires.

PB96-109574 01,402 PC A03/MF A01

Effect of Swelling on the Elasticity of Rubber: Localization

Model Description.
PB94-211034

01,216 Not available NTIS

Effect of Thermal Noise on Shapiro Steps in High-Tc Josephson Weak Links

04,506 Not available NTIS

Effect of Three Sterilization Techniques on Finger Pluggers.
PB94-216090

Effect of Training Dynamics on Neural Network PerformPB95-267845

01,852 PC A03/MF A01

Effect of Transformation of Alloy on Transient and Residual Stresses in a Porcelain-Metal Strip

PB94-198397

00,143 Not available NTIS

Effect of Two Initiator/Stabilizer Concentrations in a Metal Primer on Bond Strengths of a Composite to a Base Metal PB94-172723

00,141 Not available NTIS

Effect of 1-Hydroxyethylidene-1,1-Bisphosphonate on Membrane-Mediated Calcium Phosphate Formation in Model Liposomal Suspensions.

PB95-169223

03,469 Not avallable NTIS

Effective Measurement Techniques for Heat, Smoke, and Toxic Fire Gases.

01,369 Not available NTIS

PB94-198439

te-Loaded Materials.

Effeclive Me

01,893 PC A03/MF A01

Effectiveness of a Heat Recovery Ventilator, an Outdoor Air Intake Damper and an Electrostatic Particulate Filter at Controlling Indoor Air Quality in Residential Buildings.

PB96-14664

02,564 Not available NTIS

Effectiveness of Halon Alternatives in Suppressing Dynamic Combustion Process.

PB96-175732

03,288 Not available NTIS

Effects of Adhesive Thickness, Open Time, and Surface Eleanness on the Peel Strength of Adhesive-Bonded

\begin{tabular}{l} 
PB95-151338 00,372 Not available NTIS \\
\hline
\end{tabular}

Effects of Aluminum Oxalate/Glycine Pretreatment Solutions on Dentin Permeability.

03,565 Not available NTIS Effects of Anneal Time and Cooling Rate on the Formation
and Texture of $\mathrm{Bi2S} 2 \mathrm{CaCu} 2 \mathrm{O}$ Films.

and Texture of Bi2Sr2CaCu2O8 Films.
PB95-161600 Not available NTIS

Effects of Calcium Phosphate Solutions on Dentin Permeability.

00,157 Not available NTIS

Effects of Copper, Nickel and Boron on Mechanical PropEffects of Copper, Nickel and Boron on Mechanical Prop-
erties of Low-Alloy Steel Weld Metals Deposited at High erties of Low-Alloy Steel Weld Metals Deposited at High
Heat Input.
PB96-135231 03,363 Not available NTIS

03,363 Not available NTIS

Effects of Critical Current Density, Equilibrium Magnetization and Surface Barrier on Magnetization of High Temperature Superconductors.

PB94-185162

04,446 Not available NTIS

Effects of Critical Current Density, Equilibrium Magnetization and Surface Barrier on Magnetization of High Temperature Superconductors.

PB95-153060

04,588 Not available NTIS

Effects of Crystalline Anisotropy and Buoyancy-Driven Convection on Morphological Stability.
PB94-200441 Not available NTIS

Effects of Elastic Stress on Phase Equilibrium in the Ni-V

System.

03,313 Not available NTIS
Effects of Elastic Stress on the Stability of a Solid-Liquid Interface.

03,350 Not available NTIS

Effects of Etching on the Morphology and Surtace Resistance of YBa2Cu3O7-delta Films.

PB96- 135355

02,410 Not available NTIS Effects of Heavy Doping on Numerical Simulations of
Gallium Arsenide Bipolar Transistors.

PB95-150975

02,334 Not available NTIS

Effects of Hurnidity and Elevated Temperature on the Density and Thermal Conductivity of a Rigid Polyisocyanurate

PB95-152021

00,373 Not available NTIS

Effects of Humidity and Elevated Temperature on the Density and Thermal Conductivity of a Rigid Polyisocyanurate
Foam Co-Blown with $\mathrm{CCl} 3 \mathrm{~F}$ and $\mathrm{CO} 2$.

PB95-150462 Wo, 00 Not available NTIS

Effects of Interfacial Roughness on the Magnetoresistance of Magnetic Metallic Multilayers.

PB95-150017

04,556 Not available NTIS

Effects of Molecular Weight and Thermal Stability on Polymer Gasification

01,228 Not available NTIS

Effects of Nonmedel Errors on Model-Based Testing

PB96-155577

03,420 Not available NTIS

Effects of Nonmodel Errors on Model-Based Testing

PB96-123146

02,604 Not available NTIS

Effects of Pipe Elbows and Tube Bundles on Selected Types of Flowmeters.

PB96-160999

01,135 Not available NTIS

Effects of Sample Mounting on Flammability Properties of Intumescent Polymers.

03,389 Not available NTIS

Effects of Soxhlet Extraction on the Surface Oxide Layer of Silicon Nitride Powders.

03,057 Not available NTIS

Effects of Specimen Edge Conditions on Heat Release PB95-152864

00,375 Not available NTIS

Effects of Spindle Dynamic Characteristics on Hard Turning. 96-122981 02,699 Not available NTIS

Effects of Substrate Surface Steps on the Microstructure of Epitaxial Ba2YCu3O7-x Thin Films on (001) LaAlO3.

04,774 Not available NTIS

Effects of Surface-Active Resins on Dentin/Composite Bonds. 9405 - 140448

00,156 Not available NTIS

Effects of Target Shape and Neutron Scattering on Element Sensitivities for Neutron-Capture Prompt Gamma-ray Activation Analysis.

00,558 Not available NTIS

Effects of Target Temperature on Analytical Sensitivities of Cold-Neutron Capture Prompt gamma-ray Activation AnalyPBis. 112131

00,616 Not available NTIS

Effects of Testing Variables on the Measured Compressive Strength of High-Strength (90 MPa) Concrete.
PB95-179040 P07/MF A02 Effects of Testing Variables on the Strength of HighStrength (90 Mpa) Concrete Cylinders.
PB96-112198 Not available NTIS

Effects of Variable Excluded Volume on the Dimensions of Off-Lattice Polymer Chains.

01,229 Not available NTIS

Effects on Whole Saliva of Chewing Gums Containing Cal-

cium Phosphates
PB95-153169

03,563 Not available NTIS

Efficiency of Electric Motors. National Voluntary Lab. Accreditation Program (NVLAP)

02,107 PC A05/MF A01

Efficient $\mathrm{Br}\left({ }^{*}\right)$ Laser Pumped by Frequency-Doubled $\mathrm{Nd}$ : YAG and Electronic-to-Vibrational Transter-Pumped $\mathrm{CO} 2$ and HCN Lasers.

PB95-108684

04,248 Not available NTIS

Efficient Experiment to Study Superconducting Ceramics PB94-212578 04,505 Not available NTIS

Efficient Method to Compute the Maximum Transient Drain Current Overshoot in Silicon on Insulator Devices.

PB94-172483 02,300 Not available NTIS

Efficient Way of Identilying New Active Stars: A VLA Survey

of X-ray Selected Active Stellar Candidates.
PB96-122882
00,099 Not available NTIS

Elastic Constants and Debye Temperature of $\mathrm{Y} 1 \mathrm{Ba2Cu3Ox}$ : Effect of Oxygen Content.

04,515 Not available NTIS 


\section{TITLE INDEX}

Elastic Constants and Internal Friction of Polycrystalline Copper. 03,364 Not available NTIS

Elastic Constants and Microcracks in YBa2Cu3O7.

PB96-200761 03,005 Not available NTIS

Elastic Constants of a Material with Orthorhombic Symme-

try: An Alternative Measurement Approach.
PB95-180527 04,684 Not available NTIS

Elastic Constants of Isotropic Cylinders Using Resonant Ultrasound.

PB94-211919

04,497 Not available NTIS

Elastic Constants of Polycrystalline $\mathrm{Y} 1 \mathrm{Ba} 2 \mathrm{Cu} 3 \mathrm{OX}$

PB94-213196 04,513 Not available NTIS

Elastic Deformation of a Monolithic Perfect Crystal Inter ferometer: Implications for Gravitational Phase Shitt Experi-

PB94-213154

03,858 Not available NTIS

Elastic-Electron-Scattering Effects on Angular Distributions in $X$-ray Photoelectron Spectroscopy.

PB95-175758

01,000 Not available NTIS

Elastic Green's Function for a Bimaterial Composite Solid Containıng a Free Surface Normal to the Interface.

03,162 Not available NTIS

Elastic Properties of Al2O3/Al Composites: Measurements and Modeling.

03,157 Not available NTIS

Elastic Properties of Central-Force Networks with BondLength Mismatch.

PB95-163366

04,623 Not available NTIS

Elastic Properties of Uniaxial-Fiber Reinforced Composites: General Features.

PB94-200649

03,140 Not available NTIS

Elastic Scattering from Spheres under Non Plane-Wave Illumination.
PB96-163688

04,370 Not available NTIS

Elastic Scattering of Polymer Networks.

PB95-161816 01,255 Not available NTIS

Electric Dipole Excitation of a Long Conductor in a Lossy Medium.
PB96-146675

04,058 Not available NTIS

Electric Field Dependent Dielectric Breakdown of Intrinsic SiO2 Films Under Dynamic Stress.

PB96-204102 02,449 Not available NTIS

Electric Field Effects on a Near-Critical Fluid in Microgravity PB96-161880 04,217 Not available NTIS

Electric Field Effects on Crack Growth in a Lead Magnesium Niobate.

03,339 Not available NTIS

Electric-Field Strengths Measured Near Personal Transceivers.

01,564 Not available NTIS

Electrical Breakdown in Transformer Oil in Large Gaps.

PB95-150579 01,889 Not available NTIS

Electrical Characteristics of Argon Radio Frequency Glow Discharges in an Asymmetric Cell.

PB96-176490 04,109 Not available NTIS

Electrical Characterization of Integrated Circuit Metal Line Thickness.

PB96-138433

02,414 Not available NTIS

Electrical Characterization of Liquid-Phase Epitaxially Grown Single-Crystal Films of Mercury Cadmium Telluride by Variable-Magnetic-Field Hall Measurements.

PB95-175782 02,177 Not available NTIS

Electrical Characterization of Narrow Gap n-Type Bulk $\mathrm{HgCdTe}$ Single Crystals by Variable-Magnetic-Field $\mathrm{Ha}$ Measurements and Reduced-Conductivity-Tensor Analyses.

Electrical Characterization of Radio-Frequency Discharges

in the Gaseous Electronics Conference Reference Call.
PB95-164612 01,905 Not available NTIS

Electrical Measurements for Monitoring and Control of Plasma Processing.

04,369 Not available NTIS

Electrical Measurements of Microwave Flip-Chip Interconnections.

02,436 Not available NTIS

Electrical Method for Determining the Thickness of Metal Films and the Cross-Sectional Area of Metal Lines.

PB95-203170 02,370 Not available NTIS

Electrical Product Requirements (Especially Quality Requirements) in the United States.
PB96-119235

Electrical Sensors for Monitoring if Plasma Sheaths.

PB95-162962 04,412 Not available NTIS
Electrical Test Structure for Improved Measurement of Feature Placement and Overlay in Integrated Circuit Fabrication

02,355 Not available NTIS

PB95-164273

Electrical Test Structure for Overlay Metrology Referenced
to Absolute Length Standards. to Absolute Length Standards.

02,336 Not available NTIS

Electrical Test Structures Replicated in Silicon-on-Insulator Material.

02,454 Not available NTIS

PB97-111827 Electrically Calibrated Pyroelectric Detector-Refinements for Improved Optical

02,164 Not available NTIS

Electro-Optic-Based RMS Voltage Measurement Technique. PB96-138490 02,194 Not available NTIS

Electro-Optical Sensor for Surface Displacement Measurements of Compliant Coatings.

02,123 Not available NTIS

Electro-Thermal Simulation of an IGBT PWM Inverter.

PB94-185592 02,303 Not available NTIS

Electroacoustic Characterization of Particle Size and Zeta Potential in Moderately Concentrated Suspensions.

PB96-119425 01,079 Not available NTIS

Electroacoustics for Characterization of Particulates and Suspensions. Proceedings of a Workshop. Held in Gaithersburg, Maryland on February $3-4,1993$.
PB94-112695 00,725 PC A15/MF A03

Electrochemical Synthesis of Metal and Intermetallic Com-

posites.

03,304 PC A04/MF A01

Electrode Extension Model for Gas Metal Arc Welding PB96-135074 02,871 Not available NTIS

Electrodeposited Cobalt-Tungsten as a Diffusion Barrier between Graphite Fibers and Nickel.

PB96-146881

03,176 Not available NTIS

Electrodeposition

PB94-172517

00,760 Not available NTIS

Electrohydrodynamic Instability and Electrical Discharge Ini-

tiation in Hexane.
PB96-186119

02,244 Not available NTIS

Electrokinetic Sonic Analysis of Silicon Nitride Suspensions. PB96-123575 03,073 Not available NTIS

Electrolytes Constrained on Fractal Structures: DebyeHuckel Theory.

01,174 Not available NTIS

Electromagnetic Coupling Character of (001) Twist Boundaries in Sintered Bi2Sr2CaCu2O8+x Bicrystals.

PB96-176573 01,963 Not available NTIS

Electromagnetic Properties of Materials: The NIST MetrolOgy Program.

01,933 Not available NTIS

Electromagnetic Scattering by a Periodic Surface with a Wedge Profile.

PB94-211950

04,421 Not available NTIS

Electromagnetic Scattering from a Dielectric Wedge and the

Single Hypersingular Integral Equation.
PB97-110530

Electromagnetic Shielding Characterization of Gaskets

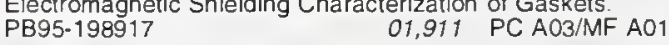

Electromechanical properties of superconductors for DOE fusion applications

DE95015476

04,432 PC A04/MF A01

Electromechanical Properties of Superconductors for DOE Fusion Applications.

02,250 PC A06/MF A02

Electron and Hole Trapping in Irradiated SIMOX, ZMR and BESOI Buried Oxides.

PB96-160320

01,956 Not available NTIS

Electron and Proton Dosimetry with Custom-Developed Radiochromic Dye Films.

PB95-151106

03.713 Not avallable NTIS

Electron-atom collision studies using optically state selected beams. Progress report, May 15, 1987--May 14, 1988. DE95004446

03,776 PC A03/MF A01

Electron-atom collision studies using optically state selected beams. Progress report, May 15, 1988--May 14, 1991. DE 95004447

$03,777 \mathrm{PC} \mathrm{A03/MF} \mathrm{AO1}$

Electron Attachment to Excited Molecules(1)

PB96-122809

01,087 Not available NTIS

Electron Beam Crosslinking of Poly(vinylmethyl ether).

PB94-185550 01,205 Not available NTIS

Electron-electron Interactions, Coupled-Plasmon-Phonon Modes, and Mobility in n-Type GaAs.
Electron-Impact Excitation of Si $(3+)(3 S$ yields $3 \mathrm{P})$ Using a PB95-151239 Electron-Energy-Loss 03,904 Not available NTIS

Electron-impact Ionization of in $(+)$ and $\mathrm{Xe}(+)$

PB94-185089 00,770 Not available NTIS

Electron-impact Ionization of $\ln (+)$ and $\mathrm{Xe}(+)$

PB95-152906 03.918 Not available NTIS

Electron-Ion Collisions in the Plasma Edge.

PB96-111885 04,037 Not available NTIS

Electron-Ion-X-ray Spectrometer System.

PB95-176137 03,958 Not available NTIS

Electron-Photon Monte Carlo Calculations: The ETRAN Code.

PB97-110407 04,138 Not available NTIS

Electron Probe X-Ray Microanalysis.

PB95-107165 00,562 Not available NTIS

Electron Scattering and Dissociative Attachment by SF6 and Its Electrical-Discharge By-Products.

PB95-151288

02,256 Not available NTIS

Electron Screening Correction to the Self Energy in High-Z

Atoms.
PB94-172376

03,789 Not available NTIS

Electron Transfer Reaction Rates and Equilibria of the Carbonate and Sulfate Radical Anions.

PB94-212180

00,829 Not available NTIS

Electron transport calculations with biomedical and environmental applications. Final report, December 23, 1992.-January 31,1994

DE95007065

$03,613 \mathrm{PC} \mathrm{A01/MF} \mathrm{A01}$

Electron Traps, Structural Change, and Hydrogen Related SIMOX Defects.
PB94-200391

02,312 Not available NTIS

Electronic Access: Blueprint for the National Archives and Records Administration.

PB95-219218

02,731 PC A04/MF A01

Electronic Access to Standards on the Information Highway.
PB96-131578

Electronic Balance and Some Gravimetric Applications. (The Density of Solids and Liquids, Pycnometry and Mass). Electronic Correlations and Satellites in Superconducting Oxides.

PB94-200045

04,477 Not available NTIS

Electronic Implementors' Workshop.

PB95-210936

01,484 PC A03/MF A01

Electronic Microrefrigerator Based on a Normal-insulator-

Superconductor Tunnel Junction.
PB96-102827

04,718 Not available NTIS

Electronic Mode Stirring for Reverberation Chambers.

PB95-180329

01,908 Not available NTIS

Electronic Publishing and the Journals of the American Chemical Society
PB97-109185

(Order as PB97-109011, PC A11/MF A03)

Electronic Spectra of $\mathrm{CF}_{2} \mathrm{Cl}$ and $\mathrm{CFCl} 2$ Radicals Observed by Resonance Enhanced Multiphoton Ionization. PB95.151023

00,927 Not available NTIS

Electronic Structure and Phase Equilibria in Ternary Substitutional Alloys.

03,378 Not available NTIS

Electronically Tunable Fiber Laser for Optical Pumping of (3) $\mathrm{He}$ and (4) $\mathrm{He}$

PB96-201165

04,381 Not available NTIS

Electronics and Electrical Engineering Laboratory Technical Progress Bulletin Covering Laboratory Programs, April to June 1991, with 1992 EEEL Events Calendar.

PB95-209821

01,916 PC A03/MF A01

Electronics and Electrical Engineering Laboratory Technical Progress Bulletin Covering Laboratory Programs, April to June 1994 with 1994/1995 EEEL Events Calendar.

PB95- 143186

$02,329 \mathrm{PC} \mathrm{A03/MF} \mathrm{A01}$

Electronics and Electrical Engineering Laboratory Technical Progress Bulletin Covering Laboratory Programs, April to June 1995 with 1995 EEEL Events Calendar.

PB96-106455

01,923 PC A03/MF A01

Electronics and Electrical Engineering Laboratory Technical Progress Bulletın Covering Laboratory Programs, April to June 1996 with 1996-1998 EEEL Events Calendar. PB97-113880

01,994 PC A04/MF A01

Electronics and Electrical Engineering Laboratory Technical Progress Bulletin Covering Laboratory Programs, January to March 1991, with 1991 EEEL Events Calendar.

PB94.145968

$02,113 \mathrm{PC} \mathrm{A03/MF} \mathrm{A01}$ 


\section{TITLE INDEX}

Electronics and Electrica! Engineering Laboratory Technical Progress Bulletin Covering Laboratory Programs, January to March 1992, with 1992/1993 EEEL Events Calendar.
PB95-210480
01.917 PC A03/MF A01

Electronics and Electrical Engineering Laboratory Technical Progress Bulletin Covering Laboratory Programs, January to March 1994 with 1994/1995 EEEL Events Calendar.
PB94-193810
02,308 PC A03/MF A01

Electronics and Electrical Engineering Laboratory Technica Progress Bulletin Covering Laboratory Programs, January to March 1996, with 1996 EEEL Events Calendar.

PB96-191390

01,969 PC A04/MF A01

Electronics and Electrical Engineering Laboratory Technical Progress Bulletin Covering Laboratory Programs, July to September 1993 with 1994 EEEL Events Calendar.
PB94-194354

Electronics and Electrical Engineering Laboratory Technica Progress Bulletin Covering Laboratory Programs, July to Sember 1994 with 1994/1995 EEEL Events Ca, July PB95-170395 with 1994/1995 EEEL Events Calendar.
02,360 PC A03/MF A0 1

Electronics and Electrical Engineering Laboratory Technica Progress Bulletin Covering Laboratory Programs, July to PB96-147905

01,942 PC A04/MF A01

Electronics and Electrical Engineering Laboratory Technical Progress Bulletin Covering Laboratory Programs, October to December 1992, with 1992/1993 EEEL Events Calendar.

Electronics and Electrical Engineering Laboratory Technica Progress Bulletin Covering Laboratory Programs October PB95-208724
02,372 PC A04/MF A01

Electronics and Electrical Engineering Laboratory Technical Progress Bulletin Covering Laboratory Programs, October to December 1995 with 1996 EEEL Events Calendar.

Electronics and Electrical Engineering Laboratory Technical Progress Bulletin Covering Programs, October to December 993 with 1994/1995 EFEL Events Calendar.

$\begin{array}{ll}\text { PB94-154341 } & 02,115 \text { PC A03/MF A01 }\end{array}$

Electronics and Electrical Engineering Laboratory Technical Publication Announcements Covering Laboratory Programs, April to June 1995 with 1995 EEEL Events Calendar.
PB96-137187

Electronics and Electrical Engineering Laboratory Technical Publication Announcements Covering Laboratory Programs January to March 1994 with 1994/1995 EEEL Events Cal-

PB94-213774

$01,883 \mathrm{PC} \mathrm{A03/MF} \mathrm{A01}$

Electronics and Electrical Engineering Laboratory Technical Publication Announcements Covering Laboratory Programs, January to March 1995 with 1995 EEEI Events Calendar.
PB95-242277

Electronics and Electrical Engineering Laboratory Technical Publication Announcements Covering Laboratory Programs, January to March 1996, with 1996 EEEL Events Calendar.
PB96-214622

Electronics and Electrical Engineering Laboratory Technica Publication Announcements Covering Laboratory Programs, July to September 1994 with 1995 EEEL Events Calendar.
PB95-198925

Electronics and Electrical Engineering Laboratory Technica Publication Announcements Covering Laboratory Programs, July to September 1995 with 1996 EEEL Events Calendar. PB96-183066 01,965 PC A03/MF A01

Electronics and Electrical Engineering Laboratory Technical Publication Announcements Covering Laboratory Programs, October to December 1993 with 1994/1995 EEEL Events PB94-193752

02,307 PC A03/MF A01

Electronics and Electrical Engineering Laboratory Technical Publication Announcements Covering Laboratory Programs,
October to December 1994 with 1995 EEEL Events CalPB95-231841

01,918 PC A03/MF A01

Electronics and Electrical Engineering Laboratory Technica Publication Announcements Covering Laboratory Programs October to December 1995, with 1996 EEEL Events Calenda

PB96-202346

$01,978 \mathrm{PC} \mathrm{A04/MF} \mathrm{A01}$

Electronics and Electrical Engineering Laboratory $1994 \mathrm{Pro}-$ gram Plan: Supporting Technology for U.S. Competitiveness in Electronics.

PB94-126901

$01,873 \mathrm{PC}$ A09/MF A02

Electronics and Electrical Engineering Laboratory: 1994 Strategic Plan. Supporting Technology for U.S. Competitiveness in Electronics.

PB94-161320

$01,874 \mathrm{PC} \mathrm{A04/MF} \mathrm{A01}$ Electronics and Electrical Engineering Laboratory 1994 Competitiveness in Electronics. PB95-144309

01,887 PC A06/MF A02
Electronics and Electrical Engineering Laboratory 1995 Program Plan. Supporting Technology for U.S. Competitiveness in Electronics.

01,894 PC A09/MF A03

PB95-159885 Electronics and Electrical Engineering Laboratory 1995 Electrotechnology to Support the U.S. Economy.

Electronics and Electrical Engineering Laboratory: 1996 Program Plan. Supporting Technology for U.S. Competitiveness in Electronics.

01,962 PC A10/MF A03

Electronics Packaging inaterials Research at NIST.

PB96-122692

02,405 Not available NTIS

Electropermeabilization of Cell Membranes: Effect of the Resting Membrane Potential.

PB95-163291

03,537 Not available NTIS

Electrophoretic Separations of Polymerase Chain Reaction: Amplified DNA Fragments in DNA Typing Using a Capillary

Electrophoresis-Lased Induced Fluorescence System.
PB95-163036 03,536 Not available NTIS

Electrostatic Modes as a Diagnostic in Penning-Trap Experiments.

PB95-176244

03,959 Not available NTIS

Electrostatic Mo

Plasmas.

Electrostatic Rigidity of Polyelectrolytes from Reparametrization Invariance. 04,113 Not available NTIS
PB96-180062

Elements of a Framework for Fire Safety Engineering.

PB96-151402 00,214 Not available NTIS

ELF Electric and Magnetic Field Measurement Method

PB95-161675

04,423 Not available NTIS

Embossable Grating Couplers for Planar Waveguide Optical Sensors.

PB96-190277

00,641 Not available NTIS

EMISS: A Program for Estimating Local Air Pollution Emis sion Factors Related to Energy Use in Buildings: User's Guide and Reference Manual.

PB96-109566

02,560 PC A03/MF A01

Emission Linewidth Measurements of Two-Dimensional Array Josephson Oscillators.

PB95-175139

02,055 Not available NTIS

Empirical Linear Prediction Applied to a NIST Calibration Service.
PB97-112353

02,287 Not available NTIS

Empirical Validation of a Transient Computer Model fo Combined Heat and Moisture Transfer.
PB97-111991 00,416 Not available NTIS Encouraging Environmentally-Aware Inventions.

Encouraging Environmentally-Aware Inventions.
PB95-161394 02,521 Not available NTIS Energy and Migration of Grair, Boundaries in Polycrystals.
03,332 Not available NTIS

Energy-Based Method for Liquefaction Potential Evaluation Phase 1. Feasibility Study.

PB96-214747

$03,691 \mathrm{PC} \mathrm{A13/MF} \mathrm{A03}$

Energy Calibration of $X$-ray Photoelectron Spectrometers: Results of an Interlaboratory Comparison to Evaluate a Proposed Calibration Procedure.

PB96-102918

04,027 Not available NTIS

Energy Dependence of Collision Characteristics in Molecule-Surface Collisions.

PB94-198504

00,786 Not available NTIS

Energy Dependences of Absorption in Beryllium Windows and Argon Gas
PB96-102124

04,020 Not available NTIS

Energy Distributions of Neutrons Scattered from Solid C60 by the Beryllium Detector Method

PB96-176631

03,740 Not available NTIS

Energy Flows in an Orifice Pulse Tube Refrigerator

PB95-169082
02,752 Not available NTIS

Energy Levels of Germanium, Ge I through Ge XxXII

PB94-162351 00,747 Not available NTIS

Energy Levels of Zinc, Zn I through $\mathrm{Zn} X X X$

PB96-145982 01,122 Not available NTIS

Energy-Pooling Collisions in Barium.

PB95-203030 03,997 Not available NTIS

Energy Price Indices and Discount Factors for Life-Cycle Cost Analysis 1995. Annual Supplement to NIST Handbook 135 and NBS Special Publication 709. (Revised).
PB95-105011 02,509 PC A04/MF A01

Energy Price Indices and Discount Factors for Life-Cycle Cost Analysis 1996. Annual Supplement to NIST Handbook 135 and NBS Special Publication 709. (Revised).
PB96-162441
Energy Price Indices and Discount Factors for Life-Cycle Cost Analysis 1997. Annual Supplement to NIST Handbook 135 and NBS Special Publication 709. (Revised). PB96-210745 02.512 PC A05/MF A0

Energy Prices and Discount Factors for Life-Cycle Cost Analysis 1994. Annual Supplement to NIST Handbook 135 and NBS Special Publication 709 .

PB94-206018

$02,508 \mathrm{PC}$ A04/MF A01

Energy Transduction between a Concentration Gradient and an Alternating Electric Field.

PB94-216363

03,461 Not available NTIS

Enhanced Curie Temperatures and Magnetoelastic Domains in Dy/Lu Super Lattices and Films.

PB94-172665 04,443 Not available NTIS

Enhanced Detection of PCR Products Through Use of TOTO and YOYO Intercalating Dyes with Laser Induced 年

PB95-164653

00,599 Not available NTIS

Enhanced Flux Pinning via Chemical Substitution in Bulk

Superconducting T1-2212.
PB95-169033

04,647 Not available NTIS

Enhanced Machine Controller Architecture Overview.

PB94-142460
02,802 PC A03/MF A0

Enhanced Magnetocaloric Effect in Gd3Ga5-xFexO12.

PB94-185659 04,450 Not available NTIS

Enhanced Voltage-Dividing Potentiometer for High-Precision Feature Placement Metrology.

PB96-164025

02,428 Not available NTIS

Enhancement of EXIT89 and Analysis of World Trade Cen ter Data.

PB96-202247

$00,231 \mathrm{PC} \mathrm{A04/MF} \mathrm{AO1}$

Enhancement of R123 Pool Boiling by the Addition of $\mathrm{N}$ -

$\begin{array}{ll}\text { Hexane. } & 02,605 \text { PC A04/MF A01 }\end{array}$

Enhancements to Program IDARC: Modeling Inelastic Behavior of Welded Connections in Steel Moment-Pesisting

PB95-231601

00,452 PC A05/MF A01

Ensuring Accuracy and Traceability of Weighing Instruments.

02,638 Not available NTIS

Enthalpy Increment Measurements from $4.5 \mathrm{~K}$ to $350 \mathrm{~K}$ and the Thermodynamic Properties of the Titanium Silicide Ti5Si3(cr).

PB96-204037

00,679 Not available NTIS

Enthalpy Increment Measurements from 4.5 to $318 \mathrm{~K}$ to Bismuth(cr). Thermodynamic Properties from $0 \mathrm{~K}$ to the Melting Point

01,166 Not available NTIS

Enthalpy Increment Measurements from 4.5 to $350 \mathrm{~K}$ and the Thermodynamic Properties of Titanium Disilicide(cr) to

$1700 \mathrm{~K}$.
PB96-204029

00,678 Not available NTIS

Environment Simulation for a Continuous Mining Machine.

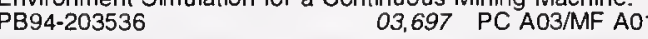

Environmental Aspects of Halon Replacements: Consider ations for Advanced Agents and the Ozone Depletion Potential of CF3I

03,301 Not available NTIS

Environmental Durability of Glass-Fiber Composites.

PB95-203220 03, 166 Not available NTIS

Environmental Evaluation of a New Federal Office Building.
PB94-199874 02,538 Not available NTIS

Environmental Scanning Electron Microscope Imaging Examples Related to Particle Analysis.

$\begin{array}{ll}\text { PB94-172822 } & 00,766 \text { Not available NTIS }\end{array}$

Environmental Sensitivities of Quartz Oscillators

PB96-103148 02,271 Not available NTIS

Environmentally Enhanced Fracture of Ceramics.

PB95-125746 03,046 Not available NTIS

Enzyme and Protein Mass Transfer Coefficient in Aqueous Two Phase Systems. 1. Spray Extraction Columns.

PB95-161162 00,594 Not available NTIS

Epitaxial Growth and Characterization of the Ordered Va cancy Compound Culn3Se5 on GaAs (100) Fabricated by Molecular Beam Epitaxy.

04,687 Not available NTIS

Epitaxial Growth of BaTiO3 Thin Films at $600 \mathrm{C}$ by Metalorganic Chemical Vapor Deposition.
PB96-122510

Epitaxial Growth of Sb/GaSb Structures: An Example of $\mathrm{V}$ III-V Heteroepitaxy.

PB95-202560

04,693 Not available NTIS

Epitaxial Integration of Single Crystal $\mathrm{C} 60$

PB95-153490 04,592 Not available NTIS 


\section{TITLE INDEX}

Epitaxial Nucleation and Growth of Chemically Derived Ba2YCu3O7-x Thin Films on (001) SrTiO3.

04,787 Not available NTIS

EPR Bone Dosimetry: A New Approach to Spectral Deconvolution Problems.

03,616 Not available NTIS

EPR Dosimetry of Cortical Bone and Tooth Enamel Irradiated with $X$ and Gamma Rays: Study of Energy Dependence.
$P B 97-110373$

03,639 Not available NTIS

Equation of State Formulation of the Thermodynamic Properties of R134a $(1,1,1,2$-Tetrafluoroethane).

PB94-212081 03,256 Not available NTIS

Equilibrium and Calorimetric Investigation of the Hydrolysis of L-Tryptophan to (Indole + Pyruvate + Ammonia)

PB95-163317 00,661 Not available NTIS

Equilibrium Pair Distribution Function of a Gas: Aspects Associated with the Presence of Bound States

01,004 Not available NTIS

Equipment for Investigation of Cryogenic Compaction of

Nanosize Silicon Nitride Powders. 1993 Report.
DE94013593
03,028 PC A03/MF A01

Error Analysis of the NIST Optically Pumped Primary Frequency Standard.
PB95-153482

01,535 Not available NTIS

Error-Bounding in Level-Index Computer Arithmetic

PB96-109582 01,742 PC A02/MF A01

Error Propagation Biases in the Calculation of Indentation Fracture Toughness for Ceramics.
PB94-172434

Error Propagation in Laser Beam Spatial Parameters.

PB95-180394 04,310 Not available NTIS

Error Protecting Characteristics of CDMA and Impacts on PB96-122452

01,491 Not available NTIS

Escherichis coli Cyclic AMP Receptor Protein Mutants Provide Evidence for Ligand Contacts Important in Activation.
PB96-201017 03,592 Not available NTIS

Escrowed Encryption Standard (EES); Category: Computer Security; Subcategory: Cryptography.
FIPS PUB 185

ESR-Based Analysis in Radiation Processing.

PB95-161634 03,937 Not available NTIS

Establishing Quality Measurements for Inorganic Analysis of Biomaterials.
PB94-199726

00,548 Not available NTIS

Estimate of Flame Radiance via a Single Location Measurement in Liquid Pool Flames.

02,476 Not available NTIS

Estimate of the Effect of Scale on Radiative Heat Loss Fraction and Combustion Efficiency.
PB95-150447
02,486 Not available NTIS

Estimates of Hurricane Wind Speeds by the 'Peaks Over Threshold' Method.

00,471 PC A04/MF A01

Estimating the Virial Coefficients of Small Polar Molecules.
PB95-176236 03,276 Not available NTiS

Estimation of Measurement Uncertainty of Small Circular Features Measured by CMMs.
PB95-267928

02,918 PC A03/MF A01

Estimation of System Damping at the Lotung Site by Application of System Identification

01,351 PC A10/MF A03

Estimation of the Absorbed Dose in Radiation-Processed Food. 1. Test of the EPR Response Function by a Linear Food. 1. Test of the EPR Response Function by a Linear
Regression Analysis.
PB94-199718

00,039 Not available NTIS

Estimation of the Absorbed Dose in Radiation-Processed Food. 2. Test of the EPR Response Function by an Exponential Fitting Analysis.

00,036 Not available NTIS

Estimation of the Absorbed Dose in Radiation-Processed Food. 3. The Effect of Time of Evaluation on the Accuracy of the Estimate.
PB94-199684

00,037 Not available NTIS

Estimation of the Absorbed Dose in Radiation-Processed

Food. 4. EPR Measurements on Eggshell.
PB94-199692 00,038 Not available NTIS

Estimation of the Heat Capacities of Organic Liquids as a Function of Temperature Using Group Additivity. I. Hydrocarbon Compounds.

00,739 Not available NTIS

Estimation of the Heat Capacities of Organic Liquids as a Function of Temperature Using Group Additivity. II. Com pounds of Carbon, Hydrogen, Halogens, Nitrogen, Oxygen, and Sulfur.

00,740 Not available NTIS
Estimation of the Orientation Distribution of Short-Fiber Composites Using Ultrasonic Velocities.

$\begin{array}{ll}\text { PB96-176656 03,178 Not available NTIS } & 0\end{array}$

Estimation of the Thermodynamic Properties of C-H-N-O-S Halogen Compounds at $298.15 \mathrm{~K}$

PB94-162328 O0,744 Not available NTIS

Evaluated Bimolecular Ion-Molecule Gas Phase Kinetics of Positive lons for Use in Modeling Planetary Atmospheres,

Cometary Comae, and Interstellar Clouds.
PB94-168598 00,753 Not available NTIS

Evaluating Form Designs for Optical Character Recognition.
PB94-168044 01,830 PC A06/MF A02

Evaluating Investments in Law Enforcement Equipment: An Annotated Bibliography.

PB95-151379

04,867 Not available NTIS

Evaluating Small Board and Care Homes: Sprinklered vs. Nonsprinklered Fire Protection.

PB94-206356

00,195 PC A04/MF A01

Evaluating the Seismic Performance of Lightly-Reinforced

Circular Concrete Bridge Columns.
PB95-163259 01,335 Not available NTIS

Evaluation and Accreditation of State Calibration Labora.

PB97-110183

00,486 Not available NTIS

Evaluation and Qualification Standards for an X-Ray Laminography System.

02,029 Not available NTIS

Evaluation and Retrofit Standards for Existing Federally Owned and Leased Buildings.

PB95-150918

00,434 Not available NTIS

Evaluation and Strengthening Guidelines for Federal Buildings: Assessment of Current Federal Agency Evaluation ings: Assessment of Current Federal Agency Evaluation

Ty94-181856

$00,425 \mathrm{PC} \mathrm{A08/MF} \mathrm{A02}$

Evaluation and Strengthening Guidelines for Federal Build-

ings: Identification of Current Federal Agency Programs.
PB94-176278
00,424 PC A08/MF A02 -176278

Evaluation of a Tapered Roller Bearing Spindle for HighPrecision Hard Turning Applications.
PB96-160494 Not available NTIS Evaluation of Alternative In-Flight Fire Suppressants for Evaluation of Alternative In-Flight Fire Suppressants for
Full-Scale Testing in Simulated Aircraft Engine Nacelles Full-Scale Testing in Simulated Aircraft Engine Nacelles
and Dry Bays. and Dry Bays.

00,023 PC A99/MF E08

Evaluation of Corrosion Data: A Review.

PBo

03,187 Not available NTIS

Evaluation of Crystallographic Data with the Program DIA. MOND.

PB97-109045

00,649

(Order as PB97-109011, PC A11/MF A03)

Evaluation of Fracture Toughness and Residual Stress in Dental Porcelain by Indentation-Microfracture Method.

PB95-125613

00,154 Not available NTIS

Evaluation of Fracture Toughness and Residual Stress in Dental Porcelain by Indentation-Microfracture Method

PB95-152831

00,159 Not available NTIS

Evaluation of GSA Maintenance Practices of Large Centrifugal Chillers and Review of GSA Refrigerant Management Practices.

02,502 PC A04/MF A01

PB94-143344

Evaluation of Methylene Lactone Monomers in Dental Resins.

00,164 Not available NTIS

Evaluation of Sprinkler Activation Prediction Methods.
PB96-141056 00,304 Not available NTIS

Evaluation of Survey Procedures for Determining Occupant Load Factors in Contemporary Office Buildings. Load Factors in Contemporary Office Buildings.
PB97-116222 00,238 PC A03/MF A01

Evaluation of the Economic Impacts Associated with the Evaluation of the Economic Impacts Associated with the
NIST Power and Energy Calibration Services.
PB95-188850 02,461 PC A03/MF A01

Evaluation of the Electrochemical Behavior of Ductile Nickel Aluminide and Nickel in a pH 7.9 Solution. Technical Report Number 3, April-June 1991

DE94017351

03,307 PC A02/MF A01

Evaluation of the Environmentally Induced Fracture Resistance of Ductile Nickel Aluminide. Technical Report Number 1. Final report. October-December 1990

DE94017331

03,306 PC A03/MF A01

Evaluation of Thermal Wave Imaging for Detection of Machining Damage in Ceramics.

03,062 PC A05/MF A02

Evaluation of Two-Photon Exchange Graphs for HighlyCharged Heliumlike lons.

03,808 Not available NTIS
Evaluation of Uncertainties of the Null-Balanced TotalPower Radiometer System NCS

PB94-169794

02,023 Not available NTIS

Evaluation of Wear Resistant Ceramic Valve Seats in GasFueled Power Generation Engines. Topical Report, December 1991-April 1994.

02,466 PC A07/MF A02

Even-Odd Asymmetry of a Superconductor Revealed by the Coulomb Blockade of Andreev Reflection.

PB95-153540

04,593 Not available NTIS

Evidence for a Deep Electron Trap and Charge Compensa-

tion in Separation by Implanted Oxygen Oxides.
PB95-175337 02,362 Not available NTIS

Evidence for Inelastic Processes for $\mathrm{N}(+) 3$ and $\mathrm{N}(+) 4$ from Ion Energy Distributions in He/N2 Radio Frequency Glow Discharges.
PB96-146683 04,059 Not available NTIS

Evidence for Parallel Junctions Within High-Tc GrainBoundary Junctions
PB95-175410

04,657 Not available NTIS

Evidence for Significant Backscattering in Near-Threshold Electron-Impact Excitation of $\operatorname{Ar}(7+)(3$ s yields $3 p)$.
PB95-126405

Evidence for Tunneling and Magnetic Scatttering at 'In situ' YBCO/Noble-Metal Interfaces.
PB96- 141098

04,752 Not available NTIS

Evidence of Crosslinking in Methyl Pendent PBZT Fiber.

PB97-112486 03,393 Not available NTIS

Evidence of Film-Induced Cleavage by Electrodeposited Rhodium.
PB95-162327

03,191 Not available NTIS

Evidence That Voltage Rather Than Resistance is Quantized in Breakdown of the Quantum Hall Effect.

PB96-179163

01,868

(Order as PB96-177381, PC A07/MF A02)

Evolution Equations for Phase Separation and Ordering in Binary Alloys.
PB96-119243

02,979 Not available NTIS

Evolution of a Turbulent Boundary Layer Induced by a Three-Dimensional Roughness Element.
PB94-212818

Evolution of a United States Information System.

Evolution of a United States Information System.
PB96-157896 Not

Evolution of Automatic Line Scale Measurement at the National Institute of Standards and Technology.

PB95-108809 02,897 Not available NTIS

Evolution of Porosity and Calcium Hydroxide in Laboratory Concretes Containing Silica Fume.
PB95-175063

Evolution of the Flight Telerobotic Servicer.

Evolution of the Flight Telerobotic Servicer.
PB94-216082 04,832 Not available NTIS

Evolution of the Pore Size Distribution in Final-Stage Sintering of Alumina Measured by Small-Angle X-ray Scattering. (Reannouncement with New Availability Information)

AD-A249 178/5

$03,023 \mathrm{PC} \mathrm{A03/MF} \mathrm{A01}$

Evolution of $X$-ray Resonance Raman Scattering into $X$-ray Fluorescence from the Excitation of Xenon Near the L3 Edge. 04,025 Not available NTIS

Exact Recursion Relation Solution for the Steady-State Sur-

face Temperature of a General Multilayer Structure.
PB96-102017 376 Not available NTIS

Exact Series Solution to the Epstein-Hubbell Generalized Elliptic Type Integral Using Complex Variable Residue ThePBy. 03,423 Not available NTIS

Exact Solution of the Steady-State Surface Temperature for a General Multilayer Structure.
PB95-152773 02,337 Not available NTIS Examination of Objects Made of Wood Using Air-Coupled Ultrasound.

PB95-125712

03,404 Not available NTIS

Examination of Parameters That Can Cause Error in Mass Determinations.
PB94-163037

03,784 PC A03/MF A01

Examination of the Correlation between Cone Calorimeter

Data and Full-Scale Furniture Mock-Up Fires.
PB96-148200

Examination of the $1 / d$ Expansion Method from Exact Enu-

meration for a Self-Interacting Self-Avoiding Walk.
PB95-175733
01,266 Not available NTIS

Examination Procedure Outlines: Keys to Solving the Handbook 44 Puzzle
PB97-110217

02,690 Not available NTIS

Exchange Coupling in Magnetic Heterostructures

PB95-150314 04,566 Not available NTIS 


\section{TITLE INDEX}

Excitation of Balmer Lines in Low-Current Discharges of Hydrogen and Deuterium.

03,893 Not available NTIS

Excitation Transfer in Barium by Collisions with Noble Gases.
PB96-200274

01,163 Not available NTIS

Excitons in Complex Quantum Nanostructures.

PB97-118343 01,184 Not available NTIS

Executive Order 12941. Seismic Safety of Existing Federally Owned or Leased Buildings: It's History, Content and Objectives.

00,465 Not available NTIS

Executive Summary: Proceedings of the Workshop on the Measurement of Transient Pressure and Temperature.
PB96-160841 NTIS Not available NTIS

Existence and Nonexistence Theorems of Finite Diameter Sequential Confidence Regions for Errors-in-Variables ModPB95-180352

03,441 Not available NTIS

Exits in Multistable Systems Excited by Coin-Toss SquareWave Dichotomous Noise: A Chaotic Dynamics Approach.
PB96-160650 Not, Not available NTIS

EXITT: A Simulation Model of Occupant Decisions and ACtions in Residential Fires.

tions in Resident

00,351 Not available NTIS

Expansion of Cementitious Materials Exposed to Sulfate PB94-185782

02,577 Not available NTIS

Experience with MPI: 'Converting Pvmmake to Mpimake under LAM' and 'MPI and Parallel Genetic Programming'.

Experimental and Abinitio Studies of Electronic Structures of the CCI3 Radical and Cation.

PB94-21213

00,826 Not available NTIS

Experimental and Numerical Buming Rates of Premixed Methane-Air Inhibited by Fluoromethanes.
PB95-180592

Experimental and Numerical Chaos in Continuous Systems: Two Case Studies.

00,219 Not available NTIS

Experimental and Numerical Studies of Refractory Particle Formation in Flames: Application to Silica Growth

PB95-152005 00,673 Not available NTIS

Experimental and Numerical Studies on Two-Dimensional Gravity Currents in a Horizontal Channel. PB94-165941

01,359 PC A12/MF A03

Experimental Aspects and Z-Dependent Systematics in One-and Two-Electron lons and Single Vacancy Systems.
PB94-199627

Experimental Aspects of Flux Expulsion in Type-II $\begin{array}{ll}\text { Superconductors. } & 04,660 \text { Not available NTIS }\end{array}$ Experimental assessment of absorbed dose to mineralized bone tissue from internal emitters: An electron paramagnetic resonance study.
DE 96007979

03,614 PC A02/MF A01

Experimental Assessment of Crack-Tip Dislocation Emission Models for an Al67Cr8Ti25 Intermetallic Alloy. PB96-204466 an Al677 Not available NTIS

Experimental Constraints on Some Mechanisms for High-

Temperature Superconductivity. 04,463 Not available NTIS
PB94-198553

Experimental Data and Theoretical Modeling of Gas Flows Through Metal Capillary Leaks. 04,206 Not available NTIS
PB95-150892 Experimental Determination of the Ionization Energy of IO(X(sup 2)\|(sub 3/2)) and Estimations of Delta(sub f) H(sup deg)(sub 0$)(10$ (sup -1$)$ ) and $P A(10)$

PB96-146899

00,694 Not available NTIS

Experimental Determination of the Rate Constant for the Reaction of $\mathrm{C}_{2} \mathrm{H}_{3}$ with $\mathrm{H} 2$ and Implications for the Partitioning of Hydrocarbons in Atmospheres of the Outer Planets.
PB97-122295 00,112 Not available NTIS

Experimental Evaluation of Specification Techniques for Improving Functional Testing

01,779 Not available NTIS

Experimental Investigation of the Validity of TDDB Voltage Acceleration Models.

02,304 Not available NTIS

Experimental Method for Obtaining Critical Densities of Binary Mixtures: Application to Ethane $+n$-Butane

PB95-151148 00,930 Not available NTIS

Experimental Models for Software Diagnosis.

PB97-113906 01,783 PC A04/MF A01

Experimental Optimization of Peak Shape with Application

to Aerosol Generation
PB94-185535

00,501 Not availabie NTIS
Experimental plan to determine the performance of the Oak Ridge National Laboratory Cold Neutron Moderator. Fina report, September 1, 1993--November 30, 1993. DE95011352 03,778 PC A03/MF A01

Experimental Results on Normal Modes in Cold, Pure Ion

Plasmas.
PB95-175105

03,956 Not available NTIS

Experimental Results on Single Flux Quantum Logic.

PB95-175071 02,053 Not available NTIS

Experimental Studies of Line Shapes from a Balle-Flygare Spectrometer.
PB94-199452

00,796 Not available NTIS

Experimental Study of Reverse-Bias Failure Mechanisms in Bipolar Mode JFET (BMFET)

PB95-152997

02,340 Not available NTIS

Experimental Study of the Stabilization Region of Lifted Turbulent-Jet Diffusion Flames.

PB96-122676

01,405 Not available NTIS

Experimental Vatidation of Radiopharmaceutical Absorbed Dose to Mineralized Bone Tissue.

PB94-199668

03,617 Not available NTIS

Experimental Verification of a Micromagnetic Model of DualLayer Magnetic Films.

04,553 Not available NTIS

Experimental Verification of a Moisture and Heat Transfer Model in the Hygroscopic Regime.

PB97-111546

00,309 Not available NTIS

Experimental Verification of a Vector Preisach Model

PB95-163564

04,626 Not available NTIS

Experimentally Measured Total X-ray Attenuation Coefficients Extracted from Previously Unprocessed Documents Held by the NIST Photon and Charged Particle Data CenPB97-114474

04,165 PC A04/MF A01

Expert Control System Shell Version 1.0 User's Guide. PB95-198859

01,790 PC A03/MF A01

Exploring the Low-Frequency Performance of Thermal Converters Using Circuit Models and a Digitally Synthesized Source.
PB97-112551 02,848 Not available NTIS

and Buckling in Coherently Exponentially Rapid Coarse PB95-202347

04,821 Not available NTIS

Exposure: An Expert System Fire Code

PB95-162913

04,868 Not available NTIS

Exposure-to-Absorbed-Dose Conversion for Human Adult Cortical Bone.

03,640 Not available NTIS

B97-110381

MA.

Expression Forn

01,735 PC A03/MF A01

Extended $\mathrm{CO}(7$ yields 6$)$ Emission from Warm Gas in Orion.
PB96-102504

00,090 Not available NTIS

Extending the Angular Range of Neutron Reflectivity Measurements from Planar Lipid Bilayers: Applications to a

Model Biological Membrane.
PB96-122569 PB96-122569

Extension of Heterodyne Frequency Measurements on OCS to $87 \mathrm{THz}(2900 \mathrm{~cm}(-1))$

to $87 \mathrm{THz}(2900$
$\mathrm{PB} 94-200680$

00,811 Not available NTIS

Extensions of the Prototype Application Protocol of Readyto-Wear Apparel Pattern Making.

B96-128194

03, 198 PC A03/MF A01

External Gamma-ray Counting of Selected Tissues from a Thorotrast Patient.

03,637 Not available NTIS

B96-160254

on $(90) \mathrm{Sr}+(90) \mathrm{Y}$

Extrapolation Chamber Measurements on

Beta-Particle

03,626 Not available NTIS

Extrapolation of the Heat Capacity in Liquid and Amorphous Phases

PB97-111421

04,147 Not available NTIS

Extreme Value Theory and Applications: Proceedings of the Conference on Extreme Value Theory and Applications, Vonference on Extreme 3 . Held in Gaithersburg, Maryland in May 1993.
PB95-104956
03,432 PC A1 1/MF A03

Extreme Wind Distribution Tails: A 'Peaks Over Threshold' Approach.

00,127 PC A05/MF A01

Extreme Wind Estimates by the Conditional Mean Exceedance Procedure.

00,120 PC A03/MF A01

PB95-220471

OD, 120 PC AOBMF AO

Extreme Winds Estir

PB96-159686

00,468 Not available NTIS
Fabrication Issues for the Prototype National Institute of Standards and Technology SRM 2090A Scanning Electron Microscope Magnification Calibration Standard.

PB96-160585 01,131 Not available NTIS

Fabrication Issues in Optimizing YBa2Cu307-x Flux Transformers for Low l/f Noise.

PB95-175857

02,059 Not available NTIS

Fabrication of Flaw-Tolerant Aluminum-Titanate-Reinforced Alumina

PB95-162533

03,161 Not available NTIS

Fabrication of Optics by Diamond Turning

PB96-111695 02,954 Not available NTIS

Fabrication of Platinum-Gold Alloys in Pre-Hispanic South America: Issues of Temperature and Microstructure Control PB94-211646 03,333 Not available NTIS

Fabrication of Transparent gamma-Al2O3 from Nanosize Particles.
PB95-175493

03,054 Not available NTIS

Face Recognition Technology for Law Enforcement Applications.
PB94-207768

01,837 PC A05/MF A01

Facile Synthesis of Novel Fluorinated Multifunctiona Acrylates.
PB94-198389

01,207 Not available NTIS

Factorial Design Techniques Applied to Optimization of AMS Graphite Target Preparation.
PB95-151197 00,584 Not available NTIS

Factors Affecting the Energy Consumption of Two Refrigerator-Freezers

PB97-112312

00,311 Not available NTIS

Factors Significant to Precracking of Fracture Specimens.
PB96-109558
03,358 PC A06/MF AO

PB96-109558 03,358 PC A06/MF AO2

Failure of All-Ceramic Fixed Partial Dentures 'In vitro' and 'In vivo': Analysis and Modeling. 00,175 Not available NTIS

Failures of the Four-Wave Mixing Model for Cone Emission. PB95-202636 04,318 Not available NTIS

Far-Infrared Kinetic Inductance Detector

PB95-126348 02,142 Not available NTIS

Far Infrared Laser Frequencies of $\mathrm{CH} 3 \mathrm{OD}$ and N2H4

PB96-119623 04,341 Not available NTIS

Far Infrared Laser Frequencies of (13) $\mathrm{CD} 3 \mathrm{OH}$.

PB95-169363 04,292 Not available NTIS

Far-Ultraviolet Flare on a Pleiades G Dwart

PB96-102033 00,086 Not available NTIS

Faraday Constant.

PB96-159793

01,955 Not available NTIS

Faraday Effect Current Sensor with Improved SensitivityBandwidth Product.

PB95-203154

02,180 Not available NTIS

Faraday Effect Current Sensors.

PB94-200698 02,127 Not available NTIS

Faraday Effect Sensors: A Review of Recent Progress

PB94-200706

02,128 Not available NTIS

Faraday Effect Sensors for Magnet Field and Electric CurPB96-119664

04,736 Not available NTIS

Fast Computer Evaluation of Radiative Properties of Hydrogenic Systems.

03,894 Not available NTIS

Fast-lon Conducting Y2(ZryTi1-y)2O7 Pyrochlores: Neutron Rietveld Analysis of Disorder Induced by Zr Substitution.

Fast-Ion Conduction and Disorder in Cation and Anion Arrays in Y2(ZryTi(1-y))2O7 Pyrochlores Induced by $\mathrm{Zr}$ Substitution: A Neutron Rietveld Analysis.
PB94-211869 04,496 Not available NTIS

Faster BKL Monte Carlo Simulations.

PB95-136370

01,706 PC A03/MF A01

Faster Monte Carlo Simulations.

PB96-102074

04,018 Not available NTIS

Fatigue Crack Thresholds of a Nickel-Iron Alloy for PB96-190343

03,223 Not available NTIS

Fault Diagnosis of an Air-Handling Unit Using Artificial Neural Networks

00,283 Not available NTIS

Feasibility and Design Considerations of Emergency Evacuation by Elevators.

00,287 PC A07/MF AO2

Feasibility of Fire Evacuation by Elevators at FAA Control Towers.
PB94-213857

$04,844 \mathrm{PC} \mathrm{AO6/MF} \mathrm{AO2}$ 


\section{TITLE INDEX}

Feasibility of Fluorescence Detection of Tetracycline in Media Mixtures Employing a Fiber Optic Probe.

PB96-163654

00,511 Not available NTIS

Feasibility Study: Reference Architecture for Machine Conrol Systems Integration.

02,804 PC A12/MF A03 Federal Basis lor Weights and Measures: A Historical Re-
view of Federal Legislative Effort, Statutes, and Administraview of Federal Legislative Effort, Statutes, and AdministraUnited States. United States.

02,616 PC A03/MF A01

Federal Building Grounding and Bonding Requirements for Telecommunications. Category: Telecommunications Stand ard; Subcategory: Grounding and Bondirig.

01,802 PC E18

Federal Certification Authority Liability and Policy: Law and Policy of Certificate-Based Public Key and Digital Signatures.

$01,578 \mathrm{PC} \mathrm{A21/MF} \mathrm{A04}$

Federal Government and Information Technology Standards: Building the National Information Intrastructure. PB95-180840 01,812 Not available NTIS

Federal Implementaion Guideline for Eletronic Data Interchange. ASC X12 003050 Transaction Set 865 Purchase Order Change Acknowledgement/Request - Seller Initiated. implementation Convention.

$01,825 \mathrm{PC} \mathrm{A09/MF} \mathrm{A02}$

Federal Implementation Guideline for Electronic Data Interchange: ASC X12 003040 Transaction Set 838 Trading Partner Profile (Confirmation of Vendor Registration). Implementation Convention.

PB96-111190

$01,813 \mathrm{PC}$ A03/MF A01

Federal Implementation Guideline for Electronic Data Interchange: ASC X12 003040 Transaction Set 838 Trading Partner Profile (Vendor Registration). Implementation Convention

PB96-112651

$03,674 \mathrm{PC} \mathrm{A04/MF} \mathrm{A01}$

Federal Implementation Guideline for Electronic Data Interchange: ASC $\times 12003050$ Transaction Set 836 Procurement Notices. Implementation Convention. PB96-178892

01,827 PC A04/MF AO

Federal Implementation Guideline for Electronic Data Interchange. ASC X12 003050 Transaction Set 840 Request for Quotation. Implementation Convention. PB96-172531

$01.824 \mathrm{PC}$ A $10 / \mathrm{MF}$ A03

Federal Implementation Guideline for Electronic Data Interchange. ASC X12 003050 Transaction Set 843 Response to Request for Quotation. Implementation Convention. to Request for Quotation. Implementation Convention.
PB96-168984 01,822 PC A09/MF A02

Federal Implementation Guideline for Electronic Data Interchange. ASC $\times 12003050$ Transaction Set 850 Award Instrument. Implementation Convention. Strument. Implementation Convention.
PB96-114913 PC A11/MF A03

Federal Implementation Guideline for Electronic Data Interchange. ASC X12 003050 Transaction Set 855 Purchase Order Acknowledgment: Implemen

PB96-172374 01,823 PC A04/MF A01

Federal Implementation Guideline for Electronic Data Interchange. ASC X12 003050 Transaction Set 860 Modifications to Award Instrument. Implementation Convention

PB96-114921 01,815 PC A11/MF A03

Federal Labs Have Key Role in Metrication.

PB96-123401 02,920 Not available NTIS

Federal Metric Progress in 1993.

PB94-196029

02,600 PC A03/MF A01

Femtosecond Time-Resolved Molecular Multiphoton Ionization: The Na2 System

PB95-202305

03,975 Not available NTIS

Femtosecond Time-Resolved Wave Packet Motion in Mo-

lecular Multiphoton Ionization and Fragmentation.
PB94-198611 00,790 Not available NTIS

Ferric Ion Assisted Photooxidation of Halocetates.

PB97-112460 00,521 Not available NTIS

Ferroelectric Thin Film Characterization Using Superconducting Microstrip Resonators.
PB96-102389 02,270 Not available NTIS

Few Caveats on Carbon Dioxide Monitoring

PB96-122650 02,562 Not available NTIS
Norbon Dioxide Monitoring.

Fiber Coating Diameter: Toward a Glass Artifact Standard.

PB96-140389 Diameter: Toward a Glass Artifact Standard.

Fiber-Optic Faraday-Effect Magnetic-Field Sensor Based on Flux Concentrators.

02,201 Not available NTIS

Fiber Spot Size: A Simple Method of Calculation

PB95-125936 04,250 Not available NTIS

Fibre Splice Loss: A Simple Method of Calculation.

PB95-175519 04,299 Not available NTIS
Field and Temperature Acceleration of Time-Dependent Dielectric Breakdown in Intrinsic Thin $\mathrm{SiO} 2$ PB94-185956

02,305 Not available NTIS

Field Dependence of the Magnetic Ordering of $\mathrm{Cu}$ in $\mathrm{R} 2 \mathrm{CuO} 4(\mathrm{R}=\mathrm{Nd}, \mathrm{Sm})$

04,633 Not available NTIS

Field Evaluation of the System for Calibration of the Marshall Compaction Hammer.

01,323 PC A05/MF A01

PB95-190674

ion and Ventilation Effectiveness in an Office/Library Building.

PB95-108833

00,247 Not available NTIS

Field Modeling: Simulating the Effect of Sloped Beamed Ceilings on Detector and Sprinkler Response.

PB96-122866

01,406 Not available NTIS

Filter Transmite

(Order as PB94-140555, PC A06/MF A02)

Financing Tomorrow's Infrastructure: Challenges and Issues. Proceedings of a Colloquium. Held in Washington DC. on October 20, 1995.

00,481 PC A07/MF A02

Findings and Recommendations from a Software Reengineering Case Study

PB96-155791

01,752 Not available NTIS

Fine-Structure Constant.

PB94-172996

03,795 Not available NTIS

Fine-Structure Intervals of $(14) \mathrm{N}(+)$ By Far-Infrared Laser Magnetic Resonance.

00,993 Not available NTIS

Fine Structure of Negative lons of Alkaline-Earth-Metal Atoms.
PB94-211182

03,837 Not available NTIS

FIPS PUB 8-6, Metropolitan Areas (for Microcomputers)

PB95-503280 04,874 Diskette $\$ 59.00$

Fire and Smoke Control: An Historical Perspective.

PB95-175808 00,202 Not available NTIS

Fire Codes for Global Practice.

PB96-102108

00,205 Not available NTIS

Fire Data Management System, FDMS 2.0, Technical Documentation.
PB94-164019

$01,358 \mathrm{PC}$ A05/MF A01

Fire Growth Analysis of the Fire of March 20, 1990, Pulask Building, 20 Massachusetts Avenue, N.W., Washington, PB.

00, $194 \mathrm{PC}$ A03/MF A01

Fire Growth Models for Materials.

PB94-195856

$01,367 \mathrm{PC} \mathrm{A03/MF} \mathrm{A01}$

Fire Hazard and Risk: Evaluating Alternative Technologies.
PB94-173077

Fire Hazard Model Developments and Research Efforts at NIST

PB96-159652

00,407 Not available NTIS

Fire Induced Thermal Fields in Window Glass I: Theory.

PB94-139722

00,328 PC AO3/MF A01

Fire Performance of an Interstitial Space Construction System.

00,390 PC A04/MF A01

Fire-Plume-Generated Ceiling Jet Characteristics and Convective Heat Transfer to Ceiling and Wall Surfaces in a Two-Layer Fire Environment: Uniform Temperature Ceiling and Walls.

PB95-164711

00,382 Not available NTIS

Fire Propagation in Concurrent Flows.

PB94-193844

$01,365 \mathrm{PC} \mathrm{A04/MF} \mathrm{A01}$

Fire Protection Engineering Tools. Simple Tools: The EquaPB96-156179

00,221 Not available NTIS Fire Protection Foam Behavior in a Radiative Environment.
PB97-116131. 00,237 PC A10/MF A02

Fire Safety Engineering in the Pursuit of PerformanceBased Codes: Collected Papers. 00,235 PC A07/MF A02
PB97-114482

Fire Safety Engineering Research in the United States. PB96-151394

00,213 Not available NTIS

Fire Safety of Passenger Trains: A Review of Current Approaches and of New Concepts.

$04,848 \mathrm{PC} \mathrm{A11/MF} \mathrm{A03}$

Fire Service and Fire Sciences: A Winning Combination PB95-150264

01,383 Not available NTIS

Fire Suppression System Performance of Alternative Agents in Aircraft Engine and Dry Bay Laboratory Simulations. SP890: Volume 1.

PB96-117775

03,277 PC A99/MF E08

Fire Suppression System Performance of Alternative Agents in Aircraft Engine and Dry Bay Laboratory Simulations. SP 890: Volume 2 .

890: Volume

03.278 PC A99/MF A06

FIREDOC Users Manual, 3rd Edition

PB95-128674

01,378 PC A03/MF A01

First Phase Formation Kinetics in the Reaction of Nb/Al. PB95-168456 03,353 Not available NTIS

First Results from a Coordinated ROSAT, IUE, and VLA Study of RS CVn Systems.

00,069 Not available NTIS

First Results from the Goddard High-Resolution Spectrograph: The Chromosphere of Taur 00.054 Not available NTIS

First VAMAS USA Interlaboratory Comparison of High Temperature Superconductor Critical Current Measurements. PB96-147178 04,768 Not available NTIS

Five Q's (Cues) of the U.S. GOSIP Testing Program.

PB96-175716 01,826 Not available NTIS

Flame Heights and Heat Release Rates of 1991 Kuwait Oil Field Fires.

PB96-119342

01,404 Not available NTIS

Flame Retardants - Overview.

PB94-185287

01,363 Not available NTIS

Flame Synthesis of High TC Superconductors.

PB95-151981

00,659 Not available NTIS

Flammability Characterization with the Lift Apparatus and the Cone Calorimeter.

01,435 Not available NTIS

Flat and Curved Crystal Spectrography for Mammographic X-ray Sources.

03,642 Not available NTIS

Flat and Rising R-Curves for Elliptical Surface Cracks from Indentation and Superposed Flexure.

PB95-161295

03,156 Not available NTIS

Flaw-Insensitive Ceramics

PB97-110027

03,095 Not available NTIS

Flaw-Tolerance and Crack-Resistance Properties of Alumina-Aluminum Titanate Composites with Tailored Microstructures.

03,101 Not available NTIS PB97-110324 Flaw Tolerance and Toughness Curves in Two-Phase Particulate Composites: SiC/Glass System.
PB96-179460 Not available NTIS

Flexible-Diaphragm Force Microscope

PB95-180915 03,966 Not available NTIS

Flow Conditioner Tests for Three Orifice Flowmeter Sizes. PB95-105540

Orifice Flowmeter Sizes.
04,201 PC A05/MF A01

Flow Immunoassay Using Solid-Phase Entrapment

PB96-200951 00,642 Not available NTIS

Flow-Induced Structure in Polymer. Chapter 1. An Introducion to Flow-Induced Structurner. Chapter 1.

in Polymers.

Flow-Induced Structure in Polymers: Chapter 16. Shear-Induced Changes in the Order-Disorder Transition Temperature and the Morphology of a Triblock Copolymer.

PB96-123237 03,127 Not available NTIS
0

Flow-Induced Structure in Polymers. Chapter 17. PhaseSeparation Kinetics of a Polymer Blend Solution Studied by a Two-Step Shear Quench.

PB96-123377

03,388 Not available NTIS

Flow of Alternative Agents in Piping

PB95-202420

00,022 Not available NTIS

Flow of Microemulsions through Microscopic Pores.

PB95-140463 00,905 Not available NTIS

Flowmeter Installation Effects Due to a Generic Header.

$\begin{array}{ll}\text { PB96-210893 } & 02,606 \text { PC A07/MF A02 }\end{array}$

Fluctuation Dominated Recombination Kinetics with Traps.

PB95-107264 00,875 Not available NTIS

Fluctuations in Probability Distribution on Chaotic Attractors. PB96-102330 04,022 Not available NTIS

Fluorescence Anisotropy Measurements on a Polymer Melt as a Function of Applied Shear Stress.

PB94-199296 Applied Shear Stress. Not available NTIS

Fluorescence Measurements of Tetracycline in High Cell Mass for Fermentation Monitoring.

PB95-175709

00.601 Not available NTIS

Fluorescence Monitoring of Polarity Change and Gelation during Epoxy Cure.

01,204 Not available NTIS

Fluoride Analytical Methods.

PB96-180237 


\section{TITLE INDEX}

Fluoride Elimination Upon Reaction of Pentafluoroaniline with $e$ (sub eq')(sup -), H, and OH Radicals in Aqueous Solution.

01,177 Not available NTIS Fluorinated Hydrocarbon Flame Suppression Chemistry. PB94-185113 01,362 Not available NTIS Flux Expulsion at Intermediate Fields in Type-ll $\begin{array}{ll}\text { Superconductors. } & 04,502 \text { Not available NTIS } \\ \text { PB94-212230 }\end{array}$ Flux-Locked Current Source Reference. PB95-150785 02,039 Not available NTIS Foias-Temam Approximations of Attractors for Galloping PBcillators.

04,817 PC A01

Fokker-Planck Description of Multivalent Interactions.
PB95-108478 00,879 Not available NTIS

Food Irradiation Dosimetry.

C0,879 Not available NTIS

PB95-180675

00,041 Not available NTIS

Force Calibrations in the Nanonewton Regime.

PB95-168696 03,949 Not available NTIS

Formal Methods in Conformance Testing: Result and Perspectives.

01,710 Not available NTIS Formal Multi-Layer Test Methodology and Its Application to

PB94-172194

02,718 Not available NTIS

Formal Specification and Verification of Control Software for Cryptographic Equipment.

01,585 Not available NTIS

Formalism and Parameters for Quantitative Surface Analysis by Auger Electron Spectroscopy and $X$-Ray Photoelectron Spectroscopy.

Formation of DNA-Protein Cross-Links in Cultured Mamma-

lian Cells Upon Treatment with Iron lons.
PB96-137724 03,651 Not available NTIS

Formation of Hydroxyapatite in a Polymeric Calcium Phosphate Cement.

00,173 Not available NTIS

Formation of Hydroxyapatite in Cement Systems.

PB95-175261 00,170 Not available NTIS

Formation of Technical Committee 201 on Surface Chemical Analysis by the International Organization for Standardization.

00,568 Not available NTIS

Formulation of Position on U.S. Standards Role in Enterprise Integration

$02,773 \mathrm{PC} \mathrm{A03/MF} \mathrm{A01}$

Formulation of the Refractive Index of Water and Steam

PB95-140133 00,900 Not available NTIS

FORTRAN Compiler Validation System, Version 2.1

PB94-500691 01,698 CP T99

Forum for International Cooperation on Fire Research

PB95-162939 04,869 Not available NTIS

Forward Scaftering of a Gaussian Beam by a Nonabsorbing Sphere.
PB97-112288

04,395 Not available NTIS

Four Years of Monitoring alpha Orionis with the VLA: Where Have All the Flares Gone.
PB94-185212 00,048 Not available NTIS Fourier Transform Atomic Emission Studies Using a Glow Discharge as the Emission Source.
PB94-185980 00,533 Not available NTIS FPETOOL: Fire Protection Tools for Hazard Estimation. An Overview of Features.

00,367 Not available NTIS

Fracture Behavior of Large-Scale Thin-Sheet Aluminum Alloy.

03,311

(Order as N95-19468/4, PC A24/MF A04)

Fracture in Multilayers.

PB96-163613

02,988 Not available NTIS

Fracture Mechanics Analysis of Near-Surface Cracks.

PB94-172400 03,230 Not available NTIS

Fracture Mechanism Maps: Their Applicability to Silicon Nitride.
PB96-204532

03,094 Not available NTIS

Fracture of Silicon Nitride and Silicon Carbide at Elevated Temperatures.
PB96-180260

03,179 Not available NTIS

Fracture Testing of Large-Scale Thin-Sheet Aluminum Alloy. AD-A306 625/5 03,305 PC A04/MF A01

Fracture Testing of Large-Scale Thin-Sheet Aluminum Alloy. PB95-242368 00,024 PC A03/MF A01
Fracture Toughness of Advanced Ceramics at Room Temperature: A vamas Round-Robin

03,160 Not available NTIS

Fragment Energy and Vector Correlations in the OvertonePumped Dissociation of HN3X(1)A

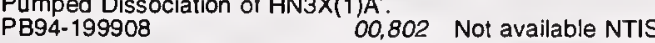

Fragment State Correlations in the Dissociation of NO.HF $(V=1)$.
PB95-164430

00,982 Not available NTIS

Framework for Information Technology Integration in Process Plant and Related Industries.

PB94-219086

02,772 PC A04/MF A01 Framework for National Information Infrastructure Services.
PB95-103719 02,723 PC A07/MF A02

Framework for the Development and Assurance of High Integrity Software.
PB95-173084

01,716 PC A05/MF A01

Free Radical Chemistry of the Atmospheric Aqueous Phase.
PB96-148101 00,117 Not available NTIS

Frequency Dependence of the Emission from 2D Array Josephson Oscillators.
PB95-175147

02,056 Not available NTIS

Frequency Extension of the NIST AC-DC Difference Calibration Service for Current.

PB95-161253

01,895 Not available NTIS

Frequency Shifting of Pulsed Narrow-Band Laser Light in a Multipass Raman Cell.

PB95-203352

04,321 Not available NTIS

Frequency Stabilization of a Fiber Laser to Rubidium: A High-Accuracy $1.53 \mathrm{mu} \mathrm{m}$ Wavelength Standard.

PB95-126082 04,252 Not available NTIS

Frequency-Stabilized Lasers: A Driving Force for New Spectroscopies.
PB96-135199

04,350 Not available NTIS

Frequency Stabilized Lasers: A Parochial Review.

PB95-153771 04,269 Not available NTIS

Frequency-Stabilized LNA Laser at 1.083 mum: Application

to the Manipulation of Helium 4 Atoms.
PB95-176186 04,304 Not available NTIS

Frequency Synthesis and Metrology at 10(-17) and Beyond.
PB97-113187 02,101 Not available NTIS

Fresh Look at Strategies for Fire Safety.

PB95-162947 04,870 Not available NTIS

Friction and Oxidative Wear of $440 \mathrm{C}$ Ball Bearing Steels Under High Load and Extreme Bulk Temperatures.
PB95-175253

Friction Processes in Brittle Fracture.

PB96-161765

03,076 Not available NTIS

From Superconductivity to Supernovae: The Ginzburg Symposium. Report on the Symposium Held in Honor of Vitaly L. Ginzburg. Held in Gaithersburg, Maryland on May 22

04,649 PC A05/MF A01

Frozen Human Serum Reference Material for Standardization of Sodium and Potassium Measurements in Serum or Plasma by lon-Selective Electrode Analyzers.

00,532 Not available NTIS

Frozen Orbits for Satellites Close to an Earth-Like Planet.

PB96-102165 04,839 Not available NTIS

FTS Infrared Measurements of the Rotational and Vibra-

tional Spectrum of $\mathrm{LiH}$ and $\mathrm{LiD}$.
PB94-216231

00,856 Not available NTIS

Fugacity Coefficients of Hydrogen in (Hydrogen + Butane). 02,491 Not available NTIS

Full-Scale Room Fire Experiments Conducted at the University of Maryland

00,236 PC A04/MF A01

Full Scale Smoke Control Tests at the Plaza Hotel Building PB94-212859 00,347 Not available NTIS

Function of DnaJ and DnaK as Chaperones in Origin-Specific DNA Binding by RepA. $\quad 03,533$ Not available NTIS
PB95-151544

B95-151544 03,533 Not availabe

Functional Security Criteria for Distributed Systems.
PB96-123492 01,607 Not available NTIS

Functions of Technology Infrastructure in a Competitive Economy.

00,478 Not available NTIS

Fundamental Computer Simulation Models for CementBased Materials.

PB95-126009

00,364 Not available NTIS

Fundamental Limits on the Frequency Stabilities of Crystal Oscillators.
PB96-176565

02,277 Not available NTIS
Fundamental Mechanisms for $\mathrm{CO}$ and Soot Formation. PB95-143160

$01,380 \mathrm{PC}$ A08/MF A02

Fundamental Processes in Gas Discharges.

PB96-123450 01,089 Not available NTIS

Fundamental Studies of Gas Sensor Response Mechanisms: Palladium on $\mathrm{SnO} 2(110)$

PB95-162731

00,963 Not available NTIS

Fundamental Torsion Band in Acetaldehyde.

00,840 Not available NTIS

Fundamentals and Problems of Fiber Current Sensors.

PB97-111835 02,205 Not available NTIS

Fundamentals of Fracture: A 1993 Prologue, and Othe Comments.

PB96-16197

03,218 Not available NTIS

Further Calculations of $X$-ray Diffusion in an Infinite Me-

AD-A295 314/9

$03,772 \mathrm{PC} \mathrm{A01/MF} \mathrm{A01}$

Further Development of the N-Gas Mathematical Model: An Approach for Predicting the Toxic Potency of Complex Combustion Mixtures. PB96-123260

03,650 Not available NTIS

FUSE: The Far Ultraviolet Spectrograph Explore

PB94-213410 00,063 Not available NTIS

Future of Time and Frequency Dissemination.

AD-P009 138/9 01,520 PC A

Future of Time and Frequency Dissemination. N94-30684/2

(Order as N94-30639/6, PC A25/MF A06)

Fuzed-Quartz Fibers. A Survey of Properties, Applications and Production Methods.

$00,656 \mathrm{PC} \mathrm{A03/MF} \mathrm{A01}$

G203.2-12.3: A New Optical Supernova Remnant in Orion. PB95-203535

00,085 Not available NTIS

Gage Block Handbook

PB95-251716

$02,667 \mathrm{PC} \mathrm{A08/MF} \mathrm{A02}$

Gage Block Standards, Measurement Capabilities and Laboratory Accreditation.

PB96-163621

02,757 Not available NTIS

Gas Absorption during Ion-Irradiation of a Polymer Target. PB96-161864

04,099 Not available NTIS

Gas-Coupled, Pulse-Echo Ultrasonic Crack Detection and Thickness Gaging

04,847 Not available NTIS

Gas Phase Oxygen Effect on Chain Scission and Monomer Content in Bulk Poly(methyl methacrylate) Degraded by External Thermal Radiation.

PB96-204078

01,293 Not available NTIS

Gas Phase Reactions Relevant to Chemical Vapor Deposition: Numerical Modeling.

PB94-199346

03,117 Not available NTIS

Gas Phase Reactions Relevant to Chemical Vapor Deposition: Optical Diagnostics.

PB94-199338

03,116 Not available NTIS

Gas Phase Reactivity Study of $\mathrm{OH}$ Radicals with 1,1Dichloroethene and cis-1,1-Dichloroethene and Trans-1,2 Dichloroethene over the Temperature Range 240-400 K. PB95-152146

00,939 Not available NTIS

Gas Transport Properties of Solution-Cast Perfluorosulfonic

Acid lonomer Films Containing lonic Surfactants.
PB95-175998 01,267 Not available NTIS

Gaseous Dielectrics Research: Possible SF6 Substitutes. PB96-119268

02,228 Not available NTIS

Gaseous Electronics Conference Radio-Frequency Reference Cell: A Defined Parallel-Plate Radio-Frequency System for Experimental and Theoretical Studies of Plasma Processing Discharges.

PB94-172327

04,404 Not available NTIS

Gaseous Electronics Conference RF Reference Cell: An Introduction.

PB96-113329

02,387

(Order as PB96-113311, PC A09/MF A03)

GATT Standards Code Activities of the National Institute of Standards and Technology 1994

PB96-106935

$00,497 \mathrm{PC} \mathrm{A03/MF} \mathrm{A01}$

General Motion Model and Spatio-Temporal Filters for Computing Optical Flow.

$01,847 \mathrm{PC} \mathrm{A03/MF} \mathrm{A01}$

General Motion Model and Spatio-Temporal Filters for 3-D Motion Interpretations.

PB96-210703

$01,861 \mathrm{PC} \mathrm{A07/MF} \mathrm{A02}$

General Order ' $N$ ' Analytic Correction of Probe-Position Errors in Planar Near-Field Measurements.

PB96-200688 


\section{TITLE INDEX}

General Procedures for Registering Computer Security Obects.

$01,573 \mathrm{PC} \mathrm{A03/MF} \mathrm{A01}$

General Types of Information Services.

PB96-147053

02,735 Not available NTIS

Generalized Form Registration Using Structure-Based Techniques.

01,858 PC A03/MF A01

Generalized Optical Theorem for On-Axis Gaussian Beams. PB97-122345

04,177 Not available NTIS

Generalized Plane Strain Analysis of a Bimaterial Composite Containing a Free Surface Normal to the Interface.
PB95-163341 03,164 Not available NTIS

Generalized Stokes-Einstein Equation for Spherical Particle Suspensions.

01,031 Not available NTIS

Generating and Measuring Displacements Up to $0.1 \mathrm{~m}$ to an Accuracy of $0.1 \mathrm{~nm}$ : Is it Possible.

PB96-160700 04,090 Not available NTIS

Generation and Characterization of Acetylene Smokes.

PB94-200292 01,372 Not available NTIS

Generation Rate and Distribution of Products of Combustion in Two-Layer Fire Environments: A Model and Applications.
PB96-102173 01,398 Not available NTIS PB96-102173

Generic Manufacturing Controllers

PB94-199940

02,818 Not available NTIS

Generic Model for Creep Rupture Lifetime Estimation on Fibrous Ceramic Composites.

PB94-200235

03,137 Not available NTIS

Genetically Engineered Pore as a Metal Ion Biosensor.

PB96.161658 03,551 Not available NTIS

Genetically Engineered Pores as Metal Ion Biosensors.

PB96-167408 03,553 Not available NTIS

Genetically Engineered Pores for New Materials.

PB96-161641 03,550 Not available NTIS

Geographic Information Systems Standards: A Federal PerPBective.

03,678 Not available NTIS

Geometric Characterization of Rockwell Diamond Indenters. PB95-203287 02,950 Not available NTIS

Geometrical Percolation Threshold of Overlapping Ellipsoids.

PB96-102397

03,167 Not available NTIS

Germanium Detector Optimization of MDA for Efficiency vs Low Intrinsic Background.

PB94-199155

00,543 Not available NTIS

Getting Started on Mosaic

PB95-180360

01,721 Not available NTIS

GHRS Observations of Cool, Low-Gravity Stars. 1. The FarUltraviolet Spectrum of alpha Orions (M2 lab)

PB96-112016

00,094 Not available NTIS

Glass Temperature of Polymer Blends: Comparison of Both the Free Volume and the Entropy Predictions with Data.

Glass Transition of Organic Liquids Confined to Small Pores.

PB94-212305

00,833 Not available NTIS

Glasses for Waveguide Lasers.

PB96-111950

04,335 Not available NTIS

Glimpse of Materials Research in China: A Report from an Interagency Study Team on Materials Visiting China from June 19, 1995 to June 30,1995

PB96-112677

02,978 PC A11/MF A03

Global Climatic Effects of Aerosols: The AAAR Symposium.
PB95-108791

Global Density Effects on the Self-Preservation Behavior of Turbulent Free Jets.

PB95-162301

04,207 Not available NTIS

Global Equivalence Ratio Concept and the Formation Mechanisms of Carbon Monoxide in Enclosure Fires. Mechanisms of Carbon Monoxide in Enclosure Fires.
PB96-146790 NTIS

Global Equivalence Ratio Concept and the Prediction of Carbon Monoxide Formation in Enclosure Fires.
PB94-207511 00,313 PC A08/MF A02 Global Information Infrastructure: Agenda for Cooperation. PB95-178604 01,482 PC\$9.00

Global Thermodynamic Behavior of Fluid Mixtures in the Critical Region.
PB94-212420

04,199 Not available NTIS

Glossary of Software Reuse Terms.

PB95-178992 01,720 PC A03/MF A01
Glucose Permease of Bacillus Subtilis is a Single Polypeptide Chain That Functions to Energize the Sucrose Permease.

PB95-163192

03,466 Not available NTIS

Goddard High Resolution Spectrograph: Instrument, Goals, and Science Results.

00,044 Not available NTIS

PB96-123278 Goddard High-Resolution Spectrograph Observations of the Ratio along the Line of Sight Toward Capella.

PB94-213444

00,066 Not available NTIS

Good Security Practices for Electronic Commerce, Including Electronic Data Interchange.

$01,463 \mathrm{PC} \mathrm{A04/MF} \mathrm{A01}$

PB94-139045

Gordon Research Conference on the Physics and Chem-
istry of Laser Diagnostics in Combustion Held in Plymouth, istry of Laser Diagnostics in Combusti

New Hampshire

01,353 PC A03/MF A01

GOSIP Testing Program

PB96-161229

01,504 Not available NTIS

Graded and Nongraded Regenerator Performance.

PB95-169090 02,753 Not available NTIS

Gradiometer Antennas for Detection of Long Subsurface Conductors.
PB95-175667

01,862 Not available NTIS

Grafted Interpenetrating Polymer Networks

PB94-185055 01,200 Not available NTIS

Grain Alignment and Transport Properties Bi2Sr2CaCu2O8 Grown by Laser Heated Float Zone MethPB95-161451

04,602 Not available NTIS

Graphical Analysis of the CCRL Portland Cement Proficiency Sample Database (Samples 1-72). (Part 1. PB94-196557

01,308 PC A06/MF A02

Graphical Conceptual Navigation as a Presentation Technique for a Graphics Standard.

PB94-200573

01,692 Not available NTIS

Graphical Kemel System (GKS). Category: Software Standard. Subcategory: Graphics. Intemational Standard: Information Technology; Computer Graphics; Graphical Kernel System (GKS) Language Bindings. Part 4: C. Gravitational Sisyphus Cooling of (87)Rb in a Magnetic Trap.
PB96-200704 04,379 Not available NTIS

\section{Gravity-Current Transport in Building Fires.}

Gravity-Current Transport in Building Fires.
PB96-147046

Gravity Dependent Processes and Intracellular Motion.

03,538 Not ava

Grazing Angle X-Ray Photoemission System for Depth-De-

pendent Analysis.
PB95-161154

04,600 Not available NTIS

Grazing Incidence Prompt Gamma Emissions and Resonance-Enhanced Neutron Standing Waves in a Thin-Film. $\begin{array}{ll}\text { PB95-150470 03,892 Not available NTIS } & \end{array}$

Grazing-Incidence X-Ray Photoelectron Spectroscopy: A

Novel Approach to Thin Film Characterization.
PB95-153128 04,589 Not available NTIS

Grazing Incidence $X$-ray Photoemission and Its Implementation on Synchrotron Light Source $X$-ray Beamlines.

PB95-175766 01,001 Not available NTIS
0

Grazing-Incidence X-Ray Photoemission Spectroscopy Investigation of Oxidized GaAs(100): A Novel Approach to Nondestructive Depth Profiling.

PB94-200151

04,480 Not available NTIS

Greatly Enhanced Soot Scattering in Flickering Ch4/Air Diffusion Flames

01,361 Not available NTIS

Green's Function for Generalized Hilbert Problem for Cracks and Free Surfaces in Composite Materials.

PB95-163333

03,163 Not available NTIS

Greenspan Acoustic Viscometer for Gases.

PB96-204417 04,220 Not available NTIS

Ground-Based Smoke Sampling Techniques Training Course and Coliaborative Local Smoke Sampling in Saud Arabia.

PB94-143542

02,532 PC A05/MF A01

Ground Improvement Techniques for Liquefaction Remediation Near Existing Lifelines.

PB96-128111

01,350 PC A05/MF A01

Ground Vehicle Control at NIST: From Teleoperation to AUtonomy.

03,758

(Order as N94-34019/7, PC A21/MF A04)
Group 1 for the Plant Spatial Configuration STEP Application Protocol.

02,789 PC A11/MF A03

Group 1 for the Process Engineering Data STEP Application Protocol.

02,797 PC A99/MF A06

Growing Significance of $\mathrm{ClB}$.

PB95-126306

00,314 Not available NTIS

Growth and Nucleation of Hydrogenated Amorphous Silicon on Silicon (100) Surfaces.

02,991 Not available NTIS

Growth Characteristics of Fiber Gratings. Not available NTIS
PB96-122957

Growth of Bragg Gratings Produced by Continuous-Wave Ultraviolet Light in Optical Fiber.

Growth of Epitaxial $\mathrm{KNbO} 3$ Thin Films

PB96-135181 02,409 Not available NTIS

Growth of Iron on Iron Whiskers

PB95-150322 04,567 Not available NTIS

Growth of Laser Ablated YBa2Cu307-delia Films as Examined by Rheed and Scanning Tunneling Microscopy.
PB95-162541

Growth Surface for the Slopes at the Boundary of a Polyp̧on.

$03,408 \mathrm{PC} \mathrm{A03/MF} \mathrm{A01}$

Guarding Against Transients.

PB94-216470

01,623 Not available NTIS

Guidance of the Legality of Keystroke Monitoring.

PB96-161237 00,005 Not available NTIS

Guidance to Federal Agencies on the Use of Trusted Sys-

PB95-163440

01,597 Not available NTIS

Guide for the Use of the International System of Units (SI).
PB95-226692 02,747 PC A05/MF A01

Guide on Open System Environment (OSE) Procurements.
PB95-169496 01.626 PC A08/MF A02

Guide to a Format for Data on Chemical Admixtures in a Materials Property Database.

PB96-165394

01,327 PC A04/MF A01

Guide to a Format for Data on Chemical Admixtures in a Materials Property Database. (Reannouncement with new

PB96-186192

01,328 PC A04/MF A01

Guide to Configuration Management and the Revision Con-

trol System for Testbed Users.
PB94-150919

01,678 PC AO3/MF A01

Guide to Instrumentation Literature.

AD-A280 278/3

02,617 PC A08/MF A02

Guide to Locating and Accessing Computerized Numeric Materials Databases.

03,007 Not available NTIS

Guide to Software Engineering Environment Assessment and Evaluation

$01,676 \mathrm{PC} \mathrm{A04/MF} \mathrm{A01}$

Guideline: Codes for Named Populatd Places. Primary County Divisions, and Other Locational Entities of the United States, Puerto Rico, and the Outlying Areas. Category: Data Standards and Guidelines; Subcategory: Representation and Codes.

FIPS PUB 55-DC3

04,866 PC E99

Guideline: Codes for Named Populated Places, Primary County Divisions, and Other Locational Entities of the United States, Puerto Rico, and the Outlying Areas. Category: Data Standards and Guidelines. Subcategory: Representations and Codes.

FIPS PUB 55-3

04,865 PC E04

Guideline for the Analysis of Local Area Network Security. Category: Computer Security; Subcategory: Risk Analysis and Contingency Planning.

01,799 PC A04/MF A01

Guideline for the Use of Advanced Authentication Technology Alternatives. Category: Computer Security. Subcategory: Access Control.

FIPS PUB 190

$01,798 \mathrm{PC} \mathrm{A04/MF} \mathrm{A01}$

Guidelines for Evaluating and Expressing the Uncertainty of

NIST Measurement Results. 1994 Edition.
PB95-143087 649 PC A03/MF A01

Guidelines for Pre-Oualification, Prototype and Ouality Con-

trol Testing of Seismic Isolation Systems.
PB96-193685

Guidelines for Refractive Index Measurements of Asbestos. PB95-151189 02,543 Not available NTIS

Guidelines for Reporting Resulis of Computational Experiments. Report of the Ad hoc Committee.
PB94-212347 03,427 Not available NTIS 


\section{TITLE INDEX}

Guidelines for the Development of Mapping Tables.

Guidelines for
PB96-154539

M2,786 PC A03/MF A01
Papping Tables.

Guidelines for the Evaluation of Electronic Data Interchange Products.

Products.
PB96-172325

01,506 PC A05/MF A01

Guidelines for the Evaluation of X.500 Directory Products.
PB95-231908
02,732 PC A04/MF A01

Gust Factors Applied to Hurricane Winds.

PB95-180469 00,446 Not available NTIS

Hair Analysis for Drugs of Abuse: Evaluation of Analytica Methods, Environmental Issues, and Development of Reference Materials

03,501 Not available NTIS

Hair Testing for Drugs of Abuse: International Research on Standards and Technology.

03,504 PC A18/MF A04

Half-Integral Constant Voltage Steps in High-Tc Grain Boundary Junctions.

PB94-211216

04,489 Not available NTIS

Halon Thermochemistry: 'Ab Initio'. Calculations of the Enthalpies of Formation of Fluoromethanes.

PB96-175740

03,289 Not available NTIS

Handbook Preferred Circuits Navy Aeronautical Electronic Equipment. Supplement Number 1.

AD-A278 784/4

$00,028 \mathrm{PC} \mathrm{A05/MF} \mathrm{A01}$

Handbook Preferred Circuits Navy Aeronautical Electronic Equipment. Supplement Number 2 .

AD-A278 783/6

00,027 PC A03/MF A01

Handbook Preferred Circuits Navy Aeronautical Electronic

Equipment. Supplement Number 3.
$\mathrm{AD}-\mathrm{A} 278$ 782/8
$00,026 \mathrm{PC} \mathrm{A03/MF} \mathrm{A01}$

Handling Passwords with Security and Reliability in Background Processes.
PB95-180550

01,722 Not available NTIS

Hardware Measurement Techniques for High-Speed Net-

works.
PB96-160551

01,500 Not available NTIS

Harmonic and Static Susceptibilities of $\mathrm{YBa} 2 \mathrm{Cu} 3 \mathrm{O} 7$

PB95-161139 04,599 Not available NTIS

HAZARD I Fire Hazard Assessment Method (Version 1.2) (for Microcomputers)

00,196 Diskette $\$ 250.00$

HAZARD I Fire Hazard Assessment Method, Version 1.2 (Upgrade Package) (for Microcomputers).
PB94-501996 00,197 CP D99 Head Start on Assurance: Proceedings of an Invitational
Workshop on Information Technology (IT) Assurance and Workshop on Information Technology (IT) Assurance and Trustworthiness. Held in Williamsburg, Virginia on March
$21-23,1994$.
PB94-215746
01,586 PC A05/MF A01

Heap of Data.

03,424 Not available NTIS

Heat and Moisture Transfer in Wood-Based Wall Construction: Measured versus Predicted.
PB95-200655 00,391 PC A05/MF A01

Heat Flux from Flames to Vertical Surfaces.
PB97-110357 Heat Transfer in an Intumescent Material Using a Three-Dimensional Lagrangian Model.
PB96-164066 Heat Transfer in Thin, Compact Heat Exchangers with Circular, Rectangular, or Pin-Fin Flow Passages.
PB95-140943 Not available NTIS

02,751 Not available NTIS

Heavily Accumulated Surfaces of Mercury Cadmium Telluride Detectors: Theory and Experiment.
PB9 $134-216074$

Heights of Wall-Fire Flames.

Heights of Wall
PB96-148192

00,212 Not available NTIS

Helium Refrigeration and Liquefactlon Using a Liquid Hydrogen Refrigerator for Precooling.
AD-A286 683/8

Helping to Reduce Technical Barriers to Trade.
PB96-190046 00,491 Not available NTIS

Hemispherical Test Fixture for Measuring the Wavefields Generated in an Anisotropic Solid.
PB96-190087 Heterodyne Measurement of the Fluorescence Spectrum of Optical Molasses. PB95-108411

03,873 Not available NTIS Heterodyne Mixing and Direct Detection in High Temperature Josephson Junctions.
PB96-147202 Hg1-xCdxTe Characterization Measurements: Current Practice and Future Needs.

PB95-164299
Hierarchical Ada Robot Programming System (HARPS): A Complete and Working Telerobot Control System Based on the NASREM Model

02,934 Not available NTIS

Hierarchical Interaction between Sensory Processing and World Modeling in Intelligent Systems.
PB94-198256 Not available NTIS

Hierarchical Real-Time Control System for Use with Coal Mining Automation.

03,698 Not available NTIS

High Accuracy Measurement of Aperture Area Relative to a Standard Known Aperture.

01,919

(Order as PB95-261897, PC A07/MF A02)

High-Accuracy Principal-Angle Scanning Spectroscopic Ellipsometry of Semiconductor Interfaces.
PB96-163787 02,427 Not available NTIS High Critical Temperature Superconductor Tunneling Spectroscopy Using Squeezable Electron Tunneling Junctions. PB95-163721 Squeezable Electron Tunneling Junctions.
04,627 Not available NTIS

High Current Pressure Contacts to Ag Pads on Thin Film Superconductors
PB95-168621

04,639 Not available NTIS

High-Current Thin Film Multijunction Thermal Converters

and Multi-Converter Modules.
PB97-112379

01,989 Not available NTIS

High-Efficiency, High-Power Difference-Frequency Generation of $0.9-1.5 \mathrm{mu} \mathrm{m}$ Light in BBO.

PB95-202255

04,317 Not available NTIS

High-Energy Behavior of the Double Photoionization of Helium from 2 to $12 \mathrm{keV}$

03,860 Not available NTIS

High-Energy Phonon Dispersion in La1.85Sro.15CuO4.

PB96-138458 04,748 Not available NTIS

High-Frequency Linear Response of Anisotropic Type-ll Superconductors in the Mixed State.
PB94-200359 Not available NTIS

High Frequency Magnetic Field Sensors Based on the Far-

aday Effect in Garnet Thick Films.
PB96-190384

High-Frequency Oscillators Using Phase-Locked Arrays of Josephson Junctions.

PB96-135157

02,080 Not available NTIS

High Integrity Software Standards Activities at NIST

PB96-112214 01,744 Not available NTIS

High-Level CAD Melds Micromachined Devices with Foundries.

02,321 Not available NTIS

High-Order Harmonic Mixing with GaAs Schottky Diodes

PB95-108585

01,528 Not available NTIS

High-Order Multipole Excitation of a Bound Electron. PB96-119789 04,044 Not available NTIS

High-Performance Concrete: Research Needs to Enhance Its Use.

PB95-180147

01,322 Not available NTIS

High-Performance Liquid Chromatography of Phytoplankton Pigments Using a Polymeric Reversed-Phase C18 Column.

High Power Generation with Distributed Josephson-Junction

Arrays.

02,099 Not available NTIS

High-Power, High-Frequency Oscillators Using Distributed Josephson-Junction Arrays.

PB96-200266

02,095 Not available NTIS

High-Precision Calculations of Cross Sections for Low-Energy Electron Scattering by Ground and Excited State of Sodium

PB95-152161

03,914 Not available NTIS

High-Pressure Equilibrium Cell for Solubility Measurements in Supercritical Fluids.

PB95-175634

00,998 Not available NTIS

High Resolution Angle Resolved Photoelectron Spectros-

copy Study of N2.

03,907 Not available NTIS

High-Resolution Atomic Spectroscopy of Laser-Cooled lons. PB95-169330

03,953 Not available NTIS

High-Resolution Diode-Laser Spectroscopy of Calcium.

PB95-181244 03,969 Not available NTIS

High-Resolution, Direct Infrared Laser Absorption Spectroscopy in Slit Supersonic Jets: Intermolecular Forces and Unimolecular Vibrational Dynamics in Clusters.

PB95-203006 01,040 Not available NTIS

High Resolution Hard X-Ray Microscope

PB94-213055
High Resolution Inelastic Neutron Scattering Study of Phonon Self-Energy Effects in YBCO.
PB95-180881 High-Resolution Infrared Overtone Spectroscopy of ArHF via Nd:YAG/Dye Laser Difference Frequency Generation.
PB94-211448 00,816 Not available NTIS

High-Resolution Infrared Overtone Spectroscopy of N2-HF Vibrational Red Shifts and Predissociation Rate as a Function of HF Stretching Quanta.

PB96-102298

01,061 Not available NTIS

High-Resolution Infrared Spectroscopy of DF Trimer: A Cyclic Ground State Structure and DF Stretch Induced Intramolecular Vibrational Coupling.
PB95-150678 00,920 Not available NTIS

High-Resolution IR Laser-Driven Vibrational Dynamics in Supersonic Jets: Weakly Bound Complexes and Intramolecular Energy Flow. $\quad 00,862$ Not available NTIS
PB94-216751

High Resolution IR Studies of Polymolecular Clusters: Micromatrices and Unimolecular Ring Opening. PB94-185691 00,777 Not available NTIS

High Resolution, Jet-Cooled Infrared Spectroscopy of ( $\mathrm{HCl}$ 2: Analysis of nu1 and nu2 $\mathrm{HCl}$ Stretching Fundamentals, Interconversion Tunneling, and Mode-Specific
Predissociation Lifetimes.

PB95-203196

01,046 Not available NTIS

High-Resolution Measurements of the nu2 and 2nu2-nu2 Bands of (34)S(16)O2.

PB94-216223

00,855 Not available NTIS

High Resolution Near Infrared Spectroscopy of HCl-DCl and $\mathrm{DCI}-\mathrm{HCl}$ : Relative Binding Energies, Isomer Interconversion Rates, and Mode Specific Vibrational Predissociation.

PB95-203212 01,048 Not available NTIS

High-Resolution Optical Multiplex Spectroscopy.

PB95-203543

04,323 Not available NTIS

High-Resolution Spectroscopy of Laser-Cooled Rubidium in a Vapor-Cell Trap.

5-153714

04,268 Not available NTIS

High Resolution Time Interval Counter

PB96-138607

01,495 Not available NTIS

High-Sensitivity Acoustic Emission Sensor/Preamplifier Subsystems.
PB95-125704

02,900 Not available NTIS

High-Sensitivity Determination of lodine Isotopic Ratios by Thermal and Fast Neutron Activation. PB94-213386

00,555 Not available NTIS

High-Sensitivity Optical Sampling Using an Erbium-Doped

Fiber Laser Strobe.

04,302 Not available NTIS

High-Sensitivity Spectroscopy with Diode Lasers

PB95-175477 04,297 Not available NTIS

High Sensitivity Survey of Radio Continuum Emission in

Herbig Ae/Be Stars
PB94-185915

00,051 Not available NTIS

High-Spatial-Resolution Resistivity Mapping Applied to Mercury Cadmium Telluride.

PB94-212917

02,131 Not available NTIS

High Spectral Purity $X$-Band Source.

PB95-163713 02,045 Not available NTIS

High Speed, High Sensitivity Magnetic Field Sensors Based on the Faraday Eftect in Iron Garnets.

PB95-153391 02,151 Not available NTIS

High-Speed Interconnection Characterization Using Time Domain Network Analysis.

PB96-148176

04,061 Not available NTIS

High-Speed Spatial Scanning Pyrometer

PB94-200003

02,636 Not available NTIS

High-Tc Multilayer Step-Edge Josephson Junctions and PB96-200183

04,790 Not available NTIS

High-Tc Supercanducting Antenna-Coupled Microbolomete on Silicon.

02,166 Not available NTIS

High Temperature.

PB94-211737

03,844 Not available NTIS

High-Temperature Adiabatic Calorimeter for Constant-Volume Heat Capacity Measurements of Compressed Gases and Liquids.

PB95-168860

00,989 Not available NTIS

PB94-172848

of Structural Composites.

High-Temperature Furnace for In situ Small-Angte Neutron Scattering during Ceramic Processing.

PB96-148127

03,743 Not available NTIS 


\section{TITLE INDEX}

High-Temperature High-Pressure Oscillating Tube Densimeter.

01,123 Not available NTIS

High-Temperature Laser-Pulse Thermal Diffusivity ApparaPBS.

02,631 Not available NTIS

High Temperature Reactions of Uranium Dioxide with Various Metal Oxides.
AD-A286 648/1

$00,717 \mathrm{PC} \mathrm{A03/MF} \mathrm{A01}$

High Temperature Reliability of Thin Film $\mathrm{SiO} 2$

PB95-150348 02,333 Not available NTIS

High Temperature Silicide Thin-Film Thermocouples.

PB94-185501 02,252 Not available NTIS

High Temperature Structural Reliability of Silicon Nitride

PB97-110456 03,104 Not available NTIS

High-Temperature Superconductor Cryogenic Current Comparator.

02,074 Not available NTIS

High Temperature Superconductor-Normal MetalSuperconductor Josephson Junctions with High Characteristic Voltages.

04,666 Not available NTIS

High Velocity Plasm in the Transition Region of Au Mic: A

Stellar Analog of Solar Explosive Events.
PB96-123294 00,102 Not available NTIS

High-Velocity Plasma in the Transition Region of $\mathrm{AU}$ Microscopii: Evidence for Magnetic Reconnection and Saturated Heating during Ouiescent and Flaring Conditions.

PB96-102694 00,091 Not available NTIS

Higher-Order Approximations in lonospheric Wave-PropagaAD-A292 471/0

04,420 PC A02/MF A01

Highway Concrete (HWYCON) Expert System User Reference and Enhancement Guide.
PB94-215670 01,316 PC A08/MF A02

Histopathology, Blood Chemistry, and Physiological Status of Normal and Moribund Striped Bass ('Morone saxatilis') Involved in Summer Mortality ('Die-Oft') in the Sacramento San Joaquin Delta of California.

PB94-198157

00,034 PC A03/MF A01

History of NIST's Contributions to Development of Standard Reference Materials and Reference and Definitive Methods for Clinical Chemistry.

PB96-119706

03,503 Not available NTIS

Hole Dispersion and Enhancement of Antiferromagnetic Interaction of Localized Spins in High-Tc Superconductors.
PB95-202602

Hollow Clay Tile Prism Tests for Martin Marietta Energy Systems: Task 2 Testing.
PB94-217486

00,352 PC A14/MF A03

Holographic Properties of Triton X-100-Treated Bacteriorhodopsin Embedded in Gelatin Films.
PB96-119284

Homoepitaxial Growth of Iron and a Real Space View of

Reflection-High-Energy-Electron Diffraction.
PB94-173069

Homogeneous Gas Phase Decyclization of Tetralin and Benzocyclobutene.

00,928 Not available NTIS

Hot Carrier Excitation of Adlayers: Time-Resolved Measurement of Adsorbate-Lattice Coupling.
PB94-172285 00,758 Not available NTIS

Hot-Deformation Apparatus for Thermomechanical Processing Simulation

03,207 Not available NTIS

Hot-Electron Microcalorimeter for $X$-ray Detection Using a Superconducting Transition Edge Sensor with Electrothermal Feedback.
PB96-200399

Hot-Electron Microcalorimeters as High-Resolution $X$-ray Detectors.
PB96-123641

04,739 Not available NTIS

Hot-Electron Microcalorimeters for $X$-ray and Phonon Detection.

04,644 Not available NTIS

Hot-Electron-Microcalorimeters with $0.25 \mathrm{~mm}$ (2) Area.

PB96-200670 04,793 Not available NTIS

How Accurate Are the Josephson and Quantum Hall Effects and OED.
PB95-163283

03,942 Not available NTIS

How Far Is Far from Critical Point in Polymer Blends. Lattice Cluster Theory Computations for Structured Monomer Compressible Systems.

01,217 Not available NTIS

How the Cambridge Crystallographic Data Centre Obtains Its Information
PB97-109193

04,808
(Order as PB97-109011, PC A11/MF A03)

How to Evaluate Alternative Designs Based on Fire Modeling. 102116

00,206 Not available NTIS

How to Get NIST-Traceable Time on Your Computer.

PB96-200647 01,559 Not available NTIS

How-To Suggestions for Implementing Executive Order 12941 on Seismic Safety of Existing Federal Buildings, A Handbook.

00,461 PC A10/MF A03

PB96-131552 as.

How to Verity
PB95-151486

03,497 Not available NTIS

Human and Machine Recognition of Faces: A Survey.

PBg6-111687

01,854 Not available NTIS

Human Factors Considerations for the Potential Use of Elevators for Fire Evacuation of FAA Air Traftic Control Tow-

PB94-217163

01,300 PC A03/MF A01

HVAC CAD Layout Tools: A Case Study of University/lndustry Collaboration.

00,281 Not available NTIS

Hybrid Digital/Analog Servo for the NIST-7 Frequency Standard.

01,544 Not available NTIS

Hybrid Gauss-Trapezoidal Ouadrature Rules.

PB96-193750 03,422 PC A04/MF A01

Hybrid Method for Determining Material Properties from Instrumented Micro-Indentation Experiments.

PB95-152229 03,348 Not available NTIS

Hybrid Optical-Electrical Overlay Test Structure.

PB96-204136 02,450 Not available NTIS

Hybrid Undulator for the NIST-NRL Free-Electron Laser.

PB94-212529 04,238 Not available NTIS

Hydration in Semicrystalline Polymers: Small-Angle Neutron Scattering Studies of the Effect of Drawing in Nylon-6 Fibers. 202990

03,385 Not available NTIS

Hydraulic Radius and Transport in Reconstructed Model Three-Dimensional Porous Media.

PB96-123419

00,403 Not available NTIS

Hydrodynamic Friction of Arbitrarily Shaped Brownian Particles.

04,136 Not available NTIS

Hydrodynamic Similarity in an Oscillating-Body Viscometer.

Hydrogen Atom Attack on Perchloroethylene.

PB95-163473 00,969 Not available NTIS

Hydrogen Balmer Alpha Line Shapes for Hydrogen-Argon Mixtures in a Low-Pressure if Discharge.

PB95-153433

03,924 Not available NTIS

Hydrogen in YBa2Cu3Ox: A Neutron Spectroscopy and a Nuclear Magnetic Resonance Study.

PB95-161279

04,601 Not available NTIS

Hydrogen Lyman-alpha Emission of Capella.

PB95-202263 00,075 Not available NTIS

Hydrolysis of Proteins by Microwave Energy

PB94-216322 03,528 Not available NTIS

Hygrothermal Effects on the Performance of Polymers and Polymeric Composites: A Workshop Report. Held in Gaithersburg, Maryland on September 21-22, 1995.
PB96-183207
03,180 PC A04/MF A01

Hypercubic Lattice SAW Exponents nu and gamma : 3.99 Dimensions Revisited.

PB94-211026

01,215 Not available NTIS

Hyperfine Effects and Associative Ionization of Ultracold Sodium. 151221

03,903 Not available NTIS Hyperfine Structure Investigations and Identification of New
Energy Levels in the Ionic Spectrum of (147)Pm.
PB96-180203 04,117 Not available NTIS PB96-180203

04,117 Not available NTIS

Hyperfine-Structure Studies of $\mathrm{Zr}$ II: Experimental and Relativistic Configuration-Interaction Results.

PB95-203824 04,011 Not available NTIS

Hypersingular Single Integral Equation and the Dielectric

04,428 Not available NTIS

Hysteresis Measurements of Remanent Polarization and Coercive Field in Polymers.

04,475 Not available NTIS

I: Improvement of Resonance Ionization Spectroscopy (RIS) I: Improvement of Resonance lonization Spectroscopy (RIS) Techniques; 11: Atomic Data for RIS; III: Standards for
Ultratrace Analysis. Progress Report.
DE 94018565
ICSSC Guidance on Implementing Executive Order 12941 on Seismic Safety of Existing Federally Owned or Leased PBildings.

$00,459 \mathrm{PC} \mathrm{A03/MF} \mathrm{A0}$

Ideal Gas Thermodynamic Properties of Sulphur Heterocyclic Compounds.

01,110 Not available NTIS

IEEE's POSIX: Making Progress.

PB96-160924

01,757 Not available NTIS

Ignition and Subsequent Flame Spread Over a Thin Celulosic Material.

PB96-160270

04,836 Not available NTIS

Ignition and Subsequent Transition to Flame Spread in a Microgravity Environment.

04,828

(Order as N96-15552, PC A2O/MF AO4)

Ignition and Transition to Flame Spread Over a Thermally Thin Cellulosic Sheet in a Microgravity Environment. PB96-160288

04,837 Not available NTIS

IGOSS-Industry/Government Open Systems Specification. PB94-207453

01,806 PC A07/MF A02

II-3: Critical Current Measurement Methods: Ouantitative Evaluation.

PB96-147160

04,767 Not available NTIS

II-5: Thermal Contraction of Materials Used in Nb3Sn Critical Current Measurements.

PB96-147186

04,769 Not available NTIS

IIW Commission V Ouality Control and Ouality Assurance of Welded Products Annual Report 1994/95.

PB95-198743

02,866 PC AO3/MF A01

IIW Commission V Quality Control and Ouality Assurance of Welded Products, Annual Report 1995/96.

PB96-191366

2,880 PC A04/MF A01

Image Depth Profiling SIMS: An Evaluation for the Analysis of Light Element Diffusion in YBa2Cu3O7-x Single Crystal Superconductors.

PB95-126116

04,530 Not available NTIS

Image Gradient Evolution: A Visual Cue for Danger

PB96-154562

02,939 PC A03/MF A01

Image Information Transfer Properties of X-Ray Intensifying

Screens in the Energy Range from 17 to $320 \mathrm{keV}$.
PB95-126173 00,155 Not available NTIS

Image Restoration and Diffusion Processes.

PB95-153003

01,843 Not available NTIS

Imaging of Fine Porosity in a Colloidal Silica: Potassium Silcate $\mathrm{Gel}$ by Defocus Contrast Microscopy.

PB94-212750

03,039 Not available NTIS

IML++ V.1.2 lterative Methods Library Reference Guide.

PB96-195219 01,776 PC A04/MF A01

Impact of Computer-Aided Acquisition and Logistic Support (CALS) in the Application of Standards.

PB96-160908

01,756 Not available NTIS

Impact of the FCC's Open Network Architecture on NS/NP Telecommunications Security.

01,483 PC A03/MF A01

Implementation of a Standard Format for GPS Common View Data. View Data.

03,779

(Order as N95-32319, PC A2O/MF A04)

Implementation of a Standard Format for GPS Cornmon View Data.

01,555 Not available NTIS

Implementation of Executive Order 12699: Seismic Safety of Federal and Federally Assisted or Regulated New Building Construction.

PB95-151809

00,436 Not available NTIS

Implementation of the Fastener Ouality $A C$

PB96-160676

02,876 Not available NTIS

Implementing a Transition Manager in the AMRF Cell Controller.

02,817 Not available NTIS

Importance of Bound-Free Correlation Effects for Vibrational Excitation of Molecules by Electron Impact: A Sensitivity Analysis.

PB95-202974

03,996 Not available NTIS

Importance of Chemometrics in Biomedical Measurements. PB94-200599

00,550 Not available NTIS

Importance of Measurement in Technology-Based CompetiPB94-211844

02,929 Not available NTIS

Importance of Unraveling Memory Propagation Effects in Interpreting Data on Partial Discharge Statistics.

PB95-163572

01,901 Not available NTIS 


\section{TITLE INDEX}

Important Link in Entire-House Protection: Surge Reference Equalizers.
PB94-216504

02,219 Not available NTIS

Important Papers in the History of Document Preparation Systems: Basic Sources.

02,712 Not available NTIS

Imposed Oscillations of Kinetic Barriers Can Cause an Enzyme to Drive a Chemical Reaction Away from Equilibrium.
PB96-161625

Improved Annealing Technique for Optical Fiber.

PB96-119680 04,343 Not available NTIS

Improved Automated Current Control for Standard Lamps.
PB94-219367 (Order as PB94-219326, PC A05/MF A02)

Improved Crystallographic Data for Aluminum Niobate (AlNbO4).

04,523 Not available NTIS

Improved Dose Metrology in Optical Lithography.

PB96-179510 02,439 Not available NTIS

Improved Eddy-Current Decay Method for Resistivity Characterization.

02,265 Not available NTIS

Improved Gas Flow Measurements for Next-Generation Processes.
PB96-156013

04,216 Not available NTIS

Improved Hyperfine Measurements of the Na NP Excited State Through Frequency-Controlled Dopplerless Spectros-

copy in a Zeeman Magneto-Optic Laser Trap.
PB95-203840 04,012 Not available NTIS

Improved Molecular Constants and Frequencies for the $\mathrm{CO} 2$ Laser from New High-J Regular and Hot-Band Frequency Measurements.

04,312 Not available NTIS

Improved Reflectometry Facility at the Nationa! Institute of Standards and Technology.

04,087 Not available NTIS

Improved Rubidium Frequency Standards Using Diode La-

sers with AM and FM Noise Control.
PB95-176152 04,303 Not available NTIS

Improved Uniaxial Strain Tolerance of the Critical Current Measured in Ag-Sheathed Bi2Sr2Ca1Cu2O8+x Superconductors

PB95-153565

04,594 Not available NTIS

Improved Variational Analysis of Inhomogeneous Optical Waveguides Using Airy Function

PB95-168639 04,285 Not available NTIS

Improved Wavelengths for Prominent Lines of $\mathrm{Cr} X \mathrm{XI}$ to $\mathrm{Cr}$ XXII.

03,895 Not available NTIS

Improved Wavelengths for Prominent Lines of $\mathrm{Fe} X X$ to $\mathrm{Fe}$ XXIII.

04,334 Not available NTIS

Improvement in Predicting Smoke Movement in Compartmented Structures.

PB94-172418

00,332 Not available NTIS

Improvement of Ultrasensitive Techniques Isotopic Biasing in the RIS Process lonization Efficiencies and Selectivities.
DE94018563 00,522 PC A02/MF A01

Improvements in Computation of Form Factors.

PB94-200078 03,820 Not available NTIS

Improving Color Measurements of Displays.

PB96-204441 02,203 Not available NTIS

Improving measurement quality assurance for photon irradiations at Department of Energy facilities. Final technical report.

03,711 PC A03/MF A01

Improving Neural Network Performance for Character and Fingerprint Classification by Altering Network Dynamics.
PB95-267803 01,851 PC A03/MF A01

Improving Neural Network Performance for Character and Fingerprint Classification by Altering Network Dynamics. PB96-123195 01,856 Not available NTIS

Improving Photomask Linewidth Measurement Accuracy via Emulated Stepper Aerial Image Measurement.

PB95-180816

02,911 Not available NTIS

Improving the Design Process by Predicting Downstream Values of Design Attributes.

PB97-113096

02,795 Not available NTIS

Improving the Evaluation of Building Ventilation.

PB96-138508 00,271 Not available NTIS

In-Line Optical Monitoring of Injection Molding

PB94-185105 01,201 Not available NTIS

In Search of Altemative Fire Suppressants.

PB $96 \cdot 164165$

03,285 Not available NTIS
In situ Buming of Oil Spills: Mesoscale Experiments. PB94-142973 01,355 PC A04/MF A01

In situ Burning of Oil Spills: Mesoscale Experiments and Analysis.

PB95-163747

02,587 Not avallable NTIS

In Situ Burning Oil Spill Workshop Proceedings. Held in Or-

PB95-104907
02,583 PC A06/MF A02

In situ Characterization of Vapor Phase Growth of Iron Oxide-Silica Nanocomposites: Part 1.2-D Planar Laser-Induced Fluorescence and Mie Imaging.
PB97-112478 03,185 Not available NTIS In situ Fluorescence Cell Mass Measurements of 'Saccharomyces cerevisiae' Using Cellular Tryptophan.
PB96-135041

In-situ Fume Particle Size and Number Density Measurements from a Synthetic Smelt. 03,334 Not available NTIS
PB94-212040

In situ Measurements of Chloride Ion and Corrosion Poten-

tial at the Coating/Metal Interface

03,122 Not available NTIS

In-situ Monitoring of Molecular Beam Epitaxial Growth Using Single Photon Ionization. 01,023 Not available NTIS
PB95-202222

In-situ Neutron Reflectivity of MBE Grown and Chemically Processed Surfaces and Interfaces. 02,416 Not available NTIS

In situ Noble Metal YBa2Cu3O7 Thin-Film Contacts.

PB94-211323 04,494 Not available NTIS

In situ Observation of Surface Morphology of InP Grown on Singular and Vicinal (001) Substrates.

PB95-168431 04,636 Not available NTIS

In situ On-Line Optical Fiber Sensor for Fluorescence Monitoring in Bioreactors.

03,587 Not available NTIS

In-situ Studies of a Novel Sodium Flame Process for Synthesis of Fine Particles.

PB97-113047

00,681 Not available NTIS

In situ-Toughened Silicon Carbide.

PB97-110332 03,102 Not available NTIS

In-Space Welding: VIsıons and Realities

PB95-163234 04,830 Not available NTIS

In vitro Fracture Behavior of Ceramic and Metal-Ceramic Restorations.

PB96-119722

03,569 Not available NTIS

In vitro Inhibition of Membrane-Mediated Calcification by Novel Phosphonates

PB96-201140

03,595 Not available NTIS

Incinerability of Perchloroethylene and Chlorobenzene.

PB95-163457 01,388 Not available NTIS

Incommensurate Magnetic Order in UPtGe.

PB95-140513 04,542 Not available NTIS

Incorporation of Gold into YBa2Cu3O7: Structure and Tc Enhancement.
PB94-200276

04,481 Not available NTIS

Increased Pinning Energies and Critical Current Densities in Heavy-Ion-Irradiated Bi2Sr2CaCu2O8 Single Crystals.

PB95-175352 04,654 Not available NTIS

Increased Transition Temperature in In situ Coevaporated YBa2Cu307-delta Thin Films by Low Temperature Post-An-

PB95-180071

04,672 Not available NTIS

Increasing the Value of Atomic Force Microscopy Process Metrology Using a High-Accuracy Scanner, Tip Characterization, and Morphological Image Analysis.

PB96-190293 02,758 Not available NTIS

Indentation Fatigue: A Simple Cyclic Hertzian Test for Measuring Damage Accumulation in Polycrystalline Ceramics. 180013

03,084 Not available NTIS

India: Environmental Technologies Export Market Plan

PB97-114359 02,529 PC A05/MF A01

Individual Carotenoid Content of SRM 1548 Total Diet and Influence of Storage Temperature, Lyophilization, and Irradiation on Dietary Carotenoids.

PB94-200524

00,033 Not available NTIS

Indoor Air Ouality Commissioning of a New Office Building.
PB95-182309 00,262 PC A04/MF A01

Indoor Air Ouality Commissioning of a New Office Building.
PB97-110142 00,279 Not available NTIS

Indoor Air Ouality Impacts of Residential HVAC Systems

Phase II.B Report: IAO Control Retrofit Simulations and Analysis.
PB96-106877

02,559 PC A05/MF A01
Indoor Air Ouality Impacts of Residentıal HVAC Systems, Phase 1 Report: Computer Simulation Plan. PB95-135596

$00,249 \mathrm{PC} \mathrm{A06/MF} \mathrm{A02}$

Indoor Air Ouality Impacts of Residential HVAC Systems

Phase 2.A Report: Baseline and Preliminary Simulations.
PB95- 178893 PB95-178893

Inductive Voltage Divider Calibration for the NASA Flight Experiment.

02,042 Not available NTIS

Inductively Coupled Plasma Source for the Gaseous Electronics Conference RF Reference Cell.

PB96-113394

(Order as PB96-113311, PC A09/MF A03)

Industry and Government-Laboratory Cooperative R and D An Idea Whose Time Has Come.

PB94-172939

02,970 Not available NTIS

Industry/Government Open Systems Specification Testing Framework. Version 1.0.

01,809 PC A06/MF A02

Industry/Government Open Systems Specification: The Development of GOSIP Version 3.

PB96-161245

01,505 Not available NTIS

Inelastic Interactions of Electrons with Surfaces: Application to Auger-Electron Spectroscopy and X-ray Photoelectron PBectroscopy.

00,764 Not available NTIS

Inelastic Neutron Scattering Measurements of Phonons in Icosahedral Al-Li-Cu.

PB95-126215

04,532 Not available NTIS

Inelastic Neutron Scattering Studies of Nonlinear Optica Materials: p-Nitroaniline Adsorbed in ALPO-5.

PB95-107223

00,874 Not available NTIS

Inelastic-Neutron-Scattering Studies of Poly( $p$-phenylene vinylene

PB95-180766

01,014 Not available NTIS

Inelastic Neutron Scattering Studies of Rotational Excitations and the Orientational Potential in $\mathrm{C} 60$ and $\mathrm{A} 3 \mathrm{C} 60$ Compounds.

00,763 Not available NTIS

Inelastic Neutron Scattering Study of Hydrogen in Nanocrystalline

03,366 Not available NTIS

Inexpensive Laser Cooling and Trapping Experiment for Undergraduate Laboratories.

PB96-140371

04,353 Not available NTIS

Infinite Divisibility and the Identification of Singular Waveforms.

02,701 Not available NTIS

Influence of an Impenetrable Interface on a Polymer GlassTransition Temperature.

PB96-146873

03,175 Not available NTIS

Influence of Coadsorbed Potassium on the Electron-Stimulated Desorption of $F(+), F(-)$, and $F\left({ }^{*}\right)$ from PF3 on Ru(0001).

B96-157946

04,072 Not available NTIS

Influence of $\mathrm{Cr}$ Growth on Exchange Coupling in $\mathrm{Fe} / \mathrm{Cr}$ / $\mathrm{Fe}(100)$.
$\mathrm{PB} 95-150181$

04,562 Not available NTIS

Influence of Deposition Parameters on Properties of Laser Ablated YBa2Cu307-Delta Films.

PB95-140539

04,544 Not available NTIS

Influence of Electrical Isolation on the Structure and Reflectivity of Multilayer Coatings Deposited on Dielectric Sub-

PB96-159736

04,365 Not available NTIS

Influence of Electrode Material on Measured Ion Kinetic-Energy Distributions in Radio-Frequency Discharges

PB96-123179 01,935 Not available NTIS

Influence of Envelopes Geometry on the Sensitivity of 'Nude' lonization Gauges.

PB97-119077

04,174 Not available NTIS

Influence of Films' Thickness and Air Gaps in Surface Impedance Measurements of High Temperature Superconductors Using the Dielectric Resonator Technique. PB96-157862 01,946 Not available NTIS

Influence of Ignition Source on the Flaming Fire Hazard of Upholstered Furniture. (NIST Reprint) PB95-180162

00,297 Not available NTIS

Influence of Lattice Mismatch on Indium Phosphide Based High Electron Mobility Transistor (HEMT) Structures Observed in High Resolution Monochromatic Synchrotron XRadiation Diffraction Imaging.

PB95-164679

02,357 Not available NTIS

Influence of Natural and Synthetic Inhibitors on the Cryslallization of Calcium Oxalate Hydrates.

PB95-150967

03,560 Not available NTIS 


\section{TITLE INDEX}

Influence of Physical Aging on the Yield Response of Model

DGEBA + Poly(propylene oxide) Epoxy Glasses.

Influence of Shear on the Ordering Temperature of a Triblock Copolymer Melt. $\quad 01,288$ Not available NTIS
PB96-163753

Influence of Specimen Absorbed Energy in LOX Mechanical-Impact Tests.

03,341 Not available NTIS

Influence of Stationary Phase Chemistry on Shape Recognition in Liquid Chromatography.
PB96-123682

Influence of Surface Charge on the Stochastic Behavior of Partial Discharge in Dielectrics.

PB96-122767

01,931 Not available NTIS

Influence of Surface Interaction and Chain Stiffness on Polymer-Induced Entropic Forces and the Dimensions of Confined Polymers.

01,203 Not available NTIS

Influence of Tempering Method on Residual Stress in Dental Porcelain.

00,138 Not available NTIS

Influence of the Filament Potential Wave Form on the Sen-

sitivity of Glass-Envelope Bayard-Alpert Gages.
PB95-175014 02,657 Not available NTIS

Influence of Thickness Fluctuations on Exchange Coupling in $\mathrm{Fe} / \mathrm{Cr} / \mathrm{Fe}$ Structures.

04,745 Not available NTIS

Information Infrastructure: Reaching Society's Goals. A Report of the Information Infrastructure Task Force Committee on Applicasions and Technology.

ED-376 823

00,131 Not available NTIS

Information Infrastructure: Reaching Society's Goals. Report of the Information Infrastructure Task Force Committee on Applications and Technology.

$01,469 \mathrm{PC}$ A08/MF A02

Information Resource Dictionary System (IRDS): A Status Report

01,810 Not available NTIS

Information Resources for the Fire Community.

PB96-148119 00,211 Not available NTIS

Information Retrieval Using Key Words and a Structured Review.
PB95-161121

03,724 Not available NTIS

Information Technologies Make Business Sense for the Custom Therapeutic Footwear Industry.
PB95-251708 PC A03/MF A01

Information Technology Engineering and Measurement Model: Adding Lane Markings to the Information Superhighway.
PB95-143145

01,474 PC A03/MF AO1

Information Technology Standards in a Changing World: The Rose of the Users.

02,737 Not available NTIS

Information Technology Standards in Federal Acquisitions.

PB96-161252 01,636 Not available NTIS

Information Transfer in the 21st Century.
PB96-157904 02,714 Not available NTIS

Infrared and Microwave Spectroscopy of the Argon Propyne Dimer.
PB94-198892

00,794 Not available NTIS

Infrared and Near-Intrared Spectra of HCC and DCC Trapped in Solid Neon.

03,773 PC A03/MF A01

Infrared Spectra of van der Waals Complexes of Importance in Planetary Atmospheres.

PB95-125738

00,071 Not available NTIS

Infrared Spectrum of OCIO in the $2000 / \mathrm{cm}(-1)$ Region: The

2(nu sub 1) and (nu sub $1+$ nu sub 3 ) Bands.
PB95-141032 00,908 Not available NTIS

Infratechnologies: Tools for Innovation.

PB94-185998 00,317 Not available NTIS

Inhibition of Premixed Methane-Air Flames by Halon Alter-

natives.
PB96-146741

01,414 Not available NTIS

Inhibition of Premixed Methane-Air Flames by Iron Pentacarbonyl.
PB96-163712

00,513 Not available NTIS

Initial and Final Orbital Alignment Probing of the Fine-Structure-Changing Collisions among the $\mathrm{Ca}(4 \mathrm{~s})(1)(4 \mathrm{p})(1)$, (3)PJ States with He: Determination of Coherence and Conventional Cross-Sections.

PB95-203279

04,004 Not available NTIS

Initial Graphics Exchange Specification (IGES): Procedures for the NIST IGES Validation Test Service.

02,780 PC A03/MF A01
Initial NIST Testing Policy for STEP: Beta Testing Program for AP 203 Implementations. National PDES Testbed Re-

PB95- 154688

$02,779 \mathrm{PC} \mathrm{AO} / \mathrm{MF}$ A01

Inner-Valence States $\mathrm{CO}(+)$ between $22 \mathrm{eV}$ and $46 \mathrm{eV}$ Studied by High Resolution Photoelectron Spectroscopy and ab Initio $\mathrm{Cl}$ Calculations.

03,961 Not available NTIS

Innovation in the Japanese Construction Industry: A 1995 Appraisal.
PB96-177373

$00,225 \mathrm{PC} \mathrm{A13/MF} \mathrm{A03}$

Inorganic Crystal Structure Database (ICSD) and Standardized Data and Crystal Chemical Characterization of Inorganic Structure Types (TYPIX): Two Tools for Inorganic Chemists and Crystallographers.

Order as PB97-109011, PC A11/MF A03)

Inserting Line Segments into Triangulations and PBg5-198933

03,415 PC A03/MF A01

Instrument for Evaluating Phase Behavior of Mixtures for

Supercritical Fluid Experiments.

00,606 Not available NTIS

Instrumental Smearing Effects in Radially Symmetric SmallAngle Neutron Scattering by Numerical and Analytical Methods.

02,984 Not available NTIS

Insulating Boundary Layer and Magnetic Scattering in YBa2Cu307-delta/Ag Interfaces Over a Contact Resistivity Range of $10(-8) \cdot 10(-3)$ Omega cm(2)

PB95-169157 04,648 Not available NTIS

Insulating Nanoparticles on YBa2Cu3O7-delta Thin Films Revealed by Comparison of Atomic Force and Scanning Tunneling Microscopy.

04,575 Not available NTIS

Integral Occurring in Coherence Theory.

PB95-203550 04,324. Not available NTIS

Integrated Inspection System for Improved Machine Performance.

PB96-160569

02,959 Not available NTIS

Integrated Laser Doppler Method for Measuring Planetary Gravity Fields

03,681 Not available NTIS

Integrated Mobile Robot System for Testing Vision Algorithms.

PB95-164133

02,936 Not available NTIS

Integrated Network Management

PB94-199247

01,583 Not available NTIS

Integrated Optic Laser Emitting at 905, 1057, $1356 \mathrm{~nm}$

PB94-216298

Integrated Optic Laser Emitting at 906, 1057, and $1358 \mathrm{~nm}$. PB94-216280 02,135 Not available NTIS

Integrated-Optical Devices in Rare-Earth-Doped Glass.

PB95-202909

02,179 Not available NTIS

Integrated Optical Polarization-Discriminating Receiver in Glass.
PB97-113179

02,206 Not available NTIS

Integrated Services Digital Network (ISDN); Category: Telecommunications Standard; Subcategory: Integrated Services Digital Network.

FIPS PUB 182

01,460 PC E03

Integrated Thin-Film Micropotentiometers

PB96-146709

02,109 Not available NTIS

Integrated Vision Touch-Probe System for Dimensional Inspection Tasks.

02,917 PC A03/MF A01

Integrating Automated Systems with Modular Architecture. PB95-150231

00,577 Not available NTIS

Integrating Sphere Simulation: Application to Total Flux Scale Realization.

04,261 Not available NTIS

Integration Definition for Function Modeling (IDEFO); Category: Software Standard; Subcategory: Modeling Techniques. 02,800 PC E07

Integration Definition for Information Modeling (IDEF 1X); Category: Software Standard; Subcategory: Modeling Tech-

FIPSPUB184

01,673 PC E10

Integration of Real-Time Process Planning for Small-Batch Flexible Manufacturing.

PB95-151908

02,822 Not available NTIS

Integration of Scanning Tunneling Microscope Nanolithography and Electronics Device Processing.
PB95-153359
02,341 Not available NTIS
Integration of Servo Control into a Large-Scale Control System Design: An Example from Coal Mining.
PB94-203429
03,696 PC AO2/MF A01 Intelligent Control for Multiple Autonomous Undersea VehjPB94-211877 03,747 Not available NTIS Intelligent Control of an Inert Gas Aiomization Process. PB95-141057 03,344 Not available NTIS

Intelligent Processing of Hot Isostatic Pressing.

PB94-172913 03,315 Not available NTIS Intelligent Processing of Materials.

PB94-172780

02,811 Not available NTIS

Intelligent Processing of Materials, Technical Activities 1993

(NAS-NRC Assessment Panel, April 21-22, 1994).
02,809 PC AO4/MF A01

Intelligent Processing of Materials, Technical Activities 1994 (NAS-NRC Assessment Panel, April 6-7, 1995).

Intensities and Dipole Moment Derivatives of the Fundamental Bands of (35) $\mathrm{ClO} 2$ and an Intensity Analysis of he nu1 Band.

PB95-141040

00,909 Not available NTIS

Intensity-Dependent Scattering Rings in High Order Above-

04,032 Not available NTIS

Intensive Swimming: Can It Affect Your Patients' Smiles.

PB96-123666 03,570 Not available NTIS

Inter-Laboratory Trials of the EPR Method for the Detection of Irradiated Meats Containing $B$ One

PB96-161690

00,042 Not available NTIS

Interaction between Dislocations and Intergranular Cracks.
03,190 Not available NTIS

Interaction between Micro and Macroscopic Flow in RTM Preforms.

PB95-162012

03,159 Not available NTIS

Interaction between Naphthalene Sulfonate and Silica Fume in Portland Ceme
PB94-199759

01,315 Not available NTIS

Interaction Coefficients for 15 Mixtures of Flammable and Non-Flammable Components.

PB96-146626

03,281 Not available NTIS

Interaction of an Isolated Sprinkler Spray and a Two-Laye Compartment Fire Environment. Phenomena and Model Simulations.

01,437 Not available NTIS

Interaction of Chlorhexidine Digluconate with and Adsorp-

tion of Chlorhexidine on Hydroxyapatite.
PB95-175907 03,566 Not available NTIS

Interaction of Citric Acid with Hydroxyapatite: Surface Ex-

change of lons and Precipitation of Calcium Citrate.
PB97-119309 03,584 Not available NTIS

Interaction of HFC-125, FC-218 and CF3l with High Speed Combustion Waves.

03,290 Not available NTIS

Interaction of Rayleigh Waves with a Rib Attached 10 a

Plate.

04, 184 Not available NTIS

Interaction of Some Coupling Agents and Organic Compounds with Hydroxyapatite: Hydrogen Bonding. Adsorption and Adhesion

00,140 Not available NTIS

Interaction of Stoichiometry, Mechanical Stress, and Interface Trap Density in LPCVD Si-rich SiNx-Si Structures $\begin{array}{ll}P B 95-176301 & 02,366 \\ \text { Not available NTIS }\end{array}$

Interatomic Potential of Argon.

PB95-141180

00,912 Not available NTIS

Interatomic Potential of Argon.

PB95-152989

00,945 Not available NTIS

Intercomparison between NPL (India) and NIST (USA) Pressure Standards in the Hydraulic Pressure Region Up to $26 \mathrm{MPa}$

PB96-113543

04,211

(Order as PB96-113535, PC A05/MF A01)

Intercomparison of DNA Sizing Ladders in Electrophoretic Separation Matrices and Their Potential for Accurate Typing of the D1S80 Locus.

PB96-200928

03,485 Not available NTIS

Intercomparison of Intemal Proportional Gas Counting of (85) $\mathrm{Kr}$ and (3) $\mathrm{H}$

03,800 Not available NTIS

Intercomparison of NIST, NPL, PTB, and VSL Thermal Voltage Converters from $100 \mathrm{kHz}$ 10 $1 \mathrm{MHz}$.

Intercomparison of Photometric Units Maintained at NIST USA) and PTB (Germany), 1993.

04,329 


\section{TITLE INDEX}

(Order as PB95-261897, PC A07/MF A02)

Intercomparison of the Effective Areas of a Pneumatic Piston Gauge Determined by Different Techniques.

PB94-212370 02,640 Not available NTIS

Intercomparison of the ITS-90 Radiance Temperature Scales of the National Physical Laboratory (U.K.) and the National Institute of Standards and Technology.

02,674

(Order as PB96-113535, PC A05/MF A01)

Intercomparison of Thermal Converters at NIM, NIST, PTB SIRI and VSL from 10 to $100 \mathrm{MHz}$

PB94-172459 02,027 Not available NTIS

Intercomparison Study of (237)Np Determination in Artificial Urine Samples.

03,633 Not available NTIS

Interconnection Transmission Line Parameter Characterization.
PB94-216397 02,218 Not available NTIS Interdigitated Stacked P-I-N Multiple Quantum Well ModulaPB97-112296

02,455 Not available NTIS

Interface-Filter Characterization of Spectraradiometers and Colorimeters.

04,399 Not available NTIS

Interface Modification and Characterization of Silicon Carbide Platelets Coated with Alumina Particles.

PB95-108734 03,121 Not available NTIS

Interface Properties for Ceramic Composites from a Single Fiber Pull-Out Test

03,135 Not available NTIS

Interface Roughness, Composition, and Alloying of LowOrder AlAs/GaAs Superlattices Studies by X-ray Diffraction. PB96-160262 02,983 Not available NTIS

Interface Roughness-Induced Changes in the Near-E(sub 0 ) Spectroscopic Behavior of Short-Period AlAs/GaAs Superlattices.

02,118 Not available NTIS

Interface Roughness of Short-Period AIAs/GaAs Superlattices Studied by Spectroscopic Ellipsometry.

PB95-107215 137 Not available NTIS
PB

Interface Sharpness during the initial Stages of Growth of Thin, Short-Period III-V Superlattices.

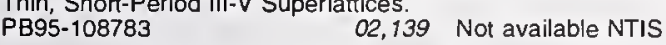

Interface Sharpness in Low-Order III-V Superlattices.

PB95-108775 02,138 Not available NTIS

Interfaces in $\mathrm{Mo} / \mathrm{Si}$ Multilayers.

PB96-160668

02,423 Not available NTIS

Interfacial Crack in a Two-Dimensional Hexagonal Lattice. PB96-161989 04,100 Not available NTIS

Interfacial Free Energies from Substrate Curvature Measurements of the Creep of Multilayer Thin Films.

PB94-185428 04,448 Not available NTIS

Interfacial Transport in Porous Media: Application to dc Electrical Conductivity of Mortars.

PB96-146816

01,326 Not available NTIS

Interference in the Resonance Fluorescence of Two Trapped Atoms

03,948 Not available NTIS

Interim Testing Artifact (ITA): A Performance Evaluation System for Coordinate Measuring Machines (CMMs). User Manual.

PB95-210589

02,914 PC A04/MF A01

Interior-Point Method for Linear and Quadratic Programming Problems. (NIST Reprint).

PB95-180089

03,429 Not available NTIS

Interlaboratory Comparison of Autoradiographic DNA

Profiling Measurements. 1. Data and Summary Statistics.
PB95-175923

Interlaboratory Comparison of Autoradiographic DNA Profiling Measurements. 2. Measurement Uncertainty and Its Propagation.

PB96-112123

03,545 Not available NTIS

Interlaboratory Comparison of Polarization-Holding ParamInterlaboratory Comparison
eter Measurements on High-Birefringence Optical Fiber.
PB95-168464

Interlaboratory Comparison Studies on the Analysis of Hair for Drugs of Abuse

03,500 Not available NTIS

Interlaboratory Studies on the Analysis of Hair for Drugs of Abuse: Results from the Fitth Exercise. Not available NTIS
PB97-110449 Interlaboratory Studies on the Analysis of Hair for Drugs of Abuse: Results from the Fourth Exercise.
PB97.111322
Intermediate Coupling in L2-L3 Core Excitons of $\mathrm{MgO}$ $\mathrm{Al} 2 \mathrm{O} 3$, and $\mathrm{SiO} 2$.

04,079 Not available NTIS

Intermediate Structure in the Neutron-Induced Fission Cross Section of $236 \mathrm{U}$

03,802 Not available NTIS

Intermolecular HF Motion in $\operatorname{Ar}($ sub $n) H F$ Micromatrices $(n=1,2,3,4$, : Classical and Quantum Calculations on a Pairwise Additive Potential Surface.

PB95-107025

03,871 Not available NTIS

Internal Droplet Circulation Induced by Surface-Driven Rotation.

02,500 Not available NTIS

Internal Waves in Xenon Near the Critical Point.

PB97-111504 04,221 Not available NTIS

International Challenges in Defining the Public and Private Interest in Standards.

00,498 Not available NTIS

International Conference on Chemical Kinetics (2nd). Held in Gaithersburg, Maryland on July 24-27, 1989

PB94-211901 00,822 Not available NTIS

International Equations for the Saturation Properties of Ordinary Water Substance. Revised According to the International Temperature Scale of 1990. Addendum to Journa of Physical and Chemical Reference Data 16, 893 (1987).
PB94-162302 00,742 Not available NTIS

International Green Building Conference and Exposition (2nd). Held in Big Sky, Montana on August 13-15, 1995.
PB95-253605

02,525 PC AOAMF A01

International Green Building Conference and Exposition
(3rd). Held in San Diego, California on November 17-19, 1996. (Reannouncement with new abstract)

PB97-121826

$02,539^{\circ} \mathrm{PC} \mathrm{A} 12 / \mathrm{MF} \mathrm{A03}$

International Institute of Welding: Report on 1992 Actions. PB94-185873 02,856 Not available NTIS

International Institute of Welding: Report on 1993 Actions. PB94-185881 02,857 Not available NTIS

International Institute of Welding: Report on 1995 Actions. PB96-158076 02,874 Not available NTIS

International Intercomparison of Detector Responsivity at 1300 and $1550 \mathrm{~nm}$

02,140 Not available NTIS

International Intercomparison of Detector Responsivity at 1300 and $1550 \mathrm{~nm}$

02,141 Not available NTIS

International Marine-Atmospheric (222)Rn Measurement Intercomparison in Bermuda. Part 1. NIST Calibration and Methodology for Standardized Sample Additions.

00.114

(Order as PB96-175666, PC A07/MF A02)

International Marine-Atmospheric (222)Rn Measurement Intercomparison in Bermuda. Part 2. Pesults for the Participating Laboraiories.

00,115

(Order as PB96-175666, PC A07/MF A02)

International Organization for Standardization: Current ACtivities in Fire Safely Engineering

PB96-159660

00,223 Not available NTIS

International Radon-in-Air Measurement Intercomparison Using a New Transfer Standard.

PBing a New Transfer Standard. 03,708 Not available NTIS

International Standard Equation of State for the Thermodynamic Properties of Refrigerant 123 (2,2-Dichloro- 1,1,1 Trifluaric Prope

PB96-176805

03,294 Not available NTIS

International Standards and Reference Materials.

PB97-113120 00,188 Not available NTIS

Internationalization of Fire Safety Engineering Research and Strategy.

00,220 Not available NTIS

Interoperability Experiments with CORBA and Persistent Object Base Systems.

01,772 PC A04/MF A01

PB96-183140

PC A04/MF AO

Interoperability Requirements for CAD Data Transfer in the AutoSTEP Project

02,796 PC A03/MF A01

Interpreting the Readings of Multi-Element Personnel Dosimeters in Terms of the Personal Dose Equivalent. Dosimeters in Terms of the Personal 00se Equivalent.
PB95-175428
03,631 Not available NTIS

Interstellar Disk-Halo Connection in Galaxies: Review of
Observational Aspects. Observational Aspects.

PB94-211802

00,058 Not available NTIS

Intersystem Crossing in Collisions of Aligned $\mathrm{Ca}(455 \mathrm{p}(1) \mathrm{P}$ + He: A Half Collision Analysis Using Multichannel Quantum Defect Theory.

00,813 Not available NTIS
Intra-Laboratory Comparison of a Line-Heat-Source Guarded Hot Plate and Heat-Flow-Meter Apparatus.

PB95-181202 00,387 Not available NTIS

Intracomparison Tests of the FG5 Absolute Gravity Meters. PB96-102991 03,688 Not available NTIS

Intracycle Evaporative Cooling in a Vapor Compression Cycle.
P'997-116107

02,762 PC A03/MF A01

Intrinsic Conductivity of Objects Having Arbitrary Shape and Conductivity.
PB97-111934

04,150 Not available NTIS

Intrinsic Stress in DC Sputtered Niobium.

PB94-199031 04,468 Not available NTIS

Intrinsic Viscosity and the Polarizability of Particles Having a Wide Range of Shapes.

03,170 Not available NTIS

Introduction of a NIST Instrument Sensitivity Standard Reference Material for X-Ray Powder Diffraction

erence Material for X-Ray Powder Diffraction.
PB94-200318 00,807 Not available NTIS

Introduction to ASTM 1199 'Wear Test Selection for Design and Application

03,238 Not available NTIS

Introduction to Frequency Calibration. Part 1

PBg6-200654

01,560 Not available NTIS

Introduction to Phase-Stable Optical Sources.

Introduction to Secure Telephone Terminals.

Introduction to Secure Telephone Terminals.
PB97-110498 01,512 Not available NTIS

Introduction to the P1003.1g and CPI-C Network Application Programming Interfaces.

01,731 PC A03/MF A01

Introduction to Traffic Management for Broadband ISDN.
PB94-142494
01,464 PC A03/MF AO

Inversion of the Phase Diagram from UCST to LCST in Deuterated Polybutadiene and Protonated Polybutadiene Blends.

PB94-212446

01,226 Not available NTIS

Investigating the 3.3 Micron Infrared Fluorescence from Naphthalene Following Ultraviolet Excitation.

N95-15839/0

00,724

(Order as N95-15827/5, PC A07/MF A02)

Investigation into a Practical Grain Growth Model for Ho

Isostatic Pressing
PB95-151684

03.347 Not available NTIS

Investigation into the Flammability Properties of Honeycomb Composites.
PB95-143293

03,152 PC A03/MF A01

Investigation of Applicability of Alanine and Radiochromic

Detectors to Dosimetry of Proton Clinical Beams.
PB96-146782 03,636 Not available NTIS

Investigation of High-Temperature Platinum Resistance Thermometers at Temperatures Up to $962 \mathrm{C}$, and, in Some Cases, $1064 \mathrm{C}$

00,629 Not available NTIS

Investigation of LS Coupling in Boronlike lons.

PB94-185295 03,797 Not available NTIS

Investigation of Mercury Interstitials in $\mathrm{Hg}(1-\mathrm{x}) \mathrm{CdxTe}$ Alloys

Using Resonant Impact-Ionization Spectroscopy.
PB94-213485 02,133 Not available NTIS

Investigation of Oil and Gas Well Fires and Flares.

PB94-193976 03,695 PC A04/MF A01

Investigation of S2F10 Production and Mitigation in Compressed SF6-Insulated Power Systems.
PB94-212388

Investigation of S2F10 Production and Mitigation in Compressed SF6- Insulated Power Systems.
PB96-155528

Investigation of the Drive Circuit Requirements for the Power Insulated Gate Bipolar Transistor (IGBT).
PB94-211794 02,316 Not available NTIS

Investigation of the Effects of Aging on the Calibration of a Kerr-Cell Measuring System for High Voltage Impulses. 02,025 Not available NTIS Investigation of the ITS-90 Subrange Inconsistencies for 25.5 Omega SPRTs

00,630 Not available NTIS

Investigation of the Thermal Stability and Char-Forming Tendency of Cross-linked Poly(methyl methacrylate).
PB94-213501 03,380 Not available NTIS

Investigations of $\mathrm{AM}$ and $\mathrm{PM}$ Noise in X-Band Devices.

PB95-180022 02,062 Not available NTIS

Investigatlons of Sulfur Interferences in the Extraction of Methylmercury from Marine Tissues.
PB96. 190020 Not available NTIS 


\section{TITLE INDEX}

Investigations of the Systematics of Crystal Packing Using the Cambridge Structural Database.

00,519

(Order as PB97-109011, PC A11/MF A03)

Investing in Education to Meet a National Need for a Tech nical-Professional Workforce in a Post-Industrial Economy.

Atoms and lodomethane Radical Cations: Their Formation in the Pulse Radiolysis of lodomethane in Organic Solvents. Their Complexes, and Their Reactivity with OrSolvents, Their Complexes, and Their Reactivity with Or-
ganic Reductants. ganic Reductan
PB 95- 162764

00,965 Not available NTIS

Ion Broadening Parameters for Several Argon and Carbon

Lines.

03,843 Not available NTIS

Ion Kinetic-Energy Distributions and Balmer-alpha (Halpha) Excitation in Ar-H2 Radio-Frequency Discharges.

04,029 Not available NTIS

Ion Kinetic-Energy Distributions in Argon if Glow Discharges.
PB95-141008

04,409 Not available NTIS

Ion Kinetics and Symmetric Charge-Transfer Collisions in Low-Current, Diffuse (Townsend) Discharges in Argon and Nitrogen,
PB96-123658

04,051 Not available NTIS

Ionization Energies, Appearance Energies and Thermochemistry of CF2O and FCO.
PB97-111538 01,178 Not available NTIS Ionization Energy of Sulfur Pentafluoride and the Sulfur Pentafluoride-Fluorine Atom Bond Dissociation Energy.
PB95-162814 00,966 Not available NTIS

lonizing Radiation Causes Greater DNA Base Damage in Radiation-Sensitive Mutant M10 Cells That in Parent Mouse Lymphoma L5178Y Cells.

03,632 Not available NTIS

lonizing radiation-induced DNA damage and its repair in human cells. Progress report, (April 1, 1993--February 28 , DE94014709

03,612 PC A01/MF A01

lonospheric Radio Propagation.
AD-A286 619/2

IRAS Spectroscopic Observations of Young Planetary NebUlae. 152070

00,072 Not available NTIS

Iridium Oxide Thin-Film Stability in High-Temperature Corrosive Solutions
PB94-213014

03,234 Not available NTIS

Irradiance of Horizontal Ouartz-Halogen Standard Lamps. PB96-179130 01,866

(Order as PB96-177381, PC A07/MF A02)

Irradiances of Spectral Lines in Mercury Pencil Lamps.

PB96-176466 04,375 Not available NTIS

Irradiation Damage in Inorganic Insulation Materials for ITER Magnets: A Review.

03,705 PC A18/MF A04

ISDN Conformance Testing.

PB95-163176

01,478 Not available NTIS

ISDN Conformance Testing Guidelines: Guidelines for Implementors of ISDN Customer Premises Equipment to Conform to Both National ISDN-1 and North American ISDN Users' Forum Layer 3 Basic Rate Interface Basic Call Control Abstract Test Suites.

PB94-219094

01,471 PC A03/MF A0

ISDN in North America.

PB96-160767

01,502 Not available NTIS

ISDN LAN Bridging.

01,477 PC A03/MF A01

ISO Environmental Management Standardization Efforts.
PB95-220513
$02,524 \quad$ PC A03/MF A01

ISO Environmental Management Standardization Efforts.
PB96-158662
02,528 PC A03/MF A01

ISO/IEC Workshop on Worldwide Recognition of OSI Test Results Regional Progress - North America.
PB94-172202

ISO TC 184/SC4 Reference Manual.
PB95-242293 02,663 PC A04/MF A01

Isochoric (p-p-T) Measurements on Liquid and Gaseous Air

from 67 to $400 \mathrm{~K}$ at Pressures to $35 \mathrm{MPa}$.
PB96-167390 01,154 Not available NTIS

Isolated Spin Pairs and Two-Dimensional Magnetism in

SrCr(sub 9p)Ga(sub 12-9p)O19.

04,154 Not available NTIS

Isolation and Structural Elucidation of the Predominant Geometrical Isomers of alpha-Carotene.
PB96-190061
Isopiestic Investigation of the Osmotic and Activity Coefticients of Aqueous $\mathrm{NaBr}$ and the Solubility of $\mathrm{NaBr} 2 \mathrm{H} 2 \mathrm{O}(\mathrm{cr})$ at $298.15 \mathrm{~K}$ : Thermodynamic Properties of the $\mathrm{NaBr}+\mathrm{H} 2 \mathrm{O}$ System over Wide Ranges of Temperature and Pressure.
PB97-110365 Isotope Dilution Mass Spectrometry as a Candidate Definitive Method for Determining Total Glycerides and Triglycerides in Serum.

03,519 Not available NTIS

Isotope Shifts and Hyperfine Splittings of the 398.8-nm $\mathrm{Yb}$ |

Line.

03,814 Not available NTIS

Isotopic and Nuclear Analytical Techniques in Biologica Systems: A Critical Study. 10. Elemental Isotopic Dilution Analysis with Radioactive and Stable Isotopes (Technical Report)

PB96-164157

00,696 Not available NTIS

Issues and Recommendations for a STEP Application ProTramework. National PDES Testbed.

PB94-160868

02,770 PC A05/MF A01

Issues Concerning Material Removal Shape Element Volumes (MRSEVs)

02,882 Not available NTIS

Issues Concerning Material Removal Shape Element Volumes (MRSEVs)

02,885 PC A03/MF A01

PB95-210167

02,693 Not available NTIS

PB97-118368

Issues in the Field Measurement of VOC Emission Rates.
PB97-118806

ITS-90 Calibration Facility

PB96-160916

00,627 Not available NTIS

IUE Observations of Solar-Type Stars in the Pleiades and the Hyades.
PB94-199437

00,053 Not available NTIS

Jacket Thickness Requirements for Seismic Retrofitting of Circular Bridge Columns.

01,336 Not available NTIS

PB95-163267 January 17, 1995 Hyogoken-Nanbu (Kobe) Earthquake.
Performance of Structures, Lifelines and Fire Protection Systems. Executive Summary and Paper.

PB97-104160

00,475 PC A99/MF A06

Japan Technology Program Assessment: Precision Engineering/Precision Optics in Japan.

neering/Precisior

02,884 PC A03/MF A01

Japan Technology Program Assessment. Simulation: Stateof the-Art in Japan

$02,827 \mathrm{PC} \mathrm{A03/MF} \mathrm{A01}$

PB95-217097

eriment: Lessons Learned. PB95-203188 02,371 Not available NTIS

Joint DoD/NIST Workshop on International Manufacturing Systems Research and Development. Held in Rockville,

Maryland on November 3-5, 1992. Proceedings.
PB96-109491 PB96-109491

PB96-148044

02,418 Not available NTIS

Josephson Voltage Standard Based on Single-Flux-Ouantum Voltage Multipliers.

PB95-175600

02,058 Not available NTIS

Journal of Physical and Chemical Reference Data, Volume 22, No. 1, January/February 1993.

PB94-160975

00,729 Not available NTIS

Journal of Physical and Chemical Reference Data, Volume 22, No. 2, March/April 1993

PB94-162211

00,733 Not available NTIS

Journal of Physical and Chemical Reference Data, Volume 22, No. 3, May/June 1993

PB94-162260

00,738 Not available NTIS

Journal of Physical and Chemical Reference Data, Volume 22, No. 4, July/August 1993

00,743 Not available NTIS

Joumal of Physical and Chemical Reference Data, Volume

22, No. 5, September/October 1993.
PB94-162336 00,745 Not available NTIS

Journal of Physical and Chemical Reference Data, Volume

22, No. 6, November/December 1993 .

Journal of Physical and Chemical Reference Data, Volume

24, No. 1, January/February 1995

01,101 Not available NTIS

Journal of Physical and Chemical Reference Data, Volume

24, No. 2, March/April 1995.

01,105 Not available NTIS

Journal of Physical and Chemical Reference Data, Volume

24, No. 3, May/June 1995.

Journal of Physical and Chemical Reference Data, Volume 24, No. 4, July/August 1995

PB96-145883

01,112 Not available NTIS

Journal of Physical and Chemical Reference Data, Volume 24, No. 5, September/October 1995

PB96-145925

01,116 Not available NTIS

Journal of Physical and Chemical Reference Data, Volume 24, No. 6, November/December 1995 .

PB96-145966

01,120 Not available NTIS

Journal of Research of the National Institute of Standards and Technology. January/February 1994. Volume 99, Number 1

PB94-169737

02,019 PC A07/MF A02

Journal of Research of the National Institute of Standards and Technology, January/February 1996. Volume 101, Number 1.

PB96-175666

$00,113 \mathrm{PC} \mathrm{A07/MF} \mathrm{AO2}$

Journal of Research of the National Institute of Standards and Technology, July/August 1994. Volume 99, Number 4. Special Issue: Extreme Value Theory and Applications. ProApplications, Volume 2. Held at Gaithersburg, Maryland, in May 1993.

May 1993.

03,440 PC A13/MF A03

Journal of Research of the National Institute of Standards 列 and Technology, July/August 1995. Volume 100, Number 4. Frequency Reference Cell.

Frequency Re

$02,386 \mathrm{PC} \mathrm{A09/MF} \mathrm{AO3}$

Journal of Research of the National Institute of Standards and Technology. March/April 1994. Volume 99, Number 2. PB94-219219

02,000 PC A06/MF A02

Journal of Research of the National Institute of Standards and Technology, March/April 1995. Volume 100, Number 2. PB96-126180 04,349 PC A05/MF A01

Journal of Research of the National Institute of Standards and Technology, March/April 1996. Volume 101, Number 2. PB96-177381 01,863 PC A07/MF A02

Journal of Research of the National Institute of Standards and Technology. May/June 1994. Volume 99, Number 3.
PB94-219326

Journal of Research of the National Institute of Standards and Technology, May/June 1995. Volume 100, Number 3. PB95-261897
02,670 PC A07/MF A02

Journal of Research of the National Institute of Standards and Technology, May/June 1996. Volume 101, Number 3. Special Issue: NIST Workshop on Crystallographic Databases.

04,798 PC A11/MF A03

Journal of Research of the National Institute of Standards and Technology, November-December 1993. Volume 98,

Number 6.
PB94-140555

04,223 PC A06/MF AO2

Journal of Research of the National Institute of Standards and Technology, November/December 1994. Volume 99. Number 6.

04,336 PC A05/MF A01

Journal of Research of the National Institute of Standards and Technology, November/December 1995. Volume 100 , Number 6.

PB96-159215

01,949 PC A08/MF A02

Journal of Research of the National Institute of Standards and Technology, September-October 1993. Volume 98, Number 5.

03,362 PC A05/MF A01

Journal of Research of the National Institute of Standards and Technology, September/October 1993. Volume 98 Number 5.

PB96-169057

03,368 PC A06/MF A01

Journal of Research of the National Institute of Standards and Technology, September/October 1994. Volume 99 Number 5.

PB95-16937

04,293 PC A07/MF A02

Journal of Research of the National Institute of Standards and Technology, September/October 1995. Volume 100, Number 5 .

PBumber 5.

01,927 PC A08/MF A02

Joy of Acceptance Diagrams. PB94-200433

03,828 Not available NTIS

Junction Locations by Scanning Tunneling Microscopy: InAir-Ambient Investigation of Passivated GaAs pn Junctions.
02,306 Not available NTIS 02,306 Not available

Kalpha Transitions in Few-Electron lons and in Atoms.
PB94-212248 Keeping Time on Your PC. 01,537 Not available NTIS PB95-161410 Keeping Up with the Reality of Today's Surge Environment.
PB96-123633 
Keeping Your Site Comfortably Secure: An Introduction to Internet Firewalls.

02,730 PC A05/MF A01 Kibitz-Connecting Multiple Interactive Programs Together.
PB94-213311 Kinetic Energy Distribution of lons Produced from Townsend Discharges in Neon and Argon.
PB96-111927 Not available NTIS Kinetic Energy Distributions of $\mathrm{H}(+), \mathrm{H} 2(+)$, and $\mathrm{H} 3(+)$ from
a Diffuse Townsend Discharge in $\mathrm{H} 2$ at High $\mathrm{E} / \mathrm{N}$. a Diffuse Townsend Discharge in $\mathrm{H} 2$ at High E/N.
PB96-123351 04,415 Not available NTIS Kinetic-Energy Distributions of lons Sampled from Argon Plasmas in a Parallel-Plate, Radio-Frequency Reference

03,935 Not available NTIS

Kinetic-Energy Distributions of lons Sampled from RadioFrequency Discharges in Helium, Nitrogen, and Oxygen. PB96-123732 01,092 Not available NTIS Kinetic-Energy Distributions of $\mathrm{K}(+)$ in Argon and Neon in Uniform Electric Fields.

03,902 Not available NTIS

Kinetic-Energy-Enhanced Neutral Etching

PB96-200613 00,665 Not available NTIS

Kinetic-Inductance Infrared Detector Based on an AntennaCoupled High-TC SQUID.

PB95-169116

02,165 Not available NTIS

Kinetics and Dynamics of Vibrationally State Resolved IonMolecule Reactions: $(14) \mathrm{N} 2(+)(\mathrm{v}=1$ and 2$)$ and $(15) \mathrm{N} 2(+)(\mathrm{v}=0,1$ and 2$)$ with $(14) \mathrm{N} 2$

PB96-102348 04,023 Not available NTIS

Kinetics and Mechanism of the Collision-Activated Dissociation of the Acetone Cation.

00,859 Not available NTIS

Kinetics of the Reaction $\mathrm{C} 2 \mathrm{H}+\mathrm{O} 2$ from 193 to $350 \mathrm{KU}$ Using Laser Flash Kinetic Infrared Absorption Spectroscopy.

PB95-203055

01,043 Not available NTIS

Kinetics of the Reaction of $\mathrm{CCl}_{3}-8 \mathrm{r}-2$ and the Thermochemistry of $\mathrm{CCl} 3$ Radical and Cation.

PB94-212115 00,824 Not available NTIS

Kinetics of the Reaction of the Sulfate Radical with the Oxalate Anion.

PB97-119127 01,186 Not available NTIS

Kinetics of the Self-Reaction of Hydroxymethylperoxyl Radi-

cals.
PB94-212164

00,827 Not available NTIS

Knowledge-Based Approach for Automating a Design Method for Concurrent and Real-Time Systems.

PB97-112502

01,780 Not available NTIS

L-threo-beta-Hydroxyhistidine, an Unprecedented Iron(III) lon-Binding Amino Acid in a Pyoverdine-type Siderophore from Pseudomonas fluorescens 244

PB94-211620 00,553 Not available NTIS

Lab Report Special Section: Natural Language Processing and Information Retrieval Group Information Access and User Interfaces Division, National Institute of Standards and User Interfaces Division, National Institute of Standards and
Technology.
PB97-118665
02,742 Not available NTIS

Labeling Conventions in Isoelectronic Sequences - Reply.

P895-162574 03,937 Not available NTIS

Laboratory Accreditation for Testing Energy Efficient Lighting.

00,270 Not available NTIS

Laboratory Measurements for the Astrophysical Identification of $\mathrm{MgH}$.
PB95-152195

00,073 Not available NTIS

Laboratory Studies of Low-Temperature Reactions of $\mathrm{C} 2 \mathrm{H}$ with $\mathrm{C} 2 \mathrm{H} 2$ and Implications for Atmospheric Models of

PB95-108726

00,690 Not available NTIS

Lake Erie Water Temperature Data, Put-in-Bay, Ohio, 1918.

03,692 PC A04/MF A01

Langevin Approach to Hysteresis and Barkhausen Modeling in Sieel.

PB94-185675

03,206 Not available NTIS

Langmuir Probe Measurements in the Gaseous Electronics

Conference RF Reterence Cell.
PB96-113386

02,393

(Order as PB96-113311, PC A09/MF A03)

Laplace's Equation and the Dirichlet-Neumann Map in Multiply Connected Domains. (NIST Reprint).
PB95-180295 Not available NTIS

Large-Amplitude Shapiro Steps and Self-Field Effects in High-Tc Josephson Weak Links.
PB95-180410 10,682 Not availabie NTIS Large Amplitude Skeletal Isomerization as a Promoter of
Intramolecular Vibrational Relaxation in $\mathrm{CH}$ Stretch Excited Hydrocarbons.

01,036 Not available NTIS

Large Eddy Simulations of Smoke Movement in Three DiPB96-190012

01,426 Not available NTiS

Large Fire Experiments for Fire Model Evaluations.

(19)

01,427 Not available NTIS

Large Local-Field Corrections in Optical Rotatory Power of

04,400 Not available NTIS

PB97-122378

as a Free Atom Source for

aser Ablation

00,540 Not available NTIS

PB94-198710

acold Temperatures.

PB95.161220

03,929 Not available NTIS

Laser Bandwidth Effects in Quantitative Cavity Ring-Down PBg7-112254

04,394 Not available NTIS

Laser-Beam Analysis Pinpoints Critical Parameters.

P894-212552 04,240 Not available NTIS

Laser-Cooled Neutral Atom Frequency Standards.

PB96-160312

04,086 Not available NTIS

Laser-Cooled Positron Source.

PB95-169348

03,954 Not available NTIS

Laser Cooling

03,908 Not available NTIS

Laser Cooling and the Recoil Lim

PB97-111280 04,391 Not available NTIS

Laser Cooling and Trapping for the Masses.

P895-126090 04,253 Not available NTIS

Laser Cooling of Trapped Ions

PB95-168746

03,950 Not available NTIS

Laser Doppler Velocimeter Studies of the Pipeflow Produced by a Generic Header

02,602 PC A05/MF A01

Laser Double Resonance Measurements of the Quenching Rates of $\mathrm{Br}((2) \mathrm{P} 1 / 2)$ with $\mathrm{H} 20, \mathrm{D} 20, \mathrm{HDO}$, and $\mathrm{O} 2$

P895-150694 00,921 Not available NTIS

Laser Flash Photolysis, Time-Resolved Fourier Transform Infrared Emission Study of the Reaction $\mathrm{Cl}+\mathrm{C} 2 \mathrm{H} 5$ yields HCl(v) + $\mathrm{C} 2 \mathrm{H} 4$

01,049 Not available NTIS Laser Flash Photolysis/Time-Resolved FTIR Emission Study
of a New Channel in the Reaction of $\mathrm{CH} 3+\mathrm{O}$ : Production of of a New Channel in the Reaction of $\mathrm{CH} 3+\mathrm{O}$ : Production of
$\mathrm{CO}(\mathrm{v})$. $\mathrm{CO}(v)$
P895-164281

00,974 Not available NTIS

Laser-Focused Atomic Deposition.

PB95-161618 04,604 Not available NTIS

Laser Focused Atomic Deposition.

P895-180659 04,685 Not available NTIS

Laser Focusing of Atoms: A Particle Optics Approach.

03,870 Not available NTIS

Laser Gas Ionization Technique Monitors MEB Crystal Growth.

01,076 Not available NTIS

Laser Imaging of Chemistry-Flowfield Interactions: En-

hanced Soot Formation in Time-Varying Diffusion Flames.
PB94-185352 01,364 Not available NTIS

Laser-Induced Desorption of In and $\mathrm{Ga}$ from $\mathrm{Si}(100)$ and

Adsorbate Enhanced Surface Damage.
PB95-203311 PB95.203311

Laser-Induced Desorption of NO from Si(111): Effects of Coverage on NO Vibrational Populations.

P895-162319

00,959 Not available NTIS

Laser-Induced Fluorescence Measurements of Formaldehyde in a Methane/Air Diffusion Flame.

Laser-Induced Fluorescence Measurements of $\mathrm{OH}$. Concentrations in the Oxidation Region of Laminar, Hydrocarbon Diffusion Flames.
PB95-162160 $\quad 01,387$ Not available NTIS

Not available NTIS

Laser-Induced Fluorescence Measurements of $\mathrm{OH}$ in Lam-

inar Diffusion Flames in the Presence of Soot Particles.
P896-123120 07,409 Not available NTIS

Laser-Induced Fluorescence Measurements of Rotationally Resolved Velocity Distributions for $\mathrm{CO}(+)$ Drifted in $\mathrm{He}$.

PB94-213139

00,848 Not available NTIS

Laser Magnetic-Resonance Measurement of the (3)P1 .

(3) P2 Fine-Structure Splittings in (17)O and (18)O

PB95-175170 00,994 Not available NTIS

Laser Melting of Thin Silicon Films.

PB94-199239 04,471 Not available NTIS aser Modification of Ultracold Collisions: Experiment. P896-157987

04,075 Not available NTIS

Laser Photoionization Measurements of Pressure in VacuPB95-180600

03,964 Not available NTIS

Laser Preparation and Probing of Initial and Final Orbita Alignment in Collision-Induced Energy Transfe PB95-203261 + He yields Ca(4s5p,(3)P

Laser-Produced and Tokamak Spectra of Lithiumlike Iron, $\mathrm{Fe}(23+)$.

$95-180857$

04,314 Not available NTIS

Laser Spectroscopy of Carbon Monoxide: A Frequency Reference for the Far Infrared.

-163606

04,277 Not available NTIS

Laser-Synchrotron Hybrid Experiments: A Photon to Tickle A Photon to Poke.

03,704 Not available NTIS

Laser Vacuum Ultraviolet Single Photon Ionization Probing of III-V Semiconductor Growth. 04,691 Not available NTIS
PB95-202370 attice Dynamics of $\mathrm{Ba} 1-\mathrm{xK} \times \mathrm{BiO}$

PB96-102421 04,706 Not available NTIS

Lattice Dynamics of Semiconducting. Metallic, and Superconducting $\mathrm{Ba}+\times \mathrm{KK} \times \mathrm{BiO} 3$ Studied by Inelastic Neutron Scattering. 96-1024

Lattice Imperfections Studied by Use of Lattice Green's Functions.
PB95-150850

04,576 Not available NTIS

Lattice Model of a Hydrogen-Bonded Polymer Blend.

PB97-112262

03,391 Not available NTIS

Lattice Position of Si in GaAs Determined by X-Ray Standing Wave Measurements.

PB95-164406

04,632 Not available NTIS

Lattice Statics of Interfaces and Interfacial Cracks in Bimaterial Solids.

02,985 Not available NTIS

Lead Abatement in Buildings and Related Structures.

P894-172038 03,601 Not available NTIS

Leady Axisymmetric Modes in Infinite Clad Rods. Part 1

P895-162905 04,187 Not available NTIS

Leakage Current Detection in Cryogenic Current Comparator Bridges.

02,024 Not available NTIS

Lean flammability limit as a fundamental refrigerant property. Phase 1, Interim technical report, 1 October 1994--31 March 1995

03,248 PC A03/MF A01

E95011238

Learning to Change: Opportunities to Improve the Performance of Smaller Manufacturers.

PB94-166212

00,010 PC A08/MF A02

Least-Cost Energy Decisions for Buildings: Part 2. Uncertainty and Risk

00,240 PC A04/MF A01

Least-Cost Energy Decisions for Buildings. Part 3. Choosing Economic Evaluation Methods. Video Training WorkPB95-253597

$00,265 \mathrm{PC} \mathrm{A04/MF} \mathrm{A01}$

Length Metrology of Complimentary Small Plastic Rulers. PB96-161724

04,096 Not available NTIS

Length Scales for Fragile Glass-Forming Liquids.

P896-102801 07,065 Not available NTIS

Lessons from the Establishment of the U.S. GOSIP Testing

PB96-119359

01,817 Not available NTIS

Lessons trom the Loma Prieta Earthquake.

PB95-164091

00,442 Not available NTIS

Lessons Learned by a Wing Engineer

00,429 Not available NTIS

Letter Report on Flame Spread Testing of a Composite MaP897-110068

01,436 Not available NTIS

Life-Cycle Costing Manual for the Federal Energy Management Program. 1995 Edition.

P896-172317

$02,511 \mathrm{PC} \mathrm{A10/MF} \mathrm{A02}$

Life-Cycle Costing Workshop for Energy Conservation in Buidings: Student Manual.

00,257 PC A11/MF A03

Life Prediction of a Continuous Fiber Reinforced Ceramic Composite Under Creep Conditions.

P896-204128 03,091 Not available NTIS

Light Scattered by Coated Paper.

P894-216546 


\section{TITLE INDEX}

Light Scattered from Two Atoms. PB95-168753

04,286 Not available NTIS

Light Scattering by Sinusoidal Surfaces: Illumination Windows and Harmonics in Standards.

PB97.110548

04,387 Not available NTIS

Light Scattering from Glossy Coatings on Paper.

PB94-213246 04,242 Not available NTIS

Light-Scattering Studies on Phase Separation in a Binary Blend with Addition of Diblock Copolymers.

PB96-146865 01,286 Not available NTIS

Light-Weight Alloys for Aerospace Applications II

PB96-190244 03,375 Not available NTIS

Lighting and HVAC.

PB.250 Not available NTIS

Lighting Quality and Light Source Size.

$\infty, 252$ No: available NTIS

Lighting Research and Theory Can Create Business ProsPects.

00,253 Not available NTIS

Lightwave Standards Development at NIST.

PB̈95-168563 01,480 Not available NTIS

Limits of $\mathrm{CO} 2$ Monitoring in Determining Ventilation Rates.

PB95-176004 02,552 Not available NTIS

Line-Heat-Source Guarded-Hot-Plate Apparatus.

PB97-118996 00,417 Not available NTIS

Line-Reflect-Match Calibrations with Nonideal Microstrip Siandards.

PB96-176599

02,242 No: available NTIS

Lineshape Analysis of the Raman Spectrum of Diamond Films Grown by Hot-Flament and Microwave-Plasma Chemical-Vapor Deposition PB95-162392 03,016 Not available NTIS

Linewidth Narrowing in an Imbalanced Y-Branch Waveguide Laser.
PB95-140844

04,258 Not available NTIS

Linking Anisotropic Sharp and Diffuse Surface Motion Laws via Gradient Flow

PB95-203378

04,698 Not available NTIS

Liposome-Based Flow-Injection Immunoassay for Determining Theophylline in Serum.

03,494 Not available NTIS

Liquid and Solid Aiomic Ion Plasmas.

PB94-198991 03,809 Not available NTIS

Liquid Chromatographic Determination of Carotenoids in Human Serum Using an Engineered C30 and a C18 Stationary Phase.
PB97-119333

03,512 Not available NTIS

Liquid Chromatographic Determination of Polycyclic Aro matic Hydrocarbon Isomers of Molecular Weight 278 and 302 in Environmental Standard Reference Materials.
PB95-164042

Liquid Chromatographic Method for the Determination of Carotenoids, Retinoids, and Tocopherols in Human Serum and in Food.

00,593 Not available NTIS

Liquid Chromatography: Laser-Enhanced Ionization Spec. trometry for the Speciation of Organo'ead Compounds.
P894-185253

Liquid-Hydrogen Cold Neutron Source for the NBSR.

PB95-151619 03,729 Not available NTIS

Liouid-Nitrogen-Cooled High Tc Electrical Substitution RadiOmeter as a Broadband IR Transfer Standard.

Liquid-Scintillation Counting Technigues for the Standard

ization of Radionuclides Used in Therapy.
PB97-110084 03,709 Not available NTIS

Literatura Review on Seismic Petormance of Building Cladding Systems
PB96-106901

00,455 PC AO9MF AO2

Lithium-Drift Technique for Making Submicron Thick Silicon Membranes.
PB95-161386

02,260 Not available NTIS

LMTO/CVM and LAPW/CVM Calculations of the Nickel AluminideNickel Titanium Pseudobinary Phase Diagram.
PB94-199353 03,323 Not available NTIS

Loading Davice for Fracture Testing of Compact Tension Specimens in the Scanning Electron Microscope.

PB95-162434 02,652 Not available NTIS

Loading Effects in Resistance Scaling.
PB97-111884 Not available NTIS

Local Area Networks in NAA: Advantages and Pittalls.

PB94-172095 00,527 Not available NTIS
Local-Mode Dynamics in YH2 and YD2 by 1sotope-Dilution Neutron Spectroscopy.

01,017 Nor available NTIS

Local Oscillator Requirements and Strategies for the Next Generation of High-Stability Frequency Standards. PB96-112230 01,551 Not available NTIS

Local Partial Densities of States in $\mathrm{Ni}$ and Co Silicides Studied by Soft X-Ray Emission Spectroscopy.
PB94-212412

Localization Model of Rubber Elasticity: Comparison with Torsional Data for Natural Rubber Networks in the Dry State.

03,195 Not available NTIS

Localization of a Homopolymer Dissolved in a Lamellar Structure of a Block Copolymer Studied by Small-Angle Neutron Scattering

01,253 Not available NTIS

Localization of Atoms in a Three-Dimensional Standing

PB95-163887

03,944 Not available NTIS

Locating Fire Engineering Information

PB95-161188

00,198 Not available NTIS

Locating Fire Information.

00,227 Not available NTIS

Location of a (1)A(sub g) State in Bithiophene.

PB95-202313 01,025 Not available NTIS

Long-Lived Structures in Fragile Glass-Forming Liquids.

PB90-119565

04,212 Not available NTIS

Lono-Range Parity-Nonconserving Interaction

Long-Term Performance of Engineered Concrete Barriers.
PB95-260816 03.727 PC A03MF A01

Long-Term Stability of Bayard-Alper Gauge Performance: National Institute of Standards and Technology Priman echnology Primary Vacuum Siandard.

02,676 Not available NTIS

Long-Term Stability of Carrier-Free Polonium Solution PB94-185170

00,685 Not available NTIS

Longterm Changes of Silicon Photodiodes and Their Use PB94-211349

04,234 Not available NTIS

Look at Uncertainties over Twenty Decades of Pressure Measurement.

02,655 Not available NTIS

Loss-Free Counting at IRI and NIST.

PB96-167119 04,105 Not available NTIS

Low-Coherence Interferometric Measurement of Group

Transit Times in Precision Optical Fibre Delay Lines.
PB95-168480
02,158 Not available NTIS PB95-168480

Low Electrolytic Conductivity Standards.

PB96-122098

01,081

(Order as PB96-117767, PC A08/MF A02)

Low-Energy-Electron Collisions with Sodium: Elaśtic and InElastic Scattering from the Ground State.
PB96-103106 Low-Energy Electron Scattering from Caesium Atoms: Comparison of a Semirelativistic Breit-Pauli and a Full Relativisic Direc Treatment

PB94-185030

0, 769 Not available NTIS

Low-Energy Vibrations and Octahedral Site Occupation in No95V5H(D)y.
PB96-160734

01,133 Not available NTIS

Low-Frequency, Active Vioration Isolation System

P895-203303 02,710 Not available NTIS

Low-Frequency Excitations of Oriented DNA.

PB96-137799 03,548 Not availab!e NTIS

Low-Frequency Modal for Radio-Frequency Absorbers.

PB95-261939 0.424

(Order as PB95-261897, PC A07MF A02)

Low Heat-Flux Veasurements: Some Precautions.

PB96-201116 02,685 Not available NTIS

Low Magnetostriction in Annealed NiFelAg Giant Magnetoresistive Multilayers.

PB96-14669

04,762 Not available NTIS

Low-Noise High-Speed Diode Laser Current Controllar.
PB95-202826
02,178 Not available NTIS

Low Noise YBa2Cu307-x-SrTiO3-YBa2Cu307-x Mulïlayers

for Improved Superconducting Magnetometers.
PB96-138417
04,747 Not available NTIS

Low-Temperature Elastic Constants of $\mathrm{Y} 1 \mathrm{Ba} 2 \mathrm{Cu} 3 \mathrm{O} 7$

PB95-168837 04,642 Not available NTIS
Low temperature tabncation from nano-size ceramic powDE 95013505

$03,029 \mathrm{PC} \mathrm{AO5/MF} \mathrm{AO}$

Low Temperature H(sub 2)S Separation Using Membrane Reactor with Redox Catalyst.

$02,471 \mathrm{PC} \mathrm{A02/MF} \mathrm{AO1}$

Low-Temperature Performance of Radiosonde Electric Hygrometer Elements
AD-A295 $319 / 8$

00,121 PC AO2MF AO1

Low-Temperature Properties of Silver.

PB96-126198

03,361

(Order as PB96-126180, PC A05/MF A01)

Low Thermal Guarded Scanner for High Resistance Measurament Systems.

02,288 Not available NTIS

Low Voltage Standards in the $10 \mathrm{~Hz}$ to $1 \mathrm{MHz}$ Range.

PB97-112569 02,100 Not available NTIS

Lowest Excited Singlet State of Isolated 1-phenyl-1,3-butadiene and 1-phenyl-1,3,5-hexatriene.
PB95-202339 Not available NTIS LP11-Mode Leakage Loss in Coated Depressed Clad Fi. PBS. 02,145 Not available NTIS

LRM Probe-Tip Calibrations Using Nonideal Standards. PB96-135389 02,411 Not available NTIS LRM Probe-Tip Calibrations with Imperfect Resistors and Lossy Lines.
PB95-163952

02,353 Not available NTIS

Lubrication Theory for Reactive Spreading of a Thin Drop.
PB95-189460 Lubrication Theory for Reactive Sprea ding of a Thin Drop.
PB96-123427

Lunar Laser Ranging: A Continuing Legacy of the Apollo Program.

03,683 Not available NTIS

M (also known as MUMPS) Validation Test Suite. Version 8.3 (for Microcomputers)

01,699 Diskette $\$ 250.00$

Machine Performance Standard Provides Opportunity $10 \mathrm{~lm}$ prove Quality and Productivity.
PB96-154521

02,837 PC AO2MF AO1

Machining Process Planning Activity Model for Systems Integration.
PB96-165428

$02.841 \mathrm{PC} \mathrm{A03/MF} \mathrm{AO1}$

Macro- and Microreactions in Mechanical-Impact Tests of Aluminum Alloys.

03,340 Not available NTIS

Magnetic and Magnetoresistive Properties of Inhomogeneous Magnetic Dual-Laye Films.
PB95-169025 646 Not available NTIS

Magnetic and Structural Properties of Electrodeposited Copper-Nickel Microlayered Alloys.

PB94-213121

04,512 Not available NTIS

Magnetic Characteristics and Neasurements of Filamentary ND-Ti Wire for the Superconducling Super Collider.

Magnetic Dead Layer in Fe/Si Muttilayer. Profile Refinement of Polarizeo Neutron Reftectivity Data.

PBOA-198363 utron Retiectivity Data. Not available NTIS

Magnetic Dielectric Oxides: Subsolidus Phase Relations in the BaO: Fe203: TiO2 System.

PBe BaO. Fe203: TIO2 System. 01,156 Not available NTIS

Magnetic Dipole Line from $U$ LXXI Ground-Term Levels Predicted at 3200 Angstroms.

PB94-211497

04,407 Not available NT1S

Magnetic Field Dependence of the Critical Current Arisotropy in Normal Metal-YBa2Cu307-delta Thin Film Bilayers.
PB95-141024

Nagnetic Fields in Star-Forming Ragions: Observations. PB96-123005

Ragions: Observations.

Nagnetic Flux Pinning in Epitaxial YBa2Cu307-delta Thin Films.

04,795 Not available NTIS

Nagnetic Force Microscopy Images of Magnetic Garnet with Thin-Film Magnetic Tip.

0.4,669 Not available NTIS

Viagnetic Force Microscopy of Flux in Superconductors.

P895-161733 04,608 Not available NTIS

Magnetic Measurement of Transport Crtical Current Density of Granular Superconductors.

P895-126199

04,531 Not available NTIS

Magnetic Woments in $\mathrm{Cr}$ Thin Films on Fe(100).

PB95-108429 04,525 Not available NTIS 


\section{TITLE INDEX}

Magnetic Neutron Scattering (Invited)

PB95-150074

04,557 Not available NTIS

Magnetic Ordering of the $\mathrm{Cu}$ Spins in PrBa2Cu3O6+x

PB95-140547 04,545 Not available NTIS

Magnetic Properties of Pd/Co Multilayers.
PB94-198751

Magnetic Properties of Single-Crystalline UCu3AI2.
PB95-180717

Magnetic Rare Earth Artificial Metallic Superlattices.

PB95-162293 04,611 Not available NTIS

Magnetic Structure and Spin Dynamics of the $\mathrm{Pr}$ and $\mathrm{Cu}$ in PBO 11183

04,036 Not available NTIS

Magnetic Structure Determination for Annealed Ni80Fe20/

Ag Multilayers Using Polarized-Neutron Reflectivity.
PB96-176615 03,739 Not available NTIS

Magnetic Susceptibility of $\mathrm{Pr} 2-\mathrm{xCexCuO} 4$ Monocrystals and Polycrystals.

04,677 Not available NTIS

Magneto-Optic Magnetic Field Sensor with $1.4 \mathrm{pT} /$ square root of $1(\mathrm{~Hz})$ Minimum Detectable Field at $1 \mathrm{kHz}$.

PB94-199551 02,125 Not available NTIS

Magneto-Optic Magnetic Field Sensors Based on Uniaxial Iron Garnet Films in Optical Waveguide Geometry.

PB95-153409 02,152 Not available NTIS

Magneto-Optic Magnetic Field Sensors Based on Uniaxial

Iron Garnet Films in Optical Waveguide Geometry.
PB95-168498

Magneto-Optic Rotation Sensor Using a Laser Diode as Both Source and Detector

04,390 Not available NTIS

Magneto-Optical Trapping of Metastable Xenon: IsotopeShift Measurements.

03,905 Not available NTIS

Magneto-Transport Properties of $\mathrm{HgCdTe}$

PB95-175840 04,661 Not available NTIS

Magnetocaloric Effect in Nanocomposites

PB95-162798 04,618 Not available NTIS

Magnetocaloric Effect in Rapidly Solidified Nd-Fe-Al-B Materials.

04,451 Not available NTIS

Magnetocaloric Effect of Ferromagnetic Particles.

PB94-185857 04,453 Not available NTIS

Magnetoelasticity in Rare-Earth Multilayers and Films.

Magnetoelasticity in Rare-Earth Multilayers and Films.
PB94-211356 04,495 Not available NTIS

Magnetometer Calibration Services

PB97-113252

01,993 Not available NTIS

Magnetooptic Effects.

PB96-119292

04,338 Not available NTIS

Magnetoresistance of Thin-Film NiFe Devices Exhibiting Single-Domain Behavior.

04,766 Not available NTIS

Magnetostriction and Giant Magnetoresistance in Annealed $\mathrm{NiFe/Ag} \mathrm{Multilayers.}$

PB96-102603

04,716 Not available NTIS

Majority and Minority Electron and Hole Mobilities in Heavily Doped Gallium Aluminum Arsenide.

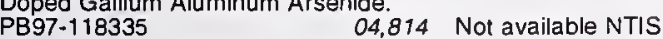

Majority and Minority Mobilities in Heavily Doped Silicon for Device Simulations.

PB94-198728

02,311 Not available NTIS

Making Connections.

PB97-119044

01,785 Not avallable NTIS

Making Displays

Measure. Not available NTIS

Making Sense of Software Engineering Environment Framework Standards.

01,701 PC A03/MF A01

Manager's Guide for Monitoring Data Integrity in Financial

Systems.
PB96-165915

00,003 PC A05/MF A01

Manipulation of Adsorbed Atoms and Creation of New Manipulation of Adsorbed Atoms and Creation of New
Structures on Room-Temperature Surfaces with a Scanning Structures on Room-Tem

PB95-151536

04,578 Not available NTIS

Manual for Ventilation Assessment in Mechanically Ventilated Commercial Buildings.

00,239 PC A07/MF A02 Manufactured Homes: Probability of Failure and the Need
for Better Windstorm Protection through Improved Anchoring Systems.

00,432 PC A04/MF A01
Manufactured Housing Walls That Provide Satisfactory Moisture Performance in All Climates.
PB95-178885 00,383 PC A03/MF A01 Mapping Domains in Proteins: Dissection and Expression of 'Escherichia coli' Adenylyl Cyclase.

03,478 Not available NTIS

Mapping Integration Definition for Function Modeling (IDEFO) Model into CASE Data Interchange Format (CDIF) Transfer File.

$01,741 \mathrm{PC} \mathrm{A07/MF} \mathrm{A02}$

PB96-109533 Mapping integration Definition for Information Modeling
(IDEF 1X) Model into CASE D.1ta Interchange Format CDIF) Transfer File.

01,711 PC A06/MF A02

PB95-154670

Mapping Processes to Processors for Space-Based Robot Systems.

PB95-151510

04,833 Not available NTIS

Mapping the Droplet Transfer Modes for an ER100S-1

$\begin{array}{ll}\text { GMAW Electrode. } & 03,295 \text { Not available NTIS } \\ \text { PB96-190095 }\end{array}$

MasPar MP-1 as a Computer Arithmetic Laboratory.

PB95- 189437 01,627 PC A03/MF A01

MasPar MP-1 as a Ccmputer Arithmetic Laboratory.

PB96-179155 01,617

(Order as PB96-177381, PC A07/MF A02)

Mass and Density Determinations.
PB94-200672 00,504 Not available NTIS

Mass Assay and Uniformity Test of Boron Targets by Neutron Beam Methods.

04,175 Not available NTIS

Mass Unit Disseminated to Surrogated Laboratories Using the NIST Portable Mass Calibration Package.
PB94-142486 03,781 PC A03/MF A01

Material Characterization By a Time-Resolved and Polariza-

PB $97-122576$ Ultrasonic Technique. 764 Not available NTIS

Materials and Fire Threat

PB97-12231

01,442 Not available NTIS

Materials Aspects of Fiber-Reinforced Polymer Composites in Infrastructure.

03,184 PC A04/MF A01

Materials Reliability. Technical Activities, 1995

PB96-183082

02,999 PC A07/MF A02

Materials Science and Engineering Laboratory Annual Report, December 1993.

02,668 PC A03/MF A01

Materials Science and Engineering Laboratory Annual Report, 1993. NAS-NRC Assessment Panel, April 21-22, 1994.
PB94-162534

Materials Science and Engineering Laboratory Annual Re port, 1994. NA.S-NRC Assessment Panel, April 6-7, 1995.
PB95-196697
02,976 PC A06/MF A02

Materials Science and Engineering Laboratory Annual Report, 1995. Technical Activities.
PB96-214754 03,009 PC A05/MF A01

Materlals-Science Based Approach to Phenol Emissions

from a Flooring Material in an Office Building.
PB97-118749

Materials Science with SR Using X-Ray Imaging: SpatialResolution/Source Size

04,510 Not available NTIS

Mathematical Analysis of Practices to Control Moisture in the Roof Cavities of Manufactured Houses. PB97-106843

00,278 PC A05/MF A01

Mathematical Aspects of Rietveld Refinement

PB95-1086CI 04,526 Not available NTIS

Mathematical Modeling and Computer Simulation of Fire Phenomena.

00,384 Not available NTIS

Mathematical Modeling of Human Egress from Fires in Residential Buildings.

00,337 PC A05/MF A02

Mathematical Models of Transport Phenomena Associated with Arc-Welding Processes: A Survey. Not available NTIS
PB96-135058

Matrix Grain Bridging Contribution to the Toughness of Whisker Reinforced Ceramics.
PB94-198645 03,134 Not available NTIS

Matrix Isolation Study of the Interaction of Excited Neon Matrix Isolation Siudy of the Interaction of Excited Neon BCl(sub 2, sup +), and BCl(sub 3, sup - ). PB97-119143

01,187 Not available NTIS

Matrix Isolation Study of the Interaction of Excited Neon Atoms with 03: Intrared Spectrum of O((sub 3)(-)) and Evidence for the Stabilization of O2... $(($ sub 4$)(+))$.
PB97-112403
Maturity Functions for Concrete Made with Various $\mathrm{Ce}$ ments and Adm

01,314 Not available NTIS

Maturity Method

01,313 Not available NTIS

Maximum Entropy as a Tool for the Determination of the C. Axis Profile of Layered Compounds. Axis Profile of

00,800 Not available NTIS

Maximum Permissible Amounts of Radioisotopes in the Human Body and Maximum Permissible Concentrations in Air and Water.

03,609 PC A04/MF A0

Maximum Permissible Body Burdens and Maximum Permissible Concentrations of Radionuclides in Air and in Water for Occupational Exposure. AD-A280 282/5

03,610 PC A06/MF A02

MCNP Model of the National Bureau of Standards Reactor (NBSA) Core.

03,733 Not available NTIS

Measured Stopping Powers of Hydrogen and Helium in Polystyrene Near Their Maximum Values. (5ityrene Near

04.729 Not available NTIS

Measurement Accuracies for Various Techniques for Measuring Amplifier Noise.

PB95-163671

02,350 Not available NTIS

Measurement and Calibration of Large-Area Alpha-Particle Sources at NIST.

PB94-172855

03,791 Not available NTIS

Measurement and Determination of Radon Source Potential: A Literature Review.

PB94-165602

$02,576 \mathrm{PC} \mathrm{A09/MF} \mathrm{A03}$

Measurement and Interpretation of Tidal Tilts in a Small Array.

B96-102611

03,686 Not available NTIS

Measurement and Reduction of Alignment Errors of the NIST Watt Experiment.

PB97-111959

01,987 Not available NTIS

Measurement and Simulation of Photoluminescence Spectra from Vertical-Cavity Quantum-Well Laser Structures.

PBa5-169181

Measurement and Uncertainty of a Calibration Standard for the Scanning Electron Microscope.

00,560

(Order as PB94-219219, PC A06/MF A02)

Measurement Comparability, Traceability, and Measurement Assurance Programs.

02,692 Not available NTIS

Measurement Methods and Algorithms for Comparison of Local and Remote Clocks.

PB96-102652

01,549 Not available NTIS

Measurement Methods and Standards for Processing and Application of Thermal Barrier Coatings.

Order as N95-26119, PC A03/MF A01)

Measurement of Absorbed Dose of Neutrons, and of Mixtures of Neutrons and gamma rays. AD-A286 647/3

$03,710 \mathrm{PC}$ A05/MF A01

Measurement of Ascorbic Acid in Human Plasma and Serum: Stability, Intralaboratory Repeatability, and Interlaboratory Reproducibility.

Interlaboratory
PB97-112445

03,511 Not available NTIS

Measurement of Atmospheric Methyl Bromide Using Gravimetric Gas Standards.

PB96-155494

02,497 Not available NTIS

Measurement of Boron at Silicon Wafer Surfaces by Neutron Depth Profiling.

04,487 Not available NTIS

Measurement of CO Pressures in the Ultrahigh Vacuum Regime Using Resonance-Enhanced Multiphoton-Ionization Time-of-Flight Mass Spectroscopy.
PB94-216041

Measurement of Diffusion in Fluid Systems: Applications to the Supercritical Fluid Region.

PB95-175188

02,490 Not available NTIS

Measurement of Diffusion in Supercritical Fluid Systems: A

PB94-199189

00,795 Not available NTIS

Measurement of Patterned Film Linewidth for Interconnect Characterization.

PB96-148168

02,420 Not available NTIS

Measurement

01,781 Not available NTIS

Measurement of Radial Dose Distributions Around Small Beta Particle Emitters Using High Resolution Radiochromic Foil Dosimetry.

PB95-164604

03,518 Not available NTIS 


\section{TITLE INDEX}

Measurement of Radiative Feedback to the Fuel Surface of a Pool Fire.

02,477 Not available NTIS

Measurement of Rheological Properties of High Perform-

ance Concrete: State of the Art Report.
PB96-202338
00,414 PC A04/MF A01

Measurement of Room Conditions and Response of Sprinklers and Smoke Detectors during a Simulated Two-Bed Hospital Patient Room Fire.

00,292 PC A07/MF A02

Measurement of S2OF10, and S2O2F10 Production Rates from Spark and Negative Glow Corona Discharge in SF6/ $\mathrm{O} 2$ Gas Mixtures.

PB96-123740

01,093 Not available NTIS

Meașurement of Surface Tension of Tantalum by a Dy-

namic Technique in a Microgravity Environment.
PB95-161667
032 Not available NTIS

Measurement of the Atomic Na(3P) Lifetime and of Retardation in the Interaction between Two Atoms Bound in a

PB97-122360

04,178 Not available NTIS

Measurement of the Heat of Fusion of Tungsten by a

Microsecond-Resolution Transient Technique.
PB94-216686 03,400 Not available NTIS

Measurement of the $\mathrm{J}=2$ less than 1 Fine-Structure Interval for (28) Si and (29)Si in the Ground (3)P State.

PB94-185097 00,771 Not available NTIS

Measurement of the Neutron Lifetıme.
PB96-161708 04,094 Not available NTIS

Measurement of the Self Broadening of the $\mathrm{H} 2 \mathrm{O}(0-5)$

Raman Transitions from 295 to $1000 \mathrm{~K}$.
PB95-108627
00,884

Measurement of the Thermal Properties of Electrically Conducting Fluids Using Coated Transient Hot Wires.

DE94017816 00,722 PC A02/MF A01

Measurement of the Thermal Properties of Electrically Conducting Fluids Using Coated Transient Hot Wires.

03,269 Not available NTIS

Measurement of the Uniformity of Particle Deposition of Fil-

ter Cassette Sampling in a Low Velocity Wind Tunnel.
PB95-163754 02,549 Not available NTIS

Measurement of the Weak-Localization Complex Conductivity at $1 \mathrm{Ghz}$ in Disordered Ag Wires.

04,731 Not available NTIS

Measurement of the (10)B(n, alpha1gamma)(7)Li Cross Section in the 0.3 to $4 \mathrm{MeV}$ Neutron Energy Interval.

PB96-161799 04,098 Not available NTIS

Measurement of the $(93) \mathrm{Nb}(\mathrm{n}, 2 \mathrm{n})(92 \mathrm{~m}) \mathrm{Nb}$ Cross Section in a (235) U Fission Spectrum.

03,945 Not available NTIS

Measurement of the (235)U(n,i) Reaction from Thermal to 1 keV.
PB95-140422

03,886 Not available NTIS

Measurement of Very Low Frequency Vibrations.

03,687 Not available NTIS

Measurement of Very-Low Partial Pressures.

PB95-180998 02,659 Not available NTIS

Measurements of Fluorescence from Cold Atoms: Localization in Three-Dimensional Standing Waves.

03 Not available NTIS

Measurements of Indoor Pollutant Emissions from EPA Phase II Wood Stoves.

02,556 PC A04/MF A01

Measurements of Moisture Diffusivity for Porous Building Materials.
PB95-107397

00,356 Not available NTIS

Measurements of Molar Heat Capacity at Constant Volume (Cv) for 1,1,1,2-Tetrafluoroethane (R134a).

03,264 Not available NTIS

Measurements of Outdoor Air Distribution in an Office BuildPB95-210944

00,264 PC A04/MF A01

Measurements of Permittivity and the Dielectric Loss Tangent of Low Loss Dielectric Materials with a Dielectric Resogent of Low Loss Dielectric Materials with a Dielectric Reso-
nator Operating on the Higher Order Te(sub 0 gamma delta) Modes.

02.273 Not available NTIS

Measurements of Properties of Materials in Electronic PackPBing.

01,973 Not available NTIS

Measurements of quantum electrodynamic sensitive transitions in Na-like and Cu-like ions: Final report, 1 October $1993-29$ Septen

04,403 PC A02/MF A01

Measurements of Shielding Effectiveness and Cavity Characteristics of Airplanes.

$00,030 \quad \mathrm{PC}$ A04/MF A01
Measurements of the Characteristic Impedance of Coaxial Air Line Standards.

02,221 Not available NTIS

Measurements of the Relative Permittivity of Liquid Water at Frequencies in the Range of 0.1 to $10 \mathrm{kHz}$ and at Temperatures between 273.1 and $373.2 \mathrm{~K}$ at Ambient Pressure.

PB96-119375 01,078 Not available NTIS

Measurements of the Resonance Lines of (6) Li and (7) Li by Doppler-Free Frequency-Modulation Spectroscopy.

PB96-180211

04,118 Not available NTIS

Measurements of the Vapor Pressures of Difluoromethane, 1-Chloro-1,2,2,2-Tetrafluoroethane, and Pentafluoroethane. PB95-169272 03,270 Not available NTIS Measurements of the Virial Coefficients and Equation of
State of the Carbon Dioxide + Ethane System in the Supercritical Region.

PB95-151353

03,906 Not available NTIS

Measurements of the Viscosities of Saturated and Compressed Fluid 1-chloro-1,2,2,2-tetrafluoroethane (R124) and Pentafluoroethane (R125) at Temperatures between 120

PB94-199791

03,254 Not available NTIS

Measurements of the Viscosities of Saturated and Compressed Liquid 1,1,1,2-Tetrafluoroethane (R134a), 2,2Dichloro-1,1,1-Trifluoroethane (R123) and 1,1-Dichloro-1 Fluoroethane (R141b)

PB95-175386

03,273 Not available NTIS

Measurements of the $(235) \cup(n, f)$ Cross Section in the 3 to $30 \mathrm{MeV}$ Neutron Energy Region.

PB97-119051

04,172 Not available NTIS

Measurements of the (237) Np(n,f) Cross Section.

PB97-119069 04,173 Not available NTIS

Measurements of Thermophysical Properties of Nickel Near Its Melting Temperature by a Microsecond-Resolution Transient Technique.

Sient Technique.

04,210 Not available NTIS

Measuring Contact Charge Transfer at Interfaces: A New Experimental Technique.

Experimental

03,053 Not available NTIS

Measuring Hydrogen by Cold-Neutron Prompt-Gamma Activation Analysis.

PB96-111877

00,612 Not available NTIS

Measuring Long Gage Blocks with the NIST Line Scale Interferometer.
PB95-242400

02,665 PC A03/MF AO

Measuring Matching Wear Scars on Balls and Flats.

PB95-151528

03,153 Not available NTIS

Measuring Nondipolar Asymmetries of Photoelectron Angular Distributions.

01,193 Not available NTIS

Measuring Pertormance of Parallel Computers. Final Re-

port.

01,665 PC A03/MF A01

Measuring Performance of Parallel Computers. Progress Report, 1989

01,666 PC A03/MF A01

Measuring the Stability of Three Copper Alloys.

PB94-199866

03,326 Not available NTIS

Mechanical Properties and Warm Prestress of Ultra-Low Carbon Steel at $4 \mathrm{~K}$

PB96-190350

03,224 Not available NTIS

Mechanism and Rate Constants for the Reactions of Hydrogen Atoms with Isobutene at High Temperatures.

PB95-151064 00,929 Not available NTIS

Mechanism of Defect Formation in Low-Dose Oxygen Implanted Silicon-on-Insulator Material

PB97-111462

02,453 Not available NTIS

Mechanism of Mild to Severe Wear Transition in Alpha-Alu-

PBina.

03,233 Not available NTIS

Mechano-Chemical Model: Reaction Temperatures in a Concentrated Contact.

03,227 Not available NTIS

PB96-119460

Loss Behavior in Single-Crystal YBa2C Shielding

PB94-198744

04,464 Not available NTIS

Melnikov Function and Homoclinic Chaos Induced by Weak Perturbations.

03,414 Not available NTIS

Melting Behavior of Organic Materials Confined in Porous Solids.

00,834 Not available NTIS

Membrane Gas Separation for a Fluidized-Bed Incinerator. PB95-169041 02,550 Not available NTIS
Membrane-Mediated Precipitation of Calcium Phosphate in Model Liposomes with Matrix Vesicle-Like Lipid ComposiPB95-164547 03,468 Not available NTIS

Memory Function Approach to the Shape of Pressure Broadened Molecular Bands.

PB95-152930

00,944 Not available NTIS

MEMS in Standard CMOS VLSI Technology.

PB96-102363 02,377 Not available NTIS Merged-Beams Energy-Loss Technique for Electron-Ion Excitation: Absolute Total Cross Sections for $\mathrm{O}(5+)$ (2s yields

PB). 102058 04,017 Not available NTIS

Mesoscopic Conductance Fluctuations in Large Devices. PB96-119656 04,735 Not available NTIS

Metallic-Barrier Junctions for Programmable Josephson Voltage Standards.

02,089 Not available NTIS

Metalloporphyrin Sensitized Photooxidation of Water to Oxygen on the Surface of Colloidal Iridium Oxides - Photochemical and Pulse Radiolytic Studies.

PB95-107082 00,868 Not available NTIS

Metallurgy Technical Activities 1994 (NAS-NRC Assessment Panel, April 6-7, 1995).
PB96-136981

$02,981 \mathrm{PC} \mathrm{A08/MF} \mathrm{A02}$

Metallurgy. Technical Activities, 1995

PB96-195284 03,003 PC A09/MF A02

Method and Evaluation of Character Stroke Preservation on Handprint Recognition

01,850 PC A03/MF A01

Method for Determining Both Magnetostriction and Elastic Modulus by Ferromagnetic Resonance.
PB95-150108 Not available NTIS

Method for the Assay of Hydrolytic Enzymes Using Dynamic Light Scattering

03,531 Not available NTIS

Method of Estimating the Parameters of Tuned Mass Dampers for Seismic Applications.

PB96-167820

00,473 PC A04/MF A01

Method of Predicting Smoke Movement in Atria with Application to Smoke Management.

00,376 PC A05/MF A01

Method of Realizing Spectral Irradiance Based on an Absolute Cryogenic Radiometer.

04,270 Not available NTIS

Method of Sale for CNG Paves Way to Greater Public Acceptance.
PB95-168449

02,489 Not available NTIS

Method to Determine a Basis Set of Paths to Perform Program Testing.

01,747 PC A03MF A01

Method to Determine the Calorimetric Equivalence Correction for a Coaxial Microwave Microcalorimeter.

PB95-202404

01,913 Not available NTIS

Methodologies for Predicting the Service Lives of Coating Systerns.
PB95-146387

03,124 PC A05/MF A01

Methodology for Developing and Implementing Altemative Temperature-Time Curves for Testing the Fire Resistance ( Barriers for Nuclar Power Plant Applications. of Barriers for Nuclear Power Plant Applications. Methodology for Electromagnetic Interference Measurements.
PB96-200126

02,014 Not available NTIS

Methodology for the Certification of Reference Specimens for Determination of Oxygen Concentration in Semiconducor Silicon by Infrared Spectrophotomition

tor Silicon by

02,421 Not available NTIS

Methods for Aligning the NIST Watt-Balance.

PB96-123153 01,934 Not available NTIS

Methods for Analysis of Cancer Chemopreventive Agents in Human Serum.

03,502 PC A07/MF A02 Methods of Measuring Humidity and Testing Hygrometers.
AD-A278 $851 / 1$
00,123 PC A03/MF A01

Methods to Improve the Accuracy of On-Line Ultrasonic Measurement of Steel Sheet Formability. PB96-186051

02,281 Not available NTIS

Methyl Torsional Levels of Solid Acetonitrile ( $\mathrm{CH} 3 \mathrm{CN}$ ): A Neutron Scattering Siudy.

00,926 Not available NTIS

Metric for Success.

PB94-187630

02,633 PC A03/MF A01

Metric Path to Global Markets and New Jobs: A Questionand-Answer and Thematic Discussion.
PB94-206307 02,601 PC A02/MF A01 


\section{TITLE INDEX}

Metrication.
PB94-172079

03,676 Not available NTIS

METRICATION: An Economic Wake-Up Call for Surveyors and Mappers.
PB96-159629

03,680 Not available NTIS

Metrological Accuracy of the Electron Pump.

PB95-168910 03,951 Not available NTIS

Metrological Measurement Accuracy: Discussion of 'Meas-

Urement Error Models' by Leon Jay Gleser.
PB94-200607

Metrology.

PB95-164497

03,946 Not available NTIS

Metrology.
PB96-160759

02,925 Not available NTIS

Metrology and Data for Microelectronic Packaging and Interconnection: Results of a Joint Workshop on Materials Metrology and Data for Commercial Electrical and Optica Gaithersburg, Maryland on May 5-6, 1994. Volume 1. ReGalts.
sulsourg, Maryland on May 56, 1994. Volume 1 . Ro PB95-143111

02,328 PC A05/MF A01

Metrology and Data for Microelectronic Packaging and Interconnection: Results of a Joint Workshop on Materials Metrology and Data for Commercial Electrical and Optical Packaging and Interconnection Technologies. Held in
Gaithersburg, Maryland on May 5-6, 1994. Volume 2. PresGaithersburg, Maryland on May 5-6, 1994. Volume 2. Pres-
entation Material.

02,330 PC A08/MF A02

Metrology and Regional Trade Pacts.

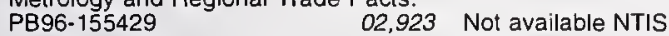

Metrology Applications of Mode-Locked Erbium Fiber LaPers. 140158

04,256 Not available NTIS

Metrology Approach to Unitying Rockwell C Hardness Scales.

02,957 Not available NTIS

Metrology for Electromagnetic Technology: A Bibliography of NIST Publications.

02,116 PC A04/MF A01

Metrology for Electromagnetic Technology: A Bibliography of NIST Publications.

02,143 PC A05/MF A01

Metrology for Electromagnetic Technology: A Bibliography of NIST Publications

04,396 PC A06/MF A01

Metrology for Electromagnetic Technology: A Bibliography of NIST Publications, September 1995.
PB96-128269 PC A05/MF A01

Metrology Issues in Terahertz Physics and Technology.
PB96-128277

Metrology Model for Submicrometer Dimensional Measurements.

02,647 Not available NTIS

Metrology Requirements of Future Space Power Systems. PB95-140984 04,840 Not available NTIS

Metrology Standards for Advanced Semiconductor Lithography Reterenced to Atomic Spacings and Geometry.

Meropolian Areas (Including MSAs, CMSAs PMASs, and NECMAS). Category: Data Standards and Guidelines; Subcategory: Representations and Codes.

04,873 PC E09

FIPS PUB $8 \cdot 6$

On

Michaelis-Men

PB95-140489

00,906 Not available NTIS

Micro-Mechanical Aspects of Asperity-Controlled Friction in Fiber-Toughened Ceramic Composites.

PB94-199536

03,136 Not available NTIS

Microanalysis to Nanoanalysis: Measuring Composition at High Spatial Resolution.

PBg Spatial Res

00,563 Not available NTIS

Microbial Degradation of Polysulfides and Insights into Their Possible Occurrence in Coal.

PB95-163374

02,488 Not available NTIS

Microdosimetry and Cellular Radiation Effects of Radon Progency in Human Bronchial Airways.
PB95-152344 Not available NTIS

Microelectronic Test Structures for Feature Placement and Electrical Linewidth Metrology. 02,367 Not available NTIS

02,367 Not available

Microelectronic Test Structures for
PB96-164249 02,430 Not available NTIS

Microform Calibration Uncertainties of Rockwell Diamond Indenters.

(Order as PB96-117767, PC A08/MF A02)
Microform Calibrations in Surface Metrology. PB95-203295 02,951 Not available NTIS Microlithography by Using Neutral Metastable Atoms and Self-Assembled Monolayers.

PB96-190038

02,441 Not available NTIS

Micromachined Coplanar Waveguides in CMOS Technology.
PB97-119283

02,456 Not available NTIS

Micromachined Display Output for a Cellular Neural Network.

02,422 Not available NTIS

Micromagnetic Model of Dual-Layer Magnetic-Recording Thin Films.

PB95-141065

04,551 Not available NTIS

Micromagnetic Scanning Microprobe System.

PB95-176178 02,224 Not available NTIS

Micromagnetic Simulations of Tunneling Stabilized Magnetic Force Microscopy.

PB95-141073

04,552 Not available NTIS

Micromagnetic Structure of Domains in Co/Pt Multilayers. 1.

Investigations of Wall Structure.
PB95-162111 $04,610 \quad$ Not available NTIS

Micromechanics of Densification and Distortion

$\begin{array}{lll}\text { PB94-200326 03,327 Not available NTIS } & \text { 20 }\end{array}$

Micromechanics of Fracture in Rubber-Toughened Epoxies. PB94-212222 03,011 Not available NTIS

Microstrains and Domain Sizes in Bi-Cu-O Microstrains and Domain Sizes in Bi-Cu-O Study.
PB94-198520 04,461 Not available NTIS 04,461 Nol available NTIS Microstructural Characterization of Cobalt-Tungsten Coated
Graphite Fibers.
PB96-159231

01,951

(Order as PB96-159215, PC A07/MF A02)

Microstructural Evolution in Two-Dimensional Two-Phase Polycrystals.
PB94-211992

04,498 Not available NTIS

Microstructural Features of Some Low Water/Solids, Silica

Fume Mortars Cured at Different Temperatures.
PB94-160777 00,330 PC A03/MF A01

Microstructure and Ferroelectric Properties of Lead Zircon ate-Titanate Films Produced by Laser Evaporation. PB94-199148 $04,470 \quad$ Not available NTIS
PBo

Microstructure and Tensile Properties of Microalloyed Stee Forgings.
PB94-172715

03,204 Not available NTIS

Microstructure Effect on the Phase Behavior of Blends of Deuterated Polybutadiene and Protonated Polyisopene.
PB97-113146

Microstructure Study of Mclybdenum Liners by Neutron Diffraction.

PB95-202396

03,756 Not available NTIS

Microwave and Submillimeter Spectroscopy of Ar-NH3 States Correlating with $\mathrm{Ar}+\mathrm{NH} 3(\mathrm{j}=1, \mathrm{k}=1)$.
$\mathrm{PB95} 152211$
00,942

Microwave Characterization of Flip-Chip MMIC Components.

PB96-176722

02,434 Not available NTIS

Microwave Characterization of Flip-Chip MMIC Interconnec-

tions.

02,435 Not available NTIS

Microwave Characterization of Printed Circuit Transmission Lines.

PB96-122585

02,077 Not available NTIS

Microwave Diagnostic Results from the Gaseous Electronics Conterence RF Reference Cell.

02,392

(Order as PB96-113311, PC A09/MF A03)

Microwave Dielectric Properties of Anisotropic Materials at Cryogenic Temperatures
PB96-137765

02,412 Not available NTIS

Microwave Leakage as a Source of Frequency Error and Long-Term Instability in Cesium Atomic-Beam Frequency Standards.

01,541 Not available NTIS

Microwave Noise in High-Tc Josephson Junctions.

PB96-148010 04,771 Not available NTIS

Microwave Properties of Voltage-Tunable YBa2Cu3O7delta/SrTiO3 Coplanar Waveguide Transmission Lines. PB96-141262

02,235 Not available NTIS

Microwave Properties of $\mathrm{YBa} 2 \mathrm{Cu} 3 \mathrm{O} 7-\mathrm{x}$ Films at $35 \mathrm{GHz}$ from Magnetotransmission and Magnetoreflection Measurements.
PB95-168977

04,643 Not available NTIS
Microwave Spectra of van der Waals Complexes of Impor tance in Planetary Atmospheres.
PB95-150611 O0,919 Not available NTIS Microwave Spectrum and Structure of $\mathrm{CH}_{2} \mathrm{O}-\mathrm{H} 2 \mathrm{O}$. PB97-118723 04,168 Not available NTIS Microwave Spectrum and Structure of $\mathrm{CH} 3 \mathrm{NO}_{2}-\mathrm{H} 2 \mathrm{O}$. AD-A296 377/5 00,719 PC A03/MF A01 Mid- and Near-Infrared Spectra of Water and Water Dimer Isolated in Solid Neon

00,888 Not available NTIS

Millimeter- and Submillimeter-Wave Spectrum of trans-Ethyl Alcohol.

PB96-145578

01,102 Not available NTIS

Millimeter-Resolution Optical Time-Domain Reflectometry

Using a Four-Wave Mixing Sampling Gate.
PB96-122700 02,190 Not available NTIS

Millisecond-Resolution Pulse Heating System for Specific-

Heat Measurements at High Temperatures.
PB94-199999

Milliwatt Mixer for Small Fluid Samples.

PB94-198819 02,634 Not available NTIS

Minimum Mass Flux Requirements to Suppress Burning

Surfaces with Water Sprays.
PB96-183181

01,425 PC A05/MF A01

Minutes of the CAALS Workshop on modularity and communications standards.

DE95005780

02,621 PC A03/MF A01

Mixed Diet Reference Materials for Nutrient Analysis of Foods: Preparation of SRM-1548 Total Diet.

PB95-151692 03,593 Not available NTIS

Mixed Phospholipid Liposome Calcification.

PB94-211190 03,457 Not available NTIS

Mixing and Radiation Properties of Buoyant Luminous

Flame Environments.
PB96-202254

01,432 PC A06/MF A01

Mixing and Radiation Properties of Buoyant Turbulent Diffusion Flames.
PB95-242327

01,396 PC A05/MF A02

Mixing Plate-Like and Rod-Like Molecules with Solvent: $A$ Test of Flory-Huggins Lattice Statistics.
PB96-126206

03,173

(Order as PB96-126180, PC A05/MF A01)

Modal Characteristics of Bent Dual Mode Planar Optical Waveguide.

04,311 Not available NTIS

Modal Properties of Circular and Noncircular Optica Waveguides.

04,251 Not available NTIS

Mode Coupling and Loss on Tapered Optical Waveguides.

PB95-168571 04,282 Not available NTIS

Mode-Locked Lasers for High-Accuracy Radiometry.

PB96-204201 04,134 Not available NTIS

Mode Specific Vibrational Predissociation Dynamics in Fragile Molecules.

PB95-107132

00,871 Not available NTIS

Model for Calculating Virial Coefficients of Natural Gas Hydrocarbons with Impurities.

PB96-156047

04,064 Not available NTIS

Model for Determining the Density and Mobility of Carriers in Thin Semiconducting Layers with Only Two Contacts.

Model for Microcrack Initiation and Propagation beneath Hertzian Contacts in Polycrystalline Ceramics.

PB96-163704 03,077 Not available NTIS
PBrtzian Contacts in Polycrystalline Ceramics.

Model for Toughness Curves in Two-Phase Ceramics. 1 Basic Fracture Mechanics.

PB96-180088

03,085 Not available NTIS

Model for Toughness Curves in Two-Phase Ceramics. 2. Microstructural Variables.

PB96-163795

03,078 Not available NTIS

Model Minimum Performance Specifications for Lidar Speed Measurement Devices.

PB95-197455

$04,861 \mathrm{PC} \mathrm{A03/MF} \mathrm{A01}$

Model of an Optical Roughness-Measuring Instrument.

PB97-110209

04,384 Not available NTIS

Model Precast Concrete Beam-to-Column Connections Subject to Cyclic Loading.

00,438 Not available NTIS

Modeling and Test Point Selection for a Thermal Transfer PB95-161287

01,896 Not available NTIS

Modeling Buffer Layer 1GBT's for Circuit Simulation.

PB96-164173 Layer IGBT's for Circuit Simulation. 


\section{TITLE INDEX}

Modeling Buffer Layer IGBTs for Circuit Simulation.

02,344 Not available NTIS

Modeling Ceramic Sub-Micron Particle Formation from the Vapor Using Detailed Chemical Kinetics: Comparison with In-situ Laser Diagnostics.

In-situ Laser

00,671 Not available NTIS

Modeling Detector Response for Neutron Depth Profiling.

Modeling Etfects of Temperature Annealing on Giant Magnetoresistive Response in Discontinuous Multilayer PB97-112585

04,157 Not available NTIS

Modeling of Extreme Loading by 'Peaks Over Threshold" Methods.

00,469 Not available NTIS

Modeling Polarization Curves and impedance Spectra for Simple Electrode Systems

03,188 Not available NTIS

Modeling Radon Transport in Multistory Residential BuildPB95-162087

00.256 Not available NTIS

Modeling the Evolution of Structure in Unstable Solid Solu-

tion Phases by Diffusional Mechanisms.
PB94-199403 03,324 Not available NTIS

Modelling Drying Shrinkage of Cement Paste and Mortar.

Part 1. Structural Models from Angstroms to Millimeters.
PB96-135132 03,074 Not available NTIS

Modelling the Leaching of Calcium Hydroxide from Cement Paste: Effects on Pore Space Percolation and Ditfusivity.
PB94-198793

Models and Interactions.

01,311 Not available NTIS

PB94-216306

02,641 Not available NTIS

Models, Managing Models, Ouality Models: An Example of Ouality Management.

PB94-163466

02,891 PC A03/MF A01

Models of Granualr Giant Magnetoresistance Multilayer Thin Films.

01,968 Not available NTIS

Modern Test Methods for Flammability.

PB94-198447 01,370 Not available NTIS

Modification of a Commercial SEM with a Computer Con-

trolled Cathode Stabilized Power Supply.
PB96-201066 04,129 Not available NTIS

Modification of Cast Epoxy Resin Surfaces during Exposure to Partial Discharges.

PB96-122734

01,086 Not available NTIS

Modification of Deoxyribose-Phosphate Residues by Ex racts of Ataxia Telangiectasia Cells.

PB94-212602 03,458 Not available NTIS

Modification of DNA Bases in Chromatin of Intact Target Human Cells by Activated Human Polymorphonuclear Leu-

PB94-199833

03,526 Not available NTIS

Modification of the Phase Stability of Polymer Blends by Diblock Copolymer Additivies.

03,172 Not available NTIS

Modified Airy Function Method for the Analysis of Tunneling Problems in Optical Waveguides and Ouantum Well Struc-

PB94-185824

02,120 Not available NTIS

Modified Effective Range Theory as an Alternative to Low-

Energy Close-Coupling Calculations.
PB95-202701 Not available NTIS

Modified Leung-Gritfiths Model of Vapor-Liquid Equilibrium Extended Scaling and Binary Mixtures of Dissimilar Fluids. PB94-216108 00,851 Not available NTIS

Modified Optimal Algorithm for Active Structural Control. PB96-165949 00,472 PC A04/MF A01

Modified Surface-Active Monomers for Adhesive Bonding to Dentin.

PB95-151163

00,158 Not available NTIS

Modified Surface Layers and Coatings.

PB95-176087

03,125 Not available NTIS

Modulation of Fossil Fuel Production by Global Tempera ture Variations, 2.

PB94-146636

02,533 PC A04/MF A01

Moisfure and Water-Induced Crack Growth in Optical Materials.

04,267 Not available NTIS

Molar Absorptivities of Bilirubin (NIST SRM 916A) and Its Neutral and Alkaline Azopigments.

PB94-211042 03,456 Not available NTIS

Molar Heat Capacity at Constant Volume for Air from 67 to $300 \mathrm{~K}$ at Pressures $1035 \mathrm{MPa}$.

PB96-163738
Molar Heat Capacity at Constant Volume for $(x \mathrm{CO} 2+1$ (1x) $\mathrm{C} 2 \mathrm{H6}$ ) from 220 to $340 \mathrm{~K}$ at Pressures to $35 \mathrm{MPa}$. PB96-167135 01,148 Not available NTIS

Molecular-Beam Optothermal Spectrum of the $\mathrm{OH}$ Stretching Band of Methanol.

00,839 Not available NTIS

Molecular Dynamics Investigation of the Surface/Bulk Equilibrium in an Ethanol-Water Solution.
PB97-113112 Not available NTIS

Molecular Ion Imaging and Dynamic Secondary Ion Mass Spectrometry of Organic Compounds.
PB95-126124 00,571 Not available NTIS

Molecular Microwave Spectra Tables.

AD-A296 498/9 $00,720 \quad$ PC A03/MF A01

Molecular Orbital Calculations of Bond Rupture in Brittle Solids.

00,973 Not available NTIS

Molecular Orbital Study of Water Enhanced Crack Growth Process.

PB95-164067

03,240 Not available NTIS

Molecular Spectroscopy.

PB94-213337

03,861 Not available NTIS

Molecular Weight Dependence of the Lamellar Comain Spacing of ABC Triblock Copolymers and Their Chain Conformation in Lamellar Domains.

PB95-16169

01,254 Not available NTIS

Moments of the Ouartic Assignment Statistic with an Application to Multiple Regression.

PB95-181103

03,442 Not available NTIS

Monitoring Polymer Cure by Fluorescence Recovery After Photobleaching
PB94-211422

01,218 Not available NTIS

MONSEL-II: Monte Carlo Simulation of SEM Signals for

Linewidth Metrology
PB96-102710

02,379 Not available NTIS

Monte Carlo and Analytic Methods in the Transport of Electrons, Neutrons, and Alpha Particles.

PB96-111612
$04,033 \quad$ Not available NTIS

Monte Carlo and Mean-Field Calculations of the Magnetocaloric Effect of Ferromagnetically Interacting Clusters.
PB94-172087

03,201 Not available NTIS

Monte Carlo Approach to the Approximation of Invariant Measures.

PB94-172053

03,409 Not available NTIS

Monte Carlo Electron Trajectory Simulation of X-Ray Emis-

sion from Films Supported on Substrates.
PB95-107207

Monte Carlo Model for SEM Linewidth Metrology.

PB95-150058 02,331 Not available NTIS

Monte Carlo Simulation of Scanning Electron Microscope Signals.
PB96-200969

02,444 Not available NTIS

Montgomery Education Connection and Resource Education Awareness Partnership Making Connections between Local Schools and NIST Volunteers.

PB96-159769

00.134 Not available NTIS

Morphological Development of Second-Phase Particles in Elastically-Stressed Solids.

03,355 Not available NTIS

Morphological Estimation of Tip Geometry for Scanned Probe Microscopy.

02,662 Not available NTIS

Morphological Stability.

PB95-153318

04,591 Not available NTIS

Morphology and Phase Separation Kinetics of a Compatibilized Blend.

PB97-119135

01,297 Not available NTIS

Morphology of Symmetric Diblock Copolymers as Revealed by Neutron Reflectivity.

PB95-140075

01,234 Not available NTIS

PBo5-189502

01,849 PC A03/MF A01

Move-to-Root Rule for Self-Organizing Trees with Markov Dependent Requests.

PB96-179528

03,431 Not available NTIS

Multi-Agency Certification and Accreditation ( $C$ and $A$ ) Process: A Worked Example.

01,601 PC A05/MF A01 Multi-Scale Picture of Concrete and Its Transport Prop-
erties: Introduction for Non-Cement Researchers. erties: Introductior

03, 107 PC A05/MF A01

Multi-Stage, Position Stabilized Vibration Isolation System for Neutron Interferometry.
Multi-State Two-Port: An Altemative Transfer Standard B95-168530 02,049 Not available NTIS

Multiattribute Decision Analysis Method for Evaluating Buildings and Building Systems.

$00,325 \mathrm{PC} \mathrm{A06/MF} \mathrm{A01}$

Multicarrier Characterization Method for Extracting Mobilities and Carrier Densities of Semiconductors from Variable Magnetic Field Measurements. Magnetic Field
PB94-212776

02,317 Not available NTIS

Multichannel Quantum Defect Half Collision Analysis of K2 Photodissociation Through the B1Pi(sub u) State.
PB94-211125 00,812 Not available NTIS

Multijunction Thermal Converters by Commercial CMOS Fabrication.

02,343 Not available NTIS

Multimedia Tutorial on Phase Equilibria Diagrams.
PB96-200829

Multiphoton Ionization of $\mathrm{SiH} 3$ and $\mathrm{SiD} 3$ Radicals: Electronic Spectra, Vibrational Analyses of the Ground and Pydberg States,
PB94-212503

00,837 Not available NTIS

Multiphoton Ionization of $\mathrm{SiH} 3$ and $\mathrm{SiD} 3$ Radicals. 2. Three 460 and $610 \mathrm{~nm}$. 460 and $610 \mathrm{~nm}$.

Multiphoton Ionization Spectroscopy Measurements of Silicon Atoms during Vapor Phase Synthesis of Ceramic Particles.

03,913 Not available NTIS

Multiwavelength Birefringent-Cavity Mode-Locked Fibre Laser.
PB95-150496

04,262 Not available NTIS

Multizone Modeling of Three Residential Indoor Air Ouality Control Options.

02,565 Not available NTIS

Multizone Modeling of Three Residential Indoor Air Ouality Control Options.

$02,567 \mathrm{PC} \mathrm{A08/MF} \mathrm{A02}$

Mutual Phase Locking in Systems of High-Tc Superconductor-Normal Metal-Superconductor Junctions.
PB96-135348 Not available NTIS MVtrix/Vector Class Reference Guide. 15 a Matr

MV++ v. 1.5a Matrix/Vector Class Reference Guide.
PB96-195326
01,777 PC A03/MF A01 $\mathrm{n}$-Value and Second Derivative of the Superconductor Voltage-Current Characteristic.

04,533 Not available NTIS

$n-V e c t o r$ Correlations in Collision Dynamics with Atomic Orbital Alignment: The Importance of Coherence Denoting Azimuthal Structure for $n$ (greater than or equal to) 3 .

imuthal Structure for $n$ (greater than or equal to) 3 .
PB95-202545 NT,983 Not available NTIS

N2(a'(sup 1)Sigma(sub g)(sup +)) Metastable Collisional Destruction and Rotational Excitation Transfer by N2.
PB95-151395 No,933 Not available NTIS

Nano-Defects in Commercial Bonded SOI and SIMOX

Nano-Detects in Commercial Bonded SOI and SIMOX.
PB96-123674 NTIS

Nanocomposite Magnetic Materials.

Nanocomposite Magnetic Materials.
PB95-162780 Not available NTIS

Nanofabrication of a Two-Dimensional Array Using LaserFocused Atomic Deposition.

04,732 Not available NTIS

Nanoindentation and Instrumented Scratching Measurements on Hard Coatings.

PB97-122477

03,111 Not available NTIS

Nanometrology

02,425 Not available NTIS

Nanoscale Study of the As-Grown Hydrogenated Amorphous Silicon Surface.

PB95-150595

04,573 Not available NTIS

Nanoscale Study of the Hydrogenated Amorphous Silicon Surface.
PB96-103056

04,720 Not available NTIS

Nanostructure Fabrication via Direct Writing with Atoms Fo-

cused in Laser Fields. 04,564 Not available NTIS

Nanostructure Fabrication via Laser-Focused Atomic Deposition (Invited)

04,132 Not available NTIS

Narrow-Band Tunable Diode Laser System with Grating Feedback, and a Saturated Absorption Spectrometer for Cs and $\mathrm{Rb}$. 04,319 Not available NTIS

NASA Fire Detector Study

B96-183108

$01,423 \mathrm{PC} \mathrm{A04/MF} \mathrm{A01}$

National Center for Standards and Certification Information: Service and Programs.

02,717 


\section{TITLE INDEX}

(Order as N95-15919/0, PC A11/MF A03) National Construction Sector Goals: Industry Strategies for Implementation
PB95-269817

00,204 PC A03/MF AO National Information Infrastructure and Advanced Digital Video.

01,488 Not available NTIS

National Institute of Standards and Technology High-Accuracy Cryogenic Radiometer.
PB96-179585 National Institute of Standards and Technology Resonance Ionization Spectroscopy/Resonance Ionization Mass Spectroscopy Data Service.

03,793 Not available NTIS

National Measurement System for Radiometry, Photometry, and Pyrometry Base Upon Absolute Detectors.
PB97-108559 04.382 PC A04/MF A01

National PDES Testbed: An Overview.

PB95-125829 02,775 Not available NTIS

National Planning for Construction and Building $R$ and $D$.

PB96-137104 00,324 PC A06/MF A02

National Quality Assurance Program for Personnel Radiation Dosimetry: A Case History.
PB94-211273 National Semiconductor Metrology Program, Project Portfolio FY 1996

$04.789 \mathrm{PC} \mathrm{AO3/MF} \mathrm{AO}$

National Standard Petroleum Oil Tables.

AD-A279 952/6 02,469 PC A09/MF A02

National Status and Trends Program Specimen Bank: Sampling Protocols. Analytical Methods, Results, and Archive PB97-119226

02,598 Not available NTIS

National Type Evaluation Program: Index of Device Evalua tions by Company. NCWM Publication 5 Part A (Second Edition).

02,889 PC A05/MF A01

National Voluntary Laboratory Accreditation Program Acoustical Testing Services. 04,188 PC A05/MF A01

04,188 PC A05/MF AO National Voluntary Laboratory Accreditation Program: Bulk
Asbestos Analysis. Asbestos Analysis
PB95-138129

02,541 PC A04/MF AO1

National Voluntary Laboratory Accreditation Prograrn: Carpet and Carpet Cushion.

pet and Carpot

00,295 PC A04/MF A01

National Voluntary Laboratory Accreditation Program: Construction Materials Testing.

01,319 PC A05/MF A01

National Voluntary Laboratory Accreditation Program: Electromagnetic Compatibility and Telecommunications. FCC PB95-242376

02,664 PC A06/MF AO2

National Voluntary Laboratory Accreditation Prograrn: Energy Efficient Lighting Products.
PB94-219060

National Voluntary Laboratory Accreditation Program. GOSIP: Government Open Systems Interconnection Profile.
PB95-267993 01,486 PC A06/MF A02 National Voluntary Laboratory Accreditation Program: Ionizing Radiation Dosimetry.

03,623 PC A06/MF AO2

National Voluntary Laboratory Accreditation Program National Voluntary Laboratory Accreditation Program
(NVLAP): Commercial Products Testing.
PB95-267944
02,671 PC A02/MF A01 National Voluntary Laboratory Accreditation Program (NVLAP): Fasteners and Metals.

PB97-114185

02,881 PC A06/MF AO

National Voluntary Laboratory Accreditation Program (NVLAP): Wood Based Products.
PB95-170429

National Voluntary Laboratory Accreditation Program POSIX. Portable Operating System Interface.
PB95-189478
02,661 National Voluntary Laboratory Accreditation Program: Procedures and General Requirements.

PB94-178225

$02,630 \mathrm{PC} \mathrm{A03/MF} \mathrm{AO}$

National Voluntary Laboratory Accreditation Program: Thermal Insulation Materials.

02,977 PC A05/MF A01

National Voluntary Laboratory Accreditation Program 1994 Directory.
PB94-178969

00,482 PC A08/MF AO2

National Voluntary Laboratory Accreditation Program 1995 Directory.
PB95-174454

00,483 PC AO8/MF AO2
National Voluntary Laboratory Accreditation Program 1996 Directory.
PB96-162714

$00,485 \mathrm{PC} \mathrm{A10/MF} \mathrm{A03}$

Natural Convection from an Array of Electronic Packages Mounted on a Horizontal Board in a Narrow Aspect Ratio Enclosure.

02,087 Not available NTIS

Nature of $(001)$ Tilt Grain Boundaries in YBa2Cu3O6+x PB95-126033 04,528 Not available NTIS NBS/NIST Peltier-Effect Microcalorimeter: A Four-Decade Review.

01,064 Not available NTIS

Nd:LiTaO3 Waveguide Laser.

PB95-140851

04,259 Not available NTIS

Near Critical Fluid Interfaces: A Comparison of Theory and Experiment.

00,901 Not available NTIS

Necessary Condition for Homoclinic Chaos Induces by $\mathrm{Ad}$. ditive Noise.
PB96-155775

04,063 Not available NTIS

Need for Advanced Characterization Techniques in Product Manufacturing: A Case Study on Ceramic Matrix Composites.

03,089 Not available NTIS

Need for, and Availability of, Working Fluid Property Data:

Results from Annexes XIII and XVIII.
PB95-168969 Nol available NTIS

Needs for Brachytherapy Source Calibrations in the United SBates.

03,521 Not available NTIS

Neighbor Tables for Molecular Dynamics Simulations.
PB95-171948 00,991 PC A03/MF A01

Network Brokers Handbook: An Entrepreneurial Guide to Cooperative Strategies for Manufacturing Competitiveness.
PB95-219325 00,490 PC A08/MF A02

Neutron and Raman Spectroscopies of 134 and 134a Hydrofluorocarbons Encaged in Na-X Zeolite.
P896-186168 03,001 Not available NTIS

Neutron and X-Ray Scattering Cross Sections of Orientationally Disordered Soild C60.
PB95-153300

Neutron Capture Prompt Gamma-Ray Activation Analysis at the NIST Cold Neutron Research Facility.
PB94-213394 00,556 Not available NTIS

Neutron Diffraction Texture Study of Deformed Uranium

Plates.
PB97-111587

03,010 Not available NTIS

Neutron Energy Deposition on the Nanometer Scale. PB95-152260 03,624 Not available NTIS

Neutron Focusing Lens Using Polycapillary Fibers

PB95-141206 03,889 Not available NTIS

Neutron Focusing Lens Using Polycapillary Fibers

PB95-153078 03,922 Not available NTIS

Neutron Leakage Benchmark for Criticality Safety ReSearch.

03,723 Not available NTIS

Neutron Measurement Intercomparisons Sponsored by CCEMRI, Section 3 (Neutron Measurements)

PB94-199916 03,819 Not available NTIS

Neutron Powder Diffraction Study of a Na, Cs-Rho Zeolite.
PB94-198629 00,791 Not available NTIS

Neutron Powder Diffraction Study of the Crystal Structure of YSr2AICu2O7.

PB94-212073

04,499 Not available NTIS

Neutron-Powder-Diffraction Study of the Long-Range Order

in the Octahedral Sublattice of LaD2.25.
PB96-141155 04,753 Not available NTIS

Neutron Powder Diffraction Study of the Nuclear and Magnetic Structures of the Oxygen-Deficient Perovskite YBaCuCOO5.

PB95-161097

00,954 Not available NTIS

Neutron Powder Diffraction Study of the Structures of La1.9Ca1.1 Cu2O6 and La1.9Sr1. 1Cu2O6+Delta.

PB95-140042 04,538 Not available NTIS

Neutron Reflectivity of End-Graffed Polymers: Concentration and Solvent Quality Dependence in Equilibrium Conditions.
PB94-185758 01,206 Not available NTIS

Neutron Reflectivity Study of the Density Profile of a Model End-Graffed Polymer Brush: Influence of Solvent Quality. PB95-202735 01,274 Not available NTIS

Neutron Reflectometry Studies of Surface Oxidation.

PB95-150421 00,917 Not available NTIS

Neutron Scattering by Hydrogen in Cold Neutron Prompt Gamma-Activation Analysis. 00,603 Not available NTIS
PB95-175972
Neutron Scattering by Multiblock Copolymers of Structure (A-B) N-A.

PB94-211547

01,219 Not available NTIS

Neutron Scattering Structural Study of AICuFe Quasicrystals Using Double Isotopic Substitution.
PB94-200458 04,485 Not available NTIS

Neutron Scattering Studies of Surfeces and Interfaces.

PB94-216207 04,517 Not available NTIS

Neutron-Scattering Studies of the Two Magnetic Correlation Lengths in Terbium.

PB95-152328

04,586 Not available NTIS

Neutron Scattering Study of Antiferromagnetic Order in the Magnetic Superconductors RNi2B2C.
PB97-112411 04,812 Not available NTIS

Neutron-Scattering Study of $\mathrm{C} 60(n-)(n=3,6)$ Librations in Alkali-Metal Fullerides.

PB94-200219

00,806 Not available NTIS

Neutron-Scattering Study of Librations and Intramolecular Phonons in Rb2.6K0.4C60.

PB95-162269

00,958 Not available NTIS

Neutron Scattering Study of Shear Induced Turbidity in Polystyrene/Dioctyl Phthalate Solutions at High Shear Rates.
01,197 Not available NTIS

Neutron Scattering Study of Shear Induced Turbidity in Polystyrene Dissolved in Dioctyl Phthalate.
PB95-161865 Not available NTIS

Neutron Scattering Study of the Lattice Modes of Solid Cubane

PB96-147452 01,126 Not available NTIS

Neutron Scattering Study of the Orientation of a Liquid Crystalline Polymer by Shear Flow.
PB95-180196 01,270 Not available NTIS Neutron Spectroscopic Comparison of beta-Phase Rare Earth Hydrides
PB96-160742

01,134 Not available NTIS

Neutron Spectroscopic Comparison of Rare-Earth/Hydrogen

alpha-Phase Systems
PB95-163523

00,970 Not available NTIS

Neutron Spectroscopic Evidence of Concentration-Dependent Hydrogen Ordering in the Octahedral Sublattice of betaTbH2+x.
PB95-181020

01,018 Not available NTIS

Neutron-Spectroscopy Study of the Hydrogen Vibrations in Hydrogen-Doped YBa2Cu3Ox.
PB94-172475 04,441 Not available NTIS

Neutron Standard Cross Sections in Reactor Physics: Need and Status.

PB94-199510

03,813 Not available NTIS

Neutron Techniques in Materials Science and Related Disciplines. 02,980 Not available NTIS

New Alloys Show Extraordinary Resistance to Fracture and Wear.
PB95-151346 03,346 Not available NTIS

New and Revised Half-Life Measurement Results.

PB96-160346

00,695 Not available NTIS

New Approach for Reducing the Toxicity of the Combustion Products from Flexible Polyurethane Foam.

PB96-123625

01,411 Not available NTIS

New Coaxial Microwave Microcalorimeter Evaluation Technique.
PB95-153227

01,892 Not available NTIS

New Concepts for Fire Protection of Passenger Rail Trans-

portation Vehicles.
PB95-162046

04,850 Not available NTIS

New Concepts for Fire Protection of Passenger Rail Transportation Vehicles. (NIST Reprint)

04,863 Not available NTIS

New Concepts of Precision Dimensional Measurement for

Modern Manufacturing.
PB96-160684

02,924 Not available NTIS

New Critical Review of Experimental Stark Widths and Shifts

PB94-172830 03,790 Not available NTIS

New cw CO2 Laser Lines: The 9-mu $m$ Hot Band

PB95-168548 04,281 Not available NTIS

New Data and Correlations for the Custody Transfer of Nat-

ural Gas Liquids.
PB96-176664

02,499 Not available NTIS

New Electronic States of NH and ND Observed from 258 to $288 \mathrm{~nm}$ by Resonance Enhanced Multiphoton Ionization PBectroscopy. 00,836 Not available NTIS

New EPR Dosimeter Based on Polyvinylalcohol

PB94-199700 03,619 Not available NTIS 


\section{TITLE INDEX}

New Exact Solution of the One-Dimensional Schrodinger Equation and Its Application to Polarized Neutron Reflectometry.
PB95-161832

04,609 Not available NTIS

New Expressions of Uncertainties for Humidity Calibrations at the National Institute of Standards and Technology. PB95-103826 02,645 PC A02/MF A0 1

New Extrapolation/Spherical/Cylindrical Measurement Facility at the National Institute of Standards and Technology. PB95.153755

02,004 Not available NTIS

New Generation of Fire Resistant Polymers. Part 1. Computer-Aided Molecular Design.

PB96-160593

01,419 Not available NTIS

New High-Redshitt Damped Lyman-alpha Absorption Systems and the Redshift Evolution of Damped Absorbers.

tems and the Redshift Evolution of Damped Absorbers.
PB95-203501 00,083 Not available NTIS

New International Representations of the Volt and Ohm Effective January 1, 1990.

fective January
PB95-150777

01,890 Not available NTIS

New IUPAC Guidelines for the Reporting of Stable Hyd:ogen, Carbon, and Oxygen Isotope-Ratio Data. Letter to the

PB95-261962

01,058

(Order as PB95-261897, PC A07/MF A02)

New Mass Transport Elements and Components for the

02,558 PC A05/MF A01

New Materials, Advanced Ceramics and Standards.

New Materials, Advanced Ceramics and Standards.
PB95-140208

New Mechanisms for Laser Cooling

PB95-153268

03,923 Not available NTIS

New Method for Achieving Accurate Thickness Control for Uniform and Graded Multulayer Coatings on Large Flat Substrates.

PB96-159744

04,366 Not available NTIS

New Method for Realizing a Luminous Flux Scale Using an Integrating Sphere with an External Source.

PB96-102843 04,333 Not available NTIS

New Method for Shielding Electron Beams Used for Head and Neck Cancer Treatment.

PB94-211430

03,621 Not available NTIS

New Method for the Detection and Measurement of Polyaromatic Carcinogens and Related Compounds by ONA Intercalation

03,481 Not available NTIS

New Method to Calculate Looming for Autonomous Obsta. cle Avoidance.
PB95- 171435

$01,600 \mathrm{PC} \mathrm{A03/MF} \mathrm{A01}$

New Method to Evaluate Deposit Forming Tendencies of

Liquid Lubricants by Differential Scanning Calorimetry.
PB 95-152120

New Model of $1 / F$ Noise in Baw Ouartz Resonators.

PB96-1 12248 02,383 Not available NTIS

New NIST/ARPA National Soft X-ray Reflectometry Facility.
PB96-158092

New NIST Rapid Pneumatic Tube System.

PB96-167259 03,738 Not available NTIS
O

New Oxide Degradation Mechanism for Stresses in the Fowler-Nordheim Tunneling Regime.

PB96-200985 04,128 Not available NTIS

New Physics-Based Model for Time-Dependent- DielectricBreakdown.

02,440 Not available NTIS

New Physics-Based Model for Time-Dependent- DielectricBreakdown.

02,448 Not available NTIS

New Refractometer by Combining a Variable Length Vacuum Cell and a Double-Pass Michelson Interferometer.

01,986 Not available NTIS

New Rydberg States of Aluminum Monofluoride Observed by Resonance-Enhanced Multiphoton Ionization SpectrosPB94-199544

00,797 Not available NTIS

New Surface-Active Comonomer for Adhesive Bonding.

PB96-204052 03,579 Not available NTIS

New Test Structure for Nanometer-Level Overlay and Feature-Placement Metrology.

PB95-175345

02,363 Not available NTIS

New Values for Silicon Reference Materials, Certified for sotope Abundance Ratios. Letter to the Editor.

00,863

(Order as PB94-219219, PC A06/MF A02)

New 5 and $10 \mathrm{MHz}$ High Isolation Distribution Amplifier

PB96-190202 01,510 Not available NTIS teristics of Dental Resins and Composites.
Next Generation Computer Resources: Reference Model for Project Support Environments (Version 2.0).
PB94-143401 Nickel(II)-Mediated Oxidative DNA Base Damage in Renal and Hepatic Chromatin of Pregnant Rats and Their

PB94-212628 03,646 Not available NTIS

Nickel-63 Standardization: 1968-1995.

PB97-111819 00,701 Not available NTIS

Niobium Microbolometers for Far-Infrared Detection

PB96-111729 02,184 Not available NTIS

NIOSH Comments to DOL on Risk Estimates from the Cadmium Cohort Study by L. Stayner, February 7, 1992.
PB95-267779

NIOSH Pocket Guide to Chemical Hazards.

PB95-100368 03,603 PC $\$ 14.00 / \mathrm{MF}$ A04

NIR-Spectroscopic Investigation of Water Sorption Charac-

PB95-151171 00,189 Not available NTIS

NIST and the Navy: Past, Present and Future.

PB96-119649 03,655 Not available NTIS

NIST Assessment of ITS-90 Non-Uniqueness for $25.5 \mathrm{Ohm}$ SPRTS at Gallium, Indium and Cadmium Fixed Points.

PB96-161310 00,631 Not available NTIS

NIST ATM Network Simulator: Operation and Programming. Version 1.0.
PB96-106851

01,487 PC A04/MF A01

NIST Calibration of ASTM E127-Type Ultrasonic Reference Blocks.

PB94-191640

$02,702 \mathrm{PC} \mathrm{A03/MF} \mathrm{AO1}$

NIST Capacitance Measurement Assurance Program (MAP).

02,254 Not available NTIS

NIST Comparison of the Ouantized Hall Resistance and the Realization of the SI Ohm Through the Calculable Capaci-

PB97-119184

02,291 Not available NTIS

NIST Comparison of the Ouantized Hall Resistance and the Realization of the SI Ohm Through the Calculable Capacitor. Conference Proceedings, June 17-20, 1996.

PB97-119192 02,292 Not available NTIS

NIST Construction Automation Program Report No. 2. Proceedings of the NIST Construction Automation Workshop.

Held in Gaithersburg, Maryland on March 30-31, 1995.
PB96-202239 PB96-202239

NIST Cooperative Laboratory for OSI Routing Technology.
01,791 PC AO3/MF A01
DE 94015308

NIST-Coordinated Standard for Fingerprint Data Inter-

PBa4-216645

01,808 Not available NTIS

NIST Crystallographic Databases for Research and AnalyPB97-109094

04,801 Corder as PB97-109011, PC
NIST Detector-Based Luminous Intensity Scale. PB96-179114

Order as PB96-177381, PC A07/MF A02)

NIST Form-Based Handprint Recognition System. PB94-217106

01,838 PC A04/MF A01

NIST Frequency Measurement Service.

PB96-200662

01,561 Not available NTIS

NIST Handbook 44, 1994: Specifications, Tolerances and Other Technical Requirements for Weighing and Measuring Devices as Adopted by the 78th National Conference on Weights and Measures 1993.

PB94-136009

$02,888 \mathrm{PC} \mathrm{A11/MF} \mathrm{A03}$

NIST Handbook 44, 1995: Specifications, Tolerances and Other Technical Requirements for Weighing and Measuring Devices as Adopted by the 79 .h National Conference Devices as Adopted by the

PB $95-146379$

$02,903 \mathrm{PC} \mathrm{A11/MF} \mathrm{A03}$

NIST Handbook 44, 1996: Specifications, Tolerances, and Other Technical Requirements for Weighing and Measuring Devices as Adopted by the 80th National Conference on Devices as Adopted by the

Weights and

$02,926 \mathrm{PC} \mathrm{A13/MF} \mathrm{A03}$

NIST High-Accuracy Sampling Wattmete

NIST High-Accur
PB97-108575

$02,689 \mathrm{PC} \mathrm{AO4/MF} \mathrm{AO}$

NIST High Accuracy Scale for Absolute Spectral Response from $406 \mathrm{~nm}$ to $920 \mathrm{~nm}$

01,865

(Order as PB96-177381, PC A07/MF A02)

NIST Implementation and Realization of the ITS-90 Over the Range $83 \mathrm{~K}$ to $1235 \mathrm{~K}$ : Reproducibility, Stability, and Uncertainties.
PB96-161328

00,632 Not available NTIS
NIST Industrial Impacts: A Sampling of Successful PartnerShips.

00,488 PC A05/MF A01

NIST Industrial Impacts: A Sampling of Successful Partnerships (Revision, March 1995).

PB95-209193

00,489 PC A05/MF A01

NIST Internet Time Service.

AD-P009 132/2

$01,519 \mathrm{PC} \mathrm{A02/MF} \mathrm{A01}$

NIST Lighting and HVAC Interaction Test Facility

PB95-151007 00,251 Not available NTIS

NIST List of Publications, LP 103, March 1996. National Semiconductor Metrology Program.

PB96-175856

02,432 PC AO7/MF AO2

NIST Measurement Assurance of SPRT Calibrations on the ST-90: A Ouantitative Approach.

PB96-161336

00,633 Not available NTIS

NIST Measurement Assurance Program for Capacitance Slandards at $1 \mathrm{kHz}$

PB96-172333

02,276 PC A03/MF A01

NIST Measurement Services: NIST Pressure Calibration PB94-164043

02,892 PC AO6/MF A02

NIST Metrology for Soft X-ray Multilayer Optics.

PB96-160379 04,088 Not available NTIS

NIST Metrology Program on Electromagnetic Characterizaion of Materials.

01,944 Not available NTIS

rement System for $1 \mathrm{~mW}$ at $1 \mathrm{GHz}$

PB94-135803

02.018 PC A03/MF A01

NIST/NCMS Program on Electronic Packaging: First Up-

PB96-204086

03,008 Not available NTIS

NIST-NRL Free-Electron Laser Facility.

PB94-212511

04,237 Not available NTIS

NIST Optically Pumped Cesium Frequency Standard.

PB94-211.117

NIST POSIX Testing Program.

PB96-160973

01,821 Not available NTIS

NIST Power Reference Source.

PB94-211513

00,148 Not available NTIS

NIST Program for Investigating Error Reporting Capabilities of Optical Disk Drives.

PB96-160627

01,635 Not available NTIS

NIST Reactor: Summary of Activities October 1992 through September 1993

PB94-161502

04,437 PC A08/MF A02

NIST Reactor: Summary of Activities, October 1993 through September 1994.

PB95-220430

04,700 PC A09/MF AO2

NIST Reference Matenals to Support Accuracy in Drug PBsting. 03,505 Not available NTIS

NIST Research on Less-Flammable Materials.

PB97-118632

01,439 Not available NTIS

NIST Research Program on the Seismic Resistance of Parfially-Grouted Masonry Shear Walls.

PB94-219052

00,354 PC A06/MF AO2

NIST Response to the Fifth CORM Report on the Pressing Problems and Projected Needs in Optical Radiation Meas-

PQ94-188240

04.227 PC AO3/MF AO

NIST RS274KT Interpreter.

PB96-147954

$02,835 \quad P C$ A05/MF AO

NIST RS274/NGC Interpreter Version 1 PB94-187788

02,814 PC A03/MF AO

NIST Serial Holdings, 1994

PB94-178068

$02,745 \mathrm{PC} \mathrm{A12/MF} \mathrm{AO3}$

NIST Serial Holdings, 1995

PB95-188926

02746 PC A12/MF AO3

NIST Serial Holdings, 1996

PB96-172523

02,748 PC A13/MF A03

NIST SIMA Interactive Management Workshop. Held in For Belvoir, Virginia on November 14-16, 1994

PB96-154877

02,838 PC AO8/MF AO2

NIST SRM 9983 High-Pigidity Ball-Bar Stand. User Manual. PB95-255840

02,669 PC AO3/MF AO

NIST Standard Reference Data Products Catalog, 1994. PB94-151842

00,727 PC A06/MF A02

NIST Standard Reference Data Products Catalog, 1995-96. Achieve with Standard Reference Data.

PB95-260808

01,057

PC A03/MF AO 


\section{TITLE INDEX}

NIST Standard Reference Materials (SRMs) for Polychlorinated Biphenyl (PCB) Determinations and Their Applicability to Toxaphene Measurements.

PB95-140109

02,585 Not available NTIS

NIST Standard Reference Materials (Trade Name) Catalog 1995- 1996.
PB95-232518

$00.508 \mathrm{PC} \mathrm{A03/MF} \mathrm{A01}$

NIST Strategies for Reducing Testing Requirements.

PB95-180444 01,909 Not available NTIS

NIST Support to the Next Generation Controller Program: 1991 Final Technical Report.

02,808 PC A09/MF A02

NIST Thermal Infrared Transfer Standard Radiometer for

the Earth Observing System (EOS) Program.
PB97-113013

NIST Traceable Reference Material Program for Gas Stand-

ards: Standard Reference Materials
PB96-210786

00.644 PC A04/MF A01

NIST Watt Balance: Progress Toward Monitoring the Kilogram.

01,991 Not available NTIS

NIST Watt Experiment: Monitoring the Kilogram.
PB97-122329

NIST Workshop on Gas Sensors: Strategies for Future Technologies. Proceedings of a Workshop. Held in Gaithersburg, Maryland on September 8-9, 1993.
PB95-210225 NIST Workshop on Nanostructured Material (1st): Peport of an Industrial Workshop Conducted by the National Institute of Standards and Technology. Held in Gaithersburg. Maryland on May 14-15, 1992.

NIST Workshop on the Computer Interface to Flat Panel Displays. Held in San Jose, California on January 13-14, PB95-136388

$01,625 \mathrm{PC} \mathrm{A11/MF} \mathrm{A03}$

NIST-7, the New US Primary Frequency Standard

PB95-153458 01,534 Not available NTIS

NIST $30 \mathrm{MHz}$ Linear Measurement System.

PB94- 169752 02,020 Not available NTIS

NIST 60-Millimeter Diameter Cylindrical Cavity Resonator: Performance Evaluation for Permittivity Measurements.
PB94-151776 02,251 PC A11/MF A03 Nitrogen Effect on Elastic Constants of f.c.c. Fe-18Cr-19Mn Alloys.

Alloys.
PB94-172541

03,203 Not available NTIS

NMR Characterization of Injection-Moulded Alumina Green Compacts. Part 2. T2-Weighted Proton Imaging

PB96-201181 01,165 Not available NTIS

NO Production and Destruction in a Methane/Air Diffusion Flame

01,443 Not available NTIS

Noise Analysis of Ionization Kinetics in a Protein Ion Channel.
PB96-161682

04,093 Not available NTIS

Noise Characteristics Below $1 \mathrm{~Hz}$ of Zener Diode-Based Voltage Reference

PB96-123476

04,049 Not available NTIS

Noise in the Coulomb Blockade Electrometer

PB95-176327 04,670 Not available NTIS

Noise-Induced Chaos and Phase Space Flux.

PB95-125761 03,433 Not available NTIS

Noise-Induced Transitions to Chaos.

PB96-156120

00,217 Not available NTIS

Noise Modeling and Reliability of Behavior Prediction for Multi-Stable Hydroelastic Systems.

PB96-111943

04,822 Not available NTIS

Noise Reduction in Low-Frequency SOUID Measurements with Laser-Driven Switching

02,081 Not available NTIS

Non-Equilibrium Thermodynamic Theory of Viscoplastic Materials.
PB94-198868

04,467 Not available NTIS

Non-Gaussian Noise Effects on Reliability of Multistable Systems.
PB96-122726

04,213 Not available NTIS

Non-Neutral Ion Plasmas and Crystals, Laser Cooling, and Atomic Clocks
PB95-175113

03,957 Not available NTIS

Non-Newtonian Flow between Concentric Cylinders Calculated from Thermophysical Properties Obtained from Simulations.

04, 103 Not available NTIS

Non-Osmotic, Defect-Controlled Cathodic Disbondment of a Coating from a Steel Substrate.
PB94-216447
Non-Perturbative Relation between the Mutual Diffusion Coefficient, Suspension Viscosity, and Osmotic Compressibility: Applicatıon to Concentrated Protein Solutions.

PB96-102355 01,062 Not available NTIS

Nonadiabatic Effects in the Photoassociation of H2S.

PB95-151437 00,934 Not available NTIS

Noncontact Ultrasonic Inspection of Train Rails for Stress.

04,851 Not available NTIS

Nondestructive Evaluation and Materials Processing

PB95-140455 02,902 Not available NTIS

Nondestructive Testing of Concrete: History and Challenges.
PB95-180139 00,385 Not available NTIS

Nonequilibrium Statistical Mechanics.

PB96-161781 St,097 Not available NTIS
PBistical Mechanics.

Nonequilibrium Thermodynamic Theory of Viscoplastic Materials.

PB96-11166

04,034 Not available NTIS

Nonequiiibrium Total-Dielectric-Function Approach to the Electron Boltzmann Equation for Inelastic Scattering in

Doped Polar Semiconductors.
PB96-138532 PB96-138532

Noninvasive High-Voltage Measurement in Mammography by Crystal Diffraction Spectroscopy.
PB95-153417 00,160 Not available NTIS Nonlinear Color Transformations in Real Time Using a Video Supercomputer.

02,191 Not available NTIS

B 96- 123021

Pressure Vapor-Liquid Equilibrium Data for Ethylene + n-Butane Showing Inconsist-

encies in Experimental Compositions.
PB96-161906

Nonlinear Dynan
PB96-122478

01,278 Not available NTIS

Nonlinear Response of Type-II Superconductors in the Mixed State in Slab Geometry.

PB94-200367 04,484 Not available NTIS

Nonlocal Effects of Existing Dislocations on Crack-Tip Emission and Cleavage.

PB96-161807

03,367 Not available NTIS

Nonstatıonary Behavior of Partial Discharge during Insulation Aging.

01,902 Not available NTIS

Nonstationary Behaviour of Partial Discharge during Dis-

charge Induced Ageing of Dielectrics.
PB96-103114 01,922 Not available NTIS

Normal-Incidence Complex-Index Refractometry.

PB94-213097 04,241 Not available NTIS

Normal Modes and Structure Factor for a Canted Spin Sys-

tem: The Generalized Villain Model.
PB95-140067 04,539 Not available NTIS

North American Agreements on ISDN.

PB96-160775 01,503 Not available NTIS

North American ISDN Users' Forum Agreements on Integrated Services Digital Network. 01,466 PC A16/MF A03
PB94-162559 Northridge Earthquake, 1994. Performance of Structures, Lifelines and Fire Protection Systems.
PB94-161114 00,421 PC A09/MF A02 00,421
Northridge Earthquake 1994: Performance of Structures, Northridge Earthquake 1994: Performance of Structures,
Lifelines, and Fire Protection Systems.
PB94-207461 04,825 PC A09/MF A02

Notion of a xi-Vector and a Stress Tensor for a General Class of Anisotropic Diffuse Interface Models.
PB96-193776 04,788 PC A03/MF A01

Novel Active-Vision-Based Motion Cues for Local Navigation. 193727

02,941 PC A05/MF A01

Novel Activities of Human Uracil DNA N-glycosylase for Cytosine-Derived Products of Oxidative DNA Damage.
PB96-164132 03,479 Not available NTIS

Novel Activity of E. coli uracil DNA N-glycosylase Excision of Isodialuric Acid (5,6-dihydroxyuracil), a Major Product of

Oxidative DNA Damage, from DNA

03,543 Not available NTIS

Novel Amperometric Immunosensor for Procainamide Employing Light Activated Labels.

00,512 Not available NTIS

Novel Bulk Iron Garnets for Magneto-Optic Magnetic Field Sensing.

04,675 Not available NTIS

Novel DNA N-Glycosylase Activity of $\mathrm{E}$. coli T4 Endonuclease $V$ That Excises 4,6-Diamino-5 Formamidopyrimidine from DNA, a UV-Radiation- and Hydroxyl Radical-Induced Product of Adenine.
PB96-160478
0349 Not available NTIS
Novel Hot-Electron Microbolometer.

PB96-201025 01,977 Not available NTIS

Novel Magnetic Field Characterization Techniques for Compound Semiconductor Materials and Devices.

PB96-176458 02,433 Not available NTIS

Novel Method for Determining Thin Film Density by EnergyDispersive $\mathrm{X}$-ray Reflectivity.

PB96-122783

04,737 Not available NTIS

Novel Polydiacetylenes Derived from Liquid Crystalline Monomers.

01,235 Not available NTIS

Novel Radiochromic Films for Clinical Dosimetry

PB97- 119259

03,641 Not available NTIS

Novel Vortex Dynamics in Two-Dimensional Josephson Arrays.

02,091 Not available NTIS

Novel YBa2Cu3O7-x and YBa2Cu3O7-xY4Ba3O9 Multilayer Films by Bias-Masked 'On-Axis' Magnetron Sputtering PB95-181186

04,690 Not available NTIS

Nuclear Heat Load Calculations for the NBSR Cold Neutron Source Using MCNP.

PB95-152955

03,730 Not available NTIS

Nucleic Acid Database: Present and Future.

PB97-109078

00,518

(Order as PB97-109011, PC A11/MF A03)

Null-Balanced Total-Power Radiometer System NCS1.

PB94-169778

02,021 Not available NTIS

Numeric Data Distribution: The Vital Role of Data Exchange in Today's World

02,622

(Order as N95-15919/0, PC A11/MF A03)

Numerical Analysis Support for Compartment Fire Modeling and Incorporation of Heat Conduction into a Zone Fire

PB94-156965

01,357 PC A04/MF AO

Numerical Evaluation of Hypersingular Integrals for Scattering by a Dielectric Wedge.

PB97-110555

02,017 Not available NTIS

Numerical Evaluation of Special Functions.

PB96-119557 03,417 Not available NTIS

Numerical Micromagnetic Techniques and Their Applica-

tions to Magnetic Force Microscopy Calculations.
PB95-175931 04,664 Not available NTIS

Numerical Reference Models for Optical Metrology Simula

tion.

04,392 Not available NTIS

Numerical Simulation of Rapid Combustion in an Under ground Enclosure.
PB96-183132

$01,424 \mathrm{PC} \mathrm{A03/MF} \mathrm{AO1}$

Numerical Simulation of Submicron Photolithographic Processing.

02,310 Not available NTIS

Numerical Solution of the Nonlinear Schroedinger Equation for Small Samples of Trapped Neutral Atoms.

PB95-202578 03,985 Not available NTIS

Nutritional Status and Growth in Juvenile Rheumatoid Arthritis.

PB94-198470

03,515 Not available NTIS

NVLAP Procedures U.S. Code of Federal Regulations. Title 15. Subtitle A, Chapter 2, Part 7. (Effective December 1984; Amended September 1990)

PB94-160850

02,627 PC A03/MF A01

Object Finder for Digital Images Based on Multiple Thresholds, Connectivity, and Internal Structures.

01,833 Not available NTIS

Object-Oriented Technology Research Areas.

PB95-199329

01,726 PC A03/MF A01

Object-Oriented Tel/Tk Binding for Interpreted Control of the NIST EXPRESS Toolkit in the NIST STEP Application Pro-

tocol Development Environmen

02,785 Not available NTIS

Object SOL: Language Extensions for Object Data ManagePB95-125902

01,704 Not available NTIS

Objective Evaluation of Short-Crack Toughness Curves Using Indentation Flaws: Case Study on Alumina-Based Ceramics.

PB96-179429

03,079 Not available NTIS

Observation and Visible and uv Magnetic Dipole Transitions Obshan Chand

PB96-138441

04,056 Not available NTIS

Observation of a Stable Methoxy Intermediate on $\operatorname{Cr}(110)$

PB95-164422 00,981 Not available NTIS 


\section{TITLE INDEX}

Observation of Hot-Electron Shot Noise in a Metallic Resistor.

01,988 Not available NTIS

Observation of Insulating Nanoparticles on YBCO ThinFilms by Atomic Force Microscopy.
PB95-163358 Observation of Noncollinear Magnetic Structure for the $\mathrm{Cu}$ Spins in Nd2CuO4-Type Systems.
PB95-164539 04,634 Not available NTIS Observation of Oscillatory Magnetic Order in the Antiferromagnetic Superconductor HoNi2B2C.
PB95-180303 34,679 Not available NTIS Observation of Pd-Like Resonance Lines Through Pt(32+) and $\mathrm{Zn}$-Like Resonance Lines of Er(38+) and $\mathrm{Hf}(42+)$.
$\mathrm{PB} 95+150637$
03,896 Not available NTIS

Observation of the Transverse Second Harmonic MagnetoOptic Kert Effect from Ni81Fe19 Thin Film Structures. Optic Kert Effect from Ni81Fe19 Thin Film Structures.
PB96-200332 01,971 Not available NTIS Observation of Two Length Scales Above ( $T$ sub $N$ ) in a Holmium Thin Film

04, 151 Not available NTIS

Observation of Vortex Dynamics in Two-Dimensional Jo-

sephson-Junction Arrays.
PB95-168811

02,050 Not available NTIS

Observations of Partial Discharges in Hexane Under High Magnification.
PB95-163127

01,900 Not available NTIS

Observations of Shear Induced Molecular Orientation in a Polymer Melt Using Fluorescence Anisotropy Measurements.

01,210 Not available NTIS

Observations of Shear Stress and Molecular Orientation Using Fluorescence Anisotropy Measurements.

PB94-199312 01,211 Not available NTIS

Observations of $3 \mathrm{C} 273$ with the Goddard High Resolution

Spectrograph on the Hubble Space Telescope.
PB95-202321 00.076 Not available NTIS

Observed and Theoretical Creep Rates for an Alumina $\mathrm{Ce}$ ramic and a Silicon Nitride Ceramic in Flexure.

PB94-212958 03.040 Not available NTIS

Observed Frustration in Confined Block Copolymers.

PB95.150033 01,238 Not available NTIS

Observing Stellar Coronae with the Goddard High Resolution Spectrograph. I. The dMe Star AU Microscopi.

PB96-102777 00,092 Not available NTIS

Obtaining and Installing a Public Domain TEX.

PB95-175741 01,719 Not available NTIS

Octacalcium Phosphate Carboxylates IV. Kinetics of Forma-

tion and Solubility of Octacalcium Phosphate Succinate.
PB94-185600 00,776 Not available NTIS

Octacalcium Phosphate Carboxylates. 1. Preparation and Identification.
PB95-161535

00,660 Not available NTIS

Octacalcium Phosphate Carboxylates. 2. Characterization

and Structural Consideration.
PB95-161543

00,955 Not available NT1S

Octacalcium Phosphate Carboxylates. 5. Incorporation of Excess Succinate and Ammonium Ions in the Octacalcium Phosphate Succinate Structure.
PB95-168894 00,166 Not available NTIS

Octacalcium Phosphate. 3. Infrared and Raman Vibrational Spectra.

PB94-172244

00.756 Not available NTIS

Off-Diagonal Orthorhombic-Symmetry Elastic Constants.

PB95-202768 04,695 Not available NTIS

Office Work Station Heat Release Rate Study: Fufl Scale versus Bench Scale.

PB96-190178

01,428 Not available NTIS

OHset Susceptibility of Superconductors.

PB94-212263 04,503 Not available NTIS

On-Water Impedance Measurement on Lossy Substrates.

PB95-176285 02,365 Not available NTIS

One- and Two-Sided Buming of Themally Thin Materials.
PB95-140935

One-Dimensional Modeling Studies of the Gaseous Electronics Conference RF Reference Cell.

PB96-113428

02,397

(Order as PB96-113311, PC A09/MF A03)

One-Electron Oxidation of Metalloporphycenes as Studied by Radiolytic Methods.

01,179 Not available NTIS

One-Electron Oxidation of Nickel Porphyrins. Effect of Structure and Medium on Formation of Nickel(III) Porphyrin

Or Nickel(II) Porphyrin pi-Radical Cation.
PB95-107058 00,865 Not available NTIS
One Gigarad Passivating Nitrided Oxides for $100 \%$ Internal Quantum Efficiency Silicon Photodiodes.
PB94-185485 Not available NTIS One-Sided beta-Content Tolerance Intervals for Mixed ModPB96-141171 03,449 Not available NTIS

Opacity Project and the Practical Utilization of Atomic Data.
PB94-212214 00,059 Not available NTIS

Open Architectures for Machine Control.
PB94-135621 PC A03/MF A01

Open Document Architecture (ODA) Raster Document ApOpen Document Architecture (ODA) Raster Document Ap-
plication Profile (DAP). Category: Software Standard; SubCategory: Graphics.

01,669 PC E04

Open-Ended Coaxial Probes for Nondestructive Testing of Substrates and Circuit Boards. 02,078 Not available NTIS
PB96-122825 Open Issues in OSI Protocol Development and Conformance Testing.

01,818 Not available NTIS

Open System Environment Implementors Workshop (OIW): Standardization Role Defined.

01,828 Not available NTIS

Open System Environment (OSE): Architectural Framework for Information Infrastructure.

00,002 PC A04/MF A01

Open System Environment Procurement.
N94-36858/6

(Order as N94-36853/7, PC A10/MF A03)

Open System Environments.
N94-36857/8

01,674

(Order as N94-36853/7, PC A10/MF A03)

Open Systems Software Standards in Concurrent Engineering.

01,758 Not available NTIS

Operating Principles of Muiltikron II Performance Instrumentation for MIMD Computers.

01,628 PC A03/MF A01

Operating Principles of MultiKron Virtual Counter Performance Instrumentation for MIMD Computers.
PB96-131529

Operating Principles of the SBus MultiKron Interface Board. PB95-231783 01,630 PC A03/MF A01

Operating Procedures and Life Cycle Documentation for the Initial Graphics Exchange Specification.

Operational Mode and Gas Species Effects on Rotational Drag in Pneumatic Dead Weight Pressure Gages.

PB95-140182 00,903 Not available NTIS

Operator Experience with a Hierarchical Real-Time Control System (RCS).

03,751 PC A03/MF A01

Opportunities for Innovation: Advanced Manufacturing Technology.

02,801 PC AO7/MF A02

Opportunities for Innovation: Advanced Surface Engineering.

02.697 PC AO9/MF AO2

Opportunities

00,009 PC A12MF AO3

Opportunities for Innovation: Optoelectronics.

PB96-118039

01,928 PC A19/MF AO4

Opportunities for Innovation: Pollution Prevention.

PB95-147146 02,520 PC AO9/MF AO2

Opportunities for Innovation: Software for Manufacturing
PB95-155578 02,851 PC A09/MF A03

Optical and Mass Spectrometric Investigations of lons and Neutral Species in SF6 Radio-Frequency Discharges.
01,985 Not available NTIS

Optical and Modeling Studies of Sodium/Halide Reactions for the Formation of Titanium and Boron Nanoparticles.
PB 97.113054

Optical Biosensor Using a Fluorescent, Swelling Sensing Element.

PB95-175899

03,541 Not available NTIS

Optical Characterization in Microelectronics Manufacturing.
PB95-169397

(Order as PB95-169371, PC A07/MF A02)

Optical Characterization of Materials and Devices for the Semiconductor Industry: Trends and Needs.
PB96-167192

Optical Conductivity of Single Crystals of Ba1$x M \times B i O 3(M=K, R b, x=0.04,0.37)$.
$P B 94-185329$
Optical Control of Enzymatic Conversion of Sucrose 10 Glucose by Bacteriorhodopsin Incorporated into Self-AssemPB96-123344 03,477 Not available NTIS
P. Optical Current Transducer for Calibration Studies.

PB94-185907 02,121 Not available NTIS Optical Density Measurements of Laser Eye Protection Materials. 00,190 Not available NTIS

Optical Detector Nonlinearity: A Comparison of Five MethPB95-169355

04,291 Not available NTIS

Optical Detector Nonlinearity: Simulation

02,199 PC AOAMF AO1

Optical Diagnostics in the Gaseous Electronics Conference Reterence Ceil.
PB96-113352

02,390

(Order as PB96-113311, PC A09/MF A03)

Optical Emission Spectroscopy on the Gaseous Electronics Conference RF Reference Cell.

PB96- 113345

(Order as PB96-113311, PC A09/MF A03)

Optical Fiber, Fiber Coating, and Connector Ferrule Geometry: Results of Interlaboratory Measurement Comparisons.
PB96-154422 04,360 PC A05/MF A01

Optical Fiber Geometry: Accurate Measurement of Cladding Diameter.
PB95-151940

04,266 Not available NTIS

Optical Fiber Geometry by Gray-Scale Analysis with Robust Regression.
PB95-161519

04,272 Not available NTIS

Optical Fiber Sensors: Accelerating Applications in Navy Ships.
PB94-186848

02,632 PC A06/MF A02

Optical Measurements of Atomic Hydrogen, Hydroxyl, and Carbon Monoxide in Hydrocarbon Diffusion Flames.
PB95-150900 02,487 Not available NTIS

Optical Metrology and More. Programs and Services of the

Radiometric Physics Division, Physics Laboratory.
PB94-191707
04,228 PC A03/MF A01

Optical Molasses: Cold Atoms for Precision Measurements.
PB,877 Not available NTIS $95-108890$

Optical Molasses: The Coldest Atoms Ever.

PB95-108908 03,878 Not available NTIS

Optical Performance of Commercial Windows.
PB95-208757 00,392 PC A09/MF A02

Optical Performance of Photoinductive Mixers at Terahertz Frequencies.

02,161 Not available NTIS

Optical Power Meter Calibration Using Tunable Laser Di-

PB95-169256

04,290 Not available NTIS

Optical Probing of Cold Trapped Atoms.

PB95-175469 04,296 Not available NTIS

Optical Properties of Triton X-100-Treated Purple MernPranes Embed

03,546 Not available NTIS

Optical Sampling Using Nondegenerate Four-Wave Mixing in a Semiconduc

02,076 Not available NTIS

Optical Sampling Using Nondegenerate Four-Wave Mixing in a Semiconductor Laser Amplitier.

PB96-123609

04,348 Not available NTIS

Optical Scattering from Moderately Rough Surfaces.

Optical Storage Media Data Integrity Studies.

N95-24130/3

(Order as N95-24108, PC A17/MF A04)

Optically Linked Three-Loop Antenna System for Determining the Radiation Characteristics of an Electrically Small Source.
PB95-161915

02,005 Not available NTIS

Optically Sensed EM-Field Probes for Pulsed Fields

PB94-212594 02,130 Not available NTIS

Optically Stabilized Tunable Diode-Laser System for Saturation Spectroscopy.

04,717 Not available NTIS

Optimal Control of Building and HVAC Systems.

PB96-141353 00,272 Not available NTIS

Optimization of ECR-Based PECVD Oxide Films for Superconducting Integrated Circuit Fabrication.
PB95-169165 -169165

Optimization of Highway Concrete Technology.
PB94-182995 


\section{TITLE INDEX}

Optimization of Inert Gas Atomization

PB95-107405

01,377 Not available NTIS

Optimizing Time

Analysis

02,085 Not available NTIS

Optoelectronics and Optomechanics Manufacturing: An ATP Focused Program Development. Workshop Proceedings Held in Gaithersburg, Maryland on February 15, 1995. PB97-104186 02,204 PCA11/MF A03

Optoelectronics at NIST.

PB96-200860

02,202 Not available NTIS

Orbital Alignment and Vector Correlations in Inelastic Atomic Collisions.

04,047 Not available NTIS

Orbital Stereochemistry: Discovering the Symmetries of Colision Processes.

01,034 Not available NTIS

Ordered omega-Derivatives in a Ti-37.5Al-2ONb at \% Alloy. PB94-211091 03,331 Not available NTIS Organization and Implementation of Calibration in the EOS Project. Part

PB96-179437

04,841 Not available NTIS

Orientation Effects on ESR Analysis of Alanine-Polymer Dosimeters.

03,720 Not available NTIS

Orientational Fluctuatıons, Diffuse Scattering, and Orientational Order in Solid C60

PB97-119275

04,176 Not available NTIS

Origin of $/ / \mathrm{PM}$ and $\mathrm{AM}$ Noise in Bipolar Junction Transistor Amplifiers.

PB96-200787

02,096 Not available NTIS

Origin of the Second Length Scale Above the Magnetic Spiral Phase of Tb
PB95-153698

04,596 Not available NTIS

Orthotropic Elastic Constants of a Boron-Aluminum FiberReinforced Composite: An Acoustic-Resonance-Spectroscopy Study.
PB96-200175

03,182 Not available NTIS

Oscillator Strengths and Radiative Branching Ratios in
Atomic Sr. Atomic Sr.

04,008 Not available NTIS

Oscillatory Exchange Coupling in $\mathrm{Fe} / \mathrm{Au} / \mathrm{Fe}(100)$.

PB95-150371 04,569 Not available NTIS

Oscillometric and Conductometric Analysis of Aqueous and Organic Dosimeter Solutions. $\quad 04,054$ Not available NTIS
PB96-135256

Ouch Those Programs are Painful.

PB96-102678 01,739 Not available NTIS

Outlier-Resistant Methods for Estimation and Model Fitting.
PB95-444 Not available NTIS

Outline of a Multiple Dimensional Reference Model Architecture and a Knowledge Engineering Methodology for Intelligent Systems Control.

$03,703 \mathrm{PC} \mathrm{A03/MF} \mathrm{A01}$

Overcoming Hoelder Continuity in III-Posed Continuation Problems

PB95-202354

03,416 Not available NTIS

Overview of a Radiation Accident at an Industrial Accelerator Facility.

02,612 Not available NTIS

Overview of Bioelectrical Impedance Analyzers.

PB96-122635 00,176 Not available NTIS

Overview of Bioelectrical Impedance Analyzers.

PB97-118780 00,181 Not available NTIS

Overview of NASREM: The NASANBS Standard Reference

Model for Telerobot Control System Architecture.
PB94-194560 04,831 PC A03/MF A01

Overview of Radiometric Program of the NIST Thermal Imaging Laboratory.

04,230 Not available NTIS

Overview of Reference Materials Prepared for Standardization of DNA Typing Procedures.

PB96-111653

00,611 Not available NTIS

Overview of Smoke Control Technology.

PB94-212867

00,348 Not available NTIS

Overview of the Manufacturing Engineering Toolkit ProtoPBg6- 128228

02,833 PC A03/MF A01

Overview of the Text REtrieval Conference (3rd) (TREC-3) Held in Gaithersburg, Maryland on November $2-4.1994$.
PB95-216883

Overview of U.S. Government Advanced Packaging Programs. 02,443 Not avallable NTIS
Oxidation of Caffeic Acid and Related Hydroxycinnamic

PB97-111975

00,651 Not available NTIS

Oxidation of Ferrous and Ferrocyanide Ions by Peroxy Radicals.

PB97-122402

01,191 Not available NTIS

Oxidation of SiC.

02,401 Not available NTIS

PB96-119516

on Monoxide in Hydrocarbon

Oxidation of Soot

01,382 Not available NTIS

PB95-150215 Oxidation of 10-Methylacridan, a Synthetic Analogue of Application of Laser Flash Photolysis and Direct and Redox Catalyzed Electrochemistry to the Kinetics of Deprotonation of the Cation Radical.

PB94-198371

00,785 Not available NTIS

Oxidative Damage to DNA in Mammalian Chromatin.

PB94-199825

03,525 Not available NTIS

Oxidative DNA Base Damage in Renal, Hepatic, and Pulmonary Chromatin of Rats After Intraperitoneal Injection of Cobalt (II) Acetate.

PB95-150025

03,647 Not available NTIS

Oxygen Annealin

$\mathrm{BCO} / \mathrm{Ag}$ Thin-Film Interfaces.

Oxygen Dependance of the Crystal Structure of $\mathrm{HgBa} 2 \mathrm{CuO} 4+$ and Its Relation to Superconductivity. PB96-102512 04,711 Not available NTIS
PBgation Relation to Superconductivity

P-Type Doubling in the Infrared Spectrum of NO-HF.

P-Type Doubling in the Intrared Spectrum of NO-HF.
PB94-211463

Paffenbarger Research Center: The Cutting Edge of Dental

Science.
PB94-216355

00,151 Not available NTIS

Pairwise and Nonpairwise Additive Forces in Weakly Bound Complexes: High Resolution Infrared Spectroscopy of Complexes: High Resolution Infrared Spectroscopy of
ArnDF $(n=1,2,3)$.
PB96-200357

04,125 Not available NTIS

Panel: Building and Using Test Collections.

PB97.118673 02,743 Not available NTIS

Panel Discussion on Units in Magnetism

PB96-137773 04,746 Not available NTIS

Papers on the Symposium on Collision Phenomena in Astrophysics, Geophysics, and Masers.

AD-A280 291/6 00,047 PC A03/MF A01

Papers Presentations Shine.

PB94-200383

00,244 Not available NTIS

Parallel and Serial Implementations of SLI Arithmetic.

PB95-242335 01,732 PC A03/MF A01

Parallel Monte Carlo Simulation of MBE Growth

PB96.122841 02,406 Not available NTIS

Parametric Investigation of Metal Powder Atomization Using Laser Diffraction.

03,342 Not available NTIS

Parametric Study of Hydrogen Fluoride Formation in Suppressed Fires.

00,514 Not available NTIS

Parametric Study of Wall Moisture Contents Using a Revised Variable Indoor Relative Humidity Version of the 'Moist' Transient Heat and Moisture Transter Model. 'Moist' Transient Heat and Moisture Transfer Model.
PB97-122535 00,419 Not available NTIS Partial Discharge: Induced Aging of Cast Epoxies and Related Nonstationary Behavior of the Discharge Statistics.
01,903 Not available NTIS
PB95-163598 Partial Pressure Analysis in Space Testing N95-14084/4 (Order as N95-14062/0, PC A20/MF A04)

Partial Pressure Analysis in Space Testing.

PB96-103072 04,829 Not available NTIS

Partial Scattered Intensities from a Binary Suspension of Polystyrene and Silica.

00,996 Not available NTIS

Partially Coherent Transmittance of Dielectric Lamellae.

PB96-148028 04,359 Not available NTIS

Partially Prestressed and Debonded Precast Concrete Beam-Column Joints.

PB95-153102

00,439 Not available NTIS

Particle Size Standards and Their Certification at NIST.

PB94-211695

2,639 Not available NTIS

Passively O-Switched Nd-Doped Waveguide Laser.

PB95-180048 04,308 Not available NTIS

Pattern-Recognition Analysis of Low-Resolution X-Ray Fluorescence Spectra.

00,588 Not available NTIS
PC-Based Prototype Expert System for Data Management and Analysis of Creep and Fatigue of Selected Materials at Elevated Temperatures.

PB94-172251

03,202 Not available NTIS

PC-Based Spinning Rotor Gage Controller.

PB95-175832 02,609 Not available NTIS

PCASYS: A Pattern-Level Classification Automation System for Fingerprints.

$01,853 \mathrm{PC} \mathrm{A03/MF} \mathrm{A01}$

Peeking Through the Picket Fence: What Astrophysical Surprises May Be Present in the 100-1200 Angstrom Region.
PB95-202859

Peeling a Polymer from a Surface or from a Line.

PB94-199809 01,213 Not available NTIS

Penetration and Diffusion of Hard X-rays through Thick Barriers. Ill. Studies of Spectral Distributions. AD-A292 $502 / 2$

$03,77 i \mathrm{PC}$ A01/MF A01

Perception of Clamp Noise in Television Receivers.

PB96-119433 01,489 Not available NTIS

Percolation and Pore Structure in Mortars and Concrete.

PB95-150439 00,370 Not available NTIS

Performance and Reliability of NIST 10-V Josephson ArPB96-148051 02,419 Not available NTIS

Performance Approach to the Development of Criteria for Low-Sloped Roof Membranes.

PB94-160751

$00,329 \mathrm{PC} \mathrm{A03/MF} \mathrm{A01}$

Performance Characteristics of Fast Elliptic Solvers on Parallel Platiorms.

01,723 Not available NTIS

Performance Evaluation of a New Digital Partial Discharge Recording and Analysis System.
PB95-150389

01,888 Not available NTIS

Performance Measures for Geometric Fitting in the NIST Algorithm Testing and Evaluation Program for Coordinate Measurement Systems.

01,745

(Order as PB96-117767, PC A08/MF A02)

Performance of a Reflectron Energy Compensating Mirror.
PB94-199460 00,547 Not available NTIS

Performance of Commercial CMOS Foundry-Compatible

Multijunction Thermal Converters.
PB95-153656
02,342 Not available NTIS

Performance of Compact Fluorescent Lamps at Different Ambient Temperatures.

PB95-175329

00,258 Not available NTIS

Performance of Federal Buildings in the January 17, 1994 Northridge Earthquake.

PB95-231775

$00,453 \mathrm{PC} \mathrm{A03/MF} \mathrm{A01}$

Performance of HUD-Affiliated Properties during the January 17, 1994 Northridge Earthquake.

PB95-174488

$00,443 \mathrm{PC} \mathrm{A04/MF} \mathrm{A01}$

Performance of Multilayer Thin-Film Multijunction Therma Ponverters.

02,084 Not available NTIS

Performance of Plastic Packaging for Hazardous Materials

Transportation. Part 1. Mechanical Properties.
AD-A301 258/0
$02,580 \quad$ PC A04/MF A01

Performance of Tape-Bonded Seams of EPDM Membranes: Comparison of the Peel Creep-Rupture Response of TapeBonded and Liquid-Adhesive-Bonded Seams.

PB96-183249

03,012 PC A05/MF A01

Performance of the Electron Pump with Stray Capacitances.

Performance of $1 / 3$-Scale Model Precast Concrete BeamColumn Connections Subjected to Cyclic Inelastic Loads. Report No. 4.
PB95-179024

00,444 PC A04/MF A01

Performance Parameters of Fire Detection Systems

PB94-194339

$00,288 \mathrm{PC} \mathrm{A03/MF} \mathrm{A01}$

Performance Standards: The Pro's and Con's.

PB94-216132

02,896 Not availabje NTIS

Performance Testing of a Family of Type I Combination Ap-

PB95-22052

02,505 PC A03/MF A01

Periapical Tissue Reactions to a Calcium Phosphate $\mathrm{Ce}$ ment in the Teeth of Monkeys.

PB94-212008

00,149 Not available NTIS

Permeation Tube Approach to Long-Term Use of Automatic

Sampler Retention Index Standards.
PB96-167291 Not available NTIS

Permissible Dose from External Sources of lonizing Radi-

AD-A279 281/0

03,608 PC A05/MF A01 


\section{TITLE INDEX}

Perpendicular C-H Stretching Band nu9/nu13 and the Torsional Potential of Dimethylacetylene.
PB97-122451 Not available NTIS Persistent Object Base System Testing and Evaluation PB95-220588 01,730 PC A05/MF A01 Perspective on Fiber Coating Technology PB94-200540 03,118 Not available NTIS Perspective on Software Engineering Standards PB95-171377 01,811 PC A03/MF A01 Perturbative Calculation of the AC Stark Effect by the Complex Rotation Method

04,260 Not available NTIS

PET and DINGO Tools for Deriving Distributed Implementations from Estelle.

01,727 Not available NTIS

Phase Behavior of a Hydrogen Bonding Molecular Composite.

01,202 Not available NTIS

Phase Composition, Viscosities, and Densities for Aqueous Two-Phase Systems Composed of Polyethylene Glycol and Various Salts at $25 \mathrm{C}$

PB95-164596

00,986 Not available NTIS

Phase Equilibria in the Systems $\mathrm{CaO}-\mathrm{CuO}$ and $\mathrm{CaO}-\mathrm{Bi} 2 \mathrm{O} 3$ PB95-140570 03,048 Not available NTIS

Phase-Field Model for Solidification of a Eutectic Alloy. PB95-147914

$03,345 \mathrm{PC}$ A04/MF A01

Phase Identification in a Scanning Electron Microscope Using Backscattered Electron Kikuchi Patterns. PB97-109128

(Order as PB97-109011, PC A11/MF A03)

Phase-Locked Oscillator Optimization for Arrays of Josephson Junctions.
PB95-169314

02,052 Not available NTIS

Phase Locking in Two-Dimensional Arrays of Josephson Junctions: Effect of Critical-Current Nonuniformity.

PB96-102587 04,714 Not available NTIS

Phase Locking in Two-Junction Systems of High-Temperature Superconductor-Normal Metal-Superconductor Junctions.

02,060 Not available NTIS

Phase Separation in Thin Film Polymer Blends With and Without Block Copolymer Additives.
PB96-204482 Not available NTIS

Phase Shifts and Intensity Dependence in Frequency-Modulation Spectroscopy.

01,071 Not available NTIS

PB96-103205

70: Supercooling, Metastable Phase Transitions in Solid

PB95-150090

00,914 Not available NTIS

Phonon Density of States in $\mathrm{R} 2 \mathrm{CuO} 4$ and Superconducting $\mathrm{R} 1.85 \mathrm{Ce} 0.15 \mathrm{CuO} 4(\mathrm{R}=\mathrm{Nd}, \mathrm{Pr}$ )

PB95-150686

04,574 Not available NTIS

Phonon Relaxation in Soft-X-ray Emission of Insulators.

PB96-160296

04,085 Not available NTIS

Phospholipid/Alkanethiol Bilayers for Cell-Surface Receptor

Studies by Surface Plasmon Resonance.
PB96-102900 03,472 Not available NTIS

Photodecomposition Dynamics of Mo(CO)6/Si(111) $7 \times 7$ : CO Internal State and Translational Energy Distributions--Trans-

00,687 Not available NTIS

Photodecomposition of $\mathrm{Mo}(\mathrm{CO}) 6 / \mathrm{Si}(111) 7 \times 7$ : CO State-Resolved Evidence for Excited State Relaxation and OuenchPBg5-180154

01,009 Not available NTIS

Photodesorption Dynamics of CO from Si(111): The Role of Surface Defects.

PB96-111646

03,066 Not available NTIS

Photodetector Frequency Response Measurements at NIST, US, and NPL, UK: Preliminary Results of a Standards Laboratory Comparison.

02,175 Not available NTIS

Photodissociation Dynamics in Ouantum State-Selected Clusters: A Test of the One-Atom Cage Effect in Ar-H2O.

PB95-203121 01,044 Not available NTIS

Photodissociation of Ammonia at 193.3nm: Rovibrational State Distribution of the NH2(A(2)A1) Fragment.

PB95-151775 00,937 Not available NTIS

Photoelectron Spectroscopic Study of the Valence and Core-Level Electronic Structure of $\mathrm{BaTiO}$.

PB94-212149 04,500 Not available NTIS

Photoelectron Spectroscopy of Negatively Charged Bismuth Clusters: $\mathrm{Bi}(-) 2, \mathrm{Bi}(-) 3$, and $\mathrm{Bi}(-) 4$

PB95-108494

00,880 Not available NTIS
Photoelectron Spectroscopy of Small Antimony Cluster Anions: $\mathrm{Sb}(-), \mathrm{Sb} 2(-), \mathrm{Sb} 3(-)$, and $\mathrm{Sb} 4(-)$.
PB95-203139 Photoelectron Study of Electronic Autoionization in Rotationally Cooled N2: The $n=6$ Member of the Hopfield Series. PB95-163531

00,971 Not available NTIS

Photographic Response to X-Ray Irradiation. 1. Estimation of the Photographic Error Statistic and Development of AnaIytic Density-Intensity Equations. 03,821 Not available NTIS Photographic Response to X-Ray Irradiation. 2. Correlated PB94-200094

03,822 Not available NTIS

Photographic Response to X-Ray Irradiation. 3. Photo-

graphic Linearization of Beam-Foil Spectra.
PB 84,823 Not available NTIS

Photoionization of Small Molecules Using Synchrotron RadiPB94-211505 03,841 Not available NTIS

Photoluminescence Spectra of Epitaxial Single Crystal C60. PB96-141205

04,754 Not available NTIS

Photonic Band-Structure Effects for Low-Index-Contrast Two-Dimensional Lattices in the Near Infrared.

PB97-122469

04,401 Not available NTIS

Photonic Materials: A Report on the Results of a Workshop Held in Gaithersburg, Maryland on August 26-27, 1992, Vol-

Ume 1. 152733

02, 114 PC A05/MF A01

Physical and Chemical Properties of Resin-Reinforced Calcium Phosphate Cements.

PB95-180212

00,171 Not available NTIS

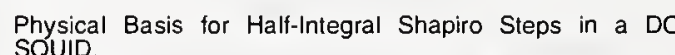
PB96-102264

04,704 Not available NTIS

Physical Characterization of Herparin by Light Scattering.
PB96-119383

Physical Limit to the Stability of Superheated and Stretched Water

PB96-122551

01,083 Not available NTIS

Physical Properties of Alternatives to the Fully Halogenated Ch!orofluorocarbons.
PB96-119573

03,279 Not available NTIS

Physical Properties of Some Purified Aliphatic Hydrocarbons. $265 / 1$

00,657 PC A03/MF A01

Physicochemical Characterization of Low Molecular Weigh Heparin.

PB96-112040

03,474 Not available NTIS

Physicochemical Characterization of Natural and Bioprosthetic Heart Valve Calcific Deposits: Implications fo Prevention.
PB96-156039

00,187 Not available NTIS

Physicochemical Properties of Calcific Deposits Isolated from Porcine Bioprosthetic Heart Valves Removed from Patients Following 2-13 Years Function.

PB94-172863 00,184 Not available NTIS

Physics and Chemistry of Partial Discharge and Corona

Recent Advances and Future Challenges.
PB95-181046

Physics and Chemistry of Partial Discharge and Corona Recent Advances and Future Challenges.
PB96-123757 Physics and Prospects of Inertial Confinement Fusion. PB94-185048 04,405 Not available NTIS Physics-Based Vision: Principles and Practice, Shape Recovery (Book Review)

PB95-164075 01,846 Not available NTIS

Physics for Device Simulations and lts Verification by Measurements.

02,327 Not available NTIS

Physics for Device Simulations and Its Verification by Measurements.

PB95-152914

02,339 Not available NTIS

Physics Laboratory Technical Activities, 1993

PB94-176088 03,796 PC A10/MF A03

Physics Required for Prediction of Long Term Performance of Polymers and Their Composites.

03,146

(Order as PB94-219219, PC A06/MF A02)

Picosecond Measurement of Substrate-to-Adsorbate Energy Transfer: The Frustrated Translation of CO/Pt(111)--TransPB95-126041

00,895 Not available NTIS

PIECS: A Sottware Program for Machine Tool ProcessIntermittent Error Compensation.

PB96-165980
Piezoelectric and Pyroelectric Polymers

01,250 Not available NTIS

Piggyback Balance Experiment: An Illustration of Archimedes Principles and Newton's Third Law.
PB94-163060
03,786 PC A03/MF A01 Pilot Studies for Improving Sampling Protocols.

PB97-118715 02,530 Not available NTIS

Pinch Effect in Commensurate Vortex-Pin Lattices.

PB96-147079 01,125 Not available NTIS

Planar Lenses for Field-Emitter Arrays.

PB96-103064

02,112 Not available NTIS

Planar Near-Field Alignment.

PB94-172491

01,998 Not available NTIS

Planar Near-Field Measurements and Microwave Holography for Measuring Aperture Distribution on a $60 \mathrm{GHz}$ Active Array Antenna.

PB96-167366

03,762 Not available NTIS

Planar Near-Field Measurements of Low-Sidelobe AntenPB94-219235

02,001 (Order as PB94-219219, PC A06/MF A02) Planar Resistors for Probe Station Calibration PB95-163697 02,351 Not available NTIS Planar Waveguide Optical Sensors

PB94-200185 03,586 Not available NTIS Planning for the Fiber Distributed Data Interface (FDDI) PB94-135761 01,621 PC A06/MF A02 Planning the Infrastructure for Global Electronic Commerce. PB94-185832 00,494 Not available NTIS Plasma Chemical Model for Decomposition of SF6 in a Negative Glow Corona Discharge.
PB95-181053 No20 Not available NTIS

Plasma Chemistry in Disilane Discharges.
PB94-211075 Plasma Chemistry in Silane/Germane and Disilane/Germane Mixtures.

01,027 Not available i IIS

Point Charges, Radiation Reaction, and Ouantum Mechanics. 04,139 Not available NTIS

Point Probe Decision Trees for Geometric Concept Classes. Polarimetric Calibration of Reciprocal-Anenn Radas.

Polarimetric Calibration of Reciprocal-Antenna Radars.
PB95-216925 01,872 PC A03/MF A01

Polarimetric Calibration of Reciprocal-Antenna Radars.

PB96-200696 02,016 Not available NTIS
PBion of Reciprocal-Antenna Radars.

Polarizability of the Nucleon.

PB94-211760

03,846 Not available NTIS

Polarization Analysis of the Magnetic Excitations in Fe65Ni35 Invar.

04,558 Not available NTIS

Polarization Analysis of the Magnetic Excitations in Invar and Non-Invar Amorphous Alloys.

Polarization Dependence of Response Functions in $3 \times 3$ Sagnac Optical Fiber Current Sensors.

Polarization Dependence of Response Functions in $3 \times 3$ Sagnac Optical Fiber Current Sensors. Polarization Effects on Multiple Scattering Gamma Transport.
PB95-153615 03,926 Not available NTIS

Polarization Insensitive $3 \times 3$ Sagnac Current Sensor Using Polarizing Spun High-Birefringence Fiber.
02,187 Not available NTIS

Polarization Measurements on a Magnetic Ouadrupole Line in Ne-Like Barium.

PB97-113104

04,161 Not available NTIS

Polarization of Light Emitted After Positron Impact Excitation of Alkali Atoms.

03,816 Not available NTIS

Polarized Transient Hot Wire Thermal Conductivity Measurements.

00,886 Not available NTIS

Polarized X-Ray Emission Spectroscopy.
PB94-213360

Polyelectrolytes as Dispersants in Colloidal Processing of

Silicon Nitride Ceramics
PB95-175568

03,055 Not available NTIS

Polyethylene Crystallized from an Entangled Solution Observed by Scanning Tunneling Microscopy
PB95-107389 


\section{TITLE INDEX}

Polymer Combustion and Flammability: Role of the Condensed Phase.

01,279 Not available NTIS

Polymer Composites Workshop. Held in Winona, Minnesota on April 29-30, 1992 (Video).

03,147 AV E99

PB94-780129

Polymer Liquid Crystalline Materials.

PB94-212339 01,224 Not available NTIS

Polymeric Calcium Phosphate Cements Derived from Poly(methyl vinyl ether-maleic acid). 00,180 Not available NTIS PB96-164264 Polymeric Calcium Phosphate Composites with Remineralization Potential. $\quad 03,575$ Not available NTIS
PB96-155544 Polymerization Initiation by $\mathrm{N}$-p-Tolylglycine: Free-Radical Reactions Studied by Pulse and Steady-State Radiolysis. PB95-180014 01,269 Not available NTIS

Polymers Technical Activities 1994. NAC-NRC Assessment Panel. April 6-7, 1995.

PB95-209896

01,275 PC A07/MF A02

Polymers Technical Activities, 1995.

PB96-193719

01,291 PC A10/MF A02

Polyvinyl Chloride (PVC) Plastic Drain, Waste, and Vent Pipe and Fittings.
AD-A310 426/2

$00,326 \mathrm{PC} \mathrm{A03/MF} \mathrm{A01}$

Population Distributions and Intralaboratory Reproducibility for Fat-Soluble Vitamin-Related Compounds in Human

PB96-155536

00.624 Not available NTIS

Population Trapping in Short-Pulse Multiphoton Ionization. PB96-164140 04.371 Not available NTIS

Pore-Forming Protein with a Metal-Actuated Switch

PB96-176557 03,554 Not available NTIS

Portable Operating System Interface (POSIX). Part 2. Shell and Utilities. Category: Software Standard; Subcategory: Operating Systems

FIPS PUB 189

01,797 PC A03/MF A01

Portable Vectorized Software for Bessel Function Evaluation.

PB94-198975

01,690 Not available NTIS

Porting Multımedia Applications to the Open System Environment.

PB94-172921

02,721 Not available NTIS

Portsmouth Fastener Manufacturing Workstation. User's Manual.

PB95-147922

02,860 PC A06/MF A02

Positive and Negative Cooperativities at Subsequent Steps of Oxygenation Regulate the Allosteric Behavior of Multistate Sebacylhemoglobin.
PB97-119374 $03,486 \quad$ Not available NTIS

Positronium in Relativistic Ouantum Mechanics.

PB97-110571 04,140 Not available NTIS

Post-Earthquake Fire and Lifelines Workshop. Held in Long Beach, California on January 30-31, 1995. Proceedings.

PB96-117916 00,209. PC A04/MF A01
O P

Post-Flame Soot

PB96-193701

$01,430 \mathrm{PC} \mathrm{A04/MF} \mathrm{A01}$

Post-Occupancy Evaluation of the Forrestal Building.

PB97-111298 00,280 Not available NTIS

Post-Process Control of Machine Tools.

PB95-203451 02,952 Not available NTIS

Posterior Restorative Materials Research

PB97-118624 03,582 Not available NTIS

Postfailure Subsidiary Cracking from Indentation Flaws in Brittle Materials.
PB97-110340

03,103 Not available NTIS

Potential and Current Distributions Calculated Across a Ouantum Hall Effect Sample at Low and High Currents. PB96-122106

(Order as PB96-117767, PC A08/MF A02)

Potential Drop in the Center-Cracked Panel with Asymmetric Crack Extension.

04,819 Not available NTIS

Potential Surfaces and Dynamics of Weakly Bound Trimers: Perspectives from High Resolution IR Spectroscopy.
PB96-176508 01,155 Not available NTIS

Potentiometric Enzyme-Amplified Flow Injection Analysis Detection System: Behavior of Free and Liposome-Released Peroxidase.

PB95-151833

03,534 Not available NTIS

Powder Diffraction File: Past, Present, and Future.

PB97-109086

04,800

(Order as PB97-109011, PC A11/MF A03)
Powder Neutron Diffraction Investigation of Structure and Cation Ordering in Ba2+xBi2-xO6-y.
PB95-180865 01,015 Not available NTIS Powder X-ray Diffraction Data for $\mathrm{Ca} 2 \mathrm{Bi2O}$ and PB96-161278

04,777 Not available NTIS

Power Characteristics in GMAW: Experimental and Numerical Investigation.

PB96-190145

03,296 Not available NTIS

Practical Applications of the ITS-90: Inherent Uncertainties. PB95-161527 03,930 Not available NTIS

Practical Photomask Linewidth Measurements.

PB95-108510 02,324 Not available NTIS

Practical Standards for PM and AM Noise at 5, 10 and 100 PB95-181129

01,546 Not available NTIS

Precise Identification of Computer Viruses.

PB96-160825 01,613 Not available NTIS

Precise Laser-Based Measurements of Zero-Dispersion Wavelength in Single-Mode Fibers.

PB96-201124

01,511 Not available NTIS

Precise Measurement of Heat of Combustion with a Bomb Calorimeter.

$03,770 \mathrm{PC} \mathrm{A03/MF} \mathrm{A01}$

AD-A286 701/8 quency Measurements with Diode Lasers.

PB95-176228 04,305 Not available NTIS
PBents with Diode Lasers.

Precise Spectroscopy for Fundamental Physics.

PB96-112164 04,040 Not available NTIS

Precision, Accuracy, Uncertainty and Traceability and Their Application to Submicrometer Dimensional Metrology.
PB94-213105 02,319 Not available NTIS

02,319 Not available NTIS

Precision and Accuracy in Tandem Mass Spectrometry Measurements: A Kinetics-Based Protocol for Instrument-
Independent Measurements of Collision-Activated
Dissociation in RF-Only Ouadrupoles. Dissociation in RF-Only Ouadrupoles.
PB94-216454 Not available NTIS

Precision Comparison of the Lattice Parameters of Silicon Monocrystals.

04,438 Not available NTIS

Precision High Temperature Blackbodies.

PB95-140059 03,885 Not available NTIS

Precision in Machining: Research Challenges.

Precision in Machining: Research Challenges.
PB95-242301

Precision Laboratory Standards of Mass and Laboratory

Weights.
AD-A280 562/0

02,618 PC A03/MF A01

Precision Lifetime Measurements of Cs $6 \mathrm{p}(2) \mathrm{P} 1 / 2$ and $6 \mathrm{p}$ (2) P3/2 Levels by Single-Photon Counting.
PB95-203816

04,010 Not available NTIS

Precision Nuclear Orientation Measurements for Determining Mixed Magnetic Dipole/Electric Ouadrupole Hyperfine Interactions.

03,810 Not available NTIS

Precision of Marchall Stability and Flow Test Using 6-in (152.4-mm) Diameter Specimens.
PB96-200910

03,006 Not available NTIS

Precision Oscillators: Dependence of Frequency on Temperature, Humidity and Pressure.

$02,031 \mathrm{PC} \mathrm{A03}$

Precision Resistors and Their Measurement

$\begin{array}{lll}\text { AD-A284 623/6 } 02,249 & \text { PC A03/MF A01 }\end{array}$

Precision Tests of a Ouantum Hall Effect Device DC Equivalent Circuit Using Double-Series and Triple-Series ConnecPB96-159256

01,953

(Order as PB96-159215, PC A07/MF A02)

Predicate Differences and the Analysis of Dependencies in Formal Specifications.

01,759 Not available NTIS

Predicting the Energy Performance Ratings of a Family of Type I Combination Appliances.

PB95-105524

02,504 PC A03/MF A01

Predicting the Fire Performance of Buildings: Establishing Appropriate Calculation Methods for Regulatory Applica.

PB96-141239

00,316 Not available NTIS

Predicting the Ignition Time and Burning Rate of Thermoplastics in the Cone Calorimeter.

PB96-154794

$01,418 \mathrm{PC} \mathrm{A10/MF} \mathrm{A03}$

Prediction of Cracking in Reinforced Concrete Structures.
PB95-220448
03,725 PC A04/MF A01 PB95-220448

Prediction of Fire Dynamics.

PB94-193620

Prediction of Geometric-
Artificial Neural Networks.

PB94-186673

02,943 PC A07/MF AQ2

Prediction of Potential Concrete Strength at Later Ages

PB96-112180

01,324 Not available NTIS

Prediction of Strengthening Due to $V$ Additions in DirectCooled Ferrite-Pearlite Forging Steels.

Cong 190251

03,220 Not available NTIS

Prediction of the Strength Properties for Plain-Carbon and Vanadium Micro-Alloyed Ferrite-Pearlite Steel.

Vanadium Micro-Alloyed Ferrite-Pearlite Steel.
PB96-123393 Not available NTIS

Prediction of the Thermal Conductivity of Refrigerants and

Refrigerant Mixtures.

03,258 Not available NTIS

Prediction of Viscosity of Refrigerants and Refrigerant Mixtures.

PB94-212099

03,257 Not available NTIS

Predictive Extended Corresponding States Model for Pure and Mixed Refrigerants Including an Equation of State for

PB95-175717

03,275 Not available NTIS

Preferential In-Plane Rotational Excitation of $\mathrm{H} 2 \mathrm{O}(001)$ by Translational-to-Vibrational Transfer from $2.2 \mathrm{eV} H$ Atoms. PB95-202875 01,035 Not available NTIS

Preliminary Comparison of Time Transfers via LASSO, GPS and Two-Way Satellite.

PB95-151098

01,529 Not available NTIS

Preliminary Functional Specifications of a Prototype Electronic Research Notebook for NIST.

PB94-207750

$00,012 \mathrm{PC} \mathrm{A04/MF} \mathrm{A01}$

Preliminary Investigation of Oleoresin Capsicum

PB96-179486 03,520 Not available NTIS

Preliminary List of References Containing Compilations of

Data on Properties of Materials
$\mathrm{AD} \cdot \mathrm{A} 302670 / 5$

$03,243 \mathrm{PC} \mathrm{A05/MF} \mathrm{A01}$

Preliminary Processing of the Lotung LSST Data.

PB96-165972 03,690 PC A05/MF A01

Preliminary Results of a Comparison of Water Triple-Point

Cells Prepared by Different Methods.

00,634 Not available NTIS

Preliminary Subject and Authors Index to Compilations of

Data on Properties of Materials.
$\mathrm{AD}-\mathrm{A} 302669 / 7$

03,242 PC A04/MF AO

Preparation and Certification of a Rhodium Standard Reference Material Solution.

PB94-18507

00,529 Not available NTIS

Preparation and Characterization of Cyclopolyr erizable Resin Formulations

PB96-146840

01,285 Not available NTIS

Preparation and Characterization of (6) LiF and (10)B Reference Deposits for the Measurement of the Neutron Life-

PB95-108692

03,874 Not available NTIS

Preparation and Crystal Structure of Sr5TiNb4O17.

PB96-167341 04,107 Not available NTIS

Preparation and Monitoring of Lead Acetate Containing Drinking Water Solutions for Toxicity Studies.

PB94-193885 00,538 PC A03/MF A01

Preparation, Crystal Structure, Dielectric Properties, and Magnetic Behavior of Ba2Fe2Ti4O13.

PB96-186176

01,162 Not available NTIS

Preparation of Immobilized Proteins Covalently Coupled Through Silane Coupling Agents to Inorganic Supports.
PB95-151403
03,530 Not available NTIS

Preparation of Low Resistivity Contacts for High-Tc Superconductors

PB95-153557

02,258 Not available NTIS

Preparation of 2-Dimensional Ultra Thin Polystyrene Film by Water Casting Method.

PB95-162806

04,619 Not available NTIS

Present and Future Standard Specimens for Surface Finish Metrology.

02,928 Not available NTIS

Present Worth Factors for Life-Cycle Cost Studies in the Department of Defense (1995)

PB95-105029

$03,664 \mathrm{PC} \mathrm{A04/MF} \mathrm{AO1}$

Present Worth Factors for Life-Cycle Cost Studies in the Department of Defense (1996). PB96-106869

$03,673 \mathrm{PC} \mathrm{A04/MF} \mathrm{AO}$

President's Column 'Editorial'

PB96-164090

02,239 Not available NTIS

President's Column for Dielectrics and Electrical Insulation 


\section{TITLE INDEX}

Pressure Dependencies of Standard Resistors. PB95-153516 02,257 Not available NTIS

Pressure Equations in Zone-Fire Modeling PB96-102967

00,208 Not available NTIS

Pressure Measurements with the Mercury Melting Line Referred to ITS-90.

01,136 Not available NTIS

Pressure-Volume-Temperature Relations in Liquid and Solid Tritium

PB94-140571

00,726

(Order as PB94-140555, PC A06/MF A02)

Pressurized Internal Lenticular Cracks at Healed Mica Inter. taces

PB96-180252

02,997 Not available NTIS

Preview of ASHRAE's Revised Smoke Control Manual.

PB94-212875

00,349 Not available NTIS

Principal Points and Self-Consistent Points of Symmetric Multivariate Distributions.

PB96-135090

03,446 Not available NTIS

Principle of Congruence and Its Application to Compressible States.

01,068 Not available NTIS

Probabilistic Computation of Poiseuille Flow Velocity Fields. PB96-102520 04,209 Not available NTIS

Probabilistic Estimates of Design Load Factors for WindSensitive Structures Using the 'Peaks Over Threshold' Approach.

00,474 PC A04/MF A01

Probing Bose-Einstein Condensed Atoms with Short Laser Pulses.

03,991 Not available NTIS

Probing Potential-Energy Surfaces via High-Resolution IR Laser Spectroscopy.

PB96. 102835

01,066 Not available NTIS

Problem of Convection in the Water Absorbed Dose Calorimeter.

PB97-110159

03,523 Not available NTIS

Problems Related to the Determination of Mass Densities of Evaporated Reference Deposits.

PB95-163226

03,941 Not available NTIS

Procedure for Measuring Trace Ouantities of S2F10 S2OF10, and S2O2F10 in SF6 Using a Gas Chromatograph-Mass Spectrometer. matograph-Mass

02,513 Not available NTIS

Procedure for Product Data Exchange Using STEP Developed in the AutoSTEP Pilot.

PB96-183058

$02,843 \mathrm{PC} \mathrm{AO4/MF} \mathrm{A01}$

Procedure for the Study of Acidic Calcium Phosphate Precursor Phases in Enamel Mineral Formation.

PB95-164448 03,564 Not available NTIS

Procedures for Document Facsimile Transmission Issued by General Services Administration, April 14, 1982. Federal Standard 1063

FIPS PUB 148

01,516 PC E99

Proceedings of a Workshop on Developing and Adopting Seismic Design and Construction Standards for Lifelines. Held in Denver, Colorado on September 25-27, 1991. PB97.115794 01.302 PC A16/MF A03

Proceedings of NIST Workshop: Industry Needs in Welding Research and Standards Development. Held on August 15 . $16,1995$.

PB'66-183124

02,877 PC A04/MF A01

Proceedings of the Annual Manufacturing Technology Conference (2nd): Toward a Common Agenda. Held in Gaithersburg, Maryland on April 18-20, 1995. PB96-112693 02,887 PC A99/MF E08

Proceedings of the Applied Diamond Conference 1995: Applications of Diamond Films and Related Materials International Conference (3rd). Held in Gaithersburg, Maryland,

national Conference (3rd). Held in Gaithersburg, Maryland,
PB August 21-24, 1995.
PB95-255204 04,701 PC A99/MF E11

04,701 PC A99/MF E11

Proceedings of the Digital Systems Reliability and Nuclear Satety Workshop. Held in Rockville, Maryland on September 13-14, 1993

$03,728 \mathrm{PC} \mathrm{A} 16 / \mathrm{MF} \mathrm{A03}$

Proceedings of the Manufacturing Technology Needs and Issues: Establishing National Priorities and Strategies Conference. Held in Gaithersburg. Maryland on April 26-28,

PB95-206181

02,930 PC A22/MF A04

Proceedings of the Meeting of the Intergovernmental U.S. Russian Business Development Committee's Standard Working Group (4th). Held in New York City, New York on March 27-29, 1995 and in Northbrook. Illinois on March 30. 31, 1995

PBं95-255881

$00,496 \mathrm{PC} \mathrm{A11/MF} \mathrm{A03}$
Proceedings of the Open Forum on Laboratory Accreditation at the National Institute of Standards and Technology, October 13,1995

02,686 PC A10/MF A03

Proceedings of the Workshop on the Federal Criteria for Information Technology Security. Held in Ellicot City, Maryland on June 2-3, 1993.

01,575 PC A04/MF A01

Proceedings of the 1995 Workshop on Fire Detector Re. search. Held on February 6-7, 1995.

PB95-270062

$02,611 \mathrm{PC} \mathrm{A03/MF} \mathrm{A01}$

Proceedings Report of the International Invitation Workshop on Development Assurance. Held in Ellicott City, Maryland on June 16-17, 1994

PB95-189494

02,912 PC AO3/MF A01

Proceedings: Workshop on Research Needs in Wind Engineering. Held in Gaithersburg, Maryland on September 12 13,1994

PB'95-189528

00,448 PC A05/MF A01

Process for Selecting Standard Reterence Algorithms for Evaluating Coordinate Measurement Software.

PB94-173754 02,629 PC A03/MF A01

Process Gas Chromatography Detector for Hydrocarbons

Based on Catalytic Cracking.
PB95-141099

02,485 Not available NTIS

Process Modeling and Control of Inert Gas Atomization.

PB95.162343 02,824 Not available NTIS

Process Monitoring with Residual Gas Analyzers (RGAs) Limiting Factors.

02,660 Not available NTIS

Product Kinetic Energies, Correlations, and Scattering Anisotropy in the Bimolecular Reactor $\mathrm{O}((1) \mathrm{D})+\mathrm{H} 2 \mathrm{O}$ yields $2 \mathrm{OH}$

PB94-212792

00.838 Not available NTIS

Product Models and Virtual Prototypes in Mechanical Engineering.

02,783 PC AO3/MF AO1

Product Realization Process Modeling: A Study of Requirements. Methods and Research Issues.
PB96-147962 02,836 PC A04/MF A01

Product State Correlations in the Reaction of $O((1) D)$ and $\mathrm{H} 2 \mathrm{O}$ in Bimolecular Collisions and in $\mathrm{O} 3 . \mathrm{H} 2 \mathrm{O}$ ClustersTranslation.

00,946 Not available NTIS

Production and Characterization of Ion Beam Sputtered Multilayers.

PB95-162053

03,936 Not available NTIS

Production and Spectroscopy of Small Polyatomic Molecular Ions Isolated in Solid Neon. (Reannouncement with New Availability Information)

AD-A234 043/8

$00,704 \mathrm{PC} \mathrm{A03/MF} \mathrm{A01}$

Production Management Information Model for Discrete Manufacturing
PB96-112008

02,830 Not available NTIS

Proficiency Testing as a Component of Ouality Assurance

in Construction Materials Laboratories.
PB94-185774 00.334 Not available NTIS

Proficiency Tests for the NIST Airborne Asbestos Program, 1990.

PB94-188836

00,535 PC A07/MF A02

Proficiency Tests for the NIST Airborne Asbestos Program -

PB94-193828

00,537 PC A04/MF A01

Proficiency Tests for the NIST Airborne Asbestos Program 1992

PB94-194362

00,539 PC A03/MF A01

Proficiency Tests for the NIST Airborne Asbestos Program

PB96-106463

00,610 PC A03/MF A01

Profile Fitting of X-Ray Diffraction Lines and Fourier Analysis of Broadening

04,460 Not available NTIS

Program Handbook: Requirements for Obtaining NIST Approval/Recognition of a Laboratory Accreditation Body Under P.L. 101.592. The Fastener Ouality ACI.

PB94-210143

02,859 PC A03/MF A01

Program of the Manufacturing Engineering Laboratory, 1995. Infrastructural Technology, Measurements, and Standards for the U.S. Manufacturing Industries. PB95-188835

02,754 PC A11/MF A02

Program of the Manufacturing Engineering Laboratory 1996. Infrastructural Technology, Measurements, and Standards for the U.S. Manufacturing Industries.

PB96-195276

02,760 PC A11/MF A03

Program of the Subcommittee on Construction and Building.
PB94-193646 00,319 PC A03/MF A01

Program of the Subcommittee on Construction and Building (July 1994).

$00,321 \mathrm{PC}$ A03/MF A01

Program Requirements to Advance the Technology of Custom Footwear Manufacturing.

02,883 PC A04/MF A01

Program Slicing.

PB96-160981

01,761 Not available NTIS

Programmable Guarded Coaxial Connector Panel

PB96-122544

02, 108 Not available NTIS

Programmer's Hierarchical Interactive Graphics System (PHIGS). Category: Sottware Standard; Subcategory: Graphics.

01,668 PC E99

Progress in the Development of a Chemical Kinetic Database for Combustion Chemistry.
PB95-151056 01,384 Not available NTIS Progress on a Cryogenic Linear Trap for $(199) \mathrm{Hg}(+)$ lons. PB95-180790 03,965 Not available NTIS Progress on the Ouantized Hall Resistance Recommended Intrinsic/Derived Standards Practice.

PB96-122460

02,403 Not available NTIS

Progress Report to National Aeronautics and Space Admin. istration on Cryogenic Research and Development.
AD-A286 612/7
01,458 PC A05/MF A01

Progress Toward Accurate Metrology Using Atomic Force Microscopy.
PB96-14677

02,417 Not available NTIS

Project Summaries 1994: NIST Building and Fire Research Laboratory.

00.343 PC A08/MF A02

Project Summaries 1995: NIST Building and Fire Research Laboratory.

00,400 PC A10/MF A03

Promise into Practice: Implementing TA2 on Real Clocks at NIST. 01,533 Not available NTIS

Properties and Interactions of Oral Structures and Restora. tive Materials. Annual Report for Period October 1, 1990 to tive Materials. Annual

September 30 ,
PB94-160843

03,558 PC A06/MF A01

Properties and Mechanisms of Fast-Setting Calcium Phosphate Cements.

00,178 Not available NTIS

Properties of a Bose-Einstein Condensate in an Anisotropic Harmonic Potentia

PB96-204144

04,133 Not available NTIS

Properties of Working Fluids for Thermoacoustic RefrigAD-A297 420/2

04,864 PC A06/MF A02

Proposed Analysis of RCS Measurement Uncertainty. PB95-203568 01,871 Not available NTIS

Proposed Antiferromagnetically Coupled Dual-Layer Magnetic Force Microscope Tips.

PB95-169017

04,645 Not available NTIS

Proposed Changes to Charpy V-Notch Machine Certification Requirements.
PB96-135363

02,955 Not available NTIS

Proposed Coating Technology Consortium. (National Coil Coaters Association Fall Conference). Held in Rosemont, IIlinois in September 1992

PB97-110431

03,129 Not available NTIS

Proposed High-Accuracy Superconducting Power Meter for Millimeter Waves.

02,034 Not available NTIS

Proposed International Interactive Courseware Standard

PB96-123161

00,137 Not available NTIS

Proposed Tests to Evaluate the Frequency-Dependent $\mathrm{Ca}$ pacitor Ratio for Single Electron Tunneling Experiment.
PB97-111454 NTIS

Protecting Your Family from Fire.

PB96-156187

00,307 Not available NTIS

Protection Against Neutron Radiation Up to 30 Million Electron Volts.

AD-A286 681/2

03,611 PC A05/MF A01

Protection Against Radiations from Radium, Cobalt-60, and Cesium-137.

AD-A279 261/2

03,607 PC A04/MF AO1

Protection of Data Processing Equipment with Fine Water Sprays. 


\section{TITLE INDEX}

Protein Extraction in a Spray Column Using a Polyethylene Glycol Maltodextrin Two-Phase Polymer System.

PB95-162228

00,595 Not available NTIS

Proteoglycan Inhibition of Calcium Phosphate Precipitation Liposomal Suspensions.

00,658 Not available NTIS

Proton Aftinity Ladders from Variable-Temperature Equilibrium Measurements. 1. A Re-Evaluation of the Upper Proton Affinity Range.

00,861 Not available NTIS

Protonation Dynamics in an Ion Channel Pore.

PB96-161757 03,589 Not available NTIS

Protonation Dynamics of the alpha-Toxin Ion Channel from Spectral Analysis of $\mathrm{pH}$-Dependent Current Fluctuations.

PB96-161740 03,652 Not available NTIS

Prototype Information Retrieval System to Perform a BestMatch Search for Names.

PB95-181152

02,740 Not available NTIS

Prototyping a Graphical User Interface for DHCP

PB96-160544 02,599 Not available NTIS

Provision of Isochronous Service on IEEE 802.6.

PB96-160635 01,501 Not available NTIS

Psychological Aspects of Lighting: A Review of the Work of CIE TC 3.16 .

00,241 Not available NTIS

Psychological Aspects of Lighting: A Review of the Work of CIE TC 3.16.

00,254 Not available NTIS

Public Key Infrastructure Invitational Workshop. Held in McLean, Virginia on September 28, 1995 . 01,616 PC A08/MF A02

Publication and Presentation Abstracts, 1993. (Published by Paffenbarger Research Center and Center ol Excellence for Materials Science Research).

PB95-153052 03,562 Not available NTIS

Publication and Presentation Abstracts, 1994

PB96-176623 03,577 Not available NTIS

Publication and Presentation Abstracts, 1995

PB96-164082 03.576 Not available NTIS

Publication and Presentation Abstracts, 1996

PB97-122238 03,585 Not available NTIS

Publications and Presentation Abstracts, 1995. (Published by Paffenbarger Research Center and Center of Excellence for Materials Science Research).

PB96-119250 03,568 Not available NTIS

Publications of the Intelligent Systems Division (Previously Robot Systems Division) Covering the Period January 1971-April 1994

PB94-217098

02,935 PC A05/MF A01

Publications of the Manufacturing Engineering Laboratory Covering the Period January 1989-September 1992.

Publications of the National Institute of Standards and Technology 1992 Catalog

00,014 PC A17/MF A04

Publications of the National Institute of Standards and Technology 1993 Catalog.

00,017 PC A11/MF A03

Publications 1995: NIST Building and Fire Research LabPratory.

00,226 PC A08/MF A02

Pulse-Driven Programmable Josephson Voltage Standard. PB97-111496

04, 148 Not available NTIS

Pulse-Echo Ultrasonic Evaluation of the Integrity of Seams

of Single-Ply Roof Membranes.
PB95-163804 00,381 Not available NTIS

Pulsed Laser Irradiation at $532 \mathrm{~nm}$ of $\mathrm{In}$ and Ga Adsorbed on Si(100): Desorption, Incorporation, and Damage.
PB95-203329 01,051 Not available NTIS

Pulsed Laser Photolysis Time-Resolved FT-IR Emission Studies of Molecular Dynamics.
PB95-203162 04,002 Not available NTIS

Pump-Induced Dispersion of Erbium-Doped Fiber Measured by Fourier-Transform Spectroscopy.
PB94-211935

Pure Element Sputtering Yield Data: Appendix 4.
PB94-200037 00,805 Not available NTIS

Pure Rotational Spectra ol CuH and CuD in Their Ground States Measured by Tunable Far-Infrared Spectroscopy.
PB95-176194 01,005 Not available NTIS

Putting the Information Inlrastructure to Work: Report of the Putting the Information Inlrastructure to Work: Report of the
Information Infrastructure Task Force Committee on Applications and Technology.

N94-31228/7

02,715 PC A06/MF A02
Putting the Information Infrastructure to Work: Report of the Information Inlrastructure Task Force Committee on Applications and Technology.

00,001 PC A06/MF A02

$\mathrm{Q}$ Branch Lineshape Functions for CARS Thermometry. PB96-160643 01,132 Not available NTIS

q Dependence of Sell-Energy Elfects of the Plane Oxygen Vibration in YBa2Cu3Q7.

PB96-138516

01,096 Not available NTIS

Quadratic Response of a Chemical Reaction to External Qscillations.

PB96-161633

01,138 Not available NTIS

Quality Assurance of Contaminant Measurements in Marine Mammal Tissues.

PB95-164034

02,588 Not available NTIS

Quality Characteristics and Metrics for Reusable Software (Preliminary Report)

PB94-203437

$01,693 \mathrm{PC} \mathrm{A03/MF} \mathrm{A01}$

Quality in Automated Manufacturing

PB96-160437

02,839 Not available NTIS

Quantifying the Ignition Propensity of Cigarettes.

PB96-155411 00,306 Not available NTIS

Quantitative Analysis of Selected PCB Congeners in Marine Matrix Reference Materials Using a Novel Cyanobiphenyl Stationary Phase

Stationary Phase.

02,591 Not available NTIS

Quantitative Determination of Qxidative Base Damage in DNA by Stable Isotope-Dilution Mass Spectrometry.

PB96-200886

03,483 Not available NTIS

Quantitative Evaluation of Building Fire Safety: New Tools for Assessing Fire and Building Code Provisions

for Assessing Fire and Building Code Provisions.
PB95-164588 O0,199 Not available NTIS

Quantitative Measure of Efficiency of Monte Carlo Simulations.

01.011 Not available NTIS

Quantitative Measurements of Enhanced Soot Production in a Flickering Methane/Air Diffusion Flame.

PB95-203246 01,393 Not available NTIS

Quantitative Phase Abundance Analysis of Three Cement Clinker Reference Materials by Scanning Electron MicrosCopy.

00,333 Not available NTIS

Quantitative X-Ray Powder Diffraction Methods for Clinker and Cement.

$01,317 \mathrm{PC} \mathrm{A03/MF} \mathrm{A01}$

Quantized Dissipation of the Quantum Hall Effect at High Currents.

PB94-199395

04,472 Not available NTIS

Quantum Collisional Transfer Contributions to the Density

Dependence of Gaseous Viscosity.
PB96-161914 Not available NTIS

Quantum Conductance Fluctuations in the Larger-Size Scale Regime
PB97-111264

04,144 Not available NTIS

Quantum Dots in Quantum Well Structures.

PB97-118350 01,185 Not available NTIS

Quantum Dynamics of Renner-Teller Vibronic Coupling: The Predissociation HCQ.

PB94-185303

00,773 Not available NTIS

Quantum Hall Effect-Based Resistance Standard: Capabilities and Implementation.

PB96-180096

04,114 Not available NTIS

Quantum Hall Effect-Based Resistance Standard (Quantum Hall Res).

PB96-200944

04,127 Not available NTIS

Quantum-Limited Cooling and Detection of Radio-Frequency Qsciflations by Laser-Cooled lons.

PB96-112073

04,039 Not available NTIS

Quantum Measurements of Trapped Ions.

PB95-161147

03,928 Not available NTIS

Quantum Mechanics of a Solid-State Bar Gravitational AnPB95-202628

03,987 Not available NTIS

Quantum Projection Noise: Population Fluctuations in TwoLevel Systems.

03,850 Not available NTIS

Quantum Yields for the Photosensitized Formation of the owest Electronically Excited Singlet State of Molecular Qx ygen in Solution.

00,732 Not available NTIS Quasielastic and Inelastic Neutron-Scattering Studies of
((CD3)3ND)FeCl3.2D2Q: A Qne-Dimensional Ising Ferromagnet.

04,547 Not available NTIS
Quasipotential and the Stability of Phase Lock in Nonhysteretic Josephson Junctions.
PB95-180402 04,681 Not available NTIS Quench Energy and Fatigue Degradation Properties of Cuand Al/Cu-Stablized Nb-Ti Epoxy-Impregnated Superconductor Coils.

B96-14121

04,755 Not available NTIS

Quest to Understand and Reduce $1 / f$ Noise in Ampliliers and Baw Quartz Qscillators.

PB96-200795

02,097 Not available NTIS

Questions and Answers on Quality, the ISQ 9000 Standard Series, Quality System Registration, and Related Issues. More Questions and Answers on the ISQ 9000 Standard Series and Related Issues.

PB95-103461

00,495 PC A03/MF AO

Radiance Temperature (in the Wavelength Range 519-906 $\mathrm{nm})$ ol Tungsten at Its Melting Point by a Pulse-Heating Technique.

PB94-172500

03,397 Not available NTIS

Radiance Temperatures at $1500 \mathrm{~nm}$ of Niobium and Molybdenum at Their Melting Points by a Pulse-Heating Tech nique.

PB97-118699

04,167 Not available NTIS

Radiance Temperatures (in the Wavelength Range 523-907 $\mathrm{nm}$ ) ol Group IV B Transition Metals Titanium, Zirconium, and Hafnium at Their Melting Points by a Pulse-Heating Technique.

PB96-102207

03,356 Not available NTIS

Radiance Temperatures (in the Wavelength Range 523-907 $\mathrm{nm}$ ) of Group IVB Transition Metals Titanium, Zirconium, and Hafnium at Their Melting Points by a Pulse-Heating Technique.

PB96-135025

02,677 Nol available NTIS

Radiated Emissions and Immunity ol Microstrip Transmission Lines: Theory and Measurements.

02,238 PC AO4MF AO1

Radiation Accident at an Industrial Accelerator Facility.

PB95-140117

02,575 Not available NTIS

Radiation and Mixing Properties of Buoyant Turbulent Diffusion Flames.
PB94-165974

$01,360 \mathrm{PC} \mathrm{A04/MF} \mathrm{A01}$

Radiation-Chemical Reaction of 2,3,5-Triphenyl-Tetrazolium Chloride in Liquid and Solid State.
PB96-146733 01,124 Not available NTIS

Radiation Chemistry of Cyanine Dyes: Qxidation and Reduction of Merocyanine 540

PB94-21166

00,818 Not available NTIS

Radiation Doses.

PB94-199676

03,618 Not aivailable NTIS

Radiation-Driven Winds of Hot Luminous Stars $X$. The Determination of Stellar Masses Radii and Distances from Ter-

minal Velocities and Mass-Loss Rates.
PB94-213022 00,060 Not avallable NTIS

Radiation Process Data: Collection, Analysis, and Interpretation.

PB95-162632

03,628 Not available NTIS

Radio and X-ray Emissions from Chemically Peculiar B and A-Type Stars: Qbservations and a Model.

PB96-123302 00,103 Not available NTIS

Radio Continuum and X-Ray Properties of the Coronae of RS Canum Venaticorum and Related Active Binary Sys PB94-211083

00,057 Not available NTIS

Radio Emission from Chemically Peculiar Stars.

PB94-213469

00,068 Not available NTIS

Radioassays of Yttrium-90 Used in Nuclear Medicine. PB97-110100

03,522 Not available NTIS

Radiocarbon Measurements of Atmospheric Volatile Organic Compounds: Quantifying the Biogenic Contribution.

Radiochromic Solid-State Polymerization Reaction.

01,271 Not available NTIS

Radiochromic Solid-State Polymerization Reaction

PB96-180146 01,290 Not available NTIS

Radiometer Equation for Noise Comparison Radiometers.

PB96-140363 02,195 Not available NTIS

Radiometric Model of the Transmission Cell-Reciprocal Nephelometer.

00,124 Not available NTIS

PB95-150132

03,638 Not available NTIS

Radon in the Lung.

Raman and Fluorescence Spectra Qbserved in Laser Microprobe Measurements ol Several Compositions in the Ln-Ba-Cu-Q System.

PB94-172210

04,440 Not available NTIS 


\section{TITLE INDEX}

Range Statistics and Rutherford Backscattering Studies on Fe-Implanted In0.53Ga0.47As.

PB95-126397

04,535 Not available NTIS

RangeCAD and the NIST RCS Uncertainty Analysis.

RangeCAD and the NIST RCS Uncertainty Analysis.
PB94.218591 01,870 PC A03/MF A01

Ranges of Confidence Coefficients for Confiedence Inter-

vals on Variance Components.
PB95-151312 03,437 Not available NTIS

Rapid Decline in the Optical Emission from SN $1957 D$ in PB94. 216033

00,070 Not available NTIS

Rapid Evaluation of Mode-Stirred Chambers Using Impulsive Waveforms.

$01,979 \mathrm{PC} \mathrm{A04/MF} \mathrm{A01}$

Rapid Hot Pressing of Ultra-Fine PSZ Powders

PB94-216587 03,045 Not available NTIS

Rapid Method for the Isolation of Genomic DNA from 'As-

$\begin{array}{ll}\text { pergillus fumigatus'. } & 03,488 \text { Not available NTIS } \\ \text { PB96-147061 }\end{array}$

Rapid pH Change Due to Bacteriorhodopsin Measured with a Tin-Oxide Electrode.

PB96-112081

03,544 Not available NTIS

Rapid Post-Polishing of Diamond-Turned Optics

PB95-175949 04,301 Not available NTIS

Rare-Earth-Doped Waveguide Devices: The Potential for Compact Blue-Green Lasers.

PB95-140836

04,257 Not available NTIS

Rare-Earth Isotopes as Tracers of Particulate Emissions: An Urban Scale Test

PB94-161635

$02,535 \mathrm{PC} \mathrm{A03/MF} \mathrm{A01}$

Rate Constants for Hydrogem Atom Attack on Some Chlorinated Benzenes at High Temperature.
PB94-200581 00,810 Not available NTIS

00,810 Not available NTIS

Rate Constants for the Decay and Reactions of the Lowest Electronically Excited Singlet State of Molecular Oxygen in Solution. An Expanded and Revised Compilation.

07, 106 Not available NTIS

Rate Constants for the Gas Phase Reactions of the $\mathrm{OH}$ Radical with $\mathrm{CF}_{3} \mathrm{CF}_{2} \mathrm{CHCl} 2$ (HCFC-225ca) and

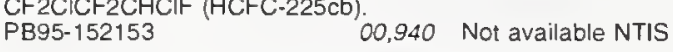

Rate of Heat Release of Wood Products.

PB94-212404 03,403 Not available NTIS

Rationale and Prelimınary Plan for Federal Research for Construction and Building.

00,322 PC A07/MF A02

Rayleigh Instability for a Cylindrical Crystal-Melt Interface.

PB95-180667 01,010 Not available NTIS

Rayleigh Scattering Limits for Low-Level Bidirectional Reflectance Distribution Function Measurements.

PB95-180030 04,307 Not available NTIS

Rb-Like Spectra: PdX 10 Nd XXIV

PB95-150645 03,897 Not available NTIS

RDFs and $\mathrm{Fe}-\mathrm{Fe}$ Pair Correlations in an AICuFe Icosahedral Alloy by Double Isotopic Substitution.
PB94-172129 04,439 Not available NTIS RDI-SIM ECMA Inter-Domain Routing Protocol Simulation

PB94-172301

01,683 Not available NTIS

Reaction of Nitric Oxide with Organic Peroxyl Radicals.

PB95-141107 00,910 Not available NTIS

Reaction of NO with Superoxide.

PB94-212198 00,830 Not available NTIS

Reaction Rate Determinations of Vinyl Radical Reactions with Vinyl, Methyl, and Hydrogen Atoms.

PB94-211398 00.815 Not available NTIS
PBith

Reaction Sensitivities of Al-Li Alloys and Alloy 2219 in Mechanical-Impact Tests.

PB94-172764

03,314 Not available NTIS

Reactive Coevaporation of DyBaCuO Superconducting Films: The Segregation of Bulk Impurities on Annealed Films: The Segregation of Bulk Impurities on Annealed
$\mathrm{MgO}(100)$ Substrates.
PB95-164562

04,635 Not available NTIS

Reactive Ion Etching in the Gaseous Electronics Conference RF Reference Cell.

02,395

(Order as PB96-113311, PC A09/MF A03)

Reactivity of $\mathrm{Pd}$ and $\mathrm{Sn}$ Adsorbates on Plasma and Thermally Oxidized $\mathrm{SnO} 2(110)$

PB94-199973

00,804 Not available NTIS

Reactivity of Product Gases Generated in Idealized Enclosure Fire Environments.

01,386 Not available NTIS
Reactor Radiation Technical Activities, 1994. NAS-NRC ASsessment Panel, April 6-7, 1995.

sessment Pane

03,732 PC A08/MF AO2

Reactor Radiation Technical Activities, 1995. PB96-193644

03,741 PC A07/MF A02

Real Time Compensation for Tool Form Errors in Tuming Using Computer Vision.

02,945 Not available NTIS

Real Time Differential Range Estimation Based on TimeSpace Imagery Using PIPE.

PB95-161808

01,844 Not available NTIS

Real-Time Implementation of a Differential Range Finder

PB95-108650

01,839 Not available NTIS

Real Time Monitoring of Electron Processors.

PB96-135306 03,719 Not available NTIS

Real-Time Obstacle Avoidance Using Central Flow Divergence and Peripheral Flow.

02,937 PC A03/MF A01

Real-Time Small-Angle $X$-Ray Scattering Study of the Early

Stage of Phase Separation in the SiO2-BaO-K20 System.
PB95-163069
03,052 Not available NTIS PB95-163069

Real-Time Vision for Autonomous and Teleoperated Control of Unmanned Vehicles.

03,701 Not available NTIS

B94-211885

Real-Time Vision for Unmanned Vehicles.
PB94-211893 03,702 Not available NTIS

Realization of a Scale of Absolute Spectral Response Using the NIST High Accuracy Cryogenic Radiometer.

PB97-118640

04,397 Not available NTIS

Realization of New NIST Radiation Temperature Scales for the $1000 \mathrm{~K}$ to $3000 \mathrm{~K}$ Region, Using Absolute Radiometric Techniques.

03,794 Not available NTIS

Realization of NIST 1995 Luminous Flux Scale Using the Integrating Sphere Method.

04,374 Not available NTIS

Realizing Suspended Structures on Chips Fabricated by CMOS Foundry Processes Through the MOSIS Service.

Reanalysis of the (010), (020), (100), and (001) Rotational Levels of (32)S(16)O2.

00,887 Not available NTIS

Recalibration for the Final Archive of the International Ultra violet Explorer (IUE) Satellite.

PB96-135264

00,106 Not available NTIS

Recent Approaches to Extreme Value Estimation with Application to Wind Speeds. Part 1. The Pickands Method. Recent Development in Nondestructive Testing of Concrete. Recent Developments at NIST on Optical Current Sensors and Partial Discharge Diagnostics.

PB95-151114 02,147 Not available NTIS

Recent Developments in NIST Botanical SRMs.

PB96-167267 03,489 Not available NTIS

Recent Experimental and Modeling Developments in High Temperature Thermochemistry. 00,759 Not available NTIS
PB94-172343

Recent Experiments on Trapped lons at the National Institute of Standards and Technology.
PB95-169322 Not available NTIS

Recent Results in Magnetic Force Microscopy.

PB96-103130 04,721 Not available NTIS

Recent VAMAS Activity in Ceramics

PB95-162681

03,051 Not available NTIS

Recently Developed NIST Food Related Standard Reference Materials

00,035 Not available NTIS

Recertification of the Standard Reference Material 1475A, a Linear Polyethylene Resin.

$02,628 \mathrm{PC} \mathrm{A03/MF} \mathrm{A01}$

Rechargeable Batteries for Personal/Portable.

PB96-164231 02,459 Not available NTIS

Reciprocity Relations in Waveguide Junctions.

PB94-172814 02,213 Not available NTIS

Recombination Line Intensities for Hydrogenic Ions-III. Effects of Finite Optical Depth and Dust. 00,079 Not available NTIS

, 079 Not available NTIS

Recommendations for the Disposal of Carbon-14 Wastes.
AD-A279 $133 / 3$

Recommendations on Selection of Vehicle-to-Roadside Communications Standards for Commercial Vehicle Operations.

PB94-195914

$04,859 \mathrm{PC} \mathrm{A05/MF} \mathrm{A01}$
Recommended Changes in ASTM Test Methods D2512-82 and G86-84 for Oxygen-Compatibility Mechanical Impact $\begin{array}{ll}\text { Tests on Metals. } & 03,338 \text { Not available NTIS } \\ \text { PB94-216694 } & \end{array}$

Recommended Performance-Based Criteria for the Design of Manufactured Home Foundation Systems to Resist Wind and Seismic Loads.

and Seismic Loac

00,460 PC A05/MF A01

Reconstructing Stratified Fluid Flow from Reciprocal Scattering Measurements.

04,202 Not available NTIS

Reconstruction during Camera Fixation

PB95-162236

01,593 Not available NTIS

Redshifts in Stellar Transition Regions.

PB96-123310

00,104 Not available NTIS

Reducing Errors, Complexity, and Measurement Time of PB96-119771

02,075 Not available NTIS

Reducing the Effect of Local Oscillator Phase Noise on the Frequency Stability of Passive Frequency Standards.
PB95-180972

Reducing the $1 / f$ AM and PM Noise in Electronics for Precision Frequency Metrology.

02,102 Not available NTIS

Reduction of Dinitrogen to Ammonia in Aqueous Solution

Mediated by Colloidal Metals.
PB95- 107074

00,867 Not available NTIS

Reduction of Interfilament Contact Loss in Nb3Sn Superconductor Wires.

02,223 Not available NTIS

Reduction of Light-Assisted Collisional Loss Rate from a Low-Pressure Vapor-Cell Trap.

03,971 Not available NTIS

Reduction of Marginal Gaps in Composite Restorations by Use of Glass-Ceramic Inserts.

00,174 Not available NTIS

Reference Architecture for Machine Control Systerns Integration: Interim Report.

02,820 PC AO6/MF A02

Reference Data for the Thermophysical Properties of Cryogenic Fluids.

03,263 Not available NTIS

Reference Information for the Software Verification and Validation Process
PB96-188164

$01,773 \mathrm{PC}$ A06/MF A01

Reference Manual for the Algorithm Testing System Version 2.0.
PB96-128244 02,922 PC A03/MF A01

Reference Materials by Isotope Dilution Mass Spectrometry.
PB 95.153383
00,592 Not available NTIS

Reference Model Architecture for Intelligent Systems Design.
PB̈95-143137 01,789 PC A03/MF A01

Reference Relations for the Evaluation of the Materials Properties of Orthorhombic YBa2Cu3Ox Superconductors.
PB96-176763 04,782 Not available NTIS Reference Tables for Thermocouples. AD-A279 948/4

02,614 PC A05/MF A01

REFPROP Refnigerant Properties Database: Capabilities, Limitations, and Future Directions.
PB96-167150 Refraction of Light by Graded Birefringent Media PB96-123716 02,192 Not available NTIS Refractive Indices of Fluids Related to Alternative Refrig-

PBants. 03,260

(Order as PB94-219326, PC A05/MF A02)

Regimes of Surface Roughness Measurable with Light Scattering.

04,265 Not available NTIS

Regulation of Lithium and Boron Levels in Normal Human Blood: Environmental and Genetic Considerations. PB94-198579 03,491 Not available NTIS

Reinforcement of Cancellous Bone Screws with Calcium Phosphate Cement

00,179 Not available NTIS

Relating Bench-Scale and Full-Scale Toxicity Data PB95-125977

00,361 Not available NTIS

Relation between AC Impedance Data and Degradation of Coated Steel. 1. Effects of Surface Roughness and Conamination on the Corrosion Behavior of Epoxy Coated Steel.

PB94-213345

03,189 Not available NTIS

Relationship between Bulk-Modulus Temperature Dependence and Thermal Expansivity. 04,641 Not available NTIS 


\section{TITLE INDEX}

Relationship between Indoor Air Quality and Carbon Dioxide. PB97-111249 02,569 Not available NTIS Relationship between Radiative and Magnetic Fluxes for Three Active Solar-Type Dwarfs.
PB96-119540 00,097 Not available NTIS Relationship of AM to PM Noise in Selected RF Oscillators.
PB95-169009 02,262 . Not available NTIS Relationship of Silver with Selenium and Mercury in the Liver of Two Species of Toothed Whales (Odontocetes).
PB96-167275 Relative Accuracy of Isolated and Unisolated Noise Comparison Radiometers.

PB96-111851

01,924 Not available NTIS

Relative Photoionization and Photodetachment Cross Sections for Particular Fine-Structure Transitions with Application to $\mathrm{Cl} 35$-subshell Photoionization.
PB95-203097 03,998 Not available NTIS

Relative Sensitivity Factors and Useful Yields for a Microfocused Gallium Ion Beam and Time-of-Flight Secondary Ion Mass Spectrometer

00,541 Not available NTIS

Relativistic and Quantum Electrodynamic Effects in HighlyCharged lons.
PB94-212784

03,854 Not available NTIS

Relativistic Effects in Spin-Polarization Parameters for LowEnergy Electron-Cs Scattering.
PB95-150868

Relativistic Modifications of Charge Expansion Theory.
PB96-123799 04,052 Not available NTIS

PB96-123799 04,052 Not available NTI

Relativistic Quantum Mechanics of Interacting Particles.
PB97-110589 04,141 Not available NTIS

Relativistic R-Matrix Calculations for Electron - Alkali-MetalAtom Scattering: Cs as a Test Case.
PB95-203410 Not available NTIS

Relaxation After a Temperature Jump Within the One Phase Region of a Polymer Mixture.
PB97-112494 Not available NTIS

Relıable Optical Flow Algorithm Using 3-D Hermite Polynomials.
PB94-145620

01,829 PC A03/MF A01

Remineralization of Root Lesions with Concentrated Calcium and Phosphate Solutions.
PB96-102140

Remineralizing Dental Composites Based on Amorphous Calcium Phosphate.
PB96-147020 03,573 Not available NTIS Repair of Products of Oxidative DNA Base Damage in
Human Cells. PB96-190129

03,555 Not available NTIS

Replicate Measurements for Data Quality and Environmental Modeling.
PB94-172533 02,515 Not available NTIS Reply to Professor Wolf's Comments on My Paper on Wolf PB94-219409

04,247

(Order as PB94-219326, PC A05/MF A02)

Report of a Workshop on Requalification of Tubular Steel Joints in Offshore Structures. Held in Houston, Texas on September 5-6, 1995.

PB96-210760
PBtem

03,699 PC A07/MF A02

Report of a Workshop on the Assurance of High Integrity Soltware.
PB96-161377

01,763 Not available NTIS

Report of Density Intercomparisons Undertaken by the

Working Group on Density of the CCM.
PB94-200664 Not available NTIS

Report of the Federal Internetworking Requirements Panel.
DE95017761 03,407 PC A03/MF A01

03,407 PC A03/MF A01

Report of the International Commission on Radiological Units and Measurements (ICRU), 1956.
AD-A279 120/0

Report of the National Conference on Weight and Measures (78th). Held in Kansas City, MO. on July 18-22, 1993 PB94-138989
PBansas City, MO. on July
02,623 PC A18/MF A04 Report of the National Conference on Weights and Meas-
ures (79th). Held in San Diego. California on July 17-21, 1994.

PB95-169819

02,656 PC A2O/MF A04 Report of the National Conference on Weights and Meas-
ures (80th) as Adopted by the 80th National Conference on Weights and Measures, 1995. Held in Portland, Maine on July 16-20, 1995 .

02,681 PC A15/MF A03

Report of the NIST Workshop on Digital Signature Certificate Management. Held on December 10-11, 1992. cate Managem
PB94-135001

PBine Measurem
Report of the NIST Workshop on Key Escrow Encryption PB94-209459
Held

Report of the Refrigeration, Air Conditioning and Heat Pumps Technical Options Commitiee

PB96-176755

03,293 Not available NTIS

Report on Application Integration Architectures (AIA) Workshop. Held in Dallas, Texas on February 8-12, 1993.
PB94-142536

Report on the Advanced Sottware Technology Workshop. Held on February 1, 1994.

PB95-136610

$01,707 \mathrm{PC} \mathrm{A03/MF} \mathrm{A01}$

Report on the Meeting of the CCU (10th) (of the International Committee of Weights and Measures). Held on July $10-11,1990$.

03,792 Not available NTIS

Report on the NIST Low Accelerating Voltage SEM Magnification Standard Interlaboratory Study.
PB96-201074 02,445 Not available NTIS Report on the Workshop on Advanced Digital Video in the National Information Infrastructure. Held in Washington D.C. On May 10-11, 1994.

01,472 PC A09/MF A03

Report on the Workshop on Manufacturing Polymer Composites by Liquid Molding. Held in Gaithersburg, Maryland on September 20-22, 1993

PB94-160066

$03,131 \mathrm{PC} \mathrm{A13/MF} \mathrm{A03}$

Report on USDA U/traviolet Spectroradiometers.

PB96-214648 00,125 PC A04/MF A01

Report on 1994 Actions of the International Institute of Welding.
PB96-138540

02,873 Not available NTIS

Representation of Axes for Geometric Fitting.
PB97-113799

Representing a Large Collection of Curves: A Case for Principal Points.
PB95-152286

03,438 Not available NTIS

Representing Designs with Logic Formulations of Spatial Relations.
PB97-111561

02,792 Not available NTIS

Reproducibility of JEDEC Standard Current and Voltage Ramp Test Procedures for Thin-Dielectric Breakdown Characterization.

01,879 Not available NTIS

Reproducibility of Tests on Energy Management and Control Systems Using Building Emulators.
PB95-175980 00,260 Not available NTIS

Reproducibility of the Temperature of the Ice Point in Routine Measurements.

04,015 PC A03/MF A01

Requisite Elements, Rationale, and Technology Overview for the Systems Intergration for Manufacturing Applications (SIMA) Program. Background Study. 231 PC A07/MF A02

Activities in Electron Research and Development Activities in Electron
Paramagnetic Resonance Dosimetry. PB96-141288

03,635 Not available NTIS

Research on Methods for Determining Optical Disk Media
Life Expectancy Estimates. 01,633 Not available NTIS

a Vision-Based Coordinate Residual Error Compensation of a Vision-Based Coordinate
Measuring Machine.
PB96-161617 04,091 Not available NTIS

04,091 Not available NTIS

Residual Stress in Induction-Heated Railroad Wheels: Ultra sonic and Saw Cut Measurements. Report No. 28
PB96-106992

04,854 PC AO4/MF A01

Residual Stresses in Aluminum-Mullite (alpha-Alumina) Composites.
PB95-152880

03, 155 Not available NTIS

Resistance Measurements from $10 \mathrm{M}$ Omega to $1 \mathrm{~T}$ Omega at NIST
PB97-119168

02,290 Not available NTIS

Resistance Thermometers with Fast Response for Use in

Rapidly Oscillating Gas Flows.
PB95-107298

03,261 Not available NTIS

Resistors.

PB97-111876

02,284 Not available NTIS

Resolution of Discrepant Analytical Data in the Certification of Platinum in Two Automobile Catalyst SRMs.

PB96-167283 00,638 Not available NTIS

Resolution of DNA in the Presence of Mobility Modifying Polar and Nonpolar Compounds by Discontinuous Electrophoresis on Rehydratable Polyacrylamide Gels. PB95-152799 00,590 Not available NTIS

Resonance and Threshold Effects in Polarized X-Ray Emission from Atoms and Molecules.

PB95-150298

03,891 Not available NTIS
Resonance Enhanced Multiphoton Ionization Detection of $\mathrm{GeF}$ and $\mathrm{GeCl}$ Radicals.

PB94-212123

00,825 Not available NTIS

Resonance Enhanced Multiphoton Ionization Spectroscopy of the PF Radical.

PB97-119119

00,702 Not available NTIS

Resonance Enhanced Multiphoton Ionization Spectroscopy of the SnF Radical.

PB97-111223

01,176 Not available NTIS

Resonance Enhanced Multiphoton Ionization Spectroscopy of 2-Butene-1-yl (C4H7) between 455-485 nm.

PB95-151031 00,670 Not available NTIS

Resonance Fluorescence with Squeezed-Light Excitation

PB95-203469

04,322 Not available NTIS

Resonance Ionization Spectroscopy/Pesonance Ionization Mass Spectrometry Data Service. V-Data Sheets for Ga $\mathrm{Mn}, \mathrm{Sc}$, and $\mathrm{TI}$

00,625 Not available NTIS

Resonance Structure and Absolute Cross Sections in Near Threshold Electron-Impact Excitation of the $4 \mathrm{~s}$ (2) (1)S Threshold Electron-Impact Excitation of the $4 \mathrm{~s}(2)$ (1)
yields $4 \mathrm{~s} 4 \mathrm{p}$ (3) $\mathrm{P}$ Intercombination Transition in $\mathrm{Kr}(6+)$. yields 4s4p (3)P Intercombination Transition in $\mathrm{Kr}(6+)$.
PB95-202271 03,972 Not available NTIS

Resonances in Two-Dimensional Array Oscillator Circuits.

PB96-102082 02,066 Not available NTIS

Resonant-Photoemission Investigation of the Heusler Alloys $\mathrm{Ni} \mathrm{MnSb}$ and $\mathrm{NiMnSb}$

PB95-162384

04.612 Not available NTIS

Resonant Two-Color Detachment of $\mathrm{H}(-)$ with Excitation of $H(n=2)$

PB95-202552

03,984 Not available NTIS

Response Comparison of Electret Ion Chambers, LiF TLD and HPIC.

Response of a Terminally Ano ple Shear Flow.

PB95-108668

02,578 Not available NTIS

Response of Buildings to Ambient Vibration and the Loma Prieta Earthquake: A Comparison.

PB96-119607

00,457 Not available NTIS

Response to Comments on the NIST Proposed Digital Signature Standard.

PB96-161815

01,615 Not available NTIS

Response to 'Draining in Dilute Polymer Solutions and Renormalizatio

PB96-146667

01,283 Not available NTIS

Results of a NIST/NNIIOFI Comparison of Spectral-Padiance Measurements.

PB97-113021

04,159 Not available NTIS

Results of Capacitance Ratio Measurements for the Single

Electron Pump-Capacitor Charging Experiment.
PB97-113286

Results of the ASTM Nuclear Methods Intercomparison on NIST Apple and Peach Leaves Standard Reference Materials.
PB97-119036

03,490 Not available NTIS

Retention of Halocarbons on a Hexafluoropropylene Epoxide Modified Graphitized Carbon Black. Part 1. MethaneBased Compounds.

PB5-175196

03,272 Not available NTIS

Retention of Halocarbons on a Hexafluoropropylene Epoxide-Modified Graphitized Carbon Black. 3. Ethene-Based Compounds. ide-Modified Graphitized Carbon Black. 4. Propane-Based Compounds.

Retinal-Protein Complexes as Optoelectronic Components. PB95-150397 02,146 Not available NTIS

Retrieving Articles from the Internet (without a UNIX Workstation). Part 1. File Formats and Software Tools.

PB95-168720 02,728 Not available NTIS

Retrieving Articles from the Internet (without a UNIX Workstation). Part 2. An Example.

PB95-168738

02,729 Not available NTIS

Review and Upgrading of Military Fastener Test Standard MIL-STD-1312
PB95-154720

02,947 PC AO7/MF AO2

Review of Corrosion Behavior of Ceramic Heat Exchanger Materals: Corrosion Characteristics of Silicon Carbide and Silicon Nitride. Final Report, September 11, 1992*-March

11, 1993.
DE 93041307

03,228 PC A06/MF A02

Review of Cure Monitoring Techniques for On-Line Process

Control.
PB94-216728

03,145 Not available NTIS 


\section{TITLE INDEX}

Review of Flows Driven By Natural Convection in Adiabatic Shafts.
PB96-147897

$01,416 \mathrm{PC} \mathrm{A04/MF} \mathrm{A01}$

Review of Internatioanl Fire Risk Predictions Methods

PB96-156195

00,222 Not available NTIS

Review of Mathematical Function Library for MicrosoftFORTRAN, John Wiley and Sons, 1989.

01,679 PC A03/MF A01

Review of Measurements and Candidate Signatures for Early Fire Detection.

00,300 PC A03/MF A01

Review of Semiconductor Microelectronic Test Structures with Applications to Infrared Detector Materials and Processes.

02,132 Not available NTIS

Review of the USCEANIST Measurement Assurance Program for the Nuclear Power Industry.
PB95-126272 Not available NTIS

Revised Uncertainty Analysis for the NIST 30-MHz Attenuation Calibration System.

PB95.168761

01,907 Not available NTIS

Rh I Isoelectronic Sequence Observed from $\operatorname{Er}(23+)$ to $\mathrm{Pt}(33+)$
$\mathrm{PB} 95-150652$

03,898 Not available NTIS

Rheology of Fresh Cement Paste.

PBg5-163150

00.378 Not available NTIS

Riass Coronathon: Joint $\mathrm{X}$-ray and Ultraviolet Observations of Normal F-K Stars.

PB96-200217

00,109 Not available NTIS

Rietveld Analysis of $\mathrm{NaxWO}+x / 2 . y H 2 O$, Which Has the Hexagonal Tungsten Bronze Structure.
PB95-107371 Not available NTIS

Rigid Bender Analysis of van der Waals Complexes: The Intermolecular Bending Potential of a Hydrogen Bond.
PB95-203022

FII Spectroscopy of Trap Levels in Bulk and LPE $\mathrm{Hg} 1$ -

xCdxTe.
PB96-160247

04,084 Not available NTIS

Ring-Opening Dental Resin Systems Based on Cyclic Acetals.

00,162 Not available NTIS

Ring-Opening Polymerication of a 2-Methylene Spiro Orthocarbonate Bearing a Pendant Methacrylate Group.
PB95-176145

RIS Measurement of AC Stark Shifts and Photoionization Cross Sections in Calcium.

PB96-157953

04,073 Not available NTIS

RIS Measurements of the AC Stark Shift.

PB96-158035 04,078 Not available NTIS

RIS Studies of Autoionization in Calcium

PB94-213295 00,849 Not available NTIS

Risk Analysis for the Fire Safety of Airline Passengers.

PB94-194065 04,862 PC A03/MF A01

Roadmap for the Computer Integrated Manufacturing (CIM) Application Framework.

02,832 Not available NTIS

Robotics Application to Highway Transportation. Volume 1. Final Report.

03,654 PC A03/MF A01

Robotics Application to Highway Transportation. Volume 2. Literature Search.

PB95-170551

$01,337 \mathrm{PC}$ A06/MF A02

Robotics Application to Highway Transportation. Volume 3. Proposed Research Topics and Cost/Benefit Evaluations by

PB95-171633

$01,338 \mathrm{PC} \mathrm{A20/MF} \mathrm{A04}$

Robotics Application to Highway Transportation. Volume 4.

Proposals for Potential Research
PB95-193173

$01,339 \mathrm{PC} \mathrm{A07/MF} \mathrm{A02}$

Role of Adsorbed Alkalis in Desorption Induced by Elec-

tronic Transitions
PB94-172574

00,762 Not available NTIS

Role of Certified Reference Materials in Trace Analysis Quality Assurance.

PB97-110019

00,650 Not available NTIS

Role of Combustion on Droplet Transport in Pressure-Atom-

ized Spray Flames.
PB96-204433

01,434 Not available NTIS

Role of Corrosion in a Material Selector Expert System for Advanced Structural Ceramics.

PB97-110308

03,099 Not available NTIS

Role of Journals in Maintaining Data Integrity: Checking of Crystal Structure Data in 'Acta Crystallographica'.
PB97-109177 (Order as PB97-109011, PC A11/MF A03)
Role of R22 in Refrigerating and Air Conditioning Equipment.
PB94-199783

03,253 Not available NTIS

Role of Refrigerant Mixtures as Alternatives to CFCs.

PB94-199775 03,252 Not available NTIS

Role of the Oftice of Radiation Measurement in Quality Assurance.

00,689 Not available NTIS Role of World Modeling and Value Judgment in Perception.
PB94-198264

Roles of Copper in Applied Superconductivity

PB94-211521 02,255 Not available NTIS

Roles of Local Classical Acceleration and Spatial Separation in the Neutral Particle Analogs of the Aharonov-Bohm

PB95-202362

03,976 Not available NTIS

Room-Temperature Flexure Fixture for Advanced Ceramics. PB95-210498

03,061 PC A03/MF A01

Room-Temperature Thermal Conductivity of Expanded Polystyrene Board for a Standard Reference Material.
OB 90,412 PC A04/MF A0

Room Temperature Thermal Conductivity of Fumed-Silica Insulation for a Standard Reference Material. 00,374 Not available NTIS ROSAT All-Sky Survey of Active Binary Coronae. 1. Quiescent Fluxes for the RS Canum Venaticorum Systems.
PB95-202479 00,077 Not available NTIS ROSAT All-Sky Survey of Active Binary Coronae. 2. Coronal Temperatures of the RS Canum Venaticorum Systems. -19960 00,055 Not available NTIS

Rotational Dynamics of $\mathrm{C} 60$ in $\mathrm{Na2RbC60}$

00,948 Not available NTIS

Rotational Dynamics of Solid C70: A Neutron-Scattering Study.
PB94-172178

00,755 Not available NTIS

Rotational Dynamics of Solid C70: A Neutron-Scattering Study.
PB95-153219

00,949 Not available NTIS

Rotational Far Infrared Spectrum of (13) CO

PB95-152187 00,941 Not available NTIS

Rotational Modulation and Flares on RS Canum Venaticorum and BY Draconis Stars. XVI. IUE Spectroscopy and VLA Observations of C1182(=V 1005 Orionis) in copy and 1983 . Observations of C1182(=V 1005 Orionis) in
October 1983 .

00,050 Not available NTIS

Rotational Modulation and Flares on RS Canum Slars. XVIll. Coordinated VLA, ROSAT, and IUE Observations of RS CVn Binaries.

00,089 Not available NTIS

Rotational-RKR Inversion of Intermolecular Stretching Po tentials: Extension to Linear Hydrogen Bonded Complexes.

Rotational Spectra of $\mathrm{CH} 3 \mathrm{CCH}-\mathrm{NH} 3, \mathrm{NCCCH}-\mathrm{NH}_{3}$, and $\mathrm{NCCCH}-\mathrm{OH} 2$

04,170 Not available NTIS

PB97-118798

$\mathrm{CoH}$ Radical in Its Ground Rotational Spectroscopy of the CoH Radical in Resonance: Determination of Molecular Parameters. Not available NTIS
PB95-175048 00,992 Not

Rotational Spectrum and Structure of a Weakly Bound

Complex of Ketene and Acetylene.
PB95-126140

Rotational Spectrum of Copper Hydride Using Tunable Far Infrared Radiation.

PB94-198637

00,792 Not available NTIS

Rotational Spectrum of $\mathrm{OH}$ in the $\mathrm{v}=0-3$ Levels of Its Ground State.

01,006 Not available NTIS

Safety Assessment of Railroad Wheels by Residual Stress Measurements

04,855 Not available NTIS

Safety Assessment of Railroad Wheels Through Roll-by Detection of Tread Cracks.

PB96-141254

04,856 Not available NTIS

Salt-PEG Two-Phase Aqueous Systems to Purify Proteins and Nucleic Acid Mixtures.

03,527 Not available NTIS

SANS and LS Studies of Polymer Mixtures Under Shear Flow.

01,231 Not available NTIS

SANS Studies of Space-Time Organization of Structure in Polymer Blends.

01,251 Not available NTIS

SANS Study of the Plastic Deformation Mechanism in Polyethylene.

01,242 Not available NTIS
Santa Ana Fire Department Experiment at 1315 South Bristol, July 14, 1994

00,389 PC A03/MF A01

Santa Ana Fire Department Experiment at 1315 South Bristol, July 14, 1994. (Reprint)

00,207 Not available NTIS

Santa Ana Fire Department Experiments at South Bristol PB96-154810

$00,305 \mathrm{PC} \mathrm{A11/MF} \mathrm{A03}$

Satellite Two-Way Time Transfer: Fundamentals and Recent Progress.
PB95-161089

01536 Not available NTIS

SC4 Short Names Registry

PB97-122410

02,799 Not available NTIS

Scalability Test for Parallel Code

PB96-146758

01,749 Not available NTIS

Scale-Space-Based Visual-Motion-Cue for Autonomous Navigation.

PB96-183173

02,940 PC A06/MF A01

Scaling Compartment Fires: Reduced- and Full-Scale Enclosure Burns

PB96-175708

00,224 Not available NTIS

Scaling of Diffusion-Mediated Island Growth in Iron-on-Iron Homoepitaxy.
PB94-185923

04,455 Not available NTIS

Scaling of the Nonlinear Optical Cross Sections of GaAs AIGaAs Multiple Quantum-Well Hetero n-i-p-i's.

PB96-102793 02,183 Not available NTIS

Scanned Probe Microscope Tip Characterization Withou Calibrated Tip Characterizers.

PB96-190368

02,759 Not available NTIS

Scanned Probe Microscopies: Opportunities and Issues in Metrology.
PB96-160783

02,426 Not available NTIS

Scanning Capacitance Microscopy Measurements and Modeling for Dopant Profiling of Silicon.

PB96-164207

04,781 Not available NTIS

Scanning Capacitance Microscopy Measurements and Modeling: Progress Towards Dopant Profiling of Silicon.

PB96-148150

04,773 Not available NTIS

Scanning Capacitance Microscopy Measurements and Modeling. Progress Towards Dopant Profiling of Silicon.

PB96-180070

01,964 Not available NTIS

Scanning Electron Microscope Magnification Calibration Interlaboratory Study

01,164 Not available NTIS

Scanning Electron Microscope Metrology.

PB96-201090

02,446 Not available NTIS

Scanning Electron Microscopy Observations of Misfit Dislocations in Epitaxial In0.25Ga0.75As on GaAs(001).

PB96-200159

03,004 Not available NTIS

Scanning Tunneling Microscopy and Fabrication of

Nanometer Scale Structures at the Liquid-Gold Interface.
PB95-140414 00,904 Not available NTIS PB95-140414

Scanning Tunneling Microscopy of the Charge-DensityWave Structure in $1 \mathrm{~T}$-TaS2.

04,689 Not available NTIS

Scanning Tunneling Microscopy Study of the Growth of $\mathrm{Cr} /$ Fe(001): Correlation with Exchange Coupling of Magnetic

PB95- 150330

04,568 Not available NTIS

Scattered Fractions of Dose from 18 and 25 MV X-ray Ra-

diotherapy Linear Accelerators.

04,120 Not available NTIS

Scattering and Absorption Effects in Neutron Beam Activation Analysis Experiments.

PB94-216140

00,557 Not available NTIS

Scattering Properties of the Leveled-Wave Model of Random Morphologies.

PB94-198835

03,807 Not available NTIS

Science, Technology, and Competitiveness: Retrospective on a Symposium in Ćelebration of NIST's 90th Anniversary and the 25 th Anniversary of the Gaithersburg Laboratories, November 14-15, 1991.

PB97-121610

$02,696 \mathrm{PC} \mathrm{A08/MF} \mathrm{A02}$

Scientific Protocols in Statistical Standards for Environmental Studies.

PB94-185527

02,517 Not available NTIS

Scientific Rationale and Present Implementation Strategy for the Far Uitraviolet Spectrograph Explorer (FUSE). PB96-123328

00,045 Not available NTIS

Screened-Room Measurements on the NIST SphericalDipole Standard Radiator

PB96-113568

(Order as PB96-113535, PC A05/MF A01) 


\section{TITLE INDEX}

Screw-Thread Standards for Federal Services, 1957. Handbook H28 (1957), Part 2. Revised.
AD-A280 082/9 Screw-Thread Standards for Federal Services, 1957. Part 1.
AD-A279 290/1 02,855 PC A10/MF A03 Screw-Thread Standards for Federal Services, 1957. Part 3.
AD-A279 121/8 02,854 PC A04/MF A01 SDNS Security Management.

PB95-161170

01,592 Not available NTIS Sealed Water Calorimeter for Measuring Absorbed Dose.
PB94-219227 (Order as PB94-219219, PC A06/MF A02)

Search for Radio Emission from the 'Non-Magnetic' Chemically Peculiar Stars.

00,087 Not avallable NTIS

Search for Small Violations of the Symmetrization Postulate in an Excited State of Helium. 04,050 Not available NTIS
PB96-123518 PB96-123518 Conference. Conference.
PB94-188711

$01,832 \mathrm{PC} \mathrm{A12/MF} \mathrm{A03}$

Second Text REtrieval Conterence (TREC-2). Held in Gaithersburg, Maryland on August 31-September 2, 1993.
PB94-178407 01,686 PC A21/MF A04

PB94-178407 Secondary Standard for PM and AM Noise at 5, 10, and
$100 \mathrm{MHz}$. PB96-123187

01,554 Not available NTIS

Secondary Target X-Ray Excitation for In vivo Measurement of Lead in Bone.
PB95-108767 03,496 Not available NTIS Secure Hash Standard. Category: Computer Security.
FIPS PUB $180-1$ Security Considerations for SOL-Based Implementations of PB94-139649 $02,766 \mathrm{PC} \mathrm{A03/MF} \mathrm{A01}$

Security in Open Systems.

$01,473 \mathrm{PC} \mathrm{A13/MF} \mathrm{A03}$

PB95-105383

01,610 Not available NTIS

Security Prog
PB96-156112

01,610 Not available NTIS Security Requirements for Cryptographic Modules; Cat-
egory: Computer Security; Subcategory: Cryptography.
FIPS PUB 140-1
01,567 PC E05 egory: Computer
FIPS PUB 140-1

Segmental Concentration Profiles of End-Tethered Polymers with Excluded-Volume and Surface Interactions.
PB97-119002 00,654 Not available NTIS Seismic Instrumentation of Existing Buildings. PB94-159779 00,420 PC A04/MF A01 Seismic Performance Behavior of Precast Concrete BeamColumn Joints.

00,440 Not available NTIS

Seismic Performance of Circular Bridge Columns Designed in Accordance with AASHTO/CALTRANS Standards.
PB96-146352 01,346 PC A07/MF A02 Seismic Safety of Federal Buildings. Initial Program: How Much Will it Cost.

$00,447 \mathrm{PC} \mathrm{A03/MF} \mathrm{A01}$

Seismic Strengthening of Reintorced Concrete Frame BuildPBg5-108841

00,430 Not available NTIS

Selected Ion Flow Tube-Laser Induced Fluorescence Instrument for Vibrationally State-Specific Ion-Molecule Reactions.

PB94-185444

00,774 Not available NTIS

Selection of Appropriate UItrasonic System Components for NDE of Thick Polymer-Composites

PB94-185279

03,133 Not available NTIS

Selective Inhibition of Crystal Growth on Octacalcium Phosphate and Nonstoichiometric Hydroxyapatite by Pyrophosphate
PBg4-211257 00,147 Not available NTIS

Selectivity Trends in Packed Column Supercritical Fluid Chromatography with $\mathrm{C} 18$ Stationary Phases. PB96-138581 00.622 Not available NTIS

Self-Assembled Phospholipid/Alkanethiol Biomimetic Bilayers on Gold.
PB95-108460 00,878 Not available NTIS Self-Avoiding Surfaces, Topology, and Lattice Animals.
PB95-150512 Self-Avoiding-Walk Contacts and Random-Walk Self-Intersections in Variable Dimensionality.
PB96-102231 Not available NTIS Self-Biasing Cryogenic Particle Detector Utilizing Electrothermal Feedback and a SQUID Readout.
PB96-102538
Self Broadening in the nu1 Band of $\mathrm{NH3}$. PB94-216371

00,857 Not available NTIS

Selt-Calibrated Intelligent Optical Sensors and Systems. PB96-200738 04,380 Not available NTIS
Polis Self-Calibrating Fiber Optic Sensors: Potential Design Methods.
PB95-169298 02,172 Not available NTIS Self Calibrating Fiber Optic Sensors: Potential Design MethSelf Calibrating Fiber Optic Sensors: Potential Design Meth-
ods.
PB95-169306 02,173 Not available NTIS -Order Calculations of ElecSelf-Consistent 'GW' and Higher-Order Calculations of Elec-
tron States in Metals.
PB97-119341 01,189 Not available NTIS Self-Heating in the Coulomb-Blockade Electrometer.
PB94-212685 04,507 Not available NTIS Self Monitoring Accounting Systems.

Self Monitoring Accounting Systems.
PB95-216602 00,007 PC A03/MF A01 Self-, N2- and Ar-Broadening and Line Mixing in $\mathrm{HCN}$ and $\mathrm{C} 2 \mathrm{H} 2$.
$\mathrm{PB} 95-108445$ 00,876 Not available NTIS

Self-Organizing Neural Network Character Recognition on a Massively Parallel Computer. PB95-163994

01,845 Not available NTIS

Self-Organizing Neural Network Character Recognition Using Adaptive Filtering and Feature Extraction. PB96-119797 01,855 Not available NTIS Self-Reciprocal Fourier Functions.

PB96-200852

01,974 Not available NTIS

SEM Linewidth Metrology of X-ray Lithography Masks PB96-201108

02,447 Not available NTIS

Semiclassical Explanation of the Generalized RamsauerTownsend Minima in Electron-Atom Scattering

PB95-153532

03,925 Not available NTIS

Semiconductor Measurement Technology: Design and Testing Guides for the CMOS and Lateral Bipolar-On-SOI Test $\begin{array}{ll}\text { Library. } & 02,301 \quad \text { PC A07/MF A02 }\end{array}$

Semiconductor Measurement Technology: HOTPAC. Programs for Thermal Analysis Including Version 3.0 of the TXYZ Program, TXYZ30, and the Thermal MultiLayer Pro-

gram, TML.
PB95-260766
02,374 PC A05/MF A01 B95-260766

Semiconductor Measurement Technology: Improved Characterization and Evaluation Measurements for $\mathrm{HgCdTe} \mathrm{De}-$ GOES and TIROS Satellites. PB94-188810

02,122 PC A09/MF A02

Semiconductor Measurement Technology: Survey of Optical Characterization Methods for Materials, Processing, and Manufacturing in the Semiconductor Industry.
PB96-154596 02,706 PC A05/MF A01 Semiconductor Measurement Technology: Test Structure Implementation Document: DC Parametric Test Structures and Test Methods for Monolithic Microwave Integrated Cir cuits (MMICs)
PB96-117692

02,399 PC A05/MF A01

SEMPA Studies of Exchange Coupling in Magnetic Multilayers.
PB96-164074

04,780 Not available NTIS

SEMPA Studies of Oscillatory Exchange Coupling. PB95-163556

04,625 Not available NTIS

Sensing Droplet Detachment and Electrode Extension for Control of Gas Metal Arc Welding.
PB96-190160 3,297 Not available NTIS Sensitivity Analysis for Mathematical Modeling of Fires in
Residential Buildings.
PB96-154968
00,215 PC A03/MF A01 PB96-154968

Sensitivity of Three-Point Circle Fitting.
PB95-136354 02,901 PC A03/MF A01 Sensor System for Intelligent Processing of Hot-Rolled Steel.
PB96-186069 03,373 Not available NTIS

Separation and Identification of Organic Gunshot and Explosive Constituents by Micellar Electrokinetic Capillary Electrophoresis.

PB95-107249

00,566 Not available NTIS

Serial Sectioning of Hardened Cement Paste for Scanning Electron Microscopy.

PB94-172640

01,305 Not available NTIS

Series Array of DC SOUIDs.

PB95-16386

02,046 Not available NTIS

SF6 Insulation: Possible Greenhouse Problems and Solutions. 02,269 PC A03/MF A01
SF6/N2 Mixtures: Basic and High-Voltage-Insulation Properties.

PB96-123468

02,230 Not available NTIS

SGML Environment for STEP.

PB95-143103

$02,778 \mathrm{PC}$ A03/MF A01

SGML Parser Validation Procedures.

PB95-174959

$01,717 \mathrm{PC} \mathrm{A03/MF} \mathrm{A01}$

Shape of the Temperature-Entropy Saturation Boundary

PBg6-135068

02,506 Not available NTIS

Shape-Resonance-Enhanced Continuum-Continuum Coupling in Photoionization of $\mathrm{CO}$.

PB95-16447

00,983 Not available NTIS

Shape Selectivity Assessment of Stationary Phases in Gas Chromatography.

00,579 Not available NTIS

Shape Selectivity in Reversed-Phase Liquid Chromatography for the Separation of Planar and Non-Planar

PB95-162608

00,596 Not available NTIS

Shapiro Steps in Large-Area Metallic-Barrier Josephson Junctions.

PB96-200142

02,090 Not available NTIS

Sharing Information via the Internet: An Infoserver Case

Study.
PB96-13151

01,493 PC A03/MF A01

Shear Dependence of Critical Fluctuations in Binary Polymer Mixtures by Small Angle Neutron Scattering.

PB94-211612 01,220 Not available NTIS

Shear Design of High-Strength Concrete Beams: A Review of the State-of-the-Ant

PB96-214713

$01,330 \mathrm{PC} \mathrm{A11/MF} \mathrm{A03}$

Shear-Excited Morphological States in a Triblock CopolyPB94-172392

01,196 Not available NTIS

Shear-Induced Changes in the Order-Disorder Transition Temperature and the Morphology of a Triblock Copolymer. PB97-118772

03,130 Not available NTIS

Shear-Induced Martensitic-Like Transformation in a Block Copolymer Melt.

01,277 Not available NTIS

Shear-Induced Melting of Two-Dimensional Solids.

PB96-1 12057

01,075 Not available NTIS

Shear-Induced Mixing in Polymer Blends.

PB96-148085

01,287 Not available NTIS

Shear Suppression of Critical Fluctuations in a Diluted Polymer Blend.

04,418 Not available NTIS

Shielding of Cracks in a Plastically Polarizable Material.

PB95-164257 04,631 Not available NTIS

Shock Tube Techniques in Chemical Kinetics.

Shock Tube Techniques in Chemical Kinetics.
PB95-163465 00,968 Not available NTIS

Short-Pulse Detachment of $\mathrm{H}(-)$ in the Presence of a Static Electric Field

04,007 Not available NTIS

Short-Range Correlation and Relaxation Effects on the $(6 p(2))(1)$ SO Autoionizing State of Atomic Barium. 03,973 Not available NTIS

Should NIST Accredit U.S. Calibration Laboratories.

PB95-107280 02,646 Not available NTIS

Sitting Through Nine Years of NIST Clock Data with TA2.

PB95-181137 01,547 Not available NTIS

sigma+-sigma- Optical Molasses in a Longitudinal Magnetic PB95-161840

03,934 Not available NTIS

Signatures of Large Amplitude Motion in a Weakly Bound Complex: High-Resolution IR Spectroscopy and Quantum Calculations lor HeCO2.

PB95-203485

01,054 Not available NTIS

Significant Contributions of IAPWS to the Power Industry, Science and Technology

01,088 Not available NTIS

Silicon Nitride Boundary Lubrication: Effect of Oxygenates. PB96-111711 03,068 Not available NTIS

Silicon Nitride Boundary Lubrication: Lubrication Mechanism of Alcohols.

PB96-111703

03,067 Not available NTIS

Silicon Photodiodes Optimized for EUV and Solt X-Ray Regions.

02, 124 Not available NTIS

Silicon Surface Chemistry by IR Spectroscopy in the Mid- to Far-IR Region: $\mathrm{H} 2 \mathrm{O}$ and Ethanol on $\mathrm{Si}(100)$.

PB96-138565

01,097 Not available NTIS 


\section{TITLE INDEX}

Silver Metalization of Octadecanethiol Monolayers Self-Assembled on Gold.

00,923 Not available NTIS

Simple and Efticient Low-Temperature Sample Cell for Infrared Spectrophotometry.
PB94-199197

00,545 Not available NTIS

Simple and Efficient Methane-Marker Devices for Chromatographic Samples. $\quad 00,635$ Not available NTIS
PB96-164041

Simple and Repeatable Technique for Measuring the Critical Current of Nb3Sn Wires.

PB96-119409

02,229 Not available NTIS

Simple, Compact, High-Purity Cr Evaporator for Ultrahigh Vacuum.
PB94-216678

04,520 Not available NTIS

Simple, Inexpensive Apparatus for Sample Concentration.
PB94-199205

Simple Method of Composition Shifting with a Distillation Column for a Heat Pump Employing a Zeotropic Refrigeran

Mixture.
PB95-255824

$02,603 \mathrm{PC} \mathrm{A03/MF} \mathrm{A01}$

Simple Scalability Test for MIMD Code.

PB94-193638

01,688 PC A03/MF A01

Simple Variable Line Space Grating Monochromator for Synchrotron Light Source Beamlines.
PB96-156203 No65 Novalable NTIS

Simplified Cycle Simulation Model for the Performance Rat-

ing of Refrigerants and Refrigerant Mixtures.
PB94-199890 03,255 Not available NTIS

Simplified Design Procedure for Hybrid Precast Concrete Connections.
PB96-154836

00,405 PC A06/MF A01

Simulating Device Size Effects on Magnetization Pinning Mechanisms in Spin Valves.

PB97.112593

04,158 Not available NTIS

Simulating Smoke Movement through Long Vertical Shafts

in Zone-Type Compartment Fire Models.
PB95-143152 00,368 PC A03/MF A01

Simulating the Dynamic Electro Thermal Behavior of Power Electronic Circuits and Systems.

PB95-161014

02,345 Not available NrIS

Simulation and SANS Studies of Gelation Under Shear.

PB96-167176 01,150 Not available NTIS

Simulation of C60 Through the Plastic Transition Tempera-

tures.

04,713 Not available NTIS

Simulation of Ceramic Particle Formation: Comparison with In-situ Measurements.

PB95-152013

00,674 Not available NTIS

Simulation Studies of Supercooled and Glass Forming Liquids.

01,085 Not available NTIS

Simulations of Glass Forming Liquids: What Has Been Learned.
PB95-150124

00,915 Not available NTIS

Simulations of Neutron Focusing with Curved Mirrors.

PB96-176649 02,200 Not available NTIS

Simultaneous Forward-Backward Raman Scattering Studies of D2 Broadened by D2, He, and Ar.
PB95-162459 00,961 Not available NTIS

Simultaneous Laser-Diode Emission and Detection for Fiber-Optic Sensor Applications.
PB96-155502 No62 Not available NTIS

Simultaneous Measurement of Normal Spectral Emissivity by Spectral Radiometry and Laser Polarimetry at High Temperatures in Pulse-Heating Experiments: Application to Molybdenum and Tungsten.

B $97-118376$

02,694 Not available NTIS

Simultaneous Optical Measurement of Soot Volume Fraction, Temperature, and CO2 in Heptane Pool Fire.

PB96-102132
01,397 Not available NTIS

Simultaneous Visual and Calorimetric Measurements of R11, R123, and R123/Alkylbenzene Nucleate Flow Boiling.
PB94-172426 03,251 Not available NTIS

Single-Atom Point Source for Electrons: Field-Emission Resonance Tunneling in Scanning Tunneling Microscopy.
PB95-125860

Single-Phase Heat Transfer and Pressure Drop Character-

istics of an Integral-Spine-Fin Within an Annulus.
PB94-194073 03,805 PC A03/MF A01

Single-Phase Heat Transfer and Pressure Drop Character-

istics of an Integral-Spine Fin Within an Annulus.
PB97-122386
04,179 Not available NTIS

Single-Photon Ionization and Detection of $\mathrm{Ga}$, In, and As(sub n) Species in GaAs Growth.
PB95-152815 00,591 Not available NTIS
Single Photon Ionization, Laser Optical Probe Technique for Semiconductor Growth.

01,032 Not available NTIS

Single Photon Laser Ionization as an In-situ Diagnostic for MBE growth

01,059 Not available NTIS

Single-Photon Laser Ionization Time-of-Flight Mass Spectroscopy Detection in Molecular-Beam Epitaxy: Application to $\mathrm{As} 4, \mathrm{As} 2$, and $\mathrm{Ga}$.

PB95-203337

01,052 Not available NTIS

Single-Port Technique for Adaptor Efficiency Evaluation.

PB96-176441 02,088 Not available NTIS

Singularities in Minimum Surface Energy Problems and Their Influence in Surface Motion.
PB94-199411 04,473 Not available NTIS

Sinusoidal Surfaces as Standards for BRDF Instruments.
PB97-110597 04,388 Not available NTIS

Site of One-Electron Reduction of Ni(II) Porphyrins. Formation of Ni(I) Porphyrin of Ni(II) Porphyrin pi-Radical Anion. PB95-107066 00,866 Not available NTIS

Size and Self-Field Effects in Giant Magnetoresistive ThinFilm Devices.

04,674 Not available NTIS

Size Effects and Giant Magnetoresistance in Unannealed NiFe/Ag Multilayer Stripes.

PB97-111306

04,145 Not available NTIS

Size Effects in Submicron NiFe/Ag GMR Devices.

PB96-155510

02,237 Not available NTIS

Slab Transmission and Reflection for Point Source and Point Detector

03,838 Not available NTIS

Slant Path Atmospheric Refraction Calibrator: An Instrument to Measure the Microwave Propagation Delays Induced by Atmospheric Water Vapor.

Atmospheric Wat
PB95-151270

01,476 Not available NTIS

Sleuthing the Dynamo: HST/FOS Observations of UV Emissions of Solar-Type Stars in Young Clusters.

PB96-122817 00,098 Not available NTIS

Slicing in the Presence of Parameter Aliasing

PB96-160858 01,755 Not available NTIS

Sliding Vane Flow Conditioner Tests in a 100 Diameter Long 10 inch Natural Gas Orifice Meter at Pacific Gas and Electric. Topical Report, 1990-1992.

PB95-256335

02,493 PC A04/MF A01

Slit Jet Infrared Spectroscopy of Hydrogen Bonded N2HF Isotopomers: Rotational Rydberg-Klein-Rees Analysis and H/D Dependent Vibrational Predissociation Rates

PB95-161873

00,956 Not available NTIS

Slit-Jet Near-Intrared Diode Laser Spectroscopy of (DCl)2: nu1, nu2 DCl Stretching Fundamentals. Tunneling Dynamics, and the Influence of Large Amplitude 'Geared' Intermolecular Rotation.

01,047 Not available NTIS

Slit-Jet Near-Intrared Spectroscopy and Internal Rotor Dynamics of the ArH2O van der Waals Complex: An Angular Potential-Energy Surface for Internal H2O Rotation.

PB95-202792 01,033 Not available NTIS

Slow Dynamics of Segregation in Hydrogen-Bonded Poly-

mer Blends.
PB96-12359

01,281 Not available NTIS

Slow-Electron Collisions with CO Molecules in an Exact-Exchange Plus Parameter-Free Polarization Model.

change Plus Par

03,989 Not available NTIS

Slow Evolution from the Boundary: A New Stabilizing Constraint in Ill-Posed Continuation Problems.

PB96-122858

03,418 Not available NTIS

Slowly Divergen Space Marching Schemes in the Inverse Heat Conduction Problem.

03,812 Not available NTIS Small-Angle Neutron Scattering Characterization of Processing/Microstructure Relationships in the Sintering of Crys-
talline and Glassy Ceramics. (Reannouncement with New talline and Glassy Ceram

Availability Inform

03,025 PC A03/MF A01

Small-Angle Neutron Scattering of Poly(vinyl alcohol) Gels. PB95-164117 01,260 Not available NTIS

Small-Angle Neutron Scattering (SANS) Study of WormLike Micelles Under Shear.

PB96-176698

04,111 Not available NTIS

Small Angle Neutron Scattering Studies of Structural Characteristics of Argarose Gels. PB96-112305

03,475 Not available NTIS

Small Angle Neutron Scattering Studies on Chain Asymmetry of Coextruded Poly(Vinyl Alcohol) Film

PB95-164372
Small Angle Neutron Scattering Study of a Clay Suspension Under Shear.
PB96.167374

00,663 Not available NTIS

Small-Angle Neutron-Scattering Study of Dense Sheared Silica Gels.

01,151 Not available NTIS

Small Angle Neutron Scattering Study of the Structure and Formation of MCM-41 Mesoporous Molecular Sieves.

PB97-122337

03,110 Not available NTIS

Small Angle Neutron Scattering Study of the Structure and Formation of Ordered Mesopores in Silica.

PB96-111919

03,069 Not available NTIS

Small Angle Neutron Scattering Study on Poly(N-Isopropyl Acrylamide) Gels Near Their Volume-Phase Transition Temperature.

01,263 Not available NTIS

Small-Angle Neutron Scattering Study on Weakly Charged Temperature Sensitive Polymer Gels.

PB95-164398

01,264 Not available NTIS

Small Angle Neutrons Scattering from Nanocrystalline Palla dium as a Function of Annealing.

PB95-176103

03,354 Not available NTIS

Small-Angle X-Ray and Neutron Scattering Study of Block Copolymer/Homopolymer Mixtures.

PB94-211729

01,221 Not available NTIS

Small genomes: New initiatives in mapping and sequencing Workshop summary report.

03,451 PC A05/MF A01

Smart Clock: A New Time.

PB95-151445

01,530 Not available NTIS

Smoke Control

vator Fire Evacuation

Bm-212883

00,291 Not available NTIS

Smoke Emission from Burning Crude Oil.

PB96-122890

01,407 Not available NTIS

Smoke Plume Trajectory from In situ Burning of Crude Oil: Field Experiments.

PB96-200993

02,597 Not available NTIS

Smoke Plume Trajectory from In situ Burning of Crude Oil in Alaska: Field Experiments.

PB96-131560

02,594 PC A03/MF A01

Sobolev Approximation for Line Formation with Partial Frequency Redistribution.

B95-202669

00,078 Not available NTIS

Soft-X-ray Damage to $\mathrm{p}$-terphenyl Coatings for Detectors. PB96-159611

04,364 Not available NTIS

Soft-X-ray-Emission Investigation of Cobalt Implanted Silicon Crystals.

04,069 Not available NTIS

Soft-X-ray-Emission Spectra of Solid $\mathrm{Kr}$ and $\mathrm{Xe}$

PB96-157920

04,070 Not available NTIS

Soft-X-ray-Emission Studies of Bulk Fe3Si, FeSi, and FeSi2, and Implanted Iron Silicides.

PB96-157938

Soft X-ray Reflectometry Program at the National Institute of Standards and Technology.

Software Libraries, Numerical and Statistical.
PB94-198967 Software Needs in Special Functions.

$\begin{array}{ll}\text { PB95-105045 } 01,702 & \text { PC A03/MF A01 }\end{array}$

Software Needs in Special Functions.

PB96-200977 01,778 Not available NTIS

Software Safety and Program Slicing

Solid Propellant Gas Generators: Proceedings of the 1995 Workshop. Held in Gaithersburg, Maryland on June 28-29.

1995.
PB96- 131479

01,412 PC A11/MF A03

Solid State (13)C NMR and Raman Studies of Cellulose Triacetate: Oligomers, Polymorphism, and Inferences about Chain Polarity

PB96-176532

01,289 Not available NTIS

Solubilities of Copper(II) and Chromium(III) betaDiketonates in Supercritical Carbon Dioxide.
PB96-164215 01,147 Not available NTIS

Solubility Measurement by Direct Injection of SupercriticalFluid Solutions into a HPLC System. PB95-175626

00,997 Not available NTIS

Solvent Effects in the Reactions of Peroxyl Radicals with Organic Reductants. Evidence for Proton Transfer Mediated Electron Transfer

PB95-107157

00,873 Not available NTIS

Some Aspects of Fundamental Neutron Physics.

PB95-126298 03,882 Not available NTIS 


\section{TITLE INDEX}

Some Basics on Who's Who and What's What in Seismic Pafety.

00,435 Not available NTIS

Some Considerations for Interim Testing of Coordinate Measuring Machine Performance Using a Specilic Artifact.
PB95-108858
02,898 Not available NTIS

Some Factors Affecting Design ol a Furniture Calorimeter Hood and Exhaust.

$00,285 \mathrm{PC} \mathrm{A03/MF} \mathrm{A01}$

Some Factors Affecting the Design of a Calorimeter Hood and Exhaust.

00,302 Not available NTIS

Some Notable Hurricanes Revisited.

PB96-122601 O0,458 Not available NTIS

Sorption ol Moisture on Epoxy and Alkyd Free Films and Coated Steel Panels.

03,192 Not available NTIS

PB95-162475 Office Building

03,600 PC A04/MF A01

Sources of Strain-Measurement Error in Flag-Based Extensometry.
PB97-118731

03, 108 Not available NTIS

Sources of Uncertainty in a DVM-Based Measurement System for a Ouantized Hall Resistance Standard.

(Order as PB94-219326, PC A05/MF A02)

Sources of Urban Contemporary Carbon Aerosol.

PB95-175659 02,551 Not available NTIS

Sparse Water Sprays in Fire Protection.
PB96-202304

SparseLib++ v. 1.5 Sparse Matrix Class Library. Reference Guide. 193636

$01,775 \mathrm{PC} \mathrm{A03/MF} \mathrm{A01}$

Spatial Correlation Function for Fields in a Reverberation PB96-148077

04,427 Not available NTIS

Spatial Data Transfer Standard (SDTS): Category: Software Standard; Subcategory: Information interchange.
FIPS PUB 173-1
01,794 PC A04/MF A01

Spatial Data Transfer Standard (SDTS). Category: Sottware Standard; Subcategory: Information Interchange. (FIPS PUB 173-1A).

FIPS PUB 173-1A

$01,795 \quad P C \$ 44.50$

Spatial Data Transfer Standard (SDTS). Category: Software Standard; Subcategory: information interchange. (FIPS PUB 173-1B).
FIPS PUB 173.1B

01.796 PC E99

Spatial Dependence of Electrical Fields Due to Space Charges in Films of Organic Dielectrics Used for Insulation of Power Cables.

PB94-199130

02,214 Not available NTIS

Spatial Information and Technology Standards Evolving.

PB96-135108

03,679 Not available NTIS

Spatial Uniformity of Optical Detector Responsivity.

PB95-168845 02, 162 Not available NTIS

SPC Artifact for Automated Solder Joint Inspection.

PB96-161716 04,095 Not available NTIS

Specification for Interoperability between Ballistic Imaging Systems. Part 1. Cartridge Cases.

PB96-195524 01,860 PC A04/MF A01

Specifications and Tolerances for Reference Standards and Field Standard Weights and Measures. 2. Specifications and Tolerances for Field Standard Measuring Flasks.

PB96-178926 02,682 PC A03/MF A01

Spectra of $\mathrm{Ag} I$ Isoelectronic Sequence Observed from

Er(21+) to Au(32+)
PB95-150660

03,899 Not available NTIS

Spectral Data and Grotrian Diagrams for Highly lonized Chromium, Cr V through Cr XXIV

PB94-162369 00,748 Not available NTIS

Spectral Data for Highly lonized Krypton, $K r V$ through $K_{r}$ XXXVI.

PB96-145917

01,115 Not available NTIS

Spectral Interference in the Determination of Arsenic in High-Purity Lead and Lead-Base Alloys Using Electrothermal Atomic Absorption Spectrometry and Zeeman-EHect Background Correction.
PB96-112099 Not available NTIS

Spectrally Smooth Reflectances That Match.

PB95-176319 04,306 Not available NTIS

Spectroscopic Constants for the 2.5 and 3.0 micrometer Bands of Acetylene.

00,847 Not available NTIS

Spectroscopic Data for Fusion Edge Plasmas.

PB95-151569
Spectroscopic Data Tables for Highly-lonized Atoms.

P895-151585 03,910 Not available NTIS

Spectroscopic Diagnostics of Low Temperature Plasmas: Techniques and Required Data. 04,411 Not available NTIS
PB95-151577

Spectroscopic Ellipsometry Determination of the Properties of the Thin Underlying Strained Si Layer and the Roughness at SiO2/Si Interface.

04,560 Not available NTIS

Spectroscopic Puzzle in ArHF Solved: The Test of a New PB94-216058

00,850 Not available NTIS

Spectroscopic Study ol Ouantized Breakdown Voltage States of the Ouantum Hall Effect.

04,730

(Order as PB96-113535, PC A05/MF A01)

Spectroscopic Study of Reaction Intermediates and Mecha nisms in Nitramine becomposition and Combustion.
AD-A296 061/5 03,774 PC A03/MF A01

Spectroscopy and Structure of the Lithium Hydride Diatomic Molecules and lons.

00,731 Not available NTIS

Spectrum and Energy Levels of Five-Times-Ionized Niobium (Nb VI).

04,226 Not available NTIS

Spectrum and Energy Levels of Triply Ionized Barium (Ba PB) $95-140877$

03,888 Not available NTIS

Spectrum of the Stochastically Forced Dutfing-Holmes OsPillator.

00,216 Not available NTIS

Specular and Diffuse Reflection Measurements of Electronic Displays.

02,208 Not available NTIS

Speed-of-Sound Measurements in Liquid and Gaseous Air.
PB95-151957 04,186 Not available NTiS

95-151957

Spherical-Wave Source-Scattering Matrix Analysis of Antennas and Antenna-Antenna Interactions.
PB96-111166 PC A08/MF A02

Spin-Dependent Interface Transmission and Reflection in Magnetic Multilayers (Invited). 04,130 Not available NTIS Spin-Resolved Elastic Scattering of Electrons from Sodium.
PB95-161774 03,933 Not available NTiS

Spin Squeezing and Reduced Ouantum Noise in SpectrosPB95-151635

03,912 Not available NTIS

Spinor Equation
P897-110605

04,142 Not available NTiS

Spot-Prolile-Analyzing LEED Siudy of the Epitaxial Growth

Of Fe, $\mathrm{Co}$, and $\mathrm{Cu}$ on $\mathrm{Cu}(100)$.
PB95-150165

Sprinkler Fire Suppression Algorithm.

PB94-216181 00,293 Not available NTIS

Sputtered High Temperature Thin Film Thermocouples.

PB95-161311 02,259 Not available NTIS

SOA and TOM in Software Ouality Improvement.

PB96-160791 01,754 Not available NTIS

SOA Standards and Total Ouality Management

PB96-111844 01,743 Not available NTIS

SOL Environments. Category: Software Standard; Sub. category: Database.

$01,801 \mathrm{PC} \mathrm{A04/MF} \mathrm{A01}$

Squeezed Atomic States and Projection Noise in Spectros. PB95-176293

03,960 Not available NTIS

SSME LOX Duct Flowmeter Design and Test Results.

04,826 Not available NTIS

Stability and Surface Energies of Wetted Grain Boundaries in Aluminum Oxide.

03,059 Not available NTIS

Stability/Instability of Gas Mixtures Containing 1,3-Butadiene in Treated Aluminum Gas Cylinders.
PB95-162285

Stability, Microstructural Evolution, Grain Growth, and Coarsening in a Two-Dimensional Two-Phase Microstructure.

03,325 Not available NTIS

Stability of Compressed Gas Mixtures Containing Low Level Volatile Organic Compounds in Aluminum Cylinders.
PB96-111968

Stabilization and Precise Calibration of a Continuous-Wave Difference Frequency Spectrometer by Use ol a Simple Transfer Cavity.
Stabilization of Optical Phase/Frequency of a Laser System Application to a Commercial Dye Laser with an Externa Stabinizer.

04,327 Not available NTIS

Stabilization of 3.3 and $5.1 \mathrm{~m}$ Lead-Salt Diode Lasers by Optical Feedback

04,313 Not available NTIS

Stable Phase Locking in a Two-Cell Ladder Array of Jo sephson Junctions.

04,722 Not available NTIS

Stable Silicon Photodiodes lor Absolute Intensity Measurements in the VUV and Soft X-ray Regions.

04,135 Not available NTIS

Stable, Tightly Confining Magnetic Trap for Evaporative Cooling of Neutral Atoms.

04,126 Not available NTIS

Stacked Series Arrays of High-Tc Trilayer Josephson JuncPB96-102272

04,705 Not available NTIS

Stacking Fault Pyramid Formation and Energetics in Siliconon-Insulator Material Formed by Multiple Cycles of Oxygen Implantation and Annealing. PB96-160221

Stacking the Cards in Europe: One Company's Story.
PB97-110126

Stagnant Film Model of the Etfect of Natural Convection on the Dendrite Operating State.

B96-146832

04,765 Not available NTiS

Standard Antennas for Electromagnetic Interference MeasSrements and Methods to Calibrate Them.

PB96-102561 02,007 Not available NTIS

STandard for the Exchange of Product Model Data (STEP) Procedures for NIST STEP Validat

PB96-154976 02,787 PC A03/MF A01

Standard Generalized Markup Language Test Suite Evalua tion Report.

$01,751 \mathrm{PC} \mathrm{A03/MF} \mathrm{AO}$

Standard Materials. A Descriptive List with Prices.

AD-A278 140/9 $\quad 00,500 \quad$ PC A03/MF AO

Standard of Attenuation for Microwave Measurements.

AD-A297 905/2 01,517 PC A02/MF A01

Standard Polarization Components: Progress Toward an

Optical Retardance Standard.
PB96-119672

04,342 Not available NTIS

Standard Probes for Electromagnetic Field Measurements.
PB94-185436

Standard Reference Data for the Thermal Conductivity of

Water.

01,111 Not available NTIS

Standard Reference Devices for High Temperature Superconductor Critical Current Measurements.
PB95-175543 04,659 Not available NTIS

Standard Reference Material for the Measurement of Par-

ticle Mobility by Electrophoretic Light Scattering.
PB96- $102488 \quad 00,609$ Not available NTIS

Standard Relerence Material 1744: Aluminum FreezingPoint Standard

$01,055 \mathrm{PC} \mathrm{A03/MF} \mathrm{A01}$

Standard Reference Materials: Certification of a Standard Reference Material lor the Determination of Interstitial OxyReference Material lor the Determination of Interstitial Oxy-
gen Concentration in Semiconductor Silicon by Infrared gen Concentration

Spectrophotom

02,326 PC A05/MF A01

Standard Reference Materials for Dioxins and Other Environmental Pollutants.

02,518 Not available NTIS

Standard Reference Materials for Optical Fibers and Connectors.

04,344 Not available NTIS

Standard Reference Materials for the Determination of Polycyclic Aromatic Hydrocarbons in Environmental Samples - Current Activities.

PB95-151668

00,586 Not available NTIS

Standard Reference Materials for the Determination of Trace Organic Constituents in Environmental Samples.

Trace Organic Constituents in Environmental Samples.
PB95-164026 Not available NTIS

Standard Reference Materials: Glass Filters as a Standard Reference Material for Spectrophotometry - Selection, Preparation, Certification, and Use of SRM 930 and SRM 1930.

Standard Reference Materials: Polystyrene Films for Calibrating the Wavelength Scale of Intrared Spectrophotometers - SRM 1921 . Scale of Intrared
Spe

03,386 PC A03/MF AO

Standard Reference Materials (SRM's) for Measuring Genetic Damage.

03,516 Not available NTIS 


\section{TITLE INDEX}

Standard Samples and Reference Standards Issued by the National Bureau of Standards.

AD-A279 240/6

$02,613 \mathrm{PC} \mathrm{AO3/MF} \mathrm{A01}$

Standard Security Label for Information Transfer; Category: Computer Security: Subcategory: Security Labels.

Standard Source Method for Reducing Antenna Factor Er-

rors in Shielded Room Measurements.
PB96-183157 02,013 PC A04/MF A01

Standard States, Reference States and Finite-Concentration Effects in Near-Critical Mixtures with Applications to Aqueous Solutions.

00,979 Not available NTIS

Standardised Computer Data File Format for Storage, Transport, and Off-Line Processing of Partial Discharge Data.

PB96-122486

01.930 Not available NTIS

Standardization and Decay Scheme of Rhenium-186

PB94-200490

03,830 Not available NTIS

Standardization for ATM and Related B-ISDN Technologies. 01,499 Not available NTIS

Standardization of Formats and Presentation of Fire Data The FDMS.

PB94-198462

01,371 Not available NTIS

Standardization of Testing Methods for Optical Disk Media Characteristics and Related Activities at NIST.

PB95-108486

01,624 Not available NTIS

Standards: A Cardinal Direction for Geographic Information Systems.
PB95-150942

03,677 Not available NTIS

Standards Activities of Organizations in the United States.

00,006 PC A99/MF E08

Standards and Linkages: What Data Sharing Needs.

PB95-161881 01,713 Not available NTIS

Standards Development in Nonh America for Performance

of Whole Buildings and Facilities.

00,312 Not available NTIS

Standards for Atmospheric Measurements.

PB95-163622 02,547 Not available NTIS

Standards for Corrected Fluorescence Spectra.

PB95-150835 00,581 Not available NTIS

Standards for High Integrity Software.

PB96-161385 01,764 Not available NTIS

Standards in Building Economics: Why We Need Them and

How to Write Them.
PB94-216405

00.320 Not available NTIS

Standards of Seismic Safety for Existing Federally Owned or Leased Buildings and Commentary.
PB95-130209 00,431 PC A06/MF A02

Standards Policy and Information Infrastructure.

PB95-231882 01,485 PC A20/MF A04

Standards Promote Credibility and Technology Transfer: The Need for Greater Industry Support of Technical Committees.

02,961 PC A03/MF A01

Standards Setting in the European Union: Standards Organization and Officials in EU Standards Activities.

PB96-115019

02,919 PC A04/MF A01

Stars, Atmospheres, Radiative Transter.

PB96-119474 00,095 Not available NTIS

State of the Art Report on Seismic Design Requirements for Nonstructural Building Components.

PB96-193800

00,308 PC A06/MF A01

State-of-the-Art Survey of Methodologies for Representing Manufacturing Process Capabilities.

PB94-187655 02,812 PC A03/MF A01

State-Resolved Rotational Energy Transfer in Open Shell Collisions: $\mathrm{Cl}((2) \mathrm{P} 3 / 2)+\mathrm{HCl}$

PB96-176607

01,157 Not available NTIS

State Weights and Measures Laboratories: Program Hand-

book.

02,687 PC A07/MF A02

State Weights and Measures Laboratories: State Standards Program Description and Directory. 1994 Edition.

PB94-207727 02,895 PC AO7/MF A02
0

Static Dielectric Constant of Water and Steam.

PB96-123559 01,090 Not available NTIS

Static Structural Analysis of a Reconfigurable Rigid Plattorm Supported by Elastic Legs.

02.960 PC A05/MF A01

Statistical Analysis of Parameters Affecting the Measurement of Particle-Size Distribution of Silicon Nitride Powders by Sedigraph (Trade Name).

03,042 Not available NTIS
Statistical Aspects of the Certification of Chemical Batch SRMs. Standard Reference Materials.

PB96-210877

00.645 PC A05/MF A01

Statistical Descriptors in Crystallography. 2. Repon of a Working Group on Expression of Uncertainty in MeasurePB96-146824

04,764 Not available NTIS

Statistical Quality Control Technology in Japan.

PB94-199064 02,708 Not available NTIS

Statistical Thermodynamics of Phase Separation and Ion Partitioning in Aqueous Two-Phase Systems.

PB94-199387 01,212 Not available NTIS

Status and Trends in Power Semiconductor Devices.

PB95-175097 02.361 Not available NTIS

Status of a Silicon Lattice Measurement and Dissemination Exercise.

PB94-199635

04,474 Not available NTIS

Status of Construction and Construction Technologies.

PB94-186004 00,318 Not available NTIS

Status of Electrocomposites.

PB94-212453

03,143 Not available NTIS

Status of Emerging Standards for Removable Computer Storage Media and Related Contributions of NIST.

PB96-160619

01,634 Not available NTIS

Status of the Round Robin on the Transpor Properties of PB96-167218

01,152 Not available NTIS

Status Report: AWS Standards for Identifying Arc Welds Status Report: AWS Standards for Identifying Arc Welds
(A91.1) and Recording Weld Data (A9.2).
PB95-162855 02,861 Not available NTIS

Stellar Coronal Structures

PB95-202834

00,080 Not available NTIS

Step-Edge and Stacked-Heterostructure High-Tc Josephson Junctions for Voltage-Standard Arrays.
PB96-102066 Not available NTIS

STEP On-Line Information Service (SOLIS). The IGES/ PDES Organization.
PB95-137790

02,777 PC A02/MF A01

Stochastic Modeling of a New Spectrometer

PB96-157870 04,068 Not available NTIS

Stranding Experiments on Double Hull Tanker Structures.

PB96-123112 03,749 Not available NTIS

Strangeness Flow Difference in Nuclear Collisions at $15 \mathrm{~A}$ and $200 \mathrm{~A} \mathrm{GeV}$.

04,042 Not available NTIS

Strategy to Support Multipoint Communication Service Over Native ATM Service.

PB96-176672

01,507 Not available NTIS

Strengthening Methodology for Lightly Reinforced Concrete PB96-158050

00,466 Not available NTIS

Strengthening Methodology for Lightly Reinforced Concrete Frames-II. Recommended Calculation Techniques for the Design of Infill Walls.

PB94-187648

00,426 PC A03/MF A01

Strengthening Methodology for Lightly Reinforced Concrete Frames: Recommended Design Guidelines for Strengthening with Infill Walls.

ing with Infill Wall
PB95-260725

00.454 PC A04/MF A01

Strong Hydrogen Bond in the Formic Acid-Formate Anion System.
PB94-198595

00,788 Not available NTIS

Structural Analysis in Context
PB

00,437 Not available NTIS

Structural Analysis of Heparin by Raman Spectroscopy.

PB96-167226

03,480 Not available NTIS

Structural and Chemical Investigations of Na3(ABO4)3.4H2O-Type Sodalite Phases.
PB95-180733 01,012 Not available NTIS

01,012 Not avalable NTIS

Structural and Magnetic Ordering in Iron Oxide/Nickel Oxide Multilayers by X-ray and Neutron Diffraction (Invited).
PB94-172558

Structural and Magnetic Properties of $\mathrm{CuCl} 2$ Graphite Intercalation Compounds.

03,020 Not available NTIS

Structural Ceramics Database. Topical Report, June 1989May 1991.

PB95-203758

03,060 PC A04/MF A01

Structural EXPRESS Editor.

PB94-159795

02,769 PC A04/MF A01

Structural Heterogeneity in Epoxies.

PB95-151866

Structural Stabilization of Phase Separating PC/Polyester Blends through Interfacial Modification by Transesterification Reaction.

PB95-150454

01,239 Not available NTIS

Structure and Conductivity of Layered Oxides

(Ba, Sr) $n+1$ ( $\mathrm{Sn}, \mathrm{Sb}) \mathrm{nO} n+1$.

04,707 Not available NT1S

Structure and Dynamics of Buckyballs.
PB95-153292

Structure and Radiation Properties of Pool Fires.
PB94-193802 02,473 PC A08/MF A02

Structure and Rheology of Hard-Sphere Systems.

PB96-167333 00,662 Not available NTIS

Structure of a Swirl-Stabilized Kerosene Spray Flame.

PB95-108569 02,480 Not available NTIS

Structure of a Triglyceride Microemulsion: A Small Angle Neutron Scattering Study.

PB96-112255

01,077 Not available NTIS

Structure of Glycine-Water H-Bonded Complexes.

PB94-198603 00,789 Not available NTIS

Structure of Molecules on Surfaces as Determined Using

Electron-Stimulated Desorption
$\mathrm{PB} 94-216165$

00,852 Not available NTIS

Structure of the Vapor-Liquid Interface Near the Critical Point

PB95-140174

00,902 Not available NTIS

Structured Testing: A Testing Methodology Using the Cyclomatic Complexity Metric.

PB97-114169

01,784 PC A07/MF A02

Structures of Sodium Metal.

PB94-198850

03.319 Not available NTIS

Structures of Vapor-Deposited Yttria and Zirconia Thin PB94-216025

03,041 Not available NTIS

Studies Assess Performance of Residential Detectors.

PB94-199262

00.290 Not available NTIS

Studies of Defects in Diamond Films and Particles by Raman and Luminescence Spectroscopies. PB95-162400

03,017 Not available NTIS

Studies of Ion Kinetic-Energy Distributions in the Gaseous Electronics Cont
PB96-113360

(Order as PB96-113311, PC A09/MF A03)

Studies of the Higher Order Smectic Phase of the Large Electroclinic Effect Material W317.

PB95-151601

00.935 Not available NTIS

Study of Diffusion Zones with Electron Microprobe Compositional Mapping.

00,559 Not available NTIS

PB94-216348

Alcohol-Based Spray Flames

Study of Droplet Transpon in

Using Phase/Do

02479 Not available NTIS

Study of Federal Agency Needs for Information Technology PB94-193653

01,579 PC A07/MF A02

Study of Heat Pump Pertormance Using Mixtures of R32/ R134a and R32/R125/R134a as 'Drop-In' Working Fluids for R22 with and Without a Liquid-Suction Heat Exchanger. PB94-218559

02,503 PC A04/MF A01

Study of Laser Resonance Ionization Mass Spectrometry Using a Glow Discharge Source. DE94018566

03,308 PC A01/MF A01

Study of Laser Resonance Ionization Mass Spectrometry Using a Glow Discharge Source PB96-123203 03,360 Not available NTIS

Study of Multiple Scattering Background in Compton Scatter Imaging. 04,425 Not available NTIS

Study of Potential Applications of Automation and Robotics Technology in Construction, Maintenance and Operation of Highway Systems: A Final Report. Volume 1.

PB95-251682

01,341 PC A06/MF AO2

Study of Potential Applications of Automation and Robotics Technology in Construction, Maintenance and Operation of Highway Systems: A Final Report. Volume 2 .

PB95-255865 01,343 PC A19/MF A04

Study of Potential Applications of Automation and Robotics Technology in Construction. Maintenance and Operation of Highway Systems: A Final Report. Volume 3 PB95-251690

01,342 PC A15/MF A03

Study of Potential Applications of Automation and Robotics Technology in Construction. Maintenance and Operation of Highway Systems: A Final Report. Volume 4.

PB95-251641 


\section{TITLE INDEX}

Study of Technology for Detecting Pre-Ignition Conditions of Cooking-Related Fires Associated with Electric and Gas Ranges and Cooktops, Phase 1 Report.
PB96-128095 PC A06/MF A02 Study of the Hydroxycarbonate Precursor Route to the YBa2C
PB95-140471 04,540 Not available NTIS Study of Ventilation and Carbon Dioxide in an Office BuildPBg5-150140 02,542 Not available NTIS Study of Ventilation Measurement in an Office Building PB96-155593 00,274 Not available NTIS

Study on Hazard Analysis in High Integrity Software Standards and Guidelines.

PB95-198727

01,725 PC A04/MF A01

Study on the Reuse of Plastic Concrete Using Extended Set-Retarding Admixtures.

00,402

(Order as PB96-117767, PC A08/MF A02)

Study to Determine the Existence of an Azeotropic R-22 'Drop-ln' Substitute.

02,568 PC A04/MF A01

Study to Determine the Most Important Parameters for Evaluating the Resistance of Soft Body Armor to Penetration by Edged Weapons.

03,757 PC A03/MF A01

Stylus Flight in Surface Profiling

PB96-123138 02,675 Not available NTIS

Stylus Technique for the Direct Verification of Rockwell Diamond Intenders.

02,958 Not available NTIS

Sub-Doppler Frequency Measurements on OCS at 87 Thz (3.4 micrometers) with the CO Overtone Laser: Considerations and Details

04,255 PC A03/MF A01

Sub-Doppler Frequency Measurements on OCS at $87 \mathrm{THz}$

$(3.4 \mathrm{mu}$ m) with the CO Overtone Laser.
PB96-102215

Sub-Doppler, Infrared Laser Spectroscopy of the Propyne 2nu1 Band: Evidence of $z$-Axis Coriolis Dominated Intramolecular State Mixing in the Acetylenic $\mathrm{CH}$ Stretch PBertone.202941

01,037 Not available NTIS

Submarine Automation: Demonstration No. 5.
PB95-251633

Submicroampere-Per-Root-Hertz Current Sensor Based on

the Faraday Effect in Ga: YIG.
PB95-162467

02,155 Not available NTIS

Submissions to a Planned Encyclopedia of Operations Research on Computational Geometry and the Voronoil Delaunay Construct.

03,425 PC A03/MF A01 Substitution-Induced Midgap States in the Mixed Oxides
AXBa1-ChiTiO3-Delta, with $\mathrm{A}=\mathrm{Y}$, La, and Nd. AxBa1-ChiTiO3-Delta, with $\mathrm{A}=\mathrm{Y}$, La, and $\mathrm{Nd}$.
PB95-140505 Not available NTIS

Substrate and Thin Film Measurements.
PB96-112297 Not available NTIS

Substrate Specificity of the Escherichia coli Endonuclease III: Exicision of Thymine- and Cytosine-Derived Lesions in DNA Produced by Radiation-Generated Free Radicals. DNA Produced by Radiation-Generated Free Radicals.
PB95-153425 NTIS Not available NTIS

Suggestions for a Logically-Consistent Structure for Service Life Prediction Standards.

00,358 Not available NTIS

Suitability of Metalorganic Chemical Vapor Deposition-Derived PrGaO3 Films as Buffer Layers for YBa2Cu307-x

PB Plsed-168670 Deposition. 04,640 Not available NTIS

Sulfate Attack of Cementitious Materials: Volumetric Relations and Expansions

03,232 PC A03/MF A01

Sulfur Dioxide Capture in the Combustion of Mixtures of Lime, Refuse-Derived Fuel, and Coal. 02,534 PC A04/MF A01 SUM and MEAN: Standard Programs for ACtivation Analysis.
PBg6-112149 Summaries of BFRL Fire Research In-House Projects and Summaries of BFRL Fire Research In-House Projects and
Grants, 1994.
PB95-130845 00,366 PC A10/MF A03

Summaries of Center for Fire Research In-House Projects and Grants: 1990

00,286 PC A10/MF A03

Summary and Notes of the Joint ISO/IGES/PDES Organization Technical Committee Meeting. Held in Albuquerque, tion Mechnico on October 15-20, 1989.
New Mot Met available NTIS
PB95-107314
Summary and Results of the NIST Workshop on Proposed Guidelines for Testing and Evaluation of Seismic Isolation Systems. Held in San Francisco. Califomia on July 25, PB96-154901

00,463 PC A04/MF A01

Summary of Federal Construction and Building $R$ and $D$ in PB97.114250

00,234 PC A04/MF A01 Summary of the Apparent Standard Partial Molal Gibbs
Free Energies of Formation of Aqueous Species Minerals, Free Energies of Formation of Aqueous Species, Minerals,
and Gases at Pressures 1 to 5000 Bars and Temperatures $\begin{array}{ll}25 \text { to } 1000 \mathrm{C} \text {. } & 01,113 \text { Not available NTIS } \\ \text { PB96-145891 }\end{array}$

01,113 , Not availa

Summary of the Patent Literature of Supercritical Fluid Pechnology.

00,502 Not available NTIS

Summary of the Proceedings of the Workshop on Standard Phantoms for In-vivo Radioactivity Measurement.

Not available NTIS

Summary Report of NIST's Industry-Government Consortium Research Program on Flowmeter Installation Effects: The 45 Degree Elbow.

04,204 PC A06/MF A02

Summary Report of NIST's Industry-Government Consortium Research Program on Flowmeter Installation Effects tium Research Program on Flowmeter Installation Effects
with Emphasis on the Research Period, January-September with Emphasis on the
1991: The Reducer.

1991: The Reduce

04,196 PC A04/MF A01

Summary Report on the Workshop on Advanced Digital Video in the National Information Infrastructure.

PB96-141320

01,497 Not available NTIS

Summary Report: Workshop on Industrial Applications of Scanned Probe Microscopy (2nd). A Workshop Co-SponY NIST, SEMATECH, ASTM E42.14, and the American Vacuum Society. Held in Gaithersburg, Maryland on
May 2-3.1995.
PB96-131602 00,509 PC AO7/MF A02

$00,509 \mathrm{PC} \mathrm{AO7/MF} \mathrm{A02}$

Superconducting Energy Gap of Bulk UBe13.

PB95-150116 O4,559 Not available NTIS

Superconducting Integrated Circuit Fabrication with Low Temperature ECR-Based PECVD SiO2 Dielectric Films.
PB96-103015 04,719 Not available NTIS

Oediometer.

Superconducting Kinetic Inductance Radiometer.
PB 145 Not available NTIS

Superconducting Materials: Specification.

PB94-211299 Materials: Specification. Not available NTIS

Superconducting Resonator and a Cryogenic GaAs FieldEffect Transistor Amplifier as a Single-lon Detection SysPB95-202727 03,990 Not available NTIS

Superconductor Critical Current Standards for Fusion Applications. Final Progress Report, October 1993-July 1994.
02,222 PC A05/MF A02

Superconductor- Normal-Superconductor Junctions for Digital/Analog Converters.

02,092 Not available NTIS

Superconductor- Normal-Superconductor Junctions for Programmable Voltage Standards. 02,093 Not available NTIS

Supercritical Fluid Extraction-Immunoassay for the Rapid Screening of Cocaine in Hair. 00,637 Not available NTIS
PB96-167168

Supercritical Fluid Extraction of Biological Products.

PB95-175204 00,040 Not available NTIS

Supercritical Solubility of Solids from Near-Critical DiluteMixture Theory

00,819 Not available NTIS

Supermirror Transmission Polarizers for Neutrons.

PB94-216215 03,866 Not available NTIS

Supplement to Stable Implementation Agreements for Open Systems Interconnection Protocols. Version 3, September 1990. Change Page Index, Version 3, June 1990 (Stable) Change Pages Issued December 1990; Output from Sep177).

PB94-164035

01,467 PC A06/MF A02

Supported Phospholipid/Alkanthiol Biomimetic Membranes: Insulating Properties.

PB95-180782

03,470 Not available NTIS

Suppression Effectiveness of Extinguishing Agents under Highly Dynamic Conditions. 00,020 Not available NTIS
PB95-180279

Suppression of Elevated Temperature Hydraulic Fluid and JP-8 Spray Flames.

PB95-181095

00,021 Not available NTIS

Suppression of High-Speed C2H4/Air Flames with $\mathrm{C}_{1}$ Halocarbons.
PB96-175724

03,287 Not available NTIS
Suppression of High Speed Turbulent Flames in a Detona-

tion/Deflagration Tube.
PB95-231817

01,395 PC A04/MF A01

Suppression of Ignition Over a Heated Metal Surface.

PB96-176425 03,291 Not available NTIS

Suppression of Ionization in One- and Two-Dimensional

Model Calculations.
PB95-203600

04,009 Not available NTIS

Suppression Research: Strategies.

PB94-211372 00,346 Not available NTIS

Surface Barrier and Lower Critical Field in YBa2Cu3O7delta Superconductors.

PB94-200128

04,478 Not available NTIS

Surface Chemical Interactions of Si3N4 with Polyelectrolyte Deflocculants.

03,056 Not available NTIS

Surface Chemistry of Silicon Nitride Powder in the Presence of Dissolved lons.

PB96-111760

01,073 Not available NTIS

Surface Core-Level Shitts of Barium Observed in Photoemission of Vacuum-Fractured BaTiO3 (100).

PB94-212156

04,501 Not available NTIS

Surface Degradation of Superconducting YBa2Cu307-delta Thin Films.

04,667 Not available NTIS

Surface Energy Reduction in Fibrous Monotectic Structures. PB95-140828

03,150 Not available NTIS

Surface Forces and Adhesion between Dissimilar Materials Measured in Various Environments.

PB94-172970

03,033 Not available NTIS

Surface Geometry of $\mathrm{BaO}$ on W(100): A Surface-Extended $X$-Ray-Absorption Fine-Structure Study.

PB95-164414

00,980 Not available NTIS

Surface Magnetic Microstructural Analysis Using Scanning Electron Microscopy with Polarization Analysis (SEMPA)
PB95-162657 03,938 Not available NTIS

Surface Modification of YBa2Cu307-delta Thin Films Using the Scanning Tunneling Microscope: Five Methods.

PB95-203394

04,699 Not available NTIS

Surface Plasmon Microscopy of Biotin-Streptavidin Binding Reactions on UV-Photopatterned Alkanethiol Self-Assembled Monolayers.

PB96-176771

01,158 Not available NTIS

Surface Roughness Evaluation of Diamond Films Grown on Substrates with a High Density of Nucleation Sites.

PB95-162418 03,018 Not available NTIS

Surface Roughness of Glass-Ceramic Insert. Composite Restorations: Assessing Several Polishing Techniques. PB97-119010 03,583 Not available NTIS

Surface Texture

PB95-164620

03,351 Not available NTIS

Surface Topography and Ordering-Variant Segregation in GB95-153649

04,595 Not available NTIS

Surface Transverse Wave Oscillators with Extremely Low Thermal Noise Floors

PB96-186010

01,967 Not available NTIS

Surging the Upside-Down House: Looking into Upsetting

Reference Voltages.
PB96-112313

02,385 Not available NTIS

Surging the Upside-Down House: Measurements and ModPBing Results.

02,243 Not available NTIS

Survey of Concrete Transport Properties and Their Meas-

PB95-220489

00,396 PC A04/MF A01

Survey of Fuel Loads in Contemporary Office Buildings. PB97-114235

00,233 PC A04/MF A01

Survey of Recent Cementitious Materials Research in WestPB94-218583

00,353 PC A03/MF A01

Survey of Standards for the U.S. Fiber/Textile/Apparel Industry. 193792

03,199 PC A06/MF A01

Survey of Steel Moment-Resisting Frame Buildings Affected by the 1994 Northridge Earthquake.
PB95-211918 PC AO9/MF AO2

Survey of the Components of Display-Measurement StandPB96-122528

02,188 Not avellable NTIS

Survey of the Components of Display-Measurement StandPB97.122394

02,209 Not avallable NTIS 


\section{TITLE INDEX}

Survey of the Literature on Heat Transfer from Solid Surlaces to Cryogenic Fluids.

04,193 PC A04/MF AO1

Survey on the Implementation of ISO/IEC Guide 25 by National Laboratory Accreditation Programs.
PB94-210150 00,479 PC A04/MF A01

SUSAN: SUperconducting Systems ANalysis by Low Temperature Scanning Electron Microscopy (LTSEM). $\begin{array}{ll}\text { PB96-112065 04,728 Not available NTIS } & \end{array}$

Susceptibility Critical Exponent for a Nonaqueous lonic Binary Mixture Near a Consolute Point.
PB95-152112 00,938 Not available NTIS

Swelling and Growth of Polymers, Membranes and Sponges.

03,396 Not available NTIS

Symbolic Programming with Series Expansions: Applications to Optical Waveguides.

PB95-168589

04,283 Not available NTIS

Synthesis and Polymerization of Difunctional and Multifunctional Monomers Capable of Cyclopolymerization.

01,257 Not available NTIS

Synthesis of Hybrid Organic-Inorganic Materials from

Interpenetrating Polymer Network Chemistry.
PB96-180054 02,994 Not available NTIS

Synthesis of Thermally Stable Elastomers

$\begin{array}{lll}\text { AD-A307 789/8 } & 03,194 & \text { PC A03/MF A01 }\end{array}$

Synthetic-Perturbation Tuning of MIMD Programs.

PB94-185568 01,687 Not available NTIS

System for Calibration of the Marshall Compaction Hammer. 145661

$01,303 \mathrm{PC} \mathrm{A08/MF} \mathrm{A02}$

System for Intercomparing Standard Solutions of Beta-Particle Emitting Radionuclides.

03,707 Not available NTIS

PB96-159637

Systematic Correction in Bragg X-ray Diffraction of Flat and
Curved Crystals.
PB97-112239
04,152 Not available NTIS PB97-112239

4,152 Not available NTIS

Systematic Studies of the Effect of a Bandpass Filter on a Josephson-Junction Noise Thermometer.

PB95-162970

03,939 Not available NTIS

Systematic Studies of the Effect of a Post-Detection Filter on a Josephson-Junction Noise Thermometer.

PB95-162988 03,940 Not available NTIS

Systematics of Alpha-Particle Energy Spectra and Lineal Energy (Y) Spectra for Radon Daughters.
PB94-185139

Systems Integration for Manufacturing Applications Program 1995 Annual Report.

02,844 PC A05/MF A01

Table of Dielectric Constants of Pure Liquids.

AD-A278 956/8 00,712 PC A04/MF AO

Tables of Chemical Kinetics Homogeneous Reactions.

AD-A280 293/2 00,715 PC A2O/MF A04

Tables of $X$-ray Mass Attenuation Coefficients and Mass Energy-Absorption Coefficients $1 \mathrm{keV}$ 10 $20 \mathrm{MeV}$ for Elements $Z=1$ to 92 and 48 Additional Substances of Dosimetric Interest.

04,013 PC A06/MF AO2

Tabulation of Data on Receiving Tubes. Handbook 68. AD-A285 495/8 02,111 PC A06/MF A02

Tabulation of the Thermodynamic Properties of Normal Hydrogen from Low Temperatures to $300 \mathrm{~K}$ and from 1 to 100 Atmospheres.

AD-A279 951/8

00,713 PC A05/MF A01

Taguchi's Parameter Design: A Panel Discussion.

PB96-111802 03,445 Not available NTIS

Tapered Cross-Pin Attachments for Fixed Bridges.

PB94-185238 00,185 Not available NTIS

Task Decomposition Methodology for the Design of a Coal Mining Automation Hierarchical Real-Time Control System.
PB94-185386

Taxonomy for Security Standards.

PB95-180386

01,602 Not available NTIS

TBT Agreement Activities of the National Institute of Standards and Technology, 1995

00.499 PC A04/MF A01

PB97-104178

SiO2 Films with Bimodal TDDB Characterizatio PB96-102926

02,381 Not available NTIS

Technical Digest: Symposium on Optical Fiber Measurements (8th), 1994. Held in Boulder, Colorado on September $13-15,1994$.

04,231 PC A10/MF A03
Technical Digest: Symposium on Optical Fiber Measurements (9th), 1996. Held in Boulder, Colorado on October 1

04,383 PC A12/MF A03

Technical Impact of the NIST Calibration Service for Electrical Power and Energy.

02,462 PC A05/MF A01

Technical Program Description Systems Integration for Manufacturing (SIMA)

02,819 PC A05/MF A01

Technical Program of the Factory Automation Systems Division 1993.
PB94-160819

02,805 PC A03/MF A01

Technique for Analyzing the Effects of Changes in Formal Specifications
PB96-160957

01,760 Not available NTIS

Technique to Evaluate Benchmarks: A Case Study Using

the Livermore Loops.
PB95-151320 04.577 Not available NTIS

Technologic Papers of the Bureau of Standards: Number 170. Pyrometric Practice.

AD-A279 282/8

$03,766 \mathrm{PC} \mathrm{A15/MF} \mathrm{A03}$

Technology Trends in Telecommunications: An Overview.
PB94-123080 01,462 PC A03/MF A01

Telecommunications Security Guidelines for Telecommunications Management Network. Computer Security.

PB96-139415 01,496 PC A03/MF A01

Telegraph Noise in Silver-Permalloy Giant Magnetoresistance Test Structures.
PB96-146717 04.763 Not available NTIS

TEM/Reverberating Chamber Electromagnetic Radiation Test Facility at Rome Laboratory.
PB96-155023 03,675 PC A05/MF A01

Temperature and Field Dependence of Flux Pinning in NbTi with Aritificial Pinning Centers.

PB96-112024

04,726 Not available NTIS

Temperature and Frequency Dependence of Anelasticity in a Nickel Oscillator
PB $96-137732$

03,689 Not available NTIS

Temperature and Relative Humidity Dependence of Radiochromic Film Dosimeter Response to Gamma and Electron Radiation

03,718 Not available NTIS

Temperature Dependence and Anharmonicity of Phonons on $\mathrm{Ni}(110)$ and $\mathrm{Cu}(110)$ Using Molecular Dynamics Simulations.
PB94-185477

04.449 Not available NTIS

Temperature Dependence and Magnetic Field Modulation of Critical Currents in Step-Edge SNS YBCO/Au Junctions.
PB96-111745

Temperature Dependence of the Gas and Liquid Phase UItraviolet Absorption Cross Sections of HCFC-123 (CF3CHCl2) and $\mathrm{HCFC}-142 \mathrm{~b}(\mathrm{CH}$

PB96-201033

03,298 Not available NTIS

Temperature Dependence of the Magnetic Excitations in Ordered and Disordered Fe72Pt28.

PB95-150223

04.563 Not available NTIS

Temperature Dependence of the Morphology of Thin Diblock Copolymer Films as Revealed by Neutron ReflectivPB94-172756

01,199 Not available NTIS

Temperature Dependence of the Rate Constants for Reac tion of Dihalide and Azide Radicals with Inorganic Reduc

PB95-162756

00.964 Not available NTIS

Temperature Dependence of the Rate Constants for Reac tion of Inorganic Radicals with Organic Reductants. PB94-198280

00,783 PC A02

Temperature Dependence of the Rate Constants for Reactions of the Carbonate Radical with Organic and Inorganic Reductants.

00,831 Not available NTIS

Temperature Dependence of the Rate Constants for Reactions of the Suliate Radical, SO4-, with Anions.

PB94-212172

00,828 Not available NTIS

Temperature Dependence of the Ultraviolet Absorption Cross Section of CF3I.

01,168 Not available NTIS

Temperature Dependence of Vortex Twin Boundary Interaction in Yitrium Banium Copper Oxide ( $\mathrm{YBa}$ Cu3O6+x).
$\mathrm{PB} 95-162442$

Temperature Dependent Ultraviolet Absorption Cross Sections of Propylene, Methylacetylene and Vinylacetylene.
PB96-204177
01,169 Not available NTIS

Temperature Increases in Aluminum Alloys during Mechani-

cal-Impact Tests for Oxygen Compatibility.
PB94-172962

03,316 Not available NTIS
Temperature-Induced Transition in Ductile Fracture Appearance of a Nitrogen-Strengthened Austenitic Stainless Steel. PB96-190269 03.221 Not available NTIS

Temperature of Optical Molasses for Two Different Atomic Angular Momenta.

03,881 Not available NTIS

Template-Driven Systems Development with IDEF: Enterprise Standards for Reuse.

PB96-160965

02,788 Not available NTIS

Tensile Creep of a Silicon Nitride Ceramic.

PB95-161303 03,049 Not available NTIS

Tensile Creep of Silicide Composites.

PB96-200803 03,183 Not available NTIS

Tensile Creep of Whisker Reinforced Silicon Nitride.

PB94-211984 03,142 Not available NTIS

Tensile Creep Testing of Structural Ceramics.

PB97-110464 03,105 Not available NTIS

Tensile Deformation-Induced Microstructures in Free-Standing Copper Thin Films.

04.715 Not available NTIS

Tension/Compression Creep Asymmetry in Si3N4

PB97-110258 03,096 Not available NTIS

Terahertz Detectors Based on Superconducting Kinetic In-

ductance.

02,160 Not available NTIS

Terahertz Shapiro Steps in High Temperature SNS Josephson Junctions.
PB95-169140

02,168 Not available NTIS

Terminal Invariant Description of Amplifier Noise.

PB95-153573 02,043 Not available NTIS

Terminally Anchored Chain Interphases: The Effect of Multicomponent, Polydisperse Solvents on Their Equilibrium Properties.

PB95-181079

01,273 Not available NTIS

Terminally Anchored Chain Interphases: Their PB95-18106

01,272 Not available NTIS

Tert-Butyl Hydroperoxide-Mediated DNA Base Damage in Cultured Mammalian Cells.

PB94-182003

03,644 Not available NTIS

Test of a Slow Off-Axis Parabola at lis Center of Curvature.
PB96-138482 04,352 Not available NTIS PB96-138482

Test of Newton's Inverse Square Law of Gravitation Using the 300-m Tower at Erie, Colorado.
PB95-202446

Test Optics Error Removal.

PB96-179536

04,377 Not available NTIS

Test Procedures for Advanced Insulation Panels.

PB97-111892

00,415 Not available NTIS

Test Structures for Determining Design Rules for Microelectromechanical-Based Sensors and Actuators. PB95-150488

02,105 Not available NTIS

Test Structures for the In-Plane Locations of Projected Features with Nanometer-Level Accuracy Traceable to a Coordinate Measurement System.

PB94-200565

02,313 Not available NTIS

Testability of Object-Oriented Systems.

PB95-242418

01,733 PC A05/MF A01

Testers Open Dialogue at Inaugural NIST Workshop.

PB95-175550 01,718 Not available NTIS

Testing Conformance and Interoperability of BACnet (Trade Name) Building Automation Products.

PB97-111553

00,310 Not available NTIS

Testing for Metrological Accuracy of the Electron Pump.

PB95-175873

04,663 Not available NTIS

Testing of Selected Self-Leveling Compounds for Floors.

PB95-220455

00,395 PC A03/MF A01

Testing the Sensitivity of Accelerometers Using Mechanical Shock Pulses Under NIST Special Publication 250 Test No. 24040 S

PB96-179544

02,683 Not available NTIS

Tetrahedral-Framework Lithium Zinc Phosphate Phases: Location of Light-Atom Positions in LiZnPO4 $\mathrm{H} 2 \mathrm{O}$ by Powder Neutron Diffraction and Structure Determination of LiZnPO 4 by ab Initio Methods.

PB96-160510

01,129 Not available NTIS

Text REtrieval Conference (4th) (TREC-4). Held in Gaithersburg, Maryland on November 1-3, 1995 . $99 /$ MF E08

Texture-Independent Vision-Based Closed-Loop Fuzzy Controllers for Navigation Tasks.

PB95-220505

00,183 PC A03/MF A01 


\section{TITLE INDEX}

Texture Measurement of Sintered Alumina Using the March-Dollase Function. $\quad 04,784 \quad$ Not available NTIS
PB96-179494 Texture Study of Two Molybdenum Shaped Charge Liners by Neutron Diffraction.

03,754 Not available NTIS

Textures of Tantalum Metal Sheets by Neutron Diffraction Textures of Tantalum Metal Sheets by Neutron Diffraction.
PB94-200169 03,399 Not available NTIS

Theoretical Analysis of the Coherence-Induced Spectral Shift Experiments of Kandpal, Vaishya, and Joshi. PB94-219383

00,561 (Order as PB94-219326, PC A05/MF A02) Theoretical Aspects of Tagged Photon Facilities.

PB94-216611 03,868 Not available NTIS

Theoretical Evaluation of the Vapor Compression Cycle with a Liquid-Line/Suction-Line Heat Exchanger, Economizer, and Ejector.
PB95-216917

02,607 PC A03/MF A01

Theoretical Form Factor, Attenuation and Scattering Tabulation for $Z=1-92$ from $E=1-10$ eV to $E=0.4-1.0 \mathrm{MeV}$.
PB96-145594 01,104 Not available NTIS

Theory for Quantum-Dot Quantum Wells: Pair Correlation
and Internal Quantum Confinement in and Internal
Nanoheterostructures. Quantum Confinement in
PB96-179445
02,437 Not available NTIS

tracold Temperatures. Theory of Atomic Collisions at Ultracold Temperatures.
PB94-212560 03,851 Not available NTIS Theory of Electron Beam Moire

(Order as PB96-175666, PC A07/MF A02)

Theory of Intelligent Systems.

$01,582 \mathrm{PC} \mathrm{A02}$

Theory of the Magneto-Optic Kerr Effect in the Near Field.
PB96-141387 04,761 Not available NTIS Thermal and Nonequilibrium Responses of Superconductors for Radiation Delectors.
PB $155-164232$

Thermal Anemonetry for Mass Flow Measurement in Oscillating Cryogenic Gas Flows.

04,218 Not available NTIS

Thermal Behavior of 4-Maleimidophenyl Glycidyl Ether Resins.

01,249 Not available NTIS

Thermal Behaviour of Methyl Methacrylate and N-Phenyl Maleimide Copolymers

01,246 Not available NTIS

Thermal Component Models for Electro-Thermal Network Simulation.

PB95-161022

02,346 Not available NTIS

Thermal Conductivity of Metals and Alloys at Low Temperatures. A Review of the Literature.
AD-A279 180/4 03,302 PC A04/MF A01

Thermal Conductivity of Polypyromellitimide Film with Alu-

mina Filler Particles from 4.2 10 $300 \mathrm{~K}$.
PB96-200753 Not available NTIS

Thermal Conductivity of R134a

PB94-213063

03,857 Not available NTIS

Thermal Decomposition of Hydroxy- and Methoxy-Substituted Anisoles.

00,767 Not available NTIS

Thermal Decomposition Pathways in Nitramine Propellants. AD-A295 896/5 03,753 PC A03/MF A01

Thermal Diffusivity of POCO AXM-5Q1 Graphite in the Range 1500 to $2500 \mathrm{~K}$ Measured by a Laser-Pulse Technique.
PB94-185022 03,013 Not available NTIS

Thermal Enhancement of Cotunneling in Ultra-Small Tunnel Junctions.

04,658 Not available NTIS

Thermal Equilibration Near the Critical Point: Effects Due to Three Dimensions and Gravity.
PB95-152922

Thermal Expansion of an SiC Particle-Reinforced Aluminum Composite.

03,144 Not available NTIS

Thermal Hydraulic Tests of a Liquid Hydrogen Cold Neutron Source. 03,884 PC A03/MF A01

Thermal Isolation of High-Temperature Superconducting Thin Films Using Silicon Wafer Bonding and $\begin{array}{ll}\text { Micromachining. } & 02,408 \text { Not available NTIS } \\ \text { PB96-135017 }\end{array}$

Thermal Modeling and Analysis of Laser Calorimeters. PB96-140405 04,354 Not available NTIS
Thermal Modeling of Absolute Cryogenic Radiometers. PB95-181236 04,316 Not available NTIS

Thermal Noise in High-Temperature Superconducting-Normal-Superconducting Step-Edge Josephson Junctions. PB95-175089

04,650 Not available NTIS

Thermal Pulse Study of the Polarization Distributions Produced in Polyvinylidene Fluoride by Corona Poling at ConStant Current.

01,195 Not available NTIS

Thermal Stability of Internal Electric Field and Polarization Distribution in Blend of Polyvinylidene Fluoride and

01,240 Not available NTIS

PB95-151072

ced Materials Using Mirage

Thermal Wave

PB96-204516

04,191 Not available NTIS

Thermal Wave Propagation in Diamond Films.

PB94-211489 03,015 Not available NTIS

Thermally Activated Hopping of a Single Abrikosov Vortex.
PB95-140810 04,548 Not available NTIS

95-140810

Thermoacoustic Technique for Determining the Interface

and/or Interply Strength in Polymeric Composites.
PB95-161824

Thermochemical and Chemical Kinetic Data for Fluorinated Hydrocarbons.

01,056 PC A09/MF A02

Thermochemical Studies of Inorganic Chalocogenides by Fluorine-Combustion Calorimetry: Binary Compounds of Germanium and Silicon with Sulfur, Selenium and Tellurium.

PB97-112528

01,181 Not available NTIS

Thermochemistry of the Hydrolysis of L-arginine to (Lcitrulline + Ammonia) and of the Hydrolysis of L-arginine to (L-ornithine + Urea)

03,463 Not available NTIS

PB95-150801

Thermochemistry of the Reactions between Adenosine,
Adenosine 5 '-monophosphate, Inosine, and Inosine $5^{\prime}$. monophosphate; the Conversion of L-histidine to (Urocanic Acid+Ammonia)

PB94-213113

03,460 Not available NTIS

Thermodynamic Analysis of Heparin Binding to Human Antithrombin.

03,455 Not available NTIS

Thermodynamic and NMR Study of the Interactions of Cyclodextrins with Cyclohexane Derivatives.

Pyclodextrins

00,781 Not available NTIS

Thermodynamic and Thermophysical Properties of Organic Nitrogen Compounds. Part II. 1- and 2-Butanamine, 2-Meth yl-1-Propanamine, 2-Methyl-2-Propanamine, Pyrrole, 1-, 2 and 3-Methylpyrrole, Pyridine, 2-, 3-, and 4-Methylpyridine Pyrrolidine, Piperidine, Indole, Quinoline, Isoquinoline, Acridine, Carbazole, Phenanthridine, i- and 2 dine, Carbazole, , Phenanthridine,

PB94-162294

00,741 Not available NTIS

Thermodynamic Assessment and Calculation of the Ti-Al System.
PB94-212644

03,337 Not available NTIS

Thermodynamic Behavior of the $\mathrm{CO} 2-\mathrm{H} 2 \mathrm{O}$ System from 400 to $1000 \mathrm{~K}$, up to $100 \mathrm{MPa}$ and $30 \%$ Mole Fraction of $\mathrm{CO} 2$

00,736 Not available NTIS

Thermodynamic Calculation of the Ternary Ti-Al-Nb System.

PB94-212636

03,336 Not available NTIS

Thermodynamic Constraints on Non-Equilibrium Solidification of Ordered Intermetallic Compounds.

PB94-198934 03,321 Not available NTIS

Thermodynamic Interactions and Correlations in Mixtures of Two Homopolymers and a Block Copolymers by Small Angle Neutron Scattering.

PB95-152872

01,247 Not available NTIS

Thermodynamic Interactions in Model Polyolefin Blends Obtained by Small-Angle Neutron Scattering.

PB94-198496 01,208 Not available NTIS

Thermodynamic Properties of Alkenes (Mono-Olefins Larger Than C4)

PB94-162237

00,735 Not available NTIS

Thermodynamic Properties of CF3-CHF-CHF2, 1,1,1,2,3,3Hexafluoropropane

PB97-118384

03,299 Not available NTIS

Thermodynamic Properties of CHF2-CF2-CHF, 1,1,2,2,3Pentafluoropropane.

PB97-118392

03,300 Not available NTIS

Thermodynamic Properties of CHF2-OCHF2,Bis(difluoromethyl) Ether.
PB94-199569 00,798 Not available NTIS Thermodynamic Properties of Difluoromethane. PB94-185204 Properties of Difluoromethane.
00,772 Not available NTIS
Thermodynamic Properties of Dilute and Semidilute Solutions of Regular Star Polymers.

PB96-146808

01,284 Not available NTIS

Thermodynamic Properties of Gas Phase Species of Imporance to Ozone Depletion.

00,126 Not available NTIS

Thermodynamic Properties of Gaseous Silicon Monotelluride and the Bond Dissociation Enthalpy $\mathrm{D}$ (sub m)(SiTe) at T approaches 0.

00,751 Not available NTIS

Thermodynamic Properties of $\mathrm{R} 134 \mathrm{a}(1,1,1,2$ Tetrafluoroethane). $\quad 00,988$ Not available NTIS
PB95-168704

Thermodynamic Properties of Silicides. 5. Standard Mola Enthalpy of Formation at the Temperature $298.15 \mathrm{~K}$ of Trimolybdenum Monosilicide Mo3Si Determined by Fluorine-Combustion Calorimetry.

PB97-119358

01,190 Not available NTIS

Thermodynamic Properties of Synthetic Otavite, $\mathrm{CdCO} 3(\mathrm{cr})$ : Enthalpy Increment Measurements from $4.5 \mathrm{~K}$ to $350 \mathrm{~K}$.
PB97-111447 00,680 Not available NTIS

Thermodynamic Properties of Synthetic Sapphire (alphaAl2O3), Standard Reference Material 720 and the Effect of Temperature-Scale Differences on Thermodynamic Prop-

Prties.
PB94-168564

00,750 Not available NTIS

Thermodynamic Properties of the Aqueous $\mathrm{Ba}(\sup 2+$ ) Ion and the Key Compounds of Barium.

PB96-145834 01,107 Not available NTIS

Thermodynamic Properties of the Aqueous lons $(2+$ and $3+$ ) of Iron and the Key Compounds of Iron.

PB96-145958 01,119 Not available NTIS

Thermodynamic Properties of the Group IIA Elements.

PB94-160983 00,730 Not available NTIS

Thermodynamic Properties of the Methane-Ethane System.
PB95-125779 00,891 Not available NTIS

Thermodynamic Properties of Two Gaseous Halogenated Ethers from Speed-ot-Sound Measurements Difluoromethoxy-Difluoromethane and 2-Difluoromethoxy 1,1,1-Trifluoroethane.

04,189 Not available NTIS

Thermodynamic Study of the Reactions of Cyclodextrins with Primary and Secondary Aliphatic Alcohols, with D- and L.Phenylalanine, and with L-Phenylalanineamide.

PB95-180873

01,016 Not available NTIS

Thermodynamics of Enzyme-Catalyzed Reactions: Part 1. Oxidoreductase

00,737 Not available NTIS

Thermodynamics of Enzyme-Catalyzed Reactions. Part 4 Lyases.

01,118 Not available NTIS

Thermodynamics of Enzyme-Catalyzed Reactions. Part 5. Isomerases and Ligases.

01,121 Not available NTIS

Thermodynamics of (Germanium + Selenium): A Review and Critical Assessment.

01,182 Not available NTIS

Thermodynamics of the Binding of Galactopyranoside Derivatives to the Basic Lectin from Winged Bean (Psophocarpus Tetrogonolobus). 03,465 Not available NTIS Thermodynamics of the Hydrolysis of N-Acetyl-L. phenylalanine Ethyl Ester in Water and in Organic Solvents.

Thermodynamics of the Hydrolysis of Penicillin $G$ and Ampi-

PBillin. 172467

03,596 Not available NTIS

Thermodynamics of the Hydrolysis of 3,4,53,4,5-Trinydroxybenzoic Acid (Gallic Acid) and Propan-1-o

in Aqueous Media and in Toluene.
PB96-186143 160 Not available NTIS

Thermophysical Properties of $\mathrm{CO} 2$ and $\mathrm{CO} 2-$ Rich Mixtures.

Thermophysical Properties of Fluids for the Gas Industry Thermophysical Properties of Fluids for the Gas Industry.
PB96-122437 02,494 Not available NTIS

Thermophysical Properties of Fluids for the Gas Industry. Final Report, February 1, 1988-August 31, 1993 .
PB94-146677
02,472 PC A03/MF A01

Thermophysical properties of HCFC alternatives. Quarterly report, April 1--June 30, 1995

DE96010579

03,250 PC A05/MF AO

Thermophysical properties of HCFC alternatives. Quarterly

report, October 1--December 31, 1995.
DE96010433
03,249 PC A04/MF A01

Thermophysical properties of HCFC alternatives. Quarterly report, 1 July 1994--30 September 1994.
DE95002261
03,247 PC A02/MF A01 


\section{TITLE INDEX}

Thermophysical Properties of HFC-143a and HFC-152a. Quarterly Report, 1 July 1993--30 September 1993. DE94004236

$03,245 \mathrm{PC} \mathrm{A03/MF} \mathrm{A01}$

Thermophysical Property Computer Packages from NIST. PB95-125787 04,203 Not available NTIS

Thermophysical Property Data for Supercritical Fluid Extraction Design.

00,668 Not available NTIS

Thermophysical Property Standard Reference Data from NIST.

01,153 Not available NTIS

Thin Dyed-Plastic Dosimeter for Large Radiation Doses PB95-107363 03,872 Not available NTIS

Thin Film Reaction Kinetics of Niobium/Aluminum Multilayers.
PB95-175295

04,651 Not available NTIS

Thin-Film Ruthenium Oxide - Iridium Oxide Thermocouples. PB97-110225 00,520 Not available NTIS

Thin Film Thermocouple Research at NIST.

PB97-110233 02,283 Not available NTIS

Thin Film Thermocouples for Measurement of Wall Tem-

peratures in Internal Combustion Engines.
PB94-172103 01,449 Not available NTIS

Thin Film Transparent Thermocouples.

PB94-185519 02,253 Not available NTIS

Think Metric.

PB95-151825

01,298 Not available NTIS

Third Generation Water Bath Based Blackbody Source.

PB96-122148 04,046

(Order as PB96-117767, PC A08/MF A02)

Three-Axis Coil Probe Dimensions and Uncertainties during Measurement of Magnetic Fields from Appliances. PB94-219359

01,885

(Order as PB94-219326, PC A05/MF A02)

Three Dimensional Position Determination from Motion

PB95-107108

01,788 Not available NTIS

Three-Vector Correlation Study of Orientation and Coherence Effects in $\mathrm{Na}\left(3 \mathrm{p},(2) \mathrm{P}_{1} / 2\right.$ inversely maps (2) $\left.\mathrm{P} 3 / 2\right)+\mathrm{He}$ Serniclassical and Quantum Calculations.

PB,979 Not available NTIS

Three-Vector Correlation Theory for Orientation/Alignment Studies in Atomic and Molecular Collision

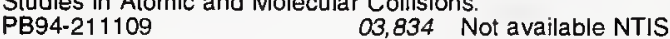

Threshold Electron Excitation of $\mathrm{Na}$

PB95-202917 03,994 Not available NTIS

Through-the-Arc Sensing for Measuring Gas Metal Arc Weld Quality in Real Time.

02,908 Not available NTIS

Through-the-Arc Sensing for Monitoring Arc Welding

PB94-185899 02,858 Not available NTIS

Through-the-Arc Sensing for Real-Time Measurement of Gas Meta! Arc Weld Quality.

PB95-162871

02,863 Not available NTIS

Tilt Effects in Optical Angle Measurements.

PB95-169389

04,294

(Order as PB95-169371, PC A07/MF A02)

Time and Frequency: Bibliography of NIST Publications, March 1995.

01,548 PC A06/MF A02

Time and Frequency Metrology.

PB96-190319 01,556 Not available NTIS

Time and Frequency Technology at NIST.

N94-30641/2

01,522

(Order as N94-30639/6, PC A25/MF A06)

Time-Dependent Dielectric Breakdown of Intrinsic $\mathrm{SiO} 2$ Films under Dynamic Stress.

02,438 Not available NTIS

Time Dependent Small Angle Neutron Scattering Behavio

in Triblock Copolymers Under Steady Shear.
PB94-172632 01,198 Not available NTIS

Time Dependent Vector Dynamic Programming Algorithm for the Path Planning Problem.

PB94-215688

03,428 PC A04/MF A01

Time-Domain Antenna Characterizations.

PB95-152781 02,003 Not available NTIS

Time-Domain Measurements of the Electromagnetic Backscatter of Pyramidal Absorbers and Metallic Plates.

PB94-185410 01,877 Not available NTIS

Time Domain Network Analysis Using the Multiline TRL Calibration.

02,065 Not available NTIS
Time Generation and Distribution

PB96-103049

01,550 Not available NTIS

Time-Perturbation Tuning of MIMD Programs.

01,681 PC A03/MF A01

Time-Perturbation Tuning of MIMD Programs.
PB94-172566

Time-Resolved Balmer-Alpha Emission from Fast Hydrogen

Atoms in Low Pressure, Radio-Frequency Discharges in

Hydrogen.

04,028 Not available NTIS

Time-Resolved Measurements of Energy Transfer at Sur-

faces.

00,913 Not available NTIS

Time-Resolved Measurements of Energy Transfer at SurPBas-153037

00,947 Not available NTIS

Time-Resolved Measurements of the Polarization State of Four-Wave Mixing Signals from GaAs Multiple Quantum Wells. PB96-201058

04,796 Not available NTIS

Time-Resolved Probes of Surface Dynamics.

PB94-199957 00,803 Not available NTIS

Time-Resolved Small-Angle Neutron Scattering Study of Spinodal Decomposition in Deuterated and Protonated Polybutadiene Blends. 1. Effect of Initial Thermal Fluctuations.

01,252 Not available NTIS

Time Scale Algorithm for Post-Processing: AT1 Plus Frequency Variance.

01,525 Not available NTIS

Tin Oxide Gas Sensor Fabricated Using CMOS MicroHotplates and In-situ Processing.
PB95-150603 00,580 Not available NTIS

TMACH Experiment Phase 1. Preliminary Developmental Evaluation.

01,618 PC A03/MF A01

Tolerance Intervals for the Distribution of True Values in the Presence of Measurement Errors.
PB95-150405 03,434 Not available NTIS

Not available NTIS

Tomographic Decoding Algorithm for a Nonoverlapping Redundant Array.

01,842 Not available NTIS

Tomographic Reconstruction of the Moments of Local Probability Density Functions in Turbulent Flow Fields.

ability Density Functions in Turbulent Flow Fields.
PB96-180195

Topological Influences on Polymer Adsorption and Desorption Dynamics.

PBesorption Dyna

01,227 Not available NTIS

Torsion Modulus and Internal Friction of a Fiber-Reinforced Composite.

03,070 Not available NTIS

Torsional Dilatometer for Volume Change Measurements on Deformed Glasses: Instrument Description and Measure-

ments on Equilibrated Glasses.
PB94-211166 03,379 Not available NTIS

Torsional Relaxation and Volume Response during Physical Aging in Epoxy Glasses Subjected to Large Torsional De-

PB95-140026

03,383 Not available NTIS

Total-Dielectric-Function Approach to Electron and Phonon Response in Solids.

01,067 Not available NTIS

Total Surface Areas of Group IVA Organometallic Compounds: Predictors of Toxicity to Algae and Bacteria.

PB94-211331

00,814 Not available NTIS

Toward a Reference Model Architecture for Real-Time Intelligent Control Systems (ARTICS).

PB94-172046

02,932 Not available NTIS

Poxicity, Fire

00,289 Not available NTIS

Trace Detection in Conducting Solids Using Laser-Induced Fluorescence in a Cathodic Sputtering Cell.

PB95-163424

00,598 Not available NTIS

Trace Element Concentrations in Cetacean Liver Tissues Archived in the National Marine Mammal Tissue Bank.

PB96-167127

02,595 Not available NTIS

Trace Elements Associated with Proteins. Neutron Activation Analysis Combined with Biological Isolation Technigues.

PB95-16310

00,597 Not available NTIS

Traceability to the Mole: A New Initiative by CIPM.

PB95-180246 00,605 Not available NTIS

Transfer of Technology from Defense to Civilian Sectors.

PB94-185360

00,011 Not available NTIS

Transformation of BCC and B2 High Tempere 'hases to HCP and Orthorhombic Structures in the i. ib Sys- tem. Part 1. Microstructural Predictions Based on a Subgroup Relation between Phases.

03,370

(Order as PB96-169057, PC A05/MF A01)

Transiormation of BCC and B2 High Temperature Phases to HCP and Orthorhombic Structures in the Ti-Al-Nb System. Part 2. Experimental TEM Study of Microstructures. PB96-169073

03,371

(Order as PB96-169057, PC A05/MF A01)

Transient Analysis of a Line-Focus Transducer Probing a Liquid/Solid Interface.

PB97-11868

02,763 Not available NTIS

Transient Cooling of a Hot Surface by Droplets Evaporation. PB94-156957

$03,783 \mathrm{PC} \mathrm{A08/MF} \mathrm{A02}$ Transient Cooling of a Hot Surface by Droplets Evaporation.
PB95-143194 03,890 PC A09/MF A02

Transient Creep Behaviour of Hot Isostatically Pressed Silicon Nitride.

03,086 Not available NTIS

Transient Errors in a Precision Resistive Divider.

PB97-111512 01,983 Not available NTIS

Transient Methods for Thermal Conductivity.

PB94-198405

04,197 Not available NTIS

Transient Subcritical Crack-Growth Behavior in Transformation-Toughened Ceramics.

PB94-200656

03,038 Not available NTIS

Transition from Localized Ignition to Flame Spread Over a Thin Cellulosic Material in Microgravity.
PB96-155809 04,835 Not available NTIS

04,835 Not ava

Transition Metal Implants in InO.53GA0.47As.
PB95-126389

Transition Regions of Capella.

Transition Regions of Capella.

00, 105 Not available NTIS

Transition Regions of Capella (1995)

PB96-176714

00,108 Not available NTIS

Transitions to Chaos Induced by Additive and Multiplicative PB96-155759

03,750 Not available NTIS

Transmission Properties of Short Curved Neutron Guides. Part 1. Acceptance Diagram Analysis and Calculations PB96-102199 04,021 Not available NTIS

Transmission/Reflection and Short-Circuit Line Methods for

Measuring Permittivity and Permeability.
PB94-165537 02,211 PC A06/MF A02

Transport and Diffusion in Three-Dimensional Composite Media.

PB95-176129 04,668 Not available NTIS

Transport by Gravity Currents in Building Fires.

PB97-119325 01,441 Not available NTIS

Transport Critical Current of Aligned Polycrystalline Yttrium

Barium Copper Oxide (YBa2Cu3O7-delta).
PB94-211307 04,492 Not available NTIS

Transverse Magnetoresistance: A Novel Two-Terminal Method for Measuring the Carrier Density and Mobility of a Semiconductor Layer.

PB95-150066

02,332 Not available NTIS

Transverse stress effect on the critical current of internal tin and bronze process $\mathrm{Nb}$ (sub 3)Sn superconductors.
DE95016659 04,434 PC A01/MF A01

Transverse Thermomagnetic Effects in the Mixed State and Lower Critical Field of High-Tc Superconductors.
PB95- 153250
04,590 Not available NTIS Trapped Atoms and Laser Cooling. PB95-151627

03,911 Not available NTIS

Trapped Ions and Laser Cooling 4: Selected Publications of the Ion Storage Group of the Time and Frequency Division, NIST, Boulder, Colorado.

PB96-172358

04,108 PC A11/MF A03

Trapped Vortices in a Superconducting Microbridge. PB95-141149

04,554 Not available NTIS

Treatment of Wistar Rats with a Renal Carcinogen, Ferric Nitrilotriacetate, Causes DNA-Protein Cross-Linking be-

tween Thymine and Tyrosine in Their Renal Chromatin.
PB96- 112115
03,649 Not available NTIS

Tree-Lookup for Partial Sums Or: How Can I Find This Stuff Ouickly.

PB96-179411

01,770 Not available NTIS

Tribochemical Reaction of Stearic Acid on Copper Surface PB94-212057

Tribological Behavior of 440/Diamond-Like-Carbon Film

Couples.

03,019 Not available NTIS 


\section{TITLE INDEX}

Tribological Characteristics of Alpha-Alumina at Elevated Temperatures
PB94-211018

02,963 Not available NTIS

Tribological Data: Needs and Opportunities.

PB95-140596 03,237 Not available NTIS Tribology Education: Present Status and Future Challenges.
PB94-212362 Tribometer for Measurements in Hostile Environments. PB95-180949 02,967 Not available NTIS Troublesome Crystal Structures: Prevention, Detection, and Resolution.
PB97-109151

01,172

(Order as PB97-109011, PC A11/MF A03)

Tunable High Temperature Superconductor Microstrip Res$\begin{array}{ll}\text { onators. } & 02,048 \text { Not available NTIS } \\ \text { PB95-168423 }\end{array}$ Tunneling Measurement of the Zero-Bias Conductance
Peak and the Bi-Sr-Ca-Cu-O Thin-Film Energy Gap.
PB95-163739 04,628 Not available NTIS
04, PB95-163739

04,628 Not available NTIS

Tunneling-Rotation Spectrum of the Hydrogen Fluoride

Dimer.
PB94- 198678

00,793 Not available NTIS

Tunneling Spectroscopy of bcc(001) Surface States.

PB96-155585 04,775 Not available NTIS

Tunneling Spectroscopy of Thallium-Based High-Temperaiure Superconductors.

PB95- 161709

04,606 Not available NTIS

Tunneling Stabilized Magnetic-Force Microscopy.

PB95-161717 04,607 Not available NTIS

Turbulent Flame Spread on Vertical Corner Walls

PB96-114764 01,403 PC A08/MF A02

Turbulent Spray Burner for Assessing Halon Alternative Fire Suppressants.
PB95-153748

01,385 Not available NTIS

Turbulent Upward Flame Spread on a Vertical Wall under External Radiation.

PB94-207388

00.341 PC A05/MF A01

Two-Dimensional POMMIE Carbon-Proton Chemical Shift Correlated (13)C NMR Spectrum Editing.
PB94-200508 00,809 Not available NTIS

Two-Dimensional Self-Consistent Radio Frequency Plasma Simulations Relevant to the Gaseous Electronics Con erence RF Reference Cell.

02,398

(Order as PB96-11331१, PC A09/MF A03)

Two New Probes for a Coordinate Measuring Machine.
PB95-163093 02,653 Not available NTIS

Two Numerical Techniques for Light Scattering by Dielectric Agglomerated Structures.

04,225

(Order as PB94-140555, PC A06/MF A02)

Two Phase Aqueous Extraction: Rheological Properties of Dextran, Polyethylene Glycol, Bovine Serum Albumin and Their Mixtures.
PB95-161998
Po,676 Not available NTIS

00,676 Not available NTIS

Two Principal Points of Symmetric, Strongly Unimodal Distributions.
PB95-203360

03.443 Not available NTIS

Two-Stage Integrated SQUID Amplifier with Series Array PB95-176277

02,061 Not available NTIS

Two-Tier Multiline TRL for Calibration of Low-Cost Network Analyzers.
PB96- 157888 01,947 Not available NTIS

A-type and Chemically Peculiar Stars.
PB96-123286
$00,101 \quad$ Not available NTIS

U.S. GOSIP Testing Program.

PB94-211455

01,807 Not available NTIS

U.S. Government Accreditation and Conformity Assessment System Evaluation.

02,678 Not available NTIS

U.S. Green Building Conference, 1994

PB94-206364

02,519 PC A08/MF A02

U.S. Navy Coordinate Measuring Machines: A Study of PB94-162831

02,807 PC A04/MF AO

Ultra-High Stability Synthesizer for Diode. Laser Pumped Rubidium.

01,527 Not available NTIS

Ultracold Collisions: Associative lonization in a Laser Trap.
PB94-213238

Ultrafast Time-Resolved Infrared Probing of Energy Transfer at Surfaces.
PB96-123443

00,620 Not available NTIS
Ultrafine Combustion Aerosol Generator.
PB94-200300 Not available NTIS

Ultralinear Small-Angle Phase Modulator.

PB95-168852 02,261 Not available NTIS

Ultrasensitive-Hot-Electron Microbolometer.

PB95-168985 02,163 Not available NTIS

Ultrasonic Measurement of Residual Stress in Cast Steel Railroad Wheels.

04,852 Not available NTIS

Ultrasonic Measurement of Residual Stress in Railroad Wheel Rims.

04,849 Not available NTIS

Ultrasonic Measurement of Sheet Anisotropy and Formability.

03,213 Not available NTIS

Ultrasonic Measurements of Surface Roughness

PB94-172137 04,181 Not available NTIS

Ultrasonic Method for Reconstructing the Two-Dimensiona

Liquid-Solid Interface in Solidifying Bodies.
PB95-16 1782

Ulirasonic Methods

PB96-190327

02,707 Not available NTIS

Ultrasonic NDE of Sprayed Ceramic Coatings

PB96-201157 02,761 Not available NTIS

Ultrasonic-Resonance Spectroscopy of Bulk and Layered

Solids.
PB96- 141338

04,759 Not available NTIS

Uitrasonic Sensing of GMAW: Laser/EMAT Defect Detection System.
PB96-186028

02,878 Not available NTIS

Uitrasonic Spectroscopy of Metallic Spheres Using Electro-

magnetic-Acoustic Transduction. 04,185 Not available NTIS
PB94-212537

Uitrasonic Technique for Sizing Voids Using Area FuncPB95-151916 02,904 Not available NTIS Ultrasound Power Measurement Techniques at NIST PB96-179569 02,684 Not available NTIS Ultraviolet Multiplet Table. AD-A278 446/0

00,710 PC A08/MF A02

Ultraviolet Multiplet Table. Finding List for Spectra of the Elements Molybdenum to Lanthanum $(Z=42$ to 57$)$; Hafnium to Radium ( $Z=72$ to 88 )

AD-A278 131/8

00,709 PC A03/MF A0

Ultraviolet Observations of Stellar Coronae: Early Results from HST.

PB94-213428

00,064 Not available NTIS

Uncertainties in Dimensional Measurements Made at Non-

standard Temperatures
PB94-169760

02,893 Not available NTIS

Uncertainties of Frequency Response Estimates Derived from Responses to Uncertain Step-Like Inputs.

01,984 Not available NTIS

Uncertainties of the NIST Coaxial Noise Calibration System PB96-111984 02,070 Not available NTIS

Uncertainty Analysis of the NIST Nitrogen Flow Facility. PB95-128906 02,608 PC A04/MF A01

Uncertainty Intervals for Polarized Beam Scattering Asymmetry Statistics.

03,825 Not available NTIS

Unconstrained Handprint Recognition Using a Limited LexiPB94-168051

$01,831 \mathrm{PC} \mathrm{A03/MF} \mathrm{A01}$

Unconventional Ferromagnetic Transition in La(sub 1 .

x) $\mathrm{Ca}(\mathrm{sub}$ x) $\mathrm{MnO} 3$
$\mathrm{PB} 97-112429$

04,156 Not available NTIS

Underflow-Induced Graphics Failure Solved by SLI Arithmetic.

01,712 Not available NTIS

Unexpected Effects of Gold on the Structure, Superconductivity, and Normal State of $\mathrm{YBa} 2 \mathrm{Cu} 307$. PB94-200284 04,482 Not available NTIS

Unified Approach to Camera Fixation and Vision-Based Road Following.

01,594 Not available NTIS

Unified Process Specification Language: Requirements for

Modeling Process.
PB97-116123

02,850 PC A06/MF A01

Unified Telerobotic Architecture Project (UTAP) Standard Interface Environment (SIE), May 1995.

PB95-242350

02,938 PC A09/MF A02

Uniform Laws and Regulations in the Areas of Legal Metrology and Motor Fuel Quality as Adopted by the 80 th Na- tional Conference on Weights and Measures 1995. 1996 Edition.

PB96-172309

02,927 PC A12/MF A03

Uniform Laws and Regulations in the Areas of Legal Metrology and Motor Fuel Quality, 1994 as Adopted by the 79th National Conference on Weights and Measures 1994.
PB95-174470 02,909 PC A09/MF A02

Unique Ouality Assurance Aspects of INAA for Reference Material Homogeneity and Certification.

PB96-200811 00,699. Not available NTIS Unpredictable Certainty. Information Infrastructure through
2000 .

PB96-182266

00,016 PC A14/MF A03

Unravel: A CASE Tool to Assist Evaluation of High Integrity Software. Volume 1. Requirements and Design.

PB95-267886 01,736 PC A05/MF A01

Unravel: A CASE Tool to Assist Evaluation of High Integrity Software. Volume 2. User Manual. PB95-267894

01.737 PC A04/MF A01

Unreacted Cement Content in Macro-Defect-Free Composites: Impact on Processing-Structure-Property Relations. PB96-141270 03,174 Not available NTIS

Unusual Spin-Trap Chemistry for the Reaction of Hydroxyl Radical with the Carcinogen N-Nitrosodimethylamine.
PB95-15 1643
00,692 Not available NTIS

Update on the Low Background IR Calibration Facility at the National Institute of Standards and Technology.

PB94-211224 04,232 Not available NTIS

Updated Calculations for Routine Space-Shielding Radiation

Dose Estimates: SHIELDOSE-2.
PB95-171039

04,838 PC A04/MF A01

Upgrade and Modernization Projects at the NBSR

PB96-161872 03,737 Not available NTIS Upgraded Facility for Multilayer Mirror Characterization at
NIST.

PB96-160387

04,367 Not available NTIS

Upward Flame Spread along the Vertical Corner Walls (October 1993)

PB94-206299

00,340 PC A03/MF A01

USA Interlaboratory Comparison of Superconductor Simulator Critical Current Measurements.

PB96-147194 04,770 Not available NTIS

Usability Engineering: Industry-Government Collaboration for System Effectiveness and Efficiency.
PB97-122287 Not available NTIS

USCEANIST Measurement Assurance Programs for the Radiopharmaceutical and Nuclear Power Industries

PB97-110514 03,721 Not available NTIS

Use of a Naphthylethylcarbamoylated- beta-Cyclodextrin Chiral Stationary Phase for the Separation of Drug Enantiomers and Related Compounds by Sub- and

Supercritical Fluid Chromatography.
PB97-113260

00,653 Not available NTIS

Use of a Radiochromic Detector for the Determination of

Stereotactic Radiosurgery Dose Characteristics.
PB94-185642
03,514 Not available NTIS

Use of an Environment Classification Model.

PB95-152062 01,709 Not available NTIS

Use of an Ion Energy Analyzer: Mass Spectrometer to Measure Ion Kinetic-Energy Distributions from RF Discharges in Argon-Helium Gas Mixtures.

PB94-185717 04,406 Not available NTIS

Use of Building Emulators to Evaluate the Performance of Building Energy Management Systems.

PB96- $111901 \quad 00,269$ Not available NTIS

Use of Computer Models to Predict Temperature and Smoke Movement in High Bay Spaces.
PB94-145976 00,191 PC A04/MF A01

Use of Extended Permutation-Inversion Groups in Constructing Hyperfine Hamiltonians for Symmetrical-Top Internal Rotor Molecules Like $\mathrm{H} 3 \mathrm{C}-\mathrm{SiH} 3$.

PB94-212032 00,823 Not available NTIS

Use of Ion Scattering Spectroscopy to Monitor the Nb Target Nitridation during Reactive Sputtering.
PB94-172525

Use of lonospheric Data in GPS Time Transfer.

PB95-163853 01,540 Not available NTIS

Use of Kinetic Energy Distributions to Determine the Relative Contributions of Gas Phase and Surface Fragmentation in KeV Ion Sputtering of a Ouaternary Ammonium Salt. PB95-126108 00,570 Not available NTIS

Use of Monte Carlo Modeling for Interpreting Scanning Electron Microscope Linewidth Measurements.

PB96-137807 02,413 Not available NTIS

Use of Neutron Beams for Chemical Analysis at NIST.

PB97- $112437 \quad 00,652$ Not available NTIS 


\section{TITLE INDEX}

Use of Pressure for Ouantum-Well Band-Structure CharacPBrization. 164058

04,779 Not available NTIS

Use of Sum Rules on the Energy-Loss Function for the Evaluation of Experimental Optical Data.
PB95-150736 04,264 Not available NTIS

Use of the Electronic Balance for Highly Accurate Direct Use of the Electronic Balance for Highly Accurate Direct
Mass Measurements Without the Use of External Mass Standards.

$03,803 \mathrm{PC} \mathrm{A03/MF} \mathrm{A01}$

Use of Thermal Mechanical Analysis to Characterize Ethylene-Propylene-Diene Terpolymer (EPDM) Roofing Membrane Materials.

00,360 Not available NTIS

User Interface Component of the Applications Portability Profile Category: Sottware Standard; Subcategory: Application Program Interface.

01,793 PC E01

User Profile for Researchers Studying Objects: Implications for Computer Systems.

00,133 PC A03/MF A01

User's Guide for the Algorithm Testing System Version 2.0.

02,916 PC A03MF AO

User's Guide for the PHIGS Validation Tests (Version 2.1).

PB94-165206 01,682 PC A08/MF A02

User's Guide to NIST SRM 2084: CMM Probe Performance Standard.

02,709 PC A03/MF A01

User's Guide to 'SuperFit' Modeling Software for CMM Probe Lobing.

02,921 PC A03/MF A01

User's Manual for the Program MONSEL-1: Monte Carlo Simulation of SEM Signals for Linewidth Metrology. PB95-111522

02,325 PC A03/MF A01
Powidth Metrology.

User Study: Informational Needs of Remote National Archives and Records Administration Customers.
PB95-154738 02,725 PC A06/MF A02

Using a Multi-Layered Approach to Representing Tort Law Cases for Case-Based Reasoning.
PB96-160874 Not available NTIS

Using Archie to Find Files on the INTERNET

PB95-168605 02,727 Not available NTIS

Using Atom Optics to Fabricate Nanostructures.

PB96-141247 04,757 Not available NTIS

Using Collocation in Three Dimensions and Solving a Model Semiconductor Problem.

01,952

(Order as PB96-159215, PC A07/MF A02)

Using Emulator/Testers for Commissioning EMCS Software, Operator Training, Algorithm Development, and Tuning Local Control Loops.

PB94-212735

00,245 Not available NTIS

Using Emulators to Evaluate the Performance of Building Energy Management Systerns.

00,259 Not available NTIS

Using Expect to Automate System Administration Tasks.

PB94-213329 01,697 Not available NTIS

Using Grafcet to Design Generic Controllers.

PB95-150827 02,821 Not available NTIS

Using Information Technology Standards in Federal Acquisitions. 161260

01,637 Not available NTIS

Using Logic to Specify Shapes and Spatial Relations in Design Grammars.

02,793 Not available NTIS

Using LORAN-C Broadcasts for Automated Frequency CaliPB94-216017

01,526 Not available NTIS

Using NIST Crystal Data within Siemens Sottware for FourCircle and SMART CCD Diffractometers.

PB97-109110

04,803

(Order as PB97-109011, PC A11/MF A03)

Using Ouantized Breakdown Voltage Signals to Determine the Maximum Electric Fields in a Ouantum Hall Effect SamPle.

02,375

(Order as PB95-261897, PC A07/MF A02)

Using S-Check, Alpha Release 1.0

PB96-165964

01,767 PC A05/MF A01

Using Secondary Ion Mass Spectrometry (SIMS) to Characterize Optical Waveguide Materials.

$$
04,340 \text { Not available NTIS }
$$

Using Synthetic-Perturbation Techniques for Tuning Shared Mermory Programs (Extended Abstract).
PB94-172657
Using Synthetic Perturbations and Statistical Screening to Assay Shared-Memory Programs.
PB96-103031 01,740 Not available NTIS Using Technology to Manage and Protect Intellectual PropPB97-112395 01,513 Not available NTIS Using Torsional Dilatometry to Measure the Effects of Deformations on Physical Aging. 03,384 Not available NTIS

Utc Dissemination to the Real-Time User.

(Order as N19960042616, PC A20/MF A04)

UV Absorption Cross Sections of Methylchloroform: Temperature-Dependent Gas and Liquid Phase Measurements.
PB96-201041 00,643 Not available NTIS

UV-Photopatterning of Alkylthiolate Monolayers Self-Assembled on Gold and Silver.

00,924 Not available NTIS

V-6: Effects of Temperature Variation.
PB96-148143 Not available NTIS

Vacuum Gauges and Partial Pressure Analyzers

PB95-150884 02,650 Not available NTIS
O

Validated Products List (Cobol, Fortran, ADA, Pascal, MUMPS, SOL).
PB94-937300

01,700 Standing Order

Validated Products List (Cobol, Fortran, ADA, Pascal, MUMPS, SOL).
PB95-937300

01,738 Standing Order

Validation of a Turbulent Spray Flame Facility for the Assessment of Halon Alternatives.
PB96-159728 03,283 Not available NTIS Validation of the Inverse Square Law of Gravitation Using the Tower at Erie, Colorado, USA.
PB95-164646 947 Not available NTIS

Validation Testing System Requirements. National PDES Testbed Report Series

PB94-163482

02,771 PC A03/MF A01

VAMAS Intercomparison of Critical Current Measurements

on NB3Sn Superconductors: A Summary Report.
PB96-119763 Not available NTIS

VAMAS interlaboratory comparisons of critical current vs. strain in $\mathrm{Nb}$ (sub 3) Sn

DE95016656

04,433 PC A01/MF A01

Van der Waals Bond Lengths and Electronic Spectral Shitts

of the Benzene-Kr and Benzene-Xe Complexes.
PB95-151387 00,932 Not available NTIS

Vapor-Liquid Equilibria of Mixtures of Propane and Isomeric Hexanes.
PB95-175287

00.995 Not available NTIS

Vapor-Liquid Equilibria of Ternary Mixtures in the Critical Region on Paths of Constant Temperature and Overall Composition.

01,139 Not available NTIS

Vapor Pressure of Pentafluorodimethyl Ether.
PB96-201199 00,677 Not available NTIS

Vapor Pressure of 1,1,1,2,2-Pentafluoropropane

PB97-113237 00,684 Not available NTIS

Vapor Pressure of 1,1-dichloro-2,2,2-trifluoroethane (R123).

PB95-126231 00,899 Not available NTIS

Vapor Pressures and Gas-Phase PVT Data for 1-Chloro1,2,2,2-Tetrafluoroethane (R124).
PB95-175154 271 Not available NTIS

Vapor Transport in Materials and Process Chemistry.
PB94-211745 00,669 Not available NTIS

Vapour Pressure Measurements on 1,1,1,2Tetrafluoroethane (R134a) from 180 to $350 \mathrm{~K}$.
PB95.168886
03,265 Not available NTIS

Vapour Pressures and Gas-Phase $(p$, rho $n, T)$ Values for CF3CHF2(R125)

PB96-102090

04,019 Not available NTIS

Variances in the Measurement of Ceramic Powder PropPrties.

03,100 Not available NTIS

Variant Design for Mechanical Artifacts-A State of the Art Survey.
PB94-154358

02,768 PC A03/MF A01

Variation in Magnetic Properties of Cu/Fcc (001) Sandwich Structures.

04,555 Not available NTIS

Variationally Stable Treatment of Two- and Three-Photon Detachment of $\mathrm{H}(-)$ Including Electron-Correlation Etfects.

Variations in Size Measurements by Indicating Gagıng Systems.

02,864 Not available NTIS
Vector and Ouasi-Vector Solutions for Optical Waveguide Modes Using Efficient Galerkin's Method with HermiteGauss Basis Functions.

04,357 Not available NTIS

Vector Theory of Diffraction by a Single-Mode Fiber: Application to Mode-Field Diameter Measurements.
PB95-164182 04,279 Not available NTIS

PB95-164182 04,279 Not available NTIS
Vehicle-Command Center Communications in a Robotic Ve-
hicle System. hicle System.
PB95-162723

03,665 Not available NTIS

Vehicle-to-Roadside Communications for Commercial Vehicle Operations: Requirements and Approaches.
PB95-188827 04,860 PC A05/MF A01

Velocity Distribution of Atomic Beams by Gated Optical Pumping.

01,542 Not available NTIS

VENTCF2: An Algorithm and Associated FORTRAN 77 Subroutine for Calculating Flow through a Horizontal Ceiling/Floor Vent in a Zone-Type Compartment Fire Model.
PB94-210127

Ventilation, Carbon Dioxide and ASHRAE Standard 62 . PB95-162095

02,544 Not available NTIS

Ventilation Effectiveness Measurements in Two Modern Office Buildings.

02,553 Not available NTIS

Ventilation Rates in Office Buildings.
PB95-108825

Verification and Validation.

PBg6-161393

01,765 Not available NTIS

Verification and Validation of Reengineered Software

PB96-161401 01,766 Not available NTIS

Verification of a Model of Fire and Smoke Transport.

PB95-108718 00,357 Not available NTIS

Verification of Commercial Probe-Tip Calibrations

PB95-161576

02,347 Not available NTIS

Verification of Revised Water Vapour Correction to the Index of Refraction of Air.

02,680 Not available NTIS

Verification of Scattering Parameter Measurements.

PB95.163960

01,904 Not available NTIS

Verification of the Ponderomotive Approximation for the ac Stark Shift in Xe Rydberg Levels.

PB94-185709

03,801 Not available NTIS

Vertical-Cavity Optoelectronic Structures: CAD, Growth, and Structural Characterization.

02,148 Not available NTIS

PB95-153177

asers: Structural CharacterVertical-Cavity Semiconductor Laser

PB95-153193

02,150 Not available NTIS

Vertical-Cavity Semiconductor Structures: Materials Charac-

terization.

02,149 Not available NTIS

Vibration Laboratory Automation at NIST with Personal Computers.

02,648 Not available NTIS

Vibration, Rotation, and Parity Specific Predissociation Dynamics in Asymmetric $\mathrm{OH}$ Stretch Excited ArH2O: A Halt Collision Study of Resonant V-V Energy Transfer in a Weakly Bound Complex.

00,872 Not available NTIS

Vibrational Branching Ra. tios ational Autoionization in $v(+)=3$ Threshold

$v(+)=3$ Threshold
PB94-199577

00,799 Not available NTIS

Vibrational Dependence of the Anisotropic Intermolecular Potential of Ar-HCl

PB95-202685

01,029 Not available NTIS

Vibrational Dependence of the Anisotropic Intermolecular Potential of Ar-HF.

PB95-202693

01,030 Not available NTIS

Vibrational Distributions of As2 in the Cracking of As4 on Si $(100)$ and Si(111).

PB94-198314

00,784 Not available NTIS

Vibrational Energy Transfer in S1 p-Difluorobenzene. A Comparison of Low and Room Temperature Collisions. PB95-108619

00,883 Not available NTIS

Vibrational Excitations and the Position of Hydrogen in Nanocrystalline Palladium.

PB96-111828

04,035 Not available NTIS

Vibrational Predissociation Dynamics of Overtone-Excited

HN3. 125720

00.691 Not available NTIS

Vibrational Relaxation Measurements of Carbon Monoxide on Metal Clusters.

PB94-211810

00,820 Not available NTIS 


\section{TITLE INDEX}

Vibrational Spectra of Molecular Ions Isolated in Solid Neon: HCCH+ and HCC-. (Reannouncement with New Availability Information).

00,707 PC A03/MF A01

Vibrational Spectra of Molecular Ions Isolated in Solid Neon. X. $\mathrm{H} 2 \mathrm{O}(+), \mathrm{HDO}(+)$, and $\mathrm{D} 2 \mathrm{O}(+)$.
PB95-125670 Vibrational Spectra of Molecular lons Isolated in Solid Neon. XI. NO2 (t), NO2(-), and NO3(-).
PB95-125688 Vibrational Spectra of Molecular lons Isolated in Solid Neon. 6. $\mathrm{CO} 4(-)$. (Reannouncement with New Availability Information)
AD-A238 $415 / 4$

$00,705 \mathrm{PC} \mathrm{A02/MF} \mathrm{A01}$

Vibrational Spectra of Molecular lons Isolated in Solid Neon. 7. $\mathrm{CO}(+), \mathrm{C} 2 \mathrm{O} 2(+)$, and $\mathrm{C} 2 \mathrm{O} 2(-)$. (Reannouncement with New Availability Information)

AD-A239 729/7

$00,706 \mathrm{PC} \mathrm{A03/MF} \mathrm{A01}$

Vibrational Spectra of Molecular lons Isolated in Solid $\mathrm{Neon} .11 . \mathrm{NO} 2(+), \mathrm{NO} 2(-)$, and $\mathrm{NO} 3(-)$.
$\mathrm{AD}-\mathrm{A} 275828 / 2$ Vibrational Spectra of Molecular lons Isolated in Solid Neon. 13. Ions Derived from $\mathrm{HBr}$ and $\mathrm{HI}$.
PB97-119234 Vibrationally Resolved Photoelectron Angular Distributions and Branching Ratios for the Carbon Dioxide Molecule in the Wavelength Region 685-795 Angstrom.

PB96-201207 04,131 Not available NTIS

Vibrations of Hydrogen and Deuterium in Solid Solution with Lutetium.
PB95-181038

01,019 Not available NTIS

Vibrations of $S 1((1) B 2 U)$ p-Difluorobenzene - d4. S1-So Fluorescence Spectroscopy and ab Initio Calculations.
PB95-202586 1,028 Not available NTIS PB95-202586 01,028 Not available NTIS Vibronic Coupling and Other Many-Body Effects in the 4sigmag(-1) Photoionization Channel of $\mathrm{CO} 2$.
PB95-162509 00,962 Not available NTIS

Video Microscopy Applied to Optical Fiber Geometry Meas$\begin{array}{lll}\text { urements. } & \text { 04,295 } & \text { PC A04/MF A01 }\end{array}$

Videoconferencing Procurement and Usage Guide.

PB94-217023
P1, 70 PC A04/MF A01

Viewpoint: Nanocrystalline and Nanophase Materials.

PB94-185865 04,454 Not available NTIS

Virial Coefficients of Five Binary Mixtures of Fluorinated Methanes and Ethanes.

PB96-156054

01,128 Not available NTIS

Virtual Environments for Health Care. A White Paper for the Advanced Technology Program (ATP), the National Institute of Standards and Technology. 03,594 PC A05/MF A01 Virtual Software Repository System.

PB94-198983

01,691 Not available NTIS

Viscosity of Ammonia.

PB96-145933

01,117 Not available NTIS

Viscosity of Defined and Undefined Hydrocarbon Liquids Calculated Using an Extended Corresponding-States Model.
PB96-167234

Viscosity of the Saturated Liquid Phase of Six Halogenated Compounds and Three Mixtures.

PB95-162368

00,960 Not available NTIS

Viscosity of 1,1,1,2,3,3-Hexafluoropropane and 1,1,1,3,3,3 Hexafluoropropane at Saturated-Liquid Conditions from 262 K to $353 \mathrm{~K}$.
PB96-176680

03,292 Not available NTIS

Visible and UV Light from Highly Charged Ions: Exotic Matter Advancing Technology.
PB96-119391

04,414 Not available NTIS

Visual Measurement Technique for Analysis of Nucleate Flow Boiling.
PB95-143301

03,262 PC A05/MF A02

Visual-Motion Fixation Invariant

PB94-206281

$01,836 \quad P C$ A03/MF A01

Visual Pursuit Systems.

PB95-143285

01,841 PC A03/MF A01

Visual Road Following without 3-D Reconstruction. PB95-161030 01,591 Not available NTIS

Visualization Applications for Manufacturing: A State-of-theArt Survey. Final Report.
PB94-194552

02,816 PC A03/MF A01

Visualization of Surface Figure by the Use of Zernike Polynomials.

04,351 Not available NTIS

Vitrification and Crystallization of Organic Liquids Confined

to Nanoscale Pores.
PB97-112304

03,392 Not available NTIS
VLSI Architectures for Template Matchıng and Block MatchPB94-200029 01,834 Not available NTIS Void Shape in Sintered Titanium. PB96-14 1023 02,705 Not available NTIS Voigt-Function Modeling in Fourier Analysis of Size- and Strain-Broadened X-Ray Diffraction Peaks.

PB94-198538 04,462 Not available NTIS
Nifraction Peaks.

Volatile Corrosion Inhibitors.

AD-A310 087/2

03,114 PC A03/MF A01

Voltage Gain in the Single-Electron Transistor.

PB95-176335 04,671 Not available NTIS

Voltage Ratio Measurements of a Zener Reference Using a Digital Voltmeter.

PB95-164695

01,906 Not available NTIS

Voltage-Standard Devices

PB96-102496

01,920 Not available NTIS

Volume-Limited ROSAT Survey of Extreme Ultraviolet Emission from all Nondegenerate Stars within 10 Parsecs.
PB96-103189 00,093 Not available NTIS

Volume Magnetic Hysteresis Loss of Nb3Sn Superconductors as a Function of Wire Length.
PB95-153722
04,597 Not available NTIS Volume Recovery in Epoxy Glasses Subjected to Torsional
Deformations: The Ouestion of Rejuvenation.

PB95-140018 03,382 Not available NTIS

Voluntary Product Standard PS 1-95 Construction and Industrial Plywood

03,406 PC A04/MF A01

Voluntary Product Standard PS 20-94. American Softwood Lumber Standard

$03,402 \mathrm{PC} \mathrm{A03/MF} \mathrm{A01}$

Vortex Dynamics and Melting in Niobium.

PB96-112362

02,073 Not available NTIS

Vortex Images in Thin Films of $\mathrm{YBa} 2 \mathrm{Cu} 3 \mathrm{O}$ (sub 7-x) and Bi2Sr2Ca1Cu2O (sub $8+x$ ) Obtained by Low-Temperature Magnetic Force Microscopy.

PB97-119408

04,815 Not available NTIS

Vortex Shedding Flowmeters for SSME Ducts.

PB95-169215 01,453 Not available NTIS

Wall Flame Heights with External Radiation.

PB95-163481 00,380 Not available NTIS

Warping of Terrace Pavers at the U.S. Capitol Building.
PB96-193651 00,411 PC A03/MF AO

Water Adsorption at a Polyimide/Silicon Wafer Interface

PB96-103197 01,070 Not available NTIS

Water Adsorption at Polymer/Silicon Waier Interfaces.

PB95-181178 01,022 Not available NTIS

Water Droplet Evaporation from Radiantly Heated Solids.

PB95-217147 00,394 PC A08/MF AO2

Water Efficient Plumbing Fixtures through Standards and Test Methods

00,248 Not available NTIS

Water Mist Fire Suppression Workshop Summary

PB95-161907

02,853 Not available NTIS

Water Permeability and Chloride Ion Diffusion in Portland Cement Mortars: Relationship to Sand Content and Critical Pore Diameter.

PB96-148036

03,193 Not available NTIS

Water-Vapor Measurements of Low-Slope Roofing Mate-

rials.
PB95-251617

00,399 PC A03/MF A01

Waveguide Polarizers Processed by Localized Plasma Etching.

PB95-169264

02,171 Not available NTIS Wavelength Dependence of Normal Spectral Emissivity of
High-Temperature Metals at Their Melting Point. PB94-200011

03,398 Not available NTIS

Wavelengths and Energy Level Classifications for the Spectra of Sulfur (S I through S XVI).
PB94-162229 00,734 Not available NTIS

Wavelengths and Energy Levels of Neutral $\operatorname{Kr}(84)$ and Level Shifts in All Kr Even Isotopes.

PB94-140605

03,780

(Order as PB94-140555, PC A06/MF A02)

Wavelengths and Isotope Shifts for Lines of Astrophysical Interest in the Spectrum of Doubly lonized Mercury (Hg III).
PB95-140869

Wavelengths of Spectral Lines in Mercury Pencil Lamps PB96-176474 04,376 Not available NTIS Wavelet Analysis for Synchronization and Timekeeping PB96-20038
Wavelet Variance, Allan Variance, and Leakage.

PB96-190111 01,509 Not available NTIS

Weak-Link-Free Behavior of High Angle $\mathrm{YBa} 2 \mathrm{Cu} 3 \mathrm{O} 7-x$ Grain Boundaries in High Magnetic Fields.

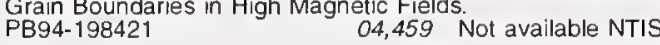

Wear Mechanism Maps of Ceramics.

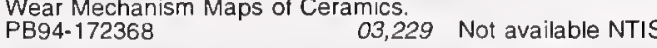

Wear Mechanism Maps of $440 \mathrm{C}$ Martensitic Stainless Steel PB96-111810 04,834 Not available NTIS

Wear Model for Alumina Sliding Wear.

PB95-163796 03,239 Not available NTIS

Wear Modeling of Si-Based Ceramics.

PB97-122501 03,112 Not available NTIS

Wear of Enamel against Glass-Ceramic, Porcelain, ano Amalgam.
PB96-179593

03,082 Not available NTIS

Wear of Human Enamel against a Commercial Castable Ceramic Restorative Material.
PB95-161972 00,161 Not available NTIS Wear of Selected Materials and Composites Sliding against
MoS2 Films.
PB94-172749 03,231 Not available NTIS PB94-172749

03,231 Not available NTIS
Alumina and Zirconia-Alu-

Wear Transitions in Monolithic Alumina and Zirconia-Alu-
mina Composites. mina Composites.
PB96-103163

03,168 Not available NTIS

Weatherability of Plastic Materials.

Welding for Cryogenic Service.
PB95-162889 2,852 Not available NTIS

$02,968 \mathrm{PC} \mathrm{A14/MF} \mathrm{A03}$

Well-Shielded EMAT for On-Line Ultrasonic Monitoring of GMA Welding

02,879 Not available NTIS

What Is a 'Standard Reference Material' - What Is Any RefPB96-186135

03,000 Not available NTIS

What's Available

02,875 Not available NTIS

White Papers Prepared for the White House: Construction Industry Workshop on National Construction Goals. Held on December 14-16, 1994.

PB95-216891

01,299 PC A08/MF A02

Whither Computational Materials Science. Some Thoughts from the Mechanical Prcperties Front.
PB96-161997 Not available NTIS

Wide Band Active Current Transformer and Shunt

PB95-126371 02,036 Not available NTIS

Wideband Current and Magnetic Field Sensors Based on Iron Garnets.
PB96-200878

01,975 Not available NTIS

Wideband Sampling Voltmeter

PB97-113039

01,990 Not available NTIS

Widths and Propagation of a Truncated Gaussian Beam. PB95-168779 04,287 Not available NTIS

Wind and Seismic Effects: Proceedings of the Joint Meeting of the U.S.-Japan Cooperative Program in Natural Re sources Panel on Wind and Seismic Effects (28th). Held in

Gaithersburg, Maryland on May $14-17,1996$.
PB97-104376 00,476 PC A99/MF A06

Wind and Seismic Effects. Proceedings of the U.S.-Japan Cooperative Program in Natural Resources Panel on Wind and Seismic Effects (26th). Held in Gaithersburg, Maryland on May 17-20, 1994

PB95-147385

00,433 PC A99/MF A06

Wind Load Provisions of the Manufactured Home Construction and Safety Standards: A Review and Recommendations for Improvement.

PB94-206125

00,428 PC A06/MF A02

Windowing Effects on Light Scattering by Sinusoidal Sur-

PB97-111215

04,389 Not available NTIS

Wire Bond Testing

PB94-211653

02,314 Not available NTIS

Wire Bonding to Multichip Modules and Other Soft SubStrates.

02,079 Not available NTIS

Wire Bonding to Multichip Modules and Other Sott Substrates.

02,082 Not available NTIS

Workgroup Summary Report: Plastic Hinge-Based Techniques for Advanced Analysis. $00,470 \quad$ Not available NTIS
PB96-159702 Working Conference on Global Growth of Technology: is America Prepared. Held in Gaithersburg. Maryland on December 7, 1995

PB96-210059

00,018 PC A07/MF AO2 
TITLE INDEX

Workplan to Analyze the Energy Impacts of Envelope Airtightness in Office Buildings. PB 96-154463

$00,273 \mathrm{PC} \mathrm{A04/MF} \mathrm{A01}$

Workshop Highlights. PB97-109227

04,811

(Order as PB97-109011, PC A11/MF A03)

Workshop on Characterizing Diamond Films (3rd). Held in Gaithersburg, Maryland on February 23-24, 1994. PB94-187663

$04,456 \mathrm{PC} \mathrm{A04/MF} \mathrm{A01}$

Workshop on Characterizing Diamond Films (4th). Held in Gaithersburg, Maryland on March 4-5, 1996. PB96-183090

Workshop on the Application of Virtual turing. Final Report. Held in Gaithersburg, Maryland on August 9,1994 PB95-173555

$02,825 \mathrm{PC} \mathrm{A11/MF} \mathrm{A03}$

Workshop Summary Report: Industrial Applications of Scanned Probe Microscopy. A Workshop Cosponsored by NIST, SEMATECH, ASTM, E42.14, and the American Vacum Society. Held in Gaithersburg, Maryland on March 24 25,1994

00,506 PC A07/MF A02

World Model Registration for Effective Off-Line Programming of Robots.

PB94-173010

02,933 Not available NTIS

World of Building Codes. PB 95-203428

$\infty, 449$ Not available NTIS

World Wide Web and Mosaic: User's Guide.

PB 94-207354 02,722 PC A03/MF A01

World Wide Web for Crystallography. PB97-109219

$X$-ray Attenuation Coefficients from $10 \mathrm{Kev}$ to $100 \mathrm{Mev}$.

AD-A278 139/1
$X$-Ray Attenuation Coefficients from $10 \mathrm{kev}$ to $100 \mathrm{Mev}$. 03,767 PC AO3/MF AQ

$X$-Ray Attenuation Coefficients from $10 \mathrm{KEV}$ to $100 \mathrm{MEV}$. AD-A280 290/8 03,768 PC A04/MF A01

$X-$ Ray Characterization of the Crystallization Process of High-Tc Superconducting Oxides in the Sr-Bi-Pb-Ca-Cu-O System.

04,579 Not available NTIS

$X$-Ray Diffraction from Anodic TiO2 Films: In situ and Ex situ Comparison of the Ti(0001) Face.

PB94-185972 00,782 Not available NTIS

$X$-Ray-Diffraction Study of a Thermomechanically Detwinned Single Crystal of YBa2Cu3O6+x.
PB95-151726 04,581 Not available NTIS

$X$-ray Emission from Chemically Peculiar Stars.

PB96-102256 00,088 Not available NTIS

$X$-Ray Image Quality Indicator Designed for Easy Alignmerit. 02,907 Not available NTIS

$X$-ray Mask Metrology: The Development of Linewidth Standards for X-ray Lithography. PB95-162129 02,348 Not available NTIS

$X$-ray Observation of Electroclinic Layer Constriction and Rearrangement in a Chiral Smectic-A Liquid Crystal. PB96-141080 01,100 Not available NTIS

$X$-ray Photoelectron and Auger Electron Forward Scattering: A Structural Diagnostic for Epitaxial Thin Films.
PB95-180220 04,676 Not available NTIS

$X$-Ray Photoelectron and Auger Electron Spectroscopy Study of Ultraviolet/Ozone Oxidized P2S5/(NH4)2S Treated GaAs(100) Surfaces. PB94-200144 04,479 Not available NTIS X-Ray Powder Diffraction Data for $\mathrm{BaCu}\left(\mathrm{C}_{2} \mathrm{O} 4\right) 2.6 \mathrm{H} 2 \mathrm{O}$. PB95-151767 04,583 Not available NTIS

X-ray Powder Diffraction from Carbon Nanotube and Nanoparticles. 03,064 Not available NTIS

$X$-ray Protection.

AD-A279 132/5

03,605 PC A03/MF A01

$X$-ray Protection Design.

AD-A279 181/2

03,606 PC A03/MF A01

X-ray Reflectivity Determination of Interface Roughness Correlated with Transport Properties of (AlGa)As/GaAs High Electron Mobility Transistor Devices. PB97-111868 04,149 Not available NTIS

$X$-rays from Stellar Flares.

PB94-213436

00,065 Not available NTIS

$X$ Window System, Version 11, Release 5 . PB96-169099

X.500 Directory Schema Design Handbook.

PB96-183041 02,739 PC A03/MF A01

xi-Vector Formulation of Anisotropic Phase-Field Models: 3D Asymptotic.

04,536 PC A03/MF A01

Xi-Vector Formulation of Anisotropic Phase-Field Models: 3D Asymptotic.

04,816 Not available NTIS

XUV Characterization Comparison of Mo/Si Multilayer Coatlings.
PB95-164000 04,278 Not available NTIS

YBa2Cu3O7-x to $\mathrm{Si}$ Interconnection for Hybrid Superconductor/Semiconductor Integration. PB94-211711 02,315 Not available NTIS

Z39.50 Implementation Experiences.

PB96-114939 01,816 PC A07/MF A02

Rim Plot and Its Analogs as Indicators of Vesicle and Micell Size Polydispersity.

01,094 Not available NTIS

TI -58 


\section{SAMPLE ENTRY}

\section{NISTIR-5439}

Performance Parameters of Fire Detection Systems

PB94-194339

00,123

PC A03/MF A01

\section{PB94-194339}

Performance Parameters of Fire Detection Systems

PB94-194339
00,123

PC A03/MF A01
Report or series number

Title

NTIS order number Abstract number

Availability

Price Code

\section{Report or series number}

Title

NTIS order number

Abstract number

Availability

Price code

\section{AD-A234 043/8}

Production and Spectroscopy of Small Polyatomic Molecular lons Isolated in Solid Neon. (Reannouncement with New Availability Information).

AD-A234 043/8

00,704 PC A03/MF A01

AD-A238 415/4

Vibrational Spectra of Molecular Ions Isolated in Solid Neon. 6. $\operatorname{CO} 4(-)$. (Reannouncement with New Availability Information)

AD-A238 415/4

00,705 PC A02/MF A01

AD-A239 729/7

Vibrational Spectra of Molecular Ions Isolated in Solid Neon. 7. $\mathrm{CO}(+), \mathrm{C} 2 \mathrm{O} 2(+)$, and $\mathrm{C} 2 \mathrm{O} 2(-)$. (Reannouncement with New Availability Information). AD-A239 729/7

00,706 PC A03/MF A01

AD-A244 582/3

Etfect of Green Density and the Role of Magnesium Oxide Additive on the Densitication of Alumina Measured by Additive on the Densilication of Aleannouncement with New Avalabily lntom

AD-A244 582/3

03,022 PC A02/MF A01

AD-A249 178/5

Evolution of the Pore Size Distribution in Final-Stage Sintering of Alumina Measured by Small-Angle X-ray Scattering g o Alumina Measured by Availability Information

AD-A249 178/5

03,023 PC A03/MF A01

\section{AD-A249 179/3}

Characterization of the Densification of Alumina by Multiple Small-Angle Neutron Scattering. (Reannouncement with New Availability Information) AD-A249 179/3

AD-A249 510/9

Small-Angle Neutron Scattering Characterization of Processing/Microstructure Relationships in the Sintering of Crystalline and Glassy Ceramics. (Reannouncement with New Availability Information).

AD-A249 $510 / 9$

$03,025 \mathrm{PC} \mathrm{A03/MF} \mathrm{A01}$ AD-A253 551/6

Vibrational Spectra of Molecular lons Isolated in Solid Neon: $\mathrm{HCCH}+$ and HCC-. (Reannouncement with New Availability Information).

AD-A253 $551 / 6$

$00,707 \mathrm{PC} \mathrm{A03/MF} \mathrm{AO}$

\section{AD-A274 609/7}

Gordon Research Conference on the Physics and Chemistry of Laser Diagnostics in Combustion Held in Plymouth, 12-16 July 1993.

AD-A274 609/7

$01,353 \mathrm{PC} \mathrm{A03/MF} \mathrm{A01}$

AD-A274 872/1

Ada Compiler Validation Summary Report: Certificate Number: 931029S1.11330, Digital Equipment Corporation, DEC Ada for DEC OSF/1 AXP Systems, Version 3.1, DEC 3000 Model 400 AXP Workstation, DEC 3000 Model 400 AXP Workstation.

AD-A274 872/1

$01,639 \mathrm{PC} \mathrm{A04/MF} \mathrm{A01}$
AD-A275 828/2

Vibrational Spectra of Molecular Ions Isolated in Solid $\mathrm{Neon}$ 11. $\mathrm{NO} 2(+)$. NO2(-), and $\mathrm{NO}_{3}(-)$. AD-A275 828/2 AD-A275 977/7

Ada Compiler Validation Summary Report: Certificate Number: 93121751.11336 Control Data Systems, Inc. NOSNE Ada, Version 1.4 Cyber 180-930-31 => Cyber 180-930-31. AD-A275 977/7 01,640 PC AO3/MF AO AD-A276 181/5

Ada Compiler Validation Summary Report. Certificate Number: 931119 S1.11332 DDC-1, Inc. DACS MIPS R3000 BamAd Cross Compiler System, Version 47.1 Sun

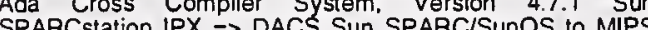
R3000 Bare Instruction Set Architecture Simulator. Version 4.7.1.
AD-A276 181/5

$01,641 \mathrm{PC} \mathrm{A04/MF} \mathrm{A01}$ AD-A276 283/9

Ada Compiler Validation Summary Report: Certificate Number: 93111951.11331 DDC-l. Inc. DACS Sun SPARC SunOS to 80386 PM Bare Ada Cross Compiler System Version 4.6.4 Sun Sparcstation 1+ $\Rightarrow>$ Bare Board iSBC $386 / 116$

AD-A276 283/9

AD-A277 981/7

01,642 PC AOB/MF A02

Ada Compiler Validation Summary Report. Certificate Number: $930927 S 1.11328$ Green Hills Software C Ada, Version 1.1 ZENY 386 => ZENY 386

$A D-A 277981 / 7$

01,643 PC A03/MF AO 
AD-A278 130\%

Atomic Energy Levels As Derived from the Analyses of Optical Spectra. Volume 1. Section 1. The Spectra of Hydro gen, Deuterium, Helium, Lithium Beryllium, Boron, Carbon, Nitrogen, Oxygen, and Fluorine.

$A D-A 278130 \%$

$03,764 \mathrm{PC}$ A05/MF A01

\section{AD-A278 131/8}

Ultraviolet Multiplet Table. Finding List for Spectra of the Elements Molybdenum to Lanthanum ( $Z=42$ to 57 ); Haf nium to Radium ( $Z=72$ to 88 )

AD-A278 131/8

$00,709 \mathrm{PC} \mathrm{A03/MF} \mathrm{A01}$

AD-A278 138/3

Bibliography of Books and Published Reports on Gas Tur(

AD-A278 138/3

$01.445 \mathrm{PC} \mathrm{A04/MF} \mathrm{A01}$

AD-A278 139/1

$X$-ray Attenuation Coefficients from $10 \mathrm{Kev}$ to $100 \mathrm{Mev}$

AD-A278 139/1

$03,765 \mathrm{PC} \mathrm{A04/MF} \mathrm{A01}$

AD-A278 140/9

Standard Materials. A Descriptive List with Prices.

AD-A278 140/9

00,500 PC A03/MF A0

AD-A278 213/4

Bibliography of Books and Published Reports on Gas Turbines, Jet Propulsion, and Rocket Power Plants, January 1950 through December 1953.

AD-A278 213/4

01,446 PC A06/MF A02

AD-A278 $249 / 8$

Characteristics of Turbulence in a Boundary Layer with Zero Pressure Gradient.

AD-A278 249/8

AD-A278 446/0

Ultraviolet Multiplet Table.

AD-A278 446/0

04,192 PC A03/MF A01

AD-A278 517/8

Density of Solids and Liquids.

AD-A278 517/8

$00,710 \mathrm{PC} \mathrm{A08/MF} \mathrm{A02}$

AD-A278 521/0

Aerodynamic Phenomena in Stellar Atmospheres - A BibliADraphy.

AD-A278 782/8

00,046 PC A05/MF A02

Handbook Preferred Circuits Navy Aeronautical Electronic Equipment. Supplement Number 3 . 00,026 PC A03/MF A01

\section{AD-A278 783/6}

Handbook Preferred Circuits Navy Aeronautical Electronic Equipment. Supplement Number

AD-A278 $783 / 6$

$00,027 \mathrm{PC} \mathrm{A03/MF} \mathrm{A01}$

$A D-A 278784 / 4$

Handbook Preferred Circuits Navy Aeronautical Electronic Equipment. Supplement Number 1 .
AD-A278 784/4 028 PC A05/MF A01

AD-A278 851/1

Methods of Measuring Humidity and Testing Hygrometers.
AD-A278 851/1 00,123 PC A03/MF A01

AD-A278 956/8

Table of Dielectric Constants of Pure Liquids.
AD-A278 956/8 00,712 PC A04/MF A0

$A D-A 279120 / 0$

Report of the International Commission on Radiological Units and Measurements (ICRU), 1956.
03,513 PC A04/MF A01 AD-A279 $120 / 0$

(1957 Part 3

Screw-Thread Standards for Federal Services, 1957. Part 3

AD-A279 121/8

AD-A279 132/5

X-ray Protection

AD-A279 133/3

Recommendations for the Disposal of Carbon-14 Wastes.

AD-A279 133/3

AD-A279 180/4

Thermal Conductivity of Metals and Alloys at Low Temperaures. A Review of the Literature.

AD-A279 $180 / 4$

$A D-A 279181 / 2$

$X$-ray Protection Design.

AD-A279 181/2

03,302 PC A04/MF A01

AD-A279 240/6

Standard Samples and Reference Standards Issued by the National Bureau of Standards.

AD.A279 240/6

AD-A279 261/2

Protection Against Radiations from Radium, Cobalt-60, and Cesium-137.

AD-A279 261/2

AD-A279 281/0

Permissible Dose from External Sources of Ionizing RadiAD-A279 281/0

AD-A279 282/8

03,608 PC A05/MF A0

Technologic Papers of the Bureau of Standards: Number metric Practice.

AD-A279 282/8

AD-A279 289/3

$03,766 \mathrm{PC} \mathrm{A15/MF} \mathrm{A03}$

$X$-Ray Attenuation Coefficients from $10 \mathrm{kev}$ to $100 \mathrm{Mev}$

AD-A279 289/3 03.767 PC A03/MF A01

\section{AD-A279 290/}

Screw-Thread Standards for Federal Services, 1957. Part 1. AD-A279 642/3

Ada Compiler Validation Summary Report: Certificate Num940325 S1.11348 DDC-I. DACS Sun SPARC/Solaris to (B)

Sun SPARCclassic $=>$ Intel iSBC $386 / 116$ (Bare Machine).

\section{AD-A279 643/1}

Ada Compiler Validation Summary Report: Certificate NumAer: 940325S1.11341 DDC-1, DACS Sun SPARC/SunOS to 80186 Bare Ada Cross Compiler System, Version 4.6.4 Sun SPARCstation IPX $\Rightarrow$ Intel iSBC 186100 (Bare 4 act AD-A279 643/1

Ada Compiler Validation Summary Report: Certificate Number: 940325S1.11349 DDC-I, DACS Sun SPARC/Solaris to 80386 PM Bare Ada Cross Compiler System with Rale Monotonic Scheduling, Version 4.6.4 Sun SPARCclassic $=>$ Intel iSBC 386/116 (Bare Machine)

AD-A279 644/9

AD-A279 645/6

Ada Compiler Validation Summary Report: Certificate Number: 940325 S1.11354 DDC-I. DACS Sun SPARC/Solaris Native Ada Compiler System, Version 4.6.2 Sun SPARCclassic $\Rightarrow$ Sun SPARCclassic.

AD-A279 645/6

$01,647 \mathrm{PC} \mathrm{A04/MF} \mathrm{A01}$

AD-A279 646/4

Ada Compiler Validation Summary Report: Certificate Number: $940325 S 1.11346$ DDC-I, DACS Sun SPARC/SunOS to 680x0 Bare Ada Cross Compiler System (BASIC MODE),
Version 4.6.9 Sun SPARCstation IPX $=>$ Lynwood j435TU Version 4.6.9 Sun SPARC

(68030) (Bare
AD-A279 $646 / 4$

AD-A279 757/9

01,648 PC A05/MF A02

Ada Compiler Validation Summary Report: Certificate Number: $940325 S 1.11343$ DDC-I. DACS Sun SPARC/Solaris to 80186 Bare Ada Cross Compiler System, Version 4.6.4 Sun SPARCclassic $\Rightarrow$ Intel iSBC $186 / 100$ (Bare Machine)

AD-A279 757/9

AD-A279 758/7

Ada Compiler Validation Summary Report: Certificate Number: 940325 S1.11344 DDC-I, DACS Sun SPARC/Solaris to 80186 Bare Ada Cross Compiler System with Rate Monotonic Scheduling, Version 4.6.4 Sun SPARCclassic $\Rightarrow$ ntel iSBC 186/100 (Bare Machine).

AD-A279 758/7

01,650 PC A06/MF A02

AD-A279 759/5

Characterization of the Hydrogen Induced Cold Cracking Susceptibility at Simulated Weld Zones in HSLA-100 Steel.

AD-A279 759/5

03,200 PC A04/MF AO

Ada Compiler Validation Summary Report: Certificate Number: 940325 S1.11347 DDC-I, DACS Sun SPARC/SunOS to $680 \times 0$ Bare Ada Cross Compiler System (SECURE MODE) Version 4.6.9 Sun SPARCstation IPX $\Rightarrow$ Lynwood j435TU 68030) (Bare Machine).

AD-A279 $778 / 5$

01,651 PC A06/MF A02

Ada Compiler Validation Summary Report: Certificate Number: 940325 S1.11342 DDC-I, DACS Sun SPARC/SunOS to 80186 Bare Ada Cross Compiler System with Rate Monotonic Scheduling Version 4.6.4 Sun SPARCstation IPX $\Rightarrow>$ Intel iSBC 186/100 (Bare Machine)

AD-A279 $779 / 3$

AD-A279 804/9

Ada Compiler Validation Summary Report: Certificate Number: 940325 S1.11351 DDC-I, DACS Sun SPARC/SunOS to Pentium PM Bare Ada Cross Compiler System with Rate Monotonic Scheduling, Version 4.6.4 Sun SPARCstation IPX $\Rightarrow>$ Intel Pentium (operated as Bare Machine) based in Xpress Desktop (Intel product number: XBASE6E4F-B

AD-A279 804/9

AD-A279 805/6

Ada Compiler Validation Summary Report: Certificate Number: 940325S1.11353 DDC-I, DACS Sun SPARC/Solaris to Pentium PM Bare Ada Cross Compiler System with Rate Monotonic Scheduling, Version 4.6.4 Sun SPARCclassic => Intel Pentium (operated as Bare Machine) based in Xpress Intel Pentium (operated as Bare Machine) bas)

Desktop (Intel product number: XBASE6E4F-B).
AD-A279 805/6 01,654 PC A06/MF A02 AD-A279 864/3

Ada Compiler Validation Summary Report: Certificate Number: 940325 S1.11350 DDC-1, DACS Sun SPARC/SunOS to Pentium PM Bare Ada Cross Compiler System, Version
4.6.4 Sun SPARCstation IPX $\Rightarrow$ Intel Pentium (Operated as 4.6.4 Sun SPARCstation IPX $\Rightarrow>$ Intel Pentium (Operated as
Bare Machine) Based in Xpress Desktop (Intel Product Bare Machine) Based in Xpress Desktop (Intel Product
Number: XBASE6E4F-B).
AD-A279 864/3 01,655 PC A06/MF A02

.

Refor Thermocouples.

$\begin{array}{ll}\text { RD-A279 948/4 } & 02,614 \\ \text { PC A05/MF A01 }\end{array}$

AD-A279 948/4

Corrected Optical Pyrometer Readings.
AD-A279 949/2 02,615 PC A05/MF A01

AD-A279 951/8

Tabulation of the Thermodynamic Properties of Normal Hydrogen from Low Temperatures to $300 \mathrm{~K}$ and from 1 to 100 Atmospheres.

AD-A279 95216

00,713 PC A05/MF A01

National Standard Petroleum Oil Tables.

AD-A279 952/6

02,469 PC A09/MF A02

AD-A280 082/9

Screw-Thread Standards for Federal Services, 1957. Handbook H28 (1957), Part 2. Revised.

$\mathrm{AD}-\mathrm{A} 280$ 082/9

03,599 PC A06/MF A02

AD-A280 086/0

Federal Basis for Weights and Measures: A Historical Review of Federal Legislative Effort, Statutes, and Administrative Action in the Field of Weights and Measures in the United States.

AD-A280 086/0

AD-A280 145/4

02,616 PC A03/MF A01

Ada Compiler Validation Summary Report: Certificate Number 940325 S1.11345 DDC-I. DACS Sun SPARC/SunOS to $680 \times 0$ Bare Ada Cross Compiler System, Version 4.6.9 Sun SPARCstation IPX $\Rightarrow$ Motorola MVME143 68030/6888? (Bare Machine).

AD-A280 150/4

Atomic Energy Levels in Crystals.

AD-A280 150/4

01,656 PC A05/MF A02

AD-A280 223/9

1950 Supplement to Screw-Thread Standards for Federal Services. 1944.

AD-A280 278/3

Guide to Instrumentation Literature.

AD-A280 278/3

AD-A280 279/1

Atomic Energy Levels. As Derived From the Analyses of Optical Spectra. Volume 3.

AD-A280 279/1

00,714 PC A13/MF A03

$A D-A 2802817$

Maximum Permissible Amounts of Radioisotopes in the Human Body and Maximum Permissible Concentrations in Air and Water.

AD-A280 282/5

$03,609 \mathrm{PC} \mathrm{A04/MF} \mathrm{A01}$

Maximum Permissible Body Burdens and Maximum Permissible Concentrations of Radionuclides in Air and in Water for Occupational Exposure.

AD-A280 282/5

03.610 PC A06/MF A02

AD-A280 290/8

X-Ray Attenuation Coefficients from $10 \mathrm{KEV}$ to $100 \mathrm{MEV}$.
AD-A280 290/8 03,768 PC A04/MF A01 AD-A2 $80290 / 8$

AD-A280 291/6

Papers on the Symposium on Collision Phenomena in Astrophysics, Geophysics, and Masers.

AD-A280 293/2

Tables of Chemical Kinetics Homogeneous Reactions. AD-A280 293/2

$00,047 \mathrm{PC}$ A03/MF A01

$A D-A 280$

Ada Compiler Validation Summary Report: Certificate Number: $940325 S 1.11352$ DDC-I DACS Sun SPARC/Solaries to Pentium PM Bare Ada Cross Compiler System, Version 4.6.4 Sun SPARCclassic $\Rightarrow$ Intel Pentium (Operated as Bare Machine) Based in Xpress Desktop (Intel Product Number: XBASE6E4F-B).

AD-A280 295/7

$01,657 \mathrm{PC} \mathrm{A06/MF} \mathrm{A02}$

AD-A280 398/9

Cryogenic Research and Development (Quarterly Report Number 2 for Period Ending December 31, 1960)

AD-A280 398/9

01,454 PCA05/MF A01

AD-A280 39977

Cryogenic Research and Development (Progress Report Number 4 for Period Ending December 31, 1961).

AD-A280 399/7

01,455 PC A04/MF A01

AD-A280 401/1

Cryogenic Research and Development (Quarterly Report Number 1 for Period Ending September 30,1960 ).
AD-A280 401/1 01,456 PC A04/MF A01 
AD-A286 619/2

lonospheric Radio Propagation.

AD-A286 619/2

$01,459 \mathrm{PC} \mathrm{A1O/MF} \mathrm{A03}$

Fuzed-Ouartz Fibers. A Survey of Properties, Applications and Production Methods.

AD-A286 62010

$00,656 \mathrm{PC}$ A03/MF A01

AD-A286 647/3

Measurement of Absorbed Dose of Neutrons, and of Mixtures of Neutrons and gamma rays.

AD-A286 647/3

03,710 PC A05/MF A01

AD-A286 648/1

High Temperature Reactions of Uranium Dioxide with Various Metal Oxides.

00,717 PC A03/MF A01

AD-A286 675/4

Cryogenic Materials Data Handbook.

AD-A286 675/4

$03,303 \mathrm{PC}$ A04/MF A01

AD-A286 680/4

Survey of the Literature on Heat Transfer from Solid Surfaces to Cryogenic Fluids

AD-A286 680\%

$04,193 \mathrm{PC} \mathrm{A04/MF} \mathrm{A01}$

AD-A286 681/2

Protection Against Neutron Radiation Up to 30 Million Electron Volts

AD-A286 683/8

$03,611 \mathrm{PC}$ A05/MF A01

Helium Refrigeration and Liquefaction Using a Liquid Hydrogen Refrigerator for Precooling.

AD-A286 701/8

02,749 PC A03/MF A01

Precise Measurement of Heat of Combustion with a Bomb Calorimeter.

AD-A288 571/3

$03,770 \mathrm{PC} \mathrm{A03/MF} \mathrm{A01}$

Ada Compiler Validation Summary Report: Certificate Number 940902 S1.11377 UNISYS Corporation. IntegrAda for Windows NT Version 10. Intel Deskside Server with Intel $80486 \mathrm{D} \times 266 \Rightarrow$ Intel Deskside Server with Intel $80486 \mathrm{D} \times 266$

$\mathrm{AD}-\mathrm{A} 288571 / 3$

AD-A288 572/1

$01,658 \mathrm{PC} \mathrm{A03/MF} \mathrm{A01}$

Ada Compiler Validation Summary Report: Certificate Number $940902 S 1.11376$. UNISYS Corporation IntegrAda for Windows NT, Version 1.0. Intel Deskside Server for Intel Pentium $60 \mathrm{MHz} \Rightarrow$. Intel Deskside Server with Intel Pentium $60 \mathrm{MHz}$

AD-A288 572/

$01,659 \mathrm{PC} \mathrm{A03/MF} \mathrm{A01}$

AD-A288 573/9

Ada Compiler Validation Summary Report: Certificate Number 94101251.11379 TISOFT, Inc. Green Hills Optimizing Ada Compileer, Version 1.8.7 with PATCK ID 1 COMPAO ProLiiant 200

AD-A288 573/9

$01,660 \quad P C$ A03/MF A01

AD-A288 574/7

Ada Complier Validation Summary Report: Certificate Number: $940929 S 1.11378$. Digital Equipment Corporation DEC Model 400 AXP Workstation $\Rightarrow$ DEC 3000 Model 400 AXP Workstation.

AD-A288 $574 / 7$

01,661 PC A05/MF A01

AD-A289 895/5

Ada Compiler Validation Summary Report. Certificate Number $941117 \mathrm{~S} 1.11380$. Electronic Data Systems Corp. Com-
piler: OC Systems Legacy Ada/370, Release 1.4.1 (without piler: OC Systen

AD-A289 895/5

01,662 PC A05/MF A01

AD-A292 039/5

Comparison of Meteor Activity with Occurrence of Sporadic E Reflections.

AD-A292 471/0

$00,116 \mathrm{PC} \mathrm{A01/MF} \mathrm{A01}$

Higher-Order Approximations in lonospheric Wave-Propagaion

AD-A292 471/0

04,420 PC A02/MF A01

AD-A292 502/2

Penetration and Diffusion of Hard X-rays through Thick Barriers. Ill. Studies of Spectral Distributions.
AD-A292 502/2 03,771 PC A01/MF A01

\section{AD-A293 709/2}

ADA Compiler Validation Summary Report, VC Number 950303S1.11381. Digital Equipment Corporation - Compiler Name: DEC Ada for OpenVMS Alpha Systems, Version 3.2. D.A294 088/0

Electrochemical Synthesis of Metal and Intermetallic Composites.

AD-A294 088/0

03,304 PC A04/MF A01

Further Calculations of $X$-ray Diffusion in an Infinite Medium.

AD-A295 314/9

$03,772 \mathrm{PC} \mathrm{A01/MF} \mathrm{A01}$

AD-A295 319/8

Low-Temperature Performance of Radiosonde Electric Hygrometer Elements

AD-A295 411/3

00,121 PC A02/MF A01

Arc Spectra of Gallium, Indium, and Thallium

AD-A295 411/3 00,718 PC A03/MF A01
AD-A295 578/9

Infrared and Near-Infrared Spectra of $\mathrm{HCC}$ and DCC Trapped in Solid Neon

AD-A295 578/9

$03,773 \mathrm{PC} \mathrm{A03/MF} \mathrm{A01}$

AD-A295 896/5

Thermal Decomposition Pathways in Nitramine Propellants.

AD-A295 896/5

03,753 PC A03/MF A01

AD-A296 061/5

Spectroscopic Study of Reaction Intermediates and Mechanisms in Nitramine Decomposition and Combustion.

AD-A296 061/5 03,774 PC A03/MF A01

AD-A296 $377 / 5$

Microwave Spectrum and Structure of $\mathrm{CH} 3 \mathrm{NO} 2-\mathrm{H} 2 \mathrm{O}$

AD-A296 377/5

$00,719 \mathrm{PC}$ A03/MF A01

AD-A296 498/9

Molecular Microwave Spectra Tables.

AD-A296 498/9

$00,720 \mathrm{PC}$ A03/MF A01

AD-A296 794/1

Ada Compiler Validation Summary Report, VC No 950609S1.11390 Digital Equipment Corporation - Compile Name: DEC Ada Version 3.2 for OpenVMS VAX Systems.

Physical Properties of Some Purified Aliphatic Hydrocarbons.

AD-A297 265/1

00,657 PC A03/MF A01

AD-A297 391/5

Air Flow in the Boundary Layer of an Elliptic Cylinder.

AD-A297 391/5

$04,194 \mathrm{PC} \mathrm{A03/MF} \mathrm{A01}$

AD-A297 420/2

Properties of Working Fluids for Thermoacoustic Refrig-

AD-A297 420/2

04,864 PC A06/MF A02

D-A297 905/2

Standard of Attenuation for Microwave Measurements.

AD-A297 905/2

$01,517 \mathrm{PC} \mathrm{A02/MF} \mathrm{A01}$

AD-A297 943/3

Crystal Diffraction Spectrometry for Accurate, Non-Invasive kV/Spectral Measurement for Improvement of Mammographic Image Ouality

00,721 PC A03/MF A01

$A D-A 301$ 258/0

Performance of Plastic Packaging for Hazardous Materials Transportation. Part 1. Mechanical Properties.

AD-A301 258/0 02,580 PC A04/MF A01

AD-A301 675/5

Weatherability of Plastic Materials.

AD-A302 669/7

Preliminary Subject and Authors Index to Compilations of Data on Properties of Materials.

AD-A302 669/7

AD-A302 670/5

$03,242 \mathrm{PC} \mathrm{A04/MF} \mathrm{A01}$

Preliminary List of References Containing Compilations of ata on Properties of Materials.

AD-A302 670/5

03,243 PC A05/MF A01

AD-A306 625/5

Fracture Testing of Large-Scale Thin-Sheet Aluminum Alloy. AD-A306 625/5

03,305 PC A04/MF A01

AD-A307 789/8

Synthesis of Thermally Stable Elastomers

AD-A307 789/8

03, $194 \mathrm{PC}$ A03/MF A01

AD-A310 087/2

Volatile Corrosion Inhibitors

AD-A310 087/2

$03,114 \mathrm{PC} \mathrm{A03/MF} \mathrm{A01}$

AD-A310 426/2

Polyvinyl Chloride (PVC) Plastic Drain, Waste, and Vent Pipe and Fittings.

$A D-A 310724 / 0$

00,326 PC A03/MF A01

Acrylonitrile-Butadiene-Styrene (ABS) Plastic Drain, Waste, and Vent Pipe and Fittings.

$A D-A 310724 / 0$

00,327 PC A03/MF A01

D-P009 114/0

Comparison of GPS Broadcast and DMA Precise Ephemerides.

AD-P009 132/2

NIST Internet Time Service.

AD-P009 132/2

$01,518 \mathrm{PC} \mathrm{A03/MF} \mathrm{A01}$

A-P009 138/9

Future of Time and Frequency Dissemination.

AD-P009 138/9

ANSUISO/EC-8652-1995

ADA; Category: Software Standard; Subcategory: Program

ming Language.

ARO-25664. 2- $\mathrm{CH}$

01,667 PC $\$ 278.00$

Production and Spectroscopy of Small Polyatomic Molecular lons Isolated in Solid Neon. (Reannouncement with New Availability Information).

AD-A234 043/8

$00,704 \mathrm{PC} \mathrm{A03/MF} \mathrm{AO}$

ARO-25664.9-CH

Vibrational Spectra of Molecular Ions Isolated in Solid Neon. 6. $\operatorname{CO} 4(-)$. (Reannouncement with New Availability AD-A238 415/4

00,705 PC A02/MF A01

ARO-25664.10- $\mathrm{CH}$

Vibrational Spectra of Molecular Ions Isolated in Solid Neon. 7. $\mathrm{CO}(+), \mathrm{C}_{2} \mathrm{O} 2(+)$, and $\mathrm{C}_{2} \mathrm{O} 2(-)$. (Reannouncemen with New Availability Information)

AD-A239 729/7

ARO-25664.12- $\mathrm{CH}$

00,706 PC A03/MF A01

Vibrational Spectra of Molecular Ions Isolated in Solid Neon: $\mathrm{HCCH}+$ and HCC-. (Reannouncement with New Availability Information)

AD-A253 551/6

00,707 PC A03/MF A01

ARO-261232-MS

Characterization of the Densification of Alumina by Multiple Small-Angle Neutron Scattering. (Reannouncement with New Avallability Information).

AD-A249 179/3

$03,024 \mathrm{PC} \mathrm{A02/MF} \mathrm{A01}$

-26123.3-MS

Effect of Green Density and the Role of Magnesium Oxide Additive on the Densification of Alumina Measured by Small-Angle Neutron Scattering. (Reannouncement with New Availability Information).

AD-A244 582/3

ARO-26123.4-MS

03,022 PC A02/MF AO

Evolution of the Pore Size Distribution in Final-Stage Sintering of Alumina Measured by Small-Angle X-ray Scattering. Reannouncement with New Availability Information)

AD-A249 178/5

ARO-26123.5-MS

Small-Angle Neutron Scattering Characterization of Processing/Microstructure Relationships in the Sintering of Crystalline and Glassy Ceramics. (Reannouncement with New Availability Information)

AD-A249 510/9

03,025 PC A03/MF A01

ARO-29596.1-CH

Microwave Spectrum and Structure of $\mathrm{CH} 3 \mathrm{NO} 2-\mathrm{H} 2 \mathrm{O}$

ARO-29596.2-CH

$00,719 \mathrm{PC} \mathrm{A03/MF} \mathrm{AO}$

Thermal Decomposition Pathways in Nitramine Propellants. AD-A295 896/5

ARO-30094.3-CH

$03,753 \mathrm{PC} \mathrm{A03/MF} \mathrm{AO}$

Vibrational Spectra of Molecular Ions Isolated in Solid Neon. 11. NO2(+), NO2(-), and NO3(-)

AD-A275 828/2

ARO-30094.4-CH

Infrared and Near-Infrared Spectra of HCC and DCC rapped in Solid Neon.

AD-A295 578/9

03,773 PC A03/MF AO

ARO-30094.5- $\mathrm{CH}$

Spectroscopic Study of Reaction Intermediates and Mechanisms in Nitramine Decomposition and Combustion.

AD-A296 061/5 03,774 PC A03/MF A01 ARPA-7829-2

Submarine Automation: Demonstration No. 5

PB95-251633
BUAER-16-1-519-SUPP-1

03,748 PC A03/MF AO

Handbook Preferred Circuits Navy Aeronautical Electronic Equipment. Supplement Number 1 .

AD-A278 784/4

BUAER-16-1-519-SUPP.2

Handbook Preferred Circuits Navy Aeronautical Electronic Equipment. Supplement Number 2 .

AD-A278 783/6

$00,027 \mathrm{PC} \mathrm{A03/MF} \mathrm{A01}$

A AM-A278

Structural Ceramics Database. Topical Report, June 1989- 


\section{CONF-9404137-5}

Ebulliometers for Measuring the Thermodynamic Properties of Fluids and Fluid Mixtures.

E94017817

04,195 PC A02/MF A01

CONF-9404137-6

Measurement of the Thermal Properties of Electrically Conducting Fluids Using Coated Transient Hot Wires.

DE94017816 00,722 PC A02/MF A01

CRPL-9-8

Standard of Attenuation for Microwave Measurements.

AD-A297 905'2

01,517 PC A02/MF A0

CS270-65

Acrylonitrile-Butadiene-Styrene (ABS) Plastic Drain, Waste, and Vent Pipe and Fittings.

AD-A310 724/0

$00,327 \mathrm{PC} \mathrm{A03/MF} \mathrm{A0}$

DE94-017162

Economic, Energy, and Environmental Impacts of the En-

ergy-Related Inventions Program
DE94-017162

00,008 PC A05/MF A02

DE93019682

Development of a Dual-Sinker Densimeter for High-Accuracy Fluid P.V-T Measurements. Appendix A.

DE93019682 02,620 PC A02/MF A01

DE30196

Dielectric Studies of Fluids with Reentrant Resonators. Appendix $\mathrm{B}$.

DE93019683

$03,244 \mathrm{PC} \mathrm{A02/MF} \mathrm{A01}$

DE93041307

Review of Corrosion Behavior of Ceramic Heat Exchanger Corrosion Characteristics of Silicon Silicon Nitride. Final Report, September 11, 1992--March 11,1993

DE93041307

03,228 PC A06/MF A02

DE 94004236

Thermophysical Properties of HFC-143a and HFC-152a. Quarterly Report, 1 July 1993--30 September 1993.

DE 94004236

DE94004399

$03,245 \mathrm{PC} \mathrm{A03/MF} \mathrm{A01}$

Development of Measurement Capabilities for the Thermophysical Properties of Energy-Related Fluids. Annual Report, December 1, 1990--November 30, 1991. DE 94004399

02,470 PC A02/MF A01
PO

DE94004400

Determination of Atomic Data Pertinent to the Fusion Energy Program. Progress Report for FY 92

DE 94004400

$04,402 \mathrm{PC} \mathrm{A02/MF} \mathrm{A01}$

DE94005988

Magnetic Characteristics and Measurements of Filamentary $\mathrm{Nb}-\mathrm{Ti}$ Wire for the Superconducting Super Collider.

DE94005988

03,775 PC AO2/MF AO1

DE94008991

Low Temperature H(sub 2)S Separation Using Membrane Reactor with Redox Catalyst

DE 94013170

$02,471 \mathrm{PC} \mathrm{A02/MF} \mathrm{A01}$

Ceramic Characterization.

DE94013170

DE94013486

Densification of Nano-Size Powders. 1994 Report

DE 94013486

DE94013593

Equipment for Investigation of Cryogenic Compaction of Nanosize Silicon Nitride Powders. 1993 Report

DE94013593

DE94014586

Measuring Performance of Parallel Computers. Final Report. 9014586

$01,665 \mathrm{PC} \mathrm{A03/MF} \mathrm{A01}$

DE94014587

Measuring Performance of Parallel Computers. Progress Report, 1989
DE94014587

DE94014709

$01,666 \mathrm{PC} \mathrm{A03/MF} \mathrm{AO}$

lonizing radiation-induced DNA damage and its repair in human cells. Progress report, (April 1, 1993--February 28, DE94014709

DE 94015308

$03,612 \mathrm{PC}$ A01/MF A01

DE94015308

DE94017331

Evaluation of the Environmentally Induced Fracture Resistance of Ductile Nickel Aluminide. Technical Report Number 1.Final report. Qclober-Decemb

DE94017331

03,306 PC A03/MF A01

DE94017332

Comparison of the Corrosion Rates of $\mathrm{FeAl}, \mathrm{Fe}$ (sub 3)Al and Steel in Distilled Water and $0.5 \mathrm{M}$ Sodium Chloride. Technical Report Number 2, January--March 1991.

DE94017332

03,186 PC AO2/MF A01

DE94017351

Evaluation of the Electrochemical Behavior of Ductile Nickel Aluminide and Nickel in a pH 7.9 Solution. Technical Report Number 3, April-June 1991.

DE9401735

$03,307 \mathrm{PC} \mathrm{A02/MF} \mathrm{A01}$

Development of Measurement Capabilities for the Thermophysical Properties of Energy-Related Fluids. Annual Report, December 1, 1993--November 30, 1994 .
DE94017738
03,246 PC A03/MF A01

\section{DE94017816}

Measurement of the Thermal Properties of Electrically Conducting Fluids Using Coated Transient Hot Wires.

DE 94017816

$00,722 \mathrm{PC} \mathrm{A02/MF} \mathrm{A01}$ DE94017817

Ebulliometers for Measuring the Thermodynamic Properties of Fluids and Fluid Mixtures.

DE94017817

\section{(2)}

04,195 PC AO2/MF A01

Atom-counting standards and Doppler-free resonance ionization mass spectroscopy. (Progress report)

00,723 PC AO2/MF AO

DE94018563

Improvement of Ultrasensitive Techniques Isotopic Biasing in the RIS Process Ionization Efficiencies and Selectivities. DE 94018563

$00,522 \mathrm{PC} \mathrm{A02/MF} \mathrm{AO}$

DE94018565

I: Improvement of Resonance lonization Spectroscopy (RIS) Techniques; II: Atomic Data for RIS; III: Standards for Ultratrace Analysis. Progress Report DE94018565

00,523 PC AO2/MF AO

DE94018566

Study of Laser Resonance Ionization Mass Spectrometry Using a Glow Discharge Source.

03,308 PC A01/MF A01

DE95002261

Thermophysical properties of HCFC alternatives. Quarterly report, 1 July 1994--30 September 1994.

DE 95002261

03,247 PC A02/MF A01

DE95004446

Electron-atom collision studies using optically state selected

beams. Progress report, May 15, 1987--May 14, 1988

DE $95004446 \quad 03,776$ PC AO3/MF A0

DE95004447

Electron-atom collision studies using optically state selected eams. Progress report, May 15, 1988--May 14, 1991.

DE 95004447

03,777 PC AO3/MF AO

DE95005780

Minutes of the CAALS Workshop on modularity and communications standards.

DE95005780

$02,621 \mathrm{PC}$ A03/MF A01

DE95007065

Electron transport calculations with biomedical and environmental applications. Final report, December 23, $1992-$-January $31,1994$.

DE95009287

$03,613 \mathrm{PC} \mathrm{A01/MF} \mathrm{AO}$

Atomic-scale characterization of hydrogenated amorphous silicon films and devices. Annual subcontract report, 14 February 1994--14 April 1995.

DE95009287

$02,294 \mathrm{PC}$ A03/MF A0

DE95011238

Lean flammability limit as a fundamental refrigerant prop erty. Phase 1, Interim technical report, 1 Qctober 1994--31 DE95011238

DE95011352

$03,248 \mathrm{PC} \mathrm{A03/MF} \mathrm{AO}$

Experimental plan to determine the performance of the Qak Ridge National Laboratory Cold Neutron Moderator. Final eport, September 1, 1993--November 30, 1993.

DE95011352

$03,778 \mathrm{PC} \mathrm{AO3/MF} \mathrm{A01}$

DE95011593

Measurements of quantum electrodynamic sensitive transitions in Na-like and $\mathrm{Cu}$-like ions: Final report, 1 October 1993--29 September 1994

DE95011593

04,403 PC A02/MF A01

DE95013079

Distributed measurements of tracer response on packed bed flows using a fiberoptic probe array. Final report. DE95013079 00,667 PC A03/MF A01 DE95013505

Low temperature fabrication from nano-size ceramic powDE 95013505

DE95015476

03,029 PC A05/MF A01

Electromechanical properties of superconductors for DQE fusion applications.

DE501

04,432 PC A04/MF A01

VAMAS interlaboratory comparisons of critical current vs. strain in Nb(sub 3)Sn.

DE95016656

DE95016659

04,433 PC A01/MF A01

Transverse stress effect on the critical current of internal tin and bronze process $\mathrm{Nb}$ (sub 3)Sn superconductors.

DE9501665

$04,434 \mathrm{PC}$ AO1/MF A0

\section{DE95017761}

Report of the Federal Internetworking Requirements Panel. DE9501776

03,407 PC A03/MF A01

DE96007979

Experimental assessment of absorbed dose to mineralized bone tissue from internal emitters: An electron paramagnetic resonance study.
DE96007979

DE96010065

03,614 PC A02/MF A01

Improving measurement quality assurance for photon irradiations at Department of Energy facilities. Final technical

report.
DE96010065

03,711 PC A03/MF A01

DE96010433

Thermophysical properties of HCFC alternatives. Quarterly Teport, October 1--December 31, 1995.

DE96010433

03,249 PC A04/MF A01

DE96010579

Thermophysical properties of HCFC alternatives. Quarterly report, April 1 1--June 30, 1995.

DE96010579

03,250 PC A05/MF AO

96014476

Small genomes: New initiatives in mapping and sequencing. Workshop summary report.

DOE/CE/23810-22A

$0.3,451$ PC A05/MF A01

Thermophysical Properties of HFC-143a and HFC-152a. Quarterly Report, 1 July 1993--30 September 1993.

DE94004236

$03.245 \mathrm{PC} \mathrm{A03/MF} \mathrm{A01}$

Thermophysical properties of HCFC alternatives. Quarterly report, 1 July 1994--30 September 1994.

\section{DOE/CE/23810-58}

$03,247 \mathrm{PC}$ AO2/MF AO

Lean flammability limit as a fundamental refrigerant property. Phase 1, Interim technical report, 1 Qctober 1994--31 March 1995.

DOE/CE/23810-61A

03,248 PC A03/MF A01

Thermophysical properties of HCFC alternatives. Quarterly report, April 1--June 30, 1995

DE96010579

03,250 PC A05/MF A01

DOE/CE/23810-66A

Thermophysical properties of HCFC alternatives. Quarterly report, Qctober 1--December 31, 1995.

DE96010433

03,249 PC A04/MF A01

DEE

Improving measurement quality assurance for photon irradiations at Department of Energy facilities. Fina! technical

DE96010065

03,711 PC A03/MF A01

DOE/EH/89334-T1

Experimental assessment of absorbed dose to mineralized bone tissue from internal emitters: An electron paramagnetic resonance study.

03,614 PC A02/MF A01

DOE/ER/13520-T

Electron-atom collision studies using optically state selected beams. Progress

DOE/ER/13520-T2

03,776 PC A03/MF A01

Electron-atom collision studies using optically state selected beams. Progress report, May 15, 1988--May 14, 1991

DE95004447

Distributed measurements of tracer response on packed 
DOE/ER/60826-T1

lonizing radiation-induced DNA damage and its repair in human cells. Progress report, (April 1, 1993--February 28 1994).

$03,612 \mathrm{PC} \mathrm{A01/MF} \mathrm{A01}$

DOE/ER/61529-T1

Electron transport calculations with biomedical and environmental applications. Final report, December 23, 1992--January 31,1994

DOEJEW/50009-T

$03,613 \mathrm{PC} \mathrm{A01/MF} \mathrm{A01}$

Minutes of the CAALS Workshop on modularity and communications slandards.

DE95005780

$02,621 \mathrm{PC} \mathrm{A03/MF} \mathrm{A01}$

DOE/OR/21941-T1

Evaluation of the Environmentally Induced Fracture Resistance of Ductile Nickel Aluminide. Technical Report Numbe 1. Final report. October-December 1990.

DE94017331

03,306 PC A03/MF A01

DOE/OR/21941-T2

Comparison of the Corrosion Rates of $\mathrm{FeAl}, \mathrm{Fe}(\mathrm{sub} 3) \mathrm{Al}$ and Steel in Distilled Water and $0.5 \mathrm{M}$ Sodium Chloride. Technical Report Number 2, January--March 1991.

DE94017332

03, 186 PC A02/MF A01

DOE/OR/21941-T3

Evaluation of the Electrochemical Behavior of Ductile Nicke Aluminide and Nickel in a pH 7.9 Solution. Technical Report Number 3, April-June 199

DE94017351

03,307 PC A02/MF A0

DOE/OR/22041-2

Densification of Nano-Size Powders. 1994 Report. DE94013486

03,027 PC A03/MF A01 DOE/OR/22041-T

Equipment for Investigation of Cryogenic Compaction of Nanosize Silicon Nitride Powders. 1993 Report.

DE94013593

03,028 PC A03/MF AO

DOE/OR/22041-T2

Low temperature fabrication from nano-size ceramic powders. 135013505

03,029 PC A05/MF A01

DOEIOR/22121-T1

Experimental plan to determine the performance of the Oak Ridge National Laboratory Cold Neutron Moderator. Fina report, September 1, 1993--November 30, 1993.

DE95011352

03,778 PC A03/MF A01

DOE/PC/92179-T1

Review of Corrosion Behavior of Ceramic Heat Exchanger Materals: Corrosion Characteristics of Silicon Carbide and Silicon Nitride. Final Report, September 11, 1992--March $11,1993$.

DOE/SF/20143-T2

$03,228 \mathrm{PC} \mathrm{A06/MF} \mathrm{A02}$

Measurements of quantum electrodynamic sensitive transivions in Na-like and Cu-like ions: Final report, 1 October 1993--29 September 1994.

DE95011593

04,403 PC A02/MF A01

DOT/FAAAR-95/11

Fracture Testing of Large-Scale Thin-Sheet Aluminum Alloy AD-A306 625/5

DOT-HS-808 214

$03,305 \mathrm{PC} \mathrm{A04/MF} \mathrm{AO}$

Model Minimum Perfo

Measurement Devices.

DOT/MTB/OHMO-76/4

04,861 PC A03/MF A01

Performance of Plastic Packaging for Hazardous Materials Transportation. Part 1. Mechanical Properties.

AD-A301 258/0

02,580 PC A04/MF A01

ED-376 823

Information Infrastructure: Reaching Society's Goals. A Report of the Information Infrastructure Task Force Committee on Applications and Technology.

ED-376 823

00,131 Not available NTIS

EIA-AS-466

Procedures for Document Facsimile Transmission Issued by General Services Administration, April 14, 1982. Federal Standard 1063.

EPA $600 / J-94 / 339$

01,516 PC E99

Histopathology, Blood Chemistry, and Physiological Status of Normal and Moribund Striped Bass ('Morone saxa Involved in Summer Mortality ("Die-Off") in the SacramentoSan Joaquin Delta of California.

PB94-198157

00,034 PC A03/MF A01

FEMA-202

Earthquake Resistant Construction of Electric Transmission and Telecommunication Facilities Serving the Federal Government Report

PB94-161817

FEMA-233

02,460 PC A03/MF A01

Earthquake Resistant Construction of Gas and Liquid Fuel Pipeline Systems Serving, or Regulated By, the Federal Government.

FHWA/RD-94/002

04,846 PC A05/MF A01

System for Calibration of the Marshall Compaction Ham-

mer.

01,303 PC A08/MF A02

FHWA/RD-94/053

Robotics Application to Highway Transportation. Volume 1 Final Report.

PB95-203790

03,654 PC A03/MF A01
FHWAVRD-94/054

Robotics Application 10 Highway Transportation. Volume 2. Literature Search.
PB95-170551

FHWARD-94/094

01,337 PC A06/MF A02

Robotics Application to Highway Transportation. Volume 3 Proposed Research Topics and CostBenefit Evaluations by

PB95-171633

01,338 PC A20/MF A04

FHWARD-94/095

Robotics Application to Highway Transportation. Volume 4 Proposals for Polential Research.

PB95-193173

FHWARD-94/174

01,339 PC A07/MF A02

Degradation of Powder Epoxy Coated Panels Immersed in a Saturated Calcium Hydroxide Solution Containing Sodium Chloride.

101050

01,344 PC A03/MF A01

FHWARD-95/063

Field Evaluation of the System for Calibration of the MarShall Compaction Hammer.

PB95-190674

01,323 PC A05/MF A01

Metropolitan Areas (Including MSAs, CMSAs, PMASs, and NECMAs). Category: Data Standards and Guidelines; Subcategory: Representations and Codes.

FIPS PUB 8-6

04,873 PC E09

FIPS PUB 10-4

Countries, Dependencies, Areas of Special Sovereignty, and Their Principal Administrative Divisions. Category: Dat

and Codes.

FIPS PUB 21.4

00,128 PC E05

COBOL. Category: Software Standard; Subcategory: Programming Language. Includes ANSI'S X3.23-1985, X3.23A 1989 and $\times 3.23 \mathrm{~B}-1993$

FIPS PUB 21-4

01,670 PC E99

FIPS PUB 21-4A

COBOL. Category: Software Standard; Subcategory: Programming Language. Part A

FIPS PUB $21-4 B$

01,671 PC E99

COBOL. Category: Software Standard; Subcategory: Programming Language. Part B.

01,672 PC E99

FIPS PUB 46-2

Data Encryption Standard (DES); Category: Computer Security; Subcategory: Cryplography.

FIPS PUB $46-2$

01,572 PC E04

Guideline: Codes for Named Populated Places, Primary County Divisions, and Other Locational Entities of the United States, Puerto Rico, and the Outlying Areas. Category: Data Standards and Guidelines. Subcategory: Representations and Codes.

FIPS PUB 55-DC3

$04.865 \mathrm{PCEO}$

Guideline: Cudes for Named Populatd Places, Primary County Divisions, and Other Locational Entities of the UnitCounty Divisions, and Other Locational Entities of the UnitData Standards and Guidelines; Subcategory: RepresentaData Standards

tion and Codes.

FIPS PUB 119-1

04,866 PC E99

ADA; Category: Software Standard; Subcategory: Programming Language.

FIPS PUB 120-1C

01,667 PC $\$ 278.00$

Graphical Kernel System (GKS). Category: Software Standard. Subcategory: Graphics. International Standard: Information Technology; Computer Graphics; Graphical Kernel mation Technology; Computer Graphics; Graph

System (GKS) Language Bindings. Part 4: C.
FIPS PUB 120-1C

FIPS PUB 140-1

Security Requirements for Cryptographic Modules; Category: Computer Security; Subcategory: Cryptography.
FIPS PUB 140-1

01,567 PC E05

PS PUB 148

Procedures for Document Facsimile Transmission Issued by General Services Administration, April 14, 1982. Federal Standard 1063
FIPS PUB 148

FIPS PUB 148
FIPS PUB 153-1

01,516 PC E99

Programmer's Hierarchical Interactive Graphics System (PHIGS). Category: Sottware Standard; Subcategory: Graphics.

FIPS PUB 153-1

01,668 PC E99

FIPS PUB 158-1

User Interface Component of the Applications Portability Profile Category: Software Standard; Subcategory: Application Program Interface.

FIPS PUB 158-1

01,793 PC E01

FIPS PUB 173-1

Spatial Data Transfer Standard (SDTS): Category: Software Standard; Subcategory: Information Interchange.
FIPS PUB 173-1 01,794 PC A04/MF A01

FIPS PUB 173-1A

01,794 PC A04/MF A01

Spatial Data Transfer Standard (SDTS). Category: Software Standard; Subcategory: Information Interchange. (FIPS PUB 173-1A

FIPS PUB $173-1 A$

01,795 PC $\$ 44.50$

FIPS PUB 173-18 Spatial Data Transfer Standard (SDTS). Category: Sottware
Standard; Subcategory: Information Interchange. (FIPS FIPS PUB 173-1B

FIPS PUB 180-1

Secure Hash Standard. Category: Computer Security.
FIPS PUB 180-1 01,568 PC E04 FIPS PUB 182

Integrated Services Digital Network (ISDN); Category: Telecommunications Standard; Subcategory: Integrated Services Digital Network.

FIPS PUB 182

$01,460 \mathrm{PCE}$ E3

Escrowed Encryption Standard (EES); Category: Computer Security; Subcategory: Cryptography.

$01,569 \mathrm{PCE} 03$

FIPS PUB 186

Digital Signature Standard (DSS). Category: Computer Security; Subcategory: Cryptography.

FIPS PUB 186
FIPS PUB 187

01,570 PC E04

Administration Standard for the Telecommunications Infrastructure of Federal Buildings. Category: Telecommunications Standard; Subcategory: Telecommunications Administration.

FIPS PUB 188

01,461 PC A02/MF A01

Standard Security Label for Information Transfer; Category: Computer Security; Subcategory: Security Labels.

FIPS PUB 188

FIPS PUB 189

Portable Operating System Interface (POSIX). Part 2. Shell and Utilities. Category: Software Standard; Subcategory: Operating Systems
FIPS PUB 189

FIPS PUB 190

01.797 PC A03/MF A01

Guideline for the Use of Advanced Authentication Technology Alternatives. Category: Computer Security. Subcategory: Access Control

01,798 PC A04/MF A01

FIPS PUB 191 Guideline for the Analysis of Local Area Network Security.
Category: Computer Security; Subcategory: Risk Analysis and Contingency Planning.

FIPS PUB 192

01,799 PC A04/MF A01

Application Profile for the Government Information Locator Service (GILS). Category: Software Standard; Subcategory: Information Interchange.

$01,800 \mathrm{PC}$ A03/MF A01

FIPS PUB 193

SQL Environments. Category: Software Standard; Subcategory: Database

FIPS PUB 194

01,801 PC A04/MF A01

Open Document Architecture (ODA) Raster Document Application Profile (DAP). Category: Software Standard; Subcategory: Graphics.

FIPS PUB 195 
GRI-94/0274

Constituents and Physical Properties of the $\mathrm{C} 6+$ Fraction of Natural Gas. Topical Report, April-June 1994 .

PB95-136644 02,483 PC A03/MF A01 GRI-94/0411

Evaluation of Wear Resistant Ceramic Valve Seats in GasFueled Power Generation Engines. Topical Report, December 1991-April 1994

PB95-2002 18

02,466 PC A07/MF A02

HANDBOOK-52

Maximum Permissible Amounts of Radioisotopes in the Human Body and Maximum Permissible Concentrations in Air and Water.

HANDBOOK-69

03,609 PC A04/MF A01

Maximum Permissible Body Burdens and Maximum Permis sible Concentrations of Radionuclides in Air and in Water for Occupational Exposure.

AD-A280 282/5

HUD-0006080

Controlling Moisture in the Walls of Manufactured Housing. PB95-105136

IIW-V-1046-95

00,355 PC A03/MF A0

IIW Commission V Ouality Control and Ouality Assurance of Welded Products Annual Report 1994/95.

PB95-198743 02,866 PC A03/MF A01

IIW-V-1059-9

IIW Commission V Ouality Control and Ouality Assurance of

Welded Products, Annual Report 1995/96.

PB96-191366

ISBN-0-16-043188-3

Putting the Information Infrastructure to Work: Report of the Information Infrastructure Task Force Committee on Applications and Technolog

N94-31228/7

02,715 PC A06/MF A02 Putting the Information Infrastructure to Work: Report of the Information Infrastructure Task Force Committee on Applications and Technology

PB94-163383

00,001 PC A06/MF A02

ISBN-0-16-045384-4

Checking the Net Contents of Packaged Goods as Adopted by the 79th National Conference on Weights and Measures,

1994, Third Edition, Supplement 4.
PB95-182226 00,484 PC A04/MF A01

ISBN-0-309-04982-2

Learning to Change: Opportunities to Improve the Performance of Smaller Manufacturers.

PB94-166212

00,010 PC A08/MF A02

ISBN-0-309-05252-1

Binder Characterization and Evaluation by Nuclear Magnetic Resonance Spectroscopy. 01,334 PC A08/MF A02
PB94-193471

ISBN-0-309-05432-X

Unpredictable Certainty. Information Infrastructure through 2000.

00,016 PC A14/MF A03

B96-182266

ISBN-0-309-05543-1

Financing Tomorrow's Infrastructure: Challenges and is sues. Proceedings of a Colloquium. Held in Washington, DC. On October $20,1995$.

PB96-189444

00,481 PC A07/MF A02

ISBN-0-309-05751-5

Optimization of Highway Concrete Technology.
PB94-182995 01,333 PC A13/MF A03

ISBN-1-886843-01-5

Proceedings of the Applied Diamond Conference 1995: Applications of Diamond Films and Related Materials International Conference (3rd). Held in Gaithersburg, Maryland,

on August 21-24, 1995
PB95-255204

04,701 PC A99/MF E11

N94-23605/

Activities of NIST (National Inst. Of Standards and Tech-

nology).

(Order as N94-23595/9, PC A21/MF A04)

\section{N94-25124/6}

Dynamic Measurements of Thermophysical Properties of Metals and Alloys at High Temperatures by Subsecond Pulse Heating Techniques.

03,309
(Order as N94-25120/4, PC A11/MF A03)

\section{N94-30641/2}

Time and Frequency Technology at NIST.

N94-30641/2 01,522

\section{N94-30660/2}

Comparison of GPS Broadcast and DMA Precise N94-30660/2

Corder as NG4-30639/6, PC A25

\section{N94-30684/2}

Future of Time and Frequency Dissemination.

N94-30684/2

(Order as N94-30639/6, PC A25/MF A06)

N94-31228/7

Putting the Information Infrastructure to Work: Report of the Information Infrastructure Task Force Committee on Applications and Technology.

02,715 PC A06/MF A02
N94-34037/9

Ground Vehicle Control at NIST: From Teleoperation to AuNonomy.

03,758

N94-35335/6

Classification of Advanced Technical Ceramics

N94-35335/6

$03,030 \mathrm{PC} \mathrm{A07/MF} \mathrm{A02}$

N94-36857/8

Open System Environments.

(Order as $\mathrm{N} 94-36853 /$, PC A10/MF A03)

N94-36858/6

Open System Environment Procurement.

N94-36858/6

02,716
(Order as $\mathrm{N} 94-36853 / 7, \mathrm{PC}$ A10/MF A03)

N95-14084/4

Partial Pressure Analysis in Space Testing.

N95-14084/4

(Order as N95-14062/0, PC A20/MF,827

N95-14548/8

Convection and Morphological Stability During Directional Solidification.
N95-14548/8

(Order as N95-14522/3, PC A20/MF,310

N95-15839/0

Investigating the 3.3 Micron Infrared Fluorescence from Naphthalene Following Ultraviolet Excitation.

N95-15839/0

(Order as N95-15827/5, PC A07/MF 724

N95-15937/2

Numeric Data Distribution: The Vital Role of Data Exchange in Today's World.

N95-15937/2

(Order as N95-15919/0, PC A11/MF A03)

N95-15938/0

National Center for Standards and Certification Information: Service and Programs.

N95-15938/0

(Order as N95-15919/0, PC A11/MF A03)

N95-19494/0

Fracture Behavior of Large-Scale Thin-Sheet Aluminum Alloy.

N95-19494/0

(Order as N95-19468/4,PC A24/MF A04)

N95-24130/3

Optical Storage Media Data Integrity Studies.

N95-24130/3

(Order as N95-24108, PC A17/MF 620

N95-26123/6

Measurement Methods and Standards for Processing and Application of Thermal Barrier Coatings.

N95-26123/6

01,447

N95-32323/4

Implementation of a Standard Format for GPS Common View Data.

N95-32323/4

(Order as N95-32319, PC A20/MF A04)

N96-15569/2

Combustion of a Polymer (PMMA) Sphere in Microgravity. N96-15569/2

(Order as N96-15552, PC A20/MF A04)

N96-15584/1

Ignition and Subsequent Transition to Flame Spread in a Microgravity Environment.

N96-15584/1

(Order as N96-15552, PC A20/MF A04)

\section{N19960042622}

Utc Dissemination to the Real-Time User.

N19960042622

(Order as N19960042616, PC A20/MF A04)

NASA-L-1247

Characteristics of Turbulence in a Boundary Layer with Zero Pressure Gradient.

AD-A278 249/8

04,192 PC A03/MF A01

NBS-CIRC-487

Density of Solid

NBS-CIRC-509-SUPPL

Bibliography of Books and Published Reports on Gas Turbines, Jet Propulsion, and Rocket Power Plants, January 1950 through December 1953

AD-A278 213/4

01,446 PC A06/MF A02

NBS-CIRC-552

Standard Samples and Reference Standards Issued by the National Bureau of Standards.

AD-A279 240/6

$02,613 \mathrm{PC} \mathrm{A03/MF} \mathrm{A01}$

NBS-CIRC-568

High Temperature Reactions of Uranium Dioxide with Various Metal Oxides.

NBS-CIRC-583-SUPP

$X$-Ray Attenuation Coefficients from $10 \mathrm{kev}$ to $100 \mathrm{Mev}$ AD-A279 289/3

$00,717 \mathrm{PC} \mathrm{A03/MF} \mathrm{A01}$

NBS-CIRCULAR-467

03,767 PC A03/MF A0

Atomic Energy Levels As Derived from the Analyses of Optical Spectra. Volume 1. Section 1. The Spectra of Hydro-

gen, Deuterium, Helium, Lithium Beryllium, Boron, Carbon, Nitrogen, Oxygen, and Fluorine.

03.764 PC A05/MF A01

NBS-CIRCULAR-509

Bibliography of Books and Published Reports on Gas Turbines, Jet Propulsion, and Rocket Power Plants.

AD-A278 138/3

01,445 PC A04/MF A01

NBS-CIRCULAR-583

$X$-ray Attenuation Coefficients from $10 \mathrm{Kev}$ to $100 \mathrm{Mev}$.

AD-A278 139/1

03,765 PC A04/MF A01

NBS-HB-H28-PT- 1

Screw-Thread Standards for Federal Services, 1957. Part 1. AD-A279 290/1

02,855 PC A 10/MF A03

NBS-HB-54

Protection Against Radiations from Radium, Cobalt-60, and Cesium-137.

AD-A279 261/2

03,607 PC A04/MF A01

Measurement of Absorbed Dose of Neutrons, and of Mixtures of Neutrons and gamma rays.

AD-A286 647/3

03,710 PC A05/MF A01

NBS-MR-241

Standard Materials. A Descriptive List with Prices. AD-A278 140/9

00,500 PC A03/MF A01

NBS-RP-2107

Molecular Microwave Spectra Tables.

AD-A296 498/9

00,720 PC A03/MF A01

NBS-TN-56

Bibliography of the Physical Equilibria and Related PropBome Cryogenic Systems.

AD-A281 167/7

$03,769 \mathrm{PC} \mathrm{A07/MF} \mathrm{A02}$

NBS-TN-124

Papers on the Symposium on Collision Phienomena in Astrophysics, Geophysics, and Masers.

AD-A280 291/6

00,047 PC A03/MF A01

NBS-34

Tables of Chemical Kinetics Homogeneous Reactions.

AD-A280 293/2 00,715 PC A20/MF A04

NBS-462

Ionospheric Radio Propagation.

AD-A286 619/2

NBS-470

Precision Resistors and Their Measurement.

AD-A284 623/6

02,249 PC A03/MF AO

NBS-512

Methods of Measuring Humidity and Testing Hygrometers.

AD-A278 851/1

$00,123 \mathrm{PC}$ A03/MF A01

NBS-514

Table of Dielectric Constants of Pure Liquids.

AD-A278 956/8

00,712 PC A04/MF A01

ABS-567

Guide to Instrumentation Literature.

AD-A280 278/3

$02,617 \mathrm{PC}$ A08/MF A02

NBS-569

Fuzed-Ouartz Fibers. A Survey of Properties, Applications and Production Methods.

AD-A286 620/0

NBS-652

00,656 PC A03/MF A01

Air Flow in the Boundary Layer of an Elliptic Cylinder.

AD-A297 391/5

04,194 PC A03/MF AO

NBS-6728

Cryogenic Research and Development (Ouarterly Report Number 1 for P

mber 30, 1960).

NBS-6736

Cryogenic Research and Development (Ouarterly Report Number 2 for Period Ending December 31, 1960)

AD-A280 $398 / 9$ 
NIST/BSS-172

Methodologies for Predicting the Service Lives of Coating Systems.

PB95-146387

03,124 PC A05/MF A01

NIST/BSS-173

Heat and Moisture Transfer in Wood-Based Wall Construction: Measured versus Predicted.

PB95-200655

NIST/BSS-175

Performance of Tape-Bonded Seams of EPDM Membranes: Comparison of the Peel Creep-Rupture Response of TapeBonded and Liquid-Adhesive-Bonded Seams.

$\begin{array}{ll}\text { PB96-183249 03,012 PC A05/MF A01 } & 0\end{array}$ NIST/GCR-92/603

Concurrent Flow Flame Spread Study.
PB 1,356 PC A08/MF A02

NIST/GCR-92/605

Numerical Analysis Support for Compartment Fire Modeling and Incorporation of Heat Conduction into a Zone Fire

PB94-156965

$01,357 \mathrm{PC} \mathrm{A04/MF} \mathrm{A01}$

NIST/GCR-92/610-1

Opportunities for Innovation: Advanced Manufacturing Technology.

NIST/GCR-93/6

02,801 PC A07/MF A02

Transient Cooling of a Hot Surface by Droplets Evaporation. PB94-156957

NIST/GCR-93/630

03,783 PC A08/MF A02

Experimental and Numerical Studies on Two-Dimensional Gravity Currents in a Horizontal Channel.
PB94-165941 PC A12/MF A03

01.359 PC A12/MF A03

NIST/GCR-93/631

Radiation and Mixing Properties of Buoyant Turbulent DiffuSion Flames.

NIST/GCR-93/633

Opportunities for Innovation: Biotechnology.
PB9 00,009 PC A12/MF A03

NIST-GCR-93-634

Fire Induced Thermal Fields in Window Glass I: Theory.

NIST/GCR-93/636

Time Dependent Vector Dynamic Programming Algorithm for the Path Planning Problem.

03,428 PC A04/MF A01

IST-GCR-93-637

Computational Model for the Rise and Dispersion of WindBlown, Buoyancy-Driven Plumes. Part 2. Linearly Stratified Atmosphere.

NIST/GCR-93/659

00.119 PC A03/MF A01

Opportunities for Innovation: Pollution Prevention

PB95-147146

02,520 PC A09/MF A02

NIST/GCR-94/638

Turbulent Upward Flame Spread on a Vertical Wall under Exernal Radiation.

PB94-207388

00,341 PC A05/MF A01

NIST/GCR-94/639

Development of the Fire Data Management System

PB94-206091

00,339 PC A03/MF A01

Opportunities for Innovation: Advanced Surface EngineerPBg4-176666

NIST/GCR-94/642

Prediction of Fire Dynamics

PB94-193620

NIST/GCR-94/643

02,697 PC A09/MF A02

Mathematical Modeling of Human Egress from Fires in Residential Buildings
PB94-193778

NIST/GCR-94/644

Fire Propagation in Concurrent Flows.

PB94-193844

NIST/GCR-94/645

Behavior of Charring Materials in Simulated Fire Environ. ments.

01,368 PC A99/MF A06

NIST/GCR-94/646

Backdraft Phenomena.

PB94-193927

NIST/GCR-94/647

Fire Growth Models for Materials. 01,367 PC A03/MF A01
PB94-195856

PB94-195856

NIST/GCR-94/648

Upward Flame Spread along the Vertical Corner Walls (October 1993).

NIST/GCR-94/649

00,340 PC A03/MF A01

Evaluation and Strengthening Guidelines for Federal Buildings: Identification of Current Federal Agency Programs.
PB94-176278 00.424 PC AO8/MF A02 NIST/GCR-94/650

Evaluation and Strengthening Guidelines for Federal Buildings: Assessment of Current Federal Agency Evaluation
Programs and Rehabilitation Criteria and Development of Typical Costs for Seismic Rehabilitation. 00,425 PC A08/MF A02
PB94-181856 NIST/GCR-94/651

Structure and Radiation Properties of Pool Fires.

PB94-193802

02,473 PC A08/MF A02

NIST/GCR-94/653

Investigation of Oil and Gas Well Fires and Flares.

PB94-193976

03,695 PC A04/MF A01

NIST/GCR-94/654

Federal Certification Authority Liability and Policy: Law and Policy of Certificate-Based Public Key and Digital SignaPB94-191202

NIST/GCR-94/656

01,578 PC A21/MF A04

Human Factors Considerations for the Potential Use of Elevators for Fire Evacuation of FAA Air Traffic Control TowPBs.

01,300 PC A03/MF A01 NIST/GCR-94/657

Framework for Information Technology Integration in Process Plant and Related Industries.

PB94-219086

Opportunities for Innovation: Software for Manufacturing

PB95-155578

NIST/GCR-94/661

Fundamental Mechanisms for $\mathrm{CO}$ and Soot Formation.

PB95-143160

01,380 PC A08/MF A02

NIST/GCR-94/662

Transient Cooling of a Hot Surface by Droplets Evaporation.
PB95-143194 IIST/GCR-94/663

Network Brokers Handbook: An Entrepreneurial Guide to Cooperative Strategies for Manufacturing Competitiveness.
PB95-219325 00,490 PC A08/MF A02

NIST/GCR-95/664

Burning Rate and Flame Heat Flux For PMMA in the Cone Calorimeter.

NIST/GCR-95/665

Water Droplet Evaporation from Radiantly Heated Solids.
PB95-217147

NIST/GCR-95/666

00,394 PC AO8/MF A02

Compartment Fire Combustion Dynamics. Annual Report September 1, 1993-September 1, 1994.
00,203 PC A04/MF A01 NIST/GCR-95/667

Assessment of Site Response Analysis Procedures.
PB95-210928
00,450 PC A07/MF A02 NISTIGCR-

Turbulent Flame Spread on Vertical Corner Walls. PB96-114764

01,403 PC A08/MF A02

NIST/GCR-95/670

Standards Policy and Information Infrastructure. PB95-231882

NIST/GCR-95/671

Mixing and Radiation Properties of Buoyant Turbulent Diffusion Flames.

NIST/GCR-95/672

Opportunities for Innovation: Optoelectronics

PB96-118039

NIST/GCR-95/673

Enhancements to Program IDARC: Modeling Inelastic Behavior of Welded Connections in Steel Moment-Resisting

PB95-231601

00,452 PC A05/MF A01

NIST/GCR-95/674

Comparison of the Seismic Provisions of Model Building Codes and Standards to the 1991 NEHRP Recommended rovisions.

PB95-231858

00,315 PC A05/MF A02

NIST/GCR-95/675

Testability of Object-Oriented Systems.

PB95-242418

01,733 PC A05/MF A01

NIST/GCR-95/676

New Mass Transport Elements and Components for the NIST IAO Model.

PB95-255899

NIST/GCR-95/677

02,558 PC A05/MF A01

Predicting the Ignition Time and Burning Rate of Thermo-

plastics in the Cone Calorimeter.

NIST/GCR-95/679

Review of Flows Driven By Natural Convection in Adiabatic

Shafts. 147897

NIST/GCR-95/681

Literature Review on Seismic Performance of Building Cladding Systems.

NIST/GCR-95/683

00,455 PC A09/MF A02

Sensitivity Analysis for Mathematical Modeling of Fires in Residential Buildings.

00,215 PC A03/MF A01

NIST/GCR-95/684

Enhancement of EXIT89 and Analysis of World Trade Center Data

PB96-202247

$00,231 \mathrm{PC} \mathrm{AO4/MF} \mathrm{A01}$

NIST/GCR-95/685

Chemical Inhibition of Methane-Air Diffusion Flame.

PB96-195532

01.431 PC AO4/MF AO

NIST/GCR-96/687

Sparse Water Sprays in Fire Protection.

PB96-202304

01,433 PC A13/MF A03

NIST/GCR-96/688

Dynamics, Transport and Chemical Kinetics of Compart

ment Fire Exhaust Gases

PB96-195508

$00,229 \mathrm{PC} \mathrm{A06/MF} \mathrm{AO}$

/GCR-96/690

Preliminary Processing of the Lotung LSST Data

PB96.165972

03,690 PC A05/MF AO

NIST/GCR-96/691

Mixing and Radiation Properties of Buoyant Luminous Flame Environments.

PB96-202254

01,432 PC A06/MF A01

IST/GCR-96/694

Post-Flame Soot.

PB96-193701

NIST/GCR-96/695

Development of an Economical Video Based Fire Detection and Location System.

NIST/GCR-96/697

Survey of Fue

NIST/GCR-96/698

Evaluation of Survey Procedures for Determining Occupan Load Factors in Contemporary Office Buildings.

PB97-116222

NIST/GCR-96/700

Estimation of System Damping at the Lotung Site by Appli-

cation of System Identification.

PB96-214697

NIST/GCR-96/701

$01,351 \mathrm{PC} \mathrm{A10/MF} \mathrm{A03}$

Energy-Based Method for Liquefaction Potential Evaluation. Phase 1. Feasibility Study

NIST/GCR-1

$03,691 \mathrm{PC} \mathrm{A13/MF} \mathrm{A03}$

Fire Protection Foam Behavior in a Radiative Environment.

PB97-116131

NIST/GCR-96/703

Full-Scale Room Fire Experiments Conducted at the University of Maryland.

NIST/HB-44

$00,236 \mathrm{PC} \mathrm{A04/MF} \mathrm{A01}$

NIST Handbook 44, 1994: Specifications, Tolerances and Other Technical Requirements for Weighing and Measuring Devices as Adopted by the 78th National Conference on Weights and Measures 1993.

PB94-136009

$02,888 \mathrm{PC} \mathrm{A11}$ /MF A03

NIST Handbook 44, 1995: Specifications, Tolerances and Other Technical Requirements for Weighing and Measuring Devices as Adopted by the 79th National Conference on Weights and Measures 1994

PB95-146379

$02,903 \mathrm{PC} \mathrm{A11/MF} \mathrm{A03}$

NIST/HB-105/2

Specifications and Tolerances for Reference Standards and Field Standard Weights and Measures. 2. Specification and Tolerances for Field Standard Measuring Flasks.

PB96-178926

NIST/HB-130-1995

Uniform Laws and Regulations in the Areas of Legal Metrology and Motor Fuel Ouality, 1994 as Adopted by the 79th National Conference on Weights and Measures 1994 . National Conference on Weights and Measures 1994.
PB95-174470 NIST/HB-130-1996

Uniform Laws and Regulations in the Areas of Legal Metrology and Motor Fuel Ouality as Adopted by the 80th $\mathrm{Na}$ tional Conference on Weights and Measures 1995. 1996 Edition.

PB96-172309

NIST/HB-133-ED-3-SUP-4

$02,927 \mathrm{PC} \mathrm{A12/MF} \mathrm{A03}$

Checking the Net Contents of Packaged Goods as Adopted by the 79 th National Conference on Weights and Measures, 1994, Third Edition, Supplement 4.

PB95-182226 
NIST/HB-150/3

National Voluntary Laboratory Accreditation Program: Bulk Asbestos Analysis.

PB95-138129

$02,541 \mathrm{PC} \mathrm{A04/MF} \mathrm{AO}$

NIST/HB-150-4

National Voluntary Laboratory Accreditation Program: Ionizing Radiation Dosimetry.

NIST/HB-150/5

03,623 PC A06/MF A02

National Voluntary Laboratory Accreditation Program: Construction Materials Testing.

$01,319 \mathrm{PC} \mathrm{A05/MF} \mathrm{A01}$

NIST/HB-150/6

National Voluntary Laboratory Accreditation Program: Carpet and Carpet Cushion.

NIST/HB-150/7

00,295 PC A04/MF A01

National Voluntary Laboratory Accreditation Program: POSIX. Portable Operating System Interface.
PB95-189478 02,661 PC A05/MF A01 PB95-189478
NIST/HB-150-8

National Voluntary Laboratory Accreditation Program Acoustical Testing Services.

PB95-182234

04,188 PC A05/MF A01

NIST/HB-150-9

National Voluntary Laboratory Accreditation Program (NVLAP): Wood Based Products.

PB95-170429

NIST/HB-150/10

03,405 PC A04/MF A01

Efficiency of Electric Motors. National Voluntary Lab. Ac-

creditation Program (NVLAP)

$02,107 \mathrm{PC} \mathrm{A05/MF} \mathrm{A01}$

National Voluntary Laboratory Accreditation Program: Electromagnetic Compatibility and Telecommunications. FCC Methods.

PB95-242376

NIST/HB-150/12

02,664 PC A06/MF A02

National Voluntary Laboratory Accreditation Program.

GOSIP: Government Open Systems Interconnection Profile.

NIST

01,486 PC A06/MF A02

Airborne Asbestos Analysis: National Voluntary Laboratory Accreditation Program.

PB96-147392

NIST/HB-150-15

02,566 PC A05/MF A01

National Voluntary Laboratory Accreditation Program: Thermal Insulation Materials.

NIST

02,977 PC A05/MF A01

National Voluntary Laboratory Accreditation Program

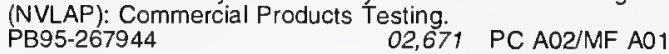

NIST/HB-150-18

National Voluntary Laboratory Accreditation Program

(NVLAP): Fasteners and Metals. NIST/MONO-179

Global Equivalence Ratio Concept and the Prediction of Carbon Monoxide Formation in Enclosure Fires.

PB94-207511 00,313 PC A08/MF A02

NIST/MONO-180

Gage Block Handbook

$02,667 \mathrm{PC} \mathrm{A08/MF} \mathrm{A02}$

NIST/PS-20/94

Voluntary Product Standard PS 20-94. American Softwood Lumber Standard.

PB94-162500

NIST-S-PUB-868

03,402 PC A03/MF A01

Information Infrastructure: Reaching Society's Goals. A Re-

port of the Information Infrastructure Task Force Committee

on Applications and Technology.

ED-376 823

NIST-SP-250-39

NIST Measurement Services: NIST Pressure Calibration

PB94-164043

NIST/SP-260

02,892 PC A06/MF A02

NIST Standard Reference Materials (Trade Name) Catalog $1995-1996$.
PB95-2325

NIST/SP-260/116

00,508 PC A03/MF A01

Standard Reference Materials: Glass Filters as a Standard Reference Material for Spectrophotometry - Selection Preparation Certification, and Use of SRM 930 and SRM 1930 D0 536 aC A10/MF A0

NIST-SP-260-120

User's Guide to NIST SRM 2084: CMM Probe Performance

PB94-206109

NIST/SP-260/121

$02,709 \mathrm{PC}$ A03/MF A01

Standard Reference Materials: Certification of a Standard Reference Material for the Determination of Interstitial Oxy gen Concentration in Semiconductor Silicon by Infrared Spectrophotometry.

NIST/SP-260-122

02,326 PC A05/MF A01

Standard Reference Materials: Polystyrene Films for Calibrating the Wavelength Scale of Intrared Spectrophotometers - SRM 1921.
PB95-226866
NIST/SP-260-124

Standard Reference Material 1744: Aluminum Freezing Point Standard

NIST-SP-260-125

$01,055 \mathrm{PC} \mathrm{A03/MF} \mathrm{A01}$

Statistical Aspects of the Certification of Chemical Batch SRMs. Standard Reference Materials.

PB96-210877

00,645 PC A05/MF A01

T-SP-260-126

NIST Traceable Reference Material Program for Gas Stand $\begin{array}{ll}\text { ards: Standard Reference Materials. } & \\ \text { PB96-210786 } & \text { PC A04/MF A0 } 1\end{array}$ PB96-21078

NIST/SP-305/2

Publications of the National Institute of Standards and Technology 1992 Catalog.

PB95-200747

00,014 PC A17/MF A04

NIST/SP-305/25

Publications of the National Institute of Standards and Technology 1993 Catalog

PB96-183215

00,017 PC A11/MF A03

NIST/SP-400-93

Semiconductor Measurement Technology: Design and Testing Guides for the CMOS and Lateral Bipolar-on-SOI Test Library.

$02,301 \mathrm{PC} \mathrm{A07/MF} \mathrm{A02}$

Semiconductor Measurement Technology: Improved Char acterization and Evaluation Measurements for $\mathrm{HgCdTe} \mathrm{De}$ tector Materials, Processes, and Devices Used on the GOES and TIROS Satellites.

02,122 PC A09/MF A02

NIST/SP-400-95

User's Manual for the Program MONSEL-1: Monte Carlo Simulation of SEM Signals for Linewidth Metrology.

PB95-111522

$02,325 \mathrm{PC} \mathrm{AO3/MF} \mathrm{A01}$

NIST/SP-400-96

Semiconductor Measurement Technology: HOTPAC. Programs for Thermal Analysis Including Version 3.0 of the TXYZ Program, TXYZ30, and the Thermal MultiLayer Program, TML.

NIST/SP-400-97

02,374 PC A05/MF A01

Semiconductor Measurement Technology: Test Structure Implementation Document: DC Parametric Test Structures and Test Methods for Monolithic Microwave Integrated Circuits (MMICs)

PBP6-11

02,399 PC A05/MF A01

Semiconductor Measurement Technology: Survey of Optica Characterization Methods for Materials, Processing, and Manufacturing in the Semiconductor Industry.

PB96-154596

02,706 PC A05/MF A01

NIST/SP-480/20-ED-1996

Directory of Law Enforcement and Criminal Justice Associations and Research Centers.

PB96-178918

NIST/SP-500/212

Planning for the Fiber Distributed Data Interface (FDD). PB94-135761

NIST/SP-500/213

Next Generation Computer Resources: Reference Model for Project Support Environments (Version 2.0).
01,677 PC A06/MF A02

NIST/SP-500-215

Second Text REtrieval Conference (TREC-2). Held in Gaithersburg, Maryland on August 31-September 2, 1993. PB94-178407

$01,686 \mathrm{PC} \mathrm{A21/MF} \mathrm{A04}$

NIST-SP-500-216

Proceedings of the Digital Systems Reliability and Nuclea Safety Workshop. Held in Rockville, Maryland on Septem ber 13-14, 1993.

NUREG/CP-013

NIST/SP-500/217

03,728 PC A16/MF A03

IGOSS-Industry/

NIST/SP-500/218

Systems Specification.

Analyzing Electror

PB94-219102

NIST/SP-500/219

NIST Workshop on the Computer Interface to Flat Pane Displays. Held in San Jose, California on January 13-14

PB95-136388

NIST/SP-500/220

Guide on Open System Environment (OSE) Procurements. PB95-169496

NIST/SP-500/221

User Study: Informational Needs of Remote National Archives and Records Administration Customers.

Chives and Records Administration Customers.
PB95-154738
02,725 PC A06/MF A02 NIST/SP-500/222

Glossary of Software Reuse Terms.

PB95-178992

01, 720 PC A03/MF A01

NIST/SP-500/223

Framework for the Development and Assurance of High Integrity Software

NIST/SP-500/225

$01,716 \mathrm{PC} \mathrm{A05/MF} \mathrm{A01}$

Overview of the Text REtrieval Conference (3rd) (TREC-3) Held in Gaithersburg, Maryland on November $2-4,1994$.

NIST/SP-500/226

Self Monitoring Accounting Systems

PB95-216602

NIST/SP-500/227

Electronic Access: Blueprint for the National Archives and Records Administration

NIST/SP-500/22

$02,731 \mathrm{PC} \mathrm{A04/MF} \mathrm{A01}$

Guidelines for the Evaluation of X.500 Directory Products.

PB95-231908

NIST/SP-500/229

Z39.50 Implementation Experiences.

02,732 PC A04/MF A01

NIST/SP-500/230

Application Portability Profile (APP): The U.S. Government's

Open System

NIST/SP-500/231

Guidelines for the Evaluation of Electronic Data Interchange Products.

PB96-172325

$01,506 \mathrm{PC}$ A05/MF A01

NIST/SP-500/232

Open System Environment (OSE): Architectural Framework for Information Infrastructure.

PB96-146360

00,002 PC A04/MF A01

NIST/SP-500-233

Manager's Guide for Monitoring Data Integrity in Financial

Systems.

NIST/SP-500-2

00,003 PC A05/MF A01

Reference Information for the Software Verification and $\mathrm{Val}$ idation Process.

PB96-188164

NIST/SP-500/235

$01,773 \mathrm{PC}$ A06/MF A01

Structured Testing: A Testing Methodology Using the Cyclomatic Complexity Metric.

01,784 PC A07/MF A02

NIST/SP-500-236

Text REtrieval Conference (4th) (TREC-4). Held in Gaithersburg,

01,786 PC A99/MF E08

NIST/SP-777-94-ED

NIST Serial Holdings, 1994

PB94-178068

NIST/SP-777-95

NIST Serial Holdings, 1995

PB95-188926

NIST/SP-782

NIST Standard Reference Data Products Catalog, 1995-96. Achieve with Sta

NIST/SP-782-1994ED

NIST Standard Reference Data Products Catalog, 1994.

PB94-151842

NIST/SP-791

State Weights and Measures Laboratories: State Standards Program Description and Directory. 1994 Edition.

NIST/SP-800/7

Security in Open Systems.

PB95-105383

01,473 PC A13/MF A03

$8-800 / 9$

Good Security Practices for Electronic Commerce, Including Electronic Data Interchange.

PB94-139045 
NIST-SP-811

Guide for the Use of the International System of Units (SI). PB95-22669

$02,747 \mathrm{PC} \mathrm{A05/MF} \mathrm{AO}$

NIST/SP-823/5

North American ISDN Users' Forum Agreements on Integrated Services Digital Network.

NIST/SP.823/6

01,466 PC A16/MF A03

ISDN Conformance Testing Guidelines: Guidelines for mplementors of ISDN Customer Premises Equipment to Conform to Both National ISDN-1 and North American
ISDN Users' Forum Layer 3 Basic Rate Interface Basic Call Control Abstract Test Suites.

Control Abstract

01,471 PC A03/MF A01 NIST/SP-837

Science, Technology, and Competitiveness: Retrospective on a Symposium in Celebration of NIST's 90th Anniversary and the 25th Anniversary of the Gaithersburg Laboratories November 14-15, 1991.

PB97-121610

02,696 PC A08/MF A02

NIST/SP-838/5

Project Summaries 1994: NIST Building and Fire Research Laboratory.

NIST/SP-838/6

00,343 PC A08/MF A02

Building and Fire Research Laboratory Publications, 1993

PB95-143202

NIST/SP-838/7

00,369 PC A06/MF A02

Building and Fire Research Laboratory Publications, 1994.

PB95-22668

00,398 PC A07/MF A02

NIST/SP-838/8

Project Summaries 1995: NIST Building and Fire Research

Laboratory.

NIST/SP-838/9

$00,400 \mathrm{PC} \mathrm{A10/MF} \mathrm{A03}$

Publications 1995: NIST Building and Fire Research Laboratory.

PB96-183074
NIST/SP-854

00,226 PC A08/MF AO2

Report of the National Conference on Weight and Measures (78th). Held in Kansas City. MO. on July 18-22, 1993.

PB94-138989

02,623 PC A18/MF A04

NIST/SP-856

Electroacoustics for Characterization of Particulates and Suspensions. Proceedings of a Workshop. Held in Gaithersburg, Maryland on February 3-4, 1993 .
PB94-112695 00,725 PC A15/MF A03 NIST/SP-857

Putting the Information Infrastructure to Work: Report of the Information Infrastructure Task Force Committee on Applications and Technology.

N94-31228/7

02,715 PC A06/MF A02 Putting the Information Infrastructure to Work: Report of the Information Infrastructure Task Force Committee on Applications and Technology.

NIST-SP-859

$00,001 \mathrm{PC} \mathrm{A06/MF} \mathrm{AO2}$

Abstract and Index Collection in the Research Information Center of the National Institute of Standards and TechPBoly-152204

NIST/SP-860

02,744 PC A03/MF A01

Advanced Components for Electric and Hybrid Electric Vehicles. Workshop Proceedings. Held in Gaithersburg. Maryland on October 27-28, 1993. PB94-177060

04,858 PC A10/MF A03

NIST/SP-861

Evaluation of Alternative In-Flight Fire Suppressants for Full-Scale Testing in Simulated Aircraft Engine Nacelles and Dry Bays.

NIST/SP-862

00,023 PC A99/MF E08

Northridge Earthquake 1994: Performance of Structures, Lifelines, and Fire Protection Systems.

PB94-207461 04.825 PC A09/MF AO2 NIST/SP-863

U.S. Green Building Conference, 1994.
PB94-206364 PB94-206364
NIST/SP-864

Technical Digest: Symposium on Optical Fiber Measurements (8th), 1994. Held in Boulder, Colorado on September 13-15, 1994 .

NIST/SP-865

$04,231 \mathrm{PC} \mathrm{A10/MF} \mathrm{A03}$

NIST Workshop on Gas Sensors: Strategies for Future Technologies. Proceedings of a Workshop. Held in Gaithersburg, Maryland on September 8-9, 1993 PB95-210225

00,507 PC AO8/MF AO2 NIST/SP-866

Extreme Value Theory and Applications: Proceedings of the Conference on Extreme Value Theory and Applications, Volume 3. Held in Gaithersburg, Maryland in May 1993.

PB95-10495

$03,432 \mathrm{PC} \mathrm{A} 11 / \mathrm{MF}$ A03

In Situ Burning Oil Spill Workshop Proceedings. Held in Orlando. Florida on January 26-28, 1994

PB95-104907

$02,583 \mathrm{PC} \mathrm{A06/MF} \mathrm{AO2}$

Intormation Infrastructure: Reaching Society's Goals Report of the Information Infrastructure Task Force Committee on Applications and Technology.

PB94-214756

$01,469 \mathrm{PC} \mathrm{A08/MF} \mathrm{AO2}$
NIST/SP-869

Databases Available in the Research Information Center of the National Institute of Standards and Technology.
PB95-128641
02,724 PC A08/MF A01 PB95-128641

Report of the National Conference on Weights and Measures (79th). Held in San Diego, California on July 17-21, PB95-169819

02,656 PC A2O/MF A04

NIST/SP-871

Wind and Seismic Effects. Proceedings of the U.S.-Japan Cooperative Program in Natural Resources Panel on Wind and Seismic Effects (26th). Held in Gaithersburg, Maryland on May 17-20, 1994.

NIST/SP-872

00,433 PC A99/MF A06

NIST Industrial Impacts: A Sampling of Successful Partnerships.

(111514

00,488 PC A05/MF A0

PITSP-872-REV-1-95

NIST Industrial Impacts: A Sampling of Successful Partnerships (Revision, March 1995).

PB95-209193

00,489 PC A05/MF A01

NIST/SP.873

Joint DoD/NIST Workshop on International Manufacturing Systems Research and Development. Held in Rockville. Maryland on November 3-5, 1992. Proceedings.

Maryland on

02,931 PC A11/MF A03

NIST/SP-874

Methods for Analysis of Cancer Chemopreventive Agents in Human Serum.
PB95-200648

NIST/SP-876

03,502 PC A07/MF A02

Boundary Lubrication of Silicon Nitride.

PB95-213583

03,226 PC A18/MF A04

Proceedings of the Manufacturing Technology Needs and Issues: Establishing National Priorities and Strategies Conference. Held in Gaithersburg. Maryland on April 26-28,

PB95-206181

NIST/SP-879

02,930 PC A22/MF AO4

Ceramic Powders Characterization: Results of an International Laboratory Study.

02,672 PC A22/MF AO4

NIST/SP-881/1

Federal Implementation Guideline for Electronic Data Interchange: ASC X12 003040 Transaction Set 838 Trading Partner Profile (Vendor Registration), Implementation Con-

PB96-11265

03,674 PC A04/MF AO

NIST/SP-881/2

Federal Implementation Guideline for Electronic Data Interchange: ASC $\times 12003040$ Transaction Set 838 Trading Partner Profile (Confirmation of Vendor Registration). Implementation Convention.

PB96-111190

$01.813 \mathrm{PC} \mathrm{A03/MF} \mathrm{A01}$

NIST/SP-881/3

Federal Implementation Guideline for Electronic Data Interchange. ASC X12 003050 Transaction Set 850 Award Instrument. Implementation Convention.

PB96-114913

$01,814 \mathrm{PC} \mathrm{A11/MF} \mathrm{A03}$

NIST/SP-881-4

Federal Implementation Guideline for Electronic Data Interchange. ASC X12 003050 Transaction Set 860 Modifications to Award Instrument. Implementation Convention.
PB96-114921

PB96-114921

01,815 PC A11/MF A03

Federal Implementaion Guideline for Eletronic Data Interchange. ASC X12 003050 Transaction Set 865 Purchase Order Change Acknowledgement/Request - Seller Initiated. Implementation Convention.

PB96-172549

$01,825 \mathrm{PC} \mathrm{A09/MF} \mathrm{A02}$

NIST/SP-881/6

Federal Implementation Guideline for Electronic Data Interchange. ASC $\times 12003050$ Transaction Set 855 Purchase Order Acknowledgment: Implementation Convention.

PB96-17237

NIST/SP-881-7

Federal Implementation Guideline for Electronic Data Interchange. ASC X12 003050 Transaction Set 843 Response to Request for Quotation. Implementation Convention.

PB96-16898

01,822 PC A09/MF AO2

NIST/SP-881-8

Federal Implementation Guideline for Electronic Data Interchange. ASC $\times 12003050$ Transaction Set 840 Request for Quotation. Implementation Convention.

PB96-17253

$01,824 \mathrm{PC} \mathrm{A10/MF} \mathrm{A03}$

NIST/SP-881/9

Federal Implementation Guideline for Electronic Data Interchange: ASC X12 003050 Transaction Sel 836 Procurement Notices. Implementation Convention.

ment Notices.

01,827 PC A04/MF A01

NIST/SP-882

Conceptual Design Plan for the National Advanced Manufacturing Testbed
PB95-231866

NIST/SP-884

02,828 PC A05/MF AO

Abstract and Index Collection in the Research Information Center of the National Institute of Standards and Technology

$02,741 \mathrm{PC} \mathrm{A03/MF} \mathrm{AO}$

NIST/SP-885

Proceedings of the Applied Diamond Conference 1995: Applications of Diamond Films and Related Materials International Conference (3rd). Held in Gaithersburg. Maryland, on August 21-24, 1995

NIST/SP-886

04,701 PC A99/MF E11

Proceedings of the Annual Manufacturing Technology Con-

terence (2nd): Toward a Common Agenda. Held in

Gaithersburg, Maryland on April 18-20, 1995

PB96-112693

02,887 PC A99/MF E08

Composite Materials for Offshore Operations: Proceedings of the International Workshop (1st). Held in Houston, Texas on October 26-28, 1993

NIST/SP-888

$03,169 \mathrm{PC} \mathrm{A17/MF} \mathrm{A04}$

International Green Building Conference and Exposition (2nd). Held in Big Sky, Montana on August 13-15, 1995

PB95-253605

$02,525 \mathrm{PC} \mathrm{AO} 4 / \mathrm{MF}$ A01

\section{NIST/SP-889}

Post-Earthquake Fire and Lifelines Workshop. Held in Long Beach, California on January 30-31, 1995. Proceedings.
PB96-117916 00,209 PC A04/MF A01

NIST/SP-890-V1

Fire Suppression System Performance of Alternative Agents in Aircraft Engine and Dry Bay Laboratory Simulations. SP890: Volume 1.

NIST/SP-890-

03,277 PC A99/MF E08

Fire Suppression System Performance of Alternative Agents in Aircraft Engine and Dry Bay Laboratory Simulations. SP 890: Volume 2

PB96-117783

$03,278 \mathrm{PC}$ A99/MF A06

(1)

Standards Setting in the European Union: Standards Organization and Officials in EU Standards Activities.

PB96-115019

02,919 PC A04/MF A01

NIST/SP-893

Glimpse of Materials Research in China: A Report from an

Interagency Study Team on Materials Visiting China from June 19, 1995 to June 30, 1995.

PB96-112677

02,978 PC A11/MF A03

NIST/SP-894

Report of the National Conference on Weights and Measures ( 80 th) as Adopted by the 80 th National Conference on Weights and Measures, 1995. Held in Portland, Maine on July 16-20, 1995

NIST/SP-895

02,681 PC A15/MF A03

Databases Available in the Research Information Center of the National Institute of Standards and Technology (December 1995).

NIST/SP-896

02,734 PC AO7/MF AO2

Conference Proceedings: Intemational Workshop on Instrumented Indentation. Held in San Diego, California on April 22-23, 1995

NIST/SP-897

01.948 PC A06/MF A01

Working Conference on Global Growth of Technology: Is

America Prepared. Held in Gaithersburg. Maryland on De-

cember 7, 1995 


\section{NIST/SW/DK-94/002}

HAZARD I Fire Hazard Assessment Method, Version 1.2 (Upgrade Package) (for Microcomputers).

PB94-501996

\section{NIST/SW/DK-94/003}

Building Life Cycle Cost Computer Program (BLCC), Version 4.2 .95 (for Microcomputers).

PB95-501953

00,266 CP D02

NIST/SW/DK-95/002

Building Life Cycle Cost Computer Program (BLCC) Version 4.21-95 (for Microcomputers)

00,267 CP D02

Building Life Cycle Cost Computer Program (BLCC) Version

4.22-95 (for Microcomputers)

PB95-503397

00,268 CP DO2

NIST/SW/MT-93/008

FORTRAN Compiler Validation System, Version 2.1

PB94-500691

$01,698^{\circ}$ CP T99

NIST/TN-1297

Guidelines for Evaluating and Expressing the Uncertainty of NIST Measurement Results. 1994 Edition.

$\begin{array}{lll}\text { PB95-143087 } & 02,649 & \text { PC A03/MF A01 }\end{array}$ NIST/TN-1

NIST 60-Millimeter Diameter Cylindrical Cavity Resonator: Performance Evaluation for Permittivity Measurements.

PB94-151776 02,251 PC A11/MF A03

NIST/TN-1355-R

Transmission/Reflection and Short-Circuit Line Methods for Measuring Permittivity and Permeability.
PB94-165537 2,211 PC A06/MF A02 PB94-165537

Coaxial Reference Standard for Microwave Power.
PB94-193786 01,880 PC A04/MF A01

NIST/TN-19

Aperture Excitation of Electrically Large, Lossy Cavities.
PB94-145711 00,029 PC A05/MF A01

NIST/TN-1363

Cryogenic Properties of Silver

PB94-203593

$03,330 \mathrm{PC} \mathrm{A03}$

NIST/TN-1364

Uncertainty Analysis of the NIST Nitrogen Flow Facility.

PB95-128906

02,608 PC A04/MF A01

NIST/TN-1365

Sub-Doppler Frequency Measurements on OCS at $87 \mathrm{Thz}$ (3.4 micrometers) with the CO Overtone Laser: Considerations and Details.

PB95-128633

NIST/TN-1366

04,255 PC A03/MF A01

Analytical Estimation of Carrier Multipath Bias on GPS Position Measurements

PB94-215712

04,845 PC A04/MF A01

NIST/TN-1367

Flow Conditioner Tests for Three Orifice Flowmeter Sizes.

PB95-105540

04,201 PC A05/MF A01

NIST/TN-1369

Video Microscopy Applied to Optical Fiber Geometry Measurements.

PB95-173068

NIST/TN-1371

04,295 PC A04/MF A01

Effective Medium Theory for Ferrite-Loaded Materials.

PB95-154662

01,893 PC A03/MF A01

NIST/TN-1372

Assessment of Data by a Second-Order Transfer Function.

PB95-182390

$03,760 \mathrm{PC} \mathrm{A05/MF} \mathrm{A01}$

NIST/TN-1373

Spherical-Wave Source-Scattering Matrix Analysis of Antennas and Antenna-Antenna Interactions.

PB96-111166

02,008 PC A08/MF A02

\section{ST/TN-1374}

Calibration Service for Coaxial Reference Standards for Microwave Power.

PB96-16272

01,958 PC A07/MF A02

NIST/TN-1375

Band-Limited, White Gaussian Noise Excitation for Reverberation Chambers and Applications to Radiated Susceptibility Testing.

NIST/TN-1376

01,960 PC A07/MF A02

Optical Detector Nonlinearity: Simulation

PB96-165378

02,199 PC A04/MF A01

NIST/TN-1377

Radiated Emissions and Immunity of Microstrip Transmission Lines: Theory and Measurements.
PB96-162649 02,238 PC A04/MF A01

Connector Ferrule Geom

Optical Fiber, Fiber Coating, and Connector Ferrule Geom-

etry: Results of Interlaboratory Measurement Comparisons.
PB96-154422 NIST/TN-13

Direct Comparison Transfer of Microwave Power Sensor Calibrations.
PB96-158654

NIST/TN-1380

$02,086 \mathrm{PC}$ A03/MF A01

Trapped Ions and Laser Cooling 4: Selected Publications of the lon Storage Group of the Time and Frequency Division, NIST, Boulder, Colorado.

04,108 PC A11/MF A03
NIST/TN-1381

Rapid Evaluation of Mode-Stirred Chambers Using Impulsive Waveforms.

01,979 PC A04/MF A01

NIST/TN-1382

Standard Source Method for Reducing Antenna Factor Erors in Shielded Room Measurements.

PB96-183157

NIST/TN-1406

$02,013 \mathrm{PC}$ A04/MF AO

Fire Safety of Passenger Trains: A Review of Current Approaches and of New Concepts.

PB94-152006

NIST/TN-1407

04,848 PC A11/MF A03

Fire Data Management System, FDMS 2.0, Technical DocUmentation.

01,358 PC A05/MF A01

Summary Report of NIST's Industry-Government Consortium Research Program on Flowmeter Installation Effects: The 45 Degree Elbow.

PB95-143061

04,204 PC A06/MF A02

NIST/TN-1409

aser Doppler Velocimeter Studies of the Pipeflow Pro duced by a Generic Header.

PB95-226916

02,602 PC A05/MF A01

NIST/TN-1410

Measuring Long Gage Blocks with the NIST Line Scale Interferometer.

NIST/TN-1411

$02,665 \mathrm{PC} \mathrm{A03/MF} \mathrm{A01}$

Reproducibility of the Temperature of the Ice Point in Rouine Measurements.

PB95-255923

04,015 PC A03/MF A01

NIST/TN-1412

Thermochemical and Chemical Kinetic Data for Fluorinated Hydrocarbons.

NIST/TN-1413

$01,056 \mathrm{PC} \mathrm{A09/MF} \mathrm{A02}$

$45 \mathrm{deg} / 0 \mathrm{deg}$ Reflectance Factors of Pressed Polytetra fluoroethylene (PTFE) Powder.
PB95-260758 NIST/TN-1414

Liquid-Nitrogen-Cooled High Tc Electrical Substitution Radiometer as a Broadband IR Transfer Standard.

PB96-158704

NIST/TN-1416

Estimates of Hurricane Wind Speeds by the 'Peaks Over Threshold' Method.

PB96-162540

NIST/TN-1417

NIST Measurement Assurance Program for Capacitance Standards at $1 \mathrm{kHz}$.

PB96-172333

02,276 PC A03/MF AO

NIST/TN-1418

Probabilistic Estimates of Design Load Factors for Wind Sensitive Structures Using the 'Peaks Over Threshold' Approach

PB96-183223
NIST/TN-1419

00,474 PC A04/MF A01

Flowmeter Installation Effects Due to a Generic Header.

NIST/TN-1420

NIST High-Accuracy Sampling Wattmeter.

PB97-108575

02,689 PC A04/MF A01

NIST/TN-1421

National Measurement System for Radiometry. Photometry and Pyrometry Base Upon Absolute Detectors.

PB97-108559

04,382 PC A04/MF A01

NIST92DDI510-5-1.11

Ada Compiler Validation Summary Report. Certificate Number: 931119S1.11332, DDC-I, Inc. DACS MIPS R3000 Bar Ada Cross Compiler System, Version 4.7.1 Sun R3000 Bare Instruction Set Architecture Simulator, Version R3000 Bare Instruction Set Architecture Simulator, Version

AD-A276 181/5

$01,641 \mathrm{PC} \mathrm{A04/MF} \mathrm{A01}$

NISTIR/GCR-95/680

National Construction Sector Goals: Industry Strategies for Implementation.

NISTIR-85-3273-8

00,204 PC A03/MF A01

Energy Prices and Discount Factors for Life-Cycle Cos Analysis 1994. Annual Supplement to NIST Handbook 135 and NBS Special Publication 709.

PB94-206018

NISTIR-85/3273-9

$02,508 \mathrm{PC}$ A04/MF AO

Energy Price Indices and Discount Factors for Life-Cycle Cost Analysis 1995. Annual Supplement to NIST Handbook 35 and NBS Special Publication 709 . (Revised). PB95-105011

02,509 PC AO4/MF A01

NISTIR-85/3273-10

Energy Price Indices and Discount Factors for Life-Cycle Cost Analysis 1996. Annual Supplement to NIST Handbook 135 and NBS Special Publication 709. (Revised)

PB96-162441 02,510 PC A05/MF A01

NISTIR-85-3273-1

Energy Price Indices and Discount Factors for Life-Cycle Cost Analysis 1997. Annual Supplement to NIST Handbook 135 and NBS Special Publication 709. (Revised).

PB96-210745 02,512 PC A05/MF A01

NISTIR-89-4157

Development and Characterization of Insulating Layers on Silicon Carbide: Annual Report for February 14, 1988 to February 14, 1989.

PB94-155579

02,295 PC AO3/MF A01

NISTIR-89/4213

Earthquake Resistant Construction of Electric Transmission and Telecommunication Facilities Serving the Federal Gov-

PB94-161817

NISTIR-89/4214

02,460 PC A03/MF AO1

Factors Significant to Precracking of Fracture Specimens.

PB96-109558

of Fracture Specimens.
03,358 PC A06/MF A02

NISTIR-3976

Droplet Transfer Modes for a MIL 1005-1 GMAW Electrode.
PB95-209300 D95-209300

PTIR-3977

Alternating-Field Susceptometry and Magnetic Susceptibility of Supercondoctors. Presented at Office of Naval Research Workshop on Magnetic Susceptibility of Superconductors and Other Spin Systems. Held in Berkeley Springs, West Virginia on 20 May 1991.

PB94-145984

04,435 PC A01/MF A01

NISTIR-3993

Bibliography of the NIST Electromagnetic Fields Division Publications.

NISTIR-3994

$01,875 \mathrm{PC}$ A06/MF A02

Metrology for Electromagnetic Technology: A Bibliography of NIST Publications.

PB94-15976

02,116 PC A04/MF A01

R.4333

National Type Evaluation Program: Index of Device Evaluations by Company. NCWM Publication 5 Part A (Second

PB94-160835

02,889 PC A05/MF A01

NISTIR-4419

Seismic Instrumentation of Existing Buildings.

PB94-159779

00,420 PC A04/MF A01

NISTIR-4440

Summaries of Center for Fire Research In-House Projects and Grants: 1990

PB94-160876

$00,286 \mathrm{PC} \mathrm{A10/MF} \mathrm{A03}$

NISTIR-4443

Sulfur Dioxide Capture in the Combustion of Mixtures of Lime Refuse-Derived Fuel, and Coal

PB94-155587

$02,534 \mathrm{PC} \mathrm{A04/MF} \mathrm{A01}$

NISTIR-4489

Fire Growth Analysis of the Fire of March 20, 1990, Pulaski Building, 20 Massachusetts Avenue, N.W., Washington, PB. 905952

$00,194 \mathrm{PC}$ A03/MF A01

NISTIR-4490

Review of Mathematical Function Library for Microsoft$\begin{array}{ll}\text { FORTRAN; John Wiley and Sons, } 1989 . & \\ 01,679 \text { PC A03/MF A01 }\end{array}$ PB94-160793
NISTIR-4493

NVLAP Procedures U.S. Code of Federal Regulations. Title 15, Subtitle A, Chapter 2, Part 7. (Effective December 1984; Amended September 1990). 
NISTIR-4721

Ouestions and Answers on Ouality, the ISO 9000 Standard Series, Ouality System Registration, and Related Issues. More Ouestions and Answers on the ISO 9000 Standard Series and Related Issues.
PB95-103461

NISTIR-4738

00,495 PC A03/MF A01

Models, Managing Models, Ouality Models: An Example of Ouality Management

PB94-163465

02,891 PC A03/MF A01

NISTIR-4755

Issues and Recommendations for a STEP Application Protocol Framework. National PDES Testbed.

PB94-160868

02,770 PC A05/MF A01

NISTIR-4779

Summary Report of NIST's Industry-Government Consortium Research Program on Flowmeter Installation Effects with Emphasis on the Research Period, January-September 1991: The Reducer.

PB94-160736

04,196 PC A04/MF A01

NISTIR-4790

Microstructural Features of Some Low Water/Solids, Silica Fume Mortars Cured at Different Temperatures.

Fume Mortars Cured at Different Temperatures.
PB94-160777 00,330 PC A03/MF A01

NISTIR-4795

Earthquake Resistant Construction of Gas and Liquid Fuel

Pipeline Systems Serving, or Regulated By, the Federal Government.

NISTIR-4804

04,846 PC A05/MF A01

Issues Concerning Material Removal Shape Element Volumes (MRSEVs)

PB95-210167
NISTIR-4841

02,885 PC A03/MF A01

Properties and Interactions of Oral Structures and Restora tive Materials. Annual Report for Period October 1, 1990 to September 30, 1991

PB94-160843

$03,558 \mathrm{PC}$ A06/MF A01

NISTIR-4859

Time-Perturbation Tuning of MIMD Programs.
PB94-164399 01,681 PC A03/MF A01

NISTIR-4870

Feasibility and Design Considerations of Emergency Evacuation by Elevators.

PB94-163441
NISTIR-4877

00,287 PC A07/MF A02

Room-Temperature Flexure Fixture for Advanced Ceramics.

PB95-210498 03,061 PC A03/MF A01

NISTIR-4888

NIST Support to the Next Generation Controller Program:

1991 Final Technical Report. $02,808 \quad$ PC A09/MF A02
PB94-163490 PB94- 163490

Design Guide for CMOS-On-SIMOX. Test Chips NIST3 and NIST4.

PB94- 163458

NISTIR-4894

02.297 PC A05/MF A01

Computation of Dendrites Using a Phase Field Model.

PB94-160744

NISTIR-4895

04,436 PC A03/MF A01

Study to Determine the Most Important Parameters for Evaluating the Resistance of Soft Body Armor to Penetra. tion by Edged Weapons.

PB94-158573

$03.757 \mathrm{PC} \mathrm{A03/MF} \mathrm{A01}$

NISTIR-4901

Electronics and Electrical Engineering Laboratory Technical Progress Bulletin Covering Laboratory Programs, Januany to March 1992, with 1992/1993 EEEL Events Calendar.
PB95-210480 01,917 PC A03/MF A01

NISTIR-4903

Structural EXPRESS Editor.

PB94-159795

NISTIR-4904

02,769 PC A04/MF A01

Compatibility

PB94-163474

NISTIR-4942-2

Present Worth Factors for Life-Cycle Cost Studies in the Department of Defense (1995)

PB95-105029

NISTIR-4942-3

Present Worth Factors for Life-Cycle Cost Studies in the Department of Defense (1996)

PB96-106869

$03,673 \mathrm{PC}$ A04/MF A01

Publications of the Manufacturing Engineering Laboratory Covering the Period January 1989-September 1992 . NISTIR-4981

Controlling Moisture in the Walls of Manufactured Housing. PB95-105136

00,355 PC A03/MF A01

NISTIR-5002

TEM/Reverberating Chamber Electromagnetic Radiation Test Facility at Rome Laboratory.

PB96-155023

03,675 PC A05/MF A01

Electromechanical properties of superconductors for DOE fusion applications.

04,432 PC A04/MF A01
Electromechanical Properties of Superconductors for DOE Fusion Applications PB94-13967

NISTIR-5015

Crosstalk between Microstrip Transmission Lines.

PB94-135639

02,250 PC A06/MF A02

NISTIR-5016

02,210 PC A03/MF A01

NIST Model PM2 Power Measurement System for $1 \mathrm{~mW}$ at $1 \mathrm{GHz}$

PB94-135803

$02,018 \mathrm{PC} \mathrm{A03/MF} \mathrm{A01}$

NISTIR-5018

Optical Fiber Sensors: Accelerating Applications in Navy

PB94-186848

02,632 PC A06/MF A02

NISTIR-5022

RangeCAD and the NIST RCS Uncertainty Analysis. PB94-218591

NISTIR-5023

01,870 PC A03/MF A01

Measurements of Shielding Effectiveness and Cavity Characteristics of Airplanes.

PB94-21005

$00,030 \quad$ PC A04/MF AO

NISTIR-5024

Composite Struts for SMES Plants.

PB95-155586

02,507 PC A99/MF A06

ISTIR-5025

irradiation Damage in Inorganic Insulation Materials for TER Magnets: A Review.

PB95-147351

$03,705 \mathrm{PC} \mathrm{A18/MF} \mathrm{A04}$ STIR-502

Thermal Hydraulic Tests of a Liquid Hydrogen Cold Neutron

Source.

PB95-135570

$03,884 \mathrm{PC} \mathrm{A03/MF} \mathrm{A01}$

NISTIR-5027

Superconductor Critical Current Standards for Fusion Applications. Final Progress Report, October 1993-July 1994.

PB95-169538

\section{NISTIR-5028}

Bibliography of the NIST Electromagnetic Fields Division Publications.

\section{NISTIR-5029}

Metrology for Electromagnetic Technology: A Bibliography of NIST Publications.

PB95-135588

NISTIR-5030

02,143 PC A05/MF A01

Cryogenic Properties of Inorganic Insulation Materials for ITER Magnets: A Review.

NISTIR-5032

$03,706 \mathrm{PC} \mathrm{A10/MF} \mathrm{A03}$

Electromagnetic Shielding Characterization of Gaskets

PB95-198917 01,911 PC A03/MF A01 NISTIR-5033

Polarimetric Calibration of Reciprocal-Antenna Radars. PB95-216925

01,872 PC A03/MF A01 NISTIR-5034

IIW Commission V Ouality Control and Ouality Assurance of Welded Products Annual Report 1994/95.

PB95-198743

NISTIR-5035

02,866 PC AO3/MF AO

Time and Frec

March 1995.

NISTIR-5037

01,548 PC A06/MF AO2

Control of Gas-Metal-Arc Welding Using Arc-Light Sensing. PB96-131461

NISTIR-5038

$02,869 \mathrm{PC}$ A06/MF A02

Residual Stress in Induction-Heated Railroad Wheels: Ultra

sonic and Saw Cut Measurements. Report No. 28.
PB96-106992
04.854 PC A04/MF A01 NISTIR-50

Metrology for Electromagnetic Technology: A Bibliography of NIST Publications, September 1995.

PB96-128269

$01,938 \mathrm{PC}$ A05/MF AO

NISTIR-5041

Bibliography of the NIST Optoelectronics Division.

PB96-128210

$02,193 \mathrm{PC} \mathrm{AO4/MF} \mathrm{A01}$ NISTIR-5043

Dynamoneter-Induced Residual Stress in Railroad Wheels: Uitrasonic and Saw Cut Measurements. Report No. 30

PB96-183199 04,857 PC A04/MF A0

NISTIR-5044

IIW Commission V Ouality Control and Ouality Assurance of Welded Products, Annual Report 1995/96.

PB96-19136

$02880 \mathrm{PC} \mathrm{A04/MF} \mathrm{A01}$

NISTIR-5045

Dielectric and Magnetic Measurements from $-50 \mathrm{C}$ to $200 \mathrm{C}$ and in the Frequency Band $50 \mathrm{MHz}$ to $2 \mathrm{GHz}$

PB96-191382

$02,245 \mathrm{PC} \mathrm{A03/MF} \mathrm{A01}$

NISTIR-50

Hybrid Gauss-Trapezoidal Quadrature Rules.

PB96-193750

NISTIR-5050

$03,422 \mathrm{PC}$ A04/MF A01

Bibliography

Publications.

NISTIR-5051

01080 PC A08/MF A02

Metrology for Electromagnetic Technology: A Bibliography of NIST Publications.

PB97-116057

04,396 PC A06/MF AO

NISTIR-5052

Bibliography of the NIST Optoelectronics Division

PB97-116040

$02,207 \mathrm{PC}$ A06/MF A01

STIR-5102

Diffusion of Cations Beneath Organic Coatings on Steel Substrate.

PB94-215704

$03,119 \mathrm{PC}$ AO3/MF A01

Ouestions and Answers on Ouality, the ISO 9000 Standard Series, Ouality System Registration, and Related Issues. More Ouestions and Answers on the ISO 9000 Standard

Series and Related Issues.

00,495 PC A03/MF A01

NISTIR-5145

Electronics and Electrical Engineering Laboratory Technical Progress Bulletin Covering Laboratory Programs, October to December 1992, with 1992/1993 EEEL Events Calendar. PB94-165958 02,299 PC A03/MF A01 NISTIR-5165

Life-Cycle Costing Workshop for Energy Conservation in Buildings: Student Manual.

PB95-17500

00,257 PC A11/MF A03

NISTIR-5178

Least-Cost Energy Decisions for Buildings: Part 2. Uncertainty and Risk Video Training Workbook

PB94-165982

00,240 PC A04/MF A01

NISTIR-5184

Highway Concrete (HWYCON) Expert System User Reference and Enhancement Guide.

PB94-215670

01,316 PC A08/MF A02

PTIR-5185-2

BLCC: The NIST 'Building Life-Cycle Cost' Program, Version 4.21. User's Guide and Reference Manual.
PB95-190682 00,263 PC A06/MF A02 NISTIR-5189

Wind Load Provisions of the Manufactured Home Construction and Safety Standards: A Review and Recommendations for Improvement

PB94-206125

00,428 PC A06/MF A02

NISTIR-5199

Recertification of the Standard Reference Material 1475A, a Linear Polyethylene Resin

PB94-161932

02,628 PC AO3/MF AO

NISTIR-5212

Constituents and Physical Properties of the $\mathrm{C} 6+$ Fraction of Natural Gas. Topical Report, April-June 1994

PB95-136644

$02,483 \mathrm{PC} \mathrm{AO} / \mathrm{MF} \mathrm{AO}$

NISTIR-5214

Introduction to Traffic Management for Broadband ISDN PB94-142494

$01,464 \mathrm{PC} \mathrm{AO} / \mathrm{N}^{\prime} \overline{\mathrm{N}} \mathrm{AO}$

NISTIR-5225

Technical Program of the Factory Automation Systems Division 1993.

NISTIR-5234

02,805 PC A03/MF AO

Report of the NIST Workshop on Digital Signature Certificate Management. Held on December 10-11 1992

PB94-13500 
NISTIR-5295

Guide to Software Engineering Environment Assessment and Evaluation.

NISTIR-5296

$01,676 \mathrm{PC}$ A04/MF A0

Computing Effects and Error for Large Synthetic Perturbation Screenings.

NISTIR-5297

Feasibility Study: Reference Architecture for Machine Control Systems Integration.

PB94-14279

$01,675 \mathrm{PC} \mathrm{A03/MF} \mathrm{A01}$

NISTIR-5298

Some Factors Affecting Design of a Furniture Calorimeter Hood and Exhaust.

PB94-139193

NISTIR-5299

$00,285 \mathrm{PC}$ A03/MF A01

Photonic Materials: A Report on the Results of a Workshop. Held in Gaithersburg, Maryland on August 26-27, 1992, Volume 1.

PB94-152733

02,114 PC A05/MF A01

NISTIR-5302

Evaluating Small Board and Care Homes: Sprinklered vs. Nonsprinklered Fire Protection.

PB94-206356

00,195 PC A04/MF A01

NISTIR-5303 CSTL Technical Activities, $1993.00,953$ PC A17/MF A04
PB95-160602

NISTIR-5304

Use of Computer Models to Predict Temperature and Smoke Movement in High Bay Spaces.

PB94-145976

$00,191 \mathrm{PC}$ A04/MF A01

NISTIR-5305

FIREDOC Users Manual, 3rd Edition.

PB95-128674

$01,378 \mathrm{PC} \mathrm{A03/MF} \mathrm{AO}$

NISTIR-5306

Ground-Based Smoke Sampling Techniques Training Course and Collaborative Local Smoke Sampling in Saud

B94-143542

02,532 PC A05/MF A01

NISTIR-5307

open Architectures for Machine Control.

PB94-13562

$02,942 \mathrm{PC} \mathrm{A03/MF} \mathrm{AO}$

NISTIR-5308

General Procedures for Registering Computer Security Objects.

PB94-134897

$01,573 \mathrm{PC} \mathrm{A03/MF} \mathrm{A01}$

NISTIR-5309

Context Analysis of the Network Management Domain

Conducted as Part of the Domain Analysis Case Study.
PB94-142528

NISTIR-5310

Unconstrained Handprint Recognition Using a Limited LexiPB94-168051

$01,831 \mathrm{PC} \mathrm{A03/MF} \mathrm{AO1}$

NISTIR-5311

Materials Science and Engineering Laboratory Annual Report, 1993. NAS-NRC Assessment Panel, April 21-22, 1994 PB94-162534 02,969 PC AO5MF AO1

NISTIR-5312

Intelligent Processing of Materials, Technical Activities 1993 (NAS-NRC Assessment Panel, April 21-22, 1994)

PB94-164183 02,809 PC A04/MF A01 NISTIR-5313

Ceramics Technical Activities, 1993 (NAS-NRC Assessmen Panel April 21-22, 1994)

PB94-162591

03,031 PC A10/MF A03

NISTIR-5318

APDE Demonstration System Architecture. National PDES Testbed Report Series.

PB94-154325

$02,767 \mathrm{PC} \mathrm{A03/MF} \mathrm{A01}$

NISTIR-5319

Assessment of Uncertainties of Calibration of Resistance Thermometers at the National Institute of Standards and Technology.

NISTIR-5320

02,624 PC A03/MF A0

Measurements of Outdoor Air Distribution in an Office Build PBg5-210944

NISTIR-5321

00,264 PC A04/MF A01

Study of Heat Pump Performance Using Mixtures of R32 R134a and R32/R125/R134a as 'Drop-In' Working Fluids for R22 with and Without a Liquid-Suction Heat Exchanger.

PB94-218559
02,503 PC A04/MF A01

PB94-2185

Computer Programs for Simulation of Lighting/HVAC Interactions.

PB94-140407

NISTIR-5324

02,501 PC AOB/MF AO2

Color Supplement to NIST Special Publication 400-93 Semiconductor Measurement Technology: Design and Testing Guides for the CMOS and Lateral Bipolar-on-SOI Test Library

02,298 PC A03/MF A01

NISTIR-5325

Conformance Assessment of Transport Layer Security ImPB94-164373

01,576 PC A03/MF A01
NISTIR-5326

Report on Application Integration Architectures (AIA) Workshop. Held in Dallas, Texas on February 8-12, 1993.

PB94-142536

01,803 PC A07/MF AO2

NISTIR-5328

Hollow Clay Tile Prism Tests for Martin Marietta Energy Systems: Task 2 Testing.

PB94-217486

00,352 PC A14/MF A03

STIR-5329

Manual for Ventilation Assessment in Mechanically Ventiated Commercial Buildings.

PB94-145653

00,239 PC A07/MF A02

-5331

Enhanced Machine Controller Architecture Overview.

PB94-142460

02,802 PC A03/MF A01

NISTIR-5332

Modulation of Fossil Fuel Production by Global Tempera. ture Variations, 2.

NISTIR-5333

02,533 PC A04/MF A01

Reliable Optical Flow Algorithm Using 3-D Hermite Poly. nomials.

PB94-145620

01,829 PC A03/MF A01

NISTIR-5336

Evaluation of GSA Maintenance Practices of Large Centrifugal Chillers and Review of GSA Refrigerant Management Practices.

PB94-143344

02,502 PC A04/MF A01

Electronics and Electrical Engineering Laboratory 1994 Program Plan: Supporting Technology for U.S. Competitiveness in Electronics.

PB94- 126901

01,873 PC A09/MF A02

NISTIR-5338

System for Calibration of the Marshall Compaction Hammer.

NISTIR-5339

$01,303 \quad \mathrm{PC} \mathrm{A08/MF} \mathrm{A02}$

Comparison of Fire Sprinkler Piping Materials: Steel, Copper, Chlorinated Polyvinyl Chloride and Polybutylene, in Residential and Light Hazard Installations.

PB95-182267

00,299 PC A03/MF A01

NISTIR-5340

Assessmen

at NIST.

NISTIR-5341

03,782 PC A03/MF A01

Assessment of Uncertainties of Liquid-in-Glass Thermometer Calibrations at the National Institute of Standards and Technology.

PB94-14251

NISTIR-5342

02,625 PC A03/MF A01

Computer Systems Laboratory Annual Report 1993.

PB94-162518

01,622 PC A05/MF A02

NISTIR-5343

Applying Virtual Environments to Manufacturing

PB94-142502

02,803 PC A03/MF A01

NISTIR-5344

Growth Surface for the Slopes at the Boundary of a Polygon

PB94-15272

03,408 PC A03/MF A01

TSTIR-5345

Draft Guidelines for Quality Control Testing of Elastomeric Seismic Isolation Systems.

PB94-161734

00,422 PC A03/MF A01

NISTIR-5346

Indoor Air Quality Impacts of Residential HVAC Systems, Phase 1 Report Computer Simulation Plan.

PB95-135596

00,249 PC A06/MF A02

NISTIR-5349

Submissions to a Planned Encyclopedia of Operations Research on Computational Geometry and the Voronoi/ Delaunay Construct.

PB94-152709

03,425 PC A03/MF A01

NISTIR-5350

Airbome Asbestos Method: Standard Test Method for High Precision Counting of Asbestos Collected on Filters. Version 1.0

PB94-163003

$00,525 \mathrm{PC}$ A02/MF A01

NISTIR-5351

Airbome Asbestos Method: Standard Test Method for Verified Analysis of Asbestos by Transmission Electron Microscopy. Version 2.0 .

NISTIR-5352

Boundary Conforming Grid Generation System for Interface Tracking.

NISTIR-5353

Source of Phenol Emissions Affecting the Indoor Air of an Office Building.

NISTIR-535

03,600 PC A04/MF A01

Variant Design for Mechanical Artifacts-A State of the Art Survey.

PB94-154358

02,768 PC A03/MF A01

NISTIR-5357

Electronics and Electrical Engineering Laboratory Technical Progress Bulletin Covering Programs, October to December 1993, with 1994/1995 EEEL Events Calendar.

PB94-154341

02,115 PC A03/MF A01

NISTIR-5358

Airborne Asbestos Method: Standard Practice for Recording Transmission Electron Microscopy Data for the Analysis of Asbestos Collected onto Filters. Version 1.0

PB94-210168

00,552 PC A03/MF A03

NISTIR-5359

Draft Guidelines for Pre-Qualification and Prototype Testing of Seismic Isolation Systems.

PB94-161940

01,331 PC A06/MF A01

NISTIR-5360

Corrosion Resistance of Materials for Renovation of the

United States Botanic Garden Conservatory.
PB94-154390 00,032 PC A03/MF A01

NISTIR-5362

NIST Reactor: Summary of Activities October 1992 through September 1993.

PB94-161502

04,437 PC AO8/MF AO2

NISTIR-5363

Physics Laboratory Technical Activities, 1993. PB94-176088

03,796 PC A10/MF A03

NISTIR-5364

Evaluating Form Designs for Optical Character Recognition. PB94-168044

NISTIR-5365 01,830 PC A06MF AO2

Bibliography on

NISTIR-5366

Concept for an Algorithm Testing and Evaluation Program at NIST.

PB94-163029

NISTIR-5367

$02,890 \mathrm{PC}$ A03/MF A01

Prediction of Geometric-Thermal Machine Tool Errors by Artificial Neural Networks.

PB94-186673

$02,943 \mathrm{PC}$ A07/MF A02

NISTIR-5370

Manufactured Homes: Probability of Failure and the Need for Better Windstorm Protection through Improved Anchoring Systems.

NISTIR-5371

00,432 PC A04/MF A01

Draft Guidelines for Quality Control Testing of Sliding Seismic Isolation Systems.

PB94-161957

01,332 PC A03/MF A01

NISTIR-5372

Computer Graphics Metafile (CGM): Procedures for NIST CGM Validation Test Service.

PB94-161809

01,804 PC AO3/MF AO1

NISTIR-5373

Report on the Workshop on Manufacturing Polymer Composites by Liquid Molding. Held in Gaithersburg, Maryland on September 20-22, 1993.

PB94-160066

$03,131 \mathrm{PC} \mathrm{A13/MF} \mathrm{A03}$

NISTIR-5374 
NISTIR-5387

Graphical Analysis of the CCRL Portland Cement Proficiency Sample Database (Samples 1-72). (Part 1.

Univariant Analysis of Portland Cement).
PB94-196557 01,308 PC A06/MF A02 NISTIR-5388

Preparation and Monitoring of Lead Acetate Containing Drinking Water Solutions for Toxicity Studies.
PB94-193885 00,538 PC A03/MF A01 PISTIR-5389

$00,538 \mathrm{PC}$ A03/MF A01

Analysis of Failed Dry Pipe Fire Suppression System Couplings from the Filene Center at Wolf Trap Farm Park for

the Performing Arts.
PB94-164407

00,331 PC A03/MF A01

\section{NISTIR-5390}

Sulfate Attack of Cementitious Materials: Volumetric Relations and Expansions.

NISTIR-5391

$03,232 \mathrm{PC}$ A03/MF A01

State-of-the-Art Survey of Methodologies for Representing Manufacturing Process Capabilities.

PB94-187655

NISTIR-5392

02,812 PC A03/MF A01

Calculating Flame Spread on Horizontal and Vertical Surfaces.

PB94-187283

$00,335 \mathrm{PC} \mathrm{A04/MF} \mathrm{A01}$

NISTIR-5394

Design and Development of an Information Retrieval Sys tem for the EAMATE Data. Volume 2 of 2. Appendices. PB94-168390

00,487 PC A19/MF A04

NISTIR-5395

Preliminary Functional Specifications of a Prototype Electronic Research Notebook for NIST.
PB94-207750 00,012 PC A04/MF A01

NISTIR-5396

tormance of Structures,

Northridge Earthquake, 1994. Pertormance of Structures,
Lifelines and Fire Protection Systems. PB94-161114

00,421 PC A09/MF A02

NISTIR-5397

BFAL Fire Publications, 1993

PB94-164191

00,192 PC A03/MF A01

NISTIR-5398

User's Guide for the PHIGS Validation Tests (Version 2.1).
PB94-165206 PB94-165206

NISTIR-5399

Measurement and Determination of Radon Source Potential: A Literature Review.

PB94-165602

02,576 PC A09/MF A03

NISTIR-5402

Realizing Suspended Structures on Chips Fabricated by CMOS Foundry Processes Through the MOSIS Service.

NISTIR-5403

Quantitative X-Ray Powder Diffraction Methods for Clinker and Cement.

PB95-143079

NISTIR-5405

$01,317 \mathrm{PC} \mathrm{A03/MF} \mathrm{A01}$

Effects of Testing Variables on the Measured Compressive Strength of High-Strength ( $90 \mathrm{MPa}$ ) Concrete.

PB95-179040

00,445 PC A07/MF A02

NISTIR-5408

Characterization of the Hydrogen Induced Cold Cracking

Susceptibility at Simulated Weld Zones in HSLA-100 Steel.
AD-A279 759/5 03,200 PC A04/MF A01

Characterization of the Hydrogen Induced Cold Cracking

Susceptibility at Simulated Weld Zones in HSLA-100 Steel.

PB94-174505

NISTIR-5409

03,746 PC A04/MF A01

Electronics and Electrical Engineering Laboratory: 1994 Strategic Plan. Supporting Technology for U.S. Competitiveness in Electronics.

PB94-161320

01,874 PC A04/MF A01

NISTIR-5411

Body Dimensions for Apparel.
PB94-187739

NISTIR-5412

Overview of NASREM: The NASANBS Standard Reference

Model for Telerobot Control System Architecture.
PB94-194560 04,831 PC A03/MF A01 NISTIR-5413

Federal Metric Progress in 1993

PB94-196029

NISTIR-5415

$02,600 \mathrm{PC} \mathrm{A03/MF} \mathrm{A01}$

User Profile for Researchers Studying Objects: Implications for Computer Systems.

PB94-188463

$00,133 \mathrm{PC} \mathrm{AO3/MF} \mathrm{AO}$

NISTIR-5416

NIST RS274/NGC Interpreter Version 1

PB94-187788

$02,814 \mathrm{PC}$ A03/MF A01

NISTIR-5417

Simple Scalability Test for MIMD Cocte.
PB94-193638 PB94-193638
NISTIR-5418

01,688 PC A03/MFAO

Workshop on Characterizing Diamond Films (3rd). Held in

Gaithersburg, Maryland on February 23-24, 1994.
PB94-187663 04,456 PC A04/MF A01

NISTIR-5419

Seismic Safety of Federal Buildings. Initial Program: How Much Will It Cost.

PB95-182291

00,447 PC A03/MF A01
NISTIR-5420

NIST Response to the Fifth CORM Report on the Pressing Problems and Projected Needs in Optical Radiation Measurements.

NISTIR-5421

04,227 PC A03/MF A01

Strengthening Methodology for Lightly Reinforced Concrete Frames-II. Recommended Calculation Techniques for the Frames-ll. Recommended Calculation Techniques for the
Design of Intill Walls.

PB94-187648

$00,426 \mathrm{PC} \mathrm{A03/MF} \mathrm{A01}$

Application of the Electronic Balance in High Precision Pycnometry.

NISTIR-5423

Use of the Electronic Balance for Highly Accurate Direct Mass Measurements Without the Use of External Mass Standards.

PB94-187713

$03,803 \mathrm{PC} \mathrm{A03/MF} \mathrm{A01}$

NISTIR-5424

Study of Federal Agency Needs for Information Technology

Security.

NISTIR-5425

$01,579 \mathrm{PC} \mathrm{A07/MF} \mathrm{A02}$

Metric for Success.

PB94-197630

NISTIR-5427

02,633 PC A03/MF AO

Visualization Applications for Manufacturing: A State-of-theArt Survey. Final Report

02,816 PC A03/MF A01

\section{NISTIR-5428}

Program Handbook: Requirements for Obtaining NIST Approval/Recognition of a Laboratory Accreditation Body Under P.L. 101-592. The Fastener Ouality Act. PB94-210143

02,859 PC A03/MF A01 $-5429$

Optical Metrology and More. Programs and Services of the Radiometric Physics Division. Physics Laboratory.
PB94-191707 04,228 PC A03/MF A01 NISTIR-5430

NIST Calibration of ASTM E127-Type Ultrasonic Reference Blocks. PB94-191640

$02,702 \mathrm{PC} \mathrm{A03/MF} \mathrm{A01}$ NISTIR-5431

Proficiency Tests for the NIST Airborne Asbestos Program,

PB94-188836

00,535 PC A07/MF A02

NISTIR-5432

Proficiency Tests for the NIST Airborne Asbestos Program 1991.

PB94-19382

$00.537 \mathrm{PC} \mathrm{A04/MF} \mathrm{A01}$

NISTIR-5433

Proficiency Tests for the NIST Airborne Asbestos Program -

PB94-194362

00,539 PC A03/MF A01

NISTIR-5434

Electronics and Electrical Engineering Laboratory Technical Progress Bulletin Covering Laboratory Programs, January to March 1994 with 1994/1995 EEEL Events Calendar. PB94-193810

02,308 PC A03/MF A01 NISTIR-5435

Electronics and Electrical Engineering Laboratory Technica Publication Announcements Covering Laboratory Programs, October to

PBalendar.

NISTIR-5436

02,307 PC A03/MF A01

Performance of $1 / 3$-Scale Model Precast Concrete BeamColumn Connections Subjected to Cyclic Inelastic Loads. Report No. 4.
PB95-179024

NISTIR-5437

$00,444 \mathrm{PC} \mathrm{A04/MF} \mathrm{A01}$

Bibliography of Photon Total Cross Section (Attenuation Cosurements $10 \mathrm{eV}$ to

PB94-193760

03,804 PC AO6/MF A02

NISTIR-5438

Industry/Government Open Systems Specification Testing Framework. Version 1.0

01,809 PC A06/MF A02

PB94-21911
NISTIR-5439

Performance Parameters of Fire Detection Systems. PB94-194339

00,288 PC A03/MF A0

NISTIR-5440

CONTAM88 Building Input Files for Multi-Zone Airflow and Contaminant Dispersal Modeling.

PB94-194388

$02,537 \mathrm{PC} \mathrm{A04/MF} \mathrm{AO}$

NISTIR-5441

Risk Analysis for the Fire Safety of Airline Passengers.

PB94-194065

NISTIR-5442

Visual-Motion Fixation Invariant

PB94-206281

04,862 PC AO3/MF AO

NISTIR-5443

Program of the

NISTIR-5443-A

Program of the Subcommittee on Construction and Building (July 1994).

PB95-122537

$00,321 \mathrm{PC} \mathrm{A03/MF} \mathrm{AO}$

NISTIR-5444

Recommendations on Selection of Vehicle-to-Roadside Communications Standards for Commercial Vehicle Oper-

PB94-195914

04,859 PC A05/MF A0

NISTIR-5445

Feasibility of Fire Evacuation by Elevators at FAA Control Towers.

04,844 PC A06/MF A02

NISTIR-5446

Integration of Servo Control into a Large-Scale Control System Design: An Example from Coal Mining.

PB94-203420

03,696 PC A02/MF A01

NISTIR-5447

Certainty Grid to Object Boundary Algorithm

PB94-203510

$01,835 \mathrm{PC}$ A03/MF A0

NISTIR-5448

Continuous Mining Machine Control Using the Real-Time Control System

PB94-203528
NISTIR-5449

03,700 PC A02/MF AO

Environment

NISTIR-5452

Second Census Optical Character Recognition Systems Conference.

NISTIR-5453

World Wide Web and Mosaic: User's Guide

PB94-207354

02,722 PC A03/MF AO

NISTIR-5454

Single-Phase Heat Transfer and Pressure Drop Characteristics of an Integral-Spine-Fin Within an Annulus.

PB94-194073

03,805 PC A03/MF A01

\section{NISTIR-5455}

New Expressions of Uncertainties for Humidity Calibrations at the National Institute of Standards and Technology.

PB95-103826

$02,645 \mathrm{PC} \mathrm{A02/MF} \mathrm{A01}$

NISTIR-5456

NIST Workshop on Nanostructured Material (1st): Report of an Industrial Workshop Conducted by the National Institute of Standards and Technology. Held in Gaithersburg, Maryland on May 14-15, 1992

PB94-218567

$02,973 \mathrm{PC} \mathrm{A04/MF} \mathrm{A01}$

STIR-545

Report on the Workshop on Advanced Digital Video in the National Information infrastructure. Held in Washington D.C. on May 10-11, 1994

NISTIR-5459

$01,472 \mathrm{PC} \mathrm{A09/MF} \mathrm{A03}$

Quality Characteristics and Metrics for Reusable Software (Preliminary Report).

(P)-203437

01,693 PC A03/MF A01 PTIR-5460

Adaptive, Predictive 2-D Feature Tracking Algorithm for Finding the Focus of Expansion.

PB94-218575

01,588 PC A03/MF AO 
Trustworthiness. Held in Williamsburg, Virginia on March $21-23,1994$.
PB94-215746

NISTIR-5473

01,586 PC A05/MF A01

Survey on the Implementation of ISO/IEC Guide 25 by National Laboratory Accreditation Programs.

00,479 PC A04/MF A01 NISTIR-5

Publications of the Intelligent Systems Division (Previously Robot Systems Division) Covering the Period January 1971-April 1994

PB94-217098

NISTIR-5476

02,935 PC A05/MF A01

Technical Program Description Systems Integration for Manufacturing (SIMA)

PB94-213758
NISTIR-5477

Updated Calculations for Routine Space-Shielding Radiation Dose Estimates: SHIELDOSE-2.

PB95-171039

04,838 PC A04/MF A01

NISTIR-5478

Framework for National Information Infrastructure Services. PB95-103719 02,723 PC A07/MF A02

NISTIR-5480

Survey of Recent Cementitious Materials Research in Western Europe.

NISTIR-5481

$00,353 \mathrm{PC} \mathrm{A03/MF} \mathrm{A01}$

NIST Research Program on the Seismic Resistance of Partially-Grouted Masonry Shear Wals.

PB94-219052

$00,354 \mathrm{PC}$ A06/MF A02

NISTIR-5483

Electronics and Electrical Engineering Laboratory Technical Progress Bulletin Covering Laboratory Programs, April to June 1994 with 1994/1995 EEEL Events Calenda

PB95-143186 02,329 PC A03/MF A01

NISTIR-5484

Formulation of Position on U.S. Standards Role in Enterprise Integration

NISTIR-5485

02,773 PC A03/MF A01

Videoconferencing Procurement and Usage Guide.

PB94-217023

NISTIR-5487

Making Sense of Software Engineering Environment Framework Standards.

PB95-10503

01,701 PC A03/MF A01

NISTIR-5488

Performance of HUD-Affiliated Properties during the January 17, 1994 Northridge Earthquake. 43 PC A04/MF A01 NISTIR-5489

Faster BKL Monte Carlo Simulations. PB95-136370

01,706 PC A03/MF A01

NISTIR-5490

Software Needs in Special Functions.

PB95-105045

$01,702 \mathrm{PC} \mathrm{A03/MF} \mathrm{A01}$

NISTIR-5491

Japan Technology Program Assessment: Precision Engi-

neering/Precision Optics in Japan.
PB95-171112

$02,884 \mathrm{PC}$ A03/MF A01 NISTIR-5492

Diagnosis of Causes of Concrete Deterioration in the MLP7 A Parking Garage.

PB95-143095

01,318 PC A03/MF A01

NISTIR-5493

Comparison of FFT Fingerprint Filtering Methods for Neura Network Classification

$01,840 \quad P C$ A03/MF A01

NISTIR-5494

Domain Analysis of the Alarm Surveillance Domain. Version

1.0. Conducted as Part of the Domain Analysis Case Study

PBoject.

01,705 PC A10/MF A03

NISTIR-5495

Computer Security Training and Awareness Course Compendium.

PB95-130985

$01,589 \mathrm{PC} \mathrm{A09/MF} \mathrm{A02}$

NISTIR-5498

Challenges to the National Information Infrastructure: The Barriers to Product Data Sharing. National PDES Testbed Report Series.

NISTIR-5499

02,776 PC A03/MF A01

Annual Conference on Fire Research: Book of Abstracts, October 17-20, 1994

PB95-104964

NISTIR-5500

01,376 PC A09/MF A02

Report on the Advanced Software Technology Workshop. Held on February 1, 1994.

PB95-1366

NISTIR-5501

$01,707 \mathrm{PC} \mathrm{A03/MF} \mathrm{A01}$

Sensitivity of Three-Point Circle Fitting

PB95-136354

02,901 PC A03/MF AO NISTIR-5502

Reference Model Architecture for Intelligent Systems DePBgn. 143137

01,789 PC A03/MF A01
NISTIR-5503

Channel Coding for Code Excited Linear Prediction (CELP) Encoded Speech in Mobile Radio Applications.

PB95-143178 01,475 PC A03/MF A01 NISTIR-5504

Summaries of BFRL Fire Research In-House Projects and Grants, 1994

NISTIR-5505

00,366 PC A10/MF A03

xi-Vector Formulation of Anisotropic Phase-Field Models: 3 D Asymptotics.

NISTIR-5507

04,536 PC A03/MF A01

Portsmouth Fastener Manufacturing Workstation. User's Manual.

PB95-147922

NISTIR-5509

$02,860 \quad P C$ A06/MF AO2

nvestigation in

PB95-143293

NISTIR-5511

$03,152 \mathrm{PC}$ A03/MF AO

STEP On-Line Information Service (SOLIS). The IGES PDES Organization.

PB95-137790

$02,777 \mathrm{PC} \mathrm{A02/MFAO}$

NISTIR-5512

New Method to Calculate Looming for Autonomous Obstacle Avoidance.

NISTIR-5513

Visual Pursuit Systems.

PB95-143285

$01,600 \mathrm{PC}$ A03/MF A0

STIR-5514

01,841 PC A03/MF AO

Protection of Data Processing Equipment with Fine Water Sprays

PB95-174975

$02,610 \mathrm{PC} \mathrm{A04/MF} \mathrm{A0}$

NISTIR-5515

SGML Environment for STEP.

PB95-143103

$02,778 \mathrm{PC}$ A03/MF A01 NISTIR-5516

Method of Predicting Smoke Movement in Atria with Application to Smoke Management.

PB95-154746

00,376 PC A05/MF AO

NISTIR-5517

Reference Architecture for Machine Control Systems Integration: Interim Report.

PB95-14454

$02,820 \mathrm{PC}$ A06/MF A02

ISTIR-5519

Visual Measurement Technique for Analysis of Nucleate Flow Boiling.

\section{NISTIR-5520}

$03,262 \mathrm{PC} \mathrm{A05/MF} \mathrm{A02}$

Metrology and Data for Microelectronic Packaging and interconnection: Results of a Joint Workshop on Materia Metrology and Data for Commercial Electrical and Optical Gackaging and Interconnection Technologies. Held in Gaither

sults.

PB95-143111

NISTIR-5520-V2

02,328 PC A05/MF A01

Metrology and Data for Microelectronic Packaging and Interconnection: Results of a Joint Workshop on Materials Metrology and Data for Commercial Electrical and Optical Packaging and Interconnection Technologies. Held in Gaithersburg, Maryland on May 5-6, 1994. Volume 2. Presentation Material.

PB95-143327

PB95-14332

$02,330 \mathrm{PC}$ A08/MF A02

Program Requirements to Advance the Technology of Custom Footwear Manufacturing

PB95-147906

$02,883 \mathrm{PC} \mathrm{A04/MF} \mathrm{AO}$

NISTIR-5522

Information Technology Engineering and Measurement Model: Adding Lane Markings to the Information Superhighway

PB95-143145

$01,474 \mathrm{PC} \mathrm{A03/MF} \mathrm{A01}$

NISTIR-5523

Phase-Field Model for Solidification of a Eutectic Alloy. PB95-147914

$03,345 \mathrm{PC} \mathrm{A04/MF} \mathrm{A01}$

NISTIR-5524

Review and Upgrading of Military Fastener Test Standard MIL-STD-1312.

PB95-1547
NISTIR-5526

02,947 PC A07/MF A02

Simulating Smoke Movement through Long Vertical Shafts in Zone-Type Compartment Fire Models.

in Zone- Type

00,368 PC A03/MF A01

NISTIR-5529

Electronics and Electrical Engineering Laboratory Technica Progress Bulletin Covering Laboratory Programs, July to September 1994 with 1994/1995 EEEL Events Calendar. PB95-170395

NISTIR-5530

Mapping Integration Definition for Information Modeling (IDEF1X) Model into CASE Data Interchange Forma (CDIF) Transfer File.

01,711 PC A06/MF A02

NISTIR-5531

Extreme Wind Estimates by the Conditional Mean

Exceedance Procedure.

00,120 PC A03/MF A01

NISTIR-5532

ISDN LAN Bridging

PB95-154696

01,477 PC A03/MF A01

NISTIR-5533

Design Challenges in a Commercial Ouantum Hall EffectBased Resistance Standard.

PB95-171419

$02,263 \mathrm{PC} \mathrm{A03/MF} \mathrm{A01}$

NISTIR-5535

Initial NIST Testing Policy for STEP: Beta Testing Program for AP 203 Implementations. National PDES Testbed Re-

PB95-154688

$02,779 \mathrm{PC} \mathrm{A03/MF} \mathrm{A01}$

NISTIR-5536

Rationale and Preliminary Plan for Federal Research for Construction and Building.

PB95-154704

00,322 PC A07/MF A02

NISTIR-5538

SGML Parser Validation Procedures.

PB95-174959

01,717 PC A03/MF A01

PISTR-5539

General Motion Model and Spatio-Temporal Filters for Computing Optical Flow.

NISTIR-5540

01,847 PC A03/MF A01

Multi-Agency Certification and Accreditation ( $\mathrm{C}$ and $\mathrm{A})$ Process: A Worked Example.

PB95-171955

01,601 PC A05/MF A01

NISTIR-5541

Initial Graphics Exchange Specification (IGES): Procedures for the NIST IGES Validation Test Service.

PB95-171427

02,780 PC A03/MF A01

NISTIR-5542

Binary Decision Clustering for Neural Network Based Optical Character Recognition.

PB95-171971

01,848 PC A03/MF A01

NISTIR-5543

Workshop on the Application of Virtual Reality to Manufacturing. Final Report. Held in Gaithersburg, Maryland on August 9, 1994.

NISTIR-5545

02,825 PC A11/MF A03

Neighbor Tables for Molecular Dynamics Simulations.

PB95-171948

00,991 PC A03/MF A01

NISTIR-5546

Perspective on Software Engineering Standards. PB95-171377

01,811 PC A03/MF A01

NISTIR-5547

Algorithm to Describe the Spread of a Wall Fire under a Ceiling.

PB95-182259

00,261 PC A04/MF A01

NISTIR-5550

Workshop Summary Report: Industrial Applications of Scanned Probe Microscopy. A Workshop Co-sponsored by uum Society. Held in Gaithersburg, Maryland on March 24 25, 1994. 
NISTIR-5564

Technical Impact of the NIST Calibration Service for Electrical Power and Energy.

PB96-147913

02,462 PC A05/MF A01

NISTIR-5565

Evaluation of the Economic Impacts Associated with the NIST Power and Energy Calibration Services.

PB95-188850 02,461 PC A03/MF A01 NISTIR-5566

10 kV DC Resistive Divider Calibration.

PB95-198685

02,063 PC A04/MF A01

NISTIR-5568

Carbon Monoxide Production in Compartment Fires: Reduced-Scale Enclosure Test Facility.

PB95-231700

$01,394 \mathrm{PC} \mathrm{A10/MF} \mathrm{A03}$

NISTIR-5569

MasPar MP-1 as a Computer Arithmetic Laboratory.

PB95-189437 01,627 PC A03/MF A01

NISTIR-5570

Assessment of the DOD Goal Security Architecture (DGSA) for Non-Military Use.

PB95-189510

$03.653 \mathrm{PC} \mathrm{A03/MF} \mathrm{A01}$

NISTIR-557

Operating Principles of MultiKron II Performance Instrumentation for MIMD Computers.

PB95-189486

$01,628 \mathrm{PC} \mathrm{A03/MF} \mathrm{A01}$

NISTIR-5572

Apparel Manufacturing Glossary for Application Protocol Development.

NISTIR- 5573

02,755 PC A04/MF A01

Agile Manufacturing from a Statistical Perspective.

PB96-109525 02,886 PC A03/MF A01

NISTIR-5574

Periormance of Federal Buildings in the January 17, 1994

Northridge Earthquake.
PB95-231775

00,453 PC A03/MF A01

NISTIR- 5575

Measurements of Indoor Pollutant Emissions from EPA Phase II Wood Stoves.

PB95-198735

NISTIR-5576

Computer Systems Laboratory Annual Report 1994

PB95-209920

NISTIR-5577

Materials Science and Engineering Laboratory Annual Report, 1994. NAS-NRC Assessment Panel, April 6-7, 1995.

PB95-196697

02,976 PC A06/MF A02

NISTIR-5578

Intelligent Processing of Materials, Technical Activities 1994 (NAS-NRC Assessment Panel, April 6-7, 1995).

03,359 PC A04/MF A01

NISTIR-5581

Polymers Technical Activities 1994. NAC-NRC Assessment Panel, April 6-7, 1995

PB95-209896

01,275 PC A07/MF A02

NISTIR-5582

Metallurgy Technical Activities 1994 (NAS-NRC Assessment Panel, April 6-7, 1995

PB96-136981

$02,981 \mathrm{PC} \mathrm{A08/MF} \mathrm{A02}$

NISTIR-5583

Reactor Radiation Technical Activities, 1994. NAS-NRC Assessment Panel, April 6-7, $1995.03,732$ PC A08/MF A02
PB95-20988 NISTIR-5584

CSTL Technical Activities, 1994.00608 PC A16/MF A03
PB95-242319

NISTIR-5586

Indoor Air Ouality Commissioning of a New Office Building.

PB95-182309 00,262 PC A04/MF A01

NISTIR-5587

Motion-Model-Based Boundary Extraction.

PB95-189502

01,849 PC A03/MF A01

NISTIR- 5589

Study on Hazard Analysis in High Integrity Software Standards and Guidelines.

PB95-198727

01,725 PC A04/MF A01

PTIR.5590

Proceedings Report of the International Invitation Workshop on Development Assurance. Held in Ellicott City, Maryland on June 16-17, 1994

NISTIR-5592

02,912 PC A03/MF A01

Survey of Concrete Transport Properties and Their MeasPB95-220489

NISTIR-5593

00,396 PC A04/MF A01

Vehicle-to-Roadside Communications for Commercial Vehicle Operations: Requirements and Approaches.

PB95-18882

04,860 PC A05/MF A01

NISTIR-5594

NIST Reactor: Summary of Activities, October 1993 through September 1994.

NISTIR-5595

04,700 PC A09/MF A02

Application Software Interface: ISDN Services for an Open Systems Environment.

01,492 PC A03/MF A01
NISTIR-5596

Inserting Line Segments into Triangulations and Tetrahedralizations.

PB95-198933

03,415 PC A03/MF A01

NISTIR-5597

Proceedings: Workshop on Research Needs in Wind Engineering. Held in Gaithersburg, Maryland on September 12 13,1994

PB95-189528

NISTIR-5598

00,448 PC A05/MF A0

Compositional Analysis of Beneficiated Fly Ashes.

PB95-220497

00,397 PC A03/MF A01

NISTIR-5599

Program of the Manufacturing Engineering Laboratory 1995. Infrastructural Technology. Measurements, and Standards for the U.S. Manufacturing Industries. PB95-188835

NISTIR-5600

02,754 PC A11/MF A02

Object-Oriented Technology Research Areas.
PB95-199329
01,726 PC A03/MF A01

NISTIR-5601

Expert Control System Shell Version 1.0 User's Guide.
PB95-198859 01,790 PC A03/MF A01 NISTIR-5602

Interim Testing Artifact (ITA): A Performance Evaluation System for Coordinate Measuring Machines (CMMS). User Manual.

PB95-210589

02,914 PC A04/MF A01

NISTIR-5604

Least-Cost Energy Decisions for Buildings. Part 3. Choos ing Economic Evaluation Methods. Video Training Work

PB95-253597

NISTIR-5605

$00,265 \mathrm{PC} \mathrm{A04/MF} \mathrm{A01}$

Real-Time Obstacle Avoidance Using Central Flow Divergence and Peripheral Flow.

PB95-198677

02,937 PC A03/MF A01

NISTIR-5606

Theoretical Evaluation of the Vapor Compression Cycle with a Liquid-Line/Suction-Line Heat Exchanger, Economizer and Ejector.

NISTIR-5607

02,607 PC A03/MF A01 Electronics and Electrical Engineering Laboratory Technical
Publication Announcements Covering Laboratory Programs, July to September 1994 with 1995 EEEL Events Calendar. July to September 1994 with 1995 EEEL Events Calendar.
PB95-198925 NISTIR-5608

Electronics and Electrical Engineering Laboratory Technical Progress Bulletin Covering Laboratory Programs, Octobe to December 1994 with 1995 EEEL Events Calendar. PB95-208724

NISTIR-5609

02,372 PC A04/MF A01

Calculating Time-to-Contact Using Real-Time Ouantized Optical Flow.

NISTIR-5610

01,604 PC A03/MF A01

White Papers Prepared for the White House: Construction Industry Workshop on National Construction Goals. Held on December 14-16, 1994

PB95-216891

$01,299 \mathrm{PC} \mathrm{A08/MF} \mathrm{A02}$

NISTIR-5611

Computer Simulations of Aurflow and Radon Transport in Four Large Buildings.

NISTIR-5612

02,557 PC A03/MF A01

4SIGHT Manual: A Computer Program for Modelling Degradation of Underground Low Level Waste Concrete Vaults PB95-231593 03,726 PC A05/MF A01 NISTIR-5613

Automated Manufacturing Research Facility 1994 Annual Report

PB95-209854

00,015 PC A05/MF A01

NISTIR-5614

Japan Technology Program Assessment. Simulation: Stateof the-Art in Japan.

PB95-217097

NISTIR-5615

$02,827 \mathrm{PC} \mathrm{A03/MF} \mathrm{AO}$

Design, Specification and Tolerancing of Micrometer-Tolerance Assemblies.

PB95-209862

02,913 PC A03/MF A01

Expression Formatter for MACSYMA

PB95-267829

01,735 PC A03/MF A01

NISTIR-5620

Concentrations of Chlorinated Hydrocarbons, Heavy Metals and Other Elements in Tissues Banked by the Alaska Marine Mammal Tissue Archival Project. PB95-209870

$02,590 \mathrm{PC} \mathrm{A06/MF} \mathrm{A02}$ NISTIR-5622

DETAN 95: Computer Code for Calculating Spectrum-Averaged Cross Sections and Detector Responses in Neutron Spectra.

PB95-242384

NISTIR-5623

Electronic Implementors' Workshop.

PB95-210936

04,014 PC A04/MF A01 NISTIR-5625

Survey of Steel Moment-Resisting Frame Buildings Affected by the 1994 Northridge Earthquake.

PB95-211918

$00,451 \mathrm{PC}$ A09/MF A02

NISTIR-5626

Performance Testing of a Family of Type I Combination Ap

pliance.

$02,505 \mathrm{PC} \mathrm{A03/MF} \mathrm{A01}$

NISTIR-5628

Precision in Machining: Research Challenges.

PB95-242301

$02,953 \mathrm{PC} \mathrm{A04/MF} \mathrm{A01}$

-5631

Analysis of ANSI ASC $\times 12$ and UN/EDIFACT Electronic Data Interchange (EDI) Standards.

PB95-22055

$01,729 \mathrm{PC}$ A03/MF AO

NISTIR-5632

Tables of X-ray Mass Attenuation Coefficients and Mass Energy-Absorption Coetficients $1 \mathrm{keV}$ to $20 \mathrm{MeV}$ for Elements $Z=1$ to 92 and 48 Additional Substances of Dosimetric Interest.

95-220539

04,013 PC A06/MF A02

NISTIR-5633

Testing of Selected Self-Leveling Compounds for Floors.

PB95-220455

$00,395 \mathrm{PC} \mathrm{A03/MF} \mathrm{AO}$

NISTIR-5634

Prediction of Cracking in Reinforced Concrete Structures.
PB95-220448
$03,725 \mathrm{PC} \mathrm{A04/MF} \mathrm{AO}$

NISTIR-5636

Persistent Object Base System Testing and Evaluation

PB95-220588

esting and Evaluation.
01,730 PC A05/MF A0

NISTIR-5637

Texture-Independent Vision-Based Closed-Loop Fuzzy Conrollers for Navigation Tasks.

PB95-220505

$00,183 \mathrm{PC} \mathrm{A03/MF} \mathrm{AO1}$

NISTIR-5638

SO Environmental Management Standardization Efforts.

PB95-220513

$02,524 \mathrm{PC} \mathrm{A03/MF} \mathrm{AO}$

TIR-5638-1

ISO Environmental Management Standardization Efforts.

NISTIR-5639

Certification of the Standard Reference Material 1473a, a Low Density Polyethylene Resin

$01,282 \mathrm{PC} \mathrm{A03/MF} \mathrm{A01}$

NISTIR-5641

Anisotropy of Interfaces in an Ordered Alloy: A Multiple Order-Parameter Model.

PB96-131594

04,741 PC A04/MF AO

TIR-5642

Suppression of High Speed Turbulent Flames in a Detona tion/Deflagration Tube

PB95-2318

$01,395 \mathrm{PC}$ A04/MF AO

NISTIR-5643

Outline of a Multiple Dimensional Reference Model Architecture and a Knowledge Engineering Methodology for Intelligent Systems Control.

$03,703 \mathrm{PC} \mathrm{A03/MF} \mathrm{A01}$

Evaluation of Thermal Wave Imaging for Detection of Ma chining Damage in Ceramics.

PB95-220547 
NISTIR-5660

Parallel and Serial Implementations of SLI Arithmetic PB95-242335

01,732 PC A03/MF A01 NISTIR-5661

Fracture Testing of Large-Scale Thin-Sheet Aluminum Alloy PB95-242368

00,024 PC A03/MF A01 NISTIR-5662

Requisite Elements, Rationale, and Technology Overview for the Systems Intergration for Manufacturing Applications SIMA) Program. Background Study. PB96-112685

NISTIR-5663

Multiattribute Decision Analysis Method for Evaluating Buildings and Building Systems.

00,325 PC A06/MF A01

NISTIR-5664

Recommended Pertormance-Based Criteria for the Design of Manufactured Home Foundation Systems to Resist Wind and Seismic Loads.

PB96-12828

00,460 PC A05/MF A01

NISTIR-5665

SO TC 184/SC4 Reference Manual.

PB95-242293

$02,663 \mathrm{PC} \mathrm{A04/MF} \mathrm{A01}$ NISTIR-5666

Operating Procedures and Life Cycle Documentation for the nitial Graphics Exchange Specification.

PB95-242285

02,782 PC A04/MF AO

NISTIR-5667-V

Study of Potential Applications of Automation and Robotics Technology in Construction, Maintenance and Operation of Highway Systems: A Final Report Volume 1

01,341 PC AOQTF A02

NISTIR-5667-V2

$01.341 \mathrm{PC} \mathrm{A06/MF} \mathrm{A02}$

Study of Potential Applications of Automation and Robotics Technology in Construction, Maintenance and Operation of

$\begin{array}{ll}\text { Highway Systems: A Final Report. Volume } 2 . \\ \text { PB95-255865 } & 01,343 \text { PC A19/MF A04 }\end{array}$ NISTIR-5667-V3

Study of Potential Applications of Automation and Robotics Technology in Construction, Maintenance and Operation of Highway Systems: A Final Report. Volume 3 .

PB95-251690

01,342 PC A15/MF A03

NISTIR-5667-V4

Study of Potential Applications of Automation and Robotics Technology in Construction. Maintenance and Operation of Highway Systems: A Final Report Volume 4.

PB95-251641
01,340 PC A06/MF A02 NISTIR-5669

Electronics and Electrical Engineering Laboratory Technica Publication Announcements Covering Laboratory Programs, January to March 1995 with 1995 EEEl Events Calendar.

PB95-242277 02,373 PC A03/MF A01

NISTIR-5671

Determination of the Residual Stresses Near the Ends of Skip Welds Using Neutron Diffraction and X-ray Diffraction Procedures.

PB95-253589

02,868 PC A03/MF A01

NISTIR-5672

Advanced Mass Calibration and Measurement Assurance Program ior State Calibration Laboratories. $P$ PC A03/MF A0 NISTIR-5673

Information Technologies Make Business Sense for the Custom Therapeutic Footwear Industry.
PB95-251708
02,829 PC A03/MF A01

NISTIR-5674

User's Guide for the Algorithm Testing System Version 2.0. PB95-251666

NISTIR-5675

Group 1 for the Plant Spatial Configuration STEP Applicaion Protocol.

PB96-16540

NISTIR-5676

02,789 PC A11/MF A03

Submarine Automation: Demonstration No. 5

PB95-251633

03,748 PC A03/MF AO1

NISTIR-5677

Center for High Integrity Software System Assurance: Initia Goals and Activities.

PB95-251674

$01,734 \mathrm{PC} \mathrm{A03/MF} \mathrm{A01}$

NISTIR-5678

Integrated Vision Touch-Probe System for Dimensional Inspection Tasks.

NISTIR-5680

02.917 PC A03/MF AO

Proficiency Tests for the NIST Airborne Asbestos Program

PB96-106463

NISTIR-5681

00,610 PC A03/MF A01

Water-Vapor Measurements of Low-Slope Roofing Materials.

NISTIR-5682

00,399 PC A03/MF A01

Strengthening Methodology for Lightly Reinforced Concrete Frames: Recommended Design Guidelines for Strengthening with Infill Walls.

PB95-260725

00,454 PC A04/MF A01 (1)

SF6 Insulation: Possible Greenhouse Problems and Solutions.

$02,269 \mathrm{PC} \mathrm{A03/MF} \mathrm{A01}$
NISTIR-5686

Algorithm Testing and Evaluation Program for Coordinate Measuring Systems: Testing Methods.

PB95-251658

02,666 PC A03/MF A01

NISTIR-5687

Method and Evaluation of Character Stroke Preservation on Handprint Recognition.

PB95-251724

01,850 PC A03/MF A01

\section{STIR-5688}

Proceedings of the Meeting of the Intergovernmental U.S.Russian Business Development Committee's Standard Working Group (4th). Held in New York City, New York on March 27-29, 1995 and in Northbrook, Illinois on March 30 $31,1995$.

PB95-25588

$00,496 \mathrm{PC} \mathrm{A11/MF} \mathrm{A03}$

NISTIR-5689

Simple Method of Composition Shifting with a Distillation Column for a Heat Pump Employing a Zeotropic Refrigerant Mixture.

PB95-255824

$02,603 \mathrm{PC} \mathrm{A03/MF} \mathrm{A01}$

NISTIR-5690

Long-Term P

red Concrete Barriers.

ISTIR-5691-V1

03,727 PC A03/MF A01

Unravel: A CASE Tool to Assist Evaluation of High Integrity Software. Volume 1. Requirements and Design.
PB95-267886
01,736 PC A05/MF A01 NISTIR-5691-V2

Unravel: A CASE Tool to Assist Evaluation of High Integrity Software. Volume 2. User Manual.

PB95-267894

01,737 PC A04/MF A01

NISTIR-5692-SUP

Applications of Diamond Films and Related Materials: International Conference (3rd). Held in Gaithersburg, Maryland on August

PB95-256053

03,063 PC A04/MF A01

NISTIR-5695

Improving Neural Network Performance for Character and

Fingerprint Classification by Altering Network Dynamics.
PB95-267803 01,851 PC A03/MF A01

NISTIR-5696

Effect of Training Dynamics on Neural Network Performance.

01,852 PC A03/MF A01

NISTIR-5697

GATT Standards Code Activities of the National Institute of

Standards and Technology 1994

NISTIR-5698

Estimation of Measurement Uncertainty of Small Circular Features Measured by CMMs.

PB95-267928

02,918 PC A03/MF A01

NISTIR-5699

Computer-Aided Manufacturing Engıneering Forum (1st). Technical Meeting Proceedings. Held in Gaithersburg, Maryland on March 21-22, 1995.

PB96-136965

$02,834 \mathrm{PC} \mathrm{A10/MF} \mathrm{A03}$

NISTIR-5700

Proceedings of the 1995 Workshop on Fire Detector Research. Held on February 6-7, 1995.
PB95-270062

NISTIR-5701

Metrology Issues in Terahertz Physics and Technology.

PB96-128277

01,939 PC A08/MF A02

NISTIR-5703

NIST ATM Network Simulator: Operation and Programming. Version 1.0.
PB96-106851

01,487 PC A04/MF A01

NISTIR-5704

EMISS: A Program for Estimating Local Air Pollution Emission Factors Related to Energy Use in Buildings: User's Guide and Refere

$02,560 \mathrm{PC} \mathrm{A03/MF} \mathrm{A01}$

NISTIR-5705

CFAST Output Comparison Method and Its Use in Comparing Different CFAST Versions.

PB96-109541

00,401 PC A04/MF A01

NISTIR-5708 Electronic Access to Standards on the Information Highway.
PB96-131578 01,494 PC A03/MF A01

01,494 PC A03/MF A01

NISTIR-5709

Electronics and Electrical Engineering Laboratory Technical Progress Bulletin Covering Laboratory Programs, April to June 1995 with 1995 EEEL Events Calendar.

PB96-106455

$01,923 \mathrm{PC}$ A03/MF A01

NISTIR-5710

Effect of Suppressants on Metal Fires.

PB96-109574

01,402 PC A03/MF A01

NISTIR-5712 Indoor Air Quality Impacts of Residential HVAC Systems
Phase II.B Report: IAQ Control Retrofit Simulations and

Analysis.

02,559 PC A05/MF A01

NISTIR-5714

Ground Improvement Techniques for Liquefaction Remediation Near Existing Lifelines.

01,350 PC A05/MF A01

NISTIR-5715

Optoelectronics and Optomechanics Manufacturing: An ATP Focused Program Development. Workshop Proceedings Held in Gaithersburg, Maryland on February 15, 1995

PB97.104186

02,204 PC A $11 / \mathrm{MF}$ AO3

NISTIR-5716

Guidelines for the Development of Mapping Tables.

PB96-154539

02,786 PC A03/MF A01

NISTIR-5717

NIST SIMA Interactive Management Workshop. Held in Fort

Belvoir, Virginia on November 14-16, 1994

PB96- 154877

02,838 PC A08/MF A02

NISTIR-5719 Mapping Integration Definition for Function Modeling
(IDEFO) Model into CASE Data Interchange Format (CDIF) Transfer File.

NISTIR-5720

01,741 PC A07/MF A02

User's Guide to 'SuperFit' Modeling Software for CMM Probe Lobing.

$02,921 \mathrm{PC} \mathrm{A03/MF} \mathrm{AO1}$

NISTIR-5722

Reference Manual for the Algorithm Testing System Version 2.0.

PB96-128244

02,922 PC A03/MF A01

NISTIR-5723

Airborne Asbestos Method: Bootstrap Method for Determining the Uncertainty of Asbestos Concentration. Version 1.0.
00,646 PC A04/MF A01 NISTIR-5724

Error-Bounding in Level-Index Computer Arithmetic.

PB96-109582

01,742 PC A02/MF A01

NISTIR-5726

Generalized Form Registration Using Structure-Based Techniques.

NISTIR-5727

01,858 PC A03/MF A01

Extensions of the Prototype Application Protocol of Readyto-Wear Apparel Pattern Making

PB96-128194

$03,198 \mathrm{PC} \mathrm{A03/MF} \mathrm{A01}$

NISTIR-5728

Image Gradient Evolution: A Visual Cue for Danger.

PB96-154562

02,939 PC A03/MF A01

NISTIR-5729

Study of Technology for Detecting Pre-Ignition Conditions of Cooking-Related Fires Associated with Electric and Gas Ranges and Cooktops, Phase 1 Report.

PB96-128095

$00,303 \mathrm{PC} \mathrm{A06/MF} \mathrm{A02}$

NISTIR-5730

Overview of the Manufacturing Engineering Toolkit Prototype.

NISTIR-5732

02,833 PC A03/MF A01

Calorimetric and Visual Measurements of R123 Pool Boiling Cour Enhanced Surtaces.

PB96-128129

NISTIR-5734 
NISTIR-5748

Materials Reliability. Technical Activities, 1995 PB96-183082

02,999 PC A07/MF A02

Polymers Technical Activities, 1995.

PB96-193719

01,291 PC A10/MF A02

NISTIR-5750 Metallurgy. Technical Activities, 1995.
PB96-195284 03,003 PC A09/MF A02

NISTIR-5751

Reactor Radiation Technical Activities, 1995.
PB96-193644

NISTIR-5752

Summary Report: Workshop on Industrial Applications of Scanned Probe Microscopy (2nd). A Workshop Co-Sponsored by NIST, SEMATECH, ASTM E42.14, and the American Vacuum Society. Held in Gaithersburg, Maryland on May 2-3, 1995

PB96-131602

00,509 PC A07/MF A02

NISTIR-5757

Sharing Information via the Internet: An Infoserver Case Study.

01,493 PC A03/MF A01

NISTIR-5758

Workplan to Analyze the Energy Impacts of Envelope Airtightness in Office Buildings.

PB96-154463

$00,273 \mathrm{PC} \mathrm{A04/MF} \mathrm{A01}$

NISTIR-5759

National Planning for Construction and Building $\mathrm{R}$ and $\mathrm{D}$.

$\begin{array}{lll}\text { PB96-137104 00,324 PC A06/MF A02 } & 0\end{array}$

NISTIR-5760

Classified Bibliography: Insulation Condition Monitoring Methods, 1989-1995.
PB96-131586

PB96-131586
PISTIR-5762

02,232 PC A05/MF A01

Standard Generalized Markup Language Test Suite Evalua-

Sion Report.
PB96-154992
P1,751

NISTIR-5763

General Motion Model and Spatio-Temporal Filters for 3-D Motion Interpretations

PB96-210703

$01,861 \mathrm{PC}$ A07/MF A02

NISTIR-5764

Smoke Plume Trajectory from In situ Burning of Crude Oil

in Alaska: Field Experiments.

PB96-131560

02,594 PC A03/MF A01

Simplified Design Procedure for Hybrid Precast Concrete Connections.

NISTIR-5766

00,405 PC A06/MF A01

Solid Propellant Gas Generators: Proceedings of the 1995 Workshop. Held in Gaithersburg. Maryland on June 28-29,

PB96-131479

01,412 PC A11/MF A03

NISTIR-5767

Effect of Inclination on the Performance of a Compact

Brazed Plate Condenser and Evaporator.
PB96-136973 02,756 PC A03/MF A01

NISTIR-5769

C++ in Safety Critical Systems

$01,750 \mathrm{PC} \mathrm{A04/MF} \mathrm{A01}$

NISTIR-5770

How-To Suggestions for Implementing Executive Order 12941 on Seismic Safety of Existing Federal Buildings, A Handbook.

PB96-131552

NISTIR-5771

00,461 PC A10/MF A03

STandard for the Exchange of Product Model Data (STEP): $\begin{array}{lll}\text { Procedures for NIST STEP Validation. } & \\ \text { PB96-154976 } & 02,787 & \text { PC A03/MF A01 }\end{array}$

Electronics and Electrical Engineering Laboratory Technical Publication Announcements Covering Laboratory Programs, April to June 1995 with 1995 EEEL Events Calendar.

PB96-137187 01,941 PC A03/MF A01

NISTIR-5774

Electronics and Electrical Engineering Laboratory Technical Progress Bulletin Covering Laboratory Programs, July to September 1995 with 1996 EEEL Events Calendar.

PB96-147905

01,942 PC A04/MF A01

NISTIR-5775

Machine Performance Standard Provides Opportunity to Improve Quality and Productivity.

PB96-15452

$02,837 \mathrm{PC} \mathrm{A02/MF} \mathrm{A01}$

Santa Ana Fire Department Experiments at South Bristol

PB96-154810

$00,305 \mathrm{PC} \mathrm{A11/MF} \mathrm{A03}$

NISTIR-5777

Beyond the Technology Roadmaps: An Assessment of Electronic Materials Research and Development.

Electronic Materials Research and Development.
PB96-165998 01,961 PC A05/MF A01 NISTIR-5778

Collection of Results for the SPC/E Water Model.

PB96-147889

01,127 PC A03/MF A01

NISTIR-5780

Enhancement of R123 Pool Boiling by the Addition of NHexane.

PB96-165956

02,605 PC A04/MF A01
Electronics and Electrical Engineering Laboratory Technical
Progress Bulletin Covering Laboratory Programs, October

NISTIR-5782

Modified Optimal Algorithm for Active Structural Control.

PB96-165949

NISTIR-5783

$00,472 \mathrm{PC} \mathrm{A04/MF} \mathrm{A01}$

Development of a Test Method for Leaching of Lead from Lead-Based Paints Through Encapsulants.

PB96-154984

$03,128 \mathrm{PC} \mathrm{A04/MF} \mathrm{A01}$

R-5784

Study to Determine the Existence of an Azeotropic R-22 'Drop-In' Substitute

PB96-167812

$02,568 \mathrm{PC} \mathrm{A04/MF} \mathrm{A01}$

Summary and Results of the NIST Workshop on Proposed Guidelines for Testing and Evaluation of Seismic Isolation Systems. Held in San Francisco, California on July 25,

00,463 PC A04/MF A01

$-154901$

AutoBid 2.0: The Microcomputer System for Police Patrol
Vehicle Selection.
PB96-154570 04,871 PC A03/MF A01

PB96-154570

NISTIR-5788

Public Key Infrastructure Invitational Workshop. Held in McLean, Virginia on September 28, 1995.
PB96-166004 NISTIR-5789

Using S-Check, Alpha Release 1.0.

PB96-165964

01,767 PC A05/MF A01

NISTIR-5790

Scale-Space-Based Visual-Motion-Cue for Autonomous Navigation.

NISTIR-5791

$02,940 \mathrm{PC} \mathrm{A06/MF} \mathrm{A01}$

Novel Active-Vision-Based Motion Cues for Local Navigation.

PBפ6-19:

$02,941 \mathrm{PC}$ A05/MF A01

Data Communications Strategy.
PB96-167846

\section{IR-5795}

Minimum Mass Flux Requirements to Suppress Burning

$01,425 \mathrm{PC} \mathrm{A05/MF} \mathrm{A01}$

Guide to a Format for Data on Chemical Admixtures in a Materials Property Database.

01,327 PC A04/MF A01 Guide to a Format for Data on Chemical Admixtures in a Materials Property Database. (Reannouncement with new abstract).

B96-186192

$01,328 \mathrm{PC} \mathrm{A04/MF} \mathrm{A01}$

ISTIR-5797

PIECS: A Software Program for Machine Tool ProcessIntermittent Error Compensation.

PB96-165980

$02,842 \mathrm{PC} \mathrm{A08/MF} \mathrm{A02}$

PTIR-5798

NASA Fire Detector Study.

PB96-183108

$01,423 \mathrm{PC} \mathrm{A04/MF} \mathrm{A01}$ NISTIR-5799

Application of the Pointer State Subgraph to Static Program

PB96-167838

01,768 PC A03/MF A01

Guidelines for Pre-Oualification, Prototype and Quality Con-

trol Testing of Seismic Isolation Systems.
PB96-193685 01,347 PC A08/MF A02

NISTIR-5801

Multizone Modeling of Three Residential Indoor Air Ouality Control Options.

NISTIR-5806

$02,567 \mathrm{PC}$ A08/MF A02

Method of Estimating the Parameters of Tuned Mass Dampers for Seismic Applications.

PB96-167820

NISTIR-5808

Machining Process Planning Activity Model for Systems Integration.

NISTIR-5809

$02,841 \mathrm{PC} \mathrm{A03/MF} \mathrm{A01}$

Numerical Simulation of Rapid Combustion in an Underground Enclosure.

NISTIR-5810

TMACH Experiment Phase 1. Preliminary Developmental Evaluation.
PB96-195318

NISTIR-5811

$01,618 \mathrm{PC} \mathrm{A03/MF} \mathrm{A01}$

Basic Linear Algebra Operations in SLI Arithmetic PB96-165931

$03,421 \mathrm{PC} \mathrm{A03/MF} \mathrm{A01}$

NISTIR-5815 Progress Bulletin Covering Laboratory Programs,
to December 1995 with 1996 EEEL Events Calendar. PB96-183116

01,966 PC A04/MF A01

NISTIR-5816

Electronics and Electrical Engineering Laboratory Technical

Publication Announcements Covering Laboratory Programs,

July to September 1995 with 1996 EEEL Events Calendar.
PB96-183066 Surfaces with Water Sprays.

NISTIR-5818

Electronics and Electrical Engineering Laboratory 1995 Technical Accomplishments: Advancing Metrology for Electrotechnology to Support the U.S. Economy.
PB96-164520 NISTIR-5819

X.500 Directory Schema Design Handbook

PB96-183041

$02,739 \mathrm{PC} \mathrm{A03/MF} \mathrm{A01}$

NISTIR-5820

Distributed Communication Methods and Role-Based ACcess Control for Use in Health Care Applications.
PB96-183165 01,508 PC A05/MF A01

NISTIR-5821

Aspects of a Product Model Supporting Apparel Virtual Enterprises.

NISTIR-5822

$02,790 \quad \mathrm{PC} \mathrm{A03/MF} \mathrm{A01}$

Proceedings of NIST Workshop: Industry Needs in Welding Research and Standards Development. Held on August 15$16,1995$.

PB96-183124

02,877 PC A04/MF A01

NISTIR-5823

Survey of Standards for the U.S. Fiber/Textile/Apparel Industry.

PB96-193792
NISTIR-5824

03,199 PC A06/MF A01

Interoperability Experiments with CORBA and Persistent Object Base Systems.
PB96-183140

NISTIR-5826

01,772 PC A04/MF A0

Hygrothermal Effects on the Pertormance of Polymers and Polymeric Composites: A Workshop Report. Held in Gaithersburg, Maryland on September $21-22,1905$

$03,180 \mathrm{PC}$ A04/MF A01

NISTIR-5828

CSTL Technical
PB96-214630

NISTIR-5832

$00,647 \mathrm{PC} \mathrm{A10/MF} \mathrm{A03}$

Electronics and Electrical Engineering Laboratory: 1996 Program Plan. Supporting Technology for U.S. Competitiveness in Electronic

$01,962 \mathrm{PC} \mathrm{A10/MF} \mathrm{A03}$

NISTIR-5833

Procedure for Product Data Exchange Using STEP Developed in the AutoSTEP Pilot.

PB96-183058

02,843 PC A04/MF A01

NISTIR-5837

Workshop on Characterizing Diamond Films (4th). Held in Gaithersburg, Maryland on March 4-5, 1996. PB96-183090

NISTIR-5838

Room-Temperature Thermal Conductivity of Expanded Polystyrene Board for a Standard Reference Material.

PB96-193693

00,412 PC A04/MF A0

NISTIR-5839

Systems Integration for Manufacturing Applications Program 1995 Annual Report

PB96-193735

02,844 PC A05/MF A01

Benefits and Costs of Research: Two Case Studies in Building Technology

PB96-20222

00,230 PC A07/MF A02

Methodology for Developing and Implementing Alternative Temperature-Time Curves for Testing the Fire Resistance of Barriers for Nuclear Power Plant Applications.

PB96-193784

03,742 PC A07/MF A02 
NISTIR-5853

Electronics and Electrical Engineering Laboratory Technical Progress Bulletin Covering Laboratory Programs, January o March 1996, with 1996 EEEL Event

01,969 PC A04/MF A01 NISTIR-5854

Computer Systems Laboratory Computing and Applied Mathematics Laboratory Technical Accomplishments, October 1994-March 1996

PB96-193768

$01,638 \mathrm{PC} \mathrm{A05/MF} \mathrm{A01}$

NISTIR-5855

Specification for Interoperability between Ballistic Imaging Systems. Part 1. Cartridge Cases.

P1,860 PC A04/MF A01 NISTIR-5856

NIST Construction Automation Program Report No. 2. Proceedings of the NIST Construction Automation Workshop.

Held in Gaithersburg, Maryland on March 30-31, 1995.
PB96-202239 00,413 PC A09/MF A02 NISTIR-5857

State of the Art Report on Seismic Design Requirements for Nonstructural Building Components.
DB96-193800 PC A06/MF A01 PB96-193800

NISTIR-5859

MV++ V. 1.5a Matrix/Nector Class Reference Guide.
PB96-195326 NISTIR-

IML++ v.1.2 Iterative Methods Library Reference Guide

PB96-195219 01,776 PC A04/MF A0

NISTIR-5861

SparseLib++ v. 1.5 Sparse Matrix Class Library. Reference Guide.

$01,775 \mathrm{PC}$ A03/MF A01

NISTIR-5862

Operator Experience with a Hierarchical Real-Time Control System (RCS).

NISTIR-5863

Benefits and Costs of Research: A Case Study of the Fire Satety Evaluation System.

PB96-20228

$00,232 \mathrm{PC} \mathrm{A06/MF} \mathrm{A01}$

NISTIR-5864

Economics of New-Technology Materials: A Case Study of FRP Bridge Decking

PB96-202353

01,349 PC A06/MF AO

NISTIR-5865

Development of a Method for Measuring Water-Stripping

Resistance of Asphalt/Siliceous Aggregate Mixtures.
PB96-202296 NISTIR-5866

Developing Quality System Documentation Based on ANSI/ NCSL Z540-1-1994: The Optical Technology Division's Effort.

PB96-202122

01,869 PC A05/MF A01

NISTIR-5868

Application Protocol Information Base World Wide Web Gateway.

NISTIR-5869

$02,791 \mathrm{PC} \mathrm{A03/MF} \mathrm{AO}$

Measurement of Rheological Properties of High Perform-

ance Concrete: State of the Art Report.
PB96-202338
00,414 PC A04/MF A01 NISTIR-5870

Shear Design of High-Strength Concrete Beams: A Review of the State-of-the-Art

PB96-214713

$01,330 \quad \mathrm{PC} \mathrm{A11/MF} \mathrm{A03}$

NISTIR-5871

Report on USDA Ultraviolet Spectroradiometers

PB96-214648

$00,125 \mathrm{PC} \mathrm{A04/MF} \mathrm{A01}$

R-5872

Electronics and Electrical Engineering Laboratory Technica Publication Announcements Covering Laboratory Programs, October to December 1995, with 1996 EEEL Events Calendar.

PB96-202346

$01,978 \mathrm{PC} \mathrm{A04/MF} \mathrm{A01}$

NISTIR-5873

Intracycle Evaporative Cooling in a Vapor Compression

Pycle.

02,762 PC A03/MF A01

NISTIR-5877

Report of a Workshop on Requalification of Tubular Steel Joints in Offshore Structures. Held in Houston, Texas on September 5-6, 1995.

PB96-210760

03,699 PC A07/MF A02

NISTIR-5878

Fire Safety Engineering in the Pursuit of PerformanceBased Codes: Collected Papers.

PB97-114482

$00,235 \mathrm{PC} \mathrm{A07/MF} \mathrm{A02}$

NISTIR-5879

Conformance Testing and Specification Managemen

PB97-113781

NISTIR-5880

02,849 PC A03/MF A01

Mathematical Analysis of Practices to Control Moisture in the Roof Cavities of Manufactured Houses.
PB97-106843 00,278 PC A05/MF A01 PB97-106843

Static Structural Analysis of a Reconfigurable Rigid Platform Supported by Elastic Legs.

02,960 PC A05/MF A01
NISTIR-5887

Diffuse-Interface Description of Fluid Systems.

PB96-210711

01,170 PC A04/MF A01

NISTIR-5888

Materials Aspects of Fiber-Reinforced Polymer Composites in Infrastructure.

NISTIR-5889

Experimental Models for Software Diagnosis.

PB97-113906

01,783 PC A04/MF A01

NISTIR-5892

Electronics and Electrical Engineering Laboratory Technical Publication Announcements Covering Laboratory Programs, January to March 1996, with 1996 EEEL Events Calendar.

NISTIR-5893

Experimentally Measured Total X-ray Attenuation Coefficients Extracted from Previously Unprocessed Documents Held by the NIST Photon and Charged Particle Data Center.

PB97-114474

04,165 PC A04/MF A01

NISTIR-5895

Electronics and Electrical Engineering Laboratory Technical Progress Bulletin Covering Laboratory Programs, April to June 1996 with 1996-1998 EEEL Even

PB97-113880

01,994 PC A04/MF A01

NISTIR-5897

Representation of Axes for Geometric Fitting PB97-113799

01,782 PC A03/MF A01

NISTIR-5898

TBT Agreement Activities of the National Institute of Standards and Technology, 1995

PB97-104178

00,499 PC A04/MF A01

NISTIR-5899

Standards Promote Credibility and Technology Transfer: The Need for Greater Industry Support of Technical Committees.

PB97-116206

$02,961 \mathrm{PC} \mathrm{A03/MF} \mathrm{A01}$

NISTIR-5900

Multi-Scale Picture of Concrete and Its Transport Prop-

erties: Introduction for Non-Cement Researchers.
PB97-115802 03,107 PC A05/MF A01 NISTIR-5906

Carbon Monoxide Dispersion in Residential Buildings: Literature Review and Technical Analysis.
PB97-114227 02,571 PC A05/MF A01

NISTIR-5907

Proceedings of a Workshop on Developing and Adopting Seismic Design and Construction Standards for Lifelines. Held in Denver, Colorado on September 25-27, 1991

PB97-115794

01,302 PC A16 MF A03

NISTIR-5909

Group 1 for the Process Engineering Data STEP Application Protocol

NISTIR-5910

02,797 PC A99/MF A06

Unified Process Specification Language: Requirements for Modeling Process.

NOAA-TM-ERL-GLERL-97

$02,850 \mathrm{PC} \mathrm{A06/MF} \mathrm{A01}$

Lake Erie Water Temperature Data, Put-in-Bay, Ohio, 1918 Lake Erie Water Temperature Data, Put-in-Bay, Ohio, 1918-
1992.
PB96-202452 03,692 PC A04/MF A01

NRELTP-411-8246

Atomic-scale characterization of hydrogenated amorphoussilicon films and devices. Annual subcontract report, 14 February 1994--14 April 1995. DE95009287

NUREG/CP-0136

$02,294 \mathrm{PC} \mathrm{A03/MF} \mathrm{A01}$

Proceedings of the Digital Systems Reliability and Nuclear Safety Workshop. Held in Rockville, Maryland on September 13-14, 1993.

ORNUCON-381

Economic, Energy, and Environmental Impacts of the En-

ergy-Pelated Inventions Program.
DE94-017162

00,008 PC A05/MF A02

ORNLSUB/83-21322/03

Abrasive Wear by Diesel Engine Coal-Fuel and Related Particles.

PB95-104915

01,450 PC A04/MF A01

PB94-100278

Opportunities for Innovation: Advanced Manufacturing Technology.

PB94-112695

02,801 PC A07/MF A02

Electroacoustics for Characterization of Particulates and Suspensions. Proceedings of a Workshop. Held in Gaithersburg, Maryland on February 3-4, 1993 .
PB94-112695 PB94-123080

Technology Trends in Telecommunications: An Overview. PB94-123080

01,462 PC A03/MF A01

PB94-126901

Electronics and Electrical Engineering Laboratory 1994 Program Plan: Supporting Technology for U.S. Competitiveness in Electronics.

$01.873 \mathrm{PC} \mathrm{A09/MF} \mathrm{A02}$

PB94-134897

General Procedures for Registering Computer Security Obects.

PB94-134897

01,573 PC A03/MF A01

PB94-135001

Report of the NIST Workshop on Digital Signature Certifi-

cate Management. Held on December 10-11, 1992.
PB94-135001 PB94-135621

Open Architectures for Machine Control.

PB94-135621

02,942 PC A03/MF A01

PB94-135639

Crosstalk between Microstrip Transmission Lines.

PB94-135639

02,210 PC A03/MF A01

PB94-135761

Planning for the Fiber Distributed Data Interface (FDDI).

PB94-135761

PB94-135803

01,621 PC A06/MF A02

NIST Model PM2 Power Measurement System for $1 \mathrm{~mW}$ at $1 \mathrm{GHz}$

$-135803$

02,018 PC A03/MF A01

PB94-136009

NIST Handbook 44, 1994: Specifications, Tolerances and Other Technical Requirements for Weighing and Measuring Devices as Adopted by the 78th National Conference on Weights and Measures 1993

PB94-136009

PB94-138989

$02,888 \mathrm{PC} \mathrm{A11/MF} \mathrm{A03}$

Report of the National Conference on Weight and Measures 78th). Held in Kansas City, MO. on July 18-22, 1993 PB94-13898

PB94-139045

$02,623 \mathrm{PCA}$ A $18 / \mathrm{MF}$ A04

Good Security Practices for Electronic Commerce, Including Electronic Data Interchange.

PB94-139045

$01,463 \mathrm{PC} \mathrm{A04/MF} \mathrm{A01}$

PB94-139193

Some Factors Affecting Design of a Furniture Calorimeter Hood and Exhaust.

PB94-139193

$00,285 \mathrm{PC} \mathrm{A03/MF} \mathrm{A01}$

PB94-139623

Computing Effects and Error for Large Synthetic Perturbation Screenings.

PB94-139649

$01,675 \mathrm{PC} \mathrm{A03/MF} \mathrm{A0}$

Security Considerations for SQL-Based Implementations of STEP.

PB94-139649

PB94-139672

02,766 PC A03/MF A01

Electromechanical Properties of Superconductors for DOE Fusion Applications

PB94-139672

$02,250 \mathrm{PC}$ A06/MF A02

Fire Induced Thermal Fields in Window Glass I: Theory

PB94-139722

PB94-140167

Guide to Software Engineering Environment Assessment and Evaluation.

$01,676 \mathrm{PC}$ A04/MF A01

B94-140407

Computer Programs for Simulation of Lighting/HVAC Inter-

PB94-14040

02,501 PC A08/MF A02

PB94-140555

Journal of Research of the National Institute of Standards and Technology, November-December 1993. Volume 98 Number 6 .

PB94-140555 
PB94-142486

Mass Unit Disseminated to Surrogated Laboratories Using the NIST Portable Mass Calibration Package.
PB94-142486 03,781 PC A03/MF A01

PB94-142494

Introduction to Traffic Management for Broadband ISDN.

PB94-142494

PB94-142502

Applying Virtual Environments to Manufacturing

PB94-14250

PB94-142510

Assessment of Uncertainties of Liquid-in-Glass Thermometer Calibrations at the National Institute of Standards and Technology.

PB94-142528

$02,625 \mathrm{PC} \mathrm{A03/MF} \mathrm{A01}$

Context Analysis of the Network Management Domain. Conducted as Part of the Domain Analysis Case Study.

PB94-142528

01,465 PC A05/MF A01

PB94-142536

Report on Application Integration Architectures (AIA) Workshop. Held in Dallas. Texas on February 8-12, 1993 .
PB94-142536 PB94-142791

Feasibility Study: Reference Architecture for Machine Control Systems Integration.

PB94-14279

02,804 PC A12/MF A03

PB94-142973

In situ Buming of Oil Spills: Mesoscale Experiments.

PB94-142973

01,355 PC A04/MF A01

PB94-143344

Evaluation of GSA Maintenance Practices of Large Centrifugal Chillers and Review of GSA Refrigerant Management Practices.

PB94-143344

$02,502 \mathrm{PC} \mathrm{A04/MF} \mathrm{A01}$

PB94-143401

Next Generation Computer Resources: Reference Model for Project Support Environments (Version 2.0).

PB94-14340 01,677 PC A06/MF A02

and Tor the Rise and Dispersion of WindComputational Model for the Rise and Dispersion of Wind-
Blown, Buoyancy-Driven Plumes. Part 2. Linearly Stratified Blown, Buoyancy-Driven Plumes. Part 2. Linearly Stratified
Atmosphere. Atmosphere.

00,119 PC A03/MF A01

PB94-143542 Ground-Based Smoke Sampling Techniques Training
Course and Collaborative Local Smoke Sampling in Saudi

PB94-143542

02,532 PC A05/MF A01

PB94.145620

Reliable Optical Flow Algorithm Using 3-D Hermite Polynomials.
PB94-145620

01,829 PC A03/MF A01

PB94-145653

Manual for Ventilation Assessment in Mechanically Ventilated Commercial Buildings.

PB94-145653

00,239 PC A07/MF A02

PB94-145661

System for Calibration of the Marshall Compaction Ham.

PB94-14566

01,303 PC A08/MF A02

PB94-145711

Aperture Excitation of Electrically Large, Lossy Cavities.

PB94-145711

Large, Lossy Cavities.
00,029 PC A05/MF A01

PB94-145968

Electronics and Electrical Engineering Laboratory Technica

Progress Bulletin Coverıng Laboratory Programs, January

to March 1991, with 1991 EEEL Events Calendar.

PB94-145968 W2, 113 PC A03/MF A01

PB94-145976

Use of Computer Models to Predict Temperature and

Smoke Movement in High Bay Spaces.
PB94-145976 PB94-14597

PB94-145984

Alternating-Field Susceptometry and Magnetic Susceptibility of Supercondoctors. Presented at Office of Naval Research Workshop on Magnetic Susceptibility of Superconductors and Other Spin Systems. Held in Berkeley Springs, West Virginia on 20 May 1991.

PB94-145984

$04,435 \mathrm{PC} \mathrm{A01/MF} \mathrm{A01}$

PB94-146636

Modulation of Fossil Fuel Production by Global Tempera ture Variations, 2

PB94-146636

PB94-146677

02,533 PC A04/MF AO

Thermophysical Properties of Fluids for the Gas Industry.

Final Report, February 1, 1988-August 31, 1993 .
PB94-146677
O2,472 PC A03/MF A01

PB94-150919

02,472 PC A03/MF A0

Guide to Configuration Management and the Revision Con trol System for Testbed Users.

PB94-150919

$01,678 \mathrm{PC} \mathrm{A03/MF} \mathrm{A01}$

PB94-151776

NIST 60-Millimeter Diameter Cylindrical Cavity Resonator: Performance Evaluation for Permittivity Measurements.
PB94-151776 02,251 PC A11/MF A03 PB94-151842

NIST Standard Reference Data Products Catalog, 1994.

PB94-151842

00,727 PC A06/MF A02
PB94-152006

Fire Safety of Passenger Trains: A Review of Current Aproaches and of New Concepts.

PB94-152006

04,848 PC A11/MF A03

PB94-152204

Abstract and Index Collection in the Research Information Center of the National Institute of Standards and Tech-

nology.
PB94.152204

PB94-152691

02,744 PC A03/MF A01

Assessment of Uncertainties of Thermocouple Calibrations at NIST.

4-152691

$03,782 \mathrm{PC} \mathrm{A03/MF} \mathrm{A01}$

PB94-152709

Submissions to a Planned Encyclopedia of Operations Research on Computational Geometry and the Voronoi/ Delaunay Construct.

PB94-152709

\section{P94-152725}

03,425 PC A03/MF A01

Growth Surface for the Slopes at the Boundary of a Polygon.

PB94-152733

$03,408 \mathrm{PC} \mathrm{A03/MF} \mathrm{A01}$

Photonic Materials: A Report on the Results of a Workshop. Held in Gaithersburg, Maryland on August 26-27, 1992, Vol-

PB94-152733

02,114 PC A05/MF A01

PB94-154325

APDE Demonstration System Architecture. National PDES Testbed Report Series

PB94-154325

$02,767 \mathrm{PC} \mathrm{A03/MF} \mathrm{A01}$

PB94-154341

Electronics and Electrical Engineering Laboratory Technical Progress Bulletin Covering Programs, October to December 1993, with 1994/1995 EEEL Events Calendar.

1993, with 1994/1995 EEEL Events Calendar.
PB94-154341
02,115 PC A03/MF A01

PB94-154358

Variant Design for Mechanical Artifacts-A State of the Art Survey

PB94-154358

PB94-154382

02,768 PC A03/MF A01

Source of Phenol Emissions Affecting the Indoor Air of an Office Building

PBg4-154390

03,600 PC A04/MF A01

Corrosion Resistance of Materials for Renovation of the United States Botanic Garden Conservatory.

PB94-154390

00,032 PC A03/MF A01

PB94-155579

Development and Characterization of Insulating Layers on Silicon Carbide: Annual Report for February 14, 1988 to February 14, 1989

PB94-15557

$02,295 \mathrm{PC} \mathrm{A03/MF} \mathrm{A01}$

Sulfur Dioxide Capture in the Combustion of Mixtures of

Lime, Refuse-Derived Fuel, and Coa

PB94-156866

02,534 PC A04/MF A01

Concurrent Flow Flame Spread Stud

PB94-156866

PB94-156957

ransient Cooling of a Hot Surface by Droplets Evaporation. PB94-156957

PB94-156965

Numerical Analysis Support for Compartment Fire Modeling and Incorporation of Heat Conduction into a Zone Fire PBo4-15

01,357 PC A04/MF A01

PB94-157831

PB94-157831

B94-158268

00,009 PC A12/MF A03

Boundary Conforming Grid Generation System for Interface Tracking.

PB94-158268

PB94-158573

03,312 PC A03/MF A01

Study io Determine the Most Important Parameters for Evaluating the Resistance of Soft Body Armor to Penetration by Edged Weapons.

PB94-158573

03,757 PC A03/MF A01

P94-159761

Metrology for Electromagnetic Technology: A Bibliography of NIST Publications.

PB94-15976

PB94-159779

Seismic Instrumentation of Existing Buildings.

PB94-159779

02,116 PC A04/MF A01

PB94-159787

00,420 PC A04/MF A01

Center for Electronics and Electrical Engineering Technical

Progress Bulletin Covering Center Programs, October to

December, with 1991 CEEE Events Calendar.

PB94-159787

02,296 PC A03/MF A01

PB94-159795

Structural EXPRESS Editor

$02,769 \mathrm{PC} \mathrm{A04/MF} \mathrm{A01}$

PB94-160066 Report on the Workshop on Manufacturing Polymer Com-
posites by Liquid Molding. Held in Gaithersburg, Maryland on September 20-22, 1993.

$03,131 \mathrm{PC} \mathrm{A13/MF} \mathrm{A03}$

PB94-160736

Summary Report of NIST's Industry-Government ConsorWrogram on Flowmeter Installation Eltects with Emphasis on the Research Period, January-September 1991: The Reducer.

PB94-160736

04,196 PC A04/MF A0

PB94-160744

Computation of Dendrites Using a Phase Field Model

PB94-160744

PB94-160751

$04,436 \mathrm{PC} \mathrm{A03/MF} \mathrm{AO}$

Performance Approach to the Development of Criteria for Low-Sloped Roof Membranes.

$00,329 \mathrm{PC} \mathrm{A03/MF} \mathrm{AO}$

PB94-160769

CSTL Technical Activities 199

PB94-160769

PB94-160777

00,728 PC A16/MF A03

Microstructural Features of Some Low Water/Solids, Silica

Fume Mortars Cured at Different Temperatures.
PB94-160777 00,330 PC A03/MF AO PB94-160785

Comparison of Finite Element and Analylic Calculations of the Resonant Modes and Frequencies of a Thick Shell Sphere.

PB94-160785

PB94-160793

02,626 PC A03/MF A0

Review of Mathematical Function Library for Microsoft-

(1960)

PB94-160793

PB94-160801

01,679 PC A03/MF A01

Cylinder Wipe Air-Drying Intaglio Ink Vehicles for U.S. Cur-

PB94-160801

PB94-160819

Technical Program of the Factory Automation Systems Division 1993.

$02,805 \mathrm{PC} \mathrm{A03/MF} \mathrm{AO}$

PB94-160835

National Type Evaluation Program: Index of Device Evaluations by Company. NCWM Publication 5 Part A (Second Edition).

PB94-160835

PB94-160843

02,889 PC A05/MF A0

Properties and Interactions of Oral Structures and Restorative Materials. Annual Report for Period October 1, 1990 to September 30, 1991.

PB94-160843

03,558 PC A06/MF AO

PB94-160850

NVLAP Procedures U.S. Code of Federal Regulations. Title 15, Subtitle A, Chapter 2, Part 7. (Effective December 1984; Amended September 1990)

PB94-160850

PB94-160868 


\section{PB94-161817}

Earthquake Resistant Construction of Electric Transmission and Telecommunication Facilities Serving the Federal Government Report

PB94-161924

Bibliography on Apparel Sizing and Related Issues.

PB94-16192

PB94-161932

Recertification of the Standard Reíerence Material 1475A a Linear Polyethylene Resin.

PB94-161932

02,628 PC A03/MF A01

PB94-161940

Draft Guidelines for Pre-Oualification and Prototype Testing of Seismic Isolation Systems.

PB94-161940

01,331 PC A06/MF A01

PB94-161957

Draft Guidelines for Ouality Control Testing of Sliding Seismic Isolation Systems.

PB94-161957

$01,332 \mathrm{PC} \mathrm{A03/MF} \mathrm{A01}$

PB94-161999

Earthquake Resistant Construction of Gas and Liquid Fuel Pipeline Systems Serving, or Regulated By, the Federal Government.

PB94-161999

04,846 PC A05/MF A01

PB94-162211

Journal of Physical and Chemical Reference Data, Volume 22, No. 2, March/April 1993

PB94-162211

00,733 Not available NTIS

PB94-162229

Wavelengths and Energy Level Classifications for the Spectra of Sulfur (S I through S XVI).
PB94-162229 00,734 Not available NTIS

PB94-162237

Thermodynamic Properties of Alkenes (Mono-Olefins Larger Than C4)

PB94-162245

00,735 Not available NTIS

Thermodynamic Behavior of the $\mathrm{CO} 2-\mathrm{H} 2 \mathrm{O}$ System from 400 to $1000 \mathrm{~K}$, up to $100 \mathrm{MPa}$ and $30 \%$ Mole Fraction of $\mathrm{CO}$

PB94-162245

00,736 Not available NTIS

PB94-162252

Thermodynamics of Enzyme-Catalyzed Reactions: Part 1. Oxidoreductases
PB94-162252

PB94-162260

$\infty, 737$ Not available NTIS

Journal of Physical and Chemical Reference Data, Volume 22, No. 3, May/June 1993

PB94-162260

00,738 Not available NTIS

PB94-162278

Estimation of the Heat Capacities of Organic Liquids as a Function of Temperature Using Group Additivity. I. Hydrocarbon Compounds.

PB94-162278

00,739 Not available NTIS

PB94-162286

Estimation of the Heat Capacities of Organic Liquids as a Function of Temperature Using Group Additivity. II. Compounds of Carbon, Hydrogen, Halogens, Nitrogen, Oxygen, and Sulfur.

PB94-162286

00,740 Not available NTIS

PB94-162294

Thermodynamic and Thermophysical Properties of Organic Nitrogen Compounds. Part 11. 1- and 2-Butanamine, 2-Methyl-1-Propanamine, 2-Methyl-2-Propanamine, Pyrrole, 1-, 2and 3-Methylpyrrole, Pyridine, 2-, 3-, and 4-Methylpyridine, Pyrrolidine, Piperidine, Indole, Quinoline, Isoquinoline, Acridine, Carbazole, Phenanthridine, 1- and 2antenamine, and 9-Methyloarbazole.

PB94-162294 00,741 Not available NTIS

\section{PB94-162302}

International Equations for the Saturation Properties of Ordinary Water Substance. Revised According to the International Temperature Scale of 1990 . Addendum to Journal of Physical and Chemical Reference Data 16, 893 (1987). PB94-162302

00,742 Not available NTIS

PB94-162310

Journal of Physical and Chemical Reference Data, Volume 22, No. 4, July/August 1993

PB94-162310

00,743 Not available NTIS

PB94-162328

Estimation of the Thermodynamic Properties of C-H-N-O-SHalogen Compounds at $298.15 \mathrm{~K}$

PB94-162328

00,744 Not available NTIS

PB94-162336

Joumal of Physical and Chemical Reference Data, Volume 22, No. 5, September/October 1993

PB94-162336 00,745 Not available NTIS

PB94-162344

Compilation of Energy Levels and Wavelengths for the Spectrum of Singly-lonized Oxygen (O II).

PB94-162344 00,746 Not available NTIS

PB94-162351

Energy Levels of Germanium, Ge I through Ge XXXII.

PB94-162351

00,747 Not available NTIS

PB94-162369

Spectral Data and Grotrian Diagrams for Highly lonized Chromium, Cr V through Cr XXIV

PB94-162369

0,748 Not available NTIS
PB94-162500

Voluntary Product Standard PS 20-94. American Softwood Lumber Standard.

PB94-162500

$03,402 \mathrm{PC} \mathrm{A03/MF} \mathrm{A01}$

Computer Systems Laboratory Annual Report 1993

PB94-162518

01,622 PC A05/MF A02
P.

PB94-162534

Materials Science and Engineering Laboratory Annual Report, 1993. NAS-NRC Assessment Panel, Aprif 21-22, 1994. PB94-162534

$02,969 \mathrm{PC}$ A05/MF AO

PB94-162559

North American ISDN Users' Forum Agreements on Integrated Services Digital Network. 01,466 PC A16/MF A03
PB94-162559

PB94-162583

Proceedings of the Workshop on the Federal Criteria for Information Technology Security. Held in Ellicol City, Maryformation Technology Security. Held in Ellicol City, Mary
land on June $2-3,1993$.

$01,575 \mathrm{PC} \mathrm{A04/MF} \mathrm{A01}$

\section{PB94-162591}

Ceramics Technical Activities, 1993 (NAS-NRC Assessment Panel April 21-22, 1994)

PB94-162591

$03,031 \mathrm{PC} \mathrm{A10/MF} \mathrm{A03}$

PB94-162831

U.S. Navy Coordinate Measuring Machines: A Study of Needs.

PB94-162831

PB94-163003

02,807 PC A04/MF A01

Airborne Asbestos Method: Standard Test Method for High Precision Counting of Asbestos Collected on Filters. Version 1.0

PB94-163003

$00,525 \mathrm{PC} \mathrm{A02/MF} \mathrm{A01}$

PB94-163029

Concept for an Algorithm Testing and Evaluation Program at NIST.

PB94-163029

02,890 PC A03/MF A01

Examination of Parameters That Can Cause Error in Mass Determinations.

PB94-163037

PB94-163045

$03,784 \mathrm{PC} \mathrm{A03/MF} \mathrm{A01}$

Airborne Asbestos Method: Standard Test Method for Verified Analysis of Asbestos by Transmission Electron Microscopy. Version 2.0

$00,526 \mathrm{PC} \mathrm{A03/MF} \mathrm{A01}$

PB94-163052

Electronic Balance and Some Gravimetric Applications. (The Density of Solids and Liquids, Pycnometry and Mass). PB94-163052

03,785 PC A03/MF A01 PB94-163060

Piggyback Balance Experiment: An Illustration of Archimedes' Principles and Newton's Third Law.

PB94-163060

03,786 PC A03/MF A01

PB94-163078

Determination of Density of Mass Standards: Requirement and Method.

PB94-163086

$03,787 \mathrm{PC} \mathrm{A03/MF} \mathrm{A01}$

Distributed Supercomputing Software: Experiences with the Parallel Virtual Machine - PVM.

PB94-163086

$01,680 \quad P C$ A03/MF A01

PB94-163383

Putting the Information Infrastructure to Work: Report of the Information Infrastructure Task Force Committee on Applications and Technology.

PB94-163383

00,001 PC A06/MF A02

PB94-163441

Feasibility and Design Considerations of Emergency Evacuation by Elevators.

$00,287 \mathrm{PC}$ A07/MF A02

PB94-163458

Design Guide for CMOS-On-SIMOX. Test Chips NIST3 and

PB94-163458

02,297 PC A05/MF AO

PB94-163466

Models, Managing Models, Ouality Models: An Example of Ouality Management

PB94-163466

$02,891 \mathrm{PC} \mathrm{A03/MF} \mathrm{A0}$

PB94-163474

Compatibility Analysis of the ANSI and ISO IRDS Services PB94-163474

PB94-163482

01,805 PC A06/MF A02

Validation Testing System Requirements. National PDES Testbed Report Series.

PB94-163482

$02,771 \mathrm{PC} \mathrm{A03/MF} \mathrm{AO}$

PB94-163490

NIST Support to the Next Generation Controller Program 1991 Final Technical Report

PB94-163490

PB94-164019

02,808 PC A09/MF A02

Fire Data Management System, FDMS 2.0, Technical Documentation.

PB94-164035

$01,358 \mathrm{PC} \mathrm{A05/MF} \mathrm{A01}$

Supplement to Stable Implementation Agreements for Open Systems Interconnection Protocols. Version 3, September

1990. Change Page Index, Version 3, June 1990 (Stable) Ver 1990; Output from Sep1990 OSI Workshop (NIST Special Publication 500

-164035

01,467 PC A06/MF A02

PB94-164043

NIST Measurement Services: NIST Pressure Calibration Service.

PB94-164043

02,892 PC A06/MF A02

4-164183

ntelligent Processing of Materials, Technical Activities 1993 (NAS-NRC Assessment Panel, April 21-22, 1994).

PB94-164183

$02,809 \mathrm{PC} \mathrm{A04/MF} \mathrm{A01}$

PFR. Fire Publications, 1993.

PB94-164191

$00,192 \mathrm{PC} \mathrm{A03/MF} \mathrm{A01}$

PB94-164316

Color Supplement to NIST Special Publication 400-93 Semiconductor Measurement Technology: Design and Testing Guides for the CMOS and Lateral Bipolar-on-SOI Test

PB94-164316

$02,298 \mathrm{PC} \mathrm{A03/MF} \mathrm{A01}$

PB94-164373

Conformance Assessment of Transport Layer Security Implementations.

$01,576 \mathrm{PC} \mathrm{A03/MF} \mathrm{A01}$

PB94-164381

CONTAM93 User Manual.

PB94-164381

$02,536 \mathrm{PC}$ A05/MF A01

PB94-16439

Time-Perturbation Tuning of MIMD Programs.

PB94-164399

01,681 PC A03/MF A01

PB94-164407

Analysis of Failed Dry Pipe Fire Suppression System Couplings from the Filene Center at Wolf Trap Farm Park for he Performing Arts.

PB94-164407

00,331 PC A03/MF A01

PB94-165206

User's Guide for the PHIGS Validation Tests (Version 2.1).
PB94-165206

PB94-165537

Transmission/Reflection and Short-Circuit Line Methods for Measuring Permittivity and Permeability.
PB94-165537 $02,211 \quad$ PC A06/MF A02

Measurement and Determination of Radon Source Potential: A Literature Review.

PB94-165602

$02,576 \mathrm{PC} \mathrm{A09/MF} \mathrm{A03}$

PB94-16594

Experimental and Numerical Studies on Two-Dimensional Gravity Currents in a Horizontal Channel

PB94-16594

01,359 PC A12/MF A03

PB94-165958 
Temperature-Scale Differences on Thermodynamic Prop00.750 Not available NTIS

\section{PB94-168572}

Thermodynamic Properties of Gaseous Silicon Monotelluride and the Bond Dissociation Enthalpy D(sub m)(SiTe) at T approaches 0 . 00,751 Not available NTIS PB94-168580

Disilicides of Tungsten, Molybdenum, Tantalum, Titanium Cobalt, and Nickel, and Platinum Monosilicide: A Survey of Their Thermodynamic Properties.

PB94-168580

00.752 Not available NTIS

PB94-168598

Evaluated Bimolecular Ion-Molecule Gas Phase Kinetics of Positive Ions for Use in Modeling Planetary Atmospheres, Cometary Comae, and Interstellar Clouds.

PB94-168598

0,753 Not available NTIS

PB94-168606

Atomic Weights of the Elements 1991.

PB94-168606

00,754 Not available NTIS

PB94-169737

Journal of Research of the National Institute of Standards and Technology. January/February 1994. Volume 99, Num-

PBer 1. 169737

PB94.169745

Precision Comparison of the Lattice Parameters of Silicon Monocrystals.

PB94-169752

04,438 Not available NTIS

NIST $30 \mathrm{MHz}$ Linear Measurement System

PB94-169752 02,020 Not available NTIS

PB94-169760

Uncertainties in Dimensional Measurements Made at Nonstandard Temperatures.

PB94-169760

02,893 Not available NTIS

Null-Balanced Total-Power Radiometer System NCS1.

PB94-169778

02,021 Not available NTIS

PB94-169786

Derivation of the System Equation for Null-Balanced TotalPower Radiometer System NCS1.

PB94-169786

02,022 Not available NTIS

Evaluation of Uncertainties of the Null-Balanced TotalPower Radiometer System NCS1.

PB94-169794

02,023 Not available NTIS

PB94-169802 Cryogenic Blackbody Calibrations at the National Institute of
Standards and Technology Low Background Infrared Calibration Facility.
PB94-169802

PB94-172012

02,117 Not available NTIS

Influence of Tempering Method on Residual Stress in Dental Porcelain.

PB94-17201

00,138 Not available NTIS

Electric-Field Strengths Measured Near Personal

Transceivers. $\quad 01,564$ Not available NTIS

PB94-172038

Lead Abatement in Buildings and Related Structures.

PB94-172038

03,601 Not available NTIS

Toward a Reference Model Architecture for Real-Time Intelligent Control Systems (ARTICS)

PB94-172046

02,932 Not available NTIS

PB94-172053

Monte Carlo Approach to the Approximation of Invariant Measures.

PB94-172061

03,409 Not available NTIS

Coordinating Cascaded Surge Protection Devices: HighLow versus Low-High. $\quad 02,463$ Not available NTIS

PB94-172079

Metrication.

PB94-172079

03,676 Not available NTIS

Monte Carlo and Mean-Field Calculations of the Magnetocaloric Effect of Ferromagnetically Interacting ClusPB94-172087

PB94-172095

03,201 Not available NTIS

Local Area Networks in NAA: Advantages and Pitfalls.

PB94-172095

00,527 Not available NTIS

Thin Film Thermocouples for Measurement of Wall Tem-

peratures in Intemal Combustion Engines.

PB94-172103

01,449 Not available NTIS

Infinite Divisibility and the Identification of Singular Wave-

forms.

PB94-17211

02,701 Not available NTIS

RDFs and $\mathrm{Fe}-\mathrm{Fe}$ Pair Correlations in an AlCuFe

Icosahedral Alloy by Double Isotopic Substitution.
PB94-172129
PB94-172137

Ultrasonic Measurements of Surface Roughness.

PB94-172137

04,181 Not available NTIS

PB94-172145

Calcium Phosphate Precipitation in Liposomal Suspensions.

PB94-172145 03,452 Not available NTIS

PB94-172152

Complete Reduction of the Euler-Poinsot Problem.

PB94-172152

03.788 Not available NTIS

PB94-172160

Psychological Aspects of Lighting: A Review of the Work of CIE TC 3.16 .

PB94-172160

PB94-172178

00,241 Not available NTIS

Rotational Dynamics of Solid C70: A Neutron-Scattering Study.

PB94-172178

00,755 Not available NTIS

PB94-172186

Dielectric Properties Measurements and Data.

PB94-172186

01,876 Not available NTIS

PB94-172194

Formal Multi-Layer Test Methodology and Its Application to OSI.

PB94-172194

02,718 Not available NTIS

B94-172202

ISO/IEC Workshop on Worldwide Recognition of OSI Test Results Regional Progress - North America.

PB94-172202 02,719 Not available NTIS PB94-172210

Raman and Fluorescence Spectra Observed in Laser Microprobe Measurements of Several Compositions in the Microprobe Measurem

Ln-Ba-Cu-O System

PB94-172228

04,440 Not available NTIS

Leakage Current Detection in Cryogenic Current Comparator Bridges.

PB94-172236

Digitized Simulation of Mercury Intrusion Porosimetry.

PB94-172236

PB94-172244

Octacalcium Phosphate. 3. Infrared and Raman Vibrational

Spectra.
PB94-172244

PB94-172251

PC-Based Prototype Expert System for Data Management and Analysis of Creep and Fatigue of Selected Materials a Elevated Temperatures.

PB94-17225

03,202 Not available NTIS

PB94-172269

Complementary Molecular Information on Phthalocyanine Compounds Derived from Laser Microprobe Mass Specrometry and Micro-Raman Spect

PB94-172269

PB94-172277

00,757 Not available NTIS

Analysis of a Biologically Motivated Neural Network for Character Recognition

PB94-17227

00,182 Not available NTIS

P84-172285

Hot Carrier Excitation of Adiayers: Time-Resolved Measure ment of Adsorba

PB94-172285

00,758 Not available NTIS

Thermal Pulse Study of the Polarization Distributions Produced in Polyvinylidene Fluoride by Corona Poling at Constant Current.

PB94-172293

PB94-172301

01,195 Not available NTIS

RDI-SIM ECMA Inter-Domain Routing Protocol Simulation Tool.

PB94-172301

01,683 Not available NTIS

PB94-172319

beta-D-Glucosyl-Hydroxymethyluracil: A Novel Modified Base Present in the DNA of the Parastic Protozoan T. brucel.

PB94-172319

PB94-172327

03,524 Not available NTIS

Gaseous Electronics Conference Radio-Frequency Reference Cell: A Defined Parallel-Plate Radio-Frequency Sys tem for Experimental and Theoretical Studies of PlasmaProcessing Discharges.

PB94-172327

04,404 Not available NTIS

B94-172335

Bringing Natural Language Information Retrieval Out of the

Closet.
PB94-172335

PB94-172343

02,720 Not available NTIS

Recent Experimental and Modeling Developments in High Temperature Thermochemistry. 00,759 Not available NTIS PBg4-1

Effect of DC Tests on Induced Space Charge.
PB94-172350 PB94-172368

Wear Mechanism Maps of Ceramics. PB94-172376

Electron Screening Correction to the Self Energy in High-Z Atoms

PB94-172376

03,789 Not available NTIS

PB94-172384

Investigation of the Effects of Aging on the Calibration of Kerr-Cell Measuring System for High Voltage Impulses.

PB94-172384

Shear-Excited Morphological States in a Triblock Copoly PB94-172392

PB94-172400

01,196 Not available NTIS

Fracture Mechanics Analysis of Near-Surface Cracks.

PB94-172400

03,230 Not available NTIS

PB94-172418

Improvement in Predicting Smoke Movement in Compartmented Structures.

PB94-172418

00,332 Not available NTIS

94-172426

Simultaneous Visual and Calorimetric Measurements of R11, R123, and R123/Alkylbenzene Nucleate Flow Boiling.
PB94-172426 03,251 Not available NTis

Error Propagation Biases in the Calculation of Indentation

Fracture Toughness for Ceramics.
PB94-172434

03,032 Not available NTIS

172442

Intercomparison of NIST, NPL, PTB, and VSL. Thermal Voltage Converters from $100 \mathrm{kHz}$ to $1 \mathrm{MHz}$

PB94-172442
PB94-172459

Intercomparison of Thermal Converters at NIM, NIST, PTB, SIRI and VSL from 10 to $100 \mathrm{MHz}$

PB94-172459

2,027 Not available NTIS

PB94-172467

Thermodynamics of the Hydrolysis of Penicillin G and Ampicillin

PB94-172467

03,596 Not available NTIS

PB94-172475

Neutron-Spectroscopy Study of the Hydrogen Vibrations in Hydrogen-Doped YBa2Cu3Ox.

PB94-172475

04,441 Not available NTIS

PB94-172483

Efficient Method to Compute the Maximum Transient Drain Current Overshoot in Silicon on Insulator Devices.

PB94-172483 02,300 Not available NTIS

PB94-172491

Planar Near-Field Alignment.

01,998 Not available NTIS

PB94-172509

Characterization of the Binding of Gallium, Platinum, and Uranium to Pseudomonas Fluorescens by Small-Angle $X$ ray Scattering and Transmission Electron Microscopy.

PB94-172509

03,453 Not available NTIS

4-172517

Electrodeposition

PB94-172525

00,760 Not available NTIS

Use of Ion Scattering Spectroscopy to Monitor the $\mathrm{Nb}$ Target Nitridation during Reactive Sputtering.

PB94-172525

PB94-172533

Replicate Measurements for Data Ouality and Environmental Modeling.

PB94-172533

PB94-172541

02,515 Not available NTIS

Nitrogen Effect on Elastic Constants of f.c.c. Fe-18Cr-19Mn

Alloys.

PB94-172541 
PB94-172632

Time Dependent Small Angle Neutron Scattering Behavior

in Triblock Copolymers Under Steady Shear.
PB 94.198 Not available NTIS

PB94-172640

Serial Sectioning of Hardened Cement Paste for Scanning Electron Microscopy

PB94-172640

01,305 Not available NTIS

\section{PB94-172657}

Using Synthetic-Perturbation Techniques for Tuning Shared Memory Programs (Extended Abstract)

PB94-172657

01,685 Not available NTIS

PB94-172665

Enhanced Curie Temperatures and Magnetoelastic Domains in Dy/Lu Super Lattices and Films.

PB94-172665

PB94-172673

Inelastic Neutron Scattering Studies of Rotational Excitations and the Orientational Potential in $\mathrm{C} 60$ and $\mathrm{A} 3 \mathrm{C} 60$ Compounds.

B94-172681

AMRF Composite Fabrication Workstation.

PB94-172681 02,810 Not available NTIS

PB94-172699

Inelastic Interactions of Electrons with Surfaces: Application to Auger-Electron Spectroscopy and X-ray Photoelectron Spectroscopy.

PB94-172707

00,764 Not available NTIS

Effects of Elastic Stress on Phase Equilibrium in the Ni-V

PB94-172707

03,313 Not available NTIS

PB94-172715

Microstructure and Tensile Properties of Microalloyed Steel Forgings

PB94-172715

03,204 Not available NTIS

PB94-172723

Effect of Two Initiator/Stabilizer Concentrations in a Metal Primer on Bond Strengths of a Composite to a Base Metal Alloy.

PB94-172723

00,141 Not available NTIS

PB94-172731

Considerations on Data Requirements for Tribological ModPBing.

02,962 Not available NTIS

PB94-172749

Wear of Selected Materials and Composites Sliding against MoS2 Films.

PB94-172756

Temperature Dependence of the Morphology of Thin Diblock Copolymer Films as Revealed by Neutron Reflectivity.

01,199 Not available NTIS

PB94-172764

Reaction Sensitivities of Al-Li Alloys and Alloy 2219 in Mechanical-Impact Tests.

PB94-172764

03,314 Not available NTIS

PB94-172772

Time Scale Algorithm for Post-Processing: AT1 Plus Frequency Variance.

PB94-172780

01,525 Not available NTIS

Intelligent Processing of Materials.

PB94-172780 02,811 Not available NTIS

PB94-172798

Absolute Determination of Electrolytic Conductivity for Primary Standard $\mathrm{KC} 1$ Solutions from 0 to $50 \mathrm{C}$

PB94-172798 00,765 Not available NTIS

PB94-172806

Current Activities Within the National Biomonitoring Specimen Bank.

PB94-172806

02,516 Not available NTIS

PB94-172814

Reciprocity Relations in Waveguide Junctions

PB94-172814 02,213 Not available NTIS

PB94-172822

Environmental Scanning Electron Microscope Imaging Examples Related to Particle Analysis.
PB94-172822 00,766 Not available NTIS

PB94-172830

New Critical Review of Experimental Stark Widths and PB94-172830

03,790 Not available NTIS

PB94-172848

High Temperature Degradation of Structural Composites.

PB94-172848

03,132 Not available NTIS

PB94-172855

Measurement and Calibration of Large-Area Alpha-Particle Sources at NIST

PB94-172863

03,791 Not available NTIS

Physicochemical Properties of Calcific Deposits Isolated from Porcine Bioprosthetic Hean Valves Removed from $\mathrm{Pa}$ tients Following 2-13 Years Function.

PB94-172863

00,184 Not available NTIS
PB94-172871

Dental Materials.

PB94-172871

00,142 Not available NTIS

PB94-172889

Report on the Meeting of the CCU (10th) (of the International Committee of Weights and Measures). Held on July $10-11,1990$.

PB94-172889

03,792 No: available NTIS

PB94-172897

National Institute of Standards and Technology Resonance Ionization Spectroscopy/Resonance Ionization Mass Spectroscopy Data Service.

PB94-172897

03,793 Not available NTIS 994-172905

Realization of New NIST Radiation Temperature Scales for the $1000 \mathrm{~K}$ to $3000 \mathrm{~K}$ Region, Using Absolute Radiometric Techniques.

PB94-172913

03,794 Not available NTIS

Intelligent Processing of Hot Isostatic Pressing

PB,315 Not available NTIS

PB94-172921

Porting Multimedia Applications to the Open System Envi-

PB94-172921

PB94-172939

02,721 Not available NTIS

Industry and Government-Laboratory Cooperative $\mathrm{R}$ and $\mathrm{D}$ An Idea Whose Time Has Come.

PB94-172939

02,970 Not available NTIS

PB94-172947

Draft Guideline for Testing and Evaluation of Seismic Isolation Systems

PB94-172954

00,423 Not available NTIS

Evaluation and Qualification Standards for an X-Ray Laminography System.

PB94-172962

02,029 Not available NTIS

Temperature Increases in Aluminum Alloys during Mechanical-Impact Tests for Oxygen Compatibility.

PB94-172962

03,316 Not available NTIS

PB94-172970

Surface Forces and Adhesion between Dissimilar Materials Measured in Various Environments.

PB94-172970 03,033 Not available NTIS

PB94-17298

Greatly Enhanced Soot Scattering in Flickering Ch4/Air Diffusion Flames.

PB94-172988

PB94-172996

01,361 Not available NTIS

Fine-Structure Constant

PB94-172996

03,795 Not available NTIS

PB94-173002

Functions of Technology Infrastructure in a Competitive Economy.

PB94-173010

00,478 Not available NTIS

World Model Registration for Effective Off-Line Programming of Robots.

PB94-173028

Investing in Education to Meet a National Need for a Technical-Professional Workforce in a Post-Industrial Economy. PB94-173028

PB94-173036

Thermal Decomposition of Hydroxy- and Methoxy-Substituted Anisoles.

PB94-173036

00,767 Not available NTIS

PB94-173044

Dispersions of Magnetic Excitations of the $\mathrm{Pr}$ lons in Pr2CuO4.

PB94-173044

04,444 Not available NTIS

PB94-173051

Quantitative Phase Abundance Analysis of Three Cemen Clinker Reference Materials by Scanning Electron MicrosPB94-173051

PB94-173069

00,333 Not available NTIS

Homoepitaxial Growth of Iron and a Real Space View of

Reflection-High-Energy-Electron Diffraction. PB94-17306

B 94-173077

Fire Hazard and Risk: Evaluating Alternative Technologies.
PB94-173077 PB94-173077

PB94-173754

Process for Selecting Standard Reference Algorithms for Evaluating Coordinate Measurement Software

PB94-173754 02,629 PC A03/MF A01

PB94-174505

Characterization of the Hydrogen Induced Cold Cracking Susceptibility at Simulated Weld Zones in HSLA-100 Steel. PB94-1745

Physics Laboratory Technical Activities, 1993

PB94-176088

PB94-176278

$03,796 \mathrm{PC}$ A 10/MF A03

Evaluation and Strengthening Guidelines for Federal Buildings: Identification of Current Federal Agency Programs.
PB94-176278

\section{PB94-176666}

Opportunities for Innovation: Advanced Surface Engineering. $94-176666$

02,697 PC A09/MF A02

PB94-177060

Advanced Components for Electric and Hybrid Electric Vehicles. Workshop Proceedings. Held in Gaithersburg, Maryland on October $27 \cdot 28,1993$.

PB94-177060

04,858 PC A10/MF A03

PB94-178019

Semiconductor Measurement Technology: Design and Testing Guides for the CMOS and Lateral Bipolar-on-SOI Test Librar

94-178019

$02,301 \mathrm{PC}$ A07/MF A02

PB94-178068

NIST Serial Holdings, 1994.

PB94-178068

02,745 PC A12/MF A03

17822

National Voluntary Laboratory Accreditation Program: Procedures and General Requirements.

PB94-17822

22,630 PC A03/MF A01

PB94-178407

Second Text REtrieval Conference (TREC-2). Held in Gaithersburg, Maryland on August 31-September 2, 1993.

PB94-178407

$01,686 \mathrm{PC}$ A21/MF A04

PB94-178969

National Voluntary Laboratory Accreditation Program 1994 Directory.

PB94-178969

$00,482 \mathrm{PC} \mathrm{A08/MF} \mathrm{A02}$

PB94-181856

Evaluation and Strengthening Guidelines for Federal Buildings: Assessment of Current Federal Agency Evaluation Programs and Rehabilitation Criteria and Development of Pypical Costs tor

00,425 PC A08/MF A02

PB94-181997

Application of the Modified Voltage-Dividing Potentiometer to Overlay Metrology in a CMOS/Bulk Process.

PB94-181997

02,302 Not available NTIS

PB94-182003

Tert-Butyl Hydroperoxide-Mediated DNA Base Damage in Cultured Mammalian Cells.

PB94-182003

03,644 Not available NTIS

PB94-182995

Optimization of Highway Concrete Technology

PB94-182995

Technology.
01,333 PC A13/MF A03

PB94-185014

Can Ouantum Mechanical Description of Electron-Sodium Collisions Be Considered Complete. Present Status and Future Prospects for $3 s<->3 p$ Transitions.

PB94-185014

00,768 Not available NTIS

PB94-185022

Thermal Diffusivity of POCO AXM-501 Graphıte in the Range 1500 to $2500 \mathrm{~K}$ Measured by a Laser-Pulse Tech-

B94-185022

03,013 Not available NTIS

PB94-185030

Low-Energy Electron Scattering from Caesium Atoms: Comparison of a Semirelativistic Breit-Pauli and a Full Relativistic Direc Treatment.

PB94-185030

00,769 Not available NTIS

PB94-185048

Physics and Prospects of Inertial Confinement Fusion.

PB94-185048 
PB94-185154

Interface Roughness-Induced Changes in the Near-E(sub 0 ) Spectroscopic Behavior of Short-Period AIAs/GaAs Superlattices.

02,118 Not available NTIS

PB94-185162

Effects of Critical Current Density, Equilibrium Magnetization and Surface Barrier on Magnetization of High TemperaSuperconductors.

PBe Supercond

04,446 Not available NTIS

PB94-185170

Long-Term Stability of Carrier-Free Polonium Solution Standards.

PB94-185170

PB94-185188

00,685 Not available NTIS

Phase Behavior of a Hydrogen Bonding Molecular Composite. 9 B 185188

01,202 Not available NTIS

PB94-185196

Standards Development in North America for Pertormance of Whole Buildings and Facilities.

PB94-185196

00,312 Not available NTIS

PB94-185204

Thermodynamic Properties of Difluoromethane.

PB94-185204 Po, 772 Not available NTIS

PB94-185212

Four Years of Monitoring alpha Orionis with the VLA Where Have All the Flares Gone.

PB94-185212

00,048 Not available NTIS PB94-185220

Distant Future of Solar Activity: A Case Study of Beta Hydri. 3. Transition Region, Corona, and Stellar Wind

PB94-185220 OO,049 Not available NTIS

PB94-185238

Tapered Cross-Pin Attachments for Fixed Bridges.

PB94-185238

00,185 Not available NTIS

PB94-185246

Spectrum and Energy Levels of Five-Times-Ionized Niobium (Nb VI)

PB94-185246

PB94-185253

04,226 Not available NTIS

Liquid Chromatography: Laser-Enhanced Ionization Spectrometry for the Speciation of Organolead Compounds.
00,530 Not available NTIS

PB94-185261

Comparative Strategies for Correction of Interferences in Isotope Dilution Mass Spectrometric Determination of Vana-

PB94-185261

00,531 Not available NTIS

PB94-185279

Selection of Appropriate Ultrasonic System Components for

NDE of Thick Polymer-Composites.
PB94-185279
03, 133 Not available NTIS PB94-18527

Flame Retardants - Overview.

PB94-185287

01,363 Not available NTIS

PB94-185295

Investigation of LS Coupling in Boronlike lons.

PB94-185295

03,797 Not available NTIS

PB94-185303

Quantum Dynamics of Renner-Teller Vibronic Coupling: The Predissociation $\mathrm{HCO}$

PB94-185303

00,773 Not available NTIS

PB94-185311

Anharmonic Oscillator Analysis Using Modified Airy Func-

tions.

PB94-185311

03,798 Not available NTIS

PB94-185329

Optical Conductivity of Single Crystals of Ba1XMXBiO $3(M=K, R b, x=0.04,0.37)$.
PB94-185329

Frozen Human Serum Reference Material for Standardization of Sodium and Potassium Measurements in Serum or Plasma by Ion-Selective Electrode Analyzers.

PB94-185337 00,532 Not available NTIS

PB94-185345

Backscattering in Electron-Impact Excitation of Multiply Charged lons.

PB94-18534

PB94-185352

03,799 Not available NTIS

Laser Imaging of Chemistry-Flowfield Interactions: Enhanced Soot Formation in Time-Varying Diffusion Flames.

PB94-185352

01,364 Not available NTIS

Transfer of Technology from Defense to Civilian Sectors.

PB94-185360

00,011 Not available NTIS

PB94-185378

Contact Electrification Induced by Monolayer Modification of Surface and Relation to Acid-Base Interactions.

PB94-185378

03,034 Not available NTIS

PB94-185386

Task Decomposition Methodology for the Design of a Coa Mining Automation Hierarchical Real-Time Control System. PB94-185394

Effect of Backfill and Atomizing Gas on the Powder Porosity and Mechanical Properties of 304L Stainless Steel.
PB93-205 Not available NTIS
PB94-185402

Conformance Testing of a Lower Layer Security Protocol

PB94-185402

01,577 Not available NTIS

PB94-185410

Time-Domain Measurements of the Electromagnetic Backscatter of Pyramidal Absorbers and Metallic Plates.

PB94-185410

01.877 Not available NTIS

Interfacial Free Energies from Substrate Curvature Measurements of the Creep of Multilayer Thin Films

PB94-185428 04,448 Not available NTIS

PB94-185436

Standard Probes for Electromagnetic Field Measurements. PB94-185436

01,999 Not available NTIS

PB94-185444

Selected Ion Flow Tube-Laser Induced Fluorescence Instrument for Vibrationally State-Specific Ion-Molecule Reac-

PB94-185444

00,774 Not available NTIS

PB94-18545

Design and Operation of Series-Array Josephson Voltage Standards.

PB94-185469

02,030 Not available NTIS

Influence of Surface Interaction and Chain Stiffness on Polymer-Induced Entropic Forces and the Dimensions of Confined Polymers.

PB94-185469

01,203 Not available NTIS

PB94-185477

Temperature Dependence and Anharmonicity of Phonons on Ni(110) and $\mathrm{Cu}(110)$ Using Molecular Dynamics Simula-

PB94-185477

04,449 Not available NTIS

PB94-185485

One Gigarad Passivating Nitrided Oxides for $100 \%$ Internal Quantum Efficiency Silicon Photodiodes.

PB94-185485

02,119 Not available NTIS

PB94-185493

Issues Concerning Material Removal Shape Element VolUmes (MPSEVS).

PB94-185501

02,882 Not available NTIS

High Temperature Silicide Thin-Film Thermocouples.

PB94-185501

02,252 Not available NTIS

PB94-185519

Thin Film Transparent Thermocouples.

PB94-185519

02,253 Not available NTIS

PB94-18552

Scientific Protocols in Statistical Standards for Environmental Studies

PB94-185535

02,517 Not available NTIS

Experimental Optimization of Peak Shape with Application to Aerosol Generation.

PB94-185535

00,501 Not available NTIS

PB94-185543

Fluorescence Monitoring of Polarity Change and Gelation during Epoxy Cure.

PB94-185543

01,204 Not available NTIS

P4-185550

Electron Beam Crosslinking of Poly(vinylmethyl ether).

PB94-185550

01,205 Not available NTIS

PB94-185568

Synthetic-Perturbation Tuning of MIMD Programs.

PB94-185568

01,687 Not available NTIS

$94-185576$

Intercomparison of Internal Proportional Gas Counting of (85) Kr and (3)

PB94-185584

03,800 Not available NTIS

Charge Transfer and Collision-Induced Dissociation Reactions of $\mathrm{CF}(2+)$ and $\mathrm{CF} 2(2+)$ with the Rare Gases at a Laboratory Collision Energy of $49 \mathrm{eV}$

PB94-185584

00,775 Not available NTIS

PB94-185592

Electro-Thermal Simulation of an IGBT PWM Inverter. PB94-185592

PB94-185600

Octacalcium Phosphate Carboxylates IV. Kinetics of Formation and Solubility of Octacalcium Phosphate Succinate.

PB94-185600

00,776 Not available NTIS

PB94-185618

Corrosion Resistant Epoxy-Coated Reinforcing Steel.

PB94-185618

01,307 Not available NTIS

P94-18562

Rotational Modulation and Flares on RS Canum Venaticorum and $B Y$ Draconis Stars. XVI. IUE Spectroscopy and VLA Observations of $\mathrm{C1182}$ (=V 1005 Orionis) in October 1983

00,050 Not available NTIS

PB94-185634

Approach to Selting Performance Requirements for Automated Evaluation of the Parameters of High-Voltage Im-

PBlses. 185634

01,878 Not available NTIS

PB94-185642

Use of a Radiochromic Detector for the Determination of Stereotactic Radiosurgery Dose Characteristics.

PB94-185642

03,514 Not available NTIS

PB94-185659

Enhanced Magnetocaloric Effect in Gd3Ga5-xFexO12.

PB94-185659 04,450 Not available NTIS

PB94-185667

Magnetocaloric Effect in Rapidly Solidified Nd-Fe-Al-B Ma-

PB94-185675

04,451 Not available NTIS

Langevin Approach to Hysteresis and Barkhausen Modeling

PB94-185675

PB94-185683

Development of a Calibrated Atomic Force Microscope.

PB94-185683

PB94-185691

High Resolution IR Studies of Polymolecular Clusters:

Micromatrices and Unimolecular Ring Opening.

PB94-185691

00.777 Not available NTIS

PB94-185709

Verification of the Ponderomotive Approxımation for the ac Stark Shift in Xe Rydberg Levels.

PB94-185709

03,801 Not available NTIS

B94-185717

Use of an Ion Energy Analyzer: Mass Spectrometer to

Measure Ion Kinetic-Energy Distributions from RF Discharges in Argon-Helium Gas Mixtures.

PB94-1857

04,406 Not available NTIS

B94-185725

Angular Distributions for Near-Threshold $(e, 2 e)$ Processes for $\mathrm{Li}$ and $\mathrm{Mg}$.

PB94-185733

00,778 Not available NTIS

Application of Photochemical Reaction in Electrochemical Detection of DNA Intercalation.

PB94-185733

00,686 Not available NTIS

Intermediate Structure in the Neutron-Induced Fission Cross Section of 236

PB94-185758

03,802 Not available NTIS

Neutron Reflectivity of End-Grafted Polymers: Concentration and Solvent Quality Dependence in Equilibrium Conditions.
PB94-185758 01.206 Not available NTIS

PB94-185766

Air Change Effectiveness Measurements in Two Modern Otfice Buildings.

PB94-18576

PB94-185774

00,243 Not available NTIS

Proficiency Testing as a Component of Quality Assurance in Construction Materials Laboratories.

PB94-185774

00,334 Not available NTIS

PB94-185782

Expansion of Cementitious Materials Exposed to Sulfate

PB94-185782

02,577 Not available NTIS 
PB94-185915

High Sensitivity Survey of Radio Continuum Emission in Herbig Ae/Be Stars.

PB94-185923

$\infty, 051$ Not available NTIS

Scaling of Diffusion-Mediated Island Growth in Iron-on-Iron Homoepitaxy

PB94-18592
B $94-185931$

04,455 Not available NTIS

Reproducibility of JEDEC Slandard Current and Voltage Ramo Test Procedures for Thin-Dielectric Breakdown Characterization.

01,879 Not available NTIS

PB94-185949

Experimental Investigation of the Validity of TDDB Voltage Acceleration Models.
PB94-185949

PB94-185956

02,304 Not available NTIS

Field and Temperature Acceleration of Time-Dependent Dielectric Breakdown in Intrinsic Thin SiO?

electric Breakdown in Intrinsic Thin $\mathrm{SiO}_{0}$. Not available NTIS
PB94-185956

PB94-185964

Junction Locations by Scanning Tunneling Microsccpy: InAir-Ambient Investigation of Passivaled GaAs on Junctions. PB94-185964 02,306 Not available NTIS PB94-185972

$X$-Ray Diffraction from Arodic TiO2 Films: In situ and Ex situ Comparison of the Ti(0001) Face.

PB94-185972

PB94-185980

00,782 Not available NTIS

Fourier Transform Alomic Emission Studies Using a Glow Discherge as the Emission Source.

00,533 Not available NTIS

PB94-185998

Infratechnologies: Tools for Innovation

PB94-185998 00,317 Not available NTIS

PB94-186004

Status of Construction and Construction Technologies.

PBO4-186004

00,318 Not available NTIS

PB94-186673

Prediction of Geomatric-Thermal Machine Tool Errors by Artificial Neural Networks.

PB94-180673

02,943 PC A07/MF AO2

Optical Fiber Sensors: Accelerating Aoplications in Navy Ships

PB94-186848

PB94-187283

02,632 PC A06/MF A02

Calculating Flame Spread on Horizontal and Vertical Surfeces.

00.335 PC A04/MF A0

PB94-187317

Sulfale Aitack of Cementitious Materials: Volumetric Relations and Expansions.

PB94-187317

03,232 PC A03/NF A01

PB94-187564

Aoplication of the Electronic Balance in High Precision Pycnometry.

PB94-187630

00,534 PC A03/MF A01

Metric for Success

PB94-187630

PB94-187648

$02,633 \mathrm{PC} \mathrm{A03/MiF} \mathrm{AO}$

Strengthening Methodology for Lightly Reinforced Concrete Frames-II. Recommended Calculation Techniques for the Design of Infill Walls

$00,426 \mathrm{PC} \mathrm{A03/MF} \mathrm{A01}$

\section{PB94-187655}

State-of-the-Art Survey of Methodologies for Represenling Manufacturing Process Capabilities.

PB94-187653

02812 PC A03/MF A01

Workshop on Characterizing Diamond Films (3rd). Held in Gaithersburg, Maryland on February 23-24, 1994.

04,456 PC AO4MF A01

PB94-187713

Use of the Electronic Balance for Highly Accurete Direc Mass Measurements Without the Use of Extemal Mass Standards.

03,803 PC A03MF A01

Body Dimensions for Apparel.

PB94-187739

PB94-187788

02,813 PC AO3/MF AO

NIST RS274/NGC Interpreter Version

PB94-188240

02,814 PC AO3MF AO

NIST Response 10 the Fifth CORM Report on the Pressing Problems and Projected Nesos in Optical Radiation MeasUiements.

PB94-188240

PB94-188463

04,227 PC A03/MF A01

User Profile for Researchers Studying Objects: Implications for Computer Systems.

PBg4-188463

00,133 PC A03/MF A01

Second Census Optical Character Recognition Systems Conterence.

$01,832 \mathrm{PCA} 12 \mathrm{MF}$ A03

\section{PB94-188810}

Semiconductor Measurement Technology: Improved Characterization and Evaluation Measuremenls for $\mathrm{HgCdTe} \mathrm{De}$ ector Materials, Processes, and Devices Used on the GOES and TIROS Satellites.

$02,122 \mathrm{PC} \mathrm{A09/MF} \mathrm{A02}$

PB94-188810

PB94-188836

Proficiency Tests for the NIST Airborne Asbestos Program, 1990.

PB94-188836

00,535 PC A07/MF A02

PB94-188844

Standard Reference Materials: Glass Filters as a Standard Reference Material for Spectrophotometry - Selection, Preparation, Cerification, and Use of SRM 930 and SRM 1930.
PB94-188844 PB94-191202

$00,536 \mathrm{PC} \mathrm{A10/MF} \mathrm{A03}$

Federal Certification Authority Liability and Policy. Law and Policy of Certificate-Based Public Key and Digital SignaPB94

94-191202

01,578 PC A21/MF A04

PB94-191640

NIST Calibration of ASTM E127-Type Uitrasonic Reference

PB94-191640

02,702 PC A03/MF A01

PB94-191707

Optical Metrology and More. Programs and Services of the Radiometric Physics Division, Physics Laboralory.
PB94-191707 228 PC A03/MF A01

PB94-191715

Control Enlity Interface Specification

PB94.191715

02,815 PC A06MF A02

PB94-19347

Binder Cheracterization and Evaluation by Nuclear Magnetic Resonance Spectroscopy.
PB94-193471

PB94-193620

$01,334 \mathrm{PC} \mathrm{AO8/MF} \mathrm{AO2}$

Prediction of Fire Dynamics.

PB94-193620

$00,336 \mathrm{PC} \mathrm{A03/MF} \mathrm{A01}$

PB94-193638

Simple Scalability Test for MIMD Code

B 94-193638

$01,688 \mathrm{PC}$ A03/MF A01

PB94-193646

Program of the Subcommittee on Construction and Building.
PB94-193646 00,319 PC A03/MF A01

PB94-193653

Siudy of Federal Agency Needs for Informalion Technology Security.

01,579 PC A07/MF A02

PB94-193752

Electronics and Electrical Engineering Laboratory Technical Publication Announcements Covering Laboratory Programs, October to December 1993 with 1994/1995 EEEL Events Calendar.

B 94.193752

02,307 PC A03/MF A01

PB94-193760

Bibliography of Photon Total Cross Section (Attenuation Co

efficient) Measurements $10 \mathrm{eV} 1013.5 \mathrm{GeV}, 1907-1993$.
PB94-193760 03,804 PC AO6/MF A02

PB94-193778

Mathematical Modeling of Human Egress from Fires in Residential Buildings

PB94-193778
B 94-193786

00,337 PC A05/MF A02

Coaxial Reference Standard for Microwave Power.

PB94-193786 01,880 PC A04/MF A01 PB94-193802

Siructure and Radiation Properties of Pool Fires. PB94-193802

02,473 PC AO8MF AO2

PB94-193810

Electronics and Electrical Engineering Laboratory Technical Progress Bulletin Covering Laboratory Programs, January O March 1994 with $1994 / 1995$ EEEL Events Calenda

PB94-193810

02,308 PC A03/MF AO1

PB94-193828

Proficiency Tests for the NIST Airborne Asbestos Program PB94-193828

$00.537 \mathrm{PC} \mathrm{A04/MF} \mathrm{A01}$

PB94-193844

Fire Propagation in Concurrent Flows.

PB94-193844

01,365 PC A04/MF A01

PB94-193885

Preparation and Monitoring of Lead Acetate Containing Drinking Water Solutions for Toxicity Studies.

PB 94-193885 00,538 PC A03/NF A01

PB94-193927

Beckdraft Phenomena.

B94-193976

01,366 PC A11/MF A03

Investigation of Oil and Gas Well Fires and Flares. PB94-193976

03,695 PC A04/MF A01

PB94-193984

Reelizing Suspended Siructures on Chips Fabricated by

CMOS Foundry Processes Through the MOSIS Service.
PB94-193984 01,881 PC A04/MF A01 PB94-19398

PB94-194065

Risk Analysis for the Fire Safety of Airline Passengers.
PB94-194065

PB94-194073

Single-Phase Heat Transfer and Pressure Drop Characteristics of an Integral-Spine-Fin Within an Annulus.

PB94-194339

Performance Parameters of Fire Detection Systems

PB94-194339

PB94-194354

Electronics and Electrical Engineering Laboratory Technica Progress Bulletin Covering Laboratory Programs, July 10 September 1993 with 1994 EEEL Evenls Calendar.
PB94-194354 02,309 PC A03/MF A01

PB94-194362

Proficiency Tests for the NIST Airbome Asbestos Program 1992.

PB94-194388

$00,539 \mathrm{PC} \mathrm{A03/MF} \mathrm{A01}$

CONTAM88 Building Input Files for Multi-Zone Airflow and Conlaminanl Dispersal Modeling.

PB94-194388

02,537 PC AO4/MF A01

B94-194552

Visualization Applications for Manufacturing: A State-of-theArt Survey. Final Report

PB94-194552

02,816 PC A03MF AO

PB94-194560

Overview of NASREM: The NASANBS Slandard Reference Tedel for Telerobot Control System Architecture.

PBO4 Terobot Control System Architecture.

PB94-195047

Documentation for Immediately Dangerous to Life or Health Concentrations (IDIHS).

PB94-195047

$03,602 \mathrm{PC}$ A22/MF A04

PB94-195856

Fire Growth Models for Materials.

PB94-195856

PB94-195914

01,367 PC A03/MF A01

Recommendations on Selection of Vehicle-10-Roadside Communications Standards for Commercial Vehicle Oper-

PB94-195914

04,859 PC A05/MF AO

PB94-196029

Federal Metric Progress in 1993.

PB94-196029

$02,600 \mathrm{PC} \mathrm{A03/MF} \mathrm{A01}$

PB94-196045

Behavior of Charring Materials in Simulated Fire Environ-

PB94-196045

PB94-196557

Graphical Analysis of the CCRL Portland Cement Proficiency Sample Database (Samples 1-72). (Pant 1 Univariant Analysis of Portland Cement).
PB94-196557 01,308 PC A06MF A02

PB94-198157

Histopathology. Blood Chemistry, and Physiological Status of Normel and Moribund Striped Bass (Morone saxatilis') Involved in Summer Mortality ("Die-Off') in the SacramentoSan Joan Delta of Califity

PB94-198157

PB94-198215

$00,034 \mathrm{PC} \mathrm{A03/MF} \mathrm{A01}$

Thermodynamic Properties of Gas Phase Species of Imporance to Ozone Depletion.

PB94-198215

PB94-198223

00,126 Not available NTIS

Electro-Optical Sensor for Surface Displacement Measurements of Compliant Coatings.

PB94-198223

02,123 Not available NTIS 
PB94-198314

Vibrational Distributions of As2 in the Cracking of As4 on Si(100) and Si(111).

B $94-198322$

00,784 Not available NTIS

Recently Developed NIST Food Related Standard Reterence Materials.

PB94-198322

\$0,035 Not available NTIS

PB94-198330

Standard Reference Materials for Dioxins and Other Environmental Pollutants.

PB94-198330

02,518 Not available NTIS

PB94-198348

Evaluation of Corrosion Data: A Review.

PB94-198348

03,187 Not available NTIS

PB94-198355

Combined Low- and High-Angle X-Ray Structural Refinement of a Co/Pt(111) Multilayer Exhibiting Perpendicular Magnetic Anisotropy.

04,457 Not available NTIS

PB94-198363

Magnetic Dead Layer in Fe/Si Multilayer: Profile Refinement of Polarized Neutron Retlectivity Data.

of Polarized Neutron Reflectivity Data.

PB94-198363

Oxidation of 10-Methylacridan, a Synthetic Analogue of $\mathrm{NADH}$ and Deprotonation of Its Cation Radical. Convergent Application of Laser Flash Photolysis and Direct and Redox Catalyzed Electrochemistry to the Kinetics of Deprotonation of the Cation Radical.

PB94-198371

00,785 Not available NTIS

PB94-198389

Facile Synthesis of Novel Fluorinated Multifunctional Acrylates.

PB94-198389

01,207 Not available NTIS

PB94-198397

Effect of Transformation of Alloy on Transient and Residual Stresses in a Porcelain-Metal Strip.
PB94-198397 Not available NTIS

PB94-198405

Transient Methods for Thermal Conductivity

PB94-198405

04,197 Not available NTIS

PB94-198413

Automatic Inductive Voltage Divider Bridge Operates from $10 \mathrm{~Hz}$ to $100 \mathrm{kHz}$

PB94-198413

02,032 Not available NTIS

PB94-198421

Weak-Link-Free Behavior of High Angle YBa2Cu3O7-x Grain Boundaries in High Magnetic Fields.

PB94-198439

Effective Measurement Techniques for Heat, Smoke, and Toxic Fire Gases.

PB94-198447

01,369 Not available NTIS

Modern Test Methods for Flammability

PB94-198447 01,370 Not available NTIS

PB94-198454

Toxicity, Fire Hazard and Upholstered Fumiture.

PB94-198454

00,289 Not available NTIS

PB94-198462

Standardization of Formats and Presentation of Fire Data PB94-198462

PB94-198470

01,371 Not available NTIS

Nutritional Status and Growth in Juvenile Rheumatoid Arthritis.

PB94-198470

03,515 Not available NTIS

PB94-198488

Dilemma-Preservation versus Access.
PB94-198488 02,711 Not available NTIS

PB94-198496

Thermodynamic Interactions in Model Polyolefin Blends Obtained by Small-Angle Neutron Scattering. ${ }_{\text {PB }}$ a a a 198496 ailable NTIS PB94-198504

Energy Dependence of Collision Characteristics in Molecule-Surface Collisions.

PB94-198504

00,786 Not available NTIS

PB94-198512

Profile Fitting of X-Ray Diffraction Lines and Fourier AnalySis of Broadening.

PB94-198512

04,460 Not available NTIS

Microstrains and Domain Sizes in Bi-Cu-O Superconductors: An X-Ray Diffraction Peak-Broadening Study. 04,461 Not available NTIS

PB94-198538

04,461 Not available NTIS

Voigt-Function Modeling in Fourier Analysis of Size- and Strain-Broadened X-Ray Diffraction Peaks.
PB94-198538 PB94-198546

Density Matrix Calculation of Population Transfer between Vibrational Levels of $\mathrm{Na} 2$ by Stimulated Raman Scattering with Temporally Shifted Laser Beam

PB94-198546

00,787 Not available NTIS
PB94-198553

Experimental Constraints on Some Mechanisms for HighTemperature Superconductivity

PQ94-198561

Numerical Simulation of Submicron Photolithographic Processing.

PB94-19856

02,310 Not available NTIS

PB94-198579

Regulation of Lithium and Boron Levels in Normal Human Rlood: Environmental and Genetic Considerations.

PB94.198579 PBg4-198

Characteristics of Light Emission After Low-Energy Electron Cxpact Extion of Caesium Atoms.

PB94-198587

03.806 Not available NTIS

B94-198595

Strong Hydrogen Bond in the Formic Acid-Formate Anion Pystem.

00,788 Not available NTIS

PB94-198603

Structure of Glycine-Water H-Bonded Complexes.

PB94-198603

00,789 Not available NTIS

PB94-198611

Femtosecond Time-Resolved Wave Packet Motion in Molecular Multiphoton Ionization and Fragmentation.

PB94-198611 00,790 Not available NTIS

PB94-198629

Neutron Powder Diffraction Study of a Na, Cs-Rho Zeolite.

PB94-198629

00,791 Not available NTIS

PB94-198637

Rotational Spectrum of Copper Hydride Using Tunable Far Infrared Radiation

00,792 Not available NTIS

PB94-198645

Matrix Grain Bridging Contribution to the Toughness of Whisker Reinforced Ceramics.

(198645

03,134 Not available NTIS

PB94-198652

Discovery of an X-Ray Selected, Radio-Loud Quasar at

$z=3.9$.

00,052 Not available NTIS

PB94-198660

Computing S(alpha) Using Symbolic Monte Carlo.

PB94-198660

03,410 Not available NTIS

PB94-198678

Tunneling-Rotation Spectrum of the Hydrogen Fluoride

Dimer.

00,793 Not available NTIS

PB94-1986

od for Measuring Planetary

Integrated Laser Doppler Method for Measuring Planetary
Gravity Fields.
PB94-198686
03,681 Not available NTIS

PB94-198694

Coherent Precipitates in the BCC/Orthorhombic Two Phase Field of the Ti-Al-Nb System.

03,317 Not available NTIS

PB94-198702

Crystallographic Characterization of Some Intermetallic Compounds in the Al-Cr System.

PB94-198702

PB94-198710

Laser Ablation of Thin Films as a Free Atom Source for Pulsed RIMS.

\section{PB94-198728}

Majority and Minority Mobilities in Heavily Doped Silicon for Device Simulations.

PB94-198728

PB94-198736

Relative Sensitivity Factors and Useful Yields for a Microfocused Gallium Ion Beam and Time-ot-Flight Secondary lon Mass Spectrometer.

PB94-198744

Meissner, Shielding, and Flux Loss Behavior in Single-Crystal YBa2Cu3O6+x

PB94-198744

PB94-198751

Magnetic Properties of $\mathrm{Pd} / \mathrm{Co}$ Multilayers.

PB94-198751 04,465 Not available NTIS

PB94-198769

Concurrent Enhancement of Kerr Rotation and

Antiferromagnetic Coupling in Epitaxial $\mathrm{Fe} / \mathrm{Cu} / \mathrm{Fe}$ Structures.
PB94-198769 04,466 Not available NTIS

PB94-198777

Digitized Direct Simulation Model of the Microstructural Development of Cement Paste.

velopment of

01,309 Not available NTIS

PB94-198785

Digitized Simulation Model for Microstructural Development.
PB94-198785 PB94-198793

Modelling the Leaching of Calcium Hydroxide from Cement Paste: Effects on Pore Space Percolation and Diffusivity.

PB94-198801

Diffusion Studies in a Digital-Image-Based Cement Paste Microstructural Model.

PB94-198801

01,312 Not available NTIS

PB94-198819

Milliwatt Mixer for Small Fluid Samples.

PB94-198819 02,634 Not available NTIS

PB94-198827

Standard Reference Materials (SRM's) for Measuring Genetic Damage

PB94-198835

Scattering Properties of the Leveled-Wave Model of Random Morphologies.

PB94-198835

03,807 Not available NTIS

PB94-198843

Analysis of SANS from Controlled Pore Glasses.

PB94-198843

03,035 Not available NTIS

PB94-198850

Structures of Sodium Metal.

PB94-198850

03,319 Not available NTIS

PB94-198868

Non-Equilibrium Thermodynamic Theory of Viscoplastic Ma terials.

PB94-198868

PB94-198876

04,467 Not available NTIS

Modeling Polarization Curves and Impedance Spectra for Simple Electrode Systems.

PB94-198876

03,188 Not available NTIS

PB94-198884

Diffraction Imaging of Polycrystalline Materials

PB94-198884

02,971 Not available NTIS

PB94-198892

Intrared and Microwave Spectroscopy of the Argon Propyne Dimer.

PB94-198900

00,794 Not available NTIS

Dynamic Shear Modulus Measurements with Four Independent Techniques in Nickel-Based Alloys.

PBendent Techni

03,320 Not available NTIS

PB94-198918

Evaluation of Two-Photon Exchange Graphs for HighlyCharged Heliumlike lons.

PB94-198918

PB94-198934

03,808 Not available NTIS

Thermodynamic Constraints on Non-Equilibrium Solidification of Ordered Intermetallic Compounds.

PB94-198934

3.321 Not available NTIS

PB94-198942

Disorder Trapping in Ni2TiA

PB94-198942

03,322 Not available NTIS PB4-198959

Algorithmic Enhancements to the Method of Centers for Lin ear Programming Problems.

PB94-198959

03,426 Not available NTIS

4-198967

Software Libraries, Numerical and Statistical.

PB94-198967

01,689 Not available NTIS

PB94-198975

Portable Vectorized Sottware for Bessel Function Evaluation.

PB94-198975

01,690 Not available NTIS

PB94-198983

Virtual Software Repository System

PB94-198983

01,691 Not available NTIS

PB94-198991

Liquid and Solid Atomic Ion Plasmas.

PB94-19899

03,809 Not available NTIS

PB94-199007

Absorption of Ultrasonic Waves in Air at High Frequencies 
PB94-199080

Precision Nuclear Orientation Measurements for Determining Mixed Magnetic Dipole/Electric Ouadrupole Hyperfine Interactions.

PB94-199098

03,810 Not available NTIS

Development and Calibration of UVNUV Radiometric Sources.

PB94-199098

04,229 Not available NTIS

PB94-199106

Object Finder for Digital Images Based on Multiple Thresholds Connectivity, and internal Structures.

PB94-199106

01,833 Not available NTIS

PB94-199114

Concentration Histogram Imaging: A Scatter Diagram Technique for Viewing Two or Three Related Images.

PB94-199114

00,542 Not available NTIS

PB94-199122

Atmospheric and Marine Trace Chemistry: Interfacial Biomediation and Monitoring.

PB94-199122

03,752 Not available NTIS

PB94-199130

Spatial Dependence of Electrical Fields Due to Space Charges in Films of Organic Dielectrics Used for Insulation of Power Cables.

02,214 Not available NTIS

\section{PB94-199148}

Microstructure and Ferroelectric Properties of Lead Zirconate-Titanate Films Produced by Laser Evaporation.

PB94-199148

04,470 Not available NTIS

PB94-199155

Germanium Detector Optimization of MDA for Efficiency vs Low Intrinsic Background.

PB94-199155

00,543 Not available NTIS

PB94-199163

Overview of Radiometric Program of the NIST Thermal Imaging Laboratory

PB94-199171

04,230 Not available NTIS

Applications of the Vortex Tube in Chemical Analysis.

PB94-199171 00,544 Not available NTIS

PB94-199189

Measurement of Diffusion in Supercritical Fluid Systems: A Review.

PB94-199189

00,795 Not available NTIS

PB94-199197

Simple and Efficient Low-Temperature Sample Cell for infrared Spectrophotometry.

00,545 Not available NTIS

Simple, Inexpensive Apparatus for Sample Concentration

PB94-199205 00,546 Not available NTIS

PB94-199213

Summary of the Patent Literature of Supercritical Fluid Technology.

00,502 Not available NTIS

PB94-199221

Thermophysical Property Data for Supercritical Fluid Extraction Design.

PB94-199221

PB94-199239

00,668 Not available NTIS

Laser Meltıng of Thin Silicon Films.

PB94-199239

04,471 Not available NTIS

PB94-199247

Integrated Network Management.

PB94-199247

01,583 Not available NTIS

PB94-199254

Calculation of Higher Heating Values of Biomass Materials

and Waste Components from Elemental Analyses.
PB94-199254 N74 Not available NTIS

PB94-199262

Studies Assess Performance of Residential Detectors

PB94-199262

00,290 Not available NTIS

PB94-199270

Analysis of the Happyland Social Club Fire with HAZARD I.

PB94-199270

00,193 Not available NTIS

PB94-19928

Photodecomposition Dynamics of $\mathrm{Mo}(\mathrm{CO}) 6 / \mathrm{Si}(111) 7 \times 7$ : CO Internal State and Translational Energy Distributions--Trans-

00,687 Not available NTIS

PB94-199296

Fluorescence Anisotropy Measurements on a Polymer Mell

as a Function of Applied Shear Stress.

PB94-199296

01,209 Not available NTIS

PB94. 199304

Observations of Shear Induced Molecular Orientation in a Polymer Melt Using Fluorescence Anisotropy Measure-

ments.

PB94-199304

PB94-199312

01,210 Not available NTIS

Observations of Shear Stress and Molecular Orientation Using Fluorescence Anisotropy Measurements.

PB94-199320

Analysis of Moisture Accumulation in a Wood-Frame Wal Subjected to Winter Climate.

PB94-199320

00,338 Not available NTIS
PB94-199338

Gas Phase Reactions Relevant to Chemical Vapor Deposition: Optical Diagnostics.

PB94-199338

03,116 Not available NTIS

B94-199346

Gas Phase Reactions Relevant to Chemical Vapor Deposition: Numerical Modeling.

PB94-199346

PB94-199353

03,117 Not available NTIS

LMTO/CVM and LAPW/CVM Calculations of the Nickel Aluminide/Nickel Titanium Pseudobinary Phase Diagram.

PB94-199353

03,323 Not available NTIS

PB94-199361

Interface Properties for Ceramic Composites from a Single Fiber Pull-Out Test

PB94-19936

03,135 Not available NTIS

PB94-199379

Determination of 3-Ouinuclidinyl Benzilate (Onb) and its Major Metabolites in Urine by Isotope Dilution Gas Chroma-

tography Mass Spectrometry.
PB94-199379

03,492 Not available NTIS

PB94-199387

Statistical Thermodynamics of Phase Separation and Ion Partitioning in Aqueous Two-Phase Systems.

PB94-19938

01,212 Not available NTiS

PB94-199395

Ouantized Dissipation of the Ouantum Hall Effect at High Currents.

PB94-199403

04,472 Not available NTIS

Modeling the Evolution of Structure in Unstable Solid Solution Phases by Diffusional Mechanisms.

PB94-199403

03,324 Not available NTIS

PB94-199411

Singularties in Minimum Surface Energy Problems and Their Influence in Surface Motion.

PB94-199411

04,473 Not available NTIS

\section{4-199429}

Stability, Microstructural Evolution, Grain Growth, and Coarsening in a Two-Dimensional Two-Phase Microstructure

PB94-19942

03,325 Not available NTIS

PB94-199437

IUE Observations of Solar-Type Stars in the Pleiades and the Hyades.

PB94-199445

00,053 Not available NTIS

Collision-Induced Emission in the Fundamental VibrationRotation Band of $\mathrm{H} 2$.

PB94-199445

PB94-199452

Experimental Studies of Line Shapes from a Balle-Flygare Spectrometer.
PB94-199452

PB94-199460

00,796 Not available NTIS

Performance of a Reflectron Energy Compensating Mirror

PB94-199460

00547 Not available NTIS

PB94-199478

Silicon Photodiodes Optimized for EUV and Soft X-Ray Re-

PB94-199478

02,124 Not available NTIS

PB94-199486

Slowly Divergent Space Marching Schemes in the Inverse Heat Conduction Problem.

PB94-199486

PB94-199494

Maturity Method

PB94-199502

03,812 Not available NTIS

Maturity Functions for Concrete Made with Various Cements and Admixtures.

PB94-199502

01,314 Not available NTIS

PB94-199510

Neutron Standard Cross Sections in Reactor Physics: Need and Status.

PB94-199510

PB94-199528

03,813 Not available NTIS

First Results from the Goddard High-Resolution Spectro-

graph: The Chromosphere of Tauri.
PB94-199528 00,054 Not available NTIS PB94-199536

Micro-Mechanical Aspects of Asperity-Controlled Friction in Fiber-Toughened Ceramic Composites.

PB94-199536

03,136 Not available NTIS

PB94-199544

New Rydberg States of Aluminum Monofluoride Observed by Resonance-Enhanced Multiphoton Ionization Spectros. PB94.

PB94-199551

00,797 Not available NTIS

Magneto-Optic Magnetic Field Sensor with 1.4pT/square root of $1(\mathrm{~Hz})$ Minimum Detectable Field at $1 \mathrm{kHz}$

PB94-19955

02,125 Not available NTIS

PB94-199569

Thermodynamic Properties of $\mathrm{CHF}_{2}-\mathrm{O}$ CHF2,Bis(difluor Properties

00,798 Not available NTIS

PB94-199577

Vibrational Autoionization in $\mathrm{H} 2$ : Vibrational Branching Ra. tios and Photoelectron Angular Distributions Near the $\mathrm{V}(+)=3$ Threshold

PB94-199585

00,799 Not available NTIS

Isotope Shitts and Hyperfine Splittings of the 398.8-nm Yb I Line.

PB94-199585

03,814 Not available NTIS

PB94-199593

Thermodynamic Analysis of Heparin Binding to Human PB94-199593

03,455 Not available NTIS

PB94-199601

ROSAT All-Sky Survey of Active Binary Coronae. 2. Coronal Temperatures of the RS Canum Venaticorum Systems. PB94-199601

00,055 Not available NTIS

PB94-199619

Maximum Entropy as a Tool for the Determination of the CAxis Profile of Layered Compounds.

PB94-199619

00,800 Not available NTIS

PB94-199627

Experimental Aspects and Z-Dependent Systematics in One- and Two-Electron lons and Single Vacancy Systems. PB94-199627

PB94-199635

Status of a Silicon Lattice Measurement and Dissemination Exercise.

PB94-199643

04,474 Not available NTIS

EPR Bone Dosimetry: A New Approach to Spectral Deconvolution Problems.

PB94-199643

03,616 1:ot available NTIS

PB94-199650

Estimation of the Absorbed Dose in Radiation-Processed Food. 2. Test of the EPR Response Function by an Exponential Fitting Analysis.

PB94-199650

00,036 Not available NTIS

PB94-199668

Experımental Validation of Radiopharmaceutical Absorbed Dose to Mineralized Bone Tissue.

PB94-199668

03,617 Not available NTIS

PB94-199676

Radiation Doses.

PB94-19967

03,618 Not available NTIS

PB94-199684

Estimation of the Absorbed Dose in Radiation-Processed Food. 3. The Effect of Time of Evaluation on the Accuracy of the Estimate.

PB94-199684

00,037 Not available NTIS

PB94-199692

Estimation of the Absorbed Dose in Radiation-Processed Food. 4. EPR Measurements on Eggshell.

PB94-199692

00,038 Not available NTIS 
PB94-199817

Calibration Standards for Differential Scanning Calorimetry 1. Zinc Absolute Calorimetric Measurement of Enthalpy of Fusion and Temperature of Fusion $\mathrm{HM}$.

PB94-199817 00,801 Not available NTIS PB94-199825

Oxidative Damage to DNA in Mammalian Chromatin.
PB94-199825 03,525 Not available NTIS

PB94-199833

Modification of DNA Bases in Chromatin of Intact Target Human Cells by Activated Human Polymorphonuclear Leukocytes.

03,526 Not available NTIS

PB94-199841

Absolute Cross-Section Measurements for Electron-Impact Ionization of $\mathrm{C} 1(+1)$

03,818 Not available NTIS

Computer Vision Based Tool Setting Station

PB94-199858 02,944 Not available NTIS

PB94-199866

Measuring the Stability of Three Copper Alloys.

PB94-199866

03,326 Not available NTIS

PB94-199874

Environmental Evaluation of a New Federal Office Building.
PB94-199874 02,538 Not available NTIS

PB94-199882

Assessing the Credibility of the Calorific Content of Muncipal Solid Waste.

PB94-19988

02,581 Not available NTIS

PB94-199890

Simplified Cycle Simulation Model for the Performance Rat ing of Refrigerants and Refrigerant Mixtures.

PB94-199890

03,255 Not available NTIS

PB94-199908

Fragment Energy and Vector Correlations in the OvertonePumped Dissociation of $\mathrm{HN} 3 \times(1) \mathrm{A}^{\prime}$.

PB94-199908 00,802 Not available NTIS PB94-199916

Neutron Measurement Intercomparisons Sponsored by

CCEMRI, Section 3 (Neutron Measurements).
PB94-199916 03,819 Not available NTIS PB94-1999

B94-199924

Diffraction of X-rays at the Far Tails of the Bragg Peaks.

PB94-199924

04,476 Not available NTIS

B94-199932

Implementing a Transition Manager in the AMRF Cell Conroller.

PB94-199932

02,817 Not available NT1S

B94-199940

Generic Manufacturing Controllers.
PB94-199940 PB94-199940

Time-Resolved Probes of Surface Dynamics.

PB94-199957 00,803 Not available NTIS

PB94-199965

Dynamics of Nonthermal Reactions: Femtosecond Surface Chemistry.

PB94-199973

00,688 Not available NTIS

Reactivity of Pd and Sn Adsorbates on Plasma and Thermally Oxidized $\mathrm{SnO} 2(110)$

B94-199973

00,804 Not available NTIS

PB94-199981

Dynamic Characteristics of Five Tall Buildings during Strong and Low-Amplitude Motions.

PB94-199981

00,427 Not available NTIS

PB94-199999

Millisecond-Resolution Pulse Heating System for SpecificHeat Measurements at High Temperatures.
PB94-19999 PB94-200003

High-Speed Spatial Scanning Pyrometer.

PB94-200003

02,636 Not available NTIS

PB94-200011

Wavelength Dependence of Normal Spectral Emissivity of High-Temperature Metals at Their Melting Point.

PB94-200011 03,398 Not available NTIS

PB94-200029

VLSI Architectures for Template Matching and Block Matching.

01,834 Not available NTIS

PB94-200037

Pure Element Sputtering Yield Data: Appendix 4.

PB94-200037 00,805 Not available NTIS

PB94-200045

Electronic Correlations and Satellites in Superconducting Oxides.

PB94-200045

04,477 Nol available NTIS

PB94-200052

Effect of Curing History on Ultimate Glass Transition Tem-

perature and Network Structure of Crosslinking Polymers.

PB94-200052

01,214 Not available NTIS

PB94-200060

NIST Capacitance Measurement Assurance Program

(MB94-200060

02,254 Nol available NTIS
PB94-200078

mprovements in Computation of Form Factors.

PB94-200078 03,820 Not available NTIS

PB94-200086

03,820 Not avallable NTIS

Photographic Response to X-Ray Irradiation. 1. Estimation of the Photographic Error Statistic and Development of Anaytic Density-Intensity Equations.

PB94-200086

03,821 Not available NTIS

PB94-200094

Photographic Response to X-Ray Irradiation. 2. Correlated

PBg 4-200

03,822 Not available NTIS

PB94-200102

Photographic Response to X-Ray Irradiation. 3. Photo-

graphic Linearızation of Beam-Foil Spectra.

PB94-200102

03,823 Not available NTIS

PB94-200110

Cavitation Damage During Flexural Creep of SiAION-YAG Ceramics.

PB94-200128

03,036 Not available NTIS

Surface Barrier and Lower Critical Field in YBa2Cu3O7 delta Superconductors.

PB94-200128

04,478 Not available NTIS

Hot-Deformation Apparatus for Thermomechanical Processing Simulation

PB94-200144

03,207 Not available NTIS

$X$-Ray Photoelectron and Auger Electron Spectroscopy Study of UltraviolevOzone Oxidized P2S5/(NH4)2S Treated GaAs(100) Surfaces.

PB94-200144

04,479 Not available NTIS

PB94-200151

Grazing-Incidence X-Ray Photoemission Spectroscopy Investigation of Oxidized GaAs(100): A Novel Approach to Nondestructive Depth Profiling.

PB94-20015

04,480 Not available NTIS

PB94-200169

Textures of Tantalum Metal Sheets by Neutron Diffraction.

PB94-200169

03,399 Not available NTIS

PB94-200177

Texture Study of Two Molybdenum Shaped Charge Liners by Neutron Diffraction

PB94-200185

03,754 Not available NTIS

Planar Waveguide Optical Sensors.

PB94-200185

03,586 Not available NTIS

PB94-200193

Characterization of Vertical-Cavity Semiconductor Struc-

PB94-200193

PB94-200201

Determination of Oltipraz in Serum by High-Performanace Liquid Chromatography with Optical Absorbance and Mass Spectrometric Detection.

03,493 Not available NTIS

PB94-200219

Neutron-Scattering Study of C60(n-) $(n=3,6)$ Librations in Alkali-Metal Fullerides.

B94-200219

00,806 Not available NTIS

200227

Crack Growth Resistance of Strain-Softening Materials under Flexural Loading.

PB94-200227

02,972 Not available NTIS

PB94-200235

Generic Model for Creep Rupture Lifetime Estimation on Fibrous Ceramic Composites.

PB94-200235

03,137 Not available NTIS

PB94-200243

Asymmetric Tip Morphology of Creep Microcracks Growing Along Bimaterial Intertaces.

PB94-200250

03,138 Not available NTIS

Application of a Simple Technique for Estimating Errors of Finite-Element Solutions Using a General-Purpose Code. PB94-200250

PB94-200268

C-Ring by Finite Element

Analysis of Creep in a $\mathrm{Si}-\mathrm{SiC} \mathrm{C}$-Ring by Finite Element

PB94-200268

03,037 Not available NTIS

PB94-200276

Incorporation of Gold into $\mathrm{YBa} 2 \mathrm{Cu} 3 \mathrm{O} 7$ : Structure and $\mathrm{Tc}$ Enhancement
PB94-200276

PB94-20027
PB94-200284

04,481 Not available NTIS

Unexpected Effects of Gold on the Structure

Superconductivity, and Normal State of $\mathrm{YBa} 2 \mathrm{Cu} 307$.

PB94-200284 04,482 Not available NTIS

PB94-200292

Generation and Characterization of Acetylene Smokes.

PB94-200292 01,372 Not available NTIS

PB94-200300

Ultrafine Combustion Aerosol Generator

PB94-200300

01,373 Not available NTIS

PB94-200318

Introduction of a NIST Instrument Sensitivity Standard Reference Material for X-Ray Powder Diffraction.

PB94-200318

00,807 Not available NTIS

PB94-200326

Micromechanics of Densification and Distortion.

PB94-200326

03,327 Not available NTIS

PB94-200334

Analysis of Scattering Asymmetry Statistics When Back-

ground Corrected Counts Are Negative.

PB94-200334

03,824 Not available NTIS

P94-200342

Uncertainty Intervals for Polarized Beam Scattering Asymmetry Statistics.

PB94-200342
PB94-200359

03,825 Not available NTIS

High-Frequency Linear Response of Anisotropic Type-II Superconductors in the Mixed State.
PB94-200359 Not available NTIS

PB94-200367

Nonlinear Response of Type-ll Superconductors in the Mixed State in Slab Geometry

PB94-200367

04,484 Not available NTIS

PB94-200375

Salt-PEG Two-Phase Aqueous Systems to Purify Proteins and Nucleic Acid Mixtures

PB94-200375

03,527 Not available NTIS

994-200383

Papers Presentations Shine.

PB94-200383

00,244 Not available NTIS

PB94-200391

Electron Traps, Structural Change, and Hydrogen Related SIMOX Defects.

PB94-200409

02,312 Not available NTIS

President's Column for Dielectrics and Electrical Insulation Society Newsletter.

PB94-200409

PB94-200417

Acceptance Diagram Analysis of the Performance of Multidisk Neutron Velocity Selecto

PB94-200417

03,826 Not available NTIS

PB94-200425

Acceptance Diagram Analysis of the Performance of Vertically Curved Neutron Monochromators.

PB94-200425

03,827 Not available NTIS

PB94-200433

Joy of Acceptance Diagrams.

PB94-200441

03,828 Not available NTIS

Effects of Crystalline Anisotropy and Buoyancy-Driven Convection on Morphological Stability.

PB94-200441

3,328 Not available NTIS

$994-200458$

Neutron Scattering Structural Study of AICuFe Quasicrystals Using Double Isotopic Substitution.
PB94-200458 04,485 Not available NTIS PB94-200466

2-Tunneling Path Internal-Axis-Method-Like Treatment of the Microwave Spectrum of Divinyl Ether.

PB94-200466 00,808 Not available NTIS

PB94-200474

Assessment of the Al-Sb System

PB94-200474 
PB94-200573

Graphical Conceptual Navigation as a Presentation Technique for a Graphics Standard. 01,692 Not available NTIS
PB94-200573 PB94-200573

PB94-200581

Rate Constants for Hydrogem Atom Attack on Some

Chlorinated Benzenes at High Temperature.
00,810 Not available NTIS PB94-200599

Importance of Chemometrics in Biomedical Measurements. PB9 4-200599

PB94-200607

00,550 Not available NTIS

Metrological Measurement Accuracy: Discussion of 'Measurement Error Models' by Leon Jay Gleser. PB94-200607

PB94-200615

Accuracy-Weighted Variational Principle for Degenerate Continuum States.

PB94-200615

PB94-200623

03,831 Not available NTIS

Comprehensive Theory of Nuclear Effects on the Intrinsic Sticking Probability. 1

PB94-200623

03,832 Not available NTIS

B94-200631

Comprehensive Theory of Nuclear Effects on the Intrinsic Sticking Probability. 2.

PB94-200631

03.833 Not available NTIS

PB94-200649

Elastic Properties of Uniaxial-Fiber Reinforced Composites: General Features.

PB94-20064

03,140 Not available NTIS

PB94-200656

Transient Subcritical Crack-Growth Behavior in Transformation-Toughened Ceramics

PB94-200656

PB94-200664

Report of Density Intercomparisons Undertaken by the Working Group on Density of the CCM.

PB94-200664 00,503 Not available NTIS

PB94-200672

Mass and Density Determinations.
PB94-200672 00,504 Not available NTIS PB94-200672

Extension of Heterodyne Frequency Measurements on OCS

$1087 \mathrm{THz}(2900 \mathrm{~cm}(-1))$.

PB94-200680

00,811 Not available NTIS

Faraday Effect Current Sensors.

PB94-200698

02,127 Not available NTIS

PB94-200706

Faraday Effect Sensors: A Review of Recent Progress.

PB94-200706 02,128 Not available NTIS

PB94-200714

Correlation of $\mathrm{HgCdTe}$ Epilayer Defects with Underlying Substrate Defects by Synchrotron X-Ray Topography.
PB94-200714 02,129 Not available NTIS

PB94-203403

Evaluation of Altemative In-Flight Fire Suppressants for Full-Scale Testing in Simulated Aircraft Engine Nacelles and Dry Bays.

PB94-203429

00,023 PC A99/MF E08

Integration of Servo Control into a Large-Scale Control Sys-

tem Design: An Example from Coal Mining.
PB94-203429 03,696 PC A02/MF A01 PB94-203437

Ouality Characteristics and Metrics for Reusable Software (Preliminary Report)

PB94-203437

$01,693 \mathrm{PC} \mathrm{A03/MF} \mathrm{A01}$

Certainty Grid to Object Boundary Algorithm

PB94-203510 01,835 PC A03/MF A0

PB94-203528

Continuous Mining Machine Control Using the Real-Time Control System

PB94-203536

$03,700 \quad P C$ A02/MF AO

Environment Simulation for a Continuous Mining Machine

PB94-203536

PB94-203593

Cryogenic Properties of Silver.

03,697 PC A03/MF AO

PB94-205952

03,330 PC A03

Fire Growth Analysis of the Fire of March 20, 1990, Pulask Building. 20 Massachusetts Avenue, N.W., Washington, PB. 4 -205952

PB94-206018

$00,194 \mathrm{PC} \mathrm{A03/MF} \mathrm{A01}$

Energy Prices and Discount Factors for Life-Cycle Cost Analysis 1994. Annual Supplement to NIST Handbook 135 and NBS Special Publication 709. 02,508 PC A04/MF A0 PB94-206091

Development of the Fire Data Management System. PB94-206091

PB94-206109

00,339 PC A03/MF A01

User's Guide to NIST SRM 2084: CMM Probe Performance Standard.

02,709 PC AO3/MF A0
PB94-206125

Wind Load Provisions of the Manufactured Home Construction and Safety Standards: A Review and Recommendations for Improvement.

PB94-206125

00,428 PC AO6/MF AO2

PB94-206281

Visual-Motion Fixation Invariant

PB94-20628

PB94-206299

Upward Flame Spread along the Vertical Corner Walls (October 1993).

PB94-20629

PB94-206307

Metric Path to Global Markets and New Jobs: A Ouestionand-Answer and Thematic Discussion.

PB94-20630

$02,601 \mathrm{PC}$ A02/MF A01

PB94-206356

Evaluating Small Board and Care Homes: Sprinklered vs. Nonsprinklered Fire Protection.

PB94-206356

$00.195 \mathrm{PC} \mathrm{A04/MF} \mathrm{A01}$

PB94-206364

U.S. Green Building Conference, 1994.

PB94-206364

02,519 PC AOB/MF AO2

PB94-206646

Assessment of Technology for Detection of Stress Corrosion Cracking in Gas Pipelines. Final Repor, July 1993March 1994

PB94-206646

PB94-207354

02,475 PC A05/MF A02

World Wide Web and Mosaic: User's Guide

PB94-207354

02,722 PC A03/MF A01

PB94-207388

Turbulent Upward Flame Spread on a Vertical Wall under External Radiation.

PB94-207388

00,341 PC A05/MF A01

PB94-207404

Comparison of Wall-Fire Behavior With and Without a Ceiling.

00,342 PC A03/MF A01

PB94-207453

IGOSS-Industry/Government Open Systems Specification.

PB94-207453

01,806 PC A07/MF A02

PB94-207461

Northridge Earthquake 1994: Performance of Structures, Lifelines, and Fire Protection Systems.

PB94-207461

04,825 PC A09MF A02

PB94-207495

Project Summaries 1994: NIST Building and Fire Research PB94-207495

00,343 PC AO8/MF AO2

PB94-207511

Global Equivalence Ratio Concept and the Prediction of Carbon Monoxide Formation in Enclosure Fires

PB94-207511

00,313 PC A08/MF AO2

PB94-207636

Technical Digest: Symposium on Optical Fiber Measurements (8th), 1994. Held in Boulder, Colorado on September $13-15,1994$

PB94-207636

PB94-207727

$04,231 \mathrm{PC} \mathrm{A10/MF} \mathrm{A03}$

State Weights and Measures Laboratories: State Standards Program Description and Directory. 1994 Edition.

PB94-207727

$02,895 \mathrm{PC}$ A07/MF A02

PB94-207750

Preliminary Functional Specifications of a Prototype Electronic Research Notebook for NIST

PB94-207750

$00,012 \mathrm{PC} \mathrm{A04/MF} \mathrm{A01}$

PB94-207768

Face Recognition Technology for Law Enforcement Applications.

01,837 PC AO5/MF A01

PB94-209459

Report of the NIST Workshop on Key Escrow Encryption. Held in Gaithersburg. Maryland on June $8-10,1994$

PB94-209459

01,584 PC A08/MF A02

PB94-210051

Measurements of Shielding Effectiveness and Cavity Characteristics of Airplanes.

PB94-210051

PB94-210077

Combined Buoyancy- and Pressure-Driven Flow Through a Shallow, Horizontal, Circular Vent.

PB94-210077

00,344 PC A03/MF A01

PB94-210127

VENTCF2: An Algorithm and Associated FORTRAN 77 Subroutine for Calculating Flow through a Horizontal Ceiling/Flor Vent in a Zone-Type Compartment Fire Model.

PB94-210127

$00,345 \mathrm{PC} \mathrm{A04/MF} \mathrm{A01}$

PB94-210143

Program Handbook: Requirements for Obtaining NIST Approval/Recognition of a Laboratory Accreditation Body Under P.L. 101-592. The Fastener Ouality Act.

PB94-210143

02,859 PC AO3/MF A01

PB94-210150

Survey on the Implementation of ISO/IEC Guide 25 by National Laboratory Accreditation Programs.

PB94-210150

PC AO4/MF A01

PB94-210168

Airborne Asbestos Method: Standard Practice for Recording Transmission Electron Microscopy Data for the Analysis of Asbestos Collected onto Fil

PB94-210168

$00.552 \mathrm{PC} \mathrm{A03/MF} \mathrm{A03}$

PB94-211018

Tribological Characteristics of Alpha-Alumina at Elevated

Temperatures.

PB94-211026

02,963 Not available NTIS

Hypercubic Lattice SAW Exponents nu and gamma : 3.99 Dimensions Revisited.

PB94-211026

PB94-211034

Effect of Swelling on the Elasticity of Rubber: Localization Model Description.

PB94-211034

01,216 Not available NTIS

PB94-211042

Molar Absorptivities of Bilirubin (NIST SRM 916A) and Its

PB94-211042

03,456 Not available NTIS

PB94-211059

Measurement of Boron at Silicon Wafer Surfaces by Neutron Depth Profiling.

PB94-211059

04,487 Not available NTIS

Dynamic Phenomena on the RS Canum Venaticorum Binary II Pegasi in August 1989. 1. Observational Data.

PB9 4-211067 00,056 Not available NTIS

PB94-211075

Plasma Chemistry in Disilane Discharges.

PB94-211075

02,514 Not available NTIS

PB94-211083

Radio Continuum and X-Ray Properties of the Coronae of RS Canum Venaticorum and Related Active Binary Sys-

PB94-211083

00,057 Not available NTIS

PB94-21109

Ordered omega-Derivatives in a Ti-37.5Al-2ONb at\% Alloy.

PB94-211109

Three-Vector Correlation Theory for Orientation/Alignment Studies in Atomic and Molecular Collisions.

PB94-211109

PB94-211117

NIST Optically Pumped Cesium Frequency Standard.

PB94-211117

Frequency Standard.

PB94-211125

Multichanne! Ouantum Defect Half Collision Analysis of K2 Photodissociation Through the B1Pi(sub u) State.

PB94-211125

00,812 Not available NTIS

PB94-211133

Intersystem Crossing in Collisions of Aligned $\mathrm{Ca}(4 \mathrm{~s} 5 \mathrm{p}(1) \mathrm{P})$ + He: A Half Collision Analysis Using Multichannel Ouantum Defect Theory.

PB94-211133

PB94-211141

00,813 Not available NTIS

How Far Is Far trom Critical Point in Polymer Blends. Lattice Cluster Theory Computations for Structured Monomer. Compressible Systems.

PB94-211141 
PB94-211240

Clinical Perspective on Dentin Adhesives.

PB94-211240

00, 146 Not available NT!S

PB94-211257

Selective Inhibition of Crystal Growth on Octacalcium Phos phate and Nonstoichiometric Hydroxyapatite by Pyrophosphate at Physiological Concentration

PB94-211257 00,147 Not available NTIS

PB94-211265

Slab Transmission and Reflection for Point Source and PB94-211265

03,838 Not available NTIS

PB94-211273

National Quality Assurance Program for Personnel Radiation Dosimetry: A Case History.
PB94-211273 620 Not available NT!S

B 94-211281

Critical Magnetic-Field Angle for High-Field Current Transport in $\mathrm{YBa2Cu} 3 \mathrm{O} 7$ at $76 \mathrm{~K} . \quad 04,490 \quad$ Not available NT!S

PB94-211299

Superconducting Materials: Specification

PB94-211299

04,491 Not available NTIS

PB94-211307

Transport Critical Current of Aligned Polycrystalline Yttrium Barium Copper Oxide (YBa2Cu3O7-delta

PB94-21130

04,492 Not available NT1S

PB94-211315

Effect of Axial Strain on the Critical Current of Ag-Sheathed Bi-Based Superconductors in Magnetic Fields Up to $25 \mathrm{~T}$

PB94-211315 04,493 Not available NTIS

PB94-211323

In situ Noble Metal YBa2Cu3O7 Thin-Film Contacts.

PB94-211323 04,494 Not available NTIS

PB94-211331

Total Surface Areas of Group IVA Organometallic Compounds: Predictors of Toxicity to Algae and Bacteria.

PB94-211331

00,814 Not available NTIS

PB94-211349

Longterm Changes of Silicon Photodiodes and Their Use

for Photometric Standardization.

PB94-211349

04,234 Not available NTIS

PB94-211356

Magnetoelasticity in Rare-Earth Multilayers and Films.

PB94-21135

04,495 Not available NTIS

PB94-211364

Advanced Angle Metrology System.

PB94-211364

02,637 Not available NTIS

PB94-211372

Suppression Research: Strategies.

PB94-211372

PB94-211380 Sheared Atomic Fluid.

PB94-211380

B94-211398

Reaction Rate Determinations of Vinyl Radical Reactions with Vinyl, Methyl, and Hydrogen Atom

PB94-21139

PB94-211406

Anomalous Odd- to Even-Mass Isotope Ratios in Reso-

nance lonization with Broad-Band Lasers.

PB94-211406

03,839 Not available NTIS

PB94-211414

Earth-Based Gravitational Experiments

PB94-211414

03,840 Not available NTIS

PB94-211422

Monitoring Polymer Cure by Fluorescence Recovery After PB94-211422

PB94-211430

01,218 Not available NTIS

New Method for Shielding Electron Beams Used for Head and Neck Cancer Treatment.

PB94-211430

PB94-211448

03,621 Not available NTIS

High-Resolution Infrared Overtone Spectroscopy of ArHF via Nd:YAG/Dye Laser Difference Frequency Generation.

PB94-211448 00,816 Not available NTIS

U.S. GOSIP Testing Program.

PB94-211463

01,807 Not available NTIS

P-Type Doubling in the Infrared Spectrum of NO-HF

PB94-211463 00,817 Not available NTIS

PB94-211471

Analysis of Thermal Wave Propagation in Diamond Films.

PB94-211471

03,014 Not available NTIS

PB94-211489

Thermal Wave Propagation in Diamond Films

PB94-211489

03,015 Not available NTIS

PB94-211497

Magnetic Dipole Line from $U$ LXXI Ground-Term Levels Predicted at 3200 Angstroms.

B94-211505

04,407 Not available NTIS

Photoionization of Small Molecules Using Synchrotron Radi-

ation.

03,841 Not available NTIS
PB94-211513

NIST Power Reference Source.

PB94-211513

PB94-211521

Roles of Copper in Applied Superconductivity.

PB94-211521 02,255 Not available NTIS

PB94-211539

Benchmarks for the Evaluation of Speech Recognizers

PB94-211539

01,566 Not available NTIS

PB94-211547

Neutron Scattering by Multiblock Copolymers of Structure (A-B) N-A.

PB94-211547

PB94-211554

01,219 Not available NTIS

Atomic Transition Probability Ratios between Some Ar I 45$4 \mathrm{p}$ and $4 \mathrm{~s}-5 \mathrm{p}$ Transitions.

PB94-211554

03,842 Not available NTIS

PB94-211562

Ion Broadening Parameters for Several Argon and Carbon Lines.

PB94-2

03,843 Not available NTIS

P94-211570

Dreams About the Next Generation of Super-Stable Lasers.
PB94-211570 04,235 Not available NTIS

PB94-211588

$24 \mathrm{GHz}$ Josephson Array Voltage Standard.

PB94-211588

02,033 Not available NTIS

PB94-211596

Estimate of Flame Radiance via a Single Location Measurement in Liquid Pool Flames.

PB94-211596

PB94-211604

Measurement of Radiative Feedback to the Fuel Surface of a Pool Fire.

PB94-211612

02,477 Not available NTIS

Shear Dependence of Critical Fluctuations in Binary Polymer Mixtures by Small Angle Neutron Scattering.

PB94-211612 Sy Small Angle Nev 220 Not available NTIS

PB94-211620

01,220 Not available NTIS

L-threo-beta-Hydroxyhistidine, an Unprecedented Iron(III) lon-Binding Amino Acid in a Pyoverdine-type Siderophore from Pseudomonas fluorescens 244.

PB94-211620 00,553 Not available NTIS

PB94-211638

Energy and Migration of Grain Boundaries in Polycrystals.

03,332 Not available NTIS

PB94-211646

Fabrication of Platinum-Gold Alloys in Pre-Hispanic South America: Issues of Temperature and Microstructure Control. PB94-211646

PB94-211653

Wire Bond Testing

PB94-211653

03,333 Not available NTIS

PB94-211661

02,314 Not available NTIS

Radiation Chemistry of Cyanine Dyes: Oxidation and Reduction of Merocyanine 540 .

PB94-21166

00,818 Not available NTIS

PB94-211679

Laser-Induced Fluorescence Measurements of Formalde-

hyde in a Methane/Air Diffusion Flame.
PB94-211679 01,374 Not available NTIS

PB94-211687

Ensuring Accuracy and Traceability of Weighing Instru-

ments. 11687

PB94-211695

Particle Size Standards and Their Certification at NIST.

PB94-211695

02,638 Not available NTIS

PB94-211703

Supercritical Solubility of Solids from Near-Critical DiluteMixture Theory
PB94-211703

PB94-211711

00,819 Not available NTIS

YBa2Cu3O7-x to $\mathrm{Si}$ Interconnection for Hybrid

Superconductor/Semiconductor Integration.
PB94-211711

02,315 Not available NTIS

Small-Angle $X$-Ray and Neutron Scattering Study of Block Copolymer/Homopolymer Mixtures.
PB94-211729 01,221 Not available NTIS

PB94-211737

High Temperature

PB94-211737

03,844 Not available NTIS

PB94-211745

Vapor Transport in Materials and Process Chemistry.

PB94-211745

00,669 Not available NTIS PB94-211752

Drill-Hearn-Gerasimov Sum Rule.

PB94-211752

03,845 Not available NTIS

4-211760

Polarizability of the Nucleon.

PB94-211778

Effect of Microstructure on the Wear Transition of ZirconiaPoughened Alumina.

03,141 Not available NTIS

PB94-211786

Asymptotic Wave Function Splitting Procedure for Propagating Spatially Extended Wave Functions: Application to In tense Field Photodissociation of $\mathrm{H} 2(+)$

PB94-211786

03,847 Not available NTIS

PB94-211794

Investigation of the Drive Circuit Requirements for the Power Insulated Gate Bipolar Transistor (IGBT).

PB94-211794

PB 94-211802

Interstellar Disk-Halo Connection in Galaxies: Review of

Observational Aspects.

B94-211802

00,058 Not available NTIS

PB94-211810

Vibrational Relaxation Measurements of Carbon Monoxide on Metal Clusters.

PB94-211810

PB94-211828

Discontinuous Volume Change at the Orientational-Ordering Transition in Solid C60.

4-211828

00,821 Not available NTIS

PB94-211836

Differences in Competitive Strategies between the United States and Japan.

PB94-211836

00,013 Not available NTIS

PB94-211844

Importance of Measurement in Technology-Based Competiion.

PB94-211844

PB94-211851

Design and Characterization of X-Ray Multilayer Analyzers or the $50-1000 \mathrm{eV}$ Region.

PB94-211851

03,848 Not available NTIS

PB94-211869

Fast-Ion Conduction and Disorder in Cation and Anion $\mathrm{Ar}$ rays in Y2(ZryTi(1-y))2O7 Pyrochlores Induced by Zr Substitution: A Neutron Rietveld Analysis.

PB94-211869

04,496 Not available NTIS

PB94-211877

Intelligent Control for Multiple Autonomous Undersea Vehicles.

94-211877

03,747 Not available NTIS

PB94-211885

Real-Time Vision for Autonomous and Teleoperated Control of Unmanned Vehicles.

PB94-211885

03,701 Not available NTIS

PB94-211893

Real-Time Vision for Unmanned Vehicles.

PB94-211893

PB94-211901

03,702 Not available NTIS

International Conference on Chemical Kinetics (2nd). Held in Gaithersburg, Maryland on July 24-27, 1989.

PB94-211901

PB94-211919

Elastic Constants of Isotropic Cylinders Using Resonan Ultrasound.

PB94-211919

PB94-211927

04,497 Not available NTIS

Determination of the Prior-Austenitic Grain Size of Selected Steels Using a Molten Glass Etch.

PB94-211927

PB94-211935 
PB94-212032

Use of Extended Permutation-Inversion Groups in Constructing Hyperfine Hamiltonians for Symmetrical-Top Internal Rotor Molecules Like H3C-SiH3

\section{PB94-212040}

In-situ Fume Particle Size and Number Density Measure-

ments from a Synthetic Smelt.

03,334 Not available NTIS

PB94-212057

Tribochemical Reaction of Stearic Acid on Copper Surface Studied by Surface Enhanced Raman Spectroscopy.

PB94-212057 02,964 Not available NTIS

PB94-212065

Hierarchical Real-Time Control System for Use with Coal Mining Automation.

PB94-212065

03.698 Not available NTIS

PB94-212073

Neutron Powder Diffraction Study of the Crystal Structure of YSr2AICu2O7.

PB94-212081

04,499 Not available NTIS

Equation of State Formulation of the Thermodynamic Properties of R134a (1,1,1,2-Tetrafluoroethane).

PB94-212081 03,256 Not available NTIS

PB94-212099

Prediction of Viscosity of Refrigerants and Refrigerant Mixtures.

PB94-212099

PB94-212107

03,257 Not available NTIS

Prediction of the Thermal Conductivity of Refrigerants and Refrigerant Mixtures.

PB94-212115

Kinetics of the Reaction of CCl3-Br-2 and the Thermochemistry of CCl3 Radical and Cation.

PB94-212115

PB94-212123

Resonance Enhanced Multiphoton Ionization Detection of

GeF and GeCl Radicals.
PB94-212123

PB94-212131

00,825 Not available NTIS

Experimental and Abinitio Studies of Electronic Structures of the $\mathrm{CCl} 3$ Radical and Cation.

PB94-212131

PB94-212149

Photoelectron Spectroscopic Study of the Valence and Core-Level Electronic Structure of $\mathrm{BaTiO}_{3}$

PB94-212149 04,500 Not available NTIS

B94-212156

Surface Core-Level Shifts of Barium Observed in Photoemission of Vacuum-Fractured $\mathrm{BaTiO}_{3}(100)$

PB94-212156 04,501 Not available NTIS

PB94-212164

Kinetics of the Self-Reaction of Hydroxymethylperoxyl Radicals.

PB94-21216

00,827 Not available NTIS

PB94-212172

Temperature Dependence of the Rate Constants for Reac-

tions of the Sulfate Radical, SO4-, with Anions.
PB94-212172 00,828 Not available NTIS

PB94-212180

Electron Transfer Reaction Rates and Equilibria of the Car bonate and Sulfate Radical Anions.

PB94-212180

0,829 Not available NTIS

PB94-212198

Reaction of NO with Superoxide.

PB94-212198

00,830 Not available NTIS

PB94-212206

Temperature Dependence of the Rate Constants for Reactions of the Carbonate Radical with Organic and Inorganic Reductants.

PB94-212214

00,831 Not available NTIS

Opacity Project and the Practical Utilization of Atomic Data.

PB94-212214

PB94-212222

Micromechanics of Fracture in Rubber-Toughened Epoxies.

PB94-212222

PB94-212230

Flux Expulsion at Intermediate Fields in Type-II Superconductors.

PB94-212248

$\mathrm{K}$ alpha Transitions in Few-Electron lons and in Atoms. PB94-212248 00,059 Not available NTIS

PB94-212255

Role of the Office of Radiation Measurement in Quality Assurance.

PB94-212263

00,689 Not available NTIS

Offset Susceptibility of Superconductors.

PB94-212263 04,503 Not available NTIS

PB94-212271

Ouantum Projection Noise: Population Fluctuations in TwoLevel Systems
PB94-212271

03,850 Not available NTIS
PB94-212289

Determination of Boron and Lithium in Diverse Biological Matrices Using Neutron Activation - Mass Spectrometry PB94-21228

00,554 Not available NTIS

PB94-212297

Formalism and Parameters for Quantitative Surface Analysis by Auger Electron Spectroscopy and $X$-Ray Photoelectron Spectroscopy.

PB94-212297

00,832 Not available NTIS

PB94-212305

Glass Transition of Organic Liquids Confined to Small Pores

PB94-212305

PB94-212313

00,833 Not available NTIS

Melting Behavior of Organic Materials Confined in Porous

Solids.

PB94-212313

00,834 Not available NTIS

PB94-212321

Anomalous Freezing and Melting of Solvent Crystals in Swollen Gels of Natural Rubber.

PB94-212321

PB94-212339

Polymer Liquid Crystalline Materials.

PB94-212339

01,223 Not available NTIS

PB94-212347

Guidelines for Reporting Results of Computational Experiments. Report of the Ad hoc Committee.

PB94-212347

03,427 Not available NTIS

PB94-21235

Mechanism of Mild to Severe Wear Transition in Alpha-Alu-

mina.

PB94-212362

03,233 Not available NTIS

Tribology Education: Present Status and Future Challenges.

PB94-212362

02,965 Not available NTIS

PB94-212370

Intercomparison of the Effective Areas of a Pneumatic Pis-

ton Gauge Determined by Different Techniques.

PB94-212370

02,640 Not available NTIS

94-212388

Investigation of S2F10 Production and Mitigation in Compressed SF6-Insulated Power Systems.
PB94-212388 02,467 Not available NTIS

PB94-212396

Beneficial Effects of Nitrogen Atomization on an Austenitic Stainless Steef.

PB94-212404

03,209 Not available NTIS

Rate of Heat Release of Wood Products.

PB94-212404

03,403 Not available NTIS

PB94-212412

Local Partial Densities of States in $\mathrm{Ni}$ and $\mathrm{Co}$ Silicides

Studied by Soft X-Ray Emission Spectroscopy.
PB94-212412 04,504 Not avallable NTIS PB94-212420

Global Thermodynamic Behavior of Fluid Mixtures in the Critical Region
PB94-212420

PB94-212438

04,199 Not available NTIS

Effect of Cross-Links on the Miscibility of a Deuterated Polybutadiene and Protonated Polybutadiene Blend.

PB94-212438

PB94-212446

Inversion of the Phase Diagram from UCST to LCST in Deuterated Polybutadiene and Protonated Polybutadiene Blends.

PB94-212446

PB94-212453

Status of Electrocomposites.

PB94-212453

PB94-212461

Aid for Smaller Businesses.

PB94-212461

01,226 Not available NTIS

PB94-212479

Topological Influences on Polymer Adsorption and Desorption Dynamics.

PB94-212487

Multiphoton Ionization of $\mathrm{SiH} 3$ and SiD3 Radicals. 2. Three Photon Resonance-Enhanced Spectra Observed between 460 and $610 \mathrm{~nm}$.

460 and 610 n

PB94-212495

00,835 Not available NTIS

New Electronic States of NH and ND Observed from 258 to $288 \mathrm{~nm}$ by Resonance Enhanced Multiphoton Ionization Spectroscopy.

00,836 Not available NTIS

Multiphoton Ionization of $\mathrm{SiH} 3$ and $\mathrm{SiD} 3$ Radicals: Electronic Spectra, Vibrational Analyses of the Ground and Rydberg States, and Ionization Potential.

PB94-212503 00,837 Not available NTIS

PB94-212511

NIST-NRL Free-Electron Laser Facility,
PB94-212511 04,237 Not available NTIS

PB94-212529

Hybrid Undulator for the NIST-NRL Free-Electron Laser

PB94-212529 04,238 Not available NTIS

PB94-212537

Ultrasonic Spectroscopy of Metallic Spheres Using Electromagnetic-Acoustic Transduction.

PB94-212537

04,185 Not available NTIS

PB94-212545

Beam Analysis Round Robin

PB94-212545

04,239 Not available NTIS

PB94-212552

Laser-Beam Analysis Pinpoints Critical Parameters.

PB94-212552

04.240 Not available NTIS

PB94-212560

Theory of Atomic Collisions at Ultracold Temperatures.

PB9 4-212560

03,851 Not available NTIS

PB94-212578

Efficient Experiment to Study Superconducting Ceramics.

PB94-212578

04,505 Not available NTIS

PB94-212586

Development in the Theory and Analysis of Eddy Current Sensing of Velocity in Liquid Metals.

PB94-212586

03.335 Not available NTIS

PB94-212594

Optically Sensed EM-Field Probes for Pulsed Fields.

PB94-212594

02,130 Not available NTIS

PB94-212602

Modification of Deoxyribose-Phosphate Residues by Exracts of Ataxia Telangiectasia Cells.

PB94-212602

03,458 Not available NTIS

PB94-212610

Effects of Molecular Weight and Thermal Stability on Polymer Gasification.

PB94-212628

01,228 Not available NTIS

Nickel(II)-Mediated Oxidative DNA Base Damage in Renal and Hepatic Chromatin of Pregnant Rats and Their Fetuses. Possible Relevance to Carcinogenesis.

PB94-212628 03,646 Not available NTIS

PB94-212636

Thermodynamic Calculation of the Ternary Ti-Al-Nb Sys. tem. 212636

PB94-212644

Thermodynamic Assessment and Calculation of the Ti-A System.
PB94-212644

PB94-212651

Chaos in a Computer-Animated Pendulum

PB94-212651

PB94-212669

Proposed High-Accuracy Superconducting Power Meter for Millimeter Waves.

PB94-212677

Effect of Thermal Noise on Shapiro Steps in High-Te Josephson Weak Links

PB94-212677

04,506 Not available NTIS

PB94-212685

Self-Heating in the Coulomb-Blockade Electrometer.

PB94-212685

04,507 Not available NTIS

PB94-212693

Application of Boundary Element Methods to a Transient AxIs-Symmetric Heat Conduction Problem.

PB94-212693

01,375 Not available NTIS

PB94-212701

Causes of the Apparent Heat Transfer Degradation for Re frigerant Mixtures.

PB94-212701

PB94-212719

03,259 Not available NTIS

Attempts at Extending the Unified Theory to Include ManyBody Effects.

PB94-212727

04,408 Not available NTIS 
PB94-212784

Relativistic and Quantum Electrodynamic Effects in HighlyCharged lons

03,854 Not available NTIS

PB94-212792

Product Kinetic Energies, Correlations, and Scattering Anisotropy in the Bimolecular Reactor $\mathrm{O}((1) \mathrm{D})+\mathrm{H} 2 \mathrm{O}$ yields

00,838 Not available NTIS

PB94-212792

Apparent Molar Heat Capacities and Apparent Molar Vol-

Apparent Molar Heat Capacities and Apparent Molar Vol-
umes of Aqueous Glucose at Temperatures from $298.15 \mathrm{~K}$ umes of Aqueous Glucose at Temperatures from $298.15 \mathrm{~K}$
to $327.01 \mathrm{~K}$. to $327.01 \mathrm{~K}$.

03,459 Not available NTIS

PB94-212818

Evolution of a Turbulent Boundary Layer Induced by a Three-Dimensional Roughness Element.

PB94-212818

04,200 Not available NTIS

PB94-212826

Molecular-Beam Optothermal Spectrum of the $\mathrm{OH}$ Stretching Band of Methanol.

PB94-21282

00,839 Not available NTIS

PB94-212834

Fundamental Torsion Band in Acetaldehyde.

PB94-212834

00,840 Not available NTIS

PB94-212842

Atomic Branching Ratio Data for Carbon-Like Ions.

PB94-212842 03,855 Not available NTIS

PB94-212859

Full Scale Smoke Control Tests at the Plaza Hotel Building

PB94-212859 00,347 Not available NTIS

PB94-212867

Overview of Smoke Control Technology.

PB94-212867

00,348 Not available NTIS

PB94-212875

Preview of ASHRAE's Revised Smoke Control Manual.

PB94-212875 00,349 Not available NTIS

PB94-212883

Smoke Control Systems for Elevator Fire Evacuation

PB94-212883 00,291 Not available NTIS

PB94-212891

Determination of Osmotic Pressure and Fouling Resistances and Their Effects on Performance of Ultrafiltration Membranes.

PB94-21289

PB94-212909

Characterization of the Adsorption-Fouling Layer Using Globular Proteins on Ultrafiltration Membrangs

PB94-212909

00,842 Not available NTIS

PB94-212917

High-Spatial-Resolution Resistivity Mapping Applied to Mercury Cadmium Telluride.

PB94-212917

02,131 Not available NTIS

PB94-212925

Review of Semiconductor Microelectronic Test Structures with Applications to Infrared Detector Materials and Proc-

PB94-212925

02,132 Not available NTIS

PB94-212933

Summary of the Proceedings of the Workshop on Standard Phantoms for In-vivo Radioactivity Measurement

PB94-212933 03,622 Not available NTIS

PB94-212941

Effects of Variable Excluded Volume on the Dimensions of Off-Lattice Polymer Chains.

PB94-212941

PB94-212958

Observed and Theoretical Creep Rates for an Alumina Ceramic and a Silicon Nitride Ceramic in Flexure.

PB94-212958

03,040 Not available NTIS

Defect of Thermal Ramping and Annealing Conditions on Defect Formation in Oxygen Implanted Silicon-On-Insulator Material.

PB94-212966

02,318 Not available NTIS

PB94-212974

Active Site lonicity and the Mechanism of Carbonic Anhy-

drase.

PB94-212982

Dipole Moments in Rare Gas Interactions

PB94-212982 00,844 Not available NTIS PB94-212990

Degut Nivity in a Cluster

Analysis 0

PB94-212990

00.845 Not available NTIS

PB94-213006

Cs Cluster Binding to a GaAs Surface.
PB94-213006 No, Navailable NTIS

PB94-213014

O 846 Not avalabie NTIS

Iridium Oxide Thin-Film Stability in High-Temperature CorroSive Solutions.

PB94-213022

03,234 Not available NTIS

Radiation-Driven Winds of Hot Luminous Stars $X$. The Determination of Stellar Masses Radii and Distances from Terminal Velocities and Mass-Loss Rates.

PB94-213022

00,060 Not available NTIS

\section{PB94-213030}

Formal Specification and Verification of Control Software for Cryptographic Equipment.

Cryptographic

PB94-213048

Materials Science with SR Using X-Ray Imaging: SpatialResolution/Source Size.

PB94-213048

PB94-213055

High Resolution Hard X-Ray Microscope

PB94-213055

04,510 Not available NTIS

PB94-213063

Thermal Conductivity of R134

PB94-213063

03,856 Not available NTIS

PB94 190

03.857 Not available NTIS

Spectroscopic Constants for the 2.5 and 3.0 micrometer Bands of Acetylene.

PB94-21307

00,847 Not available NTIS

PB94-213089

Analysis of Boron in CVD Diamond Surfaces Using Neutron Depth Profiling

PB94-213097

Normal-Incidence Complex-Index Refractometry.

PB94-213097

04,511 Not available NTIS

PB94-21310

04,241 Not available NTIS

Precision, Accuracy, Uncertainty and Traceability and Their Application to Submicrometer Dimensional Metrology.

PB94-213105 02,319 Not available NTIS

PB94-213113

Thermochemistry of the Reactions between Adenosine, 5' Inosine, and Inosine 5' monophosphate; the Conversion of L-histidine to (Urocanic Acid+Ammonia)

$-213113$

03,460 Not available NTIS

PB94-213121

Magnetic and Structural Properties of Electrodeposited Copper-Nickel Microlayered Alloys.

PB94-213121

04,512 Not available NTIS

PB94-213139

Laser-Induced Fluorescence Measurements of Rotationally Resolved Velocity Distributions for $\mathrm{CO}(+)$ Drifted in $\mathrm{He}$

PB94-213139

00,848 Not available NTIS

PB94-213147

Custom Integrated Circuit Comparator for High-Performance Sampling Application

PB94-213154

02,320 Not available NTIS

Elastic Deformation of a Monolithic Perfect Crystal Interferometer: Implications for Gravitational Phase Shift Experiments.

PB94-213154

03,858 Not available NTIS

PB94-21316

Hierarchical Ada Robot Programming System (HARPS): A Complete and Working Telerobot Control System Based on the NASREM Mode

PB94-21316

02,934 Not available NTIS

PB94-213170

Recent Approaches 10 Extreme Value Estimation with Application to Wind Speeds. Part 1. The Pickands Method

00,019 Not available NTIS

PB94-213188

Dynamics vs. Static Young's Moduli: A Case Study

PB94-213188

PB94-213196

Elastic Constants of Polycrystalline $\mathrm{Y} 1 \mathrm{Ba} 2 \mathrm{Cu} 3 \mathrm{Ox}$

PB94-213196

04,513 Not available NTIS

PB94-213204

Thermal Expansion of an SiC Particle-Reinforced Aluminum Composite.

03,144 Not available NTIS

PB94-213212

Cast-Iron Elastic Constants: Effect of Graphite Aspect

PB94-213212

03,211 Not available NTIS

PB94-213220

Correlation between TC and Elastic Constants of (La$\mathrm{M}) 2 \mathrm{CuO} 4$.
$\mathrm{PB} 94-21322$

PB94-213220
$P B 94-213238$

04,514 Not available NTIS

Ultracold Collisic

PB94-213238

PB94-213246

Light Scattering from Glossy Coatings on Paper.

PB94-213246

PB94-213253

Development of a New Small-Scale Smoke Toxicity Test Method and its Comparison with Real-Scale Fire Tests.

PB94-213253

00,350 Not available NTIS

PB94-213261

EXITT: A Simulation Model of Occupant Decisions and ACtions in Residential Fires.

PB94-213261

00,351 Not available NTIS

PB94-213279

High-Energy Behavior of the Double Photoionization of Helium from 2 to $12 \mathrm{keV}$.

03,860 Not available NTIS

PB94-213287

Astrophysical Aspects of Neutral Atom Line Broadening

PB94-213287

00,061 Not available NTIS

PB94-213295

RIS Studies of Autoionization in Calcium

PB94-213295 00,849 Not available NTIS

PB94-213303

Debugger for Tcl Applications.

PB94-213303

01,695 Not available NTIS

PB94-213311

Kibitz-Connecting Multiple Interactive Programs Together.

PB94-213311

01,696 Not available NTIS

BB94-213329

Using Expect to Automate System Administration Tasks.

PB94-213329

01,697 Not available NTIS

(1)

Molecular Spectroscopy.

03,861 Not available NTIS

PB94-213345

Relation between AC Impedance Data and Degradation of

Coated Steel. 1. Effects of Surface Roughness and Con

tam

PB94-213345

03,189 Not available NTIS

PB94-213352

Elastic Constants and Debye Temperature of $\mathrm{Y} 1 \mathrm{Ba2Cu} 3 \mathrm{Ox}$

Effect of Oxygen Content

PB94-213352

213360

04,515 Not available NTIS

Polarized X-Ray Emission Spectroscopy

PB94-213360

Atomic, Molecular, and Optical Physics with $X$-rays

PB94-213378

03,863 Not available NTIS

PB94-213386

High-Sensitivity Determination of lodine Isotopic Ratios by

Thermal and Fast Neutron Activation.

PB94-213386

PB94-213394

Neutron Capture Prompt Gamma-Ray Activation Analysis at the NIST Cold Neutron Research Facility.

PB94-213394

00.556 Not available NTIS

PB94-213402

Atomic Data Needed for Far Ultraviolet Astronomy with HUT and FUSE

PB94-213410

FUSE: The Far Ultraviolet Spectrograph Explorer

PB94-213410

00,063 Not available NTIS

PB94-213428

Ultraviolet Observations of Stellar Coronae: Early Results PB94-213428

00.064 Not available NTIS

PB94-213436

$X$-rays from Stellar Flares.

PB94-213436

00,065 Not available NTIS

PB94-213444

Goddard High-Resolution Spectrograph Observations of the

Local Interstellar Medium and the Deuterium/Hydrogen

Ratio along the Line of Sight Toward Capella.

PB94-213444

PB94-213451 
January to March 1994 with 1994/1995 EEEL Events Calendar.

94-213774

PB94-213857

Feasibility of Fire Evacuation by Elevators at FAA Control

Towers.

PB94-214756

Information Infrastructure: Reaching Society's Goals. Report of the Information Infrastructure Task Force Committee on Applications and Technology

PB94-214756

01,469 PC A08/MF A02

PB94-215670

Highway Concrete (HWYCON) Expert System User Reference and Enhancement Guide.

PB94-215670

PB94-215688

Time Dependent Vector Dynamic Programming Algorithm for the Path Planning Problem.

PB94-215688

$03,428 \mathrm{PC} \mathrm{AO4/MF} \mathrm{AO1}$

PB94-215704

Diffusion of Cations Beneath Organic Coatings on Steel Substrate.

PB94-215704

$03,119 \mathrm{PC}$ A03/MF A01

PB94-215712

Analytical Estimation of Carrier Multipath Bias on GPS Posiion Measurements.

PB94-215712

$04,845 \mathrm{PC} \mathrm{A04/MF} \mathrm{A01}$

PB94-215746

Head Start on Assurance: Proceedings of an Invitational Workshop on Information Technology (IT) Assurance and Trustworthiness. Held in Williamsburg. Virginia on March 21-23, 1994.

PB94-215746

01,586 PC A05/MF A01

PB94-216017

Using LORAN-C Broadcasts for Automated Frequency Cali-

PB94-216017

PB94-216025

Structures of Vapor-Deposited Yttria and Zirconia Thin

PB94-216025

03,041 Not available NTIS

PB94-216033

Rapid Decline in the Optical Emission from SN 1957D in M83.

00,070 Not available NTIS

PB94-216033

PB94-216041

Measurement of CO Pressures in the Ultrahigh Vacuum Regime Using Resonance-Enhanced Multiphoton-Ionization Time-of-Flight Mass Spectroscop

PB94-21604

PB94-216058

Spectroscopic Puzzle in ArHF Solved: The Test of a New Potential.

PB94-216058

00.850 Not available NTIS

PB94-216066

Uitra-High Stability Synthesizer for Diode Laser Pumped

Rubidium.

PB94-216066

01,527 Not available NTIS

PB94-216074

Heavily Accumulated Surfaces of Mercury Cadmium Telluride Detectors: Theory and Experiment.

PB94-216074 02,134 Not available NTIS

PB94-216082

Evolution of the Flight Telerobotic Servicer.

PB94-216082 04,832 Not available NTIS

PB94-216090

Effect of Three Sterilization Techniques on Finger Pluggers.

PB94-216090

0,150 Not available NTIS
00,19 ob

PB94-216108

Modified Leung-Griffiths Model of Vapor-Liquid Equilibrium Extended Scaling and Binary Mixtures of Dissimilar Fluids.
PB94-216108 00,851 Not available NTIS

PB94-216116

00,851 Not available NTIS

Polarization Analysis of the Magnetic Excitations in Invar and Non-Invar Amorphous Alloys.

PB94-216116

04.516 Not available NTIS

PB94-216124

Analytical Method of Determining the Heat Capacity at High Temperatures from the Surface Temperature of a Cooling Sphere.

PB94-216124

03,865 Not available NTIS

PB94-216132

Performance Standards: The Pro's and Con's

PB94-216132

02,896 Not available NTIS

PB94-216140

Scattering and Absorption Effects in Neutron Beam Activation Analysis Experiments.

PB94-216140

0.557 Not available NTIS

PB94-216157

Effects of Target Shape and Neutron Scattering on Element Sensitivities for Neutron-Capture Prompt Gamma-ray Activation Analysis.

PB94-216165

00.558 Not available NTIS

Structure of Molecules on Surfaces as Determined Using Electron-Stimulated Desorption.

PB94-216165

00,852 Not available NTIS
PB94-216173

Desorption Induced by Electronic Transitions

PB94-216181

Sprınkler Fire Suppression Algorithm.

PB94-216181

PB94-216199

Thermophysical Properties of $\mathrm{CO} 2$ and $\mathrm{CO} 2-$ Rich Mixtures.

00,854 Not available NTIS

PB94-216207

Neutron Scattering Studies of Surfaces and Interfaces.

PB94-216207

04,517 Not available NTIS

PB94-216215

Supermirror Transmission Polarizers for Neutrons

PB94-216215

03,866 Not available NTIS

PB94-216223

High-Resolution Measurements of the nu2 and 2nu2-nu2 Bands of (34) S(16)O2.

PB९4-216223

00,855 Not available NTIS

PB94-216231

FTS Infrared Measurements of the Rotational and Vibrational Spectrum of $\mathrm{LiH}$ and $\mathrm{LiD}$

PB94-216231

\section{PB94-216249}

Statistical Analysis of Parameters Affecting the Measurement of Particle-Size Distribution of Silicon Nitride Powders by Sedigraph (Trade Name)

PB94-216256

03,042 Not available NTIS

Coating of Fibers by Colloidal Techniques in Ceramic Com-

posites.

PB94-216256

03,196 Not available NTIS

PB94-216264

Characterization of Phase and Surface Composition of Silicon Carbide Platelets.

03,043 Not available NTIS

B94-216272

Deposition of Colloidal Sintering-Aid Particles on Silicon Nitride.

PB94-216272

PB94-216280

Integrated Optic Laser Emitting at 906, 1057, and $1358 \mathrm{~nm}$.

PB94-21628

PB94-216298

Integrated Optic Laser Emitting at 905, 1057, $1356 \mathrm{~nm}$. PB94-216298

PB94-216306

Models and Interactions.

PB94-216306

03,044 Not available NTIS

B94-216314

Crystal Structure of Pb2Sr2YCu3O8+delta with delta $=1.32$ $1.46,1.61,1.71$, by Powder Neutron Diffraction.
PB94-216314 PB94-216322

Hydrolysis of Proteins by Microwave Energy

PB94-216322

03,528 Not available NTIS

PB94-216330

Ascorbic and Dehydroascorbic Acids Measured in Plasma Preserved with Dithiothreitol or Metaphorphoric Acid.

PB94-216330 03,495 Not available NTIS

PB94-216348

Study of Diffusion Zones with Electron Microprobe Compositional Mapping.

$-2163$

PB94-216355

Paffenbarger Research Center: The Cutting Edge of Dental

Science.

PB94-216363

00.151 Not available NTIS

Energy Transduction between a Concentration Gradient and an Alternating Electric Field.

PB94-216363

PB94-216371

Self Broadening in the nu1 Band of $\mathrm{NH}_{3}$.

PB94-216371

03,461 Not available NTIS

PB94-216389

Accurate Experimental Characterization of Interconnects: A Discussion of "Experimental Electrical Characterization of Interconnects and Discontinuities in High-Speed Digital Sys-

PB94-216389

02,217 Not available NTIS

PB94-216397

Interconnection Transmission Line Parameter Characteriza. PB94-216397

PB94-216

02,218 Not available NTIS

Standards in Building Economics: Why We Need Them and How to Write Them.

PB94-216405

PB94-216413

00,320 Not available NTIS

High-Level CAD Melds Micromachined Devices with Found-

ries.

PB94-216421

02,321 Not available NTIS

Lessons Learned by a Wing Engineer.
PB94-216421

PB94-216439

Atomic Beam Splitters and Mirrors by Adiabatic Passage in Multilevel Systems.

03,867 Not available NTIS

PB94-216447

Non-Osmotic, Defect-Controlled Cathodic Disbondment of a Coating from a Steel Substrate.

PB94-216447

PB94-216454

Precision and Accuracy in Tandem Mass Spectrometry

Measurements: A Kinetics-Based Protocol for Instrument-

Independent Measurements of Collision-Activated

Dissociation in RF-Only Quadrupoles.

PB94-216454

00.858 Not available NTIS

B94-216462

Kinetics and Mechanism of the Collision-Activated

Dissociation of the Acetone Cation. 00 Not available NTIS

PB94-216470

Guarding Against Transients

PB94-216488

01,623 Not available NTIS

Cascading Surge-Protective Devices: Options for Effective Implementation

PB94-21648

02,464 Not available NTIS

PB94-216496

Coordinating Cascaded Surge-Protective Devices: An Elusive Goal.

PB94-216504

02,465 Not available NTIS

Important Link in Entire-House Protection: Surge Reference Equalizers.

PB94-216512

02,219 Not available NTIS

Alternative Single Integral Equation for Scattering by a Di-

electric.

PB94-216512

04.422 Not available NTIS

PB94-216520

Determination of Surface Roughness from Scattered Light. PB94-216520

Autocorrelation Functions from Optical Scattering for One-

Dimensionally Rough Surfaces

PB94-216546

Light Scattered by Coated Paper.

PB94-216546

PB94-216553

Atomic Theory of Fracture of Brittle Materials: Application to Covalent Semiconductors.

PB94-21655

04,519 Not available NTIS

PB94-21656

Crystal Structure of Dicalcium Potassium Trihydrogen Bis(pyrophospha

00,152 Not available NTIS

PB94-216579

Crystal Structure of Calcium Adipate Monohydrate.

PB94-216579

00,153 Not available NTIS

B94-216587

Rapid Hot Pressing of Ultra-Fine PSZ Powders.

PB94-216587

03,045 Not available NTIS

PB94-216595

Carbon Acidities of Aromatic Compounds. 1. Effects of In Ring Aza and External Electron-Withdrawing Groups.

PB94-216595

PB94-216603

00,860 Not available NTIS 
PB94-216694

Recommended Changes in ASTM Test Methods D2512-82 and G86-84 for Oxygen-Compatibility Mechanical Impact Tests on Metals.

PB94-216694
PB94-216702

03,338 Not available NTIS

Digital Techniques in HV Tests - Summary of 1989 Panel Session.

PB94-216702

PB94.216710

Bibliography of Computer Security Glossaries.

01,587 Not available NTIS

PB94-216728

On-Line Process

Review

PB94-216728

03,145 Not available NTIS

PB94-216736

Effect of Modulated Taylor-Couette Flows on Crystal-Melt Interfaces: Theory and Initial Experiments.
PB94-216736 04,521 Not available NTIS

PB94-216744

Boundary Integral Method for the Simulation of Two-DimenSional Particle Coarsening.

PB94-216751

03;411 Not available NTIS

High-Resolution IR Laser-Driven Vibrational Dynamics in Supersonic Jets: Weakly Bound Complexes and Intramolecular Energy Flow.

PB94-21675

$\infty, 862$ Not available NTIS

PB94-217023

Videoconferencing Procurement and Usage Guide.

PB94-217023

01,470 PC A04/MF A01

PB94-217031

Determination of the Weight Average Molecular Weight of Two Poly(Ethylene Oxides), SRM 1923 and SRM 1924

PB94-21703

$01,230 \mathrm{PC} \mathrm{A03/MF} \mathrm{A01}$

B94-217098

Publications of the Intelligent Systems Division (Previously Robot Systems Division) Covering the Period January 1971-April 1994

PB94-217098

PB94-217106

02,935 PC A05/MF A01

NIST Form-Based Handprint Recognition System.

PB94-217106

01.838 PC AO4/MF AO

PB94-217163

Human Factors Considerations for the Potential Use of Elevators for Fire Evacuation of FAA Air Traffic Control Tow-

PBS. 217163

P894.217486

$01,300 \mathrm{PC} \mathrm{A03/MF} \mathrm{A01}$

Hollow Clay Tile Prism Tests for Martin Marielta Energy Systems: Task 2 Testing

PB94-21748

$00,352 \mathrm{PC} \mathrm{A14/MF} \mathrm{A03}$

PB94-218559

Study of Hea: Pump Performance Using Mixtures of R32/ R134a and R32/R 125/R134a as 'Drop-In' Working Fluids for R22 with and Without a Liquid-Suction Heat Exchanger. PB94-218559

PB94-218567

02,503 PC A04/MF A01

NIST Workshop on Nanostructured Material (1st): Report of an Industrial Workshop Conducted by the National Institute of Standards and Technology. Held in Gaithersburg, Maryand on May 14-15, 1992.

PB94-218567

02,973 PC AO4/MF AO

PB94-218575

Adaptive, Predictive 2-D Feature Tracking Algorithm for Finding the Focus of Expansion.

PB94-218575

$01,588 \mathrm{PC} \mathrm{A03/MF} \mathrm{A0}$

Survey of Recent Cementitjous Materials Research in Western Europe.

PB94-218583

PB94-218591

RangeCAD and the NIST RCS Uncertainty Analysis.

PB94-218591

PBg4-219052

$01,870 \mathrm{PC} \mathrm{AO3/MF} \mathrm{AO1}$

NIST Research Program on the Seismic Resistance of Partially-Grouted Masonry Shear Walls.

PB94-21905

00,354 PC A06/MF AO2

PB94-219060

National Voluntary Laboratory Accreditation Program: Energy Efficient Lighting Products.

PB94-21906

$02,642 \mathrm{PC} \mathrm{A04/MF} \mathrm{AO}$

Framework for Information Technology Integration in Process Plant and Related Industries.

PB94-219086

$02,772 \mathrm{PC} \mathrm{AO4/MF} \mathrm{AO}$

PB94-219094

ISDN Conformance Testing Guidelines: Guidelines for Implementors of ISDN Customer Premises Equipment to ISDN Users' Forum Lational ISDN-1 and North American Con Users Forum Layer 3

Control Abstract

$01,471 \mathrm{PC} \mathrm{A03/MF} \mathrm{A0}$

PB94-219102

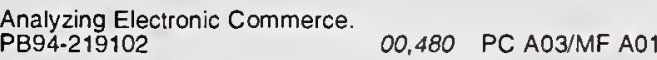

PB94-219102

Specification Testing

Industry/Government Open Systems Specification Testing
Framework. Version 1.0.
PB94-219110 01,809 PC A06/MF A02

PB94-219110
PB94-219219

Journal of Research of the National Institute of Standards and Technology. March/April 1994. Volume 99. Number 2. PB94-219219

PB94-219227

Sealed Water Calorimeter for Measuring Absorbed Dose.

03,517

PB94-219235

Planar Near-Field Measurements of Low-Sidelobe Anten-

PB94-219235

02,001

PB94-219243

Physics Required for Prediction of Long Term Performance of Polymers and Their Composites.

PB94-219243 03,146

PB94-219250

Measurement and Uncertainty of a Calibration Standard for the Scianning Electron Microscope.

PB94-219250

(Order as PB94-219219, PC AO6/MF A02)

PB94-219268

New Values for Silicon Reference Materials, Certified for .

PB94-219268

(Order as PB94-219219, PC A06/MF AO2)

\section{PB94-219326}

Joumal of Research of the National Institute of Standards

and Technology. May/June 1994. Volume 99, Number 3 .

PB94-219326

02,643 PC A05/MF A02

Sources of Uncertainty in a DVM-Based Measurement System for a Ouantized Hall Resistance Standard.

(Order as PB94-219326, PC AO5/MF A02) PB94-219334

P894-219342

dc Method for the Absolute Determination of Conductivities of the Primary Standard $\mathrm{KCI}$ Solutions from OC to $50 \mathrm{C}$

PB94-219342 02,644

Three-Axis Coil Probe Dimensions and Uncertainties during A

PB94-219359

01,885
(Order as PB94-219326, PC A05/MF A02)

PB94-219367

Improved Automated Current Control for Standard Lamps.

PB94-219367 00,246

PB94-219375

Refractive Indices of Fluids Related to Alternative Refrigerants.

PB94-219375

Order as PB94-219326, PC A05/MF AO2)

PB94-219383

Theoretical Analysis of the Coherence-Induced Spectral Shift Experiments of Kandpal, Vaishya, and Joshi.

PB94-219383

(Order as PB94-219326, PC A05/MF A02)

PB94-219391

Comments on the Paper 'Wolf Shifts and Their Physical Interpretation under Laboratory Conditions'

PB94-219391 04,246

PB94-219409

Reply to Professor Wolf's Comments on My Paper on Wolf Shitts.

PB94-219409

(Order as PB94-219326, PC A05/MF A02)

PB94-500691

FORTRAN Compiler Validation System, Version 2.1

PB94-50069

PB94-501988

HAZARD I Fire Hazard Assessment Method (Version 1.2) (for Microcomputers)

PB94-501988

PB94-501996

HAZARD I Fire Hazard Assessment Method, Version 1.2 (Upgrade Package) (for Microcomputers)

PB94-50 1996

00,197 CP D99

PB94-502077

M (also known as MUMPS) Validation Test Suite, Version 8.3 (for Microcomputers)

PB94-502077

01,699 Diskette $\$ 250.00$

PB94-780129

Polymer Composites Workshop. Held in Winona, Minnesota on April 29-30, 1992 (Video).

PB94-937300

03,147 AV E99

Validated Products List (Cobol, Fortran, ADA, Pascal, MUMPS, SQL

PB94-962000

01,700 Standing Order

CALS-Automated Interchange of Technical Information.

PB94-962000

PB94-962100

CALS-Digital Representation for Communication of Product Data: IGES Application Subsets.

PB94-962100

PB94-962200

03,658 Standing Orde

CALS-Markup Requirements and Generic Style Specifications for Electronic Printed Output and Exchange of Text.

PB94-962200 03,659 Standing Orde

PB94-962300

CALS-Raster Graphics Representation Binary Format Requirements.
PB94-962300

PB94-962400

03,660 Standing Order

CALS-Digital Representation for Communication of Illustra-

tion Date: CGM Application Profile.

PB94-962500

CALS-Department of Defense Computer Aided Acquisition Logistic Support (CALS).

PB94-962500
PB94-962600

03,662 Standing Orde

CALS-Contractor Integrated Technical Information Service (CITIS), Functional Requirements.

PB94-962600

03,663 Standing Order

PB95-100368

NIOSH Pocket Guide to Chemical Hazards.

PB95-100368

PB95-103461

Ouestions and Answers on Quality, the ISO 9000 Standard Series, Ouality System Registration, and Related Issues. More Ouestions and Answers on the ISO 9000 Standard Series and Related Issues.

PB95.103461

00,495 PC A03/MF A01

PB95-103677

Report on the Workshop on Advanced Digital Video in the National Information Infrastructure. Held in Washington, D.C. on May 10-11, 1994.
PB95-103677

PB95-103719

$01,472 \mathrm{PC} \mathrm{A09/MF} \mathrm{A03}$

Framework for National Information Infrastructure Services.

PB95-103719

02,723 PC A07/MF A02
0.7 Infrastructure Services.

PB95-103826

New Expressions of Uncertainties for Humidity Calibrations at the National Institute of Standards and Technology.
PB95-103826
02,645 PC A02/MF A01

PB95-104907

In Situ Burning Oil Spill Workshop Proceedings. Held in Orlando, Florida on January 26-28, $1994.02,583$ PC A06/MF A02 PB95-104915

Abrasive Wear by Diesel Engine Coal-Fuel and Related Particles.

PB95-104915

PB95-104956

$01,450 \mathrm{PC}$ A04/MF AO

Extreme Value Theory and Applications: Proceedings of the

Conference on Extreme Value Theory and Applications,

Volume 3. Held in Gaithersburg, Maryland in May 1993.

PB95-10495

$03,432 \mathrm{PC} \mathrm{A11/MF} \mathrm{A03}$

Annual Conference on Fire Research: Book of Abstracts, October $17-20,1994$.

PB95-104964

PB95-105011

01,376 PC A09/MF A02

Energy Price Indices and Discount Factors for Life-Cycle Cost Analysis 1995. Annual Supplement to NIST Handbook 135 and NBS Special Publication 709. (Revised)

PB95.10501

PB95-105029

Present Worth Factors for Life-Cycle Cost Studies in the Department of Defense (1995).

PB95-105029

PB95-105037

Making Sense of Software Engineering Environment Framework Standards.

PB95-105037
PB95-105045 
PB95-107025

Intermolecular HF Motion in $\operatorname{Ar}($ sub $n) H F$ Micromatrices $(n=1,2,3,4)$ : Classical and Quantum Calculations on a Pairwise Additive Potential Surface.

PB95-107025

PB95-107033

Localization Model of Rubber Elasticity: Comparison with Torsional Data for Natural Rubber Networks in the Dry State.

PB95-107033

03,195 Not available NTIS

PB95-107041

Aging in Glasses Subjected to Large Stresses and Deformations.

PB95-107041

PB95-107058

03,235 Not available NTIS

One-Electron Oxidation of Nickel Porphyrins. Effect of Structure and Medium on Formation of Nickel(IIi) Porphyrin

or Nickel(II) Porphyrin pi-Radical Cation.
PB95.107058 00,865 Not available NTIS PB95-107058

00,865 Not available NTIS

Site of One-Electron Reduction of Ni(II) Porphyrins. Formation of Ni(I) Porphyrin of Ni(II) Porphyrin pi-Radical Anion. PB95-107066

PB95-107074

00,866 Not available NTIS

Reduction of Dinitrogen to Ammonia in Aqueous Solution Mediated by Colloidal Metals.

00,867 Not available NTIS

B95-107082

Metalloporphyrin Sensitized Photooxidation of Water to Oxygen on the Surface of Colloidal Iridium Oxides - Photochemical and Pulse Radiolytic Studies.

PB95-107082

PB95-107090

00,868 Not available NTIS

SANS and LS Studies of Polymer Mixtures Under Shear Flow.

PB95-107090

01,231 Not available NTIS

B95-107108

Three Dimensional Position Determination from Motion.

PB95-107108

01,788 Not available NTIS

B95-107116

Determination of the Molecular Parameters of $\mathrm{NiH}$ in Its (2)Delta Ground State by Laser Magnetic Resonance.
PB95-107116 No,869 Not available NTIS PB95-1071

Crystal Structure of a New Sodium Zinc Arsenate Phase Solved by 'Simulated Annealing'.

PB95-107124

PB95-107132

00,870 Not available NTIS

Mode Specific Vibrational Predissociation Dynamics in Fragile Molecules.

PB95-107132

PB95-107140

00,871 Not available NTIS

Vibration, Rotation, and Parity Specific Predissociation Dynamics in Asymmetric $\mathrm{OH}$ Stretch Excited ArH2O: A Half Collision Study of Resonant V-V Energy Transter in a Weakly Bound Complex

PB95-107140

PB95-107157

00,872 Not available NTIS

Solvent Effects in the Reactions of Peroxyl Radicals with Organic Reductants. Evidence for Proton Transfer Mediated Electron Transfer.

PB95-107157

00,873 Not available NTIS

PB95-107165

Electron Probe $X$-Ray Microanalysis.

PB95-107165

00,562 Not available NTIS

Microanalysis to Nanoanalysis: Measuring Composition at High Spatial Resolution.

PB95-107173

PB95-107181

00,563 Not available NTIS

Design of a Protoco

Value Round Robin.

PB95-10718

00,564 Not available NTIS

PB95-107199

Compositional Mapping of the Microstructure of Materials.

PB95-107199

PB95-107207

00,565 Not available NTIS

Monte Carlo Electron Trajectory Simulation of X-Ray Emission from Films Supported on Substrates.

PB95-107207 Supported on Substrates.
04,522 Not available NTIS

PB95-1072

Interface Roughness of Short-Period AlAs/GaAs Superlattices Studied by Spectroscopic Ellipsometry.
PB95-107215 02,137 Not available NTIS

PB95-107223

02,137 Not available NTIS

Inelastic Neutron Scattering Studies of Nonlinear Optical Materials: $p$-Nitroaniline Adsorbed in ALPO-5.

Materials: p-Nitroaniline Adsorbed in ALPO-5.
PB95-107223 00,874 Not available NTIS

PB95-107223

Real Time Compensation for Tool Form Errors in Turning Using Computer Vision

PB95-10723

02,945 Not available NTIS

PB95-107249

Separation and Identification of Organic Gunshot and Explosive Constituents by Micellar Electrokinetic Capillary Electrophoresis.

PBg5-107256

00,566 Not available NTIS

Reconstructing Stratified Fluid Flow from Reciprocal Scattering Measurements

04,202 Not available NTIS
PB95-107264

Fluctuation Dominated Recombination Kinetics with Traps. PB95-107264

00,875 Not available NTIS

PB95-107272

Certification, Development and Use of Standard Reference

Materials.
PB95-107272

00,567 Not available NTIS

PB95-107280

Should NIST Accredit U.S. Calibration Laboratories

PB95-107280 02,646 Not available NTIS

PB95-107298

Resistance Thermometers with Fast Response for Use in Rapidly Oscillating Gas Flows.

PB95-107298

03,261 Not available NTIS

PB95-107306

Improved Crystallographic Data for Aluminum Niobate (AINbO4)

PB95-107306

04,523 Not available NTIS

B95-107314

Summary and Notes of the Joint ISO/IGES/PDES Organization Technical Committee Meeting. Held in Albuquerque, New Mexico on October 15-20, 1989

PB95-107314

02,774 Not available NTIS 107322

Electric Field Effects on Crack Growth in a Lead Magnesium Niobate.

PB95-107322
PB95-107330

03,339 Not available NTIS

Potential Drop in the Center-Cracked Panel with Asymmetric Crack Extension.

ric Crack Exten

04,819 Not available NTIS

PB95-107348

Macro- and Microreactions in Mechanical-Impact Tests of Aluminum Alloys.
PB95-107348

03,340 Not available NTIS

Influence of Specimen Absorbed Energy in LOX Mechanical-Impact Tests.

PB95-107355

03,341 Not available NTIS

PB95-107363

Thin Dyed-Plastic Dosimeter for Large Radiation Doses.

PB95-107363

Large Radiation Doses.
Not available NTIS P95-107371

Rietveld Analysis of NaxWO3+x/2.yH2O, Which Has the Hexagonal Tungsten Bronze Structure.

PB95.107371

04,524 Not available NTIS

PB95-10738

Polyethylene Crystallized from an Entangled Solution Observed by Scanning Tunneling Microscopy.
01,232 Not available NTIS PB95-10738

PB95-107397

Measurements of Moisture Diffusivity for Porous Building

Materials.
PB95-107397

PB95-107405

00,356 Not available NTIS

Optimization of Inert Gas Atomization

PB95-107405

PB95-108411

Heterodyne Measurement of the Fluorescence Spectrum of Optical Molasses.

PB95-10841

03,873 Not available NTIS

PB95-108429

Magnetic Moments in $\mathrm{Cr}$ Thin Films on $\mathrm{Fe}(100)$

PB95-108429

04,525 Not available NTIS

PB95-108437

1,4-Dinitrocubane and Cubane under High Pressure.

PB95-108437

03,755 Not available NTIS

PB95-108445

Self-, N2- and Ar-Broadening and Line Mixing in HCN and P2H2. 108445

00,876 Not available NTIS

PB95-108452

Decoupling in the Line Mixing of Acetylene Infrared $O$ Branches.

PB95-108452

00,877 Not available NTIS

PB95-108460

Self-Assembled Phospholipid/Alkanethiol Biomimetic Bilayers on Gold

PB95-108460

PB95-108478

Fokker-Planck Description of Multivalent Interactions.

PB95-108478

00,879 Not available NTIS

PB95-108486

Standardization of Testing Methods for Optical Disk Media Characteristics and Related Activities at NIST.

PB95-108486

01,624 Not available NTIS

PB95-108494

Photoelectron Spectroscopy of Negatively Charged Bismuth Clusters: $\mathrm{Bi}(\cdot) 2, \mathrm{Bi}(-) 3$, and $\mathrm{Bi}(-) 4$. 00,880 Not available NTIS
PB95-108494

PB95-108502

Metrology Model for Submicrometer Dimensional Measurements.

PB95-10851

02,647 Not available NTIS

Practical Photomask Linewidth Measurements.
PB95-108510 02,324 Not available NTIS

PB95-108528

Activities of the ASTM Committee E.42 on Surface Analysis. $05-108528$

PB95-108536

00,881 Not available NTIS

Formation of Technical Committee 201 on Surface Chemical Analysis by the International Organization for Standard-

ization.

PB95. 108

00,568 Not available NTIS

Combustion of Methanol and Methanol/Dodecanol Spray

Flames.

PB95-10854

PB95-108551

02,478 Not available NTIS

Study of Droplet Transport in Alcohol-Based Spray Flames

Using Phase/Doppler Interferometry.

PB95-108569

Structure of a Swirl-Stabilized Kerosene Spray Flame.

PB95-108569

PB95-108577

02,480 Not available NTIS

Parametric Investigation of Metal Powder Atomization Using Laser Diffraction

PB95-108585

High-Order Harmonic Mixing with GaAs Schottky Diodes.

PB95-108585

03,342 Not available NTIS

PB95-108593

Construction of Maximum-Entropy Density Maps, and Their Use in Phase Determination and Extension.

PB95-108593

PB95-108601

Mathematical Aspects of Rietveld Refinement.

PB95-108601

04,526 Not available NTIS

PB95-108619

Vibrational Energy Transfer in S1 p-Difluorobenzene. A Comparison of Low and Room Temperature Collisions.

PB95-108619

00,883 Not available NTIS

PB95-108627

Measurement of the Self Broadening of the $\mathrm{H} 2 \mathrm{Q}(0-5)$ Raman Transitions from 295 to $1000 \mathrm{~K}$

PB95-108627

00,884 Not available NTIS

PB95-108635

Calculation of Enthalpy and Entropy Differences of Near-

Critical Binary Mixtures with the Modified Leung-Griffiths

PB95-108635

PB95-108643

00,885 Not available NTIS

Correction Factor for Nonplanar Incident Field in Monopole Calibrations

PB95-108650

Real-Time Implementation of a Differential Range Finder.

PB95-108650 
PB95-108775

Interface Sharpness in Low-Order III-V Superlattices. PB95-108775 02,138 Not available NTIS PB95-108783

\section{2,138 Not avalable NiS}

Interface Sharpness during the Initial Stages of Growh of Thin, Short-Period III-V Superlattices.

PB95-108783

02,139 Not available NTIS

Global Clim

PB95-108791

PB95-108809

Evolution of Automatic Line Scale Measurement at the National Institute of Standards and Technology.
PB95-108809 02,897 Not available NTIS PB95-108817

Polarized Transient Hot Wire Thermal Conductivity Measurements.

PB95-108817

PB95-108825

Ventilation Rates in Office Buildings.

PB95-108825

00,886 Not available NTIS

PB95-108833

Field Measurements of Ventilation and Ventilation Effective. ness in an Office/Library Building.

PB95-108833

00,247 Not available NTIS

PB95-108841

Seismic Strengthening of Reinforced Concrete Frame Buildings.

PB95-108858

00,430 Not available NTIS

Some Considerations for Interim Testing of Coordinate Measuring Machine Performance Using a Specific Artifact.

PB95-108858

02,898 Not available NTIS

PB95-108866

Development of an Automated Part Inspection System Using the DMIS Standard.

PB95-108866

02,899 Not available NTIS

Atoms in Optical Molasses.

PB95-108874

03,875 Not available NTIS

PB95-108882

Atoms in Optical Molasses: Applications to Frequency Standards

PB95-108882

PB95-108890

03,876 Not available NTIS

Optical Molasses: Cold Atoms for Precision Measurements. PB95-108890

03,877 Not available NTIS

PB95-108908

Optical Molasses: The Coldest Atoms Ever.

PB95-108908 03,878 Not available NTIS

PB95-111514

NIST Industrial Impacts: A Sampling of Successful Partnerships.

PB95-111514

00,488 PC A05/MF A01

PB95-111522

User's Manual for the Program MONSEL-1: Monte Carlo Simulation of SEM Signals for Linewidth Metrology.
PB95-111522 PB95-122537

Program of the Subcommittee on Construction and Building (July 1994).

PB95-125076

00,321 PC A03/MF A01

Standard Reference Materials: Certification of a Standard Reference Material for the Determination of Interstitial Oxy. gen Concentration in Semiconductor Silicon by Infrared Spectrophotometry

PB95-125076

PB95-125613

02,326 PC A05/MF A01

Evaluation of Fracture Toughness and Residual Stress in Dental Porcelain by Indentation-Microfracture Method.

PB95-125613

00,154 Not available NTIS

Reanalysis of the $(010),(020),(100)$, and (001) Rotationa Levels of (32)S(16)O2

PB95-125621

00,887 Not available NTIS

Development of a Temperature Scale below $0.5 \mathrm{~K}$.

PB95.125639

PB95-125647

03,879 Not available NTIS

Asymmetry between Flux Penetration and Flux Expulsion in Tl-2212 Superconductors.

PB95-125647

04,527 Not available NTIS

Application of Expert System to Select Data Sources from Chemical Information Databases.
PB95-125654 00,505 Not available NTIS PB95-125662

00,505 Not available NTIS

Mid- and Near-Infrared Spectra of Water and Water Dimer Isolated in Solid Neon.

PB95-125670

00,888 Not available NTIS

Vibrational Spectra of Molecular lons Isolated in Solid $\mathrm{Neon}$ X. $\mathrm{H}_{2} \mathrm{O}(+), \mathrm{HDO}(+)$, and $\mathrm{D} 2 \mathrm{O}(+)$.

PB95-125670

00.889 Not available NTIS

PB95-125688

Vibrational Spectra of Molecular Ions Isolated in Solid Neon. XI. NO2(+), NO2(-), and $\mathrm{NO} 3(-)$.
PB95-125688
PB95-125696

Appropriate Ultrasonic System Components for NDE of Thick Polymer Composites. PB95-125696

03,148 Not available NTIS

PB95-125704

High-Sensitivity Acoustic Emission Sensor/Preamplifier Subsystems.

PB95-125704

02,900 Not available NTIS

PB95-125712

Examination of Objects Made of Wood Using Air-Coupled Ultrasound.

PB95-125720

Vibrational Predissociation Dynamics of Overtone-Excited HN3.

PB95-125720

00,691 Not available NTIS

PB95-125738

Infrared Spectra of van der Waals Complexes of Impor-

tance in Planetary Atmospheres.
PB95-125738 00,071 Not available NTIS

Environmentally Enhanced Fracture of Ceramics.

PB95-125746 03,046 Not available NTIS

PB95-125753

Capacity of the Lp Norm-Constrained Poisson Channel.

PB95-125753

01,515 Not available NTIS

PB95-125761

Noise-Induced Chaos and Phase Space Flux.

PB95-125761

03,433 Not available NTIS

PB5-125779

Thermodynamic Properties of the Methane-Ethane System.
PB95-125779 00,891 Not available NTIS

PB95-125787

Thermophysical Property Computer Packages from NIST.

PB95-125787

04,203 Not available NTIS
04 . Nockages from NIST

PB95-125795

Suggestions for a Logically-Consistent Structure for Service Life Prediction Standards.

PB95-125795

PB95-125803

Conductance Response of Pd/SnO2(110) Model Gas Sensors to $\mathrm{H} 2$ and $\mathrm{O} 2$.

PB95-125803

00,892 Not available NTIS

PB95-125811

Determination of Fiber-Matrix Interfacial Properties of Impor-

tance to Ceramic Composite Toughening.
PB95-125811 PB95-125829

National PDES Testbed: An Overview.
PB95-125829 Not available NTIS PB95-125837

Important Papers in the History of Document Preparation Systems: Basic Sources.

PB95-125837

02,712 Not available NTIS

PB95-125845

Application of Thermal Analysis Techniques to the Characterization of EPDM Roofing Membrane Materials.

PB95-125845

00,359 Not available NTIS

PB95-125852

Use of Thermal Mechanical Analysis to Characterize Ethyl ene-Propylene-Diene Terpolymer (EPDM) Roofing Membrane Materials.
PB95-125852

PB95-125860

00,360 Not available NTIS

Single-Atom Point Source for Electrons: Field-Emission Resonance Tunneling in Scanning Tunneling Microscopy.
PB95-125860
00,893 Not available NTIS PB95-125860

PB95-125878

Aggregation Kinetics of Colloidal Particles Destabilized by Enzymes.

PB95-125886

Associative Ionization in Collisions of Slowed and Trapped Sodium.

PB95-125886

PB95-125894

03,880 Not available NTIS

Software Safety and Program Slicing

PB95-125894

01,703 Not available NTIS

PB95-125902

Object SQL: Language Extensions for Object Data Manage-

mB95-125902

01,704 Not available NTIS

5-125910

Complex Propagation Constants for Nonuniform Optical Waveguides: Calculations.

PB95-125910

04,249 Not available NTIS

PB95-125928

International Intercomparison of Detector Responsivity at 1300 and $1550 \mathrm{~nm}$

PB95-125928

02,140 Not available NTIS

PB95-125936

Fiber Spot Size: A Simple Method of Calculation

PB95-125936

04,250 Not available NTIS

B95-125944

Modal Properties of Circular and Noncircular Optical Waveguides.
PB95-125944

04,251 Not available NTIS

PB95-125951

Water Efficient Plumbing Fixtures through Standards and Test Methods.

PB95-12595

PB95-125969

00,248 Not available NTIS

Control of Friction and Wear of Alpha-Alumina with a Composite Solid-Lubricant Coating.

PB95-125969

03,225 Not available NTIS

P95-125977

Relating Bench-Scale and Full-Scale Toxicity Data

PB95-12597

00,361 Not available NTIS

PB95-125985

Computer Simulation of the Diffusivity of Cement-Based Materials.

PB95-12598
PB95-125993

00,362 Not available NTIS

Digital Simulation of the Aggregate-Cement Paste Interfacial Zone in Concrete.

PB95-125993

PB95-126009

Fundamental Computer Simulation Models for CementBased Materials.

PB95-126009

00,364 Not avalable NTIS

International Intercomparison of Detector Responsivity at 1300 and $1550 \mathrm{~nm}$

PB95-126017

PB95-126025

02,141 Not available NTIS

Development and Validation of Multicriteria Ratings: A Case Study

PB95-126025

00,004 Not available NTIS

995-126033

Nature of (001) Tilt Grain Boundaries in YBa2Cu3O6+X.

PB95-126033

95-12604

Picosecond Measurement of Substrate-to-Adsorbate Energy Transter: The Frustrated Translation of CO/Pt(111)--Trans-

PB95-126041

00,895 Not available NTIS

PB95-126058

Temperature of Optical Molasses for Two Different Atomic Angular Momenta.

PB95-126066

Dispersion and Deposition of Smoke Plumes Generated in Massive Fires

PB95-12606

02,540 Not available NTIS

Cryogenic Precision Capacitance Bridge Using a Single Electron Tunneling Electrometer.

PB95-126074

PB95-126082

Frequency Stabilization of a Fiber Laser to Rubidium: A High-Accuracy $1.53 \mathrm{mu} \mathrm{m}$ Wavelength Standard.

PB95-12608

PB95-126090

Laser Cooling and Trapping for the Masses.

PB95-126090

04,253 Not available NTIS

Use of Kinetic Energy Distributions to Determine the Relative Contributions of Gas Phase and Surface Fragmentation in $\mathrm{KeV}$ lon Sputtering of a Ouaternary Ammonium Salt. PB95-126108

PB95-126116

Image Depth Profiling SIMS: An Evaluation for the Analysis of Light Element Diffusion in YBa2Cu307-x Single Crysta 
PB 95-126207

Information Resource Dictionary System (IRDS): A Status Report.

PB95-126207

PB95-126215

Inelastic Neutron Scattering Measurements of Phonons in Icosahedral Al-Li-Cu.

PB95-126215

PB95-126223

$n-V a l u e$ and Second Derivative of the Superconductor Volt-

age-Current Characteristic.
PB95-126223

PB95-126231

Vapor Pressure of 1,1-dichloro-2,2,2-trifluoroethane (R123) PB95-126231

PB95-126249

Accuracy of Eigenvalues: A Comparison of Two Methods.

PB95-126249

03,413 Not available NTIS

PB95-126256

Approximate Solution to the Scalar Wave Equation for Optical Waveguides.

PB95-126256

04,254 Not available NTIS

PB95-126264

Comparison of Heat-Flow-Meter Tests from Four Labora-

tories.

PB95-126272

00,365 Not available NTIS

Review of the USCEA/NIST Measurement Assurance Program for the Nuclear Power Industry.
PB95-126272 Not available NTIS

(a)

Dissolution Problems with Botanical Reference Materials.

PB95-126280

03,487 Not available NTIS

PB95-126298

Some Aspects of Fundamental Neutron Physics.

PB95-126298 03,882 Not available NTIS

PB95-126306

Growing Significance of $\mathrm{CiB}$

PB95-126306

$\infty 0,314$ Not available NTiS

PB95-126314

Ashland Tank Collapse Investigation

PB95-126314

02,481 Not available NTIS

PB95-126322

Ashland Tank-Collapse Investigation: Closure by Authors

PB95-126322 02,482 Not available NTIS

PB95-126330

Assessment of Technologies for Advanced Fire Detection

PB95-126330

00,294 Not available NTIS

PB95-126348

Far-Infrared Kinetic Inductance Detector.

PB95-126348

02,142 Not available NTIS

PB95-126355

Effect of Mn Content on the Microstructure of Al-Mn Alloys Electrodeposited at $150 \mathrm{C}$.
$\mathrm{PB} 95-126355$

PB95-126363

03,343 Not available NTIS

Influence of Physical Aging on the Yield Response of Model DGEBA + Poly(propylene oxide) Epoxy Glasses.

PB95-126363

PB95-126371

Wide Band Active Current Transformer and Shunt

PB95-126371

02,036 Not available NTIS

PB95-126389

Transition Metal Implants in in $0.53 \mathrm{GA0.47As.}$

PB95-126389

04,534 Not available NTIS

PB95-126397

Range Statistics and Rutherford Backscattering Studies on Fe-Implanted In0.53Ga0.47As.

PB95-126397

PB95-126405

Evidence for Significant Backscattering in Near-Threshold Electron-Impact Excitation of $\mathrm{Ar}(7+)(3 s$ yields $3 p)$.

PB95-126405

03,883 Not available NTIS

PB95-128633

Sub-Doppler Frequency Measurements on OCS at 87 Thz (3.4 micrometers) with the CO Overtone Laser: Considerations and Details.

PB95-128633

PB95-128641

04,255 PC A03/MF A01

Databases Available in the Research Information Center of the National Institute of Standards and Technology.
PB95-128641 02,724 PC A08/MF A01 PB95-12864

National Voluntary Laboratory Accreditation Program: Ioniz-

ing Radiation Dosimetry.
PB95-128658

$03,623 \mathrm{PC} \mathrm{A06/MF} \mathrm{A02}$

PB95-128674

FIREDOC Users Manual, 3rd Edition

PB95-128674

01,378 PC A03/MF A01

PB95-128906

Uncertainty Analysis of the NiST Nitrogen Flow Facility

PB95-128906

Nitrogen Flow Facility.
02,608 PC A04/MF A01

PB95-130209

Standards of Seismic Safety for Existing Federally Owned or Leased Buildings and Commentary.
PB95-130209 00,431 PC A06/MF A02
PB95-130845

Summaries of BFRL Fire Research In-House Projects and Grants, 1994.

PB95-130845

PB95-130985

$00,366 \mathrm{PC} \mathrm{A10/MF} \mathrm{A03}$

Computer Security Training and Awareness Course Compendium.
PB95-130985

PB95-135562

$01,589 \mathrm{PC} \mathrm{A09/MF} \mathrm{A02}$

Bibliography of the NIST Electromagnetic Fields Division Publications.

PB95-135570

01,886 PC A06/MF A02

Thermal Hydraulic Tests of a Liquid Hydrogen Cold Neutron

PB95-135570

03,884 PC A03/MF A01

PB95-135588

Metrology for Electromagnetic Technology: A Bibliography of NIST Publications

PB95-13558

$02,143 \mathrm{PC} \mathrm{A05/MF} \mathrm{A01}$

PB95-135596

Indoor Air Quality Impacts of Residential HVAC Systems, Phase 1 Report: Computer Simulation Plan.

00,249 PC A06/MF A02

PB95-136339

Domain Analysis of the Alarm Surveillance Domain. Version 1.0. Conducted as Part of the Domain Analysis Case Study Project.

PB95-136339

01,705 PC A10/MF A03

PB95-136347

Challenges to the National Information Infrastructure: The Barriers to Product Data Sharing. National PDES Testbed Report Series.
PB95-136347

PB95-136354

$02,776 \mathrm{PC} \mathrm{A03/MF} \mathrm{A01}$

Sensitivity of Three-Point Circle Fitting

PB95-136354

$02,901 \mathrm{PC} \mathrm{A03/MF} \mathrm{AO1}$

PB95-136362

Comparison of FFT Fingerprint Filtering Methods for Neural Network Classification.

PB95-136362

$01,840 \mathrm{PC} \mathrm{A03/MF} \mathrm{A01}$

PB95-136370

Faster BKL Monte Carlo Simulations.

PB95-136370

$01,706 \mathrm{PC} \mathrm{A03/MF} \mathrm{A01}$

B95-136388

NIST Workshop on the Computer Interface to Flat Pane Displays. Held in San Jose, California on January 13-14,

PB95-136388

$01,625 \mathrm{PC} \mathrm{A11/MF} \mathrm{A03}$

B95-136610

Report on the Advanced Software Technology Workshop. Held on February 1, 1994

PB95-136628

$01,707 \mathrm{PC} \mathrm{A03/MF} \mathrm{A01}$

xi-Vector Formulation of Anisotropic Phase-Field Models: 3 D Asymptotics.

PB95-136644

$04,536 \mathrm{PC} \mathrm{A03/MF} \mathrm{A01}$

Constituents and Physical Properties of the C6+ Fraction of Natural Gas. Topical Report, April-June 1994

PB95-136644

PB95-137790

$02,483 \mathrm{PC}$ A03/MF A01

STEP On-Line Information Service (SOLIS). The IGES/ PDES Organization.

PB95-138129

02,777 PC A02/MF A01

National Voluntary Laboratory Accreditation Program: Bulk Asbestos Analysis.

PB95-1381:

$02,541 \mathrm{PC}$ A04/MF A01

Volume Recovery in Epoxy Glasses Subjected to Torsional Deformations: The Ouestion of Rejuvenation. PB95-140018

PB95-140026

Torsional Relaxation and Volume Response during Physical Aging in Epoxy Glasses Subjected to Large Torsional Deformations.

PB95-140026
PB95-140034

03,383 Not available NTIS

Defective Structures of Barium Yttrium Copper Oxide Defective Structures of Barium Ytrium Copper Oxide
(Ba2YCu3Ox) and Ba2YCu3-yMyOz ( $\mathrm{M}=\mathrm{Fe}$, Co, Al, Ga, .).
PB95-140034 04,537 Not available NTiS PB95-140042

Neutron Powder Diffraction Study of the Structures of La1.9Ca1.1Cu2O6 and La1.9Sr1.1Cu2O6+Delta. PB95-140042

04,538 Not available NTIS

PB95-140059

Precision High Temperature Blackbodies.

PB95-140059 03,885 Not available NTiS

PB95-140067

Normal Modes and Structure Factor for a Canted Spin System: The Generalized Villain Model. PB95-140067

04,539 Not available NTIS

PB95-140075

Morphology of Symmetric Diblock Copolymers as Revealed by Neutron Reflectivity.

01,234 Not available NTIS

PB95-140083

Superconducting Kinetic inductance Radiometer

PB95-140083

02,144 Not available NTIS

PB95-140091

Comparison of Methods for Gas Chromatographic Determination of PCBs and Chlorinated Pesticides in Marine Ref rence Materials.

PB95-140091

02,584 Not available NTIS

PB95-140109

NIST Standard Reference Materials (SRMs) for Polychlorinated Biphenyl (PCB) Determinations and Their Appli-

$\begin{array}{ll}\text { PB95-140109 } 02,585 & \text { Not available NTIS }\end{array}$ PB95-140117

Radiation Accident at an industrial Accelerator Facility.

PB95-140125

02,575 Not available NTIS

Novel Polydiacetylenes Derived from Liquid Crystalline Monomers.

PB95-14

01,235 Not available NTIS

Formulation of the Refractive Index of Water and Steam.

PB95-140133

00,900 Not available NTIS

PB95-140141

Combining Data from Independent Chemical Analysis Methods.

PB95-14014

00,572 Not available NTIS

PB95-140158

Metrology Applications of Mode-Locked Erbium Fiber La-

PBS. 140158

PB95-140166

Near Critical Fluid Interfaces: A Comparison of Theory and Experiment.

PB95-140166

PB95-140174

00,901 Not available NTIS

Structure of the Vapor-Liquid Interface Near the Critical Point.

PB95-140174

00,902 Not available NTIS

PB95-140182

Operational Mode and Gas Species Effects on Rotational Drag in Pneumatic Dead Weight Pressure Gages.

PB95-140182

00,903 Not available NTIS

(140190

Glass Temperature of Polymer Blends: Comparison of Both the Free Volume and the Entropy Predictions with Data:

PB95-140190

PB95-140208

New Materials, Advanced Ceramics and Standards.

PB95-140208

03,047 Not available NTIS

PB95-140414

Scanning Tunneling Microscopy and Fabrication of Nanometer Scale Structures at the Liquid-Gold interface. PB95-140414

PB95-140422

Measurement of the $(235) \mathrm{U}(n, f)$ Reaction from Thermal to

PB95-140422

03,886 Not available NTIS

PB95-140430

Ultrasonic Measurement of Residual Stress in Railroad Wheel Rims.

PB95-140430

PB95-140448

Effects of Surface-Active Resins on Dentin/Composite Bonds. 
PB95-140547

Magnetic Ordering of the $\mathrm{Cu}$ Spins in PrBa2Cu3O6+x PB95-140547 04.545 Not available NTIS
0.510 in PrBa2Cu3O6+X. PB95-140554

Crystal Structure and Magnetic Ordering of the Rare-Earth and $\mathrm{Cu}$ Moments in $\mathrm{RBa} 2 \mathrm{Cu} 2 \mathrm{NbO} 8(\mathrm{R}=\mathrm{Nd}, \mathrm{Pr})$. PB95-140554 04,546 Not available NTIS PB95-140562

Quasielastic and Inelastic Neutron-Scattering Studies of ((CD3)3ND)FeCl3.2D2O: A One-Dimensional Ising Ferromagnet.

PB95-140570

04.547 Not available NTIS

Phase Equilibria in the Systems $\mathrm{CaO}-\mathrm{CuO}$ and $\mathrm{CaO}-\mathrm{Bi} 2 \mathrm{O} 3$ PB95-140570 03,048 Not available NTIS

PB95-14058

Critical Factors in Non-Lubricated, Non-Abrasive Wear Testing.

PB95-140596

03,236 Not available NTIS

Tribological Data: Needs and Opportunities.

PB95-140596

03,237 Not available NTIS

PB95-140604

Device for Subambient Temperature Control in Liquid Chromatography.

PB95-140810

Thermally Activated Hopping of a Single Abrikosov Vortex. PB95-140810

PB95-140828

Surface Energy Reduction in Fibrous Monotectic Structures. PB95-140828

PB95-140836

Rare-Earth-Doped Waveguide Devices: The Potential for Compact Blue-Green Lasers.

PB95-140836

04,257 Not available NTIS

PB95-140844

Linewidth Narrowing in an Imbalanced $Y$-Branch Waveguide Laser.

PB95-140844

04,258 Not available NTIS

PB95-140851

Nd:LiTaO3 Waveguide Laser. $\quad 04,259$ Not available NTIS
PB95-140851

PB95-140869

Wavelengths and Isotope Shifts for Lines of Astrophysical Interest in the Spectrum of Doubly lonized Mercury ( $\mathrm{Hg}$ III).

PB95-140869

PB95-140877

Spectrum and Energy Levels of Triply Ionized Barium ( $\mathrm{Ba}$ PB95-140877

PB95-140885

FPETOOL: Fire Protection Tools for Hazard Estimation. An Overview of Features.

PB95-140885

PB95-140893

$\infty, 367$ Not available NTIS

In situ Measurements of Chloride lon and Corrosion Potential at the Coating/Metal Interface.

PBial at the Coatin

03,122 Not available NTIS

PB95-140901

Using Torsional Dilatometry to Measure the Effects of Deformations on Physical Aging.

PB95-140901

PB95-140919

03,384 Not available NTIS

Comparison of Experimental and Computed Species Concentration and Temperature Profiles in Laminar, Two-D1mensional Methane/Air Diffusion Flames

PB95-140927

Aging Effects on XRF Measurements of Solder Coatings.

PB95-140935

03.123 Not available NTIS

One- and Two-Sided Burning of Thermally Thin Materials PB95-140935

03,151 Not available NTIS

PB95-140943

Heat Transfer in Thin, Compact Heat Exchangers with Cir-

cular, Rectangular, or Pin-Fin Flow Passages.
PB95-140943

PB95-140950

Chemical and Microbiological Problems Associated with Research on the Biodesulfurization of Coal.

PB95-140950 02,484 Not available NTIS

PB95-140968

Bioleaching of Cobalt from Smelter Wastes by 'Thiobacillus 'ferrooxidans'.

PB95-140976

02,582 Not available NTIS

Comparison of the Liquid Chromatographic Behavior of Selected Steroid Isomers Using Different Reversed-Phase Ma-

terials and Mobile Phase Compositions.
PB95-140976 00.574 Not available NTIS

PB95-140976

Metrology Requirements of Future Space Power Systems.

PB95-140984

04,840 Not available NTIS

PB95-140992

Associative Electron Attachment to S2F10, S2OF10, and $\mathrm{S} 2 \mathrm{O} 2 \mathrm{~F} 10$.

$\infty 0,907$ Not available NTIS
Infrared Spectrum of $\mathrm{OClO}$ in the $2000 / \mathrm{cm}(-1)$ Region: The

2(nu sub 1) and (nu sub $1+$ nu sub 3 ) Bands. PB95-141032

PB95-141008

Ion Kinetic-Energy Distributions in Argon if Glow Discharges.

PB95-141016

04,409 Not available NTIS

Critical Current Behavior of Ag-Coated YBa2Cu3O7-x Thin

04,549 Not available NTIS

PB95-141024

Magnetic Field Dependence of the Critical Current Anisotropy in Normal Metal-YBa2Cu3O7-delta Thin Film Bilayers. PB95-141024

B95-141040

Intensities and Dipole Moment Derivatives of the Fundamental Bands of (35) $\mathrm{ClO} 2$ and an Intensity Analysis of the nut Band.

Intelligent Control of an Inert Gas Atomization Process.

PB95-141057

03,344 Not available NTIS

PB95-141065

Micromagnetic Model of Dual-Layer Magnetic-Recording Thin Films.

PB95-141073

04,551 Not available NTIS

Micromagnetic Simulations of Tunneling Stabilized Magnetic Force Microscopy.

04,552 Not available NTIS

Experimental Verification of a Micromagnetic Model of DualLayer Magnetic Films.

PB95-14108

04,553 Not available NTIS

PB95-141099

Process Gas Chromatography Detector for Hydrocarbons

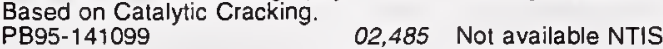
PB95-141107

Reaction of Nitric Oxide with Organic Peroxyl Radicals.

PB95-141107 00,910 Not available NTIS

PB95-141115

LP11-Mode Leakage Loss in Coated Depressed Clad Fibers.

PB95-141115

02,145 Not available NTIS

PB95-141123

Perturbative Calculation of the AC Stark Effect by the Complex Rotation Method.

PB95-141123

PB95-141131

Ca4Bi6013: A Compound Containing an Unusually Low Bismuth Coordination Number and Short Bi Bi Contacts. PBg5-141149

Trapped Vortices in a Superconducting Microbridge

PB95-141149

04554 Not available NTIS

PB95-141156

Effect of Hydrodynamic Interactions on a Terminally An-

chored Bead-Rod Model Chain

01,237 Not available NTIS

PB95-141164

Variation in Magnetic Properties of Cu/Fcc (001) Sandwich Structures.

PB95-141164

PB95-141172

Physics for Device Simulations and Its Verification by Measurements.

PB95-141180

02,327 Not available NTIS

Interatomic Potential of Argon

PB95-141180

00,912 Not available NTIS

PB95-141198

Time-Resolved Measurements of Energy Transfer at Sur-

faces.

PB95-141206

Neutron Focusing Lens Using Polycapillary Fibers.

PB95-141206

PB95-143061

Summary Report of NIST's Industry-Government Consortium Research Program on Flowmeter Installation Effects: The 45 Degree Elbow.

PB95-143061

04,204 PC A06/MF A02

PB95-143079

Quantitative X-Ray Powder Diffraction Methods for Clinker and Cement.

PB95-143087

Guidelines for Evaluating and Expressing the Uncertainty of NIST Measurement Results. 1994 Edition.
PB95-143087 02,649 PC A03/MF A01

PB95-143095

Diagnosis of Causes of Concrete Deterioration in the MLP-

7A Parking Garage.
PB95-143095

$01,318 \mathrm{PC}$ A03/MF A01
PB95-141057

PB95-143103

SGML. Environment for STEP.

PB95-143103

$02,778 \mathrm{PC} \mathrm{AO3/MF} \mathrm{A01}$

PB95-143111

Metrology and Data for Microelectronic Packaging and Interconnection: Results of a Joint Workshop on Materials Metrology and Data for Commercial Electrical and Optical Gaithersburg. Maryland on May 5-6, 1994. Volume 1. Re-

PB95-143111

$02,328 \mathrm{PC} \mathrm{A05/MF} \mathrm{A01}$

PB95-143129

Manufactured Homes: Probability of Failure and the Need for Better Windstorm Protection through Improved Anchoring Systems.

PB95-143137

00,432 PC A04/MF A01

Reference Model Architecture for Intelligent Systems Design. 143137

$01,789 \mathrm{PC} \mathrm{A03/MF} \mathrm{AO1}$

PB95-143145

Information Technology Engineering and Measurement Model: Adding Lane Markings to the Information Superhighway.

PBighway.

PB95-143152

01,474 PC A03/MF AO1

Simulating Smoke Movement through Long Vertical Shafts in Zone-Type Compartment Fire Models. 00,368 PC A03/MF A0 PB95-143160

Fundamental Mechanisms for $\mathrm{CO}$ and Soot Formation. PB95-143160

$01,380 \mathrm{PC} \mathrm{A08/MF} \mathrm{A02}$

PB95-143178

Channel Coding for Code Excited Linear Prediction (CELP) Encoded Speech in Mobile Radio Applications.

PB95-143186

Electronics and Electrical Engineering Laboratory Technical Progress Bulletin Covering Laboratory Programs, April to June 1994 with 1994/1995 EEEL Events Calendar.

PB 95-143186

PB95-143194

Transient Cooling of a Hot Surface by Droplets Evaporation PB95-143194

PB95-143202

Building and Fire Research Laboratory Publications, 1993.

PB95-14320

Visual Pursuit Systems.

PB95-143285

00,369 PC A06/MF A02

PB95-143285

$01,841 \mathrm{PC} \mathrm{A03/MF} \mathrm{A01}$

Investigation into the Flammability Properties of Honeycomb Composites.

PB95-143301

03,152 PC A03/MF AO

Visual Measurement Technique for Analysis of Nucleate Flow Boiling.

PB95-143327

03,262 PC A05/MF A02

Metrology and Data for Microelectronic Packaging and Interconnection: Results of a Joint Workshop on Materials Metrology and Data for Commercial Electrical and Optical Packaging and Interconnection Technologies. Held in entation Material.

PB $95-143327$

$02,330 \mathrm{PC}$ AO8/MF AO2

PB95-144309

Electronics and Electrical Engineering Laboratory 1994 Technical Accomplishments Supporting Technology for U.S. Competitiveness in Electronics.
PB95-144309

$01,887 \mathrm{PC} \mathrm{A06/MF} \mathrm{A02}$

Reference Architecture for Machine Control Systems Integration: Interim Report

PB95-144549

02,820 PC A06/MF A02

PB95-146379

NIST Handbook 44, 1995: Specifications, Tolerances and Other Technical Requirements for Weighing and Measuring Devices as Adopted by the 79th National Conference on Weights and Measures 1994 
PB95-147906

Program Requirements to Advance the Technology of Custom Footwear Manufacturing. PB95-147906

02,883 PC A04/MF A01

PB95-147914

Phase-Field Model for Solidification of a Eutectic Alloy

PB95-147914

03,345 PC A04/MF A01

PB95-147922

Portsmouth Fastener Manufacturing Workstation. User's Manual.

PB95-147922

02,860 PC A06/MF A02

PB95-150017

Effects of Interfacial Roughness on the Magnetoresistance of Magnetic Metallic Multilayers.

PB95-150017

PB95-150025

Oxidative DNA Base Damage in Renal, Hepatic, and Pulmonary Chromatin of Rats After Intraperitoneal Injection of

Cobalt (II) Acetate

PB95-150033

03,647 Not available NTIS

Observed Frustration in Confined Block Copolymers.

PB95-150033 01,238 Not available NTIS

PB95-150041

Airborne Smoke Sampling Package for Field Measurements of Fires.

-15004

PB95-150058

Monte Carlo Model for SEM Linewidth Metrology

PB95-150058

01,381 Not available NTIS

PB95-1500

02,331 Not available NTIS

Transverse Magnetoresistance: A Novel Two-Termina Method for Measuring the Carrier Density and Mobility of a Semiconductor Layer.

PB95-150066

02,332 Not available NTIS

PB95-150074

Magnetic Neutron Scattering (Invited)

PB95-150074

04.557 Not available NTIS

PB95-150082

Polarization Analysis of the Magnetic Excitations in Fe65Ni35 Invar.
PB95-150082

PB95-150090

04,558 Not available NTIS

Phase Transitions in Solid C70: Supercooling. Metastable Phases, and Impurity Effect.

PB95-150090

00,914 Not available NTIS

PB95-150108

Method for Determining Both Magnetostriction and Elastic Modulus by Ferromagnetic Resonance.

02,974 Not available NTIS

PB95-150116

Superconducting Energy Gap of Bulk UBe13.

PB95-150116 04,559 Not available NTIS

PB95-150124

Simulations of Glass Forming Liquids: What Has Been Learned.

PB95-150132

00,915 Not available NTIS

Radiometric Model of the Transmission Cell-Reciprocal Nephelometer

00,124 Not available NTIS

PB95-150140

Study of Ventilation and Carbon Dioxide in an Office Build. ing.

PB95-150157

02,542 Not available NTIS

Spectroscopic Ellipsometry Determination of the Properties of the Thin Underlying Strained Si Layer and the Roughness at $\mathrm{SiO} / \mathrm{Si}$ Interface.

PB95-150157

04,560 Not available NTIS

PB95-150165

Spot-Profile-Analyzing LEED Study of the Epitaxial Growth of $\mathrm{Fe}, \mathrm{Co}$, and $\mathrm{Cu}$ on $\mathrm{Cu}(100)$.

PB95-150165

PB95-150173

04,561 Not available NTIS

Integrating Sphere Simulation: Application to Total Flux Scale Realization

PB95-150173

PB95-150181

04,261 Not available NTIS

Influence of $\mathrm{Cr}$ Growth on Exchange Coupling in $\mathrm{Fe} / \mathrm{Cr}$ / $\mathrm{Fe}(100)$.

PB95-150199

04562 Not available NTIS

Automated, High-Precision Coulometric Titrimetry. Part 1 Engineering and Implementation.

PB95-150199

PB95-150207

00,575 Not available NTIS

Automated, High Precision Coulometric Titrimetry. Part 2. Strong and Weak Acids and Bases.

PB95-150207

PB95-150215

00,576 Not available NTIS

Oxidation of Soot and Carbon Monoxide in Hydrocarbon Difusion Flames.

PB95-150223

01,382 Not available NTIS

Temperature Dependence of the Magnetic Excitations in Ordered and Disordered Fe72Pt28.

PB95-150223

04,563 Not available NTIS
Integrating Automated Systems with Modular Architecture PB95-150231

PB95-150249

Development of Engineered Stationary Phases for the Sep-

aration of Carotenoid Isomers.

PB95-150256

00,578 Not availabte NTIS

Shape Selectivity Assessment of Stationary Phases in Gas Chromatography.

PB95-150264

Fire Service and Fire Sciences: A Winning Combination. PB95-150264

PB95-150272

Nanostructure Fabrication via Direct Writing with Atoms Focused in Laser Fields.

PB95-150272

04,564 Not available NTIS

\section{PB95-150280}

Cross-Property Relations and Permeability Estimation in Model Porous Media

PB95-15028

04,205 Not available NTIS

PB95-150298

Resonance and Threshold Effects in Polarized X-Ray Emission from Atoms and Molecules.

PB95-150298

PB95-150306

Effect of a Crystal Monochromator on the Local Angular Divergence of an X-Ray Beam.

PB95-150306

PB95-150314

04,565 Not available NTIS

Exchange Coupling in Magnetic Heterostructures.

PB95-150314 Not available NTIS

PB95-150322

Growth of Iron on Iron Whiskers.

PB95-150322

04,567 Not available NTIS

PB95-150330

Scanning Tunneling Microscopy Study of the Growth of $\mathrm{Cr} /$ Fe(001): Correlation with Exchange Coupling of Magnetic PB95-150330

PB95-150348

High Temperature Reliability of Thin Film SiO2.

PB95-150348

04.568 Not available NTIS

PB95-

02,333 Not available NTIS

Calculations of Electron Inelastic Mean Free Paths. 5. Data for 14 Organic Compounds over the 50-2000 eV Range. PB95-150355

PB95-150363

DNA Base Modifications in Renal Chromatin of Wistar Rats Treated with a Renal Carcinogen. Ferric Nitrilotriacetate.

PB95-150363

PB95-150371

Oscillatory Exchange Coupling in Fe/Au/Fe(100).

PB95-150371

04,569 Not available NTIS

PB95-150389

Performance Evaluation of a New Digital Partial Discharge Recording and Analysis System.

PB95-150389

01,888 Not available NTIS

PB95-150397

Retinal-Protein Complexes as Optoelectronic Components.

02,146 Not available NTIS

PB95-150405

Tolerance Intervals for the Distribution of True Values in the Presence of Measurement Errors.

PB95-150405

03,434 Not available NTIS

\section{3}

Band-to-Band Photoluminescence and Luminescence Excitation in Extremely Heavily Carbon-Doped Epitaxial GaAs.
PB95.150413

PB95-150421

Neutron Reflectometry Studies of Surface Oxidation.

PB95-150421

00,917 Not available NTIS

PB95-150439

Percolation and Pore Structure in Mortars and Concrete

PB95-150439

00,370 Not available NTIS

B95-150447

Estimate of the Effect of Scale on Radiative Heat Loss Fraction and Combustion Efficiency.

PB95-15044

PB95-150454

Structural Stabilization of Phase Separating PC/Polyester Blends through Interfacial Modification by Transesterification Reaction

PB95-150454

PB95-150462

01,239 Not available NTIS

Effects of Humidity and Elevated Temperature on the Density and Thermal Conductivity of a Rigid Polyisocyanurate Foam Co-Blown with $\mathrm{CCl} 3 \mathrm{~F}$ and $\mathrm{CO} 2$.

PB95-15046

00,371 Not available NTIS

PB95-150470

Grazing Incidence Prompt Gamma Emissions and Resonance-Enhanced Neutron Standing Waves in a Thin-Film.

PB95-150470

03,892 Not available NTIS

PB95-150488

Test Structures for Determining Design Rules for Microelectromechanical-Based Sensors and Actuators.
PB95.150488

PB95-150496

Multiwavelength Birefringent-Cavity Mode-Locked Fibre

Laser.

04,262 Not available NTIS

PB95-150512

Self-Avoiding Surfaces, Topology, and Lattice Animals.

PB95-150512 04,571 Not available NTIS

PB95-150520

Developing Linear Error Models for Analog Devices.

PB95- 150520

02,037 Not available NTIS

(1505-15058

Diakoptic and Large Change Sensitivity Analysis.

PB95-150538

02,038 Not available NTIS
Notivity Analysis.

PB95-150546

Excitation of Balmer Lines in Low-Current Discharges of Hydrogen and Deuterium.

PB95-150553

03,893 Not available NTIS

Fast Computer Evaluation of Radiative Properties of Hydrogenic Systems.

03,894 Not available NTIS

PB95-150561

Contrast Matched Studies of a Sheared Binary Colloidal Suspension.

PB95-150579

00,918 Not available NTIS

Electrical Breakdown in Transformer Oil in Large Gaps.

PB95-150579

PB95-150587

01,889 Not available NTIS

Atomic Manipulation of Polarizable Atoms by Electric Field Directional Diffusion

B95-150587

04,572 Not available NTIS

Nanoscale Study of the As-Grown Hydrogenated Amorphous Silicon Surface.

PB95-150595

04,573 Not available NTIS

PB95-150603

Tin Oxide Gas Sensor Fabricated Using CMOS MicroTh In-situ Processing

PB95-150603

00,580 Not available NTIS PB95-150611

Microwave Spectra of van der Waals Complexes of Importance in Planetary Atmospheres.

PB95-150611

PB95-150629

Improved Wavelengths for Prominent Lines of $\mathrm{Cr} X \mathrm{VI}$ to $\mathrm{C}$ XXII.

PB95-150629

PB95-150637

Observation of Pd-Like Resonance Lines Through Pt(32+)

and $\mathrm{Zn}$-Like Resonance Lines of $\mathrm{Er}(38+)$ and $\mathrm{Hf}(42+)$.

PB95-150637

Rb-Like Spectra: Pd X to Nd XXIV

PB95-150645

03,897 Not available NTIS

PB95-150652

Rh I Isoelectronic Sequence Observed from $\operatorname{Er}(23+)$ to 
PB95-150751

UV-Photopatterning of Alkylthiolate Monolayers Self-Assembled on Gold and Silver.

PB95-15075

00,924 Not available NTIS

PB95-150769

Analysis of the $5 s(2) 5 p(2)-(5 s 5 p(3)+5 s(2) 5 p 5 d+5 s(2) 5 p 6 s)$ Transitions of Four-Times lonized Xenon ( $\mathrm{XeV}$ ).

Transitions of Four-Times lonized
PB95-150769

PB95-150777

New International Representations of the Volt and Onm Eftective January 1, 1990.

PB95-150777

01,890 Not available NTIS

PB95-150785

Flux-Locked Current Source Reference.

PB95-150785

02,039 Not available NTIS

PB95-150793

Application of Thermodynamics to Biotechnology.

PB95-150793 03,529 Not available NTIS

PB95-150801

Thermochemistry of the Hydrolysis of L-arginine to (Lcitrulline + Ammonia) and of the Hydrolysis of L-arginine to (L-ornithine + Urea)

PB95-150801

03,463 Not available NTIS

Activated Dynamics, Loss of Ergodicity, and Transport in Supercooled Liquids.

PB95-150819

00,925 Not available NTIS

PB95-150827

Using Grafcet to Design Generic Controllers.

PB95-150827 02,821 Not available NTIS

PB95-150835

Standards for Corrected Fluorescence Spectra.

PB95-150835 00,581 Not available NTIS

PB95-150843

Insulating Nanoparticles on YBa2Cu307-delta Thin Films Revealed by Comparison of Atomic Force and Scanning Tunneling Microscopy.

PB95-150843

04,575 Not available NTIS

PB95-150850

Lattice Imperfections Studied by Use of Lattice Green's Functions.

04,576 Not available NTIS

PB95-150868

Relativistic Effects in Spin-Polarization Parameters for LowEnergy Electron-Cs Scattering.

PB95-150868

03,901 Not available NTIS

PB95-150876

Characteristics of Partial Pressure Analyzers

PB95-150876 00,582 Not available NTIS

PB95-150884

Vacuum Gauges and Partial Pressure Analyzers.

PB95-150884 02,650 Not available NTIS

PB95-150892

Experimental Data and Theoretical Modeling of Gas Flows Through Metal Capillary Leaks. 04,206 Not available NTIS PB95-150892

PB95-150900

Optical Measurements of Atomic Hydrogen, Hydroxyl, and

Carbon Monoxide in Hydrocarbon Diffusion Flames.
PB 2,487 Not available NTIS

PB95-150918

Evaluation and Retrofit Standards for Existing Federally Owned and Leased Buildings.
PB95-150918

PB95-150918

00,434 Not available NTIS

Some Basics on Who's Who and What's What in Seismic

Satety.

00,435 Not available NTIS

PB95-150934

Bistatic Scattering of Absorbing Materials from 30101000

PB95-150934

PB95-150942

01,891 Not available NTIS

Standards: A Cardinal Direction for Geographic Intormation Systems.

PB95-150942

PB95-150959

03,677 Not available NTIS

Critical Evaluation of the Puritication of Biominerals by $\mathrm{Hy}$ pochlorite Treatment.

PB95-150959

00,186 Not available NTIS

PB95-150967

Influence of Natural and Synthetic Inhibitors on the Crystallization of Calcium Oxalate Hydrates.

PB95-150967

03,560 Not available NTIS

PB95-150975

Effects of Heavy Doping on Numerical Simulations of

Gallium Arsenide Bipolar Transistors.
PB95-150975 Not available NTIS PB95-150975

PB95-150983

Compensation of Markov Estimator Errors in Time-Jittered Sampling of Nonmonotonic Signals.

PB95-150983

01,590 Not available NTIS

PB95-150991

Lighting and HVAC

PB95-15099

00,250 Not available NTIS

PB95-151007

NIST Lighting and HVAC Interaction Test Facility.
PB95-151007
00,251 Not available NTIS
PB95-151015

Methyl Torsional Levels of Solid Acetonitrile ( $\mathrm{CH} 3 \mathrm{CN}$ ): A Neutron Scattering Study.

PB95- 151015

00,926 Not avạilable NTIS

B 95-151023

Electronic Spectra of $\mathrm{CF} 2 \mathrm{Cl}$ and $\mathrm{CFC} / 2$ Radicals Observed by Resonance Enhanced Multiphoton lonization.

PB95-151023

00,927 Not available NTIS

PB95-151031

Resonance Enhanced Multiphoton Ionization Spectroscopy

of 2-Butene-1-yl (C4H7) between $455-485 \mathrm{~nm}$.
PB95-151031 00,670 Not available NTIS

\section{(51049}

Homogeneous Gas Phase Decyclization of Tetralin and Benzocyclobutene.

PB95-151049

00,928 Not available NTIS

Progress in the Development of a Chemical Kinetic Database for Combustion Chemistry.
PB95-151056 Not available NTIS

PBg

PB95-151064

Mechanism and Rate Constants for the Reactions of Hydrogen Atoms with Isobutene at High Temperatures.

PB95-151064

PB95-151072

Thermal Stability of Internal Electric Field and Polarization Distribution in Blend of Polyvinylidene Fluoride and Polymethylmethacrylate.

01,240 Not available NTIS

PB95-151080

Effects of Calcium Phosphate Solutions on Dentin Permeability.

PB95-151098

Preliminary Comparison of Time Transfers va LASSO, GPS and Two-Way Satellite.

01,529 Not available NTIS

B95-151106

Electron and Proton Dosimetry with Custom-Developed Radiochromic Dye Films.

PB95-151106

03,713 Not available NTIS

PB95-151114

Recent Developments at NIST on Optical Current Sensors and Partial Discharge Diagnostics.

2,147 Not available NTIS

PB95-151122

Kinetic-Energy Distributions of $\mathrm{K}(+)$ in Argon and Neon in Uniform Electric Fields.

PB95-151122

03,902 Not available NTIS

PB95-151130

High-Performance Liquid Chromatography of Phytoplankton Pigments Using a Polymeric Reversed-Phase C18 Column.
PB95-151130

PB95-151148

Experimental Method for Obtaining Critical Densities of Bi-

nary Mixtures: Application to Ethane $+n$-Butane.
PB95-151148
00,930 Not available NTIS

PB95-151155

Effect of Electrode-Polymer Interfacial Layers on Polymer Conduction. Part 2. Device Summary.

PB95-151155

02,335 Not available NTIS

PB95-151163

Moditied Surface-Active Monomers for Adhesive Bonding to Dentin

PB95-151163

00,158 Not available NTIS

PB95-151171

NIR-Spectroscopic Investigation of Water Sorption Characteristics of Dental Resins and Composites.

PB95-151171

PB95-151189

Guidelines for Refractive Index Measurements of Asbestos.
PB95-151189 PB95-151189

PB95-151197

Factorial Design Techniques Applied to Optimization of AMS Graphite Target Preparation.
PB95-151197 Not available NTIS

\section{PB95-151205}

Distribution of Fluoride in Saliva and Plaque Fluid After a $0.048 \mathrm{~mol} / \mathrm{L} \mathrm{NaF}$ Rinse

PB95-151205

03,561 Not available NTIS

PB95-151213

Regimes of Surface Roughness Measurable with Light

PCattering.

04,265 Not available NTIS

PB95-151221

Hyperfine Effects and Associative Ionization of Ultracold So. PB95-151221

03,903 Not available NTIS

PB95-151239

Electron-Impact Excitation of Si(3+)(3S yields 3P) Using a Merged-Beam Electron-Energy-Loss Technique.

PB95-151239

PB95-151247

Anisotropic Phase Separation Kinetics in a Polymer Blend Solution Following Cessation of Shear Studied by Light

Scattering.

01,241 Not available NTIS

PB95-151254

Magneto-Optical Trapping of Metastable Xenon: IsotopeShift Measurements.

PB95-151254

PB95-151262

Analysis of Selected Software Safety Standards.

PB95-151262

03,905 Not available NTIS

Slant Path Atmospheric Refraction Calibrator: An Instrumen to Measure the Microwave Propagation Delays Induced by Atmospheric Water Vapor.

PB95-151288

Electron Scattering and Dissociative Attachment by SF6 and Its Electrical-Discharge By-Products.
PB95-151288 02,256 Not available NTIS

PB95-151296

Approximate Confidence Intervals on Linear Combinations of Expected Mean Squares.

PB95-151296

03,435 Not available NTIS

PB95-151304

Approximate Confidence Intervals on Positive Linear Com binations of Expected Mean Squares

PB95-151304

PB95-151312

Ranges of Confidence Coefficients for Confiedence Intervals on Variance Components.

PB95-151320

Technique to Evaluate Benchmarks: A Case Study Using the Livermore Loops

PB95.151320

04,577 Not available NTIS

PB95-151338

Effects of Adhesive Thickness, Open Time, and Surface Cleanness on the Peel Strength of Adhesive-Bonded

Seams of EPDM Rubber Roofing Membrane.
PB95-151338 00,372 Not available NTIS

PB95-151346

New Alloys Show Extraordinary Resistance to Fracture and

B95-151346

03,346 Not available NTIS

PB95-151353

Measurements of the Virial Coefficients and Equation of State of the Carbon Dioxide + Ethane System in the Supercritical Region

(151353

03,906 Not available NTIS

PB95-151361

Ebulliometric Measurement of the Vapor Pressure of Difluoromethane.

PB95-15136

Evaluating Investments in Law Entorcement Equipment: An Annotated Bibliography.

PB95-151379

00,931 Not available NTIS

P95-151387

Van der Waals Bond Lengths and Electronic Spectral Shifts of the Benzene- $K r$ and Benzene-Xe Complexes.

PB95-151387

PB95-151395

N2(a'(sup 1)Sigma(sub g)(sup +)) Metastable Collisiona Destruction and Rotational Excitation Transfer by N2

PB95-151395

00,933 Not available NTIS

PB95-151403

Preparation of Immobilized Proteins Covalently Coupled Through Silane Coupling Agents to Inorganic Supports.

PB95-151403 
PB95-151502

Laser Cooling

(1)

03,908 Not available NTIS

Mapping Processes to Processors for Space-Based Robot Systems.

PB95-151510

PB95-151528

Measuring Matching Wear Scars on Balls and Flats.

PB95-151528

04,833 Not available NTIS

PB95-151536

Manipulation of Adsorbed Atoms and Creation of New Structures on Room-Temperature Surfaces with a Scanning Tunneling Microscope.

PB95-151536

PB95-151544

04,578 Not available NTIS

Function of DnaJ and DnaK as Chaperones in Origin-Specific DNA Binding by RepA PB95-151544

03,533 Not available NTIS

PB95-151551

Comprehensive Spectroscopic Data Tabulations and Progress in the Compilation of Atomic Transition Probabilities

5-15155

03,909 Not available NTIS

PB95-151569

Spectroscopic Data for Fusion Edge Plasmas.

PB95-151569 04,410 Not available NTIS

PB95-151577

Spectroscopic Diagnostics of Low Temperature Plasmas: Techniques and Required Data.

04,411 Not available NTIS

PB95-151585

Spectroscopic Data Tables for Highly-Ionized Atoms.

PB95-151585 03,910 Not available NTIS

PB95-151593

Accurate Transmission Line Characterization

PB95-151593 02,220 Not available NTIS

PB95-151601

Studies of the Higher Order Smectic Phase of the Large Electroclinic Effect Material W317.

PB95-151601 00,935 Not available NTIS

PB95-151619

Liquid-Hydrogen Cold Neutron Source for the NBSF

PB95-151619 03,729 Not available NTIS

PB95-151627

Trapped Atoms and Laser Cooling.

03,911 Not available NTIS

PB95-151635

Spin Squeezing and Reduced Ouantum Noise in SpectrosPB95-151635

03,912 Not available NTIS

PB95-151643

Unusual Spin-Trap Chemistry for the Reaction of Hydroxyl Radical with the Carcinogen N-Nitrosodimethylamine.

PB95-151643

00,692 Not available NTIS

PB95-151650

Determination of Polycyclic Aromatic Hydrocarbons by Liquid Chromatography

PB95-151650

00,585 Not available NTIS

Standard Reference Materials for the Determination of Polycyclic Aromatic Hydrocarbons in Environmental Samples - Current Activities.

PB95-151668

00,586 Not available NTIS

PB95-151676

Development of Frozen Whale Blubber and Liver Reference Materials for the Measurement of Organic and Inorganic Contaminants

00,587 Not available NTIS

PB95-151684

Investigation into a Practical Grain Growth Model for Hot Isostatic Pressing.

PB95-15168

03,347 Not available NTIS

\section{PB95-151692}

Mixed Diet Reference Materials for Nutrient Analysis of Foods: Preparation of SRM-1548 Total Diet.

PB95-151692

03,593 Not available NTIS

PB95-151700

$X$-Ray Characterization of the Crystallization Process of High-Tc Superconducting Oxides in the Sr-Bi-Pb-Ca-Cu-O System

PB95-151700

04,579 Not available NTIS

B95-151718

Crystal Chemistry and Phase Equilibrium Studies of the $\mathrm{BaO}(\mathrm{BaCO} 3)-\mathrm{R} 2 \mathrm{O} 3-\mathrm{CuO}$ Systems. 5. Melting Relations in $\mathrm{Ba2}(\mathrm{Y}, \mathrm{Nd}, \mathrm{Eu}) \mathrm{Cu} 3 \mathrm{O} 6+\mathrm{x}$

PB95-151718

PB95-151726

04,580 Not available NTIS

X-Pay-Diffraction Study of a Thermomechanically

Detwinned Single Crystal of YBa2Cu306+X.
PB95-151726 04,581 Not available NTIS 0 B 5 -151734

Crystal Chemistry and Phase Equilibrium Studies of the $\mathrm{BaO}-\mathrm{R} 2 \mathrm{O} 3-\mathrm{CuO}$ Systems. 2. X-Ray Characterization and Standard Patterns of BaR2CuO4, R=Lanthanides.

PB95-151734 04,582 Not available NTIS PB95-151759

Crystal Chemistry and Phase Equilibria Studies of the $\mathrm{BaO}(\mathrm{BaCO})-\mathrm{R} 2 \mathrm{O} 3-\mathrm{CuO}$ Systems. 4. Crystal Chemistry and Subsolidus Phase Relationship Studies of the CuO Rich Region of the Ternary Diagrams, R=Lanthanides.

PB95-151759 00,936 Not available NTIS

PB95-151767

X-Ray Powder Diffraction Data for $\mathrm{BaCu}(\mathrm{C} 2 \mathrm{O} 4) 2.6 \mathrm{H} 2 \mathrm{O}$.
PB95-151767 04,583 Not available NTIS PB95-151

Photodissociation of Ammonia at $193.3 \mathrm{~nm}$ : Rovibrational

State Distribution of the $\mathrm{NH}$ 2(A(2)A1) Fragment.

PB95-151775 00,937 Not available NTIS

PB95-151783

Lighting Ouality and Light Source Size.

PB95-151783

00,252 Not available NTIS

PB95-151791

Lighting Research and Theory Can Create Business Pros-

Pects.

PB95-151809

00,253 Not available NTIS

Implementation of Executive Order 12699: Seismic Safety of Federal and Federally Assisted or Regulated New Build ing Construction

00,436 Not available NTIS

B 95-151817

Structural Analysis in Context.

PB95-151825

00,437 Not available NTIS

Think Metric.

PB95-151825

PB95-151833

01,298 Not available NTIS

Potentiometric Enzyme-Amplified Flow Injection Analysis Detection System: Behavior of Free and Liposome-Released Peroxidase.

PB95-151833

PB95-151841

03,534 Not available NTIS

SANS Study of the Plastic Deformation Mechanism in Polyethylene.

01,242 Not available NTIS

PB95-151866

Structural Heterogeneity in Epoxies.

PB95-151866

01,243 Not available NTIS

Characterization of Polyquinoline Block Copolymer Using Small Angle Scattering.

PB95-15188

01,244 Not available NTIS

B95-151890

Dynamic Light-Scattering Study of a Diluted Polymer Blend Near Its Critical Point.

PB95-151890

01,245 Not available NTIS

Integration of Real-Time Process Planning for Small-Batch Flexible Manufacturing.

PB95-15190

02,822 Not available NTIS

B 95-151916

Ultrasonic Technique for Sizing Voids Using Area Functions.

-151916

02,904 Not available NTIS

Pattern-Recognition Analysis of Low-Resolution X-Ray Fluorescence Spectra.

PB95-151924

00,588 Not available NTIS

PB95-151932

Tomographic Decoding Algorithm for a Nonoverlapping Redundant Array.

PB95-151940

01,842 Not available NTIS

Optical Fiber Geomerry: Accurate Measurement of Cladding Diameter.

PB95-151957

04,266 Not available NTIS

Speed-of-Sound Measurements in Liquid and Gaseous Air. PB95-151957

04,186 Not available NTIS

PB95-151

Modeling Ceramic Sub-Micron Particle Formation from the Vapor Using Detailed Chemical Kinetics: Comparison with In-situ Laser Diagnostics.

PB95-151965

00,671 Not available NTIS

PB95-151973

Controlled Nucleation in Aerosol Reactors for Supression of Agglomerate Formation

PB95-1519

00,672 Not available NTIS

Flame Synthesis of High Tc Superconductors.

PB95-151981

PB95-151999

ultiphoton Ionization Spectroscopy Measurements of Silicon Atoms during Vapor Phase Synthesis of Ceramic Par-

PB95-151999

03,913 Not available NTIS

PB95-152005

Experimental and Numerical Studies of Refractory Particle Formation in Flames: Application to Silica Growth

PB95-152005

PB95-152013

00,673 Not available NTIS

Simulation of Ceramic Particle Formation: Comparison with In-situ Measurements.

PB95-152013

00,674 Not available NTIS

Effects of Humidity and Elevated Temperature on the Density and Thermal Conductivity of a Rigid Polyisocyanurate

PB95-152021

00,373 Not available NTIS

PB95-152039

Room Temperature Thermal Conductivity of Fumed-Silica Insulation for a Standard Reference Material.

PB95-152039

00,374 Not available NTIS

PB95-152047

Determination of Inorganic Constituents in Marine Mammal

Tissues.

PB95-152047

00,589 Not available NTIS

PB95-152062

Use of an Environment Classification Model.

PB95-152062

01,709 Not available NTIS

B95-152070

IRAS Spectroscopic Observations of Young Planetary Neb-

ulae.

-152070

00,072 Not available NTIS

PB95-152088

Displacement Method for Machine Geometry Calibration

PB95-152088

02,946 Not available NTIS

PB95-152096

Interaction between Dislocations and Intergranular Cracks.
PB95-152096 03,190 Not available NTIS

PB95-152104

Coupled-Bilayer Two-Dimensional Magnetic Order of the Dy lons in Dy2Ba4Cu7O15.

PBS5-1 100

04,584 Not available NTIS

PB95-152112

Susceptibility Critical Exponent for a Nonaqueous Ionic Binary Mixture Near a Consolute Point.

PB95-152112

00,938 Not available NTIS

PB95-152120

New Method to Evaluate Deposit Forming Tendencies of Liquid Lubricants by Differential Scanning Calorimetry.
PB95-152120

PB95-152138

Deposit Forming Tendencies of Diesel Engine Oils-Correlation between the Two-Peak Method and Engine Tests.

PB95-152138

01,452 Not available NTIS

PB95-152146

Gas Phase Reactivity Study of $\mathrm{OH}$ Radicals with 1,1Dichloroethene and cis-1,1-Dichloroethene and Trans-1,2Dichloroethene over the Temperature Range $240-400 \mathrm{~K}$.

PB95-15214

Rate Constants for the Gas Phase Reactions of the $\mathrm{OH}$ Radical with $\mathrm{CF}_{3} \mathrm{CF} 2 \mathrm{CHCl} 2$ (HCFC-225ca) and CF2CICF2CHCIF (HCFC-225cb) PB95-152153

00,940 Not available NTIS

PB95-152161

High-Precision Calculations of Cross Sections for Low-Energy Electron Scattering by Ground and Excited State of PB95-1521

PB95-152179

03,914 Not available NTIS

Automated Optical Roughness Inspection. 
PB95-152294

Creep Rupture of $\mathrm{MoSi} / \mathrm{SiCp}$ Composites. PB95-152294

03,154 Not available NTIS

PB95-152302

Anomalous Dispersion and Thermal Expansion in LightlyDoped KTa1-xNbxO3.

04,585 Not available NTIS

PB95-152310

Cryogenic Precision Capacitance Bridge Using a Single Electron Tunneling Electrometer.
PB95-152310 $040 \quad$ Not available NTIS PB95-152328

Neutron-Scattering Studies of the Two Magnetic Correlation Lengths in Terbium.

B95-152344

04,586 Not available NTIS

Microdosimetry and Cellular Radiation EHects of Radon Progency in Human Bronchial Airways.
PB95-152344 Not available NTIS

PB95-152773

Exact Solution of the Steady-State Surface Temperature for

a General Multilayer Structure.
PB95-152773

02,337 Not available NTIS

PB95-152781

Time-Domain Antenna Characterizations.

PB95-152781 02,003 Not available NTIS

PB95-152799

Resolution of DNA in the Presence of Mobility Modifying Polar and Nonpolar Compounds by Discontinuous Electrophoresis on Rehydratable Polyacrylamide Gels.

PB95-152799

00,590 Not available NTIS

Comparisons of Measured Linewidths of Sub-Micrometer Lines Using Optical, Electrical, and SEM Metrologies.

PB95-152807 02,338 Not available NTIS

PB95-152807

Single-Photon Ionization and Detection of $\mathrm{Ga}, \ln$, and As(sub $n$ ) Species in GaAs Growth.
PB95-152815 Not available NTIS

PB95-152823

Crystal Packing Interactions of Two Different Crystal Forms of Bovine Ribonuclease $A$.

PB95-152823

00,943 Not available NTIS

Evaluation of Fracture Toughness and Residual Stress in Dental Porcelain by Indentation-Microfracture Method. PB95-152831

00,159 Not available NTIS

PB95-152849

Automatic Calibration of Inductive Voltage Dividers for the NASA Zeno Experiment

PB95-152849

02,041 Not available NTIS

PB95-152856

Inductive Voltage Divider Calibration for the NASA Flight Experiment.

PB95-152864

02,042 Not available NTIS

Effects of Specimen Edge Conditions on Heat Release PB95

00,375 Not available NTIS

PB95-152872

Thermodynamic Interactions and Correlations in Mixtures of Two Homopolymers and a Block Copolymers by Small Angle Neutron Scattering.

PB95-152872

01,247 Not available NTIS

95-152880

Residual Stresses in Aluminum-Mullite (alpha-Alumina) Composites.

03,155 Not available NTIS

PB95-152906

Electron-Impact lonization of $\operatorname{In}(+)$ and $\mathrm{Xe}(+)$.

PB95-152906

03,918 Not available NTIS

PB95-152914

Physics for Device Simulations and Its Verification by Meas-

urements.

PB95-152922

Thermal Equilibration Near the Critical Point: Effects Due to Three Dimensions and Gravity.

PB95-152922

PB95-152930

Memory Function Approach to the Shape of Pressure Broadened Molecular Bands.

00,944 Not available NTIS

PB95-152955

Nuclear Heat Load Calculations for the NBSR Cold Neutron Source Using MCNP.

PB95-152955

PB95-152963

Electrostatic Modes of Ion-Trap Plasmas.

PB95-152963

PB95-152971

Comment on 'Phase Transitions in Antiferromagnetic

Superlattices'

PB95-152989

Interatomic Potential of Argon.

PB95-152989

00,945 Not available NTIS
PB95-152997

Experimental Study of Reverse-Bias Failure Mechanisms in Bipolar Mode JFET (BMFET).

PB95-152997

PB95-153003

Image Restoration and Diffusion Processes

PB95.153003

01,843 Not available NTIS

PB95-153011

Product State Correlations in the Reaction of $O((1) D)$ and $\mathrm{H} 2 \mathrm{O}$ in Bimolecular Collisions and in 03. H2O Clusters-Translation.

PB95-153029

00,946 Not available NTIS

Formal Methods in Conformance Testing: Result and Per-

Spectives.

PB95-153037

01,710 Not available NTIS

Time-Resolved Measurements of Energy Transfer at Sur-

PB95-153037

00,947 Not available NTIS

PB95-153045

Dynamic Technique for Measuring Normal Spectral Emissivity of Electrically Conducting Solids at High Temperares with a High-Speed Spatial Scanning Pyrometer PB95-1530

PB95-153052

Publication and Presentation Abstracts, 1993. (Published by Paffenbarger Research Center and Center of Excellence for Materials Science Research).

PB95-153052

03,562 Not available NTIS

PB95-153060

Effects of Critical Current Density, Equilibrium Magnetization and Surface Barrier on Magnetization of High Temperalure Superconductors.

PB95-153060

PB95-153078

Neutron Focusing Lens Using Polycapillary Fibers.

PB95-153078

PB95-153094

Model Precast Concrete Beam-to-Column Connections Subject to Cyclic Loading

PB95-153094

00,438 Not available NTIS

PB95-153102

Partially Prestressed and Debonded Precast Concrete Beam-Column Joints.

PB95-153102

00,439 Not available NTIS

\section{B95-153110}

Seismic Performance Behavior of Precast Concrete BeamColumn Joints.

PB95-153110

00,440 Not available NTIS

Grazing-Incidence X-Ray Photoelectron Spectroscopy: A Novel Approach to Thin Film Characterization.

PB95.153128 04,589 Not available NTIS PB95-1531

Copolymerization of $\mathrm{N}$-Phenyl Maleimide and gamma. Methacryloxypropyl Trimethoxysilan

PB95-153144 01,248 Not available NTIS PB95-153151

Thermal Behavior of 4-Maleimidophenyl Glycidyl Ether Resins.

01,249 Not available NTIS

PB95-153169

Effects on Whole Saliva of Chewing Gums Containing Calcium Phosphates.

PB95-153169

03,563 Not available NTIS

PB95-153177

Vertical-Cavity Optoelectronic Structures: CAD, Growth, and Structural Characterization.

02,148 Not available NTIS

Vertical-Cavity Semiconductor Structures: Materials Characterization.

PB95-153185

PB95-153193

02,149 Not available NTIS

Vertical-Cavity Semiconductor Lasers: Structural Characterzation, CAD, and DFB Structures.

ization, CAD,

02,150 Not available NTIS

PB95-153201

Rotational Dynamics of $\mathrm{C} 60$ in Na2RbC60

PB95-153201

00,948 Not available NTIS

PB95-153219

Rotational Dynamics of Solid C70: A Neutron-Scattering Study.

PB95-153227

00,949 Not available NTIS

New Coaxial Microwave Microcalorimeter Evaluation Technique

PB95-153227

PB95-153235

Ultrasonic Measurement of Sheet Anisotropy and Formability.

PB95-153250

03,213 Not available NTIS

Transverse Thermomagnetic Eflects in the Mixed State and Lower Critical Field of High-Tc Superconductors.

PB95-153250

PB95-153268

New Mechanisms for Laser Cooling

PB95-153268

03,923 Not available NTIS

PB95-153276

Psychological Aspects of Lighting: A Review of the Work of CIE TC 3.16

PB95.153276

PB95-153292

00,254 Not available NTIS

Structure and Dynamics of Buckyballs.

PB95-153292

00,950 Not available NTIS

PB95-153300

Neutron and X-Ray Scattering Cross Sections of

Orientationally Disordered Soild C60.

PB95-153300

00,951 Not available NTIS

PB95-153318

Morphological Stability

PB95-153318

04,591 Not available NTIS

PB95-153334

Moisture and Water-Induced Crack Growth in Optical Materials.

95.153334

04,267 Not available NTIS

PB95-153359

Integration of Scanning Tunneling Microscope

Nanolithography and Electronics Device Processing.
PB95.153359

PB.5 153367

Piezoelectric and Pyroelectric Polymers

PB95-153367

01,250 Not available NTIS

PB95-153375

Extrapolation Chamber Measurements on $(90) \mathrm{Sr}+(90) \mathrm{Y}$

Beta-Particle Ophthalmic Applicator Dose Rates.

PB95-153375

03,626 Not available NTIS

PB95-153383

Reference Materials by Isotope Dilution Mass Spectrometry.
PB95.153383 00,592 Not available NTIS

High Speed, High Sensitivity Magnetic Field Sensors Based on the Faraday Effect in Iron Gamets

PB95-15339

PB95-153409

Magneto-Optic Magnetic Field Sensors Based on Uniaxial Iron Garnet Films in Optical Waveguide Geos on

PB95-153409

02,152 Not available NTIS

PB95-153417

Noninvasive High-Voltage Measurement in Mammography

by Crystal Diffraction Spectroscopy. 00,160 Not available NTIS PB95-153425

Substrate Specificity of the Escherichia coli Endonuclease III: Exicision of Thymine- and Cytosine-Derived Lesions in DNA Produced by Radiation-Generated Free Radicals.

PB95-153425 03,535 Not available NTIS

PB95-153433

Hydrogen Balmer Alpha Line Shapes for Hydrogen-Argon Mixtures in a Low-Pressure if Discharge.

PB95-153441

Distant Future of Solar Activity: A Case Study of beta Hydri. 3. Transition Region, Corona, and Stellar Wind.

PB95-15344

00,074 Not available NTIS

PB95-153458

NIST-7, the New US Primary Frequency Standard

PB95-153458 01,534 Not available NTIS

PB95-153482

Error Analysis of the NIST Optically Pumped Primary Frequency Standard.

PB95-153490

Epitaxial Integration of Single Crystal C60

PB95-153490

PB95-153516

Pressure Dependencies of Standard Resistors.

PB95-153516

B95-153524 


\section{PB95-153599}

Liquid Chromatographic Method for the Determination of Carotenoids. Retinoids, and Tocopherols in Human Serum and in Food

PB95-153599

PB95-153615

Polarization Effects on Multiple Scattering Gamma Trans

PB95-153615

03,926 Not available NTIS

PB95-153649

Surface Topography and Ordering-Variant Segregation in GainP2

PB95-153649

04,595 Not available NTIS

PB95-153656

Performance of Commercial CMOS Foundry-Compatible Multijunction Thermal Converters.

PB95-153656

02,342 Not available NTIS

PB95-153664

Multijunction Thermal Converters by Commercial CMOS Fabrication.

PB95-153664

02,343 Not available NTIS

PB95-153698

Origin of the Second Length Scale Above the Magnetic Spiral Phase of Tb.

PB95-153698

PB95-153714

04,596 Not available NTIS

High-Resolution Spectroscopy of Laser-Cooled Rubidium in a Vapor-Cell Trap.

PB95-1537

04,268 Not available NTIS

PB95-153722

Volume Magnetic Hysteresis Loss of Nb3Sn Superconductors as a Function of Wire Length.

PB95-153730

Dielectric Studies of Fluids with Reentrant Resonators. PB95-153730

PB95-153748

Turbulent Spray Burner for Assessing Halon Alternative Fire Suppressants

PB95-153755

01,385 Not available NTIS

New Extrapolation/Spherical/Cylindrical Measurement Facility at the National Institute of Standards and Technology. PB95-153755 02,004 Not available NTIS

PB95-153763

Accurate Characterization of High Speed Photodectors.

PB95-153763 02,153 Not available NTIS

PB95-153771

( A

Frequency Stabilized Lasers: A Parochial Review.

PB95-153771 04,269 Not available NTIS

PB95-153789

SANS Studies of Space-Time Organization of Structure in Polymer Blends.

PB95-1537

01,251 Not available NTIS

B95-153797

Directions in MEMS Research Application Development

PB95-153797

02, 106 Not available NTIS

PB95-153805

Modeling Buffer Layer IGBTs for Circuit Simulation PB95-153805

02,344 Not available NTIS

PB95-154662

Effective Medium Theory for Ferrite-Loaded Materials

PB95-154662

$01,893 \mathrm{PC} \mathrm{A03/MF} \mathrm{A01}$

PB95-154670

Mapping Integration Definition for Information Modeling IDEF1X) Model into CASE Data interchange Format CDIF) Transfer File.

95-154670

01,711 PC A06/MF A02

PB95-154688

Initial NIST Testing Policy for STEP: Beta Testing Program for AP 203 Implementations. National PDES Testbed Re port Series.

B95-154696

ISDN LAN Bridging

PB95-154696

PB95-154704

Rationale and Preliminary Plan for Federal Research for Construction and Building.

PB95-154704

PB95-154720

00,322 PC A07/MF A02

Review and Upgrading of Military Fastener Test Standard MIL-STD-1312.

PB95-154738

02,947 PC A07/MF A02

User Study: Informational Needs of Remote National Archives and Records Administration Customers.

PB95-154738 02,725 PC A06/MF A02

PB95-154746

Method of Predicting Smoke Movement in Atria with Application to Smoke Management

PB95-154746

PB95-155552

00,376 PC A05/MF A01

National Voluntary Laboratory Accreditation Program: Construction Materials Testing.

01,319 PC A05/MF A01

\section{PB95-155560}

National Voluntary Laboratory Accreditation Program: Carpet and Carpet Cushion.

PB95-155560

PB95-155578

Opportunities for Innovation: Software for Manufacturing.

PB95-155578

22,851 PC A09/MF ${ }_{\text {AO3 }}$

PB95-155586

Composite Struts for SMES Plants.

PB95-155586

02,507 PC A99/MF A06

PB95-159885

Electronics and Electrical Engineering Laboratory 1995 Program Plan. Supporting Technology for U.S. Competitiveness in Electronics.

PB95-159885

$01,894 \mathrm{PC} \mathrm{A09/MF} \mathrm{A03}$

PB95-160594

Journal of Research of the National Institute of Standards and Technology, July/August 1994. Volume 99, Number 4. Special Issue: Extreme Value Theory and Applications. Proceedings of the Conference on Extreme Value Theory and Applications, Volume 2. Held at Gaithersburg, Maryland, in

PB95-160594

03,440 PC A13/MF A03

PB95-160602

CSTL Technical Activities, 1993

PB95-160602

00,953 PC A17/MF A04

PB95-161014

Simulating the Dynamic Electro Thermal Behavior of Power Electronic Circuits and Systems.

PB95-161014

02,345 Not available NTIS

B95-161022

Thermal Component Models for Electro-Thermal Network Simulation

PB95-161022

02,346 Not available NTIS

PB95-161030

Visual Road Following without 3-D Reconstruction

PB95-161030

PB95-161089

Satellite Two-Way Time Transfer: Fundamentals and Recent Progress.

P95-161097

Neutron Powder Diffraction Study of the Nuclear and Magnetic Structures of the Oxygen-Deficient Perovskite YBaCuCoO5.

PB95-161105

00,954 Not available NTIS

Crystal Structure of Annealed and As-Prepared $\mathrm{HgBa2CaC}$ (g2O6+delta Superconductors.
PB95-161105 Not available NTIS

PB95-161105

Calibration of High-Energy Electron Beams by Use of Graphite Calorimeters

PB95-161113

04,598 Not available NTIS

PB95-161121

Information Retrieval Using Key Words and a Structured

Review.

PB95-161139

03,724 Not available NTIS

Harmonic and Static Susceptibilities of $\mathrm{YBa} 2 \mathrm{Cu} 3 \mathrm{O} 7$

PB95-161139

04,599 Not available NTIS

PB95-161147

Ouantum Measurements of Trapped lons.

PB95-161147

03,928 Not available NTIS

PB95-161154

Grazing Angle X-Ray Photoemission System for Depth-Dependent Analysis.

PB95-161154

04,600 Not available NTIS

Enzyme and Protein Mass Transfer Coefficient in Aqueous Two Phase Systems. 1. Spray Extraction Columns

PB95-161162 00,594 Not available NTIS

PB95-161170

SDNS Security Management.

PB95-161170

01,592 Not available NTIS

PB95-161188

Locating Fire Engineering Information.
PB95-161188
00,198 Not available NTIS

PB95-161196

Time-Resolved Small-Angle Neutron Scattering Study of Spinodal Decomposition in Deuterated and Protonated

PB95-161196

01,252 Not available NTIS

PB95-161204

Method of Realizing Spectral Irradiance Based on an Abso. lute Cryogenic Radiometer.

PB95-161204

PB95-161212

04,270 Not available NTIS

Continuous Counter-Current Two Phase Aqueous Extraction.

PBפ5-16.

00,675 Not available NTIS

B95-161220

Laser Assisted Collisions at Ultracold Temperatures

PB95-161220

03,929 Not available NTIS

B95-161253

Frequency Extension of the NIST AC-DC Difference Calibration Service for Current.

01,895 Not available NTIS

PB95-161261

Cryogenic Toughness of Austenitic Stainless Steel Weld PB95-161261

B95-161279

03,214 Not available NTIS

Hydrogen in YBa2Cu3Ox: A Neutron Spectroscopy and a Nuclear Magnetic Resonance Study. 04,601 Not available NTIS

PB95-161287

Modeling and Test Point Selection for a Thermal Transfer

Standard.

PB95-161287

01,896 Not available NTIS

Flat and Rising R-Curves for Elliptical Surface Cracks from

Indentation and Superposed Flexure.
PB95-161295 03,156 Not available NTIS

PB95-161303

Tensile Creep of a Silicon Nitride Ceramic

PB95-161303

03,049 Not available NTIS

PB95-161311

Sputtered High Temperature Thin Film Thermocouples. PB95-16131

02,259 Not available NTIS

PB95-161329

Bending-Induced Phase Shifts in Dual-Mode Planar Optical Waveguides.

PB95-161360

04,271 Not available NTIS

Assessment of 'Peaks Over Threshold' Methods for Estimating Extreme Value Distribution Tails.

mating Extreme Value Distribution Tails. Not available NTIS PB95-161378

Elastic Properties of Al2O3/Al Composites: Measurements and Modeling.

PB95-161386

03,157 Not available NTIS

Lithium-Drift Technique for Making Submicron Thick Silicon Membranes.

PB95-161394

02,260 Not available NTIS

Encouraging Environmentally-Aware Inventions.

PB95-161394

02,521 Not available NTIS

PB95-161402

Development of the National Marine Mammal Tissue Bank.

PB95-161402 02,586 Not available NTIS

95-161410

Keeping Time on Your PC.

B95-161410

01,537 Not available NTIS

PB95-161444

Underflow-Induced Graphics Failure Solved by SLI Arith-

PB95-161444

PB95-161451

Grain Alignment and Transport Properties of Bi2Sr2CaCu2O8 Grown by Laser Heated Float Zone Method.

PB95-16145

04,602 Not available NTIS

PB95-161485

Characterization of Unknown Linear Systems Based on Measured CW Amplitude.

PB95-161485

01,897 Not available NTIS

PB95-161501 
PB95-161634

ESR-Based Analysis in Radiation Processing PB95-161634

03.931 Not available NTIS PB95-161659

Comparison of Elastic and Plastic Contact Models for the Prediction of Thermal Contact Conductance.

PB95-161659 04,605 Not available NTIS

PB95-161667

Measurement of Surface Tension of Tantalum by a Dynamic Technique in a Microgravity Environment.

PB95-161667 03,932 Not available NTIS

PB95-161675

ELF Electric and Magnetic Field Measurement Methods.

PB95-161675

04,423 Not available NTIS

PB95-161691

Molecular Weight Dependence of the Lamellar Domain Spacing of ABC Triblock Copolymers and Their Chain Conformation in Lamellar Domains.

PB95-161691

01,254 Not available NTIS

PB95-161709

Tunneling Spectroscopy of Thallium-Based High-Temperature Superconductors.

PB95-161717

04,606 Not available NTIS

Tunneling Stabilized Magnetic-Force Microscopy.

PB95-161717 04,607 Not available NTIS

PB95-161733

Magnetic Force Microscopy of Flux in Superconductors.

PB95-161733

04,608 Not available NTIS

PB95-161741

DNA Base Damage Generated In vivo in Hepatic Chromatin of Mice upon Whole Body y-lrradiation.

03,627 Not available NTIS

PB95-161774

Spin-Resolved Elastic Scattering of Electrons from Sodium.
PB95-161774

PB95-16177

PB95-161782

Ultrasonic Method for Reconstructing the Two-Dimensional Liquid-Solid Interface in Solidifying Bodies.

PB95-16178

03,349 Not available NTIS

PB95-161790

Reactivity of Product Gases Generated in Idealized Enclosure Fire Environments.

PB95-161790

01,386 Not available NTIS

PB95-161808

Real Time Differential Range Estimation Based on TimeSpace Imagery Using PIPE

P95-161816

01,844 Not available NTIS

Elastic Scattering of Polymer Networks

PB95-16181

01,255 Not available NTIS

Thermoacoustic Technique for Determining the Interface and/or Interply Strength in Polymeric Composites.

PB95-161824

03,158 Not available NTIS

PB95-161832

New Exact Solution of the One-Dimensional Schrodinger Equation and Its Application to Polarized Neutron Reflec-

PB95-161832

PB95-161840

sigma+-sigma- Optical Molasses in a Longitudinal Magnetic PB95-161840

03,934 Not available NTIS

PB95-161865

Neutron Scattering Study of Shear Induced Turbidity in Polystyrene Dissolved in Diotyl Phthalate.

ystyrene Dissolved in Dioctyl Phthalate. Not available NTIS
PB95-161865

PB95-161873

01,256 Not available NTIS

Slit Jet Infrared Spectroscopy of Hydrogen Bonded N2HF sotopomers: Rotational Rydberg-Klein-Rees Analysis and H/D Dependent Vibrational Predissociation Rates.

PB95-161873 00,956 Not available NTIS

PB95-161881

Standards and Linkages: What Data Sharing Needs.

PB95-161881 01,713 Not available NTIS

PB95-161907

Water Mist Fire Suppression Workshop Summary.

PB95-161907

02,853 Not available NTIS

PB95-161915

Optically Linked Three-Loop Antenna System for Determining the Radiation Characteristics of an Electrically Small Source.

PB95-161915

02,005 Not available NTIS

PB95-161949

Detector-Based Candela Scale and Related Photometric Calibration Procedures at NIST

PB95-161949

04,273 Not available NTIS

PB95-161964

Kinetic-Energy Distributions of Ions Sampled from Argon Plasmas in a Parallel-Plate, Radio-Frequency Reference Call

PB95-16196

03,935 Not available NTIS

PB95-161972

Wear of Human Enamel against a Commercial Castable Ceramic Restoratıve Materia!

PB95-161972

00,161 Not available NTIS
PB95-161980

Amperometric Flow-Injection Analysis Biosensor for Glucose Based on Graphite Paste Modified with Tetracyanoquinodimethane.

PB95-161980

03,498 Not available NTIS

PB95-161998

Two Phase Aqueous Extraction: Rheological Properties of Dextran, Polyethylene Glycol, Bovine Serum Albumin and Their Mixtures.

PB95-161998

PB95-162004

00,676 Not available NTIS

Comparison of the Unidirectional and Radial In-Plane Flow of Fluids Through Woven Composite Reinforcements.

02,698 Not available NTIS

PB95-162012

Interaction between Micro and Macroscopic Flow in RTM Preforms.

PB95-162012

PB95-162020

03,159 Not available NTIS

Bragg Gratings in Optical Fibers Produced by a Continuous-Wave Ultraviolet Source.

PB95-162020

04.274 Not available NTIS

PB95-162038

Growth of Bragg Gratings Produced by Continuous-Wave Ultraviolet Light in Optical Fiber.

PB95-162038

04,275 Not available NTIS

B95-162046

New Concepts for Fire Protection of Passenger Rail Transportation Vehicles.

PB95-162046

PB95-162053

04,850 Not available NTIS

Production

Multilayers.

PB95-162061

Broadband Mismatch Error in Noise Measurement Systems PB95-162061

PB95-162079

Assessing Ventilation Effectiveness in Mechanically Ventiated Office Buildings.

PB95-162079

00,255 Not available NTIS

PB95-162087

Modeling Radon Transport in Multistory Residential Buildings.
PB95-162087

PB95-162095

00,256 Not available NTIS

Ventilation, Carbon Dioxide and ASHRAE Standard 621989

PB95-162095

02,544 Not available NTIS

\section{PBO5-162111}

Micromagnetic Structure of Domains in Co/Pt Multilayers. 1 Investigations of Wall Structure.

PB95-162111

PB95-162129

X-ray Mask Metrology: The Development of Linewidth Standards for $X$-ray Lithography.
PB95-162129 02,348 Not available NTIS

PB95-162152

ating

Diffusion of Copp
PB95- 162152

00,957 Not available NTIS

PBg 1

Laser-Induced Fluorescence Measurements of $\mathrm{OH}$. Concentrations in the Oxidation Region of Laminar, Hydrocarbon Diffusion Flames.

PB95-162160

01,387 Not available NTIS

PB95-162194

Fracture Toughness of Advanced Ceramics at Room Tem.

perature: A Vamas Round-Robin.

Dimensional Characterization of Small Bores: A Survey.

PB95-162202

PB95-162210

Condensed Catalogue of Electromagnetic Environment Measurements, $30-300 \mathrm{~Hz}$

PB95-162210

PB95-162228

Protein Extraction in a Spray Column Using a Polyethylene Glycol Maltodextrin Two-Phase Polymer System.

PB95-162228

PB95-162236

Reconstruction during Camera Fixation.

PB95-162236

PB95-162244

Unified Approach to Camera Fixation and Vision-Based Road Following.

PB95-1622

01,594 Not available NTIS

Ring-Opening Dental Resin Systems Based on Cyclic Acetals.

PB95-162251

PB95-162269

00,162 Not available NTIS

Neutron-Scattering Study of Librations and Intramolecular Phonons in Rb2.6K0.4C60.

PB95-162269

PB95-162277

Development of a Gas Standard Reference Material Containing Eighteen Volatile Organic Compounds.

PB95-162277 02,545 No: available NTIS

PB95-162285

Stability/Instability of Gas Mixtures Containing 1,3-Butadiene in Treated Aluminum Gas Cylinders.

diene in Treated Aluminum Gas Cylinders.

PB $95-162293$

Magnetic Rare Earth Artificial Metallic Superlattices.

PB95-162293

04,611 Not available NTIS

PB95-162301

Global Density Effects on the Self-Preservation Behavior of Turbulent Free Jets.

PB95-162301

04,207 Nol avallable NTIS

Laser-Induced Desorption of NO from Si(111): Effects o Coverage on NO Vibrational Populations.

PB95-162319

PB95-162327

Evidence of Film-Induced Cleavage by Electrodeposited Rhodium.

PB95-162327

03,191 Not available NTIS

PB95-162335

Development of Adaptive Control Strategies for Inert Gas Atomization.

PB95-162343

Process Modeling and Control of Inert Gas Atomization

PB95-162343

PB95-162350

Stabilization and Precise Calibration of a Continuous-Wave Difference Frequency Spectrometer by Use of a Simple Transfer Cavity.

PB95-162350

04.276 Not available NTIS

Viscosity of the Saturated Liquid Phase of Six Halogenated Compounds and Three Mixtures.

PB95-162368

00,960 Not available NTIS

PB95-162376

Data Encryption Standard

01,595 Not available NTIS

PB95-162384

Resonant-Photoemission Investigation of the Heusler Alloys $\mathrm{Ni2MnSb}$ and NiMnSb.

PB95-162384

04,612 Not available NTIS

PB95-162392

Lineshape Analysis of the Raman Spectrum of Diamond

Films Grown by Hot-Filament and Microwave-Plasma

Chemical-Vapor Deposition.

03,016 Not available NTIS

PB95-162400

Studies of Defects in Diamond Films and Particles by Raman and Luminescence Spectroscopies.

PB95-162400

03,017 Not available NTIS

162418

Surface Roughness Evaluation of Diamond Films Grown on Substrates with a High Density of Nucleation Sites.

PB95-162418
PB95-162426

Polarization Dependence of Response Functions in $3 \times 3$ Sagnac Optical Fiber Current Sensors.

PB95-162426

02,154 Not available NTIS

PB95-162434

Loading Device for Fracture Testing of Compact Tension 
PB95-162541

Growth of Laser Ablated YBa2Cu3O7-delta Films as Exam-

ined by Rheed and Scanning Tunneling Microscopy.
PB95-162541 PB95-162574

Labeling Conventions in Isoelectronic Sequences - Reply. PB95-162574

03,937 Not available NTIS
.

PB95-162608

Shape Selectivity in Reversed-Phase Liquid Chromatography for the Separation of Planar and Non-Planar Solutes.

PB95-162608

00,596 Not available NTIS

PB95-162624

Description of Layered Structures: Applications to High Tc Superconductors.

PB95-162624

PB95-162632

Radiation Process Data: Collection, Analysis, and Interpretation.

PB95-162632

03,628 Not available NTIS

PB95-162640

Determination of PCBs and Chlorinated Hydrocarbons in Marine Mammal Tissues.

PB95-162640

03,744 Not available NTIS

PB95-162657

Surface Magnetic Microstructural Analysis Using Scanning Electron Microscopy with Polarization Analysis (SEMPA).
PB95-162657 03,938 Not available NTIS

PB95-162673

Noncontact Ultrasonic Inspection of Train Rails for Stress.

PB95-162673 04,851 Not available NTIS

PB95-162681

Recent VAMAS Activity in Ceramics.

PB95-162681

03,051 Not available NTIS

PB95-162707

Biological Thermodynamic Data for the Calibration of Differential Scanning Calorimeters: Dynamic Temperature Data on the Gel to Liquid Crystal Phase Transition of Dialkylphosphatidylcholine in Water Suspensions

Dialkylphosphatidylcholine in Water Suspensions.
PB95-162707

PB95-162715

Thermodynamics of the Binding of Galactopyranoside Derivatives to the Basic Lectin from Winged Bean (Psophocarpus Tetrogonolobus)

PB95-162715

03,465 Not available NTIS

PB95-162723

Vehicle-Command Center Communications in a Robotic Vehicle System.
PB95-162723

PB95-162731

03,665 Not available NTIS

Fundamental Studies of Gas Sensor Response Mechanisms: Palladium on SnO2(110)

PB95-162731

PB95-162749

Critical Current Density, Irreversibility Line, and Flux Creep Activation Energy in Silver-Sheathed Bi2Sr2Ca2Cu2Ox Superconducting Tapes.

PB95-162749

04,616 Not available NTIS

PB95-162756

Temperature Dependence of the Rate Constants for Reaction of Dihalide and Azide Radicals with Inorganic Reductants.

PB95-162756

00.964 Not available NTIS

PB95-162764

lodine Aloms and lodomethane Radical Cations: Their For mation in the Pulse Radiolysis of lodomethane in Organic mation in the Pulse Radiolysis of lodomethane in Organic
Solvents, Their Complexes, and Their Reactivity with OrSolvents, Their Complexes, and Their Reactivity with Or-
ganic Reductants. ganic Reductan

PB95-162780

Nanocomposite Magnetic Materials.

PB95-162780

00,965 Not available NTIS

B95-162798

Magnetocaloric Effect in Nanocomposites

PB95-162798

04,618 Not available NTIS

Preparation of 2-Dimensional Ultra Thin Polystyrene Film by Water Casting Method.

PB95-162806

04,619 Not available NTIS

PB95-162814

Ionization Energy of Sulfur Pentafluoride and the Sulfur Pentafluoride-Fluorine Atom Bond Dissociation Energy.

PB95-162814

00,966 Not available NTIS

PB95-162822

Ca1-xCuO2, a NaCuO2-Type Related Structure.

PB95-162822

04,620 Not available NTIS

B95-162855

Status Report: AWS Standards for Identifyıng Arc Welds (A91.1) and Recording Weld Data (A9.2)

PB95-162855

PB95-162863

Computers in Welding: A Prime

PB95-162863

02,861 Not available NTIS

PB95-162871

02,862 Not available NTIS

Through-the-Arc Sensing for Real-Time Measurement of Gas Metal Arc Weld Quality.

PB95-162871

02,863 Not available NTIS
PB95-162889 Welding for Cryogenic Service.
PB95-162889 02,852 Not available NTIS

PB95-162897

Algebraic Approximation of Altractors for Galloping Oscillators.

04,820 Not available NTIS

PB95-162905

Leady Axisymmetric Modes in Infinite Clad Rods. Part 1.

PB95.162905

04,187 Not available NTIS

PB95-162913

Exposure: An Expert System Fire Code.
PB95-162913 04,868 Not available NTIS

PB95-162939

Forum for International Cooperation on Fire Research.

PB95-162939 04,869 Not available NTIS

PB95-162947

Fresh Look at Strategies for Fire Safety.

PB95-162947

04,870 Not available NTIS

PB95-162954

Dose Mapping of Radioactive Hot Particles Using Radiochromic Film.

PB95-162954

PB95-162962

Electrical Sensors for Monitoring if Plasma Sheaths.

PB95-162962

03,714 Not available NTIS

PB95-162962
PB95-162970

Systematic Studies of the Effect of a Bandpass Filter on a Josephson-Junction Noise Thermometer.

PB95-162970

03,939 Not available NTIS

PB95-162988

Systematic Studies of the Effect of a Post-Detection Filter on a Josephson-Junction Noise Thermometer.

PB95-162988

03,940 Not available NTIS

PB95-163002

Anisotropy of Polarized X-ray Emission from Atoms and Molecules.

PB95-163028

04,621 Not available NTIS

Effects of Elastic Stress on the Stability of a Solid-Liquid

Interface.

03,350 Not available NTIS

PB95-163036

Electrophoretic Separations of Polymerase Chain Reaction: Amplified DNA Fragments in DNA Typing Using a Capillary Electrophoresis-Lased Induced Fluorescence System.

PB95-163036

03,536 Not available NTIS

PB95-163044

Synthesis and Polymerization of Difunctional and Multifunctional Monomers Capable of Cyclopolymerization.
PB95-163044 01,257 Not available NTIS PB95-163051

Diamond and Graphite Precursors: Comments.

PB95-163051

00,967 Not available NTIS

PB95-163069

Real-Time Small-Angle X-Ray Scattering Study of the Early Stage of Phase Separation in the SiO2-BaO-K20 System. PB95-163069

03,052 Not available NTIS

PB95-163077

Automated Inspection: The Integration of National Stand-

ards and Commercial Products at NIST.
PB95-163077 02,906 Not available NTIS

PB95-163093

Two New Probes for a Coordinate Measuring Machine.

PB95-163093

02,653 Not available NTIS
Note

PB95-163101

Trace Elements Associated with Proteins. Neutron Activation Analysis Combined with Biological Isolation Techniques.

PB95-16310

00,597 Not available NTIS

PB95-163127

Observations of Partial Discharges in Hexane Under High Magnification

PB95-163150

Rheology of Fresh Cement Paste.

PB95-163150

01,900 Not available NTIS

PB95-163168

Cement and Concrete Characterization by Scanning Electron Microscopy.

PB95-163176

ISDN Conformance Testing.

PB95-163176

PB95-163192

Glucose Permease of Bacillus Subtilis Is a Single Polypeptide Chain That Functions to Energize the Sucrose PB95-163192

PB95-163200

03,466 Not available NTIS

Control System Architecture for a Remotely Operated Unmanned Land Vehicle.

PB95-163200

03,759 Not available NTIS

PB95-163218

Diode-Laser Pumped, Rubidium Cell Frequency Standards.

PB95-163226

Problems Related to the Determination of Mass Densities of Evaporated Reference Deposits. PB95-163226

03,941 Not available NTIS

PB95-163234

In-Space Welding: Visions and Realities.

PB95-163234

04,830 Not available NTIS

B95-163259

Evaluating the Seismic Performance of Lightly-Reinforced Circular Concrete Bridge Columns.

PB95-163259

01,335 Not available NTIS

PB95-163267

Jacket Thickness Requirements for Seismic Retrofitting of Circular Bridge Columns.

PB95-163267

01,336 Not available NTIS

PB95-163283

How Accurate Are the Josephson and Quantum Hall Effects and QED

PB95-163283

PB95-163291

03,942 Not available NTIS

Electropermeabilization of Cell Membranes: Effect of the Resting Membrane Potential

03,537 Not available NTIS

PB95-163309

Data-Parallel Algorithm for Three-Dimensional Delaunay Triangulation and its Implementa

PB95-163309

01,714 Not available NTIS

PB95-163317

Equilibrium and Calorimetric Investigation of the Hydrolysis of L-Tryptophan to (Indole + Pyruvate + Ammonia

PB95-163317

PB95-163325

Elastic Green's Function for a Bimaterial Composite Solid

PB95.163325

03,162 Not available NTIS

PB95-163333

Green's Function for Generalized Hilbert Problem for Cracks and Free Surfaces in Composite Materials.

PB95-163333

03,163 Not available NTIS

PB95-163341

Generalized Plane Strain Analysis of a Bimaterial Composite Containing a Free Surface Normal to the Interface.
PB
03,164 Not available NTIS PB95-163341

PB95-163358

Observation of Insulating Nanoparticles on YBCO ThinFilms by Atomic Force Microscopy.

PB95-163358

P895-163366

04,622 Not available NTIS

Elastic Properties of Central-Force Networks with BondLength Mismatch.

PB95-163366

04,623 Not available NTIS

PB95-163374

Microbial Degradation of Polysulfides and Insights into Their Possible Occurrence in Coal.

PB95-163374

02,488 Not available NTIS

PB95-163382

Gravity Dependent Processes and Intracellular Motion

PB95-163382

03,538 Not available NTIS

PB95-163390

Geographic Information Systems Standards: A Federal Per-

Spective. 
PB95-163531

Photoelectron Study of Electronic Autoionization in Rotationally Cooled N2: The $n=6$ Member of the Hopfield Rotation

PB95-163531

00,971 Not available NTIS

PB95-163556

SEMPA Studies of Oscillatory Exchange Coupling

PB95-163556

04,625 Not available NTIS

PB95-163564

Experimental Verification of a Vector Preisach Model

PB95-163564

04,626 Not available NTIS

PB95-163572

Importance of Unraveling Memory Propagation Effects in In terpreting Data on Partial Discharge Statistics.

PB95.163572 01,901 Not available NTIS

PB $95-163580$

1,901 Not available NTIS

Nonstationary Behavior of Partial Discharge during Insula tion Aging.

PB95-163598

01,902 Not available NTIS

Partial Discharge: Induced Aging of Cast Epoxies and Related Nonstationary Behavior of the Discharge Statistics.

PB95-163598

01,903 Not available NTIS

PB95-163606

Laser Spectroscopy of Carbon Monoxide: A Frequency Ref-

erence for the Far Infrared.

B 95-163606

04,277 Not available NTIS

PB95-163614

Variations in Size Measurements by Indicating Gaging Sys

PB95-163614

02,864 Not available NTIS

PB95-163622

Standards for Atmospheric Measurements.

PB95-163622

02,547 Not available NTIS

PB95-163655

Computer Virus Altacks

PB95-163655

01,715 Not available NTIS

PB95-163663

Comparison of Three Techniques for the Precision Measurement of Amplifier Noise.

PB95-163663

02,349 Not available NTIS

PB95-16367

Measurement Accuracies for Various Techniques for Measuring Amplifier Noise.

02,350 Not available NTIS

PB95-163689

Application Profile for ISDN.

PB95-163689

PB95-163697

Planar Resistors for Probe Station Calibration

PB95-163697

PB95-163705

Atmospheric Reactivity of alpha-Methyl-Tetrahydrofuran

PB95-163705

-Methyl-Tetrahydrofuran.
02548 Not available NTIS

PB95-163713

High Spectral Purity X-Band Source.

PB95-163713

02,045 Not available NTIS

PB95-163721

High Critical Temperature Superconductor Tunneling Spectroscopy Using Squeezable Electron Tunneling Junctions.

PB95-163721 04,627 Not available NTIS

PB95-163739

Tunneling Measurement of the Zero-Bias Conductance Peak and the Bi-Sr-Ca-Cu-O Thin-Film Energy Gap.

PB95-163739 04,628 Not available NTIS

PB95-163747

In situ Burning of Oil Spills: Mesoscale Experiments and Analysis.

PB95-163747

02,587 Not available NTIS

PB95-163754

Measurement of the Uniformity of Particle Deposition of Filter Cassette Sampling in a Low Velocity Wind Tunne

PB95-16375

PB95-163770

Applications of Fluorescence Spectroscopy in Polymer Science and Technology.

PB95-163770

01,258 Not available NTIS

PB95-163796

Wear Model for Alumina Sliding Wea

PB95.163796 03,239 Not available NTIS

PB95-163804

Pulse-Echo Uitrasonic Evaluation of the Integrity of Seams of Single-Ply Roof Membranes. 00,381 Not available NTIS
PB95-163804 PB95-16380

PB95-163812

Criteria for Establishing Accurate Vapor Pressure Curves.

PB95-163812

00,972 Not available NTIS

PB95-163820

Affinity Chromatography on Inorganic Support Materials.
PB95-163820 03,467 Not available NTIS

PB95-163838

Confidence on the Three-Point Estimator of Frequency Drift.

PB95-163838

PB95-163853

PB95-163853

01,540 No: available NTIS
PB95-163861

Series Array of DC SOUIDs.

PB95-163861

PB95-163879

02,046 Not available NTIS

Measurements of Fluorescence from Cold Atoms: Localization in Three-Dimensional Standing Waves

$\begin{array}{ll}\text { PB95-163879 03,943 Not available NTIS } & \end{array}$

PB95-163887

Localization of Atoms in a Three-Dimensional Standing

Wave.

PB95-163

03.944 Not available NTIS

B95-163895

Configuration and Performance Evaluation of a Real-Time Robot Control System: A Skeleton Approach

PB95-163895

01,598 Not available NTIS

\section{1}

Deletion Analysis of the Mini-P1 Plasmid Origin of Replica-

tion and the Role of E.coli DnaA Protein.
PB95-163911 03,539 Not available NTIS

PB95-163929

Creep and Creep Rupture of Ceramic Matrix Composites.
PB95-163929
03,165 Not available NTIS

PB95-163945

Calibrating On-Wafer Probes to the Probe Tips.

PB95-163945

22,352 Not avallable NTIS

PB95-163952

LRM Probe-Tip Calibrations with Imperfect Resistors and Lossy Lines.

PB95-163960

Verification of Scattering Parameter Measurements.

PB95-163960

PB95-163978

Cold Neutron Gain Calculations for the NBSR Using MCNP.

PB95-163978

03,731 Not available NTIS

B95-163986

Measurement of the $(93) \mathrm{Nb}(n, 2 n)(92 \mathrm{~m}) \mathrm{Nb}$ Cross Section in a (235)U Fission Spectrum.

PB95-163986

03,945 Not available NTIS

PB95-163994

Self-Organizing Neural Network Character Recognition on a Massively Parallel Computer.

PB95-16399

01,845 Not available NTIS

XUV Characterization Comparison of Mo/Si Multilayer Coatings.

PB95-164026

04,278 Not available NTIS

Standard Reference Materials for the Determination of

Trace Organic Constituents in Environmental Samples.
PB95-164026

PB95-164034

Ouality Assurance of Contaminant Measurements in Marine Mammal Tissues.
PB95-164034

PB95-164042

02,588 Not available NTIS

Liquid Chromatographic Determination of Polycyclic Aromatic Hydrocarbon Isomers of Molecular Weight 278 and 302 in Environmental Standard Reference

PB95.164042

PB95-164059

Molecular Orbital Calculations of Bond Rupture in Brittle

Solids.

PB95-164059

PB95-164067

00,973 Not available NTIS

Molecular Orbital Study of Water Enhanced Crack Growth Process.

PB95-164067

PB95-164075

03,240 Not available NTIS

Physics-Based Vision: Principles and Practice, Shape Recovery (Book Review)

PB95-164091

01,846 Not available NTIS

Lessons from the Loma Prieta Earthquake.

PB95-16409

PB95-164109

00,442 Not available NTIS

Characterization of Molecular Network of Thermosets Using Neutron Scattering

PB95-164117

01,259 Not available NTIS

Small-Angle Neutron Scattering of Poly(vinyl alcohol) Gels. PB95-164117

PB95-164125

Not available NTIS

Characteriz

Scattering.
PB95-164125

PB95-164133

1,261 Not available NTIS

Integrated Mobile Robot System for Testing Vision Algo-

PB95-164133

PB95-164158

02,936 Not available Ni1S

Asperity-Asperity Contact Mechanisms Simulated by a TwoBall Collision Apparatus.

PB95-164158

02,966 Not available NTIS

PB95-164174

Anomalous Behavior of a Ouantized Hall Plateau in a High-

PB95-164174

02,354 Not available NTIS

PB95-164182

Vector Theory of Diffraction by a Single-Mode Fiber: Application to Mode-Field Diameter Measurements.

PB95-164182

04,279 Not avaikable NTIS

PB95-164224

Analytic Calculation of Polarized Neutron Reflectivity from Superconductors.

PB95-164224

PB95-164232

04,629 Not available NTIS

Thermal and Nonequilibrium Responses of

Superconductors for Radiation Detectors.
02,156 Not available NTIS

PB95-1642

Dislocation Emission at Ledges on Cracks.

PB95.164240

PB95-164257

Shielding of Cracks in a Plastically Polarizable Material.

PB95-164257

04,631 Not available NTIS

PB95-164273

Electrical Test Structure for Improved Measurement of Feature Placement and Overlay in Integrated Circuit Fabrication Processes.

PB95-164273

PB95-164281

Laser Flash Photolysis/Time-Resolved FTIR Emission Study of a New Channel in the Reaction of $\mathrm{CH}_{3}+\mathrm{O}$ : Production of

PB95-164281

00,974 Not available NTIS

PB95-164299

Hg1-xCdxTe Characterization Measurements: Current Practice and Future Needs.

164299

02,157 Not available NTIS

Brownian Diffusion of Hard Spheres at Finite Concentra-

tions.

00,975 Not available NTIS

PB95-164315

Bonding in Doubly Charged Diatomics.

PB95. 164315

00,976 Not available NTIS

PB95-164323

Application of the Taylor Dispersion Method in Supercritical

PB95-164323

PB95-164331

00,977 Not available NTIS

Critical Behavior of Ionic Fluids.

PB95-164331

00,978 Not available NTIS

PB95-164349

Standard States, Reference States and Finite-Concentration Effects in Near-Critical Mixtures with Applications to AqueOus Solutions

PB95-164356

00,979 Not available NTIS

Effect of Annealing Ambient on the Removal of Oxide Precipitates in High-Dose Oxygen Implanted Silicon

PB95-16435

PB95-164372

Small Angle Neutron Scattering Studies on Chain Asym- 
PB95-164489

Ebulliometric Measurement of the Vapor Pressure of 1Chloro-1,1-Difluoroethane and 1,1-Difluoroethane.

PB95-164489

00,984 Not available NTIS

PB95-164497

Metrology.
PB95-164497

PB95-164505

03,946 Not available NTIS

Effects of Aluminum Oxalate/Glycine Pretreatment Solutions on Dentin Permeability.

PB95-164505

03,565 Not available NTIS

2nu9 Band of Propyne-d3.

PB95-164513

00,985 Not available NTIS

PB95-164521

Field Dependence of the Magnetic Ordering of $\mathrm{Cu}$ in $\mathrm{R} 2 \mathrm{CuO} 4(\mathrm{R}=\mathrm{Nd}, \mathrm{Sm})$

PB95-164521

04,633 Not available NTIS

PB95-164539

Observation of Noncollinear Magnetic Structure for the $\mathrm{Cu}$ Spins in Nd2CuO 4-Type Systems.
PB95-164539 04,634 Not available NTIS

PB95-164547

Membrane-Mediated Precipitation of Calcium Phosphate in Model Liposomes with Matrix Vesicle-Like Lipid Composition.

PB95-164547

03,468 Not available NTIS

PB95-164554

Characterization of a Health Physics Instrument Calibration

PB95-164554

03,629 Not available NTIS

PB95-164562

Reactive Coevaporation of DyBaCuO Superconducting Films: The Segregation of Bulk Impurities on Annealed $\mathrm{MgO}(100)$ Substrates

PB95-164562

04,635 Not available NTIS

PB95-164570

Measuring Contact Charge Transter at Interfaces: A New Experimental Technique.

PB95-164570

03,053 Not available NTIS

PB95-164588

Ouantitative Evaluation of Building Fire Safety: New Tools for Assessing Fire and Building Code Provisions.

PB95-164588 00.199 Not available NTIS

PB95-164596

Phase Composition, Viscosities, and Densities for Aqueous Two-Phase Systems Composed of Polyethylene Glycol and Various Salts at $25 \mathrm{C}$

PB95-164596

PB95-164604

00,986 Not available NTIS

Measurement of Radial Dose Distributions Around Small Beta Particle Emitters Using High Resolution Radiochromic Foil Dosimetry

PB95-164604

03,518 Not available NTIS

PB95-164612

Electrical Characterization of Radio-Frequency Discharges

in the Gaseous Electronics Conference Reference Call.

PB95-164612

01,905 Not available NTIS

PB95-164620

Surface Texture

PB95- 164620

PB95-164638

03,351 Not available NTIS

Characterization of Cytochrome c/Alkanethiolate Structures Prepared by Selt-Assembly on Gold

PB95-164638

$00,987 \mathrm{Nct}$ available NTIS

PB95-164646

Validation of the Inverse Square Law of Gravitation Using the Tower at Erie, Colorado, USA

PB95-164646

PB95-164653

Enhanced Detection of PCR Products Through Use of TOTO and YOYO Intercalating Dyes with Laser Induced Fluorescence - Capillary Electrophoresis.

00,599 Not available NTIS

Evaluation of Methylene Lactone Monomers in Dental Resins.

PB95-164679

00,164 Not available NTIS

Influence of Lattice Mismatch on Indium Phosphide Based High Electron Mobility Transistor (HEMT) Structures Observed in High Resolution Monochromatic Synchrotron XRadiation Diffraction Imaging.

02,357 Not avallable NTIS

PB95-164687

Accuracy Comparisons of Josephson Array Systems.

PB95-164687

02,047 Not available NTIS

PB95-164695

Voltage Ratio Measurements of a Zener Reference Using a Digital Volimeter.

01,906 Not available NTIS

B95-164703

Cryogenics.

Big5-164703

PB95-164711

Fire-Plume-Generated Ceiling Jet Characteristics and Convective Heat Transfer to Ceiling and Wall Surfaces in a
Two-Layer Fire Environment: Uniform Temperature Ceiling and Walls

PB95-164711

PB95-164810

00,382 Not available NTIS

Acoustic Emission of Structural Materials Exposed to Open Flames.

PB95-164810

00,296 Not available NTIS

PB95-168415

Effect of Microstructure on Phase Formation in the Reaction of Nb/Al Multilayer Thin Films.

PB95-168415

PB95-168423

03,352 Not available NTIS

Tunable High Temperature Superconductor Microstrip ResPB95-168423

02,048 Not available NTIS

PB95-168431

In situ Observation of Surface Morphology of inP Grown on Singular and Vicinal (001) Substrates.

PB95-168431

04,636 Not available NTIS

PB95-168449

Method of Sale for CNG Paves Way to Greater Public Acceptance.

PB95-168449

PB95-168456

02,489 Not available NTIS

First Phase For

in the Reaction of $\mathrm{Nb} / \mathrm{Al}$

PB95-168456

03,353 Not available NTIS

PB95-168464

Interlaboratory Comparison of Polarization-Holding Parameter Measurements on High-Biretringence Optical Fiber.

PB95-168464

04,280 Not available NTIS

\section{(68472}

Angular Variation of the Personal Dose Equivalent, $\mathrm{Hp}(0.07)$, for Beta Radiation and Nearly Monoenergetic Electron Beams: Preliminary Results.

PB95-168472

03,630 Not available NTIS

PB95-168480

Low-Coherence Interferometric Measurement of Group Transit Times in Precision Optical Fibre Delay Lines.

PB95-168480

02,158 Not available NTIS

PB95-168498

Magneto-Optic Magnetic Field Sensors Based on Uniaxial Iron Garnet Films in Optical Waveguide Geometry.

PB95-168498

PB95-168506

Look at Uncertainties over Twenty Decades of Pressure Measurement.

PB95-168506

02,655 Not available NTIS

Interference in the Resonance Fluorescence of Two Trapped Atoms.

PB95-168522

03,948 Not available NTIS

Combined Josephson and Charging Behavior of the Supercurrent in the Superconducting Single-Electron Transistor.

PB95-168530

04.637 Not available NTIS

Multi-State Two-Port: An Alternative Transter Standard. PB95-168530 02,049 Not available NTIS

PB95-168548

New cw CO2 Laser Lines: The 9-mu $m$ Hot Band.

PB95-168548

PB95-168555

Not 88 available NTIS

Coping with Different Retrieval Methods in Next Generation Networks.

PB95- 168555

PB95-168563

02,726 Not available NTIS

Lightwave Standards Development at NIST

PB95-168563

01,480 Not available NTIS

PB95-16857

Mode Coupling and Loss on Tapered Optical Waveguides.

PB95-168571

04,282 Not available NTIS

PB95-168589

Symbolic Programming with Series Expansions: Applica-

tions to Optical Waveguides.
PB95-168589

PB95-168597

04,283 Not available NTIS

Comparison of UV-Induced Fluorescence and Bragg Grat-

ing Growth in Optical Fiber.

04,284 Not available NTIS

PB95-168605

Using Archie to Find Files on the INTERNET.

PB95-168605

02.727 Not available NTIS

PB95-168613

Alternating-Field Susceptometry and Magnetic Susceptibility of Superconductors.

PB95-168613

04,638 Not available NTIS

High Current Pressure Contacts to Ag Pads on Thin Film Superconductors

PB95-168621

04,639 Not available NTIS

PB95-168639

Improved Variational Analysis of Inhomogeneous Optical Waveguides Using Airy Functions.

PB95-168639

04,285 Not available NTIS

PB95-168647

Terahertz Detectors Based on Superconducting Kinetic Inductance.

02,160 Not available NTIS

PB95-168662

Optical Performance of Photoinductive Mixers at Terahertz Frequencies.

PB95-168670

02,161 Not available NTIS

Suitability of Metalorganic Chemical Vapor Deposition-Derived PrGaO3 Films as Buffer Layers for YBa2Cu3O7-X Pulsed Laser Deposition.

PB95-168670

PB95-168688

Reference Data for the Thermophysical Properties of Cryogenic Fluids.
PB95-168688

PB95-168696

03,263 Not available NTIS

Force Calibrations in the Nanonewton Regime.

PB95-168696

03.949 Not available NTIS

PB95-168704

Thermodynamic Properties of R134a(1,1,1,2-

PB95-168704

PB95-168712

Behavior of a Calcium Phosphate Cernent in Simulated Blood Plasma In vitro.

PB95-168712

00,165 Not available NTIS

PB95-168720

Retrieving Articles from the Internet (without a UNIX Workstation). Part 1. File Formats and Software Tools.

PB95-16872

PB95-168738

Retrieving Articles from the Internet (without a UNIX Workstation). Part 2 . An Example.

PB95-168738

PB95-168746

Laser Cooling of Trapped Jons

Light Scattered from Two Atoms.

PB95-168753

PB95-168761

04,286 Not available NTIS

Revised Uncertainty Analysis for the NIST 30-MHz Attenuation Calibration System.

PB95-168761

01,907 Not available ITIS

PB95-168779

Widths and Propagation of a Truncated Gaussian Beam.

PB95-168779

04,287 Not available NTIS

PB95-168787

Measurements of the Characteristic Impedance of Coaxia Air Line Standards.

PB95-168787

02,221 Not available NTIS

PB95-168795

Bending-Induced Loss in Dual-Mode Rectangular PBav-168795

PB95-168803

04,288 Not available NTIS

Bent Rectangula 
PB95-168910

Metrological Accuracy of the Electron Pump.

Metrological Acc

PB95-168928

Crystal Structure of Calcium Succinate Monohydrate.

PB95-168928

00,167 Not available NTIS

PB95-168936

Annex 18: An International Study of Refrigerant Properties.

PB95-16893

PB95-168951

Development of a Dual-Sinker Densimeter for High-Accuracy Fluid P.V-T Measurements.

PB95-168951

03,267 Not available NTIS

PB95-168969

Need for, and Availability of, Working Fluid Property Data

Results from Annexes XIII and XVIII

PB95-168969

03,268 Not available NTIS

PB95-168977

Microwave Properties of YBa2Cu307-x Films at $35 \mathrm{GHz}$ from Magnetotransmission and Magnetoreflection Measure-

PB95-168977

04,643 Not available NTIS

PB95-168985

Ultrasensitive-Hot-Electron Microbolometer.

PB95-168985

02,163 Not available NTIS

PB95-168993

Hot-Electron Microcalorimeters for X-ray and Phonon De-

PB95-168993

04,644 Not available NTIS

PB95-169009

Relationship of AM to PM Noise in Selected RF Oscillators PB95-169009

PB95-169017

Proposed Antiferromagnetically Coupled Dual-Layer Magnetic Force Microscope Tips.

PB95-169017

04,645 Not available NTIS

PB95-169025

Magnetic and Magnetoresistive Properties of Inhomogeneous Magnetic Dual-Layer Films.
PB95-169025 04,646 Not available NTIS

PB95-169033

Enhanced Flux Pinning via Chemical Substitution in Bulk Superconducting $T 1-2212$

PB95-169033

04,647 Not available NTIS

Membrane Gas Separation for a Fluidized-Bed Incinerator.

PB95-169041

02,550 Not availabie NTIS

PB95-169058

Measurement of the Thermal Properties of Electrically Conducting Fluids Using Coated Transient Hot Wires.

PB95-169058

03,269 Not available NTIS

PB95-169066

Electrically Calibrated Pyroelectric Detector-Refinements for Improved Optical Power Measurements.

PB95-169066

02, 164 Not available NTIS

PB95-169074

Ambient Temperature Synthesis of Bulk Intermetallics.

PB95-169074

00,168 Not available NTIS

PB95-169082

Energy Flows in an Orifice Pulse Tube Refrigerator.

PB95-169082

02,752 Not available NTIS

95-169090

Graded and Nongraded Regenerator Performance.

PB95-169090

02,753 Not available NTIS

PB95-169108

Approaches Using Virtual Environments with Mosaic

PB95-169108

01,599 Not available NTIS

PB95-169116

Kinetic-Inductance Infrared Detector Based on an AntennaCoupled High-Tc SOUID.

PB95-169116

02,165 Not available NTIS

PB95-169124

High-Tc Superconducting Antenna-Coupled Microbolometer on Silicon.

PB95-169132

02, 166 Not available NTIS

Effect of Semiconductor Laser Characteristics on Optical Fiber Sensor Performance.

PB95-169132

02,167 Not available NTIS

PB95-169140

Terahertz Shapiro Steps in High Temperature SNS Joseph. PBon Junctions.

PB95-169157

02,168 Not available NTIS

Insulating Boundary Layer and Magnetic Scattering in YBa2Cu307-delta/Ag Interfaces Over a Contact Resistivity Range of $10(-8)-10(-3)$ Omega $\mathrm{cm}(2)$.

PB95-16915

04,648 Not available NTIS

Optimization of ECR-Based PECVD Oxide Films for Superconducting Integrated Circuit Fabrication.

PB95-169165

02,051 Not available NTIS

PB95-169173

Comparative Photoluminescence Measurement and Simulation of Vertical-Cavity Semiconductor Laser Structures.

PB95-169173

02,169 Not available NTIS
PB95-169181

Measurement and Simulation of Photoluminescence Specra from Vertical-Cavity Ouantum-Well Laser Structures.

PB95-169181 02,170 Not available NTIS PB95-16919

Ultrasonic Measurement of Residual Stress in Cast Steel Railroad Wheels.

PB95-169207

04,852 Not available NTIS

Comparing NIST-B $50 \mathrm{~mm}$ Orifice Meter Gas Data to the ANSI Equation.

PB95-169207

Vortex Shedding Flowmeters for SSME Ducts.

PB95-169215

02,949 Not available NTIS

PB95-1692

01,453 Not available NTIS

Effect of 1-Hydroxyethylidene-1,1-Bisphosphonate on Membrane-Mediated Calcium Phosphate Formation in Mode Liposomal Suspensions.

PB95-169223

03,469 Not available NTIS

PB95-169231

Effect of Ethanol on the Solubility of Hydroxyapatite in the System $\mathrm{Ca}(\mathrm{OH}) 2-\mathrm{H} 3 \mathrm{PO} 4-\mathrm{H} 2 \mathrm{O}$ at $25 \mathrm{C}$ and $33 \mathrm{C}$

PB95-169249

Critical Scaling Laws and a Classical Equation of State. PB95-169249

00,169 Not available NTIS

PB95-169249

00,990 Not available NTIS

Optical Power Meter Calibration Usıng Tunable Laser DiOdes.

PB95-169256

04,290 Not available NTIS

PB95-169264

Waveguide Polarizers Processed by Localized Plasma Etching.

PB95-169264

02,171 Not available NTIS

PB95-169272

Measurements of the Vapor Pressures of Difluoromethane

-Chloro-1,2,2,2-Tetrafluoroethane, and Pentafluoroethane. PB95-169272

03,270 Not available NTIS

PB95-169298

Self-Calibrating Fiber Optic Sensors: Potential Design MethPB95-169298

02,172 Not available NTIS

PB95-169306

Self Calibrating Fiber Optic Sensors: Potential Design Methods.

02,173 Not available NTIS

PB95-169314

Phase-Locked Oscillator Optimization for Arrays of Josephson Junctions.

PB95-169322

02,052 Not available NTIS

Recent Experiments on Trapped Ions at the National Institute of Standards and Technology.

PB95-169330

High-Resolution Atomic Spectroscopy of Laser-Cooled Ions.

Laser-Cooled Positron Source.

PB95-169348

PB95-169355

Optical Detector Nonlinearity: A Comparison of Five MethPB95-169355

PB95-169363

Far Infrared Laser Frequencies of (13) CD3OH

PB95-169363

PB95-169371

Journal of Research of the National Institute of Standards and Technology, September/October 1994. Volume 99 Number 5 .

PB95-169389

Tilt Effects in Optical Angle Measurements.

PB95-169389

Order as PB95.169371, PC A07/MF A02)

PB95-169397

Optical Characterization in Microelectronics Manufacturing.

PB95-169397

(Order as PB95-169371, PC A07/MF A02)

PB95-169405

Critical Issues in Scanning Electron Microscope Metrology.

PB95-169405
(Order as PB95-169371, PC A07/MF A02)

PB95-169496

Guide on Open System Environment (OSE) Procurements. PB95-169496

PB95-169538

$01,626 \mathrm{PC} \mathrm{A08/MF} \mathrm{A02}$

Superconductor Critical Current Standards for Fusion Applications. Final Progress Report, October 1993-July 1994.

PB95- 169538

PB95-169819

Report of the National Conference on Weights and Measures (79th). Held in San Diego, California on July 17-21, 1994

PB95-169819

02,656 PC A20/MF A04

PB95-170387

Workshop Summary Report: Industrial Applications of NIST SEMATECH ASTM E 42.14 and the American uum Society. Held in Gaithersburg, Maryland on March 24 25,1994 .

PB95.170387

PB95-170395

$00,506 \mathrm{PC}$ A07/MF A02

Electronics and Electrical Engineering Laboratory Technical Progress Bulletin Covering Laboratory Programs, July to September 1994 with 1994/1995 EEEL Events Calendar.
PB95-170395 02,360 PC A03/MF A0

PB95-170429

National Voluntary Laboratory Accreditation Program (NVLAP): Wood Based Products.

PB95-170551

Robotics Application to Highway Transportation. Volume 2 Literature Search

$01,337 \mathrm{PC}$ A06/MF A02

Updated Calculations for Routine Space-Shielding Radiation Dose Estimates: SHIELDOSE-2

PB95-171039

04,838 PC A04/MF AO

PB95-171096

General Motion Model and Spatio-Temporal Filters for Computing Optical Flow

$01,847 \mathrm{PC} \mathrm{A03/MF} \mathrm{A01}$

B95-171112

Japan Technology Program Assessment: Precision Engineering/Precision Optics in Japan.

PB95-171112

PB95-171344

Alaska Marine Mammal Tissue Archival Project: Specimen Inventory.

PB95-171344

02,589 PC A05/MF A0

Perspective on Software Engineering Standards.

PB95-171377

01,811 PC A03/MF A01

PB95-171419

Design Challenges in a Commercial Quantum Hall EffectBased Resistance Standard.

PB95-171419

02,263 PC A03/MF A0

PB95-171427

Initial Graphics Exchange Specification (IGES): Procedures for the NIST IGES Validation Test Service.

New Method to Calculate Looming for Autonomous Obstacle Avoidance.
PB95-171435

PB95-171633

01,600 PC A03/MF AO

Robotics Application to Highway Transportation. Volume 3 Proposed Research Topics and Cost/Benefit Evaluations by

PB95-171633

01,338 PC A20/MF A04

PB95-171948

Neighbor Tables for Molecular Dynamics Simulations.

PB95-171948

$00,991 \mathrm{PC} \mathrm{A03/MF} \mathrm{A01}$

PB95-171955

Multi-Agency Certification and Accreditation ( $\mathrm{C}$ and $\mathrm{A}$ ) Process: A Worked Example.

PB95-171955

01,601 PC A05/MF AO

PB95-171963

From Superconductivity to Supernovae: The Ginzburg Symposium. Report on the Symposium Held in Honor of Vitaly $\mathrm{L}$. Ginzburg. Held in Gaithersburg, Maryland on May 22

PB95-171963

04,649 PC A05/MF A01 
PB95-174959

SGML Parser Validation Procedures

SGML Parser
PB95-174959

01,717 PC A03/MF A01

PB95-174967

Asynchronous Transfer Mode Procurement and Usage

PB95-174967

01,481 PC A03/MF A01

PB95-174975

Protection of Data Processing Equipment with Fine Water

Sprays.

PB95-175006

Life-Cycle Costing Workshop for Energy Conservation in Buildings: Student Manual.

PB95-175006

$00,257 \mathrm{PCA} 11 / \mathrm{MF} \mathrm{A03}$

PB95-175014

Influence of the Filament Potential Wave Form on the Sen-

sitivity of Glass-Envelope Bayard-Alpert Gages.

PB95-175022

Multi-Stage, Position Stabilized Vibration Isolation System for Neutron Interferometry.

PB95-175022

03,955 Not available NTIS

PB95-175030

Crystal Structure and Compressibility of 3:2 Mullite.

PB95-175030 03,682 Not available NTIS

\section{PB95-175048}

Rotational Spectroscopy of the $\mathrm{CoH}$ Radical in Its Ground (3)Phi State by Far-Infrared Laser Magnetic Resonance Determination of Molecular Parameters.

PB95-175048

00,992 Not available NTIS

PB95-175055

Cellular Automaton Simulations of Cement Hydration and Microstructure Development.

01,320 Not available NTIS

PB95-175063

Evolution of Porosity and Calcium Hydroxide in Laboratory Concretes Containing Silica Fume.

PB95-175063 01,321 Not available NTIS

PB95-17507

Experimental Results on Single Flux Ouantum Logic.

PB95-175071

PB95-175089

02,053 Not available NTIS

Thermal Noise in High-Temperature Superconducting-Normal-Superconducting Step-Edge Josephson Junctions.

PB95-175089

04,650 Not available NTIS

PB95-175097

Status and Trends in Power Semiconductor Devices

PB95-175097

02,361 Not available NTIS

PB95-175105

Experimental Results on Normal Modes in Cold, Pure Ion Plasmas.

PB95-175105

03,956 Not available NTIS

PB95-175113

Non-Neutral Ion Plasmas and Crystals, Laser Cooling, and Alomic Clocks.

PB95-175113

03,957 Not available NTIS

$5 \cdot 175121$

Characterization of the Emission from 2D Array Josephson Oscillators.

PB95-175139

02,054 Not available NTIS

Emission Linewidth Measurements of Two-Dimensional Array Josephson Oscillators.

PB95-175139

02,055 Not available NTIS

PB95-175147

Frequency Dependence of the Emission from 2D Array Josephson Oscillators.

PB95-175147

02,056 Not available NTIS

PB95-175154

Vapor Pressures and Gas-Phase PVT Data for 1-Chloro1,2,2,2-Tetrafluoroethane (R124)

PB95-175154

03,271 Not available NTIS

PB95-175162

Fine-Structure Intervals of $(14) \mathrm{N}(+)$ By Far-Infrared Laser Magnetic Resonance

17516

00,993 Not available NTIS

PB95-175170

Laser Magnetic-Resonance Measurement of the (3)P1 (3) P2 Fine-Structure Splittings in (17) O and (18)O.

PB95-175170 00,994 Not available NTIS

PB95-17

Measurement of Diffusion in Fluid Systems: Applications to

the Supercritical Fluid Region.
PB95-175188

02,490 Not available NTIS

PB95-175196

Retention of Halocarbons on a Hexafluoropropylene Epoxide Modified Graphitized Carbon Black. Part 1. MethaneBased Compounds.

PB95-175204

03,272 Not available NTIS

Supercritical Fluid Extraction of Biological Products. PB95-175204 00,040 Not available NTIS
P PB95-175212

Fugacity Coefficients of Hydrogen in (Hydrogen + Butane).
PB95-175212 02,491 Not available NTiS
PB95-175220

Developing Rational Performance-Based Fire Safety Requirements in Model Building Codes.

PB95-175220

00,200 Not available NTIS

PB95-175238

Earthquake and Fire in Japan: When the Threat Became a Reality

PB95-175238

00,201 Not available NTIS

PB95-175246

Automated Josephson Integrated Circuit Test System.

PB95-175246

02,057 Not available NTIS

PB95-175253

Friction and Oxidative Wear of $440 \mathrm{C}$ Ball Bearing Steels Under High Load and Extreme Bulk Temperatures

PB95-175253 03,215 Not available NTIS

PB95-175261

Formation of Hydroxyapatite in Cement Systems

PB95-175261 00,170 Not available NTIS

PB95-175279

Correlation of Optical, X-ray, and Electron Microscopy Measurements of Semiconductor Multilayer Structures.

Measurements of Semiconductor Multilayer Structures.
PB95-175279 02,174 Not available NTIS

PB95-175287

02,174 Not available NTIS

Vapor-Liquid Equilibria of Mixtures of Propane and Isomeric Hexanes.

PB95-175295

00,995 Not available NTIS

Thin Film Reaction Kinetics of Niobium/Aluminum Multilayers.

PB95-175303

04,651 Not available NTIS

Aspects of a Deformable Superconductor Model for the Vortex Mass.

tex Mass.

PB95-175311

Deformable Superconductor Model for the Fluxon Mass.

PB95- 175311

PB95-175329

04,653 Not available NTIS

Performance of Compact Fluorescent Lamps at Different Ambient Temperatures.

PB95-17532

00,258 Not available NTIS

PB95-175337

Evidence for a Deep Electron Trap and Charge Compensation in Separation by Implanted Oxygen Oxides.

PB95-175337

02,362 Not available NTIS

PB95-175345

New Test Structure for Nanometer-Level Overlay and Feature-Placement Metrology.

PB95-175345

02,363 Not available NTIS

PB95-175352

Increased Pinning Energies and Critical Current Densities in Heavy-Ion-Irradiated Bi2Sr2CaCu2O8 Single Crystals.

Heavy-lon-lrradiated Bi2Sr2CaCu2O8 Single Crystals.
PB95-175352

PB95-175360

Characterization of the $\mathrm{ZnSe} / \mathrm{GaAs}$ Interface Layer by TEM

and Spectroscopic Ellipsometry
PB95-175360

PB95-175378

Determination of the Optical Constants of ZnSe Films by Spectroscopic Ellipsometry

04,656 Not available NTIS

PB95-175386

Measurements of the Viscosities of Saturated and Compressed Liquid 1,1,1,2-Tetrafluoroethane (R134a), 2,2Dichloro-1,1,1-Trifluoroethane (R123) and 1,1-Dichloro-1Fluoroethane (R141b)

PB95-175386

03,273 Not available NTIS

PB95-175394

Chemical Determination of Oxidative DNA Damage by Gas Chromatography-Mass Spectrometry.
PB95-175394
03,540 Not available NTIS PB95-175402

Competition between Hydrodynamic Screening ('Draining') and Excluded Volume Interactions in an Isolated Polyme

PB95-175402

01,265 Not available NTIS

PB95-175410

Evidence for Parallel Junctions Within High-Tc GrainBoundary Junctions.

PB95-175410

PB95-175428

Interpreting the Readings of Multi-Element Personnel Dosimeters in Terms of the Personal Dose Equivalent.

PB95-175428

03,631 Not available NTIS

PB95-175436

Thermal Enhancement of Cotunneling in Ultra-Small Tunnel

Junctions.

04,658 Not available NTIS

PB95-175444

Determination of Amphetamine and Methamphetamine in a

Lyophilized Human Urine Reference Material.

PB95-175444

03,597 Not available NTIS

PB95-175451

Dimensional Inspection Planning Based on Product Data Standards.

PB95-175469

02,910 Not available NTIS

Optical Probing of Cold Trapped Atoms.

PB95-175469
04,296. Not available NTIS

PB95-175477

High-Sensitivity Spectroscopy with Diode Lasers.

PB95-175477 04,297 Not available NTIS

PB95-175485

Diode Laser as a Spectroscopic Tool.

PB95-175485 00,600 Not available NTIS

PB95-175493

Fabrication of Transparent gamma-Al2O3 from Nanosize

PB95-175493

03,054 Not available NTIS

\section{5-175501}

Calculated Fiber Attenuation: A General Method Yielding Stationary Values.

PB95-175519

Fibre Splice Loss: A Simple Method of Calculation.

PB95-175519

PB95-175527

Characterization of a Tunable Thin Film Microwave

YBa2Cu3O7-x/SrTiO3 Coplanar Capacitor.

PB95-175527

02,264 Not available NTIS

PB95-175535

Reduction of Interfilament Contact Loss in Nb3Sn Superconductor Wires.

02,223 Not available NTIS

B95-175543

Standard Reference Devices for High Temperature Superconductor Critical Current Measurements.

PB95-175543

PB95-175550

Testers Open Dialogue at Inaugural NIST Workshop.

PB95-175550

PB95-175568

Polyelectrolytes as Dispersants in Colloidal Processing of Silicon Nitride Ceramics.

PB95-175568

PB95-175576

Surface Chemical Interactions of Si3N4 with Polyelectrolyte Deflocculants.

PB95-175584

03,056 Not available NTIS

Effects of Soxhlet Extraction on the Surface Oxide Layer of Silicon Nitride Powders.

PB95-175584

03,057 Not available NTIS

PB95-175592

Photodetector Frequency Response Measurements at NIST, US, and NPL, UK: Preliminary Results of a Standards Laboratory Comparison.

PB95-175592

02,175 Not available NTIS

PB95-175600

Josephson Voltage Standard Based on Single-Flux-Quanturn Voltage Multipliers.

PB95-175600

02,058 Not available NTIS

PB95-175618

Partial Scattered Intensities from a Binary Suspension of Polystyrene and Silica.

PB95-175618

00,996 Not available NTIS 
PB95-175725

Experimental Aspects of Flux Expulsion in Type-ll Superconductors.

PB95-175725

PB95-175733

04,660 Not available NTIS

Examination of the 1/d Expansion Method from Exact Enu Exeration for a Self-Interacting Self-Avoiding Walk.

PB95-175733

01,266 Not available NTIS

PB95-175741

Obtaining and Installing a Public Domain TEX.

PB95-175741

01,719 Not available NTIS

PB95-175758

Elastic-Electron-Scattering Effects on Angular Distributions in X-ray Photoelectron Spectroscopy.
PB95-175758 01,000 Not available NTIS

PB95-175766

Grazing Incidence X-ray Photoemission and Its Implementation on Synchrotron Light Source $X$-ray Beamlines.

PB95-175766
P1,001 Not available NTIS PB95-175774

Using Emulators to Evaluate the Performance of Building Energy Management Systems.

PB95-175774

00,259 Not available NTIS

PB95-175782

Electrical Characterization of "Liquid-Phase Epitaxially Grown Single-Crystal Films of Mercury Cadmium Telluride by Variable-Magnetic-Field Hall Measur

PB95-175782

02,177 Not available NTIS

PB95-175790

Comparative Study of Fe-C Bead and Graphite Target Perormance with the National Ocean Sciences AMS (NOSAMS) Facility Recombinator Ion Source.

PB95-175790 00,693 Not available NTIS PB $95-175808$

Fire and Smoke Control: An Historical Perspective.

PB95-175808 00,202 Not available NTIS

PB95-175816

Correlation of the Ideal Gas Properties of Five Aromatic Hydrocarbons.

PB95-175824

01,002 Not available NTIS

Defect Pair Formation by Implantation-Induced Stresses in High-Dose Oxygen Implanted Silicon-on-Insulator Material. PB95-175824 02,364 Not available NTIS

PB95-175832

PC-Based Spinning Rotor Gage Controller

PB95-175832 02,609 Not available NTIS

PB95-175840

Magneto-Transport Properties of $\mathrm{HgCdTe}$

PB95-175840 04,661 Not available NTIS

PB95-175857

Fabrication Issues in Optimizing $\mathrm{YBa} 2 \mathrm{Cu} 307-x$ Flux Transformers for Low $1 / f$ Noise.

PB95-175857

PB95-175865

02,059 Not available NTIS

Effect in Environmental Noise on the Accuracy of CoulombBlockade Devices.

PB95-175865

04,662 Not available NTIS

Testing for Metrological Accuracy of the Electron Pump.

PB95-175873 04,663 Not available NTIS

PB95-175881

Absolute Frequency Measurements of Methanol from 1.5 to $6.5 \mathrm{THz}$.

PB95-17588

04,300 Not available NTIS

PB95-175899

Optical Biosensor Using a Fluorescent, Swelling Sensing Element.

PB95-175899

03,541 Not available NTIS

PB95-175907

Interaction of Chlorhexidine Digluconate with and Adsorption of Chlorhexidine on Hydroxyapatite.

PB95-175907

03,566 Not available NTIS

PB95-175915

Ionizing Radiation Causes Greater DNA Base Damage in Radiation-Sensitive Mutant M10 Cells That in Parent Mouse Lymphoma L5178Y Cells.

03,632 Not available NTIS

PB95-175923

Interlaboratory Comparison of Autoradiographic DNA Protiling Measurements. 1. Data and Summary Statistics.

PB95-175923

03,542 Not available NTIS

Numerical Micromagnetic Techniques and Their Applications to Magnetic Force Microscopy Calculations

PB95-175931 04,664 Not available NTIS

PB95-175949

Rapid Post-Polishing of Diamond-Turned Optics

PB95-175949

04,301 Not available NTIS

PB95-175956

Determination of Hydrogen in Titanium Alloy Jet Engine Compressor Blades by Cold Neutron Capture Promp Gamma-ray Activation Analysis.

PB95-175956

PB95-175964

Cold Neutron Prompt Gamma Activation Analysis at NIST A Progress Repor

00,602 Not available NTIS
PB95-175972

Neutron Scattering by Hydrogen in Cold Neutron Promp Gamma-Activation Analysis.

PB95-175972

00,603 Not available NTIS

PB95-175980

Reproducibility of Tests on Energy Management and Control Systems Using Building Emulators.

PB95-175980

PB95-175998

Gas Transport Properties of Solution-Cast Perfluorosulfonic Acid lonomer Films Containing lonic Surfactants.

PB95-175998

PB95-176004

Limits of $\mathrm{CO} 2$ Monitoring in Determining Ventilation Rates.

PB95-176004

PB95-176012

Ventilation Effectiveness Measurements in Two Modern Office Bujldings

PB95-176020

02,553 Not available NTIS

Effect of Dodecanol Content on the Combustion of Meth anol Spray Flames.

PB95-176038

01,389 Not available NTIS

Composition Dependence of a Field Variable Along the Binary Fluid Mixture Critical Locus.

PB95-17603

01.003 Not available NTIS

PB95-176046

Equilibrium Pair Distribution Function of a Gas: Aspects Associated with the Presence of Bound States.

PB95-176046

01,004 Not available NTIS

PB95-176053

Phase Locking in Two-Junction Systems of High-Tempera ture Superconductor-Normal Metal-Superconductor Junc-

PB95-176053

02,060 Not available NTIS

PB95-176061

dc Magnetic Force Microscopy Imaging of Thin-Film Recording Head

P895-17606

04.665 Not available NTIS

High Temperature Superconductor-Normal MetalSuperconductor Josephson Junctions with High Characteristic Voltages.

PB95-176079

PB95-176087

04,666 Not available NTIS

Modified Surface Layers and Coatings.

PB95-176087

03,125 Not available NTIS

PB95-176095

Surface Degradation of Superconducting YBa2Cu3O7-delta Thin Films.

PB $95-1$

04,667 Not available NTIS

Small Angle Neutrons Scattering from Nanocrystalline Palladium as a Function of Annealing

PB95-176103

03,354 Not available NTIS

PB95-176111

High-Sensitivity Optical Sampling Using an Erbium-Doped Fiber Laser Strob

PB95-176129

Transport and Diffusion in Three-Dimensional Composite Media.

PB95-176129

04,668 Not available NTIS

PB95-176137

Electron-Ion-X-ray Spectrometer System.

PB95-176137

03958 Not available NTIS

PB95-176145

Ring-Opening Polymerication of a 2-Methylene Spiro Orthocarbonate Bearing a Pendant Methacrylate Group.

PB95-176145

Improved Rubidium Frequency Standards Using Diode Lasers with $\mathrm{AM}$ and FM Noise Control.

PB95-176152 04,303 Not available NTIS

PB95-176160

Certification of Morphine and Codeine in a Human Urine Standard Reference Material. 03,499 Not available NTIS

PB95-176160

Micromagnetic Scanning Microprobe System.

PB95-176178

02,224 Not available NTIS

PB95-176186

Frequency-Stabilized LNA Laser at 1.083 mum: Application to the Manipulation of Helium 4 Atoms.

PB95-176186

04,304 Not available NTIS

B95-176194

Pure Rotational Spectra of CuH and CuD in Their Ground States Measured by Tunable Far-Infrared Spectroscopy.

PB95-176194 01,005 Not available NTIS

PB95-176202

Rotational Spectrum of $\mathrm{OH}$ in the $\mathrm{v}=0-3$ Levels of Its PB95-176202

PB95-176210

01,006 Not available NTIS

Magnetic Force Microscopy Images of Magnetic Garnet with Thin-Film Magnetic Tip.

04,669 Not available NTIS

PB95-176228

Precise Optical Frequency References and Difference Frequency Measurements with Diode Lasers.
PB95-176228 04,305 Not available NTIS

PB95-176236

Estimating the Virial Coefficients of Small Poiar Molecules. PB95-176236

03,276 Not available NTiS

PB95-176244

Electrostatic Modes as a Diagnostic in Penning-Trap Experiments.

PB95-17624

PB95-176251

03,959 Not available NTIS

Interlaboratory Comparison Studies on the Analysis of Hair for Drugs of Abuse.

03,500 Not available NTIS

PB95-176269

Hair Analysis for Drugs of Abuse: Evaluation of Analytical Methods, Environmental Issues, and Development of Reference Materials.

PB95-176269

PB95-176277

Two-Stage Integrated SOUID Amplifier with Series Array PB95-176277

PB95-176285

On-Water Impedance Measurement on Lossy Substrates.

PB95-176285

02,365 Not available NTIS

395-176293

Squeezed Atomic States and Projection Noise in SpectrosPBPy. 176293

PB95-176301

03,960 Not available NTIS

Interaction of Stoichiometry, Mechanical Stress, and Interface Trap Density in LPCVD Si-rich SiNx-Si Structures.

PB95-176301 02,366 Not available NTIS

PB95-176319

Spectrally Smooth Reflectances That Match.

PB95-176319

PB95-176327

Noise in the Coulomb Blockade Electrometer.

PB95-176327

04,670 Not available NTIS

PB95-176335

Voltage Gain in the Single-Electron Transistor.

PB95-176335

04,671 Not available NTIS

Global Information Infrastructure: Agenda for Cooperation.

PB95-178604

PB95-178885

Manufactured Housing Walls That Provide Satisfactory Moisture Performance in All Climates.
PB95-178885 00,383 PC A03/MF A01

PB95-178893

Indoor Air Quality Impacts of Residential HVAC Systems. Phase 2.A Report: Baseline and Preliminary Simulations.

PBg

Glossary of Software Reuse Terms.

PB95-178992

PB95-179024

01,720 PC A03/MF A01

Performance of $1 / 3$-Scale Model Precast Concrete BeamColumn Connections Subjected to Cyclic Inelastic Loads. Report No. 4.

PB95-179040

00,444 PC A04/MF AO

Effects of Testing Variables on the Measured Compressive Strength of High

PB95-180014

Polymerization Initiation by $\mathrm{N}$-p-Toly/glycine: Free-Radical Reactions Studied by Pulse and Steady-State Radiolysis.

PB95-180014 
PB95-180097

Convergence Properties of a Class of Rank-Two Updates (NIST Reprint)

PB95-180097
PB95-180105

Atomic Sulfur: Frequency Measurement of the $\mathrm{J}=0$ inversely maps 1 Fine-Structure ransition at 56.3 Microns by Laser Magnetic Resonance.

PB95-180105

01,007 Not available NTIS

PB95-180113

Chromatographic Cryofocusing and Cryotrapping with the Vortex Tube.
PB95-180113

PB95-180121

00,604 Not available NTIS

Cyclic Polyamine Ionophore for Use in a Dibasic Phosphate-Selective Electrode.

PB95-180121

01,008 Not available NTIS

PB95-180139

Nondestructive Testing of Concrete: History and Challenges.

00,385 Not available NTIS

PB95-180139

High-Performance Concrete: Research Needs to Enhance Its Us

PB95-180147

01,322 Not available NTIS

PB95-180154

Photodecomposition of Mo(CO)6/Si(111) 7x7: CO State-Resolved Evidence for Excited State Relaxation and Ouenching.

01,009 Not available NTIS

PB95-180162

Influence of Ignition Source on the Flaming Fire Hazard of Upholstered Furniture. (NIST Reprint).

PB95-180162

00,297 Not available NTIS

PB95-180170

Crossover in the Pinning Mechanism of Anisotropic Fluxon Cores.

PB95-180170

PB95-180188

04,673 Not available NTIS

Size and Self-Field Effects in Giant Magnetoresistive ThinPBilm Devices.

PB95-180196

04,674 Not available NTIS

Neutron Scattering Study of the Orientation of a Liquid Crystalline Polymer by Shear Flow.

PB95-180196 01,270 Not available NTIS

PB95-180204

Novel Bulk Iron Garnets for Magneto-Optic Magnetic Field Sensing.

04,675 Not available NTIS

B95-180212

Physical and Chemical Properties of Resin-Reinforced Calcium Phosphate Cements.

PB95-180212

PB95-180220

00,171 Not available NTIS

$X$-ray Photoelectron and Auger Electron Forward Scattering A Structural Diagnostic for Epitaxial Thin Films.

PB95-180220 04,676 Not available NTIS PB95-180238

Application of a Multizone Airflow and Contaminant Dispersal Model to Indoor Air Ouality Control in Residential Build-

ings.
PB95-180238

PB95-180246

02,555 Not available NTIS

Traceability to the Mole: A New Initiative by CIPM

PB95-180246 00,605 Not available NTIS

PB95-180253

Magnetic Susceptibility of Pr2-xCexCuO4 Monocrystals and Polycrystals.

PB95-180261

04,677 Not available NTIS

Broadband High-Optical-Density Filters in the Infrared.

PB95-180261

PB95-180279

Suppression Effectiveness of Extinguishing Agents under Highly Dynamic Conditions.

PB5-180287

Crystal Structure and Magnetic Properties of $\mathrm{CuGeO} 3$. PB95-180287

PB95-180295

04,678 Not available NTIS

Laplace's Equation and the Dirichlet-Neumann Map in Muliply Connected Domains. (NIST Reprint)

03,962 Not available NTIS

PB95-180303

Observation of Oscillatory Magnetic Order in the Antiferromagnetic Superconductor HoNi2B2C

PB95-180303 04,679 Not available NTIS

PB95-180311

Early Detection of Room Fires Through Acoustic Emission. (NIST Reprint).
PB95-180311

PB95-180329

00,298 Not available NTIS

Electronic Mode Stirring for Reverberation Chambers.

PB95-180329

01,908 Not available NTIS

PB95-180337

Crosstalk between Microstrip Transmission Lines. (NIST Reprint).

02,225 Not available NTIS
PB95-180345

Analytical Expressions for Barkhausen Jump Size Distributions.

PB95-180345

04,680 Not available NTIS

PB95-180352

Existence and Nonexistence Theorems of Finite Diameter Sequential Confidence Regions for Errors-in-Variables Models.

395-18035

03,441 Not available NTIS

PB95-180360

Getting Started on Mosaic
PB95-180360

01,721 Not available NTIS

PB95-180378

Dual Frequency mm-Wave Radiometer Antenna for Airborne Remote Sensing of Atmosphere and Ocean.

PB95-180378

02,006 Not available NTIS

PB95-180386

Taxonomy for Security Standards.

PB95-180386

01,602 Not available NTIS

PB95-180394

Error Propagation in Laser Beam Spatial Parameters.

PB95-180394

04,310 Not available NTIS

PB95-180402

Ouasipotential and the Stability of Phase Lock in Nonhysteretic Josephson Junctions. 04,681 Not available NTIS

PB95-180402

B95-180410

Large-Amplitude Shapiro Steps and Self-Field Effects in High-Tc Josephson Weak Links.

PB95-180410

Design and Machining of Copper Specimens with Micro Holes for Accurate Heat Transfer Measurements.

02,658 Not available NTIS

PB95-180436

Atomic Branching Ratio Data for Oxygen-Like Species.

PB95.180436

03,963 Not available NTIS

PB95-180444

NIST Strategies for Reducing Testing Requirements.

PB95-180444

01,909 Not available NTIS

PB95-180451

Improved Eddy-Current Decay Method for Resistivity Characterization.

PB95-180469

02,265 Not available NTIS PB95-180469

PB95-180477

Dielectric Properties of Single Crystals of Al2O3, LaAlO3, $\mathrm{SrTiO3}$, and $\mathrm{MgO}$ at Cryogenic Temperatures.

PB95-180477

PB95-180485

Modal Characteristics of Bent Dual Mode Planar Optical Waveguide.

PB95-180493

04,311 Not available NTIS

Dependence of Tc on Debye Temperature Theta(sub D) for Various Cuprates.

PB95-180493

PB95-180501

04,683 Not available NTIS

Microwave Leakage as a Source of Frequency Error and Long-Term Instability in Cesium Atomic-Beam Frequency Standards.

PB5-180519

Velocity Distribution of Atomic Beams by Gated Optical Pumping.

01,541 Not available NTIS

01,542 Not available NTIS

PB-180527

Elastic Constants of a Material with Orthorhombic Symmetry: An Alternative Measurement Approach.

PB95-180527

PB95-180535

Cross-Correlation Analysis Improves Time Domain Measurements.

PB95-180535

01,543 Not available NTIS

PB95-180543

Concepts of the NIST EXPRESS Server. PB95-180543

PB95-180550

Handling Passwords with Security and Reliability in Background Processes.

PB95-180568

01,722 Not available NTIS

Microelectronic Test Structures for Feature Placement and Electrical Linewidth Metrology.

PB95.180568

PB95-180576

Acid Gas Production in Inhibited Diffusion Flames.

PB95-180576

01,390 Not available NTIS

PB95-180584

Burning Rate of Premixed Methane-Air Flames Inhibited by Fluorinated Hydrocarbons. 18058

01,391 Not available NTIS

PB95-180592

Experimental and Numerical Burning Rates of Premixed Methane-Air Inhibited by Fluoromethanes.

PB95-180592

01,392 Not available NTIS

PB95-180600

Laser Photoionization Measurements of Pressure in VacuUm. 950600

03,964 Not available NTIS

PB95-180618

Hybrid Digital/Analog Servo for the NIST-7 Frequency

Standard

PB95-180618

01,544 Not available NTIS

PB95-180626

Diagnosis and Treatment of an Oral Base-Metal Contact Lesion Following Negative Dermatologic Patch Tests.

PB95-180626

00,172 Not available NTIS

PB95-180634

Improved Molecular Constants and Frequencies for the $\mathrm{CO} 2$ Laser from New High-J Regular and Hot-Band Frequency Measurements.

PB95-180634

04,312 Not available NTIS

PB95-180642

Formation of Hydroxyapatite in a Polymeric Calcium Phosphate Cement

00,173 Not available NTIS

PB95-180659

Laser Focused Atomic Deposition.

PB95-180659

04,685 Not available NTIS

PB95-180667

Rayleigh Instability for a Cylindrical Crystal-Melt Interface.

PB95-180667

01,010 Not available NTIS

PB95-180675

Food Irradiation Dosimetry

PB95-180675

00,041 Not available NTIS

PB95-180683

Radiochromic Solid-State Polymerization Reaction.

PB95.180683

01,271 Not available NTIS

PB95-180691

Ouantitative Measure of Efficiency of Monte Carlo Simula.

tions.

PB95-18

01,011 Not available NTIS

Stabilization of 3.3 and $5.1 \mathrm{~m}$ Lead-Salt Diode Lasers by Optical Feedback

PB95-180709

PB95-180717

04,313 Not available NTIS

Magnetic Properties of Single-Crystalline UCu3AI2.

PB95-180717

04,686 Not available NTIS

PB95-180725

Epitaxial Growth and Characterization of the Ordered Vacancy Compound Culn3Ses on GaAs (100) Fabricated by Molecular Beam Epitaxy.

PB95-180725

04,687 Not available NTIS

PB95-180733

Structural and Chemical Investigations of PB95-180733

PB95-18074

01,012 Not available NTIS

Characterization of the Interaction of Hydrogen with Iridium Clusters in Zeolites by Inelastic Neutron Scattering Spectroscopy.

PB95-18074

PB95-180758

01,013 Not available NTIS

Instrument for Evaluating Phase Behavior of Mixtures for 
PB95-180857

Laser-Produced and Tokamak Spectra of Lithumlike Iron, $\mathrm{Fe}(23+)$

PB95-18085

04,314 Not available NTIS

PB95-180865

Powder Neutron Diffraction Investigation of Structure and Cation Ordering in $\mathrm{Ba2}+\mathrm{xBi2}-\mathrm{x} \mathrm{O} 6 \mathrm{-y}$

PB95-180865

01,015 Not available NTIS

PB95-180873

Thermodynamic Study of the Reactions of Cyclodextrins with Primary and Secondary Aliphatic Alcohols, with D- and L-Phenylalanine, and with L-Phenylalanineamide. PB95-180873

01,016 Not available NTIS

PB95-180881

High Resolution Inelastic Neutron Scattering Study of

Phonon Self-Energy Effects in YBCO.
PB95-180881 PB95-180881

PB95-180899

Antenna-Coupled High-Tc Air-Bridge Microbolometer on Silicon.

04,315 Not available NTIS

PB95-180907

Comparison of Magnetic Fields of Thin-Film Heads and Their Corresponding Bit Patterns Using Magnetic Force Microscopy.

PB95-180915

Flexible-Diaphragm Force Microscope.

PB95-180915

03,763 Not available NTIS

PB95-180923

Melnikov Function and Homoclinic Chaos Induced by Weak Perturbations.

PB95-180923

03,414 Not available NTIS

PB95-180931

Calculation of the Thermal Conductivity and Gas Permeability in a Uniaxial Bundle of Fibers.

PB95-180931

03,058 Not available NTIS

PB95-180949

Tribometer for Measurements in Hostile Environments.

PB95-180949

02,967 Not available NTIS

PB95-180956

Comparison of NIST and ISO Filtered Bremsstrahlung Calibration Beams.

PB95-180956

03,967 Not available NTIS

PB95-180964

Calibration of Dosimeters for the Cryogenic Irradiation of Composite Materials Using an Electron Beam.

PB95-180964

03.968 Not available NTIS

PB95-180972

Reducing the Effect of Local Oscillator Phase Noise on the Frequency Stability of Passive Frequency Standards.

PB95-180972

01,545 Not available NTIS

PB95-180980

Scanning Tunneling Microscopy of the Charge-DensityWave Structure in $1 \mathrm{~T}-\mathrm{TaS} 2$.

PB95-180980

04,689 Not available NTIS

PB95-180998

Measurement of Very-Low Partial Pressures.

PB95-180998

02,659 Not available NTIS

PB95-181004

Process Monitoring with Residual Gas Analyzers (RGAs): Limiting Factors.

PB95-181012

02,660 Not available NTIS

Local-Mode Dynamics in $\mathrm{YH} 2$ and $\mathrm{YD} 2$ by Isotope-Dilution Neutron Spectroscopy.

PB95-181012

01,017 Not available NTIS

PB95-181020

Neutron Spectroscopic Evidence of Concentration-Dependent Hydrogen Ordering in the Octahedral Sublattice of beta$\mathrm{TbH} 2+\mathrm{x}$

PB95-181020

PB95-181038

Vibrations of Hydrogen and Deuterium in Solid Solution with Lutetium.

PB95-181038

PB95-181046

01,019 Not available NTIS

Physics and Chemistry of Partial Discharge and Corona: Recent Advances and Future Challenges. PB95-181046

Plasma Chemical Model for Decomposition of SF6 in a Negative Glow Corona Discharge.
PB95-181053

B95-181061

Terminally Anchored Chain Interphases: Their Chromatographic Properties. $\quad 01,272$ Not available NTIS
PB95-181061 PB95-181061

PB95-181079

Terminally Anchored Chain Interphases: The Effect of Multicomponent, Polydisperse Solvents on Their Equilibrium Properties.

PB95-181079

01,273 Not available NTIS

PB95-181087

Detection of OH+ in its a(1)Delta State by Far Infrared Laser Magnetic Resonance.

PB95-181087

01,021 Not available NTIS
PB95-181095

Suppression of Elevated Temperature Hydraulic Fluid and JP-8 Spray Flames.

00,021 Not available NTIS

PB95-181103

Moments of the Ouartic Assignment Statistic with an Application to Multiple Regression.

PB95-181103

PB95-181111

Morphological Development of Second-Phase Particles in Elastically-Stressed Solids.

PB95-181111

03,355 Not available NTIS

95-181129

Practical Standards for PM and AM Noise at 5, 10 and 100 $\mathrm{MHz}$

P95-181137

01,546 Not available NTIS

Sifting Through Nine Years of NIST Clock Data with TA

PB95-181137

01,547 Not available NTIS

PB95-181145

Convective Stability in the Rayleigh-Benard and Directiona

Solidification Problems: High-Frequency Gravity Modulation.
PB95-181145 04,208 Not available NTIS PB95-181145

Prototype Information Retrieval System to Perform a BestMatch Search for Names.

PB95-181152

02,740 Not available NTIS

PB95-181160

Dielectric Properties of Thin Film $\mathrm{SrTiO}_{3}$ Grown on LaAlO3 with YBa2Cu3O7-x Electodes.

PB95-181160

PB95-181178

02,267 Not available NTIS

Water Adsorption at Polymer/Silicon Wafer Interfaces.

PB95-181178

01,022 Not available NTIS

PB95-181186

Novel YBa2Cu3O7-x and YBa2Cu3O7-x/Y4Ba3O9 Multilayer Films by Bias-Masked 'On-Axis' Magnetron Sputtering. PB95-181194

Control Stability of a Heat-Flow-Meter Appartus.

PB95-181194

PB95-181202

Intra-Laboratory Comparison of a Line-Heat-Source Guarded Hot Plate and Heat-Flow-Meter Apparatus.

PB95-181202

PB95-181210

00,387 Not available NTIS

Effect of Environmentally Exposures on the Properties of Polyisocyanurate Foam Insulation: Thermal Conductivity Measurements.

PB95-1812
PB95-181228

Analysis of Autocorrelations in Dynamic Processes
PB95-181228

PB95-181236

Thermal Modeling of Absolute Cryogenic Radiometers.

PB95-181236

PB95-181244

High-Resolution Diode-Laser Spectroscopy of Calcium. PB95-181244

03,969 Not available NTIS

PB95-182226

Checking the Net Contents of Packaged Goods as Adopted by the 79 th National Conference on Weights and Measures, 1994, Third Edition, Supplement 4 . PB95-182226

PB95-182234

National Voluntary Laboratory Accreditation Program Acoustical Testing Services.

PB95-182234

PB95-182259

04,188 PC A05/MF A01

Algorithm to Describe the Spread of a Wall Fire under a Ceiling.

PB95-182259

00,261 PC A04/MF A01

B95-182267

Comparison of Fire Sprinkler Piping Materials: Steel, Copper, Chlorinated Polyvinyl Chloride and Polybutylene, in Residential and Light Hazard Installations.

PB95-182267

PB95-182275

00,299 PC A03/MF A01

Keeping Your Site Comfortably Secure: An Introduction to Internet Firewals.

PB95-1822

02,730 PC A05/MF A01

Seismic Safety of Federal Buildings. Initial Program: How Much Will It Cost.

PB95-182291

PB95-182309

$00,447 \mathrm{PC} \mathrm{A03/MF} \mathrm{A01}$

Indoor Air Ouality Commissioning of a New Office Building

PB95-182309

PB95-182390

Assessment of Data by a Second-Order Transfer Function. PB95-182390

PB95-188827

03,760 PC A05/MF A0

Vehicle-to-Roadside Communications for Commercial Vehicle Operations: Requirements and Approaches.
04,860 PC A05/MF A01 PB95-188827

Program of the Manufacturing Engineering Laboratory,
1995. Infrastructural Technology, Measurements, and Standards for the U.S. Manufacturing Industries.

PB95-188835

02,754 PC A11/MF A02

PB95-188850

Evaluation of the Economic Impacts Associated with the NIST Power and Energy Calibration Services.

PB95-188850

02.461 PC A03/MF A01

PB95-188868

Santa Ana Fire Department Experiment at 1315 South Bristol, July 14,1994
PB95-188868

PB95-18891

$00,389 \mathrm{PC}$ A03/MF AO

Fire Performance of an Interstitial Space Construction Sys PB95-188918

PB95-188926

NIST Serial Holdings, 1995.

PB95-188926

$00,390 \mathrm{PC} \mathrm{A04/MF} \mathrm{A01}$

PB95-188926

02,746 PC A12/MF A03

В95-189437

MasPar MP-1 as a Computer Arithmetic Laboratory.

PB95-189437

01,627 PC AO3/MF AO

Impact of the FCC's Open Network Architecture on NS/NP Telecommunications Security.

$01,483 \mathrm{PC} \mathrm{A03/MF} \mathrm{A01}$

PB95-189452

Review of Measurements and Candidate Signatures for Early Fire Detection

PB95-189460

00,300 PC A03/MF A0

Lubrication Theory for Reactive Spreading of a Thin Drop.

PB95-189460

PB95-189478

National Voluntary Laboratory Accreditation Program POSIX. Portable Operating System Interface.

PB95-189478

02,661 PC A05/MF A01

PB95-189486

Operating Principles of Multikron II Performance Instrumentation for MIMD Computers.

PB95-189486

$01,628 \mathrm{PC} \mathrm{A03/MF} \mathrm{AO}$

189494

Proceedings Report of the International Invitation Workshop on Development Assurance. Held in Ellicott City, Maryland on June 16-17, 1994

$5-189494$

02,912 PC A03/MF AO

PB95-18950?

Motion-Mode

PB95-18951

01,849 PC A03/MF AO

Assessment of the DOD Goal Security Architecture (DGSA) for Non-Military Use.

PB95-189510

$03,653 \mathrm{PC} \mathrm{A03/MF} \mathrm{AO}$

PB95-189528

Proceedings: Workshop on Research Needs in Wind Engineering. Held in Gaithersburg, Maryland on September 12 13. 1994.

PB95-189528

00,448 PC A05/MF A01

PB95-190674

Field Evaluation of the System for Calibration of the Marshall Compaction Hamme.

PB95-190674

PB95-190682 
PB95-198750

Apparel Manufacturing Glossary for Application Protocol Development.

PB95-198768

02,755 PC A04/MF A01

Cryogenic Properties of Inorganic Insulation Materials for ITER Magnets: A Review

PB95-198768

03,706 PC A10/MF A03

PB95-198859

Expert Control System Shell Version 1.0 User's Guide.

PB95-198859 01,790 PC A03/MF A01

PB95-198917

Electromagnetic Shielding Characterization of Gaskets

PB95-198917 01,911 PC A03/MF A01

PB95-198925

Electronics and Electrical Engineering Laboratory Technical Publication Announcements Covering Laboratory Programs, July to September 1994 with 1995 EEEL Events Calendar.
PB95-198925

PB95-198933

Inserting Line Segments into Triangulations and Tetrahedralizations.

PB95-199329

03,415 PC A03/MF A01

Object-Oriented Technology Research Areas.

PB95-199329 01,726 PC A03/MF A01

\section{PB95-200218}

Evaluation of Wear Resistant Ceramic Valve Seats in GasFueled Power Generation Engines. Topical Report, December 1991-April 1994

PB95-200218

02,466 PC A07/MF A02

PB95-200648

Methods for Analysis of Cancer Chemopreventive Agents in Human Serum.

PB95-200655

$03.502 \mathrm{PC} \mathrm{A07/MF} \mathrm{A02}$

Heat and Moisture Transfer in Wood-Based Wall Construction: Measured versus Predicted.

PB95-200655

00,391 PC A05/MF A01

PB95-200747

Publications of the National Institute of Standards and Technology 1992 Catalog.
PB95-200747

PB95-202214

Core Potentials for Quasi-One-Electron Systems.

PB95-202214

00,014 PC A17/MF A04

PB95-202222

In-situ Monitoring of Molecular Beam Epitaxial Growth

Using Single Photon lonization. 01,023 Not available NTIS
PB95-202222

PB95-202230

Direct Detection of Atomic Arsenic Desorption from Si( 100$)$.
PB95-202230

(0)

B95-20224

Reduction of Light-Assisted Collisional Loss Rate from a Low-Pressure Vapor-Cell Trap.

PB95-202248

03,971 Not available NTIS

PB95-202255

High-Efficiency, High-Power Difference-Frequency Genera-

tion of 0.9-1.5 mu m Light in BBO.

PB95-202263

Hydrogen Lyman-alpha Emission of Capella.

PB95-202263

00,075 Not available NTIS

PB95-20227

Resonance Structure and Absolute Cross Sections in NearThreshold Electron-Impact Excitation of the 4s(2) (1)S yields 4 s4p (3)P Intercombination Transition in $\mathrm{Kr}(6+)$.
PB95-202271 NTIS

PB95-202289

Short-Range Correlation and Relaxation Effects on the $(6 p(2))(1)$ SO Autoionizing State of Atomic Barium

03,973 Not available NTIS

PB95-202297

Connection between Superelastic and Inelastic Electron-

Atom Collisions Involving Polarized Collision Partners.

PB95-202297 Involving Polarized Colis Not available NTIS

PB95-202305

Femtosecond Time-Resolved Molecular Multiphoton Ionization: The Na2 System.

PB95-202305

PB95-202313

Location of a (1)A(sub g) State in Bithiophene.

PB95-202313 01,025 Not available NTIS

P895-202321

Observations of $3 \mathrm{C} 273$ with the Goddard High Resolution

Spectrograph on the Hubble Space Telescope.
PB95-202321 00,076 Not available NTIS

PB95-202339

Lowest Excited Singlet State of Isolated 1-phenyl-1,3-butadiene and 1-phenyl-1,3,5-hexatriene.

PB95-202339

01,026 Not available NTIS

PB95-202347

Exponentially Rapid Coarsening and Buckling in Coherently Self-Stressed Thin Plates.

04,821 Not available NTIS
PB95-202354

Overcoming Hoelder Continuity in III-Posed Continuation Problems.

PB95-202362

03,416 Not available NTIS

Roles of Local Classical Acceleration and Spatial Separation in the Neutral Particle Analogs of the Aharonov-Bohm PBases.

202362

03,976 Not available NTIS

B95-202370

Laser Vacuum Ultraviolet Single Photon Ionization Probing of III-V Semiconductor Growth.

PB95-202370

04,691 Not available NTIS

PB95-202388

Application of ODF to the Rietveld Profile Refinement of Polycrystalline Solid.

PB95-202388

03,401 Not available NTIS

PB95-202396

Microstructure Study of Molybdenum Liners by Neutron Dif-

PB95-202396

PB95-202404

Method to Determine the Calorimetric Equivalence Correction for a Coaxial Microwave Microcalorimeter

PB95-20240

01,913 Not available NTIS

PB95-202412

Developing a NIST Coaxial Microwave Power Standard at 1 $\mathrm{mW}$.

01,914 Not available NTIS

PB95-202412

iping.

Flow of Alternati

PB95-202420

PB95-202438

Analyses of Recent Experimental and Theoretical Determinations of e-H2 Vibrational Excitation Cross Sections: As sessing a Long-Standing Controversy.
PB95-202438

PB95-202446

Test of Newton's Inverse Square Law of Gravitation Using the 300-m Tower at Erie, Colorado.

PB95-202446 PB95-20

Three-Vector Correlation Study of Orientation and Coherence Effects in $\mathrm{Na}(3 \mathrm{p},(2) \mathrm{P} 1 / 2$ inversely maps (2) $\mathrm{P} 3 / 2)+\mathrm{He}$

Semiclassical and Quantum Calculations.
PB95-202453 Not available NTIS

PB95-202461

Domain Effects in Faraday Effect Sensors Based on Iron Garnets.

PB95-202461

PB95-202479

ROSAT All-Sky Survey of Active Binary Coronae. 1. Quiescent Fluxes for the RS Canum Venaticorum Systems.

PB95-202479

PB95-202487

Early History and Future Outlook for the $\mathrm{X}$-ray Crystal Density Method.
PB95-202487

PB95-202495

04,692 Not available NTIS

Lunar Laser Ranging: A Continuing Legacy of the Apollo Program.

PB95-202495

03,683 Not available NTIS

PB95-202503

Absolute Cross-Section Measurements for Electron-Impact Single lonization of $\mathrm{Se}(+)$ and $\mathrm{Te}(+)$.

PB95-202503

03,980 Not available NTIS

PB95-202511

Crossed-Beams Measurements of Absolute Cross Sections for Electron Impact Ionization of S(+).
PB95-202511
03,981 Not available NTIS

PB95-202529

Absolute Cross Sections for Electron-Impact Single Ionization of $\mathrm{Si}(+)$ and $\mathrm{Si}(2+)$

PB95-202529

03,982 Not available NTIS

PB95-202537

Plasma Chemistry in Silane/Germane and Disilane/Germane Mixtures.
PB95-202537

PB95-202545

01,027 Not available NTIS

n-Vector Correlations in Collision Dynamics with Atomic Orbital Alignment: The Importance of Coherence Denoting AZbital Alignment: The Importance of Coherence Denot

imuthal Structure for $n$ (greater than or equal to) 3 .
PB95-202545 NTIS

PB95-202552

Resonant Two-Color Detachment of $\mathrm{H}(-)$ with Excitation of $\mathrm{H}(\mathrm{n}=2)$

PB95-202552

PB95-202560

03,984 Not available NTIS

Epitaxial Growth of $\mathrm{Sb} / \mathrm{GaSb}$ Structures: An Example of V/ III-V Heteroepitaxy.

PB95-202560

04,693 Not available NTIS

PB95-202578

Numerical Solution of the Nonlinear Schroedinger Equation for Small Samples of Trapped Neutral Atoms.

PB95-202578

03,985 Not available NTIS

PB95-202586

Vibrations of $S_{1}((1) B 2 u)$ p-Difluorobenzene - d4. S1-SO Flu-

orescence Spectroscopy and ab Initio Calculations.
PB95-202586
01,028 Not available NTIS

PB95-202594

Long-Range Parity-Nonconserving Interaction.

PB95-202594 03,986 Not available NTIS

P895-202602

Hole Dispersion and Enhancement of Antiferromagnetic Interaction of Localized Spins in High-Tc Superconductors.

PB95-202602

04,694 Not available NTIS

PB95-202610

Dielectric Properties of Materials at Cryogenic Temperatures and Microwave Frequencies.

PB95-202610

02,369 Not available NTIS

PB95-202628

Quantum Mechanics of a Solid-State Bar Gravitational AnPB95-202628

03,987 Not available NTIS

PB95-202636

Failures of the Four-Wave Mixing Model for Cone Emission.

PB95-202636

g Model for Cone Emission.

PB95-202644

Accuracy and Repeatability in Time Domain Network AnalyPB.

02,064 Not available NTIS

PB95-202651

Calibration of a Superconducting Gravimeter Using Absolute Gravity Measurements.

PB95-202651

03,684 Not available NTIS

PB95-202669

Sobolev Approximation for Line Formation with Partial Frequency Redistribution.

PB95-202669

00,078 Not available NTIS

PB95-202677

Recombination Line Intensities for Hydrogenic lons-III. Effects of Finite Optical Depth and Dust.

PB95-202677

P895-202685

Vibrational Dependence of the Anisotropic Intermolecular Potential of Ar- $\mathrm{HCl}$

PB95-202685

01,029 Not available NTIS

PB95-202693

Vibrational Dependence of the Anisotropic Intermolecular Potential of Ar-HF.

PB95-202693

01,030 Not available NTIS

PB95-202701

Modified Effective Range Theory as an Alternative to LowEnergy Close-Coupling Calculations.

PB95-202701

03,988 Not available NTIS

PB95-202719

Slow-Electron Collisions with CO Molecules in an Exact-Exchange Plus Parameter-Free Polarization Model.

PB95-202719

03,989 Not available NTIS

PB95-202727

Superconducting Resonator and a Cryogenic GaAs FieldEffect Transistor Amplifier as a Single-Ion Detection Sys-

PB95-202727

03,990 Not available NTIS

PB95-202735

Neutron Reflectivity Study of the Density Profile of a Model End-Grafted Polymer Brush: Influence of Solvent Quality.

PB95-202743

Generalized Stokes-Einstein Equation for Spherical Particle Suspensions.

PB95-202750

01,031 Not available NTIS

Stability and Surface Energies of Wetted Grain Boundaries in Aluminum Oxide.

PB95-202750

03,059 Not available NTIS

PB95-202768

Off-Diagonal Orhorhombic-Symmetry Elastic Constants.

PB95-202768

04,695 Not available NTIS

P895-202776

Single Photon Ionization, Laser Optical Probe Technique for 
PB95-202842

Deuterium in the Local Interstellar Medium: Its Cosmological Significance. PB95-202842

00,081 Not available NTIS

\section{PB95-202859}

Peeking Through the Picket Fence: What Astrophysical Surprises May Be Present in the 100-1200 Angstrom Region. PB95-202859

00,082 Not available NTIS

Variationally Stable Treatment of Two- and Three-Photon Detachment of $\mathrm{H}(-)$ Including Electron-Correlation Effects. PB95-202867

03,992 Not available NTIS

\section{PB95-202875}

Preferential in-Plane Rotational Excitation of $\mathrm{H} 2 \mathrm{O}(001)$ by Translational-to-Vibrational Transfer from $2.2 \mathrm{eV} \mathrm{H}$ Aloms. PB95-202875

01,035 Not available NTIS

Alignment in Two-Step Pulsed Laser Excitation of Rydberg Levels in Light Atoms: The Example of Sodium

PB95-202883

03,993 Not available NTIS

Narrow-Band Tunable Diode Laser System with Grating Feedback, and a Saturated Absorption Spectrometer for CS and $\mathrm{Rb}$.

PB95-202891

PB95-202909

04,319 Not available NTIS

Integrated-Optical Devices in Rare-Earth-Doped Glass

PB95-202909

PB95-202917

02,179 Not available NTIS

Threshold Electron Excitation of $\mathrm{Na}$

PB95-202917

03,994 Not available NTIS

Time Domain Network Analysis Using the Multiline TRL Calibration.

PB95-202933

02,065 Not available NTIS

Large Amplitude Skeletal Isomerization as a Promoter of Intramolecular Vibrational Relaxation in $\mathrm{CH}$ Stretch Excited Hydrocarbons

PB95-202941

01,036 Not available NTIS

Sub-Doppler, Infrared Laser Spectroscopy of the Propyne 2nu1 Band: Evidence of Z-Axis Coriolis Dominated Intramolecular State Mixing in the Acetylenic $\mathrm{CH}$ Stretch Overtone.

PB95-202941

PB95-202958

01,037 Not available NTIS

Collisional Energy Transfer between Excited-State Strontium and Noble-Gas Atoms.

PB5-202966

03,995 Not available NTIS

Determination of Complex Structures from Powder Diffraction Data: The Crystal Structure of La3Ti5Al15O37.

PB95-202966 01,038 Not available NTIS

PB95-202974

Importance of Bound-Free Correlation Effects for Vibrational Excitation of Molecules by Electron Impact: A Sensitivity

PB95-202974

PB95-202982

03,996 Not available NTIS

Acceleration of Intramolecular Vibrational Redistribution by methyl Internal Rotation. A Chemical Timing Study of pfluorotoluene and p-fluorotoluene-d3.

PB95-202982

01,039 Not available NTIS

Hydration in Semicrystalline Polymers: Small-Angle Neutron Scattering Studies of the Effect of Drawing in Nylon-6 Fibers.

202990

03,385 Not available NTIS

B95-203006

High-Resolution, Direct Infrared Laser Absorption Spectroscopy in Slit Supersonic Jets: Intermolecular Forces and Unimolecular Vibrational Dynamics in Clusters.

PB95-203006

01,040 Not available NTIS

Rotational-RKR Inversion of Intermolecular Stretching Potentials: Extension to Linear Hydrogen Bonded Complexes.

PB95-203014 01,041 Not available NTIS
tentials: Extension to Linear Hydrogen Bonded Complexes.

PB95-203022

Rigid Bender Analysis of van der Waals Complexes: The intermolecular Bending Potential of a Hydrogen Bond.

PB95-203022

01,042 Not available NTIS

Energy-Pooling Collisions in Barium.

PB95-203030

03,997 Not available NTIS B95-203048

Continuous Gravity Observations Using Joint Institute for Laboratory Astrophysics Absolute Gravimeters.

PB95-203048

03,685 Not available NTIS

(205-203055

Kinetics of the Reaction $\mathrm{C} 2 \mathrm{H}+\mathrm{O} 2$ from 193 to $350 \mathrm{~K}$ Using Laser Flash Kinetic Infrared Absorption Spectroscopy.

PB95-203055

01,043 Not available NTIS

PB95-203063

Construction of Silicon Nanocolumns with the Scanning Tunneling Microscope.

PB95-203063

04,696 Not available NTIS

Decrease of Fluorescence in Optical Fiber during Exposure to Pulsed or Continuous-Wave Ultraviolet Light.
04,320 Not available NTIS
PB95-203089

Casual Regularizing Deconvolution Filter for Optimal Waveform Reconstruction

PB95-20308

01,603 Not available NTIS

PB95-203097

Relative Photoionization and Photodetachment Cross Sections for Particular Fine-Structure Transitions with Applica-

tion to $\mathrm{Cl} 35$-subshell Photoionization.
PB95-203097 03,998 Not available NTIS

Behavior of Atoms in a Compressed Magneto-Optical Trap.
PB95-203105 03,999 Not available NTIS

PB95-203113

Dielectronic Capture Processes in the Electron-Impact Ionization of $\mathrm{Sc}(2+)$

PB95-20312

04,000 Not available NTIS

Photodissociation Dynamics in Ouantum State-Selected Clusters: A Test of the One-Atom Cage Effect in Ar-H2O.
PB95-203121 01,044 Not available NT

01,044 Not available NTIS

203139

Photoelectron Spectroscopy of Small Antimony Cluster Anions: $\mathrm{Sb}(-), \mathrm{Sb} 2(-), \mathrm{Sb} 3(-)$, and $\mathrm{Sb} 4(-)$.
PB95-203139 PB95-203147

Heavy Atoms After Excitation Charge Cloud Distribution of Heavy Atoms Atter Excitation
by Polarized Electrons.
PB95-203147 04,001 Not available NTIS

PB95-203154

Faraday Effect Current Sensor with Improved SensitivityBandwidth Product.

PB95-203154

02,180 Not available NTIS

Pulsed Laser Photolysis Time-Resolved FT-IR Emission Studies of Molecular Dynamics.

PB95-203162

04,002 Not available NTIS

PB95-203170

Electrical Method for Determining the Thickness of Meta Films and the Cross-Sectional Area of Metal Lines.

PB95-203170

02,370 Not available NTIS

PB95-203188

JEDEC 'TCR' Interlaboratory Experiment: Lessons Learned

PB95-20319

02,371 Not available NTIS

High Resolution, Jet-Cooled Infrared Spectroscopy of ( $\mathrm{HCl}$ 2: Analysis of nu1 and nu2 $\mathrm{HCl}$ Stretching Fundamentals, Interconversion Tunneling, and Mode-Specific Predissociation Lifetimes.

01,046 Not available NTIS

PB95-203204

Slit-Jet Near-Infrared Diode Laser Spectroscopy of (DCI)2 nu1, nu2 DCl Stretching Fundamentals, Tunneling Dynamics, and the Influence of Large Amplitude 'Geared' Intermolecular Rotation.

PB95-203204

01,047 Not available NTIS

PB95-203212

High Resolution Near Infrared Spectroscopy of $\mathrm{HCl}-\mathrm{DCl}$ and $\mathrm{DCl}-\mathrm{HCl}$ : Relative Binding Energies, Isomer Interconversion Rates, and Mode Specific Vibrational Predissociation.

PB95-203212

01,048 Not available NTIS

PB95-203220

Environmental Durability of Glass-Fiber Composites.

PB95-203220

03,166 Not available NTIS

PB95-203238

Laser Flash Photolysis, Time-Resolved Fourier Transform Infrared Emission Study of the Reaction $\mathrm{Cl}+\mathrm{C} 2 \mathrm{H} 5$ yields $\mathrm{HCl}(\mathrm{V})+\mathrm{C} 2 \mathrm{H} 4$
$\mathrm{~PB} 95-203238$

PB95-203246

01,049 Not available NTIS

Quantitative Measurements of Enhanced Soot Production in

a Flickering Methane/Air Diffusion Flame.
PB95-203246 01,393 Not available NTIS

PB95-203253

PET and DINGO Tools for Deriving Distributed Implementations from Estelle.

PB95-203261

01,727 Not available NTIS

Laser Preparation and Probing of Initial and Final Orbital Alignment in Collision-Induced Energy Transfe Ca(4s5p,(1)P 1) + He yields Ca(4s5p.(3)P2) + He. PB95-203261

PB95-203279

Initial and Final Orbital Alignment Probing of the Fine-Structure-Changing Collisions among the $\mathrm{Ca}(4 s)(1)(4 p)(1)$, (3)PJ States with He: Determination of Coherence and Conven tional Cross-Sections.

PB95-203279

04,004 Not available NTIS

PB95-203287

Geometric Characterization of Rockwell Diamond Indenters. PB95-203287

02,950 Not available NTIS

Microform Calibrations in Surface Metrology

PB95-203295 02,951 Not available NTIS PB95-203303

Low-Frequency, Active Vibration Isolation System.

PB95-203303 02,710 Not available NTIS

PB95-203311

Laser-Induced Desorption of In and $\mathrm{Ga}$ from Si(100) and Adsorbate Enhanced Surface Damage.
PB95-203311

PB95-203329

Pulsed Laser Irradiation at $532 \mathrm{~nm}$ of In and Ga Adsorbed on Si(100): Desorption, Incorporation, and Damage.

01,051 Not available NTIS

PB95-203337

Single-Photon Laser Ionization Time-of-Flight Mass Spectroscopy Detection in Molecular-Beam Epitaxy: Application to As4, As2, and Ga.

(1)

01,052 Not available NTIS

Deposition Rates in Direct Current Diode Sputtering.

PB95-203345 04,697 Not available NTIS

PB95-203352

Frequency Shitting of Pulsed Narrow-Band Laser Light in a Multipass Raman Cell

PB95-20335

04,321 Not available NTIS

PB95-203360

Two Principal Points of Symmetric, Strongly Unimodal Dis. tributions.

PB95-203360

PB95-203378

03,443 Not available NTIS

Linking Anisotropic Sharp and Diffuse Surface Motion Laws via Gradient Flows.

04,698 Not available NTIS

B95-203386

Thermodynamics of the Hydrolysis of N-Acetyl-L phenylalanine Ethyl Ester in Water and in Organic Solvents.
PB95-203386

PB95-203394

Surface Modification of YBa2Cu3O7-delta Thin Films Using the Scanning Tunneling Microscope: Five Methods.

PB95-203394

04,699 Not available NTIS

PB95-203402

Angle-Differential and Momentum-Transfer Cross Sections for Low-Energy Electron-Cs Scattering.

PB95-203402

B95-203410

Relativistic R-Matrix Calculations for Electron - Alkali-Metal Atom Scattering: $\mathrm{Cs}$ as a Test Case.

PB95-203410

PB95-203428

World of Building Codes.

PB95-203428

04,006 Not available NTIS

P95-203436

Outlier-Resistant Methods for Estimation and Model Fitting

PB95-203436

Estimation and Model Fitting.

PB95-203444

Morphological Estimation of Tip Geometry for Scanned Probe Microscopy.

PB95-20345

02,662 Not available NTIS

Post-Process Control of Machine Tools.

PB95-203451

02,952 Not available NTIS

PB95-203469

Resonance Fluorescence with Squeezed-Light Excitation

PB95-203469

PB95-203477

Short-Pulse Detachment of $\mathrm{H}(-)$ in the Presence of a Static Electric Field

PB95-203485

04,007 Not available NTIS

Signatures of Large Amplitude Motion in a Weakly Bound Complex: High-Resolution IR Spectroscopy and Ouantum Calculations for $\mathrm{HeCO}$.

PB95-203485 
PB95-203592

Cone Emission from Laser-Pumped Two-Level Atoms. 2

Analytical Model Studies.
PB95-203592

PB95-203600

Suppression of Ionization in One- and Two-Dimensional Model Calculations.

PB95-203600

PB95-203758

Structural Ceramics Database. Topical Report, June 1989 May 1991.

PB95-203758

03,060 PC A04/MF AO

PB95-203790

Robotics Application to Highway Transportation. Volume 1 Final Report.

PB95-203816

03,654 PC A03/MF A01

Precision Lifetime Measurements of $\mathrm{Cs} 6 p(2) \mathrm{P}_{1 / 2}$ and $6 p$

(2)P3/2 Levels by Single-Photon Counting.
PB95-203816 04,010 Not available NTIS PB95-203816

PB95-203824

Hyperfine-Structure Studies of $\mathrm{Zr}$ II: Experimental and Relativistic Configuration-Interaction Results.

PB95-203824 04,011 Not available NTIS

PB95-203832

Stabilization of Optical Phase/Frequency of a Laser System: Application to a Commercial Dye Laser with an External Stabilizer.

04,327 Not available NTIS

PB95-203840

Improved Hyperfine Measurements of the Na NP Excited State Through Frequency-Controlled Dopplerless Spectros-

copy in a Zeeman Magneto-Optic Laser Trap.
PB95-203840 04,012 Not available NTIS PB95-206181

Proceedings of the Manufacturing Technology Needs and Issues: Establishing National Priorities and Strategies Conference. Held in Gaithersburg, Maryland on April 26-28, 1994.

PB95-20618

PB95-208724

$02,930 \mathrm{PC} \mathrm{A22/MF} \mathrm{A04}$

Electronics and Electrical Engineering Laboratory Technical Progress Bulletin Covering Laboratory Programs, Octobe to December 1994 with 1995 EEEL Events Calendar

PB95-208724

$02,372 \mathrm{PC} \mathrm{A04/MF} \mathrm{AO1}$

PB95-208757

Optical Performance of Commercial Windows

PB95-208757

$00,392 \mathrm{PC}$ A09/MF A02

PB95-209193

NIST Industrial Impacts: A Sampling of Successful Partnerships (Revision, March 1995)

PB95-209193

PB95-209300

$00,489 \mathrm{PC}$ A05/MF AO

Droplet Transfer Modes for a MIL 100S-1 GMAW Electrode PB95-209300

PB95-209821

2,867 PC A04/MF AO

Electronics and Electrical Engineering Laboratory Technical

Progress Bulletin Covering Laboratory Programs, April to

June 1991, with 1992 EEEL Events Calendar.

PB95-209821

01,916 PC A03/MF A01

PB95-209854

Automated Manufacturing Research Facility 1994 Annual Report

PB95-209854

$00,015 \mathrm{PC}$ A05/MF A01

PB95-209862

Design, Specification and Tolerancing of Micrometer-Tolerance Assemblies.

PB95-20986

$02,913 \mathrm{PC} \mathrm{A03/MF} \mathrm{A01}$

PB95-209870

Concentrations of Chlorinated Hydrocarbons, Heavy Metals and Other Elements in Tissues Banked by the Alaska Maand Other Elements in Tissues Banked by the Alaska Maammal Tissue Archival Project.

PB95-209870

$02,590 \quad P C$ A06/MF AO2

Reactor Radiation Technical Activities, 1994. NAS-NRC Assessment Panel, April 6-7, 1995.

$03,732 \mathrm{PC} \mathrm{A08/MF} \mathrm{A02}$

Polymers Technical Activities 1994. NAC-NRC Assessment Panel, April 6-7, 1995.

PB95-209896

PB95-209920

01,275 PC A07/MF A02

Computer Systems Laboratory Annual Report 1994

PB95-209920

$01,629 \mathrm{PC} \mathrm{A07/MF} \mathrm{A02}$

PB95-210167

Issues Concerning Material Removal Shape Element Volumes (MRSEVs).

PB95-210225

$02,885 \mathrm{PC} \mathrm{A03/MF} \mathrm{AO}$

NIST Workshop on Gas Sensors: Strategies for Future Technologies. Proceedings of a Workshop. Held in Gaithersburg, Maryland on September 8-9, 1993 .
PB95-210225 00,507 PC A08/MF A02 BB95-210480

Electronics and Electrical Engineering Laboratory Technical Progress Bulletin Covering Laboratory Programs, January to March 1992, with 1992/1993 EEEL Events Calendar.

PB95-210480 01,917 PC A03/MF A01
PB95-210498

Room-Temperature Flexure Fixture for Advanced Ceramics. PB95-210498 03,061 PC A03/MF AO1

PB95-210522

Calculating Time-to-Contact Using Real-Time Quantized Optical Flow.
PB95-210522

PB95-210589

01,604 PC A03/MF A01

Interim Testing Artifact (ITA): A Performance Evaluation System for Coordinate Measuring Machines (CMMs). User PBg

$02,914 \mathrm{PC} \mathrm{A04/MF} \mathrm{A01}$

PB95-210928

Assessment of Site Response Analysis Procedures. PB95-210928

PB95-210936

$00,450 \quad \mathrm{PC}$ A07/MF A02

Electronic Implementors' Workshop

PB95-210936

$01,484 \mathrm{PC} \mathrm{A03/MF} \mathrm{A01}$

PB95-210944

Measurements of Outdoor Air Distribution in an Office Build-

ing. 5 - 10944

$00,264 \mathrm{PC} \mathrm{A04/MF} \mathrm{A01}$

PB95-211918

Survey of Steel Moment-Resisting Frame Buildings Affected by the 1994 Northridge Earthquake.

PB95-211918

$00,451 \mathrm{PC} \mathrm{A09/MF} \mathrm{A02}$

PB95-213583

Boundary Lubrication of Silicon Nitride.

PB95-213583

$03,226 \mathrm{PC} \mathrm{A18/MF} \mathrm{A04}$

PB95-216602

Self Monitoring Accounting Systems.

PB95-216602

$00,007 \mathrm{PC} \mathrm{A03/MF} \mathrm{A01}$

\section{(1)}

Overview of the Text REtrieval Conference (3rd) (TREC-3). Held in Gaithersburg, Maryland on November $2-4,1994$.
PB95-216883 PB95-216883

PB95-216891

White Papers Prepared for the White House: Construction Industry Workshop on National Construction Goals. Held on December 14-16, 1994.

PB95-216891

$01,299 \mathrm{PC}$ A08/MF A02

PB95-216917

Theoretical Evaluation of the Vapor Compression Cycle with a Liquid-Line/Suction-Line Heat Exchanger, Economizer and Ejector.

PB95-216925

02,607 PC A03/MF A01

Polarimetric Calibration of Reciprocal-Antenna Radars.

PB95-216925

01,872 PC A03/MF A01

PB95-216990

Burning Rate and Flame Heat Flux For PMMA in the Cone Calorimeter.

PB95-217097

00,393 PC A07/MF A02

Japan Technology Program Assessment. Simulation: Stateof the-Art in Japan

$02,827 \mathrm{PC} \mathrm{A03/MF} \mathrm{A01}$

PB95-217147

Water Droplet Evaporation from Radiantly Heated Solids.

PB95-217147

PB95-217162

$00,394 \mathrm{PC} \mathrm{A08/MF} \mathrm{A02}$

Compartment Fire Combustion Dynamics. Annual Report, September 1, 1993-September 1, 1994.
PB95-217162

\section{PB95-219218}

Electronic Access: Blueprint for the National Archives and Records Administration.

02,731 PC A04/MF A01

$-219325$

Network Brokers Handbook: An Entrepreneurial Guide to Cooperative Strategies for Manufacturing Competitiveness.
PB95-219325 00,490 PC A08/MF A02 PB95-219325

PB95-219416

Extreme Wind Distribution Tails: A 'Peaks Over Threshold' Approach.

PB95-219416

00,127 PC A05/MF A01

PB95-220414

Outline of a Multiple Dimensional Reference Model Architecture and a Knowledge Engineering Methodology for Intelligent Systems Control.

PB95-220414

$03,703 \mathrm{PC} \mathrm{A03/MF} \mathrm{A01}$

PB95-220422

Computer Simulations of Airflow and Radon Transport in Four Large Buildings.

PB95-2204

$02,557 \mathrm{PC} \mathrm{A03/MF} \mathrm{A01}$

B95-220430

NIST Reactor: Summary of Activities, October 1993 through September 1994

PB95-220448

Prediction of Cracking in Reinforced Concrete Structures. PB95-220448

PB95-220455

Testing of Selected Self-Leveling Compounds for Floors. PB95-220455

$00,395 \mathrm{PC} \mathrm{A03/MF} \mathrm{A01}$

PB95-220463

Time and Frequency: Bibliography of NIST Publications, March 1995.

01,548 PC A06/MF A02

PB95-22047

Extreme Wind Estimates by the Conditional Mean Exceedance Procedure

PB95-220471

$00,120 \quad$ PC A03/MF A01

Survey of Concrete Transport Properties and Their Meas-

PB95-220489

00,396 PC A04/MF A01

-220497

Compositional Analysis of Beneficiated Fly Ashes.

PB95-220497

0,397 PC A03/MF A01
0.006

PB95-220505

Texture-Independent Vision-Based Closed-Loop Fuzzy Controllers for Navigation Tasks.

PB95-220505

00,183 PC A03/MF A01

PB95-220513

ISO Environmental Management Standardization Efforts.

PB95-220513

02,524 PC A03/MF A01

PB95-220521

Performance Testing of a Family of Type I Combination Ap-

PB95-220521

PB95-220539

02,505 PC A03/MF A01

Tables of $X$-ray Mass Attenuation Coefficients and Mass Energy-Absorption Coefficients $1 \mathrm{keV}$ to $20 \mathrm{MeV}$ for Elements $Z=1$ to 92 and 48 Additional Substances of Dosimetric Interest.

PB95-220539

04,013 PC A06/MF A02

PB95-220547

Evaluation of Thermal Wave Imaging for Detection of Machining Damage in Ceramics.

PB95-220547

03,062 PC A05/MF A02

-

Analysis of ANSI ASC $\times 12$ and UN/EDIFACT Electronic Data Interchange (EDI) Standards.

PB95-220554

PB95-220588

Persistent Object Base System Testing and Evaluation PB95-220588

$01,729 \mathrm{PC} \mathrm{A03/MF} \mathrm{A01}$

PB95-226684

01,730 PC A05/MF A01

Building and Fire Research Laboratory Publications, 1994

PB95-226684

PB95-226692

00,398 PC AO7/MF A02

PB95-226692

PB95-226866

nal System of Units (SI).

Standard Reference Materials: Polystyrene Films for Calibrating the Wavelength Scale of Infrared Spectrophotom
PB95-226866

PB95-226916

$03,386 \quad \mathrm{PC}$ A03/MF A01

Laser Doppler Velocimeter Studies of the Pipeflow Produced by a Generic Header.

PB95-226916 
PB95-231866

Conceptual Design Plan for the National Advanced Manu-

$\begin{array}{ll}\text { facturing Testbed. } \\ \text { PB95-231866 } & 02,828 \text { PC A05/MF A01 }\end{array}$ PB95-231866

Standards Policy and Information Infrastructure.

PB95-231882
01,485 PC A20/MF A04 PB95-231908

Guidelines for the Evaluation of X.500 Directory Products. PB95-231908

.500 Directory Products.
02,732 PC A04/MF A01

PB95-232518

NIST Standard Reference Materials (Trade Name) Catalog

1995-1996.

PB95-232518

PB95-232633

$00,508 \mathrm{PC} \mathrm{A03/MF} \mathrm{A0}$

Abstract and Index Collection in the Research Information Center of the National Institute of Standards and Technology

PB 95-232633

02,741 PC A03/MF A01

PB95-242277

Electronics and Electrical Engineering Laboratory Technical Publication Announcements Covering Laboratory Programs, January lo March 1995 with 1995 EEEI Events Calendar.
02,373 PC A03/MF A0

PB95-242285

Operating Procedures and Life Cycle Documentation for the

Initial Graphics Exchange Specification.
PB95-242285 02,782 PC A04/MF A01 PB95-242285

ISO TC $184 / \mathrm{SC} 4$ Reference Manual.

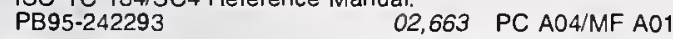

PB95-242301

Precision in Machining: Research Challenges.

PB95-242301 02,953 PC A04/MF A01

PB95-242319

CSTL Technical Activities, 1994

PB95-242319

$00,608 \mathrm{PC} \mathrm{A16/MF} \mathrm{A03}$

PB95-242327

Mixing and Radiation Properties of Buoyant Turbulent Diffusion Flames.

PB95-242335

$01,396 \mathrm{PC} \mathrm{A05/MF} \mathrm{A02}$

Parallel and Serial Implementations of SLI Arithmetic

PB 95-242335 01,732 PC A03/MF A01

PB95-242350

Unified Telerobotic Architecture Project (UTAP) Standard Interface Environment (SIE), May 1995.
PB95-242350 PB95-242368

Fracture Testing of Large-Scale Thin-Sheet Aluminum Alloy PB95-242368

PB95-242376

$00,024 \mathrm{PC} \mathrm{A03/MF} \mathrm{A01}$

National Voluntary Laboratory Accreditation Program: Electromagnetic Compatibility and Telecommunications. FCC Methods.

PB95-242376

$02,664 \mathrm{PC} \mathrm{A06/MF} \mathrm{A02}$

PB95-242384

DETAN 95: Computer Code for Calculating Spectrum-Averaged Cross Sections and Detector Responses in Neutron

PB95-242384

PB95-242400

04,014 PC A04/MF A01

Measuring Long Gage Blocks with the NIST Line Scale Interferometer.

PB95-242400

02,665 PC A03/MF A01

Testability of Object-Oriented Systems.

PB95-242418

PB95-251617

$01,733 \mathrm{PC} \mathrm{A05/MF} \mathrm{A01}$

Water-Vapor Measurements of Low-Slope Roofing Materials.

PB95-251617

00,399 PC A03/MF A01

PB95-251625

SF6 Insulation: Possible Greenhouse Problems and Solu-

tions.

PB95-251625

$02,269 \mathrm{PC} \mathrm{A03/MF} \mathrm{A01}$

Submarine Automation: Demonstration No 5

PB95-251633

PB95-251641

03,748 PC A03/MF A01

Study of Potential Applications of Automation and Robotics Technology in Construction, Maintenance and Operation of Highway Systems: A Final Report. Volume 4.

PB95-251641

01,340 PC A06/MF A02

PB95-251658

Algorithm Testing and Evaluation Program for Coordinate Measuring Systems: Testing Methods.
PB95-251658 02,666 PC A03/MF A01

User's Guide for the Algorithm Testing System Version 2.0 PB95-251666

PB95-251674

02,916 PC A03/MF A01

Center for High Integrity Software System Assurance: Initial Goals and Activities

PB95-251674

01.734 PC A03/MF A01

PB95-251682

Study of Potential Applications of Automation and Robotics Technology in Construction, Maintenance and Operation of Highway Systems: A Final Report. Volume 1

PB95-251682

01,341 PC A06/MF A02
PB95-251690

Study of Potential Applications of Automation and Robotics Technology in Construction Maintenance and Operation of Highway Systems: A Final Report Volume 3 .

Highwal A Final Rort

01342 PC A15/MF A03

Information Technologies Make Business Sense for the Custom Therapeutic Footwear Industry. $P C$ A03/MF A0

2,829 PC A03/MF A01

Gage Block Handbook.

PB95-251716

02,667 PC A08/MF A02

PB95-251724

Method and Evaluation of Character Stroke Preservation on Handprint Recognition

$01,850 \quad \mathrm{PC} \mathrm{A03/MF} \mathrm{A01}$

PB95-251732

Standard Reference Material 1744: Aluminum FreezingPBint Standard.

PB95-253563

01,055 PC A03/MF A01

Product Models and Virtual Prototypes in Mechanical Engineering.

PB95-253563

$02,783 \mathrm{PC} \mathrm{A03/MF} \mathrm{AO}$

PB95-253571

Advanced Mass Calibration and Measurement Assurance Program for State Calibration Laboratories.

PB95-253571

02,492 PC A03/MF A01

PB95-253589

Determination of the Residual Stresses Near the Ends of Skip Welds Using Neutron Diffraction and $\mathrm{X}$-ray Diffraction Procedures.

PB95-253589

PB95-253597

$02,868 \mathrm{PC} \mathrm{A03/MF} \mathrm{AO}$

Least-Cost Energy Decisions for Buildings. Part 3. Choosing Economic Evaluation Methods. Video Training Work

PB95-253597

$00,265 \mathrm{PC} \mathrm{A04/MF} \mathrm{A01}$

PB95-253605

International Green Building Conference and Exposition (2nd). Held in Big Sky, Montana on August 13-15, 1995.

PB95-253605

$02,525 \mathrm{PC} \mathrm{A04/MF} \mathrm{A0}$

Materials Science and Engineering Laboratory Annual Report, December 1993.

PB95-255204

$02,668 \mathrm{PC} \mathrm{A03/MF} \mathrm{AO}$

Proceedings of the Applied Diamond Conference 1995: Ap plications of Diamond Films and Related Materials International Conference (3rd). Held in Gaithersburg, Maryland on August 21-24, 1995.

PB95-25520

04,701 PC A99/MF E1

PB95-255824

Simple Method of Composition Shifting with a Distillation Column for a Heat Pump Employing a Zeotropic Refrigerant Mixture.

PB95-255824

02.603 PC A03/MF A01

PB95-255832

Integrated Vision Touch-Probe System for Dimensional In spection Tasks.
PB95-255832

PB95-255840

$02,917 \mathrm{PC} \mathrm{A03/MF} \mathrm{AO}$

NIST SRM 9983 Hıgh-Rıgidity Ball-Bar Stand. User Manual.

PB95-255840

02.669 PC A03/MF A01

PB95-255865

Study of Potential Applications of Automation and Robotics Technology in Construction Maintenance and Operation of Highway Systems: A Final Report Volume

PB95-255865

01.343 PC A19/MF A04

PB95-255881

Proceedings of the Meeting of the Intergovernmental U.S. Russian Business Development Committee's Standard Working Group (4th). Held in New York City, New York on March 27-29, 1995 and in Northbrook, Illinois on March 30 31,1995

PBं95-25588

PB95-255899

$00.496 \mathrm{PC} \mathrm{A11/MF} \mathrm{A03}$

New Mass Transport Elements and Components for the NIST IAQ Mod

PB95-2

02,558 PC A05/MF A01

PB95-255923

Reproducibility of the Temperature of the Ice Point in Routine Measurements.

PB95-255923

04,015 PC A03/MF A01

PB95-256053

Applications of Diamond Films and Related Materials: International Conference (3rd). Held in Gaithersburg. Maryland on August 21-24, 1995. Supplement to NIST Special Publication 885 .

PB95-256053

PB95-256335

03,063 PC A04/MF AO

Sliding Vane Flow Conditioner Tests in a 100 Diameter Long 10 inch Natural Gas Orifice Meter at Pacific Gas and
Electric. Topical Report, 1990-1992. PB95-256335

02.493 PC A04/MF A01

PB95-260618

Thermochemical and Chemical Kinetic Data for Fluorinated Hydrocarbons
PB95-260618

01,056 PC A09/MF A02

\section{PB95-260725}

Strengthening Methodology for Lightly Reinforced Concrete Frames: Recommended Design Guidelines for Strengthening with Infill Walls.

PB95-260758

00,454 PC A04/MF A01

$45 \mathrm{deg} / 0$ deg Retlectance Factors of Pressed Polytetrafluorothylene (PTFE) Powder.

B95-260758

$04,328 \mathrm{PC} \mathrm{A06/MF} \mathrm{A02}$

Semiconductor Measurement Technology: HOTPAC. Programs for Thermal Analysis Including Version 3.0 of the TXYZ Program, TXYZ30, and the Thermal MultiLayer Program, TML.

02,374 PC A05/MF A01

PB95-260808

NIST Standard Reference Data Products Catalog, 1995-96. Achieve with Standard Reference Data.

PB95-260808

395-260816

Long-Term Performance of Engineered Concrete Barriers.

PB95-260816

03,727 PC A03/MF A01

PB95-261897

Journal of Research of the National Institute of Standards and Technology, May/June 1995. Volume 100, Number 3.

PB95-261897

$02,670 \mathrm{PC}$ A07/MF A02

Determining the Magnetic Properties of $1 \mathrm{~kg}$ Mass Stand-

PB95-261905

O4,016
(Order as PB95-261897, PC A07/MF A02)

PB95-261913

Intercomparison of Photometric Units Maintained at NIST (USA) and PTB (Germany), 1993.

(Order as PB95-261897, PC A07/MF A029)

\section{PB95-261921}

Determination of the Transmittance Uniformity of Optical Filter Standard Reference Materials.

PB95-261921

(Order as PB95-261897, PC A07/MF A02)

PB95-261939

Low-Frequency Model for Radio-Frequency Absorbers.

PB95-261939 04,424

PB95-261947

(Order as PB95-261897, PC A07/MF A02)

Using Ouantized Breakdown Voltage Signals to Determine the Maximum Electric Fields in a Ouantum Hall Effect Sample

-261947

(Order as PB95-261897, PC A07/MF 02,375

PB95-261954

High Accuracy Measurement of Aperture Area Relative to a Standard Known Aperture.

PB95-261954

(Order as PB95-261897, PC A07/MF A02)

PB95-261962

New IUPAC Guidelines for the Reporting of Stable Hydro-

gen, Carbon, and Oxygen Isotope-Ratio Data. Letter to the 


\section{PB95-267985}

National Voluntary Laboratory Accreditation Program: Thermal Insulation Materials.

PB95-267985

02,977 PC A05/MF AO

PB95-267993

National Voluntary Laboratory Accreditation Program. GOSIP: Government Open Systems Interconnection Profile.

PB95-267993

$01,486 \mathrm{PC}$ A06/MF A02

National Construction Sector Goals: Industry Strategies for Implementation

PB95-270039

$00,204 \mathrm{PC} \mathrm{A03/MF} \mathrm{A0}$

Ceramic Powders Characterization: Results of an International Laboratory Study.

PB95-270039

02,672 PC A22/MF A04

\section{PB95-270047}

Project Summaries 1995: NIST Building and Fire Research Laboratory.

PB95-270062

$00,400 \mathrm{PC} \mathrm{A10/MF} \mathrm{A03}$

Proceedings of the 1995 Workshop on Fire Detector Research. Held on February 6-7, 1995

PB95-27006

$02,611 \mathrm{PC}$ A03/MF A01

PB95-501953

Building Life Cycle Cost Computer Program (BLCC), Version 4.2-95 (for Microcomputers).

PB95-501953

00,266 CP D02

PB95-502563

Codes for Named Populated Places, Primary County Divi sions, and Other Locational Entities of the United States (FIPS PUB 55-3) (on Magnetic Tape)

PB95-502563

00,129 CP T05

PB95-502779

Building Life Cycle Cost Computer Program (BLCC) Version 4.21-95 (for Microcomputers)

PB95.50277

00,267 CP D02

FIPS PUB 8-6, Metropolitan Areas (for Microcomputers)

PB95.503280, 04,874 Diskette $\$ 59.00$

PB95-503397

Building Life Cycle Cost Computer Program (BLCC) Version 4.22-95 (for Microcomputers)

00,268 CP D02

PB95-503504 Countries, Dependencies, Areas of Special Sovereignty,
and Their Principal Administrative Divisions (for MicroComputers).

PB95-937300

00,130 Diskette $\$ 30.00$

Validated Products List (Cobol, Fortran, ADA, Pascal, MUMPS, SOL)

PB95-937300
PB95-962000

CALS-Automated Interchange of Technical Information. PB95-962000

01,738 Standing Orde (1)

03,666 Standing Order

CALS-Digital Representation for Communication of Producl Data: IGES Application Subsets.

PB95-96210

03,667 Standing Order

PB95-962200

CALS-Markup Requirements and Generic Style Specifications for Electronic Printed Output and Exchange of Text.

03,668 Standing Order

PB95-962300

CALS-Raster Graphics Representation Binary Format Requirements.
PB95-962300

PB95-962400

03,669 Standing Orde

CALS-Digital Representation for Communication of Illustration Date: CGM Application Profile.

03,670 Standing Order B95-962500

CALS-Department of Defense Computer Aided Acquisition Logistic Support (CALS).

PB95-962600

03,671 Standing Orde

CALS-Contractor Integrated Technical Information Service (CITIS), Functional Requirements.

03,672 Standing Order

PB96-101050

Degradation of Powder Epoxy Coated Panels Immersed in a Saturated Calcium Hydroxide Solution Containing Sodium Chloride.

PB96-101050

PB96-102017

$01,344 \mathrm{PC} \mathrm{A03/MF} \mathrm{A01}$

Exact Recursion Relation Solution for the Steady-State Surface Temperature of a General Multilayer Structure.

PB96-102017 02,376 Not available NTIS PB96-102025

Single Photon Laser Ionization as an In-situ Diagnostic for MBE growth.

PB96-102033

01,059 Not available NTIS

Far-Ultraviolet Flare on a Pleiades G Dwarf

00,086 Not available NTIS PB96-102041

Ab initio Calculations for Helium: A Standard for Transport Property Measurements.

01,060 Not available NTIS
PB96-102058

Merged-Beams Energy-Loss Technique for Electron-Ion Excitation: Absolute Total Cross Sections for $O(5+)(2 s$ yields $2 p)$.

PB96-10205

04,017 Not available NTIS

PB96-102066

Step-Edge and Stacked-Heterostructure High-Tc Josephson Junctions for Voltage-Standard Arrays. PB96-102066

Faster Monte Carlo Simulations.

PB96-102074

PB96-102082

Resonances in Two-Dimensional Array Oscillator Circuits.

PB96-102082

PB96-102090

Vapour Pressures and Gas-Phase ( $p$, rho $n, T)$ Values for CF3CHF2(R125)

PB96-102108

04,019 Not available NTIS

Fire Codes for Global Practice.

PB96-102108

PB96-102116

How to Evaluate Alternative Designs Based on Fire Model-

PB96-102116

00.206 Not available NTIS

PB96-102124

Energy Dependences of Absorption in Beryllium Windows and Argon Gas.

PB96-102132

04,020 Not available NTIS

Simultaneous Optical Measurement of Soot Volume Fraction. Temperature, and $\mathrm{CO} 2$ in Heptane Pool Fire.

PB96-102132

PB96-102140

01,397 Not available NTIS

Remineralization of Root Lesions with Concentrated Calcium and Phosphate Solutions.

PB96-102140

03,567 Not available NTIS

PB96-102157

Application of Single Electron Tunneling: Precision Capacitance Ratio Measurements.

PB96-102157

PB96-102165

Frozen Orbits for Satellites Close to an Earth-Like Planet. PB96-102165

PB96-102173

04,839 Not available NTIS

Generation Rate and Distribution of Products of Combustion in Two-Layer Fire Environments: A Model and Applications.
PB96-102173 398 Not available NTIS PB96-10

\section{PB96-102181}

Some Factors Affecting the Design of a Calorimeter Hood and Exhaust.

PB96-102199

00,302 Not available NTIS

Transmission Properties of Short Curved Neutron Guides. Part 1. Acceptance Diagram Analysis and Calculations.

PB96-102199

PB96-102207

Radiance Temperatures (in the Wavelength Range 523-907 $\mathrm{nm}$ ) of Group IV B Transition Metals Titanium, Zirconium, and Hafnium at Their Melting Points by a Pulse-Heating Technique.

PB96-102215

03,356 Not available NTIS

Sub-Doppler Frequency Measurements on OCS at $87 \mathrm{THz}$ ( $3.4 \mathrm{mu} \mathrm{m}$ ) with the CO Overtone Laser.

PB96-102215 04,330 Not available NTIS

PB96-102223

Direct Observation of Vortex Dynamics in Two-Dimensional Josephson-Junction Arrays

02,067 Not available NTIS

B96-102231

Self-Avoiding-Walk Contacts and Random-Walk Selt-Intersections in Variable Dimensionality.

PB96-102231

PB96-102249

01,276 Not available NTIS

Search for Radio Emission from the 'Non-Magnetic' Chemically Peculiar Stars.

PB96-102249

00,087 Not available NTIS

$X$-ray Emission from Chemically Peculiar Stars. PB96-102256

00,088 Not available NTIS

PB96-102264

Physical Basis for Half-Integral Shapiro Steps in a DC SOUID

PB96-10226

04,704 Not available NTIS

PB96-102272

Stacked Series Arrays of High-Tc Trilayer Josephson Junctions. 102272

04,705 Not available NTIS

PB96-102280

Isotope Dilution Mass Spectrometry as a Candidate Definitive Method for Determining Total Glycerides and Triglycerides in Serum. PB96-10228

03,519 Not available NTIS

PB96-102298

High-Resolution Infrared Overtone Spectroscopy of N2-HF Vibrational Red Shifts and Predissociation Rate as a Function of HF Stretching Ouanta.

PB96-102298

PB96-102306

Computing Radiative Heat Transfer Occurring in a Zone Fire Model.

PB96-102306

PB96-102314

01,399 Not available NTIS

Analyzing and Exploiting Numerical Characteristics of Zone PB96-102314

PB96-102322

01,400 Not available NTIS

Rotational Modulation and Flares on RS Canum Venaticorum and BY Draconis Stars. XVIII. Coordinated VLA, ROSAT, and IUE Observations of RS CVn Binaries. PB96-102322 00,089 Not available NTIS

PB96-102330 Fluctuations in Probability Distribution on Chaotic Attractors.
PB96-102330 04,022 Not available NTIS

PB96-102348

Kinetics and Dynamics of Vibrationally State Resolved lonMolecule Reactions: (14)N2(t)(v=1 and 2) and $(15) \mathrm{N} 2(+)(\mathrm{v}=0,1$ and 2$)$ with $(14) \mathrm{N} 2$. PB96-10234

04,023 Not available NTIS

PB96-102355

Non-Perturbative Relation between the Mutual Diffusion Coefficient, Suspension Viscosity, and Osmotic Compress bility: Application to Concentrated Protein Solutions.

PB96- 102355

01,062 Not available NTIS

PB96-102363

MEMS in Standard CMOS VLSI Technology.

PB96-102363

02,377 Not available NTIS

PB96-102371

Critical Lines for Type-III Aqueous Mixtures by Generalized Corresponding-States Models.

PB96-10237

01,063 Not available NTIS

Ferroelectric Thin Film Characterization Using Superconducting Microstrip Resonators.
PB96-102389

PB96-102397

Geometrical Percolation Threshold of Overlapping

Ellipsoids.

PB96-102397

03,167 Not available NTIS

PB96-102405

Reduction of Marginal Gaps in Composite Restorations by Use of Glass-Ceramic Inserts.

PB96-102405

00,174 Not available NTIS

PB96-102413

Thermodynamic Properties of Two Gaseous Halogenated Ethers from Speed-of-Sound Measurements: Difluoromethoxy-Difluoromethane and 2-Difluoromethoxy1.1,1-Trifluoroethane.

PB96-102413

04,189 Not available NTIS

PB96-102421

Lattice Dynamics of $\mathrm{Ba1}$-xKxBiO3.

PB96-10242

PB96-102439 $(\mathrm{Ba}, \mathrm{Sr}) \mathrm{n}+1(\mathrm{Sn}, \mathrm{Sb}) \mathrm{nO} \mathrm{O} n+1$

PB96-102439

04,706 Not available NTIS

96-102447

04,707 Not available NTIS

Lattice Dynamics of Semiconducting, Metallic, and 
PB96-102546

Simulation of $\mathrm{C} 60$ Through the Plastic Transition Tempera-

PB96-102546

04,713 Not available NTIS

PB96-102553

Characterization of a Clipped Gaussian Beam.

PB96-102553

04,331 Not available NTIS

PB96-102561

Standard Antennas for Electromagnetic Interference Meas-

urements and Methods to Calibrate Them.

PB96-102561

02,007 Not available NTIS

PB96-102579

Measurements of Thermophysical Properties of Nickel Near Its Melting Temperature by a Microsecond-Resolution Transient Technique.

PB96-102579
PB96-102587

04,210 Not available NTIS

Phase Locking in Two-Dimensional Arrays of Josephson

Junctions: Effect of Critical-Current Nonuniformity.
PB96-102587 04,714 Not available NTIS

PB96-102595

Tensile Deformation-Induced Microstructures in Free-Standing Copper Thin Films.

PB96-102595

04,715 Not available NTIS

PB96-102603

Magnetostriction and Giant Magnetoresistance in Annealed NiFe/Ag Multilayers.

PB96-102603

04,716 Not available NTIS

PB96-102611

Measurement and Interpretation of Tidal Tilts in a Smal Array.

PB96-10261

PB96-102629

100 Ampere, $100 \mathrm{kHZ}$ Transconductance Amplifier.

PB96-102629

03,686 Not available NTIS

PB96-102629

02068 Not available NTIS

Bonding Wires to Quantized Hall Resistors.

PB96-102637 01,921 Not available NTIS

PB96-102645

Intercomparison Study of (237)Np Determination in Artificial Urine Samples

PB96-102652

03,633 Not available NTIS

Measurement Methods and Algorithms for Comparison of Local and Remote Clocks.

PB96-102652

01,549 Not available NTIS

PB96-102660

Measurement of Very Low Frequency Vibrations.

PB96-102660 03,687 Not available NTIS

PB96-102678

Ouch Those Programs are Painful.

PB96-102678

01,739 Not available NTIS

PB96-102686

Conformance Testing for OSI Protocols.

PB96-102686

01,631 Not available NTIS

PB96-102694

High-Velocity Plasma in the Transition Region of $\mathrm{AU}$ Microscopii: Evidence for Magnetic Reconnection and Saturated Heating during Quiescent and Flaring Conditions.

rated Heating during Quiescent and Flaring Conditions.
PB96-102694 00,091 Not available NTIS

PB96-102702

Model for Determining the Density and Mobility of Carriers in Thin Semiconducting Layers with Only Two Contacts.

PB96-102702 02,378 Not available NTIS

PB96-102710

MONSEL-11: Monte Carlo Simulation of SEM Signals for Linewidth Metrology.

PB96-1027

02,379 Not available NTIS

PB96-102728

Bacteriorhodopsin Retains Its Light-Induced Proton-Pumping Function After Being Heated to $140 \mathrm{C}$.

PB96-102728

PB96-102736

03,471 Not available NTIS

Delivering the Same Optical Frequency at Two Places: Accurate Cancellation of Phase Noise Introduced by an Optical Fiber or Other Time-Varying Path

PB96-102736

04,332 Not available NTIS

PB96-102744

Analytical Method for Determining Thermal Conductivity from Dynamic Experiments.

PB96-10274

04,024 Not available NTIS

Evolution of $X$-ray Resonance Raman Scattering into $X$-ray Fluorescence from the Excitation of Xenon Near the L. Edge.

PB96-10275

04,025 Not available NTIS

PB96-102769

NBS/NIST Peltier-Effect Microcalorimeter: A Four-Decade

Review.

01,064 Not available NTIS

PB96-102777

Observing Stellar Coronae with the Goddard High Resolution Spectrograph. I. The dMe Star AU Microscopii.

PB96-102777

00,092 Not available NTIS

PB96-102785

Comments on 'Conversions between S, Z, Y, h, ABCD, and Parameters Which Are Valid for Complex Source and Load Impedances'.

PB96-102785

02,069 Not available NTIS
PB96-102793

Scaling of the Nonlinear Optical Cross Sections of GaAsAlGaAs Multiple Quantum-Well Hetero n-i-p-i's.

PB96-102793 02,183 Not available NTIS

PB96-10280

Length Scales for Fragile Glass-Forming Liquids.

PB96-10280

01,065 Not available NTIS

PB96-102819

Optically Stabilized Tunable Diode-Laser System for Saturation Spectroscopy.

PB96-102827

04,717 Not available NTIS

Electronic Microrefrigerator Based on a Normal-InsulatorSuperconductor Tunnel Junction.

PB96-102827

PB96-102835

Probing Potential-Energy Surfaces via High-Resolution IR Laser Spectroscopy

PB96-102843

01,066 Not available NTIS

New Method for Realizing a Luminous Flux Scale Using an Integrating Sphere with an External Source.

PB96-102843

04,333 Not available NTIS

PB96-102850

Effect of Electrode Material on Measured Ion Energy Distributions in Radio-Frequency Discharges.

PB96-102850 04,026 Not available NTIS

PB96-102868

Concepts for Fire Protection of Passenger Rail Transportation Vehicles: Past, Present, and Futur

PB96-102868

PB96-102876

Buffer Layer Modulation-Doped Field-Effect-Transistor Interactions in the Al0.33Ga0.67As/GaAs Superlattice System.

PB96-102876

02,380 Not available NTIS

PB96-102884

Total-Dielectric-Function Approach to Electron and Phonon Response in Solids.

PB96-102884

01,067 Not available NTIS

PB96-102892

Principle of Congruence and Its Application to Compressible States.

01,068 Not available NTIS

PB96-102892

B96-102900

Phospholipid/Alkanethiol Bilayers for Cell-Surface Recepto Studies by Surface Plasmon Resonance.

PB96-102900

03,472 Not available NTIS

PB96-102918

Energy Calibration of X-ray Photoelectron Spectrometers: Results of an Interlaboratory Comparison to Evaluate a Proposed Calibration Procedure.

PB96-102918

04,027 Not available NTIS

PB96-102926

TDDB Characterization of Thin SiO2 Films with Bimodal Failure Populations.

PB96-102926

02,381 Not available NTIS

PB96-102934

Santa Ana Fire Department Experiment at 1315 South Bristol, July 14, 1994. (Reprint).

PB96-102934

00,207 Not available NTIS

PB96-102942

Time-Resolved Balmer-Alpha Emission from Fast Hydrogen Atoms in Low Pressure, Radio-Frequency Discharges in Hydrogen.

PB96-102959

04,028 Not available NTIS

Ion Kinetic-Energy Distributions and Balmer-alpha (Halpha) Excitation in $\mathrm{Ar}-\mathrm{H} 2$ Radio-Frequency Discharges.

Excitation in Ar-H2 Radio-Frequency Discharges.
PB96-102959 029 Not available NTIS

PB96-102967

Pressure Equations in Zone-Fire Modeling.

PB96-102967

00,208 Not available NTIS

B96-102975

$X$-ray Powder Diffraction from Carbon Nanotubes and Nanoparticles.

PBos-102983

03,064 Not available NTIS

Damage Processes in Ceramics Resulting from Diamond Tool Indentation and Scratching in Various Environments.

PB96-102983 a 03,065 Not available NTIS

PB96-102991

Intracomparison Tests of the FG5 Absolute Gravity Meters. PB96-102991

03,688 Not available NTIS

PB96-103007

Boundary Conforming Grid Generation System for Interface Tracking.

PB96-103015

03,357 Not available NTIS

Superconducting Integrated Circuit Fabrication with Low Temperature ECR-Based PECVD SiO2 Dielectric Films.

PB96-103015

04,719 Not available NTIS

PB96-103023

Certification of Polychlorinated Biphenyl Congeners and Chlorinated Pesticides in a Whale Blubber Standard Reference Material.

PB96-103023

PB96-103031

03,745 Not available NTIS

Using Synthetic Perturbations and Statistical Screening to Assay Shared-Memory Programs.
PB96-103031

PB96-103049

Time Generation and Distribution

PB96-103049

PB96-103056

Nanoscale Study of the Hydrogenated Amorphous Silicon

Surface.

PB96-103056
PB96-103064

04.720 Not available NTIS

Planar Lenses for Field-Emitter Arrays.

PB96-103064

PB96-103072

Partial Pressure Analysis in Space Testing

PB96-103072

PB96-103080

Comments on the Stability of Bayard-Alpert Ionization

Gages.

PB96-103080

PB96-103098

02.673 Not available NTIS

Comparison of Techniques for Nondestructive Composition

Measurements in CdZnTe Substrates.

PB96-103098

02,703 Not available NTIS

P896-103106

Low-Energy-Electron Collisions with Sodium: Elastic and In-

elastic Scattering from the Ground State.

PB96-103106

04,030 Not available NTIS

Nonstationary Behaviour of Partial Discharge during Dis-

charge Induced Ageing of Dielectrics.

PB96-103114

01,922 Not available NTIS

Aging, Warm-Up Time and Retrace; Important Characteristics of Standard Frequency Generators.

PB96-103122

PB96-103130

Recent Results in Magnetic Force Microscopy.

PB96-103130

04,721 Not available NTIS

PB96-103148

Environmental Sensitivities of Quartz Oscillators.

PB96-103148

PB96-103155

Digital Impedance Bridge

02,271 Not available NTIS

PB96-103163

Wear Transitions in Monolithic Alumina and Zirconia-Alumina Composites.

PB96-103163

PB96-103171

03,168 Not available NTIS

Collisional Alignment of $\mathrm{CO} 2$ Rotational Angular Momentum

States in a Supersonic Expansion.

PB96-103171

PB96-103189

01,069 Not available NTIS

Volume-Limited ROSAT Survey of Extreme Ultraviolet

Emission from all Nondegenerate Stars within 10 Parsecs.
PB96-103189 00,093 Not available NTIS

PB96-103197

Water Adsorption at a Polyimide/Silicon Wafer Interface.

PB96-103197

01,070 Not available NTIS 
PB96-109509

Composite Materials for Offshore Operations: Proceedings of the International Workshop (1st). Held in Houston, Texas on October $26-28,1993$.

PB96-109509

$03,169 \mathrm{PC} \mathrm{A17/MF} \mathrm{AO4}$

PB96-109525

Agile Manufacturing from a Statistical Perspective.

PB96-109525

PB96-109533

Mapping Integration Definition for Function Modeling (IDEFO) Model into CASE Data Interchange Format (CDIF) Transfer File.

PB96-109541

$01,741 \mathrm{PC} \mathrm{A07/MF} \mathrm{A02}$

CFAST Output Comparison Method and Its Use in Comparing Different CFAST Versions.

PB96-109541

$00,401 \mathrm{PC} \mathrm{A04/MF} \mathrm{A01}$

PB96-109558

Factors Significant to Precracking of Fracture Specimens.

of Fracture Specimens.
03,358 PC A06/MF A02

PB96-109566

EMISS: A Program for Estimating Local Air Pollution Emission Factors Related to Energy Use in Buildings: User's Guide and Reference Manual.

PB96-109566

$02,560 \mathrm{PC}$ AO3/MF A01

PB96-109574

Effect of Suppressants on Metal Fires.
PB96-109574 01,402 PC A03/MF A01

PB96-109582

Error-Bounding in Level-Index Computer Arithmetic.

PB96-109582

PB96-110739

Intensity-Dependent Scattering Rings in High Order AboveThreshold lonization

PB96-110747

04,032 Not available NTIS

Novel Activity of E. coli uracil DNA N-glycosylase Excision of Isodialuric Acid (5,6-dihydroxyuracil), a Major Product of Oxidative DNA Damage, from DNA.

PB96-110747

03,543 Not available NTIS

PB96-111166

Spherical-Wave Source-Scattering Matrix Analysis of Antennas and Antenna-Antenna Interactions. 02,008 PC A08/MF A02 PB96-11116

Efficiency of Electric Motors. National Voluntary Lab. ACcreditation Program (NVLAP)

PB96-111174

02,107 PC A05/MF A01

\section{PB96-111190}

Federal Implementation Guideline for Electronic Data Interchange: ASC X12 003040 Transaction Set 838 Trading Partner Profile (Confirmation of Vendor Registration). Implementation Convention.

PB96-111190

$01,813 \mathrm{PC} \mathrm{A03/MF} \mathrm{A01}$

PB96-111612

Monte Carlo and Analytic Methods in the Transport of Electrons, Neutrons, and Alpha Particles.

PBO6-111612 and Alpha Particles. 04,033 Not available NTIS PB96-111620

Comparison of a Fixed-Charge and a Polarizable Wate Model.

PB96-111620

PB96-111638

Improved Wavelengths for Prominent Lines of $\mathrm{Fe} X X$ to $\mathrm{Fe}$ XXIII.

PB96-111638

PB96-111646

04,334 Not available NTIS

Photodesorption

Surface Defects.

PB96-111653

Overview of Reterence Materials Prepared for Standardization of DNA Typing Procedures.

PB96-111653

PB96-111661

Nonequilibrium Thermodynamic Theory of Viscoplastic Materials.

PB96-111679

Stable Phase Locking in a Two-Cell Ladder Array of Josephson Junctions.

PB96-111679

04,722 Not available NTIS

B96-111687

Human and Machine Recognition of Faces: A Survey.

PB96-111687

01,854 Not available NTIS

PB96-111695

Fabrication of Optics by Diamond Turning

PB96-111695

02,954 Not available NTIS

PB96-111703

Silicon Nitride Boundary Lubrication: Lubrication Mechanism of Alcohols.

PB96-111703

PB96-111711

Siticon Nitride Boundary Lubrication: Effect of Oxygenates.

PB96-111711

03,067 Not available NTIS

PB96-111729

Niobium Microbolometers for Far-Infrared Detection.

PB96-111729
PB96-111737

Quantitative Analysis of Selected PCB Congeners in Marine Matrix Reference Materials Using a Novel Cyanobiphenyl Stationary Phase.

PB96-111737

PB95-111745

02,591 Not available NTIS

Temperature Dependence and Magnetic Field Modulation of Critical Currents in Step-Edge SNS YBCO/Au Junctions.

PB96-111745 04,723 Not available NTIS

PB96-111752

Critical Current and Normal Resistance of High-Tc StepEdge SNS Junctions.

PBg6-111

04,724 Not available NTIS

Surface Chemistry of Silicon Nitride Powder in the Presence of Dissolved lons.

PB96-111760

01,073 Not available NTIS

(111778

Certification of Polycyclic Aromatic Hydrocarbons in a Marine Sediment Standard Reference Material.

PB96-111778

02,592 Not available NTIS

PB96-111786

Contributions of Out-of-Plane Material to a Scanned-Beam Laminography Image.

PBg6-11

02,704 Not available NTIS

PB96-111794

Crystal Structure of a New Monoclinic Form of Potassium Dihydrogen Phosphate Containing Orthophosphacidium Ion, (H4PO4)(sup+1).

PB96-111802

Taguchi's Parameter Design: A Panel Discussion.

PB96-111802

03,445 Not available NTIS

PB96-111810

Wear Mechanism Maps of $440 \mathrm{C}$ Martensitic Stainless Steel.

PB96-111810

04,834 Not available NTIS

PB96-111828

Vibrational Excitations and the Position of Hydrogen in Nanocrystalline Palladium.

PB96-111828

04,035 Not available NTIS

PB96-111836

Magnetic Structure and Spin Dynamics of the Pr and $\mathrm{Cu}$ in Pr2CuO4.

PB96-111836

04,036 Not available NTIS

PB96-111844

SOA Standards and Total Quality Management.

PB96-111844

01,743 Not available NTIS

PB96-111851

Relative Accuracy of Isolated and Unisolated Noise Comparison Radiometers.

PB96-111851

PB96-111869

01,924 Not available NTIS

Measurements of Permittivity and the Dielectric Loss Tangent of Low Loss Dielectric Materials with a Dielectric Resonator Operating on the Higher Order Te(sub 0 gamma delta) Modes

PB96-111869

PB96-111877

02,273 Not available NTIS

Measuring Hydrogen by Cold-Neutron Prompt-Gamma Activation Analysis.

PB96-111885

Electron-Ion Collisions in the Plasma Edge.

PB96-111885

00,612 Not available NTIS

B96-111893

Crystal Structure of Calcium Glutarate Monohydrate.

PB96-111893

01,074 Not available NTIS

PB96-111901

Use of Building Emulators to Evaluate the Performance of Building Energy Management Systems.

PB96-111901

00,269 Not available NTIS

PB96-111919

Small Angle Neutron Scattering Study of the Structure and Formation of Ordered Mesopores in Silica.

PB96-111919 03,069 Not available NTIS

PB96-111927

Kinetic Energy Distribution of Ions Produced from Townsend Discharges in Neon and Argon.

PB96-111927

04,413 Not available NTIS

PB96-111935

Can Displays Deliver a Full Measure: Manufacturing

PB96-111935

02,185 Not available NTIS

PB96-111943

Noise Modeling and Reliability of Behavior Prediction for Multi-Stable Hydroelastic Systems.

04,822 Not available NTIS

PB96-111950

Glasses for Waveguide Lasers.

PB96-111950

04,335 Not available NTIS

PB96-111968

Stability of Compressed Gas Mixtures Containing Low Level Volatile Organic Compounds in Aluminum Cylinders.

PB96-111968

Alu 13 Not available NTIS

PB96-111976

Detection of Aromatic Compounds Based on DNA PB96-111976

3,473 Not available NTIS

PB96-111984

Uncertainties of the NIST Coaxial Noise Calibration System. PB96-111984

PB96-111992

Comparison of Photodiode Frequency Response Measurements to $40 \mathrm{GHz}$ between NPL and NIST.

PB96-111992

04,038 Not available NT1S

PB96-112008

Production Management information Model for Discrete Manufacturing

PB96-112016

02,830 Not available NTIS

GHAS Observations of Cool, Low-Gravity Stars. 1. The Far-

Ultraviolet Spectrum of alpha Orions (M2 lab).

PB96-112016

PB96-112024

Temperature and Field Dependence of Flux Pinning in NbTi with Aritificial Pinning Centers.

B96-112024

04,726 Not available NTIS

PB96-112032

Distribution of Dielectric Relaxation Times and the Momen

Problem.

PB96-112040

04,727 Not available NTIS

Physicochemical Characterization of Low Molecular Weight

Heparin

PB96-112040

03,474 Not available NTIS

PB96-112057

Shear-Induced Melting of Two-Dimensional Solids.

PB96-112057

PB96-112065

01,075 Not available NTIS

SUSAN: SUperconducting Systems ANalysis by Low Temperature Scanning Electron Microscopy (LTSEM).

04,728 Not available NTIS

PB96-112073

Quantum-Limited Cooling and Detection of Radio-Frequency Oscillations by Laser-Cooled Ions.

PB96-112073

04,039 Not available NTIS

PB96-112081

Rapid $\mathrm{pH}$ Change Due to Bacteriorhodopsin Measured with a Tin-Oxide Electrode.

PB96-11208

03,544 Not available NTIS

PB96-112099

Spectral Interference in the Determination of Arsenic in Head and Lead-Base Alloys Using Zeeman-Effect Background Correction. Zeeman-Etfect PB96-112107

Applications of the Vortex Tube in Chemical Analysis. Part 2. Applications.

PB96-1 12115

00,615 Not available NTIS

Treatment of Wistar Rats with a Renal Carcinogen, Ferric Nitrilotriacetate, Causes DNA-Protein Cross-Linking between Thymine and Tyrosine in Their Renal Chromatin.

PB96-112115

PB96-112123

Interlaboratory Comparison of Autoradiographic DNA Profiling Measure

Its Propagation

PBg6-112

03,545 Not available NTIS 
PB96-112230

Local Oscillator Requirements and Strategies for the Next Generation of High-Stability Frequency Standards.
PB96-112230 PB96-112248

New Model of 1/F Noise in Baw Quartz Resonators. PB96-112248 02,383 Not available NTIS PB96-112255

Structure of a Triglyceride Microemulsion: A Small Angle Neutron Scattering Study.

PB96-112255

01,077 Not available NTIS

PB96-112263

Binary versus Decade Inductive Voltage Divider CompariSB96-112263 Decomposition. 02,071 Not available NTIS

PB96-112271

Cryogenic Precision Capacitance Bridge Using a Single

Electron Tunneling Electrometer. 02,072 Not available NTIS
PB96-112271 PB96-11227

PB96-112289

Dual-Frequency Millimeter-Wave Radiometer Antenna for Airbome Remote Sensing of Atmosphere and Ocean.

PB96-112289 02,009 Not available NTIS

PB96-112297

Substrate and Thin Film Measurements.

PB96-112297 02,384 Not available NTIS

PB96-112305

Small Angle Neutron Scattering Studies of Structural Characteristics of Argarose Gels. 03,475 Not available NTIS PB96-11230

PB96-112313

Surging the Upside-Down House: Looking into Upsetting Reference Voltages.

PB96-112321

02,385 Not available NTIS

Measured Stopping Powers of Hydrogen and Helium in Pol-

ystyrene Near Their Maximum Values.
PB96-112321

PB96-112339

Torsion Modulus and Internal Friction of a Fiber-Reinforced Composite.

03,070 Not available NTIS

PB96-112347

Analytical Applications of Guided Neutron Beams.

PB96-112347

04,041 Not available NTIS

PB96-112354

Application of a Novel Slurry Fumace AAS Protocol for Rapid Assessment of Lead Environmental Contamination.

PB96-112354 02,526 Not available NTIS

PB96-112362

Vortex Dynamics and Melting in Niobium

PB96-112362

02,073 Not available NTIS

PB96-112370

Considerations in the Design of an Environmental Specimen Bank: Experiences of the National Biomonitoring Specimen Bank Program.

PB96.112370

02,527 Not available NTIS

PB96-112651

Federal Implementation Guideline for Electronic Data Interchange: ASC $X_{12} 003040$ Transaction Set 838 Trading Partner Profile (Vendor Registration), Implementation Convention.

PB96-11265

03,674 PC A04/MF A01

PB96-112677

Glimpse of Materials Research in China: A Report from an Interagency Study Team on Materials Visiting China from June 19, 1995 to June 30,1995

PB96-112677

02,978 PC A11/MF A03

PB96-112685

Requisite Elements, Rationale, and Technology Overview for the Systems Intergration for Manufacturing Applications (SIMA) Program. Background Study.

B96-112693

Proceedings of the Annual Manufacturing Technology Conference (2nd): Toward a Common Agenda. Held in Gaithersburg, Maryland on April 18-20, 1995.

PB96-112693

02,887 PC A99/MF E08

PB96-113311

Joumal of Research of the National Institute of Standards and Technology, July/August 1995. Volume 100, Number 4 Special Issue: The Gaseous Electronics Conference RadioFrequency Reference Cell.

PB96-113311

$02,386 \mathrm{PC} \mathrm{A09/MF} \mathrm{A03}$

PB96-113329

Gaseous Electronics Conference RF Reference Cell: An Introduction.

PB96-113329

02,387
(Order as PB96-113311, PC A09/MF A03)

PB96-113337

Current and Voltage Measurements in the Gaseous Electronics Conference RF Reference Cell.

PB96-113337

02,388
(Order as PB96-113311, PC A09/MF A03)

PB96-113345

Optical Emission Spectroscopy on the Gaseous Electronics Conference RF Reference Cel.

02,389
(Order as PB96-113311, PC A09/MF A03)

PB96-113352

Optical Diagnostics in the Gaseous Electronics Conference PB96-113352

(Order as PB96-113311, PC A09/MF,390

PB96-113360

Studies of Ion Kinetic-Energy Distributions in the Gaseous Electronics Conference RF Reference Cell.

PB96-113360

Order as PB96-113311, PC A09/MF A03)

PB96-113378

Microwave Diagnostic Results from the Gaseous Electronics Conference RF Reterence Cell.

PB96-113378

(Order as PB96-113311, PC A09/MF A03)

\section{PB96-113386}

Langmuir Probe Measurements in the Gaseous Electronics Conference RF Reterence Cell.

PB96-113386

(Order as PB96-113311, PC A09/MF A03)

\section{PB96-113394}

Inductively Coupled Plasma Source for the Gaseous Electronics Conference RF Reference Cell.

PB96-113394

(Order as PB96-113311, PC AO9MF A03)

\section{PB96-113402}

Reactive Ion Etching in the Gaseous Electronics Conference RF Reference Cell.

PB96-113402

(Order as PB96-113311, PC A09/MF A03)

PB96-113410

Dusty Plasma Studies in the Gaseous Electronics Conference Reference Cell.

PB96-113410

(Order as PB96-113311, PC A09/MF A03)

\section{PB96-113428}

One-Dimensional Modeling Siudies of the Gaseous Electronics Conference RF Reference Cel.

PB96-113428

(Order as PB96-113311, PC A09/MF A03)

\section{PB96-113436}

Two-Dimensional Self-Consistent Radio Frequency Plasma Simulations Relevant to the Gaseous Electronics Conference RF Reference Cell.

PB96-113436

(Order as PB96-113311, PC AOg/MF A03)

PB96-113535

Journal of Research of the National Institute of Standards and Technology. November/December 1994. Volume 99,

Number 6.
PB96-113535

PB96-113543

$04.336 \mathrm{PC} \mathrm{A05/MF} \mathrm{A01}$

Intercomparison between NPL (India) and NIST (USA) Pressure Standards in the Hydraulic Pressure Region Up to $26 \mathrm{MPa}$

PB96-113543

(Order as PB96-113535, PC A05/MF A01)

\section{PB96-113550}

Intercomparison of the ITS-90 Radiance Temperature Scales of the National Physical Laboratory (U.K.) and the National Institute of Standards and Technology. PB96-113550

(Order as PB96-113535, PC A05/MF A01)

\section{PB96-113568}

Screened-Room Measurements on the NIST SphericalDipole Standard Radiator.

PB96-113568

(Order as PB96-113535, PC A05/MF A01)

PB96-113576

Beamcon III, a Linearity Measurement Instrument for Optical Detectors.
PB96-113576

\section{PB96-113584}

(Order as PB96-113535, PC A05/MF A01)

Spectroscopic Study of Quantized Breakdown Voltage States of the Quantum Hall Effect.

04.730

\section{PB96-114764}

Spread on Vertical Corner Walls.

Turbulent Flame Spread on Vertical Corner Walls. PB.66-114913

Federal Implementation Guideline for Electronic Data Inter-
change. ASC X12 003050 Transaction Set 850 Award Inchange. ASC X12 003050 Transaction Set 850 Award Instrument. Implementation Convention.

PB96-114913

$01,814 \mathrm{PC} \mathrm{A11/MF} \mathrm{A03}$

PB96-114921

Federal Implementation Guideline for Electronic Data Interchange. ASC X12 003050 Transaction Set 860 Modifications to Award Instrument. Implementation Convention. tions to Award Instrument. Implementation Convention.
PB96-114921 01,815 PC A11/MF A03 PB96-114939

Z39.50 Implementation Experiences. PB96-114939

$01,816 \mathrm{PC} \mathrm{A07/MF} \mathrm{A02}$

PB96-115019

Standards Setting in the European Union: Standards Organization and Officials in EU Standards Activities.
PB96-115019 02,919 PC A04/MF A01

PB96-115050

Intelligent Processing of Materials, Technical Activities 1994 (NAS-NRC Assessment Panel. April 6-7, 1995

PB96-115050 03,359 PC A04/MF A0 PB96-117221

Analysis by a Combination of Gas Chromatography and Tandem Mass Spectrometry: Development of Ouantitative Tandilu(ion Ouantfication of 11-Nor-Delta-9-Tetrahydro cannabinol9-Carboxylic Acid.

PB96-117221

02.561 Not available NTIS

Measurement of the Weak-Localization Complex Conductivity at $1 \mathrm{Ghz}$ in Disordered $\mathrm{Ag}$ Wires.

PB96-117239

04,731 Not available NTIS

Semiconductor Measurement Technology: Test Structure Implementation Document: DC Parametric Test Structures and Test Methods for Monolithic Microwave Integrated Circuits (MMICs)

PB96-117692
PB96-117767

02,399 PC A05/MF A01

Joumal of Research of the National Institute of Standards and Technology. September/October 1995. Volume 100 Number 5 .

PB96-117767

PB96-117775

$01,927 \mathrm{PC} \mathrm{A08/MF} \mathrm{A02}$

Fire Suppression System Performance of Alternative Agents in Aircraft Engine and Dry Bay Laboratory Simulations. SP890: Volume 1.

PB96-117775

03,277 PC A99/MF E08

PB96-117783 Fire Suppression System Performance of Alternative Agents
in Aircraft Engine and Dry Bay Laboratory Simulations. SP 890: Volume

890: Volume

PB96-117916

$03,278 \mathrm{PC}$ A99/MF A06

Post-Earthquake Fire and Lifelines Workshop. Held in Long Beach, Calitornia on January $30-31,1995$. Proceedings.
PB96-117916 00,209 PC A04/MF A01 PB96-118039

Opportunities for Innovation: Optoelectronics.

PB96-118039

01,928 PC A19/MF A04

PB96-119219

Comparing the Accuracy of Critical-Current Measurements Using the Voltage-Current Simulato

PB96-119219

PB96-119227

Display-Measurement Round-Robin

PB96-119227

02,186 Not available NTIS

PB96-119235

Electrical Product Requirements (Especially Ouality Requirements) in the United States.

PB96-119235

PB96-119243

01,929 Not available NTIS

Evolution Equations for Phase Separation and Ordering in Binary Alloys.
PB96-119243

PB96-119250

02,979 Not available NTIS

Publications and Presentation Abstracts, 1995. (Published by Paffenbarger Research Center and Center of Excellence for Materials Science Research).

PB96-119250

03,568 Not available NTIS

PB96-119268

Gaseous Dielectrics Research: Possible SF6 Substitutes.

PB96-119268

Possible SF6 Substitutes.
02,228 Not available NTIS

PB96-119276

Polarization Insensitive $3 \times 3$ Sagnac Current Sensor Using Polarizing Spun High-Birefringence Fiber.

PB96-119276 02,187 Not available NTIS 
PB96-119367

National Information Infrastructure and Advanced Digital

Video.

01,488 Not available NTIS

PB96-119375

Measurements of the Relative Permittivity of Liquid Water at Frequencies in the Range of 0.1 to $10 \mathrm{kHz}$ and at Temperatures between 273.1 and $373.2 \mathrm{~K}$ at Ambient Pressure.
PB96-119375 PB96-119383

Physical Characterization of Herparin by Light Scattering

PB96-119383 03,598 Not available NTIS

PB96-119391

Visible and UV Light from Highly Charged Ions: Exotic Mat-

ter Advancing Technology.
PB96-119391

04,414 Not available NTIS

PB96-119409

Simple and Repeatable Technique for Measuring the Critical Current of Nb3Sn Wires.

PB96-119417

02,229 Not available NTIS

Nanofabrication of a Two-Dimensional Array Using LaserFocused Atomic Deposition.

PB96-119417

04,732 Not available NTIS

PB96-119425

Electroacoustic Characterization of Particle Size and Zeta Potential in Moderately Concentrated Suspensions.

PB96-119425 01,079 Not available NTIS

PB96-119433

Perception of Clamp Noise in Television Receivers.

PB96-119433 01,489 Not available NTIS

PB96-119441

Effect of Sm2BaCuO5 on the Properties of Sintered (Bulk) YBa2Cu3O6+x

PB96-119458

04,733 Not available NTIS

Comparison of Selectivities for PCBs in Gas Chromatography for a Series of Cyanobiphenyl Stationary Phases. PB96-119458 00,618 Not available NTis PB96-119466

Mechano-Chemical Model: Reaction Temperatures in a Concentrated Contact

PB96-1 19466

PB96-119474

03,227 Not available NTIS

Stars, Atmospheres, Radiative Transfer.

PB96-119474

Transfer. Not available NTIS

PB96-119482

Accurate Measurement of Time.

01,552 Not available NTIS

Alomic Clock

PB96-1 19490

PB96-119508

01,553 Not available NTIS

Shear-Induced Martensitic-Like Transformation in a Block Copolymer Melt

PB96-119516

Oxidation of $\mathrm{SiC}$.
PB96-119516

PB96-119524

Defect Formation Mechanism Causing Increasing Defect Density during Decreasing Implant Dose in Low-Dose Simox

PB96- 119524

PB96-119532

02,402 Not available NTIS

Discussion: Statistical Signal Processing of

$\begin{array}{ll}\text { Quasiperiodicities. } & \\ \text { PB96-119532 } & 00,096 \text { Not available NTIS }\end{array}$

PB96-119540

Relationship between Radiative and Magnetic Fluxes for

Three Active Solar-Type Dwarts.
PB96-119540 00,097 Not available NTIS

PB96-119557

Numerical Evaluation of Special Functions

PB96-119557 03,417 Not available NTIS

PB96-119565

Long-Lived Structures in Fragile Glass-Forming Liquids.

PB96-119565

04,212 Not available NTIS

PB96-119573

Physical Properties of Alternatives to the Fully Halogenated Chlorofluorocarbons.

PB96- 119573

PB96-119581

03,279 Not available NTIS

Doppler-Free Spectroscopy of Large Polyatomic Molecules and van der Waals Complexes.
PB96- 119581

PB96-119599

Using Secondary Ion Mass Spectrometry (SIMS) to Characterize Optical Waveguide Materials.

PB96-119599 04,340 Not available NTIS PB96-119607

Response of Buildings to Ambient Vibration and the Loma Prieta Earthquake: A Comparison.
PB96-119607 00,457 Not available NTIS

PB96-119615

Building a Better Crycooler.

PB96-119615

04,734 Not available NTIS
PB96-119623

Far Infrared Laser Frequencies of $\mathrm{CH} 3 \mathrm{OD}$ and $\mathrm{N} 2 \mathrm{H} 4$ PB96-119623

04,341 Not available NTIS

PB96-119631

Strangeness Flow Difference in Nuclear Collisions at $15 \mathrm{~A}$ and $200 \mathrm{~A} \mathrm{GeV}$.

PB96-11963

PB96-119649

04,042 Not available NTIS

NIST and the Navy: Past, Present and Future.

PB96-119649

03,655 Not available NTIS

PB96-119656

Mesoscopic Conductance Fluctuations in Large Devices.

PB96-119656

04,735 Not available NTIS

PB96-119664

Faraday Effect Sensors for Magnet Field and Electric Cur-

PB96-119664

04,736 Not available NTIS

PB96-119672

Standard Polarization Components: Progress Toward an Optical Retardance Standard. 04,342 Not available NTIS
PB96-119672

PB96-119680

Improved Annealing Technique for Optical Fiber.

PB96-119680 04,343 Not available NTIS

PB96-11

04.343 Not available NTIS

Neutron Techniques in Materials Science and Related Dis-

PB96-119698

02,980 Not available NTIS

PB96-119706

History of NIST's Contributions to Development of Standard Reference Materials and Reference and Definitive Methods for Clinical Chemistry.

PB96-119706

03,503 Not available NTIS

PB96-119714

Tribological Behavior of 440/Diamond-Like-Carbon Film

Couples.

PB96-119722

03,019 Not available NTIS

In vitro Fracture Behavior of Ceramic and Metal-Ceramic Restorations.

PB96-119730

03,569 Not available NTIS

Appearance Potentials of lons Produced by Electron-Impact Induced Dissociative Ionization of SF6, SF4, SF5Cl, S2F 10 $\mathrm{SO} 2$, SO2F2, SOF2, and SOF

PB96-119730

01,080 Not available NTIS

PB96-119748

Structural and Magnetic Properties of $\mathrm{CuCl} 2$ Graphite Intercalation Compounds.

03,020 Not available NTIS

PB96-119755

Procedure for Measuring Trace Quantities of $\mathrm{S}_{2} \mathrm{~F}_{10}$, S2OFto, and $\mathrm{S}_{2} \mathrm{O} 2 \mathrm{~F}_{10}$ in SF6 Using a Gas Chromatograph-Mass Spectrometer.

PB96-119755

02,513 Not available NTIS

PB96-119763

VAMAS Intercomparison of Critical Current Measurements on NB3Sn Superconductors: A Summary Report.

PB96-119763 04,043 Not available NTIS

PB96-119771

Reducing Errors, Complexity, and Measurement Time of PM Noise Measurements.
PB96-119771

PB96-119789

High-Order Multipole Excitation of a Bound Electron.

PB96-119789

04,044 Not available NTIS

PB96-119797

Self-Organizing Neural Network Character Recognition Using Adaptive Filtering and Feature Extraction.

PB96-119797 01,855 Not available NTIS

PB96-119805

Standard Reference Materials for Optical Fibers and Connectors.

PB96-119805

04,344 Not available NTIS

PB96-120555

Hair Testing for Drugs of Abuse: International Research on Standards and Technology.

PB96-120555

$03.504 \mathrm{PC} \mathrm{A18/MF} \mathrm{A04}$

PB96-122098

Low Electrolytic Conductivity Standards.

PB96-122098

(Order as PB96-117767, PC A08/MF A02)

PB96-122106

Potential and Current Distributions Calculated Across a Quantum Hall Effect Sample at Low and High Currents.
PB96-122106

(Order as PB96-117767, PC A08/MF A02)

PB96-122114

Microform Calibration Uncertainties of Rockwell Diamond

Indenters.

(Order as PB96-117767, PC A08/MF A02)

PB96-122122

Performance Measures for Geometric Fitting in the NIST Algorithm Testing and Evaluation Program for Coordinate gorithm Testing and

PB96-122122

01,745

(Order as PB96-117767. PC A08/MF A02)

PB96-122130

Study on the Reuse of Plastic Concrete Using Extended Set-Relarding Admixtures

PB96-122130

(Order as PB96-117767, PC A08/MF A02)

PB96-122148

Third Generation Water Bath Based Blackbody Source.

PB96-122148 (Order as PB96-117767, PC A0B/MF,046

PB96-122411

Making Displays Deliver a Full Measure.
PB96-122411 01,490 Not available NTIS

PB96-122429

Hydrodynamic Similarity in an Oscillating-Body Viscometer. PB96-122429

PB96-122437

Thermophysical Properties of Fluids for the Gas Industry.

PB96-122437

02,494 Not available NTIS

PB96-122445

Recent Development in Nondestructive Testing of Concrete PB96-122445

01,325 Not available NTIS
Noting of Concrete.

PB96-122452

Error Protecting Characteristics of CDMA and Impacts on

PB96-122452

01,491 Not available NTIS

B96-122460

Progress on the Quantized Hall Resistance Recommended Intrinsic/Derived Standards Practice.

PB96-122460

02,403 Not available NTIS

B96-122478

Nonlinear Dynamics of Stiff Polymers.

PB96-122478

01,278 Not available NTIS

PB96-122486

Standardised Computer Data File Format for Storage, Transport, and Off-Line Processing of Partial Discharge Data

PB96-122486

01,930 Not available NTIS

PB96-122494

Constant Temperature and Humidity Chamber for Standard

PB96-122494

02,275 Not available NTIS

122502

Optical Sampling Using Nondegenerate Four-Wave Mixing in a Semiconductor Laser Amplifie

PB96-12250

02,076 Not available NTIS

PB96-122510

Epitaxial Growth of $\mathrm{BaTiO} 3$ Thin Films at $600 \mathrm{C}$ by Metalorganic Chemical Vapor Deposition. PB96-12251

Survey of the Components of Display-Measurement Stand-

PB96-122528

02,188 Not available NTIS

PB96-122536

Failure of All-Ceramic Fixed Partial Dentures 'In vitro' and 'In vivo': Analysis and Modeling.

PB96-122536 0,175 Not available NTIS

PB96-122544

Programmable Guarded Coaxial Connector Panel.

PB96-122544

02,108 Not available NTIS

PB96-122551

Physical Limit to the Stability of Superheated and Stretched

PB96-122551

01,083 Not available NTIS

PB96-122569

Extending the Angular Range of Neutron Reflectivity Measurements from Planar Lipid Bilayers: Applications to a 
PB96-122643

Decay of Bragg Gratings in Hydrogen-Loaded Optical Fibers.

PB96-122643

04,345 Not available NTIS

PB96-122650

Few Caveats on Carbon Dioxide Monitoring

PB96-122650

02,562 Not available NTIS

PB96-122668

Development of a Standard Reference Material for Measurement of Interstitial Oxygen Concentration in Semiconductor Silicon by Infrared Absorption.

PB96-122668 02,404 Not available NTIS

PB96-122676

Experimental Study of the Stabilization Region of Lifted Tur bulent-Jet Diffusion Flames.

PB96-122676

01,405 Not available NTIS

PB96-122684

Polarization Dependence of Response Functions in $3 \times 3$

Sagnac Optical Fiber Current Sensors.
PB96-122684 Not available NTIS

PB96-122692

Electronics Packaging Materials Research at NIST

PB96-122692 02,405 Not available NTIS

PB96-122700

Millimeter-Resolution Optical Time-Domain Reflectometry Using a Four-Wave Mixing Sampling Gate.

PB96-122700

02,190 Not available NTIS

PB96-122718

Chaotic Motions of Coupled Galloping Oscillators and Their Modeling as Diffusion Progresses.
PB96-122718 PB96-122718

PB96-122726

Non-Gaussian Noise Effects on Reliability of Multistable

Systems.
PB96-122726

04,213 Not available NTIS

PB96-122734

Modification of Cast Epoxy Resin Surfaces during Exposure to Partial Discharges.

PB96-122734

01,086 Not available NTIS

PB96-122742

Orbital Alignment and Vector Correlations in Inelastic Atomic Collisions.

PB96-122742

04,047 Not available NTIS

PB96-122759

Roadmap for the Computer Integrated Manufacturing (CIM)

PB96-122759

02,832 Not available NTIS

PB96-122767

Influence of Surface Charge on the Stochastic Behavior of Partial Discharge in Dielectrics.

PB96-122767

PB96-122775

Comment and Discussion on Digital Processing of PD

Pulses.

PB96-122783

01,932 Not available NTIS

Novel Method for Determining Thin Film Density by EnergyDispersive X-ray Reflectivity. 04,737 Not available NTIS
PB96-122783 PB96-122783

PB96-122791

Electromagnetic Properties of Materials: The NIST Metrology Program.

PB96-122809

01,933 Not available NTIS

Electron Attachment to Excited Molecules(1)

PB96-122809 01,087 Not available NTIS

PB96-122817

Sleuthing the Dynamo: HST/FOS Observations of UV Emissions of Solar-Type Stars in Young Clusters.

PB96-122817 00,098 Not available NTIS

PB96-122825

Open-Ended Coaxial Probes for Nondestructive Testing of Substrates and Circuit Boards.

PB96-122825

PB96-122833

EDI and EFT Security Standards.

PB96-122833

PB96-122841

Parallel Monte Carlo Simulation of MBE Growth.

PB96-122841 02,406 Not available NTIS

PB96-122858

Slow Evolution from the Boundary: A New Stabilizing ConStraint in III-Posed Continuation Problems.

PB96-122858

03,418 Not available NTIS

PB96-122866

Field Modeling: Simulating the Effect of Sloped Beamed Ceilings on Detector and Sprinkler Response.

PB96-122866 01,406 Not available NTIS

PB96-122874

Bounds on Frequency Response Estimates Derived from Uncertain Step Response Data.

PB96-122874

03,419 Not available NTIS

PB96-122882

Efficient Way of Identifying New Active Stars: A VLA Survey of X-ray Selected Active Stellar Candidates.
PB96-122882 00,099 Not available NTIS
PB96-122890

Smoke Emission from Burning Crude Oil

PB96-122890

PB96-122908

01,407 Not available NTIS

Open Issues

PB96-122908

PB96-122916

Atom Cooling and Trapping, and Collisions of Trapped

Atoms

PB96- 122916

PB96-122924

Database Management Standards: Status and Applicability PB96-122924

PB96-122932

Laboratory Accreditation for Testing Energy Efficient Light-

PB96-122932

00.270 Not available NTIS

PB96-122940

Dental Applications of Ceramics

PB96-122940

00,177 Not available NTIS

PB96-122957

Growth Characteristics of Fiber Grating

PB96-122957

04,346 Not available NTIS

PB96-122965

Development of Hazard Assessment and Suppression Technology for Oil and Gas Well Blowout and Diverter

PB96-122965

01,408 Not available NTIS

PB96-122973

Introduction to Phase-Stable Optical Sources.

PB96-122973

04,347 Not available NTIS

PB96-122981

Effects of Spindle Dynamic Characteristics on Hard Turning.
PB96-122981

(12298

PB96-122999

Chemically Assisted Machining of Si3N4

PB96-122999

03,072 Not available NTIS

- 123005

Magnetic Fields in Star-Forming Regions: Observations

PB96. 123005

00,700 Not available NTIS

PB96-123021

Nonlinear Color Transformations in Real Time Using a Video Supercomputer.

PB96-123021

02,191 Not available NTIS

PB96-123112

Stranding Experiments on Double Hull Tanker Structures.

PB96-123112

03,749 Not available NTIS

PB96-123120

Laser-Induced Fluorescence Measurements of $\mathrm{OH}$ in Laminar Diffusion Flames in the Presence of Soot Particles.

PB96-123120 01,409 Not available NTIS

PB96-123

Stylus Flight in Surface Profiling

PB96-123138

02,675 Not available NTIS

PB96-123146

Effects of Nonmodel Errors on Model-Based Testing

PB96-123146

02,604 Not available NTIS

PB96-123153

Methods for Aligning the NIST Watt-Balance.

PB96-123153 01,934 Not available NTIS

PB96-12316

Proposed International Interactive Courseware Standard.

PB96-123161

00,137 Not available NTIS

PB96-123179

Influence of Electrode Material on Measured Ion Kinetic-Energy Distributions in Radio-Frequency Discharges.

PB96-123179

01,935 Not available NTIS

PB96-123187

Secondary Standard for PM and AM Noise at 5, 10, and $100 \mathrm{MHz}$.
PB96-123187

PB96-123195

01,554 Not available NTIS

Improving Neural Network Performance for Character and Fingerprint Classification by Altering Network Dynamics.

PB96-123195 01,856 Not available NTIS

PB96-123203

Study of Laser Resonance Ionization Mass Spectrometry Using a Glow Discharge Source. PB96-123203

PB96-12321

03,360 Not available NTIS

Anisotropy of the Surfaces of Pores in Plasma Sprayed Alumina Deposits.

PB96-123229

03,126 Not available NTIS

Properties and Mechanisms of Fast-Setting Calcium Phosphate Cements.

PB96-123237

00,178 Not available NTIS

Flow-Induced Structure in Polymers: Chapter 16. Shear-Induced Changes in the Order-Disorder Transition Temperature and the Morphology of a Triblock Copolymer.

PB96-123237

03,127 Not available NTIS

PB96-123245

Polymer Combustion and Flammability: Role of the Condensed Phase.

01,279 Not available NTIS

PB96-123252

Significant Contributions of IAPWS to the Power Industry, Science and Technology

PB96-123252

01,088 Not available NTIS

PB96-123260

Further Development of the N-Gas Mathematical Model: An

Approach for Predicting the Toxic Potency of Complex

Combustion Mixtures.

PB96-123260

03,650 Not available NTIS

PB96-123278

Goddard High Resolution Spectrograph: Instrument, Goals, and Science Results.

PB96-123278

00,044 Not available NTIS

PB96-123286

A-type and Chemically Peculiar Stars.

PB96-123286

00,101 Not available NTIS

High Velocity Plasm in the Transition Region of Au Mic: A Stellar Analog of Solar Explosive Events.

PB96-123302

Radio and X-ray Emissions from Chemically Peculiar Band A-Type Stars: Observations and a Model.

PB96-12330

00,103 Not available NTIS

PB96-123310

Redshifts in Stellar Transition Regions.

PB96-123310

00, 104 Not available NTIS

Scientific Rationale and Present Implementation Strategy for the Far Ultraviolet Spectrograph Explorer (FUSE).

PB96-123328

PB96-123336

Transition Regions of Capella.

00,105 Not available NTIS

PB96-123344

Optical Control of Enzymatic Conversion of Sucrose to Glucose by Bacteriorhodopsin Incorporated into Self-Assembled Phosphatidylcholine Vesicles.

PB96-123344

03,477 No: available NTIS

B96-123351

Kinetic Energy Distributions of $\mathrm{H}(+), \mathrm{H} 2(+)$, and $\mathrm{H} 3(+)$ from a Diffuse Townsend Discharge in $\mathrm{H} 2$ at High $\mathrm{E} / \mathrm{N}$.

PB96-123351 04,415 Not available NTIS

PB96-123369

Flow-Induced Structure in Polymer. Chapter 1. An Introduction to Flow-Induced Structures in Polymers

PB96-123369

PB96-123377

Flow-Induced Structure in Polymers. Chapter 17. PhaseSeparation Kinetics of a Polymer Blend Solution Siudied by a Two-Step Shear Ouench.

PB96-123377

03,388 Not available NTIS

PB96-123385

Chemical Stability of Upper-Layer Fire Gases.

PB96-12338

PB96-123393

Prediction of the Strength Properties for Plain-Carbon and

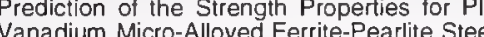

PB96-123393

03,216 Not available NTIS

PB96-123401

Federal Labs Have Key Role in Metrication.

PB96-123401

02,920 Not available NTIS

PB96-123419 


\section{PB96-123500}

Optical Properties of Triton X-100-Treated Purple Membranes Embedded in Gelatin Films. PB96-123500

PB96-123518

Search for Small Violations of the Symmetrization Postulate in an Excited State of Helium.

PB96-123518

04,050 Not available NTIS

PB96-123526

Book Review: Statistical Physics of Macromolecules.

PB96-123526

01,280 Not available NTIS

PB96-123534

Book Review: Aspects and Applications of the Random

PBalk. 123534

04,215 Not available NTIS

\section{PB96-12354}

Modification of the Phase Stability of Polymer Blends by Diblock Copolymer Additivies.

PB96-12354

03,172 Not available NTIS

PB96-123559

Static Dielectric Constant of Water and Steam.

PB96-123559

01,090 Not available NTIS

\section{PB96-123567}

Long-Term Stability of Bayard-Alpert Gauge Performance: Results Obtained from Repeated Calibrations against the National Institute of Standards and Technology Primary Vacuum Standard.

PB96-123567

02,676 Not available NTIS

PB96-123575

Electrokinetic Sonic Analysis of Silicon Nitride Suspensions. PB96-123575

PB96-123583

Wire Bonding to Multichip Modules and Other Soft SubStrates.

02,079 Not available NTIS

PB96-123591

Slow Dynamics of Segregation in Hydrogen-Bonded Polymer Blends.

PB96-123609

01,281 Not available NTIS

Optical Sampling Using Nondegenerate Four-Wave Mixing in a Semiconductor Laser Amplifier.

PB96-123609

04,348 Not available NTIS

PB96-123617

Characterization of Liquid-Phase Epitaxially Grown $\mathrm{HgCdTe}$

Films by Magnetoresistance Measurements.
PB96-123617 04,738 Not available NTIS

PB96-123625

New Approach for Reducing the Toxicity of the Combustion Products from Flexible Polyurethane Foam.

PB96-123625 01,411 Not available NTIS

PB96-12365

Keeping Up with the Reality of Today's Surge Environment.
PB96-123633

PB96-123641

Hot-Electron Microcalorimeters as High-Resolution X-ray PB'tectors.

PB96-123658

04,739 Not available NTIS

Ion Kinetics and Symmetric Charge-Transfer Collisions in Low-Current, Diffuse (Townsend) Discharges in Argon and Nitrogen.

PB96-123658

04,051 Not available NTIS

PB96-123666

Intensive Swimming: Can It Affect Your Patients' Smiles

PB96-123666 03,570 Not available NTIS

PB96-123674

Nano-Defects in Commercial Bonded SOI and SIMOX.

PB96-123674

02,407 Not available NTIS

PB96-123682

Influence of Stationary Phase Chemistry on Shape Rec-

ognition in Liquid Chromatography.
PB96-123682 00,621 Not available NTIS

PB96-123690

Certification of Standard Reference Material (SRM) 1941a, Organics in Marine Sediment.

PB96-123690

02,593 Not available NTIS

PB96-123708

Comparison of NIST and Manufacturer Calibrations of $(90) \mathrm{Sr}+(90) \mathrm{Y}$ Ophthalmic Applicators.
PB96-123708 03,634 Not available NTIS

PB96-123716

Refraction of Light by Graded Birefringent Media.
PB96-123716
02,192 Not available NTIS

PB96-123724

02,192 Not available NTIS

Characterization of the Vibrational Dynamics in the Octahedral Sublattices of LaD2.25 and $\mathrm{LaH} 2.25$.

PB96-123724 01,091 Not available NTIS

PB96-123732

Kinetic-Energy Distributions of Ions Sampled from RadioFrequency Discharges in Helium, Nitrogen, and Oxygen.

PB96-123732 01,092 Not available NTIS

PB96-123740

Measurement of S2OF10, and S2O2F10 Production Rates from Spark and Negative Glow Corona Discharge in SF6/ O2 Gas Mixtures.

01,093 Not available NTIS
PB96-123757

Physics and Chemistry of Partial Discharge and Corona Recent Advances and Future Challenges PB96-123757

01.936 Not available NTIS PB96-123765

Zimm Plot and Its Analogs as Indicators of Vesicle and Micelle Size Polydispersity.

PB96-123765

01,094 Not available NTIS

B96-123773

Distributed Feedback Lasers in Rare-Earth-Doped Phosphate Glass.

PB96-123781

04,740 Not available NTIS

Behavior of Surface Partial Discharge on Aluminum Oxide Dielectrics.

PB96-12378
PB96-123799

01,937 Not available NTIS

Relativistic Modifications of Charge Expansion Theory.

PB96-123799

PB96-123807

04,052 Not available NTIS

NIST Reference Materials to Support Accuracy in Drug Testing.

03,505 Not available NTIS

PB96-126180

Journal of Research of the National Institute of Standards and Technology, March/April 1995. Volume 100, Number 2. PB96-126180

PB96-126198

Low-Temperature Properties of Silver

PB96-126198 Order as PB96-126180, PC A05 03,361

PB96-126206

Mixing Plate-Like and Rod-Like Molecules with Solvent: A Test of Flory-Huggins Lattice Statistics.

PB96-126206

(Order as PB96-126180, PC A05/MF 03,173

PB96-128095

Study of Technology for Detecting Pre-Ignition Conditions of Cooking-Related Fires Associated with Electric and Gas Ranges and Cooktops. Phase 1 Report.
PB96-128095 PB96-128103

ICSSC Guidance on Implementing Executive Order 12941 on Seismic Safety of Existing Federally Owned or Leased Buildings.

PB96-128103

$00,459 \mathrm{PC} \mathrm{A03/MF} \mathrm{AO}$

Ground Improvement Techniques for Liquefaction Remediation Near Existing Lifelines.

PB96-128111

01,350 PC A05/MF A0

Calorimetric and Visual Measurements of R123 Pool Boiling on Four Enhanced Surfaces.

On Four Enhanc

PB96-128194

04,053 PC A04/MF A01

Extensions of the Prototype Application Protocol of Ready to-Wear Apparel Pattern Making.

PB96-128202

$03,198 \mathrm{PC} \mathrm{A03/MF} \mathrm{A0}$

Distributed Systems: Survey of Open Management Approaches

PB96-128202

01,746 PC A03/MF AO

PB96-128210

Bibliography of the NIST Optoelectronics Division.

PB96-128210

PB96-128228

02,193 PC AO4/MF AO

Overview of the Manufacturing Engineering Toolkit Proto-

type.

$02,833 \mathrm{PC} \mathrm{A03/MF} \mathrm{A0}$

PB96-128236

User's Guide to 'SuperFit' Modeling Software for CMM Probe Lobing.

PB96-128244

$02,921 \mathrm{PC} \mathrm{A03/MF} \mathrm{AO}$

Reference Manual for the Algorithm Testing System Version 2.0.

PB96-128244

$02,922 \mathrm{PC} \mathrm{A03/MF} \mathrm{A01}$

PB96-128251

Certification of the Standard Reference Material 1473a, a Low Density Polyethylene Resin.

PB96-128251

PB96-128269

$01,282 \mathrm{PC} \mathrm{A03/MF} \mathrm{AO}$

Metrology for Electromagnetic Technology: A Bibliography of NIST Publications, September 1995.01 .938 PC A05/MF A0 PB96-128269
PB96-128277

Metrology Issues in Terahertz Physics and Technology.
PB96-128277 01,939 PC AO8/MF AO2 PB96-128285

Recommended Performance-Based Criteria for the Design of Manufactured Home Foundation Systems to Resist Wind and Seismic Loads.

PB96-12828

$00,460 \quad \mathrm{PC}$ A05/MF A01

Control of Gas-Metal-Arc Welding Using Arc-Light Sensing PB96-131461

$02,869 \mathrm{PC} \mathrm{A06/MF} \mathrm{A02}$

PB96-131479

Solid Propellant Gas Generators: Proceedings of the 1995 Workshop. Held in Gaithersburg, Maryland on June 28-29, 1995

$01.412 \mathrm{PC}$ A11/MF A03

PB96-131487

Application Software Interface: ISDN Services for an Open Systems Environment.

PB96-131495

01,492 PC A03/MF A01

Comparison of POSIX Open System Environment (OSE) and Open Distributed Processing (ODP) Reference Models. PB96-131495

PB96-131503

Method to Determine a Basis Set of Paths to Perform Program Testing.

PB96-131511

01,747 PC A03/MF A01

Sharing Information via the Internet: An Infoserver Case

Study,

01,493 PC A03/MF A01

PB96-131529

Operating Principles of MultiKron Virtual Counter Performance Instrumentation for MIMD Computers.

PB96-131529

01,632 PC A03/MF A01

PB96-131537

Alkali-Silica Reaction and High Performance Concrete.

PB96-131537

$01,345 \mathrm{PC} \mathrm{A03/MF} \mathrm{A01}$

PB96-131545

Defining Environment Integration Requirements.

PB96-131545

02,733 PC A03/MF A01

PB96-131552

How-To Suggestions for Implementing Executive Order 12941 on Seismic Satety of Existing Federal Buildings, A Handook.

PB96-131560

$00,461 \mathrm{PC} \mathrm{A10/MF} \mathrm{A03}$

Smoke Plume Trajectory from In situ Burning of Crude Oil in Alaska: Field Experiments.

PB96-131560

$02,594 \mathrm{PC} \mathrm{A03/MF} \mathrm{A01}$

PB96-131578

Electronic Access to Standards on the Information Highway. PB96-131578

PB96-131586

Classified Bibliography: Insulation Condition Monitoring Methods, 1989-1995.

PB96-131586

02.232 PC A05/MF A01

PB96-131594

Anisotropy of Interfaces in an Ordered Alloy: A MultipleOrder-Parameter Model.

PB96-131594

$04,741 \mathrm{PC}$ A04/MF A01

PB96-131602

Summary Report: Workshop on Industrial Applications of Scanned Probe Microscopy (2nd). A Workshop Co-Sponican Vacuum Society. Held in Gaithersburg, Maryland on May 2-3, 1995

PB96-131610

00,509 PC A07/MF A02

Computer Security: An Introduction to Computer Security. The NIST Handbook.

PB96-131610

01,608 PC A13/MF A03

PB96-134954

Joumal of Research of the National Institute of Standards and Technology. September-October 1993. Volume 98, Number 5.

PB96-134954

PB96-135017

03,362 PC A05/MF A01

Thermal Isolation of High-Temperature Superconducting Thin Films Using Silicon Wafer Bonding and Micromachining. 
PB96-135108

Spatial Information and Technology Standards Evolving PB96-135108

03,679 Not available NTIS

PB96-135116

Analysis of an Open-Ended Coaxial Probe with Litt-Off for Nondestructive Testing.

PB96-135116

01,940 Not available NTIS

PB96-135124

Distinguishing the Contributions of Residential Wood Combustion and Mobile Source Emissions Using Relative Concentrations of Dimethylphenanthrene Isomers.

PB96-135124 02.563 Not available NTIS

PB96-135132

Modelling Drying Shrinkage of Cement Paste and Mortar. Part 1. Structural Models from Angstroms to Millimeters. PB96- 135132

PB96-135140

Catastrophic Failures Propagate Field of Fracture Mechanics. 135140

03,217 Not available NTIS

PB96-135157

High-Frequency Oscillators Using Phase-Locked Arrays of Josephson Junctions.

PB96-135157

02,080 Not available NT1S

PB96-135165

Noise Reduction in Low-Frequency SQUID Measurements with Laser-Driven Switching.

PB96-135165

02,081 Not available NTIS

PB96-135173

Anionic Triphenylmethane Dye Solutions for Low-Dose Food Irradiation Dosimetry.

PB96-135173

03,715 No: available NTIS

PB96-135181

Growth of Epitaxial KNbO3 Thin Films.
PB96-135181 02,409 Not available NTIS

PB96-135199

Frequency-Stabilized Lasers: A Driving Force for New Spectroscopies

PB96-135207

04,350 Not available NTIS

Wire Bonding to Multichip Modules and Other Soft Sub-

strates.
PB96- 135207

02,082 Not available NTIS

PB96-135215

Effect of Supersaturation on Apatite Crystal Formation in

Aqueous Solutions at Physiologic $\mathrm{pH}$ and Temperature.
PB96-135215
03,571 Not available NTIS

PB96-135223

Accuracy of the Electron Pump.

PB96-135223

04,743 Not available NTIS

PB96-135231

Effects of Copper, Nickel and Boron on Mechanical Properties of Low-Alloy Steel Weld Metals Deposited at High Heat Input.

PB96-135231

PB96-135249

Alcohol Solutions of Triphenyl-Tetrazolium Chloride as High-Dose Radiochromic Dosimeters.
PB96-135249 03.716 Not available NTIS

03.716 Not available NTIS

Oscillometric and Conductometric Analysis of Aqueous and Organic Dosimeter Solutions. 04,054 Not available NTIS PB96-135256

PB96-135264

Recalibration for the Final Archive of the International Ultraviolet Explorer (IUE) Satellite.

PB96-135264

00,106 Not available NTIS

PB96-135272

Calorimeters for Calibration of High-Dose Dosimeters in High-Energy Electron Beams. 04,055 Not available NTIS
PB96-135272

PB96-135280

Dosimetry Systems for Radiation Processing.
PB96-135280 03,717 Not available NTIS

PB96-135298

Temperature and Relative Humidity Dependence of Radiochromic Film Dosimeter Response to Gamma and Electron Radiation.

PB96-135298

PB96-135306

Real Time Monitoring of Electron Processors.

PB96-135306 03,719 Not available NTIS

PB96-135314

Decomposition of Sulfur Hexafluoride by X-rays.

Decomposition of Sulfur Hexafluoride by X-rays.
PB96-135314 01,095 Not available NTIS

PB96-135322

Application of Thermodynamic and Detailed Chemical Kinetic Modeling to Understanding Combustion Product Generation in Enclosure Fires.

PB96-135322

01,413 Not available NTIS

PB96-135330

Contact Tube Wear Detection in Gas Metal Arc Welding.

PB96-135330

02,872 Not available NTIS

PB96-135348

Mutual Phase Locking in Systems of High-TC PB96-135348 04,744 Not available NTIS
PB96-135355

Effects of Etching on the Morphology and Surface Resistance of YBa2Cu3O7-delta Films.

PB96-135355

02,410 Not available NTIS

B96-135363

Proposed Changes to Charpy V-Notch Machine Certification PB96-135363

PB96-135371

02,955 Not available NTIS

Influence of Thickness Fluctuations on Exchange Coupling in $\mathrm{Fe} / \mathrm{Cr} / \mathrm{Fe}$ Structures.

PB96-135371

PB96-135389

LRM Probe-Tip Calibrations Using Nonideal Standards.

PB96- 135389

PB96-136965

Computer-Aided Manufacturing Engineering Forum (tst). Technical Meeting Proceedings. Held in Gaithersburg Maryland on March 21-22, 1995.

PB96-136965

$02,834 \mathrm{PC} \mathrm{A10/MF} \mathrm{A03}$

PB96-136973

Effect of Inclination on the Performance of a Compac Brazed Plate Condenser and Evaporator.

PB96-136973

02,756 PC A03/MF AO

-136981

Metallurgy Technical Activities 1994 (NAS-NRC Assessment Panel, April 6-7, 1995)

PB96-13698

National Planning for Construction and Building $R$ and $D$.

PB96-137104 00,324 PC A06/MF A02

PB96-137187

Electronics and Electrical Engineering Laboratory Technical
Publication Announcements Covering Laboratory Programs, Publication Announcements Covering Laboratory Programs,
April to June 1995 with 1995 EEEL Events Calendar.
PB96-137187 01,941 PC A03/MF A01

$01,941 \mathrm{PC} \mathrm{A03/MF} \mathrm{AO}$

Formation of DNA-Protein Cross-Links in Cultured Mammalian Cells Upon Treatment with Iron lons.
PB96-137724 03,651 Not available NTIS

PB96-137732

Temperature and Frequency Dependence of Anelasticity in a Nickel Oscillator.

PB96-13

03,689 Not available NTIS

Churchill Eisenhart, 1913-1994

PB96-137740

PB96-137757

03,447 Not available NTIS

Visualization of Surface Figure by the Use of Zernike Poly-

nomials.

PB96-137757

04,351 Not available NTIS

PB96-137765

Microwave Dielectric Properties of Anisotropic Materials at Cryogenic Temperatures.

PB96-137765

B 96-137773

Panel Discussion on Units in Magnetism.

PB96-137773

2,412 Not available NTIS

PB96-137781

Anomalous Switching Phenomenon in Critical-Current Measurements When Using Conductive Mandrels.

PB96-137781 When Using Conductive Mand available NTIS
PB

PB96-137799

Low-Frequency Excitations of Oriented DNA.

PB96-137799

03,548 Not available NTIS

PB96-137807

Use of Monte Carlo Modeling for Interpreting Scanning Electron Microscope Linewidth Measurements.

PB96-13780

02,413 Not available NTIS

PB96-138417

Low Noise YBa2Cu3O7-x-SrTiO3-YBa2Cu3O7-x Mulitlayers for Improved Superconducting Magnetometers.

PB96-138417 04,747 Not available NTIS PB96-138425

Determination of Vitamin $\mathrm{K} 1$ in Serum Using Catalytic-Reduction Liquid Chromatography with Fluorescence Detection.

PB96-138433

03,506 Not available NTIS

Electrical Characterization of Integrated Circuit Metal Line Thickness.

PB96-138433

02,414 Not available NTIS

PB96-138441

Observation and Visible and uv Magnetic Dipole Transitions

in Highly Charged Xenon and Barium.

PB96-138441

PB96-138458

High-Energy Phonon Dispersion in La 1.85Sro.15CuO4.

PB96-138458 04,748 Not available NTIS

PB96-138466

Atomic Transition Probabilities and Tests of the Spectroscopic Coupling Scheme for N I.
04,057 Not available NTIS PB96-138466

PB96-138474

Accurate Computations of Radar Cross Sections of Simple

Objects.
PB96-138474

04,426 Not available NTIS

PB96-138482

Test of a Slow Off-Axis Parabola at Its Center of Curvature PB96-138482

PB96-138490

Electro-Optic-Based RMS Voltage Measurement Technique PB96-138490

PB96-1385

02,194 Not available NTIS

Improving the Evaluation of Building Ventilation.

PB96-13850

00,271 Not available NTIS

PB96-138516

q Dependence of Self-En

PB96-138516

Eftects of the Plane Oxygen

PB96-138524

Electron-electron Interactions, Coupled-Plasmon-Phonon Modes, and Mobility in n-Type GaAs.

PB96-138524

PB96-138532

Nonequilibrium Total-Dielectric-Function Approach to the Electron Boltzmann Equation for Inelastic Scattering in Doped Polar Semiconductors.

PB96-138532

PB96-138540

04,416 Not available NTIS

Report on 1994 Actions of the International Institute of Welding.

PB96- 138540

PB96-138557

02,873 No: available NTIS

Dependence of Contrast on Probe/Sample Spacing with the Magneto-Optic Kerr-Effect Scanning Near-Field Optical Microscope (MOKE-SNOM)

PB96-138557

04.750 Not available NTIS

PB96-138565

Silicon Surface Chemistry by IR Spectroscopy in the Mid. to Far-IR Region: $\mathrm{H} 2 \mathrm{O}$ and Ethanol on Si(100).

PB96-138565

01,097 Not available NTIS

966-138573

Development of Highly Conductive Cantilevers for Atomic Force Microscopy Point Contact Measurements.

PB96-138573

04,751 Not available NTIS

PB96-13858

Selectivity Trends in Packed Column Supercritical Fluid Chromatography with $\mathrm{C} 18$ Stationary Phases.

PB96-138581 00,622 Not available NTIS

PB96-138599

MCNP Model of the National Bureau of Standards Reactor (NBSR) Core.

PB96-138607

03,733 Not available NTIS

High Resolution Time Interval Counter.

PB96-138607

01,495 Not avallable NTIS

PB96-139407

Databases Available in the Research Information Center of the National Institute of Standards and Technology (Decem-

PB96-13940

02,734 PC A07/MF A02

PB96-139415

Telecommunications Security Guidelines for Telecommunications Management Network. Computer Security.

PB96-139415

$01,496 \mathrm{PC} \mathrm{AO} / \mathrm{MF} \mathrm{A01}$

PB96-140363

Radiometer Equation for Noise Comparison Radiometers

PB96-140363

PB96-140371

02, 195 Not available NTIS

Inexpensive Laser Cooling

dergraduate Laboratories.

PB96-140371

PB96-140389

Fiber Coating Diameter: Toward a Glass Artifact Standard. 
PB96-141072

Atom-Optical Properties of a Standing-Wave Light Field. PB96-141072

PB96-141080

X-ray Observation of Electroclinic Layer Constriction and Rearrangement in a Chiral Smectic-A Liquid Crystal.

PB96-141080

01,100 Not available NTIS

PB96-141098

Evidence for Tunneling and Magnetic Scattering at 'In situ' YBCO/Noble-Metal Interfaces.
PB96-141098

PB9-141106

04,752 Not available NTIS

Cross-Sectional Photoluminescence and Its Application to Buried-Layer Semiconductor Structures.
PB96-141106

PB96-141114

Safety Assessment of Railroad Wheels by Residual Stress Measurements.

PB96-141114

04,855 Not available NTIS

PB96-141122

Effect of Charpy V-Notch Striker Radii on the Absorbed Energy.

03,365 Not available NTIS

PB96-14113

Apparatus for Resistance Measurement of Short, Small-Diameter Conductors.

PB96-141130

04,417 Not available NTIS

PB96-141148

De Facto Microzonation through the Use of Soils Factors in Design Triggers

PB96-141155

00,462 Not available NTIS

Neutron-Powder-Diffraction Study of the Long-Range Order in the Octahedral Sublattice of LaD2.25.

PB96-141155

04,753 Not available NTIS

PB96-141163

Anova Estimates of Variance Components for a Class of Mixed Models.
PB96-141163

PB96-141171

03,448 Not available NTIS

One-Sided beta-Content Tolerance Intervals for Mixed Models.

PB96-14117

03,449 Not available NTIS

PB96-141189

Effect of Magnetic Field Orientation on the Critical Current of HTS Conductor and Coils.

PB96-141189

PB96-141197

Vector and Quasi-Vector Solutions for Optical Waveguide Modes Using Efficient Galerkin's Method with HermiteGauss Basis Functions.

PB96-141197

PB96-141205

Photoluminescence Spectra ol Epitaxial Single Crystal C60 PB96-141205

PB96-141213

Quench Energy and Fatigue Degradation Properties of $\mathrm{Cu}$ and $\mathrm{Al} / \mathrm{Cu}$-Stablized $\mathrm{Nb}-\mathrm{Ti}$ Epoxy-Impregnated Superconductor Coils. PB96-141213

04,755 Not available NTIS

PB96-141221

Atomic Iron in Its (5)D Ground State: A Direct Measuremen of the $\mathrm{J}=0$ inverted arrow 1 and $\mathrm{J}=1$ inverted arrow Fine-Structure Intervals (1.2).

PB96-141221

04,756 Not available NTIS

PB96-141239

Predicting the Fire Performance of Buildings: Establishing Appropriate Calculation Methods for Regulatory Applica-

tions.
PB96-141239

PB96-141247

Using Atom Optics to Fabricate Nanostructures.

PB96-141247

00,316 Not available NTIS

PB96-141254

04,757 Not available NTIS

Safety Assessment of Railroad Wheels Through Roll-by De ection of Tread Cracks.

PB96-14125

04,856 Not available NTIS

PB96-141262

Microwave Properties of Voltage-Tunable YBa2Cu3O7.

delta/SrTiO3 Coplanar Waveguide Transmission Lines.
PB96-141262 02,235 Not available NTIS

PB96-141270

Unreacted Cement Content in Macro-Defect-Free Composites: Impact on Processing-Structure-Property Relations.

PB96-141270 03,174 Not available NTIS

PB96-141288

Research and Development Activities in Electron Paramagnetic Resonance Dosimetry.
PB96-14128 PB96-14128

PB96-141296

Experience with MPI: 'Converting Pvmmake to Mpimake under LAM' and 'MPI and Parallel Genetic Programming'. PBg6-141304

Bounds on Least-Squares Four-Parameter Sine-Fit Errors Due to Harmonic Distortion and Noise.
PB96-141304 Not available NTIS
PB96-141312

Oxygen Annealing of Ex-situ YBCO/Ag Thin-Film Interfaces.

PB96-141312

04.758 Not available NTIS

PB96-141320

Summary Report on the Workshop on Advanced Digital Video in the National Information Infrastructure.

PB96-141320 01,497 Not available NTIS

PB96-141338

Ultrasonic-Resonance Spectroscopy of Bulk and Layered

$-14133$

04,759 Not available NTIS

PB96-141346

Domain Structures in Magneforesistive Granular Metals.

PB96-141346

04,760 Not available NTIS

PB96-141353

Optimal Control of Building and HVAC Systems.

PB96-141353

00,272 Not available NTIS

B96-141361

Alternative Contour Technique for the Efficient Computation of the Effective Length of an Antenna.

PB96-14136

02,011 Not available NTIS

PB96-141379

Determination of Sulfur in Fossil Fuels by Isotope Dilution Thermal lonization Mass Spectrometry.
PB96-141379 Not available NTIS

PB96-141387

Theory of the Magneto-Optic Kerr Effect in the Near Field. PB96-141387

PB96-141395

Artificial Crack in Steel: An Ultrasonic-Resonance-Spectroscopy and Modeling Study.

PB96-141395

03,241 Not available NTIS

PB96-145560

Journal of Physical and Chemical Reference Data, Volume 24 , No. 1, January/February 1995

PB'96-145560

01,101 Not available NTIS

PB96-145578

Millimeter- and Submillimeter-Wave Spectrum of trans-Ethyl Alcohol.

PB96-145578

01,102 Not available NTIS

PB96-145586

Database for the Static Dielectric Constant of Water and

SBeam.

01,103 Not available NTIS

PB96-145594

Theoretical Form Factor, Attenuation and Scattering Tabulaion for $Z=1-92$ from $E=1-10$ eV to $E=0.4-1.0 \mathrm{MeV}$

PB96-145594

PB96-145818

Journal of Physical and Chemical Reference Data, Volume 24, No. 2, March/April 1995.

PB'96-145818

01,105 Not available NTIS

PB96-145826

Rate Constants for the Decay and Reactions of the Lowest Electronically Excited Singlet State of Molecular Oxygen in Solution. An Expanded and Revised Compilation.

PB96-145826

01,106 Not available NTIS

PB96-145834

Thermodynamic Properties of the Aqueous $\mathrm{Ba}($ sup 2+) Ion and the Key Compounds of Barium.

PB96-145834

01,107 Not available NTIS

PB96-145842

Journal of Physical and Chemical Reference Data, Volume 24, No. 3, May/June 1995.

PB96-145842

01,108 Not available NTIS

\section{-145859}

Critical Review of Rate Constants for Reactions of Transients from Metal Ions and Metal Complexes in Aqueous

PB96-145859

PB96-145867

01,109 Not available NTIS

Ideal Gas Thermod

cyclic Compounds.

PB96-14587

Properties of Sulphur Hetero-

Standard Reference Data for the Thermal Conductivity of

Water.
PB96-145875

PB96-145883

Journal of Physical and Chemical Reference Data, Volume 24. No. 4, July/August 1995

PB96-145883

PB96-145891

Summary of the Apparent Standard Partial Molal Gibbs Free Energies of Formation of Aqueous Species, Minerals and Gases at Pressures 1105000 Bars and Temperatures 25 to $1000 \mathrm{C}$.

PB96-145909

01,113 Not available NTIS

Atomic Weights of the Elements, 1993

PB96-145909

PB96-145917

01,114 Not available NTIS

Spectral Data for Highly Ionized Krypton, $\mathrm{Kr} V$ through $\mathrm{Kr}_{\mathrm{r}}$ XXXVI.

PB96-145917

01,115 Not available NTIS

PB96-145925

Journal of Physical and Chemical Reference Data, Volume 24 , No. 5, September/October 1995

PB96-145925

01,116 Not available NTIS

PB96-145933

Viscosity of Ammonia

PB96-145933

PB96-145941

Thermodynamics of Enzyme-Catalyzed Reactions. Part 4.

PB96-145941

PB96-145958

Thermodynamic Properties of the Aqueous Ions $(2+$ and $3+$ ) of Iron and the Key Compounds of Iron.

PB96-145958

01,119 Not available NTIS

PB96-145966

Journal of Physical and Chemical Reference Data, Volume

24. No. 6. November/December 1995

PB96-145966

01,120 Not available NTIS

PB96-145974

Thermodynamics of Enzyme-Catalyzed Reactions. Part 5 Isomerases and Ligases.

PB96-145974

01,121 Not available NTIS

PB96-145982

Energy Levels of Zinc, $\mathrm{Zn} I$ through $\mathrm{Zn} \mathrm{XXX.}$

PB96-145982
PB96-146352

Seismic Performance of Circular Bridge Columns Designed in Accordance with AASHTO/CALTRANS Standards.

PB96-146352

01,346 PC A07/MF A02

PB96-146360

Open System Environment (OSE): Architectural Framework or Information Infrastructure.

PB96-146360

PB96-146618

High-Temperature High-Pressure Oscillating Tube DensimPBer.

PB96-146626

01,123 Not available NTIS

Interaction Coefficients for 15 Mixtures of Flammable and Non-Flammable Components.

PB96-146626

03,281 Not available NTIS

PB96-146634

In-situ Neutron Reflectivity of MBE Grown and Chemically Processed Surfaces and Interfaces.
PB96-146634 Not available NTIS

PB96-146642

Effectiveness of a Heat Recovery Ventilator, an Outdoor Air Intake Damper and an Electrostatic Particulate Filter at Intake Damper and an Electrostatic Particulate Filter at Controlling Indoor Air Quality in Residential Buildings.
PB96-146642

PB96-146659

Multizone Modeling of Three Residential Indoor Air Quality Control Options.

PB96-146659

PB96-146667

02,565 Not available NTIS

Response to 'Draining in Dilute Polymer Solutions and Renormalization'.

PB96-146667

PB96-146675

01,283 Not available NTIS

Electric Dipole Excitation of a Long Conductor in a Lossy

04,058 Not available NTIS

PB96-146683

Evidence for Inelastic Processes for $\mathrm{N}(+) 3$ and $\mathrm{N}(+) 4$ from on Energy Distributions in He/N2 Radio Frequency Glow Discharges.

PB96-146683

PB96-146691 
PB96-146782

Investigation of Applicability of Alanine and Radiochromic Detectors to Dosimetry of Proton Clinical Beams.

PB96-146782

03,636 Not available NTIS

PB96-146790

Global Equivalence Ratio Concept and the Formation Mechanisms of Carbon Monoxide in Enclosure Fires.

PB96-146790 00,210 Not available NTIS

PB96-146808

Thermodynamic Properties of Dilute and Semidilute Solutions of Regular Star Polymers.

PB96-146808

01,284 Not available NTIS

PB96-146816

Interfacial Transport in Porous Media: Application to dc Electrical Conductivity of Mortars.

PB96-146816

01,326 Not available NTIS

PB96-146824

Statistical Descriptors in Crystallography. 2. Report of a Working Group on Expression of Uncertainty in Measure-

PB96-146824

04,764 Not available NTIS

PB96-146832

Stagnant Film Model of the Effect of Natural Convection on the Dendrite Operating State.

PB96-146832

04,765 Not available NTIS

PB96-146840

Preparation and Characterization of Cyclopolymerizable Resin Formulations.

PB96-146840

PB96-146857

Inelastic Neutron Scattering Study of Hydrogen in Nanocrystalline $\mathrm{Pd}$

PB96-14685

03,366 Not available NTIS

PB96-146865

Light-Scattering Studies on Phase Separation in a Binary Blend with Addition of Diblock Copolymers.

PB96-146865

01,286 Not available NTIS

PB96-146873

Influence of an Impenetrable Interface on a Polymer GlassTransition Temperature.

PB96-146873

03,175 Not available NTIS

PB96-146881

Electrodeposited Cobalt-Tungsten as a Diffusion Barrier between Graphite Fibers and Nickel.
PB96-146881 03,176 Not available NTIS PB96-146881

PB96-146899

Experimental Determination of the Ionization Energy of $1 O(X$ (sup 2) 11 (sub 3/2)) and Estimations of Delta(sub f) $\mathrm{H}$ (sup deg)(sub 0$)(10$ (sup - 1$)$ and $P A(10)$

PB96-146899

00.694 Not available NTIS

PB96-147012

Bioactive Polymeric Dental Materials Based on Amorphous Calcium Phosphate.

PB96-147012

03,572 Not available NTIS

PB96-147020

Remineralizing Dental Composites Based on Amorphous Calcium Phosphate.

PB96-147020

03,573 Not available NTIS

PB96-147038

Dielectric Measurements on Printed-Wiring and Circuit

Boards. Thin Films, and Substrates: An Overview.

PB96-147038

PB96-147046

02,236 Not available NTIS

Gravity-Current Transport in Building Fires.

PB96-147046

01,415 Not available NTIS

PB96-147053

General Types of Information Services

PB96-147053 02,735 Not available NTIS

PB96-147061

Rapid Method for the Isolation of Genomic DNA from 'Aspergillus fumigatus'

PB96-14706

03,488 Not available NTIS

PB96-147079

Pinch Effect in Commensurate Vortex-Pin Lattices.

PB96-147079

01,125 Not available NTIS

PB96-147087

Magnetoresistance of Thin-Film NiFe Devices Exhibiting Single-Domain Behavior.

PB96-147087

PB96-147095

Dynamics of Calcium Phosphate Precipitation

PB96-147095

04,766 Not available NTIS

PB96-147103 Cryogenic Bolometer.

PB96-147103

04,358 Not available NTIS

PB96-147111

Computing the Effect of Sprinkler Sprays on Fire Induced Gas Flow.

PB96-14711

PB96-147129

00,404 Not available NTIS

Gas-Coupled, Pulse-Echo Ultrasonic Crack Detection and Thickness Gaging.

PB96-147137

04,847 Not available NTIS

Comparison of k-Correction and Taylor-Series Correction for Probe-Position Errors in Planar Near-Field Scanning. PB96-147137 02,012 Not available NTIS
PB96-147152

Neutron Scattering Study of the Lattice Modes of Solid Cubane.

PB96-147152

01,126 Not available NTIS

PB96-147160

II-3: Critical Current Measurement Methods: Quantitative PBa6-147160

PB96-147178

04,767 Not available NTIS

First VAMAS USA Interlaboratory Comparison of High Temperature Superconductor Critical Current Measurements.
PB96-147178 04,768 Not available NTIS

PB96-147186

II-5: Thermal Contraction of Materials Used in Nb3Sn Critical Current Measurements.

PB96-147186

04,769 Not available NTIS

PB96-147194

USA Interlaboratory Comparison of Superconductor Simula tor Critical Current Measurements.

PB96-147194

04,770 Not available NTIS

PB96-147202

Heterodyne Mixing and Direct Detection in High Temperaure Josephson Junctions.

PB96-147202

PB96-147392

01,565 Not available NTIS

Airbome Asbestos Analysis: National Voluntary Laboratory Accreditation Program.

PB96-14739

02,566 PC A05/MF A01

PB96-147814

Virtual Environments for Health Care. A White Paper for the Advanced Technology Program (ATP), the National Institute f Standards and Tect

of Standards

03,594 PC A05/MF A01

PB96-147889

Collection of Results for the SPC/E Water Model. PB96-147889

PB96-147897

01,127 PC A03/MF A01

Review of Flows Driven By Natural Convection in Adiabatic Shafts.

PB96-147897

01,416 PC A04/MF A01

PB96-147905

Electronics and Electrical Engineering Laboratory Technical Progress Bulletin Covering Laboratory Programs, July to PB96-147905

01,942 PC A04/MF A01

PB96-147913

Technical Impact of the NIST Calibration Service for Electrical Power and Energy

96-147913

02,462 PC A05/MF A01

PB96-147954

NIST RS274KT interpreter

PB96-147954

02,835 PC A05/MF A01

Product Realization Process Modeling: A Study of Requirements, Methods and Research Issues.
PB96-147962

PB96-148010

Microwave Noise in High-Tc Josephson Junctions. PB96-148010

04,771 Not available NTIS

PB96-148028

Partially Coherent Transmittance of Dielectric Lamellae.

PB96-148028 04,359 Not available NTIS

PB96-148036

Water Permeability and Chloride Ion Diffusion in Portland Cement Mortars: Relationship to Sand Content and Critical Pore Diameter

PB96-148036

PB96-148044

Josephson D/A Converter with Fundamental Acculacy. PB96-148044

03,193 Not available NTIS

02,418 Not available NTIS

Performance and Reliability of NIST 10-V Josephson ArPB96-148051

02,419 Not available NTIS

PB96-148069

Accuracy in Time Domain Transmission Line Measurements.

PB96-148077

04,060 Not available NTIS

Spatial Correlation Function for Fields in a Reverberation Chamber.

PB96-148077

04,427 Not available NTIS

PB96-148085

Shear-Induced Mixing in Polymer Blends.

PB96-148085 01,287 Not available NTIS

PB96-148093

AC-DC Difference Characteristics of High-Voltage Thermal Converters.

PB96-148093

02,083 Not available NTIS

Free Radical Chemistry of the Atmospheric Aqueous

Phase.

0,117 Not available NTIS

Fire Community

Information R

00,211 Not available NTIS

PB96-148127

High-Temperature Fumace for In situ Small-Angle Neutron Scattering during Ceramic Processing.

PB96-148135

Performance of Multilayer Thin-Film Multijunction Thermal Converters.

PB96-148143

$\mathrm{V}-6$ : Effects of Temperature Variation

PB96-148143 04,772 Not available NTIS

PB96-148150

Scanning Capacitance Microscopy Measurements and Modeling: Progress Towards Dopant Profiling of Silicon.

PB96-148150

04,773 Not available NTIS

PB96-148168

Measurement of Patterned Film Linewidth for Interconnect Characterization.

PB96-148168

PB96-148176

High-Speed Interconnection Characterization Using Time Domain Network Analysis.

PB96-148176

04,061 Not available NTIS

Effects of Substrate Surface Steps on the Microstructure of Epitaxial BazYCu3O7-x Thin Films on (001) LaAlO3.

PB96-148184

04,774 Not available NTIS

PB96-148192

Heights of Wall-Fire Flames.

PB96-148192

00,212 Not available NTIS

PB96-148200

Examination of the Correlation between Cone Calorimete Data and Full-Scale Furniture Mock-Up Fires.

PB96-148200

PB96-151394

Fire Safety Engineering Research in the United States.

PB96-151394

00,213 Not available NTIS

PB96-151402

Elements of a Framework for Fire Safety Engineering.

PB96-151402

00,214 Not available NTIS

PB96-154422

Optical Fiber, Fiber Coating, and Connector Ferrule Geometry: Results

PB96-15442

PB96-154463

Workplan to Analyze the Energy Impacts of Envelope Airtightness in Office Buildings

PB96-154463

00,273 PC A04/MF A01

PB96-154521

Machine Performance Standard Provides Opportunity to Im-

prove Quality and Productivity.

PB96-154521

$02,837 \mathrm{PC} \mathrm{A02/MF} \mathrm{AO}$

PB96-154539

Guidelines for the Development of Mapping Tables.

PB96-154539

PB96-154562

Image Gradient Evolution: A Visual Cue for Dange

PB96-154562 
PB96-154976

STandard for the Exchange of Product Model Data (STEP) Procedures for NIST STEP Validation. PB96-154976 $02,787 \mathrm{PC} \mathrm{A03/MF} \mathrm{A01}$ PB96-154984

Development of a Test Method for Leaching of Lead from Lead-Based Paints Through Encapsulants.
PB96-154984 03,128 PC A04/MF A01

PB96-154992

Standard Generalized Markup Language Test Suite Evaluation Report.

PB96-155023

01,751 PC A03/MF A01

TEM/Reverberating Chamber Electromagnetic Radiation

Test Facility at Rome Laboratory.
PB96- 155023

$03,675 \mathrm{PC} \mathrm{A05/MF} \mathrm{A01}$

PB96-155411

Quantifying the Ignition Propensity of Cigarettes.

PB96-155411 00,306 Not available NTIS

PB96-155429

Metrology and Regional Trade Pacts

PB96-155429

02,923 Not available NTIS

PB96-155437

Annealing of Bragg Gratings in Hydrogen-Loaded Optical

Fiber.

PB96-155437

04,361 Not available NTIS

PB96-155445

Comparison of UV Photosensitivity and Fluorescence during Fiber Grating Formation

PB96-155445

04,362 Not available NTIS

PB96-155452

Catalogue of Electromagnetic Environment Measurements, $30-300 \mathrm{~Hz}$.

PB96-155452

01,943 Not available NTIS

PB96-155460

Mapping Domains in Proteins: Dissection and Expression of 'Escherichia coli' Adenylyl Cyclase.
PB96-155460 03,478 Not available NTIS

PB96-155478

Methodology for the Certification of Reference Specimens for Determination of Oxygen Concentration in Semiconductor Silicon by Infrared Spectrophotometry. 155478

PB96-155486

Development of Gas Standards from Solid 1,4dichlorobenzene.

02,496 Not available NTIS

Measurement of Atmospheric Methyl Bromide Using Gravimetric Gas Standards.

PB96-155494

02,497 Not available NTIS

Simultaneous Laser-Diode Emission and Detection for Simultaneous Laser-Diode Emission and Detection for
Fiber-Optic Sensor Applications.
PB96-155502 04,062 Not available NTIS

PB96-155510

Size Effects in Submicron NiFe/Ag GMR Devices.

PB96-155510 02,237 Not available NTIS

PB96-155528

Investigation of S2F10 Production and Mitigation in Com-

pressed SF6- Insulated Power Systems.
PB96-155528

PB96-155536

Population Distributions and Intralaboratory Reproducibility for Fat-Soluble Vitamin-Related Compounds in Human

PB96-155536

00,624 Not available NTIS

PB96-155544

Polymeric Calcium Phosphate Composites with

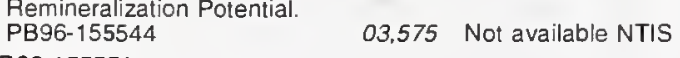

PB96-155551

Metrology Approach to Unifying Rockwell C Hardness PB96-155551

02,957 Not available NTIS

PB96-155569

Stylus Technique for the Direct Verification of Rockwell Diamond Intenders.

PB96-155577

Effects of Nonmedel Errors on Model-Based Testing.

PB96-155577

02,958 Not available NTIS

PB96-15557

Tunneling Spectroscopy of bcc(001) Surface States

PB96-155585 04,775 Not available NTIS

PB96-155593

Protecting Your Family from Fire.

Study of Ventilation Measurement in an Office Building.

PB96-155593 00,274 Not available NTIS

PB96-155601

Development and Application of an Indoor Air Ouality Com-

missioning Program in a New Office Building.
PB96-155601 00,275 Not available NTIS PB96-155759

Transitions to Chaos Induced by Additive and Multiplicative

PB96-155759

03,750 Not available NTIS
PB96-155767

Spectrum of the Stochastically Forced Duffing-Holmes Os-

PB96-155767

00,216 Not available NTIS

B96-155775

Necessary Condition for Homoclinic Chaos Induces by Additive Noise.
PB96-155775

PB96-155783

04,063 Not available NTIS

Development of Computer-Based Models of Standards and Attendant Knowledge-Base and Procedural Systems.

PB96-155783

00.464 Not available NTIS

PB96-155791

Findings and Recommendations from a Software Reengineering Case Study.

01,752 Not available NTIS

Transition from Localized Ignition to Flame Spread Over a Thin Cellulosic Material in Microgravity. Not available NTIS
PB96-155809 04,835 No9

PB96-156013

Improved Gas Flow Measurements for Next-Generation Processes.

PB96-156021

04,216 Not available NTIS

Executive Order 12941. Seismic Safety of Existing Federally Owned or Leased Buildings: It's History, Content and Objectives.

PB96-156021

PB96-156039

00,465 Not available NTIS

Physicochemical Characterization of Natural and Bioprosthetic Heart Valve Calcific Deposits: Implications for Prevention.

PB96-156039

PB96-156047

00,187 Not available NTIS

Model for Calculating Virial Coefficients of Natural Gas Hydrocarbons with Impurities.

04,064 Not available NTIS

PB96-156054

Virial Coefficients of Five Binary Mixtures of Fluorinated Methanes and Ethanes.

PB96-156054

01,128 Not available NTIS

PB96-156062

NIST Metrology Program on Electromagnetic Characterization of Materials.
PB96-156062

PB96-156070

01,944 Not available NTIS

Micromachined Display Output for a Cellular Neural Network

02,422 Not available NTIS

PB96-156088

CMOS Circuit Design for Controlling Temperature in Micromachined Devices.

PB96-156088

02,196 Not available NTIS

PB96-156104

Fast-Ion Conducting Y2(ZryTi1-y)2O7 Pyrochlores: Neutron Rietveld Analysis of Disorder Induced by $\mathrm{Zr}$ Substitution.

PB96-156104 04,776 Not available NTIS

PB96-156112

Security Program Management.

01,610 Not available NTIS

PB96-156120

Noise-Induced Transitions to Chaos.

PB96-156120

00,217 Not available NTIS

B96-156138

Deterministic and Stochastic Chaos.

PB96-156138

Experimental and Numerical Chaos in Continuous Systems: Two Case Studies.

PB96-156153

00,219 Not available NTIS

Internationalization of Fire Safety Engineering Research and Strategy.

PB96-156161

00,220 Not available NTIS

Application of Digital-Image-Based Models to Microstructure, Transport Properties, and Degradation of PB96-156161 Materials.

\section{B96-156179}

00,406 Not available NTIS

Fire Protection Engineering Tools. Simple Tools: The Equations.

PB96-15617

00,221 Not available NTIS

PB96-156187

00,307 Not available NTIS

PB96-156195

Review of Internatioanl Fire Risk Predictions Methods.

PB96-156195

00,222 Not available NTIS

B96-156203

Simple Variable Line Space Grating Monochromator for Synchrotron Light Source Beamlines.
PB96-156203 Not available NTIS PB96-157813

4,065 Not availabie NTIS

Modeling Detector Response for Neutron Depth Profing.
PB96-157813 04,066 Not available NTIS

PB96-157821

Optimizing Time-Domain Network Analysis.

PB96-157821

02,085 Not available NTIS

-157839

Al L2,3 Core Excitons in AlxGa1-x as Studied by Soft-X-ray Reflection and Emission.

PB96-157839

04,067 Not available NTIS

PB96-157847

Laser-Synchrotron Hybrid Experiments: A Photon to Tickle, A Photon to Poke.

PB96-157847

03,704 Not available NTIS

PB96-157854

Applicability of Effective Medium Theory to Ferroelectricl Ferrimagnetic Composites with Composition and Frequency-Depende

PB96-157854

PB96-157862 pedance Measurements of High Temperature Superconductors Using the Dielectric Resonator Technique.
PB96-157862

PB96-157870

Stochastic Modeling of a New Spectrometer

PB96-157870

04,068 Not available NTIS

PB96-157888

Two-Tier Mulitine TRL for Calibration of Low-Cost Network

Analyzers.

PB96-157896

01,947 Not available NTIS

Evolution of a United States Information System

PB96-157896

02,713 Not available NTIS

PB96-157904

Information Transfer in the 21st Century.

PB96-157904

Century. Not available NTIS

PB96-157912

Soft-X-ray-Emission Investigation of Cobalt Implanted Silicon Crystals.

PB96-157920

04,069 Not available NTIS

Sott-X-ray-Emission Spectra of Solid $\mathrm{Kr}$ and $X e$.

PB96-157920

04,070 Not available NTIS

B96-15793

Sott-X-ray-Emission Studies of Bulk Fe3Si, FeSi, and

FeSi2, and Implanted Iron Silicides.
PB96-157938 Not available NTIS

PB96-157946

Influence of Coadsorbed Potassium on the Electron-Stimulated Desorption of $F(+), F(-)$, and $F\left({ }^{*}\right)$ from $P F 3$ on Ru(0001)

PB96-157946

04,072 Not available NTIS

PB96-157953

RIS Measurement of AC Stark Shifts and Photoionization Cross Sections in Calcium

PB96-157953

04,073 Not available NTIS

6-157961

Characterization of LPE HgCdTe Film by Magnetoresistance
PB96-157961

PB96-157979

02,197 Not available NTIS

Comment On: Two-Photon Absorption Series of Calcium.

PB96-157979

PB96-157987

Laser Modification of Ultracold Collisions: Experiment.

PB96-157987

BB96-157995

Configuration-Dependent AC Stark Shifts in Calcium

PB96-157995 
PB96-158076

International Institute of Welding: Report on 1995 Actions. PB96-158076

PB96-158084

02874 Not available NTIS

What's Available in Weiding Software

PB96-158084

02,875 Not available NTIS

PB96-158092

New NIST/ARPA National Soft X-ray Reflectometry Facility.
PB96-158092

PB96-158092

B96-158654

Direct Comparison Transter of Microwave Power Sensor Calibrations.

PB96-15865

02,086 PC A03/MF A01

PB96-158662

SO Environmental Management Standardization Eftorts.

PB96-158662

02,528 PC AO3/MF AO

PB96-158670

Multiattribute Decision Analysis Method for Evaluating Build ings and Building Systems.

PB96-158670

00,325 PC A06/MF AO

PB96-158688

Conference Proceedings: International Workshop on instrumented Indentation. Held in San Diego, Calitornia on April

PB96-158688

01,948 PC A06/MF A01

PB96-158704

Liquid-Nitrogen-Cooled High Tc Electrical Substitution Radiometer as a Broadband IR Transfer Standard.

PB96-158704

02,198 PC AO3/MF AO

PB96-158712

Application Portability Profile (APP): The U.S. Government's Open System Environment Profile Version 3.0.

PB96-158712

01,753 PC A07/MF AO2

PB96-159215

Journal of Research of the National institute of Standards and Technology, November/December 1995. Volume 100 Number 6.

PB96-159215

PB96-159223

01,949 PC A08/MF A02

Calibration of Electret-Based Integral Radon Monitors Using NIST Polyethylene-Encapsulated (226)Ra/(222)Rn Emanation (PERE) Standards.

PB96-159223

01.950

PB96-159231

(Order as PB96-159215, PC A07/MF A02)

Microstructural Characterization of Cobalt-Tungsten Coated Graphite Fibers.

PB96-159231

(Order as PB96-159215, PC A07/MF A02)

PB96-15924

Using Collocation in Three Dimensions and Solving a Model Semiconductor Problem.

PB96-159249

(Order as PB96-159215, PC A07/MF A02)

PB96-159256

Precision Tests of a Ouantum Hall Eftect Device DC Equivalent Circuit Using Double-Series and Triple-Series Connec-

PB96-159256

(Order as PB96-159215, PC A07/MF A02)

PB96-159264

Analysis of the (5d(2)+5d6s)-5d6p Transition Arrays of Os Vil and Ir Vili, and the 6s (2)S-6P (2)P Transitions of Ir IX.

PB96-159264 Order as PB96-159215, PC A07/MF A02)

PB96-159611

Soft-X-ray Damage to $p$-terphenyl Coatings for Detectors

PB96-159611 04,364 Not available NTIS

PB96-159629

METRICATION: An Economic Wake-Up Call for Surveyors and Mappers.

PB96-159637

03,680 Not available NTIS

System for Intercomparing Standard Solutions of Beta-Paricle Emitting Radionuclides.

PB96-159637

03,707 Not available NTIS

PB96-159645

Comparison of Responses of a Select Number of Buildings to the 10/17/1989 Loma Prieta (California) Earthquake and Low-Level Amplitude Test Results.

PB96-159645

00,467 Not available NTIS

PB96-159652

Fire Hazard Model Developments and Research Efforts at

PB96-159652

00,407 Not available NTIS

PB96-159660

International Organization for Standardization: Current ACtivities in Fire Satety Engineering.

PB96-159660

00,223 Not available NTIS

PB96-159678

Boron-Implanted 6H-SiC Diodes

PB96-159678

04,081 Not available NTIS

PB96-159686

Extreme Winds Estimation by 'Peaks Over Threshold' and Epochal Methods.

00,468 Not available NTIS

B96-159694

Modeling of Extreme Loading by 'Peaks Over Threshold' Methods.

PB96-159694

00,469 Not available NTIS
PB96-159702

Workgroup Summary Report: Plastic Hinge-Based Techniques for Advanced Analysis.

PB96-159702

00,470 Not available NTIS

PB96-159710

Agent Screening for Halon 1301 Aviation Replacement. PB96-159710

03,282 Not available NTIS

\section{8}

Validation of a Turbulent Spray Flame Facility for the Assessment of Halon Alternatives.

PB96-15972

03,283 No: available NTIS

PB96-159736

Influence of Electrical Isolation on the Structure and Reflectivity of Multilayer Coatings Deposited on Dielectric SubStrates.

PB96-159744

04,365 Not available NTIS

New Method for Achieving Accurate Thickness Control for Uniform and Graded Multilayer Coatings on Large Flat Sub-

PB96-159744

PB96-159751

04,366 Not available NTIS

International Radon-in-Air Measurement Intercomparison Using a New Transter Standard.

PB96-159751

03,708 Not available NTIS

PB96-159769

Montgomery Education Connection and Resource Education Awareness Partnership Making Connections between Local Schools and NIST Volunteers. PBo6-159769

00,134 Not available NTIS

PB96-159777

Effects of Sample Mounting on Flammability Properties of Intumescent Polymers.

PB96-159777

03,389 Not available NTIS

\section{-159785}

Development of a Standard Reference Material for ISE Measurements of Sodium and Potassium.

PB96-159785

03,507 Not available NTIS

PB96-159793

Faraday Constant.

PB96-159801

01,955 Not available NTIS

Dynamics of Hydrogen Interactions with $\mathrm{Si}(100)$ and $\mathrm{Si}(111)$ Surtaces.

PB96-159801

04,082 Not available NTIS

PB96-160213

Effect of Intermediate Thermal Processing on Microstructural Changes of Oxygen Implanted Silicon-on-Insulator Materia.

PB96-16021

PB96-160221

02,982 Not available NTIS

Stacking Fault Pyramid Formation and Energetics in Siliconon-Insulator Material Formed by Multiple Cycles of Oxygen implantation and Annealing.

PB96-16022

04,083 Not available NTIS

PB96-160239

U.S. Government Accreditation and Conformity Assessment System Evaluation

PB96-160247

02,678 Not available NTIS

RII Spectroscopy of Trap Levels in Bulk and LPE Hg1.

xCdxTe.
PB96-160247

PB96-160254

External Gamma-ray Counting of Selected Tissues from a Thorotrast Patient

PB96-160262

Interface Roughness, Composition, and Alloying of LowOrder AIAs/GaAs Superlattices Studies by X-ray Diffraction.

PB96-160270

Ignition and Subsequent Flame Spread Over a Thin Cellulosic Material.

PB96-160270

PB96-160288

Ignition and Transition 10 Flame Spread Over a Thermally Thin Cellulosic Sheet in a Microgravity Environment.

PB96-160288

P896-160296

Phonon Relaxation in Soft-X-ray Emission of Insulators.

PB96-160296

04,085 Not available NTIS

PB96-160304

Research on Methods for Determining Optical Disk Media Life Expectancy Estimates.

PB96-160304

01,633 Not available NTIS

\section{B96-160312}

Laser-Cooled Neutral Atom Frequency Standards.

PB96-160312

04,086 Not available NTIS

PB96-160320

Electron and Hole Trapping in Irradiated SIMOX, ZMR and BESOI Buried Oxides.

PB96-160320

01,956 Not available NTIS

PB96-160338

Improved Reflectometry Facility at the National Institute of Standards and Technology.

PB96-160338

PB96-160346

New and Revised Half-Life Measurement Results. PB96-160346

PB96-160353

00,695 Not available NTIS

Effect of Single versus Multiple Implant Processing on De ect Types and Densities in SIMOX

PB96-160353

PB96-160361

International Challenges in Defining the Public and Private Interest in Standards.

PB96-160361

00,498 Not available NTIS

PB96-160379

NIST Metrology for Soft X-ray Multilayer Optics.

PB96-160379

04,088 Not available NTIS

PB96-160387

Upgraded Facility for Multilayer Mirror Characterization at PB96-160387

PB96-160395

04,367 Not available NTIS

Soft X-ray Reflectometry Program at the National Institute of Standards and Technology.

PB96-160395

04,368 Not available NTIS

Analysis of Small-Angle Scattering Data Dominated by Multiple Scattering for Systems Containing Eccentrically Shaped Particles or Pores.

PB96-160411

03,075 Not available NTIS

PB96-160429

Instrumental Smearing Effects in Radially Symmetric SmallAngle Neutron Scattering by Numerical and Analytical Meth ods.

B96-160429

02,984 Not available NTIS

PB96-160437

Ouality in Automated Manufacturing.

PB96-160437

PB96-160445

02,839 Not available NTIS

Appearance intensities for Multiply Charged Ions in a Strong Laser Field.

PB96-160445

04,089 Not available NTIS

PB96-160452

Comparison of FDDI Asynchronous Mode and DODB Cotropolitan Area Network Applications.

01,498 Not available NTIS

PB96-160460

Standardization for ATM and Related B-ISDN Technologies.
PB96-160460 01,499 Not available NTIS

PB96-160478

Novel DNA N-Glycosylase Activity of E. coli T4 Endonuclease $V$ That Excises 4,6-Diamino-5 ormamidopyrimidine from DNA, a UV-Radiation- and PB96-160478

PB96-160486 
PB96-160601

Computer-Aided Molecular Design of Fire Resistant Aircraft Materials.

PB96-160601

PB96-160619

00,025 Not available NTIS

Status of Emerging Standards for Removable Computer Storage Media and Related Contributions of NIST. PB96-160627

NIST Program for Investigating Error Reporting Capabilities of Optical Disk Drives.

PB96-160627

PB96-160635

Provision of Isochronous Service on IEEE 802.6.

PB96-160635 01,501 Not available NTIS

PB96-160643

Branch Lineshape Functions for CARS Thermometry

PB96-160643

for CARS Thermometry.
01,132 Not available NTIS

PB96-160650

Exits in Multistable Systems Excited by Coin-Toss SquareWave Dichotomous Noise: A Chaotic Dynamics Approach.

PB96-160650

04,824 Not available NTIS

PB96-160668

interfaces in Mo/Si Multilayers.

PB96-160668

02,423 Not available NTIS

PB96-160676

Implementation of the Fastener Quality Act.

PB96-160676

PB96-160684

02,876 Not available NTIS

New Concepts of Precision Dimensional Measurement for Modern Manufacturing.

PB96-160684

02.924 Not available NTIS

PB96-160692

Certification of Phencyclidine in Lyophilized Human Urine Reference Materials.
PB96-160692

PB96-160700

03,508 Not available NTIS

Generating and Measuring Displacements Up to $0.1 \mathrm{~m}$ to an Accuracy of $0.1 \mathrm{~nm}$ : Is it Possible.

PB96-160700

04,090 Not available NTIS

PB96-160718

Metrology Standards for Advanced Semiconductor Lithog-

raphy Referenced to Atomic Spacings and Geometry.
PB96-1607 18

PB96.160726

Nanometrology

PB96-160726

PB96-160734

Low-Energy Vibrations and Octahedral Site Occupation in $\mathrm{Nb95V5H(D)y}$

PB96-160742

Neutron Spectroscopic Comparison of beta-Phase Rare Earth Hydrides.

PB96-160759

Metrology.
PB96-160759

PB96-160767

ISDN in North America.

PB96-160767

PB96-160775

North American Agreements on ISDN

PB96-160775

01,133 Not available NTIS

B 96-160783

Scanned Probe Microscopies: Opportunities and Issues in Metrology.
PB96-160783

PB96-160791

SOA and TQM in Software Ouality Improvement.

PB96-160791

PB96-160817

Point Probe Decision Trees for Geometric Concept Classes. PB96-160817

01,134 Not available NTIS

02,925 Not available NTIS

PBg6-16

01,612 Not available NTIS

Precise Identification of Computer Viruses

PB96-160825 01,613 Not available NTIS

PB96-160833

Development of the NIST Transient Pressure and Temperature Calibration Facility.

PB96-160833

00,626 Not available NTIS

PB96-160841

Executive Summary: Proceedings of the Workshop on the Measurement of Transient Pressure and Temperature.

Measurement of Transient Pressure and Temperature.
PB96-160841 NTIS

PB96-160858

Slicing in the Presence of Parameter Aliasing.

PB96-160858

PB96-160866

01,755 Not available NTIS

Information Technology Standards in a Changing World: The Rose of the Users.

PB96-160866

02,737 Not available NTIS

PB96-160874

Using a Multi-Layered Approach to Representing Tort Law PB96-160874
PB96

00,135 Not available NTIS
PB96-160882

Concept Paper: Ari Overview of the Proposed Trust Technology Assessment Program.

PB96-160882

01,614 Not available NTIS

6-160890

Assessing Functional Diversity by Program Slicing

PB96- 160890

03,734 Not available NTIS

PB96-160908

Impact of Computer-Aided Acquisition and Logistic Support (CALS) in the Application of Standards.
PB96-160908

PB96.160916

ITS-90 Calibration Facility.

PB96-1609

00,627 Not available NTIS

PB96-160924

PEEE's POSIX: Making Progress.

PB96-160924

01,757 Not available NTIS

96-160932

Open Systems Software Standards in Concurrent Engineering.

PB96-160940

01,758 Not available NTIS

Predicate Differences and the Analysis of Dependencies in Formal Specifications.

60940

01,759 Not available NTIS

Technique for Analyzing the Effects of Changes in Formal Specifications.

PB96-160965

01,760 Not available NTIS

Template-Driven Systems Development with IDEF: Enterprise Standards for Reuse.

PB96-160965

02,788 Not available NTIS

6-160973

NIST POSIX Testing Program

PB96-160973

01,821 Not available NTIS

Program Slicing

PB96-160981

PB96-160999

Effects of Pipe Elbows and Tube Bundles on Selected Types of Flowmeters.

PB96-160999

01,135 Not available NTIS

PB96-161005

Pressure Measurements with the Mercury Melting Line Referred to ITS-90.

PB96-161211

01,136 Not available NTIS

Conference Report: International Conference on the Appli-

cation of Standards for Open Systems (6th).
PB96-161211 01,762 Not available NTIS

PB96-161229

GOSIP Testing Program.

PB96- 161229

01,504 Not available NTIS

PB96-161237

Guidance of the Legality of Keystroke Monitoring

PB96-161237

00,005 Not available NTIS

Industry/Government Open Systems Specification: The De-

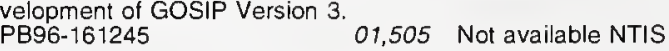

PB96-161252

Information Technology Standards in Federal Acquisitions.

PB96-161252 01,636 Not available NTIS

PB96-161260

Using Information Technology Standards in Federal Acquisi-

PB96-161260

PB96-161278

Powder X-ray Diffraction Data for $\mathrm{Ca} 2 \mathrm{Bi} 2 \mathrm{O} 5$ and PB96-161278

PB96-161286

Direct Comparison of Three PTB Silver Fixed-Point Cells with the NIST Silver Fixed-Point Cell.

PB96-161286

00,628 Not available NTIS

PB96-161294

Investigation of High-Temperature Platinum Resistance Thermometers at Temperatures Up to $962 \mathrm{C}$, and, in Some Cases, $1064 \mathrm{C}$

PB96-161294

PB96-161302

00,629 Not available NTIS

Investigation of the ITS-90 Subrange Inconsistencies for 255 Omega SPRTs.

PB96-161302

00,630 Not available NTIS

PB96-161310

NIST Assessment of ITS-90 Non-Uniqueness for $25.5 \mathrm{Ohm}$ SPRTs at Gallium, Indium and Cadmium Fixed Points.

PB96-161310

00,631 Not available NTIS

PB96-161328

NIST Implementation and Realization of the ITS-90 Over the Range $83 \mathrm{~K}$ to $1235 \mathrm{~K}$ : Reproducibility, Stability, and Uncertainties.

PB96-161328

PB96-161336

00,632 Not available NTIS

NIST Measurement Assurance of SPRT Calibrations on the

ITS-90: A Ouantitative Approach

PB96-161336

PB96-161344

Preliminary Results of a Comparison of Water Triple-Point

Cells Prepared by Different Methods.

PB96-161351

00,634 Not available NTIS

Analysis of Standards for the Assurance of High Integrity

Software.

03,735 Not available NTIS

PB96-161369

Control and Instrumentation: Standards for High-Integrity Software.

PB96-161369

PB96-161377

03,736 Not available NTIS

Report of a Workshop on the Assurance of High Integrity Software.

PB96-161377

PB96-161385

01.763 Not available NTIS

Standards for High Integrity Software.

PB96-161385

01,764 Not available NTIS

PB96-161393

Verification and Validation.

PB96-161393

PB96-16140

01,765 Not available NTIS

Verification and Validation of Reengineered Software

PB96-161401

PB96-161617

01,766 Not available NTIS

Residual Error Compensation of a Vision-Based Coordinate Measuring Machine

PBg -18

04,091 Not available NTIS

PB96-161625

imposed Oscillations of Kinetic Barriers Can Cause an Enzyme to Drive a Chemical Reaction Away from Equilibrium.

PB96-161633

Ouadratic Response of a Chemical Reaction to External Oscillations.

PB96-161641

Genetically Engineered Pores for New Materials.

PB96-161641

03,550 Not available NTIS

PB96-161658

Genetically Engineered Pore as a Metal Ion Biosensor.

PB96-161658

PB96-161666

03,551 Not available NTIS

Verification of Revised Water Vapour Correction to the Index of Retraction of Air.

PB96-161666

02,680 Not available NTIS

PB96-161674

Current Noise Reveals Protonation Kinetics and Number of Ionizable Sites in an Open Protein lon Channel.

PB96-161674

PB96-161682

Noise Analysis of Ionization Kinetics in a Protein Ion Chan-

PB96-161682

PB96-161690

04,093 Not available NTIS

Inter-Laboratory Trials of the EPR Method for the Detection of Irradiated Meats Containing Bone.

Of Irradiated Me

00,042 Not available NTIS

PB96-161708

Measurement of the Neutron Lifetime

PB96-161708

PB96-161716

04,094 Not available NTIS

SPC Artifact for Automated Solder Joint Inspection

PB96-161716

04,095 Not available NTIS 
PB96-161815

Response to Comments on the NIST Proposed Digital Signature Standard.

01,615 Not available NTIS

PB96-161823

Lattice Statics of Interfaces and Interfacial Cracks in Bimaterial Solids.

PB96-161823

02,985 Not available NTIS

PB96-161831

Effect of CF3H and CF3Br on Laminar Diffusion Flames in Normal and Microgravity.

PB96-16183

01,420 Not available NTIS

PB96-161849

Effect of CF3H and CF3Br on Laminar Diffusion Flames in Normal and Microgravity.

01,421 Not available NTIS

PB96-161856

Vapor-Liquid Equilibria of Temary Mixtures in the Critica Region on Paths of Constant Temperature and Overall Composition.

PB96-161864

01,139 Not available NTIS

Gas Absorption during Ion-Irradiation of a Polymer Targe

PB96-161864 04,099 Not available NTIS

PB96-161872

Upgrade and Modernization Projects at the NBSR

PB96-161872 03,737 Not available NTIS

PB96-161880

Electric Field Effects on a Near-Critical Fluid in Microgravity
PB96-161880

PB96-161880

PB96-161898

Density Dependence of Fluid Properties and Non-Newtonian Flows: The Weisenberg Effect.

PB96-161898

01,140 Not available NTIS

PB96-161906

Nonlinear Correlation of High-Pressure Vapor-Liquid Equilibrium Data for Ethylene $+n$-Butane Showing Inconsistencies in Experimental Compositions.

PB96-16 190

01,141 Not available NTIS

P896-161914

Quantum Collisional Transfer Contributions to the Density Dependence of Gaseous Viscosity

PB96-161914

P896-161922

Correlations between Flaw Tolerance and Reliability in

Zirconia.

02,986 Not available NTIS

P896-161930

Cryogenic Flow Calibration in NIST.

- 161930

01,143 Not available NTIS

PB96-161948

Business and Manufacturing Motivations for the Developing of Analytical Technology and Metrology for Semiconductors

PB96-161948 04,778 Not available NTIS

P896-161955

SSME LOX Duct Flowmeter Design and Test Results.

PB96-161955

04,826 Not available NTIS

PB96-161963

Electrical Measurements for Monitoring and Control of if Plasma Processing.

PB96-161971

04,369 Not available NTIS

Fundamentals of Fracture: A 1993 Prologue, and Other

Comments.

PB96-161989

03,218 Not available NTIS

Interfacial Crack in a Two-Dimensional Hexagonal Lattice.

PB96-161989 04,100 Not available NTIS

PB96-161997

Whither Computational Materials Science. Some Thoughts

PB96-161997

02,987 Not available NTIS

P896-162003

Dislocation Core-Core Interaction and Peierls Stress in a Model Hexagonal Lattice.

PB96-162003

04,101 Not available NTIS

PB96-16244

Energy Price Indices and Discount Factors for Life-Cycle Cost Analysis 1996. Annual Supplement to NIST Handbook

135 and NBS Special Publication 709. (Revised).
PB96-162441
02,510 PC A05/MF A01

P896-162540

Estimates of Hurricane Wind Speeds by the 'Peaks Over Threshold' Method.

PB96-162540

$00,471 \mathrm{PC}$ A04/MF A01

PB96-162649

Radiated Emissions and Immunity of Microstrip Transmission Lines: Theory and Measurements.

PB96-162649

02,238 PC A04/MF A01

P896-162714

National Voluntary Laboratory Accreditation Program 1996 Directory.

PB96-162714

00,485 PC A10/MF A03

Calibration Service for Coaxial Reference Standards for

Calibration Service for Coaxial Reference Standards to
Microwave Power.
PB96-162722
01,958 PC A07/MF A02

01,958 PC A07/MF A02
P896-163613

Fracture in Multilayers.

PB96-163613

PB96-163621

02,988 Not available NTIS

Gage Block Standards, Measurement Capabilities and LabOratory Accreditation.

02,757 Not available NTIS

\section{PB96-163639}

Autofluorescence Detection of 'Escherichia coli' on Silver Membrane Filters.

PB96-163647

03,590 Not available NTIS

Bacterial Enumeration in Storage Water.

PB96-163647 03,591 Not available NTIS

PB96-163654

Feasibility of Fluorescence Detection of Tetracycline in edia Mixtures Employing a Fiber Optic Probe.

PB96-16365

00,511 Not available NTIS

PB96-163662

Novel Amperometric Immunosensor for Procainamide Employing Light Activated Labels.

B96-163662

00,512 Not available NTIS

Effect of Finite Beam Width on Elastic Light Scattering from

Droplets.

PB96-163688

01,144 Not available NTIS

Elastic Scattering from Spheres under Non Plane-Wave Illumination.

PB96-163688

PB96-163696

04,370 Not available NTIS

Cracks and Dislocations in Face-Centered Cubic Metallic Multilayers.

PB96-163704

02,989 Not available NTIS

Model for Microcrack Initiation and Propagation beneath Hertzian Contacts in Polycrystalline Ceramics.

PB96-163704 03,077 Not available NTIS

PB96-163712

Inhibition of Premixed Methane-Air Flames by Iron Pentacarbonyl

PB96-163720

00,513 Not available NTIS

Parametric Study of Hydrogen Fluoride Formation in Suppressed Fires.

00,514 Not available NTIS

PB96-163738

Molar Heat Capacity at Constant Volume for Air from 67 to $300 \mathrm{~K}$ at Pressures to $35 \mathrm{MPa}$.

PB96-163738

00,515 Not available NTIS

PB96-163746

Charge-Transfer-Induced Multiplet Structure in the N4,5O2,3 Soft-X-ray Emission Spectrum of Lanthanum.
PB96-163746

PB96-163753

Influence of Shear on the Ordering Temperature of a Triblock Copolymer Melt.

PB96-163753

01,288 Not available NTIS

PB96-163761

Non-Newtonian Flow between Concentric Cylinders Calculated from Thermophysical Properties Obtained from Sim-

PB96-16376

PB96-163779

04,103 Not available NTIS

2D-Scanning Capacitance Microscopy Measurements of

Cross-Sectioned VLSI Teststructures.
PB96-163779 04,104 Not available NTIS

PB96-163787

High-Accuracy Principal-Angle Scanning Spectroscopic

Ellipsometry of Semiconductor Interfaces.
PB96-163787 02,427 Not available NTIS

PB96-163787

PB96-163795

Model for Toughness Curves in Two-Phase Ceramics. 2. Microstructural Variables.

PB96-163795

03,078 Not available NTIS

B96-163803

$\mathrm{CO} 2 / \mathrm{CH} 4$ Transport in Polyperfluorosulfonate Ionomers: Effects of Polar Solvents on Permeation and Solubility.

PB96-163803

01,145 Not available NTIS

PB96-164017

Natural Convection from an Array of Electronic Packages Mounted on a Horizontal Board in a Narrow Aspect Ratio Enclosure.

PB96-164017

02,087 Not available NTIS

PB96-164025

Enhanced Voltage-Dividing Potentiometer for High-Precision Feature Placement Metrology.

996-164025

02,428 Not available NTIS

PB96-164033

Retention of Halocarbons on a Hexafluoropropylene Epoxide-Modified Graphitized Carbon Black. 4. Propane-Based Compounds.

PB96-164033

PB96-164041

03,284 Not available NTIS

Simple and Efficient Methane-Marker Devices for Chromatographic Samples. $\quad 00,635$ Not available NTIS
PB96-164041

P896-164058

Use of Pressure for Quantum-Well Band-Structure Characerization.

PB96-164058

04,779 Not available NTIS

PB96-164066

Heat Transfer in an Intumescent Material Using a Three-Dimensional Lagrangian Model.

PB96-164066

00,408 Not available NTIS

P896-164074

SEMPA Situdies of Exchange Coupling in Magnetic Multilayers.
PB96-164074

P896-164082

Publication and Presentation Abstracts, 1995

PB96-164082

164090

President's Column 'Editorial'

PB96-164090

02,239 Not available NTIS

896-164108

Calculating Combined Buoyancy- and Pressure-Driven Flow Through a Shallow, Horizontal, Circular Vent; Application to Problem of Steady Burning in a Ceiling-Vented Enclosure.
PB96-164108

PB96-164116

Combined Buoyancy and Pressure-Driven Flow Through a Shallow, Horizontal, Circular Ven

PB96-164116

00,410 Not available NTIS

PB96-164124

Commentary on 'Optimization of Experimental Parameters for the EPR Detection of the Cellulosic Radical in Irradiated

PB96-164124

00,043 Not available NTIS

P896-164132

Novel Activities of Human Uracil DNA N-glycosylase for Cylosine-Derived Products of Oxidative DNA Damage. PB96-164132

P896-164140

Population Trapping in Short-Pulse Multiphoton Ionization. PB96-164140

P896-164157

Isotopic and Nuclear Analytical Techniques in Biological Systems: A Critical Study. 10. Elemental Isotopic Dilution Analysis with Radioactive and Stable Isotopes (Technical Report).

PB96-164157

00,696 Not available NTIS

PB96-164165

In Search of Alternative Fire Suppressants.

PB96-164165

03,285 Not available NTIS

Modeling Buffer Layer IGBT's for Circuit Simulation.

PB96-164173

02,429 Not available NTIS

P896-164181

Distributed Architecture for Standards Processing

PB96-164181

00,276 Not available NTIS

P896-164199

Electrical Characterization of Narrow Gap n-Type Bulk $\mathrm{HgCdTe}$ Single Crystals by Variable-Magnetic-Field $\mathrm{Ha}$ Measurements and Reduced-Conductivity-Tensor Analyses.
PB96-164199 P896-164207

Scanning Capacitance Microscopy Measurements and Modeling for Dopant Profiling of Silicon.
PB96-164207 04,781 Not available NTIS

P896-164215 


\section{PB96-165394}

Guide to a Format for Data on Chemical Admixtures in a Materials Property Database.

PB96-165394

$01,327 \mathrm{PC} \mathrm{A04/MF} \mathrm{A01}$

PB96-165402

Group 1 for the Plant Spatial Configuration STEP Application Protocol.

PB96-165410

02,789 PC A11/MF A03

Band-Limited, White Gaussian Noise Excitation for ReverBand-Limited, White Gaussian Noise Excitation for Rever-
beration Chambers and Applications to Radiated Susceptibility Testing

PB96-165410

PB96-165428

$01,960 \mathrm{PC} \mathrm{A07/MF} \mathrm{A02}$

Machining Process Planning Activity Model for Systems Integration.

PB96-165428

Multizone Modeling of Three Residential Indoor Air Ouality Control Options.

$02,567 \mathrm{PC} \mathrm{A08/MF} \mathrm{A02}$

PB96-165840

Report of the National Conference on Weights and Measures ( 80 th) as Adopted by the 80 th National Conference on Weights and Measures, 1995. Held in Portland, Maine on July 16-20, 1995

PB96-165840

PB96-165915

02,681 PC A15/MF A03

Manager's Guide for Monitoring Data Integrity in Financial Systems.
PB96-165915

00,003 PC A05/MF A01

PB96-165931

Basic Linear Algebra Operations in SLI Arithmetic

PB96-165931 03,421 PC A03/MF A01

PB96-165949

3,427 PC AO3/MF AO1

Modified Optimal Algorithm for Active Structural Control.

PB96-165949

00,472 PC AO4/MF A01

PB96-165956

Enhancement of R123 Pool Boiling by the Addition of NHexane.

PB96-165956

02,605 PC AO4/MF AO

PB96-165964

Using S-Check, Alpha Release 10

PB96-165964

01,767 PC A05/MF A01

PB96-165972

Preliminary Processing of the Lotung LSST Data.

PB96-165972

03,690 PC A05/MF A01

PB96-165980

PIECS: A Software Program for Machine Tool ProcessIntermittent Error Compensation.

PB96-165980

02,842 PC A08/MF AO2

PB96-165998

Beyond the Technology Roadmaps: An Assessment of Electronic Materials Research and Development.
PB96-165998 01,961 PC A05/MF A01

PB96-166004

Public Key Infrastructure Invitational Workshop. Held in McLean, Virginia on September 28, 1995.
PB96-166004 PBg6-166

NIST Handbook 44, 1996: Specifications, Tolerances, and Other Technical Requirements for Weighing and Measuring Devices as Adopted by the 80th National Conference on Weights and Measures, 1995.

PB96-166616

PB96-167093

02,926 PC A13/MF A03

Determination of Total Protein Adsorbed on Solid (Membrane) Surface by a Hydrolysis Technique: Single Protein PB96-167093

03,552 Not available NTIS

PB96-167101

Dead Time, Pileup, and Accurate Gamma-Ray Spectrom-

etry.

00,697 Not available NTIS

PB96-167119

Loss-Free Counting at IRI and NIST.

PB96-167119 04,105 Not available NTIS

PB96-167127

Trace Element Concentrations in Cetacean Liver Tissues Archived in the National Marine Mammal Tissue Bank.

PB96-167127 02,595 Not available NTIS

PB96-167135

Molar Heat Capacity at Constant Volume for $(x \mathrm{CO} 2+(1-$ x) $\mathrm{C} 2 \mathrm{H} 6$ ) from 220 to $340 \mathrm{~K}$ at Pressures to $35 \mathrm{MPa}$

PB96-167135

01,148 Not available NTIS

PB96-167143

Accurate Electrical Characterization of High-Speed Interconnections.

PB96-167150

02,240 Not available NTIS

REFPROP Refrigerant Properties Database: Capabilities, Limitations, and Future Directions.

PB96-167150

01,149 Not available NTIS

B96-167168

Supercritical Fluid Extraction-Immunoassay for the Rapid Screening of Cocaine in Hair.

00,637 Not available NTIS
PB96-167176

Simulation and SANS Studies of Gelation Under Shear. PB96-167176

01,150 Not available NTIS

PB96-167184

Small-Angle Neutron-Scattering Study of Dense Sheared Silica Gels.

PB96-167184

PB96-167192

01,151 Not available NTIS

Optical Characterization of Materials and Devices for the Semiconductor Industry: Trends and Needs.

PB96-167192

PB96-167200

Analysis of Transverse Flow in Aligned Fibrous Porous Media.

03,177 Not available NTIS

PB96-167218

Status of the Round Robin on the Transport Properties of R134a.

PB96-167218

01,152 Not available NTIS

PB96-167226

Structural Analysis of Heparin by Raman Spectroscopy

PB96-167226 03,480 Not available NTIS

PB96-167234

Viscosity of Defined and Undefined Hydrocarbon Liquids Calculated Using an Extended Corresponding-States Model.
PB96-167234

PB96-167242

Determination of 21 Elements by INAA for Certification of SRM 1570a, Spinach.

New NIST Rapid Pneumatic Tube System

PB96-167259

PB96-167267

Recent Developments in NIST Botanical SRMs.

PB96-167267

03,489 Not available NTIS

PB96-167275

Relationship of Silver with Selenium and Mercury in the Liver of Two Species of Toothed Whales (Odontocetes).

Resolution of Discrepant Analytical Data in the Certification of Platinum in Two Automobile Catalyst SRMs.

PB96-167283

PB96-167291

Permeation Tube Approach to Long-Term Use of Automatic Sampler Retention Index Standards.

Sampler Retention. Index Standards. PBo6-1e

Retention of Halocarbons on a Hexafluoropropylene Epoxde-Modified Graphitized Carbon Black. 3. Ethene-Based Compounds.

03,286 Not available NTIS

Dynamic Scaling in an Aggregating 2D Lennard-Jones SysPB96-167317

PB96-167325

Double-Modulation and Selective Excitation Photoreflectance for Wafer-Level Characterization of Quantum-Well Laser Structures.

PB96-167325

PB96-167333

Structure and Rheology of Hard-Sphere Systems.

PB96-167333 00,662 Not available NTIS

PB96-167

Preparation and Crystal Structure of Sr5TiNb4017.

PB96-167341

PB96-167358

04,107 Not available NTIS

Thermophysical Property Standard Reference Data from NIST

PB96-167358

01,153 Not available NTIS

PB96-167366

Planar Near-Field Measurements and Microwave Holography for Measuring Aperture Distribution on a $60 \mathrm{GHz} \mathrm{Ac}-$ tive Array Antenna.

PB96-167366

03,762 Not available NTIS

PB96-167374

Small Angle Neutron Scattering Study of a Clay Suspension Under Shear.

PB96-167382

00,663 Not available NTIS

New Method for the Detection and Measurement of Polyaromatic Carcinogens and Related Compounds by DNA Intercalation.

PB96-167382

03,481 Not available NTIS

PB96-167390

sochoric (p-p-T) Measurements on Liquid and Gaseous Ai from 67 to $400 \mathrm{~K}$ at Pressures to $35 \mathrm{MPa}$.

PB96-167390 01,154 Not available NTIS

PB96-16

Genetically Engineered Pores as Metal Ion Biosensors.

PB96-167408

03,553 Not available NTIS

PB96-167812

Study to Determine the Existence of an Azeotropic R-22 'Drop-ln' Substitute.

02,568 PC A04/MF A01

PB96-167820

Method of Estimating the Parameters of Tuned Mass Dampers for Seismic Applications. PB96-167820

PB96-167838

Application of the Pointer State Subgraph to Static Program

PB96-167838

01,768 PC A03/MF A01

$-16784$

Data Communications Strategy

PB96-167846

02,738 PC A05/MF A01

PB96-167978

Application of Electromagnetic-Acoustic Transducers for Nondestructive Evaluation of Stresses in Steel Bridge Struc-

96-167978

$01,301 \mathrm{PC} \mathrm{A04/MF} \mathrm{A01}$

PB96-168984

Federal Implementation Guideline for Electronic Data Interchange. ASC X12 003050 Transaction Set 843 Response to Request for Ouotation. Implementation Convention.

PB96-168984

01,822 PC A09/MF A02

PB96-169057

Journal of Research of the National Institute of Standards and Technology, September/October 1993. Volume 98 Number 5.

PB96-16905

03,368 PC AO6/MF A01

PB96-169065

Transformation of BCC and B2 High Temperature Phases to HCP and Orthorhombic Structures in the Ti-Al-Nb Sysem Part 1. Microstructural Predictions Based on a Sub group Relation between Phases.

PB96-169065 (Order as PB96-169057, PC A05/MF A01)

PB96-169073

Transformation of $\mathrm{BCC}$ and $\mathrm{B} 2$ High Temperature Phases to HCP and Orthorhombic Structures in the Ti-Al-Nb System. Part 2. Experimental TEM Study of Microstructures. PB96-169073 03,371
0

PB96-16908

Corrosion Characteristics of Silicon Carbide and Silicon Nitride.

PB96-169081

(Order as PB96-169057, PC A05/MF A01)

PB96-169099

$X$ Window System, Version 11, Release 5

PB96-169099

Uniform Laws and Regulations in the Areas of Legal Metrology and Motor Fuel Ouality as Adopted by the 80th National Conference on Weights and Measures 1995. 1996 PB96-1723

PB96-17 2317

02,927 PC A12/MF A03

Life-Cycle Costing Manual for the Federal Energy Management Program. 1995 Edition.

PB96-172317

$02,511 \mathrm{PC}$ A10/MF A02

PB96-172325

Guidelines for the Evaluation of Electronic Data Interchange Products.

PB96-172333

NIST Measurement Assurance Program for Capacitance Standards at $1 \mathrm{kHz}$

PB96-172333

02,276 PC A03/MF A01

PB96-172358

Trapped Ions and Laser Cooling 4: Selected Publications of the Ion Storage Group of the Time and Frequency Division, NIST, Boulder, Colorado.

PB96-172358

04,108 PC A11/MF A03

PB96-172374

Federal Implementation Guideline for Electronic Data Interchange. ASC X12 003050 Transaction Set 855 Purchase Order Acknowledgment: Implementation Convention. 
PB96-175674

International Marine-Atmospheric (222)Rn Measurement Intercomparison in Bermuda. Part 1. NIST Calibration and Methodology for Standardized Sample Additions.

PB96-175674

Order as PB96-175666, PC A07/MF A02)

PB96-175682

International Marine-Atmospheric (222)Rn Measuremen Intercomparison in Bermuda. Part 2. Results for the Partici-

pating Laboratories
PB96-175682

(Order as PB96-175666, PC A07/MF A02)

PB96-175690

Theory of Electron Beam Moire.

PB96-175690 04,373

PB96-175708

Scaling Compartment Fires: Reduced- and Full-Scale Enclosure Burns

PB96-175716

00,224 Not available NTIS

Five O's (Cues) of the U.S. GOSIP Testing Program

PB96-175716 01,826 Not available NTIS

PB96-175724

Suppression of High-Speed C2H4/Air Flames with C1Halocarbons.

PB96-175732

03,287 Not available NTIS

Effectiveness of Halon Alternatives in Suppressing Dynamic Combustion Process.

PB96-175732

03,288 Not available NTIS

PB96-175740

Halon Thermochemistry: 'Ab Initio' Calculations of the Enthalpies of Formation of Fluoromethanes.

PB96-175740 03,289 Not available NTIS

PB96-175856

NIST List of Publications, LP 103, March 1996. National Semiconductor Metrology Program.

PB96-175856

02,432 PC A07/MF A02

PB96-176417

Interaction of HFC-125, FC-218 and CF3I with High Speed Combustion Waves.

PB96-1764 17

03,290 Not available NTIS

Suppression of Ignition Over a Heated Metal Surface.

PB96-176425 03,291 Not available NTIS

PB96-176433

Realization of NIST 1995 Luminous Flux Scale Using the Integrating Sphere Method.

04,374 Not available NTIS

PB96-176441

Single-Port Technique for Adaptor Efficiency Evaluation PB96-176441

02,088 Not available NTIS

PB96-176458

Novel Magnetic Field Characterization Techniques for Compound Semiconductor Materials and Devices.

PB96-17

02,433 Not available NTIS

Irradiances of Spectral Lines in Mercury Pencil Lamps. PB96-176466

PB96-176474

Wavelengths of Spectral Lines in Mercury Pencil Lamps PB96-176474

PB96-176482

Dimensional Characterization of Precision Coaxial Transmission Line Standards.

PB96-176490

02,241 Not available NTIS

Electrical Characteristics of Argon Radio Frequency Glow Discharges in an Asymmetric Cell.
PB96-176490 04,109 Not available NTIS

PB96-176508

Potential Surfaces and Dynamics of Weakly Bound Trimers: Perspectives from High Resolution IR Spectroscopy.

PB96-176508

01,155 Not available NTIS

PB96-176516

Growth and Nucleation of Hydrogenated Amorphous Silicon on Silicon (100) Surfaces.

PB96-176516

02,991 Not available NTIS

PB96-176524

Magnetic Dielectric Oxides: Subsolidus Phase Relations in the BaO: Fe2O3: TiO2 System.

PB96-176524

01,156 Not available NTIS

PB96-176532

Solid State (13)C NMR and Raman Studies of Cellulose Triacetate: Oligomers, Polymorphism, and Inferences about Chain Polarity.
PB96-176532

PB96-176540

01,289 Not available NTIS

Automated Measurement of Nonlinearity of Optical Fiber PB96-176540

PB96- 176557

04,110 Not available NTIS

Pore-Forming Protein with a Metal-Actuated Switch

PB96-176557

PB96-176565

03,554 Not available NTIS

Fundamental Limits on the Frequency Stabilities of Crystal Oscillators.

PB96-176565

02,277 Not available NTIS
PB96-176573

Electromagnetic Coupling Character of (001) Twist Boundaries in Sintered Bi2Sr2CaCu2O8+x Bicrystals. PB96-176573

01,963 Not available NTIS

PB96-176581

Implementation of a Standard Format for GPS Common View Data.
PB96-176581

PB6-176599

01,555 Not available NTIS

Line-Reflect-Match Calibrations with Nonideal Microstrip Standards.

02,242 Not available NTIS

PB96-176607

State-Resolved Rotational Energy Transfer in Open Shell Collisions: $\mathrm{Cl}((2) \mathrm{P} 3 / 2)+\mathrm{HCl}$

PB96-17660

01,157 Not available NTIS

PB96-176615

Magnetic Structure Determination for Annealed Ni80Fe2O Ag Multilayers Using Polarized-Neutron Reflectivity.

PB96-176615

03,739 Not available NTIS PB96-176623

Publication and Presentation Abstracts, 1994

PB96-176623 03,577 Not available NTIS

PB96-176631

Energy Distributions of Neutrons Scattered from Solid C60

by the Beryllium Detector Method.
PB96-176631 740 Not available NTIS

B96-176649

Simulations of Neutron Focusing with Curved Mirrors. PB96-176649

PB96-176656

02,200 Not available NTIS

Estimation of the Orientation Distribution of Short-Fiber Composites Using Ultrasonic Velocities.

PB96-17665

03,178 Not available NTIS

PB96-176664

New Data and Correlations for the Custody Transfer of Natural Gas Liquids.

PB96-176672

02,499 Not available NTIS

Strategy to Support Multipoint Communication Service Over Native ATM Service.

PB96-176672

PB96- 176680

01,507 Not available NTIS

Viscosity of 1,1,1,2,3,3-Hexafluoropropane and 1,1,1,3,3,3Hexafluoropropane at Saturated-Liquid Conditions from 262 $\mathrm{K}$ to $353 \mathrm{~K}$.

PB96-176680

PB96-176698

03,292 Not available NTIS

Small-Angle Neutron Scattering (SANS) Study of WormLike Micelles Under Shear.

PB96-176698

04,111 Not available NTIS

PB96-176706

Developments in Stellar Coronae.

PB96-176706

00,107 Not available NTIS

PB96-176714

Transition Regions of Capella (1995)

PB96-176714

00,108 Not available NTIS

PB96-176722

Microwave Characterization of Flip-Chip MMIC Components.

PB96-176722

02,434 Not available NTIS

B96- 176730

Microwave Characterization of Flip-Chip MMIC Interconnec-

PB96-176730

PB96-176748

02,435 Not available NTIS

Electrical Measurements of Microwave Flip-Chip Interconnections.

PB96-176755

02,436 Not available NTIS

Report of the Refrigeration. Air Conditioning and Heat Pumps Technical Options Committee.

PB96-176755

03,293 Not available NTIS

PB96-176763

Reference Relations for the Evaluation of the Materials Properties of Orthorhombic YBa2Cu3Ox Superconductors.
PB96-176763 04,782 Not available NTIS

PB96-176771

Surface Plasmon Microscopy of Biotin-Streptavidin Binding Reactions on UV-Photopatterned Alkanethiol Self-Assembled Monolayers.

PB96-176771

01,158 Not available NTIS PB6-176789

Thermal Anemonetry for Mass Flow Measurement in Oscillating Cryogenic Gas Flows.
PB96- 176789

PB96-176797

04,218 Not available NTIS

Characterization of the Structure of LaD2.50 by Neutron Powder Diffraction

PB96-1767

04,783 Not available NTIS

B 96-176805

International Standard Equation of State for the Thermodynamic Properties

PB96-176805

PB96-177373

Innovation in the Japanese Construction Industry: A 1995 Appraisa.

PB96-177373

$00,225 \mathrm{PC} \mathrm{A13/MF} \mathrm{A03}$

PB96-17738

Journal of Research of the National Institute of Standards and Technology, March/April 1996. Volume 101, Number 2. PB96-177381

PB96-178892

Federal Implementation Guideline for Electronic Data Interchange: ASC X12 003050 Transaction Set 836 Procurement Notices. Implementation Convention.

PB96-17889

$01,827 \mathrm{PC} \mathrm{A04/MF} \mathrm{A01}$

PB96-178900

Voluntary Product Standard PS 1-95 Construction and Industrial Plywood.

PB96-178918

$03,406 \mathrm{PC}$ A04/MF A01

Directory of Law Enforcement and Criminal Justice Associations and Research Centers.

PB96-178918

04,872 PC A05/MF A01

PB96-178926

Specifications and Tolerances for Reference Standards and Field Standard Weights and Measures. 2. Specifications and Tolerances for Field Standard Measuring Flasks.

PB96-178926
02,682 PC A03/MF A01

PB96-178926

NIST Detector-Based Luminous Intensity Scale.

$\begin{array}{rr}\text { PB96-179114 } & \text { O1.864 } \\ & \text { (Order as PB96-177381, PC A07/MF A02) }\end{array}$

\section{PB96-179122}

NIST High Accuracy Scale for Absolute Spectral Response from $406 \mathrm{~nm}$ to $920 \mathrm{~nm}$

PB96-179122 01.865

\section{PB96- 179130}

Irradiance of Horizontal Ouartz-Halogen Standard Lamps.

PB96-179130 01,866

\section{PB96-179148}

Development of the Ion Exchange-Gravimetric Method for Sodium in Serum as a Definitive Method.

PB96-179148 (Order as PB96-177381, PC A07/MF A02)
(O)

PB96-179155

MasPar MP-1 as a Computer Arithmetic Laboratory.

PB96-179155 (Order as PB96-177381, PC A07/MF A02)
(O)

PB96-179163

Evidence That Voltage Rather Than Resistance is Ouan-

tized in Breakdown of the Ouantum Hall Effect.

PB96-179163

(Order as PB96-177381, PC A07/MF A02)

PB96-179411

Tree-Lookup for Partial Sums Or: How Can I Find This Stuff

PB96-179411

PB5-179429

01,770 Not available NTIS

Objective Evaluation of Short-Crack Toughness Curves Using Indentation Flaws: Case Study on Alumina-Based Ceramics.

PB96-179437

03,079 Not available NTIS

Organization and Implementation of Calibration in the EOS Project. Part 1.
PB96-179437

PB96-179445

Theory for Ouantum-Dot Ouantum Wells: Pair Correlation and Internal Ouantum Confinement in Nanoheterostr 
PB96-179544

Testing the Sensitivity of Accelerometers Using Mechanica Shock Pulses Under NIST Special Publication 250 Test No. 24040 S. PB96-179544

PB96-179551

Dielectric Behavior of a Polycarbonate/Polyester Mixture Upon Transesterification.

PB96-179551

04,785 Not available NTIS

PB96-179569

Ultrasound Power Measurement Techniques at NIST.

PB96-179569

02684 Not available NTIS

PB96-179577

Design of a High-Flux Backscattering Spectrometer for Ultra-High Resolution Inelastic Neutron Measurements.

PB96-179577 02,992 Not available NTIS

PB96-179585

National Institute of Standards and Technology High-Accuracy Cryogenic Radiometer.

PB96-179585

04,378 Not available NTIS

PB96-179593

Wear of Enamel against Glass-Ceramic, Porcelain, and Amalgam.

PB96-179601

Effect of Grain Size on Hertzian Contact Damage in Alu-

PB96-179601

03,083 Not available NTIS

PB96-180013

Indentation Fatigue: A Simple Cyclic Hertzian Test for Measuring Damage Accumulation in Polycrystalline Ceramics.

03,084 Not available NTIS

PB96-180021

Dynamics of $\mathrm{Mu}(+)$ in $\mathrm{Sc}$ and $\mathrm{ScHx}$.

PB96-180039

Diffusion of Water along 'Closed' Mica Interfaces.

PB96-180039 02,993 Not available NTIS

PB96-18004

Open System Environment Implementors Workshop (OIW); Standardization Role Defined.

PB96-180047

PB96-180054

07,828 Not available NTIS

Synthesis of Hybrid Organic-Inorganic Materials from Interpenetrating Polymer Network Chemistry.
PB96-180054 No9 PB96-180054

PB96-180062

Electrostatic Rigidity of Polyelectrolytes from

Reparametrization Invariance.
PB96-180062

PB96-18007

Scanning Capacitance Microscopy Measurements and Modeling: Progress Towards Dopant Profiling of Silicon.

PB96-180070 01,964 Not available NTIS

PB96-180088

Model for Toughness Curves in Two-Phase Ceramics. 1 Basic Fracture Mechanics.

PB96-180088

03,085 Not available NTIS

PB96-180096

Quantum Hall Effect-Based Resistance Standard: Capabilities and Implementation.

04,114 Not available NTIS

PB96-180104

Composition and Solubility Product of a Synthetic Calcium Hydroxyapatite.

PB96-180112

02,995 Not available NTIS

Assessing MOS Gate Oxide Reliability on Wafer Level with Ramped/Constant Voltage and Current Stress.

PB96-18011

04,115 Not available NTIS

396-180120

Designations of ds(2)p Energy Levels in Neutral Zirconium, Hafinium, and Rutherfordium $(Z=104)$.

PB96-180120

04,116 Not available NTIS

PB96-180138

Surging the Upside-Down House: Measurements and ModPling Results.

PB96-180146

02,243 Not available NTIS

Radiochromic Solid-State Polymerization Reaction

PB96-180146

PB96-180153

Distributions of Measurement Error for Three-Axis Magnetic

Field Meters during Measurements Near Appliances.

PB96-180153

PB96-180161

Adsorption of Potassium N-phenylglycinate on Hydroxyapatite: Role of Solvents and lonic Charge.

PB96-180161

01,159 Not available NTIS

PB96-180179

Complicated Cases and Shielded Rooms: Audiometric Booths Shielded to Attenuate Electromagnetic Interference.
PB96-180179 02,278 Not available NTIS

PB96-18017
PB96-180187

Application of Metadata Standards.

PB96-180187 01,771 Not available NTIS
PB96-180195

Tomographic Reconstruction of the Moments of Local Prob-

ability Density Functions in Turbulent Flow Fields.
PB96-180195 PB96-180203

Hyperfine Structure Investigations and Identification of New Energy Levels in the Ionic Spectrum of (147) Pm.

PB96-180203

04,117 Not available NTIS

PB96-180211

Measurements of the Resonance Lines of (6) Li and (7) Li by Doppler-Free Frequency-Modulation Spectroscopy.

PB96-180211

04,118 Not available NTIS

PB96-180229

Advances in the Measurement of Polymer CTE Micrometer- to Atomic-Scale Measurements.
PB96-180229
03,390 Not available NTIS

PB96-180237

Fluoride Analytical Methods.

PB96-180237

03,578 Not available NTIS

PB96-180245

Effect of Chemical Interaction on Barenblatt Crack Profiles in Brittle Solids.

PB96-180252

02,996 Not available NTIS

Pressurized Internal Lenticular Cracks at Healed Mica Interfaces.

PB96-180252

PB96-180260

02,997 Not available NTIS

Fracture of Silicon Nitride and Silicon Carbide at Elevated Temperatures.

PB96-18027

03,179 Not available NTIS

$96-180278$

Transient Creep Behaviour of Hot Isostatically Pressed Silicon Nitride.

PB96-180286

03,086 Not available NTIS

Development of Neutral-Density Infrared Filters Using Metallic Thin Films.

PB96-180286

02,998 Not available NTIS

PB96-182266

Unpredictable Certainty. Information Infrastructure through

PB96-182266

00,016 PC A14/MF A03

PB96-183041

X.500 Directory Schema Design Handbook

PB96-183041

02,739 PC A03/MF AO

PB96-183058

Procedure for Product Data Exchange Using STEP Developed in the AutoSTEP Pilot.

PB96-183058

$02,843 \mathrm{PC}$ A04/MF A01

-183066

Electronics and Electrical Engineering Laboratory Technical Publication Announcements Covering Laboratory Programs, July to September 1995 with 1996 EEEL Events Calendar. PB96-183066

01,965 PC A03/MF AO

PB96-183074

Publications 1995: NIST Building and Fire Research Laboratory.

B96-183074

$00,226 \mathrm{PC} \mathrm{A08/MF} \mathrm{AO2}$

PB96-183082

Materials Reliability. Technical Activities, 1995. PB96-183082

PB96-183090

Workshop on Characterizing Diamond Films (4th). Held in Gaithersburg, Maryland on March 4-5, 1996.
PB96-183090 PB96-183090

PB96-183108

NASA Fire Detector Study

PB $96-183108$

01,423 PC A04/MF A01

PB96-183116

Electronics and Electrical Engineering Laboratory Technical Progress Bulletin Covering Laboratory Programs, October to December 1995 with 1996 EEEL Events Calendar.

PB96-183116

01,966 PC A04/MF A0

PB96-183124

Proceedings of NIST Workshop: Industry Needs in Welding Research and Standards Development. Held on August 15$16,1995$.

PB96-183124

$02,877 \mathrm{PC} \mathrm{A04/MF} \mathrm{A01}$

PB96-183132

Numerical Simulation of Rapid Combustion in an Underground Enclosure.

PB96-18314

$01,424 \mathrm{PC} \mathrm{A03/MF} \mathrm{AO}$

Interoperability Experiments with CORBA and Persistent Object Base Systems

PB96-183140

$01,772 \mathrm{PC} \mathrm{A04/MF} \mathrm{A01}$

PB96-183157

Standard Source Method for Reducing Antenna Factor Er-

rors in Shielded Room Measurements. PB96-183165

Distributed Communication Methods and Role-Based Access Control for Use in Health Care Applications. PB96-183165

PB96-183173

01,508 PC A05/MF AO

Scale-Space-Based Visual-Motion-Cue for Autonomous Navigation.
PB96-183173

02,940 PC A06/MF A01

PB96-183181

Minimum Mass Flux Requirements to Suppress Burning Surfaces with Water Sprays.

PB96-18318

01,425 PC A05/MF A01

P86-183199

Dynamometer-Induced Residual Stress in Railroad Wheels: Utrasonic and Saw Cut Measurements. Report No. 30.

PB96-18319

PB96-183207

04,857 PC A04/MF A01

Hygrothermal Effects on the Performance of Polymers and Polymeric Composites: A Workshop Report. Held in Gaithersburg, Maryland on September 21-22, 1995. PB96-183207

03,180 PC AO4/MF A01

PB96-183215

Publications of the National Institute of Standards and Technology 1993 Catalog

PB96-183215

00,017 PC A11/MF A03

PB96-183223

Probabilistic Estimates of Design Load Factors for WindSensitive Structures Using the 'Peaks Over Threshold' Ap-

PB96-183223

00,474 PC A04/MF A01

PB96-183231

Aspects of a Product Model Supporting Apparel Virtual En-

PB96-18323

02,790 PC AO3/MF A01

PB96-183249

Performance of Tape-Bonded Seams of EPDM Membranes:

Comparison of the Peel Creep-Rupture Response of TapeBonded and Liquid-Adhesive-Bonded Seams

PB96-183249

03,012 PC A05/MF A01

PB96-186010

Surface Transverse Wave Oscillators with Extremely Low Thermal Noise Floors.

-186010

01,967 Not available NTIS

-186028

Ultrasonic Sensing of GMAW: Laser/EMAT Defect Detection System.

PB96-186036

02,878 Not available NTIS

Determination of Sheet Steel Formability Using Wide Band Electromagnetic-Acoustic Transducers.

PB96-186036

02,279 Not available NTIS

PB96-186044

Effect of Liftoff on Accuracu of Phase Velocity Measure-

ments Made with Electromagnetic-Acoustic Transducers.
PB96-186044
02,280 Not available NTIS

PB96-186051

Methods to Improve the Accuracy of On-Line Ultrasonic

Mteel Sheet Formability

PB96-18605

02,281 Not available NTIS

PB96-186069

Sensor System for Intelligent Processing of Hot-Rolled

PB96-186069

PB96-186077

03,373 Not available NTIS

Well-Shielded EMAT for On-Line Ultrasonic Monitoring of GMA Welding

PB96-186085

Alpha-Particle and Electron Capture Decay of (209)Po. PB96-186085

02,879 Not available NTIS

PBg 
PB96-186184

Binary Decision Clustering for Neural-Network-Based Optical Character Recognition

01,857 Not available NTIS

PB96-186192

Guide to a Format for Data on Chemical Admixtures in a Materials Property Database. (Reannouncement with new abstract)

PB96-186192

$01,328 \mathrm{PC} \mathrm{A04/MF} \mathrm{AO}$

PB96-188164

Reference Information for the Software Verification and Validation Process

PB96-189444

Financing Tomorrow's Infrastructure: Challenges and issues. Proceedings of a Colloquium. Held in Washington,

DC. on Oclober 20, 1995.
PB96-189444

00,481 PC A07/MF AO2

PB96-190012

Large Eddy Simulations of Smoke Movement in Three Di-

PB96-190012

01,426 Not available NTIS

PB96-190020

Investigations of Sulfur Interferences in the Extraction of Methylmercury from Marine Tissues.

PB96-190020

03,482 Not available NTIS

PB96-190038

Microlithography by Using Neutral Metastable Atoms and Self-Assembled Monolayers.

PB96-190038

02,441 Not available NTIS

PB96-190046

Helping to Reduce Technical Barriers to Trade

PB96-190046

00,491 Not available NTIS

PB96-190053

Dynamic Objects and Meta-Level Programming of an EXPRESS Language Environment.

PB96-190053

01,774 Not available NTIS

PB96-190061

Isolation and Structural Elucidation of the Predominant Geo metrical Isomers of alpha-Carotene.
PB96-19006 1
00,640 Not available NTIS

PB96-190079

Large Fire Experiments for Fire Model Evaluations.

PB96-190079 01.427 Not available NTIS

PB96-190087

Hemispherical Test Fixture for Measuring the Wavefields Generated in an Anisotropic Solid.

PB96-190087 Anisotropic Solid. 181 Not available NTIS

PBg6-190095

Mapping the Droplet Transfer Modes for an ER100S-1 GMAW Electrode.

PB96-190095

PB96-190103

Response Comparison of Electret Ion Chambers, LiF TLD, and $\mathrm{HPIC}$.

PB96-190103
PB96-190111

02,578 Not available NTIS

Wavelet Variance, Allan Variance, and Leakage.

PB96-190111 01,509 Not available NTIS

PB96-190129

Repair of Products of Oxidative DNA Base Damage in Human Cells.

PB96-190129

PB96-190137

Locating Fire Information

PB96-190137

03,555 Not available NTIS

PB96-190145

00,227 Not available NTIS

Power Characteristics in GMAW: Experimental and Numerical Investigation.

PB96-190145

03,296 Not available NT!S

PB96-190152

Atomic Branching Ratio Data for Nitrogen-Like Species.

PB96-190152

04,122 Not available NTIS

PB96-190160

Sensing Droplet Detachment and Electrode Extension for Control of Gas Metal Arc Welding.

PB96-190160

3,297 Not available NTIS

PB96-190178

Office Work Station Heat Release Rate Study: Full Scale versus Bench Scale.

PB96-190178

01,428 Not available NTIS

PB96-190186

Epitaxial Nucleation and Growth of Chemically Derived $\mathrm{Ba} 2 \mathrm{YCu}_{3 \mathrm{O}} 7-\mathrm{x}$ Thin Films on (001) $\mathrm{SrTiO}_{3}$

PB96-190186

04,787 Not available NTIS

PB96-190194

Charpy Impact Test as an Evaluation of $4 \mathrm{~K}$ Fracture Toughness.

PB96-190202

03,219 Not available NTIS

New 5 and $10 \mathrm{MHz}$ High Isolation Distribution Amplifier. PB96-190202 01510 Not available NTIS

PB96-190210

Analysis of High Bay Hangar Facilities for Detector Sensitivity and Placement

PB96-190210

01,429 Not available NTIS
PB96-190228

Models of Granualr Giant Magnetoresistance Multilayer Thin FBilms.

01,968 Not available NTIS

PB96-190236

Aluminum-Lithium Alloys: Evaluation of Fracture Toughness by Two Test Standards, ASTM Method E 813 and E 1304 . PB96-19023

03,374 Not available NTIS

PB96-190244

Light-Weight Alloys for Aerospace Applications II. PB96-190244

03,375 Not available NTIS

PB96-190251

Prediction of Strengthening Due to $V$ Additions in DirectCooled Ferrite-Pearlite Forging Steels.

PB96-19025

03,220 Not available NTIS

PB96-190269

Temperature-Induced Transition in Ductile Fracture Appearance of a Nitrogen-Strengthened Austenitic Stainless Steel.

PB96-190269

03,221 Not available NTIS

PB96-19027

Embossable Grating Couplers for Planar Waveguide Optical Sensors.

PB96-190277

00,641 Not available NTIS

PB96-190285

Ductile Fracture and Tempered Martensite Embrittlement of 4140 Sieel.

PB96- 190285

03,222 Not available NTIS

PB96-190293

Increasing the Value of Atomic Force Microscopy Process Metrology Using a High-Accuracy Scanner, Tip Characterization, and Morphological Image Analysis.

PB96-190301

Optical Density Measurements of Laser Eye Protection Materials.

PB96-190301

00,190 Not available NTIS

PB96-190319

Time and Frequency Metrology. 01,556 Not available NTIS

PB96-190327

Ultrasonic Methods

PB96-190327

02,707 Not available NTIS

PB96-190335

Charpy Specimen Tests at $4 \mathrm{~K}$

B96-190343

03,002 Not available NTIS

Fatigue Crack Thresholds of a Nickel-Iron Alloy for Superconductor Sheaths at $4 \mathrm{~K}$.

PB96-190343

PB96-190350

03,223 Not available NTIS

Mechanical Properties and Warm Prestress of Ultra-Low Carbon Steel at $4 \mathrm{~K}$

PB96-190350

03,224 Not available NTIS

PB96-190368

Scanned Probe Microscope Tip Characterization Without Calibrated Tip Characterizers.

PB96-190368

02,759 Not available NTIS

PB96-190376

Confidence on the Modified Allan Varıance and the Time Variance.

PB96-190384

01,557 Not available NTIS

High Frequency Magnetic Field Sensors Based on the Faraday Effect in Garnet Thick Films.

PB96-190384

02,282 Not available NTIS

PB96-191366

IIW Commission V Ouality Contro! and Ouality Assurance of Welded Products, Annual Report 1995/96.

PB96-191366

PB96-191374

Generalized Form Registration Using Structure-Based Pechniques.

01,858 PC A03/MF A01

PB96-191382

Dielectric and Magnetic Measurements from $.50 \mathrm{C}$ to $200 \mathrm{C}$ and in the Frequency Band $50 \mathrm{MHz}$ to $2 \mathrm{GHz}$

PB96.19138

$02,245 \mathrm{PC}$ A03/MF AO

PB96-191390

Electronics and Electrical Engineering Laboratory Technical Progress Bulletin Covering Laboratory Programs, January Progress Bulletin Covering Laboratory Programs,

to March 1996, with 1996 EEEL Events Calendar.
01.969 PC A04/MF A01

PB96-193636

SparseLib++ v. 1.5 Sparse Matrix Class Library. Reference

PB96-193636

$01,775 \mathrm{PC} \mathrm{A03/MF} \mathrm{A01}$

PB96-193644

Reactor Radiation Technical Activities, 1995

PB96-193644

03,741 PC A07/MF A02

PB96-193651

Warping of Terrace Pavers at the U.S. Capitol Building

PB96-193651

U.S. Capitol Building.
00,411 PC A03/MF A01

PB96-193669

Component-Based Handprint Segmentation Using Adaptive

Writing Style Model

01,859 PC A03/MF A01

PB96-193677

Ceramics Technical Activities, 1995

PB96-193677

$03,087 \mathrm{PC} \mathrm{A11/MF} \mathrm{A03}$

PB96-193685

Guidelines for Pre-Oualification, Prototype and Quality Con-

Olesting of Seismic Isolation Systems.

PB96-193685

01,347 PC A08/MF A02

PB96-193693

Room-Temperature Thermal Conductivity of Expanded Polystyrene Board for a Standard Reference Materia. PB96-193693

\section{B96-193701}

Post-Flame Soot

PB96-193701

$00,412 \mathrm{PC} \mathrm{A04/MF} \mathrm{A01}$

PB96-193719

Polymers Technical Activities, 1995

PBg6-193719

01,430 PC A04/MF A01

PB96-193727

Novel Active-Vision-Based Motion Cues for Local Naviga-

PB96-193727

$02,941 \mathrm{PC}$ A05/MF A01

PB96-193735

Systems Integration for Manufacturing Applications Program 995 Annual Report

PB96-193735

02,844 PC A05/MF A01

PB96-193743

Development of an Economical Video Based Fire Detection and Location System.

PB96-193750

Hybrid Gauss-Trapezoidal Ouadrature Rules.

PB96-193750

00,228 PC A05/MF A01

PB96-193768

Computer Systems Laboratory Computing and Applied

Mathematics Laboratory Technical Accomplishments, Octo-

ber 1994-March 1996.

PB96-193768

01,638 PC A05/MF A01

\section{6-193776}

Notion of a xi-Vector and a Stress Tensor for a General Class of Anisotropic Diffuse Interface Models.

PB96-193776

04,788 PC A03/MF A01

B96-193784

Methodology for Developing and Implementing Alternative Temperature-Time Curves for Testing the Fire Resistance

of Barriers for Nuclear Power Plant Applications.

of Barriers for Nuclear Power Plant Applications.
PB96-193784 03,742 PC A07/MF A02

PB96-193792

Survey of Standards for the U.S. Fiber/Textile/Apparel In-

PB96-193792

$03,199 \mathrm{PC}$ A06/MF A01

\section{B96-193800}

State of the Art Report on Seismic Design Requirements for Nonstructural Building Components. PB96-193800

$00,308 \mathrm{PC} \mathrm{A06/MF} \mathrm{A01}$

PB96-195219

IML++ v.1.2 Iterative Methods Library Reference Guide.

PB96-195219 
PB96-197249

Development of a Method for Measuring Water-Stripping Resistance of Asphalt/Siliceous Aggregate Mixtures.
PB96-197249

PB96-200118

Coaxial Line-Reflect-Match Calibration. PB96-200118

02,246 Not available NTIS

PB96-200126

Methodology for Electromagnetic Interference Measurements.

02,014 Not available NTIS

PB96-200126

PB96-200134

Metallic-Barrier Junctions for Programmable Josephson Voltage Standards.

PB96-20013

02,089 Not available NTIS

PB96-200142

Shapiro Steps in Large-Area Metallic-Barrier Josephson Junctions.

PB96-200159

02,090 Not available NTIS

Scanning Electron Microscopy Observations of Mistit Dis Incations in Epitaxial In0.25Ga0 75As on GaAs(001).

PB96-200159

03,004 Not available NTIS

PB96-200167

Novel Vortex Dynamics in Two-Dimensional Josephson Ar-

PB96-200167

PB96-200175

02,091 Not available NTIS

Orthotropic Elastic Constants of a Boron-Aluminum FiberReinforced Composite: An Acoustic-Resonance-Spectros copy Study.
PB96-200175

PB96-200183

03,182 Not available NTIS

High-Tc Multilayer Step-Edge Josephson Junctions and SQUIDS.

PB96-200183

04,790 Not available NTIS

PB96-200217

Riass Coronathon: Joint X-ray and Ultraviolet Observations of Normal F-K Stars.

PB96-200217

PB96-200225

00,109 Not available NTIS

Accurate Modeling of Size and Strain Broadening in the Rietveld Refinement: The 'Double-Voigt' Approach.

PB96-200225

00,664 Not available NTIS

PB96-200233

Superconductor- Normal-Superconductor Junctions for Digital/Analog Converters.

PB96-200233

PB96-200241

02,092 Not available NTis

Superconductor- Normal-Superconductor Junctions for Programmable Voltage Standards.

PB96-20024

02,093 Not available NTIS

PB96-200258

Design of High-Frequency, High-Power Oscillators Using Josephson-Junction Arrays.

PB96-200258

02,094 Not available NTIS

PB96-200266

High-Power, High-Frequency Oscillators Using Distributed Josephson-Junction Arrays.

PB96-200266

PB96-200274

Excitation Transfer in Barium by Collisions with Noble Gases.

PB96-200274

PB96-200282

Data Evaluation of a Linear System by a Second-Order Transfer Function.

PB96-200282

PB96-200290

Comment on $\ll<A$ Dynamic Electric Trap for Ground-State Atoms $>>$.

PB96-200290

PB96-200308

04,123 Not available NTIS

Fiber-Optic Faraday-Effect Magnetic-Field Sensor Based on Flux Concentrators.

PB96-200308

PB96-200316

02,201 Not available NTIS

Alignment Probing of Rydberg States by Stimulated Emis-

PB96-200316

PB96-200324

Alternative EMC Compliance Test Facilities.

PB96-200324

PB96-200332

02,247 Not available NTIS

Observation of the Transverse Second Harmonic MagnetoOptic Kerr Effect from Ni81Fe19 Thin Film Structures.

PB96-200332 01,971 Not available NTIS

PB96-200340

Collisions of Electrons with Highly-Charged lons.

PB96-200340

PB96-200357

04,791 Not available NTIS

Pairwise and Nonpairwise Additive Forces in Weakly Bound Complexes: High Resolution Infrared Spectroscopy of ArnDF $(n=1,2,3)$

04,125 Not available NTIS

PB96-200365

Design Criteria for BJT Amplifiers with Low $\mathrm{I} / \mathrm{f}$ AM and PM Noise

02,442 Not available NTIS
PB96-200373

Comparison of Uitralow-Sidelobe-Antenna Far-Field Patterns Using the Planar-Near-Field Method and the Far-Field

PB96-200373

02,015 Not available NTIS

PB96-200381

Wavelet Analysis for Synchronization and Timekeeping PB96-200381

01,558 Not available NTIS

PB96-200399

Hot-Electron Microcalorimeter for X-ray Detection Using a Superconducting Transition Edge Sensor with Electrothermal Feedback. $\quad 04,792$ Not available NTIS
PB96-200399

PB96-200407

$30 \mathrm{MHz}$ Comparison Receiver.
$\mathrm{PB} 96-200407$

PB96-200613

Kinetic-Energy-Enhanced Neutral Etching

PB96-200613

PB96-200621

Accurate Measurements of the Local Deuterium Abundance from HST Spectra.

PB96-200621

00,110 Not available NTIS

PB96-200639

Deuterium and the Local Interstellar Medium: Properties for the Procyon and Capella Lines of Sight.

PB96-200639

00,111 Not available NTIS

B96-200647

How to Get NIST-Traceable Time on Your Computer.

PB96-200647

01,559 Not available NTIS

396-200654

Introduction to Frequency Calibration. Part 1

PB96-200654

01,560 Not available NTIS

PB96-200662

NIST Frequency Measurement Service.

PB96-200662

01,561 Not available NTIS

396-200670

Hot-Electron-Microcalorimeters with $0.25 \mathrm{~mm}(2)$ Area

PB96-200670

PB96-200688

04,793 Not available NTIS

General Order 'N' Analytic Correction of Probe-Position Errors in Planar Near-Field Measurements.

PB96-200688 01,562 Not available NTIS

PB96-200696

Polarimetric Calibration of Reciprocal-Antenna Radars.

PB96-200696

PB96-200704

02,016 Not available NTIS

Trap.

PB96-200704

PB96-200712

Controlling the Critical Current Density of High-Temperature SNS Josephson Junctions.

PB96-200712

PB96-200720

04,794 Not available NTIS

Stable, Tightly Confining Magnetic Trap for Evaporative

Cooling of Neutral Atoms.

PB96-200720

04,126 Not available NTIS

PB96-200738

Self-Calibrated Intelligent Optical Sensors and Systems.

PB96-200738

PB96-200746

04,380 Not available NTIS

Magnetic Flux Pinning in Epitaxial YBa2Cu3O7-delta Thin

PB96-200746

04,795 Not available NTIS

PB96-200753

Thermal Conductivity of Polypyromellitimide Film with Alumina Filler Particles from 4.2 to $300 \mathrm{~K}$

PB96-200753

01,292 Not available NTIS

PB96-200761

Elastic Constants and Microcracks in $\mathrm{YBa} 2 \mathrm{Cu} 3 \mathrm{O} 7$

PB96-20076

03,005 Not available NTIS

96-20077

Effect of Harmonic Distortion on Phase Errors in Frequency Distribution and Synthesis.

PB96-200779

PB96-200787

01,563 Not available NTIS

Origin of $1 / 4 \mathrm{PM}$ and $\mathrm{AM}$ Noise in Bipolar Junction Transistor Amplifiers.

PB96-200795

02,096 Not available NTIS

Quest to Understand and Reduce $1 / f$ Noise in Amplifiers and Baw Ouartz Oscillators.

PB96-200795

02,097 Not available NTIS

PB96-200803

Tensile Creep of Silicide Composites.

PB96-200803

03,183 Not available NTIS

PB96-200811

Unique Quality Assurance Aspects of INAA for Reference Material Homogeneity and Certification.

PB96-200811

PB96-200829

00,699 Not available NTIS

Multimedia Tutorial on Phase Equilibria Diagrams

PB96-200829

03,088 Not available NTIS

PB96-200837

Measurements of Properties of Materials in Electronic Pack-

aging.
PB96-200837

01,973 Not available NTIS

PB96-200845

Overview of U.S. Government Advanced Packaging Pro grams.

PB96-200845

02,443 Not available NTIS

PB96-200852

Self-Reciprocal Fourier Function

PB96-200852

01,974 Not available NTIS

PB96-200860

Optoelectronics at NIST.

PB96-200860

02,202 Not available NTIS

PB96-200878

Wideband Current and Magnetic Field Sensors Based on Iron Garnets.

PB96-20087

PB96-200886

01,975 Not available NTIS

Quantitative Determination of Oxidative Base Damage in DNA by Stable Isotope-Dilution Mass Spectrometry.
PB96-200886 03,483 Not available NTIS PB96-20088

PB96-200894

Commentary: The Measurement of Oxidative Damage to DNA by HPLC and GC/MS Techniques.

.

PB96-200894

03,484 Not available NTIS

PB96-200902

Performance of the Electron Pump with Stray Capacitances.
PB96-200902 PB96-200902

PB96-200910

Precision of Marchall Stability and Flow Test Using 6-in (152.4-mm) Diameter Specimens.

PB96-200910

03,006 Not available NTIS

PB96-200928

Intercomparison of DNA Sizing Ladders in Electrophoretic Separation Matrices and Their Potential for Accurate Typing of the D1S80 Locus.

PB96-200928

03,485 Not available NTIS

PB96-200936

$100 \mathrm{~A}, 100 \mathrm{kHz}$ Transconductance Amplifier

PB96-200936

02,098 Not available NTIS

PB96-200944

Quantum Hall Effect-Based Resistance Standard (Quantum Hall Res).

PB96-200944

PB96-200951

04,127 Not available NTIS

Flow Immunoassay Using Solid-Phase Entrapment

PB96-200951

00,642 Not available NTIS

PB96-200969

Monte Carlo Simulation of Scanning Electron Microscope Signals.

PB96-200969

02,444 Not available NTIS 


\section{PB96-201090}

Scanning Electron Microscope Metrology PB96-201090

02.446 Not available NTIS

PB96-201108

SEM Linewidth Metrology of X-ray Lithography Masks.

PB96-201108

02,447 Not available NTIS

PB96-201116

Low Heat-Flux Measurements: Some Precaution

PB96-201116

02,685 Not available NTIS

PB96-201124

Precise Laser-Based Measurements of Zero-Dispersion Wavelength in Single-Mode Fibers.

PB96-201124

01,511 Not available NTIS

PB96-201132

New Physics-Based Model for Time-Dependent- DielectricBreakdown.

PB96-201140

02,448 Not available NTIS

In vitro Inhibition of Membrane-Mediated Calcification by Novel Phosphonates.

PB96-201140

03,595 Not available NTIS

PB96-201157

Ultrasonic NDE of Sprayed Ceramic Coatings

PB96-201157

02,761 Not available NTIS

PB96-201165

Electronically Tunable Fiber Laser for Optical Pumping of (3) $\mathrm{He}$ and (4) $\mathrm{He}$

PB96-201173

04,381 Not available NTIS

Spin-Dependent Interface Transmission and Reflection in Magnetic Multilayers (Invited)

PB96-201173

04,130 Not available NTIS

NMR Characterization of Injection-Moulded Alumina Green

Compacts. Part 2. T2-Weighted Proton Imaging.
PB96-201181
01,165 Not available NTIS

PBg6-201109

01,165 Not available NTIS

Vapor Pressure of Pentafluorodimethyl Ether

PB96-201199 00,677 Not available NTIS

PB96-201207

Vibrationally Resolved Photoelectron Angular Distributions and Branching Ratios for the Carbon Dioxide Molecule in the Wavelength Region 685-795 Angstrom.

the Wavelength Region 685-795 Angstrom. 04.131 Not available NTIS

PB96-202122

Developing Quality System Documentation Based on ANSI/ NCSL 2540-1-1994: The Optical Technology Division's EfPB96-202122

01,869 PC A05/MF A01

PB96-202221

Benefits and Costs of Research: Two Case Studies in Building Technology

00.230 PC A07/MF A02

PB96-202239

NIST Construction Automation Program Report No. 2. Proceedings of the NIST Construction Automation Workshop. Held in Gaithersburg, Maryland on March 30-31, 1995. PB96-202239

$00.413 \mathrm{PC} \mathrm{A09/MF} \mathrm{A02}$

PB96-202247

Enhancement of EXIT89 and Analysis of World Trade Center Data.

PB96-202247

PB96-202254

00,231 PC A04/MF A01

Mixing and Radiation Properties of Buoyant Luminous Flame Environments

PB96-20225

01,432 PC A06/MF A01

P896-202288

Benefits and Costs of Research: A Case Study of the Fire Safety Evaluation System.

PB96-202288

PB96-202296

00,232 PC A06/MF A01

Development of a Method for Measuring Water-Stripping Resistance of Asphalt/Siliceous Aggregate Mixtures.

PB96-2022

01.329 PC AO9/MF A02

Sparse Water Sprays in Fire Protection

PB96-202304

01,433 PC A13/MF A03

Application Protocol Information Base World Wide Web GBateway.

PB96-202338

02,791 PC A03/MF A01

Measurement of Rheological Properies of High Performance Concrete: State of the Art Report

PB96-202338

00,414 PC A04/MF A01

PB96-202346

Electronics and Electrical Engineering Laboratory Technical Publication Announcements Covering Laboratory Programs, October to December 1995, with 1996 EEEL Events Cal-

PB96-202346

PB96-202353

01,978 PC A04/MF A01

Economics of New-Technology Materials: A Case Study of FRP Bridge Decking.

PB96-202452

01,349 PC A06/MF A01

Lake Erie Water Temperature Data, Put-in-Bay, Ohio, 19181992.

PB96-202452

03,692 PC A04/MF A01
P896-204011

Enthalpy Increment Measurements from 4.5 to $318 \mathrm{~K}$ for Bismuth(cr). Thermodynamic Properties from $0 \mathrm{~K}$ to the Melting Point.

PB96-20401

01,166 Not available NTIS

96-204029

Enthalpy Increment Measurements from 4.5 to $350 \mathrm{~K}$ and the Thermodynamic Properties of Titanium Disilicide(cr) to

PB96-204029

00,678 Not available NTIS

PB96-204037

Enthalpy Increment Measurements from $4.5 \mathrm{~K}$ to $350 \mathrm{~K}$ and the Thermodynamic Properties of the Titanium Silicide $\mathrm{Ti} 5 \mathrm{Si}(\mathrm{cr})$.

PB96-204037

00,679 Not available NTIS

PB96-204045

Guide 10 Locating and Accessing Computerized Numeric Materials Databases.

PB96-204045

P896-204052

03,007 Not available NTIS

New Surface-Active Comonomer for Adhesive Bonding PB96-204052

03,579 Not available NTIS

P896-204060

Need for Advanced Characterization Techniques in Product Manufacturing: A Case Study on Ceramic Matrix Compos-

PB96-204060

03.089 Not available NTIS

PB96-204078

Gas Phase Oxygen Effect on Chain Scission and Monomer Content in Bulk Poly(methyl methacrylate) Degraded by External Thermal Radiation.

PB96-204078

PB96-204086

01,293 Not available NTIS

NIST/NCMS Program on Electronic Packaging: First Update.

03,008 Not available NTIS

PB96-204094

Nanostructure Fabrication via Laser-Focused Atomic Deposition (Invited).

PB96-204102

04,132 Not available NTIS

Electric Field Dependent Dielectric Breakdown of Intrinsic $\mathrm{SiO} 2$ Films Under Dynamic Stress.

PB96-204102

02,449 Not available NTIS

PB96-204110

Diffusive Crack Growth at a Bimaterial Interface.

PB96-204110

03,090 Not available NTIS

PB96-204128

Life Prediction of a Continuous Fiber Reinforced Ceramic Composite Under Creep Conditions.

PB96-204128

03.091 Not available NTIS

PB96-204136

Hybrid Optical-Electrical Overlay Test Structure.

PB96-204136

02,450 Not available NTIS

PB96-204144

Properties of a Bose-Einstein Condensate in an Anisotropic Harmonic Potential.

PBimo

04,133 Not avaiłable NTIS

96-20415

Deuterium Isotope Effect in Vinyl Radical Combination Dispropor tionationReactions.

PB96-204151

PB96-204169

01,167 Not available NTIS

Temperature Dependence of the Ultraviolet Absorption Cross Section of CF 31 .

PB96-204169

01,168 Not available NTIS

PB96-204177

Temperature Dependent Ultraviolet Absorption Cross Sections of Propylene, Methylacetylene and Vinylacetylene.

B86-204185

Bulk Modulus and Young's Modulus of Nanocrystalline gamma-Alumina.

PB96-204185

Cavity Evolution during Tensil Creep of Si3N4

PB96-204193 03,376 Not available NTIS PB96-204201

Mode-Locked Lasers for High-Accuracy Radiometry.

PB96-204201

PB96-204417

Greenspan Acoustic Viscometer for Gases.

PB96-204417

PB96-204425

Adhesion, Contact Electrification, and Acid-Base Properties of Surfaces.

PB96-204433

Role of Combustion on Droplet Transport in Pressure-Atomized Spray Flames.

PB96-204433

01,434 Not available NTIS

Improving Color Measurements of Displays.

Improving Color Measurements of Displays.
PB96-204441 Not available NTIS PB96-204458

Shear Suppression of Critical Fluctuations in a Diluted Polymer Blend.

04,418 Not available NTIS

\section{PB96-204466}

Experimental Assessment of Crack-Tip Dislocation Emission Models for an Al67Cr8Ti25 Intermetallic Alloy.

PB96-204466

03,377 Not available NTIS

\section{PB96-204474}

Alvin Van Valkenburg and the Diamond Anvil Cell.

PB96-20447

04,797 Not available NTIS

Phase Separation in Thin Film Polymer Blends With and Without Block Copolymer Additives.

PB96-204482

01,294 Not available NTIS

PB96-204490

Correlations between Electrical and Acoustic Detection of Partial Discharge in Liquids and Implications for Continuous Data Recording.

PB96-204490

PB96-204508

02,248 Not available NTIS

Determination of Acoustic Center Correction for Type LS2aP Condensor Microphones.

PB96-204508

04,190 Not available NTIS

PB96-204516

Thermal Wave NDE of Advanced Materials Using Mirage Effect Detection.

PB96-204516

PB96-204524

04,191 Not available NTIS

Creep and Creep Rupture of Structural Ceramics.

- 204524

03,093 Not available NTIS

PB96-204532

Fracture Mechanism Maps: Their Applicability to Silicon

PB96-204532

03,094 Not available NTIS

B96-210026

Rapid Evaluation of Mode-Stirred Chambers Using Impulsive Waveforms.

$01.979 \mathrm{PC}$ A04/MF A0

PB96-210059

Working Conference on Global Growth of Technology: Is America Prepared. Held in Gaithersburg, Maryland on December 7, 1995

PB96-210059

PB96-210141

00,018 PC A07/MF A02

Proceedings of the Open Forum on Laboratory Accredita tion at the National Institute of Standards and Technology, October 13, 1995.

02,686 PC A10/MF A03

PB96-210695

Materials Aspects of Fiber-Reinforced Polymer Composites in Intrastructure.

PB96-210703

$03.184 \mathrm{PC} \mathrm{A04/MF} \mathrm{AO}$ 


\section{PB96-214705}

State Weights and Measures Laboratories: Program Handbook.

B96-214705

02,687 PC A07/MF A02

PB96-214713

Shear Design of High-Strength Concrete Beams: A Review of the State-ot-the-Art

PB96-214713

$01,330 \mathrm{PC} \mathrm{A11/MF} \mathrm{A03}$

PB96-214747

Energy-Based Method for Liquefaction Potential Evaluation. Phase 1. Feasibility Study.

PB96-214747

03,691 PC A13/MF A03

PB96-214754

Materials Science and Engineering Laboratory Annual Re. port, 1995. Technical Activities.

PB96-214754

03,009 PC A05/MF A01

PB96-215074

Directory of U.S. Private Sector Product Certification Pro-

grams.

02,688 PC A2O/MF AO4

PB96-502794

Building Life Cycle Cost Computer Program (BLCC) Version 4.22-95 (for Micrcomputers)

00,277 CP DO2

PB97-104160

January 17, 1995 Hyogoken-Nanbu (Kobe) Earthquake.
Pertormance of Structures, Litelines and Fire Protection

Systems. Executive Summary and Paper.
Sire Protection PB97-104160

00.475 PC A99/MF A06

PB97-104178

TBT Agreement Activities of the National Institute of Standards and Technology, 1995

PB97-104178

00,499 PC A04/MF A01

PB97-104186

Optoelectronics and Optomechanics Manufacturing: An ATP Focused Program Development. Workshop Proceedings. Held in Gaithersburg. Maryland on February 15, 1995

PB97-104186 02,204 PC A11/MF A03

PB97-104376

Wind and Seismic Effects: Proceedings of the Joint Meeting of the U.S.-Japan Cooperative Program in Natural Resources Panel on Wind and Seismic Effects (28th). Held in Gaithersburg, Maryland on May 14-17, 1996.

PB97.104376

00,476 PC A99/MF A06

PB97-106843

Mathematical Analysis of Practices to Control Moisture in the Roof Cavities of Manufactured Houses.

PB97-106843 00.278 PC A05/MF A01

PB97-108559

National Measurement System for Radiometry, Photometry.

and Pyrometry Base Upon Absolute Detectors.
PB97-108559 04,382 PC A04/MF A01

PB97-108575

NIST High-Accuracy Sampling Wattmeter.
PB97-108575 02,689 PC A04/MF A01

Technical Digest: Symposium on Optical Fiber Measure ments (9th), 1996. Held in Boulder, Colorado on October 1. 3. 1996 .

PB97-108583

$04.383 \mathrm{PC} \mathrm{A12/MF} \mathrm{A03}$

PB97-109011

Journal of Research of the National Institute of Standards and Technology. May/June 1996. Volume 101, Number 3. Special Issue: NIST Workshop on Crystallographic Databases.

PB97-109011

PB97-109029

CRYSTMET: The NRCC Metals Crystallographic Data File.

PB 97.799
(Order as PB97-109011, PC A11/MF A03)

PB97-109037

Inorganic Crystal Structure Database (ICSD) and Standardized Data and Crystal Chemical Characterization of Inorganic Structure Types (TYPIX): Two Tools for Inorganic Chemists and Crystallographers.

Chemists and

00,648

PB97-109045

(Order as PB97-109011, PC A11/MF A03)

Evaluation of Crystallographic Data with the Program DIAMOND.

PB97-109045

00,649

PB97-109052

Cambridge Structural Database (CSD): Current Activities and Future Plans.

PB97-109052

(Order as PB97-109011, PC A11/MF A03)

PB97-109060

Protein Data Bank: Current Status and Future Challenges. PB97-109060

(Order as PB97-109011, PC A11/MF A03)

\section{PB97-109078}

Nucleic Acid Database: Present and Future.

PB97-109078

(Order as PB97-109011, PC A11/MF A03)

PB97-109086

Powder Diffraction File: Past, Present, and Future. PB97-109086
(Order as PB97-109011, PC A11/MF A03)

PB97-109094

NIST Crystallographic Databases for Research and AnalyPBis. 109094

(Order as PB97-109011, PC A11/MF A03)

PB97-109102

Conventional and Eccentric Uses of Crystallographic Databases in Practical Materials Identification Problems. PB97-109102 (Order as PB97-109011, PC A11/MF A03)

PB97-109110

Using NIST Crystal Data within Siemens Software for FourCircle and SMAR

B97-109110

(Order as PB97-109011, PC A11/MF A03)

\section{PB97-109128}

Phase Identification in a Scanning Electron Microscope PBing Backscatt

(Order as PB97-109011, PC A11/MF A03)

PB97-109136

Biological Macromolecule Crystallization Database and Mrowtal Growth Archive.

PB97-109136 01.171

PB97-109144

(Order as PB97-109011, PC A11/MF AO3)

Investigations of the Systematics of Crystal Packing Using .

PB97.109144

(Order as PB97-109011, PC A11/MF A03)

\section{PB97-109151}

Troublesome Crystal Structures: Prevention, Detection, and Resolution.

PB97-109151

Order as PB97-109011, PC A11MF A03)

PB97-109169

CIF Crystallographic Information File: A Standard for Crystallographic Data Interchange.

PB97-109169

Order as PB97-109011, PC A11/MF A03)

PB97-109177

Role of Journals in Maintaining Data Integrity: Checking of Crystal Structure Data in 'Acta Crystallographica'

PB97-109177

Order as PB97-109011, PC A11/MF A03)

PB97-109185

Electronic Publishing and the Joumals of the American

Chemical Society.
PB97-109185

(Order as PB97-109011, PC A11/MF A03)

PB97-109193

How the Cambridge Crystallographic Data Centre Obtains Its Information

PB97-109193

(Order as PB97-109011, PC A11/MF A03)

PB97-109201

Data Import and Validation in the Inorganic Crystal Struc-

ture Database
PB97-109201

(Order as PB97-109011, PC A11/MF A03)

PB97-109219

World Wide Web for Crystallography.

PB97-109219

(Order as PB97-109011, PC A11/MF A03)

PB97-109227

Workshop Highlights.

04,811

\section{PB97-110019}

Role of Certified Reference Materials in Trace Analysis Quality Assurance.

PB97-110027

Flaw-Insensitive Ceramics

PB97-110027

PB97-110035

Radon in the Lung

PB97-110035

PB97-110043

Effect of Fuel Tank Rupture Mode on the Ignitability of Expelled Fuel.

PB97-110050

01,444 Not available NTIS

Flammability Characterization with the Lift Apparatus and the Cone Calorimeter.

PB97-110050

01,435 Not available NTIS

\section{PB97-110068}

Letter Report on Flame Spread Testing of a Composite Ma-

PB97-110068

01,436 Not available NTIS

PB97-110076

Interaction of an Isolated Sprinkler Spray and a Two-Laye Compartment Fire Environment. Phenomena and Model Simulations.

01,437 Not available NTIS

\section{PB97-110084}

Liquid-Scintillation Counting Techniques for the StandardLation of Radionuclides Used in Therapy.

PB97-1 10084

03,709 Not available NTIS

PB97-110092

Needs for Brachytherapy Source Calibrations in the United

PB $97-110092$

03,521 Not available NTIS

97-110100

Radioassays of Yttrium-90 Used in Nuclear Medicine.

PB97-110100

03,522 Not available NTIS

PB97-110118

Compressed Liquid Densities, Saturated Liquid Densities, and Vapor Pressures of 1,1-Difluoroethane.

PB97-110118

01,173 Not available NTIS

PB97-110126

Stacking the Cards in Europe: One Company's Story

PB97-110126

00,493 Not available NTIS

PB97-110134

Characterization of Two-Dimensional Dopant Profiles: Stalus and Review.
PB97-110134

PB97-110142

02,451 Not available NTIS

Indoor Air Quality Commissioning of a New Office Building

PB97-110142

PB97-110159

Problem of Convection in the Water Absorbed Dose Calo-

rimeter.

PB97-110159

03,523 Not available NTIS

PB97-110167

Exact Series Solution to the Epstein-Hubbell Generalized Elliptic Type Integral Using Complex Variable Residue ThePBg7-110167

03,423 Not available NTIS

PB97-110175

Stable Silicon Photodiodes for Absolute Intensity Measurements in the VUV and Soft $X$-ray Regions.

PB97-110175

04,135 Not available NTIS

97-110183

Evaluation and Accreditation of State Calibration LaboraPB97-110183

00,486 Not available NTIS

PB97-110191

Hydrodynamic Friction of Arbitrarily Shaped Brownian ParPB97-11019

04,136 Not available NTIS

PB97-110209

Model of an Optical Roughness-Measuring Instrument

PB97-110209

04,384 Not available NTIS

PB97-110217

Examination Procedure Outlines: Keys to Solving the Handbook 44 Puzzle.
PB97-110217

PB97-110225

02,690 Not available NTIS

Thin-Film Ruthenium Oxide - Iridium Oxide Thermocouples.

PB97-110225

00,520 Not available NTIS

PB97-110233

Thin Film Thermocouple Research at NIST.

PB97-110233

02,283 Not available NTIS

PB97-110241

Electrolytes Constrained on Fractal Structures: DebyeHuckel Theory.

PB97-11024

PB97-110258

01,174 Not available NTIS

Tension/Compression Creep Asymmetry in Si3N4

PB97-110258

03,096 Not available NTIS

PB97-110266

Current Status and Trends in Temperature Measurements at NIST, Cooperative Projects and New Mutual Agreement Cetween NIST and IMGC.

PB97-110266

02,691 Not available NTIS 
PB97-110332

In situ-Toughened Silicon Carbide PB97-110332

03,102 Not available NTIS

PB97-110340

Postfailure Subsidiary Cracking from Indentation Flaws in Brittle Materials.

PB97-110357

03,103 Not available NTIS

Heat Flux from Flames to Vertical Surfaces.

PB97-110357 01,438 Not available NTIS

PB97-110365

Isopiestic Investigation of the Qsmotic and Activity Coefficients of Aqueous $\mathrm{NaBr}$ and the Solubility of $\mathrm{NaBr} 2 \mathrm{H} 2 \mathrm{O}(\mathrm{cr})$ at $298.15 \mathrm{~K}$ : Thermodynamic Properties of the $\mathrm{NaBr}+\mathrm{H} 2 \mathrm{O}$ System over Wide Ranges of Temperature and Pressure.
PB97-110365 01,175 Not available NTIS

PB97-110365
PB97-110373

EPR Dosimetry of Cortical Bone and Tooth Enamel Irradiated with $X$ and Gamma Rays: Study of Energy Depend-

ence.
PB97-110373 03,639 Not available NTIS

PB97-110373

PB97-110381

Exposure-to-Absorbed-Dose Conversion for Human Adult Cortical Bone.
PB97-110381

PB97-110399

03,640 Not available NTIS

Calculation of Photon Mass Energy-Transfer and Mass Energy-Absorption Coefficients. 04,137 Not available NTIS
PB97-110399

PB97-110407

Electron-Photon Monte Carlo Calculations: The ETRAN Code.

PB97-110407

04, 138 Not available NTIS

PB97-110415

Optical Scattering from Moderately Rough Surfaces.

PB97-110415

04,385 Not available NTIS

PB97-110423

Present and Future Standard Specimens for Surface Finish Metrology.
PB97-110423

PB97-110431

Proposed Coating Technology Consortium. (National Coil Coaters Association Fall Conference). Held in Rosemont, IIlinois in September 1992

PB97-11043

03,129 Not available NTIS

PB97-110449

Interlaboratory Studies on the Analysis of Hair for Drugs of

Abuse: Results from the Fifth Exercise.
PB97-110449

PB97-110456

High Temperature Structural Reliability of Silicon Nitride.

PB97-110456

03,104 Not available NTIS

PB97-110464

Tensile Creep Testing of Structural Ceramics.

PB97-110464 03,105 Not available NTIS

PB97-110472

Compensation of Errors Detected by Process-Intermittent Gauging.

PB97-110480

Data Management for Error Compensation and Process

Control.

PB97-110480

02,847 Not available NTIS

PB97-110498

Introduction to Secure Telephone Terminals.

PB97-110498 01,512 Not available NTIS

PB97-110506

Compensation for Errors Introduced by Nonzero Fringe Densities in Phase-Measuring Interferometers.

PB97-110514

USCEANIST Measurement Assurance Programs for the Radiopharmaceutical and Nuclear Power Industries

PB97-110514

03,721 Not available NTIS

PB97-110522

Causality and Maxwell's Equations.
PB97-110522 04,429 Not available NTIS

PB97-110530

Electromagnetic Scattering from a Dielectric Wedge and the

Single Hypersingular Integral Equation.
PB97-110530 04,430 Not available NTIS PB97-110548

Light Scattering by Sinusoidal Surfaces: Illumination Windows and Harmonics in Standards.
PB97-110548 PB97-110548

Numerical Evaluation of Hypersingular Integrals for Scattering by a Dielectric Wedge.

PB97-110563

02,017 Not available NTIS

Point Charges, Radiation Reaction, and Ouantum Mechanics. $97-110563$

PB97-110571

04,139 Not available NTIS

Positronium in Relativistic Quantum Mechanics.

PB97-110571

04,140 Not available NTIS
PB97-110589

Relativistic Ouantum Mechanics of Interacting Particles. PB97-110589

04,141 Not available NTIS

PB97-110597

Sinusoidal Surfaces as Standards for BRDF Instruments.

PB97-110597 04,388 Not available NTIS

PB97-110605

Spinor Equations in Relativistic Quantum Mechanics.

PB97-110605 04,142 Not available NTIS

PB97-110811

Computer Security: Generally Accepted Principles and Practices for Securing Information Technology Systems.
PB97-110811 01,619 PC A05/MF A01 PB97-110811
PB97-111215

01,619 PC A05/MF A01

Windowing Effects on Light Scattering by Sinusoidal Sur-

faces.
PB97-111215

04,389 Not available NTIS

PB97-111223

Resonance Enhanced Multiphoton Ionization Spectroscopy of the SnF Radical.

PB97-111231

01,176 Not available NTIS

Characterization and Processing of Spray-Dried Zirconia Powders for Plasma Spray Application.
PB97-111231 PB97-111249

Relationship between Indoor Air Quality and Carbon Dioxide.

02,569 Not available NTIS

PB97-111256

Beam Line for Highly Charged lons

PB97-111256 04,143 Not available NTIS PB97-111264

Quantum Conductance Fluctuations in the Larger-Size-
Scale Regime.
PB97-111264
04,144 Not available NTIS

PB97-111264

PB97-111272

04,144 Not available NTIS

Magneto-Qptic Rotation Sensor Using a Laser Diode as Both Source and Detector.
PB97-111272

PB97-111280

Laser Cooling and the Recoil Limit.

PB97-111280

$$
\text { imit. }
$$

PB97-111298

Post-Occupancy Evaluation of the Forrestal Building.

PB97-111298

00,280 Not available NTIS

PB97-111306

Size Effects and Giant Magnetoresistance in Unannealed NiFe/Ag Multilayer Stripes.

PB97-111306

04, 145 Not available NTIS

PB97-111314

Fluoride Elimination Upon Reaction of Pentafluoroaniline with e (sub eq') (sup -), H, and OH Radicals in Aqueous So lution.

PB97-111314

01,177 Not available NTIS

PB97-111322

Interlaboratory Studies on the Analysis of Hair for Drugs of

Abuse: Results from the Fourth Exercise. PB97-111330

Numerical Reference Models for Qptical Metrology Simulation.

04,392 Not available NTIS

PB97-111413

Commercial Helium Permeation Leak Standards: Their Properties and Reliability.

PB97-111413

04,146 Not available NTIS

PB97-111421

Extrapolation of the Heat Capacity in Liquid and Amorphous Phases.

PB97-111421

04, 147 Not available NTIS

PB97-111439

Double Modulation and Selective Excitation Photoreflectance for Characterizing Highly Luminescen Semiconductor Structures and Samples with Poor Surface Morphology.

PB97-111447

02,452 Not available NTIS

Thermodynamic Properties of Synthetic Otavite, $\mathrm{CdCO} 3(\mathrm{cr})$ Enthalpy Increment Measurements from $4.5 \mathrm{~K}$ to $350 \mathrm{~K}$. PB97-111447 00,680 Not available NTIS PB97-111454

Proposed Tests to Evaluate the Frequency-Dependent Capacitor Ratio for Single Electron Tunneling Experiment. PB97-111454

01,982 Not available NTIS

PB97-111462

Mechanism of Defect Formation in Low-Dose Oxygen Implanted Silicon-on-Insulator Material.
PB97-111462 02,453 Not available NTIS

02,453 Not available

Closed Loop Controller for Electron-Beam Evaporators. B97-111488

03,424 Not available NTIS

Heap of Data.
PB97-111488

PB97-111496

Sephson Voltage Standard.

Pulse-Driven Programmable Josephson Voltage Standard.
PB97-111496 04,148 Not available NTIS

PB97-111504

Internal Waves in Xenon Near the Critical Point.

PB97-11 1504

PB97-111512

Transient Errors in a Precision Resistive Divider.

PB97-111512

04,221 Not available NTIS

PB97-111520

High Power Generation with Distributed Josephson-Junction Arrays.

PB97-111520

02,099 Not available NTIS

PB97-111538

Ionization Energies, Appearance Energies and Thermochemistry of CF2Q and FCO.
PB97-111538 01,178 Not available NTIS

PB97-111546

Experimental Verification of a Moisture and Heat Transfer Model in the Hygroscopic Regime.

PB97-111546

PB97-111553

Testing Conformance and Interoperability of BACnet (Trade Name) Building Automation Products.

PB97-111553

00,310 Not available NTIS

PB97-111561

Representing Designs with Logic Formulations of Spatial Relations.

PB97-11156
PB97-111579

02,792 Not available NTIS

Using Logic to Specify Shapes and Spatial Relations in Design Grammars
PB97-111579

PB97-111587

Neutron Diffraction Texture Study of Deformed Uranium Plates.

PB97-111587

03,010 Not available NTIS

PB97-111595

Calcium Phospirate Cements.

PB97-111595

03,580 Not available NTIS

PB97-111603

63Ni Half-Life: A New Experimental Determination and Critical Review.

PB97-111603

00,700 Not available NTIS

PB97-111819

Nickel-63 Standardization: 1968-1995.

PB97-111819

00,701 Not available NTIS

PB97-111827

Electrical Test Structures Replicated in Silicon-on-Insulator Material.

PB97-111835

02,454 Not available NTIS

Fundamentals and Problems of Fiber Current Sensors.

PB97-111835

02,205 Not available NTIS

PB97-111843

Uncertanties of Frequency Response Estimates Derived from Responses to Uncertain Step-Like Inputs.

PB97-111843

01,984 Not available NTIS

PB97-111850

Measurement Comparability, Traceability, and Measurement Assurance Programs.

PB97-111850

02,692 Not available NTIS

\section{PB97-111868}

$X$-ray Reflectivity Determination of Interface Roughness Correlated with Transport Properties of (AIGa)As/GaAs High Electron Mobility Transistor Devices.

PB97-111868

4,149 Not available NTIS

Resistors

PB97-111876

02,284 Not available NTIS

PB97-111884

Loading Effects in Resistance Scaling.
PB97-111884 Not available NTIS

PB97-111892

Test Procedures for Advanced Insulation Panels.

PB97-111892

00,415 Not available NTIS

PB97-111900

Compatibilizatıon of Polymer Blends by Complexation. 2. Kinetics of Interfacial Mixing.

PB97-111900 
PB97-111967

One-Electron Oxidation of Metalloporphycenes as Studied by Radiolytic Methods.

PB97-111975

01,179 Not available NTIS

Oxidation of Caffeic Acid and Related Hydroxycinnamic Acids.

PB97-111975

PB97-111983

DNA Damage and DNA Sequence Retrieval from Ancient Tissues.
PB97-111983

PB97-111991

03,556 Not available NTIS

Empirical Validation of a Transient Computer Model for Combined Heat and Moisture Transfer. PB97-111991

00,416 Not available NTIS PB97-112007

Observation of Hot-Electron Shot Noise in a Metaltic Resis-

PB97-112007

01,988 Not available NTIS

PB97-112213

Calibration in the Earth Observing System (EOS) Project. Part 2. Implementation

PB97-112213

04,842 Not available NTIS

B97-112221

HVAC CAD Layout Tools: A Case Study of University/lndustry Collaboration.

PB97-112221

00,281 Not available NTIS

Systematic Correction in Bragg $X$-ray Diffraction of Flat and Curved Crystals

PB97-11223
PB97-112247

04,152 Not available NTIS

Chip Morphology, Tool Wear and Cutting Mechanics in Finish Hard Turning.

PB97-112254

03,106 Not available NTIS

Laser Bandwidth Effects in Ouantitative Cavity Ring-Down Spectroscopy.

PB97-112262

04,394 Not available NTIS

Lattice Model

PB97-112270

Analysis of Droplet Arrival Statistics in a Pressure-Atomized Spray Flame.

PB97-112288

01,352 Not available NTIS

Forward Scattering of a Gaussian Beam by a Nonabsorbing

PB97-112288

PB97-112296

04,395 Not available NTIS

Interdigitated Stacked P-I-N Multiple Ouantum Well Modulator.

PB97-112304

02,455 Not available NTIS

Vitrification and Crystallization of Organic Liquids Confined to Nanoscale Pores.

PB97-112304

PB97-112312

03,392 Not available NTIS

Factors Affecting the Energy Consumption of Two Retrigerator-Freezers

PB97-112320

Combining Interactive Exploration and Optimization for AsSembly Design

PB97-112338

02,794 Not available NTIS

Development of Thin-Film Multijunction Thermal Converters at NIST

PB97-112338

PB97-112346

02,286 Not available NTIS

Bias Current Dependent Resistance Peaks in NiFe/Ag Giant

Magnetoresistance Multilayers.
PB97-112346

04,153 Not available NTIS

PB97-112353

Empirical Linear Prediction Applied to a NIST Calibration Service.

PB97-112353

PB97-112361

02,287 Not available NTIS

Absorption Cross Sections, Kinetics of Formation, and SelfReaction of the IO Radical Produced via the Laser Photolysis of $\mathrm{N} 2 \mathrm{O} / 2 / 2 / \mathrm{N} 2$ Mixtures.

PB97-112361

PB97-112379

High-Current Thin Film Multijunction Thermal Converters and Multi-Converter Modules.

PB97-112379

PB97-112387

01,989 Not available NTIS

solated Spin Pairs and Two-Dimensional Magnetism in SrCr(sub 9p)Ga(sub 12-9p)O19.

PB97-11239

04,154 Not available NTIS

Using Technology to Manage and Protect Intellectual Property.

PB97-112403

01,513 Not available NTIS

Matrix Isolation Study of the Interaction of Excited Neon Atoms with O3: Intrared Spectrum of O((sub 3)(-)) and Evi-

dence for the Stabilization of O2... $(($ (sub 4$)(+))$.
PB97.112403
PB97-112411

Neutron Scattering Study of Antiferromagnetic Order in the Magnetic Superconductors RNi2B2C.

PB97-112411 04,812 Not available NTIS PB97-112429

Unconventional Ferromagnetic Transition in La(sub 1 . x) $\mathrm{Ca}($ sub x) $\mathrm{MnO} 3$

PB97-112429

04,156 Not available NTIS

PB97-112437

Use of Neutron Beams for Chemical Analysis at NIST.

PB97-112437

00,652 Not available NTIS

B97-112445

Measurement of Ascorbic Acid in Human Plasma and
Serum: Stability, Intralaboratory Repeatability, and Interlaboratory Reproducibility

PB97-112445

03,511 Not available NTIS

7-112452

Low Thermal Guarded Scanner for High Resistance Measurement Systems.

PB97-11245

02,288 Not available NTIS

Ferric lon Assisted Photooxidation of Halocetates.

PB97-112460 00,521 Not available NTIS

PB97-112478

In situ Characterization of Vapor Phase Growth of Iron Oxide-Silica Nanocomposites: Part 1. 2-D Planar Laser-Induced Fluorescence and Mie Imaging.
PB97-112478 Not available NTIS PB97-112486

Evidence of Crosslinking in Methyl Pendent PBZT Fiber. PB97.112486

PB97-112494

03,393 Not available NTIS

Relaxation After a Temperature Jump Within the One Phase Region of a Polymer Mixture.

PB97-112494

03,394 Not available NTIS

PB97-112502

Knowledge-Based Approach for Automating a Design Method for Concurrent and Real-Time Systems.

PB97-112502 01,780 Not available NTIS

PB97-112510

Adsorption of Polyacrylic Acids and Their Sodium Salts on Hydroxyapatite: Effect of Relative Molar Mass.

PB97-112510

03,581 Not available NTIS

PB97-112528

Thermochemical Studies of Inorganic Chalocogenides by Fluorine-Combustion Calorimetry: Binary Compounds of Germanium and Silicon with Sulfur, Selenium and Tellu-
rium. PB97.112528

01,181 Not available NTIS

PB97-112536

Thermodynamics of (Germanium + Selenium): A Review and Critical Assessment.

PB97-112536

PB97-112544

01,182 Not available NTIS

Characterization of Modified FEL Ouartz-Halogen Lamps for Photometric Standards.

PB97-112544

00,282 Not available NTIS

PB97-112551

Exploring the Low-Frequency Performance of Thermal Converters Using Circuit Models and a Digitally Synthesized Source

PB97-11255
PB97-112569

02,848 Not available NTIS

Low Voltage Standards in the $10 \mathrm{~Hz}$ to $1 \mathrm{MHz}$ Range.

PB97-112569

02,100 Not available NTIS

PB97-112577

Atmospheric Lifetimes of HFC-143a and HFC-245fa: Flash Photolysis Resonance Fluorescence Measurements of the $\mathrm{OH}$ Reaction Rate Constants.

PB97-112577

00.118 Not available NTIS

PB97-112585

Modeling Effects of Temperature Annealing on Giant Magnetoresistive Response in Discontinuous Multilayer

NiFe/Ag Films.

PB97-112593

04,157 Not available NTIS

Simulating Device Size Effects on Magnetization Pinning Mechanisms in Spin Valves.

PB97-112593

04,158 Not available NTIS

Chemical Aspects of Tool Wear in Single Point Diamond Turning.
PB97-112601

03,021 Not available NTIS

PB97-113013

NIST Thermal Infrared Transter Standard Radiometer for the Earth Observing System (EOS) Program.

the Earth Observing System (EOS) Program.
PB,843 Not available NTIS PB97-113021

Results of a NISTNNIIOFI Comparison of Spectral-Radiance Measurements.

04,159 Not available NTIS

B97-113021

Wideband Sampling Voltmeter

PB97-113039

01,990 Not available NTIS

PB97-113047

In-situ Studies of a Novel Sodium Flame Process for Synthesis of Fine Particles.

PB97-113047

00,681 Not available NTIS

PB97-113054

Optical and Modeling Studies of Sodium/Halide Reactions for the Formation of Titanium and Boron Nanoparticles.

PB97-113054 00,682 Not available NTIS

PB97-113062

NIST Watt Balance: Progress Toward Monitoring the Kilogram.

PB97-113070

01,991 Not available NTIS

Colossal Magnetoresistance without $\mathrm{Mn}(3+) / \mathrm{Mn}(4-)$ Double Exchange in the Stoichiometric Pyrochlore Tl2Mn2O7.

PB97-113070 Stoichiometric Pyrochlore T2Mn2O
P4,160 Not available NTIS

PB97-113088

Dimensional Crossover in the Phase Separation Kinetics of Thin Polymer Blend Films.

PB97-113088

PB97-113096

Improving the Design Process by Predicting Downstream Values of Design Attributes.

PB97-113096

02,795 Not available NTIS

PB97-113104

Polarization Measurements on a Magnetic Ouadrupole Line in Ne-Like Barium.

PB97-113104

04,161 Not available NTIS

PB97-113112

Molecular Dynamics Investigation of the Surface/Bulk Equi-

librium in an Ethanol-Water Solution.

PB97-113112

01,183 Not available NTIS

B97-113120

International Standards and Reference Materials.

PB97-113120

00,188 Not available NTIS

PB97-113138

Measurement of Process Complexity.

PB97-113138

01,781 Not available NTIS

PB97-113146

Microstructure Effect on the Phase Behavior of Blends of

Deuterated Polybutadiene and Protonated Polyisopene.

PB97-113146

01,296 Not available NTIS

PB97-113153

Critical Evaluation of Thermal Mass Flow Meters.

PB97-113153

00,683 Not available NTIS

PB97-113161

Comparison of Filter Radiometer Spectral Responsivity with the NIST Spectral-Irradiance and Illuminance Scales.

PB97-113161

04,162 Not available NTIS

PB97-113179

Integrated Optical Polarization-Discriminating Receiver in

Glass.
PB97-113179

PB97-113187

02,206 Not available NTIS

Frequency Synthesis and Metrology at $10(-17)$ and Beyond

PB97-113187

PB97-113195

Reducing the $1 / 4 \mathrm{AM}$ and PM Noise in Electronics for Precision Frequency Metrology.

$97-113195$

02,102 Not available NTIS

PB97-113203

CONTAM94: A Multizone Airflow and Contaminant Disper- 
PB97-113880

Electronics and Electrical Engineering Laboratory Technica Progress Bulletin Covering Laboratory Programs, April to
June 1996 with 1996-1998 EEEL Events Calendar.

PB97-113880 01,994 PC A04/MF A01 PB97-113898

Static Structural Analysis of a Reconfigurable Rigid Platform Supported by Elastic Legs.

$02,960 \mathrm{PC} \mathrm{A05/MF} \mathrm{A01}$

PB97-113906

Experimental Models for Soltware Diagnosis.

PB97-113906

$01,783 \mathrm{PC}$ A04/MF A01

PB97-114169

Structured Testing: A Testing Methodology Using the Cyclomatic Complexity Metric.

PB97-114169

$01,784 \mathrm{PC} \mathrm{A07/MF} \mathrm{A02}$

PB97-114185

National Voluntary Laboratory Accreditation Program NVLAP): Fasteners and Metals.

PB97-114185

$02,881 \mathrm{PC}$ A06/MF A01

PB97-114227

Carbon Monoxide Dispersion in Residential Buildings: Literature Review and Technical Analysis.

PB97-114227

02,571 PC A05/MF A01

PB97-114235

Survey of Fuel Loads in Contemporary Office Buildings.

PB97-114235

$00,233 \mathrm{PC} \mathrm{A04/MF} \mathrm{A01}$

PB97-114250

Summary of Federal Construction and Building $R$ and $D$ in 1994.

PB97-114250

$00,234 \mathrm{PC} \mathrm{A04/MF} \mathrm{A0}$

PB97-114268

Interoperability Requirements for CAD Data Transfer in the AutoSTEP Projec

PB97-1

$02,796 \mathrm{PC} \mathrm{A03/MF} \mathrm{A01}$

India: Environmental Technologies Export Market Plan.

PB97-114359 02,529 PC A05/MF A01

PB97-114474

Experımentally Measured Total X-ray Attenuation Coefficients Extracted from Previously Unprocessed Documents Held by the NIST Photon and Charged Particle Data Cener.

PB97.114474

$04,165 \mathrm{PC} \mathrm{A04/MF} \mathrm{A01}$

PB97-114482

Fire Safety Engineering in the Pursuit of PerformanceBased Codes: Collected Papers.

PB97-114482

$00,235 \mathrm{PC} \mathrm{A07/MF} \mathrm{A02}$

PB97-115794

Proceedings of a Workshop on Developing and Adopting Seismic Design and Construction Standards for Lifelines. Held in Denver, Colorado on September 25-27, 1991

01,302 PC A16/MF A03

PB97-115802

Multi-Scale Picture of Concrete and Its Transport Properties: Introduction for Non-Cement Researchers.

PB97-115802 03,107 PC A05/MF A01

PB97-116040

Bibliography of the NIST Optoelectronics Division

$\begin{array}{lll}\text { PB97-116040 02,207 PC A06/MF A01 } & \end{array}$

PB97-116057

Metrology for Electromagnetic Technology: A Bibliography of NIST Publications.

PB97-116057

04,396 PC A06/MF A01

Group 1 for the Process Engineering Data STEP Application Protocol.

PB97-116073

PB97-116081

02,797 PC A99/MF A06

Full-Scale Room Fire Experiments Conducted at the University of Maryland.

$00,236 \mathrm{PC} \mathrm{A04/MF} \mathrm{A01}$

PB97-116107

Cycle.

PB97-116107

PB97-116123

Unified Process Specification Language: Requirements for Modeling Process.

PB97-116123

PB97-116131

Fire Protection Foam Behavior in a Radiative Environment. PB97-116131

a vapor Compression

$00,237 \mathrm{PC} \mathrm{A} 10 / \mathrm{MF}$ A02

Standards Promote Credibility and Technology Transfer: The Need for Greater Industry Support of Technical Com

mittees.

$02,961 \mathrm{PC} \mathrm{A03/MF} \mathrm{AO}$

PB97-116222

Evaluation of Survey Procedures for Determining Occupant

Load Factors in Contemporary Office Buildings.
PB97-116222 00,238 PC A03/MF A01

PB97-118335

Majority and Minority Electron and Hole Mobilities in Heavily Doped Gallum Aluminum Arsenide.

PB97-118335 04,814 Not available NTIS

PB97.118343

Excitons in Complex Ouantum Nanostructures

PB97-118343

01,184 Not available NTIS
PB97-118350

Quantum Dots in Quantum Well Structures.

PB97-118350

PB97-118368

ssues in High-Speed Pyrometry

PB97-118368

01,185 Not available NTIS

PB97-118376

02,693 Not available NTIS

Simultaneous Measurement of Normal Spectral Emissivity by Spectral Radiometry and Laser Polarimetry at High Temybdenum and Tungsten.

yBdenum and

02,694 Not available NTIS

PB97.118384

Thermodynamic Properties of CF3-CHF-CHF2, 1,1,1,2,3,3Hexafluoropropane.

PB97-11838

03,299 Not available NTIS

PB97-118392

Thermodynamic Properties of CHF2-CF2-CHF, 1,1,2,2,3 Pentafluoropropane.

PB97.118392

03,300 Not available NTIS

PB97-118400

Swelling and Growth of Polymers, Membranes and PB97-118400

03,396 Not available NTIS

PB97.118616

Effect of Beam Voltage on the Properties of Aluminum Nitride Prepared by Ion Beam Assisted Deposition.

PB97-118616 01,995 Not available NTIS

PB97-118624

Posterior Restorative Materials Research

PB97-118624 03,582 Not available NTIS

PB97-118632

NIST Research on Less-Flammable Materials.

PB97-118632

01,439 Not available NTIS

PB97-118640

Realization of a Scale of Absolute Spectral Response Using he NIST High Accuracy Cryogenic Radiometer.

PB97-118640

04,397 Not available NTIS

PB97-118657

Bimolecular Interactions in (Et) $3 \mathrm{SiOH}: \mathrm{Base}: \mathrm{CCl} 4$ HydrogenBonded Solutions Studied by Deactivation of the Free $\mathrm{OH}$ Stretch Vibration.

PB97-11865

04, 166 Not available NTIS

PB97-118665

Lab Report Special Section: Natural Language Processing and Information Retrieval Group Information Access an User Interfaces Division, National Institute of Standards and PB97-118665

02,742 Not available NTIS

PB97-118673

Panel: Building and Using Test Collections.

PB97.118673

02,743 Not available NTIS PB97-11868

Transient Analysis of a Line-Focus Transducer Probing a Liquid/Solid Interface.

PB97-118681

02,763 Not available NTIS

PB97-118699

Radiance Temperatures at $1500 \mathrm{~nm}$ of Niobium and Molybdenum at Their Melting Points by a Pulse-Heating Technique.

PB97-118699

04,167 Not available NTIS

PB97-118707

Developing Measurement for Experimentation.

PB97-118707

03,450 Not available NTIS

PB97-118715

Pilot Studies for Improving Sampling Protocols.

PB97-118715

02,530 Not available NTIS

PB97-118723

Microwave Spectrum and Structure of $\mathrm{CH} 2 \mathrm{O}-\mathrm{H} 2 \mathrm{O}$

PB97-118723

04,168 Not available NTIS

PB97.118731

Sources of Strain-Measurement Error in Flag-Based Extensometry.

B97.118749

Materials-Science Based Approach to Phenol Emissions from a Flooring Material in an Office Building.

PB97-118749

PB97-118756

Colour Centres in LiF for Measurement of Absorbed Doses Up to $100 \mathrm{MGy}$

PB97-118756

PB97-118764

04,169 Not available NTIS

Capabilities

PB97-118764
PB97-118772

02,798 Not available NTIS

Shear-Induced Changes in the Order-Disorder Transition

Temperature and the Morphology of a Triblock Copolymer.
PB $97-118772$
03,130 Not available NTIS PB97-118772

PB97-118780

Overview of Bioelectrical Impedance Analyzers.

PB97-118780

00,181 Not available NTIS

PB97-118798

Rotational Spectra of $\mathrm{CH} 3 \mathrm{CCH}-\mathrm{NH} 3, \mathrm{NCCCH}-\mathrm{NH} 3$, and

$\mathrm{NCCCH}-\mathrm{OH} 2$

04,170 Not available NTIS

PB97-118806

Issues in the Field Measurement of VOC Emission Rates. PB97-118806 02,573 Not available NTIS

PB97-118996

Line-Heat-Source Guarded-Hot-Plate Apparatus.

PB97-119002

00,417 Not available NTfS

Segmental Concentration Profiles of End-Tethered Poly-

mers with Excluded-Volume and Surface Interactions.

PB97-119002

00,654 Not available NTIS

PB97-119010

Surface Roughness of Glass-Ceramic Insert. Composite Restorations: Assessing Several Polishing Techniques.

PB97-119010

PB97-119028

Effect of Formulation Changes on the Response to lonizing Radiation of Radiochromic Dye Films.

PB97-11902

04,171 Not available NTIS

PB97-119036

Results of the ASTM Nuclear Methods Intercomparison on NIST Apple and Peach Leaves Standard Reference Mate. rials.

B97-119036

03,490 Not available NTIS

PB97-119044

Making Connections.

PB97-119044

PB97-11905

Measurements of the $(235) \mathrm{U}(\mathrm{n}, \mathrm{f})$ Cross Section in the 3 to $30 \mathrm{MeV}$ Neutron Energy Region

PB97-11905

PB97-119069

Measurements of the $(237) \mathrm{Np}(n, f)$ Cross Section.

PB97-119069

PB97-119077

Influence of Envelopes Geometry on the Sensitivity of Nude Ionization Gauges.

PB97-119077

04,174 Not available NTIS

\section{-119085}

Mass Assay and Uniformity Test of Boron Targets by Neu tron Beam Methods.

PB97-119085

04,175 Not available NTfS

PB97-119101

Design of Technically Complex Facilities.

PB97-119101

02,695 Not available NTIS

PB97.119119

Resonance Enhanced Multiphoton Ionization Spectroscopy of the PF Radical.

PB97-119119

PB97-119127

Kinetics of the Reaction of the Sulfate Radical with the Oxalate Anion.

PB97-119127

01,186 Not available NTIS

$97-11913$

Morphology and Phase Separation Kinetics of a Compatibilized Blend 
PB97-119234

Vibrational Spectra of Molecular Ions Isolated in Solid Neon. 13. Ions Derived from $\mathrm{HBr}$ and $\mathrm{HI}$. Not available NTIS
PB97-119234 01,188 Not PB97-119242

Comparisons of Some NIST Fixed-Point Cells with Similar Cells of Other Standards Laboratories. PB97-119242

00,655 Not available NTIS

PB97-119259

Novel Radiochromic Films for Clinical Dosimetry.

PB97-119259 03,641 Not available NTIS

PB97-119267

Internal Droplet Circulation Induced by Surface-Driven Rotation

PB97-119267

02,500 Not available NTIS

PB97-119275

Orientational Fluctuations, Diffuse Scattering, and Orientational Order in Solid C60.

04.176 Not available NTIS

PB97-119283

Micromachined Coplanar Waveguides in CMOS Technology.
PB97-119283

02,456 Not available NTIS

PB97-119291

Calibration and Performance of GafChromic DM-100 Radiochromic Dosemeters

PB97-119309

00,703 Not available NTIS

Interaction of Citric Acid with Hydroxyapatite: Surface Exchange of lons and Precipitation of Calcium Citrate.

PB97-119309 03,584 Not available NTIS

PB97-119317

Active High Voltage Divider with 20-PPM Uncertainty.

PB97-119317

02,104 Not available NTIS

PB97-119325

Transport by Gravity Currents in Building Fires.

PB97-119325

01,441 Not available NTIS

PB97-119333

Liquid Chromatographic Determination of Carotenoids in Human Serum Using an Engineered C30 and a C18 Stationary Phase
PB97-119333

PB97-119341

03,512 Not available NTIS

Self-Consistent 'GW' and Higher-Order Calculations of Electron States in Metals.

PB97-11934

01,189 Not available NTIS

\section{PB97-119358}

Thermodynamic Properties of Silicides. 5. Standard Molar Enthalpy of Formation at the Temperature 298.15 K of Trimolybdenum Monosilicide Mo3Si Determined by Fluo-

rine-Combustion Calorimetry
PB97-119358

PB97-119366

01,190 Not available NTIS

Electronic Structure and Phase Equilibria in Ternary Substitutional Alloys.
PB97-119366

PB97-119374

03,378 Not available NTIS

Positive and Negative Cooperativities at Subsequent Steps of Oxygenation Regulate the Allosteric Behavior of Multistate Sebacylhemoglobin. PB97-119374

PB97-119382

DnaJ, DnaK, and GrpE Heat Shock Proteins are Required in 'ori'P1 DNA Replication Solely at the RepA Monomerization Step.

PB97-119382

03,557 Not available NTIS

PB97-119390

Anomalous Relation between Time and Frequency Domain

PMD Measurements.
PB97-119390 04,398 Not available NTIS

PB97-119408

Vortex Images in Thin Films of $\mathrm{YBa} C \mathrm{Cu} 3 \mathrm{O}$ (sub 7-x) and Bi2Sr2Ca1Cu2O(sub $8+x$ ) Obtained by Low-Temperature Magnetic Force Microscopy

PB97-11940

04,815 Not available NTIS

PB97-121321

Fault Diagnosis of an Air-Handling Unit Using Artificial Neural Networks.

PB97-121339

00,283 Not available NTIS

Computer Simulations of Binder Removal from 2-D and 3-D Model Particulate Bodies.

PB97-121339

00,418 Not available NTIS

PB97-121610

Science, Technology, and Competitiveness: Retrospective on a Symposium in Celebration of NIST's 90th Anniversary and the 25th Anniversary of the Gaithersburg Laboratories, November 14-15, 1991.

PB97-121610

02,696 PC A08/MF A02

PB97-121636

Text REtrieval Conference (4th) (TREC-4). Held in Gaithersburg, Maryland on November 1-3, 1995 .
PB97-121636
01,786 PC A99/MF E08 PB97-121826

International Green Building Conference and Exposition (3rd). Held in San Diego, California on November 17-19,

1996. (Reannouncement with new abstract).
PB97-121826 02,531 PC A12/MF A03
PB97-122212

Interface-Filter Characterization of Spectraradiometers and Colorimeters.

PB97-122212

PB97-122220

Antiferromagnetic Interlayer Correlations in Annealed Ni80Fe20/Ag Multilayers.

PB97-122220

04,399 Not available NTIS

PB97-122238

Publication and Presentation Abstracts, 1996

PB97-122238

03,585 Not available NTIS

(122246

Flat and Curved Crystal Spectrography for Mammographic $X-$ ray Sources
PB97-122246

PB97-122253

03,642 Not available NTIS

Dependence of the Thermal Electron Attachment Rate Constant in Gases and Liquids on the Energy Position of the Electron Altaching State.

PB97-122253

01,996 Not available NTIS

PB97-122261

Environmental Aspects of Halon Replacements: Considerations for Advanced Agents and the Ozone Depletion Potential of CF3l.

PB $97-122279$

Conference Report: Calorimetry Conference (50th)

PB97-122279

PB97-122287

Usability Engineering: Industry-Government Collaboration for System Effectiveness and Efficiency.

PB97-122287

01.514 Not available NTIS

PB97-122295

Experimental Determination of the Rate Constant for the Reaction of $\mathrm{C}_{2} \mathrm{H}_{3}$ with $\mathrm{H} 2$ and Implications for the Partitioning of Hydrocarbons in Atmospheres of the Outer Planets. PB97-122303

CSL View of Applications Portability, Scalability, and InterOperability.

P897-122311

01,787 Not available NTIS

Materials and Fire Threat.

PB97.122311

01,442 Not available NTIS

PB97-122329

NIST Watt Experiment: Monitorıng the Kilogram.

PB97-122329

01,997 Not available NTIS

PB97-122337

Small Angle Neutron Scattering Study of the Structure and

Formation of MCM-41 Mesoporous Molecular Sieves.
PB97-122337 03,110 Not available NTIS PB97-122337

PB97-122345

Generalized Optical Theorem for On-Axis Gaussian Beams.
PB97-122345 04,177 Not available NTIS

Radiocarbon Measurements of Atmospheric Volatile Or ganic Compounds: Quantifying the Biogenic Contribution.
02,574 Not available NTIS

\section{PB97-122360}

Measurement of the Atomic $\mathrm{Na}(3 \mathrm{P})$ Lifetime and of Retardation in the Interaction between Two Atoms Bound in Molecule

PB97-122360

04,178 Not available NTIS

PB97-122378

Large Local-Field Corrections in Optical Rotatory Power of Quartz and Selenium.

04,400 Not available NTIS

Single-Phase Heat Transfer and Pressure Drop Character

istics of an Integral-Spine Fin Within an Annulus.
PB97.122386
04,179 Not available NT!S

PB97-122394

Survey of the Components of Display-Measurement Standards.

PB97-122394

PB97-122402

Oxidation of Ferrous and Ferrocyanide lons by Peroxyl Radicals.

PB97-122402

PB97-122410

SC4 Short Names Registry.

PB97-122410

01,191 Not available NTIS

PB97-122428

02,799 Not available NTIS

Application of the Collocation Method in Three Dimensions to a Model Semiconductor Problem.

PB97-122428

PB97.122436

Complex Time Dependence of the EPR Signal of Irradiated L-alpha-alanine
PB97-122436

PB97-122444

DNA Base Damage in Lymphocytes of Cancer Patients Undergoing Radiation Therapy.

PB97-122444

PB97-122451

Perpendicular C-H Stretching Band nu9/nu13 and the Torsional Potential of Dimethylacelylene.

PB97-122451 01,192 Not available NTIS

PB97-122469

Photonic Band-Structure Effects for Low-Index-Contrast Two-Dimensional Lattices in the Near Infrared.

PB97-12246

04,401 Not available NTIS

PB97-122477

Nanoindentation and Instrumented Scratching Measurements on Hard Coatings.

PB97-122485

03,111 Not available NTIS

Overview of a Radiation Accident at an Industrial Accelerator Facility.

PB97-122493

Measuring Nondipolar Asymmetries of Photoelectron Angular Distributions.

PB97-122501

Wear Modeling of Si-Based Ceramics

PB97-122501

03,112 Not available NTIS

PB97-122519

NO Production and Destruction in a Methane/Air Diffusion

PB97-122519

01,443 Not available NTIS

PB97-122527

Characterization of Time-Dependent Dielectric Breakdown in Intrinsic Thin $\mathrm{SiO} 2$

PB97-12252

02,458 Not available NTIS

PB97-122535

Parametric Study of Wall Moisture Contents Using a Revised Variable Indoor Relative Humidity Version of the Transient Heat and Moisture Trant

PB97-122535

00,419 Not available NTIS

PB97-122543

Absence of Quantum-Mechanical Effects on the Mobility of Argon lons in Helium Gas at $4.35 \mathrm{~K}$

Argon lons in Helium Gas at $4.35 \mathrm{~K}$.

PB97-122550

Xi-Vector Formulation of Anisotropic Phase-Field Models: $3-$ D Asymptotics

PB97-122568

Determination of Anomalous Superexchange in $\mathrm{MnCl} 2$ and Its Graphite Intercalation Compound.
PB97-122568 00,666 Not available NTIS PB97-122576

Material Characterization By a Time-Resolved and Polariza. tion-Sensitive Ultrasonic Technique.

PB97-122576

02,764 Not available NTIS

PB97-122584

Design, Construction and Application of a Large Aperture Lens-Less Lin

PB97-122592

02,765 Not available NTIS

Chemical Effect in Ceramics Grinding

PB97-122592

PB97-122600

Capacitors with Very Low Loss: Cryogenic Vacuum-Gap PB97-122600

PB97-124135

Standards Activities of Organizations in the United States.

PB97-124135

00.006 PC A99/MF EO8

PB97-500342

Building Life Cycle Cost Computer Program (BLCC) Version 4.4-97 (for Microcomputers)

PB97-50034

00,284 CP DO2

RP2003

Low-Temperature Performance of Radiosonde Electric Hygrometer Elemen

SHRP-A-335

$00,121 \mathrm{PC}$ A02/MF A01

Binder Characterization and Evaluation by Nuclear Magnetic Resonance Spectroscopy.

PB94-193471

01,334 PC A08/MF A02

SHRP-C-373 


\section{APPENDIX A}

\section{List of Depository Libraries in the United States}

\section{ALABAMA}

\section{Auburn}

Auburn University Ralph Draughon Library

\section{Birmingham}

Birmingham Public Library

Birmingham-Southern College Library

Jefferson State Community College James B. Allen Library

Samford University Harwell G. Davis Library

\section{Enterprise}

Enterprise State Junior College Learning Resources Center

\section{Fayette}

Bevill State Community College at Brewer Learning Resources Center

\section{Florence}

University of North Alabama Collier Library

\section{Gadsden}

Gadsden Public Library

\section{Huntsville}

University of Alabama in Huntsville Library

\section{Jacksonville}

Jacksonville State University Houston Cole Library

\section{Maxwell Air Base}

Air University Library/LSAS

\section{Mobile}

Mobile Public Library

Spring Hill College Thomas Byrne Memorial Library

University of South Alabama University Library

\section{Montgomery}

Alabama Public Library Service

Alabama Supreme Court and State Law Library

Auburn University at Montgomery Library REGIONAL

\section{Normal}

\section{Alabama Agricultural and Mechanical University J. F. Drake} Memorial Library

Troy

Troy State University Library

\section{Tuscaloosa}

University of Alabama Amelia Gayle Gorgas Library REGIONAL

University of Alabama School of Law Library

\section{Tuskegee}

Tuskegee University Hollis Burke Frissell Library

\section{ALASKA}

\section{Anchorage}

Anchorage Law Library

Anchorage Municipal Libraries Z. J. Loussac Public Library

Department of the Interior Alaska Resources Library

University of Alaska at Anchorage Consortium Library

\section{Fairbanks}

University of Alaska Elmer E. Rasmuson Library

\section{Juneau}

Alaska State Library

University of Alaska Southeast William A. Egan Library

\section{Ketchikan}

University of Alaska Southeast Ketchikan College Library

\section{AMERICAN SAMOA}

\section{Pago Pago}

American Samoa Community College Learning Resources Center

\section{ARIZONA}

\section{Apache Junction}

Apache Junction Public Library

\section{Coolidge}

Central Arizona College Learning Resources Center

\section{Flagstaff}

Northern Arizona University Cline Library 
Glendale Public LIbrary

\section{Mesa}

Mesa Public Library

\section{Phoenix}

Arizona Department of Library Archives and Public Records REGIONAL

Grand Canyon University Fleming Library

Maricopa County Library District

Phoenix Public Library

\section{Prescott}

Yavapai College Library

\section{Tempe}

Arizona State University Hayden Library/Government Documents Arizona State University Ross-Blakley Law Library

\section{Tucson}

Tucson-Pima Public Library

University of Arizona College of Law Library

University of Arizona Main Library

\section{Winslow}

Northland Pioneer College Winslow Center Learning Resources Center

\section{Yuma}

Yuma County District Library

\section{ARKANSAS}

Arkadelphia

Ouachita Baptist University Riley-Hickingbotham Library

\section{Batesville}

Lyon College Mabee-Simpson Library

\section{Clarksville}

University of the Ozarks Dobson Memorial Library

\section{Conway}

University of Central Arkansas Torreyson Library
University of Arkansas Library

University of Arkansas School of Law Young Law Library

\section{Little Rock}

Arkansas State Library REGIONAL Arkansas Supreme Court Library Central Arkansas Library System Main Library University of Arkansas at Little Rock Ottenheimer Library University of Arkansas at Little Rock Pulaski County Law Library

\section{Magnolia}

Southern Arkansas University Magale Library

\section{Monticello}

University of Arkansas at Monticello Library

\section{Pine Bluff}

University of Arkansas at Pine Bluff Watson Memorial Library

\section{Russellville}

Arkansas Technical University Tomlinson Library

\section{Searcy}

Harding University Brackett Library

\section{State University}

Arkansas State University_Jonesboro Dean B. Ellis Library

\section{Walnut Ridge}

Williams Baptist College Felix Goodson Library

\section{CALIFORNIA}

\section{Anaheim}

Anaheim Public Library

\section{Arcadia}

Arcadia Public Library

\section{Arcata}

Humboldt State University Library

\section{Bakersfield}

California State University Walter W. Stiern Library Kern County, Beale Memorial Library 


\section{Berkeley}

University of California Main Library

University of California School of Law Library

\section{Carson}

California State University at Dominguez Library

Carson Regional Library Los Angeles County Public Library

\section{Chico}

California State University at Chico Meriam Library

\section{Claremont}

Claremont Colleges Honnold/Mouth Library

\section{Culver City}

Culver City Library Los Angeles County Public Library

\section{Davis}

University of California at Davis Shields Library

University of California at Davis Law Library

\section{Downey}

Downey City Library

\section{Fresno}

California State University at Fresno Henry Madden Library Fresno County Free Library

\section{Fullerton}

California State University at Fullerton University Library

\section{Garden Grove}

Orange County Public Library

\section{Hayward}

California State University at Hayward Library

\section{Inglewood}

Inglewood Public Library

\section{Irvine}

University of California at Irvine Main Library

\section{La Jolla}

University of California at San Diego Geisel Library

\section{Lakewood}

Angelo M. lacoboni Public Library. Los Angeles County Public Library

\section{Lancaster}

Lancaster Public Library

\section{La Verne}

University of La Verne College of Law Library

\section{Long Beach}

California State University at Long Beach Library Long Beach Public Library

\section{Los Angeles}

California State University at Los Angeles Kennedy Memorial Library Los Angeles County Law Library

Los Angeles Public Library

Loyola Law School William M. Rains Law Library

Occidental College Mary Norton Clapp Library

Southwestern University School of Law Library

University of California at Los Angeles University Research Library

University of California at Los Angeles Hugh \& Hazel Darling Law

Library

University of Southern California Doheny Memorial Library

University of Southern California Law Library

U.S. Court of Appeals Ninth Circuit Library

Whittier College School of Law Library

\section{Malibu}

Pepperdine University Payson Library

\section{Menlo Park}

U.S. Geological Survey Library

\section{Montebello}

Montebello Regional Library

\section{Monterey}

U.S. Naval Postgraduate School Dudley Knox Library

\section{Monterey Park}

Bruggemeyer Memorial Library

\section{Northridge}

California State University at Northridge Delmar T. Oviatt Library

\section{Norwalk}

Norwalk Regional Library Los Angeles County Public Library

\section{Oakland}

Oakland Public Library

\section{Ontario}

Ontario City Library 


\section{Palm Springs}

Palm Springs Public Library

\section{Pasadena}

California Institute of Technology Millikan Memorial Library Pasadena Public Library

\section{Pleasant Hill}

Contra Costa County Public Library

\section{Redding}

Shasta County Library

\section{Redlands}

University of Redlands Armacost Library

\section{Redwood City}

Redwood City Public Library

\section{Reseda}

West Valley Regional Branch Library Los Angeles Public Library

\section{Richmond}

Richmond Public Library

\section{Riverside}

Riverside City and County Public Library

University of California at Riverside Library/Government Publications

\section{Sacramento}

California State Library/Government Publications REGIONAL California State University at Sacramento Library

Sacramento County Law Library

Sacramento Public Library Central Library/Federal Documents University of the Pacific Schaber Law Library

\section{San Bernardino}

San Bernardino County Law Library

San Bernardino County Library

\section{San Diego}

San Diego County Law Library

San Diego County Library

San Diego Public Library Central Library

San Diego State University Library

University of San Diego Malcolm A. Love Library School of Law Library

\section{San Francisco}

California Supreme Court Library

Golden Gate University Law Library

San Francisco Public Library/Government Document Department

San Francisco State University J. Paul Leonard Library

University of California Hastings College of Law
University of San Francisco Richard A. Gleeson Library

U.S. Court of Appeals Ninth Circuit Library

San Jose

San Jose State University Clark Library

San Leandro

San Leandro Public Library Community Library Center

\section{San Luis Obispo}

California Polytechnic State University Robert F. Kennedy Library

\section{San Marcos}

California State University at San Marcos Library \& Information Services

\section{San Mateo}

College of San Mateo Library

\section{San Rafael}

Marin County Free Library

\section{Santa Ana}

Orange County Law Library

Santa Ana Public Library

\section{Santa Barbara}

University of California at Santa Barbara Davidson Library

\section{Santa Clara}

Santa Clara University Orradre Library

\section{Santa Cruz}

University of California at Santa Cruz McHenry Library

\section{Santa Rosa}

Sonoma County Public Library

\section{Stanford}

Stanford University Jonsson Library (1895)

Stanford University Robert Crown Law Library

\section{Stockton}

Public Library of Stockton and San Joaquin County

\section{Thousand Oaks}

California Lutheran University Pearson Library

\section{Torrance}

Torrance Public Library 


\section{Turlock}

California State University, Stanislaus Library

\section{Valencia}

Valencia Library Los Angeles County Public Library

Vallejo

Solano County Library System John F. Kennedy Library

\section{Ventura}

Ventura County Library E. P. Foster Library

\section{Visalia}

Tulare County Free Library

\section{Walnut}

Mount San Antonio College Learning Resources Library

\section{West Covina}

West Covina Regional Library

Whittier

Whittier College Wardman Library

\section{COLORADO}

\section{Alamosa}

Adams State College Library

\section{Aurora}

Aurora Public Library

\section{Boulder}

University of Colorado at Boulder Libraries Government Publications REGIONAL

University of Colorado at Boulder School of Law Library

\section{Broomfield}

Mamie Doud Eisenhower Public Library

\section{Colorado Springs}

Colorado College Tutt Library

University of Colorado at Colorado Springs Library

\section{Denver}

Regis University Dayton Memorial Library

University of Denver College of Law Library Westminister Law Library

University of Denver Penrose Library

U.S. Courts Library

\section{Fort Collins}

Colorado State University Libraries/Documents Department

\section{Golden}

Colorado School of Mines Arthur Lakes Library

\section{Grand Junction}

Mesa County Public Library District

Mesa State College John U. Tomlinson Library

\section{Greeley}

University of Northern Colorado James A. Michener Library

\section{Gunnison}

Western State College Leslie J. Savage Library

\section{La Junta}

Otero Junior College Wheeler Library

\section{Lakewood}

Jefferson County Public Library Lakewood Branch

\section{Pueblo}

Pueblo Library District McClelland Library University of Southern Colorado Library

\section{USAF Academy}

U.S. Air Force Academy Library

\section{CONNECTICUT}

\section{Bridgeport}

Bridgeport Public Library/Reference Department

\section{Danbury}

Western Connecticut State University Ruth A. Haas Library

\section{Hamden}

Quinnipiac College School of Law Library

\section{Hartford}

Connecticut State Library REGIONAL Hartford Public Library 
Trinity College Library

University of Connecticut School of Law Library

\section{Middletown}

Wesleyan University Olin Library

\section{New Britain}

Central Connecticut State University Elihu Burritt Library

\section{New Haven}

Southern Connecticut State University Hilton C. Buley Library Yale University Lillian Goldman Law Library

Yale University Seeley G. Mudd Library

\section{New London}

Connecticut College C. E. Shain Library

U.S. Coast Guard Academy Library

\section{Stamford}

Ferguson Library

\section{Storrs}

University of Connecticut Homer Babbidge Library

\section{Waterbury}

Silas Bronson Public Library

Teikyo Post University Traurig Library

\section{West Haven}

University of New Haven Marvin K. Peterson Library

\section{Willimantic}

Eastern Connecticut State University J. Eugene Smith Library

\section{DELAWARE}

\section{Dover}

Delaware Division of Libraries/Government Documents

Delaware State University William C. Jason Library

\section{Georgetown}

Delaware Technical and Community College Stephen J. Betez Library

\section{Newark}

University of Delaware Library

\section{Wilmington}

Widener University School of Law Library

\section{DISTRICT OF COLUMBIA}

\section{Washington}

American University Washington College of Law Library Board of Governors of the Federal Reserve System Law Library Catholic University of America Judge Kathryn J. Dufour Law Library

Comptroller of the Currency Library

Department of Commerce Library

Department of Education National Library of Education

Department of Housing and Urban Development Library

Department of Justice Main Library

Department of Labor Library

Department of State Law Library

Department of State Library

Department of the Army Pentagon Library

Department of the Interior Departmental Library

Department of the Navy Library Naval Historical Center

Department of the Treasury Library

Department of Transportation Main Library

Department of Transportation U.S. Coast Guard Law Library

Department of Veterans' Headquarters Library

District of Columbia Court of Appeals Library

District of Columbia Public Library/Document Department

Equal Employment Opportunity Library

Executive Office of the President Library

Federal Election Commission Law Library

Federal Energy Regulatory Commission Library

Federal Mine Safety, Health Review Library

General Accounting Office Information Services Center

General Services Administration Library

George Washington University Melvin Gelman Library

George Washington University National Law Center Jacob Burns Law Library

Georgetown University Law Center E. B. Williams Law Library

Georgetown University Lavinger Library

Library of Congress Congressional Research Service

Library of Congress Serial and Government Publications

National Defense University Library Fort Lesley J. McNair

Pension Benefit Guaranty Corporation Office of General Counsel Library

U.S. Court of Appeals Federal Circuit Library

U.S. Court of Appeals Judges' Library

U.S. Information Agency Information Resource Center

U.S. Postal Service Library

U.S. Senate Library

U.S. Supreme Court Library

\section{FLORIDA}

\section{Boca Raton}

Florida Atlantic University S. E. Wimberly Library

\section{Bradenton}

Manatee County Public Library Information Service Department 


\section{Clearwater}

Clearwater Public Library System U.S. Government Documents

\section{Cocoa}

Brevard County Library System Central Brevard Library

\section{Coral Gables}

University of Miami Otto G. Richter Library

\section{Daytona Beach}

Volusia County Public Library Volusia County Library Center

\section{De Land}

Stetson University duPont-Ball Library

\section{Fort Lauderdale}

Broward County Main Library/Government Documents

Nova Southeastern University Shepard Broad Law Center Library

\section{Fort Pierce}

Indian River Community College Miley Learning Resource Center

\section{Gainesville}

University of Florida College of Law Library

University of Florida Libraries REGIONAL

\section{Jacksonville}

Jacksonville Public Library

Jacksonville University Carl S. Swisher Library

University of North Florida Thomas G. Carpenter Library

\section{Key West}

Florida Keys Community College Key West Campus Learning Resources Center

\section{Lakeland}

Lakeland Public Library

\section{Leesburg}

Lake-Sumter Community College Library

\section{Melbourne}

Florida Institute of Technology Evans Library

\section{Miami}

Florida International University University Park Campus Library Miami-Dade Public Library Documents Division

St. Thomas University Library Documents Department

\section{North Miami}

Florida International University North Miami Campus Library

\section{Orlando}

University of Central Florida Library/Documents

\section{Palatka}

Saint Johns River Community College Library B. C. Pearce Learning Resources

\section{Panama City}

Bay County Public Library

\section{Pensacola}

University of West Florida John C. Pace Library

\section{Saint Petersburg}

Saint Petersburg Public Library Reference Department Stetson University College of Law Charles A. Dana Law Library

\section{Sarasota}

Selby Public Library

University of South Florida at Sarasota Jane Bancroft Cook Library

\section{Tallahassee}

Florida Agricultural and Mechanical University Coleman Memorial Library

Florida State University College of Law Library

Florida State University Strozier Library

Florida Supreme Court Library

State Library of Florida

\section{Tampa}

Tampa-Hillsborough County Public Library University of South Florida Library

University of Tampa Merl Kelce Library

\section{Winter Park}

Rollins College Olin Library

\section{GEORGIA}

Dougherty County Public Library

\section{Americus}

Georgia Southwestern College James Earl Carter Library

\section{Athens}

University of Georgia Libraries/Government Documents REGIONAL University of Georgia School of Law Library 
Atlanta

Atlanta University Center Robert W. Woodruff Library Atlanta-Fulton Public Library (1880)

Emory University Robert W. Woodruff Library

Emory University School of Law McMillan Law Library

Georgia Institute of Technology Library and Information Center Georgia State Law Library

Georgia State University College of Law Library

Georgia State University William Russell Pullen Library

U.S. Court of Appeals Eleventh Circuit Library

\section{Augusta}

Augusta College Reese Library

Medical College of Georgia Greenblatt Library

\section{Brunswick}

Brunswick-Glynn County Regional Library

\section{Carrollton}

State University of West Georgia Irvine Sullivan Ingram Library

\section{Columbus}

Columbus State University Simon Schwob Memorial Library

\section{Dahlonega}

North Georgia College Stewart Library

\section{Dalton}

Dalton College Library Resources Center

\section{Kennesaw}

Kennesaw State University Horace W. Sturgis Library

\section{Macon}

Mercer University Main Library

Mercer University School of Law Library

\section{Milledgeville}

Georgia College Ina Dillard Russell Library

\section{Mount Berry}

Berry College Memorial Library

\section{Savannah}

Chatham-Effingham-Liberty Regional Library

\section{Smyrna}

Smyrna Public Library

\section{Statesboro}

Georgia Southern University Zoch S. Henderson Library (1939)
Valdosta

Valdosta State University Odum Library

GUAM

Agana

Nieves M. Flores Memorial Library

\section{Mangilao}

University of Guam Kennedy Memorial Library

\section{HAWAII}

\section{Hilo}

University of Hawaii at Hilo Edwin $\mathrm{H}$. Mookini Library

\section{Honolulu}

Hawaii Medical Library Incorporated

Hawaii State Library/Federal Documents

Municipal Reference \& Records Center

Supreme Court Law Library (1973)

University of Hawaii Hamilton Library REGIONAL

University of Hawaii School of Law Library

\section{Laie}

Brigham Young University Hawaii Campus Joseph F. Smith Library

\section{Lihue}

Lihue Public Library

\section{Pearl City}

Leeward Community College Library

\section{Wailuku}

Wailuku Public Library

\section{IDAHO}

\section{Boise}

Boise Public Library

Boise State University Albertsons Library Idaho State Library

Idaho Supreme Court State Law Library

\section{Caldwell}

Albertson College of Idaho N. L. Terteling Library 


\section{Lewiston}

Lewis-Clark State College The Library

\section{Moscow}

University of Idaho College of Law Library

University of Idaho Library/Government Documents Department REGIONAL

\section{Nampa}

Northwest Nazarene College John E. Riley Library

\section{Pocatello}

Idaho State University Eli Oboler Library

\section{Rexburg}

Ricks College David O. McKay Learning Resources Center

\section{Twin Falls}

College of Southern Idaho Library

\section{ILLINOIS}

\section{Bloomington}

Illinois Wesleyan University Sheean Library

\section{Bourbonnais}

Olivet Nazarene University Benner Library and Resource Center

\section{Carbondale}

Southern Illinois University at Carbondale Morris Library Southern Illinois University at Carbondale School of Law Library

\section{Carlinville}

Blackburn College Lumpkin Library

\section{Carterville}

John A. Logan College Learning Resources Center

\section{Champaign}

University of Illinois Law Library

\section{Charleston}

Eastern Illinois University Booth Library/Documents

\section{Chicago}

Chicago Public Library Harold Washington Library/Government Publications Department Chicago State University Paul and Emily Douglas Library DePaul University Law Library

Field Museum of Natural History Library

Illinois Institute of Technology Chicago-Kent College of Law Library
Illinois Institute of Technology Paul V. Galvin Library

John Marshall Law School Library

Loyola University of Chicago E. M. Cudahy Memorial Library

Loyola University of Chicago School of Law Library

Northeastern Illinois University Ronald Williams Library

Northwestern University School of Law Library

University of Chicago D'Angelo Law Library

University of Chicago Regenstein Library

University of Illinois at Chicago Library/Documents and Maps

William J. Campbell Library of the U.S. Courts

\section{Decatur}

Decatur Public Library/Government Documents

\section{De Kalb}

Northern Illinois University College of Law Library

Northern Illinois University Founders' Memorial Library

\section{Des Plaines}

Oakton Community College Library

\section{Edwardsville}

Southern Illinois University at Edwardsville Lovejoy Memorial Library

\section{Elsah}

Principia College Marshall Brooks Library

Evanston

Northwestern University Library/Government Publications

\section{Freeport}

Freeport Public Library

\section{Galesburg}

Galesburg Public Library

\section{Jacksonville}

MacMurray College Henry Pfeiffer Library

\section{Lake Forest}

Lake Forest College Donnelley Library

\section{Lebanon}

McKendree College Holman Library

Lisle

Illinois Benedictine College Theodore F. Lownik Library

Macomb

Western Illinois University Library/Government Publications

\section{Moline}

Black Hawk College Library (1970) 
Monmouth College Hewes Library

\section{Mount Carmel}

Wabash Valley College Bauer Media Center

\section{Mount Prospect}

Mount Prospect Public Library/Government Information Center

\section{Normal}

Illinois State University Milner Library

\section{Oak Park}

Oak Park Public Library

$$
\text { Oglesby }
$$

Illinois Valley Community College Jacobs Library

\section{Palos Hills}

Moraine Valley Community College Robert E. Turner Library

\section{Peoria}

Bradley University Cullom-Davis Library

Peoria Public Library

\section{River Forest}

Rosary College Rebecca Crown Library

\section{Rockford}

Rockford Public Library

\section{Romeoville}

Lewis University Library

\section{South Holland}

South Suburban College Library

\section{Springfield}

Illinois State Library/Federal Documents Department REGIONAL

\section{Streamwood}

Poplar Creek Public Library District

\section{University Park}

Governors State University Library

\section{Urbana}

University of Illinois/Documents Library

\section{Wheaton}

Wheaton College Buswell Memorial Library
Woodstock Public Library

\section{INDIANA}

\section{Anderson}

Anderson Public Library/Government Publications Anderson University Robert A. Nicholson Library

\section{Bloomington}

Indiana University Library

Indiana University School of Law Library

\section{Crawfordsville}

Wabash College Lilly Library

\section{Evansville}

Evansville-Vanderburgh County Public Library University of Southern Indiana David L. Rice Library

\section{Fort Wayne}

Allen County Public Library Indiana University-Purdue University at Fort Wayne Helmke Library

\section{Franklin}

Franklin College Library

\section{Gary}

Gary Public Library Main Library Indiana University Northwest Campus Library

\section{Greencastle}

DePauw University Roy O. West Library

\section{Hammond}

Hammond Public Library

\section{Hanover}

Hanover College Duggan Library

\section{Huntington}

Huntington College Richlyn Library

\section{Indianapolis}

Butler University Irwin Library-Serials/Documents Section Indiana State Library REGIONAL

Indiana Supreme Court Law Library

Indiana University at Indianapolis School of Law Library

Indiana University-Purdue University Libraries

Indianapolis-Marion County Public Central Library 


\section{Kokomo}

Indiana University Kokomo Learning Resource Center

\section{Muncie}

Ball State University Alexander M. Bracken Library Muncie Public Library

\section{New Albany}

Indiana University Southeast Library

\section{Notre Dame}

University of Notre Dame Kresge Law Library

University of Notre Dame Theodore M. Hesburgh Library

\section{Rensselaer}

Saint Joseph's College Robinson Memorial Library

\section{Richmond}

Earlham College Lilly Library

Morrisson-Reeves Library

\section{South Bend}

Indiana University at South Bend Franklin D. Schurz Library

\section{Terre Haute}

Indiana State University Cunningham Memorial Library

\section{Valparaiso}

Valparaiso University Law Library

Valparaiso University Moellering Memorial Library

\section{West Lafayette}

Purdue University HSSE Library

\section{IOWA}

\section{Ames}

lowa State University Parks Library

\section{Cedar Falls}

University of Northern lowa Donald O. Rod Library

\section{Cedar Rapids}

Cedar Rapids Public Library

\section{Council Bluffs}

Council Bluffs Public Library

\section{Davenport}

\section{Des Moines}

Drake University Cowles Library

Drake University Law Library

Public Library of Des Moines/Documents Department

State Library of lowa/Documents Section

\section{Dubuque}

Carnegie-Stout Public Library

Loras College Wahlert Memorial Library

Fayette

Upper lowa University Henderson-Wilder Library

\section{Grinnell}

Grinnell College Burling Library

\section{lowa City}

University of lowa College of Law Library

University of lowa Libraries/Government Publications REGIONAL

\section{Lamoni}

Graceland College Frederick Madison Smith Library

\section{Mason City}

North lowa Area Community College Library

\section{Mount Vernon}

Cornell College Russell D. Cole Library

\section{Orange City}

Northwestern College Ramaker Library

\section{Sioux City}

Sioux City Public Library Wilbur Aalfs Library

\section{KANSAS}

\section{Atchison}

Benedictine College Library

\section{Baldwin City}

Baker University Collins Library

\section{Colby}

Colby Community College H. F. Davis Memorial Library

\section{Dodge City}

Dodge City Community College Learning Resources Center 


\section{Emporia}

Emporia State University William Allen White Library

\section{Hays}

Fort Hays State University Forsyth Library

\section{Hutchinson}

Hutchinson Public Library/Depository Section

\section{Kansas City}

Kansas City Community College Library

\section{Lawrence}

University of Kansas Government Documents and Maps Library REGIONAL

University of Kansas Law School Library

\section{Manhattan}

Kansas State University Farrell Library

\section{Overland Park}

Johnson County Library/Documents Department

\section{Pittsburg}

Pittsburg State University Leonard H. Axe Library

\section{Salina}

Kansas Wesleyan University Memorial Library

\section{Topeka}

Kansas State Historical Society Library/Government Documents Kansas State Library

Kansas Supreme Court Law Library

Washburn University of Topeka School of Law Library

\section{Wichita}

Wichita State University Ablah Library (1901)

\section{KENTUCKY}

\section{Ashland}

Ashland Community College Library

\section{Barbourville}

Union College Weeks-Townsend Memorial Library

\section{Bowling Green}

Western Kentucky University Helm-Cravens Library

\section{Columbia}

Lindsey Wilson College Katie Murrell Library

\section{Crestview Hills}

Thomas More College Library

\section{Danville}

Centre College Grace Doherty Library

\section{Frankfort}

Kentucky Department of Libraries State Library Service Division Kentucky State Law Library

Kentucky State University Paul G. Blazer Library

\section{Hazard}

Hazard Community College Library

\section{Highland Heights}

Nor thern Kentucky University W. Frank Steely Library

\section{Lexington}

University of Kentucky King Library South REGIONAL University of Kentucky Law Library

\section{Louisville}

Louisville Free Public Library/Government Publications University of Louisville Belknap Campus Ekstrom Library University of Louisville School of Law Library

\section{Morehead}

Morehead State University Camden-Carroll Library

\section{Murray}

Murray State University Waterfield Library

\section{Owensboro}

Kentucky-Wesleyan College Library Learning Center

\section{Richmond}

Eastern Kentucky University John Grant Crabbe Library

\section{Williamsburg}

Cumberland College Hagan Memorial Library

\section{LOUISIANA}

\section{Baton Rouge}

Louisiana State University Middleton Library REGIONAL Louisiana State University Paul M. Herbert Law Center Library 
Southern University John B. Cade Library

Southern University Law Center Library

State Library of Louisiana

\section{Eunice}

Louisiana State University at Eunice Arnold LeDoux Library

\section{Hammond}

Southeastern Louisiana University Sims Memorial Library

\section{Lafayette}

University of Southwestern Louisiana Edith Garland Dupre Library

\section{Lake Charles}

McNeese State University Lether E. Frazar Memorial Library

\section{Leesville}

Vernon Parish Library

\section{Monroe}

Northeast Louisiana University Sandel Library

\section{Natchitoches}

Northwestern State University Watson Memorial Library

\section{New Orleans}

Law Library of Louisiana

Loyola University Law Library

Loyola University Library

New Orleans Public Library/Government Documents

Our Lady of Holy Cross College Blaine S. Kern Library

Southern University at New Orleans Leonard S. Washington Library

Tulane University Howard-Tilton Memorial Library

Tulane University School of Law Library

U.S. Court of Appeals Fifth Circuit Library

University of New Orleans Earl K. Long Library

Xavier University Library

\section{Pineville}

Louisiana College Norton Memorial Library

\section{Ruston}

Louisiana Tech University Prescott Memorial Library REGIONAL

\section{Shreveport}

Louisiana State University in Shreveport Noel Memorial Library Shreve Memorial Library

\section{Thibodaux}

Nicholls State University Ellender Memorial Library

\section{MAINE}

\section{Augusta}

Maine Law and Legislative Reference Library Maine State Library/Documents

\section{Bangor}

Bangor Public Library

\section{Brunswick}

Bowdoin College Hawthorne-Longfellow Library

\section{Castine}

Maine Maritime Academy Nutting Memorial Library

\section{Lewiston}

Bates College George and Helen Ladd Library

\section{Orono}

University of Maine Raymond H. Fogler Library REGIONAL

\section{Portland}

Portland Public Library

University of Maine School of Law Garbrecht Law Library

\section{Presque Isle}

University of Maine at Presque Isle Library

\section{Sanford}

Louis B. Goodall Memorial Library

\section{Waterville}

Colby College Miller Library

\section{MARYLAND}

\section{Annapolis}

Maryland State Law Library

U.S. Naval Academy Nimitz Library

\section{Baltimore}

Enoch Pratt Free Library/Documents Division

Goucher College Julia Rogers Library

Johns Hopkins University Government/Publication/Maps Law Library

Morgan State University Soper Library

University of Baltimore Langsdale Library

University of Baltimore Law Library

University of Maryland School of Law Thurgood Marshall Law Library

U.S. Court of Appeals Fourth Circuit Library 
Harford Community College Library

\section{Beltsville}

Department of Agriculture National Agricultural Library

\section{Bethesda}

Depar tment of Health and Human Services National Library of Medicine Uniformed Services University of Health Sciences Learning Resources Center

\section{Catonsville}

University of Maryland Baltimore County Albin O. Kuhn Library \& Gallery

\section{Chestertown}

Washington College Clifton M. Miller Library

\section{College Park}

University of Maryland at College Park McKeldin Library REGIONAL

\section{Cumberland}

Allegany College of Maryland Library

\section{Frostburg}

Frostburg State University Lewis J. Ort Library

\section{Patuxent River}

Naval Air Warfare Center Central Library

\section{Rockville}

Montgomery County Department of Public Libraries Rockville Regional Library

\section{Salisbury}

Salisbury State University Blackwell Library

\section{Silver Spring}

Department of Commerce NOAA Central Library

\section{Towson}

Towson State University Albert S. Cook Library

\section{Westminster}

Western Maryland College Hoover Library

\section{MASSACHUSETTS}

\section{Amherst}

Boston Athenaeum Library

Boston Public Library/Government Documents REGIONAL Boston University School of Law Pappas Law Library

Nor theastern University Snell Library

State Library of Massachusetts/Documents Department

Suffolk University Law Library

Supreme Judicial Court Social Law Library

U.S. Court of Appeals First Circuit Library

\section{Brookline}

Public Library of Brookline

\section{Cambridge}

Harvard College Lamont Library

Harvard Law School Library

Massachusetts Institute of Technology Libraries

\section{Chestnut Hill}

Boston College Thomas P. O'Neill Jr. Library

Chicopee

College of Our Lady of the Elms Alumnae Library

\section{Lowell}

University of Massachusetts South Campus Lowell O'Leary Library

\section{Medford}

Tufts University Tisch Library

\section{Milton}

Curry College Levin Library

New Bedford

New Bedford Free Public Library

\section{Newton Center}

Boston College Law School Library

\section{North Dartmouth}

University of Massachusetts Dartmouth Library/Government Publications

\section{North Easton}

Stonehill College Cushing-Martin Library

\section{Springfield}

Massachusetts Trial Court Hampden Law Library Springfield City Library/Documents Section Western New England College School of Law Library

\section{Waltham}

Brandeis University Goldfarb Library 
Wellesley

Wellesley College Margaret Clapp Library

\section{Wenham}

Gordon College Jenks Learning Resource Center

\section{Williamstown}

Williams College Sawyer Library

\section{Worcester}

American Antiquarian Society Library

University of Massachusetts Medical Center Lamar Soutter Medical Library

Worcester Public Library

\section{MICHIGAN}

\section{Albion}

Albion College Stockwell-Mudd Library

\section{Allendale}

Grand Valley State University Zumberge Library

\section{Alma}

Alma College Monteith Library

\section{Ann Arbor}

University of Michigan Harlan Hatcher Graduate Library University of Michigan Law Library

\section{Benton Harbor}

Benton Harbor Public Library

\section{Clinton Township}

Macomb County L.ibrary

\section{Dearborn}

Henry Ford Community College Eshleman Library

\section{Detroit}

Detroit College of Law Library

Detroit Public Library REGIONAL

Marygrove College Library

University of Detroit Mercy School of Law Library

University of Detroit Mercy McNichols Campus Library

Wayne State University Purdy/Kresge Library

Wayne State University Arthur Neef Law Library

\section{Dowagiac}

Southwestern Michigan College Fred L. Mathews Library

\section{East Lansing}

Michigan State University Main Library

\section{Farmington Hills}

Oakland Community College M. L. King Learning Resources Center

Flint

Flint Public Library (1967)

University of Michigan at Flint Francis Wilson Thompson Library

\section{Grand Rapids}

Calvin College \& Theological Seminary Heckman Library Grand Rapids Public Library/Government Documents

\section{Houghton}

Michigan Technological University J. Robert Van Pelt Library

$$
\text { Jackson }
$$

Jackson District Library

\section{Kalamazoo}

Kalamazoo Public Library

Western Michigan University Dwight B. Waldo Library

\section{Lansing}

Library of Michigan/Government Documents REGIONAL Thomas M. Cooley Law School Library

\section{Livonia}

Livonia Public Library

Schoolcraft College Eric J. Bradner Library

\section{Madison Heights}

Madison Heights Public Library

\section{Marquette}

Northern Michigan University Lydia M. Olson Library

\section{Monroe}

Monroe County Library System

\section{Mount Pleasant}

Central Michigan University Charles V. Park Library

\section{Muskegon}

Hackley Public Library

\section{Petoskey}

North Central Michigan College Library

\section{Pontiac}

Oakland County Research Library/Reference 
Port Huron

Saint Clair County Library

\section{Rochester}

Oakland University Kresge Library

\section{Royal Oak}

Royal Oak Public Library/Reference Department

\section{Saginaw}

Hoyt Public Library

\section{Sault Ste. Marie}

Lake Superior State University Kenneth Shouldice Library

\section{Traverse City}

Northwestern Michigan College Osterlin Library

\section{University Center}

Delta College Library

\section{Warren}

Warren Public Library Arthur J. Miller Branch

\section{Ypsilanti}

Eastern Michigan University Library

\section{MINNESOTA}

\section{Bemidji}

Bemidji State University A. C. Clark Library

\section{Blaine}

Anoka County Library

\section{Collegeville}

Saint John's University Alcuin Library

\section{Duluth}

Duluth Public Library/Documents Section University of Minnesota at Duluth Library
Eagan

Dakota County Library-Westcott Branch

\section{Edina}

Hennepin County Library Southdale-Hennepin Library

\section{Mankato}

Mankato State University Memorial Library

\section{Marshall}

Southwest State University Library

\section{Minneapolis}

Minneapolis Public Library/Technology/Science/Government University of Minnesota Law School Library University of Minnesota Wilson Library REGIONAL

\section{Moorhead}

Moorhead State University Livingston Lord Library

\section{Morris}

University of Minnesota at Morris Rodney A. Briggs Library

\section{Northfield}

Carleton College The Library

Saint Olaf College Rolvaag Memorial Library

\section{Roseville}

Ramsey County Public Library Roseville Library

\section{Saint Cloud}

Saint Cloud State University, Learning Resources Center

\section{Saint Paul}

Hamline University School of Law Library Minnesota State Law Library

Saint Paul Public Library/Government Publications University of Minnesota Saint Paul Campus Library William Mitchell College of Law Library Warren E. Burger Library

\section{Saint Peter}

Gustavus Adolphus College Folke Bernadotte Memorial Library

\section{Winona}

Winona State University Maxwell Library 


\section{MISSISSIPPI}

\section{Cleveland}

Delta State University W. B. Roberts Library

\section{Columbus}

Mississippi University for Women Fant Memorial Library

\section{Hattiesburg}

University of Southern Mississippi Cook Memorial Library

\section{Jackson}

Jackson State University Henry Thomas Sampson Library Millsaps College Millsaps-Wilson Library

Mississippi College School of Law Library

Mississippi Library Commission/Documents Section

Supreme Court of Mississippi State Law Library

\section{Lorman}

Alcorn State University J. D. Boyd Library

\section{Mississippi State}

Mississippi State University Mitchell Memorial Library

\section{University}

University of Mississippi J. D. Williams Library REGIONAL University of Mississippi James O. Eastland Law Library

\section{MISSOURI}

\section{Cape Girardeau}

Southeast Missouri State University Kent Library

\section{Columbia}

University of Missouri at Columbia Ellis Library REGIONAL University of Missouri at Columbia School of Law Library

\section{Fulton}

Westminster College Reeves Library

\section{Hillsboro}

Jefferson College Library (1984)

\section{Jefferson City}

Lincoln University Inman E. Page Library

Missouri State Library

Missouri Supreme Court Library

\section{Joplin}

Missouri Southern State College George A. Spiva Library

\section{Kansas City}

Kansas City Missouri Public Library/Documents Division Rockhurst College Greenlease Library

University of Missouri at Kansas City Leon E. Bloch Law Library

University of Missouri at Kansas City Miller Nichols Library

\section{Kirksville}

Truman State University Pickler Memorial Library

\section{Liberty}

William Jewell College Charles F. Curry Library

\section{Maryville}

Northwest Missouri State University B. D. Owens Library

\section{O'Fallon}

Saint Charles City Middendorf-Kredell Branch County Library

\section{Rolla}

University of Missouri at Rolla Curtis Laws Wilson Library

\section{Saint Charles}

Lindenwood College Butler Library

Saint Charles City/County Library District Kisker Road Branch Library

\section{Saint Joseph}

River Bluffs Regional Library Central Library

\section{Saint Louis}

Maryville University Library

Saint Louis County Library

Saint Louis Public Library/Government Information Department

Saint Louis University Law Library

Saint Louis University Pius XII Memorial Library

U.S. Court of Appeals Eighth Circuit Library

University of Missouri at Saint Louis Thomas Jefferson Library

Washington University John M. Olin Library

Washington University Freund Law Library

\section{Springfield}

Drury College F. W. Olin Library

Southwest Missouri State University Duane G. Meyer Library

\section{Warrensburg}

Central Missouri State University Ward Edwards Library 


\section{Scottsbluff}

\section{MONTANA}

\section{Billings}

Montana State University Billings Library

\section{Bozeman}

Montana State University at Bozeman Renne Library

\section{Butte}

Montana Tech of the University of Montana Library

\section{Havre}

Montana State University at Havre Northern Vande Bogart Library

\section{Helena}

Carroll College Corette Library

Montana State Library

State Law Library of Montana

\section{Missoula}

University of Montana Mansfield Library REGIONAL

\section{NEBRASKA}

\section{Blair}

Dana College C. A. Dana Life Library

\section{Crete}

Doane College Perkins Library

\section{Fremont}

Midland Lutheran College Luther Library

\section{Kearney}

University of Nebraska at Kearney Calvin T. Ryan Library

\section{Lincoln}

Nebraska Library Commission

Nebraska State Library

University of Nebraska at Lincoln D. L. Love Memorial Library REGIONAL

University of Nebraska at Lincoln Schmid Law Library

\section{Omaha}

Creighton University Reinert/Alumni Library

Creighton University Klutznick Law Library

Omaha Public Library W. Dale Clark Library

University of Nebraska at Omaha University Library
Scottsbluff Public Library

\section{Wayne}

Wayne State College Conn Library

\section{NEVADA}

\section{Carson City}

Nevada State Library and Archives Nevada Supreme Court Library

\section{Elko}

Elko County Library

Great Basin College Learning Resources Center

\section{Las Vegas}

Clark County Law Library

Las Vegas-Clark County Library District

University of Nevada at Las Vegas James R. Dickinson Library

\section{Reno}

University of Nevada National Judicial College Law Library Nevada Historical Society Library

University of Nevada at Reno Getchell Library REGIONAL Washoe County Library/Government Documents Department

\section{NEW HAMPSHIRE}

\section{Concord}

Franklin Pierce Law Center Library New Hampshire Law Library

New Hampshire State Library/Reference and Information

\section{Durham}

University of New Hampshire Dimond Library

\section{Hanover}

Dartmouth College Baker Library

\section{Henniker}

New England College Danforth Library

\section{Manchester}

Manchester City Library

New Hampshire College Shapiro Memorial Library

Saint Anselm College Geisel Library 
Nashua

Nashua Public Library

\section{NEW JERSEY}

\section{Bayonne}

Bayonne Free Public Library/Government Documents Department

\section{Bridgeton}

Cumberland County Library

\section{Camden}

Rutgers University Law School Library

Rutgers University Paul Robeson Library

\section{East Brunswick}

East Brunswick Public Library

\section{East Orange}

East Orange Public Library

\section{Elizabeth}

Free Public Library of Elizabeth

\section{Glassboro}

Rowan College of New Jersey Savitz Library

\section{Hackensack}

Johnson Free Public Library

\section{Irvington}

Irvington Public Library

\section{Jersey City}

Jersey City Public Library/U.S. Documents Department Jersey City State College Forrest A. Irwin Library

\section{Lawrenceville}

Rider University Franklin F. Moore Library

\section{Madison}

\section{Mahwah}

Ramapo College George T. Potter Library

\section{Morristown}

College of Saint Elizabeth Mahoney Library

\section{Mount Holly}

Burlington County Library

\section{New Brunswick}

Rutgers University Alexander Library

\section{Newark}

Newark Public Library/U.S. Documents REGIONAL Rutgers University John Cotton Dana Library

Rutgers University Ackerson Law Library

Seton Hall University School of Law Peter W. Rodino Jr. Law Library

\section{Newton}

Sussex County Library

\section{Phillipsburg}

Phillipsburg Free Public Library

\section{Plainfield}

Plainfield Public Library

\section{Pomona}

Stockton State College of New Jersey Library/Documents

\section{Princeton}

Princeton University Firestone Library

\section{Randolph}

County College of Morris Masten Learning Resources Center

\section{Shrewsbury}

Monmouth County Library-Eastern Branch

\section{South Orange}

Seton Hall University Walsh Library

\section{Teaneck}

Fairleigh Dickinson University Weiner Library 
Toms River

Ocean County College Library

\section{Trenton}

New Jersey State Library/U.S. Documents Trenton Free Public Library

Upper Montclair

Montclair State College Harry A. Sprague Library

\section{West Long Branch}

Monmouth College Guggenheim Memorial Library

\section{Woodbridge}

Free Public Library of Woodbridge Main Library

\section{NEW MEXICO}

\section{Albuquerque}

University of New Mexico Health Science Center Library University of New Mexico School of Law Library

University of New Mexico General Library REGIONAL

\section{Hobbs}

New Mexico Junior College Pannell Library

\section{Las Cruces}

New Mexico State University Branson Library

\section{Las Vegas}

New Mexico Highlands University Thomas C. Donnelly Library

\section{Portales}

Eastern New Mexico University Golden Library

\section{Santa Fe}

New Mexico State Library REGIONAL New Mexico Supreme Court Law Library

\section{Silver City}

Western New Mexico University Miller Library
New Mexico Institute of Mining and Technology Library

\section{NEW YORK}

\section{Albany}

Albany Law School Schaffer Law Library

New York State Library/Cultural Education Center REGIONAL State University of New York at Albany University Library (1964)

\section{Binghamton}

Binghamton University Glenn G. Bartle Library

\section{Brockport}

State University of New York at Brockport Drake Memorial Library

\section{Bronx}

Fordham University Library/Public Documents Section Herbert $\mathrm{H}$. Lehman College Library

State University of New York Maritime College Stephen B. Luce Library

\section{Bronxville}

Sarah Lawrence College Esther Raushenbush Library

\section{Brooklyn}

Brooklyn College Library/Government Documents Division Brooklyn Law School Library

Brooklyn Public Library Business Library

Brooklyn Public Library Central Library

Pratt Institute Library

\section{Brookville}

Long Island University Schwartz Memorial Library

\section{Buffalo}

Buffalo and Erie County Public Library/Documents Division State University of New York at Buffalo Charles B. Sears Law Library State University of New York at Buffalo Lockwood Memorial Library

\section{Canton}

Saint Lawrence University Owen D. Young Library

\section{Corning}

Corning Community College Arthur A. Houghton Jr. Library

\section{Cortland}

State University College Cortland Memorial Library 


\section{Delhi}

State University of New York College of Technology Resnick Library

\section{East Islip}

East Islip Public Library Suffolk Cooperative Library System

Elmira

Elmira College Gannett-Tripp Library

\section{Farmingdale}

State University of New York at Farmingdale Greenley Library

\section{Flushing}

Queens College Benjamin S. Rosenthal Library

Queens College of City University of New York Law School Library

\section{Garden City}

Adelphi University Swirbul Library

\section{Geneseo}

State University of New York at Geneseo Milne Library

\section{Hamilton}

Colgate University Everett Needham Case Library

\section{Hempstead}

Hofstra University Axinn Library

Hofstra University School of Law Library

\section{Huntington}

Touro College School of Law Library

\section{Ithaca}

Cornell University Albert R. Mann Library

Cornell University Law School Library

Cornell University Olin Library

\section{Jamaica}

Queens Borough Public Library/Magazine Documents Department Saint John's University Library St. Augustine Hall

Saint John's University School of Law Library

\section{Kings Point}

U.S. Merchant Marine Academy Bland Memorial Library
LaGuardia Community College Library

\section{Middletown}

Middletown Thrall Library

\section{Mount Vernon}

Mount Vernon Public Library

\section{New Paltz}

State University College at New Paltz Sojourner Truth Library

\section{New York City}

City College of City University of New York Cohen Library College of Insurance Library Columbia University Lehman Library Columbia University School of Law Library

Cooper Union for the Advancement of Science and Arts Library Fordham University Leo T. Kissam Memorial Law Library Medical Library Center of New York/Technical Services New York Law Institute Library

New York Law School Library

New York Public Library Astor Branch

New York Public Library Hunt's Points Regional Branch New York Public Library Lenox Branch

New York University Elmer Holmes Bobst Library

New York University Law Library

U.S. Court of Appeals Second Circuit Library

Yeshiva University Chutick Law Library

Yeshiva University Pollack Library

\section{Newburgh}

Newburgh Free Library

\section{Niagara Falls}

Niagara Falls Public Library

\section{Oakdale}

Dowling College Library/Government Documents

\section{Oneonta}

State University College at Oneonta James M. Milne Library

\section{Oswego}

State University of New York at Oswego Penfield Library

\section{Plattsburgh}

State University College at Plattsburgh Benjamin F. Feinberg Library 
Potsdam

Clarkson University Burnap Memorial Library/Schuler Educational Resources Center

State University College at Potsdam Crumb Memorial Library

\section{Poughkeepsie}

Vassar College Thompson Library

\section{Purchase}

State University of New York at Purchase Library

\section{Rochester}

Rochester/Monroe County Public Library

University of Rochester Rush Rhees Library

\section{Saint Bonaventure}

Saint Bonaventure University Friedsam Memorial Library

\section{Saratoga Springs}

Skidmore College Lucy Scribner Library

\section{Schenectady}

Union College Schaffer Library

\section{Southampton}

Long Island University Southhampton Campus Library

\section{Sparkill}

St. Thomas Aquinas College Lougheed Library

\section{Staten Island}

Wagner College Horrmann Library

\section{Stony Brook}

State University of New York at Stony Brook Melville Library

\section{Syracuse}

Onondaga County Public Library/The Galleries of Syracuse Syracuse University E. S. Byrd Library

Syracuse University College of Law H. Douglas Barclay Law Library

\section{Troy}

Troy Public Library/Government Documents

\section{Uniondale}

Nassau Library System/Documents Collections

\section{Utica}

State University of New York, Institute of Technology at Utica Library Utica Public Library

\section{West Point}

U.S. Military Academy Library

\section{White Plains}

Pace University School of Law Library

\section{Yonkers}

Yonkers Public Library Getty Square Branch

\section{Yorktown Heights}

Mercy College Library Yorktown Branch Campus

\section{NORTH CAROLINA}

\section{Asheville}

University of North Carolina at Asheville D. Hiden Ramsey Library

\section{Boiling Springs}

Gardner-Webb University Dover Memorial Library

\section{Boone}

Appalachian State University Carol Grotnes Belk Library

\section{Buies Creek}

Campbell University Carrie Rich Memorial Library

\section{Chapel Hill}

University of North Carolina at Chapel Hill Everel Law Library/ Documents

University of North Carolina at Chapel Hill Walter Royal Davis Library REGIONAL

\section{Charlotte}

Public Library of Charlotte and Mecklenburg County Queens College Everett Library

University of North Carolina at Charlotte J. Murrey Atkins Library

\section{Cullowhee}

Western Carolina University Hunter Library

\section{Davidson}

Davidson College E. H. Little Library 


\section{Durham}

Duke University School of Law Library

Duke University William R. Perkins Library

North Carolina Central University Law School Library

North Carolina Central University James E. Shepard Library

\section{Elon College}

Elon College Iris Holt McEwen Library

\section{Fayetteville}

Fayetteville State University Charles W. Chesnutt Library

\section{Greensboro}

North Carolina Agricultural and Technical State University F. D. Bluford Library

University of North Carolina at Greensboro Walter Clinton Jackson Library

\section{Greenville}

East Carolina University J. Y. Joyner Library

\section{Laurinburg}

Saint Andrews Presbyterian College DeTamble Library

\section{Lexington}

Davidson County Public Library

\section{Mount Olive}

Mount Olive College Moye Library

\section{Pembroke}

Pembroke State University Mary Livermore Library

\section{Raleigh}

North Carolina State University D. H. Hill Library

North Carolina Supreme Court Library

State Library of North Carolina/Department of Cultural Resources

\section{Rocky Mount}

North Carolina Wesleyan College Pearsall Library

\section{Salisbury}

Catawba College Corriher-Linn-Black Library

\section{Wilmington}

University of North Carolina at Wilmington William M. Randall Library

\section{Wilson}

Barton College Hackney Library
Forsyth County Public Library Main Library

Wake Forest University Professional Center Library

Wake Forest University Z. Smith Reynolds Library

\section{NORTH DAKOTA}

\section{Bismarck}

Bismarck Veterans' Memorial Public Library North Dakota State Library

North Dakota Supreme Court Law Library

State Historical Society of North Dakota State Archives \& Historical Research Library

\section{Dickinson}

Dickinson State University Stoxen Library

\section{Fargo}

North Dakota State University Libraries REGIONAL

\section{Grand Forks}

University of North Dakota Chester Fritz Library REGIONAL

Minot

Minot State University Gordon B. Olson Library

\section{Valley City}

Valley City State University Allen Memorial Library

NORTHERN MARIANA ISLANDS

\section{Saipan}

Northern Marianas College Borja Memorial Library

\section{OHIO}

\section{Ada}

Ohio Northern University Jay P. Taggart Law Library

\section{Akron}

Akron-Summit County Public Library University of Akron Bierce Library University of Akron School of Law Library 
Ashland

Ashland University Library

\section{Athens}

Ohio University Alden Library

\section{Bluffton}

Bluffton College Musselman Library

\section{Bowling Green}

Bowling Green State University Jerome Library

\section{Canton}

Malone College Everett L. Cattel Library

\section{Chardon}

Chardon Public Library

\section{Cincinnati}

Public Library of Cincinnati and Hamilton County Main Library University of Cincinnati College of Law Robert Marx Law Library University of Cincinnati Langsam Library

U.S. Court of Appeals Sixth Circuit Library

\section{Cleveland}

Case Western Reserve University Kelvin Smith Library Case Western Reserve University School of Law Library Cleveland Public Library/Government Documents

Cleveland State University Joseph W. Bartunek III Law Library Cleveland State University University Library

Municipal Reference Library (1970)

\section{Columbus}

Capital University Law School Library

Capital University Library

Columbus Metropolitan Main Library

Ohio State University College of Law Library

Ohio State University Main Library

Ohio Supreme Court Law Library

State Library of Ohio/Documents Department REGIONAL

\section{Dayton}

Dayton and Montgomery County Public Library

University of Dayton Roesch Library

Wright State University Paul Laurence Dunbar Library

\section{Delaware}

Ohio Wesleyan University L. A. Beeghly Library

\section{Findlay}

University of Findlay Shafer Library

\section{Gambier}

Kenyon College Olin/Chalmers Libraries

\section{Granville}

Denison University William Howard Doane Library

\section{Hiram}

Hiram College Teachout-Price Memorial Library

\section{Kent}

Kent State University Libraries and Media Services

\section{Marietta}

Marietta College Dawes Memorial Library

\section{Marion}

Marion Public Library/Federal Documents

Middletown

Miami University Middletown Gardner-Harvey Library

\section{New Concord}

Muskingum College Library/Documents

\section{Oberlin}

Oberlin College Library/Documents Department

\section{Oxford}

Miami University King Library

\section{Portsmouth}

Shawnee State University Library

\section{Rio Grande}

University of Rio Grande Jeanette Albiez Davis Library

\section{Springfield}

Clark County Public Library/Government Documents 


\section{Steubenville}

Franciscan University of Steubenville John Paul II Library

Public Library of Steubenville and Jefferson County

Tiffin

Heidelberg College Beeghly Library

\section{Toledo}

Toledo-Lucus County Public Library/Social Sciences Department University of Toledo College of Law Library

University of Toledo William S. Carlson Library

\section{University Heights}

John Carroll University Grasselli Library

\section{Westerville}

Otterbein College Courtright Memorial Library

\section{Westlake}

Westlake Porter Public Library

\section{Wilmington}

Wilmington College S. Arthur Watson Library

\section{Wooster}

College of Wooster Andrews Library

\section{Worthington}

Worthington Public Library

\section{Youngstown}

Public Library of Youngstown and Mahoning County Main Library Youngstown State University William F. Maag Library

\section{OKLAHOMA}

\section{Ada}

East Central University Linscheid Library

\section{Alva}

Northwestern Oklahoma State University J. W. Martin Library

\section{Bethany}

Southern Nazarene University Williams Learning Resources Center

\section{Durant}

Southeastern Oklahoma State University Henry G. Bennett Memorial Library

\section{Edmond}

University of Central Oklahoma Chambers Library

\section{Enid}

Public Library of Enid and Garfield County

\section{Langston}

Langston University G. Lamar Harrison Library

\section{Lawton}

Lawton Public Library

\section{Norman}

University of Oklahoma Bizzell Memorial Library University of Oklahoma Law Library

\section{Oklahoma City}

Metropolitan Library System Downtown Library Oklahoma City University Dulaney Browne Library

Oklahoma Department of Libraries-U.S. Government Information Division REGIONAL

\section{Shawnee}

Oklahoma Baptist University Mabee Learning Center

\section{Stillwater}

Oklahoma State University Edmon Low Library REGIONAL

\section{Tahlequah}

Northeastern State University John Vaughan Library

\section{Tulsa}

Tulsa City-County Library System

University of Tulsa College of Law Library

University of Tulsa McFarlin Library

\section{Weatherford}

Southwestern Oklahoma State University AI Harris Library

\section{OREGON}

\section{Ashland}

Southern Oregon State College Library

\section{Bend}

Central Oregon Community College Library/Media Center 
Corvallis

Oregon State University Kerr Library

\section{Eugene}

University of Oregon Law Library

University of Oregon Library

\section{Forest Grove}

Pacific University Harvey Scott Memorial Library

\section{Klamath Falls}

Oregon Institute of Technology Library

\section{La Grande}

Eastern Oregon State College Walter M. Pierce Library

\section{McMinnville}

Linfield College Northup Library

\section{Monmouth}

Western Oregon State College Library

\section{Pendleton}

Blue Mountain Community College Library

\section{Portland}

Lewis and Clark College Aubrey R. Watzek Library

Multnomah County Library/Science and Business Section

Northwestern School of Law Paul L. Boley Law Library

Portland State University Branford P. Millar Library REGIONAL

Reed College Eric V. Houser Library

U.S. Department of Energy Bonneville Power Administration (BPA) Library

\section{Salem}

Oregon State Library

Oregon Supreme Court Law Library

Willamette University College of Law Library

Willamette University Mark O. Hatfield Library

\section{PANAMA CANAL}

\section{Balboa Heights}

Panama Canal Commission Technical Resources Center

\section{PENNSYLVANIA}

\section{Allentown}

Muhlenberg College Trexler Library
Altoona Area Public Library

\section{Bethel Park}

Bethel Park Public Library/Depository Library Office

\section{Bethlehem}

Lehigh University Fairchild-Martindale Library

\section{Bloomsburg}

Bloomsburg University of Pennsylvania Harvey A. Andruss Library

\section{Blue Bell}

Montgomery County Community College Learning Resources Center

\section{Bradford}

University of Pittsburgh at Bradford Hanley Library

\section{Broomall}

Marple Public Library

\section{Californla}

California University of Pennsylvania Louis L. Manderino Library

\section{Carlisle}

Dickinson College Boyd Lee Spahr Library

Dickinson School of Law Sheeley-Lee Law Library

\section{Cheyney}

Cheyney University Leslie Pinckney Hill Library

\section{Collegeville}

Ursinus College Myrin Library

\section{Coraopolls}

Robert Morris College Library

Doylestown

Bucks County Free Library/Bucks County Library Center

\section{East Stroudsburg}

East Stroudsburg University Kemp Library

\section{Erle}

Erie County Library System

\section{Greenville}

Thiel College Langenheim Memorial Library 


\section{Harrisburg}

State Library of Pennsylvania/Law-Government Publications REGIONAL

Widener University Harrisburg Campus School of Law Library

\section{Haverford}

Haverford College Magill Library

\section{Indiana}

Indiana University of Pennsylvania Stapleton Library

\section{Johnstown}

Cambria County Library System Glosser Memorial Library

\section{Lancaster}

Franklin and Marshall College Shadek-Fackenthal Library

\section{Lewisburg}

Bucknell University Ellen Clarke Bertrand Library

\section{Mansfield}

Mansfield University Library

\section{Meadville}

Allegheny College Lawrence Lee Pelletier Library

\section{Millersville}

Millersville University Helen A. Ganser Library

\section{Monessen}

Monessen Public Library

\section{New Castle}

New Castle Public Library

\section{Newton}

Bucks County Community College Library

\section{Norristown}

Montgomery County-Norristown Public Library

\section{Philadelphia}

Free Library of Philadelphia/Government Publications Department Saint Joseph's University Francis A. Drexel Library Temple University Paley Library Temple University School of Law Library U.S. Court of Appeals Third Circuit Library
University of Pennsylvania Biddle Law Library

University of Pennsylvania Library

\section{Pittsburgh}

Allegheny County Law Library

Carnegie Library of Pittsburgh Allegheny Regional Branch

Carnegie Library of Pittsburgh/Government Documents Department Duquesne University School of Law Library

La Roche College John J. Wright Library

University of Pittsburgh Hillman Library

University of Pittsburgh School of Law Barco Law Library

U.S. Department of Energy Bureau of Mines Pittsburgh Research Center Library

\section{Pottsville}

Pottsville Free Public Library

\section{Reading}

Reading Public Library/Government Documents Department

\section{Scranton}

Scranton Public Library

\section{Shippensburg}

Shippensburg University Ezra Lehman Memorial Library

\section{Slippery Rock}

Slippery Rock University Bailey Library

\section{Swarthmore}

Swarthmore College McCabe Library

\section{University Park}

Pennsylvania State University Pattee Library

\section{Villanova}

Villanova University Law School Library

\section{Warren}

Warren Library Association Warren Public Library

\section{West Chester}

West Chester University Francis Harvey Green Library

\section{Williamsport}

Lycoming College Snowden Memorial Library 


\section{PUERTO RICO}

\section{Mayaguez}

University of Puerto Rico Mayaguez Campus Library

\section{Ponce}

Pontifical Catholic University of Puerto Rico/Encarnacion Valdes Library

Pontifical Catholic University of Puerto Rico/School of Law Library

\section{San Juan}

University of Puerto Rico Jose M. Lazaro Library

University of Puerto Rico Law Library

\section{RHODE ISLAND}

\section{Barrington}

Barrington Public Library

\section{Kingston}

University of Rhode Island Library

\section{Newport}

U.S. Naval War College Library

\section{Providence}

Brown University John D. Rockefeller Jr. Library Providence College Phillips Memorial Library Providence Public Library

Rhode Island College James P. Adams Library

Rhode Island State Law Library

Rhode Island State Library

\section{Warwick}

Warwick Public Library

\section{Westerly}

Westerly Public Library

\section{Woonsocket}

Woonsocket Harris Public Library
Aiken
University of South Carolina at Aiken-Gregg-Graniteville Library

\section{Charleston}

Charleston Southern University L. Mendel Rivers Library The Citadel Military College Daniel Library

College of Charleston Robert Scott Small Library

\section{Clemson}

Clemson University Robert Muldrow Cooper Library REGIONAL

\section{Columbia}

Benedict College Payton Learning Resources Center South Carolina State Library/Documents Department University of South Carolina Coleman Karesh Law Library University of South Carolina Thomas Cooper Library REGIONAL

\section{Conway}

Coastal Carolina University Kimbel Library

\section{Due West}

Erskine College McCain Library

\section{Florence}

Florence County Library

Francis Marion University James A. Rogers Library

\section{Greenville}

Furman University James B. Duke Library Greenville County Library

\section{Greenwood}

Lander University Jackson Library

\section{Lancaster}

University of South Carolina at Lancaster Medford Library

\section{Orangeburg}

South Carolina State University Miller F. Whittaker Library

\section{Rock Hill}

Winthrop University Dacus Library 


\section{Spartanburg}

Spartanburg County Public Library

\section{SOUTH DAKOTA}

\section{Aberdeen}

Northern State University Williams Library

\section{Brookings}

South Dakota State University Hilton M. Briggs Library

\section{Pierre}

South Dakota State Library/Federal Documents Department

South Dakota Supreme Court Library

\section{Rapid City}

Rapid City Public Library

South Dakota School of Mines and Technology Devereaux Library

\section{Sioux Falls}

Augustana College Mikkelsen Library

Sioux Falls Public Library Siouxland Libraries

\section{Spearfish}

Black Hills State University E. Y. Berry Library

\section{Vermillion}

University of South Dakota I. D. Weeks Library

\section{TENNESSEE}

\section{Bristol}

King College E. W. King Library

\section{Chattanooga}

Chattanooga-Hamilton County Bicentennial Library

U.S. Tennessee Valley Authority Corporate Library

\section{Clarksville}

Austin Peay State University Felix G. Woodward Library

\section{Cleveland}

Cleveland State Community College Library

\section{Columbia}

Columbia State Community College John Finney Memorial Library
Tennessee Technological University Library

\section{Jackson}

Lambuth University Luther L. Gobbel Library

\section{Jefferson City}

Carson-Newman College Stephens-Burnett Library

\section{Johnson City}

East Tennessee State University Sherrod Library

\section{Knoxville}

Knox County Public Library System Lawson-McGhee Library University of Tennessee at Knoxville John C. Hodges Library University of Tennessee at Knoxville Law Library

\section{Martin}

University of Tennessee at Martin Paul Meek Library

\section{Memphis}

Memphis-Shelby County Public Library University of Memphis Humphreys School of Law Library University of Memphis University Libraries

\section{Murfreesboro}

Middle Tennessee State University Andrew L. Todd Library

\section{Nashville}

Fisk University Library

Public Library of Nashville and Davidson County Ben West Library Tennessee State Library and Archives/State Library Division Tennessee State University Brown-Daniel Library Vanderbilt University Massey Law Library Vanderbilt University Central Library

\section{Sewanee}

University of the South Jessie Ball duPont Library

\section{TEXAS}

\section{Abilene}

Abilene Christian University Brown Library Hardin-Simmons University Richardson Library

\section{Arlington}

Arlington Public Library University of Texas at Arlington Library 
Texas State Law Library

Texas State Library/United States Documents REGIONAL

University of Texas at Austin Wasserman Library

University of Texas at Austin Perry-Castaneda Library

University of Texas at Austin Tarlton Law Library (1965)

\section{Baytown}

Lee College Carlson Learning Resources Center

\section{Beaumont}

Lamar University Gray Library

\section{Brownwood}

Howard Payne University Walker Memorial Library

\section{Canyon}

West Texas A\&M University Cornette Library/Documents

\section{College Station}

Texas A\&M University Sterling C. Evans Library

\section{Commerce}

East Texas State University James Gilliam Gee Library

\section{Corpus Christi}

Texas A\&M University at Corpus Christi Jeff and Mary Bell Library

\section{Corsicana}

Navarro College Learning Resources Center

\section{Dallas}

Dallas Baptist University Vance Memorial Library

Dallas Public Library/Government Publications Department

Southern Methodist University Fondren Library

\section{Denton}

University of North Texas Libraries

\section{Edinburg}

University of Texas Pan American Library

\section{El Paso}

El Paso Public Library/Government Documents Section University of Texas at El Paso Library

\section{Fort Worth}

Fort Worth Public Library

Texas Christian University Mary Couts Burnett Library
Rosenberg Library

\section{Garland}

Nicholson Memorial Library System

\section{Houston}

Houston Public Library/Government Documents Section North Harris College Learning Resources Center

Rice University Fondren Library

South Texas College of Law Library/Government Documents Department

Texas Southern University Marshall School of Law Library

University of Houston at Clear Lake Alfred R. Neumann Library

University of Houston O'Quinn Law Library

University of Houston M. D. Anderson Library

\section{Huntsville}

Sam Houston State University Newton Gresham Library

$$
\text { Irving }
$$

Irving Public Library System

\section{Kingsville}

Texas A\&M University at Kingsville James C. Jernigan Library

\section{Laredo}

Laredo Community College Harold R. Yeary Library

\section{Longview}

Longview Public Library

\section{Lubbock}

Texas Tech University Libraries REGIONAL Texas Tech University School of Law Library

\section{Nacogdoches}

Stephen F. Austin State University Steen Library

\section{Richardson}

University of Texas at Dallas Eugene McDermott Library

\section{San Angelo}

Angelo State University Porter Henderson Library

\section{San Antonio}

Palo Alto College Learning Resources Center Saint Mary's University Academic Library Saint Mary's University Sarita Kenedy East Law Library San Antonio College Library San Antonio Public Library Central Library Trinity University Maddux Library University of Texas at San Antonio Library 


\section{San Marcos}

Southwest Texas State University Albert B. Alkek Library

\section{Seguin}

Texas Lutheran College Blumberg Memorial Library

\section{Sherman}

Austin College Gladys Abell Library Center

\section{Texarkana}

Texarkana College Palmer Memorial Library

\section{Victoria}

University of Houston at Victoria Library

\section{Waco}

Baylor University Caston Law Library

Baylor University Moody Memorial Library

\section{Wichita Falls}

Midwestern State University Moffett Library

\section{UTAH}

\section{Cedar City}

Southern Utah University Library

\section{Ephraim}

Snow College Lucy A. Phillips Library

\section{Logan}

Utah State University Merrill Library REGIONAL

\section{Ogden}

Weber State University Stewart Library

\section{Provo}

Brigham Young University Harold B. Lee Library Brigham Young University Howard W. Hunter Law Library

\section{Salt Lake City}

University of Utah Eccles Health Science Library

University of Utah Law Library

University of Utah Marriott Library

Utah State Library

Utah State Supreme Court Law Library

\section{VERMONT}

\section{Burlington}

University of Vermont Bailey/Howe Library

\section{Castleton}

Castleton State College Calvin Coolidge Library

\section{Johnson}

Johnson State College John Dewey Library

\section{Lyndonville}

Lyndon State College Samuel Reed Hall Library

\section{Middlebury}

Middlebury College Egbert Starr Library

\section{Montpelier}

Vermont Department of Libraries/Reference and Law Services

\section{Northfield}

Norwich University Kreitzberg Library

\section{South Royalton}

Vermont Law School Cornell Library

\section{VIRGIN ISLANDS}

\section{Saint Croix}

Virgin Islands Division of Llbraries c/o Florence Williams Public Library

\section{Saint Thomas}

University of the Virgin Islands Ralph M. Paiewonsky Library

\section{VIRGINIA}

\section{Alexandria}

Dept. of the Navy Office of Judge Advocate General Law Library

\section{Arlington}

George Mason University School of Law Library

U.S. Patent \& Trademark Office Scientific Technology Library 


\section{Blacksburg}

Virginia Polytechnic Institute and State University Newman Library

\section{Bridgewater}

Bridgewater College Alexander Mack Memorial Library

\section{Charlottesville}

University of Virginia Alderman Library REGIONAL

University of Virginia Arthur J. Morris Law Library

\section{Chesapeake}

Chesapeake Public Library System Chesapeake Central Library

\section{Danville}

Danville Community College Learning Resources Center

\section{Emory}

Emory and Henry College Kelly Library

\section{Fairfax}

George Mason University Fenwick Library

\section{Fredericksburg}

Mary Washington College Simpson Library

\section{Hampden-Sydney}

Hampden-Sydney College Eggleston Library

\section{Hampton}

Hampton University Harvey Library

\section{Harrisonburg}

James Madison University Carrier Library

\section{Lexington}

Virginia Military Institute Preston Library Washington and Lee University James B. Leyburn Library Washington and Lee University Wilbur C. Hall Law Library

\section{Martinsville}

Patrick Henry Community College Learning Resources Center

\section{Norfolk}

Norfolk Public Library System Kirn Memorial Library

Old Dominion University Library

U.S. Armed Forces Staff College Library

\section{Petersburg}

Virginia State University Johnston Memorial Library
Federal Bureau of Investigation FBI Academy Library Marine Corps Research Center Marine Corps University Library

\section{Reston}

Department of the Interior U.S. Geological Survey Library

\section{Richmond}

Library of Virginia

University of Richmond Boatwright Memorial Library

University of "Richmond Law School Library

U.S. Court of Appeals Fourth Circuit Library

Virginia Commonwealth University James Branch Cabell Library

Virginia State Law Library

\section{Roanoke}

Hollins College Fishburn Library

\section{Salem}

Roanoke College Fintel Library

\section{Williamsburg}

College of William and Mary Earl Gregg Swem Library College of William and Mary Marshall-Wythe Law Library

\section{Wise}

Clinch Valley College John Cook Wyllie Library

\section{WASHINGTON}

\section{Bellevue}

King County Library System Bellevue Regional Library

\section{Bellingham}

Western Washington University Mable Zoe Wilson Library

\section{Cheney}

Eastern Washington University John F. Kennedy Memorial Library

\section{Des Moines}

Highline Community College Library

\section{Ellensburg}

Central Washington University Library

\section{Everett}

Everett Public Library/Documents Section 


\section{Olympia}

Evergreen State College Daniel J. Evans Library

Washington State Law Library

Washington State Library/Government Publications REGIONAL

\section{Port Angeles}

North Olympic Library System Port Angeles Branch

\section{Pullman}

Washington State University Holland Library TSD

\section{Seattle}

Seattle Public Library/Government Publications University of Washington Gallagher Law Library University of Washington Suzzallo Library

U.S. Court of Appeals Ninth Circuit Library

\section{Spokane}

Gonzaga University School of Law Library Spokane Public Library/Documents Department

\section{Tacoma}

Tacoma Public Library/Documents Division University of Puget Sound Collins Memorial Library

Seattle University School of Law Library

\section{Vancouver}

Fort Vancouver Regional Library

\section{Walla Walla}

Whitman College Penrose Memorial Library

\section{WEST VIRGINIA}

\section{Athens}

Concord College J. Frank Marsh Library

\section{Bluefield}

Bluefield State College Hardway Library

\section{Charleston}

Kanawha County Public Library

West Virginia Library Commission/Reference Library

West Virginia Supreme Court Law Library

\section{Elkins}

Davis and Elkins College Booth Library

\section{Fairmont}

Fairmont State College Musick Library

\section{Huntington}

Marshall University James E. Morrow Library

\section{Institute}

West Virginia State College Drain-Jordan Library

\section{Montgomery}

West Virginia Institute of Technology Vining Library

\section{Morgantown}

West Virginia University Library/Government Documents REGIONAL

\section{Salem}

Salem-Teikyo University Benedum Library

\section{Shepherdstown}

Shepherd College Ruth Scarborough Library

$$
\text { Weirton }
$$

Mary H. Weir Public Library

\section{Wheeling}

Wheeling Jesuit College Hodges Library

\section{WISCONSIN}

\section{Appleton}

Lawrence University Seeley G. Mudd Library

\section{Beloit}

Beloit College Col. Robert H. Morse Library

\section{Eau Claire}

University of Wisconsin-Eau Claire William D. McIntyre Library

\section{Fond du Lac}

Fond du Lac Public Library

\section{Green Bay}

University of Wisconsin at Green Bay Cofrin Library

\section{La Crosse}

La Crosse Public Library

University of Wisconsin at La Crosse Murphy Library

\section{Madison}

Madison Public Library/Adult Services Division State Historical Society of Wisconsin Library/Government Publications REGIONAL

University of Wisconsin at Madison Law Library

University of Wisconsin at Madison Memorial Library

Wisconsin State Law Library 


\section{Milwaukee}

Alverno College Library/Media Center Marquette University Law Library

Medical College of Wisconsin Libraries Todd Wehr Library

Milwaukee Public Library/Documents Division REGIONAL

Mount Mary College Haggerty Library

University of Wisconsin at Milwaukee Golda Meir Library

\section{Oshkosh}

University of Wisconsin at Oshkosh Polk Memorial Library

\section{Platteville}

University of Wisconsin at Platteville Karrmann Library

\section{Racine}

Racine Public Library/Documents

\section{Ripon}

Ripon College Lane Library

River Falls

University of Wisconsin at River Falls Chalmer Davee Library

\section{Sheboygan}

Mead Public Library

\section{Stevens Point}

University of Wisconsin at Stevens Point Learning Resources Center

\section{Superior}

Superior Public Library

University of Wisconsin at Superior Jim Dan Hill Library

\section{Waukesha}

Waukesha Public Library

\section{Wausau}

Marathon County Public Library

\section{Whitewater}

University of Wisconsin at Whitewater Harold G. Anderson Library

\section{WYOMING}

\section{Casper}

Natrona County Public Library
Cheyenne

Wyoming State Law Library

Wyoming State Library

\section{Gillette}

Campbell County Public Library/Documents Division

\section{Laramie}

University of Wyoming Coe Library

University of Wyoming George W. Hopper Law Library

\section{Powell}

Northwest College John Taggart Hinckley Library

\section{Riverton}

Central Wyoming College Library

\section{Rock Springs}

Western Wyoming Community College Library

\section{Sheridan}

Sheridan College Griffith Memorial Library 


\section{APPENDIX B \\ Export Assistance Center Directory of the U.S. Department of Commerce}

\section{ALABAMA}

BIrmIngham-Medical Forum Building, 7th Floor, 950 22nd Street North, 35203, Area Code 205 Tel 731-1331, FAX 205-731-0076

\section{ALASKA}

Anchorage-421 W. First Street, World Trade Center, 99501, Area Code 907 Tel 271-6237, FAX 907-271-6242

\section{ARIZONA}

Phoenlx-Phoenix Plaza, Suite 970, 2901 N. Central Avenue, 85012, Area Code 602 Tel 640-2513, FAX 602-640-2518

\section{ARKANSAS}

LIttle Rock-TCBY Tower Building, Suite 700, 425 W. Capitol Avenue, 72201, Area Code 501 Tel 324-5794, FAX 501-324-7380

\section{CALIFORNIA}

Long Beach-One World Trade Center, Suite 1670, 90831, Area Code 310 Tel 980-4550, FAX 310-980-4561

Los Angeles-11000 Wilshire Blvd., Room 9200, 90024, Area Code 310 Tel 235-7104, FAX 310-235-7220

Monterey-c/o Center for Trade and Commercial Diplomacy, 411 Pacific Street, Suite 200, 93940, Area Code 408 Tel 641-9850, FAX 408-6419849

Newport Beach-3300 Invine Avenue, Suite 305, 92660, Area Code 714 Tel 660-1688, FAX 714-660-8039

Novato-330 Ignacio Boulevard, Suite 102, 94949, Area Code 415 Tel 883-1966, FAX 415-883-2711

Oakland-530 Water Street, Suite 740, 94607, Area Code 510 Tel 2737350, FAX 510-251-7352

Ontario-2940 Inland Empire Boulevard, Suite 121, 91764, Area Code $909 \mathrm{Tel}$ 466-4134, FAX 909-466-4140

Oxnard-300 Esplanade Drive, Suite 290, 93030, Area Code 805 Tel 988-4106, FAX 805-988-1862

Sacramento-917 7th Street, 2nd Floor, 95814, Area Code 916 Tel 4985155, FAX 916-498-5923

San Dlego-6363 Greenwich Drive, Suite 230, 92122, Area Code 619 Tel 557-5395, FAX 619-557-6176

San Francisco-250 Montgomery Street, 14th Floor, 94104, Area Code 415 Tel 705-2300, FAX 415-705-2297

San Jose-101 Park Center Plaza, Suite 1001, 95113, Area Code 408 Tel 271-7300, FAX 408-271-7307

Santa Clara-5201 Great American Parkway, \#456, 95054, Area Code 408 Tel $970-4610$, FAX 408-970-4618

\section{COLORADO}

Denver-1625 Broadway, Suite 680, 80202, Area Code 303 Tel 8446622, FAX 303-844-5651

\section{CONNECTICUT}

Middletown-213 Court Street, Suite 903, 06457-3346, Area Code 860 Tel 638-6950, FAX 860-638-6970

\section{DELAWARE}

Served by the Philadelphia, PA, Export Assistance Center

\section{DISTRICT OF COLUMBIA}

Served by the Baltimore, MD, EAC

FLORIDA

Clearwater-128 N. Osceola Avenue, 34615, Area Code 813 Tel 4610011, FAX 813-449-2889

MlamI-P.O. Box 590570, 5600 Northwest 36th Street, Suite 617, 33159, Area Code 305 Tel 526-7425, FAX 305-526-7434

Orlando-Eola Park Centre, 200 E. Robinson Street, Suite 1270, 32801, Area Code 407 Tel 648-6235, FAX 407-648-6756

Tallahassee-107 W. Gaines Street, Room G-01, 32399, Area Code 904 Tel 488-6469, FAX 904-487-1407

\section{GEORGIA}

Atlanta-Marquis Two Tower, 285 Peachtree Center Avenue, N.E., Suite 200, 30303-1229, Area Code 404 Tel 657-1900, FAX 404-657-1970

Savannah-120 Barnard Street, Room A-107, 31401, Area Code 912 Tel 652-4204, FAX 912-652-4241

\section{HAWAII}

Honolulu-P.O. Box 50026, 300 Ala Moana Blvd., Room 4106, 96850 , Area Code 808 Tel 541-1782, FAX 808-541-3435

\section{IDAHO}

Bolse-700 W. State Street, 2nd Floor, 83720, Area Code 208 Tel 3343857, FAX 208-334-2783

\section{ILLINOIS}

Chlcago-Xerox Center, 55 W. Monroe Street, Suite 2440, 60603, Area Code 312 Tel 353-8040, FAX 312-353-8098

Highland Park-610 Central Avenue, Suite 150, 60035, Area Code 847 Tel 681-8010, FAX 847-681-8012

Rockford-P.O. Box 1747, 515 N. Court Street, 61110, Area Code 815 Tel 987-8123, FAX 815-963-7943

Wheaton-Illinois Institute of Technology, 201 E. Loop Road, 60187, Area Code 312 Tel 353-4332, FAX 312-353-4336

\section{INDIANA}

Indianapolis-Penwood One, Suite 106, 11405 N. Pennsylvania Street, Carmel, IN, 46032, Area Code 317 Tel 582-2300, FAX 317-582-2301

\section{IOWA}

Des Molnes-Room 817, Federal Building, 210 Walnut Street, 50309, Area Code 515 Tel 284-4222, FAX 515-284-4021 


\section{KANSAS}

Wichita-151 North Volutsia, 67214, Area Code 316 Tel 269-6160, FAX 316-683-7326

\section{KENTUCKY}

Louisville-601 W. Broadway, Room 634B, 40202, Area Code 502 Tel 582-5066, FAX 502-582-6573

Somerset-2292 S. Highway 27, Suite 320, 42501, Area Code 606 Tel 677-6160, FAX 606-677-6161

\section{LOUISIANA}

New Orleans-One Canal Place, 365 Canal Street, Suite 2150, 70130, Area Code 504 Tel 589-6546, FAX 504-589-2337

\section{MAINE}

Portland-511 Congress Street, 04104, Area Code 207 Tel 541-7430, FAX 207-541-7420

\section{MARYLAND}

Baitimore-World Trade Center, 401 E. Pratt Street, Suite 2432, 21202, Area Code 410 Tel 962-4539, FAX 410-962-4529

\section{MASSACHUSETTS}

Boston-World Trade Center, 164 Northern Avenue, Suite 307, 02210, Area Code 617 Tel 424-5990, FAX 617-424-5992

Marlborough-100 Granger Boulevard, Unit 102, 01752, Area Code 508 Tel 624-6000, FAX 508-624-7145

\section{MICHIGAN}

Ann Arbor-425 S. Main Street, Suite 103, 48104, Area Code 313 Tel 741-2430, FAX 313-741-2432

Detroit-211 W. Fort Street, Suite 2220, 48226, Area Code 313 Tel 2263650, FAX 313-226-3657

Grand Raplds-301 W. Fulton Street, Suite 718-S, 49504, Area Code 616 Tel 458-3564, FAX 616-458-3872

Pontiac-Oakland Pointe Office Building, 250 Elizabeth Lake Road, 48341, Area Code 810 Tel 975-9600, FAX 810-975-9606

\section{MINNESOTA}

Minneapolis-110 South 4th Street, Room 108, 55401, Area Code 612 Tel 348-1638, FAX 612-348-1650

\section{MISSISSIPPI}

Jackson-201 W. Capitol Street, Suite 310, 39201, Area Code 601 Tel 965-4388, FAX 601-965-5386

\section{MISSOURI}

Kansas City-601 E. 12th Street, Room 635, 64106, Area Code 816 Tel 426-3141, FAX 816-426-3140

St. Louis-8182 Maryland Avenue, Suite 303, 63105, Area Code 314 Tel 425-3302, FAX 314-425-3381

\section{MONTANA}

Served by the Boise, ID, Export Assistance Center
NEBRASKA

Omaha-11135 “O” Street, 68137, Area Code 402 Tel 221-3664, FAX 402-221-3668

\section{NEVADA}

Reno-1755 E. Plumb Lane, Suite 152, 89502, Area Code 702 Tel 7845203, FAX 702-784-5343

\section{NEW HAMPSHIRE}

Portsmouth-601 Spaulding Turnpike, Suite 29, 03801, Area Code 603 Tel 334-6074, FAX 603-334-6110

\section{NEW JERSEY}

Newark-7-45 Raymond Plaza, W., 9th Floor, 07102, Area Code 201 Tel 645-4682, FAX 201-645-4783

Trenton-3131 Princeton Pike, Building \#6, Suite 100, 08648, Area Code 609 Tel 989-2100, FAX 609-989-2395

\section{NEW MEXICO}

Santa Fe-c/o New Mexico Dept. of Economic Development, P.O. Box 20003, 87504-5003, Area Code 505 Tel 827-0350, FAX 505-827-0263

\section{NEW YORK}

Buffalo-111 W. Huron Street, Room 1304, 14202, Area Code 716 Tel 551-4191 FAX 716-551-5290

Harlem-163 W. 125th Street, Suite 904, New York, New York, 10027, Area Code 212 Tel 860-6200, FAX 212-860-6203

Long Island-1550 Franklin Avenue, Room 207, Mineola, 11501, Area Code 516 Tel 739-1765, FAX 516-571-4161

New York-6 World Trade Center, Room 635, 10048, Area Code 212 Tel 264-0635, FAX 212-264-1356

Rochester-111 E. Avenue, Suite 220, 14604, Area Code 716 Tel 2636480, FAX 716-325-6505

Westchester-707 West Chester Avenue, White Plains, 10604, Area Code 914 Tel 682-6218 FAX 914-682-6698

\section{NORTH CAROLINA}

Carolinas-521 E. Morehead Street, Suite 435, Charlotte, 28202, Area Code 704 Tel 333-4886, FAX 704-332-2681

Greensboro-400 W. Market Street, Suite 400, 27401, Area Code 910 Tel 333-5345, FAX 910-333-5158

\section{NORTH DAKOTA}

Served by the Minneapolis, MN, Export Assistance Center

\section{OHIO}

Cincinnati-36 E. 7th Street, Suite 2650, 45202, Area Code 513 Tel 684-2944, FAX 513-684-3227

Cleveland-Bank One Center, 600 Superior Avenue, E., Suite 700, 44114, Area Code 216 Tel 522-4750, FAX 216-522-2235

Columbus-37 N. High Street, 4th Floor, 43215, Area Code 614 Tel 3659510, FAX 614-365-9598 
Toledo-300 Madison Avenue, 43604, Area Code 419 Tel 241-0683, FAX 419-241-0684

\section{OKLAHOMA}

Oklahoma Clty-301 Northwest 63rd Street, Suite 330, 73116, Area Code 405 Tel 231-5302, FAX 405-231-4211

Tulsa-440 S. Houston Steet, Room 505, 74127, Area Code 918 Tel 581-7650, FAX 918-581-2844

\section{OREGON}

Eugene-1401 Willamette Street, 97401-4003, Area Code 541 Tel 4656575, FAX 541-465-6704

Portland-One World Trade Center, 121 S.W. Salmon Street, Suite 242 , 97204, Area Code 503 Tel 326-3001, FAX 503-326-6351

\section{PENNSYLVANIA}

Harrisburg-One Commerce Square, 417 Walnut Street, 3rd Floor, 17101, Area Code 717 Tel 232-0051, FAX 717-232-0054

Phlladelphia-615 Chestnut Street, Suite 1501, 19106, Area Code 215 Tel 597-6101, FAX 215-597-6123

Plttsburgh-2002 Federal Building, 1000 Liberty Avenue, 15222, Area Code 412 Tel 644-2850, FAX 412-644-4875

Scranton-One Montage Mountain Road, Suite B, 18507, Area Code 717 Tel 969-2530, FAX 717-969-2539

\section{PUERTO RICO}

San Juan (Hato Rey)-Federal Building, Chardon Avenue, Room G-55, 00918, Area Code 787 Tel 766-5555, FAX 787-766-5692

\section{RHODE ISLAND}

Providence-One West Exchange Street, 02903, Area Code 401 Tel 528-5104, FAX 401-528-5067

\section{SOUTH CAROLINA}

Charleston-P.O. Box 975, 29402 or 81 Mary Street, 29403, Area Code 803 Tel 727-4051, FAX 803-727-4052

Columbla-Strom Thurmond Federal Building, 1835 Assemb'y Street, Suite 172, 29201, Area Code 803 Tel 765-5345, FAX 803-253-3614

Upstate-Park Central Office Park, 555 N. Pleasantburg Drive, Building 1, Suite 109, Greenville, SC 29607, Area Code 864 Tel 271-1976, FAX 864-271-4171

\section{SOUTH DAKOTA}

Slouxland-Augustana College, 2001 S. Summit Avenue, Room SS29A, Sioux Falls, 57197, Area Code 605 Tel 330-4264, FAX 605-3304266

\section{TENNESSEE}

Knoxville-301 E. Church Avenue, 37915, Area Code 423 Tel 545-4637, FAX 615-545-4435

Memphis-22 N. Front Street, Suite 200, 38103, Area Code 901 Tel 544-4137, FAX 901-544-3646

Nashville-Parkway Towers, 404 James Robertson Parkway, Suite 114 , 37219, Area Code 615 Tel 736-5161, FAX 615-736-2454

\section{TEXAS}

Austin-P.O. Box 12728, 1700 Congress, 2nd Floor, Suite 300R, 78701, Area Code 512 Tel 916-5939, FAX 512-916-5940

Dallas-P.O. Box 420069, 2050 N. Stemmons Freeway, Suite 170, 75207, Area Code 214 Tel 767-0542, FAX 214-767-8240

Houston-\#1 Allen Center, 500 Dallas, Suite 1160, 77002, Area Code 713 Tel 718-3062, FAX 713-718-3060

San Antonio-1222 N. Main, Suite 450, 78212, Area Code 210 Tel 2289878, FAX 210-228-9874

\section{UTAH}

Salt Lake City-324 S. State Street, Suite 105, 84111, Area Code 801 Tel 524-5116, FAX 801-524-5886

\section{VERMONT}

Montpeller-109 State Street, 4th Floor, 05609, Area Code 802 Tel 8284508, FAX 802-828-3258

\section{VIRGINIA}

Richmond-700 Centre, 704 E. Franklin Street, Suite 550, 23219, Area Code 804 Tel 771-2246, FAX 804-771-2390

\section{WASHINGTON}

Seattle-Westin Building, 2001 6th Avenue, Suite 650, 98121, Area Code 206 Tel 553-5615, FAX 206-553-7253

Spokane-1020 W. Riverside, 99210, Area Code 509 Tel 459-4125, FAX 509-747-0077

\section{WEST VIRGINIA}

Charleston-405 Capitol Street, Suite 807, 25301, Area Code 304 Tel 347-5123, FAX 304-347-5408

Wheeling-1310 Market Street, 2nd Floor, 26003, Area Code 304 Tel 233-7472, FAX 304-233-7492

\section{WISCONSIN}

Milwaukee-517 E. Wisconsin Avenue, Room 596, 53202, Area Code 414 Tel 297-3473, FAX 414-297-3470

\section{WYOMING}

Served by the Denver, CO, Export Assistance Center 



\section{Superintendent of Documents Publications and Subscriptions Order Form}

Charge your order.

It's Easy!

To fax your orders (202) 512-2250

Publications

Please Type or Print (Form is aligned for typewriter use.)

\begin{tabular}{|c|c|c|c|c|}
\hline Qty. & Stock Number & Title & $\begin{array}{c}\text { Price } \\
\text { Each }\end{array}$ & $\begin{array}{c}\text { Total } \\
\text { Price }\end{array}$ \\
\hline & - & & & \\
\hline & & & & \\
\hline & & & & \\
\hline & & & & \\
\hline & & & & \\
\hline & & \multicolumn{2}{|c|}{ Total for Publications } & \multicolumn{2}{|c|}{} \\
\cline { 2 - 5 }
\end{tabular}

Subscriptions

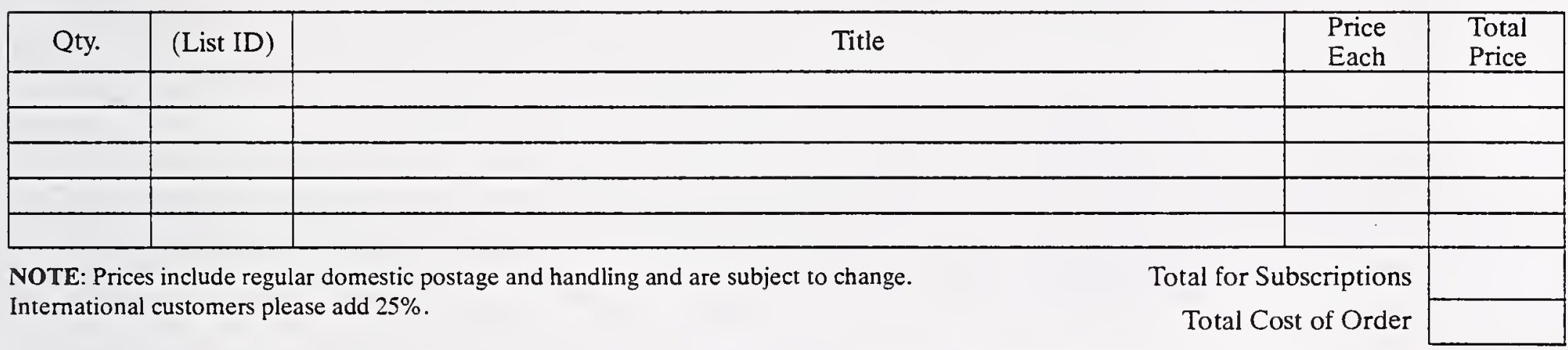

(Company or Personal Name)

(Please type or print)

(Additional address/attention line)

(Street address)

(City, State, ZIP Code)

(Daytime phone including area code)

(Purchase Order No.)

May we make your name/address available to other mailers?
YES NO ?

\section{Please Choose Method of Payment:}

$\square$ Check Payable to the Superintendent of Documents

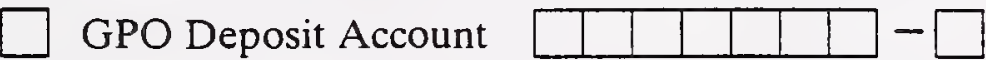

$\square$ VISA or MasterCard Account

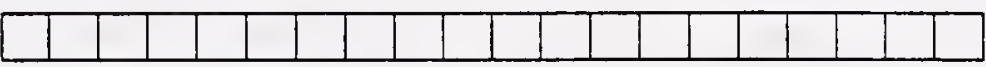

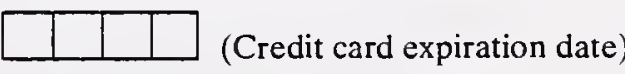

Thank you for your order?

(Authorizing Signature)

Mail To: New Orders, Superintendent of Documents P.O. Box 371954, Pittsburgh, PA 15250-7954 


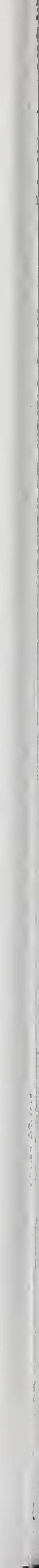


SHIP TO ADDRESS (please print or type)

\begin{tabular}{l|l}
\hline CUSTOMER MASTER NUMBER (IF KNOWN) & DATE \\
\hline
\end{tabular}

ATTENTIONINAME

\begin{tabular}{l|l}
\hline ORGANIZATION & DNISION / ROOM NUMBER \\
\hline
\end{tabular}

STREET ADDRESS

\begin{tabular}{l|l|l}
\hline CITY & STATE & ZIP CODE \\
\hline PROVINCE / TERRITORY & INTERNATIONAL POSTAL CODE \\
\hline
\end{tabular}

COUNTRY

\begin{tabular}{l|l}
\hline PHONE NUMBER & $\begin{array}{l}\text { FAX NUMBER } \\
(\end{array}$ \\
\hline CONTACT NAME & INTERNET E-MAIL ADDRESS \\
\hline
\end{tabular}

METHOD OF PAYMENT (please print or type)

\begin{tabular}{l|l}
\hline$\square$ VISA & $\square$ MasterCard \\
\hline CREDIT CARD NUMBER & EXPIRATION DATE \\
\hline CARDHOLDER'S NAME & \\
\hline
\end{tabular}

CARDHOLDER'S NAME

DTIS Deposit Account Number:

$\square$ Check / Money Order enclosed for \$

(PAYABLE TO NTIS IN U.S DOLLARS)
ORDER BY PHONE (ELIMINATE MAIL TIME)

8:30 a.m. - 5:00 p.m. Eastern Time, M - F.

Sales Desk: (703) 487-4650

TDD: (703) $487-4639$

\section{ORDER BY FAX}

24 hours/7 days a week: (703) 321-8547

To verify receipt of fax: call (703) $487-4679$

7:00 a.m. - 5:00 p.m., Eastern Time, M -F.

\section{ORDER BY MAIL}

National Technical Information Service

5285 Port Royal Road

Springfield, VA 22161

\section{RUSH SERVICE}

RUSH service is available for an additional fee. 1-800-553-NTIS

\section{NTIS ORDERNOWTM ONLINE}

Order the most recent additions to the NTIS collection at NTIS Web site http://wnw.ntis.gov/ordernow.

\section{ORDER VIA E.MAIL}

Order via E-mail 24 hours a day.

orders@ntis.fedworld.gov

If concemed about Intemel security, you may register your credit card at NTIS. Simply call (703) 487-4682.

\section{BILL ME}

(U.S., Canada, and Mexico only.)

NTIS will gladly bill your order, for an additional fee of $\$ 7.50$. A request to be billed must be on a purchase order or company letterhead. An authorizing signature, contact name, and telephone number should be included with this request. Requests may be mailed or faxed.

PRODUCT SELECTION (please print or type)

\begin{tabular}{l} 
NTIS PRODUCT NUMBER \\
\hline
\end{tabular}

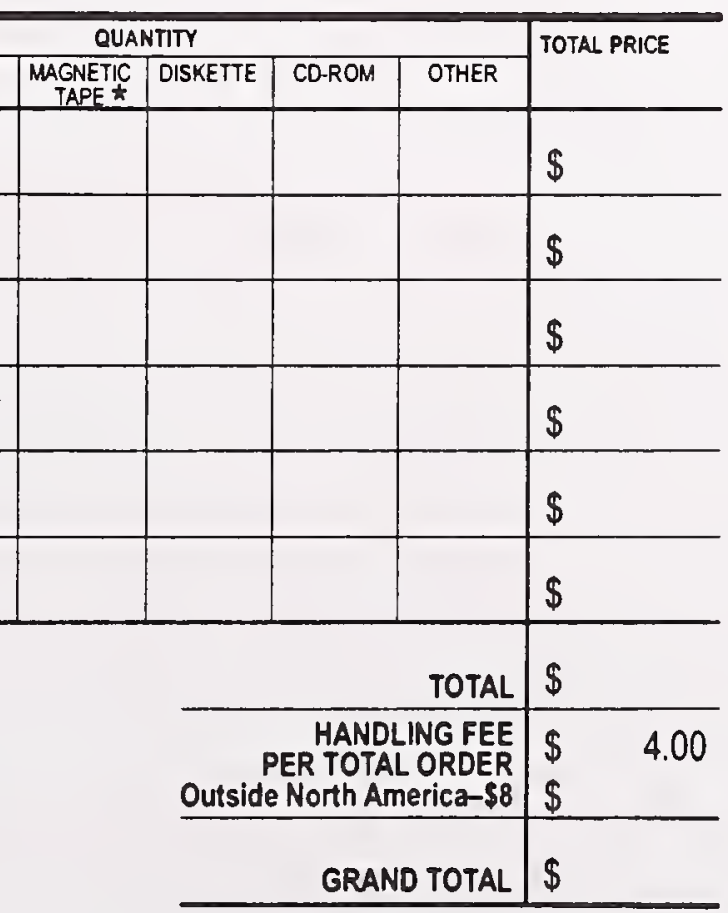

Unless microfiche or other is specifed, paper copy will be sent.

Please call the Sales Desk at (703) $487-4650$ for information on multiple copy discounts available for certain documents. 


\section{NIST Technical Publications}

\section{Periodical}

Journal of Research of the National Institute of Standards and Technology-Reports NIST research and development in those disciplines of the physical and engineering sciences in which the Institute is active. These include physics, chemistry, engineering, mathematics, and computer sciences. Papers cover a broad range of subjects, with major emphasis on measurement methodology and the basic technology underlying standardization. Also included from time to time are survey articles on topics closely related to the Institute's technical and scientific programs. Issued six times a year.

\section{Nonperiodicals}

Monographs-Major contributions to the technical literature on various subjects related to the Institute's scientific and technical activities.

Handbooks-Recommended codes of engineering and industrial practice (including safety codes) developed in cooperation with interested industries, professional organizations, and regulatory bodies.

Special Publications-Include proceedings of conferences sponsored by NIST, NIST annual reports, and other special publications appropriate to this grouping such as wall charts, pocket cards, and bibliographies.

National Standard Reference Data Series-Provides quantitative data on the physical and chemical properties of materials, compiled from the world's literature and critically evaluated. Developed under a worldwide program coordinated by NIST under the authority of the National Standard Data Act (Public Law 90-396). NOTE: The Journal of Physical and Chemical Reference Data (JPCRD) is published bimonthly for NIST by the American Chemical Society (ACS) and the American Institute of Physics (AIP). Subscriptions, reprints, and supplements are available from ACS, 1155 Sixteenth St., NW, Washington, DC 20056.

Building Science Series-Disseminates technical information developed at the Institute on building materials, components, systems, and whole structures. The series presents research results, test methods, and performance criteria related to the structural and environmental functions and the durability and safety characteristics of building elements and systems.

Technical Notes-Studies or reports which are complete in themselves but restrictive in their treatment of a subject. Analogous to monographs but not so comprehensive in scope or definitive in treatment of the subject area. Often serve as a vehicle for final reports of work performed at NIST under the sponsorship of other government agencies.

Juntary Product Standards-Developed under procedures published by the Department of Commerce in Part 10. Title 15, of the Code of Federal Regulations. The standards establish nationally recognized requiremeni ior products, and provide all concerned interests with a basis for common understanding of the characteristics of the products. NIST administers this program in support of the efforts of private-sector standardizing organizations.

Order the following NIST publications-FIPS and NISTIRs—-from the National Technical Information Service, Springfield, VA 22161.

Federal Information Processing Standards Publications (FIPS PUB)-Publications in this series collectively constitute the Federal Information Processing Standards Register. The Register serves as the official source of information in the Federal Government regarding standards issued by NIST pursuant to the Federal Property and Administrative Services Act of 1949 as amended, Public Law 89-306 (79 Stat. 1127), and as implemented by Executive Order 11717 (38 FR 12315, dated May 11, 1973) and Part 6 of Title 15 CFR (Code of Federal Regulations).

NIST Interagency Reports (NISTIR)-A special series of interim or final reports on work performed by NIST for outside sponsors (both government and nongovernment). In general, initial distribution is handled by the sponsor; public distribution is by the National Technical Information Service, Springfield, VA 22161, in paper copy or microfiche form. 
ADMINISTRATION \& MANAGEMENT

AERONAUTICS \& AERODYNAMICS

AGRICULTURE \& FOOD

ASTRONOMY \& ASTROPHYSICS

ATMOSPHERIC SCIENCES

BEHAVIOR \& SOCIETY

BIOMEDICAL TECHNOLOGY \& HUMAN FACTORS ENGINEERING

BUILDING INDUSTRY TECHNOLOGY

BUSINESS \& ECONOMICS

CHEMISTRY

CIVIL ENGINEERING

COMBUSTION, ENGINES, \& PROPELLANTS

COMMUNICATION

COMPUTERS, CONTROL \& INFORMATION THEORY

DETECTION \& COUNTERMEASURES

ELECTROTECHNOLOGY

ENERGY

ENVIRONMENTAL POLLUTION \& CONTROL

HEALTH CARE

INDUSTRIAL \& MECHANICAL ENGINEERING

LIBRARY \& INFORMATION SCIENCES

MANUFACTURING TECHNOLOGY

MATERIALS SCIENCES

MATHEMATICAL SCIENCES

MEDICINE \& BIOLOGY

MILITARY SCIENCES

NATURAL RESOURCES \& EARTH SCIENCES

NAVIGATION, GUIDANCE, \& CONTROL

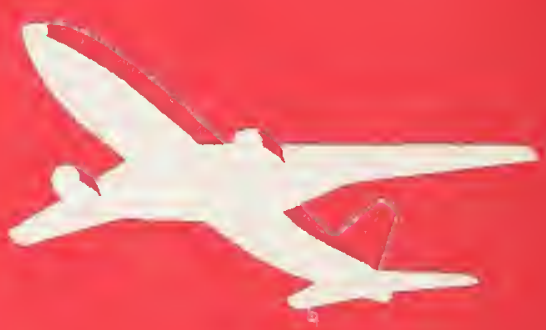

NUCLEAR SCIENCE \& TECHNOLOGY

OCEAN SCIENCES \& TECHNOLOGY

ORDNANCE

PHOTOGRAPHY \& RECORDING DEVICES

PHYSICS ,

PROBLEM-SOLVING INFORMATION FOR STATE \& LOCAL GOVERNMENTS

SPACE TECHNOLOGY.

TRANSPORTATION

URBAN \& REGIONAL TECHNOLOGY \& DEVELOPMENT 